Supporting Information

\title{
Unraveling the Importance of Noncovalent Interactions in Asymmetric Hydroformylation Reactions
}

\author{
Yuvraj Dangat,* Sahil Popli, and Raghavan B. Sunoj* \\ Department of Chemistry, Indian Institute of Technology Bombay, Powai, Mumbai 400076, \\ India
}

E-mail: sunoj@chem.iitb.ac.in

and

yuvamscphy@gmail.com 


\section{Part-A}

\section{Table of Contents}

details

page

S1. Details of Branched and Linear Pathways for AHF of $\alpha$-Methylstyrene

S3

S2. Conformational Space of Pre-Catalytic Species 1

$\mathrm{S} 12$

S3. Heat Map of the RMSD of All Atoms of Various Conformers of $\mathbf{1}$

$\mathrm{S} 12$

S4. Important NCIs in the ea1 and ea2 Coordination Modes in $\mathbf{1}$

S13

S5. Conformational Space of 2

S14-S16

S6. Conformational Space of $\mathbf{3}$

S17-S18

S7. Conformational Space of the Migratory Insertion Transition State (TS1)

S19-S23

S8. Activation Strain Analysis on TS1

S23-S24

S9. Energetics and Other Details of the Migratory Insertion (TS1)

$\mathrm{S} 25-\mathrm{S} 30$

S10. Conformational Aspects of 7 and TS4

S31-S32

S11. Energetic Span Analysis for Linear and Branched Pathways

S33-S36

S12. Predicted Enantioselectivites for Additional Substrates

S36-S39

\section{Part-B}

S41-S1928

Optimized Cartesian Coordinates and Energies of Reactants, Intermediates, Transition State, and Products 


\section{S1. Details of Branched and Linear Pathways for AHF of $\alpha$-Methylstyrene}

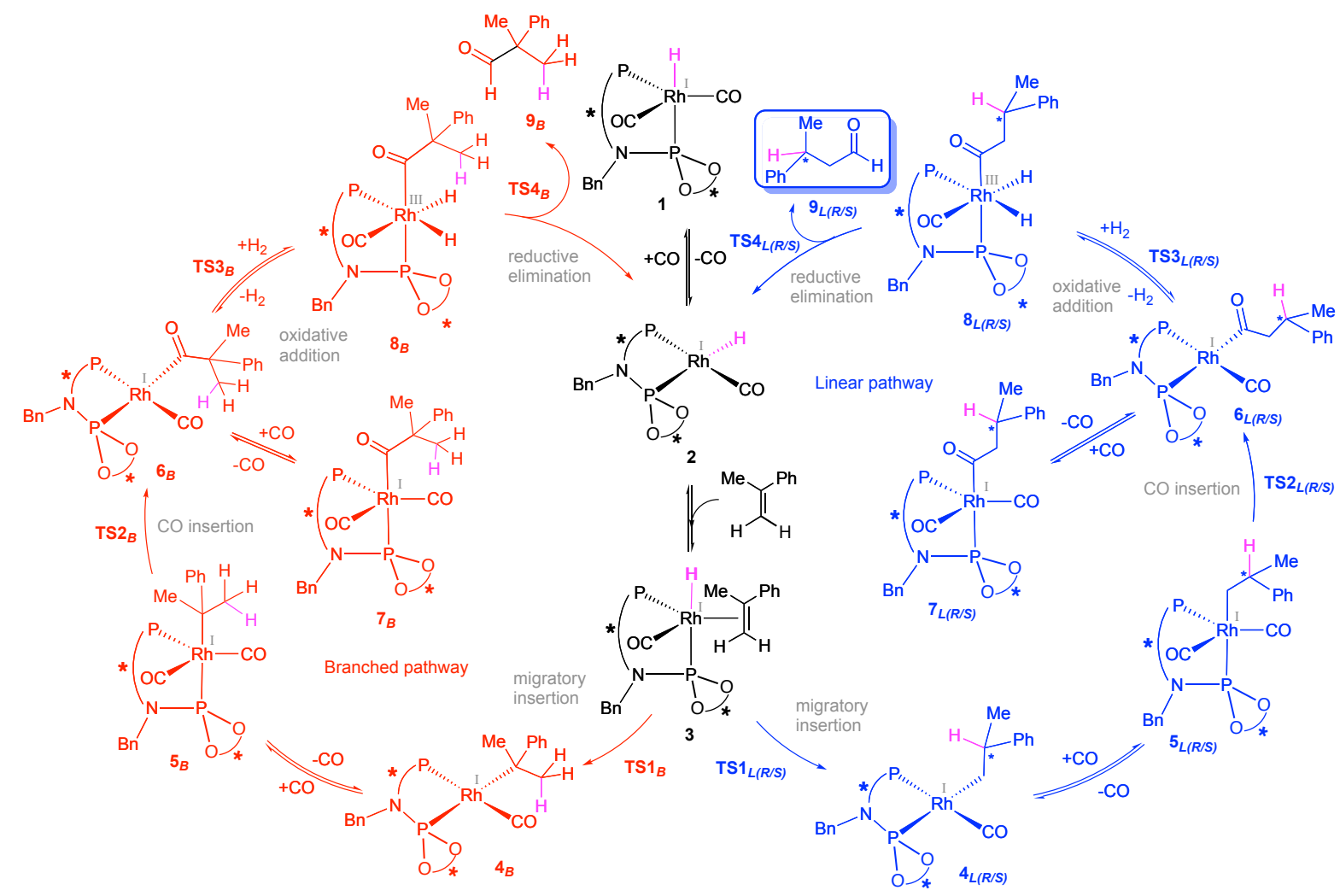

Scheme S1. Full catalytic cycle for $(S, S)$-YanPhos Rh-catalyzed AHF of $\alpha$-methylstyrene.

Here, blue colored pathway represents chiral linear aldehyde production path and red pathway corresponds to the production of achiral branch aldehyde product 2-Me-2-Phpropionaldehyde. Further, $B$ represent the branch, and $\mathrm{L}(\mathrm{R} / \mathrm{S})$ represent linear $\mathrm{R} / \mathrm{S}$ intermediates leading to the formation of $(R / S)$ 3-Me-3-Ph-propionaldehyde respectively. 
S2. Conformational Space of Pre-Catalytic Species 1

\section{S2.1. Ligand Coordination Modes}
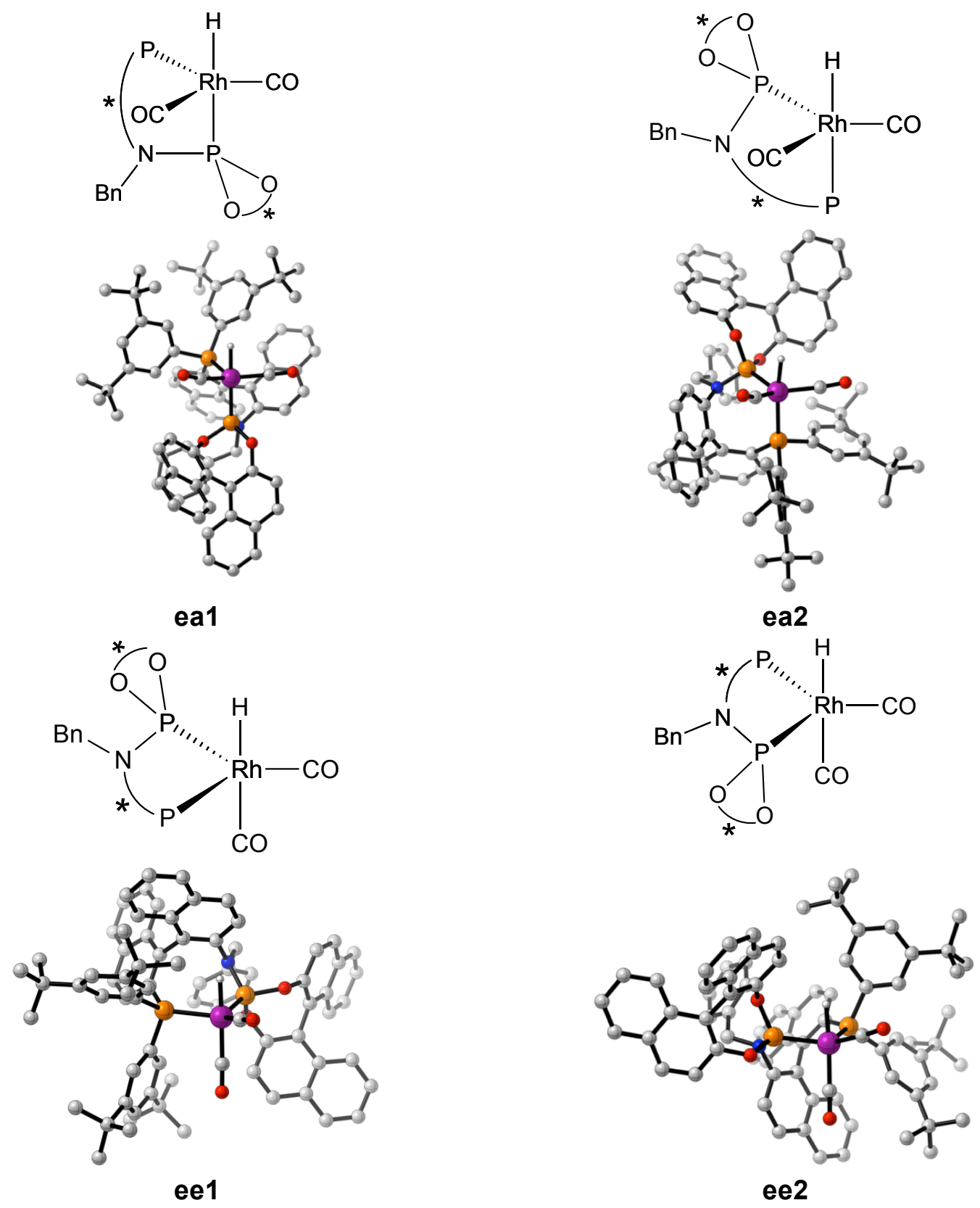

Figure S1. Schematic representation of four coordination modes of YanPhos ligand to the Rh center and the corresponding optimized geometries of complex $\mathbf{1 .}$ 


\section{S2.2. Key Dihedral Angels in the Ligand Backbone}

Here, dihedral angles are denoted as $\phi=\mathrm{P}^{1}-\mathrm{N}^{2}-\mathrm{C}^{3}-\mathrm{C}^{4}, \boldsymbol{\psi} \mathbf{1}=\mathrm{C}^{5}-\mathrm{C}^{6}-\mathrm{C}^{7}-\mathrm{C}^{8}, \boldsymbol{\psi} \mathbf{2}=\mathrm{C}^{5}-\mathrm{C}^{9}-\mathrm{C}^{10}-\mathrm{C}^{11}$, $\boldsymbol{\psi} \mathbf{3}=\mathrm{C}^{12}-\mathrm{C}^{13}-\mathrm{C}^{14}-\mathrm{C}^{15}$ and $\boldsymbol{\psi} \mathbf{4}=\mathrm{C}^{12}-\mathrm{C}^{16}-\mathrm{C}^{17}-\mathrm{C}^{18}$
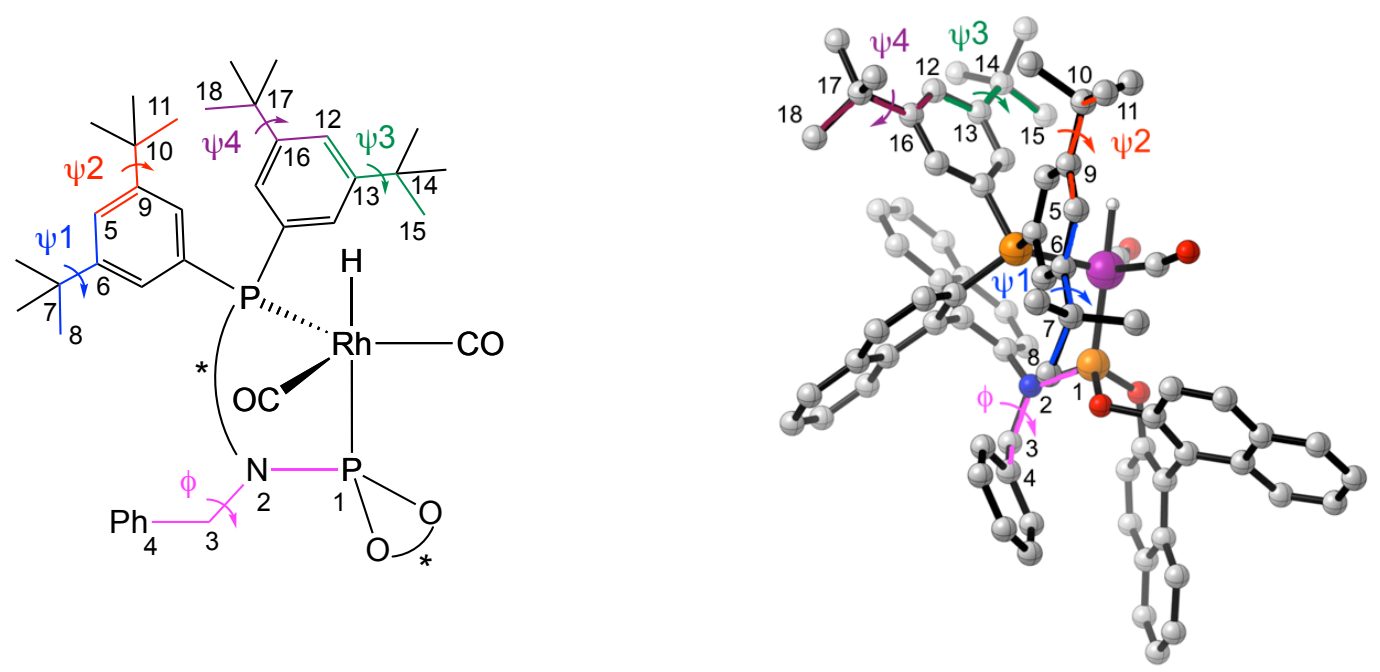

Figure S2. Five key dihedrals in the YanPhos ligand that would generate an array of different conformers. Here ea1 coordination mode is shown as a representative case.

\section{S2.3. Conformers due to the Rotations Around the $\mathrm{C}^{3}-\mathrm{N}^{2}$ Bond}

For the ea1 coordination mode, three distinct conformers denoted as $\mathbf{1}_{\mathbf{C} 1}, \mathbf{1}_{\mathbf{C} 2}$ and $\mathbf{1}_{\mathbf{C} 3}$ were identified, on the basis of the rotation around the $\mathrm{C}^{3}-\mathrm{N}^{2}$ bond characterized by the dihedral $\phi\left(\mathrm{P}^{1}-\right.$ $\left.\mathrm{N}^{2}-\mathrm{C}^{3}-\mathrm{C}^{4}\right)$. In $\mathbf{1}_{\mathrm{C} 1}, \mathbf{1}_{\mathrm{C} 2}$ and $\mathbf{1}_{\mathrm{C} 3}$ conformers, the value of the optimized dihedral $\phi$ were found to be $60.7,111.5$ and $-147.4^{\circ}$ receptively (Figure S3). Similarly, three different conformers were identified in the ea $\mathbf{2}$ coordination mode and are denoted as $\mathbf{1}_{\mathrm{C} 4}, \mathbf{1}_{\mathrm{C} 5}, \mathbf{1}_{\mathrm{C} 6}$. Conformers $\mathbf{1}_{\mathrm{C} 7}, \mathbf{1}_{\mathrm{C} 8}, \mathbf{1}_{\mathrm{C} 9}$ were identified for the ee1 coordination mode and $\mathbf{1}_{\mathbf{C 1 0}}, \mathbf{1}_{\mathbf{C 1 1}}, \mathbf{1}_{\mathbf{C 1 2}}$ for the ee2 mode. Thus, twelve distinct conformers were obtained for $\mathbf{1}$ due to the rotation around the $\mathrm{C}^{3}-\mathrm{N}^{2}$ bond in each of the four coordination modes of the YanPhos ligand. The other flexible dihedrals in the ligand, arising due to the t-butyl substituents are denoted as $\psi \mathbf{1}, \psi \mathbf{2}, \psi \mathbf{3}$ and $\psi \mathbf{4}$. Details of these conformers are presented in the next section. 

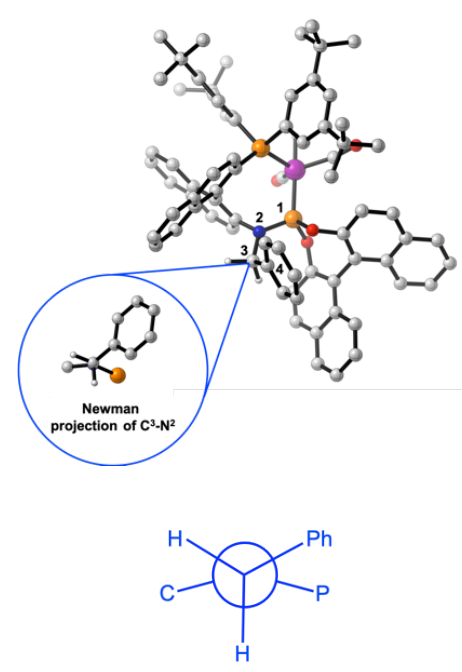

$$
\phi=60.7^{\circ}
$$

$1_{\mathrm{C} 1}$
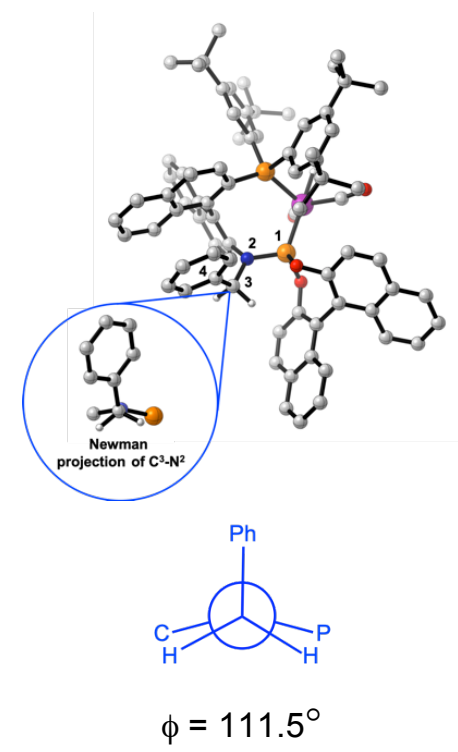

$1_{\mathrm{C} 2}$
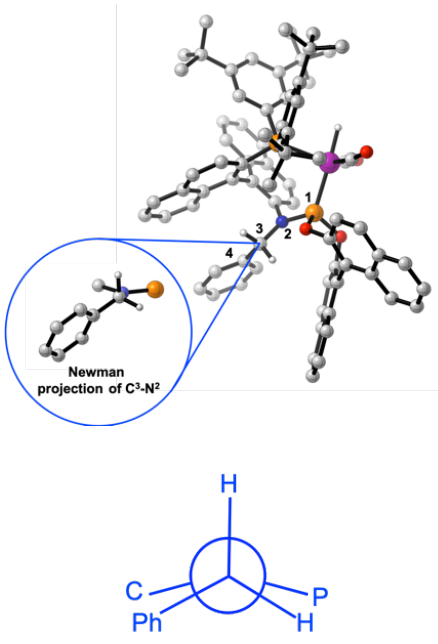

$\phi=-147.4^{\circ}$

$1_{\mathrm{C} 3}$

Figure S3. Optimized geometries and the corresponding values of $\phi\left(\mathrm{P}^{1}-\mathrm{N}^{2}-\mathrm{C}^{3}-\mathrm{C}^{4}\right)$ dihedral angle for the complexes $\mathbf{1}_{\mathbf{C} \mathbf{1}}, \mathbf{1}_{\mathbf{C} 2}$ and $\mathbf{1}_{\mathbf{C} 3}$ in the ea1 coordination mode of the YanPhos ligand.

\section{S2.4. Conformers due to the Rotations of the tert-Butyl Substituents}

Two distinct conformers were identified for a given tert-butyl dihedral such as $\psi \mathbf{1}\left(C^{5}-C^{6}-C^{7}-C^{8}\right)$ that represents the rotation around the $C^{7}-C^{6}$ bond (Figure S4). These two conformers, denoted as $\psi 1$ a and $\psi 1$ s, respectively have the value of dihedral $C^{5}-C^{6}-C^{7}-C^{8}$ as 173.6 and $-7.1^{\circ}$. The descriptors $\mathbf{a} / \mathbf{s}$ respectively convey the syn/anti orientations of the tert-butyl group, with respect to the chosen aryl $\mathrm{C}=\mathrm{C}$ bond (Figure $\mathrm{S} 2$ ). Similar conformers were identified for the other tertbutyl substituents on the diaryl groups of the phosphine region of the YanPhos ligand, whose dihedrals are denoted as $\boldsymbol{\psi} \mathbf{2}, \boldsymbol{\psi 3}$, and $\boldsymbol{\psi} \mathbf{4}$. Thus, the important conformational possibilities due to $\psi \mathbf{1}, \psi \mathbf{2}, \psi \mathbf{3}$ and $\psi \mathbf{4}$ dihedrals of tert-butyl is sixteen for a given value of $\phi\left(\mathrm{P}^{1}-\mathrm{N}^{2}-\mathrm{C}^{3}-\mathrm{C}^{4}\right)$ dihedral complex ( etc., and their relative Gibbs free energies are provided in Tables S1 and S2. The conformers $\mathbf{1}_{\mathbf{C} 1}$, $\mathbf{1}_{\mathbf{C} 2}$ and $\mathbf{1}_{\mathbf{C} 3}$ correspond to ligand coordination mode ea1 i.e., total 48 conformers were obtained within the ea1 mode. Likewise, distinct 48 conformers were also identified for the other ligand 
coordination modes such as ea2, ee1 and ee2. Thus, overall 192 distinct conformers are found for the pre-catalytic complex 1.

$\psi 1 \mathrm{a}$

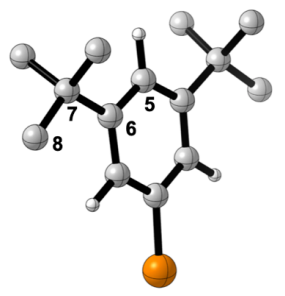

$$
\psi=173.6^{\circ}
$$

$\psi 1 \mathrm{~s}$

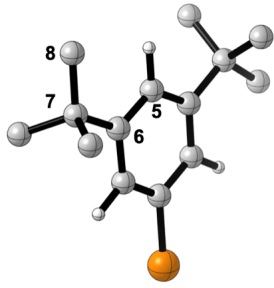

Figure S4. A truncated model for illustrating the conformational possibilities due to a typical tert-butyl dihedral $\psi$ (e.g., $\mathrm{C}^{5}-\mathrm{C}^{6}-\mathrm{C}^{7}-\mathrm{C}^{8}$ ).

\section{S2.5. Gibbs Free Energies of Conformers of 1}

Table S1. Gibbs Free Energies (in kcal/mol) for 192 Conformers of Complex 1. ${ }^{a}$ Keeping the First Entry as the Reference, the Dihedral Variations are Shown Using Blue Color for Better Comprehension

\begin{tabular}{|c|c|c|c|c|c|c|c|c|c|c|c|c|}
\hline & \multicolumn{3}{|c|}{ ea1 } & \multicolumn{3}{|c|}{ ea2 } & \multicolumn{3}{|c|}{ ee1 } & \multicolumn{3}{|c|}{ ee2 } \\
\hline & $1_{\mathrm{C} 1}$ & $1_{C 2}$ & $1_{C 3}$ & $1_{\mathrm{C} 4}$ & $\mathbf{1}_{\mathrm{C} 5}$ & $1_{\mathrm{C} 6}$ & $\mathbf{1}_{\mathrm{C} 7}$ & $1_{\mathrm{C} 8}$ & $1_{\mathrm{C} 9}$ & $1_{\mathrm{C} 10}$ & $\mathbf{1}_{\mathrm{C} 11}$ & $\mathbf{1}_{\mathrm{C} 12}$ \\
\hline$\psi-1 \mathbf{a} 2 \mathbf{a} 3 \mathbf{a} 4 \mathbf{a}$ & 0.4 & 0.7 & 3.0 & 0.7 & 0.5 & 3.0 & 3.4 & 5.2 & 6.2 & 4.2 & 4.6 & 8.2 \\
\hline$\psi-1 a_{2}$ & 0.1 & 0.8 & 2.7 & 0.5 & 0.6 & 2.4 & 3.3 & 3.2 & 6.2 & 4.7 & 3.9 & 7.8 \\
\hline$\psi-1 \mathrm{a}$ & 0.6 & 1.3 & 3.5 & -0.2 & 0.3 & 2.8 & 3.4 & 3.5 & 6.3 & 5.4 & 4.4 & 8.2 \\
\hline & 0.3 & 0.6 & 2.7 & 0.8 & 2.2 & 3.8 & 5.0 & 2.8 & 6.3 & 5.2 & 4.0 & 7.7 \\
\hline$\psi-1$ & $0.0^{\mathrm{b}}$ & 0.3 & 2.2 & 0.4 & 2.5 & 3.1 & 2.8 & 3.1 & 5.9 & 4.6 & 4.0 & 7.6 \\
\hline a4s & $0.0^{\mathrm{b}}$ & 0.1 & 2.6 & 0.5 & 0.1 & 3.0 & 3.2 & 2.7 & 6.2 & 4.2 & 5.1 & 9.8 \\
\hline & 0.2 & 1.2 & 2.9 & 0.3 & 1.1 & 3.1 & 4.7 & 3.3 & 6.0 & 6.2 & 4.0 & 8.0 \\
\hline & 0.2 & 0.6 & 3.1 & 0.7 & 2.1 & 2.7 & 2.9 & 2.7 & 8.7 & 5.5 & 4.2 & 7.8 \\
\hline$\psi-1$ & 1.9 & 2.0 & 4.4 & 1.9 & 1.9 & 4.6 & 4.3 & 5.7 & 7.6 & 6.5 & 6.0 & 9.0 \\
\hline & 1.3 & 2.5 & 3.7 & 1.7 & 1.7 & 4.5 & 4.0 & 6.3 & 7.5 & 5.5 & 4.9 & 8.9 \\
\hline & 2.1 & 2.7 & 4.6 & 1.1 & 2.7 & 4.6 & 5.5 & 3.3 & 8.4 & 5.6 & 5.0 & 8.7 \\
\hline & 1.7 & 2.2 & 3.5 & 1.0 & 1.4 & 4.2 & 4.0 & 4.2 & 8.0 & 5.7 & 5.8 & 8.8 \\
\hline & 1.9 & 2.0 & 3.6 & 1.6 & 1.9 & 4.6 & 3.7 & 3.6 & 7.2 & 6.4 & 5.9 & 8.6 \\
\hline & 1.9 & 2.1 & 4.1 & 1.7 & 1.8 & 4.8 & 3.8 & 4.1 & 7.2 & 5.5 & 5.8 & 8.6 \\
\hline$\psi-1 \mathrm{~s}$ & 2.3 & 2.5 & 4.1 & 1.3 & 1.9 & 4.5 & 4.1 & 3.4 & 7.8 & 5.3 & 5.8 & 8.5 \\
\hline$\psi-1 \mathrm{~s} 2 \mathrm{~s} 3 \mathrm{~s} 4 \mathrm{~s}$ & 1.9 & 2.1 & 4.1 & 1.6 & 1.8 & 4.7 & 4.0 & 4.0 & 7.9 & 5.3 & 5.0 & 8.3 \\
\hline
\end{tabular}

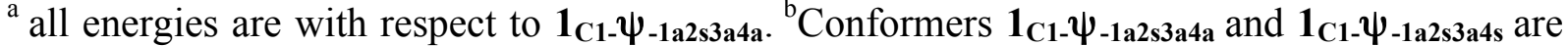

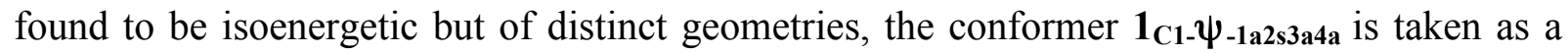
reference. 


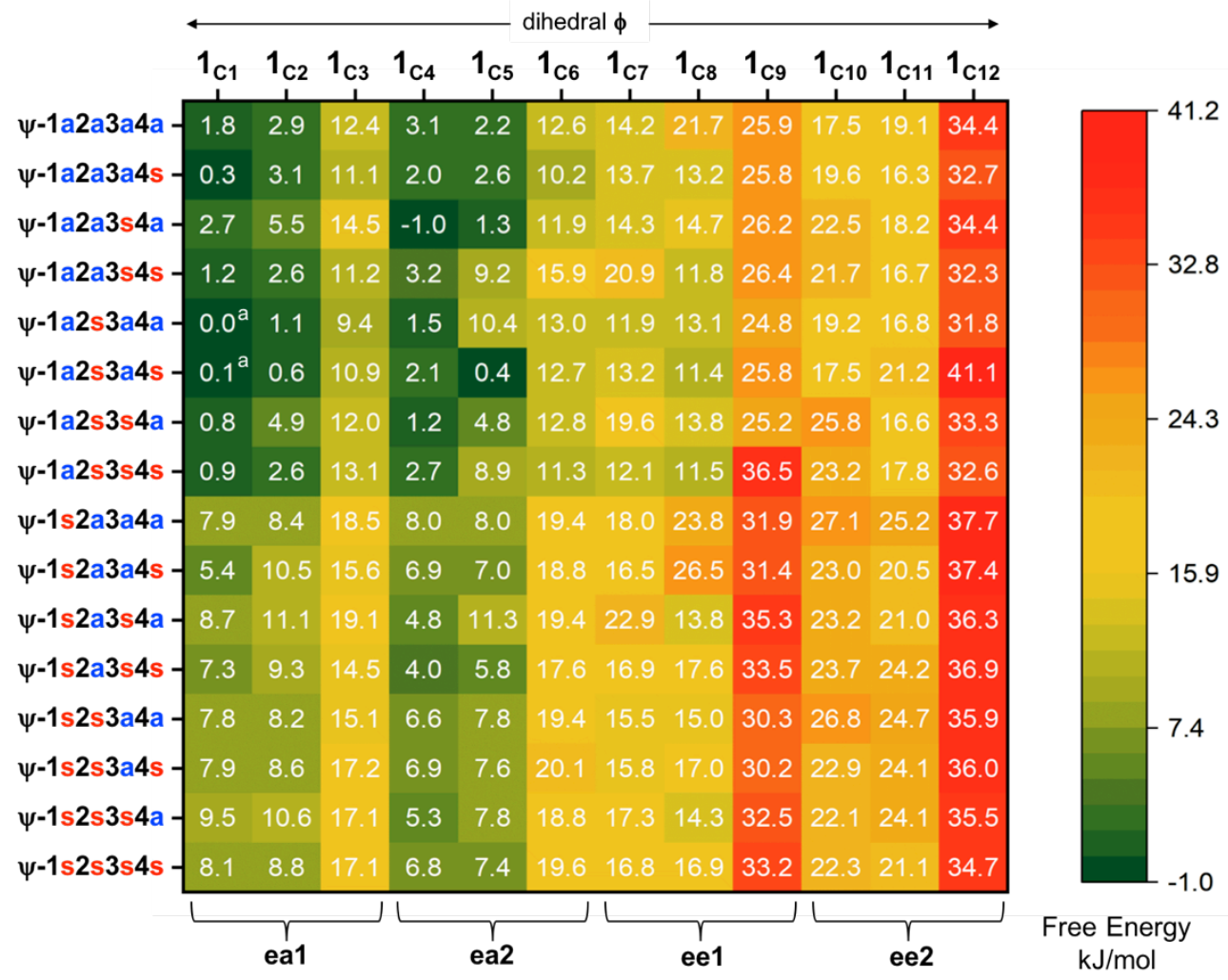

Figure S5. Gibbs free energies (in $\mathrm{kJ} / \mathrm{mol}$ ) of all 192 conformers of complex 1 with respect to

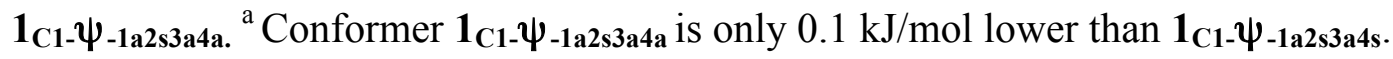

\section{S2.6. Boltzmann Distribution of Conformers of the Pre-Catalytic Complex 1}

It can be noted from Figure S5 that the energy difference between various conformers in columns $\mathbf{1}_{\mathrm{C} 1}$ to $\mathbf{1}_{\mathrm{C} 2}$ and $\mathbf{1}_{\mathrm{C} 4}$ to $\mathbf{1}_{\mathrm{C} 5}$ are marginal. This situation suggests a likely equilibrium between the ea1 and ea2 configurations of $\mathbf{1}$. To find the most favorable and more populated mode of coordination (ea1 or ea2) under the experimental conditions, we have applied Boltzmann weighting of the conformers. The probability $p_{i}$ of state $i$ with energy $\varepsilon_{i}$ at temperature $T$ across $M$ number of states, can be expressed as,

$$
p_{i}=\frac{e^{-\varepsilon_{i} / k T}}{\sum_{j=1}^{M} e^{-\varepsilon_{j} / k T}}
$$


The Boltzmann weighted Gibbs free energies were calculated for the coordination modes ea1 and ea2. For the ea1 mode, the conformers in $\mathbf{1}_{\mathbf{C} 1}$ and $\mathbf{1}_{\mathbf{C} 2}$ columns within a window of 2 $\mathrm{kcal} / \mathrm{mol}$ with respect to the lowest energy conformer $\mathbf{1}_{\mathbf{C 1} \mathbf{-}}-\boldsymbol{\psi}_{-\mathbf{1} \text { a2s3a4s }}$ were chosen for the Boltzmann weighted free energies (Table S3). Similarly, for the ea 2 mode, conformers in $\mathbf{1}_{\mathbf{C} 4}$ and $\mathbf{1}_{\mathbf{C} 5}$ columns are considered (Table S4). The comparison of the sum of the Boltzmann weighted free energies suggest that the coordination mode ea1 would be lower in energy by 0.3 $\mathrm{kcal} / \mathrm{mol}$ in comparison to ea2 coordination mode. Similar approach is used for the Boltzmann weighted free energies of the transition state conformers.

Table S2. Boltzmann Weighted Gibbs Free Energies of Conformers in the ea1 Configuration ${ }^{\mathrm{a}}$

\begin{tabular}{|c|c|c|c|}
\hline conformer identity ${ }^{b}$ & $\begin{array}{c}\text { free energy of } \\
\text { contributing } \\
\text { conformers } G_{i}\end{array}$ & probability $P_{i}$ & $\begin{array}{l}\text { Boltzmann weighted free energy } \\
\qquad P_{i}^{*} G_{i}\end{array}$ \\
\hline $1_{C 1}-\psi-1 a 2 a 3 a 4 a$ & -4126.533023 & 0.045457 & -187.580681 \\
\hline $1_{C 1}-\psi-1 a 2 a 3 a 4 s$ & -4126.533572 & 0.045460 & -187.590206 \\
\hline $1_{C 1}-\psi-1 a 2 a 3 s 4 a$ & -4126.532680 & 0.045456 & -187.574736 \\
\hline $1_{C 1}-\psi-1 a 2 a 3 s 4 s$ & -4126.533247 & 0.045458 & -187.584570 \\
\hline $1_{C 1}-\psi-1 a 2 s 3 a 4 a$ & -4126.533694 & 0.045460 & -187.592326 \\
\hline $1_{C 1}-\psi-1 \mathrm{a} 2 \mathrm{~s} 3 \mathrm{a} 4 \mathrm{~s}$ & -4126.533658 & 0.045460 & -187.591710 \\
\hline $1_{C 1}-\psi-1 a 2 s 3 s 4 a$ & -4126.533400 & 0.045459 & -187.587229 \\
\hline $1_{\mathrm{C} 1}-\psi-1 \mathrm{a} 2 \mathrm{~s} 3 \mathrm{~s} 4 \mathrm{~s}$ & -4126.533350 & 0.045459 & -187.586352 \\
\hline $1_{C 1}-\psi-1$ s2a3a4a & -4126.530687 & 0.045447 & -187.540149 \\
\hline $1_{C 1}-\psi-1 \mathrm{~s} 2 \mathrm{a} 3 \mathrm{a} 4 \mathrm{~s}$ & -4126.531629 & 0.045451 & -187.556491 \\
\hline $1_{C 1}-\psi-1 s 2 a 3 s 4 s$ & -4126.530924 & 0.045448 & -187.544250 \\
\hline $1_{C 1}-\psi-1 s 2 s 3 a 4 a$ & -4126.530709 & 0.045448 & -187.540525 \\
\hline $1_{C 1}-\psi-1 \mathrm{~s} 2 \mathrm{~s} 3 \mathrm{a} 4 \mathrm{~s}$ & -4126.530685 & 0.045447 & -187.540116 \\
\hline $1_{\mathrm{C} 1}-\psi-1 \mathrm{~s} 2 \mathrm{~s} 3 \mathrm{~s} 4 \mathrm{~s}$ & -4126.530595 & 0.045447 & -187.538544 \\
\hline $1_{C 2}-\psi-1 a 2 a 3 a 4 a$ & -4126.532595 & 0.045455 & -187.573254 \\
\hline $1_{\mathrm{C} 2}-\psi-1 \mathrm{a} 2 \mathrm{a} 3 \mathrm{a} 4 \mathrm{~s}$ & -4126.532495 & 0.045455 & -187.571511 \\
\hline $1_{C 2}-\psi-1 a 2 a 3 s 4 a$ & -4126.531595 & 0.045451 & -187.555909 \\
\hline $1_{C 2}-\psi-1 a 2 a 3 s 4 s$ & -4126.532716 & 0.045456 & -187.575350 \\
\hline $1_{C 2}-\psi-1 a 2 s 3 a 4 a$ & -4126.533258 & 0.045458 & -187.584754 \\
\hline $1_{C 2}-\psi-1 a 2 s 3 a 4 s$ & -4126.533472 & 0.045459 & -187.588481 \\
\hline $1_{C 2}-\psi-1 a 2 s 3 s 4 a$ & -4126.531825 & 0.045452 & -187.559888 \\
\hline \multirow[t]{2}{*}{$1_{C 2}-\psi-1 \mathrm{a} 2 \mathrm{~s} 3 \mathrm{~s} 4 \mathrm{~s}$} & -4126.532715 & 0.045456 & -187.575342 \\
\hline & Sum & 1 & -4126.532388 \\
\hline
\end{tabular}

${ }^{a}$ Energies in hartree/particle. ${ }^{b}$ All the conformers with free energies within $2.0 \mathrm{kcal} / \mathrm{mol}$ and below (of the lowest energy conformer $\mathbf{1}_{\mathbf{C 1}-} \psi_{-\mathbf{- 1} \mathbf{2} \mathbf{2 s} 3 \mathbf{3} 4 \mathbf{a}}$ ) are considered. 
Table S3. Boltzmann Weighted Gibbs Free Energies of Conformers in the ea2 Configuration ${ }^{\mathrm{a}}$

\begin{tabular}{|c|c|c|c|}
\hline conformer identity ${ }^{\mathrm{b}}$ & $\begin{array}{c}\text { free energy of } \\
\text { contributing } \\
\text { conformers } G_{i}\end{array}$ & probability $P_{i}$ & $\begin{array}{l}\text { Boltzmann weighted free energy } \\
\qquad P_{i}^{*} G_{i}\end{array}$ \\
\hline $1_{C 4}-\psi-1 a 2 a 3 a 4 a$ & -4126.532507 & 0.035716 & -147.383503 \\
\hline $1_{C 4}-\psi-1 a 2 a 3 a 4 s$ & -4126.532921 & 0.035717 & -147.389159 \\
\hline $1_{C 4}-\psi-1 a 2 a 3 s 4 a$ & -4126.534074 & 0.035721 & -147.404871 \\
\hline $1_{C 4}-\psi-1 \mathrm{a} 2 \mathrm{a} 3 \mathrm{~s} 4 \mathrm{~s}$ & -4126.532458 & 0.035715 & -147.382835 \\
\hline $1_{C 4}-\psi-1 \mathrm{a} 2 \mathrm{~s} 3 \mathrm{a} 4 \mathrm{a}$ & -4126.533135 & 0.035718 & -147.392073 \\
\hline $1_{C 4}-\psi-1 \mathrm{a} 2 \mathrm{~s} 3 \mathrm{a} 4 \mathrm{~s}$ & -4126.532911 & 0.035717 & -147.389013 \\
\hline $1_{C 4}-\psi-1 \mathrm{a} 2 \mathrm{~s} 3 \mathrm{~s} 4 \mathrm{a}$ & -4126.533221 & 0.035718 & -147.393248 \\
\hline $1_{C 4}-\psi-1 a 2 s 3 s 4 s$ & -4126.532650 & 0.035716 & -147.385453 \\
\hline $1_{C 4}-\psi-1$ s2a3a4a & -4126.530652 & 0.035709 & -147.358218 \\
\hline $1_{C 4}-\psi-1 \mathrm{~s} 2 \mathrm{a} 3 \mathrm{a} 4 \mathrm{~s}$ & -4126.531057 & 0.035711 & -147.363740 \\
\hline $1_{C 4}-\psi-1 s 2 a 3 s 4 a$ & -4126.531875 & 0.035713 & -147.374890 \\
\hline $1_{C 4}-\psi-1 s 2 a 3 s 4 s$ & -4126.532155 & 0.035714 & -147.378706 \\
\hline $1_{C 4}-\psi-1 \mathrm{~s} 2 \mathrm{~s} 3 \mathrm{a} 4 \mathrm{a}$ & -4126.531163 & 0.035711 & -147.365176 \\
\hline $1_{C 4}-\psi-1 s 2 s 3 a 4 s$ & -4126.531059 & 0.035711 & -147.363757 \\
\hline $1_{\mathrm{C} 4}-\psi-1 \mathrm{~s} 2 \mathrm{~s} 3 \mathrm{~s} 4 \mathrm{a}$ & -4126.531682 & 0.035713 & -147.372262 \\
\hline $1_{\mathrm{C} 4}-\psi-1 \mathrm{~s} 2 \mathrm{~s} 3 \mathrm{~s} 4 \mathrm{~s}$ & -4126.531106 & 0.035711 & -147.364398 \\
\hline $1_{C 5}-\psi-1 a 2 a 3 a 4 a$ & -4126.532845 & 0.035717 & -147.388114 \\
\hline $1_{C 5}-\psi-1 a 2 a 3 a 4 s$ & -4126.532715 & 0.035716 & -147.386344 \\
\hline $1_{C 5-} \psi-1 a 2 a 3 s 4 a$ & -4126.533195 & 0.035718 & -147.392882 \\
\hline $1_{C 5}-\psi-1 a 2 s 3 a 4 s$ & -4126.533555 & 0.035719 & -147.397793 \\
\hline $1_{C 5}-\psi-1 a 2 s 3 s 4 a$ & -4126.531867 & 0.035713 & -147.374778 \\
\hline $1_{C 5-} \psi-1 \mathrm{~s} 2 a 3 a 4 a$ & -4126.530644 & 0.035709 & -147.358101 \\
\hline $1_{C 5-\psi-1 s 2 a 3 a 4 s}$ & -4126.531022 & 0.035711 & -147.363256 \\
\hline $1_{\mathrm{C} 5}-\psi 1 \mathrm{~s} 2 \mathrm{a} 3 \mathrm{~s} 4 \mathrm{~s}$ & -4126.531478 & 0.035712 & -147.369482 \\
\hline $1_{C 5-\psi-1 s 2 s 3 a 4 a}$ & -4126.530715 & 0.035710 & -147.359077 \\
\hline $1_{C 5}-\psi-1 s 2 s 3 a 4 s$ & -4126.530813 & 0.035710 & -147.360416 \\
\hline $1_{C 5}-\psi-1 \mathrm{~s} 2 \mathrm{~s} 3 \mathrm{~s} 4 \mathrm{a}$ & -4126.530720 & 0.035710 & -147.359139 \\
\hline \multirow{2}{*}{$1_{\mathrm{C} 5}-\psi-1 \mathrm{~s} 2 \mathrm{~s} 3 \mathrm{~s} 4 \mathrm{~s}$} & -4126.530876 & 0.035710 & -147.361269 \\
\hline & Sum & 1 & -4126.531967 \\
\hline
\end{tabular}

${ }^{\mathrm{a}}$ Energies in hartree/particle. ${ }^{\mathrm{b}}$ All the conformers with free energies within $2.0 \mathrm{kcal} / \mathrm{mol}$ and below (of the lowest energy conformer $\mathbf{1}_{\mathbf{C 1}-} \psi_{-\mathbf{- 1} \mathbf{2} 2 \mathbf{s} 3 \mathbf{3} 4 \mathbf{a}}$ ) are considered.

\section{S2.7. Probing the Likely flexibility of the Rh-YanPhos Chelate Ring in 1}

Most of the dihedral angles, shown highlighted in Figure S6, in the YanPhos ligand in 1 are highly restricted due to its chelate binding with the $\mathrm{Rh}$ center. Larger deviations in these dihedral angles would result in the de-coordination of the ligand and hence only a limited conformational space is likely for the Rh-P-N-C-C-C-C-P-(Rh) eight-membered ring as shown in Figure S6. The 
inter-ring rotations along the binapthyl region, within the axially chiral $S$ configuration, are also likely to remain less flexible due to the presence of the 2,2'-bulky substituents (P(bis-(3,5-dit-Buphenyl)(binapthyl)) phosphine and $\mathrm{N}(\mathrm{Bn})$ phosphoramidite). We have explored rotations using $\omega(\mathrm{C} 21-\mathrm{C} 22-\mathrm{C} 23-\mathrm{C} 24)$ as the key dihedral to examine whether additional conformers could be identified. The variations in $\omega$ from its most preferred value of $86^{\circ}$ are considered in the interval of 66 to $126^{\circ}$ for various initial guess geometries. The range of dihedral $\omega$ is chosen such that the chelate binding is either maintained or could be regained during the geometry optimization as shown in Table SY. These results indicate a restricted conformational feature of the Rh-P-N-CC-C-C-P-(Rh) eight-membered chelate ring.

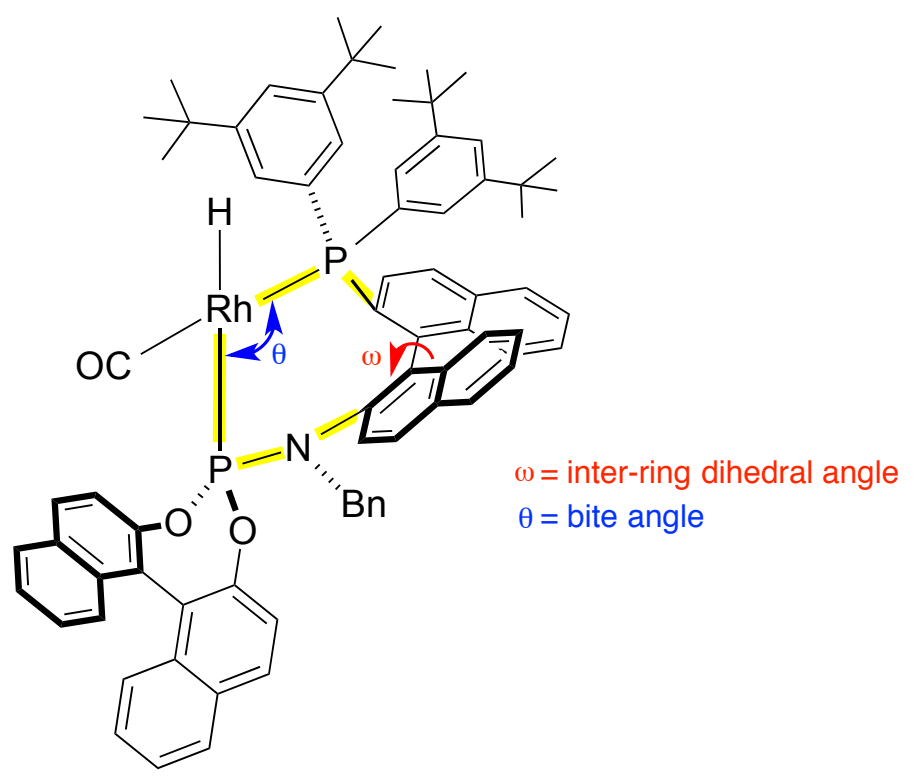

Figure S6. A schematic representation of inter-ring dihedral in the bi-napthyl region of the YanPhos chelate considered for examining the likely conformers arising due to the Rh-P-CC-C-C-N-P-(Rh) eight membered ring in intermediate 1. 
Table S4. A Comparison of the Key Geometric and Energetic Changes During the Geometry Optimization Starting from Various Initial Guess Values of the Dihedral angles $\omega$ in intermediate 1 (as shown in Figure S6)

\begin{tabular}{|c|c|c|c|c|c|c|c|c|c|}
\hline \multicolumn{4}{|c|}{ initial guess } & \multicolumn{4}{|c|}{ at chelation } & \multicolumn{3}{|c|}{ final (fully optimized) } \\
\hline$\omega$ & $\theta$ & $\mathrm{E}_{\mathrm{t}}{ }^{\mathrm{b}}$ & step $\#^{\mathrm{c}}$ & $\omega$ & $\theta$ & $\mathrm{E}_{\mathrm{t}}$ & $\omega$ & $\theta$ & $\mathrm{E}_{\mathrm{t}}$ \\
\hline 66.0 & 77.0 & -4125.192112 & 10 & 85.9 & 92.4 & -4125.459168 & 86.5 & 96.6 & -4125.470744 \\
\hline 76.0 & 88.2 & -4125.428656 & 5 & 84.1 & 94.7 & -4125.468992 & 86.5 & 96.6 & -4125.470744 \\
\hline 81.0 & 93.0 & -4125.461749 & 8 & 86.7 & 96.7 & -4125.470679 & 86.1 & 96.9 & -4125.470745 \\
\hline${ }^{\mathrm{a}} \mathbf{8 6 . 0}$ & $\mathbf{9 6 . 8}$ & $\mathbf{- 4 1 2 5 . 4 2 6 5 7 5}$ & $\mathbf{1}$ & $\mathbf{8 6 . 0}$ & $\mathbf{8 6 . 0}$ & $\mathbf{- 4 1 2 5 . 4 2 6 5 7 5}$ & $\mathbf{8 6 . 0}$ & $\mathbf{9 6 . 8}$ & $\mathbf{- 4 1 2 5 . 4 7 0 7 5 2}$ \\
\hline 91.0 & 99.8 & -4125.464694 & 8 & 84.8 & 96.6 & -4125.470531 & 85.7 & 96.7 & -4125.470735 \\
\hline 96.0 & 102.1 & -4125.447358 & 4 & 89.5 & 96.3 & -4125.469614 & 86.0 & 96.7 & -4125.470752 \\
\hline 106.0 & 104.8 & -4125.339615 & 9 & 87.4 & 96.6 & -4125.469051 & 85.8 & 96.7 & -4125.470638 \\
\hline 116.0 & 105.5 & -4125.024690 & 12 & 96.5 & 100.3 & -4125.446962 & 86.5 & 96.6 & -4125.470744 \\
\hline 126.0 & 104.7 & -4124.393438 & 25 & 91.6 & 96.9 & -4125.453816 & 85.4 & 96.6 & -4125.470725 \\
\hline
\end{tabular}

${ }^{a}$ The most preferred conformer towards which all the initial guess geometries were found to converge to. ${ }^{b} \mathrm{E}_{\mathrm{t}}=$ Electronic energy (in hartree/particle). ${ }^{\mathrm{c}}$ The step during the geometry optimization when the Rh-P chelation was regained as compared to the opened-up initial guess geometry.

\section{S3. Heat Map of the RMSD (Å) of All Atoms of Various Conformers of 1}

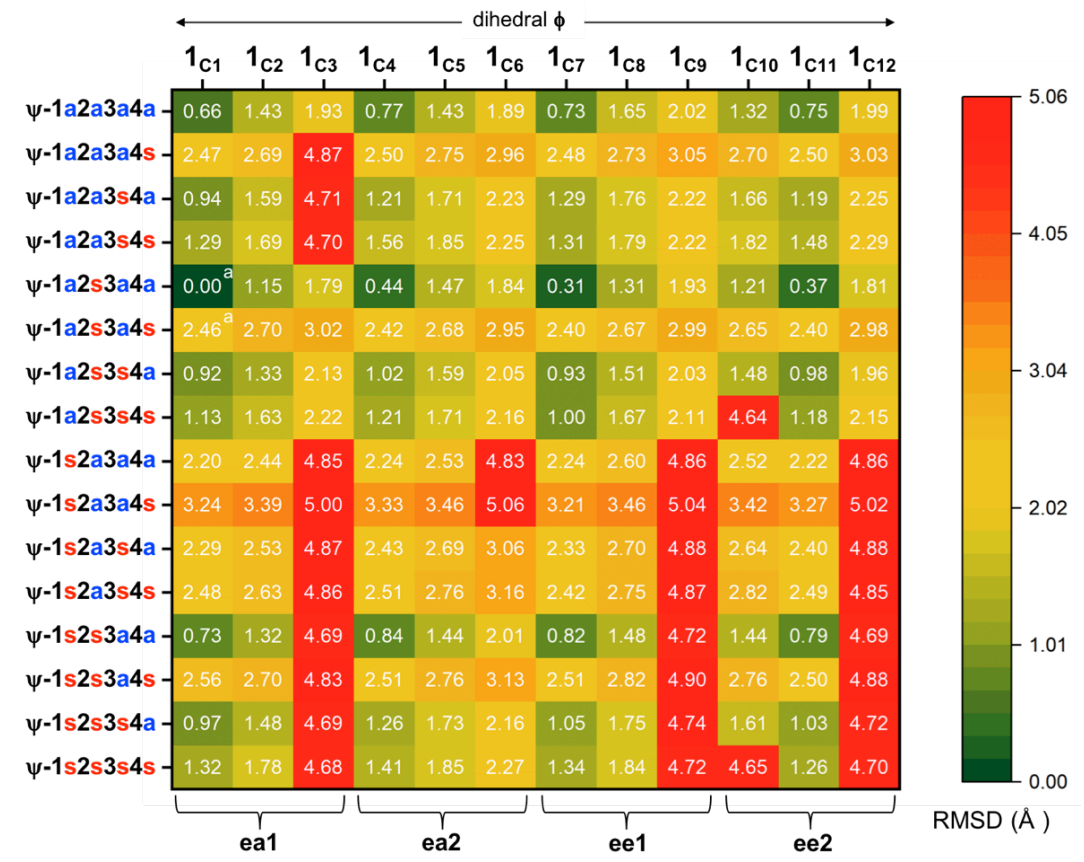

Figure S7. RMSD $(\AA)$ of All Atoms of Various Conformers of 1 calculated with respect to the

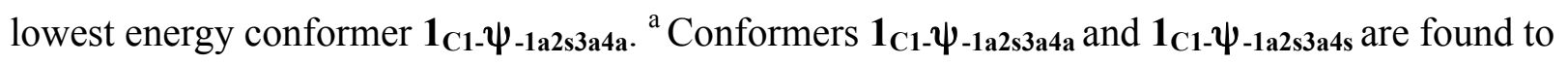
be isoenergetic having an energy difference of only $0.1 \mathrm{~kJ} / \mathrm{mol}$ and distinct in geometry. 


\section{S4. Important NCIs in the ea1 and ea2 coordination modes of complex 1}
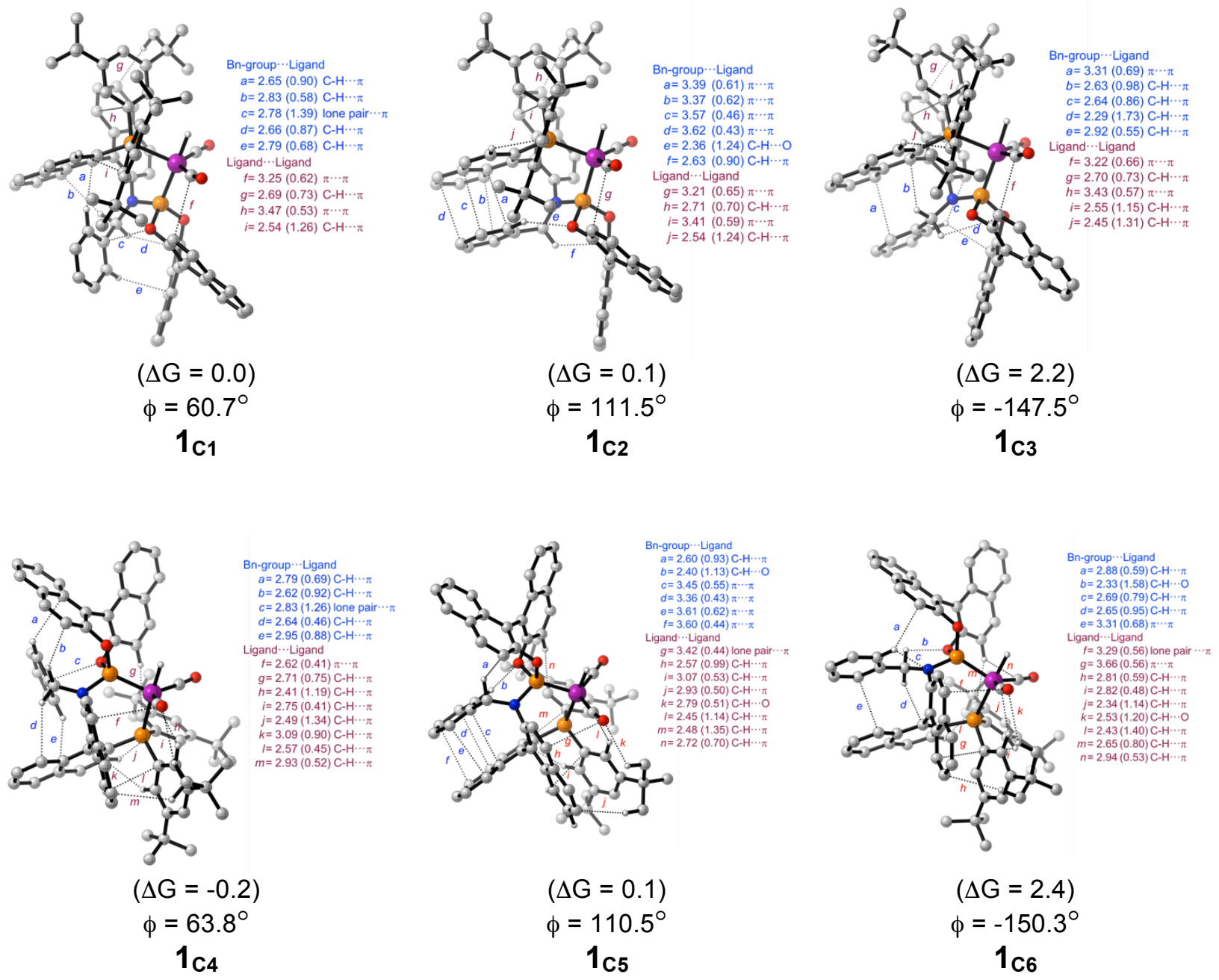

Figure S8. Noncovalent Interactions in the low energy conformers of 1. The interatomic contacts for the NCIs are in $\AA$ and the corresponding electron densities $\left(\rho \times 10^{2}\right.$ au) at the bcps are given in parentheses. Hydrogen atoms are omitted wherever possible, to ensure better clarity. Energies are in $\mathrm{kcal} / \mathrm{mol}$ with respect to $\mathbf{1}_{\mathrm{C} 1-} \boldsymbol{\psi}_{-\mathbf{1 a 2 s 3 a 4 a}}$. 


\section{S5. Conformational space for complex 2}

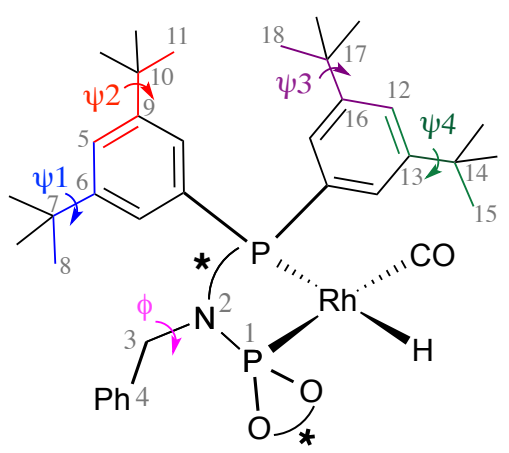

$2 \mathrm{c}$

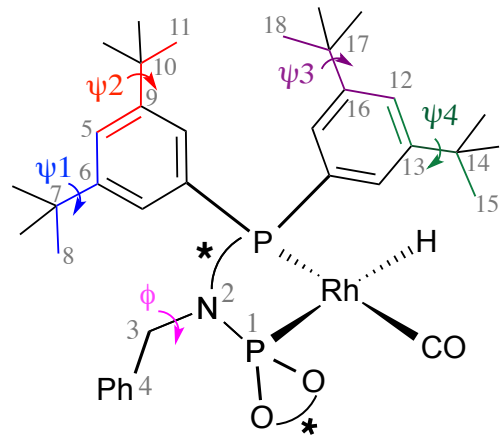

$2_{\mathrm{t}}$

Figure S9. Representation of two possible configurations of complex 2.

For each configuration shown in Figure S9, three important conformers arising due to the rotations around the dihedral $\phi(\mathrm{P} 1-\mathrm{N} 2-\mathrm{C} 3-\mathrm{C} 4)$ are identified. In the cis $\mathbf{2}_{\mathbf{c}}$ configuration (P of phosphoramidite and the hydride), the conformers $\mathbf{2}_{\mathrm{C} 1-\mathrm{c}}, \mathbf{2}_{\mathrm{C} 2-\mathrm{c}}$ and $\mathbf{2}_{\mathrm{C} 3-\mathrm{c}}$ are optimized with the $\phi$ values of around 60,111 and $-147^{\circ}$ respectively. Similarly, in the trans $\mathbf{2}_{\mathbf{t}}$ the conformers $\mathbf{2}_{\mathrm{C1}-\mathrm{t}}$, $\mathbf{2}_{\mathrm{C2}-\mathrm{t}}$ and $\mathbf{2}_{\mathrm{C} 3-\mathrm{t}}$ are identified. The other conformers due to the tert-butyl groups are considered using $\psi 1, \psi 2, \psi 3$, and $\psi \mathbf{4}$ dihedrals. The dihedral $\psi 1$ is considered as $\psi \mathbf{1 a}$ as was learnt from the rigorous conformational sampling of intermediate $\mathbf{1}$, where it was the most favorable tertbutyl orientation. Thus, the conformers with $\psi \mathbf{1}$ s dihedral have been excluded for $\mathbf{2}_{\mathrm{C2}-\mathrm{c}}, \mathbf{2}_{\mathrm{C2}-\mathrm{t}}$, $\mathbf{2}_{\mathrm{C} 3-\mathrm{c}}$, and $\mathbf{2}_{\mathrm{C} 2-\mathrm{t}}$ conformer groups while it has been explicitly considered for $\mathbf{2}_{\mathrm{C} 1-\mathrm{c}}$ and $\mathbf{2}_{\mathrm{C} 1-\mathrm{t}}$ as shown in the heat map. The results of this conformational search are shown in Figure S10. 


\section{S5.1. Heat Map of Gibbs Free Energies of Various Conformers of Complex 2}
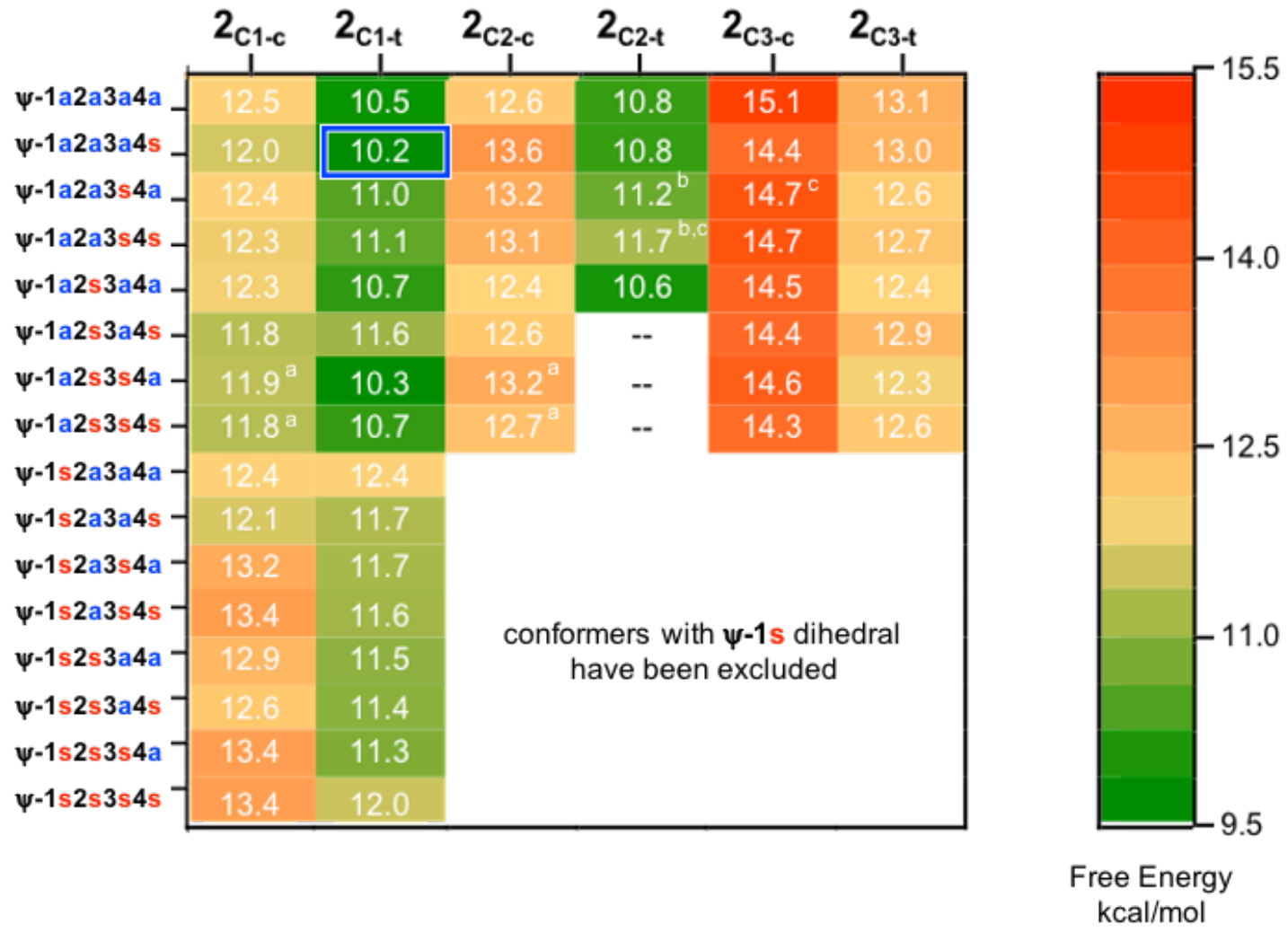

Figure S10. Relative Gibbs free energies $(\mathrm{kcal} / \mathrm{mol})$ of various conformers of complex 2 with respect to the lowest energy conformer $\mathbf{1}_{\mathbf{C 1} 1-\psi_{-12} \mathbf{2 s 3 a 4 a} .}{ }^{a} \psi_{3 \mathbf{s}}$ dihedral converged to $\psi_{\mathbf{3 a}}$ during the geometry optimization. ${ }^{\mathrm{b}}$ A low amplitude imaginary frequency could not be eliminated. ${ }^{\mathrm{c}}$ Could be optimized only using the ultrafine integration grid. All the Gibbs free energies are reported with the default value of the integration grid. The geometries pertaining to the four empty cells, shown using dashed lines, could not be optimized even after repeated attempts. It shall be noted that for sake of brevity a simpler notation of $\mathbf{2}$ is used in the main manuscript, which refers to the lowest energy conformer $\mathbf{2}_{\mathbf{C 1} \mathbf{t}_{\mathbf{t}}-\boldsymbol{\psi}_{-\mathbf{1 a 2}} \mathbf{a} \mathbf{3} \mathbf{4} \mathbf{s}}$ shown here using an additional blue border (row 2, column 2) in the heat map. 


\section{S5.2. Optimized Geometries and Mapping of NCIs in complex $\boldsymbol{2}_{t}$}

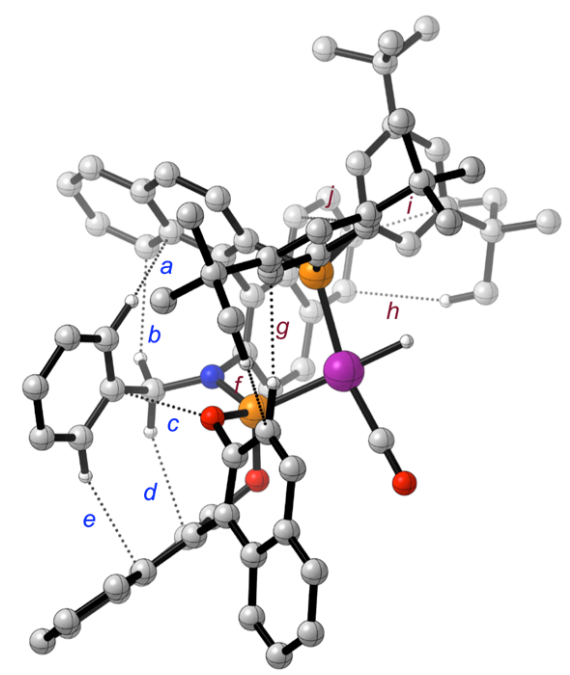

Bn-group $\cdots$ Ligand

$a=2.87(0.67) \mathrm{C}-\mathrm{H} \cdots \pi$

$b=3.12(0.59) \mathrm{C}-\mathrm{H} \cdots \pi$

$c=2.89(1.14)$ lone pair $\cdots \pi$

$d=2.68(0.80) \mathrm{C}-\mathrm{H} \cdots \pi$

$e=2.81(0.69) \mathrm{C}-\mathrm{H} \cdots \pi$

Ligand $\cdots$ Ligand

$f=2.97(0.47) \mathrm{C}-\mathrm{H} \cdots \pi$

$g=2.57(0.90) \mathrm{C}-\mathrm{H} \cdots \pi$

$h=3.00(0.43) \mathrm{C}-\mathrm{H} \cdots \pi$

$i=2.75(0.66) \mathrm{C}-\mathrm{H} \cdots \pi$

$j=3.50(0.51) \pi \cdots \pi$

Figure S11. Geometry and mapping of noncovalent interactions in the low energy conformer

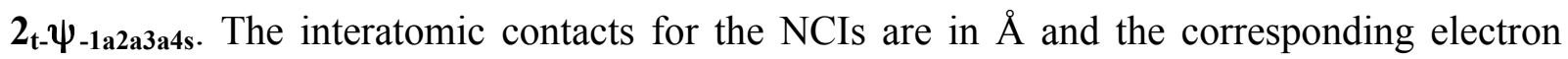
densities $\left(\rho \times 10^{2} \mathrm{au}\right)$ at the bcps are given in parentheses. Hydrogen atoms are omitted wherever possible, to ensure better clarity. 


\section{S6. Conformational Space for Complex 3}

\section{S6.1. Coordination of $\alpha$-Methylstyrene to the Active Catalyst 2 through Sites I and II}
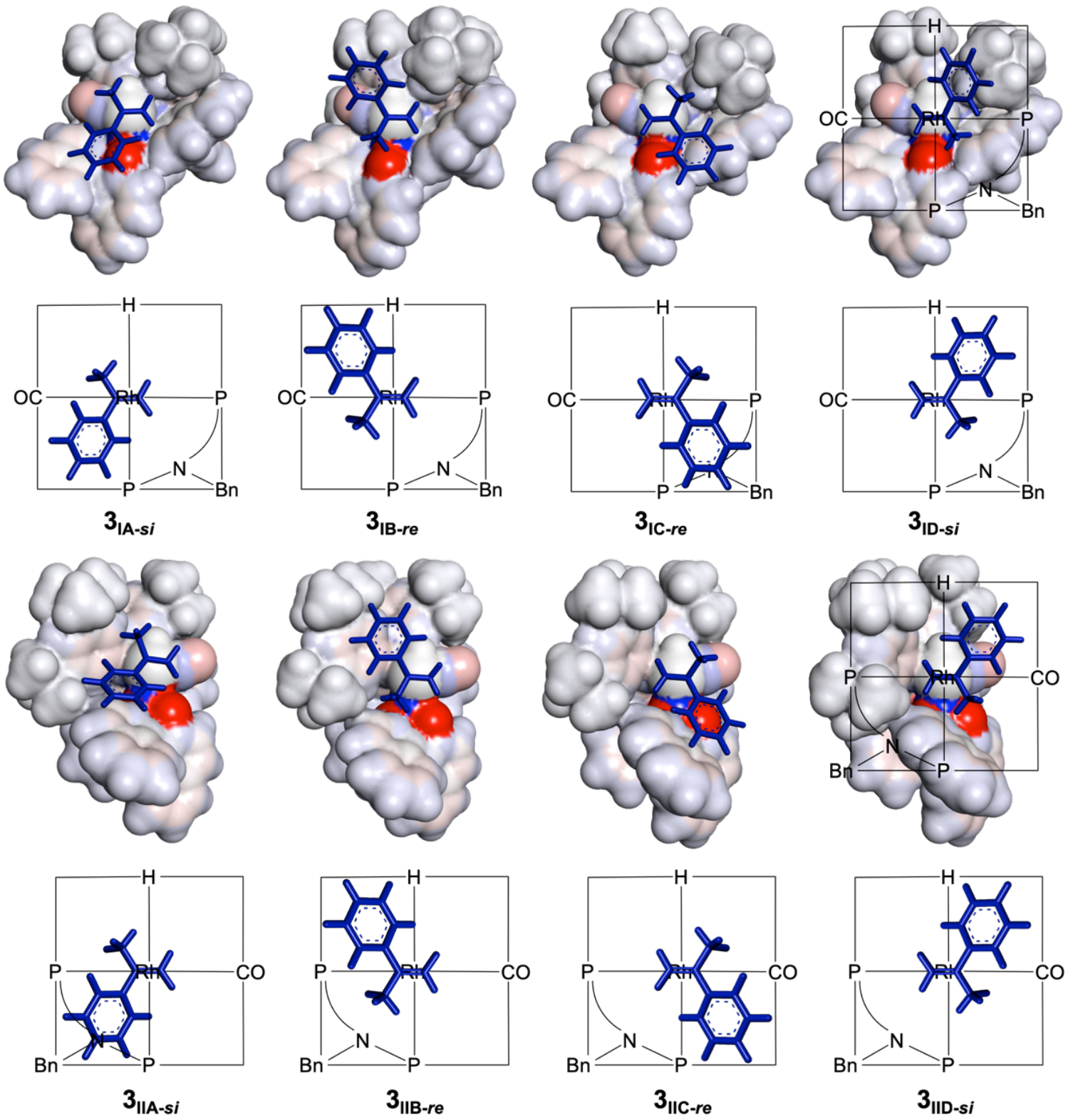

Figure S12. Space-filling representation of the catalyst backbone and stick representation of $\alpha$-methylstyrene depicting the eight possible coordination modes to the active catalyst $\mathbf{2}$ through sites I and II. 
S6.2 Heat Map of Gibbs Free Energies for Various Conformers of 3 in the ea1

\section{Coordination of the Yanphos Ligand}

\begin{tabular}{|c|c|c|c|c|c|c|c|c|c|}
\hline & $3_{\text {IA-si }}$ & $3_{\text {IB-re }}$ & $3_{\text {IC-re }}$ & $3_{\text {ID-si }}$ & $3_{\| A-s i}$ & $3_{\text {IIB-re }}$ & $3_{\text {IIIC-re }}$ & $3_{\text {IID-si }}$ & \\
\hline$\psi-1 a 2 a 3 a 4 a-$ & 15.3 & 19.2 & 25.7 & 24.9 & 22.3 & 21.5 & 12.8 & 15.2 & -30.0 \\
\hline$\psi-1 \mathrm{a} 2 \mathrm{a} 3 \mathrm{a} 4 \mathrm{~s}$ & 14.7 & 21.4 & 26.0 & 24.7 & 22.0 & 22.4 & 12.6 & 14.7 & \\
\hline$\psi-1 \mathrm{a} 2 \mathrm{a} 3 \mathrm{~s} 4 \mathrm{a}$ & 14.3 & 19.7 & 25.4 & 24.8 & 25.0 & 20.8 & 12.2 & 14.8 & \\
\hline$\psi-1 \mathrm{a} 2 \mathrm{a} 3 \mathrm{~s} 4 \mathrm{~s}$ & 14.7 & 19.7 & 25.5 & 24.9 & 24.4 & 20.6 & 12.9 & 15.0 & -26.4 \\
\hline$\psi-1 \mathrm{a} 2 \mathrm{~s} 3 \mathrm{a} 4 \mathrm{a}$ & 14.4 & 18.7 & 25.8 & 24.6 & 22.0 & 19.4 & 11.9 & 14.3 & \\
\hline$\psi-1 a 2 s 3 a 4 s$ & 14.5 & 18.6 & 25.5 & 24.5 & 21.4 & 19.4 & 11.9 & 14.0 & \\
\hline$\psi-1 a 2 s 3 s 4 a-$ & 14.1 & 20.3 & 24.9 & 24.7 & 22.6 & 21.2 & 12.0 & 14.2 & -22.7 \\
\hline$\psi-1 \mathrm{a} 2 \mathrm{~s} 3 \mathrm{~s} 4 \mathrm{~s}$ & 14.4 & 21.0 & 25.0 & 24.4 & 22.8 & 19.4 & 12.2 & 14.3 & \\
\hline$\psi-1$ sa $a \mathrm{a} 4 \mathrm{a}-$ & 15.3 & 20.1 & 30.0 & 25.5 & 23.6 & 18.7 & 14.0 & 15.1 & \\
\hline$\psi-1 \mathrm{~s} 2 \mathrm{a} 3 \mathrm{a} 4 \mathrm{~s}$ & 15.3 & 20.4 & 29.7 & 25.1 & 23.3 & 18.2 & 13.5 & 15.3 & -19.1 \\
\hline$\psi-1 s 2 a 3 s 4 a-$ & 15.7 & 19.9 & 25.9 & 26.3 & 23.4 & 18.2 & 14.1 & 16.7 & \\
\hline$\psi-1 \mathrm{~s} 2 \mathrm{a} 3 \mathrm{~s} 4 \mathrm{~s}$ & 15.5 & 20.7 & 25.4 & 25.8 & 23.5 & 18.6 & 13.6 & 17.0 & \\
\hline$\psi-1 s 2 s 3 a 4 a$ & 15.7 & 20.5 & 26.3 & 26.3 & 23.5 & 18.9 & 14.0 & 14.9 & 15.5 \\
\hline$\psi-1 s 2 s 3 a 4 s$ & 16.4 & 21.1 & 29.6 & 25.4 & 23.6 & 20.0 & 14.1 & 15.2 & \\
\hline$\psi-1 \mathrm{~s} 2 \mathrm{~s} 3 \mathrm{~s} 4 \mathrm{a}$ & 17.6 & 20.6 & 25.9 & 25.9 & 24.0 & 18.9 & 14.0 & 15.3 & \\
\hline$\psi-1 \mathrm{~s} 2 \mathrm{~s} 3 \mathrm{~s} 4 \mathrm{~s}$ & 15.7 & 20.9 & 25.8 & 25.6 & 24.0 & 19.5 & 14.0 & 15.8 & , \\
\hline
\end{tabular}

Figure S13. Gibbs free energies $(\mathrm{kcal} / \mathrm{mol})$ of 128 conformers of the catalyst-substrate complex 3 with respect to the lowest energy pre-catalytic species $\mathbf{1}_{\mathbf{C 1}-} \boldsymbol{\psi}_{-\mathbf{1} \text { a2s3a4a. }}$ 
S7. Conformational Aspects of the Migratory Insertion Transition State (TS1)

a.

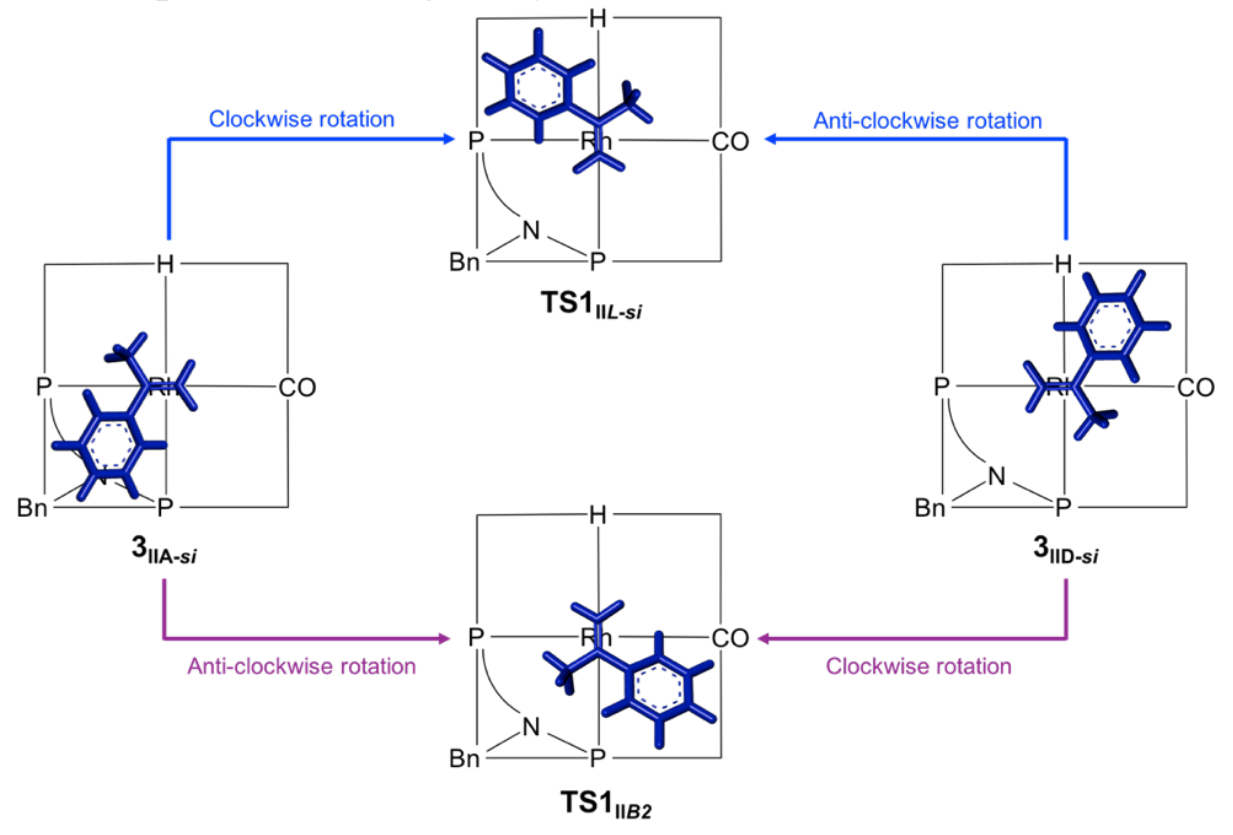

b.

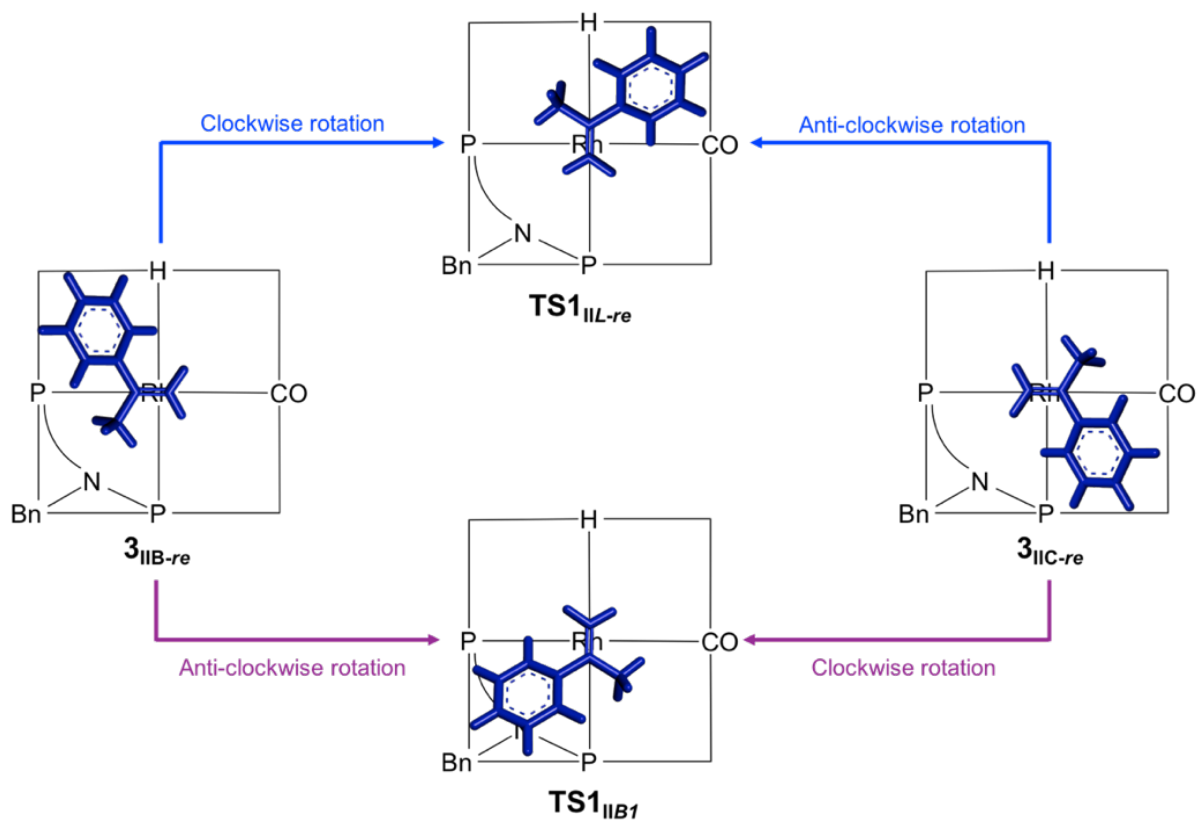

Figure S14. Schematic representation of the migratory insertion of $\alpha$-methylstyrene into the $\mathrm{Rh}-\mathrm{H}$ bond in catalyst-substrate $\pi$-complexes such as $\mathbf{3}_{\text {IIA-si }}, \mathbf{3}_{\text {IID-si } i}$ and $\mathbf{b} . \mathbf{3}_{\text {IIA-re }}, \mathbf{3}_{\text {IID-re }}$ to illustrate how a given catalyst-substrate complex is connected to the Rh-alkyl intermediate (4) of the linear or the branched pathway. 

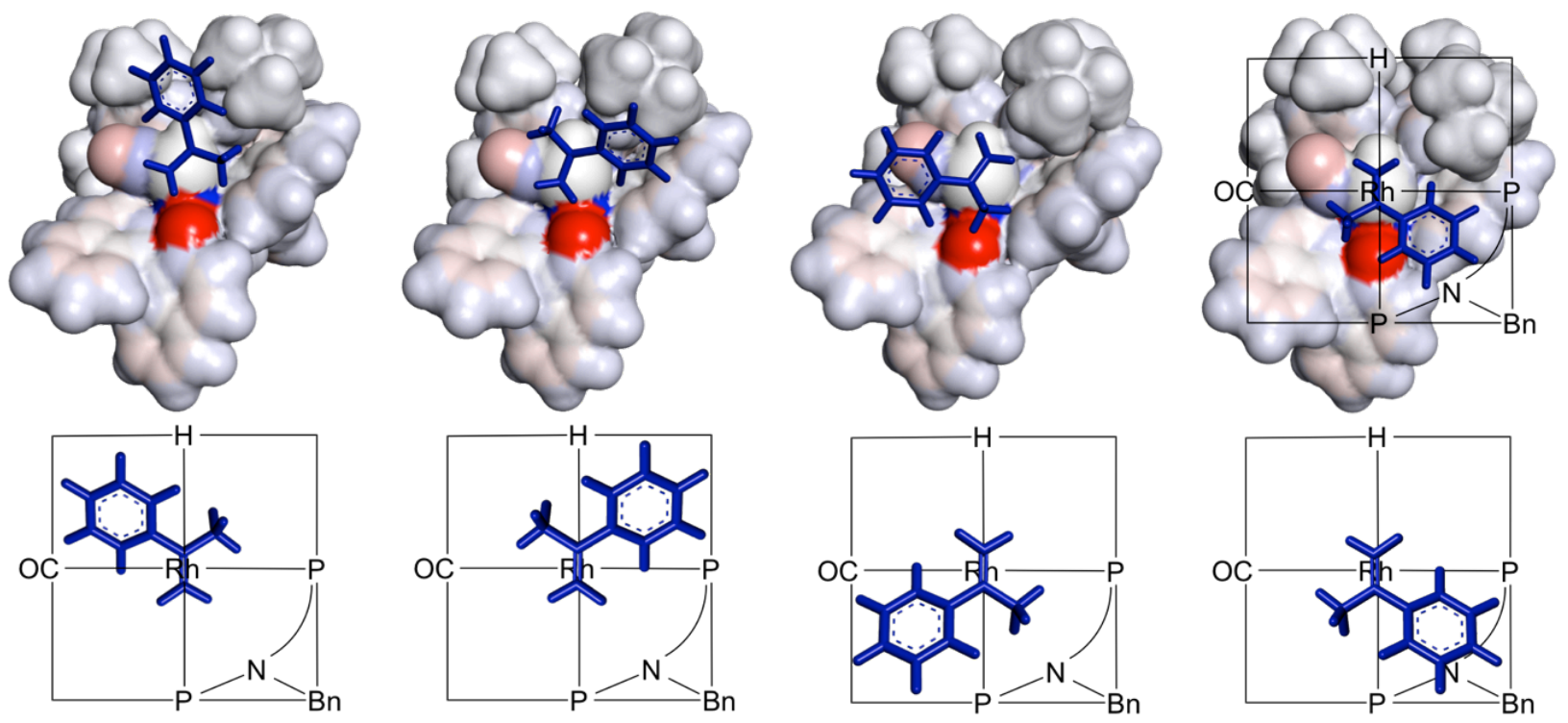

TS1 $1_{\text {IL-si }}$
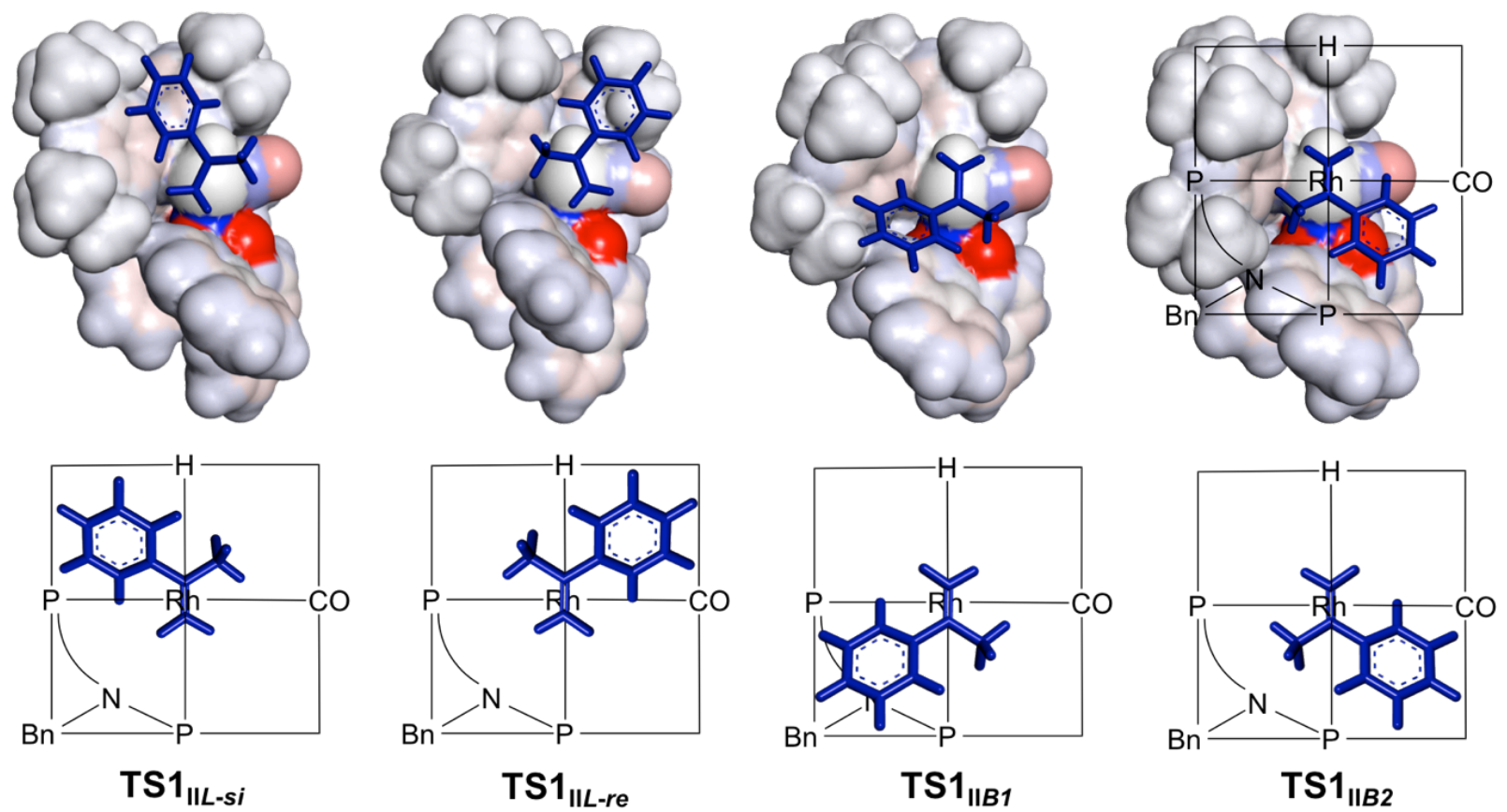

Figure S15. Space-filling representation of the catalyst backbone and stick representation of $\alpha$ methylstyrene depicting eight possible migratory insertion transition states (TS1) at binding sites I and II. 
Table S5. Gibbs Free Energies (in kcal/mol) of Various Conformers of Complex TS1 in the ea1 Coordination Mode ${ }^{\mathrm{a}}$

\begin{tabular}{|c|c|c|c|c|c|c|c|c|}
\hline & \multicolumn{4}{|c|}{ Site I } & \multicolumn{4}{|c|}{ Site II } \\
\hline & TS1 $_{I L-s i}$ & TS1 $1_{\text {IL-re }}$ & $\mathrm{TS1}_{\mathrm{IBI}}$ & $\mathrm{TS1}_{I B 2}$ & TS1 $1_{I L-s i}$ & TS1 $1_{\text {IIL-re }}$ & TS1 $1_{I I B 1}$ & TS1 $_{I I B 2}$ \\
\hline$\psi-1 \mathbf{a} 2 \mathrm{a} 3 \mathrm{a} 4 \mathrm{a}$ & 35.6 & 35.0 & 32.7 & 36.9 & 27.7 & 28.5 & 31.6 & 29.7 \\
\hline$\psi-1 \mathrm{a} 2 \mathrm{a} 3 \mathrm{a} 4 \mathrm{~s}$ & 35.3 & 34.9 & 32.7 & 35.8 & 28.0 & 28.3 & 31.3 & 29.0 \\
\hline$\psi-1 \mathbf{a} 2 \mathrm{a} 3 \mathrm{~s} 4 \mathrm{a}$ & 35.5 & 33.7 & 33.0 & 35.5 & 27.6 & 27.8 & 31.7 & 30.7 \\
\hline$\psi-1 \mathrm{a} 2 \mathrm{a} 3 \mathrm{~s} 4 \mathrm{~s}$ & 35.1 & 33.9 & 32.4 & 35.6 & 27.7 & 27.6 & 31.6 & 29.6 \\
\hline$\psi-1 \mathrm{a} 2 \mathrm{~s} 3 \mathrm{a} 4 \mathrm{a}$ & 33.9 & 34.9 & 33.0 & 35.6 & $27.7^{\mathrm{b}}$ & 28.0 & 31.8 & 32.6 \\
\hline$\psi-1 \mathrm{a} 2 \mathrm{~s} 3 \mathrm{a} 4 \mathrm{~s}$ & 34.3 & 35.0 & 33.2 & 35.7 & 30.9 & 28.0 & 31.8 & 29.5 \\
\hline$\psi-1 \mathrm{a} 2 \mathrm{~s} 3 \mathrm{~s} 4 \mathrm{a}$ & 34.9 & 32.7 & 32.3 & 35.7 & $27.6^{\mathrm{b}}$ & 26.9 & 31.9 & 29.3 \\
\hline$\psi-1 \mathrm{a} 2 \mathrm{~s} 3 \mathrm{~s} 4 \mathrm{~s}$ & 35.0 & 33.5 & 33.4 & 35.8 & 30.9 & 27.1 & 31.9 & 29.2 \\
\hline$\psi-1 \mathrm{~s} 2 \mathrm{a} 3 \mathrm{a} 4 \mathrm{a}$ & 36.3 & 35.7 & 33.7 & 36.5 & 27.7 & 29.7 & $33.3^{\mathrm{c}}$ & 31.1 \\
\hline$\psi-1 \mathrm{~s} 2 \mathrm{a} 3 \mathrm{a} 4 \mathrm{~s}$ & 36.6 & 35.3 & 33.8 & 36.0 & 25.4 & 29.7 & 33.3 & 30.8 \\
\hline$\psi-1 \mathrm{~s} 2 \mathrm{a} 3 \mathrm{~s} 4 \mathrm{a}$ & 37.3 & 34.5 & 34.9 & 36.3 & 25.4 & 29.4 & 33.1 & 30.4 \\
\hline$\psi-1 \mathrm{~s} 2 \mathrm{a} 3 \mathrm{~s} 4 \mathrm{~s}$ & 37.1 & 34.4 & 34.2 & 35.8 & 25.4 & 29.6 & 33.4 & 30.6 \\
\hline$\psi-1 \mathrm{~s} 2 \mathrm{~s} 3 \mathrm{a} 4 \mathrm{a}$ & 33.2 & 35.4 & 33.7 & 36.7 & $27.7^{\mathrm{b}}$ & 29.4 & 33.3 & 30.4 \\
\hline$\psi-1 \mathrm{~s} 2 \mathrm{~s} 3 \mathrm{a} 4 \mathrm{~s}$ & 33.4 & 35.4 & 34.2 & 36.6 & 29.7 & 28.9 & 34.1 & 30.6 \\
\hline$\psi-1 \mathrm{~s} 2 \mathrm{~s} 3 \mathrm{~s} 4 \mathrm{a}$ & 37.2 & 34.4 & 34.2 & 36.0 & $25.4^{\mathrm{b}}$ & 29.3 & 33.5 & 30.2 \\
\hline$\psi-1 \mathrm{~s} 2 \mathrm{~s} 3 \mathrm{~s} 4 \mathrm{~s}$ & 37.6 & 34.6 & 34.1 & 36.2 & 28.3 & 29.3 & 33.7 & 30.6 \\
\hline
\end{tabular}

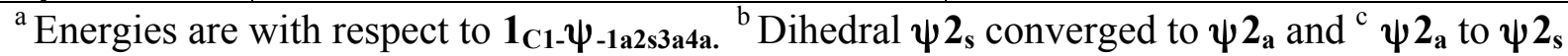
during geometry optimization.

Table S6. Boltzmann Weighted Gibbs Free Energies ${ }^{a}$ of Various Conformers of the Enantiocontrolling Migratory Insertion Transition State

\begin{tabular}{|c|c|c|c|}
\hline conformer identity & $\begin{array}{c}\text { free energy of } \\
\text { contributing } \\
\text { conformer } G i\end{array}$ & probability $P i$ & $\begin{array}{c}\text { Boltzmann weighted } \\
\text { free energy } P i^{*} G i\end{array}$ \\
\hline${\text { (i) } \text { TS1 }_{\text {IL-si }}}$ & & & \\
\hline$\psi$-1a2a3a4a & -4361.979955 & 0.062500 & -272.623752 \\
\hline$\psi$-1a2a3a4s & -4361.979449 & 0.062497 & -272.610981 \\
\hline$\psi$-1a2a3s4a & -4361.980161 & 0.062501 & -272.628952 \\
\hline$\psi$-1a2a3s4s & -4361.979874 & 0.062499 & -272.621709 \\
\hline$\psi$-1a2s3a4a & -4361.979955 & 0.062500 & -272.623752 \\
\hline$\psi$-1a2s3a4s & -4361.974862 & 0.062470 & -272.495349 \\
\hline$\psi$-1a2s3s4a & -4361.980161 & 0.062501 & -272.628952 \\
\hline$\psi-1 \mathbf{1 a 2 s 3 s 4 s}$ & -4361.974910 & 0.062470 & -272.496553 \\
\hline
\end{tabular}




\begin{tabular}{|c|c|c|c|}
\hline Table S6 Contd... & & & \\
\hline$\psi-1 \mathrm{~s} 2 \mathrm{a} 3 \mathrm{a} 4 \mathrm{a}$ & -4361.979931 & 0.062499 & -272.623157 \\
\hline$\psi$-1s2a3a4s & -4361.983529 & 0.062520 & -272.713896 \\
\hline$\psi-1 \mathrm{~s} 2 \mathrm{a} 3 \mathrm{~s} 4 \mathrm{a}$ & -4361.983606 & 0.062521 & -272.715835 \\
\hline$\psi-1 \mathrm{~s} 2 \mathrm{a} 3 \mathrm{~s} 4 \mathrm{~s}$ & -4361.983630 & 0.062521 & -272.716452 \\
\hline$\psi-1 \mathrm{~s} 2 \mathrm{~s} 3 \mathrm{a} 4 \mathrm{a}$ & -4361.979931 & 0.062499 & -272.623157 \\
\hline$\psi-1 \mathrm{~s} 2 \mathrm{~s} 3 \mathrm{a} 4 \mathrm{~s}$ & -4361.976722 & 0.062481 & -272.542235 \\
\hline$\psi-1 \mathrm{~s} 2 \mathrm{~s} 3 \mathrm{~s} 4 \mathrm{a}$ & -4361.983606 & 0.062521 & -272.715835 \\
\hline \multirow{2}{*}{$\psi-1 \mathrm{~s} 2 \mathrm{~s} 3 \mathrm{~s} 4 \mathrm{~s}$} & -4361.978989 & 0.062494 & -272.599380 \\
\hline & Sum & 1 & -4361.979955 \\
\hline \multicolumn{4}{|l|}{ (ii) $\mathrm{TS1} 1_{\text {IIL-re }}$} \\
\hline$\psi-1 \mathrm{a} 2 \mathrm{a} 3 \mathrm{a} 4 \mathrm{a}$ & -4361.978715 & 0.062501 & -272.629376 \\
\hline$\psi-1 \mathrm{a} 2 \mathrm{a} 3 \mathrm{a} 4 \mathrm{~s}$ & -4361.978911 & 0.062502 & -272.634328 \\
\hline$\psi-1 a 2 a 3 s 4 a$ & -4361.979715 & 0.062507 & -272.654584 \\
\hline$\psi-1 \mathrm{a} 2 \mathrm{a} 3 \mathrm{~s} 4 \mathrm{~s}$ & -4361.980095 & 0.062509 & -272.664188 \\
\hline$\psi-1 \mathrm{a} 2 \mathrm{~s} 3 \mathrm{a} 4 \mathrm{a}$ & -4361.979391 & 0.062505 & -272.646425 \\
\hline$\psi-1 \mathrm{a} 2 \mathrm{~s} 3 \mathrm{a} 4 \mathrm{~s}$ & -4361.979442 & 0.062505 & -272.647717 \\
\hline$\psi-1 \mathrm{a} 2 \mathrm{~s} 3 \mathrm{~s} 4 \mathrm{a}$ & -4361.981138 & 0.062515 & -272.690483 \\
\hline$\psi-1 \mathrm{a} 2 \mathrm{~s} 3 \mathrm{~s} 4 \mathrm{~s}$ & -4361.980904 & 0.062513 & -272.684597 \\
\hline$\psi$-1s2a3a4a & -4361.976707 & 0.062489 & -272.578746 \\
\hline$\psi$-1s2a3a4s & -4361.976796 & 0.062490 & -272.580979 \\
\hline$\psi-1 \mathrm{~s} 2 \mathrm{a} 3 \mathrm{~s} 4 \mathrm{a}$ & -4361.977180 & 0.062492 & -272.590660 \\
\hline$\psi-1 \mathrm{~s} 2 \mathrm{a} 3 \mathrm{~s} 4 \mathrm{~s}$ & -4361.976841 & 0.062490 & -272.582129 \\
\hline$\psi-1 \mathrm{~s} 2 \mathrm{~s} 3 \mathrm{a} 4 \mathrm{a}$ & -4361.977204 & 0.062492 & -272.591276 \\
\hline$\psi-1 \mathrm{~s} 2 \mathrm{~s} 3 \mathrm{a} 4 \mathrm{~s}$ & -4361.978066 & 0.062497 & -272.613016 \\
\hline$\psi-1 s 2 s 3 s 4 a$ & -4361.977376 & 0.062493 & -272.595607 \\
\hline \multirow[t]{2}{*}{$\psi-1 \mathrm{~s} 2 \mathrm{~s} 3 \mathrm{~s} 4 \mathrm{~s}$} & -4361.977327 & 0.062493 & -272.594372 \\
\hline & Sum & 1 & -4361.978488 \\
\hline \multicolumn{4}{|l|}{ (iii) $\mathrm{TS1} 1_{\mathrm{IIB2}}$} \\
\hline$\psi-1 a 2 a 3 a 4 a$ & -4361.976787 & 0.062505 & -272.647799 \\
\hline$\psi-1 \mathrm{a} 2 \mathrm{a} 3 \mathrm{a} 4 \mathrm{~s}$ & -4361.977817 & 0.062511 & -272.673793 \\
\hline$\psi-1 \mathrm{a} 2 \mathrm{a} 3 \mathrm{~s} 4 \mathrm{a}$ & -4361.975112 & 0.062495 & -272.605549 \\
\hline$\psi-1 \mathrm{a} 2 \mathrm{a} 3 \mathrm{~s} 4 \mathrm{~s}$ & -4361.976899 & 0.062506 & -272.650626 \\
\hline$\psi-1 a 2 s 3 a 4 a$ & -4361.972136 & 0.062478 & -272.530518 \\
\hline$\psi-1 \mathrm{a} 2 \mathrm{~s} 3 \mathrm{a} 4 \mathrm{~s}$ & -4361.977025 & 0.062506 & -272.653802 \\
\hline$\psi-1 \mathrm{a} 2 \mathrm{~s} 3 \mathrm{~s} 4 \mathrm{a}$ & -4361.977301 & 0.062508 & -272.660771 \\
\hline$\psi-1 \mathrm{a} 2 \mathrm{~s} 3 \mathrm{~s} 4 \mathrm{~s}$ & -4361.977467 & 0.062509 & -272.664960 \\
\hline$\psi-1 \mathrm{~s} 2 \mathrm{a} 3 \mathrm{a} 4 \mathrm{a}$ & -4361.974589 & 0.062492 & -272.592362 \\
\hline$\psi-1 \mathrm{~s} 2 \mathrm{a} 3 \mathrm{a} 4 \mathrm{~s}$ & -4361.974928 & 0.062494 & -272.600906 \\
\hline
\end{tabular}




\begin{tabular}{|c|c|c|c|}
\hline Table S6 Contd... & & & \\
\hline$\psi$-1s2a3s4a & -4361.975552 & 0.062498 & -272.616649 \\
\hline$\psi$-1s2a3s4s & -4361.975319 & 0.062497 & -272.610785 \\
\hline$\psi$-1s2s3a4a & -4361.975696 & 0.062499 & -272.620285 \\
\hline$\psi$-1s2s3a4s & -4361.975287 & 0.062496 & -272.609971 \\
\hline$\psi$-1s2s3s4a & -4361.976004 & 0.062501 & -272.628052 \\
\hline$\psi$-1s2s3s4s & -4361.975248 & 0.062496 & -272.608986 \\
\hline & Sum & 1 & -4361.975823 \\
\hline
\end{tabular}

${ }^{\mathrm{a}}$ Energies in hartree/particle.

Table S7. Boltzmann Weighted Gibbs Free Energies ${ }^{\mathrm{a}}$ of Lower Conformers of the Enantiocontrolling Migratory Insertion Transition State that are within $2 \mathrm{kcal} / \mathrm{mol}$ of the Lowest Energy Conformer $\left(\mathbf{T S 1}_{\mathrm{II} L \text {-si- }} \psi_{-1 \mathrm{~s} 2 \mathrm{a} 3 \mathrm{a} 4 \mathrm{~s}}\right)^{\mathrm{b}}$

\begin{tabular}{|c|c|c|c|}
\hline conformer identity & $\begin{array}{c}\text { free energy of } \\
\text { contributing } \\
\text { conformers } G i\end{array}$ & probability $P i$ & $\begin{array}{c}\text { Boltzmann weighted } \\
\text { free energy } P i^{*} G i\end{array}$ \\
\hline \multicolumn{4}{|l|}{ (i) $\mathrm{TS1}_{\mathrm{IIL} L \mathrm{si}}$} \\
\hline$\psi-1 \mathrm{~s} 2 \mathrm{a} 3 \mathrm{a} 4 \mathrm{~s}$ & -4361.983529 & 0.249998 & -1090.489465 \\
\hline$\psi-1 \mathrm{~s} 2 \mathrm{a} 3 \mathrm{~s} 4 \mathrm{a}$ & -4361.983606 & 0.250000 & -1090.497218 \\
\hline$\psi-1 \mathrm{~s} 2 \mathrm{a} 3 \mathrm{~s} 4 \mathrm{~s}$ & -4361.983630 & 0.250000 & -1090.499689 \\
\hline \multirow[t]{2}{*}{$\psi-1 \mathrm{~s} 2 \mathrm{~s} 3 \mathrm{~s} 4 \mathrm{a}$} & -4361.983606 & 0.250000 & -1090.497218 \\
\hline & Sum & 1 & -4361.983593 \\
\hline \multicolumn{4}{|l|}{ (ii) $\mathrm{TS1} 1_{\text {IL-re }}$} \\
\hline$\psi-1 \mathrm{a} 2 \mathrm{~s} 3 \mathrm{~s} 4 \mathrm{a}$ & -4361.981138 & 0.500005 & -2181.014049 \\
\hline \multirow[t]{2}{*}{$\psi-1 \mathrm{a} 2 \mathrm{~s} 3 \mathrm{~s} 4 \mathrm{~s}$} & -4361.980904 & 0.499994 & -2180.966972 \\
\hline & Sum & 1 & -4361.981021 \\
\hline
\end{tabular}

${ }^{\mathrm{a}}$ Energies in hartree/particle.

${ }^{b}$ All other conformers for $\mathbf{T S 1}_{\mathbf{I I} \boldsymbol{B} 2}$ are found to be more than $2.0 \mathrm{kcal} / \mathrm{mol}$ higher in energy than the lowest energy conformer.

\section{S8. Activation Strain Analysis}

The activation barrier $\left(\Delta \mathrm{E}\left(\zeta^{\mathrm{TS}}\right)\right)$, is partitioned into (i) total destabilizing distortion energy in the reactants and catalyst $\left(\Delta \mathrm{E}_{\mathrm{dist}}(\zeta)\right)$ and (ii) stabilizing interaction energy between such distorted species $\left(\Delta \mathrm{E}_{\mathrm{int}}(\zeta)\right)$, and is expressed as the sum of the distortion and interaction $\Delta \mathrm{E}\left(\zeta^{\mathrm{TS}}\right)=\Delta \mathrm{E}_{\mathrm{int}}(\zeta)$ $+\Delta \mathrm{E}_{\text {dist }}(\zeta)$. Here, the distortion energy is calculated with respect to the undistorted ground state 
geometry of the catalyst $\left(\mathrm{E}_{1}\right)$ and the substrate $\left(\mathrm{E}_{\text {sub }}\right)$ and the corresponding distorted geometries of the catalyst $\left(\mathrm{E}_{\text {cat-F1 }}\right)$ and the substrate $\left(\mathrm{E}_{\text {sub-F2 }}\right)$ in the transition state, $\Delta \mathrm{E}_{\text {dist }}(\zeta)=\left[\left(\mathrm{E}_{\text {cat- } \mathrm{F} 1}+\mathrm{E}_{\text {sub- }}\right.\right.$ $\left.\left.{ }_{\mathrm{F} 2}+\mathrm{E}_{\mathrm{CO}}\right)-\left(\mathrm{E}_{1}+\mathrm{E}_{\mathrm{sub}}\right)\right]$. The $\Delta \mathrm{E}_{\mathrm{int}}(\zeta)$ is calculated as the difference between the activation energy $\left(\Delta \mathrm{E}\left(\zeta^{\mathrm{TS}}\right)\right)$ and the distortion energy $\left(\Delta \mathrm{E}_{\text {dist }}(\zeta)\right), \quad \Delta \mathrm{E}_{\text {int }}(\zeta)=\Delta \mathrm{E}\left(\zeta^{\mathrm{TS}}\right)-\Delta \mathrm{E}_{\text {dist }}(\zeta)$. A schematic representation of fragments considered in the computation of the distortion and interaction energies are shown in Figure S14 and the corresponding values are given in Table S9.

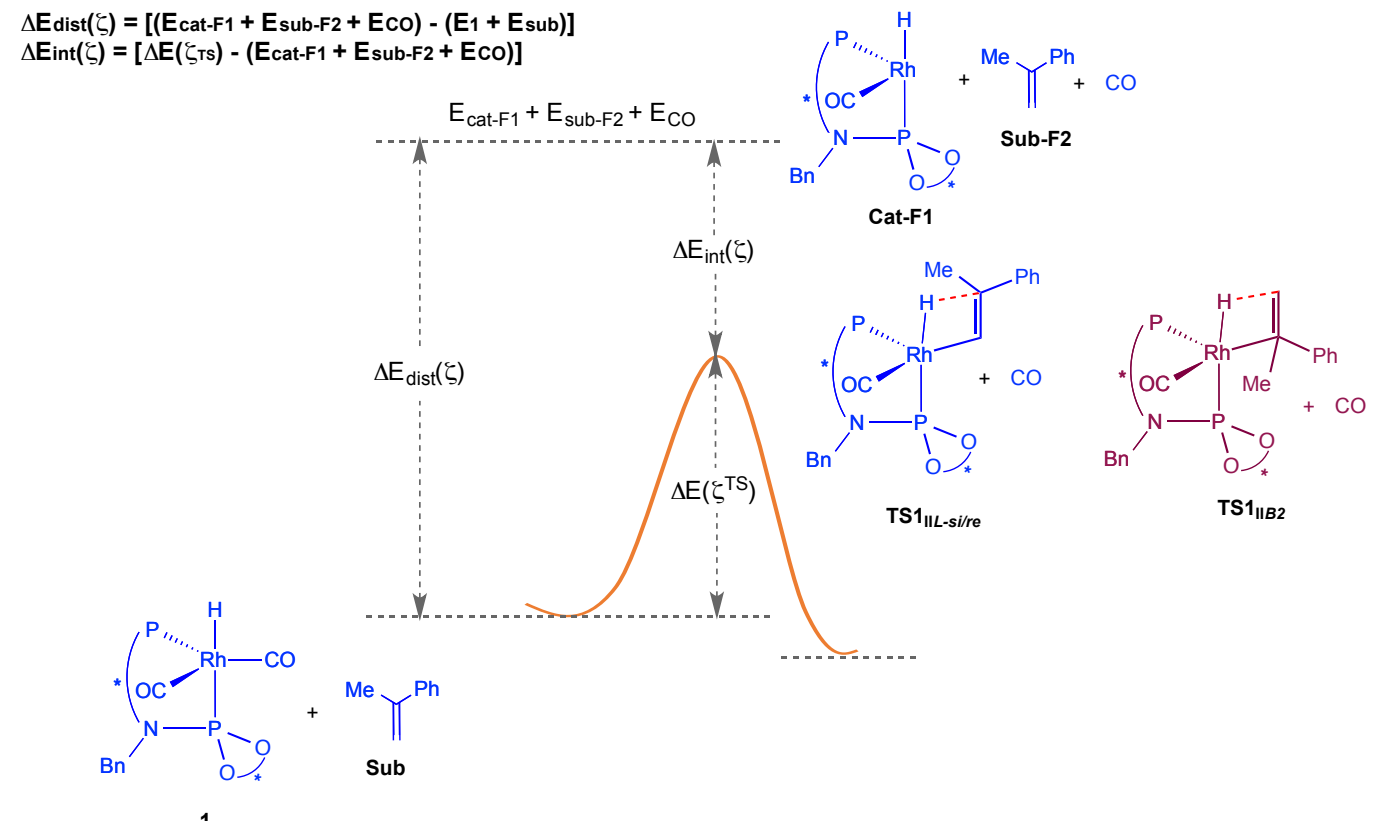

Figure S16. The partition scheme used in the activation strain model for the migratory insertion transition state $(\mathbf{T S} 1)$.

Table S8. Summary of Distortion-Interaction/Activation-Strain (DI-AS) Analysis for the Migratory Insertion Transition States (TS1). Energies are in $\mathrm{kcal} / \mathrm{mol}$

\begin{tabular}{|c|c|c|c|c|c|}
\hline \multirow{2}{*}{$\begin{array}{l}\text { transition } \\
\text { state }\end{array}$} & \multicolumn{3}{|c|}{ distortion energy $\left(\Delta \mathrm{E}_{\mathrm{dis}}(\zeta)\right)$} & \multirow{2}{*}{$\begin{array}{l}\text { interaction } \\
\text { energy } \\
\left(\Delta \mathrm{E}_{\text {int }}(\mathcal{\zeta})\right) \\
\text { (d) }\end{array}$} & \multirow{2}{*}{$\begin{array}{c}\text { activation } \\
\text { energy } \\
\Delta \mathrm{E}\left(\xi^{\mathrm{TS}}\right) \\
(\mathrm{e})=(\mathrm{c})+(\mathrm{d})\end{array}$} \\
\hline & $\begin{array}{l}\text { catalyst } \\
\text { (a) }\end{array}$ & $\begin{array}{l}\text { substrate } \\
\text { (b) }\end{array}$ & $\begin{array}{l}\text { in the TS } \\
(\mathrm{c})=(\mathrm{a})+(\mathrm{b})\end{array}$ & & \\
\hline TS1 $_{\text {IIL-si }}$ & 44.6 & 16.8 & 61.4 & -39.7 & 21.7 \\
\hline TS1 $_{\text {ILL-re }}$ & 50.7 & 14.1 & 64.8 & -40.5 & 24.3 \\
\hline TS1 & 44.2 & 12.6 & 56.8 & -31.1 & 25.7 \\
\hline
\end{tabular}




\section{S9. Additional Analysis of the Enantio/Regio-Controlling Migratory Insertion Transition}

\section{States}

\section{S9.1. Single Point Energies}

Table S9. Comparison of the Single Point Energies Across Different Functionals ${ }^{a}$ Obtained using the Optimized Geometries at the SMD(toluene)/M06/6-31G**,SDD(Rh) Level of Theory

\begin{tabular}{|c|c|c|c|c|c|c|c|c|}
\hline & \multicolumn{2}{|c|}{ L1 } & \multicolumn{2}{|c|}{ L2 } & \multicolumn{2}{|c|}{ L3 } & \multicolumn{2}{|c|}{ L4 } \\
\hline & TS1 $_{\text {IIL-si }}$ & TS1 $1_{I I L-r e}$ & TS1 $_{I I L-s i}$ & TS1 $_{\text {IIL-re }}$ & TS1 $_{\text {IIL-si }}$ & TS1 $_{\text {IIL-re }}$ & TS1 $_{\text {IIL-si }}$ & TS1 $_{\text {IIL-re }}$ \\
\hline$\psi-1 \mathrm{a} 2 \mathrm{a} 3 \mathrm{a} 4 \mathrm{a}$ & 1.2 & 1.0 & 2.0 & 1.2 & 1.6 & 1.6 & 2.2 & 3.0 \\
\hline$\psi-1 a 2 a 3 a 4 s$ & 1.6 & 0.6 & 2.5 & 1.1 & 2.0 & 0.9 & 2.5 & 2.9 \\
\hline$\psi-1 \mathrm{a} 2 \mathrm{a} 3 \mathrm{~s} 4 \mathrm{a}$ & 1.5 & 1.0 & 1.9 & 0.5 & 1.9 & 1.9 & 2.2 & 2.5 \\
\hline$\psi-1 \mathrm{a} 2 \mathrm{a} 3 \mathrm{~s} 4 \mathrm{~s}$ & 1.6 & 0.5 & 2.0 & 0.3 & 2.0 & 1.1 & 2.3 & 2.2 \\
\hline$\psi-1 \mathrm{a} 2 \mathrm{~s} 3 \mathrm{a} 4 \mathrm{a}$ & - & 0.5 & - & 0.9 & - & 1.2 & - & 2.5 \\
\hline$\psi-1 \mathrm{a} 2 \mathrm{~s} 3 \mathrm{a} 4 \mathrm{~s}$ & - & 0.8 & - & 0.8 & - & 1.4 & - & 2.6 \\
\hline$\psi-1 \mathrm{a} 2 \mathrm{~s} 3 \mathrm{~s} 4 \mathrm{a}$ & - & 0.2 & - & -0.4 & - & 1.2 & - & 1.5 \\
\hline$\psi-1 \mathrm{a} 2 \mathrm{~s} 3 \mathrm{~s} 4 \mathrm{~s}$ & - & 0.3 & - & -0.3 & - & 1.2 & - & 1.6 \\
\hline$\psi-1$ s2a3a4a & 2.2 & 1.8 & 2.3 & 2.6 & 2.3 & 2.7 & 2.3 & 4.3 \\
\hline$\psi-1 \mathrm{~s} 2 \mathrm{a} 3 \mathrm{a} 4 \mathrm{~s}$ & 0.0 & 1.4 & 0.1 & 2.5 & 0.0 & 2.0 & 0.0 & 4.2 \\
\hline$\psi-1 s 2 a 3 s 4 a$ & 0.3 & 2.0 & 0.1 & 2.3 & 0.4 & 3.0 & 0.0 & 4.1 \\
\hline$\psi-1 \mathrm{~s} 2 \mathrm{a} 3 \mathrm{~s} 4 \mathrm{~s}$ & 0.2 & 1.9 & 0.0 & 2.5 & 0.2 & 2.7 & 0.0 & 4.2 \\
\hline$\psi-1 \mathrm{~s} 2 \mathrm{~s} 3 \mathrm{a} 4 \mathrm{a}$ & - & 1.5 & - & 2.2 & - & 2.6 & - & 3.9 \\
\hline$\psi-1 \mathrm{~s} 2 \mathrm{~s} 3 \mathrm{a} 4 \mathrm{~s}$ & - & 1.0 & - & 1.8 & - & 2.1 & - & 3.4 \\
\hline$\psi-1 \mathrm{~s} 2 \mathrm{~s} 3 \mathrm{~s} 4 \mathrm{a}$ & - & 1.7 & - & 2.1 & - & 2.9 & - & 3.9 \\
\hline$\psi-1 \mathrm{~s} 2 \mathrm{~s} 3 \mathrm{~s} 4 \mathrm{~s}$ & - & 1.6 & - & 2.1 & - & 2.8 & - & 3.9 \\
\hline
\end{tabular}

\section{S9.2. Geometry Optimization of the Lowest Energy Transition States}

Table S10. Gibbs Free Energies of the Lowest Energy Enantiocontrolling Migratory Insertion

Transition States Obtained using Various Density Functionals and Basis Sets Combinations in the SMD Solvation Model for Toluene as the Condensed Phase

\begin{tabular}{|c|c|}
\hline \multicolumn{2}{|c|}{ A. Fully Optimized Geometries at the B3LYP-D3 Level } \\
\hline & $\Delta \Delta \mathrm{G}$ \\
\hline (i) SMD(toluene)/B3LYP-D3/6-31G**,SDD(Rh) \\
\hline TS1 $_{\text {II } L-s i}-\boldsymbol{\psi}-\mathbf{1 s 2 a 3 s 4 s}$ & 0.0 \\
\hline
\end{tabular}




\begin{tabular}{|c|c|}
\hline TS1 IIL-re $-\psi-1 \mathrm{a} 2 \mathrm{~s} 3 \mathrm{~s} 4 \mathrm{a}$ & -0.8 \\
\hline \multicolumn{2}{|c|}{ (ii) Single Point Calculation ${ }^{\text {a }}$ at the SMD(toluene)/ $\omega-\mathrm{B} 97 \mathrm{XD} / 6-31 \mathrm{G}^{* *}, \mathrm{SDD}(\mathrm{Rh})$ Level } \\
\hline$T S 1_{I I L-s i}-\psi-1 \mathrm{~s} 2 \mathrm{a} 3 \mathrm{~s} 4 \mathrm{~s}$ & 0.0 \\
\hline TS1 $_{\text {IIL-re }}-\psi-1 \mathrm{a} 2 \mathrm{~s} 3 \mathrm{~s} 4 \mathrm{a}$ & 1.5 \\
\hline \multicolumn{2}{|c|}{ (iii) Single Point Calculation at the SMD(toluene) $/ \omega-B 97 \mathrm{XD} / 6-311 \mathrm{G}^{* *}, \mathrm{SDD}(\mathrm{Rh})$ Level } \\
\hline $\mathrm{TS1}_{\mathrm{IIL-si}-}-\psi-1 \mathrm{~s} 2 \mathrm{a} 3 \mathrm{~s} 4 \mathrm{~s}$ & 0.0 \\
\hline TS1 $_{\text {IIL-re }}-\psi-1 \mathrm{a} 2 \mathrm{~s} 3 \mathrm{~s} 4 \mathrm{a}$ & 1.6 \\
\hline \multicolumn{2}{|c|}{ B. Fully Optimized Geometries at the M06-D3 Level } \\
\hline \multicolumn{2}{|c|}{ (iv) SMD(toluene)/M06-D3/6- 31G**,SDD(Rh) } \\
\hline TS1 $_{\text {IIL-si }}-\psi-1 \mathrm{~s} 2 \mathrm{a} 3 \mathrm{~s} 4 \mathrm{~s}$ & 0.0 \\
\hline$T S 1_{\text {IIL-re }-\psi-1 a 2 s 3 s 4 a}$ & 1.2 \\
\hline \multicolumn{2}{|c|}{ (v) Single Point Calculation at the SMD(toluene)/ $\omega-\mathrm{B} 97 \mathrm{XD} / 6-31 \mathrm{G}^{* *}, \mathrm{SDD}(\mathrm{Rh})$ Level } \\
\hline TS1 IIL-si $-\psi-1 \mathrm{~s} 2 \mathrm{a} 3 \mathrm{~s} 4 \mathrm{~s}$ & 0.0 \\
\hline$T S 1_{I I L-r e}-\psi-1 \mathrm{a} 2 \mathrm{~s} 3 \mathrm{~s} 4 \mathrm{a}$ & 2.2 \\
\hline \multicolumn{2}{|c|}{ (vi) Single Point Calculation at the SMD(toluene) $/ \omega-\mathrm{B} 97 \mathrm{XD} / 6-311 \mathrm{G}^{* *}, \mathrm{SDD}(\mathrm{Rh})$ Level } \\
\hline TS1 $_{I I L-s i}-\psi-1 \mathrm{~s} 2 \mathrm{a} 3 \mathrm{~s} 4 \mathrm{~s}$ & 0.0 \\
\hline TS1 $_{I I L-r e}-\psi-1 \mathrm{a} 2 \mathrm{~s} 3 \mathrm{~s} 4 \mathrm{a}$ & 2.3 \\
\hline \multicolumn{2}{|c|}{ C. Fully Optimized Geometries $\omega$-B97XD Level } \\
\hline \multicolumn{2}{|c|}{ (vii) $\mathrm{SMD}\left(\right.$ toluene) $/ \omega-\mathrm{B} 97 \mathrm{XD} / 6-31 \mathrm{G}^{* *}, \mathrm{SDD}(\mathrm{Rh})$} \\
\hline TS1 $_{\text {IIL-si } i}-\psi-1 \mathrm{~s} 2 \mathrm{a} 3 \mathrm{~s} 4 \mathrm{~s}$ & 0.0 \\
\hline TS1 $_{\text {IIL-re }}-\psi-1 \mathrm{a} 2 \mathrm{~s} 3 \mathrm{~s} 4 \mathrm{a}$ & 1.4 \\
\hline \multicolumn{2}{|c|}{ (viii) Single Point Calculation at the SMD(toluene)/ $\omega-\mathrm{B} 97 \mathrm{XD} / 6-311 \mathrm{G}^{* *}, \mathrm{SDD}(\mathrm{Rh})$ Level } \\
\hline$T_{S 1} 1_{I L-s i}-\psi-1 \mathrm{~s} 2 \mathrm{a} 3 \mathrm{~s} 4 \mathrm{~s}$ & 0.0 \\
\hline TS1 $_{I I L-r e}-\psi-1 \mathrm{a} 2 \mathrm{~s} 3 \mathrm{~s} 4 \mathrm{a}$ & 1.6 \\
\hline
\end{tabular}

${ }^{a}$ Thermal and entropic corrections (incorporating the quasi-harmonic rigid-rotor approximation obtained through the frequency calculations at the same level of theory as geometry optimization) are added to the electronic energies to obtain the Gibbs free energies (in $\mathrm{kcal} / \mathrm{mol}$ ).

Table S11. Gibbs Free Energies of the Lowest Energy Regiocontrolling Migratory Insertion Transition States Leading to the Linear Chiral or the Branched Achiral Aldehyde Product as Obtained using Various Density Functionals and Basis Sets Combinations in the SMD Solvation Model for Toluene as the Condensed Phase

\begin{tabular}{|c|c|}
\hline \multicolumn{2}{|c|}{ A. Fully Optimized Geometries at the B3LYP-D3 Level } \\
\hline & $\Delta \Delta \mathrm{G}$ \\
\hline \multicolumn{2}{|c|}{ (i) $\mathrm{SMD}$ (toluene)/B3LYP-D3/6-31G**,SDD(Rh) } \\
\hline TS1 $1_{I L-s i}-\psi-1 \mathrm{~s} 2 \mathrm{a} 3 \mathrm{~s} 4 \mathrm{~s}$ & 0.0 \\
\hline $\mathrm{TS1}_{I I B 2}-\psi-1 \mathrm{a} 2 \mathrm{a} 3 \mathrm{a} 4 \mathrm{~s}$ & 2.0 \\
\hline
\end{tabular}




\begin{tabular}{|c|c|}
\hline $\mathrm{TS1}_{\mathrm{IIL}-\mathrm{si}-\psi} \psi-1 \mathrm{~s} 2 \mathrm{a} 3 \mathrm{~s} 4 \mathrm{~s}$ & 0.0 \\
\hline $\mathrm{TS1}_{\text {IIB } 2-\psi-1 \mathrm{a} 2 \mathrm{a3a} \mathbf{a} \mathrm{s}}$ & 4.5 \\
\hline \multicolumn{2}{|c|}{ (iii) Single Point Calculation at the SMD(toluene) $/ \omega-\mathrm{B} 97 \mathrm{XD} / 6-311 \mathrm{G}^{* *}, \mathrm{SDD}(\mathrm{Rh})$ Level } \\
\hline $\mathrm{TS1}_{\text {IIL-si }}-\psi-1 \mathrm{~s} 2 \mathrm{a3s} 4 \mathrm{~s}$ & 0.0 \\
\hline$T S 1_{I I B 2}-\psi-1 \mathrm{a} 2 \mathrm{a} 3 \mathrm{a} 4 \mathrm{~s}$ & 3.8 \\
\hline \multicolumn{2}{|c|}{ B. Fully Optimized Geometries at the M06-D3 Level } \\
\hline \multicolumn{2}{|c|}{ (iv) SMD(toluene)/M06-D3/6- 31G**,SDD(Rh) } \\
\hline TS1 $1_{I L-s i}-\psi-1 \mathrm{~s} 2 \mathrm{a} 3 \mathrm{~s} 4 \mathrm{~s}$ & 0.0 \\
\hline $\mathrm{TS1}_{\text {IIB } 2-\psi-1 \mathrm{a} 2 \mathrm{a} 3 \mathrm{a} 4 \mathrm{~s}}$ & 2.2 \\
\hline \multicolumn{2}{|c|}{ (v) Single Point Calculations at the SMD(toluene)/ $\omega-\mathrm{B} 97 \mathrm{XD} / 6-31 \mathrm{G}^{* *}, \mathrm{SDD}(\mathrm{Rh})$ Level } \\
\hline $\mathrm{TS1}_{I I L-s i}-\psi-1 \mathrm{~s} 2 \mathrm{a} 3 \mathrm{~s} 4 \mathrm{~s}$ & 0.0 \\
\hline TS1 $_{I I B 2}-\psi-1 \mathrm{a} 2 \mathrm{a} 3 \mathrm{a} 4 \mathrm{~s}$ & 4.3 \\
\hline \multicolumn{2}{|c|}{ (vi) Single Point Calculation at the SMD(toluene)/ $\omega-\mathrm{B} 97 \mathrm{XD} / 6-311 \mathrm{G}{ }^{* *}, \mathrm{SDD}(\mathrm{Rh}) \mathrm{Level}$} \\
\hline$T_{S S 1} I L-s i-\psi-1 \mathrm{~s} 2 \mathrm{a} 3 \mathrm{~s} 4 \mathrm{~s}$ & 0.0 \\
\hline $\mathrm{TS1}_{I I B 2}-\psi-1 \mathrm{a} 2 \mathrm{a} 3 \mathrm{a} 4 \mathrm{~s}$ & 3.6 \\
\hline \multicolumn{2}{|c|}{ C. Fully Optimized Geometries $\omega$-B97XD Level } \\
\hline \multicolumn{2}{|c|}{ (vii) $\mathrm{SMD}\left(\right.$ toluene) $/ \omega-\mathrm{B} 97 \mathrm{XD} / 6-31 \mathrm{G}^{* *}, \mathrm{SDD}(\mathrm{Rh})$} \\
\hline TS1 $_{I I L-s i}-\psi-1 \mathrm{~s} 2 \mathrm{a} 3 \mathrm{~s} 4 \mathrm{~s}$ & 0.0 \\
\hline $\mathrm{TS1}_{\text {IIB2}}-\psi-1 \mathrm{a} 2 \mathrm{a3a4}$ & 4.8 \\
\hline \multicolumn{2}{|c|}{ (viii) Single Point Calculation at the SMD(toluene)/ $\omega$-B97XD/6-311G ${ }^{* *}, \mathrm{SDD}(\mathrm{Rh})$ Level } \\
\hline $\mathrm{TS1}_{\text {IIL-si }}-\psi-1 \mathrm{~s} 2 \mathrm{a3s} 4 \mathrm{~s}$ & 0.0 \\
\hline TS1 $_{I I B 2}-\psi-1 \mathrm{a} 2 \mathrm{a} 3 \mathrm{a} 4 \mathrm{~s}$ & 4.1 \\
\hline
\end{tabular}

${ }^{a}$ Thermal and entropic corrections (incorporating the quasi-harmonic rigid-rotor approximation obtained through the frequency calculations at the same level of theory as geometry optimization) are added to the electronic energies to obtain the Gibbs free energies (in $\mathrm{kcal} / \mathrm{mol}$ ).

\section{S9.3. Topological Analysis of the Electron Density Using the AIM Formalism in the}

\section{Enantiocontrolling Transition States}

Various noncovalent interactions were identified the AIM formalism, wherein the wave function generated for a given optmized geometry (say, that of a transition state) is subjected to topological analysis of the electron density distribution to obtain topological maps as shown in Figure S17. Important aspects of these topological maps are the identification of bond paths and the bond critical points along such bond paths. The dotted lines, designated as $a-p$, in the simplified representation of molecular geometry (shown in the right side in each row), denote the 
noncovalent interactions. The corresponding bond paths for each such noncovalent interaction can be noted in the topological maps provided on the left side.

Characterization of a given bond critical point $(b c p)$ as representing a $\mathrm{C}-\mathrm{H} \cdots \pi$ interaction is done based on the presence of a bond path connecting the hydrogen to one or more atoms of the $\pi$ framework of an aryl ring. For instance, the $b c p$ s shown as $d$ and $e$ in the case of TS1 ${ }_{\text {IIA-si- }}$ $\psi_{\text {-1s2a3a4s }}$ (chosen here as a representative case for ease visualization) are the ones that manifest between an alkyl/aryl $\mathrm{C}-\mathrm{H}$ bonds of the $\mathrm{N}-\mathrm{Bn}$ group and the $\pi$ face of the binaphthyl ring of the phosphoramidite region of the YanPhos ligand. Similarly, the bcps $h$ and $i$ both denote the $\pi \cdots \pi$ noncovalent interaction between the phenyl ring of styrene and the aryl ring of the $-\mathrm{P}(3,5-\mathrm{t}-\mathrm{Bu}-$ $\left.\mathrm{C}_{6} \mathrm{H}_{3}\right)_{2}$ group in the catalyst backbone.

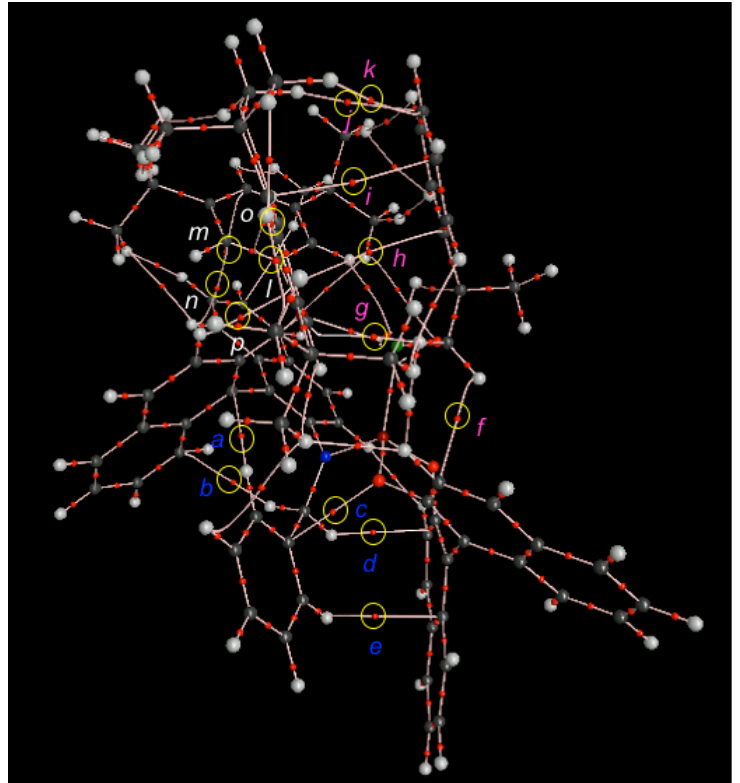

Bn-group *'Ligand $a=2.66(0.90) \mathrm{C}-\mathrm{H} \cdots \pi$ $b=2.79(0.62) \mathrm{C}-\mathrm{H} \cdots \pi$ $c=2.76(1.46)$ lone pair $\cdots \pi$ $d=2.69(0.88) \mathrm{C}-\mathrm{H} \cdots \pi$ $e=2.83(0.64) \mathrm{C}-\mathrm{H} \cdots \pi$

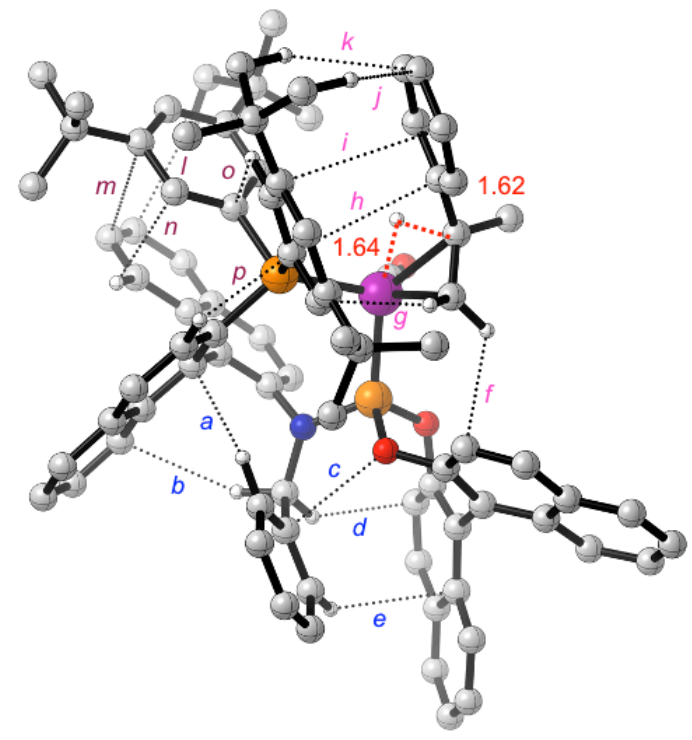

$$
\begin{aligned}
& \text { Ligand } \cdots \text { Ligand } \\
& \qquad \begin{aligned}
I & =2.79(0.66) \mathrm{C}-\mathrm{H} \cdots \pi \\
m & =3.73(0.41) \pi \cdots \pi \\
n & =2.87(0.67) \mathrm{C}-\mathrm{H} \cdots \pi \\
o & =2.60(1.14) \mathrm{C}-\mathrm{H} \cdots \pi \\
p & =2.41(1.48) \mathrm{C}-\mathrm{H} \cdots \pi
\end{aligned}
\end{aligned}
$$

TS1 $_{\text {IIA-si- }} \psi_{-1 \mathbf{s} 2 \mathbf{a 3 a 4 s}}(\Delta \mathrm{G}=25.4 \mathrm{kcal} / \mathrm{mol})$ 


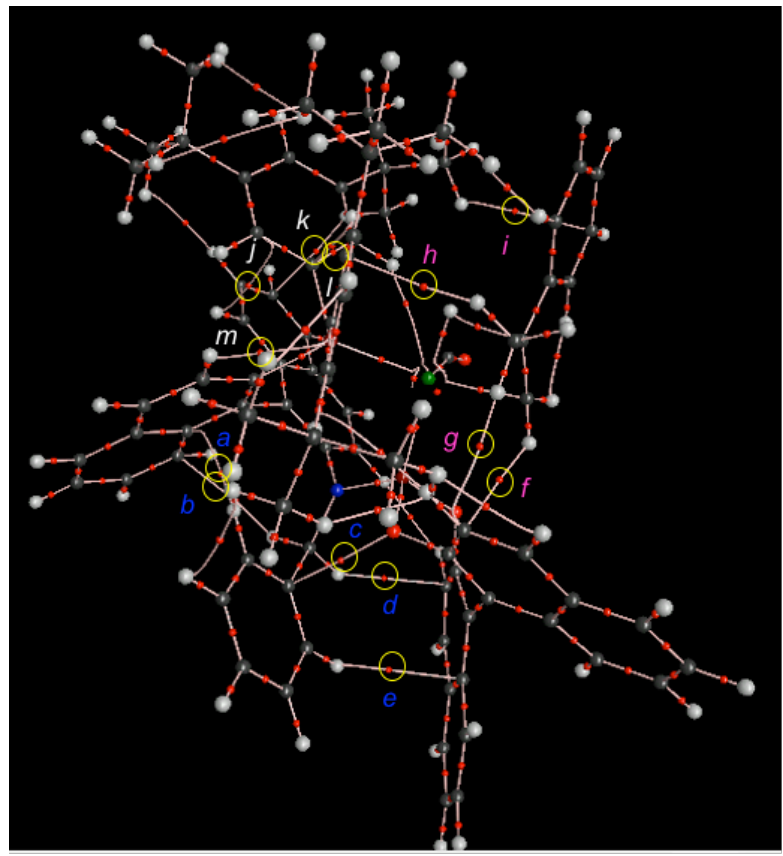

Bn-group $\cdots$ Ligand

$a=2.65(0.87) \mathrm{C}-\mathrm{H} \cdots \pi$ $b=2.98(0.44) \mathrm{C}-\mathrm{H} \cdots \pi$ $c=2.75$ (1.45) lone pair $\cdots \pi$ $d=2.69(0.84) \mathrm{C}-\mathrm{H} \cdots \pi$ $e=2.77(0.69) \mathrm{C}-\mathrm{H} \cdots \pi$

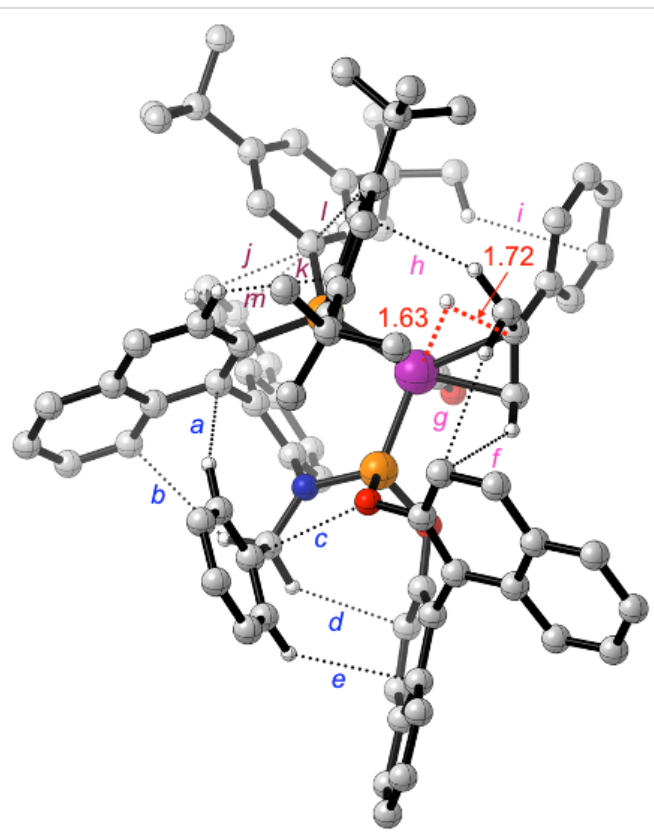

Ligand '. Ligand

$j=2.92(0.51) \mathrm{C}-\mathrm{H} \cdots \pi$

$k=3.01(0.49) \pi \cdots \pi$

$I=2.50(1.12) \mathrm{C}-\mathrm{H} \cdots \pi$

$m=2.56(1.33) \mathrm{C}-\mathrm{H} \cdots \pi$

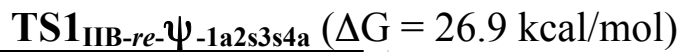

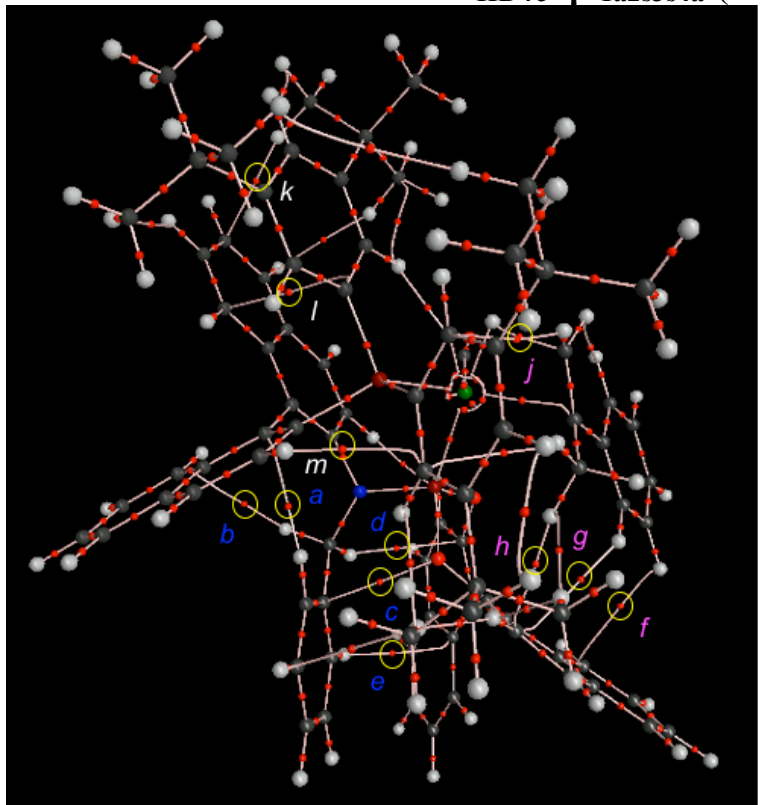

Bn-group '..'Ligand $a=2.62(0.87) \mathrm{C}-\mathrm{H} \cdots \pi$ $b=2.87(0.44) \mathrm{C}-\mathrm{H} \cdots \pi$ $c=2.75$ (1.45) lone pair $\cdots \pi$ $d=2.67(0.84) \mathrm{C}-\mathrm{H} \cdots \pi$ $e=2.80(0.69) \mathrm{C}-\mathrm{H} \cdots \pi$

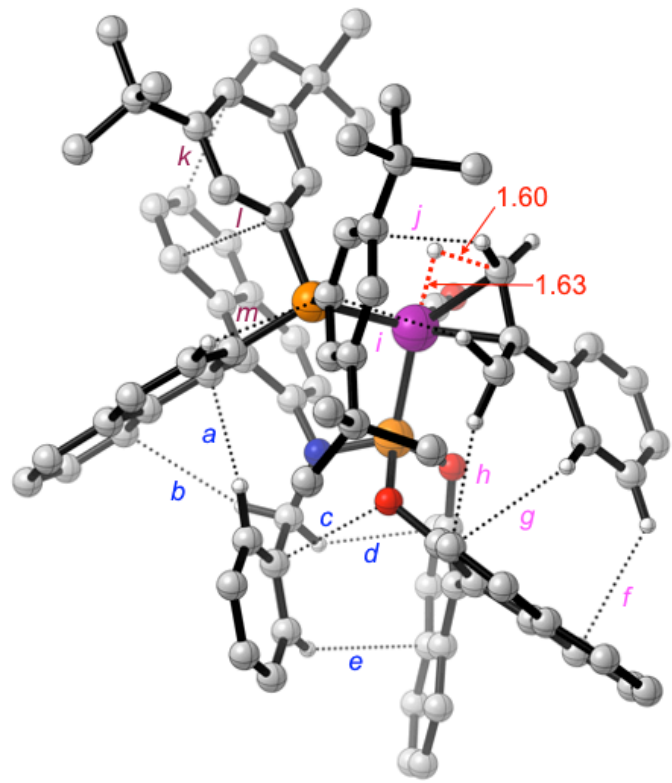

Ligand $\cdots$ Ligand

$k=2.68(0.49) \mathrm{C}-\mathrm{H} \cdots \pi$

$I=3.41(1.12) \pi \cdots \pi$ $m=2.99$ (1.33) C-H $\cdots \pi$ $h=2.50(0.88) \mathrm{C}-\mathrm{H} \cdots \tau$ $j=2.75(0.47) \mathrm{C}-\mathrm{H} \cdots \pi$ $j=3.01(0.51) \mathrm{C}-\mathrm{H} \cdots \pi$

TS1 $_{\text {IID- }} \psi_{\text {-1a2a3a4s }}(\Delta \mathrm{G}=29.0 \mathrm{kcal} / \mathrm{mol})$ 
Figure S17. The bond critical points and the bond paths obtained from the topogical analysis of the electron densities obtained using the Atoms-In-Molecule formalism on the

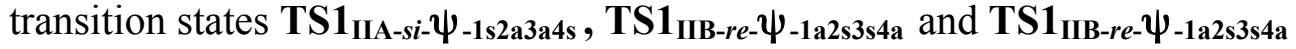




\section{S10. Additional Conformers of the Dicarbonyl Rh-Keto Alkyl Intermediate 7 and TS4}

A. Three key conformers of intermediate $7_{S}$ are identified that differ in the dihedral $\beta$ as shown in Figure S18 below.

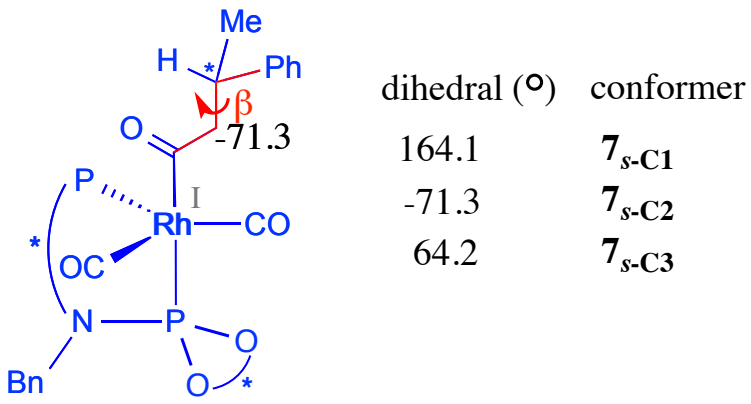

(a) Different orientations of the keto alkyl chain with respect the catalyst
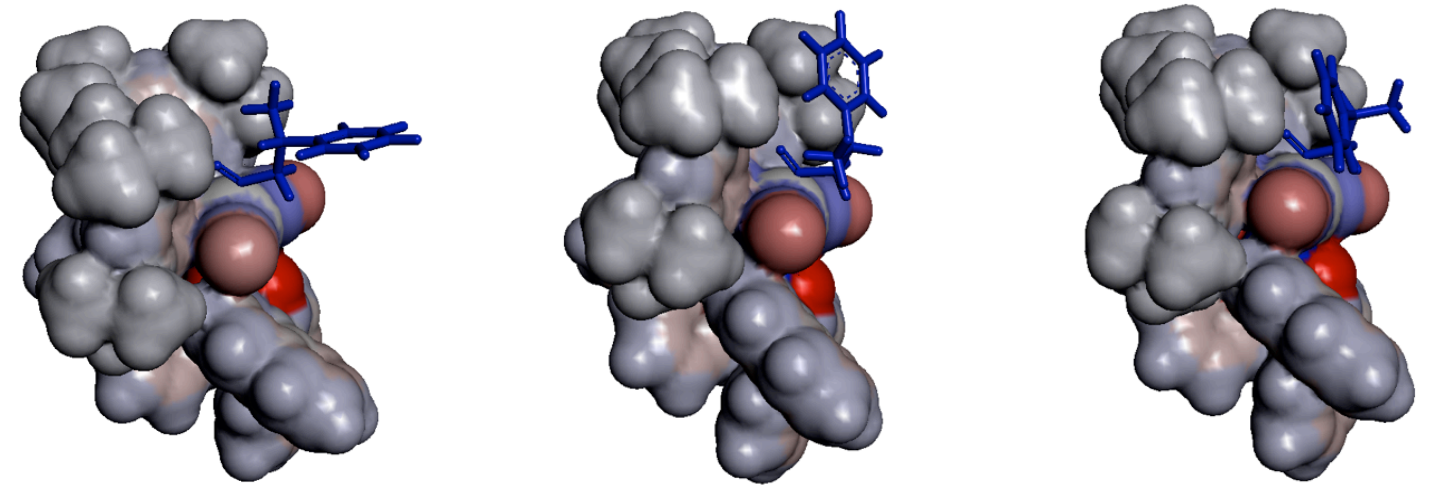

(b) Different orientations of the keto alkyl chain with respect the catalyst showing the protrusion of the product away from the catalyst

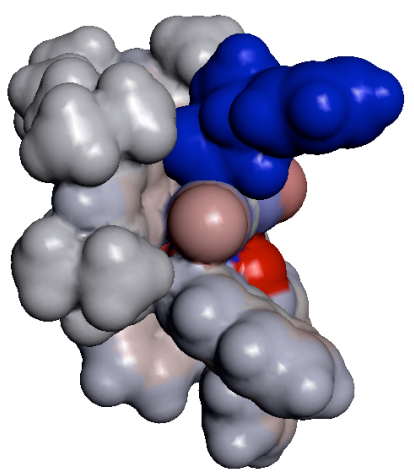

$7_{S-\mathrm{C} 1}(-5.4)$

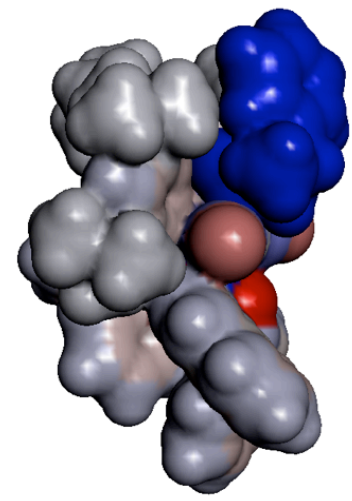

$7_{S-\mathrm{C} 2}(-6.3)$

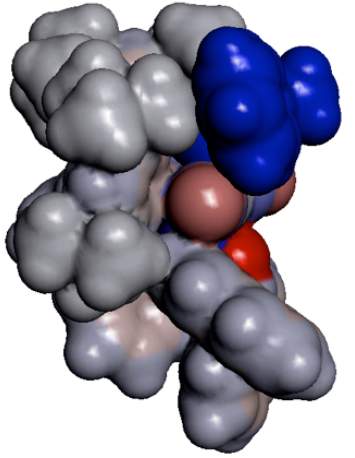

$7_{S-\mathrm{C} 3}(-6.2)$

Figure S18. Three key conformers of $7_{s}$ and the corresponding relative Gibbs free energies (in $\mathrm{kcal} / \mathrm{mol}$ ) with respect to the reference conformer $\mathbf{1}_{\mathrm{C1}-} \boldsymbol{\psi}$-1a2s3a4a. The transition states (TS4) corresponding to the $\mathbf{c 1}, \mathbf{c 2}$, and $\mathbf{c 3}$ conformers shown above are found to be 20.7, 19.9, and 20.1 $\mathrm{kcal} / \mathrm{mol}$. 
B. Depiction of the orientation of the alkyl region of the developing product in TS4

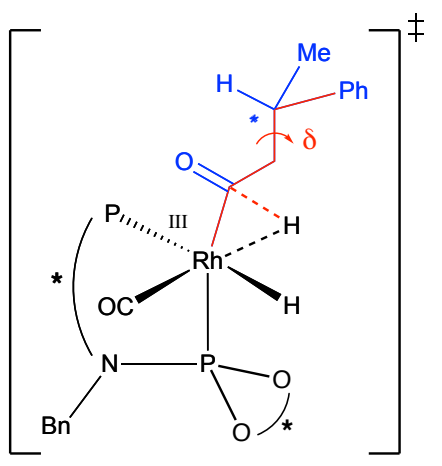

TS4
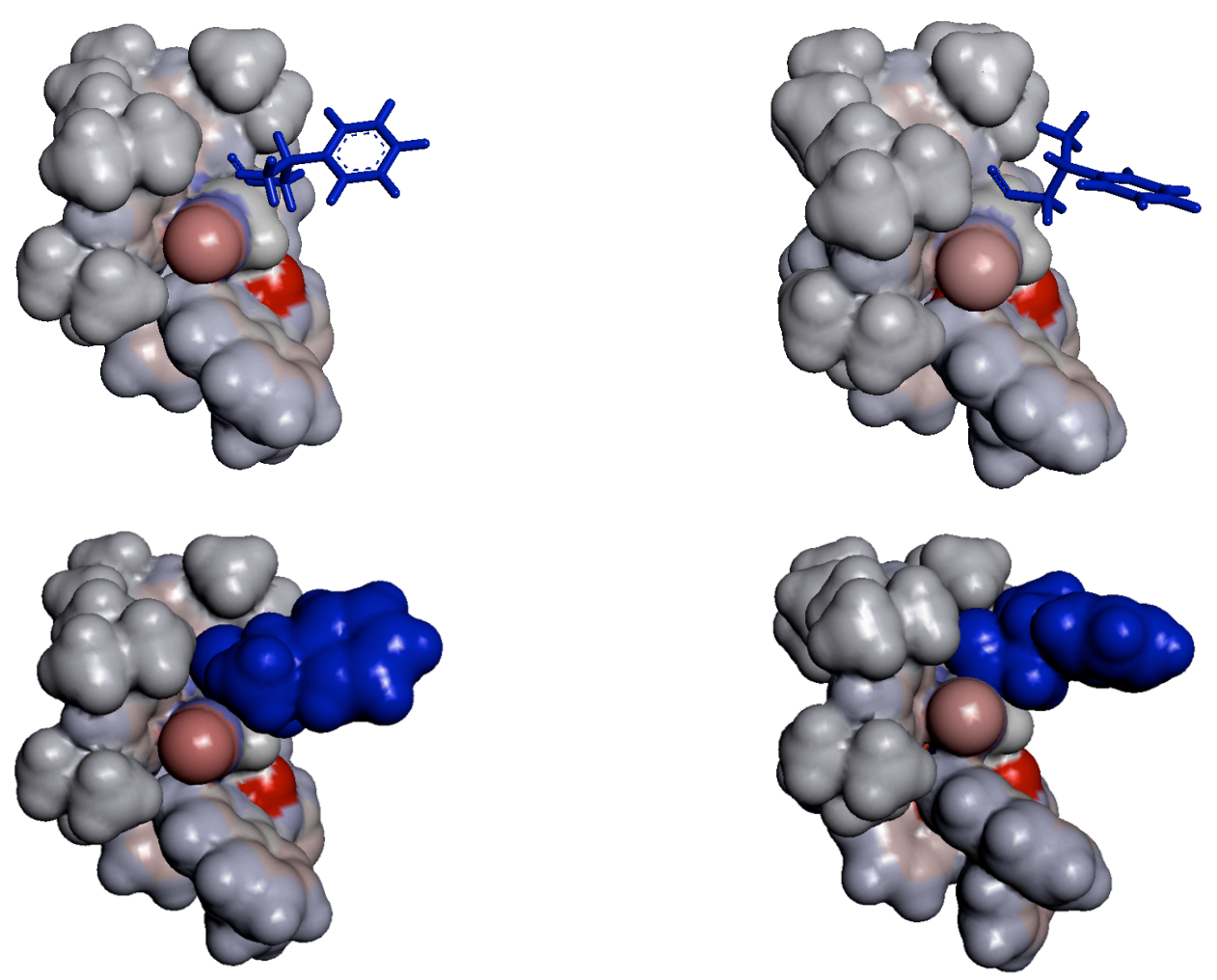

$\operatorname{TS}_{R}(20.5)$

$\mathrm{TS}_{S}(20.7)$

Figure S19. Space filling representation of TS4 responsible for the formation of the linear $S$ and linear $R$ chiral aldehydes and the corresponding relative Gibbs free energies (in $\mathrm{kcal} / \mathrm{mol}$ ) with

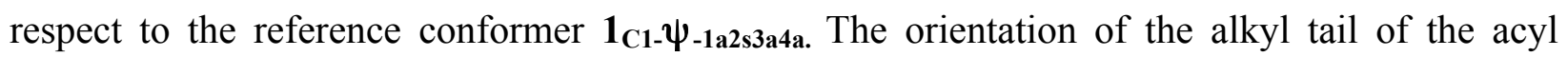
ligand (-CO-CH2-CH(Me)(Ph), shown in blue color) conveys that it occupies the space well outside the chiral environment of the YanPhos ligand. 


\section{S11. Energetic Span of the Catalytic Cycle}

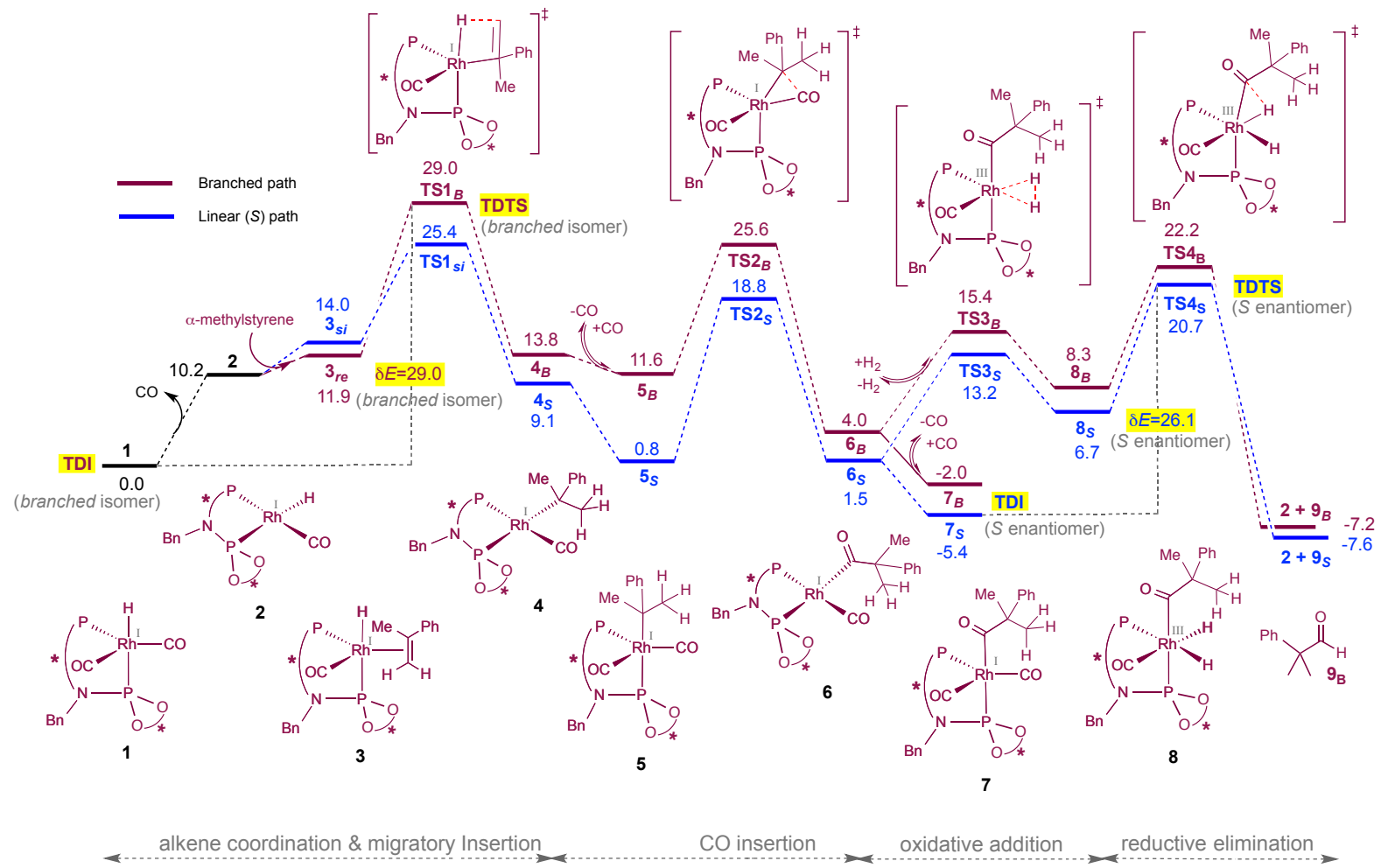

Figure S20. Gibbs free energy profile $(\mathrm{kcal} / \mathrm{mol})$ for the catalytic asymmetric hydroformylation of $\alpha$-methylstyrene. The blue and brown lines respectively represent the chiral linear $S$-aldehyde $\left(\mathbf{9}_{\mathbf{S}}\right)$ and achiral branched aldehyde product $\left(\mathbf{9}_{\mathbf{B}}\right)$. 
Table S12. Calculation of Energetic Span $(\delta \mathrm{E}$ in $\mathrm{kcal} / \mathrm{mol})$ by using Different Likely Combinations of TDI and TDTS. The Ones with Maximum Span is Highlighted

\begin{tabular}{|c|c|c|c|c|}
\hline \multicolumn{5}{|c|}{ Linear $(S)$ path $(\Delta \mathrm{Gr}=-7.6 \mathrm{kcal} / \mathrm{mol})$} \\
\hline \multicolumn{2}{|c|}{ TDI } & \multicolumn{2}{|c|}{ TDTS } & $\delta \mathrm{E}$ \\
\hline 1 & 0.0 & TS1 & 25.4 & 25.4 \\
\hline & & TS2 & 18.8 & 18.8 \\
\hline & & TS3 & 13.2 & 13.2 \\
\hline & & TS4 & 20.7 & 20.7 \\
\hline \multirow[t]{4}{*}{2} & 10.2 & TS1 & 25.4 & 15.2 \\
\hline & & TS2 & 18.8 & 8.6 \\
\hline & & TS3 & 13.2 & 3.0 \\
\hline & & TS4 & 20.7 & 10.5 \\
\hline \multirow[t]{4}{*}{3} & 14.0 & TS1 & 25.4 & 11.4 \\
\hline & & TS2 & 18.8 & 4.8 \\
\hline & & TS3 & 13.2 & -0.8 \\
\hline & & TS4 & 20.7 & 6.7 \\
\hline \multirow[t]{4}{*}{4} & 9.1 & TS1 & 25.4 & 8.7 \\
\hline & & TS2 & 18.8 & 9.7 \\
\hline & & TS3 & 13.2 & 4.1 \\
\hline & & TS4 & 20.7 & 11.6 \\
\hline \multirow[t]{4}{*}{5} & 0.8 & TS1 & 25.4 & 17.0 \\
\hline & & TS2 & 18.8 & 18.0 \\
\hline & & TS3 & 13.2 & 12.4 \\
\hline & & TS4 & 20.7 & 19.9 \\
\hline \multirow[t]{4}{*}{6} & 1.5 & TS1 & 25.4 & 16.3 \\
\hline & & TS2 & 18.8 & 9.7 \\
\hline & & TS3 & 13.2 & 11.7 \\
\hline & & TS4 & 20.7 & 19.2 \\
\hline \multirow[t]{4}{*}{7} & -5.4 & TS1 & 25.4 & 23.2 \\
\hline & & TS2 & 18.8 & 16.6 \\
\hline & & TS3 & 13.2 & 18.6 \\
\hline & & TS4 & 20.7 & 26.1 \\
\hline \multirow[t]{4}{*}{8} & 6.7 & TS1 & 25.4 & 11.1 \\
\hline & & TS2 & 18.8 & 4.5 \\
\hline & & TS3 & 13.2 & -1.1 \\
\hline & & TS4 & 20.7 & 14.0 \\
\hline \multicolumn{5}{|c|}{ Linear $(\boldsymbol{R})$ path $(\Delta \mathrm{Gr}=-7.6 \mathrm{kcal} / \mathrm{mol})$} \\
\hline \multicolumn{2}{|c|}{ TDI } & \multicolumn{2}{|c|}{ TDTS } & $\delta \mathrm{E}$ \\
\hline 1 & 0.0 & TS1 & 26.9 & 26.9 \\
\hline & & TS2 & 16.3 & 16.3 \\
\hline & & TS3 & 11.3 & 11.3 \\
\hline & & TS4 & 20.5 & 20.5 \\
\hline 2 & 10.2 & TS1 & 26.9 & 16.7 \\
\hline
\end{tabular}




\begin{tabular}{|c|c|c|c|c|}
\hline \multicolumn{2}{|c|}{ Table S12 Continued... } & \multirow[b]{2}{*}{ TS2 } & \multirow[b]{2}{*}{16.3} & \multirow[b]{2}{*}{6.1} \\
\hline & & & & \\
\hline & & TS3 & 11.3 & 1.1 \\
\hline & & TS4 & 20.5 & 10.3 \\
\hline \multirow[t]{4}{*}{3} & 11.9 & TS1 & 26.9 & 15.0 \\
\hline & & TS2 & 16.3 & 4.4 \\
\hline & & TS3 & 11.3 & -0.6 \\
\hline & & TS4 & 20.5 & 8.6 \\
\hline \multirow[t]{4}{*}{4} & 4.1 & TS1 & 26.9 & 15.2 \\
\hline & & TS2 & 16.3 & 12.2 \\
\hline & & TS3 & 11.3 & 7.2 \\
\hline & & TS4 & 20.5 & 16.4 \\
\hline \multirow[t]{4}{*}{5} & 2.5 & TS1 & 26.9 & 16.8 \\
\hline & & TS2 & 16.3 & 13.8 \\
\hline & & TS3 & 11.3 & 8.8 \\
\hline & & TS4 & 20.5 & 18.0 \\
\hline \multirow[t]{4}{*}{6} & 1.1 & TS1 & 26.9 & 18.2 \\
\hline & & TS2 & 16.3 & 7.6 \\
\hline & & TS3 & 11.3 & 10.2 \\
\hline & & TS4 & 20.5 & 19.4 \\
\hline \multirow[t]{4}{*}{7} & -5.8 & TS1 & 26.9 & 25.1 \\
\hline & & TS2 & 16.3 & 14.5 \\
\hline & & TS3 & 11.3 & 17.1 \\
\hline & & TS4 & 20.5 & 26.3 \\
\hline \multirow[t]{4}{*}{8} & 4.2 & TS1 & 26.9 & 15.1 \\
\hline & & TS2 & 16.3 & 4.5 \\
\hline & & TS3 & 11.3 & -0.5 \\
\hline & & TS4 & 20.5 & 16.3 \\
\hline \multicolumn{5}{|c|}{ Branched path $(\Delta \mathrm{Gr}=-7.2 \mathrm{kcal} / \mathrm{mol})$} \\
\hline \multicolumn{2}{|c|}{ TDI } & \multicolumn{2}{|c|}{ TDTS } & $\delta \mathrm{E}$ \\
\hline \multirow[t]{4}{*}{1} & 0.0 & TS1 & 29.0 & 29.0 \\
\hline & & TS2 & 25.6 & 25.6 \\
\hline & & TS3 & 15.4 & 15.4 \\
\hline & & TS4 & 22.2 & 22.2 \\
\hline \multirow[t]{4}{*}{2} & 10.2 & TS1 & 29.0 & 18.8 \\
\hline & & TS2 & 25.6 & 15.4 \\
\hline & & TS3 & 15.4 & 5.2 \\
\hline & & TS4 & 22.2 & 12.0 \\
\hline \multirow[t]{4}{*}{3} & 11.9 & TS1 & 29.0 & 17.1 \\
\hline & & TS2 & 25.6 & 13.7 \\
\hline & & TS3 & 15.4 & 3.5 \\
\hline & & TS4 & 22.2 & 10.3 \\
\hline \multirow[t]{3}{*}{4} & 13.8 & TS1 & 29.0 & 8.0 \\
\hline & & TS2 & 25.6 & 11.8 \\
\hline & & TS3 & 15.4 & 1.6 \\
\hline
\end{tabular}




\begin{tabular}{|c|c|c|c|c|}
\hline \multicolumn{2}{|l|}{ Table S12 Continued... } & & & \\
\hline & & & & \\
\hline & & TS4 & 22.2 & 8.4 \\
\hline $\mathbf{5}$ & 11.6 & TS1 & 29.0 & 10.2 \\
\hline & & TS2 & 25.6 & 14.0 \\
\hline & & TS3 & 15.4 & 3.8 \\
\hline $\mathbf{6}$ & & TS4 & 22.2 & 10.6 \\
\hline & & TS1 & 29.0 & 17.8 \\
\hline & & TS2 & 25.6 & 14.4 \\
\hline & & TS3 & 15.4 & 11.4 \\
\hline $\mathbf{7}$ & -2.0 & TS4 & 22.2 & 18.2 \\
\hline & & TS1 & 29.0 & 23.8 \\
\hline & & TS2 & 25.6 & 20.4 \\
\hline $\mathbf{8}$ & & TS3 & 15.4 & 17.4 \\
\hline & 8.3 & TS4 & 22.2 & 24.2 \\
\hline & & TS1 & 29.0 & 13.5 \\
\hline & & TS2 & 25.6 & 10.1 \\
\hline & & TS3 & 15.4 & -0.1 \\
\hline
\end{tabular}

${ }^{a}$ The energy span calculations were done using the Gibbs free energy profile diagram shown in Figure 4 in main text and Figure S15 in SI.

\section{S12. Application of the Enantiocontrolling TS Model to Other Substrates}

Table S13. Relative Gibbs Free Energies (in $\mathrm{kcal} / \mathrm{mol}$ ) of the Enantiocontrolling Migratory Insertion Transition States with Different para-Substituted $\alpha$-Methylstyrenes

\begin{tabular}{|c|c|c|c|}
\hline \multicolumn{4}{|c|}{$\mathbf{S 2}\left(\right.$ para $\left.-\mathrm{CH}_{3}\right)$} \\
\hline & TS1 $_{\text {IIL-si }}$ & & TS1 IIL-re \\
\hline$\psi-1 \mathrm{~s} 2 \mathrm{a} 3 \mathrm{a} 4 \mathrm{a}$ & 0.2 & $\psi-1 \mathbf{a} 2 \mathrm{~s} 3 \mathrm{a} 4 \mathrm{a}$ & 2.4 \\
\hline$\psi-1 \mathrm{~s} 2 \mathrm{a} 3 \mathrm{a} 4 \mathrm{~s}$ & 2.3 & $\psi-1 \mathrm{a} 2 \mathrm{~s} 3 \mathrm{a} 4 \mathrm{~s}$ & 2.7 \\
\hline$\psi-1 \mathrm{~s} 2 \mathrm{a} 3 \mathrm{~s} 4 \mathrm{a}$ & 0.0 & $\psi-1 \mathrm{a} 2 \mathrm{~s} 3 \mathrm{~s} 4 \mathrm{a}$ & 2.6 \\
\hline$\psi-1 \mathrm{~s} 2 \mathrm{a} 3 \mathrm{~s} 4 \mathrm{~s}$ & 0.4 & $\psi-1 \mathrm{a} 2 \mathrm{~s} 3 \mathrm{~s} 4 \mathrm{~s}$ & 2.4 \\
\hline \multicolumn{4}{|c|}{$\mathbf{S 3}\left(\right.$ para $\left.-\mathrm{CF}_{3}\right)$} \\
\hline & TS1 $_{\text {IIL-si }}$ & & TS1 IIL-re \\
\hline$\psi-1 \mathrm{~s} 2 \mathrm{a} 3 \mathrm{a} 4 \mathrm{a}$ & 0.3 & $\psi-1 \mathbf{a} 2 \mathrm{~s} 3 \mathrm{a} 4 \mathrm{a}$ & 1.8 \\
\hline$\psi-1 \mathrm{~s} 2 \mathrm{a} 3 \mathrm{a} 4 \mathrm{~s}$ & 0.3 & $\psi-1 \mathrm{a} 2 \mathrm{~s} 3 \mathrm{a} 4 \mathrm{~s}$ & 2.0 \\
\hline$\psi-1 \mathrm{~s} 2 \mathrm{a} 3 \mathrm{~s} 4 \mathrm{a}$ & 0.1 & $\psi-1 \mathrm{a} 2 \mathrm{~s} 3 \mathrm{~s} 4 \mathrm{a}$ & 1.6 \\
\hline$\psi-1 \mathrm{~s} 2 \mathrm{a} 3 \mathrm{~s} 4 \mathrm{~s}$ & 0.0 & $\psi-1 \mathrm{a} 2 \mathrm{~s} 3 \mathrm{~s} 4 \mathrm{~s}$ & 1.1 \\
\hline \multicolumn{4}{|c|}{ S4 (para-F) } \\
\hline & TS1 $1_{I I L-s i}$ & & TS1 IIL-re \\
\hline$\psi$-1s2a3a4a & 0.6 & $\psi-1 \mathrm{a} 2 \mathrm{~s} 3 \mathrm{a} 4 \mathrm{a}$ & 3.6 \\
\hline$\psi-1 \mathrm{~s} 2 \mathrm{a} 3 \mathrm{a} 4 \mathrm{~s}$ & 0.4 & $\psi-1 \mathrm{a} 2 \mathrm{~s} 3 \mathrm{a} 4 \mathrm{~s}$ & 3.3 \\
\hline
\end{tabular}




\begin{tabular}{|c|c|c|c|}
\hline$\psi-1 \mathrm{~s} 2 \mathrm{a} 3 \mathrm{~s} 4 \mathrm{a}$ & 0.1 & $\psi-1 a 2 s 3 s 4 a$ & 3.1 \\
\hline$\psi-1 \mathrm{~s} 2 \mathrm{a} 3 \mathrm{~s} 4 \mathrm{~s}$ & 0.0 & $\psi-1 \mathrm{a} 2 \mathrm{~s} 3 \mathrm{~s} 4 \mathrm{~s}$ & 3.2 \\
\hline \multicolumn{4}{|c|}{$\mathbf{S 5}$ (para-Cl) } \\
\hline & TS1 $_{\text {IIL-si }}$ & & TS1 $_{\text {IIL-re }}$ \\
\hline$\psi-1 s 2 a 3 a 4 a$ & 0.4 & $\psi-1 a 2 s 3 a 4 a$ & 1.9 \\
\hline$\psi-1 \mathrm{~s} 2 \mathrm{a} 3 \mathrm{a} 4 \mathrm{~s}$ & 0.9 & $\psi-1 a 2 s 3 a 4 s$ & 2.0 \\
\hline$\psi-1 \mathrm{~s} 2 \mathrm{a} 3 \mathrm{~s} 4 \mathrm{a}$ & 0.0 & $\psi-1 \mathrm{a} 2 \mathrm{~s} 3 \mathrm{~s} 4 \mathrm{a}$ & 1.7 \\
\hline$\psi-1 s 2 a 3 s 4 s$ & 0.5 & $\psi-1 \mathrm{a} 2 \mathrm{~s} 3 \mathrm{~s} 4 \mathrm{~s}$ & 1.8 \\
\hline
\end{tabular}

Table S14. Boltzmann Weighted Gibbs Free Energies ${ }^{\mathrm{a}}$ of Important Conformers of the Migratory Insertion Transition State of Other Substrates

\begin{tabular}{|c|c|c|c|}
\hline conformer identity $^{\mathrm{b}}$ & $\begin{array}{l}\text { free energy of } \\
\text { contributing } \\
\text { conformers } G i\end{array}$ & probability $P i$ & $\begin{array}{l}\text { Boltzmann weighted } \\
\text { free energy } P i^{*} G i\end{array}$ \\
\hline \multicolumn{4}{|l|}{ S1 } \\
\hline \multicolumn{4}{|l|}{ TS1 $_{\text {IIL-si }}$} \\
\hline$\psi-1 \mathrm{~s} 2 \mathrm{a} 3 \mathrm{a} 4 \mathrm{a}$ & -4361.979931 & 0.249936 & -1090.219027 \\
\hline$\psi$-1s2a3a4s & -4361.983529 & 0.250019 & -1090.581889 \\
\hline$\psi-1 \mathrm{~s} 2 \mathrm{a} 3 \mathrm{~s} 4 \mathrm{a}$ & -4361.983606 & 0.250021 & -1090.589643 \\
\hline \multirow[t]{2}{*}{$\psi-1 \mathrm{~s} 2 \mathrm{a} 3 \mathrm{~s} 4 \mathrm{~s}$} & -4361.983630 & 0.250022 & -1090.592114 \\
\hline & Sum & 1 & -4361.982674 \\
\hline \multicolumn{4}{|l|}{ TS1 $_{\text {IIL-re }}$} \\
\hline$\psi-1 \mathrm{a} 2 \mathrm{~s} 3 \mathrm{a} 4 \mathrm{a}$ & -4361.979391 & 0.249980 & -1090.411547 \\
\hline$\psi-1 \mathrm{a} 2 \mathrm{~s} 3 \mathrm{a} 4 \mathrm{a}$ & -4361.979442 & 0.249982 & -1090.416713 \\
\hline$\psi-1 \mathrm{a} 2 \mathrm{~s} 3 \mathrm{a} 4 \mathrm{a}$ & -4361.981138 & 0.250021 & -1090.587749 \\
\hline \multirow[t]{2}{*}{$\psi-1 a 2 s 3 a 4 a$} & -4361.980904 & 0.250015 & -1090.564209 \\
\hline & Sum & 1 & -4361.980219 \\
\hline \multicolumn{4}{|l|}{ S2 } \\
\hline \multicolumn{4}{|l|}{$\mathrm{TS1}_{\mathrm{IL} L-s i}$} \\
\hline$\psi-1 \mathrm{~s} 2 \mathrm{a} 3 \mathrm{a} 4 \mathrm{a}$ & -4401.274554 & 0.250020 & -1100.407021 \\
\hline$\psi$-1s2a3a4s & -4401.271181 & 0.249942 & -1100.063791 \\
\hline$\psi-1 \mathrm{~s} 2 \mathrm{a} 3 \mathrm{~s} 4 \mathrm{a}$ & -4401.274847 & 0.250026 & -1100.436879 \\
\hline \multirow[t]{2}{*}{$\psi-1 \mathrm{~s} 2 \mathrm{a} 3 \mathrm{~s} 4 \mathrm{~s}$} & -4401.274151 & 0.250010 & -1100.365990 \\
\hline & Sum & 1 & -4401.273683 \\
\hline \multicolumn{4}{|l|}{ TS1 $_{\text {IIL-re }}$} \\
\hline$\psi-1 \mathrm{a} 2 \mathrm{~s} 3 \mathrm{a} 4 \mathrm{a}$ & -4401.271048 & 0.250004 & -1100.337855 \\
\hline
\end{tabular}




\begin{tabular}{|c|c|c|c|}
\hline \multicolumn{4}{|l|}{ Table S14 Continued... } \\
\hline$\psi-1 \mathrm{a} 2 \mathrm{~s} 3 \mathrm{a} 4 \mathrm{a}$ & -4401.270598 & 0.249994 & -1100.292088 \\
\hline$\psi-1 \mathrm{a} 2 \mathrm{~s} 3 \mathrm{a} 4 \mathrm{a}$ & -4401.270699 & 0.249996 & -1100.302368 \\
\hline \multirow[t]{2}{*}{$\psi-1 a 2 s 3 a 4 a$} & -4401.271054 & 0.250004 & -1100.338538 \\
\hline & Sum & 1 & -4401.270850 \\
\hline \multicolumn{4}{|l|}{ S3 } \\
\hline \multicolumn{4}{|l|}{ TS1 $_{\text {IL } L-s i}$} \\
\hline$\psi-1 \mathrm{~s} 2 a 3 a 4 a$ & -4699.033072 & 0.249996 & -1174.739961 \\
\hline$\psi$-1s2a3a4s & -4699.033021 & 0.249994 & -1174.734423 \\
\hline$\psi-1 s 2 a 3 s 4 a$ & -4699.033320 & 0.250001 & -1174.766912 \\
\hline \multirow[t]{2}{*}{$\psi-1 \mathrm{~s} 2 \mathrm{a} 3 \mathrm{~s} 4 \mathrm{~s}$} & -4699.033550 & 0.250007 & -1174.791944 \\
\hline & Sum & 1 & -4699.033241 \\
\hline \multicolumn{4}{|l|}{ TS1 $1_{\text {IIL-re }}$} \\
\hline$\psi-1 \mathrm{a} 2 \mathrm{~s} 3 \mathrm{a} 4 \mathrm{a}$ & -4699.030679 & 0.249993 & -1174.726540 \\
\hline$\psi-1 \mathrm{a} 2 \mathrm{~s} 3 \mathrm{a} 4 \mathrm{a}$ & -4699.030337 & 0.249985 & -1174.689331 \\
\hline$\psi-1 \mathrm{a} 2 \mathrm{~s} 3 \mathrm{a} 4 \mathrm{a}$ & -4699.031043 & 0.250001 & -1174.766092 \\
\hline \multirow[t]{2}{*}{$\psi-1 \mathrm{a} 2 \mathrm{~s} 3 \mathrm{a} 4 \mathrm{a}$} & -4699.031806 & 0.250019 & -1174.849002 \\
\hline & Sum & 1 & -4699.030967 \\
\hline \multicolumn{4}{|l|}{ S4 } \\
\hline \multicolumn{4}{|l|}{ TS1 $_{I I L-s i}$} \\
\hline$\psi-1 \mathrm{~s} 2 \mathrm{a} 3 \mathrm{a} 4 \mathrm{a}$ & -4461.230973 & 0.249987 & -1115.253635 \\
\hline$\psi$-1s2a3a4s & -4461.231341 & 0.249996 & -1115.291665 \\
\hline$\psi-1 \mathrm{~s} 2 \mathrm{a} 3 \mathrm{~s} 4 \mathrm{a}$ & -4461.231775 & 0.250006 & -1115.336439 \\
\hline \multirow[t]{2}{*}{$\psi-1 \mathrm{~s} 2 \mathrm{a} 3 \mathrm{~s} 4 \mathrm{~s}$} & -4461.231904 & 0.250009 & -1115.349758 \\
\hline & Sum & 1 & -4461.231499 \\
\hline \multicolumn{4}{|l|}{ TS1 $1_{\text {IIL-re }}$} \\
\hline$\psi-1 \mathrm{a} 2 \mathrm{~s} 3 \mathrm{a} 4 \mathrm{a}$ & -4461.226107 & 0.249988 & -1115.255138 \\
\hline$\psi-1 \mathrm{a} 2 \mathrm{~s} 3 \mathrm{a} 4 \mathrm{a}$ & -4461.226630 & 0.250001 & -1115.309079 \\
\hline$\psi-1 a 2 s 3 a 4 a$ & -4461.226912 & 0.250007 & -1115.338172 \\
\hline \multirow[t]{2}{*}{$\psi-1 a 2 s 3 a 4 a$} & -4461.226777 & 0.250003 & -1115.324216 \\
\hline & Sum & 1 & -4461.226606 \\
\hline \multicolumn{4}{|l|}{ S5 } \\
\hline \multicolumn{4}{|l|}{ TS1 $_{I L-s i}$} \\
\hline$\psi-1 \mathrm{~s} 2 \mathrm{a} 3 \mathrm{a} 4 \mathrm{a}$ & -4821.602341 & 0.250002 & -1205.413283 \\
\hline$\psi-1 \mathrm{~s} 2 \mathrm{a} 3 \mathrm{a} 4 \mathrm{~s}$ & -4821.601471 & 0.249982 & -1205.316283 \\
\hline$\psi-1 \mathrm{~s} 2 \mathrm{a} 3 \mathrm{~s} 4 \mathrm{a}$ & -4821.602964 & 0.250017 & -1205.482719 \\
\hline$\psi-1 \mathrm{~s} 2 \mathrm{a} 3 \mathrm{~s} 4 \mathrm{~s}$ & -4821.602132 & 0.249997 & -1205.389940 \\
\hline
\end{tabular}




\begin{tabular}{|c|c|c|c|}
\hline & Sum & 1 & -4821.602227 \\
\hline Table S14 Continued... & & & \\
\hline TS1 $_{\text {IIL-re }}$ & & & \\
\hline$\psi-1 \mathbf{a 2 s 3 a 4 a}$ & -4821.599876 & 0.249998 & -1205.390785 \\
\hline$\psi-1 \mathbf{a 2 s 3 a 4 a}$ & -4821.599701 & 0.249994 & -1205.371274 \\
\hline$\psi-1 \mathbf{a 2 s 3 a 4 a}$ & -4821.600210 & 0.250005 & -1205.428047 \\
\hline$\psi-1 \mathbf{a 2 s 3 a 4 a}$ & -4821.600047 & 0.250002 & -1205.409851 \\
\hline & Sum & 1 & -4821.599959 \\
\hline
\end{tabular}

${ }^{a}$ Energies in hartree/particle. ${ }^{b}$ All the obtained conformers are considered.

-----End of Part-A----- 


\section{Part-B}

\section{Table of Contents}

\begin{tabular}{lr}
\hline \multicolumn{1}{c}{ details } & page \\
\hline Cartesian Coordinates of Reactants & S41 \\
Cartesian Coordinates of $\mathbf{1}$ & S42 \\
Cartesian Coordinates of $\mathbf{2}$ & S646 \\
Cartesian Coordinates of 3 & S841 \\
Cartesian Coordinates of TS1 & S1282 \\
Cartesian Coordinates of 4-9, TS2, TS3, and TS4 & S1706 \\
Cartesian Coordinates of TS1 for Additional Substrates & S1807 \\
Single Point Energies, Quasi-Harmonic Corrections, and Gibbs Free & S1919 \\
Energies of Various Stationary Points & \\
\hline
\end{tabular}


The optimized cartesian coordinates of all the stationary points obtained at the $\mathrm{SMD}_{(\mathrm{toluene})} / \mathrm{M06} / 6-$

$31 G * *, S D D(R h)$ level of theory

1. Cartesian coordinates for reactants

$\alpha$-methyl styrene

$\begin{array}{lcc}\text { Number of imaginary frequencies : } 0 & & \\ \text { The smallest frequencies are }: 55.8750 & 148.4294 & 226.5359 \mathrm{~cm}(-1) \\ \text { Electronic energy : } \mathrm{HF}=-348.7009588 & \\ \text { Zero-point correction= } & 0.160973 & \text { (Hartree/Particle) } \\ \text { Thermal correction to Energy= } & 0.169000 \\ \text { Thermal correction to Enthalpy= } & 0.169944 \\ \text { Thermal correction to Gibbs Free Energy= } & 0.128261 \\ \text { Sum of electronic and zero-point Energies= } & -348.539986 \\ \text { Sum of electronic and thermal Energies= } & -348.531959 \\ \text { Sum of electronic and thermal Enthalpies }= & -348.531015 \\ \text { Sum of electronic and thermal Free Energies }= & -348.572698\end{array}$

Cartesian Coordinates

$\begin{array}{lrrr}6 & 0.572192 & 1.207912 & -0.184291 \\ 6 & -0.206209 & 0.057961 & 0.008736 \\ 6 & 0.461756 & -1.161384 & 0.176350 \\ 6 & 1.850597 & -1.225759 & 0.181266 \\ 6 & 2.606144 & -0.072702 & 0.005408 \\ 6 & 1.958168 & 1.145409 & -0.181522 \\ 6 & -1.686165 & 0.122855 & 0.021502 \\ 6 & -2.439729 & -1.129648 & -0.317234 \\ 6 & -2.343647 & 1.244320 & 0.339594 \\ 1 & -3.431010 & 1.278043 & 0.326529 \\ 1 & -1.834922 & 2.155650 & 0.645005 \\ 1 & -0.109557 & -2.076102 & 0.318050 \\ 1 & 2.343523 & -2.185180 & 0.322276 \\ 1 & 3.692499 & -0.122434 & 0.003179 \\ 1 & 2.537390 & 2.052610 & -0.339371 \\ 1 & 0.080560 & 2.161434 & -0.365937 \\ 1 & -2.315012 & -1.897887 & 0.457514 \\ 1 & -3.511747 & -0.929139 & -0.406722 \\ 1 & -2.090371 & -1.570774 & -1.259384\end{array}$

\section{CO}

Number of imaginary frequencies : 0

The smallest frequencies are : 2222.2175

Electronic energy : $\quad H F=-113.247326$

Zero-point correction $=\quad 0.005063$ (Hartree/Particle)

Thermal correction to Energy $=\quad 0.007423$

Thermal correction to Enthalpy= $\quad 0.008367$

Thermal correction to Gibbs Free Energy $=\quad-0.014075$

Sum of electronic and zero-point Energies $=\quad-113.242263$ 
Sum of electronic and thermal Energies=

Sum of electronic and thermal Enthalpies=

Sum of electronic and thermal Free Energies=
$-113.239903$

$-113.238959$

$-113.261401$

Cartesian Coordinates

$\begin{array}{llll}6 & 0.000000 & 0.000000 & 0.081426\end{array}$

$\begin{array}{llll}8 & 0.000000 & 0.000000 & 1.218574\end{array}$

$\mathbf{H}_{2}$

Number of imaginary frequencies : 0

The smallest frequencies are : 4442.8830

Electronic energy : $\quad H F=-1.1690875$

Zero-point correction $=$

Thermal correction to Energy=

Thermal correction to Enthalpy=

0.010122 (Hartree/Particle)

0.013426

Thermal correction to Gibbs Free Energy $=\quad-0.001365$

Sum of electronic and zero-point Energies $=\quad-1.158966$

Sum of electronic and thermal Energies $=\quad-1.156605$

Sum of electronic and thermal Enthalpies $=\quad-1.155661$

Sum of electronic and thermal Free Energies $=\quad-1.170452$

Cartesian Coordinates

$1 \quad 0.000000 \quad 0.000000 \quad 0.028770$

$1 \quad 0.000000 \quad 0.000000 \quad 0.771230$

\section{Cartesian coordinates for intermediate 1}

$1_{\mathrm{C} 1-} \psi_{-1 \mathbf{a} 2 \mathbf{a} 3 \mathrm{a} 4 \mathrm{a}}$

Number of imaginary frequencies : 0

The smallest frequencies are : $15.6483 \quad 18.6820 \quad 20.3657 \mathrm{~cm}(-1)$

Electronic energy : $\quad H F=-4125.4699915$

Zero-point correction $=\quad 1.299138$ (Hartree/Particle)

Thermal correction to Energy $=\quad 1.377555$

Thermal correction to Enthalpy= $\quad 1.378499$

Thermal correction to Gibbs Free Energy $=\quad 1.187437$

Sum of electronic and zero-point Energies $=\quad-4124.170853$

Sum of electronic and thermal Energies $=\quad-4124.092436$

Sum of electronic and thermal Enthalpies= $\quad-4124.091492$

Sum of electronic and thermal Free Energies $=\quad-4124.282555$

\begin{tabular}{cccc}
\multicolumn{4}{c}{ Cartesian Coordinates } \\
$\ldots \ldots \ldots \ldots \ldots \ldots \ldots \ldots \ldots \ldots \ldots \ldots \ldots \ldots \ldots \ldots \ldots \ldots \ldots$ \\
6 & 3.971551 & -0.005799 & 4.156609 \\
6 & 4.786786 & -0.819097 & 3.377455 \\
6 & 4.227226 & -1.634679 & 2.396616 \\
6 & 2.851268 & -1.641152 & 2.177923 \\
6 & 2.040075 & -0.818576 & 2.961510 \\
6 & 2.593503 & -0.011179 & 3.945900
\end{tabular}




\begin{tabular}{|c|c|c|c|}
\hline 6 & 2.259099 & -2.552540 & 1.135708 \\
\hline 7 & 1.313715 & -1.914507 & 0.187117 \\
\hline 6 & 0.210999 & -2.731280 & -0.243219 \\
\hline 6 & -1.007381 & -2.671465 & 0.410267 \\
\hline 6 & -2.105586 & -3.441357 & -0.083166 \\
\hline 5 & -1.924912 & -4.267243 & -1.232411 \\
\hline 6 & -0.655470 & -4.320044 & -1.851984 \\
\hline 6 & 0.383079 & -3.570831 & -1.368160 \\
\hline 6 & -3.388465 & -3.400679 & 0.523160 \\
\hline 6 & -4.428983 & -4.140170 & 0.020259 \\
\hline 6 & -4.241835 & -4.969101 & -1.107862 \\
\hline 6 & -3.017102 & -5.025249 & -1.722508 \\
\hline 6 & -1.180975 & -1.875713 & 1.656736 \\
\hline 6 & -1.489418 & -0.523305 & 1.634760 \\
\hline 6 & -1.710282 & 0.156770 & 2.863947 \\
\hline 6 & -1.602471 & -0.487789 & 4.066884 \\
\hline 6 & -1.270042 & -1.860631 & 4.124146 \\
\hline 6 & -1.067278 & -2.569823 & 2.904714 \\
\hline 6 & -1.130483 & -2.538653 & 5.358488 \\
\hline 6 & -0.803326 & -3.869945 & 5.395465 \\
\hline 6 & -0.606445 & -4.579515 & 4.192152 \\
\hline 6 & -0.738443 & -3.948971 & 2.979663 \\
\hline 15 & -1.672747 & 0.353022 & 0.005091 \\
\hline 45 & -0.155369 & 0.324712 & -1.955538 \\
\hline 6 & 0.411629 & 2.174864 & -2.043232 \\
\hline 8 & 0.550084 & 3.311036 & -2.204017 \\
\hline 15 & 1.668122 & -0.657825 & -0.885347 \\
\hline 8 & 2.627499 & 0.221397 & 0.119362 \\
\hline 6 & 3.507566 & 1.127979 & -0.429834 \\
\hline 6 & 4.688233 & 0.684863 & -0.993967 \\
\hline 6 & 5.553434 & 1.659749 & -1.590616 \\
\hline 6 & 5.216534 & 3.044779 & -1.498826 \\
\hline 6 & 4.015847 & 3.432051 & -0.857519 \\
\hline 6 & 3.165319 & 2.490126 & -0.347365 \\
\hline 6 & 6.074603 & 4.011512 & -2.077219 \\
\hline 6 & 7.210897 & 3.636974 & -2.746369 \\
\hline 6 & 7.527263 & 2.268318 & -2.873030 \\
\hline 6 & 6.722655 & 1.307764 & -2.312274 \\
\hline 6 & 4.981867 & -0.766283 & -1.026694 \\
\hline 6 & 4.050480 & -1.639719 & -1.559383 \\
\hline 6 & 4.326111 & -3.011172 & -1.745370 \\
\hline 6 & 5.513754 & -3.533159 & -1.312116 \\
\hline 6 & 6.455339 & -2.714680 & -0.641426 \\
\hline 6 & 6.188825 & -1.320977 & -0.487583 \\
\hline 6 & 7.107344 & -0.547081 & 0.266346 \\
\hline 6 & 8.232448 & -1.114872 & 0.812096 \\
\hline 6 & 8.509950 & -2.485390 & 0.626951 \\
\hline 6 & 7.635382 & -3.265511 & -0.085275 \\
\hline 8 & 2.817942 & -1.188030 & -1.977492 \\
\hline 6 & -3.435634 & -0.012517 & -0.370439 \\
\hline 6 & -4.440279 & 0.263605 & 0.560780 \\
\hline 6 & -5.776629 & -0.004600 & 0.270417 \\
\hline 6 & -6.071701 & -0.565451 & -0.978597 \\
\hline 6 & -5.092406 & -0.862346 & -1.927858 \\
\hline 6 & -3.763830 & -0.579599 & -1.597605 \\
\hline 6 & -6.898216 & 0.264838 & 1.272266 \\
\hline
\end{tabular}




\begin{tabular}{|c|c|c|c|}
\hline & -7.463751 & -1.077008 & 1.751115 \\
\hline & -5.485207 & -1.417805 & -3.295623 \\
\hline & -4.266216 & -1.860227 & -4.103574 \\
\hline & -1.682415 & 2.119367 & 0.500459 \\
\hline & -2.632918 & 3.001354 & -0.008043 \\
\hline & -2.543415 & 4.373266 & 0.242992 \\
\hline & -1.489154 & 4.824980 & 1.039043 \\
\hline & -0.523654 & 3.965205 & 1.574813 \\
\hline & -0.617436 & 2.609614 & 1.262408 \\
\hline & -3.556678 & 5.371139 & -0.314770 \\
\hline & 0.580331 & 4.521375 & 2.473283 \\
\hline & -0.314510 & -0.975729 & -3.358657 \\
\hline & -0.408985 & -1.666109 & -4.283297 \\
\hline & -6.404682 & 1.041189 & 2.491874 \\
\hline & -8.014310 & 1.082498 & 0.614446 \\
\hline & -6.210994 & -0.316710 & -4.077793 \\
\hline & -6.414610 & -2.624511 & -3.140520 \\
\hline & 6.897326 & 0.506260 & 0.434269 \\
\hline & -1.968202 & 1.212693 & 2.851675 \\
\hline & 7.823301 & -4.329525 & -0.223290 \\
\hline & -1.286579 & -1.977382 & 6.278677 \\
\hline & 5.801173 & 5.062026 & -1.987842 \\
\hline & -3.547016 & -2.758663 & 1.387773 \\
\hline & -5.409117 & -4.083653 & 0.489520 \\
\hline & 3.765983 & 4.490783 & -0.801345 \\
\hline & -1.775612 & 0.050881 & 4.997835 \\
\hline & 5.738585 & -4.589180 & -1.451837 \\
\hline & 8.414094 & 1.969278 & -3.427334 \\
\hline & 3.577802 & -3.627437 & -2.238783 \\
\hline - & -2.860139 & -5.654447 & -2.597576 \\
\hline & -0.521204 & -4.954305 & -2.726747 \\
\hline & -0.350850 & -5.636100 & 4.225792 \\
\hline & 6.975862 & 0.257754 & -2.432193 \\
\hline & -0.590908 & -4.510818 & 2.059483 \\
\hline 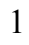 & 1.356162 & -3.590458 & -1.855796 \\
\hline & 7.859553 & 4.387711 & -3.191620 \\
\hline 1 & 8.913415 & -0.501577 & 1.398051 \\
\hline & -5.073050 & -5.559018 & -1.488669 \\
\hline 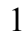 & -0.695331 & -4.381796 & 6.348869 \\
\hline 1 & 9.407898 & -2.920642 & 1.059168 \\
\hline & 2.217517 & 2.756734 & 0.117129 \\
\hline 1 & -4.167200 & 0.706000 & 1.516176 \\
\hline & -2.957189 & -0.797932 & -2.293389 \\
\hline 1 & -7.113500 & -0.775751 & -1.220447 \\
\hline & -3.443546 & 2.607717 & -0.616256 \\
\hline 1 & 0.135662 & 1.909596 & 1.626391 \\
\hline l & -1.415871 & 5.891357 & 1.252681 \\
\hline 1 & 1.671297 & -3.332905 & 1.639799 \\
\hline 1 & -1.430004 & 0.977498 & -2.681671 \\
\hline & -5.653364 & 0.481090 & 3.063816 \\
\hline 1 & -5.968523 & 2.009075 & 2.208679 \\
\hline l & -7.245928 & 1.240407 & 3.167606 \\
\hline 1 & -8.477385 & 0.557795 & -0.229278 \\
\hline 1 & -8.808662 & 1.291799 & 1.343142 \\
\hline 1 & -7.634783 & 2.043385 & 0.243619 \\
\hline 1 & -7.862015 & -1.671832 & 0.919433 \\
\hline
\end{tabular}




$\begin{array}{lrrr}1 & -6.686002 & -1.672812 & 2.247530 \\ 1 & -8.278703 & -0.917622 & 2.470376 \\ 1 & -5.566202 & 0.562305 & -4.207743 \\ 1 & -6.496285 & -0.678666 & -5.075029 \\ 1 & -7.124419 & 0.011172 & -3.565566 \\ 1 & -7.348529 & -2.373360 & -2.623473 \\ 1 & -6.685932 & -3.020231 & -4.128274 \\ 1 & -5.919704 & -3.426530 & -2.577613 \\ 1 & -3.598741 & -1.020620 & -4.338439 \\ 1 & -3.681326 & -2.625188 & -3.573051 \\ 1 & -4.593267 & -2.291120 & -5.058341 \\ 1 & 3.065663 & -3.069722 & 0.593592 \\ 1 & 4.873835 & -2.262974 & 1.781748 \\ 1 & 0.969101 & -0.792427 & 2.772284 \\ 1 & 5.865207 & -0.818571 & 3.523779 \\ 1 & 1.942351 & 0.618458 & 4.55114 \\ 1 & 4.406037 & 0.632275 & 4.923291 \\ 6 & 1.439505 & 3.413202 & 3.081179 \\ 6 & 1.479737 & 5.454891 & 1.656893 \\ 6 & -0.046500 & 5.307062 & 3.631037 \\ 6 & -4.651645 & 4.688384 & -1.131529 \\ 6 & -4.226111 & 6.124299 & 0.839907 \\ 6 & -2.831165 & 6.367777 & -1.225251 \\ 1 & 2.181568 & 3.850004 & 3.761648 \\ 1 & 0.830034 & 2.706018 & 3.661986 \\ 1 & 1.993454 & 2.839576 & 2.326605 \\ 1 & 0.738866 & 5.700418 & 4.290246 \\ 1 & -0.641598 & 6.160199 & 3.284836 \\ 1 & -0.701931 & 4.664277 & 4.233176 \\ 1 & 2.293563 & 5.847946 & 2.281168 \\ 1 & 1.929334 & 4.925452 & 0.805969 \\ 1 & 0.921991 & 6.309801 & 1.255436 \\ 1 & -5.362327 & 5.440884 & -1.495827 \\ 1 & -4.246737 & 4.167007 & -2.008207 \\ 1 & -5.218434 & 3.961300 & -0.533504 \\ 1 & -3.544711 & 7.085607 & -1.652118 \\ 1 & -2.068340 & 6.939892 & -0.683273 \\ 1 & -2.332144 & 5.848860 & -2.053971 \\ 1 & -4.958031 & 6.844792 & 0.450713 \\ 1 & -4.755851 & 5.431058 & 1.506518 \\ 1 & -3.502849 & 6.683347 & 1.445668\end{array}$

$1_{\mathrm{C} 1-} \psi_{-1 \mathrm{a} 2 \mathrm{a} 3 \mathrm{a} 4 \mathrm{~s}}$

Number of imaginary frequencies : 0

The smallest frequencies are : $6.5220 \quad 16.6131 \quad 18.9492 \mathrm{~cm}(-1)$

Electronic energy : $\quad H F=-4125.4705291$

Zero-point correction $=\quad 1.298997($ Hartree/Particle $)$

Thermal correction to Energy $=\quad 1.377594$

Thermal correction to Enthalpy= $\quad 1.378538$

Thermal correction to Gibbs Free Energy= $\quad 1.185175$

Sum of electronic and zero-point Energies $=\quad-4124.171532$

Sum of electronic and thermal Energies $=\quad-4124.092935$

Sum of electronic and thermal Enthalpies $=\quad-4124.091991$

Sum of electronic and thermal Free Energies $=\quad-4124.285354$ 
Cartesian Coordinates

\begin{tabular}{|c|c|c|c|}
\hline 6 & 2.047953 & -0.775731 & 2.941155 \\
\hline 6 & 2.864051 & -1.611413 & 2.176634 \\
\hline 6 & 4.237319 & -1.609045 & 2.411693 \\
\hline 6 & 4.789101 & -0.785955 & 3.390799 \\
\hline 6 & 3.968865 & 0.040129 & 4.150883 \\
\hline 6 & 2.593554 & 0.040319 & 3.922642 \\
\hline 6 & 2.281199 & -2.531653 & 1.136616 \\
\hline 7 & 1.338848 & -1.905096 & 0.177576 \\
\hline 15 & 1.688512 & -0.646112 & -0.893531 \\
\hline 45 & -0.136062 & 0.331596 & -1.964662 \\
\hline 6 & 0.426482 & 2.183491 & -2.049627 \\
\hline 8 & 0.561090 & 3.319965 & -2.211659 \\
\hline 6 & 0.236530 & -2.724321 & -0.248265 \\
\hline 6 & -0.977744 & -2.670424 & 0.413351 \\
\hline 6 & -2.077932 & -3.439936 & -0.075506 \\
\hline 6 & -1.905443 & -4.256086 & -1.232672 \\
\hline 6 & -0.640050 & -4.303494 & -1.861142 \\
\hline 6 & 0.401829 & -3.557413 & -1.379057 \\
\hline 6 & -3.356213 & -3.403382 & 0.540802 \\
\hline 6 & -4.401352 & -4.135866 & 0.037604 \\
\hline 6 & -4.222973 & -4.954524 & -1.099448 \\
\hline 6 & -3.002099 & -5.008113 & -1.722118 \\
\hline 6 & -1.151204 & -1.870560 & 1.656719 \\
\hline 6 & -1.467599 & -0.520203 & 1.624573 \\
\hline 6 & -1.695003 & 0.166936 & 2.848499 \\
\hline 6 & -1.590398 & -0.470562 & 4.055804 \\
\hline 6 & -1.251229 & -1.841279 & 4.122989 \\
\hline 6 & -1.038032 & -2.556294 & 2.908816 \\
\hline 6 & -1.115945 & -2.512054 & 5.361794 \\
\hline 6 & -0.783141 & -3.841669 & 5.407695 \\
\hline 6 & -0.576031 & -4.556945 & 4.209384 \\
\hline 6 & -0.703669 & -3.933530 & 2.992820 \\
\hline 15 & -1.659103 & 0.339394 & -0.013535 \\
\hline 6 & -3.411208 & -0.069418 & -0.393393 \\
\hline 6 & -4.411229 & 0.159961 & 0.546504 \\
\hline 6 & -5.749523 & -0.123181 & 0.262056 \\
\hline 6 & -6.049385 & -0.642670 & -0.998113 \\
\hline 6 & -5.065681 & -0.900160 & -1.962399 \\
\hline 6 & -3.741482 & -0.610519 & -1.635619 \\
\hline 6 & -6.802594 & 0.146592 & 1.334368 \\
\hline 6 & -5.461498 & -1.427209 & -3.340458 \\
\hline 6 & -1.727881 & 2.107538 & 0.471525 \\
\hline 6 & -0.668315 & 2.641178 & 1.211190 \\
\hline 6 & -0.625078 & 3.999263 & 1.524129 \\
\hline 6 & -1.639549 & 4.817262 & 1.014009 \\
\hline 6 & -2.689046 & 4.322835 & 0.236929 \\
\hline 6 & -2.725723 & 2.948803 & -0.014712 \\
\hline 6 & -3.767866 & 5.272610 & -0.280813 \\
\hline 6 & 0.489079 & 4.602950 & 2.378514 \\
\hline 8 & 2.845640 & -1.166833 & -1.981851 \\
\hline 6 & 4.075314 & -1.618843 & -1.555348 \\
\hline 6 & 5.001980 & -0.745784 & -1.013840 \\
\hline 6 & 6.204178 & -1.301376 & -0.464912 \\
\hline 6 & 6.471330 & -2.695160 & -0.617338 \\
\hline
\end{tabular}




\begin{tabular}{|c|c|c|c|}
\hline ) & 5.535452 & -3.512923 & -1.296851 \\
\hline & 4.351782 & -2.990222 & -1.740001 \\
\hline 6 & 7.645727 & -3.247150 & -0.050430 \\
\hline & 8.513989 & -2.468147 & 0.670676 \\
\hline & 8.235761 & -1.097529 & 0.853943 \\
\hline & 7.116269 & -0.528612 & 0.297946 \\
\hline & 4.707167 & 0.705259 & -0.979176 \\
\hline & 3.521861 & 1.145788 & -0.422762 \\
\hline & 3.180047 & 2.507642 & -0.334192 \\
\hline 6 & 4.035564 & 3.452433 & -0.830187 \\
\hline 6 & 5.240911 & 3.068176 & -1.464732 \\
\hline & 5.577422 & 1.683410 & -1.562943 \\
\hline 6 & 6.752414 & 1.335124 & -2.276908 \\
\hline 6 & 7.562538 & 2.298598 & -2.824587 \\
\hline 6 & 7.246264 & 3.666665 & -2.691559 \\
\hline U & 6.104713 & 4.037846 & -2.029465 \\
\hline 8 & 2.637857 & 0.237712 & 0.116162 \\
\hline 6 & -0.289463 & -0.961477 & -3.375382 \\
\hline 8 & -0.383084 & -1.647201 & -4.303536 \\
\hline 1 & 6.905283 & 0.524659 & 0.464995 \\
\hline 1 & -1.957511 & 1.221624 & 2.828642 \\
\hline 1 & 7.834214 & -4.311209 & -0.187314 \\
\hline 1 & -1.280062 & -1.946699 & 6.278072 \\
\hline 1 & 5.831568 & 5.088020 & -1.935312 \\
\hline 1 & -3.507483 & -2.769625 & 1.413205 \\
\hline 1 & -5.378341 & -4.081022 & 0.513747 \\
\hline 1 & 3.786541 & 4.510977 & -0.767034 \\
\hline 1 & -1.770306 & 0.072820 & 4.982721 \\
\hline 1 & 5.761030 & -4.568994 & -1.434977 \\
\hline 1 & 8.453786 & 2.002410 & -3.373311 \\
\hline 1 & 3.606908 & -3.605647 & -2.239676 \\
\hline 1 & -2.852114 & -5.630103 & -2.603558 \\
\hline 1 & -0.511431 & -4.931056 & -2.741591 \\
\hline 1 & -0.316160 & -5.612228 & 4.250357 \\
\hline 1 & 7.005707 & 0.285638 & -2.401347 \\
\hline 1 & -0.548867 & -4.499361 & 2.076269 \\
\hline 1 & 1.371737 & -3.573654 & -1.873285 \\
\hline 1 & 7.899328 & 4.419717 & -3.126329 \\
\hline 1 & 8.911525 & -0.485079 & 1.446775 \\
\hline 1 & -5.057676 & -5.539531 & -1.480202 \\
\hline 1 & -0.678656 & -4.347994 & 6.364433 \\
\hline 1 & 9.407408 & -2.904350 & 1.111248 \\
\hline 1 & 2.229169 & 2.772015 & 0.125187 \\
\hline 1 & -4.144970 & 0.578111 & 1.517143 \\
\hline 1 & -2.936539 & -0.799529 & -2.341808 \\
\hline 1 & -7.087036 & -0.856055 & -1.246458 \\
\hline 1 & -3.533324 & 2.521333 & -0.603882 \\
\hline 1 & 0.120861 & 1.972201 & 1.557704 \\
\hline 1 & -1.609437 & 5.884452 & 1.233114 \\
\hline 1 & 1.694272 & -3.311515 & 1.642445 \\
\hline 1 & -1.413657 & 0.982646 & -2.687433 \\
\hline 1 & 3.093168 & -3.048813 & 0.602664 \\
\hline 1 & 4.888379 & -2.246321 & 1.810867 \\
\hline 1 & 0.979956 & -0.744058 & 2.736861 \\
\hline 1 & 5.865643 & -0.789296 & 3.550277 \\
\hline 1 & 1.938365 & 0.680835 & 4.511807 \\
\hline
\end{tabular}




$\begin{array}{rrrr}1 & 4.397448 & 0.684469 & 4.915631 \\ 6 & 1.363596 & 3.528038 & 3.024201 \\ 6 & 1.366595 & 5.498640 & 1.497348 \\ 6 & -0.115027 & 5.444104 & 3.508554 \\ 1 & 2.103647 & 3.999054 & 3.683564 \\ 1 & 0.763337 & 2.837573 & 3.633861 \\ 1 & 1.922061 & 2.931401 & 2.291411 \\ 1 & 0.683799 & 5.866273 & 4.132606 \\ 1 & -0.713238 & 6.282818 & 3.134066 \\ 1 & -0.760005 & 4.833283 & 4.153636 \\ 1 & 2.197493 & 5.918814 & 2.080298 \\ 1 & 1.791022 & 4.933905 & 0.656058 \\ 1 & 0.795061 & 6.334290 & 1.074824 \\ 6 & -4.791694 & 4.559845 & -1.162168 \\ 6 & -4.509914 & 5.887873 & 0.910883 \\ 6 & -3.120028 & 6.385197 & -1.111424 \\ 1 & -5.544071 & 5.280127 & -1.507502 \\ 1 & -4.327569 & 4.114405 & -2.051590 \\ 1 & -5.321915 & 3.764784 & -0.620403 \\ 1 & -5.288102 & 6.579803 & 0.561230 \\ 1 & -4.996076 & 5.109250 & 1.513946 \\ 1 & -3.836243 & 6.449629 & 1.569479 \\ 1 & -3.889262 & 7.065648 & -1.500818 \\ 1 & -2.415753 & 6.986162 & -0.523926 \\ 1 & -2.571607 & 5.968124 & -1.965812 \\ 6 & -4.243801 & -1.838552 & -4.166568 \\ 6 & -6.376082 & -2.648227 & -3.207995 \\ 6 & -6.204789 & -0.316271 & -4.091699 \\ 6 & -8.206688 & -0.251394 & 0.887568 \\ 6 & -6.457295 & -0.659864 & 2.592322 \\ 6 & -6.812582 & 1.643155 & 1.668461 \\ 1 & -7.534098 & 1.852683 & 2.469768 \\ 1 & -5.829675 & 1.999836 & 2.002895 \\ 1 & -7.103038 & 2.235955 & 0.790935 \\ 1 & -8.923161 & -0.042684 & 1.692109 \\ 1 & -8.532560 & 0.312681 & 0.004265 \\ 1 & -8.274026 & -1.322505 & 0.656258 \\ 1 & -7.202382 & -0.479549 & 3.378884 \\ 1 & -6.449693 & -1.736508 & 2.375115 \\ 1 & -5.473247 & -0.397368 & 3.001368 \\ 1 & -4.573183 & -2.244525 & -5.131419 \\ 1 & -3.581129 & -0.989736 & -4.381135 \\ 1 & -3.652995 & -2.615159 & -3.660213 \\ 1 & -6.495036 & -0.658597 & -5.094438 \\ 1 & -7.117224 & -0.009318 & -3.564942 \\ 1 & -5.569757 & 0.571889 & -4.207016 \\ 1 & -6.644085 & -3.027485 & -4.203105 \\ 1 & -5.870267 & -3.455113 & -2.661728 \\ 1 & -7.312206 & -2.419280 & -2.684873\end{array}$

$1_{\mathrm{C} 1-} \psi_{-1 \mathrm{a} 2 \mathrm{a3s4a}}$

Number of imaginary frequencies: 0

The smallest frequencies are: $15.8095 \quad 18.8157 \quad 19.5155 \mathrm{~cm}(-1)$

Electronic energy:

$\mathrm{HF}=-4125.4693978$

Zero-point correction $=$

1.299028 (Hartree/Particle) 
Thermal correction to Energy= $\quad 1.377591$

Thermal correction to Enthalpy= $\quad 1.378535$

Thermal correction to Gibbs Free Energy= $\quad 1.186261$

Sum of electronic and zero-point Energies $=\quad-4124.170370$

Sum of electronic and thermal Energies $=\quad-4124.091807$

Sum of electronic and thermal Enthalpies $=\quad-4124.090863$

Sum of electronic and thermal Free Energies $=\quad-4124.283137$

Cartesian Coordinates

$\begin{array}{cccc}0 & 2.043032 & -0.780851 & 2.953306 \\ 6 & 2.862366 & -1.605047 & 2.179950 \\ 6 & 4.236511 & -1.594702 & 2.409772 \\ 6 & 4.786276 & -0.774235 & 3.392094 \\ 6 & 3.962989 & 0.040531 & 4.161149 \\ 6 & 2.586764 & 0.031924 & 3.938665 \\ 6 & 2.281816 & -2.521499 & 1.135590 \\ 7 & 1.328971 & -1.892954 & 0.187640 \\ 15 & 1.675776 & -0.635431 & -0.885811 \\ 45 & -0.145304 & 0.357930 & -1.949088 \\ 6 & 0.435002 & 2.202967 & -2.030626 \\ 8 & 0.576015 & 3.339589 & -2.186737 \\ 6 & 0.236393 & -2.725114 & -0.239400 \\ 6 & -0.987480 & -2.667889 & 0.403984 \\ 6 & -2.067779 & -3.472675 & -0.074669 \\ 6 & -1.855997 & -4.350613 & -1.179227 \\ 6 & -0.583205 & -4.391841 & -1.793256 \\ 6 & 0.431830 & -3.594121 & -1.337944 \\ 6 & -3.358474 & -3.431335 & 0.514323 \\ 6 & -4.372922 & -4.227796 & 0.045313 \\ 6 & -4.149407 & -5.119967 & -1.026002 \\ 6 & -2.918700 & -5.172535 & -1.628276 \\ 6 & -1.172258 & -1.866102 & 1.645552 \\ 6 & -1.488547 & -0.515598 & 1.623843 \\ 6 & -1.710173 & 0.164757 & 2.852842 \\ 6 & -1.597794 & -0.478201 & 4.056118 \\ 6 & -1.256665 & -1.848762 & 4.113883 \\ 6 & -1.050917 & -2.557889 & 2.894837 \\ 6 & -1.109941 & -2.523636 & 5.349151 \\ 6 & -0.771534 & -3.851968 & 5.387974 \\ 6 & -0.569955 & -4.561346 & 4.185379 \\ 6 & -0.709415 & -3.934043 & 2.972065 \\ 15 & -1.676092 & 0.360127 & -0.004320 \\ 6 & -3.427308 & -0.036835 & -0.398281 \\ 6 & -4.441598 & 0.147190 & 0.550763 \\ 6 & -5.767582 & -0.131730 & 0.239841 \\ 6 & -6.055735 & -0.603040 & -1.052984 \\ 6 & -5.072128 & -0.793445 & -2.018435 \\ 6 & -3.746602 & -0.512914 & -1.660213 \\ 6 & -6.892185 & 0.024176 & 1.262160 \\ 6 & -5.364944 & -1.273326 & -3.437572 \\ 6 & -1.732412 & 2.123760 & 0.498517 \\ 6 & -0.664373 & 2.642171 & 1.237014 \\ 6 & -0.610027 & 3.995945 & 1.565531 \\ 6 & -1.624681 & 4.825607 & 1.074926 \\ 6 & -2.686929 & 4.345761 & 0.306401 \\ & & & \\ 6 & & & \end{array}$




\begin{tabular}{|c|c|c|c|}
\hline & -2.731105 & 2.975713 & 0.033629 \\
\hline & -3.764230 & 5.309441 & -0.188591 \\
\hline & 0.513940 & 4.582421 & 2.418976 \\
\hline & 2.825247 & -1.158729 & -1.981741 \\
\hline & 4.057760 & -1.613153 & -1.566408 \\
\hline & 4.988516 & -0.744126 & -1.025342 \\
\hline & 6.192463 & -1.304037 & -0.484637 \\
\hline & 6.458078 & -2.696950 & -0.646677 \\
\hline & 5.519672 & -3.509172 & -1.329241 \\
\hline & 4.334327 & -2.982929 & -1.763532 \\
\hline & 7.634218 & -3.253277 & -0.087723 \\
\hline & 8.505588 & -2.479404 & 0.635142 \\
\hline & 8.228838 & -1.109821 & 0.828223 \\
\hline ) & 7.107849 & -0.536602 & 0.279711 \\
\hline & 4.698279 & 0.707504 & -0.984237 \\
\hline & 3.517697 & 1.150360 & -0.420040 \\
\hline & 3.182142 & 2.513216 & -0.324129 \\
\hline & 4.038295 & 3.456545 & -0.821679 \\
\hline D & 5.238247 & 3.069996 & -1.464980 \\
\hline & 5.568959 & 1.684317 & -1.569713 \\
\hline & 6.738465 & 1.333991 & -2.291642 \\
\hline & 7.548838 & 2.296225 & -2.841131 \\
\hline & 7.238390 & 3.665005 & -2.701939 \\
\hline & 6.102227 & 4.038280 & -2.031802 \\
\hline & 2.633317 & 0.243730 & 0.120344 \\
\hline 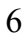 & -0.340138 & -0.933718 & -3.357069 \\
\hline & -0.483672 & -1.609276 & -4.286544 \\
\hline & 6.898091 & 0.515732 & 0.454180 \\
\hline & -1.974891 & 1.218947 & 2.839521 \\
\hline & 7.821548 & -4.316539 & -0.232184 \\
\hline & -1.269055 & -1.961908 & 6.268544 \\
\hline ] & 5.833545 & 5.089147 & -1.932675 \\
\hline & -3.543025 & -2.747385 & 1.341136 \\
\hline & -5.358169 & -4.171949 & 0.503104 \\
\hline & 3.793658 & 4.515780 & -0.753123 \\
\hline & -1.772801 & 0.060237 & 4.986840 \\
\hline ] & 5.744753 & -4.564020 & -1.477118 \\
\hline & 8.435691 & 1.998512 & -3.396118 \\
\hline 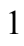 & 3.588860 & -3.594587 & -2.266773 \\
\hline ] & -2.735791 & -5.843566 & -2.466491 \\
\hline & -0.426440 & -5.061703 & -2.637223 \\
\hline l & -0.304561 & -5.615478 & 4.220070 \\
\hline & 6.987131 & 0.283998 & -2.420981 \\
\hline 1 & -0.557455 & -4.496744 & 2.053147 \\
\hline & 1.408185 & -3.608132 & -1.818878 \\
\hline 1 & 7.891574 & 4.417028 & -3.138311 \\
\hline 1 & 8.907101 & -0.501604 & 1.422554 \\
\hline 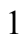 & -4.959323 & -5.754855 & -1.378834 \\
\hline 1 & -0.657745 & -4.361376 & 6.342009 \\
\hline 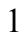 & 9.400345 & -2.918937 & 1.069640 \\
\hline 1 & 2.235310 & 2.779497 & 0.142178 \\
\hline ( & -4.178024 & 0.524639 & 1.536271 \\
\hline 1 & -2.941620 & -0.660764 & -2.378090 \\
\hline 1 & -7.091508 & -0.822839 & -1.303416 \\
\hline 1 & -3.546268 & 2.560154 & -0.553531 \\
\hline 1 & 0.122554 & 1.964021 & 1.570430 \\
\hline
\end{tabular}




\begin{tabular}{|c|c|c|c|}
\hline & -1.584988 & 5.890467 & 1.303840 \\
\hline ] & 1.703063 & -3.309840 & 1.637808 \\
\hline & -1.410141 & 1.033832 & -2.671228 \\
\hline 1 & 3.094856 & -3.028337 & 0.593406 \\
\hline & 4.889618 & -2.223355 & 1.802106 \\
\hline [ & 0.973797 & -0.756301 & 2.753832 \\
\hline 1 & 5.863509 & -0.770705 & 3.546978 \\
\hline 1 & 1.929463 & 0.663131 & 4.535439 \\
\hline 1 & 4.389851 & 0.682778 & 4.928623 \\
\hline 6 & 1.387433 & 3.493856 & 3.042752 \\
\hline & 1.388696 & 5.486576 & 1.543824 \\
\hline & -0.077165 & 5.410337 & 3.565562 \\
\hline 1 & 2.135937 & 3.951104 & 3.702315 \\
\hline$\frac{1}{1}$ & 0.787741 & 2.798783 & 3.647824 \\
\hline & 1.935849 & 2.903484 & 2.297458 \\
\hline 1 & 0.728625 & 5.819491 & 4.189352 \\
\hline & -0.673947 & 6.257206 & 3.207537 \\
\hline 1 & -0.720356 & 4.793608 & 4.206787 \\
\hline & 2.226288 & 5.894326 & 2.126015 \\
\hline 1 & 1.803515 & 4.932462 & 0.690819 \\
\hline 1 & 0.817769 & 6.331066 & 1.138340 \\
\hline 6 & -4.848143 & 4.599671 & -0.997129 \\
\hline 5 & -4.434910 & 5.985383 & 1.012581 \\
\hline 0 & -3.121322 & 6.375142 & -1.082410 \\
\hline 1 & -5.602179 & 5.328318 & -1.320545 \\
\hline 1 & -4.442923 & 4.122943 & -1.898785 \\
\hline 1 & -5.364836 & 3.830236 & -0.406771 \\
\hline 1 & -5.215332 & 6.679392 & 0.672447 \\
\hline & -4.906264 & 5.241913 & 1.668904 \\
\hline 1 & -3.721922 & 6.559816 & 1.616193 \\
\hline 1 & -3.884377 & 7.071852 & -1.455142 \\
\hline 1 & -2.368569 & 6.964374 & -0.544927 \\
\hline 1 & -2.628623 & 5.915076 & -1.948665 \\
\hline 6 & -6.857529 & -1.471997 & -3.686583 \\
\hline 6 & -4.843803 & -0.236865 & -4.440873 \\
\hline 6 & -4.654618 & -2.608981 & -3.676216 \\
\hline 6 & -6.426052 & 0.750765 & 2.522775 \\
\hline 6 & -8.055005 & 0.822247 & 0.664015 \\
\hline 6 & -7.383759 & -1.371111 & 1.663708 \\
\hline 1 & -8.201863 & -1.297834 & 2.393297 \\
\hline 1 & -7.753844 & -1.936761 & 0.799245 \\
\hline 1 & -6.571500 & -1.950893 & 2.122969 \\
\hline 1 & -7.271223 & 0.874186 & 3.211544 \\
\hline 1 & -5.648052 & 0.193261 & 3.060486 \\
\hline 1 & -6.033909 & 1.751132 & 2.293266 \\
\hline 1 & -8.847421 & 0.951863 & 1.412988 \\
\hline 1 & -7.727128 & 1.819783 & 0.343870 \\
\hline 1 & -8.505989 & 0.324111 & -0.201976 \\
\hline 1 & -7.017578 & -1.806784 & -4.719194 \\
\hline 1 & -7.285915 & -2.236278 & -3.024948 \\
\hline 1 & -7.424056 & -0.541411 & -3.550814 \\
\hline 1 & -4.847161 & -2.966502 & -4.696975 \\
\hline 1 & -3.567156 & -2.524107 & -3.553638 \\
\hline 1 & -5.009272 & -3.371916 & -2.971364 \\
\hline 1 & -5.060188 & -0.561598 & -5.467462 \\
\hline 1 & -5.322228 & 0.739040 & -4.285916 \\
\hline
\end{tabular}


$1_{\mathrm{C} 1-} \psi_{-1 \mathrm{a} 2 \mathrm{a} 3 \mathrm{~s} 4 \mathrm{~s}}$

Number of imaginary frequencies : 0

The smallest frequencies are : $16.6329 \quad 17.5136 \quad 18.9426 \mathrm{~cm}(-1)$

Electronic energy : $\quad H F=-4125.4697601$

Zero-point correction $=\quad 1.298685($ Hartree/Particle $)$

Thermal correction to Energy= $\quad 1.377415$

Thermal correction to Enthalpy= $\quad 1.378359$

Thermal correction to Gibbs Free Energy $=\quad 1.184866$

Sum of electronic and zero-point Energies $=\quad-4124.171075$

Sum of electronic and thermal Energies $=\quad-4124.092346$

Sum of electronic and thermal Enthalpies $=\quad-4124.091401$

Sum of electronic and thermal Free Energies $=\quad-4124.284894$

Cartesian Coordinates

$\begin{array}{cccc}6 & 2.044896 & -0.778029 & 2.940991 \\ 6 & 2.869337 & -1.600333 & 2.170978 \\ 6 & 4.242837 & -1.584236 & 2.404298 \\ 6 & 4.786840 & -0.760171 & 3.386889 \\ 6 & 3.958473 & 0.052739 & 4.152390 \\ 6 & 2.582890 & 0.038696 & 3.926174 \\ 6 & 2.295531 & -2.520163 & 1.125708 \\ 7 & 1.342943 & -1.897314 & 0.173740 \\ 15 & 1.684573 & -0.634586 & -0.895119 \\ 45 & -0.141709 & 0.349492 & -1.956609 \\ 6 & 0.424022 & 2.199461 & -2.034625 \\ 8 & 0.553092 & 3.337642 & -2.189657 \\ 6 & 0.251865 & -2.731886 & -0.252173 \\ 6 & -0.966585 & -2.687823 & 0.402361 \\ 6 & -2.046563 & -3.495496 & -0.071522 \\ 6 & -1.842478 & -4.357703 & -1.189549 \\ 6 & -0.576047 & -4.383837 & -1.817633 \\ 6 & 0.440527 & -3.587862 & -1.362169 \\ 6 & -3.329026 & -3.472059 & 0.536331 \\ 6 & -4.342795 & -4.272203 & 0.072429 \\ 6 & -4.128445 & -5.145064 & -1.016584 \\ 6 & -2.905985 & -5.179636 & -1.636821 \\ 6 & -1.152591 & -1.888834 & 1.644875 \\ 6 & -1.480684 & -0.541300 & 1.619942 \\ 6 & -1.715123 & 0.137626 & 2.847329 \\ 6 & -1.604845 & -0.504297 & 4.051556 \\ 6 & -1.250283 & -1.871338 & 4.112185 \\ 6 & -1.030126 & -2.578643 & 2.894730 \\ 6 & -1.104736 & -2.544757 & 5.348391 \\ 6 & -0.753525 & -3.869716 & 5.389233 \\ 6 & -0.537649 & -4.577245 & 4.187972 \\ 6 & -0.676106 & -3.951409 & 2.973827 \\ 15 & -1.668519 & 0.330607 & -0.010939 \\ 6 & -3.414583 & -0.079437 & -0.411837 \\ 6 & -4.425984 & 0.098784 & 0.532457 \\ 6 & -5.757998 & -0.167791 & 0.220750 \\ 6 & -6.052819 & -0.614091 & -1.073980 \\ 6 & -5.063488 & -0.805822 & -2.040988\end{array}$




\begin{tabular}{|c|c|c|c|}
\hline & -3.738468 & -0.543870 & -1.680782 \\
\hline & -6.823443 & 0.056231 & 1.292348 \\
\hline & -5.359521 & -1.280746 & -3.461604 \\
\hline & -1.752420 & 2.093032 & 0.492787 \\
\hline & -0.692071 & 2.627356 & 1.230799 \\
\hline & -0.661553 & 3.980499 & 1.565310 \\
\hline & -1.690742 & 4.794390 & 1.078179 \\
\hline & -2.743192 & 4.299405 & 0.305557 \\
\hline & -2.766155 & 2.929173 & 0.031783 \\
\hline & -3.841364 & 5.243087 & -0.181681 \\
\hline & 0.454366 & 4.582634 & 2.418616 \\
\hline & 2.842578 & -1.145842 & -1.987219 \\
\hline & 4.077582 & -1.589275 & -1.567194 \\
\hline & 4.998881 & -0.711629 & -1.023849 \\
\hline & 6.206651 & -1.260534 & -0.480242 \\
\hline & 6.485007 & -2.651142 & -0.640829 \\
\hline & 5.554787 & -3.472465 & -1.323741 \\
\hline & 4.365870 & -2.957034 & -1.761250 \\
\hline & 7.665094 & -3.196544 & -0.079406 \\
\hline & 8.528331 & -2.414411 & 0.644343 \\
\hline & 8.239003 & -1.047243 & 0.835975 \\
\hline & 7.113803 & -0.484504 & 0.285230 \\
\hline & 4.694180 & 0.737072 & -0.980576 \\
\hline & 3.508186 & 1.166646 & -0.417273 \\
\hline & 3.159741 & 2.525952 & -0.316044 \\
\hline & 4.008203 & 3.479253 & -0.807705 \\
\hline & 5.212752 & 3.106480 & -1.450560 \\
\hline & 5.556580 & 1.724409 & -1.560544 \\
\hline 6 & 6.730532 & 1.388081 & -2.281883 \\
\hline f & 7.532782 & 2.360162 & -2.825996 \\
\hline & 7.209356 & 3.725389 & -2.681526 \\
\hline 6 & 6.068646 & 4.085174 & -2.011735 \\
\hline & 2.631087 & 0.249541 & 0.116973 \\
\hline 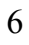 & -0.334378 & -0.937835 & -3.368676 \\
\hline & -0.481165 & -1.611696 & -4.298872 \\
\hline & 6.894502 & 0.566068 & 0.458454 \\
\hline 1 & -1.988194 & 1.189672 & 2.832193 \\
\hline & 7.862102 & -4.258210 & -0.222727 \\
\hline 1 & -1.275112 & -1.984761 & 6.266813 \\
\hline & 5.789931 & 5.133038 & -1.908572 \\
\hline 1 & -3.506532 & -2.800650 & 1.375177 \\
\hline & -5.320940 & -4.232990 & 0.546882 \\
\hline 1 & 3.753863 & 4.535911 & -0.734904 \\
\hline L & -1.790838 & 0.032788 & 4.980925 \\
\hline & 5.789031 & -4.525654 & -1.469242 \\
\hline 1 & 8.423258 & 2.073081 & -3.380782 \\
\hline & 3.626040 & -3.575796 & -2.264164 \\
\hline 1 & -2.730296 & -5.836804 & -2.487478 \\
\hline ] & -0.424881 & -5.041655 & -2.672032 \\
\hline & -0.262098 & -5.628699 & 4.224484 \\
\hline 1 & 6.989096 & 0.340976 & -2.415216 \\
\hline & -0.513593 & -4.512297 & 2.055591 \\
\hline 1 & 1.411833 & -3.591680 & -1.853583 \\
\hline & 7.856207 & 4.485303 & -3.113642 \\
\hline 1 & 8.910806 & -0.432525 & 1.430959 \\
\hline 1 & -4.938557 & -5.780774 & -1.367426 \\
\hline
\end{tabular}




\begin{tabular}{|c|c|c|c|}
\hline . & -0.640677 & -4.378014 & 6.343970 \\
\hline & 9.426264 & -2.845606 & 1.080638 \\
\hline . & 2.209515 & 2.781634 & 0.149451 \\
\hline & -4.168114 & 0.466877 & 1.525436 \\
\hline & -2.933825 & -0.693632 & -2.398641 \\
\hline & -7.087994 & -0.815115 & -1.334819 \\
\hline & -3.575900 & 2.501576 & -0.554382 \\
\hline & 0.107022 & 1.961542 & 1.560355 \\
\hline & -1.671289 & 5.858268 & 1.313856 \\
\hline & 1.719170 & -3.310813 & 1.626935 \\
\hline & -1.413325 & 1.018865 & -2.672910 \\
\hline 1 & 3.112408 & -3.023661 & 0.586277 \\
\hline 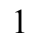 & 4.900079 & -2.210780 & 1.798863 \\
\hline & 0.976221 & -0.757298 & 2.738244 \\
\hline 1 & 5.863625 & -0.752197 & 3.544626 \\
\hline 1 & 1.921537 & 0.668804 & 4.519636 \\
\hline 1 & 4.380920 & 0.697952 & 4.919819 \\
\hline U & 1.338440 & 3.506243 & 3.048847 \\
\hline 6 & 1.321470 & 5.491141 & 1.540234 \\
\hline 6 & -0.146410 & 5.410119 & 3.560385 \\
\hline 1 & 2.077992 & 3.974464 & 3.710787 \\
\hline 1 & 0.744905 & 2.804782 & 3.652570 \\
\hline & 1.898178 & 2.921673 & 2.307406 \\
\hline 1 & 0.654629 & 5.830318 & 4.182896 \\
\hline 1 & -0.750964 & 6.249523 & 3.197953 \\
\hline 1 & -0.784017 & 4.790187 & 4.204106 \\
\hline 1 & 2.153195 & 5.911334 & 2.122018 \\
\hline 1 & 1.744535 & 4.936235 & 0.691699 \\
\hline 1 & 0.742132 & 6.326761 & 1.128314 \\
\hline 6 & -4.865816 & 4.532137 & -1.063753 \\
\hline v & -4.577356 & 5.825254 & 1.030191 \\
\hline 6 & -3.219580 & 6.379990 & -0.999106 \\
\hline 1 & -5.631771 & 5.248150 & -1.387478 \\
\hline 1 & -4.405936 & 4.108951 & -1.966100 \\
\hline 1 & -5.379086 & 3.720457 & -0.530095 \\
\hline 1 & -5.369737 & 6.512065 & 0.703028 \\
\hline 1 & -5.044707 & 5.028427 & 1.624291 \\
\hline 1 & -3.903700 & 6.384523 & 1.690924 \\
\hline 1 & -4.003045 & 7.055934 & -1.367409 \\
\hline 1 & -2.517529 & 6.980640 & -0.408618 \\
\hline 1 & -2.674800 & 5.986427 & -1.866811 \\
\hline 6 & -6.853660 & -1.463598 & -3.714244 \\
\hline 6 & -4.825807 & -0.250756 & -4.464798 \\
\hline 6 & -4.663209 & -2.624745 & -3.697944 \\
\hline 6 & -8.220513 & -0.338190 & 0.820510 \\
\hline 6 & -6.485508 & -0.783986 & 2.529761 \\
\hline 6 & -6.847966 & 1.542210 & 1.671198 \\
\hline 1 & -7.579487 & 1.723389 & 2.470448 \\
\hline 1 & -5.870363 & 1.893925 & 2.026153 \\
\hline 1 & -7.132342 & 2.159122 & 0.808378 \\
\hline 1 & -8.944263 & -0.167345 & 1.627513 \\
\hline 1 & -8.546946 & 0.256075 & -0.042447 \\
\hline 1 & -8.275639 & -1.400160 & 0.547702 \\
\hline 1 & -7.241049 & -0.632394 & 3.312385 \\
\hline 1 & -6.466368 & -1.853686 & 2.282023 \\
\hline 1 & -5.508829 & -0.525595 & 2.958359 \\
\hline
\end{tabular}




$\begin{array}{llrrr}1 & -7.014573 & -1.794475 & -4.748001 \\ 1 & -7.291816 & -2.224605 & -3.055341 \\ 1 & -7.411046 & -0.527560 & -3.578176 \\ 1 & -4.863307 & -2.984425 & -4.716538 \\ 1 & -3.574488 & -2.550025 & -3.579648 \\ 1 & -5.022466 & -3.381574 & -2.988523 \\ 1 & -5.040780 & -0.575321 & -5.491762 \\ 1 & -5.296909 & 0.729263 & -4.313407 \\ 1 & -3.739280 & -0.116426 & -4.383503\end{array}$

$1_{\mathrm{C} 1-} \Psi-1 \mathrm{a2s3a4a}$

Number of imaginary frequencies : 0

The smallest frequencies are : $16.0540 \quad 19.477120 .8191 \mathrm{~cm}(-1)$

Electronic energy : $\quad H F=-4125.4707528$

Zero-point correction $=\quad 1.299143($ Hartree/Particle $)$

Thermal correction to Energy= $\quad 1.377609$

Thermal correction to Enthalpy= $\quad 1.378553$

Thermal correction to Gibbs Free Energy $=\quad 1.187101$

Sum of electronic and zero-point Energies $=\quad-4124.171610$

Sum of electronic and thermal Energies $=\quad-4124.093144$

Sum of electronic and thermal Enthalpies $=\quad-4124.092199$

Sum of electronic and thermal Free Energies $=\quad-4124.283652$

Cartesian Coordinates

$\begin{array}{crrr}6 & 3.978878 & -0.051762 & 4.152921 \\ 6 & 4.791849 & -0.856120 & 3.362198 \\ 6 & 4.229261 & -1.661945 & 2.375058 \\ 6 & 2.852498 & -1.667482 & 2.161729 \\ 6 & 2.043561 & -0.854306 & 2.957343 \\ 6 & 2.599983 & -0.056535 & 3.947842 \\ 6 & 2.256988 & -2.566911 & 1.111063 \\ 7 & 1.313102 & -1.916615 & 0.169164 \\ 6 & 0.208470 & -2.726180 & -0.269491 \\ 6 & -1.008590 & -2.673238 & 0.387220 \\ 6 & -2.109552 & -3.433302 & -0.115289 \\ 6 & -1.932546 & -4.242950 & -1.276710 \\ 6 & -0.664115 & -4.290337 & -1.898745 \\ 6 & 0.376933 & -3.550300 & -1.406291 \\ 6 & -3.391973 & -3.397301 & 0.492509 \\ 6 & -4.435126 & -4.126129 & -0.020426 \\ 6 & -4.251619 & -4.939047 & -1.160704 \\ 6 & -3.027500 & -4.990263 & -1.776919 \\ 6 & -1.178741 & -1.892102 & 1.643381 \\ 6 & -1.481443 & -0.538248 & 1.636530 \\ 6 & -1.699816 & 0.129438 & 2.872908 \\ 6 & -1.595713 & -0.529151 & 4.068627 \\ 6 & -1.269102 & -1.903953 & 4.110585 \\ 6 & -1.068168 & -2.600438 & 2.883457 \\ 6 & -1.133402 & -2.596390 & 5.337351 \\ 6 & -0.811416 & -3.929244 & 5.359696 \\ 6 & -0.615841 & -4.626015 & 4.148684 \\ 6 & -0.744341 & -3.981467 & 2.943203 \\ 15 & -1.663128 & 0.354909 & 0.016665 \\ 45 & -0.152115 & 0.350349 & -1.947145\end{array}$




\begin{tabular}{|c|c|c|c|}
\hline 6 & 0.420005 & 2.199909 & -2.016761 \\
\hline 8 & 0.561801 & 3.337032 & -2.167273 \\
\hline 15 & 1.669755 & -0.648314 & -0.888942 \\
\hline 8 & 2.630020 & 0.217603 & 0.126307 \\
\hline 6 & 3.509483 & 1.131304 & -0.411965 \\
\hline 6 & 4.689089 & 0.695670 & -0.984268 \\
\hline 6 & 5.552697 & 1.678445 & -1.570216 \\
\hline 6 & 5.216250 & 3.062123 & -1.458796 \\
\hline 6 & 4.017351 & 3.440833 & -0.809150 \\
\hline 6 & 3.167676 & 2.492268 & -0.310035 \\
\hline 6 & 6.072883 & 4.036564 & -2.026275 \\
\hline 6 & 7.207203 & 3.671076 & -2.703712 \\
\hline 6 & 7.522835 & 2.304219 & -2.850101 \\
\hline 6 & 6.719709 & 1.336216 & -2.300090 \\
\hline 6 & 4.983755 & -0.754785 & -1.034862 \\
\hline 6 & 4.051760 & -1.623046 & -1.574871 \\
\hline 6 & 4.327132 & -2.992468 & -1.775354 \\
\hline 6 & 5.516324 & -3.518337 & -1.351201 \\
\hline 6 & 6.459691 & -2.706300 & -0.675230 \\
\hline 6 & 6.192588 & -1.314593 & -0.505097 \\
\hline 6 & 7.113096 & -0.548472 & 0.254367 \\
\hline 6 & 8.240829 & -1.121271 & 0.789378 \\
\hline 6 & 8.519052 & -2.489276 & 0.587590 \\
\hline 6 & 7.642357 & -3.262201 & -0.129811 \\
\hline 8 & 2.818669 & -1.167378 & -1.986931 \\
\hline 6 & -3.429006 & 0.003264 & -0.357982 \\
\hline 6 & -4.429136 & 0.272588 & 0.580544 \\
\hline 6 & -5.766970 & 0.010188 & 0.292897 \\
\hline 6 & -6.069304 & -0.529854 & -0.963857 \\
\hline 6 & -5.095046 & -0.818653 & -1.920462 \\
\hline 6 & -3.763700 & -0.547517 & -1.590539 \\
\hline 6 & -6.885230 & 0.264998 & 1.301929 \\
\hline 6 & -7.501829 & -1.078915 & 1.706215 \\
\hline 6 & -5.494808 & -1.357114 & -3.293062 \\
\hline 6 & -4.280574 & -1.796232 & -4.110042 \\
\hline 6 & -1.668543 & 2.115467 & 0.528026 \\
\hline 6 & -2.608853 & 2.999656 & 0.020406 \\
\hline 6 & -2.522865 & 4.374950 & 0.277088 \\
\hline 6 & -1.480630 & 4.824237 & 1.081949 \\
\hline 6 & -0.515757 & 3.954943 & 1.618796 \\
\hline 6 & -0.605250 & 2.604210 & 1.300499 \\
\hline 6 & -3.573919 & 5.299717 & -0.330771 \\
\hline 6 & 0.583287 & 4.508033 & 2.525008 \\
\hline 6 & -0.318304 & -0.933104 & -3.365391 \\
\hline 8 & -0.417872 & -1.612334 & -4.297700 \\
\hline 6 & -6.375924 & 0.955633 & 2.565306 \\
\hline 6 & -7.963994 & 1.156619 & 0.678712 \\
\hline 6 & -6.218568 & -0.244659 & -4.060825 \\
\hline 6 & -6.428873 & -2.561557 & -3.147814 \\
\hline 1 & 6.902868 & 0.502740 & 0.434868 \\
\hline 1 & -1.953352 & 1.186555 & 2.872179 \\
\hline 1 & 7.830632 & -4.324435 & -0.280462 \\
\hline 1 & -1.288185 & -2.044841 & 6.263620 \\
\hline 1 & 5.799850 & 5.085780 & -1.921668 \\
\hline 1 & -3.548479 & -2.767455 & 1.366439 \\
\hline 1 & -5.414924 & -4.073389 & 0.450105 \\
\hline
\end{tabular}




\begin{tabular}{|c|c|c|c|}
\hline & 3.768008 & 4.498783 & -0.737880 \\
\hline & -1.766979 & -0.000229 & 5.005477 \\
\hline & 5.741287 & -4.572715 & -1.502602 \\
\hline & 8.407891 & 2.012616 & -3.411166 \\
\hline & 3.577498 & -3.603920 & -2.272738 \\
\hline & -2.873217 & -5.606992 & -2.661289 \\
\hline & -0.532792 & -4.912437 & -2.782646 \\
\hline & -0.363990 & -5.683799 & 4.170763 \\
\hline & 6.972190 & 0.287909 & -2.435317 \\
\hline & -0.597800 & -4.533366 & 2.016856 \\
\hline & 1.349112 & -3.564782 & -1.895978 \\
\hline & 7.854755 & 4.427709 & -3.140511 \\
\hline & 8.923414 & -0.513922 & 1.379624 \\
\hline & -5.085057 & -5.520553 & -1.549483 \\
\hline & -0.706344 & -4.452151 & 6.307410 \\
\hline & 9.419151 & -2.928450 & 1.011279 \\
\hline & 2.220918 & 2.752763 & 0.160085 \\
\hline & -4.150981 & 0.702095 & 1.540352 \\
\hline & -2.960112 & -0.762628 & -2.290871 \\
\hline & -7.113165 & -0.731451 & -1.204919 \\
\hline & -3.417518 & 2.616812 & -0.600845 \\
\hline & 0.145942 & 1.901943 & 1.663990 \\
\hline & -1.404085 & 5.886035 & 1.306325 \\
\hline & 1.667012 & -3.350553 & 1.607501 \\
\hline 1 & -1.426529 & 1.016520 & -2.662588 \\
\hline & -5.637008 & 0.345471 & 3.101354 \\
\hline 1 & -5.919227 & 1.929529 & 2.342466 \\
\hline & -7.213447 & 1.132668 & 3.251741 \\
\hline & -8.426028 & 0.699239 & -0.204099 \\
\hline 1 & -8.764681 & 1.347401 & 1.405703 \\
\hline & -7.546825 & 2.125564 & 0.374489 \\
\hline ] & -7.919740 & -1.614185 & 0.844549 \\
\hline ] & -6.748136 & -1.728903 & 2.170772 \\
\hline 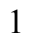 & -8.312464 & -0.927819 & 2.431993 \\
\hline 1 & -5.569546 & 0.631902 & -4.186174 \\
\hline 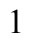 & -6.511125 & -0.595512 & -5.059917 \\
\hline 1 & -7.127499 & 0.083301 & -3.540673 \\
\hline & -7.360731 & -2.311899 & -2.626362 \\
\hline 1 & -6.703817 & -2.946421 & -4.138849 \\
\hline 1 & -5.936064 & -3.371026 & -2.593818 \\
\hline ] & -3.608887 & -0.957633 & -4.336388 \\
\hline 1 & -3.698533 & -2.571398 & -3.591205 \\
\hline & -4.612972 & -2.213103 & -5.069184 \\
\hline 1 & 3.061754 & -3.080927 & 0.563250 \\
\hline & 4.874089 & -2.282936 & 1.750945 \\
\hline 1 & 0.971825 & -0.827578 & 2.772952 \\
\hline 1 & 5.870827 & -0.856019 & 3.504368 \\
\hline 1 & 1.950566 & 0.565976 & 4.562197 \\
\hline 1 & 4.415818 & 0.578789 & 4.924417 \\
\hline 5 & 1.443110 & 3.398324 & 3.129176 \\
\hline 6 & 1.484045 & 5.448488 & 1.717926 \\
\hline 5 & -0.049184 & 5.285316 & 3.685419 \\
\hline 6 & -3.359235 & 6.760569 & 0.055269 \\
\hline 6 & -3.508491 & 5.193428 & -1.859030 \\
\hline 6 & -4.965654 & 4.875814 & 0.154373 \\
\hline . & 2.181723 & 3.832921 & 3.814773 \\
\hline
\end{tabular}




$\begin{array}{lrrr}1 & 0.833279 & 2.686285 & 3.703581 \\ 1 & 2.000999 & 2.830805 & 2.372879 \\ 1 & 0.733205 & 5.674814 & 4.350444 \\ 1 & -0.643287 & 6.140373 & 3.342613 \\ 1 & -0.706318 & 4.637802 & 4.280631 \\ 1 & 2.295452 & 5.837976 & 2.347575 \\ 1 & 1.936872 & 4.925175 & 0.864876 \\ 1 & 0.926874 & 6.305750 & 1.320883 \\ 1 & -4.140190 & 7.381211 & -0.401946 \\ 1 & -3.413713 & 6.911390 & 1.141363 \\ 1 & -2.391214 & 7.139005 & -0.297491 \\ 1 & -5.736998 & 5.517678 & -0.292197 \\ 1 & -5.200780 & 3.837572 & -0.114173 \\ 1 & -5.043350 & 4.963446 & 1.246318 \\ 1 & -4.247064 & 5.862788 & -2.320640 \\ 1 & -2.514025 & 5.476532 & -2.227816 \\ 1 & -3.715385 & 4.175873 & -2.211985\end{array}$

$1_{\mathrm{C} 1-} \psi_{-1 \mathrm{a} 253 \mathrm{ats}}$

Number of imaginary frequencies : 0

The smallest frequencies are : $16.4234 \quad 18.8933 \quad 21.3214 \mathrm{~cm}(-1)$

Electronic energy : $\quad H F=-4125.4710372$

Zero-point correction $=\quad 1.299538($ Hartree/Particle $)$

Thermal correction to Energy= $\quad 1.377885$

Thermal correction to Enthalpy= $\quad 1.378830$

Thermal correction to Gibbs Free Energy $=\quad 1.187867$

Sum of electronic and zero-point Energies $=\quad-4124.171499$

Sum of electronic and thermal Energies $=\quad-4124.093152$

Sum of electronic and thermal Enthalpies $=\quad-4124.092208$

Sum of electronic and thermal Free Energies $=\quad-4124.283171$

\section{Cartesian Coordinates}

$\begin{array}{cccc}6 & 2.028915 & -0.896134 & 2.945091 \\ 6 & 2.840240 & -1.701931 & 2.144364 \\ 6 & 4.216424 & -1.697623 & 2.360982 \\ 6 & 4.776197 & -0.899749 & 3.356147 \\ 6 & 3.960997 & -0.101814 & 4.151057 \\ 6 & 2.582552 & -0.105679 & 3.942918 \\ 6 & 2.247351 & -2.589965 & 1.082635 \\ 7 & 1.313306 & -1.926594 & 0.140222 \\ 15 & 1.675554 & -0.642645 & -0.896830 \\ 45 & -0.140648 & 0.372952 & -1.950185 \\ 6 & 0.435288 & 2.222553 & -1.993270 \\ 8 & 0.579503 & 3.361249 & -2.128653 \\ 6 & 0.209256 & -2.726467 & -0.316615 \\ 6 & -1.010917 & -2.679198 & 0.334467 \\ 6 & -2.114587 & -3.419561 & -0.190033 \\ 6 & -1.937044 & -4.204876 & -1.367950 \\ 6 & -0.664363 & -4.251814 & -1.981234 \\ 6 & 0.379387 & -3.530062 & -1.467641 \\ 6 & -3.400894 & -3.383285 & 0.409482 \\ 6 & -4.447582 & -4.089292 & -0.127247 \\ 6 & -4.263775 & -4.878909 & -1.283853 \\ 6 & -3.035866 & -4.929409 & -1.892432\end{array}$




\begin{tabular}{|c|c|c|c|}
\hline 6 & -1.184070 & -1.918480 & 1.602352 \\
\hline 6 & -1.480311 & -0.563405 & 1.613927 \\
\hline & -1.704301 & 0.086241 & 2.858900 \\
\hline & -1.617301 & -0.592709 & 4.044835 \\
\hline & -1.299122 & -1.969925 & 4.067766 \\
\hline & -1.087653 & -2.646996 & 2.831626 \\
\hline 0 & -1.181928 & -2.683576 & 5.284247 \\
\hline 0 & -0.867204 & -4.018332 & 5.287931 \\
\hline 0 & -0.660161 & -4.695615 & 4.067742 \\
\hline 0 & -0.770965 & -4.030289 & 2.871825 \\
\hline & -1.658422 & 0.349053 & 0.004535 \\
\hline 6 & -3.424841 & -0.003265 & -0.369523 \\
\hline 6 & -4.406527 & 0.201105 & 0.595581 \\
\hline 6 & -5.752481 & -0.058836 & 0.324465 \\
\hline 6 & -6.079869 & -0.517928 & -0.952124 \\
\hline 6 & -5.116592 & -0.740270 & -1.945246 \\
\hline 6 & -3.782716 & -0.484774 & -1.628805 \\
\hline 6 & -6.783260 & 0.158560 & 1.430289 \\
\hline 6 & -5.546115 & -1.209063 & -3.334110 \\
\hline 6 & -1.676978 & 2.103602 & 0.536754 \\
\hline 6 & -0.618364 & 2.590976 & 1.316216 \\
\hline 6 & -0.538672 & 3.938978 & 1.648782 \\
\hline 6 & -1.506365 & 4.807606 & 1.116021 \\
\hline 6 & -2.543687 & 4.360158 & 0.303588 \\
\hline 6 & -2.622990 & 2.986369 & 0.036826 \\
\hline 6 & -3.587537 & 5.287809 & -0.312607 \\
\hline 6 & 0.554485 & 4.489906 & 2.563457 \\
\hline 8 & 2.830072 & -1.145939 & -1.995892 \\
\hline 6 & 4.060469 & -1.607577 & -1.582313 \\
\hline 6 & 4.990137 & -0.746096 & -1.027716 \\
\hline 6 & 6.196776 & -1.312426 & -0.499746 \\
\hline 6 & 6.463964 & -2.702233 & -0.684877 \\
\hline 6 & 5.522587 & -3.506282 & -1.373018 \\
\hline 6 & 4.335465 & -2.974969 & -1.796173 \\
\hline 6 & 7.644532 & -3.264891 & -0.141855 \\
\hline 6 & 8.519199 & -2.500638 & 0.587192 \\
\hline 6 & 8.240791 & -1.134946 & 0.803759 \\
\hline 6 & 7.115017 & -0.555557 & 0.271688 \\
\hline 6 & 4.694353 & 0.703413 & -0.958578 \\
\hline 6 & 3.512376 & 1.130324 & -0.384409 \\
\hline 6 & 3.170524 & 2.489646 & -0.262366 \\
\hline 6 & 4.022218 & 3.445664 & -0.743471 \\
\hline 6 & 5.223180 & 3.076792 & -1.394973 \\
\hline 6 & 5.559866 & 1.694961 & -1.526542 \\
\hline 6 & 6.729123 & 1.363791 & -2.257893 \\
\hline 6 & 7.534170 & 2.339976 & -2.790382 \\
\hline 6 & 7.218322 & 3.704479 & -2.623841 \\
\hline 6 & 6.081847 & 4.059686 & -1.944535 \\
\hline 8 & 2.630372 & 0.208728 & 0.135779 \\
\hline 6 & -0.295425 & -0.887230 & -3.391094 \\
\hline 8 & -0.383295 & -1.548682 & -4.337128 \\
\hline 1 & 6.904773 & 0.493634 & 0.463563 \\
\hline 1 & -1.950504 & 1.145086 & 2.872619 \\
\hline 1 & 7.832796 & -4.325438 & -0.303970 \\
\hline 1 & -1.344970 & -2.146719 & 6.217708 \\
\hline 1 & 5.808598 & 5.107205 & -1.824606 \\
\hline
\end{tabular}




\begin{tabular}{|c|c|c|c|}
\hline 1 & -3.556747 & -2.770113 & 1.295890 \\
\hline 1 & -5.430624 & -4.034775 & 0.336347 \\
\hline & 3.772829 & 4.502420 & -0.656460 \\
\hline & -1.794436 & -0.077776 & 4.988347 \\
\hline & 5.747396 & -4.559138 & -1.534866 \\
\hline & 8.420888 & 2.056837 & -3.353155 \\
\hline & 3.587326 & -3.580538 & -2.302932 \\
\hline & -2.881461 & -5.528495 & -2.788782 \\
\hline & -0.532172 & -4.857304 & -2.876474 \\
\hline & -0.413717 & -5.754869 & 4.075093 \\
\hline & 6.981639 & 0.317640 & -2.408729 \\
\hline & -0.616098 & -4.567083 & 1.937975 \\
\hline & 1.354517 & -3.541989 & -1.951569 \\
\hline & 7.867427 & 4.467596 & -3.046841 \\
\hline & 8.921657 & -0.534679 & 1.403165 \\
\hline & -5.100194 & -5.443195 & -1.691113 \\
\hline & -0.776649 & -4.557723 & 6.227871 \\
\hline & 9.417708 & -2.945044 & 1.008798 \\
\hline & 2.222172 & 2.743083 & 0.208443 \\
\hline & -4.119210 & 0.573370 & 1.578803 \\
\hline & -2.992136 & -0.655126 & -2.355800 \\
\hline & -7.123655 & -0.712149 & -1.190646 \\
\hline & -3.427987 & 2.604262 & -0.589891 \\
\hline & 0.136815 & 1.889598 & 1.673392 \\
\hline & -1.435786 & 5.867885 & 1.349346 \\
\hline & 1.649650 & -3.374634 & 1.568281 \\
\hline & -1.411277 & 1.050663 & -2.661714 \\
\hline & 3.053071 & -3.103389 & 0.535602 \\
\hline & 4.863127 & -2.312862 & 1.733103 \\
\hline & 0.957584 & -0.868682 & 2.758448 \\
\hline & 5.854777 & -0.900445 & 3.501261 \\
\hline 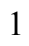 & 1.931454 & 0.511971 & 4.560391 \\
\hline ] & 4.395910 & 0.522926 & 4.928404 \\
\hline 6 & 1.413559 & 3.379027 & 3.166731 \\
\hline 6 & 1.457452 & 5.435892 & 1.765285 \\
\hline 6 & -0.084979 & 5.260567 & 3.724412 \\
\hline 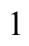 & 2.145607 & 3.811698 & 3.860511 \\
\hline & 0.801830 & 2.661299 & 3.731915 \\
\hline 1 & 1.979232 & 2.818408 & 2.411091 \\
\hline ] & 0.693595 & 5.648621 & 4.394715 \\
\hline 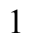 & -0.679160 & 6.116057 & 3.382898 \\
\hline 1 & -0.743501 & 4.609127 & 4.313758 \\
\hline & 2.265096 & 5.823848 & 2.400713 \\
\hline 1 & 1.915212 & 4.917483 & 0.911885 \\
\hline & 0.900494 & 6.293935 & 1.369599 \\
\hline 6 & -3.403480 & 6.740609 & 0.117101 \\
\hline 6 & -3.468319 & 5.219818 & -1.839971 \\
\hline 6 & -4.990427 & 4.836981 & 0.111484 \\
\hline 1 & -4.179885 & 7.362905 & -0.345558 \\
\hline 1 & -3.489530 & 6.861379 & 1.204952 \\
\hline 1 & -2.431817 & 7.141440 & -0.198751 \\
\hline 1 & -4.202938 & 5.886735 & -2.311486 \\
\hline 1 & -2.467233 & 5.528953 & -2.167591 \\
\hline 1 & -3.643764 & 4.206567 & -2.221881 \\
\hline 1 & -5.751186 & 5.490162 & -0.336764 \\
\hline 1 & -5.209178 & 3.808853 & -0.204512 \\
\hline
\end{tabular}




$\begin{array}{rrrr}1 & -5.105532 & 4.882757 & 1.202676 \\ 6 & -4.347763 & -1.541353 & -4.221117 \\ 6 & -6.418681 & -2.463279 & -3.227670 \\ 6 & -6.348029 & -0.088168 & -4.005643 \\ 6 & -8.200610 & -0.190759 & 0.984538 \\ 6 & -6.427043 & -0.732422 & 2.626776 \\ 6 & -6.766575 & 1.629247 & 1.862599 \\ 1 & -7.480728 & 1.795206 & 2.680539 \\ 1 & -5.777113 & 1.946828 & 2.215155 \\ 1 & -7.050434 & 2.285619 & 1.029282 \\ 1 & -8.900043 & -0.022069 & 1.813115 \\ 1 & -8.532250 & 0.432074 & 0.143806 \\ 1 & -8.289385 & -1.244274 & 0.688768 \\ 1 & -7.152454 & -0.590443 & 3.439236 \\ 1 & -6.442516 & -1.792940 & 2.341594 \\ 1 & -5.429768 & -0.512167 & 3.029118 \\ 1 & -4.699189 & -1.905744 & -5.194731 \\ 1 & -3.718739 & -0.661451 & -4.410972 \\ 1 & -3.718250 & -2.325410 & -3.777836 \\ 1 & -6.663227 & -0.393113 & -5.012855 \\ 1 & -7.250356 & 0.166756 & -3.435726 \\ 1 & -5.743403 & 0.823180 & -4.101493 \\ 1 & -6.719397 & -2.799563 & -4.228991 \\ 1 & -5.866364 & -3.280926 & -2.746081 \\ 1 & -7.336302 & -2.289538 & -2.652880\end{array}$

$1_{\mathrm{C} 1-} \psi_{-12 \mathrm{a} 33 \mathrm{~s} 4 \mathrm{a}}$

Number of imaginary frequencies : 0

The smallest frequencies are : $17.0584 \quad 18.7856 \quad 19.6115 \mathrm{~cm}(-1)$

Electronic energy : $\quad H F=-4125.4701944$

Zero-point correction $=\quad 1.299127$ (Hartree/Particle)

Thermal correction to Energy= $\quad 1.377634$

Thermal correction to Enthalpy= $\quad 1.378578$

Thermal correction to Gibbs Free Energy= $\quad 1.186879$

Sum of electronic and zero-point Energies $=\quad-4124.171067$

Sum of electronic and thermal Energies $=\quad-4124.092561$

Sum of electronic and thermal Enthalpies $=\quad-4124.091616$

Sum of electronic and thermal Free Energies $=\quad-4124.283316$

Cartesian Coordinates

$\begin{array}{cccc}6 & 2.020123 & -0.928740 & 2.954619 \\ 6 & 2.829845 & -1.720864 & 2.138928 \\ 6 & 4.207783 & -1.710121 & 2.343630 \\ 6 & 4.771162 & -0.917594 & 3.340903 \\ 6 & 3.957725 & -0.132399 & 4.150340 \\ 6 & 2.577425 & -0.144193 & 3.955119 \\ 6 & 2.232510 & -2.599287 & 1.072026 \\ 7 & 1.291724 & -1.925641 & 0.142783 \\ 15 & 1.659078 & -0.641116 & -0.891568 \\ 45 & -0.148737 & 0.404542 & -1.930199 \\ 6 & 0.454464 & 2.244490 & -1.963824 \\ 8 & 0.603895 & 3.383950 & -2.087171 \\ 6 & 0.191152 & -2.730164 & -0.315416 \\ 6 & -1.035113 & -2.676730 & 0.323798\end{array}$




\begin{tabular}{|c|c|c|c|}
\hline 6 & -2.124097 & -3.449651 & -0.187221 \\
\hline & -1.916745 & -4.297410 & -1.316103 \\
\hline & -0.640667 & -4.338403 & -1.923209 \\
\hline & 0.381744 & -3.567512 & -1.439144 \\
\hline & -3.419225 & -3.406068 & 0.392030 \\
\hline & -4.440800 & -4.175001 & -0.106675 \\
\hline & -4.221276 & -5.039959 & -1.200858 \\
\hline & -2.987235 & -5.091321 & -1.796160 \\
\hline & -1.213033 & -1.915678 & 1.592084 \\
\hline & -1.500155 & -0.558745 & 1.617848 \\
\hline & -1.718517 & 0.081360 & 2.868886 \\
\hline & -1.631766 & -0.607404 & 4.048746 \\
\hline & -1.318914 & -1.985909 & 4.058942 \\
\hline & -1.114960 & -2.654146 & 2.816607 \\
\hline & -1.197583 & -2.708036 & 5.270010 \\
\hline & -0.883777 & -4.042932 & 5.263642 \\
\hline & -0.681458 & -4.711242 & 4.037872 \\
\hline & -0.797468 & -4.037756 & 2.846986 \\
\hline & -1.666350 & 0.375422 & 0.021867 \\
\hline & -3.432062 & 0.043891 & -0.368566 \\
\hline & -4.431301 & 0.216993 & 0.598801 \\
\hline & -5.763975 & -0.041534 & 0.299957 \\
\hline & -6.076595 & -0.465162 & -1.004039 \\
\hline & -5.109301 & -0.635817 & -1.989327 \\
\hline & -3.774743 & -0.387837 & -1.639952 \\
\hline & -6.873143 & 0.083443 & 1.343274 \\
\hline & -5.426499 & -1.069729 & -3.418031 \\
\hline 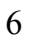 & -1.664886 & 2.124137 & 0.571821 \\
\hline & -0.601405 & 2.591682 & 1.356882 \\
\hline 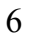 & -0.505573 & 3.935789 & 1.700550 \\
\hline ) & -1.461671 & 4.820661 & 1.173530 \\
\hline 5 & -2.503315 & 4.392837 & 0.356368 \\
\hline f & -2.599214 & 3.022360 & 0.077910 \\
\hline 5 & -3.541275 & 5.337272 & -0.243863 \\
\hline 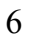 & 0.591790 & 4.466208 & 2.622128 \\
\hline & 2.806887 & -1.148311 & -1.996012 \\
\hline 5 & 4.040525 & -1.611141 & -1.594105 \\
\hline 6 & 4.974372 & -0.752628 & -1.041999 \\
\hline 5 & 6.185614 & -1.321460 & -0.527056 \\
\hline 6 & 6.453202 & -2.709431 & -0.724341 \\
\hline 6 & 5.507188 & -3.509522 & -1.410662 \\
\hline 6 & 4.316012 & -2.976640 & -1.820081 \\
\hline 6 & 7.639521 & -3.274115 & -0.196217 \\
\hline 6 & 8.519533 & -2.513798 & 0.530495 \\
\hline 6 & 8.240448 & -1.150423 & 0.760286 \\
\hline 6 & 7.109071 & -0.568982 & 0.242504 \\
\hline 5 & 4.680829 & 0.696576 & -0.961476 \\
\hline 6 & 3.504946 & 1.122091 & -0.373917 \\
\hline 6 & 3.171036 & 2.481633 & -0.233423 \\
\hline 6 & 4.022467 & 3.438979 & -0.712271 \\
\hline 6 & 5.214922 & 3.071976 & -1.380122 \\
\hline 6 & 5.544649 & 1.690242 & -1.528490 \\
\hline 6 & 6.704996 & 1.361673 & -2.275052 \\
\hline 6 & 7.508191 & 2.339875 & -2.806670 \\
\hline U & 7.199533 & 3.703920 & -2.623678 \\
\hline 6 & 6.071689 & 4.056899 & -1.929017 \\
\hline
\end{tabular}




\begin{tabular}{|c|c|c|c|}
\hline 8 & 2.622670 & 0.199061 & 0.142799 \\
\hline 6 & -0.381203 & -0.847004 & -3.368955 \\
\hline & -0.557870 & -1.491744 & -4.314436 \\
\hline & 6.898829 & 0.478387 & 0.444100 \\
\hline & -1.960494 & 1.141012 & 2.891617 \\
\hline & 7.828046 & -4.333088 & -0.368011 \\
\hline 1 & -1.355757 & -2.176961 & 6.207616 \\
\hline & 5.803758 & 5.104231 & -1.796140 \\
\hline & -3.601918 & -2.742938 & 1.236052 \\
\hline & -5.428612 & -4.118422 & 0.345436 \\
\hline & 3.779029 & 4.495905 & -0.611259 \\
\hline 1 & -1.804497 & -0.099691 & 4.996952 \\
\hline & 5.732109 & -4.560740 & -1.582673 \\
\hline 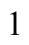 & 8.387708 & 2.058785 & -3.381632 \\
\hline & 3.565527 & -3.579692 & -2.326243 \\
\hline 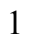 & -2.807100 & -5.740262 & -2.652151 \\
\hline 1 & -0.487781 & -4.984877 & -2.785892 \\
\hline 1 & -0.434035 & -5.770295 & 4.036492 \\
\hline 1 & 6.951565 & 0.316096 & -2.438996 \\
\hline 1 & -0.644578 & -4.569246 & 1.909841 \\
\hline & 1.360263 & -3.578729 & -1.915843 \\
\hline 1 & 7.847113 & 4.468584 & -3.046226 \\
\hline 1 & 8.925443 & -0.553524 & 1.358343 \\
\hline 1 & -5.036932 & -5.653673 & -1.577144 \\
\hline 1 & -0.789412 & -4.588761 & 6.199477 \\
\hline 1 & 9.422675 & -2.959699 & 0.940446 \\
\hline 1 & 2.228825 & 2.734506 & 0.249768 \\
\hline 1 & -4.149581 & 0.561118 & 1.591406 \\
\hline | & -2.980842 & -0.530723 & -2.371141 \\
\hline ] & -7.118527 & -0.665802 & -1.245679 \\
\hline & -3.408658 & 2.655106 & -0.551931 \\
\hline I & 0.144591 & 1.877737 & 1.708403 \\
\hline [ & -1.378164 & 5.877995 & 1.415639 \\
\hline 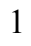 & 1.638021 & -3.389326 & 1.552867 \\
\hline ] & -1.404377 & 1.119065 & -2.631469 \\
\hline 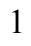 & 3.035508 & -3.106389 & 0.515120 \\
\hline 1 & 4.852651 & -2.315281 & 1.704200 \\
\hline & 0.946863 & -0.907730 & 2.778181 \\
\hline 1 & 5.851019 & -0.912253 & 3.476211 \\
\hline 1 & 1.927901 & 0.462986 & 4.584513 \\
\hline 1 & 4.395452 & 0.488326 & 4.929334 \\
\hline 6 & 1.440126 & 3.340537 & 3.212814 \\
\hline 6 & 1.503630 & 5.413867 & 1.836143 \\
\hline 6 & -0.042071 & 5.228759 & 3.791533 \\
\hline ] & 2.176034 & 3.757986 & 3.911830 \\
\hline 1 & 0.821172 & 2.622111 & 3.769148 \\
\hline$\frac{1}{1}$ & 2.000396 & 2.783451 & 2.450597 \\
\hline 1 & 0.739226 & 5.602335 & 4.466889 \\
\hline 1 & -0.628580 & 6.093170 & 3.459319 \\
\hline 1 & -0.706679 & 4.576195 & 4.372724 \\
\hline 1 & 2.315049 & 5.785705 & 2.476358 \\
\hline 1 & 1.956239 & 4.902456 & 0.975835 \\
\hline 1 & 0.954951 & 6.282397 & 1.451936 \\
\hline 6 & -3.312293 & 6.790379 & 0.162778 \\
\hline 6 & -3.468903 & 5.251054 & -1.773158 \\
\hline 6 & -4.939991 & 4.922736 & 0.228188 \\
\hline
\end{tabular}




$\begin{array}{lrrr}1 & -4.083221 & 7.425872 & -0.291035 \\ 1 & -3.372023 & 6.927534 & 1.250410 \\ 1 & -2.337788 & 7.162284 & -0.178882 \\ 1 & -4.198010 & 5.934401 & -2.229326 \\ 1 & -2.469626 & 5.528632 & -2.133046 \\ 1 & -3.684577 & 4.240621 & -2.141236 \\ 1 & -5.701307 & 5.581298 & -0.211182 \\ 1 & -5.186203 & 3.892564 & -0.059341 \\ 1 & -5.021880 & 4.992855 & 1.320968 \\ 6 & -6.925658 & -1.224526 & -3.658198 \\ 6 & -4.888495 & -0.020511 & -4.398938 \\ 6 & -4.751664 & -2.415965 & -3.697862 \\ 6 & -6.369591 & 0.691370 & 2.651027 \\ 6 & -8.005052 & 0.970807 & 0.815452 \\ 6 & -7.420031 & -1.316205 & 1.646669 \\ 1 & -8.229045 & -1.261729 & 2.387852 \\ 1 & -7.820360 & -1.803098 & 0.748657 \\ 1 & -6.629945 & -1.960723 & 2.055541 \\ 1 & -7.201826 & 0.779329 & 3.360708 \\ 1 & -5.599490 & 0.070171 & 3.127415 \\ 1 & -5.954066 & 1.696844 & 2.500684 \\ 1 & -8.793275 & 1.068504 & 1.573785 \\ 1 & -7.639475 & 1.978213 & 0.578063 \\ 1 & -8.471862 & 0.562470 & -0.088476 \\ 1 & -7.103503 & -1.521693 & -4.699367 \\ 1 & -7.366409 & -1.999562 & -3.017560 \\ 1 & -7.468036 & -0.285533 & -3.486399 \\ 1 & -4.957769 & -2.739381 & -4.727316 \\ 1 & -3.661816 & -2.363018 & -3.577591 \\ 1 & -5.123337 & -3.189234 & -3.013323 \\ 1 & -5.122888 & -0.312433 & -5.431375 \\ 1 & -5.340609 & 0.962693 & -4.213898 \\ 1 & -3.798676 & 0.092639 & -4.329691\end{array}$

$1_{\mathrm{C} 1-} \psi_{-1 \mathrm{a} 25354 \mathrm{~s}}$

Number of imaginary frequencies : 0

The smallest frequencies are : $16.5133 \quad 17.3629 \quad 19.1686 \mathrm{~cm}(-1)$

Electronic energy : $\quad \mathrm{HF}=-4125.4703583$

Zero-point correction $=\quad 1.299236($ Hartree/Particle $)$

Thermal correction to Energy= $\quad 1.377662$

Thermal correction to Enthalpy= $\quad 1.378606$

Thermal correction to Gibbs Free Energy= 1.186943

Sum of electronic and zero-point Energies $=\quad-4124.171122$

Sum of electronic and thermal Energies $=\quad-4124.092696$

Sum of electronic and thermal Enthalpies $=\quad-4124.091752$

Sum of electronic and thermal Free Energies $=\quad-4124.283415$

\begin{tabular}{cccc}
\multicolumn{4}{c}{ Cartesian Coordinates } \\
$\ldots \ldots \ldots \ldots \ldots \ldots \ldots \ldots \ldots \ldots \ldots \ldots \ldots \ldots \ldots \ldots \ldots \ldots \ldots \ldots$ \\
6 & 2.023113 & -0.922541 & 2.945065 \\
6 & 2.838465 & -1.713609 & 2.133884 \\
6 & 4.215956 & -1.694550 & 2.340887 \\
6 & 4.773328 & -0.894136 & 3.335297 \\
6 & 3.954389 & -0.109290 & 4.139450 \\
6 & 2.574457 & -0.129683 & 3.942232
\end{tabular}




\begin{tabular}{|c|c|c|c|}
\hline 6 & 2.247992 & -2.597331 & 1.067403 \\
\hline 7 & 1.307122 & -1.930064 & 0.133603 \\
\hline 1 & 1.667647 & -0.640259 & -0.896454 \\
\hline & -0.145006 & 0.397110 & -1.933740 \\
\hline 6 & 0.444758 & 2.241930 & -1.963302 \\
\hline 8 & 0.582404 & 3.383155 & -2.083996 \\
\hline 6 & 0.208931 & -2.737961 & -0.323847 \\
\hline 6 & -1.014482 & -2.693704 & 0.321335 \\
\hline 6 & -2.103248 & -3.467131 & -0.188498 \\
\hline 6 & -1.899646 & -4.304695 & -1.325431 \\
\hline 6 & -0.626289 & -4.337188 & -1.938970 \\
\hline 6 & 0.396654 & -3.567203 & -1.454083 \\
\hline 6 & -3.394763 & -3.431704 & 0.399284 \\
\hline 6 & -4.417116 & -4.199014 & -0.099980 \\
\hline 6 & -4.202042 & -5.052847 & -1.203825 \\
\hline 6 & -2.971260 & -5.096433 & -1.806615 \\
\hline 6 & -1.194602 & -1.935690 & 1.590525 \\
\hline 6 & -1.491358 & -0.580990 & 1.614481 \\
\hline 6 & -1.719701 & 0.057536 & 2.864473 \\
\hline 6 & -1.634919 & -0.631348 & 4.044604 \\
\hline 6 & -1.312076 & -2.007546 & 4.056279 \\
\hline 6 & -1.096492 & -2.673752 & 2.814926 \\
\hline 6 & -1.192612 & -2.729554 & 5.267603 \\
\hline 6 & -0.869690 & -4.062281 & 5.262065 \\
\hline 6 & -0.656114 & -4.728588 & 4.037056 \\
\hline 6 & -0.770125 & -4.055206 & 2.845945 \\
\hline 15 & -1.660014 & 0.349002 & 0.016068 \\
\hline 6 & -3.420661 & -0.000358 & -0.380234 \\
\hline 6 & -4.417027 & 0.166772 & 0.582191 \\
\hline 6 & -5.755939 & -0.080081 & 0.283629 \\
\hline 6 & -6.074143 & -0.484673 & -1.019457 \\
\hline 6 & -5.100739 & -0.658482 & -2.005975 \\
\hline 6 & -3.767529 & -0.423671 & -1.657291 \\
\hline 6 & -6.801959 & 0.103946 & 1.382109 \\
\hline 6 & -5.422088 & -1.085745 & -3.436363 \\
\hline 6 & -1.688825 & 2.097684 & 0.566065 \\
\hline 6 & -0.633543 & 2.583783 & 1.350750 \\
\hline 6 & -0.563739 & 3.928724 & 1.697928 \\
\hline 6 & -1.537235 & 4.796176 & 1.173696 \\
\hline 6 & -2.571124 & 4.350130 & 0.356209 \\
\hline 6 & -2.641317 & 2.978734 & 0.075777 \\
\hline 6 & -3.618172 & 5.277048 & -0.255382 \\
\hline 6 & 0.525979 & 4.478163 & 2.617613 \\
\hline 8 & 2.822072 & -1.135197 & -1.999014 \\
\hline 6 & 4.058013 & -1.589029 & -1.593791 \\
\hline 6 & 4.983903 & -0.723641 & -1.038987 \\
\hline 6 & 6.198578 & -1.283633 & -0.522270 \\
\hline 6 & 6.476814 & -2.669541 & -0.719528 \\
\hline 6 & 5.537660 & -3.476785 & -1.406895 \\
\hline 6 & 4.343393 & -2.952617 & -1.818573 \\
\hline 6 & 7.666616 & -3.225503 & -0.189955 \\
\hline 6 & 8.540056 & -2.458864 & 0.538034 \\
\hline 6 & 8.250459 & -1.097704 & 0.767843 \\
\hline 6 & 7.115380 & -0.524615 & 0.248832 \\
\hline 6 & 4.677936 & 0.722955 & -0.955135 \\
\hline 6 & 3.497651 & 1.136494 & -0.367746 \\
\hline
\end{tabular}




\begin{tabular}{|c|c|c|c|}
\hline 6 & 3.152696 & 2.492656 & -0.220935 \\
\hline 6 & 3.997080 & 3.459023 & -0.694068 \\
\hline & 5.193116 & 3.104550 & -1.362387 \\
\hline & 5.534258 & 1.726206 & -1.516576 \\
\hline & 6.698141 & 1.410388 & -2.263086 \\
\hline & 7.493948 & 2.397405 & -2.789533 \\
\hline & 7.173937 & 3.758065 & -2.600951 \\
\hline & 6.042494 & 4.098776 & -1.905988 \\
\hline & 2.621806 & 0.204308 & 0.142781 \\
\hline & -0.372654 & -0.850532 & -3.376510 \\
\hline & -0.550302 & -1.493425 & -4.323055 \\
\hline & 6.897134 & 0.521081 & 0.450555 \\
\hline & -1.968207 & 1.115680 & 2.886279 \\
\hline 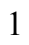 & 7.863181 & -4.283020 & -0.361754 \\
\hline & -1.359682 & -2.200249 & 6.204659 \\
\hline & 5.765871 & 5.143291 & -1.768820 \\
\hline 1 & -3.573283 & -2.775256 & 1.249809 \\
\hline & -5.402362 & -4.149047 & 0.358497 \\
\hline 1 & 3.745083 & 4.513444 & -0.588204 \\
\hline & -1.816086 & -0.125122 & 4.992032 \\
\hline 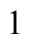 & 5.770391 & -4.526469 & -1.577909 \\
\hline 1 & 8.376358 & 2.125987 & -3.364704 \\
\hline 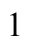 & 3.597650 & -3.561032 & -2.325392 \\
\hline 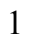 & -2.794974 & -5.737119 & -2.669600 \\
\hline 1 & -0.475827 & -4.976335 & -2.807524 \\
\hline | & -0.401679 & -5.785973 & 4.036559 \\
\hline 1 & 6.953295 & 0.367554 & -2.431324 \\
\hline 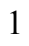 & -0.609016 & -4.584805 & 1.909078 \\
\hline 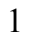 & 1.372797 & -3.572026 & -1.935910 \\
\hline 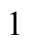 & 7.815676 & 4.529833 & -3.019473 \\
\hline 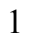 & 8.930190 & -0.495837 & 1.366930 \\
\hline ] & -5.018588 & -5.664712 & -1.581195 \\
\hline 1 & -0.776914 & -4.608077 & 6.198076 \\
\hline D & 9.445986 & -2.898115 & 0.949012 \\
\hline 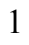 & 2.207723 & 2.735484 & 0.262163 \\
\hline 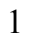 & -4.140853 & 0.501499 & 1.581991 \\
\hline 1 & -2.974301 & -0.566183 & -2.389320 \\
\hline 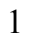 & -7.114926 & -0.668468 & -1.270345 \\
\hline 1 & -3.443475 & 2.597816 & -0.555250 \\
\hline 1 & 0.126403 & 1.883346 & 1.699977 \\
\hline D & -1.473774 & 5.854532 & 1.417574 \\
\hline 1 & 1.655715 & -3.389121 & 1.548058 \\
\hline ] & -1.406729 & 1.105284 & -2.630811 \\
\hline 1 & 3.054809 & -3.101826 & 0.513666 \\
\hline 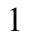 & 4.865351 & -2.298525 & 1.704895 \\
\hline 1 & 0.950109 & -0.907872 & 2.766635 \\
\hline 1 & 5.852904 & -0.882226 & 3.472414 \\
\hline 1 & 1.920529 & 0.477322 & 4.567230 \\
\hline 1 & 4.387556 & 0.517897 & 4.915811 \\
\hline 6 & 1.387350 & 3.366753 & 3.216636 \\
\hline 6 & 1.427399 & 5.430564 & 1.825320 \\
\hline 6 & -0.117135 & 5.241242 & 3.781521 \\
\hline 1 & 2.114897 & 3.797736 & 3.916176 \\
\hline 1 & 0.776424 & 2.642486 & 3.774225 \\
\hline 1 & 1.958600 & 2.814087 & 2.459382 \\
\hline 1 & 0.659576 & 5.627350 & 4.455076 \\
\hline
\end{tabular}




$\begin{array}{lrrr}1 & -0.712424 & 6.097421 & 3.443764 \\ 1 & -0.775240 & 4.585387 & 4.366391 \\ 1 & 2.233017 & 5.817946 & 2.463680 \\ 1 & 1.887696 & 4.917305 & 0.970146 \\ 1 & 0.868345 & 6.288687 & 1.432733 \\ 6 & -3.450589 & 6.725185 & 0.196126 \\ 6 & -3.483717 & 5.230600 & -1.782432 \\ 6 & -5.021061 & 4.808659 & 0.149273 \\ 1 & -4.230027 & 7.346756 & -0.262379 \\ 1 & -3.544268 & 6.829188 & 1.285083 \\ 1 & -2.480975 & 7.139795 & -0.107938 \\ 1 & -4.217640 & 5.899445 & -2.252336 \\ 1 & -2.481094 & 5.549383 & -2.096067 \\ 1 & -3.649160 & 4.221313 & -2.179254 \\ 1 & -5.782972 & 5.461541 & -0.297435 \\ 1 & -5.228689 & 3.783145 & -0.182384 \\ 1 & -5.147003 & 4.838709 & 1.239826 \\ 6 & -6.922003 & -1.235850 & -3.675659 \\ 6 & -4.882771 & -0.034977 & -4.414854 \\ 6 & -4.751775 & -2.433301 & -3.722193 \\ 6 & -8.211738 & -0.244974 & 0.912316 \\ 6 & -6.455841 & -0.808119 & 2.565517 \\ 6 & -6.799950 & 1.565773 & 1.844465 \\ 1 & -7.524912 & 1.711414 & 2.656838 \\ 1 & -5.816079 & 1.881023 & 2.214480 \\ 1 & -7.077259 & 2.237206 & 1.020920 \\ 1 & -8.920675 & -0.105525 & 1.738274 \\ 1 & -8.541216 & 0.398316 & 0.086351 \\ 1 & -8.288186 & -1.290360 & 0.585929 \\ 1 & -7.193087 & -0.686326 & 3.370574 \\ 1 & -6.461100 & -1.862936 & 2.260133 \\ 1 & -5.465792 & -0.589937 & 2.986258 \\ 1 & -7.101002 & -1.531966 & -4.716953 \\ 1 & -7.365131 & -2.009552 & -3.035127 \\ 1 & -7.461736 & -0.295367 & -3.503776 \\ 1 & -4.964977 & -2.755004 & -4.750771 \\ 1 & -3.661123 & -2.382562 & -3.608657 \\ 1 & -5.120414 & -3.206965 & -3.036218 \\ 1 & -5.118818 & -0.322971 & -5.448066 \\ 1 & -5.332379 & 0.948705 & -4.226220 \\ 1 & -3.792681 & 0.075583 & -4.345932 \\ & & & \\ 1 & & \end{array}$

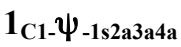

Number of imaginary frequencies : 0

The smallest frequencies are : $12.3629 \quad 17.548322 .0676 \mathrm{~cm}(-1)$

Electronic energy : $\quad H F=-4125.4687872$

Zero-point correction $=\quad 1.299402($ Hartree/Particle $)$

Thermal correction to Energy= $\quad 1.377804$

Thermal correction to Enthalpy= $\quad 1.378748$

Thermal correction to Gibbs Free Energy $=\quad 1.186958$

Sum of electronic and zero-point Energies $=\quad-4124.169385$

Sum of electronic and thermal Energies $=\quad-4124.090983$

Sum of electronic and thermal Enthalpies $=\quad-4124.090039$

Sum of electronic and thermal Free Energies $=\quad-4124.281829$ 
Cartesian Coordinates

$\begin{array}{cccc}6 & 3.900058 & 0.407410 & 4.111979 \\ 6 & 4.753321 & -0.442643 & 3.417409 \\ 6 & 4.237191 & -1.350722 & 2.495652 \\ 6 & 2.867021 & -1.414240 & 2.252599 \\ 6 & 2.016639 & -0.558923 & 2.955449 \\ 6 & 2.526847 & 0.343173 & 3.879191 \\ 6 & 2.320228 & -2.407963 & 1.262174 \\ 7 & 1.381620 & -1.848181 & 0.259643 \\ 6 & 0.304073 & -2.707387 & -0.151766 \\ 6 & -0.923123 & -2.655521 & 0.485669 \\ 6 & -1.998166 & -3.465126 & 0.004187 \\ 6 & -1.786574 & -4.320624 & -1.117671 \\ 6 & -0.509480 & -4.362929 & -1.721653 \\ 6 & 0.506532 & -3.576859 & -1.248841 \\ 6 & -3.288454 & -3.435299 & 0.595248 \\ 6 & -4.306777 & -4.212153 & 0.103458 \\ 6 & -4.088580 & -5.070211 & -0.997010 \\ 6 & -2.856038 & -5.117231 & -1.596488 \\ 6 & -1.132517 & -1.827682 & 1.705366 \\ 6 & -1.479305 & -0.485927 & 1.639462 \\ 6 & -1.751228 & 0.217429 & 2.845108 \\ 6 & -1.646382 & -0.389927 & 4.067618 \\ 6 & -1.270087 & -1.748706 & 4.169924 \\ 6 & -1.021775 & -2.483474 & 2.974390 \\ 6 & -1.137038 & -2.389234 & 5.424819 \\ 6 & -0.772579 & -3.709011 & 5.504401 \\ 6 & -0.530201 & -4.444029 & 4.324886 \\ 6 & -0.655235 & -3.849944 & 3.093390 \\ 15 & -1.650256 & 0.340727 & -0.018402 \\ 45 & -0.113446 & 0.283166 & -1.968492 \\ 6 & 0.407414 & 2.142974 & -2.106821 \\ 8 & 0.512004 & 3.277609 & -2.300856 \\ 15 & 1.725553 & -0.638174 & -0.870056 \\ 8 & 2.678510 & 0.297354 & 0.086823 \\ 6 & 3.557845 & 1.175010 & -0.509020 \\ 6 & 4.744035 & 0.704673 & -1.036939 \\ 6 & 5.614085 & 1.647516 & -1.676139 \\ 6 & 5.272117 & 3.034413 & -1.664985 \\ 6 & 4.062669 & 3.452480 & -1.060089 \\ 6 & 3.209572 & 2.537243 & -0.507378 \\ 6 & 6.135211 & 3.970289 & -2.285214 \\ 6 & 7.281291 & 3.563128 & -2.917722 \\ 6 & 7.603442 & 2.190764 & -2.963795 \\ 6 & 6.793900 & 1.260016 & -2.361397 \\ 6 & 5.036135 & -0.745873 & -0.988613 \\ 6 & 4.109665 & -1.644939 & -1.486472 \\ 6 & 4.388879 & -3.023691 & -1.599111 \\ 6 & 5.569076 & -3.522819 & -1.120429 \\ 6 & 6.500068 & -2.671569 & -0.476499 \\ 6 & 6.233774 & -1.271501 & -0.402895 \\ 6 & 7.140261 & -0.457952 & 0.323438 \\ 6 & 8.254220 & -0.996178 & 0.919494 \\ 6 & 8.532221 & -2.375171 & 0.813905 \\ 6 & 7.669285 & -3.192276 & 0.129429\end{array}$




\begin{tabular}{|c|c|c|c|}
\hline & 2.879777 & -1.214793 & -1.936130 \\
\hline & -3.397750 & -0.087552 & -0.410175 \\
\hline & -4.422851 & 0.190190 & 0.497897 \\
\hline & -5.748919 & -0.112635 & 0.194451 \\
\hline & -6.012584 & -0.713132 & -1.042978 \\
\hline & -5.011965 & -1.014891 & -1.968168 \\
\hline & -3.694988 & -0.694069 & -1.626274 \\
\hline & -6.889957 & 0.159507 & 1.173554 \\
\hline & -7.410172 & -1.180580 & 1.705834 \\
\hline & -5.372310 & -1.615941 & -3.325536 \\
\hline & -4.131973 & -2.047492 & -4.105925 \\
\hline & -1.739723 & 2.120388 & 0.427702 \\
\hline & -2.708145 & 2.943174 & -0.153168 \\
\hline b & -2.721320 & 4.314482 & 0.090522 \\
\hline & -1.755700 & 4.839069 & 0.959821 \\
\hline & -0.775075 & 4.047286 & 1.556944 \\
\hline ) & -0.757301 & 2.683223 & 1.240871 \\
\hline & -3.760761 & 5.242432 & -0.534852 \\
\hline ) & 0.201288 & 4.590766 & 2.600571 \\
\hline & -0.233549 & -1.058754 & -3.335107 \\
\hline & -0.297549 & -1.776845 & -4.240943 \\
\hline & -6.436235 & 1.007352 & 2.360706 \\
\hline & -8.030650 & 0.905011 & 0.473381 \\
\hline & -6.116099 & -0.555611 & -4.146064 \\
\hline & -6.271837 & -2.842171 & -3.148958 \\
\hline & 6.928469 & 0.603134 & 0.429710 \\
\hline & -2.047463 & 1.262464 & 2.800138 \\
\hline & 7.858121 & -4.262268 & 0.052802 \\
\hline & -1.328986 & -1.808599 & 6.326018 \\
\hline 1 & 5.858073 & 5.023265 & -2.257918 \\
\hline & -3.470492 & -2.771651 & 1.438474 \\
\hline ] & -5.293053 & -4.164394 & 0.560657 \\
\hline & 3.808937 & 4.511584 & -1.064688 \\
\hline & -1.857805 & 0.168921 & 4.978794 \\
\hline 1 & 5.794893 & -4.584675 & -1.202536 \\
\hline & 8.498720 & 1.864625 & -3.488440 \\
\hline ] & 3.650487 & -3.665757 & -2.073729 \\
\hline & -2.674909 & -5.768778 & -2.450285 \\
\hline 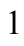 & -0.350745 & -5.019844 & -2.575353 \\
\hline 1 & -0.244934 & -5.491370 & 4.392156 \\
\hline & 7.051900 & 0.205887 & -2.419084 \\
\hline 1 & -0.472162 & -4.431348 & 2.191989 \\
\hline & 1.484385 & -3.588866 & -1.726118 \\
\hline 1 & 7.933639 & 4.290406 & -3.395369 \\
\hline & 8.925963 & -0.352759 & 1.483370 \\
\hline 1 & -4.902081 & -5.690076 & -1.368376 \\
\hline l & -0.670240 & -4.192196 & 6.473253 \\
\hline 1 & 9.421436 & -2.787062 & 1.285274 \\
\hline 1 & 2.260717 & 2.826050 & -0.062679 \\
\hline 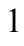 & -4.175054 & 0.663827 & 1.445105 \\
\hline 1 & -2.873760 & -0.911379 & -2.305025 \\
\hline ( & -7.045639 & -0.952063 & -1.295296 \\
\hline 1 & -3.453747 & 2.495310 & -0.805493 \\
\hline ] & 0.013763 & 2.038397 & 1.669840 \\
\hline 1 & -1.779870 & 5.904651 & 1.176593 \\
\hline 1 & 1.739300 & -3.168898 & 1.802693 \\
\hline
\end{tabular}




$\begin{array}{rrrr}1 & -1.397338 & 0.888264 & -2.718692 \\ 1 & -5.677279 & 0.498017 & 2.968815 \\ 1 & -6.023661 & 1.972517 & 2.035494 \\ 1 & -7.292098 & 1.214007 & 3.015511 \\ 1 & -8.465070 & 0.328989 & -0.351845 \\ 1 & -8.839605 & 1.111207 & 1.186704 \\ 1 & -7.686505 & 1.865012 & 0.067433 \\ 1 & -7.782557 & -1.822049 & 0.897041 \\ 1 & -6.614610 & -1.728087 & 2.229005 \\ 1 & -8.234138 & -1.020928 & 2.414734 \\ 1 & -5.492728 & 0.336390 & -4.291222 \\ 1 & -6.378221 & -0.951271 & -5.136822 \\ 1 & -7.044693 & -0.238419 & -3.654858 \\ 1 & -7.215861 & -2.602345 & -2.645053 \\ 1 & -6.524725 & -3.268308 & -4.128941 \\ 1 & -5.761292 & -3.617560 & -2.563001 \\ 1 & -3.484274 & -1.196777 & -4.355716 \\ 1 & -3.534496 & -2.781218 & -3.546356 \\ 1 & -4.434745 & -2.513544 & -5.052144 \\ 1 & 3.149458 & -2.938300 & 0.769115 \\ 1 & 4.913811 & -2.004565 & 1.942783 \\ 1 & 0.949381 & -0.580967 & 2.745469 \\ 1 & 5.828224 & -0.397853 & 3.581902 \\ 1 & 1.846530 & 1.000632 & 4.419919 \\ 1 & 4.301233 & 1.119314 & 4.830217 \\ 6 & 0.089745 & 6.103794 & 2.772691 \\ 6 & -0.122734 & 3.930665 & 3.947168 \\ 6 & 1.646443 & 4.264960 & 2.217316 \\ 6 & -4.743516 & 4.489202 & -1.428602 \\ 6 & -4.564387 & 5.935227 & 0.571285 \\ 6 & -3.048174 & 6.296691 & -1.388497 \\ 1 & 0.815880 & 6.442870 & 3.522198 \\ 1 & 0.305159 & 6.638774 & 1.838667 \\ 1 & -0.904830 & 6.408267 & 3.122611 \\ 1 & 2.337141 & 4.659302 & 2.974622 \\ 1 & 1.830047 & 3.185151 & 2.145511 \\ 1 & 1.908530 & 4.723481 & 1.254183 \\ 1 & 0.553034 & 4.301884 & 4.729822 \\ 1 & -1.153325 & 4.152540 & 4.255321 \\ 1 & -0.014952 & 2.839018 & 3.901196 \\ 1 & -5.476560 & 5.192041 & -1.844105 \\ 1 & -4.241015 & 3.999864 & -2.272716 \\ 1 & -5.300165 & 3.722599 & -0.871751 \\ 1 & -3.780764 & 6.965381 & -1.860491 \\ 1 & -2.367689 & 6.917501 & -0.793267 \\ 1 & -2.458070 & 5.822538 & -2.183456 \\ 1 & -5.318906 & 6.601871 & 0.132403 \\ 1 & -5.087007 & 5.199084 & 1.196505 \\ 1 & -3.930808 & 6.542793 & 1.228510\end{array}$

$1_{\mathrm{C} 1-} \psi_{-1 \mathrm{~s} 2 \mathrm{a} a \mathrm{ats}}$

Number of imaginary frequencies : 0

The smallest frequencies are : $12.2162 \quad 15.502920 .5903 \mathrm{~cm}(-1)$

Electronic energy :

Zero-point correction $=$

$\mathrm{HF}=-4125.4694367$

1.299094 (Hartree/Particle) 


$\begin{array}{lc}\text { Thermal correction to Energy= } & 1.377610 \\ \text { Thermal correction to Enthalpy= } & 1.378554 \\ \text { Thermal correction to Gibbs Free Energy= } & 1.185742 \\ \text { Sum of electronic and zero-point Energies }= & -4124.170343 \\ \text { Sum of electronic and thermal Energies }= & -4124.091826 \\ \text { Sum of electronic and thermal Enthalpies }= & -4124.090882 \\ \text { Sum of electronic and thermal Free Energies }= & -4124.283694\end{array}$

Cartesian Coordinates

$\begin{array}{ccccc}6 & 2.015320 & -0.524406 & 2.951605 \\ 6 & 2.860695 & -1.395331 & 2.261731 \\ 6 & 4.232353 & -1.328673 & 2.494915 \\ 6 & 4.755737 & -0.398398 & 3.389998 \\ 6 & 3.908181 & 0.471190 & 4.067031 \\ 6 & 2.533120 & 0.401353 & 3.847170 \\ 6 & 2.307071 & -2.399500 & 1.286046 \\ 7 & 1.387135 & -1.844165 & 0.263636 \\ 15 & 1.741274 & -0.633735 & -0.862550 \\ 45 & -0.089522 & 0.297011 & -1.966278 \\ 6 & 0.428477 & 2.159652 & -2.083522 \\ 8 & 0.529726 & 3.296105 & -2.268359 \\ 6 & 0.309061 & -2.699834 & -0.153364 \\ 6 & -0.916454 & -2.654432 & 0.488025 \\ 6 & -1.994718 & -3.456092 & 0.000922 \\ 6 & -1.789241 & -4.296438 & -1.133221 \\ 6 & -0.514380 & -4.332590 & -1.742231 \\ 6 & 0.505456 & -3.555350 & -1.262524 \\ 6 & -3.284086 & -3.427270 & 0.594511 \\ 6 & -4.307018 & -4.191781 & 0.093235 \\ 6 & -4.094608 & -5.036405 & -1.018714 \\ 6 & -2.863186 & -5.081762 & -1.620618 \\ 6 & -1.130056 & -1.828463 & 1.707634 \\ 6 & -1.478557 & -0.487634 & 1.634908 \\ 6 & -1.767270 & 0.216999 & 2.835785 \\ 6 & -1.681424 & -0.390155 & 4.060133 \\ 6 & -1.304838 & -1.748311 & 4.169159 \\ 6 & -1.035716 & -2.482949 & 2.978139 \\ 6 & -1.193860 & -2.389308 & 5.426002 \\ 6 & -0.831269 & -3.709231 & 5.511415 \\ 6 & -0.568391 & -4.444043 & 4.336051 \\ 6 & -0.671693 & -3.849495 & 3.102807 \\ 15 & -1.639494 & 0.330892 & -0.028639 \\ 6 & -3.376887 & -0.121986 & -0.435868 \\ 6 & -4.400832 & 0.111307 & 0.476861 \\ 6 & -5.727557 & -0.203857 & 0.172552 \\ 6 & -5.991913 & -0.757211 & -1.081167 \\ 6 & -4.983460 & -1.019926 & -2.017893 \\ 6 & -3.671544 & -0.699505 & -1.670638 \\ 6 & -6.806937 & 0.068993 & 1.217664 \\ 6 & -5.341706 & -1.588182 & -3.389873 \\ 6 & -1.768028 & 2.109005 & 0.414092 \\ 6 & -0.793379 & 2.695322 & 1.219892 \\ 6 & -0.842769 & 4.058366 & 1.536612 \\ 6 & -1.849137 & 4.825467 & 0.949324 \\ 6 & -2.807498 & 4.277702 & 0.086487\end{array}$




\begin{tabular}{|c|c|c|c|}
\hline 6 & -2.762443 & 2.907028 & -0.156862 \\
\hline & -3.878149 & 5.177605 & -0.527679 \\
\hline & 0.133776 & 4.627416 & 2.566211 \\
\hline & 2.899748 & -1.212272 & -1.922260 \\
\hline & 4.125780 & -1.644760 & -1.464185 \\
\hline & 5.053014 & -0.745717 & -0.967769 \\
\hline & 6.249728 & -1.271448 & -0.379965 \\
\hline & 6.512626 & -2.672528 & -0.447154 \\
\hline & 5.578669 & -3.524933 & -1.085077 \\
\hline & 4.400280 & -3.025068 & -1.567415 \\
\hline & 7.681837 & -3.193179 & 0.158853 \\
\hline & 8.548400 & -2.374962 & 0.837305 \\
\hline & 8.273614 & -0.994926 & 0.937225 \\
\hline & 7.159466 & -0.456942 & 0.341270 \\
\hline & 4.761949 & 0.705052 & -1.017409 \\
\hline & 3.575510 & 1.176516 & -0.490630 \\
\hline & 3.231711 & 2.539909 & -0.483219 \\
\hline & 4.088020 & 3.455075 & -1.030937 \\
\hline & 5.296440 & 3.035801 & -1.637088 \\
\hline 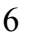 & 5.634574 & 1.647994 & -1.652962 \\
\hline & 6.813437 & 1.259631 & -2.339385 \\
\hline & 7.625632 & 2.190201 & -2.938473 \\
\hline & 7.307354 & 3.563302 & -2.887560 \\
\hline & 6.162315 & 3.971438 & -2.253789 \\
\hline & 2.690773 & 0.300461 & 0.098683 \\
\hline & -0.198676 & -1.028270 & -3.349454 \\
\hline & -0.255764 & -1.735893 & -4.263953 \\
\hline & 6.950516 & 0.605108 & 0.443275 \\
\hline & -2.063379 & 1.261857 & 2.785463 \\
\hline & 7.867879 & -4.263982 & 0.086850 \\
\hline & -1.401668 & -1.809015 & 6.323895 \\
\hline & 5.888110 & 5.025085 & -2.222871 \\
\hline & -3.462279 & -2.773052 & 1.446520 \\
\hline & -5.292754 & -4.143566 & 0.551672 \\
\hline & 3.837643 & 4.514987 & -1.030826 \\
\hline & -1.907571 & 0.168862 & 4.967619 \\
\hline & 5.801289 & -4.587947 & -1.160683 \\
\hline 1 & 8.519965 & 1.863383 & -3.464304 \\
\hline & 3.660473 & -3.667675 & -2.039049 \\
\hline ] & -2.686848 & -5.722367 & -2.483673 \\
\hline & -0.360126 & -4.977322 & -2.606006 \\
\hline l & -0.284845 & -5.491538 & 4.408039 \\
\hline ] & 7.068355 & 0.204953 & -2.400816 \\
\hline & -0.473553 & -4.430357 & 2.204224 \\
\hline ] & 1.480770 & -3.562505 & -1.745036 \\
\hline & 7.961789 & 4.290425 & -3.362578 \\
\hline 1 & 8.948060 & -0.350385 & 1.496578 \\
\hline & -4.911803 & -5.647055 & -1.397154 \\
\hline 1 & -0.746367 & -4.192888 & 6.481709 \\
\hline 1 & 9.437832 & -2.786679 & 1.308412 \\
\hline 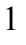 & 2.283114 & 2.829533 & -0.038580 \\
\hline 1 & -4.163366 & 0.557817 & 1.442142 \\
\hline 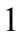 & -2.849064 & -0.890572 & -2.355641 \\
\hline 1 & -7.019946 & -0.995950 & -1.346129 \\
\hline & -3.503105 & 2.440922 & -0.802008 \\
\hline 1 & -0.001900 & 2.069180 & 1.639934 \\
\hline
\end{tabular}




\begin{tabular}{|c|c|c|c|}
\hline 1 & -1.900100 & 5.889628 & 1.168855 \\
\hline & 1.709280 & -3.141224 & 1.834525 \\
\hline & -1.370428 & 0.908680 & -2.716636 \\
\hline & 3.131754 & -2.951797 & 0.809624 \\
\hline & 4.904753 & -1.995681 & 1.952761 \\
\hline & 0.946251 & -0.551469 & 2.750552 \\
\hline & 5.831573 & -0.351445 & 3.547463 \\
\hline & 1.858298 & 1.073753 & 4.376138 \\
\hline & 4.315089 & 1.201875 & 4.762836 \\
\hline & -0.003957 & 6.139396 & 2.728276 \\
\hline & -0.163104 & 3.971051 & 3.920761 \\
\hline & 1.580139 & 4.324544 & 2.168084 \\
\hline & 0.725027 & 6.497902 & 3.465878 \\
\hline & 0.189212 & 6.670782 & 1.787420 \\
\hline & -0.999574 & 6.427742 & 3.088670 \\
\hline & 2.273014 & 4.743217 & 2.910164 \\
\hline & 1.783393 & 3.247561 & 2.110281 \\
\hline & 1.820230 & 4.773137 & 1.194365 \\
\hline & 0.515323 & 4.358582 & 4.693140 \\
\hline & -1.193672 & 4.177487 & 4.239463 \\
\hline 1 & -0.037495 & 2.881096 & 3.880089 \\
\hline & -4.819853 & 4.406973 & -1.450655 \\
\hline D & -4.717252 & 5.807692 & 0.589637 \\
\hline ) & -3.205590 & 6.283279 & -1.348145 \\
\hline 1 & -5.574970 & 5.090414 & -1.859081 \\
\hline & -4.286334 & 3.959355 & -2.298990 \\
\hline & -5.352350 & 3.604847 & -0.921266 \\
\hline 1 & -5.490989 & 6.460414 & 0.163330 \\
\hline & -5.219289 & 5.035020 & 1.187150 \\
\hline I & -4.109861 & 6.415890 & 1.270785 \\
\hline & -3.963997 & 6.929591 & -1.810286 \\
\hline 1 & -2.559234 & 6.920219 & -0.732433 \\
\hline 1 & -2.588693 & 5.856484 & -2.149483 \\
\hline & -4.099765 & -1.986003 & -4.185547 \\
\hline 6 & -6.225960 & -2.829730 & -3.242910 \\
\hline 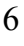 & -6.101015 & -0.515853 & -4.179979 \\
\hline 6 & -8.191904 & -0.377553 & 0.757322 \\
\hline f & -6.465904 & -0.692403 & 2.504708 \\
\hline 5 & -6.860562 & 1.573497 & 1.508437 \\
\hline ] & -7.598456 & 1.786708 & 2.293739 \\
\hline . & -5.891915 & 1.965517 & 1.844641 \\
\hline 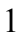 & -7.154245 & 2.133279 & 0.610511 \\
\hline ] & -8.927668 & -0.167830 & 1.543997 \\
\hline 1 & -8.517013 & 0.155461 & -0.145312 \\
\hline ] & -8.226891 & -1.455540 & 0.552177 \\
\hline 1 & -7.231747 & -0.510539 & 3.270691 \\
\hline 1 & -6.424775 & -1.774126 & 2.318807 \\
\hline 1 & -5.498108 & -0.391701 & 2.926057 \\
\hline 1 & -4.401311 & -2.423455 & -5.145715 \\
\hline 1 & -3.456050 & -1.124905 & -4.408846 \\
\hline 1 & -3.498078 & -2.734181 & -3.650373 \\
\hline 1 & -6.365160 & -0.890023 & -5.178516 \\
\hline 1 & -7.030037 & -0.220251 & -3.676312 \\
\hline 1 & -5.487405 & 0.385582 & -4.307168 \\
\hline 1 & -6.472623 & -3.235842 & -4.232963 \\
\hline & -5.705792 & -3.612066 & -2.674703 \\
\hline
\end{tabular}


$1_{\mathrm{C} 1-}-\mathbf{- 1 s 2 a 3 s 4 a}$

Number of imaginary frequencies : 0

The smallest frequencies are : $12.3229 \quad 16.9678 \quad 21.0630 \mathrm{~cm}(-1)$

Electronic energy : $\quad \mathrm{HF}=-4125.4684651$

Zero-point correction $=\quad 1.299359($ Hartree/Particle $)$

Thermal correction to Energy= $\quad 1.377867$

Thermal correction to Enthalpy= $\quad 1.378811$

Thermal correction to Gibbs Free Energy= $\quad 1.186033$

Sum of electronic and zero-point Energies $=\quad-4124.169106$

Sum of electronic and thermal Energies $=\quad-4124.090598$

Sum of electronic and thermal Enthalpies $=\quad-4124.089654$

Sum of electronic and thermal Free Energies $=\quad-4124.282433$

\begin{tabular}{|c|c|c|c|}
\hline & \multicolumn{3}{|c|}{ Cartesian Coordinates } \\
\hline 6 & 1.940664 & -0.513648 & 2.988705 \\
\hline 6 & 2.784134 & -1.384808 & 2.295754 \\
\hline 6 & 4.160061 & -1.289262 & 2.491134 \\
\hline 6 & 4.691101 & -0.324806 & 3.344400 \\
\hline 6 & 3.846542 & 0.549562 & 4.018726 \\
\hline 6 & 2.467226 & 0.445444 & 3.844080 \\
\hline 6 & 2.222586 & -2.417969 & 1.355954 \\
\hline 7 & 1.315836 & -1.887067 & 0.308449 \\
\hline 15 & 1.683919 & -0.719228 & $-0.85805 \mathrm{C}$ \\
\hline 45 & -0.128914 & 0.193628 & -2.010243 \\
\hline 6 & 0.437805 & 2.028229 & -2.235027 \\
\hline 8 & 0.550546 & 3.152598 & -2.479290 \\
\hline 6 & 0.251487 & -2.763526 & -0.101914 \\
\hline 6 & -0.998425 & -2.674657 & 0.483656 \\
\hline 6 & -2.061130 & -3.496771 & -0.003657 \\
\hline 6 & -1.803449 & -4.437434 & -1.044622 \\
\hline 6 & -0.500486 & -4.524037 & -1.586563 \\
\hline 6 & 0.494770 & -3.699470 & -1.134220 \\
\hline 6 & -3.381263 & -3.403485 & 0.509900 \\
\hline 6 & -4.381836 & -4.211610 & 0.031731 \\
\hline 6 & -4.113771 & -5.168011 & -0.972371 \\
\hline 6 & -2.853428 & -5.271181 & -1.502549 \\
\hline 6 & -1.239032 & -1.826793 & 1.683070 \\
\hline 6 & -1.557081 & -0.480497 & 1.600359 \\
\hline 6 & -1.873336 & 0.229440 & 2.792485 \\
\hline 6 & -1.842548 & -0.378580 & 4.018160 \\
\hline 6 & -1.490580 & -1.742588 & 4.139589 \\
\hline 6 & -1.192549 & -2.481563 & 2.958530 \\
\hline 6 & -1.430566 & -2.382858 & 5.400006 \\
\hline 6 & -1.086822 & -3.706952 & 5.499493 \\
\hline 6 & -0.790896 & -4.445303 & 4.334686 \\
\hline 6 & -0.845848 & -3.851341 & 3.097745 \\
\hline 15 & -1.651987 & 0.352960 & -0.06051 \\
\hline 6 & -3.421328 & 0.044493 & -0.465456 \\
\hline 6 & -4.438442 & 0.331317 & 0.455039 \\
\hline 6 & -5.768771 & 0.066279 & 0.153399 \\
\hline 6 & -6.062925 & -0.483464 & -1.106769 \\
\hline 6 & -5.078743 & -0.768725 & -2.047171 \\
\hline
\end{tabular}




\begin{tabular}{|c|c|c|c|}
\hline 6 & -3.747269 & -0.510166 & -1.693167 \\
\hline 6 & -6.898255 & 0.331138 & 1.146684 \\
\hline 6 & -5.373162 & -1.337825 & -3.432573 \\
\hline 6 & -1.607380 & 2.125723 & 0.410225 \\
\hline 6 & -0.594712 & 2.584772 & 1.250355 \\
\hline 6 & -0.526523 & 3.930360 & 1.630046 \\
\hline 6 & -1.446575 & 4.811997 & 1.061884 \\
\hline 6 & -2.436071 & 4.392016 & 0.162413 \\
\hline 6 & -2.513475 & 3.034133 & -0.140715 \\
\hline 6 & -3.406096 & 5.413603 & -0.427901 \\
\hline 6 & 0.453016 & 4.348725 & 2.727707 \\
\hline 8 & 2.849860 & -1.337424 & -1.885730 \\
\hline 6 & 4.080796 & -1.737539 & -1.412840 \\
\hline 6 & 5.000334 & -0.809415 & -0.957139 \\
\hline 6 & 6.208609 & -1.298592 & -0.360598 \\
\hline 6 & 6.488469 & -2.697967 & -0.378016 \\
\hline 6 & 5.558809 & -3.585107 & -0.973237 \\
\hline 6 & 4.371022 & -3.117608 & -1.464549 \\
\hline 6 & 7.671170 & -3.181450 & 0.232480 \\
\hline 6 & 8.536049 & -2.328027 & 0.868269 \\
\hline 6 & 8.244685 & -0.948803 & 0.920470 \\
\hline 6 & 7.116291 & -0.447152 & 0.319454 \\
\hline 6 & 4.692290 & 0.635231 & -1.057403 \\
\hline 6 & 3.503628 & 1.113943 & -0.541153 \\
\hline 6 & 3.149334 & 2.474471 & -0.577055 \\
\hline 6 & 3.996501 & 3.377855 & -1.157678 \\
\hline 6 & 5.204628 & 2.948584 & -1.756812 \\
\hline 6 & 5.553351 & 1.563706 & -1.729442 \\
\hline 6 & 6.730678 & 1.162380 & -2.411158 \\
\hline 6 & 7.532012 & 2.079162 & -3.045121 \\
\hline 6 & 7.203903 & 3.450874 & -3.03 \\
\hline 6 & 6.059592 & 3.870489 & -2.408505 \\
\hline 8 & 2.626354 & 0.249734 & \\
\hline 6 & -0.344573 & -1.197178 & -3.319323 \\
\hline 8 & -0.507589 & -1.923400 & -4.206180 \\
\hline 1 & 6.895746 & 0.615422 & 0.384353 \\
\hline 1 & -2.143850 & 1.281040 & 2.734184 \\
\hline 1 & 7.869354 & -4.251952 & 0.198409 \\
\hline 1 & -1.661526 & -1.798385 & 6.289499 \\
\hline 1 & 5.777128 & 4.922395 & -2.409763 \\
\hline 1 & -3.598686 & -2.669901 & 1.284455 \\
\hline 1 & -5.390577 & -4.110901 & 0.426982 \\
\hline 1 & 3.737253 & 4.435125 & -1.190203 \\
\hline 1 & -2.090158 & 0.184772 & 4.917368 \\
\hline 1 & 5.793629 & -4.647512 & -1.010689 \\
\hline 1 & 8.425288 & 1.742109 & -3.566265 \\
\hline 1 & 3.636818 & -3.786330 & -1.907758 \\
\hline 1 & -2.636860 & -5.991768 & -2.289942 \\
\hline 1 & -0.305719 & -5.245046 & -2.378852 \\
\hline 1 & -0.520029 & -5.495397 & 4.416930 \\
\hline 1 & 6.993202 & 0.108250 & -2.440634 \\
\hline 1 & -0.621034 & -4.436816 & 2.208476 \\
\hline 1 & 1.491676 & -3.741849 & -1.568254 \\
\hline 1 & 7.849928 & 4.167211 & -3.538089 \\
\hline 1 & 8.917705 & -0.275473 & 1.446642 \\
\hline 1 & -4.913439 & -5.811368 & -1.333226 \\
\hline
\end{tabular}




\begin{tabular}{|c|c|c|c|}
\hline t & -1.041353 & -4.190349 & 6.472550 \\
\hline & 9.436481 & -2.710924 & 1.342740 \\
\hline & 2.201629 & 2.772799 & -0.135202 \\
\hline & -4.174244 & 0.768829 & 1.414382 \\
\hline & -2.942496 & -0.742509 & -2.387998 \\
\hline & -7.103827 & -0.686151 & -1.351812 \\
\hline & -3.284441 & 2.663766 & -0.812297 \\
\hline & 0.125065 & 1.870277 & 1.657707 \\
\hline & -1.404788 & 5.865266 & 1.330909 \\
\hline & 1.609402 & -3.129359 & 1.927492 \\
\hline & -1.383974 & 0.821358 & -2.787187 \\
\hline & 3.042025 & -2.999603 & 0.905938 \\
\hline & 4.829143 & -1.960678 & 1.950537 \\
\hline & 0.865455 & -0.570118 & 2.825169 \\
\hline & 5.769588 & -0.255219 & 3.472177 \\
\hline & 1.797974 & 1.120693 & 4.377048 \\
\hline & 4.257863 & 1.309310 & 4.680243 \\
\hline & 0.454470 & 5.856478 & 2.967054 \\
\hline & 0.017350 & 3.657475 & 4.027334 \\
\hline & 1.883142 & 3.922842 & 2.387974 \\
\hline & 1.185726 & 6.103969 & 3.746946 \\
\hline & 0.733778 & 6.413180 & 2.063148 \\
\hline 1 & -0.522151 & 6.222233 & 3.308948 \\
\hline & 2.556005 & 4.161553 & 3.223453 \\
\hline 1 & 1.974003 & 2.845210 & 2.201265 \\
\hline & 2.252115 & 4.455482 & 1.501291 \\
\hline 1 & 0.688564 & 3.931044 & 4.853385 \\
\hline 1 & -1.003037 & 3.953817 & 4.306197 \\
\hline ] & 0.030428 & 2.563737 & 3.929309 \\
\hline 6 & -4.445662 & 4.763759 & -1.338881 \\
\hline 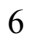 & -4.152420 & 6.130515 & 0.702651 \\
\hline 6 & -2.617661 & 6.436584 & -1.252469 \\
\hline 1 & -5.124845 & 5.532498 & -1.728544 \\
\hline 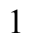 & -3.983982 & 4.265808 & -2.201018 \\
\hline 1 & -5.056866 & 4.023841 & -0.803501 \\
\hline 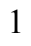 & -4.860648 & 6.860067 & 0.287409 \\
\hline 1 & -4.721904 & 5.415868 & 1.311675 \\
\hline t & -3.474194 & 6.674519 & 1.370770 \\
\hline 1 & -3.298157 & 7.173322 & -1.700575 \\
\hline 1 & -1.890619 & 6.984295 & -0.640682 \\
\hline 1 & -2.066877 & 5.943341 & -2.063923 \\
\hline 6 & -6.869251 & -1.496985 & -3.688730 \\
\hline 5 & -4.800644 & -0.395836 & -4.499103 \\
\hline 6 & -4.712042 & -2.713041 & -3.565918 \\
\hline 6 & -6.399295 & 1.012779 & 2.419265 \\
\hline 6 & -7.955742 & 1.238066 & 0.508891 \\
\hline 6 & -7.537347 & -1.006023 & 1.538343 \\
\hline 1 & -8.352953 & -0.846882 & 2.256768 \\
\hline 1 & -7.955242 & -1.530956 & 0.670492 \\
\hline 1 & -6.797454 & -1.669155 & 2.006824 \\
\hline 1 & -7.243381 & 1.189863 & 3.097623 \\
\hline 1 & -5.668284 & 0.396529 & 2.959765 \\
\hline 1 & -5.936677 & 1.985902 & 2.205770 \\
\hline 1 & -8.763416 & 1.441565 & 1.224696 \\
\hline 1 & -7.519152 & 2.199814 & 0.209203 \\
\hline 1 & -8.411873 & 0.786646 & -0.379998 \\
\hline
\end{tabular}




$\begin{array}{lrrr}1 & -7.029661 & -1.899675 & -4.696758 \\ 1 & -7.335431 & -2.194163 & -2.980231 \\ 1 & -7.400503 & -0.538035 & -3.628937 \\ 1 & -4.895635 & -3.129148 & -4.565929 \\ 1 & -3.625113 & -2.662887 & -3.420869 \\ 1 & -5.113160 & -3.414615 & -2.823050 \\ 1 & -5.021177 & -0.782225 & -5.503200 \\ 1 & -5.239600 & 0.607140 & -4.417636 \\ 1 & -3.710621 & -0.292556 & -4.419548\end{array}$

$1_{\mathrm{C} 1-} \psi_{-1 \mathrm{~s} 2 \mathrm{a} \text { s4s }}$

Number of imaginary frequencies : 0

The smallest frequencies are : $12.5548 \quad 17.0682 \quad 20.9244 \mathrm{~cm}(-1)$

Electronic energy : $\quad H F=-4125.4687874$

Zero-point correction $=\quad 1.299150($ Hartree/Particle $)$

Thermal correction to Energy= $\quad 1.377674$

Thermal correction to Enthalpy= $\quad 1.378618$

Thermal correction to Gibbs Free Energy $=\quad 1.185575$

Sum of electronic and zero-point Energies $=\quad-4124.169637$

Sum of electronic and thermal Energies $=\quad-4124.091114$

Sum of electronic and thermal Enthalpies $=\quad-4124.090170$

Sum of electronic and thermal Free Energies $=\quad-4124.283212$

Cartesian Coordinates

$\begin{array}{cccc}6 & 2.029276 & -0.468538 & 2.921034 \\ 6 & 2.889692 & -1.336666 & 2.246818 \\ 6 & 4.257583 & -1.258814 & 2.497718 \\ 6 & 4.762206 & -0.319956 & 3.394693 \\ 6 & 3.899635 & 0.548118 & 4.054710 \\ 6 & 2.528131 & 0.467071 & 3.816885 \\ 6 & 2.358372 & -2.343765 & 1.261408 \\ 7 & 1.423354 & -1.798298 & 0.246597 \\ 15 & 1.758844 & -0.570286 & -0.865724 \\ 45 & -0.081887 & 0.381572 & -1.928962 \\ 6 & 0.436769 & 2.247079 & -1.976835 \\ 8 & 0.545788 & 3.388952 & -2.119034 \\ 6 & 0.360183 & -2.672089 & -0.170823 \\ 6 & -0.857915 & -2.670116 & 0.487200 \\ 6 & -1.909528 & -3.519402 & 0.020768 \\ 6 & -1.684476 & -4.363471 & -1.107051 \\ 6 & -0.423749 & -4.338413 & -1.745431 \\ 6 & 0.569400 & -3.515361 & -1.287242 \\ 6 & -3.186694 & -3.551212 & 0.639722 \\ 6 & -4.173769 & -4.384388 & 0.176353 \\ 6 & -3.939068 & -5.234355 & -0.926256 \\ 6 & -2.721521 & -5.217260 & -1.556373 \\ 6 & -1.080695 & -1.853716 & 1.712231 \\ 6 & -1.459620 & -0.519949 & 1.649052 \\ 6 & -1.742679 & 0.176131 & 2.855855 \\ 6 & -1.624268 & -0.431682 & 4.077217 \\ 6 & -1.218669 & -1.781919 & 4.176674 \\ 6 & -0.953430 & -2.508544 & 2.979686 \\ 6 & -1.074899 & -2.422823 & 5.430407 \\ 6 & -0.683562 & -3.734871 & 5.507444\end{array}$




\begin{tabular}{|c|c|c|c|}
\hline 6 & -0.423630 & -4.461767 & 4.326425 \\
\hline 6 & -0.558710 & -3.867504 & 3.096257 \\
\hline 15 & -1.649002 & 0.304263 & -0.007975 \\
\hline 6 & -3.359111 & -0.217053 & -0.442000 \\
\hline 6 & -4.398818 & -0.076704 & 0.477685 \\
\hline 6 & -5.713130 & -0.387059 & 0.134046 \\
\hline 6 & -5.960706 & -0.842455 & -1.167470 \\
\hline 6 & -4.941584 & -1.002523 & -2.108903 \\
\hline 6 & -3.634948 & -0.695025 & -1.717175 \\
\hline 6 & -6.814707 & -0.179253 & 1.172064 \\
\hline 6 & -5.188927 & -1.478764 & -3.538397 \\
\hline 6 & -1.876358 & 2.071138 & 0.445211 \\
\hline 6 & -0.912103 & 2.714886 & 1.218852 \\
\hline 6 & -1.031279 & 4.073081 & 1.537139 \\
\hline 6 & -2.105343 & 4.777146 & 0.991688 \\
\hline 6 & -3.059498 & 4.172006 & 0.163955 \\
\hline 6 & -2.937362 & 2.807517 & -0.086979 \\
\hline 6 & -4.201896 & 5.004242 & -0.415219 \\
\hline 6 & -0.037910 & 4.709310 & 2.508970 \\
\hline 8 & 2.910193 & -1.124217 & -1.946357 \\
\hline 6 & 4.136313 & -1.572304 & -1.503227 \\
\hline 6 & 5.067039 & -0.689573 & -0.984749 \\
\hline 6 & 6.259594 & -1.234907 & -0.406348 \\
\hline 6 & 6.516355 & -2.635096 & -0.506936 \\
\hline 6 & 5.582060 & -3.466745 & -1.171127 \\
\hline 6 & 4.406852 & -2.950086 & -1.643446 \\
\hline 6 & 7.680719 & -3.175994 & 0.090646 \\
\hline 6 & 8.547944 & -2.378842 & 0.792848 \\
\hline 6 & 8.278953 & -1.000427 & 0.925780 \\
\hline 6 & 7.170173 & -0.442551 & 0.338271 \\
\hline 6 & 4.785367 & 0.763710 & -1.001157 \\
\hline 6 & 3.603545 & 1.231796 & -0.461849 \\
\hline 6 & 3.275466 & 2.597915 & -0.412077 \\
\hline 6 & 4.138877 & 3.519833 & -0.936389 \\
\hline 6 & 5.340605 & 3.105324 & -1.559313 \\
\hline 6 & 5.665484 & 1.715224 & -1.613094 \\
\hline 6 & 6.839468 & 1.333876 & -2.311601 \\
\hline 6 & 7.658803 & 2.272142 & -2.888561 \\
\hline 6 & 7.353134 & 3.646286 & -2.801483 \\
\hline 6 & 6.213671 & 4.048495 & -2.153889 \\
\hline 8 & 2.710337 & 0.350984 & 0.104817 \\
\hline 6 & -0.212058 & -0.891953 & -3.358280 \\
\hline 8 & -0.308047 & -1.563835 & -4.296407 \\
\hline 1 & 6.965083 & 0.617388 & 0.466566 \\
\hline 1 & -2.062982 & 1.213994 & 2.812396 \\
\hline 1 & 7.862319 & -4.245512 & -0.007375 \\
\hline 1 & -1.280568 & -1.848542 & 6.332639 \\
\hline 1 & 5.949749 & 5.103565 & -2.094519 \\
\hline 1 & -3.382675 & -2.894880 & 1.486090 \\
\hline 1 & -5.147922 & -4.388685 & 0.660281 \\
\hline 1 & 3.900298 & 4.581978 & -0.902929 \\
\hline 1 & -1.847266 & 0.120527 & 4.989572 \\
\hline 1 & 5.801492 & -4.528030 & -1.274984 \\
\hline 1 & 8.548957 & 1.950942 & -3.424855 \\
\hline 1 & 3.667633 & -3.577301 & -2.135870 \\
\hline 1 & -2.529455 & -5.856352 & -2.417281 \\
\hline
\end{tabular}




\begin{tabular}{|c|c|c|c|}
\hline 1 & -0.256287 & -4.981513 & -2.607989 \\
\hline & -0.116713 & -5.503093 & 4.391658 \\
\hline & 7.084393 & 0.278711 & -2.400497 \\
\hline & -0.362121 & -4.442867 & 2.193809 \\
\hline & 1.535885 & -3.484188 & -1.785636 \\
\hline & 8.013036 & 4.379400 & -3.259425 \\
\hline & 8.953741 & -0.373145 & 1.504019 \\
\hline & -4.729090 & -5.894142 & -1.278346 \\
\hline & -0.573352 & -4.218419 & 6.475245 \\
\hline & 9.433441 & -2.806228 & 1.257382 \\
\hline & 2.332297 & 2.882850 & 0.046087 \\
\hline & -4.179234 & 0.304761 & 1.474680 \\
\hline & -2.809246 & -0.813631 & -2.416990 \\
\hline & -6.981807 & -1.077326 & -1.454150 \\
\hline & -3.669218 & 2.300837 & -0.710370 \\
\hline & -0.066625 & 2.137630 & 1.603229 \\
\hline & -2.210871 & 5.837078 & 1.212038 \\
\hline & 1.780532 & -3.106716 & 1.801886 \\
\hline & -1.366387 & 1.023552 & -2.648895 \\
\hline & 3.195639 & -2.869818 & 0.776985 \\
\hline & 4.942172 & -1.922447 & 1.966670 \\
\hline & 0.963834 & -0.501827 & 2.703331 \\
\hline 1 & 5.835670 & -0.263892 & 3.565226 \\
\hline & 1.840371 & 1.137597 & 4.331467 \\
\hline 1 & 4.292406 & 1.286479 & 4.750541 \\
\hline & -0.237076 & 6.217196 & 2.642414 \\
\hline & -0.238321 & 4.073012 & 3.890175 \\
\hline & 1.399882 & 4.459121 & 2.046208 \\
\hline & 0.506233 & 6.626011 & 3.338314 \\
\hline 1 & -0.110188 & 6.732228 & 1.681368 \\
\hline & -1.227889 & 6.470054 & 3.040549 \\
\hline 1 & 2.108215 & 4.967725 & 2.713682 \\
\hline 1 & 1.659527 & 3.393009 & 2.057067 \\
\hline & 1.557164 & 4.844512 & 1.028872 \\
\hline 1 & 0.462403 & 4.503969 & 4.618390 \\
\hline & -1.258730 & 4.244988 & 4.257960 \\
\hline 1 & -0.069736 & 2.988157 & 3.864854 \\
\hline b & -5.135722 & 4.171756 & -1.291709 \\
\hline 6 & -5.029661 & 5.603704 & 0.726958 \\
\hline 5 & -3.622324 & 6.134168 & -1.272966 \\
\hline & -5.945676 & 4.806648 & -1.672573 \\
\hline 1 & -4.614370 & 3.746047 & -2.158847 \\
\hline & -5.597880 & 3.345423 & -0.733253 \\
\hline 1 & -5.853488 & 6.208155 & 0.323992 \\
\hline & -5.465869 & 4.814301 & 1.353566 \\
\hline 1 & -4.429925 & 6.253279 & 1.375925 \\
\hline 1 & -4.431606 & 6.734412 & -1.710373 \\
\hline 1 & -2.986545 & 6.810470 & -0.689043 \\
\hline 1 & -3.015608 & 5.731126 & -2.094057 \\
\hline 5 & -6.668175 & -1.728002 & -3.820854 \\
\hline 6 & -4.686528 & -0.412644 & -4.519986 \\
\hline 6 & -4.427409 & -2.786687 & -3.776655 \\
\hline 6 & -8.174399 & -0.677784 & 0.689662 \\
\hline 6 & -6.462890 & -0.934214 & 2.459308 \\
\hline 6 & -6.931449 & 1.319942 & 1.474455 \\
\hline 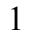 & -7.682573 & 1.498779 & 2.256003 \\
\hline
\end{tabular}




$\begin{array}{rrrr}1 & -5.978990 & 1.744376 & 1.818487 \\ 1 & -7.240335 & 1.874699 & 0.578131 \\ 1 & -8.924197 & -0.523746 & 1.476016 \\ 1 & -8.520117 & -0.136871 & -0.200321 \\ 1 & -8.156209 & -1.750165 & 0.454861 \\ 1 & -7.249856 & -0.790159 & 3.211651 \\ 1 & -6.371592 & -2.011704 & 2.269394 \\ 1 & -5.518291 & -0.594149 & 2.902094 \\ 1 & -6.793831 & -2.060747 & -4.858891 \\ 1 & -7.083856 & -2.511046 & -3.173324 \\ 1 & -7.270161 & -0.818924 & -3.692518 \\ 1 & -4.594421 & -3.143289 & -4.802229 \\ 1 & -3.345174 & -2.664858 & -3.639933 \\ 1 & -4.763133 & -3.567459 & -3.081625 \\ 1 & -4.868672 & -0.732786 & -5.554677 \\ 1 & -5.204823 & 0.542691 & -4.363978 \\ 1 & -3.609156 & -0.229225 & -4.417018\end{array}$

$1_{\mathrm{C} 1-}-\mathbf{- 1 s 2 3 3 a 4 a}$

Number of imaginary frequencies : 0

The smallest frequencies are : $13.9636 \quad 20.2045 \quad 24.7817 \mathrm{~cm}(-1)$

Electronic energy : $\quad H F=-4125.4697604$

Zero-point correction $=\quad 1.299813($ Hartree/Particle)

Thermal correction to Energy= $\quad 1.377890$

Thermal correction to Enthalpy= $\quad 1.378834$

Thermal correction to Gibbs Free Energy= 1.189234

Sum of electronic and zero-point Energies $=\quad-4124.169947$

Sum of electronic and thermal Energies $=\quad-4124.091871$

Sum of electronic and thermal Enthalpies $=\quad-4124.090927$

Sum of electronic and thermal Free Energies $=\quad-4124.280527$

\section{Cartesian Coordinates}

$\begin{array}{lrrr}6 & 3.867141 & 0.467420 & 4.018820 \\ 6 & 4.695726 & -0.414310 & 3.334383 \\ 6 & 4.146478 & -1.366789 & 2.479196 \\ 6 & 2.768203 & -1.442874 & 2.292366 \\ 6 & 1.940746 & -0.564741 & 2.995893 \\ 6 & 2.485431 & 0.382565 & 3.852929 \\ 6 & 2.186632 & -2.461829 & 1.349514 \\ 7 & 1.293115 & -1.909530 & 0.302320 \\ 6 & 0.211718 & -2.759584 & -0.118170 \\ 6 & -1.033092 & -2.659704 & 0.476550 \\ 6 & -2.120209 & -3.432288 & -0.036095 \\ 6 & -1.898655 & -4.314891 & -1.134700 \\ 6 & -0.599048 & -4.423558 & -1.680058 \\ 6 & 0.425818 & -3.662265 & -1.185669 \\ 6 & -3.432842 & -3.331116 & 0.495146 \\ 6 & -4.464360 & -4.066287 & -0.031897 \\ 6 & -4.237888 & -4.951400 & -1.109359 \\ 6 & -2.982454 & -5.068884 & -1.649170 \\ 6 & -1.255892 & -1.820494 & 1.684834 \\ 6 & -1.553470 & -0.469193 & 1.605615 \\ 6 & -1.861325 & 0.241122 & 2.799598 \\ 6 & -1.840221 & -0.371729 & 4.023272\end{array}$




\begin{tabular}{|c|c|c|c|}
\hline & -1.510772 & -1.741901 & 4.140206 \\
\hline & -1.223486 & -2.481185 & 2.956728 \\
\hline & -1.464573 & -2.389236 & 5.397578 \\
\hline 6 & -1.146645 & -3.720316 & 5.490932 \\
\hline & -0.863820 & -4.459243 & 4.323129 \\
\hline & -0.904628 & -3.858175 & 3.089082 \\
\hline & -1.638916 & 0.369212 & -0.054397 \\
\hline & -0.127520 & 0.181818 & -2.014922 \\
\hline & 0.449400 & 2.011665 & -2.254620 \\
\hline & 0.578365 & 3.130760 & -2.514369 \\
\hline & 1.677785 & -0.737153 & -0.854866 \\
\hline & 2.625433 & 0.219735 & 0.087685 \\
\hline 5 & 3.503356 & 1.083822 & -0.531399 \\
\hline & 4.690193 & 0.603483 & -1.050798 \\
\hline & 5.550813 & 1.531134 & -1.724488 \\
\hline & 5.203579 & 2.916412 & -1.750699 \\
\hline & 3.996782 & 3.346838 & -1.149779 \\
\hline & 3.149431 & 2.444449 & -0.567743 \\
\hline f & 6.058218 & 3.837545 & -2.403960 \\
\hline 6 & 7.200480 & 3.416780 & -3.034316 \\
\hline & 7.526690 & 2.044613 & -3.045150 \\
\hline 6 & 6.725755 & 1.128578 & -2.409633 \\
\hline & 4.994945 & -0.842182 & -0.953412 \\
\hline 6 & 4.070370 & -1.766375 & -1.406885 \\
\hline 6 & 4.351877 & -3.148227 & -1.455522 \\
\hline 6 & 5.539401 & -3.620981 & -0.968579 \\
\hline 6 & 6.477157 & -2.737348 & -0.380816 \\
\hline ) & 6.203756 & -1.336609 & -0.362308 \\
\hline 6 & 7.119802 & -0.489140 & 0.311427 \\
\hline 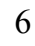 & 8.250366 & -0.995555 & 0.904364 \\
\hline 6 & 8.535143 & -2.376082 & 0.850852 \\
\hline 6 & 7.661747 & -3.225901 & 0.221934 \\
\hline 8 & 2.841995 & -1.360039 & -1.880934 \\
\hline 6 & -3.418719 & 0.097202 & -0.443982 \\
\hline 6 & -4.419123 & 0.446219 & 0.467833 \\
\hline 6 & -5.759148 & 0.181176 & 0.194433 \\
\hline 6 & -6.067529 & -0.428890 & -1.028384 \\
\hline 6 & -5.095093 & -0.790829 & -1.961098 \\
\hline 6 & -3.760143 & -0.524690 & -1.640699 \\
\hline 6 & -6.873139 & 0.491568 & 1.192530 \\
\hline 6 & -7.502973 & -0.829549 & 1.649961 \\
\hline 6 & -5.496015 & -1.413228 & -3.297528 \\
\hline 6 & -4.287364 & -1.954663 & -4.059787 \\
\hline 6 & -1.546877 & 2.138995 & 0.417767 \\
\hline 6 & -2.405178 & 3.071440 & -0.155569 \\
\hline 6 & -2.289931 & 4.433445 & 0.136277 \\
\hline 6 & -1.313763 & 4.829400 & 1.053687 \\
\hline 6 & -0.432125 & 3.916961 & 1.646502 \\
\hline 6 & -0.533267 & 2.574866 & 1.275550 \\
\hline 6 & -3.251117 & 5.413320 & -0.532938 \\
\hline 6 & 0.542579 & 4.313872 & 2.757199 \\
\hline 6 & -0.314466 & -1.218056 & -3.317910 \\
\hline 8 & -0.427158 & -1.959385 & -4.199862 \\
\hline 6 & -6.353691 & 1.224440 & 2.427895 \\
\hline 6 & -7.944277 & 1.368046 & 0.536033 \\
\hline 0 & -6.167402 & -0.333411 & -4.154108 \\
\hline
\end{tabular}




\begin{tabular}{|c|c|c|c|}
\hline 6 & -6.476192 & -2.570096 & -3.084061 \\
\hline & 6.904964 & 0.574549 & 0.376949 \\
\hline & -2.116061 & 1.296918 & 2.745118 \\
\hline & 7.854789 & -4.297317 & 0.187098 \\
\hline & -1.686041 & -1.805165 & 6.289739 \\
\hline & 5.777033 & 4.889794 & -2.404165 \\
\hline & -3.621212 & -2.643703 & 1.318072 \\
\hline & -5.467479 & -3.959434 & 0.376958 \\
\hline & 3.738283 & 4.404283 & -1.182712 \\
\hline & -2.079627 & 0.192188 & 4.924369 \\
\hline & 5.768260 & -4.684748 & -1.004245 \\
\hline 1 & 8.418121 & 1.706636 & -3.568847 \\
\hline & 3.610848 & -3.813806 & -1.892312 \\
\hline & -2.795017 & -5.742350 & -2.484353 \\
\hline & -0.431278 & -5.105758 & -2.511917 \\
\hline & -0.614731 & -5.515054 & 4.400966 \\
\hline & 6.986722 & 0.074104 & -2.440466 \\
\hline & -0.691215 & -4.442785 & 2.196436 \\
\hline & 1.420179 & -3.718214 & -1.624381 \\
\hline & 7.846219 & 4.132530 & -3.537666 \\
\hline & 8.930273 & -0.324950 & 1.425125 \\
\hline & -5.063135 & -5.535673 & -1.511339 \\
\hline & -1.112254 & -4.209140 & 6.461733 \\
\hline & 9.437293 & -2.762792 & 1.318926 \\
\hline & 2.201961 & 2.743698 & -0.125431 \\
\hline & -4.139552 & 0.928626 & 1.401122 \\
\hline & -2.958745 & -0.805328 & -2.319492 \\
\hline & -7.113615 & -0.632513 & -1.257789 \\
\hline & -3.175540 & 2.727161 & -0.845205 \\
\hline 1 & 0.157161 & 1.841964 & 1.700185 \\
\hline & -1.239810 & 5.879089 & 1.321291 \\
\hline & 1.558167 & -3.162052 & 1.918154 \\
\hline & -1.384115 & 0.800168 & -2.797973 \\
\hline & -5.616651 & 0.628139 & 2.982536 \\
\hline & -5.891722 & 2.186351 & 2.168111 \\
\hline & -7.187360 & 1.431856 & 3.110560 \\
\hline & -8.406339 & 0.882608 & -0.331680 \\
\hline & -8.745779 & 1.588867 & 1.253614 \\
\hline 1 & -7.521147 & 2.323277 & 0.198961 \\
\hline & -7.940452 & -1.386951 & 0.812458 \\
\hline & -6.752418 & -1.475336 & 2.125737 \\
\hline & -8.301828 & -0.642542 & 2.380382 \\
\hline & -5.483537 & 0.506577 & -4.333052 \\
\hline 1 & -6.461883 & -0.744377 & -5.129448 \\
\hline & -7.068561 & 0.065345 & -3.671027 \\
\hline 1 & -7.408175 & -2.250351 & -2.602884 \\
\hline & -6.746448 & -3.016142 & -4.050381 \\
\hline 1 & -6.024103 & -3.354801 & -2.463425 \\
\hline & -3.584174 & -1.159106 & -4.338931 \\
\hline & -3.740329 & -2.707545 & -3.473956 \\
\hline 1 & -4.621679 & -2.431675 & -4.989807 \\
\hline & 2.994523 & -3.058583 & 0.898403 \\
\hline 1 & 4.802957 & -2.044039 & 1.930508 \\
\hline & 0.863797 & -0.606432 & 2.839587 \\
\hline & 5.775729 & -0.359812 & 3.456264 \\
\hline & 1.828751 & 1.063967 & 4.393633 \\
\hline
\end{tabular}




$\begin{array}{lrrr}1 & 4.292791 & 1.218119 & 4.681624 \\ 6 & 0.573206 & 5.821000 & 2.999299 \\ 6 & 0.079132 & 3.631387 & 4.051879 \\ 6 & 1.967467 & 3.859266 & 2.432261 \\ 6 & -3.012912 & 6.856541 & -0.096582 \\ 6 & -3.068848 & 5.337633 & -2.053379 \\ 6 & -4.692791 & 5.036200 & -0.170358 \\ 1 & 1.298321 & 6.050945 & 3.790251 \\ 1 & 0.878220 & 6.373465 & 2.101162 \\ 1 & -0.400105 & 6.207544 & 3.327474 \\ 1 & 2.634680 & 4.075998 & 3.278340 \\ 1 & 2.036676 & 2.781716 & 2.236154 \\ 1 & 2.359628 & 4.390929 & 1.555093 \\ 1 & 0.744356 & 3.894970 & 4.885988 \\ 1 & -0.939524 & 3.945090 & 4.317894 \\ 1 & 0.075204 & 2.537428 & 3.955893 \\ 1 & -3.723428 & 7.517688 & -0.608608 \\ 1 & -3.160210 & 6.990501 & 0.983043 \\ 1 & -2.002327 & 7.200169 & -0.352057 \\ 1 & -5.400221 & 5.726845 & -0.648875 \\ 1 & -4.948912 & 4.020344 & -0.497265 \\ 1 & -4.850913 & 5.088298 & 0.915254 \\ 1 & -3.748233 & 6.040459 & -2.554499 \\ 1 & -2.040787 & 5.595878 & -2.338432 \\ 1 & -3.279176 & 4.335255 & -2.445542\end{array}$

$1_{\mathrm{C} 1-} \psi_{\text {-1s2s3a4s }}$

Number of imaginary frequencies : 0

The smallest frequencies are : $11.2526 \quad 17.8890 \quad 23.2702 \mathrm{~cm}(-1)$

Electronic energy : $\quad H F=-4125.4699014$

Zero-point correction $=\quad 1.299612($ Hartree/Particle $)$

Thermal correction to Energy= $\quad 1.377820$

Thermal correction to Enthalpy= $\quad 1.378764$

Thermal correction to Gibbs Free Energy $=1.187606$

Sum of electronic and zero-point Energies $=\quad-4124.170289$

Sum of electronic and thermal Energies $=\quad-4124.092082$

Sum of electronic and thermal Enthalpies $=\quad-4124.091138$

Sum of electronic and thermal Free Energies $=\quad-4124.282295$

Cartesian Coordinates

$\begin{array}{cccc}6 & 1.942228 & -0.530582 & 2.966591 \\ 6 & 2.779926 & -1.411537 & 2.279170 \\ 6 & 4.155525 & -1.331921 & 2.483423 \\ 6 & 4.691728 & -0.373870 & 3.340635 \\ 6 & 3.852930 & 0.510932 & 4.008754 \\ 6 & 2.473697 & 0.423353 & 3.824491 \\ 6 & 2.213387 & -2.431705 & 1.328046 \\ 7 & 1.321492 & -1.881200 & 0.278521 \\ 15 & 1.702413 & -0.690014 & -0.860780 \\ 45 & -0.114275 & 0.252229 & -1.984019 \\ 6 & 0.435805 & 2.100551 & -2.141515 \\ 8 & 0.555605 & 3.230277 & -2.354901 \\ 6 & 0.240734 & -2.728537 & -0.149214 \\ 6 & -0.996330 & -2.659702 & 0.466981\end{array}$




\begin{tabular}{|c|c|c|c|}
\hline 6 & -2.082724 & -3.432108 & -0.047601 \\
\hline 6 & -1.870644 & -4.279107 & -1.175574 \\
\hline & -0.581276 & -4.350944 & -1.749818 \\
\hline & 0.443874 & -3.593855 & -1.249501 \\
\hline & -3.386930 & -3.363013 & 0.509416 \\
\hline & -4.417882 & -4.097197 & -0.019878 \\
\hline & -4.199409 & -4.950349 & -1.124375 \\
\hline & -2.953262 & -5.034072 & -1.691114 \\
\hline & -1.218964 & -1.842799 & 1.690151 \\
\hline & -1.543475 & -0.496718 & 1.626473 \\
\hline & -1.855231 & 0.195814 & 2.829386 \\
\hline & -1.813741 & -0.429369 & 4.046610 \\
\hline & -1.459178 & -1.794431 & 4.146997 \\
\hline & -1.167336 & -2.516327 & 2.953832 \\
\hline & -1.394061 & -2.454506 & 5.397005 \\
\hline & -1.054406 & -3.781187 & 5.473550 \\
\hline & -0.768843 & -4.503335 & 4.295810 \\
\hline 6 & -0.827484 & -3.889851 & 3.068774 \\
\hline 15 & -1.650052 & 0.350238 & -0.027555 \\
\hline 6 & -3.413939 & 0.015396 & -0.438693 \\
\hline 6 & -4.428360 & 0.308222 & 0.468292 \\
\hline 6 & -5.765791 & 0.032499 & 0.171011 \\
\hline 6 & -6.052337 & -0.531905 & -1.072894 \\
\hline 6 & -5.055943 & -0.847145 & -2.005830 \\
\hline 6 & -3.732569 & -0.572261 & -1.663067 \\
\hline 6 & -6.832169 & 0.328197 & 1.223417 \\
\hline 6 & -5.434525 & -1.430548 & -3.365938 \\
\hline 6 & -1.641703 & 2.121443 & 0.450604 \\
\hline 6 & -0.648587 & 2.601847 & 1.307909 \\
\hline 6 & -0.587530 & 3.951860 & 1.656311 \\
\hline 6 & -1.494427 & 4.827997 & 1.047330 \\
\hline 6 & -2.457710 & 4.387173 & 0.136648 \\
\hline 6 & -2.530228 & 3.017915 & -0.134100 \\
\hline 6 & -3.434961 & 5.327449 & -0.565428 \\
\hline 6 & 0.385179 & 4.396043 & 2.750329 \\
\hline 8 & 2.859178 & -1.299321 & -1.904960 \\
\hline 6 & 4.082815 & -1.727108 & -1.436195 \\
\hline 6 & 5.015882 & -0.821075 & -0.963589 \\
\hline 6 & 6.214224 & -1.338468 & -0.371274 \\
\hline 6 & 6.471086 & -2.741852 & -0.408099 \\
\hline 6 & 5.528371 & -3.604948 & -1.017861 \\
\hline 6 & 4.349806 & -3.110959 & -1.505750 \\
\hline 6 & 7.643984 & -3.253317 & 0.198587 \\
\hline 6 & 8.520575 & -2.423425 & 0.849289 \\
\hline 6 & 8.251516 & -1.040528 & 0.920848 \\
\hline 6 & 7.133480 & -0.511824 & 0.323772 \\
\hline 6 & 4.728291 & 0.629346 & -1.037679 \\
\hline 6 & 3.545736 & 1.112002 & -0.511792 \\
\hline 6 & 3.206847 & 2.476770 & -0.520184 \\
\hline 6 & 4.064753 & 3.381180 & -1.083356 \\
\hline 6 & 5.268107 & 2.949433 & -1.690661 \\
\hline 6 & 5.600884 & 1.560282 & -1.690449 \\
\hline 6 & 6.774364 & 1.158376 & -2.378307 \\
\hline 6 & 7.587007 & 2.077959 & -2.993525 \\
\hline 0 & 7.274516 & 3.452912 & -2.958172 \\
\hline 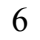 & 6.134474 & 3.873685 & -2.323696 \\
\hline
\end{tabular}




\begin{tabular}{|c|c|c|c|}
\hline 5 & 2.660815 & 0.244985 & 0.090921 \\
\hline & -0.265083 & -1.097866 & -3.340119 \\
\hline & -0.350097 & -1.814693 & -4.245167 \\
\hline & 6.930141 & 0.553211 & 0.403238 \\
\hline & -2.130709 & 1.246831 & 2.787029 \\
\hline 1 & 7.825060 & -4.326247 & 0.149298 \\
\hline 1 & -1.618934 & -1.883800 & 6.296910 \\
\hline & 5.864611 & 4.928730 & -2.304729 \\
\hline & -3.571226 & -2.700208 & 1.353612 \\
\hline & -5.414798 & -4.014238 & 0.408934 \\
\hline & 3.818490 & 4.441990 & -1.094902 \\
\hline & -2.056896 & 0.120557 & 4.955266 \\
\hline & 5.745382 & -4.670574 & -1.068961 \\
\hline & 8.477144 & 1.740914 & -3.520016 \\
\hline & 3.605244 & -3.761443 & -1.958617 \\
\hline & -2.772285 & -5.681079 & -2.548369 \\
\hline & -0.420779 & -5.002977 & -2.606932 \\
\hline & -0.503686 & -5.556103 & 4.360604 \\
\hline 1 & 7.024568 & 0.101924 & -2.427600 \\
\hline & -0.612623 & -4.461241 & 2.167958 \\
\hline l & 1.429039 & -3.623185 & -1.710395 \\
\hline & 7.929347 & 4.171313 & -3.445752 \\
\hline 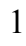 & 8.933763 & -0.386045 & 1.458778 \\
\hline ] & -5.023700 & -5.536213 & -1.525973 \\
\hline & -1.005532 & -4.279884 & 6.438693 \\
\hline 1 & 9.413239 & -2.827789 & 1.320634 \\
\hline & 2.262387 & 2.776884 & -0.072624 \\
\hline 1 & -4.174277 & 0.756858 & 1.428233 \\
\hline & -2.918782 & -0.812984 & -2.342526 \\
\hline 1 & -7.088717 & -0.739385 & -1.331114 \\
\hline 1 & -3.285567 & 2.640968 & -0.822932 \\
\hline & 0.061468 & 1.897912 & 1.747541 \\
\hline 1 & -1.449654 & 5.884291 & 1.294858 \\
\hline | & 1.588998 & -3.141718 & 1.888702 \\
\hline 1 & -1.380685 & 0.879673 & -2.745283 \\
\hline | & 3.029580 & -3.017707 & 0.877846 \\
\hline 1 & 4.820544 & -2.009315 & 1.945066 \\
\hline 1 & 0.868085 & -0.572288 & 2.793495 \\
\hline 1 & 5.770105 & -0.316444 & 3.475289 \\
\hline 1 & 1.808067 & 1.106852 & 4.351811 \\
\hline 1 & 4.269112 & 1.265715 & 4.672828 \\
\hline 6 & 0.369067 & 5.906795 & 2.969898 \\
\hline 6 & -0.036301 & 3.717951 & 4.061246 \\
\hline 6 & 1.819648 & 3.983492 & 2.410011 \\
\hline 1 & 1.092827 & 6.172247 & 3.750897 \\
\hline 1 & 0.648181 & 6.455117 & 2.060876 \\
\hline 1 & -0.613806 & 6.266133 & 3.300508 \\
\hline 1 & 2.495016 & 4.248381 & 3.235385 \\
\hline 1 & 1.923834 & 2.903628 & 2.242912 \\
\hline 1 & 2.173924 & 4.503850 & 1.509947 \\
\hline 1 & 0.633069 & 4.014261 & 4.880768 \\
\hline 1 & -1.059921 & 4.003501 & 4.339259 \\
\hline 1 & -0.007087 & 2.623073 & 3.981405 \\
\hline 6 & -3.260060 & 6.781159 & -0.134312 \\
\hline 6 & -3.202153 & 5.245490 & -2.078937 \\
\hline 6 & -4.873591 & 4.904126 & -0.245011 \\
\hline
\end{tabular}




\begin{tabular}{|c|c|c|c|}
\hline & -3.984475 & 7.411942 & -0.664857 \\
\hline & -3.433915 & 6.915242 & 0.941352 \\
\hline & -2.258457 & 7.161730 & -0.372043 \\
\hline & -3.885483 & 5.923492 & -2.608162 \\
\hline & -2.173187 & 5.531022 & -2.333089 \\
\hline & -3.369356 & 4.233365 & -2.467315 \\
\hline & -5.587332 & 5.559243 & -0.762545 \\
\hline & -5.082701 & 3.872924 & -0.557797 \\
\hline & -5.071835 & 4.973172 & 0.832938 \\
\hline & -4.208431 & -1.906284 & -4.14361 \\
\hline & -6.378557 & -2.624306 & -3.19619 \\
\hline & -6.137440 & -0.342406 & -4.185872 \\
\hline & -8.240137 & -0.008803 & 0.74004 \\
\hline & -6.539655 & -0.5 & \\
\hline & -6.798164 & & \\
\hline & & & \\
\hline & -5.81 & 2.12 & $1.9^{\prime}$ \\
\hline & -7.028120 & 2.44 & 0.719777 \\
\hline & -8.966316 & 0.224611 & 1.528928 \\
\hline & -8.519979 & 0.573835 & -0.14714 \\
\hline & -8.348171 & -1.074593 & 0.50053 \\
\hline & -7.289468 & -0.327 & 3.24861 \\
\hline & -6.5 & -1.58 & \\
\hline & -5.5 & -0.3( & 2.895 \\
\hline & -4.525 & -2.35 & -5.0 \\
\hline & -3.526480 & -1.080860 & -4.38669 \\
\hline & -3.642943 & -2.666110 & $-3.58527 c$ \\
\hline & -6.417210 & -0.728089 & -5.17574 \\
\hline & -7.051879 & 0.011265 & -3.69297 \\
\hline & -5.479533 & 0.523953 & -4.33352 \\
\hline & -6.633313 & -3.044907 & -4.178052 \\
\hline & -5.902937 & -3.415151 & -2.60136 \\
\hline & -7.320488 & -2.350473 & -2.70604 \\
\hline
\end{tabular}

$1_{\mathrm{C} 1-} \psi_{-1 \mathrm{~s} 2 \mathrm{2} 3 \mathrm{~s} 4 \mathrm{a}}$

Number of imaginary frequencies : 0

The smallest frequencies are : $11.7879 \quad 18.5351 \quad 24.1770 \mathrm{~cm}(-1)$

Electronic energy : $\quad H F=-4125.4691053$

Zero-point correction $=\quad 1.299893($ Hartree/Particle $)$

Thermal correction to Energy= $\quad 1.378089$

Thermal correction to Enthalpy= $\quad 1.379033$

Thermal correction to Gibbs Free Energy= 1.188143

Sum of electronic and zero-point Energies $=\quad-4124.169212$

Sum of electronic and thermal Energies $=\quad-4124.091016$

Sum of electronic and thermal Enthalpies $=\quad-4124.090072$

Sum of electronic and thermal Free Energies $=\quad-4124.280963$

\begin{tabular}{|c|c|c|c|}
\hline & \multicolumn{3}{|c|}{ Cartesian Coordinates } \\
\hline 6 & 1.939349 & -0.556482 & 2.989170 \\
\hline 6 & 2.779265 & -1.420107 & 2.282548 \\
\hline 6 & 4.155896 & -1.329682 & 2.475260 \\
\hline 6 & 4.691232 & -0.377442 & 3.339461 \\
\hline 6 & 3.850176 & 0.489245 & 4.027899 \\
\hline 6 & 2.470163 & 0.390008 & 3.855886 \\
\hline
\end{tabular}




\begin{tabular}{|c|c|c|c|}
\hline 6 & 2.213447 & -2.440699 & 1.331762 \\
\hline 7 & 1.307456 & -1.895376 & 0.291045 \\
\hline 1 & 1.678867 & -0.717945 & -0.864503 \\
\hline & -0.131498 & 0.208796 & -2.010788 \\
\hline 6 & 0.449040 & 2.038916 & -2.232387 \\
\hline 8 & 0.570958 & 3.162455 & -2.475803 \\
\hline 6 & 0.239833 & -2.764139 & -0.126705 \\
\hline 6 & -1.010025 & -2.673673 & 0.458487 \\
\hline 6 & -2.075569 & -3.488413 & -0.034898 \\
\hline 6 & -1.820522 & -4.425046 & -1.080087 \\
\hline 6 & -0.517461 & -4.513685 & -1.621527 \\
\hline 6 & 0.480392 & -3.694929 & -1.164304 \\
\hline 6 & -3.395673 & -3.392325 & 0.478077 \\
\hline 6 & -4.398699 & -4.195403 & -0.003451 \\
\hline 6 & -4.133226 & -5.148475 & -1.011457 \\
\hline 6 & -2.873193 & -5.253152 & -1.542113 \\
\hline 6 & -1.247638 & -1.834792 & 1.664897 \\
\hline 6 & -1.558677 & -0.486214 & 1.594756 \\
\hline 6 & -1.873447 & 0.213995 & 2.793079 \\
\hline 6 & -1.847027 & -0.405519 & 4.013018 \\
\hline 6 & -1.501569 & -1.772323 & 4.121983 \\
\hline 6 & -1.205754 & -2.501685 & 2.934366 \\
\hline 6 & -1.445721 & -2.424554 & 5.376441 \\
\hline 6 & -1.108242 & -3.751110 & 5.463918 \\
\hline 6 & -0.814618 & -4.479991 & 4.292618 \\
\hline 6 & -0.865556 & -3.874317 & 3.061221 \\
\hline 15 & -1.647786 & 0.364972 & -0.057005 \\
\hline 6 & -3.422987 & 0.083014 & -0.459007 \\
\hline 6 & -4.435193 & 0.374590 & 0.465836 \\
\hline 6 & -5.768057 & 0.112755 & 0.170781 \\
\hline 6 & -6.069863 & -0.432472 & -1.089591 \\
\hline 6 & -5.091326 & -0.717185 & -2.036132 \\
\hline 6 & -3.757072 & -0.466286 & -1.687079 \\
\hline 6 & -6.890671 & 0.365152 & 1.175784 \\
\hline 6 & -5.394514 & -1.281758 & -3.421550 \\
\hline 6 & -1.579232 & 2.131615 & 0.431363 \\
\hline 6 & -0.565994 & 2.573522 & 1.286506 \\
\hline 6 & -0.487522 & 3.911211 & 1.677830 \\
\hline 6 & -1.394969 & 4.814869 & 1.110679 \\
\hline 6 & -2.373605 & 4.414104 & 0.198250 \\
\hline 6 & -2.462214 & 3.055300 & -0.117596 \\
\hline 6 & -3.368031 & 5.383443 & -0.436949 \\
\hline 6 & 0.489947 & 4.310234 & 2.785224 \\
\hline 8 & 2.845147 & -1.328225 & -1.896069 \\
\hline 6 & 4.076445 & -1.731523 & -1.426980 \\
\hline 6 & 4.996044 & -0.806627 & -0.964904 \\
\hline 6 & 6.205899 & -1.300016 & -0.374973 \\
\hline 6 & 6.486539 & -2.699053 & -0.404575 \\
\hline 6 & 5.555811 & -3.581941 & -1.004458 \\
\hline 6 & 4.366863 & -3.111094 & -1.489728 \\
\hline 6 & 7.671161 & -3.186818 & 0.198748 \\
\hline 6 & 8.537391 & -2.338116 & 0.838990 \\
\hline 6 & 8.245405 & -0.959544 & 0.903203 \\
\hline 6 & 7.114990 & -0.453676 & 0.309563 \\
\hline 6 & 4.686568 & 0.638597 & -1.051782 \\
\hline 6 & 3.498088 & 1.111691 & -0.529751 \\
\hline
\end{tabular}




\begin{tabular}{|c|c|c|c|}
\hline & 3.142767 & 2.472252 & -0.552524 \\
\hline & 3.988853 & 3.381542 & -1.125470 \\
\hline & 5.196543 & 2.958742 & -1.730038 \\
\hline & 5.546153 & 1.573894 & -1.716203 \\
\hline & 6.722704 & 1.179697 & -2.403431 \\
\hline & 7.522563 & 2.102889 & -3.029883 \\
\hline & 7.193698 & 3.474274 & -3.007205 \\
\hline & 6.050065 & 3.887277 & -2.374177 \\
\hline & 2.621634 & 0.241005 & 0.081095 \\
\hline & -0.361716 & -1.174414 & -3.326208 \\
\hline & -0.534525 & -1.893561 & -4.216908 \\
\hline & 6.894342 & 0.608288 & 0.383435 \\
\hline & -2.138873 & 1.267428 & 2.744375 \\
\hline & 7.869842 & -4.256892 & 0.155343 \\
\hline & -1.674808 & -1.847352 & 6.271137 \\
\hline & 5.766987 & 4.938979 & -2.365151 \\
\hline & -3.610041 & -2.660597 & 1.255202 \\
\hline & -5.407220 & -4.092889 & 0.391806 \\
\hline & 3.728885 & 4.438892 & -1.147958 \\
\hline & -2.092781 & 0.150588 & 4.917232 \\
\hline & 5.790936 & -4.643941 & -1.050570 \\
\hline & 8.415233 & 1.771313 & -3.555562 \\
\hline & 3.631755 & -3.776562 & -1.936403 \\
\hline & -2.658812 & -5.970953 & -2.332641 \\
\hline ] & -0.324700 & -5.231427 & -2.417268 \\
\hline & -0.548712 & -5.532052 & 4.365343 \\
\hline & 6.985794 & 0.126036 & -2.443263 \\
\hline & -0.642591 & -4.452652 & 2.166841 \\
\hline & 1.477550 & -3.738833 & -1.597742 \\
\hline 1 & 7.838577 & 4.195716 & -3.503485 \\
\hline & 8.919580 & -0.290098 & 1.432837 \\
\hline 1 & -4.934814 & -5.787781 & -1.375238 \\
\hline & -1.065991 & -4.243748 & 6.432474 \\
\hline & 9.439407 & -2.724336 & 1.307726 \\
\hline & 2.194959 & 2.765765 & -0.107432 \\
\hline & -4.165157 & 0.810646 & 1.424298 \\
\hline ] & -2.956417 & -0.701851 & -2.385580 \\
\hline & -7.112305 & -0.633398 & -1.329317 \\
\hline 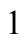 & -3.233601 & 2.706793 & -0.803884 \\
\hline & 0.141248 & 1.846790 & 1.693952 \\
\hline & -1.340532 & 5.861045 & 1.396190 \\
\hline 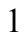 & 1.598401 & -3.156378 & 1.895933 \\
\hline & -1.383757 & 0.849958 & -2.781674 \\
\hline 1 & 3.030634 & -3.019827 & 0.874497 \\
\hline & 4.822037 & -1.995433 & 1.924170 \\
\hline 1 & 0.863526 & -0.608824 & 2.828234 \\
\hline & 5.770183 & -0.311555 & 3.465161 \\
\hline & 1.803760 & 1.059239 & 4.399830 \\
\hline 1 & 4.264722 & 1.239429 & 4.698269 \\
\hline 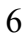 & 0.502938 & 5.815235 & 3.041845 \\
\hline 6 & 0.043795 & 3.608853 & 4.075826 \\
\hline 6 & 1.918329 & 3.877407 & 2.446300 \\
\hline 1 & 1.232348 & 6.047094 & 3.828275 \\
\hline . & 0.791899 & 6.380248 & 2.146203 \\
\hline 1 & -0.472082 & 6.185737 & 3.383078 \\
\hline 1 & 2.589034 & 4.097631 & 3.288648 \\
\hline
\end{tabular}




$\begin{array}{lrrr}1 & 2.001101 & 2.802245 & 2.242580 \\ 1 & 2.296235 & 4.420402 & 1.569746 \\ 1 & 0.712813 & 3.869729 & 4.907780 \\ 1 & -0.975923 & 3.909134 & 4.353068 \\ 1 & 0.050022 & 2.516179 & 3.966799 \\ 6 & -3.164605 & 6.821703 & 0.031972 \\ 6 & -3.201120 & 5.349469 & -1.960390 \\ 6 & -4.794898 & 4.958399 & -0.068291 \\ 1 & -3.899434 & 7.474821 & -0.455467 \\ 1 & -3.302393 & 6.925134 & 1.116206 \\ 1 & -2.167242 & 7.199825 & -0.226654 \\ 1 & -3.910613 & 6.038979 & -2.437771 \\ 1 & -2.186515 & 5.652263 & -2.249465 \\ 1 & -3.379152 & 4.349447 & -2.373811 \\ 1 & -5.525952 & 5.640896 & -0.522340 \\ 1 & -5.027481 & 3.944083 & -0.416767 \\ 1 & -4.942014 & 4.980268 & 1.019875 \\ 6 & -6.892453 & -1.432945 & -3.671651 \\ 6 & -4.821774 & -0.340989 & -4.488983 \\ 6 & -4.740896 & -2.660039 & -3.560342 \\ 6 & -6.388700 & 1.063749 & 2.438068 \\ 6 & -7.977516 & 1.244531 & 0.548930 \\ 6 & -7.498246 & -0.982271 & 1.583228 \\ 1 & -8.310517 & -0.834400 & 2.307875 \\ 1 & -7.912807 & -1.521714 & 0.722642 \\ 1 & -6.741269 & -1.626674 & 2.050351 \\ 1 & -7.228471 & 1.233970 & 3.123516 \\ 1 & -5.644409 & 0.461544 & 2.976272 \\ 1 & -5.941558 & 2.041372 & 2.212669 \\ 1 & -8.778593 & 1.432089 & 1.276334 \\ 1 & -7.570628 & 2.215525 & 0.238231 \\ 1 & -8.437481 & 0.777847 & -0.330013 \\ 1 & -7.059172 & -1.831666 & -4.680230 \\ 1 & -7.358899 & -2.130197 & -2.963410 \\ 1 & -7.418862 & -0.471678 & -3.606435 \\ 1 & -4.931628 & -3.073648 & -4.560048 \\ 1 & -3.652993 & -2.615567 & -3.420834 \\ 1 & -5.141694 & -3.360776 & -2.816539 \\ 1 & -5.046556 & -0.726001 & -5.492653 \\ 1 & -5.257252 & 0.663341 & -4.405970 \\ 1 & -3.731160 & -0.241527 & -4.412593\end{array}$

$1_{\mathrm{C} 1-} \psi_{-1 \mathrm{~s} 2 \mathrm{2} 3 \mathrm{~s} 4 \mathrm{~s}}$

Number of imaginary frequencies : 0

The smallest frequencies are : $12.0740 \quad 19.1667 \quad 21.4604 \mathrm{~cm}(-1)$

Electronic energy : $\quad H F=-4125.4691396$

Zero-point correction $=\quad 1.299411($ Hartree/Particle $)$

Thermal correction to Energy $=\quad 1.377821$

Thermal correction to Enthalpy= $\quad 1.378765$

Thermal correction to Gibbs Free Energy $=\quad 1.186875$

Sum of electronic and zero-point Energies $=\quad-4124.169728$

Sum of electronic and thermal Energies $=\quad-4124.091318$

Sum of electronic and thermal Enthalpies $=\quad-4124.090374$

Sum of electronic and thermal Free Energies $=\quad-4124.282264$ 
Cartesian Coordinates

\begin{tabular}{|c|c|c|c|}
\hline 6 & 1.937437 & -0.555088 & 2.954681 \\
\hline 6 & 2.787682 & -1.416459 & 2.258000 \\
\hline 6 & 4.161495 & -1.323669 & 2.468487 \\
\hline 6 & 4.683663 & -0.371635 & 3.340914 \\
\hline 6 & 3.832313 & 0.493478 & 4.018927 \\
\hline 6 & 2.454800 & 0.392349 & 3.828385 \\
\hline 6 & 2.237373 & -2.429373 & 1.289480 \\
\hline 7 & 1.330755 & -1.875877 & 0.253763 \\
\hline 15 & 1.697188 & -0.668898 & -0.872698 \\
\hline 45 & -0.122804 & 0.294467 & -1.971026 \\
\hline 6 & 0.439315 & 2.140253 & -2.089806 \\
\hline 8 & 0.559161 & 3.275181 & -2.273189 \\
\hline 6 & 0.261216 & -2.735905 & -0.176967 \\
\hline 6 & -0.979891 & -2.678408 & 0.431868 \\
\hline 6 & -2.046950 & -3.485440 & -0.071283 \\
\hline 6 & -1.805640 & -4.372525 & -1.161917 \\
\hline 6 & -0.513578 & -4.424278 & -1.732998 \\
\hline 6 & 0.487635 & -3.620294 & -1.257559 \\
\hline 6 & -3.356071 & -3.428703 & 0.475133 \\
\hline 6 & -4.360349 & -4.222218 & -0.019367 \\
\hline 6 & -4.108858 & -5.123272 & -1.077394 \\
\hline 6 & -2.859939 & -5.189474 & -1.639506 \\
\hline 6 & -1.214512 & -1.871311 & 1.660065 \\
\hline 6 & -1.553105 & -0.527938 & 1.616689 \\
\hline 6 & -1.870053 & 0.144416 & 2.829835 \\
\hline 6 & -1.820344 & -0.496607 & 4.038205 \\
\hline 6 & -1.448561 & -1.858293 & 4.119162 \\
\hline 6 & -1.150626 & -2.560587 & 2.915754 \\
\hline 6 & -1.370223 & -2.532703 & 5.360773 \\
\hline 6 & -1.009975 & -3.854692 & 5.420206 \\
\hline 6 & -0.716629 & -4.557380 & 4.232802 \\
\hline 6 & -0.789005 & -3.930122 & 3.013502 \\
\hline 15 & -1.663019 & 0.347472 & -0.020417 \\
\hline 6 & -3.423671 & 0.017160 & -0.446828 \\
\hline 6 & -4.446961 & 0.234896 & 0.477208 \\
\hline 6 & -5.776454 & -0.038123 & 0.157932 \\
\hline 6 & -6.059409 & -0.510366 & -1.130211 \\
\hline 6 & -5.060965 & -0.726175 & -2.081922 \\
\hline 6 & -3.737884 & -0.473662 & -1.708624 \\
\hline 6 & -6.851153 & 0.175540 & 1.223015 \\
\hline 6 & -6.872005 & 1.649869 & 1.641635 \\
\hline 6 & -5.343510 & -1.229475 & -3.495370 \\
\hline 6 & -4.675522 & -2.595181 & -3.684192 \\
\hline 6 & -1.667469 & 2.110844 & 0.487144 \\
\hline 6 & -0.678579 & 2.587474 & 1.351425 \\
\hline 6 & -0.631171 & 3.932533 & 1.720796 \\
\hline 6 & -1.548514 & 4.808575 & 1.127178 \\
\hline 6 & -2.508644 & 4.372236 & 0.211135 \\
\hline 6 & -2.566194 & 3.006913 & -0.082047 \\
\hline 6 & -3.498477 & 5.312374 & -0.473151 \\
\hline 6 & -4.931002 & 4.851560 & -0.177951 \\
\hline 6 & 0.338147 & 4.370342 & 2.820386 \\
\hline 6 & 1.775614 & 3.972093 & 2.475741 \\
\hline 6 & -8.246687 & -0.197817 & 0.729701 \\
\hline
\end{tabular}




\begin{tabular}{|c|c|c|c|}
\hline 6 & -6.528453 & -0.699540 & 2.440808 \\
\hline 6 & -6.837024 & -1.383014 & -3.770472 \\
\hline & -4.767771 & -0.238665 & -4.514633 \\
\hline & -3.356337 & 6.756506 & -0.000134 \\
\hline & -3.252416 & 5.276405 & -1.986424 \\
\hline & 0.311331 & 5.878196 & 3.058220 \\
\hline & -0.078680 & 3.673283 & 4.122781 \\
\hline & 2.852576 & -1.254427 & -1.931889 \\
\hline & 4.079525 & -1.686260 & -1.475909 \\
\hline & 5.009650 & -0.786841 & -0.985276 \\
\hline & 6.209120 & -1.312322 & -0.402376 \\
\hline & 6.471158 & -2.713635 & -0.468180 \\
\hline & 5.533413 & -3.566697 & -1.099374 \\
\hline & 4.353252 & -3.066881 & -1.577387 \\
\hline & 7.644473 & -3.233548 & 0.130486 \\
\hline & 8.516152 & -2.414464 & 0.801180 \\
\hline y & 8.241810 & -1.034376 & 0.901329 \\
\hline & 7.123660 & -0.497143 & 0.312217 \\
\hline & 4.719743 & 0.664248 & -1.031027 \\
\hline & 3.537600 & 1.135503 & -0.494267 \\
\hline & 3.200489 & 2.500525 & -0.470049 \\
\hline & 4.057775 & 3.416634 & -1.014866 \\
\hline & 5.259287 & 2.997639 & -1.634754 \\
\hline 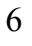 & 5.591656 & 1.608710 & -1.664935 \\
\hline ) & 6.764114 & 1.221212 & -2.362713 \\
\hline & 7.575666 & 2.153454 & -2.960044 \\
\hline & 7.263208 & 3.527363 & -2.895473 \\
\hline & 6.124527 & 3.934875 & -2.249937 \\
\hline & 2.653341 & 0.256486 & 0.090335 \\
\hline & -0.322985 & -1.028976 & -3.348311 \\
\hline & -0.470598 & -1.717956 & -4.267043 \\
\hline & 6.915921 & 0.565156 & 0.413894 \\
\hline & -2.157014 & 1.192820 & 2.801804 \\
\hline & 7.829737 & -4.304506 & 0.058734 \\
\hline & -1.600700 & -1.976203 & 6.268122 \\
\hline & 5.854956 & 4.989351 & -2.208196 \\
\hline ] & -3.561656 & -2.734854 & 1.289055 \\
\hline & -5.359504 & -4.154809 & 0.405857 \\
\hline 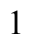 & 3.812566 & 4.477673 & -1.001779 \\
\hline ] & -2.068277 & 0.038043 & 4.954634 \\
\hline & 5.755301 & -4.629875 & -1.174783 \\
\hline 1 & 8.464865 & 1.827547 & -3.495074 \\
\hline & 3.612818 & -3.709823 & -2.047363 \\
\hline ] & -2.655648 & -5.867763 & -2.466811 \\
\hline & -0.330987 & -5.103571 & -2.564152 \\
\hline 1 & -0.434167 & -5.606398 & 4.283620 \\
\hline 1 & 7.014135 & 0.165964 & -2.434514 \\
\hline t & -0.567444 & -4.488037 & 2.105882 \\
\hline 1 & 1.475986 & -3.638365 & -1.711979 \\
\hline & 7.917086 & 4.255784 & -3.369282 \\
\hline 1 & 8.920084 & -0.389007 & 1.455065 \\
\hline & -4.911861 & -5.754336 & -1.452221 \\
\hline 1 & -0.950307 & -4.364386 & 6.378982 \\
\hline 1 & 9.409023 & -2.825492 & 1.266334 \\
\hline ] & 2.257460 & 2.790958 & -0.013257 \\
\hline & -4.201045 & 0.620937 & 1.466092 \\
\hline
\end{tabular}




\begin{tabular}{|c|c|c|c|}
\hline 1 & -2.925914 & -0.656720 & -2.409846 \\
\hline & -7.092355 & -0.713702 & -1.397795 \\
\hline & -3.318027 & 2.633093 & -0.776301 \\
\hline & 0.038668 & 1.883505 & 1.779368 \\
\hline & -1.515061 & 5.861119 & 1.391967 \\
\hline & 1.628670 & -3.161851 & 1.838545 \\
\hline 1 & -1.379748 & 0.958276 & -2.717058 \\
\hline 1 & 3.063307 & -2.990742 & 0.825679 \\
\hline 1 & 4.836267 & -1.985285 & 1.922723 \\
\hline 1 & 0.864532 & -0.606308 & 2.776705 \\
\hline 1 & 5.760997 & -0.303304 & 3.479095 \\
\hline 1 & 1.779186 & 1.060155 & 4.363131 \\
\hline 1 & 4.237294 & 1.243693 & 4.694994 \\
\hline 1 & -6.987897 & -1.732263 & -4.799697 \\
\hline 1 & -7.304452 & -2.119540 & -3.103940 \\
\hline 1 & -7.374353 & -0.431551 & -3.663536 \\
\hline 1 & -4.974159 & -0.583698 & -5.536633 \\
\hline 1 & -5.216495 & 0.756291 & -4.395953 \\
\hline 1 & -3.679712 & -0.129300 & -4.418851 \\
\hline 1 & -4.858351 & -2.973151 & -4.699416 \\
\hline 1 & -3.588794 & -2.544191 & -3.538043 \\
\hline 1 & -5.071746 & -3.326807 & -2.968042 \\
\hline 1 & -7.288052 & -0.563201 & 3.222452 \\
\hline 1 & -6.514544 & -1.762505 & 2.164688 \\
\hline 1 & -5.551967 & -0.459030 & 2.880667 \\
\hline 1 & -8.977321 & -0.031689 & 1.531462 \\
\hline 1 & -8.556976 & 0.412800 & -0.127877 \\
\hline 1 & -8.310697 & -1.254926 & \\
\hline 1 & -7.614592 & 1.811446 & 2.434816 \\
\hline 1 & -5.899462 & 1.986950 & 2.021464 \\
\hline 1 & -7.139864 & 2.293545 & 0.793455 \\
\hline 1 & -5.653500 & 5.513776 & -0.673756 \\
\hline 1 & -5.119234 & 3.830675 & -0.533754 \\
\hline 1 & -5.136981 & 4.873138 & 0.900529 \\
\hline 1 & -3.954043 & 5.944888 & -2.503819 \\
\hline 1 & -2.232042 & 5.602815 & -2.226175 \\
\hline 1 & -3.383310 & 4.269128 & -2.400853 \\
\hline 1 & -4.089811 & 7.387339 & -0.518020 \\
\hline 1 & -3.540053 & 6.855978 & 1.077687 \\
\hline 1 & -2.361348 & 7.164333 & -0.219623 \\
\hline 1 & 1.032740 & 6.139198 & 3.842880 \\
\hline 1 & 0.587034 & 6.439365 & 2.156017 \\
\hline 1 & -0.674120 & 6.226704 & 3.392663 \\
\hline 1 & 2.449706 & 4.237756 & 3.301887 \\
\hline 1 & 1.888606 & 2.894042 & 2.302799 \\
\hline 1 & 2.124388 & 4.500679 & 1.578291 \\
\hline 1 & 0.589977 & 3.962151 & 4.945539 \\
\hline 1 & -1.103450 & 3.950045 & 4.405342 \\
\hline 1 & -0.044069 & 2.579775 & 4.028314 \\
\hline
\end{tabular}

$1_{\mathrm{C} 2-} \psi_{-1 \mathrm{a2a3a4a}}$

Number of imaginary frequencies : 0

The smallest frequencies are : $13.3534 \quad 17.3666 \quad 21.2441 \quad \mathrm{~cm}(-1)$

Electronic energy : $\quad H F=-4125.4703825$ 
Zero-point correction $=$

Thermal correction to Energy=

Thermal correction to Enthalpy=

Thermal correction to Gibbs Free Energy=

Sum of electronic and zero-point Energies=

Sum of electronic and thermal Energies $=$

Sum of electronic and thermal Enthalpies=

Sum of electronic and thermal Free Energies=
1.299460 (Hartree/Particle)

1.377679

1.378623

1.187994

$-4124.170922$

$-4124.092703$

$-4124.091759$

$-4124.282388$

Cartesian Coordinates

$\begin{array}{cccc}6 & 1.628318 & -2.285516 & 5.151772 \\ 6 & 1.868764 & -3.525430 & 4.570751 \\ 6 & 2.064950 & -3.622979 & 3.195711 \\ 6 & 2.025859 & -2.488948 & 2.385599 \\ 6 & 1.781423 & -1.247104 & 2.977557 \\ 6 & 1.584394 & -1.147227 & 4.349517 \\ 6 & 2.328633 & -2.616072 & 0.913608 \\ 7 & 1.398142 & -1.899961 & 0.016927 \\ 6 & 0.308002 & -2.644063 & -0.555934 \\ 6 & -0.965887 & -2.605388 & -0.017281 \\ 6 & -2.038031 & -3.249378 & -0.714665 \\ 6 & -1.772817 & -3.946395 & -1.930212 \\ 6 & -0.445966 & -4.008072 & -2.413984 \\ 6 & 0.561308 & -3.367696 & -1.746231 \\ 6 & -3.378350 & -3.195932 & -0.249931 \\ 6 & -4.393079 & -3.796791 & -0.951634 \\ 6 & -4.122056 & -4.502584 & -2.144372 \\ 6 & -2.838601 & -4.571819 & -2.622752 \\ 6 & -1.246653 & -1.986883 & 1.307461 \\ 6 & -1.493774 & -0.631416 & 1.463197 \\ 6 & -1.838119 & -0.131316 & 2.748689 \\ 6 & -1.948278 & -0.962717 & 3.830134 \\ 6 & -1.717954 & -2.351186 & 3.701640 \\ 6 & -1.353099 & -2.873491 & 2.428049 \\ 6 & -1.842984 & -3.226269 & 4.807031 \\ 6 & -1.630433 & -4.573807 & 4.664734 \\ 6 & -1.265418 & -5.097313 & 3.406917 \\ 6 & -1.124654 & -4.270167 & 2.320323 \\ 15 & -1.541660 & 0.470252 & -0.031776 \\ 45 & 0.079547 & 0.651033 & -1.885924 \\ 6 & 0.682454 & 2.485553 & -1.718189 \\ 8 & 0.835126 & 3.631125 & -1.734521 \\ 15 & 1.814989 & -0.526496 & -0.870628 \\ 8 & 2.778715 & 0.167136 & 0.282314 \\ 6 & 3.654284 & 1.151860 & -0.136977 \\ 6 & 4.831923 & 0.797573 & -0.766098 \\ 6 & 5.680809 & 1.849975 & -1.240308 \\ 6 & 5.335090 & 3.207530 & -0.961974 \\ 6 & 4.142699 & 3.495490 & -0.256602 \\ 6 & 3.304021 & 2.486853 & 0.132986 \\ 6 & 6.177709 & 4.249479 & -1.420772 \\ 6 & 7.305461 & 3.975354 & -2.150114 \\ 6 & 7.629590 & 2.637910 & -2.460236 \\ 6 & 6.840906 & 1.604966 & -2.018565 \\ 6 & 5.140794 & -0.636116 & -0.974178\end{array}$




\begin{tabular}{|c|c|c|c|}
\hline & 4.212176 & -1.446260 & -1.604598 \\
\hline & 4.482552 & -2.793018 & -1.923439 \\
\hline & 5.668876 & -3.357577 & -1.542725 \\
\hline & 6.616898 & -2.610934 & -0.802972 \\
\hline & 6.351997 & -1.240442 & -0.503027 \\
\hline & 7.293609 & -0.541068 & 0.294680 \\
\hline & 8.440378 & -1.151697 & 0.737759 \\
\hline & 8.715071 & -2.495421 & 0.407976 \\
\hline & 7.816116 & -3.207384 & -0.343426 \\
\hline & 2.988213 & -0.943155 & -1.986387 \\
\hline & -3.292278 & 0.211301 & -0.539541 \\
\hline & -4.330087 & 0.318169 & 0.391127 \\
\hline & -5.650132 & 0.074997 & 0.020456 \\
\hline & -5.899204 & -0.271526 & -1.314472 \\
\hline & -4.888364 & -0.387501 & -2.268663 \\
\hline & -3.575001 & -0.143280 & -1.853959 \\
\hline & -6.802811 & 0.133315 & 1.021283 \\
\hline & -5.223484 & -0.707680 & -3.724740 \\
\hline & -1.536749 & 2.150606 & 0.702703 \\
\hline & -2.423986 & 3.128912 & 0.260625 \\
\hline & -2.310365 & 4.449913 & 0.702974 \\
\hline & -1.300711 & 4.748129 & 1.620240 \\
\hline & -0.399853 & 3.787616 & 2.092938 \\
\hline & -0.513527 & 2.491741 & 1.591866 \\
\hline & -3.247579 & 5.555552 & 0.220619 \\
\hline & 0.656181 & 4.175714 & 3.126819 \\
\hline & -0.002584 & -0.427947 & -3.475720 \\
\hline & -0.041229 & -0.958987 & -4.503567 \\
\hline & 7.092660 & 0.491549 & 0.567896 \\
\hline & -2.031897 & 0.931128 & 2.875398 \\
\hline . & 8.002098 & -4.251043 & -0.593576 \\
\hline & -2.116397 & -2.803407 & 5.773089 \\
\hline . & 5.898560 & 5.276771 & -1.190758 \\
\hline & -3.602392 & -2.649875 & 0.664690 \\
\hline & -5.416865 & -3.726618 & -0.588295 \\
\hline & 3.886619 & 4.535122 & -0.056608 \\
\hline & -2.225519 & -0.563931 & 4.805423 \\
\hline . & 5.891759 & -4.394784 & -1.787460 \\
\hline & 8.509498 & 2.422388 & -3.062189 \\
\hline . & 3.736943 & -3.355740 & -2.480337 \\
\hline & -2.614429 & -5.106665 & -3.544606 \\
\hline ] & -0.243899 & -4.552334 & -3.335078 \\
\hline & -1.088537 & -6.165165 & 3.298259 \\
\hline & 7.098215 & 0.581521 & -2.278403 \\
\hline 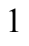 & -0.827988 & -4.687986 & 1.360242 \\
\hline & 1.579633 & -3.383588 & -2.129621 \\
\hline d & 7.941775 & 4.783644 & -2.502743 \\
\hline & 9.141520 & -0.594342 & 1.354802 \\
\hline & -4.933889 & -4.987992 & -2.682335 \\
\hline & -1.736521 & -5.239489 & 5.518400 \\
\hline & 9.630595 & -2.964585 & 0.760385 \\
\hline 1 & 2.360655 & 2.682572 & 0.640876 \\
\hline & -4.093324 & 0.592250 & 1.416390 \\
\hline 1 & -2.744803 & -0.239577 & -2.549502 \\
\hline & -6.928543 & -0.461324 & -1.618046 \\
\hline 1 & -3.203206 & 2.852029 & -0.445817 \\
\hline
\end{tabular}




\begin{tabular}{|c|c|c|c|}
\hline 1 & 0.189269 & 1.718792 & 1.903721 \\
\hline & -1.211760 & 5.771724 & 1.983814 \\
\hline & -1.136277 & 1.425137 & -2.587512 \\
\hline & 2.334011 & -3.677152 & 0.623126 \\
\hline & 3.344295 & -2.242616 & 0.731050 \\
\hline & 2.243386 & -4.597772 & 2.740733 \\
\hline & 1.744430 & -0.355465 & 2.353771 \\
\hline & 1.890867 & -4.423332 & 5.185260 \\
\hline & 1.393480 & -0.172616 & 4.796037 \\
\hline & 1.468446 & -2.205785 & 6.225155 \\
\hline & -3.982821 & -1.118841 & -4.517391 \\
\hline & -6.240723 & -1.848270 & -3.816263 \\
\hline & -5.819249 & 0.550328 & -4.367813 \\
\hline & -6.348393 & 0.617336 & 2.396986 \\
\hline & -7.891059 & 1.087390 & 0.519126 \\
\hline & -7.388357 & -1.274541 & 1.180469 \\
\hline y & -4.302658 & 5.036168 & -0.753596 \\
\hline & -3.973494 & 6.175339 & 1.419630 \\
\hline & -2.425314 & 6.633050 & -0.494761 \\
\hline & 1.447482 & 2.966616 & 3.624965 \\
\hline & 1.629833 & 5.183656 & 2.507979 \\
\hline ) & -0.026412 & 4.812820 & 4.342964 \\
\hline 1 & -4.272681 & -1.395927 & -5.538887 \\
\hline 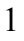 & -3.252413 & -0.303591 & -4.600280 \\
\hline 1 & -3.480740 & -1.984597 & -4.062405 \\
\hline & -6.065263 & 0.364480 & -5.422344 \\
\hline & -6.737646 & 0.867409 & -3.857193 \\
\hline 1 & -5.108066 & 1.385820 & -4.327520 \\
\hline & -6.445136 & -2.083569 & -4.869000 \\
\hline I & -5.855487 & -2.756614 & -3.334712 \\
\hline & -7.201116 & -1.596626 & -3.350880 \\
\hline ] & -8.715993 & 1.136330 & 1.242402 \\
\hline ] & -7.495163 & 2.102925 & 0.389544 \\
\hline & -8.315602 & 0.769388 & -0.440364 \\
\hline 1 & -8.221834 & -1.266398 & 1.896000 \\
\hline & -7.768157 & -1.670810 & 0.230376 \\
\hline 1 & -6.627082 & -1.971736 & 1.556443 \\
\hline ] & -7.208461 & 0.652133 & 3.077545 \\
\hline ] & -5.602679 & -0.052632 & 2.845867 \\
\hline 1 & -5.919846 & 1.627581 & 2.353229 \\
\hline & 2.410623 & 5.457928 & 3.230196 \\
\hline 1 & 2.120813 & 4.764004 & 1.619564 \\
\hline[ & 1.120972 & 6.104733 & 2.198690 \\
\hline 1 & 0.723124 & 5.088879 & 5.096622 \\
\hline 1 & -0.580080 & 5.722271 & 4.082117 \\
\hline 1 & -0.732570 & 4.113284 & 4.809027 \\
\hline 1 & 2.151145 & 3.281796 & 4.405503 \\
\hline 1 & 0.783311 & 2.206635 & 4.060807 \\
\hline 1 & 2.038762 & 2.490056 & 2.831475 \\
\hline 1 & -4.956632 & 5.861852 & -1.061183 \\
\hline 1 & -3.852485 & 4.617454 & -1.662692 \\
\hline 1 & -4.937951 & 4.263839 & -0.299342 \\
\hline 1 & -3.082108 & 7.431090 & -0.866791 \\
\hline 1 & -1.684844 & 7.094796 & 0.169648 \\
\hline 1 & -1.885566 & 6.208448 & -1.351303 \\
\hline 1 & -4.650853 & 6.972025 & 1.083849 \\
\hline
\end{tabular}


$1_{\text {C2- }- \text {-1a2a3a4s }}$

Number of imaginary frequencies : 0

The smallest frequencies are : $13.7927 \quad 17.7433 \quad 20.0255 \mathrm{~cm}(-1)$

Electronic energy :

$\mathrm{HF}=-4125.4709428$

Zero-point correction $=$

1.299778 (Hartree/Particle)

Thermal correction to Energy= $\quad 1.377919$

Thermal correction to Enthalpy $=\quad 1.378863$

Thermal correction to Gibbs Free Energy= $\quad 1.188479$

Sum of electronic and zero-point Energies $=\quad-4124.171164$

Sum of electronic and thermal Energies $=\quad-4124.093024$

Sum of electronic and thermal Enthalpies $=\quad-4124.092080$

Sum of electronic and thermal Free Energies $=\quad-4124.282463$

\begin{tabular}{cccc} 
& \multicolumn{3}{c}{ Cartesian Coordinates } \\
\hline 6 & 1.654499 & -2.247510 & 5.168516 \\
6 & 1.907286 & -3.487012 & 4.592072 \\
6 & 2.106054 & -3.587234 & 3.217667 \\
6 & 2.056604 & -2.456486 & 2.403632 \\
6 & 1.798523 & -1.214994 & 2.990582 \\
6 & 1.599546 & -1.112512 & 4.362110 \\
6 & 2.363476 & -2.586453 & 0.932919 \\
7 & 1.427623 & -1.883920 & 0.031226 \\
6 & 0.342675 & -2.640951 & -0.534741 \\
6 & -0.929413 & -2.614647 & 0.009194 \\
6 & -1.997210 & -3.275236 & -0.679095 \\
6 & -1.730113 & -3.972046 & -1.894113 \\
6 & -0.405148 & -4.019244 & -2.384708 \\
6 & 0.598392 & -3.365966 & -1.723742 \\
6 & -3.335590 & -3.236117 & -0.206716 \\
6 & -4.346520 & -3.850832 & -0.901569 \\
6 & -4.073340 & -4.557102 & -2.093459 \\
6 & -2.792156 & -4.611462 & -2.579494 \\
6 & -1.215841 & -1.984447 & 1.326703 \\
6 & -1.475634 & -0.629179 & 1.463057 \\
6 & -1.824369 & -0.114540 & 2.741392 \\
6 & -1.931192 & -0.932940 & 3.833399 \\
6 & -1.693313 & -2.321534 & 3.723128 \\
6 & -1.319675 & -2.857276 & 2.457842 \\
6 & -1.819066 & -3.183865 & 4.838461 \\
6 & -1.598487 & -4.531789 & 4.713489 \\
6 & -1.224270 & -5.068490 & 3.463826 \\
6 & -1.082574 & -4.253748 & 2.368101 \\
15 & -1.531723 & 0.451137 & -0.048104 \\
45 & 0.095855 & 0.644349 & -1.891403 \\
6 & 0.689757 & 2.482429 & -1.745916 \\
8 & 0.836813 & 3.628541 & -1.777019 \\
15 & 1.836008 & -0.513244 & -0.864274 \\
8 & 2.789355 & 0.197397 & 0.287196 \\
6 & 3.656991 & 1.187087 & -0.137031 \\
6 & 4.840609 & 0.839402 & -0.758671 \\
6 & 5.681118 & 1.896524 & -1.237538
\end{tabular}




\begin{tabular}{|c|c|c|c|}
\hline 6 & 5.320848 & 3.252827 & -0.971459 \\
\hline & 4.122499 & 3.534670 & -0.273784 \\
\hline & 3.292380 & 2.520817 & 0.120078 \\
\hline & 6.155187 & 4.299464 & -1.434672 \\
\hline & 7.288872 & 4.030869 & -2.156801 \\
\hline & 7.627521 & 2.694322 & -2.454985 \\
\hline & 6.847055 & 1.657076 & -2.008813 \\
\hline & 5.163705 & -0.592848 & -0.955206 \\
\hline & 4.244552 & -1.416192 & -1.582487 \\
\hline & 4.527678 & -2.762874 & -1.890135 \\
\hline & 5.718204 & -3.313576 & -1.502380 \\
\hline & 6.657532 & -2.552647 & -0.766090 \\
\hline & 6.379132 & -1.182642 & -0.476380 \\
\hline b & 7.312057 & -0.469108 & 0.319040 \\
\hline & 8.463456 & -1.065894 & 0.768976 \\
\hline & 8.751694 & -2.409092 & 0.448848 \\
\hline ) & 7.861273 & -3.134600 & -0.299734 \\
\hline & 3.017356 & -0.927300 & -1.972154 \\
\hline D & -3.267880 & 0.143418 & -0.578075 \\
\hline & -4.315335 & 0.222644 & 0.335282 \\
\hline & -5.633295 & -0.032497 & -0.051775 \\
\hline & -5.866062 & -0.360946 & -1.388570 \\
\hline 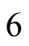 & -4.835742 & -0.453255 & -2.332959 \\
\hline 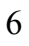 & -3.533531 & -0.200764 & -1.903099 \\
\hline ) & -6.740364 & 0.056793 & 0.996566 \\
\hline b & -5.155055 & -0.761759 & -3.794904 \\
\hline & -1.587347 & 2.137708 & 0.671206 \\
\hline & -2.536460 & 3.070331 & 0.260306 \\
\hline & -2.478773 & 4.393702 & 0.706980 \\
\hline 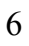 & -1.463681 & 4.738665 & 1.601204 \\
\hline & -0.502189 & 3.823456 & 2.044500 \\
\hline 6 & -0.560216 & 2.526816 & 1.536062 \\
\hline 6 & -3.499506 & 5.444638 & 0.273557 \\
\hline & 0.565298 & 4.260606 & 3.046679 \\
\hline & 0.014357 & -0.450694 & -3.471215 \\
\hline & -0.029104 & -0.992795 & -4.492985 \\
\hline 1 & 7.100691 & 0.563391 & 0.584810 \\
\hline & -2.025642 & 0.948028 & 2.854020 \\
\hline 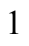 & 8.057605 & -4.178148 & -0.542378 \\
\hline . & -2.099698 & -2.750939 & 5.797963 \\
\hline & 5.864872 & 5.325694 & -1.213953 \\
\hline 1 & -3.561011 & -2.688857 & 0.707204 \\
\hline & -5.369686 & -3.790096 & -0.535012 \\
\hline ] & 3.855082 & 4.573271 & -0.083430 \\
\hline & -2.212873 & -0.523383 & 4.802934 \\
\hline 1 & 5.951167 & -4.350548 & -1.738539 \\
\hline & 8.512227 & 2.482813 & -3.051298 \\
\hline 1 & 3.788026 & -3.336809 & -2.443566 \\
\hline 1 & -2.566797 & -5.146277 & -3.501073 \\
\hline 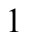 & -0.201649 & -4.562887 & -3.305863 \\
\hline 1 & -1.041041 & -6.136597 & 3.369043 \\
\hline t & 7.115729 & 0.634309 & -2.259590 \\
\hline 1 & -0.779389 & -4.681201 & 1.414314 \\
\hline . & 1.614753 & -3.371035 & -2.112792 \\
\hline 1 & 7.918783 & 4.842648 & -2.512893 \\
\hline 1 & 9.157722 & -0.497777 & 1.383974 \\
\hline
\end{tabular}




\begin{tabular}{|c|c|c|c|}
\hline 1 & -4.882221 & -5.054448 & -2.624860 \\
\hline & -1.705404 & -5.187720 & 5.574563 \\
\hline & 9.670818 & -2.867120 & 0.806499 \\
\hline & 2.344655 & 2.711621 & 0.621411 \\
\hline & -4.101534 & 0.488324 & 1.370436 \\
\hline & -2.695388 & -0.275846 & -2.591176 \\
\hline & -6.886343 & -0.553693 & -1.713499 \\
\hline & -3.321719 & 2.756926 & -0.423598 \\
\hline & 0.189578 & 1.789003 & 1.824703 \\
\hline & -1.420019 & 5.762944 & 1.970850 \\
\hline & -1.124364 & 1.407656 & -2.597256 \\
\hline 1 & 2.381181 & -3.648594 & 0.646783 \\
\hline & 3.375365 & -2.202669 & 0.751016 \\
\hline & 2.294696 & -4.561731 & 2.766174 \\
\hline & 1.751505 & -0.326032 & 2.363564 \\
\hline & 1.936980 & -4.382596 & 5.209644 \\
\hline & 1.397638 & -0.138714 & 4.805461 \\
\hline & 1.492712 & -2.165527 & 6.241438 \\
\hline & -3.895404 & -1.078579 & -4.600386 \\
\hline & -6.099108 & -1.962117 & -3.904744 \\
\hline & -5.830210 & 0.469360 & -4.411408 \\
\hline & -8.108252 & -0.320556 & 0.434371 \\
\hline & -6.423201 & -0.900412 & 2.152107 \\
\hline & -6.818173 & 1.493908 & 1.524903 \\
\hline & -4.495233 & 4.897790 & -0.746987 \\
\hline & -4.285777 & 5.919293 & 1.500674 \\
\hline & -2.774138 & 6.635374 & -0.361948 \\
\hline 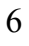 & 1.387951 & & 3.564220 \\
\hline & 1.506733 & 5.265196 & 2.374305 \\
\hline b & -0.099284 & 4.923101 & 4.258985 \\
\hline & -4.171973 & -1.337280 & -5.630375 \\
\hline 1 & -3.210143 & -0.222543 & -4.655217 \\
\hline & -3.345488 & -1.930655 & -4.175946 \\
\hline 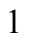 & -6.065596 & 0.289711 & -5.469399 \\
\hline 1 & -6.766625 & 0.718464 & -3.896057 \\
\hline 1 & -5.172916 & 1.346933 & -4.354226 \\
\hline 1 & -6.308195 & -2.179749 & -4.960501 \\
\hline & -5.646326 & -2.856581 & -3.457319 \\
\hline ] & -7.063545 & -1.786929 & -3.413301 \\
\hline & -7.208016 & -0.846270 & 2.918608 \\
\hline 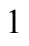 & -6.371178 & -1.938339 & 1.796927 \\
\hline & -5.467945 & -0.669065 & 2.640005 \\
\hline ] & -7.576625 & 1.572469 & 2.315605 \\
\hline 1 & -5.861728 & 1.833349 & 1.943142 \\
\hline & -7.095870 & 2.188796 & 0.721554 \\
\hline 1 & -8.864666 & -0.252688 & 1.226488 \\
\hline & -8.421043 & 0.351721 & -0.374803 \\
\hline 1 & -8.122481 & -1.349781 & 0.051922 \\
\hline 1 & 2.296492 & 5.579523 & 3.070068 \\
\hline 1 & 1.987383 & 4.824788 & 1.489939 \\
\hline 1 & 0.971585 & 6.163967 & 2.044128 \\
\hline 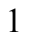 & 0.663502 & 5.228040 & 4.987644 \\
\hline 1 & -0.668177 & 5.819828 & 3.987615 \\
\hline & -0.786508 & 4.228878 & 4.759914 \\
\hline & 2.091159 & 3.429037 & 4.330889 \\
\hline & 0.745604 & 2.314770 & 4.021982 \\
\hline
\end{tabular}




$\begin{array}{lrrr}1 & 1.984600 & 2.600151 & 2.777458 \\ 1 & -5.203953 & 5.686883 & -1.028267 \\ 1 & -3.997423 & 4.557736 & -1.664204 \\ 1 & -5.079746 & 4.059467 & -0.344954 \\ 1 & -3.500229 & 7.391057 & -0.690773 \\ 1 & -2.084621 & 7.123480 & 0.337249 \\ 1 & -2.193392 & 6.318892 & -1.237898 \\ 1 & -5.024778 & 6.678661 & 1.211250 \\ 1 & -4.824436 & 5.084176 & 1.968182 \\ 1 & -3.632511 & 6.363542 & 2.261663\end{array}$

$1_{\mathrm{C} 2-} \psi_{-1 \mathbf{a 2 a 3 s 4 a}}$

Number of imaginary frequencies : 0

The smallest frequencies are : $10.0892 \quad 17.339124 .3358 \mathrm{~cm}(-1)$

Electronic energy : $\quad H F=-4125.4699878$

Zero-point correction $=\quad 1.300084$ (Hartree/Particle)

Thermal correction to Energy $=\quad 1.378140$

Thermal correction to Enthalpy= $\quad 1.379085$

Thermal correction to Gibbs Free Energy= 1.189219

Sum of electronic and zero-point Energies $=\quad-4124.169904$

Sum of electronic and thermal Energies $=\quad-4124.091847$

Sum of electronic and thermal Enthalpies $=\quad-4124.090903$

Sum of electronic and thermal Free Energies $=\quad-4124.280769$

Cartesian Coordinates

$\begin{array}{cccc}6 & 1.601143 & -2.324001 & 5.130539 \\ 6 & 1.870835 & -3.552708 & 4.539431 \\ 6 & 2.092557 & -3.630434 & 3.166936 \\ 6 & 2.050426 & -2.487251 & 2.370457 \\ 6 & 1.777101 & -1.256026 & 2.972353 \\ 6 & 1.553990 & -1.176292 & 4.341474 \\ 6 & 2.371633 & -2.589161 & 0.901169 \\ 7 & 1.418247 & -1.897865 & 0.008533 \\ 6 & 0.347035 & -2.676237 & -0.554620 \\ 6 & -0.934763 & -2.644019 & -0.034127 \\ 6 & -1.981697 & -3.341590 & -0.719569 \\ 6 & -1.678808 & -4.109903 & -1.882094 \\ 6 & -0.344765 & -4.155703 & -2.348335 \\ 6 & 0.633478 & -3.445777 & -1.708271 \\ 6 & -3.330588 & -3.293776 & -0.279546 \\ 6 & -4.314111 & -3.985180 & -0.941651 \\ 6 & -4.002368 & -4.773006 & -2.070947 \\ 6 & -2.712943 & -4.825179 & -2.534250 \\ 6 & -1.243533 & -2.021791 & 1.283647 \\ 6 & -1.513130 & -0.671436 & 1.442550 \\ 6 & -1.883059 & -0.181883 & 2.725647 \\ 6 & -1.997059 & -1.018219 & 3.802175 \\ 6 & -1.742209 & -2.402291 & 3.671936 \\ 6 & -1.349506 & -2.913735 & 2.402211 \\ 6 & -1.869486 & -3.282420 & 4.773074 \\ 6 & -1.629475 & -4.625392 & 4.631456 \\ 6 & -1.232327 & -5.137810 & 3.379070 \\ 6 & -1.090479 & -4.305383 & 2.296625 \\ 15 & -1.553473 & 0.444678 & -0.039849\end{array}$




\begin{tabular}{|c|c|c|c|}
\hline 45 & 0.047228 & 0.626986 & -1.899556 \\
\hline 6 & 0.652375 & 2.460506 & -1.770466 \\
\hline 8 & 0.795122 & 3.607410 & -1.792381 \\
\hline 15 & 1.802620 & -0.519880 & -0.886718 \\
\hline 8 & 2.750928 & 0.198376 & 0.264191 \\
\hline 6 & 3.612701 & 1.195496 & -0.153894 \\
\hline 6 & 4.797946 & 0.858239 & -0.778476 \\
\hline 6 & 5.633468 & 1.922704 & -1.249908 \\
\hline 6 & 5.267173 & 3.275206 & -0.973352 \\
\hline 6 & 4.068127 & 3.546011 & -0.272659 \\
\hline 6 & 3.242631 & 2.525669 & 0.114666 \\
\hline 6 & 6.096280 & 4.329286 & -1.428999 \\
\hline 6 & 7.230898 & 4.071568 & -2.153584 \\
\hline 6 & 7.575642 & 2.738924 & -2.461999 \\
\hline 6 & 6.800251 & 1.694638 & -2.023454 \\
\hline 6 & 5.129250 & -0.570460 & -0.987093 \\
\hline 6 & 4.215842 & -1.393766 & -1.622615 \\
\hline 6 & 4.510428 & -2.733483 & -1.949138 \\
\hline 6 & 5.705012 & -3.279956 & -1.567902 \\
\hline 6 & 6.637420 & -2.521448 & -0.820346 \\
\hline 6 & 6.348891 & -1.156951 & -0.514831 \\
\hline 6 & 7.276268 & -0.445836 & 0.289186 \\
\hline 6 & 8.431536 & -1.039553 & 0.733244 \\
\hline 6 & 8.729535 & -2.376926 & 0.398011 \\
\hline 6 & 7.844976 & -3.100082 & -0.359754 \\
\hline 8 & 2.983445 & -0.911371 & -2.003046 \\
\hline 6 & -3.307665 & 0.225213 & -0.551546 \\
\hline 6 & -4.352164 & 0.338396 & 0.375933 \\
\hline 6 & -5.673186 & 0.149351 & -0.011488 \\
\hline 6 & -5.929087 & -0.143203 & -1.362908 \\
\hline 6 & -4.916410 & -0.252555 & -2.309988 \\
\hline 6 & -3.595908 & -0.078847 & -1.872738 \\
\hline 6 & -6.833961 & 0.235003 & \\
\hline 6 & -5.170015 & -0.543330 & -3.786995 \\
\hline 6 & -1.523504 & 2.113450 & 0.719203 \\
\hline 6 & -2.401542 & 3.109573 & 0.300383 \\
\hline 6 & -2.277263 & 4.418440 & 0.774795 \\
\hline 6 & -1.265568 & 4.686336 & 1.699341 \\
\hline 6 & -0.375626 & 3.705376 & 2.151661 \\
\hline 6 & -0.499033 & 2.423660 & 1.617937 \\
\hline 6 & -3.208213 & 5.540198 & 0.318236 \\
\hline 6 & 0.663460 & 4.049243 & 3.218376 \\
\hline 6 & -0.126957 & -0.505264 & -3.447747 \\
\hline 8 & -0.276890 & -1.063505 & -4.450721 \\
\hline 1 & 7.057249 & 0.582004 & 0.566583 \\
\hline 1 & -2.091430 & 0.877593 & 2.854068 \\
\hline 1 & 8.049061 & -4.139270 & -0.614406 \\
\hline 1 & -2.166834 & -2.867061 & 5.735288 \\
\hline 1 & 5.801237 & 5.352418 & -1.200335 \\
\hline 1 & -3.585878 & -2.686815 & 0.587659 \\
\hline 1 & -5.343538 & -3.923027 & -0.594521 \\
\hline 1 & 3.795990 & 4.581901 & -0.074751 \\
\hline 1 & -2.295232 & -0.627424 & 4.774488 \\
\hline 1 & 5.947013 & -4.311396 & -1.818710 \\
\hline 1 & 8.461098 & 2.536052 & -3.060208 \\
\hline 1 & 3.776868 & -3.303600 & -2.514518 \\
\hline
\end{tabular}




\begin{tabular}{|c|c|c|c|}
\hline 1 & -2.459333 & -5.414802 & -3.414078 \\
\hline & -0.113987 & -4.747275 & -3.232774 \\
\hline & -1.031322 & -6.201433 & 3.270874 \\
\hline & 7.073819 & 0.675145 & -2.282144 \\
\hline & -0.768143 & -4.716234 & 1.341859 \\
\hline & 1.657729 & -3.454168 & -2.075962 \\
\hline & 7.856858 & 4.888973 & -2.503726 \\
\hline & 9.121292 & -0.473611 & 1.355280 \\
\hline & -4.789886 & -5.325497 & -2.579065 \\
\hline & -1.737501 & -5.295286 & 5.481578 \\
\hline & 9.651626 & -2.832473 & 0.751164 \\
\hline & 2.295490 & 2.709911 & 0.619831 \\
\hline & -4.115414 & 0.578090 & 1.409586 \\
\hline & -2.770059 & -0.181547 & -2.574337 \\
\hline & -6.962539 & -0.284577 & -1.673663 \\
\hline & -3.183347 & 2.856084 & -0.411748 \\
\hline & 0.193494 & 1.636292 & 1.916101 \\
\hline & -1.167933 & 5.699737 & 2.088750 \\
\hline & -1.169067 & 1.402649 & -2.598416 \\
\hline & 2.418920 & -3.646391 & 0.600822 \\
\hline & 3.372817 & -2.174559 & 0.729339 \\
\hline & 2.293438 & -4.596671 & 2.703155 \\
\hline & 1.738336 & -0.356907 & 2.358674 \\
\hline & 1.896173 & -4.457445 & 5.143672 \\
\hline & 1.340673 & -0.210368 & 4.797058 \\
\hline & 1.420795 & -2.259819 & 6.201714 \\
\hline & -6.658347 & -0.612835 & -4.117620 \\
\hline & -4.541301 & 0.566674 & -4.638559 \\
\hline & -4.531553 & -1.886226 & -4.154591 \\
\hline 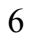 & -6.369445 & 0.622393 & 2.380040 \\
\hline 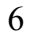 & -7.844216 & 1.286165 & 0.506029 \\
\hline 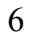 & -7.519194 & -1.133377 & 1.062118 \\
\hline b & -4.283195 & 5.043022 & -0.646320 \\
\hline & -3.911368 & 6.155856 & 1.532814 \\
\hline 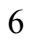 & -2.382025 & 6.614957 & -0.396625 \\
\hline & 1.461820 & 2.823719 & 3.662079 \\
\hline 5 & 1.636392 & 5.101126 & 2.677580 \\
\hline ) & -0.050063 & 4.609042 & 4.455067 \\
\hline ] & -6.788997 & -0.810407 & -5.189028 \\
\hline 1 & -7.163120 & -1.421376 & -3.572959 \\
\hline & -7.173657 & 0.329770 & -3.890868 \\
\hline 1 & -4.677032 & -2.098089 & -5.222691 \\
\hline & -3.451957 & -1.897561 & -3.955927 \\
\hline 1 & -4.982221 & -2.704433 & -3.578263 \\
\hline & -4.736274 & 0.382631 & -5.703540 \\
\hline 1 & -4.960950 & 1.548083 & -4.381472 \\
\hline 1 & -3.452601 & 0.623223 & -4.510052 \\
\hline 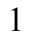 & -8.675180 & 1.362225 & 1.220112 \\
\hline 1 & -7.374076 & 2.275197 & 0.427678 \\
\hline 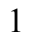 & -8.273467 & 1.041974 & -0.472883 \\
\hline 1 & -8.360966 & -1.098768 & 1.766952 \\
\hline 1 & -7.911855 & -1.457511 & 0.090548 \\
\hline 1 & -6.815684 & -1.900405 & 1.412973 \\
\hline 1 & -7.233965 & 0.670470 & 3.054121 \\
\hline 1 & -5.665824 & -0.109242 & 2.799732 \\
\hline & -5.886237 & 1.608611 & 2.391889 \\
\hline
\end{tabular}




$\begin{array}{rrrr}1 & 2.383843 & 5.359223 & 3.439823 \\ 1 & 2.170983 & 4.728236 & 1.793769 \\ 1 & 1.120798 & 6.025065 & 2.388848 \\ 1 & 0.680440 & 4.847675 & 5.239612 \\ 1 & -0.607742 & 5.526644 & 4.233811 \\ 1 & -0.759028 & 3.877488 & 4.864356 \\ 1 & 2.159018 & 3.106107 & 4.460787 \\ 1 & 0.801646 & 2.038387 & 4.057417 \\ 1 & 2.060221 & 2.391745 & 2.848285 \\ 1 & -4.936135 & 5.877914 & -0.930340 \\ 1 & -3.851628 & 4.635369 & -1.569391 \\ 1 & -4.916242 & 4.267505 & -0.193366 \\ 1 & -3.033089 & 7.425183 & -0.752095 \\ 1 & -1.627622 & 7.059593 & 0.263872 \\ 1 & -1.858113 & 6.192544 & -1.264087 \\ 1 & -4.584191 & 6.963056 & 1.213516 \\ 1 & -4.512551 & 5.404304 & 2.061349 \\ 1 & -3.202569 & 6.583418 & 2.252066\end{array}$

$1_{\mathrm{C} 2-} \psi_{-1 \mathrm{a} 2 \mathrm{a3} 34 \mathrm{~s}}$

Number of imaginary frequencies : 0

The smallest frequencies are : $13.7328 \quad 18.8329 \quad 19.3132 \mathrm{~cm}(-1)$

Electronic energy : $\quad H F=-4125.4703755$

Zero-point correction $=\quad 1.299211$ (Hartree/Particle)

Thermal correction to Energy $=\quad 1.377474$

Thermal correction to Enthalpy= $\quad 1.378418$

Thermal correction to Gibbs Free Energy $=\quad 1.187437$

Sum of electronic and zero-point Energies $=\quad-4124.171164$

Sum of electronic and thermal Energies $=\quad-4124.092902$

Sum of electronic and thermal Enthalpies $=\quad-4124.091958$

Sum of electronic and thermal Free Energies $=\quad-4124.282939$

Cartesian Coordinates

$\begin{array}{llll}6 & 1.687764 & -2.145870 & 5.168451 \\ 6 & 1.973882 & -3.388103 & 4.613715 \\ 6 & 2.179831 & -3.506173 & 3.241953 \\ 6 & 2.103328 & -2.391338 & 2.407946 \\ 6 & 1.809342 & -1.147560 & 2.973295 \\ 6 & 1.605024 & -1.027011 & 4.342641 \\ 6 & 2.419778 & -2.542937 & 0.940700 \\ 7 & 1.479340 & -1.870009 & 0.021692 \\ 6 & 0.414931 & -2.654909 & -0.544297 \\ 6 & -0.858838 & -2.658092 & -0.003768 \\ 6 & -1.902519 & -3.367150 & -0.681142 \\ 6 & -1.606020 & -4.100914 & -1.867246 \\ 6 & -0.280697 & -4.105413 & -2.359891 \\ 6 & 0.695928 & -3.393062 & -1.719517 \\ 6 & -3.242645 & -3.359708 & -0.213608 \\ 6 & -4.225022 & -4.052499 & -0.875588 \\ 6 & -3.919213 & -4.805969 & -2.029674 \\ 6 & -2.637558 & -4.822236 & -2.516910 \\ 6 & -1.162381 & -2.040645 & 1.316381 \\ 6 & -1.458665 & -0.694346 & 1.466104 \\ 6 & -1.821332 & -0.200757 & 2.748692\end{array}$




\begin{tabular}{|c|c|c|c|}
\hline 6 & -1.906013 & -1.031803 & 3.833059 \\
\hline 6 & -1.626522 & -2.411771 & 3.711038 \\
\hline 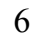 & -1.238597 & -2.925623 & 2.440775 \\
\hline & -1.725445 & -3.286698 & 4.819284 \\
\hline & -1.463069 & -4.626028 & 4.683603 \\
\hline & -1.071280 & -5.140439 & 3.430031 \\
\hline 6 & -0.956402 & -4.313191 & 2.340596 \\
\hline & -1.536370 & 0.396381 & -0.033691 \\
\hline 4 & 0.090294 & 0.655455 & -1.863583 \\
\hline 6 & 0.667329 & 2.495345 & -1.695835 \\
\hline 8 & 0.802244 & 3.643405 & -1.704175 \\
\hline 15 & 1.856627 & -0.484960 & -0.863662 \\
\hline 8 & 2.803960 & 0.224313 & 0.292752 \\
\hline 6 & 3.654482 & 1.235744 & -0.110374 \\
\hline 6 & 4.839631 & 0.920817 & -0.746463 \\
\hline 6 & 5.662233 & 2.002588 & -1.200650 \\
\hline 6 & 5.286787 & 3.345648 & -0.891419 \\
\hline 6 & 4.090875 & 3.591698 & -0.175775 \\
\hline 6 & 3.275475 & 2.556178 & 0.190994 \\
\hline 6 & 6.104739 & 4.416044 & -1.328734 \\
\hline 6 & 7.236263 & 4.183573 & -2.066646 \\
\hline 6 & 7.589477 & 2.861046 & -2.407189 \\
\hline 6 & 6.825537 & 1.801051 & -1.986605 \\
\hline 6 & 5.185507 & -0.501962 & -0.972732 \\
\hline 6 & 4.276438 & -1.331260 & -1.607100 \\
\hline 6 & 4.579689 & -2.668252 & -1.936754 \\
\hline 6 & 5.781279 & -3.205352 & -1.564622 \\
\hline 6 & 6.712397 & -2.440192 & -0.822491 \\
\hline 6 & 6.413634 & -1.079371 & -0.510278 \\
\hline 6 & 7.340016 & -0.363759 & 0.290989 \\
\hline 6 & 8.503540 & -0.948877 & 0.724863 \\
\hline 6 & 8.811534 & -2.281996 & 0.382054 \\
\hline 6 & 7.928176 & -3.009889 & -0.372476 \\
\hline 8 & 3.039335 & -0.857744 & -1.983941 \\
\hline 6 & -3.262074 & 0.061765 & -0.579018 \\
\hline 6 & -4.319098 & 0.117162 & 0.330122 \\
\hline 6 & -5.635585 & -0.085815 & -0.079261 \\
\hline 6 & -5.869145 & -0.338336 & -1.437288 \\
\hline 6 & -4.833389 & -0.405219 & -2.371703 \\
\hline 6 & -3.525848 & -0.214517 & -1.914813 \\
\hline 6 & -6.750334 & -0.008785 & 0.962499 \\
\hline 6 & -5.062733 & -0.658241 & -3.860135 \\
\hline 6 & -1.659871 & 2.078169 & 0.689933 \\
\hline 6 & -2.643957 & 2.968087 & 0.266488 \\
\hline 6 & -2.652180 & 4.292498 & 0.710250 \\
\hline 6 & -1.658251 & 4.687441 & 1.607790 \\
\hline 6 & -0.660181 & 3.817180 & 2.061138 \\
\hline 6 & -0.660133 & 2.514605 & 1.563074 \\
\hline 6 & -3.724619 & 5.287231 & 0.269637 \\
\hline 6 & 0.394807 & 4.308911 & 3.051197 \\
\hline 6 & -0.039353 & -0.439466 & -3.440697 \\
\hline 8 & -0.157047 & -0.982022 & -4.456523 \\
\hline 1 & 7.114072 & 0.660770 & 0.574651 \\
\hline 1 & -2.051666 & 0.855064 & 2.870005 \\
\hline 1 & 8.139453 & -4.046280 & -0.632589 \\
\hline 1 & -2.019155 & -2.870384 & 5.782225 \\
\hline
\end{tabular}




\begin{tabular}{|c|c|c|c|}
\hline & 5.803666 & 5.431526 & -1.075181 \\
\hline & -3.492199 & -2.780464 & 0.674066 \\
\hline 1 & -5.248475 & -4.020029 & -0.507424 \\
\hline & 3.814851 & 4.620577 & 0.051604 \\
\hline & -2.199098 & -0.639128 & 4.806178 \\
\hline & 6.030012 & -4.234428 & -1.818512 \\
\hline & 8.472325 & 2.678762 & -3.015780 \\
\hline & 3.847371 & -3.244333 & -2.497531 \\
\hline & -2.389107 & -5.385439 & -3.415370 \\
\hline 1 & -0.054546 & -4.670389 & -3.262741 \\
\hline 1 & -0.854147 & -6.201376 & 3.326756 \\
\hline 1 & 7.105149 & 0.789518 & -2.269290 \\
\hline 1 & -0.640991 & -4.724385 & 1.383603 \\
\hline 1 & 1.712861 & -3.372706 & -2.106238 \\
\hline 1 & 7.853293 & 5.013627 & -2.402569 \\
\hline 1 & 9.192135 & -0.379218 & 1.344787 \\
\hline & -4.705097 & -5.361721 & -2.536808 \\
\hline 1 & -1.549653 & -5.291891 & 5.539328 \\
\hline & 9.740095 & -2.730628 & 0.727007 \\
\hline 1 & 2.329601 & 2.718928 & 0.705886 \\
\hline & -4.109585 & 0.334850 & 1.377172 \\
\hline & -2.688533 & -0.274174 & -2.608011 \\
\hline ] & -6.891205 & -0.485793 & -1.774331 \\
\hline 1 & -3.406139 & 2.619821 & -0.425682 \\
\hline 1 & 0.115154 & 1.809850 & 1.866648 \\
\hline 1 & -1.661210 & 5.714684 & 1.971402 \\
\hline 1 & -1.139299 & 1.420515 & -2.551953 \\
\hline 1 & 2.451657 & -3.609528 & 0.673367 \\
\hline 1 & 3.428072 & -2.150671 & 0.756267 \\
\hline 1 & 2.395919 & -4.482938 & 2.808037 \\
\hline 1 & 1.741465 & -0.269664 & 2.332936 \\
\hline 1 & 2.024220 & -4.272217 & 5.246312 \\
\hline 1 & 1.375871 & -0.051238 & 4.768132 \\
\hline 1 & 1.521135 & -2.049868 & 6.239486 \\
\hline 6 & -6.544450 & -0.762983 & -4.211333 \\
\hline 6 & -4.459935 & 0.497121 & -4.669393 \\
\hline 6 & -4.379515 & -1.969010 & -4.261880 \\
\hline 6 & -8.124894 & -0.312823 & 0.372438 \\
\hline 6 & -6.476421 & -1.027287 & 2.075817 \\
\hline 6 & -6.786759 & 1.405004 & 1.555716 \\
\hline 6 & -4.682713 & 4.686916 & -0.757558 \\
\hline 6 & -4.544327 & 5.716024 & 1.491788 \\
\hline 6 & -3.061008 & 6.516158 & -0.360167 \\
\hline 6 & 1.235325 & 3.161872 & 3.613069 \\
\hline 6 & 1.321216 & 5.299914 & 2.338331 \\
\hline 6 & -0.278096 & 5.008017 & 4.237475 \\
\hline 1 & -6.655802 & -0.936049 & -5.289160 \\
\hline 1 & -7.031816 & -1.599722 & -3.694131 \\
\hline 1 & -7.090624 & 0.157755 & -3.967687 \\
\hline 1 & -4.511810 & -2.154539 & -5.336600 \\
\hline 1 & -3.301484 & -1.951672 & -4.056249 \\
\hline 1 & -4.806613 & -2.817610 & -3.711842 \\
\hline 1 & -4.639634 & 0.342997 & -5.741841 \\
\hline 1 & -4.911077 & 1.456640 & -4.384019 \\
\hline 1 & -3.374735 & 0.582134 & -4.527521 \\
\hline 1 & -7.270373 & -0.987347 & 2.833726 \\
\hline
\end{tabular}




$\begin{array}{rrrr}1 & -6.446274 & -2.048289 & 1.672371 \\ 1 & -5.522314 & -0.845010 & 2.586550 \\ 1 & -7.549926 & 1.472328 & 2.342981 \\ 1 & -5.823859 & 1.693594 & 1.997053 \\ 1 & -7.035028 & 2.144635 & 0.782848 \\ 1 & -8.886101 & -0.252772 & 1.160612 \\ 1 & -8.406053 & 0.405108 & -0.408593 \\ 1 & -8.172317 & -1.322815 & -0.055297 \\ 1 & 2.112987 & 5.646207 & 3.016542 \\ 1 & 1.797610 & 4.833816 & 1.464765 \\ 1 & 0.772965 & 6.180493 & 1.981436 \\ 1 & 0.481172 & 5.348093 & 4.954055 \\ 1 & -0.855768 & 5.888669 & 3.934300 \\ 1 & -0.957840 & 4.325695 & 4.764190 \\ 1 & 1.924390 & 3.547329 & 4.374865 \\ 1 & 0.603679 & 2.397395 & 4.087376 \\ 1 & 1.849379 & 2.669960 & 2.846977 \\ 1 & -5.433870 & 5.435233 & -1.040330 \\ 1 & -4.162120 & 4.378065 & -1.673338 \\ 1 & -5.220252 & 3.814562 & -0.360895 \\ 1 & -3.824848 & 7.229725 & -0.697382 \\ 1 & -2.407485 & 7.042822 & 0.345521 \\ 1 & -2.454126 & 6.231839 & -1.229402 \\ 1 & -5.319581 & 6.436883 & 1.198653 \\ 1 & -5.042716 & 4.851964 & 1.951648 \\ 1 & -3.921043 & 6.190928 & 2.259394\end{array}$

$1_{\text {C2- }- \text {-1a2s3a4a }}$

Number of imaginary frequencies : 0

The smallest frequencies are : $8.1297 \quad 15.5336 \quad 20.4983 \mathrm{~cm}(-1)$

Electronic energy : $\quad \mathrm{HF}=-4125.4712301$

Zero-point correction $=\quad 1.299403($ Hartree/Particle $)$

Thermal correction to Energy $=\quad 1.377642$

Thermal correction to Enthalpy= $\quad 1.378586$

Thermal correction to Gibbs Free Energy= $\quad 1.187289$

Sum of electronic and zero-point Energies $=\quad-4124.171827$

Sum of electronic and thermal Energies $=\quad-4124.093588$

Sum of electronic and thermal Enthalpies $=\quad-4124.092644$

Sum of electronic and thermal Free Energies $=\quad-4124.283942$

Cartesian Coordinates

$\begin{array}{lrrr}6 & 1.650242 & -2.324631 & 5.129704 \\ 6 & 1.893511 & -3.558814 & 4.537781 \\ 6 & 2.086835 & -3.644310 & 3.161536 \\ 6 & 2.041978 & -2.503777 & 2.360888 \\ 6 & 1.794471 & -1.267814 & 2.963766 \\ 6 & 1.600280 & -1.179964 & 4.336916 \\ 6 & 2.341950 & -2.617678 & 0.887209 \\ 7 & 1.408394 & -1.895605 & -0.001467 \\ 6 & 0.317839 & -2.636148 & -0.577918 \\ 6 & -0.953941 & -2.606778 & -0.033601 \\ 6 & -2.027948 & -3.243562 & -0.734589 \\ 6 & -1.766381 & -3.925067 & -1.959605 \\ 6 & -0.441142 & -3.980037 & -2.448480\end{array}$




\begin{tabular}{|c|c|c|c|}
\hline 6 & 0.567757 & -3.345807 & -1.777305 \\
\hline 6 & -3.366973 & -3.196018 & -0.265062 \\
\hline & -4.383479 & -3.788714 & -0.970973 \\
\hline & -4.115735 & -4.480031 & -2.172852 \\
\hline & -2.834092 & -4.541830 & -2.656808 \\
\hline & -1.232101 & -2.003789 & 1.298695 \\
\hline & -1.483184 & -0.650892 & 1.468710 \\
\hline p & -1.827627 & -0.164790 & 2.759508 \\
\hline & -1.933702 & -1.007829 & 3.832425 \\
\hline 6 & -1.698789 & -2.394177 & 3.689401 \\
\hline 6 & -1.334115 & -2.902232 & 2.409961 \\
\hline 6 & -1.819297 & -3.281280 & 4.785778 \\
\hline 6 & -1.602253 & -4.626492 & 4.629024 \\
\hline 6 & -1.237251 & -5.135710 & 3.365332 \\
\hline 6 & -1.101178 & -4.296891 & 2.287177 \\
\hline & -1.533525 & 0.465004 & -0.014517 \\
\hline 45 & 0.078758 & 0.674503 & -1.872009 \\
\hline 6 & 0.680465 & 2.507246 & -1.687690 \\
\hline 8 & 0.829236 & 3.653464 & -1.691244 \\
\hline 15 & 1.819704 & -0.511835 & -0.875788 \\
\hline 8 & 2.783921 & 0.171665 & 0.282776 \\
\hline 6 & 3.655842 & 1.163637 & -0.126601 \\
\hline 6 & 4.832745 & 0.820115 & -0.763073 \\
\hline 6 & 5.677683 & 1.880523 & -1.226342 \\
\hline 6 & 5.329420 & 3.233597 & -0.929881 \\
\hline 6 & 4.138315 & 3.509715 & -0.217603 \\
\hline 6 & 3.303105 & 2.494223 & 0.161393 \\
\hline 6 & 6.168436 & 4.283321 & -1.377425 \\
\hline 6 & 7.294984 & 4.021216 & -2.112993 \\
\hline 6 & 7.621480 & 2.688628 & -2.440990 \\
\hline 6 & 6.836404 & 1.648225 & -2.010554 \\
\hline 6 & 5.145471 & -0.610239 & -0.988035 \\
\hline 6 & 4.217736 & -1.416581 & -1.624613 \\
\hline 6 & 4.491011 & -2.759331 & -1.957641 \\
\hline 6 & 5.679880 & -3.324344 & -1.585653 \\
\hline 6 & 6.627658 & -2.582520 & -0.840765 \\
\hline 6 & 6.359583 & -1.215926 & -0.526104 \\
\hline 6 & 7.301160 & -0.522218 & 0.276568 \\
\hline 6 & 8.450685 & -1.134121 & 0.710636 \\
\hline 6 & 8.728382 & -2.473586 & 0.366372 \\
\hline 6 & 7.829631 & -3.180274 & -0.390228 \\
\hline 8 & 2.991502 & -0.913005 & -1.998459 \\
\hline 6 & -3.283858 & 0.212449 & -0.526964 \\
\hline 6 & -4.323506 & 0.309512 & 0.402592 \\
\hline 6 & -5.643346 & 0.072894 & 0.025797 \\
\hline 6 & -5.890342 & -0.251825 & -1.314946 \\
\hline 6 & -4.877656 & -0.353613 & -2.269139 \\
\hline 6 & -3.564790 & -0.121370 & -1.847257 \\
\hline 6 & -6.797281 & 0.115022 & 1.026119 \\
\hline 6 & -5.212238 & -0.648984 & -3.730473 \\
\hline 6 & -1.534917 & 2.136480 & 0.737508 \\
\hline 6 & -2.424508 & 3.110108 & 0.308687 \\
\hline 6 & -2.324435 & 4.432711 & 0.762737 \\
\hline 6 & -1.322136 & 4.733498 & 1.679967 \\
\hline 6 & -0.409720 & 3.769734 & 2.141239 \\
\hline 6 & -0.511292 & 2.480250 & 1.631479 \\
\hline
\end{tabular}




\begin{tabular}{|c|c|c|c|}
\hline 6 & -3.314848 & 5.466586 & 0.233520 \\
\hline 6 & 0.646998 & 4.159564 & 3.173630 \\
\hline 6 & -0.024958 & -0.392698 & -3.468971 \\
\hline 8 & -0.085337 & -0.916838 & -4.499267 \\
\hline 1 & 7.098005 & 0.506885 & 0.561058 \\
\hline 1 & -2.024678 & 0.895752 & 2.897217 \\
\hline 1 & 8.017906 & -4.220785 & -0.651506 \\
\hline 1 & -2.092931 & -2.869598 & 5.756585 \\
\hline 1 & 5.887563 & 5.306923 & -1.133495 \\
\hline 1 & -3.589098 & -2.659210 & 0.655566 \\
\hline 1 & -5.406591 & -3.722511 & -0.605138 \\
\hline 1 & 3.880496 & 4.546087 & -0.003468 \\
\hline 1 & -2.211030 & -0.620095 & 4.812131 \\
\hline 1 & 5.905033 & -4.358410 & -1.841360 \\
\hline 1 & 8.500418 & 2.482914 & -3.047778 \\
\hline 1 & 3.745409 & -3.318603 & -2.518042 \\
\hline 1 & -2.612711 & -5.064992 & -3.585999 \\
\hline 1 & -0.241865 & -4.513039 & -3.376736 \\
\hline 1 & -1.056939 & -6.201775 & 3.245359 \\
\hline 1 & 7.095545 & 0.628724 & -2.283847 \\
\hline 1 & -0.804839 & -4.703539 & 1.322197 \\
\hline 1 & 1.584651 & -3.355854 & -2.164719 \\
\hline 1 & 7.928539 & 4.835460 & -2.456773 \\
\hline 1 & 9.151695 & -0.581179 & 1.331786 \\
\hline 1 & -4.928948 & -4.959957 & -2.713610 \\
\hline 1 & -1.704883 & -5.301423 & 5.475830 \\
\hline 1 & 9.646038 & -2.943791 & 0.711779 \\
\hline 1 & 2.360801 & 2.681272 & 0.674532 \\
\hline 1 & -4.088673 & 0.569558 & 1.432003 \\
\hline 1 & -2.733775 & -0.209017 & -2.542645 \\
\hline 1 & -6.919500 & -0.434738 & -1.624021 \\
\hline 1 & -3.203734 & 2.840692 & -0.403481 \\
\hline 1 & 0.197179 & 1.709791 & \\
\hline 1 & -1.236800 & 5.749984 & 2.057871 \\
\hline 1 & -1.139056 & 1.459126 & -2.559230 \\
\hline 1 & 2.348730 & -3.676206 & 0.587509 \\
\hline 1 & 3.356552 & -2.240789 & 0.705859 \\
\hline 1 & 2.267523 & -4.614692 & 2.698092 \\
\hline 1 & 1.753066 & -0.371045 & 2.347778 \\
\hline 1 & 1.920061 & -4.461735 & 5.144708 \\
\hline 1 & 1.406869 & -0.209738 & 4.791836 \\
\hline 1 & 1.492633 & -2.254375 & 6.204078 \\
\hline 6 & -3.964760 & -0.994280 & -4.543440 \\
\hline 6 & -6.188908 & -1.822746 & -3.840465 \\
\hline 6 & -5.857849 & 0.602100 & -4.338427 \\
\hline 6 & -6.343374 & 0.571523 & 2.411330 \\
\hline 6 & -7.883803 & 1.079788 & 0.541049 \\
\hline 6 & -7.385445 & -1.294595 & 1.158582 \\
\hline 6 & -3.095571 & 6.849581 & 0.840499 \\
\hline 6 & -3.152410 & 5.578992 & -1.287111 \\
\hline 6 & -4.743945 & 5.020352 & 0.565235 \\
\hline 6 & 1.447874 & 2.953253 & 3.663051 \\
\hline 6 & 1.612349 & 5.176137 & 2.555557 \\
\hline 6 & -0.033966 & 4.787050 & 4.395698 \\
\hline 1 & -4.253559 & -1.249333 & -5.570959 \\
\hline 1 & -3.264018 & -0.151478 & -4.605212 \\
\hline
\end{tabular}




$\begin{array}{rrrr}1 & -3.427659 & -1.855732 & -4.121229 \\ 1 & -6.102991 & 0.433480 & -5.396006 \\ 1 & -6.784538 & 0.873119 & -3.816495 \\ 1 & -5.177482 & 1.461928 & -4.281106 \\ 1 & -6.408721 & -2.030060 & -4.896062 \\ 1 & -5.759168 & -2.731288 & -3.398957 \\ 1 & -7.146216 & -1.622908 & -3.344374 \\ 1 & -8.710679 & 1.113691 & 1.262959 \\ 1 & -7.487854 & 2.097975 & 0.434172 \\ 1 & -8.305748 & 0.781996 & -0.426081 \\ 1 & -8.220211 & -1.297711 & 1.872636 \\ 1 & -7.764217 & -1.673271 & 0.200929 \\ 1 & -6.626109 & -1.999652 & 1.523652 \\ 1 & -7.204014 & 0.594836 & 3.091653 \\ 1 & -5.599183 & -0.108439 & 2.847563 \\ 1 & -5.912619 & 1.581539 & 2.388051 \\ 1 & 2.393490 & 5.452741 & 3.276531 \\ 1 & 2.103306 & 4.762572 & 1.664201 \\ 1 & 1.096759 & 6.095078 & 2.251256 \\ 1 & 0.717118 & 5.059229 & 5.149208 \\ 1 & -0.589441 & 5.697496 & 4.142837 \\ 1 & -0.737451 & 4.082941 & 4.858932 \\ 1 & 2.152968 & 3.269342 & 4.441926 \\ 1 & 0.790169 & 2.187362 & 4.098300 \\ 1 & 2.038622 & 2.483764 & 2.865011 \\ 1 & -3.833027 & 7.552209 & 0.432426 \\ 1 & -3.216621 & 6.843299 & 1.931632 \\ 1 & -2.099702 & 7.246839 & 0.605767 \\ 1 & -5.469722 & 5.745593 & 0.173379 \\ 1 & -4.985365 & 4.041980 & 0.130531 \\ 1 & -4.890521 & 4.948470 & 1.651106 \\ 1 & -3.846252 & 6.328612 & -1.691160 \\ 1 & -2.130779 & 5.883258 & -1.549559 \\ 1 & -3.356040 & 4.628908 & -1.795901\end{array}$

$1_{\mathrm{C} 2-} \psi_{-1 \mathbf{a} 233 \mathrm{a} 4 \mathrm{~s}}$

Number of imaginary frequencies : 0

The smallest frequencies are : $14.1576 \quad 17.5035 \quad 21.8396 \mathrm{~cm}(-1)$

Electronic energy : $\quad H F=-4125.4715318$

Zero-point correction $=\quad 1.299263$ (Hartree/Particle)

Thermal correction to Energy $=\quad 1.377520$

Thermal correction to Enthalpy= $\quad 1.378465$

Thermal correction to Gibbs Free Energy $=\quad 1.187290$

Sum of electronic and zero-point Energies $=\quad-4124.172269$

Sum of electronic and thermal Energies $=\quad-4124.094011$

Sum of electronic and thermal Enthalpies $=\quad-4124.093067$

Sum of electronic and thermal Free Energies $=\quad-4124.284242$

Cartesian Coordinates

$\begin{array}{llll}6 & 1.651909 & -2.352722 & 5.114353 \\ 6 & 1.904214 & -3.583435 & 4.519063 \\ 6 & 2.098616 & -3.663739 & 3.142714 \\ 6 & 2.045077 & -2.521473 & 2.345065 \\ 6 & 1.787518 & -1.289183 & 2.951116\end{array}$




\begin{tabular}{|c|c|c|c|}
\hline 6 & 1.593051 & -1.206396 & 4.324555 \\
\hline & 2.345706 & -2.630146 & 0.871007 \\
\hline 7 & 1.415597 & -1.900792 & -0.014941 \\
\hline 6 & 0.324150 & -2.634597 & -0.598026 \\
\hline ב & -0.946012 & -2.614042 & -0.049541 \\
\hline 6 & -2.021050 & -3.246346 & -0.752902 \\
\hline 6 & -1.762505 & -3.912648 & -1.986836 \\
\hline 6 & -0.439171 & -3.957038 & -2.482029 \\
\hline 6 & 0.571060 & -3.328804 & -1.807065 \\
\hline 6 & -3.358036 & -3.209120 & -0.276235 \\
\hline 6 & -4.374900 & -3.799584 & -0.983436 \\
\hline 6 & -4.110319 & -4.475827 & -2.194487 \\
\hline 6 & -2.831018 & -4.525888 & -2.685937 \\
\hline 6 & -1.224625 & -2.023493 & 1.288043 \\
\hline 6 & -1.484762 & -0.673478 & 1.467547 \\
\hline 6 & -1.836361 & -0.200002 & 2.761024 \\
\hline 6 & -1.939904 & -1.052388 & 3.827076 \\
\hline 6 & -1.695902 & -2.436031 & 3.673921 \\
\hline 6 & -1.324040 & -2.931282 & 2.391591 \\
\hline 6 & -1.813678 & -3.332807 & 4.762814 \\
\hline 6 & -1.587145 & -4.675171 & 4.595662 \\
\hline 6 & -1.215520 & -5.171737 & 3.328821 \\
\hline 6 & -1.082384 & -4.323421 & 2.257841 \\
\hline 15 & -1.535021 & 0.452123 & -0.008234 \\
\hline 45 & 0.085979 & 0.688965 & -1.855261 \\
\hline 6 & 0.683122 & 2.520797 & -1.648663 \\
\hline 8 & 0.829894 & 3.667202 & -1.639098 \\
\hline 15 & 1.827741 & -0.506730 & -0.871728 \\
\hline 8 & 2.793199 & 0.160137 & 0.295137 \\
\hline 6 & 3.657509 & 1.164130 & -0.100017 \\
\hline 6 & 4.833862 & 0.837575 & -0.746376 \\
\hline 6 & 5.670640 & 1.910300 & -1.195634 \\
\hline 6 & 5.315741 & 3.256241 & -0.875316 \\
\hline 6 & 4.126367 & 3.513820 & -0.153132 \\
\hline 6 & 3.298444 & 2.487560 & 0.212442 \\
\hline 6 & 6.146869 & 4.318056 & -1.308814 \\
\hline 6 & 7.271831 & 4.074691 & -2.053178 \\
\hline 6 & 7.604663 & 2.749670 & -2.404574 \\
\hline 6 & 6.827435 & 1.697707 & -1.988233 \\
\hline 6 & 5.154574 & -0.587960 & -0.990762 \\
\hline 6 & 4.229087 & -1.393226 & -1.632135 \\
\hline 6 & 4.507444 & -2.731930 & -1.977060 \\
\hline 6 & 5.700467 & -3.294214 & -1.614422 \\
\hline 6 & 6.647492 & -2.553980 & -0.867017 \\
\hline 6 & 6.373537 & -1.191705 & -0.538834 \\
\hline 6 & 7.314442 & -0.500918 & 0.267165 \\
\hline 6 & 8.468620 & -1.110916 & 0.691451 \\
\hline 6 & 8.752002 & -2.445608 & 0.333642 \\
\hline 6 & 7.854108 & -3.149733 & -0.426349 \\
\hline 8 & 2.999721 & -0.892415 & -1.999375 \\
\hline 6 & -3.278844 & 0.185693 & -0.537278 \\
\hline 6 & -4.321251 & 0.257791 & 0.382753 \\
\hline 6 & -5.643136 & 0.020003 & -0.002323 \\
\hline 6 & -5.885855 & -0.272313 & -1.346036 \\
\hline 6 & -4.861695 & -0.350140 & -2.298348 \\
\hline 6 & -3.554428 & -0.124612 & -1.868414 \\
\hline
\end{tabular}




\begin{tabular}{|c|c|c|c|}
\hline 6 & -6.742384 & 0.074570 & 1.057007 \\
\hline 6 & -5.190737 & -0.615468 & -3.766596 \\
\hline & -1.566758 & 2.120248 & 0.751340 \\
\hline & -2.459700 & 3.085074 & 0.308834 \\
\hline & -2.377095 & 4.409596 & 0.760470 \\
\hline & -1.388774 & 4.721990 & 1.689060 \\
\hline & -0.474686 & 3.767447 & 2.165391 \\
\hline & -0.560083 & 2.475125 & 1.659833 \\
\hline 6 & -3.361853 & 5.436291 & 0.207153 \\
\hline o & 0.571230 & 4.170370 & 3.203631 \\
\hline 0 & -0.012622 & -0.363932 & -3.461619 \\
\hline 8 & -0.068647 & -0.880909 & -4.495720 \\
\hline 1 & 7.107194 & 0.524313 & 0.562306 \\
\hline 1 & -2.040601 & 0.858143 & 2.906451 \\
\hline 1 & 8.046649 & -4.186823 & -0.697892 \\
\hline 1 & -2.093031 & -2.930958 & 5.736096 \\
\hline 1 & 5.861313 & 5.335857 & -1.046737 \\
\hline 1 & -3.578147 & -2.681156 & 0.650498 \\
\hline 1 & -5.396690 & -3.741755 & -0.612906 \\
\hline 1 & 3.864041 & 4.545034 & 0.079669 \\
\hline 1 & -2.222544 & -0.674183 & 4.808991 \\
\hline 1 & 5.929601 & -4.325053 & -1.879453 \\
\hline 1 & 8.482269 & 2.559194 & -3.018224 \\
\hline 1 & 3.761961 & -3.290223 & -2.538643 \\
\hline 1 & -2.612157 & -5.037181 & -3.622308 \\
\hline 1 & -0.242246 & -4.477862 & -3.417677 \\
\hline 1 & -1.028196 & -6.235640 & 3.200688 \\
\hline 1 & 7.091221 & & -2.279338 \\
\hline 1 & -0.781731 & -4.720277 & 1.290109 \\
\hline 1 & 1.586379 & -3.331631 & -2.198921 \\
\hline 1 & 7.899268 & 4.898202 & -2.385916 \\
\hline 1 & 9.168910 & -0.560192 & 1.315382 \\
\hline 1 & -4.924274 & -4.953092 & -2.736451 \\
\hline 1 & -1.687677 & -5.357666 & 5.436638 \\
\hline 1 & 9.673260 & -2.914326 & 0.671401 \\
\hline 1 & 2.357421 & 2.661208 & 0.732825 \\
\hline 1 & -4.099212 & 0.496165 & 1.422804 \\
\hline 1 & -2.720121 & -0.195080 & -2.561679 \\
\hline 1 & -6.909393 & -0.448646 & -1.669933 \\
\hline 1 & -3.225426 & 2.808182 & -0.415078 \\
\hline 1 & 0.147597 & 1.711160 & 1.981713 \\
\hline 1 & -1.315998 & 5.740699 & 2.063546 \\
\hline 1 & -1.131299 & 1.479228 & -2.537480 \\
\hline 1 & 2.348642 & -3.687293 & 0.566386 \\
\hline 1 & 3.362028 & -2.256615 & 0.691941 \\
\hline 1 & 2.286453 & -4.631532 & 2.676706 \\
\hline 1 & 1.738369 & -0.391283 & 2.337533 \\
\hline 1 & 1.937003 & -4.487832 & 5.123486 \\
\hline 1 & 1.391724 & -0.238897 & 4.781882 \\
\hline 1 & 1.493634 & -2.286564 & 6.188886 \\
\hline 6 & -3.938891 & -0.933063 & -4.583955 \\
\hline 6 & -6.156437 & -1.795201 & -3.905018 \\
\hline 6 & -5.845709 & 0.642976 & -4.348795 \\
\hline 6 & -8.118552 & -0.259329 & 0.487638 \\
\hline 6 & -6.426961 & -0.941431 & 2.161980 \\
\hline 6 & -6.801159 & 1.483158 & 1.658394 \\
\hline
\end{tabular}




$\begin{array}{rrrr}6 & -3.194006 & 6.811058 & 0.848396 \\ 6 & -3.130526 & 5.578113 & -1.302121 \\ 6 & -4.797265 & 4.961534 & 0.461613 \\ 6 & 1.368465 & 2.970720 & 3.715089 \\ 6 & 1.542092 & 5.178458 & 2.579998 \\ 6 & -0.119778 & 4.813695 & 4.411527 \\ 1 & -4.222987 & -1.164269 & -5.618406 \\ 1 & -3.242233 & -0.085477 & -4.622390 \\ 1 & -3.399085 & -1.802191 & -4.181205 \\ 1 & -6.087919 & 0.494797 & -5.410107 \\ 1 & -6.775669 & 0.895290 & -3.823346 \\ 1 & -5.172420 & 1.506831 & -4.272081 \\ 1 & -6.371215 & -1.981922 & -4.965491 \\ 1 & -5.718589 & -2.708465 & -3.481913 \\ 1 & -7.116876 & -1.615655 & -3.407345 \\ 1 & -7.202771 & -0.913557 & 2.939022 \\ 1 & -6.392949 & -1.961908 & 1.757679 \\ 1 & -5.463064 & -0.745721 & 2.648767 \\ 1 & -7.559255 & 1.529239 & 2.451944 \\ 1 & -5.841546 & 1.786873 & 2.095470 \\ 1 & -7.069266 & 2.223660 & 0.893511 \\ 1 & -8.869073 & -0.215950 & 1.287027 \\ 1 & -8.426450 & 0.452230 & -0.289267 \\ 1 & -8.150226 & -1.270507 & 0.060828 \\ 1 & 2.316522 & 5.465817 & 3.304038 \\ 1 & 2.041220 & 4.751800 & 1.699191 \\ 1 & 1.028949 & 6.092484 & 2.257339 \\ 1 & 0.625053 & 5.095658 & 5.167615 \\ 1 & -0.672934 & 5.720894 & 4.142452 \\ 1 & -0.827197 & 4.115832 & 4.878162 \\ 1 & 2.066070 & 3.297051 & 4.496488 \\ 1 & 0.708054 & 2.209056 & 4.153519 \\ 1 & 1.967356 & 2.492470 & 2.928270 \\ 1 & -3.929920 & 7.506837 & 0.426039 \\ 1 & -3.355126 & 6.779949 & 1.933980 \\ 1 & -2.198962 & 7.233790 & 0.659223 \\ 1 & -5.514857 & 5.690071 & 0.061010 \\ 1 & -5.004933 & 3.996027 & -0.016591 \\ 1 & -4.991629 & 4.851099 & 1.536760 \\ 1 & -3.823298 & 6.316691 & -1.727950 \\ 1 & -2.105761 & 5.911996 & -1.510469 \\ 1 & -3.284554 & 4.630493 & -1.832881\end{array}$

$1_{\mathrm{C} 2-} \psi_{-1 \mathbf{a} 25354 a}$

Number of imaginary frequencies : 0

The smallest frequencies are : $9.6538 \quad 17.653921 .3393 \mathrm{~cm}(-1)$

Electronic energy : $\quad H F=-4125.4708313$

Zero-point correction $=\quad 1.300420($ Hartree/Particle $)$

Thermal correction to Energy $=\quad 1.378373$

Thermal correction to Enthalpy= $\quad 1.379318$

Thermal correction to Gibbs Free Energy= $\quad 1.189613$

Sum of electronic and zero-point Energies $=\quad-4124.170411$

Sum of electronic and thermal Energies $=\quad-4124.092458$

Sum of electronic and thermal Enthalpies $=\quad-4124.091514$

Sum of electronic and thermal Free Energies $=\quad-4124.281218$ 
Cartesian Coordinates

$\begin{array}{cccc}\cdots & 1.585780 & -2.331889 & 5.116063 \\ 6 & 1.849166 & -3.560895 & 4.522730 \\ 6 & 2.071843 & -3.636881 & 3.150251 \\ 6 & 2.037104 & -2.491760 & 2.356175 \\ 6 & 1.770172 & -1.260151 & 2.960369 \\ 6 & 1.546224 & -1.182281 & 4.329453 \\ 6 & 2.359990 & -2.591833 & 0.887156 \\ 7 & 1.407391 & -1.899628 & -0.005570 \\ 6 & 0.334138 & -2.675698 & -0.567721 \\ 6 & -0.948900 & -2.633061 & -0.051356 \\ 6 & -1.998607 & -3.324432 & -0.738505 \\ 6 & -1.696933 & -4.101597 & -1.895399 \\ 6 & -0.361113 & -4.161116 & -2.355146 \\ 6 & 0.619529 & -3.453302 & -1.716213 \\ 6 & -3.348849 & -3.262245 & -0.304619 \\ 6 & -4.335372 & -3.949040 & -0.966993 \\ 6 & -4.025346 & -4.745591 & -2.090689 \\ 6 & -2.734487 & -4.811331 & -2.548233 \\ 6 & -1.255867 & -2.012591 & 1.267629 \\ 6 & -1.518808 & -0.661903 & 1.432727 \\ 6 & -1.886877 & -0.176651 & 2.718207 \\ 6 & -2.004861 & -1.017218 & 3.790869 \\ 6 & -1.756183 & -2.401925 & 3.654395 \\ 6 & -1.365874 & -2.909317 & 2.382352 \\ 6 & -1.886270 & -3.286059 & 4.751925 \\ 6 & -1.651434 & -4.629357 & 4.604613 \\ 6 & -1.256641 & -5.137901 & 3.349987 \\ 6 & -1.112073 & -4.301478 & 2.270938 \\ 15 & -1.551873 & 0.463817 & -0.042443 \\ 45 & 0.036802 & 0.625152 & -1.914550 \\ 6 & 0.662598 & 2.451871 & -1.815433 \\ 8 & 0.821108 & 3.596046 & -1.859069 \\ 15 & 1.792336 & -0.522248 & -0.901468 \\ 8 & 2.738324 & 0.197778 & 0.250220 \\ 6 & 3.604469 & 1.191699 & -0.166603 \\ 6 & 4.791633 & 0.849331 & -0.784790 \\ 6 & 5.633775 & 1.910063 & -1.252832 \\ 6 & 5.270916 & 3.264203 & -0.979887 \\ 6 & 4.068527 & 3.540327 & -0.287051 \\ 6 & 3.236866 & 2.523653 & 0.097150 \\ 6 & 6.106742 & 4.314673 & -1.431597 \\ 6 & 7.244780 & 4.051950 & -2.148989 \\ 6 & 7.586231 & 2.717704 & -2.454130 \\ 6 & 6.804174 & 1.676830 & -2.019336 \\ 6 & 5.118192 & -0.580744 & -0.991752 \\ 6 & 4.204637 & -1.400219 & -1.632008 \\ 6 & 4.496006 & -2.740442 & -1.959275 \\ 6 & 5.686804 & -3.291743 & -1.573179 \\ 6 & 6.618179 & -2.537654 & -0.819861 \\ 6 & 6.333257 & -1.172409 & -0.514203 \\ 6 & 7.259089 & -0.465753 & 0.295488 \\ 6 & 8.409721 & -1.064414 & 0.744913 \\ 6 & 8.704401 & -2.402511 & 0.409588 \\ & & & \\ 6\end{array}$




\begin{tabular}{|c|c|c|c|}
\hline 6 & 7.821127 & -3.121399 & -0.353715 \\
\hline 8 & 2.974845 & -0.913680 & -2.015745 \\
\hline & -3.314608 & 0.277166 & -0.540733 \\
\hline & -4.352005 & 0.402590 & 0.393683 \\
\hline & -5.676563 & 0.210929 & 0.018464 \\
\hline & -5.943871 & -0.086485 & -1.329742 \\
\hline & -4.939436 & -0.201267 & -2.284787 \\
\hline & -3.614823 & -0.032599 & -1.858058 \\
\hline & -6.828063 & 0.285105 & 1.019731 \\
\hline & -5.205751 & -0.499368 & -3.758168 \\
\hline & -1.482617 & 2.124418 & 0.729992 \\
\hline 0 & -2.333033 & 3.141627 & 0.323413 \\
\hline & -2.185094 & 4.447297 & 0.813539 \\
\hline & -1.175678 & 4.686099 & 1.741339 \\
\hline & -0.303525 & 3.675890 & 2.181486 \\
\hline & -0.450751 & 2.405810 & 1.636038 \\
\hline 0 & -3.144051 & 5.528480 & 0.321688 \\
\hline & 0.736725 & 3.985440 & 3.257421 \\
\hline 0 & -0.164798 & -0.521820 & -3.449413 \\
\hline & -0.332097 & -1.084832 & -4.446862 \\
\hline & 7.042384 & 0.562535 & 0.573061 \\
\hline & -2.089299 & 0.883379 & 2.852063 \\
\hline & 8.022608 & -4.161050 & -0.608561 \\
\hline & -2.181581 & -2.873534 & 5.715980 \\
\hline & 5.814166 & 5.339136 & -1.205730 \\
\hline & -3.601839 & -2.648397 & 0.558469 \\
\hline & -5.365849 & -3.875347 & -0.625067 \\
\hline & 3.798751 & 4.577633 & -0.093262 \\
\hline & -2.300806 & -0.629392 & 4.765053 \\
\hline 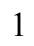 & 5.926370 & -4.323661 & -1.824362 \\
\hline & 8.474489 & 2.510863 & -3.046799 \\
\hline 1 & 3.762981 & -3.306789 & -2.529127 \\
\hline & -2.482316 & -5.407879 & -3.423787 \\
\hline & -0.131123 & -4.759912 & -3.234916 \\
\hline & -1.059549 & -6.201777 & 3.237192 \\
\hline & 7.075408 & 0.656096 & -2.275505 \\
\hline 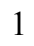 & -0.791163 & -4.709823 & 1.314634 \\
\hline & 1.645075 & -3.470409 & -2.080147 \\
\hline 1 & 7.876017 & 4.866612 & -2.496031 \\
\hline 1 & 9.098305 & -0.501917 & 1.371352 \\
\hline 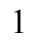 & -4.815420 & -5.293830 & -2.599456 \\
\hline 1 & -1.761626 & -5.302398 & 5.451966 \\
\hline & 9.622856 & -2.862017 & 0.767064 \\
\hline 1 & 2.287019 & 2.711882 & 0.596109 \\
\hline & -4.106784 & 0.648875 & 1.423812 \\
\hline 1 & -2.794899 & -0.145722 & -2.564871 \\
\hline 1 & -6.979749 & -0.229518 & -1.631319 \\
\hline 1 & -3.121183 & 2.919317 & -0.395394 \\
\hline 1 & 0.222803 & 1.600300 & 1.928707 \\
\hline 1 & -1.056092 & 5.687541 & 2.149411 \\
\hline 1 & -1.176667 & 1.407798 & -2.611290 \\
\hline 1 & 2.408067 & -3.648667 & 0.585569 \\
\hline 1 & 3.361071 & -2.176450 & 0.717035 \\
\hline 1 & 2.268023 & -4.603179 & 2.684586 \\
\hline 1 & 1.737327 & -0.359192 & 2.348663 \\
\hline 1 & 1.869224 & -4.466976 & 5.125139 \\
\hline
\end{tabular}




\begin{tabular}{lrrr}
1 & 1.339087 & -0.216032 & 4.787213 \\
1 & 1.404976 & -2.268854 & 6.187231 \\
6 & -6.696808 & -0.549352 & -4.080154 \\
6 & -4.566186 & 0.594233 & -4.622683 \\
6 & -4.589579 & -1.854766 & -4.118044 \\
6 & -6.354784 & 0.691330 & 2.413952 \\
6 & -7.867821 & 1.309815 & 0.554705 \\
6 & -7.484198 & -1.096675 & 1.122107 \\
6 & -2.872551 & 6.886429 & 0.963284 \\
6 & -2.996722 & 5.678414 & -1.196957 \\
6 & -4.583185 & 5.119907 & 0.659077 \\
6 & 1.526029 & 2.743409 & 3.669915 \\
6 & 1.719322 & 5.044109 & 2.748438 \\
6 & 0.023107 & 4.517284 & 4.506445 \\
1 & -6.836202 & -0.753242 & -5.149277 \\
1 & -7.210741 & -1.346170 & -3.526942 \\
1 & -7.196671 & 0.402566 & -3.857710 \\
1 & -4.730882 & -2.066573 & -5.186704 \\
1 & -3.512194 & -1.887219 & -3.909398 \\
1 & -5.060922 & -2.662561 & -3.543612 \\
1 & -4.771898 & 0.404902 & -5.684667 \\
1 & -4.969045 & 1.583898 & -4.370624 \\
1 & -3.475903 & 0.635303 & -4.503017 \\
1 & -8.693153 & 1.366712 & 1.276985 \\
1 & -7.424995 & 2.310544 & 0.469968 \\
1 & -8.301503 & 1.052537 & -0.418826 \\
1 & -8.318061 & -1.073817 & 1.836782 \\
1 & -7.881679 & -1.435231 & 0.157366 \\
1 & -6.761531 & -1.847053 & 1.469984 \\
1 & -7.213069 & 0.734228 & 3.096286 \\
1 & -5.637928 & -0.027639 & 2.833065 \\
1 & -5.884054 & 1.683663 & 2.412369 \\
1 & 2.460433 & 5.281155 & 3.523546 \\
1 & 2.261608 & 4.686869 & 1.863020 \\
1 & 1.212017 & 5.977331 & 2.475632 \\
1 & 0.752431 & 4.727163 & 5.300274 \\
1 & -0.524922 & 5.445825 & 4.308071 \\
1 & -0.694173 & 3.780987 & 4.892245 \\
1 & 2.227424 & 3.000902 & 4.473386 \\
1 & 0.859671 & 1.954915 & 4.048385 \\
1 & 2.118156 & 2.325905 & 2.843796 \\
1 & -3.586601 & 7.625029 & 0.577708 \\
1 & -2.988600 & 6.856382 & 2.054555 \\
1 & -1.864276 & 7.253984 & 0.733133 \\
1 & -5.289336 & 5.880040 & 0.298735 \\
1 & -4.861581 & 4.163389 & 0.198716 \\
1 & -4.718476 & 5.019819 & 1.744196 \\
1 & -3.678512 & 6.453869 & -1.571696 \\
1 & -1.972297 & 5.968438 & -1.463928 \\
1 & -3.227312 & 4.747647 & -1.729093 \\
& & & \\
\hline & & & \\
6 & &
\end{tabular}

$1_{\mathrm{C} 2-} \psi_{-12-25354 s}$

Number of imaginary frequencies : 0

The smallest frequencies are : $13.6194 \quad 17.3726 \quad 22.5749 \mathrm{~cm}(-1)$

Electronic energy : $\quad H F=-4125.4709726$ 
Zero-point correction $=$

Thermal correction to Energy=

Thermal correction to Enthalpy=

Thermal correction to Gibbs Free Energy=

Sum of electronic and zero-point Energies=

Sum of electronic and thermal Energies $=$

Sum of electronic and thermal Enthalpies=

Sum of electronic and thermal Free Energies=
1.299719 (Hartree/Particle)

1.377757

1.378701

1.189157

$-4124.171254$

$-4124.093216$

$-4124.092271$

$-4124.281816$

Cartesian Coordinates

$\begin{array}{cccc}6 & 1.679483 & -2.389822 & 5.087017 \\ 6 & 1.952767 & -3.610305 & 4.480263 \\ 6 & 2.153003 & -3.673931 & 3.103967 \\ 6 & 2.083939 & -2.524717 & 2.317879 \\ 6 & 1.804164 & -1.302859 & 2.935125 \\ 6 & 1.604289 & -1.236672 & 4.308531 \\ 6 & 2.388871 & -2.612703 & 0.843695 \\ 7 & 1.440434 & -1.896324 & -0.033835 \\ 6 & 0.361737 & -2.653234 & -0.610859 \\ 6 & -0.910369 & -2.646710 & -0.066453 \\ 6 & -1.966976 & -3.325141 & -0.755596 \\ 6 & -1.683493 & -4.047320 & -1.951911 \\ 6 & -0.359304 & -4.064580 & -2.447412 \\ 6 & 0.628663 & -3.375312 & -1.799366 \\ 6 & -3.307070 & -3.300139 & -0.287990 \\ 6 & -4.300457 & -3.970531 & -0.956692 \\ 6 & -4.006966 & -4.716529 & -2.118801 \\ 6 & -2.726560 & -4.745155 & -2.608724 \\ 6 & -1.202588 & -2.060270 & 1.270787 \\ 6 & -1.483692 & -0.715895 & 1.459658 \\ 6 & -1.844927 & -0.256297 & 2.755707 \\ 6 & -1.938112 & -1.116760 & 3.815901 \\ 6 & -1.671380 & -2.495291 & 3.654610 \\ 6 & -1.288596 & -2.976357 & 2.369998 \\ 6 & -1.778569 & -3.400085 & 4.738172 \\ 6 & -1.529195 & -4.737456 & 4.564286 \\ 6 & -1.143421 & -5.219815 & 3.296266 \\ 6 & -1.021139 & -4.363391 & 2.230481 \\ 15 & -1.543540 & 0.421831 & -0.004396 \\ 45 & 0.066364 & 0.691766 & -1.849062 \\ 6 & 0.654776 & 2.525086 & -1.638459 \\ 8 & 0.787370 & 3.673098 & -1.615958 \\ 15 & 1.825632 & -0.490400 & -0.883188 \\ 8 & 2.780283 & 0.183259 & 0.288712 \\ 6 & 3.631497 & 1.202853 & -0.093163 \\ 6 & 4.813069 & 0.900128 & -0.741759 \\ 6 & 5.636708 & 1.989733 & -1.174770 \\ 6 & 5.264704 & 3.326314 & -0.835321 \\ 6 & 4.071501 & 3.558590 & -0.110940 \\ 6 & 3.255830 & 2.517064 & 0.238314 \\ 6 & 6.083036 & 4.404727 & -1.251735 \\ 6 & 7.212058 & 4.186535 & -1.997746 \\ 6 & 7.561765 & 2.871148 & -2.368283 \\ 6 & 6.797128 & 1.803527 & -1.968778 \\ 6 & 5.153743 & -0.517671 & -1.003846\end{array}$




\begin{tabular}{|c|c|c|c|}
\hline & 4.240858 & -1.328419 & -1.656311 \\
\hline & 4.541045 & -2.656667 & -2.022297 \\
\hline & 5.742300 & -3.205907 & -1.667118 \\
\hline & 6.676371 & -2.462705 & -0.906446 \\
\hline & 6.381216 & -1.109694 & -0.558857 \\
\hline & 7.310214 & -0.416862 & 0.259132 \\
\hline & 8.472619 & -1.015666 & 0.676896 \\
\hline & 8.776877 & -2.340422 & 0.299907 \\
\hline & 7.891132 & -3.046620 & -0.472320 \\
\hline & 3.002863 & -0.843781 & -2.015579 \\
\hline & -3.285106 & 0.160878 & -0.541449 \\
\hline & -4.335443 & 0.223399 & 0.375363 \\
\hline & -5.657023 & 0.038045 & -0.027507 \\
\hline & -5.902612 & -0.194607 & -1.387122 \\
\hline & -4.874745 & -0.260662 & -2.330069 \\
\hline & -3.561976 & -0.094735 & -1.879002 \\
\hline & -6.763895 & 0.092679 & 1.024393 \\
\hline & -5.117091 & -0.496607 & -3.819442 \\
\hline & -1.592391 & 2.083030 & 0.770250 \\
\hline & -2.498244 & 3.040055 & 0.337236 \\
\hline & -2.434467 & 4.360636 & 0.802276 \\
\hline & -1.452313 & 4.677030 & 1.735942 \\
\hline & -0.525706 & 3.730072 & 2.203439 \\
\hline & -0.591714 & 2.442121 & 1.683591 \\
\hline & -3.432827 & 5.377972 & 0.256448 \\
\hline & 0.512125 & 4.136417 & 3.248462 \\
\hline & -0.087942 & -0.369762 & -3.446041 \\
\hline & -0.220966 & -0.890384 & -4.471469 \\
\hline & 7.086909 & 0.600546 & 0.569195 \\
\hline & -2.065354 & 0.797734 & 2.907212 \\
\hline & 8.099794 & -4.076518 & -0.758883 \\
\hline & -2.068372 & -3.008292 & 5.712482 \\
\hline & 5.784442 & 5.414904 & -0.975047 \\
\hline & -3.547835 & -2.724443 & 0.604619 \\
\hline & -5.323265 & -3.925026 & -0.588106 \\
\hline & 3.796743 & 4.582955 & 0.137215 \\
\hline . & -2.229621 & -0.749316 & 4.799302 \\
\hline & 5.988336 & -4.228448 & -1.948593 \\
\hline & 8.442449 & 2.700692 & -2.983397 \\
\hline & 3.805471 & -3.215745 & -2.595828 \\
\hline & -2.487770 & -5.300961 & -3.514367 \\
\hline 1 & -0.143423 & -4.619467 & -3.358979 \\
\hline & -0.936386 & -6.279398 & 3.162788 \\
\hline . & 7.074098 & 0.798172 & -2.274929 \\
\hline & -0.708873 & -4.750725 & 1.262558 \\
\hline & 1.644875 & -3.363699 & -2.188736 \\
\hline & 7.829668 & 5.022657 & -2.317138 \\
\hline & 9.163201 & -0.463786 & 1.310545 \\
\hline 1 & -4.801593 & -5.254871 & -2.631047 \\
\hline & -1.621842 & -5.426539 & 5.400792 \\
\hline & 9.704504 & -2.800128 & 0.632588 \\
\hline & 2.312423 & 2.671641 & 0.760523 \\
\hline & -4.115613 & 0.421009 & 1.424283 \\
\hline 1 & -2.729948 & -0.161145 & -2.577819 \\
\hline 1 & -6.928523 & -0.327467 & -1.718431 \\
\hline 1 & -3.259778 & 2.760801 & -0.390055 \\
\hline
\end{tabular}




\begin{tabular}{|c|c|c|c|}
\hline & 0.126004 & 1.684466 & 1.998285 \\
\hline & -1.394106 & 5.692653 & 2.121254 \\
\hline & -1.155538 & 1.487072 & -2.517328 \\
\hline & 2.416813 & -3.666633 & 0.529348 \\
\hline & 3.395735 & -2.213037 & 0.668665 \\
\hline & 2.357578 & -4.633871 & 2.628897 \\
\hline & 1.742492 & -0.400004 & 2.330062 \\
\hline 1 & 1.997429 & -4.520063 & 5.075841 \\
\hline & 1.386016 & -0.277207 & 4.774840 \\
\hline 1 & 1.516806 & -2.337081 & 6.161634 \\
\hline 6 & -6.602409 & -0.573023 & -4.162589 \\
\hline & -4.499165 & 0.653598 & -4.624331 \\
\hline & -4.459141 & -1.815885 & -4.235425 \\
\hline f & -8.148411 & -0.153130 & 0.430502 \\
\hline & -6.502796 & -0.985477 & 2.083456 \\
\hline 6 & -6.768645 & 1.474180 & 1.689171 \\
\hline f & -3.282735 & 6.750167 & 0.907476 \\
\hline 6 & -3.203099 & 5.533448 & -1.251823 \\
\hline & -4.861895 & 4.882298 & 0.508022 \\
\hline 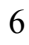 & 1.324447 & 2.942484 & 3.749250 \\
\hline & 1.470568 & 5.164100 & 2.637765 \\
\hline & -0.190430 & 4.757609 & 4.461283 \\
\hline$\frac{1}{1}$ & -6.723098 & -0.732314 & -5.241543 \\
\hline & -7.100379 & -1.407413 & -3.651767 \\
\hline 1 & -7.131882 & 0.353757 & -3.905323 \\
\hline ] & -4.599617 & -1.989865 & -5.311030 \\
\hline & -3.380010 & -1.819511 & -4.034510 \\
\hline 1 & -4.899016 & -2.661228 & -3.690723 \\
\hline & -4.682260 & 0.506121 & -5.697115 \\
\hline 1 & -4.937058 & 1.618042 & -4.335443 \\
\hline 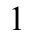 & -3.412731 & 0.723169 & -4.483762 \\
\hline | & -7.286468 & -0.964188 & 2.852797 \\
\hline 1 & -6.501822 & -1.985934 & 1.630328 \\
\hline 1 & -5.537807 & -0.849773 & 2.588471 \\
\hline 1 & -7.538861 & 1.519693 & 2.471025 \\
\hline & -5.804959 & 1.713217 & 2.156425 \\
\hline 1 & -6.987037 & 2.261123 & 0.955032 \\
\hline 1 & -8.903457 & -0.108382 & 1.225560 \\
\hline 1 & -8.415500 & 0.604207 & -0.317686 \\
\hline 1 & -8.223942 & -1.142607 & -0.038849 \\
\hline 1 & 2.239522 & 5.454164 & 3.366549 \\
\hline 1 & 1.977126 & 4.753515 & 1.753592 \\
\hline 1 & 0.945697 & 6.074497 & 2.323778 \\
\hline 1 & 0.548711 & 5.041662 & 5.222159 \\
\hline 1 & -0.755153 & 5.659999 & 4.200268 \\
\hline 1 & -0.889418 & 4.045393 & 4.918838 \\
\hline 1 & 2.015246 & 3.269846 & 4.536240 \\
\hline 1 & 0.673281 & 2.167128 & 4.177347 \\
\hline 1 & 1.932078 & 2.481332 & 2.958975 \\
\hline 1 & -4.027415 & 7.439578 & 0.490072 \\
\hline 1 & -3.443404 & 6.709248 & 1.992792 \\
\hline 1 & -2.293050 & 7.186672 & 0.721441 \\
\hline 1 & -5.589224 & 5.602134 & 0.109154 \\
\hline 1 & -5.056385 & 3.914851 & 0.027375 \\
\hline 1 & -5.055154 & 4.766217 & 1.582842 \\
\hline 1 & -3.901956 & 6.269644 & -1.671768 \\
\hline
\end{tabular}


$1_{\mathrm{C} 2-}-$-1s2a3a4a

Number of imaginary frequencies : 0

The smallest frequencies are : $11.1667 \quad 16.1816 \quad 19.2991 \mathrm{~cm}(-1)$

Electronic energy : $\quad H F=-4125.4693997$

Zero-point correction $=\quad 1.299678($ Hartree/Particle $)$

Thermal correction to Energy= $\quad 1.377889$

Thermal correction to Enthalpy= $\quad 1.378833$

Thermal correction to Gibbs Free Energy= 1.187566

Sum of electronic and zero-point Energies $=\quad-4124.169721$

Sum of electronic and thermal Energies $=\quad-4124.091510$

Sum of electronic and thermal Enthalpies $=\quad-4124.090566$

Sum of electronic and thermal Free Energies $=\quad-4124.281834$

\begin{tabular}{cccc} 
& \multicolumn{3}{c}{ Cartesian Coordinates } \\
\hline 6 & 1.611733 & -1.841866 & 5.239198 \\
6 & 1.916604 & -3.112847 & 4.765150 \\
6 & 2.148149 & -3.312550 & 3.406913 \\
6 & 2.082158 & -2.249751 & 2.506877 \\
6 & 1.776252 & -0.975870 & 2.992176 \\
6 & 1.542374 & -0.774224 & 4.346993 \\
6 & 2.414996 & -2.481144 & 1.053718 \\
7 & 1.494675 & -1.838551 & 0.094343 \\
6 & 0.434812 & -2.632881 & -0.467033 \\
6 & -0.842267 & -2.630152 & 0.064454 \\
6 & -1.886319 & -3.328414 & -0.623161 \\
6 & -1.591696 & -4.035183 & -1.825919 \\
6 & -0.261577 & -4.056447 & -2.303618 \\
6 & 0.719100 & -3.369473 & -1.642424 \\
6 & -3.228782 & -3.317294 & -0.161847 \\
6 & -4.219108 & -3.963829 & -0.857360 \\
6 & -3.918806 & -4.677568 & -2.038212 \\
6 & -2.632074 & -4.708924 & -2.511689 \\
6 & -1.165162 & -1.991886 & 1.369659 \\
6 & -1.499122 & -0.650649 & 1.481095 \\
6 & -1.920112 & -0.145016 & 2.741497 \\
6 & -1.998223 & -0.951363 & 3.844611 \\
6 & -1.665906 & -2.322646 & 3.763336 \\
6 & -1.241704 & -2.853476 & 2.511849 \\
6 & -1.756308 & -3.174365 & 4.889916 \\
6 & -1.456477 & -4.509190 & 4.788073 \\
6 & -1.035801 & -5.041472 & 3.551484 \\
6 & -0.925562 & -4.235335 & 2.445529 \\
15 & -1.539897 & 0.417933 & -0.039455 \\
45 & 0.118055 & 0.627435 & -1.870621 \\
6 & 0.654706 & 2.480954 & -1.709720 \\
8 & 0.766954 & 3.631144 & -1.731182 \\
15 & 1.884962 & -0.487742 & -0.838133 \\
8 & 2.846850 & 0.254324 & 0.283142 \\
6 & 3.699888 & 1.241888 & -0.173942 \\
6 & 4.881007 & 0.889535 & -0.796930 \\
6 & 5.708078 & 1.943245 & -1.305481
\end{tabular}




\begin{tabular}{|c|c|c|c|}
\hline 6 & 5.337408 & 3.301922 & -1.065620 \\
\hline 6 & 4.141395 & 3.588847 & -0.365366 \\
\hline & 3.323262 & 2.576329 & 0.056165 \\
\hline & 6.159750 & 4.345123 & -1.557167 \\
\hline & 7.290589 & 4.070800 & -2.281720 \\
\hline & 7.638742 & 2.731178 & -2.554066 \\
\hline & 6.870384 & 1.697238 & -2.080070 \\
\hline & 5.212070 & -0.545043 & -0.960399 \\
\hline & 4.291461 & -1.390725 & -1.555922 \\
\hline & 4.580449 & -2.743644 & -1.828523 \\
\hline & 5.778090 & -3.276653 & -1.437810 \\
\hline & 6.719815 & -2.490336 & -0.732353 \\
\hline & 6.435706 & -1.114570 & -0.477692 \\
\hline f & 7.371675 & -0.374464 & 0.289521 \\
\hline & 8.531038 & -0.951994 & 0.744142 \\
\hline & 8.824926 & -2.301459 & 0.457188 \\
\hline b & 7.931803 & -3.052237 & -0.262585 \\
\hline & 3.057653 & -0.920424 & -1.949037 \\
\hline 6 & -3.263919 & 0.088223 & -0.602561 \\
\hline & -4.339002 & 0.200676 & 0.283712 \\
\hline & -5.640778 & -0.068303 & -0.132312 \\
\hline ) & -5.833004 & -0.448056 & -1.466963 \\
\hline 6 & -4.783906 & -0.569079 & -2.378727 \\
\hline ) & -3.490798 & -0.301010 & -1.918466 \\
\hline 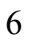 & -6.835718 & 0.016854 & 0.815624 \\
\hline$b$ & -5.064546 & -0.923622 & -3.838048 \\
\hline ) & -1.641422 & 2.111941 & 0.662172 \\
\hline & -2.540801 & 3.038866 & 0.129496 \\
\hline 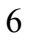 & -2.551676 & 4.360069 & 0.570493 \\
\hline 6 & -1.654885 & 4.724547 & 1.583758 \\
\hline ) & -0.744815 & 3.824915 & 2.138572 \\
\hline 6 & -0.727722 & 2.520244 & 1.631447 \\
\hline 6 & -3.510242 & 5.402060 & -0.002645 \\
\hline 6 & 0.147589 & 4.180249 & 3.328009 \\
\hline & 0.078794 & -0.481397 & -3.441162 \\
\hline & 0.066348 & -1.035653 & -4.457184 \\
\hline 1 & 7.156234 & 0.663329 & 0.530086 \\
\hline & -2.194502 & 0.903093 & 2.834181 \\
\hline 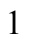 & 8.132222 & -4.100768 & -0.479118 \\
\hline 1 & -2.076338 & -2.745120 & 5.838664 \\
\hline . & 5.862584 & 5.373451 & -1.356084 \\
\hline 1 & -3.474074 & -2.767660 & 0.744903 \\
\hline & -5.246362 & -3.920722 & -0.500297 \\
\hline 1 & 3.866558 & 4.628677 & -0.194219 \\
\hline & -2.329573 & -0.545283 & 4.799962 \\
\hline 1 & 6.015138 & -4.318162 & -1.648539 \\
\hline l & 8.521011 & 2.514550 & -3.152154 \\
\hline 1 & 3.841501 & -3.336712 & -2.362000 \\
\hline 1 & -2.385818 & -5.250452 & -3.423953 \\
\hline 1 & -0.035477 & -4.608413 & -3.214505 \\
\hline 1 & -0.792745 & -6.099026 & 3.474651 \\
\hline ( & 7.145933 & 0.671526 & -2.310687 \\
\hline 1 & -0.588806 & -4.659044 & 1.501290 \\
\hline 1 & 1.738826 & -3.354839 & -2.021112 \\
\hline 1 & 7.910945 & 4.880184 & -2.659428 \\
\hline 1 & 9.227363 & -0.363342 & 1.337103 \\
\hline
\end{tabular}




\begin{tabular}{|c|c|c|c|}
\hline 1 & -4.711179 & -5.199685 & -2.570735 \\
\hline & -1.537863 & -5.157852 & 5.657412 \\
\hline & 9.750468 & -2.743995 & 0.817803 \\
\hline & 2.380705 & 2.767374 & 0.564394 \\
\hline & -4.148496 & 0.505609 & 1.309789 \\
\hline & -2.633976 & -0.396724 & -2.580701 \\
\hline & -6.847896 & -0.653358 & -1.808055 \\
\hline & -3.234044 & 2.712755 & -0.642132 \\
\hline & -0.015455 & 1.794099 & 2.029399 \\
\hline & -1.677224 & 5.748278 & 1.950785 \\
\hline & -1.110494 & 1.359489 & -2.594828 \\
\hline 1 & 2.432240 & -3.559775 & 0.838275 \\
\hline 1 & 3.431616 & -2.114047 & 0.862046 \\
\hline & 2.375840 & -4.312598 & 3.036745 \\
\hline 1 & 1.723207 & -0.139060 & 2.298226 \\
\hline & 1.959898 & -3.956926 & 5.450738 \\
\hline & 1.306312 & 0.225401 & 4.709873 \\
\hline & 1.422876 & -1.683559 & 6.299038 \\
\hline & -3.780960 & -1.226659 & -4.609518 \\
\hline & -5.973492 & -2.152098 & -3.931158 \\
\hline y & -5.759275 & 0.271303 & -4.501897 \\
\hline & -6.436784 & 0.514112 & 2.203627 \\
\hline 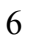 & -7.883284 & 0.981595 & 0.250344 \\
\hline & -7.452605 & -1.378147 & 0.966777 \\
\hline & -4.450735 & 4.808288 & -1.049275 \\
\hline & -4.367874 & 5.992061 & 1.122250 \\
\hline & -2.696667 & 6.518494 & -0.666178 \\
\hline ) & 0.075632 & & \\
\hline & -0.328844 & 3.367853 & 4.539419 \\
\hline b & 1.610614 & 3.837124 & 3.036996 \\
\hline & -4.028636 & -1.514263 & -5.639163 \\
\hline 1 & -3.115543 & -0.355155 & -4.667820 \\
\hline & -3.220713 & -2.055845 & -4.155019 \\
\hline 1 & -5.966797 & 0.056410 & -5.559120 \\
\hline 1 & -6.712825 & 0.509361 & -4.013622 \\
\hline 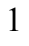 & -5.126933 & 1.167683 & -4.456044 \\
\hline 1 & -6.161389 & -2.402305 & -4.983729 \\
\hline & -5.502775 & -3.021667 & -3.454271 \\
\hline 1 & -6.948995 & -1.991466 & -3.456671 \\
\hline & -8.739610 & 1.055980 & 0.933964 \\
\hline 1 & -7.463376 & 1.988058 & 0.124087 \\
\hline 1 & -8.267999 & 0.655684 & -0.723259 \\
\hline 1 & -8.323265 & -1.343128 & 1.635649 \\
\hline 1 & -7.787176 & -1.786051 & 0.004829 \\
\hline 1 & -6.726015 & -2.081304 & 1.396033 \\
\hline 1 & -7.324313 & 0.560486 & 2.847239 \\
\hline & -5.712964 & -0.153581 & 2.690198 \\
\hline 1 & -6.002347 & 1.522125 & 2.166222 \\
\hline 1 & 0.280564 & 3.600641 & 5.423365 \\
\hline 1 & -1.375634 & 3.598397 & 4.778364 \\
\hline 1 & -0.259796 & 2.286917 & 4.357957 \\
\hline 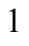 & 2.237176 & 4.087746 & 3.903306 \\
\hline 1 & 1.760240 & 2.769458 & 2.829642 \\
\hline & 1.984958 & 4.409306 & 2.177380 \\
\hline & 0.745029 & 5.867846 & 4.533945 \\
\hline & 0.389697 & 6.301871 & 2.854411 \\
\hline
\end{tabular}




$\begin{array}{lllr}1 & -0.934359 & 5.964192 & 3.993725 \\ 1 & -5.128621 & 5.587993 & -1.418884 \\ 1 & -3.905426 & 4.410709 & -1.914700 \\ 1 & -5.070205 & 4.000823 & -0.634947 \\ 1 & -3.365859 & 7.273824 & -1.100166 \\ 1 & -2.039210 & 7.027766 & 0.048888 \\ 1 & -2.066975 & 6.117831 & -1.471299 \\ 1 & -5.066302 & 6.736146 & 0.716354 \\ 1 & -4.958168 & 5.210089 & 1.617889 \\ 1 & -3.763938 & 6.492580 & 1.888489\end{array}$

$1_{\mathrm{C} 2-} \psi_{-1 \mathrm{~s} 2 \mathrm{aza}} \mathrm{s}$

Number of imaginary frequencies : 0

The smallest frequencies are : $10.9534 \quad 15.6867 \quad 21.6048 \mathrm{~cm}(-1)$

Electronic energy : $\quad H F=-4125.4701503$

Zero-point correction $=\quad 1.300426($ Hartree/Particle $)$

Thermal correction to Energy= $\quad 1.378176$

Thermal correction to Enthalpy= $\quad 1.379120$

Thermal correction to Gibbs Free Energy= 1.189816

Sum of electronic and zero-point Energies $=\quad-4124.169725$

Sum of electronic and thermal Energies $=\quad-4124.091975$

Sum of electronic and thermal Enthalpies $=\quad-4124.091030$

Sum of electronic and thermal Free Energies $=\quad-4124.280334$

\section{Cartesian Coordinates}

$\begin{array}{cccc}6 & 1.723662 & -1.665228 & 5.266292 \\ 6 & 2.078194 & -2.938904 & 4.834293 \\ 6 & 2.278747 & -3.183890 & 3.478533 \\ 6 & 2.133515 & -2.163901 & 2.538452 \\ 6 & 1.777656 & -0.888689 & 2.981858 \\ 6 & 1.574089 & -0.641321 & 4.334112 \\ 6 & 2.438628 & -2.443262 & 1.086878 \\ 7 & 1.520464 & -1.811390 & 0.118306 \\ 6 & 0.463583 & -2.613689 & -0.437592 \\ 6 & -0.804640 & -2.638752 & 0.114697 \\ 6 & -1.841886 & -3.371268 & -0.547604 \\ 6 & -1.552292 & -4.070255 & -1.756098 \\ 6 & -0.234693 & -4.045393 & -2.267086 \\ 6 & 0.741929 & -3.336879 & -1.622767 \\ 6 & -3.171918 & -3.409752 & -0.051822 \\ 6 & -4.152286 & -4.103825 & -0.715043 \\ 6 & -3.856560 & -4.806817 & -1.903273 \\ 6 & -2.583998 & -4.784018 & -2.413994 \\ 6 & -1.125149 & -1.985903 & 1.413154 \\ 6 & -1.488909 & -0.649742 & 1.498647 \\ 6 & -1.912222 & -0.127888 & 2.751177 \\ 6 & -1.954487 & -0.910674 & 3.873371 \\ 6 & -1.580860 & -2.272259 & 3.820640 \\ 6 & -1.164670 & -2.822670 & 2.574667 \\ 6 & -1.615195 & -3.093587 & 4.972350 \\ 6 & -1.267599 & -4.418505 & 4.900222 \\ 6 & -0.862790 & -4.971838 & 3.667745 \\ 6 & -0.810735 & -4.196457 & 2.536280 \\ 15 & -1.546902 & 0.389915 & -0.043015\end{array}$




\begin{tabular}{|c|c|c|c|}
\hline 45 & 0.135468 & 0.644798 & -1.844728 \\
\hline 6 & 0.643715 & 2.504624 & -1.653085 \\
\hline 8 & 0.743144 & 3.656038 & -1.656803 \\
\hline 15 & 1.909262 & -0.461894 & -0.816632 \\
\hline 8 & 2.878188 & 0.276039 & 0.300193 \\
\hline 6 & 3.720832 & 1.272239 & -0.155956 \\
\hline 6 & 4.895812 & 0.932522 & -0.797178 \\
\hline 6 & 5.708803 & 1.996195 & -1.307560 \\
\hline 6 & 5.333559 & 3.350253 & -1.049009 \\
\hline 6 & 4.147865 & 3.623382 & -0.325768 \\
\hline 6 & 3.342024 & 2.601843 & 0.097305 \\
\hline 6 & 6.142301 & 4.403026 & -1.542625 \\
\hline 6 & 7.263842 & 4.142453 & -2.286464 \\
\hline 6 & 7.616139 & 2.807664 & -2.576517 \\
\hline 6 & 6.861220 & 1.764532 & -2.101018 \\
\hline 6 & 5.237383 & -0.498931 & -0.967077 \\
\hline 6 & 4.316601 & -1.354275 & -1.548381 \\
\hline 6 & 4.611442 & -2.706938 & -1.815859 \\
\hline 6 & 5.817865 & -3.229012 & -1.437788 \\
\hline 6 & 6.762992 & -2.431737 & -0.749478 \\
\hline 6 & 6.471426 & -1.057134 & -0.497072 \\
\hline 6 & 7.411227 & -0.307117 & 0.255744 \\
\hline 6 & 8.580817 & -0.873789 & 0.697711 \\
\hline 6 & 8.881805 & -2.221895 & 0.412005 \\
\hline 6 & 7.985290 & -2.982357 & -0.293206 \\
\hline 8 & 3.076055 & -0.894661 & -1.933270 \\
\hline 6 & -3.242245 & -0.020620 & -0.635975 \\
\hline 6 & -4.331464 & 0.067777 & 0.225611 \\
\hline 6 & -5.626158 & -0.223522 & -0.211138 \\
\hline 6 & -5.791206 & -0.602691 & -1.544023 \\
\hline 6 & -4.716898 & -0.707774 & -2.437446 \\
\hline 6 & -3.439657 & -0.417909 & -1.958265 \\
\hline 6 & -6.782600 & -0.103351 & 0.779160 \\
\hline 6 & -4.969443 & -1.075647 & -3.898532 \\
\hline 6 & -1.748975 & 2.090529 & 0.623812 \\
\hline 6 & -2.700911 & 2.955748 & 0.078138 \\
\hline 6 & -2.784490 & 4.282465 & 0.494939 \\
\hline 6 & -1.910937 & 4.714092 & 1.502055 \\
\hline 6 & -0.948438 & 3.878292 & 2.067171 \\
\hline 6 & -0.858132 & 2.568420 & 1.582543 \\
\hline 6 & -3.807827 & 5.256013 & -0.086771 \\
\hline 6 & -0.061163 & 4.309974 & 3.234315 \\
\hline 6 & 0.130141 & -0.450239 & -3.423948 \\
\hline 8 & 0.140485 & -1.004075 & -4.440189 \\
\hline 1 & 7.190938 & 0.729739 & 0.495636 \\
\hline 1 & -2.217107 & 0.913174 & 2.823100 \\
\hline 1 & 8.190909 & -4.030214 & -0.508112 \\
\hline 1 & -1.926877 & -2.648530 & 5.916511 \\
\hline 1 & 5.842366 & 5.427609 & -1.327096 \\
\hline 1 & -3.415739 & -2.864608 & 0.858417 \\
\hline 1 & -5.168973 & -4.107627 & -0.327174 \\
\hline 1 & 3.871819 & 4.659809 & -0.136924 \\
\hline 1 & -2.285132 & -0.491881 & 4.823477 \\
\hline 1 & 6.059562 & -4.270127 & -1.645149 \\
\hline 1 & 8.490891 & 2.602190 & -3.189395 \\
\hline 1 & 3.869505 & -3.308992 & -2.334830 \\
\hline
\end{tabular}




\begin{tabular}{|c|c|c|c|}
\hline 1 & -2.341449 & -5.316070 & -3.332872 \\
\hline & -0.013391 & -4.587085 & -3.185312 \\
\hline & -0.586435 & -6.022672 & 3.614393 \\
\hline & 7.139483 & 0.742600 & -2.345017 \\
\hline & -0.486222 & -4.636344 & 1.595267 \\
\hline & 1.752411 & -3.294753 & -2.023414 \\
\hline & 7.873634 & 4.959205 & -2.665504 \\
\hline & 9.279783 & -0.277362 & 1.279703 \\
\hline & -4.641225 & -5.363138 & -2.411883 \\
\hline & -1.301285 & -5.043933 & 5.789528 \\
\hline & 9.815401 & -2.655727 & 0.762283 \\
\hline & 2.409048 & 2.780987 & 0.627134 \\
\hline & -4.171536 & 0.378268 & 1.257802 \\
\hline & -2.569800 & -0.494803 & -2.605972 \\
\hline & -6.792344 & -0.822635 & -1.909331 \\
\hline & -3.376740 & 2.577831 & -0.685232 \\
\hline & -0.101511 & 1.893498 & 1.988783 \\
\hline & -1.993453 & 5.740232 & 1.853337 \\
\hline & -1.099739 & 1.363598 & -2.571888 \\
\hline & 2.431024 & -3.527610 & 0.902572 \\
\hline & 3.461327 & -2.105821 & 0.872365 \\
\hline & 2.544640 & -4.186576 & 3.142346 \\
\hline & 1.656972 & -0.087448 & 2.256472 \\
\hline & 2.185457 & -3.750123 & 5.551719 \\
\hline ] & 1.295529 & 0.359883 & 4.661290 \\
\hline & 1.558703 & -1.472159 & 6.324335 \\
\hline 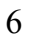 & -4.645254 & 4.620733 & -1.194579 \\
\hline f & -4.758409 & 5.717887 & 1.023392 \\
\hline & -3.080670 & 6.469013 & -0.676778 \\
\hline f & -0.206232 & 5.794787 & 3.559343 \\
\hline 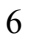 & -0.472789 & 3.504377 & 4.473345 \\
\hline 6 & 1.412500 & 4.036244 & 2.922345 \\
\hline 6 & -3.668089 & -1.324557 & -4.658968 \\
\hline 6 & -5.822145 & -2.343913 & -3.994203 \\
\hline 5 & -5.710151 & 0.085082 & -4.573132 \\
\hline & -8.117868 & -0.522624 & 0.170472 \\
\hline 6 & -6.511927 & -1.001916 & 1.992075 \\
\hline 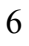 & -6.899967 & 1.355342 & 1.237173 \\
\hline 1 & 2.046080 & 4.379248 & 3.751005 \\
\hline 1 & 1.618400 & 2.967121 & 2.782961 \\
\hline 1 & 1.726868 & 4.569730 & 2.014680 \\
\hline 1 & 0.459900 & 6.056275 & 4.391108 \\
\hline ] & 0.065363 & 6.428370 & 2.705112 \\
\hline 1 & -1.227298 & 6.051921 & 3.868134 \\
\hline 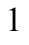 & 0.145002 & 3.783548 & 5.337824 \\
\hline 1 & -1.523267 & 3.693163 & 4.731855 \\
\hline 1 & -0.358761 & 2.423805 & 4.313069 \\
\hline 1 & -5.499870 & 6.422084 & 0.622404 \\
\hline 1 & -5.300722 & 4.865906 & 1.454850 \\
\hline 1 & -4.227406 & 6.224343 & 1.838521 \\
\hline 1 & -3.803871 & 7.169404 & -1.115930 \\
\hline 1 & -2.507694 & 7.017764 & 0.080276 \\
\hline 1 & -2.382743 & 6.161406 & -1.466226 \\
\hline 1 & -5.366344 & 5.354097 & -1.577011 \\
\hline 1 & -4.026443 & 4.293665 & -2.040062 \\
\hline 1 & -5.216369 & 3.754270 & -0.834550 \\
\hline
\end{tabular}




$\begin{array}{rrrr}1 & -3.894918 & -1.617067 & -5.692025 \\ 1 & -3.037024 & -0.427202 & -4.706912 \\ 1 & -3.080357 & -2.133562 & -4.203098 \\ 1 & -5.990861 & -2.605865 & -5.047257 \\ 1 & -5.316219 & -3.189745 & -3.510355 \\ 1 & -6.807032 & -2.224875 & -3.526911 \\ 1 & -5.897760 & -0.142816 & -5.631325 \\ 1 & -6.678458 & 0.282728 & -4.096214 \\ 1 & -5.118374 & 1.008601 & -4.525176 \\ 1 & -7.332252 & -0.919631 & 2.717767 \\ 1 & -6.434521 & -2.054664 & 1.689566 \\ 1 & -5.582594 & -0.737756 & 2.512484 \\ 1 & -7.698183 & 1.460700 & 1.984466 \\ 1 & -5.968607 & 1.722740 & 1.687210 \\ 1 & -7.142431 & 2.011238 & 0.390603 \\ 1 & -8.911928 & -0.430306 & 0.922258 \\ 1 & -8.398961 & 0.109887 & -0.681237 \\ 1 & -8.102536 & -1.567303 & -0.167040\end{array}$

$1_{\mathrm{C} 2-} \psi_{-1 \mathrm{~s} 223 \mathrm{ss} 4 \mathrm{a}}$

Number of imaginary frequencies : 0

The smallest frequencies are : $11.2835 \quad 14.9910 \quad 17.9040 \mathrm{~cm}(-1)$

Electronic energy : $\quad H F=-4125.4690054$

Zero-point correction $=\quad 1.300285($ Hartree/Particle)

Thermal correction to Energy= $\quad 1.378259$

Thermal correction to Enthalpy= $\quad 1.379203$

Thermal correction to Gibbs Free Energy $=\quad 1.188845$

Sum of electronic and zero-point Energies $=\quad-4124.168720$

Sum of electronic and thermal Energies $=\quad-4124.090746$

Sum of electronic and thermal Enthalpies $=\quad-4124.089802$

Sum of electronic and thermal Free Energies $=\quad-4124.280161$

Cartesian Coordinates

$\begin{array}{lrrr}6 & 1.587894 & -1.933022 & 5.212309 \\ 6 & 1.900241 & -3.192493 & 4.713280 \\ 6 & 2.143320 & -3.361632 & 3.352839 \\ 6 & 2.080948 & -2.279571 & 2.476100 \\ 6 & 1.766896 & -1.017304 & 2.986232 \\ 6 & 1.522108 & -0.845970 & 4.343245 \\ 6 & 2.423554 & -2.476746 & 1.020633 \\ 7 & 1.490525 & -1.834119 & 0.072867 \\ 6 & 0.442111 & -2.642605 & -0.490247 \\ 6 & -0.840316 & -2.646842 & 0.028611 \\ 6 & -1.863977 & -3.384870 & -0.649782 \\ 6 & -1.537972 & -4.146855 & -1.810243 \\ 6 & -0.204857 & -4.146634 & -2.280834 \\ 6 & 0.751069 & -3.405648 & -1.642396 \\ 6 & -3.212096 & -3.384118 & -0.205046 \\ 6 & -4.172411 & -4.112950 & -0.860986 \\ 6 & -3.836590 & -4.894148 & -1.987881 \\ 6 & -2.547750 & -4.901880 & -2.455604 \\ 6 & -1.177597 & -2.014670 & 1.334101 \\ 6 & -1.510819 & -0.674887 & 1.462152 \\ 6 & -1.936661 & -0.184195 & 2.727066\end{array}$




\begin{tabular}{|c|c|c|c|}
\hline 6 & -2.021074 & -1.003360 & 3.819753 \\
\hline 6 & -1.687973 & -2.373419 & 3.723244 \\
\hline 6 & -1.257093 & -2.889291 & 2.467718 \\
\hline 6 & -1.782665 & -3.237230 & 4.840240 \\
\hline 6 & -1.477989 & -4.569851 & 4.726565 \\
\hline 6 & -1.046808 & -5.086978 & 3.487359 \\
\hline 6 & -0.933583 & -4.269138 & 2.390378 \\
\hline 15 & -1.546308 & 0.416742 & -0.040023 \\
\hline & 0.097127 & 0.639418 & -1.873153 \\
\hline 6 & 0.642895 & 2.489728 & -1.722843 \\
\hline 8 & 0.752814 & 3.640298 & -1.740587 \\
\hline 15 & 1.869290 & -0.474257 & -0.850820 \\
\hline 8 & 2.821746 & 0.268431 & 0.278586 \\
\hline 6 & 3.672617 & 1.262719 & -0.167758 \\
\hline 6 & 4.858464 & 0.920347 & -0.787420 \\
\hline 6 & 5.684279 & 1.981464 & -1.282673 \\
\hline 6 & 5.307015 & 3.336668 & -1.033762 \\
\hline 6 & 4.105796 & 3.613055 & -0.338359 \\
\hline 6 & 3.289511 & 2.593907 & 0.070493 \\
\hline 6 & 6.128239 & 4.387183 & -1.511402 \\
\hline 6 & 7.264637 & 4.123378 & -2.231095 \\
\hline 6 & 7.619549 & 2.787452 & -2.512613 \\
\hline 6 & 6.852231 & 1.746574 & -2.052315 \\
\hline 6 & 5.196623 & -0.511007 & -0.963589 \\
\hline 6 & 4.283354 & -1.354062 & -1.573887 \\
\hline 6 & 4.582711 & -2.700786 & -1.865725 \\
\hline 6 & 5.781272 & -3.232244 & -1.475678 \\
\hline 6 & 6.713851 & -2.450818 & -0.752798 \\
\hline 6 & 6.420811 & -1.080092 & -0.481751 \\
\hline 6 & 7.348437 & -0.344865 & 0.300137 \\
\hline 6 & 8.508064 & -0.922217 & 0.754340 \\
\hline 6 & 8.810596 & -2.266424 & 0.452078 \\
\hline 6 & 7.925903 & -3.012457 & -0.282908 \\
\hline 8 & 3.047605 & -0.886315 & -1.963618 \\
\hline 6 & -3.272822 & 0.112031 & -0.606372 \\
\hline 6 & -4.351800 & 0.213404 & 0.282297 \\
\hline 6 & -5.652880 & -0.023868 & -0.144169 \\
\hline 6 & -5.853528 & -0.354095 & -1.496095 \\
\hline 6 & -4.805941 & -0.453584 & -2.405770 \\
\hline 6 & -3.506419 & -0.230955 & -1.928727 \\
\hline 6 & -6.848737 & 0.050785 & 0.803540 \\
\hline 6 & -5.001584 & -0.773717 & -3.885600 \\
\hline 6 & -1.634061 & 2.101171 & 0.686612 \\
\hline 6 & -2.525601 & 3.042467 & 0.166079 \\
\hline 6 & -2.526332 & 4.357502 & 0.625177 \\
\hline 6 & -1.627719 & 4.700799 & 1.644154 \\
\hline 6 & -0.725839 & 3.786230 & 2.187971 \\
\hline 6 & -0.718468 & 2.488476 & 1.662842 \\
\hline 6 & -3.477263 & 5.414381 & 0.066745 \\
\hline 6 & 0.164436 & 4.117794 & 3.385973 \\
\hline 6 & -0.007531 & -0.491855 & -3.425937 \\
\hline 8 & -0.101568 & -1.060199 & -4.429966 \\
\hline 1 & 7.126134 & 0.688742 & 0.552318 \\
\hline 1 & -2.209644 & 0.863211 & 2.831048 \\
\hline 1 & 8.133209 & -4.057018 & -0.511790 \\
\hline 1 & -2.109360 & -2.818634 & 5.791469 \\
\hline
\end{tabular}




\begin{tabular}{|c|c|c|c|}
\hline 1 & 5.825802 & 5.412604 & -1.303492 \\
\hline & -3.485052 & -2.786190 & 0.662754 \\
\hline & -5.201729 & -4.088884 & -0.509394 \\
\hline & 3.825638 & 4.650310 & -0.160396 \\
\hline & -2.356885 & -0.608740 & 4.778322 \\
\hline & 6.026219 & -4.268901 & -1.700788 \\
\hline & 8.506359 & 2.579224 & -3.106962 \\
\hline & 3.851537 & -3.289669 & -2.414521 \\
\hline & -2.276076 & -5.485667 & -3.333961 \\
\hline & 0.043958 & -4.731830 & -3.164623 \\
\hline & -0.797573 & -6.142353 & 3.400978 \\
\hline & 7.133327 & 0.724010 & -2.290052 \\
\hline & -0.587534 & -4.682761 & 1.445034 \\
\hline & 1.774020 & -3.381140 & -2.011550 \\
\hline & 7.884266 & 4.938292 & -2.597949 \\
\hline & 9.197830 & -0.337628 & 1.358875 \\
\hline & -4.605360 & -5.477352 & -2.490352 \\
\hline & -1.562506 & -5.227926 & 5.588499 \\
\hline & 9.736198 & -2.708806 & 0.812728 \\
\hline & 2.343606 & 2.777537 & 0.575032 \\
\hline & -4.159037 & 0.486248 & 1.316834 \\
\hline & -2.654820 & -0.321126 & -2.600838 \\
\hline 1 & -6.870931 & -0.532482 & -1.839176 \\
\hline & -3.220443 & 2.732890 & -0.610864 \\
\hline ] & -0.013220 & 1.750637 & 2.051789 \\
\hline & -1.642432 & 5.719436 & 2.025479 \\
\hline & -1.129711 & 1.383392 & -2.588386 \\
\hline & 2.463826 & -3.550568 & 0.785380 \\
\hline & 3.432205 & -2.084150 & 0.838599 \\
\hline 1 & 2.377375 & -4.352457 & 2.962431 \\
\hline & 1.715295 & -0.166644 & 2.308773 \\
\hline ] & 1.940330 & -4.051234 & 5.380613 \\
\hline ] & 1.280204 & 0.144702 & 4.726296 \\
\hline 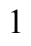 & 1.390230 & -1.798419 & 6.273833 \\
\hline f & -4.419780 & 4.842787 & -0.990347 \\
\hline & -4.332677 & 5.992161 & 1.199645 \\
\hline 6 & -2.656257 & 6.535947 & -0.578583 \\
\hline 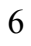 & 0.102332 & 5.594930 & 3.767681 \\
\hline 6 & -0.324830 & 3.292333 & 4.583347 \\
\hline 6 & 1.626409 & 3.767810 & 3.098277 \\
\hline 5 & -6.474876 & -0.910093 & -4.260189 \\
\hline 6 & -4.395255 & 0.353033 & -4.731488 \\
\hline 5 & -4.296942 & -2.092284 & -4.217810 \\
\hline 6 & -6.445694 & 0.501097 & 2.206340 \\
\hline 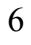 & -7.881530 & 1.046999 & 0.266048 \\
\hline 6 & -7.486598 & -1.338510 & 0.912977 \\
\hline 1 & 2.248312 & 3.994222 & 3.974547 \\
\hline 1 & 1.769004 & 2.703890 & 2.868208 \\
\hline 1 & 2.012637 & 4.355194 & 2.254387 \\
\hline 1 & 0.771947 & 5.784153 & 4.616121 \\
\hline 1 & 0.422229 & 6.244050 & 2.942347 \\
\hline 1 & -0.905952 & 5.900134 & 4.074826 \\
\hline 1 & 0.281104 & 3.508377 & 5.473900 \\
\hline 1 & -1.371317 & 3.527104 & 4.819434 \\
\hline 1 & -0.262549 & 2.213606 & 4.387054 \\
\hline 1 & -5.025662 & 6.747079 & 0.804567 \\
\hline
\end{tabular}




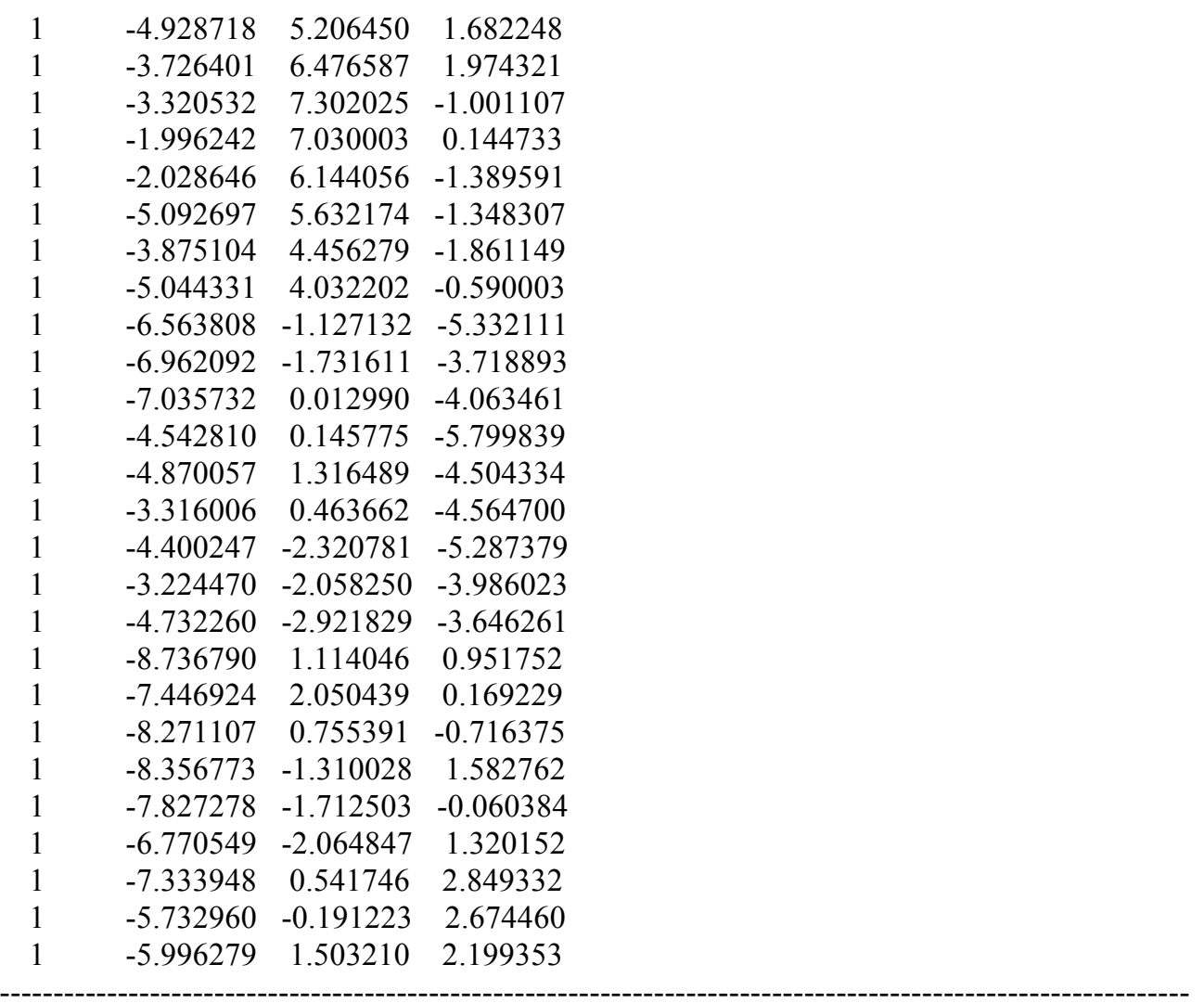

$1_{\mathrm{C} 2-} \psi_{-1 \mathrm{~s} 2 \mathrm{a} 3 \mathrm{~s} 4 \mathrm{~s}}$

Number of imaginary frequencies : 0

The smallest frequencies are : $13.4390 \quad 15.6137 \quad 18.5078 \mathrm{~cm}(-1)$

Electronic energy : $\quad H F=-4125.4694236$

Zero-point correction $=\quad 1.299911$ (Hartree/Particle)

Thermal correction to Energy $=\quad 1.378010$

Thermal correction to Enthalpy $=\quad 1.378954$

Thermal correction to Gibbs Free Energy= $\quad 1.188559$

Sum of electronic and zero-point Energies $=\quad-4124.169513$

Sum of electronic and thermal Energies $=\quad-4124.091414$

Sum of electronic and thermal Enthalpies= $\quad-4124.090470$

Sum of electronic and thermal Free Energies $=\quad-4124.280865$

Cartesian Coordinates

$\begin{array}{llll}6 & 1.589244 & -1.954174 & 5.199195 \\ 6 & 1.905532 & -3.211250 & 4.696486 \\ 6 & 2.146071 & -3.376298 & 3.335191 \\ 6 & 2.077410 & -2.292698 & 2.460635 \\ 6 & 1.759310 & -1.032963 & 2.974482 \\ 6 & 1.517011 & -0.865753 & 4.332534 \\ 6 & 2.418406 & -2.488212 & 1.004266 \\ 7 & 1.492549 & -1.835406 & 0.056761 \\ 6 & 0.441351 & -2.632700 & -0.516570 \\ 6 & -0.838443 & -2.645249 & 0.008435 \\ 6 & -1.867106 & -3.368623 & -0.677525 \\ 6 & -1.549121 & -4.106791 & -1.855383 \\ 6 & -0.218766 & -4.098343 & -2.333835\end{array}$




\begin{tabular}{|c|c|c|c|}
\hline 6 & 0.742606 & -3.372730 & -1.685672 \\
\hline 6 & -3.212775 & -3.373165 & -0.225553 \\
\hline 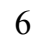 & -4.178798 & -4.083833 & -0.892800 \\
\hline & -3.851001 & -4.842696 & -2.037158 \\
\hline & -2.564254 & -4.845691 & -2.510838 \\
\hline & -1.172068 & -2.028430 & 1.321643 \\
\hline & -1.509888 & -0.690910 & 1.460196 \\
\hline 5 & -1.938277 & -0.212548 & 2.728697 \\
\hline & -2.020487 & -1.041657 & 3.814360 \\
\hline 6 & -1.682479 & -2.409647 & 3.706651 \\
\hline 6 & -1.249488 & -2.913193 & 2.446852 \\
\hline 6 & -1.773725 & -3.283414 & 4.816232 \\
\hline 6 & -1.464459 & -4.613881 & 4.690706 \\
\hline 6 & -1.032688 & -5.119036 & 3.446700 \\
\hline 6 & -0.922572 & -4.291435 & 2.356804 \\
\hline & -1.546337 & 0.409227 & -0.035705 \\
\hline 45 & 0.108019 & 0.667628 & -1.855821 \\
\hline 6 & 0.649976 & 2.515708 & -1.661911 \\
\hline 8 & 0.759688 & 3.666329 & -1.655530 \\
\hline 15 & 1.876527 & -0.464905 & -0.848277 \\
\hline 8 & 2.831420 & 0.257575 & 0.291797 \\
\hline 6 & 3.681823 & 1.259022 & -0.138198 \\
\hline 6 & 4.865723 & 0.926826 & -0.766964 \\
\hline 6 & 5.690228 & 1.996170 & -1.246210 \\
\hline 6 & 5.314109 & 3.346903 & -0.972299 \\
\hline 6 & 4.115762 & 3.611616 & -0.267286 \\
\hline 6 & 3.300422 & 2.585759 & 0.126272 \\
\hline 6 & 6.134099 & 4.405369 & -1.434245 \\
\hline 6 & 7.268090 & 4.153851 & -2.162082 \\
\hline 6 & 7.621879 & 2.822975 & -2.467832 \\
\hline 6 & 6.855787 & 1.774359 & -2.023308 \\
\hline 6 & 5.204089 & -0.501903 & -0.963467 \\
\hline 6 & 4.289468 & -1.338323 & -1.580894 \\
\hline 6 & 4.586576 & -2.682902 & -1.884614 \\
\hline 6 & 5.785592 & -3.218829 & -1.502169 \\
\hline 6 & 6.720769 & -2.444553 & -0.774977 \\
\hline 6 & 6.429135 & -1.076329 & -0.489999 \\
\hline 6 & 7.359038 & -0.349437 & 0.296992 \\
\hline 6 & 8.519438 & -0.931785 & 0.742721 \\
\hline 6 & 8.820537 & -2.273108 & 0.426590 \\
\hline 6 & 7.933620 & -3.011416 & -0.313494 \\
\hline 8 & 3.054045 & -0.865457 & -1.965813 \\
\hline 6 & -3.263926 & 0.084811 & -0.618921 \\
\hline 6 & -4.343311 & 0.141179 & 0.264009 \\
\hline 6 & -5.645475 & -0.100495 & -0.170549 \\
\hline 6 & -5.843297 & -0.379729 & -1.528952 \\
\hline 6 & -4.785957 & -0.439913 & -2.439120 \\
\hline 6 & -3.492688 & -0.221196 & -1.955023 \\
\hline 6 & -6.785596 & -0.044312 & 0.845249 \\
\hline 6 & -4.976962 & -0.719370 & -3.928317 \\
\hline 6 & -1.667727 & 2.088825 & 0.697232 \\
\hline 6 & -2.564600 & 3.020373 & 0.167768 \\
\hline 6 & -2.578687 & 4.337112 & 0.621678 \\
\hline 6 & -1.697839 & 4.688583 & 1.653566 \\
\hline 6 & -0.793155 & 3.783251 & 2.207746 \\
\hline 6 & -0.765517 & 2.486292 & 1.681477 \\
\hline
\end{tabular}




\begin{tabular}{|c|c|c|c|}
\hline 6 & -3.529654 & 5.386198 & 0.048920 \\
\hline 6 & 0.087215 & 4.124714 & 3.410075 \\
\hline & 0.015145 & -0.427973 & -3.433443 \\
\hline & -0.069126 & -0.974908 & -4.450177 \\
\hline & 7.138112 & 0.681751 & 0.559904 \\
\hline & -2.216007 & 0.832693 & 2.841067 \\
\hline & 8.139701 & -4.053845 & -0.552897 \\
\hline & -2.101557 & -2.874367 & 5.771202 \\
\hline & 5.832764 & 5.427108 & -1.207485 \\
\hline & -3.479382 & -2.791484 & 0.655772 \\
\hline & -5.206454 & -4.062681 & -0.536191 \\
\hline & 3.837306 & 4.645767 & -0.069706 \\
\hline & -2.358245 & -0.656421 & 4.776064 \\
\hline & 6.028866 & -4.253829 & -1.736528 \\
\hline & 8.506862 & 2.624990 & -3.068371 \\
\hline & 3.853068 & -3.266816 & -2.435574 \\
\hline & -2.298895 & -5.412096 & -3.402430 \\
\hline & 0.023810 & -4.665148 & -3.231250 \\
\hline & -0.780734 & -6.172962 & 3.351032 \\
\hline & 7.135890 & 0.755891 & -2.279335 \\
\hline & -0.576581 & -4.695482 & 1.407273 \\
\hline & 1.763103 & -3.341466 & -2.061268 \\
\hline & 7.886763 & 4.974912 & -2.516632 \\
\hline & 9.210923 & -0.353480 & 1.351324 \\
\hline & -4.624226 & -5.412605 & -2.548002 \\
\hline & -1.546260 & -5.279730 & 5.546911 \\
\hline & 9.746742 & -2.719507 & 0.780677 \\
\hline & 2.356471 & 2.760097 & 0.638029 \\
\hline & -4.162988 & 0.380169 & 1.311785 \\
\hline 1 & -2.638491 & -0.283667 & -2.626993 \\
\hline & -6.854105 & -0.554985 & -1.885946 \\
\hline ] & -3.248104 & 2.703534 & -0.616433 \\
\hline & -0.053607 & 1.757395 & 2.074930 \\
\hline & -1.727427 & 5.707189 & 2.034183 \\
\hline & -1.116567 & 1.423360 & -2.563133 \\
\hline & 2.449031 & -3.561641 & 0.765814 \\
\hline 1 & 3.430983 & -2.104895 & 0.823695 \\
\hline & 2.382952 & -4.365427 & 2.942170 \\
\hline 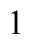 & 1.701982 & -0.180275 & 2.300217 \\
\hline & 1.950427 & -4.071416 & 5.361670 \\
\hline & 1.270975 & 0.123126 & 4.717711 \\
\hline 1 & 1.393307 & -1.822756 & 6.261435 \\
\hline & -4.383153 & 4.832474 & -1.090006 \\
\hline 6 & -4.470746 & 5.879563 & 1.153444 \\
\hline & -2.716174 & 6.564472 & -0.497451 \\
\hline 6 & 0.003365 & 5.600215 & 3.793567 \\
\hline 5 & -0.392919 & 3.290639 & 4.605118 \\
\hline 6 & 1.554536 & 3.794802 & 3.124598 \\
\hline 6 & -6.448436 & -0.853414 & -4.311450 \\
\hline 5 & -4.374399 & 0.432859 & -4.742052 \\
\hline 6 & -4.264534 & -2.024955 & -4.294272 \\
\hline 5 & -8.138369 & -0.385507 & 0.225711 \\
\hline 6 & -6.511991 & -1.050808 & 1.969528 \\
\hline 5 & -6.871771 & 1.369393 & 1.431910 \\
\hline 1 & 2.173804 & 4.039613 & 3.997791 \\
\hline . & 1.712730 & 2.730529 & 2.906079 \\
\hline
\end{tabular}




$\begin{array}{rrrr}1 & 1.930341 & 4.379378 & 2.273987 \\ 1 & 0.662570 & 5.796397 & 4.648548 \\ 1 & 0.322722 & 6.254679 & 2.972242 \\ 1 & -1.011538 & 5.892168 & 4.091666 \\ 1 & 0.207986 & 3.514316 & 5.497184 \\ 1 & -1.443077 & 3.510227 & 4.839485 \\ 1 & -0.314244 & 2.213077 & 4.408267 \\ 1 & -5.160640 & 6.636656 & 0.756792 \\ 1 & -5.071421 & 5.052542 & 1.554940 \\ 1 & -3.925588 & 6.334225 & 1.989494 \\ 1 & -3.384565 & 7.317321 & -0.936632 \\ 1 & -2.128917 & 7.060545 & 0.284504 \\ 1 & -2.019756 & 6.230611 & -1.277731 \\ 1 & -5.048262 & 5.619581 & -1.467101 \\ 1 & -3.769040 & 4.487502 & -1.931908 \\ 1 & -5.015672 & 3.996232 & -0.763517 \\ 1 & -6.532215 & -1.042247 & -5.389135 \\ 1 & -6.933709 & -1.691456 & -3.794359 \\ 1 & -7.015026 & 0.061144 & -4.092526 \\ 1 & -4.520921 & 0.255565 & -5.815964 \\ 1 & -4.852236 & 1.388069 & -4.487714 \\ 1 & -3.295515 & 0.542238 & -4.571749 \\ 1 & -4.369455 & -2.228885 & -5.368685 \\ 1 & -3.191809 & -1.988042 & -4.064703 \\ 1 & -4.691319 & -2.871286 & -3.740806 \\ 1 & -7.321355 & -1.023907 & 2.711598 \\ 1 & -6.451814 & -2.073098 & 1.572986 \\ 1 & -5.571974 & -0.844722 & 2.497005 \\ 1 & -7.665430 & 1.422683 & 2.189471 \\ 1 & -5.932898 & 1.677121 & 1.909154 \\ 1 & -7.103248 & 2.103157 & 0.648834 \\ 1 & -8.918230 & -0.341742 & 0.996492 \\ 1 & -8.419946 & 0.321946 & -0.564705 \\ 1 & -8.151028 & -1.398135 & -0.198234\end{array}$

$1_{\mathrm{C} 2-} \psi_{-1 \mathrm{~s} 2 \mathrm{~s} 3 \mathrm{ata}}$

Number of imaginary frequencies : 0

The smallest frequencies are : $11.4067 \quad 15.4999 \quad 19.4758 \mathrm{~cm}(-1)$

Electronic energy : $\quad H F=-4125.4700216$

Zero-point correction $=\quad 1.299930($ Hartree/Particle $)$

Thermal correction to Energy= $\quad 1.378036$

Thermal correction to Enthalpy= $\quad 1.378981$

Thermal correction to Gibbs Free Energy $=\quad 1.188390$

Sum of electronic and zero-point Energies $=\quad-4124.170091$

Sum of electronic and thermal Energies $=\quad-4124.091985$

Sum of electronic and thermal Enthalpies $=\quad-4124.091041$

Sum of electronic and thermal Free Energies $=\quad-4124.281631$

Cartesian Coordinates

$\begin{array}{llll}6 & 1.590176 & -1.915563 & 5.219267 \\ 6 & 1.882522 & -3.182653 & 4.727145 \\ 6 & 2.114309 & -3.364751 & 3.366458 \\ 6 & 2.061155 & -2.288184 & 2.482111 \\ 6 & 1.766961 & -1.018488 & 2.985372\end{array}$




\begin{tabular}{|c|c|c|c|}
\hline 6 & 1.532926 & -0.834265 & 4.342650 \\
\hline 6 & 2.395584 & -2.501627 & 1.026620 \\
\hline & 1.480159 & -1.841512 & 0.074502 \\
\hline & 0.416462 & -2.622850 & -0.497646 \\
\hline & -0.863036 & -2.614259 & 0.028464 \\
\hline & -1.909865 & -3.296701 & -0.671102 \\
\hline & -1.614027 & -3.996738 & -1.877779 \\
\hline & -0.281542 & -4.026735 & -2.348140 \\
\hline & 0.701134 & -3.352650 & -1.677019 \\
\hline 6 & -3.255504 & -3.277863 & -0.218654 \\
\hline 6 & -4.246079 & -3.912004 & -0.925355 \\
\hline 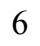 & -3.944313 & -4.619714 & -2.109370 \\
\hline 6 & -2.655144 & -4.657059 & -2.575254 \\
\hline 6 & -1.181740 & -1.989411 & 1.341419 \\
\hline 6 & -1.495437 & -0.645165 & 1.472749 \\
\hline 6 & -1.906775 & -0.150693 & 2.740659 \\
\hline 6 & -1.996296 & -0.971191 & 3.832321 \\
\hline 6 & -1.684778 & -2.346032 & 3.731028 \\
\hline 6 & -1.268889 & -2.865488 & 2.471884 \\
\hline 6 & -1.786965 & -3.211950 & 4.845725 \\
\hline 6 & -1.504858 & -4.549034 & 4.725659 \\
\hline 6 & -1.090779 & -5.069800 & 3.482001 \\
\hline 6 & -0.970392 & -4.250344 & 2.386974 \\
\hline & -1.528907 & 0.442724 & -0.033009 \\
\hline 45 & 0.123253 & 0.655940 & -1.864958 \\
\hline 6 & 0.681341 & 2.501779 & -1.691156 \\
\hline 8 & 0.807842 & 3.650513 & -1.704485 \\
\hline 15 & 1.880953 & -0.483559 & -0.843100 \\
\hline 8 & 2.845070 & 0.240143 & 0.288289 \\
\hline 6 & 3.705316 & 1.227529 & -0.155575 \\
\hline 6 & 4.884767 & 0.874854 & -0.781504 \\
\hline 6 & 5.719372 & 1.928893 & -1.276821 \\
\hline 6 & 5.357632 & 3.287045 & -1.021107 \\
\hline 6 & 4.163144 & 3.573532 & -0.318126 \\
\hline 6 & 3.337759 & 2.561649 & 0.090640 \\
\hline 6 & 6.187344 & 4.330480 & -1.499616 \\
\hline 6 & 7.316967 & 4.057159 & -2.226436 \\
\hline 6 & 7.656421 & 2.718550 & -2.514300 \\
\hline 6 & 6.880727 & 1.684227 & -2.053263 \\
\hline 6 & 5.207503 & -0.559701 & -0.960462 \\
\hline 6 & 4.283186 & -1.393437 & -1.566835 \\
\hline 6 & 4.565250 & -2.744761 & -1.854260 \\
\hline 6 & 5.759015 & -3.288915 & -1.467016 \\
\hline 6 & 6.703664 & -2.515951 & -0.750737 \\
\hline 6 & 6.426945 & -1.141400 & -0.481734 \\
\hline 6 & 7.365645 & -0.414894 & 0.295066 \\
\hline 6 & 8.520642 & -1.003975 & 0.745897 \\
\hline 6 & 8.807167 & -2.352068 & 0.445318 \\
\hline 6 & 7.911297 & -3.089907 & -0.284387 \\
\hline 8 & 3.052695 & -0.911691 & -1.956615 \\
\hline 6 & -3.259242 & 0.134376 & -0.589752 \\
\hline 6 & -4.326474 & 0.231835 & 0.308011 \\
\hline 6 & -5.630280 & -0.043839 & -0.097191 \\
\hline 6 & -5.833967 & -0.405744 & -1.435329 \\
\hline 6 & -4.793664 & -0.506152 & -2.359547 \\
\hline 6 & -3.497299 & -0.237449 & -1.908467 \\
\hline
\end{tabular}




\begin{tabular}{|c|c|c|c|}
\hline 6 & -6.810877 & -0.005558 & 0.871812 \\
\hline 6 & -5.085071 & -0.841362 & -3.821505 \\
\hline & -1.613515 & 2.129100 & 0.686498 \\
\hline & -2.495628 & 3.066510 & 0.158531 \\
\hline & -2.497524 & 4.390259 & 0.605099 \\
\hline & -1.609607 & 4.741424 & 1.624037 \\
\hline & -0.708214 & 3.823988 & 2.177980 \\
\hline & -0.698645 & 2.525520 & 1.665261 \\
\hline 6 & -3.481242 & 5.375839 & -0.021523 \\
\hline 0 & 0.181782 & 4.166862 & 3.373661 \\
\hline 6 & 0.067933 & -0.438272 & -3.445510 \\
\hline 8 & 0.045184 & -0.982927 & -4.466510 \\
\hline 1 & 7.155878 & 0.621623 & 0.545936 \\
\hline 1 & -2.165891 & 0.899924 & 2.848142 \\
\hline 1 & 8.106149 & -4.137210 & -0.511607 \\
\hline 1 & -2.100925 & -2.791445 & 5.800399 \\
\hline 1 & 5.896863 & 5.358325 & -1.286651 \\
\hline 1 & -3.503815 & -2.732463 & 0.690130 \\
\hline 1 & -5.275177 & -3.866616 & -0.573795 \\
\hline 1 & 3.895216 & 4.613073 & -0.134735 \\
\hline 1 & -2.320056 & -0.573656 & 4.793848 \\
\hline 1 & 5.990658 & -4.329304 & -1.688993 \\
\hline 1 & 8.537780 & 2.503125 & -3.114161 \\
\hline 1 & 3.824285 & -3.327383 & -2.396384 \\
\hline 1 & -2.407149 & -5.193569 & -3.489989 \\
\hline 1 & -0.055361 & -4.574638 & -3.261443 \\
\hline 1 & -0.861076 & -6.129195 & 3.390838 \\
\hline 1 & 7.149586 & & -2.295786 \\
\hline 1 & -0.639002 & -4.665597 & 1.437102 \\
\hline 1 & 1.723220 & -3.344444 & -2.049418 \\
\hline 1 & 7.943078 & 4.866775 & -2.594016 \\
\hline 1 & 9.219206 & -0.425726 & 1.346415 \\
\hline 1 & -4.737396 & -5.132179 & -2.650140 \\
\hline 1 & -1.594843 & -5.208407 & 5.586044 \\
\hline 1 & 9.729273 & -2.803872 & 0.803224 \\
\hline 1 & 2.396209 & 2.753279 & 0.600542 \\
\hline 1 & -4.127596 & 0.524174 & 1.336133 \\
\hline 1 & -2.646480 & -0.322285 & -2.579867 \\
\hline 1 & -6.850428 & -0.617131 & -1.767685 \\
\hline 1 & -3.190791 & 2.758374 & -0.621716 \\
\hline 1 & 0.004584 & 1.790055 & 2.061991 \\
\hline 1 & -1.620623 & 5.759283 & 2.001592 \\
\hline 1 & -1.099421 & 1.407959 & -2.579940 \\
\hline 1 & 2.407574 & -3.577407 & 0.796984 \\
\hline 1 & 3.414367 & -2.137213 & 0.841540 \\
\hline 1 & 2.332583 & -4.361470 & 2.981954 \\
\hline 1 & 1.723097 & -0.171310 & 2.303299 \\
\hline 1 & 1.916026 & -4.037050 & 5.400374 \\
\hline 1 & 1.305713 & 0.162366 & 4.719347 \\
\hline 1 & 1.401344 & -1.770794 & 6.281056 \\
\hline 6 & -3.361980 & 6.777748 & 0.570427 \\
\hline 6 & -3.209985 & 5.469281 & -1.527619 \\
\hline 6 & -4.912975 & 4.878349 & 0.211973 \\
\hline 6 & 0.124072 & 5.648365 & 3.739346 \\
\hline 6 & -0.308598 & 3.356216 & 4.580728 \\
\hline 6 & 1.642962 & 3.809743 & 3.089729 \\
\hline
\end{tabular}




$\begin{array}{lrrr}6 & -3.808217 & -1.149101 & -4.602631 \\ 6 & -6.007944 & -2.058392 & -3.926818 \\ 6 & -5.769914 & 0.369303 & -4.466998 \\ 6 & -6.405196 & 0.496918 & 2.255998 \\ 6 & -7.908633 & 0.917928 & 0.334615 \\ 6 & -7.366645 & -1.426140 & 1.025330 \\ 1 & 2.267137 & 4.051763 & 3.960228 \\ 1 & 1.783272 & 2.741274 & 2.880043 \\ 1 & 2.027829 & 4.380578 & 2.233898 \\ 1 & 0.793096 & 5.844178 & 4.586782 \\ 1 & 0.447371 & 6.287778 & 2.907791 \\ 1 & -0.883379 & 5.960604 & 4.041973 \\ 1 & 0.296094 & 3.583892 & 5.469266 \\ 1 & -1.355457 & 3.593335 & 4.812855 \\ 1 & -0.244975 & 2.275075 & 4.398384 \\ 1 & -3.903180 & 6.180658 & -1.996673 \\ 1 & -2.186128 & 5.815173 & -1.719798 \\ 1 & -3.333888 & 4.503211 & -2.031930 \\ 1 & -5.635577 & 5.562788 & -0.252663 \\ 1 & -5.079115 & 3.880000 & -0.212635 \\ 1 & -5.138875 & 4.826015 & 1.285328 \\ 1 & -4.085219 & 7.444868 & 0.084585 \\ 1 & -3.576767 & 6.790484 & 1.646946 \\ 1 & -2.363428 & 7.205337 & 0.412951 \\ 1 & -4.064187 & -1.426594 & -5.633030 \\ 1 & -3.136080 & -0.282700 & -4.658605 \\ 1 & -3.251907 & -1.986267 & -4.157703 \\ 1 & -6.202968 & -2.291717 & -4.981928 \\ 1 & -5.544950 & -2.939891 & -3.464486 \\ 1 & -6.979890 & -1.893802 & -3.446539 \\ 1 & -5.985084 & 0.170172 & -5.525785 \\ 1 & -6.718244 & 0.611067 & -3.970321 \\ 1 & -5.127642 & 1.258175 & -4.413130 \\ 1 & -8.756289 & 0.945007 & 1.032329 \\ 1 & -7.537743 & 1.944137 & 0.215392 \\ 1 & -8.294726 & 0.587056 & -0.636708 \\ 1 & -8.219578 & -1.432984 & 1.717595 \\ 1 & -7.710204 & -1.838286 & 0.068235 \\ 1 & -6.599553 & -2.101731 & 1.427849 \\ 1 & -7.284099 & 0.517046 & 2.912668 \\ 1 & -5.657397 & -0.153683 & 2.729309 \\ 1 & -5.997400 & 1.515854 & 2.217272\end{array}$

$1_{\mathrm{C} 2}-\psi_{-1 \mathrm{~s} 2 \mathrm{~s} 3 \mathrm{a} 4 \mathrm{~s}}$

Number of imaginary frequencies : 0

The smallest frequencies are : $13.9017 \quad 16.193122 .5901 \mathrm{~cm}(-1)$

Electronic energy : $\quad H F=-4125.4704253$

Zero-point correction $=\quad 1.300244($ Hartree/Particle)

Thermal correction to Energy $=\quad 1.378123$

Thermal correction to Enthalpy= $\quad 1.379068$

Thermal correction to Gibbs Free Energy= $\quad 1.189808$

Sum of electronic and zero-point Energies $=\quad-4124.170181$

Sum of electronic and thermal Energies = $\quad-4124.092302$

Sum of electronic and thermal Enthalpies $=\quad-4124.091358$

Sum of electronic and thermal Free Energies $=\quad-4124.280617$ 
Cartesian Coordinates

\begin{tabular}{|c|c|c|c|}
\hline 6 & 1.609356 & -1.917668 & 5.206999 \\
\hline 6 & 1.914782 & -3.180694 & 4.712526 \\
\hline 6 & 2.145178 & -3.358347 & 3.351091 \\
\hline 6 & 2.077331 & -2.281520 & 2.468011 \\
\hline 6 & 1.771161 & -1.015653 & 2.973818 \\
\hline 6 & 1.538455 & -0.835995 & 4.331983 \\
\hline 6 & 2.409074 & -2.491492 & 1.011376 \\
\hline 7 & 1.489563 & -1.835016 & 0.060523 \\
\hline 6 & 0.427731 & -2.620497 & -0.509343 \\
\hline 6 & -0.845296 & -2.633943 & 0.031787 \\
\hline 6 & -1.889830 & -3.331590 & -0.656111 \\
\hline 6 & -1.599787 & -4.020389 & -1.870409 \\
\hline 6 & -0.274944 & -4.018828 & -2.363045 \\
\hline 6 & 0.706257 & -3.334253 & -1.700223 \\
\hline 6 & -3.227160 & -3.343802 & -0.179884 \\
\hline 6 & -4.214340 & -4.004329 & -0.866927 \\
\hline 6 & -3.918384 & -4.699761 & -2.059503 \\
\hline 6 & -2.638240 & -4.701708 & -2.551517 \\
\hline 6 & -1.165437 & -2.012342 & 1.345543 \\
\hline 6 & -1.500991 & -0.672732 & 1.471914 \\
\hline 6 & -1.924515 & -0.182263 & 2.737293 \\
\hline 6 & -2.004253 & -1.001789 & 3.830724 \\
\hline 6 & -1.670501 & -2.371746 & 3.734049 \\
\hline 6 & -1.243058 & -2.887279 & 2.477306 \\
\hline 6 & -1.762303 & -3.236846 & 4.850343 \\
\hline 6 & -1.460799 & -4.569969 & 4.733293 \\
\hline 6 & -1.036833 & -5.087148 & 3.491414 \\
\hline 6 & -0.925196 & -4.267925 & 2.395372 \\
\hline 15 & -1.537291 & 0.415058 & -0.034730 \\
\hline 45 & 0.115858 & 0.658055 & -1.867232 \\
\hline 6 & 0.653593 & 2.508112 & -1.678256 \\
\hline 8 & 0.770258 & 3.657920 & -1.683205 \\
\hline 15 & 1.882133 & -0.470839 & -0.850687 \\
\hline 8 & 2.840443 & 0.254202 & 0.284665 \\
\hline 6 & 3.694757 & 1.248974 & -0.153826 \\
\hline 6 & 4.877511 & 0.906842 & -0.779447 \\
\hline 6 & 5.705890 & 1.968765 & -1.268344 \\
\hline 6 & 5.334517 & 3.323316 & -1.007068 \\
\hline 6 & 4.136675 & 3.598744 & -0.305311 \\
\hline 6 & 3.317520 & 2.579455 & 0.097434 \\
\hline 6 & 6.158377 & 4.374487 & -1.478695 \\
\hline 6 & 7.291484 & 4.112146 & -2.204108 \\
\hline 6 & 7.640454 & 2.777221 & -2.497578 \\
\hline 6 & 6.870575 & 1.735561 & -2.043360 \\
\hline 6 & 5.209562 & -0.524942 & -0.964129 \\
\hline 6 & 4.290748 & -1.362078 & -1.574234 \\
\hline 6 & 4.579696 & -2.711446 & -1.863821 \\
\hline 6 & 5.776148 & -3.250044 & -1.477183 \\
\hline 6 & 6.716412 & -2.473505 & -0.759024 \\
\hline 6 & 6.431907 & -1.101300 & -0.486169 \\
\hline 6 & 7.366075 & -0.372063 & 0.293556 \\
\hline 6 & 8.524142 & -0.956065 & 0.743160 \\
\hline 6 & 8.818428 & -2.301559 & 0.438524 \\
\hline
\end{tabular}




\begin{tabular}{|c|c|c|c|}
\hline 6 & 7.927025 & -3.042133 & -0.293848 \\
\hline 8 & 3.058154 & -0.885860 & -1.964478 \\
\hline & -3.261801 & 0.100614 & -0.606107 \\
\hline & -4.335456 & 0.205290 & 0.273935 \\
\hline & -5.644287 & -0.044237 & -0.147797 \\
\hline & -5.840711 & -0.392950 & -1.484988 \\
\hline & -4.783413 & -0.514403 & -2.395834 \\
\hline & -3.491668 & -0.268504 & -1.931448 \\
\hline & -6.780031 & 0.054926 & 0.869108 \\
\hline & -5.064553 & -0.865891 & -3.855696 \\
\hline & -1.639954 & 2.099581 & 0.688694 \\
\hline O & -2.532253 & 3.030939 & 0.166576 \\
\hline & -2.544743 & 4.353133 & 0.618095 \\
\hline ס & -1.660711 & 4.707187 & 1.639385 \\
\hline & -0.751475 & 3.795277 & 2.189470 \\
\hline & -0.728567 & 2.500023 & 1.669070 \\
\hline 0 & -3.526654 & 5.337178 & -0.013983 \\
\hline & 0.133562 & 4.139637 & 3.388359 \\
\hline ס & 0.075167 & -0.426852 & -3.454070 \\
\hline & 0.064648 & -0.966459 & -4.477945 \\
\hline & 7.150439 & 0.662477 & 0.547552 \\
\hline & -2.198982 & 0.864742 & 2.841951 \\
\hline & 8.127734 & -4.087663 & -0.524135 \\
\hline & -2.085029 & -2.819376 & 5.803402 \\
\hline & 5.860658 & 5.399356 & -1.261462 \\
\hline & -3.471710 & -2.801993 & 0.732358 \\
\hline 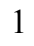 & -5.236743 & -3.985537 & -0.494541 \\
\hline & 3.861322 & 4.635613 & -0.117874 \\
\hline & -2.337610 & -0.607216 & 4.790181 \\
\hline 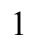 & 6.013207 & -4.288876 & -1.700718 \\
\hline & 8.524650 & 2.570510 & -3.096330 \\
\hline 1 & 3.841254 & -3.297581 & -2.405616 \\
\hline & -2.395373 & -5.227570 & -3.473794 \\
\hline J & -0.052760 & -4.554564 & -3.284524 \\
\hline 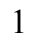 & -0.792340 & -6.143432 & 3.402624 \\
\hline & 7.146866 & 0.713787 & -2.290115 \\
\hline 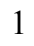 & -0.585988 & -4.679955 & 1.446834 \\
\hline & 1.721860 & -3.304738 & -2.089124 \\
\hline 1 & 7.913113 & 4.927647 & -2.566241 \\
\hline ] & 9.219106 & -0.375772 & 1.345876 \\
\hline 1 & -4.709058 & -5.229305 & -2.587087 \\
\hline 1 & -1.543434 & -5.229035 & 5.594649 \\
\hline l & 9.742899 & -2.749278 & 0.795463 \\
\hline 1 & 2.373779 & 2.762211 & 0.606624 \\
\hline 1 & -4.151390 & 0.484490 & 1.311084 \\
\hline 1 & -2.634257 & -0.363591 & -2.592818 \\
\hline 1 & -6.852865 & -0.579316 & -1.838160 \\
\hline 1 & -3.224392 & 2.720602 & -0.615516 \\
\hline 1 & -0.017499 & 1.769563 & 2.061132 \\
\hline 1 & -1.681091 & 5.722995 & 2.022005 \\
\hline 1 & -1.113333 & 1.402760 & -2.579196 \\
\hline 1 & 2.425923 & -3.566928 & 0.780459 \\
\hline 1 & 3.425846 & -2.122321 & 0.824556 \\
\hline 1 & 2.373211 & -4.352190 & 2.964762 \\
\hline 1 & 1.716306 & -0.167880 & 2.293332 \\
\hline 1 & 1.959130 & -4.035657 & 5.384426 \\
\hline
\end{tabular}




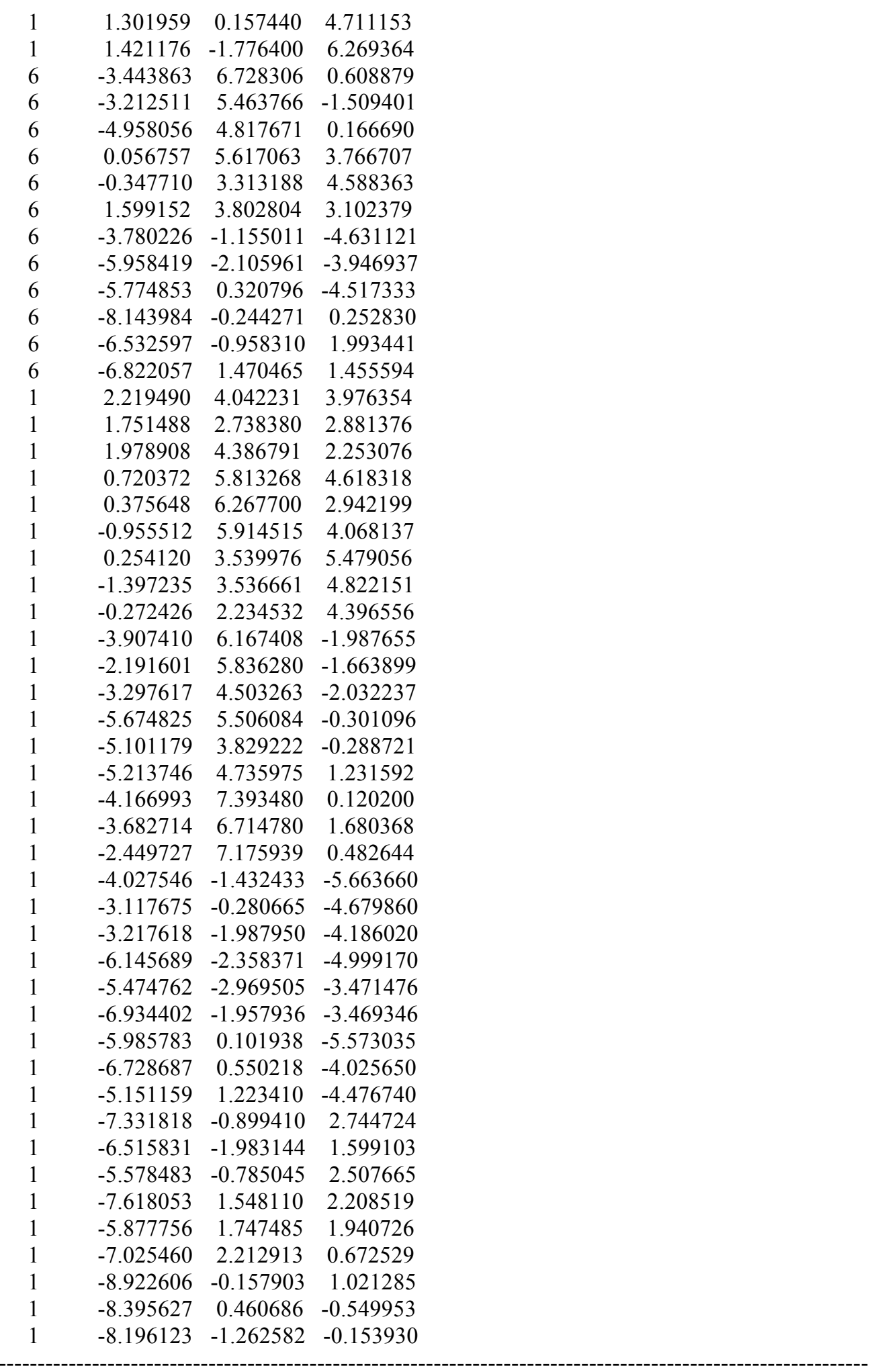

$1_{\text {C2- }} \psi_{-1 \text {-1s2s3s4a }}$

Number of imaginary frequencies : 0

The smallest frequencies are : $12.3864 \quad 16.7200 \quad 22.7292 \mathrm{~cm}(-1)$

Electronic energy : $\quad H F=-4125.4696361$ 
Zero-point correction $=$

Thermal correction to Energy=

Thermal correction to Enthalpy=

Thermal correction to Gibbs Free Energy=

Sum of electronic and zero-point Energies=

Sum of electronic and thermal Energies $=$

Sum of electronic and thermal Enthalpies=

Sum of electronic and thermal Free Energies=
1.300402 (Hartree/Particle)

1.378240

1.379184

1.190171

$-4124.169235$

$-4124.091396$

$-4124.090452$

$-4124.279465$

Cartesian Coordinates

$\begin{array}{cccc}6 & 1.610458 & -1.955830 & 5.201618 \\ 6 & 1.926635 & -3.211068 & 4.694431 \\ 6 & 2.167491 & -3.371091 & 3.332523 \\ 6 & 2.099133 & -2.284153 & 2.462250 \\ 6 & 1.781172 & -1.026157 & 2.980556 \\ 6 & 1.538585 & -0.863982 & 4.339125 \\ 6 & 2.439798 & -2.472638 & 1.005148 \\ 7 & 1.503547 & -1.828210 & 0.061776 \\ 6 & 0.456334 & -2.636694 & -0.503522 \\ 6 & -0.824374 & -2.649345 & 0.019559 \\ 6 & -1.848247 & -3.384865 & -0.661142 \\ 6 & -1.523392 & -4.138818 & -1.827097 \\ 6 & -0.191689 & -4.131223 & -2.301752 \\ 6 & 0.763877 & -3.390804 & -1.661938 \\ 6 & -3.195834 & -3.387455 & -0.214605 \\ 6 & -4.156504 & -4.113118 & -0.873487 \\ 6 & -3.821257 & -4.888644 & -2.004504 \\ 6 & -2.533159 & -4.892220 & -2.474430 \\ 6 & -1.160941 & -2.026463 & 1.329523 \\ 6 & -1.499216 & -0.688775 & 1.464549 \\ 6 & -1.925725 & -0.205685 & 2.732115 \\ 6 & -2.005208 & -1.030556 & 3.820906 \\ 6 & -1.667031 & -2.398901 & 3.717245 \\ 6 & -1.236178 & -2.907142 & 2.458515 \\ 6 & -1.756610 & -3.268605 & 4.830128 \\ 6 & -1.447258 & -4.599521 & 4.709272 \\ 6 & -1.016530 & -5.109063 & 3.466758 \\ 6 & -0.908144 & -4.285465 & 2.373629 \\ 15 & -1.537636 & 0.409458 & -0.032073 \\ 45 & 0.100476 & 0.654618 & -1.865344 \\ 6 & 0.634984 & 2.507076 & -1.696322 \\ 8 & 0.736375 & 3.658465 & -1.700991 \\ 15 & 1.876853 & -0.460693 & -0.852874 \\ 8 & 2.827984 & 0.276256 & 0.281368 \\ 6 & 3.673715 & 1.278084 & -0.157506 \\ 6 & 4.859605 & 0.946263 & -0.782841 \\ 6 & 5.679479 & 2.015342 & -1.270733 \\ 6 & 5.296787 & 3.366599 & -1.008897 \\ 6 & 4.096347 & 3.631614 & -0.307679 \\ 6 & 3.285542 & 2.605374 & 0.094218 \\ 6 & 6.112097 & 4.424948 & -1.479303 \\ 6 & 7.247843 & 4.172595 & -2.204097 \\ 6 & 7.608096 & 2.840824 & -2.498157 \\ 6 & 6.846632 & 1.792460 & -2.045171 \\ 6 & 5.204149 & -0.482167 & -0.970588\end{array}$




\begin{tabular}{|c|c|c|c|}
\hline ) & 4.293503 & -1.325326 & -1.584645 \\
\hline & 4.597611 & -2.669123 & -1.884873 \\
\hline 6 & 5.799186 & -3.197865 & -1.500473 \\
\hline & 6.730124 & -2.416750 & -0.775139 \\
\hline & 6.431884 & -1.048971 & -0.494993 \\
\hline & 7.358233 & -0.314680 & 0.289296 \\
\hline & 8.521370 & -0.889868 & 0.737236 \\
\hline & 8.828871 & -2.230897 & 0.426054 \\
\hline & 7.945617 & -2.976127 & -0.311467 \\
\hline 8 & 3.055316 & -0.860205 & -1.969784 \\
\hline & -3.263692 & 0.106858 & -0.600689 \\
\hline & -4.343236 & 0.215647 & 0.286320 \\
\hline U & -5.645265 & -0.013930 & -0.141643 \\
\hline & -5.845629 & -0.345726 & -1.493260 \\
\hline 6 & -4.797357 & -0.451617 & -2.401488 \\
\hline U & -3.497163 & -0.235414 & -1.923191 \\
\hline 6 & -6.840871 & 0.066067 & 0.806050 \\
\hline 6 & -4.993421 & -0.765197 & -3.882643 \\
\hline 6 & -1.636368 & 2.087859 & 0.704429 \\
\hline 6 & -2.533976 & 3.019983 & 0.193969 \\
\hline 6 & -2.556526 & 4.335325 & 0.663510 \\
\hline 6 & -1.670050 & 4.684391 & 1.684274 \\
\hline 6 & -0.751117 & 3.772764 & 2.218952 \\
\hline 6 & -0.723798 & 2.482666 & 1.685989 \\
\hline 6 & -3.559374 & 5.313431 & 0.056292 \\
\hline 6 & 0.136674 & 4.110444 & 3.417751 \\
\hline 6 & -0.000266 & -0.464005 & -3.427148 \\
\hline 8 & -0.092203 & -1.026094 & -4.434882 \\
\hline 1 & 7.132250 & 0.716425 & 0.548302 \\
\hline 1 & -2.202847 & 0.840099 & 2.841653 \\
\hline 1 & 8.156759 & -4.018390 & -0.547198 \\
\hline 1 & -2.083225 & -2.856008 & 5.783996 \\
\hline 1 & 5.805685 & 5.447150 & -1.261619 \\
\hline 1 & -3.468724 & -2.793356 & 0.655889 \\
\hline 1 & -5.185609 & -4.090435 & -0.521066 \\
\hline 1 & 3.812493 & 4.666068 & -0.119583 \\
\hline 1 & -2.341128 & -0.641924 & 4.781882 \\
\hline 1 & 6.047933 & -4.232197 & -1.732011 \\
\hline 1 & 8.494402 & 2.641835 & -3.096411 \\
\hline 1 & 3.867495 & -3.257940 & -2.435192 \\
\hline 1 & -2.262317 & -5.470759 & -3.356513 \\
\hline 1 & 0.056249 & -4.709930 & -3.190050 \\
\hline 1 & -0.763834 & -6.163137 & 3.374758 \\
\hline 1 & 7.131805 & 0.773271 & -2.292462 \\
\hline 1 & -0.562502 & -4.693126 & 1.425527 \\
\hline 1 & 1.785381 & -3.360112 & -2.034756 \\
\hline 1 & 7.862878 & 4.993496 & -2.565287 \\
\hline 1 & 9.210074 & -0.306045 & 1.343718 \\
\hline 1 & -4.590091 & -5.470133 & -2.508870 \\
\hline 1 & -1.527855 & -5.262143 & 5.568096 \\
\hline 1 & 9.757179 & -2.671548 & 0.781833 \\
\hline 1 & 2.340393 & 2.780320 & 0.603458 \\
\hline 1 & -4.150387 & 0.490912 & 1.320173 \\
\hline 1 & -2.645490 & -0.327929 & -2.595019 \\
\hline 1 & -6.863344 & -0.519260 & -1.837729 \\
\hline 1 & -3.226682 & 2.714251 & -0.589293 \\
\hline
\end{tabular}




\begin{tabular}{|c|c|c|c|}
\hline 1 & -0.009511 & 1.750875 & 2.069799 \\
\hline 1 & -1.697090 & 5.695136 & 2.079717 \\
\hline & -1.130247 & 1.400081 & -2.573855 \\
\hline 1 & 2.482762 & -3.545145 & 0.764472 \\
\hline 1 & 3.447076 & -2.076406 & 0.823307 \\
\hline 1 & 2.404593 & -4.358663 & 2.935755 \\
\hline 1 & 1.724501 & -0.171332 & 2.308759 \\
\hline 1 & 1.971345 & -4.073652 & 5.356491 \\
\hline 1 & 1.293446 & 0.123428 & 4.728445 \\
\hline 1 & 1.414463 & -1.828288 & 6.264323 \\
\hline 6 & -3.483869 & 6.698976 & 0.692147 \\
\hline 6 & -3.272264 & 5.458458 & -1.442818 \\
\hline 6 & -4.980657 & 4.772192 & 0.256380 \\
\hline 6 & 0.061961 & 5.586131 & 3.803433 \\
\hline 6 & -0.343295 & 3.277962 & 4.614041 \\
\hline 6 & 1.601755 & 3.774894 & 3.127867 \\
\hline 6 & -6.466420 & -0.907219 & -4.256201 \\
\hline 6 & -4.393846 & 0.370661 & -4.721347 \\
\hline 6 & -4.282390 & -2.077687 & -4.224875 \\
\hline 6 & -6.439626 & 0.542064 & 2.200997 \\
\hline 6 & -7.884959 & 1.043904 & 0.256987 \\
\hline 6 & -7.464749 & -1.327947 & 0.936168 \\
\hline 1 & 2.223210 & 4.010040 & 4.002167 \\
\hline 1 & 1.754949 & 2.711850 & 2.900417 \\
\hline 1 & 1.979920 & 4.363986 & 2.281484 \\
\hline 1 & 0.730843 & 5.778613 & 4.651749 \\
\hline 1 & 0.375239 & 6.240405 & 2.979640 \\
\hline 1 & -0.948266 & 5.882201 & 4.112879 \\
\hline 1 & 0.261297 & 3.497968 & 5.504581 \\
\hline 1 & -1.391775 & 3.502125 & 4.851753 \\
\hline 1 & -0.270849 & 2.200361 & 4.415171 \\
\hline 1 & -3.980409 & 6.161896 & -1.901471 \\
\hline 1 & -2.256611 & 5.839505 & -1.610979 \\
\hline 1 & -3.359511 & 4.503271 & -1.974981 \\
\hline 1 & -5.716102 & 5.459606 & -0.182961 \\
\hline 1 & -5.120523 & 3.791215 & -0.215795 \\
\hline 1 & -5.213349 & 4.665414 & 1.324448 \\
\hline 1 & -4.220756 & 7.361070 & 0.220169 \\
\hline 1 & -3.707927 & 6.671924 & 1.766559 \\
\hline 1 & -2.496371 & 7.158627 & 0.557246 \\
\hline 1 & -6.555238 & -1.123677 & -5.328248 \\
\hline 1 & -6.949813 & -1.731091 & -3.715010 \\
\hline 1 & -7.031050 & 0.013376 & -4.058588 \\
\hline 1 & -4.542239 & 0.170980 & -5.791047 \\
\hline 1 & -4.872335 & 1.330359 & -4.485888 \\
\hline 1 & -3.314806 & 0.484655 & -4.555076 \\
\hline 1 & -4.387840 & -2.300147 & -5.295487 \\
\hline 1 & -3.209352 & -2.039298 & -3.996753 \\
\hline 1 & -4.711058 & -2.913298 & -3.657159 \\
\hline 1 & -8.740292 & 1.108969 & 0.942751 \\
\hline 1 & -7.462888 & 2.051689 & 0.148685 \\
\hline 1 & -8.272695 & 0.736636 & -0.721333 \\
\hline 1 & -8.331969 & -1.299884 & 1.609843 \\
\hline 1 & -7.806149 & -1.716631 & -0.031290 \\
\hline 1 & -6.739469 & -2.042452 & 1.348037 \\
\hline 1 & -7.328437 & 0.592203 & 2.842561 \\
\hline
\end{tabular}


$1_{\mathrm{C} 2-} \psi_{-1 \mathrm{~s} 22334 \mathrm{~s}}$

Number of imaginary frequencies : 0

The smallest frequencies are : $13.9510 \quad 16.5666 \quad 20.8132 \mathrm{~cm}(-1)$

Electronic energy : $\quad \mathrm{HF}=-4125.4698236$

Zero-point correction $=\quad 1.300002($ Hartree/Particle $)$

Thermal correction to Energy= $\quad 1.377962$

Thermal correction to Enthalpy= $\quad 1.378906$

Thermal correction to Gibbs Free Energy= 1.189279

Sum of electronic and zero-point Energies $=\quad-4124.169821$

Sum of electronic and thermal Energies $=\quad-4124.091862$

Sum of electronic and thermal Enthalpies $=\quad-4124.090918$

Sum of electronic and thermal Free Energies $=\quad-4124.280545$

\begin{tabular}{cccc} 
& \multicolumn{3}{c}{ Cartesian Coordinates } \\
6 & 1.611574 & -2.053140 & 5.179791 \\
6 & 1.940016 & -3.296778 & 4.651893 \\
6 & 2.179732 & -3.432380 & 3.287195 \\
6 & 2.097164 & -2.332486 & 2.434538 \\
6 & 1.764661 & -1.087170 & 2.973084 \\
6 & 1.523974 & -0.949015 & 4.334690 \\
6 & 2.436947 & -2.495537 & 0.974107 \\
7 & 1.507570 & -1.824387 & 0.043089 \\
6 & 0.456595 & -2.613597 & -0.541746 \\
6 & -0.822351 & -2.639230 & -0.014517 \\
6 & -1.848437 & -3.359384 & -0.708412 \\
6 & -1.527766 & -4.081460 & -1.895642 \\
6 & -0.198883 & -4.057967 & -2.377423 \\
6 & 0.759429 & -3.335284 & -1.721842 \\
6 & -3.193644 & -3.378410 & -0.255000 \\
6 & -4.155090 & -4.089389 & -0.928747 \\
6 & -3.823899 & -4.833127 & -2.081896 \\
6 & -2.538237 & -4.820476 & -2.558008 \\
6 & -1.155775 & -2.040625 & 1.307530 \\
6 & -1.490467 & -0.704153 & 1.465509 \\
6 & -1.913961 & -0.241500 & 2.741226 \\
6 & -1.998352 & -1.085941 & 3.815001 \\
6 & -1.665193 & -2.453369 & 3.687513 \\
6 & -1.233679 & -2.940399 & 2.420561 \\
6 & -1.756793 & -3.342165 & 4.785233 \\
6 & -1.448353 & -4.670865 & 4.641669 \\
6 & -1.017248 & -5.159452 & 3.390775 \\
6 & -0.907399 & -4.317546 & 2.312033 \\
15 & -1.533320 & 0.412694 & -0.016760 \\
45 & 0.122625 & 0.710821 & -1.825483 \\
6 & 0.658651 & 2.557006 & -1.595805 \\
8 & 0.763441 & 3.707623 & -1.566378 \\
15 & 1.891798 & -0.438768 & -0.838565 \\
8 & 2.849498 & 0.262051 & 0.312574 \\
6 & 3.696601 & 1.273185 & -0.101462 \\
6 & 4.877977 & 0.955416 & -0.742292 \\
6 & 5.697653 & 2.035353 & -1.205909
\end{tabular}




\begin{tabular}{|c|c|c|c|}
\hline & 5.320316 & 3.379877 & -0.904723 \\
\hline & 4.125649 & 3.628727 & -0.187836 \\
\hline & 3.314580 & 2.593934 & 0.190644 \\
\hline & 6.135430 & 4.448613 & -1.351349 \\
\hline & 7.265772 & 4.213234 & -2.090155 \\
\hline & 7.620614 & 2.889096 & -2.422697 \\
\hline & 6.859327 & 1.830642 & -1.993475 \\
\hline & 5.219349 & -0.468675 & -0.965109 \\
\hline & 4.304420 & -1.297312 & -1.592418 \\
\hline & 4.603971 & -2.635964 & -1.919230 \\
\hline & 5.806089 & -3.174667 & -1.550651 \\
\hline & 6.742314 & -2.409753 & -0.814923 \\
\hline & 6.448057 & -1.047223 & -0.506372 \\
\hline & 7.379387 & -0.330561 & 0.288255 \\
\hline & 8.543345 & -0.916710 & 0.719434 \\
\hline & 8.846774 & -2.251870 & 0.380278 \\
\hline & 7.958707 & -2.980580 & -0.367919 \\
\hline & 3.066515 & -0.821240 & -1.965285 \\
\hline & -3.245299 & 0.074240 & -0.608715 \\
\hline & -4.325794 & 0.111856 & 0.273901 \\
\hline & -5.626409 & -0.129242 & -0.165415 \\
\hline & -5.821305 & -0.388706 & -1.528257 \\
\hline 0 & -4.762545 & -0.430652 & -2.437913 \\
\hline & -3.470660 & -0.212114 & -1.949532 \\
\hline 6 & -6.767115 & -0.097032 & 0.850829 \\
\hline 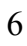 & -4.950568 & -0.690440 & -3.931016 \\
\hline ( & -1.690509 & 2.084003 & 0.728338 \\
\hline 6 & -2.608865 & 2.990498 & 0.207354 \\
\hline & -2.663212 & 4.309500 & 0.663575 \\
\hline 6 & -1.794834 & 4.685765 & 1.690230 \\
\hline 6 & -0.860711 & 3.799409 & 2.240029 \\
\hline 6 & -0.796119 & 2.507046 & 1.715025 \\
\hline 6 & -3.666675 & 5.266608 & 0.024385 \\
\hline & 0.022969 & 4.173585 & 3.430786 \\
\hline 6 & 0.035064 & -0.358369 & -3.421158 \\
\hline & -0.044824 & -0.891607 & -4.445471 \\
\hline 1 & 7.156813 & 0.695548 & 0.568918 \\
\hline 1 & -2.189091 & 0.802823 & 2.868080 \\
\hline & 8.166690 & -4.018370 & -0.625080 \\
\hline 1 & -2.083469 & -2.945741 & 5.745910 \\
\hline & 5.833335 & 5.465280 & -1.103742 \\
\hline 1 & -3.464389 & -2.807568 & 0.632278 \\
\hline & -5.182277 & -4.081516 & -0.570664 \\
\hline 1 & 3.846589 & 4.658366 & 0.031177 \\
\hline | & -2.333667 & -0.713404 & 4.782523 \\
\hline & 6.051230 & -4.204997 & -1.802950 \\
\hline 1 & 8.502590 & 2.704333 & -3.031808 \\
\hline & 3.869771 & -3.213068 & -2.476430 \\
\hline 1 & -2.269960 & -5.374407 & -3.456540 \\
\hline l & 0.044992 & -4.611546 & -3.282702 \\
\hline 1 & -0.765304 & -6.212001 & 3.280996 \\
\hline 1 & 7.140147 & 0.817704 & -2.269928 \\
\hline 1 & -0.561575 & -4.708978 & 1.357179 \\
\hline 1 & 1.778887 & -3.292942 & -2.099151 \\
\hline 1 & 7.880686 & 5.042147 & -2.432763 \\
\hline 1 & 9.235874 & -0.346367 & 1.334332 \\
\hline
\end{tabular}




\begin{tabular}{|c|c|c|c|}
\hline . & -4.593774 & -5.403360 & -2.597397 \\
\hline & -1.529890 & -5.348107 & 5.488917 \\
\hline 1 & 9.775753 & -2.701318 & 0.723056 \\
\hline & 2.373557 & 2.757130 & 0.711121 \\
\hline & -4.147389 & 0.336726 & 1.325143 \\
\hline & -2.615531 & -0.258385 & -2.621777 \\
\hline & -6.830878 & -0.563300 & -1.889033 \\
\hline & -3.287651 & 2.663884 & -0.579627 \\
\hline & -0.062692 & 1.797702 & 2.105700 \\
\hline 1 & -1.846501 & 5.699279 & 2.075729 \\
\hline 1 & -1.102815 & 1.477810 & -2.521008 \\
\hline 1 & 2.470278 & -3.563492 & 0.712549 \\
\hline 1 & 3.448206 & -2.105604 & 0.800368 \\
\hline 1 & 2.426190 & -4.410949 & 2.874084 \\
\hline 1 & 1.691596 & -0.223712 & 2.314467 \\
\hline 1 & 1.994920 & -4.169339 & 5.299949 \\
\hline 1 & 1.262743 & 0.028400 & 4.738390 \\
\hline 1 & 1.416168 & -1.944867 & 6.244755 \\
\hline 6 & -3.631082 & 6.656957 & 0.653136 \\
\hline 6 & -3.337920 & 5.406567 & -1.466906 \\
\hline 6 & -5.084688 & 4.705657 & 0.185668 \\
\hline 6 & -0.122359 & 5.640860 & 3.828025 \\
\hline 6 & -0.390071 & 3.310686 & 4.630182 \\
\hline 6 & 1.497558 & 3.916425 & 3.110352 \\
\hline 6 & -6.420658 & -0.830836 & -4.317150 \\
\hline 6 & -4.356644 & 0.477829 & -4.728069 \\
\hline 6 & -4.227557 & -1.985234 & -4.314371 \\
\hline 6 & -8.118298 & -0.430384 & 0.223782 \\
\hline 6 & -6.489770 & -1.124708 & 1.954947 \\
\hline 6 & -6.858158 & 1.303493 & 1.467387 \\
\hline 1 & 2.127513 & 4.204416 & 3.962484 \\
\hline 1 & 1.701176 & 2.858171 & 2.902696 \\
\hline 1 & 1.818374 & 4.505591 & 2.240436 \\
\hline 1 & 0.536713 & 5.858222 & 4.677962 \\
\hline 1 & 0.159687 & 6.316380 & 3.010148 \\
\hline 1 & -1.145928 & 5.885212 & 4.138954 \\
\hline 1 & 0.216336 & 3.561248 & 5.511291 \\
\hline 1 & -1.445070 & 3.475166 & 4.886832 \\
\hline 1 & -0.258998 & 2.239444 & 4.427264 \\
\hline 1 & -4.041415 & 6.098009 & -1.950382 \\
\hline 1 & -2.322748 & 5.799689 & -1.608051 \\
\hline 1 & -3.397565 & 4.446743 & -1.994754 \\
\hline 1 & -5.814922 & 5.375353 & -0.288423 \\
\hline 1 & -5.194528 & 3.715488 & -0.275424 \\
\hline 1 & -5.351089 & 4.612236 & 1.246963 \\
\hline 1 & -4.368992 & 7.302586 & 0.160403 \\
\hline 1 & -3.879273 & 6.631499 & 1.722272 \\
\hline 1 & -2.649525 & 7.134334 & 0.537996 \\
\hline 1 & -6.502162 & -1.005649 & -5.397366 \\
\hline 1 & -6.899663 & -1.679597 & -3.811817 \\
\hline 1 & -6.994665 & 0.076155 & -4.086353 \\
\hline 1 & -4.499196 & 0.313572 & -5.804581 \\
\hline 1 & -4.843865 & 1.425233 & -4.462401 \\
\hline 1 & -3.279226 & 0.594944 & -4.553814 \\
\hline 1 & -4.332090 & -2.176435 & -5.391152 \\
\hline 1 & -3.154893 & -1.941916 & -4.085759 \\
\hline
\end{tabular}




$\begin{array}{rrrr}1 & -4.646098 & -2.842254 & -3.771055 \\ 1 & -7.298127 & -1.113912 & 2.698531 \\ 1 & -6.428037 & -2.139196 & 1.539292 \\ 1 & -5.549407 & -0.926422 & 2.484897 \\ 1 & -7.654770 & 1.338311 & 2.222962 \\ 1 & -5.921471 & 1.600946 & 1.955490 \\ 1 & -7.087817 & 2.054981 & 0.700229 \\ 1 & -8.898844 & -0.404565 & 0.994655 \\ 1 & -8.401496 & 0.291780 & -0.552662 \\ 1 & -8.127326 & -1.434407 & -0.220257\end{array}$

$1_{\mathrm{C} 3-} \psi_{-1 \mathrm{a} 2 \mathrm{za} a 4 \mathrm{a}}$

Number of imaginary frequencies : 0

The smallest frequencies are : $13.0067 \quad 16.4588 \quad 19.3304 \mathrm{~cm}(-1)$

Electronic energy : $\quad H F=-4125.4676425$

Zero-point correction $=\quad 1.299576($ Hartree/Particle $)$

Thermal correction to Energy= $\quad 1.377979$

Thermal correction to Enthalpy= $\quad 1.378923$

Thermal correction to Gibbs Free Energy= $\quad 1.186923$

Sum of electronic and zero-point Energies $=\quad-4124.168067$

Sum of electronic and thermal Energies $=\quad-4124.089664$

Sum of electronic and thermal Enthalpies $=\quad-4124.088720$

Sum of electronic and thermal Free Energies $=\quad-4124.280720$

Cartesian Coordinates

\begin{tabular}{|c|c|c|c|}
\hline 6 & 3.176996 & -6.163289 & 1.746399 \\
\hline 6 & 2.998771 & -5.276082 & 2.804073 \\
\hline 6 & 2.667337 & -3.950971 & 2.551056 \\
\hline 6 & 2.526716 & -3.481783 & 1.242811 \\
\hline 6 & 2.729880 & -4.372551 & 0.188997 \\
\hline 6 & 3.042738 & -5.705750 & 0.439522 \\
\hline 6 & 2.219486 & -2.019426 & 1.058748 \\
\hline 7 & 1.553105 & -1.646729 & -0.201120 \\
\hline 6 & 0.437632 & -2.384629 & -0.717102 \\
\hline 6 & -0.756876 & -2.524954 & -0.029072 \\
\hline 6 & -1.847627 & -3.213945 & -0.64919 \\
\hline 6 & -1.702403 & -3.717783 & -1.97516 \\
\hline 6 & -0.473239 & -3.537376 & -2.64857 \\
\hline 6 & & & \\
\hline 6 & -3.099401 & -3.383873 & -0.00171 \\
\hline 6 & -4.137276 & -4.027580 & -0.62818 \\
\hline 6 & -3.979878 & -4.545642 & -1.93190 \\
\hline 6 & -2.788573 & -4.385007 & -2.59223 \\
\hline 6 & -0.926906 & -1.971960 & 1.343026 \\
\hline 6 & -1.205095 & -0.626146 & 1.554407 \\
\hline 6 & -1.371166 & -0.156569 & 2.885053 \\
\hline 6 & & & \\
\hline 6 & -1.013007 & -2.378120 & 3.776357 \\
\hline 6 & -0.845287 & -2.875858 & 2.451245 \\
\hline 6 & -0.935357 & -3.270115 & 4.873307 \\
\hline 6 & -0.717471 & -4.609783 & 4.673603 \\
\hline 6 & -0.558332 & -5.109534 & 3.363845 \\
\hline 6 & & -4.265717 & \\
\hline
\end{tabular}




\begin{tabular}{|c|c|c|c|}
\hline 15 & -1.517273 & 0.484749 & 0.091880 \\
\hline & -0.069286 & 0.907849 & -1.882192 \\
\hline 6 & 0.412078 & 2.773404 & -1.681456 \\
\hline 8 & 0.491249 & 3.926449 & -1.689329 \\
\hline & 1.792211 & -0.149263 & -0.963454 \\
\hline & 2.583027 & 0.601153 & 0.282344 \\
\hline 6 & 3.424572 & 1.654058 & -0.025356 \\
\hline 6 & 4.677237 & 1.399689 & -0.551491 \\
\hline 6 & 5.499913 & 2.519597 & -0.898316 \\
\hline 6 & 5.045605 & 3.840727 & -0.601905 \\
\hline 6 & 3.774104 & 4.026166 & -0.009758 \\
\hline 6 & 2.965993 & 2.953818 & 0.255106 \\
\hline 6 & 5.862430 & 4.949332 & -0.933004 \\
\hline 6 & 7.070182 & 4.774239 & -1.557258 \\
\hline 6 & 7.505329 & 3.473133 & -1.886133 \\
\hline 6 & 6.742895 & 2.377404 & -1.566498 \\
\hline 6 & 5.085580 & -0.004418 & -0.791516 \\
\hline 6 & 4.262464 & -0.821040 & -1.546579 \\
\hline 6 & 4.604614 & -2.146534 & -1.873911 \\
\hline 6 & 5.765824 & -2.685468 & -1.390862 \\
\hline 6 & 6.611919 & -1.930310 & -0.544672 \\
\hline 6 & 6.271415 & -0.579494 & -0.229923 \\
\hline 6 & 7.109996 & 0.124916 & 0.671865 \\
\hline 6 & 8.233594 & -0.460673 & 1.199913 \\
\hline 6 & 8.584965 & -1.783602 & 0.858625 \\
\hline 6 & 7.784627 & -2.500839 & 0.007743 \\
\hline 8 & 3.059882 & -0.340635 & -2.023440 \\
\hline 6 & -3.249966 & -0.001478 & -0.296637 \\
\hline 6 & -4.196441 & -0.128981 & 0.723518 \\
\hline 6 & -5.516416 & -0.466442 & 0.434156 \\
\hline 6 & -5.860902 & -0.655094 & -0.910568 \\
\hline 6 & -4.940180 & -0.539447 & -1.952878 \\
\hline 6 & -3.620981 & -0.218084 & -1.618730 \\
\hline 6 & -6.571772 & -0.651293 & 1.523320 \\
\hline 6 & -5.385944 & -0.706531 & -3.404395 \\
\hline 6 & -1.737567 & 2.154483 & 0.832237 \\
\hline 6 & -2.811699 & 2.947060 & 0.429286 \\
\hline 6 & -2.919647 & 4.276870 & 0.840596 \\
\hline 6 & -1.934395 & 4.778449 & 1.693276 \\
\hline 6 & -0.849632 & 4.010655 & 2.128182 \\
\hline 6 & -0.747328 & 2.701606 & 1.654141 \\
\hline 6 & -4.075198 & 5.174182 & 0.400840 \\
\hline 6 & 0.181480 & 4.623866 & 3.075348 \\
\hline 6 & -0.187006 & -0.096320 & -3.512856 \\
\hline 8 & -0.256658 & -0.584105 & -4.560775 \\
\hline 1 & 6.847934 & 1.141166 & 0.954687 \\
\hline 1 & -1.604252 & 0.890907 & 3.057812 \\
\hline 1 & 8.029431 & -3.529593 & -0.253091 \\
\hline 1 & -1.061245 & -2.868613 & 5.877990 \\
\hline 1 & 5.499468 & 5.947360 & -0.691322 \\
\hline 1 & -3.237221 & -2.983066 & 1.000994 \\
\hline 1 & -5.092203 & -4.137524 & -0.117570 \\
\hline 1 & 3.435068 & 5.039062 & 0.204350 \\
\hline 1 & -1.404977 & -0.620232 & 4.970317 \\
\hline 1 & 6.041145 & -3.711274 & -1.630414 \\
\hline 1 & 8.451261 & 3.336488 & -2.405327 \\
\hline
\end{tabular}




\begin{tabular}{|c|c|c|c|}
\hline & 3.930567 & -2.711949 & -2.513248 \\
\hline & -2.653875 & -4.769327 & -3.602525 \\
\hline & -0.366873 & -3.913334 & -3.664900 \\
\hline & -0.372441 & -6.170008 & 3.208649 \\
\hline & 7.087038 & 1.383213 & -1.839364 \\
\hline & -0.456046 & -4.663121 & 1.281619 \\
\hline & 1.509909 & -2.748110 & -2.554235 \\
\hline & 7.686170 & 5.633175 & -1.812928 \\
\hline & 8.856010 & 0.100100 & 1.893619 \\
\hline 1 & -4.806274 & -5.066987 & -2.410558 \\
\hline 1 & -0.667604 & -5.289017 & 5.521731 \\
\hline 1 & 9.480879 & -2.233077 & 1.280377 \\
\hline 1 & 1.966564 & 3.073621 & 0.669989 \\
\hline 1 & -3.888536 & 0.038998 & 1.753395 \\
\hline 1 & -2.856865 & -0.130465 & -2.387237 \\
\hline 1 & -6.894129 & -0.906155 & -1.151281 \\
\hline & -3.565334 & 2.517549 & -0.225708 \\
\hline 1 & 0.106343 & 2.085472 & 1.935952 \\
\hline & -2.013762 & 5.810921 & 2.032801 \\
\hline 1 & 3.162586 & -1.462667 & 1.097193 \\
\hline 1 & 1.630414 & -1.668254 & 1.921128 \\
\hline 1 & -1.390192 & 1.611165 & -2.458031 \\
\hline 1 & 2.499908 & -3.266164 & 3.383263 \\
\hline 1 & 2.637765 & -4.023744 & -0.836420 \\
\hline 1 & 3.096160 & -5.620995 & 3.831591 \\
\hline 1 & 3.183581 & -6.390115 & -0.394664 \\
\hline 1 & 3.420431 & -7.205848 & 1.939462 \\
\hline 5 & 1.171192 & 3.588795 & 3.608547 \\
\hline 6 & 0.958654 & 5.711628 & 2.326061 \\
\hline 6 & -0.524820 & 5.247402 & 4.284712 \\
\hline 6 & -5.054085 & 4.449214 & -0.520895 \\
\hline 6 & -4.848237 & 5.653312 & 1.634477 \\
\hline 5 & -3.516758 & 6.384718 & -0.354763 \\
\hline 6 & -4.197361 & -0.786044 & -4.361973 \\
\hline 6 & -6.212717 & -1.984155 & -3.572150 \\
\hline 6 & -6.241744 & 0.505757 & -3.790211 \\
\hline 6 & -6.013222 & -0.383940 & 2.919388 \\
\hline 6 & -7.738759 & 0.311956 & 1.283033 \\
\hline 6 & -7.084392 & -2.095386 & 1.489101 \\
\hline 1 & 1.849050 & 4.065949 & 4.327430 \\
\hline 1 & 0.660288 & 2.767391 & 4.129405 \\
\hline 1 & 1.797215 & 3.153896 & 2.819533 \\
\hline 1 & 0.215083 & 5.676280 & 4.973477 \\
\hline 1 & -1.211747 & 6.053081 & 4.001516 \\
\hline 1 & -1.101537 & 4.493408 & 4.836061 \\
\hline 1 & 1.732305 & 6.148856 & 2.971910 \\
\hline 1 & 1.449120 & 5.300753 & 1.432987 \\
\hline 1 & 0.300143 & 6.523155 & 1.992763 \\
\hline 1 & -5.684741 & 6.298063 & 1.333038 \\
\hline 1 & -5.260866 & 4.803775 & 2.194278 \\
\hline 1 & -4.216420 & 6.230597 & 2.320393 \\
\hline 1 & -5.868278 & 5.129934 & -0.799831 \\
\hline 1 & -4.572743 & 4.114644 & -1.448964 \\
\hline 1 & -5.508079 & 3.573809 & -0.036895 \\
\hline 1 & -4.335085 & 7.037709 & -0.687179 \\
\hline 1 & -2.845121 & 6.986559 & 0.269267 \\
\hline
\end{tabular}




$\begin{array}{rrrr}1 & -2.953125 & 6.067402 & -1.241610 \\ 1 & -4.558806 & -0.953121 & -5.384556 \\ 1 & -3.610457 & 0.142028 & -4.374147 \\ 1 & -3.523178 & -1.615177 & -4.104891 \\ 1 & -6.521458 & -2.098182 & -4.619766 \\ 1 & -5.623663 & -2.867277 & -3.292259 \\ 1 & -7.125068 & -1.978629 & -2.963810 \\ 1 & -6.569511 & 0.428448 & -4.835942 \\ 1 & -7.137949 & 0.585094 & -3.161639 \\ 1 & -5.672983 & 1.438621 & -3.680974 \\ 1 & -6.800056 & -0.542338 & 3.667659 \\ 1 & -5.182895 & -1.059834 & 3.166527 \\ 1 & -5.657534 & 0.648925 & 3.028144 \\ 1 & -8.499258 & 0.192676 & 2.066458 \\ 1 & -7.396685 & 1.354905 & 1.300200 \\ 1 & -8.229422 & 0.137449 & 0.318017 \\ 1 & -7.868299 & -2.241421 & 2.244626 \\ 1 & -7.508235 & -2.361738 & 0.512952 \\ 1 & -6.272091 & -2.801729 & 1.709084\end{array}$

$1_{\mathrm{C} 3-} \psi_{-1 \mathrm{a} 2 \mathrm{a3a} \mathbf{5}}$

Number of imaginary frequencies : 0

The smallest frequencies are : $13.2890 \quad 16.6555 \quad 17.2281 \mathrm{~cm}(-1)$

Electronic energy : $\quad H F=-4125.4683125$

Zero-point correction $=\quad 1.299285($ Hartree/Particle $)$

Thermal correction to Energy= $\quad 1.377815$

Thermal correction to Enthalpy= $\quad 1.378760$

Thermal correction to Gibbs Free Energy $=\quad 1.185930$

Sum of electronic and zero-point Energies $=\quad-4124.169027$

Sum of electronic and thermal Energies $=\quad-4124.090497$

Sum of electronic and thermal Enthalpies $=\quad-4124.089553$

Sum of electronic and thermal Free Energies $=\quad-4124.282383$

Cartesian Coordinates

$\begin{array}{lrrr}6 & 3.366015 & -6.089614 & 1.734698 \\ 6 & 3.172846 & -5.206735 & 2.793397 \\ 6 & 2.805077 & -3.890751 & 2.542639 \\ 6 & 2.641692 & -3.426124 & 1.235412 \\ 6 & 2.859137 & -4.312189 & 0.180574 \\ 6 & 3.209169 & -5.636521 & 0.428853 \\ 6 & 2.293023 & -1.972771 & 1.052699 \\ 7 & 1.615820 & -1.619737 & -0.207012 \\ 6 & 0.515534 & -2.386061 & -0.714306 \\ 6 & -0.672394 & -2.549165 & -0.019893 \\ 6 & -1.749432 & -3.267728 & -0.630180 \\ 6 & -1.598706 & -3.777284 & -1.953265 \\ 6 & -0.376391 & -3.574287 & -2.632918 \\ 6 & 0.649244 & -2.903760 & -2.026593 \\ 6 & -2.994418 & -3.459874 & 0.023890 \\ 6 & -4.020373 & -4.131139 & -0.592968 \\ 6 & -3.857751 & -4.653596 & -1.894215 \\ 6 & -2.672775 & -4.472363 & -2.560626 \\ 6 & -0.849774 & -1.984810 & 1.346651 \\ 6 & -1.154504 & -0.642283 & 1.541217\end{array}$




\begin{tabular}{|c|c|c|c|}
\hline 6 & -1.319688 & -0.156286 & 2.865625 \\
\hline 6 & -1.194967 & -0.983305 & 3.950046 \\
\hline 6 & -0.913884 & -2.358195 & 3.785395 \\
\hline 6 & -0.743189 & -2.871258 & 2.466367 \\
\hline 6 & -0.810782 & -3.232624 & 4.894332 \\
\hline 6 & -0.564166 & -4.569734 & 4.712299 \\
\hline 6 & -0.400872 & -5.084525 & 3.408841 \\
\hline 6 & -0.476920 & -4.257813 & 2.316032 \\
\hline 15 & -1.499087 & 0.438227 & 0.063591 \\
\hline 45 & -0.064045 & 0.884486 & -1.908506 \\
\hline 6 & 0.392675 & 2.759777 & -1.756909 \\
\hline 8 & 0.458403 & 3.912984 & -1.802423 \\
\hline 15 & 1.820673 & -0.121434 & -0.977357 \\
\hline 8 & 2.576072 & 0.661312 & 0.271074 \\
\hline 6 & 3.393652 & 1.733006 & -0.035399 \\
\hline 0 & 4.658677 & 1.506905 & -0.544427 \\
\hline 0 & 5.457834 & 2.644515 & -0.888196 \\
\hline 6 & 4.967899 & 3.955886 & -0.605451 \\
\hline 6 & 3.684265 & 4.113511 & -0.031651 \\
\hline 6 & 2.899449 & 3.023027 & 0.229253 \\
\hline 6 & 5.762159 & 5.082080 & -0.932286 \\
\hline 6 & 6.981863 & 4.932624 & -1.539727 \\
\hline 6 & 7.452397 & 3.640627 & -1.855494 \\
\hline 6 & 6.712476 & 2.528590 & -1.539432 \\
\hline 6 & 5.105311 & 0.111886 & -0.768452 \\
\hline 6 & 4.313235 & -0.731134 & -1.527594 \\
\hline 6 & 4.692377 & -2.050690 & -1.838345 \\
\hline 6 & 5.858275 & -2.557314 & -1.331879 \\
\hline 6 & 6.672366 & -1.774930 & -0.478925 \\
\hline 6 & 6.295745 & -0.429224 & -0.183731 \\
\hline 6 & 7.102677 & 0.303599 & 0.724148 \\
\hline 6 & 8.229522 & -0.250922 & 1.278040 \\
\hline 6 & 8.616123 & -1.569260 & 0.957576 \\
\hline 6 & 7.847583 & -2.313041 & 0.100154 \\
\hline 8 & 3.104729 & -0.284527 & -2.022235 \\
\hline 6 & -3.207504 & -0.120470 & -0.329710 \\
\hline 6 & -4.148827 & -0.256970 & 0.685069 \\
\hline 6 & -5.475922 & -0.587987 & 0.400992 \\
\hline 6 & -5.827560 & -0.764490 & -0.938386 \\
\hline 6 & -4.902479 & -0.640601 & -1.984019 \\
\hline 6 & -3.584288 & -0.328597 & -1.655018 \\
\hline 6 & -6.469974 & -0.702549 & 1.555133 \\
\hline 6 & -5.357274 & -0.794577 & -3.433959 \\
\hline 6 & -1.820566 & 2.099104 & 0.790634 \\
\hline 6 & -2.971382 & 2.798992 & 0.430122 \\
\hline 6 & -3.179897 & 4.112114 & 0.853098 \\
\hline 6 & -2.211305 & 4.695933 & 1.671925 \\
\hline 6 & -1.048915 & 4.023148 & 2.063349 \\
\hline 6 & -0.853040 & 2.727443 & 1.580329 \\
\hline 6 & -4.435977 & 4.893335 & 0.471152 \\
\hline 6 & -0.030982 & 4.719280 & 2.967746 \\
\hline 6 & -0.183878 & -0.142859 & -3.525515 \\
\hline 8 & -0.268310 & -0.642648 & -4.566596 \\
\hline 1 & 6.813168 & 1.316638 & 0.991192 \\
\hline 1 & -1.570761 & 0.889133 & 3.025064 \\
\hline 1 & 8.120120 & -3.338607 & -0.145166 \\
\hline
\end{tabular}




\begin{tabular}{|c|c|c|c|}
\hline 1 & -0.939707 & -2.819719 & 5.893993 \\
\hline & 5.372252 & 6.072375 & -0.701026 \\
\hline & -3.135173 & -3.055444 & 1.024587 \\
\hline & -4.971337 & -4.256055 & -0.078482 \\
\hline & 3.316999 & 5.118912 & 0.170723 \\
\hline & -1.330741 & -0.591591 & 4.957478 \\
\hline & 6.160861 & -3.578801 & -1.556643 \\
\hline & 8.408079 & 3.524044 & -2.361565 \\
\hline & 4.041012 & -2.637936 & -2.481735 \\
\hline & -2.534527 & -4.860318 & -3.569032 \\
\hline & -0.265569 & -3.955226 & -3.646915 \\
\hline 1 & -0.191856 & -6.142664 & 3.267737 \\
\hline & 7.083997 & 1.541454 & -1.801812 \\
\hline & -0.321500 & -4.665889 & 1.319483 \\
\hline & 1.588807 & -2.738558 & -2.552752 \\
\hline & 7.580418 & 5.804711 & -1.792267 \\
\hline & 8.827025 & 0.330979 & 1.976230 \\
\hline & -4.675495 & -5.193865 & -2.366589 \\
\hline & -0.494351 & -5.235536 & 5.569591 \\
\hline & 9.513967 & -1.994177 & 1.400168 \\
\hline & 1.890893 & 3.121173 & 0.626833 \\
\hline & -3.846584 & -0.083758 & 1.717937 \\
\hline & -2.824412 & -0.230803 & -2.425937 \\
\hline & -6.859970 & -1.004754 & -1.185438 \\
\hline & -3.710028 & 2.310543 & -0.198711 \\
\hline & 0.056929 & 2.183400 & 1.834401 \\
\hline & -2.368977 & 5.716069 & 2.020490 \\
\hline & 3.219187 & -1.388274 & 1.092195 \\
\hline & 1.693718 & -1.639453 & 1.915034 \\
\hline & -1.402320 & 1.555480 & -2.481345 \\
\hline & 2.626667 & -3.210054 & 3.376002 \\
\hline ] & 2.748931 & -3.966625 & -0.844082 \\
\hline & 3.287769 & -5.548295 & 3.820207 \\
\hline & 3.360866 & -6.317424 & -0.406272 \\
\hline 1 & 3.638232 & -7.125298 & 1.926255 \\
\hline 6 & 0.998054 & 3.744485 & 3.540579 \\
\hline 6 & 0.702353 & 5.789920 & 2.152345 \\
\hline & -0.738144 & 5.385107 & 4.153553 \\
\hline 6 & -5.350683 & 4.098264 & -0.459686 \\
\hline 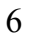 & -5.225652 & 5.232007 & 1.740387 \\
\hline 6 & -4.038523 & 6.188278 & -0.244761 \\
\hline$b$ & -4.183655 & -0.744165 & -4.411376 \\
\hline 5 & -6.076237 & -2.132503 & -3.628080 \\
\hline 6 & -6.315525 & 0.353916 & -3.771249 \\
\hline & -7.840273 & -1.194109 & 1.096720 \\
\hline 6 & -5.940379 & -1.689914 & 2.602215 \\
\hline & -6.641381 & 0.679316 & 2.197806 \\
\hline 1 & 1.657630 & 4.275413 & 4.238444 \\
\hline l & 0.518309 & 2.925236 & 4.093416 \\
\hline t & 1.641373 & 3.303108 & 2.769471 \\
\hline 1 & 0.001389 & 5.856757 & 4.813890 \\
\hline ] & -1.438803 & 6.168202 & 3.842607 \\
\hline 1 & -1.297196 & 4.648696 & 4.745300 \\
\hline & 1.458685 & 6.295306 & 2.768514 \\
\hline & 1.209166 & 5.345478 & 1.284837 \\
\hline & 0.010447 & 6.551923 & 1.772564 \\
\hline
\end{tabular}


$1_{\mathrm{C} 3-} \psi_{-1 \text {-1a2a3s4a }}$

Number of imaginary frequencies : 0

The smallest frequencies are : $11.9229 \quad 16.6150 \quad 21.8120 \mathrm{~cm}(-1)$

Electronic energy :

$\mathrm{HF}=-4125.4671459$

Zero-point correction $=$

1.299822 (Hartree/Particle)

Thermal correction to Energy $=\quad 1.378060$

Thermal correction to Enthalpy= $\quad 1.379005$

Thermal correction to Gibbs Free Energy= $\quad 1.187659$

Sum of electronic and zero-point Energies $=\quad-4124.167324$

Sum of electronic and thermal Energies $=\quad-4124.089085$

Sum of electronic and thermal Enthalpies $=\quad-4124.088141$

Sum of electronic and thermal Free Energies $=\quad-4124.279487$

\begin{tabular}{|c|c|c|c|}
\hline & \multicolumn{3}{|c|}{ Cartesian Coordinates } \\
\hline 6 & -0.840460 & 2.697278 & 1.681315 \\
\hline 6 & -1.791895 & 2.096594 & 0.851436 \\
\hline 6 & -2.888273 & 2.839245 & 0.414550 \\
\hline 6 & -3.055488 & 4.171307 & 0.792406 \\
\hline 6 & -2.112069 & 4.725945 & 1.659614 \\
\hline 6 & -1.007027 & 4.009385 & 2.130541 \\
\hline 15 & -1.504918 & 0.435724 & 0.112066 \\
\hline 6 & -3.227150 & -0.093328 & -0.263693 \\
\hline 6 & -4.142939 & -0.309161 & 0.773703 \\
\hline 6 & -5.465446 & -0.635097 & 0.500641 \\
\hline 6 & -5.858493 & -0.713944 & -0.846705 \\
\hline 6 & -4.975021 & -0.496106 & -1.899527 \\
\hline
\end{tabular}




\begin{tabular}{|c|c|c|c|}
\hline 6 & -3.641399 & -0.202259 & -1.580957 \\
\hline 6 & -6.484171 & -0.909377 & 1.605519 \\
\hline y & -7.004923 & -2.344528 & 1.471233 \\
\hline & -5.393912 & -0.512381 & -3.367251 \\
\hline$b$ & -4.547555 & -1.526317 & -4.143173 \\
\hline p & -4.240964 & 5.004311 & 0.308416 \\
\hline D & -3.732584 & 6.290300 & -0.351330 \\
\hline 6 & -0.018871 & 4.681409 & 3.084299 \\
\hline 6 & -1.149318 & -0.676904 & 1.559782 \\
\hline 6 & -0.864244 & -2.018486 & 1.329934 \\
\hline 6 & -0.761432 & -2.931718 & 2.428676 \\
\hline 6 & -0.905311 & -2.445509 & 3.761136 \\
\hline 6 & -1.164308 & -1.070877 & 3.960610 \\
\hline 6 & -1.294078 & -0.218920 & 2.896515 \\
\hline 6 & -0.527655 & -4.319875 & 2.244946 \\
\hline 6 & -0.455901 & -5.172572 & 3.318075 \\
\hline 6 & -0.587994 & -4.683736 & 4.634773 \\
\hline 6 & -0.803144 & -3.345813 & 4.849226 \\
\hline 6 & -0.702844 & -2.553345 & -0.051196 \\
\hline 6 & 0.481695 & -2.383862 & -0.750683 \\
\hline 6 & 0.605514 & -2.876644 & -2.072946 \\
\hline 6 & -0.426711 & -3.531238 & -2.685351 \\
\hline 6 & -1.646468 & -3.739279 & -2.002625 \\
\hline 6 & -1.788390 & -3.251760 & -0.669947 \\
\hline 6 & -2.726866 & -4.416784 & -2.617640 \\
\hline 6 & -3.913826 & -4.594332 & -1.954245 \\
\hline 6 & -4.067391 & -4.094211 & -0.643639 \\
\hline 6 & -3.032837 & -3.446309 & -0.015727 \\
\hline 7 & 1.589796 & -1.635771 & -0.233195 \\
\hline 15 & 1.797581 & -0.116231 & -0.956364 \\
\hline 8 & 3.072235 & -0.242004 & -2.017703 \\
\hline 6 & 4.286922 & -0.707212 & -1.558195 \\
\hline 6 & 5.087203 & 0.098788 & -0.767709 \\
\hline 6 & 6.284772 & -0.470019 & -0.224624 \\
\hline 6 & 6.661308 & -1.796830 & -0.595464 \\
\hline 6 & 5.839887 & -2.535489 & -1.479632 \\
\hline 6 & 4.666564 & -2.006480 & -1.943863 \\
\hline 6 & 7.100735 & 0.215616 & 0.711724 \\
\hline 6 & 8.235848 & -0.364056 & 1.221422 \\
\hline 6 & 8.622443 & -1.661721 & 0.825590 \\
\hline 6 & 7.845226 & -2.361120 & -0.060800 \\
\hline 6 & 4.647865 & 1.484722 & -0.480836 \\
\hline 6 & 3.385620 & 1.697048 & 0.041226 \\
\hline 6 & 2.896729 & 2.978454 & 0.353818 \\
\hline 6 & 3.685442 & 4.074619 & 0.131842 \\
\hline 6 & 4.967160 & 3.933434 & -0.450224 \\
\hline 6 & 5.450661 & 2.631778 & -0.784358 \\
\hline 6 & 5.764701 & 5.068424 & -0.736136 \\
\hline 6 & 6.981481 & 4.938015 & -1.353607 \\
\hline 6 & 7.445154 & 3.657387 & -1.721181 \\
\hline 6 & 6.702156 & 2.536736 & -1.445133 \\
\hline 8 & 2.564988 & 0.618625 & 0.313275 \\
\hline 6 & 2.283640 & -2.044313 & 1.001222 \\
\hline 6 & 2.624792 & -3.506572 & 1.109288 \\
\hline 6 & 2.716062 & -4.064137 & 2.386216 \\
\hline 6 & 3.088022 & -5.391884 & 2.561217 \\
\hline
\end{tabular}




\begin{tabular}{|c|c|c|c|}
\hline 6 & 3.357617 & -6.191730 & 1.454938 \\
\hline 6 & 3.265201 & -5.647220 & 0.177617 \\
\hline & 2.908282 & -4.313348 & 0.006976 \\
\hline & -0.080474 & 0.925868 & -1.856540 \\
\hline 0 & -0.198321 & -0.052807 & -3.501625 \\
\hline 8 & -0.281790 & -0.521708 & -4.557352 \\
\hline 6 & 0.377994 & 2.794816 & -1.633303 \\
\hline & 0.443286 & 3.948798 & -1.629878 \\
\hline & -5.878697 & -0.758095 & 2.999535 \\
\hline 6 & -7.654368 & 0.072505 & 1.483954 \\
\hline 6 & -6.863032 & -0.884306 & -3.547773 \\
\hline & -5.175025 & 0.888902 & -3.952168 \\
\hline 6 & -5.094381 & 4.255836 & -0.715133 \\
\hline & -5.128041 & 5.358505 & 1.506946 \\
\hline 1 & 6.811574 & 1.211988 & 1.035967 \\
\hline & -1.536863 & 0.824204 & 3.081607 \\
\hline & 8.117495 & -3.371103 & -0.364332 \\
\hline 1 & -0.909697 & -2.952256 & 5.859244 \\
\hline & 5.379342 & 6.050396 & -0.465168 \\
\hline 1 & -3.168403 & -3.060672 & 0.993320 \\
\hline & -5.017479 & -4.219944 & -0.127649 \\
\hline 1 & 3.322597 & 5.073411 & 0.371849 \\
\hline 1 & -1.283468 & -0.700438 & 4.978092 \\
\hline 1 & 6.143373 & -3.542150 & -1.762799 \\
\hline 1 & 8.398150 & 3.556620 & -2.235657 \\
\hline 1 & 4.008472 & -2.559965 & -2.610200 \\
\hline 1 & -2.595510 & -4.784405 & -3.634634 \\
\hline 1 & -0.323805 & -3.894711 & -3.706542 \\
\hline 1 & -0.273624 & -6.231755 & 3.150462 \\
\hline 1 & 7.069126 & 1.559542 & -1.747703 \\
\hline 1 & -0.394542 & -4.709991 & 1.238099 \\
\hline 1 & 1.543394 & -2.705659 & -2.600106 \\
\hline 1 & 7.582597 & 5.816863 & -1.574662 \\
\hline 1 & 8.839993 & 0.181427 & 1.942924 \\
\hline 1 & -4.740078 & -5.111851 & -2.437026 \\
\hline 1 & -0.520323 & -5.369605 & 5.476261 \\
\hline 1 & 9.527029 & -2.106527 & 1.233542 \\
\hline & 1.888675 & 3.066313 & 0.755364 \\
\hline 1 & -3.803521 & -0.217134 & 1.802996 \\
\hline 1 & -2.909776 & -0.037910 & -2.370825 \\
\hline 1 & -6.896704 & -0.955031 & -1.067411 \\
\hline 1 & -3.610240 & 2.370803 & -0.248781 \\
\hline 1 & 0.036824 & 2.125201 & 1.983546 \\
\hline 1 & -2.240278 & 5.759384 & 1.980442 \\
\hline 1 & 3.213078 & -1.465853 & 1.052072 \\
\hline 1 & 1.696970 & -1.746687 & 1.884859 \\
\hline 1 & -1.413026 & 1.620879 & -2.416748 \\
\hline 1 & 2.471936 & -3.451168 & 3.254745 \\
\hline 1 & 2.848862 & -3.892309 & -0.993475 \\
\hline 1 & 3.146178 & -5.806968 & 3.565577 \\
\hline 1 & 3.472912 & -6.264025 & -0.694475 \\
\hline 1 & 3.635689 & -7.235147 & 1.586894 \\
\hline 1 & -5.988660 & 5.959397 & 1.183023 \\
\hline 1 & -5.511916 & 4.451134 & 1.991919 \\
\hline 1 & -4.583356 & 5.936789 & 2.263499 \\
\hline 1 & -5.920017 & 4.899202 & -1.044697 \\
\hline
\end{tabular}




$\begin{array}{rrrr}1 & -4.515115 & 3.977222 & -1.605749 \\ 1 & -5.538700 & 3.341153 & -0.299236 \\ 1 & -4.577640 & 6.889162 & -0.716686 \\ 1 & -3.161826 & 6.917540 & 0.343707 \\ 1 & -3.082991 & 6.062203 & -1.206259 \\ 1 & -7.113365 & -0.890749 & -4.616101 \\ 1 & -7.081824 & -1.884853 & -3.151615 \\ 1 & -7.533435 & -0.165385 & -3.059164 \\ 1 & -5.480587 & 0.915314 & -5.006893 \\ 1 & -5.766047 & 1.638009 & -3.408050 \\ 1 & -4.121485 & 1.194112 & -3.903358 \\ 1 & -4.835006 & -1.526093 & -5.203274 \\ 1 & -3.474216 & -1.302801 & -4.092515 \\ 1 & -4.693500 & -2.539643 & -3.747418 \\ 1 & -6.640394 & -0.979045 & 3.757909 \\ 1 & -5.041417 & -1.451246 & 3.162300 \\ 1 & -5.517929 & 0.262531 & 3.181825 \\ 1 & -8.388209 & -0.107764 & 2.280996 \\ 1 & -7.306606 & 1.110019 & 1.573053 \\ 1 & -8.178133 & -0.023506 & 0.525342 \\ 1 & -7.767283 & -2.547840 & 2.235457 \\ 1 & -7.458743 & -2.531328 & 0.490099 \\ 1 & -6.190385 & -3.068570 & 1.610879 \\ 6 & 0.988570 & 3.693726 & 3.671718 \\ 6 & 0.746082 & 5.769096 & 2.322414 \\ 6 & -0.772867 & 5.319269 & 4.256816 \\ 1 & 1.482234 & 6.254790 & 2.977511 \\ 1 & 1.281466 & 5.343722 & 1.462439 \\ 1 & 0.072738 & 6.545060 & 1.938031 \\ 1 & -0.062754 & 5.789445 & 4.949904 \\ 1 & -1.474605 & 6.096492 & 3.933321 \\ 1 & -1.341765 & 4.565563 & 4.816578 \\ 1 & 1.628366 & 4.211024 & 4.397716 \\ 1 & 0.490949 & 2.867595 & 4.197739 \\ 1 & 1.652708 & 3.263168 & 2.911939\end{array}$

$1_{\mathrm{C} 3-} \Psi_{-1 \mathrm{a} 2 \mathrm{2} 3 \mathrm{~s} 4 \mathrm{~s}}$

Number of imaginary frequencies : 0

The smallest frequencies are : $10.7516 \quad 14.0283 \quad 15.0236 \mathrm{~cm}(-1)$

Electronic energy : $\quad H F=-4125.4675688$

Zero-point correction $=\quad 1.299107($ Hartree/Particle $)$

Thermal correction to Energy $=\quad 1.377767$

Thermal correction to Enthalpy= $\quad 1.378711$

Thermal correction to Gibbs Free Energy $=\quad 1.184555$

Sum of electronic and zero-point Energies $=\quad-4124.168462$

Sum of electronic and thermal Energies $=\quad-4124.089802$

Sum of electronic and thermal Enthalpies $=\quad-4124.088858$

Sum of electronic and thermal Free Energies $=\quad-4124.283014$

Cartesian Coordinates

$\begin{array}{llll}6 & 3.482616 & -6.107764 & 1.576078 \\ 6 & 3.213870 & -5.292660 & 2.671642 \\ 6 & 2.813413 & -3.975898 & 2.478020 \\ 6 & 2.690100 & -3.444376 & 1.192457\end{array}$




\begin{tabular}{|c|c|c|c|}
\hline 6 & 2.972601 & -4.266223 & 0.101316 \\
\hline 6 & 3.359774 & -5.589236 & 0.290804 \\
\hline 6 & 2.315956 & -1.991372 & 1.064151 \\
\hline 7 & 1.614727 & -1.613658 & -0.175700 \\
\hline 6 & 0.511279 & -2.387283 & -0.665998 \\
\hline o & -0.666331 & -2.548516 & 0.045936 \\
\hline 6 & -1.739956 & -3.297743 & -0.533073 \\
\hline 6 & -1.592202 & -3.850546 & -1.838754 \\
\hline 6 & -0.384212 & -3.639199 & -2.541773 \\
\hline 6 & 0.635016 & -2.930399 & -1.968864 \\
\hline 6 & -2.977383 & -3.482200 & 0.136471 \\
\hline 6 & -3.998861 & -4.187895 & -0.448910 \\
\hline 6 & -3.836816 & -4.758944 & -1.729055 \\
\hline 6 & -2.657487 & -4.588545 & -2.408258 \\
\hline 6 & -0.843490 & -1.954957 & 1.399999 \\
\hline 6 & -1.170102 & -0.613666 & 1.564226 \\
\hline 6 & -1.352747 & -0.103452 & 2.877491 \\
\hline 6 & -1.216760 & -0.903217 & 3.980207 \\
\hline 6 & -0.909582 & -2.275813 & 3.846969 \\
\hline 6 & -0.728582 & -2.815378 & 2.539891 \\
\hline 6 & -0.797650 & -3.122846 & 4.975749 \\
\hline 6 & -0.535674 & -4.460988 & 4.824929 \\
\hline 6 & -0.364719 & -5.002963 & 3.533839 \\
\hline 6 & -0.447462 & -4.202050 & 2.422055 \\
\hline 15 & -1.512952 & 0.437943 & 0.064563 \\
\hline 45 & -0.055245 & 0.890180 & -1.883672 \\
\hline 6 & 0.403587 & 2.766330 & -1.740665 \\
\hline 8 & 0.471934 & 3.918335 & -1.805255 \\
\hline 15 & 1.820495 & -0.115716 & -0.941603 \\
\hline 8 & 2.576010 & 0.661673 & 0.310296 \\
\hline 6 & 3.383379 & 1.743164 & 0.011852 \\
\hline 6 & 4.647590 & 1.533634 & -0.506214 \\
\hline 6 & 5.435616 & 2.682298 & -0.840725 \\
\hline 6 & 4.937785 & 3.985936 & -0.537048 \\
\hline 6 & 3.656764 & 4.125854 & 0.046857 \\
\hline 6 & 2.881599 & 3.026143 & 0.296689 \\
\hline 6 & 5.721162 & 5.122812 & -0.853003 \\
\hline 6 & 6.937769 & 4.991395 & -1.470633 \\
\hline 6 & 7.415541 & 3.707620 & -1.807984 \\
\hline 6 & 6.686469 & 2.585500 & -1.502406 \\
\hline 6 & 5.107788 & 0.146611 & -0.750729 \\
\hline 6 & 4.322241 & -0.696570 & -1.516637 \\
\hline 6 & 4.725731 & -2.000152 & -1.862101 \\
\hline 6 & 5.906311 & -2.494986 & -1.378786 \\
\hline 6 & 6.712218 & -1.716627 & -0.514643 \\
\hline 6 & 6.312538 & -0.385255 & -0.186774 \\
\hline 6 & 7.113385 & 0.342696 & 0.730359 \\
\hline 6 & 8.255231 & -0.202236 & 1.262642 \\
\hline 6 & 8.664253 & -1.505418 & 0.909651 \\
\hline 6 & 7.902543 & -2.244711 & 0.042351 \\
\hline 8 & 3.101566 & -0.265149 & -1.992659 \\
\hline 6 & -3.202282 & -0.155719 & -0.358084 \\
\hline 6 & -4.161387 & -0.314620 & 0.642050 \\
\hline 6 & -5.472624 & -0.670754 & 0.334223 \\
\hline 6 & -5.803668 & -0.846801 & -1.015624 \\
\hline 6 & -4.867302 & -0.693835 & -2.040996 \\
\hline
\end{tabular}




\begin{tabular}{|c|c|c|c|}
\hline 6 & -3.555913 & -0.363016 & -1.685328 \\
\hline 6 & -6.485965 & -0.810812 & 1.469297 \\
\hline & -5.218986 & -0.824402 & -3.522086 \\
\hline & -1.876083 & 2.100395 & 0.768204 \\
\hline & -3.038564 & 2.773062 & 0.394458 \\
\hline & -3.276177 & 4.085248 & 0.805556 \\
\hline & -2.326322 & 4.694557 & 1.627857 \\
\hline & -1.154319 & 4.048104 & 2.034531 \\
\hline & -0.927824 & 2.754149 & 1.560536 \\
\hline & -4.545135 & 4.838058 & 0.409618 \\
\hline & -0.157745 & 4.767606 & 2.944704 \\
\hline & -0.149136 & -0.119735 & -3.511049 \\
\hline & -0.221994 & -0.600781 & -4.562163 \\
\hline & 6.806991 & 1.343882 & 1.022179 \\
\hline & -1.626343 & 0.939251 & 3.013975 \\
\hline & 8.192360 & -3.259161 & -0.228172 \\
\hline & -0.934001 & -2.688561 & 5.965266 \\
\hline & 5.325094 & 6.106608 & -0.605107 \\
\hline & -3.118091 & -3.042425 & 1.122043 \\
\hline & -4.944900 & -4.304084 & 0.077237 \\
\hline & 3.283684 & 5.125466 & 0.266449 \\
\hline & -1.365356 & -0.492336 & 4.978132 \\
\hline & 6.227173 & -3.504529 & -1.630794 \\
\hline & 8.368315 & 3.605406 & -2.322581 \\
\hline & 4.080193 & -2.584632 & -2.514010 \\
\hline & -2.522792 & -5.006481 & -3.405308 \\
\hline & -0.278942 & -4.046574 & -3.546080 \\
\hline & -0.145972 & -6.062067 & 3.416815 \\
\hline & 7.063932 & 1.605404 & -1.781864 \\
\hline 1 & -0.286481 & -4.632292 & 1.435684 \\
\hline & 1.563777 & -2.759119 & -2.511683 \\
\hline & 7.528044 & 5.871464 & -1.714817 \\
\hline & 8.847388 & 0.375381 & 1.968895 \\
\hline & -4.651584 & -5.322805 & -2.178301 \\
\hline 1 & -0.460347 & -5.106390 & 5.697194 \\
\hline & 9.573854 & -1.922639 & 1.335225 \\
\hline ] & 1.875473 & 3.112940 & 0.702205 \\
\hline & -3.879040 & -0.138581 & 1.680192 \\
\hline & -2.790297 & -0.247661 & -2.451107 \\
\hline ] & -6.825719 & -1.108633 & -1.274205 \\
\hline & -3.762425 & 2.264664 & -0.235936 \\
\hline 1 & -0.008799 & 2.230137 & 1.825193 \\
\hline & -2.507618 & 5.713622 & 1.967730 \\
\hline ] & 3.231550 & -1.391001 & 1.110255 \\
\hline & 1.720012 & -1.695618 & 1.942241 \\
\hline & -1.390830 & 1.560397 & -2.465545 \\
\hline & 2.574686 & -3.349834 & 3.338786 \\
\hline & 2.889063 & -3.866034 & -0.905786 \\
\hline 1 & 3.297846 & -5.687336 & 3.682312 \\
\hline & 3.566554 & -6.217802 & -0.573077 \\
\hline 1 & 3.783717 & -7.142744 & 1.722966 \\
\hline 5 & 0.859991 & 3.807422 & 3.561166 \\
\hline 6 & 0.591555 & 5.820884 & 2.121453 \\
\hline 6 & -0.889771 & 5.458970 & 4.100368 \\
\hline 6 & -5.430339 & 4.022292 & -0.531471 \\
\hline & -5.356181 & 5.158179 & 1.670162 \\
\hline
\end{tabular}




$\begin{array}{rrrr}6 & -4.169996 & 6.141759 & -0.302435 \\ 6 & -6.690020 & -1.167422 & -3.741936 \\ 6 & -4.927206 & 0.510624 & -4.219471 \\ 6 & -4.371190 & -1.924643 & -4.167750 \\ 6 & -7.831134 & -1.347541 & 0.986918 \\ 6 & -5.949098 & -1.775728 & 2.533399 \\ 6 & -6.712542 & 0.568712 & 2.099936 \\ 1 & 1.502363 & 4.353972 & 4.263024 \\ 1 & 0.369306 & 2.998094 & 4.118984 \\ 1 & 1.522143 & 3.351826 & 2.814830 \\ 1 & -0.163682 & 5.941507 & 4.767615 \\ 1 & -1.580059 & 6.238340 & 3.758415 \\ 1 & -1.464825 & 4.736500 & 4.693945 \\ 1 & 1.328329 & 6.346775 & 2.744227 \\ 1 & 1.124250 & 5.356812 & 1.280109 \\ 1 & -0.094305 & 6.568126 & 1.703243 \\ 1 & -6.271430 & 5.705494 & 1.406881 \\ 1 & -5.650839 & 4.237495 & 2.191304 \\ 1 & -4.790738 & 5.776779 & 2.377780 \\ 1 & -6.329486 & 4.599049 & -0.782419 \\ 1 & -4.916880 & 3.783421 & -1.471988 \\ 1 & -5.760813 & 3.078199 & -0.075752 \\ 1 & -5.075900 & 6.684519 & -0.604322 \\ 1 & -3.583543 & 6.810859 & 0.338487 \\ 1 & -3.578530 & 5.939888 & -1.204619 \\ 1 & -6.892105 & -1.251035 & -4.817222 \\ 1 & -6.959718 & -2.126974 & -3.281394 \\ 1 & -7.357899 & -0.393267 & -3.342167 \\ 1 & -5.194854 & 0.451203 & -5.283094 \\ 1 & -5.507715 & 1.326277 & -3.768435 \\ 1 & -3.865021 & 0.782115 & -4.160587 \\ 1 & -4.599840 & -1.997663 & -5.239708 \\ 1 & -3.295108 & -1.730989 & -4.070914 \\ 1 & -4.574534 & -2.899150 & -3.705643 \\ 1 & -8.512249 & -1.459310 & 1.840017 \\ 1 & -8.314520 & -0.670141 & 0.271659 \\ 1 & -7.728771 & -2.332832 & 0.512508 \\ 1 & -6.670917 & -1.872211 & 3.355515 \\ 1 & -5.788377 & -2.776964 & 2.111376 \\ 1 & -4.999367 & -1.440882 & 2.969529 \\ 1 & -7.409735 & 0.497416 & 2.945998 \\ 1 & -5.776467 & 1.006768 & 2.470288 \\ 1 & -7.141096 & 1.266757 & 1.368120\end{array}$

$1_{\mathrm{C} 3-}-$-1a2s3a4a

Number of imaginary frequencies : 0

The smallest frequencies are : $11.9236 \quad 15.0022 \quad 18.4920 \mathrm{~cm}(-1)$

Electronic energy : $\quad \mathrm{HF}=-4125.4684158$

Zero-point correction $=\quad 1.298987$ (Hartree/Particle)

Thermal correction to Energy= $\quad 1.377541$

Thermal correction to Enthalpy= $\quad 1.378485$

Thermal correction to Gibbs Free Energy $=\quad 1.185844$

Sum of electronic and zero-point Energies $=\quad-4124.169429$

Sum of electronic and thermal Energies $=\quad-4124.090875$

Sum of electronic and thermal Enthalpies $=\quad-4124.089931$ 
Cartesian Coordinates

\begin{tabular}{|c|c|c|c|}
\hline 6 & 3.200979 & -6.142275 & 1.756863 \\
\hline 6 & 3.036546 & -5.250086 & 2.812698 \\
\hline 6 & 2.695798 & -3.927730 & 2.558188 \\
\hline 6 & 2.531871 & -3.465874 & 1.249941 \\
\hline 6 & 2.720810 & -4.361485 & 0.197730 \\
\hline 6 & 3.043078 & -5.692203 & 0.450143 \\
\hline 6 & 2.215717 & -2.005404 & 1.065289 \\
\hline 7 & 1.544008 & -1.635839 & -0.192613 \\
\hline 6 & 0.426491 & -2.376307 & -0.700260 \\
\hline 6 & -0.761063 & -2.524101 & -0.001886 \\
\hline 6 & -1.850831 & -3.225661 & -0.609686 \\
\hline 6 & -1.710945 & -3.738681 & -1.932538 \\
\hline 6 & -0.491468 & -3.544107 & -2.619778 \\
\hline 6 & 0.545032 & -2.887467 & -2.016837 \\
\hline 6 & -3.095622 & -3.402688 & 0.049153 \\
\hline 6 & -4.128481 & -4.070918 & -0.559648 \\
\hline 6 & -3.973939 & -4.603460 & -1.857947 \\
\hline 6 & -2.792117 & -4.429779 & -2.531962 \\
\hline 6 & -0.926300 & -1.967904 & 1.369257 \\
\hline 6 & -1.212619 & -0.623267 & 1.575547 \\
\hline 6 & -1.378107 & -0.148950 & 2.904682 \\
\hline 6 & -1.261368 & -0.986452 & 3.981601 \\
\hline 6 & -0.993169 & -2.362505 & 3.804781 \\
\hline 6 & -0.831696 & -2.866028 & 2.481094 \\
\hline 6 & -0.898371 & -3.247733 & 4.905787 \\
\hline 6 & -0.670455 & -4.586544 & 4.711477 \\
\hline 6 & -0.518749 & -5.092239 & 3.403159 \\
\hline 6 & -0.585929 & -4.254794 & 2.317934 \\
\hline 15 & -1.520481 & 0.483186 & 0.108915 \\
\hline 45 & -0.080362 & 0.914573 & -1.874387 \\
\hline 6 & 0.389716 & 2.783034 & -1.672038 \\
\hline 8 & 0.462121 & 3.936531 & -1.682773 \\
\hline 15 & 1.783655 & -0.140943 & -0.960683 \\
\hline 8 & 2.580193 & 0.611466 & 0.280134 \\
\hline 6 & 3.420779 & 1.663242 & -0.034214 \\
\hline 6 & 4.670600 & 1.406694 & -0.565991 \\
\hline 6 & 5.491577 & 2.525280 & -0.920859 \\
\hline 6 & 5.039061 & 3.847465 & -0.626348 \\
\hline 6 & 3.771205 & 4.035128 & -0.026994 \\
\hline 6 & 2.964237 & 2.963864 & 0.245722 \\
\hline 6 & 5.854149 & 4.954755 & -0.966010 \\
\hline 6 & 7.058335 & 4.777298 & -1.596481 \\
\hline 6 & 7.491609 & 3.474997 & -1.923080 \\
\hline 6 & 6.730868 & 2.380509 & -1.595282 \\
\hline 6 & 5.077628 & 0.001556 & -0.802691 \\
\hline 6 & 4.250957 & -0.817990 & -1.550631 \\
\hline 6 & 4.590655 & -2.145276 & -1.873206 \\
\hline 6 & 5.753524 & -2.682735 & -1.392527 \\
\hline 6 & 6.603840 & -1.924154 & -0.553676 \\
\hline 6 & 6.265656 & -0.571624 & -0.243784 \\
\hline 6 & 7.108771 & 0.136465 & 0.650856 \\
\hline 6 & 8.234229 & -0.447473 & 1.176776 \\
\hline
\end{tabular}




\begin{tabular}{|c|c|c|c|}
\hline & 8.583029 & -1.772336 & 0.840395 \\
\hline & 7.778475 & -2.492978 & -0.003612 \\
\hline & 3.046791 & -0.338601 & -2.024663 \\
\hline & -3.252058 & 0.002819 & -0.290232 \\
\hline & -4.224146 & -0.066228 & 0.711576 \\
\hline & -5.542185 & -0.397458 & 0.404262 \\
\hline & -5.854506 & -0.652674 & -0.937772 \\
\hline & -4.907955 & -0.594274 & -1.961578 \\
\hline & -3.594482 & -0.269340 & -1.609730 \\
\hline & -6.626668 & -0.513801 & 1.474668 \\
\hline & -5.319297 & -0.813052 & -3.416609 \\
\hline & -1.732972 & 2.152798 & 0.848563 \\
\hline & -2.796564 & 2.950021 & 0.447747 \\
\hline & -2.901759 & 4.284692 & 0.858834 \\
\hline & -1.920622 & 4.783978 & 1.709503 \\
\hline & -0.835556 & 4.005500 & 2.143296 \\
\hline & -0.738116 & 2.699035 & 1.672733 \\
\hline & -4.084235 & 5.112091 & 0.362037 \\
\hline & 0.198222 & 4.616319 & 3.088841 \\
\hline & -0.193392 & -0.087530 & -3.505887 \\
\hline & -0.257289 & -0.575953 & -4.553921 \\
\hline & 6.848827 & 1.154324 & 0.929828 \\
\hline & -1.618801 & 0.897343 & 3.074116 \\
\hline & 8.021306 & -3.523176 & -0.260563 \\
\hline & -1.019543 & -2.841930 & 5.909325 \\
\hline & 5.492717 & 5.953702 & -0.725835 \\
\hline & -3.230657 & -2.990745 & 1.047355 \\
\hline & -5.076802 & -4.189589 & -0.039097 \\
\hline & 3.434179 & 5.048746 & 0.186775 \\
\hline & -1.395097 & -0.602493 & 4.992287 \\
\hline & 6.026897 & -3.709982 & -1.628092 \\
\hline & 8.434664 & 3.336396 & -2.446969 \\
\hline & 3.913404 & -2.713571 & -2.506585 \\
\hline & -2.661094 & -4.822162 & -3.539694 \\
\hline & -0.390408 & -3.923151 & -3.635541 \\
\hline & -0.325511 & -6.152001 & 3.252110 \\
\hline & 7.073378 & 1.385253 & -1.866362 \\
\hline & -0.438501 & -4.656281 & 1.317593 \\
\hline 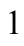 & 1.483490 & -2.732212 & -2.547782 \\
\hline & 7.672936 & 5.635260 & -1.858675 \\
\hline & 8.860148 & 0.116155 & 1.864993 \\
\hline l & -4.795678 & -5.144596 & -2.322428 \\
\hline & -0.607309 & -5.260601 & 5.562832 \\
\hline ] & 9.480386 & -2.220528 & 1.260445 \\
\hline & 1.968078 & 3.084899 & 0.668149 \\
\hline 1 & -3.940097 & 0.151782 & 1.739064 \\
\hline & -2.811128 & -0.222578 & -2.362097 \\
\hline & -6.883968 & -0.905924 & -1.191689 \\
\hline 1 & -3.555111 & 2.532234 & -0.212498 \\
\hline & 0.111726 & 2.078586 & 1.956197 \\
\hline 1 & -1.989561 & 5.814128 & 2.051681 \\
\hline & 3.155928 & -1.443804 & 1.101423 \\
\hline 1 & 1.627220 & -1.656985 & 1.929186 \\
\hline ] & -1.406376 & 1.613635 & -2.445697 \\
\hline 1 & 2.538692 & -3.239401 & 3.389574 \\
\hline 1 & 2.609795 & -4.019191 & -0.827957 \\
\hline
\end{tabular}




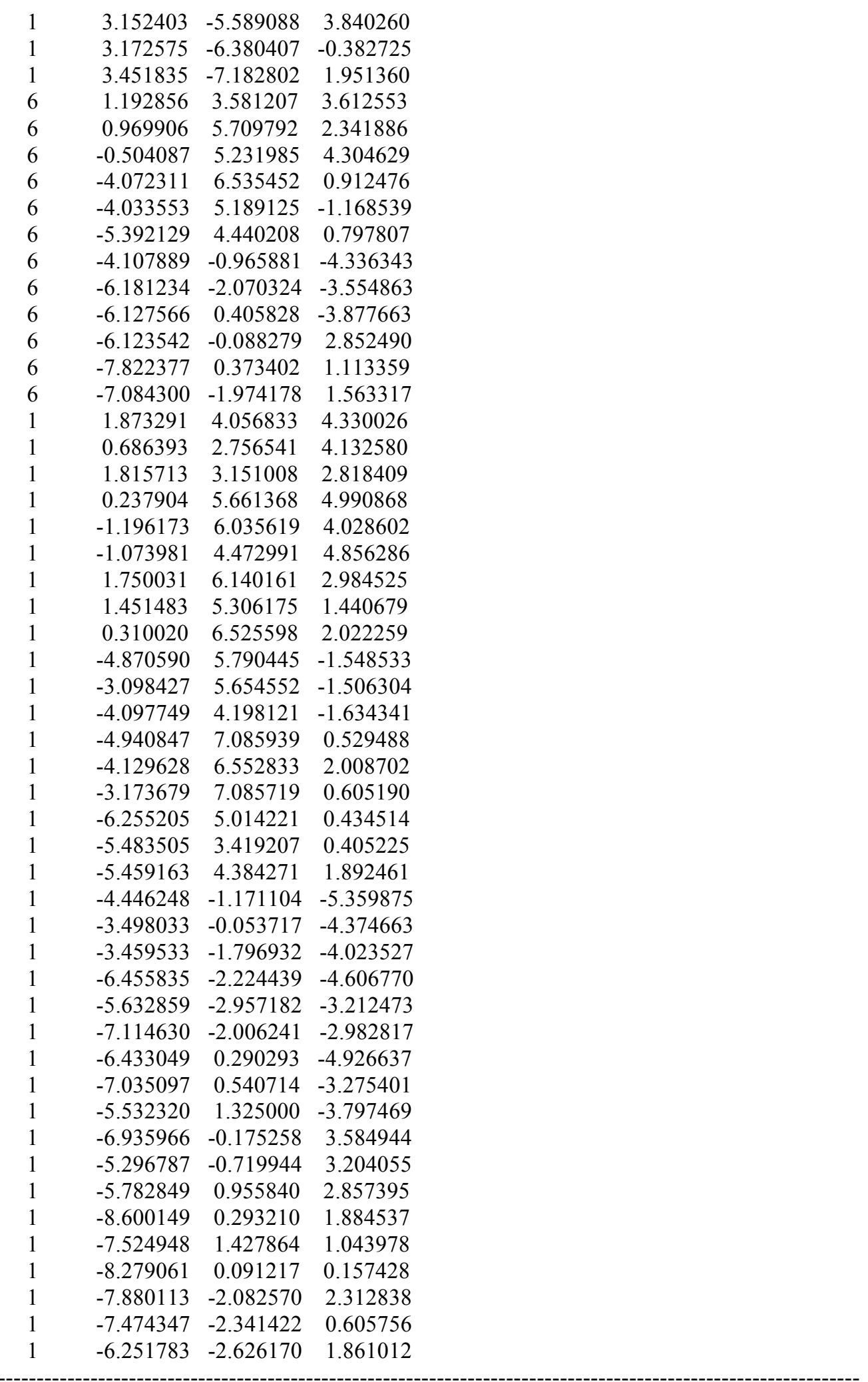

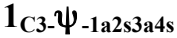

Number of imaginary frequencies : 0

The smallest frequencies are : $13.3346 \quad 15.9738 \quad 19.1173 \mathrm{~cm}(-1)$ 
Electronic energy : $\quad H F=-4125.4689178$

Zero-point correction $=$

1.299679 (Hartree/Particle)

Thermal correction to Energy $=\quad 1.377953$

Thermal correction to Enthalpy= $\quad 1.378897$

Thermal correction to Gibbs Free Energy= $\quad 1.187483$

Sum of electronic and zero-point Energies $=\quad-4124.169239$

Sum of electronic and thermal Energies $=\quad-4124.090965$

Sum of electronic and thermal Enthalpies $=\quad-4124.090021$

Sum of electronic and thermal Free Energies $=\quad-4124.281435$

\begin{tabular}{|c|c|c|c|}
\hline & \multicolumn{3}{|c|}{ Cartesian Coordinates } \\
\hline 6 & 3.187606 & -6.151803 & 1.737457 \\
\hline 6 & 3.004599 & -5.266545 & 2.795937 \\
\hline 6 & 2.669245 & -3.942212 & 2.544027 \\
\hline 6 & 2.529634 & -3.471800 & 1.236105 \\
\hline 6 & 2.737199 & -4.360623 & 0.181498 \\
\hline 6 & 3.053918 & -5.693121 & 0.430905 \\
\hline 6 & 2.218642 & -2.010070 & 1.053360 \\
\hline 7 & 1.553657 & -1.637756 & -0.207518 \\
\hline 6 & 0.441081 & -2.379089 & -0.724658 \\
\hline 6 & -0.750971 & -2.529868 & -0.034563 \\
\hline 6 & -1.838380 & -3.224867 & -0.653610 \\
\hline 6 & -1.690939 & -3.730647 & -1.978377 \\
\hline 6 & -0.465271 & -3.537905 & -2.654975 \\
\hline 6 & 0.568366 & -2.885775 & -2.042054 \\
\hline 6 & -3.089002 & -3.398891 & -0.004950 \\
\hline 6 & -4.121558 & -4.055511 & -0.626549 \\
\hline 6 & -3.960433 & -4.579969 & -1.927339 \\
\hline 6 & -2.771817 & -4.410858 & -2.590574 \\
\hline 6 & -0.924702 & -1.983023 & 1.339123 \\
\hline 6 & -1.208477 & -0.639225 & 1.553101 \\
\hline 6 & -1.383983 & -0.174233 & 2.884191 \\
\hline 6 & -1.284663 & -1.021448 & 3.955434 \\
\hline 6 & -1.021766 & -2.397448 & 3.770238 \\
\hline 6 & -0.844990 & -2.890318 & 2.444558 \\
\hline 6 & -0.944478 & -3.292269 & 4.864871 \\
\hline 6 & -0.718554 & -4.630138 & 4.662114 \\
\hline 6 & -0.551031 & -5.125153 & 3.351604 \\
\hline 6 & -0.601344 & -4.278392 & 2.272773 \\
\hline 15 & -1.513063 & 0.475275 & 0.092025 \\
\hline 45 & -0.073125 & 0.917636 & -1.882054 \\
\hline 6 & 0.394717 & 2.786533 & -1.675082 \\
\hline 8 & 0.463141 & 3.940338 & -1.679846 \\
\hline 15 & 1.791100 & -0.138143 & -0.967074 \\
\hline 8 & 2.577725 & 0.611708 & 0.281674 \\
\hline 6 & 3.415859 & 1.668162 & -0.023313 \\
\hline 6 & 4.669753 & 1.418739 & -0.548853 \\
\hline 6 & 5.488711 & 2.541877 & -0.893748 \\
\hline 6 & 5.029596 & 3.861048 & -0.595966 \\
\hline 6 & 3.757251 & 4.041356 & -0.003953 \\
\hline 6 & 2.952612 & 2.965886 & 0.259059 \\
\hline 6 & 5.842764 & 4.972832 & -0.925428 \\
\hline 6 & 7.051409 & 4.802614 & -1.549315 \\
\hline 6 & 7.491319 & 3.503406 & -1.879373 \\
\hline 6 & 6.732555 & 2.404677 & -1.561329 \\
\hline
\end{tabular}




\begin{tabular}{|c|c|c|c|}
\hline & 5.083239 & 0.016136 & -0.789190 \\
\hline & 4.263867 & -0.803100 & -1.545444 \\
\hline & 4.609747 & -2.128118 & -1.870768 \\
\hline & 5.771223 & -2.663800 & -1.384726 \\
\hline & 6.613935 & -1.905716 & -0.537788 \\
\hline & 6.269640 & -0.555361 & -0.225125 \\
\hline & 7.104942 & 0.152022 & 0.677379 \\
\hline & 8.228811 & -0.430417 & 1.208318 \\
\hline & 8.583769 & -1.752987 & 0.869387 \\
\hline & 7.786792 & -2.472981 & 0.017687 \\
\hline & 3.060915 & -0.325874 & -2.024830 \\
\hline & -3.243386 & -0.009535 & -0.307066 \\
\hline & -4.195885 & -0.110532 & 0.702024 \\
\hline & -5.521616 & -0.444117 & 0.413445 \\
\hline & -5.861476 & -0.653900 & -0.924263 \\
\hline & -4.927221 & -0.560438 & -1.964570 \\
\hline & -3.610179 & -0.246786 & -1.630875 \\
\hline & -6.521740 & -0.553607 & 1.563037 \\
\hline & -5.369568 & -0.744707 & -3.415147 \\
\hline & -1.742378 & 2.138287 & 0.842286 \\
\hline & -2.815335 & 2.926427 & 0.447910 \\
\hline & -2.932230 & 4.258456 & 0.864102 \\
\hline & -1.955748 & 4.762532 & 1.717423 \\
\hline & -0.863533 & 3.992129 & 2.147736 \\
\hline 6 & -0.752869 & 2.689468 & 1.669394 \\
\hline & -4.114032 & 5.081943 & 0.359040 \\
\hline 6 & 0.165089 & 4.607935 & 3.095700 \\
\hline & -0.189005 & -0.081935 & -3.515511 \\
\hline & -0.258687 & -0.568815 & -4.563780 \\
\hline 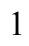 & 6.840169 & 1.168060 & 0.958402 \\
\hline & -1.620097 & 0.872198 & 3.059362 \\
\hline 1 & 8.034441 & -3.501484 & -0.241444 \\
\hline & -1.076928 & -2.894455 & 5.870174 \\
\hline & 5.476256 & 5.969332 & -0.682801 \\
\hline 1 & -3.229071 & -2.991860 & 0.994928 \\
\hline & -5.076176 & -4.167793 & -0.115818 \\
\hline 1 & 3.414861 & 5.052772 & 0.211723 \\
\hline & -1.427133 & -0.645025 & 4.967766 \\
\hline 1 & 6.049218 & -3.689342 & -1.622333 \\
\hline & 8.438014 & 3.370652 & -2.398187 \\
\hline 1 & 3.938077 & -2.696025 & -2.510412 \\
\hline 1 & -2.635640 & -4.797841 & -3.599644 \\
\hline & -0.357613 & -3.913120 & -3.671452 \\
\hline 1 & -0.358867 & -6.184176 & 3.194139 \\
\hline & 7.080273 & 1.411950 & -1.835037 \\
\hline 1 & -0.441452 & -4.671820 & 1.271151 \\
\hline & 1.510732 & -2.730126 & -2.565974 \\
\hline 1 & 7.664507 & 5.663974 & -1.803771 \\
\hline 1 & 8.848631 & 0.132599 & 1.902530 \\
\hline & -4.782525 & -5.111309 & -2.402381 \\
\hline 1 & -0.668802 & -5.311626 & 5.508434 \\
\hline & 9.479797 & -2.199985 & 1.293524 \\
\hline 1 & 1.952933 & 3.081434 & 0.674606 \\
\hline 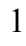 & -3.901283 & 0.080254 & 1.734081 \\
\hline 1 & -2.841049 & -0.177056 & -2.396056 \\
\hline 1 & -6.891643 & -0.899200 & -1.174416 \\
\hline
\end{tabular}




\begin{tabular}{|c|c|c|c|}
\hline 1 & -3.570455 & 2.504930 & -0.213870 \\
\hline & 0.103244 & 2.075855 & 1.949089 \\
\hline & -2.034030 & 5.790450 & 2.064280 \\
\hline & 3.160010 & -1.450620 & 1.094604 \\
\hline & 1.626837 & -1.661830 & 1.915022 \\
\hline & -1.399784 & 1.616783 & -2.451562 \\
\hline & 2.497070 & -3.259282 & 3.376831 \\
\hline & 2.645539 & -4.010799 & -0.843646 \\
\hline & 3.101055 & -5.612494 & 3.823195 \\
\hline & 3.198222 & -6.376050 & -0.403866 \\
\hline & 3.434132 & -7.193796 & 1.929645 \\
\hline & 1.164815 & 3.577969 & 3.619939 \\
\hline & 0.931928 & 5.705930 & 2.350321 \\
\hline & -0.541927 & 5.218976 & 4.311034 \\
\hline & -4.135772 & 6.490843 & 0.945296 \\
\hline & -4.020907 & 5.197368 & -1.167286 \\
\hline & -5.425570 & 4.384365 & 0.739454 \\
\hline & -4.179163 & -0.827379 & -4.369965 \\
\hline & -6.190301 & -2.027830 & -3.570695 \\
\hline & -6.230969 & 0.458374 & -3.817144 \\
\hline 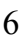 & -7.899759 & -1.012775 & 1.094362 \\
\hline ) & -6.010004 & -1.569194 & 2.592220 \\
\hline & -6.672039 & 0.818566 & 2.230017 \\
\hline & 1.841294 & 4.056720 & 4.339077 \\
\hline 1 & 0.662210 & 2.749758 & 4.138056 \\
\hline & 1.791648 & 3.152429 & 2.826421 \\
\hline & 0.196968 & 5.652982 & 4.997689 \\
\hline 1 & -1.238901 & 6.018320 & 4.034909 \\
\hline & -1.107255 & 4.456345 & 4.862368 \\
\hline 1 & 1.707802 & 6.141279 & 2.994760 \\
\hline & 1.418335 & 5.304787 & 1.450550 \\
\hline 1 & 0.267739 & 6.517480 & 2.028695 \\
\hline 1 & -4.856525 & 5.794203 & -1.557501 \\
\hline & -3.084134 & 5.685418 & -1.466424 \\
\hline 1 & -4.054236 & 4.216218 & -1.656862 \\
\hline t & -5.005194 & 7.037459 & 0.558794 \\
\hline 1 & -4.214518 & 6.478787 & 2.040305 \\
\hline ] & -3.240709 & 7.063328 & 0.669997 \\
\hline 1 & -6.284029 & 4.962731 & 0.372171 \\
\hline 1 & -5.498738 & 3.376112 & 0.311286 \\
\hline ] & -5.520588 & 4.292572 & 1.829675 \\
\hline 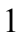 & -4.538250 & -1.000194 & -5.392419 \\
\hline d & -3.593559 & 0.101458 & -4.385206 \\
\hline 1 & -3.503753 & -1.653775 & -4.107442 \\
\hline ] & -6.486290 & -2.160857 & -4.619761 \\
\hline 1 & -5.601891 & -2.903959 & -3.269004 \\
\hline 1 & -7.109686 & -2.014365 & -2.973181 \\
\hline 1 & -6.562413 & 0.362124 & -4.860144 \\
\hline 1 & -7.125508 & 0.545940 & -3.187466 \\
\hline 1 & -5.664337 & 1.394525 & -3.726848 \\
\hline 1 & -8.575343 & -1.092889 & 1.955384 \\
\hline 1 & -8.352525 & -0.304158 & 0.389104 \\
\hline 1 & -7.858858 & -1.999088 & 0.613183 \\
\hline 1 & -6.716271 & -1.650071 & 3.429482 \\
\hline 1 & -5.908013 & -2.565948 & 2.142698 \\
\hline 1 & -5.033855 & -1.291477 & 3.010342 \\
\hline
\end{tabular}




$\begin{array}{llll}1 & -7.359566 & 0.757072 & 3.084574 \\ 1 & -5.712816 & 1.204457 & 2.598239 \\ 1 & -7.077920 & 1.554339 & 1.522979\end{array}$

$1_{\mathrm{C} 3-} \psi-1 \mathrm{a} 2 \mathrm{~s} 3 \mathrm{~s} 4 \mathrm{a}$

Number of imaginary frequencies : 0

The smallest frequencies are : $11.9291 \quad 15.7529 \quad 19.3075 \mathrm{~cm}(-1)$

Electronic energy : $\quad H F=-4125.4678062$

Zero-point correction $=\quad 1.299347($ Hartree/Particle $)$

Thermal correction to Energy= $\quad 1.377743$

Thermal correction to Enthalpy= $\quad 1.378687$

Thermal correction to Gibbs Free Energy= 1.186266

Sum of electronic and zero-point Energies $=\quad-4124.168459$

Sum of electronic and thermal Energies $=\quad-4124.090064$

Sum of electronic and thermal Enthalpies $=\quad-4124.089119$

Sum of electronic and thermal Free Energies $=\quad-4124.281540$

Cartesian Coordinates

$\begin{array}{cccc}6 & 3.364708 & -6.202903 & 1.394965 \\ 6 & 3.100292 & -5.413528 & 2.509981 \\ 6 & 2.726294 & -4.084544 & 2.349201 \\ 6 & 2.627582 & -3.515418 & 1.077911 \\ 6 & 2.905513 & -4.311817 & -0.033261 \\ 6 & 3.264739 & -5.646838 & 0.123208 \\ 6 & 2.284970 & -2.052453 & 0.985215 \\ 7 & 1.586166 & -1.632598 & -0.242617 \\ 6 & 0.474411 & -2.375187 & -0.760053 \\ 6 & -0.706112 & -2.550238 & -0.055080 \\ 6 & -1.796028 & -3.241929 & -0.673594 \\ 6 & -1.661894 & -3.718998 & -2.010834 \\ 6 & -0.445942 & -3.505984 & -2.698814 \\ 6 & 0.590166 & -2.856853 & -2.087059 \\ 6 & -3.037439 & -3.439409 & -0.014443 \\ 6 & -4.076075 & -4.081520 & -0.641576 \\ 6 & -3.930083 & -4.571753 & -1.956849 \\ 6 & -2.746534 & -4.390292 & -2.625281 \\ 6 & -0.860254 & -2.025854 & 1.330785 \\ 6 & -1.144339 & -0.685881 & 1.570213 \\ 6 & -1.284294 & -0.235893 & 2.910153 \\ 6 & -1.149289 & -1.094482 & 3.968325 \\ 6 & -0.890267 & -2.467753 & 3.759133 \\ 6 & -0.752739 & -2.945907 & 2.423093 \\ 6 & -0.782809 & -3.374769 & 4.841072 \\ 6 & -0.568786 & -4.711386 & 4.617265 \\ 6 & -0.443356 & -5.192195 & 3.296947 \\ 6 & -0.520285 & -4.332864 & 2.229453 \\ 15 & -1.500710 & 0.435447 & 0.130494 \\ 45 & -0.084834 & 0.946237 & -1.839339 \\ 6 & 0.375756 & 2.813015 & -1.604264 \\ 8 & 0.442514 & 3.966885 & -1.595129 \\ 15 & 1.793816 & -0.107250 & -0.953902 \\ 8 & 2.565498 & 0.615382 & 0.319994 \\ 6 & 3.386560 & 1.695340 & 0.055565 \\ 6 & 4.647152 & 1.486282 & -0.471819\end{array}$




\begin{tabular}{|c|c|c|c|}
\hline 6 & 5.450540 & 2.635261 & -0.766523 \\
\hline & 4.969550 & 3.934259 & -0.418656 \\
\hline & 3.689601 & 4.071498 & 0.168249 \\
\hline & 2.899969 & 2.974285 & 0.381672 \\
\hline & 5.767792 & 5.070939 & -0.695784 \\
\hline & 6.982741 & 4.944957 & -1.317756 \\
\hline & 7.443807 & 3.667353 & -1.698841 \\
\hline & 6.700172 & 2.545021 & -1.431474 \\
\hline & 5.084191 & 0.102589 & -0.773010 \\
\hline & 4.280875 & -0.695305 & -1.568679 \\
\hline & 4.658476 & -1.990851 & -1.968559 \\
\hline & 5.833044 & -2.525037 & -1.513565 \\
\hline & 6.657653 & -1.795649 & -0.624690 \\
\hline b & 6.283097 & -0.472429 & -0.239354 \\
\hline & 7.102448 & 0.203269 & 0.701247 \\
\hline & 8.238764 & -0.382206 & 1.201585 \\
\hline b & 8.623325 & -1.676037 & 0.791456 \\
\hline & 7.842928 & -2.365966 & -0.099564 \\
\hline ) & 3.064967 & -0.225220 & -2.020003 \\
\hline & -3.225596 & -0.084237 & -0.246999 \\
\hline & -4.145394 & -0.291930 & 0.788465 \\
\hline ) & -5.470189 & -0.608071 & 0.512716 \\
\hline 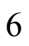 & -5.860455 & -0.684273 & -0.835635 \\
\hline ) & -4.973362 & -0.470622 & -1.886424 \\
\hline 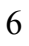 & -3.638056 & -0.188657 & -1.565166 \\
\hline$b$ & -6.486315 & -0.897582 & 1.616514 \\
\hline 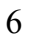 & -5.391002 & -0.472758 & -3.354531 \\
\hline & -1.784730 & 2.091290 & 0.879673 \\
\hline 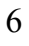 & -2.873791 & 2.834653 & 0.444283 \\
\hline ) & -3.045869 & 4.169649 & 0.823908 \\
\hline 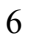 & -2.108257 & 4.726124 & 1.688467 \\
\hline 6 & -1.001722 & 4.002602 & 2.162087 \\
\hline 6 & -0.834010 & 2.693425 & 1.716168 \\
\hline 6 & -4.239957 & 4.936192 & 0.261880 \\
\hline & -0.016882 & 4.675648 & 3.118388 \\
\hline & -0.207999 & -0.019723 & -3.491673 \\
\hline & -0.291857 & -0.480806 & -4.550783 \\
\hline & 6.814956 & 1.196566 & 1.036241 \\
\hline 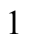 & -1.526704 & 0.806027 & 3.102664 \\
\hline . & 8.113617 & -3.372980 & -0.414168 \\
\hline . & -0.884531 & -2.987503 & 5.854013 \\
\hline 1 & 5.384419 & 6.050734 & -0.414314 \\
\hline & -3.167734 & -3.060971 & 0.998041 \\
\hline 1 & -5.023227 & -4.210311 & -0.120804 \\
\hline & 3.328855 & 5.068393 & 0.419097 \\
\hline 1 & -1.264134 & -0.730516 & 4.988632 \\
\hline 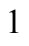 & 6.135188 & -3.528924 & -1.807802 \\
\hline 1 & 8.395312 & 3.570351 & -2.216788 \\
\hline 1 & 3.997987 & -2.537273 & -2.638343 \\
\hline 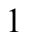 & -2.621143 & -4.749997 & -3.645865 \\
\hline 1 & -0.349070 & -3.861141 & -3.723521 \\
\hline . & -0.262251 & -6.250386 & 3.121969 \\
\hline 1 & 7.065164 & 1.570272 & -1.744143 \\
\hline . & -0.392537 & -4.716658 & 1.219477 \\
\hline 1 & 1.525158 & -2.681874 & -2.618070 \\
\hline 1 & 7.584367 & 5.825145 & -1.531988 \\
\hline
\end{tabular}




\begin{tabular}{|c|c|c|c|}
\hline & 8.845459 & 0.155593 & 1.926703 \\
\hline ] & -4.759433 & -5.084725 & -2.439163 \\
\hline & -0.497051 & -5.402416 & 5.454177 \\
\hline 1 & 9.528887 & -2.125482 & 1.192083 \\
\hline & 1.893174 & 3.059568 & 0.787016 \\
\hline | & -3.807287 & -0.200251 & 1.818249 \\
\hline & -2.904216 & -0.027127 & -2.353503 \\
\hline ] & -6.899302 & -0.918510 & -1.060117 \\
\hline 1 & -3.596740 & 2.375570 & -0.228012 \\
\hline 1 & 0.041098 & 2.119948 & 2.021825 \\
\hline 1 & -2.228820 & 5.758637 & 2.008780 \\
\hline 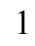 & 3.214149 & -1.473845 & 1.038268 \\
\hline & 1.701313 & -1.763724 & 1.873752 \\
\hline ] & -1.418125 & 1.650751 & -2.387867 \\
\hline & 2.486604 & -3.479709 & 3.224636 \\
\hline 1 & 2.839962 & -3.881678 & -1.029464 \\
\hline 1 & 3.164376 & -5.837741 & 3.510152 \\
\hline 1 & 3.468191 & -6.255450 & -0.755617 \\
\hline 1 & 3.644507 & -7.247200 & 1.515835 \\
\hline 6 & 0.994632 & 3.690840 & 3.703418 \\
\hline & 0.744547 & 5.768965 & 2.360956 \\
\hline 6 & -0.774418 & 5.306282 & 4.292705 \\
\hline 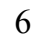 & -4.332955 & 6.355018 & 0.815812 \\
\hline ? & -4.098187 & 5.020936 & -1.263129 \\
\hline 6 & -5.537673 & 4.197293 & 0.611269 \\
\hline ? & -6.862939 & -0.831238 & -3.539195 \\
\hline 6 & -5.160219 & 0.932221 & -3.926310 \\
\hline 6 & -4.552186 & -1.486184 & -4.139047 \\
\hline 6 & -5.908067 & -0.656251 & 3.009481 \\
\hline 6 & -7.717813 & -0.001237 & 1.452556 \\
\hline f & -6.911238 & -2.367850 & 1.527635 \\
\hline 1 & 1.633059 & 4.209407 & 4.429732 \\
\hline 1 & 0.500464 & 2.862097 & 4.228551 \\
\hline 1 & 1.659702 & 3.263969 & 2.942382 \\
\hline 1 & -0.066545 & 5.775621 & 4.988676 \\
\hline 1 & -1.478321 & 6.082494 & 3.971729 \\
\hline 1 & -1.341373 & 4.548197 & 4.848564 \\
\hline 1 & 1.477094 & 6.256066 & 3.019045 \\
\hline 1 & 1.283774 & 5.347951 & 1.501171 \\
\hline 1 & 0.069089 & 6.543420 & 1.977434 \\
\hline 1 & -4.943608 & 5.573098 & -1.695864 \\
\hline 1 & -3.172698 & 5.541534 & -1.541816 \\
\hline 1 & -4.073612 & 4.027885 & -1.730111 \\
\hline 1 & -5.206337 & 6.862574 & 0.387235 \\
\hline 1 & -4.450266 & 6.361947 & 1.907459 \\
\hline 1 & -3.449043 & 6.953318 & 0.560194 \\
\hline 1 & -6.403843 & 4.745578 & 0.217059 \\
\hline 1 & -5.568044 & 3.185006 & 0.187220 \\
\hline 1 & -5.660283 & 4.107611 & 1.698899 \\
\hline 1 & -7.112393 & -0.827445 & -4.607730 \\
\hline 1 & -7.090199 & -1.832954 & -3.150858 \\
\hline 1 & -7.527942 & -0.110716 & -3.045599 \\
\hline 1 & -5.468003 & 0.972451 & -4.979943 \\
\hline 1 & -5.743300 & 1.681126 & -3.373165 \\
\hline 1 & -4.103662 & 1.226921 & -3.876806 \\
\hline 1 & -4.839751 & -1.474886 & -5.199045 \\
\hline
\end{tabular}




$\begin{array}{rrrr}1 & -3.477242 & -1.271032 & -4.087007 \\ 1 & -4.705579 & -2.501656 & -3.751902 \\ 1 & -6.672020 & -0.869984 & 3.767621 \\ 1 & -5.048287 & -1.307668 & 3.218762 \\ 1 & -5.589314 & 0.385845 & 3.144016 \\ 1 & -8.443727 & -0.205392 & 2.250934 \\ 1 & -7.443670 & 1.060051 & 1.510376 \\ 1 & -8.229081 & -0.164040 & 0.496491 \\ 1 & -7.659988 & -2.598129 & 2.297807 \\ 1 & -7.349488 & -2.612087 & 0.551780 \\ 1 & -6.050159 & -3.031466 & 1.688194\end{array}$

$1_{\mathrm{C} 3-} \psi_{-1 \mathrm{a} 23354 \mathrm{~s}}$

Number of imaginary frequencies : 0

The smallest frequencies are : $12.9739 \quad 16.2888 \quad 20.5014 \mathrm{~cm}(-1)$

Electronic energy : $\quad H F=-4125.4681254$

Zero-point correction $=\quad 1.299692($ Hartree/Particle $)$

Thermal correction to Energy $=\quad 1.377910$

Thermal correction to Enthalpy= $\quad 1.378855$

Thermal correction to Gibbs Free Energy= $\quad 1.187677$

Sum of electronic and zero-point Energies $=\quad-4124.168434$

Sum of electronic and thermal Energies= $\quad-4124.090215$

Sum of electronic and thermal Enthalpies $=\quad-4124.089271$

Sum of electronic and thermal Free Energies $=\quad-4124.280448$

Cartesian Coordinates

$\begin{array}{lrrrr}6 & 3.370502 & -6.196882 & 1.401377 \\ 6 & 3.101820 & -5.407287 & 2.515192 \\ 6 & 2.727580 & -4.078538 & 2.352685 \\ 6 & 2.632756 & -3.510022 & 1.080860 \\ 6 & 2.915141 & -4.306621 & -0.029066 \\ 6 & 3.274657 & -5.641331 & 0.129052 \\ 6 & 2.289472 & -2.047405 & 0.985750 \\ 7 & 1.591319 & -1.630334 & -0.243569 \\ 6 & 0.481498 & -2.376595 & -0.760011 \\ 6 & -0.698080 & -2.554159 & -0.054335 \\ 6 & -1.785366 & -3.252111 & -0.670364 \\ 6 & -1.650016 & -3.734520 & -2.005318 \\ 6 & -0.435253 & -3.518216 & -2.694636 \\ 6 & 0.598254 & -2.862042 & -2.085671 \\ 6 & -3.025802 & -3.449204 & -0.009859 \\ 6 & -4.062944 & -4.097494 & -0.632728 \\ 6 & -3.915831 & -4.594345 & -1.945434 \\ 6 & -2.732893 & -4.412745 & -2.615384 \\ 6 & -0.856819 & -2.026213 & 1.329505 \\ 6 & -1.145254 & -0.686388 & 1.563719 \\ 6 & -1.293627 & -0.233195 & 2.901677 \\ 6 & -1.165214 & -1.089264 & 3.962762 \\ 6 & -0.902846 & -2.462641 & 3.758576 \\ 6 & -0.753931 & -2.943361 & 2.424717 \\ 6 & -0.801486 & -3.366915 & 4.843396 \\ 6 & -0.581966 & -4.703357 & 4.624255 \\ 6 & -0.444469 & -5.186670 & 3.306050 \\ 6 & -0.515797 & -4.330041 & 2.236011\end{array}$




\begin{tabular}{|c|c|c|c|}
\hline 15 & -1.498560 & 0.430574 & 0.119421 \\
\hline & -0.081380 & 0.942722 & -1.846555 \\
\hline 6 & 0.367794 & 2.813390 & -1.615390 \\
\hline 8 & 0.423589 & 3.967877 & -1.609961 \\
\hline & 1.797407 & -0.105063 & -0.955526 \\
\hline 8 & 2.562759 & 0.621225 & 0.320069 \\
\hline 6 & 3.379259 & 1.704697 & 0.055866 \\
\hline 6 & 4.642311 & 1.500567 & -0.467453 \\
\hline 6 & 5.441166 & 2.652763 & -0.761886 \\
\hline 6 & 4.952881 & 3.950174 & -0.418127 \\
\hline 6 & 3.670324 & 4.082565 & 0.164219 \\
\hline 6 & 2.885335 & 2.981983 & 0.377356 \\
\hline 6 & 5.746658 & 5.090080 & -0.694866 \\
\hline 6 & 6.964239 & 4.968609 & -1.312558 \\
\hline 6 & 7.432621 & 3.692464 & -1.689571 \\
\hline 6 & 6.693421 & 2.567140 & -1.422511 \\
\hline 6 & 5.086370 & 0.118156 & -0.764348 \\
\hline 6 & 4.288804 & -0.685152 & -1.560425 \\
\hline 6 & 4.672583 & -1.980530 & -1.955010 \\
\hline 6 & 5.847475 & -2.508980 & -1.494184 \\
\hline 6 & 6.666371 & -1.773747 & -0.604833 \\
\hline 6 & 6.285675 & -0.450664 & -0.225002 \\
\hline 6 & 7.099358 & 0.230987 & 0.716236 \\
\hline 6 & 8.236059 & -0.348760 & 1.222321 \\
\hline 6 & 8.626658 & -1.642522 & 0.817764 \\
\hline 6 & 7.851836 & -2.338117 & -0.073700 \\
\hline 8 & 3.072554 & -0.221002 & -2.017039 \\
\hline 6 & -3.216613 & -0.103218 & -0.268312 \\
\hline 6 & -4.136241 & -0.314131 & 0.758818 \\
\hline 6 & -5.461112 & -0.645973 & 0.483318 \\
\hline 6 & -5.850852 & -0.724106 & -0.860043 \\
\hline 6 & -4.957428 & -0.504246 & -1.910997 \\
\hline 6 & -3.627566 & -0.214552 & -1.590146 \\
\hline 6 & -6.424631 & -0.878219 & 1.646402 \\
\hline 6 & -5.374571 & -0.513192 & -3.379865 \\
\hline 6 & -1.802403 & 2.083069 & 0.868081 \\
\hline 6 & -2.903495 & 2.812633 & 0.439600 \\
\hline 6 & -3.087612 & 4.146608 & 0.817705 \\
\hline 6 & -2.151678 & 4.714558 & 1.676484 \\
\hline 6 & -1.035449 & 4.003630 & 2.146608 \\
\hline 6 & -0.854776 & 2.696460 & 1.700029 \\
\hline 6 & -4.290676 & 4.900554 & 0.257820 \\
\hline 6 & -0.054550 & 4.687713 & 3.099207 \\
\hline 6 & -0.195823 & -0.026458 & -3.497392 \\
\hline 8 & -0.274278 & -0.491095 & -4.555352 \\
\hline 1 & 6.807170 & 1.224285 & 1.047126 \\
\hline 1 & -1.537405 & 0.809129 & 3.090216 \\
\hline 1 & 8.127224 & -3.345172 & -0.384079 \\
\hline 1 & -0.911906 & -2.977641 & 5.854652 \\
\hline 1 & 5.357751 & 6.068600 & -0.416580 \\
\hline 1 & -3.156622 & -3.064480 & 0.999817 \\
\hline 1 & -5.010508 & -4.223369 & -0.111516 \\
\hline 1 & 3.303934 & 5.078233 & 0.411747 \\
\hline 1 & -1.286901 & -0.722988 & 4.981461 \\
\hline 1 & 6.154197 & -3.512734 & -1.784105 \\
\hline 1 & 8.386311 & 3.598974 & -2.204131 \\
\hline
\end{tabular}




\begin{tabular}{|c|c|c|c|}
\hline & 4.016375 & -2.531523 & -2.625243 \\
\hline & -2.607315 & -4.777154 & -3.634282 \\
\hline & -0.337020 & -3.876465 & -3.718146 \\
\hline & -0.258685 & -6.244678 & 3.134892 \\
\hline & 7.064063 & 1.593514 & -1.732024 \\
\hline & -0.378666 & -4.715594 & 1.227914 \\
\hline ] & 1.532171 & -2.684663 & -2.617832 \\
\hline & 7.562425 & 5.851204 & -1.526511 \\
\hline & 8.838330 & 0.193577 & 1.947749 \\
\hline 1 & -4.743751 & -5.112022 & -2.425179 \\
\hline 1 & -0.514823 & -5.392349 & 5.463222 \\
\hline 1 & 9.532423 & -2.087417 & 1.222990 \\
\hline 1 & 1.876704 & 3.063274 & 0.778817 \\
\hline 1 & -3.809894 & -0.212279 & 1.793708 \\
\hline & -2.892824 & -0.051355 & -2.377243 \\
\hline 1 & -6.884735 & -0.963840 & -1.091586 \\
\hline & -3.625371 & 2.344390 & -0.227447 \\
\hline 1 & 0.027968 & 2.132728 & 2.002212 \\
\hline & -2.281751 & 5.746256 & 1.995528 \\
\hline 1 & 3.218271 & -1.468104 & 1.038531 \\
\hline & 1.704842 & -1.757570 & 1.873294 \\
\hline ] & -1.417298 & 1.639772 & -2.398378 \\
\hline$\frac{1}{1}$ & 2.484918 & -3.473401 & 3.227099 \\
\hline & 2.852597 & -3.876689 & -1.025554 \\
\hline 1 & 3.162858 & -5.831090 & 3.515722 \\
\hline 1 & 3.481553 & -6.250127 & -0.748833 \\
\hline 1 & 3.650484 & -7.240972 & 1.523592 \\
\hline 6 & 0.964535 & 3.712395 & 3.687119 \\
\hline 6 & 0.698405 & 5.783567 & 2.336994 \\
\hline 6 & -0.815096 & 5.317696 & 4.271916 \\
\hline 6 & -4.397094 & 6.318736 & 0.811011 \\
\hline 6 & -4.151643 & 4.985580 & -1.267496 \\
\hline 6 & -5.580231 & 4.148859 & 0.609904 \\
\hline 6 & -6.843689 & -0.883145 & -3.565687 \\
\hline 5 & -5.154266 & 0.891709 & -3.955900 \\
\hline 6 & -4.528163 & -1.522508 & -4.161526 \\
\hline 6 & -7.782523 & -1.398808 & 1.181611 \\
\hline 6 & -5.833320 & -1.910130 & 2.614766 \\
\hline 6 & -6.642024 & 0.448512 & 2.383380 \\
\hline 1 & 1.597840 & 4.237598 & 4.413149 \\
\hline 1 & 0.476688 & 2.880512 & 4.213188 \\
\hline 1 & 1.634209 & 3.289731 & 2.927778 \\
\hline 1 & -0.109524 & 5.794319 & 4.965236 \\
\hline 1 & -1.524329 & 6.088189 & 3.948929 \\
\hline 1 & -1.376428 & 4.558065 & 4.831355 \\
\hline 1 & 1.427331 & 6.278855 & 2.992997 \\
\hline 1 & 1.240887 & 5.363241 & 1.478947 \\
\hline 1 & 0.017041 & 6.551326 & 1.950447 \\
\hline 1 & -5.001578 & 5.531378 & -1.699454 \\
\hline 1 & -3.230139 & 5.512485 & -1.547705 \\
\hline 1 & -4.120408 & 3.992411 & -1.733819 \\
\hline 1 & -5.277512 & 6.816411 & 0.385260 \\
\hline 1 & -4.510434 & 6.325206 & 1.903068 \\
\hline 1 & -3.520730 & 6.926474 & 0.551683 \\
\hline 1 & -6.452389 & 4.686525 & 0.214241 \\
\hline 1 & -5.598894 & 3.135041 & 0.188272 \\
\hline
\end{tabular}




$\begin{array}{rrrr}1 & -5.701256 & 4.060417 & 1.697718 \\ 1 & -7.091379 & -0.883078 & -4.634662 \\ 1 & -7.064250 & -1.885810 & -3.176017 \\ 1 & -7.515134 & -0.166894 & -3.074676 \\ 1 & -5.459734 & 0.925918 & -5.010452 \\ 1 & -5.745203 & 1.637468 & -3.406883 \\ 1 & -4.100463 & 1.195646 & -3.904415 \\ 1 & -4.815525 & -1.516473 & -5.221641 \\ 1 & -3.454940 & -1.299113 & -4.109619 \\ 1 & -4.674119 & -2.537933 & -3.771404 \\ 1 & -8.425940 & -1.581709 & 2.051553 \\ 1 & -8.303438 & -0.679028 & 0.537671 \\ 1 & -7.688973 & -2.345823 & 0.633030 \\ 1 & -6.518493 & -2.074108 & 3.457309 \\ 1 & -5.681684 & -2.876997 & 2.115667 \\ 1 & -4.868794 & -1.596045 & 3.033975 \\ 1 & -7.303831 & 0.304975 & 3.248480 \\ 1 & -5.698367 & 0.874724 & 2.747229 \\ 1 & -7.108242 & 1.190188 & 1.721248\end{array}$

$1_{\mathrm{C} 3-} \psi_{-1 \text { s2a3a4a }}$

Number of imaginary frequencies : 0

The smallest frequencies are : $13.7345 \quad 14.5879 \quad 16.0039 \mathrm{~cm}(-1)$

Electronic energy : $\quad H F=-4125.4663959$

Zero-point correction $=\quad 1.299855($ Hartree/Particle $)$

Thermal correction to Energy $=\quad 1.378100$

Thermal correction to Enthalpy= $\quad 1.379045$

Thermal correction to Gibbs Free Energy $=1.187448$

Sum of electronic and zero-point Energies $=\quad-4124.166540$

Sum of electronic and thermal Energies $=\quad-4124.088296$

Sum of electronic and thermal Enthalpies $=\quad-4124.087351$

Sum of electronic and thermal Free Energies $=\quad-4124.278947$

Cartesian Coordinates

$\begin{array}{lrrrr}6 & 3.523470 & -5.964690 & 1.761381 \\ 6 & 3.337354 & -5.085072 & 2.824368 \\ 6 & 2.934348 & -3.778083 & 2.582273 \\ 6 & 2.728536 & -3.318208 & 1.279065 \\ 6 & 2.936245 & -4.200816 & 0.219808 \\ 6 & 3.321820 & -5.516993 & 0.460073 \\ 6 & 2.346727 & -1.871465 & 1.109734 \\ 7 & 1.666938 & -1.514727 & -0.147074 \\ 6 & 0.577934 & -2.292417 & -0.659375 \\ 6 & -0.598474 & -2.498272 & 0.043279 \\ 6 & -1.648927 & -3.264706 & -0.556933 \\ 6 & -1.492095 & -3.760921 & -1.884304 \\ 6 & -0.291094 & -3.494767 & -2.580164 \\ 6 & 0.714981 & -2.791351 & -1.979142 \\ 6 & -2.871668 & -3.527622 & 0.113978 \\ 6 & -3.867875 & -4.253243 & -0.490670 \\ 6 & -3.699300 & -4.757713 & -1.797490 \\ 6 & -2.536767 & -4.507546 & -2.480894 \\ 6 & -0.797406 & -1.926082 & 1.404096 \\ 6 & -1.160159 & -0.594329 & 1.579556\end{array}$




\begin{tabular}{|c|c|c|c|}
\hline 6 & -1.364574 & -0.103137 & 2.896289 \\
\hline 6 & -1.202450 & -0.907329 & 3.992738 \\
\hline 6 & -0.851905 & -2.268306 & 3.848232 \\
\hline 6 & -0.659556 & -2.792287 & 2.536283 \\
\hline 6 & -0.711494 & -3.121350 & 4.969540 \\
\hline 6 & -0.413246 & -4.450357 & 4.806564 \\
\hline 6 & -0.233647 & -4.977566 & 3.510311 \\
\hline 6 & -0.341150 & -4.169952 & 2.405789 \\
\hline 15 & -1.509493 & 0.462546 & 0.085171 \\
\hline 45 & -0.018519 & 0.997046 & -1.825641 \\
\hline 6 & 0.380855 & 2.875834 & -1.567839 \\
\hline 8 & 0.421421 & 4.030666 & -1.539539 \\
\hline 15 & 1.872120 & -0.014191 & -0.914198 \\
\hline 8 & 2.662105 & 0.749923 & 0.322046 \\
\hline 6 & 3.488586 & 1.811935 & 0.004480 \\
\hline 6 & 4.741068 & 1.573990 & -0.528682 \\
\hline 6 & 5.545435 & 2.706115 & -0.879652 \\
\hline 6 & 5.073117 & 4.020968 & -0.581848 \\
\hline 6 & 3.801186 & 4.188882 & 0.015293 \\
\hline 6 & 3.012712 & 3.103866 & 0.285625 \\
\hline 6 & 5.873109 & 5.140790 & -0.916330 \\
\hline 6 & 7.080977 & 4.982843 & -1.544970 \\
\hline 6 & 7.533235 & 3.688088 & -1.875545 \\
\hline 6 & 6.787543 & 2.581741 & -1.552991 \\
\hline 6 & 5.165285 & 0.175744 & -0.774324 \\
\hline 6 & 4.346339 & -0.649030 & -1.525205 \\
\hline 6 & 4.705586 & -1.967002 & -1.864070 \\
\hline 6 & 5.878146 & -2.492194 & -1.393476 \\
\hline 6 & 6.719450 & -1.730412 & -0.548564 \\
\hline 6 & 6.363126 & -0.385872 & -0.224768 \\
\hline 6 & 7.198562 & 0.325528 & 0.674410 \\
\hline 6 & 8.333147 & -0.247758 & 1.192417 \\
\hline 6 & 8.699436 & -1.564443 & 0.842710 \\
\hline 6 & 7.903167 & -2.288065 & -0.006584 \\
\hline 8 & 3.131830 & -0.184285 & -1.987895 \\
\hline 6 & -3.169590 & -0.190664 & -0.372216 \\
\hline 6 & -4.147134 & -0.374721 & 0.608652 \\
\hline 6 & -5.438000 & -0.771468 & 0.266605 \\
\hline 6 & -5.717018 & -0.972553 & -1.091149 \\
\hline 6 & -4.762399 & -0.802483 & -2.095704 \\
\hline 6 & -3.475823 & -0.415517 & -1.709303 \\
\hline 6 & -6.521757 & -1.016156 & 1.316042 \\
\hline 6 & -5.148301 & -0.981326 & -3.562904 \\
\hline 6 & -1.963722 & 2.109514 & 0.779553 \\
\hline 6 & -3.124354 & 2.735172 & 0.314782 \\
\hline 6 & -3.468016 & 4.019237 & 0.724544 \\
\hline 6 & -2.628172 & 4.661773 & 1.642524 \\
\hline 6 & -1.452027 & 4.079585 & 2.114567 \\
\hline 6 & -1.114239 & 2.804498 & 1.639588 \\
\hline 6 & -4.750629 & 4.699015 & 0.248336 \\
\hline 6 & -0.570649 & 4.757562 & 3.164213 \\
\hline 6 & -0.062914 & 0.013317 & -3.470905 \\
\hline 8 & -0.090771 & -0.473728 & -4.521002 \\
\hline 1 & 6.925063 & 1.337040 & 0.963446 \\
\hline 1 & -1.680070 & 0.926662 & 3.040847 \\
\hline 1 & 8.159746 & -3.312259 & -0.273946 \\
\hline
\end{tabular}




\begin{tabular}{|c|c|c|c|}
\hline 1 & -0.856356 & -2.699328 & 5.963185 \\
\hline & 5.497132 & 6.133638 & -0.673177 \\
\hline & -3.018756 & -3.140239 & 1.120596 \\
\hline & -4.797966 & -4.441873 & 0.041988 \\
\hline & 3.447209 & 5.196101 & 0.230377 \\
\hline & -1.366505 & -0.509535 & 4.993672 \\
\hline & 6.165843 & -3.512700 & -1.641117 \\
\hline & 8.479074 & 3.564746 & -2.398236 \\
\hline & 4.034900 & -2.537844 & -2.502222 \\
\hline & -2.393703 & -4.880235 & -3.494558 \\
\hline & -0.177541 & -3.861568 & -3.599140 \\
\hline & 0.013334 & -6.029347 & 3.383750 \\
\hline & 7.144818 & 1.592585 & -1.827225 \\
\hline & -0.170757 & -4.586619 & 1.415264 \\
\hline & 1.643054 & -2.591092 & -2.513186 \\
\hline & 7.683880 & 5.850428 & -1.802658 \\
\hline & 8.952738 & 0.317861 & 1.884719 \\
\hline & -4.494479 & -5.338046 & -2.260643 \\
\hline & -0.316509 & -5.100378 & 5.673274 \\
\hline & 9.603747 & -2.004177 & 1.256746 \\
\hline & 2.016603 & 3.206909 & 0.707603 \\
\hline & -3.889901 & -0.189436 & 1.649445 \\
\hline 1 & -2.690032 & -0.273133 & -2.447221 \\
\hline & -6.725195 & -1.274474 & -1.376072 \\
\hline 1 & -3.763773 & 2.203724 & -0.383595 \\
\hline & -0.183403 & 2.335078 & 1.965864 \\
\hline & -2.907534 & 5.651479 & 1.995688 \\
\hline 1 & 3.260133 & -1.268823 & 1.159960 \\
\hline & 1.737619 & -1.561235 & 1.973852 \\
\hline 1 & -1.357637 & 1.653599 & -2.415353 \\
\hline & 2.757416 & -3.101427 & 3.419604 \\
\hline 1 & 2.790280 & -3.860465 & -0.802007 \\
\hline 1 & 3.486027 & -5.422946 & 3.848050 \\
\hline & 3.465764 & -6.195606 & -0.378296 \\
\hline 1 & 3.823957 & -6.993688 & 1.946556 \\
\hline ) & -1.023041 & 6.183469 & 3.471550 \\
\hline 6 & -0.639128 & 3.942666 & 4.461662 \\
\hline$b$ & 0.882825 & 4.815707 & 2.686288 \\
\hline 6 & -5.436296 & 3.918132 & -0.872509 \\
\hline 6 & -5.722856 & 4.799348 & 1.429498 \\
\hline 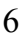 & -4.439844 & 6.103348 & -0.278688 \\
\hline 6 & -3.941419 & -0.861663 & -4.492247 \\
\hline ) & -5.782126 & -2.357487 & -3.782985 \\
\hline 6 & -6.155826 & 0.110929 & -3.941032 \\
\hline j & -6.064511 & -0.620285 & 2.718677 \\
\hline 6 & -7.777501 & -0.204652 & 0.982461 \\
\hline 6 & -6.870437 & -2.508725 & 1.331785 \\
\hline 1 & -0.351227 & 6.628611 & 4.216045 \\
\hline 1 & -0.998104 & 6.821591 & 2.578766 \\
\hline 1 & -2.036947 & 6.215247 & 3.890020 \\
\hline 1 & 1.496139 & 5.380765 & 3.400709 \\
\hline 1 & 1.327695 & 3.817175 & 2.604578 \\
\hline 1 & 0.957366 & 5.309669 & 1.707702 \\
\hline 1 & -0.023128 & 4.410014 & 5.242087 \\
\hline 1 & -1.670286 & 3.882751 & 4.834243 \\
\hline 1 & -0.272238 & 2.917865 & 4.318859 \\
\hline
\end{tabular}




$\begin{array}{rrrr}1 & -6.658436 & 5.283078 & 1.117170 \\ 1 & -5.969978 & 3.802112 & 1.818251 \\ 1 & -5.302784 & 5.386094 & 2.255934 \\ 1 & -6.338159 & 4.453907 & -1.194582 \\ 1 & -4.784171 & 3.807265 & -1.748804 \\ 1 & -5.749519 & 2.914446 & -0.554020 \\ 1 & -5.359200 & 6.583910 & -0.638981 \\ 1 & -4.012207 & 6.754746 & 0.492573 \\ 1 & -3.729501 & 6.061689 & -1.114206 \\ 1 & -4.257696 & -1.027893 & -5.529814 \\ 1 & -3.483934 & 0.135755 & -4.447384 \\ 1 & -3.166519 & -1.604579 & -4.257344 \\ 1 & -6.070367 & -2.477710 & -4.835940 \\ 1 & -5.071858 & -3.155096 & -3.530080 \\ 1 & -6.684365 & -2.505178 & -3.176965 \\ 1 & -6.437719 & 0.022696 & -4.999113 \\ 1 & -7.074015 & 0.045778 & -3.343918 \\ 1 & -5.726995 & 1.110011 & -3.786771 \\ 1 & -6.875241 & -0.801197 & 3.435668 \\ 1 & -5.196846 & -1.205911 & 3.051577 \\ 1 & -5.801690 & 0.444596 & 2.775885 \\ 1 & -8.553215 & -0.379328 & 1.739903 \\ 1 & -7.559517 & 0.871109 & 0.967024 \\ 1 & -8.204804 & -0.474032 & 0.009400 \\ 1 & -7.667309 & -2.709728 & 2.060763 \\ 1 & -7.216164 & -2.859931 & 0.351260 \\ 1 & -5.995293 & -3.108863 & 1.616629\end{array}$

$1_{\mathrm{C} 3-} \Psi_{-1 \mathrm{~s} 2 \mathrm{aza} 4 \mathrm{~s}}$

Number of imaginary frequencies : 0

The smallest frequencies are : $10.5539 \quad 14.2615 \quad 15.8651 \mathrm{~cm}(-1)$

Electronic energy : $\quad H F=-4125.4673782$

Zero-point correction $=\quad 1.299725($ Hartree/Particle $)$

Thermal correction to Energy= $\quad 1.377985$

Thermal correction to Enthalpy $=\quad 1.378929$

Thermal correction to Gibbs Free Energy= 1.186497

Sum of electronic and zero-point Energies $=\quad-4124.167653$

Sum of electronic and thermal Energies $=\quad-4124.089393$

Sum of electronic and thermal Enthalpies $=\quad-4124.088449$

Sum of electronic and thermal Free Energies $=\quad-4124.280881$

Cartesian Coordinates

$\begin{array}{lrrr}6 & 3.620429 & -5.985071 & 1.692773 \\ 6 & 3.340960 & -5.167309 & 2.783889 \\ 6 & 2.915501 & -3.859679 & 2.582768 \\ 6 & 2.776278 & -3.339688 & 1.293825 \\ 6 & 3.067700 & -4.164318 & 0.207436 \\ 6 & 3.481148 & -5.478420 & 0.404677 \\ 6 & 2.378891 & -1.893134 & 1.160957 \\ 7 & 1.680012 & -1.523019 & -0.082298 \\ 6 & 0.586121 & -2.309065 & -0.572270 \\ 6 & -0.590701 & -2.481133 & 0.138546 \\ 6 & -1.638713 & -3.277492 & -0.425840 \\ 6 & -1.479103 & -3.837571 & -1.727237\end{array}$




$\begin{array}{cccc}6 & -0.280117 & -3.598023 & -2.436630 \\ 6 & 0.723360 & -2.863420 & -1.869305 \\ 6 & -2.861272 & -3.508179 & 0.255752 \\ 6 & -3.861315 & -4.253664 & -0.317508 \\ 6 & -3.689811 & -4.823263 & -1.596888 \\ 6 & -2.522848 & -4.613487 & -2.286990 \\ 6 & -0.793650 & -1.844378 & 1.470482 \\ 6 & -1.161146 & -0.506587 & 1.584153 \\ 6 & -1.368249 & 0.043628 & 2.876821 \\ 6 & -1.211826 & -0.709502 & 4.009415 \\ 6 & -0.863099 & -2.075934 & 3.928479 \\ 6 & -0.662495 & -2.657841 & 2.642558 \\ 6 & -0.733847 & -2.877460 & 5.088174 \\ 6 & -0.438569 & -4.213467 & 4.988105 \\ 6 & -0.249454 & -4.798125 & 3.718234 \\ 6 & -0.346621 & -4.040708 & 2.577425 \\ 15 & -1.511128 & 0.490643 & 0.046482 \\ 45 & 0.026053 & 0.966725 & -1.825734 \\ 6 & 0.481796 & 2.843400 & -1.675244 \\ 8 & 0.570728 & 3.994151 & -1.736903 \\ 15 & 1.893130 & -0.034803 & -0.863502 \\ 8 & 2.675320 & 0.737418 & 0.372377 \\ 6 & 3.484443 & 1.812736 & 0.057355 \\ 6 & 4.738074 & 1.597373 & -0.482754 \\ 6 & 5.521689 & 2.744725 & -0.832884 \\ 6 & 5.031742 & 4.049999 & -0.521672 \\ 6 & -3.402017 & -0.514844 & -1.773730 \\ 6 & 3.762421 & 4.193955 & 0.086960 \\ 6 & 2.991601 & 3.095258 & 0.352074 \\ 6 & -5.014630 & -1.196024 & -3.639482 \\ 6 & -2.054154 & 2.135954 & 0.686417 \\ 6 & -3.811521 & 5.184718 & -0.853832 \\ 6 & -3.277266 & 2.667700 & 0.265980 \\ 6 & 7.016151 & 5.050308 & -1.493927 \\ 6 & -3.6867307 & 3.938142 & 0.659688 \\ 6 & 7.485091 & 3.765253 & -1.838575 \\ 6 & -2.847682 & 4.663241 & 1.513583 \\ 6 & 6.759661 & 2.644965 & -1.517884 \\ 6 & 5.189210 & 0.208299 & -0.731985 \\ 6 & 4.385027 & -0.635604 & -1.477746 \\ 6 & 4.779138 & -1.940180 & -1.830009 \\ 6 & 5.970334 & -2.435393 & -1.374150 \\ 6 & 6.796723 & -1.656536 & -0.530161 \\ 6 & 6.405657 & -0.324683 & -0.194249 \\ 6 & 7.228075 & 0.403096 & 0.703774 \\ 6 & -1.613652 & 4.174993 & 1.943804\end{array}$




\begin{tabular}{|c|c|c|c|}
\hline 6 & -1.215204 & 2.910900 & 1.487749 \\
\hline & -5.020155 & 4.533876 & 0.211546 \\
\hline & -0.741720 & 4.940661 & 2.939848 \\
\hline & 0.019216 & -0.053562 & -3.451076 \\
\hline & 0.029332 & -0.558590 & -4.492901 \\
\hline & 6.929171 & 1.404613 & 1.002213 \\
\hline & -1.683268 & 1.079012 & 2.973502 \\
\hline & 8.282198 & -3.199816 & -0.277777 \\
\hline & -0.885200 & -2.410238 & 6.060321 \\
\hline & 5.422111 & 6.169648 & -0.599910 \\
\hline & -3.007897 & -3.074785 & 1.243060 \\
\hline & -4.795163 & -4.409561 & 0.220687 \\
\hline & 3.395639 & 5.193902 & 0.314464 \\
\hline & -1.379840 & -0.266231 & 4.990353 \\
\hline & 6.284870 & -3.445239 & -1.632861 \\
\hline & 8.427785 & 3.660556 & -2.370924 \\
\hline & 4.118419 & -2.524322 & -2.466932 \\
\hline & -2.378633 & -5.036493 & -3.280670 \\
\hline & -0.166603 & -4.010265 & -3.438124 \\
\hline & -0.005539 & -5.855376 & 3.640807 \\
\hline & 7.129814 & 1.663818 & -1.803381 \\
\hline & -0.170332 & -4.503191 & 1.608524 \\
\hline & 1.649921 & -2.680167 & -2.411974 \\
\hline & 7.603403 & 5.929003 & -1.750097 \\
\hline & 8.990393 & 0.435336 & 1.901704 \\
\hline & -4.486343 & -5.420977 & -2.035001 \\
\hline & -0.350985 & -4.824080 & 5.883935 \\
\hline 1 & 9.700925 & -1.863244 & 1.252071 \\
\hline & 1.996945 & 3.180158 & 0.781994 \\
\hline & -3.899069 & -0.206348 & 1.568034 \\
\hline & -2.605588 & -0.364805 & -2.498818 \\
\hline & -6.625651 & -1.479357 & -1.481993 \\
\hline & -3.913969 & 2.075063 & -0.383518 \\
\hline & -0.244656 & 2.508003 & 1.786980 \\
\hline 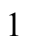 & -3.174837 & 5.645213 & 1.847607 \\
\hline & 3.284578 & -1.279127 & 1.213780 \\
\hline & 1.772410 & -1.606794 & 2.034752 \\
\hline & -1.301062 & 1.619907 & -2.447437 \\
\hline & 2.669734 & -3.231202 & 3.440009 \\
\hline ] & 2.971897 & -3.774234 & -0.802409 \\
\hline & 3.438026 & -5.552953 & 3.796867 \\
\hline ] & 3.695647 & -6.109096 & -0.455792 \\
\hline & 3.942240 & -7.012919 & 1.845653 \\
\hline 6 & -1.226379 & 6.372924 & 3.157200 \\
\hline 6 & -0.788404 & 4.206765 & 4.285840 \\
\hline 6 & 0.709979 & 5.002065 & 2.457208 \\
\hline 6 & -5.773822 & 3.610506 & -0.744231 \\
\hline & -5.906436 & 4.774764 & 1.438563 \\
\hline 6 & -4.771793 & 5.863555 & -0.508305 \\
\hline 6 & -3.790965 & -1.086045 & -4.547357 \\
\hline 6 & -5.619920 & -2.589632 & -3.830483 \\
\hline 6 & -6.033484 & -0.135853 & -4.074373 \\
\hline 6 & -7.729583 & -1.695574 & 0.738354 \\
\hline 6 & -5.898905 & -1.951966 & 2.382127 \\
\hline 5 & -6.786921 & 0.323400 & 1.835007 \\
\hline 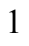 & -0.553007 & 6.885188 & 3.855657 \\
\hline
\end{tabular}




$\begin{array}{rrrr}1 & -1.235350 & 6.947188 & 2.221782 \\ 1 & -2.231832 & 6.409994 & 3.594497 \\ 1 & 1.303414 & 5.644071 & 3.121493 \\ 1 & 1.185275 & 4.014669 & 2.465487 \\ 1 & 0.775221 & 5.411360 & 1.439799 \\ 1 & -0.176592 & 4.731491 & 5.032445 \\ 1 & -1.816375 & 4.151120 & 4.667665 \\ 1 & -0.404167 & 3.181682 & 4.200797 \\ 1 & -6.867097 & 5.212444 & 1.135167 \\ 1 & -6.115389 & 3.832649 & 1.962859 \\ 1 & -5.440962 & 5.461779 & 2.155768 \\ 1 & -6.724143 & 4.076950 & -1.033139 \\ 1 & -5.206017 & 3.419410 & -1.664109 \\ 1 & -6.008793 & 2.640782 & -0.283121 \\ 1 & -5.722849 & 6.296592 & -0.846460 \\ 1 & -4.285390 & 6.601687 & 0.140571 \\ 1 & -4.132544 & 5.719754 & -1.388741 \\ 1 & -4.081999 & -1.293542 & -5.584889 \\ 1 & -3.351450 & -0.079894 & -4.528260 \\ 1 & -3.010418 & -1.807743 & -4.269486 \\ 1 & -5.888886 & -2.742079 & -4.884351 \\ 1 & -4.900363 & -3.368410 & -3.546594 \\ 1 & -6.529592 & -2.738185 & -3.236005 \\ 1 & -6.294621 & -0.266937 & -5.133343 \\ 1 & -6.960986 & -0.196098 & -3.491339 \\ 1 & -5.624184 & 0.875196 & -3.949064 \\ 1 & -8.444295 & -1.820725 & 1.561561 \\ 1 & -8.217340 & -1.075659 & -0.024535 \\ 1 & -7.542958 & -2.688400 & 0.306855 \\ 1 & -6.653990 & -2.077630 & 3.169602 \\ 1 & -5.645101 & -2.950203 & 2.000028 \\ 1 & -4.999933 & -1.536518 & 2.854802 \\ 1 & -7.511091 & 0.233948 & 2.656349 \\ 1 & -5.896198 & 0.833750 & 2.224594 \\ 1 & -7.231098 & 0.966747 & 1.063043 \\ ---------------------------------------------------------------------------- \\ & & \end{array}$

Number of imaginary frequencies : 0

The smallest frequencies are : $9.8114 \quad 14.6038 \quad 18.4085 \mathrm{~cm}(-1)$

Electronic energy : $\quad H F=-4125.4660159$

Zero-point correction $=\quad 1.300016($ Hartree/Particle $)$

Thermal correction to Energy= $\quad 1.378242$

Thermal correction to Enthalpy= $\quad 1.379187$

Thermal correction to Gibbs Free Energy= $\quad 1.187176$

Sum of electronic and zero-point Energies $=\quad-4124.166000$

Sum of electronic and thermal Energies $=\quad-4124.087773$

Sum of electronic and thermal Enthalpies $=\quad-4124.086829$

Sum of electronic and thermal Free Energies $=\quad-4124.278840$

Cartesian Coordinates

$\begin{array}{llll}6 & 3.631474 & -5.981319 & 1.602075 \\ 6 & 3.378164 & -5.161963 & 2.698457 \\ 6 & 2.948194 & -3.854925 & 2.505293 \\ 6 & 2.780013 & -3.335994 & 1.219286\end{array}$




\begin{tabular}{|c|c|c|c|}
\hline 6 & 3.050309 & -4.160223 & 0.127196 \\
\hline 6 & 3.465809 & -5.474996 & 0.317010 \\
\hline 6 & 2.371269 & -1.891597 & 1.098927 \\
\hline & 1.674932 & -1.512180 & -0.142564 \\
\hline & 0.593047 & -2.298836 & -0.656885 \\
\hline & -0.579819 & -2.517892 & 0.048189 \\
\hline & -1.621982 & -3.297767 & -0.549655 \\
\hline & -1.455159 & -3.805194 & -1.871434 \\
\hline & -0.258958 & -3.524640 & -2.569910 \\
\hline 6 & 0.735244 & -2.799177 & -1.975281 \\
\hline 6 & -2.845634 & -3.565442 & 0.117970 \\
\hline & -3.832164 & -4.305792 & -0.484802 \\
\hline 6 & -3.652305 & -4.823777 & -1.784564 \\
\hline 6 & -2.487190 & -4.572728 & -2.462500 \\
\hline 6 & -0.785907 & -1.952129 & 1.410962 \\
\hline 6 & -1.165066 & -0.625875 & 1.592242 \\
\hline 6 & -1.386966 & -0.145015 & 2.910095 \\
\hline 6 & -1.226047 & -0.953927 & 4.002939 \\
\hline 6 & -0.857851 & -2.309631 & 3.853093 \\
\hline 6 & -0.647035 & -2.823224 & 2.539766 \\
\hline 6 & -0.720060 & -3.167765 & 4.970724 \\
\hline 6 & -0.406221 & -4.492609 & 4.803120 \\
\hline 6 & -0.207492 & -5.009624 & 3.505703 \\
\hline 6 & -0.313036 & -4.196669 & 2.404702 \\
\hline 15 & -1.518738 & 0.434820 & 0.103112 \\
\hline 45 & -0.029994 & 1.009066 & -1.790280 \\
\hline 6 & 0.360065 & 2.887039 & -1.512558 \\
\hline 8 & 0.390244 & 4.041896 & -1.474553 \\
\hline 15 & 1.866357 & -0.002123 & -0.891335 \\
\hline 8 & 2.653251 & 0.749842 & 0.354472 \\
\hline 6 & 3.466403 & 1.826607 & 0.053965 \\
\hline 6 & 4.719592 & 1.612910 & -0.487930 \\
\hline 6 & 5.510209 & 2.760824 & -0.819455 \\
\hline 6 & 5.024903 & 4.064141 & -0.493250 \\
\hline 6 & 3.753390 & 4.206141 & 0.111273 \\
\hline 6 & 2.977494 & 3.107579 & 0.362373 \\
\hline 6 & 5.811386 & 5.199459 & -0.807097 \\
\hline 6 & 7.018393 & 5.067847 & -1.443296 \\
\hline 6 & 7.482954 & 3.785257 & -1.802715 \\
\hline 6 & 6.750733 & 2.664293 & -1.500351 \\
\hline 6 & 5.159640 & 0.225438 & -0.765038 \\
\hline 6 & 4.348295 & -0.593779 & -1.530316 \\
\hline 6 & 4.728841 & -1.894695 & -1.909381 \\
\hline 6 & 5.914162 & -2.411799 & -1.462475 \\
\hline 6 & 6.747344 & -1.659555 & -0.601249 \\
\hline 6 & 6.369981 & -0.331035 & -0.237761 \\
\hline 6 & 7.198646 & 0.369107 & 0.676390 \\
\hline 6 & 8.345759 & -0.199016 & 1.172079 \\
\hline 6 & 8.732468 & -1.498791 & 0.783274 \\
\hline 6 & 7.943683 & -2.211753 & -0.081849 \\
\hline 8 & 3.123124 & -0.139440 & -1.973288 \\
\hline 6 & -3.169710 & -0.229800 & -0.367936 \\
\hline 6 & -4.147436 & -0.445315 & 0.611714 \\
\hline 6 & -5.433967 & -0.836096 & 0.262150 \\
\hline 6 & -5.724250 & -0.989607 & -1.104906 \\
\hline 6 & -4.777058 & -0.777589 & -2.102360 \\
\hline
\end{tabular}




\begin{tabular}{|c|c|c|c|}
\hline 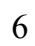 & -3.482521 & -0.413079 & -1.704866 \\
\hline & -6.514592 & -1.114289 & 1.306274 \\
\hline & -5.091795 & -0.868753 & -3.593812 \\
\hline & -1.997604 & 2.073740 & 0.799285 \\
\hline & -3.151893 & 2.692647 & 0.310126 \\
\hline & -3.506235 & 3.977703 & 0.705925 \\
\hline & -2.688245 & 4.626434 & 1.639567 \\
\hline 6 & -1.521116 & 4.049272 & 2.139041 \\
\hline 6 & -1.168596 & 2.774306 & 1.674143 \\
\hline 6 & -4.770552 & 4.659466 & 0.186778 \\
\hline 6 & -0.662971 & 4.732926 & 3.204063 \\
\hline 6 & -0.084498 & 0.055945 & -3.452407 \\
\hline 8 & -0.141386 & -0.400244 & -4.515443 \\
\hline 1 & 6.909547 & 1.367237 & 0.995273 \\
\hline 1 & -1.717450 & 0.879619 & 3.057551 \\
\hline 1 & 8.216033 & -3.223496 & -0.379366 \\
\hline 1 & -0.879600 & -2.753074 & 5.965196 \\
\hline 1 & 5.425517 & 6.182768 & -0.541731 \\
\hline 1 & -3.002809 & -3.168497 & 1.119461 \\
\hline 1 & -4.763772 & -4.496188 & 0.044533 \\
\hline 1 & 3.389695 & 5.205079 & 0.347507 \\
\hline 1 & -1.405282 & -0.564890 & 5.004683 \\
\hline 1 & 6.218109 & -3.419641 & -1.740967 \\
\hline 1 & 8.427775 & 3.683099 & -2.331793 \\
\hline 1 & 4.062463 & -2.459449 & -2.557821 \\
\hline 1 & -2.336310 & -4.951453 & -3.472845 \\
\hline 1 & -0.140279 & -3.898005 & -3.585928 \\
\hline 1 & 0.051118 & -6.058111 & 3.375307 \\
\hline 1 & 7.117763 & 1.685311 & -1.797067 \\
\hline 1 & -0.129587 & -4.606481 & 1.413581 \\
\hline 1 & 1.658652 & -2.584620 & -2.511763 \\
\hline 1 & 7.610972 & 5.947056 & -1.685030 \\
\hline 1 & 8.959474 & 0.357348 & 1.877003 \\
\hline 1 & -4.441119 & -5.412771 & -2.247479 \\
\hline 1 & -0.311800 & -5.146943 & 5.666817 \\
\hline 1 & 9.646456 & -1.934426 & 1.180023 \\
\hline 1 & 1.981714 & 3.191845 & 0.789555 \\
\hline 1 & -3.887052 & -0.289331 & 1.656428 \\
\hline 1 & -2.704558 & -0.246818 & -2.448995 \\
\hline 1 & -6.733454 & -1.280715 & -1.389497 \\
\hline 1 & -3.774538 & 2.156320 & -0.399873 \\
\hline 1 & -0.241059 & 2.311211 & 2.017976 \\
\hline 1 & -2.977611 & 5.617578 & 1.980933 \\
\hline 1 & 3.272220 & -1.271789 & 1.161909 \\
\hline 1 & 1.760466 & -1.619972 & 1.974607 \\
\hline 1 & -1.372174 & 1.669507 & -2.369079 \\
\hline 1 & 2.721101 & -3.226002 & 3.367293 \\
\hline 1 & 2.935404 & -3.770853 & -0.880993 \\
\hline 1 & 3.496882 & -5.546764 & 3.709438 \\
\hline 1 & 3.661626 & -6.106402 & -0.547379 \\
\hline 1 & 3.954974 & -7.009574 & 1.748575 \\
\hline 6 & -1.130111 & 6.155822 & 3.502881 \\
\hline 6 & -0.748983 & 3.918128 & 4.500509 \\
\hline 6 & 0.798379 & 4.800407 & 2.751619 \\
\hline 6 & -5.453508 & 3.847691 & -0.913605 \\
\hline 6 & -5.759510 & 4.824952 & 1.346182 \\
\hline
\end{tabular}




$\begin{array}{rrrr}6 & -4.421695 & 6.035291 & -0.389790 \\ 6 & -6.548493 & -1.240106 & -3.858010 \\ 6 & -4.819196 & 0.496187 & -4.239189 \\ 6 & -4.202847 & -1.927297 & -4.252811 \\ 6 & -6.046286 & -0.785847 & 2.722577 \\ 6 & -7.761405 & -0.273320 & 1.013624 \\ 6 & -6.882097 & -2.601624 & 1.262296 \\ 1 & -0.476071 & 6.604860 & 4.260731 \\ 1 & -1.091376 & 6.794557 & 2.611038 \\ 1 & -2.152392 & 6.180820 & 3.900921 \\ 1 & 1.395522 & 5.368890 & 3.476976 \\ 1 & 1.251048 & 3.804756 & 2.676755 \\ 1 & 0.886445 & 5.295833 & 1.774976 \\ 1 & -0.149531 & 4.389691 & 5.291206 \\ 1 & -1.786007 & 3.852072 & 4.855252 \\ 1 & -0.373243 & 2.895576 & 4.364809 \\ 1 & -6.680082 & 5.313185 & 0.998529 \\ 1 & -6.032507 & 3.849006 & 1.769833 \\ 1 & -5.342901 & 5.436659 & 2.155972 \\ 1 & -6.345012 & 4.382589 & -1.264719 \\ 1 & -4.793726 & 3.697325 & -1.778565 \\ 1 & -5.781364 & 2.859565 & -0.562345 \\ 1 & -5.325465 & 6.524104 & -0.777613 \\ 1 & -3.983912 & 6.703803 & 0.360919 \\ 1 & -3.704502 & 5.944071 & -1.215536 \\ 1 & -6.725499 & -1.291409 & -4.939709 \\ 1 & -6.803397 & -2.221472 & -3.436755 \\ 1 & -7.243769 & -0.496758 & -3.446656 \\ 1 & -5.062338 & 0.466894 & -5.309881 \\ 1 & -5.430844 & 1.280436 & -3.773304 \\ 1 & -3.766152 & 0.793219 & -4.147541 \\ 1 & -4.402329 & -1.970707 & -5.332112 \\ 1 & -3.134240 & -1.714388 & -4.121098 \\ 1 & -4.398074 & -2.919545 & -3.826677 \\ 1 & -6.852455 & -0.999182 & 3.435823 \\ 1 & -5.177626 & -1.388759 & 3.020776 \\ 1 & -5.780764 & 0.274244 & 2.829587 \\ 1 & -8.533353 & -0.464107 & 1.771055 \\ 1 & -7.527268 & 0.798999 & 1.034933 \\ 1 & -8.200305 & -0.501450 & 0.035222 \\ 1 & -7.686190 & -2.819542 & 1.978340 \\ 1 & -7.225492 & -2.912721 & 0.267671 \\ 1 & -6.016406 & -3.222505 & 1.531033\end{array}$

$1_{\mathrm{C} 3-} \psi_{-1 \mathrm{~s} 2 \mathrm{azs} 4 \mathrm{~s}}$

Number of imaginary frequencies : 0

The smallest frequencies are : $6.9610 \quad 12.9252 \quad 13.9085 \mathrm{~cm}(-1)$

Electronic energy : $\quad H F=-4125.4668151$

Zero-point correction $=\quad 1.299085($ Hartree/Particle)

Thermal correction to Energy= $\quad 1.377686$

Thermal correction to Enthalpy= $\quad 1.378630$

Thermal correction to Gibbs Free Energy= $\quad 1.183607$

Sum of electronic and zero-point Energies $=\quad-4124.167730$

Sum of electronic and thermal Energies $=\quad-4124.089129$

Sum of electronic and thermal Enthalpies $=\quad-4124.088185$ 
Cartesian Coordinates

\begin{tabular}{|c|c|c|c|}
\hline 6 & 3.631072 & -5.991804 & 1.622485 \\
\hline 6 & 3.359551 & -5.184693 & 2.723453 \\
\hline 6 & 2.932746 & -3.875176 & 2.538081 \\
\hline 6 & 2.783966 & -3.342771 & 1.255280 \\
\hline 6 & 3.067398 & -4.156824 & 0.158818 \\
\hline 6 & 3.482341 & -5.472715 & 0.340384 \\
\hline 6 & 2.387132 & -1.894599 & 1.140341 \\
\hline 7 & 1.677346 & -1.509999 & -0.092767 \\
\hline 6 & 0.581729 & -2.294601 & -0.581132 \\
\hline 6 & -0.584851 & -2.485260 & 0.142212 \\
\hline 6 & -1.627188 & -3.296163 & -0.413168 \\
\hline 6 & -1.470932 & -3.853959 & -1.715672 \\
\hline 6 & -0.287099 & -3.587734 & -2.440992 \\
\hline 6 & 0.710282 & -2.836991 & -1.884229 \\
\hline 6 & -2.839874 & -3.547289 & 0.278629 \\
\hline 6 & -3.832937 & -4.307570 & -0.287002 \\
\hline 6 & -3.665567 & -4.873544 & -1.568314 \\
\hline 6 & -2.504557 & -4.650767 & -2.263823 \\
\hline 6 & -0.780318 & -1.853122 & 1.477656 \\
\hline 6 & -1.146181 & -0.515080 & 1.597046 \\
\hline 6 & -1.341185 & 0.033901 & 2.891790 \\
\hline 6 & -1.176008 & -0.721016 & 4.021951 \\
\hline 6 & -0.829250 & -2.087542 & 3.936015 \\
\hline 6 & -0.639410 & -2.667930 & 2.647650 \\
\hline 6 & -0.690797 & -2.890503 & 5.093722 \\
\hline 6 & -0.396251 & -4.226301 & 4.989780 \\
\hline 6 & -0.216864 & -4.809304 & 3.717705 \\
\hline 6 & -0.323288 & -4.050624 & 2.578590 \\
\hline 15 & -1.505681 & 0.486535 & 0.065315 \\
\hline 45 & 0.020831 & 1.007298 & -1.796884 \\
\hline 6 & 0.494518 & 2.878253 & -1.640844 \\
\hline 8 & 0.591374 & 4.028308 & -1.704468 \\
\hline 15 & 1.888507 & -0.013233 & -0.857471 \\
\hline 8 & 2.673193 & 0.742937 & 0.387241 \\
\hline 6 & 3.482013 & 1.822520 & 0.087752 \\
\hline 6 & 4.733541 & 1.615040 & -0.460728 \\
\hline 6 & 5.516243 & 2.767363 & -0.796144 \\
\hline 6 & 5.028810 & 4.067740 & -0.461529 \\
\hline 6 & 3.763267 & 4.202619 & 0.156961 \\
\hline 6 & 2.992468 & 3.100550 & 0.407884 \\
\hline 6 & 5.807558 & 5.207188 & -0.779699 \\
\hline 6 & 7.008717 & 5.082296 & -1.428190 \\
\hline 6 & 7.475116 & 3.802621 & -1.795545 \\
\hline 6 & 6.750712 & 2.677769 & -1.488844 \\
\hline 6 & 5.184761 & 0.229736 & -0.730932 \\
\hline 6 & 4.378965 & -0.604912 & -1.485222 \\
\hline 6 & 4.771677 & -1.905065 & -1.854775 \\
\hline 6 & 5.964165 & -2.405912 & -1.408755 \\
\hline 6 & 6.793092 & -1.637423 & -0.557810 \\
\hline 6 & 6.402996 & -0.310007 & -0.203652 \\
\hline 6 & 7.228235 & 0.406012 & 0.701248 \\
\hline 6 & 8.383572 & -0.145790 & 1.196299 \\
\hline
\end{tabular}




\begin{tabular}{|c|c|c|c|}
\hline & 8.782656 & -1.444346 & 0.816211 \\
\hline & 7.997570 & -2.172617 & -0.039456 \\
\hline 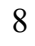 & 3.149697 & -0.167001 & -1.931593 \\
\hline & -3.105075 & -0.272502 & -0.433956 \\
\hline & -4.096586 & -0.506465 & 0.518874 \\
\hline & -5.357290 & -0.968426 & 0.148594 \\
\hline & -5.603634 & -1.178441 & -1.214456 \\
\hline & -4.632839 & -0.952638 & -2.193257 \\
\hline & -3.373916 & -0.509021 & -1.775437 \\
\hline & -6.419173 & -1.170406 & 1.228953 \\
\hline & -4.901587 & -1.108002 & -3.689178 \\
\hline 6 & -2.086998 & 2.117599 & 0.707758 \\
\hline 6 & -3.321491 & 2.620908 & 0.286747 \\
\hline 6 & -3.755560 & 3.885904 & 0.671501 \\
\hline 6 & -2.930235 & 4.634189 & 1.518363 \\
\hline 6 & -1.684862 & 4.174617 & 1.948664 \\
\hline 6 & -1.262773 & 2.915070 & 1.502304 \\
\hline 6 & -5.101556 & 4.449440 & 0.219194 \\
\hline 6 & -0.828664 & 4.965375 & 2.938815 \\
\hline 6 & -0.040168 & 0.009721 & -3.434291 \\
\hline 8 & -0.099560 & -0.474400 & -4.484795 \\
\hline 1 & 6.930163 & 1.403456 & 1.013769 \\
\hline 1 & -1.655752 & 1.069001 & 2.992129 \\
\hline 1 & 8.279338 & -3.183762 & -0.330178 \\
\hline 1 & -0.834359 & -2.424338 & 6.067558 \\
\hline 1 & 5.420147 & 6.188162 & -0.508035 \\
\hline 1 & -2.984302 & -3.118661 & 1.268180 \\
\hline 1 & -4.759210 & -4.478062 & 0.259806 \\
\hline 1 & 3.399412 & 5.199076 & 0.403574 \\
\hline 1 & -1.335602 & -0.279254 & 5.004952 \\
\hline 1 & 6.277895 & -3.412530 & -1.680701 \\
\hline 1 & 8.414950 & 3.705926 & -2.334432 \\
\hline 1 & 4.108705 & -2.481436 & -2.496400 \\
\hline 1 & -2.359821 & -5.071969 & -3.258228 \\
\hline 1 & -0.180412 & -3.993376 & -3.446003 \\
\hline 1 & 0.026889 & -5.866363 & 3.637070 \\
\hline 1 & 7.118684 & 1.701027 & -1.791792 \\
\hline 1 & -0.154315 & -4.511784 & 1.607780 \\
\hline 1 & 1.626404 & -2.634713 & -2.437851 \\
\hline 1 & 7.595117 & 5.964623 & -1.673596 \\
\hline 1 & 8.994312 & 0.422884 & 1.893941 \\
\hline 1 & -4.458177 & -5.479492 & -2.002040 \\
\hline 1 & -0.301442 & -4.837970 & 5.884153 \\
\hline 1 & 9.703114 & -1.866907 & 1.212137 \\
\hline 1 & 2.000549 & 3.179498 & 0.845559 \\
\hline 1 & -3.882242 & -0.302019 & 1.568177 \\
\hline 1 & -2.585167 & -0.323911 & -2.503145 \\
\hline 1 & -6.585829 & -1.526709 & -1.520689 \\
\hline 1 & -3.946039 & 2.012980 & -0.360225 \\
\hline 1 & -0.282958 & 2.535261 & 1.800683 \\
\hline 1 & -3.276006 & 5.612436 & 1.844812 \\
\hline 1 & 3.294346 & -1.282835 & 1.191516 \\
\hline 1 & 1.789165 & -1.616222 & 2.022465 \\
\hline 1 & -1.307764 & 1.679858 & -2.394158 \\
\hline 1 & 2.693783 & -3.254841 & 3.403144 \\
\hline 1 & 2.963745 & -3.757079 & -0.846504 \\
\hline
\end{tabular}




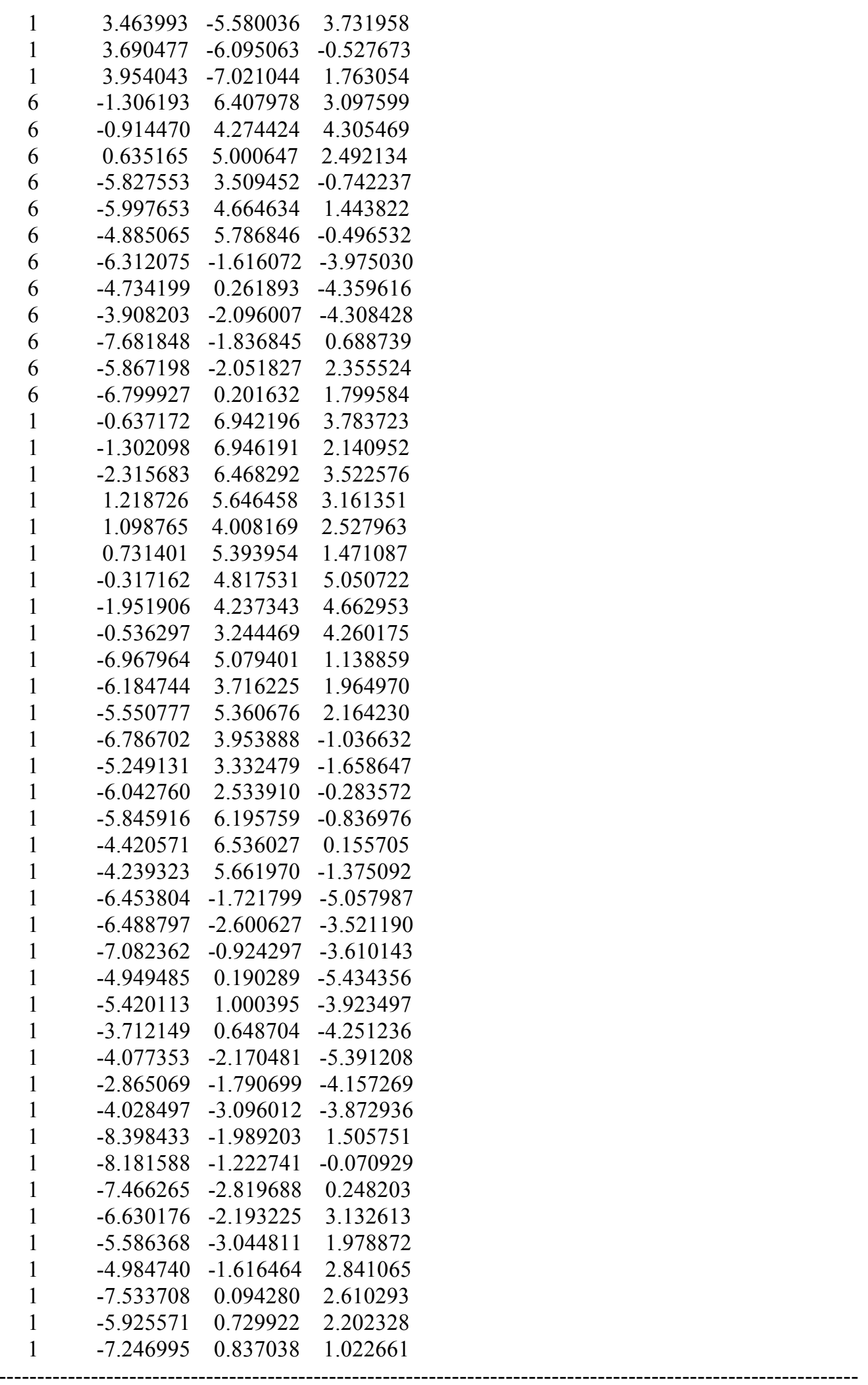

$1_{\text {C3- }} \psi_{-1 \mathrm{~s} 233 \mathrm{a} a \mathrm{a}}$

Number of imaginary frequencies : 0

The smallest frequencies are : $10.2483 \quad 14.7128 \quad 17.0434 \mathrm{~cm}(-1)$ 
Electronic energy : $\quad \mathrm{HF}=-4125.4668234$

Zero-point correction $=\quad 1.299354($ Hartree/Particle $)$

Thermal correction to Energy= $\quad 1.377847$

Thermal correction to Enthalpy= $\quad 1.378791$

Thermal correction to Gibbs Free Energy $=\quad 1.185549$

Sum of electronic and zero-point Energies $=\quad-4124.167469$

Sum of electronic and thermal Energies $=\quad-4124.088976$

Sum of electronic and thermal Enthalpies $=\quad-4124.088032$

Sum of electronic and thermal Free Energies $=\quad-4124.281274$

Cartesian Coordinates

$\begin{array}{cccc}0 & 3.602110 & -5.969866 & 1.676201 \\ 6 & 3.347579 & -5.142788 & 2.766503 \\ 6 & 2.920947 & -3.836057 & 2.563701 \\ 6 & 2.757046 & -3.325368 & 1.273851 \\ 6 & 3.026956 & -4.157848 & 0.188040 \\ 6 & 3.439805 & -5.472025 & 0.387433 \\ 6 & 2.353050 & -1.880575 & 1.141532 \\ 7 & 1.660587 & -1.509545 & -0.104502 \\ 6 & 0.572074 & -2.293716 & -0.608612 \\ 6 & -0.602279 & -2.495059 & 0.099345 \\ 6 & -1.649697 & -3.275460 & -0.488527 \\ 6 & -1.488875 & -3.797305 & -1.805526 \\ 6 & -0.290485 & -3.535047 & -2.507317 \\ 6 & 0.709981 & -2.811805 & -1.920652 \\ 6 & -2.873621 & -3.527658 & 0.184289 \\ 6 & -3.866852 & -4.267278 & -0.408014 \\ 6 & -3.693347 & -4.799018 & -1.703398 \\ 6 & -2.529371 & -4.560453 & -2.388474 \\ 6 & -0.805650 & -1.905937 & 1.452730 \\ 6 & -1.178238 & -0.574760 & 1.610632 \\ 6 & -1.394127 & -0.068979 & 2.920109 \\ 6 & -1.233097 & -0.858062 & 4.027196 \\ 6 & -0.873017 & -2.218336 & 3.901221 \\ 6 & -0.669391 & -2.757242 & 2.596880 \\ 6 & -0.736976 & -3.056391 & 5.034093 \\ 6 & -0.431932 & -4.386096 & 4.890194 \\ 6 & -0.240064 & -4.928041 & 3.601992 \\ 6 & -0.343723 & -4.134895 & 2.486428 \\ 15 & -1.519544 & 0.467185 & 0.104358 \\ 45 & -0.015519 & 1.002529 & -1.791756 \\ 6 & 0.390780 & 2.880773 & -1.543699 \\ 8 & 0.434336 & 4.035733 & -1.527935 \\ 15 & 1.869787 & -0.013592 & -0.876790 \\ 8 & 2.670110 & 0.746813 & 0.354766 \\ 6 & 3.490671 & 1.812427 & 0.034381 \\ 6 & 4.739198 & 1.580335 & -0.510386 \\ 6 & 5.536874 & 2.716846 & -0.863928 \\ 6 & 5.063434 & 4.028826 & -0.555453 \\ 6 & 3.796555 & 4.190179 & 0.053996 \\ 6 & 3.013572 & 3.101672 & 0.325872 \\ 6 & 5.856571 & 5.152922 & -0.891910 \\ 6 & 7.058746 & 5.002115 & -1.533022 \\ 6 & 7.511481 & 3.710488 & -1.874756 \\ 6 & 6.772546 & 2.600115 & -1.550393 \\ & & & \\ 6\end{array}$




\begin{tabular}{|c|c|c|c|}
\hline 6 & 5.166986 & 0.185550 & -0.769022 \\
\hline & 4.345747 & -0.638693 & -1.518264 \\
\hline & 4.717765 & -1.945713 & -1.884877 \\
\hline & 5.903558 & -2.463498 & -1.439985 \\
\hline & 6.745904 & -1.705740 & -0.592625 \\
\hline & 6.377451 & -0.371217 & -0.242377 \\
\hline & 7.215503 & 0.335611 & 0.657933 \\
\hline & 8.363051 & -0.232300 & 1.152857 \\
\hline & 8.740860 & -1.538460 & 0.776889 \\
\hline & 7.942935 & -2.257684 & -0.074589 \\
\hline & 3.121169 & -0.182827 & -1.960939 \\
\hline & -3.166520 & -0.201685 & -0.375299 \\
\hline & -4.169496 & -0.363661 & 0.583746 \\
\hline & -5.451632 & -0.766428 & 0.215709 \\
\hline & -5.694026 & -1.000664 & -1.143939 \\
\hline & -4.712503 & -0.856275 & -2.126104 \\
\hline & -3.436924 & -0.459348 & -1.714127 \\
\hline & -6.567031 & -0.975256 & 1.239395 \\
\hline & -5.060492 & -1.058443 & -3.599791 \\
\hline & -1.992166 & 2.114409 & 0.785059 \\
\hline & -3.153692 & 2.728213 & 0.322811 \\
\hline ) & -3.506768 & 4.017672 & 0.722631 \\
\hline & -2.670146 & 4.676296 & 1.624526 \\
\hline & -1.486830 & 4.099567 & 2.101007 \\
\hline & -1.143971 & 2.825102 & 1.640260 \\
\hline & -4.788385 & 4.632178 & 0.163882 \\
\hline & -0.611211 & 4.792885 & 3.146234 \\
\hline & -0.058639 & 0.018814 & -3.437497 \\
\hline & -0.089371 & -0.466542 & -4.488345 \\
\hline & 6.933297 & 1.338839 & 0.966891 \\
\hline & -1.717672 & 0.960176 & 3.050299 \\
\hline & 8.208454 & -3.274148 & -0.361998 \\
\hline & -0.891064 & -2.622376 & 6.021137 \\
\hline & 5.479629 & 6.143261 & -0.640182 \\
\hline 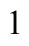 & -3.024386 & -3.119740 & 1.182113 \\
\hline & -4.798331 & -4.445924 & 0.125677 \\
\hline & 3.441931 & 5.195402 & 0.277139 \\
\hline & -1.406496 & -0.449404 & 5.022144 \\
\hline & 6.201079 & -3.475653 & -1.709598 \\
\hline 1 & 8.452301 & 3.592690 & -2.407689 \\
\hline & 4.045401 & -2.513990 & -2.523961 \\
\hline 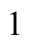 & -2.382946 & -4.952943 & -3.394196 \\
\hline & -0.175294 & -3.919737 & -3.519522 \\
\hline & 0.011847 & -5.980317 & 3.490278 \\
\hline D & 7.130349 & 1.613808 & -1.833787 \\
\hline & -0.165040 & -4.564161 & 1.502692 \\
\hline 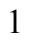 & 1.635290 & -2.611878 & -2.459281 \\
\hline & 7.656404 & 5.872885 & -1.792173 \\
\hline ] & 8.984105 & 0.329304 & 1.847114 \\
\hline & -4.485916 & -5.390601 & -2.156700 \\
\hline 1 & -0.339026 & -5.024916 & 5.765590 \\
\hline 1 & 9.655352 & -1.973874 & 1.172721 \\
\hline ] & 2.020984 & 3.200013 & 0.757136 \\
\hline 1 & -3.940962 & -0.151024 & 1.626161 \\
\hline l & -2.630803 & -0.334054 & -2.432980 \\
\hline & -6.695017 & -1.306986 & -1.448321 \\
\hline
\end{tabular}




\begin{tabular}{|c|c|c|c|}
\hline & -3.798161 & 2.192496 & -0.371366 \\
\hline & -0.211347 & 2.362218 & 1.970575 \\
\hline & -2.944384 & 5.669929 & 1.964592 \\
\hline & 3.255449 & -1.262651 & 1.201476 \\
\hline & 1.740848 & -1.599969 & 2.013322 \\
\hline & -1.350927 & 1.664899 & -2.385421 \\
\hline & 2.692817 & -3.201011 & 3.420930 \\
\hline & 2.914391 & -3.775007 & -0.822880 \\
\hline & 3.463314 & -5.521082 & 3.780287 \\
\hline & 3.636050 & -6.109640 & -0.472296 \\
\hline & 3.923488 & -6.997663 & 1.830375 \\
\hline & -1.058947 & 6.226266 & 3.425085 \\
\hline & -0.696767 & 4.000491 & 4.456681 \\
\hline & 0.847563 & 4.836567 & 2.683683 \\
\hline & -5.073024 & 6.015009 & 0.743424 \\
\hline & -4.656888 & 4.765470 & -1.357946 \\
\hline & -5.977648 & 3.720544 & 0.491721 \\
\hline & -3.824477 & -0.996475 & -4.495807 \\
\hline & -5.730048 & -2.418917 & -3.810364 \\
\hline & -6.022429 & 0.055756 & -4.029312 \\
\hline & -6.157146 & -0.516772 & 2.637387 \\
\hline ) & -7.815923 & -0.186273 & 0.833186 \\
\hline & -6.906478 & -2.468514 & 1.305763 \\
\hline & -0.389750 & 6.681135 & 4.166067 \\
\hline 1 & -1.024945 & 6.848424 & 2.521429 \\
\hline & -2.075144 & 6.271038 & 3.836456 \\
\hline & 1.455650 & 5.409210 & 3.396504 \\
\hline 1 & 1.288448 & 3.834860 & 2.622199 \\
\hline & 0.935410 & 5.315777 & 1.699031 \\
\hline 1 & -0.086980 & 4.478145 & 5.235794 \\
\hline & -1.732049 & 3.951131 & 4.819217 \\
\hline 1 & -0.333108 & 2.971882 & 4.333977 \\
\hline ] & -5.567276 & 5.210246 & -1.782397 \\
\hline & -3.808277 & 5.408997 & -1.623564 \\
\hline 1 & -4.502835 & 3.793908 & -1.843938 \\
\hline & -6.003327 & 6.409679 & 0.315764 \\
\hline 1 & -5.198641 & 5.985500 & 1.833674 \\
\hline ] & -4.275022 & 6.730694 & 0.507655 \\
\hline 1 & -6.907058 & 4.147965 & 0.091707 \\
\hline 1 & -5.860804 & 2.719094 & 0.057020 \\
\hline t & -6.098036 & 3.604467 & 1.577225 \\
\hline 1 & -4.116660 & -1.172828 & -5.538738 \\
\hline & -3.330375 & -0.016424 & -4.457539 \\
\hline 1 & -3.084091 & -1.761104 & -4.223771 \\
\hline ] & -5.980196 & -2.557261 & -4.870734 \\
\hline 1 & -5.057299 & -3.232332 & -3.509320 \\
\hline 1 & -6.660489 & -2.521727 & -3.238882 \\
\hline 1 & -6.279391 & -0.047875 & -5.092350 \\
\hline 1 & -6.957103 & 0.032548 & -3.455009 \\
\hline 1 & -5.565918 & 1.044202 & -3.885715 \\
\hline 1 & -6.991985 & -0.664753 & 3.333950 \\
\hline 1 & -5.302568 & -1.086754 & 3.025962 \\
\hline 1 & -5.894287 & 0.549577 & 2.654112 \\
\hline 1 & -8.613568 & -0.334495 & 1.573279 \\
\hline 1 & -7.602903 & 0.889773 & 0.779770 \\
\hline & -8.211970 & -0.498627 & -0.140240 \\
\hline
\end{tabular}




$\begin{array}{llll}1 & -7.721799 & -2.646575 & 2.020205 \\ 1 & -7.222728 & -2.860757 & 0.330820 \\ 1 & -6.035428 & -3.050303 & 1.637783\end{array}$

$1_{\mathrm{C} 3-} \psi_{-1 \mathrm{~s} 2 \mathrm{~s} 3 \mathrm{a} 4 \mathrm{~s}}$

Number of imaginary frequencies : 0

The smallest frequencies are : $13.8531 \quad 16.5219 \quad 18.6477 \mathrm{~cm}(-1)$

Electronic energy : $\quad H F=-4125.4673019$

Zero-point correction $=\quad 1.300174($ Hartree/Particle $)$

Thermal correction to Energy= $\quad 1.378254$

Thermal correction to Enthalpy= $\quad 1.379198$

Thermal correction to Gibbs Free Energy= $\quad 1.188121$

Sum of electronic and zero-point Energies $=\quad-4124.167128$

Sum of electronic and thermal Energies $=\quad-4124.089048$

Sum of electronic and thermal Enthalpies $=\quad-4124.088104$

Sum of electronic and thermal Free Energies $=\quad-4124.279181$

Cartesian Coordinates

$\begin{array}{cccc}6 & 3.505850 & -6.025938 & 1.617009 \\ 6 & 3.247090 & -5.211572 & 2.715744 \\ 6 & 2.843024 & -3.895567 & 2.526473 \\ 6 & 2.706504 & -3.363316 & 1.242246 \\ 6 & 2.979937 & -4.183698 & 0.148008 \\ 6 & 3.370202 & -5.506648 & 0.333516 \\ 6 & 2.328182 & -1.910495 & 1.123847 \\ 7 & 1.643861 & -1.517134 & -0.120072 \\ 6 & 0.547219 & -2.282667 & -0.634722 \\ 6 & -0.630816 & -2.476591 & 0.068629 \\ 6 & -1.687542 & -3.235094 & -0.531061 \\ 6 & -1.534372 & -3.738174 & -1.856082 \\ 6 & -0.330490 & -3.485043 & -2.551862 \\ 6 & 0.680545 & -2.787035 & -1.952590 \\ 6 & -2.914484 & -3.479497 & 0.138370 \\ 6 & -3.921440 & -4.188900 & -0.466829 \\ 6 & -3.756614 & -4.702411 & -1.770601 \\ 6 & -2.587497 & -4.474354 & -2.450997 \\ 6 & -0.833918 & -1.897698 & 1.426522 \\ 6 & -1.191822 & -0.563739 & 1.594582 \\ 6 & -1.420023 & -0.070038 & 2.906604 \\ 6 & -1.288241 & -0.874515 & 4.006561 \\ 6 & -0.942623 & -2.237534 & 3.870030 \\ 6 & -0.723790 & -2.763200 & 2.562819 \\ 6 & -0.834739 & -3.090463 & 4.994837 \\ 6 & -0.543276 & -4.421906 & 4.839801 \\ 6 & -0.337709 & -4.950991 & 3.548358 \\ 6 & -0.413854 & -4.143483 & 2.440929 \\ 15 & -1.518832 & 0.495317 & 0.096886 \\ 45 & -0.002114 & 1.030579 & -1.784917 \\ 6 & 0.422370 & 2.903724 & -1.523030 \\ 8 & 0.480526 & 4.057888 & -1.502259 \\ 15 & 1.869209 & -0.013315 & -0.872039 \\ 8 & 2.674118 & 0.722974 & 0.370850 \\ 6 & 3.507243 & 1.783401 & 0.065857 \\ 6 & 4.755291 & 1.543887 & -0.476820\end{array}$




\begin{tabular}{|c|c|c|c|}
\hline 6 & 5.567320 & 2.675625 & -0.812771 \\
\hline 6 & 5.107107 & 3.988906 & -0.490261 \\
\hline & 3.839002 & 4.157127 & 0.114723 \\
\hline & 3.042573 & 3.074349 & 0.369907 \\
\hline & 5.914444 & 5.108115 & -0.808689 \\
\hline & 7.117986 & 4.951861 & -1.445916 \\
\hline & 7.557738 & 3.659608 & -1.801907 \\
\hline & 6.804814 & 2.553655 & -1.495040 \\
\hline & 5.167726 & 0.147877 & -0.753553 \\
\hline & 4.339633 & -0.655992 & -1.517321 \\
\hline & 4.698927 & -1.960836 & -1.903787 \\
\hline 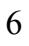 & 5.878331 & -2.497887 & -1.464791 \\
\hline & 6.726300 & -1.762521 & -0.603400 \\
\hline b & 6.370710 & -0.430080 & -0.232608 \\
\hline & 7.213471 & 0.253399 & 0.681201 \\
\hline & 8.353786 & -0.334216 & 1.169791 \\
\hline$b$ & 8.719330 & -1.637844 & 0.773378 \\
\hline & 7.916351 & -2.334953 & -0.091662 \\
\hline 8 & 3.120973 & -0.180740 & -1.955870 \\
\hline & -3.165899 & -0.169604 & -0.388055 \\
\hline & -4.141663 & -0.396184 & 0.577218 \\
\hline ) & -5.415542 & -0.848215 & 0.224659 \\
\hline 6 & -5.685270 & -1.033183 & -1.132315 \\
\hline 5 & -4.728160 & -0.806057 & -2.130595 \\
\hline 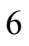 & -3.458943 & -0.386612 & -1.733344 \\
\hline$b$ & -6.444350 & -1.095709 & 1.326743 \\
\hline 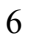 & -5.104514 & -0.961501 & -3.603163 \\
\hline & -1.987871 & 2.138576 & 0.788755 \\
\hline 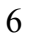 & -3.141658 & 2.763794 & 0.321903 \\
\hline 6 & -3.474656 & 4.062796 & 0.709414 \\
\hline ) & -2.636285 & 4.710786 & 1.617486 \\
\hline 6 & -1.467282 & 4.117201 & 2.108181 \\
\hline 6 & -1.136624 & 2.839016 & 1.649366 \\
\hline 6 & -4.731474 & 4.704277 & 0.124747 \\
\hline 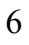 & -0.594078 & 4.797735 & 3.163695 \\
\hline & -0.032241 & 0.063201 & -3.440350 \\
\hline & -0.046044 & -0.413129 & -4.495595 \\
\hline & 6.940662 & 1.254372 & 1.005524 \\
\hline 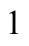 & -1.731738 & 0.961946 & 3.043696 \\
\hline | & 8.172249 & -3.349265 & -0.394943 \\
\hline 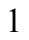 & -0.999269 & -2.666294 & 5.984466 \\
\hline 1 & 5.547427 & 6.099379 & -0.546162 \\
\hline & -3.058075 & -3.086907 & 1.143047 \\
\hline 1 & -4.856768 & -4.359619 & 0.064147 \\
\hline & 3.494651 & 5.163637 & 0.347832 \\
\hline 1 & -1.472279 & -0.475531 & 5.003503 \\
\hline l & 6.166483 & -3.508369 & -1.750366 \\
\hline 1 & 8.499697 & 3.537863 & -2.331930 \\
\hline 1 & 4.022476 & -2.511664 & -2.553754 \\
\hline 1 & -2.447519 & -4.853984 & -3.462589 \\
\hline 1 & -0.219011 & -3.856912 & -3.569246 \\
\hline ( & -0.097259 & -6.004996 & 3.427975 \\
\hline 1 & 7.152946 & 1.567099 & -1.789336 \\
\hline . & -0.225737 & -4.563227 & 1.454817 \\
\hline 1 & 1.610387 & -2.594709 & -2.486162 \\
\hline 1 & 7.726642 & 5.819013 & -1.691220 \\
\hline
\end{tabular}




\begin{tabular}{|c|c|c|c|}
\hline & 8.978596 & 0.209527 & 1.874807 \\
\hline ] & -4.559544 & -5.271488 & -2.234350 \\
\hline & -0.471908 & -5.072117 & 5.708810 \\
\hline & 9.628310 & -2.088974 & 1.164255 \\
\hline & 2.048973 & 3.178674 & 0.797576 \\
\hline & -3.905796 & -0.214468 & 1.626002 \\
\hline & -2.671427 & -0.214569 & -2.463024 \\
\hline ] & -6.677104 & -1.365610 & -1.432292 \\
\hline & -3.790462 & 2.233489 & -0.372733 \\
\hline & -0.210848 & 2.365638 & 1.984007 \\
\hline 1 & -2.896392 & 5.710799 & 1.949793 \\
\hline & 3.241234 & -1.308984 & 1.190135 \\
\hline & 1.719542 & -1.627375 & 1.997329 \\
\hline ] & -1.329444 & 1.706947 & -2.381948 \\
\hline & 2.610714 & -3.270296 & 3.389788 \\
\hline ] & 2.887994 & -3.783815 & -0.858436 \\
\hline & 3.341684 & -5.606647 & 3.725308 \\
\hline 1 & 3.569790 & -6.134316 & -0.532729 \\
\hline ] & 3.809635 & -7.060617 & 1.760402 \\
\hline 6 & -1.021899 & 6.238735 & 3.434617 \\
\hline & -0.710567 & 4.008576 & 4.473767 \\
\hline 6 & 0.871314 & 4.816440 & 2.721116 \\
\hline 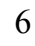 & -4.984537 & 6.103375 & 0.679903 \\
\hline & -4.574081 & 4.812460 & -1.396844 \\
\hline 6 & -5.951531 & 3.833572 & 0.447716 \\
\hline ? & -3.894138 & -0.811733 & -4.523401 \\
\hline 6 & -5.724934 & -2.337371 & -3.861052 \\
\hline 0 & -6.120269 & 0.130307 & -3.961290 \\
\hline 6 & -7.729280 & -1.723323 & 0.792862 \\
\hline 6 & -5.858443 & -2.043432 & 2.380945 \\
\hline ) & -6.797476 & 0.241852 & 1.987311 \\
\hline ] & -0.353456 & 6.684859 & 4.181579 \\
\hline 1 & -0.968658 & 6.858140 & 2.529993 \\
\hline 1 & -2.041567 & 6.300516 & 3.835105 \\
\hline 1 & 1.479688 & 5.376752 & 3.443431 \\
\hline 1 & 1.295409 & 3.807296 & 2.662969 \\
\hline 1 & 0.980617 & 5.296500 & 1.739024 \\
\hline 1 & -0.105252 & 4.478331 & 5.261135 \\
\hline 1 & -1.751603 & 3.974876 & 4.821218 \\
\hline 1 & -0.360136 & 2.974640 & 4.357359 \\
\hline 1 & -5.464330 & 5.278908 & -1.840357 \\
\hline 1 & -3.702448 & 5.426098 & -1.658807 \\
\hline 1 & -4.442954 & 3.830104 & -1.867800 \\
\hline 1 & -5.896494 & 6.517909 & 0.231988 \\
\hline 1 & -5.128172 & 6.093768 & 1.768294 \\
\hline 1 & -4.162822 & 6.792515 & 0.446515 \\
\hline 1 & -6.861167 & 4.283263 & 0.027157 \\
\hline 1 & -5.860101 & 2.821943 & 0.030901 \\
\hline 1 & -6.091171 & 3.739289 & 1.532818 \\
\hline 1 & -4.203252 & -0.960811 & -5.565718 \\
\hline 1 & -3.445102 & 0.188275 & -4.457429 \\
\hline 1 & -3.115184 & -1.553026 & -4.297310 \\
\hline 1 & -6.013220 & -2.428692 & -4.916878 \\
\hline 1 & -5.006663 & -3.135562 & -3.634043 \\
\hline 1 & -6.625350 & -2.513082 & -3.259984 \\
\hline 1 & -6.394093 & 0.066277 & -5.023231 \\
\hline
\end{tabular}




$\begin{array}{lrrr}1 & -7.041693 & 0.040458 & -3.372344 \\ 1 & -5.702237 & 1.129373 & -3.778598 \\ 1 & -8.422878 & -1.909176 & 1.622661 \\ 1 & -8.243845 & -1.066902 & 0.079694 \\ 1 & -7.538511 & -2.685322 & 0.298339 \\ 1 & -6.591206 & -2.220161 & 3.179650 \\ 1 & -5.604685 & -3.017146 & 1.939715 \\ 1 & -4.950959 & -1.643957 & 2.851974 \\ 1 & -7.508764 & 0.088832 & 2.810492 \\ 1 & -5.910545 & 0.742068 & 2.396980 \\ 1 & -7.261305 & 0.924662 & 1.262922\end{array}$

$1_{\mathrm{C} 3-} \psi_{-1 \mathrm{~s} 2 \mathrm{~s} 3 \mathrm{~s} 4 \mathrm{a}}$

Number of imaginary frequencies : 0

The smallest frequencies are : 7.9565 $13.4623 \quad 14.3077 \mathrm{~cm}(-1)$

Electronic energy : $\quad H F=-4125.4663673$

Zero-point correction $=\quad 1.299518$ (Hartree/Particle)

Thermal correction to Energy= $\quad 1.378039$

Thermal correction to Enthalpy= $\quad 1.378983$

Thermal correction to Gibbs Free Energy= $\quad 1.184371$

Sum of electronic and zero-point Energies $=\quad-4124.166849$

Sum of electronic and thermal Energies $=\quad-4124.088329$

Sum of electronic and thermal Enthalpies $=\quad-4124.087384$

Sum of electronic and thermal Free Energies= $\quad-4124.281996$

Cartesian Coordinates

$\begin{array}{lrrr}6 & 3.673657 & -6.021969 & 1.491628 \\ 6 & 3.415901 & -5.229710 & 2.606609 \\ 6 & 2.979592 & -3.920411 & 2.443938 \\ 6 & 2.808171 & -3.372988 & 1.170289 \\ 6 & 3.078689 & -4.171780 & 0.059514 \\ 6 & 3.502434 & -5.487909 & 0.218506 \\ 6 & 2.399342 & -1.926390 & 1.080841 \\ 7 & 1.678021 & -1.527491 & -0.140604 \\ 6 & 0.583255 & -2.308187 & -0.636405 \\ 6 & -0.576968 & -2.523533 & 0.090532 \\ 6 & -1.625546 & -3.313545 & -0.482727 \\ 6 & -1.478777 & -3.831133 & -1.803051 \\ 6 & -0.298588 & -3.546714 & -2.526915 \\ 6 & 0.703147 & -2.813721 & -1.954769 \\ 6 & -2.835096 & -3.583795 & 0.208764 \\ 6 & -3.827215 & -4.336060 & -0.369711 \\ 6 & -3.667327 & -4.863282 & -1.668486 \\ 6 & -2.516119 & -4.610333 & -2.369077 \\ 6 & -0.762871 & -1.938415 & 1.448487 \\ 6 & -1.138452 & -0.608865 & 1.614828 \\ 6 & -1.335224 & -0.105401 & 2.928030 \\ 6 & -1.151569 & -0.894683 & 4.031462 \\ 6 & -0.787779 & -2.253111 & 3.897721 \\ 6 & -0.604966 & -2.790153 & 2.589432 \\ 6 & -0.628778 & -3.091584 & 5.027294 \\ 6 & -0.321828 & -4.420102 & 4.876936 \\ 6 & -0.151321 & -4.960384 & 3.585041 \\ 6 & -0.277398 & -4.166751 & 2.472080\end{array}$




$\begin{array}{cccc}15 & -1.506867 & 0.432542 & 0.115982 \\ 45 & -0.023075 & 1.003174 & -1.777232 \\ 6 & 0.376660 & 2.882970 & -1.522441 \\ 8 & 0.408573 & 4.038229 & -1.507880 \\ 15 & 1.870258 & -0.014601 & -0.880654 \\ 8 & 2.656726 & 0.727293 & 0.371637 \\ 6 & 3.451265 & 1.819776 & 0.079492 \\ 6 & 4.702613 & 1.632350 & -0.476161 \\ 6 & 5.471209 & 2.796861 & -0.801773 \\ 6 & 4.968802 & 4.088231 & -0.454369 \\ 6 & 3.702729 & 4.202379 & 0.167207 \\ 6 & 2.946530 & 3.088661 & 0.410959 \\ 6 & 5.733361 & 5.239827 & -0.763194 \\ 6 & 6.935093 & 5.135241 & -1.414181 \\ 6 & 7.416332 & 3.864670 & -1.793756 \\ 6 & 6.705736 & 2.728453 & -1.496810 \\ 6 & 5.164794 & 0.254850 & -0.766286 \\ 6 & 4.361696 & -0.575577 & -1.528247 \\ 6 & 4.761228 & -1.868818 & -1.914374 \\ 6 & 5.958101 & -2.367526 & -1.477714 \\ 6 & 6.785111 & -1.603930 & -0.620490 \\ 6 & 6.388283 & -0.283059 & -0.249833 \\ 6 & 7.211950 & 0.427591 & 0.660732 \\ 6 & 8.371914 & -0.123030 & 1.146205 \\ 6 & 8.777330 & -1.414893 & 0.750284 \\ 6 & 7.994158 & -2.137918 & -0.11580 \\ 8 & 3.127130 & -0.140607 & -1.963654 \\ 6 & -3.145353 & -0.257994 & -0.359447 \\ 6 & -4.135643 & -0.453276 & 0.611619 \\ 6 & -5.423713 & -0.830349 & 0.251029 \\ 6 & -5.698195 & -1.002019 & -1.117054 \\ 6 & -4.736868 & -0.814131 & -2.105884 \\ 6 & -3.443922 & -0.456337 & -1.697606 \\ 6 & -6.523331 & -1.066762 & 1.285587 \\ 6 & -5.037787 & -0.912537 & -3.599577 \\ 6 & -2.016023 & 2.069943 & 0.797419 \\ 6 & -3.190516 & 2.653387 & 0.328095 \\ 6 & -3.574595 & 3.936929 & 0.715746 \\ 6 & -2.758850 & 4.621040 & 1.617694 \\ 6 & -1.564796 & 4.075185 & 2.103499 \\ 6 & -1.188465 & 2.806813 & 1.650783 \\ 6 & -4.862859 & 4.517912 & 0.137623 \\ 6 & -0.710900 & 4.795821 & 3.148224 \\ 6 & -0.080552 & 0.050390 & -3.439115 \\ 8 & -0.137873 & -0.407181 & -4.501626 \\ 1 & 6.908773 & 1.419615 & 0.985422 \\ 1 & -1.664016 & 0.921232 & 3.064285 \\ 1 & 8.280995 & -3.144043 & -0.414523 \\ 1 & -0.767090 & -2.658906 & 6.017249 \\ 1 & 5.334642 & 6.213528 & -0.481877 \\ 1 & -2.976126 & -3.180495 & 1.209953 \\ 1 & -4.747100 & -4.529141 & 0.178786 \\ 1 & 3.326697 & 5.191977 & 0.422784 \\ 1 & -1.310311 & -0.488042 & 5.029652 \\ 1 & 6.276809 & -3.369229 & -1.761782 \\ 1 & 8.356736 & 3.784256 & -2.334323\end{array}$




\begin{tabular}{|c|c|c|c|}
\hline ] & 4.099522 & -2.442211 & -2.559927 \\
\hline & -2.380109 & -4.997082 & -3.378546 \\
\hline & -0.197941 & -3.924385 & -3.543335 \\
\hline 1 & 0.101105 & -6.011966 & 3.467986 \\
\hline 1 & 7.085021 & 1.758949 & -1.808894 \\
\hline & -0.115371 & -4.594542 & 1.484847 \\
\hline & 1.615684 & -2.596209 & -2.508510 \\
\hline & 7.510666 & 6.026714 & -1.652052 \\
\hline & 8.981355 & 0.441212 & 1.848573 \\
\hline & -4.460109 & -5.461543 & -2.112288 \\
\hline & -0.211220 & -5.059297 & 5.749987 \\
\hline & 9.701255 & -1.836605 & 1.138983 \\
\hline & 1.954710 & 3.151020 & 0.851156 \\
\hline & -3.886910 & -0.280381 & 1.656725 \\
\hline & -2.657191 & -0.299407 & -2.434559 \\
\hline & -6.707418 & -1.284323 & -1.409471 \\
\hline ] & -3.819367 & 2.100351 & -0.366710 \\
\hline & -0.244712 & 2.370771 & 1.986298 \\
\hline 1 & -3.058326 & 5.610193 & 1.949365 \\
\hline & 3.301816 & -1.307913 & 1.135968 \\
\hline ] & 1.805605 & -1.667661 & 1.971787 \\
\hline & -1.366802 & 1.666083 & -2.350812 \\
\hline & 2.750557 & -3.312331 & 3.320282 \\
\hline 1 & 2.956780 & -3.760308 & -0.939039 \\
\hline & 3.537677 & -5.636855 & 3.608453 \\
\hline 1 & 3.699711 & -6.098666 & -0.660244 \\
\hline & 4.003646 & -7.051273 & 1.614347 \\
\hline 6 & -1.211819 & 6.209483 & 3.437464 \\
\hline 6 & -0.758418 & 3.995692 & 4.455753 \\
\hline 6 & 0.742270 & 4.897726 & 2.676549 \\
\hline 6 & -5.194955 & 5.890298 & 0.716475 \\
\hline 6 & -4.705016 & 4.659564 & -1.381087 \\
\hline 6 & -6.033114 & 3.573123 & 0.439637 \\
\hline 5 & -6.495017 & -1.272504 & -3.875909 \\
\hline 6 & -4.746158 & 0.446376 & -4.249814 \\
\hline 6 & -4.151870 & -1.982523 & -4.243242 \\
\hline 6 & -6.096967 & -0.627844 & 2.685025 \\
\hline 5 & -7.785581 & -0.282098 & 0.913041 \\
\hline 6 & -6.849747 & -2.563479 & 1.330131 \\
\hline 1 & -0.560432 & 6.682638 & 4.182779 \\
\hline 1 & -1.199354 & 6.839953 & 2.539081 \\
\hline 1 & -2.229513 & 6.212757 & 3.847551 \\
\hline 1 & 1.328755 & 5.504647 & 3.379053 \\
\hline 1 & 1.224540 & 3.914894 & 2.624651 \\
\hline 1 & 0.803705 & 5.368566 & 1.685875 \\
\hline 1 & -0.162603 & 4.493627 & 5.232917 \\
\hline 1 & -1.788841 & 3.905795 & 4.824207 \\
\hline 1 & -0.356478 & 2.982220 & 4.327580 \\
\hline 1 & -5.621538 & 5.072309 & -1.824298 \\
\hline 1 & -3.874358 & 5.333467 & -1.627523 \\
\hline 1 & -4.504362 & 3.694744 & -1.864241 \\
\hline 1 & -6.127795 & 6.261563 & 0.273732 \\
\hline 1 & -5.338398 & 5.852925 & 1.804302 \\
\hline 1 & -4.412584 & 6.627983 & 0.497082 \\
\hline 1 & -6.968178 & 3.984277 & 0.035877 \\
\hline 1 & -5.888429 & 2.581223 & -0.008947 \\
\hline
\end{tabular}




$\begin{array}{lrrr}1 & -6.161761 & 3.437895 & 1.522057 \\ 1 & -6.660242 & -1.334271 & -4.958895 \\ 1 & -6.764183 & -2.246443 & -3.446513 \\ 1 & -7.187339 & -0.517747 & -3.480781 \\ 1 & -4.985735 & 0.415483 & -5.321230 \\ 1 & -5.349519 & 1.240188 & -3.789324 \\ 1 & -3.689777 & 0.730527 & -4.155404 \\ 1 & -4.337875 & -2.028156 & -5.324797 \\ 1 & -3.083344 & -1.778967 & -4.098064 \\ 1 & -4.363727 & -2.970693 & -3.815709 \\ 1 & -6.922156 & -0.789157 & 3.390051 \\ 1 & -5.235741 & -1.200270 & 3.054576 \\ 1 & -5.837318 & 0.439203 & 2.712436 \\ 1 & -8.563623 & -0.438645 & 1.671919 \\ 1 & -7.579604 & 0.795364 & 0.859636 \\ 1 & -8.204909 & -0.592561 & -0.050971 \\ 1 & -7.660615 & -2.759670 & 2.044914 \\ 1 & -7.166312 & -2.942162 & 0.349888 \\ 1 & -5.972130 & -3.142156 & 1.649713\end{array}$

$1_{\mathrm{C} 3-} \psi_{-1 \mathrm{~s} 2 \mathrm{2} 3 \mathrm{~s} 4 \mathrm{~s}}$

Number of imaginary frequencies : 0

The smallest frequencies are : $14.0596 \quad 15.9413 \quad 18.3556 \mathrm{~cm}(-1)$

Electronic energy : $\quad H F=-4125.4667864$

Zero-point correction $=\quad 1.299824$ (Hartree/Particle)

Thermal correction to Energy $=\quad 1.378086$

Thermal correction to Enthalpy= $\quad 1.379030$

Thermal correction to Gibbs Free Energy= 1.186833

Sum of electronic and zero-point Energies $=\quad-4124.166962$

Sum of electronic and thermal Energies $=\quad-4124.088700$

Sum of electronic and thermal Enthalpies $=\quad-4124.087756$

Sum of electronic and thermal Free Energies= $\quad-4124.279954$

\section{Cartesian Coordinates}

$\begin{array}{lrrr}6 & 3.543727 & -6.026992 & 1.561612 \\ 6 & 3.285099 & -5.226422 & 2.670487 \\ 6 & 2.868107 & -3.912167 & 2.497698 \\ 6 & 2.717693 & -3.368069 & 1.219941 \\ 6 & 2.989145 & -4.175159 & 0.115441 \\ 6 & 3.393254 & -5.496177 & 0.284527 \\ 6 & 2.329082 & -1.916583 & 1.119886 \\ 7 & 1.630596 & -1.513240 & -0.113227 \\ 6 & 0.530834 & -2.281106 & -0.618114 \\ 6 & -0.636670 & -2.484333 & 0.100120 \\ 6 & -1.689418 & -3.263959 & -0.479981 \\ 6 & -1.538969 & -3.786782 & -1.797352 \\ 6 & -0.350388 & -3.515533 & -2.512868 \\ 6 & 0.654211 & -2.790486 & -1.935137 \\ 6 & -2.908224 & -3.514090 & 0.201847 \\ 6 & -3.910804 & -4.244413 & -0.385952 \\ 6 & -3.748011 & -4.779058 & -1.681174 \\ 6 & -2.582476 & -4.553862 & -2.368426 \\ 6 & -0.833186 & -1.897802 & 1.455520 \\ 6 & -1.200893 & -0.565665 & 1.617346\end{array}$




\begin{tabular}{|c|c|c|c|}
\hline 6 & -1.427670 & -0.066723 & 2.927689 \\
\hline 6 & -1.278821 & -0.861969 & 4.032007 \\
\hline 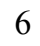 & -0.918958 & -2.221891 & 3.902266 \\
\hline & -0.707427 & -2.754813 & 2.596830 \\
\hline & -0.792286 & -3.065307 & 5.032189 \\
\hline & -0.490269 & -4.395202 & 4.884174 \\
\hline & -0.293033 & -4.931945 & 3.594664 \\
\hline & -0.387186 & -4.133375 & 2.482059 \\
\hline & -1.526692 & 0.489699 & 0.116562 \\
\hline 45 & -0.012769 & 1.051016 & -1.757431 \\
\hline 6 & 0.421580 & 2.920496 & -1.489365 \\
\hline 8 & 0.479727 & 4.074652 & -1.468663 \\
\hline 15 & 1.856584 & -0.007816 & -0.860395 \\
\hline 8 & 2.667145 & 0.720030 & 0.384163 \\
\hline 6 & 3.494314 & 1.786225 & 0.084593 \\
\hline 6 & 4.738571 & 1.556952 & -0.471246 \\
\hline 6 & 5.542817 & 2.695377 & -0.803455 \\
\hline 6 & 5.081103 & 4.003409 & -0.462243 \\
\hline 6 & 3.819158 & 4.160301 & 0.158465 \\
\hline 6 & 3.028856 & 3.072025 & 0.409155 \\
\hline 6 & 5.880877 & 5.129038 & -0.777158 \\
\hline 6 & 7.078179 & 4.984151 & -1.428647 \\
\hline 6 & 7.518977 & 3.697432 & -1.802918 \\
\hline 6 & 6.773596 & 2.585364 & -1.499732 \\
\hline 6 & 5.156580 & 0.165134 & -0.760941 \\
\hline 6 & 4.327519 & -0.639764 & -1.522559 \\
\hline 6 & 4.691848 & -1.940041 & -1.919622 \\
\hline 6 & 5.878207 & -2.472079 & -1.493578 \\
\hline 6 & 6.728455 & -1.736314 & -0.634791 \\
\hline 6 & 6.367325 & -0.408489 & -0.252975 \\
\hline 6 & 7.213116 & 0.274668 & 0.658261 \\
\hline 6 & 8.361060 & -0.308597 & 1.134058 \\
\hline 6 & 8.731750 & -1.607356 & 0.726646 \\
\hline 6 & 7.926225 & -2.304233 & -0.136212 \\
\hline 8 & 3.103305 & -0.169645 & -1.950463 \\
\hline 6 & -3.157714 & -0.191313 & -0.395216 \\
\hline 6 & -4.152409 & -0.431125 & 0.552948 \\
\hline 6 & -5.411687 & -0.892090 & 0.174227 \\
\hline 6 & -5.658497 & -1.075009 & -1.192718 \\
\hline 6 & -4.688840 & -0.829404 & -2.167816 \\
\hline 6 & -3.426996 & -0.404199 & -1.741181 \\
\hline 6 & -6.466414 & -1.143348 & 1.251844 \\
\hline 6 & -4.960219 & -0.947697 & -3.666779 \\
\hline 6 & -2.016003 & 2.128085 & 0.804620 \\
\hline 6 & -3.170455 & 2.745286 & 0.329418 \\
\hline 6 & -3.510785 & 4.044716 & 0.709530 \\
\hline 6 & -2.681050 & 4.699991 & 1.620389 \\
\hline 6 & -1.512189 & 4.113652 & 2.120266 \\
\hline 6 & -1.172936 & 2.836004 & 1.667174 \\
\hline 6 & -4.761419 & 4.681886 & 0.107365 \\
\hline 6 & -0.648238 & 4.800686 & 3.179136 \\
\hline 6 & -0.082206 & 0.100644 & -3.420330 \\
\hline 8 & -0.148137 & -0.358020 & -4.481921 \\
\hline 1 & 6.936598 & 1.271819 & 0.991029 \\
\hline 1 & -1.751258 & 0.962021 & 3.060401 \\
\hline 1 & 8.186044 & -3.315018 & -0.447825 \\
\hline
\end{tabular}




\begin{tabular}{|c|c|c|c|}
\hline & -0.951336 & -2.635272 & 6.020171 \\
\hline & 5.512931 & 6.116031 & -0.500230 \\
\hline & -3.049933 & -3.108425 & 1.201520 \\
\hline & -4.840737 & -4.415911 & 0.154191 \\
\hline & 3.474159 & 5.162826 & 0.407300 \\
\hline & -1.460924 & -0.458296 & 5.027430 \\
\hline & 6.170486 & -3.478981 & -1.787512 \\
\hline & 8.455678 & 3.584991 & -2.344197 \\
\hline & 4.013400 & -2.491356 & -2.567127 \\
\hline & -2.442108 & -4.949487 & -3.373904 \\
\hline & -0.245928 & -3.895837 & -3.527977 \\
\hline & -0.045252 & -5.984840 & 3.479513 \\
\hline & 7.122226 & 1.603256 & -1.808025 \\
\hline & -0.205195 & -4.559069 & 1.497407 \\
\hline & 1.572821 & -2.584318 & -2.482975 \\
\hline & 7.680933 & 5.856184 & -1.671208 \\
\hline & 8.988001 & 0.234842 & 1.837414 \\
\hline & -4.547956 & -5.362026 & -2.132589 \\
\hline & -0.404523 & -5.038276 & 5.757171 \\
\hline & 9.646713 & -2.055028 & 1.107403 \\
\hline & 2.039680 & 3.168104 & 0.849177 \\
\hline & -3.936798 & -0.249163 & 1.606160 \\
\hline & -2.634513 & -0.217359 & -2.464344 \\
\hline & -6.640261 & -1.418862 & -1.505147 \\
\hline 1 & -3.810925 & 2.210072 & -0.369414 \\
\hline & -0.245811 & 2.369353 & 2.007114 \\
\hline ] & -2.947590 & 5.700275 & 1.946837 \\
\hline L & 3.238907 & -1.310244 & 1.184955 \\
\hline & 1.726268 & -1.646526 & 2.001361 \\
\hline 1 & -1.339278 & 1.744677 & -2.336808 \\
\hline & 2.636609 & -3.297637 & 3.368973 \\
\hline 1 & 2.884738 & -3.766261 & -0.886167 \\
\hline & 3.390823 & -5.630885 & 3.675222 \\
\hline 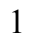 & 3.591800 & -6.113228 & -0.589555 \\
\hline ] & 3.858406 & -7.060110 & 1.692205 \\
\hline 6 & -1.080915 & 6.242030 & 3.440135 \\
\hline 6 & -0.773897 & 4.016810 & 4.491615 \\
\hline 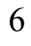 & 0.820925 & 4.819723 & 2.748961 \\
\hline 6 & -5.033763 & 6.075600 & 0.666915 \\
\hline 5 & -4.573850 & 4.801324 & -1.410030 \\
\hline 6 & -5.982564 & 3.802379 & 0.400481 \\
\hline 5 & -6.375594 & -1.435850 & -3.963155 \\
\hline 5 & -4.781645 & 0.436846 & -4.303706 \\
\hline 5 & -3.977161 & -1.929022 & -4.312585 \\
\hline 6 & -7.731006 & -1.789023 & 0.690864 \\
\hline 6 & -5.901799 & -2.074788 & 2.331311 \\
\hline 6 & -6.850933 & 0.196464 & 1.890384 \\
\hline 1 & -0.419102 & 6.693119 & 4.190018 \\
\hline 1 & -1.022258 & 6.857217 & 2.532970 \\
\hline 1 & -2.103649 & 6.303491 & 3.832735 \\
\hline 1 & 1.422554 & 5.380924 & 3.476235 \\
\hline 1 & 1.245874 & 3.810613 & 2.695671 \\
\hline 1 & 0.939004 & 5.299108 & 1.767648 \\
\hline & -0.175321 & 4.490585 & 5.281727 \\
\hline 1 & -1.817545 & 3.983141 & 4.831107 \\
\hline 1 & -0.421283 & 2.982819 & 4.382046 \\
\hline
\end{tabular}


$1_{\mathrm{C} 4-} \psi_{-1 \text {-1a2a3a4a }}$

Number of imaginary frequencies : 0

The smallest frequencies are : $15.2331 \quad 20.2484 \quad 22.2519 \mathrm{~cm}(-1)$

Electronic energy : $\quad H F=-4125.4689173$

Zero-point correction $=\quad 1.299405($ Hartree/Particle $)$

Thermal correction to Energy= $\quad 1.377833$

Thermal correction to Enthalpy= $\quad 1.378777$

Thermal correction to Gibbs Free Energy= 1.187582

Sum of electronic and zero-point Energies $=\quad-4124.169512$

Sum of electronic and thermal Energies $=\quad-4124.091084$

Sum of electronic and thermal Enthalpies $=\quad-4124.090140$

Sum of electronic and thermal Free Energies $=\quad-4124.281335$

\begin{tabular}{cccc}
\multicolumn{4}{c}{ Cartesian Coordinates } \\
\hline $6 \ldots \ldots \ldots \ldots \ldots \ldots \ldots \ldots \ldots \ldots \ldots \ldots \ldots . . \ldots \ldots \ldots \ldots \ldots \ldots \ldots \ldots \ldots \ldots$ \\
6 & -0.406775 & 2.478576 & 1.242567 \\
6 & -1.506944 & 2.101861 & 0.466814 \\
6 & -2.423068 & 3.061573 & 0.046289 \\
6 & -2.259543 & 4.405583 & 0.393280 \\
6 & -1.166992 & 4.744384 & 1.193686 \\
6 & -0.240412 & 3.801167 & 1.652568 \\
15 & -1.636312 & 0.358323 & -0.092371 \\
6 & -3.433469 & 0.137297 & -0.433071 \\
6 & -4.372364 & 0.420295 & 0.563882 \\
6 & -5.732521 & 0.220377 & 0.345326 \\
6 & -6.123959 & -0.281367 & -0.902208 \\
6 & -5.215962 & -0.581272 & -1.917651
\end{tabular}




\begin{tabular}{|c|c|c|c|}
\hline 6 & -3.858507 & -0.354882 & -1.662349 \\
\hline 6 & -6.778555 & 0.485643 & 1.426242 \\
\hline 6 & -7.873640 & 1.411869 & 0.887945 \\
\hline 6 & -5.714357 & -1.108964 & -3.262465 \\
\hline 6 & -4.568451 & -1.614527 & -4.135930 \\
\hline 6 & -3.235462 & 5.488550 & -0.062786 \\
\hline 6 & -3.812814 & 6.212969 & 1.158158 \\
\hline 6 & 0.871450 & 4.228088 & 2.611722 \\
\hline 6 & 0.241047 & 4.902221 & 3.836982 \\
\hline 6 & -1.501300 & -0.558925 & 1.522195 \\
\hline 6 & -1.245021 & -1.919948 & 1.550515 \\
\hline 6 & -1.214971 & -2.623154 & 2.800394 \\
\hline 6 & -1.454501 & -1.915058 & 4.013280 \\
\hline 6 & -1.731661 & -0.530570 & 3.948722 \\
\hline 6 & -1.753914 & 0.122085 & 2.746516 \\
\hline 6 & -0.932644 & -4.012259 & 2.882870 \\
\hline 6 & -0.881764 & -4.652946 & 4.096234 \\
\hline 6 & -1.116547 & -3.944163 & 5.292955 \\
\hline 6 & -1.398750 & -2.602863 & 5.248686 \\
\hline 6 & -1.023948 & -2.731671 & 0.324055 \\
\hline 6 & 0.240610 & -2.864607 & -0.217413 \\
\hline 6 & 0.474435 & -3.766175 & -1.282868 \\
\hline 6 & -0.551062 & -4.495172 & -1.822092 \\
\hline 6 & -1.871157 & -4.346042 & -1.334971 \\
\hline 6 & -2.112855 & -3.464658 & -0.239913 \\
\hline 6 & -2.956242 & -5.053044 & -1.908259 \\
\hline 6 & -4.230282 & -4.898420 & -1.422531 \\
\hline 6 & -4.472485 & -4.026801 & -0.337984 \\
\hline 6 & -3.440708 & -3.331936 & 0.241954 \\
\hline 7 & 1.324680 & -2.050306 & 0.261881 \\
\hline 15 & 1.683148 & -0.797443 & -0.827609 \\
\hline 8 & 2.876552 & -1.363628 & -1.870467 \\
\hline 6 & 4.142330 & -1.690990 & -1.448922 \\
\hline 6 & 5.018900 & -0.719654 & -0.995771 \\
\hline 6 & 6.280699 & -1.143992 & -0.466359 \\
\hline 6 & 6.656570 & -2.518232 & -0.550649 \\
\hline 6 & 5.761982 & -3.447103 & -1.137190 \\
\hline 6 & 4.524141 & -3.046339 & -1.559091 \\
\hline 6 & 7.156980 & -0.254268 & 0.205648 \\
\hline 6 & 8.345790 & -0.693988 & 0.734447 \\
\hline 6 & 8.729941 & -2.046077 & 0.616343 \\
\hline 6 & 7.897354 & -2.936706 & -0.012079 \\
\hline 6 & 4.607604 & 0.701758 & -1.036925 \\
\hline 6 & 3.408909 & 1.068762 & -0.456733 \\
\hline 6 & 2.924955 & 2.390979 & -0.480490 \\
\hline 6 & 3.660544 & 3.366793 & -1.096946 \\
\hline 6 & 4.881896 & 3.052820 & -1.739486 \\
\hline 6 & 5.357473 & 1.706048 & -1.732143 \\
\hline 6 & 5.622752 & 4.050679 & -2.418290 \\
\hline 6 & 6.775844 & 3.739166 & -3.091186 \\
\hline 6 & 7.227556 & 2.403296 & -3.120712 \\
\hline 6 & 6.538606 & 1.415429 & -2.461619 \\
\hline 8 & 2.643488 & 0.109886 & 0.172918 \\
\hline 6 & 2.277995 & -2.718752 & 1.178891 \\
\hline 6 & 2.875165 & -1.820510 & 2.228233 \\
\hline 0 & 4.257255 & -1.716556 & 2.367601 \\
\hline
\end{tabular}




\begin{tabular}{|c|c|c|c|}
\hline 6 & 4.811420 & -0.888598 & 3.340485 \\
\hline 6 & 3.984645 & -0.166596 & 4.194663 \\
\hline & 2.600713 & -0.279688 & 4.071038 \\
\hline & 2.051908 & -1.095619 & 3.090841 \\
\hline & -0.080134 & 0.167410 & -1.992812 \\
\hline 6 & -0.851708 & -1.177474 & -3.171906 \\
\hline & -1.108895 & -1.919620 & -4.020921 \\
\hline & -0.067583 & 2.080161 & -2.245547 \\
\hline & 0.013621 & 3.208215 & -2.488823 \\
\hline 6 & -7.400688 & -0.850933 & 1.846801 \\
\hline 6 & -6.171684 & 1.143559 & 2.664348 \\
\hline J & -6.687318 & -2.273772 & -3.055149 \\
\hline 6 & -6.431095 & 0.027013 & -4.000673 \\
\hline 0 & -2.491769 & 6.491944 & -0.950510 \\
\hline 6 & -4.401670 & 4.913258 & -0.863725 \\
\hline 6 & 1.688701 & 3.037278 & 3.109978 \\
\hline & 1.813195 & 5.218306 & 1.920738 \\
\hline 1 & 6.866426 & 0.787341 & 0.318394 \\
\hline 1 & -1.966568 & 1.187706 & 2.734016 \\
\hline 1 & 8.168380 & -3.988459 & -0.095706 \\
\hline 1 & -1.581802 & -2.040954 & 6.163478 \\
\hline 1 & 5.245061 & 5.072188 & -2.403191 \\
\hline 1 & -3.639125 & -2.654263 & 1.070849 \\
\hline 1 & -5.486844 & -3.898730 & 0.036113 \\
\hline 1 & 3.297963 & 4.393729 & -1.126723 \\
\hline 1 & -1.927582 & 0.011816 & 4.872869 \\
\hline 1 & 6.065772 & -4.489194 & -1.222287 \\
\hline 1 & 8.127286 & 2.150791 & -3.677447 \\
\hline 1 & 3.812069 & -3.749357 & -1.986721 \\
\hline 1 & -2.755738 & -5.720653 & -2.745041 \\
\hline 1 & -0.370636 & -5.177188 & -2.651485 \\
\hline 1 & -0.660462 & -5.717022 & 4.135190 \\
\hline 1 & 6.894879 & 0.389741 & -2.506980 \\
\hline 1 & -0.753728 & -4.574559 & 1.968743 \\
\hline 1 & 1.485373 & -3.847904 & -1.678832 \\
\hline 1 & 7.333742 & 4.512907 & -3.613477 \\
\hline 1 & 8.994735 & 0.007134 & 1.254574 \\
\hline 1 & -5.056872 & -5.445579 & -1.871169 \\
\hline 1 & -1.072577 & -4.464262 & 6.246980 \\
\hline 1 & 9.676800 & -2.379940 & 1.034204 \\
\hline 1 & 1.965528 & 2.603407 & -0.011111 \\
\hline 1 & -4.029043 & 0.802286 & 1.521249 \\
\hline 1 & -3.109686 & -0.569589 & -2.419922 \\
\hline 1 & -7.185452 & -0.447254 & -1.084990 \\
\hline 1 & -3.267479 & 2.752330 & -0.564695 \\
\hline 1 & 0.305076 & 1.714257 & 1.555676 \\
\hline 1 & -1.037577 & 5.786252 & 1.486843 \\
\hline 1 & 1.696880 & -3.510248 & 1.673340 \\
\hline 1 & 0.948690 & 0.243793 & -3.197854 \\
\hline 1 & 2.587233 & 5.559158 & 2.621462 \\
\hline 1 & 2.320028 & 4.751916 & 1.065974 \\
\hline 1 & 1.280633 & 6.104736 & 1.554958 \\
\hline 1 & 1.021891 & 5.189807 & 4.553596 \\
\hline 1 & -0.315175 & 5.810067 & 3.574807 \\
\hline 1 & -0.451840 & 4.220388 & 4.347623 \\
\hline 1 & 1.052773 & 2.296774 & 3.615363 \\
\hline
\end{tabular}




$\begin{array}{lrrr}1 & 2.229141 & 2.523113 & 2.304393 \\ 1 & 2.439698 & 3.379305 & 3.833940 \\ 1 & -5.086386 & 5.721694 & -1.149125 \\ 1 & -4.065889 & 4.426134 & -1.787948 \\ 1 & -4.978480 & 4.181284 & -0.281270 \\ 1 & -4.349743 & 5.514012 & 1.812947 \\ 1 & -3.036636 & 6.703843 & 1.757407 \\ 1 & -4.521467 & 6.988745 & 0.838720 \\ 1 & -1.670718 & 6.983816 & -0.414799 \\ 1 & -2.064831 & 5.994047 & -1.830691 \\ 1 & -3.177934 & 7.274859 & -1.300982 \\ 1 & -5.423258 & 0.502891 & 3.149870 \\ 1 & -5.696357 & 2.104467 & 2.423241 \\ 1 & -6.960586 & 1.339590 & 3.401233 \\ 1 & -8.403507 & 0.979837 & 0.030958 \\ 1 & -8.620437 & 1.607921 & 1.668760 \\ 1 & -7.455480 & 2.376065 & 0.571409 \\ 1 & -7.900586 & -1.351112 & 1.007960 \\ 1 & -6.633179 & -1.534461 & 2.234154 \\ 1 & -8.147927 & -0.696216 & 2.636929 \\ 1 & -5.752444 & 0.872543 & -4.172905 \\ 1 & -6.795443 & -0.319433 & -4.977420 \\ 1 & -7.293250 & 0.399905 & -3.433199 \\ 1 & -4.000994 & -2.411191 & -3.634359 \\ 1 & -4.970943 & -2.027465 & -5.069537 \\ 1 & -3.869845 & -0.814566 & -4.411297 \\ 1 & -6.197378 & -3.099208 & -2.521797 \\ 1 & -7.581265 & -1.986133 & -2.489366 \\ 1 & -7.027424 & -2.654520 & -4.027257 \\ 1 & 3.080462 & -3.221566 & 0.617427 \\ 1 & 4.910137 & -2.273506 & 1.694085 \\ 1 & 0.971981 & -1.164913 & 2.975602 \\ 1 & 5.893592 & -0.807027 & 3.422915 \\ 1 & 1.942728 & 0.272070 & 4.741236 \\ 1 & 4.414745 & 0.481381 & 4.955717\end{array}$

$\mathbf{1}_{\mathrm{C} 4-\psi_{-12 a z a 4 s}}$

Number of imaginary frequencies : 0

The smallest frequencies are : $14.1636 \quad 19.9461 \quad 20.7202 \mathrm{~cm}(-1)$

Electronic energy : $\quad H F=-4125.4693254$

Zero-point correction $=\quad 1.299213($ Hartree/Particle $)$

Thermal correction to Energy= $\quad 1.377757$

Thermal correction to Enthalpy= $\quad 1.378702$

Thermal correction to Gibbs Free Energy $=\quad 1.186506$

Sum of electronic and zero-point Energies $=\quad-4124.170112$

Sum of electronic and thermal Energies $=\quad-4124.091568$

Sum of electronic and thermal Enthalpies $=\quad-4124.090624$

Sum of electronic and thermal Free Energies $=\quad-4124.282819$

Cartesian Coordinates

$\begin{array}{lllll}6 & 2.049183 & -1.114029 & 3.076565 \\ 6 & 2.871234 & -1.839105 & 2.212943 \\ 6 & 4.253417 & -1.740049 & 2.354555 \\ 6 & 4.808854 & -0.916929 & 3.330847\end{array}$




\begin{tabular}{|c|c|c|c|}
\hline 6 & 3.983266 & -0.194734 & 4.185975 \\
\hline 6 & 2.599146 & -0.302760 & 4.059905 \\
\hline 6 & 2.272657 & -2.731442 & 1.159310 \\
\hline 7 & 1.322228 & -2.056714 & 0.244037 \\
\hline 15 & 1.681719 & -0.794689 & -0.834276 \\
\hline & -0.080241 & 0.179624 & -1.992401 \\
\hline ( & -0.076816 & 2.093692 & -2.233601 \\
\hline & -0.003562 & 3.223455 & -2.471582 \\
\hline 6 & 0.235417 & -2.864825 & -0.239625 \\
\hline & -1.026720 & -2.735650 & 0.308225 \\
\hline & -2.120299 & -3.458012 & -0.259602 \\
\hline 0 & -1.886173 & -4.324814 & -1.367716 \\
\hline 0 & -0.568207 & -4.472486 & -1.861296 \\
\hline 0 & 0.462280 & -3.754449 & -1.316515 \\
\hline 6 & -3.445526 & -3.326098 & 0.229901 \\
\hline 0 & -4.482454 & -4.007683 & -0.356354 \\
\hline 6 & -4.248129 & -4.864971 & -1.454034 \\
\hline 6 & -2.976598 & -5.018926 & -1.946666 \\
\hline 6 & -1.243615 & -1.933572 & 1.541080 \\
\hline 6 & -1.500630 & -0.572729 & 1.520203 \\
\hline 6 & -1.752114 & 0.100950 & 2.748706 \\
\hline 6 & -1.728868 & -0.559151 & 3.947108 \\
\hline 6 & -1.452264 & -1.944065 & 4.003373 \\
\hline 6 & -1.213086 & -2.644639 & 2.786153 \\
\hline 6 & -1.396454 & -2.639581 & 5.234472 \\
\hline 6 & -1.114784 & -3.981211 & 5.270103 \\
\hline 6 & -0.880700 & -4.682632 & 4.068868 \\
\hline 6 & -0.931579 & -4.034369 & 2.859559 \\
\hline 15 & -1.637395 & 0.350440 & -0.091087 \\
\hline 6 & -3.432220 & 0.121120 & -0.437673 \\
\hline 6 & -4.369598 & 0.378542 & 0.559365 \\
\hline 6 & -5.733985 & 0.177994 & 0.340705 \\
\hline 6 & -6.128409 & -0.291420 & -0.912725 \\
\hline 6 & -5.213736 & -0.570911 & -1.935607 \\
\hline 6 & -3.859694 & -0.350214 & -1.678602 \\
\hline 6 & -6.710741 & 0.456660 & 1.480615 \\
\hline 6 & -8.158001 & 0.163884 & 1.094396 \\
\hline 6 & -5.713667 & -1.074745 & -3.288802 \\
\hline 6 & -6.433682 & 0.072864 & -4.005584 \\
\hline 6 & -1.521031 & 2.092150 & 0.476380 \\
\hline 6 & -0.422802 & 2.469792 & 1.254368 \\
\hline 6 & -0.262449 & 3.790715 & 1.671774 \\
\hline 6 & -1.189670 & 4.732906 & 1.212368 \\
\hline 6 & -2.275346 & 4.394887 & 0.402110 \\
\hline 6 & -2.439101 & 3.050483 & 0.056321 \\
\hline 6 & -3.245235 & 5.479993 & -0.061567 \\
\hline 6 & -4.360676 & 4.919756 & -0.941110 \\
\hline 6 & 0.845976 & 4.216495 & 2.635295 \\
\hline 6 & 1.785930 & 5.212912 & 1.950747 \\
\hline 6 & -6.614372 & 1.932281 & 1.885044 \\
\hline 6 & -6.347298 & -0.433479 & 2.675634 \\
\hline 6 & -4.568497 & -1.563375 & -4.172726 \\
\hline 6 & -6.684422 & -2.244837 & -3.100737 \\
\hline 6 & -3.890500 & 6.145573 & 1.158790 \\
\hline 6 & -2.479406 & 6.528746 & -0.875221 \\
\hline 6 & 0.210759 & 4.882611 & 3.862368 \\
\hline
\end{tabular}




\begin{tabular}{|c|c|c|c|}
\hline 6 & 1.666568 & 3.026051 & 3.129169 \\
\hline 8 & 2.877029 & -1.351109 & -1.879777 \\
\hline & 4.142290 & -1.680327 & -1.458041 \\
\hline & 5.017283 & -0.711032 & -0.997512 \\
\hline & 6.278936 & -1.137559 & -0.469562 \\
\hline & 6.655836 & -2.511044 & -0.561647 \\
\hline & 5.762419 & -3.437204 & -1.154239 \\
\hline & 4.524812 & -3.034811 & -1.575311 \\
\hline & 7.896489 & -2.931821 & -0.024556 \\
\hline & 8.728088 & -2.044291 & 0.609528 \\
\hline & 8.343007 & -0.693134 & 0.735094 \\
\hline y & 7.154196 & -0.251189 & 0.208171 \\
\hline & 4.603811 & 0.710091 & -1.028767 \\
\hline & 3.404091 & 1.070735 & -0.446669 \\
\hline & 2.917650 & 2.392201 & -0.461250 \\
\hline & 3.651934 & 3.373867 & -1.069914 \\
\hline & 4.874398 & 3.066805 & -1.713715 \\
\hline & 5.352354 & 1.720840 & -1.715967 \\
\hline & 6.534473 & 1.437674 & -2.446746 \\
\hline & 7.222213 & 2.431575 & -3.097982 \\
\hline & 6.768196 & 3.766415 & -3.058844 \\
\hline & 5.614061 & 4.070937 & -2.384530 \\
\hline & 2.639641 & 0.105811 & 0.174830 \\
\hline 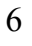 & -0.851294 & -1.151953 & -3.187591 \\
\hline & -1.107971 & -1.881502 & -4.047569 \\
\hline & 6.862874 & 0.789535 & 0.326841 \\
\hline & -1.963505 & 1.166805 & 2.742863 \\
\hline & 8.168265 & -3.982895 & -0.114092 \\
\hline & -1.579182 & -2.083396 & 6.152810 \\
\hline & 5.234618 & 5.091664 & -2.362078 \\
\hline & -3.638344 & -2.658922 & 1.069076 \\
\hline & -5.494802 & -3.878040 & 0.022351 \\
\hline & 3.287514 & 4.400331 & -1.092609 \\
\hline & -1.923563 & -0.022170 & 4.874654 \\
\hline 1 & 6.066944 & -4.478628 & -1.244749 \\
\hline & 8.122772 & 2.184745 & -3.655914 \\
\hline ] & 3.813277 & -3.735701 & -2.007336 \\
\hline & -2.782444 & -5.675824 & -2.793363 \\
\hline . & -0.393336 & -5.144172 & -2.700264 \\
\hline 1 & -0.660075 & -5.747067 & 4.101157 \\
\hline & 6.892466 & 0.412950 & -2.499537 \\
\hline 1 & -0.753569 & -4.590840 & 1.941686 \\
\hline & 1.471438 & -3.834843 & -1.717396 \\
\hline 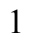 & 7.325152 & 4.544979 & -3.574930 \\
\hline & 8.991186 & 0.005412 & 1.259622 \\
\hline ] & -5.078810 & -5.402180 & -1.907169 \\
\hline 1 & -1.070885 & -4.507392 & 6.220789 \\
\hline t & 9.674894 & -2.379943 & 1.026079 \\
\hline 1 & 1.957264 & 2.599265 & 0.008559 \\
\hline & -4.033347 & 0.739262 & 1.530310 \\
\hline 1 & -3.110691 & -0.551154 & -2.439658 \\
\hline t & -7.187171 & -0.451318 & -1.105123 \\
\hline 1 & -3.282169 & 2.740955 & -0.556764 \\
\hline 1 & 0.292263 & 1.706875 & 1.563552 \\
\hline & -1.065649 & 5.773975 & 1.510985 \\
\hline & 1.689007 & -3.523511 & 1.649842 \\
\hline
\end{tabular}




$\begin{array}{rrrr}1 & 0.951202 & 0.268076 & -3.194208 \\ 1 & 2.557596 & 5.552910 & 2.654487 \\ 1 & 2.295780 & 4.751941 & 1.094780 \\ 1 & 1.251353 & 6.099320 & 1.587895 \\ 1 & 0.989050 & 5.169711 & 4.581939 \\ 1 & -0.348169 & 5.789645 & 3.603174 \\ 1 & -0.480702 & 4.195904 & 4.368421 \\ 1 & 1.032669 & 2.280945 & 3.630333 \\ 1 & 2.209947 & 2.517591 & 2.321896 \\ 1 & 2.415388 & 3.367310 & 3.855722 \\ 1 & -5.034660 & 5.731660 & -1.241847 \\ 1 & -3.968575 & 4.460171 & -1.857442 \\ 1 & -4.964301 & 4.169232 & -0.412919 \\ 1 & -4.453521 & 5.413377 & 1.752982 \\ 1 & -3.148290 & 6.611550 & 1.818312 \\ 1 & -4.589333 & 6.930489 & 0.839769 \\ 1 & -1.697731 & 7.020989 & -0.284250 \\ 1 & -1.998378 & 6.070626 & -1.749131 \\ 1 & -3.165101 & 7.308537 & -1.233195 \\ 1 & -8.492294 & 0.784394 & 0.253022 \\ 1 & -8.305609 & -0.890057 & 0.825140 \\ 1 & -8.817399 & 0.381178 & 1.944160 \\ 1 & -6.919928 & 2.583632 & 1.055753 \\ 1 & -7.275603 & 2.139600 & 2.737215 \\ 1 & -5.595619 & 2.219712 & 2.175465 \\ 1 & -6.409815 & -1.495701 & 2.402391 \\ 1 & -5.330302 & -0.245973 & 3.043366 \\ 1 & -7.040355 & -0.257222 & 3.509262 \\ 1 & -5.756146 & 0.921632 & -4.165904 \\ 1 & -6.801047 & -0.257574 & -4.986744 \\ 1 & -7.294513 & 0.435491 & -3.429523 \\ 1 & -4.001335 & -2.369618 & -3.686252 \\ 1 & -4.971534 & -1.958208 & -5.113914 \\ 1 & -3.869524 & -0.758655 & -4.432905 \\ 1 & -6.193375 & -3.077036 & -2.578930 \\ 1 & -7.580007 & -1.967720 & -2.532411 \\ 1 & -7.021842 & -2.611688 & -4.079115 \\ 1 & 3.074292 & -3.233999 & 0.596407 \\ 1 & 4.905537 & -2.296848 & 1.680146 \\ 1 & 0.969284 & -1.178706 & 2.958976 \\ 1 & 5.891157 & -0.839280 & 3.415276 \\ 1 & 1.941956 & 0.249262 & 4.730684 \\ 1 & 4.414465 & 0.449432 & 4.949629\end{array}$

\section{$1_{\mathrm{C} 4-} \psi_{-1 \mathbf{a} 2 a 3 s 4 a}$}

Number of imaginary frequencies : 0

The smallest frequencies are : $12.7086 \quad 17.9966 \quad 18.9949 \mathrm{~cm}(-1)$

Electronic energy : $\quad \mathrm{HF}=-4125.4692495$

Zero-point correction $=\quad 1.298738($ Hartree/Particle $)$

Thermal correction to Energy $=\quad 1.377506$

Thermal correction to Enthalpy= $\quad 1.378450$

Thermal correction to Gibbs Free Energy= 1.184923

Sum of electronic and zero-point Energies $=\quad-4124.170512$

Sum of electronic and thermal Energies $=\quad-4124.091744$

Sum of electronic and thermal Enthalpies $=\quad-4124.090800$ 
Cartesian Coordinates

\begin{tabular}{|c|c|c|c|}
\hline 6 & 2.041813 & -1.178252 & 3.056312 \\
\hline 6 & 2.873492 & -1.869753 & 2.174723 \\
\hline 6 & 4.254191 & -1.754363 & 2.318229 \\
\hline 6 & 4.798306 & -0.946976 & 3.313804 \\
\hline 6 & 3.963056 & -0.257597 & 4.186504 \\
\hline 6 & 2.580589 & -0.383264 & 4.058980 \\
\hline 6 & 2.286249 & -2.743350 & 1.099301 \\
\hline 7 & 1.327837 & -2.057045 & 0.200857 \\
\hline 15 & 1.678557 & -0.773362 & -0.854201 \\
\hline 45 & -0.105415 & 0.208005 & -1.980617 \\
\hline 6 & -0.082771 & 2.128232 & -2.182052 \\
\hline 8 & -0.009784 & 3.262852 & -2.394417 \\
\hline 6 & 0.249476 & -2.865616 & -0.300772 \\
\hline 6 & -1.017662 & -2.754758 & 0.240492 \\
\hline 6 & -2.097865 & -3.487510 & -0.341017 \\
\hline 6 & -1.841858 & -4.358859 & -1.441076 \\
\hline 6 & -0.521016 & -4.477783 & -1.934164 \\
\hline 6 & 0.493757 & -3.741291 & -1.385043 \\
\hline 6 & -3.429698 & -3.373923 & 0.135467 \\
\hline 6 & -4.447613 & -4.089151 & -0.444359 \\
\hline 6 & -4.188345 & -4.961247 & -1.524033 \\
\hline 6 & -2.913079 & -5.088060 & -2.012571 \\
\hline 6 & -1.241824 & -1.984932 & 1.494014 \\
\hline 6 & -1.492700 & -0.622540 & 1.518127 \\
\hline 6 & -1.733619 & 0.015360 & 2.767648 \\
\hline 6 & -1.712597 & -0.681702 & 3.944786 \\
\hline 6 & -1.443868 & -2.069155 & 3.956678 \\
\hline 6 & -1.209893 & -2.733248 & 2.717850 \\
\hline 6 & -1.388613 & -2.801630 & 5.166326 \\
\hline 6 & -1.110491 & -4.144330 & 5.161566 \\
\hline 6 & -0.878606 & -4.809247 & 3.939393 \\
\hline 6 & -0.929740 & -4.124985 & 2.750084 \\
\hline 15 & -1.635726 & 0.350648 & -0.059062 \\
\hline 6 & -3.433512 & 0.150151 & -0.403191 \\
\hline 6 & -4.374063 & 0.295017 & 0.626260 \\
\hline 6 & -5.731041 & 0.124856 & 0.382331 \\
\hline 6 & -6.129695 & -0.198035 & -0.926113 \\
\hline 6 & -5.224879 & -0.332795 & -1.973563 \\
\hline 6 & -3.864009 & -0.153071 & -1.685047 \\
\hline 6 & -6.780545 & 0.253469 & 1.484670 \\
\hline 6 & -6.169015 & 0.686883 & 2.815647 \\
\hline 6 & -5.638527 & -0.716548 & -3.391849 \\
\hline 6 & -5.003741 & 0.242031 & -4.405528 \\
\hline 6 & -1.507508 & 2.077132 & 0.549580 \\
\hline 6 & -0.405229 & 2.439378 & 1.328771 \\
\hline 6 & -0.242580 & 3.753120 & 1.768305 \\
\hline 6 & -1.175575 & 4.702693 & 1.336263 \\
\hline 6 & -2.270533 & 4.378271 & 0.532990 \\
\hline 6 & -2.430495 & 3.041965 & 0.156620 \\
\hline 6 & -3.251925 & 5.467627 & 0.104548 \\
\hline 6 & -4.413074 & 4.908440 & -0.715266 \\
\hline 6 & 0.874118 & 4.163593 & 2.728836 \\
\hline
\end{tabular}




\begin{tabular}{|c|c|c|c|}
\hline 6 & 1.803191 & 5.177358 & 2.055026 \\
\hline & -7.830021 & 1.296017 & 1.084893 \\
\hline & -7.460788 & -1.104615 & 1.689180 \\
\hline & -7.152390 & -0.676229 & -3.586193 \\
\hline & -5.153088 & -2.144399 & -3.666938 \\
\hline & -3.835883 & 6.154098 & 1.344106 \\
\hline & -2.512287 & 6.499300 & -0.753703 \\
\hline & 0.249499 & 4.802578 & 3.975655 \\
\hline & 1.702960 & 2.965943 & 3.189569 \\
\hline & 2.873191 & -1.303159 & -1.914984 \\
\hline & 4.140198 & -1.639470 & -1.504175 \\
\hline & 5.013772 & -0.680017 & -1.020764 \\
\hline & 6.275945 & -1.117072 & -0.502860 \\
\hline & 6.656013 & -2.486934 & -0.629188 \\
\hline & 5.765066 & -3.399524 & -1.245981 \\
\hline & 4.526452 & -2.989520 & -1.656546 \\
\hline ) & 7.897052 & -2.918434 & -0.101631 \\
\hline & 8.725677 & -2.045503 & 0.556182 \\
\hline D & 8.336922 & -0.699028 & 0.716320 \\
\hline & 7.147964 & -0.246377 & 0.198890 \\
\hline & 4.599151 & 0.741090 & -1.020324 \\
\hline & 3.398759 & 1.088000 & -0.431484 \\
\hline & 2.909973 & 2.408575 & -0.420020 \\
\hline & 3.643179 & 3.403607 & -1.007930 \\
\hline & 4.866862 & 3.111786 & -1.656480 \\
\hline b & 5.346830 & 1.766867 & -1.685898 \\
\hline & 6.530017 & 1.500615 & -2.421277 \\
\hline & 7.216883 & 2.508697 & -3.051257 \\
\hline & 6.760833 & 3.841763 & -2.985185 \\
\hline 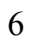 & 5.605590 & 4.130596 & -2.305882 \\
\hline & 2.636074 & 0.109730 & 0.170804 \\
\hline ) & -0.948512 & -1.091596 & -3.152234 \\
\hline & -1.302647 & -1.806458 & -3.990272 \\
\hline & 6.853394 & 0.789943 & 0.344919 \\
\hline & -1.939461 & 1.082162 & 2.793673 \\
\hline & 8.171395 & -3.966229 & -0.217812 \\
\hline 1 & -1.568035 & -2.272086 & 6.100934 \\
\hline & 5.224493 & 5.150047 & -2.262787 \\
\hline 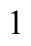 & -3.641937 & -2.697467 & 0.962184 \\
\hline . & -5.464793 & -3.976179 & -0.074306 \\
\hline & 3.277075 & 4.429700 & -1.010404 \\
\hline ] & -1.901571 & -0.172664 & 4.889074 \\
\hline & 6.072168 & -4.437475 & -1.363623 \\
\hline 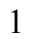 & 8.118354 & 2.274680 & -3.613220 \\
\hline & 3.817368 & -3.680992 & -2.107331 \\
\hline 1 & -2.700187 & -5.749439 & -2.851230 \\
\hline & -0.330670 & -5.147240 & -2.771546 \\
\hline 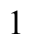 & -0.659076 & -5.874390 & 3.939181 \\
\hline 1 & 6.889638 & 0.477791 & -2.494878 \\
\hline 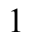 & -0.751805 & -4.654848 & 1.816705 \\
\hline 1 & 1.505668 & -3.801676 & -1.782029 \\
\hline ( & 7.317039 & 4.631563 & -3.484722 \\
\hline 1 & 8.982416 & -0.012880 & 1.260172 \\
\hline ] & -5.004554 & -5.523645 & -1.972347 \\
\hline 1 & -1.066883 & -4.698954 & 6.095957 \\
\hline 1 & 9.672711 & -2.389486 & 0.965353 \\
\hline
\end{tabular}




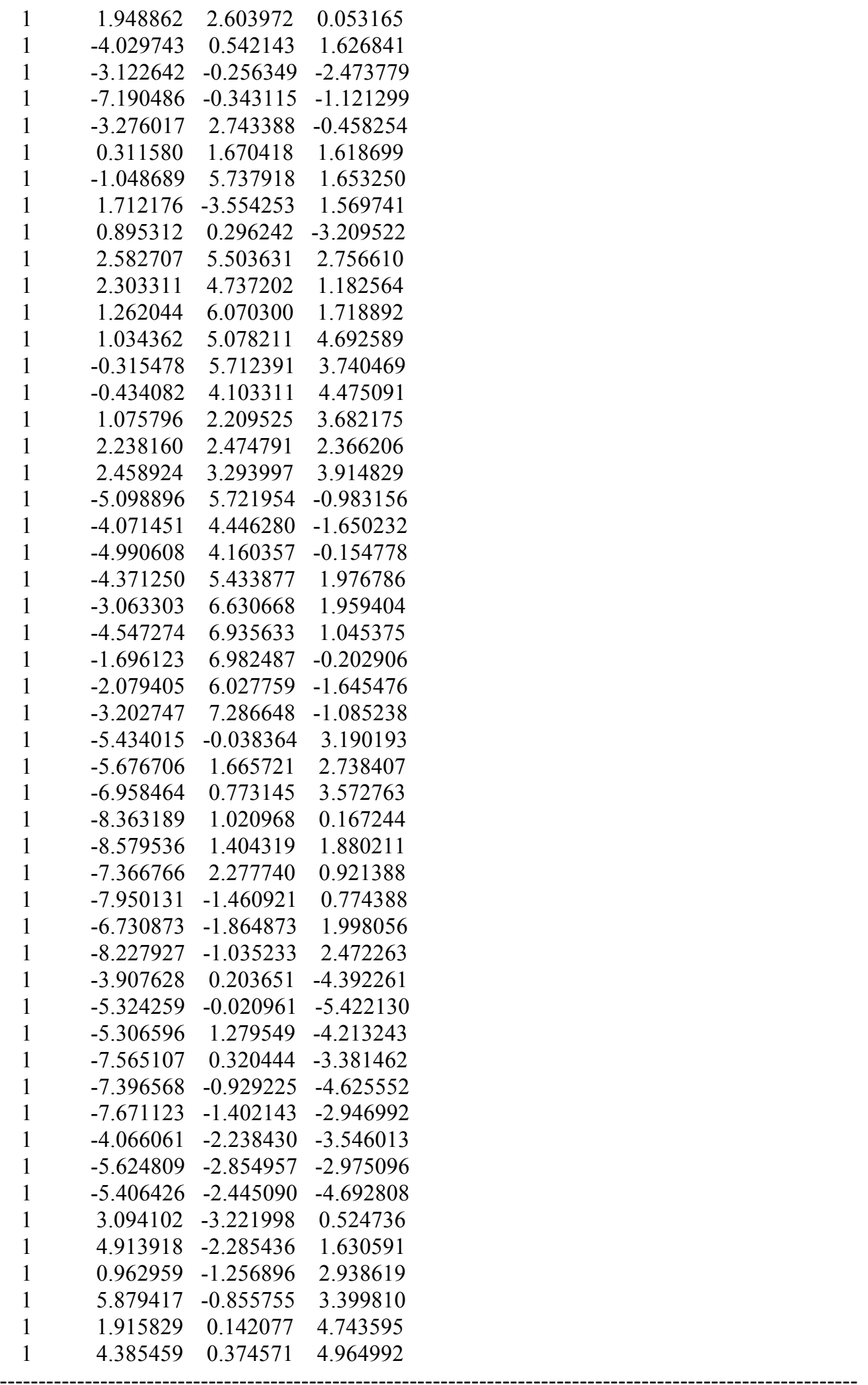

$1_{\mathrm{C} 4-\psi_{-1 \mathrm{a} 2 \mathrm{a3} 4 \mathrm{~s}}}$

Number of imaginary frequencies : 0

The smallest frequencies are : $12.2106 \quad 15.073421 .5118 \mathrm{~cm}(-1)$ 
Electronic energy : $\quad H F=-4125.4695906$

$\begin{array}{lc}\text { Zero-point correction }= & 1.299170(\text { Hartree/Particle }) \\ \text { Thermal correction to Energy }= & 1.377749\end{array}$

Thermal correction to Enthalpy= $\quad 1.378693$

Thermal correction to Gibbs Free Energy $=1.185995$

Sum of electronic and zero-point Energies $=\quad-4124.170420$

Sum of electronic and thermal Energies $=\quad-4124.091842$

Sum of electronic and thermal Enthalpies $=\quad-4124.090898$

Sum of electronic and thermal Free Energies $=\quad-4124.283595$

Cartesian Coordinates

$\begin{array}{ccccc}6 & -3.858211 & -0.081937 & -1.710197 \\ 6 & -3.416915 & 0.127229 & -0.409006 \\ 6 & -4.347614 & 0.184006 & 0.629002 \\ 6 & -5.710217 & 0.021113 & 0.387029 \\ 6 & -6.120538 & -0.195364 & -0.933733 \\ 6 & -5.216965 & -0.245996 & -1.997468 \\ 15 & -1.621814 & 0.339932 & -0.060402 \\ 45 & -0.096057 & 0.235341 & -1.985402 \\ 6 & -0.072506 & 2.159077 & -2.153172 \\ 8 & -0.005171 & 3.297584 & -2.345442 \\ 6 & -6.680051 & 0.086719 & 1.565597 \\ 6 & -6.607200 & 1.481223 & 2.199045 \\ 6 & -5.645537 & -0.539323 & -3.433402 \\ 6 & -7.157434 & -0.433457 & -3.622158 \\ 6 & -1.473580 & -0.651072 & 1.504951 \\ 6 & -1.233704 & -2.014931 & 1.459988 \\ 6 & -1.210920 & -2.783315 & 2.670942 \\ 6 & -1.440932 & -2.137255 & 3.919874 \\ 6 & -1.692344 & -0.746544 & 3.930220 \\ 6 & -1.706208 & -0.029951 & 2.764220 \\ 6 & -0.947843 & -4.178672 & 2.680112 \\ 6 & -0.910719 & -4.883795 & 3.857650 \\ 6 & -1.139441 & -4.236905 & 5.090189 \\ 6 & -1.399797 & -2.890914 & 5.117057 \\ 6 & -1.013561 & -2.761833 & 0.192495 \\ 6 & 0.252142 & -2.860389 & -0.354724 \\ 6 & 0.491950 & -3.709023 & -1.461244 \\ 6 & -0.526969 & -4.425582 & -2.028959 \\ 6 & -1.846845 & -4.314340 & -1.531387 \\ 6 & -2.097315 & -3.475842 & -0.404925 \\ 6 & -2.923120 & -5.016561 & -2.126448 \\ 6 & -4.197504 & -4.896454 & -1.633874 \\ 6 & -4.450156 & -4.060909 & -0.524140 \\ 6 & -3.427537 & -3.371624 & 0.078479 \\ 7 & 1.331350 & -2.062041 & 0.161509 \\ 6 & 2.277637 & -2.759201 & 1.063743 \\ 6 & 2.868209 & -1.893361 & 2.143512 \\ 6 & 2.039345 & -1.190159 & 3.018597 \\ 6 & 2.581329 & -0.400817 & 4.023802 \\ 6 & 3.964399 & -0.293379 & 4.161280 \\ 6 & 4.796697 & -0.994688 & 3.295389 \\ 6 & 4.249178 & -1.795564 & 2.296383 \\ 15 & 1.687792 & -0.762851 & -0.872308 \\ 8 & 2.636978 & 0.109612 & 0.170243\end{array}$




\begin{tabular}{|c|c|c|c|}
\hline & 3.401764 & 1.096076 & -0.415845 \\
\hline & 4.606489 & 0.758290 & -1.001372 \\
\hline & 5.355728 & 1.792962 & -1.651095 \\
\hline & 4.872747 & 3.136509 & -1.609643 \\
\hline & 3.644581 & 3.418574 & -0.965243 \\
\hline & 2.909960 & 2.415442 & -0.393112 \\
\hline & 6.543585 & 1.537213 & -2.382701 \\
\hline & 7.231824 & 2.553492 & -2.997774 \\
\hline & 6.772591 & 3.884823 & -2.919697 \\
\hline & 5.613004 & 4.163860 & -2.243711 \\
\hline & 5.023569 & -0.662121 & -1.015132 \\
\hline & 4.153649 & -1.616908 & -1.514055 \\
\hline & 4.539748 & -2.965871 & -1.674744 \\
\hline & 5.775638 & -3.379835 & -1.259846 \\
\hline & 6.663454 & -2.472560 & -0.630842 \\
\hline & 6.282939 & -1.103749 & -0.494452 \\
\hline & 7.152033 & -0.239212 & 0.218397 \\
\hline & 8.338832 & -0.696283 & 0.736864 \\
\hline & 8.727870 & -2.041565 & 0.567509 \\
\hline & 7.901896 & -2.908819 & -0.101043 \\
\hline & 2.890240 & -1.275387 & -1.931967 \\
\hline & -1.520707 & 2.061012 & 0.567803 \\
\hline & -0.420354 & 2.434052 & 1.344360 \\
\hline & -0.281768 & 3.743961 & 1.803170 \\
\hline & -1.236316 & 4.680711 & 1.390522 \\
\hline & -2.326656 & 4.346885 & 0.584585 \\
\hline & -2.465010 & 3.012534 & 0.193679 \\
\hline & 0.835584 & 4.162115 & 2.759585 \\
\hline & 0.213646 & 4.790862 & 4.012794 \\
\hline & -3.339055 & 5.419358 & 0.187159 \\
\hline & -4.021202 & 5.960150 & 1.448707 \\
\hline & 1.678758 & 2.970709 & 3.210976 \\
\hline & 1.750533 & 5.186709 & 2.082666 \\
\hline & -2.618955 & 6.560156 & -0.539106 \\
\hline & -4.422347 & 4.875882 & -0.742392 \\
\hline & -0.960795 & -1.034603 & -3.173125 \\
\hline & -1.339875 & -1.727215 & -4.018817 \\
\hline & -6.290205 & -0.971572 & 2.604962 \\
\hline & -8.125230 & -0.171979 & 1.148036 \\
\hline & -5.212566 & -1.969672 & -3.777482 \\
\hline & -4.978396 & 0.442252 & -4.403163 \\
\hline & 6.856886 & 0.795804 & 0.372060 \\
\hline & -1.902264 & 1.038141 & 2.806562 \\
\hline & 8.176495 & -3.955692 & -0.224712 \\
\hline & -1.575962 & -2.375093 & 6.059918 \\
\hline & 5.229473 & 5.181988 & -2.191672 \\
\hline & -3.635584 & -2.719992 & 0.926686 \\
\hline & -5.466179 & -3.956003 & -0.148479 \\
\hline & 3.276335 & 4.443911 & -0.958677 \\
\hline & -1.875360 & -0.250691 & 4.882665 \\
\hline & 6.082980 & -4.416974 & -1.383889 \\
\hline & 8.136881 & 2.327470 & -3.557243 \\
\hline & 3.832693 & -3.653172 & -2.134988 \\
\hline & -2.715100 & -5.650983 & -2.986867 \\
\hline & -0.340939 & -5.071144 & -2.885846 \\
\hline & -0.704774 & -5.951503 & 3.840158 \\
\hline
\end{tabular}




\begin{tabular}{|c|c|c|c|}
\hline & 6.905700 & 0.515968 & -2.465421 \\
\hline & -0.772531 & -4.694411 & 1.738399 \\
\hline ] & 1.502871 & -3.762227 & -1.861926 \\
\hline 1 & 7.329831 & 4.681125 & -3.407618 \\
\hline & 8.982207 & -0.014766 & 1.288987 \\
\hline & -5.017868 & -5.437129 & -2.100871 \\
\hline & -1.107228 & -4.807993 & 6.015069 \\
\hline & 9.673048 & -2.389163 & 0.977927 \\
\hline & 1.945168 & 2.603342 & 0.075626 \\
\hline & -4.002379 & 0.355944 & 1.647396 \\
\hline & -3.122066 & -0.119935 & -2.509544 \\
\hline & -7.179323 & -0.327220 & -1.137760 \\
\hline & -3.308770 & 2.706565 & -0.419985 \\
\hline & 0.313333 & 1.675130 & 1.618157 \\
\hline & -1.131336 & 5.712902 & 1.724948 \\
\hline & 1.692874 & -3.564822 & 1.530104 \\
\hline & 0.900349 & 0.339523 & -3.216886 \\
\hline & 2.532835 & 5.517126 & 2.779217 \\
\hline & 2.247279 & 4.754209 & 1.204360 \\
\hline & 1.198520 & 6.075853 & 1.754129 \\
\hline & 1.000972 & 5.070777 & 4.725308 \\
\hline & -0.360600 & 5.696784 & 3.785429 \\
\hline$\frac{1}{1}$ & -0.460235 & 4.083798 & 4.514395 \\
\hline & 1.060696 & 2.205286 & 3.701218 \\
\hline ] & 2.216731 & 2.489342 & 2.383588 \\
\hline & 2.433510 & 3.302599 & 3.935690 \\
\hline & -5.120743 & 5.681357 & -1.002059 \\
\hline ] & -4.002053 & 4.487856 & -1.679350 \\
\hline & -5.006901 & 4.073970 & -0.271741 \\
\hline 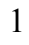 & -4.554798 & 5.159303 & 1.978147 \\
\hline & -3.302075 & 6.403011 & 2.148743 \\
\hline ] & -4.751619 & 6.737138 & 1.185528 \\
\hline$\frac{1}{1}$ & -1.868619 & 7.048028 & 0.094477 \\
\hline & -2.108710 & 6.191172 & -1.438305 \\
\hline 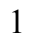 & -3.339739 & 7.329030 & -0.848225 \\
\hline & -8.487041 & 0.576019 & 0.431067 \\
\hline 1 & -8.250503 & -1.166528 & 0.700392 \\
\hline & -8.777758 & -0.123149 & 2.028915 \\
\hline ] & -6.915936 & 2.252450 & 1.481279 \\
\hline 1 & -7.275608 & 1.542630 & 3.068509 \\
\hline & -5.593592 & 1.729597 & 2.538167 \\
\hline 1 & -6.346936 & -1.980873 & 2.175489 \\
\hline & -5.271408 & -0.829799 & 2.988207 \\
\hline 1 & -6.974441 & -0.933170 & 3.463266 \\
\hline & -3.885236 & 0.354506 & -4.407632 \\
\hline 1 & -5.320981 & 0.247409 & -5.427801 \\
\hline 1 & -5.231892 & 1.480919 & -4.153468 \\
\hline 1 & -7.533956 & 0.562871 & -3.355364 \\
\hline 1 & -7.409877 & -0.613301 & -4.674695 \\
\hline 1 & -7.703456 & -1.177830 & -3.028934 \\
\hline 1 & -4.128773 & -2.105939 & -3.666649 \\
\hline 1 & -5.706441 & -2.694004 & -3.115737 \\
\hline 1 & -5.481444 & -2.214133 & -4.814361 \\
\hline 1 & 3.083264 & -3.245398 & 0.492250 \\
\hline 1 & 4.906822 & -2.335204 & 1.613369 \\
\hline 1 & 0.960452 & -1.252343 & 2.891753 \\
\hline
\end{tabular}




$\begin{array}{rrrr}1 & 5.878227 & -0.917739 & 3.389553 \\ 1 & 1.918743 & 0.134308 & 4.702920 \\ 1 & 4.389563 & 0.333868 & 4.942223\end{array}$

$1_{C 4-\psi-125 s 3 a 4 a}$

Number of imaginary frequencies : 0

The smallest frequencies are : $14.5513 \quad 20.2983 \quad 21.4328 \mathrm{~cm}(-1)$

Electronic energy : $\quad H F=-4125.4697917$

Zero-point correction $=\quad 1.299407($ Hartree/Particle $)$

Thermal correction to Energy $=\quad 1.377786$

Thermal correction to Enthalpy= $\quad 1.378731$

Thermal correction to Gibbs Free Energy= $\quad 1.187407$

Sum of electronic and zero-point Energies $=\quad-4124.170384$

Sum of electronic and thermal Energies $=\quad-4124.092005$

Sum of electronic and thermal Enthalpies $=\quad-4124.091061$

Sum of electronic and thermal Free Energies $=\quad-4124.282385$

Cartesian Coordinates

$\begin{array}{cccc}6 & 3.984451 & -0.215406 & 4.189472 \\ 6 & 4.812986 & -0.921816 & 3.324011 \\ 6 & 4.260996 & -1.740721 & 2.342280 \\ 6 & 2.879187 & -1.850676 & 2.205241 \\ 6 & 2.054241 & -1.142232 & 3.079712 \\ 6 & 2.600878 & -0.335550 & 4.068695 \\ 6 & 2.283381 & -2.735813 & 1.144042 \\ 7 & 1.327992 & -2.055669 & 0.237787 \\ 6 & 0.242608 & -2.862500 & -0.251013 \\ 6 & -1.023552 & -2.727956 & 0.286479 \\ 6 & -2.114995 & -3.445086 & -0.292640 \\ 6 & -1.872886 & -4.319112 & -1.393487 \\ 6 & -0.550746 & -4.474648 & -1.873134 \\ 6 & 0.476368 & -3.756010 & -1.323096 \\ 6 & -3.445896 & -3.301187 & 0.177726 \\ 6 & -4.480361 & -3.975882 & -0.421046 \\ 6 & -4.237099 & -4.842332 & -1.509497 \\ 6 & -2.960079 & -5.009246 & -1.982803 \\ 6 & -1.242681 & -1.933399 & 1.524589 \\ 6 & -1.492098 & -0.570927 & 1.518132 \\ 6 & -1.738255 & 0.092752 & 2.753188 \\ 6 & -1.718695 & -0.578819 & 3.945012 \\ 6 & -1.450282 & -1.965863 & 3.987387 \\ 6 & -1.215552 & -2.656335 & 2.763324 \\ 6 & -1.397418 & -2.673043 & 5.212001 \\ 6 & -1.122197 & -4.016247 & 5.235284 \\ 6 & -0.891717 & -4.707491 & 4.027540 \\ 6 & -0.940101 & -4.047974 & 2.824204 \\ 15 & -1.627705 & 0.368955 & -0.081749 \\ 45 & -0.080890 & 0.189899 & -1.988048 \\ 6 & -0.067570 & 2.104601 & -2.224592 \\ 8 & 0.007691 & 3.235419 & -2.456209 \\ 15 & 1.685241 & -0.788614 & -0.835993 \\ 8 & 2.646474 & 0.105335 & 0.175753 \\ 6 & 3.410582 & 1.073177 & -0.441586 \\ 6 & 4.608427 & 0.714796 & -1.028810\end{array}$




\begin{tabular}{|c|c|c|c|}
\hline & 5.356836 & 1.729209 & -1.710872 \\
\hline & 4.880945 & 3.075831 & -1.698143 \\
\hline & 3.660373 & 3.380144 & -1.049493 \\
\hline & 2.925919 & 2.395337 & -0.446162 \\
\hline & 5.620640 & 4.083579 & -2.363473 \\
\hline & 6.772865 & 3.782146 & -3.042424 \\
\hline & 7.224711 & 2.446931 & -3.092016 \\
\hline & 6.536872 & 1.449485 & -2.446316 \\
\hline & 5.020657 & -0.706846 & -1.008008 \\
\hline & 4.144251 & -1.672825 & -1.472924 \\
\hline & 4.527426 & -3.026107 & -1.602570 \\
\hline & 5.766408 & -3.431332 & -1.188374 \\
\hline & 6.660916 & -2.509786 & -0.590283 \\
\hline & 6.283632 & -1.137276 & -0.486297 \\
\hline & 7.159717 & -0.256187 & 0.197245 \\
\hline & 8.349725 & -0.701953 & 0.718226 \\
\hline & 8.735437 & -2.051723 & 0.580264 \\
\hline & 7.902972 & -2.934361 & -0.059491 \\
\hline & 2.877283 & -1.341384 & -1.887559 \\
\hline & -3.427488 & 0.163860 & -0.419882 \\
\hline & -4.361722 & 0.396493 & 0.594535 \\
\hline & -5.721906 & 0.193114 & 0.376739 \\
\hline & -6.118595 & -0.247306 & -0.892009 \\
\hline & -5.215754 & -0.487033 & -1.927914 \\
\hline & -3.857893 & -0.269541 & -1.669343 \\
\hline & -6.760974 & 0.378605 & 1.480922 \\
\hline & -7.356305 & -0.992018 & 1.824430 \\
\hline & -5.722924 & -0.954000 & -3.291350 \\
\hline & -6.623095 & -2.182957 & -3.129052 \\
\hline 6 & -1.489892 & 2.103775 & 0.498882 \\
\hline 0 & -2.395994 & 3.069355 & 0.088222 \\
\hline & -2.236458 & 4.412598 & 0.457599 \\
\hline 6 & -1.155265 & 4.739857 & 1.270090 \\
\hline ) & -0.227084 & 3.783554 & 1.715425 \\
\hline 6 & -0.389976 & 2.470465 & 1.286721 \\
\hline f & -3.258850 & 5.436337 & -0.029359 \\
\hline 6 & -3.218216 & 5.497302 & -1.560412 \\
\hline 6 & 0.884187 & 4.197628 & 2.680561 \\
\hline 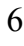 & 1.705174 & 3.001071 & 3.158162 \\
\hline 6 & -0.870861 & -1.142217 & -3.168406 \\
\hline & -1.151417 & -1.877664 & -4.015751 \\
\hline 5 & 1.822760 & 5.200805 & 2.003840 \\
\hline 5 & 0.254590 & 4.850642 & 3.917594 \\
\hline 6 & -2.985035 & 6.837210 & 0.511190 \\
\hline 6 & -4.659478 & 5.012938 & 0.429915 \\
\hline 6 & -6.152854 & 0.967545 & 2.752710 \\
\hline 6 & -7.876130 & 1.317524 & 1.010498 \\
\hline 6 & -6.522347 & 0.182167 & -3.938391 \\
\hline 6 & -4.577491 & -1.338410 & -4.224405 \\
\hline 1 & 6.867911 & 0.783241 & 0.325676 \\
\hline 1 & -1.944797 & 1.159699 & 2.757372 \\
\hline 1 & 8.175072 & -3.984511 & -0.158400 \\
\hline 1 & -1.576761 & -2.124212 & 6.135425 \\
\hline 1 & 5.242758 & 5.104678 & -2.332928 \\
\hline & -3.645028 & -2.628437 & 1.010648 \\
\hline 1 & -5.497652 & -3.834245 & -0.060073 \\
\hline
\end{tabular}




\begin{tabular}{|c|c|c|c|}
\hline & 3.297461 & 4.407271 & -1.064202 \\
\hline & -1.909825 & -0.049711 & 4.877800 \\
\hline & 6.071215 & -4.471779 & -1.288638 \\
\hline & 8.123661 & 2.202668 & -3.653659 \\
\hline & 3.815498 & -3.723856 & -2.039014 \\
\hline & -2.759002 & -5.672122 & -2.823193 \\
\hline & -0.370298 & -5.151028 & -2.707130 \\
\hline & -0.675608 & -5.773106 & 4.049872 \\
\hline & 6.893194 & 0.424651 & -2.507253 \\
\hline & -0.763918 & -4.597097 & 1.901586 \\
\hline & 1.488746 & -3.839666 & -1.714950 \\
\hline & 7.329885 & 4.563514 & -3.554183 \\
\hline & 8.998425 & -0.007559 & 1.247607 \\
\hline & -5.065743 & -5.375065 & -1.971567 \\
\hline & -1.080358 & -4.551342 & 6.181078 \\
\hline & 9.683288 & -2.390313 & 0.992032 \\
\hline & 1.966970 & 2.600390 & 0.027302 \\
\hline & -4.015278 & 0.735876 & 1.566943 \\
\hline & -3.112306 & -0.445461 & -2.439836 \\
\hline & -7.179662 & -0.415866 & -1.075764 \\
\hline & -3.238709 & 2.776864 & -0.537223 \\
\hline & 0.319352 & 1.700545 & 1.591513 \\
\hline & -1.025444 & 5.772234 & 1.587919 \\
\hline & 1.704301 & -3.535529 & 1.627423 \\
\hline & 0.942071 & 0.270942 & -3.198107 \\
\hline & 2.598037 & 5.531117 & 2.708240 \\
\hline & 2.328064 & 4.749303 & 1.140109 \\
\hline & 1.288100 & 6.092425 & 1.654252 \\
\hline & 1.036094 & 5.125283 & 4.638547 \\
\hline & -0.300913 & 5.763319 & 3.671848 \\
\hline & -0.438054 & 4.160112 & 4.416819 \\
\hline & 1.072115 & 2.251976 & 3.654371 \\
\hline & 2.243766 & 2.500049 & 2.343066 \\
\hline & 2.457940 & 3.333453 & 3.884789 \\
\hline & -3.747583 & 7.531677 & 0.136719 \\
\hline & -3.021108 & 6.869261 & 1.608006 \\
\hline & -2.008332 & 7.218336 & 0.186670 \\
\hline & -4.946471 & 4.030446 & 0.034213 \\
\hline & -4.715399 & 4.962733 & 1.525541 \\
\hline & -5.408299 & 5.738872 & 0.085845 \\
\hline & -2.227837 & 5.812516 & -1.913168 \\
\hline & -3.440536 & 4.526007 & -2.018767 \\
\hline & -3.957160 & 6.219429 & -1.933322 \\
\hline & -5.387978 & 0.310953 & 3.188808 \\
\hline & -5.698415 & 1.950788 & 2.570482 \\
\hline & -6.937375 & 1.100176 & 3.508238 \\
\hline & -8.406993 & 0.931149 & 0.132583 \\
\hline & -8.618752 & 1.451660 & 1.808211 \\
\hline & -7.477546 & 2.307060 & 0.751988 \\
\hline & -7.846252 & -1.454486 & 0.958377 \\
\hline & -6.574238 & -1.679814 & 2.174131 \\
\hline & -8.105569 & -0.897582 & 2.622149 \\
\hline & -5.897034 & 1.074184 & -4.073977 \\
\hline & -6.893219 & -0.124360 & -4.925872 \\
\hline & -7.388778 & 0.470129 & -3.329653 \\
\hline & -3.956795 & -2.136372 & -3.793818 \\
\hline
\end{tabular}




$\begin{array}{llll}1 & -4.983445 & -1.707171 & -5.174830 \\ 1 & -3.927237 & -0.485538 & -4.458015 \\ 1 & -6.064558 & -3.014936 & -2.679912 \\ 1 & -7.501047 & -1.985122 & -2.502362 \\ 1 & -6.989578 & -2.512550 & -4.110348 \\ 1 & 3.086629 & -3.228551 & 0.574885 \\ 1 & 4.915301 & -2.285192 & 1.660015 \\ 1 & 0.974512 & -1.217064 & 2.967009 \\ 1 & 5.894874 & -0.835040 & 3.404763 \\ 1 & 1.941461 & 0.203361 & 4.747893 \\ 1 & 4.412941 & 0.425745 & 4.957202\end{array}$

$1_{\mathrm{C} 4-\psi_{-1 \mathrm{a} 253 a 4 s}}$

Number of imaginary frequencies : 0

The smallest frequencies are : $15.6531 \quad 19.3240 \quad 22.5666 \mathrm{~cm}(-1)$

Electronic energy : $\quad H F=-4125.4700233$

Zero-point correction $=\quad 1.299766($ Hartree/Particle $)$

Thermal correction to Energy $=\quad 1.377995$

Thermal correction to Enthalpy= $\quad 1.378939$

Thermal correction to Gibbs Free Energy= $\quad 1.188343$

Sum of electronic and zero-point Energies $=\quad-4124.170258$

Sum of electronic and thermal Energies $=\quad-4124.092029$

Sum of electronic and thermal Enthalpies $=\quad-4124.091085$

Sum of electronic and thermal Free Energies $=\quad-4124.281680$

Cartesian Coordinates

$\begin{array}{cccc}6 & -0.412283 & 2.457450 & 1.295228 \\ 6 & -1.511684 & 2.084385 & 0.509637 \\ 6 & -2.426594 & 3.044305 & 0.104214 \\ 6 & -2.273081 & 4.388855 & 0.472241 \\ 6 & -1.192161 & 4.721842 & 1.282935 \\ 6 & -0.257672 & 3.770757 & 1.726286 \\ 15 & -1.631988 & 0.350267 & -0.078678 \\ 6 & -3.430059 & 0.135461 & -0.420374 \\ 6 & -4.362977 & 0.348142 & 0.591919 \\ 6 & -5.728785 & 0.155791 & 0.370967 \\ 6 & -6.128150 & -0.253239 & -0.902007 \\ 6 & -5.218509 & -0.480199 & -1.942186 \\ 6 & -3.863352 & -0.274628 & -1.680863 \\ 6 & -6.702173 & 0.368463 & 1.528711 \\ 6 & -6.585641 & 1.808087 & 2.041698 \\ 6 & -5.727545 & -0.917827 & -3.314493 \\ 6 & -4.584399 & -1.301535 & -4.250802 \\ 6 & -3.296105 & 5.409705 & -0.020430 \\ 6 & -4.699940 & 4.981333 & 0.423422 \\ 6 & 0.852371 & 4.190298 & 2.690481 \\ 6 & 0.220433 & 4.838756 & 3.928712 \\ 6 & -1.491139 & -0.597590 & 1.516063 \\ 6 & -1.236419 & -1.959236 & 1.514106 \\ 6 & -1.208528 & -2.690292 & 2.747799 \\ 6 & -1.447114 & -2.008873 & 3.976152 \\ 6 & -1.717765 & -0.622092 & 3.942856 \\ 6 & -1.738404 & 0.057485 & 2.755340 \\ 6 & -0.929911 & -4.081659 & 2.799515\end{array}$




\begin{tabular}{|c|c|c|c|}
\hline 6 & -0.882397 & -4.749364 & 3.998327 \\
\hline 6 & -1.116753 & -4.067043 & 5.210450 \\
\hline & -1.394851 & -2.724362 & 5.196012 \\
\hline & -1.013063 & -2.743012 & 0.270037 \\
\hline & 0.253356 & -2.865117 & -0.269845 \\
\hline & 0.490153 & -3.743700 & -1.353526 \\
\hline & -0.534810 & -4.456904 & -1.914437 \\
\hline & -1.857204 & -4.313185 & -1.431959 \\
\hline & -2.101512 & -3.458163 & -0.316773 \\
\hline & -2.942406 & -4.996832 & -2.032222 \\
\hline & -4.219158 & -4.844511 & -1.553357 \\
\hline & -4.463686 & -4.000744 & -0.447612 \\
\hline & -3.431666 & -3.330305 & 0.160050 \\
\hline & 1.334847 & -2.057464 & 0.226197 \\
\hline 15 & 1.688132 & -0.782773 & -0.839632 \\
\hline & 2.883949 & -1.323871 & -1.892908 \\
\hline & 4.151079 & -1.653431 & -1.477136 \\
\hline U & 5.023357 & -0.686535 & -1.006450 \\
\hline 6 & 6.287260 & -1.114737 & -0.485288 \\
\hline 6 & 6.669368 & -2.485502 & -0.594693 \\
\hline 6 & 5.778530 & -3.407693 & -1.197224 \\
\hline 6 & 4.538710 & -3.004952 & -1.611404 \\
\hline 6 & 7.159795 & -0.233458 & 0.202530 \\
\hline 6 & 8.351099 & -0.677186 & 0.722266 \\
\hline 6 & 8.741641 & -2.025012 & 0.578880 \\
\hline 6 & 7.912594 & -2.907927 & -0.064903 \\
\hline 6 & 4.605462 & 0.733543 & -1.021099 \\
\hline 6 & 3.405291 & 1.084172 & -0.433887 \\
\hline 6 & 2.914936 & 2.404229 & -0.433518 \\
\hline 6 & 3.645939 & 3.394835 & -1.031528 \\
\hline 6 & 4.868637 & 3.098599 & -1.679919 \\
\hline 6 & 5.350302 & 1.754089 & -1.697833 \\
\hline 6 & 5.604740 & 4.112279 & -2.340228 \\
\hline 6 & 6.758894 & 3.818602 & -3.019304 \\
\hline 6 & 7.216466 & 2.485538 & -3.074078 \\
\hline 6 & 6.532247 & 1.482488 & -2.433260 \\
\hline 8 & 2.644196 & 0.110368 & 0.177826 \\
\hline 6 & 2.288994 & -2.737809 & 1.133464 \\
\hline 6 & 2.880426 & -1.853312 & 2.197594 \\
\hline 6 & 4.261615 & -1.737576 & 2.335886 \\
\hline 6 & 4.809199 & -0.917203 & 3.318862 \\
\hline 6 & 3.976893 & -0.215170 & 4.184256 \\
\hline 6 & 2.593952 & -0.341524 & 4.062537 \\
\hline 6 & 2.051695 & -1.149795 & 3.072469 \\
\hline 45 & -0.083220 & 0.194198 & -1.985396 \\
\hline 6 & -0.873629 & -1.126662 & -3.177389 \\
\hline 8 & -1.158383 & -1.851817 & -4.032186 \\
\hline 6 & -0.074807 & 2.110645 & -2.207829 \\
\hline 8 & -0.001139 & 3.242758 & -2.433228 \\
\hline 6 & -6.351098 & -0.608312 & 2.657846 \\
\hline 6 & -8.153432 & 0.124837 & 1.123595 \\
\hline 6 & -6.644324 & -2.137244 & -3.174968 \\
\hline 6 & -6.510571 & 0.238660 & -3.945497 \\
\hline 6 & -3.241351 & 5.474307 & -1.550949 \\
\hline 6 & -3.032341 & 6.810425 & 0.525565 \\
\hline o & 1.680655 & 2.997960 & 3.166241 \\
\hline
\end{tabular}




\begin{tabular}{|c|c|c|c|}
\hline 6 & 1.784575 & 5.199387 & 2.013706 \\
\hline & 6.864194 & 0.804360 & 0.335212 \\
\hline & -1.945442 & 1.124267 & 2.766208 \\
\hline & 8.188435 & -3.956695 & -0.168003 \\
\hline & -1.577129 & -2.182272 & 6.122832 \\
\hline & 5.222497 & 5.131630 & -2.305769 \\
\hline & -3.631231 & -2.674397 & 1.006497 \\
\hline & -5.480073 & -3.875423 & -0.078971 \\
\hline & 3.278627 & 4.420454 & -1.042383 \\
\hline & -1.909523 & -0.099491 & 4.879159 \\
\hline & 6.086911 & -4.446730 & -1.301144 \\
\hline & 8.116966 & 2.247445 & -3.635885 \\
\hline & 3.829614 & -3.703425 & -2.051273 \\
\hline & -2.739920 & -5.643360 & -2.884911 \\
\hline & -0.352407 & -5.119787 & -2.758777 \\
\hline & -0.664056 & -5.814643 & 4.013714 \\
\hline & 6.892916 & 0.459433 & -2.498230 \\
\hline & -0.750985 & -4.623912 & 1.873392 \\
\hline & 1.502727 & -3.818582 & -1.746524 \\
\hline & 7.313053 & 4.604463 & -3.527267 \\
\hline & 8.997092 & 0.017264 & 1.254872 \\
\hline & -5.046383 & -5.372485 & -2.023267 \\
\hline 1 & -1.075637 & -4.608640 & 6.152565 \\
\hline & 9.690482 & -2.361965 & 0.989707 \\
\hline ] & 1.954334 & 2.602898 & 0.039321 \\
\hline & -4.021878 & 0.662451 & 1.577355 \\
\hline 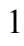 & -3.117964 & -0.438429 & -2.454114 \\
\hline 1 & -7.187392 & -0.405496 & -1.098282 \\
\hline & -3.269719 & 2.747252 & -0.518566 \\
\hline 1 & 0.303669 & 1.691984 & 1.595813 \\
\hline & -1.067130 & 5.754928 & 1.600351 \\
\hline 1 & 1.710057 & -3.539456 & 1.613791 \\
\hline$\frac{1}{1}$ & 0.939093 & 0.287196 & -3.195042 \\
\hline 1 & 2.558783 & 5.533270 & 2.717594 \\
\hline 1 & 2.291412 & 4.751586 & 1.148959 \\
\hline 1 & 1.244607 & 6.088401 & 1.665636 \\
\hline 1 & 1.001179 & 5.117555 & 4.648887 \\
\hline & -0.340732 & 5.748276 & 3.684158 \\
\hline 1 & -0.467528 & 4.143880 & 4.428371 \\
\hline 1 & 1.052293 & 2.244691 & 3.662116 \\
\hline 1 & 2.221622 & 2.500736 & 2.350379 \\
\hline 1 & 2.431981 & 3.333941 & 3.892692 \\
\hline 1 & -3.796294 & 7.502027 & 0.148636 \\
\hline 1 & -3.074792 & 6.839112 & 1.622249 \\
\hline & -2.055885 & 7.197641 & 0.207622 \\
\hline 1 & -4.977137 & 3.994758 & 0.030411 \\
\hline l & -4.769574 & 4.938120 & 1.518489 \\
\hline 1 & -5.447524 & 5.701822 & 0.065389 \\
\hline 1 & -2.249402 & 5.795189 & -1.893900 \\
\hline 1 & -3.455315 & 4.502896 & -2.013035 \\
\hline 1 & -3.980080 & 6.194036 & -1.928946 \\
\hline l & -8.477670 & 0.807376 & 0.327452 \\
\hline 1 & -8.317566 & -0.905471 & 0.782326 \\
\hline & -8.809796 & 0.292156 & 1.986872 \\
\hline 1 & -6.853458 & 2.527572 & 1.256527 \\
\hline 1 & -7.265642 & 1.966341 & 2.889557 \\
\hline
\end{tabular}




$\begin{array}{lrrr}1 & -5.570497 & 2.047332 & 2.382205 \\ 1 & -6.444645 & -1.648194 & 2.317074 \\ 1 & -5.325467 & -0.470007 & 3.024021 \\ 1 & -7.031010 & -0.469775 & 3.509292 \\ 1 & -5.872590 & 1.123541 & -4.068145 \\ 1 & -6.885100 & -0.048552 & -4.937410 \\ 1 & -7.373469 & 0.529809 & -3.333321 \\ 1 & -3.974715 & -2.113922 & -3.831705 \\ 1 & -4.992489 & -1.649441 & -5.208158 \\ 1 & -3.922829 & -0.453370 & -4.469161 \\ 1 & -6.102705 & -2.978711 & -2.723083 \\ 1 & -7.529548 & -1.933533 & -2.560785 \\ 1 & -6.999580 & -2.455575 & -4.164035 \\ 1 & 3.094398 & -3.228011 & 0.565135 \\ 1 & 4.918846 & -2.278311 & 1.653452 \\ 1 & 0.972394 & -1.229340 & 2.958938 \\ 1 & 5.890618 & -0.825811 & 3.400772 \\ 1 & 1.931657 & 0.193679 & 4.741865 \\ 1 & 4.401987 & 0.427286 & 4.952785\end{array}$

$1_{\mathrm{C} 4-} \psi_{-1 \mathbf{a} 25354 a}$

Number of imaginary frequencies : 0

The smallest frequencies are : $13.5054 \quad 18.3245 \quad 19.2983 \mathrm{~cm}(-1)$

Electronic energy : $\quad \mathrm{HF}=-4125.4701587$

Zero-point correction $=\quad 1.299798($ Hartree/Particle $)$

Thermal correction to Energy= $\quad 1.378127$

Thermal correction to Enthalpy= $\quad 1.379072$

Thermal correction to Gibbs Free Energy $=1.187507$

Sum of electronic and zero-point Energies $=\quad-4124.170361$

Sum of electronic and thermal Energies $=\quad-4124.092031$

Sum of electronic and thermal Enthalpies $=\quad-4124.091087$

Sum of electronic and thermal Free Energies $=\quad-4124.282652$

\section{Cartesian Coordinates}

$\begin{array}{cccc}6 & 2.043507 & -1.191332 & 3.043061 \\ 6 & 2.870134 & -1.885059 & 2.158441 \\ 6 & 4.251610 & -1.781749 & 2.303458 \\ 6 & 4.801610 & -0.984834 & 3.304205 \\ 6 & 3.971428 & -0.293457 & 4.180154 \\ 6 & 2.588070 & -0.406593 & 4.050623 \\ 6 & 2.276843 & -2.748688 & 1.078330 \\ 7 & 1.321878 & -2.051579 & 0.184654 \\ 15 & 1.678822 & -0.761839 & -0.861062 \\ 45 & -0.103278 & 0.233454 & -1.978574 \\ 6 & -0.079655 & 2.155216 & -2.165104 \\ 8 & -0.011778 & 3.292378 & -2.364664 \\ 6 & 0.238868 & -2.851245 & -0.321447 \\ 6 & -1.026774 & -2.739401 & 0.223277 \\ 6 & -2.111743 & -3.461994 & -0.362162 \\ 6 & -1.861828 & -4.324182 & -1.470848 \\ 6 & -0.542401 & -4.445383 & -1.967196 \\ 6 & 0.477073 & -3.718979 & -1.413395 \\ 6 & -3.442104 & -3.348267 & 0.118929 \\ 6 & -4.464137 & -4.054150 & -0.465232\end{array}$




\begin{tabular}{|c|c|c|c|}
\hline 6 & -4.210965 & -4.915740 & -1.554752 \\
\hline 6 & -2.937432 & -5.042579 & -2.047673 \\
\hline & -1.244861 & -1.977859 & 1.482781 \\
\hline & -1.488958 & -0.614475 & 1.516222 \\
\hline & -1.724282 & 0.016741 & 2.770179 \\
\hline & -1.704210 & -0.688225 & 3.942728 \\
\hline f & -1.442328 & -2.077047 & 3.945037 \\
\hline o & -1.214241 & -2.734275 & 2.701482 \\
\hline 6 & -1.388346 & -2.817726 & 5.149741 \\
\hline 6 & -1.117078 & -4.161775 & 5.135592 \\
\hline 6 & -0.891033 & -4.819860 & 3.908609 \\
\hline 6 & -0.941122 & -4.127552 & 2.723924 \\
\hline & -1.627822 & 0.369624 & -0.053954 \\
\hline 6 & -3.426203 & 0.180600 & -0.401205 \\
\hline 6 & -4.366808 & 0.296562 & 0.631673 \\
\hline 6 & -5.722497 & 0.116320 & 0.386012 \\
\hline 6 & -6.120102 & -0.179417 & -0.929212 \\
\hline 6 & -5.215265 & -0.280984 & -1.980553 \\
\hline 6 & -3.855943 & -0.096080 & -1.689305 \\
\hline 6 & -6.765781 & 0.178476 & 1.500052 \\
\hline 6 & -6.163278 & 0.627318 & 2.830080 \\
\hline 6 & -5.625078 & -0.646038 & -3.404784 \\
\hline 6 & -4.995644 & 0.330436 & -4.404552 \\
\hline 6 & -1.492219 & 2.089795 & 0.568013 \\
\hline 6 & -0.385959 & 2.446655 & 1.351341 \\
\hline 6 & -0.225632 & 3.752513 & 1.802950 \\
\hline 6 & -1.163663 & 4.712131 & 1.385741 \\
\hline 6 & -2.252937 & 4.393999 & 0.580545 \\
\hline 6 & -2.409781 & 3.057047 & 0.188608 \\
\hline 6 & -3.289745 & 5.418516 & 0.127272 \\
\hline 6 & -2.996155 & 6.819293 & 0.657528 \\
\hline 6 & 0.894947 & 4.155211 & 2.762055 \\
\hline 6 & 1.820604 & 5.174656 & 2.091844 \\
\hline 6 & -7.879194 & 1.163598 & 1.130090 \\
\hline 6 & -7.362242 & -1.220679 & 1.692420 \\
\hline 6 & -7.139033 & -0.613680 & -3.599582 \\
\hline 6 & -5.127984 & -2.066380 & -3.699113 \\
\hline 6 & -4.673341 & 4.995176 & 0.636098 \\
\hline 6 & -3.301962 & 5.480001 & -1.404428 \\
\hline 6 & 0.277387 & 4.783901 & 4.017616 \\
\hline 6 & 1.726322 & 2.954055 & 3.208877 \\
\hline 8 & 2.871044 & -1.288911 & -1.925622 \\
\hline 6 & 4.136329 & -1.634246 & -1.516906 \\
\hline 6 & 5.014342 & -0.682435 & -1.026503 \\
\hline 6 & 6.274276 & -1.129323 & -0.511485 \\
\hline 6 & 6.647529 & -2.500173 & -0.647209 \\
\hline 6 & 5.752289 & -3.404048 & -1.270557 \\
\hline 6 & 4.515880 & -2.985055 & -1.678632 \\
\hline 6 & 7.886187 & -2.941591 & -0.122259 \\
\hline 6 & 8.719059 & -2.077402 & 0.541702 \\
\hline 6 & 8.337117 & -0.730076 & 0.710862 \\
\hline 6 & 7.150556 & -0.267897 & 0.196335 \\
\hline 6 & 4.606237 & 0.740557 & -1.015861 \\
\hline 6 & 3.407335 & 1.088753 & -0.424698 \\
\hline 6 & 2.924611 & 2.411455 & -0.403722 \\
\hline 6 & 3.662456 & 3.407315 & -0.984357 \\
\hline
\end{tabular}




\begin{tabular}{|c|c|c|c|}
\hline & 4.884819 & 3.114554 & -1.634982 \\
\hline & 5.358667 & 1.767706 & -1.673942 \\
\hline & 6.540778 & 1.501359 & -2.411015 \\
\hline & 7.232311 & 2.510766 & -3.033721 \\
\hline & 6.782284 & 3.845375 & -2.958251 \\
\hline & 5.628264 & 4.134591 & -2.277031 \\
\hline & 2.639988 & 0.109745 & 0.170474 \\
\hline & -0.960931 & -1.054561 & -3.152248 \\
\hline & -1.332637 & -1.762188 & -3.988867 \\
\hline & 6.861164 & 0.768825 & 0.349572 \\
\hline & -1.925868 & 1.084187 & 2.803423 \\
\hline & 8.155190 & -3.989957 & -0.245595 \\
\hline & -1.563138 & -2.293412 & 6.088166 \\
\hline & 5.251759 & 5.155424 & -2.226716 \\
\hline & -3.650148 & -2.679559 & 0.953274 \\
\hline & -5.479803 & -3.943594 & -0.090056 \\
\hline & 3.301201 & 4.435118 & -0.979206 \\
\hline & -1.888781 & -0.184441 & 4.890691 \\
\hline & 6.054241 & -4.442674 & -1.395313 \\
\hline & 8.132822 & 2.276673 & -3.597190 \\
\hline & 3.803395 & -3.669725 & -2.134394 \\
\hline & -2.729153 & -5.696272 & -2.893485 \\
\hline & -0.356977 & -5.108209 & -2.810931 \\
\hline & -0.676855 & -5.886064 & 3.901004 \\
\hline & 6.895807 & 0.477472 & -2.491757 \\
\hline & -0.767684 & -4.652118 & 1.786717 \\
\hline & 1.488018 & -3.781065 & -1.812589 \\
\hline & 7.342121 & 4.636167 & -3.452127 \\
\hline & 8.985939 & -0.050925 & 1.259515 \\
\hline 1 & -5.030432 & -5.470468 & -2.006646 \\
\hline & -1.074388 & -4.722701 & 6.066253 \\
\hline 1 & 9.664217 & -2.428967 & 0.948767 \\
\hline & 1.964651 & 2.607887 & 0.071338 \\
\hline & -4.023411 & 0.522516 & 1.637612 \\
\hline & -3.114577 & -0.177360 & -2.480399 \\
\hline & -7.178854 & -0.335945 & -1.126480 \\
\hline 1 & -3.258341 & 2.772076 & -0.432328 \\
\hline & 0.330789 & 1.674430 & 1.632427 \\
\hline & -1.034781 & 5.739480 & 1.719980 \\
\hline & 1.698461 & -3.558974 & 1.544553 \\
\hline & 0.894384 & 0.325472 & -3.209904 \\
\hline & 2.604141 & 5.494570 & 2.791889 \\
\hline & 2.315668 & 4.741912 & 1.212742 \\
\hline 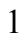 & 1.277858 & 6.070635 & 1.766678 \\
\hline & 1.066337 & 5.049615 & 4.733789 \\
\hline 1 & -0.284853 & 5.698201 & 3.794305 \\
\hline & -0.406247 & 4.081882 & 4.513142 \\
\hline & 1.101639 & 2.194030 & 3.699040 \\
\hline 1 & 2.257010 & 2.468834 & 2.379084 \\
\hline 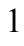 & 2.486290 & 3.276435 & 3.932492 \\
\hline 1 & -3.767764 & 7.515410 & 0.305317 \\
\hline & -2.999676 & 6.853275 & 1.754837 \\
\hline 1 & -2.028182 & 7.196682 & 0.303555 \\
\hline & -4.968398 & 4.004887 & 0.265995 \\
\hline 1 & -4.693203 & 4.960173 & 1.733515 \\
\hline 1 & -5.436161 & 5.713506 & 0.306905 \\
\hline
\end{tabular}


$1_{\mathrm{C} 4-} \psi_{-1 \mathrm{a} 25344 \mathrm{~s}}$

Number of imaginary frequencies : 0

The smallest frequencies are : $12.9238 \quad 19.1949 \quad 21.1713 \mathrm{~cm}(-1)$

Electronic energy : $\quad \mathrm{HF}=-4125.4703015$

Zero-point correction $=\quad 1.300167($ Hartree/Particle $)$

Thermal correction to Energy $=\quad 1.378322$

Thermal correction to Enthalpy= $\quad 1.379266$

Thermal correction to Gibbs Free Energy= $\quad 1.188266$

Sum of electronic and zero-point Energies $=\quad-4124.170135$

Sum of electronic and thermal Energies $=\quad-4124.091980$

Sum of electronic and thermal Enthalpies $=\quad-4124.091036$

Sum of electronic and thermal Free Energies $=\quad-4124.282035$

Cartesian Coordinates

$\begin{array}{cccc}6 & -0.405134 & 2.443031 & 1.351429 \\ 6 & -1.507729 & 2.079117 & 0.566461 \\ 6 & -2.434820 & 3.038866 & 0.191499 \\ 6 & -2.290774 & 4.375425 & 0.589226 \\ 6 & -1.205292 & 4.700394 & 1.397043 \\ 6 & -0.258088 & 3.747964 & 1.810087 \\ 15 & -1.627053 & 0.359597 & -0.060069 \\ 6 & -3.423439 & 0.161309 & -0.413204 \\ 6 & -4.361355 & 0.259163 & 0.615933 \\ 6 & -5.721650 & 0.078385 & 0.372465 \\ 6 & -6.123037 & -0.192568 & -0.941271 \\ 6 & -5.213053 & -0.280741 & -1.996636\end{array}$




\begin{tabular}{|c|c|c|c|}
\hline 6 & -3.856625 & -0.100588 & -1.707428 \\
\hline 6 & -6.697664 & 0.163368 & 1.544780 \\
\hline y & -6.631075 & 1.565075 & 2.161719 \\
\hline 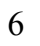 & -5.628665 & -0.634133 & -3.422727 \\
\hline D & -7.142350 & -0.581879 & -3.617471 \\
\hline p & -3.327411 & 5.394993 & 0.124921 \\
\hline f & -4.726684 & 4.930347 & 0.547049 \\
\hline 6 & 0.859226 & 4.155860 & 2.770805 \\
\hline 0 & 0.237909 & 4.780826 & 4.026311 \\
\hline 6 & -1.488050 & -0.625359 & 1.509785 \\
\hline 6 & -1.242291 & -1.988156 & 1.473542 \\
\hline 6 & -1.214814 & -2.748601 & 2.689509 \\
\hline 6 & -1.446871 & -2.095509 & 3.934378 \\
\hline 6 & -1.707842 & -0.706490 & 3.935614 \\
\hline 6 & -1.725768 & 0.002252 & 2.765072 \\
\hline 6 & -0.942496 & -4.142040 & 2.707781 \\
\hline 6 & -0.896919 & -4.838508 & 3.890182 \\
\hline 6 & -1.126786 & -4.184516 & 5.118685 \\
\hline 6 & -1.397265 & -2.840355 & 5.136706 \\
\hline 6 & -1.023901 & -2.744079 & 0.211272 \\
\hline 6 & 0.240760 & -2.852338 & -0.336217 \\
\hline 6 & 0.476341 & -3.713410 & -1.434026 \\
\hline 6 & -0.544796 & -4.435970 & -1.990192 \\
\hline 6 & -1.863396 & -4.317258 & -1.490768 \\
\hline 6 & -2.110433 & -3.461871 & -0.376370 \\
\hline 6 & -2.941197 & -5.030831 & -2.069554 \\
\hline 6 & -4.213813 & -4.903831 & -1.574070 \\
\hline 6 & -4.463823 & -4.047544 & -0.479561 \\
\hline 6 & -3.439582 & -3.347784 & 0.107890 \\
\hline 7 & 1.324125 & -2.055307 & 0.173328 \\
\hline 15 & 1.682048 & -0.759844 & -0.864802 \\
\hline 8 & 2.878592 & -1.279627 & -1.927635 \\
\hline 6 & 4.143436 & -1.623134 & -1.515982 \\
\hline 6 & 5.017752 & -0.670243 & -1.021096 \\
\hline 6 & 6.278140 & -1.115025 & -0.505374 \\
\hline 6 & 6.655250 & -2.484543 & -0.644023 \\
\hline 6 & 5.763102 & -3.389491 & -1.270253 \\
\hline 6 & 4.526286 & -2.972751 & -1.679439 \\
\hline 6 & 7.151501 & -0.252945 & 0.205244 \\
\hline 6 & 8.339041 & -0.713015 & 0.719381 \\
\hline 6 & 8.724792 & -2.058892 & 0.547349 \\
\hline 6 & 7.894694 & -2.923816 & -0.119113 \\
\hline 6 & 4.605048 & 0.751446 & -1.006260 \\
\hline 6 & 3.403865 & 1.093593 & -0.416104 \\
\hline 6 & 2.916890 & 2.414691 & -0.391131 \\
\hline 6 & 3.652771 & 3.415206 & -0.966186 \\
\hline 6 & 4.877357 & 3.128811 & -1.615485 \\
\hline 6 & 5.355426 & 1.783573 & -1.658880 \\
\hline 6 & 5.618920 & 4.153675 & -2.252015 \\
\hline 6 & 6.775105 & 3.870719 & -2.932190 \\
\hline 6 & 7.229311 & 2.537792 & -3.012203 \\
\hline 6 & 6.539690 & 1.523808 & -2.394855 \\
\hline 8 & 2.638024 & 0.109930 & 0.173341 \\
\hline 6 & 2.275685 & -2.755081 & 1.068440 \\
\hline 6 & 2.868768 & -1.893162 & 2.150002 \\
\hline 6 & 4.250172 & -1.792424 & 2.297229 \\
\hline
\end{tabular}




\begin{tabular}{|c|c|c|c|}
\hline 5 & 4.800000 & -0.995448 & 3.298026 \\
\hline 5 & 3.969660 & -0.301138 & 4.171474 \\
\hline 6 & 2.586295 & -0.411754 & 4.039781 \\
\hline 6 & 2.041946 & -1.197098 & 3.032632 \\
\hline 45 & -0.097804 & 0.239905 & -1.981125 \\
\hline 6 & -0.946493 & -1.042272 & -3.169032 \\
\hline 8 & -1.307896 & -1.742471 & -4.016198 \\
\hline 5 & -0.083395 & 2.162342 & -2.157567 \\
\hline 8 & -0.021634 & 3.300972 & -2.350836 \\
\hline o & -6.309399 & -0.881867 & 2.598197 \\
\hline 6 & -8.140656 & -0.103150 & 1.124520 \\
\hline 6 & -5.150270 & -2.060265 & -3.721545 \\
\hline 6 & -4.988462 & 0.336912 & -4.420840 \\
\hline 6 & -3.264638 & 5.505632 & -1.403004 \\
\hline 6 & -3.087327 & 6.781392 & 0.716052 \\
\hline 6 & 1.696265 & 2.958505 & 3.217749 \\
\hline 6 & 1.780344 & 5.180001 & 2.101463 \\
\hline 1 & 6.859156 & 0.782592 & 0.360760 \\
\hline 1 & -1.926756 & 1.069663 & 2.801120 \\
\hline 1 & 8.166651 & -3.971150 & -0.244709 \\
\hline 1 & -1.574956 & -2.319269 & 6.076377 \\
\hline 1 & 5.239198 & 5.173146 & -2.198315 \\
\hline 1 & -3.645154 & -2.682328 & 0.945918 \\
\hline 1 & -5.478880 & -3.934815 & -0.103438 \\
\hline 1 & 3.288293 & 4.441858 & -0.957759 \\
\hline 1 & -1.893868 & -0.205561 & 4.884802 \\
\hline 1 & 6.067844 & -4.427123 & -1.396519 \\
\hline 1 & 8.131561 & 2.308693 & -3.574938 \\
\hline 1 & 3.815887 & -3.658243 & -2.137244 \\
\hline 1 & -2.735731 & -5.680148 & -2.919417 \\
\hline 1 & -0.361283 & -5.093376 & -2.838577 \\
\hline 1 & -0.683467 & -5.904831 & 3.879634 \\
\hline 1 & 6.897944 & 0.501330 & -2.479073 \\
\hline 1 & -0.766468 & -4.663301 & 1.769206 \\
\hline 1 & 1.486367 & -3.773231 & -1.836059 \\
\hline 1 & 7.333447 & 4.665182 & -3.421846 \\
\hline 1 & 8.985649 & -0.033353 & 1.270010 \\
\hline 1 & -5.035084 & -5.454192 & -2.028051 \\
\hline 1 & -1.087714 & -4.748757 & 6.047496 \\
\hline 1 & 9.670674 & -2.408805 & 0.954158 \\
\hline 1 & 1.955136 & 2.606099 & 0.082314 \\
\hline 1 & -4.023000 & 0.469666 & 1.629478 \\
\hline 1 & -3.115539 & -0.172635 & -2.499817 \\
\hline 1 & -7.179652 & -0.339923 & -1.145792 \\
\hline 1 & -3.279711 & 2.748619 & -0.431798 \\
\hline 1 & 0.318823 & 1.676330 & 1.629004 \\
\hline 1 & -1.087032 & 5.727326 & 1.736491 \\
\hline 1 & 1.694614 & -3.564393 & 1.533003 \\
\hline 1 & 0.904456 & 0.343616 & -3.207619 \\
\hline 1 & 2.561952 & 5.503622 & 2.801970 \\
\hline 1 & 2.277920 & 4.749768 & 1.222492 \\
\hline 1 & 1.233260 & 6.073280 & 1.776091 \\
\hline 1 & 1.025178 & 5.049635 & 4.743162 \\
\hline 1 & -0.328333 & 5.692746 & 3.803472 \\
\hline 1 & -0.442779 & 4.075327 & 4.520959 \\
\hline 1 & 1.074482 & 2.194735 & 3.705856 \\
\hline
\end{tabular}




$\begin{array}{rrrr}1 & 2.231250 & 2.476914 & 2.388557 \\ 1 & 2.453131 & 3.284074 & 3.943154 \\ 1 & -3.862911 & 7.472073 & 0.361928 \\ 1 & -3.129418 & 6.773039 & 1.813138 \\ 1 & -2.117753 & 7.195305 & 0.410953 \\ 1 & -4.989397 & 3.956505 & 0.114536 \\ 1 & -4.799924 & 4.841225 & 1.639172 \\ 1 & -5.483035 & 5.654055 & 0.214923 \\ 1 & -2.270730 & 5.836313 & -1.731425 \\ 1 & -3.474044 & 4.547211 & -1.893790 \\ 1 & -4.002783 & 6.234564 & -1.764173 \\ 1 & -8.497529 & 0.630161 & 0.390041 \\ 1 & -8.264823 & -1.106794 & 0.697398 \\ 1 & -8.797882 & -0.035499 & 2.000637 \\ 1 & -6.946199 & 2.326715 & 1.436349 \\ 1 & -7.298672 & 1.631986 & 3.031393 \\ 1 & -5.618765 & 1.823462 & 2.496848 \\ 1 & -6.360039 & -1.895871 & 2.178992 \\ 1 & -5.292328 & -0.731798 & 2.982974 \\ 1 & -6.997475 & -0.836714 & 3.453126 \\ 1 & -3.892705 & 0.290794 & -4.410075 \\ 1 & -5.311757 & 0.093141 & -5.441321 \\ 1 & -5.283949 & 1.373229 & -4.211162 \\ 1 & -7.550658 & 0.411289 & -3.388305 \\ 1 & -7.386362 & -0.807672 & -4.663122 \\ 1 & -7.665881 & -1.320802 & -2.997580 \\ 1 & -4.062135 & -2.156963 & -3.612507 \\ 1 & -5.615453 & -2.780386 & -3.034608 \\ 1 & -5.414226 & -2.346559 & -4.748941 \\ 1 & 3.080217 & -3.236565 & 0.491515 \\ 1 & 4.906172 & -2.326816 & 1.608567 \\ 1 & 0.962670 & -1.263143 & 2.911116 \\ 1 & 5.881745 & -0.915950 & 3.387513 \\ 1 & 1.925328 & 0.117686 & 4.724893 \\ 1 & 4.396634 & 0.323071 & 4.953863\end{array}$

$1_{\mathrm{C} 4-} \psi_{-1 \text { s2a3a4a }}$

Number of imaginary frequencies : 0

The smallest frequencies are : $13.8468 \quad 19.8525 \quad 21.9671 \mathrm{~cm}(-1)$

Electronic energy : $\quad H F=-4125.4681236$

Zero-point correction $=\quad 1.299833($ Hartree/Particle $)$

Thermal correction to Energy= $\quad 1.377973$

Thermal correction to Enthalpy= $\quad 1.378917$

Thermal correction to Gibbs Free Energy $=\quad 1.188964$

Sum of electronic and zero-point Energies $=\quad-4124.168291$

Sum of electronic and thermal Energies $=\quad-4124.090151$

Sum of electronic and thermal Enthalpies $=\quad-4124.089206$

Sum of electronic and thermal Free Energies $=\quad-4124.279160$

Cartesian Coordinates

$\begin{array}{llll}6 & 2.000431 & -0.762513 & 3.090765 \\ 6 & 2.821838 & -1.591668 & 2.323696 \\ 6 & 4.204119 & -1.479845 & 2.451888 \\ 6 & 4.763535 & -0.539085 & 3.312766\end{array}$




\begin{tabular}{|c|c|c|c|}
\hline 6 & 3.940403 & 0.291901 & 4.064276 \\
\hline 6 & 2.555570 & 0.170507 & 3.957112 \\
\hline 6 & 2.224312 & -2.595781 & 1.376442 \\
\hline & 1.316141 & -2.014722 & 0.361089 \\
\hline 1 & 1.687221 & -0.836694 & -0.806227 \\
\hline & -0.075024 & 0.049704 & -2.027738 \\
\hline & -0.117499 & 1.934799 & -2.418635 \\
\hline 8 & -0.072688 & 3.042681 & -2.750449 \\
\hline 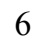 & 0.255305 & -2.872545 & -0.095498 \\
\hline 6 & -1.020733 & -2.731648 & 0.415090 \\
\hline 6 & -2.094833 & -3.487031 & -0.146516 \\
\hline 6 & -1.824317 & -4.413533 & -1.196524 \\
\hline 6 & -0.491190 & -4.580732 & -1.640707 \\
\hline 6 & 0.518107 & -3.820823 & -1.112651 \\
\hline 6 & -3.436261 & -3.326945 & 0.287662 \\
\hline 6 & -4.453842 & -4.039516 & -0.295659 \\
\hline 6 & -4.183121 & -4.957440 & -1.334546 \\
\hline 6 & -2.895426 & -5.139350 & -1.772800 \\
\hline 6 & -1.274588 & -1.881608 & 1.607005 \\
\hline 6 & -1.533667 & -0.524265 & 1.525186 \\
\hline 6 & -1.839693 & 0.191417 & 2.717941 \\
\hline 6 & -1.860341 & -0.424168 & 3.939591 \\
\hline 6 & -1.577290 & -1.804090 & 4.058466 \\
\hline 6 & -1.286912 & -2.546640 & 2.878394 \\
\hline 6 & -1.576990 & -2.455803 & 5.314312 \\
\hline 6 & -1.301069 & -3.796045 & 5.409156 \\
\hline 6 & -1.015093 & -4.538912 & 4.244750 \\
\hline 6 & -1.010591 & -3.932831 & 3.012423 \\
\hline 15 & -1.611629 & 0.345406 & -0.121116 \\
\hline 6 & -3.410751 & 0.168984 & -0.479686 \\
\hline 6 & -4.359991 & 0.528230 & 0.482138 \\
\hline 6 & -5.719650 & 0.338862 & 0.252693 \\
\hline 6 & -6.101152 & -0.230238 & -0.968789 \\
\hline 6 & -5.183449 & -0.601863 & -1.950820 \\
\hline 6 & -3.826501 & -0.384941 & -1.685757 \\
\hline 6 & -6.777504 & 0.687676 & 1.297381 \\
\hline 6 & -6.179520 & 1.412416 & 2.502112 \\
\hline 6 & -5.669308 & -1.193505 & -3.273362 \\
\hline 6 & -6.351603 & -0.086464 & -4.084634 \\
\hline 6 & -1.431490 & 2.094817 & 0.408687 \\
\hline 6 & -0.371262 & 2.449103 & 1.240577 \\
\hline 6 & -0.217530 & 3.766472 & 1.689163 \\
\hline 6 & -1.107003 & 4.724671 & 1.201874 \\
\hline 6 & -2.149611 & 4.408872 & 0.320210 \\
\hline 6 & -2.308856 & 3.076362 & -0.054554 \\
\hline 6 & -3.082002 & 5.510449 & -0.179289 \\
\hline 6 & -4.192536 & 4.963798 & -1.073895 \\
\hline 6 & 0.810219 & 4.071818 & 2.780067 \\
\hline 6 & 2.202503 & 3.561343 & 2.400549 \\
\hline 6 & -7.847577 & 1.595848 & 0.683187 \\
\hline 6 & -7.427811 & -0.610601 & 1.788848 \\
\hline 6 & -4.518940 & -1.765715 & -4.098764 \\
\hline 6 & -6.668225 & -2.326933 & -3.020358 \\
\hline 6 & -3.739641 & 6.213748 & 1.013090 \\
\hline 6 & -2.268789 & 6.525093 & -0.990216 \\
\hline 6 & 0.343059 & 3.362229 & 4.058837 \\
\hline
\end{tabular}




\begin{tabular}{|c|c|c|c|}
\hline 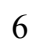 & 0.921467 & 5.565409 & 3.076761 \\
\hline 8 & 2.894966 & -1.470577 & -1.790648 \\
\hline 6 & 4.153040 & -1.761646 & -1.320579 \\
\hline & 5.023408 & -0.754213 & -0.938586 \\
\hline & 6.281020 & -1.130546 & -0.365285 \\
\hline & 6.657567 & -2.506813 & -0.329262 \\
\hline & 5.767675 & -3.483385 & -0.839863 \\
\hline ठ & 4.534503 & -3.120937 & -1.308113 \\
\hline & 7.896479 & -2.876711 & 0.248064 \\
\hline 6 & 8.727139 & -1.934830 & 0.799248 \\
\hline 6 & 8.342169 & -0.577689 & 0.799182 \\
\hline 6 & 7.154503 & -0.185960 & 0.231442 \\
\hline 6 & 4.606473 & 0.656166 & -1.106122 \\
\hline 6 & 3.394100 & 1.060490 & -0.581493 \\
\hline 6 & 2.890024 & 2.364481 & -0.743827 \\
\hline 6 & 3.626291 & 3.290975 & -1.430979 \\
\hline 6 & 4.865976 & 2.936909 & -2.015551 \\
\hline 6 & 5.357270 & 1.602764 & -1.877103 \\
\hline 6 & 6.555103 & 1.263071 & -2.556732 \\
\hline 6 & 7.246219 & 2.196455 & -3.288769 \\
\hline 6 & 6.779470 & 3.523742 & -3.387224 \\
\hline 6 & 5.609592 & 3.879165 & -2.767085 \\
\hline 8 & 2.630339 & 0.155106 & 0.127574 \\
\hline 6 & -0.831848 & -1.367449 & -3.140133 \\
\hline 8 & -1.066540 & -2.141607 & -3.966323 \\
\hline 1 & 6.862648 & 0.861132 & 0.253855 \\
\hline 1 & -2.057568 & 1.255160 & 2.666922 \\
\hline 1 & 8.168002 & -3.931598 & 0.256094 \\
\hline 1 & -1.799827 & -1.868076 & 6.203696 \\
\hline 1 & 5.220032 & 4.892838 & -2.851590 \\
\hline 1 & -3.657457 & -2.612920 & 1.079670 \\
\hline 1 & -5.478102 & -3.888390 & 0.040854 \\
\hline 1 & 3.252293 & 4.305640 & -1.560374 \\
\hline 1 & -2.095345 & 0.146621 & 4.837450 \\
\hline 1 & 6.072342 & -4.528643 & -0.832467 \\
\hline 1 & 8.159280 & 1.906443 & -3.804010 \\
\hline 1 & 3.828829 & -3.858377 & -1.684889 \\
\hline 1 & -2.673738 & -5.840910 & -2.575736 \\
\hline 1 & -0.287522 & -5.298706 & -2.433536 \\
\hline 1 & -0.798784 & -5.601813 & 4.323565 \\
\hline 1 & 6.923556 & 0.242143 & -2.503572 \\
\hline 1 & -0.793180 & -4.521464 & 2.123576 \\
\hline 1 & 1.537583 & -3.913908 & -1.482764 \\
\hline 1 & 7.338993 & 4.254756 & -3.966295 \\
\hline 1 & 8.989473 & 0.166071 & 1.258600 \\
\hline 1 & -4.999190 & -5.516683 & -1.787498 \\
\hline 1 & -1.301914 & -4.288918 & 6.378517 \\
\hline 1 & 9.673044 & -2.230728 & 1.246843 \\
\hline 1 & 1.918709 & 2.601412 & -0.314744 \\
\hline 1 & -4.026275 & 0.960875 & 1.420776 \\
\hline 1 & -3.070156 & -0.655140 & -2.417314 \\
\hline 1 & -7.162663 & -0.388067 & -1.158545 \\
\hline 1 & -3.120705 & 2.785197 & -0.716648 \\
\hline 1 & 0.313759 & 1.674333 & 1.593378 \\
\hline 1 & -0.999258 & 5.757560 & 1.525902 \\
\hline 1 & 1.605253 & -3.304641 & 1.945297 \\
\hline
\end{tabular}




$\begin{array}{lrrr}1 & 0.959245 & 0.071152 & -3.229996 \\ 1 & 2.897914 & 3.718530 & 3.236770 \\ 1 & 2.219314 & 2.488884 & 2.167951 \\ 1 & 2.598937 & 4.102341 & 1.531562 \\ 1 & 1.041336 & 3.557682 & 4.884837 \\ 1 & -0.651416 & 3.716499 & 4.363182 \\ 1 & 0.281391 & 2.274940 & 3.917940 \\ 1 & 1.217274 & 6.137390 & 2.187719 \\ 1 & -0.018355 & 5.983727 & 3.459055 \\ 1 & 1.686718 & 5.730718 & 3.845610 \\ 1 & -4.845339 & 5.785615 & -1.393740 \\ 1 & -3.794578 & 4.488827 & -1.979759 \\ 1 & -4.819273 & 4.228942 & -0.549066 \\ 1 & -4.326582 & 5.504889 & 1.612306 \\ 1 & -3.005328 & 6.685776 & 1.676584 \\ 1 & -4.418939 & 7.001632 & 0.661146 \\ 1 & -1.485115 & 6.999124 & -0.386870 \\ 1 & -1.783794 & 6.040635 & -1.847713 \\ 1 & -2.922075 & 7.320939 & -1.372901 \\ 1 & -5.450566 & 0.791031 & 3.039658 \\ 1 & -5.683386 & 2.348447 & 2.209628 \\ 1 & -6.976769 & 1.667011 & 3.211621 \\ 1 & -8.371508 & 1.118546 & -0.153297 \\ 1 & -8.602847 & 1.854388 & 1.437220 \\ 1 & -7.406703 & 2.530409 & 0.312538 \\ 1 & -7.915629 & -1.157544 & 0.972390 \\ 1 & -6.678631 & -1.277081 & 2.237115 \\ 1 & -8.190064 & -0.395939 & 2.550100 \\ 1 & -5.653702 & 0.735571 & -4.290400 \\ 1 & -6.706348 & -0.478804 & -5.047453 \\ 1 & -7.215560 & 0.332973 & -3.553530 \\ 1 & -3.972940 & -2.543728 & -3.546575 \\ 1 & -4.914238 & -2.221433 & -5.015379 \\ 1 & -3.802269 & -0.993515 & -4.405401 \\ 1 & -6.205051 & -3.129901 & -2.431693 \\ 1 & -7.567046 & -1.991197 & -2.489856 \\ 1 & -6.997222 & -2.755848 & -3.976075 \\ 1 & 3.024504 & -3.184300 & 0.900621 \\ 1 & 4.853477 & -2.119722 & 1.852783 \\ 1 & 0.918302 & -0.839280 & 2.986108 \\ 1 & 5.845961 & -0.454894 & 3.387982 \\ 1 & 1.904947 & 0.809759 & 4.552987 \\ 1 & 4.373133 & 1.031768 & 4.734793\end{array}$

$1_{\mathrm{C} 4-} \psi_{-1 \mathrm{~s} 2 \mathrm{aza}} \mathrm{s}$

Number of imaginary frequencies : 0

The smallest frequencies are : $12.7816 \quad 17.530921 .7394 \mathrm{~cm}(-1)$

Electronic energy : $\quad \mathrm{HF}=-4125.4685275$

Zero-point correction $=\quad 1.299731$ (Hartree/Particle)

Thermal correction to Energy= $\quad 1.377950$

Thermal correction to Enthalpy= $\quad 1.378894$

Thermal correction to Gibbs Free Energy $=\quad 1.188151$

Sum of electronic and zero-point Energies $=\quad-4124.168796$

Sum of electronic and thermal Energies $=\quad-4124.090578$

Sum of electronic and thermal Enthalpies $=\quad-4124.089633$ 
Cartesian Coordinates

\begin{tabular}{|c|c|c|c|}
\hline 6 & 5.586132 & 3.915685 & -2.716755 \\
\hline 6 & 4.845398 & 2.961581 & -1.977448 \\
\hline 6 & 5.340522 & 1.627095 & -1.856656 \\
\hline 6 & 6.538978 & 1.299629 & -2.541157 \\
\hline 6 & 7.227130 & 2.244446 & -3.261251 \\
\hline 6 & 6.756666 & 3.571636 & -3.342032 \\
\hline 6 & 4.592626 & 0.668422 & -1.097838 \\
\hline 6 & 3.379184 & 1.062456 & -0.567733 \\
\hline 6 & 2.871507 & 2.367141 & -0.712645 \\
\hline 6 & 3.604955 & 3.304533 & -1.387904 \\
\hline 6 & 5.013699 & -0.742750 & -0.948018 \\
\hline 6 & 4.145959 & -1.747825 & -1.341930 \\
\hline 6 & 4.530344 & -3.106319 & -1.344822 \\
\hline 6 & 5.764424 & -3.471193 & -0.880847 \\
\hline 6 & 6.652189 & -2.498363 & -0.359452 \\
\hline 6 & 6.272393 & -1.122645 & -0.379458 \\
\hline 6 & 7.892147 & -2.871964 & 0.213244 \\
\hline 6 & 8.720871 & -1.934528 & 0.774833 \\
\hline 6 & 8.332870 & -0.578349 & 0.790276 \\
\hline 6 & 7.144032 & -0.182914 & 0.227595 \\
\hline 8 & 2.887456 & -1.454225 & -1.809276 \\
\hline 15 & 1.677678 & -0.836463 & -0.817714 \\
\hline 8 & 2.617868 & 0.145755 & 0.129306 \\
\hline 7 & 1.308507 & -2.030427 & 0.333854 \\
\hline 6 & 0.245367 & -2.880729 & -0.131207 \\
\hline 6 & -1.028877 & -2.744071 & 0.384658 \\
\hline 6 & -2.106787 & -3.488467 & -0.183658 \\
\hline 6 & -1.841912 & -4.400173 & -1.247833 \\
\hline 6 & -0.510365 & -4.564843 & -1.697922 \\
\hline 6 & 0.502898 & -3.815679 & -1.161915 \\
\hline 6 & -2.916963 & -5.114120 & -1.831491 \\
\hline 6 & -4.202579 & -4.935286 & -1.385895 \\
\hline 6 & -4.467307 & -4.032359 & -0.332396 \\
\hline 6 & -3.446196 & -3.330674 & 0.257808 \\
\hline 6 & -1.278920 & -1.909089 & 1.587501 \\
\hline 6 & -1.539247 & -0.551324 & 1.521854 \\
\hline 6 & -1.844819 & 0.149454 & 2.723493 \\
\hline 6 & -1.862906 & -0.480777 & 3.937852 \\
\hline 6 & -1.578741 & -1.861860 & 4.039856 \\
\hline 6 & -1.289301 & -2.589741 & 2.850502 \\
\hline 6 & -1.011953 & -3.977316 & 2.967028 \\
\hline 6 & -1.014140 & -4.598308 & 4.191865 \\
\hline 6 & -1.298808 & -3.869823 & 5.365642 \\
\hline 6 & -1.576008 & -2.528773 & 5.287662 \\
\hline 15 & -1.617437 & 0.337066 & -0.114438 \\
\hline 6 & -1.434942 & 2.080858 & 0.433311 \\
\hline 6 & -0.379294 & 2.422149 & 1.276263 \\
\hline 6 & -0.218713 & 3.735875 & 1.733467 \\
\hline 6 & -1.090244 & 4.705176 & 1.236549 \\
\hline 6 & -2.120847 & 4.404198 & 0.335520 \\
\hline 6 & -2.296768 & 3.073209 & -0.037315 \\
\hline 6 & 0.802610 & 4.025956 & 2.834365 \\
\hline
\end{tabular}




\begin{tabular}{|c|c|c|c|}
\hline 5 & 0.912046 & 5.515302 & 3.151693 \\
\hline 6 & -3.023525 & 5.521421 & -0.183433 \\
\hline$b$ & -2.167902 & 6.569000 & -0.903837 \\
\hline & 2.217294 & -2.624537 & 1.340956 \\
\hline & 2.815013 & -1.632992 & 2.301246 \\
\hline & 1.994076 & -0.813479 & 3.079075 \\
\hline ) & 2.549641 & 0.107487 & 3.957923 \\
\hline 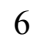 & 3.934549 & 0.226699 & 4.066854 \\
\hline ) & 4.757222 & -0.594472 & 3.304110 \\
\hline 5 & 4.197296 & -1.523652 & 2.431097 \\
\hline & -0.085445 & 0.061855 & -2.028051 \\
\hline f & -0.846977 & -1.339505 & -3.157677 \\
\hline 8 & -1.085023 & -2.099402 & -3.996028 \\
\hline 6 & -0.131650 & 1.949045 & -2.408038 \\
\hline 8 & -0.088750 & 3.057641 & -2.737870 \\
\hline 5 & -3.416893 & 0.166828 & -0.474671 \\
\hline ) & -4.361399 & 0.512039 & 0.488504 \\
\hline 6 & -5.727326 & 0.333826 & 0.260485 \\
\hline ) & -6.116307 & -0.206162 & -0.966072 \\
\hline 6 & -5.195276 & -0.570008 & -1.955788 \\
\hline 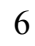 & -3.839963 & -0.368398 & -1.691104 \\
\hline 6 & -6.713316 & 0.716183 & 1.361855 \\
\hline$b$ & -6.400666 & -0.106371 & 2.617895 \\
\hline 6 & -5.688460 & -1.139768 & -3.285087 \\
\hline 6 & -6.684857 & -2.278294 & -3.044303 \\
\hline 6 & -8.162855 & 0.447194 & 0.966954 \\
\hline 6 & -6.573252 & 2.210552 & 1.674383 \\
\hline f & -6.376434 & -0.020829 & -4.074983 \\
\hline 6 & -4.542857 & -1.698168 & -4.126250 \\
\hline 6 & -4.069187 & 5.005753 & -1.169344 \\
\hline 6 & -3.756511 & 6.176704 & 0.992066 \\
\hline 6 & 2.196394 & 3.520406 & 2.453279 \\
\hline 5 & 0.330473 & 3.299320 & 4.101599 \\
\hline 1 & 6.849920 & 0.863216 & 0.261973 \\
\hline 1 & -2.062075 & 1.213748 & 2.685542 \\
\hline 1 & 8.166108 & -3.926242 & 0.209188 \\
\hline 1 & -1.798099 & -1.952211 & 6.184480 \\
\hline 1 & 5.193826 & 4.929323 & -2.787840 \\
\hline 1 & -3.663035 & -2.626404 & 1.060164 \\
\hline 1 & -5.490035 & -3.884801 & 0.010166 \\
\hline 1 & 3.228099 & 4.319758 & -1.503874 \\
\hline 1 & -2.096467 & 0.079081 & 4.842939 \\
\hline 1 & 6.071476 & -4.515770 & -0.885134 \\
\hline 1 & 8.140745 & 1.963777 & -3.780667 \\
\hline 1 & 3.825869 & -3.840965 & -1.729311 \\
\hline 1 & -2.700014 & -5.804343 & -2.645482 \\
\hline 1 & -0.310990 & -5.272087 & -2.501438 \\
\hline 1 & -0.797094 & -5.661953 & 4.257445 \\
\hline 1 & 6.910279 & 0.279094 & -2.501368 \\
\hline 1 & -0.795733 & -4.554820 & 2.070611 \\
\hline 1 & 1.521092 & -3.906938 & -1.536208 \\
\hline 1 & 7.313922 & 4.311758 & -3.911652 \\
\hline 1 & 8.978715 & 0.161637 & 1.257769 \\
\hline 1 & -5.021735 & -5.485646 & -1.844117 \\
\hline 1 & -1.297843 & -4.374554 & 6.328878 \\
\hline 1 & 9.667621 & -2.233369 & 1.218665 \\
\hline
\end{tabular}




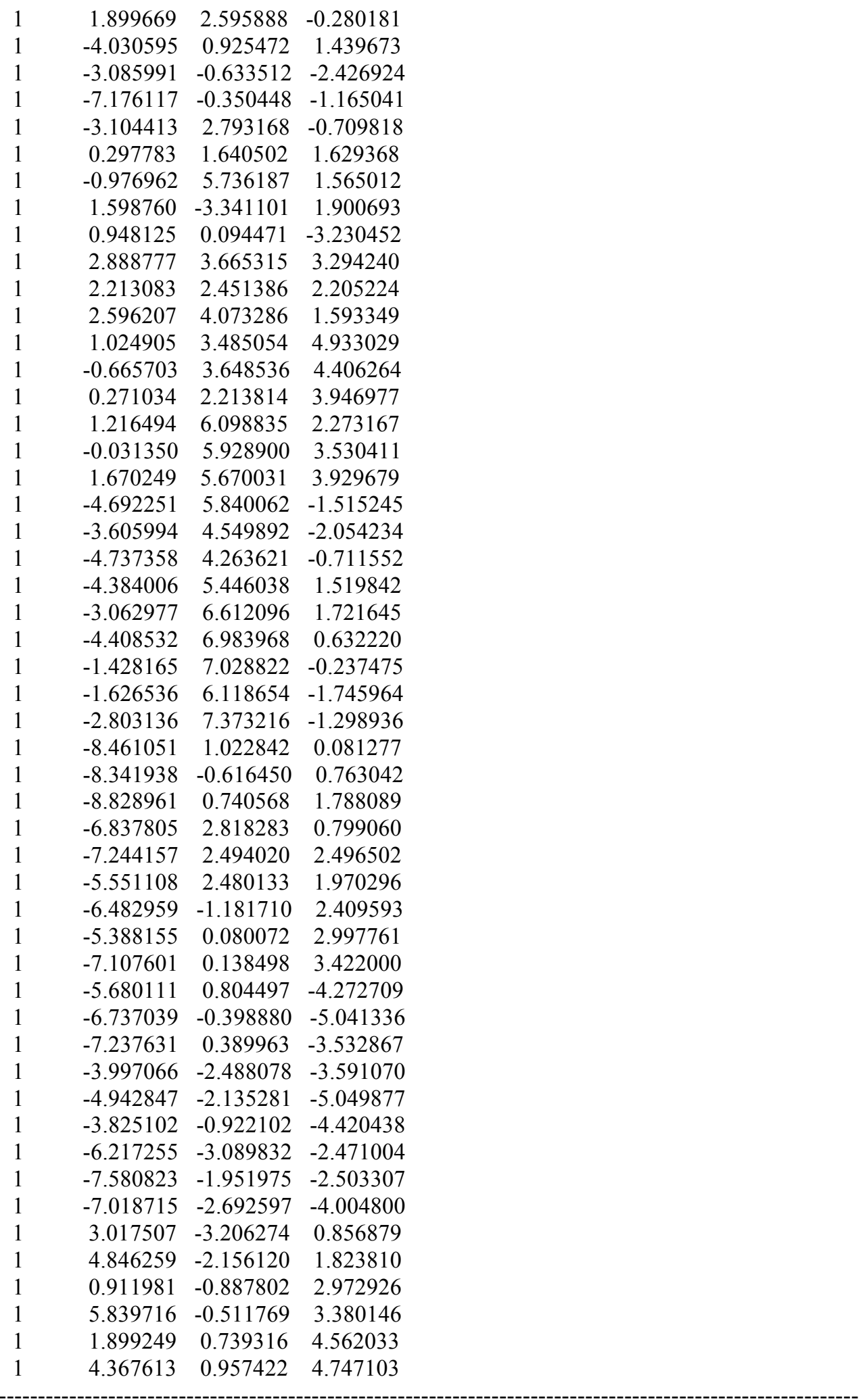

$1_{\text {C4- }- \text {-1s2a3s4a }}$

Number of imaginary frequencies : 0

The smallest frequencies are : $12.5219 \quad 18.3698 \quad 21.9088 \mathrm{~cm}(-1)$ 
Electronic energy : $\quad H F=-4125.4686506$

Zero-point correction $=\quad 1.299706($ Hartree/Particle $)$

Thermal correction to Energy= $\quad 1.377949$

Thermal correction to Enthalpy= $\quad 1.378893$

Thermal correction to Gibbs Free Energy $=\quad 1.187656$

Sum of electronic and zero-point Energies $=\quad-4124.168945$

Sum of electronic and thermal Energies $=\quad-4124.090702$

Sum of electronic and thermal Enthalpies $=\quad-4124.089758$

Sum of electronic and thermal Free Energies $=\quad-4124.280995$

Cartesian Coordinates

$\begin{array}{cccc}0 & 5.512429 & 4.029914 & -2.547957 \\ 6 & 4.783749 & 3.035344 & -1.851281 \\ 6 & 5.281398 & 1.696939 & -1.812883 \\ 6 & 6.469952 & 1.409406 & -2.531532 \\ 6 & 7.146221 & 2.393605 & -3.208960 \\ 6 & 6.673274 & 3.722453 & -3.209392 \\ 6 & 4.545266 & 0.696573 & -1.097885 \\ 6 & 3.338635 & 1.057593 & -0.530477 \\ 6 & 2.830220 & 2.369116 & -0.589480 \\ 6 & 3.553976 & 3.343524 & -1.221669 \\ 6 & 4.975234 & -0.718076 & -1.024595 \\ 6 & 4.110852 & -1.708939 & -1.459001 \\ 6 & 4.505239 & -3.062875 & -1.532781 \\ 6 & 5.745564 & -3.440973 & -1.097127 \\ 6 & 6.630062 & -2.488670 & -0.533601 \\ 6 & 6.240460 & -1.116493 & -0.483611 \\ 6 & 7.875356 & -2.881048 & 0.014300 \\ 6 & 8.699918 & -1.966480 & 0.618321 \\ 6 & 8.302592 & -0.615602 & 0.702296 \\ 6 & 7.108516 & -0.201429 & 0.164782 \\ 8 & 2.844897 & -1.403289 & -1.897146 \\ 15 & 1.641642 & -0.832851 & -0.869777 \\ 8 & 2.587746 & 0.099527 & 0.120694 \\ 7 & 1.281643 & -2.077139 & 0.230827 \\ 6 & 0.209999 & -2.900881 & -0.260890 \\ 6 & -1.064589 & -2.763407 & 0.254287 \\ 6 & -2.148767 & -3.475031 & -0.344426 \\ 6 & -1.886876 & -4.370802 & -1.422842 \\ 6 & -0.555235 & -4.538290 & -1.871598 \\ 6 & 0.462013 & -3.808635 & -1.316852 \\ 6 & -2.965033 & -5.065864 & -2.022950 \\ 6 & -4.251614 & -4.878592 & -1.585546 \\ 6 & -4.515432 & -3.982068 & -0.527083 \\ 6 & -3.490798 & -3.303091 & 0.083467 \\ 6 & -1.303810 & -1.985451 & 1.498344 \\ 6 & -1.543071 & -0.622185 & 1.513490 \\ 6 & -1.830472 & 0.014278 & 2.755017 \\ 6 & -1.854169 & -0.682913 & 3.931658 \\ 6 & -1.589777 & -2.071408 & 3.953129 \\ 6 & -1.317080 & -2.735502 & 2.722713 \\ 6 & -1.053888 & -4.130372 & 2.762226 \\ 6 & -1.054913 & -4.817239 & 3.951359 \\ 6 & -1.324398 & -4.151872 & 5.165278 \\ 6 & -1.587164 & -2.805925 & 5.162583 \\ & & & \\ 6 & & & \end{array}$




\begin{tabular}{|c|c|c|c|}
\hline 15 & -1.612707 & 0.364734 & -0.061247 \\
\hline 6 & -1.354517 & 2.067674 & 0.576179 \\
\hline 6 & -0.296735 & 2.326163 & 1.445061 \\
\hline 6 & -0.086464 & 3.611739 & 1.959715 \\
\hline 6 & -0.910647 & 4.637395 & 1.496521 \\
\hline 6 & -1.943684 & 4.419475 & 0.574265 \\
\hline 6 & -2.169318 & 3.114802 & 0.141040 \\
\hline 6 & 0.938606 & 3.811566 & 3.077182 \\
\hline 6 & 1.095952 & 5.278625 & 3.469024 \\
\hline 6 & -2.794630 & 5.595734 & 0.099725 \\
\hline 6 & -1.891910 & 6.630384 & -0.580431 \\
\hline 6 & 2.217041 & -2.727151 & 1.177182 \\
\hline 6 & 2.812010 & -1.800935 & 2.203035 \\
\hline 6 & 1.989131 & -1.053725 & 3.048457 \\
\hline 6 & 2.541866 & -0.205783 & 3.999918 \\
\hline 6 & 3.926425 & -0.085532 & 4.113087 \\
\hline 6 & 4.751278 & -0.830886 & 3.278182 \\
\hline 6 & 4.194113 & -1.689048 & 2.333623 \\
\hline 45 & -0.142722 & 0.111200 & -2.021333 \\
\hline 6 & -1.034503 & -1.225351 & -3.120132 \\
\hline 8 & -1.425714 & -1.954692 & -3.928392 \\
\hline 6 & -0.139654 & 2.009331 & -2.355154 \\
\hline 8 & -0.085686 & 3.122492 & -2.666718 \\
\hline 6 & -3.419507 & 0.280638 & -0.415023 \\
\hline 6 & -4.366169 & 0.450988 & 0.604861 \\
\hline 6 & -5.724548 & 0.313823 & 0.346905 \\
\hline 6 & -6.119863 & 0.005692 & -0.966008 \\
\hline 6 & -5.208959 & -0.149501 & -2.005028 \\
\hline 6 & -3.847630 & -0.004805 & -1.702221 \\
\hline 6 & -6.783185 & 0.463782 & 1.437582 \\
\hline 6 & -7.538412 & -0.861114 & 1.590645 \\
\hline 6 & -5.615255 & -0.533259 & -3.425381 \\
\hline 6 & -5.175255 & -1.980777 & -3.677455 \\
\hline 6 & -6.173182 & 0.821195 & 2.791427 \\
\hline 6 & -7.766614 & 1.573598 & 1.051118 \\
\hline 6 & -4.932709 & 0.390056 & -4.440774 \\
\hline 6 & -7.123937 & -0.442307 & -3.641596 \\
\hline 6 & -3.863486 & & -0.903197 \\
\hline 6 & -3.497243 & 6.238630 & 1.300384 \\
\hline 6 & 2.316307 & 3.279086 & 2.674274 \\
\hline 6 & 0.438636 & 3.037870 & 4.305176 \\
\hline 1 & 6.807803 & 0.839791 & 0.251141 \\
\hline 1 & -2.032534 & 1.082099 & 2.777572 \\
\hline 1 & 8.156775 & -3.931830 & -0.042941 \\
\hline 1 & -1.796278 & -2.276007 & 6.090769 \\
\hline 1 & 5.118853 & 5.045515 & -2.556557 \\
\hline 1 & -3.705390 & -2.607498 & 0.893640 \\
\hline 1 & -5.540204 & -3.818673 & -0.198847 \\
\hline 1 & 3.176863 & 4.363990 & -1.271693 \\
\hline 1 & -2.075326 & -0.171770 & 4.868072 \\
\hline 1 & 6.059688 & -4.481873 & -1.154798 \\
\hline 1 & 8.052199 & 2.143626 & -3.756647 \\
\hline 1 & 3.801531 & -3.783344 & -1.944839 \\
\hline 1 & -2.749304 & -5.746275 & -2.845498 \\
\hline 1 & -0.359298 & -5.230900 & -2.688634 \\
\hline 1 & -0.848107 & -5.884902 & 3.956896 \\
\hline
\end{tabular}




\begin{tabular}{|c|c|c|c|}
\hline & 6.842440 & 0.388637 & -2.553213 \\
\hline & -0.847419 & -4.661154 & 1.835246 \\
\hline & 1.480569 & -3.897957 & -1.690908 \\
\hline & 7.221149 & 4.493771 & -3.745758 \\
\hline & 8.945453 & 0.105077 & 1.202883 \\
\hline & -5.073231 & -5.411475 & -2.059335 \\
\hline & -1.322503 & -4.708664 & 6.099395 \\
\hline & 9.650686 & -2.280139 & 1.042916 \\
\hline & 1.868355 & 2.576312 & -0.123789 \\
\hline & -4.027387 & 0.681858 & 1.610814 \\
\hline & -3.100877 & -0.126784 & -2.482739 \\
\hline & -7.182360 & -0.112369 & -1.170290 \\
\hline & -2.979212 & 2.897727 & -0.552116 \\
\hline & 0.343507 & 1.502637 & 1.770385 \\
\hline & -0.757107 & 5.647678 & 1.870122 \\
\hline & 1.621730 & -3.496495 & 1.689711 \\
\hline & 0.838381 & 0.126908 & -3.268948 \\
\hline & 3.011527 & 3.367145 & 3.520727 \\
\hline & 2.298854 & 2.221161 & 2.382477 \\
\hline & 2.734552 & 3.854043 & 1.838019 \\
\hline & 1.137782 & 3.156029 & 5.144953 \\
\hline & -0.545388 & 3.405238 & 4.627299 \\
\hline & 0.342699 & 1.964499 & 4.094697 \\
\hline & 1.427488 & 5.894197 & 2.622736 \\
\hline & 0.163854 & 5.705107 & 3.861309 \\
\hline & 1.852579 & 5.368523 & 4.258643 \\
\hline & -4.442529 & 6.043829 & -1.219944 \\
\hline 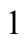 & -3.422990 & 4.720282 & -1.804163 \\
\hline & -4.569848 & 4.445543 & -0.471429 \\
\hline 1 & -4.152128 & 5.516149 & 1.805036 \\
\hline & -2.785001 & 6.620354 & 2.041844 \\
\hline 1 & -4.116587 & 7.083828 & 0.971230 \\
\hline ] & -1.131791 & 7.028172 & 0.102653 \\
\hline & -1.372116 & 6.190021 & -1.441094 \\
\hline 1 & -2.489316 & 7.478342 & -0.941586 \\
\hline & -5.477013 & 0.049761 & 3.147510 \\
\hline 1 & -5.637281 & 1.778999 & 2.759307 \\
\hline & -6.969663 & 0.915098 & 3.540258 \\
\hline 1 & -8.292376 & 1.354496 & 0.114164 \\
\hline ] & -8.526312 & 1.699287 & 1.834226 \\
\hline & -7.246803 & 2.532593 & 0.926622 \\
\hline 1 & -8.036882 & -1.162040 & 0.661193 \\
\hline & -6.853982 & -1.669273 & 1.881723 \\
\hline 1 & -8.309245 & -0.774717 & 2.368324 \\
\hline & -3.838897 & 0.311401 & -4.413404 \\
\hline 1 & -5.249939 & 0.127575 & -5.458522 \\
\hline 1 & -5.199287 & 1.440197 & -4.263677 \\
\hline 1 & -7.504435 & 0.569663 & -3.449745 \\
\hline 1 & -7.362511 & -0.694872 & -4.682355 \\
\hline 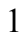 & -7.676561 & -1.144759 & -3.004630 \\
\hline 1 & -4.093113 & -2.110468 & -3.543744 \\
\hline ] & -5.677906 & -2.667492 & -2.982948 \\
\hline 1 & -5.427875 & -2.284974 & -4.702521 \\
\hline 1 & 3.020662 & -3.255151 & 0.640462 \\
\hline 1 & 4.844962 & -2.264311 & 1.673840 \\
\hline 1 & 0.907427 & -1.130416 & 2.942334 \\
\hline
\end{tabular}




$\begin{array}{rrrr}1 & 5.833553 & -0.744486 & 3.353475 \\ 1 & 1.888934 & 0.367130 & 4.657767 \\ 1 & 4.357316 & 0.588353 & 4.850960\end{array}$

$1_{\mathrm{C} 4-} \psi_{-1 \mathrm{~s} 2 \mathrm{a} s 4 \mathrm{~s}}$

Number of imaginary frequencies : 0

The smallest frequencies are : $11.6347 \quad 18.9756 \quad 19.3533 \mathrm{~cm}(-1)$

Electronic energy : $\quad \mathrm{HF}=-4125.4688293$

Zero-point correction $=\quad 1.299376($ Hartree/Particle $)$

Thermal correction to Energy= $\quad 1.377652$

Thermal correction to Enthalpy= $\quad 1.378596$

Thermal correction to Gibbs Free Energy= 1.187306

Sum of electronic and zero-point Energies $=\quad-4124.169454$

Sum of electronic and thermal Energies $=\quad-4124.091177$

Sum of electronic and thermal Enthalpies $=\quad-4124.090233$

Sum of electronic and thermal Free Energies $=\quad-4124.281523$

Cartesian Coordinates

$\begin{array}{cccc}6 & 5.493410 & 4.043221 & -2.586697 \\ 6 & 4.771192 & 3.050932 & -1.880112 \\ 6 & 5.279785 & 1.717201 & -1.824542 \\ 6 & 6.472424 & 1.431107 & -2.537005 \\ 6 & 7.142230 & 2.412813 & -3.224398 \\ 6 & 6.658397 & 3.737620 & -3.241650 \\ 6 & 4.550031 & 0.719254 & -1.099596 \\ 6 & 3.338965 & 1.077243 & -0.539576 \\ 6 & 2.819806 & 2.383599 & -0.615862 \\ 6 & 3.537037 & 3.356394 & -1.257809 \\ 6 & 4.990396 & -0.691271 & -1.009226 \\ 6 & 4.133650 & -1.693137 & -1.433615 \\ 6 & 4.536754 & -3.045233 & -1.490187 \\ 6 & 5.778981 & -3.409821 & -1.048532 \\ 6 & 6.656655 & -2.444787 & -0.496203 \\ 6 & 6.257895 & -1.074715 & -0.462660 \\ 6 & 7.904588 & -2.822290 & 0.056139 \\ 6 & 8.723284 & -1.895100 & 0.648738 \\ 6 & 8.316993 & -0.545976 & 0.716560 \\ 6 & 7.119972 & -0.146270 & 0.174605 \\ 8 & 2.866966 & -1.401235 & -1.879003 \\ 15 & 1.656869 & -0.829117 & -0.861114 \\ 8 & 2.593808 & 0.121278 & 0.121074 \\ 7 & 1.302277 & -2.065069 & 0.250942 \\ 6 & 0.237960 & -2.902418 & -0.234062 \\ 6 & -1.037084 & -2.774957 & 0.282542 \\ 6 & -2.115216 & -3.502621 & -0.307351 \\ 6 & -1.846679 & -4.402993 & -1.380223 \\ 6 & -0.514349 & -4.559270 & -1.831157 \\ 6 & 0.496775 & -3.815305 & -1.284038 \\ 6 & -2.919078 & -5.114015 & -1.971855 \\ 6 & -4.205954 & -4.939132 & -1.530041 \\ 6 & -4.476018 & -4.039262 & -0.475901 \\ 6 & -3.457449 & -3.343060 & 0.125181 \\ 6 & -1.285847 & -1.986310 & 1.517390 \\ 6 & -1.543759 & -0.626636 & 1.513210 \\ & & & \\ 6\end{array}$




\begin{tabular}{|c|c|c|c|}
\hline 6 & -1.844432 & 0.022306 & 2.745031 \\
\hline 6 & -1.862971 & -0.659920 & 3.930681 \\
\hline & -1.580676 & -2.044508 & 3.971374 \\
\hline & -1.294324 & -2.720814 & 2.750849 \\
\hline & -1.015222 & -4.111906 & 2.808640 \\
\hline & -1.013186 & -4.783749 & 4.006282 \\
\hline & -1.295317 & -4.106183 & 5.210591 \\
\hline & -1.574141 & -2.763604 & 5.190007 \\
\hline & -1.615394 & 0.338169 & -0.075478 \\
\hline 6 & -1.393807 & 2.050549 & 0.547847 \\
\hline 6 & -0.337456 & 2.338156 & 1.409200 \\
\hline 6 & -0.159010 & 3.629345 & 1.921356 \\
\hline 6 & -1.013522 & 4.632131 & 1.462291 \\
\hline 6 & -2.045818 & 4.384928 & 0.546779 \\
\hline 6 & -2.239608 & 3.074119 & 0.117554 \\
\hline 6 & 0.863870 & 3.857096 & 3.035387 \\
\hline 6 & 0.992883 & 5.329567 & 3.417177 \\
\hline 6 & -2.931600 & 5.534844 & 0.072817 \\
\hline 6 & -2.059644 & 6.594677 & -0.608762 \\
\hline 6 & 2.237202 & -2.697478 & 1.209566 \\
\hline 6 & 2.827347 & -1.752802 & 2.221128 \\
\hline 6 & 2.000822 & -0.991038 & 3.049904 \\
\hline 6 & 2.549769 & -0.122124 & 3.984471 \\
\hline 6 & 3.933803 & 0.003990 & 4.097685 \\
\hline 6 & 4.762205 & -0.757329 & 3.280929 \\
\hline 6 & 4.208907 & -1.635773 & 2.352899 \\
\hline 45 & -0.131417 & 0.092126 & -2.023932 \\
\hline 6 & -1.000433 & -1.266991 & -3.115504 \\
\hline 8 & -1.381275 & -2.009166 & -3.916842 \\
\hline 6 & -0.159664 & 1.989336 & -2.358958 \\
\hline 8 & -0.128041 & 3.106169 & -2.660682 \\
\hline 6 & -3.417292 & 0.230458 & -0.445381 \\
\hline 6 & -4.369759 & 0.421256 & 0.557030 \\
\hline 6 & -5.731755 & 0.287114 & 0.293466 \\
\hline 6 & -6.120323 & -0.040629 & -1.011275 \\
\hline 6 & -5.195286 & -0.221922 & -2.040762 \\
\hline 6 & -3.839087 & -0.078588 & -1.733055 \\
\hline 6 & -6.726479 & 0.489758 & 1.435144 \\
\hline 6 & -6.422444 & -0.517011 & 2.551411 \\
\hline 6 & -5.593672 & -0.625871 & -3.458284 \\
\hline 6 & -5.145990 & -2.074172 & -3.690931 \\
\hline 6 & -8.173516 & 0.284489 & 0.994400 \\
\hline 6 & -6.591906 & 1.918047 & 1.976448 \\
\hline 6 & -4.910575 & 0.288232 & -4.481759 \\
\hline 6 & -7.101622 & -0.545048 & -3.683779 \\
\hline 6 & -3.987081 & 5.073447 & -0.929701 \\
\hline 6 & -3.653719 & 6.154482 & 1.274022 \\
\hline 6 & 2.251215 & 3.349064 & 2.634160 \\
\hline 6 & 0.379980 & 3.081867 & 4.268973 \\
\hline 1 & 6.812380 & 0.893877 & 0.248662 \\
\hline 1 & -2.058523 & 1.087930 & 2.752997 \\
\hline 1 & 8.192985 & -3.871777 & 0.011378 \\
\hline 1 & -1.793723 & -2.224887 & 6.110667 \\
\hline 1 & 5.091463 & 5.055339 & -2.608317 \\
\hline 1 & -3.677558 & -2.643722 & 0.931082 \\
\hline 1 & -5.501021 & -3.888295 & -0.142694 \\
\hline
\end{tabular}




\begin{tabular}{|c|c|c|c|}
\hline 1 & 3.151448 & 4.372927 & -1.321439 \\
\hline 1 & -2.093357 & -0.139557 & 4.859769 \\
\hline & 6.100192 & -4.449191 & -1.093233 \\
\hline & 8.051577 & 2.163875 & -3.766955 \\
\hline & 3.838235 & -3.775102 & -1.894454 \\
\hline & -2.698854 & -5.797223 & -2.790875 \\
\hline & -0.313103 & -5.255289 & -2.643992 \\
\hline & -0.794415 & -5.848853 & 4.026192 \\
\hline & 6.853431 & 0.413298 & -2.545703 \\
\hline & -0.799705 & -4.651513 & 1.888787 \\
\hline & 1.515316 & -3.897118 & -1.659937 \\
\hline & 7.201225 & 4.507063 & -3.785786 \\
\hline & 8.955194 & 0.184965 & 1.208151 \\
\hline & -5.023117 & -5.485032 & -1.996631 \\
\hline & -1.290857 & -4.651216 & 6.151606 \\
\hline & 9.676271 & -2.197269 & 1.076678 \\
\hline & 1.854235 & 2.587736 & -0.156683 \\
\hline & -4.043619 & 0.670122 & 1.565473 \\
\hline & -3.087753 & -0.217057 & -2.506131 \\
\hline & -7.178244 & -0.154242 & -1.229874 \\
\hline & -3.048386 & 2.834262 & -0.568971 \\
\hline & 0.324721 & 1.531353 & 1.732865 \\
\hline 1 & -0.885190 & 5.646704 & 1.833804 \\
\hline & 1.642790 & -3.460142 & 1.733078 \\
\hline & 0.854984 & 0.118218 & -3.267448 \\
\hline 1 & 2.945683 & 3.455415 & 3.479136 \\
\hline & 2.254424 & 2.289319 & 2.348602 \\
\hline ] & 2.657168 & 3.927295 & 1.794050 \\
\hline & 1.077322 & 3.218800 & \\
\hline 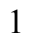 & -0.610592 & 3.432697 & 4.589591 \\
\hline 1 & 0.303673 & 2.005527 & 4.065328 \\
\hline & 1.307731 & 5.946198 & 2.565316 \\
\hline 1 & 0.054150 & 5.739500 & 3.811195 \\
\hline & 1.750990 & 5.440103 & 4.202762 \\
\hline 1 & -4.597642 & 5.930185 & -1.241382 \\
\hline & -3.533224 & 4.645360 & -1.833206 \\
\hline 1 & -4.665715 & 4.324779 & -0.498987 \\
\hline 1 & -4.290706 & 5.411975 & 1.773247 \\
\hline & -2.953484 & 6.551339 & 2.019027 \\
\hline 1 & -4.294548 & 6.984339 & 0.946957 \\
\hline . & -1.314332 & 7.018780 & 0.074893 \\
\hline 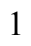 & -1.523514 & 6.166060 & -1.465679 \\
\hline 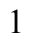 & -2.681912 & 7.422114 & -0.975511 \\
\hline 1 & -8.469913 & 0.994395 & 0.211583 \\
\hline 1 & -8.349000 & -0.732849 & 0.621299 \\
\hline 1 & -8.844056 & 0.440403 & 1.848890 \\
\hline 1 & -6.826974 & 2.654617 & 1.196846 \\
\hline 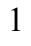 & -7.286408 & 2.077538 & 2.812297 \\
\hline 1 & -5.578868 & 2.131623 & 2.339711 \\
\hline 1 & -6.520337 & -1.547500 & 2.183812 \\
\hline 1 & -5.406899 & -0.403469 & 2.952007 \\
\hline 1 & -7.125387 & -0.387644 & 3.385397 \\
\hline 1 & -3.816471 & 0.217471 & -4.445603 \\
\hline 1 & -5.219269 & 0.010272 & -5.498084 \\
\hline 1 & -5.185160 & 1.338821 & -4.320200 \\
\hline & -7.488079 & 0.467815 & -3.509493 \\
\hline
\end{tabular}




$\begin{array}{llll}1 & -7.333047 & -0.813964 & -4.722065 \\ 1 & -7.654725 & -1.240343 & -3.039410 \\ 1 & -4.063150 & -2.196062 & -3.555582 \\ 1 & -5.645425 & -2.753767 & -2.987265 \\ 1 & -5.396928 & -2.393663 & -4.711766 \\ 1 & 3.043253 & -3.231835 & 0.682724 \\ 1 & 4.862345 & -2.222655 & 1.706024 \\ 1 & 0.919371 & -1.071790 & 2.942619 \\ 1 & 5.844118 & -0.667125 & 3.357014 \\ 1 & 1.894557 & 0.463349 & 4.628830 \\ 1 & 4.361471 & 0.694461 & 4.821973\end{array}$

$1_{\mathrm{C} 4-} \psi_{-1 \mathrm{~s} 2 \mathrm{~s} 3 \mathrm{a} 4 \mathrm{a}}$

Number of imaginary frequencies : 0

The smallest frequencies are : $14.0983 \quad 18.291924 .0911 \mathrm{~cm}(-1)$

Electronic energy : $\quad H F=-4125.4687638$

Zero-point correction $=\quad 1.299705$ (Hartree/Particle)

Thermal correction to Energy= $\quad 1.377870$

Thermal correction to Enthalpy= $\quad 1.378815$

Thermal correction to Gibbs Free Energy= $\quad 1.188437$

Sum of electronic and zero-point Energies $=\quad-4124.169059$

Sum of electronic and thermal Energies $=\quad-4124.090893$

Sum of electronic and thermal Enthalpies $=\quad-4124.089949$

Sum of electronic and thermal Free Energies $=\quad-4124.280326$

Cartesian Coordinates

$\begin{array}{cccc}6 & -2.263692 & 3.105389 & -0.009857 \\ 6 & -1.405977 & 2.107398 & 0.439498 \\ 6 & -0.342537 & 2.437814 & 1.283889 \\ 6 & -0.177956 & 3.742158 & 1.753553 \\ 6 & -1.057473 & 4.724072 & 1.280608 \\ 6 & -2.093422 & 4.434466 & 0.389788 \\ 15 & -1.608297 & 0.368278 & -0.114880 \\ 6 & -1.529660 & -0.529417 & 1.515157 \\ 6 & -1.279856 & -1.889747 & 1.573209 \\ 6 & -1.296082 & -2.575987 & 2.833586 \\ 6 & -1.578532 & -1.850970 & 4.026582 \\ 6 & -1.849693 & -0.466753 & 3.931767 \\ 6 & -1.827119 & 0.168703 & 2.720425 \\ 6 & -1.582035 & -2.523620 & 5.271326 \\ 6 & -1.317432 & -3.867513 & 5.343341 \\ 6 & -1.039440 & -4.593129 & 4.166246 \\ 6 & -1.031366 & -3.966609 & 2.944263 \\ 6 & -1.029731 & -2.723807 & 0.368775 \\ 6 & 0.247325 & -2.869703 & -0.137779 \\ 6 & 0.507739 & -3.810684 & -1.162267 \\ 6 & -0.504721 & -4.558296 & -1.701629 \\ 6 & -1.838807 & -4.383445 & -1.263259 \\ 6 & -2.107140 & -3.463977 & -0.206583 \\ 6 & -3.449340 & -3.296151 & 0.221837 \\ 6 & -4.470355 & -3.991475 & -0.376114 \\ 6 & -4.201611 & -4.902602 & -1.421473 \\ 6 & -2.913008 & -5.093652 & -1.852681 \\ 7 & 1.312021 & -2.022751 & 0.329614\end{array}$




\begin{tabular}{|c|c|c|c|}
\hline 15 & 1.682689 & -0.828069 & -0.821132 \\
\hline 8 & 2.888859 & -1.448426 & -1.815931 \\
\hline 6 & 4.146881 & -1.749320 & -1.351845 \\
\hline 6 & 5.019309 & -0.750070 & -0.953548 \\
\hline 6 & 6.276053 & -1.138254 & -0.386202 \\
\hline 6 & 6.650355 & -2.515506 & -0.373484 \\
\hline 6 & 5.759024 & -3.481671 & -0.901187 \\
\hline 6 & 4.526375 & -3.109267 & -1.362936 \\
\hline 6 & 7.150452 & -0.205413 & 0.227468 \\
\hline 6 & 8.336810 & -0.608759 & 0.789756 \\
\hline 6 & 8.719592 & -1.966300 & 0.766909 \\
\hline 6 & 7.888086 & -2.897249 & 0.198643 \\
\hline 6 & 4.605914 & 0.663778 & -1.098387 \\
\hline 6 & 3.394327 & 1.062786 & -0.567905 \\
\hline 6 & 2.894223 & 2.370824 & -0.708959 \\
\hline 6 & 3.633705 & 3.306380 & -1.380189 \\
\hline 6 & 4.872802 & 2.958533 & -1.969632 \\
\hline 6 & 5.360042 & 1.620804 & -1.853172 \\
\hline 6 & 5.619913 & 3.911128 & -2.704454 \\
\hline 6 & 6.789278 & 3.562717 & -3.329498 \\
\hline 6 & 7.251941 & 2.232538 & -3.253123 \\
\hline 6 & 6.557417 & 1.288996 & -2.537494 \\
\hline 8 & 2.628025 & 0.148371 & 0.126716 \\
\hline 6 & 0.849990 & 4.020445 & 2.852209 \\
\hline 6 & 2.237923 & 3.501370 & 2.468196 \\
\hline 6 & -3.065652 & 5.492364 & -0.128383 \\
\hline 6 & -2.763064 & 6.884111 & 0.420854 \\
\hline 6 & -3.411896 & 0.213183 & -0.466423 \\
\hline 6 & -3.833001 & -0.280371 & -1.696759 \\
\hline 6 & -5.190174 & -0.490288 & -1.964545 \\
\hline 6 & -6.102862 & -0.184174 & -0.955690 \\
\hline 6 & -5.716492 & 0.316400 & 0.293912 \\
\hline 6 & -4.356814 & 0.514888 & 957 \\
\hline 6 & -6.770836 & 0.575321 & 1.368618 \\
\hline 6 & -6.168097 & 1.176928 & 2.636703 \\
\hline 6 & -5.683542 & -1.018285 & -3.310720 \\
\hline 6 & -6.450326 & 0.097549 & -4.028955 \\
\hline 45 & -0.082038 & 0.075497 & -2.029030 \\
\hline 6 & -0.864709 & -1.323745 & -3.143845 \\
\hline 8 & -1.127387 & -2.088952 & -3.969959 \\
\hline 6 & -0.105950 & 1.963795 & -2.404555 \\
\hline 8 & -0.050441 & 3.073279 & -2.728465 \\
\hline 6 & -7.835818 & 1.542880 & 0.842867 \\
\hline 6 & -7.431088 & -0.758133 & 1.739142 \\
\hline 6 & -4.530199 & -1.470842 & -4.202664 \\
\hline 6 & -6.610058 & -2.220293 & -3.102541 \\
\hline 6 & 2.224949 & -2.623529 & 1.328985 \\
\hline 6 & 2.821842 & -1.638901 & 2.296949 \\
\hline 6 & 2.000112 & -0.826999 & 3.081777 \\
\hline 6 & 2.554702 & 0.085864 & 3.969667 \\
\hline 6 & 3.939482 & 0.204817 & 4.080508 \\
\hline 6 & 4.762987 & -0.608302 & 3.310069 \\
\hline 6 & 4.204025 & -1.529684 & 2.428234 \\
\hline 6 & 0.372871 & 3.296599 & 4.119231 \\
\hline 6 & 0.977050 & 5.508075 & 3.172402 \\
\hline 6 & -4.491907 & 5.114354 & 0.289622 \\
\hline
\end{tabular}




\begin{tabular}{|c|c|c|c|}
\hline 6 & -2.981187 & 5.553930 & -1.657375 \\
\hline & 6.860191 & 0.841589 & 0.267741 \\
\hline & -2.037425 & 1.234710 & 2.687441 \\
\hline & 8.157899 & -3.952560 & 0.189008 \\
\hline & -1.798580 & -1.948946 & 6.170713 \\
\hline & 5.233519 & 4.927267 & -2.772198 \\
\hline 1 & -3.666446 & -2.589224 & 1.021240 \\
\hline & -5.495896 & -3.834243 & -0.046080 \\
\hline 1 & 3.262840 & 4.324167 & -1.492885 \\
\hline 1 & -2.077671 & 0.090851 & 4.839664 \\
\hline 1 & 6.061898 & -4.527421 & -0.911669 \\
\hline 1 & 8.164596 & 1.948542 & -3.772420 \\
\hline 1 & 3.819255 & -3.839097 & -1.751678 \\
\hline 1 & -2.692958 & -5.790241 & -2.660381 \\
\hline 1 & -0.302849 & -5.271000 & -2.499660 \\
\hline 1 & -0.832074 & -5.658995 & 4.226870 \\
\hline 1 & 6.922837 & 0.266226 & -2.501175 \\
\hline 1 & -0.819766 & -4.542264 & 2.045590 \\
\hline 1 & 1.528214 & -3.908350 & -1.528501 \\
\hline 1 & 7.351565 & 4.301754 & -3.895569 \\
\hline 1 & 8.984704 & 0.125956 & 1.262699 \\
\hline 1 & -5.020158 & -5.449740 & -1.884584 \\
\hline 1 & -1.321161 & -4.376592 & 6.304280 \\
\hline 1 & 9.664463 & -2.271418 & 1.210470 \\
\hline 1 & 1.923567 & 2.603938 & -0.276229 \\
\hline 1 & -4.019947 & 0.900200 & 1.478222 \\
\hline 1 & -3.079255 & -0.511078 & -2.444245 \\
\hline 1 & -7.164115 & -0.345927 & -1.144833 \\
\hline 1 & -3.079942 & 2.841551 & -0.682176 \\
\hline 1 & 0.331489 & 1.649137 & 1.626727 \\
\hline 1 & -0.936526 & 5.745213 & 1.629136 \\
\hline 1 & 1.610028 & -3.347036 & 1.883629 \\
\hline 1 & 0.944413 & 0.094100 & -3.238272 \\
\hline 1 & 2.932919 & 3.638841 & 3.308227 \\
\hline 1 & 2.243942 & 2.432470 & 2.219543 \\
\hline 1 & 2.641992 & 4.051103 & 1.608258 \\
\hline 1 & 1.070476 & 3.473660 & 4.949966 \\
\hline 1 & -0.619290 & 3.655211 & 4.426127 \\
\hline 1 & 0.302068 & 2.212155 & 3.961893 \\
\hline 1 & 1.278830 & 6.091129 & 2.292643 \\
\hline 1 & 0.042244 & 5.930776 & 3.561969 \\
\hline 1 & 1.744343 & 5.652689 & 3.943427 \\
\hline 1 & -3.485630 & 7.603805 & 0.015991 \\
\hline 1 & -2.841525 & 6.921764 & 1.515177 \\
\hline 1 & -1.761243 & 7.229250 & 0.134677 \\
\hline 1 & -4.794117 & 4.137771 & -0.109583 \\
\hline 1 & -4.582261 & 5.072868 & 1.383285 \\
\hline 1 & -5.208267 & 5.859835 & -0.080981 \\
\hline 1 & -1.969077 & 5.824871 & -1.984077 \\
\hline 1 & -3.237554 & 4.595682 & -2.124827 \\
\hline 1 & -3.676818 & 6.309639 & -2.046631 \\
\hline 1 & -5.433161 & 0.506913 & 3.102953 \\
\hline 1 & -5.678976 & 2.140461 & 2.440300 \\
\hline 1 & -6.962191 & 1.353972 & 3.372885 \\
\hline 1 & -8.354224 & 1.152661 & -0.040914 \\
\hline 1 & -8.595455 & 1.725477 & 1.614576 \\
\hline
\end{tabular}




\begin{tabular}{|c|c|c|c|}
\hline & -7.392859 & 2.509708 & 0.571116 \\
\hline & -7.930698 & -1.221549 & 0.879361 \\
\hline & -6.686212 & -1.471255 & 2.117174 \\
\hline & -8.186080 & -0.608290 & 2.522726 \\
\hline & -5.806285 & 0.970223 & -4.198516 \\
\hline & -6.810155 & -0.252443 & -5.006042 \\
\hline & -7.321461 & 0.431461 & \\
\hline & -3.934834 & -2.260066 & -3.722821 \\
\hline & -4.926968 & -1.876 & -5.14 \\
\hline & -3.857384 & -0.644130 & -4.46 \\
\hline & -6.078173 & -3.036643 & -2.59601 \\
\hline & -7.497843 & -1.972558 & -2.50841 \\
\hline & -6.961005 & -2.596352 & -4.07272 \\
\hline & 3.025919 & -3.198543 & 0.83828 \\
\hline & 4.853628 & -2.156457 & 1.81577 \\
\hline & & -0.901757 & 2.97513( \\
\hline & 5.8453 & -0.525540 & 3.38716 \\
\hline & 1.903607 & 0.711363 & $4.57955^{\circ}$ \\
\hline & 4.371729 & 0.929294 & 4.76792 \\
\hline
\end{tabular}

$1_{\mathrm{C} 4-} \psi_{-1 \mathrm{~s} 2 \mathrm{~s} 3 \mathrm{a} 4 \mathrm{~s}}$

Number of imaginary frequencies : 0

The smallest frequencies are : $12.7848 \quad 16.2439 \quad 19.6743 \mathrm{~cm}(-1)$

Electronic energy : $\quad \mathrm{HF}=-4125.4688024$

Zero-point correction $=\quad 1.299570($ Hartree/Particle $)$

Thermal correction to Energy= $\quad 1.377783$

Thermal correction to Enthalpy= $\quad 1.378727$

Thermal correction to Gibbs Free Energy $=\quad 1.186993$

Sum of electronic and zero-point Energies $=\quad-4124.169233$

Sum of electronic and thermal Energies $=\quad-4124.091019$

Sum of electronic and thermal Enthalpies $=\quad-4124.090075$

Sum of electronic and thermal Free Energies $=\quad-4124.281809$

Cartesian Coordinates

$\begin{array}{cccc}6 & -3.822685 & -0.235751 & -1.742805 \\ 6 & -3.406061 & 0.182645 & -0.479053 \\ 6 & -4.353305 & 0.395641 & 0.519957 \\ 6 & -5.714297 & 0.185577 & 0.285346 \\ 6 & -6.096532 & -0.229778 & -0.990676 \\ 6 & -5.173069 & -0.452594 & -2.019256 \\ 15 & -1.607253 & 0.365659 & -0.114548 \\ 45 & -0.067904 & 0.11050 & -2.022762 \\ 6 & -0.089138 & 2.008012 & -2.351636 \\ 8 & -0.034061 & 3.127357 & -2.639809 \\ 6 & -6.698429 & 0.357388 & 1.440646 \\ 6 & -6.615022 & 1.785005 & 1.990716 \\ 6 & -5.660176 & -0.909490 & -3.393234 \\ 6 & -4.502195 & -1.312609 & -4.302955 \\ 6 & -1.522259 & -0.533942 & 1.514391 \\ 6 & -1.271806 & -1.894305 & 1.566225 \\ 6 & -1.286168 & -2.587239 & 2.822189 \\ 6 & -1.564641 & -1.867980 & 4.019578 \\ 6 & -1.832772 & -0.482680 & 3.932457 \\ 6 & -1.814248 & 0.159001 & 2.723809\end{array}$




\begin{tabular}{|c|c|c|c|}
\hline 6 & -1.024676 & -3.979139 & 2.923951 \\
\hline 6 & -1.031601 & -4.612380 & 4.142373 \\
\hline f & -1.304934 & -3.892437 & 5.324197 \\
\hline & -1.566584 & -2.547600 & 5.260620 \\
\hline$b$ & -1.025230 & -2.717087 & 0.354151 \\
\hline & 0.250378 & -2.856633 & -0.158132 \\
\hline 6 & 0.504556 & -3.778471 & -1.201241 \\
\hline 5 & -0.513833 & -4.504743 & -1.758497 \\
\hline 6 & -1.846714 & -4.329330 & -1.317037 \\
\hline 6 & -2.107572 & -3.438826 & -0.234177 \\
\hline 6 & -2.927483 & -5.007550 & -1.931470 \\
\hline 6 & -4.214999 & -4.812904 & -1.498854 \\
\hline 6 & -4.475623 & -3.933691 & -0.424497 \\
\hline 6 & -3.448470 & -3.270647 & 0.199025 \\
\hline 7 & 1.316677 & -2.016070 & 0.317115 \\
\hline 6 & 2.216501 & -2.616814 & 1.328277 \\
\hline 6 & 2.815335 & -1.629201 & 2.292173 \\
\hline 6 & 1.995565 & -0.803936 & 3.065171 \\
\hline 6 & 2.552068 & 0.117287 & 3.943172 \\
\hline 6 & 3.937065 & 0.230450 & 4.056740 \\
\hline 6 & 4.758573 & -0.597504 & 3.300052 \\
\hline 6 & 4.197630 & -1.526474 & 2.427455 \\
\hline 15 & 1.692014 & -0.814421 & -0.824755 \\
\hline 8 & 2.639566 & 0.152849 & 0.129936 \\
\hline 6 & 3.413753 & 1.066902 & -0.555773 \\
\hline 6 & 4.624858 & 0.664231 & -1.084692 \\
\hline 6 & 5.387524 & 1.621849 & -1.829953 \\
\hline 6 & 4.908964 & 2.963365 & -1.938705 \\
\hline 6 & 3.669767 & 3.314568 & -1.351531 \\
\hline 6 & 2.922107 & 2.378845 & -0.689671 \\
\hline 6 & 6.585361 & 1.287490 & -2.512262 \\
\hline 6 & 7.288360 & 2.231788 & -3.218542 \\
\hline 6 & 6.834239 & 3.565349 & -3.287066 \\
\hline 6 & 5.664718 & 3.916547 & -2.663887 \\
\hline 6 & 5.028620 & -0.753205 & -0.947995 \\
\hline 6 & 4.150796 & -1.743728 & -1.356165 \\
\hline 6 & 4.520101 & -3.106317 & -1.372810 \\
\hline 6 & 5.748164 & -3.490304 & -0.908124 \\
\hline 6 & 6.644732 & -2.533600 & -0.372214 \\
\hline 6 & 6.280459 & -1.153602 & -0.378528 \\
\hline 6 & 7.159661 & -0.230488 & 0.243051 \\
\hline 6 & 8.341469 & -0.645419 & 0.806414 \\
\hline 6 & 8.714611 & -2.005531 & 0.777017 \\
\hline 6 & 7.878038 & -2.927364 & 0.201415 \\
\hline 8 & 2.897153 & -1.430014 & -1.823836 \\
\hline 6 & -1.441587 & 2.105505 & 0.446796 \\
\hline 6 & -0.373529 & 2.456118 & 1.277211 \\
\hline 6 & -0.235460 & 3.760335 & 1.754887 \\
\hline 6 & -1.145653 & 4.723659 & 1.300783 \\
\hline 6 & -2.187089 & 4.414282 & 0.423308 \\
\hline 6 & -2.331602 & 3.083519 & 0.018870 \\
\hline 6 & 0.795574 & 4.055211 & 2.846389 \\
\hline 6 & 0.334624 & 3.326867 & 4.116935 \\
\hline 6 & -3.187975 & 5.450869 & -0.082706 \\
\hline 6 & -4.605353 & 5.024857 & 0.319200 \\
\hline 6 & 0.904340 & 5.545144 & 3.162945 \\
\hline
\end{tabular}




\begin{tabular}{|c|c|c|c|}
\hline & 2.188538 & 3.554030 & 2.457204 \\
\hline & -3.095706 & 5.535874 & -1.610392 \\
\hline & -2.928967 & 6.842116 & 0.489073 \\
\hline & -0.846414 & -1.266419 & -3.167746 \\
\hline & -1.107185 & -2.017206 & -4.007521 \\
\hline & -6.331577 & -0.642412 & 2.545100 \\
\hline & -8.142433 & 0.093939 & 1.022025 \\
\hline & -6.583020 & -2.124476 & -3.251126 \\
\hline & -6.428730 & 0.239574 & -4.054637 \\
\hline & 6.876476 & 0.818233 & 0.288932 \\
\hline & -2.024716 & 1.225066 & 2.695892 \\
\hline & 8.140278 & -3.984529 & 0.186778 \\
\hline & -1.779608 & -1.977615 & 6.163804 \\
\hline & 5.284867 & 4.935526 & -2.725764 \\
\hline & -3.660360 & -2.585571 & 1.019438 \\
\hline & -5.500549 & -3.773307 & -0.094325 \\
\hline & 3.305517 & 4.335414 & -1.458379 \\
\hline & -2.056197 & 0.070770 & 4.843996 \\
\hline & 6.043293 & -4.538215 & -0.923167 \\
\hline & 8.201155 & 1.945851 & -3.736525 \\
\hline & 3.809710 & -3.828819 & -1.769084 \\
\hline & -2.713392 & -5.681385 & -2.759824 \\
\hline & -0.317390 & -5.198712 & -2.574236 \\
\hline & -0.827065 & -5.679125 & 4.196750 \\
\hline & 6.944282 & 0.262220 & -2.481959 \\
\hline & -0.817098 & -4.549688 & 2.021089 \\
\hline & 1.524102 & -3.875395 & -1.569970 \\
\hline & 7.403234 & 4.304913 & -3.845696 \\
\hline & 8.993207 & 0.081980 & 1.285345 \\
\hline & -5.038793 & -5.334700 & -1.981554 \\
\hline & -1.307561 & -4.406971 & 6.282227 \\
\hline & 9.656028 & -2.319878 & 1.221475 \\
\hline & 1.951164 & 2.614821 & -0.259241 \\
\hline & -4.027202 & 0.711645 & 1.509613 \\
\hline 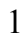 & -3.066966 & -0.399952 & -2.505902 \\
\hline & -7.151565 & -0.397199 & -1.196482 \\
\hline 1 & -3.151404 & 2.803287 & -0.642153 \\
\hline & 0.323658 & 1.680941 & 1.604354 \\
\hline & -1.044879 & 5.745245 & 1.654497 \\
\hline 1 & 1.590566 & -3.330232 & 1.883817 \\
\hline & 0.963180 & 0.154066 & -3.227568 \\
\hline 1 & 2.884835 & 3.700648 & 3.294589 \\
\hline & 2.208431 & 2.485198 & 2.208694 \\
\hline 1 & 2.582200 & 4.109106 & 1.595850 \\
\hline & 1.033478 & 3.514673 & 4.944247 \\
\hline 1 & -0.661078 & 3.671844 & 4.427975 \\
\hline | & 0.278229 & 2.241300 & 3.960835 \\
\hline 1 & 1.189035 & 6.130644 & 2.279125 \\
\hline 1 & -0.032466 & 5.955280 & 3.560939 \\
\hline ] & 1.676791 & 5.702223 & 3.926340 \\
\hline 1 & -3.673266 & 7.545724 & 0.095436 \\
\hline 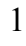 & -3.008555 & 6.858853 & 1.583859 \\
\hline 1 & -1.938378 & 7.222986 & 0.209317 \\
\hline 1 & -4.876690 & 4.046631 & -0.097640 \\
\hline 1 & -4.702638 & 4.962105 & 1.411235 \\
\hline 1 & -5.339951 & 5.755734 & -0.044745 \\
\hline
\end{tabular}




\begin{tabular}{|c|c|c|c|}
\hline & -2.090136 & 5.840715 & -1.927263 \\
\hline & -3.319321 & 4.575764 & -2.090994 \\
\hline & -3.811409 & 6.275172 & -1.994795 \\
\hline & -8.474312 & 0.783919 & 0.235478 \\
\hline & -8.285316 & -0.933238 & 0.662207 \\
\hline & -8.807101 & 0.234014 & 1.88377 \\
\hline & -6.907282 & 2.517127 & 1.22661 \\
\hline & -7.293756 & 1.903022 & 2.846066 \\
\hline & -5.604764 & 2.042529 & \\
\hline & -6.389503 & -1.673982 & 2.171587 \\
\hline & -5.314387 & -0.484595 & 2.926895 \\
\hline & -7.024416 & -0.550245 & 3.392450 \\
\hline & -5.785147 & 1.119724 & -4.182228 \\
\hline & -6.790344 & -0.061984 & -5.04710 \\
\hline & -7.298793 & 0.544173 & -3.45924 \\
\hline & -3.907996 & -2.124473 & -3.86138 \\
\hline & -4.894525 & -1.669500 & -5.263588 \\
\hline & -3.8 & -0.47 & $-4.51 c$ \\
\hline & -6.055912 & -2.957761 & -2.76725 \\
\hline & -7.483765 & -1.906249 & -2.66521 \\
\hline & -6.913476 & -2.462816 & -4.24212 \\
\hline & 3.015728 & -3.202796 & 0.84762 \\
\hline & 4.846047 & -2.163311 & 1.824090 \\
\hline & 0.913646 & -0.872339 & 2.954821 \\
\hline & 5.841107 & -0.519993 & 3.380560 \\
\hline & 1.902435 & 0.754345 & 4.542704 \\
\hline & 4.371179 & 0.961345 & 4.73611 \\
\hline
\end{tabular}

$1_{\mathrm{C} 4-} \psi_{-1 \mathrm{~s} 23354 \mathrm{a}}$

Number of imaginary frequencies : 0

The smallest frequencies are : $10.1882 \quad 14.1857 \quad 19.3295 \mathrm{~cm}(-1)$

Electronic energy : $\quad H F=-4125.4691582$

Zero-point correction $=\quad 1.299778($ Hartree/Particle $)$

Thermal correction to Energy= $\quad 1.377932$

Thermal correction to Enthalpy $=\quad 1.378876$

Thermal correction to Gibbs Free Energy= 1.187811

Sum of electronic and zero-point Energies $=\quad-4124.169380$

Sum of electronic and thermal Energies $=\quad-4124.091226$

Sum of electronic and thermal Enthalpies $=\quad-4124.090282$

Sum of electronic and thermal Free Energies $=\quad-4124.281347$

Cartesian Coordinates

$\begin{array}{cccc}6 & -3.832280 & -0.126169 & -1.719144 \\ 6 & -3.412192 & 0.258577 & -0.455676 \\ 6 & -4.366223 & 0.511395 & 0.540200 \\ 6 & -5.723293 & 0.358133 & 0.284288 \\ 6 & -6.110644 & -0.048334 & -1.004419 \\ 6 & -5.192349 & -0.285317 & -2.021195 \\ 15 & -1.608352 & 0.358014 & -0.090719 \\ 45 & -0.123637 & 0.068674 & -2.031063 \\ 6 & -0.152199 & 1.958334 & -2.401718 \\ 8 & -0.116055 & 3.069622 & -2.722049 \\ 6 & -6.784106 & 0.570940 & 1.362484 \\ 6 & -7.833484 & 1.578274 & 0.881489\end{array}$




\begin{tabular}{|c|c|c|c|}
\hline 6 & -5.587704 & -0.757489 & -3.417972 \\
\hline 6 & -7.097958 & -0.719191 & -3.636963 \\
\hline p & -1.541457 & -0.586503 & 1.510941 \\
\hline & -1.279447 & -1.945282 & 1.538491 \\
\hline y & -1.290908 & -2.658654 & 2.784830 \\
\hline & -1.584318 & -1.962828 & 3.992611 \\
\hline 6 & -1.870119 & -0.579844 & 3.927562 \\
\hline 6 & -1.849101 & 0.081879 & 2.730780 \\
\hline 6 & -1.010738 & -4.048341 & 2.867325 \\
\hline 6 & -1.016230 & -4.700895 & 4.075606 \\
\hline 6 & -1.306517 & -4.004158 & 5.266901 \\
\hline 6 & -1.584996 & -2.661987 & 5.222699 \\
\hline 6 & -1.027634 & -2.758589 & 0.319832 \\
\hline 6 & 0.247817 & -2.894975 & -0.193425 \\
\hline 6 & 0.509068 & -3.829463 & -1.223729 \\
\hline 6 & -0.498474 & -4.592533 & -1.750605 \\
\hline 6 & -1.830551 & -4.433767 & -1.299945 \\
\hline 6 & -2.102728 & -3.505355 & -0.252130 \\
\hline 6 & -2.898934 & -5.171534 & -1.865780 \\
\hline 6 & -4.185714 & -4.994889 & -1.424518 \\
\hline 6 & -4.460461 & -4.063711 & -0.399030 \\
\hline 6 & -3.445560 & -3.341407 & 0.176811 \\
\hline 7 & 1.312960 & -2.050343 & 0.276909 \\
\hline 6 & 2.243357 & -2.667357 & 1.249865 \\
\hline 6 & 2.836510 & -1.708931 & 2.246157 \\
\hline 6 & 2.012481 & -0.941442 & 3.072059 \\
\hline 6 & 2.564541 & -0.070103 & 4.002550 \\
\hline 6 & 3.948912 & 0.054949 & 4.112417 \\
\hline 6 & 4.774799 & -0.710129 & 3.296651 \\
\hline 6 & 4.218484 & -1.592662 & 2.374398 \\
\hline 15 & 1.664708 & -0.830013 & -0.852122 \\
\hline 8 & 2.600674 & 0.139021 & 0.113016 \\
\hline 6 & 3.346440 & 1.081507 & -0.566725 \\
\hline 6 & 4.558757 & 0.713329 & -1.117488 \\
\hline 6 & 5.289814 & 1.697558 & -1.859670 \\
\hline 6 & 4.780445 & 3.029541 & -1.942672 \\
\hline 6 & 3.543841 & 3.345846 & -1.330794 \\
\hline 6 & 2.825866 & 2.385336 & -0.671429 \\
\hline 6 & 6.484561 & 1.398620 & -2.563325 \\
\hline 6 & 7.155942 & 2.367460 & -3.267221 \\
\hline 6 & 6.671450 & 3.691401 & -3.311180 \\
\hline 6 & 5.504173 & 4.008626 & -2.665929 \\
\hline 6 & 4.998406 & -0.695479 & -1.001426 \\
\hline 6 & 4.140177 & -1.703100 & -1.409140 \\
\hline 6 & 4.540162 & -3.056810 & -1.439974 \\
\hline 6 & 5.780823 & -3.415701 & -0.989117 \\
\hline 6 & 6.659711 & -2.442533 & -0.453304 \\
\hline 6 & 6.264294 & -1.071074 & -0.446112 \\
\hline 6 & 7.127396 & -0.132855 & 0.175260 \\
\hline 6 & 8.322187 & -0.525321 & 0.727319 \\
\hline 6 & 8.725086 & -1.876567 & 0.685941 \\
\hline 6 & 7.905518 & -2.812660 & 0.108791 \\
\hline 8 & 2.875497 & -1.414342 & -1.862414 \\
\hline 6 & -1.372471 & 2.075441 & 0.515242 \\
\hline 6 & -0.312858 & 2.364922 & 1.378837 \\
\hline 6 & -0.133063 & 3.653324 & 1.886418 \\
\hline
\end{tabular}




\begin{tabular}{|c|c|c|c|}
\hline 6 & -0.993537 & 4.661593 & 1.434386 \\
\hline 6 & -2.028379 & 4.412113 & 0.530199 \\
\hline 6 & -2.213537 & 3.098172 & 0.090567 \\
\hline 6 & 0.896694 & 3.885676 & 2.993907 \\
\hline 6 & 0.421837 & 3.116116 & 4.234503 \\
\hline 6 & -2.987689 & 5.495326 & 0.041600 \\
\hline 6 & -4.418024 & 5.121592 & 0.450824 \\
\hline 6 & 1.028951 & 5.359455 & 3.370421 \\
\hline 6 & 2.281576 & 3.376869 & 2.584985 \\
\hline 6 & -2.902629 & 5.597740 & -1.485238 \\
\hline 6 & -2.668555 & 6.867672 & 0.628918 \\
\hline 6 & -0.982021 & -1.316029 & -3.100608 \\
\hline 8 & -1.349675 & -2.076218 & -3.891163 \\
\hline 6 & -7.462350 & -0.772566 & 1.655382 \\
\hline 6 & -6.185516 & 1.098809 & 2.665067 \\
\hline 6 & -5.108777 & -2.203045 & -3.596462 \\
\hline 6 & -4.929499 & 0.132046 & -4.478887 \\
\hline 1 & 6.822564 & 0.909384 & 0.228397 \\
\hline 1 & -2.065827 & 1.147038 & 2.720938 \\
\hline 1 & 8.191442 & -3.863482 & 0.084326 \\
\hline 1 & -1.810347 & -2.108575 & 6.133189 \\
\hline 1 & 5.101527 & 5.019800 & -2.708383 \\
\hline 1 & -3.668241 & -2.619100 & 0.961006 \\
\hline 1 & -5.486100 & -3.907348 & -0.069860 \\
\hline 1 & 3.157416 & 4.360478 & -1.415967 \\
\hline 1 & -2.105954 & -0.044497 & 4.846736 \\
\hline 1 & 6.099804 & -4.456420 & -1.013759 \\
\hline 1 & 8.067032 & 2.108779 & -3.802246 \\
\hline 1 & 3.841442 & -3.792619 & -1.832873 \\
\hline 1 & -2.675569 & -5.877466 & -2.664411 \\
\hline 1 & -0.294468 & -5.307981 & -2.545653 \\
\hline 1 & -0.797105 & -5.765426 & 4.113973 \\
\hline 1 & 6.866185 & 0.381096 & -2.55 \\
\hline 1 & -0.788809 & -4.602868 & 1.957933 \\
\hline 1 & 1.527764 & -3.915759 & -1.598161 \\
\hline 1 & 7.215538 & 4.450667 & -3.868207 \\
\hline 1 & 8.961281 & 0.213137 & 1.206354 \\
\hline 1 & -4.999551 & -5.562030 & -1.871178 \\
\hline 1 & -1.308605 & -4.534020 & 6.216549 \\
\hline 1 & 9.676339 & -2.172986 & 1.121692 \\
\hline 1 & 1.858546 & 2.596912 & -0.219291 \\
\hline 1 & -4.033978 & 0.822862 & 1.526346 \\
\hline 1 & -3.081630 & -0.311287 & -2.483143 \\
\hline 1 & -7.171671 & -0.179812 & -1.207793 \\
\hline 1 & -3.029183 & 2.865512 & -0.593862 \\
\hline 1 & 0.346461 & 1.556982 & 1.705232 \\
\hline 1 & -0.859857 & 5.670876 & 1.811805 \\
\hline 1 & 1.643768 & -3.416100 & 1.787332 \\
\hline 1 & 0.867934 & 0.075182 & -3.270741 \\
\hline 1 & 2.985836 & 3.501508 & 3.419258 \\
\hline 1 & 2.285496 & 2.311980 & 2.319012 \\
\hline 1 & 2.674459 & 3.941000 & 1.729400 \\
\hline 1 & 1.136454 & 3.239349 & 5.060509 \\
\hline 1 & -0.556377 & 3.483824 & 4.573780 \\
\hline 1 & 0.322535 & 2.041881 & 4.030604 \\
\hline 1 & 1.349704 & 5.971882 & 2.517673 \\
\hline
\end{tabular}




$\begin{array}{lrrr}1 & 0.090701 & 5.774825 & 3.759515 \\ 1 & 1.784095 & 5.469899 & 4.158917 \\ 1 & -3.380667 & 7.607358 & 0.241966 \\ 1 & -2.749363 & 6.876889 & 1.723696 \\ 1 & -1.661538 & 7.207210 & 0.354455 \\ 1 & -4.733801 & 4.162369 & 0.021238 \\ 1 & -4.506790 & 5.045057 & 1.542825 \\ 1 & -5.125759 & 5.887643 & 0.106452 \\ 1 & -1.887401 & 5.866040 & -1.804516 \\ 1 & -3.167566 & 4.655146 & -1.978970 \\ 1 & -3.590090 & 6.370966 & -1.853975 \\ 1 & -5.465912 & 0.395341 & 3.105246 \\ 1 & -5.679719 & 2.063125 & 2.520093 \\ 1 & -6.984351 & 1.251539 & 3.401570 \\ 1 & -8.354684 & 1.240154 & -0.021749 \\ 1 & -8.593293 & 1.733176 & 1.659061 \\ 1 & -7.375529 & 2.550924 & 0.659679 \\ 1 & -7.959025 & -1.182561 & 0.767353 \\ 1 & -6.728687 & -1.513357 & 2.000801 \\ 1 & -8.222740 & -0.656403 & 2.439525 \\ 1 & -3.833788 & 0.091485 & -4.440943 \\ 1 & -5.231515 & -0.194587 & -5.482679 \\ 1 & -5.231551 & 1.180688 & -4.359396 \\ 1 & -7.504118 & 0.293137 & -3.510980 \\ 1 & -7.330309 & -1.044695 & -4.658696 \\ 1 & -7.631982 & -1.391911 & -2.953595 \\ 1 & -4.024656 & -2.299412 & -3.451765 \\ 1 & -5.598613 & -2.867219 & -2.871827 \\ 1 & -5.347275 & -2.563349 & -4.606543 \\ 1 & 3.047293 & -3.216615 & 0.735265 \\ 1 & 4.869748 & -2.185726 & 1.731107 \\ 1 & 0.930558 & -1.023227 & 2.968837 \\ 1 & 5.857002 & -0.621076 & 3.370026 \\ 1 & 1.911242 & 0.516804 & 4.647161 \\ 1 & 4.378800 & 0.747429 & 4.833467\end{array}$

$1_{\mathrm{C} 4-} \psi_{-1 \mathrm{~s} 22354 \mathrm{~s}}$

Number of imaginary frequencies : 0

The smallest frequencies are : $11.8888 \quad 19.6401 \quad 23.0438 \mathrm{~cm}(-1)$

Electronic energy : $\quad H F=-4125.4691743$

Zero-point correction $=\quad 1.300164($ Hartree/Particle $)$

Thermal correction to Energy= $\quad 1.378186$

Thermal correction to Enthalpy= $\quad 1.379130$

Thermal correction to Gibbs Free Energy= 1.188799

Sum of electronic and zero-point Energies $=\quad-4124.169010$

Sum of electronic and thermal Energies= $\quad-4124.090989$

Sum of electronic and thermal Enthalpies $=\quad-4124.090044$

Sum of electronic and thermal Free Energies $=\quad-4124.280375$

Cartesian Coordinates

$\begin{array}{llll}6 & 5.583670 & 4.054659 & -2.537469 \\ 6 & 4.844356 & 3.064014 & -1.846400 \\ 6 & 5.341611 & 1.725920 & -1.791607 \\ 6 & 6.540807 & 1.434469 & -2.490889\end{array}$




\begin{tabular}{|c|c|c|c|}
\hline 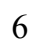 & 7.227232 & 2.414748 & -3.163757 \\
\hline & 6.754569 & 3.743604 & -3.179084 \\
\hline & 4.595186 & 0.730094 & -1.080706 \\
\hline & 3.380563 & 1.095164 & -0.533031 \\
\hline & 2.871463 & 2.404894 & -0.612413 \\
\hline & 3.603838 & 3.375563 & -1.240109 \\
\hline & 5.020816 & -0.685024 & -0.995466 \\
\hline & 4.157775 & -1.675323 & -1.434351 \\
\hline & 4.549149 & -3.030558 & -1.498243 \\
\hline & 5.784475 & -3.410205 & -1.049917 \\
\hline & 6.666717 & -2.457911 & -0.483095 \\
\hline & 6.280161 & -1.084632 & -0.441756 \\
\hline & 7.906736 & -2.851240 & 0.076065 \\
\hline & 8.728842 & -1.936370 & 0.682891 \\
\hline 0 & 8.334018 & -0.584267 & 0.758816 \\
\hline U & 7.145264 & -0.169284 & 0.210265 \\
\hline & 2.896975 & -1.367959 & -1.886774 \\
\hline & 1.684542 & -0.800041 & -0.868644 \\
\hline 3 & 2.621570 & 0.144616 & 0.118578 \\
\hline 7 & 1.327524 & -2.039066 & 0.239312 \\
\hline & 0.268124 & -2.878840 & -0.252077 \\
\hline 6 & -1.010440 & -2.758547 & 0.258869 \\
\hline 6 & -2.083567 & -3.484613 & -0.342697 \\
\hline 6 & -1.806160 & -4.374412 & -1.422335 \\
\hline 6 & -0.470823 & -4.526191 & -1.865062 \\
\hline 6 & 0.535231 & -3.784526 & -1.306062 \\
\hline 6 & -2.873106 & -5.077837 & -2.032160 \\
\hline 6 & -4.164057 & -4.905421 & -1.602038 \\
\hline 6 & -4.442851 & -4.019217 & -0.538781 \\
\hline 6 & -3.429422 & -3.332221 & 0.081914 \\
\hline 6 & -1.262455 & -1.977066 & 1.498050 \\
\hline 6 & -1.513253 & -0.615720 & 1.502271 \\
\hline 6 & -1.800434 & 0.029946 & 2.738804 \\
\hline 6 & -1.818741 & -0.658747 & 3.921029 \\
\hline 6 & -1.549728 & -2.046157 & 3.952630 \\
\hline 6 & -1.272107 & -2.718003 & 2.727455 \\
\hline 6 & -1.003817 & -4.111652 & 2.776846 \\
\hline 6 & -1.005268 & -4.790299 & 3.970546 \\
\hline 6 & -1.279522 & -4.117374 & 5.179376 \\
\hline 6 & -1.546751 & -2.772428 & 5.167154 \\
\hline 15 & -1.601701 & 0.350765 & -0.084056 \\
\hline 6 & -1.430838 & 2.067152 & 0.543423 \\
\hline 6 & -0.359982 & 2.391265 & 1.380678 \\
\hline 6 & -0.224907 & 3.678607 & 1.903833 \\
\hline 6 & -1.141343 & 4.653812 & 1.488952 \\
\hline 6 & -2.184761 & 4.372082 & 0.604616 \\
\hline 6 & -2.326542 & 3.056089 & 0.154966 \\
\hline 6 & 0.811402 & 3.939066 & 2.999267 \\
\hline 6 & 0.932189 & 5.419945 & 3.352278 \\
\hline 6 & -3.189348 & 5.421609 & 0.134347 \\
\hline 6 & -3.111633 & 5.544273 & -1.391881 \\
\hline 6 & 2.255399 & -2.664074 & 1.209957 \\
\hline 6 & 2.834785 & -1.711156 & 2.220288 \\
\hline 6 & 2.000268 & -0.933876 & 3.026134 \\
\hline 6 & 2.539613 & -0.047887 & 3.950098 \\
\hline 6 & 3.922355 & 0.078542 & 4.077060 \\
\hline
\end{tabular}




\begin{tabular}{|c|c|c|c|}
\hline 6 & 4.758759 & -0.700544 & 3.285664 \\
\hline 6 & 4.214921 & -1.594993 & 2.367285 \\
\hline & -0.104200 & 0.137633 & -2.020788 \\
\hline 6 & -0.970528 & -1.215496 & -3.121352 \\
\hline & -1.347358 & -1.962437 & -3.919707 \\
\hline 0 & -0.123107 & 2.041982 & -2.310870 \\
\hline & -0.089308 & 3.167991 & -2.575042 \\
\hline & -3.401228 & 0.199296 & -0.452613 \\
\hline & -4.348437 & 0.285389 & 0.568426 \\
\hline 6 & -5.709106 & 0.129405 & 0.307308 \\
\hline & -6.099983 & -0.104742 & -1.015770 \\
\hline & -5.180862 & -0.171950 & -2.065620 \\
\hline 0 & -3.825723 & -0.017126 & -1.759279 \\
\hline & -6.694885 & 0.200858 & 1.472865 \\
\hline 6 & -6.350256 & -0.895350 & 2.488911 \\
\hline 6 & -5.593955 & -0.493087 & -3.500464 \\
\hline 6 & -5.250923 & -1.963493 & -3.770442 \\
\hline 6 & -8.140937 & -0.000770 & 1.027833 \\
\hline 0 & -6.590889 & 1.573248 & 2.147273 \\
\hline 6 & -4.839367 & 0.392762 & -4.497486 \\
\hline 6 & -7.090400 & -0.290435 & -3.732817 \\
\hline 6 & -2.922386 & 6.798145 & 0.737201 \\
\hline 6 & -4.602818 & 4.986570 & 0.540221 \\
\hline 6 & 2.200548 & 3.437842 & 2.596026 \\
\hline 6 & 0.346785 & 3.181962 & 4.251498 \\
\hline & 6.845867 & 0.872745 & 0.291451 \\
\hline 1 & -2.010673 & 1.096371 & 2.751888 \\
\hline 1 & 8.185984 & -3.902915 & 0.025002 \\
\hline 1 & -1.759492 & -2.237105 & 6.091381 \\
\hline 1 & 5.190048 & 5.070079 & -2.558131 \\
\hline 1 & -3.656507 & -2.644312 & 0.896087 \\
\hline 1 & -5.471515 & -3.874922 & -0.214336 \\
\hline 1 & 3.226033 & 4.394926 & -1.305195 \\
\hline 1 & -2.040627 & -0.141723 & 4.854016 \\
\hline 1 & 6.096297 & -4.452146 & -1.100687 \\
\hline 1 & 8.141390 & 2.161611 & -3.696193 \\
\hline 1 & 3.847713 & -3.751000 & -1.913899 \\
\hline 1 & -2.645345 & -5.751647 & -2.856877 \\
\hline 1 & -0.263308 & -5.215538 & -2.681981 \\
\hline 1 & -0.795292 & -5.857277 & 3.983907 \\
\hline 1 & 6.913922 & 0.413784 & -2.500896 \\
\hline 1 & -0.794255 & -4.647728 & 1.853621 \\
\hline 1 & 1.556248 & -3.862413 & -1.675578 \\
\hline 1 & 7.310726 & 4.511880 & -3.711272 \\
\hline 1 & 8.974479 & 0.136732 & 1.262018 \\
\hline 1 & -4.977689 & -5.443393 & -2.083670 \\
\hline 1 & -1.277693 & -4.667870 & 6.117208 \\
\hline 1 & 9.675516 & -2.250644 & 1.116086 \\
\hline 1 & 1.900264 & 2.612103 & -0.167685 \\
\hline 1 & -4.019275 & 0.459975 & 1.591263 \\
\hline 1 & -3.077395 & -0.070006 & -2.546236 \\
\hline 1 & -7.156160 & -0.233710 & -1.234355 \\
\hline 1 & -3.148139 & 2.796384 & -0.512148 \\
\hline 1 & 0.339354 & 1.606443 & 1.679009 \\
\hline 1 & -1.043130 & 5.662730 & 1.878014 \\
\hline 1 & 1.658500 & -3.426028 & 1.731814 \\
\hline
\end{tabular}




$\begin{array}{lrrr}1 & 0.879212 & 0.179246 & -3.267017 \\ 1 & 2.898489 & 3.560893 & 3.435837 \\ 1 & 2.215134 & 2.375150 & 2.323853 \\ 1 & 2.596724 & 4.010112 & 1.746845 \\ 1 & 1.048796 & 3.341212 & 5.082146 \\ 1 & -0.645488 & 3.527305 & 4.572824 \\ 1 & 0.280521 & 2.101565 & 4.065994 \\ 1 & 1.208838 & 6.026532 & 2.480328 \\ 1 & 0.002828 & 5.824368 & 3.772962 \\ 1 & 1.715170 & 5.553374 & 4.109406 \\ 1 & -3.668003 & 7.512792 & 0.366641 \\ 1 & -2.993300 & 6.789044 & 1.832658 \\ 1 & -1.932833 & 7.182787 & 0.458828 \\ 1 & -4.876983 & 4.015038 & 0.109336 \\ 1 & -4.690215 & 4.904320 & 1.631820 \\ 1 & -5.341280 & 5.723270 & 0.196263 \\ 1 & -2.106491 & 5.847920 & -1.711317 \\ 1 & -3.348006 & 4.598812 & -1.895109 \\ 1 & -3.824544 & 6.299163 & -1.750172 \\ 1 & -8.463569 & 0.772031 & 0.318339 \\ 1 & -8.294399 & -0.982322 & 0.560956 \\ 1 & -8.805760 & 0.055039 & 1.898993 \\ 1 & -6.844891 & 2.375781 & 1.442514 \\ 1 & -7.286917 & 1.635274 & 2.994676 \\ 1 & -5.582576 & 1.772491 & 2.530839 \\ 1 & -6.428033 & -1.890876 & 2.031936 \\ 1 & -5.332499 & -0.790712 & 2.887169 \\ 1 & -7.044438 & -0.860465 & 3.339440 \\ 1 & -3.755151 & 0.230246 & -4.475294 \\ 1 & -5.174372 & 0.172196 & -5.519275 \\ 1 & -5.025073 & 1.457357 & -4.303816 \\ 1 & -7.404001 & 0.735280 & -3.498021 \\ 1 & -7.327542 & -0.478328 & -4.787452 \\ 1 & -7.702711 & -0.981376 & -3.139773 \\ 1 & -4.177640 & -2.157089 & -3.642761 \\ 1 & -5.791265 & -2.622823 & -3.078087 \\ 1 & -5.528570 & -2.242473 & -4.796285 \\ 1 & 3.067595 & -3.198150 & 0.692340 \\ 1 & 4.875236 & -2.193232 & 1.737862 \\ 1 & 0.920311 & -1.011631 & 2.905867 \\ 1 & 5.839805 & -0.610903 & 3.373507 \\ 1 & 1.878049 & 0.551822 & 4.574695 \\ & 4.342797 & 0.782548 & 4.792498\end{array}$

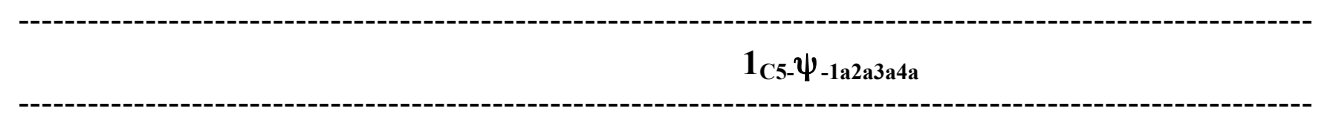

Number of imaginary frequencies : 0

The smallest frequencies are : $14.0292 \quad 16.1671 \quad 19.8633 \mathrm{~cm}(-1)$

Electronic energy : $\quad H F=-4125.4699709$

Zero-point correction $=\quad 1.299914$ (Hartree/Particle)

Thermal correction to Energy $=\quad 1.378027$

Thermal correction to Enthalpy= $\quad 1.378971$

Thermal correction to Gibbs Free Energy= 1.188593

Sum of electronic and zero-point Energies $=\quad-4124.170057$ 
Sum of electronic and thermal Energies= Sum of electronic and thermal Enthalpies= Sum of electronic and thermal Free Energies=
$-4124.091944$

$-4124.091000$

$-4124.281378$

Cartesian Coordinates

$\begin{array}{cccc}6 & 5.826304 & 4.399359 & -1.707262 \\ 6 & 5.067772 & 3.322484 & -1.186900 \\ 6 & 5.544757 & 1.985347 & -1.344567 \\ 6 & 6.746212 & 1.790264 & -2.072932 \\ 6 & 7.452359 & 2.854753 & -2.575563 \\ 6 & 6.998264 & 4.176166 & -2.382390 \\ 6 & 4.777485 & 0.900016 & -0.809676 \\ 6 & 3.564091 & 1.191291 & -0.216975 \\ 6 & 3.076455 & 2.505043 & -0.083023 \\ 6 & 3.827735 & 3.552223 & -0.544872 \\ 6 & 5.180169 & -0.518251 & -0.952303 \\ 6 & 4.305997 & -1.401898 & -1.564666 \\ 6 & 4.662716 & -2.739573 & -1.835642 \\ 6 & 5.875821 & -3.219696 & -1.424877 \\ 6 & 6.769480 & -2.393519 & -0.701954 \\ 6 & 6.419825 & -1.032138 & -0.452009 \\ 6 & 7.997322 & -2.900280 & -0.211290 \\ 6 & 8.844592 & -2.109718 & 0.521580 \\ 6 & 8.487418 & -0.773744 & 0.800181 \\ 6 & 7.310340 & -0.249335 & 0.326798 \\ 8 & 3.062191 & -0.989479 & -1.977253 \\ 15 & 1.837475 & -0.588587 & -0.896420 \\ 8 & 2.780498 & 0.156242 & 0.260246 \\ 7 & 1.470533 & -1.979468 & 0.001031 \\ 6 & 0.409551 & -2.746131 & -0.595276 \\ 6 & -0.892146 & -2.664683 & -0.134873 \\ 6 & -1.941625 & -3.290603 & -0.880512 \\ 6 & -1.625745 & -4.032838 & -2.055930 \\ 6 & -0.271617 & -4.157940 & -2.446856 \\ 6 & 0.712413 & -3.518894 & -1.743733 \\ 6 & -2.670718 & -4.620797 & -2.808807 \\ 6 & -3.979981 & -4.480075 & -2.422426 \\ 6 & -4.297269 & -3.745557 & -1.259105 \\ 6 & -3.304360 & -3.174081 & -0.502682 \\ 6 & -1.216688 & -2.046307 & 1.178362 \\ 6 & -1.444283 & -0.690731 & 1.349811 \\ 6 & -1.792475 & -0.205029 & 2.641743 \\ 6 & -1.942482 & -1.050234 & 3.706812 \\ 6 & -1.753069 & -2.442351 & 3.557004 \\ 6 & -1.369597 & -2.949307 & 2.283019 \\ 6 & -1.174170 & -4.349435 & 2.155067 \\ 6 & -1.375450 & -5.193606 & 3.218904 \\ 6 & -1.765305 & -4.684872 & 4.475135 \\ 6 & -1.938630 & -3.334337 & 4.639977 \\ 15 & -1.502012 & 0.459340 & -0.110118 \\ 6 & -1.393323 & 2.098728 & 0.705273 \\ 6 & -2.300659 & 3.111391 & 0.409230 \\ 6 & -2.153573 & 4.386046 & 0.963185 \\ 6 & -1.088649 & 4.597151 & 1.840932 \\ 6 & -0.172763 & 3.593689 & 2.178265\end{array}$




\begin{tabular}{|c|c|c|c|}
\hline 6 & -0.320415 & 2.350737 & 1.563915 \\
\hline 6 & -3.119855 & 5.526251 & 0.648040 \\
\hline 6 & -2.348062 & 6.662669 & -0.030707 \\
\hline 6 & 0.903482 & 3.864039 & 3.229510 \\
\hline 6 & 1.848508 & 4.967947 & 2.746954 \\
\hline 6 & 2.428467 & -2.678590 & 0.879955 \\
\hline 6 & 2.074376 & -2.633673 & 2.343641 \\
\hline 6 & 1.863667 & -1.412157 & 2.989834 \\
\hline 6 & 1.599060 & -1.374106 & 4.352891 \\
\hline 6 & 1.542356 & -2.555116 & 5.091099 \\
\hline 6 & 1.748913 & -3.773796 & 4.455627 \\
\hline 6 & 2.011678 & -3.809720 & 3.088284 \\
\hline 45 & 0.108720 & 0.529155 & -1.964415 \\
\hline 6 & -0.640199 & -0.662229 & -3.307859 \\
\hline 8 & -0.900123 & -1.310867 & -4.229047 \\
\hline 6 & 0.138143 & 2.458789 & -1.940054 \\
\hline 8 & 0.227536 & 3.610140 & -2.005995 \\
\hline 6 & -3.285187 & 0.312121 & -0.554488 \\
\hline 6 & -3.659608 & 0.094384 & -1.876150 \\
\hline 6 & -5.003504 & -0.077374 & -2.226104 \\
\hline 6 & -5.952157 & -0.003607 & -1.206614 \\
\hline 6 & -5.613305 & 0.224775 & 0.132663 \\
\hline 6 & -4.264593 & 0.378500 & 0.441853 \\
\hline 6 & -5.445738 & -0.317173 & -3.668642 \\
\hline 6 & -6.381530 & -1.527908 & -3.742815 \\
\hline 6 & -6.704924 & & 1.200131 \\
\hline 6 & -7.362912 & -1.126823 & 1.274006 \\
\hline 6 & -6.181698 & 0.928444 & -4.173855 \\
\hline 6 & -4.259379 & -0.592445 & -4.589572 \\
\hline 6 & -7.759457 & 1.307468 & 0.840637 \\
\hline 6 & -6.150679 & 0.596013 & 2.582124 \\
\hline 6 & 0.225278 & 4.310510 & 4.530887 \\
\hline 6 & 1.727978 & 2.616144 & 3.543633 \\
\hline 6 & -3.754474 & 6.038764 & 1.945531 \\
\hline 6 & -4.245864 & 5.087374 & -0.285827 \\
\hline 1 & 7.044275 & 0.778537 & 0.560392 \\
\hline 1 & -1.957913 & 0.859278 & 2.788493 \\
\hline 1 & 8.248030 & -3.939046 & -0.422916 \\
\hline 1 & -2.227521 & -2.922147 & 5.606064 \\
\hline 1 & 5.447046 & 5.410951 & -1.568827 \\
\hline 1 & -3.563010 & -2.598324 & 0.384742 \\
\hline 1 & -5.337666 & -3.620444 & -0.963458 \\
\hline 1 & 3.463821 & 4.574829 & -0.453162 \\
\hline 1 & -2.222768 & -0.658852 & 4.684145 \\
\hline 1 & 6.162810 & -4.249337 & -1.632196 \\
\hline 1 & 8.367919 & 2.675827 & -3.134893 \\
\hline 1 & 3.955782 & -3.362483 & -2.379142 \\
\hline 1 & -2.411182 & -5.181264 & -3.705799 \\
\hline 1 & -0.031273 & -4.737321 & -3.336840 \\
\hline 1 & -1.226639 & -6.263808 & 3.093140 \\
\hline 1 & 7.104701 & 0.778587 & -2.243115 \\
\hline 1 & -0.858183 & -4.757987 & 1.197459 \\
\hline 1 & 1.750654 & -3.572142 & -2.066291 \\
\hline 1 & 7.569682 & 5.010555 & -2.782312 \\
\hline 1 & 9.148438 & -0.153611 & 1.401522 \\
\hline 1 & -4.776116 & -4.931076 & -3.011224 \\
\hline
\end{tabular}




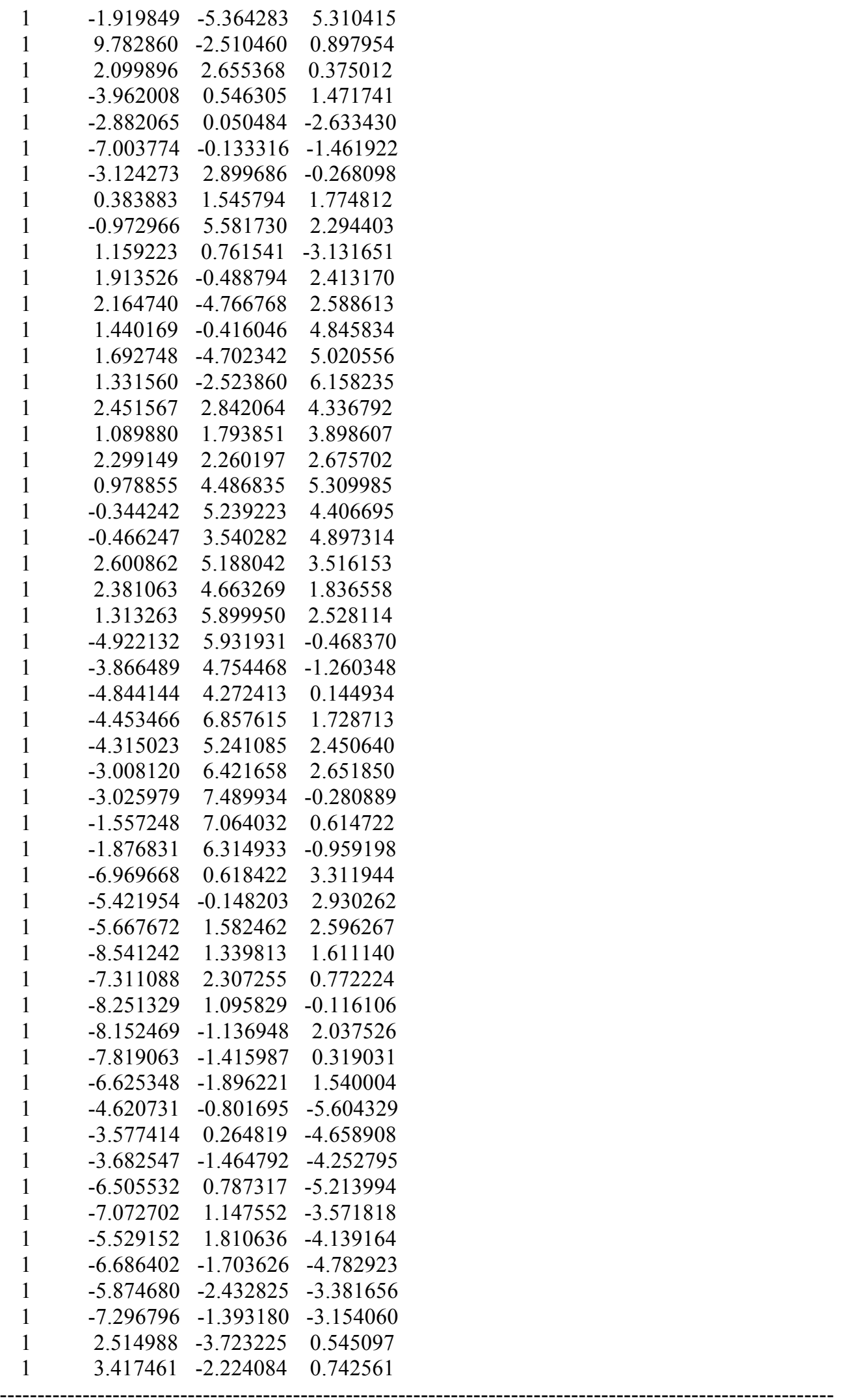

$1_{\text {C5- } \psi-1 a 2 a 3 a 4 s}$ 
Number of imaginary frequencies : 0

The smallest frequencies are : $14.2592 \quad 15.9947 \quad 21.1503 \mathrm{~cm}(-1)$

Electronic energy : $\quad H F=-4125.4703608$

Zero-point correction $=\quad 1.300161$ (Hartree/Particle)

Thermal correction to Energy $=\quad 1.378178$

Thermal correction to Enthalpy= $\quad 1.379122$

Thermal correction to Gibbs Free Energy= 1.188993

Sum of electronic and zero-point Energies $=\quad-4124.170200$

Sum of electronic and thermal Energies $=\quad-4124.092183$

Sum of electronic and thermal Enthalpies $=\quad-4124.091238$

Sum of electronic and thermal Free Energies $=\quad-4124.281368$

\section{Cartesian Coordinates}

$\begin{array}{rrrr}0 & -4.257322 & 0.338577 & 0.419579 \\ 6 & -3.279194 & 0.318653 & -0.571677 \\ 6 & -3.653886 & 0.133708 & -1.902468 \\ 6 & -4.992358 & -0.045310 & -2.254116 \\ 6 & -5.948295 & -0.015310 & -1.231462 \\ 6 & -5.608510 & 0.170654 & 0.108753 \\ 15 & -1.498794 & 0.473424 & -0.117483 \\ 45 & 0.118741 & 0.553171 & -1.966219 \\ 6 & 0.154798 & 2.482490 & -1.926781 \\ 8 & 0.248502 & 3.633963 & -1.983341 \\ 6 & -5.432043 & -0.256249 & -3.701905 \\ 6 & -4.243603 & -0.513337 & -4.625413 \\ 6 & -6.629416 & 0.153238 & 1.244028 \\ 6 & -8.057248 & -0.045180 & 0.742547 \\ 6 & -1.446611 & -0.677545 & 1.343114 \\ 6 & -1.231902 & -2.034705 & 1.168314 \\ 6 & -1.390389 & -2.939655 & 2.269973 \\ 6 & -1.765795 & -2.433013 & 3.546361 \\ 6 & -1.939456 & -1.039331 & 3.700964 \\ 6 & -1.785590 & -0.192236 & 2.637557 \\ 6 & -1.208270 & -4.341001 & 2.136627 \\ 6 & -1.414451 & -5.186657 & 3.198288 \\ 6 & -1.796343 & -4.678250 & 4.457196 \\ 6 & -1.956815 & -3.326767 & 4.627003 \\ 6 & -0.917601 & -2.647881 & -0.149104 \\ 6 & 0.380828 & -2.729913 & -0.618418 \\ 6 & 0.671432 & -3.485798 & -1.781056 \\ 6 & -0.321304 & -4.107389 & -2.487648 \\ 6 & -1.671792 & -3.983374 & -2.084046 \\ 6 & -1.975616 & -3.259123 & -0.894392 \\ 6 & -2.724675 & -4.555719 & -2.837927 \\ 6 & -4.029818 & -4.419619 & -2.436785 \\ 6 & -4.334885 & -3.704621 & -1.258047 \\ 6 & -3.334871 & -3.145291 & -0.502054 \\ 7 & 1.450092 & -1.979376 & -0.016242 \\ 6 & 2.396984 & -2.693398 & 0.862625 \\ 6 & 2.046832 & -2.644998 & 2.327402 \\ 6 & 1.850558 & -1.422520 & 2.976503 \\ 6 & 1.593964 & -1.384178 & 4.341179 \\ 6 & 1.530228 & -2.565493 & 5.078190 \\ 6 & 1.722058 & -3.785099 & 4.439858 \\ 6 & 1.977481 & -3.821346 & 3.071192\end{array}$




$\begin{array}{lrrr}15 & 1.834254 & -0.586535 & -0.902782 \\ 8 & 2.780198 & 0.141185 & 0.262284 \\ 6 & 3.573040 & 1.173738 & -0.204639 \\ 6 & 4.785777 & 0.877693 & -0.796428 \\ 6 & 5.562622 & 1.961404 & -1.320713 \\ 6 & 5.095540 & 3.300893 & -1.153342 \\ 6 & 3.855609 & 3.535129 & -0.512670 \\ 6 & 3.095098 & 2.490090 & -0.061300 \\ 6 & 6.764365 & 1.762978 & -2.047700 \\ 6 & 7.480089 & 2.826034 & -2.539708 \\ 6 & 7.035827 & 4.149312 & -2.336691 \\ 6 & 5.863844 & 4.376061 & -1.662790 \\ 6 & 5.178305 & -0.542589 & -0.947793 \\ 6 & 4.298593 & -1.415984 & -1.566955 \\ 6 & 4.644501 & -2.755405 & -1.843203 \\ 6 & 5.853207 & -3.247172 & -1.433332 \\ 6 & 6.752620 & -2.431577 & -0.705576 \\ 6 & 6.413130 & -1.068988 & -0.448306 \\ 6 & 7.308544 & -0.297771 & 0.336459 \\ 6 & 8.481061 & -0.833930 & 0.808028 \\ 6 & 8.828507 & -2.170829 & 0.521753 \\ 6 & 7.976034 & -2.950559 & -0.216641 \\ 8 & 3.059554 & -0.991139 & -1.981337 \\ 6 & -1.397731 & 2.111320 & 0.701079 \\ 6 & -2.306202 & 3.123813 & 0.407915 \\ 6 & -2.158376 & 4.397359 & 0.964959 \\ 6 & -1.099873 & 4.603025 & 1.851903 \\ 6 & -0.183248 & 3.599174 & 2.186054 \\ 6 & -0.325524 & 2.361058 & 1.561287 \\ 6 & -3.127344 & 5.537793 & 0.658630 \\ 6 & -4.185910 & 5.136857 & -0.366379 \\ 6 & 0.889965 & 3.863422 & 3.241905 \\ 6 & 1.706805 & 2.610932 & 3.558628 \\ 6 & -2.349845 & 6.732107 & 0.096112 \\ 6 & -3.845173 & 5.951369 & 1.948185 \\ 6 & 1.842375 & 4.962391 & 2.762570 \\ 6 & 0.209942 & 4.312664 & 4.541297 \\ 6 & -0.631482 & -0.615939 & -3.329536 \\ 8 & -0.887218 & -1.246337 & -4.264382 \\ 6 & -6.366147 & -1.466611 & -3.803563 \\ 6 & -6.168687 & 0.998394 & -4.183090 \\ 6 & -6.291825 & -1.004917 & 2.191576 \\ 6 & -6.572692 & 1.480785 & 2.008024 \\ 1 & 7.050095 & 0.730705 & 0.575887 \\ 1 & -1.941347 & 0.872924 & 2.787782 \\ 1 & 8.218975 & -3.989970 & -0.434079 \\ 1 & -2.239172 & -2.915172 & 5.595262 \\ 1 & 5.492114 & 5.389407 & -1.516954 \\ 1 & -3.585043 & -2.582787 & 0.396934 \\ 1 & -5.372584 & -3.588451 & -0.949802 \\ 1 & 3.499379 & 4.559760 & -0.413719 \\ 1 & -2.211484 & -0.648128 & 4.680684 \\ 1 & 6.132076 & -4.278195 & -1.644835 \\ 1 & 8.395660 & 2.644591 & -3.098207 \\ 1 & 3.932728 & -3.370719 & -2.389035 \\ 1 & -2.474410 & -5.101855 & -3.746320\end{array}$




\begin{tabular}{|c|c|c|c|}
\hline 1 & -0.090633 & -4.672206 & -3.389475 \\
\hline 1 & -1.276007 & -6.257806 & 3.068811 \\
\hline 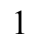 & 7.115343 & 0.749957 & -2.225388 \\
\hline 1 & -0.898910 & -4.748897 & 1.176572 \\
\hline & 1.706743 & -3.538454 & -2.112894 \\
\hline & 7.614824 & 4.982506 & -2.728119 \\
\hline & 9.145988 & -0.222442 & 1.413895 \\
\hline 1 & -4.832236 & -4.860456 & -3.024754 \\
\hline ( & -1.955007 & -5.358943 & 5.290658 \\
\hline & 9.763312 & -2.580896 & 0.896696 \\
\hline 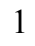 & 2.118388 & 2.644136 & 0.395349 \\
\hline & -3.963271 & 0.471381 & 1.459614 \\
\hline 1 & -2.876000 & 0.120739 & -2.660574 \\
\hline 1 & -6.994768 & -0.151411 & -1.495879 \\
\hline 1 & -3.128560 & 2.914078 & -0.271847 \\
\hline 1 & 0.381133 & 1.556689 & 1.766816 \\
\hline 1 & -0.990437 & 5.583725 & 2.315224 \\
\hline 1 & 1.175679 & 0.790539 & -3.126339 \\
\hline 1 & 1.905190 & -0.498192 & 2.401858 \\
\hline 1 & 2.119713 & -4.779096 & 2.569659 \\
\hline 1 & 1.446895 & -0.425235 & 4.836080 \\
\hline 1 & 1.660646 & -4.713964 & 5.003709 \\
\hline 1 & 1.325740 & -2.533767 & 6.146537 \\
\hline 1 & 2.426684 & 2.832136 & 4.356498 \\
\hline 1 & 1.062953 & 1.790726 & 3.908052 \\
\hline 1 & 2.282012 & 2.253825 & 2.693831 \\
\hline 1 & 0.961995 & 4.483917 & 5.322973 \\
\hline 1 & -0.353640 & 5.244879 & 4.416603 \\
\hline 1 & -0.487114 & 3.546037 & 4.904838 \\
\hline 1 & 2.593878 & 5.178288 & 3.533805 \\
\hline 1 & 2.375531 & 4.654969 & 1.853401 \\
\hline 1 & 1.312510 & 5.897088 & 2.542140 \\
\hline 1 & -4.855385 & 5.985623 & -0.554488 \\
\hline 1 & -3.738428 & 4.850008 & -1.326746 \\
\hline 1 & -4.806045 & 4.301613 & -0.014568 \\
\hline 1 & -4.545667 & 6.773499 & 1.748604 \\
\hline 1 & -4.418474 & 5.111749 & 2.363531 \\
\hline 1 & -3.143995 & 6.292324 & 2.719790 \\
\hline 1 & -3.037632 & 7.553361 & -0.146217 \\
\hline 1 & -1.611730 & 7.119636 & 0.808446 \\
\hline 1 & -1.815198 & 6.454307 & -0.821557 \\
\hline 1 & -8.749987 & -0.045667 & 1.593564 \\
\hline 1 & -8.370207 & 0.759560 & 0.064862 \\
\hline 1 & -8.179569 & -1.002827 & 0.220048 \\
\hline 1 & -7.271864 & 1.464360 & 2.854845 \\
\hline 1 & -5.572229 & 1.690157 & 2.406983 \\
\hline 1 & -6.853316 & 2.318365 & 1.356316 \\
\hline 1 & -7.012881 & -1.046704 & 3.019102 \\
\hline 1 & -6.330658 & -1.965898 & 1.660544 \\
\hline 1 & -5.289212 & -0.908023 & 2.627970 \\
\hline 1 & -4.602580 & -0.702731 & -5.644921 \\
\hline 1 & -3.560851 & 0.344494 & -4.676997 \\
\hline 1 & -3.668923 & -1.392643 & -4.303041 \\
\hline 1 & -6.493882 & 0.876944 & -5.225316 \\
\hline 1 & -7.059034 & 1.205575 & -3.575847 \\
\hline 1 & -5.516191 & 1.879851 & -4.132401 \\
\hline
\end{tabular}




$\begin{array}{lrrr}1 & -6.660685 & -1.625727 & -4.849297 \\ 1 & -5.862277 & -2.377364 & -3.451986 \\ 1 & -7.287501 & -1.341194 & -3.222497 \\ 1 & 2.466954 & -3.738946 & 0.526617 \\ 1 & 3.393055 & -2.254673 & 0.724660\end{array}$

$1_{\mathrm{C} 5-} \psi_{-1 \mathrm{a} 2 \mathrm{a3s4a}}$

Number of imaginary frequencies : 0

The smallest frequencies are : $14.7979 \quad 17.5867 \quad 19.8223 \mathrm{~cm}(-1)$

Electronic energy : $\quad H F=-4125.4704408$

Zero-point correction $=\quad 1.300206($ Hartree/Particle $)$

Thermal correction to Energy= $\quad 1.378288$

Thermal correction to Enthalpy= $\quad 1.379232$

Thermal correction to Gibbs Free Energy= 1.189169

Sum of electronic and zero-point Energies $=\quad-4124.170235$

Sum of electronic and thermal Energies $=\quad-4124.092153$

Sum of electronic and thermal Enthalpies $=\quad-4124.091209$

Sum of electronic and thermal Free Energies $=\quad-4124.281272$

\section{Cartesian Coordinates}

$\begin{array}{cccc}6 & 1.847639 & -1.432821 & 2.985899 \\ 6 & 2.068039 & -2.646781 & 2.328940 \\ 6 & 2.006511 & -3.830611 & 3.061109 \\ 6 & 1.734777 & -3.809631 & 4.427005 \\ 6 & 1.518211 & -2.598398 & 5.073244 \\ 6 & 1.574340 & -1.409573 & 4.347502 \\ 6 & 2.426851 & -2.674555 & 0.866230 \\ 7 & 1.464714 & -1.973305 & -0.006716 \\ 15 & 1.828824 & -0.578652 & -0.899382 \\ 45 & 0.089519 & 0.530833 & -1.960036 \\ 6 & 0.123130 & 2.460954 & -1.930772 \\ 8 & 0.208048 & 3.612759 & -1.991296 \\ 6 & 0.408702 & -2.744527 & -0.606237 \\ 6 & -0.895458 & -2.670430 & -0.150503 \\ 6 & -1.935686 & -3.318065 & -0.891330 \\ 6 & -1.606178 & -4.087038 & -2.045732 \\ 6 & -0.251170 & -4.190774 & -2.438962 \\ 6 & 0.722223 & -3.523391 & -1.747476 \\ 6 & -3.301617 & -3.213102 & -0.519595 \\ 6 & -4.280394 & -3.842797 & -1.247509 \\ 6 & -3.947301 & -4.617776 & -2.379467 \\ 6 & -2.637782 & -4.731175 & -2.770976 \\ 6 & -1.228592 & -2.059682 & 1.164820 \\ 6 & -1.459542 & -0.706303 & 1.348385 \\ 6 & -1.816016 & -0.232230 & 2.642378 \\ 6 & -1.970619 & -1.086864 & 3.699058 \\ 6 & -1.775098 & -2.476911 & 3.538369 \\ 6 & -1.383148 & -2.972088 & 2.262204 \\ 6 & -1.962166 & -3.377932 & 4.613650 \\ 6 & -1.780969 & -4.726256 & 4.439879 \\ 6 & -1.380965 & -5.223276 & 3.182208 \\ 6 & -1.178667 & -4.370204 & 2.125729 \\ 15 & -1.508101 & 0.459959 & -0.096370 \\ 6 & -3.287855 & 0.330923 & -0.554781\end{array}$




\begin{tabular}{|c|c|c|c|}
\hline 6 & -3.645973 & 0.192386 & -1.886336 \\
\hline 6 & -4.987283 & 0.057098 & -2.272129 \\
\hline 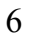 & -5.950994 & 0.071420 & -1.269816 \\
\hline & -5.627237 & 0.227247 & 0.089089 \\
\hline & -4.286066 & 0.354001 & 0.429631 \\
\hline & -5.316247 & -0.155656 & -3.747408 \\
\hline & -4.618493 & 0.905934 & -4.605132 \\
\hline & -6.737634 & 0.222314 & 1.137983 \\
\hline & -7.769358 & 1.307401 & 0.812209 \\
\hline & -1.392427 & 2.089380 & 0.737514 \\
\hline 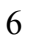 & -2.301728 & 3.105946 & 0.461478 \\
\hline ) & -2.152100 & 4.371920 & 1.034580 \\
\hline & -1.081282 & 4.570428 & 1.907982 \\
\hline f & -0.161915 & 3.563104 & 2.223553 \\
\hline & -0.314790 & 2.328388 & 1.594090 \\
\hline b & 0.923405 & 3.820976 & 3.268488 \\
\hline ) & 0.255412 & 4.238598 & 4.584615 \\
\hline & -3.128079 & 5.513038 & 0.754426 \\
\hline f & -3.776328 & 5.964660 & 2.067918 \\
\hline & 3.054473 & -0.972256 & -1.981698 \\
\hline$b$ & 4.300014 & -1.382006 & -1.571671 \\
\hline ) & 5.171325 & -0.498228 & -0.955444 \\
\hline ) & 6.412955 & -1.010150 & -0.458012 \\
\hline ) & 6.767367 & -2.369038 & -0.714657 \\
\hline 6 & 5.876704 & -3.194633 & -1.441901 \\
\hline ) & 4.661821 & -2.716805 & -1.850024 \\
\hline 6 & 7.997111 & -2.873853 & -0.226784 \\
\hline 6 & 8.841856 & -2.083820 & \\
\hline 6 & 8.480163 & -0.750405 & 0.794512 \\
\hline$b$ & 7.301068 & -0.227878 & 0.324054 \\
\hline 6 & 4.764385 & 0.918234 & -0.807296 \\
\hline 6 & 3.550068 & 1.203901 & -0.213724 \\
\hline ) & 3.058480 & 2.515842 & -0.075843 \\
\hline 6 & 3.806786 & 3.566723 & -0.534121 \\
\hline 6 & 5.047534 & 3.342770 & -1.176775 \\
\hline 6 & 5.528442 & 2.007581 & -1.338745 \\
\hline 6 & 6.730360 & 1.818427 & -2.067929 \\
\hline 5 & 7.433245 & 2.886623 & -2.567273 \\
\hline 6 & 6.975289 & 4.206053 & -2.369767 \\
\hline 5 & 5.802786 & 4.423576 & -1.693733 \\
\hline 8 & 2.769505 & 0.164854 & 0.260219 \\
\hline 5 & 1.760097 & 2.573614 & 3.550357 \\
\hline 6 & 1.855949 & 4.940313 & 2.797050 \\
\hline 6 & -4.242468 & 5.097661 & -0.203665 \\
\hline 6 & -2.368315 & 6.686326 & 0.127201 \\
\hline 6 & -6.203802 & 0.486958 & 2.544403 \\
\hline 5 & -7.420244 & -1.150129 & 1.135799 \\
\hline 6 & -6.815713 & -0.078604 & -4.024369 \\
\hline 6 & -4.817574 & -1.546734 & -4.156051 \\
\hline 6 & -0.700829 & -0.657384 & -3.277291 \\
\hline 8 & -1.022813 & -1.308919 & -4.177272 \\
\hline 1 & 7.031557 & 0.798002 & 0.562436 \\
\hline 1 & -1.985183 & 0.830511 & 2.796163 \\
\hline & 8.251409 & -3.910695 & -0.443502 \\
\hline 1 & -2.258339 & -2.974264 & 5.581125 \\
\hline & 5.420538 & 5.433588 & -1.552030 \\
\hline
\end{tabular}




\begin{tabular}{|c|c|c|c|}
\hline & -3.572455 & -2.608978 & 0.345297 \\
\hline & -5.322953 & -3.737457 & -0.953213 \\
\hline & 3.439794 & 4.587914 & -0.438984 \\
\hline & -2.258319 & -0.704930 & 4.677949 \\
\hline & 6.167556 & -4.222010 & -1.654979 \\
\hline & 8.349217 & 2.712189 & -3.127348 \\
\hline & 3.957652 & -3.339151 & -2.397687 \\
\hline & -2.366611 & -5.315009 & -3.649395 \\
\hline & -0.000965 & -4.784463 & -3.316733 \\
\hline & -1.224798 & -6.291569 & 3.049353 \\
\hline & 7.091923 & 0.808415 & -2.241440 \\
\hline & -0.854373 & -4.770611 & 1.167497 \\
\hline & 1.761274 & -3.565755 & -2.068908 \\
\hline & 7.544150 & 5.043418 & -2.767104 \\
\hline & 9.139305 & -0.130776 & 1.398427 \\
\hline & -4.733115 & -5.113494 & -2.945427 \\
\hline & -1.936581 & -5.412676 & 5.269211 \\
\hline & 9.781663 & -2.483050 & 0.883695 \\
\hline & 2.081597 & 2.662150 & 0.382667 \\
\hline & -3.999066 & 0.468222 & 1.471364 \\
\hline & -2.861859 & 0.184748 & -2.639006 \\
\hline & -6.998745 & -0.040923 & -1.541616 \\
\hline ] & -3.129326 & 2.904276 & -0.214156 \\
\hline & 0.389054 & 1.519496 & 1.790824 \\
\hline I & -0.964094 & 5.547843 & 2.376360 \\
\hline & 1.124570 & 0.757141 & -3.143081 \\
\hline 1 & 1.897008 & -0.503956 & 2.417919 \\
\hline & 2.166823 & -4.781821 & 2.552658 \\
\hline & 1.407404 & -0.457468 & 4.849300 \\
\hline ] & 1.679166 & -4.744026 & 4.982258 \\
\hline & 1.299884 & -2.578942 & 6.139145 \\
\hline l & 2.493276 & 2.791274 & 4.336972 \\
\hline ] & 1.132695 & 1.741339 & 3.900989 \\
\hline | & 2.320732 & 2.235096 & 2.668663 \\
\hline ] & 1.015573 & 4.406736 & 5.359135 \\
\hline 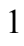 & -0.322453 & 5.164901 & 4.482578 \\
\hline 1 & -0.426458 & 3.456539 & 4.943992 \\
\hline & 2.614537 & 5.151013 & 3.562731 \\
\hline 1 & 2.381390 & 4.656441 & 1.875856 \\
\hline ] & 1.312561 & 5.872719 & 2.601268 \\
\hline l & -4.922574 & 5.943431 & -0.365150 \\
\hline 1 & -3.851352 & 4.798388 & -1.184501 \\
\hline ] & -4.839780 & 4.265972 & 0.194896 \\
\hline 1 & -4.484026 & 6.783485 & 1.881069 \\
\hline 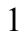 & -4.329605 & 5.140027 & 2.536525 \\
\hline 1 & -3.037266 & 6.326074 & 2.793182 \\
\hline 1 & -3.056713 & 7.513765 & -0.091628 \\
\hline 1 & -1.586254 & 7.072361 & 0.792228 \\
\hline 1 & -1.888684 & 6.384975 & -0.813103 \\
\hline 1 & -7.035389 & 0.484405 & 3.260208 \\
\hline 1 & -5.490767 & -0.283070 & 2.868036 \\
\hline 1 & -5.708990 & 1.464996 & 2.615659 \\
\hline 1 & -8.563730 & 1.315110 & 1.570408 \\
\hline 1 & -7.304498 & 2.301893 & 0.800300 \\
\hline 1 & -8.247714 & 1.150460 & -0.161696 \\
\hline 1 & -8.225460 & -1.179443 & 1.882263 \\
\hline
\end{tabular}




\begin{tabular}{|c|c|c|c|}
\hline & -7.862260 & -1.388396 & 0.160546 \\
\hline & -6.702085 & -1.943809 & 1.382429 \\
\hline & 998864 & -0.207878 & 098432 \\
\hline & -7.373588 & -0.868149 & -3.504766 \\
\hline & -7.236760 & 0.891832 & -3.730110 \\
\hline & -4.884652 & 0.768549 & -5.661375 \\
\hline & -4.922232 & 1.918381 & -4.308624 \\
\hline & -3.524983 & 0.849035 & -4.538154 \\
\hline & -5.007371 & -1.723918 & 3585 \\
\hline & -3.740000 & -1.663241 & -3.980165 \\
\hline & -5.332414 & -2.328973 & -3.582145 \\
\hline & 2.521105 & -3.715242 & 0.521389 \\
\hline & 3.412773 & -2.211648 & 0.735192 \\
\hline
\end{tabular}

$1_{\mathrm{C} 5-} \psi_{-1 \mathbf{a} 2 a 3 s 4 \mathrm{~s}}$

Number of imaginary frequencies : 0

The smallest frequencies are : $16.0174 \quad 19.0288 \quad 22.5179 \mathrm{~cm}(-1)$

Electronic energy : $\quad H F=-4125.4705835$

Zero-point correction $=\quad 1.301544($ Hartree/Particle $)$

Thermal correction to Energy= $\quad 1.379084$

Thermal correction to Enthalpy= $\quad 1.380029$

Thermal correction to Gibbs Free Energy= $\quad 1.191735$

Sum of electronic and zero-point Energies $=\quad-4124.169040$

Sum of electronic and thermal Energies $=\quad-4124.091499$

Sum of electronic and thermal Enthalpies $=\quad-4124.090555$

Sum of electronic and thermal Free Energies $=\quad-4124.278848$

Cartesian Coordinates

$\begin{array}{cccc}6 & 5.825310 & 4.428396 & -1.602718 \\ 6 & 5.058854 & 3.343080 & -1.112358 \\ 6 & 5.530588 & 2.007784 & -1.298949 \\ 6 & 6.735197 & 1.824524 & -2.025264 \\ 6 & 7.448989 & 2.897291 & -2.498688 \\ 6 & 6.999997 & 4.215883 & -2.276554 \\ 6 & 4.755822 & 0.913488 & -0.793521 \\ 6 & 3.538722 & 1.197222 & -0.204133 \\ 6 & 3.057166 & 2.509207 & -0.040177 \\ 6 & 3.816160 & 3.563579 & -0.472188 \\ 6 & 5.157976 & -0.502423 & -0.960993 \\ 6 & 4.287027 & -1.374504 & -1.593880 \\ 6 & 4.644171 & -2.706699 & -1.889643 \\ 6 & 5.855240 & -3.195168 & -1.483147 \\ 6 & 6.745638 & -2.383249 & -0.740263 \\ 6 & 6.395406 & -1.026802 & -0.465121 \\ 6 & 7.971279 & -2.900208 & -0.254818 \\ 6 & 8.815970 & -2.124771 & 0.496898 \\ 6 & 8.458139 & -0.794458 & 0.800368 \\ 6 & 7.283172 & -0.260169 & 0.332694 \\ 8 & 3.044978 & -0.955070 & -2.003953 \\ 15 & 1.814415 & -0.578957 & -0.922575 \\ 8 & 2.745854 & 0.159572 & 0.247385 \\ 7 & 1.454341 & -1.981407 & -0.040262 \\ 6 & 0.392635 & -2.746277 & -0.637445 \\ 6 & -0.908755 & -2.668852 & -0.174565\end{array}$




\begin{tabular}{|c|c|c|c|}
\hline 6 & -1.956431 & -3.305971 & -0.913502 \\
\hline 6 & -1.637418 & -4.068933 & -2.074624 \\
\hline ) & -0.284862 & -4.177623 & -2.475458 \\
\hline & 0.695941 & -3.519428 & -1.785321 \\
\hline f & -2.677046 & -4.701249 & -2.798817 \\
\hline & -3.983795 & -4.581590 & -2.399742 \\
\hline D & -4.306072 & -3.812362 & -1.260677 \\
\hline D & -3.319514 & -3.194494 & -0.533308 \\
\hline D & -1.234086 & -2.063105 & 1.144634 \\
\hline 6 & -1.456147 & -0.709297 & 1.335130 \\
\hline 6 & -1.803828 & -0.240029 & 2.633451 \\
\hline 6 & -1.962163 & -1.099529 & 3.685782 \\
\hline 6 & -1.781439 & -2.490597 & 3.516055 \\
\hline 6 & -1.394428 & -2.980947 & 2.236741 \\
\hline 6 & -1.202063 & -4.379848 & 2.091298 \\
\hline 6 & -1.412440 & -5.237919 & 3.142129 \\
\hline 6 & -1.808095 & -4.745475 & 4.402979 \\
\hline 6 & -1.976693 & -3.396694 & 4.585636 \\
\hline 15 & -1.508178 & 0.461713 & -0.106575 \\
\hline 6 & -1.378711 & 2.088926 & 0.729237 \\
\hline 6 & -0.308645 & 2.312224 & 1.599072 \\
\hline 6 & -0.148961 & 3.542762 & 2.235072 \\
\hline 6 & -1.046240 & 4.564824 & 1.904565 \\
\hline 6 & -2.100642 & 4.386009 & 1.006834 \\
\hline 6 & -2.266504 & 3.120318 & 0.437567 \\
\hline 6 & 0.918281 & 3.779495 & 3.303369 \\
\hline 6 & 1.741175 & 2.522825 & 3.584599 \\
\hline 6 & -3.045779 & 5.546357 & 0.701305 \\
\hline 6 & -4.098887 & 5.174272 & -0.340049 \\
\hline 6 & 2.418767 & -2.688748 & 0.825110 \\
\hline 6 & 2.060562 & -2.678745 & 2.288152 \\
\hline 6 & 1.988925 & -3.872131 & 3.003555 \\
\hline 6 & 1.719867 & -3.867903 & 4.370165 \\
\hline 6 & 1.516362 & -2.663975 & 5.034012 \\
\hline 6 & 1.582827 & -1.465434 & 4.325214 \\
\hline 6 & 1.853018 & -1.472072 & 2.962811 \\
\hline 45 & 0.073717 & 0.524626 & -1.981651 \\
\hline 6 & -0.742958 & -0.649816 & -3.293196 \\
\hline 8 & -1.084909 & -1.293936 & -4.191354 \\
\hline 6 & 0.133444 & 2.454076 & -1.955499 \\
\hline 8 & 0.237089 & 3.603998 & -2.021318 \\
\hline 6 & -3.292857 & 0.342995 & -0.550352 \\
\hline 6 & -4.276674 & 0.356727 & 0.440125 \\
\hline 6 & -5.627365 & 0.235591 & 0.118219 \\
\hline 6 & -5.971647 & 0.100961 & -1.232421 \\
\hline 6 & -5.014434 & 0.094095 & -2.248718 \\
\hline 6 & -3.671032 & 0.219921 & -1.881995 \\
\hline 6 & -6.658087 & 0.242762 & 1.246165 \\
\hline 6 & -8.086698 & 0.078230 & 0.734195 \\
\hline 6 & -5.365916 & -0.108333 & -3.720857 \\
\hline 6 & -6.868117 & -0.013743 & -3.978019 \\
\hline 6 & -6.361710 & -0.914633 & 2.207653 \\
\hline 6 & -6.573859 & 1.574188 & 2.001893 \\
\hline 6 & -4.888024 & -1.503539 & -4.141570 \\
\hline 6 & -4.668734 & 0.948073 & -4.585357 \\
\hline 6 & -2.241058 & 6.732433 & 0.159873 \\
\hline
\end{tabular}




\begin{tabular}{|c|c|c|c|}
\hline 6 & -3.770814 & 5.959779 & 1.986843 \\
\hline 6 & 1.867821 & 4.898680 & 2.867146 \\
\hline 6 & 0.225438 & 4.183291 & 4.610954 \\
\hline 1 & 7.016910 & 0.763172 & 0.585170 \\
\hline 1 & -1.962546 & 0.823191 & 2.794575 \\
\hline 1 & 8.222357 & -3.934691 & -0.486072 \\
\hline 1 & -2.269155 & -2.996607 & 5.555710 \\
\hline 1 & 5.449879 & 5.438202 & -1.442482 \\
\hline 1 & -3.581197 & -2.594547 & 0.337785 \\
\hline 1 & -5.346260 & -3.701783 & -0.960353 \\
\hline 1 & 3.456239 & 4.585408 & -0.358667 \\
\hline 1 & -2.242278 & -0.720753 & 4.668097 \\
\hline 1 & 6.143276 & -4.220519 & -1.709403 \\
\hline 1 & 8.366658 & 2.727269 & -3.057351 \\
\hline 1 & 3.938879 & -3.317658 & -2.448724 \\
\hline 1 & -2.414551 & -5.280701 & -3.682756 \\
\hline 1 & -0.042716 & -4.766541 & -3.358704 \\
\hline 1 & -1.266000 & -6.306696 & 3.002291 \\
\hline 1 & 7.090065 & 0.815556 & -2.217714 \\
\hline 1 & -0.881177 & -4.776923 & 1.130582 \\
\hline 1 & 1.733124 & -3.564096 & -2.113326 \\
\hline 1 & 7.577470 & 5.056866 & -2.653313 \\
\hline 1 & 9.116974 & -0.186578 & 1.416431 \\
\hline 1 & -4.775816 & -5.067868 & -2.965253 \\
\hline 1 & -1.970381 & -5.435860 & 5.227730 \\
\hline 1 & 9.752618 & -2.533307 & 0.868898 \\
\hline 1 & 2.077432 & 2.652788 & 0.413208 \\
\hline 1 & -3.983356 & 0.456596 & 1.483981 \\
\hline 1 & -2.896480 & 0.216286 & -2.644479 \\
\hline 1 & -7.020008 & -0.000432 & -1.497857 \\
\hline 1 & -3.086388 & 2.931903 & -0.251499 \\
\hline 1 & 0.382289 & 1.493941 & 1.802251 \\
\hline 1 & -0.923592 & 5.538707 & 2.378938 \\
\hline 1 & 1.101093 & 0.737561 & -3.173759 \\
\hline 1 & 1.910369 & -0.535725 & 2.407945 \\
\hline 1 & 2.139976 & -4.817482 & 2.481506 \\
\hline 1 & 1.427169 & -0.518813 & 4.840821 \\
\hline 1 & 1.657028 & -4.809451 & 4.912388 \\
\hline 1 & 1.300775 & -2.657648 & 6.100625 \\
\hline 1 & 2.462864 & 2.726226 & 4.385578 \\
\hline 1 & 1.102311 & 1.691154 & 3.915476 \\
\hline 1 & 2.313770 & 2.191150 & 2.707861 \\
\hline 1 & 0.969857 & 4.336221 & 5.403753 \\
\hline 1 & -0.344819 & 5.114325 & 4.508744 \\
\hline 1 & -0.468564 & 3.400465 & 4.944811 \\
\hline 1 & 2.611606 & 5.093027 & 3.651443 \\
\hline 1 & 2.410600 & 4.623935 & 1.953300 \\
\hline 1 & 1.335403 & 5.837826 & 2.673697 \\
\hline 1 & -4.750540 & 6.036785 & -0.528207 \\
\hline 1 & -3.644683 & 4.888063 & -1.297554 \\
\hline 1 & -4.738147 & 4.347120 & -0.003620 \\
\hline 1 & -4.453753 & 6.796748 & 1.788051 \\
\hline 1 & -4.364209 & 5.126671 & 2.386766 \\
\hline 1 & -3.072361 & 6.279620 & 2.769907 \\
\hline 1 & -2.911720 & 7.567909 & -0.081870 \\
\hline 1 & -1.504744 & 7.100014 & 0.884516 \\
\hline
\end{tabular}




$\begin{array}{rrrr}1 & -1.700834 & 6.454702 & -0.754577 \\ 1 & -8.784815 & 0.090698 & 1.580740 \\ 1 & -8.377475 & 0.891968 & 0.057532 \\ 1 & -8.227089 & -0.874489 & 0.207367 \\ 1 & -7.283967 & 1.584007 & 2.839770 \\ 1 & -5.572400 & 1.756828 & 2.411523 \\ 1 & -6.821770 & 2.414683 & 1.340661 \\ 1 & -7.088286 & -0.923051 & 3.031310 \\ 1 & -6.429657 & -1.880290 & 1.688945 \\ 1 & -5.359437 & -0.845735 & 2.649569 \\ 1 & -7.065957 & -0.133760 & -5.050566 \\ 1 & -7.428311 & -0.800520 & -3.456842 \\ 1 & -7.275161 & 0.959107 & -3.672240 \\ 1 & -4.950542 & 0.816224 & -5.638257 \\ 1 & -4.957825 & 1.962880 & -4.282298 \\ 1 & -3.575122 & 0.879640 & -4.533523 \\ 1 & -5.100357 & -1.676907 & -5.205540 \\ 1 & -3.808215 & -1.629620 & -3.986843 \\ 1 & -5.397709 & -2.283233 & -3.559269 \\ 1 & 2.518252 & -3.725041 & 0.468571 \\ 1 & 3.402240 & -2.219443 & 0.699829\end{array}$

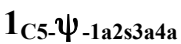

Number of imaginary frequencies : 0

The smallest frequencies are : $14.6311 \quad 16.7443 \quad 20.0211 \mathrm{~cm}(-1)$

Electronic energy : $\quad \mathrm{HF}=-4125.4707517$

Zero-point correction $=\quad 1.301097$ (Hartree/Particle)

Thermal correction to Energy= $\quad 1.378676$

Thermal correction to Enthalpy= $\quad 1.379621$

Thermal correction to Gibbs Free Energy= 1.190989

Sum of electronic and zero-point Energies $=\quad-4124.169655$

Sum of electronic and thermal Energies $=\quad-4124.092075$

Sum of electronic and thermal Enthalpies $=\quad-4124.091131$

Sum of electronic and thermal Free Energies $=\quad-4124.279763$

Cartesian Coordinates

$\begin{array}{cccc}6 & 2.009774 & -3.830065 & 3.052489 \\ 6 & 2.074868 & -2.648268 & 2.317404 \\ 6 & 1.865023 & -1.431602 & 2.972986 \\ 6 & 1.599593 & -1.403657 & 4.336050 \\ 6 & 1.540094 & -2.590540 & 5.064674 \\ 6 & 1.745332 & -3.804431 & 4.419776 \\ 6 & 2.429347 & -2.680878 & 0.853655 \\ 7 & 1.466415 & -1.982024 & -0.019984 \\ 15 & 1.825649 & -0.588169 & -0.916403 \\ 45 & 0.091430 & 0.514697 & -1.986016 \\ 6 & 0.149323 & 2.443960 & -1.971468 \\ 8 & 0.260615 & 3.592626 & -2.046240 \\ 6 & 0.403832 & -2.750310 & -0.610547 \\ 6 & -0.897065 & -2.665536 & -0.148386 \\ 6 & -1.947531 & -3.296936 & -0.888289 \\ 6 & -1.632826 & -4.050575 & -2.056860 \\ 6 & -0.279508 & -4.175787 & -2.450744 \\ 6 & 0.705064 & -3.529717 & -1.754944\end{array}$




\begin{tabular}{|c|c|c|c|}
\hline 6 & -3.309534 & -3.180547 & -0.507877 \\
\hline 6 & -4.302143 & -3.767730 & -1.252586 \\
\hline 6 & -3.986096 & -4.514132 & -2.408566 \\
\hline 6 & -2.677840 & -4.651429 & -2.799356 \\
\hline 6 & -1.220288 & -2.045298 & 1.164667 \\
\hline 6 & -1.445051 & -0.689526 & 1.338427 \\
\hline 6 & -1.793794 & -0.205656 & 2.631168 \\
\hline 6 & -1.946757 & -1.051920 & 3.694677 \\
\hline 6 & -1.760266 & -2.444270 & 3.542741 \\
\hline 6 & -1.376139 & -2.949783 & 2.268454 \\
\hline 6 & -1.949503 & -3.337302 & 4.624167 \\
\hline 6 & -1.778591 & -4.687968 & 4.457785 \\
\hline 6 & -1.387486 & -5.195471 & 3.201548 \\
\hline 6 & -1.182780 & -4.350095 & 2.139258 \\
\hline 15 & -1.499922 & 0.466577 & -0.116929 \\
\hline 6 & -3.291573 & 0.350330 & -0.538149 \\
\hline 6 & -4.257664 & 0.465411 & 0.466838 \\
\hline 6 & -5.612254 & 0.317457 & 0.180399 \\
\hline 6 & -5.971104 & 0.052653 & -1.147000 \\
\hline 6 & -5.036871 & -0.061563 & -2.176303 \\
\hline 6 & -3.685894 & 0.097511 & -1.847801 \\
\hline 6 & -6.686711 & 0.381958 & 1.264491 \\
\hline 6 & -7.324109 & -1.005386 & 1.404977 \\
\hline 6 & -5.501806 & -0.329288 & -3.606951 \\
\hline 6 & -6.444777 & -1.536125 & -3.641975 \\
\hline 6 & -1.356173 & 2.101783 & 0.701325 \\
\hline 6 & -0.290699 & 2.330457 & 1.581942 \\
\hline 6 & -0.128672 & 3.566292 & 2.199140 \\
\hline 6 & -1.019195 & 4.596110 & 1.851360 \\
\hline 6 & -2.062385 & 4.410612 & 0.949430 \\
\hline 6 & -2.224574 & 3.136121 & 0.388060 \\
\hline 6 & -3.042177 & 5.515308 & 0.562001 \\
\hline 6 & -2.930002 & 5.780454 & -0.943631 \\
\hline 6 & 0.932487 & 3.812842 & 3.271249 \\
\hline 6 & 1.890559 & 4.921262 & 2.825897 \\
\hline 8 & 3.053484 & -0.976759 & -1.996834 \\
\hline 6 & 4.296310 & -1.392241 & -1.584821 \\
\hline 6 & 5.168847 & -0.513832 & -0.962979 \\
\hline 6 & 6.406980 & -1.033182 & -0.463587 \\
\hline 6 & 6.756422 & -2.392604 & -0.724564 \\
\hline 6 & 5.864435 & -3.212107 & -1.457144 \\
\hline 6 & 4.652762 & -2.727666 & -1.866780 \\
\hline 6 & 7.982578 & -2.904676 & -0.235214 \\
\hline 6 & 8.828499 & -2.121452 & 0.507006 \\
\hline 6 & 8.471411 & -0.787871 & 0.796697 \\
\hline 6 & 7.295999 & -0.258315 & 0.324836 \\
\hline 6 & 4.767515 & 0.903555 & -0.807239 \\
\hline 6 & 3.552899 & 1.192599 & -0.215547 \\
\hline 6 & 3.074305 & 2.506046 & -0.056228 \\
\hline 6 & 3.832900 & 3.556646 & -0.498141 \\
\hline 6 & 5.072036 & 3.330503 & -1.143264 \\
\hline 6 & 5.541440 & 1.993460 & -1.323081 \\
\hline 6 & 6.742811 & 1.803813 & -2.053072 \\
\hline 6 & 7.455530 & 2.872494 & -2.537201 \\
\hline 6 & 7.008728 & 4.193063 & -2.322427 \\
\hline 6 & 5.837377 & 4.411545 & -1.644695 \\
\hline
\end{tabular}




\begin{tabular}{|c|c|c|c|}
\hline & 2.760197 & 0.158938 & 0.244580 \\
\hline & -4.469927 & 5.067533 & 0.898621 \\
\hline & -2.767934 & 6.823369 & 1.298876 \\
\hline & 0.234115 & 4.238210 & 4.569122 \\
\hline & 1.747609 & 2.556672 & 3.575435 \\
\hline & -6.239854 & 0.908917 & -4.127379 \\
\hline$y$ & -4.331246 & -0.627922 & -4.540733 \\
\hline & -7.764651 & 1.402248 & 0.885298 \\
\hline ) & -6.112358 & 0.784568 & 2.621569 \\
\hline & -0.681033 & -0.676795 & -3.313203 \\
\hline & -0.958538 & -1.330864 & -4.225645 \\
\hline & 7.030278 & 0.767614 & 0.567040 \\
\hline & -1.955467 & 0.858883 & 2.780600 \\
\hline 1 & 8.233020 & -3.941698 & -0.455536 \\
\hline & -2.239239 & -2.925786 & 5.590285 \\
\hline & 5.463770 & 5.422855 & -1.489742 \\
\hline 1 & -3.567455 & -2.596317 & 0.374202 \\
\hline & -5.341863 & -3.649725 & -0.952016 \\
\hline & 3.475216 & 4.579723 & -0.388790 \\
\hline & -2.226905 & -0.661336 & 4.672388 \\
\hline & 6.151661 & -4.239970 & -1.672787 \\
\hline & 8.370583 & 2.697677 & -3.098668 \\
\hline ] & 3.946547 & -3.344546 & -2.418103 \\
\hline & -2.419069 & -5.220113 & -3.691370 \\
\hline ] & -0.040446 & -4.762433 & -3.336305 \\
\hline & -1.240032 & -6.265703 & 3.074455 \\
\hline 1 & 7.095711 & 0.793072 & -2.239851 \\
\hline ] & -0.865450 & -4.758230 & 1.181949 \\
\hline & 1.742980 & -3.583702 & -2.079066 \\
\hline 1 & 7.585263 & 5.030777 & -2.707810 \\
\hline & 9.131125 & -0.173624 & 1.405470 \\
\hline ] & -4.782467 & -4.976151 & -2.988355 \\
\hline ] & -1.935983 & -5.368263 & 5.291819 \\
\hline | & 9.765486 & -2.526255 & 0.882224 \\
\hline ] & 2.097001 & 2.653601 & 0.401240 \\
\hline & -3.940627 & 0.665982 & 1.486367 \\
\hline ] & -2.918616 & 0.015735 & -2.612450 \\
\hline & -7.027361 & -0.071733 & -1.385274 \\
\hline 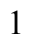 & -3.038052 & 2.953048 & -0.313026 \\
\hline 1 & 0.390984 & 1.509358 & 1.803886 \\
\hline ( & -0.889987 & 5.570066 & 2.318858 \\
\hline 1 & 1.131853 & 0.728969 & -3.165535 \\
\hline & 1.916433 & -0.504443 & 2.402553 \\
\hline 1 & 2.162114 & -4.783343 & 2.545441 \\
\hline & 1.442209 & -0.449246 & 4.836538 \\
\hline 1 & 1.687050 & -4.737273 & 4.977367 \\
\hline l & 1.328232 & -2.567483 & 6.131807 \\
\hline ] & 2.464854 & 2.767776 & 4.378419 \\
\hline 1 & 1.102386 & 1.732826 & 3.913274 \\
\hline 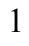 & 2.324677 & 2.210743 & 2.707278 \\
\hline 1 & 0.974767 & 4.394586 & 5.364766 \\
\hline ] & -0.327236 & 5.172754 & 4.452438 \\
\hline 1 & -0.468314 & 3.465083 & 4.907937 \\
\hline ] & 2.630157 & 5.122527 & 3.612415 \\
\hline 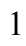 & 2.438019 & 4.630999 & 1.919658 \\
\hline 1 & 1.364596 & 5.860516 & 2.616262 \\
\hline
\end{tabular}




$\begin{array}{rrrr}1 & -3.498398 & 7.580845 & 0.988001 \\ 1 & -2.855908 & 6.708752 & 2.387194 \\ 1 & -1.769596 & 7.219534 & 1.073073 \\ 1 & -5.189002 & 5.851021 & 0.624418 \\ 1 & -4.755938 & 4.154327 & 0.361447 \\ 1 & -4.577084 & 4.871243 & 1.973841 \\ 1 & -3.636081 & 6.565440 & -1.246494 \\ 1 & -1.918031 & 6.112784 & -1.208509 \\ 1 & -3.150872 & 4.885636 & -1.538048 \\ 1 & -6.920159 & 0.836515 & 3.362282 \\ 1 & -5.376174 & 0.058326 & 2.991389 \\ 1 & -5.632591 & 1.772087 & 2.584567 \\ 1 & -8.535532 & 1.445327 & 1.666150 \\ 1 & -7.338018 & 2.407902 & 0.778337 \\ 1 & -8.267499 & 1.150349 & -0.055802 \\ 1 & -8.092962 & -0.997721 & 2.189408 \\ 1 & -7.801666 & -1.332512 & 0.472937 \\ 1 & -6.569767 & -1.756291 & 1.675868 \\ 1 & -4.709613 & -0.852363 & -5.545988 \\ 1 & -3.645778 & 0.224156 & -4.635057 \\ 1 & -3.753344 & -1.497625 & -4.199131 \\ 1 & -6.581056 & 0.746385 & -5.158796 \\ 1 & -7.120105 & 1.146064 & -3.516485 \\ 1 & -5.582535 & 1.788172 & -4.123259 \\ 1 & -6.765718 & -1.732224 & -4.673588 \\ 1 & -5.937450 & -2.435686 & -3.269013 \\ 1 & -7.350554 & -1.383938 & -3.042979 \\ 1 & 2.523177 & -3.722634 & 0.511811 \\ 1 & 3.414887 & -2.218307 & 0.719288\end{array}$

$\mathbf{1}_{\mathrm{C} 5-} \psi_{-12253 a 4 s}$

Number of imaginary frequencies : 0

The smallest frequencies are : $15.5842 \quad 16.3368 \quad 21.6355 \mathrm{~cm}(-1)$

Electronic energy : $\quad \mathrm{HF}=-4125.4710325$

Zero-point correction $=\quad 1.299940($ Hartree/Particle $)$

Thermal correction to Energy= $\quad 1.377939$

Thermal correction to Enthalpy= $\quad 1.378883$

Thermal correction to Gibbs Free Energy= $\quad 1.189297$

Sum of electronic and zero-point Energies $=\quad-4124.171093$

Sum of electronic and thermal Energies $=\quad-4124.093093$

Sum of electronic and thermal Enthalpies $=\quad-4124.092149$

Sum of electronic and thermal Free Energies $=\quad-4124.281735$

\section{Cartesian Coordinates}

$\begin{array}{llll}6 & 5.856174 & 4.405651 & -1.649064 \\ 6 & 5.091221 & 3.326528 & -1.142959 \\ 6 & 5.563113 & 1.989126 & -1.313365 \\ 6 & 6.766162 & 1.796723 & -2.039811 \\ 6 & 7.478596 & 2.863518 & -2.528506 \\ 6 & 7.029510 & 4.184707 & -2.322547 \\ 6 & 4.789646 & 0.901421 & -0.792385 \\ 6 & 3.575639 & 1.191756 & -0.200397 \\ 6 & 3.093063 & 2.506068 & -0.053888 \\ 6 & 3.850145 & 3.554828 & -0.502357\end{array}$




\begin{tabular}{|c|c|c|c|}
\hline 6 & 5.186964 & -0.517156 & -0.947221 \\
\hline 6 & 4.310543 & -1.391749 & -1.569337 \\
\hline & 4.660915 & -2.729342 & -1.848728 \\
\hline & 5.870692 & -3.218410 & -1.438774 \\
\hline & 6.766815 & -2.401878 & -0.708018 \\
\hline & 6.422973 & -1.040915 & -0.447918 \\
\hline f & 7.991197 & -2.918374 & -0.218862 \\
\hline o & 8.840514 & -2.137829 & 0.522300 \\
\hline 6 & 8.488867 & -0.802577 & 0.811130 \\
\hline 6 & 7.315299 & -0.268798 & 0.339476 \\
\hline 8 & 3.070341 & -0.969752 & -1.983288 \\
\hline 15 & 1.843419 & -0.572740 & -0.903844 \\
\hline 8 & 2.786224 & 0.155163 & 0.263513 \\
\hline 7 & 1.464224 & -1.969568 & -0.021157 \\
\hline 6 & 0.398356 & -2.723253 & -0.625461 \\
\hline 6 & -0.900789 & -2.647514 & -0.156905 \\
\hline 6 & -1.955996 & -3.260562 & -0.904706 \\
\hline 6 & -1.648283 & -3.982546 & -2.094748 \\
\hline 6 & -0.296912 & -4.101112 & -2.497048 \\
\hline 6 & 0.692769 & -3.476255 & -1.789018 \\
\hline 6 & -2.698285 & -4.557797 & -2.850438 \\
\hline 6 & -4.004446 & -4.425819 & -2.451206 \\
\hline 6 & -4.313447 & -3.712030 & -1.272766 \\
\hline 6 & -3.316244 & -3.150600 & -0.514609 \\
\hline 6 & -1.218671 & -2.040039 & 1.162229 \\
\hline 6 & -1.437977 & -0.684305 & 1.341257 \\
\hline 6 & -1.780108 & -0.203950 & 2.636720 \\
\hline 6 & -1.932963 & -1.055050 & \\
\hline 6 & -1.754809 & -2.447686 & 3.538072 \\
\hline 6 & -1.375868 & -2.949057 & 2.260610 \\
\hline 6 & -1.189083 & -4.349344 & 2.122955 \\
\hline 6 & -1.394257 & -5.199110 & 3.181516 \\
\hline 6 & -1.779765 & -4.696016 & 4.441461 \\
\hline 6 & -1.944672 & -3.345594 & 4.615474 \\
\hline 15 & -1.491788 & 0.470728 & -0.115387 \\
\hline 6 & -1.398750 & 2.104122 & 0.711535 \\
\hline 6 & -2.315476 & 3.105777 & 0.434242 \\
\hline 6 & -2.189808 & 4.378100 & 1.010374 \\
\hline 6 & -1.135141 & 4.591954 & 1.892394 \\
\hline 6 & -0.199272 & 3.591963 & 2.208540 \\
\hline 6 & -0.325402 & 2.360912 & 1.574755 \\
\hline 6 & -3.219493 & 5.448704 & 0.658218 \\
\hline 6 & -3.145010 & 5.739422 & -0.845029 \\
\hline 6 & 0.878244 & 3.863285 & 3.258069 \\
\hline 6 & 1.818752 & 4.969836 & 2.772152 \\
\hline 6 & 2.413606 & -2.681730 & 0.856645 \\
\hline 6 & 2.061074 & -2.639983 & 2.321042 \\
\hline 6 & 1.860249 & -1.420565 & 2.974558 \\
\hline 6 & 1.600932 & -1.388360 & 4.338866 \\
\hline 6 & 1.539247 & -2.572747 & 5.071148 \\
\hline 6 & 1.735767 & -3.789307 & 4.428449 \\
\hline 6 & 1.993676 & -3.819430 & 3.060110 \\
\hline 45 & 0.123589 & 0.564342 & -1.963119 \\
\hline 6 & -0.621132 & -0.605650 & -3.329188 \\
\hline 8 & -0.875508 & -1.236180 & -4.264265 \\
\hline 6 & 0.145268 & 2.493896 & -1.916046 \\
\hline
\end{tabular}




\begin{tabular}{|c|c|c|c|}
\hline & 0.227672 & 3.646537 & -1.965741 \\
\hline & -3.272292 & 0.317597 & -0.570028 \\
\hline & -3.647062 & 0.129874 & -1.900309 \\
\hline & -4.986149 & -0.045156 & -2.252014 \\
\hline & -5.942342 & -0.008232 & -1.229836 \\
\hline & -5.602408 & 0.180655 & 0.110094 \\
\hline y & -4.250737 & 0.344664 & 0.420928 \\
\hline & -5.426232 & -0.260848 & -3.699044 \\
\hline & -6.355942 & -1.475003 & -3.796130 \\
\hline & -6.622964 & 0.162113 & 1.245699 \\
\hline & -6.299190 & -1.015974 & 2.173474 \\
\hline f & -6.168209 & 0.989342 & -4.183532 \\
\hline & -4.237819 & -0.515702 & -4.623189 \\
\hline b & -6.547014 & 1.474880 & 2.033032 \\
\hline & -8.053751 & -0.007623 & 0.742234 \\
\hline & 0.206123 & 4.306453 & 4.563625 \\
\hline b & 1.706859 & 2.616686 & 3.567610 \\
\hline & -4.622654 & 4.940372 & 1.011876 \\
\hline D & -2.982760 & 6.755367 & 1.410361 \\
\hline & 7.053590 & 0.758391 & 0.580880 \\
\hline & -1.939194 & 0.860270 & 2.790285 \\
\hline & 8.237460 & -3.956564 & -0.438385 \\
\hline & -2.229843 & -2.938082 & 5.584635 \\
\hline & 5.480852 & 5.417344 & -1.500960 \\
\hline ] & -3.569931 & -2.588803 & 0.383922 \\
\hline & -5.351989 & -3.597768 & -0.966624 \\
\hline & 3.490496 & 4.578008 & -0.400767 \\
\hline & -2.207403 & -0.667938 & 4.677736 \\
\hline & 6.152951 & -4.248042 & -1.652536 \\
\hline 1 & 8.395313 & 2.686690 & -3.086604 \\
\hline & 3.951600 & -3.345426 & -2.396897 \\
\hline ] & -2.445021 & -5.102468 & -3.758877 \\
\hline & -0.063185 & -4.664255 & -3.399136 \\
\hline & -1.252228 & -6.269387 & 3.048773 \\
\hline ] & 7.120919 & 0.785392 & -2.219660 \\
\hline & -0.876893 & -4.753109 & 1.162064 \\
\hline 1 & 1.728552 & -3.524592 & -2.120028 \\
\hline & 7.605931 & 5.020918 & -2.711337 \\
\hline 1 & 9.151353 & -0.190497 & 1.419071 \\
\hline ] & -4.804687 & -4.868467 & -3.040778 \\
\hline l & -1.937622 & -5.379924 & 5.272439 \\
\hline 1 & 9.776075 & -2.546010 & 0.897417 \\
\hline ] & 2.115990 & 2.655746 & 0.403360 \\
\hline 1 & -3.956626 & 0.478778 & 1.460772 \\
\hline & -2.868968 & 0.111764 & -2.658078 \\
\hline 1 & -6.989265 & -0.140959 & -1.494282 \\
\hline 1 & -3.138907 & 2.899533 & -0.248418 \\
\hline 1 & 0.390152 & 1.562545 & 1.772469 \\
\hline 1 & -1.033399 & 5.564460 & 2.369479 \\
\hline ] & 1.179222 & 0.812482 & -3.122270 \\
\hline 1 & 1.913507 & -0.493676 & 2.403842 \\
\hline ] & 2.139542 & -4.774784 & 2.555069 \\
\hline 1 & 1.450226 & -0.431809 & 4.837290 \\
\hline ] & 1.675999 & -4.720538 & 4.988559 \\
\hline 1 & 1.332697 & -2.545747 & 6.139229 \\
\hline 1 & 2.429251 & 2.841839 & 4.362080 \\
\hline
\end{tabular}




$\begin{array}{rrrr}1 & 1.071104 & 1.790733 & 3.918383 \\ 1 & 2.280388 & 2.265732 & 2.699245 \\ 1 & 0.963788 & 4.477958 & 5.339765 \\ 1 & -0.360955 & 5.237344 & 4.446731 \\ 1 & -0.485243 & 3.536309 & 4.930556 \\ 1 & 2.574606 & 5.190948 & 3.537669 \\ 1 & 2.347046 & 4.666770 & 1.858658 \\ 1 & 1.280152 & 5.900622 & 2.556368 \\ 1 & -3.749795 & 7.487108 & 1.127188 \\ 1 & -3.042737 & 6.620256 & 2.498252 \\ 1 & -2.006366 & 7.195557 & 1.170458 \\ 1 & -5.376147 & 5.698394 & 0.759155 \\ 1 & -4.881431 & 4.022741 & 0.467898 \\ 1 & -4.704862 & 4.726227 & 2.085859 \\ 1 & -3.881349 & 6.505857 & -1.122381 \\ 1 & -2.149465 & 6.107410 & -1.124249 \\ 1 & -3.349761 & 4.846671 & -1.448243 \\ 1 & -8.746454 & -0.006576 & 1.593259 \\ 1 & -8.353105 & 0.809785 & 0.073553 \\ 1 & -8.192071 & -0.957186 & 0.209216 \\ 1 & -7.253553 & 1.456290 & 2.873671 \\ 1 & -5.546515 & 1.656615 & 2.445057 \\ 1 & -6.805562 & 2.330542 & 1.395123 \\ 1 & -7.019599 & -1.062937 & 3.001327 \\ 1 & -6.349614 & -1.967405 & 1.626456 \\ 1 & -5.294557 & -0.937427 & 2.609248 \\ 1 & -4.597149 & -0.706892 & -5.642231 \\ 1 & -3.557395 & 0.343938 & -4.675889 \\ 1 & -3.660409 & -1.393237 & -4.300897 \\ 1 & -6.495238 & 0.862319 & -5.224501 \\ 1 & -7.058041 & 1.196270 & -3.575486 \\ 1 & -5.518517 & 1.873135 & -4.138515 \\ 1 & -6.652093 & -1.637655 & -4.840870 \\ 1 & -5.847823 & -2.382941 & -3.443545 \\ 1 & -7.276398 & -1.351732 & -3.213148 \\ 1 & 2.489359 & -3.725794 & 0.517294 \\ 1 & 3.407548 & -2.237393 & 0.721504\end{array}$

$\mathbf{1}_{\mathrm{C} 5-}-\psi_{-1225354 a}$

Number of imaginary frequencies : 0

The smallest frequencies are : $15.085921 .005424 .2231 \mathrm{~cm}(-1)$

Electronic energy : $\quad H F=-4125.4712157$

Zero-point correction $=\quad 1.301714$ (Hartree/Particle)

Thermal correction to Energy= $\quad 1.379128$

Thermal correction to Enthalpy $=\quad 1.380072$

Thermal correction to Gibbs Free Energy= $\quad 1.192584$

Sum of electronic and zero-point Energies $=\quad-4124.169502$

Sum of electronic and thermal Energies $=\quad-4124.092088$

Sum of electronic and thermal Enthalpies $=\quad-4124.091144$

Sum of electronic and thermal Free Energies $=\quad-4124.278632$

Cartesian Coordinates

$\begin{array}{llll}6 & -0.273884 & 2.307675 & 1.604260 \\ 6 & -1.350357 & 2.097426 & 0.732485\end{array}$




\begin{tabular}{|c|c|c|c|}
\hline 5 & -2.229700 & 3.133530 & 0.460102 \\
\hline 6 & -2.066369 & 4.392938 & 1.054936 \\
\hline 6 & -1.010026 & 4.560497 & 1.945152 \\
\hline 6 & -0.108090 & 3.527190 & 2.251219 \\
\hline 1 & -1.500964 & 0.474876 & -0.107298 \\
\hline 6 & -1.457253 & -0.693575 & 1.336915 \\
\hline 6 & -1.235285 & -2.048170 & 1.153071 \\
\hline 6 & -1.393299 & -2.960241 & 2.250648 \\
\hline 6 & -1.779831 & -2.463268 & 3.527592 \\
\hline 6 & -1.965949 & -1.071936 & 3.688985 \\
\hline 6 & -1.808137 & -0.218068 & 2.632309 \\
\hline 6 & -1.970944 & -3.363326 & 4.602899 \\
\hline 6 & -1.798487 & -4.712732 & 4.428367 \\
\hline 6 & -1.403733 & -5.211746 & 3.169890 \\
\hline 6 & -1.197757 & -4.359501 & 2.113377 \\
\hline 6 & -0.910750 & -2.661119 & -0.163048 \\
\hline 6 & 0.390569 & -2.741844 & -0.625673 \\
\hline 6 & 0.693904 & -3.523361 & -1.767843 \\
\hline 6 & -0.286622 & -4.188375 & -2.451739 \\
\hline 6 & -1.638976 & -4.078204 & -2.050652 \\
\hline 6 & -1.958616 & -3.304043 & -0.897020 \\
\hline 6 & -3.322197 & -3.189478 & -0.518949 \\
\hline 6 & -4.307747 & -3.818111 & -1.238606 \\
\hline 6 & -3.984391 & -4.600025 & -2.368628 \\
\hline 6 & -2.677655 & -4.720558 & -2.767372 \\
\hline 7 & 1.452986 & -1.974209 & -0.033144 \\
\hline 15 & 1.815921 & -0.578259 & -0.924587 \\
\hline 8 & 3.044330 & -0.964435 & -2.004859 \\
\hline 6 & 4.286682 & -1.381924 & -1.593513 \\
\hline 6 & 5.159341 & -0.505865 & -0.968575 \\
\hline 6 & 6.397181 & -1.027455 & -0.470748 \\
\hline 6 & 6.745943 & -2.386225 & -0.736096 \\
\hline 6 & 5.853657 & -3.203021 & -1.471329 \\
\hline 6 & 4.642345 & -2.716643 & -1.879649 \\
\hline 6 & 7.286685 & -0.255551 & 0.320009 \\
\hline 6 & 8.461892 & -0.787214 & 0.790041 \\
\hline 6 & 8.818254 & -2.120062 & 0.496130 \\
\hline 6 & 7.971857 & -2.900492 & -0.248476 \\
\hline 6 & 4.758448 & 0.911319 & -0.809205 \\
\hline 6 & 3.543377 & 1.199475 & -0.217923 \\
\hline 6 & 3.064476 & 2.512799 & -0.057096 \\
\hline 6 & 3.823807 & 3.564123 & -0.496018 \\
\hline 6 & 5.063631 & 3.338959 & -1.140238 \\
\hline 6 & 5.532919 & 2.002155 & -1.322324 \\
\hline 6 & 5.829665 & 4.420786 & -1.638910 \\
\hline 6 & 7.001410 & 4.203367 & -2.316319 \\
\hline 6 & 7.447975 & 2.883128 & -2.533540 \\
\hline 6 & 6.734668 & 1.813672 & -2.051994 \\
\hline 8 & 2.750083 & 0.165024 & 0.239802 \\
\hline 6 & -3.064062 & 5.498783 & 0.720472 \\
\hline 6 & -4.476846 & 5.035651 & 1.097908 \\
\hline 6 & 0.969028 & 3.750427 & 3.312324 \\
\hline 6 & 0.289463 & 4.133625 & 4.633018 \\
\hline 6 & -3.287854 & 0.374942 & -0.545493 \\
\hline 6 & -4.273083 & 0.420631 & 0.450884 \\
\hline 6 & -5.619202 & 0.293479 & 0.130457 \\
\hline
\end{tabular}




\begin{tabular}{|c|c|c|c|}
\hline & -5.961935 & 0.123503 & -1.222006 \\
\hline & -5.011843 & 0.095029 & -2.237201 \\
\hline & -3.664449 & 0.223116 & -1.870623 \\
\hline & -5.362180 & -0.126187 & -3.706428 \\
\hline & -4.669841 & 0.925328 & -4.580788 \\
\hline & -6.710960 & 0.281347 & 1.198833 \\
\hline & -7.800951 & 1.302633 & 0.858545 \\
\hline & 0.076621 & 0.523869 & -1.986622 \\
\hline 0 & -0.740068 & -0.658154 & -3.291859 \\
\hline & -1.079876 & -1.308469 & -4.186378 \\
\hline b & 0.136439 & 2.453497 & -1.969215 \\
\hline & 0.238591 & 3.603221 & -2.040061 \\
\hline 6 & -6.864824 & -0.041514 & -3.963274 \\
\hline & -4.877750 & -1.522698 & -4.113449 \\
\hline & -6.165310 & 0.619896 & 2.585087 \\
\hline & -7.324534 & -1.122613 & 1.254573 \\
\hline & 2.416189 & -2.677388 & 0.836951 \\
\hline & 2.059630 & -2.654395 & 2.300270 \\
\hline & 1.848374 & -1.442012 & 2.963407 \\
\hline & 1.580296 & -1.423257 & 4.326090 \\
\hline & 1.519393 & -2.615018 & 5.046674 \\
\hline & 1.726100 & -3.824588 & 4.394213 \\
\hline & 1.993288 & -3.841050 & 3.027325 \\
\hline & -2.771579 & 6.794770 & 1.471476 \\
\hline & -3.005313 & 5.791907 & -0.782846 \\
\hline & 1.797782 & 2.491865 & 3.566314 \\
\hline & 1.912178 & 4.877834 & 2.882804 \\
\hline & 7.021549 & 0.769794 & 0.565348 \\
\hline & -1.969521 & 0.845555 & 2.788276 \\
\hline - & 8.221724 & -3.936938 & -0.472142 \\
\hline & -2.263001 & -2.958086 & 5.570964 \\
\hline & 5.456221 & 5.431886 & -1.482220 \\
\hline ] & -3.586624 & -2.579121 & 0.343821 \\
\hline & -5.348147 & -3.705355 & -0.939417 \\
\hline 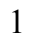 & 3.466165 & 4.587008 & -0.384837 \\
\hline & -2.248884 & -0.688194 & 4.668579 \\
\hline 1 & 6.140402 & -4.230349 & -1.690136 \\
\hline & 8.363291 & 2.709177 & -3.094850 \\
\hline 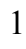 & 3.935784 & -3.331381 & -2.432928 \\
\hline l & -2.414282 & -5.308916 & -3.645141 \\
\hline & -0.044373 & -4.784965 & -3.329789 \\
\hline 1 & -1.254387 & -6.280929 & 3.036377 \\
\hline & 7.087413 & 0.803226 & -2.240644 \\
\hline 1 & -0.877358 & -4.761590 & 1.154560 \\
\hline & 1.730945 & -3.569793 & -2.095964 \\
\hline 1 & 7.578418 & 5.041704 & -2.699632 \\
\hline | & 9.122034 & -0.175224 & 1.400613 \\
\hline 1 & -4.775596 & -5.094661 & -2.927990 \\
\hline 1 & -1.957147 & -5.398461 & 5.257700 \\
\hline 1 & 9.755077 & -2.526542 & 0.869942 \\
\hline 1 & 2.086749 & 2.659793 & 0.399381 \\
\hline 1 & -3.972507 & 0.549190 & 1.486922 \\
\hline 1 & -2.890301 & 0.199038 & -2.633183 \\
\hline 1 & -7.013412 & 0.010622 & -1.478393 \\
\hline 1 & -3.055054 & 2.963665 & -0.230520 \\
\hline 1 & 0.411525 & 1.482624 & 1.798044 \\
\hline
\end{tabular}




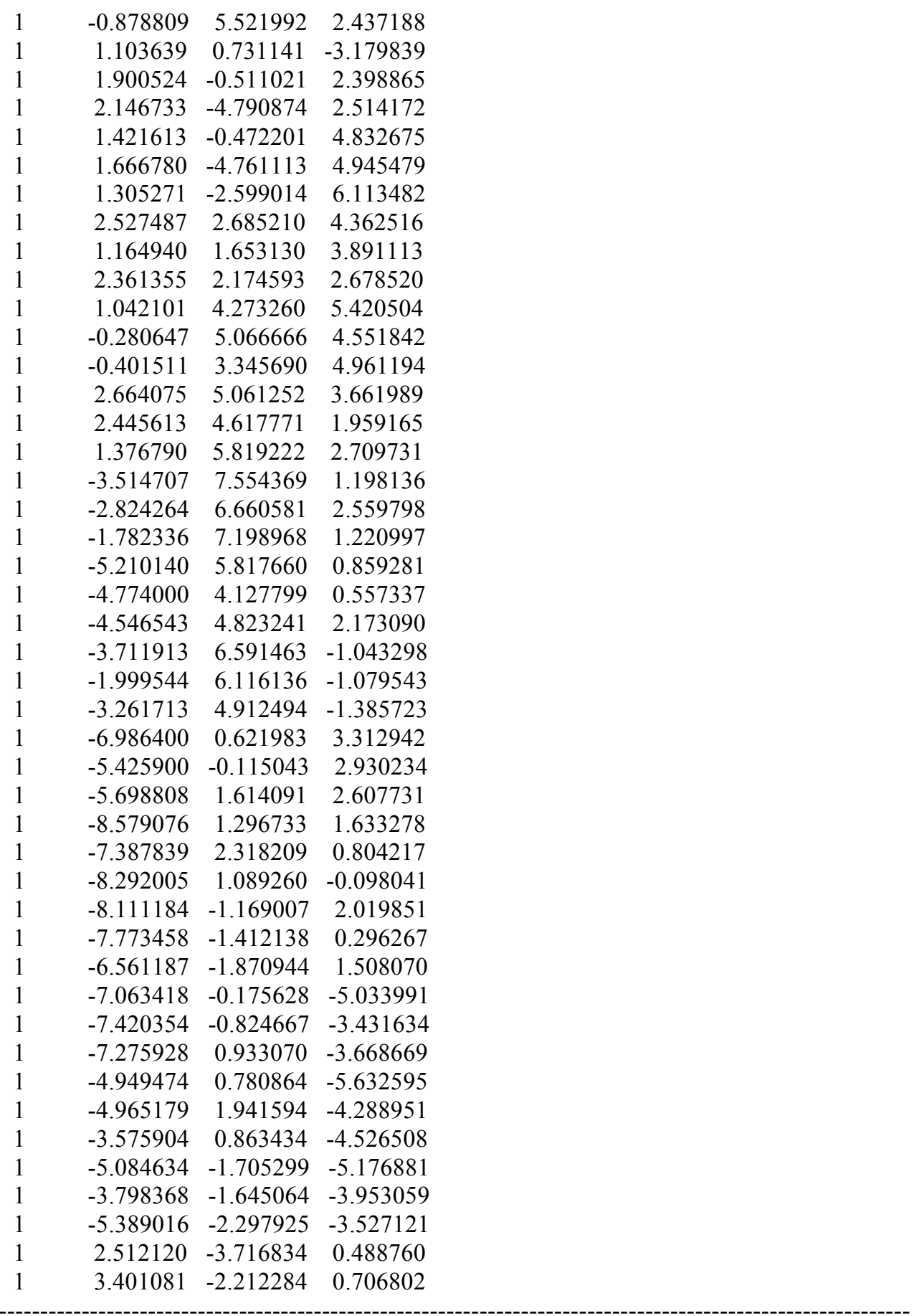

$1_{\text {C5- }} \psi_{-12 \text {-125354s }}$

Number of imaginary frequencies : 0

The smallest frequencies are : $15.4064 \quad 18.8706 \quad 21.5978 \mathrm{~cm}(-1)$

Electronic energy : $\quad H F=-4125.4712339$

Zero-point correction $=\quad 1.301732$ (Hartree/Particle)

Thermal correction to Energy $=\quad 1.379055$

Thermal correction to Enthalpy= $\quad 1.380000$

Thermal correction to Gibbs Free Energy= 1.192506

Sum of electronic and zero-point Energies $=\quad-4124.169501$ 
Sum of electronic and thermal Energies= Sum of electronic and thermal Enthalpies= Sum of electronic and thermal Free Energies=
$-4124.092178$

$-4124.091234$

$-4124.278728$

Cartesian Coordinates

$\begin{array}{cccc}6 & 1.982084 & -3.882567 & 2.985748 \\ 6 & 2.053670 & -2.685555 & 2.276507 \\ 6 & 1.842294 & -1.482497 & 2.956511 \\ 6 & 1.568838 & -1.483144 & 4.318274 \\ 6 & 1.502351 & -2.685325 & 5.020918 \\ 6 & 1.709358 & -3.885612 & 4.351639 \\ 6 & 2.414927 & -2.687902 & 0.814253 \\ 7 & 1.450304 & -1.978600 & -0.049124 \\ 15 & 1.810461 & -0.573937 & -0.928100 \\ 45 & 0.068762 & 0.530449 & -1.984416 \\ 6 & 0.125343 & 2.459807 & -1.957924 \\ 8 & 0.224803 & 3.610012 & -2.024611 \\ 6 & 0.388757 & -2.741985 & -0.648693 \\ 6 & -0.913017 & -2.664534 & -0.187072 \\ 6 & -1.960990 & -3.296618 & -0.929679 \\ 6 & -1.642026 & -4.057367 & -2.092158 \\ 6 & -0.288976 & -4.168038 & -2.490948 \\ 6 & 0.692065 & -3.512820 & -1.798176 \\ 6 & -3.324354 & -3.181322 & -0.551732 \\ 6 & -4.311581 & -3.794229 & -1.282359 \\ 6 & -3.989486 & -4.561940 & -2.422529 \\ 6 & -2.682361 & -4.684809 & -2.819581 \\ 6 & -1.240635 & -2.064571 & 1.133965 \\ 6 & -1.464178 & -0.712025 & 1.329658 \\ 6 & -1.816244 & -0.248685 & 2.629119 \\ 6 & -1.977065 & -1.112883 & 3.677119 \\ 6 & -1.794397 & -2.503097 & 3.501874 \\ 6 & -1.403380 & -2.987473 & 2.221556 \\ 6 & -1.991496 & -3.414007 & 4.566971 \\ 6 & -1.820852 & -4.761824 & 4.378903 \\ 6 & -1.421411 & -5.248359 & 3.116991 \\ 6 & -1.209280 & -4.385470 & 2.070430 \\ 15 & -1.506950 & 0.468507 & -0.104403 \\ 6 & -3.294458 & 0.377218 & -0.545065 \\ 6 & -4.279157 & 0.405592 & 0.444807 \\ 6 & -5.630347 & 0.288770 & 0.122340 \\ 6 & -5.974490 & 0.150672 & -1.228165 \\ 6 & -5.016968 & 0.133977 & -2.243884 \\ 6 & -3.672935 & 0.250396 & -1.876337 \\ 6 & -6.661821 & 0.288817 & 1.249950 \\ 6 & -6.375428 & -0.889400 & 2.189152 \\ 6 & -5.368346 & -0.072736 & -3.715519 \\ 6 & -4.895749 & -1.471350 & -4.131044 \\ 6 & -1.353118 & 2.083809 & 0.748554 \\ 6 & -0.277647 & 2.282521 & 1.624066 \\ 6 & -0.106510 & 3.496875 & 2.279236 \\ 6 & -1.000526 & 4.537811 & 1.976110 \\ 6 & -2.054216 & 4.382598 & 1.080364 \\ 6 & -2.225280 & 3.126915 & 0.479700 \\ 6 & -3.031491 & 5.502785 & 0.733472\end{array}$




\begin{tabular}{|c|c|c|c|}
\hline & -2.899876 & 5.832617 & -0.757735 \\
\hline & 0.968597 & 3.706041 & 3.345204 \\
\hline & 1.915033 & 4.836619 & 2.931554 \\
\hline & 3.041123 & -0.947709 & -2.009940 \\
\hline & 4.283526 & -1.366738 & -1.600443 \\
\hline & 5.153810 & -0.494541 & -0.966880 \\
\hline & 6.391885 & -1.018252 & -0.471944 \\
\hline & 6.743129 & -2.374179 & -0.748365 \\
\hline & 5.853160 & -3.186219 & -1.491643 \\
\hline & 4.641599 & -2.698387 & -1.897461 \\
\hline & 7.969340 & -2.890542 & -0.263723 \\
\hline & 8.813654 & -2.115022 & 0.488337 \\
\hline & 8.454887 & -0.785224 & 0.792952 \\
\hline & 7.279321 & -0.251512 & 0.326125 \\
\hline & 4.750092 & 0.920685 & -0.797535 \\
\hline & 3.533056 & 1.202305 & -0.207025 \\
\hline & 3.050197 & 2.513473 & -0.040605 \\
\hline & 3.807940 & 3.569293 & -0.471381 \\
\hline & 5.050233 & 3.351066 & -1.113153 \\
\hline & 5.523280 & 2.016545 & -1.301990 \\
\hline & 6.727517 & 1.835530 & -2.029470 \\
\hline & 7.439760 & 2.909690 & -2.502075 \\
\hline & 6.989498 & 4.227488 & -2.277805 \\
\hline & 5.815166 & 4.437838 & -1.602655 \\
\hline & 2.741244 & 0.163235 & 0.243450 \\
\hline & -4.463400 & 5.041912 & \\
\hline & -2.765885 & 6.776800 & 1.530668 \\
\hline & 0.285671 & 4.075105 & 4.668156 \\
\hline & 1.794892 & 2.443256 & 3.586246 \\
\hline & -4.666497 & 0.977469 & -4.583729 \\
\hline & -6.870006 & 0.026684 & -3.973894 \\
\hline & -6.566692 & 1.603498 & 2.032410 \\
\hline & -8.091774 & 0.147069 & 0.734880 \\
\hline & -0.750640 & -0.640454 & -3.297708 \\
\hline & -1.094555 & -1.281307 & -4.197445 \\
\hline & 7.012340 & 0.771432 & 0.579449 \\
\hline & -1.975065 & 0.813807 & 2.795041 \\
\hline & 8.221185 & -3.924636 & -0.495878 \\
\hline & -2.287080 & -3.018475 & 5.537968 \\
\hline & 5.438843 & 5.447044 & -1.440720 \\
\hline & -3.585174 & -2.583248 & 0.320846 \\
\hline & -5.352006 & -3.680108 & -0.984076 \\
\hline & 3.447313 & 4.590562 & -0.355096 \\
\hline & -2.260146 & -0.738587 & 4.660300 \\
\hline & 6.141950 & -4.211167 & -1.718763 \\
\hline & 8.357158 & 2.741412 & -3.061711 \\
\hline & 3.936564 & -3.309458 & -2.456766 \\
\hline & -2.420222 & -5.262898 & -3.704520 \\
\hline 1 & -0.046590 & -4.755396 & -3.375166 \\
\hline & -1.273459 & -6.316356 & 2.972885 \\
\hline & 7.083331 & 0.827183 & -2.223483 \\
\hline & -0.885538 & -4.778074 & 1.108823 \\
\hline & 1.729510 & -3.558200 & -2.125324 \\
\hline & 7.565734 & 5.069602 & -2.653937 \\
\hline 1 & 9.113475 & -0.177289 & 1.409226 \\
\hline & -4.781982 & -5.044249 & -2.990762 \\
\hline
\end{tabular}




$\begin{array}{rrrr}1 & -1.984534 & -5.455927 & 5.200252 \\ 1 & 9.750748 & -2.523106 & 0.859712 \\ 1 & 2.071304 & 2.655261 & 0.415125 \\ 1 & -3.986368 & 0.506977 & 1.488711 \\ 1 & -2.897745 & 0.235278 & -2.638030 \\ 1 & -7.023190 & 0.053347 & -1.493717 \\ 1 & -3.048352 & 2.966754 & -0.216010 \\ 1 & 0.403036 & 1.452748 & 1.813669 \\ 1 & -0.864845 & 5.495423 & 2.474496 \\ 1 & 1.094870 & 0.744618 & -3.177197 \\ 1 & 1.898993 & -0.543246 & 2.406047 \\ 1 & 2.135961 & -4.825042 & 2.459349 \\ 1 & 1.410242 & -0.539287 & 4.838164 \\ 1 & 1.646517 & -4.829928 & 4.889017 \\ 1 & 1.283945 & -2.684578 & 6.086972 \\ 1 & 2.524314 & 2.626689 & 4.385049 \\ 1 & 1.160577 & 1.601992 & 3.901468 \\ 1 & 2.358365 & 2.134858 & 2.695200 \\ 1 & 1.036010 & 4.205388 & 5.459449 \\ 1 & -0.283530 & 5.009427 & 4.595176 \\ 1 & -0.406989 & 3.284172 & 4.985466 \\ 1 & 2.665434 & 5.008768 & 3.714736 \\ 1 & 2.450189 & 4.586606 & 2.006145 \\ 1 & 1.382395 & 5.781399 & 2.768902 \\ 1 & -3.497933 & 7.544997 & 1.251437 \\ 1 & -2.858132 & 6.611471 & 2.612136 \\ 1 & -1.768404 & 7.187454 & 1.328152 \\ 1 & -5.178023 & 5.837404 & 0.781172 \\ 1 & -4.742124 & 4.152963 & 0.451434 \\ 1 & -4.585959 & 4.799073 & 2.095253 \\ 1 & -3.600816 & 6.631293 & -1.035869 \\ 1 & -1.883963 & 6.173872 & -0.994416 \\ 1 & -3.113880 & 4.963762 & -1.392181 \\ 1 & -8.790218 & 0.156477 & 1.581191 \\ 1 & -8.372521 & 0.972849 & 0.068585 \\ 1 & -8.243154 & -0.797108 & 0.195956 \\ 1 & -7.278251 & 1.601511 & 2.869119 \\ 1 & -5.564892 & 1.768324 & 2.448278 \\ 1 & -6.806846 & 2.460265 & 1.389170 \\ 1 & -7.101282 & -0.907114 & 3.013341 \\ 1 & -6.452060 & -1.844414 & 1.652227 \\ 1 & -5.371758 & -0.836857 & 2.630332 \\ 1 & -7.067705 & -0.096227 & -5.046137 \\ 1 & -7.433418 & -0.756185 & -3.450299 \\ 1 & -7.273607 & 1.002079 & -3.671684 \\ 1 & -4.944623 & 0.839599 & -5.636835 \\ 1 & -4.955576 & 1.994458 & -4.288231 \\ 1 & -3.573161 & 0.908050 & -4.527314 \\ 1 & -5.108984 & -1.647661 & -5.194342 \\ 1 & -3.816398 & -1.601416 & -3.976283 \\ 1 & -5.408495 & -2.246967 & -3.546017 \\ 1 & 2.517598 & -3.722308 & 0.453106 \\ 1 & 3.397429 & -2.215515 & 0.692969\end{array}$

$1_{\text {C5- }} \psi_{-1 \text { s2a3a4a }}$ 
Number of imaginary frequencies : 0

The smallest frequencies are : $14.9795 \quad 18.5853 \quad 19.8660 \mathrm{~cm}(-1)$

Electronic energy :

$\mathrm{HF}=-4125.4682048$

Zero-point correction=

Thermal correction to Energy=

Thermal correction to Enthalpy=

Thermal correction to Gibbs Free Energy=

Sum of electronic and zero-point Energies=

Sum of electronic and thermal Energies $=$

Sum of electronic and thermal Enthalpies=

Sum of electronic and thermal Free Energies=
1.299534 (Hartree/Particle)

1.377756

1.378700

1.188018

$-4124.168671$

$-4124.090449$

$-4124.089504$

$-4124.280187$

Cartesian Coordinates

$\begin{array}{cccc}6 & 1.946174 & -1.318779 & 2.982580 \\ 6 & 2.197767 & -2.549713 & 2.371524 \\ 6 & 2.193133 & -3.702503 & 3.154667 \\ 6 & 1.939762 & -3.633173 & 4.522499 \\ 6 & 1.686847 & -2.404567 & 5.121538 \\ 6 & 1.690360 & -1.246821 & 4.346060 \\ 6 & 2.531062 & -2.627960 & 0.903196 \\ 7 & 1.574606 & -1.926819 & 0.023731 \\ 15 & 1.925875 & -0.512769 & -0.842256 \\ 45 & 0.185782 & 0.627702 & -1.866783 \\ 6 & 0.142731 & 2.552747 & -1.771710 \\ 8 & 0.196025 & 3.708026 & -1.800151 \\ 6 & 0.526659 & -2.694602 & -0.593761 \\ 6 & -0.774269 & -2.660039 & -0.125045 \\ 6 & -1.809559 & -3.302349 & -0.877497 \\ 6 & -1.481011 & -3.991803 & -2.081522 \\ 6 & -0.127794 & -4.062184 & -2.487106 \\ 6 & 0.843200 & -3.423169 & -1.766733 \\ 6 & -3.171110 & -3.257141 & -0.478788 \\ 6 & -4.149641 & -3.840567 & -1.244559 \\ 6 & -3.819761 & -4.516122 & -2.439595 \\ 6 & -2.511570 & -4.590743 & -2.845693 \\ 6 & -1.110949 & -2.070677 & 1.198966 \\ 6 & -1.397402 & -0.727079 & 1.384073 \\ 6 & -1.780119 & -0.274612 & 2.677429 \\ 6 & -1.880729 & -1.135059 & 3.736786 \\ 6 & -1.614873 & -2.513245 & 3.576711 \\ 6 & -1.217265 & -2.989833 & 2.294650 \\ 6 & -1.746864 & -3.423360 & 4.652656 \\ 6 & -1.511916 & -4.762533 & 4.472457 \\ 6 & -1.112709 & -5.240866 & 3.207015 \\ 6 & -0.962589 & -4.378814 & 2.149155 \\ 15 & -1.484729 & 0.427465 & -0.071249 \\ 6 & -3.235083 & 0.144322 & -0.582473 \\ 6 & -3.557140 & -0.037766 & -1.922678 \\ 6 & -4.879036 & -0.264971 & -2.321284 \\ 6 & -5.859971 & -0.285926 & -1.330199 \\ 6 & -5.573678 & -0.096699 & 0.027206 \\ 6 & -4.245092 & 0.115620 & 0.384307 \\ 6 & -5.267642 & -0.460180 & -3.785572 \\ 6 & -6.072468 & 0.758572 & -4.250025 \\ 6 & -6.695683 & -0.166972 & 1.061008\end{array}$




\begin{tabular}{|c|c|c|c|}
\hline 6 & -7.805071 & 0.825484 & 0.698428 \\
\hline 6 & -1.575251 & 2.080744 & 0.728661 \\
\hline & -2.533307 & 3.001959 & 0.299500 \\
\hline & -2.573254 & 4.290281 & 0.825705 \\
\hline & -1.645111 & 4.622788 & 1.821067 \\
\hline & -0.679155 & 3.726133 & 2.278280 \\
\hline & -0.635254 & 2.458379 & 1.684643 \\
\hline & 0.238323 & 4.038758 & 3.460960 \\
\hline & -0.191419 & 3.152291 & 4.637612 \\
\hline & -3.597504 & 5.325657 & 0.365663 \\
\hline & -4.449466 & 5.773173 & 1.558571 \\
\hline y & 3.138108 & -0.878048 & -1.950723 \\
\hline & 4.394730 & -1.280348 & -1.567675 \\
\hline & 5.268180 & -0.397123 & -0.952774 \\
\hline & 6.524883 & -0.903447 & -0.487800 \\
\hline & 6.889283 & -2.253708 & -0.774001 \\
\hline b & 5.993875 & -3.077198 & -1.497441 \\
\hline & 4.766245 & -2.606381 & -1.874183 \\
\hline & 8.133290 & -2.753262 & -0.317848 \\
\hline & 8.983163 & -1.966801 & 0.416336 \\
\hline ) & 8.612379 & -0.642617 & 0.730931 \\
\hline ) & 7.419151 & -0.125164 & 0.291390 \\
\hline & 4.846746 & 1.010433 & -0.765352 \\
\hline 5 & 3.640681 & 1.264022 & -0.141877 \\
\hline ) & 3.134780 & 2.563102 & 0.046120 \\
\hline & 3.861666 & 3.637186 & -0.391204 \\
\hline & 5.092126 & 3.448337 & -1.064829 \\
\hline ) & 5.586617 & 2.125037 & -1.277694 \\
\hline 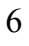 & 6.777225 & 1.973573 & -2.033654 \\
\hline$b$ & 7.458012 & 3.065501 & -2.511777 \\
\hline b & 6.987702 & 4.372211 & -2.264470 \\
\hline 6 & 5.825278 & 4.553906 & -1.560882 \\
\hline & 2.883751 & 0.201993 & 0.317001 \\
\hline & 0.143488 & 5.497723 & 3.901918 \\
\hline b & 1.700812 & 3.744167 & 3.120238 \\
\hline 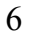 & -4.537259 & 4.773454 & -0.704379 \\
\hline 6 & -2.860889 & 6.534031 & -0.221969 \\
\hline$b$ & -6.205088 & 0.166803 & 2.468417 \\
\hline 6 & -7.263789 & -1.590617 & 1.077769 \\
\hline 6 & -4.043488 & -0.610346 & -4.685166 \\
\hline & -6.118689 & -1.724272 & -3.942637 \\
\hline 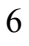 & -0.463510 & -0.538898 & -3.287861 \\
\hline & -0.642955 & -1.159625 & -4.246438 \\
\hline 1 & 7.143388 & 0.893399 & 0.552678 \\
\hline & -2.015881 & 0.775249 & 2.830968 \\
\hline 1 & 8.394397 & -3.783438 & -0.557103 \\
\hline 1 & -2.046713 & -3.034757 & 5.625167 \\
\hline 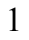 & 5.434234 & 5.554341 & -1.380283 \\
\hline 1 & -3.441250 & -2.729124 & 0.434403 \\
\hline & -5.190753 & -3.775891 & -0.932826 \\
\hline 1 & 3.486902 & 4.650440 & -0.252732 \\
\hline t & -2.183632 & -0.766090 & 4.716275 \\
\hline 1 & 6.291622 & -4.097669 & -1.733023 \\
\hline 1 & 8.365773 & 2.920354 & -3.093181 \\
\hline 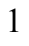 & 4.059899 & -3.228281 & -2.419243 \\
\hline & -2.240814 & -5.109403 & -3.764241 \\
\hline
\end{tabular}




\begin{tabular}{|c|c|c|c|}
\hline 1 & 0.122365 & -4.603977 & -3.397800 \\
\hline & -0.917670 & -6.302111 & 3.069109 \\
\hline & 7.147611 & 0.973771 & -2.244446 \\
\hline & -0.639756 & -4.763551 & 1.183958 \\
\hline & 1.880670 & -3.441804 & -2.093818 \\
\hline 1 & 7.539268 & 5.228746 & -2.645021 \\
\hline 1 & 9.275763 & -0.026118 & 1.333407 \\
\hline 1 & -4.605239 & -4.978517 & -3.033911 \\
\hline 1 & -1.625663 & -5.456317 & 5.302432 \\
\hline 1 & 9.933978 & -2.361973 & 0.766184 \\
\hline 1 & 2.169061 & 2.681244 & 0.533207 \\
\hline 1 & -3.982727 & 0.260059 & 1.429000 \\
\hline 1 & -2.757212 & -0.003713 & -2.656725 \\
\hline 1 & -6.895167 & -0.460348 & -1.623859 \\
\hline 1 & -3.247676 & 2.700909 & -0.462500 \\
\hline 1 & 0.113826 & 1.733819 & 2.009983 \\
\hline 1 & -1.689520 & 5.618494 & 2.256776 \\
\hline 1 & 1.259767 & 0.956283 & -2.987986 \\
\hline 1 & 1.957745 & -0.414649 & 2.377473 \\
\hline 1 & 2.379955 & -4.668611 & 2.684769 \\
\hline 1 & 1.497519 & -0.279989 & 4.808335 \\
\hline 1 & 1.929956 & -4.544319 & 5.117574 \\
\hline 1 & 1.482605 & -2.348484 & 6.188925 \\
\hline 1 & 0.834453 & 5.675134 & 4.735506 \\
\hline 1 & 0.416305 & 6.187563 & 3.092806 \\
\hline 1 & -0.862473 & 5.759533 & 4.253358 \\
\hline 1 & 0.432646 & 3.353423 & \\
\hline 1 & -1.237549 & 3.344007 & 4.911361 \\
\hline 1 & -0.099264 & 2.084717 & \\
\hline 1 & 2.341350 & 3.977592 & 3.980959 \\
\hline 1 & 1.871922 & 2.689563 & 2.870217 \\
\hline 1 & 2.041746 & 4.355502 & 2.274322 \\
\hline 1 & -5.257002 & 5.548596 & -0.996101 \\
\hline 1 & -3.995995 & 4.470131 & -1.609805 \\
\hline 1 & -5.111748 & 3.909164 & -0.343534 \\
\hline 1 & -5.192316 & 6.515585 & \\
\hline 1 & -4.989180 & 4.923220 & 1.996808 \\
\hline 1 & -3.847023 & 6.232173 & 2.351579 \\
\hline 1 & -3.580033 & 7.285965 & -0.573914 \\
\hline 1 & -2.209006 & 7.017685 & 0.515612 \\
\hline 1 & -2.236839 & 6.234385 & -1.073855 \\
\hline 1 & -7.045222 & 0.117860 & 3.172489 \\
\hline 1 & -5.442486 & -0.541257 & 2.819593 \\
\hline 1 & -5.784362 & 1.179773 & 2.524331 \\
\hline 1 & -8.608521 & 0.786279 & 1.445951 \\
\hline 1 & -7.419735 & 1.853146 & 0.670403 \\
\hline 1 & -8.255480 & 0.610734 & -0.277803 \\
\hline 1 & -8.081795 & -1.670183 & 1.806569 \\
\hline 1 & -7.659193 & -1.887063 & 0.098071 \\
\hline 1 & -6.487891 & -2.315022 & 1.361112 \\
\hline 1 & -4.364842 & -0.791639 & -5.718566 \\
\hline 1 & -3.419141 & 0.292450 & -4.693218 \\
\hline 1 & -3.418035 & -1.458486 & -4.374933 \\
\hline 1 & -6.356887 & 0.651003 & -5.305603 \\
\hline 1 & -6.992830 & 0.886324 & -3.666182 \\
\hline 1 & -5.482734 & 1.679330 & -4.150466 \\
\hline
\end{tabular}




$\begin{array}{llll}1 & -6.390394 & -1.866686 & -4.997000 \\ 1 & -5.559747 & -2.610645 & -3.613933 \\ 1 & -7.052015 & -1.679630 & -3.368677 \\ 1 & 2.589625 & -3.679767 & 0.585533 \\ 1 & 3.528572 & -2.198765 & 0.743738\end{array}$

$1_{\text {C5- }} \psi_{-1 \text { s2a3a4s }}$

Number of imaginary frequencies : 0

The smallest frequencies are : $10.7830 \quad 18.1231 \quad 19.0862 \mathrm{~cm}(-1)$

Electronic energy : $\quad H F=-4125.4688509$

Zero-point correction $=\quad 1.299588($ Hartree/Particle $)$

Thermal correction to Energy $=\quad 1.377793$

Thermal correction to Enthalpy= $\quad 1.378737$

Thermal correction to Gibbs Free Energy $=\quad 1.188060$

Sum of electronic and zero-point Energies $=\quad-4124.169263$

Sum of electronic and thermal Energies $=\quad-4124.091058$

Sum of electronic and thermal Enthalpies $=\quad-4124.090114$

Sum of electronic and thermal Free Energies $=\quad-4124.280791$

Cartesian Coordinates

$\begin{array}{cccc}6 & -2.613447 & 2.939753 & 0.290598 \\ 6 & -1.615527 & 2.056778 & 0.707528 \\ 6 & -0.683912 & 2.468089 & 1.657497 \\ 6 & -0.776891 & 3.729255 & 2.259218 \\ 6 & -1.785279 & 4.586534 & 1.817574 \\ 6 & -2.704350 & 4.221612 & 0.825401 \\ 15 & -1.472077 & 0.406240 & -0.089531 \\ 6 & -1.381500 & -0.740841 & 1.372101 \\ 6 & -1.089163 & -2.083699 & 1.191184 \\ 6 & -1.204097 & -3.002791 & 2.285251 \\ 6 & -1.614767 & -2.526998 & 3.563143 \\ 6 & -1.880963 & -1.148657 & 3.721287 \\ 6 & -1.773138 & -0.287961 & 2.662467 \\ 6 & -1.755452 & -3.437708 & 4.637576 \\ 6 & -1.515901 & -4.776284 & 4.459251 \\ 6 & -1.103595 & -5.253709 & 3.197520 \\ 6 & -0.945318 & -4.391105 & 2.141417 \\ 6 & -0.744006 & -2.666027 & -0.132947 \\ 6 & 0.556569 & -2.679070 & -0.603665 \\ 6 & 0.878094 & -3.384841 & -1.789251 \\ 6 & -0.088178 & -4.020220 & -2.519206 \\ 6 & -1.441145 & -3.971619 & -2.109639 \\ 6 & -1.774115 & -3.308066 & -0.892440 \\ 6 & -3.135248 & -3.282696 & -0.490144 \\ 6 & -4.109131 & -3.862479 & -1.264366 \\ 6 & -3.775091 & -4.513503 & -2.471818 \\ 6 & -2.467268 & -4.567332 & -2.882328 \\ 7 & 1.596920 & -1.908460 & 0.023473 \\ 15 & 1.950051 & -0.491785 & -0.837048 \\ 8 & 3.171401 & -0.853350 & -1.936588 \\ 6 & 4.427193 & -1.249385 & -1.544099 \\ 6 & 5.292382 & -0.360226 & -0.926234 \\ 6 & 6.549328 & -0.858270 & -0.453367 \\ 6 & 6.922475 & -2.207385 & -0.733680\end{array}$




\begin{tabular}{|c|c|c|c|}
\hline 6 & 6.034768 & -3.038011 & -1.458429 \\
\hline 6 & 4.806757 & -2.574821 & -1.843361 \\
\hline f & 7.435533 & -0.072694 & 0.327690 \\
\hline f & 8.629504 & -0.582173 & 0.774394 \\
\hline & 9.009042 & -1.905278 & 0.465675 \\
\hline ) & 8.166978 & -2.698703 & -0.270015 \\
\hline & 4.861494 & 1.044935 & -0.742989 \\
\hline 6 & 3.651019 & 1.291793 & -0.125385 \\
\hline 5 & 3.136713 & 2.588168 & 0.058368 \\
\hline 6 & 3.858884 & 3.666133 & -0.377159 \\
\hline 6 & 5.093379 & 3.483867 & -1.045155 \\
\hline 6 & 5.596812 & 2.163277 & -1.253685 \\
\hline 6 & 5.821668 & 4.593072 & -1.540245 \\
\hline 6 & 6.987898 & 4.417247 & -2.239012 \\
\hline 6 & 7.467234 & 3.113007 & -2.482066 \\
\hline 6 & 6.791366 & 2.017763 & -2.004555 \\
\hline 8 & 2.897103 & 0.225564 & 0.329569 \\
\hline 6 & 0.139140 & 4.073376 & 3.433984 \\
\hline 6 & -0.002843 & 5.527795 & 3.877027 \\
\hline 6 & -3.783185 & 5.208095 & 0.384174 \\
\hline 6 & -4.677950 & 4.631898 & -0.711756 \\
\hline 6 & -3.211799 & 0.090271 & -0.616236 \\
\hline 6 & -3.533015 & -0.082746 & -1.961559 \\
\hline 6 & -4.851856 & -0.303037 & -2.361084 \\
\hline 6 & -5.842144 & -0.331057 & -1.371229 \\
\hline 6 & -5.554487 & -0.160392 & -0.016748 \\
\hline 6 & -4.221610 & 0.049880 & 0.341335 \\
\hline 6 & -6.612857 & -0.204667 & 1.082841 \\
\hline 6 & -8.010384 & -0.482149 & 0.536143 \\
\hline 6 & -5.240068 & -0.485412 & -3.826970 \\
\hline 6 & -4.015969 & -0.613163 & -4.730066 \\
\hline 45 & 0.211200 & 0.646334 & -1.866307 \\
\hline 6 & -0.415801 & -0.505142 & -3.310756 \\
\hline 8 & -0.584171 & -1.110618 & -4.280986 \\
\hline 6 & 0.136334 & 2.568999 & -1.751976 \\
\hline 8 & 0.167400 & 3.725313 & -1.766871 \\
\hline 6 & -6.260410 & -1.318577 & 2.076504 \\
\hline 6 & -6.641781 & 1.144209 & 1.811163 \\
\hline 6 & -6.078435 & -1.756574 & -3.996758 \\
\hline 6 & -6.057464 & 0.730360 & -4.277409 \\
\hline 6 & 2.549486 & -2.606470 & 0.909340 \\
\hline 6 & 2.203189 & -2.537973 & 2.375131 \\
\hline 6 & 2.204981 & -3.695641 & 3.151208 \\
\hline 6 & 1.941636 & -3.637655 & 4.517596 \\
\hline 6 & 1.672219 & -2.415442 & 5.122417 \\
\hline 6 & 1.668465 & -1.253010 & 4.353991 \\
\hline 6 & 1.934163 & -1.313299 & 2.991764 \\
\hline 6 & 1.607351 & 3.829768 & 3.077954 \\
\hline 6 & -0.247026 & 3.170764 & 4.613405 \\
\hline 6 & -3.118809 & 6.477256 & -0.159457 \\
\hline 6 & -4.668553 & 5.564247 & 1.583427 \\
\hline 1 & 7.152995 & 0.945143 & 0.584494 \\
\hline 1 & -2.010993 & 0.761568 & 2.814211 \\
\hline 1 & 8.434884 & -3.728163 & -0.504808 \\
\hline 1 & -2.065031 & -3.050014 & 5.607391 \\
\hline 1 & 5.423727 & 5.591388 & -1.363013 \\
\hline
\end{tabular}




\begin{tabular}{|c|c|c|c|}
\hline & -3.409019 & -2.772360 & 0.432334 \\
\hline & -5.150285 & -3.812840 & -0.950456 \\
\hline & 3.477116 & 4.677198 & -0.242060 \\
\hline & -2.190543 & -0.779738 & 4.698704 \\
\hline & 6.338587 & -4.057971 & -1.688397 \\
\hline & 8.378106 & 2.972436 & -3.059715 \\
\hline & 4.105961 & -3.203171 & -2.388224 \\
\hline & -2.193474 & -5.066757 & -3.810584 \\
\hline & 0.165477 & -4.542158 & -3.440482 \\
\hline & -0.905065 & -6.314537 & 3.061431 \\
\hline & 7.168696 & 1.019858 & -2.212040 \\
\hline & -0.612702 & -4.774178 & 1.178871 \\
\hline & 1.914404 & -3.385543 & -2.120392 \\
\hline & 7.535627 & 5.276465 & -2.619057 \\
\hline & 9.286702 & 0.039851 & 1.377972 \\
\hline & -4.557205 & -4.973647 & -3.072315 \\
\hline & -1.636061 & -5.470506 & 5.287951 \\
\hline & 9.960369 & -2.294115 & 0.821183 \\
\hline & 2.167462 & 2.701167 & 0.539152 \\
\hline & -3.967658 & 0.185074 & 1.391656 \\
\hline & -2.731920 & -0.043417 & -2.694005 \\
\hline & -6.874223 & -0.494195 & -1.675172 \\
\hline & -3.320232 & 2.614199 & -0.467679 \\
\hline & 0.097889 & 1.773418 & 1.971677 \\
\hline & -1.871929 & 5.574972 & 2.263581 \\
\hline & 1.295099 & 1.001739 & -2.970135 \\
\hline & 1.939441 & -0.404284 & 2.393141 \\
\hline & 2.404952 & -4.656745 & 2.676495 \\
\hline & 1.460325 & -0.291775 & 4.821180 \\
\hline & 1.937433 & -4.552533 & 5.106958 \\
\hline & 1.460083 & -2.368006 & 6.188681 \\
\hline & 0.690287 & 5.729501 & 4.703272 \\
\hline & 0.236110 & 6.227047 & 3.065304 \\
\hline & -1.013496 & 5.753356 & 4.239875 \\
\hline & 0.377899 & 3.393119 & 5.489128 \\
\hline & -1.296730 & 3.324163 & 4.897498 \\
\hline & -0.118193 & 2.107588 & 4.370062 \\
\hline & 2.249414 & 4.092436 & 3.929005 \\
\hline & 1.812566 & 2.779664 & 2.834265 \\
\hline & 1.915264 & 4.446344 & 2.223137 \\
\hline 1 & -5.444721 & 5.367766 & -0.984793 \\
\hline 1 & -4.111049 & 4.393518 & -1.621096 \\
\hline 1 & -5.196662 & 3.719628 & -0.385533 \\
\hline 1 & -5.449645 & 6.275416 & 1.282675 \\
\hline 1 & -5.162574 & 4.669488 & 1.985354 \\
\hline 1 & -4.096631 & 6.025944 & 2.397382 \\
\hline & -3.880980 & 7.190506 & -0.501097 \\
\hline 1 & -2.509649 & 6.982312 & 0.599916 \\
\hline & -2.465779 & 6.243008 & -1.010209 \\
\hline 1 & -8.731555 & -0.509056 & 1.362739 \\
\hline 1 & -8.341128 & 0.297596 & -0.161901 \\
\hline 1 & -8.063578 & -1.450432 & 0.021415 \\
\hline 1 & -7.371570 & 1.121148 & 2.631706 \\
\hline 1 & -5.665751 & 1.405055 & 2.239911 \\
\hline 1 & -6.931344 & 1.951957 & 1.125921 \\
\hline 1 & -7.006610 & -1.362773 & 2.881230 \\
\hline
\end{tabular}




\begin{tabular}{|c|c|c|c|}
\hline & -6.245615 & -2.297061 & 1.577866 \\
\hline & -5.278202 & -1.169381 & 2.543331 \\
\hline & -4.337456 & -0.782418 & -5.765472 \\
\hline & -3.398770 & 0.294555 & -4.725919 \\
\hline & -3.383352 & -1.460658 & -4.432849 \\
\hline & -6.340023 & 0.632424 & -5.334420 \\
\hline & -6.979884 & 0.841715 & -3.693567 \\
\hline & -5.477241 & 1.655878 & -4.166119 \\
\hline & -6.348124 & -1.891649 & -5.052647 \\
\hline & -5.510703 & -2.640476 & -3.676082 \\
\hline & -7.012233 & -1.726613 & -3.422817 \\
\hline & 2.618765 & -3.656425 & 0.587601 \\
\hline & 3.545292 & -2.169414 & 0.76079 \\
\hline
\end{tabular}

$1_{\mathrm{C} 5-} \psi_{-1 \mathrm{~s} 2 \mathrm{a3s4a}}$

Number of imaginary frequencies : 0

The smallest frequencies are : $13.191120 .323124 .4076 \mathrm{~cm}(-1)$

Electronic energy : $\quad H F=-4125.4692248$

Zero-point correction $=\quad 1.302017($ Hartree/Particle $)$

Thermal correction to Energy= $\quad 1.379613$

Thermal correction to Enthalpy $=\quad 1.380557$

Thermal correction to Gibbs Free Energy= $\quad 1.192847$

Sum of electronic and zero-point Energies $=\quad-4124.167208$

Sum of electronic and thermal Energies $=\quad-4124.089612$

Sum of electronic and thermal Enthalpies $=\quad-4124.088668$

Sum of electronic and thermal Free Energies $=\quad-4124.276378$

Cartesian Coordinates

$\begin{array}{cccc}6 & 5.727486 & 4.468758 & -1.701759 \\ 6 & 4.991254 & 3.373369 & -1.188008 \\ 6 & 5.489583 & 2.046541 & -1.366959 \\ 6 & 6.687286 & 1.880184 & -2.108408 \\ 6 & 7.370914 & 2.962261 & -2.604573 \\ 6 & 6.896525 & 4.273336 & -2.390604 \\ 6 & 4.746634 & 0.942457 & -0.836705 \\ 6 & 3.533871 & 1.207513 & -0.231007 \\ 6 & 3.023060 & 2.509720 & -0.078165 \\ 6 & 3.753827 & 3.574945 & -0.530916 \\ 6 & 5.173292 & -0.468241 & -0.986667 \\ 6 & 4.309249 & -1.368888 & -1.589235 \\ 6 & 4.685422 & -2.702065 & -1.856289 \\ 6 & 5.909586 & -3.160568 & -1.453755 \\ 6 & 6.795705 & -2.316484 & -0.742503 \\ 6 & 6.425459 & -0.960042 & -0.494962 \\ 6 & 8.035558 & -2.801184 & -0.259771 \\ 6 & 8.875494 & -1.994170 & 0.463504 \\ 6 & 8.498380 & -0.663289 & 0.740180 \\ 6 & 7.309144 & -0.160120 & 0.274240 \\ 8 & 3.056384 & -0.979289 & -1.997225 \\ 15 & 1.832955 & -0.589252 & -0.911804 \\ 8 & 2.775037 & 0.153091 & 0.243220 \\ 7 & 1.472517 & -1.982666 & -0.016783 \\ 6 & 0.422715 & -2.758584 & -0.619799 \\ 6 & -0.883104 & -2.682362 & -0.171263\end{array}$




\begin{tabular}{|c|c|c|c|}
\hline 6 & -1.921654 & -3.325106 & -0.917850 \\
\hline 6 & -1.587770 & -4.094707 & -2.070599 \\
\hline 6 & -0.230233 & -4.205672 & -2.453620 \\
\hline 6 & 0.741592 & -3.540342 & -1.757363 \\
\hline 6 & -2.618152 & -4.731504 & -2.804031 \\
\hline 6 & -3.929862 & -4.609582 & -2.422255 \\
\hline 6 & -4.266666 & -3.834373 & -1.291484 \\
\hline 6 & -3.289362 & -3.212550 & -0.554902 \\
\hline 6 & -1.223531 & -2.084034 & 1.147313 \\
\hline 6 & -1.483615 & -0.737721 & 1.340717 \\
\hline 6 & -1.881400 & -0.290182 & 2.632875 \\
\hline 6 & -2.016454 & -1.156581 & 3.682370 \\
\hline 6 & -1.767536 & -2.537440 & 3.515849 \\
\hline 6 & -1.359301 & -3.010236 & 2.236491 \\
\hline 6 & -1.113437 & -4.400265 & 2.088191 \\
\hline 6 & -1.282006 & -5.266176 & 3.140459 \\
\hline 6 & -1.693588 & -4.791153 & 4.402725 \\
\hline 6 & -1.921002 & -3.450877 & 4.585676 \\
\hline 15 & -1.499925 & 0.453680 & -0.085547 \\
\hline 6 & -1.364507 & 2.070007 & 0.777664 \\
\hline 6 & -0.384480 & 2.261037 & 1.748485 \\
\hline 6 & -0.265588 & 3.481588 & 2.424420 \\
\hline 6 & -1.101130 & 4.524964 & 2.026190 \\
\hline 6 & -2.056790 & 4.382470 & 1.010679 \\
\hline 6 & -2.192162 & 3.133038 & 0.409776 \\
\hline 6 & 0.672311 & 3.583036 & 3.628114 \\
\hline 6 & 0.751033 & 5.000933 & 4.188914 \\
\hline 6 & -2.927528 & 5.575112 & 0.620329 \\
\hline 6 & -3.889752 & 5.239823 & -0.516939 \\
\hline 6 & 2.421056 & -2.665241 & \\
\hline 6 & 2.061197 & -2.577687 & 2.345002 \\
\hline 6 & 2.037063 & -3.728033 & 3.131070 \\
\hline 6 & 1.764030 & -3.653346 & 4.495000 \\
\hline 6 & 1.514212 & -2.421153 & 5.087517 \\
\hline 6 & 1.536018 & -1.265441 & 4.308951 \\
\hline 6 & 1.806309 & -1.342875 & 2.948333 \\
\hline 45 & 0.088374 & 0.515636 & -1.964899 \\
\hline 6 & -0.697961 & -0.679134 & -3.282402 \\
\hline 8 & -1.006157 & -1.326482 & -4.189954 \\
\hline 6 & 0.101910 & 2.444058 & -1.991406 \\
\hline 8 & 0.180513 & 3.591672 & -2.113839 \\
\hline 6 & -3.280000 & 0.360011 & -0.558451 \\
\hline 6 & -4.287941 & 0.386060 & 0.415798 \\
\hline 6 & -5.625446 & 0.256887 & 0.062324 \\
\hline 6 & -5.937261 & 0.107296 & -1.300030 \\
\hline 6 & -4.964119 & 0.094009 & -2.292950 \\
\hline 6 & -3.626313 & 0.223097 & -1.893367 \\
\hline 6 & -6.745839 & 0.242835 & 1.100316 \\
\hline 6 & -6.222705 & 0.460096 & 2.518748 \\
\hline 6 & -5.278676 & -0.118181 & -3.771391 \\
\hline 6 & -6.774603 & -0.031518 & -4.064258 \\
\hline 6 & -7.450743 & -1.117698 & 1.056514 \\
\hline 6 & -7.754638 & 1.353743 & 0.790584 \\
\hline 6 & -4.785043 & -1.513454 & -4.172641 \\
\hline 6 & -4.564812 & 0.937637 & -4.622966 \\
\hline 6 & -2.030411 & 6.728619 & 0.159246 \\
\hline
\end{tabular}




\begin{tabular}{|c|c|c|c|}
\hline 6 & -3.754900 & 6.021631 & 1.830805 \\
\hline 6 & 2.092687 & 3.140003 & 3.268048 \\
\hline 6 & 0.121576 & 2.662172 & 4.725660 \\
\hline 1 & 7.028161 & 0.863932 & 0.506754 \\
\hline 1 & -2.090030 & 0.764134 & 2.795650 \\
\hline 1 & 8.301524 & -3.836537 & -0.469531 \\
\hline 1 & -2.229256 & -3.063826 & 5.556155 \\
\hline 1 & 5.333293 & 5.472340 & -1.547389 \\
\hline 1 & -3.562779 & -2.608467 & 0.309325 \\
\hline 1 & -5.310837 & -3.721871 & -1.005417 \\
\hline 1 & 3.376134 & 4.590118 & -0.417262 \\
\hline 1 & -2.327147 & -0.789707 & 4.660256 \\
\hline 1 & 6.211368 & -4.186455 & -1.658659 \\
\hline 1 & 8.284080 & 2.805663 & -3.174423 \\
\hline 1 & 3.984122 & -3.338669 & -2.391231 \\
\hline 1 & -2.344514 & -5.315986 & -3.681252 \\
\hline 1 & 0.023356 & -4.802140 & -3.328525 \\
\hline 1 & -1.092011 & -6.327950 & 2.999794 \\
\hline 1 & 7.060854 & 0.876480 & -2.293508 \\
\hline 1 & -0.780857 & -4.784475 & 1.126117 \\
\hline 1 & 1.782801 & -3.586854 & -2.071329 \\
\hline 1 & 7.450179 & 5.122090 & -2.785306 \\
\hline 1 & 9.153650 & -0.030107 & 1.334177 \\
\hline 1 & -4.714638 & -5.098921 & -2.995142 \\
\hline 1 & -1.822537 & -5.487687 & 5.228156 \\
\hline 1 & 9.823152 & -2.378058 & 0.833887 \\
\hline 1 & 2.051122 & 2.637809 & 0.395702 \\
\hline 1 & -4.012698 & 0.498742 & 1.460557 \\
\hline 1 & -2.834377 & 0.210233 & -2.637596 \\
\hline 1 & -6.982824 & -0.002530 & -1.581494 \\
\hline 1 & -2.940389 & 2.972666 & -0.363467 \\
\hline 1 & 0.260188 & 1.424816 & 2.027042 \\
\hline 1 & -1.020412 & 5.487577 & 2.526539 \\
\hline 1 & 1.123230 & 0.734731 & -3.147762 \\
\hline 1 & 1.827326 & -0.439367 & 2.339805 \\
\hline 1 & 2.225437 & -4.696413 & 2.666627 \\
\hline 1 & 1.349521 & -0.295031 & 4.768402 \\
\hline 1 & 1.738719 & -4.562756 & 5.092225 \\
\hline 1 & 1.298172 & -2.359954 & 6.152257 \\
\hline 1 & 1.447335 & 5.022657 & 5.036613 \\
\hline 1 & 1.117922 & 5.716289 & 3.441539 \\
\hline 1 & -0.220174 & 5.354755 & 4.557362 \\
\hline 1 & 0.760560 & 2.702048 & 5.618604 \\
\hline 1 & -0.892545 & 2.964831 & 5.019687 \\
\hline 1 & 0.071947 & 1.616983 & 4.391492 \\
\hline 1 & 2.733488 & 3.180083 & 4.158852 \\
\hline 1 & 2.134736 & 2.111552 & 2.886362 \\
\hline 1 & 2.535252 & 3.797976 & 2.508958 \\
\hline 1 & -4.483746 & 6.127208 & -0.769256 \\
\hline 1 & -3.356548 & 4.930560 & -1.425612 \\
\hline 1 & -4.592564 & 4.441834 & -0.242342 \\
\hline 1 & -4.389873 & 6.876984 & 1.563546 \\
\hline 1 & -4.408625 & 5.211512 & 2.180146 \\
\hline 1 & -3.123121 & 6.329234 & 2.672894 \\
\hline 1 & -2.642047 & 7.592268 & -0.134444 \\
\hline 1 & -1.345347 & 7.061552 & 0.948208 \\
\hline
\end{tabular}




$\begin{array}{rrrr}1 & -1.425164 & 6.431212 & -0.706703 \\ 1 & -7.060874 & 0.442154 & 3.226626 \\ 1 & -5.518433 & -0.325569 & 2.823752 \\ 1 & -5.721436 & 1.431149 & 2.626006 \\ 1 & -8.558991 & 1.356590 & 1.538283 \\ 1 & -7.271526 & 2.339412 & 0.809972 \\ 1 & -8.221349 & 1.229589 & -0.193769 \\ 1 & -8.260919 & -1.153558 & 1.797246 \\ 1 & -7.891006 & -1.323007 & 0.073121 \\ 1 & -6.747014 & -1.929405 & 1.285558 \\ 1 & -6.947286 & -0.158987 & -5.140274 \\ 1 & -7.342945 & -0.817862 & -3.551200 \\ 1 & -7.192581 & 0.941360 & -3.773713 \\ 1 & -4.818644 & 0.799270 & -5.682102 \\ 1 & -4.866820 & 1.952366 & -4.332417 \\ 1 & -3.472474 & 0.875483 & -4.542462 \\ 1 & -4.965124 & -1.691176 & -5.241776 \\ 1 & -3.710158 & -1.637109 & -3.985531 \\ 1 & -5.310610 & -2.291807 & -3.602946 \\ 1 & 2.497015 & -3.719508 & 0.577932 \\ 1 & 3.415996 & -2.225736 & 0.737278\end{array}$

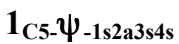

Number of imaginary frequencies : 0

The smallest frequencies are : $13.8280 \quad 17.2846 \quad 21.9812 \mathrm{~cm}(-1)$

Electronic energy : $\quad \mathrm{HF}=-4125.4694341$

Zero-point correction $=\quad 1.300067$ (Hartree/Particle)

Thermal correction to Energy= $\quad 1.378081$

Thermal correction to Enthalpy= $\quad 1.379026$

Thermal correction to Gibbs Free Energy= 1.189433

Sum of electronic and zero-point Energies $=\quad-4124.169367$

Sum of electronic and thermal Energies $=\quad-4124.091353$

Sum of electronic and thermal Enthalpies $=\quad-4124.090409$

Sum of electronic and thermal Free Energies $=\quad-4124.280001$

Cartesian Coordinates

$\begin{array}{cccc}6 & 1.807856 & -1.281835 & 2.967398 \\ 6 & 2.061468 & -2.525233 & 2.381404 \\ 6 & 2.034668 & -3.664636 & 3.183280 \\ 6 & 1.762321 & -3.570607 & 4.546094 \\ 6 & 1.514806 & -2.329805 & 5.121684 \\ 6 & 1.537533 & -1.185218 & 4.326838 \\ 6 & 2.427518 & -2.634435 & 0.922996 \\ 7 & 1.487832 & -1.960485 & 0.007060 \\ 15 & 1.858400 & -0.577015 & -0.898727 \\ 45 & 0.121851 & 0.526618 & -1.965401 \\ 6 & 0.117817 & 2.454871 & -1.964074 \\ 8 & 0.179056 & 3.606685 & -2.052018 \\ 6 & 0.442375 & -2.741913 & -0.597205 \\ 6 & -0.863972 & -2.675352 & -0.148468 \\ 6 & -1.898595 & -3.327326 & -0.892309 \\ 6 & -1.560134 & -4.091778 & -2.047206 \\ 6 & -0.202356 & -4.190832 & -2.432482 \\ 6 & 0.765649 & -3.520572 & -1.735685\end{array}$




\begin{tabular}{|c|c|c|c|}
\hline 6 & -3.266600 & -3.229760 & -0.524827 \\
\hline & -4.239155 & -3.858671 & -1.261665 \\
\hline & -3.897970 & -4.626272 & -2.396322 \\
\hline & -2.586028 & -4.735405 & -2.780932 \\
\hline & -1.209533 & -2.065455 & 1.162735 \\
\hline & -1.474889 & -0.717503 & 1.335056 \\
\hline & -1.875147 & -0.251430 & 2.619539 \\
\hline & -2.010770 & -1.103277 & 3.681175 \\
\hline & -1.760373 & -2.486073 & 3.534669 \\
\hline & -1.346168 & -2.976105 & 2.263817 \\
\hline & -1.916965 & -3.384782 & 4.616514 \\
\hline & -1.687831 & -4.727202 & 4.452880 \\
\hline & -1.270458 & -5.219365 & 3.198980 \\
\hline & -1.097795 & -4.367848 & 2.135802 \\
\hline & -1.492120 & 0.449365 & -0.111978 \\
\hline & -3.260939 & 0.312283 & -0.614142 \\
\hline & -3.592190 & 0.198725 & -1.959138 \\
\hline & -4.919532 & 0.049472 & -2.372574 \\
\hline & -5.910193 & 0.023400 & -1.389329 \\
\hline & -5.613419 & 0.146187 & -0.026634 \\
\hline & -4.277461 & 0.292856 & 0.342149 \\
\hline & -5.217825 & -0.155498 & -3.855935 \\
\hline & -4.497833 & 0.903023 & -4.698579 \\
\hline & -6.677348 & 0.100429 & 1.068421 \\
\hline 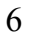 & -6.647160 & 1.410913 & 1.863477 \\
\hline & -1.413032 & 2.076271 & 0.736040 \\
\hline 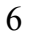 & -2.304039 & 3.095849 & 0.397861 \\
\hline f & -2.223912 & 4.343804 & 1.011944 \\
\hline & -1.251858 & 4.528727 & 2.004345 \\
\hline 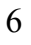 & -0.351109 & 3.528175 & 2.371227 \\
\hline ) & -0.421521 & 2.309584 & 1.686007 \\
\hline 5 & 0.599516 & 3.666270 & 3.561353 \\
\hline 6 & 0.086026 & 2.739744 & 4.672243 \\
\hline 5 & -3.178215 & 5.484519 & 0.664759 \\
\hline 6 & -3.997945 & 5.857067 & 1.905214 \\
\hline & 3.087718 & -0.984206 & -1.971843 \\
\hline 5 & 4.338724 & -1.367287 & -1.551897 \\
\hline 6 & 5.199427 & -0.456513 & -0.959930 \\
\hline 5 & 6.450252 & -0.938432 & -0.455776 \\
\hline 6 & 6.823474 & -2.298058 & -0.680292 \\
\hline 6 & 5.940634 & -3.154718 & -1.380495 \\
\hline 6 & 4.717328 & -2.704443 & -1.794962 \\
\hline 6 & 8.062373 & -2.772883 & -0.185475 \\
\hline 6 & 8.898370 & -1.952933 & 0.527782 \\
\hline 6 & 8.518042 & -0.618436 & 0.781969 \\
\hline 6 & 7.329709 & -0.124814 & 0.303805 \\
\hline 6 & 4.769525 & 0.955297 & -0.833205 \\
\hline 6 & 3.554416 & 1.226651 & -0.235325 \\
\hline 6 & 3.038961 & 2.529467 & -0.106576 \\
\hline 6 & 3.767889 & 3.589437 & -0.574238 \\
\hline 6 & 5.008078 & 3.380782 & -1.223885 \\
\hline 6 & 5.510961 & 2.052680 & -1.379077 \\
\hline 6 & 6.711563 & 1.877570 & -2.113757 \\
\hline 6 & 7.393795 & 2.953279 & -2.625426 \\
\hline 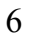 & 6.914897 & 4.266305 & -2.434757 \\
\hline & 5.742930 & 4.469676 & -1.753219 \\
\hline
\end{tabular}




\begin{tabular}{|c|c|c|c|}
\hline 8 & 2.796012 & 0.177703 & 0.252682 \\
\hline 6 & 0.646422 & 5.090973 & 4.108790 \\
\hline 6 & 2.027202 & 3.257440 & 3.190740 \\
\hline 6 & -4.150773 & 5.104886 & -0.449717 \\
\hline 0 & -2.369628 & 6.699444 & 0.198258 \\
\hline 6 & -8.085290 & -0.083563 & 0.508447 \\
\hline y & -6.379087 & -1.077298 & 2.004878 \\
\hline 6 & -6.710731 & -0.070976 & -4.166115 \\
\hline ( & -4.717186 & -1.549591 & -4.254036 \\
\hline & -0.640536 & -0.673661 & -3.292929 \\
\hline & -0.935033 & -1.323838 & -4.202904 \\
\hline 1 & 7.046056 & 0.902262 & 0.519067 \\
\hline & -2.086708 & 0.804579 & 2.766043 \\
\hline & 8.330822 & -3.811005 & -0.377649 \\
\hline & -2.229329 & -2.984736 & 5.580366 \\
\hline & 5.345325 & 5.474505 & -1.616794 \\
\hline & -3.545285 & -2.630515 & 0.341890 \\
\hline & -5.283606 & -3.759368 & -0.972204 \\
\hline & 3.387300 & 4.605361 & -0.478063 \\
\hline & -2.323190 & -0.723144 & 4.653412 \\
\hline & 6.243992 & -4.183641 & -1.567079 \\
\hline & 8.309334 & 2.789973 & -3.189560 \\
\hline & 4.018529 & -3.351783 & -2.320205 \\
\hline & -2.308725 & -5.314950 & -3.660272 \\
\hline & 0.054617 & -4.783234 & -3.309159 \\
\hline & -1.079110 & -6.282850 & 3.073862 \\
\hline & 7.088434 & 0.871982 & -2.280994 \\
\hline & -0.761009 & -4.764295 & 1.180106 \\
\hline & 1.806406 & -3.559224 & -2.051690 \\
\hline 1 & 7.467438 & 5.110030 & -2.841609 \\
\hline & 9.170141 & 0.025176 & 1.368195 \\
\hline ] & -4.679517 & -5.121092 & -2.968910 \\
\hline & -1.819684 & -5.412477 & 5.287221 \\
\hline & 9.845333 & -2.329251 & 0.907596 \\
\hline & 2.066034 & 2.660977 & 0.363516 \\
\hline & -4.022546 & 0.380790 & 1.396878 \\
\hline & -2.791791 & 0.218351 & -2.694043 \\
\hline & -6.946548 & -0.098477 & -1.690918 \\
\hline ] & -3.061739 & 2.902736 & -0.357857 \\
\hline & 0.266786 & 1.502324 & 1.945135 \\
\hline 1 & -1.212023 & 5.489249 & 2.513682 \\
\hline & 1.167023 & 0.761704 & -3.136692 \\
\hline & 1.831392 & -0.386261 & 2.347279 \\
\hline 1 & 2.222137 & -4.639639 & 2.732540 \\
\hline & 1.353171 & -0.207735 & 4.771969 \\
\hline 1 & 1.736099 & -4.471598 & 5.155927 \\
\hline & 1.299798 & -2.253434 & 6.185651 \\
\hline 1 & 1.359040 & 5.141540 & 4.941545 \\
\hline & 0.975059 & 5.810220 & 3.347457 \\
\hline & -0.326791 & 5.418676 & 4.495427 \\
\hline 1 & 0.731973 & 2.805689 & 5.558646 \\
\hline & -0.933765 & 3.015238 & 4.973376 \\
\hline 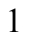 & 0.064183 & 1.690876 & 4.346323 \\
\hline & 2.676711 & 3.331083 & 4.072972 \\
\hline & 2.094641 & 2.223578 & 2.827333 \\
\hline & 2.442140 & 3.914996 & 2.415839 \\
\hline
\end{tabular}




$\begin{array}{rrrr}1 & -4.813063 & 5.953085 & -0.663997 \\ 1 & -3.627907 & 4.851129 & -1.381017 \\ 1 & -4.786504 & 4.253654 & -0.170798 \\ 1 & -4.690586 & 6.676923 & 1.671992 \\ 1 & -4.591772 & 5.001393 & 2.253554 \\ 1 & -3.363701 & 6.186285 & 2.737231 \\ 1 & -3.042781 & 7.525021 & -0.069216 \\ 1 & -1.688630 & 7.066554 & 0.975457 \\ 1 & -1.767228 & 6.452066 & -0.685478 \\ 1 & -8.809043 & -0.110801 & 1.332771 \\ 1 & -8.374854 & 0.741247 & -0.155244 \\ 1 & -8.186160 & -1.024557 & -0.047739 \\ 1 & -7.393567 & 1.387014 & 2.668852 \\ 1 & -5.668065 & 1.594360 & 2.323170 \\ 1 & -6.876795 & 2.267311 & 1.215839 \\ 1 & -7.132713 & -1.133678 & 2.801973 \\ 1 & -6.398059 & -2.027733 & 1.454522 \\ 1 & -5.394984 & -0.991589 & 2.483778 \\ 1 & -6.869438 & -0.188201 & -5.245464 \\ 1 & -7.283450 & -0.864105 & -3.668866 \\ 1 & -7.135661 & 0.897557 & -3.871196 \\ 1 & -4.740556 & 0.765437 & -5.760404 \\ 1 & -4.805551 & 1.916559 & -4.409912 \\ 1 & -3.406288 & 0.844096 & -4.607770 \\ 1 & -4.891073 & -1.729201 & -5.323953 \\ 1 & -3.642863 & -1.667971 & -4.060735 \\ 1 & -5.241688 & -2.330125 & -3.685973 \\ 1 & 2.500352 & -3.693070 & 0.631911 \\ 1 & 3.425541 & -2.201838 & 0.776172\end{array}$

\section{$1_{\text {C5- }- \text {-1s2s3a4a }}$}

Number of imaginary frequencies : 0

The smallest frequencies are : $16.0790 \quad 17.2208 \quad 22.9781 \mathrm{~cm}(-1)$

Electronic energy : $\quad H F=-4125.4693292$

Zero-point correction $=\quad 1.300296($ Hartree/Particle $)$

Thermal correction to Energy $=\quad 1.378125$

Thermal correction to Enthalpy= $\quad 1.379069$

Thermal correction to Gibbs Free Energy= $\quad 1.190519$

Sum of electronic and zero-point Energies $=\quad-4124.169033$

Sum of electronic and thermal Energies $=\quad-4124.091204$

Sum of electronic and thermal Enthalpies $=\quad-4124.090260$

Sum of electronic and thermal Free Energies $=\quad-4124.278810$

\section{Cartesian Coordinates}

$\begin{array}{cccc}6 & -0.379429 & 2.322736 & 1.660903 \\ 6 & -1.372410 & 2.093054 & 0.705349 \\ 6 & -2.238290 & 3.123101 & 0.354865 \\ 6 & -2.147572 & 4.379031 & 0.961793 \\ 6 & -1.180413 & 4.560907 & 1.952523 \\ 6 & -0.293242 & 3.544985 & 2.330016 \\ 15 & -1.482571 & 0.457903 & -0.124941 \\ 6 & -1.460616 & -0.693179 & 1.336596 \\ 6 & -1.198028 & -2.043704 & 1.180243 \\ 6 & -1.336535 & -2.941479 & 2.291816\end{array}$




\begin{tabular}{|c|c|c|c|}
\hline 6 & -1.746336 & -2.435468 & 3.557756 \\
\hline & -1.992579 & -1.050272 & 3.688452 \\
\hline & -1.857893 & -0.211669 & 2.616475 \\
\hline & -1.905271 & -3.321351 & 4.649650 \\
\hline & -1.683373 & -4.666746 & 4.500428 \\
\hline & -1.271612 & -5.174878 & 3.251145 \\
\hline & -1.096428 & -4.335896 & 2.178330 \\
\hline & -0.854939 & -2.670537 & -0.123615 \\
\hline & 0.451605 & -2.753706 & -0.567940 \\
\hline & 0.771137 & -3.547593 & -1.697135 \\
\hline & -0.202837 & -4.204503 & -2.398344 \\
\hline 0 & -1.562299 & -4.074483 & -2.027528 \\
\hline & -1.894417 & -3.310874 & -0.870335 \\
\hline & -3.262038 & -3.187548 & -0.512505 \\
\hline & -4.244701 & -3.772512 & -1.271984 \\
\hline & -3.911595 & -4.529064 & -2.416675 \\
\hline & -2.597228 & -4.677354 & -2.782580 \\
\hline 7 & 1.499091 & -1.967062 & 0.025406 \\
\hline & 1.863577 & -0.592869 & -0.897392 \\
\hline 8 & 3.092828 & -1.010584 & -1.966615 \\
\hline 6 & 4.343970 & -1.387881 & -1.541721 \\
\hline 6 & 5.203489 & -0.469438 & -0.960084 \\
\hline 6 & 6.453446 & -0.944445 & -0.447554 \\
\hline 6 & 6.827531 & -2.306707 & -0.654041 \\
\hline 6 & 5.946437 & -3.172267 & -1.345556 \\
\hline 6 & 4.723675 & -2.727695 & -1.767903 \\
\hline 6 & 7.330803 & -0.120855 & 0.303637 \\
\hline 6 & 8.518027 & -0.607981 & 0.791027 \\
\hline 6 & 8.899310 & -1.945515 & 0.554715 \\
\hline 6 & 8.065380 & -2.774760 & -0.150207 \\
\hline 6 & 4.772975 & 0.943696 & -0.852123 \\
\hline 6 & 3.557439 & 1.222395 & -0.258657 \\
\hline$b$ & 3.041164 & 2.526621 & -0.148110 \\
\hline 5 & 3.770081 & 3.580441 & -0.629578 \\
\hline 5 & 5.010890 & 3.363682 & -1.275389 \\
\hline 6 & 5.514416 & 2.033826 & -1.412191 \\
\hline 6 & 5.745809 & 4.445568 & -1.818828 \\
\hline 6 & 6.918453 & 4.233401 & -2.496521 \\
\hline 6 & 7.398028 & 2.918098 & -2.668927 \\
\hline 6 & 6.715676 & 1.849173 & -2.143410 \\
\hline 8 & 2.799808 & 0.179381 & 0.243321 \\
\hline 6 & -3.130035 & 5.472034 & 0.546294 \\
\hline 6 & -4.561554 & 4.991514 & 0.815572 \\
\hline 6 & 0.655545 & 3.685609 & 3.522187 \\
\hline 6 & 0.125570 & 2.781477 & 4.643738 \\
\hline 6 & -3.263110 & 0.330840 & -0.593396 \\
\hline 5 & -4.258687 & 0.418943 & 0.385239 \\
\hline 6 & -5.601360 & 0.244683 & 0.060418 \\
\hline 6 & -5.919189 & -0.013950 & -1.278666 \\
\hline 6 & -4.955117 & -0.100885 & -2.282358 \\
\hline 6 & -3.617268 & 0.082113 & -1.915172 \\
\hline 6 & -5.373328 & -0.366603 & -3.727624 \\
\hline 6 & -6.089653 & 0.874622 & -4.270511 \\
\hline & -6.704990 & 0.266751 & 1.116024 \\
\hline & -7.801024 & 1.260583 & 0.719479 \\
\hline S & 0.129941 & 0.499050 & -1.981668 \\
\hline
\end{tabular}




\begin{tabular}{|c|c|c|c|}
\hline 6 & -0.594495 & -0.728129 & -3.311220 \\
\hline 8 & -0.826587 & -1.391492 & -4.229092 \\
\hline & 0.123502 & 2.425750 & -2.020679 \\
\hline & 0.193131 & 3.574458 & -2.138168 \\
\hline & -4.172504 & -0.669162 & -4.620884 \\
\hline & -6.318208 & -1.570349 & -3.796365 \\
\hline & -6.178900 & 0.668766 & 2.492562 \\
\hline ठ & -7.303325 & -1.140963 & 1.223414 \\
\hline ) & 2.441653 & -2.630091 & 0.946037 \\
\hline & 2.075594 & -2.509954 & 2.403481 \\
\hline & 1.826848 & -1.261030 & 2.979672 \\
\hline 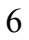 & 1.557926 & -1.152430 & 4.338462 \\
\hline & 1.531516 & -2.290735 & 5.142237 \\
\hline & 1.772644 & -3.537064 & 4.576115 \\
\hline & 2.044151 & -3.643027 & 3.213912 \\
\hline ) & -2.914186 & 6.774657 & 1.312270 \\
\hline & -2.960753 & 5.763905 & -0.948705 \\
\hline & 0.721574 & 5.116534 & 4.051884 \\
\hline & 2.079019 & 3.252981 & 3.162859 \\
\hline & 7.046186 & 0.908737 & 0.505174 \\
\hline & -2.066074 & 0.846491 & 2.752745 \\
\hline & 8.334703 & -3.815131 & -0.328457 \\
\hline & -2.213861 & -2.909173 & 5.609611 \\
\hline & 5.347718 & 5.451998 & -1.696346 \\
\hline & -3.532965 & -2.596587 & 0.361324 \\
\hline & -5.288918 & -3.641486 & -0.992688 \\
\hline & 3.389010 & 4.597391 & -0.547445 \\
\hline & -2.301996 & -0.657566 & 4.656695 \\
\hline & 6.250607 & -4.203276 & -1.518871 \\
\hline & 8.314125 & 2.747523 & -3.229995 \\
\hline & 4.026016 & -3.381584 & -2.286587 \\
\hline 1 & -2.326024 & -5.254557 & -3.665381 \\
\hline & 0.049984 & -4.801528 & -3.273065 \\
\hline & -1.086617 & -6.240746 & 3.137213 \\
\hline & 7.092864 & 0.841509 & -2.296758 \\
\hline 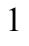 & -0.763977 & -4.744542 & 1.226276 \\
\hline 1 & 1.813412 & -3.603502 & -2.005939 \\
\hline & 7.471028 & 5.071715 & -2.914356 \\
\hline ] & 9.168493 & 0.043166 & 1.370709 \\
\hline & -4.699786 & -4.990812 & -3.007788 \\
\hline ] & -1.817041 & -5.342046 & 5.342576 \\
\hline & 9.845388 & -2.316705 & 0.941717 \\
\hline . & 2.067692 & 2.663996 & 0.319203 \\
\hline 1 & -3.974384 & 0.615264 & 1.415015 \\
\hline & -2.828017 & 0.022297 & -2.658938 \\
\hline 1 & -6.965563 & -0.158599 & -1.546730 \\
\hline & -2.998959 & 2.941630 & -0.404010 \\
\hline t & 0.293559 & 1.507198 & 1.933608 \\
\hline & -1.122303 & 5.520617 & 2.456738 \\
\hline 1 & 1.185968 & 0.720633 & -3.145043 \\
\hline 1 & 1.853906 & -0.371407 & 2.351238 \\
\hline & 2.227386 & -4.622303 & 2.770779 \\
\hline 1 & 1.377845 & -0.170930 & 4.776386 \\
\hline & 1.742733 & -4.433113 & 5.193041 \\
\hline & 1.317792 & -2.205118 & 6.205763 \\
\hline & 1.434550 & 5.167121 & 4.884348 \\
\hline
\end{tabular}




$\begin{array}{rrrr}1 & 1.060795 & 5.821897 & 3.282272 \\ 1 & -0.246805 & 5.462973 & 4.434164 \\ 1 & 0.767595 & 2.852747 & 5.532612 \\ 1 & -0.892447 & 3.073214 & 4.935547 \\ 1 & 0.092958 & 1.728428 & 4.332652 \\ 1 & 2.725456 & 3.327291 & 4.047312 \\ 1 & 2.133449 & 2.214561 & 2.811331 \\ 1 & 2.506863 & 3.895629 & 2.382531 \\ 1 & -3.638857 & 7.524941 & 0.971879 \\ 1 & -3.058617 & 6.646740 & 2.392955 \\ 1 & -1.911194 & 7.187714 & 1.144647 \\ 1 & -5.284692 & 5.763106 & 0.518853 \\ 1 & -4.804197 & 4.078293 & 0.257481 \\ 1 & -4.710674 & 4.780878 & 1.883122 \\ 1 & -3.658215 & 6.551445 & -1.264462 \\ 1 & -1.941026 & 6.104870 & -1.168604 \\ 1 & -3.156732 & 4.880166 & -1.567619 \\ 1 & -7.007184 & 0.687717 & 3.211943 \\ 1 & -5.431722 & -0.040392 & 2.873332 \\ 1 & -5.727853 & 1.670233 & 2.479827 \\ 1 & -8.591983 & 1.275305 & 1.481003 \\ 1 & -7.398469 & 2.278169 & 0.632616 \\ 1 & -8.273017 & 1.004602 & -0.236356 \\ 1 & -8.096869 & -1.165534 & 1.982512 \\ 1 & -7.739343 & -1.474707 & 0.273465 \\ 1 & -6.534270 & -1.869873 & 1.513622 \\ 1 & -4.516801 & -0.898340 & -5.637243 \\ 1 & -3.484072 & 0.182344 & -4.696653 \\ 1 & -3.607126 & -1.537231 & -4.254799 \\ 1 & -6.398258 & 0.714482 & -5.312527 \\ 1 & -6.987858 & 1.114015 & -3.687112 \\ 1 & -5.429667 & 1.751513 & -4.244259 \\ 1 & -6.602774 & -1.764864 & -4.838834 \\ 1 & -5.827384 & -2.471850 & -3.406210 \\ 1 & -7.244533 & -1.415524 & -3.230414 \\ 1 & 2.518611 & -3.690802 & 0.663579 \\ 1 & 3.437823 & -2.194676 & 0.795384\end{array}$

$1_{\mathrm{C} 5-} \psi_{-1 \mathrm{~s} 2 \mathrm{23a4s}}$

Number of imaginary frequencies : 0

The smallest frequencies are : $13.1746 \quad 18.2578 \quad 19.6741 \mathrm{~cm}(-1)$

Electronic energy : $\quad H F=-4125.4692307$

Zero-point correction $=\quad 1.299778($ Hartree/Particle)

Thermal correction to Energy= $\quad 1.377871$

Thermal correction to Enthalpy= $\quad 1.378815$

Thermal correction to Gibbs Free Energy $=\quad 1.188358$

Sum of electronic and zero-point Energies $=\quad-4124.169453$

Sum of electronic and thermal Energies $=\quad-4124.091360$

Sum of electronic and thermal Enthalpies $=\quad-4124.090415$

Sum of electronic and thermal Free Energies $=\quad-4124.280873$

Cartesian Coordinates

$\begin{array}{llll}6 & 1.930630 & -1.330939 & 2.973226 \\ 6 & 2.183650 & -2.559196 & 2.357386\end{array}$




\begin{tabular}{|c|c|c|c|}
\hline 6 & 2.178165 & -3.715195 & 3.135854 \\
\hline 6 & 1.922315 & -3.651555 & 4.503500 \\
\hline 6 & 1.667617 & -2.425540 & 5.107159 \\
\hline 6 & 1.672162 & -1.264652 & 4.336444 \\
\hline 6 & 2.520556 & -2.631546 & 0.889430 \\
\hline 7 & 1.570569 & -1.921365 & 0.010142 \\
\hline 1 & 1.929112 & -0.501806 & -0.843459 \\
\hline 45 & 0.193809 & 0.652453 & -1.860398 \\
\hline 6 & 0.141092 & 2.575624 & -1.745866 \\
\hline 8 & 0.186768 & 3.731383 & -1.760351 \\
\hline 6 & 0.521757 & -2.680924 & -0.615991 \\
\hline 0 & -0.778344 & -2.652967 & -0.144797 \\
\hline 0 & -1.814739 & -3.287905 & -0.901580 \\
\hline 0 & -1.488387 & -3.962332 & -2.114603 \\
\hline 6 & -0.136312 & -4.024458 & -2.525272 \\
\hline 6 & 0.835947 & -3.393675 & -1.799281 \\
\hline 6 & -3.175061 & -3.249143 & -0.497814 \\
\hline 6 & -4.154094 & -3.828654 & -1.265774 \\
\hline 6 & -3.826499 & -4.491271 & -2.468686 \\
\hline 6 & -2.519867 & -4.556442 & -2.881290 \\
\hline 6 & -1.115424 & -2.073754 & 1.183066 \\
\hline 6 & -1.400689 & -0.730916 & 1.374849 \\
\hline 6 & -1.785392 & -0.285410 & 2.670035 \\
\hline 6 & -1.890185 & -1.152304 & 3.723961 \\
\hline 6 & -1.628370 & -2.530350 & 3.555720 \\
\hline 6 & -1.227301 & -2.999537 & 2.272206 \\
\hline 6 & -1.765173 & -3.447065 & 4.625471 \\
\hline 6 & -1.531263 & -4.785354 & 4.437516 \\
\hline 6 & -1.128216 & -5.256249 & 3.170446 \\
\hline 6 & -0.973417 & -4.387658 & 2.118665 \\
\hline 15 & -1.482059 & 0.431980 & -0.074193 \\
\hline 6 & -3.229669 & 0.148492 & -0.596118 \\
\hline 6 & -3.552674 & -0.027289 & -1.941077 \\
\hline 6 & -4.871045 & -0.253181 & -2.338960 \\
\hline 6 & -5.859117 & -0.286389 & -1.347175 \\
\hline 6 & -5.570662 & -0.112256 & 0.006713 \\
\hline 6 & -4.238454 & 0.108279 & 0.362972 \\
\hline 6 & -5.260383 & -0.440155 & -3.804233 \\
\hline 6 & -6.074197 & 0.775876 & -4.260168 \\
\hline 6 & -6.628221 & -0.169721 & 1.106904 \\
\hline 6 & -6.667429 & 1.174207 & 1.843121 \\
\hline 6 & -1.582242 & 2.078042 & 0.739299 \\
\hline 6 & -2.540687 & 2.996704 & 0.323789 \\
\hline 6 & -2.588146 & 4.285378 & 0.861251 \\
\hline 6 & -1.667194 & 4.613908 & 1.857482 \\
\hline 6 & -0.693166 & 3.712333 & 2.304614 \\
\hline 6 & -0.640883 & 2.454343 & 1.700669 \\
\hline 6 & 0.221445 & 4.021104 & 3.491177 \\
\hline 6 & -0.215295 & 3.134412 & 4.665152 \\
\hline 6 & -3.643443 & 5.261071 & 0.344926 \\
\hline 6 & -5.038789 & 4.655560 & 0.539812 \\
\hline 8 & 3.142803 & -0.862740 & -1.951333 \\
\hline 6 & 4.397929 & -1.269691 & -1.568210 \\
\hline 6 & 5.272119 & -0.391353 & -0.947409 \\
\hline 6 & 6.528181 & -0.902060 & -0.485295 \\
\hline 6 & 6.890811 & -2.251072 & -0.779502 \\
\hline
\end{tabular}




\begin{tabular}{|c|c|c|c|}
\hline 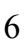 & 5.993964 & -3.069534 & -1.506816 \\
\hline & 4.767049 & -2.594889 & -1.881020 \\
\hline & 8.134448 & -2.754814 & -0.326954 \\
\hline & 8.985658 & -1.973751 & 0.411409 \\
\hline & 8.616504 & -0.651093 & 0.734180 \\
\hline & 7.423664 & -0.129625 & 0.298332 \\
\hline & 4.851854 & 1.015201 & -0.749750 \\
\hline & 3.645941 & 1.265155 & -0.124420 \\
\hline & 3.142509 & 2.563318 & 0.076189 \\
\hline & 3.871202 & 3.640138 & -0.351269 \\
\hline & 5.100993 & 3.455378 & -1.027273 \\
\hline & 5.593282 & 2.133253 & -1.252264 \\
\hline & 6.783266 & 1.986665 & -2.010139 \\
\hline & 7.465566 & 3.081767 & -2.478763 \\
\hline & 6.997514 & 4.386963 & -2.219396 \\
\hline & 5.835747 & 4.564198 & -1.513585 \\
\hline & 2.886496 & 0.200364 & 0.323765 \\
\hline & 0.130522 & 5.479887 & 3.934409 \\
\hline & 1.684415 & 3.722651 & 3.155992 \\
\hline & -3.603173 & 6.603361 & 1.070459 \\
\hline & -3.399218 & 5.515700 & -1.147065 \\
\hline 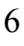 & -8.023975 & -0.455036 & 0.559525 \\
\hline 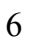 & -6.267112 & -1.286490 & 2.094253 \\
\hline & -4.036713 & -0.575886 & -4.706703 \\
\hline 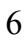 & -6.103300 & -1.708806 & -3.969926 \\
\hline & -0.450511 & -0.496535 & -3.299281 \\
\hline & -0.627284 & -1.103263 & -4.267189 \\
\hline & 7.149330 & 0.887709 & 0.565802 \\
\hline & -2.018006 & 0.764171 & 2.829876 \\
\hline ] & 8.394184 & -3.783856 & -0.572483 \\
\hline & -2.067563 & -3.064261 & 5.599488 \\
\hline | & 5.446455 & 5.563592 & -1.323658 \\
\hline & -3.443413 & -2.729544 & 0.421155 \\
\hline . & -5.194367 & -3.770181 & -0.950519 \\
\hline l & 3.498484 & 4.652754 & -0.202948 \\
\hline & -2.193432 & -0.788654 & 4.705337 \\
\hline l & 6.290039 & -4.089318 & -1.747430 \\
\hline & 8.372746 & 2.940433 & -3.062008 \\
\hline L & 4.059387 & -3.213317 & -2.428315 \\
\hline 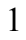 & -2.251100 & -5.064367 & -3.806395 \\
\hline | & 0.112109 & -4.554012 & -3.443617 \\
\hline d & -0.933803 & -6.316814 & 3.026600 \\
\hline & 7.151826 & 0.988181 & -2.230194 \\
\hline 1 & -0.647821 & -4.766399 & 1.152011 \\
\hline & 1.872299 & -3.406134 & -2.130121 \\
\hline 1 & 7.550302 & 5.246006 & -2.592451 \\
\hline 1 & 9.280868 & -0.038958 & 1.340017 \\
\hline 1 & -4.612727 & -4.950789 & -3.064246 \\
\hline 1 & -1.648686 & -5.484219 & 5.262700 \\
\hline 1 & 9.936234 & -2.372071 & 0.758319 \\
\hline 1 & 2.177179 & 2.678556 & 0.564738 \\
\hline 1 & -3.983947 & 0.244945 & 1.413047 \\
\hline 1 & -2.752279 & 0.011717 & -2.674302 \\
\hline 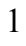 & -6.890389 & -0.457697 & -1.649064 \\
\hline 1 & -3.257370 & 2.705967 & -0.443296 \\
\hline 1 & 0.111876 & 1.730317 & 2.018524 \\
\hline
\end{tabular}




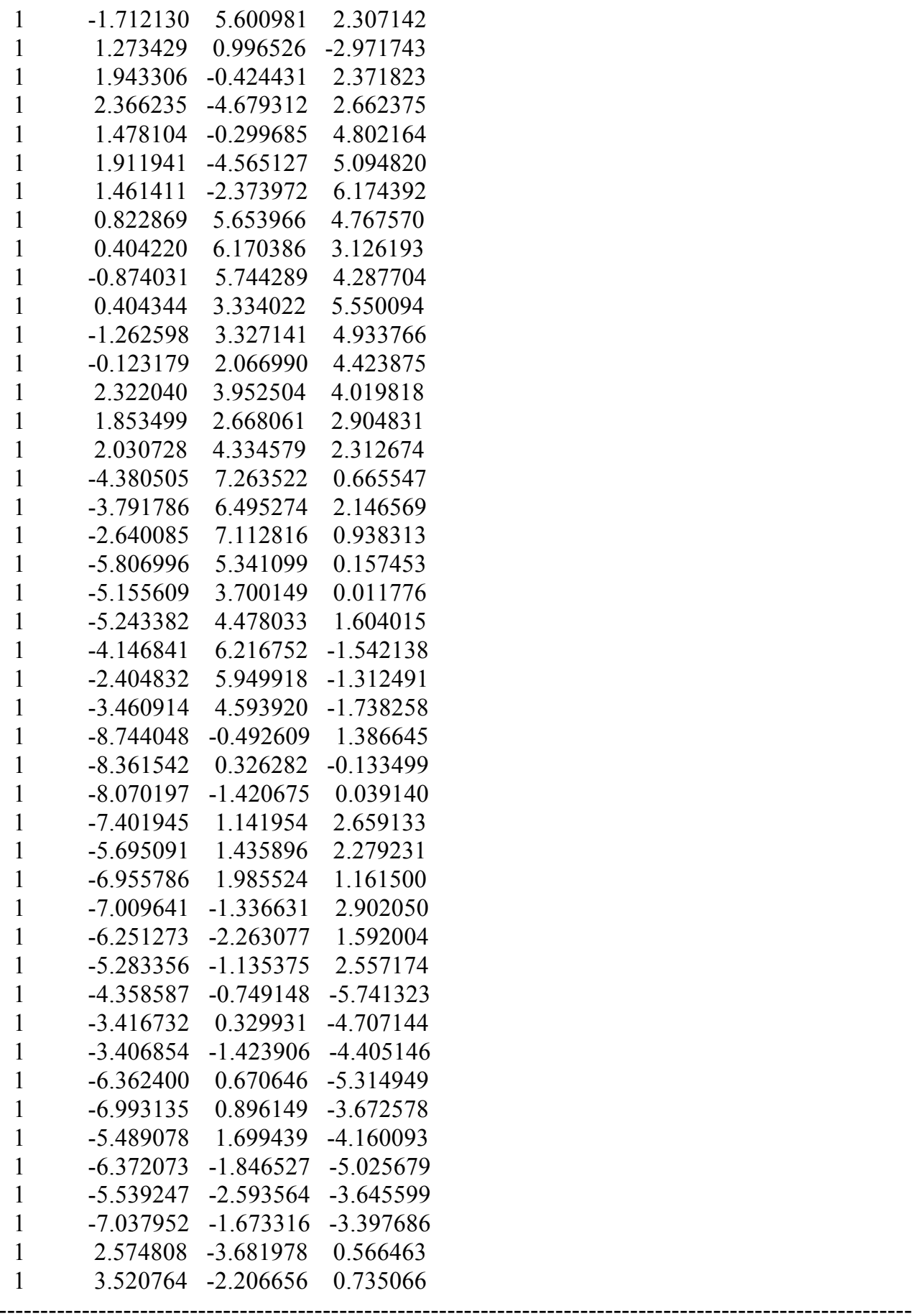

$1_{\text {C5- }} \psi_{-1 \text {-1s2s3s4a }}$

Number of imaginary frequencies : 0

The smallest frequencies are : $15.5054 \quad 18.348924 .3144 \mathrm{~cm}(-1)$

Electronic energy : $\quad H F=-4125.4698402$

Zero-point correction $=\quad 1.300847$ (Hartree/Particle)

Thermal correction to Energy= $\quad 1.378508$

Thermal correction to Enthalpy= $\quad 1.379452$

Thermal correction to Gibbs Free Energy= $\quad 1.191634$

Sum of electronic and zero-point Energies $=\quad-4124.168993$ 
Sum of electronic and thermal Energies= Sum of electronic and thermal Enthalpies= Sum of electronic and thermal Free Energies=
$-4124.091333$

$-4124.090388$

$-4124.278206$

Cartesian Coordinates

\begin{tabular}{|c|c|c|c|}
\hline 6 & -4.284848 & 0.379321 & 0.394722 \\
\hline 6 & -3.272958 & 0.349672 & -0.575887 \\
\hline 6 & -3.613838 & 0.198775 & -1.910711 \\
\hline 6 & -4.949516 & 0.057087 & -2.313556 \\
\hline 6 & -5.925796 & 0.068714 & -1.323715 \\
\hline 6 & -5.620272 & 0.234090 & 0.038310 \\
\hline 15 & -1.494300 & 0.456586 & -0.098538 \\
\hline 45 & 0.102810 & 0.517649 & -1.968006 \\
\hline 6 & 0.111199 & 2.446222 & -1.982011 \\
\hline 8 & 0.185256 & 3.595706 & -2.086330 \\
\hline 6 & -5.258604 & -0.166644 & -3.791517 \\
\hline 6 & -6.754087 & -0.089223 & -4.089437 \\
\hline 6 & -6.742718 & 0.207316 & 1.074362 \\
\hline 6 & -6.229546 & 0.471826 & 2.488289 \\
\hline 6 & -1.473414 & -0.723595 & 1.336961 \\
\hline 6 & -1.205299 & -2.070017 & 1.155229 \\
\hline 6 & -1.337299 & -2.987364 & 2.252287 \\
\hline 6 & -1.749017 & -2.505548 & 3.527198 \\
\hline 6 & -2.004259 & -1.124481 & 3.681979 \\
\hline 6 & -1.873226 & -0.266496 & 2.625061 \\
\hline 6 & -1.086408 & -4.377826 & 2.115812 \\
\hline 6 & -1.252768 & -5.235523 & 3.175105 \\
\hline 6 & -1.667132 & -4.751494 & 4.433033 \\
\hline 6 & -1.899855 & -3.410647 & 4.604497 \\
\hline 6 & -0.858709 & -2.676839 & -0.158069 \\
\hline 6 & 0.448579 & -2.748624 & -0.603236 \\
\hline 6 & 0.774616 & -3.535921 & -1.734968 \\
\hline 6 & -0.191464 & -4.211546 & -2.429121 \\
\hline 6 & -1.550324 & -4.107086 & -2.049194 \\
\hline 6 & -1.891458 & -3.331679 & -0.902388 \\
\hline 6 & -2.574797 & -4.756534 & -2.779768 \\
\hline 6 & -3.887922 & -4.641860 & -2.400739 \\
\hline 6 & -4.232029 & -3.861230 & -1.275987 \\
\hline 6 & -3.260551 & -3.226594 & -0.542577 \\
\hline 7 & 1.493462 & -1.964070 & -0.002704 \\
\hline 6 & 2.444128 & -2.638555 & 0.901458 \\
\hline 6 & 2.082562 & -2.544832 & 2.361802 \\
\hline 6 & 1.822106 & -1.307767 & 2.957964 \\
\hline 6 & 1.550822 & -1.223807 & 4.317973 \\
\hline 6 & 1.533401 & -2.375295 & 5.102931 \\
\hline 6 & 1.788009 & -3.609801 & 4.517215 \\
\hline 6 & 2.062235 & -3.691014 & 3.153906 \\
\hline 15 & 1.850656 & -0.575708 & -0.906683 \\
\hline 8 & 2.788124 & 0.179828 & 0.243873 \\
\hline 6 & 3.543176 & 1.232805 & -0.240121 \\
\hline 6 & 4.758261 & 0.967208 & -0.840654 \\
\hline 6 & 5.497620 & 2.069238 & -1.380056 \\
\hline 6 & 4.992991 & 3.395526 & -1.215474 \\
\hline 6 & 3.752855 & 3.597971 & -0.563805 \\
\hline 6 & 3.025568 & 2.533911 & -0.102865 \\
\hline
\end{tabular}




\begin{tabular}{|c|c|c|c|}
\hline 6 & 6.697948 & 1.900900 & -2.116781 \\
\hline 6 & 7.378303 & 2.981071 & -2.621520 \\
\hline & 6.897725 & 4.292089 & -2.421480 \\
\hline & 5.725927 & 4.489083 & -1.737786 \\
\hline & 5.190712 & -0.442933 & -0.977005 \\
\hline & 4.331487 & -1.351858 & -1.573983 \\
\hline & 4.714251 & -2.685472 & -1.829662 \\
\hline & 5.939462 & -3.135488 & -1.420618 \\
\hline & 6.820275 & -2.282160 & -0.713773 \\
\hline & 6.443562 & -0.925368 & -0.478222 \\
\hline & 7.321607 & -0.115285 & 0.286758 \\
\hline & 8.511629 & -0.609468 & 0.760135 \\
\hline & 8.895222 & -1.940956 & 0.495350 \\
\hline b & 8.060850 & -2.757516 & -0.223683 \\
\hline & 3.077648 & -0.970596 & -1.986976 \\
\hline & -1.376541 & 2.076642 & 0.759123 \\
\hline b & -2.234716 & 3.116898 & 0.419706 \\
\hline & -2.135564 & 4.364935 & 1.041395 \\
\hline D & -1.169241 & 4.527477 & 2.036372 \\
\hline & -0.291221 & 3.500114 & 2.404087 \\
\hline & -0.384704 & 2.286683 & 1.720088 \\
\hline y & -3.109924 & 5.469876 & 0.638317 \\
\hline 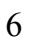 & -2.877862 & 6.765441 & 1.411497 \\
\hline 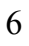 & 0.656636 & 3.618432 & 3.599208 \\
\hline ) & 0.728567 & 5.040605 & 4.151027 \\
\hline b & -2.948017 & 5.771113 & -0.855588 \\
\hline 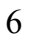 & -4.543581 & 4.999714 & 0.913639 \\
\hline & 2.078436 & 3.185142 & 3.233830 \\
\hline 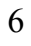 & 0.121128 & 2.699722 & 4.706216 \\
\hline 6 & -0.671790 & -0.686827 & -3.283085 \\
\hline 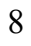 & -0.974254 & -1.342005 & -4.186989 \\
\hline 6 & -4.757149 & -1.562013 & -4.181767 \\
\hline 6 & -4.547594 & 0.886743 & -4.648390 \\
\hline & -7.400676 & -1.177309 & 1.057084 \\
\hline & -7.791132 & 1.274319 & 0.743402 \\
\hline & 7.035422 & 0.909348 & 0.510180 \\
\hline 1 & -2.087035 & 0.788223 & 2.778707 \\
\hline & 8.331937 & -3.793371 & -0.424196 \\
\hline 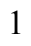 & -2.210553 & -3.016684 & 5.571405 \\
\hline . & 5.326966 & 5.492385 & -1.594222 \\
\hline & -3.539762 & -2.617903 & 0.316501 \\
\hline 1 & -5.277546 & -3.754609 & -0.992617 \\
\hline & 3.370790 & 4.612626 & -0.460681 \\
\hline 1 & -2.316655 & -0.750750 & 4.656716 \\
\hline & 6.246027 & -4.161676 & -1.616764 \\
\hline 1 & 8.293690 & 2.822927 & -3.187369 \\
\hline 1 & 4.017097 & -3.329609 & -2.361010 \\
\hline 1 & -2.295399 & -5.345086 & -3.652438 \\
\hline 1 & 0.067727 & -4.812133 & -3.299547 \\
\hline ] & -1.058925 & -6.297762 & 3.043416 \\
\hline 1 & 7.076273 & 0.897070 & -2.291153 \\
\hline I & -0.751778 & -4.768780 & 1.157162 \\
\hline 1 & 1.816734 & -3.578249 & -2.046302 \\
\hline 1 & 7.448826 & 5.139380 & -2.822843 \\
\hline 1 & 9.162506 & 0.031305 & 1.350815 \\
\hline 1 & -4.668222 & -5.141108 & -2.971186 \\
\hline
\end{tabular}




$\begin{array}{rrrr}1 & -1.794138 & -5.441587 & 5.264153 \\ 1 & 9.843453 & -2.317726 & 0.871536 \\ 1 & 2.052468 & 2.660809 & 0.368343 \\ 1 & -4.014212 & 0.505742 & 1.439219 \\ 1 & -2.819311 & 0.184479 & -2.652215 \\ 1 & -6.969007 & -0.055204 & -1.607794 \\ 1 & -2.994944 & 2.950178 & -0.343217 \\ 1 & 0.282241 & 1.463240 & 1.983651 \\ 1 & -1.104465 & 5.480826 & 2.551800 \\ 1 & 1.139402 & 0.741526 & -3.148793 \\ 1 & 1.840316 & -0.407657 & 2.344308 \\ 1 & 2.254682 & -4.661186 & 2.694899 \\ 1 & 1.360283 & -0.251528 & 4.771771 \\ 1 & 1.766068 & -4.515964 & 5.119507 \\ 1 & 1.316705 & -2.309129 & 6.167243 \\ 1 & 1.439218 & 5.074503 & 4.986343 \\ 1 & 1.074041 & 5.755952 & 3.393504 \\ 1 & -0.239223 & 5.386213 & 4.535563 \\ 1 & 0.764263 & 2.751609 & 5.595629 \\ 1 & -0.894533 & 2.994063 & 5.003528 \\ 1 & 0.080098 & 1.652249 & 4.377830 \\ 1 & 2.724186 & 3.239653 & 4.120247 \\ 1 & 2.128124 & 2.153411 & 2.862447 \\ 1 & 2.510401 & 3.840078 & 2.466070 \\ 1 & -3.596909 & 7.524819 & 1.079383 \\ 1 & -3.018516 & 6.632238 & 2.492027 \\ 1 & -1.871623 & 7.169690 & 1.241742 \\ 1 & -5.262704 & 5.774927 & 0.616556 \\ 1 & -4.793130 & 4.085073 & 0.360894 \\ 1 & -4.690051 & 4.794334 & 1.982420 \\ 1 & -3.643541 & 6.564463 & -1.160918 \\ 1 & -1.928415 & 6.109801 & -1.079147 \\ 1 & -3.152354 & 4.893425 & -1.480400 \\ 1 & -7.068804 & 0.449155 & 3.194729 \\ 1 & -5.505935 & -0.287260 & 2.814422 \\ 1 & -5.753866 & 1.458126 & 2.571740 \\ 1 & -8.598238 & 1.256036 & 1.487854 \\ 1 & -7.348415 & 2.278741 & 0.752056 \\ 1 & -8.249282 & 1.119036 & -0.240451 \\ 1 & -8.212939 & -1.224950 & 1.794913 \\ 1 & -7.828252 & -1.417976 & 0.076025 \\ 1 & -6.671084 & -1.959424 & 1.306861 \\ 1 & -6.922606 & -0.222401 & -5.165419 \\ 1 & -7.319980 & -0.876334 & -3.574880 \\ 1 & -7.178176 & 0.882670 & -3.804495 \\ 1 & -4.796666 & 0.738679 & -5.707335 \\ 1 & -4.856811 & 1.901894 & -4.367201 \\ 1 & -3.455257 & 0.831718 & -4.563389 \\ 1 & -4.932870 & -1.747992 & -5.250211 \\ 1 & -3.682381 & -1.679296 & -3.990188 \\ 1 & -5.281470 & -2.338232 & -3.608253 \\ 1 & 2.523949 & -3.694055 & 0.600989 \\ 1 & 3.437732 & -2.196465 & 0.753650\end{array}$

$1_{\text {C5- }} \psi_{-1 \mathrm{~s} 22354 s}$ 
Number of imaginary frequencies : 0

The smallest frequencies are : $15.6341 \quad 17.9733 \quad 23.0669 \mathrm{~cm}(-1)$

Electronic energy : $\quad H F=-4125.4699188$

Zero-point correction $=\quad 1.300578($ Hartree/Particle $)$

Thermal correction to Energy= $\quad 1.378280$

Thermal correction to Enthalpy= $\quad 1.379224$

Thermal correction to Gibbs Free Energy= $\quad 1.191159$

Sum of electronic and zero-point Energies $=\quad-4124.169341$

Sum of electronic and thermal Energies $=\quad-4124.091639$

Sum of electronic and thermal Enthalpies $=\quad-4124.090695$

Sum of electronic and thermal Free Energies $=\quad-4124.278760$

\section{Cartesian Coordinates}

$\begin{array}{cccc}6 & 5.746255 & 4.484413 & -1.720412 \\ 6 & 5.010718 & 3.391919 & -1.199539 \\ 6 & 5.512582 & 2.064691 & -1.365288 \\ 6 & 6.712915 & 1.894543 & -2.101584 \\ 6 & 7.395828 & 2.973766 & -2.604897 \\ 6 & 6.917947 & 4.285597 & -2.403762 \\ 6 & 4.770492 & 0.963594 & -0.827719 \\ 6 & 3.555844 & 1.231345 & -0.227258 \\ 6 & 3.041342 & 2.533461 & -0.088202 \\ 6 & 3.770839 & 3.596462 & -0.547982 \\ 6 & 5.199226 & -0.447573 & -0.965580 \\ 6 & 4.337425 & -1.353323 & -1.563698 \\ 6 & 4.715123 & -2.688592 & -1.818112 \\ 6 & 5.938545 & -3.142866 & -1.408455 \\ 6 & 6.822394 & -2.292336 & -0.702083 \\ 6 & 6.450278 & -0.934296 & -0.466442 \\ 6 & 8.061407 & -2.771931 & -0.212167 \\ 6 & 8.898613 & -1.958279 & 0.506848 \\ 6 & 8.519456 & -0.625587 & 0.771923 \\ 6 & 7.331021 & -0.127352 & 0.298768 \\ 8 & 3.086002 & -0.966834 & -1.979172 \\ 15 & 1.858176 & -0.567309 & -0.901672 \\ 8 & 2.797085 & 0.179454 & 0.253593 \\ 7 & 1.489133 & -1.956363 & -0.003549 \\ 6 & 0.442823 & -2.734371 & -0.610572 \\ 6 & -0.863913 & -2.666387 & -0.163075 \\ 6 & -1.898856 & -3.313864 & -0.910347 \\ 6 & -1.560306 & -4.077473 & -2.065783 \\ 6 & -0.202199 & -4.178761 & -2.449315 \\ 6 & 0.766055 & -3.511204 & -1.750288 \\ 6 & -2.586426 & -4.718602 & -2.801350 \\ 6 & -3.898586 & -4.607768 & -2.418000 \\ 6 & -4.239878 & -3.840151 & -1.283388 \\ 6 & -3.267167 & -3.213301 & -0.544972 \\ 6 & -1.209550 & -2.063377 & 1.151438 \\ 6 & -1.473826 & -0.716399 & 1.332520 \\ 6 & -1.872269 & -0.257832 & 2.620336 \\ 6 & -2.007244 & -1.116114 & 3.676859 \\ 6 & -1.758784 & -2.498319 & 3.521330 \\ 6 & -1.346700 & -2.980894 & 2.246945 \\ 6 & -1.099948 & -4.372002 & 2.109857 \\ 6 & -1.271855 & -5.229924 & 3.168004\end{array}$




\begin{tabular}{|c|c|c|c|}
\hline 6 & -1.687140 & -4.745204 & 4.425472 \\
\hline 6 & -1.914934 & -3.403634 & 4.597730 \\
\hline & -1.489239 & 0.460473 & -0.105874 \\
\hline 6 & -1.398797 & 2.079746 & 0.754436 \\
\hline & -2.282212 & 3.102502 & 0.429584 \\
\hline & -2.206701 & 4.347054 & 1.061324 \\
\hline & -1.238545 & 4.522750 & 2.052139 \\
\hline & -0.334709 & 3.512201 & 2.404911 \\
\hline & -0.405095 & 2.303546 & 1.710377 \\
\hline 6 & -3.201314 & 5.435267 & 0.663503 \\
\hline 5 & -3.013053 & 5.769500 & -0.820548 \\
\hline & 0.616330 & 3.640012 & 3.596507 \\
\hline & 2.041866 & 3.223853 & 3.225627 \\
\hline & 2.431175 & -2.636288 & 0.905637 \\
\hline 6 & 2.066645 & -2.538144 & 2.365147 \\
\hline 6 & 1.811847 & -1.299191 & 2.959972 \\
\hline & 1.540881 & -1.212549 & 4.319922 \\
\hline 6 & 1.518520 & -2.362910 & 5.106374 \\
\hline 6 & 1.767513 & -3.599287 & 4.522078 \\
\hline 6 & 2.040752 & -3.683312 & 3.158764 \\
\hline & 0.120448 & 0.541086 & -1.961724 \\
\hline 6 & -0.643328 & -0.655336 & -3.292073 \\
\hline 8 & -0.938614 & -1.303467 & -4.203230 \\
\hline 6 & 0.115885 & 2.469426 & -1.955343 \\
\hline 8 & 0.177163 & 3.621358 & -2.040435 \\
\hline 6 & -3.260902 & 0.337732 & -0.604347 \\
\hline 6 & -3.592679 & 0.223379 & -1.949201 \\
\hline 6 & -4.920079 & 0.074205 & -2.362488 \\
\hline 6 & -5.909893 & 0.043498 & -1.378621 \\
\hline 6 & -5.613138 & 0.165664 & -0.015712 \\
\hline 6 & -4.277491 & 0.320125 & 0.352534 \\
\hline 6 & -5.218975 & -0.129342 & -3.845988 \\
\hline 6 & -4.720649 & -1.523986 & -4.245006 \\
\hline 6 & -6.676670 & 0.100977 & 1.079387 \\
\hline 6 & -6.362311 & -1.078400 & 2.008685 \\
\hline 6 & -4.497542 & 0.928293 & -4.688488 \\
\hline 6 & -6.711831 & -0.042100 & -4.155803 \\
\hline 6 & -6.666568 & 1.405973 & 1.883191 \\
\hline 6 & -8.082005 & -0.100203 & 0.518514 \\
\hline 6 & 0.095795 & 2.712660 & 4.703479 \\
\hline 6 & 0.673152 & 5.062145 & 4.150263 \\
\hline 6 & -4.629021 & 4.925427 & 0.895999 \\
\hline 6 & -3.016726 & 6.718760 & 1.468635 \\
\hline 1 & 7.048321 & 0.898203 & 0.522398 \\
\hline 1 & -2.082344 & 0.797544 & 2.773807 \\
\hline 1 & 8.328948 & -3.808677 & -0.412853 \\
\hline 1 & -2.225746 & -3.009315 & 5.564443 \\
\hline 1 & 5.349432 & 5.488433 & -1.575957 \\
\hline 1 & -3.545616 & -2.614056 & 0.321847 \\
\hline 1 & -5.284570 & -3.739271 & -0.995335 \\
\hline 1 & 3.390943 & 4.611865 & -0.443916 \\
\hline 1 & -2.317740 & -0.741674 & 4.651925 \\
\hline 1 & 6.241295 & -4.170350 & -1.603729 \\
\hline 1 & 8.311162 & 2.814245 & -3.170443 \\
\hline 1 & 4.015518 & -3.330916 & -2.348426 \\
\hline 1 & -2.309104 & -5.297776 & -3.680928 \\
\hline
\end{tabular}




\begin{tabular}{|c|c|c|c|}
\hline & 0.054812 & -4.770498 & -3.326427 \\
\hline & -1.081613 & -6.292773 & 3.036021 \\
\hline ] & 7.089155 & 0.890069 & -2.276789 \\
\hline 1 & -0.764745 & -4.762935 & 1.151356 \\
\hline & 1.807290 & -3.551347 & -2.064652 \\
\hline & 7.471053 & 5.132123 & -2.803980 \\
\hline & 9.172512 & 0.012925 & 1.362644 \\
\hline & -4.680306 & -5.100874 & -2.991828 \\
\hline & -1.818509 & -5.435519 & 5.255725 \\
\hline & 9.845647 & -2.338254 & 0.882822 \\
\hline & 2.068659 & 2.661950 & 0.383258 \\
\hline & -4.022528 & 0.410126 & 1.407222 \\
\hline & -2.792180 & 0.241297 & -2.684080 \\
\hline & -6.945770 & -0.083066 & -1.679842 \\
\hline & -3.042320 & 2.925969 & -0.330935 \\
\hline & 0.280318 & 1.491864 & 1.962927 \\
\hline & -1.192511 & 5.472696 & 2.575946 \\
\hline & 1.164280 & 0.779686 & -3.133434 \\
\hline & 1.834398 & -0.399310 & 2.346148 \\
\hline & 2.229025 & -4.654885 & 2.701001 \\
\hline ] & 1.355188 & -0.238622 & 4.772231 \\
\hline & 1.741570 & -4.504661 & 5.125391 \\
\hline$\frac{1}{1}$ & 1.302638 & -2.294435 & 6.170703 \\
\hline & 1.389125 & 5.104453 & 4.980627 \\
\hline ] & 1.003258 & 5.783149 & 3.391257 \\
\hline & -0.296345 & 5.394135 & 4.542343 \\
\hline & 0.740475 & 2.771525 & 5.591341 \\
\hline 1 & -0.922885 & 2.993119 & 5.003919 \\
\hline & 0.067935 & 1.665295 & 4.373187 \\
\hline$\frac{1}{1}$ & 2.690230 & 3.286794 & 4.109540 \\
\hline & 2.103304 & 2.192413 & 2.854644 \\
\hline ] & 2.462713 & 3.884074 & 2.456197 \\
\hline 1 & -3.752385 & 7.465230 & 1.143752 \\
\hline & -3.167770 & 6.556027 & 2.543740 \\
\hline 1 & -2.020394 & 7.155343 & 1.322313 \\
\hline & -5.359202 & 5.692776 & 0.605588 \\
\hline 1 & -4.846762 & 4.022348 & 0.311488 \\
\hline[ & -4.793581 & 4.684882 & 1.954841 \\
\hline | & -3.712572 & 6.559594 & -1.125288 \\
\hline 1 & -1.992884 & 6.123949 & -1.016495 \\
\hline 1 & -3.192427 & 4.900590 & -1.465230 \\
\hline 1 & -8.804991 & -0.141953 & 1.342908 \\
\hline & -8.383609 & 0.723866 & -0.140705 \\
\hline 1 & -8.169659 & -1.039689 & -0.042462 \\
\hline | & -7.404187 & 1.359411 & 2.695702 \\
\hline 1 & -5.687124 & 1.608557 & 2.333993 \\
\hline 1 & -6.922979 & 2.261582 & 1.244587 \\
\hline 1 & -7.115016 & -1.149270 & 2.805502 \\
\hline 1 & -6.369392 & -2.025845 & 1.452918 \\
\hline 1 & -5.379337 & -0.982751 & 2.487893 \\
\hline 1 & -6.870797 & -0.156466 & -5.235420 \\
\hline 1 & -7.285666 & -0.835654 & -3.660556 \\
\hline 1 & -7.135447 & 0.926257 & -3.858419 \\
\hline 1 & -4.738998 & 0.789837 & -5.750476 \\
\hline 1 & -4.805513 & 1.942191 & -4.401398 \\
\hline 1 & -3.406125 & 0.869330 & -4.596391 \\
\hline
\end{tabular}




$\begin{array}{llll}1 & -4.894646 & -1.702537 & -5.315072 \\ 1 & -3.646562 & -1.644299 & -4.051425 \\ 1 & -5.246647 & -2.303976 & -3.677611 \\ 1 & 2.504539 & -3.692707 & 0.606765 \\ 1 & 3.428481 & -2.201566 & 0.760465\end{array}$

$1_{\mathrm{C6}-} \psi_{-1 \mathrm{a} 2 \mathrm{a} 3 \mathrm{a} 4 \mathrm{a}}$

Number of imaginary frequencies : 0

The smallest frequencies are : $13.0144 \quad 14.4176 \quad 19.6130 \mathrm{~cm}(-1)$

Electronic energy : $\quad H F=-4125.4667265$

Zero-point correction $=\quad 1.299624$ (Hartree/Particle)

Thermal correction to Energy= $\quad 1.378139$

Thermal correction to Enthalpy= $\quad 1.379083$

Thermal correction to Gibbs Free Energy= $\quad 1.186421$

Sum of electronic and zero-point Energies $=\quad-4124.167102$

Sum of electronic and thermal Energies $=\quad-4124.088588$

Sum of electronic and thermal Enthalpies= $\quad-4124.087644$

Sum of electronic and thermal Free Energies $=\quad-4124.280305$

Cartesian Coordinates

$\begin{array}{cccc}\ldots \ldots \ldots \ldots \ldots \ldots \ldots \ldots \ldots \ldots \ldots \ldots \ldots \\ 6 & 3.075279 & -6.167787 & 1.998078 \\ 6 & 2.783591 & -5.258637 & 3.010629 \\ 6 & 2.467250 & -3.942988 & 2.694179 \\ 6 & 2.456318 & -3.505918 & 1.367961 \\ 6 & 2.769552 & -4.419586 & 0.361174 \\ 6 & 3.067338 & -5.742616 & 0.673035 \\ 6 & 2.158322 & -2.054231 & 1.103739 \\ 7 & 1.554192 & -1.752114 & -0.202883 \\ 6 & 0.469794 & -2.515097 & -0.749927 \\ 6 & -0.774058 & -2.593347 & -0.147240 \\ 6 & -1.854489 & -3.237311 & -0.826991 \\ 6 & -1.639709 & -3.796044 & -2.120293 \\ 6 & -0.348653 & -3.721317 & -2.692367 \\ 6 & 0.669682 & -3.094321 & -2.028826 \\ 6 & -3.160412 & -3.297894 & -0.274603 \\ 6 & -4.193141 & -3.876950 & -0.968809 \\ 6 & -3.974415 & -4.436000 & -2.246854 \\ 6 & -2.722886 & -4.394516 & -2.808395 \\ 6 & -0.999853 & -2.036535 & 1.210780 \\ 6 & -1.252937 & -0.688956 & 1.419540 \\ 6 & -1.459721 & -0.228999 & 2.750901 \\ 6 & -1.416686 & -1.083127 & 3.819464 \\ 6 & -1.187015 & -2.465309 & 3.635202 \\ 6 & -0.981330 & -2.953384 & 2.313064 \\ 6 & -1.171185 & -3.368363 & 4.725242 \\ 6 & -0.979092 & -4.711254 & 4.519116 \\ 6 & -0.783753 & -5.202016 & 3.211209 \\ 6 & -0.772479 & -4.346366 & 2.138039 \\ 15 & -1.514487 & 0.466994 & -0.023528 \\ 45 & -0.068378 & 0.711429 & -2.024751 \\ 6 & -0.182079 & 2.637817 & -1.959191 \\ 8 & -0.184698 & 3.792754 & -2.024133 \\ 15 & 1.779771 & -0.279336 & -1.033944\end{array}$




\begin{tabular}{|c|c|c|c|}
\hline 8 & 2.556105 & 0.547478 & 0.191018 \\
\hline 6 & 3.322089 & 1.624460 & -0.218409 \\
\hline & 4.597780 & 1.397307 & -0.699111 \\
\hline & 5.356895 & 2.518319 & -1.163543 \\
\hline & 4.802997 & 3.830196 & -1.054620 \\
\hline & 3.500852 & 3.996602 & -0.526390 \\
\hline 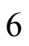 & 2.762475 & 2.913375 & -0.129062 \\
\hline & 5.553886 & 4.942836 & -1.506428 \\
\hline ) & 6.792858 & 4.776555 & -2.069181 \\
\hline y & 7.326813 & 3.478754 & -2.214233 \\
\hline & 6.628968 & 2.381077 & -1.775761 \\
\hline b & 5.073468 & -0.001856 & -0.801176 \\
\hline & 4.296416 & -0.910788 & -1.499431 \\
\hline$b$ & 4.698943 & -2.245691 & -1.699147 \\
\hline & 5.867422 & -2.693760 & -1.144838 \\
\hline 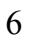 & 6.665730 & -1.834034 & -0.353522 \\
\hline$b$ & 6.266754 & -0.475202 & -0.169558 \\
\hline & 7.056805 & 0.342624 & 0.678639 \\
\hline 6 & 8.188840 & -0.146670 & 1.281171 \\
\hline & 8.597195 & -1.480902 & 1.070997 \\
\hline 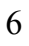 & 7.845556 & -2.304302 & 0.273057 \\
\hline & 3.093620 & -0.517411 & -2.043069 \\
\hline$b$ & -3.312372 & 0.188631 & -0.320653 \\
\hline ) & -4.206969 & 0.197720 & 0.754263 \\
\hline 6 & -5.568492 & -0.013599 & 0.554592 \\
\hline 6 & -6.006506 & -0.241486 & -0.755794 \\
\hline 6 & -5.142996 & -0.262619 & -1.850766 \\
\hline f & -3.783312 & -0.036698 & -1.609507 \\
\hline 6 & -6.569852 & -0.036613 & 1.707853 \\
\hline 6 & -5.693821 & -0.493746 & -3.256968 \\
\hline ) & -1.482610 & 2.109575 & 0.797752 \\
\hline 6 & -2.477412 & 3.049508 & 0.540546 \\
\hline 6 & -2.408755 & 4.331909 & 1.090376 \\
\hline 6 & -1.333502 & 4.624499 & 1.931756 \\
\hline 6 & -0.325893 & 3.699125 & 2.224227 \\
\hline & -0.396196 & 2.449318 & 1.608126 \\
\hline 6 & -3.471804 & 5.393682 & 0.816236 \\
\hline ) & 0.761009 & 4.053536 & 3.239314 \\
\hline 6 & -0.869446 & -0.432136 & -3.383553 \\
\hline 5 & -1.173927 & -1.008638 & -4.338886 \\
\hline . & 6.748762 & 1.369495 & 0.859383 \\
\hline 1 & -1.664138 & 0.822648 & 2.932478 \\
\hline & 8.135880 & -3.341878 & 0.113173 \\
\hline 1 & -1.324551 & -2.973512 & 5.728696 \\
\hline & 5.116119 & 5.935169 & -1.406496 \\
\hline 1 & -3.344598 & -2.856295 & 0.703202 \\
\hline 1 & -5.191416 & -3.894762 & -0.535028 \\
\hline 1 & 3.080476 & 4.999784 & -0.467199 \\
\hline 1 & -1.578003 & -0.705877 & 4.828611 \\
\hline 1 & 6.185745 & -3.725549 & -1.285646 \\
\hline 1 & 8.298335 & 3.345338 & -2.684833 \\
\hline 1 & 4.063775 & -2.894453 & -2.298529 \\
\hline 1 & -2.540421 & -4.816184 & -3.795868 \\
\hline 1 & -0.184875 & -4.147478 & -3.680798 \\
\hline 1 & -0.618175 & -6.265146 & 3.051901 \\
\hline 1 & 7.048998 & 1.387327 & -1.907252 \\
\hline
\end{tabular}




\begin{tabular}{|c|c|c|c|}
\hline & -0.592070 & -4.738628 & 1.139689 \\
\hline & 1.654541 & -3.006418 & -2.484447 \\
\hline & 7.358298 & 5.637946 & -2.416999 \\
\hline & 8.773788 & 0.499448 & 1.931981 \\
\hline & -4.800551 & -4.897214 & -2.784319 \\
\hline & -0.977387 & -5.399788 & 5.361227 \\
\hline & 9.498745 & -1.854230 & 1.551060 \\
\hline & 1.748343 & 3.019162 & 0.252006 \\
\hline & -3.828022 & 0.373936 & 1.757533 \\
\hline & -3.069999 & -0.038966 & -2.428631 \\
\hline 1 & -7.069420 & -0.412186 & -0.926415 \\
\hline 1 & -3.307560 & 2.774974 & -0.105580 \\
\hline 1 & 0.384846 & 1.709429 & 1.780634 \\
\hline 1 & -1.283706 & 5.611640 & 2.391594 \\
\hline 1 & 3.096570 & -1.488732 & 1.156081 \\
\hline 1 & 1.529657 & -1.665338 & 1.921725 \\
\hline 1 & 0.890416 & 1.034957 & -3.245343 \\
\hline 1 & 2.206096 & -3.242161 & 3.487766 \\
\hline 1 & 2.777314 & -4.092247 & -0.675685 \\
\hline 1 & 2.778212 & -5.579208 & 4.050654 \\
\hline ] & 3.296493 & -6.444788 & -0.126067 \\
\hline 1 & 3.307467 & -7.202793 & 2.239700 \\
\hline 6 & -4.578863 & -0.704127 & -4.278756 \\
\hline ? & -6.589443 & -1.736124 & -3.280871 \\
\hline 6 & -6.514396 & 0.732594 & -3.671748 \\
\hline ? & -5.923951 & 0.341511 & 3.039325 \\
\hline 6 & -7.706636 & 0.954134 & 1.436512 \\
\hline 0 & -7.144165 & -1.452002 & 1.836154 \\
\hline 6 & 1.739458 & 2.903211 & 3.470835 \\
\hline 6 & 1.552431 & 5.276060 & 2.766196 \\
\hline f & 0.089933 & 4.371244 & 4.581662 \\
\hline 6 & -4.564721 & 4.887825 & -0.122613 \\
\hline 6 & -4.132861 & 5.808059 & 2.135340 \\
\hline 6 & -2.810371 & 6.613419 & 0.165848 \\
\hline 1 & -5.306031 & 5.680386 & -0.284420 \\
\hline 1 & -4.164021 & 4.606077 & -1.104786 \\
\hline 1 & -5.095402 & 4.020103 & 0.292647 \\
\hline 1 & -3.562438 & 7.382790 & -0.055013 \\
\hline 1 & -2.052681 & 7.067645 & 0.815716 \\
\hline 1 & -2.319702 & 6.335780 & -0.776129 \\
\hline 1 & -4.899900 & 6.572754 & 1.953410 \\
\hline 1 & -4.619223 & 4.949399 & 2.617007 \\
\hline 1 & -3.411331 & 6.226526 & 2.847504 \\
\hline 1 & 2.482251 & 3.200558 & 4.221690 \\
\hline 1 & 1.232643 & 2.004027 & 3.847495 \\
\hline 1 & 2.288815 & 2.631053 & 2.560165 \\
\hline 1 & 0.848537 & 4.609802 & 5.339123 \\
\hline 1 & -0.591386 & 5.227837 & 4.512949 \\
\hline 1 & -0.489489 & 3.510831 & 4.942499 \\
\hline 1 & 2.306959 & 5.552506 & 3.514769 \\
\hline 1 & 2.077163 & 5.066399 & 1.825036 \\
\hline 1 & 0.908476 & 6.149167 & 2.606295 \\
\hline 1 & -5.015948 & -0.908163 & -5.264388 \\
\hline 1 & -3.939319 & 0.181265 & -4.385097 \\
\hline 1 & -3.940036 & -1.557600 & -4.011704 \\
\hline 1 & -6.967715 & -1.906711 & -4.297475 \\
\hline
\end{tabular}




$\begin{array}{rrrr}1 & -6.024591 & -2.626697 & -2.975552 \\ 1 & -7.460562 & -1.643070 & -2.621522 \\ 1 & -6.919273 & 0.598951 & -4.684141 \\ 1 & -7.358896 & 0.906033 & -2.992612 \\ 1 & -5.893786 & 1.638206 & -3.672396 \\ 1 & -6.680965 & 0.326304 & 3.833375 \\ 1 & -5.131084 & -0.360571 & 3.329936 \\ 1 & -5.494041 & 1.352060 & 3.010224 \\ 1 & -8.423775 & 0.944421 & 2.268096 \\ 1 & -7.321933 & 1.977127 & 1.334782 \\ 1 & -8.262285 & 0.713396 & 0.522614 \\ 1 & -7.872802 & -1.498731 & 2.656777 \\ 1 & -7.653061 & -1.772362 & 0.918576 \\ 1 & -6.348313 & -2.178322 & 2.049270\end{array}$

$1_{\mathrm{C} 6-}-$-1a2a3a4s

Number of imaginary frequencies : 0

The smallest frequencies are : $11.7410 \quad 18.2410 \quad 19.0156 \mathrm{~cm}(-1)$

Electronic energy : $\quad H F=-4125.4674055$

Zero-point correction $=\quad 1.299367($ Hartree/Particle $)$

Thermal correction to Energy= $\quad 1.377878$

Thermal correction to Enthalpy= $\quad 1.378822$

Thermal correction to Gibbs Free Energy $=\quad 1.186685$

Sum of electronic and zero-point Energies $=\quad-4124.168039$

Sum of electronic and thermal Energies $=\quad-4124.089528$

Sum of electronic and thermal Enthalpies $=\quad-4124.088584$

Sum of electronic and thermal Free Energies $=\quad-4124.280721$

\begin{tabular}{cccc} 
& \multicolumn{4}{c}{ Cartesian Coordinates } \\
\hline 6 & -0.526967 & 2.504740 & 1.572008 \\
6 & -1.589264 & 2.058290 & 0.781149 \\
6 & -2.671762 & 2.899057 & 0.535138 \\
6 & -2.715491 & 4.187083 & 1.072036 \\
6 & -1.657608 & 4.588544 & 1.889666 \\
6 & -0.564148 & 3.763002 & 2.174935 \\
15 & -1.493076 & 0.412067 & -0.030645 \\
6 & -3.271254 & 0.034952 & -0.334455 \\
6 & -4.151601 & -0.029631 & 0.742064 \\
6 & -5.519724 & -0.232854 & 0.549540 \\
6 & -5.973651 & -0.382249 & -0.761028 \\
6 & -5.114373 & -0.341287 & -1.866605 \\
6 & -3.756351 & -0.122776 & -1.632147 \\
6 & -6.437658 & -0.270363 & 1.769485 \\
6 & -6.372367 & 1.083275 & 2.487428 \\
6 & -5.685360 & -0.508380 & -3.273403 \\
6 & -6.604686 & 0.678251 & -3.583424 \\
6 & -3.884330 & 5.134363 & 0.809539 \\
6 & 0.510050 & 4.237485 & 3.154234 \\
6 & -1.175921 & -0.724688 & 1.415021 \\
6 & -0.907070 & -2.068966 & 1.201151 \\
6 & -0.863912 & -2.986468 & 2.300759 \\
6 & -1.057502 & -2.501783 & 3.626179 \\
6 & -1.294735 & -1.121794 & 3.815704 \\
6 & -1.363592 & -0.266420 & 2.748644
\end{tabular}




\begin{tabular}{|c|c|c|c|}
\hline 6 & -0.642355 & -4.376986 & 2.120671 \\
\hline 6 & -0.631957 & -5.234079 & 3.192406 \\
\hline 6 & -0.816543 & -4.747060 & 4.503596 \\
\hline 6 & -1.019252 & -3.406637 & 4.714495 \\
\hline 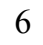 & -0.682914 & -2.612152 & -0.162713 \\
\hline b & 0.553007 & -2.494060 & -0.775833 \\
\hline 6 & 0.753809 & -3.043212 & -2.067556 \\
\hline 6 & -0.258096 & -3.676087 & -2.735247 \\
\hline 6 & -1.541634 & -3.789929 & -2.152858 \\
\hline 6 & -1.755024 & -3.267851 & -0.844053 \\
\hline 6 & -2.619146 & -4.392255 & -2.846244 \\
\hline 6 & -3.863736 & -4.473242 & -2.274377 \\
\hline 6 & -4.080892 & -3.952585 & -0.979990 \\
\hline 6 & -3.053343 & -3.371460 & -0.279941 \\
\hline 7 & 1.624655 & -1.716559 & -0.224964 \\
\hline 15 & 1.816145 & -0.227387 & -1.035007 \\
\hline 8 & 3.140630 & -0.421406 & -2.039561 \\
\hline 6 & 4.345277 & -0.804254 & -1.492362 \\
\hline 6 & 5.100313 & 0.104718 & -0.770206 \\
\hline 6 & 6.293352 & -0.361156 & -0.132823 \\
\hline 6 & 6.715462 & -1.710337 & -0.335539 \\
\hline 6 & 5.940149 & -2.568318 & -1.151373 \\
\hline 6 & 4.770930 & -2.128978 & -1.711149 \\
\hline 6 & 7.060123 & 0.453735 & 0.739238 \\
\hline 6 & 8.192274 & -0.028626 & 1.347083 \\
\hline 6 & 8.623906 & -1.352497 & 1.118567 \\
\hline 6 & 7.894972 & -2.173226 & 0.297143 \\
\hline 6 & 4.602454 & & -0.653693 \\
\hline 6 & 3.316794 & 1.696269 & -0.188058 \\
\hline 6 & 2.732791 & 2.974362 & -0.100109 \\
\hline 6 & 3.459090 & 4.073702 & -0.474232 \\
\hline 6 & 4.773098 & 3.934774 & -0.980324 \\
\hline 6 & 5.349966 & 2.633244 & -1.094741 \\
\hline 6 & 5.513105 & 5.064788 & -1.406381 \\
\hline 6 & 6.763774 & 4.925173 & -1.950105 \\
\hline 6 & 7.320753 & 3.637847 & -2.101757 \\
\hline 6 & 6.633737 & 2.523911 & -1.687986 \\
\hline 8 & 2.565229 & 0.603395 & 0.204720 \\
\hline 6 & 2.244773 & -2.024320 & 1.073044 \\
\hline 6 & 2.562650 & -3.474451 & 1.321234 \\
\hline 6 & 2.598328 & -3.919920 & 2.644261 \\
\hline 6 & 2.932774 & -5.234247 & 2.947231 \\
\hline 6 & 3.218079 & -6.133878 & 1.924385 \\
\hline 6 & 3.185630 & -5.700316 & 0.602501 \\
\hline 6 & 2.869876 & -4.378273 & 0.303873 \\
\hline 45 & -0.048445 & 0.739662 & -2.015072 \\
\hline 6 & -0.824171 & -0.391235 & -3.399596 \\
\hline 8 & -1.122970 & -0.953397 & -4.365120 \\
\hline 6 & -0.224568 & 2.659605 & -1.913574 \\
\hline 8 & -0.273060 & 3.814614 & -1.953630 \\
\hline 6 & -7.892640 & -0.542326 & 1.397164 \\
\hline 6 & -5.974700 & -1.380142 & 2.720989 \\
\hline 6 & -4.589126 & -0.562001 & -4.334354 \\
\hline 6 & -6.485813 & -1.812042 & -3.360769 \\
\hline 1 & 6.733597 & 1.472295 & 0.934177 \\
\hline 1 & -1.579523 & 0.782367 & 2.932579 \\
\hline
\end{tabular}




\begin{tabular}{|c|c|c|c|}
\hline & 8.203437 & -3.203224 & 0.122864 \\
\hline & -1.163757 & -3.014992 & 5.720506 \\
\hline & 5.057455 & 6.048596 & -1.302125 \\
\hline & -3.235334 & -2.959985 & 0.711587 \\
\hline & -5.074846 & -4.002846 & -0.539278 \\
\hline & 3.020783 & 5.069137 & -0.412598 \\
\hline & -1.442847 & -0.747249 & 4.827855 \\
\hline & 6.276204 & -3.592468 & -1.306031 \\
\hline & 8.301891 & 3.525611 & -2.557695 \\
\hline & 4.151772 & -2.776953 & -2.327939 \\
\hline & -2.437735 & -4.785126 & -3.845705 \\
\hline & -0.095906 & -4.075869 & -3.734912 \\
\hline & -0.457564 & -6.295292 & 3.029703 \\
\hline & 7.072137 & 1.538835 & -1.824368 \\
\hline & -0.470292 & -4.765282 & 1.119316 \\
\hline ] & 1.732650 & -2.925100 & -2.529605 \\
\hline & 7.321016 & 5.799575 & -2.278119 \\
\hline & 8.759105 & 0.614730 & 2.016390 \\
\hline & -4.685749 & -4.937657 & -2.815348 \\
\hline & -0.797662 & -5.436901 & 5.344415 \\
\hline & 9.525326 & -1.720308 & 1.603111 \\
\hline & 1.708977 & 3.058646 & 0.259750 \\
\hline$\frac{1}{1}$ & -3.767937 & 0.094949 & 1.753936 \\
\hline & -3.053101 & -0.069580 & -2.458411 \\
\hline 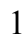 & -7.036043 & -0.538438 & -0.936796 \\
\hline & -3.485016 & 2.543532 & -0.091676 \\
\hline 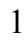 & 0.320649 & 1.840304 & 1.740010 \\
\hline 1 & -1.691120 & 5.582617 & 2.335557 \\
\hline & 3.176706 & -1.448140 & 1.123271 \\
\hline 1 & 1.619664 & -1.651185 & 1.900423 \\
\hline & 0.909319 & 1.113282 & -3.222541 \\
\hline 1 & 2.342150 & -3.226894 & 3.446270 \\
\hline ] & 2.857010 & -4.044713 & -0.730875 \\
\hline 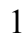 & 2.946432 & -5.561506 & 3.985087 \\
\hline 1 & 3.409402 & -6.394997 & -0.204631 \\
\hline 1 & 3.464254 & -7.168032 & 2.155581 \\
\hline 1 & -5.040924 & -0.706529 & -5.323841 \\
\hline ] & -4.005116 & 0.366697 & -4.375421 \\
\hline 1 & -3.895795 & -1.395791 & -4.159325 \\
\hline 1 & -6.889876 & -1.939659 & -4.373950 \\
\hline 1 & -5.843686 & -2.675399 & -3.140142 \\
\hline 1 & -7.333310 & -1.833559 & -2.665003 \\
\hline 1 & -7.021534 & 0.584867 & -4.595375 \\
\hline 1 & -7.445300 & 0.741160 & -2.881191 \\
\hline | & -6.052660 & 1.625896 & -3.530954 \\
\hline 1 & -8.508052 & -0.562682 & 2.305448 \\
\hline 1 & -8.302204 & 0.237143 & 0.741914 \\
\hline 1 & -8.012960 & -1.511674 & 0.896092 \\
\hline 1 & -6.620142 & -1.414525 & 3.608949 \\
\hline 1 & -6.024951 & -2.361488 & 2.230697 \\
\hline 1 & -4.943468 & -1.233936 & 3.067181 \\
\hline 1 & -6.988523 & 1.065482 & 3.396593 \\
\hline 1 & -5.348494 & 1.347213 & 2.781950 \\
\hline 1 & -6.750803 & 1.886643 & 1.841005 \\
\hline 6 & 1.544550 & 3.153244 & 3.453399 \\
\hline 6 & 1.232677 & 5.460880 & 2.582650 \\
\hline
\end{tabular}




$\begin{array}{rrrr}6 & -0.155733 & 4.618315 & 4.482746 \\ 6 & -4.938081 & 4.510743 & -0.104103 \\ 6 & -4.558338 & 5.493454 & 2.138264 \\ 6 & -3.362450 & 6.408580 & 0.137629 \\ 1 & 1.984968 & 5.826773 & 3.294309 \\ 1 & 1.749839 & 5.212678 & 1.646595 \\ 1 & 0.540406 & 6.285898 & 2.374807 \\ 1 & 0.604365 & 4.935730 & 5.208979 \\ 1 & -0.869221 & 5.443448 & 4.372235 \\ 1 & -0.695662 & 3.763368 & 4.910847 \\ 1 & 2.266114 & 3.529355 & 4.189444 \\ 1 & 1.079743 & 2.252195 & 3.876987 \\ 1 & 2.115715 & 2.857322 & 2.564076 \\ 1 & -5.766203 & 5.216580 & -0.245377 \\ 1 & -4.533011 & 4.275098 & -1.096739 \\ 1 & -5.358705 & 3.587570 & 0.319224 \\ 1 & -4.192701 & 7.095483 & -0.075148 \\ 1 & -2.643652 & 6.943632 & 0.769911 \\ 1 & -2.862742 & 6.174390 & -0.811350 \\ 1 & -5.397446 & 6.180973 & 1.966538 \\ 1 & -4.952536 & 4.596098 & 2.633559 \\ 1 & -3.866273 & 5.982882 & 2.834362\end{array}$

$1_{\mathrm{C} 6-} \psi_{-1 \mathrm{a} 2 \mathrm{2a3s4a}}$

Number of imaginary frequencies : 0

The smallest frequencies are : $7.4956 \quad 13.793019 .7276 \mathrm{~cm}(-1)$

Electronic energy : $\quad H F=-4125.4671103$

Zero-point correction $=\quad 1.299765($ Hartree/Particle $)$

Thermal correction to Energy= $\quad 1.378230$

Thermal correction to Enthalpy= $\quad 1.379174$

Thermal correction to Gibbs Free Energy $=\quad 1.185934$

Sum of electronic and zero-point Energies $=\quad-4124.167345$

Sum of electronic and thermal Energies $=\quad-4124.088880$

Sum of electronic and thermal Enthalpies $=\quad-4124.087936$

Sum of electronic and thermal Free Energies $=\quad-4124.281176$

Cartesian Coordinates

$\begin{array}{cccc}6 & 2.737903 & -4.444442 & 0.187458 \\ 6 & 2.446842 & -3.558605 & 1.225231 \\ 6 & 2.464536 & -4.037085 & 2.537076 \\ 6 & 2.765985 & -5.365963 & 2.809574 \\ 6 & 3.034715 & -6.247078 & 1.766373 \\ 6 & 3.019824 & -5.780743 & 0.455418 \\ 6 & 2.165516 & -2.095198 & 1.012628 \\ 7 & 1.556267 & -1.738381 & -0.277801 \\ 15 & 1.784041 & -0.234594 & -1.049006 \\ 45 & -0.069019 & 0.806804 & -1.978215 \\ 6 & -0.165777 & 2.728141 & -1.812353 \\ 8 & -0.176213 & 3.884725 & -1.812822 \\ 6 & 0.470414 & -2.476210 & -0.854709 \\ 6 & -0.770224 & -2.588931 & -0.249438 \\ 6 & -1.846983 & -3.218142 & -0.950205 \\ 6 & -1.630013 & -3.726856 & -2.264075 \\ 6 & -0.344872 & -3.608456 & -2.841722\end{array}$




\begin{tabular}{|c|c|c|c|}
\hline 6 & 0.668904 & -2.995225 & -2.159282 \\
\hline 6 & -3.148786 & -3.327478 & -0.394753 \\
\hline 6 & -4.170156 & -3.911477 & -1.102322 \\
\hline 6 & -3.947054 & -4.423201 & -2.398613 \\
\hline 6 & -2.702185 & -4.328466 & -2.966008 \\
\hline 6 & -0.983055 & -2.090111 & 1.134036 \\
\hline 6 & -1.203511 & -0.747137 & 1.406202 \\
\hline 6 & -1.378367 & -0.338785 & 2.757752 \\
\hline 6 & -1.345326 & -1.239838 & 3.787839 \\
\hline 6 & -1.152820 & -2.617590 & 3.540233 \\
\hline 6 & -0.969635 & -3.052700 & 2.196219 \\
\hline 6 & -1.145722 & -3.566866 & 4.590678 \\
\hline 6 & -0.978928 & -4.902630 & 4.326165 \\
\hline 6 & -0.800740 & -5.340415 & 2.996756 \\
\hline 6 & -0.784204 & -4.440410 & 1.960871 \\
\hline 15 & -1.488041 & 0.465145 & 0.019624 \\
\hline 6 & -3.281081 & 0.173841 & -0.283895 \\
\hline 6 & -4.147486 & -0.091598 & 0.784100 \\
\hline 6 & -5.508066 & -0.276144 & 0.571256 \\
\hline 6 & -5.988322 & -0.173526 & -0.744726 \\
\hline 6 & -5.155219 & 0.091669 & -1.827451 \\
\hline 6 & -3.787038 & 0.262191 & -1.571123 \\
\hline 6 & -6.472419 & -0.605552 & 1.709272 \\
\hline 6 & -7.545063 & 0.484301 & 1.804299 \\
\hline 6 & -5.651790 & 0.124712 & -3.270105 \\
\hline 6 & -5.177582 & -1.159200 & -3.962648 \\
\hline 6 & -1.497565 & 2.079868 & 0.895494 \\
\hline 6 & -0.413869 & 2.440094 & 1.700787 \\
\hline 6 & -0.379889 & 3.680488 & 2.339254 \\
\hline 6 & -1.420471 & 4.577468 & 2.072775 \\
\hline 6 & -2.496549 & 4.262513 & 1.240574 \\
\hline 6 & -2.528806 & 2.987790 & 0.671212 \\
\hline 6 & -3.595104 & 5.292606 & 0.985757 \\
\hline 6 & -4.242912 & 5.694677 & 2.315045 \\
\hline 6 & 0.710549 & 4.058750 & 3.342136 \\
\hline 6 & 1.477765 & 5.288732 & 2.848613 \\
\hline 6 & -7.139734 & -1.957788 & 1.434571 \\
\hline 6 & -5.762802 & -0.700344 & 3.058741 \\
\hline 6 & -5.082026 & 1.342712 & -4.005526 \\
\hline 6 & -7.174916 & 0.189645 & -3.359041 \\
\hline 6 & -2.978841 & 6.527595 & 0.320071 \\
\hline 6 & -4.691232 & 4.753953 & 0.067708 \\
\hline 6 & 0.051898 & 4.379084 & 4.690015 \\
\hline 6 & 1.707919 & 2.924599 & 3.573042 \\
\hline 8 & 3.098344 & -0.435332 & -2.066162 \\
\hline 6 & 4.297605 & -0.856670 & -1.535425 \\
\hline 6 & 5.077022 & 0.019615 & -0.799053 \\
\hline 6 & 6.263924 & -0.485291 & -0.180268 \\
\hline 6 & 6.654200 & -1.839270 & -0.412621 \\
\hline 6 & 5.854754 & -2.662576 & -1.240699 \\
\hline 6 & 4.692704 & -2.185596 & -1.784423 \\
\hline 6 & 7.826475 & -2.341466 & 0.203327 \\
\hline 6 & 8.579042 & -1.553954 & 1.035873 \\
\hline 6 & 8.179568 & -0.225176 & 1.292569 \\
\hline 6 & 7.054927 & 0.294705 & 0.701962 \\
\hline 6 & 4.608239 & 1.416257 & -0.644255 \\
\hline
\end{tabular}




\begin{tabular}{|c|c|c|c|}
\hline 6 & 3.331966 & 1.630319 & -0.159279 \\
\hline 6 & 2.775930 & 2.917017 & -0.027410 \\
\hline & 3.519497 & 4.011350 & -0.382121 \\
\hline & 4.824145 & 3.859753 & -0.908612 \\
\hline & 5.373726 & 2.550590 & -1.063345 \\
\hline & 6.648498 & 2.431161 & -1.673678 \\
\hline & 7.352829 & 3.541625 & -2.067233 \\
\hline & 6.822950 & 4.835245 & -1.876874 \\
\hline & 5.581533 & 4.985456 & -1.315003 \\
\hline & 2.561185 & 0.541934 & 0.208377 \\
\hline & -0.957289 & -0.226459 & -3.367717 \\
\hline & -1.358915 & -0.716107 & -4.335893 \\
\hline & 6.753470 & 1.316489 & 0.918887 \\
\hline & -1.563245 & 0.707630 & 2.983934 \\
\hline & 8.110224 & -3.374566 & 0.006956 \\
\hline & -1.283326 & -3.212047 & 5.611207 \\
\hline & 5.146770 & 5.975079 & -1.180872 \\
\hline & -3.337801 & -2.926167 & 0.600163 \\
\hline & -5.162839 & -3.980171 & -0.661638 \\
\hline & 3.101551 & 5.013117 & -0.289870 \\
\hline & -1.487695 & -0.903922 & 4.814237 \\
\hline & 6.166488 & -3.690619 & -1.418621 \\
\hline & 8.326486 & 3.421983 & -2.537117 \\
\hline & 4.056398 & -2.806756 & -2.411205 \\
\hline & -2.515369 & -4.709650 & -3.968943 \\
\hline & -0.181170 & -3.995086 & -3.846333 \\
\hline & -0.652997 & -6.398269 & 2.791401 \\
\hline & 7.065525 & 1.441310 & -1.839743 \\
\hline & -0.617900 & -4.792146 & 0.945179 \\
\hline 1 & 1.650637 & -2.878323 & -2.615019 \\
\hline & 7.393563 & 5.706688 & -2.189423 \\
\hline & 8.765408 & 0.391990 & 1.970122 \\
\hline & -4.765700 & -4.884945 & -2.946276 \\
\hline & -0.982545 & -5.626339 & 5.138206 \\
\hline 1 & 9.474689 & -1.951703 & 1.507308 \\
\hline & 1.759007 & 3.011514 & 0.348913 \\
\hline ] & -3.741468 & -0.161356 & 1.789439 \\
\hline & -3.099955 & 0.470711 & -2.388079 \\
\hline & -7.053326 & -0.316130 & -0.918582 \\
\hline & -3.358576 & 2.696345 & 0.032427 \\
\hline & 0.394220 & 1.723916 & 1.848250 \\
\hline & -1.394682 & 5.559815 & 2.544867 \\
\hline & 3.111309 & -1.543668 & 1.078811 \\
\hline & 1.547497 & -1.727730 & 1.848048 \\
\hline & 0.874340 & 1.172418 & -3.200176 \\
\hline & 2.221728 & -3.357533 & 3.354583 \\
\hline & 2.739572 & -4.085728 & -0.838989 \\
\hline & 2.766560 & -5.718442 & 3.839226 \\
\hline ] & 3.231416 & -6.460832 & -0.367297 \\
\hline & 3.254800 & -7.292207 & 1.973284 \\
\hline 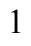 & -5.459769 & 5.523780 & -0.076340 \\
\hline & -4.303052 & 4.487650 & -0.924162 \\
\hline 1 & -5.186529 & 3.868453 & 0.488876 \\
\hline ] & -3.755069 & 7.275975 & 0.110806 \\
\hline & -2.221810 & 7.003051 & 0.955412 \\
\hline & -2.497637 & 6.260159 & -0.629641 \\
\hline
\end{tabular}




$\begin{array}{rrrr}1 & -5.032774 & 6.438346 & 2.143987 \\ 1 & -4.697928 & 4.826354 & 2.809295 \\ 1 & -3.520140 & 6.135564 & 3.012226 \\ 1 & 2.447604 & 3.234595 & 4.321844 \\ 1 & 1.215817 & 2.018054 & 3.951502 \\ 1 & 2.260698 & 2.659865 & 2.662235 \\ 1 & 0.817534 & 4.631260 & 5.435868 \\ 1 & -0.638841 & 5.228259 & 4.625294 \\ 1 & -0.513705 & 3.515684 & 5.064847 \\ 1 & 2.235699 & 5.585246 & 3.586070 \\ 1 & 1.995465 & 5.078033 & 1.903735 \\ 1 & 0.817190 & 6.148976 & 2.686273 \\ 1 & -7.479798 & 0.247907 & -4.411435 \\ 1 & -7.654248 & -0.701624 & -2.934794 \\ 1 & -7.576485 & 1.073911 & -2.846652 \\ 1 & -5.485957 & -1.164240 & -5.017165 \\ 1 & -4.085114 & -1.262122 & -3.927198 \\ 1 & -5.607089 & -2.045293 & -3.475359 \\ 1 & -5.479404 & 1.383173 & -5.028060 \\ 1 & -5.357118 & 2.277041 & -3.498212 \\ 1 & -3.988704 & 1.313034 & -4.087056 \\ 1 & -6.491395 & -0.947600 & 3.841044 \\ 1 & -4.994596 & -1.486585 & 3.064197 \\ 1 & -5.284458 & 0.246538 & 3.341452 \\ 1 & -8.244834 & 0.261672 & 2.621010 \\ 1 & -7.092339 & 1.464144 & 2.004417 \\ 1 & -8.130420 & 0.568677 & 0.880776 \\ 1 & -7.848316 & -2.203320 & 2.237038 \\ 1 & -7.694775 & -1.963432 & 0.488869 \\ 1 & -6.391250 & -2.760612 & 1.391062\end{array}$

$1_{\mathrm{C} 6-} \psi_{-1 \mathrm{a} 2 \mathrm{a} 3 \mathrm{~s} 4 \mathrm{~s}}$

Number of imaginary frequencies : 0

The smallest frequencies are : $14.9949 \quad 16.0658 \quad 23.1596 \mathrm{~cm}(-1)$

Electronic energy : $\quad H F=-4125.467434$

Zero-point correction $=\quad 1.301240($ Hartree/Particle $)$

Thermal correction to Energy $=\quad 1.379226$

Thermal correction to Enthalpy= $\quad 1.380170$

Thermal correction to Gibbs Free Energy $=\quad 1.190010$

Sum of electronic and zero-point Energies $=\quad-4124.166194$

Sum of electronic and thermal Energies $=\quad-4124.088208$

Sum of electronic and thermal Enthalpies $=\quad-4124.087264$

Sum of electronic and thermal Free Energies $=\quad-4124.277424$

Cartesian Coordinates

\begin{tabular}{|c|c|c|c|}
\hline 6 & 3.082598 & -6.192073 & 1.891069 \\
\hline 6 & 2.787492 & -5.298228 & 2.916111 \\
\hline 6 & 2.472489 & -3.977848 & 2.618418 \\
\hline 6 & 2.466499 & -3.520690 & 1.298962 \\
\hline 6 & 2.783748 & -4.419059 & 0.279755 \\
\hline 6 & 3.079870 & -5.746828 & 0.572644 \\
\hline 6 & 2.169009 & -2.065187 & 1.056370 \\
\hline 7 & 1.562564 & -1.742302 & -0.244490 \\
\hline 6 & 0.487065 & -2.505426 & -0.808720 \\
\hline
\end{tabular}




\begin{tabular}{|c|c|c|c|}
\hline 6 & -0.759566 & -2.602753 & -0.214021 \\
\hline 6 & -1.826059 & -3.259350 & -0.904090 \\
\hline 6 & -1.589679 & -3.826991 & -2.189930 \\
\hline 6 & -0.298784 & -3.722329 & -2.757720 \\
\hline 6 & 0.702079 & -3.070971 & -2.091051 \\
\hline 6 & -3.135625 & -3.340439 & -0.362606 \\
\hline 6 & -4.145476 & -3.962619 & -1.053529 \\
\hline 6 & -3.901369 & -4.542411 & -2.317281 \\
\hline 6 & -2.649804 & -4.469914 & -2.873557 \\
\hline 6 & -0.989945 & -2.076461 & 1.156014 \\
\hline 6 & -1.233474 & -0.733072 & 1.402116 \\
\hline 6 & -1.428293 & -0.305391 & 2.745598 \\
\hline 6 & -1.388444 & -1.187767 & 3.791404 \\
\hline 6 & -1.172368 & -2.566535 & 3.569545 \\
\hline 6 & -0.972718 & -3.021325 & 2.234685 \\
\hline 6 & -1.158982 & -3.497292 & 4.636209 \\
\hline 6 & -0.972254 & -4.835061 & 4.395748 \\
\hline 6 & -0.779324 & -5.292703 & 3.075362 \\
\hline 6 & -0.767311 & -4.410169 & 2.024380 \\
\hline 15 & -1.506121 & 0.455360 & -0.008450 \\
\hline 4 & -0.083430 & 0.740869 & -2.014290 \\
\hline 6 & -0.195461 & 2.666424 & -1.915378 \\
\hline 8 & -0.211819 & 3.821978 & -1.960065 \\
\hline 15 & 1.776926 & -0.252915 & -1.046431 \\
\hline 8 & 2.541640 & 0.556529 & 0.197720 \\
\hline 6 & 3.301575 & 1.646745 & -0.186278 \\
\hline 6 & 4.580437 & 1.438387 & -0.667267 \\
\hline 6 & 5.333478 & 2.574007 & -1.105293 \\
\hline 6 & 4.770716 & 3.879675 & -0.969951 \\
\hline 6 & 3.465678 & 4.026091 & -0.442920 \\
\hline 6 & 2.733141 & 2.929788 & -0.071579 \\
\hline 6 & 5.515700 & 5.006712 & -1.395144 \\
\hline 6 & 6.757495 & 4.860622 & -1.957264 \\
\hline 6 & 7.300284 & 3.569712 & -2.128631 \\
\hline 6 & 6.608316 & 2.458310 & -1.716240 \\
\hline 6 & 5.066679 & 0.045044 & -0.796745 \\
\hline 6 & 4.299225 & -0.854880 & -1.516871 \\
\hline 6 & 4.712345 & -2.182474 & -1.742692 \\
\hline 6 & 5.881810 & -2.633418 & -1.192821 \\
\hline 6 & 6.670613 & -1.784450 & -0.380560 \\
\hline 6 & 6.260938 & -0.432605 & -0.170216 \\
\hline 6 & 7.041551 & 0.373194 & 0.697975 \\
\hline 6 & 8.174738 & -0.120221 & 1.294935 \\
\hline 6 & 8.593694 & -1.446835 & 1.058983 \\
\hline 6 & 7.851332 & -2.259007 & 0.241081 \\
\hline 8 & 3.095403 & -0.459199 & -2.056372 \\
\hline 6 & -3.301040 & 0.172385 & -0.312805 \\
\hline 6 & -4.183634 & 0.015400 & 0.756882 \\
\hline 6 & -5.548486 & -0.170994 & 0.545297 \\
\hline 6 & -6.010617 & -0.193543 & -0.776145 \\
\hline 6 & -5.155481 & -0.033930 & -1.868289 \\
\hline 6 & -3.793234 & 0.150164 & -1.611753 \\
\hline 6 & -6.465412 & -0.343915 & 1.755191 \\
\hline 6 & -5.633313 & -0.114951 & -3.315788 \\
\hline 6 & -1.503269 & 2.082152 & 0.843999 \\
\hline 6 & -2.517579 & 3.004182 & 0.598314 \\
\hline
\end{tabular}




\begin{tabular}{|c|c|c|c|}
\hline 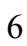 & -2.481392 & 4.277418 & 1.170806 \\
\hline & -1.414734 & 4.579800 & 2.019780 \\
\hline & -0.385707 & 3.674399 & 2.298159 \\
\hline & -0.426462 & 2.433363 & 1.662392 \\
\hline & -3.574167 & 5.314001 & 0.918608 \\
\hline & 0.697036 & 4.041800 & 3.313120 \\
\hline & -0.952443 & -0.352835 & -3.367980 \\
\hline & -1.344336 & -0.885049 & -4.317697 \\
\hline & 6.725313 & 1.393810 & 0.898784 \\
\hline & -1.626488 & 0.742431 & 2.953634 \\
\hline & 8.149938 & -3.290914 & 0.061040 \\
\hline & -1.308719 & -3.127231 & 5.649591 \\
\hline & 5.070957 & 5.993743 & -1.275370 \\
\hline & -3.338943 & -2.885980 & 0.606336 \\
\hline & -5.145237 & -4.005191 & -0.626175 \\
\hline & 3.038431 & 5.025002 & -0.363857 \\
\hline & -1.542291 & -0.836053 & 4.810858 \\
\hline & 6.208214 & -3.659734 & -1.353617 \\
\hline & 8.274088 & 3.452754 & -2.598890 \\
\hline & 4.084052 & -2.823332 & -2.357712 \\
\hline & -2.448837 & -4.900090 & -3.853679 \\
\hline & -0.120259 & -4.150300 & -3.742856 \\
\hline & -0.616413 & -6.351804 & 2.888834 \\
\hline & 7.035200 & 1.470393 & -1.868050 \\
\hline & -0.589074 & -4.777502 & 1.016288 \\
\hline & 1.687472 & -2.962592 & -2.541116 \\
\hline & 7.318428 & 5.732922 & -2.284593 \\
\hline & 8.752346 & 0.516545 & 1.961327 \\
\hline & -4.710601 & -5.035969 & -2.851193 \\
\hline l & -0.971791 & -5.544855 & 5.219999 \\
\hline & 9.496060 & -1.823447 & 1.534928 \\
\hline 1 & 1.716771 & 3.020506 & 0.307430 \\
\hline & -3.798182 & 0.037761 & 1.775219 \\
\hline & -3.095195 & 0.278211 & -2.435329 \\
\hline 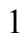 & -7.071313 & -0.340614 & -0.958187 \\
\hline & -3.340640 & 2.722134 & -0.053608 \\
\hline 1 & 0.370887 & 1.708336 & 1.823558 \\
\hline & -1.390334 & 5.559119 & 2.498236 \\
\hline 1 & 3.107619 & -1.500891 & 1.116300 \\
\hline 1 & 1.541546 & -1.688771 & 1.880933 \\
\hline 1 & 0.858080 & 1.073218 & -3.247164 \\
\hline 1 & 2.209496 & -3.288921 & 3.421683 \\
\hline & 2.794637 & -4.076198 & -0.751955 \\
\hline 1 & 2.778266 & -5.634400 & 3.951168 \\
\hline & 3.311947 & -6.436828 & -0.236158 \\
\hline 1 & 3.313531 & -7.230727 & 2.117792 \\
\hline 5 & -7.156093 & -0.122633 & -3.426758 \\
\hline & -5.104626 & 1.079569 & -4.117601 \\
\hline 6 & -5.095578 & -1.415408 & -3.924895 \\
\hline 6 & -7.920049 & -0.578393 & 1.356516 \\
\hline 6 & -5.997750 & -1.550141 & 2.578771 \\
\hline 6 & -6.404517 & 0.920415 & 2.620523 \\
\hline 6 & 1.692245 & 2.905028 & 3.540097 \\
\hline 6 & 1.469647 & 5.275613 & 2.837895 \\
\hline 6 & 0.026744 & 4.349152 & 4.658175 \\
\hline 6 & -4.631551 & 4.812090 & -0.062723 \\
\hline
\end{tabular}




$\begin{array}{lrrr}6 & -4.270688 & 5.646758 & 2.242683 \\ 6 & -2.947074 & 6.583421 & 0.333022 \\ 1 & -5.392843 & 5.588339 & -0.210599 \\ 1 & -4.200781 & 4.580613 & -1.045602 \\ 1 & -5.145454 & 3.913685 & 0.305395 \\ 1 & -3.723629 & 7.333477 & 0.131444 \\ 1 & -2.217757 & 7.038190 & 1.014132 \\ 1 & -2.431736 & 6.364949 & -0.611221 \\ 1 & -5.059185 & 6.394399 & 2.082418 \\ 1 & -4.736075 & 4.751475 & 2.676097 \\ 1 & -3.572978 & 6.053966 & 2.984698 \\ 1 & 2.428736 & 3.208721 & 4.294610 \\ 1 & 1.198158 & 1.995819 & 3.909519 \\ 1 & 2.248316 & 2.647351 & 2.629175 \\ 1 & 0.785320 & 4.595760 & 5.413073 \\ 1 & -0.664936 & 5.197720 & 4.594428 \\ 1 & -0.540787 & 3.481414 & 5.020142 \\ 1 & 2.223069 & 5.562708 & 3.583642 \\ 1 & 1.993479 & 5.073652 & 1.894449 \\ 1 & 0.811994 & 6.138962 & 2.680453 \\ 1 & -7.447382 & -0.153164 & -4.484177 \\ 1 & -7.602085 & -1.001162 & -2.943230 \\ 1 & -7.603613 & 0.777742 & -2.985457 \\ 1 & -5.472969 & 1.031077 & -5.150760 \\ 1 & -5.441899 & 2.029555 & -3.682539 \\ 1 & -4.009044 & 1.101630 & -4.166089 \\ 1 & -5.402127 & -1.502714 & -4.976359 \\ 1 & -3.999284 & -1.460172 & -3.886819 \\ 1 & -5.480183 & -2.289036 & -3.381272 \\ 1 & -8.533363 & -0.704284 & 2.257667 \\ 1 & -8.334264 & 0.268222 & 0.794158 \\ 1 & -8.037529 & -1.485401 & 0.749414 \\ 1 & -6.642185 & -1.684575 & 3.458010 \\ 1 & -6.044434 & -2.472238 & 1.984121 \\ 1 & -4.966870 & -1.437507 & 2.938915 \\ 1 & -7.048634 & 0.811005 & 3.503459 \\ 1 & -5.387635 & 1.130171 & 2.974701 \\ 1 & -6.749701 & 1.797637 & 2.057565 \\ --------------------------------------------------------------------------------- \\ - & & & \\ -------------------------------------------------------------------------------------------\end{array}$

Number of imaginary frequencies : 0

The smallest frequencies are : $13.2285 \quad 19.5086 \quad 21.4181 \mathrm{~cm}(-1)$

Electronic energy : $\quad H F=-4125.4676733$

Zero-point correction $=\quad 1.300273($ Hartree/Particle)

Thermal correction to Energy= $\quad 1.378489$

Thermal correction to Enthalpy= $\quad 1.379433$

Thermal correction to Gibbs Free Energy $=\quad 1.188374$

Sum of electronic and zero-point Energies $=\quad-4124.167400$

Sum of electronic and thermal Energies $=\quad-4124.089185$

Sum of electronic and thermal Enthalpies $=\quad-4124.088241$

Sum of electronic and thermal Free Energies $=\quad-4124.279299$

Cartesian Coordinates

$6 \quad 3.064941 \quad-6.205532 \quad 1.890871$ 


\begin{tabular}{|c|c|c|c|}
\hline 6 & 2.791218 & -5.308697 & 2.919286 \\
\hline 6 & 2.483640 & -3.985885 & 2.624910 \\
\hline f & 2.464408 & -3.529257 & 1.305363 \\
\hline & 2.760342 & -4.430679 & 0.282497 \\
\hline$b$ & 3.048709 & -5.760904 & 0.572365 \\
\hline ) & 2.175778 & -2.071272 & 1.066696 \\
\hline 7 & 1.566295 & -1.742405 & -0.230942 \\
\hline 5 & 0.475324 & -2.488999 & -0.787221 \\
\hline 6 & -0.766051 & -2.572125 & -0.179784 \\
\hline 6 & -1.854495 & -3.192582 & -0.868056 \\
\hline 6 & -1.650297 & -3.721779 & -2.175384 \\
\hline 6 & -0.361191 & -3.645505 & -2.751513 \\
\hline 6 & 0.665366 & -3.041819 & -2.079068 \\
\hline 6 & -3.158862 & -3.253457 & -0.311855 \\
\hline 6 & -4.200762 & -3.801152 & -1.017583 \\
\hline 6 & -3.991776 & -4.333260 & -2.308751 \\
\hline 6 & -2.741828 & -4.292914 & -2.873303 \\
\hline 6 & -0.979081 & -2.036123 & 1.188617 \\
\hline 6 & -1.218585 & -0.689268 & 1.418416 \\
\hline 6 & -1.403365 & -0.244671 & 2.757743 \\
\hline 6 & -1.355308 & -1.114185 & 3.814132 \\
\hline 6 & -1.143404 & -2.496123 & 3.608237 \\
\hline 6 & -0.957640 & -2.968009 & 2.277298 \\
\hline 6 & -1.125144 & -3.414834 & 4.685204 \\
\hline 6 & -0.949474 & -4.756567 & 4.457975 \\
\hline 6 & -0.773613 & -5.230979 & 3.141096 \\
\hline 6 & -0.765034 & -4.360378 & 2.080123 \\
\hline 15 & -1.497005 & 0.478686 & -0.010350 \\
\hline 45 & -0.050284 & 0.765377 & -2.001909 \\
\hline 6 & -0.163335 & 2.689108 & -1.886710 \\
\hline 8 & -0.172080 & 3.845344 & -1.915948 \\
\hline 15 & 1.793910 & -0.255185 & -1.034890 \\
\hline 8 & 2.572121 & 0.549068 & 0.203817 \\
\hline 6 & 3.341688 & 1.629634 & -0.189114 \\
\hline 6 & 4.617560 & 1.405594 & -0.670597 \\
\hline 6 & 5.381481 & 2.530860 & -1.116304 \\
\hline 6 & 4.831051 & 3.842707 & -0.990219 \\
\hline 6 & 3.527077 & 4.005158 & -0.465282 \\
\hline 6 & 2.784614 & 2.918507 & -0.085493 \\
\hline 6 & 5.586956 & 4.959540 & -1.422944 \\
\hline 6 & 6.827549 & 4.797718 & -1.983425 \\
\hline 6 & 7.358103 & 3.500549 & -2.145662 \\
\hline 6 & 6.655282 & 2.398698 & -1.726025 \\
\hline 6 & 5.087351 & 0.006208 & -0.794639 \\
\hline 6 & 4.307543 & -0.887196 & -1.509791 \\
\hline 6 & 4.703661 & -2.220853 & -1.729848 \\
\hline 6 & 5.867791 & -2.684066 & -1.178762 \\
\hline 6 & 6.668038 & -1.841561 & -0.371045 \\
\hline 6 & 6.276136 & -0.483478 & -0.167216 \\
\hline 6 & 7.067729 & 0.316542 & 0.696374 \\
\hline 6 & 8.194536 & -0.188697 & 1.295518 \\
\hline 6 & 8.595790 & -1.521976 & 1.066366 \\
\hline 6 & 7.842600 & -2.328456 & 0.252746 \\
\hline 8 & 3.107218 & -0.479117 & -2.048312 \\
\hline 6 & -3.291799 & 0.178261 & -0.309146 \\
\hline 6 & -4.174038 & 0.098848 & 0.773103 \\
\hline
\end{tabular}




\begin{tabular}{|c|c|c|c|}
\hline 6 & -5.533949 & -0.125312 & 0.573979 \\
\hline 6 & -5.982946 & -0.265414 & -0.744643 \\
\hline 6 & -5.132398 & -0.192387 & -1.847825 \\
\hline 6 & -3.773156 & 0.036053 & -1.606405 \\
\hline 6 & -6.516858 & -0.271007 & 1.734188 \\
\hline 6 & -5.700234 & -0.339525 & -3.258454 \\
\hline 6 & -1.494939 & 2.113449 & 0.826137 \\
\hline 6 & -2.510085 & 3.028012 & 0.589130 \\
\hline 6 & -2.474313 & 4.310684 & 1.153141 \\
\hline 6 & -1.404490 & 4.625642 & 1.985363 \\
\hline 6 & -0.367247 & 3.718423 & 2.258472 \\
\hline 6 & -0.408093 & 2.475946 & 1.633602 \\
\hline 6 & -3.611635 & 5.280950 & 0.844395 \\
\hline 6 & 0.722198 & 4.097676 & 3.261887 \\
\hline 6 & -0.857341 & -0.339055 & -3.390071 \\
\hline 8 & -1.164348 & -0.889200 & -4.359943 \\
\hline 1 & 6.764938 & 1.342229 & 0.892044 \\
\hline 1 & -1.598937 & 0.805739 & 2.955277 \\
\hline 1 & 8.127531 & -3.365116 & 0.077989 \\
\hline 1 & -1.263304 & -3.032738 & 5.695770 \\
\hline 1 & 5.151767 & 5.951638 & -1.310197 \\
\hline 1 & -3.333297 & -2.834918 & 0.677916 \\
\hline 1 & -5.199182 & -3.815912 & -0.583571 \\
\hline 1 & 3.108720 & 5.008477 & -0.394490 \\
\hline 1 & -1.500545 & -0.749239 & 4.830207 \\
\hline 1 & 6.180597 & -3.715374 & -1.334616 \\
\hline 1 & 8.330989 & 3.371098 & -2.614545 \\
\hline 1 & 4.066478 & -2.856841 & -2.340689 \\
\hline 1 & -2.567028 & -4.693780 & -3.870771 \\
\hline 1 & -0.205750 & -4.050138 & -3.750291 \\
\hline 1 & -0.621034 & -6.293439 & \\
\hline 1 & 7.072727 & 1.405679 & -1.870666 \\
\hline 1 & -0.600225 & -4.739759 & 1.074100 \\
\hline 1 & 1.648734 & -2.951108 & -2.537380 \\
\hline 1 & 7.396918 & 5.662385 & -2.316386 \\
\hline 1 & 8.780756 & 0.443812 & 1.958441 \\
\hline 1 & -4.824589 & -4.773290 & -2.853631 \\
\hline 1 & -0.945739 & -5.456904 & 5.290284 \\
\hline 1 & 9.493183 & -1.908027 & 1.544156 \\
\hline 1 & 1.768299 & 3.020968 & 0.290521 \\
\hline 1 & -3.785697 & 0.211234 & 1.782211 \\
\hline 1 & -3.068716 & 0.102345 & -2.430992 \\
\hline 1 & -7.044151 & -0.445686 & -0.916333 \\
\hline 1 & -3.342202 & 2.744932 & -0.054323 \\
\hline 1 & 0.391903 & 1.753582 & 1.793524 \\
\hline 1 & -1.370130 & 5.606000 & 2.455999 \\
\hline 1 & 3.118431 & -1.513467 & 1.123619 \\
\hline 1 & 1.554649 & -1.691839 & 1.894544 \\
\hline 1 & 0.913725 & 1.115486 & -3.211179 \\
\hline 1 & 2.236085 & -3.294461 & 3.430990 \\
\hline 1 & 2.761637 & -4.088512 & -0.749603 \\
\hline 1 & 2.792666 & -5.644320 & 3.954563 \\
\hline 1 & 3.264073 & -6.453381 & -0.238950 \\
\hline 1 & 3.289821 & -7.246052 & 2.115121 \\
\hline 6 & -4.599380 & -0.413139 & -4.313444 \\
\hline 6 & -6.532795 & -1.621565 & -3.360743 \\
\hline
\end{tabular}




$\begin{array}{rrrr}6 & -6.589287 & 0.871058 & -3.563842 \\ 6 & -5.862324 & 0.015647 & 3.084042 \\ 6 & -7.688694 & 0.700240 & 1.560338 \\ 6 & -7.043868 & -1.710655 & 1.753846 \\ 6 & 1.714218 & 2.960868 & 3.502528 \\ 6 & 1.496254 & 5.321574 & 2.763750 \\ 6 & 0.061669 & 4.428151 & 4.606471 \\ 6 & -3.449325 & 6.617363 & 1.563479 \\ 6 & -3.646030 & 5.550252 & -0.664384 \\ 6 & -4.942671 & 4.659190 & 1.284839 \\ 1 & -4.289678 & 7.275919 & 1.310318 \\ 1 & -3.441907 & 6.499235 & 2.654929 \\ 1 & -2.527596 & 7.133463 & 1.265668 \\ 1 & -5.774018 & 5.340290 & 1.058660 \\ 1 & -5.145528 & 3.708291 & 0.775439 \\ 1 & -4.947375 & 4.467084 & 2.366005 \\ 1 & -4.460064 & 6.246022 & -0.908844 \\ 1 & -2.703112 & 5.997914 & -1.004151 \\ 1 & -3.806653 & 4.633656 & -1.244825 \\ 1 & 2.454114 & 3.273317 & 4.250111 \\ 1 & 1.217662 & 2.059361 & 3.887161 \\ 1 & 2.267113 & 2.686763 & 2.594675 \\ 1 & 0.826994 & 4.678465 & 5.353265 \\ 1 & -0.623060 & 5.281596 & 4.536652 \\ 1 & -0.510147 & 3.569610 & 4.983333 \\ 1 & 2.254350 & 5.617817 & 3.501146 \\ 1 & 2.014731 & 5.103643 & 1.820931 \\ 1 & 0.840489 & 6.184468 & 2.596340 \\ 1 & -5.047943 & -0.549841 & -5.305497 \\ 1 & -3.998775 & 0.504901 & -4.352250 \\ 1 & -3.922152 & -1.259129 & -4.133481 \\ 1 & -6.933097 & -1.730186 & -4.377525 \\ 1 & -5.915271 & -2.503334 & -3.143300 \\ 1 & -7.386202 & -1.626739 & -2.671895 \\ 1 & -7.008798 & 0.792425 & -4.575972 \\ 1 & -7.426919 & 0.951271 & -2.859563 \\ 1 & -6.014127 & 1.804519 & -3.507961 \\ 1 & -6.607144 & -0.085450 & 3.883399 \\ 1 & -5.047155 & -0.686084 & 3.306086 \\ 1 & -5.459732 & 1.036318 & 3.132997 \\ 1 & -8.394064 & 0.595430 & 2.395392 \\ 1 & -7.339487 & 1.740769 & 1.542860 \\ 1 & -8.248349 & 0.519049 & 0.635171 \\ 1 & -7.760943 & -1.846396 & 2.574803 \\ 1 & -7.552931 & -1.974300 & 0.818268 \\ 1 & -6.222256 & -2.424707 & 1.902150\end{array}$

$1_{\mathrm{C} 6-}-\psi_{-1 \mathrm{a} 233 \mathrm{a} 4 \mathrm{~s}}$

Number of imaginary frequencies : 0

The smallest frequencies are : $13.1996 \quad 19.9947 \quad 21.2456 \mathrm{~cm}(-1)$

Electronic energy : $\quad \mathrm{HF}=-4125.468092$

Zero-point correction $=\quad 1.300491$ (Hartree/Particle)

Thermal correction to Energy $=\quad 1.378539$

Thermal correction to Enthalpy $=\quad 1.379483$

Thermal correction to Gibbs Free Energy $=\quad 1.189428$ 
Sum of electronic and zero-point Energies= Sum of electronic and thermal Energies= Sum of electronic and thermal Enthalpies= Sum of electronic and thermal Free Energies=
$-4124.167601$

$-4124.089553$

$-4124.088609$

$-4124.278664$

Cartesian Coordinates

\begin{tabular}{|c|c|c|c|}
\hline 6 & -0.426453 & 2.478269 & 1.620043 \\
\hline 6 & -1.512668 & 2.107203 & 0.815426 \\
\hline 6 & -2.537858 & 3.012261 & 0.584455 \\
\hline 6 & -2.509375 & 4.295880 & 1.147247 \\
\hline 6 & -1.439769 & 4.618920 & 1.976616 \\
\hline 6 & -0.394940 & 3.719720 & 2.247303 \\
\hline 15 & -1.497526 & 0.472455 & -0.021569 \\
\hline 6 & -3.288304 & 0.160837 & -0.330250 \\
\hline 6 & -4.169301 & 0.076982 & 0.744652 \\
\hline 6 & -5.533812 & -0.147106 & 0.548433 \\
\hline 6 & -5.985929 & -0.280842 & -0.764420 \\
\hline 6 & -5.128264 & -0.207772 & -1.869385 \\
\hline 6 & -3.772292 & 0.019449 & -1.630745 \\
\hline 6 & -6.445367 & -0.258014 & 1.768515 \\
\hline 6 & -6.400083 & 1.053877 & 2.560096 \\
\hline 6 & -5.696872 & -0.358564 & -3.279241 \\
\hline 6 & -6.593000 & 0.846480 & -3.585910 \\
\hline 6 & -3.650249 & 5.261064 & 0.835452 \\
\hline 6 & 0.693238 & 4.106801 & 3.249112 \\
\hline 6 & -1.222188 & -0.691651 & 1.411589 \\
\hline 6 & -0.980880 & -2.038767 & 1.185889 \\
\hline 6 & -0.964625 & -2.968642 & 2.276265 \\
\hline 6 & -1.158843 & -2.494539 & 3.605172 \\
\hline 6 & -1.370414 & -1.111994 & 3.807485 \\
\hline 6 & -1.412536 & -0.244303 & 2.749215 \\
\hline 6 & -0.768935 & -4.361102 & 2.082857 \\
\hline 6 & -0.783220 & -5.229722 & 3.145359 \\
\hline 6 & -0.968323 & -4.753183 & 4.460210 \\
\hline 6 & -1.146691 & -3.411262 & 4.683933 \\
\hline 6 & -0.763715 & -2.575659 & -0.181236 \\
\hline 6 & 0.476857 & -2.485902 & -0.789437 \\
\hline 6 & 0.668275 & -3.035950 & -2.082251 \\
\hline 6 & -0.356061 & -3.643702 & -2.754377 \\
\hline 6 & -1.643769 & -3.728893 & -2.176314 \\
\hline 6 & -1.849342 & -3.202560 & -0.868039 \\
\hline 6 & -2.732032 & -4.308323 & -2.872399 \\
\hline 6 & -3.979350 & -4.362974 & -2.303384 \\
\hline 6 & -4.189038 & -3.835534 & -1.010460 \\
\hline 6 & -3.151588 & -3.275294 & -0.308018 \\
\hline 7 & 1.565658 & -1.737395 & -0.231822 \\
\hline 15 & 1.795476 & -0.250785 & -1.036296 \\
\hline 8 & 3.112068 & -0.474393 & -2.045386 \\
\hline 6 & 4.311472 & -0.880427 & -1.503254 \\
\hline 6 & 5.088019 & 0.014352 & -0.786226 \\
\hline 6 & 6.275718 & -0.473557 & -0.155355 \\
\hline 6 & 6.670231 & -1.831068 & -0.357963 \\
\hline 6 & 5.873478 & -2.674910 & -1.167740 \\
\hline 6 & 4.710138 & -2.213534 & -1.722012 \\
\hline 6 & 7.063649 & 0.327710 & 0.710424 \\
\hline
\end{tabular}




\begin{tabular}{|c|c|c|c|}
\hline 6 & 8.189560 & -0.175770 & 1.312728 \\
\hline 6 & 8.593528 & -1.508425 & 1.084714 \\
\hline 6 & 7.843830 & -2.316112 & 0.269069 \\
\hline 6 & 4.616139 & 1.413257 & -0.664346 \\
\hline 6 & 3.338412 & 1.635955 & -0.187229 \\
\hline 6 & 2.778878 & 2.924049 & -0.087071 \\
\hline 6 & 3.521100 & 4.011467 & -0.465069 \\
\hline 6 & 4.827256 & 3.850462 & -0.985018 \\
\hline 6 & 5.379994 & 2.539300 & -1.108260 \\
\hline 6 & 5.583176 & 4.968101 & -1.415637 \\
\hline 6 & 6.826020 & 4.807684 & -1.971514 \\
\hline 6 & 7.358923 & 3.511159 & -2.131126 \\
\hline 6 & 6.656127 & 2.408570 & -1.713388 \\
\hline 8 & 2.569274 & 0.554604 & 0.204393 \\
\hline 6 & 2.172945 & -2.066087 & 1.066933 \\
\hline 6 & 2.461866 & -3.524059 & 1.305399 \\
\hline 6 & 2.477286 & -3.981955 & 2.624535 \\
\hline 6 & 2.784684 & -5.304922 & 2.918529 \\
\hline 6 & 3.062156 & -6.200548 & 1.890088 \\
\hline 6 & 3.049727 & -5.754642 & 0.571956 \\
\hline 6 & 2.761374 & -4.424335 & 0.282547 \\
\hline 45 & -0.047222 & 0.767150 & -2.007206 \\
\hline 6 & -0.848832 & -0.338612 & -3.398008 \\
\hline 8 & -1.154110 & -0.889225 & -4.368098 \\
\hline 6 & -0.171915 & 2.689869 & -1.889907 \\
\hline 8 & -0.190293 & 3.846074 & -1.916021 \\
\hline 6 & -7.897372 & -0.535047 & 1.388462 \\
\hline 6 & -5.956511 & -1.410926 & 2.654364 \\
\hline 6 & -4.597031 & -0.427915 & -4.335597 \\
\hline 6 & -6.522227 & -1.645702 & -3.379108 \\
\hline 1 & 6.758690 & 1.352929 & 0.905207 \\
\hline 1 & -1.607271 & 0.806745 & 2.943884 \\
\hline 1 & 8.130835 & -3.352341 & 0.095141 \\
\hline 1 & -1.291320 & -3.027471 & 5.692942 \\
\hline 1 & 5.146217 & 5.959657 & -1.304994 \\
\hline 1 & -3.328274 & -2.857690 & 0.682177 \\
\hline 1 & -5.185093 & -3.865280 & -0.572338 \\
\hline 1 & 3.101118 & 5.014260 & -0.396543 \\
\hline 1 & -1.519678 & -0.745089 & 4.822262 \\
\hline 1 & 6.188281 & -3.705755 & -1.322618 \\
\hline 1 & 8.333669 & 3.382791 & -2.596427 \\
\hline 1 & 4.075537 & -2.850555 & -2.334467 \\
\hline 1 & -2.556491 & -4.705653 & -3.871143 \\
\hline 1 & -0.199714 & -4.046098 & -3.753916 \\
\hline 1 & -0.628040 & -6.292286 & 2.972412 \\
\hline 1 & 7.075498 & 1.416066 & -1.855934 \\
\hline 1 & -0.596761 & -4.742035 & 1.078653 \\
\hline 1 & 1.650770 & -2.940066 & -2.541415 \\
\hline 1 & 7.395414 & 5.672951 & -2.302865 \\
\hline 1 & 8.772943 & 0.457655 & 1.977275 \\
\hline 1 & -4.809446 & -4.811146 & -2.845714 \\
\hline 1 & -0.969241 & -5.452058 & 5.293747 \\
\hline 1 & 9.490209 & -1.893060 & 1.564973 \\
\hline 1 & 1.760917 & 3.025208 & 0.284836 \\
\hline 1 & -3.786776 & 0.183238 & 1.758938 \\
\hline 1 & -3.068306 & 0.087104 & -2.455468 \\
\hline
\end{tabular}




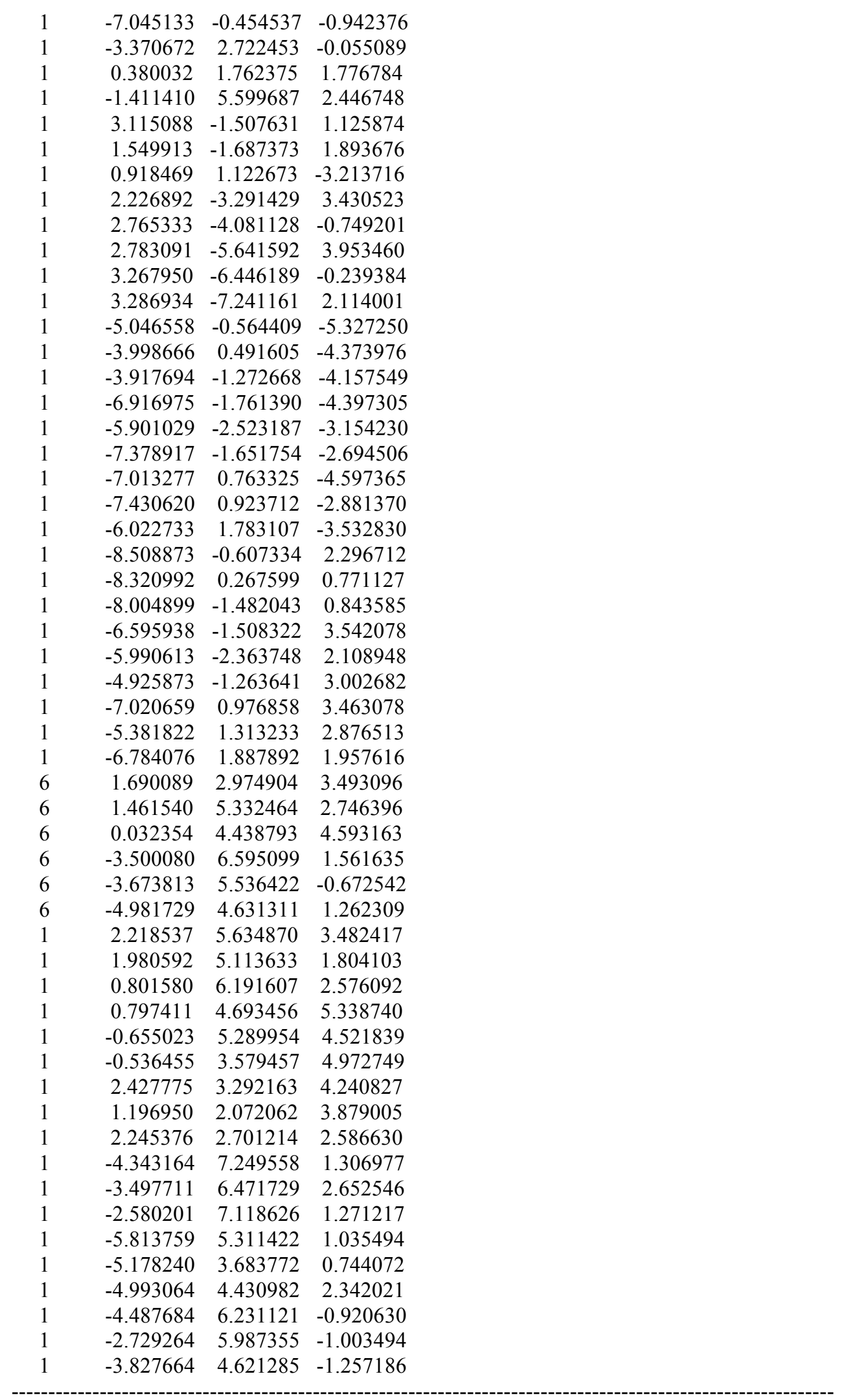

$1_{\mathrm{C} 6-} \psi_{-1 \mathrm{a} 2 \mathrm{~s} 3 \mathrm{~s} 4 \mathrm{a}}$ 
Number of imaginary frequencies : 0

The smallest frequencies are : $4.0605 \quad 17.224120 .6121 \mathrm{~cm}(-1)$

Electronic energy : $\quad \mathrm{HF}=-4125.4679087$

Zero-point correction $=\quad 1.300588($ Hartree/Particle $)$

Thermal correction to Energy $=\quad 1.378721$

Thermal correction to Enthalpy= $\quad 1.379665$

Thermal correction to Gibbs Free Energy $=1.187645$

Sum of electronic and zero-point Energies $=\quad-4124.167321$

Sum of electronic and thermal Energies $=\quad-4124.089188$

Sum of electronic and thermal Enthalpies $=\quad-4124.088243$

Sum of electronic and thermal Free Energies $=\quad-4124.280263$

Cartesian Coordinates

\begin{tabular}{cccc}
\hline 6 & 3.074254 & -6.176549 & 1.919560 \\
6 & 2.778931 & -5.278799 & 2.941148 \\
6 & 2.463554 & -3.959687 & 2.638391 \\
6 & 2.457433 & -3.507620 & 1.317173 \\
6 & 2.774814 & -4.409854 & 0.301421 \\
6 & 3.071280 & -5.736401 & 0.599434 \\
6 & 2.160151 & -2.052971 & 1.069023 \\
7 & 1.556014 & -1.734284 & -0.233907 \\
6 & 0.480601 & -2.498151 & -0.797677 \\
6 & -0.767696 & -2.591806 & -0.205805 \\
6 & -1.833229 & -3.249911 & -0.896153 \\
6 & -1.593345 & -3.826246 & -2.177478 \\
6 & -0.300971 & -3.725062 & -2.742405 \\
6 & 0.698194 & -3.069954 & -2.076883 \\
6 & -3.145020 & -3.325766 & -0.359109 \\
6 & -4.152890 & -3.953567 & -1.047960 \\
6 & -3.904383 & -4.543963 & -2.305958 \\
6 & -2.651301 & -4.474746 & -2.859257 \\
6 & -1.000410 & -2.064030 & 1.163280 \\
6 & -1.246651 & -0.721164 & 1.408386 \\
6 & -1.445950 & -0.292892 & 2.751185 \\
6 & -1.405297 & -1.174217 & 3.797641 \\
6 & -1.183341 & -2.552343 & 3.577246 \\
6 & -0.982010 & -3.008037 & 2.242909 \\
6 & -1.168033 & -3.482036 & 4.644759 \\
6 & -0.978392 & -4.819699 & 4.405685 \\
6 & -0.784576 & -5.278292 & 3.085816 \\
6 & -0.774079 & -4.396680 & 2.033982 \\
15 & -1.509338 & 0.471445 & 0.000058 \\
45 & -0.084656 & 0.749043 & -2.006897 \\
6 & -0.195746 & 2.673803 & -1.901352 \\
8 & -0.209865 & 3.829710 & -1.935689 \\
15 & 1.775594 & -0.249296 & -1.042740 \\
8 & 2.550742 & 0.560915 & 0.194052 \\
6 & 3.314561 & 1.645840 & -0.197519 \\
6 & 4.590264 & 1.428992 & -0.682887 \\
6 & 5.347581 & 2.559044 & -1.127863 \\
6 & 4.792475 & 3.868147 & -0.994370 \\
6 & 3.490487 & 4.023376 & -0.462332 \\
6 & 2.753447 & 2.932242 & -0.084632 \\
6 & 5.541911 & 4.989705 & -1.426138 \\
& & & \\
6
\end{tabular}




\begin{tabular}{|c|c|c|c|}
\hline 6 & 6.780578 & 4.835039 & -1.992861 \\
\hline & 7.315580 & 3.540643 & -2.162432 \\
\hline & 6.619198 & 2.434373 & -1.743701 \\
\hline & 5.068476 & 0.032647 & -0.808678 \\
\hline & 4.293064 & -0.866256 & -1.521569 \\
\hline & 4.698873 & -2.196722 & -1.743691 \\
\hline & 5.868425 & -2.651338 & -1.197047 \\
\hline & 6.664864 & -1.803234 & -0.391450 \\
\hline & 6.262911 & -0.448496 & -0.185092 \\
\hline & 7.051161 & 0.356835 & 0.676617 \\
\hline & 8.184133 & -0.139984 & 1.271168 \\
\hline & 8.595325 & -1.469704 & 1.039091 \\
\hline & 7.845568 & -2.281365 & 0.227463 \\
\hline & 3.088805 & -0.466766 & -2.057570 \\
\hline & -3.308131 & 0.213308 & -0.303803 \\
\hline & -4.202005 & 0.089110 & 0.768765 \\
\hline y & -5.561483 & -0.096461 & 0.546810 \\
\hline & -6.009503 & -0.154724 & -0.783824 \\
\hline D & -5.151488 & -0.019075 & -1.869993 \\
\hline & -3.787395 & 0.165546 & -1.602988 \\
\hline & -6.554498 & -0.283626 & 1.692816 \\
\hline & -5.615611 & -0.117577 & -3.320346 \\
\hline & -1.477692 & 2.092830 & 0.861007 \\
\hline 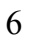 & -2.481799 & 3.024274 & 0.643718 \\
\hline & -2.430043 & 4.294463 & 1.233857 \\
\hline b & -1.355666 & 4.579532 & 2.070992 \\
\hline & -0.328528 & 3.654651 & 2.322879 \\
\hline & -0.385821 & 2.425226 & 1.674021 \\
\hline & -3.559951 & 5.281239 & 0.951499 \\
\hline 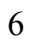 & 0.767774 & 3.998589 & 3.331245 \\
\hline & -0.946005 & -0.350808 & -3.361261 \\
\hline & -1.330052 & -0.888931 & -4.310824 \\
\hline & 6.741021 & 1.379874 & 0.874533 \\
\hline & -1.646651 & 0.754758 & 2.958234 \\
\hline & 8.138101 & -3.315542 & 0.050526 \\
\hline & -1.319059 & -3.111365 & 5.657732 \\
\hline & 5.103178 & 5.979574 & -1.307645 \\
\hline & -3.351497 & -2.863061 & 0.605036 \\
\hline 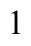 & -5.154002 & -3.992543 & -0.623277 \\
\hline & 3.069283 & 5.024914 & -0.384329 \\
\hline & -1.562480 & -0.822219 & 4.816492 \\
\hline 1 & 6.188999 & -3.679893 & -1.355263 \\
\hline & 8.286782 & 3.416876 & -2.636311 \\
\hline 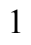 & 4.065256 & -2.837041 & -2.353745 \\
\hline & -2.447253 & -4.912236 & -3.835503 \\
\hline 1 & -0.119710 & -4.159109 & -3.724375 \\
\hline & -0.619748 & -6.337273 & 2.900290 \\
\hline & 7.039980 & 1.443625 & -1.894106 \\
\hline 1 & -0.595318 & -4.764732 & 1.026240 \\
\hline 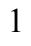 & 1.684633 & -2.964770 & -2.525242 \\
\hline 1 & 7.344939 & 5.703244 & -2.325142 \\
\hline & 8.767608 & 0.496525 & 1.932678 \\
\hline 1 & -4.711696 & -5.042304 & -2.838330 \\
\hline ] & -0.976599 & -5.528647 & 5.230671 \\
\hline 1 & 9.497577 & -1.849022 & 1.513100 \\
\hline 1 & 1.739383 & 3.029552 & 0.298724 \\
\hline
\end{tabular}




\begin{tabular}{|c|c|c|c|}
\hline & -3.819041 & 0.137418 & 1.784845 \\
\hline & -3.081973 & 0.272883 & -2.423175 \\
\hline & -7.070826 & -0.311637 & -0.965711 \\
\hline & -3.318953 & 2.764274 & -0.002988 \\
\hline & 0.404359 & 1.689290 & 1.819833 \\
\hline & -1.309515 & 5.548999 & 2.562815 \\
\hline & 3.098746 & -1.488648 & 1.128240 \\
\hline & 1.531396 & -1.673475 & 1.891369 \\
\hline & 0.857896 & 1.083698 & -3.238126 \\
\hline & 2.200345 & -3.267762 & 3.439002 \\
\hline & 2.785943 & -4.071001 & -0.731610 \\
\hline & 2.769861 & -5.610988 & 3.977492 \\
\hline & 3.303524 & -6.429437 & -0.206726 \\
\hline & 3.305541 & -7.214237 & 2.150312 \\
\hline & -7.137191 & -0.134366 & -3.443226 \\
\hline & -5.085936 & 1.073498 & -4.126852 \\
\hline & -5.065987 & -1.419764 & -3.914089 \\
\hline & -5.913774 & -0.031318 & 3.056413 \\
\hline & -7.733596 & 0.682468 & 1.539880 \\
\hline & -7.071826 & -1.726471 & 1.664876 \\
\hline & 1.758717 & 2.852306 & 3.526952 \\
\hline & 1.542484 & 5.236486 & 2.870017 \\
\hline & 0.114559 & 4.285522 & 4.689246 \\
\hline ) & -3.374207 & 6.605797 & 1.686552 \\
\hline 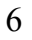 & -3.612291 & 5.571689 & -0.552900 \\
\hline & -4.891015 & 4.666205 & 1.401377 \\
\hline & -4.209270 & 7.277630 & 1.451384 \\
\hline 1 & -3.356879 & 6.472802 & 2.776160 \\
\hline & -2.449320 & 7.114698 & 1.386057 \\
\hline 1 & -5.719082 & 5.357540 & 1.194959 \\
\hline & -5.108443 & 3.723594 & 0.882829 \\
\hline I & -4.883263 & 4.459855 & 2.479913 \\
\hline 1 & -4.422096 & 6.279553 & -0.775657 \\
\hline & -2.669729 & 6.014047 & -0.900398 \\
\hline 1 & -3.791597 & 4.665557 & -1.144439 \\
\hline & 2.506870 & 3.139450 & 4.276470 \\
\hline 1 & 1.263500 & 1.940470 & 3.888455 \\
\hline & 2.300848 & 2.605452 & 2.604725 \\
\hline ] & 0.883470 & 4.511983 & 5.439986 \\
\hline 1 & -0.570768 & 5.140514 & 4.649183 \\
\hline & -0.455451 & 3.415192 & 5.041105 \\
\hline 1 & 2.306647 & 5.504244 & 3.612003 \\
\hline & 2.053862 & 5.049467 & 1.916743 \\
\hline 1 & 0.889129 & 6.107193 & 2.737630 \\
\hline & -7.420273 & -0.178631 & -4.502371 \\
\hline 1 & -7.581883 & -1.009730 & -2.952686 \\
\hline 1 & -7.592601 & 0.768660 & -3.015645 \\
\hline 1 & -5.443254 & 1.013784 & -5.163280 \\
\hline 1 & -5.433387 & 2.025157 & -3.703703 \\
\hline 1 & -3.989992 & 1.101901 & -4.164066 \\
\hline 1 & -5.361053 & -1.515624 & -4.968030 \\
\hline 1 & -3.970001 & -1.460659 & -3.864982 \\
\hline 1 & -5.453637 & -2.290350 & -3.368234 \\
\hline 1 & -6.667270 & -0.150071 & 3.845138 \\
\hline 1 & -5.102742 & -0.739927 & 3.271369 \\
\hline & -5.509462 & 0.987154 & 3.132951 \\
\hline
\end{tabular}




$\begin{array}{rrrr}1 & -8.440602 & 0.548827 & 2.369349 \\ 1 & -7.394446 & 1.726279 & 1.551951 \\ 1 & -8.289510 & 0.521688 & 0.608914 \\ 1 & -7.792083 & -1.892360 & 2.477528 \\ 1 & -7.574758 & -1.963408 & 0.719010 \\ 1 & -6.246188 & -2.439219 & 1.794973\end{array}$

$1_{\text {C6- }-\psi_{-12 a 2354 s}}$

Number of imaginary frequencies : 0

The smallest frequencies are : $13.0652 \quad 18.205920 .0453 \mathrm{~cm}(-1)$

Electronic energy : $\quad H F=-4125.4681161$

Zero-point correction $=\quad 1.300074($ Hartree/Particle)

Thermal correction to Energy= $\quad 1.378362$

Thermal correction to Enthalpy $=\quad 1.379306$

Thermal correction to Gibbs Free Energy $=\quad 1.187386$

Sum of electronic and zero-point Energies $=\quad-4124.168042$

Sum of electronic and thermal Energies $=\quad-4124.089754$

Sum of electronic and thermal Enthalpies $=\quad-4124.088810$

Sum of electronic and thermal Free Energies $=\quad-4124.280730$

Cartesian Coordinates

$\begin{array}{cccc}6 & 3.095689 & -6.173314 & 1.896681 \\ 6 & 2.800968 & -5.279239 & 2.921676 \\ 6 & 2.483592 & -3.959542 & 2.623676 \\ 6 & 2.474819 & -3.503224 & 1.303914 \\ 6 & 2.791624 & -4.401707 & 0.284693 \\ 6 & 3.090091 & -5.728907 & 0.578006 \\ 6 & 2.175011 & -2.048158 & 1.061596 \\ 7 & 1.571409 & -1.725090 & -0.240504 \\ 6 & 0.500050 & -2.490117 & -0.809977 \\ 6 & -0.746306 & -2.598511 & -0.216407 \\ 6 & -1.809425 & -3.255403 & -0.911121 \\ 6 & -1.570483 & -3.812335 & -2.201081 \\ 6 & -0.279707 & -3.698171 & -2.767244 \\ 6 & 0.718030 & -3.046789 & -2.095782 \\ 6 & -3.118763 & -3.345305 & -0.370353 \\ 6 & -4.126105 & -3.966241 & -1.065957 \\ 6 & -3.879178 & -4.536622 & -2.333520 \\ 6 & -2.627726 & -4.455464 & -2.889011 \\ 6 & -0.982294 & -2.079450 & 1.155094 \\ 6 & -1.233128 & -0.738052 & 1.403337 \\ 6 & -1.431629 & -0.313440 & 2.747160 \\ 6 & -1.389619 & -1.197999 & 3.791130 \\ 6 & -1.167845 & -2.575471 & 3.566661 \\ 6 & -0.963500 & -3.026690 & 2.231302 \\ 6 & -1.153206 & -3.508686 & 4.631184 \\ 6 & -0.961003 & -4.845176 & 4.387965 \\ 6 & -0.763627 & -5.299207 & 3.066926 \\ 6 & -0.752569 & -4.414220 & 2.018028 \\ 15 & -1.505850 & 0.452257 & -0.005097 \\ 45 & -0.073648 & 0.769363 & -1.996518 \\ 6 & -0.210997 & 2.689814 & -1.860288 \\ 8 & -0.245508 & 3.845856 & -1.867948 \\ 15 & 1.785810 & -0.233922 & -1.039711\end{array}$




\begin{tabular}{|c|c|c|c|}
\hline 8 & 2.554979 & 0.572858 & 0.202945 \\
\hline 6 & 3.313531 & 1.663163 & -0.184057 \\
\hline & 4.591781 & 1.454284 & -0.666002 \\
\hline & 5.344415 & 2.589385 & -1.105914 \\
\hline & 4.780802 & 3.894996 & -0.973159 \\
\hline & 3.475278 & 4.041777 & -0.447362 \\
\hline 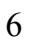 & 2.743615 & 2.945836 & -0.073258 \\
\hline & 5.525468 & 5.021645 & -1.399951 \\
\hline ) & 6.767656 & 4.875201 & -1.961114 \\
\hline f & 7.311202 & 3.584283 & -2.130002 \\
\hline & 6.619547 & 2.473240 & -1.716127 \\
\hline b & 5.076074 & 0.060352 & -0.795906 \\
\hline & 4.306313 & -0.838345 & -1.515319 \\
\hline$b$ & 4.717639 & -2.166417 & -1.741607 \\
\hline ) & 5.886978 & -2.619041 & -1.192768 \\
\hline 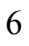 & 6.677653 & -1.771407 & -0.380994 \\
\hline 6 & 6.270160 & -0.418962 & -0.170484 \\
\hline 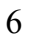 & 7.052480 & 0.385787 & 0.697156 \\
\hline 6 & 8.185157 & -0.109348 & 1.293646 \\
\hline & 8.601874 & -1.436678 & 1.057670 \\
\hline ) & 7.857921 & -2.247766 & 0.240137 \\
\hline 8 & 3.101992 & -0.440918 & -2.052945 \\
\hline$b$ & -3.296921 & 0.163779 & -0.323460 \\
\hline 6 & -4.186992 & 0.009730 & 0.740324 \\
\hline 6 & -5.550310 & -0.176991 & 0.519301 \\
\hline 6 & -6.003278 & -0.200150 & -0.805244 \\
\hline 6 & -5.140762 & -0.041600 & -1.891764 \\
\hline 6 & -3.779665 & 0.139398 & -1.626051 \\
\hline 6 & -6.475509 & -0.346787 & 1.723067 \\
\hline 6 & -5.610981 & -0.118727 & -3.342194 \\
\hline ) & -1.517560 & 2.071582 & 0.860873 \\
\hline 6 & -2.548410 & 2.975239 & 0.651850 \\
\hline 6 & -2.524758 & 4.247563 & 1.238982 \\
\hline 6 & -1.452540 & 4.561269 & 2.068528 \\
\hline 6 & -0.401154 & 3.662854 & 2.316399 \\
\hline 6 & -0.429732 & 2.432419 & 1.667525 \\
\hline 6 & -3.674133 & 5.209686 & 0.950586 \\
\hline 6 & 0.691960 & 4.037596 & 3.317379 \\
\hline 6 & -0.914463 & -0.318200 & -3.375914 \\
\hline 5 & -1.280823 & -0.844413 & -4.338827 \\
\hline | & 6.737876 & 1.406902 & 0.898034 \\
\hline 1 & -1.634012 & 0.733231 & 2.957011 \\
\hline d & 8.154823 & -3.280165 & 0.060115 \\
\hline 1 & -1.306578 & -3.141631 & 5.645115 \\
\hline & 5.080258 & 6.008689 & -1.282037 \\
\hline 1 & -3.323940 & -2.898511 & 0.601800 \\
\hline 1 & -5.125961 & -4.014245 & -0.639223 \\
\hline 1 & 3.047331 & 5.040599 & -0.371282 \\
\hline 1 & -1.546200 & -0.849143 & 4.811145 \\
\hline 1 & 6.211679 & -3.645842 & -1.353923 \\
\hline 1 & 8.285365 & 3.467040 & -2.599441 \\
\hline 1 & 4.088232 & -2.806699 & -2.356048 \\
\hline 1 & -2.424843 & -4.878544 & -3.871821 \\
\hline 1 & -0.098588 & -4.118636 & -3.755137 \\
\hline 1 & -0.596641 & -6.357323 & 2.878411 \\
\hline 1 & 7.047049 & 1.485273 & -1.865866 \\
\hline
\end{tabular}




\begin{tabular}{|c|c|c|c|}
\hline & -0.571260 & -4.778285 & 1.009294 \\
\hline & 1.703093 & -2.931991 & -2.544885 \\
\hline$\frac{1}{1}$ & 7.328368 & 5.747232 & -2.289534 \\
\hline 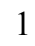 & 8.764075 & 0.526558 & 1.959722 \\
\hline & -4.686203 & -5.029949 & -2.870981 \\
\hline 1 & -0.959715 & -5.556857 & 5.210587 \\
\hline 1 & 9.503807 & -1.814676 & 1.533336 \\
\hline 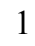 & 1.726563 & 3.036027 & 0.303898 \\
\hline & -3.808690 & 0.036001 & 1.761331 \\
\hline 1 & -3.075719 & 0.266447 & -2.444893 \\
\hline 1 & -7.062905 & -0.346327 & -0.994070 \\
\hline 1 & -3.382514 & 2.694102 & 0.010359 \\
\hline 1 & 0.380255 & 1.717087 & 1.807922 \\
\hline 1 & -1.427169 & 5.533050 & 2.557297 \\
\hline 1 & 3.112554 & -1.482384 & 1.124186 \\
\hline ] & 1.545005 & -1.673485 & 1.885034 \\
\hline & 0.875462 & 1.134203 & -3.214149 \\
\hline 1 & 2.220826 & -3.270467 & 3.426892 \\
\hline & 2.800372 & -4.059645 & -0.747325 \\
\hline 1 & 2.793914 & -5.614791 & 3.956947 \\
\hline ] & 3.321811 & -6.419087 & -0.230747 \\
\hline 1 & 3.328524 & -7.211483 & 2.123678 \\
\hline 5 & -7.133172 & -0.119984 & -3.461549 \\
\hline 6 & -5.073765 & 1.075478 & -4.138949 \\
\hline 6 & -5.075358 & -1.419926 & -3.951156 \\
\hline 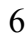 & -7.924027 & -0.601614 & 1.314673 \\
\hline 6 & -6.002467 & -1.539657 & 2.562969 \\
\hline 0 & -6.435423 & 0.928479 & 2.573335 \\
\hline 6 & 1.700320 & 2.909198 & 3.527506 \\
\hline 6 & 1.446021 & 5.280051 & 2.834657 \\
\hline 6 & 0.039635 & 4.334974 & 4.673586 \\
\hline 6 & -3.533538 & 6.527990 & 1.706625 \\
\hline 6 & -3.702144 & 5.517583 & -0.551179 \\
\hline 6 & -4.999857 & 4.559165 & 1.364763 \\
\hline 1 & -4.383436 & 7.180531 & 1.470334 \\
\hline 1 & -3.525623 & 6.379172 & 2.794351 \\
\hline 1 & -2.619542 & 7.066501 & 1.425159 \\
\hline 1 & -5.837412 & 5.238856 & 1.157833 \\
\hline 1 & -5.191508 & 3.623981 & 0.822589 \\
\hline 1 & -5.006973 & 4.330182 & 2.438830 \\
\hline 1 & -4.518333 & 6.215438 & -0.782295 \\
\hline 1 & -2.759304 & 5.977145 & -0.875223 \\
\hline 1 & -3.855466 & 4.614583 & -1.154871 \\
\hline 1 & 2.442844 & 3.216439 & 4.274633 \\
\hline 1 & 1.218407 & 1.994115 & 3.898590 \\
\hline 1 & 2.248308 & 2.660105 & 2.609439 \\
\hline 1 & 0.808446 & 4.581993 & 5.417884 \\
\hline 1 & -0.656886 & 5.180355 & 4.625339 \\
\hline 1 & -0.517372 & 3.462312 & 5.040017 \\
\hline 1 & 2.207703 & 5.571967 & 3.570076 \\
\hline 1 & 1.957724 & 5.085817 & 1.882996 \\
\hline 1 & 0.778088 & 6.137649 & 2.689679 \\
\hline 1 & -7.418609 & -0.145609 & -4.520700 \\
\hline 1 & -7.585524 & -0.998373 & -2.983727 \\
\hline 1 & -7.579565 & 0.780661 & -3.019687 \\
\hline 1 & -5.434888 & 1.028964 & -5.174727 \\
\hline
\end{tabular}




$\begin{array}{lrrr}1 & -5.412111 & 2.025775 & -3.705427 \\ 1 & -3.977859 & 1.095796 & -4.179654 \\ 1 & -5.370465 & -1.500714 & -5.006363 \\ 1 & -3.980061 & -1.471979 & -3.900592 \\ 1 & -5.471314 & -2.293452 & -3.415569 \\ 1 & -8.543801 & -0.724448 & 2.211830 \\ 1 & -8.342856 & 0.234162 & 0.739720 \\ 1 & -8.025929 & -1.516330 & 0.716271 \\ 1 & -6.656495 & -1.675177 & 3.434874 \\ 1 & -6.029693 & -2.467079 & 1.975231 \\ 1 & -4.977960 & -1.411474 & 2.935643 \\ 1 & -7.067804 & 0.814736 & 3.464248 \\ 1 & -5.419554 & 1.168379 & 2.912159 \\ 1 & -6.807616 & 1.790442 & 2.003467\end{array}$

$1_{\text {C6- }} \psi-1$ s2a3a4a

Number of imaginary frequencies : 0

The smallest frequencies are : $12.7077 \quad 14.0134 \quad 17.5925 \mathrm{~cm}(-1)$

Electronic energy : $\quad H F=-4125.4653269$

Zero-point correction $=\quad 1.299716$ (Hartree/Particle)

Thermal correction to Energy= $\quad 1.378012$

Thermal correction to Enthalpy= $\quad 1.378956$

Thermal correction to Gibbs Free Energy $=\quad 1.186997$

Sum of electronic and zero-point Energies $=\quad-4124.165611$

Sum of electronic and thermal Energies $=\quad-4124.087315$

Sum of electronic and thermal Enthalpies $=\quad-4124.086371$

Sum of electronic and thermal Free Energies= $\quad-4124.278330$

\begin{tabular}{llll}
\multicolumn{4}{c}{ Cartesian Coordinates } \\
\hline 6 & 3.415275 & -6.104510 & 1.737288 \\
6 & 3.189684 & -5.238660 & 2.803745 \\
6 & 2.834066 & -3.917518 & 2.563084 \\
6 & 2.714694 & -3.430506 & 1.259148 \\
6 & 2.966018 & -4.299354 & 0.197465 \\
6 & 3.303878 & -5.628888 & 0.434790 \\
6 & 2.371761 & -1.974600 & 1.086044 \\
7 & 1.734592 & -1.602497 & -0.186286 \\
6 & 0.658195 & -2.354524 & -0.758280 \\
6 & -0.561681 & -2.523176 & -0.124708 \\
6 & -1.616298 & -3.220265 & -0.796124 \\
6 & -1.416811 & -3.682609 & -2.129919 \\
6 & -0.161309 & -3.478809 & -2.747081 \\
6 & 0.844343 & -2.838910 & -2.078138 \\
6 & -2.884105 & -3.436466 & -0.195760 \\
6 & -3.892652 & -4.065624 & -0.882148 \\
6 & -3.688447 & -4.523609 & -2.201453 \\
6 & -2.474670 & -4.332181 & -2.810955 \\
6 & -0.785765 & -1.984325 & 1.243873 \\
6 & -1.094979 & -0.645691 & 1.459778 \\
6 & -1.299771 & -0.194589 & 2.791751 \\
6 & -1.206912 & -1.046641 & 3.859967 \\
6 & -0.922727 & -2.417163 & 3.671512 \\
6 & -0.711036 & -2.896100 & 2.346045 \\
6 & -0.857027 & -3.319787 & 4.760648
\end{tabular}




\begin{tabular}{|c|c|c|c|}
\hline 5 & -0.606615 & -4.652179 & 4.551771 \\
\hline 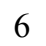 & -0.400465 & -5.133374 & 3.241410 \\
\hline 6 & -0.439282 & -4.278517 & 2.168637 \\
\hline 1 & -1.463595 & 0.474946 & 0.012701 \\
\hline 4 & 0.085321 & 0.977343 & -1.844608 \\
\hline 6 & -0.079190 & 2.883026 & -1.580004 \\
\hline 8 & -0.112257 & 4.037942 & -1.525745 \\
\hline 15 & 1.933717 & -0.083449 & -0.932130 \\
\hline 8 & 2.729413 & 0.665459 & 0.325759 \\
\hline 6 & 3.482800 & 1.769227 & -0.025009 \\
\hline 6 & 4.751632 & 1.583591 & -0.539605 \\
\hline 6 & 5.497226 & 2.740720 & -0.931375 \\
\hline 6 & 4.939272 & 4.037727 & -0.713364 \\
\hline 6 & 3.643690 & 4.157650 & -0.156420 \\
\hline 6 & 2.916941 & 3.042475 & 0.163699 \\
\hline 6 & 5.678797 & 5.185654 & -1.089696 \\
\hline 6 & 6.909160 & 5.069605 & -1.682966 \\
\hline 6 & 7.446189 & 3.789649 & -1.935484 \\
\hline 6 & 6.760246 & 2.657771 & -1.571594 \\
\hline 6 & 5.231676 & 0.196900 & -0.741679 \\
\hline 6 & 4.444684 & -0.671456 & -1.479992 \\
\hline 6 & 4.846671 & -1.991015 & -1.765173 \\
\hline 6 & 6.024751 & -2.468143 & -1.257490 \\
\hline 6 & 6.834872 & -1.654517 & -0.430390 \\
\hline 6 & 6.436979 & -0.309732 & -0.160314 \\
\hline 6 & 7.240130 & 0.458332 & 0.721529 \\
\hline 6 & 8.382762 & -0.062871 & 1.275229 \\
\hline 6 & 8.789160 & -1.381603 & \\
\hline 6 & 8.025405 & -2.158273 & 0.148219 \\
\hline 8 & 3.230939 & -0.251589 & -1.977871 \\
\hline 6 & -3.164843 & -0.121204 & -0.383613 \\
\hline 6 & -4.044802 & -0.395879 & 0.666831 \\
\hline 6 & -5.365205 & -0.759582 & 0.421710 \\
\hline 6 & -5.779579 & -0.835145 & -0.913205 \\
\hline 6 & -4.927633 & -0.575082 & -1.987327 \\
\hline 6 & -3.607359 & -0.209788 & -1.698099 \\
\hline 6 & -6.340522 & -1.099758 & 1.547051 \\
\hline 6 & -5.454301 & -0.661423 & -3.418552 \\
\hline 6 & -1.863657 & 2.100128 & 0.791337 \\
\hline 6 & -3.035385 & 2.750538 & 0.393442 \\
\hline 6 & -3.357525 & 4.015528 & 0.872846 \\
\hline 6 & -2.484660 & 4.609708 & 1.792356 \\
\hline 6 & -1.303120 & 3.997133 & 2.207855 \\
\hline 6 & -0.986502 & 2.744590 & 1.662020 \\
\hline 6 & -4.639901 & 4.734571 & 0.459496 \\
\hline 6 & -0.387318 & 4.617815 & 3.262866 \\
\hline 6 & -0.629396 & -0.031937 & -3.349738 \\
\hline 8 & -0.882242 & -0.525271 & -4.364657 \\
\hline 1 & 6.934288 & 1.472099 & 0.967643 \\
\hline 1 & -1.569427 & 0.841867 & 2.972467 \\
\hline 1 & 8.314288 & -3.184051 & -0.076962 \\
\hline 1 & -1.017128 & -2.932211 & 5.765862 \\
\hline 1 & 5.238324 & 6.165031 & -0.907347 \\
\hline 1 & -3.058768 & -3.083073 & 0.819099 \\
\hline 1 & -4.860980 & -4.212021 & -0.406588 \\
\hline 1 & 3.222054 & 5.151471 & -0.013221 \\
\hline
\end{tabular}




\begin{tabular}{|c|c|c|c|}
\hline 1 & -1.375011 & -0.676992 & 4.870840 \\
\hline 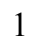 & 6.341490 & -3.489414 & -1.463374 \\
\hline 1 & 8.410723 & 3.697988 & -2.429870 \\
\hline & 4.202228 & -2.604817 & -2.390724 \\
\hline & -2.299961 & -4.678399 & -3.828723 \\
\hline & -0.011719 & -3.830091 & -3.766764 \\
\hline & -0.186973 & -6.187660 & 3.080050 \\
\hline & 7.182556 & 1.679070 & -1.784537 \\
\hline & -0.250012 & -4.661070 & 1.168046 \\
\hline 1 & 1.807512 & -2.670829 & -2.557218 \\
\hline 1 & 7.465514 & 5.958044 & -1.972573 \\
\hline 1 & 8.977617 & 0.545368 & 1.953013 \\
\hline 1 & -4.493978 & -5.029811 & -2.729404 \\
\hline 1 & -0.565523 & -5.340181 & 5.393253 \\
\hline 1 & 9.698967 & -1.780504 & 1.423160 \\
\hline 1 & 1.904076 & 3.107806 & 0.553519 \\
\hline 1 & -3.685652 & -0.320413 & 1.689922 \\
\hline 1 & -2.910577 & 0.020480 & -2.499443 \\
\hline 1 & -6.811936 & -1.115927 & -1.122042 \\
\hline 1 & -3.700648 & 2.255094 & -0.306917 \\
\hline 1 & -0.053555 & 2.252432 & 1.945848 \\
\hline 1 & -2.745905 & 5.584926 & 2.196522 \\
\hline 1 & 3.296398 & -1.389868 & 1.158411 \\
\hline 1 & 1.749917 & -1.654134 & 1.937606 \\
\hline 1 & 1.092960 & 1.457220 & -2.972449 \\
\hline 1 & 2.630888 & -3.250048 & 3.401445 \\
\hline 1 & 2.891611 & -3.934971 & -0.823924 \\
\hline 1 & 3.270044 & -5.597757 & 3.827895 \\
\hline 1 & 3.482468 & -6.295976 & -0.406178 \\
\hline 1 & 3.678153 & -7.144159 & 1.920334 \\
\hline 6 & -4.328675 & -0.579648 & -4.447287 \\
\hline 6 & -6.187803 & -1.988865 & -3.634144 \\
\hline 6 & -6.422974 & 0.502151 & -3.657412 \\
\hline 6 & -5.706432 & -0.941678 & 2.927706 \\
\hline 6 & -7.560417 & -0.175949 & 1.472287 \\
\hline 6 & -6.793038 & -2.556732 & 1.398099 \\
\hline 6 & -0.858379 & 6.002623 & 3.701084 \\
\hline 6 & -0.367040 & 3.706938 & 4.496442 \\
\hline 6 & 1.035179 & 4.752104 & 2.713564 \\
\hline 6 & -5.427367 & 3.952799 & -0.591107 \\
\hline 6 & -5.534271 & 4.914359 & 1.691389 \\
\hline 6 & -4.295579 & 6.106864 & -0.128254 \\
\hline 1 & -6.335824 & 4.507569 & -0.857369 \\
\hline 1 & -4.846750 & 3.807677 & -1.511580 \\
\hline 1 & -5.740692 & 2.964493 & -0.226811 \\
\hline 1 & -5.211268 & 6.628322 & -0.437810 \\
\hline 1 & -3.778244 & 6.750346 & 0.593305 \\
\hline 1 & -3.648620 & 6.005418 & -1.009047 \\
\hline 1 & -6.466310 & 5.425906 & 1.415578 \\
\hline 1 & -5.798032 & 3.942501 & 2.129513 \\
\hline 1 & -5.046233 & 5.511667 & 2.471209 \\
\hline 1 & -0.168133 & 6.402207 & 4.454424 \\
\hline 1 & -0.879684 & 6.712142 & 2.863809 \\
\hline 1 & -1.856665 & 5.975441 & 4.156165 \\
\hline 1 & 1.674258 & 5.280752 & 3.433176 \\
\hline 1 & 1.497358 & 3.775377 & 2.530699 \\
\hline
\end{tabular}




$\begin{array}{rrrl}1 & 1.040529 & 5.318178 & 1.771872 \\ 1 & 0.277532 & 4.133588 & 5.276896 \\ 1 & -1.375151 & 3.590275 & 4.915971 \\ 1 & 0.018168 & 2.706701 & 4.260088 \\ 1 & -4.743389 & -0.691053 & -5.457067 \\ 1 & -3.804946 & 0.384286 & -4.417487 \\ 1 & -3.586837 & -1.376355 & -4.299250 \\ 1 & -6.542265 & -2.058943 & -4.671013 \\ 1 & -5.516777 & -2.837415 & -3.445128 \\ 1 & -7.063432 & -2.098367 & -2.983282 \\ 1 & -6.816999 & 0.469991 & -4.682343 \\ 1 & -7.276386 & 0.469396 & -2.968100 \\ 1 & -5.918254 & 1.467358 & -3.518608 \\ 1 & -6.439251 & -1.202568 & 3.701614 \\ 1 & -4.838308 & -1.602668 & 3.061289 \\ 1 & -5.382006 & 0.090572 & 3.114424 \\ 1 & -8.269334 & -0.415044 & 2.276263 \\ 1 & -7.264721 & 0.875226 & 1.585355 \\ 1 & -8.096381 & -0.272000 & 0.520368 \\ 1 & -7.516795 & -2.814686 & 2.183008 \\ 1 & -7.271692 & -2.744375 & 0.428755 \\ 1 & -5.939303 & -3.242111 & 1.492318\end{array}$

$1_{\mathrm{C} 6-}-\psi_{-1 \mathrm{~s} 2 \mathrm{a} a 4 \mathrm{~s}}$

Number of imaginary frequencies : 0

The smallest frequencies are : $10.4455 \quad 13.5281 \quad 17.0505 \mathrm{~cm}(-1)$

Electronic energy :

$\mathrm{HF}=-4125.4660011$

Zero-point correction $=\quad 1.299668($ Hartree/Particle $)$

Thermal correction to Energy= $\quad 1.378073$

Thermal correction to Enthalpy= $\quad 1.379018$

Thermal correction to Gibbs Free Energy $=\quad 1.186116$

Sum of electronic and zero-point Energies $=\quad-4124.166333$

Sum of electronic and thermal Energies $=\quad-4124.087928$

Sum of electronic and thermal Enthalpies $=\quad-4124.086984$

Sum of electronic and thermal Free Energies $=\quad-4124.279885$

Cartesian Coordinates

$\begin{array}{rrrr}6 & 3.493005 & -6.081969 & 1.732138 \\ 6 & 3.260616 & -5.219299 & 2.799805 \\ 6 & 2.884392 & -3.903584 & 2.561019 \\ 6 & 2.750351 & -3.418627 & 1.257681 \\ 6 & 3.008269 & -4.283902 & 0.194819 \\ 6 & 3.367168 & -5.608278 & 0.430318 \\ 6 & 2.384292 & -1.968060 & 1.086668 \\ 7 & 1.744024 & -1.603326 & -0.186171 \\ 6 & 0.674630 & -2.366586 & -0.756812 \\ 6 & -0.543801 & -2.544725 & -0.123127 \\ 6 & -1.591766 & -3.253668 & -0.792384 \\ 6 & -1.387108 & -3.721235 & -2.123404 \\ 6 & -0.133625 & -3.506977 & -2.741315 \\ 6 & 0.865315 & -2.853614 & -2.075064 \\ 6 & -2.857795 & -3.476361 & -0.190997 \\ 6 & -3.859064 & -4.122308 & -0.872380 \\ 6 & -3.649338 & -4.587943 & -2.188214\end{array}$




\begin{tabular}{|c|c|c|c|}
\hline 6 & -2.438086 & -4.386672 & -2.799791 \\
\hline 6 & -0.772611 & -2.007650 & 1.245299 \\
\hline 6 & -1.098384 & -0.673116 & 1.460959 \\
\hline & -1.305773 & -0.223919 & 2.793235 \\
\hline & -1.196664 & -1.073563 & 3.861951 \\
\hline & -0.894741 & -2.440380 & 3.673685 \\
\hline & -0.682820 & -2.917729 & 2.347756 \\
\hline & -0.811476 & -3.340827 & 4.763333 \\
\hline & -0.543744 & -4.669872 & 4.554503 \\
\hline 6 & -0.337458 & -5.149580 & 3.243645 \\
\hline 6 & -0.393333 & -4.296452 & 2.170222 \\
\hline 5 & -1.474576 & 0.444177 & 0.013383 \\
\hline & 0.068790 & 0.959874 & -1.844840 \\
\hline ठ & -0.121066 & 2.863808 & -1.587079 \\
\hline & -0.169078 & 4.018508 & -1.538215 \\
\hline 15 & 1.927197 & -0.082169 & -0.931924 \\
\hline 8 & 2.713913 & 0.675699 & 0.326084 \\
\hline 6 & 3.451422 & 1.789791 & -0.025974 \\
\hline 6 & 4.722515 & 1.621436 & -0.540829 \\
\hline 6 & 5.451733 & 2.788752 & -0.933481 \\
\hline 0 & 4.875240 & 4.077848 & -0.716459 \\
\hline 6 & 3.577936 & 4.179907 & -0.160016 \\
\hline 6 & 2.867093 & 3.054857 & 0.160978 \\
\hline 6 & 5.598297 & 5.235997 & -1.093406 \\
\hline 6 & 6.830306 & 5.137232 & -1.686337 \\
\hline ) & 7.385667 & 3.864927 & -1.937869 \\
\hline 6 & 6.715973 & 2.723522 & -1.573368 \\
\hline 0 & 5.221235 & 0.241233 & -0.742107 \\
\hline 6 & 4.445917 & -0.638674 & -1.479206 \\
\hline 6 & 4.866195 & -1.952744 & -1.763558 \\
\hline 6 & 6.051325 & -2.412816 & -1.256472 \\
\hline 6 & 6.850482 & -1.587300 & -0.430442 \\
\hline 6 & 6.433632 & -0.248152 & -0.160887 \\
\hline 6 & 7.225843 & 0.531634 & 0.720525 \\
\hline 6 & 8.375828 & 0.027029 & 1.274295 \\
\hline 6 & 8.801034 & -1.285853 & 0.980002 \\
\hline 6 & 8.048302 & -2.073694 & 0.147951 \\
\hline & 3.226357 & -0.236025 & -1.977252 \\
\hline 6 & -3.169851 & -0.164388 & -0.386860 \\
\hline 6 & -4.056419 & -0.411472 & 0.657143 \\
\hline 6 & -5.382688 & -0.771574 & 0.414153 \\
\hline 6 & -5.791271 & -0.873810 & -0.915758 \\
\hline 6 & -4.925374 & -0.638994 & -1.991619 \\
\hline 6 & -3.607471 & -0.278361 & -1.704927 \\
\hline 6 & -6.314567 & -1.005026 & 1.601370 \\
\hline 6 & -5.448891 & -0.746251 & -3.422463 \\
\hline 6 & -1.890599 & 2.066070 & 0.791891 \\
\hline 6 & -3.065071 & 2.709965 & 0.389873 \\
\hline 6 & -3.387727 & 3.979879 & 0.856029 \\
\hline 6 & -2.518781 & 4.581695 & 1.774404 \\
\hline 6 & -1.339687 & 3.972115 & 2.200600 \\
\hline 6 & -1.017815 & 2.717857 & 1.661539 \\
\hline 6 & -4.652630 & 4.709541 & 0.409394 \\
\hline 6 & -0.430722 & 4.599237 & 3.257735 \\
\hline 6 & -0.628613 & -0.061663 & -3.350212 \\
\hline 8 & -0.868758 & -0.560039 & -4.365736 \\
\hline
\end{tabular}




\begin{tabular}{|c|c|c|c|}
\hline 1 & 6.905585 & 1.541050 & 0.966206 \\
\hline & -1.586945 & 0.809471 & 2.974075 \\
\hline & 8.351718 & -3.095355 & -0.076861 \\
\hline & -0.972182 & -2.954503 & 5.768937 \\
\hline & 5.143707 & 6.209029 & -0.911689 \\
\hline & -3.035900 & -3.115060 & 0.820225 \\
\hline & -4.827062 & -4.274016 & -0.397906 \\
\hline & 3.141889 & 5.167687 & -0.017967 \\
\hline & -1.364731 & -0.705035 & 4.873260 \\
\hline & 6.382219 & -3.429679 & -1.461901 \\
\hline & 8.351535 & 3.786783 & -2.431971 \\
\hline & 4.229965 & -2.576019 & -2.388162 \\
\hline & -2.260214 & -4.737537 & -3.815416 \\
\hline & 0.019982 & -3.861426 & -3.759303 \\
\hline & -0.110442 & -6.201031 & 3.082289 \\
\hline & 7.152439 & 1.750829 & -1.785390 \\
\hline & -0.204308 & -4.677438 & 1.168983 \\
\hline & 1.826886 & -2.677663 & -2.554708 \\
\hline & 7.373990 & 6.033329 & -1.976415 \\
\hline & 8.962012 & 0.643886 & 1.951844 \\
\hline & -4.449143 & -5.106834 & -2.712542 \\
\hline & -0.489119 & -5.356372 & 5.396425 \\
\hline & 9.716518 & -1.671585 & 1.422445 \\
\hline & 1.853049 & 3.106227 & 0.549819 \\
\hline & -3.712011 & -0.307718 & 1.685337 \\
\hline ] & -2.903424 & -0.072302 & -2.506210 \\
\hline & -6.822078 & -1.147019 & -1.131465 \\
\hline ] & -3.728677 & 2.208455 & -0.307802 \\
\hline & -0.083475 & 2.230767 & 1.949454 \\
\hline & -2.779531 & 5.562106 & 2.166292 \\
\hline & 3.299203 & -1.368735 & 1.162359 \\
\hline & 1.755340 & -1.659397 & 1.937440 \\
\hline ] & 1.071530 & 1.450507 & -2.972216 \\
\hline & 2.676345 & -3.238935 & 3.400450 \\
\hline 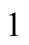 & 2.922159 & -3.920974 & -0.826093 \\
\hline & 3.352155 & -5.576975 & 3.823509 \\
\hline 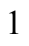 & 3.550749 & -6.272780 & -0.411623 \\
\hline 1 & 3.772240 & -7.117577 & 1.913833 \\
\hline & -4.324882 & -0.652639 & -4.451759 \\
\hline 5 & -6.160299 & -2.087378 & -3.627103 \\
\hline 6 & -6.436431 & 0.399167 & -3.672477 \\
\hline 6 & -7.679743 & -1.536029 & 1.172207 \\
\hline 5 & -5.692063 & -2.025150 & 2.562165 \\
\hline 6 & -6.520674 & 0.326637 & 2.333138 \\
\hline 6 & -0.909170 & 5.983309 & 3.690266 \\
\hline 5 & -0.412701 & 3.691684 & 4.493826 \\
\hline 6 & 0.993738 & 4.737619 & 2.714786 \\
\hline f & -5.465435 & 3.894865 & -0.595687 \\
\hline 6 & -5.540028 & 4.990521 & 1.626833 \\
\hline 5 & -4.262080 & 6.033696 & -0.256057 \\
\hline 1 & -6.366411 & 4.453562 & -0.878631 \\
\hline 1 & -4.898605 & 3.696183 & -1.514658 \\
\hline 1 & -5.791386 & 2.929970 & -0.182438 \\
\hline 1 & -5.158721 & 6.567658 & -0.598610 \\
\hline 1 & -3.723305 & 6.697391 & 0.430817 \\
\hline 1 & -3.616361 & 5.859042 & -1.126421 \\
\hline
\end{tabular}




$\begin{array}{rrrr}1 & -6.455325 & 5.513251 & 1.318242 \\ 1 & -5.834676 & 4.056402 & 2.123013 \\ 1 & -5.035915 & 5.619576 & 2.370454 \\ 1 & -0.225207 & 6.386475 & 4.447420 \\ 1 & -0.926997 & 6.691366 & 2.851669 \\ 1 & -1.910499 & 5.953642 & 4.138488 \\ 1 & 1.627536 & 5.270006 & 3.436306 \\ 1 & 1.460414 & 3.762336 & 2.535677 \\ 1 & 1.001370 & 5.302009 & 1.772027 \\ 1 & 0.226622 & 4.122896 & 5.276080 \\ 1 & -1.422275 & 3.572338 & 4.909048 \\ 1 & -0.022533 & 2.692307 & 4.261952 \\ 1 & -4.738328 & -0.774221 & -5.460903 \\ 1 & -3.816375 & 0.319576 & -4.425190 \\ 1 & -3.570877 & -1.437406 & -4.301672 \\ 1 & -6.516470 & -2.170620 & -4.662480 \\ 1 & -5.474294 & -2.922980 & -3.434434 \\ 1 & -7.031656 & -2.206849 & -2.972330 \\ 1 & -6.825430 & 0.353551 & -4.698799 \\ 1 & -7.292723 & 0.357255 & -2.987473 \\ 1 & -5.948052 & 1.373362 & -3.538220 \\ 1 & -8.303019 & -1.713045 & 2.057793 \\ 1 & -8.217337 & -0.824516 & 0.532684 \\ 1 & -7.593917 & -2.488088 & 0.631307 \\ 1 & -6.351942 & -2.182084 & 3.425869 \\ 1 & -5.551719 & -2.996696 & 2.069757 \\ 1 & -4.716922 & -1.703454 & 2.949771 \\ 1 & -7.159166 & 0.186812 & 3.216042 \\ 1 & -5.570293 & 0.760657 & 2.669853 \\ 1 & -7.008195 & 1.060091 & 1.677069\end{array}$

$1_{\mathrm{C} 6-} \psi_{-1 \mathrm{~s} 2 \mathrm{a} 3 \mathrm{~s} 4 \mathrm{a}}$

Number of imaginary frequencies : 0

The smallest frequencies are : $12.3058 \quad 15.9567 \quad 19.1592 \mathrm{~cm}(-1)$

Electronic energy : $\quad H F=-4125.4651561$

Zero-point correction $=\quad 1.300274($ Hartree/Particle)

Thermal correction to Energy= $\quad 1.378528$

Thermal correction to Enthalpy= $\quad 1.379472$

Thermal correction to Gibbs Free Energy= $\quad 1.187923$

Sum of electronic and zero-point Energies $=\quad-4124.164882$

Sum of electronic and thermal Energies $=\quad-4124.086628$

Sum of electronic and thermal Enthalpies $=\quad-4124.085684$

Sum of electronic and thermal Free Energies $=\quad-4124.277233$

Cartesian Coordinates

$\begin{array}{llll}6 & 3.199936 & -6.170416 & 1.797199 \\ 6 & 2.910795 & -5.292320 & 2.837557 \\ 6 & 2.583907 & -3.970356 & 2.561078 \\ 6 & 2.560238 & -3.495556 & 1.247954 \\ 6 & 2.871542 & -4.377819 & 0.213121 \\ 6 & 3.179134 & -5.707679 & 0.485065 \\ 6 & 2.250892 & -2.038479 & 1.031655 \\ 7 & 1.641004 & -1.694944 & -0.261870 \\ 6 & 0.576566 & -2.456018 & -0.847504\end{array}$




\begin{tabular}{|c|c|c|c|}
\hline 6 & -0.665964 & -2.595580 & -0.252332 \\
\hline 6 & -1.722164 & -3.251181 & -0.960819 \\
\hline & -1.482908 & -3.753338 & -2.273590 \\
\hline & -0.196602 & -3.604783 & -2.841384 \\
\hline f & 0.797598 & -2.969679 & -2.150496 \\
\hline & -3.025027 & -3.394267 & -0.415576 \\
\hline 6 & -4.026043 & -4.002527 & -1.131824 \\
\hline 5 & -3.780715 & -4.506939 & -2.426895 \\
\hline 6 & -2.534261 & -4.380523 & -2.984419 \\
\hline 6 & -0.902779 & -2.102368 & 1.129449 \\
\hline 6 & -1.165866 & -0.766671 & 1.402300 \\
\hline 6 & -1.382659 & -0.372044 & 2.751493 \\
\hline 6 & -1.334710 & -1.273890 & 3.780241 \\
\hline 6 & -1.090561 & -2.643181 & 3.532404 \\
\hline 6 & -0.876375 & -3.067542 & 2.189479 \\
\hline 6 & -1.068299 & -3.595280 & 4.580108 \\
\hline 6 & -0.858833 & -4.924592 & 4.313704 \\
\hline 6 & -0.652010 & -5.352452 & 2.985220 \\
\hline 6 & -0.648601 & -4.448795 & 1.952425 \\
\hline 15 & -1.460820 & 0.443734 & 0.014966 \\
\hline 45 & -0.027181 & 0.826863 & -1.961469 \\
\hline 6 & -0.187066 & 2.742428 & -1.803353 \\
\hline 8 & -0.236257 & 3.898206 & -1.809180 \\
\hline 15 & 1.838614 & -0.185523 & -1.029433 \\
\hline 8 & 2.597509 & 0.603805 & 0.228968 \\
\hline 6 & 3.334778 & 1.713326 & -0.145080 \\
\hline 6 & 4.617037 & 1.534519 & -0.628559 \\
\hline 6 & 5.348127 & & -1.051759 \\
\hline 6 & 4.757411 & 3.981833 & -0.903883 \\
\hline 6 & 3.447422 & 4.096074 & -0.381174 \\
\hline 6 & 2.737490 & 2.981024 & -0.023681 \\
\hline 6 & 5.481129 & 5.128763 & -1.312763 \\
\hline 6 & 6.728062 & 5.014895 & -1.870781 \\
\hline 6 & 7.298322 & 3.737556 & -2.054688 \\
\hline 6 & 6.627703 & 2.607390 & -1.658506 \\
\hline 6 & 5.123618 & 0.150774 & -0.780096 \\
\hline 6 & 4.367081 & -0.747661 & -1.514022 \\
\hline 6 & 4.797230 & -2.066171 & -1.760473 \\
\hline 6 & 5.972727 & -2.510503 & -1.217887 \\
\hline 6 & 6.751283 & -1.663991 & -0.393239 \\
\hline 6 & 6.324468 & -0.320822 & -0.162298 \\
\hline 6 & 7.095012 & 0.482040 & 0.717541 \\
\hline 6 & 8.234778 & -0.005552 & 1.306620 \\
\hline 6 & 8.670561 & -1.323063 & 1.050650 \\
\hline 6 & 7.938419 & -2.132450 & 0.220829 \\
\hline 8 & 3.157305 & -0.359065 & -2.045943 \\
\hline 6 & -3.237325 & 0.085079 & -0.324364 \\
\hline 6 & -4.112771 & -0.195401 & 0.732048 \\
\hline 6 & -5.466244 & -0.407654 & 0.502212 \\
\hline 6 & -5.931158 & -0.318854 & -0.820227 \\
\hline 6 & -5.088897 & -0.040203 & -1.892477 \\
\hline 6 & -3.727510 & 0.157896 & -1.618576 \\
\hline 6 & -6.439166 & -0.744472 & 1.630387 \\
\hline 6 & -5.568367 & -0.016015 & -3.341245 \\
\hline 6 & -1.578826 & 2.059482 & 0.892211 \\
\hline 6 & -2.640911 & 2.913393 & 0.584310 \\
\hline
\end{tabular}




\begin{tabular}{|c|c|c|c|}
\hline & -2.742603 & 4.171508 & 1.169047 \\
\hline & -1.777672 & 4.534480 & 2.117561 \\
\hline & -0.708690 & 3.705310 & 2.457045 \\
\hline & -0.595899 & 2.478699 & 1.786687 \\
\hline & -3.876963 & 5.135323 & 0.828412 \\
\hline & 0.261463 & 4.038510 & 3.592067 \\
\hline & -0.865944 & -0.228553 & -3.368701 \\
\hline & -1.232985 & -0.724203 & -4.347248 \\
\hline & 6.765516 & 1.495420 & 0.933333 \\
\hline & -1.616715 & 0.664187 & 2.977526 \\
\hline & 8.250357 & -3.157579 & 0.025279 \\
\hline & -1.229994 & -3.248185 & 5.599753 \\
\hline & 5.015620 & 6.104984 & -1.183403 \\
\hline & -3.232656 & -3.000146 & 0.578450 \\
\hline & -5.020579 & -4.093844 & -0.699393 \\
\hline & 3.000338 & 5.085106 & -0.290688 \\
\hline & -1.507440 & -0.944845 & 4.804458 \\
\hline & 6.312064 & -3.529980 & -1.394552 \\
\hline & 8.276574 & 3.646297 & -2.521416 \\
\hline & 4.177203 & -2.705477 & -2.385308 \\
\hline & -2.329966 & -4.756184 & -3.986010 \\
\hline & -0.016210 & -3.986853 & -3.844869 \\
\hline & -0.471494 & -6.404915 & 2.778331 \\
\hline & 7.075895 & 1.630294 & -1.819144 \\
\hline & -0.459919 & -4.792418 & 0.937921 \\
\hline & 1.780584 & -2.831144 & -2.597443 \\
\hline & 7.272399 & 5.902329 & -2.185285 \\
\hline & 8.804604 & 0.628502 & 1.982238 \\
\hline & -4.583549 & -4.987964 & -2.981331 \\
\hline & -0.851390 & -5.650885 & 5.123406 \\
\hline & 9.577930 & -1.695135 & 1.520633 \\
\hline & 1.718868 & 3.044198 & 0.353523 \\
\hline & -3.720170 & -0.250570 & 1.743452 \\
\hline & -3.035068 & 0.381733 & -2.426749 \\
\hline & -6.991063 & -0.481041 & -1.007496 \\
\hline & -3.389590 & 2.584787 & -0.131411 \\
\hline 1 & 0.244505 & 1.816878 & 2.006297 \\
\hline & -1.878001 & 5.497903 & 2.612400 \\
\hline 1 & 3.185624 & -1.468648 & 1.100973 \\
\hline 1 & 1.622192 & -1.682809 & 1.864157 \\
\hline & 0.924102 & 1.224032 & -3.167046 \\
\hline 1 & 2.324707 & -3.293943 & 3.376164 \\
\hline & 2.867241 & -4.021878 & -0.814209 \\
\hline 1 & 2.915436 & -5.642369 & 3.868020 \\
\hline & 3.406175 & -6.385390 & -0.335469 \\
\hline 1 & 3.440146 & -7.210469 & 2.007250 \\
\hline 5 & -7.091429 & 0.014258 & -3.448852 \\
\hline 5 & -5.018643 & 1.216310 & -4.067805 \\
\hline 6 & -5.057168 & -1.287040 & -4.030624 \\
\hline 6 & -5.744358 & -0.822269 & 2.988684 \\
\hline 6 & -7.526262 & 0.332599 & 1.706566 \\
\hline 6 & -7.084666 & -2.107023 & 1.355410 \\
\hline 6 & 0.013649 & 5.425470 & 4.180811 \\
\hline 6 & 0.063489 & 3.003632 & 4.708066 \\
\hline 6 & 1.712800 & 3.981436 & 3.112274 \\
\hline 6 & -4.813883 & 4.571089 & -0.238272 \\
\hline
\end{tabular}




$\begin{array}{rrrr}6 & -4.702171 & 5.420633 & 2.087838 \\ 6 & -3.283154 & 6.443700 & 0.295656 \\ 1 & -5.608763 & 5.297340 & -0.449735 \\ 1 & -4.287373 & 4.374077 & -1.181374 \\ 1 & -5.297772 & 3.638676 & 0.083875 \\ 1 & -4.084749 & 7.144675 & 0.026600 \\ 1 & -2.644703 & 6.939502 & 1.036763 \\ 1 & -2.675620 & 6.260553 & -0.600097 \\ 1 & -5.521869 & 6.114381 & 1.857420 \\ 1 & -5.142752 & 4.496970 & 2.485928 \\ 1 & -4.098895 & 5.874430 & 2.883528 \\ 1 & 0.739424 & 5.618957 & 4.980545 \\ 1 & 0.132153 & 6.216955 & 3.429526 \\ 1 & -0.987649 & 5.515018 & 4.621090 \\ 1 & 2.392805 & 4.203274 & 3.945515 \\ 1 & 1.987735 & 2.993441 & 2.723110 \\ 1 & 1.898308 & 4.720659 & 2.322318 \\ 1 & 0.711597 & 3.235737 & 5.564023 \\ 1 & -0.976465 & 2.999577 & 5.061506 \\ 1 & 0.309932 & 1.988276 & 4.371603 \\ 1 & -7.384453 & 0.070196 & -4.504739 \\ 1 & -7.555112 & -0.889789 & -3.034297 \\ 1 & -7.519831 & 0.886719 & -2.937985 \\ 1 & -5.398901 & 1.245347 & -5.097226 \\ 1 & -5.329213 & 2.142667 & -3.566628 \\ 1 & -3.923658 & 1.217441 & -4.129793 \\ 1 & -5.354781 & -1.297664 & -5.088179 \\ 1 & -3.963101 & -1.364177 & -3.984445 \\ 1 & -5.471005 & -2.183299 & -3.548777 \\ 1 & -6.478455 & -1.077370 & 3.763300 \\ 1 & -4.964137 & -1.596398 & 3.006867 \\ 1 & -5.284313 & 0.133253 & 3.272819 \\ 1 & -8.231220 & 0.107863 & 2.518228 \\ 1 & -7.087184 & 1.319637 & 1.903025 \\ 1 & -8.103441 & 0.402612 & 0.776703 \\ 1 & -7.799343 & -2.358270 & 2.150664 \\ 1 & -7.628047 & -2.125699 & 0.403128 \\ 1 & -6.324037 & -2.899037 & 1.325334\end{array}$

$1_{\mathrm{C} 6-} \psi_{-1 \mathrm{~s} 2 \mathrm{a3s} 4 \mathrm{~s}}$

Number of imaginary frequencies : 0

The smallest frequencies are : $12.1634 \quad 16.5950 \quad 19.1526 \mathrm{~cm}(-1)$

Electronic energy : $\quad H F=-4125.4655589$

Zero-point correction $=\quad 1.299757$ (Hartree/Particle)

Thermal correction to Energy= $\quad 1.378132$

Thermal correction to Enthalpy= $\quad 1.379076$

Thermal correction to Gibbs Free Energy $=\quad 1.187086$

Sum of electronic and zero-point Energies $=\quad-4124.165802$

Sum of electronic and thermal Energies $=\quad-4124.087427$

Sum of electronic and thermal Enthalpies $=\quad-4124.086483$

Sum of electronic and thermal Free Energies $=\quad-4124.278473$

Cartesian Coordinates

$6 \quad-0.677450 \quad 2.470039 \quad 1.742563$ 


\begin{tabular}{|c|c|c|c|}
\hline 6 & -1.649547 & 2.007858 & 0.857423 \\
\hline 6 & -2.754109 & 2.811485 & 0.565373 \\
\hline 6 & -2.910974 & 4.059973 & 1.158520 \\
\hline 6 & -1.950582 & 4.468146 & 2.092882 \\
\hline 6 & -0.838831 & 3.689710 & 2.415682 \\
\hline & -1.472981 & 0.390769 & -0.007795 \\
\hline 6 & -3.237108 & -0.005680 & -0.367590 \\
\hline 0 & -4.132310 & -0.222783 & 0.680211 \\
\hline 0 & -5.490046 & -0.424512 & 0.440867 \\
\hline & -5.932791 & -0.400388 & -0.887096 \\
\hline 0 & -5.064229 & -0.182856 & -1.958717 \\
\hline 6 & -3.708468 & 0.011171 & -1.674252 \\
\hline 6 & -6.424857 & -0.631986 & 1.631330 \\
\hline 6 & -6.439387 & 0.646844 & 2.477708 \\
\hline 6 & -5.523949 & -0.193201 & -3.414650 \\
\hline 6 & -4.983526 & -1.463411 & -4.081494 \\
\hline 6 & -4.101504 & 4.961468 & 0.840230 \\
\hline 6 & 0.127220 & 4.065911 & 3.540841 \\
\hline 6 & 1.575340 & 4.064258 & 3.048544 \\
\hline 6 & -1.165324 & -0.794895 & 1.397905 \\
\hline 6 & -0.857517 & -2.124251 & 1.143428 \\
\hline 6 & -0.810413 & -3.076533 & 2.214098 \\
\hline 6 & -1.046815 & -2.643611 & 3.550445 \\
\hline 6 & -1.333502 & -1.279388 & 3.780488 \\
\hline 6 & -1.403028 & -0.391140 & 2.740929 \\
\hline 6 & -0.540142 & -4.452834 & 1.993983 \\
\hline 6 & -0.523874 & -5.344619 & 3.036906 \\
\hline 6 & -0.752325 & -4.908186 & 4.359009 \\
\hline 6 & -1.003066 & -3.582788 & 4.609035 \\
\hline 6 & -0.595316 & -2.625904 & -0.230280 \\
\hline 6 & 0.649667 & -2.468251 & -0.815316 \\
\hline 6 & 0.893727 & -2.998075 & -2.107590 \\
\hline 6 & -0.079987 & -3.665583 & -2.797592 \\
\hline 6 & -1.369562 & -3.829052 & -2.241571 \\
\hline 6 & -1.631679 & -3.309982 & -0.939994 \\
\hline 6 & -2.403630 & -4.483833 & -2.953002 \\
\hline 6 & -3.655241 & -4.615872 & -2.408176 \\
\hline 6 & -3.923704 & -4.091289 & -1.125468 \\
\hline 6 & -2.938638 & -3.460141 & -0.407238 \\
\hline 7 & 1.691082 & -1.673559 & -0.232378 \\
\hline 15 & 1.856210 & -0.168713 & -1.017246 \\
\hline 8 & 3.180922 & -0.323556 & -2.028600 \\
\hline 6 & 4.399254 & -0.676088 & -1.491410 \\
\hline 6 & 5.133009 & 0.248408 & -0.766641 \\
\hline 6 & 6.346262 & -0.186710 & -0.146063 \\
\hline 6 & 6.808021 & -1.519921 & -0.366905 \\
\hline 6 & 6.050502 & -2.394003 & -1.182332 \\
\hline 6 & 4.862872 & -1.985265 & -1.726361 \\
\hline 6 & 7.096902 & 0.643378 & 0.725751 \\
\hline 6 & 8.250939 & 0.191363 & 1.315476 \\
\hline 6 & 8.721597 & -1.115724 & 1.068268 \\
\hline 6 & 8.009093 & -1.951186 & 0.247287 \\
\hline 6 & 4.592977 & 1.621182 & -0.631074 \\
\hline 6 & 3.305347 & 1.774804 & -0.153018 \\
\hline 6 & 2.676772 & 3.028780 & -0.048967 \\
\hline 6 & 3.360345 & 4.156568 & -0.417208 \\
\hline
\end{tabular}




\begin{tabular}{|c|c|c|c|}
\hline 6 & 4.674312 & 4.068023 & -0.934725 \\
\hline 6 & 5.296837 & 2.788914 & -1.066245 \\
\hline 6 & 5.370658 & 5.227666 & -1.354993 \\
\hline 6 & 6.621272 & 5.138209 & -1.909110 \\
\hline 6 & 7.222939 & 3.873190 & -2.077400 \\
\hline 6 & 6.579369 & 2.731430 & -1.669639 \\
\hline 8 & 2.593734 & 0.652762 & 0.233313 \\
\hline 6 & 2.297237 & -1.982827 & 1.071487 \\
\hline 6 & 2.645049 & -3.427474 & 1.310700 \\
\hline 6 & 2.674023 & -3.882292 & 2.630823 \\
\hline 6 & 3.036361 & -5.190286 & 2.928328 \\
\hline 6 & 3.356656 & -6.074785 & 1.902484 \\
\hline 6 & 3.330301 & -5.631985 & 0.583597 \\
\hline 6 & 2.986783 & -4.315457 & 0.290600 \\
\hline 45 & -0.027468 & 0.790562 & -1.971653 \\
\hline 6 & -0.810763 & -0.310556 & -3.376779 \\
\hline 8 & -1.147287 & -0.831803 & -4.352925 \\
\hline 6 & -0.250585 & 2.700224 & -1.830317 \\
\hline 8 & -0.336701 & 3.853828 & -1.840307 \\
\hline 6 & -7.857714 & -0.937036 & 1.202403 \\
\hline 6 & -5.925816 & -1.805135 & 2.483381 \\
\hline 6 & -7.045475 & -0.189796 & -3.544948 \\
\hline 6 & -4.983766 & 1.038671 & -4.149973 \\
\hline 6 & -0.170234 & 5.444255 & 4.126754 \\
\hline 6 & -0.020428 & 3.028078 & 4.661908 \\
\hline 1 & 6.740621 & 1.649176 & 0.934457 \\
\hline 1 & -1.664509 & 0.641429 & 2.953845 \\
\hline 1 & 8.348058 & -2.969068 & 0.058943 \\
\hline 1 & -1.182003 & -3.229571 & 5.623689 \\
\hline 1 & 4.880938 & 6.193496 & -1.237606 \\
\hline 1 & -3.161277 & -3.051144 & 0.577234 \\
\hline 1 & -4.923614 & -4.181704 & -0.705816 \\
\hline 1 & 2.888907 & 5.135351 & -0.339958 \\
\hline 1 & -1.520091 & -0.943535 & 4.800072 \\
\hline 1 & 6.416677 & -3.405483 & -1.351083 \\
\hline 1 & 8.204073 & 3.800558 & -2.541342 \\
\hline 1 & 4.259224 & -2.645252 & -2.345584 \\
\hline 1 & -2.182649 & -4.874262 & -3.945371 \\
\hline 1 & 0.119071 & -4.060416 & -3.792560 \\
\hline 1 & -0.310727 & -6.393495 & 2.843252 \\
\hline 1 & 7.051852 & 1.763958 & -1.818401 \\
\hline 1 & -0.333977 & -4.802295 & 0.984929 \\
\hline 1 & 1.877743 & -2.845724 & -2.547429 \\
\hline 1 & 7.144470 & 6.035058 & -2.232636 \\
\hline 1 & 8.805110 & 0.845814 & 1.984630 \\
\hline 1 & -4.445247 & -5.116032 & -2.964177 \\
\hline 1 & -0.728824 & -5.624815 & 5.176977 \\
\hline 1 & 9.640111 & -1.459399 & 1.538222 \\
\hline 1 & 1.655395 & 3.071491 & 0.323405 \\
\hline 1 & -3.764668 & -0.220914 & 1.705334 \\
\hline 1 & -3.002429 & 0.193371 & -2.480674 \\
\hline 1 & -6.989006 & -0.552626 & -1.089835 \\
\hline 1 & -3.495649 & 2.450575 & -0.141634 \\
\hline 1 & 0.192615 & 1.844527 & 1.954401 \\
\hline 1 & -2.088713 & 5.425181 & 2.591203 \\
\hline 1 & 3.215251 & -1.386604 & 1.141468 \\
\hline
\end{tabular}




$\begin{array}{rrrr}1 & 1.650941 & -1.633802 & 1.893375 \\ 1 & 0.923795 & 1.208780 & -3.170086 \\ 1 & 2.391010 & -3.201681 & 3.434423 \\ 1 & 2.977585 & -3.974359 & -0.741673 \\ 1 & 3.044699 & -5.525273 & 3.963758 \\ 1 & 3.581018 & -6.314790 & -0.225727 \\ 1 & 3.625140 & -7.104372 & 2.129145 \\ 1 & 0.556340 & 5.670921 & 4.916977 \\ 1 & -0.093245 & 6.236258 & 3.370597 \\ 1 & -1.168989 & 5.494969 & 4.578805 \\ 1 & 2.253271 & 4.319287 & 3.873876 \\ 1 & 1.886440 & 3.085346 & 2.663797 \\ 1 & 1.723767 & 4.804571 & 2.251675 \\ 1 & 0.626732 & 3.287232 & 5.510793 \\ 1 & -1.056039 & 2.986075 & 5.025648 \\ 1 & 0.260553 & 2.021595 & 4.325736 \\ 1 & -7.323086 & -0.164810 & -4.606194 \\ 1 & -7.500758 & -1.090505 & -3.113847 \\ 1 & -7.495779 & 0.688295 & -3.063436 \\ 1 & -5.344245 & 1.045048 & -5.186989 \\ 1 & -5.321449 & 1.966120 & -3.668796 \\ 1 & -3.887769 & 1.060006 & -4.189388 \\ 1 & -5.268860 & -1.491925 & -5.142073 \\ 1 & -3.889048 & -1.520881 & -4.022973 \\ 1 & -5.388943 & -2.360058 & -3.593891 \\ 1 & -8.483179 & -1.093424 & 2.090370 \\ 1 & -8.302114 & -0.111522 & 0.632011 \\ 1 & -7.916623 & -1.847837 & 0.592206 \\ 1 & -6.587286 & -1.955227 & 3.347171 \\ 1 & -5.917654 & -2.736711 & 1.902149 \\ 1 & -4.911442 & -1.644777 & 2.870681 \\ 1 & -7.075579 & 0.514799 & 3.363439 \\ 1 & -5.435065 & 0.923777 & 2.823230 \\ 1 & -6.837607 & 1.491644 & 1.899926 \\ 6 & -5.045246 & 4.329997 & -0.182067 \\ 6 & -4.898665 & 5.231194 & 2.121060 \\ 6 & -3.589918 & 6.286266 & 0.264657 \\ 1 & -5.891069 & 5.004116 & -0.366878 \\ 1 & -4.547915 & 4.153779 & -1.144859 \\ 1 & -5.455373 & 3.372434 & 0.168947 \\ 1 & -4.432538 & 6.944570 & 0.013486 \\ 1 & -2.949352 & 6.823381 & 0.974569 \\ 1 & -3.006388 & 6.116131 & -0.649611 \\ 1 & -5.759947 & 5.877229 & 1.904121 \\ 1 & -5.278082 & 4.294979 & 2.551728 \\ 1 & -4.295514 & 5.732529 & 2.887664\end{array}$

$1_{\mathrm{C} 6-}-\psi_{-1 \mathrm{~s} 2 s 3 a 4 a}$

Number of imaginary frequencies : 0

The smallest frequencies are : $12.0259 \quad 15.7080 \quad 18.8155 \mathrm{~cm}(-1)$

Electronic energy : $\quad \mathrm{HF}=-4125.4654639$

Zero-point correction $=\quad 1.300033$ (Hartree/Particle)

Thermal correction to Energy $=\quad 1.378294$

Thermal correction to Enthalpy $=\quad 1.379238$

Thermal correction to Gibbs Free Energy $=\quad 1.187100$ 
Sum of electronic and zero-point Energies= Sum of electronic and thermal Energies= Sum of electronic and thermal Enthalpies= Sum of electronic and thermal Free Energies=
$-4124.165431$

$-4124.087170$

$-4124.086225$

$-4124.278364$

Cartesian Coordinates

\begin{tabular}{|c|c|c|c|}
\hline 6 & 3.416604 & -6.079883 & 1.850013 \\
\hline 6 & 3.168384 & -5.195664 & 2.896148 \\
\hline 6 & 2.801051 & -3.883620 & 2.624917 \\
\hline 6 & 2.692760 & -3.423949 & 1.310208 \\
\hline 6 & 2.966909 & -4.310641 & 0.269208 \\
\hline 6 & 3.316138 & -5.631448 & 0.537005 \\
\hline 6 & 2.334410 & -1.976379 & 1.101851 \\
\hline 7 & 1.706354 & -1.641999 & -0.185046 \\
\hline 6 & 0.639343 & -2.414311 & -0.750368 \\
\hline 6 & -0.591148 & -2.560742 & -0.133148 \\
\hline 6 & -1.649573 & -3.243071 & -0.811928 \\
\hline 6 & -1.432488 & -3.744956 & -2.128141 \\
\hline 6 & -0.159489 & -3.583940 & -2.722197 \\
\hline 6 & 0.841821 & -2.935975 & -2.053458 \\
\hline 6 & -2.936942 & -3.401897 & -0.235442 \\
\hline 6 & -3.948278 & -4.020021 & -0.927604 \\
\hline 6 & -3.725161 & -4.524338 & -2.227290 \\
\hline 6 & -2.492582 & -4.386303 & -2.813559 \\
\hline 6 & -0.829436 & -2.024400 & 1.232212 \\
\hline 6 & -1.155845 & -0.691862 & 1.446729 \\
\hline 6 & -1.399036 & -0.252404 & 2.777365 \\
\hline 6 & -1.311227 & -1.107115 & 3.843294 \\
\hline 6 & -0.995925 & -2.471264 & 3.655072 \\
\hline 6 & -0.758975 & -2.941335 & 2.331114 \\
\hline 6 & -0.927763 & -3.376246 & 4.741624 \\
\hline 6 & -0.652632 & -4.703698 & 4.530893 \\
\hline 6 & -0.425472 & -5.176961 & 3.221413 \\
\hline 6 & -0.465989 & -4.318649 & 2.151147 \\
\hline 15 & -1.480148 & 0.443671 & 0.000818 \\
\hline 45 & 0.034460 & 0.874869 & -1.907230 \\
\hline 6 & -0.180919 & 2.783157 & -1.728706 \\
\hline 8 & -0.248716 & 3.937938 & -1.722926 \\
\hline 15 & 1.892337 & -0.137181 & -0.964249 \\
\hline 8 & 2.670577 & 0.657594 & 0.276855 \\
\hline 6 & 3.403338 & 1.764744 & -0.110247 \\
\hline 6 & 4.678250 & 1.584164 & -0.611560 \\
\hline 6 & 5.404006 & 2.738741 & -1.045403 \\
\hline 6 & 4.817854 & 4.031835 & -0.886362 \\
\hline 6 & 3.515855 & 4.147678 & -0.344049 \\
\hline 6 & 2.809559 & 3.032794 & 0.020599 \\
\hline 6 & 5.536790 & 5.178088 & -1.305386 \\
\hline 6 & 6.774144 & 5.062827 & -1.884084 \\
\hline 6 & 7.339209 & 3.784798 & -2.078796 \\
\hline 6 & 6.673443 & 2.655224 & -1.672805 \\
\hline 6 & 5.182268 & 0.199761 & -0.765010 \\
\hline 6 & 4.414257 & -0.703308 & -1.481402 \\
\hline 6 & 4.840295 & -2.023659 & -1.724992 \\
\hline 6 & 6.024046 & -2.464694 & -1.197935 \\
\hline 6 & 6.815643 & -1.612885 & -0.391467 \\
\hline
\end{tabular}




\begin{tabular}{|c|c|c|c|}
\hline & 6.392484 & -0.268191 & -0.162654 \\
\hline & 7.176581 & 0.539904 & 0.700323 \\
\hline & 8.325331 & 0.055943 & 1.274706 \\
\hline & 8.757141 & -1.263168 & 1.020326 \\
\hline & 8.012118 & -2.077640 & 0.207141 \\
\hline & 3.196884 & -0.318141 & -1.998345 \\
\hline & -3.219566 & -0.042955 & -0.376525 \\
\hline & -4.130981 & -0.194758 & 0.672661 \\
\hline & -5.467714 & -0.494726 & 0.424741 \\
\hline & -5.862083 & -0.643212 & -0.910352 \\
\hline & -4.980534 & -0.499456 & -1.982172 \\
\hline & -3.646357 & -0.193221 & -1.690870 \\
\hline & -6.483407 & -0.684363 & 1.550006 \\
\hline & -5.492603 & -0.641496 & -3.414264 \\
\hline & -1.738561 & 2.078185 & 0.811528 \\
\hline & -2.853557 & 2.835003 & 0.464708 \\
\hline & -3.071080 & 4.100265 & 1.012296 \\
\hline & -2.148682 & 4.577773 & 1.944530 \\
\hline & -1.012247 & 3.848293 & 2.313625 \\
\hline & -0.800305 & 2.609915 & 1.700984 \\
\hline & -4.310790 & 4.881418 & 0.583652 \\
\hline & -0.071939 & 4.315900 & 3.426637 \\
\hline & -0.666209 & -0.193222 & -3.382984 \\
\hline & -0.901686 & -0.700696 & -4.394976 \\
\hline & 6.850691 & 1.554609 & 0.915153 \\
\hline & -1.682682 & 0.780089 & 2.959672 \\
\hline & 8.320779 & -3.104102 & 0.013419 \\
\hline & -1.106792 & -2.995366 & 5.746216 \\
\hline & 5.075016 & 6.154918 & -1.167510 \\
\hline & -3.123336 & -3.009287 & 0.762611 \\
\hline & -4.933258 & -4.117984 & -0.474484 \\
\hline & 3.071870 & 5.137427 & -0.246767 \\
\hline & -1.504220 & -0.744090 & 4.852293 \\
\hline & 6.359732 & -3.485821 & -1.372134 \\
\hline & 8.309424 & 3.692525 & -2.561819 \\
\hline & 4.210183 & -2.667358 & -2.335002 \\
\hline 1 & -2.306108 & -4.766499 & -3.816983 \\
\hline & 0.004913 & -3.966716 & -3.728178 \\
\hline 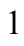 & -0.194547 & -6.227347 & 3.058680 \\
\hline & 7.117282 & 1.677507 & -1.841715 \\
\hline & -0.260659 & -4.695053 & 1.151423 \\
\hline l & 1.814434 & -2.790342 & -2.520694 \\
\hline & 7.314621 & 5.949726 & -2.206623 \\
\hline 1 & 8.905416 & 0.694203 & 1.937522 \\
\hline & -4.532623 & -5.022367 & -2.760139 \\
\hline 1 & -0.609610 & -5.393937 & 5.370457 \\
\hline & 9.671640 & -1.632395 & 1.478593 \\
\hline 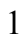 & 1.794706 & 3.095678 & 0.406172 \\
\hline 1 & -3.785715 & -0.066685 & 1.695305 \\
\hline ] & -2.922542 & -0.060901 & -2.490100 \\
\hline 1 & -6.905046 & -0.879782 & -1.121811 \\
\hline & -3.569057 & 2.431581 & -0.249912 \\
\hline 1 & 0.084365 & 2.023650 & 1.957687 \\
\hline ] & -2.322978 & 5.544990 & 2.405455 \\
\hline 1 & 3.250778 & -1.377963 & 1.170188 \\
\hline 1 & 1.699442 & -1.645460 & 1.939770 \\
\hline
\end{tabular}




$\begin{array}{rrrr}1 & 1.032482 & 1.329662 & -3.052173 \\ 1 & 2.579286 & -3.202414 & 3.447322 \\ 1 & 2.901219 & -3.966934 & -0.759955 \\ 1 & 3.239619 & -5.533592 & 3.928140 \\ 1 & 3.512494 & -6.313026 & -0.288239 \\ 1 & 3.688552 & -7.112701 & 2.056917 \\ 6 & -4.355110 & -0.633121 & -4.432308 \\ 6 & -6.255517 & -1.960211 & -3.573474 \\ 6 & -6.430787 & 0.531143 & -3.721665 \\ 6 & -5.889583 & -0.361575 & 2.919738 \\ 6 & -7.688781 & 0.235620 & 1.329940 \\ 6 & -6.950871 & -2.144002 & 1.559945 \\ 6 & -0.403653 & 5.723604 & 3.917419 \\ 6 & -0.213357 & 3.350572 & 4.611018 \\ 6 & 1.382027 & 4.312879 & 2.951560 \\ 6 & -4.454754 & 6.203909 & 1.331721 \\ 6 & -4.214449 & 5.184250 & -0.916016 \\ 6 & -5.563571 & 4.038374 & 0.854857 \\ 1 & -5.360178 & 6.722425 & 0.992105 \\ 1 & -4.547611 & 6.055247 & 2.415537 \\ 1 & -3.605468 & 6.874174 & 1.147168 \\ 1 & -6.465167 & 4.588651 & 0.553828 \\ 1 & -5.553894 & 3.091652 & 0.299172 \\ 1 & -5.653261 & 3.800392 & 1.923574 \\ 1 & -5.096354 & 5.747527 & -1.250057 \\ 1 & -3.322786 & 5.784683 & -1.137700 \\ 1 & -4.156109 & 4.268353 & -1.516840 \\ 1 & 0.309165 & 6.016408 & 4.698391 \\ 1 & -0.333991 & 6.465423 & 3.111342 \\ 1 & -1.407935 & 5.784005 & 4.355494 \\ 1 & 2.042965 & 4.660674 & 3.756496 \\ 1 & 1.724249 & 3.312119 & 2.662526 \\ 1 & 1.518159 & 4.981883 & 2.091578 \\ 1 & 0.422954 & 3.672875 & 5.446455 \\ 1 & -1.251097 & 3.316771 & 4.969144 \\ 1 & 0.084464 & 2.328857 & 4.341811 \\ 1 & -4.762305 & -0.773413 & -5.441537 \\ 1 & -3.804326 & 0.316283 & -4.433664 \\ 1 & -3.638442 & -1.444012 & -4.245002 \\ 1 & -6.610352 & -2.068446 & -4.607011 \\ 1 & -5.603910 & -2.814349 & -3.345992 \\ 1 & -7.133506 & -2.020258 & -2.919204 \\ 1 & -6.809167 & 0.459803 & -4.750445 \\ 1 & -7.296069 & 0.549960 & -3.047068 \\ 1 & -5.906237 & 1.490430 & -3.620034 \\ 1 & -6.655853 & -0.492567 & 3.694081 \\ 1 & -5.050661 & -1.023526 & 3.174263 \\ 1 & -5.536055 & 0.677163 & 2.973552 \\ 1 & -8.418077 & 0.104177 & 2.140372 \\ 1 & -7.383278 & 1.290286 & 1.319634 \\ 1 & -6.207094 & 0.027898 & 0.386524 \\ & -6.109427 & -2.822767 & 1.754722\end{array}$

$1_{\mathrm{C} 6-} \psi_{-1 \mathrm{~s} 2 \mathrm{~s} 3 \mathrm{a} 4 \mathrm{~s}}$ 
Number of imaginary frequencies : 0

The smallest frequencies are : $10.2541 \quad 15.4589 \quad 17.1620 \mathrm{~cm}(-1)$

Electronic energy : $\quad H F=-4125.4659377$

Zero-point correction $=$

1.300373 (Hartree/Particle)

Thermal correction to Energy=

1.378500

Thermal correction to Enthalpy=

1.379444

Thermal correction to Gibbs Free Energy=

Sum of electronic and zero-point Energies=

1.187759

Sum of electronic and thermal Energies=

$-4124.165565$

Sum of electronic and thermal Enthalpies=

$-4124.087438$

Sum of electronic and thermal Free Energies=

$-4124.086494$

$-4124.278179$

Cartesian Coordinates

$\begin{array}{cccc}0 & 3.362553 & -6.117528 & 1.802423 \\ 6 & 3.115254 & -5.240309 & 2.854609 \\ 6 & 2.758564 & -3.923483 & 2.592418 \\ 6 & 2.660524 & -3.452141 & 1.281074 \\ 6 & 2.933878 & -4.332054 & 0.234048 \\ 6 & 3.272136 & -5.657506 & 0.492658 \\ 6 & 2.314391 & -2.000267 & 1.082766 \\ 7 & 1.689780 & -1.652041 & -0.202137 \\ 6 & 0.621028 & -2.414373 & -0.777252 \\ 6 & -0.611071 & -2.563421 & -0.163760 \\ 6 & -1.670284 & -3.234759 & -0.852349 \\ 6 & -1.452172 & -3.721907 & -2.174026 \\ 6 & -0.177770 & -3.558137 & -2.764049 \\ 6 & 0.824325 & -2.921504 & -2.085924 \\ 6 & -2.958848 & -3.397221 & -0.279744 \\ 6 & -3.969562 & -4.006568 & -0.980632 \\ 6 & -3.745771 & -4.495829 & -2.285855 \\ 6 & -2.512289 & -4.352777 & -2.868829 \\ 6 & -0.848306 & -2.043177 & 1.208199 \\ 6 & -1.163049 & -0.710697 & 1.440654 \\ 6 & -1.401426 & -0.286343 & 2.776909 \\ 6 & -1.322007 & -1.155962 & 3.831542 \\ 6 & -1.022405 & -2.520982 & 3.624729 \\ 6 & -0.787762 & -2.975249 & 2.294923 \\ 6 & -0.965026 & -3.441123 & 4.699111 \\ 6 & -0.701959 & -4.768118 & 4.471012 \\ 6 & -0.476016 & -5.225577 & 3.155684 \\ 6 & -0.506448 & -4.352578 & 2.097085 \\ 15 & -1.485069 & 0.443990 & 0.010005 \\ 45 & 0.025092 & 0.887241 & -1.897223 \\ 6 & -0.169436 & 2.796072 & -1.696319 \\ 8 & -0.223905 & 3.951384 & -1.678520 \\ 15 & 1.880940 & -0.140501 & -0.966722 \\ 8 & 2.660979 & 0.639553 & 0.282808 \\ 6 & 3.396686 & 1.748525 & -0.093269 \\ 6 & 4.670786 & 1.569636 & -0.597300 \\ 6 & 5.398897 & 2.726403 & -1.021096 \\ 6 & 4.815782 & 4.019320 & -0.849902 \\ 6 & 3.514836 & 4.133230 & -0.304698 \\ 6 & 2.806410 & 3.016745 & 0.050819 \\ 6 & 5.536725 & 5.167626 & -1.259753\end{array}$




\begin{tabular}{|c|c|c|c|}
\hline & 6.773185 & 5.054733 & -1.840810 \\
\hline & 7.335392 & 3.777253 & -2.047110 \\
\hline & 6.667628 & 2.645599 & -1.650290 \\
\hline & 5.171521 & 0.185511 & -0.763346 \\
\hline & 4.401245 & -0.709139 & -1.487704 \\
\hline & 4.823210 & -2.028833 & -1.741807 \\
\hline & 6.005043 & -2.477965 & -1.217265 \\
\hline & 6.798945 & -1.635204 & -0.403579 \\
\hline & 6.380140 & -0.290920 & -0.164505 \\
\hline & 7.166710 & 0.507990 & 0.704726 \\
\hline & 8.313594 & 0.015777 & 1.275813 \\
\hline & 8.740900 & -1.302902 & 1.011773 \\
\hline & 7.993497 & -2.108622 & 0.192073 \\
\hline & 3.185357 & -0.315549 & -2.001878 \\
\hline & -3.227333 & -0.035262 & -0.364072 \\
\hline & -4.126802 & -0.206922 & 0.684738 \\
\hline & -5.467038 & -0.518833 & 0.447921 \\
\hline & -5.876655 & -0.644698 & -0.879754 \\
\hline & -5.000084 & -0.479365 & -1.959567 \\
\hline & -3.667638 & -0.172438 & -1.679466 \\
\hline & -6.410500 & -0.680879 & 1.638234 \\
\hline 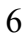 & -5.527158 & -0.601391 & -3.388001 \\
\hline & -1.732868 & 2.074626 & 0.832309 \\
\hline & -2.825748 & 2.854975 & 0.465072 \\
\hline & -3.012827 & 4.134688 & 0.990767 \\
\hline & -2.095471 & 4.593971 & 1.937593 \\
\hline & -0.988138 & 3.836015 & 2.335620 \\
\hline f & -0.795280 & 2.590077 & 1.731726 \\
\hline & -4.203785 & 4.961149 & 0.510604 \\
\hline & -0.055730 & 4.285114 & 3.462819 \\
\hline & -0.695295 & -0.158701 & -3.378228 \\
\hline & -0.946570 & -0.655232 & -4.391858 \\
\hline & 6.844347 & 1.522236 & 0.926903 \\
\hline & -1.674680 & 0.746511 & 2.972474 \\
\hline & 8.298803 & -3.134597 & -0.009358 \\
\hline & -1.142372 & -3.072095 & 5.708405 \\
\hline & 5.077200 & 6.144211 & -1.112897 \\
\hline & -3.145915 & -3.014073 & 0.722072 \\
\hline & -4.955859 & -4.108505 & -0.531706 \\
\hline & 3.073173 & 5.123077 & -0.198026 \\
\hline & -1.510458 & -0.804498 & 4.845478 \\
\hline ] & 6.337501 & -3.498742 & -1.399472 \\
\hline & 8.304968 & 3.687153 & -2.531824 \\
\hline 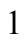 & 4.191651 & -2.665472 & -2.357682 \\
\hline l & -2.324983 & -4.721168 & -3.876472 \\
\hline & -0.012738 & -3.929993 & -3.774004 \\
\hline 1 & -0.253884 & -6.275647 & 2.979244 \\
\hline & 7.109225 & 1.668398 & -1.827986 \\
\hline 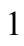 & -0.301792 & -4.717385 & 1.093001 \\
\hline & 1.798456 & -2.774168 & -2.549337 \\
\hline 1 & 7.315208 & 5.943232 & -2.156276 \\
\hline 1 & 8.895707 & 0.647080 & 1.943494 \\
\hline 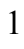 & -4.553409 & -4.986248 & -2.825450 \\
\hline 1 & -0.667189 & -5.470006 & 5.301245 \\
\hline 1 & 9.653918 & -1.678719 & 1.467633 \\
\hline & 1.792404 & 3.078309 & 0.439005 \\
\hline
\end{tabular}




\begin{tabular}{|c|c|c|c|}
\hline 1 & -3.780377 & -0.088723 & 1.710645 \\
\hline & -2.951158 & -0.029232 & -2.483416 \\
\hline & -6.917877 & -0.879667 & -1.090370 \\
\hline & -3.539846 & 2.463768 & -0.258145 \\
\hline & 0.075291 & 1.988193 & 1.999948 \\
\hline & -2.248944 & 5.571433 & 2.384115 \\
\hline & 3.235700 & -1.409901 & 1.155436 \\
\hline & 1.681959 & -1.669983 & 1.922841 \\
\hline & 1.020497 & 1.344421 & -3.043685 \\
\hline & 2.537618 & -3.247506 & 3.419292 \\
\hline & 2.876131 & -3.979234 & -0.792532 \\
\hline 1 & 3.178671 & -5.587283 & 3.884108 \\
\hline 1 & 3.468014 & -6.333625 & -0.337175 \\
\hline & 3.626062 & -7.153960 & 2.002067 \\
\hline & -4.400089 & -0.587687 & -4.417586 \\
\hline & -6.300496 & -1.912795 & -3.557967 \\
\hline & -6.460769 & 0.581295 & -3.670461 \\
\hline & -7.808268 & -1.125512 & 1.216142 \\
\hline & -5.852988 & -1.732070 & 2.605415 \\
\hline & -6.530373 & 0.666451 & 2.360268 \\
\hline f & -0.374546 & 5.694655 & 3.956552 \\
\hline & -0.225107 & 3.316541 & 4.640870 \\
\hline & 1.404000 & 4.264223 & 3.006062 \\
\hline & -4.304641 & 6.307544 & 1.222571 \\
\hline ) & -4.050062 & 5.223704 & -0.992322 \\
\hline & -5.503586 & 4.188353 & 0.764319 \\
\hline 1 & -5.174824 & 6.859011 & 0.844883 \\
\hline & -4.434557 & 6.191124 & \\
\hline & -3.419796 & 6.932397 & 1.046185 \\
\hline & -6.366311 & 4.772177 & 0.416259 \\
\hline & -5.523999 & 3.224689 & 0.238556 \\
\hline 1 & -5.639194 & 3.989836 & 1.835801 \\
\hline & -4.893165 & 5.821931 & -1.363753 \\
\hline 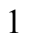 & -3.123782 & 5.774550 & -1.201510 \\
\hline 1 & -4.020703 & 4.292686 & -1.571874 \\
\hline 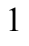 & 0.328516 & 5.971627 & 4.752004 \\
\hline 1 & -0.278785 & 6.440496 & 3.156880 \\
\hline & -1.385849 & 5.767456 & 4.376320 \\
\hline | & 2.059928 & 4.592792 & 3.823108 \\
\hline & 1.734175 & 3.261479 & 2.709639 \\
\hline 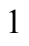 & 1.561597 & 4.940600 & 2.155602 \\
\hline 1 & 0.403557 & 3.627819 & 5.486180 \\
\hline t & -1.267891 & 3.293759 & 4.984815 \\
\hline 1 & 0.064423 & 2.292442 & 4.371790 \\
\hline & -4.818728 & -0.712708 & -5.424147 \\
\hline 1 & -3.842859 & 0.357872 & -4.413398 \\
\hline & -3.687111 & -1.405830 & -4.247674 \\
\hline 1 & -6.662709 & -2.005249 & -4.590465 \\
\hline 1 & -5.653357 & -2.774442 & -3.346161 \\
\hline 1 & -7.174858 & -1.975275 & -2.899256 \\
\hline 1 & -6.849386 & 0.526687 & -4.696464 \\
\hline 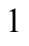 & -7.319534 & 0.595915 & -2.987502 \\
\hline 1 & -5.929019 & 1.535743 & -3.560280 \\
\hline & -8.440304 & -1.249997 & 2.104470 \\
\hline & -8.297903 & -0.387268 & 0.568347 \\
\hline & -7.787493 & -2.087795 & 0.687472 \\
\hline
\end{tabular}




$\begin{array}{rrrr}1 & -6.511861 & -1.829424 & 3.478672 \\ 1 & -5.791595 & -2.716825 & 2.123764 \\ 1 & -4.851439 & -1.478852 & 2.976095 \\ 1 & -7.178903 & 0.573784 & 3.241996 \\ 1 & -5.554693 & 1.039573 & 2.697632 \\ 1 & -6.966613 & 1.426052 & 1.697910\end{array}$

$1_{\mathrm{C6}-} \psi_{-1 \mathrm{~s} 2 \mathrm{~s} 3 \mathrm{~s} 4 \mathrm{a}}$

Number of imaginary frequencies : 0

The smallest frequencies are : $7.6278 \quad 16.7704 \quad 17.6579 \mathrm{~cm}(-1)$

Electronic energy : $\quad H F=-4125.4656146$

Zero-point correction $=\quad 1.300206($ Hartree/Particle)

Thermal correction to Energy= $\quad 1.378424$

Thermal correction to Enthalpy $=\quad 1.379368$

Thermal correction to Gibbs Free Energy $=\quad 1.187009$

Sum of electronic and zero-point Energies $=\quad-4124.165409$

Sum of electronic and thermal Energies $=\quad-4124.087191$

Sum of electronic and thermal Enthalpies $=\quad-4124.086247$

Sum of electronic and thermal Free Energies $=\quad-4124.278606$

Cartesian Coordinates

\begin{tabular}{cccc}
6 & 3.167082 & -6.113888 & 1.987764 \\
6 & 2.838217 & -5.214691 & 2.997898 \\
6 & 2.504550 & -3.903682 & 2.680448 \\
6 & 2.513169 & -3.461170 & 1.356026 \\
6 & 2.863072 & -4.364341 & 0.351970 \\
6 & 3.178164 & -5.683222 & 0.664514 \\
6 & 2.195796 & -2.013818 & 1.092124 \\
7 & 1.606855 & -1.715333 & -0.222026 \\
6 & 0.549177 & -2.495154 & -0.797201 \\
6 & -0.707222 & -2.599144 & -0.225098 \\
6 & -1.756965 & -3.265391 & -0.932155 \\
6 & -1.491538 & -3.841276 & -2.208766 \\
6 & -0.190431 & -3.732529 & -2.751631 \\
6 & 0.792439 & -3.068233 & -2.071130 \\
6 & -3.077660 & -3.349246 & -0.418020 \\
6 & -4.069840 & -3.981670 & -1.125245 \\
6 & -3.796093 & -4.570894 & -2.378577 \\
6 & -2.533560 & -4.495893 & -2.908963 \\
6 & -0.967718 & -2.081955 & 1.142844 \\
6 & -1.243120 & -0.746060 & 1.395134 \\
6 & -1.494279 & -0.340142 & 2.736264 \\
6 & -1.459908 & -1.230391 & 3.775145 \\
6 & -1.196923 & -2.600047 & 3.547650 \\
6 & -0.955436 & -3.036799 & 2.213763 \\
6 & -1.184413 & -3.540027 & 4.606046 \\
6 & -0.959369 & -4.870576 & 4.358086 \\
6 & -0.727211 & -5.311238 & 3.038302 \\
6 & -0.713873 & -4.418660 & 1.995724 \\
15 & -1.484640 & 0.462395 & -0.004672 \\
45 & -0.053912 & 0.749316 & -2.006117 \\
6 & -0.211805 & 2.668918 & -1.926397 \\
8 & -0.255212 & 3.823609 & -1.980743 \\
15 & 1.811528 & -0.230973 & -1.036097 \\
& & & \\
\hline
\end{tabular}




\begin{tabular}{|c|c|c|c|}
\hline 8 & 2.572488 & 0.594204 & 0.197215 \\
\hline 6 & 3.303791 & 1.696481 & -0.209581 \\
\hline & 4.584486 & 1.508704 & -0.693625 \\
\hline & 5.308669 & 2.654185 & -1.153382 \\
\hline & 4.714408 & 3.948036 & -1.037255 \\
\hline & 3.407698 & 4.072397 & -0.508255 \\
\hline 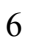 & 2.703175 & 2.965263 & -0.116989 \\
\hline & 5.431733 & 5.085521 & -1.482104 \\
\hline ) & 6.675257 & 4.960233 & -2.045418 \\
\hline f & 7.248465 & 3.680155 & -2.198285 \\
\hline & 6.584277 & 2.559292 & -1.766427 \\
\hline b & 5.097639 & 0.123363 & -0.803712 \\
\hline & 4.342751 & -0.802930 & -1.503964 \\
\hline$b$ & 4.779343 & -2.126336 & -1.709270 \\
\hline ) & 5.961108 & -2.545701 & -1.160597 \\
\hline 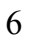 & 6.739694 & -1.667815 & -0.369526 \\
\hline 6 & 6.305051 & -0.321014 & -0.178062 \\
\hline & 7.075873 & 0.514063 & 0.671101 \\
\hline 6 & 8.223397 & 0.053140 & 1.266631 \\
\hline & 8.667169 & -1.268397 & 1.048250 \\
\hline 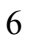 & 7.934702 & -2.108435 & 0.249831 \\
\hline 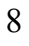 & 3.130035 & -0.438496 & -2.045919 \\
\hline$b$ & -3.277898 & 0.191929 & -0.339013 \\
\hline ) & -4.186414 & 0.053772 & 0.719114 \\
\hline 6 & -5.540650 & -0.141154 & 0.476720 \\
\hline 6 & -5.969839 & -0.194673 & -0.860287 \\
\hline 6 & -5.097011 & -0.047017 & -1.932905 \\
\hline 6 & -3.738449 & 0.147779 & -1.645021 \\
\hline 6 & -6.548544 & -0.337489 & 1.607661 \\
\hline 6 & -5.538247 & -0.145030 & -3.390490 \\
\hline ) & -1.496076 & 2.077336 & 0.878471 \\
\hline 6 & -2.502380 & 2.999526 & 0.608485 \\
\hline 6 & -2.533965 & 4.242541 & 1.244402 \\
\hline 6 & -1.543478 & 4.520921 & 2.188762 \\
\hline 6 & -0.515825 & 3.617695 & 2.485510 \\
\hline 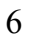 & -0.483063 & 2.410781 & 1.781614 \\
\hline 6 & -3.664568 & 5.212399 & 0.907864 \\
\hline ) & 0.483687 & 3.859041 & 3.619233 \\
\hline 6 & -0.884141 & -0.370719 & -3.368230 \\
\hline 5 & -1.242370 & -0.909995 & -4.326890 \\
\hline ( & 6.740583 & 1.531304 & 0.857812 \\
\hline 1 & -1.731913 & 0.698301 & 2.949099 \\
\hline & 8.252615 & -3.136968 & 0.083986 \\
\hline 1 & -1.366820 & -3.183375 & 5.618881 \\
\hline & 4.964055 & 6.063543 & -1.376355 \\
\hline 1 & -3.305268 & -2.888323 & 0.542326 \\
\hline 1 & -5.078476 & -4.024596 & -0.719017 \\
\hline 1 & 2.958760 & 5.062269 & -0.438620 \\
\hline 1 & -1.654415 & -0.891279 & 4.792226 \\
\hline 1 & 6.306223 & -3.568099 & -1.306566 \\
\hline 1 & 8.223666 & 3.579141 & -2.669361 \\
\hline 1 & 4.159906 & -2.789345 & -2.309585 \\
\hline 1 & -2.309595 & -4.932720 & -3.881109 \\
\hline 1 & 0.010807 & -4.167354 & -3.729347 \\
\hline 1 & -0.535315 & -6.364410 & 2.845909 \\
\hline 1 & 7.034304 & 1.579476 & -1.903540 \\
\hline
\end{tabular}




\begin{tabular}{|c|c|c|c|}
\hline 1 & -0.506262 & -4.772534 & 0.988462 \\
\hline 1 & 1.785136 & -2.955733 & -2.503380 \\
\hline 1 & 7.214439 & 5.840455 & -2.387826 \\
\hline 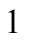 & 8.793245 & 0.711938 & 1.918126 \\
\hline 1 & -4.591427 & -5.072874 & -2.925350 \\
\hline 1 & -0.959735 & -5.587737 & 5.175939 \\
\hline 1 & 9.580747 & -1.619152 & 1.522574 \\
\hline 1 & 1.688440 & 3.035995 & 0.269929 \\
\hline 1 & -3.820283 & 0.098960 & 1.741099 \\
\hline 1 & -3.022234 & 0.266313 & -2.454150 \\
\hline 1 & -7.027495 & -0.357663 & -1.057826 \\
\hline 1 & -3.279236 & 2.742871 & -0.110902 \\
\hline 1 & 0.309462 & 1.687674 & 1.983368 \\
\hline 1 & -1.578405 & 5.466974 & 2.719959 \\
\hline 1 & 3.124199 & -1.433674 & 1.159905 \\
\hline 1 & 1.548757 & -1.639248 & 1.902533 \\
\hline 1 & 0.893418 & 1.095643 & -3.229942 \\
\hline 1 & 2.215504 & -3.210892 & 3.471343 \\
\hline 1 & 2.884628 & -4.031785 & -0.682991 \\
\hline 1 & 2.817640 & -5.540040 & 4.036234 \\
\hline 1 & 3.436247 & -6.377629 & -0.132534 \\
\hline 1 & 3.413311 & -7.145509 & 2.229928 \\
\hline 6 & -7.057702 & -0.166785 & -3.537113 \\
\hline 6 & -5.000214 & 1.048022 & -4.18 \\
\hline 6 & -4.975144 & -1.445259 & -3.976103 \\
\hline 6 & -5.921889 & -0.114092 & 2.982788 \\
\hline 6 & -7.713978 & 0.645154 & 1.454939 \\
\hline 6 & -7.079160 & -1.774518 & 1.550678 \\
\hline 6 & 0.338628 & 5.247284 & 4.238935 \\
\hline 6 & 0.219881 & 2.816900 & 4.714696 \\
\hline 6 & 1.925193 & 3.710577 & \\
\hline 6 & -3.577735 & 6.509960 & 1.706895 \\
\hline 6 & -3.602819 & 5.562425 & -0.583351 \\
\hline 6 & -5.011260 & 4.547158 & 1.219915 \\
\hline 1 & -4.407406 & 7.170861 & 1.426270 \\
\hline 1 & -3.650504 & 6.333755 & 2.788005 \\
\hline 1 & -2.644656 & 7.052464 & 1.507604 \\
\hline 1 & -5.837503 & & 0.983789 \\
\hline 1 & -5.164676 & 3.629896 & 0.636895 \\
\hline 1 & -5.083205 & 4.283853 & 2.283694 \\
\hline 1 & -4.406378 & 6.264776 & -0.843105 \\
\hline 1 & -2.644539 & 6.033106 & -0.838244 \\
\hline 1 & -3.717921 & 4.676974 & -1.220454 \\
\hline 1 & 1.086551 & 5.374250 & 5.031611 \\
\hline 1 & 0.500837 & 6.044474 & 3.502004 \\
\hline 1 & -0.647865 & 5.395876 & 4.696196 \\
\hline 1 & 2.622157 & 3.860372 & 3.963583 \\
\hline 1 & 2.127645 & 2.716432 & 2.710896 \\
\hline 1 & 2.161967 & 4.454708 & 2.357101 \\
\hline 1 & 0.889985 & 2.981990 & 5.569312 \\
\hline 1 & -0.814843 & 2.880660 & 5.078052 \\
\hline 1 & 0.387973 & 1.794323 & 4.352994 \\
\hline 1 & -7.324084 & -0.210466 & -4.600611 \\
\hline 1 & -7.507159 & -1.044389 & -3.055001 \\
\hline 1 & -7.522714 & 0.734065 & -3.115309 \\
\hline 1 & -5.343048 & 0.988315 & -5.229788 \\
\hline
\end{tabular}




$\begin{array}{rrrr}1 & -5.355900 & 1.998482 & -3.769527 \\ 1 & -3.903918 & 1.079280 & -4.210946 \\ 1 & -5.252729 & -1.541458 & -5.034766 \\ 1 & -3.879959 & -1.483153 & -3.909135 \\ 1 & -5.369124 & -2.317274 & -3.437094 \\ 1 & -6.685277 & -0.241835 & 3.760522 \\ 1 & -5.118006 & -0.832198 & 3.193436 \\ 1 & -5.511710 & 0.899989 & 3.082656 \\ 1 & -8.435020 & 0.506206 & 2.271432 \\ 1 & -7.361162 & 1.684142 & 1.489980 \\ 1 & -8.257067 & 0.507372 & 0.512709 \\ 1 & -7.807997 & -1.947576 & 2.354103 \\ 1 & -7.575773 & -1.991548 & 0.596784 \\ 1 & -6.260496 & -2.496344 & 1.675697\end{array}$

$1_{\mathrm{C} 6-}-\psi_{-1 \mathrm{~s} 2 \mathrm{~s} 344 \mathrm{~s}}$

Number of imaginary frequencies : 0

The smallest frequencies are : $9.7714 \quad 16.8915 \quad 18.5560 \mathrm{~cm}(-1)$

Electronic energy : $\quad H F=-4125.4658309$

Zero-point correction $=\quad 1.300436($ Hartree/Particle $)$

Thermal correction to Energy= $\quad 1.378504$

Thermal correction to Enthalpy $=\quad 1.379448$

Thermal correction to Gibbs Free Energy= $\quad 1.188211$

Sum of electronic and zero-point Energies $=\quad-4124.165395$

Sum of electronic and thermal Energies $=\quad-4124.087327$

Sum of electronic and thermal Enthalpies $=\quad-4124.086383$

Sum of electronic and thermal Free Energies $=\quad-4124.277620$

\begin{tabular}{rrrrr} 
& \multicolumn{4}{c}{ Cartesian Coordinates } \\
\hline 6 & 3.143899 & -6.133938 & 1.951057 \\
6 & 2.818046 & -5.239772 & 2.966596 \\
6 & 2.490856 & -3.925186 & 2.657226 \\
6 & 2.503176 & -3.474107 & 1.335728 \\
6 & 2.850168 & -4.372371 & 0.326260 \\
6 & 3.158659 & -5.694739 & 0.630664 \\
6 & 2.192170 & -2.023697 & 1.081013 \\
7 & 1.605838 & -1.714439 & -0.231779 \\
6 & 0.547463 & -2.487508 & -0.814203 \\
6 & -0.709182 & -2.595395 & -0.243463 \\
6 & -1.759058 & -3.253479 & -0.957700 \\
6 & -1.495170 & -3.813763 & -2.241500 \\
6 & -0.193820 & -3.701406 & -2.782852 \\
6 & 0.790228 & -3.047579 & -2.094045 \\
6 & -3.078777 & -3.342919 & -0.442833 \\
6 & -4.072546 & -3.964236 & -1.157518 \\
6 & -3.801338 & -4.535950 & -2.419484 \\
6 & -2.538999 & -4.456610 & -2.949732 \\
6 & -0.970852 & -2.087500 & 1.127891 \\
6 & -1.242668 & -0.752336 & 1.388794 \\
6 & -1.491508 & -0.353751 & 2.732366 \\
6 & -1.461833 & -1.251013 & 3.765500 \\
6 & -1.207080 & -2.620663 & 3.528669 \\
6 & -0.964584 & -3.049375 & 2.192364 \\
6 & -1.201180 & -3.567897 & 4.580671
\end{tabular}




\begin{tabular}{|c|c|c|c|}
\hline 6 & -0.981353 & -4.897629 & 4.324108 \\
\hline 6 & -0.747836 & -5.330277 & 3.001860 \\
\hline & -0.728267 & -4.430730 & 1.965447 \\
\hline & -1.486524 & 0.463456 & -0.003771 \\
\hline & -0.051403 & 0.766510 & -1.997966 \\
\hline & -0.198729 & 2.686444 & -1.899216 \\
\hline & -0.235261 & 3.841785 & -1.941888 \\
\hline & 1.812545 & -0.224059 & -1.034252 \\
\hline & 2.574179 & 0.590483 & 0.205692 \\
\hline & 3.308676 & 1.693764 & -0.192444 \\
\hline & 4.589526 & 1.506280 & -0.676161 \\
\hline & 5.317192 & 2.653132 & -1.126826 \\
\hline & 4.725813 & 3.947571 & -1.002720 \\
\hline & 3.418501 & 4.071375 & -0.475116 \\
\hline & 2.710864 & 2.963249 & -0.092357 \\
\hline & 5.446494 & 5.086376 & -1.438692 \\
\hline 5 & 6.690616 & 4.962006 & -2.000866 \\
\hline & 7.261070 & 3.681653 & -2.161570 \\
\hline & 6.593559 & 2.559420 & -1.738488 \\
\hline 5 & 5.098960 & 0.120406 & -0.796030 \\
\hline 0 & 4.342401 & -0.798205 & -1.504537 \\
\hline 6 & 4.775457 & -2.121314 & -1.719210 \\
\hline 6 & 5.954987 & -2.548359 & -1.171625 \\
\hline 6 & 6.734805 & -1.678938 & -0.372454 \\
\hline 6 & 6.303969 & -0.332248 & -0.171777 \\
\hline 6 & 7.075790 & 0.494141 & 0.684920 \\
\hline 6 & 8.220656 & 0.025189 & 1.279284 \\
\hline 6 & 8.660625 & -1.296153 & 1.052119 \\
\hline 6 & 7.927149 & -2.127941 & 0.246007 \\
\hline 8 & 3.131336 & -0.425937 & -2.045015 \\
\hline 6 & -3.278615 & 0.184806 & -0.342306 \\
\hline 6 & -4.181467 & 0.007994 & 0.707501 \\
\hline 6 & -5.538684 & -0.199255 & 0.466140 \\
\hline 6 & -5.974279 & -0.211873 & -0.864419 \\
\hline 6 & -5.100075 & -0.025428 & -1.936891 \\
\hline 6 & -3.745546 & 0.171767 & -1.650806 \\
\hline 6 & -6.476569 & -0.409435 & 1.654365 \\
\hline 6 & -5.547422 & -0.096968 & -3.394658 \\
\hline 6 & -1.510216 & 2.075492 & 0.884496 \\
\hline 6 & -2.518427 & 2.993865 & 0.608467 \\
\hline 6 & -2.552270 & 4.241202 & 1.235807 \\
\hline 6 & -1.565659 & 4.525541 & 2.182382 \\
\hline 6 & -0.538869 & 3.624413 & 2.488631 \\
\hline 6 & -0.500914 & 2.414705 & 1.789724 \\
\hline 6 & -3.679879 & 5.209832 & 0.885978 \\
\hline 6 & 0.454699 & 3.871849 & 3.626248 \\
\hline 6 & -0.888955 & -0.334219 & -3.370614 \\
\hline 8 & -1.253975 & -0.860845 & -4.333732 \\
\hline 1 & 6.743382 & 1.511040 & 0.878499 \\
\hline 1 & -1.723980 & 0.684570 & 2.951290 \\
\hline 1 & 8.242116 & -3.156237 & 0.073208 \\
\hline 1 & -1.384236 & -3.217334 & 5.595504 \\
\hline 1 & 4.980920 & 6.064747 & -1.327040 \\
\hline 1 & -3.302533 & -2.895178 & 0.524682 \\
\hline 1 & -5.080577 & -4.012428 & -0.750588 \\
\hline 1 & 2.971654 & 5.061801 & -0.399930 \\
\hline
\end{tabular}




\begin{tabular}{|c|c|c|c|}
\hline & -1.654098 & -0.917809 & 4.784934 \\
\hline & 6.297209 & -3.570671 & -1.324812 \\
\hline & 8.236799 & 3.581574 & -2.631749 \\
\hline & 4.155153 & -2.778043 & -2.325501 \\
\hline & -2.316735 & -4.880796 & -3.927833 \\
\hline & 0.006849 & -4.124943 & -3.765620 \\
\hline & -0.559988 & -6.382933 & 2.802788 \\
\hline & 7.041515 & 1.579511 & -1.881606 \\
\hline & -0.520043 & -4.778376 & 0.956145 \\
\hline & 1.783487 & -2.932783 & -2.524350 \\
\hline & 7.232411 & 5.843263 & -2.336417 \\
\hline & 8.791333 & 0.677368 & 1.936685 \\
\hline & -4.598168 & -5.028749 & -2.972384 \\
\hline & -0.986746 & -5.620361 & 5.137010 \\
\hline & 9.572104 & -1.653313 & 1.525699 \\
\hline & 1.695399 & 3.033580 & 0.292623 \\
\hline & -3.818699 & 0.025555 & 1.734005 \\
\hline & -3.032494 & 0.316349 & -2.458568 \\
\hline & -7.028607 & -0.374070 & -1.069159 \\
\hline & -3.292391 & 2.732801 & -0.112438 \\
\hline & 0.293407 & 1.694858 & 1.995861 \\
\hline & -1.602958 & 5.474834 & 2.707615 \\
\hline & 3.122854 & -1.447741 & 1.153531 \\
\hline & 1.545694 & -1.651936 & 1.893152 \\
\hline 1 & 0.898344 & 1.119309 & -3.218125 \\
\hline & 2.204096 & -3.236187 & 3.452249 \\
\hline & 2.874517 & -4.033426 & -0.706573 \\
\hline & 2.794664 & -5.571723 & 4.002778 \\
\hline & 3.414453 & -6.385172 & -0.170560 \\
\hline ] & 3.384907 & -7.168282 & 2.186777 \\
\hline & -7.067616 & -0.106693 & -3.537488 \\
\hline 6 & -5.004865 & 1.103608 & -4.178021 \\
\hline 6 & -4.994233 & -1.392646 & -4.000398 \\
\hline 6 & -7.913755 & -0.687884 & 1.221762 \\
\hline 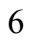 & -5.990651 & -1.606444 & 2.481077 \\
\hline & -6.476458 & 0.849774 & 2.528488 \\
\hline 6 & 0.304857 & 5.262499 & 4.239365 \\
\hline 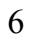 & 0.186397 & 2.834077 & 4.724775 \\
\hline 6 & 1.898879 & 3.722957 & 3.143527 \\
\hline 6 & -3.595880 & 6.513248 & 1.675702 \\
\hline 5 & -3.610131 & 5.549095 & -0.607410 \\
\hline 6 & -5.028381 & 4.548129 & 1.196351 \\
\hline 1 & -4.423063 & 7.173090 & 1.385359 \\
\hline 1 & -3.675188 & 6.345156 & 2.757640 \\
\hline 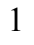 & -2.660950 & 7.053053 & 1.477778 \\
\hline 1 & -5.852978 & 5.229208 & 0.946227 \\
\hline 1 & -5.177489 & 3.622869 & 0.624712 \\
\hline 1 & -5.106722 & 4.298624 & 2.262902 \\
\hline 1 & -4.416926 & 6.243837 & -0.877459 \\
\hline 1 & -2.654026 & 6.025990 & -0.858809 \\
\hline 1 & -3.714130 & 4.658442 & -1.239247 \\
\hline 1 & 1.048269 & 5.393454 & 5.035620 \\
\hline 1 & 0.470416 & 6.056771 & 3.500051 \\
\hline 1 & -0.684290 & 5.412000 & 4.690522 \\
\hline 1 & 2.591289 & 3.876835 & 3.981864 \\
\hline 1 & 2.104583 & 2.727373 & 2.731230 \\
\hline
\end{tabular}




$\begin{array}{lrrl}1 & 2.138846 & 4.464354 & 2.370715 \\ 1 & 0.851646 & 3.003774 & 5.582271 \\ 1 & -0.850325 & 2.898041 & 5.082264 \\ 1 & 0.357947 & 1.810154 & 4.368452 \\ 1 & -7.336683 & -0.126556 & -4.601032 \\ 1 & -7.521908 & -0.991313 & -3.073240 \\ 1 & -7.526249 & 0.787909 & -3.095852 \\ 1 & -5.353825 & 1.061937 & -5.218144 \\ 1 & -5.351071 & 2.050451 & -3.743027 \\ 1 & -3.908633 & 1.127325 & -4.206574 \\ 1 & -5.276907 & -1.473406 & -5.059059 \\ 1 & -3.898976 & -1.436305 & -3.937849 \\ 1 & -5.389661 & -2.270469 & -3.471414 \\ 1 & -8.542160 & -0.840501 & 2.108300 \\ 1 & -8.343389 & 0.149111 & 0.656670 \\ 1 & -7.988120 & -1.593530 & 0.605752 \\ 1 & -6.649175 & -1.763822 & 3.345937 \\ 1 & -6.000023 & -2.526381 & 1.881425 \\ 1 & -4.971060 & -1.467638 & 2.863343 \\ 1 & -7.116805 & 0.704016 & 3.408994 \\ 1 & -5.470756 & 1.105721 & 2.884795 \\ 1 & -6.862857 & 1.712850 & 1.970539\end{array}$

\section{$\mathbf{1}_{\mathrm{C} 7-} \psi_{-1 \mathrm{a} 2 a 3 a 4 a}$}

Number of imaginary frequencies : 0

The smallest frequencies are : $16.4124 \quad 17.5992 \quad 19.8679 \mathrm{~cm}(-1)$

Electronic energy : $\quad H F=-4125.4653919$

Zero-point correction $=\quad 1.299275$ (Hartree/Particle)

Thermal correction to Energy= $\quad 1.377829$

Thermal correction to Enthalpy= $\quad 1.378773$

Thermal correction to Gibbs Free Energy $=\quad 1.186690$

Sum of electronic and zero-point Energies $=\quad-4124.166117$

Sum of electronic and thermal Energies $=\quad-4124.087563$

Sum of electronic and thermal Enthalpies $=\quad-4124.086619$

Sum of electronic and thermal Free Energies $=\quad-4124.278702$

\section{Cartesian Coordinates}

$\begin{array}{rrrr}6 & 3.974874 & -0.053258 & 4.193878 \\ 6 & 4.810196 & -0.831693 & 3.400172 \\ 6 & 4.270313 & -1.651199 & 2.411747 \\ 6 & 2.894035 & -1.695770 & 2.199536 \\ 6 & 2.062396 & -0.911365 & 3.000176 \\ 6 & 2.596242 & -0.099422 & 3.991777 \\ 6 & 2.321813 & -2.603209 & 1.142277 \\ 7 & 1.353702 & -1.968501 & 0.215628 \\ 6 & 0.263002 & -2.801750 & -0.209982 \\ 6 & -0.984666 & -2.685187 & 0.375097 \\ 6 & -2.074576 & -3.454167 & -0.139943 \\ 6 & -1.850358 & -4.350599 & -1.227117 \\ 6 & -0.547438 & -4.472917 & -1.764096 \\ 6 & 0.478349 & -3.713865 & -1.269616 \\ 6 & -3.389287 & -3.345736 & 0.383730 \\ 6 & -4.420953 & -4.080885 & -0.144130\end{array}$




\begin{tabular}{|c|c|c|c|}
\hline 6 & -4.191930 & -4.978238 & -1.210294 \\
\hline 6 & -2.932937 & -5.107336 & -1.739196 \\
\hline & -1.189453 & -1.862039 & 1.599336 \\
\hline 6 & -1.495385 & -0.509151 & 1.568932 \\
\hline & -1.740048 & 0.170109 & 2.795219 \\
\hline & -1.656599 & -0.470638 & 4.001427 \\
\hline & -1.324390 & -1.842531 & 4.068213 \\
\hline & -1.099232 & -2.552795 & 2.853613 \\
\hline & -1.203522 & -2.515599 & 5.307113 \\
\hline & -0.872421 & -3.845480 & 5.354297 \\
\hline 6 & -0.654001 & -4.557446 & 4.156414 \\
\hline & -0.767999 & -3.931185 & 2.939811 \\
\hline 15 & -1.675211 & 0.394024 & -0.051096 \\
\hline 45 & -0.126944 & 0.280533 & -1.952393 \\
\hline 6 & 0.295055 & 2.195707 & -1.928536 \\
\hline 8 & 0.431725 & 3.335325 & -2.025836 \\
\hline 15 & 1.699485 & -0.713005 & -0.875957 \\
\hline 8 & 2.668956 & 0.162290 & 0.139547 \\
\hline 6 & 3.521989 & 1.085116 & -0.418018 \\
\hline 6 & 4.709210 & 0.665845 & -0.987280 \\
\hline 6 & 5.548304 & 1.654679 & -1.597797 \\
\hline & 5.179850 & 3.032466 & -1.517813 \\
\hline 6 & 3.974331 & 3.397896 & -0.872658 \\
\hline & 3.149775 & 2.440960 & -0.347585 \\
\hline 6 & 6.011702 & 4.013151 & -2.110898 \\
\hline 5 & 7.152416 & 3.658684 & -2.783494 \\
\hline 6 & 7.499950 & 2.296516 & -2.898377 \\
\hline 6 & 6.721419 & 1.323146 & -2.323006 \\
\hline b & 5.033647 & -0.778719 & -1.010002 \\
\hline 6 & 4.118014 & -1.676349 & -1.531567 \\
\hline 5 & 4.430724 & -3.041258 & -1.714514 \\
\hline 6 & 5.632130 & -3.532679 & -1.284148 \\
\hline 5 & 6.555526 & -2.689038 & -0.619027 \\
\hline$b$ & 6.255074 & -1.301604 & -0.471810 \\
\hline 5 & 7.156836 & -0.501573 & 0.275148 \\
\hline b & 8.297724 & -1.038321 & 0.819766 \\
\hline 6 & 8.608667 & -2.402381 & 0.640430 \\
\hline 6 & 7.750719 & -3.207500 & -0.064422 \\
\hline & 2.875149 & -1.261946 & -1.946520 \\
\hline 6 & -3.430896 & 0.005255 & -0.432177 \\
\hline 6 & -4.431672 & 0.193574 & 0.526365 \\
\hline 6 & -5.753766 & -0.145061 & 0.252323 \\
\hline 5 & -6.037823 & -0.700784 & -1.003233 \\
\hline 6 & -5.062632 & -0.911441 & -1.978008 \\
\hline 6 & -3.750448 & -0.533146 & -1.672737 \\
\hline 6 & -6.870177 & 0.030307 & 1.280276 \\
\hline 6 & -5.427966 & -1.483443 & -3.347154 \\
\hline 6 & -1.702163 & 2.145688 & 0.491103 \\
\hline 6 & -2.699385 & 3.020169 & 0.065909 \\
\hline 6 & -2.642356 & 4.379210 & 0.386803 \\
\hline 6 & -1.568851 & 4.823885 & 1.161736 \\
\hline 6 & -0.557360 & 3.970142 & 1.615828 \\
\hline 6 & -0.624100 & 2.629486 & 1.238459 \\
\hline 6 & -3.719449 & 5.367051 & -0.058519 \\
\hline 6 & 0.575336 & 4.514191 & 2.485541 \\
\hline 6 & -0.545295 & 0.254894 & -3.806352 \\
\hline
\end{tabular}




\begin{tabular}{|c|c|c|c|}
\hline & -0.779698 & 0.180947 & -4.937934 \\
\hline & 6.921213 & 0.547150 & 0.438302 \\
\hline 1 & -1.999653 & 1.225157 & 2.779109 \\
\hline & 7.964258 & -4.267343 & -0.197626 \\
\hline & -1.376380 & -1.951102 & 6.222340 \\
\hline & 5.714328 & 5.057887 & -2.029981 \\
\hline & -3.580225 & -2.652604 & 1.201400 \\
\hline & -5.425606 & -3.972292 & 0.260286 \\
\hline & 3.701143 & 4.451365 & -0.823543 \\
\hline 1 & -1.848088 & 0.071132 & 4.926950 \\
\hline 1 & 5.882851 & -4.583150 & -1.422268 \\
\hline 1 & 8.390313 & 2.012661 & -3.455052 \\
\hline 1 & 3.696768 & -3.675887 & -2.206313 \\
\hline 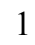 & -2.742014 & -5.792172 & -2.564379 \\
\hline & -0.378692 & -5.169346 & -2.583944 \\
\hline 1 & -0.395410 & -5.613071 & 4.196932 \\
\hline & 6.998355 & 0.278100 & -2.433597 \\
\hline 1 & -0.602048 & -4.497321 & 2.025602 \\
\hline & 1.478042 & -3.782138 & -1.694571 \\
\hline 1 & 7.780612 & 4.419874 & -3.240239 \\
\hline & 8.965249 & -0.405232 & 1.400183 \\
\hline 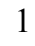 & -5.016789 & -5.564374 & -1.610479 \\
\hline 1 & -0.777729 & -4.353899 & 6.310953 \\
\hline 1 & 9.518679 & -2.813119 & 1.071452 \\
\hline 1 & 2.201116 & 2.691903 & 0.124305 \\
\hline 1 & -4.164412 & 0.612931 & 1.493504 \\
\hline 1 & -2.946762 & -0.669059 & -2.393203 \\
\hline 1 & -7.067512 & -0.981448 & -1.222721 \\
\hline 1 & -3.524941 & 2.630581 & -0.524526 \\
\hline 1 & 0.155662 & 1.932496 & 1.548323 \\
\hline & -1.520365 & 5.879399 & 1.428889 \\
\hline 1 & 1.760252 & -3.410495 & 1.634058 \\
\hline 1 & -0.614659 & -1.240040 & -2.129084 \\
\hline 1 & 3.140611 & -3.087071 & 0.588031 \\
\hline 1 & 4.932336 & -2.250038 & 1.783923 \\
\hline 1 & 0.989737 & -0.921892 & 2.820117 \\
\hline 1 & 5.889042 & -0.799620 & 3.540184 \\
\hline 1 & 1.929489 & 0.501212 & 4.609343 \\
\hline 1 & 4.394255 & 0.588841 & 4.965673 \\
\hline 6 & -4.201383 & -2.036282 & -4.074915 \\
\hline 6 & -6.444662 & -2.620667 & -3.215575 \\
\hline 6 & -6.036991 & -0.359652 & -4.193506 \\
\hline 6 & -6.389878 & 0.762503 & 2.532057 \\
\hline 6 & -8.022297 & 0.837613 & 0.673768 \\
\hline 6 & -7.379962 & -1.352786 & 1.700621 \\
\hline 1 & -4.512500 & -2.507100 & -5.016105 \\
\hline 1 & -3.478315 & -1.252905 & -4.333810 \\
\hline 1 & -3.682376 & -2.795244 & -3.472029 \\
\hline 1 & -6.307047 & -0.731202 & -5.191575 \\
\hline 1 & -6.943428 & 0.047827 & -3.727140 \\
\hline 1 & -5.324759 & 0.466108 & -4.320768 \\
\hline 1 & -6.652642 & -3.050568 & -4.204004 \\
\hline 1 & -6.056598 & -3.421705 & -2.572341 \\
\hline 1 & -7.404610 & -2.286605 & -2.804449 \\
\hline 1 & -7.227553 & 0.889513 & 3.229245 \\
\hline 1 & -5.605503 & 0.206175 & 3.062377 \\
\hline
\end{tabular}




$\begin{array}{rrrr}1 & -6.000400 & 1.762142 & 2.295547 \\ 1 & -8.189731 & -1.257216 & 2.436795 \\ 1 & -7.769754 & -1.922567 & 0.847756 \\ 1 & -6.574101 & -1.941218 & 2.160078 \\ 1 & -8.818479 & 0.978372 & 1.416882 \\ 1 & -7.681767 & 1.830253 & 0.351497 \\ 1 & -8.470227 & 0.339970 & -0.194321 \\ 6 & -4.818373 & 4.694067 & -0.877999 \\ 6 & -4.369974 & 6.002510 & 1.175516 \\ 6 & -3.081706 & 6.461522 & -0.920650 \\ 6 & 1.432071 & 3.394544 & 3.077246 \\ 6 & 1.464103 & 5.427335 & 1.634853 \\ 6 & -0.002044 & 5.318775 & 3.655448 \\ 1 & -5.152161 & 6.710982 & 0.871299 \\ 1 & -4.835151 & 5.238396 & 1.812104 \\ 1 & -3.646081 & 6.552765 & 1.788584 \\ 1 & -5.570905 & 5.438961 & -1.165798 \\ 1 & -4.428010 & 4.247298 & -1.801207 \\ 1 & -5.333833 & 3.907599 & -0.309395 \\ 1 & -3.844722 & 7.177995 & -1.253717 \\ 1 & -2.315560 & 7.023672 & -0.373165 \\ 1 & -2.606931 & 6.032015 & -1.812276 \\ 1 & 2.185297 & 3.820905 & 3.751921 \\ 1 & 0.821825 & 2.689346 & 3.659299 \\ 1 & 1.974931 & 2.820310 & 2.315367 \\ 1 & 0.810732 & 5.694121 & 4.291173 \\ 1 & -0.582372 & 6.187195 & 3.322777 \\ 1 & -0.656997 & 4.695486 & 4.278135 \\ 1 & 2.302271 & 5.814175 & 2.230189 \\ 1 & 1.879598 & 4.883588 & 0.775533 \\ 1 & 0.904291 & 6.285377 & 1.242397\end{array}$

$\mathbf{1}_{\mathrm{C} 7-\psi_{-1 \mathrm{a} 2 a 3 a 4 \mathrm{~s}}}$

Number of imaginary frequencies : 0

The smallest frequencies are : $10.0424 \quad 13.7301 \quad 17.3346 \mathrm{~cm}(-1)$

Electronic energy : $\quad H F=-4125.46557$

Zero-point correction $=\quad 1.299089($ Hartree/Particle $)$

Thermal correction to Energy= $\quad 1.377748$

Thermal correction to Enthalpy= $\quad 1.378692$

Thermal correction to Gibbs Free Energy $=\quad 1.184344$

Sum of electronic and zero-point Energies $=\quad-4124.166481$

Sum of electronic and thermal Energies $=\quad-4124.087822$

Sum of electronic and thermal Enthalpies $=\quad-4124.086878$

Sum of electronic and thermal Free Energies $=\quad-4124.281226$

Cartesian Coordinates

$\begin{array}{llll}6 & 3.966050 & -0.178628 & 4.194848 \\ 6 & 4.803910 & -0.918127 & 3.367266 \\ 6 & 4.265281 & -1.710939 & 2.356715 \\ 6 & 2.887891 & -1.765361 & 2.155140 \\ 6 & 2.053559 & -1.018109 & 2.987956 \\ 6 & 2.586274 & -0.235809 & 4.003680 \\ 6 & 2.314527 & -2.647849 & 1.078065 \\ 7 & 1.345407 & -1.989363 & 0.169722\end{array}$




$\begin{array}{cccc}6 & 0.256183 & -2.812470 & -0.279337 \\ 6 & -0.991769 & -2.710076 & 0.307064 \\ 6 & -2.085687 & -3.452338 & -0.236235 \\ 6 & -1.866590 & -4.308180 & -1.356198 \\ 6 & -0.562054 & -4.424558 & -1.890750 \\ 6 & 0.468454 & -3.691568 & -1.367019 \\ 6 & -3.401288 & -3.347522 & 0.286272 \\ 6 & -4.438886 & -4.050458 & -0.272586 \\ 6 & -4.214571 & -4.909910 & -1.370436 \\ 6 & -2.955630 & -5.031734 & -1.900784 \\ 6 & -1.202161 & -1.924047 & 1.553668 \\ 6 & -1.524975 & -0.575204 & 1.560063 \\ 6 & -1.790533 & 0.063532 & 2.804102 \\ 6 & -1.711863 & -0.612278 & 3.991430 \\ 6 & -1.361142 & -1.980946 & 4.020394 \\ 6 & -1.113744 & -2.650815 & 2.787503 \\ 6 & -1.243967 & -2.689760 & 5.239495 \\ 6 & -0.895301 & -4.015949 & 5.249785 \\ 6 & -0.654892 & -4.688139 & 4.033302 \\ 6 & -0.765107 & -4.026598 & 2.835068 \\ 15 & -1.693084 & 0.380079 & -0.030082 \\ 45 & -0.151441 & 0.302644 & -1.939288 \\ 6 & 0.262229 & 2.219567 & -1.882007 \\ 8 & 0.398185 & 3.361101 & -1.954972 \\ 6 & 1.685092 & -0.703557 & -0.888545 \\ 6 & -3.450 \\ 6 & -4.444832 & 0.125655 & 0.549861 \\ 6 & -6.076017 & -0.595822 & -1.041461 \\ 6 & -5.108738 & -0.677295 & -2.051767 \\ 6 & 3.505323 & 1.085672 & -0.394431 \\ 6 & 4.690788 & 0.684675 & -0.980236 \\ 6 & -3.795044 & -0.347591 & -1.722787 \\ 6 & 5.521491 & 1.690943 & -1.573770 \\ 6 & -6.814137 & -0.117955 & 1.378170 \\ 6 & 5.146695 & 3.064721 & -1.461315 \\ 6 & -5.510811 & -1.104644 & -3.462196 \\ 6 & 3.943295 & 3.409913 & -0.801309 \\ 6 & 3.126895 & 2.437776 & -0.291608 \\ 6 & 5.969744 & 4.062540 & -2.037887 \\ 6 & 7.107791 & 3.728985 & -2.725521 \\ 6 & 7.461168 & 2.371479 & -2.872817 \\ 6 & 6.691339 & 1.381672 & -2.313890 \\ 6 & 5.018543 & -0.758058 & -1.041527 \\ 6 & 4.101883 & -1.643808 & -1.581367 \\ 6 & 4.416102 & -3.002726 & -1.801399 \\ 6 & 5.622092 & -3.501953 & -1.393273 \\ 6 & 6.548773 & -2.673392 & -0.714006 \\ 6 & -1.679901 & 2.113198 & 0.570155\end{array}$




\begin{tabular}{|c|c|c|c|}
\hline 6 & -2.645477 & 3.027336 & 0.155435 \\
\hline 6 & -2.547640 & 4.376753 & 0.506624 \\
\hline & -1.476810 & 4.766559 & 1.314112 \\
\hline & -0.501368 & 3.869903 & 1.764433 \\
\hline & -0.599540 & 2.543566 & 1.345951 \\
\hline & -3.567600 & 5.416507 & 0.045022 \\
\hline & 0.624188 & 4.354627 & 2.677705 \\
\hline & -0.555438 & 0.287299 & -3.796681 \\
\hline & -0.775689 & 0.210560 & -4.930884 \\
\hline & 6.913941 & 0.534504 & 0.426661 \\
\hline & -2.060333 & 1.116165 & 2.816653 \\
\hline & 7.966518 & -4.257559 & -0.348630 \\
\hline & -1.434076 & -2.155747 & 6.169473 \\
\hline & 5.667561 & 5.103638 & -1.931757 \\
\hline & -3.587410 & -2.678038 & 1.125100 \\
\hline & -5.446062 & -3.936947 & 0.125109 \\
\hline & 3.664142 & 4.460568 & -0.730070 \\
\hline & -1.919931 & -0.101144 & 4.930660 \\
\hline & 5.874443 & -4.547751 & -1.560740 \\
\hline & 8.349057 & 2.104406 & -3.441607 \\
\hline & 3.679977 & -3.626340 & -2.303841 \\
\hline & -2.769698 & -5.686177 & -2.751314 \\
\hline & -0.395908 & -5.093339 & -2.733832 \\
\hline & -0.382332 & -5.740934 & 4.044564 \\
\hline & 6.972612 & 0.340872 & -2.449913 \\
\hline & -0.582124 & -4.562072 & 1.905753 \\
\hline & 1.468770 & -3.753026 & -1.791455 \\
\hline & 7.729150 & 4.503263 & -3.169431 \\
\hline & 8.970125 & -0.436512 & 1.343502 \\
\hline I & -5.044515 & -5.470076 & -1.796204 \\
\hline & -0.803939 & -4.552046 & 6.191538 \\
\hline 1 & 9.528547 & -2.832483 & 0.943989 \\
\hline & 2.179964 & 2.673311 & 0.191859 \\
\hline & -4.173815 & 0.434164 & 1.559432 \\
\hline & -2.998547 & -0.404665 & -2.461304 \\
\hline & -7.103562 & -0.855599 & -1.288061 \\
\hline 1 & -3.475531 & 2.677704 & -0.454303 \\
\hline & 0.153828 & 1.814744 & 1.646907 \\
\hline & -1.398824 & 5.813630 & 1.606971 \\
\hline & 1.751490 & -3.464121 & 1.552998 \\
\hline & -0.634748 & -1.215450 & -2.138109 \\
\hline & 3.131302 & -3.121308 & 0.511819 \\
\hline & 4.928507 & -2.281000 & 1.703881 \\
\hline 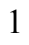 & 0.979180 & -1.037228 & 2.816820 \\
\hline & 5.883451 & -0.877511 & 3.499504 \\
\hline 1 & 1.917735 & 0.331710 & 4.649861 \\
\hline ] & 4.384183 & 0.441036 & 4.985457 \\
\hline 6 & -4.297832 & -1.272230 & -4.375992 \\
\hline 6 & -6.254989 & -2.442896 & -3.415315 \\
\hline 5 & -6.427112 & -0.032965 & -4.063989 \\
\hline 6 & -8.203139 & -0.545020 & 0.910749 \\
\hline 6 & -6.397072 & -1.040881 & 2.530033 \\
\hline 6 & -6.901995 & 1.326148 & 1.885252 \\
\hline & -4.625934 & -1.614689 & -5.365572 \\
\hline 1 & -3.756638 & -0.328558 & -4.521216 \\
\hline 1 & -3.589036 & -2.015723 & -3.983569 \\
\hline
\end{tabular}




$\begin{array}{lrrr}1 & -6.724795 & -0.312083 & -5.083902 \\ 1 & -7.342290 & 0.100691 & -3.473545 \\ 1 & -5.915913 & 0.937281 & -4.114123 \\ 1 & -6.544362 & -2.749132 & -4.429602 \\ 1 & -5.614564 & -3.228424 & -2.992771 \\ 1 & -7.171418 & -2.393420 & -2.814504 \\ 1 & -8.910688 & -0.479126 & 1.746932 \\ 1 & -8.583086 & 0.099593 & 0.107680 \\ 1 & -8.212351 & -1.583090 & 0.552682 \\ 1 & -7.626924 & 1.400142 & 2.707146 \\ 1 & -5.937351 & 1.693478 & 2.257706 \\ 1 & -7.229587 & 2.001420 & 1.084305 \\ 1 & -7.123583 & -0.976951 & 3.351389 \\ 1 & -6.357916 & -2.087507 & 2.199520 \\ 1 & -5.411702 & -0.783088 & 2.939225 \\ 6 & -4.666797 & 4.804022 & -0.820344 \\ 6 & -4.229134 & 6.063815 & 1.266755 \\ 6 & -2.852662 & 6.490401 & -0.782391 \\ 6 & 1.463820 & 3.195804 & 3.215967 \\ 6 & 1.534029 & 5.309151 & 1.897550 \\ 6 & 0.030482 & 5.093825 & 3.882680 \\ 1 & -4.963057 & 6.816499 & 0.948643 \\ 1 & -4.755192 & 5.313220 & 1.871535 \\ 1 & -3.500959 & 6.565418 & 1.915485 \\ 1 & -5.379136 & 5.584343 & -1.116534 \\ 1 & -4.265546 & 4.358597 & -1.739678 \\ 1 & -5.230386 & 4.029632 & -0.283374 \\ 1 & -3.573473 & 7.236110 & -1.144158 \\ 1 & -2.090368 & 7.021385 & -0.199579 \\ 1 & -2.355700 & 6.045976 & -1.654525 \\ 1 & 2.218248 & 3.577102 & 3.915961 \\ 1 & 0.841170 & 2.469329 & 3.757701 \\ 1 & 2.002399 & 2.653724 & 2.427551 \\ 1 & 0.833588 & 5.429308 & 4.552297 \\ 1 & -0.542720 & 5.981342 & 3.590180 \\ 1 & -0.636460 & 4.438274 & 4.457735 \\ 1 & 2.365176 & 5.650649 & 2.529293 \\ 1 & 1.960757 & 4.814705 & 1.014305 \\ 1 & 0.989746 & 6.195817 & 1.549523\end{array}$

$1_{\mathrm{C} 7} \boldsymbol{\Psi}$-112a3s4a
-

Number of imaginary frequencies : 0

The smallest frequencies are : $12.0444 \quad 16.2543 \quad 19.4482 \mathrm{~cm}(-1)$

Electronic energy : $\quad H F=-4125.4656638$

Zero-point correction $=\quad 1.299286($ Hartree/Particle $)$

Thermal correction to Energy= $\quad 1.377860$

Thermal correction to Enthalpy $=\quad 1.378804$

Thermal correction to Gibbs Free Energy= 1.185796

Sum of electronic and zero-point Energies $=\quad-4124.166378$

Sum of electronic and thermal Energies $=\quad-4124.087804$

Sum of electronic and thermal Enthalpies $=\quad-4124.086860$

Sum of electronic and thermal Free Energies $=\quad-4124.279868$

Cartesian Coordinates 


\begin{tabular}{|c|c|c|c|}
\hline 6 & 3.917240 & -0.146389 & 4.196164 \\
\hline 6 & 4.753043 & -0.898244 & 3.377404 \\
\hline 6 & 4.211982 & -1.704263 & 2.378814 \\
\hline 6 & 2.834302 & -1.758462 & 2.179105 \\
\hline 6 & 2.001907 & -1.001907 & 3.005090 \\
\hline 6 & 2.537001 & -0.206886 & 4.009683 \\
\hline 6 & 2.258094 & -2.643885 & 1.106085 \\
\hline 7 & 1.295738 & -1.982121 & 0.191582 \\
\hline 6 & 0.200659 & -2.801483 & -0.250811 \\
\hline 6 & -1.051043 & -2.679491 & 0.324222 \\
\hline 6 & -2.140795 & -3.441728 & -0.203385 \\
\hline 6 & -1.909146 & -4.353021 & -1.276344 \\
\hline 6 & -0.602688 & -4.477826 & -1.804613 \\
\hline 6 & 0.419495 & -3.714184 & -1.309646 \\
\hline 6 & -3.461018 & -3.320371 & 0.302665 \\
\hline 6 & -4.489162 & -4.065108 & -0.220403 \\
\hline 6 & -4.248924 & -4.989602 & -1.259989 \\
\hline 6 & -2.985458 & -5.125592 & -1.777055 \\
\hline 6 & -1.259902 & -1.879311 & 1.563594 \\
\hline 6 & -1.543794 & -0.521363 & 1.568659 \\
\hline 6 & -1.799237 & 0.126186 & 2.810140 \\
\hline 6 & -1.749977 & -0.550202 & 3.998307 \\
\hline 6 & -1.438299 & -1.928125 & 4.030730 \\
\hline 6 & -1.198973 & -2.606062 & 2.800391 \\
\hline 6 & -1.352452 & -2.637902 & 5.251985 \\
\hline 6 & -1.039532 & -3.972806 & 5.267949 \\
\hline 6 & -0.803381 & -4.652231 & 4.054785 \\
\hline 6 & -0.884395 & -3.990093 & 2.854586 \\
\hline 15 & -1.684368 & 0.439622 & -0.019541 \\
\hline 45 & -0.153140 & 0.320318 & -1.933690 \\
\hline 6 & 0.299995 & 2.227962 & -1.889974 \\
\hline 8 & 0.452080 & 3.367266 & -1.965984 \\
\hline 15 & 1.661648 & -0.714704 & -0.880150 \\
\hline 8 & 2.646901 & 0.128477 & 0.147283 \\
\hline 6 & 3.510770 & 1.048348 & -0.398903 \\
\hline 6 & 4.689707 & 0.622168 & -0.980043 \\
\hline 6 & 5.538429 & 1.608699 & -1.581027 \\
\hline 6 & 5.187711 & 2.989721 & -1.480300 \\
\hline 6 & 3.990434 & 3.361187 & -0.823476 \\
\hline 6 & 3.156624 & 2.407721 & -0.306680 \\
\hline 6 & 6.028516 & 3.968102 & -2.064479 \\
\hline 6 & 7.161136 & 3.608783 & -2.748065 \\
\hline 6 & 7.490965 & 2.244105 & -2.883455 \\
\hline 6 & 6.703431 & 1.272651 & -2.317161 \\
\hline 6 & 4.994612 & -0.825942 & -1.027217 \\
\hline 6 & 4.065114 & -1.702969 & -1.559200 \\
\hline 6 & 4.360003 & -3.068246 & -1.767132 \\
\hline 6 & 5.557761 & -3.581526 & -1.352327 \\
\hline 6 & 6.495212 & -2.760896 & -0.678241 \\
\hline 6 & 6.212460 & -1.372798 & -0.505025 \\
\hline 6 & 7.128031 & -0.596957 & 0.250775 \\
\hline 6 & 8.265793 & -1.156820 & 0.778410 \\
\hline 6 & 8.559746 & -2.520855 & 0.572204 \\
\hline 6 & 7.687809 & -3.303129 & -0.141061 \\
\hline 8 & 2.826125 & -1.265484 & -1.961703 \\
\hline 6 & -3.447052 & 0.121691 & -0.428788 \\
\hline
\end{tabular}




\begin{tabular}{|c|c|c|c|}
\hline 6 & -4.453557 & 0.197529 & 0.543502 \\
\hline 6 & -5.763800 & -0.151429 & 0.237660 \\
\hline ) & -6.040018 & -0.594452 & -1.068149 \\
\hline & -5.066161 & -0.665951 & -2.059078 \\
\hline 0 & -3.762583 & -0.285399 & -1.715045 \\
\hline & -6.887964 & -0.096557 & 1.271184 \\
\hline 0 & -5.344017 & -1.138438 & -3.483424 \\
\hline & -1.643367 & 2.172521 & 0.576926 \\
\hline 6 & -2.593409 & 3.101277 & 0.159339 \\
\hline 6 & -2.469362 & 4.451152 & 0.501221 \\
\hline 6 & -1.385383 & 4.827020 & 1.297702 \\
\hline 6 & -0.425506 & 3.915506 & 1.751694 \\
\hline 6 & -0.552456 & 2.587640 & 1.346672 \\
\hline 6 & -3.466439 & 5.507996 & 0.028700 \\
\hline 6 & 0.715650 & 4.387420 & 2.652140 \\
\hline 6 & -0.619906 & 0.312936 & -3.777454 \\
\hline 8 & -0.900078 & 0.241893 & -4.898887 \\
\hline 1 & 6.906241 & 0.451597 & 0.433448 \\
\hline 1 & -2.040623 & 1.185717 & 2.819257 \\
\hline 1 & 7.887981 & -4.362820 & -0.294676 \\
\hline 1 & -1.537367 & -2.097016 & 6.179043 \\
\hline 1 & 5.744701 & 5.015243 & -1.967516 \\
\hline 1 & -3.656716 & -2.610790 & 1.105256 \\
\hline 1 & -5.498163 & -3.942405 & 0.169176 \\
\hline 1 & 3.730402 & 4.417259 & -0.759780 \\
\hline 1 & -1.951625 & -0.032697 & 4.935425 \\
\hline 1 & 5.794999 & -4.632327 & -1.510277 \\
\hline 1 & 8.374711 & 1.956874 & -3.448869 \\
\hline 1 & 3.616207 & -3.685514 & -2.266084 \\
\hline 1 & -2.787246 & -5.825437 & -2.587804 \\
\hline 1 & -0.427776 & -5.181523 & -2.616946 \\
\hline 1 & -0.557148 & -5.711460 & 4.069865 \\
\hline 1 & 6.966724 & 0.225932 & -2.443779 \\
\hline 1 & -0.703922 & -4.532725 & 1.929030 \\
\hline 1 & 1.421728 & -3.785704 & -1.727895 \\
\hline 1 & 7.796397 & 4.368292 & -3.197785 \\
\hline 1 & 8.944375 & -0.542137 & 1.365738 \\
\hline 1 & -5.069767 & -5.583862 & -1.655656 \\
\hline 1 & -0.972387 & -4.509823 & 6.211210 \\
\hline 1 & 9.467717 & -2.949776 & 0.989596 \\
\hline 1 & 2.214476 & 2.664344 & 0.175346 \\
\hline 1 & -4.193868 & 0.523358 & 1.548137 \\
\hline 1 & -2.961570 & -0.329580 & -2.451424 \\
\hline 1 & -7.058808 & -0.896099 & -1.304535 \\
\hline 1 & -3.431198 & 2.762487 & -0.446041 \\
\hline 1 & 0.188048 & 1.846939 & 1.650745 \\
\hline 1 & -1.283049 & 5.875175 & 1.578241 \\
\hline 1 & 1.688904 & -3.454645 & 1.582696 \\
\hline 1 & -0.659551 & -1.193514 & -2.115113 \\
\hline 1 & 3.073531 & -3.124563 & 0.544085 \\
\hline 1 & 4.873506 & -2.283577 & 1.732536 \\
\hline 1 & 0.927208 & -1.023731 & 2.836980 \\
\hline 1 & 5.832955 & -0.856449 & 3.506402 \\
\hline 1 & 1.869910 & 0.369407 & 4.649667 \\
\hline 1 & 4.337200 & 0.484809 & 4.976656 \\
\hline 6 & -6.750512 & -1.712226 & -3.632887 \\
\hline
\end{tabular}




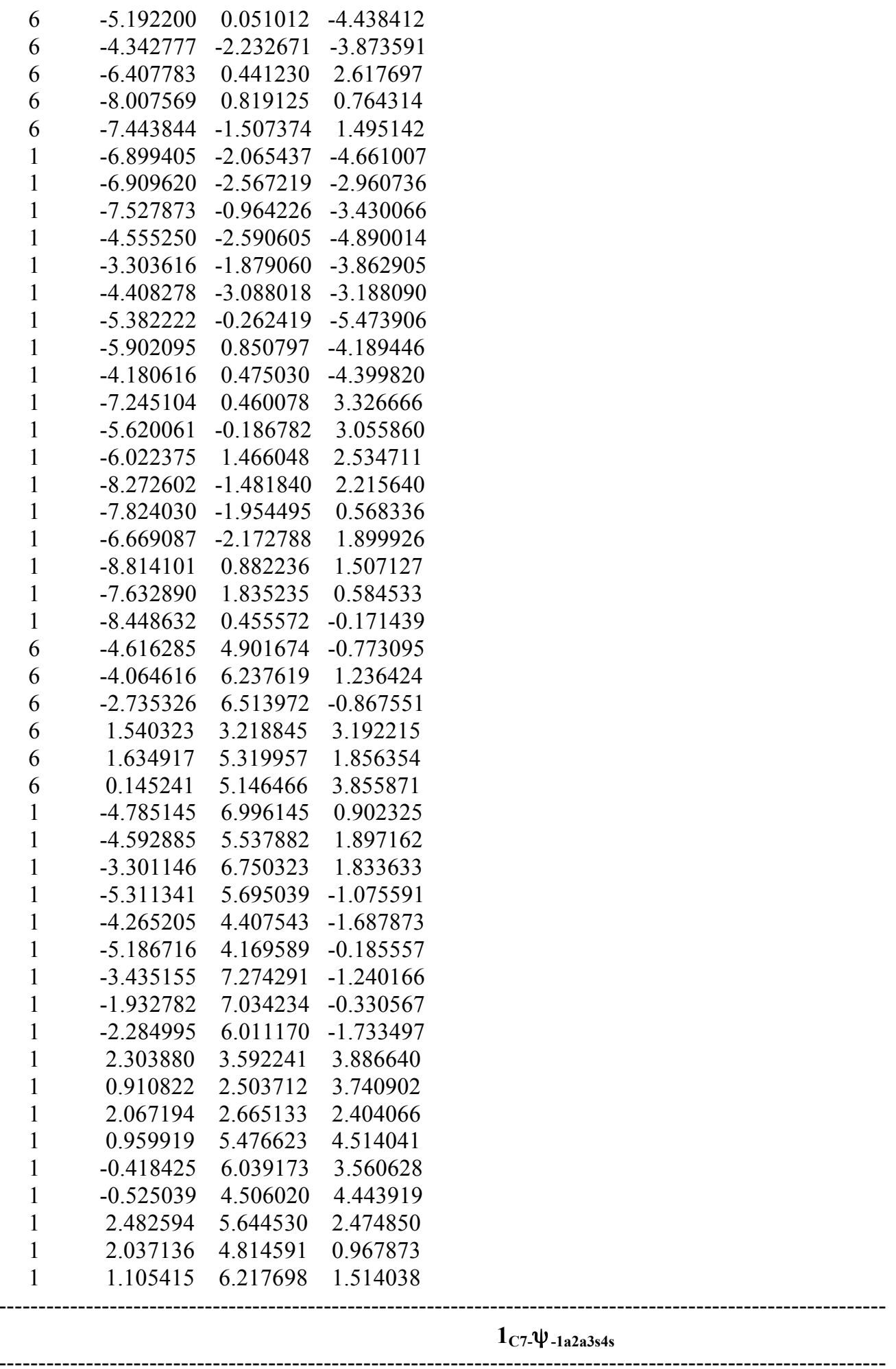

Number of imaginary frequencies : 0

The smallest frequencies are : $17.5662 \quad 18.2316 \quad 20.1964 \mathrm{~cm}(-1)$

Electronic energy : $\quad H F=-4125.4659478$

Zero-point correction $=\quad 1.301456($ Hartree/Particle $)$

Thermal correction to Energy= $\quad 1.379524$

Thermal correction to Enthalpy $=\quad 1.380468$ 
Thermal correction to Gibbs Free Energy= Sum of electronic and zero-point Energies= Sum of electronic and thermal Energies= Sum of electronic and thermal Enthalpies= Sum of electronic and thermal Free Energies=
1.189837

$-4124.164491$

$-4124.086424$

$-4124.085480$

$-4124.276111$

Cartesian Coordinates

\begin{tabular}{cccc}
\hline 6 & 3.942461 & -0.135271 & 4.192120 \\
6 & 4.770892 & -0.907877 & 3.385511 \\
6 & 4.223294 & -1.710054 & 2.387294 \\
6 & 2.846293 & -1.742718 & 2.178163 \\
6 & 2.021449 & -0.964934 & 2.992094 \\
6 & 2.562990 & -0.170375 & 3.993603 \\
6 & 2.265540 & -2.629290 & 1.108141 \\
7 & 1.306785 & -1.970113 & 0.187874 \\
6 & 0.209052 & -2.789158 & -0.247746 \\
6 & -1.038263 & -2.669266 & 0.337079 \\
6 & -2.128026 & -3.443247 & -0.173364 \\
6 & -1.901466 & -4.357087 & -1.245405 \\
6 & -0.600518 & -4.473496 & -1.789149 \\
6 & 0.422626 & -3.703416 & -1.306374 \\
6 & -3.441984 & -3.334036 & 0.350733 \\
6 & -4.469741 & -4.089671 & -0.157316 \\
6 & -4.235641 & -5.013000 & -1.199081 \\
6 & -2.977128 & -5.140404 & -1.730515 \\
6 & -1.240302 & -1.855816 & 1.568820 \\
6 & -1.526613 & -0.498011 & 1.558060 \\
6 & -1.773389 & 0.164502 & 2.793126 \\
6 & -1.713526 & -0.496842 & 3.989544 \\
6 & -1.399174 & -1.873566 & 4.037371 \\
6 & -1.168756 & -2.566637 & 2.813871 \\
6 & -1.300244 & -2.567244 & 5.266881 \\
6 & -0.983447 & -3.900954 & 5.297108 \\
6 & -0.757011 & -4.595598 & 4.090632 \\
6 & -0.850859 & -3.949314 & 2.882845 \\
15 & -1.677567 & 0.440595 & -0.044132 \\
45 & -0.134365 & 0.329070 & -1.941376 \\
6 & 0.329428 & 2.233796 & -1.899453 \\
8 & 0.487763 & 3.371975 & -1.978283 \\
15 & 1.676589 & -0.705565 & -0.884730 \\
8 & 2.654231 & 0.140078 & 0.147350 \\
6 & 3.521174 & 1.061047 & -0.391441 \\
6 & 4.703281 & 0.636837 & -0.967857 \\
6 & 5.555075 & 1.626201 & -1.559888 \\
6 & 5.205577 & 3.007060 & -1.452310 \\
6 & 4.005734 & 3.376112 & -0.798758 \\
6 & 3.168063 & 2.420204 & -0.293101 \\
6 & 6.050347 & 3.987924 & -2.026529 \\
6 & 7.185529 & 3.631369 & -2.707306 \\
6 & 7.514039 & 2.267110 & -2.849962 \\
6 & 6.722785 & 1.293229 & -2.293105 \\
6 & 5.010993 & -0.810832 & -1.014696 \\
6 & 4.085020 & -1.690221 & -1.548848 \\
6 & 4.381473 & -3.055783 & -1.752300 \\
6 & 5.578285 & -3.566966 & -1.332411
\end{tabular}




\begin{tabular}{|c|c|c|c|}
\hline & 6.512683 & -2.743724 & -0.657263 \\
\hline & 6.227573 & -1.355704 & -0.486889 \\
\hline & 7.139907 & -0.577950 & 0.270783 \\
\hline & 8.276967 & -1.135613 & 0.802278 \\
\hline & 8.573368 & -2.499471 & 0.598721 \\
\hline & 7.704346 & -3.283784 & -0.115852 \\
\hline & 2.847415 & -1.255314 & -1.958727 \\
\hline & -3.429925 & 0.075256 & -0.456249 \\
\hline & -4.435557 & 0.139140 & 0.509355 \\
\hline & -5.742447 & -0.244060 & 0.211028 \\
\hline & -6.012821 & -0.698064 & -1.086774 \\
\hline & -5.031161 & -0.760994 & -2.078401 \\
\hline & -3.738022 & -0.353951 & -1.740850 \\
\hline & -6.804999 & -0.170561 & 1.307135 \\
\hline & -5.306494 & -1.251196 & -3.497764 \\
\hline & -1.688286 & 2.181566 & 0.529172 \\
\hline & -2.672689 & 3.074322 & 0.111921 \\
\hline & -2.594439 & 4.429367 & 0.446480 \\
\hline & -1.520743 & 4.847180 & 1.236002 \\
\hline & -0.524242 & 3.973368 & 1.685065 \\
\hline & -0.606664 & 2.639674 & 1.287301 \\
\hline & -3.651408 & 5.441039 & 0.006336 \\
\hline & 0.612539 & 4.490089 & 2.565817 \\
\hline & -0.630540 & 0.335307 & -3.777334 \\
\hline & -0.934613 & 0.277359 & -4.893214 \\
\hline & 6.916207 & 0.470393 & 0.452080 \\
\hline & -2.018486 & 1.223135 & 2.790944 \\
\hline ] & 7.906177 & -4.343451 & -0.267448 \\
\hline & -1.477975 & -2.015029 & 6.188634 \\
\hline ] & 5.767474 & 5.034815 & -1.924251 \\
\hline & -3.634087 & -2.625224 & 1.155288 \\
\hline ] & -5.474698 & -3.976079 & 0.245377 \\
\hline & 3.747363 & 4.432089 & -0.727841 \\
\hline & -1.908667 & 0.032300 & 4.921559 \\
\hline l & 5.817139 & -4.617879 & -1.487146 \\
\hline & 8.399747 & 1.982203 & -3.413473 \\
\hline l & 3.639425 & -3.674686 & -2.251940 \\
\hline & -2.783076 & -5.840489 & -2.542088 \\
\hline 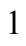 & -0.430379 & -5.177831 & -2.601935 \\
\hline . & -0.508173 & -5.654001 & 4.117165 \\
\hline . & 6.985028 & 0.246927 & -2.425270 \\
\hline d & -0.678371 & -4.503320 & 1.962495 \\
\hline & 1.421238 & -3.770404 & -1.734184 \\
\hline 1 & 7.823820 & 4.392815 & -3.149388 \\
\hline & 8.952940 & -0.519248 & 1.390850 \\
\hline 1 & -5.056652 & -5.614118 & -1.583956 \\
\hline 1 & -0.905642 & -4.425460 & 6.246564 \\
\hline 1 & 9.480768 & -2.926651 & 1.019133 \\
\hline 1 & 2.223087 & 2.674433 & 0.184338 \\
\hline & -4.188170 & 0.480581 & 1.514561 \\
\hline 1 & -2.934205 & -0.387992 & -2.474326 \\
\hline & -7.021367 & -1.021975 & -1.327059 \\
\hline 1 & -3.502002 & 2.703390 & -0.485991 \\
\hline & 0.162753 & 1.927133 & 1.587748 \\
\hline 1 & -1.458805 & 5.898178 & 1.517624 \\
\hline 1 & 1.693144 & -3.436029 & 1.587951 \\
\hline
\end{tabular}




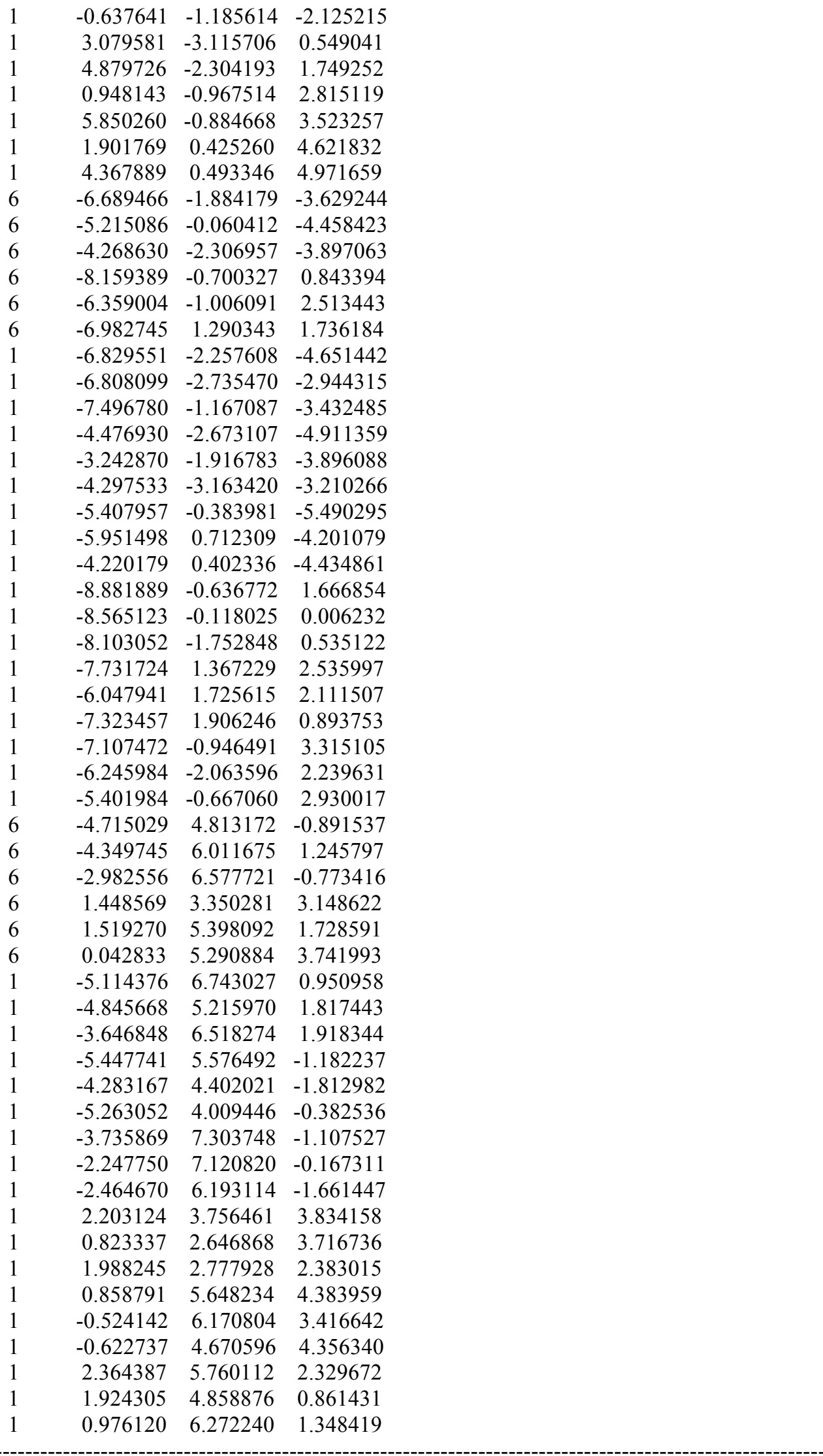


$\mathbf{1}_{\mathrm{C} 7-} \psi_{-1 \mathbf{a} 233 a 4 a}$

Number of imaginary frequencies : 0

The smallest frequencies are : $15.0027 \quad 20.0255 \quad 21.4319 \mathrm{~cm}(-1)$

Electronic energy : $\quad \mathrm{HF}=-4125.4660404$

Zero-point correction $=\quad 1.298960($ Hartree/Particle $)$

Thermal correction to Energy= $\quad 1.377692$

Thermal correction to Enthalpy= $\quad 1.378636$

Thermal correction to Gibbs Free Energy= $\quad 1.185328$

Sum of electronic and zero-point Energies $=\quad-4124.167080$

Sum of electronic and thermal Energies $=\quad-4124.088348$

Sum of electronic and thermal Enthalpies $=\quad-4124.087404$

Sum of electronic and thermal Free Energies $=\quad-4124.280713$

Cartesian Coordinates

\begin{tabular}{cccc}
\hline 6 & 3.974992 & -0.117752 & 4.189662 \\
6 & 4.807498 & -0.887932 & 3.385042 \\
6 & 4.264529 & -1.692365 & 2.386004 \\
6 & 2.888048 & -1.729791 & 2.174121 \\
6 & 2.059223 & -0.952641 & 2.984752 \\
6 & 2.596154 & -0.156386 & 3.987338 \\
6 & 2.312525 & -2.622158 & 1.105996 \\
7 & 1.346367 & -1.972518 & 0.187953 \\
6 & 0.252995 & -2.796494 & -0.248430 \\
6 & -0.993860 & -2.684545 & 0.339199 \\
6 & -2.086994 & -3.440899 & -0.187568 \\
6 & -1.866228 & -4.322685 & -1.287373 \\
6 & -0.563805 & -4.442293 & -1.826151 \\
6 & 0.464676 & -3.693757 & -1.321359 \\
6 & -3.401635 & -3.333415 & 0.336505 \\
6 & -4.435834 & -4.058163 & -0.200743 \\
6 & -4.210121 & -4.941776 & -1.278970 \\
6 & -2.951696 & -5.067950 & -1.810017 \\
6 & -1.195453 & -1.880854 & 1.576808 \\
6 & -1.498941 & -0.527213 & 1.568671 \\
6 & -1.742988 & 0.132502 & 2.805836 \\
6 & -1.660223 & -0.527878 & 4.001430 \\
6 & -1.329757 & -1.901143 & 4.045651 \\
6 & -1.105986 & -2.592086 & 2.819676 \\
6 & -1.210000 & -2.594417 & 5.273450 \\
6 & -0.881300 & -3.925460 & 5.299112 \\
6 & -0.664106 & -4.618277 & 4.089866 \\
6 & -0.777055 & -3.972200 & 2.883521 \\
15 & -1.676196 & 0.402146 & -0.035728 \\
45 & -0.134205 & 0.311767 & -1.942988 \\
6 & 0.294798 & 2.225365 & -1.889556 \\
8 & 0.437416 & 3.365717 & -1.967419 \\
15 & 1.693525 & -0.701278 & -0.884806 \\
8 & 2.667623 & 0.154716 & 0.142458 \\
6 & 3.523538 & 1.083260 & -0.400615 \\
6 & 4.708193 & 0.669178 & -0.978985 \\
6 & 5.549825 & 1.665243 & -1.574091 \\
6 & 5.186924 & 3.042846 & -1.469263 \\
6 & 3.984212 & 3.401704 & -0.815235 \\
6 & 3.156830 & 2.439025 & -0.305361
\end{tabular}




\begin{tabular}{|c|c|c|c|}
\hline 6 & 6.021446 & 4.030546 & -2.046750 \\
\hline 6 & 7.159381 & 3.683557 & -2.727902 \\
\hline & 7.501164 & 2.322262 & -2.867628 \\
\hline & 6.720005 & 1.341987 & -2.307700 \\
\hline & 5.027126 & -0.776071 & -1.026224 \\
\hline & 4.106995 & -1.661725 & -1.560244 \\
\hline & 4.413567 & -3.024802 & -1.765314 \\
\hline & 5.614145 & -3.527701 & -1.346052 \\
\hline & 6.542605 & -2.698300 & -0.670236 \\
\hline & 6.247819 & -1.312342 & -0.499492 \\
\hline & 7.154861 & -0.528110 & 0.257831 \\
\hline & 8.295582 & -1.077922 & 0.789627 \\
\hline & 8.601093 & -2.439872 & 0.586563 \\
\hline f & 7.737759 & -3.230161 & -0.128365 \\
\hline & 2.865190 & -1.235667 & -1.966545 \\
\hline & -3.438763 & 0.040934 & -0.413910 \\
\hline b & -4.433924 & 0.224046 & 0.551964 \\
\hline & -5.757837 & -0.112326 & 0.283052 \\
\hline 6 & -6.051259 & -0.648280 & -0.979068 \\
\hline & -5.083181 & -0.847271 & -1.963164 \\
\hline & -3.767203 & -0.481072 & -1.659127 \\
\hline ) & -6.866370 & 0.037873 & 1.323799 \\
\hline 6 & -5.459297 & -1.397526 & -3.338298 \\
\hline 5 & -1.681387 & 2.145034 & 0.531482 \\
\hline ) & -2.649165 & 3.038686 & 0.096543 \\
\hline 6 & -2.576095 & 4.399412 & 0.426189 \\
\hline 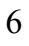 & -1.515267 & 4.821783 & 1.222028 \\
\hline & -0.523076 & 3.941764 & 1.685733 \\
\hline ) & -0.604590 & 2.607937 & 1.301073 \\
\hline 6 & -3.663075 & 5.340149 & -0.087746 \\
\hline ) & -3.661720 & 5.320331 & -1.620589 \\
\hline 6 & 0.606000 & 4.464713 & 2.572426 \\
\hline 6 & 1.453430 & 3.330988 & 3.150011 \\
\hline 6 & -0.558436 & 0.312002 & -3.795803 \\
\hline 5 & -0.797407 & 0.251977 & -4.927261 \\
\hline ) & 1.505539 & 5.387980 & 1.743902 \\
\hline 6 & 0.024024 & 5.251567 & 3.752153 \\
\hline & -3.452844 & 6.780640 & 0.370540 \\
\hline 6 & -5.027496 & 4.867913 & 0.429135 \\
\hline 1 & 6.923774 & 0.518674 & 0.438955 \\
\hline 1 & -2.001103 & 1.188089 & 2.806797 \\
\hline 1 & 7.946929 & -4.288431 & -0.279813 \\
\hline . & -1.381884 & -2.044471 & 6.197671 \\
\hline 1 & 5.728397 & 5.074848 & -1.946627 \\
\hline & -3.590467 & -2.649120 & 1.162130 \\
\hline 1 & -5.440305 & -3.950812 & 0.204621 \\
\hline l & 3.715488 & 4.455283 & -0.747046 \\
\hline 1 & -1.850754 & -0.001025 & 4.935703 \\
\hline 1 & 5.860493 & -4.576769 & -1.501592 \\
\hline 1 & 8.389121 & 2.044707 & -3.431277 \\
\hline 1 & 3.675952 & -3.648618 & -2.265372 \\
\hline 1 & -2.763445 & -5.741717 & -2.644858 \\
\hline 1 & -0.397686 & -5.127662 & -2.655792 \\
\hline 1 & -0.407265 & -5.674839 & 4.113254 \\
\hline 1 & 6.992356 & 0.297987 & -2.437615 \\
\hline 1 & -0.611923 & -4.523787 & 1.960308 \\
\hline
\end{tabular}




\begin{tabular}{|c|c|c|c|}
\hline & 1.463988 & -3.759221 & -1.747614 \\
\hline & 7.789690 & 4.450187 & -3.172478 \\
\hline ] & 8.967313 & -0.456901 & 1.378169 \\
\hline | & -5.037132 & -5.519323 & -1.687101 \\
\hline & -0.787576 & -4.449459 & 6.247421 \\
\hline & 9.511149 & -2.860884 & 1.007468 \\
\hline & 2.210198 & 2.684935 & 0.173299 \\
\hline ] & -4.160498 & 0.632667 & 1.522061 \\
\hline & -2.967269 & -0.614250 & -2.384359 \\
\hline 1 & -7.082768 & -0.924500 & -1.195495 \\
\hline 1 & -3.472419 & 2.674176 & -0.516770 \\
\hline & 0.158267 & 1.895321 & 1.617101 \\
\hline & -1.447247 & 5.871233 & 1.499862 \\
\hline ] & 1.748257 & -3.433442 & 1.588049 \\
\hline & -0.624971 & -1.205011 & -2.141820 \\
\hline 1 & 2.347282 & 5.751199 & 2.348948 \\
\hline & 1.915354 & 4.859974 & 0.872085 \\
\hline 1 & 0.956465 & 6.261774 & 1.371774 \\
\hline & 0.834032 & 5.614026 & 4.398863 \\
\hline ] & -0.552236 & 6.126713 & 3.430511 \\
\hline & -0.636022 & 4.619573 & 4.360569 \\
\hline & 0.836196 & 2.620927 & 3.718712 \\
\hline 1 & 1.995088 & 2.765106 & 2.380976 \\
\hline & 2.206532 & 3.742014 & 3.834290 \\
\hline 1 & -4.259753 & 7.413003 & -0.020669 \\
\hline 1 & -3.466340 & 6.870428 & 1.464541 \\
\hline 1 & -2.504824 & 7.193362 & 0.002236 \\
\hline 1 & -5.260704 & 3.844584 & 0.107962 \\
\hline & -5.059939 & 4.889156 & 1.526609 \\
\hline 1 & -5.825026 & 5.523545 & 0.054393 \\
\hline 1 & -2.687341 & 5.636309 & -2.015390 \\
\hline 1 & -3.874825 & 4.321909 & -2.020499 \\
\hline 1 & -4.426237 & 6.005043 & -2.011876 \\
\hline 1 & 3.129514 & -3.102139 & 0.545663 \\
\hline 1 & 4.924269 & -2.284697 & 1.749615 \\
\hline 1 & 0.986517 & -0.956168 & 2.803865 \\
\hline 1 & 5.886474 & -0.861268 & 3.525137 \\
\hline 1 & 1.931729 & 0.438021 & 4.613364 \\
\hline 1 & 4.396718 & 0.512203 & 4.970137 \\
\hline 6 & -4.239472 & -1.943896 & -4.082177 \\
\hline 6 & -6.479522 & -2.532876 & -3.217438 \\
\hline 6 & -6.069533 & -0.258763 & -4.163523 \\
\hline 6 & -6.374779 & 0.730369 & 2.593429 \\
\hline 5 & -8.021634 & 0.864286 & 0.749783 \\
\hline ) & -7.377342 & -1.355661 & 1.707475 \\
\hline 1 & -4.558308 & -2.397690 & -5.029110 \\
\hline 1 & -3.514158 & -1.159768 & -4.332413 \\
\hline 1 & -3.720697 & -2.715245 & -3.495007 \\
\hline 1 & -6.348334 & -0.614244 & -5.165030 \\
\hline 1 & -6.970886 & 0.145443 & -3.684582 \\
\hline 1 & -5.354692 & 0.565823 & -4.283776 \\
\hline 1 & -6.693750 & -2.948178 & -4.210753 \\
\hline 1 & -6.091478 & -3.344385 & -2.587370 \\
\hline 1 & -7.436406 & -2.201115 & -2.797354 \\
\hline 1 & -7.206065 & 0.834570 & 3.301959 \\
\hline 1 & -5.585128 & 0.157688 & 3.098039 \\
\hline
\end{tabular}




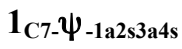

Number of imaginary frequencies : 0

The smallest frequencies are : $15.8467 \quad 21.004122 .9424 \mathrm{~cm}(-1)$

Electronic energy : $\quad H F=-4125.4662125$

Zero-point correction $=\quad 1.299541($ Hartree/Particle)

Thermal correction to Energy= $\quad 1.377952$

Thermal correction to Enthalpy $=\quad 1.378896$

Thermal correction to Gibbs Free Energy $=\quad 1.187186$

Sum of electronic and zero-point Energies $=\quad-4124.166672$

Sum of electronic and thermal Energies $=\quad-4124.088261$

Sum of electronic and thermal Enthalpies $=\quad-4124.087316$

Sum of electronic and thermal Free Energies $=\quad-4124.279026$

Cartesian Coordinates

$\begin{array}{cccc}6 & 3.978541 & -0.148010 & 4.166782 \\ 6 & 4.815820 & -0.904302 & 3.353966 \\ 6 & 4.277223 & -1.709201 & 2.352929 \\ 6 & 2.900378 & -1.759985 & 2.146918 \\ 6 & 2.066515 & -0.998512 & 2.967243 \\ 6 & 2.599133 & -0.201864 & 3.971716 \\ 6 & 2.328915 & -2.643876 & 1.069743 \\ 7 & 1.364679 & -1.984941 & 0.156468 \\ 6 & 0.274577 & -2.804476 & -0.296060 \\ 6 & -0.971762 & -2.710288 & 0.295644 \\ 6 & -2.064823 & -3.452876 & -0.249042 \\ 6 & -1.845698 & -4.302778 & -1.373680 \\ 6 & -0.543237 & -4.409028 & -1.915196 \\ 6 & 0.485948 & -3.674472 & -1.391175 \\ 6 & -3.378869 & -3.357105 & 0.278808 \\ 6 & -4.414659 & -4.063860 & -0.278485 \\ 6 & -4.190374 & -4.917665 & -1.380799 \\ 6 & -2.932841 & -5.030707 & -1.916572 \\ 6 & -1.177158 & -1.933934 & 1.549264 \\ 6 & -1.491109 & -0.582953 & 1.564760 \\ 6 & -1.741560 & 0.052589 & 2.813175 \\ 6 & -1.660459 & -0.630515 & 3.996409 \\ 6 & -1.322486 & -2.002496 & 4.015964 \\ 6 & -1.087585 & -2.668082 & 2.778200 \\ 6 & -1.206259 & -2.719234 & 5.230587 \\ 6 & -0.870219 & -4.048677 & 5.231921 \\ 6 & -0.641410 & -4.716263 & 4.010569 \\ 6 & -0.750666 & -4.047082 & 2.816543 \\ 15 & -1.673440 & 0.369635 & -0.024773 \\ 45 & -0.129946 & 0.330483 & -1.931362 \\ 6 & 0.279514 & 2.247191 & -1.841178 \\ 8 & 0.408615 & 3.390494 & -1.896788\end{array}$




\begin{tabular}{|c|c|c|c|}
\hline & 1.704418 & -0.691017 & -0.891336 \\
\hline 8 & 2.672669 & 0.153511 & 0.150961 \\
\hline 6 & 3.519009 & 1.099731 & -0.375988 \\
\hline 6 & 4.705900 & 0.707443 & -0.965008 \\
\hline 6 & 5.535668 & 1.722134 & -1.545113 \\
\hline 6 & 5.160010 & 3.094097 & -1.414320 \\
\hline 6 & 3.956522 & 3.429660 & -0.749391 \\
\hline 6 & 3.140004 & 2.450009 & -0.254573 \\
\hline 6 & 5.982671 & 4.099844 & -1.977554 \\
\hline 6 & 7.120921 & 3.775982 & -2.669519 \\
\hline 6 & 7.475066 & 2.420729 & -2.834882 \\
\hline 6 & 6.705737 & 1.423255 & -2.289092 \\
\hline 6 & 5.038723 & -0.733766 & -1.036611 \\
\hline 6 & 4.125080 & -1.619621 & -1.581332 \\
\hline 6 & 4.441394 & -2.977656 & -1.803652 \\
\hline 6 & 5.647235 & -3.476202 & -1.394422 \\
\hline 6 & 6.571506 & -2.647772 & -0.711757 \\
\hline 6 & 6.265624 & -1.266810 & -0.521041 \\
\hline 6 & 7.169116 & -0.485323 & 0.243367 \\
\hline 6 & 8.317060 & -1.032371 & 0.762376 \\
\hline 6 & 8.633593 & -2.388602 & 0.539034 \\
\hline 6 & 7.773687 & -3.176550 & -0.182546 \\
\hline 8 & 2.880076 & -1.197521 & -1.981791 \\
\hline 6 & -3.432483 & 0.004609 & -0.416601 \\
\hline 6 & -4.418264 & 0.116976 & 0.559865 \\
\hline 6 & -5.751408 & -0.191846 & 0.278740 \\
\hline 6 & -6.060388 & -0.614280 & -1.015636 \\
\hline 6 & -5.092972 & -0.740507 & -2.021078 \\
\hline 6 & -3.773987 & -0.420922 & -1.699594 \\
\hline 6 & -6.786348 & -0.081469 & 1.396742 \\
\hline 6 & -5.497550 & -1.192533 & -3.423081 \\
\hline 6 & -1.705519 & 2.102898 & 0.571261 \\
\hline 6 & -2.689977 & 2.986796 & 0.153824 \\
\hline 6 & -2.636648 & 4.343670 & 0.501999 \\
\hline 6 & -1.580667 & 4.770539 & 1.302155 \\
\hline 6 & -0.574085 & 3.899347 & 1.751470 \\
\hline 6 & -0.634535 & 2.570549 & 1.345749 \\
\hline 6 & -3.728162 & 5.279294 & -0.012065 \\
\hline 6 & -3.654916 & 5.331312 & -1.542642 \\
\hline 6 & 0.548995 & 4.426563 & 2.643395 \\
\hline 6 & 1.409452 & 3.297053 & 3.210123 \\
\hline 6 & -0.536923 & 0.355686 & -3.788015 \\
\hline 8 & -0.758974 & 0.309029 & -4.923491 \\
\hline 6 & 1.438304 & 5.367211 & 1.823368 \\
\hline 6 & -0.040920 & 5.196203 & 3.830472 \\
\hline 6 & -3.577838 & 6.700643 & 0.523080 \\
\hline 6 & -5.102766 & 4.750538 & 0.414516 \\
\hline 1 & 6.930042 & 0.556935 & 0.439622 \\
\hline 1 & -2.005218 & 1.106678 & 2.832744 \\
\hline 1 & 7.991186 & -4.230826 & -0.349380 \\
\hline 1 & -1.386782 & -2.188557 & 6.164376 \\
\hline 1 & 5.679985 & 5.139302 & -1.857673 \\
\hline 1 & -3.566155 & -2.692946 & 1.121670 \\
\hline 1 & -5.420272 & -3.957926 & 0.125081 \\
\hline 1 & 3.677872 & 4.479162 & -0.661121 \\
\hline 1 & -1.857510 & -0.122583 & 4.939745 \\
\hline
\end{tabular}




\begin{tabular}{|c|c|c|}
\hline 5.901404 & -4.521266 & -1.563736 \\
\hline 8.363051 & 2.161698 & -3.407225 \\
\hline 3.706916 & -3.600982 & -2.308943 \\
\hline-2.746644 & -5.680883 & -2.770314 \\
\hline-0.377644 & -5.071710 & -2.763177 \\
\hline-0.378551 & -5.771581 & 4.014822 \\
\hline 6.987085 & 0.384389 & -2.439131 \\
\hline-0.576925 & -4.579008 & 1.883421 \\
\hline 1.484935 & -3.728161 & -1.819847 \\
\hline 7.741835 & 4.556398 & -3.103190 \\
\hline 8.986105 & -0.413582 & 1.356305 \\
\hline-5.018743 & -5.481678 & -1.804694 \\
\hline-0.779627 & -4.590859 & 6.170257 \\
\hline 9.549293 & -2.807339 & 0.949844 \\
\hline 2.192465 & 2.677980 & 0.231054 \\
\hline-4.142485 & 0.444449 & 1.562106 \\
\hline-2.978429 & -0.507961 & -2.436381 \\
\hline-7.092903 & -0.858477 & -1.256857 \\
\hline-3.509186 & 2.618320 & -0.462577 \\
\hline 0.140250 & 1.864889 & 1.648608 \\
\hline-1.528102 & 5.816761 & 1.595110 \\
\hline 1.763738 & -3.460193 & 1.542150 \\
\hline-0.612815 & -1.184514 & -2.157853 \\
\hline 2.273808 & 5.737208 & 2.432987 \\
\hline 1.857284 & 4.850541 & 0.949097 \\
\hline 0.878597 & 6.236176 & 1.455781 \\
\hline 0.765461 & 5.560524 & 4.480636 \\
\hline-0.625689 & 6.068745 & 3.517264 \\
\hline-0.694676 & 4.552035 & 4.432866 \\
\hline 0.800389 & 2.572990 & 3.769891 \\
\hline 1.959736 & 2.746533 & 2.436064 \\
\hline 2.156300 & 3.710020 & 3.900071 \\
\hline-4.391063 & 7.327694 & 0.136422 \\
\hline-3.628396 & 6.733788 & 1.619281 \\
\hline-2.632632 & 7.160465 & 0.207337 \\
\hline-5.301260 & 3.745790 & 0.020219 \\
\hline-5.185355 & 4.703144 & 1.508526 \\
\hline-5.896009 & 5.413468 & 0.043688 \\
\hline-2.673729 & 5.694229 & -1.875442 \\
\hline-3.815529 & 4.345124 & -1.994651 \\
\hline-4.422190 & 6.009768 & -1.939698 \\
\hline 3.147523 & -3.117331 & 0.506044 \\
\hline 4.940575 & -2.289408 & 1.709155 \\
\hline 0.993042 & -1.013214 & 2.791312 \\
\hline 5.895099 & -0.865979 & 3.489015 \\
\hline 1.931117 & 0.381394 & 4.604369 \\
\hline 4.397011 & 0.482829 & 4.948305 \\
\hline-4.281776 & -1.494671 & -4.297819 \\
\hline-6.349011 & -2.463620 & -3.350890 \\
\hline-6.310057 & -0.074525 & -4.085938 \\
\hline-8.189141 & -0.463567 & 0.932583 \\
\hline-6.389505 & -1.023719 & 2.540090 \\
\hline-6.828897 & 1.360719 & 1.914354 \\
\hline-4.614037 & -1.864705 & -5.275988 \\
\hline-3.668983 & -0.602984 & -4.480566 \\
\hline-3.639352 & -2.265246 & -3.848039 \\
\hline
\end{tabular}




$\begin{array}{lrrr}1 & -6.611211 & -0.368161 & -5.100786 \\ 1 & -7.221024 & 0.156460 & -3.519186 \\ 1 & -5.719264 & 0.847654 & -4.161769 \\ 1 & -6.625080 & -2.788365 & -4.362921 \\ 1 & -5.789946 & -3.279237 & -2.873492 \\ 1 & -7.280647 & -2.317012 & -2.791638 \\ 1 & -8.892684 & -0.372671 & 1.769753 \\ 1 & -8.548457 & 0.191901 & 0.128767 \\ 1 & -8.234193 & -1.501273 & 0.576731 \\ 1 & -7.554661 & 1.451102 & 2.733839 \\ 1 & -5.854953 & 1.692760 & 2.295343 \\ 1 & -7.131016 & 2.054418 & 1.118574 \\ 1 & -7.111956 & -0.950829 & 3.364283 \\ 1 & -6.373852 & -2.067751 & 2.199008 \\ 1 & -5.396782 & -0.791242 & 2.947097\end{array}$

$1_{\mathrm{C} 7-} \psi_{-1 \mathbf{a} 25354 a}$

Number of imaginary frequencies : 0

The smallest frequencies are : $16.4575 \quad 17.7056 \quad 21.2768 \mathrm{~cm}(-1)$

Electronic energy : $\quad H F=-4125.4663451$

Zero-point correction $=\quad 1.301728($ Hartree/Particle $)$

Thermal correction to Energy= $\quad 1.379735$

Thermal correction to Enthalpy= $\quad 1.380680$

Thermal correction to Gibbs Free Energy $=\quad 1.190325$

Sum of electronic and zero-point Energies $=\quad-4124.164617$

Sum of electronic and thermal Energies $=\quad-4124.086610$

Sum of electronic and thermal Enthalpies $=\quad-4124.085665$

Sum of electronic and thermal Free Energies $=\quad-4124.276020$

\begin{tabular}{cccc}
\multicolumn{4}{c}{ Cartesian Coordinates } \\
\hline 6 & 3.969204 & -0.143507 & 4.185527 \\
6 & 4.797927 & -0.906330 & 3.369924 \\
6 & 4.249730 & -1.706631 & 2.370535 \\
6 & 2.871814 & -1.746553 & 2.168969 \\
6 & 2.046663 & -0.978330 & 2.991598 \\
6 & 2.588848 & -0.186218 & 3.994678 \\
6 & 2.290101 & -2.630002 & 1.097000 \\
7 & 1.323297 & -1.969571 & 0.186337 \\
6 & 0.227181 & -2.790515 & -0.249770 \\
6 & -1.020126 & -2.674306 & 0.335829 \\
6 & -2.109550 & -3.445353 & -0.179439 \\
6 & -1.882330 & -4.354986 & -1.254894 \\
6 & -0.580731 & -4.470190 & -1.797241 \\
6 & 0.441679 & -3.701047 & -1.311370 \\
6 & -3.424757 & -3.335373 & 0.341512 \\
6 & -4.452895 & -4.087058 & -0.171536 \\
6 & -4.217435 & -5.007928 & -1.215304 \\
6 & -2.958141 & -5.135162 & -1.744781 \\
6 & -1.222101 & -1.869052 & 1.573117 \\
6 & -1.518618 & -0.513493 & 1.572019 \\
6 & -1.764498 & 0.140214 & 2.812084 \\
6 & -1.690885 & -0.526909 & 4.004393 \\
6 & -1.364645 & -1.901235 & 4.042451 \\
6 & -1.138182 & -2.586348 & 2.813653
\end{tabular}




\begin{tabular}{|c|c|c|c|}
\hline 6 & -1.250912 & -2.600501 & 5.267487 \\
\hline 6 & -0.923963 & -3.931967 & 5.288380 \\
\hline & -0.702447 & -4.618928 & 4.076624 \\
\hline & -0.810468 & -3.967162 & 2.872925 \\
\hline & -1.678019 & 0.434419 & -0.022626 \\
\hline & -0.139535 & 0.334850 & -1.923506 \\
\hline & 0.313202 & 2.242361 & -1.873897 \\
\hline & 0.464563 & 3.381875 & -1.946966 \\
\hline 1 & 1.681326 & -0.697000 & -0.881149 \\
\hline 8 & 2.663157 & 0.146219 & 0.148911 \\
\hline 6 & 3.522671 & 1.073570 & -0.390761 \\
\hline 6 & 4.702469 & 0.657065 & -0.977356 \\
\hline & 5.546296 & 1.652893 & -1.570022 \\
\hline & 5.192039 & 3.031739 & -1.452764 \\
\hline & 3.995201 & 3.392556 & -0.789199 \\
\hline & 3.164684 & 2.430725 & -0.282968 \\
\hline 5 & 6.028882 & 4.019038 & -2.027569 \\
\hline 6 & 7.160667 & 3.670711 & -2.718189 \\
\hline 5 & 7.493523 & 2.308559 & -2.870488 \\
\hline 6 & 6.710103 & 1.328558 & -2.313237 \\
\hline 0 & 5.015083 & -0.789177 & -1.034289 \\
\hline 6 & 4.088850 & -1.668941 & -1.567440 \\
\hline 6 & 4.389510 & -3.031909 & -1.781749 \\
\hline 6 & 5.591253 & -3.540920 & -1.373500 \\
\hline 6 & 6.526913 & -2.718221 & -0.699457 \\
\hline 6 & 6.237359 & -1.332518 & -0.518205 \\
\hline 6 & 7.151836 & -0.555815 & 0.237980 \\
\hline 6 & 8.295097 & -1.112056 & 0.757535 \\
\hline 6 & 8.595811 & -2.473260 & 0.542814 \\
\hline 6 & 7.724804 & -3.256668 & -0.170327 \\
\hline 8 & 2.846747 & -1.236749 & -1.966158 \\
\hline 6 & -3.434394 & 0.083696 & -0.429814 \\
\hline 6 & -4.442907 & 0.161705 & 0.540374 \\
\hline 6 & -5.750186 & -0.198909 & 0.234901 \\
\hline 6 & -6.020724 & -0.652716 & -1.068352 \\
\hline 6 & -5.044036 & -0.729345 & -2.056299 \\
\hline 6 & -3.743390 & -0.337673 & -1.712842 \\
\hline 6 & -6.875756 & -0.150069 & 1.267035 \\
\hline 6 & -5.320746 & -1.212235 & -3.477232 \\
\hline 6 & -1.679437 & 2.170597 & 0.563036 \\
\hline 6 & -2.654464 & 3.067908 & 0.152576 \\
\hline 6 & -2.577292 & 4.424808 & 0.498068 \\
\hline 6 & -1.506768 & 4.837192 & 1.286221 \\
\hline 6 & -0.509844 & 3.951715 & 1.729127 \\
\hline 6 & -0.594789 & 2.623380 & 1.327434 \\
\hline 6 & -3.672371 & 5.372220 & 0.014030 \\
\hline 6 & -3.706389 & 5.364116 & -1.518512 \\
\hline 6 & 0.628344 & 4.462957 & 2.610943 \\
\hline 6 & 1.469843 & 3.320107 & 3.179379 \\
\hline 6 & -0.634640 & 0.342386 & -3.759538 \\
\hline 8 & -0.935902 & 0.283450 & -4.876139 \\
\hline 6 & 1.529510 & 5.382072 & 1.779642 \\
\hline 6 & 0.060677 & 5.250246 & 3.797356 \\
\hline 6 & -3.447849 & 6.808822 & 0.477942 \\
\hline 6 & -5.026160 & 4.899983 & 0.558026 \\
\hline 1 & 6.925026 & 0.490399 & 0.427593 \\
\hline
\end{tabular}




\begin{tabular}{|c|c|c|c|}
\hline 1 & -2.019056 & 1.196648 & 2.817146 \\
\hline ] & 7.929955 & -4.314469 & -0.330296 \\
\hline & -1.425487 & -2.054374 & 6.193464 \\
\hline & 5.742502 & 5.064205 & -1.917679 \\
\hline & -3.617103 & -2.628946 & 1.147609 \\
\hline & -5.458359 & -3.973096 & 0.229755 \\
\hline & 3.733222 & 4.447140 & -0.711019 \\
\hline & -1.884474 & -0.004568 & 4.940533 \\
\hline & 5.833419 & -4.589753 & -1.536958 \\
\hline & 8.376286 & 2.030172 & -3.441822 \\
\hline & 3.646815 & -3.650339 & -2.280926 \\
\hline 1 & -2.763389 & -5.832773 & -2.558311 \\
\hline 1 & -0.409731 & -5.171839 & -2.612173 \\
\hline & -0.446060 & -5.675683 & 4.095752 \\
\hline & 6.975422 & 0.284062 & -2.453124 \\
\hline & -0.641465 & -4.515285 & 1.948399 \\
\hline & 1.440523 & -3.766005 & -1.738933 \\
\hline & 7.792777 & 4.437055 & -3.160693 \\
\hline & 8.972758 & -0.496648 & 1.345161 \\
\hline & -5.038367 & -5.606879 & -1.603706 \\
\hline 1 & -0.834560 & -4.460589 & 6.234531 \\
\hline & 9.508126 & -2.899258 & 0.953678 \\
\hline & 2.222001 & 2.678596 & 0.202272 \\
\hline & -4.186831 & 0.498903 & 1.542359 \\
\hline & -2.938481 & -0.380305 & -2.444957 \\
\hline & -7.037364 & -0.961305 & -1.304814 \\
\hline 1 & -3.486490 & 2.709534 & -0.452733 \\
\hline & 0.170878 & 1.906105 & 1.625883 \\
\hline & -1.434817 & 5.883165 & 1.575534 \\
\hline 1 & 1.724093 & -3.442236 & 1.575142 \\
\hline & -0.636408 & -1.181289 & -2.111794 \\
\hline 1 & 2.377888 & 5.737191 & 2.380231 \\
\hline & 1.929505 & 4.853789 & 0.903428 \\
\hline 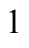 & 0.984112 & 6.260880 & 1.413824 \\
\hline & 0.878114 & 5.603134 & 4.439949 \\
\hline ] & -0.509891 & 6.131842 & 3.483271 \\
\hline 1 & -0.600696 & 4.621648 & 4.407826 \\
\hline & 0.848841 & 2.610438 & 3.744383 \\
\hline 1 & 2.006543 & 2.755468 & 2.406013 \\
\hline & 2.227041 & 3.721810 & 3.864674 \\
\hline 1 & -4.259578 & 7.446780 & 0.106328 \\
\hline 1 & -3.440748 & 6.891408 & 1.572555 \\
\hline & -2.505240 & 7.220446 & 0.094784 \\
\hline 1 & -5.271566 & 3.881739 & 0.230297 \\
\hline & -5.032640 & 4.908536 & 1.656034 \\
\hline 1 & -5.829268 & 5.563346 & 0.209732 \\
\hline & -2.740323 & 5.679479 & -1.933669 \\
\hline 1 & -3.933470 & 4.369947 & -1.921322 \\
\hline l & -4.477019 & 6.054855 & -1.886476 \\
\hline 1 & 3.103546 & -3.109259 & 0.530905 \\
\hline 1 & 4.906150 & -2.292916 & 1.725216 \\
\hline ] & 0.972439 & -0.986330 & 2.820165 \\
\hline 1 & 5.877900 & -0.876938 & 3.501656 \\
\hline & 1.927484 & 0.401612 & 4.630042 \\
\hline 1 & 4.395092 & 0.483495 & 4.966121 \\
\hline & -6.710899 & -1.828018 & -3.613146 \\
\hline
\end{tabular}




$\begin{array}{rrrr}6 & -5.213208 & -0.019018 & -4.433242 \\ 6 & -4.293445 & -2.277934 & -3.877363 \\ 6 & -6.401684 & 0.393234 & 2.613269 \\ 6 & -8.003624 & 0.753943 & 0.758149 \\ 6 & -7.419508 & -1.565313 & 1.493494 \\ 1 & -6.853508 & -2.198145 & -4.636163 \\ 1 & -6.842105 & -2.678592 & -2.929538 \\ 1 & -7.509400 & -1.100922 & -3.417060 \\ 1 & -4.504229 & -2.639192 & -4.892846 \\ 1 & -3.263724 & -1.898241 & -3.874406 \\ 1 & -4.331663 & -3.135998 & -3.193096 \\ 1 & -5.403439 & -0.337281 & -5.467202 \\ 1 & -5.943947 & 0.759423 & -4.177043 \\ 1 & -4.214284 & 0.434657 & -4.402929 \\ 1 & -7.240311 & 0.407080 & 3.320764 \\ 1 & -5.610490 & -0.228855 & 3.053474 \\ 1 & -6.023257 & 1.420610 & 2.530542 \\ 1 & -8.246140 & -1.545874 & 2.216546 \\ 1 & -7.798996 & -2.016349 & 0.568304 \\ 1 & -6.638221 & -2.224276 & 1.896216 \\ 1 & -8.814527 & 0.803902 & 1.497130 \\ 1 & -7.641325 & 1.775714 & 0.584977 \\ 1 & -8.435608 & 0.389037 & -0.181282\end{array}$

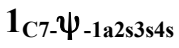

Number of imaginary frequencies : 0

The smallest frequencies are : $15.2788 \quad 18.1400 \quad 20.2567 \mathrm{~cm}(-1)$

Electronic energy : $\quad H F=-4125.4664254$

Zero-point correction $=\quad 1.299277($ Hartree/Particle)

Thermal correction to Energy= $\quad 1.377778$

Thermal correction to Enthalpy $=\quad 1.378722$

Thermal correction to Gibbs Free Energy= $\quad 1.186139$

Sum of electronic and zero-point Energies $=\quad-4124.167148$

Sum of electronic and thermal Energies $=\quad-4124.088648$

Sum of electronic and thermal Enthalpies $=\quad-4124.087703$

Sum of electronic and thermal Free Energies $=\quad-4124.280287$

Cartesian Coordinates

$\begin{array}{lrrr}6 & 3.958822 & -0.197558 & 4.160503 \\ 6 & 4.782077 & -0.967344 & 3.345972 \\ 6 & 4.228812 & -1.759834 & 2.343156 \\ 6 & 2.851244 & -1.785496 & 2.137609 \\ 6 & 2.031469 & -1.011252 & 2.960106 \\ 6 & 2.578627 & -0.226148 & 3.965843 \\ 6 & 2.263725 & -2.656267 & 1.058461 \\ 7 & 1.313354 & -1.977771 & 0.144071 \\ 6 & 0.211346 & -2.781519 & -0.308828 \\ 6 & -1.031282 & -2.678207 & 0.289300 \\ 6 & -2.128795 & -3.426564 & -0.241140 \\ 6 & -1.916385 & -4.296098 & -1.352024 \\ 6 & -0.619321 & -4.401149 & -1.907221 \\ 6 & 0.412642 & -3.658056 & -1.401066 \\ 6 & -3.438527 & -3.329115 & 0.296395 \\ 6 & -4.475168 & -4.055462 & -0.235094\end{array}$




$\begin{array}{cccc}6 & -4.255906 & -4.933827 & -1.318258 \\ 6 & -3.002220 & -5.048030 & -1.863793 \\ 6 & -1.223665 & -1.899527 & 1.544360 \\ 6 & -1.506097 & -0.541173 & 1.566886 \\ 6 & -1.744631 & 0.093554 & 2.817941 \\ 6 & -1.683092 & -0.597087 & 3.997906 \\ 6 & -1.375177 & -1.976046 & 4.011400 \\ 6 & -1.150656 & -2.640366 & 2.770947 \\ 6 & -1.278315 & -2.700598 & 5.223189 \\ 6 & -0.968967 & -4.036398 & 5.219786 \\ 6 & -0.747541 & -4.702216 & 3.996123 \\ 6 & -0.839403 & -4.025672 & 2.804841 \\ 15 & -1.662904 & 0.427899 & -0.014795 \\ 45 & -0.129606 & 0.372735 & -1.928592 \\ 6 & 0.323190 & 2.278463 & -1.838338 \\ 8 & 0.473719 & 3.419241 & -1.891992 \\ 15 & 1.683648 & -0.688187 & -0.898160 \\ 8 & 2.661298 & 0.134956 & 0.152363 \\ 6 & 3.525136 & 1.069951 & -0.366254 \\ 6 & 4.706793 & 0.661599 & -0.955143 \\ 6 & 5.554275 & 1.666040 & -1.527414 \\ 6 & 5.202097 & 3.043298 & -1.387309 \\ 6 & 4.003835 & 3.394780 & -0.721197 \\ 6 & 3.169566 & 2.425777 & -0.235141 \\ 6 & 6.042747 & 4.038741 & -1.942210 \\ 6 & 7.176158 & 3.700249 & -2.635096 \\ 6 & 7.507021 & 2.340306 & -2.809951 \\ 6 & 6.719923 & 1.352359 & -2.272270 \\ 6 & 5.018029 & -0.784181 & -1.031910 \\ 6 & 4.092141 & -1.654978 & -1.580143 \\ 6 & -2.609052 & 4.399062 & 0.549868 \\ 6 & -1.542890 & 4.817133 & 1.341111 \\ 6 & 4.387529 & -3.017475 & -1.803943 \\ 6 & -0.535038 & 3.939717 & 1.775580 \\ 6 & -0.603547 & 2.614070 & 1.361106 \\ 6 & 5.585509 & -3.534829 & -1.395034 \\ 6 & -3.707845 & 5.339589 & 0.061141 \\ 6 & 6.522022 & -2.721625 & -0.710809 \\ 6 & -3.0369099 & -1.063654 & -3.527544 \\ 6 & -3.672932 & 5.392081 & -1.470754 \\ 6 & -5.151118 & -0.571160 & 0.250758 \\ 6 & 8.290943 & -1.136521 & 0.768050 \\ 6 & 8.587761 & -2.496473 & 0.540479 \\ 6 & 7.716299 & -3.269574 & -0.183234 \\ 6 & 2.855101 & -1.212535 & -1.983736 \\ 6 & -3.416696 & 0.071826 & -0.431970 \\ 6 & -5.598281 & 4.456985 & 2.660406\end{array}$




\begin{tabular}{|c|c|c|c|}
\hline & 1.446009 & 3.318750 & 3.228944 \\
\hline & -0.607994 & 0.422277 & -3.769040 \\
\hline & -0.895276 & 0.388825 & -4.890393 \\
\hline & 1.496030 & 5.381748 & 1.831666 \\
\hline & 0.024539 & 5.240188 & 3.846555 \\
\hline & -3.538581 & 6.760143 & 0.592644 \\
\hline & -5.073252 & 4.815708 & 0.522086 \\
\hline & 6.927471 & 0.473856 & 0.450396 \\
\hline & -1.986622 & 1.152735 & 2.841659 \\
\hline & 7.918219 & -4.326453 & -0.353116 \\
\hline & -1.451648 & -2.170365 & 6.158594 \\
\hline & 5.757991 & 5.082403 & -1.815135 \\
\hline & -3.621628 & -2.650165 & 1.128311 \\
\hline & -5.476372 & -3.951235 & 0.180162 \\
\hline & 3.743617 & 4.448306 & -0.624972 \\
\hline & -1.872568 & -0.089767 & 4.943107 \\
\hline & 5.823899 & -4.583353 & -1.565889 \\
\hline & 8.391099 & 2.070135 & -3.383193 \\
\hline & 3.643809 & -3.629386 & -2.309751 \\
\hline & -2.819489 & -5.714083 & -2.706083 \\
\hline & -0.459238 & -5.074085 & -2.748173 \\
\hline & -0.504253 & -5.762231 & 3.996268 \\
\hline & 6.983465 & 0.309884 & -2.429501 \\
\hline & -0.671134 & -4.557059 & 1.870472 \\
\hline ] & 1.408014 & -3.714648 & -1.837870 \\
\hline & 7.811142 & 4.472911 & -3.062234 \\
\hline 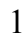 & 8.968828 & -0.529375 & 1.363958 \\
\hline ] & -5.084260 & -5.511474 & -1.722823 \\
\hline & -0.893198 & -4.584728 & 6.155854 \\
\hline 1 & 9.497308 & -2.929750 & 0.949877 \\
\hline & 2.225535 & 2.666905 & 0.250961 \\
\hline 1 & -4.159860 & 0.388695 & 1.560620 \\
\hline & -2.940390 & -0.299897 & -2.475720 \\
\hline | & -7.023218 & -0.954699 & -1.330785 \\
\hline 1 & -3.497091 & 2.682292 & -0.416705 \\
\hline | & 0.170675 & 1.902664 & 1.652196 \\
\hline 1 & -1.483535 & 5.861228 & 1.639969 \\
\hline & 1.682013 & -3.461854 & 1.529156 \\
\hline 1 & -0.633491 & -1.136446 & -2.152521 \\
\hline ] & 2.336566 & 5.747618 & 2.436877 \\
\hline ] & 1.907969 & 4.853663 & 0.960846 \\
\hline 1 & 0.944414 & 6.253430 & 1.458216 \\
\hline | & 0.839011 & 5.593959 & 4.492371 \\
\hline 1 & -0.547124 & 6.121104 & 3.532625 \\
\hline & -0.637125 & 4.608717 & 4.453718 \\
\hline 1 & 0.828370 & 2.603070 & 3.790028 \\
\hline & 1.989736 & 2.760489 & 2.455844 \\
\hline 1 & 2.197928 & 3.724382 & 3.917688 \\
\hline 1 & -4.356507 & 7.391464 & 0.223275 \\
\hline & -3.565888 & 6.793884 & 1.689642 \\
\hline 1 & -2.597671 & 7.214609 & 0.256849 \\
\hline & -5.286853 & 3.812772 & 0.131712 \\
\hline 1 & -5.127458 & 4.765915 & 1.617777 \\
\hline & -5.873136 & 5.482899 & 0.173875 \\
\hline 1 & -2.698512 & 5.749632 & -1.828347 \\
\hline 1 & -3.851327 & 4.407562 & -1.919718 \\
\hline
\end{tabular}




$\begin{array}{lrrr}1 & -4.446020 & 6.075405 & -1.847598 \\ 1 & 3.073565 & -3.144899 & 0.494958 \\ 1 & 4.881385 & -2.350524 & 1.697971 \\ 1 & 0.957900 & -1.006544 & 2.784668 \\ 1 & 5.861887 & -0.948953 & 3.480940 \\ 1 & 1.921518 & 0.367898 & 4.599889 \\ 1 & 4.388880 & 0.423944 & 4.943203 \\ 6 & -6.730604 & -1.642676 & -3.694077 \\ 6 & -5.199142 & 0.179365 & -4.415425 \\ 6 & -4.323015 & -2.123840 & -3.992116 \\ 6 & -8.136851 & -0.757342 & 0.867982 \\ 6 & -6.315207 & -1.185462 & 2.486892 \\ 6 & -6.939492 & 1.162496 & 1.881632 \\ 1 & -6.883514 & -1.947943 & -4.736855 \\ 1 & -6.877448 & -2.530034 & -3.062607 \\ 1 & -7.513489 & -0.912699 & -3.451242 \\ 1 & -4.537554 & -2.416685 & -5.028668 \\ 1 & -3.285603 & -1.767010 & -3.961744 \\ 1 & -4.383131 & -3.021138 & -3.362560 \\ 1 & -5.408143 & -0.074510 & -5.463524 \\ 1 & -5.906933 & 0.960220 & -4.107302 \\ 1 & -4.188161 & 0.604552 & -4.371220 \\ 1 & -8.849350 & -0.743963 & 1.702445 \\ 1 & -8.549074 & -0.120510 & 0.074852 \\ 1 & -8.089547 & -1.788091 & 0.491953 \\ 1 & -7.674021 & 1.184633 & 2.698040 \\ 1 & -5.997190 & 1.569173 & 2.269525 \\ 1 & -7.293771 & 1.836715 & 1.090840 \\ 1 & -7.053387 & -1.182498 & 3.300240 \\ 1 & -6.208874 & -2.221345 & 2.136512 \\ 1 & -5.352180 & -0.878108 & 2.914445\end{array}$

$\mathbf{1}_{\mathrm{C} 7-\psi_{-1} \text {-1saza4a }}$

Number of imaginary frequencies : 0

The smallest frequencies are : $15.7315 \quad 18.328622 .8520 \mathrm{~cm}(-1)$

Electronic energy : $\quad H F=-4125.4643098$

Zero-point correction $=\quad 1.299388($ Hartree/Particle)

Thermal correction to Energy= $\quad 1.377881$

Thermal correction to Enthalpy= $\quad 1.378826$

Thermal correction to Gibbs Free Energy $=\quad 1.186900$

Sum of electronic and zero-point Energies $=\quad-4124.164922$

Sum of electronic and thermal Energies $=\quad-4124.086428$

Sum of electronic and thermal Enthalpies $=\quad-4124.085484$

Sum of electronic and thermal Free Energies $=\quad-4124.277410$

Cartesian Coordinates

$\begin{array}{rrrr}6 & 3.886971 & 0.334601 & 4.196988 \\ 6 & 4.765117 & -0.500323 & 3.515238 \\ 6 & 4.285074 & -1.376096 & 2.544030 \\ 6 & 2.925993 & -1.425306 & 2.240617 \\ 6 & 2.051150 & -0.582344 & 2.928102 \\ 6 & 2.525676 & 0.288806 & 3.899518 \\ 6 & 2.421705 & -2.400431 & 1.208592 \\ 7 & 1.454209 & -1.848614 & 0.231999\end{array}$




\begin{tabular}{|c|c|c|c|}
\hline 6 & 0.395000 & -2.729292 & -0.177301 \\
\hline 6 & -0.850051 & -2.664394 & 0.421400 \\
\hline 6 & -1.912892 & -3.480219 & -0.077187 \\
\hline 6 & -1.668936 & -4.359270 & -1.173883 \\
\hline 6 & -0.370115 & -4.424980 & -1.729241 \\
\hline 6 & 0.631720 & -3.629727 & -1.242426 \\
\hline 6 & -3.221499 & -3.434494 & 0.471502 \\
\hline 6 & -4.227753 & -4.214485 & -0.040341 \\
\hline 6 & -3.977237 & -5.095818 & -1.114826 \\
\hline 6 & -2.725615 & -5.160717 & -1.671308 \\
\hline 6 & -1.085236 & -1.839910 & 1.638523 \\
\hline 6 & -1.472084 & -0.508108 & 1.589810 \\
\hline 6 & -1.767441 & 0.166779 & 2.807311 \\
\hline 6 & -1.646623 & -0.453067 & 4.021644 \\
\hline 6 & -1.225973 & -1.799388 & 4.106360 \\
\hline 6 & -0.954682 & -2.508427 & 2.900685 \\
\hline 6 & -1.071725 & -2.450932 & 5.353020 \\
\hline 6 & -0.664468 & -3.758903 & 5.415707 \\
\hline 6 & -0.400188 & -4.469970 & 4.226491 \\
\hline 6 & -0.545222 & -3.864414 & 3.002740 \\
\hline 15 & -1.674463 & 0.366795 & -0.043353 \\
\hline 45 & -0.101528 & 0.332367 & -1.933801 \\
\hline 6 & 0.254469 & 2.258774 & -1.872623 \\
\hline 8 & 0.359539 & 3.404465 & -1.931905 \\
\hline 15 & 1.765733 & -0.613168 & -0.892737 \\
\hline 8 & 2.740888 & 0.295447 & \\
\hline 6 & 3.616046 & 1.179604 & -0.500846 \\
\hline 6 & 4.801588 & 0.717412 & -1.037252 \\
\hline 6 & 5.672763 & 1.668336 & -1.662224 \\
\hline 6 & 5.331018 & 3.055270 & -1.634212 \\
\hline 6 & 4.121132 & 3.465730 & -1.024529 \\
\hline 6 & 3.269508 & 2.542749 & -0.482389 \\
\hline 6 & 6.195519 & 3.998642 & -2.240909 \\
\hline 6 & 7.343043 & 3.599397 & -2.876005 \\
\hline 6 & 7.665496 & 2.227693 & -2.938053 \\
\hline 6 & 6.854471 & 1.289567 & -2.349214 \\
\hline 6 & 5.087542 & -0.734963 & -1.015642 \\
\hline 6 & 4.157965 & -1.618120 & -1.537611 \\
\hline 6 & 4.440540 & -2.993039 & -1.691141 \\
\hline 6 & 5.616800 & -3.507420 & -1.219117 \\
\hline 6 & 6.543180 & -2.676596 & -0.542396 \\
\hline 6 & 6.279703 & -1.278017 & -0.435631 \\
\hline 6 & 7.184482 & -0.484673 & 0.314936 \\
\hline 6 & 8.292805 & -1.040411 & 0.905256 \\
\hline 6 & 8.567273 & -2.417424 & 0.768785 \\
\hline 6 & 7.706685 & -3.215169 & 0.058761 \\
\hline 8 & 2.929470 & -1.177162 & -1.970914 \\
\hline 6 & -3.400274 & -0.118130 & -0.457568 \\
\hline 6 & -4.418272 & -0.025225 & 0.495405 \\
\hline 6 & -5.721694 & -0.410035 & 0.188549 \\
\hline 6 & -5.967868 & -0.900136 & -1.100134 \\
\hline 6 & -4.977121 & -0.998007 & -2.078203 \\
\hline 6 & -3.683778 & -0.595648 & -1.732900 \\
\hline 6 & -6.855144 & -0.343735 & 1.210976 \\
\hline 6 & -5.326245 & -1.511507 & -3.473644 \\
\hline 6 & -1.828376 & 2.120689 & 0.477719 \\
\hline
\end{tabular}




\begin{tabular}{|c|c|c|c|}
\hline & -2.869128 & 2.917856 & -0.004249 \\
\hline & -2.937422 & 4.272327 & 0.314923 \\
\hline & -1.947661 & 4.802503 & 1.153479 \\
\hline & -0.894793 & 4.034997 & 1.650994 \\
\hline & -0.831490 & 2.690329 & 1.268105 \\
\hline & -4.061887 & 5.173825 & -0.191097 \\
\hline & 0.126589 & 4.582127 & 2.647213 \\
\hline & -0.527493 & 0.310460 & -3.789037 \\
\hline & -0.751545 & 0.234607 & -4.922226 \\
\hline & 6.974211 & 0.573972 & 0.446107 \\
\hline & -2.097599 & 1.201849 & 2.779106 \\
\hline & 7.893462 & -4.283502 & -0.042992 \\
\hline & -1.281678 & -1.888079 & 6.261455 \\
\hline & 5.918836 & 5.051389 & -2.200568 \\
\hline & -3.427678 & -2.753887 & 1.295996 \\
\hline & -5.229214 & -4.152539 & 0.381416 \\
\hline & 3.869077 & 4.525281 & -1.012162 \\
\hline & -1.877386 & 0.087266 & 4.939227 \\
\hline & 5.843143 & -4.566416 & -1.331985 \\
\hline & 8.562412 & 1.908170 & -3.464031 \\
\hline & 3.705896 & -3.618641 & -2.193162 \\
\hline & -2.519320 & -5.831637 & -2.504154 \\
\hline & -0.183858 & -5.110030 & -2.554889 \\
\hline & -0.081336 & -5.508395 & 4.279549 \\
\hline & 7.112756 & 0.236133 & -2.418336 \\
\hline & -0.343629 & -4.429360 & 2.094963 \\
\hline & 1.627683 & -3.658717 & -1.679129 \\
\hline ] & 7.996581 & 4.332589 & -3.342916 \\
\hline & 8.962609 & -0.412813 & 1.488983 \\
\hline & -4.781990 & -5.717098 & -1.502401 \\
\hline & -0.545339 & -4.250995 & 6.378147 \\
\hline & 9.452047 & -2.843341 & 1.236130 \\
\hline 1 & 2.324220 & 2.825994 & -0.027292 \\
\hline & -4.181030 & 0.356649 & 1.485658 \\
\hline 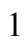 & -2.868240 & -0.653700 & -2.449924 \\
\hline & -6.981126 & -1.214621 & -1.352186 \\
\hline | & -3.627999 & 2.464055 & -0.636671 \\
\hline ] & -0.010429 & 2.061857 & 1.623426 \\
\hline & -2.011199 & 5.853502 & 1.426934 \\
\hline 1 & 1.881635 & -3.209741 & 1.721344 \\
\hline & -0.525632 & -1.206532 & -2.120779 \\
\hline 1 & 3.275574 & -2.871977 & 0.698070 \\
\hline & 4.981492 & -2.018599 & 2.002187 \\
\hline 1 & 0.994371 & -0.589709 & 2.668892 \\
\hline ] & 5.831650 & -0.468195 & 3.729876 \\
\hline l & 1.826111 & 0.934994 & 4.428900 \\
\hline 1 & 4.259945 & 1.021528 & 4.953758 \\
\hline 6 & -4.096790 & -1.599522 & -4.375438 \\
\hline 6 & -5.944762 & -2.909259 & -3.376337 \\
\hline 5 & -6.331249 & -0.552822 & -4.122346 \\
\hline 6 & -6.420630 & 0.334568 & 2.508726 \\
\hline 6 & -8.035121 & 0.447262 & 0.637128 \\
\hline & -7.310144 & -1.768888 & 1.544503 \\
\hline 1 & -4.384391 & -2.007172 & -5.352777 \\
\hline 1 & -3.646046 & -0.614484 & -4.552016 \\
\hline 1 & -3.324249 & -2.258432 & -3.954266 \\
\hline
\end{tabular}




$\begin{array}{lrrr}1 & -6.582853 & -0.891936 & -5.136327 \\ 1 & -7.265900 & -0.489303 & -3.551136 \\ 1 & -5.915403 & 0.460344 & -4.197962 \\ 1 & -6.230820 & -3.267123 & -4.374632 \\ 1 & -5.225604 & -3.622007 & -2.952205 \\ 1 & -6.844909 & -2.925618 & -2.749079 \\ 1 & -7.268415 & 0.377475 & 3.204023 \\ 1 & -5.612648 & -0.212953 & 3.012353 \\ 1 & -6.080649 & 1.364703 & 2.335620 \\ 1 & -8.140993 & -1.749224 & 2.262886 \\ 1 & -7.652118 & -2.309606 & 0.652984 \\ 1 & -6.490125 & -2.344216 & 1.995670 \\ 1 & -8.845189 & 0.508954 & 1.375969 \\ 1 & -7.735060 & 1.471073 & 0.379002 \\ 1 & -8.450192 & -0.016526 & -0.265394 \\ 6 & -5.052492 & 4.421224 & -1.076875 \\ 6 & -4.834370 & 5.745781 & 1.002964 \\ 6 & -3.463524 & 6.321809 & -1.010898 \\ 6 & -0.011056 & 6.088155 & 2.856356 \\ 6 & -0.100462 & 3.885724 & 3.994894 \\ 6 & 1.552021 & 4.297182 & 2.167823 \\ 1 & -5.651108 & 6.391689 & 0.653565 \\ 1 & -5.274569 & 4.941510 & 1.607309 \\ 1 & -4.195758 & 6.347738 & 1.660532 \\ 1 & -5.843550 & 5.105926 & -1.407670 \\ 1 & -4.573320 & 4.013356 & -1.976076 \\ 1 & -5.536280 & 3.592537 & -0.541835 \\ 1 & -4.260449 & 6.976547 & -1.388724 \\ 1 & -2.780069 & 6.940496 & -0.417032 \\ 1 & -2.902715 & 5.937367 & -1.872623 \\ 1 & 0.751754 & 6.431900 & 3.566188 \\ 1 & 0.131688 & 6.645251 & 1.921304 \\ 1 & -0.988715 & 6.360140 & 3.273948 \\ 1 & 2.280830 & 4.726692 & 2.867950 \\ 1 & 1.764752 & 3.222365 & 2.104450 \\ 1 & 1.729861 & 4.745168 & 1.180060 \\ 1 & 0.622543 & 4.243683 & 4.740597 \\ 1 & -1.110460 & 4.088112 & 4.375933 \\ 1 & 0.013179 & 2.796878 & 3.911430\end{array}$

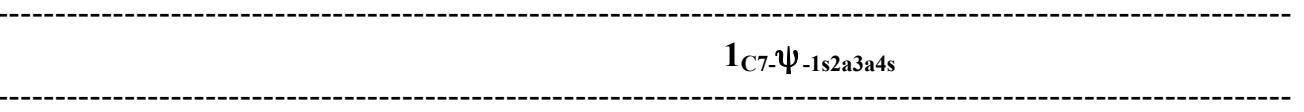

Number of imaginary frequencies : 0

The smallest frequencies are : $14.0458 \quad 17.7460 \quad 19.4744 \mathrm{~cm}(-1)$

Electronic energy : $\quad H F=-4125.4649559$

Zero-point correction $=\quad 1.299122($ Hartree/Particle $)$

Thermal correction to Energy= $\quad 1.377688$

Thermal correction to Enthalpy= $\quad 1.378633$

Thermal correction to Gibbs Free Energy $=\quad 1.185958$

Sum of electronic and zero-point Energies $=\quad-4124.165834$

Sum of electronic and thermal Energies $=\quad-4124.087268$

Sum of electronic and thermal Enthalpies $=\quad-4124.086323$

Sum of electronic and thermal Free Energies $=\quad-4124.278998$

Cartesian Coordinates 


\begin{tabular}{|c|c|c|c|}
\hline 6 & 3.894654 & 0.323321 & 4.211720 \\
\hline 6 & 4.777640 & -0.502440 & 3.524952 \\
\hline f & 4.303426 & -1.371229 & 2.544678 \\
\hline 6 & 2.945269 & -1.422791 & 2.237381 \\
\hline 6 & 2.065854 & -0.588512 & 2.929567 \\
\hline b & 2.534480 & 0.275941 & 3.909633 \\
\hline 6 & 2.447346 & -2.392045 & 1.196690 \\
\hline 1 & 1.480059 & -1.838401 & 0.220633 \\
\hline 6 & 0.423954 & -2.720320 & -0.193578 \\
\hline 6 & -0.817819 & -2.670801 & 0.413346 \\
\hline 6 & -1.878054 & -3.489236 & -0.086191 \\
\hline 6 & -1.636206 & -4.352146 & -1.195940 \\
\hline 6 & -0.340879 & -4.401687 & -1.761268 \\
\hline 6 & 0.659452 & -3.606578 & -1.270873 \\
\hline 6 & -3.182451 & -3.459377 & 0.473538 \\
\hline 6 & -4.187307 & -4.239399 & -0.040874 \\
\hline 6 & -3.939421 & -5.104068 & -1.129381 \\
\hline 6 & -2.691649 & -5.153446 & -1.696261 \\
\hline 6 & -1.055487 & -1.853136 & 1.634223 \\
\hline 6 & -1.451074 & -0.523733 & 1.587092 \\
\hline 6 & -1.750448 & 0.147800 & 2.805086 \\
\hline 6 & -1.628540 & -0.474362 & 4.018420 \\
\hline 6 & -1.200549 & -1.818424 & 4.101230 \\
\hline 6 & -0.922361 & -2.523248 & 2.894662 \\
\hline 6 & -1.044597 & -2.471876 & 5.346729 \\
\hline 6 & -0.629206 & -3.777353 & 5.407151 \\
\hline 6 & -0.358159 & -4.484192 & 4.216828 \\
\hline 6 & -0.504703 & -3.876862 & 2.994214 \\
\hline 15 & -1.662356 & 0.345519 & -0.047871 \\
\hline 45 & -0.082156 & 0.353921 & -1.929060 \\
\hline 6 & 0.256136 & 2.282608 & -1.842527 \\
\hline 8 & 0.348081 & 3.430196 & -1.885626 \\
\hline 15 & 1.787481 & -0.592142 & -0.892964 \\
\hline 8 & 2.755062 & 0.312353 & 0.095045 \\
\hline 6 & 3.625749 & 1.208204 & -0.478505 \\
\hline 6 & 4.814293 & 0.758620 & -1.019126 \\
\hline 6 & 5.679914 & 1.721326 & -1.633433 \\
\hline 6 & 5.330118 & 3.105890 & -1.590208 \\
\hline 6 & 4.118190 & 3.502879 & -0.975538 \\
\hline 6 & 3.271740 & 2.569207 & -0.443629 \\
\hline 6 & 6.189113 & 4.060720 & -2.186687 \\
\hline 6 & 7.338773 & 3.674946 & -2.826264 \\
\hline 6 & 7.669248 & 2.305880 & -2.903095 \\
\hline 6 & 6.863799 & 1.356773 & -2.324301 \\
\hline 6 & 5.109579 & -0.692233 & -1.010843 \\
\hline 6 & 4.186247 & -1.577000 & -1.541007 \\
\hline 6 & 4.475837 & -2.949457 & -1.702862 \\
\hline 6 & 5.654262 & -3.460601 & -1.232775 \\
\hline 6 & 6.575432 & -2.629180 & -0.549695 \\
\hline 6 & 6.304062 & -1.232838 & -0.433194 \\
\hline 6 & 7.203598 & -0.440034 & 0.324209 \\
\hline 6 & 8.314285 & -0.993900 & 0.911844 \\
\hline 6 & 8.596567 & -2.368329 & 0.765743 \\
\hline 6 & 7.741230 & -3.165624 & 0.048921 \\
\hline 8 & 2.956225 & -1.139707 & -1.973561 \\
\hline 6 & -3.373030 & -0.181763 & -0.471746 \\
\hline
\end{tabular}




\begin{tabular}{|c|c|c|c|}
\hline 6 & -4.388761 & -0.111978 & 0.476768 \\
\hline 6 & -5.693397 & -0.505770 & 0.169552 \\
\hline 6 & -5.941743 & -0.972689 & -1.122057 \\
\hline 6 & -4.943724 & -1.051875 & -2.102553 \\
\hline 6 & -3.654928 & -0.648808 & -1.755145 \\
\hline 6 & -6.770990 & -0.393013 & 1.245945 \\
\hline 6 & -5.292251 & -1.548121 & -3.504423 \\
\hline 6 & -1.878489 & 2.094500 & 0.468674 \\
\hline 6 & -2.952485 & 2.848007 & -0.010888 \\
\hline 6 & -3.074781 & 4.199175 & 0.304232 \\
\hline 6 & -2.105424 & 4.770847 & 1.139000 \\
\hline 6 & -1.018963 & 4.048494 & 1.632556 \\
\hline 6 & -0.902026 & 2.706223 & 1.252904 \\
\hline 6 & -4.242163 & 5.048459 & -0.194879 \\
\hline 6 & -0.008770 & 4.643736 & 2.612394 \\
\hline 6 & -0.510361 & 0.352030 & -3.783947 \\
\hline 8 & -0.737256 & 0.288686 & -4.917320 \\
\hline 1 & 6.987580 & 0.616528 & 0.462531 \\
\hline 1 & -2.086215 & 1.181040 & 2.778345 \\
\hline 1 & 7.933988 & -4.232153 & -0.060343 \\
\hline 1 & -1.259761 & -1.912492 & 6.256084 \\
\hline 1 & 5.906442 & 5.111375 & -2.134819 \\
\hline 1 & -3.386421 & -2.791295 & 1.308978 \\
\hline 1 & -5.186389 & -4.188690 & 0.388225 \\
\hline 1 & 3.860610 & 4.560844 & -0.950915 \\
\hline 1 & -1.863117 & 0.062950 & 4.936797 \\
\hline 1 & 5.886297 & -4.517672 & -1.351932 \\
\hline 1 & 8.567961 & 1.997328 & -3.432548 \\
\hline 1 & 3.744535 & -3.575820 & -2.208923 \\
\hline 1 & -2.487674 & -5.811883 & -2.539595 \\
\hline 1 & -0.155926 & -5.074577 & -2.597151 \\
\hline 1 & -0.032846 & -5.520691 & 4.268298 \\
\hline 1 & 7.128073 & 0.305583 & -2.404501 \\
\hline 1 & -0.298200 & -4.438210 & 2.085292 \\
\hline 1 & 1.652677 & -3.623451 & -1.714632 \\
\hline 1 & 7.987984 & 4.416886 & -3.285325 \\
\hline 1 & 8.979950 & -0.366817 & 1.500834 \\
\hline 1 & -4.743072 & -5.725243 & -1.519378 \\
\hline 1 & -0.508790 & -4.270879 & 6.368693 \\
\hline 1 & 9.483163 & -2.792720 & 1.231015 \\
\hline 1 & 2.324596 & 2.842171 & 0.014360 \\
\hline 1 & -4.161321 & 0.261715 & 1.474891 \\
\hline 1 & -2.839588 & -0.692082 & -2.473417 \\
\hline 1 & -6.950829 & -1.285074 & -1.384617 \\
\hline 1 & -3.695323 & 2.362810 & -0.638504 \\
\hline 1 & -0.052490 & 2.113293 & 1.602882 \\
\hline 1 & -2.212336 & 5.818414 & 1.411819 \\
\hline 1 & 1.909513 & -3.207281 & 1.702260 \\
\hline 1 & -0.491528 & -1.186384 & -2.135611 \\
\hline 1 & 3.304385 & -2.856640 & 0.685215 \\
\hline 1 & 5.003665 & -2.006367 & 1.999058 \\
\hline 1 & 1.010087 & -0.596231 & 2.666784 \\
\hline 1 & 5.843533 & -0.468319 & 3.742465 \\
\hline 1 & 1.830957 & 0.916852 & 4.440216 \\
\hline 1 & 4.263305 & 1.004939 & 4.975397 \\
\hline 6 & -4.064902 & -1.611688 & -4.411117 \\
\hline
\end{tabular}




$\begin{array}{rrrr}6 & -5.897459 & -2.952992 & -3.426610 \\ 6 & -6.308134 & -0.590015 & -4.136996 \\ 6 & -8.115190 & -0.949063 & 0.784436 \\ 6 & -6.339474 & -1.172556 & 2.493905 \\ 6 & -6.959075 & 1.085725 & 1.606277 \\ 1 & -4.353274 & -2.002128 & -5.395245 \\ 1 & -3.619910 & -0.621097 & -4.569977 \\ 1 & -3.287970 & -2.274653 & -4.004752 \\ 1 & -6.556841 & -0.915961 & -5.155971 \\ 1 & -7.243207 & -0.545709 & -3.564880 \\ 1 & -5.903073 & 0.428549 & -4.197126 \\ 1 & -6.184467 & -3.297531 & -4.429327 \\ 1 & -5.170006 & -3.665666 & -3.016920 \\ 1 & -6.794644 & -2.987302 & -2.796148 \\ 1 & -8.849180 & -0.863252 & 1.595641 \\ 1 & -8.514560 & -0.399225 & -0.077301 \\ 1 & -8.045200 & -2.010890 & 0.512915 \\ 1 & -7.708598 & 1.195728 & 2.401783 \\ 1 & -6.026369 & 1.545025 & 1.958975 \\ 1 & -7.305943 & 1.657672 & 0.735418 \\ 1 & -7.095951 & -1.073415 & 3.283954 \\ 1 & -6.229155 & -2.242006 & 2.270241 \\ 1 & -5.385634 & -0.817791 & 2.904680 \\ 6 & -5.174133 & 4.265731 & -1.117707 \\ 6 & -5.060961 & 5.535929 & 1.005806 \\ 6 & -3.705103 & 6.254567 & -0.972361 \\ 6 & -0.220536 & 6.139842 & 2.830571 \\ 6 & -0.168404 & 3.932240 & 3.961897 \\ 6 & 1.418789 & 4.434140 & 2.100513 \\ 1 & -5.906621 & 6.149680 & 0.667331 \\ 1 & -5.465264 & 4.687604 & 1.574333 \\ 1 & -4.462553 & 6.145809 & 1.693496 \\ 1 & -5.996599 & 4.914222 & -1.444881 \\ 1 & -4.656443 & 3.909756 & -2.017730 \\ 1 & -5.620288 & 3.397022 & -0.614487 \\ 1 & -4.536714 & 6.867262 & -1.345718 \\ 1 & -3.072722 & 6.900186 & -0.351383 \\ 1 & -3.107987 & 5.931297 & -1.834681 \\ 1 & 0.534336 & 6.519648 & 3.530370 \\ 1 & -0.120953 & 6.707074 & 1.896045 \\ 1 & -1.204609 & 6.359263 & 3.263790 \\ 1 & 2.139532 & 4.913180 & 2.776374 \\ 1 & 1.687125 & 3.371734 & 2.047650 \\ 1 & 1.546725 & 4.874788 & 1.101955 \\ 1 & 0.546231 & 4.331483 & 4.694511 \\ 1 & -1.180916 & 4.073180 & 4.363319 \\ 1 & 0.010251 & 2.852329 & 3.874169\end{array}$

$1_{\mathrm{C} 7-} \psi_{-1 \mathrm{~s} 2 \mathrm{a3s4a}}$

Number of imaginary frequencies : 0

The smallest frequencies are : $16.1912 \quad 19.958922 .3874 \mathrm{~cm}(-1)$

Electronic energy :

$\mathrm{HF}=-4125.4648469$

Zero-point correction $=$

1.299477 (Hartree/Particle)

Thermal correction to Energy=

1.377851

Thermal correction to Enthalpy=

1.378795 
Thermal correction to Gibbs Free Energy= Sum of electronic and zero-point Energies= Sum of electronic and thermal Energies= Sum of electronic and thermal Enthalpies= Sum of electronic and thermal Free Energies=

\author{
1.187807 \\ $-4124.165370$ \\ $-4124.086996$ \\ $-4124.086052$ \\ $-4124.277040$
}

Cartesian Coordinates

\begin{tabular}{|c|c|c|c|}
\hline 6 & -0.828603 & 2.732135 & 1.236261 \\
\hline 6 & -1.830534 & 2.155321 & 0.457150 \\
\hline 6 & -2.874679 & 2.947626 & -0.025059 \\
\hline 6 & -2.941155 & 4.305157 & 0.281749 \\
\hline 6 & -1.943605 & 4.844109 & 1.104868 \\
\hline 6 & -0.887508 & 4.081330 & 1.603363 \\
\hline 15 & -1.679736 & 0.396880 & -0.046132 \\
\hline 6 & -3.399097 & -0.094322 & -0.473544 \\
\hline 6 & -4.442076 & 0.016002 & 0.455438 \\
\hline 6 & -5.727383 & -0.405443 & 0.133755 \\
\hline 6 & -5.937099 & -0.967422 & -1.137564 \\
\hline 6 & -4.923836 & -1.085266 & -2.084382 \\
\hline 6 & -3.649771 & -0.624170 & -1.729420 \\
\hline 6 & -6.889511 & -0.309352 & 1.121139 \\
\hline 6 & -7.325950 & -1.723143 & 1.521414 \\
\hline 6 & -5.148933 & -1.651448 & -3.484257 \\
\hline 6 & -4.025551 & -2.625056 & -3.856693 \\
\hline 6 & -4.070137 & 5.201051 & -0.224080 \\
\hline 6 & -3.479436 & 6.325364 & -1.081378 \\
\hline 6 & 0.140121 & 4.640196 & 2.586494 \\
\hline 6 & 1.562596 & 4.337134 & 2.109552 \\
\hline 6 & -1.492673 & -0.464261 & 1.595048 \\
\hline 6 & -1.113214 & -1.797487 & 1.659950 \\
\hline 6 & -0.987799 & -2.450302 & 2.931703 \\
\hline 6 & -1.259035 & -1.724885 & 4.127692 \\
\hline 6 & -1.674027 & -0.378133 & 4.025232 \\
\hline 6 & -1.788548 & 0.226976 & 2.803174 \\
\hline 6 & -0.580051 & -3.805357 & 3.053543 \\
\hline 6 & -0.438341 & -4.394451 & 4.285727 \\
\hline 6 & -0.704345 & -3.667507 & 5.464824 \\
\hline 6 & -1.108999 & -2.359793 & 5.383398 \\
\hline 6 & -0.873972 & -2.645363 & 0.458963 \\
\hline 6 & 0.374929 & -2.727468 & -0.129138 \\
\hline 6 & 0.624071 & -3.671264 & -1.153632 \\
\hline 6 & -0.364682 & -4.505290 & -1.600836 \\
\hline 6 & -1.666974 & -4.428212 & -1.054357 \\
\hline 6 & -1.929047 & -3.487590 & -0.014299 \\
\hline 6 & -2.709378 & -5.274406 & -1.505707 \\
\hline 6 & -3.970569 & -5.181432 & -0.973745 \\
\hline 6 & -4.240660 & -4.233278 & 0.036746 \\
\hline 6 & -3.244629 & -3.417162 & 0.512820 \\
\hline 7 & 1.432893 & -1.836700 & 0.262425 \\
\hline 15 & 1.752694 & -0.621589 & -0.882321 \\
\hline 8 & 2.915342 & -1.208799 & -1.948956 \\
\hline 6 & 4.143136 & -1.645308 & -1.509220 \\
\hline 6 & 5.074390 & -0.756419 & -1.000283 \\
\hline 6 & 6.264819 & -1.293403 & -0.411115 \\
\hline 6 & 6.525606 & -2.693879 & -0.497082 \\
\hline
\end{tabular}




\begin{tabular}{|c|c|c|c|}
\hline 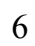 & 5.598457 & -3.532547 & -1.163054 \\
\hline & 4.423575 & -3.022736 & -1.643319 \\
\hline & 7.170064 & -0.490827 & 0.328988 \\
\hline & 8.276444 & -1.040017 & 0.929009 \\
\hline & 8.548406 & -2.419410 & 0.812974 \\
\hline & 7.687270 & -3.225815 & 0.113441 \\
\hline & 4.792601 & 0.696200 & -1.044106 \\
\hline & 3.608682 & 1.170089 & -0.514558 \\
\hline & 3.267466 & 2.534682 & -0.514397 \\
\hline & 4.122305 & 3.446903 & -1.069499 \\
\hline & 5.330271 & 3.023495 & -1.674089 \\
\hline & 5.667108 & 1.635109 & -1.682567 \\
\hline & 6.197824 & 3.955157 & -2.294388 \\
\hline & 7.343766 & 3.542968 & -2.924032 \\
\hline 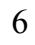 & 7.661470 & 2.169437 & -2.966575 \\
\hline & 6.847377 & 1.242570 & -2.364252 \\
\hline 3 & 2.730153 & 0.297421 & 0.082715 \\
\hline O & 2.403503 & -2.376341 & 1.243363 \\
\hline 6 & 2.906469 & -1.389297 & 2.264523 \\
\hline 6 & 4.265227 & -1.336946 & 2.568884 \\
\hline & 4.744181 & -0.450602 & 3.530936 \\
\hline 6 & 3.865207 & 0.391673 & 4.202588 \\
\hline 6 & 2.504263 & 0.342782 & 3.903942 \\
\hline & 2.030830 & -0.538658 & 2.941266 \\
\hline & -0.105847 & 0.321593 & -1.934863 \\
\hline 6 & -0.595308 & 0.288559 & -3.775544 \\
\hline 8 & -0.884671 & 0.207437 & -4.893595 \\
\hline 6 & 0.261121 & 2.245788 & -1.902298 \\
\hline 8 & 0.366022 & 3.390683 & -1.975589 \\
\hline 6 & -6.504822 & 0.448957 & 2.390064 \\
\hline 6 & -8.068959 & 0.423634 & 0.473068 \\
\hline 6 & -6.473360 & -2.404445 & -3.590355 \\
\hline 6 & -5.157667 & -0.487500 & -4.482021 \\
\hline 6 & -5.081040 & 4.432925 & -1.072910 \\
\hline 6 & -4.818390 & 5.806533 & 0.968924 \\
\hline 6 & 0.013011 & 6.150847 & 2.767646 \\
\hline 6 & -0.088584 & 3.970345 & 3.947230 \\
\hline 1 & 6.961673 & 0.570083 & 0.444017 \\
\hline 1 & -2.113452 & 1.263195 & 2.761063 \\
\hline 1 & 7.872159 & -4.295864 & 0.027625 \\
\hline 1 & -1.319646 & -1.783939 & 6.283499 \\
\hline 1 & 5.924772 & 5.009306 & -2.268972 \\
\hline 1 & -3.463749 & -2.689876 & 1.292840 \\
\hline 1 & -5.247369 & -4.149534 & 0.441851 \\
\hline 1 & 3.874255 & 4.507468 & -1.071504 \\
\hline 1 & -1.905201 & 0.174347 & 4.935425 \\
\hline 1 & 5.822975 & -4.593440 & -1.260724 \\
\hline 1 & 8.557112 & 1.839333 & -3.488164 \\
\hline 1 & 3.687910 & -3.653564 & -2.137234 \\
\hline 1 & -2.488097 & -5.993708 & -2.293163 \\
\hline 1 & -0.165382 & -5.230981 & -2.387774 \\
\hline 1 & -0.120532 & -5.432372 & 4.353175 \\
\hline 1 & 7.102007 & 0.187410 & -2.418642 \\
\hline 1 & -0.376104 & -4.383634 & 2.154726 \\
\hline 1 & 1.622334 & -3.710504 & -1.584356 \\
\hline 1 & 7.999642 & 4.267176 & -3.401555 \\
\hline
\end{tabular}




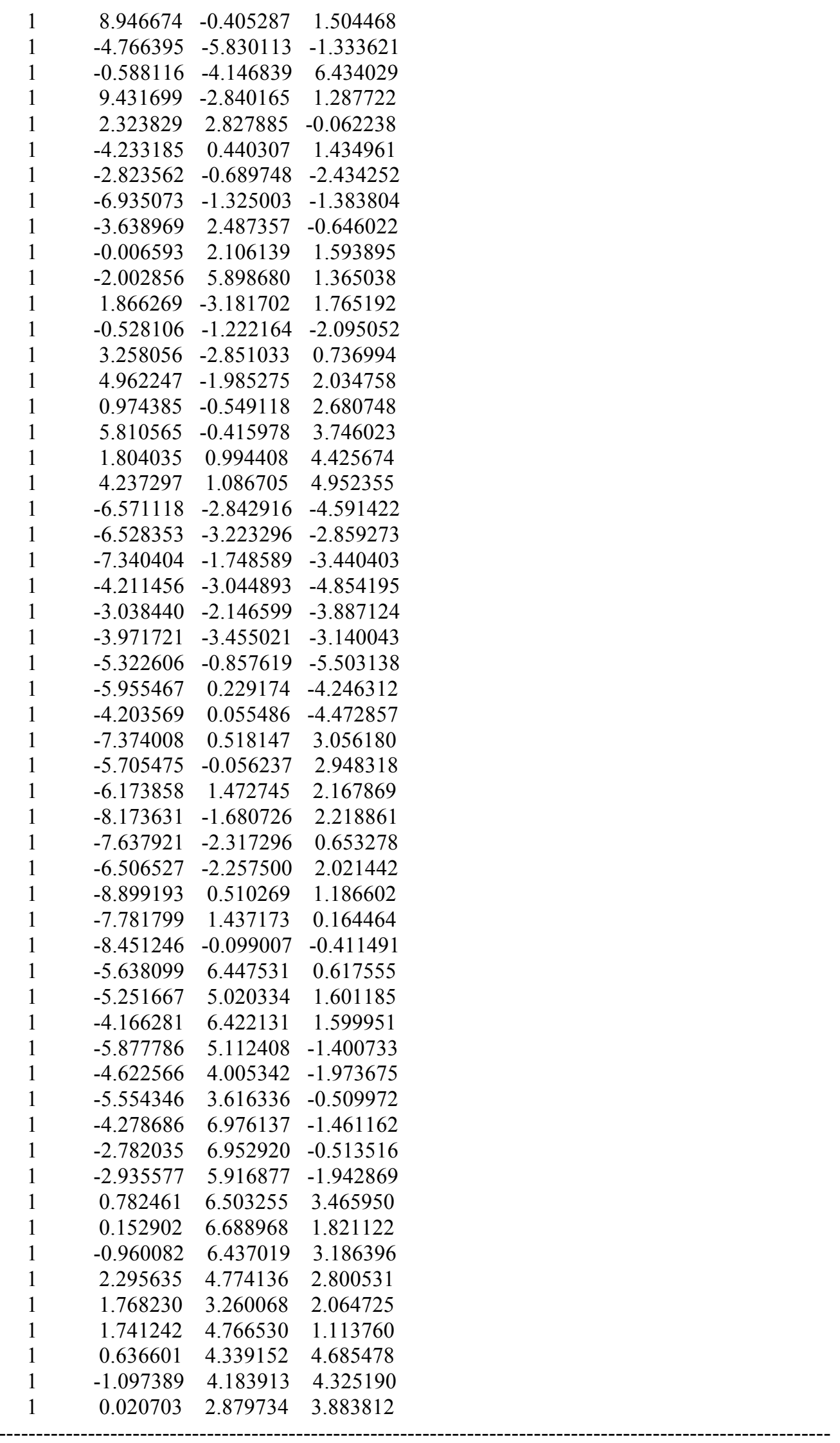


$1_{\mathrm{C} 7-} \psi_{-1 \mathrm{~s} 2 \mathrm{a} s \mathrm{~s} 4 \mathrm{~s}}$

Number of imaginary frequencies : 0

The smallest frequencies are : $11.3711 \quad 15.9065 \quad 18.9344 \mathrm{~cm}(-1)$

Electronic energy : $\quad H F=-4125.4651769$

Zero-point correction $=\quad 1.299185($ Hartree/Particle $)$

Thermal correction to Energy= $\quad 1.377704$

Thermal correction to Enthalpy= $\quad 1.378648$

Thermal correction to Gibbs Free Energy $=\quad 1.185541$

Sum of electronic and zero-point Energies $=\quad-4124.165992$

Sum of electronic and thermal Energies $=\quad-4124.087473$

Sum of electronic and thermal Enthalpies $=\quad-4124.086529$

Sum of electronic and thermal Free Energies $=\quad-4124.279636$

Cartesian Coordinates

\begin{tabular}{cccc}
\hline 6 & 3.900737 & 0.162395 & 4.212405 \\
6 & 4.766189 & -0.650120 & 3.488282 \\
6 & 4.268432 & -1.494504 & 2.498464 \\
6 & 2.903964 & -1.534215 & 2.219448 \\
6 & 2.041863 & -0.715667 & 2.950888 \\
6 & 2.534046 & 0.125172 & 3.939768 \\
6 & 2.378332 & -2.465577 & 1.158422 \\
7 & 1.420408 & -1.860227 & 0.202688 \\
6 & 0.346566 & -2.711391 & -0.230094 \\
6 & -0.895546 & -2.648665 & 0.374969 \\
6 & -1.964074 & -3.454168 & -0.131474 \\
6 & -1.727140 & -4.323049 & -1.237903 \\
6 & -0.432529 & -4.380256 & -1.804654 \\
6 & 0.573452 & -3.593370 & -1.313197 \\
6 & -3.269494 & -3.413709 & 0.423648 \\
6 & -4.281292 & -4.182142 & -0.096288 \\
6 & -4.039088 & -5.052261 & -1.180959 \\
6 & -2.786320 & -5.120792 & -1.735850 \\
6 & -1.121006 & -1.846036 & 1.609465 \\
6 & -1.483113 & -0.505851 & 1.591675 \\
6 & -1.773327 & 0.145237 & 2.822746 \\
6 & -1.673013 & -0.505910 & 4.022647 \\
6 & -1.275637 & -1.860656 & 4.077285 \\
6 & -1.008216 & -2.545434 & 2.856655 \\
6 & -1.137671 & -2.543089 & 5.309345 \\
6 & -0.748439 & -3.857397 & 5.344058 \\
6 & -0.486926 & -4.544223 & 4.139995 \\
6 & -0.617556 & -3.908969 & 2.929837 \\
15 & -1.666759 & 0.408548 & -0.020548 \\
45 & -0.079378 & 0.421354 & -1.894702 \\
6 & 0.308000 & 2.338359 & -1.768275 \\
8 & 0.426224 & 3.484038 & -1.791325 \\
15 & 1.762143 & -0.593936 & -0.877430 \\
8 & 2.749176 & 0.257593 & 0.137277 \\
6 & 3.626892 & 1.162574 & -0.409949 \\
6 & 4.803761 & 0.718474 & -0.980235 \\
6 & 5.671128 & 1.692734 & -1.573630 \\
6 & 5.338357 & 3.078842 & -1.476392 \\
6 & 4.141465 & 3.467905 & -0.828001 \\
6 & 3.291108 & 2.525516 & -0.318369
\end{tabular}




\begin{tabular}{|c|c|c|c|}
\hline 6 & 6.198957 & 4.044276 & -2.053138 \\
\hline 6 & 7.333415 & 3.668303 & -2.724872 \\
\hline & 7.646252 & 2.299136 & -2.855228 \\
\hline & 6.839229 & 1.339436 & -2.296428 \\
\hline & 5.086410 & -0.734577 & -1.017274 \\
\hline & 4.147841 & -1.598089 & -1.555737 \\
\hline & 4.419836 & -2.969803 & -1.750373 \\
\hline & 5.600280 & -3.502888 & -1.311104 \\
\hline & 6.541789 & -2.695429 & -0.627151 \\
\hline & 6.285230 & -1.299938 & -0.472217 \\
\hline & 7.206019 & -0.534426 & 0.287819 \\
\hline & 8.323154 & -1.112021 & 0.839373 \\
\hline & 8.590925 & -2.484427 & 0.653423 \\
\hline ) & 7.714185 & -3.256382 & -0.065109 \\
\hline & 2.919627 & -1.138904 & -1.969641 \\
\hline & -3.374674 & -0.100690 & -0.473315 \\
\hline b & -4.397505 & -0.113171 & 0.475532 \\
\hline & -5.676034 & -0.562633 & 0.149810 \\
\hline D & -5.902610 & -1.000324 & -1.161625 \\
\hline & -4.903748 & -0.986871 & -2.138424 \\
\hline & -3.638483 & -0.522477 & -1.770412 \\
\hline & -6.757419 & -0.564326 & 1.230047 \\
\hline & -5.141609 & -1.425749 & -3.581483 \\
\hline 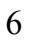 & -1.861531 & 2.151328 & 0.522708 \\
\hline 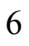 & -2.903841 & 2.931943 & 0.016202 \\
\hline b & -2.994954 & 4.287484 & 0.323668 \\
\hline & -2.031810 & 4.834250 & 1.182051 \\
\hline & -0.978682 & 4.084233 & 1.704984 \\
\hline 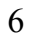 & -0.888908 & 2.738351 & 1.330616 \\
\hline 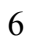 & -4.113428 & 5.174570 & -0.219891 \\
\hline 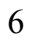 & 0.025295 & 4.657677 & 2.704103 \\
\hline 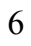 & -0.557954 & 0.479107 & -3.737101 \\
\hline & -0.844001 & 0.451739 & -4.858686 \\
\hline & 7.003109 & 0.520295 & 0.456225 \\
\hline & -2.087641 & 1.185503 & 2.815919 \\
\hline & 7.894933 & -4.321514 & -0.204503 \\
\hline 1 & -1.344579 & -1.998141 & 6.229311 \\
\hline & 5.929649 & 5.095586 & -1.959758 \\
\hline 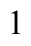 & -3.471314 & -2.743735 & 1.258170 \\
\hline | & -5.280791 & -4.122458 & 0.331144 \\
\hline & 3.897940 & 4.527408 & -0.760645 \\
\hline 1 & -1.901108 & 0.015706 & 4.951611 \\
\hline & 5.819604 & -4.559304 & -1.457003 \\
\hline 1 & 8.532287 & 1.999285 & -3.410425 \\
\hline & 3.673465 & -3.577463 & -2.257248 \\
\hline 1 & -2.584285 & -5.783211 & -2.576697 \\
\hline l & -0.252584 & -5.053849 & -2.641061 \\
\hline 1 & -0.181606 & -5.587601 & 4.170670 \\
\hline 1 & 7.089158 & 0.288718 & -2.418109 \\
\hline 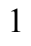 & -0.418540 & -4.455700 & 2.010400 \\
\hline 1 & 1.567121 & -3.618030 & -1.755950 \\
\hline ( & 7.983790 & 4.418525 & -3.168566 \\
\hline 1 & 9.005579 & -0.505169 & 1.430357 \\
\hline ] & -4.848211 & -5.662496 & -1.576667 \\
\hline 1 & -0.640915 & -4.373010 & 6.295481 \\
\hline 1 & 9.483010 & -2.927521 & 1.089872 \\
\hline
\end{tabular}




\begin{tabular}{|c|c|c|c|}
\hline 1 & 2.354505 & 2.791923 & 0.165017 \\
\hline & -4.186708 & 0.225707 & 1.489796 \\
\hline & -2.827397 & -0.498086 & -2.495526 \\
\hline & -6.889414 & -1.369447 & -1.426178 \\
\hline & -3.642407 & 2.465735 & -0.631319 \\
\hline & -0.062112 & 2.125131 & 1.699092 \\
\hline 1 & -2.114754 & 5.885907 & 1.447109 \\
\hline l & 1.821861 & -3.280014 & 1.644559 \\
\hline 1 & -0.513768 & -1.108281 & -2.135226 \\
\hline 1 & 3.220889 & -2.937511 & 0.629723 \\
\hline 1 & 4.954601 & -2.116978 & 1.921343 \\
\hline 1 & 0.980715 & -0.715799 & 2.711071 \\
\hline 1 & 5.836376 & -0.624148 & 3.684662 \\
\hline 1 & 1.843917 & 0.754072 & 4.501013 \\
\hline 1 & 4.288144 & 0.825388 & 4.983188 \\
\hline 6 & -6.476638 & -2.146994 & -3.750207 \\
\hline 6 & -5.134793 & -0.181477 & -4.477340 \\
\hline 6 & -4.031902 & -2.379961 & -4.036240 \\
\hline 6 & -8.064592 & -1.186510 & 0.746264 \\
\hline 6 & -6.275667 & -1.365711 & 2.445899 \\
\hline 6 & -7.040842 & 0.881568 & 1.654069 \\
\hline 1 & -6.586482 & -2.484315 & -4.788544 \\
\hline 1 & -6.538878 & -3.032962 & -3.102989 \\
\hline 1 & -7.332785 & -1.496832 & -3.529108 \\
\hline 1 & -4.215289 & -2.702960 & -5.069705 \\
\hline 1 & -3.036941 & -1.917500 & -4.011998 \\
\hline 1 & -3.999068 & -3.272342 & -3.397840 \\
\hline 1 & -5.312964 & -0.461371 & -5.524583 \\
\hline 1 & -5.918199 & 0.526667 & -4.176455 \\
\hline 1 & -4.171163 & 0.342291 & -4.431278 \\
\hline 1 & -8.800493 & -1.178795 & 1.560226 \\
\hline 1 & -8.500384 & -0.630583 & -0.093573 \\
\hline 1 & -7.929164 & -2.230632 & 0.433763 \\
\hline 1 & -7.801569 & 0.908054 & 2.446045 \\
\hline 1 & -6.141683 & 1.380960 & 2.036890 \\
\hline 1 & -7.414778 & 1.470782 & 0.806658 \\
\hline 1 & -7.040392 & -1.355217 & 3.234185 \\
\hline 1 & -6.087910 & -2.414636 & 2.179025 \\
\hline 1 & -5.351788 & -0.962026 & 2.879435 \\
\hline 6 & -5.051449 & 4.415278 & -1.155631 \\
\hline 6 & -4.943476 & 5.719371 & 0.947868 \\
\hline 6 & -3.503805 & 6.342293 & -1.002940 \\
\hline 6 & -0.141009 & 6.162926 & 2.898394 \\
\hline 6 & -0.193269 & 3.970728 & 4.057959 \\
\hline 6 & 1.457942 & 4.395000 & 2.231902 \\
\hline 1 & -5.753321 & 6.360090 & 0.573637 \\
\hline 1 & -5.397735 & 4.900830 & 1.521775 \\
\hline 1 & -4.339633 & 6.319316 & 1.639437 \\
\hline 1 & -5.836643 & 5.091459 & -1.516561 \\
\hline 1 & -4.524666 & 4.022785 & -2.034948 \\
\hline 1 & -5.547265 & 3.575248 & -0.651212 \\
\hline 1 & -4.297747 & 6.981760 & -1.411742 \\
\hline 1 & -2.862716 & 6.972493 & -0.375046 \\
\hline 1 & -2.896106 & 5.977807 & -1.841239 \\
\hline 1 & 0.609106 & 6.526380 & 3.611876 \\
\hline 1 & 0.000297 & 6.714158 & 1.959675 \\
\hline
\end{tabular}




$\begin{array}{rrrr}1 & -1.127067 & 6.421152 & 3.304789 \\ 1 & 2.176426 & 4.858197 & 2.921078 \\ 1 & 1.693644 & 3.324052 & 2.196545 \\ 1 & 1.625669 & 4.820396 & 1.232548 \\ 1 & 0.514500 & 4.357102 & 4.804003 \\ 1 & -1.210650 & 4.151224 & 4.429939 \\ 1 & -0.048665 & 2.884455 & 3.990803\end{array}$

$1_{\mathrm{C} 7-} \psi_{-1 \mathrm{~s} 2 \mathrm{~s} 3 \mathrm{a} 4 \mathrm{a}}$

Number of imaginary frequencies : 0

The smallest frequencies are : $13.4179 \quad 14.2424 \quad 16.6526 \mathrm{~cm}(-1)$

Electronic energy : $\quad H F=-4125.4647916$

Zero-point correction $=\quad 1.298775($ Hartree/Particle $)$

Thermal correction to Energy= $\quad 1.377481$

Thermal correction to Enthalpy $=\quad 1.378425$

Thermal correction to Gibbs Free Energy= $\quad 1.184633$

Sum of electronic and zero-point Energies $=\quad-4124.166017$

Sum of electronic and thermal Energies $=\quad-4124.087311$

Sum of electronic and thermal Enthalpies $=\quad-4124.086367$

Sum of electronic and thermal Free Energies $=\quad-4124.280158$

Cartesian Coordinates

$\begin{array}{cccc}6 & 3.938052 & 0.198013 & 4.230740 \\ 6 & 4.812003 & -0.600168 & 3.500972 \\ 6 & 4.322813 & -1.444505 & 2.506884 \\ 6 & 2.958558 & -1.497906 & 2.228478 \\ 6 & 2.088243 & -0.691455 & 2.963574 \\ 6 & 2.571863 & 0.148096 & 3.957814 \\ 6 & 2.442247 & -2.438940 & 1.171532 \\ 7 & 1.466453 & -1.853934 & 0.222097 \\ 6 & 0.401912 & -2.721566 & -0.200349 \\ 6 & -0.841274 & -2.661578 & 0.402572 \\ 6 & -1.906392 & -3.470369 & -0.103025 \\ 6 & -1.666842 & -4.333710 & -1.213156 \\ 6 & -0.370242 & -4.392689 & -1.774657 \\ 6 & 0.634513 & -3.606862 & -1.279011 \\ 6 & -3.211988 & -3.434548 & 0.453294 \\ 6 & -4.220383 & -4.206979 & -0.065826 \\ 6 & -3.975623 & -5.069033 & -1.157144 \\ 6 & -2.726223 & -5.125916 & -1.719634 \\ 6 & -1.072531 & -1.849649 & 1.629216 \\ 6 & -1.459595 & -0.517161 & 1.595130 \\ 6 & -1.751241 & 0.145939 & 2.819658 \\ 6 & -1.624626 & -0.485167 & 4.027688 \\ 6 & -1.201820 & -1.831632 & 4.097722 \\ 6 & -0.937041 & -2.529925 & 2.884233 \\ 6 & -1.035515 & -2.493013 & 5.337677 \\ 6 & -0.623250 & -3.799949 & 5.386433 \\ 6 & -0.367077 & -4.500803 & 4.189350 \\ 6 & -0.524147 & -3.885885 & 2.971828 \\ 15 & -1.667448 & 0.371301 & -0.029087 \\ 45 & -0.082939 & 0.374297 & -1.907430 \\ 6 & 0.270375 & 2.300252 & -1.812877 \\ 8 & 0.372160 & 3.446792 & -1.859122\end{array}$




\begin{tabular}{|c|c|c|c|}
\hline & 1.778185 & -0.594892 & -0.875592 \\
\hline 8 & 2.758259 & 0.286916 & 0.120771 \\
\hline 6 & 3.622123 & 1.192709 & -0.447835 \\
\hline 6 & 4.803908 & 0.753229 & -1.011320 \\
\hline 6 & 5.659358 & 1.727096 & -1.622585 \\
\hline 6 & 5.308487 & 3.110318 & -1.552345 \\
\hline 6 & 4.104967 & 3.495766 & -0.914330 \\
\hline 6 & 3.267025 & 2.552386 & -0.386261 \\
\hline 6 & 6.157379 & 4.075862 & -2.145996 \\
\hline 6 & 7.297872 & 3.702132 & -2.808669 \\
\hline 6 & 7.628616 & 2.334930 & -2.912620 \\
\hline 6 & 6.833083 & 1.375490 & -2.337117 \\
\hline 6 & 5.102069 & -0.696972 & -1.029184 \\
\hline 6 & 4.172632 & -1.575968 & -1.558319 \\
\hline 6 & 4.461835 & -2.945542 & -1.743747 \\
\hline 6 & 5.648795 & -3.460894 & -1.300715 \\
\hline 6 & 6.579957 & -2.637122 & -0.621915 \\
\hline 6 & 6.307137 & -1.243459 & -0.479342 \\
\hline 6 & 7.217828 & -0.460244 & 0.274708 \\
\hline 6 & 8.340458 & -1.019946 & 0.833457 \\
\hline 6 & 8.624044 & -2.390926 & 0.660481 \\
\hline 6 & 7.757782 & -3.179414 & -0.052905 \\
\hline 8 & 2.936913 & -1.135562 & -1.969955 \\
\hline 6 & -3.388059 & -0.127727 & -0.450732 \\
\hline 6 & -4.410015 & -0.036992 & 0.498825 \\
\hline 6 & -5.710666 & -0.427268 & 0.188110 \\
\hline 6 & -5.951447 & -0.916251 & -1.102411 \\
\hline 6 & -4.955935 & -1.017941 & -2.074877 \\
\hline 6 & -3.664307 & -0.611562 & -1.725066 \\
\hline 6 & -6.846578 & -0.372412 & 1.208586 \\
\hline 6 & -5.298784 & -1.523703 & -3.474926 \\
\hline 6 & -1.849229 & 2.120103 & 0.502034 \\
\hline 6 & -2.895325 & 2.898277 & \\
\hline 6 & -2.996311 & 4.254802 & 0.336053 \\
\hline 6 & -2.027151 & 4.804296 & 1.177355 \\
\hline 6 & -0.957618 & 4.052448 & 1.678947 \\
\hline 6 & -0.865389 & 2.711863 & 1.300493 \\
\hline 6 & -4.170514 & 5.058264 & -0.219173 \\
\hline 6 & 0.050924 & 4.629538 & 2.672545 \\
\hline 6 & -0.491847 & 0.390797 & -3.765970 \\
\hline 8 & -0.705586 & 0.343389 & -4.902747 \\
\hline 1 & 7.001841 & 0.593445 & 0.433331 \\
\hline 1 & -2.083701 & 1.180498 & 2.802069 \\
\hline 1 & 7.951206 & -4.243566 & -0.182416 \\
\hline 1 & -1.239708 & -1.938309 & 6.252415 \\
\hline 1 & 5.874074 & 5.125105 & -2.073087 \\
\hline 1 & -3.414510 & -2.767707 & 1.289868 \\
\hline 1 & -5.219366 & -4.153835 & 0.362972 \\
\hline 1 & 3.846268 & 4.552870 & -0.869546 \\
\hline 1 & -1.851513 & 0.046217 & 4.951422 \\
\hline 1 & 5.880961 & -4.515616 & -1.438847 \\
\hline 1 & 8.519496 & 2.036180 & -3.460615 \\
\hline 1 & 3.722848 & -3.565815 & -2.246216 \\
\hline 1 & -2.523736 & -5.783507 & -2.563972 \\
\hline 1 & -0.188186 & -5.065485 & -2.611239 \\
\hline 1 & -0.044822 & -5.538676 & 4.231441 \\
\hline
\end{tabular}




\begin{tabular}{|c|c|c|c|}
\hline 1 & 7.097129 & 0.326095 & -2.438562 \\
\hline & -0.328704 & -4.442739 & 2.057712 \\
\hline 1 & 1.629641 & -3.631014 & -1.718436 \\
\hline & 7.939262 & 4.452266 & -3.265404 \\
\hline & 9.014834 & -0.400109 & 1.420197 \\
\hline 1 & -4.781884 & -5.684849 & -1.550374 \\
\hline & -0.494047 & -4.299204 & 6.343868 \\
\hline 1 & 9.520223 & -2.819831 & 1.102678 \\
\hline & 2.326023 & 2.816320 & 0.089442 \\
\hline 1 & -4.177889 & 0.347609 & 1.489369 \\
\hline 1 & -2.845047 & -0.672022 & -2.437911 \\
\hline 1 & -6.963602 & -1.229821 & -1.359421 \\
\hline 1 & -3.645743 & 2.437495 & -0.624860 \\
\hline 1 & -0.032729 & 2.099846 & 1.657827 \\
\hline 1 & -2.105759 & 5.851211 & 1.453342 \\
\hline 1 & 1.904889 & -3.262619 & 1.663547 \\
\hline 1 & -0.504186 & -1.161202 & -2.128134 \\
\hline 1 & 3.289566 & -2.896621 & 0.638480 \\
\hline 1 & 5.015178 & -2.057641 & 1.927119 \\
\hline 1 & 1.026898 & -0.702451 & 2.724982 \\
\hline 1 & 5.882017 & -0.563649 & 3.696663 \\
\hline 1 & 1.875366 & 0.765354 & 4.523909 \\
\hline 1 & 4.318361 & 0.859919 & 5.005975 \\
\hline 6 & -4.049657 & -1.737786 & -4.327715 \\
\hline 6 & -6.046061 & -2.857632 & -3.390879 \\
\hline 6 & -6.187861 & -0.485451 & -4.168886 \\
\hline 6 & -6.412025 & 0.277606 & 2.520563 \\
\hline 6 & -8.020964 & 0.434754 & 0.646011 \\
\hline 6 & -7.309794 & & \\
\hline 1 & -4.335747 & -2.146204 & -5.305183 \\
\hline 1 & -3.510175 & -0.800556 & -4.513670 \\
\hline 1 & -3.352782 & -2.447085 & -3.859373 \\
\hline 1 & -6.441779 & -0.815761 & -5.185311 \\
\hline 1 & -7.126897 & -0.323841 & -3.624476 \\
\hline 1 & -5.674570 & 0.481973 & -4.24 \\
\hline 1 & -6.300964 & -3.210735 & -4.399011 \\
\hline 1 & -5.421611 & -3.622138 & -2.910420 \\
\hline 1 & -6.982411 & -2.781181 & -2.824887 \\
\hline 1 & -7.260106 & 0.306154 & 3.216235 \\
\hline 1 & -5.604456 & -0.281721 & 3.011797 \\
\hline 1 & -6.071142 & 1.310990 & 2.371163 \\
\hline 1 & -8.140548 & -1.789843 & 2.233914 \\
\hline 1 & -7.654745 & -2.324025 & 0.614443 \\
\hline 1 & -6.492975 & -2.389084 & 1.955935 \\
\hline 1 & -8.835634 & 0.481172 & 1.380928 \\
\hline 1 & -7.717681 & 1.464113 & 0.414287 \\
\hline 1 & -8.430858 & -0.006827 & -0.269965 \\
\hline 6 & -4.145744 & 6.516624 & 0.230763 \\
\hline 6 & -4.126477 & 5.029866 & -1.751072 \\
\hline 6 & -5.483754 & 4.430912 & 0.264865 \\
\hline 6 & -0.112646 & 6.135863 & 2.862930 \\
\hline 6 & -0.165856 & 3.947544 & 4.029309 \\
\hline 6 & 1.482642 & 4.365071 & 2.200011 \\
\hline 1 & -4.959314 & 5.612916 & -2.166885 \\
\hline 1 & -3.189320 & 5.462302 & -2.124824 \\
\hline 1 & -4.205309 & 4.010691 & -2.147742 \\
\hline
\end{tabular}




$\begin{array}{rrrr}1 & -5.006412 & 7.047431 & -0.195307 \\ 1 & -4.209723 & 6.612197 & 1.322478 \\ 1 & -3.239631 & 7.035008 & -0.108262 \\ 1 & -6.342588 & 4.988128 & -0.133075 \\ 1 & -5.586204 & 3.386898 & -0.058352 \\ 1 & -5.546989 & 4.449094 & 1.361107 \\ 1 & 0.645291 & 6.500961 & 3.567295 \\ 1 & 0.019163 & 6.683829 & 1.920943 \\ 1 & -1.093820 & 6.396969 & 3.279044 \\ 1 & 2.201993 & 4.819802 & 2.893916 \\ 1 & 1.715084 & 3.293848 & 2.155171 \\ 1 & 1.653541 & 4.799028 & 1.204873 \\ 1 & 0.539787 & 4.339549 & 4.774501 \\ 1 & -1.184331 & 4.125371 & 4.399770 \\ 1 & -0.017100 & 2.861733 & 3.965607\end{array}$

$1_{\mathrm{C} 7-\psi_{-1} \text { s2s3a4s }}$

Number of imaginary frequencies : 0

The smallest frequencies are : $13.6646 \quad 17.3660 \quad 19.8257 \mathrm{~cm}(-1)$

Electronic energy : $\quad H F=-4125.4651023$

Zero-point correction $=\quad 1.299143($ Hartree/Particle $)$

Thermal correction to Energy $=\quad 1.377583$

Thermal correction to Enthalpy= $\quad 1.378527$

Thermal correction to Gibbs Free Energy= 1.186336

Sum of electronic and zero-point Energies $=\quad-4124.165959$

Sum of electronic and thermal Energies $=\quad-4124.087520$

Sum of electronic and thermal Enthalpies $=\quad-4124.086575$

Sum of electronic and thermal Free Energies $=\quad-4124.278767$

Cartesian Coordinates

$\begin{array}{lrrr}6 & 3.927119 & 0.149961 & 4.212315 \\ 6 & 4.796155 & -0.652425 & 3.481259 \\ 6 & 4.302043 & -1.491453 & 2.485062 \\ 6 & 2.937593 & -1.535888 & 2.206536 \\ 6 & 2.072015 & -0.726776 & 2.944388 \\ 6 & 2.560579 & 0.108551 & 3.939682 \\ 6 & 2.415986 & -2.464035 & 1.140735 \\ 7 & 1.453438 & -1.859760 & 0.189588 \\ 6 & 0.383541 & -2.711886 & -0.250261 \\ 6 & -0.856606 & -2.663229 & 0.360172 \\ 6 & -1.926893 & -3.456488 & -0.159216 \\ 6 & -1.698328 & -4.286346 & -1.296810 \\ 6 & -0.405445 & -4.333292 & -1.867987 \\ 6 & 0.606127 & -3.567862 & -1.354531 \\ 6 & -3.227711 & -3.435609 & 0.409004 \\ 6 & -4.241569 & -4.191248 & -0.124160 \\ 6 & -4.007938 & -5.019884 & -1.243201 \\ 6 & -2.763577 & -5.060583 & -1.818331 \\ 6 & -1.083183 & -1.872612 & 1.601411 \\ 6 & -1.459319 & -0.536395 & 1.587933 \\ 6 & -1.750687 & 0.108540 & 2.822054 \\ 6 & -1.639213 & -0.545418 & 4.019639 \\ 6 & -1.229568 & -1.896773 & 4.068729 \\ 6 & -0.960360 & -2.575298 & 2.845072\end{array}$




\begin{tabular}{|c|c|c|c|}
\hline 6 & -1.081380 & -2.582507 & 5.297757 \\
\hline 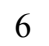 & -0.681796 & -3.893903 & 5.326084 \\
\hline b & -0.420048 & -4.574821 & 4.118640 \\
\hline 6 & -0.559849 & -3.936150 & 2.911312 \\
\hline & -1.659948 & 0.378265 & -0.022628 \\
\hline & -0.075617 & 0.416085 & -1.898089 \\
\hline f & 0.285994 & 2.338526 & -1.766415 \\
\hline 8 & 0.392119 & 3.485448 & -1.787999 \\
\hline 15 & 1.778630 & -0.582388 & -0.882647 \\
\hline 8 & 2.760828 & 0.273160 & 0.133562 \\
\hline 6 & 3.630969 & 1.186137 & -0.412877 \\
\hline 6 & 4.811442 & 0.752538 & -0.983866 \\
\hline 6 & 5.671847 & 1.735077 & -1.573861 \\
\hline 6 & 5.327943 & 3.118270 & -1.473804 \\
\hline 6 & 4.126368 & 3.496268 & -0.827621 \\
\hline 6 & 3.283001 & 2.546019 & -0.321023 \\
\hline 6 & 6.181889 & 4.092056 & -2.046358 \\
\hline 6 & 7.320478 & 3.726973 & -2.717084 \\
\hline 6 & 7.644142 & 2.360665 & -2.850782 \\
\hline 6 & 6.843750 & 1.393088 & -2.295973 \\
\hline 6 & 5.102916 & -0.698617 & -1.028914 \\
\hline 6 & 4.169213 & -1.563698 & -1.573457 \\
\hline 6 & 4.449078 & -2.932364 & -1.778026 \\
\hline 6 & 5.632845 & -3.461634 & -1.343001 \\
\hline 6 & 6.569817 & -2.653611 & -0.653515 \\
\hline 6 & 6.305204 & -1.260775 & -0.488506 \\
\hline 6 & 7.221877 & -0.495182 & 0.276404 \\
\hline 6 & 8.342603 & -1.070100 & 0.823427 \\
\hline 6 & 8.618197 & -2.439635 & 0.627800 \\
\hline 6 & 7.745702 & -3.211646 & -0.095829 \\
\hline 8 & 2.938040 & -1.108381 & -1.982683 \\
\hline 6 & -3.375109 & -0.123907 & -0.460742 \\
\hline 6 & -4.385147 & -0.111533 & 0.496760 \\
\hline 6 & -5.685166 & -0.514898 & 0.181076 \\
\hline 6 & -5.937235 & -0.920021 & -1.130744 \\
\hline 6 & -4.945775 & -0.937138 & -2.120829 \\
\hline 6 & -3.660506 & -0.532490 & -1.763173 \\
\hline 6 & -6.749355 & -0.504869 & 1.277199 \\
\hline 6 & -5.294937 & -1.372783 & -3.542432 \\
\hline 6 & -1.858612 & 2.117556 & 0.531796 \\
\hline 6 & -2.908604 & 2.892451 & 0.048859 \\
\hline 6 & -3.017488 & 4.246426 & 0.376557 \\
\hline 6 & -2.055151 & 4.794864 & 1.226578 \\
\hline 6 & -0.983523 & 4.045353 & 1.727338 \\
\hline 6 & -0.881716 & 2.708400 & 1.338968 \\
\hline 6 & -4.181480 & 5.053156 & -0.195381 \\
\hline 6 & 0.020784 & 4.621375 & 2.725735 \\
\hline 6 & -0.487393 & 0.458715 & -3.755989 \\
\hline 8 & -0.704631 & 0.419832 & -4.892408 \\
\hline 1 & 7.012724 & 0.557123 & 0.452258 \\
\hline 1 & -2.074022 & 1.146113 & 2.820114 \\
\hline 1 & 7.932625 & -4.274693 & -0.242800 \\
\hline 1 & -1.289109 & -2.042716 & 6.220568 \\
\hline 1 & 5.904013 & 5.140919 & -1.950538 \\
\hline 1 & -3.422022 & -2.793630 & 1.267050 \\
\hline 1 & -5.237075 & -4.149758 & 0.314681 \\
\hline
\end{tabular}




\begin{tabular}{|c|c|c|c|}
\hline & 3.873699 & 4.553575 & -0.759346 \\
\hline & -1.867743 & -0.028496 & 4.951120 \\
\hline & 5.858310 & -4.515671 & -1.496544 \\
\hline & 8.533380 & 2.069265 & -3.405364 \\
\hline & 3.706127 & -3.540966 & -2.288819 \\
\hline & -2.569638 & -5.692109 & -2.684318 \\
\hline & -0.231232 & -4.981287 & -2.725550 \\
\hline & -0.107386 & -5.616143 & 4.144595 \\
\hline & 7.102216 & 0.344775 & -2.420296 \\
\hline 1 & -0.361196 & -4.477603 & 1.988669 \\
\hline & 1.598518 & -3.583756 & -1.800419 \\
\hline & 7.965745 & 4.483505 & -3.157512 \\
\hline & 9.021812 & -0.463461 & 1.418324 \\
\hline 1 & -4.819066 & -5.620680 & -1.649251 \\
\hline 1 & -0.566626 & -4.412017 & 6.275252 \\
\hline 1 & 9.513014 & -2.880586 & 1.060811 \\
\hline 1 & 2.342867 & 2.803935 & 0.160017 \\
\hline 1 & -4.154601 & 0.213634 & 1.511161 \\
\hline 1 & -2.849454 & -0.533267 & -2.487564 \\
\hline 1 & -6.942663 & -1.237731 & -1.400670 \\
\hline & -3.652064 & 2.432256 & -0.600644 \\
\hline 1 & -0.046599 & 2.098646 & 1.694349 \\
\hline 1 & -2.140267 & 5.839523 & 1.509227 \\
\hline & 1.865321 & -3.284667 & 1.623086 \\
\hline 1 & -0.504048 & -1.112899 & -2.145031 \\
\hline 1 & 3.260667 & -2.927981 & 0.608531 \\
\hline 1 & 4.990966 & -2.106068 & 1.902805 \\
\hline ] & 1.010776 & -0.729842 & 2.704956 \\
\hline 1 & 5.866285 & -0.622760 & 3.677403 \\
\hline 1 & 1.867704 & 0.729978 & 4.505817 \\
\hline 1 & 4.311634 & 0.808543 & 4.988309 \\
\hline 6 & -4.063970 & -1.420064 & -4.445582 \\
\hline 6 & -5.920302 & -2.770705 & -3.523788 \\
\hline 6 & -6.293582 & -0.374680 & -4.139334 \\
\hline 5 & -8.090604 & -1.045871 & 0.789982 \\
\hline 6 & -6.284950 & -1.377653 & 2.450028 \\
\hline 6 & -6.956515 & 0.933034 & 1.766351 \\
\hline 1 & -4.352458 & -1.773211 & -5.443693 \\
\hline 1 & -3.606045 & -0.430432 & -4.569746 \\
\hline 1 & -3.297132 & -2.106105 & -4.058216 \\
\hline 1 & -6.549169 & -0.658930 & -5.169122 \\
\hline 1 & -7.226726 & -0.334175 & -3.563604 \\
\hline 1 & -5.870068 & 0.637902 & -4.163356 \\
\hline 1 & -6.191721 & -3.077514 & -4.542893 \\
\hline 1 & -5.209967 & -3.505991 & -3.124088 \\
\hline 1 & -6.830384 & -2.815030 & -2.913140 \\
\hline 1 & -8.815882 & -1.032226 & 1.613309 \\
\hline 1 & -8.508251 & -0.439983 & -0.024315 \\
\hline 1 & -8.008506 & -2.083548 & 0.439529 \\
\hline 1 & -7.708423 & 0.961151 & 2.566621 \\
\hline 1 & -6.030591 & 1.368088 & 2.163304 \\
\hline 1 & -7.307304 & 1.579091 & 0.950584 \\
\hline 1 & -7.036362 & -1.368708 & 3.251068 \\
\hline 1 & -6.145909 & -2.420401 & 2.134333 \\
\hline 1 & -5.337822 & -1.031453 & 2.883531 \\
\hline 6 & -4.194329 & 6.495313 & 0.304353 \\
\hline
\end{tabular}




$\begin{array}{rrrr}6 & -4.070672 & 5.076504 & -1.724254 \\ 6 & -5.506877 & 4.395498 & 0.207665 \\ 6 & -0.149448 & 6.125939 & 2.923740 \\ 6 & -0.194379 & 3.931039 & 4.078569 \\ 6 & 1.454378 & 4.365599 & 2.253439 \\ 1 & -4.896386 & 5.656547 & -2.158279 \\ 1 & -3.126883 & 5.538233 & -2.042360 \\ 1 & -4.110214 & 4.068386 & -2.154004 \\ 1 & -5.049140 & 7.027770 & -0.131352 \\ 1 & -4.294009 & 6.551509 & 1.396219 \\ 1 & -3.286876 & 7.039386 & 0.012578 \\ 1 & -6.353004 & 4.963930 & -0.201392 \\ 1 & -5.588545 & 3.366505 & -0.165674 \\ 1 & -5.615979 & 4.365547 & 1.299940 \\ 1 & 0.606708 & 6.490886 & 3.630111 \\ 1 & -0.019865 & 6.679202 & 1.984547 \\ 1 & -1.131855 & 6.380603 & 3.340951 \\ 1 & 2.170856 & 4.819901 & 2.950590 \\ 1 & 1.691598 & 3.295634 & 2.203826 \\ 1 & 1.624467 & 4.804853 & 1.260465 \\ 1 & 0.509747 & 4.321030 & 4.826258 \\ 1 & -1.213479 & 4.103669 & 4.449723 \\ 1 & -0.042207 & 2.845931 & 4.009078\end{array}$

$1_{\mathrm{C} 7-} \Psi_{-1} \mathbf{s 2 2 3 3 4 4 a}$

Number of imaginary frequencies : 0

The smallest frequencies are : $14.9834 \quad 17.5266 \quad 22.1171 \mathrm{~cm}(-1)$

Electronic energy : $\quad H F=-4125.4652973$

Zero-point correction $=\quad 1.299563($ Hartree/Particle)

Thermal correction to Energy= $\quad 1.377867$

Thermal correction to Enthalpy= $\quad 1.378811$

Thermal correction to Gibbs Free Energy $=\quad 1.187810$

Sum of electronic and zero-point Energies $=\quad-4124.165735$

Sum of electronic and thermal Energies $=\quad-4124.087430$

Sum of electronic and thermal Enthalpies $=\quad-4124.086486$

Sum of electronic and thermal Free Energies $=\quad-4124.277487$

\section{Cartesian Coordinates}

$\begin{array}{rrrr}6 & 3.915652 & 0.233841 & 4.239451 \\ 6 & 4.790008 & -0.570740 & 3.517219 \\ 6 & 4.301620 & -1.421600 & 2.528341 \\ 6 & 2.937838 & -1.475073 & 2.247569 \\ 6 & 2.067096 & -0.662387 & 2.975095 \\ 6 & 2.549916 & 0.183651 & 3.964275 \\ 6 & 2.422311 & -2.424140 & 1.197471 \\ 7 & 1.443974 & -1.847399 & 0.244887 \\ 6 & 0.379328 & -2.722740 & -0.162412 \\ 6 & -0.868442 & -2.643647 & 0.428594 \\ 6 & -1.927553 & -3.474208 & -0.056664 \\ 6 & -1.671361 & -4.396386 & -1.114698 \\ 6 & -0.370317 & -4.469830 & -1.664877 \\ 6 & 0.623288 & -3.649616 & -1.203463 \\ 6 & -3.241069 & -3.411213 & 0.476486 \\ 6 & -4.241347 & -4.213802 & -0.013663\end{array}$




\begin{tabular}{|c|c|c|c|}
\hline 6 & -3.977788 & -5.141907 & -1.044133 \\
\hline 6 & -2.717979 & -5.229483 & -1.580260 \\
\hline 6 & -1.102533 & -1.811351 & 1.641986 \\
\hline 6 & -1.476805 & -0.475496 & 1.596798 \\
\hline 6 & -1.765929 & 0.200803 & 2.814670 \\
\hline 6 & -1.649921 & -0.421035 & 4.028262 \\
\hline 6 & -1.239338 & -1.770490 & 4.110983 \\
\hline 6 & -0.975915 & -2.481381 & 2.904412 \\
\hline 6 & -1.082713 & -2.421519 & 5.357671 \\
\hline 6 & -0.679188 & -3.730540 & 5.420279 \\
\hline 6 & -0.421995 & -4.443338 & 4.230594 \\
\hline 6 & -0.570656 & -3.838841 & 3.006818 \\
\hline 15 & -1.668175 & 0.407123 & -0.031241 \\
\hline 45 & -0.089287 & 0.362793 & -1.910878 \\
\hline 6 & 0.275522 & 2.287114 & -1.849926 \\
\hline 8 & 0.376040 & 3.433212 & -1.908438 \\
\hline 15 & 1.763925 & -0.604386 & -0.869167 \\
\hline 8 & 2.744856 & 0.285666 & 0.119006 \\
\hline 6 & 3.611763 & 1.182591 & -0.458937 \\
\hline 6 & 4.793209 & 0.733489 & -1.015361 \\
\hline 6 & 5.652197 & 1.697894 & -1.636681 \\
\hline 6 & 5.304573 & 3.082693 & -1.583631 \\
\hline 6 & 4.101081 & 3.478724 & -0.952043 \\
\hline 6 & 3.260032 & 2.543836 & -0.413868 \\
\hline 6 & 6.156758 & 4.038958 & -2.187522 \\
\hline 6 & 7.297503 & 3.654514 & -2.843602 \\
\hline 6 & 7.625256 & 2.285398 & -2.930274 \\
\hline 6 & 6.826414 & 1.334880 & -2.344661 \\
\hline 6 & 5.087945 & -0.717493 & -1.015833 \\
\hline 6 & 4.157851 & -1.600864 & -1.536377 \\
\hline 6 & 4.446031 & -2.972425 & -1.708246 \\
\hline 6 & 5.631418 & -3.484874 & -1.257767 \\
\hline 6 & 6.561991 & -2.655549 & -0.584838 \\
\hline 6 & 6.290952 & -1.260060 & -0.457627 \\
\hline 6 & 7.200764 & -0.470251 & 0.290546 \\
\hline 6 & 8.320954 & -1.025725 & 0.858352 \\
\hline 6 & 8.602869 & -2.398885 & 0.700622 \\
\hline 6 & 7.737415 & -3.193559 & -0.006898 \\
\hline 8 & 2.922782 & -1.163996 & -1.953488 \\
\hline 6 & -3.384236 & -0.089349 & -0.466235 \\
\hline 6 & -4.423173 & -0.016341 & 0.471313 \\
\hline 6 & -5.705492 & -0.442759 & 0.145193 \\
\hline 6 & -5.918707 & -0.963485 & -1.143364 \\
\hline 6 & -4.909611 & -1.043398 & -2.098360 \\
\hline 6 & -3.637159 & -0.582757 & -1.735944 \\
\hline 6 & -6.861745 & -0.393948 & 1.142927 \\
\hline 6 & -5.131829 & -1.577692 & -3.511031 \\
\hline 6 & -1.842090 & 2.158998 & 0.488010 \\
\hline 6 & -2.892417 & 2.934133 & 0.007076 \\
\hline 6 & -2.991054 & 4.292318 & 0.320696 \\
\hline 6 & -2.013792 & 4.846781 & 1.149039 \\
\hline 6 & -0.939934 & 4.097561 & 1.645898 \\
\hline 6 & -0.851777 & 2.754622 & 1.275515 \\
\hline 6 & -4.174238 & 5.090040 & -0.223482 \\
\hline 6 & 0.074892 & 4.679941 & 2.629869 \\
\hline 6 & -0.564062 & 0.352121 & -3.755154 \\
\hline
\end{tabular}




\begin{tabular}{|c|c|c|c|}
\hline & -0.846862 & 0.281588 & -4.875654 \\
\hline & 6.985740 & 0.585312 & 0.437659 \\
\hline & -2.088424 & 1.238270 & 2.786773 \\
\hline 1 & 7.929614 & -4.259297 & -0.124609 \\
\hline & -1.286767 & -1.856768 & 6.266279 \\
\hline & 5.875850 & 5.089675 & -2.127897 \\
\hline & -3.455870 & -2.700032 & 1.272485 \\
\hline & -5.246424 & -4.136051 & 0.396949 \\
\hline & 3.844883 & 4.536902 & -0.920398 \\
\hline 1 & -1.875439 & 0.120048 & 4.946664 \\
\hline & 5.862673 & -4.541143 & -1.385197 \\
\hline & 8.516454 & 1.977934 & -3.472901 \\
\hline & 3.707042 & -3.595749 & -2.206899 \\
\hline & -2.500966 & -5.934217 & -2.381980 \\
\hline & -0.175974 & -5.182087 & -2.465238 \\
\hline 1 & -0.105433 & -5.482490 & 4.283211 \\
\hline 1 & 7.088290 & 0.283742 & -2.432812 \\
\hline 1 & -0.373509 & -4.406046 & 2.099484 \\
\hline & 1.621692 & -3.686446 & -1.634692 \\
\hline ] & 7.941433 & 4.397560 & -3.308279 \\
\hline ] & 8.994603 & -0.400794 & 1.440504 \\
\hline 1 & -4.777043 & -5.780073 & -1.415047 \\
\hline 1 & -0.557118 & -4.221898 & 6.382718 \\
\hline & 9.497100 & -2.824478 & 1.149901 \\
\hline 1 & 2.319110 & 2.815992 & 0.057477 \\
\hline 1 & -4.212809 & 0.379631 & 1.462429 \\
\hline 1 & -2.814306 & -0.621392 & -2.446780 \\
\hline ] & -6.914861 & -1.322805 & -1.394743 \\
\hline & -3.649105 & 2.469478 & -0.623456 \\
\hline 1 & -0.017804 & 2.143459 & 1.631214 \\
\hline & -2.089503 & 5.895464 & 1.419137 \\
\hline 1 & 1.887013 & -3.245325 & 1.695836 \\
\hline 1 & -0.514150 & -1.177198 & -2.096443 \\
\hline 1 & 3.270078 & -2.884099 & 0.667135 \\
\hline 1 & 4.994242 & -2.040220 & 1.954751 \\
\hline 1 & 1.006170 & -0.674552 & 2.734769 \\
\hline 1 & 5.859725 & -0.534214 & 3.714581 \\
\hline 1 & 1.853230 & 0.805627 & 4.524908 \\
\hline 1 & 4.295273 & 0.900826 & 5.010658 \\
\hline 5 & -6.460995 & -2.318174 & -3.640110 \\
\hline 6 & -5.125252 & -0.394400 & -4.485738 \\
\hline 6 & -4.013079 & -2.552840 & -3.894818 \\
\hline 6 & -6.457013 & 0.258478 & 2.463095 \\
\hline 6 & -8.024464 & 0.411202 & 0.552946 \\
\hline 5 & -7.330519 & -1.822251 & 1.442842 \\
\hline 1 & -6.558113 & -2.731631 & -4.651822 \\
\hline 1 & -6.524101 & -3.154269 & -2.929421 \\
\hline 1 & -7.323739 & -1.659683 & -3.476937 \\
\hline 1 & -4.193240 & -2.949143 & -4.903000 \\
\hline 1 & -3.021770 & -2.081905 & -3.905128 \\
\hline 1 & -3.972688 & -3.398888 & -3.195934 \\
\hline 1 & -5.287440 & -0.743223 & -5.514727 \\
\hline 1 & -5.918745 & 0.323964 & -4.240787 \\
\hline 1 & -4.166941 & 0.140589 & -4.461450 \\
\hline 1 & -7.317619 & 0.278116 & 3.143559 \\
\hline 1 & -5.652700 & -0.294026 & 2.967317 \\
\hline
\end{tabular}




$\begin{array}{rrrr}1 & -6.123772 & 1.295420 & 2.321470 \\ 1 & -8.177133 & -1.808784 & 2.142623 \\ 1 & -7.656288 & -2.348780 & 0.537131 \\ 1 & -6.524109 & -2.408571 & 1.904407 \\ 1 & -8.855330 & 0.459221 & 1.269501 \\ 1 & -7.715988 & 1.440061 & 0.325295 \\ 1 & -8.412630 & -0.033217 & -0.371109 \\ 6 & -4.143304 & 6.552457 & 0.212700 \\ 6 & -4.155390 & 5.048056 & -1.755582 \\ 6 & -5.478585 & 4.465856 & 0.288518 \\ 6 & -0.075922 & 6.190119 & 2.799526 \\ 6 & -0.147021 & 4.017990 & 3.995751 \\ 6 & 1.504122 & 4.397310 & 2.160455 \\ 1 & -4.994510 & 5.628307 & -2.162549 \\ 1 & -3.224186 & 5.476448 & -2.148320 \\ 1 & -4.241591 & 4.025830 & -2.142678 \\ 1 & -5.010461 & 7.079193 & -0.205186 \\ 1 & -4.190936 & 6.658203 & 1.304302 \\ 1 & -3.242456 & 7.067728 & -0.144635 \\ 1 & -6.344703 & 5.017023 & -0.102013 \\ 1 & -5.584423 & 3.417592 & -0.019955 \\ 1 & -5.524194 & 4.497027 & 1.385340 \\ 1 & 0.688439 & 6.559292 & 3.494763 \\ 1 & 0.055032 & 6.723298 & 1.848966 \\ 1 & -1.052694 & 6.465135 & 3.217005 \\ 1 & 2.227240 & 4.855285 & 2.848238 \\ 1 & 1.728378 & 3.323846 & 2.129628 \\ 1 & 1.678304 & 4.816877 & 1.159724 \\ 1 & 0.563226 & 4.412620 & 4.735168 \\ 1 & -1.163348 & 4.210689 & 4.364626 \\ 1 & -0.009375 & 2.929919 & 3.945766\end{array}$

$1_{\mathrm{C} 7-} \psi_{-1523344 \mathrm{~s}}$

Number of imaginary frequencies : 0

The smallest frequencies are : $12.0578 \quad 19.6816 \quad 20.9776 \mathrm{~cm}(-1)$

Electronic energy : $\quad H F=-4125.4653668$

Zero-point correction $=\quad 1.299169($ Hartree/Particle $)$

Thermal correction to Energy $=\quad 1.377681$

Thermal correction to Enthalpy= $\quad 1.378625$

Thermal correction to Gibbs Free Energy= $\quad 1.186019$

Sum of electronic and zero-point Energies $=\quad-4124.166198$

Sum of electronic and thermal Energies $=\quad-4124.087686$

Sum of electronic and thermal Enthalpies $=\quad-4124.086742$

Sum of electronic and thermal Free Energies $=\quad-4124.279348$

Cartesian Coordinates

$\begin{array}{lrrr}6 & 3.849091 & 0.260872 & 4.204273 \\ 6 & 4.719139 & -0.573310 & 3.511220 \\ 6 & 4.229257 & -1.439173 & 2.536068 \\ 6 & 2.868290 & -1.478832 & 2.240035 \\ 6 & 2.001473 & -0.637474 & 2.939470 \\ 6 & 2.485791 & 0.223824 & 3.914830 \\ 6 & 2.352139 & -2.438038 & 1.199071 \\ 7 & 1.395722 & -1.861241 & 0.224937\end{array}$




\begin{tabular}{|c|c|c|c|}
\hline 6 & 0.325776 & -2.725789 & -0.191704 \\
\hline 5 & -0.923554 & -2.639063 & 0.394678 \\
\hline 6 & -1.990673 & -3.451872 & -0.102541 \\
\hline 6 & -1.740966 & -4.367633 & -1.167503 \\
\hline 6 & -0.437995 & -4.451810 & -1.711784 \\
\hline & 0.563210 & -3.646901 & -1.239429 \\
\hline 6 & -3.306402 & -3.374415 & 0.423541 \\
\hline f & -4.314820 & -4.158558 & -0.079672 \\
\hline 6 & -4.057978 & -5.081845 & -1.116192 \\
\hline 6 & -2.796311 & -5.182213 & -1.646012 \\
\hline 6 & -1.155828 & -1.815308 & 1.613305 \\
\hline 6 & -1.508751 & -0.473923 & 1.576197 \\
\hline 6 & -1.806361 & 0.193187 & 2.797520 \\
\hline 6 & -1.721514 & -0.444268 & 4.005627 \\
\hline 6 & -1.333026 & -1.800762 & 4.080037 \\
\hline 6 & -1.057529 & -2.500923 & 2.870155 \\
\hline 6 & -1.212430 & -2.469524 & 5.321282 \\
\hline 6 & -0.831507 & -3.785693 & 5.375276 \\
\hline 6 & -0.560359 & -4.487398 & 4.182144 \\
\hline 6 & -0.674269 & -3.865366 & 2.963388 \\
\hline 15 & -1.669811 & 0.429903 & -0.044839 \\
\hline 45 & -0.100655 & 0.367598 & -1.933887 \\
\hline 6 & 0.288147 & 2.287282 & -1.868123 \\
\hline 8 & 0.402517 & 3.432199 & -1.924160 \\
\hline 15 & 1.738223 & -0.623150 & -0.887926 \\
\hline 8 & 2.719924 & 0.260274 & \\
\hline 6 & 3.603714 & 1.143975 & -0.467383 \\
\hline 6 & 4.784813 & 0.677825 & -1.010484 \\
\hline 6 & 5.662226 & 1.628627 & -1.626895 \\
\hline 6 & 5.331897 & 3.017930 & -1.582504 \\
\hline 6 & 4.127509 & 3.431672 & -0.964194 \\
\hline 6 & 3.269162 & 2.509785 & -0.43 \\
\hline 6 & 6.202283 & 3.960853 & -2.18 \\
\hline 6 & 7.344524 & 3.559281 & -2.824549 \\
\hline 6 & 7.655575 & 2.185756 & -2.902708 \\
\hline 6 & 6.838754 & 1.247841 & -2.321612 \\
\hline 6 & 5.060744 & -0.776802 & -1.001705 \\
\hline 6 & 4.124167 & -1.650101 & -1.527652 \\
\hline 6 & 4.394615 & -3.026600 & -1.687866 \\
\hline 6 & 5.568095 & -3.552640 & -1.222024 \\
\hline 6 & 6.503496 & -2.732260 & -0.544978 \\
\hline 6 & 6.250611 & -1.332424 & -0.428433 \\
\hline 6 & 7.164210 & -0.550897 & 0.323867 \\
\hline 6 & 8.271180 & -1.118495 & 0.905431 \\
\hline 6 & 8.535461 & -2.496346 & 0.758377 \\
\hline 6 & 7.665770 & -3.283120 & 0.047291 \\
\hline 8 & 2.900182 & -1.197312 & -1.960982 \\
\hline 6 & -3.397833 & -0.019323 & -0.484754 \\
\hline 6 & -4.423434 & 0.025198 & 0.460754 \\
\hline 6 & -5.709418 & -0.412604 & 0.147071 \\
\hline 6 & -5.940588 & -0.896296 & -1.147481 \\
\hline 6 & -4.939103 & -0.939999 & -2.120158 \\
\hline 6 & -3.667563 & -0.482351 & -1.766420 \\
\hline 6 & -6.787879 & -0.378403 & 1.229564 \\
\hline 6 & -5.172255 & -1.455665 & -3.538329 \\
\hline 6 & -1.788132 & 2.177387 & 0.498884 \\
\hline
\end{tabular}




\begin{tabular}{|c|c|c|c|}
\hline 6 & -2.799754 & 3.000565 & 0.015514 \\
\hline 6 & -2.843500 & 4.358324 & 0.344397 \\
\hline 6 & -1.858951 & 4.858755 & 1.198911 \\
\hline 6 & -0.826621 & 4.057643 & 1.702600 \\
\hline 6 & -0.785800 & 2.719359 & 1.308946 \\
\hline 6 & -3.961851 & 5.221588 & -0.235049 \\
\hline 6 & 0.192104 & 4.575910 & 2.717811 \\
\hline 6 & -0.571710 & 0.365581 & -3.778720 \\
\hline 8 & -0.849460 & 0.299409 & -4.900768 \\
\hline 1 & 6.962748 & 0.508446 & 0.462772 \\
\hline 1 & -2.110887 & 1.236247 & 2.776911 \\
\hline 1 & 7.844288 & -4.352084 & -0.062446 \\
\hline 1 & -1.426060 & -1.912722 & 6.232566 \\
\hline 1 & 5.934410 & 5.015330 & -2.128631 \\
\hline 1 & -3.517754 & -2.665258 & 1.222853 \\
\hline 1 & -5.321919 & -4.068685 & 0.324397 \\
\hline 1 & 3.884731 & 4.493174 & -0.938850 \\
\hline 1 & -1.954648 & 0.089956 & 4.926157 \\
\hline 1 & 5.785782 & -4.612816 & -1.340628 \\
\hline 1 & 8.548177 & 1.864782 & -3.435096 \\
\hline 1 & 3.652634 & -3.643386 & -2.190131 \\
\hline 1 & -2.584743 & -5.882731 & -2.452851 \\
\hline 1 & -0.248137 & -5.159378 & -2.517368 \\
\hline 1 & -0.260876 & -5.531920 & 4.227916 \\
\hline 1 & 7.088058 & 0.193119 & -2.403181 \\
\hline 1 & -0.467182 & -4.424188 & 2.053061 \\
\hline 1 & 1.562512 & -3.691492 & -1.667669 \\
\hline 1 & 8.002577 & 4.292224 & -3.285466 \\
\hline 1 & 8.948009 & -0.499507 & 1.490237 \\
\hline 1 & -4.863850 & -5.705352 & -1.497550 \\
\hline 1 & -0.737685 & -4.291109 & 6.333596 \\
\hline 1 & 9.419419 & -2.931618 & 1.218588 \\
\hline 1 & 2.327804 & 2.795847 & 0.031305 \\
\hline 1 & -4.207944 & 0.392493 & 1.464074 \\
\hline 1 & -2.853384 & -0.502256 & -2.488152 \\
\hline 1 & -6.932211 & -1.260955 & -1.399375 \\
\hline 1 & -3.563246 & 2.575687 & -0.635181 \\
\hline 1 & 0.015594 & 2.068493 & 1.668924 \\
\hline 1 & -1.895776 & 5.905475 & 1.484633 \\
\hline 1 & 1.797678 & -3.243404 & 1.702586 \\
\hline 1 & -0.548582 & -1.165126 & -2.124269 \\
\hline 1 & 3.199954 & -2.918052 & 0.686281 \\
\hline 1 & 4.919336 & -2.080406 & 1.984710 \\
\hline 1 & 0.943274 & -0.637715 & 2.686027 \\
\hline 1 & 5.786932 & -0.548204 & 3.720369 \\
\hline 1 & 1.792264 & 0.869046 & 4.453279 \\
\hline 1 & 4.229918 & 0.940182 & 4.964004 \\
\hline 6 & -6.516596 & -2.165997 & -3.676928 \\
\hline 6 & -5.137008 & -0.267177 & -4.505794 \\
\hline 6 & -4.073986 & -2.453820 & -3.923747 \\
\hline 6 & -8.119366 & -0.945833 & 0.744857 \\
\hline 6 & -6.328493 & -1.213036 & 2.431833 \\
\hline 6 & -7.016578 & 1.070854 & 1.673112 \\
\hline 1 & -6.624068 & -2.559362 & -4.695597 \\
\hline 1 & -6.595070 & -3.013684 & -2.981664 \\
\hline 1 & -7.364961 & -1.492728 & -3.498942 \\
\hline
\end{tabular}




$\begin{array}{lrrr}1 & -4.255696 & -2.837568 & -4.936609 \\ 1 & -3.071978 & -2.006087 & -3.921139 \\ 1 & -4.058441 & -3.306154 & -3.231328 \\ 1 & -5.305325 & -0.606268 & -5.537030 \\ 1 & -5.914709 & 0.467392 & -4.258309 \\ 1 & -4.167164 & 0.246278 & -4.477590 \\ 1 & -8.855428 & -0.905026 & 1.557670 \\ 1 & -8.528905 & -0.373767 & -0.097518 \\ 1 & -8.028384 & -1.995801 & 0.435996 \\ 1 & -7.774618 & 1.113252 & 2.466910 \\ 1 & -6.100414 & 1.532244 & 2.062504 \\ 1 & -7.370843 & 1.685892 & 0.835500 \\ 1 & -7.087939 & -1.188999 & 3.225026 \\ 1 & -6.176680 & -2.262977 & 2.146497 \\ 1 & -5.388414 & -0.846134 & 2.863456 \\ 6 & -3.906855 & 6.662720 & 0.264748 \\ 6 & -3.835014 & 5.238418 & -1.762921 \\ 6 & -5.321383 & 4.631034 & 0.157432 \\ 6 & 0.087951 & 6.083976 & 2.933532 \\ 6 & -0.072510 & 3.878664 & 4.058210 \\ 6 & 1.618458 & 4.260816 & 2.260196 \\ 1 & -4.620470 & 5.866061 & -2.205291 \\ 1 & -2.861927 & 5.642847 & -2.070746 \\ 1 & -3.928949 & 4.234247 & -2.193468 \\ 1 & -4.732374 & 7.236166 & -0.175382 \\ 1 & -4.009415 & 6.723821 & 1.356081 \\ 1 & -2.972462 & 7.161809 & -0.022293 \\ 1 & -6.134759 & 5.235788 & -0.265603 \\ 1 & -5.448216 & 3.603793 & -0.207447 \\ 1 & -5.444002 & 4.615376 & 1.248588 \\ 1 & 0.850367 & 6.405123 & 3.654307 \\ 1 & 0.255342 & 6.642599 & 2.003541 \\ 1 & -0.887539 & 6.377837 & 3.341064 \\ 1 & 2.344714 & 4.664438 & 2.978153 \\ 1 & 1.807020 & 3.182192 & 2.186689 \\ 1 & 1.824961 & 4.715934 & 1.281409 \\ 1 & 0.643301 & 4.220069 & 4.818498 \\ 1 & -1.085236 & 4.098566 & 4.421871 \\ 1 & 0.022327 & 2.788279 & 3.971870\end{array}$

$1_{\mathrm{C} 8-} \psi_{-1 \mathrm{a} 2 \mathrm{a} 3 \mathrm{a} 4 \mathrm{a}}$

Number of imaginary frequencies : 0

The smallest frequencies are : $14.0836 \quad 17.3052 \quad 18.8027 \mathrm{~cm}(-1)$

Electronic energy : $\quad H F=-4125.4660517$

Zero-point correction $=\quad 1.301949($ Hartree/Particle $)$

Thermal correction to Energy= $\quad 1.379932$

Thermal correction to Enthalpy= $\quad 1.380876$

Thermal correction to Gibbs Free Energy $=\quad 1.190370$

Sum of electronic and zero-point Energies $=\quad-4124.164103$

Sum of electronic and thermal Energies $=\quad-4124.086120$

Sum of electronic and thermal Enthalpies $=\quad-4124.085175$

Sum of electronic and thermal Free Energies $=\quad-4124.275682$

Cartesian Coordinates 


$\begin{array}{cccc}0 & 1.619399 & -2.868111 & 4.952010 \\ 6 & 1.641334 & -1.642998 & 4.287689 \\ 6 & 1.888021 & -1.591513 & 2.921616 \\ 6 & 2.115305 & -2.765891 & 2.198511 \\ 6 & 2.091130 & -3.986006 & 2.870538 \\ 6 & 1.846592 & -4.040161 & 4.240606 \\ 6 & 2.438787 & -2.719341 & 0.727634 \\ 7 & 1.466964 & -1.958219 & -0.083693 \\ 6 & 0.382869 & -2.692191 & -0.677240 \\ 6 & -0.902206 & -2.650078 & -0.165252 \\ 6 & -1.965630 & -3.268333 & -0.900970 \\ 6 & -1.681548 & -3.946485 & -2.122623 \\ 6 & -0.343883 & -4.016078 & -2.576743 \\ 6 & 0.654079 & -3.400200 & -1.874081 \\ 6 & -3.314899 & -3.214490 & -0.463111 \\ 6 & -4.319338 & -3.797833 & -1.194320 \\ 6 & -4.029557 & -4.481329 & -2.395030 \\ 6 & -2.737260 & -4.549215 & -2.848871 \\ 6 & -1.209060 & -2.086342 & 1.179965 \\ 6 & -1.466863 & -0.742426 & 1.413179 \\ 6 & -1.828802 & -0.325412 & 2.724427 \\ 6 & -1.961760 & -1.220242 & 3.750850 \\ 6 & -1.739651 & -2.599398 & 3.540060 \\ 6 & -1.344441 & -3.039770 & 2.244833 \\ 6 & -1.912893 & -3.542888 & 4.580995 \\ 6 & -1.717362 & -4.881465 & 4.355626 \\ 6 & -1.317420 & -5.325223 & 3.077961 \\ 6 & -1.127021 & -4.429956 & 2.054914 \\ 15 & -1.537382 & 0.474943 & 0.007867 \\ 45 & 0.077148 & 0.688521 & -1.820611 \\ 6 & 0.514587 & 2.565473 & -1.450052 \\ 8 & 0.665396 & 3.702536 & -1.345475 \\ 15 & 1.850200 & -0.520591 & -0.899463 \\ 8 & 2.821758 & 0.105429 & 0.301282 \\ 6 & 3.657207 & 1.141154 & -0.064235 \\ 6 & 4.841790 & 0.865287 & -0.720455 \\ 6 & 5.650137 & 1.971055 & -1.140505 \\ 6 & 5.258893 & 3.298694 & -0.787723 \\ 6 & 4.061967 & 3.505604 & -0.061922 \\ 6 & 3.263234 & 2.447845 & 0.278459 \\ 6 & 6.061461 & 4.393215 & -1.192665 \\ 6 & 7.193824 & 4.199318 & -1.940389 \\ 6 & 7.562596 & 2.892946 & -2.324381 \\ 6 & 6.813315 & 1.810340 & -1.936284 \\ 6 & 5.194666 & -0.543969 & -1.010211 \\ 6 & 4.285625 & -1.346507 & -1.679190 \\ 6 & 4.597975 & -2.664677 & -2.072858 \\ 6 & 5.803124 & -3.212681 & -1.729682 \\ 6 & 6.732377 & -2.478656 & -0.954017 \\ 6 & 6.426956 & -1.135296 & -0.578912 \\ 6 & 7.351920 & -0.451798 & 0.251459 \\ 6 & 8.519458 & -1.049713 & 0.656153 \\ 6 & 8.833164 & -2.364555 & 0.253089 \\ 6 & 7.951766 & -3.061820 & -0.532337 \\ 8 & 3.046838 & -0.865809 & -2.028713 \\ & & & \\ 6\end{array}$




\begin{tabular}{|c|c|c|c|}
\hline & -3.283217 & 0.235503 & -0.525988 \\
\hline & -4.323547 & 0.179915 & 0.405819 \\
\hline & -5.636954 & -0.041236 & -0.003032 \\
\hline & -5.873806 & -0.205092 & -1.373852 \\
\hline & -4.859628 & -0.152206 & -2.330655 \\
\hline & -3.555965 & 0.074807 & -1.880376 \\
\hline & -6.794912 & -0.148290 & 0.987834 \\
\hline & -5.188339 & -0.325572 & -3.812075 \\
\hline & -1.558808 & 2.095119 & 0.869257 \\
\hline & -2.477239 & 3.079091 & 0.509060 \\
\hline & -2.391761 & 4.367131 & 1.044064 \\
\hline & -1.376328 & 4.625970 & 1.967453 \\
\hline & -0.446383 & 3.658229 & 2.361374 \\
\hline & -0.536045 & 2.397100 & 1.772927 \\
\hline & -3.354576 & 5.483181 & 0.642910 \\
\hline & 0.626035 & 4.004527 & 3.393195 \\
\hline & -0.278106 & 0.987687 & -3.664971 \\
\hline & -0.481577 & 1.107544 & -4.798521 \\
\hline & 7.121055 & 0.557616 & 0.581710 \\
\hline & -2.027088 & 0.726432 & 2.914554 \\
\hline & 8.167936 & -4.084307 & -0.839329 \\
\hline & -2.212965 & -3.179688 & 5.563274 \\
\hline & 5.748286 & 5.396070 & -0.905360 \\
\hline & -3.554206 & -2.682090 & 0.455992 \\
\hline & -5.349689 & -3.729671 & -0.849452 \\
\hline & 3.772051 & 4.523355 & 0.196571 \\
\hline & 6.056542 & -4.227491 & -2.032173 \\
\hline & 8.445907 & 2.741604 & -2.940759 \\
\hline & 3.864788 & -3.216273 & -2.656792 \\
\hline & -2.497903 & -5.067885 & -3.776163 \\
\hline & -0.126861 & -4.549162 & -3.501034 \\
\hline & -1.151097 & -6.385966 & 2.903175 \\
\hline & 7.104998 & 0.812374 & -2.252662 \\
\hline & -0.803232 & -4.790503 & 1.080817 \\
\hline & 1.681166 & -3.420043 & -2.234149 \\
\hline & 7.799396 & 5.047582 & -2.250763 \\
\hline & 9.206738 & -0.505058 & 1.299645 \\
\hline & -4.834142 & -4.948877 & -2.958890 \\
\hline & -1.863438 & -5.600746 & 5.158439 \\
\hline & 9.764465 & -2.823924 & 0.575917 \\
\hline & 2.320665 & 2.583696 & 0.807374 \\
\hline & -4.095486 & 0.304335 & 1.461855 \\
\hline & -2.721958 & 0.116434 & -2.576920 \\
\hline & -6.896290 & -0.386830 & -1.706445 \\
\hline & -3.257755 & 2.833894 & -0.207398 \\
\hline & 0.182691 & 1.619641 & 2.030428 \\
\hline & -1.304384 & 5.624688 & 2.397640 \\
\hline & 3.429412 & -2.267160 & 0.598110 \\
\hline 1 & -0.420860 & -0.770530 & -2.270547 \\
\hline & 2.507677 & -3.741580 & 0.326057 \\
\hline & 1.908187 & -0.635113 & 2.401005 \\
\hline & 2.256314 & -4.907111 & 2.310727 \\
\hline 1 & 1.464129 & -0.721977 & 4.840089 \\
\hline & 1.816437 & -5.002746 & 4.747605 \\
\hline 1 & 1.419169 & -2.907435 & 6.020903 \\
\hline & -3.928525 & -0.375756 & -4.674872 \\
\hline
\end{tabular}




$\begin{array}{rrrr}6 & -5.961627 & -1.629565 & -4.030323 \\ 6 & -6.045187 & 0.858721 & -4.273188 \\ 6 & -6.352470 & 0.126996 & 2.423500 \\ 6 & -7.888571 & 0.861590 & 0.625537 \\ 6 & -7.370543 & -1.567911 & 0.930960 \\ 1 & -4.205929 & -0.545403 & -5.722901 \\ 1 & -3.362114 & 0.563477 & -4.636081 \\ 1 & -3.257109 & -1.191906 & -4.371199 \\ 1 & -6.285252 & 0.765467 & -5.341048 \\ 1 & -6.992019 & 0.917547 & -3.721650 \\ 1 & -5.513453 & 1.808114 & -4.127652 \\ 1 & -6.208729 & -1.748605 & -5.093784 \\ 1 & -5.358660 & -2.494069 & -3.722785 \\ 1 & -6.903838 & -1.657853 & -3.469448 \\ 1 & -7.214910 & 0.045470 & 3.096974 \\ 1 & -5.597838 & -0.592919 & 2.768335 \\ 1 & -5.938777 & 1.137873 & 2.536545 \\ 1 & -8.214648 & -1.667941 & 1.626760 \\ 1 & -7.733056 & -1.825587 & -0.072032 \\ 1 & -6.610652 & -2.308355 & 1.216278 \\ 1 & -8.721341 & 0.792110 & 1.338109 \\ 1 & -7.502195 & 1.888468 & 0.657247 \\ 1 & -8.298698 & 0.690142 & -0.376768 \\ 1 & -2.258226 & -0.879869 & 4.742477 \\ 6 & 1.436040 & 2.779856 & 3.817197 \\ 6 & 1.579770 & 5.045504 & 2.797674 \\ 6 & -0.031797 & 4.581886 & 4.651752 \\ 6 & -4.425521 & 5.001126 & -0.333240 \\ 6 & -4.060453 & 6.033378 & 1.886769 \\ 6 & -2.559247 & 6.605097 & -0.033951 \\ 1 & 2.151457 & 3.063547 & 4.599088 \\ 1 & 0.788613 & 1.991746 & 4.226419 \\ 1 & 2.017628 & 2.349468 & 2.991020 \\ 1 & 0.733588 & 4.822302 & 5.401529 \\ 1 & -0.590072 & 5.502882 & 4.447576 \\ 1 & -0.727665 & 3.860259 & 5.099000 \\ 1 & 2.374317 & 5.294384 & 3.514044 \\ 1 & 2.054316 & 4.666993 & 1.881919 \\ 1 & 1.057749 & 5.974789 & 2.538565 \\ 1 & -5.100617 & 5.831089 & -0.576916 \\ 1 & -3.992994 & 4.643093 & -1.276193 \\ 1 & -5.035805 & 4.192961 & 0.091847 \\ 1 & -4.753222 & 6.837751 & 1.605408 \\ 1 & -4.640634 & 5.248595 & 2.389482 \\ 1 & -3.354981 & 6.447471 & 2.616985 \\ 1 & -3.231905 & 7.412999 & -0.352384 \\ 1 & -1.810302 & 7.040897 & 0.638539 \\ 1 & -2.032191 & 6.230263 & -0.921271\end{array}$

$1_{\mathrm{C} 8-} \psi_{-1 \mathrm{a} 2 \mathrm{a} 3 \mathrm{a} 4 \mathrm{~s}}$

Number of imaginary frequencies : 0

The smallest frequencies are : $16.1066 \quad 18.231920 .4686 \mathrm{~cm}(-1)$

Electronic energy : $\quad H F=-4125.4667248$

Zero-point correction $=\quad 1.299670($ Hartree/Particle $)$

Thermal correction to Energy $=\quad 1.377889$ 
Thermal correction to Enthalpy=

Thermal correction to Gibbs Free Energy=

Sum of electronic and zero-point Energies=

Sum of electronic and thermal Energies=

Sum of electronic and thermal Enthalpies=

Sum of electronic and thermal Free Energies=
1.378833

1.188210

$-4124.167054$

$-4124.088836$

$-4124.087892$

$-4124.278515$

Cartesian Coordinates

$\begin{array}{cccc}6 & 1.633708 & -2.395747 & 5.083619 \\ 6 & 1.579257 & -1.243193 & 4.302064 \\ 6 & 1.813526 & -1.310920 & 2.934266 \\ 6 & 2.107451 & -2.534276 & 2.325894 \\ 6 & 2.157119 & -3.682121 & 3.115043 \\ 6 & 1.922703 & -3.616807 & 4.486119 \\ 6 & 2.434793 & -2.621285 & 0.856551 \\ 7 & 1.479246 & -1.927860 & -0.029510 \\ 6 & 0.409908 & -2.705091 & -0.594540 \\ 6 & -0.874890 & -2.668723 & -0.082520 \\ 6 & -1.929038 & -3.331631 & -0.790514 \\ 6 & -1.635243 & -4.050052 & -1.986414 \\ 6 & -0.296636 & -4.115587 & -2.438561 \\ 6 & 0.691877 & -3.455440 & -1.762425 \\ 6 & -3.279065 & -3.277169 & -0.353822 \\ 6 & -4.275853 & -3.897456 & -1.064607 \\ 6 & -3.976569 & -4.622290 & -2.238700 \\ 6 & -2.683206 & -4.692694 & -2.689319 \\ 6 & -1.193530 & -2.056548 & 1.236486 \\ 6 & -1.490606 & -0.712321 & 1.404047 \\ 6 & -1.879706 & -0.246649 & 2.691239 \\ 6 & -1.987232 & -1.095589 & 3.758727 \\ 6 & -1.709043 & -2.473495 & 3.616938 \\ 6 & -1.296506 & -2.962190 & 2.344940 \\ 6 & -1.835779 & -3.369074 & 4.705700 \\ 6 & -1.577898 & -4.706923 & 4.548834 \\ 6 & -1.163759 & -5.197894 & 3.293363 \\ 6 & -1.020979 & -4.349596 & 2.223544 \\ 15 & -1.555307 & 0.444770 & -0.053031 \\ 45 & 0.064672 & 0.617369 & -1.883558 \\ 6 & 0.466393 & 2.510117 & -1.560058 \\ 8 & 0.594263 & 3.651397 & -1.471438 \\ 15 & 1.853889 & -0.529211 & -0.913943 \\ 8 & 2.812769 & 0.165669 & 0.257542 \\ 6 & 3.651395 & 1.180998 & -0.154739 \\ 6 & 4.845478 & 0.870880 & -0.777210 \\ 6 & 5.661354 & 1.952928 & -1.242413 \\ 6 & 5.264343 & 3.297646 & -0.968956 \\ 6 & 4.054387 & 3.542990 & -0.277428 \\ 6 & 3.251013 & 2.504254 & 0.107472 \\ 6 & 6.074538 & 4.369094 & -1.418046 \\ 6 & 7.220193 & 4.135791 & -2.133524 \\ 6 & 7.595432 & 2.810796 & -2.439376 \\ 6 & 6.838678 & 1.750260 & -2.007283 \\ 6 & 5.198050 & -0.551787 & -0.991729 \\ 6 & 4.294467 & -1.383227 & -1.632769 \\ 6 & 4.608198 & -2.718974 & -1.960217\end{array}$




\begin{tabular}{|c|c|c|c|}
\hline & 5.808030 & -3.252329 & -1.577410 \\
\hline & 6.729916 & -2.484414 & -0.826385 \\
\hline & 6.423715 & -1.124373 & -0.518439 \\
\hline & 7.340860 & -0.404245 & 0.289527 \\
\hline & 8.502480 & -0.984744 & 0.734731 \\
\hline & 8.817590 & -2.317574 & 0.397146 \\
\hline & 7.943354 & -3.049387 & -0.364390 \\
\hline & 3.060579 & -0.916685 & -2.018667 \\
\hline & -3.293378 & 0.177287 & -0.594711 \\
\hline & -4.339243 & 0.174959 & 0.323397 \\
\hline & -5.655102 & -0.066387 & -0.080507 \\
\hline & -5.884872 & -0.303414 & -1.436717 \\
\hline & -4.856004 & -0.306319 & -2.387695 \\
\hline & -3.557941 & -0.060072 & -1.943612 \\
\hline & -6.762383 & -0.058761 & 0.971239 \\
\hline & -5.176662 & -0.559279 & -3.859434 \\
\hline y & -1.587238 & 2.083055 & 0.770533 \\
\hline & -2.540259 & 3.045509 & 0.446760 \\
\hline 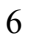 & -2.467032 & 4.332021 & 0.988925 \\
\hline & -1.431862 & 4.607202 & 1.885347 \\
\hline & -0.467035 & 3.658930 & 2.242663 \\
\hline & -0.543247 & 2.402898 & 1.643351 \\
\hline & -3.488431 & 5.417459 & 0.653893 \\
\hline & 0.623580 & 4.015201 & 3.251789 \\
\hline & -0.301613 & 0.855091 & -3.734819 \\
\hline & -0.511557 & 0.938069 & -4.870423 \\
\hline & 7.108514 & 0.620101 & 0.569227 \\
\hline & -2.111150 & 0.806128 & 2.831575 \\
\hline & 8.160450 & -4.085505 & -0.621051 \\
\hline & -2.148240 & -2.969977 & 5.669992 \\
\hline & 5.756636 & 5.385811 & -1.191135 \\
\hline ] & -3.525369 & -2.713702 & 0.545157 \\
\hline & -5.307844 & -3.824057 & -0.726274 \\
\hline & 3.758528 & 4.572873 & -0.081279 \\
\hline & 6.062733 & -4.280493 & -1.829419 \\
\hline & 8.490048 & 2.626812 & -3.030043 \\
\hline 1 & 3.882210 & -3.296630 & -2.527589 \\
\hline & -2.437588 & -5.242638 & -3.596715 \\
\hline 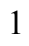 & -0.071110 & -4.680776 & -3.341485 \\
\hline . & -0.950140 & -6.257710 & 3.172690 \\
\hline & 7.136131 & 0.736748 & -2.263176 \\
\hline 1 & -0.685845 & -4.744542 & 1.266591 \\
\hline & 1.718764 & -3.472961 & -2.122053 \\
\hline ] & 7.831735 & 4.966405 & -2.478071 \\
\hline & 9.183817 & -0.411803 & 1.359710 \\
\hline 1 & -4.775380 & -5.119056 & -2.785548 \\
\hline l & -1.685974 & -5.389363 & 5.388924 \\
\hline 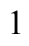 & 9.744327 & -2.762801 & 0.751392 \\
\hline 1 & 2.298977 & 2.668692 & 0.609946 \\
\hline 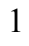 & -4.126172 & 0.361101 & 1.375773 \\
\hline 1 & -2.718951 & -0.059818 & -2.635172 \\
\hline ( & -6.901599 & -0.495815 & -1.773983 \\
\hline 1 & -3.340668 & 2.784695 & -0.241695 \\
\hline ] & 0.201458 & 1.639709 & 1.870491 \\
\hline 1 & -1.375982 & 5.601532 & 2.327896 \\
\hline & 3.433312 & -2.197196 & 0.692998 \\
\hline
\end{tabular}




\begin{tabular}{|c|c|c|c|}
\hline . & -0.404625 & -0.865231 & -2.288088 \\
\hline . & 2.492382 & -3.676202 & 0.548579 \\
\hline & 1.766474 & -0.408843 & 2.325724 \\
\hline & 2.372710 & -4.642756 & 2.646175 \\
\hline & 1.350201 & -0.283264 & 4.762633 \\
\hline & 1.953112 & -4.525575 & 5.084134 \\
\hline & 1.443505 & -2.341405 & 6.153664 \\
\hline & -3.914116 & -0.627809 & -4.716682 \\
\hline & -5.924324 & -1.887359 & -4.013523 \\
\hline 6 & -6.054650 & 0.583023 & -4.382810 \\
\hline & -8.123500 & -0.427673 & 0.387469 \\
\hline & -6.429454 & -1.072853 & 2.072436 \\
\hline 0 & -6.864706 & 1.345360 & 1.578654 \\
\hline & -4.186716 & -0.844411 & -5.757311 \\
\hline 1 & -3.360370 & 0.319795 & -4.715076 \\
\hline 1 & -3.232781 & -1.421887 & -4.378996 \\
\hline 1 & -6.289379 & 0.429237 & -5.444812 \\
\hline 1 & -7.004743 & 0.652637 & -3.838355 \\
\hline 1 & -5.541293 & 1.548558 & -4.286391 \\
\hline 1 & -6.165896 & -2.064181 & -5.070265 \\
\hline 1 & -5.305890 & -2.723930 & -3.662015 \\
\hline 1 & -6.867429 & -1.904968 & -3.453845 \\
\hline 1 & -8.880664 & -0.419372 & 1.181654 \\
\hline 1 & -8.448983 & 0.284226 & -0.381765 \\
\hline 1 & -8.119129 & -1.433219 & -0.053737 \\
\hline 1 & -7.626641 & 1.367832 & 2.369537 \\
\hline 1 & -5.914767 & 1.675460 & 2.018925 \\
\hline 1 & -7.151170 & 2.079369 & 0.814004 \\
\hline 1 & -7.215848 & -1.074074 & 2.839122 \\
\hline 1 & -6.359880 & -2.088751 & 1.661287 \\
\hline 1 & -5.479102 & -0.853334 & 2.575111 \\
\hline 1 & -2.300589 & -0.718935 & 4.731841 \\
\hline 6 & 1.454214 & 2.796396 & 3.654200 \\
\hline 6 & 1.552495 & 5.068472 & 2.639531 \\
\hline 6 & -0.012512 & 4.580780 & 4.526789 \\
\hline 6 & -4.509148 & 4.952255 & -0.382263 \\
\hline 6 & -4.246238 & 5.806726 & 1.927932 \\
\hline 6 & -2.765070 & 6.644746 & 0.089468 \\
\hline 1 & 2.178966 & 3.081566 & 4.426869 \\
\hline 1 & 0.819317 & 1.999636 & 4.067699 \\
\hline 1 & 2.026949 & 2.377071 & 2.816145 \\
\hline 1 & 0.766630 & 4.821883 & 5.261972 \\
\hline 1 & -0.580513 & 5.499609 & 4.340529 \\
\hline 1 & -0.694158 & 3.851775 & 4.983975 \\
\hline 1 & 2.355655 & 5.328987 & 3.342039 \\
\hline 1 & 2.017180 & 4.696217 & 1.716207 \\
\hline 1 & 1.012116 & 5.989721 & 2.389399 \\
\hline 1 & -5.218775 & 5.763346 & -0.588558 \\
\hline 1 & -4.033434 & 4.679953 & -1.333154 \\
\hline 1 & -5.090340 & 4.089460 & -0.030902 \\
\hline 1 & -4.984379 & 6.590256 & 1.709637 \\
\hline 1 & -4.782548 & 4.942803 & 2.343041 \\
\hline 1 & -3.574734 & 6.189434 & 2.706232 \\
\hline 1 & -3.490753 & 7.425167 & -0.176164 \\
\hline 1 & -2.061623 & 7.080396 & 0.809054 \\
\hline 1 & -2.199428 & 6.384312 & -0.814617 \\
\hline
\end{tabular}


$1_{\mathrm{C} 8-} \psi_{-1 \mathrm{a} 2 \mathrm{2} 3 \mathrm{3} 4 \mathrm{a}}$

Number of imaginary frequencies : 0

The smallest frequencies are : $14.2017 \quad 18.7850 \quad 21.8828 \mathrm{~cm}(-1)$

Electronic energy : $\quad \mathrm{HF}=-4125.4668057$

Zero-point correction $=\quad 1.300014($ Hartree/Particle $)$

Thermal correction to Energy $=\quad 1.377976$

Thermal correction to Enthalpy= $\quad 1.378920$

Thermal correction to Gibbs Free Energy= 1.189666

Sum of electronic and zero-point Energies $=\quad-4124.166792$

Sum of electronic and thermal Energies $=\quad-4124.088830$

Sum of electronic and thermal Enthalpies $=\quad-4124.087886$

Sum of electronic and thermal Free Energies $=\quad-4124.277139$

Cartesian Coordinates

\begin{tabular}{cccc}
6 & 1.558001 & -2.336764 & 5.109141 \\
6 & 1.533940 & -1.191870 & 4.315435 \\
6 & 1.765933 & -1.280750 & 2.948428 \\
6 & 2.025526 & -2.518282 & 2.353235 \\
6 & 2.044813 & -3.658552 & 3.154791 \\
6 & 1.813734 & -3.571893 & 4.525266 \\
6 & 2.351541 & -2.629603 & 0.885130 \\
7 & 1.412313 & -1.924383 & -0.009926 \\
6 & 0.334670 & -2.691185 & -0.573303 \\
6 & -0.953445 & -2.625771 & -0.072975 \\
6 & -2.007359 & -3.302771 & -0.770113 \\
6 & -1.706539 & -4.082277 & -1.925758 \\
6 & -0.366109 & -4.164271 & -2.370332 \\
6 & 0.619228 & -3.477344 & -1.716875 \\
6 & -3.360894 & -3.222037 & -0.350861 \\
6 & -4.352647 & -3.887168 & -1.028939 \\
6 & -4.044298 & -4.684216 & -2.152282 \\
6 & -2.747790 & -4.773258 & -2.591920 \\
6 & -1.263437 & -1.997566 & 1.241930 \\
6 & -1.521414 & -0.645474 & 1.413873 \\
6 & -1.895116 & -0.170666 & 2.702112 \\
6 & -2.021975 & -1.016581 & 3.769509 \\
6 & -1.775977 & -2.400425 & 3.627040 \\
6 & -1.381527 & -2.899588 & 2.353007 \\
6 & -1.913727 & -3.290467 & 4.718914 \\
6 & -1.683733 & -4.633596 & 4.564344 \\
6 & -1.286185 & -5.135256 & 3.307835 \\
6 & -1.133371 & -4.292692 & 2.234796 \\
15 & -1.557833 & 0.519473 & -0.036402 \\
45 & 0.060362 & 0.646057 & -1.874950 \\
6 & 0.516402 & 2.527489 & -1.559346 \\
8 & 0.678318 & 3.664984 & -1.478807 \\
15 & 1.819621 & -0.542664 & -0.905553 \\
8 & 2.796703 & 0.136648 & 0.259740 \\
6 & 3.663704 & 1.124588 & -0.159804 \\
6 & 4.846850 & 0.776629 & -0.782955 \\
6 & 5.691958 & 1.832078 & -1.256667 \\
6 & 5.334867 & 3.189252 & -0.989658 \\
6 & 4.134848 & 3.473193 & -0.295468 \\
& & & \\
\hline
\end{tabular}




\begin{tabular}{|c|c|c|c|}
\hline & 3.302805 & 2.460073 & 0.097094 \\
\hline & 6.174479 & 4.234055 & -1.447516 \\
\hline & 7.310539 & 3.963351 & -2.165191 \\
\hline & 7.646174 & 2.626246 & -2.464522 \\
\hline & 6.860342 & 1.590725 & -2.023817 \\
\hline & 5.160008 & -0.656757 & -0.987454 \\
\hline & 4.233825 & -1.468198 & -1.621663 \\
\hline & 4.510900 & -2.814691 & -1.938680 \\
\hline & 5.695674 & -3.377589 & -1.551113 \\
\hline & 6.638225 & -2.629285 & -0.805915 \\
\hline & 6.369472 & -1.258762 & -0.509360 \\
\hline & 7.305815 & -0.557318 & 0.292918 \\
\hline & 8.450783 & -1.165813 & 0.743702 \\
\hline & 8.729121 & -2.509674 & 0.417562 \\
\hline & 7.835396 & -3.223546 & -0.338387 \\
\hline & 3.012851 & -0.970434 & -2.009764 \\
\hline & -3.299528 & 0.279004 & -0.575951 \\
\hline & -4.356039 & 0.243393 & 0.344294 \\
\hline & -5.651115 & -0.042817 & -0.071405 \\
\hline & -5.862555 & -0.306808 & -1.436164 \\
\hline & -4.837267 & -0.268751 & -2.375648 \\
\hline 6 & -3.550378 & 0.043204 & -1.918220 \\
\hline & -6.826913 & -0.109417 & 0.901823 \\
\hline & -5.044627 & -0.545917 & -3.861950 \\
\hline 0 & -1.542413 & 2.158997 & 0.783270 \\
\hline & -2.462159 & 3.147876 & 0.442981 \\
\hline 6 & -2.347256 & 4.438985 & 0.966724 \\
\hline ? & -1.302846 & 4.692939 & 1.858513 \\
\hline 6 & -0.372080 & 3.717898 & 2.233611 \\
\hline 6 & -0.490585 & 2.455354 & 1.654975 \\
\hline 0 & -3.317939 & 5.560020 & 0.599160 \\
\hline 6 & 0.728032 & 4.054372 & 3.239075 \\
\hline o & -0.322962 & 0.903191 & -3.720814 \\
\hline 8 & -0.551499 & 1.001305 & -4.851640 \\
\hline 1 & 7.101678 & 0.475363 & 0.563803 \\
\hline & -2.100981 & 0.887461 & 2.842191 \\
\hline 1 & 8.024064 & -4.267380 & -0.586050 \\
\hline & -2.211257 & -2.882215 & 5.684104 \\
\hline 1 & 5.886891 & 5.260845 & -1.225536 \\
\hline ] & -3.613131 & -2.606293 & 0.511366 \\
\hline 1 & -5.385661 & -3.799158 & -0.697470 \\
\hline 1 & 3.869481 & 4.512117 & -0.103301 \\
\hline 1 & 5.921984 & -4.414413 & -1.794651 \\
\hline 1 & 8.533059 & 2.413011 & -3.057012 \\
\hline & 3.769601 & -3.377212 & -2.501788 \\
\hline 1 & -2.494926 & -5.372477 & -3.465556 \\
\hline 1 & -0.136143 & -4.768072 & -3.246769 \\
\hline 1 & -1.092891 & -6.199137 & 3.188639 \\
\hline 1 & 7.126898 & 0.567434 & -2.274808 \\
\hline & -0.809651 & -4.697634 & 1.278174 \\
\hline 1 & 1.648582 & -3.510919 & -2.068316 \\
\hline 1 & 7.944835 & 4.773803 & -2.516547 \\
\hline 1 & 9.147469 & -0.606694 & 1.364257 \\
\hline 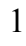 & -4.837839 & -5.215786 & -2.673069 \\
\hline 1 & -1.799673 & -5.311591 & 5.406973 \\
\hline 1 & 9.642982 & -2.977378 & 0.776256 \\
\hline
\end{tabular}




\begin{tabular}{|c|c|c|c|}
\hline & 2.357383 & 2.653303 & 0.601891 \\
\hline & -4.148054 & 0.430643 & 1.395133 \\
\hline$\frac{1}{1}$ & -2.712993 & 0.079893 & -2.612891 \\
\hline 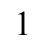 & -6.870580 & -0.556941 & -1.761950 \\
\hline & -3.267538 & 2.903803 & -0.246016 \\
\hline 1 & 0.227777 & 1.670832 & 1.894331 \\
\hline ] & -1.210015 & 5.693164 & 2.281136 \\
\hline | & 3.360188 & -2.231972 & 0.717423 \\
\hline & -0.440915 & -0.828564 & -2.274628 \\
\hline 1 & 2.383363 & -3.688944 & 0.589410 \\
\hline 1 & 1.745863 & -0.384147 & 2.330459 \\
\hline 1 & 2.234617 & -4.629633 & 2.696398 \\
\hline 1 & 1.332226 & -0.221083 & 4.765898 \\
\hline 1 & 1.821794 & -4.474233 & 5.133627 \\
\hline 1 & 1.370643 & -2.265591 & 6.178735 \\
\hline 6 & -6.436393 & -1.100513 & -4.153986 \\
\hline כ & -4.862004 & 0.762903 & -4.638516 \\
\hline 6 & -4.015894 & -1.572661 & -4.350345 \\
\hline & -6.413685 & 0.239580 & 2.330071 \\
\hline 6 & -7.913372 & 0.879109 & 0.465188 \\
\hline & -7.402230 & -1.530112 & 0.908635 \\
\hline & -6.529324 & -1.322043 & -5.224616 \\
\hline$\frac{1}{1}$ & -6.621545 & -2.033602 & -3.603624 \\
\hline & -7.230591 & -0.386521 & -3.900569 \\
\hline 1 & -4.177637 & -1.789052 & -5.414833 \\
\hline 1 & -2.981678 & -1.221301 & -4.242961 \\
\hline 1 & -4.104871 & -2.513453 & -3.791323 \\
\hline 1 & -5.004179 & 0.593353 & -5.714471 \\
\hline 1 & -5.588699 & 1.519414 & -4.314228 \\
\hline 1 & -3.856560 & 1.179701 & -4.497259 \\
\hline 1 & -7.287054 & 0.178983 & 2.991592 \\
\hline 1 & -5.656294 & -0.453476 & 2.721039 \\
\hline 1 & -6.014740 & 1.260003 & 2.402693 \\
\hline 1 & -8.264578 & -1.589761 & 1.586425 \\
\hline 1 & -7.740345 & -1.844945 & -0.086355 \\
\hline 1 & -6.652559 & -2.253569 & 1.256911 \\
\hline 1 & -8.758442 & 0.850805 & 1.166097 \\
\hline 1 & -7.525320 & 1.905826 & 0.444704 \\
\hline 1 & -8.305263 & 0.650471 & -0.533025 \\
\hline 1 & -2.323625 & -0.632596 & 4.743441 \\
\hline 6 & 1.524638 & 2.817286 & 3.653570 \\
\hline 6 & 1.686088 & 5.075685 & 2.617664 \\
\hline 6 & 0.106482 & 4.649699 & 4.507673 \\
\hline 6 & -4.406611 & 5.091142 & -0.363513 \\
\hline 6 & -4.000098 & 6.087753 & 1.865980 \\
\hline 6 & -2.540135 & 6.695748 & -0.074668 \\
\hline 1 & 2.257269 & 3.089626 & 4.423435 \\
\hline 1 & 0.867388 & 2.043121 & 4.074880 \\
\hline 1 & 2.084982 & 2.373974 & 2.819392 \\
\hline 1 & 0.891246 & 4.878933 & 5.240696 \\
\hline 1 & -0.438146 & 5.580369 & 4.310571 \\
\hline 1 & -0.593757 & 3.943335 & 4.972211 \\
\hline 1 & 2.496166 & 5.319506 & 3.318202 \\
\hline 1 & 2.140301 & 4.682233 & 1.697997 \\
\hline 1 & 1.172554 & 6.009893 & 2.359188 \\
\hline 1 & -5.082199 & 5.925972 & -0.588544 \\
\hline
\end{tabular}


$1_{\mathrm{C} 8-} \psi_{-1 \mathrm{a} 2 \mathrm{2} 3 \mathrm{3} 4 \mathrm{~s}}$

Number of imaginary frequencies : 0

The smallest frequencies are : $13.9859 \quad 16.2066 \quad 18.4819 \mathrm{~cm}(-1)$

Electronic energy : $\quad \mathrm{HF}=-4125.4669693$

Zero-point correction $=\quad 1.299039($ Hartree/Particle $)$

Thermal correction to Energy= $\quad 1.377397$

Thermal correction to Enthalpy= $\quad 1.378341$

Thermal correction to Gibbs Free Energy= $\quad 1.186973$

Sum of electronic and zero-point Energies $=\quad-4124.167931$

Sum of electronic and thermal Energies $=\quad-4124.089572$

Sum of electronic and thermal Enthalpies $=\quad-4124.088628$

Sum of electronic and thermal Free Energies $=\quad-4124.279996$

\begin{tabular}{crrr}
\multicolumn{4}{c}{ Cartesian Coordinates } \\
\hline 6 & 1.482782 & -2.460109 & 5.092885 \\
6 & 1.501568 & -1.290631 & 4.335005 \\
6 & 1.761712 & -1.342712 & 2.971403 \\
6 & 2.004985 & -2.567717 & 2.343694 \\
6 & 1.980105 & -3.732427 & 3.108578 \\
6 & 1.722459 & -3.682298 & 4.476232 \\
6 & 2.354441 & -2.635983 & 0.879170 \\
7 & 1.414858 & -1.918463 & -0.006640 \\
6 & 0.337110 & -2.680979 & -0.576677 \\
6 & -0.954405 & -2.613879 & -0.084192 \\
6 & -2.004679 & -3.290598 & -0.788011 \\
6 & -1.697540 & -4.070522 & -1.941672 \\
6 & -0.354812 & -4.152168 & -2.379208 \\
6 & 0.627028 & -3.466469 & -1.719559 \\
6 & -3.360825 & -3.210101 & -0.376793 \\
6 & -4.347940 & -3.877414 & -1.059447 \\
6 & -4.033439 & -4.674823 & -2.180696 \\
6 & -2.734543 & -4.762468 & -2.613420 \\
6 & -1.274166 & -1.981151 & 1.226371 \\
6 & -1.510095 & -0.624447 & 1.395336 \\
6 & -1.882748 & -0.141293 & 2.680572 \\
6 & -2.042815 & -0.984911 & 3.745524 \\
6 & -1.832664 & -2.374442 & 3.603123 \\
6 & -1.427803 & -2.880806 & 2.335269 \\
6 & -2.015959 & -3.262778 & 4.689743 \\
6 & -1.816951 & -4.610898 & 4.536604 \\
6 & -1.404210 & -5.119599 & 3.287719 \\
6 & -1.207357 & -4.278834 & 2.220526 \\
15 & -1.535112 & 0.537367 & -0.058299 \\
45 & 0.092126 & 0.658145 & -1.883590 \\
6 & 0.550227 & 2.538771 & -1.568753
\end{tabular}




\begin{tabular}{|c|c|c|c|}
\hline & 0.711416 & 3.676134 & -1.484700 \\
\hline & 1.836656 & -0.537806 & -0.897860 \\
\hline & 2.802147 & 0.140529 & 0.278457 \\
\hline & 3.673675 & 1.127608 & -0.134870 \\
\hline & 4.864087 & 0.778723 & -0.743482 \\
\hline & 5.714166 & 1.833540 & -1.210177 \\
\hline & 5.353126 & 3.191347 & -0.951630 \\
\hline & 4.144130 & 3.476645 & -0.273930 \\
\hline & 3.308308 & 2.463943 & 0.111206 \\
\hline & 6.197640 & 4.235435 & -1.402042 \\
\hline & 7.342552 & 3.963487 & -2.104988 \\
\hline & 7.682503 & 2.625749 & -2.396433 \\
\hline 6 & 6.891944 & 1.590962 & -1.962475 \\
\hline 6 & 5.178863 & -0.654830 & -0.943810 \\
\hline & 4.259249 & -1.466064 & -1.587817 \\
\hline 6 & 4.540104 & -2.812102 & -1.903326 \\
\hline 6 & 5.721250 & -3.374806 & -1.504615 \\
\hline 6 & 6.655938 & -2.626918 & -0.749142 \\
\hline 6 & 6.383488 & -1.256936 & -0.453788 \\
\hline 6 & 7.310991 & -0.555957 & 0.359094 \\
\hline 6 & 8.451720 & -1.164309 & 0.820726 \\
\hline 6 & 8.734250 & -2.507533 & 0.495604 \\
\hline 6 & 7.848679 & -3.221034 & -0.270249 \\
\hline 8 & 3.041927 & -0.968725 & -1.987658 \\
\hline 6 & -3.270387 & 0.279776 & -0.612172 \\
\hline 6 & -4.324261 & 0.213334 & 0.300500 \\
\hline 6 & -5.618655 & -0.093386 & -0.116080 \\
\hline 6 & -5.830203 & -0.333850 & -1.480101 \\
\hline 6 & -4.799377 & -0.263534 & -2.419630 \\
\hline 6 & -3.519677 & 0.058600 & -1.960749 \\
\hline 6 & -6.729963 & -0.178960 & 0.929322 \\
\hline 6 & -5.001372 & -0.530206 & -3.909095 \\
\hline 6 & -1.550510 & 2.179513 & 0.756662 \\
\hline 6 & -2.505370 & 3.139787 & 0.431394 \\
\hline 6 & -2.424885 & 4.432510 & 0.957243 \\
\hline 6 & -1.377796 & 4.716597 & 1.836603 \\
\hline 6 & -0.411432 & 3.770802 & 2.196687 \\
\hline 6 & -0.497381 & 2.505935 & 1.616608 \\
\hline 6 & -3.453836 & 5.512996 & 0.628462 \\
\hline 6 & 0.696675 & 4.144997 & 3.180279 \\
\hline 6 & -0.275161 & 0.908353 & -3.733685 \\
\hline 8 & -0.494763 & 1.001731 & -4.866656 \\
\hline 1 & 7.103284 & 0.476205 & 0.629313 \\
\hline 1 & -2.065524 & 0.921130 & 2.819313 \\
\hline 1 & 8.040486 & -4.264449 & -0.517279 \\
\hline 1 & -2.322890 & -2.848897 & 5.649549 \\
\hline 1 & 5.906616 & 5.262629 & -1.186507 \\
\hline 1 & -3.619909 & -2.592961 & 0.482791 \\
\hline 1 & -5.383307 & -3.788366 & -0.734915 \\
\hline 1 & 3.874729 & 4.515872 & -0.088817 \\
\hline 1 & 5.950494 & -4.411247 & -1.747016 \\
\hline 1 & 8.576665 & 2.411370 & -2.977452 \\
\hline 1 & 3.804378 & -3.374613 & -2.473663 \\
\hline 1 & -2.476331 & -5.361648 & -3.485512 \\
\hline 1 & -0.120184 & -4.755047 & -3.255041 \\
\hline 1 & -1.232900 & -6.187442 & 3.170371 \\
\hline
\end{tabular}




\begin{tabular}{|c|c|c|c|}
\hline & 7.162355 & 0.567263 & -2.207433 \\
\hline & -0.871824 & -4.689352 & 1.270451 \\
\hline & 1.657978 & -3.499986 & -2.066368 \\
\hline & 7.980615 & 4.773348 & -2.450832 \\
\hline & 9.141639 & -0.605586 & 1.449151 \\
\hline & -4.823753 & -5.207374 & -2.705342 \\
\hline & -1.968511 & -5.287660 & 5.374574 \\
\hline & 9.644750 & -2.975090 & 0.862932 \\
\hline & 2.355789 & 2.658026 & 0.601542 \\
\hline & -4.125907 & 0.387154 & 1.357931 \\
\hline & -2.682952 & 0.115606 & -2.654890 \\
\hline & -6.829471 & -0.595909 & -1.815927 \\
\hline & -3.313405 & 2.871724 & -0.245149 \\
\hline & 0.247445 & 1.742613 & 1.846289 \\
\hline & -1.313506 & 5.717030 & 2.263685 \\
\hline & 3.357464 & -2.215842 & 0.736599 \\
\hline & -0.415944 & -0.814782 & -2.280155 \\
\hline & 2.408474 & -3.686122 & 0.555078 \\
\hline & 1.775704 & -0.427930 & 2.380090 \\
\hline & 2.156671 & -4.693034 & 2.623600 \\
\hline & 1.310731 & -0.330880 & 4.812262 \\
\hline & 1.695448 & -4.602836 & 5.056152 \\
\hline 1 & 1.273619 & -2.417508 & 6.159942 \\
\hline & -6.394585 & -1.074259 & -4.213722 \\
\hline 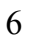 & -4.805915 & 0.780168 & -4.679790 \\
\hline 6 & -3.975700 & -1.561845 & -4.394476 \\
\hline 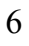 & -8.070524 & -0.587523 & 0.324275 \\
\hline & -6.353178 & -1.220682 & 1.990307 \\
\hline 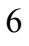 & -6.901917 & 1.190908 & 1.595423 \\
\hline 1 & -6.483864 & -1.280869 & -5.287660 \\
\hline & -6.586837 & -2.014090 & -3.677679 \\
\hline 1 & -7.186390 & -0.360025 & -3.953458 \\
\hline 1 & -4.129926 & -1.772861 & -5.461228 \\
\hline 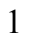 & -2.940400 & -1.217129 & -4.275835 \\
\hline 1 & -4.075369 & -2.504263 & -3.839701 \\
\hline 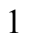 & -4.943058 & 0.615333 & -5.757129 \\
\hline 1 & -5.529792 & 1.540122 & -4.357248 \\
\hline & -3.798803 & 1.190392 & -4.531765 \\
\hline 1 & -8.830342 & -0.643349 & 1.114005 \\
\hline 1 & -8.424494 & 0.136671 & -0.420580 \\
\hline l & -8.019466 & -1.574988 & -0.153363 \\
\hline 1 & -7.683201 & 1.147470 & 2.366316 \\
\hline 1 & -5.977380 & 1.534565 & 2.076405 \\
\hline 1 & -7.196714 & 1.949227 & 0.858383 \\
\hline ] & -7.135254 & -1.281709 & 2.759182 \\
\hline 1 & -6.247106 & -2.217579 & 1.541507 \\
\hline 1 & -5.408989 & -0.983229 & 2.497045 \\
\hline 1 & -2.345901 & -0.594674 & 4.716523 \\
\hline 6 & 1.517239 & 2.929943 & 3.612833 \\
\hline 6 & 1.630716 & 5.162845 & 2.517167 \\
\hline 6 & 0.088754 & 4.763029 & 4.444499 \\
\hline 6 & -4.488459 & 5.036973 & -0.388903 \\
\hline 6 & -4.193449 & 5.908749 & 1.911398 \\
\hline 6 & -2.745335 & 6.740135 & 0.045483 \\
\hline 1 & 2.248536 & 3.230311 & 4.373463 \\
\hline 1 & 0.878440 & 2.150337 & 4.051225 \\
\hline
\end{tabular}




$\begin{array}{rrrr}1 & 2.082906 & 2.483166 & 2.784835 \\ 1 & 0.883178 & 5.017959 & 5.158270 \\ 1 & -0.469271 & 5.683281 & 4.236912 \\ 1 & -0.594956 & 4.059846 & 4.937571 \\ 1 & 2.453723 & 5.429204 & 3.194094 \\ 1 & 2.067251 & 4.755174 & 1.594911 \\ 1 & 1.100902 & 6.085854 & 2.251253 \\ 1 & -5.202020 & 5.845289 & -0.592453 \\ 1 & -4.025797 & 4.756790 & -1.343924 \\ 1 & -5.063602 & 4.176718 & -0.021922 \\ 1 & -4.939893 & 6.685749 & 1.698071 \\ 1 & -4.717332 & 5.045690 & 2.343386 \\ 1 & -3.511590 & 6.302822 & 2.674867 \\ 1 & -3.478634 & 7.518599 & -0.204453 \\ 1 & -2.026334 & 7.178685 & 0.747697 \\ 1 & -2.201001 & 6.480220 & -0.871517\end{array}$

$1_{\mathrm{C} 8-}-\psi_{-122 s 3 a 4 a}$

Number of imaginary frequencies : 0

The smallest frequencies are : $16.0592 \quad 20.231421 .9063 \mathrm{~cm}(-1)$

Electronic energy : $\quad H F=-4125.4669558$

Zero-point correction $=\quad 1.300063($ Hartree/Particle $)$

Thermal correction to Energy= $\quad 1.378139$

Thermal correction to Enthalpy= $\quad 1.379083$

Thermal correction to Gibbs Free Energy $=\quad 1.189504$

Sum of electronic and zero-point Energies $=\quad-4124.166893$

Sum of electronic and thermal Energies $=\quad-4124.088817$

Sum of electronic and thermal Enthalpies $=\quad-4124.087873$

Sum of electronic and thermal Free Energies $=\quad-4124.277452$

\begin{tabular}{rrrr} 
& \multicolumn{4}{c}{ Cartesian Coordinates } \\
\hline $6 \ldots \ldots \ldots \ldots \ldots \ldots \ldots \ldots \ldots \ldots \ldots \ldots$. \\
6 & 1.643074 & -2.457100 & 5.054646 \\
6 & 1.591555 & -1.295941 & 4.285943 \\
6 & 1.818118 & -1.350138 & 2.916212 \\
6 & 2.100868 & -2.568468 & 2.292679 \\
6 & 2.147919 & -3.725114 & 3.069334 \\
6 & 1.921764 & -3.673333 & 4.442364 \\
6 & 2.421111 & -2.641720 & 0.820721 \\
7 & 1.468868 & -1.930014 & -0.054439 \\
6 & 0.395569 & -2.691492 & -0.633189 \\
6 & -0.888966 & -2.655295 & -0.120753 \\
6 & -1.947919 & -3.295102 & -0.842582 \\
6 & -1.659444 & -3.990224 & -2.053567 \\
6 & -0.320910 & -4.059086 & -2.505328 \\
6 & 0.672580 & -3.421490 & -1.815005 \\
6 & -3.297670 & -3.239241 & -0.405761 \\
6 & -4.299683 & -3.833705 & -1.131261 \\
6 & -4.006088 & -4.533204 & -2.321989 \\
6 & -2.712652 & -4.606540 & -2.772046 \\
6 & -1.198994 & -2.067738 & 1.211580 \\
6 & -1.484454 & -0.725010 & 1.409052 \\
6 & -1.861944 & -0.282422 & 2.707783 \\
6 & -1.968080 & -1.152228 & 3.758502 \\
6 & -1.698602 & -2.528593 & 3.587189
\end{tabular}




\begin{tabular}{|c|c|c|c|}
\hline 6 & -1.299452 & -2.994606 & 2.302307 \\
\hline 6 & -1.819903 & -3.444723 & 4.659391 \\
\hline 6 & -1.569002 & -4.780334 & 4.474403 \\
\hline 6 & -1.168467 & -5.248804 & 3.205955 \\
\hline 6 & -1.032049 & -4.380680 & 2.151301 \\
\hline 15 & -1.549325 & 0.461204 & -0.022919 \\
\hline 45 & 0.062034 & 0.654858 & -1.861955 \\
\hline 6 & 0.474957 & 2.540428 & -1.509709 \\
\hline 8 & 0.607673 & 3.679915 & -1.406701 \\
\hline 15 & 1.848313 & -0.519951 & -0.918866 \\
\hline 8 & 2.816683 & 0.151518 & 0.258270 \\
\hline 6 & 3.658510 & 1.168431 & -0.143659 \\
\hline 6 & 4.847350 & 0.861070 & -0.777310 \\
\hline 6 & 5.666112 & 1.945304 & -1.232080 \\
\hline 6 & 5.277357 & 3.288038 & -0.937924 \\
\hline 6 & 4.072646 & 3.529729 & -0.236034 \\
\hline 6 & 3.266303 & 2.489772 & 0.139337 \\
\hline 6 & 6.090380 & 4.361537 & -1.376901 \\
\hline 6 & 7.230795 & 4.132413 & -2.102034 \\
\hline 6 & 7.597685 & 2.809913 & -2.428160 \\
\hline 6 & 6.838093 & 1.747297 & -2.006305 \\
\hline 6 & 5.191342 & -0.560277 & -1.013231 \\
\hline 6 & 4.280340 & -1.378046 & -1.661308 \\
\hline 6 & 4.585743 & -2.710939 & -2.008106 \\
\hline 6 & 5.784462 & -3.255650 & -1.637863 \\
\hline 6 & 6.713732 & -2.502869 & -0.880630 \\
\hline 6 & 6.415977 & -1.145479 & -0.553211 \\
\hline 6 & 7.340302 & -0.440902 & 0.260210 \\
\hline 6 & 8.500643 & -1.033294 & 0.692790 \\
\hline 6 & 8.807260 & -2.363195 & 0.336236 \\
\hline 6 & 7.926051 & -3.080258 & -0.431300 \\
\hline 8 & 3.047361 & -0.899220 & -2.035027 \\
\hline 6 & -3.296245 & 0.225732 & -0.553424 \\
\hline 6 & -4.339263 & 0.194944 & 0.376897 \\
\hline 6 & -5.651575 & -0.037529 & -0.029279 \\
\hline 6 & -5.885339 & -0.235012 & -1.396301 \\
\hline 6 & -4.868956 & -0.205565 & -2.351776 \\
\hline 6 & -3.566158 & 0.031370 & -1.904056 \\
\hline 6 & -6.810210 & -0.128391 & 0.962423 \\
\hline 6 & -5.194189 & -0.413098 & -3.829684 \\
\hline 6 & -1.553020 & 2.084218 & 0.829448 \\
\hline 6 & -2.486748 & 3.061484 & 0.518479 \\
\hline 6 & -2.404496 & 4.343588 & 1.080763 \\
\hline 6 & -1.368960 & 4.598565 & 1.975049 \\
\hline 6 & -0.411884 & 3.628823 & 2.317847 \\
\hline 6 & -0.503360 & 2.382043 & 1.709767 \\
\hline 6 & -3.453969 & 5.384457 & 0.698600 \\
\hline 6 & -3.389137 & 5.633557 & -0.812645 \\
\hline 6 & 0.684674 & 3.965342 & 3.327092 \\
\hline 6 & 1.513185 & 2.737924 & 3.706082 \\
\hline 6 & -0.303505 & 0.928734 & -3.708109 \\
\hline 8 & -0.510315 & 1.034855 & -4.842381 \\
\hline 6 & 1.614753 & 5.025162 & 2.727768 \\
\hline 6 & 0.056584 & 4.512739 & 4.614065 \\
\hline 6 & -3.240242 & 6.717471 & 1.410669 \\
\hline 6 & -4.846196 & 4.860992 & 1.071806 \\
\hline
\end{tabular}




\begin{tabular}{|c|c|c|c|}
\hline 1 & 7.114516 & 0.580776 & 0.554542 \\
\hline & -2.086529 & 0.768851 & 2.870042 \\
\hline & 8.136673 & -4.113985 & -0.702504 \\
\hline & -2.121618 & -3.063019 & 5.634110 \\
\hline & 5.778929 & 5.376603 & -1.134189 \\
\hline & -3.539242 & -2.694211 & 0.505434 \\
\hline & -5.331056 & -3.760406 & -0.790523 \\
\hline & 3.783023 & 4.558288 & -0.024252 \\
\hline & -2.272461 & -0.793452 & 4.741190 \\
\hline & 6.032546 & -4.281658 & -1.904850 \\
\hline & 8.488053 & 2.629661 & -3.026353 \\
\hline & 3.854515 & -3.277567 & -2.579909 \\
\hline & -2.471019 & -5.138079 & -3.691421 \\
\hline & -0.099402 & -4.607854 & -3.419310 \\
\hline & -0.960787 & -6.307010 & 3.062947 \\
\hline & 7.128990 & 0.735885 & -2.277630 \\
\hline & -0.707587 & -4.758661 & 1.183908 \\
\hline & 1.699613 & -3.440882 & -2.173885 \\
\hline 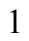 & 7.844563 & 4.964651 & -2.438605 \\
\hline & 9.187612 & -0.472316 & 1.322433 \\
\hline & -4.808872 & -5.009426 & -2.881156 \\
\hline & -1.672212 & -5.478328 & 5.302256 \\
\hline & 9.733039 & -2.817993 & 0.680688 \\
\hline & 2.318233 & 2.651567 & 0.650188 \\
\hline 1 & -4.114218 & 0.346028 & 1.430011 \\
\hline & -2.730555 & 0.053517 & -2.599503 \\
\hline 1 & -6.906878 & -0.425672 & -1.726634 \\
\hline & -3.289477 & 2.826593 & -0.179537 \\
\hline & 0.230325 & 1.605963 & 1.929235 \\
\hline 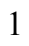 & -1.293971 & 5.583463 & 2.430435 \\
\hline 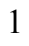 & 3.423052 & -2.225275 & 0.658087 \\
\hline 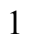 & -0.419017 & -0.816504 & -2.292026 \\
\hline D & 2.422789 & 5.269800 & 3.430384 \\
\hline 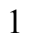 & 2.072863 & 4.666697 & 1.795754 \\
\hline ] & 1.077861 & 5.953355 & 2.496862 \\
\hline 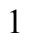 & 0.840028 & 4.738027 & 5.349725 \\
\hline 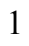 & -0.507823 & 5.437073 & 4.445301 \\
\hline & -0.626376 & 3.779292 & 5.062124 \\
\hline 1 & 0.877280 & 1.938231 & 4.112152 \\
\hline 1 & 2.078604 & 2.328767 & 2.858040 \\
\hline D & 2.244021 & 3.008804 & 4.478171 \\
\hline 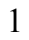 & -4.020622 & 7.426221 & 1.106289 \\
\hline$\frac{1}{1}$ & -3.297536 & 6.614976 & 2.502161 \\
\hline 1 & -2.271846 & 7.167299 & 1.156491 \\
\hline & -5.083907 & 3.917151 & 0.564661 \\
\hline 1 & -4.923488 & 4.687760 & 2.153348 \\
\hline 1 & -5.616065 & 5.591973 & 0.790286 \\
\hline 1 & -2.394438 & 5.991814 & -1.108331 \\
\hline 1 & -3.601343 & 4.725982 & -1.390159 \\
\hline 1 & -4.125971 & 6.393517 & -1.105916 \\
\hline 1 & 2.467664 & -3.693650 & 0.500795 \\
\hline 1 & 1.774622 & -0.440726 & 2.318582 \\
\hline 1 & 2.355157 & -4.681871 & 2.588935 \\
\hline 1 & 1.371239 & -0.339645 & 4.758206 \\
\hline 1 & 1.950585 & -4.588669 & 5.030339 \\
\hline 1 & 1.459266 & -2.413203 & 6.126289 \\
\hline
\end{tabular}




$\begin{array}{lrrr}6 & -3.932342 & -0.488784 & -4.687639 \\ 6 & -5.972089 & -1.718721 & -4.019085 \\ 6 & -6.045232 & 0.762905 & -4.321788 \\ 6 & -6.371900 & 0.183581 & 2.391923 \\ 6 & -7.912672 & 0.862502 & 0.575401 \\ 6 & -7.373008 & -1.554309 & 0.938186 \\ 1 & -4.208038 & -0.680652 & -5.732279 \\ 1 & -3.361495 & 0.448326 & -4.668725 \\ 1 & -3.265688 & -1.301199 & -4.364082 \\ 1 & -6.284254 & 0.643394 & -5.387254 \\ 1 & -6.992574 & 0.839814 & -3.773355 \\ 1 & -5.509508 & 1.713355 & -4.200221 \\ 1 & -6.215006 & -1.863070 & -5.080350 \\ 1 & -5.373661 & -2.577425 & -3.687789 \\ 1 & -6.916889 & -1.729496 & -3.462101 \\ 1 & -7.235207 & 0.113366 & 3.065547 \\ 1 & -5.614174 & -0.523934 & 2.755096 \\ 1 & -5.963506 & 1.198994 & 2.482652 \\ 1 & -8.215740 & -1.646470 & 1.636719 \\ 1 & -7.733529 & -1.837789 & -0.058586 \\ 1 & -6.605847 & -2.280970 & 1.239119 \\ 1 & -8.746069 & 0.800680 & 1.287904 \\ 1 & -7.537310 & 1.893997 & 0.584824 \\ 1 & -8.319437 & 0.664225 & -0.423312\end{array}$

$1_{\mathrm{C} 8-} \psi_{-1 \mathrm{a} 253 \mathrm{ats}}$

Number of imaginary frequencies : 0

The smallest frequencies are : $15.6525 \quad 18.8463 \quad 23.2778 \mathrm{~cm}(-1)$

Electronic energy : $\quad H F=-4125.4672132$

Zero-point correction $=\quad 1.299601($ Hartree/Particle $)$

Thermal correction to Energy= $\quad 1.377827$

Thermal correction to Enthalpy= $\quad 1.378771$

Thermal correction to Gibbs Free Energy $=\quad 1.188285$

Sum of electronic and zero-point Energies $=\quad-4124.167612$

Sum of electronic and thermal Energies $=\quad-4124.089387$

Sum of electronic and thermal Enthalpies $=\quad-4124.088443$

Sum of electronic and thermal Free Energies $=\quad-4124.278928$

\section{Cartesian Coordinates}

$\begin{array}{lrrr}6 & 1.650251 & -2.468405 & 5.043783 \\ 6 & 1.589056 & -1.307069 & 4.275995 \\ 6 & 1.815150 & -1.358393 & 2.906081 \\ 6 & 2.107803 & -2.573707 & 2.281362 \\ 6 & 2.164397 & -3.730552 & 3.057095 \\ 6 & 1.938236 & -3.681797 & 4.430230 \\ 6 & 2.429769 & -2.643634 & 0.809505 \\ 7 & 1.478399 & -1.931691 & -0.066130 \\ 6 & 0.405440 & -2.692527 & -0.646248 \\ 6 & -0.877352 & -2.662545 & -0.129363 \\ 6 & -1.937597 & -3.301463 & -0.849624 \\ 6 & -1.652331 & -3.989780 & -2.065074 \\ 6 & -0.315281 & -4.052305 & -2.522530 \\ 6 & 0.679613 & -3.415667 & -1.832991 \\ 6 & -3.285472 & -3.250178 & -0.406369\end{array}$




\begin{tabular}{|c|c|c|c|}
\hline 6 & -4.288946 & -3.844613 & -1.129645 \\
\hline 6 & -3.998813 & -4.538140 & -2.324754 \\
\hline & -2.707307 & -4.605546 & -2.781528 \\
\hline & -1.187613 & -2.079589 & 1.204436 \\
\hline ) & -1.481364 & -0.738881 & 1.401870 \\
\hline & -1.864810 & -0.299720 & 2.699991 \\
\hline f & -1.969186 & -1.171576 & 3.749423 \\
\hline 5 & -1.692411 & -2.546401 & 3.577241 \\
\hline 6 & -1.285919 & -3.008391 & 2.293353 \\
\hline 6 & -1.813514 & -3.465001 & 4.647355 \\
\hline 6 & -1.554279 & -4.798855 & 4.461232 \\
\hline 6 & -1.146489 & -5.263240 & 3.193574 \\
\hline 6 & -1.010954 & -4.392744 & 2.140818 \\
\hline 15 & -1.548047 & 0.445907 & -0.031357 \\
\hline 45 & 0.066887 & 0.657074 & -1.863138 \\
\hline 6 & 0.469831 & 2.542913 & -1.500394 \\
\hline 8 & 0.594521 & 3.682612 & -1.389937 \\
\hline 15 & 1.855010 & -0.515623 & -0.921685 \\
\hline 8 & 2.816716 & 0.153403 & 0.262307 \\
\hline 6 & 3.653514 & 1.177905 & -0.130560 \\
\hline 6 & 4.845649 & 0.881781 & -0.763518 \\
\hline 6 & 5.658825 & 1.973996 & -1.209209 \\
\hline 6 & 5.261617 & 3.312319 & -0.906417 \\
\hline 6 & 4.054220 & 3.542119 & -0.205149 \\
\hline 6 & 3.253008 & 2.494961 & 0.160962 \\
\hline 6 & 6.068986 & 4.393657 & -1.336454 \\
\hline 6 & 7.211958 & 4.176381 & -2.061223 \\
\hline 6 & 7.587254 & 2.858393 & -2.395918 \\
\hline 6 & 6.833294 & 1.788373 & -1.982729 \\
\hline 6 & 5.198872 & -0.536115 & -1.006912 \\
\hline 6 & 4.294104 & -1.356246 & -1.660723 \\
\hline 6 & 4.607411 & -2.685870 & -2.012634 \\
\hline 6 & 5.808651 & -3.225359 & -1.643048 \\
\hline 6 & 6.732454 & -2.470478 & -0.881210 \\
\hline 6 & 6.426211 & -1.116467 & -0.547667 \\
\hline 6 & 7.345174 & -0.410582 & 0.270694 \\
\hline 6 & 8.508415 & -0.998219 & 0.702012 \\
\hline 6 & 8.823484 & -2.324441 & 0.339260 \\
\hline 6 & 7.947566 & -3.042884 & -0.433019 \\
\hline 8 & 3.059302 & -0.883155 & -2.035640 \\
\hline 6 & -3.289522 & 0.196348 & -0.572123 \\
\hline 6 & -4.331511 & 0.160941 & 0.350097 \\
\hline 6 & -5.648757 & -0.068677 & -0.056435 \\
\hline 6 & -5.884164 & -0.257071 & -1.419409 \\
\hline 6 & -4.859452 & -0.225117 & -2.374301 \\
\hline 6 & -3.559707 & 0.007145 & -1.927313 \\
\hline 6 & -6.747895 & -0.127147 & 1.002594 \\
\hline 6 & -5.185145 & -0.426635 & -3.852893 \\
\hline 6 & -1.577469 & 2.068097 & 0.821542 \\
\hline 6 & -2.524303 & 3.032528 & 0.510178 \\
\hline 6 & -2.460879 & 4.314721 & 1.074361 \\
\hline 6 & -1.432470 & 4.581907 & 1.973589 \\
\hline 6 & -0.463922 & 3.624383 & 2.318506 \\
\hline 6 & -0.535215 & 2.378446 & 1.706072 \\
\hline 6 & -3.514793 & 5.345890 & 0.678604 \\
\hline 6 & -3.393515 & 5.629480 & -0.823257 \\
\hline
\end{tabular}




\begin{tabular}{|c|c|c|c|}
\hline 6 & 0.625134 & 3.972264 & 3.332061 \\
\hline 6 & 1.459584 & 2.751681 & 3.720441 \\
\hline 6 & -0.299428 & 0.937261 & -3.708251 \\
\hline 8 & -0.507499 & 1.046552 & -4.841984 \\
\hline 6 & 1.551521 & 5.034712 & 2.731631 \\
\hline 6 & -0.011777 & 4.521774 & 4.613713 \\
\hline 6 & -3.351062 & 6.664933 & 1.428952 \\
\hline 6 & -4.912417 & 4.792859 & 0.982211 \\
\hline 1 & 7.112949 & 0.608240 & 0.569838 \\
\hline 1 & -2.094548 & 0.750263 & 2.863172 \\
\hline 1 & 8.164657 & -4.073990 & -0.709054 \\
\hline 1 & -2.121352 & -3.086672 & 5.621469 \\
\hline 1 & 5.751088 & 5.405157 & -1.087268 \\
\hline 1 & -3.524756 & -2.708486 & 0.507691 \\
\hline 1 & -5.318889 & -3.773964 & -0.784075 \\
\hline 1 & 3.758454 & 4.567489 & 0.013466 \\
\hline 1 & -2.278143 & -0.815649 & 4.731718 \\
\hline 1 & 6.063032 & -4.248779 & -1.914006 \\
\hline 1 & 8.479640 & 2.687601 & -2.993872 \\
\hline 1 & 3.879609 & -3.253772 & -2.587486 \\
\hline 1 & -2.468798 & -5.132197 & -3.704522 \\
\hline 1 & -0.095928 & -4.595766 & -3.440204 \\
\hline 1 & -0.932688 & -6.320111 & 3.049742 \\
\hline 1 & 7.130668 & 0.780656 & -2.260679 \\
\hline 1 & -0.681459 & -4.767292 & 1.173759 \\
\hline 1 & 1.705228 & -3.430451 & -2.196411 \\
\hline 1 & 7.821282 & 5.014594 & -2.390966 \\
\hline 1 & 9.191107 & -0.436281 & 1.335442 \\
\hline 1 & -4.802757 & -5.014327 & -2.882283 \\
\hline 1 & -1.657099 & -5.498732 & 5.287540 \\
\hline 1 & 9.751507 & -2.775410 & 0.682708 \\
\hline 1 & 2.302697 & 2.647980 & 0.670461 \\
\hline 1 & -4.114138 & 0.304347 & 1.408295 \\
\hline 1 & -2.723324 & 0.032687 & -2.621643 \\
\hline 1 & -6.902220 & -0.438483 & -1.758759 \\
\hline 1 & -3.321694 & 2.788135 & -0.190678 \\
\hline 1 & 0.208526 & 1.612149 & 1.926000 \\
\hline 1 & -1.372694 & 5.566885 & 2.431097 \\
\hline 1 & 3.431515 & -2.225738 & 0.649230 \\
\hline 1 & -0.407406 & -0.814264 & -2.300355 \\
\hline 1 & 2.353605 & 5.289637 & 3.437426 \\
\hline 1 & 2.017643 & 4.673221 & 1.804695 \\
\hline 1 & 1.009229 & 5.957381 & 2.491301 \\
\hline 1 & 0.767141 & 4.753697 & 5.352080 \\
\hline 1 & -0.579654 & 5.442832 & 4.438971 \\
\hline 1 & -0.693293 & 3.786878 & 5.061598 \\
\hline 1 & 0.827179 & 1.948431 & 4.125030 \\
\hline 1 & 2.033054 & 2.343621 & 2.877387 \\
\hline 1 & 2.183848 & 3.030259 & 4.495970 \\
\hline 1 & -4.137080 & 7.364759 & 1.118491 \\
\hline 1 & -3.437892 & 6.533160 & 2.515411 \\
\hline 1 & -2.386052 & 7.142047 & 1.214988 \\
\hline 1 & -5.119124 & 3.864065 & 0.435363 \\
\hline 1 & -5.029221 & 4.582429 & 2.053696 \\
\hline 1 & -5.680508 & 5.523519 & 0.695156 \\
\hline 1 & -2.394487 & 6.011847 & -1.070227 \\
\hline
\end{tabular}




$\begin{array}{lrrr}1 & -3.563577 & 4.729464 & -1.426272 \\ 1 & -4.132329 & 6.381723 & -1.131317 \\ 1 & 2.478252 & -3.694892 & 0.487568 \\ 1 & 1.763512 & -0.449096 & 2.309045 \\ 1 & 2.379030 & -4.685252 & 2.575850 \\ 1 & 1.361007 & -0.352922 & 4.748916 \\ 1 & 1.973885 & -4.597526 & 5.017229 \\ 1 & 1.466511 & -2.426900 & 6.115524 \\ 6 & -3.923999 & -0.492992 & -4.712589 \\ 6 & -5.957792 & -1.734700 & -4.047916 \\ 6 & -6.041547 & 0.747771 & -4.339602 \\ 6 & -8.122718 & -0.405471 & 0.401231 \\ 6 & -6.427298 & -1.249954 & 1.997147 \\ 6 & -6.812185 & 1.211789 & 1.746137 \\ 1 & -4.200316 & -0.678218 & -5.758274 \\ 1 & -3.355530 & 0.445427 & -4.687614 \\ 1 & -3.255008 & -1.306024 & -4.395540 \\ 1 & -6.281991 & 0.630615 & -5.405014 \\ 1 & -6.988428 & 0.819512 & -3.789804 \\ 1 & -5.508932 & 1.699725 & -4.216118 \\ 1 & -6.195525 & -1.877887 & -5.110531 \\ 1 & -5.358036 & -2.592162 & -3.715504 \\ 1 & -6.904999 & -1.749211 & -3.495331 \\ 1 & -8.874211 & -0.444106 & 1.199884 \\ 1 & -8.430463 & 0.379639 & -0.301408 \\ 1 & -8.152066 & -1.368864 & -0.124522 \\ 1 & -7.584208 & 1.178647 & 2.526741 \\ 1 & -5.860258 & 1.462344 & 2.230908 \\ 1 & -7.063446 & 2.030268 & 1.058631 \\ 1 & -7.197935 & -1.300425 & 2.778249 \\ 1 & -6.398504 & -2.224409 & 1.491086 \\ 1 & -5.459590 & -1.104626 & 2.494653\end{array}$

$1_{\mathrm{C} 8-}-\mathbf{- 1 a 2 5 3 5 4 a}$

Number of imaginary frequencies : 0

The smallest frequencies are : $16.9655 \quad 20.6385 \quad 22.1639 \mathrm{~cm}(-1)$

Electronic energy : $\quad H F=-4125.4675161$

Zero-point correction $=\quad 1.300279($ Hartree/Particle $)$

Thermal correction to Energy= $\quad 1.378164$

Thermal correction to Enthalpy= $\quad 1.379109$

Thermal correction to Gibbs Free Energy= 1.190532

Sum of electronic and zero-point Energies $=\quad-4124.167237$

Sum of electronic and thermal Energies $=\quad-4124.089352$

Sum of electronic and thermal Enthalpies $=\quad-4124.088408$

Sum of electronic and thermal Free Energies $=\quad-4124.276984$

Cartesian Coordinates

$\begin{array}{cccc}6 & -0.484666 & 2.456655 & 1.662553 \\ 6 & -1.536306 & 2.151726 & 0.787205 \\ 6 & -2.461400 & 3.130763 & 0.456970 \\ 6 & -2.367007 & 4.423011 & 0.993466 \\ 6 & -1.329157 & 4.686023 & 1.882907 \\ 6 & -0.381826 & 3.714087 & 2.246192 \\ 15 & -1.545013 & 0.515344 & -0.036577\end{array}$




\begin{tabular}{|c|c|c|c|}
\hline 6 & -3.287494 & 0.276932 & -0.573814 \\
\hline & -4.343452 & 0.261808 & 0.347483 \\
\hline & -5.640124 & -0.024200 & -0.063222 \\
\hline & -5.853485 & -0.306956 & -1.423790 \\
\hline & -4.828768 & -0.288514 & -2.364867 \\
\hline & -3.539781 & 0.022596 & -1.912566 \\
\hline & -6.814532 & -0.074633 & 0.912439 \\
\hline & -7.897044 & 0.914849 & 0.468608 \\
\hline & -5.044668 & -0.580390 & -3.847397 \\
\hline & -3.993121 & -1.576825 & -4.348799 \\
\hline & -3.405527 & 5.466209 & 0.588386 \\
\hline & -4.804070 & 4.964756 & 0.968655 \\
\hline & 0.718451 & 4.059345 & 3.248455 \\
\hline & 0.097780 & 4.646925 & 4.521147 \\
\hline & -1.507522 & -0.650063 & 1.412825 \\
\hline & -1.248753 & -2.001535 & 1.238721 \\
\hline & -1.365320 & -2.905391 & 2.348244 \\
\hline & -1.759620 & -2.408323 & 3.623084 \\
\hline & -2.006650 & -1.024873 & 3.767853 \\
\hline & -1.880951 & -0.177082 & 2.701796 \\
\hline & -1.115358 & -4.297937 & 2.227853 \\
\hline & -1.266334 & -5.142163 & 3.299830 \\
\hline & -1.664007 & -4.642712 & 4.557197 \\
\hline & -1.895793 & -3.300117 & 4.713752 \\
\hline & -0.940481 & -2.626090 & -0.078093 \\
\hline & 0.346096 & -2.686065 & -0.583139 \\
\hline & 0.628617 & -3.469072 & -1.729466 \\
\hline ? & -0.357028 & -4.158279 & -2.380203 \\
\hline & -1.696307 & -4.080747 & -1.931156 \\
\hline 6 & -1.995312 & -3.303601 & -0.773489 \\
\hline f & -2.738449 & -4.771741 & -2.595991 \\
\hline 6 & -4.034471 & -4.683147 & -2.154448 \\
\hline b & -4.341148 & -3.887535 & -1.029670 \\
\hline & -3.348195 & -3.223802 & -0.351800 \\
\hline 1 & 1.423656 & -1.918309 & -0.020630 \\
\hline & 1.829294 & -0.533682 & -0.912878 \\
\hline 8 & 3.026446 & -0.955614 & -2.014807 \\
\hline 6 & 4.248849 & -1.448769 & -1.625164 \\
\hline 6 & 5.170512 & -0.634486 & -0.988009 \\
\hline 6 & 6.381723 & -1.232318 & -0.508953 \\
\hline 6 & 6.656773 & -2.601124 & -0.807613 \\
\hline 6 & 5.718502 & -3.352141 & -1.555469 \\
\hline 6 & 4.531957 & -2.793580 & -1.943833 \\
\hline 6 & 7.313751 & -0.528497 & 0.296275 \\
\hline 6 & 8.460581 & -1.132990 & 0.747737 \\
\hline 6 & 8.745206 & -2.474990 & 0.419412 \\
\hline 6 & 7.855732 & -3.191188 & -0.339338 \\
\hline 6 & 4.850969 & 0.797167 & -0.781158 \\
\hline 6 & 3.665226 & 1.138799 & -0.159442 \\
\hline 6 & 3.298365 & 2.472220 & 0.100069 \\
\hline 6 & 4.126989 & 3.489664 & -0.288479 \\
\hline 6 & 5.329427 & 3.212299 & -0.981154 \\
\hline 6 & 5.692631 & 1.857238 & -1.250726 \\
\hline 6 & 6.165649 & 4.261660 & -1.434761 \\
\hline 0 & 7.304269 & 3.997392 & -2.150758 \\
\hline & 7.646028 & 2.662421 & -2.452623 \\
\hline
\end{tabular}




\begin{tabular}{|c|c|c|c|}
\hline 6 & 6.863584 & 1.622588 & -2.016061 \\
\hline 8 & 2.801533 & 0.146234 & 0.256045 \\
\hline 6 & 2.364440 & -2.623434 & 0.873041 \\
\hline 6 & 2.039523 & -2.516474 & 2.341782 \\
\hline f & 1.777628 & -1.281281 & 2.940833 \\
\hline 5 & 1.547825 & -1.196682 & 4.308494 \\
\hline 6 & 1.576423 & -2.343570 & 5.099257 \\
\hline 6 & 1.834582 & -3.576395 & 4.511543 \\
\hline 6 & 2.063390 & -3.658757 & 3.140411 \\
\hline 45 & 0.067039 & 0.655590 & -1.878238 \\
\hline 6 & -0.338758 & 0.930697 & -3.716812 \\
\hline 8 & -0.588823 & 1.045635 & -4.841491 \\
\hline 6 & 0.512633 & 2.537860 & -1.553002 \\
\hline 8 & 0.662279 & 3.676577 & -1.466480 \\
\hline 6 & -6.397072 & 0.285335 & 2.336756 \\
\hline 6 & -7.395398 & -1.493021 & 0.933104 \\
\hline 6 & -6.422041 & -1.181156 & -4.117222 \\
\hline 6 & -4.912111 & 0.730617 & -4.630470 \\
\hline 6 & -3.334345 & 5.684161 & -0.927448 \\
\hline 6 & -3.179895 & 6.811020 & 1.274096 \\
\hline 6 & 1.528976 & 2.829569 & 3.657822 \\
\hline 6 & 1.664398 & 5.090269 & 2.624105 \\
\hline 1 & 7.104770 & 0.502745 & 0.568922 \\
\hline 1 & -2.087495 & 0.880680 & 2.843778 \\
\hline 1 & 8.049188 & -4.233744 & -0.588698 \\
\hline 1 & -2.193404 & -2.893654 & 5.679671 \\
\hline 1 & 5.873434 & 5.286715 & -1.210833 \\
\hline 1 & -3.599773 & -2.608895 & 0.511269 \\
\hline 1 & -5.373648 & -3.799296 & -0.696515 \\
\hline 1 & 3.857148 & 4.527021 & -0.094051 \\
\hline 1 & -2.307971 & -0.642696 & 4.742597 \\
\hline 1 & 5.949502 & -4.387618 & -1.800320 \\
\hline 1 & 8.535028 & 2.454273 & -3.043750 \\
\hline 1 & 3.793700 & -3.358322 & -2.508629 \\
\hline 1 & -2.487009 & -5.369428 & -3.471093 \\
\hline 1 & -0.128291 & -4.759864 & -3.258483 \\
\hline 1 & -1.071539 & -6.205603 & 3.179160 \\
\hline 1 & 7.134968 & 0.601021 & -2.268909 \\
\hline 1 & -0.791534 & -4.700965 & 1.270438 \\
\hline 1 & 1.656572 & -3.498686 & -2.085393 \\
\hline 1 & 7.935949 & 4.811303 & -2.498811 \\
\hline 1 & 9.153826 & -0.572119 & 1.370559 \\
\hline 1 & -4.828819 & -5.213723 & -2.675066 \\
\hline 1 & -1.778628 & -5.322016 & 5.398952 \\
\hline 1 & 9.660486 & -2.939480 & 0.778664 \\
\hline 1 & 2.351466 & 2.660521 & 0.603972 \\
\hline 1 & -4.134130 & 0.464805 & 1.395147 \\
\hline 1 & -2.701695 & 0.044452 & -2.606895 \\
\hline 1 & -6.863028 & -0.556894 & -1.744887 \\
\hline 1 & -3.266110 & 2.889450 & -0.236626 \\
\hline 1 & 0.241592 & 1.678138 & 1.897501 \\
\hline 1 & -1.244521 & 5.679359 & 2.317802 \\
\hline 1 & 3.372168 & -2.223288 & 0.705895 \\
\hline 1 & -0.426374 & -0.819560 & -2.286889 \\
\hline 1 & 2.474741 & 5.341349 & 3.321793 \\
\hline 1 & 2.118883 & 4.701254 & 1.702572 \\
\hline
\end{tabular}




$\begin{array}{lrrr}1 & 1.141028 & 6.019692 & 2.368324 \\ 1 & 0.884157 & 4.879053 & 5.251502 \\ 1 & -0.453369 & 5.574774 & 4.329644 \\ 1 & -0.595598 & 3.934385 & 4.986608 \\ 1 & 0.881211 & 2.047761 & 4.079681 \\ 1 & 2.091357 & 2.393762 & 2.821138 \\ 1 & 2.261264 & 3.107985 & 4.425839 \\ 1 & -3.952252 & 7.521434 & 0.953606 \\ 1 & -3.240649 & 6.731036 & 2.367274 \\ 1 & -2.206387 & 7.245760 & 1.013301 \\ 1 & -5.052245 & 4.013848 & 0.479903 \\ 1 & -4.885372 & 4.813895 & 2.053249 \\ 1 & -5.565185 & 5.698643 & 0.671330 \\ 1 & -2.334830 & 6.024921 & -1.227605 \\ 1 & -3.555439 & 4.767608 & -1.487162 \\ 1 & -4.061761 & 6.446311 & -1.237986 \\ 1 & 2.398550 & -3.682045 & 0.574891 \\ 1 & 1.753731 & -0.383066 & 2.325371 \\ 1 & 2.255095 & -4.628112 & 2.679157 \\ 1 & 1.343991 & -0.227632 & 4.761749 \\ 1 & 1.846113 & -4.480385 & 5.117391 \\ 1 & 1.390748 & -2.275733 & 6.169352 \\ 1 & -6.519625 & -1.419360 & -5.183855 \\ 1 & -6.569955 & -2.112168 & -3.551930 \\ 1 & -7.236083 & -0.489640 & -3.864912 \\ 1 & -4.169161 & -1.804969 & -5.408482 \\ 1 & -2.968887 & -1.192317 & -4.266297 \\ 1 & -4.040904 & -2.516490 & -3.783095 \\ 1 & -5.064046 & 0.553507 & -5.703913 \\ 1 & -5.656204 & 1.466538 & -4.298261 \\ 1 & -3.916987 & 1.176283 & -4.503445 \\ 1 & -7.269948 & 0.236574 & 2.999902 \\ 1 & -5.643406 & -0.408686 & 2.733117 \\ 1 & -5.991608 & 1.303955 & 2.399802 \\ 1 & -8.744042 & 0.891933 & 1.167390 \\ 1 & -7.506214 & 1.940602 & 0.445379 \\ 1 & -8.286701 & 0.683105 & -0.529795 \\ 1 & -8.250848 & -1.545817 & 1.620105 \\ 1 & -7.745137 & -1.811827 & -0.056567 \\ 1 & -6.644362 & -2.217434 & 1.276565\end{array}$

$1_{\mathrm{C} 8-} \psi_{-1 \mathrm{a} 23354 \mathrm{~s}}$

Number of imaginary frequencies : 0

The smallest frequencies are : $15.1910 \quad 18.5478 \quad 21.5174 \mathrm{~cm}(-1)$

Electronic energy : $\quad H F=-4125.4675389$

Zero-point correction $=\quad 1.299452$ (Hartree/Particle)

Thermal correction to Energy $=\quad 1.377608$

Thermal correction to Enthalpy= $\quad 1.378552$

Thermal correction to Gibbs Free Energy= $\quad 1.188403$

Sum of electronic and zero-point Energies $=\quad-4124.168087$

Sum of electronic and thermal Energies $=\quad-4124.089931$

Sum of electronic and thermal Enthalpies $=\quad-4124.088987$

Sum of electronic and thermal Free Energies $=\quad-4124.279136$

Cartesian Coordinates 


$\begin{array}{cccc}0 & 1.543981 & -2.351542 & 5.091735 \\ 6 & 1.525254 & -1.204002 & 4.301890 \\ 6 & 1.757063 & -1.289299 & 2.934623 \\ 6 & 2.010979 & -2.525953 & 2.335103 \\ 6 & 2.024420 & -3.669055 & 3.132922 \\ 6 & 1.793643 & -3.585954 & 4.503658 \\ 6 & 2.339984 & -2.634042 & 0.867216 \\ 7 & 1.408149 & -1.921001 & -0.029482 \\ 6 & 0.328421 & -2.679802 & -0.599944 \\ 6 & -0.959358 & -2.616115 & -0.098465 \\ 6 & -2.015737 & -3.284637 & -0.799756 \\ 6 & -1.717574 & -4.056000 & -1.961480 \\ 6 & -0.377739 & -4.136503 & -2.408247 \\ 6 & 0.609892 & -3.456721 & -1.750558 \\ 6 & -3.368950 & -3.202994 & -0.378926 \\ 6 & -4.362312 & -3.861649 & -1.060900 \\ 6 & -4.056756 & -4.651691 & -2.189950 \\ 6 & -2.760856 & -4.740140 & -2.631560 \\ 6 & -1.267640 & -1.996831 & 1.220509 \\ 6 & -1.519533 & -0.644918 & 1.400449 \\ 6 & -1.891603 & -0.176426 & 2.691467 \\ 6 & -2.022781 & -1.028940 & 3.753396 \\ 6 & -1.784850 & -2.413285 & 3.601824 \\ 6 & -1.391240 & -2.905746 & 2.324979 \\ 6 & -1.927030 & -3.309894 & 4.687747 \\ 6 & -1.702394 & -4.652882 & 4.524382 \\ 6 & -1.305769 & -5.147909 & 3.264916 \\ 6 & -1.148563 & -4.298945 & 2.197595 \\ 15 & -1.547746 & 0.527948 & -0.043643 \\ 45 & 0.070843 & 0.667328 & -1.878735 \\ 6 & 0.521226 & 2.547505 & -1.546606 \\ 8 & 0.673417 & 3.685463 & -1.454563 \\ 15 & 1.824298 & -0.534398 & -0.913619 \\ 8 & 2.796355 & 0.135579 & 0.261196 \\ 6 & 3.665105 & 1.126412 & -0.147918 \\ 6 & 4.851323 & 0.782329 & -0.767295 \\ 6 & 5.698711 & 1.840684 & -1.230349 \\ 6 & 5.339927 & 3.196212 & -0.957197 \\ 6 & 4.136269 & 3.475892 & -0.267577 \\ 6 & 3.302439 & 2.460353 & 0.114805 \\ 6 & 6.181754 & 4.243863 & -1.404355 \\ 6 & 7.321694 & 3.977622 & -2.117516 \\ 6 & 7.659203 & 2.642383 & -2.422962 \\ 6 & 6.871236 & 1.604110 & -1.992666 \\ 6 & 5.165236 & -0.649933 & -0.978445 \\ 6 & 4.241927 & -1.457815 & -1.621403 \\ 6 & 4.520031 & -2.802868 & -1.943567 \\ 6 & 5.702879 & -3.368005 & -1.553402 \\ 6 & 6.642205 & -2.623716 & -0.800184 \\ 6 & 6.372236 & -1.254756 & -0.497669 \\ 6 & 7.304782 & -0.557799 & 0.312896 \\ 6 & 8.447558 & -1.168839 & 0.765804 \\ 6 & 8.727339 & -2.510942 & 0.433713 \\ 6 & 7.837098 & -3.220637 & -0.330236 \\ 8 & 3.023099 & -0.957567 & -2.013366 \\ & & & \\ 6\end{array}$




\begin{tabular}{|c|c|c|c|}
\hline 6 & -3.288350 & 0.293937 & -0.590205 \\
\hline 6 & -4.340422 & 0.239724 & 0.325417 \\
\hline 6 & -5.637538 & -0.061895 & -0.087046 \\
\hline 6 & -5.854064 & -0.304112 & -1.449986 \\
\hline 6 & -4.825944 & -0.240851 & -2.392993 \\
\hline 6 & -3.542678 & 0.071913 & -1.937855 \\
\hline 6 & -6.745326 & -0.147521 & 0.962155 \\
\hline 6 & -5.036039 & -0.502697 & -3.882238 \\
\hline 6 & -1.539896 & 2.159889 & 0.788592 \\
\hline 6 & -2.464855 & 3.141236 & 0.464541 \\
\hline 6 & -2.366238 & 4.431545 & 1.005385 \\
\hline 6 & -1.327866 & 4.688158 & 1.896090 \\
\hline 6 & -0.383128 & 3.712507 & 2.255937 \\
\hline 6 & -0.487589 & 2.458601 & 1.665252 \\
\hline 6 & -3.395448 & 5.482924 & 0.597751 \\
\hline 6 & -3.290241 & 5.724776 & -0.912565 \\
\hline 6 & 0.716676 & 4.049986 & 3.261274 \\
\hline 6 & 1.520516 & 2.815112 & 3.668508 \\
\hline 6 & -0.310341 & 0.938951 & -3.722915 \\
\hline 8 & -0.538108 & 1.045503 & -4.853103 \\
\hline 6 & 1.668550 & 5.078335 & 2.641716 \\
\hline 6 & 0.096351 & 4.636608 & 4.534556 \\
\hline 6 & -3.181867 & 6.815716 & 3333 \\
\hline 6 & -4.803022 & 4.978967 & 749 \\
\hline 1 & 7.099409 & 0.473373 & 0.588507 \\
\hline 1 & -2.091495 & & 2.838714 \\
\hline 1 & 8.026780 & -4.263167 & -0.582568 \\
\hline 1 & -2.223439 & -2.906828 & 5.655448 \\
\hline 1 & 5.892803 & 5.269266 & -1.177795 \\
\hline 1 & -3.620326 & -2.591236 & 0.486995 \\
\hline 1 & -5.395380 & -3.772161 & -0.729315 \\
\hline 1 & 3.869753 & 4.513625 & -0.070568 \\
\hline 1 & -2.321758 & -0.649753 & 4.730035 \\
\hline 1 & 5.929956 & -4.403711 & -1.800951 \\
\hline 1 & 8.549343 & 2.432826 & -3.011871 \\
\hline 1 & 3.781046 & -3.362708 & -2.512281 \\
\hline 1 & -2.510206 & -5.333435 & -3.509857 \\
\hline 1 & -0.149878 & -4.733574 & -3.289827 \\
\hline 1 & -1.116664 & -6.211756 & 3.138944 \\
\hline 1 & 7.139450 & 0.582366 & -2.248173 \\
\hline 1 & -0.825552 & -4.698735 & 1.238566 \\
\hline 1 & 1.638492 & -3.489258 & -2.104239 \\
\hline 1 & 7.957714 & 4.790254 & -2.460609 \\
\hline 1 & 9.141310 & -0.613172 & 1.392713 \\
\hline 1 & -4.851897 & -5.177864 & -2.713747 \\
\hline 1 & -1.821779 & -5.335967 & 5.362404 \\
\hline 1 & 9.639459 & -2.980676 & 0.794179 \\
\hline 1 & 2.354476 & 2.650740 & 0.615905 \\
\hline 1 & -4.138324 & 0.414712 & 1.381971 \\
\hline 1 & -2.706916 & 0.120687 & -2.633772 \\
\hline 1 & -6.855767 & -0.561103 & -1.782394 \\
\hline 1 & -3.271028 & 2.903894 & -0.228909 \\
\hline 1 & 0.237556 & 1.677739 & 1.895863 \\
\hline 1 & -1.240883 & 5.679463 & 2.335068 \\
\hline 1 & 3.351409 & -2.241553 & 0.704012 \\
\hline 1 & -0.432520 & -0.802960 & -2.291046 \\
\hline
\end{tabular}




$\begin{array}{lrrr}1 & 2.479082 & 5.323128 & 3.341419 \\ 1 & 2.122502 & 4.690109 & 1.719575 \\ 1 & 1.150016 & 6.011119 & 2.388276 \\ 1 & 0.882394 & 4.862076 & 5.267339 \\ 1 & -0.449383 & 5.568063 & 4.345094 \\ 1 & -0.601797 & 3.926238 & 4.996193 \\ 1 & 0.867704 & 2.034869 & 4.085514 \\ 1 & 2.083254 & 2.379656 & 2.831883 \\ 1 & 2.251883 & 3.087308 & 4.439617 \\ 1 & -3.949237 & 7.531259 & 0.989277 \\ 1 & -3.260508 & 6.716397 & 2.400837 \\ 1 & -2.204778 & 7.255941 & 1.073289 \\ 1 & -5.046134 & 4.043774 & 0.418085 \\ 1 & -4.907356 & 4.798571 & 2.015928 \\ 1 & -5.554458 & 5.724645 & 0.645299 \\ 1 & -2.285224 & 6.073038 & -1.184402 \\ 1 & -3.494686 & 4.815426 & -1.490226 \\ 1 & -4.012892 & 6.489333 & -1.228422 \\ 1 & 2.367001 & -3.692588 & 0.568114 \\ 1 & 1.741533 & -0.390256 & 2.320211 \\ 1 & 2.209941 & -4.639469 & 2.671365 \\ 1 & 1.328232 & -0.233734 & 4.755561 \\ 1 & 1.797596 & -4.490364 & 5.108966 \\ 1 & 1.357214 & -2.283083 & 6.161612 \\ 6 & -6.429232 & -1.050689 & -4.180266 \\ 6 & -4.850634 & 0.812533 & -4.647330 \\ 6 & -4.010040 & -1.527860 & -4.380321 \\ 6 & -8.092067 & -0.538035 & 0.358941 \\ 6 & -6.371431 & -1.204591 & 2.009046 \\ 6 & -6.903062 & 1.215392 & 1.645435 \\ 1 & -6.523375 & -1.256651 & -5.253910 \\ 1 & -6.615887 & -1.991445 & -3.643854 \\ 1 & -7.222019 & -0.339138 & -3.915740 \\ 1 & -4.173415 & -1.735835 & -5.446290 \\ 1 & -2.975186 & -1.178892 & -4.271111 \\ 1 & -4.099813 & -2.472848 & -3.828148 \\ 1 & -4.994341 & 0.653148 & -5.724649 \\ 1 & -5.575219 & 1.568000 & -4.315860 \\ 1 & -3.844061 & 1.225638 & -4.503393 \\ 1 & -8.849050 & -0.593706 & 1.151402 \\ 1 & -8.442085 & 0.196280 & -0.377900 \\ 1 & -8.052822 & -1.521672 & -0.127618 \\ 1 & -7.682989 & 1.168846 & 2.417535 \\ 1 & -5.974918 & 1.544608 & 2.128988 \\ 1 & -7.193535 & 1.985858 & 0.919123 \\ 1 & -7.150408 & -1.269598 & 2.780770 \\ 1 & -6.273597 & -2.196512 & 1.547515 \\ 1 & -5.423131 & -0.979019 & 2.513662\end{array}$

$1_{\text {C8- }} \psi_{-1 s 2 a 3 a 4 a}$

Number of imaginary frequencies : 0

The smallest frequencies are : $14.6965 \quad 16.9357 \quad 20.9829 \mathrm{~cm}(-1)$ Electronic energy : $\quad \mathrm{HF}=-4125.464855$

Zero-point correction $=\quad 1.300967$ (Hartree/Particle)

Thermal correction to Energy= $\quad 1.378664$ 
Thermal correction to Enthalpy=

Thermal correction to Gibbs Free Energy= Sum of electronic and zero-point Energies= Sum of electronic and thermal Energies= Sum of electronic and thermal Enthalpies= Sum of electronic and thermal Free Energies=
1.379608

1.190417

$-4124.163888$

$-4124.086191$

$-4124.085247$

$-4124.274438$

Cartesian Coordinates

\begin{tabular}{cccc}
6 & 1.632666 & -2.397296 & 5.113663 \\
6 & 1.522863 & -1.254142 & 4.325461 \\
6 & 1.814679 & -1.309167 & 2.968423 \\
6 & 2.221569 & -2.506285 & 2.378552 \\
6 & 2.331072 & -3.645313 & 3.174184 \\
6 & 2.041484 & -3.593489 & 4.535036 \\
6 & 2.554033 & -2.577573 & 0.910219 \\
7 & 1.589251 & -1.869287 & 0.046183 \\
6 & 0.524468 & -2.643338 & -0.531990 \\
6 & -0.763508 & -2.636828 & -0.023972 \\
6 & -1.797586 & -3.326602 & -0.740108 \\
6 & -1.484363 & -4.022800 & -1.944263 \\
6 & -0.145786 & -4.043397 & -2.399301 \\
6 & 0.824322 & -3.370992 & -1.710353 \\
6 & -3.146999 & -3.332395 & -0.297633 \\
6 & -4.121987 & -3.987477 & -1.007587 \\
6 & -3.801932 & -4.687128 & -2.190754 \\
6 & -2.509961 & -4.697405 & -2.650267 \\
6 & -1.121575 & -2.033695 & 1.291949 \\
6 & -1.479103 & -0.701495 & 1.449356 \\
6 & -1.936986 & -0.255459 & 2.720546 \\
6 & -2.048270 & -1.108258 & 3.784669 \\
6 & -1.706465 & -2.472651 & 3.655055 \\
6 & -1.231903 & -2.942345 & 2.397735 \\
6 & -1.840922 & -3.374378 & 4.737633 \\
6 & -1.527813 & -4.701222 & 4.589468 \\
6 & -1.048827 & -5.173197 & 3.349745 \\
6 & -0.899596 & -4.318098 & 2.286360 \\
15 & -1.536729 & 0.449363 & -0.012533 \\
45 & 0.181129 & 0.729379 & -1.733096 \\
6 & 0.547640 & 2.606285 & -1.299483 \\
8 & 0.660887 & 3.741473 & -1.140301 \\
15 & 1.962043 & -0.450573 & -0.805331 \\
8 & 2.954410 & 0.192379 & 0.365041 \\
6 & 3.770860 & 1.231318 & -0.031604 \\
6 & 4.942652 & 0.960227 & -0.711314 \\
6 & 5.726702 & 2.071590 & -1.161982 \\
6 & 5.327740 & 3.398418 & -0.812967 \\
6 & 4.146545 & 3.599507 & -0.059492 \\
6 & 3.369661 & 2.535259 & 0.308546 \\
6 & 6.108048 & 4.497591 & -1.247601 \\
6 & 7.224640 & 4.309187 & -2.020147 \\
6 & 7.599565 & 3.003458 & -2.400195 \\
6 & 6.872367 & 1.916314 & -1.983682 \\
6 & 5.305724 & -0.449990 & -0.982946 \\
6 & 4.389435 & -1.278708 & -1.609833 \\
6 & 4.707438 & -2.603532 & -1.976172 \\
& & & \\
\hline
\end{tabular}




\begin{tabular}{|c|c|c|c|}
\hline & 5.927070 & -3.129427 & -1.649871 \\
\hline & 6.867199 & -2.365597 & -0.917696 \\
\hline & 6.555259 & -1.016931 & -0.568130 \\
\hline & 7.492565 & -0.303324 & 0.222341 \\
\hline & 8.677269 & -0.877730 & 0.610872 \\
\hline & 8.996929 & -2.198031 & 0.231072 \\
\hline & 8.104112 & -2.924295 & -0.514057 \\
\hline & 3.139525 & -0.818891 & -1.948000 \\
\hline & -3.214389 & 0.052391 & -0.661256 \\
\hline & -4.312017 & -0.029028 & 0.199727 \\
\hline & -5.581536 & -0.333793 & -0.286302 \\
\hline & -5.714166 & -0.558833 & -1.662129 \\
\hline & -4.640172 & -0.485205 & -2.550256 \\
\hline & -3.383875 & -0.171545 & -2.023543 \\
\hline & -6.797654 & -0.460801 & 0.629931 \\
\hline & -4.861433 & -0.728916 & -4.041739 \\
\hline & -1.770700 & 2.092581 & 0.774276 \\
\hline & -2.760411 & 2.962300 & 0.308899 \\
\hline & -2.879197 & 4.251118 & 0.823952 \\
\hline & -1.990358 & 4.642037 & 1.834425 \\
\hline & -0.988996 & 3.801183 & 2.318934 \\
\hline & -0.875411 & 2.528188 & 1.748737 \\
\hline & -3.951373 & 5.226647 & 0.342151 \\
\hline & -0.069703 & 4.190791 & 3.475413 \\
\hline & -0.133944 & 1.056793 & -3.583407 \\
\hline & -0.310522 & 1.197345 & -4.718778 \\
\hline & 7.258074 & 0.710889 & 0.534767 \\
\hline & -2.228626 & 0.783550 & 2.850201 \\
\hline & 8.324674 & -3.951344 & -0.802130 \\
\hline . & -2.204943 & -2.989204 & 5.689432 \\
\hline & 5.790518 & 5.499712 & -0.962405 \\
\hline & -3.411236 & -2.793367 & 0.610571 \\
\hline & -5.152323 & -3.966480 & -0.657078 \\
\hline & 3.851370 & 4.615501 & 0.199446 \\
\hline ] & 6.184549 & -4.148680 & -1.933375 \\
\hline & 8.469690 & 2.856244 & -3.036012 \\
\hline l & 3.968892 & -3.178540 & -2.529999 \\
\hline & -2.246599 & -5.228282 & -3.564089 \\
\hline & 0.095120 & -4.590449 & -3.309395 \\
\hline & -0.790348 & -6.223783 & 3.236374 \\
\hline & 7.167730 & 0.918387 & -2.296993 \\
\hline 1 & -0.515893 & -4.698151 & 1.341617 \\
\hline & 1.852864 & -3.361706 & -2.065086 \\
\hline | & 7.812736 & 5.161297 & -2.352860 \\
\hline & 9.373780 & -0.309728 & 1.223526 \\
\hline 1 & -4.583149 & -5.211169 & -2.737460 \\
\hline . & -1.641789 & -5.389384 & 5.424101 \\
\hline & 9.941952 & -2.638388 & 0.540244 \\
\hline 1 & 2.442674 & 2.660950 & 0.863266 \\
\hline & -4.163776 & 0.149058 & 1.262100 \\
\hline 1 & -2.509395 & -0.102198 & -2.665671 \\
\hline 1 & -6.700810 & -0.803660 & -2.056611 \\
\hline 1 & -3.439552 & 2.616835 & -0.466534 \\
\hline 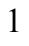 & -0.094361 & 1.847276 & 2.094700 \\
\hline 1 & -2.092322 & 5.640260 & 2.254454 \\
\hline 1 & 3.552238 & -2.152796 & 0.745561 \\
\hline
\end{tabular}




\begin{tabular}{|c|c|c|c|}
\hline & -0.242920 & -0.745315 & -2.213984 \\
\hline & 2.607912 & -3.628403 & 0.587867 \\
\hline & 1.716222 & -0.418202 & 2.350892 \\
\hline & 2.631438 & -4.589031 & 2.717828 \\
\hline & 1.198949 & -0.313564 & 4.768730 \\
\hline & 2.116416 & -4.495684 & 5.139090 \\
\hline & 1.392282 & -2.356986 & 6.174226 \\
\hline & -3.553971 & -0.685110 & -4.830097 \\
\hline & -5.496215 & -2.106407 & -4.256012 \\
\hline & -5.794623 & 0.354920 & -4.592914 \\
\hline & -6.474728 & -0.075585 & 2.072312 \\
\hline & -7.924568 & 0.451912 & 0.135774 \\
\hline & -7.280503 & -1.915642 & 0.621546 \\
\hline & -3.753699 & -0.901516 & -5.887207 \\
\hline & -3.076092 & 0.301796 & -4.783665 \\
\hline & -2.832238 & -1.431504 & -4.468609 \\
\hline & -5.957868 & 0.207873 & -5.669121 \\
\hline & -6.775809 & 0.340222 & -4.101983 \\
\hline & -5.363620 & 1.354379 & -4.449771 \\
\hline & -5.687996 & -2.273151 & -5.324591 \\
\hline & -4.825402 & -2.900137 & -3.902206 \\
\hline & -6.452003 & -2.214136 & -3.728092 \\
\hline & -7.376310 & -0.172217 & 2.690247 \\
\hline & -5.705402 & -0.725205 & 2.511711 \\
\hline 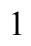 & -6.129916 & 0.964389 & 2.148932 \\
\hline & -8.171276 & -2.028204 & 1.254472 \\
\hline ] & -7.543404 & -2.255477 & -0.388267 \\
\hline [ & -6.504100 & -2.586740 & 1.014034 \\
\hline 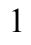 & -8.794401 & 0.375570 & 0.801751 \\
\hline ] & -7.603327 & 1.501324 & 0.118832 \\
\hline & -8.261365 & 0.188827 & -0.873927 \\
\hline 1 & -2.417167 & -0.744989 & 4.743414 \\
\hline 5 & -0.240979 & 5.650243 & 3.889688 \\
\hline 5 & -0.405836 & 3.304700 & 4.681414 \\
\hline b & 1.395847 & 3.976601 & 3.086379 \\
\hline 6 & -4.836649 & 4.622073 & -0.745401 \\
\hline 6 & -4.850374 & 5.620556 & 1.519540 \\
\hline 5 & -3.278885 & 6.479371 & -0.229325 \\
\hline 1 & 0.449225 & 5.883016 & 4.710210 \\
\hline l & -0.018358 & 6.338185 & 3.063751 \\
\hline 1 & -1.255892 & 5.860946 & 4.249747 \\
\hline 1 & 2.057750 & 4.313408 & 3.895119 \\
\hline 1 & 1.625519 & 2.918986 & 2.904818 \\
\hline 1 & 1.649277 & 4.546441 & 2.181529 \\
\hline 1 & 0.239065 & 3.555071 & 5.534753 \\
\hline 1 & -1.449670 & 3.444955 & 4.992113 \\
\hline & -0.264468 & 2.239252 & 4.456026 \\
\hline 1 & -5.594858 & 5.353902 & -1.051305 \\
\hline 1 & -4.262007 & 4.350380 & -1.640085 \\
\hline 1 & -5.366155 & 3.725853 & -0.394217 \\
\hline 1 & -5.630008 & 6.318399 & 1.185699 \\
\hline 1 & -5.346340 & 4.739080 & 1.946904 \\
\hline 1 & -4.289841 & 6.112075 & 2.323698 \\
\hline & -4.036581 & 7.188373 & -0.589427 \\
\hline 1 & -2.668658 & 6.998411 & 0.519671 \\
\hline 1 & -2.625353 & 6.223175 & -1.073208 \\
\hline
\end{tabular}


$1_{\mathrm{C} 8-} \psi_{-1 \mathrm{~s} 2 \mathrm{a3a} a \mathrm{~s}}$

Number of imaginary frequencies : 0

The smallest frequencies are : $15.1805 \quad 18.9473 \quad 23.4605 \mathrm{~cm}(-1)$

Electronic energy : $\quad \mathrm{HF}=-4125.4654859$

Zero-point correction $=\quad 1.302017($ Hartree/Particle $)$

Thermal correction to Energy $=\quad 1.379386$

Thermal correction to Enthalpy= $\quad 1.380331$

Thermal correction to Gibbs Free Energy $=\quad 1.192630$

Sum of electronic and zero-point Energies $=\quad-4124.163469$

Sum of electronic and thermal Energies $=\quad-4124.086100$

Sum of electronic and thermal Enthalpies $=\quad-4124.085155$

Sum of electronic and thermal Free Energies $=\quad-4124.272855$

Cartesian Coordinates

\begin{tabular}{cccc}
\hline 6 & 1.693117 & -2.449428 & 5.095965 \\
6 & 1.580382 & -1.299068 & 4.318803 \\
6 & 1.867092 & -1.340530 & 2.960063 \\
6 & 2.271135 & -2.532275 & 2.357977 \\
6 & 2.383149 & -3.679030 & 3.142033 \\
6 & 2.098893 & -3.640284 & 4.504341 \\
6 & 2.596925 & -2.587259 & 0.887085 \\
7 & 1.625590 & -1.873676 & 0.034084 \\
6 & 0.563288 & -2.645771 & -0.551603 \\
6 & -0.723698 & -2.650810 & -0.040868 \\
6 & -1.756473 & -3.338182 & -0.761055 \\
6 & -1.444904 & -4.013729 & -1.977134 \\
6 & -0.107395 & -4.022552 & -2.436087 \\
6 & 0.862717 & -3.357077 & -1.740174 \\
6 & -3.103542 & -3.358537 & -0.312262 \\
6 & -4.079269 & -4.003813 & -1.030093 \\
6 & -3.762199 & -4.678819 & -2.228085 \\
6 & -2.471240 & -4.678897 & -2.691091 \\
6 & -1.081398 & -2.056257 & 1.278931 \\
6 & -1.447503 & -0.726579 & 1.441858 \\
6 & -1.896880 & -0.286441 & 2.717713 \\
6 & -1.996486 & -1.143486 & 3.779985 \\
6 & -1.652368 & -2.506370 & 3.642999 \\
6 & -1.182235 & -2.969311 & 2.381523 \\
6 & -1.780263 & -3.413388 & 4.722086 \\
6 & -1.465961 & -4.738941 & 4.566101 \\
6 & -0.991699 & -5.204323 & 3.321942 \\
6 & -0.847820 & -4.343949 & 2.262219 \\
15 & -1.524977 & 0.426325 & -0.017906 \\
45 & 0.199479 & 0.737664 & -1.718154 \\
6 & 0.531213 & 2.618824 & -1.276273 \\
8 & 0.614078 & 3.756679 & -1.116895 \\
15 & 1.986105 & -0.438484 & -0.794941 \\
8 & 2.969224 & 0.197634 & 0.387222 \\
6 & 3.766742 & 1.257432 & 0.008090 \\
6 & 4.942841 & 1.018199 & -0.676198 \\
6 & 5.705980 & 2.150543 & -1.110071 \\
6 & 5.282973 & 3.464279 & -0.740342 \\
6 & 4.099247 & 3.632161 & 0.017257 \\
& & & \\
6
\end{tabular}




\begin{tabular}{|c|c|c|c|}
\hline 6 & 3.341984 & 2.548382 & 0.369025 \\
\hline 6 & 6.042341 & 4.584261 & -1.158575 \\
\hline & 7.161347 & 4.428404 & -1.934879 \\
\hline & 7.559846 & 3.135777 & -2.335357 \\
\hline & 6.853322 & 2.029077 & -1.935019 \\
\hline & 5.331072 & -0.381169 & -0.968728 \\
\hline & 4.429186 & -1.217714 & -1.606191 \\
\hline & 4.770226 & -2.531647 & -1.990262 \\
\hline & 5.999650 & -3.039837 & -1.673123 \\
\hline & 6.927277 & -2.268887 & -0.932545 \\
\hline & 6.591449 & -0.931232 & -0.563583 \\
\hline & 7.517315 & -0.212010 & 0.235281 \\
\hline & 8.713248 & -0.770068 & 0.613252 \\
\hline & 9.056324 & -2.078667 & 0.214025 \\
\hline & 8.175119 & -2.810519 & -0.539402 \\
\hline & 3.171317 & -0.776627 & -1.939058 \\
\hline ( & -3.186009 & -0.009038 & -0.682980 \\
\hline & -4.278979 & -0.139637 & 0.168529 \\
\hline 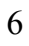 & -5.548649 & -0.447720 & -0.326436 \\
\hline & -5.683583 & -0.616715 & -1.705303 \\
\hline & -4.605916 & -0.486372 & -2.591162 \\
\hline & -3.355329 & -0.180994 & -2.056329 \\
\hline ר & -6.714706 & -0.569134 & 0.652616 \\
\hline 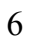 & -4.828875 & -0.668036 & -4.091009 \\
\hline 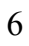 & -1.827093 & 2.060399 & 0.767185 \\
\hline 6 & -2.871278 & 2.873199 & 0.319220 \\
\hline & -3.055446 & 4.153238 & 0.835836 \\
\hline & -2.171983 & 4.596393 & 1.829031 \\
\hline & -1.115206 & 3.814435 & 2.295126 \\
\hline 6 & -0.941826 & 2.547120 & 1.726952 \\
\hline & -4.205440 & 5.051242 & 0.383488 \\
\hline 6 & -0.200205 & 4.261120 & 3.434604 \\
\hline 6 & -0.065580 & 1.064684 & -3.576906 \\
\hline & -0.200799 & 1.203643 & -4.717915 \\
\hline & 7.265149 & 0.793217 & 0.562631 \\
\hline & -2.194315 & 0.750252 & 2.852062 \\
\hline & 8.413670 & -3.829175 & -0.842581 \\
\hline & -2.140723 & -3.033340 & 5.677290 \\
\hline 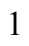 & 5.706527 & 5.575775 & -0.857638 \\
\hline & -3.365034 & -2.838370 & 0.607640 \\
\hline | & -5.108386 & -3.994290 & -0.674725 \\
\hline 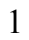 & 3.786628 & 4.638589 & 0.292540 \\
\hline & 6.274878 & -4.050478 & -1.970484 \\
\hline 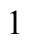 & 8.431742 & 3.014560 & -2.974208 \\
\hline & 4.040752 & -3.111997 & -2.550218 \\
\hline 1 & -2.209869 & -5.192835 & -3.615150 \\
\hline & 0.132808 & -4.554942 & -3.355041 \\
\hline 1 & -0.732354 & -6.254019 & 3.202432 \\
\hline 1 & 7.166675 & 1.041652 & -2.263761 \\
\hline ] & -0.467672 & -4.718742 & 1.313974 \\
\hline 1 & 1.889955 & -3.339464 & -2.098581 \\
\hline | & 7.733255 & 5.296206 & -2.255019 \\
\hline 1 & 9.400545 & -0.198044 & 1.232544 \\
\hline & -4.544477 & -5.192169 & -2.783306 \\
\hline 1 & -1.575393 & -5.431318 & 5.397856 \\
\hline 1 & 10.010020 & -2.505873 & 0.514893 \\
\hline
\end{tabular}




\begin{tabular}{|c|c|c|c|}
\hline 1 & 2.413291 & 2.648240 & 0.926514 \\
\hline & -4.141123 & 0.004678 & 1.239944 \\
\hline 1 & -2.483355 & -0.069500 & -2.696012 \\
\hline & -6.662603 & -0.859334 & -2.114502 \\
\hline 1 & -3.543342 & 2.491122 & -0.444136 \\
\hline 1 & -0.118667 & 1.911507 & 2.061171 \\
\hline 1 & -2.323963 & 5.586954 & 2.252091 \\
\hline 1 & 3.592546 & -2.156350 & 0.722385 \\
\hline 1 & -0.218221 & -0.735400 & -2.206820 \\
\hline 1 & 2.653726 & -3.634242 & 0.552944 \\
\hline 1 & 1.768614 & -0.443252 & 2.352390 \\
\hline 1 & 2.681234 & -4.618377 & 2.675277 \\
\hline 1 & 1.258493 & -0.363193 & 4.772915 \\
\hline 1 & 2.175727 & -4.548341 & 5.099334 \\
\hline 1 & 1.456954 & -2.418935 & 6.157800 \\
\hline 6 & -3.543217 & -0.468328 & -4.890796 \\
\hline 6 & -5.345296 & -2.083276 & -4.366939 \\
\hline 6 & -5.858656 & 0.357345 & -4.578428 \\
\hline 6 & -7.997962 & -1.038826 & -0.027227 \\
\hline 6 & -6.367733 & -1.579989 & 1.752385 \\
\hline 6 & -6.975756 & 0.803827 & 1.283975 \\
\hline 1 & -3.744013 & -0.629691 & -5.957484 \\
\hline 1 & -3.147738 & 0.549707 & -4.781530 \\
\hline 1 & -2.756389 & -1.175121 & -4.591964 \\
\hline 1 & -6.011397 & 0.258248 & -5.661536 \\
\hline 1 & -6.833989 & 0.226549 & -4.093891 \\
\hline 1 & -5.518806 & 1.381663 & -4.377158 \\
\hline 1 & -5.551562 & -2.211132 & -5.438308 \\
\hline 1 & -4.597085 & -2.830194 & -4.071965 \\
\hline 1 & -6.271579 & -2.302104 & -3.820830 \\
\hline 1 & -8.798869 & -1.131112 & 0.717193 \\
\hline 1 & -8.343208 & -0.331493 & -0.791889 \\
\hline 1 & -7.870685 & -2.021925 & -0.500309 \\
\hline 1 & -7.786130 & 0.740626 & 2.022978 \\
\hline 1 & -6.086116 & 1.196633 & 1.793602 \\
\hline 1 & -7.273276 & 1.533934 & 0.519455 \\
\hline 1 & -7.199445 & -1.665665 & 2.464459 \\
\hline 1 & -6.186868 & -2.576900 & 1.328263 \\
\hline 1 & -5.475345 & -1.294848 & 2.323744 \\
\hline 1 & -2.359830 & -0.784957 & 4.742616 \\
\hline 6 & -0.411151 & 5.725810 & 3.811186 \\
\hline 6 & -0.505012 & 3.397370 & 4.664785 \\
\hline 6 & 1.269584 & 4.079279 & 3.044706 \\
\hline 6 & -5.030209 & 4.414888 & -0.734029 \\
\hline 6 & -5.136912 & 5.311187 & 1.572938 \\
\hline 6 & -3.650747 & 6.382654 & -0.132998 \\
\hline 1 & 0.283833 & 6.002865 & 4.613694 \\
\hline 1 & -0.223870 & 6.396438 & 2.962545 \\
\hline 1 & -1.425931 & 5.915529 & 4.182776 \\
\hline 1 & 1.923643 & 4.462361 & 3.838995 \\
\hline 1 & 1.530964 & 3.023816 & 2.897922 \\
\hline 1 & 1.502423 & 4.626159 & 2.120365 \\
\hline 1 & 0.138520 & 3.685232 & 5.507288 \\
\hline 1 & -1.550148 & 3.517967 & 4.979217 \\
\hline 1 & -0.337195 & 2.331037 & 4.463615 \\
\hline 1 & -5.846676 & 5.090666 & -1.018163 \\
\hline
\end{tabular}


$1_{\mathrm{C} 8-} \psi_{-1 \mathrm{~s} 2 \mathrm{a} 3 \mathrm{~s} 4 \mathrm{a}}$

Number of imaginary frequencies : 0

The smallest frequencies are : $7.5527 \quad 15.5037 \quad 18.3574 \mathrm{~cm}(-1)$

Electronic energy : $\quad H F=-4125.4659445$

Zero-point correction $=\quad 1.298542($ Hartree/Particle $)$

Thermal correction to Energy= $\quad 1.377207$

Thermal correction to Enthalpy= $\quad 1.378152$

Thermal correction to Gibbs Free Energy= $\quad 1.183711$

Sum of electronic and zero-point Energies $=\quad-4124.167402$

Sum of electronic and thermal Energies $=\quad-4124.088737$

Sum of electronic and thermal Enthalpies $=\quad-4124.087793$

Sum of electronic and thermal Free Energies $=\quad-4124.282233$

\begin{tabular}{crrr}
\multicolumn{4}{c}{ Cartesian Coordinates } \\
\hline 6 & 1.595331 & -2.081836 & 5.188894 \\
6 & 1.528752 & -0.978380 & 4.340965 \\
6 & 1.778863 & -1.122110 & 2.981791 \\
6 & 2.101392 & -2.372146 & 2.447928 \\
6 & 2.163538 & -3.471072 & 3.303371 \\
6 & 1.912806 & -3.330256 & 4.665700 \\
6 & 2.441680 & -2.541016 & 0.987737 \\
7 & 1.520752 & -1.853759 & 0.061804 \\
6 & 0.459814 & -2.632302 & -0.515948 \\
6 & -0.830499 & -2.606325 & -0.017663 \\
6 & -1.863513 & -3.311216 & -0.719485 \\
6 & -1.541853 & -4.058381 & -1.890997 \\
6 & -0.200198 & -4.095453 & -2.336630 \\
6 & 0.766192 & -3.395934 & -1.669098 \\
6 & -3.217203 & -3.288002 & -0.293526 \\
6 & -4.190805 & -3.957231 & -0.993201 \\
6 & -3.862776 & -4.713525 & -2.138506 \\
6 & -2.563135 & -4.761827 & -2.574770 \\
6 & -1.169126 & -1.984480 & 1.293475 \\
6 & -1.496154 & -0.644427 & 1.451211 \\
6 & -1.921305 & -0.183724 & 2.727973 \\
6 & -2.015475 & -1.027246 & 3.801297 \\
6 & -1.691357 & -2.396195 & 3.674451 \\
6 & -1.258322 & -2.883618 & 2.408452 \\
6 & -1.798630 & -3.285366 & 4.770568 \\
6 & -1.504065 & -4.616954 & 4.627009 \\
6 & -1.070768 & -5.107176 & 3.377601 \\
6 & -0.945186 & -4.264614 & 2.301150 \\
15 & -1.546489 & 0.502212 & -0.014239 \\
45 & 0.160945 & 0.734571 & -1.755492 \\
6 & 0.566757 & 2.607413 & -1.343187
\end{tabular}




\begin{tabular}{|c|c|c|c|}
\hline & 0.704524 & 3.741232 & -1.194335 \\
\hline & 1.926780 & -0.463360 & -0.819996 \\
\hline & 2.928548 & 0.184775 & 0.338733 \\
\hline & 3.778250 & 1.189571 & -0.074949 \\
\hline & 4.945581 & 0.866838 & -0.739113 \\
\hline & 5.767671 & 1.942210 & -1.208120 \\
\hline & 5.408542 & 3.288421 & -0.891659 \\
\hline & 4.229574 & 3.543353 & -0.150822 \\
\hline & 3.416906 & 2.512587 & 0.235381 \\
\hline & 6.226912 & 4.352062 & -1.344480 \\
\hline & 7.342596 & 4.110734 & -2.103511 \\
\hline & 7.678782 & 2.785474 & -2.450858 \\
\hline 6 & 6.914186 & 1.731678 & -2.016095 \\
\hline 6 & 5.267064 & -0.559891 & -0.974279 \\
\hline 6 & 4.331140 & -1.374349 & -1.590672 \\
\hline 6 & 4.611398 & -2.716802 & -1.922349 \\
\hline 6 & 5.810661 & -3.272222 & -1.570033 \\
\hline 6 & 6.766819 & -2.520763 & -0.846087 \\
\hline 6 & 6.494283 & -1.154632 & -0.533141 \\
\hline 6 & 7.446021 & -0.451035 & 0.249011 \\
\hline 6 & 8.607869 & -1.052586 & 0.664463 \\
\hline 6 & 8.888977 & -2.391616 & 0.321335 \\
\hline 6 & 7.981172 & -3.107886 & -0.415256 \\
\hline 8 & 3.099008 & -0.882304 & -1.950250 \\
\hline 6 & -3.228952 & 0.107403 & -0.647342 \\
\hline 6 & -4.314856 & -0.024153 & 0.227930 \\
\hline 6 & -5.566428 & -0.403663 & -0.241234 \\
\hline 6 & -5.705829 & -0.650484 & -1.618158 \\
\hline 6 & -4.651333 & -0.512741 & -2.515907 \\
\hline 6 & -3.408194 & -0.118944 & -2.002380 \\
\hline 6 & -6.763550 & -0.589887 & 0.689529 \\
\hline 6 & -4.788714 & -0.758911 & -4.015961 \\
\hline 6 & -1.754140 & 2.156422 & 0.755147 \\
\hline 6 & -2.720770 & 3.038891 & 0.264975 \\
\hline 6 & -2.805551 & 4.344046 & 0.744880 \\
\hline 6 & -1.909964 & 4.736983 & 1.748825 \\
\hline 6 & -0.933455 & 3.882628 & 2.259465 \\
\hline 6 & -0.852194 & 2.592665 & 1.723009 \\
\hline 6 & -3.842589 & 5.339713 & 0.228117 \\
\hline 6 & -0.007553 & 4.277815 & 3.408641 \\
\hline 6 & -0.196053 & 1.053393 & -3.600687 \\
\hline 8 & -0.412023 & 1.189014 & -4.729829 \\
\hline 1 & 7.240928 & 0.577743 & 0.533243 \\
\hline 1 & -2.197617 & 0.859755 & 2.855488 \\
\hline 1 & 8.171826 & -4.148354 & -0.675198 \\
\hline 1 & -2.126668 & -2.886255 & 5.729674 \\
\hline 1 & 5.939703 & 5.369977 & -1.084337 \\
\hline 1 & -3.488301 & -2.708382 & 0.587919 \\
\hline 1 & -5.225507 & -3.906699 & -0.658838 \\
\hline 1 & 3.966327 & 4.573704 & 0.084626 \\
\hline 1 & 6.038791 & -4.305470 & -1.826729 \\
\hline 1 & 8.548917 & 2.596301 & -3.075489 \\
\hline 1 & 3.862901 & -3.282189 & -2.472734 \\
\hline 1 & -2.292841 & -5.333759 & -3.461483 \\
\hline 1 & 0.046365 & -4.677426 & -3.223246 \\
\hline 1 & -0.828559 & -6.161923 & 3.266901 \\
\hline
\end{tabular}




\begin{tabular}{|c|c|c|c|}
\hline & 7.179907 & 0.717645 & -2.303418 \\
\hline & -0.596040 & -4.659698 & 1.349222 \\
\hline & 1.796933 & -3.400111 & -2.016223 \\
\hline & 7.960197 & 4.935923 & -2.450313 \\
\hline & 9.316089 & -0.491609 & 1.270120 \\
\hline & -4.642698 & -5.249213 & -2.675603 \\
\hline & -1.597647 & -5.294766 & 5.472552 \\
\hline & 9.816199 & -2.853768 & 0.651848 \\
\hline & 2.492087 & 2.679263 & 0.783092 \\
\hline & -4.161557 & 0.159018 & 1.288685 \\
\hline & -2.550778 & -0.003766 & -2.662946 \\
\hline & -6.678711 & -0.968351 & -1.988751 \\
\hline & -3.405175 & 2.691538 & -0.505452 \\
\hline & -0.089634 & 1.901578 & 2.088793 \\
\hline & -1.984575 & 5.749139 & 2.140371 \\
\hline & 3.458949 & -2.166873 & 0.815178 \\
\hline & -0.285118 & -0.740949 & -2.216049 \\
\hline & 2.458623 & -3.610075 & 0.728078 \\
\hline & 1.722424 & -0.259353 & 2.321020 \\
\hline & 2.400579 & -4.453588 & 2.893991 \\
\hline & 1.276449 & 0.002909 & 4.740260 \\
\hline & 1.951304 & -4.202404 & 5.315599 \\
\hline ] & 1.391799 & -1.969423 & 6.251941 \\
\hline & -6.117984 & -1.421462 & -4.369001 \\
\hline 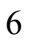 & -4.697578 & 0.586659 & -4.744664 \\
\hline 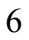 & -3.662710 & -1.677519 & -4.504074 \\
\hline & -6.417525 & -0.274845 & 2.143417 \\
\hline & -7.902461 & 0.338711 & 0.255938 \\
\hline & -7.238217 & -2.045876 & 0.625242 \\
\hline 1 & -6.157241 & -1.621189 & -5.447195 \\
\hline & -6.238793 & -2.381151 & -3.846919 \\
\hline ] & -6.978360 & -0.784802 & -4.125921 \\
\hline 1 & -3.772975 & -1.866735 & -5.580240 \\
\hline & -2.665428 & -1.246295 & -4.350198 \\
\hline ] & -3.691070 & -2.642458 & -3.980842 \\
\hline & -4.795948 & 0.443273 & -5.829300 \\
\hline 1 & -5.494248 & 1.267854 & -4.417602 \\
\hline & -3.735414 & 1.081461 & -4.559897 \\
\hline 1 & -7.301682 & -0.430136 & 2.774444 \\
\hline 1 & -5.620433 & -0.927235 & 2.526871 \\
\hline ] & -6.098951 & 0.767717 & 2.273000 \\
\hline 1 & -8.111835 & -2.191505 & 1.274931 \\
\hline & -7.527558 & -2.344888 & -0.389880 \\
\hline 1 & -6.448727 & -2.728510 & 0.969455 \\
\hline & -8.766557 & 0.221042 & 0.923573 \\
\hline 1 & -7.587367 & 1.389605 & 0.292547 \\
\hline 1 & -8.242640 & 0.127614 & -0.764913 \\
\hline 1 & -2.355080 & -0.652611 & 4.766444 \\
\hline 6 & -0.119460 & 5.758318 & 3.764820 \\
\hline 5 & -0.393200 & 3.454531 & 4.643836 \\
\hline 6 & 1.451204 & 3.987942 & 3.043298 \\
\hline 6 & -4.732975 & 4.738318 & -0.857070 \\
\hline 6 & -4.742414 & 5.788212 & 1.385198 \\
\hline 6 & -3.125413 & 6.557914 & -0.363677 \\
\hline 1 & 0.577338 & 5.994508 & 4.578793 \\
\hline 1 & 0.133470 & 6.403404 & 2.913438 \\
\hline
\end{tabular}




$\begin{array}{rrrr}1 & -1.125552 & 6.024853 & 4.112215 \\ 1 & 2.118258 & 4.319501 & 3.849923 \\ 1 & 1.638582 & 2.916854 & 2.893126 \\ 1 & 1.738610 & 4.521038 & 2.126269 \\ 1 & 0.258605 & 3.703554 & 5.492329 \\ 1 & -1.430441 & 3.657561 & 4.941438 \\ 1 & -0.305309 & 2.375954 & 4.457531 \\ 1 & -5.460802 & 5.488110 & -1.191910 \\ 1 & -4.155622 & 4.425383 & -1.736478 \\ 1 & -5.298653 & 3.870439 & -0.492638 \\ 1 & -5.494796 & 6.503378 & 1.026425 \\ 1 & -5.271911 & 4.933136 & 1.825436 \\ 1 & -4.175685 & 6.278979 & 2.185509 \\ 1 & -3.857185 & 7.279926 & -0.750466 \\ 1 & -2.509492 & 7.077109 & 0.380471 \\ 1 & -2.469425 & 6.262348 & -1.192696\end{array}$

$1_{\mathrm{C} 8-} \psi_{-1 \mathrm{~s} 2 \mathrm{a} 3 \mathrm{~s} 4 \mathrm{~s}}$

Number of imaginary frequencies : 0

The smallest frequencies are : $15.6277 \quad 18.661622 .1264 \mathrm{~cm}(-1)$

Electronic energy : $\quad H F=-4125.4663522$

Zero-point correction $=\quad 1.299756($ Hartree/Particle $)$

Thermal correction to Energy= $\quad 1.377723$

Thermal correction to Enthalpy= $\quad 1.378667$

Thermal correction to Gibbs Free Energy $=\quad 1.189132$

Sum of electronic and zero-point Energies $=\quad-4124.166596$

Sum of electronic and thermal Energies= $\quad-4124.088629$

Sum of electronic and thermal Enthalpies $=\quad-4124.087685$

Sum of electronic and thermal Free Energies $=\quad-4124.277220$

Cartesian Coordinates

$\begin{array}{lrrr}6 & 1.683913 & -2.304629 & 5.150558 \\ 6 & 1.599096 & -1.165648 & 4.352488 \\ 6 & 1.839184 & -1.246726 & 2.986308 \\ 6 & 2.171514 & -2.468755 & 2.396673 \\ 6 & 2.252973 & -3.603176 & 3.202476 \\ 6 & 2.011141 & -3.525324 & 4.571402 \\ 6 & 2.496042 & -2.570397 & 0.927220 \\ 7 & 1.551550 & -1.859089 & 0.043449 \\ 6 & 0.489082 & -2.631338 & -0.540368 \\ 6 & -0.798197 & -2.619448 & -0.033399 \\ 6 & -1.830975 & -3.323390 & -0.736797 \\ 6 & -1.514075 & -4.049267 & -1.922469 \\ 6 & -0.175826 & -4.069264 & -2.379759 \\ 6 & 0.791438 & -3.375122 & -1.707856 \\ 6 & -3.180098 & -3.319576 & -0.296403 \\ 6 & -4.154503 & -3.988003 & -0.995392 \\ 6 & -3.832135 & -4.719985 & -2.157932 \\ 6 & -2.536564 & -4.749118 & -2.608242 \\ 6 & -1.137931 & -2.010174 & 1.283720 \\ 6 & -1.477011 & -0.673693 & 1.449507 \\ 6 & -1.900245 & -0.223231 & 2.730325 \\ 6 & -1.986857 & -1.073970 & 3.798753 \\ 6 & -1.657697 & -2.440674 & 3.662179\end{array}$




\begin{tabular}{|c|c|c|c|}
\hline 6 & -1.222813 & -2.917210 & 2.392468 \\
\hline 6 & -1.763152 & -3.338694 & 4.751369 \\
\hline & -1.462244 & -4.667819 & 4.597209 \\
\hline & -1.030052 & -5.147776 & 3.343537 \\
\hline & -0.907625 & -4.296809 & 2.273668 \\
\hline & -1.542298 & 0.478802 & -0.010623 \\
\hline & 0.173858 & 0.754187 & -1.731004 \\
\hline & 0.553926 & 2.627366 & -1.294556 \\
\hline & 0.669670 & 3.762012 & -1.133301 \\
\hline & 1.943227 & -0.445084 & -0.807239 \\
\hline & 2.938096 & 0.186860 & 0.366558 \\
\hline & 3.765745 & 1.218430 & -0.025916 \\
\hline & 4.935683 & 0.936597 & -0.704245 \\
\hline & 5.730855 & 2.040884 & -1.152534 \\
\hline & 5.344435 & 3.371068 & -0.802020 \\
\hline & 4.165140 & 3.582677 & -0.048251 \\
\hline 6 & 3.377618 & 2.525484 & 0.317582 \\
\hline 6 & 6.135783 & 4.463058 & -1.234822 \\
\hline 6 & 7.251068 & 4.264646 & -2.006765 \\
\hline 6 & 7.613794 & 2.955803 & -2.387889 \\
\hline 0 & 6.875806 & 1.875286 & -1.973151 \\
\hline 6 & 5.286346 & -0.477206 & -0.974042 \\
\hline 6 & 4.363916 & -1.298426 & -1.601712 \\
\hline 6 & 4.669303 & -2.627675 & -1.962814 \\
\hline 6 & 5.882632 & -3.164749 & -1.631118 \\
\hline 6 & 6.828398 & -2.408069 & -0.898816 \\
\hline 6 & 6.529086 & -1.055215 & -0.554209 \\
\hline 6 & 7.471368 & -0.348652 & 0.236648 \\
\hline 6 & 8.649114 & -0.933774 & 0.630264 \\
\hline 6 & 8.956414 & -2.258462 & 0.255552 \\
\hline 6 & 8.058419 & -2.977985 & -0.489875 \\
\hline 8 & 3.120085 & -0.825924 & -1.945565 \\
\hline 6 & -3.210123 & 0.053789 & -0.662990 \\
\hline 6 & -4.302351 & -0.083595 & 0.194475 \\
\hline 6 & -5.554932 & -0.457414 & -0.289283 \\
\hline 6 & -5.682846 & -0.692615 & -1.664379 \\
\hline 6 & -4.612996 & -0.549333 & -2.551298 \\
\hline 6 & -3.376908 & -0.164880 & -2.024811 \\
\hline 6 & -6.717426 & -0.596751 & 0.693185 \\
\hline 6 & -4.744158 & -0.769629 & -4.056589 \\
\hline 6 & -1.802288 & 2.124651 & 0.761614 \\
\hline 6 & -2.801451 & 2.973591 & 0.277592 \\
\hline 6 & -2.931584 & 4.273294 & 0.761348 \\
\hline 6 & -2.049465 & 4.694187 & 1.765675 \\
\hline 6 & -1.038184 & 3.875701 & 2.267303 \\
\hline 6 & -0.911112 & 2.591319 & 1.725765 \\
\hline 6 & -4.015118 & 5.225370 & 0.258497 \\
\hline 6 & -0.118716 & 4.306028 & 3.409242 \\
\hline 6 & -0.175364 & 1.095717 & -3.573656 \\
\hline 8 & -0.389309 & 1.246729 & -4.701199 \\
\hline 1 & 7.246395 & 0.668842 & 0.545319 \\
\hline 1 & -2.183847 & 0.817382 & 2.864534 \\
\hline 1 & 8.269365 & -4.008146 & -0.773998 \\
\hline 1 & -2.092980 & -2.948581 & 5.713517 \\
\hline 1 & 5.827934 & 5.467925 & -0.948735 \\
\hline 1 & -3.446089 & -2.757010 & 0.597064 \\
\hline
\end{tabular}




\begin{tabular}{|c|c|c|c|}
\hline 1 & -5.186400 & -3.954294 & -0.649953 \\
\hline & 3.880356 & 4.600981 & 0.213212 \\
\hline & 6.130528 & -4.187510 & -1.910482 \\
\hline & 8.483074 & 2.800892 & -3.023029 \\
\hline 1 & 3.926820 & -3.197154 & -2.517211 \\
\hline & -2.270718 & -5.303866 & -3.507160 \\
\hline 1 & 0.067407 & -4.634507 & -3.278045 \\
\hline & -0.784934 & -6.200976 & 3.224837 \\
\hline 1 & 7.161716 & 0.874772 & -2.287055 \\
\hline 1 & -0.557927 & -4.683405 & 1.318498 \\
\hline 1 & 1.819648 & -3.367512 & -2.063369 \\
\hline 1 & 7.847644 & 5.111382 & -2.338073 \\
\hline 1 & 9.349674 & -0.370933 & 1.243059 \\
\hline 1 & -4.612533 & -5.253826 & -2.696116 \\
\hline 1 & -1.552862 & -5.352391 & 5.437649 \\
\hline 1 & 9.896010 & -2.707431 & 0.568841 \\
\hline 1 & 2.452182 & 2.658885 & 0.873323 \\
\hline 1 & -4.167821 & 0.096228 & 1.261003 \\
\hline 1 & -2.513442 & -0.043181 & -2.675936 \\
\hline 1 & -6.648818 & -1.001345 & -2.053610 \\
\hline 1 & -3.475602 & 2.605737 & -0.491886 \\
\hline 1 & -0.118304 & 1.929075 & 2.081344 \\
\hline 1 & -2.163187 & 5.699547 & 2.164938 \\
\hline 1 & 3.504735 & -2.172450 & 0.758265 \\
\hline 1 & -0.256635 & -0.719120 & -2.212236 \\
\hline 1 & 2.526871 & -3.627448 & 0.623237 \\
\hline 1 & 1.764218 & -0.357371 & 2.363289 \\
\hline 1 & 2.496170 & -4.564172 & 2.748029 \\
\hline 1 & 1.338350 & -0.206843 & 4.797565 \\
\hline 1 & 2.063486 & -4.424614 & 5.182161 \\
\hline 1 & 1.486847 & -2.241034 & 6.218841 \\
\hline 6 & -6.062252 & -1.445966 & -4.426237 \\
\hline 6 & -4.674491 & 0.592338 & -4.757046 \\
\hline 6 & -3.603355 & -1.658436 & -4.564176 \\
\hline 6 & -7.989305 & -1.110390 & 0.023411 \\
\hline 6 & -6.340955 & -1.581259 & 1.807229 \\
\hline 6 & -7.018430 & 0.775841 & 1.306989 \\
\hline 1 & -6.093518 & -1.628422 & -5.507763 \\
\hline 1 & -6.171814 & -2.415558 & -3.920570 \\
\hline 1 & -6.933378 & -0.826398 & -4.177809 \\
\hline 1 & -3.713823 & -1.830713 & -5.643119 \\
\hline 1 & -2.613911 & -1.210955 & -4.406820 \\
\hline 1 & -3.612184 & -2.632644 & -4.057943 \\
\hline 1 & -4.771597 & 0.470798 & -5.844512 \\
\hline 1 & -5.481304 & 1.254079 & -4.415059 \\
\hline 1 & -3.719850 & 1.097353 & -4.561167 \\
\hline 1 & -8.787106 & -1.206322 & 0.770726 \\
\hline 1 & -8.350996 & -0.426326 & -0.754753 \\
\hline 1 & -7.841925 & -2.099740 & -0.430075 \\
\hline 1 & -7.838074 & 0.700017 & 2.034402 \\
\hline 1 & -6.146980 & 1.193856 & 1.826828 \\
\hline 1 & -7.319951 & 1.492539 & 0.531967 \\
\hline 1 & -7.164523 & -1.671033 & 2.528367 \\
\hline 1 & -6.145001 & -2.582528 & 1.400198 \\
\hline 1 & -5.449051 & -1.268188 & 2.364648 \\
\hline 1 & -2.326656 & -0.707285 & 4.766872 \\
\hline
\end{tabular}




$\begin{array}{lrrr}6 & -0.285359 & 5.780824 & 3.767847 \\ 6 & -0.459215 & 3.467275 & 4.647139 \\ 6 & 1.347032 & 4.073865 & 3.030269 \\ 6 & -4.829995 & 4.623766 & -0.884552 \\ 6 & -4.974095 & 5.547875 & 1.410024 \\ 6 & -3.370389 & 6.519315 & -0.249535 \\ 1 & 0.408911 & 6.043294 & 4.575861 \\ 1 & -0.065374 & 6.435831 & 2.914878 \\ 1 & -1.297823 & 6.007846 & 4.124637 \\ 1 & 2.008460 & 4.444780 & 3.824273 \\ 1 & 1.576539 & 3.009402 & 2.894801 \\ 1 & 1.601590 & 4.604166 & 2.101875 \\ 1 & 0.189941 & 3.741943 & 5.489701 \\ 1 & -1.500932 & 3.627943 & 4.954781 \\ 1 & -0.328491 & 2.393522 & 4.458818 \\ 1 & -5.589681 & 5.344283 & -1.212781 \\ 1 & -4.203484 & 4.385168 & -1.753728 \\ 1 & -5.355902 & 3.708791 & -0.581345 \\ 1 & -5.762336 & 6.233261 & 1.070326 \\ 1 & -5.457226 & 4.635375 & 1.784345 \\ 1 & -4.459461 & 6.024823 & 2.253069 \\ 1 & -4.141138 & 7.203134 & -0.629661 \\ 1 & -2.821160 & 7.048599 & 0.538200 \\ 1 & -2.667271 & 6.312730 & -1.066703\end{array}$

$1_{\mathrm{C} 8-} \psi_{-1 \mathrm{~s} 2 \mathrm{~s} 3 \mathrm{a} 4 \mathrm{a}}$

Number of imaginary frequencies : 0

The smallest frequencies are : $14.1191 \quad 16.0865 \quad 19.0676 \mathrm{~cm}(-1)$

Electronic energy : $\quad \mathrm{HF}=-4125.4658734$

Zero-point correction $=\quad 1.299027($ Hartree/Particle)

Thermal correction to Energy= $\quad 1.377419$

Thermal correction to Enthalpy $=\quad 1.378364$

Thermal correction to Gibbs Free Energy $=\quad 1.186652$

Sum of electronic and zero-point Energies $=\quad-4124.166846$

Sum of electronic and thermal Energies $=\quad-4124.088454$

Sum of electronic and thermal Enthalpies $=\quad-4124.087510$

Sum of electronic and thermal Free Energies $=\quad-4124.279222$

\section{Cartesian Coordinates}

$\begin{array}{rrrr}6 & 1.659423 & -2.072467 & 5.171749 \\ 6 & 1.566337 & -0.970812 & 4.323973 \\ 6 & 1.816858 & -1.108743 & 2.964121 \\ 6 & 2.166534 & -2.351433 & 2.429820 \\ 6 & 2.255034 & -3.448560 & 3.285315 \\ 6 & 2.004033 & -3.313443 & 4.648099 \\ 6 & 2.509623 & -2.513878 & 0.969554 \\ 7 & 1.574762 & -1.848266 & 0.042529 \\ 6 & 0.528051 & -2.644398 & -0.536750 \\ 6 & -0.758817 & -2.652670 & -0.029035 \\ 6 & -1.786063 & -3.354717 & -0.739974 \\ 6 & -1.464549 & -4.053832 & -1.940462 \\ 6 & -0.125219 & -4.063741 & -2.393800 \\ 6 & 0.837789 & -3.375343 & -1.709746 \\ 6 & -3.135699 & -3.366162 & -0.299117\end{array}$




\begin{tabular}{|c|c|c|c|}
\hline 6 & -4.105559 & -4.027344 & -1.010341 \\
\hline 6 & -3.778621 & -4.728395 & -2.191257 \\
\hline 6 & -2.484973 & -4.736757 & -2.646212 \\
\hline 6 & -1.106130 & -2.049769 & 1.287567 \\
\hline 6 & -1.469737 & -0.719827 & 1.445529 \\
\hline 6 & -1.907558 & -0.271785 & 2.722721 \\
\hline 6 & -1.977061 & -1.118507 & 3.795400 \\
\hline 6 & -1.617314 & -2.478542 & 3.666878 \\
\hline 6 & -1.173682 & -2.953258 & 2.399680 \\
\hline 6 & -1.700258 & -3.372194 & 4.761364 \\
\hline 6 & -1.374444 & -4.696164 & 4.613811 \\
\hline 6 & -0.933669 & -5.173923 & 3.362190 \\
\hline 6 & -0.829746 & -4.326171 & 2.287461 \\
\hline 15 & -1.540966 & 0.426903 & -0.019072 \\
\hline 45 & 0.146515 & 0.701676 & -1.776871 \\
\hline 6 & 0.497296 & 2.587809 & -1.370164 \\
\hline 8 & 0.596207 & 3.726789 & -1.229977 \\
\hline 15 & 1.946522 & -0.449278 & -0.841211 \\
\hline 8 & 2.933376 & 0.225245 & 0.315424 \\
\hline 6 & 3.759111 & 1.248009 & -0.103181 \\
\hline 6 & 4.935254 & 0.949558 & -0.763276 \\
\hline 6 & 5.733097 & 2.041765 & -1.235418 \\
\hline 6 & 5.340636 & 3.380649 & -0.927540 \\
\hline 6 & 4.153126 & 3.611489 & -0.192516 \\
\hline 6 & 3.364333 & 2.563713 & 0.197360 \\
\hline 6 & 6.134777 & 4.461270 & -1.383201 \\
\hline 6 & 7.258803 & 4.242926 & -2.136856 \\
\hline 6 & 7.628079 & 2.924340 & -2.475797 \\
\hline 6 & 6.887331 & 1.854748 & -2.038273 \\
\hline 6 & 5.288366 & -0.470586 & -0.993285 \\
\hline 6 & 4.370630 & -1.306509 & -1.608578 \\
\hline 6 & 4.679232 & -2.643888 & -1.935271 \\
\hline 6 & 5.889676 & -3.172673 & -1.580221 \\
\hline 6 & 6.829195 & -2.398762 & -0.858213 \\
\hline 6 & 6.527562 & -1.037895 & -0.549372 \\
\hline 6 & 7.463587 & -0.312116 & 0.231559 \\
\hline 6 & 8.637788 & -0.887694 & 0.649191 \\
\hline 6 & 8.947587 & -2.221303 & 0.309764 \\
\hline 6 & 8.055615 & -2.958743 & -0.425238 \\
\hline 8 & 3.128641 & -0.841922 & -1.971097 \\
\hline 6 & -3.236453 & 0.051498 & -0.632469 \\
\hline 6 & -4.322683 & 0.031116 & 0.246863 \\
\hline 6 & -5.606030 & -0.260390 & -0.208845 \\
\hline 6 & -5.765011 & -0.537263 & -1.572876 \\
\hline 6 & -4.703157 & -0.526169 & -2.478268 \\
\hline 6 & -3.431498 & -0.222222 & -1.981839 \\
\hline 6 & -6.808882 & -0.327612 & 0.730487 \\
\hline 6 & -4.948831 & -0.811045 & -3.958863 \\
\hline 6 & -1.741958 & 2.072305 & 0.770406 \\
\hline 6 & -2.714959 & 2.954254 & 0.310281 \\
\hline 6 & -2.821395 & 4.247810 & 0.827025 \\
\hline 6 & -1.931652 & 4.628084 & 1.834008 \\
\hline 6 & -0.934636 & 3.769908 & 2.313097 \\
\hline 6 & -0.835944 & 2.498933 & 1.744532 \\
\hline 6 & -3.905206 & 5.173340 & 0.278343 \\
\hline 6 & -0.009751 & 4.150176 & 3.469007 \\
\hline
\end{tabular}




\begin{tabular}{|c|c|c|c|}
\hline & -0.200953 & 1.009880 & -3.623646 \\
\hline & -0.395724 & 1.141202 & -4.757188 \\
\hline & 7.236339 & 0.712810 & 0.512927 \\
\hline & -2.208154 & 0.764649 & 2.853456 \\
\hline & 8.268406 & -3.995618 & -0.682387 \\
\hline & -2.035847 & -2.983489 & 5.722126 \\
\hline & 5.822220 & 5.473369 & -1.129634 \\
\hline & -3.404071 & -2.825699 & 0.606999 \\
\hline & -5.137175 & -4.009141 & -0.663957 \\
\hline & 3.864450 & 4.636644 & 0.035639 \\
\hline & 6.139819 & -4.201732 & -1.833164 \\
\hline & 8.504896 & 2.752844 & -3.096163 \\
\hline & 3.943370 & -3.227631 & -2.483517 \\
\hline & -2.217015 & -5.269916 & -3.557331 \\
\hline & 0.122011 & -4.613951 & -3.300271 \\
\hline & -0.668708 & -6.222860 & 3.248518 \\
\hline & 7.178716 & 0.846024 & -2.319271 \\
\hline & -0.475752 & -4.710283 & 1.332758 \\
\hline & 1.865723 & -3.355057 & -2.064444 \\
\hline & 7.857618 & 5.080942 & -2.485843 \\
\hline & 9.333502 & -0.310098 & 1.253717 \\
\hline & -4.555712 & -5.258620 & -2.738012 \\
\hline & -1.450113 & -5.377826 & 5.458072 \\
\hline & 9.884363 & -2.662580 & 0.641859 \\
\hline 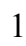 & 2.434129 & 2.711282 & 0.741335 \\
\hline & -4.155385 & 0.249652 & 1.298772 \\
\hline 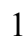 & -2.564897 & -0.202196 & -2.638173 \\
\hline & -6.763498 & -0.772192 & -1.942298 \\
\hline 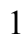 & -3.401622 & 2.625089 & -0.468462 \\
\hline ] & -0.064195 & 1.806742 & 2.089685 \\
\hline & -2.016053 & 5.622940 & 2.260251 \\
\hline 1 & 3.518343 & -2.117148 & 0.796719 \\
\hline & -0.269403 & -0.780700 & -2.241027 \\
\hline 1 & 2.550916 & -3.582516 & 0.710706 \\
\hline ] & 1.737939 & -0.247422 & 2.303247 \\
\hline 1 & 2.513569 & -4.425435 & 2.875462 \\
\hline 1 & 1.293124 & 0.004557 & 4.724539 \\
\hline & 2.062868 & -4.184521 & 5.297894 \\
\hline 1 & 1.455433 & -1.964452 & 6.235161 \\
\hline 6 & -3.642464 & -0.941233 & -4.740447 \\
\hline 6 & -5.729152 & -2.118272 & -4.126361 \\
\hline 6 & -5.758567 & 0.345118 & -4.556589 \\
\hline 6 & -6.456440 & 0.112016 & 2.150445 \\
\hline 6 & -7.929314 & 0.582479 & 0.217592 \\
\hline 6 & -7.310850 & -1.774877 & 0.789107 \\
\hline 1 & -3.861932 & -1.194855 & -5.785269 \\
\hline 1 & -3.067870 & -0.006344 & -4.748038 \\
\hline 1 & -3.000282 & -1.734092 & -4.331434 \\
\hline 1 & -5.941337 & 0.172957 & -5.626027 \\
\hline 1 & -6.732718 & 0.458283 & -4.064145 \\
\hline 1 & -5.219116 & 1.295872 & -4.454398 \\
\hline 1 & -5.901489 & -2.319832 & -5.192054 \\
\hline 1 & -5.166491 & -2.963063 & -3.707234 \\
\hline 1 & -6.710345 & -2.088962 & -3.637437 \\
\hline 1 & -7.351038 & 0.062289 & 2.783898 \\
\hline 1 & -5.695525 & -0.535043 & 2.607454 \\
\hline
\end{tabular}




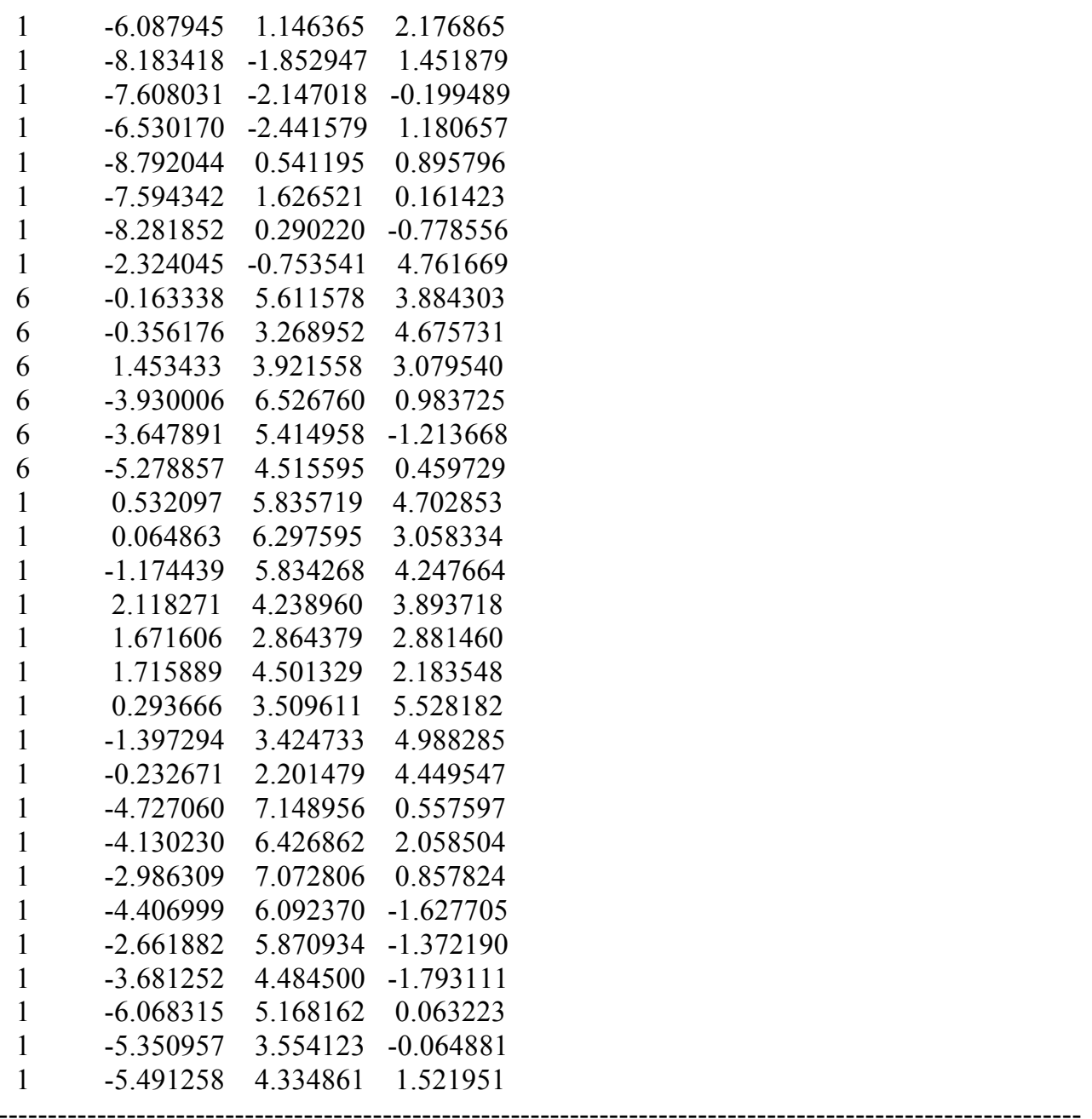

$1_{\mathrm{C} 8-} \psi_{-1 \mathrm{~s} 233 \mathrm{a} 4 \mathrm{~s}}$

Number of imaginary frequencies : 0

The smallest frequencies are : $12.9198 \quad 14.5566 \quad 17.8079 \mathrm{~cm}(-1)$

Electronic energy : $\quad \mathrm{HF}=-4125.4661583$

Zero-point correction $=\quad 1.299771$ (Hartree/Particle)

Thermal correction to Energy $=\quad 1.377855$

Thermal correction to Enthalpy= $\quad 1.378799$

Thermal correction to Gibbs Free Energy= $\quad 1.187999$

Sum of electronic and zero-point Energies= $\quad-4124.166387$

Sum of electronic and thermal Energies $=\quad-4124.088304$

Sum of electronic and thermal Enthalpies= $\quad-4124.087359$

Sum of electronic and thermal Free Energies $=\quad-4124.278160$

Cartesian Coordinates

$\begin{array}{llll}6 & 1.752411 & -2.462297 & 5.087397 \\ 6 & 1.652842 & -1.302834 & 4.320769 \\ 6 & 1.883698 & -1.345096 & 2.951294 \\ 6 & 2.221955 & -2.547628 & 2.326215 \\ 6 & 2.319038 & -3.702523 & 3.101123 \\ 6 & 2.086654 & -3.664000 & 4.473832 \\ 6 & 2.536309 & -2.604686 & 0.851610\end{array}$




\begin{tabular}{|c|c|c|c|}
\hline 7 & 1.582618 & -1.874277 & -0.006454 \\
\hline 6 & 0.519086 & -2.630368 & -0.609292 \\
\hline b & -0.764327 & -2.638153 & -0.092656 \\
\hline 6 & -1.804543 & -3.309588 & -0.814672 \\
\hline 6 & -1.503171 & -3.963930 & -2.044965 \\
\hline 6 & -0.168888 & -3.968154 & -2.513657 \\
\hline 6 & 0.809485 & -3.320310 & -1.812042 \\
\hline 6 & -3.146587 & -3.338206 & -0.352102 \\
\hline 6 & -4.128351 & -3.972550 & -1.071198 \\
\hline 6 & -3.822421 & -4.625070 & -2.284812 \\
\hline 6 & -2.536497 & -4.615423 & -2.761424 \\
\hline 6 & -1.095742 & -2.066744 & 1.242418 \\
\hline 6 & -1.444947 & -0.737261 & 1.439474 \\
\hline 6 & -1.860118 & -0.318110 & 2.733547 \\
\hline 6 & -1.931311 & -1.193308 & 3.783344 \\
\hline 6 & -1.595259 & -2.554364 & 3.612453 \\
\hline 6 & -1.167421 & -2.999448 & 2.329323 \\
\hline 6 & -1.688840 & -3.479124 & 4.679469 \\
\hline 6 & -1.383139 & -4.802868 & 4.491683 \\
\hline 6 & -0.959376 & -5.251521 & 3.223646 \\
\hline 6 & -0.848512 & -4.374350 & 2.173952 \\
\hline 15 & -1.532680 & 0.443946 & 0.003902 \\
\hline 5 & 0.170153 & 0.764950 & -1.718669 \\
\hline 6 & 0.531434 & 2.636835 & -1.257592 \\
\hline 8 & 0.635091 & 3.770588 & -1.082234 \\
\hline 15 & 1.957551 & -0.434361 & -0.821390 \\
\hline 8 & 2.948463 & 0.180667 & 0.364996 \\
\hline 6 & 3.756683 & 1.235437 & -0.006988 \\
\hline 6 & 4.928902 & 0.989129 & -0.695278 \\
\hline 6 & 5.702750 & 2.116446 & -1.122934 \\
\hline 6 & 5.293815 & 3.431788 & -0.743423 \\
\hline 6 & 4.113449 & 3.606307 & 0.017876 \\
\hline 6 & 3.345834 & 2.527937 & 0.364000 \\
\hline 6 & 6.063328 & 4.546869 & -1.156223 \\
\hline 6 & 7.178788 & 4.384900 & -1.936365 \\
\hline 6 & 7.563509 & 3.091015 & -2.346174 \\
\hline 6 & 6.846976 & 1.988824 & -1.951284 \\
\hline 6 & 5.301788 & -0.412015 & -0.998384 \\
\hline 6 & 4.390051 & -1.233683 & -1.640948 \\
\hline 6 & 4.715900 & -2.548993 & -2.033561 \\
\hline 6 & 5.939939 & -3.072898 & -1.720743 \\
\hline 6 & 6.877071 & -2.317204 & -0.976427 \\
\hline 6 & 6.556368 & -0.978299 & -0.598450 \\
\hline 6 & 7.490940 & -0.274116 & 0.203600 \\
\hline 6 & 8.681218 & -0.847405 & 0.576337 \\
\hline 6 & 9.009465 & -2.157191 & 0.168443 \\
\hline 6 & 8.119438 & -2.874791 & -0.588329 \\
\hline 8 & 3.137497 & -0.774132 & -1.970027 \\
\hline 6 & -3.210709 & 0.039337 & -0.637535 \\
\hline 6 & -4.289007 & -0.090484 & 0.232861 \\
\hline 6 & -5.567037 & -0.398342 & -0.240363 \\
\hline 6 & -5.726539 & -0.561670 & -1.617463 \\
\hline 6 & -4.663373 & -0.437205 & -2.521568 \\
\hline 6 & -3.403357 & -0.133052 & -2.007792 \\
\hline 6 & -6.710572 & -0.548237 & 0.761571 \\
\hline 6 & -4.910543 & -0.614057 & -4.018463 \\
\hline
\end{tabular}




\begin{tabular}{|c|c|c|c|}
\hline 6 & -1.792798 & 2.070783 & 0.817076 \\
\hline 6 & -2.797824 & 2.923044 & 0.368039 \\
\hline & -2.939120 & 4.212261 & 0.888040 \\
\hline & -2.055187 & 4.616274 & 1.890807 \\
\hline & -1.031298 & 3.786933 & 2.363504 \\
\hline & -0.896537 & 2.521620 & 1.789816 \\
\hline & -4.047463 & 5.111444 & 0.343522 \\
\hline & -0.111661 & 4.195177 & 3.514197 \\
\hline & -0.149358 & 1.116607 & -3.563892 \\
\hline & -0.331238 & 1.268868 & -4.696865 \\
\hline & 7.250148 & 0.731698 & 0.537579 \\
\hline & -2.149857 & 0.717155 & 2.893748 \\
\hline & 8.346703 & -3.894027 & -0.898082 \\
\hline & -2.013141 & -3.114203 & 5.653367 \\
\hline & 5.738195 & 5.539724 & -0.848072 \\
\hline & -3.398394 & -2.833918 & 0.579439 \\
\hline 1 & -5.154047 & -3.971277 & -0.706110 \\
\hline & 3.811229 & 4.613984 & 0.300078 \\
\hline 1 & 6.203392 & -4.084687 & -2.024799 \\
\hline & 8.432639 & 2.965323 & -2.987920 \\
\hline & 3.980017 & -3.118217 & -2.596558 \\
\hline & -2.284442 & -5.112761 & -3.697024 \\
\hline & 0.062767 & -4.484894 & -3.443665 \\
\hline & -0.711639 & -6.300676 & 3.077810 \\
\hline 1 & 7.149708 & 1.000447 & -2.287072 \\
\hline & -0.505530 & -4.735768 & 1.206628 \\
\hline 1 & 1.834537 & -3.301266 & -2.175912 \\
\hline & 7.758491 & 5.249035 & -2.252365 \\
\hline & 9.375641 & -0.286807 & 1.198111 \\
\hline 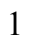 & -4.609283 & -5.131731 & -2.839726 \\
\hline & -1.464684 & -5.507285 & 5.316518 \\
\hline I & 9.958885 & -2.596610 & 0.465226 \\
\hline & 2.419001 & 2.633736 & 0.923519 \\
\hline & -4.131819 & 0.046961 & 1.302544 \\
\hline & -2.540678 & -0.029678 & -2.661343 \\
\hline & -6.713474 & -0.799116 & -2.009454 \\
\hline 1 & -3.479122 & 2.576522 & -0.407831 \\
\hline & -0.098576 & 1.855195 & 2.125099 \\
\hline 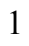 & -2.165202 & 5.608168 & 2.318045 \\
\hline & 3.541005 & -2.194290 & 0.688057 \\
\hline | & -0.264075 & -0.698574 & -2.220530 \\
\hline 1 & 2.573081 & -3.652334 & 0.516763 \\
\hline & 1.798371 & -0.439668 & 2.353360 \\
\hline 1 & 2.567043 & -4.648798 & 2.619221 \\
\hline & 1.386992 & -0.358519 & 4.793045 \\
\hline 1 & 2.151341 & -4.578961 & 5.059574 \\
\hline 1 & 1.562212 & -2.428848 & 6.158291 \\
\hline 6 & -3.613153 & -0.567562 & -4.823559 \\
\hline 6 & -5.583151 & -1.964381 & -4.284363 \\
\hline 5 & -5.823950 & 0.515020 & -4.509079 \\
\hline 6 & -8.015602 & -0.974436 & 0.094788 \\
\hline 6 & -6.343230 & -1.611707 & 1.804043 \\
\hline 6 & -6.942314 & 0.793700 & 1.465365 \\
\hline 1 & -3.832516 & -0.737660 & -5.885199 \\
\hline 1 & -3.112989 & 0.406261 & -4.747026 \\
\hline 1 & -2.902595 & -1.342494 & -4.502152 \\
\hline
\end{tabular}




$\begin{array}{lrrr}1 & -6.010533 & 0.415723 & -5.587011 \\ 1 & -6.795855 & 0.505937 & -3.999965 \\ 1 & -5.363295 & 1.496189 & -4.334759 \\ 1 & -5.765704 & -2.090023 & -5.360074 \\ 1 & -4.941374 & -2.789629 & -3.949022 \\ 1 & -6.549802 & -2.059817 & -3.774725 \\ 1 & -8.800146 & -1.084093 & 0.854119 \\ 1 & -8.367490 & -0.233465 & -0.634335 \\ 1 & -7.914610 & -1.940663 & -0.417609 \\ 1 & -7.746631 & 0.703330 & 2.208102 \\ 1 & -6.042502 & 1.142903 & 1.987634 \\ 1 & -7.233439 & 1.569814 & 0.745031 \\ 1 & -7.154897 & -1.722537 & 2.535684 \\ 1 & -6.184304 & -2.589931 & 1.330557 \\ 1 & -5.431558 & -1.359082 & 2.360436 \\ 1 & -2.264970 & -0.851098 & 4.762520 \\ 6 & -0.284287 & 5.660312 & 3.908573 \\ 6 & -0.446346 & 3.326070 & 4.732741 \\ 6 & 1.354725 & 3.979486 & 3.129003 \\ 6 & -4.111839 & 6.457977 & 1.059754 \\ 6 & -3.792767 & 5.372813 & -1.145614 \\ 6 & -5.403316 & 4.415781 & 0.513767 \\ 1 & 0.411396 & 5.906270 & 4.720593 \\ 1 & -0.069819 & 6.336966 & 3.071260 \\ 1 & -1.296485 & 5.874365 & 4.273863 \\ 1 & 2.015035 & 4.331244 & 3.932615 \\ 1 & 1.588888 & 2.920306 & 2.963795 \\ 1 & 1.606013 & 4.536361 & 2.215559 \\ 1 & 0.199444 & 3.587607 & 5.582102 \\ 1 & -1.489739 & 3.470869 & 5.042716 \\ 1 & -0.305895 & 2.257993 & 4.520646 \\ 1 & -4.926541 & 7.059949 & 0.637953 \\ 1 & -4.309599 & 6.343632 & 2.133562 \\ 1 & -3.184677 & 7.032666 & 0.938873 \\ 1 & -4.572136 & 6.028608 & -1.556891 \\ 1 & -2.821961 & 5.862263 & -1.297562 \\ 1 & -3.794406 & 4.446041 & -1.731852 \\ 1 & -6.207595 & 5.049590 & 0.116562 \\ 1 & -5.446709 & 3.455722 & -0.016504 \\ 1 & -5.616584 & 4.223456 & 1.573655\end{array}$

$1_{\mathrm{C} 8-} \psi_{-1525334 a}$

Number of imaginary frequencies : 0

The smallest frequencies are : $10.2059 \quad 14.0520 \quad 18.6433 \mathrm{~cm}(-1)$

Electronic energy : $\quad H F=-4125.4663558$

Zero-point correction $=\quad 1.299055$ (Hartree/Particle)

Thermal correction to Energy= $\quad 1.377360$

Thermal correction to Enthalpy= $\quad 1.378304$

Thermal correction to Gibbs Free Energy $=\quad 1.186597$

Sum of electronic and zero-point Energies $=\quad-4124.167301$

Sum of electronic and thermal Energies $=\quad-4124.088996$

Sum of electronic and thermal Enthalpies $=\quad-4124.088051$

Sum of electronic and thermal Free Energies $=\quad-4124.279759$

Cartesian Coordinates 


\begin{tabular}{cccc}
\hline 6 & 1.629338 & -2.201605 & 5.174906 \\
6 & 1.554305 & -1.076632 & 4.356296 \\
6 & 1.806875 & -1.182509 & 2.994087 \\
6 & 2.138882 & -2.415985 & 2.428827 \\
6 & 2.210102 & -3.536403 & 3.254788 \\
6 & 1.958008 & -3.433324 & 4.619985 \\
6 & 2.472990 & -2.545583 & 0.964102 \\
7 & 1.536962 & -1.846871 & 0.061952 \\
6 & 0.473411 & -2.625016 & -0.511763 \\
6 & -0.816176 & -2.599399 & -0.010844 \\
6 & -1.848421 & -3.310023 & -0.708704 \\
6 & -1.526900 & -4.063783 & -1.875891 \\
6 & -0.186518 & -4.097171 & -2.325767 \\
6 & 0.778829 & -3.391254 & -1.663731 \\
6 & -3.201218 & -3.288743 & -0.280016 \\
6 & -4.173313 & -3.970413 & -0.969684 \\
6 & -3.845007 & -4.734262 & -2.109842 \\
6 & -2.546757 & -4.777698 & -2.550891 \\
6 & -1.158951 & -1.972888 & 1.297692 \\
6 & -1.486733 & -0.632087 & 1.449076 \\
6 & -1.913259 & -0.165171 & 2.723274 \\
6 & -2.015031 & -1.004613 & 3.798905 \\
6 & -1.695658 & -2.375177 & 3.677871 \\
6 & -1.255979 & -2.867777 & 2.415994 \\
6 & -1.814337 & -3.260099 & 4.776319 \\
6 & -1.520954 & -4.592715 & 4.639015 \\
6 & -1.081514 & -5.088440 & 3.393927 \\
6 & -0.947112 & -4.250200 & 2.315214 \\
15 & -1.536929 & 0.510928 & -0.019176 \\
45 & 0.173872 & 0.743646 & -1.753393 \\
6 & 0.563556 & 2.621390 & -1.346263 \\
8 & 0.685616 & 3.757354 & -1.199784 \\
15 & 1.938143 & -0.450519 & -0.812937 \\
8 & 2.936415 & 0.196468 & 0.349626 \\
6 & 3.777014 & 1.209826 & -0.062579 \\
6 & 4.947023 & 0.899058 & -0.727803 \\
6 & 5.759618 & 1.983039 & -1.193961 \\
6 & 5.388033 & 3.325298 & -0.874874 \\
6 & 4.205665 & 3.567995 & -0.135434 \\
6 & 3.402365 & 2.528979 & 0.248016 \\
6 & 6.197056 & 4.397431 & -1.324409 \\
6 & 7.315552 & 4.168107 & -2.082985 \\
6 & 7.663860 & 2.846808 & -2.433372 \\
6 & 6.908465 & 1.785053 & -2.001866 \\
6 & 5.279942 & -0.524144 & -0.968684 \\
6 & 4.349671 & -1.343717 & -1.587021 \\
6 & 4.639785 & -2.682669 & -1.924304 \\
6 & 5.844134 & -3.229822 & -1.576567 \\
6 & 6.795610 & -2.473491 & -0.851376 \\
6 & 6.512485 & -1.111045 & -0.531853 \\
6 & 7.459536 & -0.403334 & 0.252265 \\
6 & 8.626931 & -0.997218 & 0.663197 \\
6 & 8.918524 & -2.332243 & 0.313351 \\
6 & 8.015456 & -3.052549 & -0.425152 \\
8 & 3.113174 & -0.860906 & -1.943522 \\
& & & \\
6
\end{tabular}




\begin{tabular}{|c|c|c|c|}
\hline & -3.216983 & 0.114763 & -0.659005 \\
\hline & -4.312088 & 0.001232 & 0.207852 \\
\hline & -5.562067 & -0.375330 & -0.268949 \\
\hline & -5.689214 & -0.641545 & -1.643550 \\
\hline & -4.625987 & -0.520611 & -2.533225 \\
\hline & -3.385815 & -0.126121 & -2.012951 \\
\hline & -6.766201 & -0.555332 & 0.654224 \\
\hline & -4.750562 & -0.785997 & -4.031171 \\
\hline & -1.755302 & 2.161409 & 0.753601 \\
\hline & -2.748395 & 3.023063 & 0.298091 \\
\hline & -2.864300 & 4.322237 & 0.799803 \\
\hline & -1.962717 & 4.728685 & 1.785447 \\
\hline & -0.948222 & 3.889843 & 2.262317 \\
\hline & -0.842675 & 2.612340 & 1.710881 \\
\hline & -3.978851 & 5.221166 & 0.268388 \\
\hline & -0.009607 & 4.299588 & 3.396740 \\
\hline & -0.171652 & 1.052931 & -3.602267 \\
\hline & -0.379158 & 1.180299 & -4.733926 \\
\hline & 7.246362 & 0.622355 & 0.541686 \\
\hline & -2.186230 & 0.879672 & 2.846883 \\
\hline & 8.214237 & -4.090212 & -0.690154 \\
\hline & -2.148279 & -2.856941 & 5.731634 \\
\hline & 5.900319 & 5.412061 & -1.062130 \\
\hline & -3.472268 & -2.702550 & 0.596912 \\
\hline & -5.206865 & -3.923440 & -0.631123 \\
\hline & 3.931728 & 4.595317 & 0.101120 \\
\hline & 6.080047 & -4.260279 & -1.837407 \\
\hline & 8.536098 & 2.667023 & -3.057837 \\
\hline & 3.893366 & -3.252000 & -2.473617 \\
\hline ] & -2.276404 & -5.354585 & -3.434375 \\
\hline & 0.059796 & -4.682135 & -3.210478 \\
\hline & -0.841178 & -6.144167 & 3.288597 \\
\hline & 7.183677 & 0.774315 & -2.291758 \\
\hline & -0.592874 & -4.649521 & 1.366935 \\
\hline 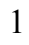 & 1.808854 & -3.393021 & -2.013693 \\
\hline & 7.925958 & 4.999703 & -2.427209 \\
\hline 1 & 9.331378 & -0.433263 & 1.270487 \\
\hline & -4.623483 & -5.279476 & -2.639349 \\
\hline 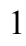 & -1.621636 & -5.267647 & 5.486060 \\
\hline 1 & 9.850056 & -2.788249 & 0.640257 \\
\hline & 2.474814 & 2.686956 & 0.793536 \\
\hline 1 & -4.168089 & 0.199113 & 1.267388 \\
\hline & -2.522359 & -0.021782 & -2.667226 \\
\hline 1 & -6.659725 & -0.961017 & -2.018508 \\
\hline & -3.443612 & 2.674005 & -0.464174 \\
\hline 1 & -0.056110 & 1.935908 & 2.053071 \\
\hline | & -2.052017 & 5.729254 & 2.197109 \\
\hline 1 & 3.484569 & -2.155779 & 0.793634 \\
\hline 1 & -0.265538 & -0.735108 & -2.209874 \\
\hline 1 & 2.500179 & -3.608214 & 0.679940 \\
\hline 1 & 1.742024 & -0.304014 & 2.354866 \\
\hline 1 & 2.453409 & -4.506073 & 2.819239 \\
\hline 1 & 1.291181 & -0.109267 & 4.781377 \\
\hline 1 & 2.002763 & -4.321668 & 5.247151 \\
\hline 1 & 1.423223 & -2.118867 & 6.240168 \\
\hline 6 & -6.076465 & -1.453933 & -4.386877 \\
\hline
\end{tabular}




$\begin{array}{rrrr}6 & -4.654683 & 0.550273 & -4.776152 \\ 6 & -3.619898 & -1.709673 & -4.498677 \\ 6 & -6.451664 & -0.156962 & 2.094689 \\ 6 & -7.936226 & 0.304443 & 0.165540 \\ 6 & -7.184465 & -2.030260 & 0.651065 \\ 1 & -6.106957 & -1.666141 & -5.462962 \\ 1 & -6.200668 & -2.407550 & -3.854642 \\ 1 & -6.939256 & -0.815252 & -4.158158 \\ 1 & -3.721120 & -1.911342 & -5.573459 \\ 1 & -2.623974 & -1.276258 & -4.341572 \\ 1 & -3.652647 & -2.668627 & -3.964736 \\ 1 & -4.741610 & 0.392679 & -5.859768 \\ 1 & -5.456457 & 1.233500 & -4.466338 \\ 1 & -3.695696 & 1.049756 & -4.587794 \\ 1 & -7.343464 & -0.295885 & 2.718683 \\ 1 & -5.649785 & -0.771473 & 2.526795 \\ 1 & -6.154632 & 0.897604 & 2.171489 \\ 1 & -8.056415 & -2.182388 & 1.301604 \\ 1 & -7.454570 & -2.379974 & -0.353359 \\ 1 & -6.371421 & -2.667297 & 1.026563 \\ 1 & -8.801332 & 0.181871 & 0.830822 \\ 1 & -7.667286 & 1.368450 & 0.155229 \\ 1 & -8.258809 & 0.030849 & -0.846030 \\ 1 & -2.357559 & -0.625501 & 4.761243 \\ 6 & -0.159507 & 5.771092 & 3.776162 \\ 6 & -0.340094 & 3.448229 & 4.628982 \\ 6 & 1.448861 & 4.060234 & 2.995190 \\ 6 & -3.989066 & 6.595092 & 0.933318 \\ 6 & -3.792406 & 5.421496 & -1.239986 \\ 6 & -5.334439 & 4.553551 & 0.531396 \\ 1 & 0.550189 & 6.018469 & 4.575493 \\ 1 & 0.051037 & 6.435343 & 2.928000 \\ 1 & -1.164203 & 6.001034 & 4.152431 \\ 1 & 2.124573 & 4.420128 & 3.782223 \\ 1 & 1.668633 & 2.995262 & 2.847847 \\ 1 & 1.693705 & 4.595608 & 2.067156 \\ 1 & 0.320338 & 3.709975 & 5.466951 \\ 1 & -1.377273 & 3.610775 & 4.950847 \\ 1 & -0.217455 & 2.375939 & 4.426689 \\ 1 & -4.808292 & 7.197031 & 0.520323 \\ 1 & -4.146868 & 6.526755 & 2.017520 \\ 1 & -3.056035 & 7.144631 & 0.754233 \\ 1 & -4.579700 & 6.076200 & -1.637472 \\ 1 & -2.822168 & 5.886888 & -1.456893 \\ 1 & -3.838957 & 4.474948 & -1.791568 \\ 1 & -6.149185 & 5.179689 & 0.143460 \\ 1 & -5.412772 & 3.570504 & 0.049821 \\ 1 & -5.499774 & 4.411469 & 1.607659\end{array}$

$1_{\mathrm{C} 8-} \psi_{-1 \mathrm{~s} 2 \mathrm{~s} 3 \mathrm{~s} 4 \mathrm{~s}}$

Number of imaginary frequencies : 0

The smallest frequencies are : $14.4018 \quad 17.6442 \quad 21.3473 \mathrm{~cm}(-1)$ Electronic energy : $\quad H F=-4125.4665759$

Zero-point correction $=\quad 1.299777$ (Hartree/Particle) Thermal correction to Energy $=\quad 1.377762$ 


$\begin{array}{lc}\text { Thermal correction to Enthalpy= } & 1.378707 \\ \text { Thermal correction to Gibbs Free Energy= } & 1.188696 \\ \text { Sum of electronic and zero-point Energies= } & -4124.166798 \\ \text { Sum of electronic and thermal Energies= } & -4124.088813 \\ \text { Sum of electronic and thermal Enthalpies }= & -4124.087869 \\ \text { Sum of electronic and thermal Free Energies }= & -4124.277880\end{array}$

Cartesian Coordinates

\begin{tabular}{ccccc}
6 & 1.692914 & -2.346024 & 5.129936 \\
6 & 1.604934 & -1.202164 & 4.339188 \\
6 & 1.839407 & -1.274852 & 2.971562 \\
6 & 2.168975 & -2.493152 & 2.372866 \\
6 & 2.253407 & -3.632543 & 3.171438 \\
6 & 2.017584 & -3.563098 & 4.541900 \\
6 & 2.489251 & -2.585707 & 0.901697 \\
7 & 1.548087 & -1.861774 & 0.024890 \\
6 & 0.479693 & -2.621153 & -0.564907 \\
6 & -0.805363 & -2.607855 & -0.052786 \\
6 & -1.846317 & -3.292310 & -0.762716 \\
6 & -1.540993 & -3.999090 & -1.962663 \\
6 & -0.204330 & -4.023929 & -2.424549 \\
6 & 0.771628 & -3.349640 & -1.744690 \\
6 & -3.193450 & -3.283055 & -0.316980 \\
6 & -4.178439 & -3.923130 & -1.027173 \\
6 & -3.868190 & -4.636143 & -2.204700 \\
6 & -2.573815 & -4.673795 & -2.658202 \\
6 & -1.137011 & -2.011637 & 1.272081 \\
6 & -1.472520 & -0.676123 & 1.451601 \\
6 & -1.889555 & -0.236469 & 2.738252 \\
6 & -1.975032 & -1.097561 & 3.798602 \\
6 & -1.650683 & -2.463914 & 3.647365 \\
6 & -1.220436 & -2.929122 & 2.371871 \\
6 & -1.755349 & -3.372862 & 4.727537 \\
6 & -1.456036 & -4.700713 & 4.559666 \\
6 & -1.029033 & -5.169289 & 3.299928 \\
6 & -0.909142 & -4.308155 & 2.237974 \\
15 & -1.537922 & 0.490479 & 0.003233 \\
45 & 0.176047 & 0.776636 & -1.717684 \\
6 & 0.559345 & 2.645824 & -1.266546 \\
8 & 0.674226 & 3.779423 & -1.097470 \\
15 & 1.943307 & -0.439364 & -0.810197 \\
8 & 2.942593 & 0.176776 & 0.368173 \\
6 & 3.770921 & 1.210968 & -0.016268 \\
6 & 4.939084 & 0.933475 & -0.699387 \\
6 & 5.735236 & 2.040352 & -1.139272 \\
6 & 5.351187 & 3.367987 & -0.776712 \\
6 & 4.173154 & 3.574744 & -0.019637 \\
6 & 3.384713 & 2.515570 & 0.338543 \\
6 & 6.143375 & 4.462579 & -1.201333 \\
6 & 7.257206 & 4.269266 & -1.976665 \\
6 & 7.617544 & 2.963245 & -2.369606 \\
6 & 6.878681 & 1.880225 & -1.963056 \\
6 & 5.285949 & -0.478563 & -0.982898 \\
6 & 4.360194 & -1.291551 & -1.616374 \\
6 & 4.661049 & -2.618695 & -1.988902 \\
\hline & & & \\
\hline
\end{tabular}




\begin{tabular}{|c|c|c|c|}
\hline 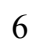 & 5.873346 & -3.162069 & -1.663634 \\
\hline & 6.822629 & -2.414296 & -0.926741 \\
\hline & 6.527710 & -1.063538 & -0.570226 \\
\hline & 7.473271 & -0.366268 & 0.224930 \\
\hline & 8.650020 & -0.957932 & 0.611603 \\
\hline & 8.952939 & -2.280318 & 0.225297 \\
\hline & 8.051684 & -2.991066 & -0.524613 \\
\hline & 3.117383 & -0.812001 & -1.954171 \\
\hline & -3.208555 & 0.079064 & -0.652207 \\
\hline 6 & -4.299836 & -0.076200 & 0.203837 \\
\hline 6 & -5.549891 & -0.453647 & -0.284440 \\
\hline & -5.678985 & -0.663214 & -1.663508 \\
\hline 0 & -4.611776 & -0.495937 & -2.549285 \\
\hline 6 & -3.376345 & -0.117169 & -2.017339 \\
\hline 6 & -6.703136 & -0.644937 & 0.700568 \\
\hline U & -4.744136 & -0.684368 & -4.058859 \\
\hline 6 & -1.792890 & 2.127067 & 0.794856 \\
\hline 6 & -2.796277 & 2.974784 & 0.335054 \\
\hline 6 & -2.934209 & 4.271330 & 0.837240 \\
\hline 6 & -2.044716 & 4.689295 & 1.829203 \\
\hline 6 & -1.020714 & 3.865003 & 2.310876 \\
\hline 6 & -0.893356 & 2.589326 & 1.759214 \\
\hline 6 & -4.045853 & 5.161633 & 0.285664 \\
\hline 6 & -0.093673 & 4.291038 & 3.449084 \\
\hline 6 & -0.166609 & 1.133533 & -3.558623 \\
\hline 8 & -0.373636 & 1.293232 & -4.686240 \\
\hline 1 & 7.251683 & 0.649189 & 0.542589 \\
\hline 1 & -2.169400 & 0.803748 & 2.883600 \\
\hline 1 & 8.259311 & -4.019410 & -0.817607 \\
\hline 1 & -2.081655 & -2.991881 & 5.694545 \\
\hline 1 & 5.837325 & 5.465368 & -0.906145 \\
\hline 1 & -3.449176 & -2.737472 & 0.589679 \\
\hline 1 & -5.208777 & -3.881859 & -0.676600 \\
\hline 1 & 3.889994 & 4.591248 & 0.250431 \\
\hline 1 & 6.117614 & -4.183300 & -1.951682 \\
\hline 1 & 8.485647 & 2.812640 & -3.007382 \\
\hline 1 & 3.915837 & -3.181622 & -2.546293 \\
\hline 1 & -2.317348 & -5.214788 & -3.568181 \\
\hline 1 & 0.030722 & -4.575811 & -3.333270 \\
\hline 1 & -0.786231 & -6.221714 & 3.170165 \\
\hline 1 & 7.162757 & 0.882038 & -2.285895 \\
\hline 1 & -0.564009 & -4.685921 & 1.277630 \\
\hline 1 & 1.798341 & -3.344786 & -2.104599 \\
\hline 1 & 7.854453 & 5.117992 & -2.301609 \\
\hline 1 & 9.353227 & -0.402232 & 1.227875 \\
\hline 1 & -4.656514 & -5.148974 & -2.751728 \\
\hline 1 & -1.545029 & -5.393210 & 5.393765 \\
\hline 1 & 9.891771 & -2.734563 & 0.533236 \\
\hline 1 & 2.459771 & 2.645754 & 0.895958 \\
\hline 1 & -4.165055 & 0.083664 & 1.273664 \\
\hline 1 & -2.513716 & 0.017301 & -2.667159 \\
\hline 1 & -6.643125 & -0.972529 & -2.056822 \\
\hline 1 & -3.479154 & 2.618520 & -0.434797 \\
\hline 1 & -0.096940 & 1.925026 & 2.102654 \\
\hline 1 & -2.150480 & 5.688295 & 2.240778 \\
\hline 1 & 3.500260 & -2.193103 & 0.733768 \\
\hline
\end{tabular}




\begin{tabular}{|c|c|c|c|}
\hline & -0.263243 & -0.689684 & -2.211192 \\
\hline & 2.512870 & -3.640604 & 0.589504 \\
\hline & 1.762913 & -0.381282 & 2.354891 \\
\hline & 2.494438 & -4.590793 & 2.710087 \\
\hline & 1.345918 & -0.246084 & 4.791094 \\
\hline & 2.072490 & -4.466168 & 5.146826 \\
\hline & 1.500418 & -2.288938 & 6.199416 \\
\hline & -6.069491 & -1.337363 & -4.443600 \\
\hline & -4.658699 & 0.690562 & -4.731950 \\
\hline & -3.612986 & -1.575479 & -4.583416 \\
\hline & -7.979233 & -1.132155 & 0.019094 \\
\hline & -6.306045 & -1.683386 & 1.757488 \\
\hline & -7.007659 & 0.690573 & 1.388726 \\
\hline & -6.102346 & -1.495563 & -5.528894 \\
\hline & -6.190719 & -2.316697 & -3.959915 \\
\hline & -6.933382 & -0.713337 & -4.181262 \\
\hline & -3.717379 & -1.714954 & -5.667711 \\
\hline & -2.617904 & -1.148894 & -4.404769 \\
\hline & -3.641173 & -2.563397 & -4.105648 \\
\hline & -4.756131 & 0.591599 & -5.821648 \\
\hline & -5.458959 & 1.353919 & -4.377961 \\
\hline & -3.698920 & 1.181716 & -4.526051 \\
\hline & -8.771455 & -1.259062 & 0.767732 \\
\hline & -8.346544 & -0.416837 & -0.727776 \\
\hline 1 & -7.835854 & -2.102146 & -0.475418 \\
\hline & -7.812011 & 0.566613 & 2.126532 \\
\hline ] & -6.132085 & 1.093508 & 1.913101 \\
\hline ] & -7.334244 & 1.441818 & 0.657759 \\
\hline & -7.124845 & -1.828059 & 2.475175 \\
\hline 1 & -6.092620 & -2.656673 & 1.294148 \\
\hline & -5.417505 & -1.384341 & 2.328219 \\
\hline 1 & -2.310292 & -0.739496 & 4.771516 \\
\hline 6 & -0.249076 & 5.767238 & 3.807972 \\
\hline 6 & -0.435614 & 3.455145 & 4.688616 \\
\hline 0 & 1.369692 & 4.050463 & 3.066527 \\
\hline 6 & -4.115725 & 6.513255 & 0.991566 \\
\hline 6 & -3.791909 & 5.411838 & -1.205544 \\
\hline & -5.398351 & 4.460653 & 0.461256 \\
\hline 1 & 0.453095 & 6.025834 & 4.610429 \\
\hline L & -0.031805 & 6.420488 & 2.952973 \\
\hline 1 & -1.256983 & 6.001270 & 4.172735 \\
\hline 1 & 2.034851 & 4.413150 & 3.861215 \\
\hline 1 & 1.592365 & 2.985378 & 2.925315 \\
\hline 1 & 1.626158 & 4.583804 & 2.140437 \\
\hline 1 & 0.217520 & 3.726089 & 5.529358 \\
\hline 1 & -1.475452 & 3.622474 & 4.999136 \\
\hline & -0.312563 & 2.380549 & 4.499850 \\
\hline 1 & -4.934058 & 7.108049 & 0.566643 \\
\hline 1 & -4.310810 & 6.405976 & 2.066600 \\
\hline 1 & -3.191713 & 7.091677 & 0.864609 \\
\hline 1 & -4.573850 & 6.060870 & -1.622646 \\
\hline 1 & -2.823001 & 5.903901 & -1.361243 \\
\hline 1 & -3.789395 & 4.480071 & -1.783984 \\
\hline & -6.206483 & 5.090224 & 0.065248 \\
\hline 1 & -5.439056 & 3.499663 & -0.067157 \\
\hline 1 & -5.608077 & 4.267997 & 1.521805 \\
\hline
\end{tabular}


$1_{\text {C9- } \Psi \text {-1a2a3a4a }}$

Number of imaginary frequencies : 0

The smallest frequencies are : $12.9230 \quad 15.5891 \quad 16.5139 \mathrm{~cm}(-1)$

Electronic energy : $\quad \mathrm{HF}=-4125.4627402$

Zero-point correction $=\quad 1.299724($ Hartree/Particle $)$

Thermal correction to Energy= $\quad 1.378200$

Thermal correction to Enthalpy= $\quad 1.379144$

Thermal correction to Gibbs Free Energy= $\quad 1.186585$

Sum of electronic and zero-point Energies $=\quad-4124.163016$

Sum of electronic and thermal Energies $=\quad-4124.084540$

Sum of electronic and thermal Enthalpies $=\quad-4124.083596$

Sum of electronic and thermal Free Energies $=\quad-4124.276155$

\begin{tabular}{|c|c|c|c|}
\hline & \multicolumn{3}{|c|}{ Cartesian Coordinates } \\
\hline 6 & 3.299423 & -6.158682 & 1.701319 \\
\hline 6 & 3.098158 & -5.269891 & 2.753675 \\
\hline 6 & 2.743965 & -3.952350 & 2.492399 \\
\hline 6 & 2.602774 & -3.491085 & 1.181201 \\
\hline 6 & 2.830350 & -4.382782 & 0.133224 \\
\hline 6 & 3.165767 & -5.709077 & 0.391711 \\
\hline 6 & 2.267700 & -2.035778 & 0.987342 \\
\hline 7 & 1.615799 & -1.680545 & -0.283746 \\
\hline 6 & 0.517626 & -2.437607 & -0.806896 \\
\hline 6 & -0.692335 & -2.571357 & -0.145334 \\
\hline 6 & -1.777625 & -3.246010 & -0.789945 \\
\hline 6 & -1.609439 & -3.751813 & -2.112284 \\
\hline 6 & -0.358455 & -3.597422 & -2.752683 \\
\hline 6 & 0.670943 & -2.962260 & -2.115278 \\
\hline 6 & -3.045615 & -3.399710 & -0.169859 \\
\hline 6 & -4.079198 & -4.025339 & -0.821223 \\
\hline 6 & -3.901112 & -4.540396 & -2.123371 \\
\hline 6 & -2.692265 & -4.398992 & -2.755508 \\
\hline 6 & -0.882739 & -2.048278 & 1.235245 \\
\hline 6 & -1.186158 & -0.715426 & 1.488840 \\
\hline 6 & -1.373886 & -0.298088 & 2.835217 \\
\hline 6 & -1.269250 & -1.176100 & 3.880257 \\
\hline 6 & -0.985008 & -2.541334 & 3.654002 \\
\hline 6 & -0.793881 & -2.987849 & 2.314600 \\
\hline 6 & -0.900253 & -3.468567 & 4.720560 \\
\hline 6 & -0.652485 & -4.795809 & 4.477358 \\
\hline 6 & -0.470558 & -5.246149 & 3.152843 \\
\hline 6 & -0.527947 & -4.366191 & 2.101135 \\
\hline 15 & -1.526386 & 0.461616 & 0.081920 \\
\hline 45 & -0.116217 & 0.879363 & -1.88499 \\
\hline 6 & 0.168584 & 2.785533 & -1.511039 \\
\hline 8 & 0.202812 & 3.931475 & -1.398134 \\
\hline 15 & 1.811446 & -0.158824 & $-1.03477 t$ \\
\hline 8 & 2.600162 & 0.587046 & 0.231478 \\
\hline 6 & 3.415857 & 1.658258 & -0.069576 \\
\hline 6 & 4.677700 & 1.434175 & -0.588714 \\
\hline 6 & 5.476663 & 2.571020 & -0.933541 \\
\hline 6 & 4.988990 & 3.882881 & -0.648635 \\
\hline
\end{tabular}




\begin{tabular}{|c|c|c|c|}
\hline 6 & 3.709495 & 4.040618 & -0.065647 \\
\hline 6 & 2.927379 & 2.949728 & 0.203162 \\
\hline & 5.782629 & 5.008518 & -0.978980 \\
\hline & 6.999914 & 4.858326 & -1.591146 \\
\hline & 7.468528 & 3.565876 & -1.908337 \\
\hline & 6.728942 & 2.454425 & -1.589483 \\
\hline & 5.114934 & 0.038138 & -0.824131 \\
\hline & 4.310511 & -0.790904 & -1.586659 \\
\hline & 4.676520 & -2.113556 & -1.902922 \\
\hline & 5.838418 & -2.634903 & -1.402410 \\
\hline & 6.664661 & -1.864626 & -0.550055 \\
\hline & 6.302623 & -0.516580 & -0.247461 \\
\hline & 7.122016 & 0.204516 & 0.658867 \\
\hline & 8.246713 & -0.363030 & 1.204040 \\
\hline & 8.618482 & -1.683874 & 0.876420 \\
\hline & 7.837581 & -2.416536 & 0.020486 \\
\hline & 3.110111 & -0.327528 & -2.077481 \\
\hline & -3.262977 & -0.012419 & -0.299340 \\
\hline D & -4.176903 & -0.238920 & 0.732484 \\
\hline & -5.501220 & -0.568192 & 0.454478 \\
\hline & -5.881084 & -0.653535 & -0.890545 \\
\hline & -4.993630 & -0.434427 & -1.945463 \\
\hline & -3.671274 & -0.112720 & -1.624924 \\
\hline & -6.521574 & -0.851146 & 1.555578 \\
\hline & -5.481210 & -0.529540 & -3.389776 \\
\hline b & -1.730936 & 2.086787 & 0.916912 \\
\hline & -2.823395 & 2.891127 & 0.595365 \\
\hline & -2.928168 & 4.192101 & 1.090549 \\
\hline & -1.918140 & 4.651367 & 1.938776 \\
\hline 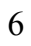 & -0.815133 & 3.868520 & 2.294076 \\
\hline & -0.720141 & 2.590021 & 1.741485 \\
\hline 6 & -4.103662 & 5.103147 & 0.741506 \\
\hline ) & 0.244159 & 4.431743 & 3.241043 \\
\hline & -0.645580 & 1.163409 & -3.689598 \\
\hline & -0.942721 & 1.273804 & -4.803167 \\
\hline & 6.843746 & 1.219309 & 0.931497 \\
\hline & -1.626847 & 0.737780 & 3.044157 \\
\hline & 8.098596 & -3.443825 & -0.230420 \\
\hline 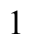 & -1.044295 & -3.104117 & 5.736867 \\
\hline & 5.394743 & 5.999156 & -0.745486 \\
\hline & -3.198304 & -3.000582 & 0.831718 \\
\hline 1 & -5.046984 & -4.123788 & -0.332566 \\
\hline & 3.345105 & 5.046256 & 0.141628 \\
\hline 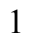 & -1.423866 & -0.832903 & 4.902558 \\
\hline & 6.129575 & -3.658618 & -1.632507 \\
\hline 1 & 8.422570 & 3.448599 & -2.417397 \\
\hline ] & 4.017956 & -2.691569 & -2.547443 \\
\hline ] & -2.540864 & -4.784675 & -3.762849 \\
\hline 1 & -0.229852 & -3.987249 & -3.761116 \\
\hline 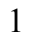 & -0.261013 & -6.296488 & 2.962798 \\
\hline 1 & 7.098687 & 1.466772 & -1.852605 \\
\hline & -0.356695 & -4.726807 & 1.089364 \\
\hline 1 & 1.629280 & -2.823748 & -2.613301 \\
\hline ] & 7.598242 & 5.730013 & -1.845713 \\
\hline 1 & 8.853966 & 0.210374 & 1.900926 \\
\hline 1 & -4.726057 & -5.043619 & -2.623393 \\
\hline
\end{tabular}




\begin{tabular}{|c|c|c|c|}
\hline & -0.596445 & -5.502831 & 5.302073 \\
\hline & 9.514596 & -2.119423 & 1.312218 \\
\hline & 1.927968 & 3.048398 & 0.623316 \\
\hline & -3.840313 & -0.157731 & 1.763425 \\
\hline & -2.932282 & 0.065816 & -2.402971 \\
\hline & -6.916078 & -0.906872 & -1.122333 \\
\hline & -3.594666 & 2.493947 & -0.059600 \\
\hline & 0.140197 & 1.960461 & 1.967782 \\
\hline & -1.993357 & 5.661202 & 2.341253 \\
\hline & 3.198489 & -1.459675 & 1.040255 \\
\hline & 1.659664 & -1.696149 & 1.841682 \\
\hline 1 & -0.540111 & -0.600071 & -2.326275 \\
\hline & 2.558490 & -3.267298 & 3.320538 \\
\hline & 2.740527 & -4.039386 & -0.894126 \\
\hline & 3.195306 & -5.608305 & 3.783413 \\
\hline 1 & 3.325391 & -6.394137 & -0.438600 \\
\hline 1 & 3.560721 & -7.195748 & 1.900685 \\
\hline 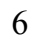 & -4.344081 & -0.341239 & -4.392028 \\
\hline 6 & -6.109201 & -1.903982 & -3.640496 \\
\hline 6 & -6.527627 & 0.563157 & -3.635909 \\
\hline 6 & -5.919307 & -0.703019 & 2.951383 \\
\hline 6 & -7.694710 & 0.126896 & 1.433267 \\
\hline 6 & -7.036246 & -2.287879 & 1.413667 \\
\hline 6 & -5.110268 & 4.421609 & -0.183554 \\
\hline 6 & -4.836411 & 5.508281 & 2.025230 \\
\hline 6 & -3.577875 & 6.356951 & 0.034497 \\
\hline 6 & 1.249884 & 3.370461 & 3.685786 \\
\hline 6 & 0.998919 & 5.558449 & 2.527167 \\
\hline 6 & -0.424011 & 4.988806 & 4.503216 \\
\hline 1 & -4.731963 & -0.444807 & -5.413274 \\
\hline 1 & -3.887371 & 0.653923 & -4.315982 \\
\hline 1 & -3.552455 & -1.092065 & -4.259205 \\
\hline 1 & -6.480154 & -1.968369 & -4.672322 \\
\hline 1 & -5.367665 & -2.700318 & -3.494557 \\
\hline 1 & -6.955725 & -2.106037 & -2.972590 \\
\hline 1 & -6.880281 & 0.528306 & -4.675580 \\
\hline 1 & -7.402191 & 0.448146 & -2.983437 \\
\hline 1 & -6.104328 & 1.560006 & -3.455364 \\
\hline 1 & -6.680835 & -0.932144 & 3.707450 \\
\hline 1 & -5.077679 & -1.391460 & 3.112121 \\
\hline 1 & -5.565380 & 0.319129 & 3.139088 \\
\hline 1 & -7.795406 & -2.499443 & 2.178794 \\
\hline 1 & -7.492754 & -2.470014 & 0.432762 \\
\hline 1 & -6.218606 & -3.009871 & 1.545425 \\
\hline 1 & -8.430861 & -0.058077 & 2.227083 \\
\hline 1 & -7.351140 & 1.165480 & 1.525865 \\
\hline 1 & -8.214201 & 0.031614 & 0.472134 \\
\hline 1 & -5.684786 & 6.164379 & 1.788421 \\
\hline 1 & -5.227102 & 4.626317 & 2.549522 \\
\hline 1 & -4.184346 & 6.049639 & 2.721318 \\
\hline 1 & -4.411285 & 7.016347 & -0.243091 \\
\hline 1 & -2.896460 & 6.934295 & 0.671040 \\
\hline 1 & -3.034250 & 6.091455 & -0.881523 \\
\hline 1 & -5.939377 & 5.109034 & -0.393453 \\
\hline 1 & -4.661515 & 4.144715 & -1.146301 \\
\hline 1 & -5.539125 & 3.515808 & 0.266328 \\
\hline
\end{tabular}




$\begin{array}{rrrr}1 & 0.337292 & 5.381860 & 5.189939 \\ 1 & -1.119557 & 5.807458 & 4.285424 \\ 1 & -0.982789 & 4.205536 & 5.031703 \\ 1 & 1.950026 & 3.808766 & 4.407987 \\ 1 & 0.755038 & 2.521161 & 4.176629 \\ 1 & 1.851347 & 2.980256 & 2.854989 \\ 1 & 1.788543 & 5.964483 & 3.173925 \\ 1 & 1.468417 & 5.193593 & 1.603207 \\ 1 & 0.330476 & 6.383811 & 2.252612\end{array}$

$1_{\mathrm{C} 9-} \psi_{-1 \mathrm{a} 2 \mathrm{a} 3 \mathrm{a} 4 \mathrm{~s}}$

Number of imaginary frequencies : 0

The smallest frequencies are : $14.2648 \quad 15.3178 \quad 19.9173 \mathrm{~cm}(-1)$

Electronic energy : $\quad H F=-4125.4633627$

Zero-point correction $=\quad 1.299918($ Hartree/Particle $)$

Thermal correction to Energy $=\quad 1.378204$

Thermal correction to Enthalpy= $\quad 1.379148$

Thermal correction to Gibbs Free Energy $=1.187976$

Sum of electronic and zero-point Energies $=\quad-4124.163445$

Sum of electronic and thermal Energies= $\quad-4124.085159$

Sum of electronic and thermal Enthalpies $=\quad-4124.084215$

Sum of electronic and thermal Free Energies $=\quad-4124.275386$

Cartesian Coordinates

$\begin{array}{cccc}6 & -0.758170 & 2.617369 & 1.701209 \\ 6 & -1.763278 & 2.086182 & 0.887378 \\ 6 & -2.880560 & 2.860623 & 0.577463 \\ 6 & -3.015728 & 4.158300 & 1.074374 \\ 6 & -2.013383 & 4.642258 & 1.918220 \\ 6 & -0.884344 & 3.890846 & 2.259305 \\ 15 & -1.524015 & 0.460204 & 0.062289 \\ 6 & -3.251108 & -0.041671 & -0.325506 \\ 6 & -4.162767 & -0.276590 & 0.698648 \\ 6 & -5.487643 & -0.622452 & 0.421880 \\ 6 & -5.866960 & -0.714131 & -0.917875 \\ 6 & -4.974153 & -0.484304 & -1.973432 \\ 6 & -3.659195 & -0.148030 & -1.654499 \\ 6 & -6.443264 & -0.875645 & 1.586266 \\ 6 & -6.633808 & 0.428912 & 2.368875 \\ 6 & -5.459589 & -0.584747 & -3.418019 \\ 6 & -6.079748 & -1.962725 & -3.668812 \\ 6 & -4.229222 & 5.028773 & 0.751659 \\ 6 & -3.766566 & 6.363228 & 0.157604 \\ 6 & 0.173665 & 4.482734 & 3.190402 \\ 6 & -0.489993 & 5.065478 & 4.443062 \\ 6 & -1.174839 & -0.702630 & 1.479866 \\ 6 & -0.867878 & -2.036993 & 1.237852 \\ 6 & -0.773634 & -2.966268 & 2.325557 \\ 6 & -0.960289 & -2.507797 & 3.661577 \\ 6 & -1.243163 & -1.140531 & 3.876198 \\ 6 & -1.355339 & -0.272790 & 2.823185 \\ 6 & -0.506286 & -4.346181 & 2.123925 \\ 6 & -0.445249 & -5.216518 & 3.183322 \\ 6 & -0.624002 & -4.754531 & 4.504292\end{array}$




\begin{tabular}{|c|c|c|c|}
\hline 5 & -0.871554 & -3.425308 & 4.736289 \\
\hline 6 & -0.676028 & -2.571338 & -0.138280 \\
\hline 6 & 0.532040 & -2.433733 & -0.802393 \\
\hline 6 & 0.686417 & -2.964538 & -2.108227 \\
\hline 5 & -0.339684 & -3.610530 & -2.740146 \\
\hline 6 & -1.587788 & -3.772088 & -2.095651 \\
\hline 6 & -1.756548 & -3.260300 & -0.775810 \\
\hline 5 & -2.667154 & -4.432173 & -2.731574 \\
\hline 6 & -3.872534 & -4.581652 & -2.094264 \\
\hline 6 & -4.050246 & -4.062342 & -0.793813 \\
\hline 6 & -3.020387 & -3.422827 & -0.150206 \\
\hline 7 & 1.627056 & -1.669281 & -0.283303 \\
\hline 15 & 1.818208 & -0.149727 & -1.039305 \\
\hline 8 & 3.122922 & -0.314877 & -2.074949 \\
\hline 6 & 4.322718 & -0.771945 & -1.576805 \\
\hline 6 & 5.119148 & 0.062620 & -0.812013 \\
\hline 6 & 6.305178 & -0.485738 & -0.225928 \\
\hline 6 & 6.674329 & -1.833340 & -0.521926 \\
\hline 6 & 5.856933 & -2.609140 & -1.377847 \\
\hline 6 & 4.696177 & -2.093929 & -1.887371 \\
\hline 6 & 7.115426 & 0.241134 & 0.683954 \\
\hline 6 & 8.238026 & -0.320733 & 1.239203 \\
\hline 6 & 8.616846 & -1.641270 & 0.918466 \\
\hline 6 & 7.844991 & -2.379285 & 0.058918 \\
\hline 6 & 4.675228 & 1.457564 & -0.582773 \\
\hline 6 & 3.409941 & & -0.070794 \\
\hline 6 & 2.917408 & 2.969393 & 0.198856 \\
\hline 6 & 3.698517 & 4.062001 & -0.065830 \\
\hline 6 & 4.981054 & 3.907022 & -0.642869 \\
\hline 6 & 5.472959 & 2.596127 & -0.924806 \\
\hline 6 & 5.773893 & 5.034251 & -0.969682 \\
\hline 6 & 6.994517 & 4.88 & -1.575761 \\
\hline 6 & 7.467457 & 3.594859 & -1.890145 \\
\hline 6 & 6.728723 & 2.481943 & -1.574474 \\
\hline 8 & 2.595779 & 0.605949 & 0.228295 \\
\hline 6 & 2.282466 & -2.018730 & 0.987815 \\
\hline 6 & 2.622030 & -3.472437 & 1.185646 \\
\hline 6 & 2.763816 & -3.930009 & 2.498033 \\
\hline 6 & 3.121499 & -5.245954 & 2.762799 \\
\hline 6 & 3.325853 & -6.136778 & 1.712809 \\
\hline 6 & 3.191868 & -5.690776 & 0.401977 \\
\hline 6 & 2.852853 & -4.366106 & 0.140042 \\
\hline 45 & -0.111568 & 0.873185 & -1.901575 \\
\hline 6 & -0.635994 & 1.137352 & -3.710960 \\
\hline 8 & -0.929397 & 1.234520 & -4.826703 \\
\hline 6 & 0.159780 & 2.784169 & -1.545585 \\
\hline 8 & 0.183491 & 3.931681 & -1.447033 \\
\hline 6 & -7.812791 & -1.361283 & 1.119208 \\
\hline 6 & -5.854379 & -1.946219 & 2.513271 \\
\hline 6 & -4.322139 & -0.391686 & -4.419063 \\
\hline 6 & -6.511406 & 0.502168 & -3.667130 \\
\hline 6 & -5.167236 & 4.365001 & -0.254823 \\
\hline 6 & -5.016902 & 5.287210 & 2.040914 \\
\hline 6 & 1.188370 & 3.437912 & 3.653863 \\
\hline 6 & 0.917217 & 5.597658 & 2.446860 \\
\hline 1 & 6.831573 & 1.255731 & 0.951440 \\
\hline
\end{tabular}




\begin{tabular}{|c|c|c|c|}
\hline . & -1.609063 & 0.764415 & 3.023867 \\
\hline . & 8.111510 & -3.406398 & -0.186839 \\
\hline & -1.012427 & -3.051736 & 5.749719 \\
\hline & 5.382767 & 6.024143 & -0.738442 \\
\hline & -3.173743 & -3.019258 & 0.849153 \\
\hline & -5.014848 & -4.167569 & -0.299852 \\
\hline & 3.331443 & 5.066746 & 0.140855 \\
\hline 1 & -1.391752 & -0.787311 & 4.895992 \\
\hline & 6.153541 & -3.632385 & -1.603034 \\
\hline 1 & 8.424236 & 3.479444 & -2.394470 \\
\hline & 4.043913 & -2.676519 & -2.534231 \\
\hline & -2.516081 & -4.821589 & -3.737534 \\
\hline 1 & -0.210342 & -4.004412 & -3.746909 \\
\hline 1 & -0.235136 & -6.268302 & 3.002112 \\
\hline 1 & 7.101867 & 1.494961 & -1.835317 \\
\hline 1 & -0.337273 & -4.715749 & 1.115012 \\
\hline 1 & 1.642796 & -2.821659 & -2.608867 \\
\hline 1 & 7.592244 & 5.759276 & -1.827703 \\
\hline 1 & 8.838075 & 0.256943 & 1.938794 \\
\hline 1 & -4.694482 & -5.094877 & -2.588997 \\
\hline 1 & -0.565260 & -5.454153 & 5.335099 \\
\hline 1 & 9.511159 & -2.072346 & 1.362335 \\
\hline 1 & 1.916216 & 3.066581 & 0.614860 \\
\hline 1 & -3.836615 & -0.187831 & 1.734770 \\
\hline 1 & -2.921459 & 0.038464 & -2.431739 \\
\hline 1 & -6.895341 & -0.977645 & -1.157600 \\
\hline 1 & -3.647487 & 2.443797 & -0.070144 \\
\hline 1 & 0.122439 & 2.012236 & 1.916465 \\
\hline 1 & -2.115953 & 5.646250 & 2.328943 \\
\hline 1 & 3.211599 & -1.439665 & 1.037423 \\
\hline 1 & 1.674916 & -1.678156 & 1.842100 \\
\hline 1 & -0.527940 & -0.611687 & -2.331597 \\
\hline 1 & 2.576045 & -3.243314 & 3.324269 \\
\hline 1 & 2.762410 & -4.025554 & -0.888198 \\
\hline 1 & 3.218864 & -5.581522 & 3.793450 \\
\hline 1 & 3.353856 & -6.377453 & -0.426537 \\
\hline 1 & 3.589781 & -7.172647 & 1.914908 \\
\hline 1 & -4.707287 & -0.502793 & -5.440554 \\
\hline 1 & -3.873538 & 0.607402 & -4.346573 \\
\hline 1 & -3.524892 & -1.135616 & -4.281178 \\
\hline 1 & -6.863521 & 0.463086 & -4.706858 \\
\hline 1 & -7.385704 & 0.384284 & -3.014911 \\
\hline 1 & -6.092820 & 1.501474 & -3.488761 \\
\hline 1 & -6.440880 & -2.032323 & -4.703765 \\
\hline 1 & -5.336467 & -2.755623 & -3.513066 \\
\hline 1 & -6.931986 & -2.165481 & -3.008701 \\
\hline 1 & -8.455306 & -1.548746 & 1.988807 \\
\hline 1 & -8.321732 & -0.619003 & 0.491213 \\
\hline 1 & -7.740344 & -2.299624 & 0.552897 \\
\hline 1 & -7.286288 & 0.264865 & 3.237252 \\
\hline 1 & -5.680724 & 0.830773 & 2.735750 \\
\hline 1 & -7.098881 & 1.197799 & 1.738173 \\
\hline 1 & -6.531008 & -2.129054 & 3.358878 \\
\hline 1 & -5.721229 & -2.897987 & 1.981056 \\
\hline 1 & -4.880633 & -1.657172 & 2.929250 \\
\hline 1 & -5.894247 & 5.914885 & 1.833901 \\
\hline
\end{tabular}




$\begin{array}{rrrr}1 & -5.370966 & 4.344388 & 2.478797 \\ 1 & -4.410440 & 5.800667 & 2.797144 \\ 1 & -4.634503 & 6.988331 & -0.091519 \\ 1 & -3.139746 & 6.934368 & 0.852609 \\ 1 & -3.187968 & 6.204590 & -0.761529 \\ 1 & -6.014621 & 5.030900 & -0.461142 \\ 1 & -4.666097 & 4.161211 & -1.210051 \\ 1 & -5.578831 & 3.419479 & 0.122772 \\ 1 & 0.274445 & 5.471344 & 5.118618 \\ 1 & -1.184247 & 5.881439 & 4.211828 \\ 1 & -1.047817 & 4.294093 & 4.989719 \\ 1 & 1.883382 & 3.893893 & 4.370045 \\ 1 & 0.700401 & 2.591790 & 4.156907 \\ 1 & 1.795055 & 3.040319 & 2.830563 \\ 1 & 1.703162 & 6.028365 & 3.082124 \\ 1 & 1.389913 & 5.213520 & 1.532421 \\ 1 & 0.239742 & 6.408240 & 2.150999\end{array}$

$1_{\text {C9- }- \text {-1a2a3s4a }}$

Number of imaginary frequencies : 0

The smallest frequencies are : $11.6673 \quad 14.1179 \quad 18.2066 \mathrm{~cm}(-1)$

Electronic energy : $\quad H F=-4125.4630976$

Zero-point correction $=\quad 1.299690($ Hartree/Particle $)$

Thermal correction to Energy $=\quad 1.378153$

Thermal correction to Enthalpy= $\quad 1.379097$

Thermal correction to Gibbs Free Energy= $\quad 1.185923$

Sum of electronic and zero-point Energies $=\quad-4124.163408$

Sum of electronic and thermal Energies $=\quad-4124.084945$

Sum of electronic and thermal Enthalpies $=\quad-4124.084001$

Sum of electronic and thermal Free Energies $=\quad-4124.277174$

\section{Cartesian Coordinates}

$\begin{array}{llll}6 & 3.146068 & -6.209223 & 1.678738 \\ 6 & 2.929509 & -5.327916 & 2.734007 \\ 6 & 2.606797 & -4.001361 & 2.476486 \\ 6 & 2.514808 & -3.524367 & 1.166868 \\ 6 & 2.755726 & -4.409715 & 0.116207 \\ 6 & 3.058818 & -5.744347 & 0.370266 \\ 6 & 2.218884 & -2.060479 & 0.976941 \\ 7 & 1.571878 & -1.686880 & -0.292285 \\ 6 & 0.463433 & -2.427703 & -0.816980 \\ 6 & -0.747273 & -2.550233 & -0.153893 \\ 6 & -1.837574 & -3.218886 & -0.798230 \\ 6 & -1.668341 & -3.742725 & -2.113707 \\ 6 & -0.416520 & -3.597621 & -2.754932 \\ 6 & 0.613799 & -2.958766 & -2.123197 \\ 6 & -3.110910 & -3.351283 & -0.185547 \\ 6 & -4.149624 & -3.966767 & -0.839277 \\ 6 & -3.968574 & -4.507755 & -2.129713 \\ 6 & -2.750613 & -4.396255 & -2.750913 \\ 6 & -0.927143 & -2.031459 & 1.230675 \\ 6 & -1.190133 & -0.692110 & 1.497107 \\ 6 & -1.359471 & -0.278559 & 2.846624 \\ 6 & -1.280553 & -1.167424 & 3.884619\end{array}$




\begin{tabular}{|c|c|c|c|}
\hline 5 & -1.043336 & -2.539511 & 3.646502 \\
\hline 5 & -0.867291 & -2.981766 & 2.303335 \\
\hline 6 & -0.991963 & -3.477037 & 4.706354 \\
\hline 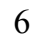 & -0.791376 & -4.810338 & 4.453749 \\
\hline 6 & -0.622728 & -5.256237 & 3.125976 \\
\hline 6 & -0.647205 & -4.366963 & 2.080923 \\
\hline 15 & -1.510504 & 0.501990 & 0.101775 \\
\hline & -0.106795 & 0.918747 & -1.872619 \\
\hline 6 & 0.224060 & 2.813658 & -1.481241 \\
\hline 8 & 0.282442 & 3.957632 & -1.359794 \\
\hline 15 & 1.798408 & -0.166444 & -1.034945 \\
\hline 8 & 2.598329 & 0.553921 & 0.238165 \\
\hline 6 & 3.433928 & 1.613020 & -0.049703 \\
\hline 6 & 4.692286 & 1.372007 & -0.569814 \\
\hline 6 & 5.511966 & 2.498570 & -0.900168 \\
\hline 6 & 5.047797 & 3.815401 & -0.599296 \\
\hline 6 & 3.770809 & 3.989234 & -0.015343 \\
\hline 6 & 2.968615 & 2.909613 & 0.238909 \\
\hline 6 & 5.861703 & 4.930593 & -0.915257 \\
\hline 6 & 7.076512 & 4.766274 & -1.528722 \\
\hline 6 & 7.521981 & 3.469656 & -1.861791 \\
\hline 6 & 6.762334 & 2.367787 & -1.557062 \\
\hline 6 & 5.105342 & -0.028656 & -0.821597 \\
\hline 6 & 4.287299 & -0.835661 & -1.593337 \\
\hline 6 & 4.633058 & -2.158936 & -1.928608 \\
\hline 6 & 5.788332 & -2.704205 & -1.438619 \\
\hline 6 & 6.627326 & -1.958048 & -0.577370 \\
\hline 6 & 6.285288 & -0.609552 & -0.254291 \\
\hline 6 & 7.116538 & 0.085829 & 0.661343 \\
\hline 6 & 8.234224 & -0.505792 & 1.195304 \\
\hline 6 & 8.586912 & -1.826400 & 0.846458 \\
\hline 6 & 7.793702 & -2.534964 & -0.018367 \\
\hline 8 & 3.093696 & -0.348865 & -2.078238 \\
\hline 6 & -3.248617 & 0.041592 & -0.285928 \\
\hline 6 & -4.161670 & -0.231607 & 0.740267 \\
\hline 6 & -5.470364 & -0.597231 & 0.451024 \\
\hline 6 & -5.847692 & -0.675359 & -0.900747 \\
\hline 6 & -4.968827 & -0.393709 & -1.942760 \\
\hline 6 & -3.656814 & -0.031848 & -1.607617 \\
\hline 6 & -6.484489 & -0.928389 & 1.544730 \\
\hline 6 & -5.369468 & -0.434257 & -3.414796 \\
\hline 6 & -1.700020 & 2.126726 & 0.939876 \\
\hline 6 & -2.779082 & 2.942855 & 0.602878 \\
\hline 6 & -2.868792 & 4.250444 & 1.082865 \\
\hline 6 & -1.860477 & 4.702899 & 1.936827 \\
\hline 6 & -0.772044 & 3.907735 & 2.309583 \\
\hline 6 & -0.689093 & 2.623389 & 1.768336 \\
\hline 6 & -4.022418 & 5.177720 & 0.704878 \\
\hline 6 & 0.286427 & 4.465738 & 3.260623 \\
\hline 6 & -0.718647 & 1.259734 & -3.639394 \\
\hline 8 & -1.094051 & 1.415542 & -4.723825 \\
\hline 1 & 6.853104 & 1.100092 & 0.950197 \\
\hline 1 & -1.581165 & 0.762807 & 3.063562 \\
\hline 1 & 8.039520 & -3.561914 & -0.285516 \\
\hline 1 & -1.124290 & -3.114883 & 5.725062 \\
\hline 1 & 5.491411 & 5.925038 & -0.669697 \\
\hline
\end{tabular}




\begin{tabular}{|c|c|c|c|}
\hline & -3.268597 & -2.936327 & 0.808804 \\
\hline & -5.123022 & -4.038112 & -0.357169 \\
\hline & 3.424842 & 4.998802 & 0.204101 \\
\hline & -1.421768 & -0.827595 & 4.909969 \\
\hline & 6.064703 & -3.728322 & -1.684554 \\
\hline & 8.473987 & 3.341538 & -2.372040 \\
\hline & 3.964980 & -2.717419 & -2.580430 \\
\hline & -2.596051 & -4.797483 & -3.751807 \\
\hline & -0.288252 & -3.997991 & -3.759261 \\
\hline 1 & -0.448754 & -6.311591 & 2.928035 \\
\hline & 7.114507 & 1.377137 & -1.832649 \\
\hline 1 & -0.484671 & -4.726473 & 1.067412 \\
\hline 1 & 1.573256 & -2.828033 & -2.621154 \\
\hline 1 & 7.690480 & 5.630188 & -1.772287 \\
\hline 1 & 8.850720 & 0.048215 & 1.899719 \\
\hline 1 & -4.797594 & -5.002807 & -2.631169 \\
\hline 1 & -0.761818 & -5.525147 & 5.273110 \\
\hline 1 & 9.477879 & -2.281009 & 1.273237 \\
\hline & 1.970161 & 3.021388 & 0.658235 \\
\hline 1 & -3.828434 & -0.166020 & 1.773328 \\
\hline & -2.930171 & 0.193521 & -2.386890 \\
\hline 1 & -6.868803 & -0.971061 & -1.134560 \\
\hline 1 & -3.549295 & 2.550623 & -0.056323 \\
\hline & 0.162581 & 1.985645 & 2.004453 \\
\hline 1 & -1.923719 & 5.718147 & 2.327674 \\
\hline 1 & 3.165191 & -1.510096 & 1.026953 \\
\hline 1 & 1.622622 & -1.705657 & 1.833284 \\
\hline ] & -0.545413 & -0.552271 & -2.332629 \\
\hline 1 & 2.405216 & -3.321653 & 3.305190 \\
\hline 1 & 2.701660 & -4.053434 & -0.909408 \\
\hline & 2.987989 & -5.678806 & 3.762531 \\
\hline 1 & 3.229828 & -6.424233 & -0.462024 \\
\hline 1 & 3.382419 & -7.252926 & 1.874639 \\
\hline 6 & -6.767062 & -1.013768 & -3.615133 \\
\hline 6 & -5.344140 & 0.995780 & -3.967714 \\
\hline 6 & -4.382781 & -1.300478 & -4.204880 \\
\hline 6 & -5.887415 & -0.794987 & 2.944457 \\
\hline 6 & -7.679494 & 0.025227 & 1.440530 \\
\hline 6 & -6.968362 & -2.372734 & 1.375800 \\
\hline 6 & -5.030341 & 4.500146 & -0.221871 \\
\hline 6 & -4.762832 & 5.620251 & 1.971546 \\
\hline 6 & -3.462131 & 6.407975 & -0.017077 \\
\hline 6 & 1.278312 & 3.397230 & 3.718736 \\
\hline 6 & 1.058533 & 5.580707 & 2.546953 \\
\hline 6 & -0.385937 & 5.036612 & 4.514441 \\
\hline 1 & -6.998656 & -1.059572 & -4.686687 \\
\hline 1 & -6.839911 & -2.034788 & -3.215100 \\
\hline 1 & -7.543418 & -0.400182 & -3.140100 \\
\hline 1 & -5.645035 & 1.003749 & -5.024108 \\
\hline 1 & -6.033001 & 1.646211 & -3.412402 \\
\hline 1 & -4.340719 & 1.436410 & -3.904604 \\
\hline 1 & -4.662515 & -1.316574 & -5.266779 \\
\hline 1 & -3.351464 & -0.930577 & -4.142633 \\
\hline 1 & -4.385819 & -2.332599 & -3.832251 \\
\hline 1 & -6.644287 & -1.058312 & 3.694074 \\
\hline 1 & -5.029318 & -1.465910 & 3.092866 \\
\hline
\end{tabular}




$\begin{array}{rrrr}1 & -5.558830 & 0.231079 & 3.154652 \\ 1 & -7.720981 & -2.614308 & 2.138483 \\ 1 & -7.424999 & -2.546121 & 0.393458 \\ 1 & -6.135473 & -3.079836 & 1.493481 \\ 1 & -8.412496 & -0.192909 & 2.228845 \\ 1 & -7.359646 & 1.069077 & 1.555498 \\ 1 & -8.194706 & -0.062155 & 0.476368 \\ 1 & -5.593990 & 6.289623 & 1.712068 \\ 1 & -5.178907 & 4.756285 & 2.505931 \\ 1 & -4.108676 & 6.160245 & 2.666720 \\ 1 & -4.278153 & 7.079609 & -0.316127 \\ 1 & -2.776262 & 6.981856 & 0.617809 \\ 1 & -2.913247 & 6.115134 & -0.921606 \\ 1 & -5.842978 & 5.199976 & -0.453991 \\ 1 & -4.575220 & 4.198088 & -1.174177 \\ 1 & -5.482350 & 3.610687 & 0.237581 \\ 1 & 0.373844 & 5.425636 & 5.205121 \\ 1 & -1.071359 & 5.860964 & 4.286400 \\ 1 & -0.956964 & 4.262027 & 5.042604 \\ 1 & 1.975230 & 3.831850 & 4.446253 \\ 1 & 0.771682 & 2.553866 & 4.207655 \\ 1 & 1.884286 & 2.999435 & 2.894820 \\ 1 & 1.842433 & 5.986451 & 3.200812 \\ 1 & 1.538122 & 5.204479 & 1.632734 \\ 1 & 0.400330 & 6.408673 & 2.256240\end{array}$

$1_{\text {C9- } \psi_{-12 a 23 s 4 s}}$

Number of imaginary frequencies : 0

The smallest frequencies are : $14.1416 \quad 16.5622 \quad 19.3520 \mathrm{~cm}(-1)$

Electronic energy : $\quad H F=-4125.4634263$

Zero-point correction $=\quad 1.299970($ Hartree/Particle $)$

Thermal correction to Energy= $\quad 1.378235$

Thermal correction to Enthalpy= $\quad 1.379179$

Thermal correction to Gibbs Free Energy= 1.187852

Sum of electronic and zero-point Energies $=\quad-4124.163456$

Sum of electronic and thermal Energies $=\quad-4124.085191$

Sum of electronic and thermal Enthalpies $=\quad-4124.084247$

Sum of electronic and thermal Free Energies $=\quad-4124.275574$

Cartesian Coordinates

$\begin{array}{rrrr}6 & 3.277294 & -6.151043 & 1.694050 \\ 6 & 3.070326 & -5.262390 & 2.745225 \\ 6 & 2.722303 & -3.943486 & 2.481901 \\ 6 & 2.593521 & -3.481118 & 1.169971 \\ 6 & 2.826486 & -4.372978 & 0.123184 \\ 6 & 3.155565 & -5.700356 & 0.383498 \\ 6 & 2.267083 & -2.024369 & 0.973806 \\ 7 & 1.607507 & -1.668633 & -0.294165 \\ 6 & 0.507456 & -2.430095 & -0.807221 \\ 6 & -0.696319 & -2.567234 & -0.134992 \\ 6 & -1.777221 & -3.266662 & -0.762117 \\ 6 & -1.609040 & -3.799437 & -2.073805 \\ 6 & -0.366527 & -3.632705 & -2.728188 \\ 6 & 0.656805 & -2.969134 & -2.110403\end{array}$




\begin{tabular}{|c|c|c|c|}
\hline 6 & -3.038948 & -3.425611 & -0.132359 \\
\hline 6 & -4.067097 & -4.080946 & -0.763612 \\
\hline f & -3.888635 & -4.625308 & -2.053155 \\
\hline 6 & -2.682671 & -4.484783 & -2.692104 \\
\hline f & -0.878319 & -2.031520 & 1.242551 \\
\hline b & -1.167635 & -0.693729 & 1.488994 \\
\hline 6 & -1.335316 & -0.262758 & 2.833293 \\
\hline 6 & -1.229243 & -1.133256 & 3.884615 \\
\hline 6 & -0.965403 & -2.503750 & 3.666648 \\
\hline 6 & -0.790097 & -2.963072 & 2.329232 \\
\hline 6 & -0.884987 & -3.423380 & 4.740301 \\
\hline 6 & -0.655676 & -4.755482 & 4.506478 \\
\hline 6 & -0.486404 & -5.218075 & 3.184480 \\
\hline 6 & -0.539704 & -4.346064 & 2.126105 \\
\hline & -1.510348 & 0.473342 & 0.074773 \\
\hline 5 & -0.110903 & 0.891700 & -1.901533 \\
\hline 6 & 0.182702 & 2.797708 & -1.538304 \\
\hline 8 & 0.213726 & 3.944807 & -1.438283 \\
\hline 15 & 1.810609 & -0.150327 & -1.047751 \\
\hline 8 & 2.587288 & 0.596692 & 0.224800 \\
\hline 6 & 3.407773 & 1.667008 & -0.064307 \\
\hline 6 & 4.672823 & 1.443440 & -0.575926 \\
\hline 6 & 5.476647 & 2.580986 & -0.907194 \\
\hline 6 & 4.991603 & 3.891814 & -0.613231 \\
\hline 6 & 3.709734 & 4.048390 & -0.035090 \\
\hline 6 & 2.922173 & 2.957689 & 0.217827 \\
\hline 6 & 5.790657 & 5.017794 & -0.928978 \\
\hline 6 & 7.010809 & & -1.535801 \\
\hline 6 & 7.477045 & 3.578074 & -1.861958 \\
\hline 6 & 6.732232 & 2.466192 & -1.557062 \\
\hline 6 & 5.111903 & 0.048072 & -0.812408 \\
\hline 6 & 4.313917 & -0.780633 & -1.581912 \\
\hline 6 & 4.682975 & -2.102492 & -1.897989 \\
\hline 6 & 5.841333 & -2.623959 & -1.389473 \\
\hline 6 & 6.660181 & -1.854786 & -0.528961 \\
\hline 6 & 6.295150 & -0.507381 & -0.226927 \\
\hline 6 & 7.106369 & 0.211937 & 0.688117 \\
\hline 6 & 8.226198 & -0.356557 & 1.242236 \\
\hline 6 & 8.601238 & -1.676588 & 0.915196 \\
\hline 6 & 7.828173 & -2.407591 & 0.050789 \\
\hline 8 & 3.116326 & -0.317799 & -2.080432 \\
\hline 6 & -3.234697 & -0.030044 & -0.318506 \\
\hline 6 & -4.151326 & -0.284605 & 0.701504 \\
\hline 6 & -5.465211 & -0.649235 & 0.414736 \\
\hline 6 & -5.838427 & -0.749180 & -0.931527 \\
\hline 6 & -4.946149 & -0.493687 & -1.975650 \\
\hline 6 & -3.638048 & -0.129978 & -1.643739 \\
\hline 6 & -6.429008 & -0.910160 & 1.571483 \\
\hline 6 & -5.343809 & -0.559595 & -3.448141 \\
\hline 6 & -1.755132 & 2.099696 & 0.896473 \\
\hline 6 & -2.874303 & 2.867365 & 0.576919 \\
\hline 6 & -3.018300 & 4.166990 & 1.065167 \\
\hline 6 & -2.022140 & 4.660873 & 1.910591 \\
\hline 6 & -0.890685 & 3.917080 & 2.260661 \\
\hline 6 & -0.755871 & 2.640511 & 1.711197 \\
\hline 6 & -4.235515 & 5.027570 & 0.730593 \\
\hline
\end{tabular}




\begin{tabular}{|c|c|c|c|}
\hline 6 & 0.160880 & 4.520855 & 3.191593 \\
\hline 6 & -0.720911 & 1.193436 & -3.676302 \\
\hline 8 & -1.090034 & 1.321138 & -4.766479 \\
\hline & 6.825537 & 1.226013 & 0.960626 \\
\hline 1 & -1.575950 & 0.777185 & 3.035999 \\
\hline 1 & 8.091558 & -3.434348 & -0.199786 \\
\hline 1 & -1.017628 & -3.048750 & 5.754446 \\
\hline 1 & 5.404663 & 6.007533 & -0.688658 \\
\hline 1 & -3.193089 & -3.006077 & 0.860220 \\
\hline 1 & -5.029761 & -4.182334 & -0.265344 \\
\hline 1 & 3.348636 & 5.053041 & 0.182136 \\
\hline 1 & -1.368655 & -0.779688 & 4.905577 \\
\hline 1 & 6.134880 & -3.647117 & -1.618983 \\
\hline 1 & 8.433471 & 3.462275 & -2.366856 \\
\hline 1 & 4.029157 & -2.679672 & -2.548123 \\
\hline 1 & -2.530865 & -4.890088 & -3.691787 \\
\hline 1 & -0.238944 & -4.038460 & -3.730441 \\
\hline 1 & -0.289328 & -6.272076 & 3.001514 \\
\hline 1 & 7.100303 & 1.479740 & -1.826975 \\
\hline 1 & -0.376848 & -4.717127 & 1.116786 \\
\hline 1 & 1.609417 & -2.823907 & -2.617603 \\
\hline 1 & 7.613282 & 5.741119 & -1.779325 \\
\hline 1 & 8.826922 & 0.215482 & 1.945867 \\
\hline 1 & -4.709477 & -5.148399 & -2.539253 \\
\hline 1 & -0.603540 & -5.456644 & 5.336427 \\
\hline 1 & 9.493442 & -2.112904 & 1.358183 \\
\hline 1 & 1.921178 & 3.056117 & 0.634166 \\
\hline 1 & -3.831167 & -0.196565 & 1.739592 \\
\hline 1 & -2.908334 & 0.082711 & -2.423553 \\
\hline 1 & -6.856896 & -1.040600 & -1.171776 \\
\hline 1 & -3.635521 & 2.443705 & -0.072723 \\
\hline 1 & 0.127523 & 2.041692 & 1.932732 \\
\hline 1 & -2.131441 & 5.666829 & 2.314758 \\
\hline 1 & 3.202199 & -1.454729 & 1.018247 \\
\hline 1 & 1.667623 & -1.677569 & 1.831156 \\
\hline 1 & -0.525700 & -0.591512 & -2.343742 \\
\hline 1 & 2.531327 & -3.258211 & 3.308526 \\
\hline 1 & 2.745338 & -4.028384 & -0.904545 \\
\hline 1 & 3.157342 & -5.601808 & 3.775558 \\
\hline 1 & 3.319471 & -6.385601 & -0.445815 \\
\hline 1 & 3.533516 & -7.189097 & 1.894872 \\
\hline 6 & -6.737389 & -1.150990 & -3.643862 \\
\hline 6 & -5.327468 & 0.862139 & -4.022392 \\
\hline 6 & -4.350263 & -1.431503 & -4.223167 \\
\hline 6 & -7.785571 & -1.423171 & 1.095341 \\
\hline 6 & -5.834616 & -1.960359 & 2.517825 \\
\hline 6 & -6.650270 & 0.399716 & 2.337556 \\
\hline 6 & -5.162277 & 4.352404 & -0.279112 \\
\hline 6 & -5.033444 & 5.285446 & 2.013623 \\
\hline 6 & -3.778348 & 6.362606 & 0.133745 \\
\hline 6 & 1.179682 & 3.484929 & 3.665546 \\
\hline 6 & 0.902107 & 5.634325 & 2.443561 \\
\hline 6 & -0.510489 & 5.108664 & 4.437748 \\
\hline 1 & -6.964120 & -1.215897 & -4.715504 \\
\hline 1 & -6.806395 & -2.165362 & -3.227073 \\
\hline 1 & -7.519742 & -0.534216 & -3.183133 \\
\hline
\end{tabular}




$\begin{array}{rrrr}1 & -5.623562 & 0.852558 & -5.080183 \\ 1 & -6.024236 & 1.514787 & -3.479573 \\ 1 & -4.328216 & 1.312190 & -3.960131 \\ 1 & -4.632446 & -1.472622 & -5.283748 \\ 1 & -3.322875 & -1.049244 & -4.171914 \\ 1 & -4.341740 & -2.455669 & -3.828861 \\ 1 & -8.432784 & -1.614741 & 1.960576 \\ 1 & -8.301606 & -0.695119 & 0.456660 \\ 1 & -7.692365 & -2.364724 & 0.537431 \\ 1 & -7.315489 & 0.235857 & 3.196305 \\ 1 & -5.708706 & 0.817612 & 2.716172 \\ 1 & -7.113951 & 1.156289 & 1.691061 \\ 1 & -6.515872 & -2.139608 & 3.360489 \\ 1 & -5.686022 & -2.917959 & 2.000583 \\ 1 & -4.867743 & -1.654162 & 2.937539 \\ 1 & -5.913756 & 5.906215 & 1.798521 \\ 1 & -5.383778 & 4.341663 & 2.452568 \\ 1 & -4.435403 & 5.805907 & 2.771795 \\ 1 & -4.648957 & 6.980498 & -0.123850 \\ 1 & -3.160164 & 6.941237 & 0.830317 \\ 1 & -3.192599 & 6.203966 & -0.780856 \\ 1 & -6.011631 & 5.012478 & -0.495812 \\ 1 & -4.653036 & 4.145120 & -1.229454 \\ 1 & -5.571721 & 3.407003 & 0.101459 \\ 1 & 0.249383 & 5.521944 & 5.113949 \\ 1 & -1.207045 & 5.920277 & 4.198432 \\ 1 & -1.067457 & 4.338439 & 4.986886 \\ 1 & 1.869153 & 3.949339 & 4.381662 \\ 1 & 0.695099 & 2.638717 & 4.171625 \\ 1 & 1.791870 & 3.086927 & 2.846562 \\ 1 & 1.680314 & 6.075940 & 3.080867 \\ 1 & 1.384649 & 5.245244 & 1.536315 \\ 1 & 0.221606 & 6.437703 & 2.135304\end{array}$

$1_{\text {C9- }- \text {-1a2s3a4a }}$

Number of imaginary frequencies : 0

The smallest frequencies are : $13.0082 \quad 15.9595 \quad 18.7682 \mathrm{~cm}(-1)$

Electronic energy : $\quad \mathrm{HF}=-4125.4632841$

Zero-point correction $=\quad 1.299770($ Hartree/Particle $)$

Thermal correction to Energy= $\quad 1.378199$

Thermal correction to Enthalpy= $\quad 1.379144$

Thermal correction to Gibbs Free Energy $=\quad 1.186906$

Sum of electronic and zero-point Energies $=\quad-4124.163514$

Sum of electronic and thermal Energies $=\quad-4124.085085$

Sum of electronic and thermal Enthalpies $=\quad-4124.084141$

Sum of electronic and thermal Free Energies $=\quad-4124.276378$

Cartesian Coordinates

$\begin{array}{llll}6 & 3.272798 & -6.170786 & 1.678491 \\ 6 & 3.073070 & -5.286463 & 2.734870 \\ 6 & 2.724506 & -3.966265 & 2.479487 \\ 6 & 2.587803 & -3.497970 & 1.170344 \\ 6 & 2.813638 & -4.385378 & 0.118316 \\ 6 & 3.143244 & -5.714243 & 0.370835\end{array}$




\begin{tabular}{|c|c|c|}
\hline 2.260256 & -2.040195 & 0.982900 \\
\hline 1.607897 & -1.676290 & -0.285581 \\
\hline 0.505926 & -2.426579 & -0.810308 \\
\hline-0.703411 & -2.559221 & -0.147384 \\
\hline-1.791224 & -3.229444 & -0.792369 \\
\hline-1.626422 & -3.731733 & -2.116472 \\
\hline-0.376348 & -3.577528 & -2.758686 \\
\hline 0.655594 & -2.947003 & -2.120836 \\
\hline-3.058034 & -3.383550 & -0.170153 \\
\hline-4.093764 & -4.005884 & -0.821190 \\
\hline-3.919154 & -4.517521 & -2.125122 \\
\hline-2.711383 & -4.375728 & -2.759311 \\
\hline-0.891928 & -2.040271 & 1.235010 \\
\hline-1.193215 & -0.707800 & 1.492293 \\
\hline-1.382042 & -0.293954 & 2.839663 \\
\hline-1.279319 & -1.175027 & 3.882273 \\
\hline-0.997250 & -2.540129 & 3.652232 \\
\hline-0.806358 & -2.983317 & 2.311664 \\
\hline-0.916051 & -3.470652 & 4.716169 \\
\hline-0.672790 & -4.798062 & 4.469272 \\
\hline-0.491693 & -5.245184 & 3.143579 \\
\hline-0.545000 & -4.361930 & 2.094394 \\
\hline-1.525360 & 0.475928 & 0.089589 \\
\hline-0.111588 & 0.899165 & -1.874058 \\
\hline 0.181058 & 2.801954 & -1.488513 \\
\hline 0.220639 & 3.946971 & -1.368510 \\
\hline 1.810381 & -0.152331 & -1.030160 \\
\hline 2.606286 & 0.582450 & 0.237905 \\
\hline 3.426653 & 1.651115 & -0.059278 \\
\hline 4.686278 & 1.423382 & -0.582269 \\
\hline 5.490089 & 2.558184 & -0.922802 \\
\hline 5.009367 & 3.870884 & -0.629943 \\
\hline 3.731777 & 4.031855 & -0.043631 \\
\hline 2.944900 & 2.943460 & 0.221229 \\
\hline 5.807749 & 4.994413 & -0.955954 \\
\hline 7.022976 & 4.841665 & -1.571569 \\
\hline 7.484560 & 3.548688 & -1.896793 \\
\hline 6.740282 & 2.439125 & -1.582271 \\
\hline 5.115784 & 0.026479 & -0.827131 \\
\hline 4.305016 & -0.793948 & -1.592340 \\
\hline 4.663880 & -2.115986 & -1.918913 \\
\hline 5.825413 & -2.645834 & -1.426677 \\
\hline 6.658291 & -1.884919 & -0.572409 \\
\hline 6.303088 & -0.537613 & -0.258627 \\
\hline 7.129116 & 0.173339 & 0.649722 \\
\hline 8.253896 & -0.402706 & 1.185783 \\
\hline 8.619126 & -1.722438 & 0.846583 \\
\hline 7.831502 & -2.445668 & -0.011190 \\
\hline 3.105406 & -0.322192 & -2.076917 \\
\hline-3.265483 & 0.016943 & -0.296618 \\
\hline-4.184908 & -0.206427 & 0.731248 \\
\hline-5.508565 & -0.536002 & 0.447616 \\
\hline-5.881509 & -0.623854 & -0.899235 \\
\hline-4.989866 & -0.401992 & -1.950196 \\
\hline-3.668991 & -0.080670 & -1.623912 \\
\hline-6.524446 & -0.848257 & 1.545616 \\
\hline
\end{tabular}




\begin{tabular}{|c|c|c|c|}
\hline 6 & -5.470987 & -0.493769 & -3.396937 \\
\hline 6 & -1.717908 & 2.097403 & 0.932786 \\
\hline & -2.799348 & 2.909363 & 0.618229 \\
\hline & -2.900944 & 4.211712 & 1.123155 \\
\hline & -1.894667 & 4.661323 & 1.973086 \\
\hline & -0.792119 & 3.864685 & 2.321722 \\
\hline & -0.702450 & 2.592611 & 1.763447 \\
\hline & -4.106884 & 5.060057 & 0.727269 \\
\hline 0 & 0.269880 & 4.417695 & 3.271513 \\
\hline U & -0.639137 & 1.198432 & -3.676688 \\
\hline 8 & -0.933624 & 1.319005 & -4.789894 \\
\hline 1 & 6.855986 & 1.187141 & 0.931133 \\
\hline 1 & -1.633832 & 0.741604 & 3.051580 \\
\hline 1 & 8.087297 & -3.472083 & -0.270927 \\
\hline 1 & -1.059679 & -3.108666 & 5.733412 \\
\hline 1 & 5.425145 & 5.985633 & -0.716292 \\
\hline 1 & -3.209346 & -2.986317 & 0.832331 \\
\hline 1 & -5.059756 & -4.105282 & -0.329078 \\
\hline 1 & 3.372811 & 5.038205 & 0.169597 \\
\hline 1 & -1.434223 & -0.834543 & 4.905436 \\
\hline 1 & 6.111472 & -3.669054 & -1.665196 \\
\hline 1 & 8.436847 & 3.429513 & -2.408691 \\
\hline 1 & 4.000384 & -2.686407 & -2.565103 \\
\hline 1 & -2.562536 & -4.758629 & -3.768099 \\
\hline 1 & -0.250263 & -3.964354 & -3.768594 \\
\hline 1 & -0.285901 & -6.295727 & 2.950577 \\
\hline 1 & 7.104642 & 1.451194 & -1.851748 \\
\hline 1 & -0.373987 & -4.720226 & 1.081747 \\
\hline 1 & 1.613537 & -2.809434 & -2.619885 \\
\hline 1 & 7.624973 & 5.711807 & -1.822747 \\
\hline 1 & 8.866335 & 0.163054 & 1.884377 \\
\hline 1 & -4.745739 & -5.018266 & -2.624910 \\
\hline 1 & -0.619830 & -5.507676 & 5.291962 \\
\hline 1 & 9.515492 & -2.164683 & 1.275059 \\
\hline 1 & 1.946796 & 3.044893 & 0.643932 \\
\hline 1 & -3.852627 & -0.124272 & 1.763587 \\
\hline 1 & -2.926874 & 0.098326 & -2.398812 \\
\hline 1 & -6.913942 & -0.881477 & -1.137043 \\
\hline 1 & -3.575530 & 2.527937 & -0.043751 \\
\hline 1 & 0.153065 & 1.955849 & 1.987573 \\
\hline 1 & -1.958901 & 5.665564 & 2.385700 \\
\hline 1 & 3.194415 & -1.469639 & 1.036001 \\
\hline 1 & 1.655819 & -1.700366 & 1.839758 \\
\hline 1 & -0.539617 & -0.576519 & -2.324379 \\
\hline 1 & 2.539881 & -3.284599 & 3.310603 \\
\hline 1 & 2.726956 & -4.036397 & -0.907444 \\
\hline 1 & 3.166821 & -5.630411 & 3.763093 \\
\hline 1 & 3.301598 & -6.395876 & -0.462531 \\
\hline 1 & 3.529650 & -7.209848 & 1.873195 \\
\hline 6 & -4.328400 & -0.307866 & -4.393485 \\
\hline 6 & -6.102603 & -1.865433 & -3.653110 \\
\hline 6 & -6.511746 & 0.603560 & -3.646474 \\
\hline 6 & -5.958618 & -0.596261 & 2.941904 \\
\hline 6 & -7.775262 & 0.019411 & 1.375669 \\
\hline 6 & -6.915731 & -2.327748 & 1.451256 \\
\hline 6 & -4.085849 & 6.441626 & 1.375250 \\
\hline
\end{tabular}




$\begin{array}{rrrr}6 & -4.114119 & 5.244225 & -0.794855 \\ 6 & -5.392869 & 4.346077 & 1.160905 \\ 6 & 1.272141 & 3.349674 & 3.707916 \\ 6 & 1.028781 & 5.546456 & 2.565283 \\ 6 & -0.394672 & 4.968902 & 4.538294 \\ 1 & -4.710918 & -0.413305 & -5.416547 \\ 1 & -3.872270 & 0.687515 & -4.316877 \\ 1 & -3.537415 & -1.058375 & -4.255157 \\ 1 & -6.466210 & -1.927500 & -4.687658 \\ 1 & -5.365265 & -2.664747 & -3.502388 \\ 1 & -6.954980 & -2.064809 & -2.991919 \\ 1 & -6.862149 & 0.569448 & -4.686930 \\ 1 & -7.388265 & 0.493468 & -2.995788 \\ 1 & -6.083752 & 1.598552 & -3.466219 \\ 1 & -6.722922 & -0.823649 & 3.695657 \\ 1 & -5.088219 & -1.231601 & 3.156778 \\ 1 & -5.659760 & 0.451614 & 3.078176 \\ 1 & -7.656240 & -2.578899 & 2.222851 \\ 1 & -7.352471 & -2.576401 & 0.475683 \\ 1 & -6.039589 & -2.973131 & 1.604528 \\ 1 & -8.502438 & -0.205785 & 2.167162 \\ 1 & -7.527959 & 1.086733 & 1.440070 \\ 1 & -8.274879 & -0.151348 & 0.414748 \\ 1 & -4.964922 & 5.868757 & -1.099090 \\ 1 & -3.192879 & 5.735005 & -1.134915 \\ 1 & -4.198158 & 4.288588 & -1.326480 \\ 1 & -6.272361 & 4.937869 & 0.873542 \\ 1 & -5.492886 & 3.356623 & 0.696831 \\ 1 & -5.418227 & 4.208898 & 2.250037 \\ 1 & -4.972221 & 7.008320 & 1.063556 \\ 1 & -4.102956 & 6.381844 & 2.471291 \\ 1 & -3.203543 & 7.021576 & 1.075239 \\ 1 & 0.369507 & 5.350794 & 5.228091 \\ 1 & -1.084230 & 5.794344 & 4.327600 \\ 1 & -0.958677 & 4.185089 & 5.060435 \\ 1 & 1.973289 & 3.779665 & 4.434124 \\ 1 & 0.774345 & 2.497802 & 4.191245 \\ 1 & 1.872633 & 2.964560 & 2.873992 \\ 1 & 1.816367 & 5.948407 & 3.217065 \\ 1 & 1.501722 & 5.184700 & 1.641850 \\ 1 & 0.362950 & 6.374217 & 2.291883\end{array}$

$1_{\text {C9- }- \text {-1a2s3a4s }}$

Number of imaginary frequencies : 0

The smallest frequencies are : $12.2143 \quad 14.179120 .0481 \mathrm{~cm}(-1)$

Electronic energy : $\quad H F=-4125.4638276$

Zero-point correction $=\quad 1.300263($ Hartree/Particle $)$

Thermal correction to Energy $=\quad 1.378440$

Thermal correction to Enthalpy= $\quad 1.379384$

Thermal correction to Gibbs Free Energy $=\quad 1.188211$

Sum of electronic and zero-point Energies $=\quad-4124.163565$

Sum of electronic and thermal Energies $=\quad-4124.085388$

Sum of electronic and thermal Enthalpies $=\quad-4124.084444$

Sum of electronic and thermal Free Energies $=\quad-4124.275616$ 
Cartesian Coordinates

\begin{tabular}{|c|c|c|c|}
\hline 6 & 3.345322 & -6.148863 & 1.669195 \\
\hline 6 & 3.136546 & -5.266957 & 2.725826 \\
\hline 6 & 2.773974 & -3.950399 & 2.470906 \\
\hline 6 & 2.631579 & -3.483345 & 1.161967 \\
\hline 6 & 2.866588 & -4.368092 & 0.109728 \\
\hline 6 & 3.210571 & -5.693380 & 0.361691 \\
\hline 6 & 2.286982 & -2.029507 & 0.974177 \\
\hline 7 & 1.629872 & -1.674706 & -0.294516 \\
\hline 6 & 0.536433 & -2.438783 & -0.817396 \\
\hline 6 & -0.671307 & -2.582167 & -0.153789 \\
\hline 6 & -1.752715 & -3.264207 & -0.796951 \\
\hline 6 & -1.582527 & -3.769919 & -2.118992 \\
\hline 6 & -0.333856 & -3.605566 & -2.761591 \\
\hline 6 & 0.691411 & -2.961528 & -2.126279 \\
\hline 6 & -3.019188 & -3.424023 & -0.175511 \\
\hline 6 & -4.049586 & -4.056594 & -0.825142 \\
\hline 6 & -3.869184 & -4.573247 & -2.126371 \\
\hline 6 & -2.661647 & -4.425678 & -2.759757 \\
\hline 6 & -0.861833 & -2.061786 & 1.227723 \\
\hline 6 & -1.169207 & -0.730277 & 1.482900 \\
\hline 6 & -1.349720 & -0.312949 & 2.830135 \\
\hline 6 & -1.236100 & -1.190681 & 3.874636 \\
\hline 6 & -0.951550 & -2.555531 & 3.646639 \\
\hline 6 & -0.765529 & -3.001340 & 2.306258 \\
\hline 6 & -0.860069 & -3.482868 & 4.712586 \\
\hline 6 & -0.610051 & -4.809393 & 4.467926 \\
\hline 6 & -0.431884 & -5.258785 & 3.142546 \\
\hline 6 & -0.496082 & -4.378772 & 2.091331 \\
\hline 15 & -1.518688 & 0.444822 & 0.077109 \\
\hline 45 & -0.119845 & 0.875062 & -1.892811 \\
\hline 6 & 0.138967 & 2.784948 & -1.519200 \\
\hline 8 & 0.151554 & 3.931254 & -1.405081 \\
\hline 15 & 1.815592 & -0.150363 & -1.042247 \\
\hline 8 & 2.590551 & 0.601094 & 0.229196 \\
\hline 6 & 3.394460 & 1.682198 & -0.067983 \\
\hline 6 & 4.661282 & 1.473182 & -0.581028 \\
\hline 6 & 5.448658 & 2.619514 & -0.920971 \\
\hline 6 & 4.943860 & 3.925394 & -0.638086 \\
\hline 6 & 3.658983 & 4.067771 & -0.062966 \\
\hline 6 & 2.888393 & 2.967560 & 0.201223 \\
\hline 6 & 5.726203 & 5.060562 & -0.962873 \\
\hline 6 & 6.948773 & 4.925107 & -1.567860 \\
\hline 6 & 7.434317 & 3.638515 & -1.883343 \\
\hline 6 & 6.706010 & 2.518171 & -1.569741 \\
\hline 6 & 5.115429 & 0.082304 & -0.815107 \\
\hline 6 & 4.324295 & -0.755491 & -1.582083 \\
\hline 6 & 4.706703 & -2.074035 & -1.896572 \\
\hline 6 & 5.871293 & -2.582676 & -1.389143 \\
\hline 6 & 6.684066 & -1.803556 & -0.531828 \\
\hline 6 & 6.305703 & -0.459419 & -0.231704 \\
\hline 6 & 7.111547 & 0.270627 & 0.679531 \\
\hline 6 & 8.238615 & -0.284787 & 1.232182 \\
\hline 6 & 8.626505 & -1.601708 & 0.907348 \\
\hline 6 & 7.859114 & -2.342792 & 0.046432 \\
\hline
\end{tabular}




\begin{tabular}{|c|c|c|c|}
\hline & 3.121214 & -0.305238 & -2.078569 \\
\hline & -3.252274 & -0.039641 & -0.303930 \\
\hline 6 & -4.166323 & -0.247052 & 0.724266 \\
\hline & -5.497497 & -0.573076 & 0.452983 \\
\hline & -5.879517 & -0.674081 & -0.885645 \\
\hline & -4.985159 & -0.468496 & -1.944831 \\
\hline & -3.663891 & -0.152329 & -1.631184 \\
\hline 6 & -6.459658 & -0.784740 & 1.620511 \\
\hline U & -5.476582 & -0.569519 & -3.387369 \\
\hline 6 & -1.744311 & 2.064936 & 0.916147 \\
\hline 6 & -2.848335 & 2.848924 & 0.607604 \\
\hline 6 & -2.978548 & 4.150024 & 1.108661 \\
\hline 6 & -1.979382 & 4.625800 & 1.952834 \\
\hline 6 & -0.855542 & 3.857656 & 2.296825 \\
\hline 6 & -0.736159 & 2.587411 & 1.739125 \\
\hline 6 & -4.199168 & 4.973317 & 0.704471 \\
\hline 6 & 0.198657 & 4.441006 & 3.237287 \\
\hline 6 & -0.650141 & 1.152327 & -3.698053 \\
\hline 8 & -0.946824 & 1.258121 & -4.812156 \\
\hline 1 & 6.820717 & 1.282464 & 0.949999 \\
\hline 1 & -1.603205 & 0.722443 & 3.040735 \\
\hline 1 & 8.132749 & -3.367264 & -0.202503 \\
\hline 1 & -1.000691 & -3.119068 & 5.729599 \\
\hline 1 & 5.325377 & 6.046374 & -0.730814 \\
\hline 1 & -3.173641 & -3.022183 & 0.824603 \\
\hline 1 & -5.017448 & -4.156973 & -0.337191 \\
\hline 1 & 3.281355 & 5.069054 & 0.141738 \\
\hline 1 & -1.384188 & -0.847569 & 4.897943 \\
\hline 1 & 6.174713 & -3.603254 & -1.617342 \\
\hline 1 & 8.392656 & 3.532889 & -2.386855 \\
\hline 1 & 4.057967 & -2.659380 & -2.544531 \\
\hline 1 & -2.509009 & -4.811807 & -3.766733 \\
\hline 1 & -0.203850 & -3.994518 & -3.770182 \\
\hline 1 & -0.219803 & -6.308412 & 2.951386 \\
\hline 1 & 7.088917 & 1.535149 & -1.831395 \\
\hline 1 & -0.327725 & -4.738583 & 1.078781 \\
\hline 1 & 1.647639 & -2.814489 & -2.626043 \\
\hline 1 & 7.538426 & 5.803924 & -1.818122 \\
\hline 1 & 8.835226 & 0.295165 & 1.932828 \\
\hline 1 & -4.691439 & -5.082147 & -2.625074 \\
\hline 1 & -0.548953 & -5.516566 & 5.292146 \\
\hline 1 & 9.524295 & -2.027647 & 1.349152 \\
\hline 1 & 1.884760 & 3.053534 & 0.614081 \\
\hline 1 & -3.837397 & -0.148368 & 1.758619 \\
\hline 1 & -2.924457 & 0.015639 & -2.411020 \\
\hline 1 & -6.912836 & -0.921420 & -1.121548 \\
\hline 1 & -3.619435 & 2.447792 & -0.048526 \\
\hline 1 & 0.137609 & 1.973836 & 1.957895 \\
\hline 1 & -2.066255 & 5.628983 & 2.363850 \\
\hline 1 & 3.214037 & -1.447310 & 1.026948 \\
\hline 1 & 1.678463 & -1.696604 & 1.830831 \\
\hline 1 & -0.533415 & -0.607759 & -2.332758 \\
\hline 1 & 2.583097 & -3.270736 & 3.302208 \\
\hline 1 & 2.775410 & -4.019727 & -0.915817 \\
\hline 1 & 3.234383 & -5.610081 & 3.753942 \\
\hline 1 & 3.375928 & -6.373041 & -0.471928 \\
\hline
\end{tabular}




$\begin{array}{rrrr}1 & 3.613223 & -7.185190 & 1.863562 \\ 6 & -4.342466 & -0.384633 & -4.393660 \\ 6 & -6.106203 & -1.944306 & -3.631513 \\ 6 & -6.522519 & 0.523332 & -3.635735 \\ 6 & -7.837278 & -1.253761 & 1.160294 \\ 6 & -5.894276 & -1.844595 & 2.573854 \\ 6 & -6.626216 & 0.540313 & 2.373707 \\ 6 & -4.230162 & 6.340370 & 1.382194 \\ 6 & -4.172606 & 5.189157 & -0.813421 \\ 6 & -5.477619 & 4.218843 & 1.088817 \\ 6 & 1.223527 & 3.397825 & 3.681157 \\ 6 & 0.932209 & 5.576243 & 2.514704 \\ 6 & -0.470686 & 4.993452 & 4.500893 \\ 1 & -4.733352 & -0.492481 & -5.413310 \\ 1 & -3.886392 & 0.611214 & -4.322554 \\ 1 & -3.549790 & -1.134193 & -4.260682 \\ 1 & -6.475477 & -2.013767 & -4.663607 \\ 1 & -5.366056 & -2.740959 & -3.480010 \\ 1 & -6.954275 & -2.140868 & -2.964138 \\ 1 & -6.878390 & 0.483318 & -4.674113 \\ 1 & -7.395492 & 0.413390 & -2.980447 \\ 1 & -6.096621 & 1.520471 & -3.462305 \\ 1 & -8.484056 & -1.413502 & 2.032237 \\ 1 & -8.331918 & -0.513686 & 0.518444 \\ 1 & -7.781470 & -2.203266 & 0.611068 \\ 1 & -7.289473 & 0.409670 & 3.239619 \\ 1 & -5.666331 & 0.926134 & 2.740483 \\ 1 & -7.067274 & 1.307334 & 1.723150 \\ 1 & -6.577006 & -1.994156 & 3.421047 \\ 1 & -5.776860 & -2.810695 & 2.065194 \\ 1 & -4.916625 & -1.564578 & 2.986482 \\ 1 & -5.033355 & 5.794642 & -1.128179 \\ 1 & -3.257548 & 5.713059 & -1.119069 \\ 1 & -4.213428 & 4.241480 & -1.364235 \\ 1 & -6.363022 & 4.799222 & 0.796417 \\ 1 & -5.546333 & 3.240520 & 0.595363 \\ 1 & -5.525414 & 4.051440 & 2.173028 \\ 1 & -5.127847 & 6.887370 & 1.067686 \\ 1 & -4.262641 & 6.255338 & 2.476268 \\ 1 & -3.361458 & 6.953287 & 1.109533 \\ 1 & 0.289361 & 5.397186 & 5.182728 \\ 1 & -1.176457 & 5.803585 & 4.284736 \\ 1 & -1.017338 & 4.205014 & 5.034416 \\ 1 & 1.915299 & 3.847989 & 4.404132 \\ 1 & 0.744372 & 2.538800 & 4.170584 \\ 1 & 1.832475 & 3.020699 & 2.849898 \\ 1 & 1.716830 & 5.999072 & 3.156792 \\ 1 & 1.404832 & 5.213689 & 1.591408 \\ 1 & 0.249185 & 6.388767 & 2.237829\end{array}$

$1_{\text {C9- } \Psi \text {-1a2s3s4a }}$

Number of imaginary frequencies : 0

The smallest frequencies are : $13.1540 \quad 16.5374 \quad 20.2248 \mathrm{~cm}(-1)$

Electronic energy :

Zero-point correction $=$

$\mathrm{HF}=-4125.4636478$

1.300020 (Hartree/Particle) 
Thermal correction to Energy $=\quad 1.378303$

Thermal correction to Enthalpy= $\quad 1.379247$

Thermal correction to Gibbs Free Energy= $\quad 1.187789$

Sum of electronic and zero-point Energies $=\quad-4124.163628$

Sum of electronic and thermal Energies $=\quad-4124.085345$

Sum of electronic and thermal Enthalpies $=\quad-4124.084401$

Sum of electronic and thermal Free Energies $=\quad-4124.275859$

Cartesian Coordinates

$\begin{array}{cccc}0 & 3.210187 & -6.179904 & 1.684420 \\ 6 & 3.010552 & -5.293520 & 2.738987 \\ 6 & 2.672530 & -3.971106 & 2.480675 \\ 6 & 2.546875 & -3.502793 & 1.170475 \\ 6 & 2.772631 & -4.392507 & 0.120302 \\ 6 & 3.091390 & -5.723421 & 0.375658 \\ 6 & 2.232794 & -2.042438 & 0.980590 \\ 7 & 1.579907 & -1.674063 & -0.287012 \\ 6 & 0.473685 & -2.421440 & -0.807449 \\ 6 & -0.733530 & -2.551008 & -0.139886 \\ 6 & -1.819765 & -3.234500 & -0.775277 \\ 6 & -1.650559 & -3.766591 & -2.087197 \\ 6 & -0.404614 & -3.607690 & -2.736748 \\ 6 & 0.621898 & -2.954555 & -2.113264 \\ 6 & -3.086418 & -3.382223 & -0.152361 \\ 6 & -4.116214 & -4.030411 & -0.788437 \\ 6 & -3.935228 & -4.577820 & -2.076350 \\ 6 & -2.725967 & -4.444627 & -2.710382 \\ 6 & -0.916025 & -2.029004 & 1.242700 \\ 6 & -1.200692 & -0.693226 & 1.502741 \\ 6 & -1.382187 & -0.277710 & 2.850379 \\ 6 & -1.289354 & -1.160846 & 3.892053 \\ 6 & -1.024095 & -2.528967 & 3.660295 \\ 6 & -0.839396 & -2.973602 & 2.319206 \\ 6 & -0.953191 & -3.460641 & 4.724034 \\ 6 & -0.724927 & -4.790597 & 4.476970 \\ 6 & -0.548255 & -5.238961 & 3.151122 \\ 6 & -0.592115 & -4.354963 & 2.102170 \\ 15 & -1.518684 & 0.495982 & 0.102823 \\ 45 & -0.111940 & 0.918846 & -1.871021 \\ 6 & 0.203152 & 2.816640 & -1.480125 \\ 8 & 0.252779 & 3.960977 & -1.358334 \\ 15 & 1.798701 & -0.153835 & -1.032972 \\ 8 & 2.598686 & 0.573102 & 0.236347 \\ 6 & 3.428489 & 1.635357 & -0.057019 \\ 6 & 4.686335 & 1.398490 & -0.580192 \\ 6 & 5.499620 & 2.527735 & -0.916875 \\ 6 & 5.029944 & 3.843412 & -0.619417 \\ 6 & 3.753991 & 4.013207 & -0.031948 \\ 6 & 2.957893 & 2.930619 & 0.228930 \\ 6 & 5.837650 & 4.961281 & -0.941798 \\ 6 & 7.051525 & 4.800465 & -1.558044 \\ 6 & 7.502320 & 3.504778 & -1.887573 \\ 6 & 6.748776 & 2.400437 & -1.576729 \\ 6 & 5.105122 & -0.001247 & -0.827746 \\ 6 & 4.288679 & -0.814784 & -1.594287\end{array}$




\begin{tabular}{|c|c|c|c|}
\hline 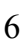 & 4.638332 & -2.138506 & -1.923679 \\
\hline & 5.796338 & -2.677332 & -1.432961 \\
\hline & 6.634329 & -1.924008 & -0.576988 \\
\hline & 6.288315 & -0.575042 & -0.260087 \\
\hline & 7.118932 & 0.127871 & 0.650354 \\
\hline & 8.239522 & -0.457272 & 1.185350 \\
\hline & 8.595859 & -1.778568 & 0.842860 \\
\hline & 7.803537 & -2.494193 & -0.016947 \\
\hline & 3.092292 & -0.334261 & -2.078702 \\
\hline & -3.256495 & 0.042263 & -0.294042 \\
\hline & -4.187114 & -0.196736 & 0.725254 \\
\hline & -5.498260 & -0.546949 & 0.425185 \\
\hline & -5.857347 & -0.650050 & -0.929858 \\
\hline & -4.959490 & -0.406753 & -1.965024 \\
\hline & -3.647767 & -0.054583 & -1.619477 \\
\hline & -6.526206 & -0.861829 & 1.511156 \\
\hline & -5.341413 & -0.474547 & -3.441081 \\
\hline & -1.707395 & 2.117858 & 0.944819 \\
\hline & -2.786166 & 2.931158 & 0.624958 \\
\hline & -2.884065 & 4.236869 & 1.121425 \\
\hline & -1.877617 & 4.688257 & 1.970162 \\
\hline 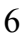 & -0.778431 & 3.889785 & 2.325581 \\
\hline & -0.691811 & 2.614184 & 1.774722 \\
\hline & -4.087549 & 5.085290 & 0.718731 \\
\hline 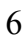 & 0.283836 & 4.445436 & 3.273575 \\
\hline & -0.712685 & 1.250167 & -3.643508 \\
\hline & -1.072996 & 1.396295 & -4.734335 \\
\hline 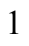 & 6.852685 & 1.142758 & 0.934395 \\
\hline & -1.621343 & 0.760713 & 3.062970 \\
\hline ] & 8.052264 & -3.521718 & -0.279145 \\
\hline & -1.092438 & -3.097079 & 5.741321 \\
\hline ] & 5.463365 & 5.954862 & -0.698806 \\
\hline . & -3.242799 & -2.960616 & 0.839370 \\
\hline . & -5.081137 & -4.124827 & -0.293509 \\
\hline l & 3.403852 & 5.021814 & 0.185313 \\
\hline | & -1.439182 & -0.819577 & 4.915705 \\
\hline l & 6.075557 & -3.701856 & -1.673938 \\
\hline & 8.453565 & 3.379346 & -2.399904 \\
\hline L & 3.970817 & -2.702715 & -2.571122 \\
\hline 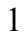 & -2.572380 & -4.850660 & -3.709491 \\
\hline | & -0.276726 & -4.011614 & -3.739702 \\
\hline 1 & -0.353054 & -6.291448 & 2.957709 \\
\hline & 7.104840 & 1.410401 & -1.849558 \\
\hline 1 & -0.423456 & -4.715253 & 1.089895 \\
\hline & 1.577194 & -2.815829 & -2.617084 \\
\hline 1 & 7.660710 & 5.666395 & -1.806416 \\
\hline 1 & 8.855493 & 0.102454 & 1.885693 \\
\hline 1 & -4.757187 & -5.096454 & -2.565280 \\
\hline 1 & -0.679906 & -5.501001 & 5.299450 \\
\hline 1 & 9.489006 & -2.228057 & 1.270511 \\
\hline 1 & 1.960762 & 3.038822 & 0.652400 \\
\hline 1 & -3.866755 & -0.111615 & 1.761151 \\
\hline 1 & -2.908363 & 0.146975 & -2.393135 \\
\hline 1 & -6.878226 & -0.938041 & -1.173291 \\
\hline 1 & -3.562749 & 2.548451 & -0.035646 \\
\hline 1 & 0.161648 & 1.976106 & 2.003097 \\
\hline
\end{tabular}




\begin{tabular}{|c|c|c|c|}
\hline & -1.938684 & 5.695516 & 2.375889 \\
\hline & 3.172710 & -1.481270 & 1.030146 \\
\hline ] & 1.633996 & -1.694951 & 1.838244 \\
\hline & -0.538692 & -0.555867 & -2.330560 \\
\hline & 2.487407 & -3.287575 & 3.310109 \\
\hline & 2.694374 & -4.043614 & -0.906191 \\
\hline . & 3.095564 & -5.637403 & 3.767999 \\
\hline . & 3.249773 & -6.406798 & -0.456269 \\
\hline & 3.458581 & -7.220614 & 1.881305 \\
\hline & -6.736038 & -1.059065 & -3.648399 \\
\hline 6 & -5.310732 & 0.945159 & -4.019539 \\
\hline & -4.344146 & -1.353917 & -4.202759 \\
\hline 6 & -5.978763 & -0.603705 & 2.913580 \\
\hline & -7.776225 & 0.003693 & 1.322321 \\
\hline & -6.915243 & -2.341836 & 1.418373 \\
\hline 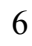 & -4.056670 & 6.475843 & 1.346747 \\
\hline & -4.101567 & 5.248133 & -0.805915 \\
\hline 6 & -5.374205 & 4.383040 & 1.169355 \\
\hline & 1.281771 & 3.377180 & 3.719033 \\
\hline & 1.048070 & 5.566932 & 2.561526 \\
\hline & -0.381321 & 5.007223 & 4.535417 \\
\hline & -6.953972 & -1.124646 & -4.721779 \\
\hline 1 & -6.813378 & -2.072383 & -3.230312 \\
\hline & -7.518968 & -0.437424 & -3.195197 \\
\hline 1 & -5.590169 & 0.932851 & -5.081770 \\
\hline 1 & -6.013682 & 1.602277 & -3.490465 \\
\hline 1 & -4.310735 & 1.391457 & -3.943765 \\
\hline ] & -4.617647 & -1.399549 & -5.265390 \\
\hline & -3.315936 & -0.974655 & -4.145409 \\
\hline ] & -4.342322 & -2.376050 & -3.803207 \\
\hline & -6.751279 & -0.832508 & 3.658483 \\
\hline 1 & -5.108136 & -1.234643 & 3.140423 \\
\hline 1 & -5.686390 & 0.445930 & 3.050641 \\
\hline 1 & -7.668108 & -2.588244 & 2.179450 \\
\hline 1 & -7.337043 & -2.596510 & 0.437993 \\
\hline 1 & -6.042042 & -2.986501 & 1.590209 \\
\hline 1 & -8.511107 & -0.215800 & 2.108305 \\
\hline . & -7.529869 & 1.071652 & 1.380816 \\
\hline l & -8.265859 & -0.174873 & 0.357735 \\
\hline 1 & -4.951057 & 5.872335 & -1.114380 \\
\hline 1 & -3.179913 & 5.730322 & -1.157041 \\
\hline 1 & -4.192475 & 4.286081 & -1.324896 \\
\hline 1 & -6.252950 & 4.973333 & 0.876647 \\
\hline 1 & -5.479498 & 3.386518 & 0.721773 \\
\hline . & -5.394952 & 4.263077 & 2.260600 \\
\hline 1 & -4.940358 & 7.043424 & 1.029117 \\
\hline 1 & -4.071528 & 6.431986 & 2.443558 \\
\hline 1 & -3.171420 & 7.045777 & 1.036354 \\
\hline 1 & 0.382811 & 5.390845 & 5.224287 \\
\hline 1 & -1.067457 & 5.833719 & 4.317902 \\
\hline 1 & -0.949216 & 4.228822 & 5.061387 \\
\hline 1 & 1.982658 & 3.809731 & 4.443969 \\
\hline 1 & 0.780372 & 2.529963 & 4.206732 \\
\hline 1 & 1.882988 & 2.985088 & 2.888942 \\
\hline 1 & 1.833555 & 5.972526 & 3.213576 \\
\hline 1 & 1.524652 & 5.196770 & 1.643239 \\
\hline
\end{tabular}


$1_{\text {C9- }-\psi_{-12} \text {-2s3s4s }}$

Number of imaginary frequencies : 0

The smallest frequencies are : $14.1558 \quad 15.5849 \quad 21.9217 \mathrm{~cm}(-1)$

Electronic energy : $\quad H F=-4125.4638309$

Zero-point correction $=\quad 1.303755($ Hartree/Particle $)$

Thermal correction to Energy= $\quad 1.381605$

Thermal correction to Enthalpy= $\quad 1.382549$

Thermal correction to Gibbs Free Energy $=\quad 1.192705$

Sum of electronic and zero-point Energies $=\quad-4124.160076$

Sum of electronic and thermal Energies $=\quad-4124.082226$

Sum of electronic and thermal Enthalpies $=\quad-4124.081282$

Sum of electronic and thermal Free Energies $=\quad-4124.271126$

Cartesian Coordinates

\begin{tabular}{cccc}
6 & 3.213009 & -6.173422 & 1.704878 \\
6 & 3.007938 & -5.284913 & 2.756584 \\
6 & 2.667805 & -3.963874 & 2.493916 \\
6 & 2.545344 & -3.499149 & 1.182162 \\
6 & 2.776460 & -4.390936 & 0.134930 \\
6 & 3.097441 & -5.720470 & 0.394587 \\
6 & 2.228591 & -2.040063 & 0.987266 \\
7 & 1.577604 & -1.676826 & -0.282900 \\
6 & 0.472815 & -2.427605 & -0.802036 \\
6 & -0.735361 & -2.555012 & -0.135953 \\
6 & -1.819802 & -3.243834 & -0.768473 \\
6 & -1.647562 & -3.786220 & -2.075549 \\
6 & -0.400817 & -3.629230 & -2.724295 \\
6 & 0.623395 & -2.968868 & -2.104299 \\
6 & -3.087725 & -3.385168 & -0.146862 \\
6 & -4.116943 & -4.036994 & -0.780027 \\
6 & -3.932830 & -4.596635 & -2.062281 \\
6 & -2.721651 & -4.470316 & -2.694456 \\
6 & -0.921992 & -2.026855 & 1.243400 \\
6 & -1.210149 & -0.690638 & 1.496296 \\
6 & -1.395499 & -0.270104 & 2.841979 \\
6 & -1.302011 & -1.148296 & 3.887821 \\
6 & -1.034655 & -2.517062 & 3.662773 \\
6 & -0.846511 & -2.966938 & 2.324004 \\
6 & -0.964175 & -3.443948 & 4.730694 \\
6 & -0.733051 & -4.774516 & 4.489755 \\
6 & -0.552764 & -5.228120 & 3.166203 \\
6 & -0.596125 & -4.348717 & 2.113372 \\
15 & -1.521666 & 0.493463 & 0.089181 \\
45 & -0.109126 & 0.910890 & -1.880699 \\
6 & 0.201111 & 2.809943 & -1.492750 \\
8 & 0.244740 & 3.954710 & -1.372488 \\
15 & 1.798794 & -0.159808 & -1.034385 \\
8 & 2.593998 & 0.573596 & 0.234390 \\
6 & 3.418811 & 1.638980 & -0.062248 \\
6 & 4.677660 & 1.406404 & -0.584851 \\
6 & 5.484713 & 2.538261 & -0.927428 \\
6 & 5.008476 & 3.852834 & -0.635603 \\
& & & \\
\hline
\end{tabular}




\begin{tabular}{|c|c|c|c|}
\hline 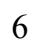 & 3.732579 & 4.018559 & -0.046875 \\
\hline & 2.942324 & 2.932991 & 0.219433 \\
\hline & 5.809836 & 4.973382 & -0.964510 \\
\hline & 7.023697 & 4.815920 & -1.581644 \\
\hline & 7.481050 & 3.521025 & -1.905261 \\
\hline & 6.733823 & 2.414237 & -1.587968 \\
\hline & 5.104444 & 0.007553 & -0.823106 \\
\hline & 4.293093 & -0.816012 & -1.584225 \\
\hline & 4.649043 & -2.141115 & -1.901244 \\
\hline 6 & 5.808475 & -2.670493 & -1.403518 \\
\hline 6 & 6.641964 & -1.905874 & -0.553143 \\
\hline & 6.289506 & -0.555661 & -0.248916 \\
\hline & 7.115669 & 0.159256 & 0.656193 \\
\hline & 8.237984 & -0.416004 & 1.198190 \\
\hline O & 8.600495 & -1.738887 & 0.868538 \\
\hline 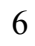 & 7.812697 & -2.465816 & 0.014057 \\
\hline 8 & 3.095780 & -0.344275 & -2.075033 \\
\hline 6 & -3.255696 & 0.035403 & -0.318310 \\
\hline 6 & -4.190649 & -0.194471 & 0.691394 \\
\hline 6 & -5.502023 & -0.557030 & 0.388929 \\
\hline 6 & -5.854431 & -0.675091 & -0.961562 \\
\hline 6 & -4.945358 & -0.438907 & -1.995620 \\
\hline 6 & -3.640305 & -0.079041 & -1.648270 \\
\hline 6 & -6.482288 & -0.811772 & 1.533382 \\
\hline 6 & -5.321477 & -0.527560 & -3.472826 \\
\hline 6 & -1.719689 & 2.114004 & 0.930455 \\
\hline 6 & -2.802412 & 2.924754 & 0.617842 \\
\hline 6 & -2.905298 & 4.224912 & 1.127720 \\
\hline 6 & -1.901285 & 4.675038 & 1.979377 \\
\hline 6 & -0.796621 & 3.879865 & 2.324700 \\
\hline 6 & -0.705190 & 2.609709 & 1.762487 \\
\hline 6 & -4.105823 & 5.073374 & 0.719896 \\
\hline 6 & 0.267147 & 4.433337 & 3.272146 \\
\hline 6 & -0.708600 & 1.240039 & -3.654119 \\
\hline 8 & -1.069313 & 1.386083 & -4.744782 \\
\hline 1 & 6.844692 & 1.175559 & 0.930473 \\
\hline 1 & -1.636614 & 0.768617 & 3.050278 \\
\hline 1 & 8.066367 & -3.494602 & -0.238242 \\
\hline 1 & -1.106022 & -3.076293 & 5.746145 \\
\hline 1 & 5.430626 & 5.966163 & -0.725934 \\
\hline 1 & -3.245950 & -2.953932 & 0.840236 \\
\hline 1 & -5.084735 & -4.123339 & -0.288817 \\
\hline 1 & 3.377564 & 5.026223 & 0.166802 \\
\hline 1 & -1.453694 & -0.802488 & 4.909693 \\
\hline 1 & 6.092288 & -3.696038 & -1.634581 \\
\hline 1 & 8.432330 & 3.398175 & -2.418146 \\
\hline 1 & 3.984935 & -2.714174 & -2.544455 \\
\hline 1 & -2.566315 & -4.885363 & -3.689589 \\
\hline 1 & -0.270419 & -4.040084 & -3.724107 \\
\hline 1 & -0.355139 & -6.281034 & 2.977666 \\
\hline 1 & 7.094741 & 1.424679 & -1.856179 \\
\hline 1 & -0.424647 & -4.713136 & 1.103052 \\
\hline 1 & 1.578920 & -2.831344 & -2.608099 \\
\hline 1 & 7.627908 & 5.683838 & -1.835170 \\
\hline 1 & 8.850516 & 0.152898 & 1.894143 \\
\hline 1 & -4.754052 & -5.118393 & -2.549142 \\
\hline
\end{tabular}




\begin{tabular}{|c|c|c|c|}
\hline 1 & -0.688426 & -5.481351 & 5.315320 \\
\hline & 9.494884 & -2.180526 & 1.301735 \\
\hline & 1.945351 & 3.037910 & 0.643970 \\
\hline & -3.885075 & -0.096361 & 1.733073 \\
\hline & -2.896360 & 0.115026 & -2.419337 \\
\hline & -6.869789 & -0.967466 & -1.213313 \\
\hline & -3.579933 & 2.552060 & -0.049564 \\
\hline & 0.151571 & 1.973839 & 1.984687 \\
\hline & -1.968329 & 5.678235 & 2.394170 \\
\hline & 3.167191 & -1.476705 & 1.037228 \\
\hline & 1.627168 & -1.691196 & 1.842500 \\
\hline 1 & -0.532905 & -0.565594 & -2.338331 \\
\hline & 2.478517 & -3.278668 & 3.321030 \\
\hline & 2.700692 & -4.044626 & -0.892643 \\
\hline & 3.090446 & -5.626032 & 3.786717 \\
\hline & 3.260131 & -6.405546 & -0.435104 \\
\hline & 3.463154 & -7.213059 & 1.905158 \\
\hline & -6.706016 & -1.136649 & -3.679378 \\
\hline & -5.311491 & 0.886227 & -4.066158 \\
\hline & -4.309360 & -1.398904 & -4.223991 \\
\hline & -7.836084 & -1.315838 & 1.040020 \\
\hline & -5.904893 & -1.868510 & 2.483272 \\
\hline & -6.705820 & 0.496403 & 2.300998 \\
\hline & -4.110692 & 6.442425 & 1.393221 \\
\hline ) & -4.067687 & 5.280785 & -0.798504 \\
\hline & -5.399627 & 4.343812 & 1.101331 \\
\hline f & 1.266369 & 3.364066 & 3.712390 \\
\hline ) & 1.029190 & 5.556475 & \\
\hline & -0.394584 & 4.992517 & 4.536835 \\
\hline 1 & -6.916740 & -1.220017 & -4.752995 \\
\hline & -6.769962 & -2.145425 & -3.248180 \\
\hline 1 & -7.501691 & -0.521798 & -3.239437 \\
\hline d & -5.588362 & 0.858499 & -5.128824 \\
\hline I & -6.025826 & 1.537965 & -3.545742 \\
\hline 1 & -4.318494 & 1.348359 & -3.992628 \\
\hline 1 & -4.580122 & -1.459050 & -5.286618 \\
\hline 1 & -3.286988 & -1.004103 & -4.168864 \\
\hline & -4.293247 & -2.416868 & -3.814400 \\
\hline ] & -8.496076 & -1.500581 & 1.897033 \\
\hline & -8.338011 & -0.585305 & 0.392942 \\
\hline 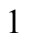 & -7.742761 & -2.259515 & 0.485767 \\
\hline 1 & -7.380748 & 0.331933 & 3.152030 \\
\hline ] & -5.767048 & 0.909488 & 2.691320 \\
\hline 1 & -7.160196 & 1.257261 & 1.652669 \\
\hline ] & -6.598321 & -2.049430 & 3.315603 \\
\hline 1 & -5.751865 & -2.824050 & 1.963373 \\
\hline & -4.942877 & -1.567183 & 2.917274 \\
\hline 1 & -4.910047 & 5.908198 & -1.120194 \\
\hline 1 & -3.137375 & 5.776890 & -1.104171 \\
\hline 1 & -4.134684 & 4.330993 & -1.343585 \\
\hline 1 & -6.273308 & 4.937785 & 0.800990 \\
\hline 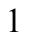 & -5.483716 & 3.363218 & 0.615487 \\
\hline 1 & -5.456359 & 4.183885 & 2.186295 \\
\hline & -4.995991 & 7.006925 & 1.074572 \\
\hline & -4.147963 & 6.361442 & 2.487429 \\
\hline & -3.228522 & 7.036137 & 1.121173 \\
\hline
\end{tabular}




$\begin{array}{rrrr}1 & 0.371548 & 5.374425 & 5.224401 \\ 1 & -1.080884 & 5.819889 & 4.323178 \\ 1 & -0.961308 & 4.213246 & 5.062808 \\ 1 & 1.967617 & 3.794156 & 4.438430 \\ 1 & 0.766054 & 2.514374 & 4.196945 \\ 1 & 1.867202 & 2.975879 & 2.880163 \\ 1 & 1.817240 & 5.960193 & 3.210340 \\ 1 & 1.502186 & 5.188526 & 1.639182 \\ 1 & 0.365303 & 6.384106 & 2.281713\end{array}$

$1_{\text {C9- } \psi-1 s 2 a 3 a 4 a}$

Number of imaginary frequencies : 0

The smallest frequencies are : $9.5538 \quad 12.6100 \quad 14.2027 \mathrm{~cm}(-1)$

Electronic energy : $\quad H F=-4125.4614277$

Zero-point correction $=\quad 1.299880($ Hartree/Particle $)$

Thermal correction to Energy= $\quad 1.378266$

Thermal correction to Enthalpy= $\quad 1.379210$

Thermal correction to Gibbs Free Energy $=\quad 1.185823$

Sum of electronic and zero-point Energies $=\quad-4124.161548$

Sum of electronic and thermal Energies $=\quad-4124.083162$

Sum of electronic and thermal Enthalpies $=\quad-4124.082218$

Sum of electronic and thermal Free Energies $=\quad-4124.275605$

Cartesian Coordinates

$\begin{array}{cccc}6 & -1.075509 & 2.675106 & 1.775386 \\ 6 & -1.936047 & 2.040571 & 0.881117 \\ 6 & -3.091076 & 2.703971 & 0.455007 \\ 6 & -3.417080 & 3.967265 & 0.935313 \\ 6 & -2.562355 & 4.551290 & 1.879358 \\ 6 & -1.394158 & 3.928944 & 2.317077 \\ 15 & -1.517901 & 0.431500 & 0.086737 \\ 6 & -3.194845 & -0.181225 & -0.364301 \\ 6 & -4.136504 & -0.446632 & 0.632858 \\ 6 & -5.435849 & -0.827146 & 0.306228 \\ 6 & -5.759526 & -0.932250 & -1.052298 \\ 6 & -4.842450 & -0.675743 & -2.073073 \\ 6 & -3.548491 & -0.296970 & -1.703549 \\ 6 & -6.485454 & -1.147318 & 1.369570 \\ 6 & -5.271688 & -0.783253 & -3.534884 \\ 6 & -4.693839 & 4.688033 & 0.506707 \\ 6 & -0.492255 & 4.539937 & 3.389585 \\ 6 & -1.141098 & -0.708212 & 1.514452 \\ 6 & -0.759233 & -2.025690 & 1.280704 \\ 6 & -0.625895 & -2.943225 & 2.373982 \\ 6 & -0.843886 & -2.488303 & 3.706774 \\ 6 & -1.212766 & -1.140297 & 3.911569 \\ 6 & -1.368195 & -0.286423 & 2.852746 \\ 6 & -0.289896 & -4.309285 & 2.180680 \\ 6 & -0.187157 & -5.169429 & 3.245268 \\ 6 & -0.391179 & -4.709307 & 4.563239 \\ 6 & -0.709358 & -3.393721 & 4.786921 \\ 6 & -0.536558 & -2.557303 & -0.092924 \\ 6 & 0.667148 & -2.375008 & -0.754283 \\ 6 & 0.840686 & -2.888829 & -2.064734\end{array}$




\begin{tabular}{|c|c|c|c|}
\hline 6 & -0.160504 & -3.566864 & -2.702288 \\
\hline 6 & -1.399769 & -3.784328 & -2.057328 \\
\hline & -1.587530 & -3.289161 & -0.733628 \\
\hline & -2.450761 & -4.484046 & -2.698152 \\
\hline 6 & -3.648084 & -4.689253 & -2.061254 \\
\hline 6 & -3.844422 & -4.190433 & -0.756013 \\
\hline 6 & -2.841810 & -3.513266 & -0.107821 \\
\hline 7 & 1.738936 & -1.585283 & -0.226162 \\
\hline & 1.896692 & -0.057183 & -0.972596 \\
\hline 8 & 3.186809 & -0.194152 & -2.030564 \\
\hline 6 & 4.406602 & -0.625725 & -1.558495 \\
\hline 6 & 5.198623 & 0.222406 & -0.803746 \\
\hline 6 & 6.410610 & -0.300523 & -0.248203 \\
\hline 6 & 6.808406 & -1.634988 & -0.565429 \\
\hline 6 & 5.992652 & -2.423946 & -1.410614 \\
\hline 6 & 4.807988 & -1.934606 & -1.889600 \\
\hline 6 & 7.221211 & 0.438696 & 0.651339 \\
\hline 6 & 8.370243 & -0.098416 & 1.176188 \\
\hline 6 & 8.776770 & -1.405181 & 0.833625 \\
\hline 6 & 8.005666 & -2.155212 & -0.016154 \\
\hline 6 & 4.726236 & 1.604009 & -0.552957 \\
\hline 6 & 3.466542 & 1.789865 & -0.014758 \\
\hline 6 & 2.947867 & 3.063194 & 0.279867 \\
\hline 6 & 3.695576 & 4.178315 & 0.014728 \\
\hline 6 & 4.970003 & 4.061385 & -0.589093 \\
\hline 6 & 5.488521 & 2.766323 & -0.896983 \\
\hline 6 & 5.728349 & 5.211740 & -0.917433 \\
\hline 6 & 6.939661 & 5.101617 & -1.549585 \\
\hline 6 & 7.437737 & 3.826143 & -1.889531 \\
\hline 6 & 6.733327 & 2.691407 & -1.572999 \\
\hline 8 & 2.682092 & 0.696425 & 0.288985 \\
\hline 6 & 2.399783 & -1.930199 & 1.042650 \\
\hline 6 & 2.802233 & -3.370812 & 1.217661 \\
\hline 6 & 2.981417 & -3.836319 & 2.522824 \\
\hline 6 & 3.398093 & -5.138592 & 2.767389 \\
\hline 6 & 3.625163 & -6.008515 & 1.704334 \\
\hline 6 & 3.451597 & -5.554970 & 0.400927 \\
\hline 6 & 3.052656 & -4.243140 & 0.159080 \\
\hline 45 & -0.055353 & 0.938822 & -1.811028 \\
\hline 6 & -0.544114 & 1.251687 & -3.624271 \\
\hline 8 & -0.812931 & 1.383325 & -4.742495 \\
\hline 6 & 0.148479 & 2.839780 & -1.372658 \\
\hline 8 & 0.134689 & 3.980358 & -1.210561 \\
\hline 1 & 6.916768 & 1.442833 & 0.935169 \\
\hline 1 & -1.697670 & 0.730889 & 3.044754 \\
\hline 1 & 8.293579 & -3.172465 & -0.278265 \\
\hline 1 & -0.874179 & -3.022789 & 5.797715 \\
\hline 1 & 5.317674 & 6.188726 & -0.666009 \\
\hline 1 & -3.010846 & -3.128348 & 0.896491 \\
\hline 1 & -4.801804 & -4.342148 & -0.260996 \\
\hline 1 & 3.307976 & 5.170458 & 0.241846 \\
\hline 1 & -1.396029 & -0.792469 & 4.927650 \\
\hline 1 & 6.310769 & -3.436813 & -1.652766 \\
\hline 1 & 8.386526 & 3.740735 & -2.414518 \\
\hline 1 & 4.158297 & -2.528546 & -2.528694 \\
\hline 1 & -2.284304 & -4.857805 & -3.707682 \\
\hline
\end{tabular}




\begin{tabular}{|c|c|c|c|}
\hline . & -0.017060 & -3.946596 & -3.712587 \\
\hline 1 & 0.074378 & -6.210677 & 3.070333 \\
\hline & 7.125244 & 1.717251 & -1.853841 \\
\hline & -0.103908 & -4.675891 & 1.173598 \\
\hline & 1.791325 & -2.707266 & -2.563710 \\
\hline & 7.510601 & 5.992000 & -1.802387 \\
\hline & 8.970085 & 0.488245 & 1.868441 \\
\hline & -4.449054 & -5.231393 & -2.559491 \\
\hline & -0.298029 & -5.400157 & 5.398205 \\
\hline 1 & 9.691838 & -1.816501 & 1.253278 \\
\hline & 1.954075 & 3.129128 & 0.715547 \\
\hline & -3.843573 & -0.345849 & 1.675624 \\
\hline 1 & -2.792284 & -0.079997 & -2.454334 \\
\hline 1 & -6.772927 & -1.228847 & -1.324151 \\
\hline 1 & -3.739525 & 2.216815 & -0.267105 \\
\hline 1 & -0.152858 & 2.174356 & 2.077802 \\
\hline 1 & -2.827327 & 5.525086 & 2.284351 \\
\hline 1 & 3.302196 & -1.312515 & 1.109751 \\
\hline 1 & 1.771793 & -1.632196 & 1.898145 \\
\hline 1 & -0.415247 & -0.546694 & -2.291190 \\
\hline 1 & 2.773995 & -3.167802 & 3.359512 \\
\hline 1 & 2.929447 & -3.896186 & -0.863438 \\
\hline 1 & 3.524812 & -5.480996 & 3.792570 \\
\hline 1 & 3.628990 & -6.225477 & -0.437633 \\
\hline 1 & 3.936206 & -7.034147 & 1.890949 \\
\hline 6 & -5.430525 & 3.945402 & -0.607522 \\
\hline 6 & -5.632390 & 4.793787 & 1.714344 \\
\hline 6 & -4.356620 & 6.092737 & -0.003095 \\
\hline 6 & -0.953562 & 5.931858 & 3.815357 \\
\hline 6 & -0.508964 & 3.631588 & 4.625117 \\
\hline 6 & 0.943467 & 4.654447 & 2.869716 \\
\hline 6 & -4.110164 & -0.527237 & -4.493394 \\
\hline 6 & -5.819056 & -2.185469 & -3.817972 \\
\hline 6 & -6.362600 & 0.257654 & -3.811138 \\
\hline 6 & -5.958761 & -0.922751 & 2.785545 \\
\hline 6 & -7.715311 & -0.254849 & 1.172995 \\
\hline 6 & -6.898053 & -2.618204 & 1.245394 \\
\hline 1 & -6.335639 & 4.501302 & -0.882853 \\
\hline 1 & -4.813154 & 3.849527 & -1.510316 \\
\hline 1 & -5.744966 & 2.937454 & -0.302997 \\
\hline 1 & -6.561401 & 5.308456 & 1.433779 \\
\hline 1 & -5.897827 & 3.797200 & 2.092163 \\
\hline 1 & -5.176936 & 5.354481 & 2.539909 \\
\hline 1 & -5.270971 & 6.608210 & -0.326078 \\
\hline 1 & -3.885345 & 6.714356 & 0.767459 \\
\hline 1 & -3.673106 & 6.046529 & -0.860520 \\
\hline 1 & 0.125579 & 4.050979 & 5.417691 \\
\hline 1 & -1.526827 & 3.529821 & 5.024286 \\
\hline 1 & -0.133685 & 2.625320 & 4.398476 \\
\hline 1 & 1.575825 & 5.169284 & 3.605191 \\
\hline 1 & 1.394209 & 3.671080 & 2.692240 \\
\hline 1 & 0.978308 & 5.225308 & 1.931478 \\
\hline 1 & -0.272073 & 6.325621 & 4.579721 \\
\hline 1 & -0.951872 & 6.638724 & 2.975569 \\
\hline 1 & -1.960013 & 5.918081 & 4.252689 \\
\hline 1 & -4.453879 & -0.645457 & -5.528770 \\
\hline
\end{tabular}




$\begin{array}{rrrr}1 & -3.712015 & 0.490993 & -4.395646 \\ 1 & -3.283742 & -1.234231 & -4.334584 \\ 1 & -6.152585 & -2.258518 & -4.861952 \\ 1 & -5.042757 & -2.944319 & -3.653974 \\ 1 & -6.675374 & -2.436492 & -3.179730 \\ 1 & -6.672584 & 0.217449 & -4.864124 \\ 1 & -7.254860 & 0.090968 & -3.194791 \\ 1 & -5.997786 & 1.272395 & -3.603940 \\ 1 & -7.681325 & -2.859335 & 1.976803 \\ 1 & -7.288401 & -2.855569 & 0.247743 \\ 1 & -6.043190 & -3.280281 & 1.440815 \\ 1 & -8.471717 & -0.475855 & 1.937943 \\ 1 & -7.448891 & 0.806667 & 1.259906 \\ 1 & -8.185465 & -0.402694 & 0.193476 \\ 1 & -6.742716 & -1.167580 & 3.513251 \\ 1 & -5.092310 & -1.560329 & 3.009787 \\ 1 & -5.667076 & 0.122596 & 2.952572\end{array}$

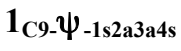

Number of imaginary frequencies : 0

The smallest frequencies are : $12.8065 \quad 14.4595 \quad 16.5093 \mathrm{~cm}(-1)$

Electronic energy : $\quad H F=-4125.4622859$

Zero-point correction $=\quad 1.300188($ Hartree/Particle $)$

Thermal correction to Energy $=\quad 1.378425$

Thermal correction to Enthalpy= $\quad 1.379369$

Thermal correction to Gibbs Free Energy $=\quad 1.187418$

Sum of electronic and zero-point Energies $=\quad-4124.162098$

Sum of electronic and thermal Energies $=\quad-4124.083861$

Sum of electronic and thermal Enthalpies $=\quad-4124.082917$

Sum of electronic and thermal Free Energies $=\quad-4124.274867$

Cartesian Coordinates

$\begin{array}{cccc}6 & -1.112510 & 2.685693 & 1.760941 \\ 6 & -1.970093 & 2.038831 & 0.872359 \\ 6 & -3.135475 & 2.687367 & 0.450610 \\ 6 & -3.466847 & 3.953335 & 0.921206 \\ 6 & -2.612206 & 4.551312 & 1.856417 \\ 6 & -1.439617 & 3.939608 & 2.297098 \\ 15 & -1.526063 & 0.434414 & 0.081866 \\ 6 & -3.188121 & -0.197027 & -0.394644 \\ 6 & -4.140594 & -0.461574 & 0.584015 \\ 6 & -5.433198 & -0.866794 & 0.244038 \\ 6 & -5.737316 & -0.991632 & -1.112647 \\ 6 & -4.801750 & -0.731672 & -2.123294 \\ 6 & -3.521284 & -0.334137 & -1.740908 \\ 6 & -6.443577 & -1.125484 & 1.360122 \\ 6 & -5.207911 & -0.854895 & -3.590482 \\ 6 & -4.735721 & 4.676464 & 0.474199 \\ 6 & -0.542211 & 4.562948 & 3.366342 \\ 6 & -1.160185 & -0.700089 & 1.517774 \\ 6 & -0.770639 & -2.017072 & 1.294660 \\ 6 & -0.643958 & -2.928761 & 2.393765 \\ 6 & -0.877379 & -2.468481 & 3.722045 \\ 6 & -1.254844 & -1.121297 & 3.916301\end{array}$




\begin{tabular}{|c|c|c|c|}
\hline 6 & -1.402751 & -0.273137 & 2.851783 \\
\hline 6 & -0.301176 & -4.294546 & 2.210585 \\
\hline p & -0.205653 & -5.148996 & 3.280444 \\
\hline & -0.424100 & -4.683241 & 4.594051 \\
\hline f & -0.749603 & -3.367857 & 4.808018 \\
\hline & -0.539993 & -2.556589 & -0.074341 \\
\hline 6 & 0.664434 & -2.375260 & -0.734427 \\
\hline 6 & 0.840481 & -2.895501 & -2.042152 \\
\hline 6 & -0.156818 & -3.583170 & -2.675702 \\
\hline 6 & -1.394834 & -3.804703 & -2.029334 \\
\hline 6 & -1.586913 & -3.298057 & -0.710813 \\
\hline 6 & -2.440072 & -4.520070 & -2.662257 \\
\hline 6 & -3.636008 & -4.727874 & -2.023237 \\
\hline 6 & -3.837150 & -4.215281 & -0.724098 \\
\hline 6 & -2.839939 & -3.523342 & -0.083344 \\
\hline 7 & 1.735598 & -1.584843 & -0.205643 \\
\hline 15 & 1.898889 & -0.057549 & -0.951977 \\
\hline 8 & 3.187609 & -0.197539 & -2.011136 \\
\hline 6 & 4.410515 & -0.623013 & -1.542194 \\
\hline 6 & 5.201993 & 0.229793 & -0.792062 \\
\hline 6 & 6.419636 & -0.286536 & -0.242717 \\
\hline 6 & 6.823190 & -1.618830 & -0.561872 \\
\hline 6 & 6.006881 & -2.412797 & -1.401790 \\
\hline 6 & 4.816971 & -1.930062 & -1.874349 \\
\hline 6 & 7.231189 & 0.457407 & 0.652043 \\
\hline 6 & 8.386328 & -0.072995 & 1.170270 \\
\hline 6 & 8.798417 & -1.377436 & 0.825489 \\
\hline 6 & 8.026606 & -2.132063 & -0.019569 \\
\hline 6 & 4.724417 & 1.609470 & -0.540195 \\
\hline 6 & 3.466235 & 1.789924 & 0.003270 \\
\hline 6 & 2.942751 & 3.060795 & 0.299739 \\
\hline 6 & 3.683920 & 4.179381 & 0.031134 \\
\hline 6 & 4.956144 & 4.068127 & -0.578450 \\
\hline 6 & 5.479554 & 2.775386 & -0.88 \\
\hline 6 & 5.707293 & 5.221995 & -0.910977 \\
\hline 6 & 6.916058 & 5.117481 & -1.548918 \\
\hline 6 & 7.418704 & 3.844286 & -1.890630 \\
\hline 6 & 6.721443 & 2.706266 & -1.570056 \\
\hline 8 & 2.687475 & 0.692996 & 0.309302 \\
\hline 6 & 2.393946 & -1.932094 & 1.063898 \\
\hline 6 & 2.798702 & -3.372499 & 1.234793 \\
\hline 6 & 2.967538 & -3.845716 & 2.538510 \\
\hline 6 & 3.386321 & -5.148299 & 2.778279 \\
\hline 6 & 3.626039 & -6.010460 & 1.711717 \\
\hline 6 & 3.462278 & -5.549266 & 0.409681 \\
\hline 6 & 3.060789 & -4.237389 & 0.172908 \\
\hline 45 & -0.045473 & 0.942096 & -1.798966 \\
\hline 6 & -0.501257 & 1.250004 & -3.621854 \\
\hline 8 & -0.745498 & 1.377084 & -4.746185 \\
\hline 6 & 0.147503 & 2.844171 & -1.363028 \\
\hline 8 & 0.126024 & 3.985271 & -1.205272 \\
\hline 1 & 6.922686 & 1.459846 & 0.937490 \\
\hline 1 & -1.737078 & 0.744078 & 3.035763 \\
\hline 1 & 8.318784 & -3.147700 & -0.283250 \\
\hline 1 & -0.925847 & -2.992602 & 5.815273 \\
\hline 1 & 5.292976 & 6.197078 & -0.658133 \\
\hline
\end{tabular}




\begin{tabular}{|c|c|c|c|}
\hline 1 & -3.012446 & -3.127921 & 0.915868 \\
\hline & -4.794159 & -4.369367 & -0.228550 \\
\hline & 3.292586 & 5.169749 & 0.259689 \\
\hline & -1.449673 & -0.769300 & 4.928792 \\
\hline & 6.329137 & -3.424017 & -1.645344 \\
\hline & 8.365276 & 3.763272 & -2.420292 \\
\hline & 4.166876 & -2.527638 & -2.509642 \\
\hline & -2.270960 & -4.903321 & -3.667804 \\
\hline & -0.010439 & -3.969246 & -3.683184 \\
\hline & 0.061136 & -6.190156 & 3.113104 \\
\hline & 7.116717 & 1.733899 & -1.852379 \\
\hline 1 & -0.104144 & -4.665791 & 1.207288 \\
\hline & 1.790561 & -2.712694 & -2.541794 \\
\hline & 7.481392 & 6.010510 & -1.804962 \\
\hline & 8.986819 & 0.517239 & 1.858911 \\
\hline & -4.432261 & -5.282042 & -2.515773 \\
\hline & -0.336433 & -5.369607 & 5.433286 \\
\hline & 9.718328 & -1.783382 & 1.239743 \\
\hline & 1.950463 & 3.121738 & 0.739485 \\
\hline & -3.874620 & -0.338529 & 1.633929 \\
\hline & -2.755886 & -0.118311 & -2.482283 \\
\hline & -6.739635 & -1.301253 & -1.402399 \\
\hline & -3.785000 & 2.188972 & -0.262912 \\
\hline & -0.184192 & 2.194696 & 2.062284 \\
\hline & -2.880432 & 5.528731 & 2.250839 \\
\hline & 3.294759 & -1.312629 & 1.135225 \\
\hline & 1.762768 & -1.638138 & 1.918406 \\
\hline & -0.401418 & -0.544328 & -2.279506 \\
\hline & 2.749716 & -3.183449 & 3.377612 \\
\hline & 2.944684 & -3.883926 & -0.848221 \\
\hline & 3.504975 & -5.497063 & 3.802278 \\
\hline ] & 3.649197 & -6.213818 & -0.431545 \\
\hline & 3.938974 & -7.036197 & 1.894546 \\
\hline 0 & -5.513447 & 3.883234 & -0.574696 \\
\hline 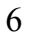 & -5.650571 & 4.890162 & 1.685381 \\
\hline 5 & -4.365335 & 6.033815 & -0.132619 \\
\hline 6 & -1.012122 & 5.954981 & 3.782265 \\
\hline & -0.556496 & 3.662232 & 4.607527 \\
\hline 5 & 0.894169 & 4.682313 & 2.849420 \\
\hline & -4.030314 & -0.613687 & -4.533445 \\
\hline 6 & -5.757652 & -2.256931 & -3.869152 \\
\hline 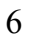 & -6.289471 & 0.188060 & -3.895178 \\
\hline 6 & -7.753145 & -1.708040 & 0.835440 \\
\hline 6 & -5.861002 & -2.115503 & 2.376276 \\
\hline & -6.750314 & 0.202476 & 2.062986 \\
\hline 1 & -6.416865 & 4.437272 & -0.859134 \\
\hline & -4.924437 & 3.720912 & -1.486833 \\
\hline 1 & -5.833325 & 2.901484 & -0.198530 \\
\hline l & -6.568667 & 5.411525 & 1.382564 \\
\hline 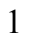 & -5.939081 & 3.930071 & 2.133441 \\
\hline 1 & -5.169373 & 5.492766 & 2.465312 \\
\hline ] & -5.269357 & 6.560858 & -0.466198 \\
\hline 1 & -3.851296 & 6.682925 & 0.586334 \\
\hline & -3.705648 & 5.908708 & -1.000851 \\
\hline & 0.074831 & 4.089500 & 5.398458 \\
\hline & -1.574466 & 3.558073 & 5.005805 \\
\hline
\end{tabular}




$\begin{array}{rrrr}1 & -0.176147 & 2.656382 & 4.387477 \\ 1 & 1.521252 & 5.205578 & 3.583459 \\ 1 & 1.351222 & 3.700337 & 2.680643 \\ 1 & 0.929025 & 5.247339 & 1.907666 \\ 1 & -0.334460 & 6.357288 & 4.545572 \\ 1 & -1.012379 & 6.656504 & 2.937998 \\ 1 & -2.019451 & 5.938467 & 4.217448 \\ 1 & -4.357488 & -0.745666 & -5.572514 \\ 1 & -3.632477 & 0.405379 & -4.443759 \\ 1 & -3.206845 & -1.318896 & -4.351604 \\ 1 & -6.069168 & -2.339443 & -4.919183 \\ 1 & -4.989589 & -3.018189 & -3.680260 \\ 1 & -6.629258 & -2.496186 & -3.247601 \\ 1 & -6.583811 & 0.137192 & -4.952228 \\ 1 & -7.191636 & 0.032702 & -3.290441 \\ 1 & -5.922338 & 1.203217 & -3.694297 \\ 1 & -7.447389 & 0.045582 & 2.897452 \\ 1 & -5.842641 & 0.670862 & 2.465148 \\ 1 & -7.212749 & 0.914758 & 1.366729 \\ 1 & -6.578945 & -2.288200 & 3.189216 \\ 1 & -5.650741 & -3.086126 & 1.907119 \\ 1 & -4.929922 & -1.756851 & 2.833456 \\ 1 & -8.434139 & -1.902742 & 1.673605 \\ 1 & -8.266729 & -1.020245 & 0.151776 \\ 1 & -7.593127 & -2.659384 & 0.310243\end{array}$

$1_{\text {C9- }} \psi_{\text {-1s2a3s4a }}$

Number of imaginary frequencies : 0

The smallest frequencies are : $13.3658 \quad 14.8268 \quad 18.8979 \mathrm{~cm}(-1)$

Electronic energy : $\quad H F=-4125.4618888$

Zero-point correction $=\quad 1.300982($ Hartree/Particle $)$

Thermal correction to Energy= $\quad 1.378906$

Thermal correction to Enthalpy= $\quad 1.379851$

Thermal correction to Gibbs Free Energy= 1.188956

Sum of electronic and zero-point Energies $=\quad-4124.160906$

Sum of electronic and thermal Energies $=\quad-4124.082982$

Sum of electronic and thermal Enthalpies $=\quad-4124.082038$

Sum of electronic and thermal Free Energies $=\quad-4124.272932$

Cartesian Coordinates

$\begin{array}{cccc}6 & -1.091561 & 2.694355 & 1.793240 \\ 6 & -1.944467 & 2.050466 & 0.898439 \\ 6 & -3.096951 & 2.708407 & 0.457486 \\ 6 & -3.427181 & 3.976370 & 0.921674 \\ 6 & -2.580212 & 4.570940 & 1.866212 \\ 6 & -1.415083 & 3.953651 & 2.319306 \\ 15 & -1.516037 & 0.442723 & 0.108765 \\ 6 & -3.184873 & -0.177852 & -0.357173 \\ 6 & -4.134192 & -0.469713 & 0.630265 \\ 6 & -5.415409 & -0.883172 & 0.286725 \\ 6 & -5.721895 & -1.006590 & -1.079710 \\ 6 & -4.803370 & -0.715314 & -2.084499 \\ 6 & -3.525599 & -0.291489 & -1.694675 \\ 6 & -6.476545 & -1.209649 & 1.337038\end{array}$




\begin{tabular}{|c|c|c|c|}
\hline 6 & -5.140792 & -0.778992 & -3.571940 \\
\hline 6 & -4.697611 & 4.693546 & 0.469547 \\
\hline 6 & -0.519881 & 4.577497 & 3.390142 \\
\hline & -1.145944 & -0.701431 & 1.533157 \\
\hline & -0.771960 & -2.020629 & 1.294892 \\
\hline & -0.638360 & -2.940021 & 2.386683 \\
\hline & -0.849388 & -2.485699 & 3.720918 \\
\hline 0 & -1.210942 & -1.136248 & 3.929332 \\
\hline 6 & -1.365553 & -0.279820 & 2.872473 \\
\hline 8 & -0.307259 & -4.307012 & 2.191055 \\
\hline 0 & -0.203555 & -5.168566 & 3.254377 \\
\hline 6 & -0.401737 & -4.709294 & 4.573538 \\
\hline 6 & -0.714444 & -3.392836 & 4.799611 \\
\hline 6 & -0.556326 & -2.551486 & -0.080633 \\
\hline 6 & 0.641965 & -2.363857 & -0.750706 \\
\hline 6 & 0.809913 & -2.878961 & -2.061574 \\
\hline 6 & -0.188923 & -3.569687 & -2.688773 \\
\hline 6 & -1.421490 & -3.796127 & -2.034012 \\
\hline 6 & -1.606943 & -3.292342 & -0.713157 \\
\hline 6 & -2.469746 & -4.507809 & -2.665536 \\
\hline 6 & -3.668806 & -4.705013 & -2.029174 \\
\hline 6 & -3.861489 & -4.199957 & -0.726259 \\
\hline 6 & -2.857836 & -3.519618 & -0.082263 \\
\hline 7 & 1.717701 & -1.576620 & -0.226710 \\
\hline 15 & 1.886028 & -0.047784 & -0.968589 \\
\hline 8 & 3.170884 & -0.186678 & -2.030880 \\
\hline 6 & 4.389889 & -0.629145 & -1.566636 \\
\hline 6 & 5.190427 & 0.208357 & -0.809117 \\
\hline 6 & 6.402206 & -0.326866 & -0.264196 \\
\hline 6 & 6.790269 & -1.660828 & -0.595295 \\
\hline 6 & 5.965207 & -2.438118 & -1.442203 \\
\hline 6 & 4.780959 & -1.937738 & -1.910409 \\
\hline 6 & 7.222380 & 0.399299 & 0.637348 \\
\hline 6 & 8.371451 & -0.149164 & 1.150383 \\
\hline 6 & 8.768490 & -1.454958 & 0.793535 \\
\hline 6 & 7.987777 & -2.192875 & -0.058017 \\
\hline 6 & 4.728335 & 1.591029 & -0.544353 \\
\hline 6 & 3.472353 & 1.781944 & 0.000938 \\
\hline 6 & 2.967195 & 3.055996 & 0.315482 \\
\hline 6 & 3.723142 & 4.167706 & 0.060035 \\
\hline 6 & 4.992633 & 4.046893 & -0.553466 \\
\hline 6 & 5.498283 & 2.751094 & -0.879328 \\
\hline 6 & 5.758766 & 5.194485 & -0.873226 \\
\hline 6 & 6.965253 & 5.081465 & -1.514060 \\
\hline 6 & 7.450441 & 3.805851 & -1.871564 \\
\hline 6 & 6.738377 & 2.673528 & -1.563598 \\
\hline 8 & 2.678477 & 0.693136 & 0.295468 \\
\hline 6 & 2.382490 & -1.929270 & 1.038439 \\
\hline 6 & 2.780619 & -3.371823 & 1.207213 \\
\hline 6 & 2.963315 & -3.841200 & 2.510532 \\
\hline 6 & 3.377774 & -5.145046 & 2.750264 \\
\hline 6 & 3.599082 & -6.012684 & 1.684134 \\
\hline 6 & 3.421995 & -5.555300 & 0.382540 \\
\hline 6 & 3.025253 & -4.241936 & 0.145452 \\
\hline 45 & -0.058625 & 0.966675 & -1.793859 \\
\hline 6 & -0.648936 & 1.322433 & -3.567405 \\
\hline
\end{tabular}




\begin{tabular}{|c|c|c|c|}
\hline & -1.007053 & 1.485671 & -4.656350 \\
\hline 5 & 0.171137 & 2.860980 & -1.341507 \\
\hline & 0.169442 & 4.000933 & -1.175701 \\
\hline & 6.925514 & 1.402495 & 0.932263 \\
\hline & -1.689471 & 0.738977 & 3.066500 \\
\hline & 8.268060 & -3.209412 & -0.330982 \\
\hline & -0.874177 & -3.022272 & 5.811359 \\
\hline & 5.357960 & 6.171943 & -0.608130 \\
\hline & -3.025456 & -3.132593 & 0.921460 \\
\hline & -4.816190 & -4.353766 & -0.227296 \\
\hline & 3.345897 & 5.160178 & 0.302726 \\
\hline & -1.388953 & -0.789451 & 4.946682 \\
\hline & 6.276121 & -3.450738 & -1.694496 \\
\hline & 8.395215 & 3.718487 & -2.403418 \\
\hline & 4.123946 & -2.522040 & -2.550892 \\
\hline & -2.304154 & -4.885969 & -3.673752 \\
\hline & -0.048915 & -3.952984 & -3.698202 \\
\hline & 0.054463 & -6.210353 & 3.077418 \\
\hline & 7.120271 & 1.699413 & -1.858045 \\
\hline & -0.125094 & -4.673188 & 1.183137 \\
\hline & 1.755831 & -2.691723 & -2.567423 \\
\hline & 7.542088 & 5.969883 & -1.760283 \\
\hline & 8.978690 & 0.427794 & 1.844338 \\
\hline & -4.471971 & -5.245581 & -2.525591 \\
\hline 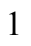 & -0.308052 & -5.401317 & 5.407473 \\
\hline & 9.683761 & -1.875205 & 1.203784 \\
\hline ] & 1.977002 & 3.124611 & 0.758969 \\
\hline 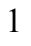 & -3.854867 & -0.364971 & 1.676403 \\
\hline 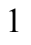 & -2.777570 & -0.038067 & -2.443739 \\
\hline L & -6.719889 & -1.341819 & -1.356224 \\
\hline & -3.738398 & 2.214110 & -0.265980 \\
\hline L & -0.169538 & 2.198736 & 2.105654 \\
\hline 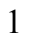 & -2.848370 & 5.549308 & 2.258037 \\
\hline L & 3.287634 & -1.315782 & 1.104601 \\
\hline . & 1.758865 & -1.631763 & 1.897239 \\
\hline 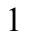 & -0.409251 & -0.515896 & -2.293904 \\
\hline 1 & 2.760653 & -3.174368 & 3.349730 \\
\hline & 2.899261 & -3.892179 & -0.875776 \\
\hline L & 3.507285 & -5.490390 & 3.774109 \\
\hline . & 3.594881 & -6.224017 & -0.458384 \\
\hline 1 & 3.908437 & -7.039509 & 1.866960 \\
\hline 5 & -5.430473 & 3.929664 & -0.632890 \\
\hline 5 & -5.644459 & 4.830610 & 1.667398 \\
\hline 5 & -4.347906 & 6.084632 & -0.068358 \\
\hline 5 & -0.986219 & 5.972867 & 3.798779 \\
\hline 6 & -0.541585 & 3.682071 & 4.634971 \\
\hline 6 & 0.918812 & 4.689842 & 2.877467 \\
\hline 6 & -6.475733 & -1.473233 & -3.829057 \\
\hline 6 & -5.218552 & 0.655803 & -4.108051 \\
\hline 6 & -4.057397 & -1.546359 & -4.337404 \\
\hline 6 & -5.966977 & -0.989268 & 2.759924 \\
\hline 6 & -7.701101 & -0.312418 & 1.126709 \\
\hline 6 & -6.893831 & -2.678394 & 1.208234 \\
\hline & -6.331135 & 4.483392 & -0.926596 \\
\hline 1 & -4.808518 & 3.809166 & -1.529759 \\
\hline 1 & -5.750983 & 2.930596 & -0.306238 \\
\hline
\end{tabular}




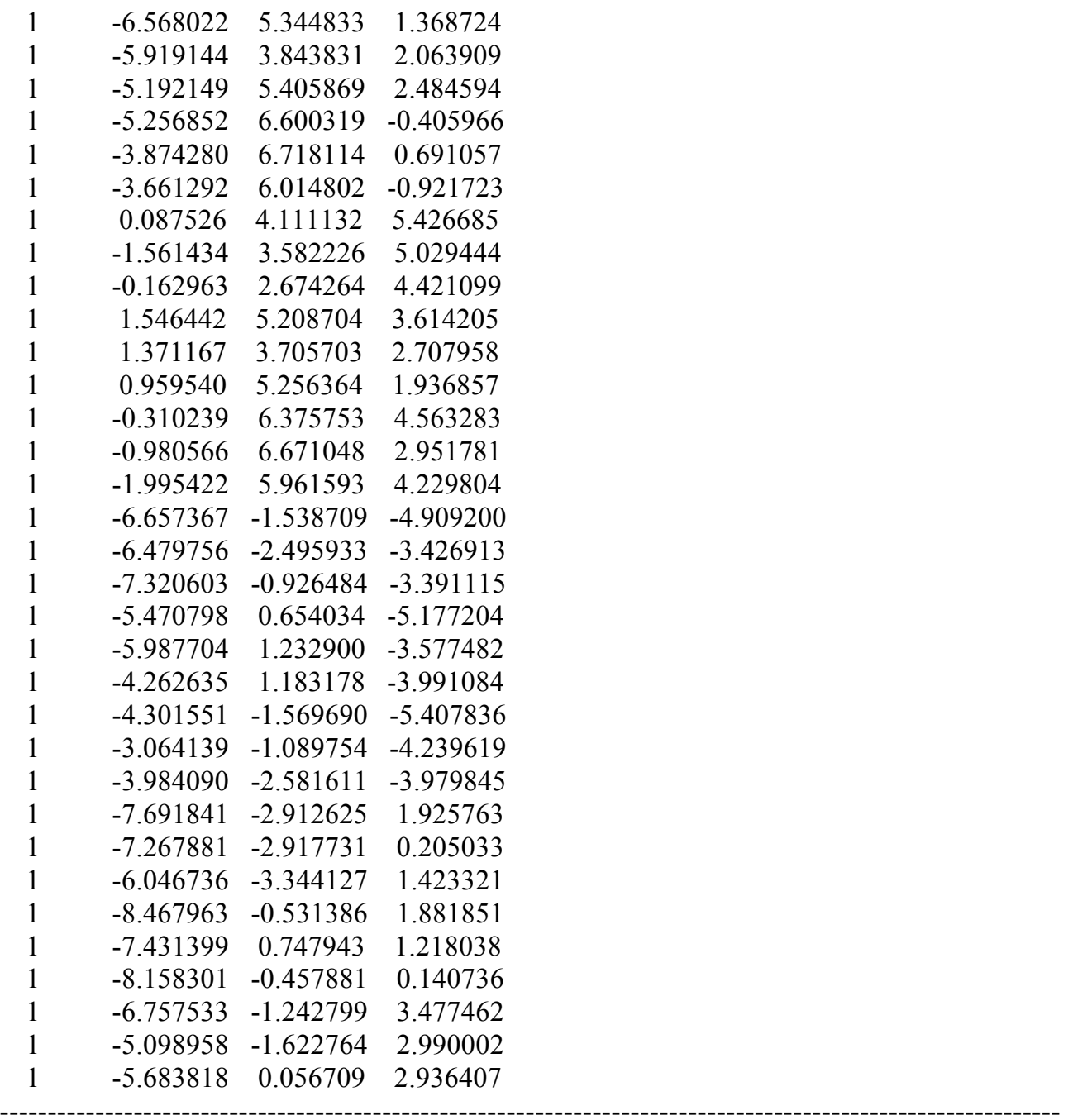

$1_{C 9-} \psi_{-1 \mathrm{~s} 2 \mathrm{a} 3 \mathrm{~s} 4 \mathrm{~s}}$

Number of imaginary frequencies : 0

The smallest frequencies are : $13.6224 \quad 13.7511 \quad 19.9228 \mathrm{~cm}(-1)$

Electronic energy : $\quad H F=-4125.4625817$

Zero-point correction $=\quad 1.300847$ (Hartree/Particle)

Thermal correction to Energy= $\quad 1.378693$

Thermal correction to Enthalpy= $\quad 1.379638$

Thermal correction to Gibbs Free Energy $=\quad 1.189860$

Sum of electronic and zero-point Energies $=\quad-4124.161735$

Sum of electronic and thermal Energies $=\quad-4124.083888$

Sum of electronic and thermal Enthalpies $=\quad-4124.082944$

Sum of electronic and thermal Free Energies= $\quad-4124.272722$

Cartesian Coordinates

$\begin{array}{llll}6 & -1.109992 & 2.714314 & 1.759842 \\ 6 & -1.966950 & 2.055046 & 0.879747 \\ 6 & -3.142839 & 2.688400 & 0.465598 \\ 6 & -3.488046 & 3.949976 & 0.937747 \\ 6 & -2.632175 & 4.561701 & 1.862806 \\ 6 & -1.447057 & 3.966437 & 2.293676\end{array}$




\begin{tabular}{|c|c|c|c|}
\hline 15 & -1.513643 & 0.453345 & 0.091132 \\
\hline 6 & -3.169532 & -0.184519 & -0.394964 \\
\hline 6 & -4.121047 & -0.489359 & 0.577766 \\
\hline 6 & -5.396112 & -0.926584 & 0.225760 \\
\hline 6 & -5.693072 & -1.051286 & -1.137261 \\
\hline 6 & -4.766153 & -0.741204 & -2.135899 \\
\hline 6 & -3.500066 & -0.302594 & -1.738588 \\
\hline 6 & -6.405871 & -1.226731 & 1.332780 \\
\hline 6 & -5.094448 & -0.807020 & -3.625829 \\
\hline 6 & -4.778033 & 4.646329 & 0.508741 \\
\hline 6 & -0.549247 & 4.603417 & 3.354769 \\
\hline 6 & -1.152562 & -0.683471 & 1.525365 \\
\hline 6 & -0.776058 & -2.004100 & 1.299845 \\
\hline 6 & -0.650164 & -2.915818 & 2.399064 \\
\hline 6 & -0.872175 & -2.452541 & 3.728369 \\
\hline 6 & -1.236219 & -1.101915 & 3.924244 \\
\hline 6 & -1.382253 & -0.252839 & 2.860203 \\
\hline 6 & -0.318307 & -4.284305 & 2.215492 \\
\hline 6 & -0.223158 & -5.138413 & 3.285602 \\
\hline 6 & -0.431020 & -4.669948 & 4.599986 \\
\hline 6 & -0.745321 & -3.351977 & 4.814452 \\
\hline 6 & -0.557511 & -2.545943 & -0.070631 \\
\hline 6 & 0.639815 & -2.363357 & -0.743310 \\
\hline 6 & 0.804222 & -2.886955 & -2.051484 \\
\hline 6 & -0.196462 & -3.581214 & -2.672533 \\
\hline 6 & -1.427898 & -3.803536 & -2.013826 \\
\hline 6 & -1.609180 & -3.290729 & -0.696356 \\
\hline 6 & -2.480798 & -4.516276 & -2.636912 \\
\hline 6 & -3.679707 & -4.703753 & -1.996504 \\
\hline 6 & -3.866838 & -4.190511 & -0.695679 \\
\hline 6 & -2.858011 & -3.510840 & -0.059809 \\
\hline 7 & 1.717927 & -1.576608 & -0.223126 \\
\hline 15 & 1.892154 & -0.049633 & -0.967553 \\
\hline 8 & 3.181345 & -0.193974 & -2.023842 \\
\hline 6 & 4.399051 & -0.632424 & -1.552563 \\
\hline 6 & 5.195694 & 0.210439 & -0.796900 \\
\hline 6 & 6.406138 & -0.319964 & -0.244537 \\
\hline 6 & 6.796787 & -1.655618 & -0.565791 \\
\hline 6 & 5.975592 & -2.439039 & -1.410859 \\
\hline 6 & 4.792689 & -1.942712 & -1.886812 \\
\hline 6 & 7.222190 & 0.412788 & 0.655394 \\
\hline 6 & 8.369752 & -0.131265 & 1.176374 \\
\hline 6 & 8.769293 & -1.439011 & 0.829557 \\
\hline 6 & 7.992651 & -2.183081 & -0.020378 \\
\hline 6 & 4.729895 & 1.593315 & -0.539745 \\
\hline 6 & 3.472314 & 1.783239 & 0.002101 \\
\hline 6 & 2.963216 & 3.057292 & 0.310306 \\
\hline 6 & 3.716890 & 4.169865 & 0.051780 \\
\hline 6 & 4.988037 & 4.049700 & -0.558462 \\
\hline 6 & 5.497784 & 2.753810 & -0.877604 \\
\hline 6 & 5.752053 & 5.197816 & -0.881402 \\
\hline 6 & 6.960368 & 5.085021 & -1.518833 \\
\hline 6 & 7.449755 & 3.809099 & -1.869490 \\
\hline 6 & 6.739812 & 2.676323 & -1.558344 \\
\hline 8 & 2.680562 & 0.693229 & 0.298034 \\
\hline 6 & 2.380232 & -1.927150 & 1.044160 \\
\hline
\end{tabular}




\begin{tabular}{|c|c|c|c|}
\hline 6 & 2.774031 & -3.370291 & 1.217440 \\
\hline 6 & 2.948319 & -3.838583 & 2.522264 \\
\hline 6 & 3.358664 & -5.143125 & 2.765623 \\
\hline 6 & 3.584195 & -6.012279 & 1.701668 \\
\hline 6 & 3.415311 & -5.555984 & 0.398558 \\
\hline 6 & 3.022597 & -4.242083 & 0.157952 \\
\hline 45 & -0.044908 & 0.969971 & -1.802530 \\
\hline 6 & -0.619299 & 1.320155 & -3.582635 \\
\hline 8 & -0.966030 & 1.480071 & -4.675679 \\
\hline 6 & 0.177824 & 2.866739 & -1.358684 \\
\hline 8 & 0.166778 & 4.007910 & -1.201682 \\
\hline 1 & 6.923345 & 1.417612 & 0.942645 \\
\hline 1 & -1.706457 & 0.767574 & 3.044938 \\
\hline 1 & 8.274970 & -3.201071 & -0.285698 \\
\hline 1 & -0.912776 & -2.974335 & 5.822319 \\
\hline 1 & 5.348171 & 6.175419 & -0.621567 \\
\hline 1 & -3.018400 & -3.119511 & 0.942788 \\
\hline 1 & -4.821197 & -4.335698 & -0.191574 \\
\hline 1 & 3.336524 & 5.162353 & 0.289585 \\
\hline 1 & -1.421892 & -0.747822 & 4.937716 \\
\hline 1 & 6.288257 & -3.453014 & -1.655421 \\
\hline 1 & 8.396108 & 3.721840 & -2.398544 \\
\hline 1 & 4.138301 & -2.531507 & -2.525849 \\
\hline 1 & -2.319798 & -4.902543 & -3.642827 \\
\hline 1 & -0.058482 & -3.970268 & -3.680052 \\
\hline 1 & 0.035468 & -6.181562 & 3.117805 \\
\hline 1 & 7.124869 & 1.701834 & -1.847441 \\
\hline 1 & -0.128736 & -4.657777 & 1.211598 \\
\hline 1 & 1.749204 & -2.703510 & -2.560574 \\
\hline 1 & 7.535547 & 5.973812 & -1.767564 \\
\hline 1 & 8.973826 & 0.450724 & 1.868888 \\
\hline 1 & -4.485991 & -5.244119 & -2.488091 \\
\hline 1 & -0.343783 & -5.356154 & 5.439395 \\
\hline 1 & 9.683282 & -1.855810 & 1.246132 \\
\hline 1 & 1.972010 & 3.125505 & 0.751685 \\
\hline 1 & -3.860450 & -0.377544 & 1.630314 \\
\hline 1 & -2.749281 & -0.039586 & -2.481433 \\
\hline 1 & -6.679799 & -1.400163 & -1.428127 \\
\hline 1 & -3.791316 & 2.180679 & -0.241966 \\
\hline 1 & -0.174087 & 2.234383 & 2.055582 \\
\hline 1 & -2.909938 & 5.535795 & 2.258754 \\
\hline 1 & 3.286993 & -1.315943 & 1.109740 \\
\hline 1 & 1.756193 & -1.625320 & 1.901120 \\
\hline 1 & -0.396219 & -0.513877 & -2.298312 \\
\hline 1 & 2.741478 & -3.170669 & 3.359644 \\
\hline 1 & 2.902860 & -3.892927 & -0.864272 \\
\hline 1 & 3.481571 & -5.487747 & 3.790524 \\
\hline 1 & 3.591387 & -6.226027 & -0.440639 \\
\hline 1 & 3.890318 & -7.039563 & 1.887319 \\
\hline 6 & -5.541157 & 3.847718 & -0.547337 \\
\hline 6 & -5.688580 & 4.814264 & 1.730441 \\
\hline 6 & -4.452332 & 6.022906 & -0.079574 \\
\hline 6 & -1.022026 & 5.998738 & 3.756372 \\
\hline 6 & -0.561604 & 3.714944 & 4.604830 \\
\hline 6 & 0.887564 & 4.720930 & 2.838177 \\
\hline 6 & -6.421488 & -1.513013 & -3.892441 \\
\hline
\end{tabular}




$\begin{array}{rrrr}6 & -5.181401 & 0.626736 & -4.163340 \\ 6 & -3.999832 & -1.565292 & -4.384079 \\ 6 & -7.704856 & -1.819869 & 0.793364 \\ 6 & -5.808013 & -2.226846 & 2.329922 \\ 6 & -6.738774 & 0.080665 & 2.062268 \\ 1 & -6.458667 & 4.383728 & -0.821014 \\ 1 & -4.951444 & 3.712102 & -1.463559 \\ 1 & -5.836489 & 2.853510 & -0.183577 \\ 1 & -6.623283 & 5.315185 & 1.444206 \\ 1 & -5.946477 & 3.838886 & 2.164104 \\ 1 & -5.216389 & 5.416475 & 2.516233 \\ 1 & -5.374167 & 6.527178 & -0.399170 \\ 1 & -3.953477 & 6.677440 & 0.645081 \\ 1 & -3.795801 & 5.930905 & -0.954235 \\ 1 & 0.068372 & 4.151354 & 5.391845 \\ 1 & -1.579449 & 3.611934 & 5.003663 \\ 1 & -0.178775 & 2.707999 & 4.394106 \\ 1 & 1.512762 & 5.248586 & 3.570677 \\ 1 & 1.346638 & 3.738850 & 2.675511 \\ 1 & 0.924307 & 5.281902 & 1.894218 \\ 1 & -0.342208 & 6.412613 & 4.511519 \\ 1 & -1.029175 & 6.689753 & 2.903504 \\ 1 & -2.026999 & 5.984132 & 4.196910 \\ 1 & -6.594980 & -1.578694 & -4.973923 \\ 1 & -6.419017 & -2.536223 & -3.491789 \\ 1 & -7.274330 & -0.974605 & -3.459680 \\ 1 & -5.435298 & 0.622209 & -5.232121 \\ 1 & -5.952637 & 1.200633 & -3.632195 \\ 1 & -4.228036 & 1.159119 & -4.048763 \\ 1 & -4.235627 & -1.590567 & -5.456354 \\ 1 & -3.010787 & -1.101476 & -4.278184 \\ 1 & -3.922223 & -2.599656 & -4.025253 \\ 1 & -7.443619 & -0.104879 & 2.884249 \\ 1 & -5.842635 & 0.550951 & 2.487132 \\ 1 & -7.201637 & 0.803595 & 1.377215 \\ 1 & -6.527477 & -2.437411 & 3.132537 \\ 1 & -5.568789 & -3.179735 & 1.838105 \\ 1 & -4.889678 & -1.855539 & 2.802962 \\ 1 & -8.386175 & -2.036025 & 1.625996 \\ 1 & -8.224914 & -1.128913 & 0.117826 \\ 1 & -7.531592 & -2.761329 & 0.255016\end{array}$

$1_{\text {C9- }} \Psi$-1s2s3a4a

Number of imaginary frequencies : 0

The smallest frequencies are : $12.2877 \quad 14.7457 \quad 18.8248 \mathrm{~cm}(-1)$

Electronic energy : $\quad H F=-4125.4616597$

Zero-point correction $=\quad 1.299796($ Hartree/Particle $)$

Thermal correction to Energy $=\quad 1.378215$

Thermal correction to Enthalpy= $\quad 1.379159$

Thermal correction to Gibbs Free Energy $=\quad 1.186430$

Sum of electronic and zero-point Energies $=\quad-4124.161863$

Sum of electronic and thermal Energies $=\quad-4124.083445$

Sum of electronic and thermal Enthalpies $=\quad-4124.082501$

Sum of electronic and thermal Free Energies $=\quad-4124.275230$ 
Cartesian Coordinates

\begin{tabular}{cccc}
6 & 3.542506 & -6.006248 & 1.763039 \\
6 & 3.319303 & -5.126635 & 2.818917 \\
6 & 2.913804 & -3.822960 & 2.563389 \\
6 & 2.742424 & -3.365591 & 1.254261 \\
6 & 2.988791 & -4.247770 & 0.202957 \\
6 & 3.376318 & -5.561010 & 0.455846 \\
6 & 2.352488 & -1.922782 & 1.068471 \\
7 & 1.700482 & -1.578589 & -0.205076 \\
6 & 0.623773 & -2.362063 & -0.732765 \\
6 & -0.582869 & -2.533399 & -0.074038 \\
6 & -1.633686 & -3.270465 & -0.709221 \\
6 & -1.440932 & -3.787370 & -2.023739 \\
6 & -0.201242 & -3.574631 & -2.669627 \\
6 & 0.798238 & -2.888248 & -2.038395 \\
6 & -2.889567 & -3.486029 & -0.083644 \\
6 & -3.886313 & -4.182516 & -0.720431 \\
6 & -3.682585 & -4.708608 & -2.013728 \\
6 & -2.485630 & -4.506997 & -2.652542 \\
6 & -0.809866 & -1.993923 & 1.295338 \\
6 & -1.189336 & -0.674748 & 1.521158 \\
6 & -1.431258 & -0.250313 & 2.856441 \\
6 & -1.286576 & -1.100872 & 3.919233 \\
6 & -0.915605 & -2.449471 & 3.722221 \\
6 & -0.688111 & -2.908862 & 2.392685 \\
6 & -0.790389 & -3.351217 & 4.806478 \\
6 & -0.473615 & -4.668294 & 4.589640 \\
6 & -0.262064 & -5.133413 & 3.274731 \\
6 & -0.354756 & -4.276547 & 2.206523 \\
15 & -1.538384 & 0.471601 & 0.089998 \\
45 & -0.057819 & 0.959901 & -1.801711 \\
6 & 0.155584 & 2.860195 & -1.365316 \\
8 & 0.148150 & 4.001299 & -1.206243 \\
15 & 1.880246 & -0.057590 & -0.961646 \\
8 & 2.686616 & 0.690590 & 0.289387 \\
6 & 3.489385 & 1.767012 & -0.028399 \\
6 & 4.744083 & 1.552940 & -0.567517 \\
6 & 5.526736 & 2.697799 & -0.924364 \\
6 & 5.032087 & 4.005098 & -0.629225 \\
6 & 3.760295 & 4.151264 & -0.026180 \\
6 & 2.993288 & 3.052433 & 0.251831 \\
6 & 5.810825 & 5.138053 & -0.969905 \\
6 & 7.019172 & 4.999700 & -1.602242 \\
6 & 7.493395 & 3.712057 & -1.930215 \\
6 & 6.768803 & 2.593605 & -1.601472 \\
6 & 5.188287 & 0.160890 & -0.811575 \\
6 & 4.376408 & -0.674816 & -1.559223 \\
6 & 4.753195 & -1.990843 & -1.890302 \\
6 & 5.932105 & -2.500042 & -1.417976 \\
6 & 6.766463 & -1.724126 & -0.579013 \\
6 & 6.394003 & -0.382442 & -0.261853 \\
6 & 7.223230 & 0.344278 & 0.630936 \\
6 & 8.366344 & -0.211732 & 1.149098 \\
6 & 8.747889 & -1.525928 & 0.806294 \\
6 & 7.958183 & -2.264063 & -0.036763 \\
& & & \\
\hline
\end{tabular}




\begin{tabular}{|c|c|c|c|}
\hline & 3.161891 & -0.223409 & -2.026277 \\
\hline & -3.218794 & -0.117729 & -0.379095 \\
\hline & -4.179567 & -0.367818 & 0.603840 \\
\hline & -5.468537 & -0.768615 & 0.258584 \\
\hline & -5.761191 & -0.912033 & -1.103640 \\
\hline & -4.825966 & -0.667137 & -2.110801 \\
\hline & -3.544702 & -0.264926 & -1.722685 \\
\hline & -6.533162 & -1.081608 & 1.309474 \\
\hline & -5.221964 & -0.809623 & -3.579288 \\
\hline & -1.919083 & 2.082701 & 0.897289 \\
\hline & -3.056369 & 2.781931 & 0.499516 \\
\hline & -3.340923 & 4.052984 & 1.000894 \\
\hline & -2.460542 & 4.601415 & 1.935280 \\
\hline ) & -1.302827 & 3.934531 & 2.351510 \\
\hline & -1.027990 & 2.683448 & 1.791892 \\
\hline & -4.600501 & 4.769798 & 0.518828 \\
\hline ) & -0.374591 & 4.506013 & 3.423893 \\
\hline & -0.518230 & 1.274879 & -3.622159 \\
\hline & -0.762907 & 1.407561 & -4.745732 \\
\hline & 6.937982 & 1.354059 & 0.914828 \\
\hline & -1.760403 & 0.767945 & 3.043505 \\
\hline & 8.226710 & -3.286595 & -0.298937 \\
\hline & -0.961854 & -2.976427 & 5.814736 \\
\hline & 5.418354 & 6.124853 & -0.727912 \\
\hline & -3.064463 & -3.080620 & 0.911528 \\
\hline & -4.844052 & -4.329512 & -0.224612 \\
\hline & 3.390353 & 5.152485 & 0.190377 \\
\hline & -1.479720 & -0.749605 & 4.932321 \\
\hline & 6.231470 & -3.518416 & -1.660933 \\
\hline 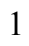 & 8.439717 & 3.604047 & -2.455483 \\
\hline & 4.090231 & -2.573947 & -2.525670 \\
\hline ] & -2.314765 & -4.899130 & -3.654352 \\
\hline & -0.054834 & -3.967836 & -3.674339 \\
\hline & -0.002557 & -6.176032 & 3.105010 \\
\hline & 7.142634 & 1.609829 & -1.873154 \\
\hline & -0.162563 & -4.647518 & 1.202241 \\
\hline 1 & 1.750096 & -2.714255 & -2.537505 \\
\hline & 7.605834 & 5.877051 & -1.864438 \\
\hline 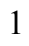 & 8.980873 & 0.365705 & 1.836207 \\
\hline & -4.478351 & -5.266846 & -2.502424 \\
\hline & -0.387902 & -5.356468 & 5.427608 \\
\hline 1 & 9.658597 & -1.952184 & 1.220491 \\
\hline & 2.001832 & 3.140184 & 0.688506 \\
\hline 1 & -3.909513 & -0.240582 & 1.649875 \\
\hline & -2.774986 & -0.058861 & -2.462410 \\
\hline 1 & -6.764274 & -1.229187 & -1.390019 \\
\hline & -3.733887 & 2.326815 & -0.221037 \\
\hline 1 & -0.119480 & 2.149096 & 2.079295 \\
\hline 1 & -2.682331 & 5.578421 & 2.352816 \\
\hline 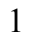 & 3.259839 & -1.312622 & 1.136125 \\
\hline 1 & 1.723014 & -1.614627 & 1.919296 \\
\hline & -0.426878 & -0.523853 & -2.281413 \\
\hline 1 & 2.707831 & -3.147054 & 3.394533 \\
\hline & 2.871919 & -3.907867 & -0.822640 \\
\hline ] & 3.440034 & -5.462439 & 3.846998 \\
\hline & 3.550660 & -6.239160 & -0.377200 \\
\hline
\end{tabular}




$\begin{array}{rrrr}1 & 3.844809 & -7.032912 & 1.958157 \\ 6 & -4.037173 & -0.582491 & -4.516695 \\ 6 & -5.771163 & -2.214884 & -3.841890 \\ 6 & -6.300760 & 0.229141 & -3.906939 \\ 6 & -6.063828 & -0.740133 & 2.722415 \\ 6 & -7.807554 & -0.279173 & 1.028052 \\ 6 & -6.854967 & -2.579573 & 1.263328 \\ 6 & -4.806089 & 6.116258 & 1.207682 \\ 6 & -4.490876 & 5.014801 & -0.990583 \\ 6 & -5.827277 & 3.894449 & 0.803505 \\ 6 & -0.784579 & 5.909746 & 3.863701 \\ 6 & -0.417185 & 3.588473 & 4.652120 \\ 6 & 1.061521 & 4.574955 & 2.898026 \\ 1 & -4.356086 & -0.730412 & -5.556165 \\ 1 & -3.640861 & 0.438175 & -4.439382 \\ 1 & -3.214616 & -1.284198 & -4.317763 \\ 1 & -6.079884 & -2.310495 & -4.891630 \\ 1 & -5.004047 & -2.973918 & -3.641349 \\ 1 & -6.644476 & -2.446217 & -3.219493 \\ 1 & -6.589063 & 0.160861 & -4.964665 \\ 1 & -7.206377 & 0.084187 & -3.304663 \\ 1 & -5.934605 & 1.247479 & -3.721424 \\ 1 & -6.862874 & -0.963681 & 3.440429 \\ 1 & -5.183878 & -1.326796 & 3.019829 \\ 1 & -5.815609 & 0.325118 & 2.822067 \\ 1 & -7.635433 & -2.827335 & 1.995634 \\ 1 & -7.212655 & -2.893122 & 0.274305 \\ 1 & -5.965040 & -3.176407 & 1.507095 \\ 1 & -8.568838 & -0.499561 & 1.788185 \\ 1 & -7.608849 & 0.800259 & 1.054434 \\ 1 & -8.244278 & -0.515800 & 0.050668 \\ 1 & -5.382604 & 5.540660 & -1.357842 \\ 1 & -3.612503 & 5.629895 & -1.225590 \\ 1 & -4.401992 & 4.077667 & -1.553368 \\ 1 & -6.741187 & 4.397344 & 0.459736 \\ 1 & -5.771119 & 2.925402 & 0.291352 \\ 1 & -5.931966 & 3.699929 & 1.879230 \\ 1 & -5.724198 & 6.585148 & 0.831914 \\ 1 & -4.912796 & 6.009928 & 2.295115 \\ 1 & -3.979464 & 6.810210 & 1.008371 \\ 1 & 1.716139 & 5.057141 & 3.636158 \\ 1 & 1.475134 & 3.578259 & 2.705370 \\ 1 & 1.111812 & 5.156113 & 1.966723 \\ 1 & -0.084405 & 6.273471 & 4.626072 \\ 1 & -0.764269 & 6.623080 & 3.029679 \\ 1 & -1.787567 & 5.927775 & 4.308550 \\ 1 & 0.233924 & 3.979550 & 5.445772 \\ 1 & -1.436485 & 3.517608 & 5.054425 \\ 1 & -0.077207 & 2.572081 & 4.415162\end{array}$

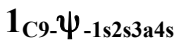

Number of imaginary frequencies : 0

The smallest frequencies are : $12.3516 \quad 13.859520 .7618 \mathrm{~cm}(-1)$

Electronic energy :

Zero-point correction=

$\mathrm{HF}=-4125.4622742$

1.300121 (Hartree/Particle) 
Thermal correction to Energy $=\quad 1.378335$

Thermal correction to Enthalpy= $\quad 1.379279$

Thermal correction to Gibbs Free Energy $=\quad 1.187653$

Sum of electronic and zero-point Energies $=\quad-4124.162153$

Sum of electronic and thermal Energies $=\quad-4124.083939$

Sum of electronic and thermal Enthalpies $=\quad-4124.082995$

Sum of electronic and thermal Free Energies $=\quad-4124.274621$

Cartesian Coordinates

$\begin{array}{ccccc}6 & 3.534381 & -6.030344 & 1.704964 \\ 6 & 3.311250 & -5.162874 & 2.770851 \\ 6 & 2.909397 & -3.855241 & 2.530148 \\ 6 & 2.741587 & -3.382110 & 1.226185 \\ 6 & 2.987334 & -4.252424 & 0.164838 \\ 6 & 3.371458 & -5.569379 & 0.402765 \\ 6 & 2.356485 & -1.936204 & 1.056085 \\ 7 & 1.702695 & -1.577496 & -0.212735 \\ 6 & 0.621068 & -2.352689 & -0.742421 \\ 6 & -0.581906 & -2.527832 & -0.078213 \\ 6 & -1.637528 & -3.257505 & -0.714021 \\ 6 & -1.456583 & -3.757424 & -2.036500 \\ 6 & -0.219909 & -3.541740 & -2.687479 \\ 6 & 0.786277 & -2.866802 & -2.054205 \\ 6 & -2.888189 & -3.478045 & -0.080596 \\ 6 & -3.894853 & -4.157417 & -0.719735 \\ 6 & -3.705098 & -4.663355 & -2.023126 \\ 6 & -2.511022 & -4.460764 & -2.667592 \\ 6 & -0.804997 & -1.993244 & 1.293859 \\ 6 & -1.182699 & -0.673984 & 1.523399 \\ 6 & -1.424630 & -0.252554 & 2.859397 \\ 6 & -1.285139 & -1.107402 & 3.919629 \\ 6 & -0.918110 & -2.456532 & 3.718769 \\ 6 & -0.687598 & -2.911840 & 2.388328 \\ 6 & -0.798732 & -3.362511 & 4.800171 \\ 6 & -0.485163 & -4.679697 & 4.579518 \\ 6 & -0.270830 & -5.140725 & 3.263582 \\ 6 & -0.357643 & -4.279812 & 2.198183 \\ 15 & -1.533948 & 0.474692 & 0.094867 \\ 45 & -0.047733 & 0.983508 & -1.784465 \\ 6 & 0.169324 & 2.879842 & -1.333753 \\ 8 & 0.163819 & 4.020280 & -1.169926 \\ 15 & 1.884244 & -0.047982 & -0.951769 \\ 8 & 2.691003 & 0.683550 & 0.308939 \\ 6 & 3.493521 & 1.763902 & 0.005071 \\ 6 & 4.747556 & 1.557187 & -0.538769 \\ 6 & 5.528739 & 2.707222 & -0.882193 \\ 6 & 5.033918 & 4.010288 & -0.568926 \\ 6 & 3.763346 & 4.147998 & 0.038756 \\ 6 & 2.997370 & 3.045285 & 0.303661 \\ 6 & 5.811061 & 5.148125 & -0.896807 \\ 6 & 7.017837 & 5.018855 & -1.534036 \\ 6 & 7.492057 & 3.735932 & -1.879913 \\ 6 & 6.769141 & 2.612765 & -1.563783 \\ 6 & 5.192430 & 0.168508 & -0.801592 \\ 6 & 4.379791 & -0.658307 & -1.558230\end{array}$




\begin{tabular}{|c|c|c|c|}
\hline 6 & 4.755529 & -1.970178 & -1.906290 \\
\hline 6 & 5.935946 & -2.484637 & -1.443702 \\
\hline & 6.772402 & -1.718458 & -0.597898 \\
\hline & 6.399944 & -0.381284 & -0.262057 \\
\hline & 7.231469 & 0.334121 & 0.637702 \\
\hline & 8.376926 & -0.227619 & 1.144467 \\
\hline & 8.758646 & -1.536663 & 0.782758 \\
\hline & 7.966526 & -2.264362 & -0.067053 \\
\hline & 3.164852 & -0.201483 & -2.018484 \\
\hline & -3.201523 & -0.136913 & -0.389237 \\
\hline & -4.152318 & -0.430345 & 0.583020 \\
\hline & -5.433233 & -0.864168 & 0.232768 \\
\hline & -5.730326 & -0.978684 & -1.126231 \\
\hline & -4.799531 & -0.680891 & -2.130738 \\
\hline & -3.529235 & -0.261470 & -1.738408 \\
\hline & -6.436893 & -1.176851 & 1.341396 \\
\hline y & -5.198836 & -0.788158 & -3.601216 \\
\hline & -1.947399 & 2.078403 & 0.900565 \\
\hline y & -3.094818 & 2.756089 & 0.494290 \\
\hline & -3.395452 & 4.030933 & 0.976162 \\
\hline & -2.525731 & 4.601682 & 1.907165 \\
\hline & -1.362682 & 3.953318 & 2.338076 \\
\hline & -1.068519 & 2.700224 & 1.792880 \\
\hline & -4.658885 & 4.727873 & 0.475065 \\
\hline & -0.449030 & 4.546659 & 3.411376 \\
\hline & -0.505779 & 1.317726 & -3.602145 \\
\hline & -0.749439 & 1.462186 & -4.724483 \\
\hline & 6.946205 & 1.339723 & 0.936030 \\
\hline & -1.751476 & 0.766114 & 3.048560 \\
\hline & 8.234987 & -3.283096 & -0.343717 \\
\hline & -0.972085 & -2.990868 & 5.809266 \\
\hline & 5.418504 & 6.131409 & -0.641073 \\
\hline & -3.050183 & -3.090295 & 0.923281 \\
\hline & -4.848713 & -4.309789 & -0.216823 \\
\hline & 3.393443 & 5.146142 & 0.269165 \\
\hline & -1.479044 & -0.759198 & 4.933614 \\
\hline 1 & 6.235242 & -3.499745 & -1.700034 \\
\hline & 8.436972 & 3.635439 & -2.409196 \\
\hline 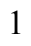 & 4.090434 & -2.545418 & -2.546610 \\
\hline & -2.350760 & -4.839277 & -3.676430 \\
\hline & -0.081213 & -3.922990 & -3.697887 \\
\hline 1 & -0.013865 & -6.183494 & 3.090952 \\
\hline & 7.142854 & 1.632921 & -1.849463 \\
\hline ] & -0.163332 & -4.647593 & 1.193105 \\
\hline & 1.736007 & -2.690813 & -2.556846 \\
\hline 1 & 7.603164 & 5.899939 & -1.786502 \\
\hline 1 & 8.993249 & 0.341238 & 1.837101 \\
\hline & -4.508407 & -5.207982 & -2.514832 \\
\hline 1 & -0.404033 & -5.371108 & 5.415269 \\
\hline ] & 9.671298 & -1.967417 & 1.187913 \\
\hline 1 & 2.006081 & 3.126836 & 0.742359 \\
\hline ( & -3.891416 & -0.318948 & 1.635542 \\
\hline 1 & -2.765780 & -0.021823 & -2.474371 \\
\hline ] & -6.723381 & -1.310709 & -1.422754 \\
\hline 1 & -3.765264 & 2.283749 & -0.221597 \\
\hline 1 & -0.152404 & 2.182494 & 2.086563 \\
\hline
\end{tabular}




\begin{tabular}{|c|c|c|c|}
\hline . & -2.759797 & 5.582009 & 2.309946 \\
\hline 1 & 3.266176 & -1.330066 & 1.127752 \\
\hline 1 & 1.730059 & -1.634162 & 1.911310 \\
\hline & -0.417623 & -0.495248 & -2.278522 \\
\hline & 2.703247 & -3.188769 & 3.368889 \\
\hline 1 & 2.872526 & -3.899769 & -0.856735 \\
\hline 1 & 3.429267 & -5.511251 & 3.795058 \\
\hline 1 & 3.545568 & -6.238116 & -0.437892 \\
\hline 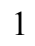 & 3.833944 & -7.059949 & 1.888458 \\
\hline 6 & -4.018572 & -0.526337 & -4.535446 \\
\hline & -5.737677 & -2.189321 & -3.903955 \\
\hline & -6.286512 & 0.251560 & -3.895311 \\
\hline U & -7.736580 & -1.766654 & 0.800294 \\
\hline & -5.830384 & -2.189568 & 2.320668 \\
\hline 6 & -6.769065 & 0.118974 & 2.090593 \\
\hline 6 & -4.869299 & 6.092896 & 1.124883 \\
\hline 6 & -4.552908 & 4.929858 & -1.041125 \\
\hline 6 & -5.881240 & 3.855761 & 0.786725 \\
\hline 6 & -0.874452 & 5.952786 & 3.828359 \\
\hline 6 & -0.498211 & 3.644585 & 4.650815 \\
\hline 6 & 0.993028 & 4.620390 & 2.902192 \\
\hline 1 & -4.338903 & -0.650792 & -5.577571 \\
\hline 1 & -3.630806 & 0.495444 & -4.433741 \\
\hline 1 & -3.189793 & -1.226061 & -4.355631 \\
\hline 1 & -6.047648 & -2.255565 & -4.955560 \\
\hline 1 & -4.963785 & -2.947450 & -3.727948 \\
\hline 1 & -6.607990 & -2.447151 & -3.288097 \\
\hline 1 & -6.576931 & 0.213370 & -4.953982 \\
\hline 1 & -7.189312 & 0.080649 & -3.295617 \\
\hline 1 & -5.927709 & 1.266594 & -3.679247 \\
\hline 1 & -8.413409 & -1.996153 & 1.632990 \\
\hline 1 & -8.261826 & -1.068125 & 0.136593 \\
\hline 1 & -7.562164 & -2.700190 & 0.248509 \\
\hline 1 & -7.466324 & -0.081577 & 2.915509 \\
\hline 1 & -5.872038 & 0.588144 & 2.514708 \\
\hline 1 & -7.240778 & 0.849002 & 1.419193 \\
\hline 1 & -6.544909 & -2.414297 & 3.123831 \\
\hline 1 & -5.590439 & -3.134145 & 1.813344 \\
\hline 1 & -4.910735 & -1.822119 & 2.794244 \\
\hline 1 & -5.445867 & 5.444952 & -1.420417 \\
\hline 1 & -3.675444 & 5.538270 & -1.296071 \\
\hline 1 & -4.465888 & 3.977312 & -1.577980 \\
\hline 1 & -6.797493 & 4.343285 & 0.427235 \\
\hline 1 & -5.820101 & 2.870734 & 0.305548 \\
\hline 1 & -5.984951 & 3.695282 & 1.868084 \\
\hline 1 & -5.788100 & 6.548337 & 0.734536 \\
\hline 1 & -4.977797 & 6.017474 & 2.214692 \\
\hline 1 & -4.043938 & 6.782858 & 0.907202 \\
\hline 1 & 1.634689 & 5.112437 & 3.645186 \\
\hline 1 & 1.416470 & 3.625314 & 2.722737 \\
\hline 1 & 1.052452 & 5.195639 & 1.967932 \\
\hline 1 & -0.184152 & 6.332619 & 4.591887 \\
\hline 1 & -0.852551 & 6.654751 & 2.984772 \\
\hline 1 & -1.881531 & 5.968628 & 4.263960 \\
\hline 1 & 0.141004 & 4.050766 & 5.446553 \\
\hline 1 & -1.521305 & 3.570720 & 5.042718 \\
\hline
\end{tabular}


$1_{C 9-} \psi_{-1 \text { s2s334a }}$

Number of imaginary frequencies : 0

The smallest frequencies are : $12.5131 \quad 14.5542 \quad 18.7156 \mathrm{~cm}(-1)$

Electronic energy : $\quad H F=-4125.4621256$

Zero-point correction $=\quad 1.300536($ Hartree/Particle $)$

Thermal correction to Energy= $\quad 1.378604$

Thermal correction to Enthalpy $=\quad 1.379548$

Thermal correction to Gibbs Free Energy $=\quad 1.188232$

Sum of electronic and zero-point Energies $=\quad-4124.161590$

Sum of electronic and thermal Energies $=\quad-4124.083522$

Sum of electronic and thermal Enthalpies $=\quad-4124.082578$

Sum of electronic and thermal Free Energies $=\quad-4124.273894$

\begin{tabular}{cccc} 
& \multicolumn{3}{c}{ Cartesian Coordinates } \\
\hline $6 \ldots \ldots \ldots \ldots \ldots \ldots \ldots \ldots \ldots \ldots \ldots \ldots \ldots \ldots$ \\
6 & 3.509400 & -6.002503 & 1.762650 \\
6 & 3.292528 & -5.119188 & 2.816722 \\
6 & 2.891252 & -3.814720 & 2.558710 \\
6 & 2.717831 & -3.360151 & 1.248835 \\
6 & 2.958421 & -4.245967 & 0.199248 \\
6 & 3.341549 & -5.560019 & 0.454736 \\
7 & 2.333951 & -1.915869 & 1.061575 \\
6 & 1.679003 & -1.569305 & -0.210482 \\
6 & 0.599922 & -2.353102 & -0.732882 \\
6 & -0.602228 & -2.528464 & -0.066639 \\
6 & -1.651207 & -3.276645 & -0.693535 \\
6 & -1.456852 & -3.809353 & -2.001515 \\
6 & -0.222644 & -3.589905 & -2.655584 \\
6 & 0.771827 & -2.885777 & -2.036415 \\
6 & -2.906391 & -3.489797 & -0.066357 \\
6 & -3.903492 & -4.190616 & -0.698699 \\
6 & -3.699488 & -4.730384 & -1.985957 \\
6 & -2.497690 & -4.542851 & -2.620374 \\
6 & -0.822825 & -1.988264 & 1.304116 \\
6 & -1.191775 & -0.666593 & 1.534154 \\
6 & -1.424386 & -0.240988 & 2.870415 \\
6 & -1.282040 & -1.093832 & 3.931644 \\
6 & -0.921471 & -2.444764 & 3.731425 \\
6 & -0.702057 & -2.904667 & 2.400555 \\
6 & -0.797247 & -3.347806 & 4.814771 \\
6 & -0.488009 & -4.666345 & 4.596310 \\
6 & -0.283031 & -5.131634 & 3.280415 \\
15 & -0.375535 & -4.273877 & 2.212949 \\
45 & -1.533465 & 0.483469 & 0.105901 \\
6 & -0.061736 & 0.984974 & -1.795425 \\
8 & 0.184491 & 2.878264 & -1.347047 \\
15 & 0.192382 & 4.018760 & -1.185076 \\
8 & 1.869304 & -0.050438 & -0.967903 \\
6 & 2.678501 & 0.690986 & 0.284659 \\
6 & 3.493104 & 1.760444 & -0.025348 \\
6 & 4.745132 & 1.537528 & -0.567249 \\
6 & 5.538542 & 2.677360 & -0.916652 \\
& 5.058350 & 3.987173 & -0.609154
\end{tabular}




\begin{tabular}{|c|c|c|c|}
\hline 6 & 3.790394 & 4.141446 & -0.000022 \\
\hline & 3.012385 & 3.048424 & 0.269899 \\
\hline & 5.847745 & 5.114782 & -0.942833 \\
\hline & 7.052803 & 4.969236 & -1.579844 \\
\hline & 7.512876 & 3.679518 & -1.919586 \\
\hline & 6.777688 & 2.565928 & -1.597890 \\
\hline & 5.178080 & 0.143047 & -0.818153 \\
\hline & 4.359946 & -0.683956 & -1.568438 \\
\hline & 4.726628 & -2.001228 & -1.905450 \\
\hline & 5.902175 & -2.520958 & -1.436362 \\
\hline & 6.742570 & -1.754653 & -0.594598 \\
\hline & 6.380084 & -0.411733 & -0.271169 \\
\hline & 7.215030 & 0.304609 & 0.624683 \\
\hline J & 8.354380 & -0.262133 & 1.139550 \\
\hline & 8.726316 & -1.577320 & 0.790203 \\
\hline & 7.930716 & -2.305735 & -0.055741 \\
\hline & 3.148177 & -0.222226 & -2.033246 \\
\hline & -3.208376 & -0.110716 & -0.373325 \\
\hline D & -4.175600 & -0.377864 & 0.603674 \\
\hline & -5.449339 & -0.805581 & 0.247557 \\
\hline & -5.727694 & -0.972044 & -1.120115 \\
\hline & -4.791776 & -0.704431 & -2.115751 \\
\hline & -3.524153 & -0.262328 & -1.713914 \\
\hline & -6.523011 & -1.121078 & 1.288668 \\
\hline & -5.102724 & -0.815468 & -3.606521 \\
\hline b & -1.919740 & 2.094626 & 0.908767 \\
\hline & -3.056374 & 2.789552 & 0.501971 \\
\hline & -3.341558 & 4.066004 & 0.988847 \\
\hline & -2.463465 & 4.623913 & 1.919692 \\
\hline 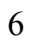 & -1.307525 & 3.960405 & 2.346730 \\
\hline & -1.031352 & 2.703649 & 1.800548 \\
\hline b & -4.600875 & 4.776132 & 0.496556 \\
\hline 6 & -0.383480 & 4.541610 & 3.417688 \\
\hline & -0.648216 & 1.348536 & -3.568849 \\
\hline & -1.002500 & 1.519016 & -4.657853 \\
\hline & 6.937293 & 1.315004 & 0.913701 \\
\hline & -1.745596 & 0.779589 & 3.058987 \\
\hline & 8.191569 & -3.328970 & -0.322859 \\
\hline 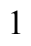 & -0.962843 & -2.972447 & 5.823806 \\
\hline & 5.466165 & 6.103540 & -0.691618 \\
\hline & -3.082720 & -3.075971 & 0.925058 \\
\hline 1 & -4.861121 & -4.333152 & -0.201646 \\
\hline & 3.432408 & 5.144461 & 0.228038 \\
\hline 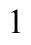 & -1.468695 & -0.742654 & 4.945967 \\
\hline & 6.194173 & -3.540333 & -1.683995 \\
\hline 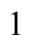 & 8.456555 & 3.566065 & -2.448439 \\
\hline & 4.058935 & -2.576483 & -2.542981 \\
\hline 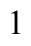 & -2.324378 & -4.945754 & -3.617653 \\
\hline 1 & -0.076887 & -3.991461 & -3.657075 \\
\hline 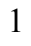 & -0.028457 & -6.175241 & 3.109268 \\
\hline 1 & 7.140545 & 1.580624 & -1.878779 \\
\hline & -0.187655 & -4.645341 & 1.208071 \\
\hline 1 & 1.719613 & -2.706844 & -2.541480 \\
\hline . & 7.647640 & 5.842651 & -1.836685 \\
\hline 1 & 8.973335 & 0.307568 & 1.829138 \\
\hline 1 & -4.496244 & -5.289088 & -2.472472 \\
\hline
\end{tabular}




\begin{tabular}{|c|c|c|c|}
\hline 1 & -0.402731 & -5.355327 & 5.433659 \\
\hline & 9.634193 & -2.012072 & 1.201766 \\
\hline & 2.023833 & 3.142554 & 0.711998 \\
\hline & -3.916303 & -0.242502 & 1.651434 \\
\hline & -2.761559 & -0.030702 & -2.454972 \\
\hline & -6.716934 & -1.324348 & -1.406243 \\
\hline & -3.732273 & 2.327361 & -0.215558 \\
\hline & -0.122848 & 2.172483 & 2.093913 \\
\hline & -2.685279 & 5.605854 & 2.325473 \\
\hline & 3.244740 & -1.310720 & 1.125721 \\
\hline & 1.708829 & -1.603191 & 1.913909 \\
\hline 1 & -0.421955 & -0.496486 & -2.293525 \\
\hline & 2.690762 & -3.135752 & 3.388662 \\
\hline & 2.840576 & -3.908617 & -0.827067 \\
\hline & 3.414817 & -5.452550 & 3.845417 \\
\hline & 3.511229 & -6.240999 & -0.376959 \\
\hline & 3.808278 & -7.029800 & 1.959698 \\
\hline & -6.415269 & -1.550897 & -3.865495 \\
\hline & -5.206230 & 0.601502 & -4.183729 \\
\hline & -3.989185 & -1.575162 & -4.334711 \\
\hline 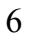 & -6.073891 & -0.762035 & 2.703808 \\
\hline & -7.799904 & -0.330705 & 0.983876 \\
\hline & -6.834840 & -2.621258 & 1.254941 \\
\hline & -4.797178 & 6.139748 & 1.153541 \\
\hline D & -4.500681 & 4.984118 & -1.019144 \\
\hline & -5.828210 & 3.911682 & 0.810795 \\
\hline f & -0.789536 & 5.953027 & 3.836128 \\
\hline ) & -0.440265 & 3.640012 & \\
\hline & 1.057419 & 4.596965 & 2.903200 \\
\hline 1 & -6.575805 & -1.652722 & -4.946163 \\
\hline & -6.400045 & -2.561090 & -3.432873 \\
\hline 1 & -7.281688 & -1.013571 & -3.459263 \\
\hline & -5.446235 & 0.563595 & -5.255032 \\
\hline ] & -5.993051 & 1.177341 & -3.678460 \\
\hline t & -4.262411 & 1.151302 & -4.072490 \\
\hline I & -4.219729 & -1.639400 & -5.406449 \\
\hline 1 & -3.010031 & -1.088401 & -4.241448 \\
\hline & -3.892697 & -2.595599 & -3.942751 \\
\hline ] & -6.878383 & -0.988945 & 3.414665 \\
\hline & -5.190519 & -1.335796 & 3.015983 \\
\hline 1 & -5.839527 & 0.306934 & 2.796920 \\
\hline & -7.625376 & -2.864431 & 1.977906 \\
\hline . & -7.176149 & -2.949013 & 0.264845 \\
\hline 1 & -5.945922 & -3.210075 & 1.520760 \\
\hline ] & -8.567388 & -0.545749 & 1.739324 \\
\hline 1 & -7.608144 & 0.750193 & 0.997469 \\
\hline & -8.224862 & -0.583603 & 0.005431 \\
\hline 1 & -5.392405 & 5.505660 & -1.392449 \\
\hline 1 & -3.621021 & 5.588909 & -1.275122 \\
\hline 1 & -4.420952 & 4.033649 & -1.560770 \\
\hline 1 & -6.743103 & 4.407765 & 0.459769 \\
\hline 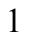 & -5.776710 & 2.929541 & 0.323542 \\
\hline 1 & -5.926730 & 3.744984 & 1.891748 \\
\hline & -5.713929 & 6.604501 & 0.769393 \\
\hline & -4.901353 & 6.060277 & 2.243484 \\
\hline & -3.967133 & 6.823871 & 0.935228 \\
\hline
\end{tabular}




$\begin{array}{rrrr}1 & 1.708602 & 5.079977 & 3.643834 \\ 1 & 1.466736 & 3.596518 & 2.720835 \\ 1 & 1.120097 & 5.172140 & 1.969029 \\ 1 & -0.091749 & 6.324210 & 4.597102 \\ 1 & -0.761889 & 6.654610 & 2.992395 \\ 1 & -1.794711 & 5.981644 & 4.275434 \\ 1 & 0.206188 & 4.038103 & 5.451098 \\ 1 & -1.463100 & 3.578813 & 5.051874 \\ 1 & -0.103186 & 2.619191 & 4.435444\end{array}$

$1_{\mathrm{C} 9-} \psi_{-1 \mathrm{~s} 2 \mathrm{3} 344 \mathrm{~s}}$

Number of imaginary frequencies : 0

The smallest frequencies are : $14.2463 \quad 15.3430 \quad 20.3555 \mathrm{~cm}(-1)$

Electronic energy : $\quad H F=-4125.4626314$

Zero-point correction $=\quad 1.300891($ Hartree/Particle $)$

Thermal correction to Energy= $\quad 1.378708$

Thermal correction to Enthalpy= $\quad 1.379652$

Thermal correction to Gibbs Free Energy $=\quad 1.189852$

Sum of electronic and zero-point Energies $=\quad-4124.161741$

Sum of electronic and thermal Energies $=\quad-4124.083923$

Sum of electronic and thermal Enthalpies $=\quad-4124.082979$

Sum of electronic and thermal Free Energies $=\quad-4124.272779$

Cartesian Coordinates

$\begin{array}{cccc}6 & 3.546736 & -6.026972 & 1.661192 \\ 6 & 3.328354 & -5.163481 & 2.731302 \\ 6 & 2.922091 & -3.856032 & 2.497373 \\ 6 & 2.745050 & -3.379020 & 1.196024 \\ 6 & 2.986062 & -4.245271 & 0.130281 \\ 6 & 3.374598 & -5.562120 & 0.361566 \\ 6 & 2.357186 & -1.932950 & 1.034006 \\ 7 & 1.697864 & -1.568888 & -0.230952 \\ 6 & 0.615666 & -2.345768 & -0.756960 \\ 6 & -0.580521 & -2.533507 & -0.083341 \\ 6 & -1.634914 & -3.269622 & -0.715141 \\ 6 & -1.457162 & -3.768375 & -2.038517 \\ 6 & -0.227191 & -3.540438 & -2.697803 \\ 6 & 0.775954 & -2.854859 & -2.071387 \\ 6 & -2.882644 & -3.495980 & -0.078443 \\ 6 & -3.892522 & -4.169526 & -0.719244 \\ 6 & -3.708883 & -4.668565 & -2.025997 \\ 6 & -2.511583 & -4.473921 & -2.667104 \\ 6 & -0.795573 & -2.007292 & 1.294009 \\ 6 & -1.168156 & -0.688328 & 1.535711 \\ 6 & -1.397844 & -0.272916 & 2.875356 \\ 6 & -1.253732 & -1.134897 & 3.929207 \\ 6 & -0.892369 & -2.483892 & 3.717122 \\ 6 & -0.672059 & -2.932190 & 2.382424 \\ 6 & -0.766961 & -3.396194 & 4.792552 \\ 6 & -0.455927 & -4.712361 & 4.562556 \\ 6 & -0.250312 & -5.166108 & 3.242662 \\ 6 & -0.344030 & -4.299308 & 2.182686 \\ 15 & -1.523797 & 0.466967 & 0.116035 \\ 45 & -0.055235 & 1.004472 & -1.774952\end{array}$




\begin{tabular}{|c|c|c|c|}
\hline 6 & 0.184031 & 2.893671 & -1.306908 \\
\hline 8 & 0.186059 & 4.032974 & -1.136481 \\
\hline 15 & 1.878499 & -0.035516 & -0.961476 \\
\hline 8 & 2.682224 & 0.687135 & 0.305781 \\
\hline 6 & 3.488429 & 1.768456 & 0.017318 \\
\hline 6 & 4.741509 & 1.566490 & -0.530659 \\
\hline 6 & 5.524562 & 2.719959 & -0.858272 \\
\hline 6 & 5.033937 & 4.019315 & -0.523589 \\
\hline 6 & 3.765979 & 4.150823 & 0.090972 \\
\hline 6 & 2.997674 & 3.046134 & 0.339902 \\
\hline 6 & 5.813087 & 5.160103 & -0.836078 \\
\hline 6 & 7.017877 & 5.037587 & -1.478383 \\
\hline 6 & 7.488095 & 3.758931 & -1.844836 \\
\hline 6 & 6.763210 & 2.632838 & -1.543983 \\
\hline 6 & 5.185864 & 0.180564 & -0.809241 \\
\hline 6 & 4.373279 & -0.639241 & -1.573339 \\
\hline 6 & 4.747885 & -1.948137 & -1.933374 \\
\hline 6 & 5.928056 & -2.467344 & -1.475626 \\
\hline 6 & 6.764847 & -1.709244 & -0.622902 \\
\hline 6 & 6.393167 & -0.375033 & -0.274608 \\
\hline 6 & 7.224914 & 0.331060 & 0.632289 \\
\hline 6 & 8.369823 & -0.236301 & 1.134056 \\
\hline 6 & 8.750893 & -1.541983 & 0.759878 \\
\hline 6 & 7.958499 & -2.260965 & -0.097045 \\
\hline 8 & 3.157964 & -0.178110 & -2.029142 \\
\hline 6 & -3.185835 & -0.153235 & -0.372615 \\
\hline 6 & -4.134911 & -0.471359 & 0.598418 \\
\hline 6 & -5.407069 & -0.915703 & 0.243600 \\
\hline 6 & -5.704776 & -1.028774 & -1.120265 \\
\hline 6 & -4.781601 & -0.701855 & -2.117081 \\
\hline 6 & -3.517528 & -0.259910 & -1.717072 \\
\hline 6 & -6.412437 & -1.238893 & 1.348516 \\
\hline 6 & -5.111956 & -0.753497 & -3.607118 \\
\hline 6 & -1.955306 & 2.066541 & 0.919565 \\
\hline 6 & -3.105533 & 2.730026 & 0.498114 \\
\hline 6 & -3.422329 & 4.006140 & 0.964392 \\
\hline 6 & -2.565862 & 4.594468 & 1.896815 \\
\hline 6 & -1.400167 & 3.961062 & 2.342864 \\
\hline 6 & -1.089839 & 2.704951 & 1.813028 \\
\hline 6 & -4.687958 & 4.683418 & 0.442729 \\
\hline 6 & -0.498098 & 4.575061 & 3.414515 \\
\hline 6 & -0.645160 & 1.386577 & -3.543160 \\
\hline 8 & -1.002259 & 1.567310 & -4.629612 \\
\hline 1 & 6.940322 & 1.333901 & 0.940272 \\
\hline 1 & -1.720415 & 0.745890 & 3.071861 \\
\hline 1 & 8.226247 & -3.277236 & -0.383282 \\
\hline 1 & -0.933033 & -3.029955 & 5.804848 \\
\hline 1 & 5.423722 & 6.140307 & -0.564176 \\
\hline 1 & -3.041337 & -3.115363 & 0.928573 \\
\hline 1 & -4.845247 & -4.321436 & -0.214222 \\
\hline 1 & 3.400091 & 5.145999 & 0.339935 \\
\hline 1 & -1.439073 & -0.792771 & 4.946835 \\
\hline 1 & 6.226808 & -3.480288 & -1.740971 \\
\hline 1 & 8.431474 & 3.664227 & -2.377906 \\
\hline 1 & 4.082086 & -2.517282 & -2.578372 \\
\hline 1 & -2.352681 & -4.849237 & -3.677493 \\
\hline
\end{tabular}




\begin{tabular}{|c|c|c|c|}
\hline 1 & -0.091983 & -3.918969 & -3.709696 \\
\hline ] & 0.005401 & -6.207923 & 3.062563 \\
\hline & 7.133809 & 1.656479 & -1.845275 \\
\hline & -0.156356 & -4.661524 & 1.174361 \\
\hline 1 & 1.720320 & -2.668261 & -2.580341 \\
\hline & 7.604709 & 5.920977 & -1.718994 \\
\hline 1 & 8.986171 & 0.325473 & 1.832424 \\
\hline 1 & -4.516285 & -5.203874 & -2.521249 \\
\hline 1 & -0.369731 & -5.408434 & 5.393920 \\
\hline 1 & 9.663112 & -1.977210 & 1.161200 \\
\hline 1 & 2.008637 & 3.122748 & 0.784648 \\
\hline 1 & -3.873761 & -0.368657 & 1.651756 \\
\hline 1 & -2.768353 & 0.011941 & -2.458433 \\
\hline 1 & -6.689076 & -1.382535 & -1.413434 \\
\hline 1 & -3.765291 & 2.246075 & -0.219670 \\
\hline 1 & -0.169781 & 2.200439 & 2.117026 \\
\hline 1 & -2.811801 & 5.576863 & 2.287451 \\
\hline 1 & 3.266658 & -1.326712 & 1.105520 \\
\hline 1 & 1.733765 & -1.635325 & 1.892885 \\
\hline 1 & -0.410604 & -0.471475 & -2.291931 \\
\hline 1 & 2.720311 & -3.192634 & 3.339576 \\
\hline 1 & 2.864052 & -3.889639 & -0.889440 \\
\hline 1 & 3.453584 & -5.514864 & 3.753624 \\
\hline 1 & 3.544970 & -6.227707 & -0.482348 \\
\hline 1 & 3.849806 & -7.056472 & 1.839442 \\
\hline 6 & -6.437292 & -1.460526 & -3.879364 \\
\hline 6 & -5.204544 & 0.685906 & -4.128777 \\
\hline 6 & & -1.500021 & -4.375436 \\
\hline 6 & -7.708302 & -1.834179 & 0.803732 \\
\hline 6 & -5.804473 & -2.248224 & 2.330139 \\
\hline 6 & -6.755318 & 0.054997 & 2.097079 \\
\hline 6 & -4.916879 & 6.055048 & 1.071884 \\
\hline 6 & -4.570541 & 4.864768 & -1.075488 \\
\hline 6 & -5.904101 & 3.802826 & 0.755730 \\
\hline 6 & -0.941782 & 5.979921 & 3.816461 \\
\hline 6 & -0.541966 & 3.684140 & 4.662157 \\
\hline 6 & 0.945405 & 4.661417 & 2.910724 \\
\hline 1 & -6.612648 & -1.514055 & -4.961207 \\
\hline 1 & -6.430860 & -2.488342 & -3.490805 \\
\hline 1 & -7.290897 & -0.929931 & -3.438499 \\
\hline 1 & -5.460893 & 0.692563 & -5.196930 \\
\hline 1 & -5.976492 & 1.251517 & -3.589746 \\
\hline 1 & -4.252833 & 1.220366 & -4.010274 \\
\hline 1 & -4.252494 & -1.511838 & -5.447829 \\
\hline 1 & -3.027940 & -1.035623 & -4.264108 \\
\hline 1 & -3.936403 & -2.539014 & -4.030344 \\
\hline 1 & -8.387080 & -2.063601 & 1.634895 \\
\hline 1 & -8.233427 & -1.138670 & 0.136866 \\
\hline 1 & -7.530777 & -2.768607 & 0.254704 \\
\hline 1 & -7.452584 & -0.150200 & 2.920876 \\
\hline 1 & -5.862604 & 0.531793 & 2.521665 \\
\hline 1 & -7.231271 & 0.781113 & 1.424326 \\
\hline 1 & -6.521150 & -2.477076 & 3.130228 \\
\hline 1 & -5.557349 & -3.191650 & 1.824306 \\
\hline 1 & -4.888908 & -1.875524 & 2.807549 \\
\hline 1 & -5.464499 & 5.366788 & -1.469646 \\
\hline
\end{tabular}




$\begin{array}{lrrr}1 & -3.696013 & 5.476982 & -1.331529 \\ 1 & -4.470886 & 3.905475 & -1.598670 \\ 1 & -6.822502 & 4.277590 & 0.384814 \\ 1 & -5.830100 & 2.813328 & 0.285394 \\ 1 & -6.013725 & 3.652405 & 1.837976 \\ 1 & -5.835938 & 6.497082 & 0.666994 \\ 1 & -5.034823 & 5.992989 & 2.161568 \\ 1 & -4.095443 & 6.749214 & 0.852723 \\ 1 & 1.578371 & 5.163942 & 3.654202 \\ 1 & 1.380114 & 3.669924 & 2.738149 \\ 1 & 1.003180 & 5.233144 & 1.974245 \\ 1 & -0.259551 & 6.374805 & 4.579607 \\ 1 & -0.924085 & 6.674211 & 2.966461 \\ 1 & -1.951114 & 5.987791 & 4.247048 \\ 1 & 0.088309 & 4.105782 & 5.456966 \\ 1 & -1.565893 & 3.601229 & 5.049921 \\ 1 & -0.177883 & 2.670000 & 4.452894\end{array}$

$1_{\text {C10- }} \psi_{-1 \text { a2a3a4a }}$

Number of imaginary frequencies : 0

The smallest frequencies are : $12.3506 \quad 15.0782 \quad 17.2060 \mathrm{~cm}(-1)$

Electronic energy : $\quad H F=-4125.4642624$

Zero-point correction $=\quad 1.299124$ (Hartree/Particle)

Thermal correction to Energy $=\quad 1.377603$

Thermal correction to Enthalpy= $\quad 1.378548$

Thermal correction to Gibbs Free Energy= $\quad 1.185887$

Sum of electronic and zero-point Energies $=\quad-4124.165138$

Sum of electronic and thermal Energies $=\quad-4124.086659$

Sum of electronic and thermal Enthalpies $=\quad-4124.085715$

Sum of electronic and thermal Free Energies= $\quad-4124.278376$

Cartesian Coordinates

$\begin{array}{llll}6 & 1.672558 & -2.162754 & 5.177826 \\ 6 & 1.971620 & -3.403044 & 4.626276 \\ 6 & 2.212528 & -3.516466 & 3.259583 \\ 6 & 2.159122 & -2.397867 & 2.430001 \\ 6 & 1.856696 & -1.154622 & 2.991908 \\ 6 & 1.615350 & -1.039324 & 4.355370 \\ 6 & 2.490585 & -2.534769 & 0.966005 \\ 7 & 1.528418 & -1.883907 & 0.055728 \\ 6 & 0.463728 & -2.685687 & -0.486004 \\ 6 & -0.828710 & -2.623507 & 0.007283 \\ 6 & -1.873170 & -3.318802 & -0.684471 \\ 6 & -1.562590 & -4.101811 & -1.835140 \\ 6 & -0.215918 & -4.203863 & -2.254198 \\ 6 & 0.762527 & -3.502323 & -1.604693 \\ 6 & -3.229202 & -3.239040 & -0.272441 \\ 6 & -4.219309 & -3.880758 & -0.973515 \\ 6 & -3.906100 & -4.656329 & -2.111165 \\ 6 & -2.604053 & -4.764590 & -2.529283 \\ 6 & -1.157529 & -1.977374 & 1.309115 \\ 6 & -1.469610 & -0.633103 & 1.442956 \\ 6 & -1.852899 & -0.134622 & 2.719183\end{array}$




\begin{tabular}{|c|c|c|c|}
\hline 6 & -1.949071 & -0.956189 & 3.808765 \\
\hline 6 & -1.662576 & -2.335979 & 3.700801 \\
\hline 6 & -1.249189 & -2.855276 & 2.440970 \\
\hline 6 & -1.782948 & -3.203904 & 4.812159 \\
\hline 6 & -1.513132 & -4.543263 & 4.690865 \\
\hline 6 & -1.094985 & -5.063701 & 3.448801 \\
\hline 6 & -0.961603 & -4.243006 & 2.356218 \\
\hline 15 & -1.537492 & 0.466554 & -0.055384 \\
\hline & 0.125591 & 0.595170 & -1.879532 \\
\hline 6 & -0.323975 & -0.731117 & -3.245099 \\
\hline 8 & -0.503427 & -1.419921 & -4.152198 \\
\hline 15 & 1.889886 & -0.531106 & -0.897968 \\
\hline 8 & 2.816085 & 0.249201 & 0.233039 \\
\hline 6 & 3.640835 & 1.259223 & -0.224753 \\
\hline 6 & 4.841176 & 0.934457 & -0.826414 \\
\hline 6 & 5.644727 & 2.003342 & -1.341525 \\
\hline 6 & 5.231777 & 3.353932 & -1.129750 \\
\hline 6 & 4.018296 & 3.616495 & -0.450132 \\
\hline 6 & 3.222407 & 2.588582 & -0.022559 \\
\hline 6 & 6.030398 & 4.412860 & -1.626324 \\
\hline 6 & 7.179423 & 4.160448 & -2.330120 \\
\hline 6 & 7.570188 & 2.827470 & -2.575226 \\
\hline 6 & 6.825206 & 1.779241 & -2.095005 \\
\hline 6 & 5.221243 & -0.491416 & -0.963983 \\
\hline 6 & 4.341141 & -1.375792 & -1.564776 \\
\hline 6 & 4.683280 & -2.720194 & -1.818836 \\
\hline 6 & 5.890519 & -3.207677 & -1.400089 \\
\hline 6 & 6.790199 & -2.381716 & -0.684666 \\
\hline 6 & 6.454327 & -1.013646 & -0.452096 \\
\hline 6 & 7.350217 & -0.233434 & 0.323232 \\
\hline 6 & 8.519129 & -0.766003 & 0.807627 \\
\hline 6 & 8.863564 & -2.108168 & 0.544018 \\
\hline 6 & 8.010535 & -2.896825 & -0.183966 \\
\hline 8 & 3.097863 & -0.957947 & -1.980026 \\
\hline 6 & -3.290214 & 0.205716 & -0.560919 \\
\hline 6 & -4.326614 & 0.266981 & 0.374773 \\
\hline 6 & -5.647166 & 0.034793 & -0.002236 \\
\hline 6 & -5.896817 & -0.270029 & -1.346237 \\
\hline 6 & -4.887467 & -0.345502 & -2.306450 \\
\hline 6 & -3.576676 & -0.093218 & -1.889116 \\
\hline 6 & -6.794874 & 0.048423 & 1.005306 \\
\hline 6 & -5.231136 & -0.677194 & -3.757529 \\
\hline 6 & -1.558677 & 2.137120 & 0.706683 \\
\hline 6 & -2.521647 & 3.083450 & 0.368408 \\
\hline 6 & -2.469844 & 4.377779 & 0.895476 \\
\hline 6 & -1.432322 & 4.684054 & 1.778168 \\
\hline 6 & -0.453751 & 3.753487 & 2.147356 \\
\hline 6 & -0.518785 & 2.485547 & 1.573230 \\
\hline 6 & -3.518218 & 5.436459 & 0.556379 \\
\hline 6 & 0.626326 & 4.135833 & 3.159289 \\
\hline 6 & -0.172575 & 2.110437 & -2.996075 \\
\hline 8 & -0.353463 & 3.074714 & -3.608721 \\
\hline 1 & 7.095409 & 0.799250 & 0.546673 \\
\hline 1 & -2.088470 & 0.920971 & 2.831585 \\
\hline 1 & 8.250102 & -3.940567 & -0.383548 \\
\hline 1 & -2.097930 & -2.782077 & 5.765917 \\
\hline
\end{tabular}




\begin{tabular}{|c|c|c|c|}
\hline & 5.700642 & 5.435192 & -1.446289 \\
\hline & -3.485560 & -2.639846 & 0.599499 \\
\hline & -5.255736 & -3.782793 & -0.654681 \\
\hline & 3.714549 & 4.651299 & -0.298179 \\
\hline & -2.259417 & -0.557081 & 4.773884 \\
\hline & 6.167717 & -4.242440 & -1.594649 \\
\hline & 8.467209 & 2.626999 & -3.156819 \\
\hline & 3.972015 & -3.341401 & -2.358271 \\
\hline & -2.346716 & -5.358752 & -3.404928 \\
\hline & 0.022803 & -4.818669 & -3.120527 \\
\hline & -0.872220 & -6.124462 & 3.356172 \\
\hline & 7.134119 & 0.758514 & -2.304406 \\
\hline & -0.625346 & -4.661365 & 1.409498 \\
\hline & 1.795551 & -3.543489 & -1.945489 \\
\hline & 7.781436 & 4.981574 & -2.712199 \\
\hline & 9.183442 & -0.146952 & 1.406408 \\
\hline & -4.699731 & -5.166862 & -2.652927 \\
\hline & -1.615276 & -5.204048 & 5.548815 \\
\hline & 9.795801 & -2.515126 & 0.928570 \\
\hline & 2.265953 & 2.765111 & 0.467143 \\
\hline & -4.089873 & 0.498117 & 1.410295 \\
\hline & -2.750332 & -0.141427 & -2.594109 \\
\hline & -6.924411 & -0.463919 & -1.653657 \\
\hline & -3.320496 & 2.801617 & -0.312882 \\
\hline 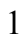 & 0.231902 & 1.734208 & 1.818017 \\
\hline & -1.387354 & 5.686532 & 2.203631 \\
\hline & 0.706173 & 1.775458 & -0.955356 \\
\hline & 2.561858 & -3.599219 & 0.696026 \\
\hline & 3.483704 & -2.103580 & 0.787549 \\
\hline l & 2.435880 & -4.491937 & 2.826389 \\
\hline & 1.807487 & -0.276259 & 2.349390 \\
\hline l & 2.003801 & -4.289449 & 5.256914 \\
\hline ] & 1.379710 & -0.064866 & 4.780591 \\
\hline 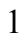 & 1.476879 & -2.070973 & 6.244325 \\
\hline 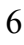 & -3.979702 & -0.925231 & -4.596976 \\
\hline & -6.092901 & -1.942264 & -3.821401 \\
\hline 6 & -6.003880 & 0.498033 & -4.365817 \\
\hline & -6.348454 & 0.543601 & 2.379942 \\
\hline 6 & -7.921172 & 0.964204 & 0.516147 \\
\hline 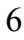 & -7.325504 & -1.381803 & 1.158913 \\
\hline l & -4.269096 & -1.199934 & -5.619163 \\
\hline 1 & -3.343133 & -0.033475 & -4.669234 \\
\hline 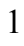 & -3.376182 & -1.747307 & -4.187630 \\
\hline 1 & -6.331187 & -2.182920 & -4.866007 \\
\hline 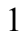 & -5.557985 & -2.798846 & -3.389409 \\
\hline 1 & -7.044306 & -1.831957 & -3.287261 \\
\hline 1 & -6.257953 & 0.289034 & -5.413914 \\
\hline 1 & -6.939519 & 0.693714 & -3.826808 \\
\hline 1 & -5.404368 & 1.417325 & -4.339745 \\
\hline 1 & -7.208240 & 0.563583 & 3.061386 \\
\hline 1 & -5.590434 & -0.110855 & 2.830806 \\
\hline 1 & -5.938084 & 1.561668 & 2.332338 \\
\hline 1 & -8.151863 & -1.411836 & 1.882077 \\
\hline 1 & -7.697815 & -1.784188 & 0.208210 \\
\hline 1 & -6.534150 & -2.052612 & 1.520764 \\
\hline 1 & -8.740064 & 0.980867 & 1.247519 \\
\hline
\end{tabular}




$\begin{array}{rrrr}1 & -7.564004 & 1.994077 & 0.385784 \\ 1 & -8.344601 & 0.633979 & -0.439579 \\ 6 & -4.593900 & 4.903398 & -0.387886 \\ 6 & -4.208238 & 5.902828 & 1.843182 \\ 6 & -2.836270 & 6.630446 & -0.119783 \\ 6 & 1.476504 & 2.934823 & 3.573405 \\ 6 & 1.544019 & 5.201564 & 2.552459 \\ 6 & -0.030353 & 4.695857 & 4.426587 \\ 1 & -5.331250 & 5.691191 & -0.587113 \\ 1 & -4.176545 & 4.592118 & -1.353926 \\ 1 & -5.132854 & 4.048054 & 0.043213 \\ 1 & -4.969502 & 6.660496 & 1.613544 \\ 1 & -4.706897 & 5.064587 & 2.347450 \\ 1 & -3.502749 & 6.348729 & 2.554542 \\ 1 & -3.577768 & 7.399534 & -0.375258 \\ 1 & -2.084894 & 7.096734 & 0.529029 \\ 1 & -2.333980 & 6.322502 & -1.045857 \\ 1 & 2.196518 & 3.239134 & 4.343277 \\ 1 & 0.854444 & 2.132043 & 3.994702 \\ 1 & 2.055499 & 2.518353 & 2.737949 \\ 1 & 0.737473 & 4.948605 & 5.169798 \\ 1 & -0.608388 & 5.606664 & 4.231559 \\ 1 & -0.707788 & 3.959100 & 4.877677 \\ 1 & 2.315501 & 5.500172 & 3.275092 \\ 1 & 2.051728 & 4.820951 & 1.656048 \\ 1 & 0.987718 & 6.101929 & 2.264100\end{array}$

$1_{\text {C10- }} \psi_{-1 \text {-1a2a3a4s }}$

Number of imaginary frequencies : 0

The smallest frequencies are : $10.3408 \quad 15.4767 \quad 16.9975 \mathrm{~cm}(-1)$

Electronic energy : $\quad H F=-4125.4647955$

Zero-point correction $=\quad 1.299735($ Hartree/Particle $)$

Thermal correction to Energy= $\quad 1.378019$

Thermal correction to Enthalpy= $\quad 1.378963$

Thermal correction to Gibbs Free Energy= $\quad 1.186673$

Sum of electronic and zero-point Energies $=\quad-4124.165060$

Sum of electronic and thermal Energies $=\quad-4124.086777$

Sum of electronic and thermal Enthalpies $=\quad-4124.085833$

Sum of electronic and thermal Free Energies $=\quad-4124.278123$

Cartesian Coordinates

$\begin{array}{llll}6 & 1.689896 & -2.183913 & 5.166855 \\ 6 & 1.998294 & -3.419302 & 4.609306 \\ 6 & 2.237590 & -3.524336 & 3.241616 \\ 6 & 2.173391 & -2.402514 & 2.417034 \\ 6 & 1.861464 & -1.164405 & 2.985076 \\ 6 & 1.621856 & -1.057319 & 4.349536 \\ 6 & 2.505583 & -2.530512 & 0.952309 \\ 7 & 1.539665 & -1.882878 & 0.043466 \\ 6 & 0.481082 & -2.690924 & -0.500755 \\ 6 & -0.810336 & -2.645196 & -0.003168 \\ 6 & -1.848906 & -3.351929 & -0.692148 \\ 6 & -1.532878 & -4.128083 & -1.846151 \\ 6 & -0.187242 & -4.209194 & -2.272953\end{array}$




\begin{tabular}{|c|c|c|c|}
\hline 6 & 0.785202 & -3.498324 & -1.624657 \\
\hline 6 & -3.203640 & -3.293660 & -0.271823 \\
\hline 6 & -4.186319 & -3.953932 & -0.966216 \\
\hline 6 & -3.867946 & -4.722572 & -2.107044 \\
\hline & -2.567398 & -4.807219 & -2.534757 \\
\hline & -1.143869 & -2.003583 & 1.299484 \\
\hline & -1.467543 & -0.662068 & 1.433160 \\
\hline 6 & -1.856013 & -0.167166 & 2.709166 \\
\hline & -1.945459 & -0.989784 & 3.798609 \\
\hline 6 & -1.647988 & -2.367182 & 3.690292 \\
\hline & -1.229348 & -2.882615 & 2.430737 \\
\hline & -1.762450 & -3.236280 & 4.801175 \\
\hline & -1.482476 & -4.573532 & 4.679399 \\
\hline 6 & -1.059321 & -5.090224 & 3.437469 \\
\hline 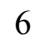 & -0.930799 & -4.267936 & 2.345533 \\
\hline 15 & -1.540411 & 0.436745 & -0.065533 \\
\hline 45 & 0.122879 & 0.590986 & -1.886702 \\
\hline 6 & -0.304243 & -0.727022 & -3.267002 \\
\hline 8 & -0.467224 & -1.407628 & -4.183394 \\
\hline 15 & 1.893418 & -0.524352 & -0.904597 \\
\hline 8 & 2.809271 & 0.258292 & 0.232983 \\
\hline 6 & 3.625875 & 1.278486 & -0.216452 \\
\hline 6 & 4.829949 & 0.968523 & -0.818573 \\
\hline 6 & 5.624226 & 2.048169 & -1.325619 \\
\hline 6 & 5.198727 & 3.393427 & -1.105112 \\
\hline 6 & 3.982540 & 3.640456 & -0.424494 \\
\hline 6 & 3.195589 & 2.602556 & -0.004674 \\
\hline 6 & 5.988124 & 4.462987 & -1.593550 \\
\hline 6 & 7.140213 & 4.225929 & -2.297672 \\
\hline 6 & 7.543517 & 2.898311 & -2.551290 \\
\hline 6 & 6.807640 & 1.840023 & -2.079112 \\
\hline 6 & 5.224406 & -0.452866 & -0.962537 \\
\hline 6 & 4.354154 & -1.343698 & -1.568021 \\
\hline 6 & 4.709167 & -2.684079 & -1.825592 \\
\hline 6 & 5.920233 & -3.161416 & -1.406314 \\
\hline 6 & 6.810905 & -2.329133 & -0.686920 \\
\hline 6 & 6.461662 & -0.965063 & -0.450414 \\
\hline 6 & 7.348940 & -0.179035 & 0.328925 \\
\hline 6 & 8.522039 & -0.702125 & 0.813533 \\
\hline 6 & 8.879532 & -2.040127 & 0.546242 \\
\hline 6 & 8.035176 & -2.834366 & -0.185774 \\
\hline 8 & 3.107828 & -0.936359 & -1.984601 \\
\hline 6 & -3.292178 & 0.169533 & -0.570465 \\
\hline 6 & -4.324060 & 0.256770 & 0.359488 \\
\hline 6 & -5.653610 & 0.040280 & -0.010169 \\
\hline 6 & -5.913095 & -0.276856 & -1.344257 \\
\hline 6 & -4.899380 & -0.384768 & -2.305036 \\
\hline 6 & -3.586737 & -0.147671 & -1.896692 \\
\hline 6 & -6.740440 & 0.144145 & 1.057386 \\
\hline 6 & -5.253647 & -0.735468 & -3.749057 \\
\hline 6 & -1.577276 & 2.108404 & 0.694276 \\
\hline 6 & -2.537908 & 3.051503 & 0.339681 \\
\hline 6 & -2.496093 & 4.347821 & 0.862484 \\
\hline 6 & -1.476923 & 4.657016 & 1.765500 \\
\hline 6 & -0.499299 & 3.731048 & 2.148254 \\
\hline 6 & -0.549264 & 2.463209 & 1.572113 \\
\hline
\end{tabular}




\begin{tabular}{|c|c|c|c|}
\hline 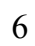 & -3.544434 & 5.401276 & 0.507331 \\
\hline & 0.565392 & 4.117474 & 3.174764 \\
\hline & -0.207587 & 2.114540 & -2.982721 \\
\hline & -0.412639 & 3.084353 & -3.578677 \\
\hline & 7.084157 & 0.850475 & 0.555292 \\
\hline & -2.098504 & 0.886743 & 2.822147 \\
\hline & 8.284871 & -3.875161 & -0.388304 \\
\hline & -2.081694 & -2.817427 & 5.754814 \\
\hline & 5.648870 & 5.481032 & -1.406994 \\
\hline & -3.465109 & -2.698504 & 0.602008 \\
\hline & -5.221491 & -3.878781 & -0.638809 \\
\hline & 3.669722 & 4.671470 & -0.265416 \\
\hline & -2.259042 & -0.593527 & 4.763844 \\
\hline & 6.207445 & -4.192965 & -1.603371 \\
\hline & 8.443055 & 2.710029 & -3.133067 \\
\hline & 4.004039 & -3.310247 & -2.367439 \\
\hline 1 & -2.305787 & -5.395361 & -3.413197 \\
\hline 1 & 0.055455 & -4.817555 & -3.142720 \\
\hline 1 & -0.828521 & -6.149241 & 3.344539 \\
\hline & 7.126303 & 0.823618 & -2.294926 \\
\hline & -0.590632 & -4.683083 & 1.398781 \\
\hline & 1.817304 & -3.525470 & -1.969811 \\
\hline 1 & 7.735083 & 5.055143 & -2.673420 \\
\hline 1 & 9.179515 & -0.078750 & 1.415370 \\
\hline 1 & -4.656636 & -5.246106 & -2.643549 \\
\hline 1 & -1.580556 & -5.235540 & 5.536881 \\
\hline 1 & 9.814908 & -2.439552 & 0.931063 \\
\hline 1 & 2.237604 & 2.766987 & 0.486385 \\
\hline & -4.089109 & 0.496993 & 1.395744 \\
\hline 1 & -2.762164 & -0.219949 & -2.601933 \\
\hline 1 & -6.941291 & -0.452918 & -1.653726 \\
\hline 1 & -3.325814 & 2.766719 & -0.353231 \\
\hline 1 & 0.203454 & 1.716661 & 1.825168 \\
\hline 1 & -1.446144 & 5.658137 & 2.195422 \\
\hline 1 & 0.695094 & 1.770526 & -0.955912 \\
\hline 1 & 2.584480 & -3.593295 & 0.677868 \\
\hline 1 & 3.495818 & -2.091835 & 0.776182 \\
\hline 1 & 2.468438 & -4.495903 & 2.803576 \\
\hline 1 & 1.803025 & -0.283497 & 2.346840 \\
\hline 1 & 2.038581 & -4.308324 & 5.235762 \\
\hline 1 & 1.379143 & -0.086702 & 4.779631 \\
\hline 1 & 1.495506 & -2.098457 & 6.234114 \\
\hline U & -4.009346 & -1.000888 & -4.593530 \\
\hline 6 & -6.120706 & -1.998110 & -3.787731 \\
\hline 6 & -6.025687 & 0.433155 & -4.371003 \\
\hline 6 & -8.133465 & -0.139822 & 0.502280 \\
\hline 6 & -6.452373 & -0.873998 & 2.167489 \\
\hline 6 & -6.740197 & 1.560534 & 1.644850 \\
\hline 1 & -4.306927 & -1.281305 & -5.611803 \\
\hline 1 & -3.364858 & -0.115826 & -4.676056 \\
\hline 1 & -3.412166 & -1.825845 & -4.180897 \\
\hline 1 & -6.359883 & -2.258946 & -4.827308 \\
\hline 1 & -5.589494 & -2.848129 & -3.338243 \\
\hline 1 & -7.071498 & -1.872507 & -3.256061 \\
\hline 1 & -6.288561 & 0.207567 & -5.413480 \\
\hline & -6.956733 & 0.642619 & -3.829412 \\
\hline
\end{tabular}




$\begin{array}{rrrr}1 & -5.421495 & 1.349706 & -4.365069 \\ 1 & -8.876205 & -0.048402 & 1.304884 \\ 1 & -8.413940 & 0.570083 & -0.286444 \\ 1 & -8.213045 & -1.156210 & 0.095135 \\ 1 & -7.485257 & 1.642456 & 2.447762 \\ 1 & -5.764662 & 1.835685 & 2.066256 \\ 1 & -6.993410 & 2.301374 & 0.874858 \\ 1 & -7.222668 & -0.816766 & 2.948383 \\ 1 & -6.452401 & -1.897258 & 1.768443 \\ 1 & -5.479765 & -0.705882 & 2.647266 \\ 6 & -4.546719 & 4.894047 & -0.527494 \\ 6 & -4.319440 & 5.785201 & 1.772901 \\ 6 & -2.855454 & 6.643047 & -0.067937 \\ 6 & 1.416018 & 2.919853 & 3.597876 \\ 6 & 1.485948 & 5.188282 & 2.581294 \\ 6 & -0.110451 & 4.672821 & 4.433940 \\ 1 & -5.279401 & 5.681159 & -0.745872 \\ 1 & -4.058685 & 4.625334 & -1.473221 \\ 1 & -5.102777 & 4.017160 & -0.168755 \\ 1 & -5.077755 & 6.545331 & 1.541187 \\ 1 & -4.834248 & 4.912486 & 2.196819 \\ 1 & -3.662500 & 6.195993 & 2.549365 \\ 1 & -3.602311 & 7.402980 & -0.334479 \\ 1 & -2.160259 & 7.100836 & 0.646045 \\ 1 & -2.288614 & 6.392761 & -0.973853 \\ 1 & 2.123591 & 3.225792 & 4.378563 \\ 1 & 0.792521 & 2.112332 & 4.007938 \\ 1 & 2.008661 & 2.509158 & 2.769036 \\ 1 & 0.646293 & 4.928850 & 5.187325 \\ 1 & -0.690528 & 5.580832 & 4.232130 \\ 1 & -0.789862 & 3.932169 & 4.875617 \\ 1 & 2.246361 & 5.489738 & 3.314416 \\ 1 & 2.007359 & 4.810997 & 1.691358 \\ 1 & 0.929161 & 6.086243 & 2.286460\end{array}$

$1_{C 10-} \psi_{-122 a 3 s 4 a}$

Number of imaginary frequencies : 0

The smallest frequencies are : $1.3563 \quad 15.1229 \quad 16.0544 \mathrm{~cm}(-1)$

Electronic energy : $\quad H F=-4125.4648607$

Zero-point correction $=\quad 1.300075($ Hartree/Particle $)$

Thermal correction to Energy $=\quad 1.378115$

Thermal correction to Enthalpy= $\quad 1.379059$

Thermal correction to Gibbs Free Energy $=\quad 1.185766$

Sum of electronic and zero-point Energies $=\quad-4124.164786$

Sum of electronic and thermal Energies $=\quad-4124.086746$

Sum of electronic and thermal Enthalpies $=\quad-4124.085802$

Sum of electronic and thermal Free Energies $=\quad-4124.279095$

Cartesian Coordinates

$\begin{array}{rrrr}6 & -3.612756 & 0.168404 & -1.857791 \\ 6 & -3.299394 & 0.322545 & -0.516066 \\ 6 & -4.326905 & 0.320250 & 0.436432 \\ 6 & -5.653035 & 0.151022 & 0.054460 \\ 6 & -5.930270 & -0.013488 & -1.313550\end{array}$




\begin{tabular}{|c|c|c|c|}
\hline 6 & -4.935878 & -0.000678 & -2.286458 \\
\hline 1 & -1.529144 & 0.484169 & -0.034295 \\
\hline & 0.057034 & 0.569661 & -1.925904 \\
\hline 6 & -0.532870 & -0.810725 & -3.178935 \\
\hline 8 & -0.845203 & -1.535891 & -4.018972 \\
\hline 6 & -6.789889 & 0.084777 & 1.072725 \\
\hline 6 & -7.353555 & -1.340973 & 1.084659 \\
\hline 6 & -5.212936 & -0.241458 & -3.768026 \\
\hline 6 & -4.443206 & 0.765308 & -4.630621 \\
\hline 6 & -1.481981 & -0.672139 & 1.419358 \\
\hline 6 & -1.208043 & -2.018371 & 1.237555 \\
\hline 6 & -1.307628 & -2.928648 & 2.344883 \\
\hline 6 & -1.683161 & -2.436141 & 3.628135 \\
\hline 6 & -1.933932 & -1.053849 & 3.781910 \\
\hline 6 & -1.835126 & -0.203240 & 2.715593 \\
\hline 6 & -1.050840 & -4.319402 & 2.217675 \\
\hline 6 & -1.179717 & -5.166593 & 3.290448 \\
\hline 6 & -1.561104 & -4.672559 & 4.554704 \\
\hline 6 & -1.797467 & -3.331468 & 4.718192 \\
\hline 6 & -0.878081 & -2.639922 & -0.076072 \\
\hline 6 & 0.422392 & -2.712919 & -0.548208 \\
\hline 6 & 0.735173 & -3.542137 & -1.653799 \\
\hline 6 & -0.234725 & -4.256280 & -2.302607 \\
\hline 6 & -1.587294 & -4.147595 & -1.903855 \\
\hline 6 & -1.915798 & -3.333173 & -0.780156 \\
\hline 6 & -2.617487 & -4.831055 & -2.594443 \\
\hline 6 & -3.925176 & -4.715139 & -2.197912 \\
\hline 6 & -4.257019 & -3.902887 & -1.092093 \\
\hline 6 & -3.279500 & -3.234424 & -0.397656 \\
\hline 7 & 1.480742 & -1.909450 & 0.004598 \\
\hline 6 & 2.461831 & -2.570155 & 0.889282 \\
\hline 6 & 2.135932 & -2.489156 & 2.358420 \\
\hline 6 & 1.875389 & -1.261857 & 2.974749 \\
\hline 6 & 1.634094 & -1.199277 & 4.341442 \\
\hline 6 & 1.652966 & -2.359632 & 5.113086 \\
\hline 6 & 1.913644 & -3.583526 & 4.508664 \\
\hline 6 & 2.152208 & -3.644565 & 3.138026 \\
\hline 15 & 1.833572 & -0.546504 & -0.940718 \\
\hline 8 & 2.745238 & 0.239462 & 0.200587 \\
\hline 6 & 3.564947 & 1.257052 & -0.251377 \\
\hline 6 & 4.772122 & 0.944029 & -0.845970 \\
\hline 6 & 5.568837 & 2.020848 & -1.355687 \\
\hline 6 & 5.141321 & 3.367344 & -1.147244 \\
\hline 6 & 3.920428 & 3.618049 & -0.476645 \\
\hline 6 & 3.131840 & 2.582560 & -0.053278 \\
\hline 6 & 6.756290 & 1.808375 & -2.101638 \\
\hline 6 & 7.494152 & 2.863825 & -2.577064 \\
\hline 6 & 7.088869 & 4.192915 & -2.334698 \\
\hline 6 & 5.932697 & 4.434040 & -1.638706 \\
\hline 6 & 5.166054 & -0.477672 & -0.988099 \\
\hline 6 & 4.297713 & -1.365574 & -1.600287 \\
\hline 6 & 4.654012 & -2.704290 & -1.864804 \\
\hline 6 & 5.863570 & -3.183728 & -1.443700 \\
\hline 6 & 6.751352 & -2.355291 & -0.716225 \\
\hline 6 & 6.401493 & -0.992301 & -0.474348 \\
\hline 6 & 7.286550 & -0.209691 & 0.310835 \\
\hline
\end{tabular}




\begin{tabular}{|c|c|c|c|}
\hline 6 & 8.458075 & -0.735182 & 0.796724 \\
\hline & 8.815991 & -2.072157 & 0.524854 \\
\hline & 7.973863 & -2.863004 & -0.213403 \\
\hline & 3.052295 & -0.956694 & -2.016800 \\
\hline & -1.451492 & 2.123428 & 0.786814 \\
\hline & -0.382798 & 2.390592 & 1.647594 \\
\hline & -0.257933 & 3.630504 & 2.272430 \\
\hline & -1.200461 & 4.616077 & 1.955035 \\
\hline & -2.259858 & 4.392564 & 1.074046 \\
\hline & -2.377263 & 3.122647 & 0.500591 \\
\hline & 0.835980 & 3.923860 & 3.299044 \\
\hline & 0.188226 & 4.384728 & 4.610408 \\
\hline & -3.263561 & 5.509768 & 0.792626 \\
\hline & -2.532405 & 6.716005 & 0.194481 \\
\hline & 1.678644 & 2.687578 & 3.609557 \\
\hline & 1.760417 & 5.027389 & 2.776168 \\
\hline y & -4.350681 & 5.076710 & -0.188652 \\
\hline & -3.944067 & 5.922646 & 2.102671 \\
\hline 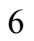 & -0.231337 & 2.012764 & -3.132610 \\
\hline & -0.405735 & 2.931925 & -3.813376 \\
\hline & -6.318895 & 0.422964 & 2.486065 \\
\hline & -7.899759 & 1.070949 & 0.695639 \\
\hline & -6.696762 & -0.117054 & -4.107351 \\
\hline & -4.751306 & -1.662926 & -4.111864 \\
\hline & 7.021247 & 0.819047 & 0.540340 \\
\hline & -2.041400 & 0.854321 & 2.862166 \\
\hline & 8.224132 & -3.902875 & -0.419939 \\
\hline & -2.081943 & -2.928995 & 5.689749 \\
\hline & 5.591581 & 5.453082 & -1.461240 \\
\hline & -3.550065 & -2.604136 & 0.448473 \\
\hline & -5.298012 & -3.801101 & -0.791274 \\
\hline 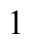 & 3.604192 & 4.649730 & -0.329014 \\
\hline & -2.216558 & -0.676227 & 4.764034 \\
\hline & 6.151923 & -4.214003 & -1.645724 \\
\hline & 8.396824 & 2.671994 & -3.152798 \\
\hline & 3.950962 & -3.327085 & -2.413242 \\
\hline 1 & -2.346491 & -5.447457 & -3.450366 \\
\hline & 0.015586 & -4.888217 & -3.153182 \\
\hline 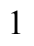 & -0.981248 & -6.228688 & 3.164110 \\
\hline & 7.076731 & 0.790862 & -2.309188 \\
\hline & -0.739860 & -4.720005 & 1.255060 \\
\hline 1 & 1.772340 & -3.586700 & -1.982105 \\
\hline & 7.685339 & 5.019863 & -2.712886 \\
\hline ] & 9.114052 & -0.114572 & 1.403029 \\
\hline & -4.710131 & -5.240530 & -2.737625 \\
\hline 1 & -1.659043 & -5.354447 & 5.396475 \\
\hline ] & 9.750077 & -2.473493 & 0.910810 \\
\hline 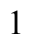 & 2.170683 & 2.750347 & 0.430849 \\
\hline 1 & -4.074308 & 0.445999 & 1.486237 \\
\hline 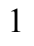 & -2.801085 & 0.170025 & -2.581773 \\
\hline 1 & -6.965240 & -0.159888 & -1.616711 \\
\hline ( & -3.197279 & 2.901987 & -0.178383 \\
\hline 1 & 0.339452 & 1.598164 & 1.847177 \\
\hline . & -1.108450 & 5.596232 & 2.423137 \\
\hline 1 & 0.682174 & 1.791043 & -1.086612 \\
\hline 1 & 2.555454 & -3.623371 & 0.584794 \\
\hline
\end{tabular}




$\begin{array}{rrrr}1 & 3.443596 & -2.110659 & 0.721400 \\ 1 & 2.345344 & -4.606448 & 2.662123 \\ 1 & 1.864079 & -0.354392 & 2.371269 \\ 1 & 1.920501 & -4.496524 & 5.100842 \\ 1 & 1.433293 & -0.237467 & 4.811101 \\ 1 & 1.459621 & -2.308182 & 6.182737 \\ 1 & -6.841407 & -0.253431 & -5.186434 \\ 1 & -7.302985 & -0.880471 & -3.603552 \\ 1 & -7.094852 & 0.870548 & -3.839677 \\ 1 & -4.908172 & -1.871626 & -5.179120 \\ 1 & -3.686021 & -1.809844 & -3.889620 \\ 1 & -5.313432 & -2.406390 & -3.530486 \\ 1 & -4.676784 & 0.604877 & -5.691181 \\ 1 & -4.716627 & 1.797687 & -4.376483 \\ 1 & -3.354830 & 0.670436 & -4.524471 \\ 1 & -7.170602 & 0.384963 & 3.176830 \\ 1 & -5.568076 & -0.288697 & 2.854739 \\ 1 & -5.890686 & 1.432783 & 2.542232 \\ 1 & -8.172575 & -1.425507 & 1.811845 \\ 1 & -7.746376 & -1.634414 & 0.103152 \\ 1 & -6.575303 & -2.063825 & 1.365853 \\ 1 & -8.711622 & 1.027287 & 1.433734 \\ 1 & -7.521051 & 2.100893 & 0.671624 \\ 1 & -8.338205 & 0.853246 & -0.285230 \\ 1 & -5.049137 & 5.906839 & -0.353331 \\ 1 & -3.935317 & 4.797215 & -1.165339 \\ 1 & -4.933364 & 4.226331 & 0.190907 \\ 1 & -4.673816 & 6.722382 & 1.917538 \\ 1 & -4.477980 & 5.074855 & 2.551853 \\ 1 & -3.225564 & 6.295172 & 2.842878 \\ 1 & -3.243108 & 7.526584 & -0.015962 \\ 1 & -1.768486 & 7.113825 & 0.873481 \\ 1 & -2.036365 & 6.447203 & -0.746943 \\ 1 & 2.410955 & 2.925220 & 4.391250 \\ 1 & 1.052839 & 1.861552 & 3.976837 \\ 1 & 2.241557 & 2.331783 & 2.735969 \\ 1 & 0.960542 & 4.579020 & 5.366543 \\ 1 & -0.391520 & 5.307264 & 4.488267 \\ 1 & -0.487114 & 3.615198 & 5.006825 \\ 1 & 2.533832 & 5.261743 & 3.519997 \\ 1 & 2.267369 & 4.713766 & 1.853999 \\ 1 & 1.212971 & 5.953026 & 2.560298\end{array}$

$1_{\mathrm{C} 10-} \psi_{-1 \mathrm{a} 2 \mathrm{a} 3 \mathrm{~s} 4 \mathrm{~s}}$

Number of imaginary frequencies : 0

The smallest frequencies are : $13.2508 \quad 16.4984 \quad 19.8879 \mathrm{~cm}(-1)$

Electronic energy : $\quad H F=-4125.4648713$

Zero-point correction $=\quad 1.300196($ Hartree/Particle $)$

Thermal correction to Energy= $\quad 1.378341$

Thermal correction to Enthalpy= $\quad 1.379285$

Thermal correction to Gibbs Free Energy $=\quad 1.187768$

Sum of electronic and zero-point Energies $=\quad-4124.164676$

Sum of electronic and thermal Energies $=\quad-4124.086531$

Sum of electronic and thermal Enthalpies $=\quad-4124.085586$

Sum of electronic and thermal Free Energies $=\quad-4124.277104$ 
Cartesian Coordinates

\begin{tabular}{|c|c|c|c|}
\hline 6 & 1.777286 & -2.180435 & 5.162239 \\
\hline 6 & 2.101248 & -3.407496 & 4.594779 \\
\hline 6 & 2.340388 & -3.499703 & 3.226201 \\
\hline 6 & 2.260768 & -2.372577 & 2.410051 \\
\hline 6 & 1.932563 & -1.143738 & 2.988043 \\
\hline 6 & 1.692290 & -1.049211 & 4.353300 \\
\hline 6 & 2.594731 & -2.483848 & 0.943816 \\
\hline 7 & 1.627514 & -1.827970 & 0.041983 \\
\hline 6 & 0.588759 & -2.639928 & -0.533964 \\
\hline 6 & -0.707473 & -2.632911 & -0.046588 \\
\hline 6 & -1.722297 & -3.356442 & -0.753942 \\
\hline 6 & -1.377014 & -4.115386 & -1.911125 \\
\hline 6 & -0.028051 & -4.148975 & -2.333263 \\
\hline 6 & 0.919698 & -3.418569 & -1.670394 \\
\hline 6 & -3.081249 & -3.339446 & -0.344206 \\
\hline 6 & -4.039311 & -4.025218 & -1.048171 \\
\hline 6 & -3.689894 & -4.784785 & -2.185603 \\
\hline 6 & -2.385424 & -4.826689 & -2.606097 \\
\hline 6 & -1.056986 & -2.030460 & 1.271282 \\
\hline 6 & -1.415306 & -0.700958 & 1.441255 \\
\hline 6 & -1.802285 & -0.246450 & 2.731847 \\
\hline 6 & -1.858514 & -1.097622 & 3.802031 \\
\hline 6 & -1.529201 & -2.463972 & 3.657098 \\
\hline 6 & -1.111001 & -2.938442 & 2.380871 \\
\hline 6 & -1.612248 & -3.362936 & 4.747376 \\
\hline 6 & -1.307661 & -4.690574 & 4.588891 \\
\hline 6 & -0.885255 & -5.166616 & 3.330310 \\
\hline 6 & -0.782019 & -4.314548 & 2.259023 \\
\hline 15 & -1.535525 & 0.422402 & -0.033306 \\
\hline 45 & 0.165237 & 0.693689 & -1.787791 \\
\hline 6 & -0.297231 & -0.591266 & -3.184435 \\
\hline 8 & -0.532097 & -1.264446 & -4.090712 \\
\hline 15 & 1.961254 & -0.434937 & -0.862830 \\
\hline 8 & 2.882757 & 0.317479 & 0.290705 \\
\hline 6 & 3.690043 & 1.356360 & -0.131028 \\
\hline 6 & 4.892721 & 1.072439 & -0.748781 \\
\hline 6 & 5.680143 & 2.171679 & -1.223357 \\
\hline 6 & 5.250125 & 3.506665 & -0.955482 \\
\hline 6 & 4.036490 & 3.725500 & -0.261047 \\
\hline 6 & 3.255613 & 2.670876 & 0.126995 \\
\hline 6 & 6.032800 & 4.595762 & -1.410420 \\
\hline 6 & 7.182739 & 4.387866 & -2.127125 \\
\hline 6 & 7.590439 & 3.071383 & -2.427532 \\
\hline 6 & 6.861076 & 1.994390 & -1.988687 \\
\hline 6 & 5.292481 & -0.341316 & -0.942380 \\
\hline 6 & 4.422825 & -1.214656 & -1.574030 \\
\hline 6 & 4.786598 & -2.541344 & -1.885302 \\
\hline 6 & 6.003224 & -3.025975 & -1.491329 \\
\hline 6 & 6.892313 & -2.215973 & -0.745339 \\
\hline 6 & 6.536201 & -0.863867 & -0.456856 \\
\hline 6 & 7.423984 & -0.101132 & 0.344851 \\
\hline 6 & 8.603208 & -0.634404 & 0.802885 \\
\hline 6 & 8.966819 & -1.959628 & 0.484992 \\
\hline
\end{tabular}




\begin{tabular}{|c|c|c|c|}
\hline 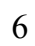 & 8.122663 & -2.731732 & -0.270513 \\
\hline 8 & 3.171273 & -0.800462 & -1.966914 \\
\hline & -3.247121 & 0.037757 & -0.592673 \\
\hline & -4.292837 & -0.074455 & 0.323737 \\
\hline & -5.599028 & -0.318747 & -0.096264 \\
\hline & -5.832667 & -0.449033 & -1.471246 \\
\hline & -4.809130 & -0.340393 & -2.415534 \\
\hline 6 & -3.513936 & -0.095851 & -1.949312 \\
\hline 6 & -6.703698 & -0.418742 & 0.954473 \\
\hline 6 & -5.036539 & -0.526563 & -3.914062 \\
\hline 6 & -1.754815 & 2.076774 & 0.736909 \\
\hline 6 & -2.823362 & 2.895656 & 0.381354 \\
\hline 6 & -2.947227 & 4.184816 & 0.906054 \\
\hline 6 & -1.978394 & 4.616077 & 1.813649 \\
\hline 6 & -0.895514 & 3.816679 & 2.199330 \\
\hline 6 & -0.783796 & 2.552622 & 1.621913 \\
\hline 6 & -4.119847 & 5.095295 & 0.544553 \\
\hline 6 & 0.115801 & 4.339318 & 3.218946 \\
\hline 6 & -0.094375 & 2.247871 & -2.865560 \\
\hline 8 & -0.252381 & 3.228085 & -3.458639 \\
\hline 1 & 7.154795 & 0.918087 & 0.609595 \\
\hline 1 & -2.077857 & 0.796201 & 2.870489 \\
\hline 1 & 8.377349 & -3.762772 & -0.512795 \\
\hline 1 & -1.929959 & -2.974668 & 5.714399 \\
\hline 1 & 5.690262 & 5.605393 & -1.187614 \\
\hline 1 & -3.365094 & -2.755525 & 0.530033 \\
\hline 1 & -5.078582 & -3.980349 & -0.728246 \\
\hline 1 & 3.722511 & 4.749146 & -0.064217 \\
\hline 1 & -2.172172 & -0.732986 & 4.779638 \\
\hline 1 & 6.296620 & -4.046815 & -1.730554 \\
\hline 1 & 8.488280 & 2.906922 & -3.019070 \\
\hline 1 & 4.085998 & -3.150952 & -2.451020 \\
\hline 1 & -2.100350 & -5.402978 & -3.485118 \\
\hline 1 & 0.237564 & -4.741042 & -3.207565 \\
\hline 1 & -0.633792 & -6.217944 & 3.208286 \\
\hline 1 & 7.183461 & 0.987378 & -2.240152 \\
\hline 1 & -0.441051 & -4.699254 & 1.299794 \\
\hline 1 & 1.954109 & -3.414221 & -2.007710 \\
\hline 1 & 7.772590 & 5.231979 & -2.476686 \\
\hline 1 & 9.260914 & -0.029260 & 1.422804 \\
\hline 1 & -4.458686 & -5.330028 & -2.729030 \\
\hline 1 & -1.383943 & -5.375971 & 5.430063 \\
\hline 1 & 9.906992 & -2.367002 & 0.849242 \\
\hline 1 & 2.300049 & 2.815354 & 0.627902 \\
\hline 1 & -4.082524 & 0.039938 & 1.386679 \\
\hline 1 & -2.688619 & -0.005690 & -2.652198 \\
\hline 1 & -6.845648 & -0.638869 & -1.814575 \\
\hline 1 & -3.565661 & 2.520157 & -0.317416 \\
\hline 1 & 0.053516 & 1.903354 & 1.878677 \\
\hline 1 & -2.070944 & 5.613219 & 2.243975 \\
\hline 1 & 0.681675 & 1.837440 & -0.781714 \\
\hline 1 & 2.674885 & -3.543226 & 0.657297 \\
\hline 1 & 3.584816 & -2.042167 & 0.773321 \\
\hline 1 & 2.583656 & -4.465017 & 2.781140 \\
\hline 1 & 1.862802 & -0.259941 & 2.356386 \\
\hline 1 & 2.154104 & -4.300556 & 5.214566 \\
\hline
\end{tabular}




$\begin{array}{rrrr}1 & 1.435074 & -0.085529 & 4.789733 \\ 1 & 1.583146 & -2.105875 & 6.230391 \\ 6 & -6.518986 & -0.533743 & -4.280308 \\ 6 & -4.359448 & 0.601721 & -4.700895 \\ 6 & -4.427413 & -1.870693 & -4.329326 \\ 6 & -8.058903 & -0.769696 & 0.346194 \\ 6 & -6.346650 & -1.507110 & 1.974194 \\ 6 & -6.833054 & 0.931007 & 1.670958 \\ 1 & -6.629198 & -0.632575 & -5.367531 \\ 1 & -7.054281 & -1.376285 & -3.824393 \\ 1 & -7.019209 & 0.395933 & -3.978636 \\ 1 & -4.556313 & 0.481052 & -5.774215 \\ 1 & -4.741761 & 1.584305 & -4.395055 \\ 1 & -3.269426 & 0.610413 & -4.573085 \\ 1 & -4.554082 & -2.033655 & -5.408516 \\ 1 & -3.354058 & -1.918405 & -4.103325 \\ 1 & -4.913966 & -2.699399 & -3.797664 \\ 1 & -8.812628 & -0.841567 & 1.140472 \\ 1 & -8.403132 & -0.005865 & -0.362660 \\ 1 & -8.034959 & -1.736301 & -0.174195 \\ 1 & -7.592857 & 0.875292 & 2.462346 \\ 1 & -5.887966 & 1.242760 & 2.134261 \\ 1 & -7.136513 & 1.718500 & 0.968173 \\ 1 & -7.128891 & -1.580203 & 2.741704 \\ 1 & -6.262501 & -2.488444 & 1.488261 \\ 1 & -5.398076 & -1.308067 & 2.489080 \\ 6 & -5.038117 & 4.466613 & -0.502034 \\ 6 & -4.949796 & 5.368574 & 1.803926 \\ 6 & -3.593686 & 6.419134 & -0.019101 \\ 6 & 1.096001 & 3.255403 & 3.665296 \\ 6 & 0.910736 & 5.490184 & 2.594507 \\ 6 & -0.614890 & 4.844696 & 4.467999 \\ 1 & -5.866040 & 5.151868 & -0.723646 \\ 1 & -4.510990 & 4.268925 & -1.444399 \\ 1 & -5.477210 & 3.521525 & -0.153139 \\ 1 & -5.796933 & 6.026980 & 1.568474 \\ 1 & -5.352497 & 4.434309 & 2.217731 \\ 1 & -4.358331 & 5.854833 & 2.589393 \\ 1 & -4.430867 & 7.075328 & -0.292632 \\ 1 & -2.973714 & 6.961380 & 0.704722 \\ 1 & -2.987891 & 6.249881 & -0.918445 \\ 1 & 1.758065 & 3.653891 & 4.444161 \\ 1 & 0.571845 & 2.384677 & 4.084061 \\ 1 & 1.738281 & 2.905799 & 2.846127 \\ 1 & 0.110490 & 5.205512 & 5.209198 \\ 1 & -1.296652 & 5.674504 & 4.248714 \\ 1 & -1.202064 & 4.042200 & 4.933127 \\ 1 & 1.650433 & 5.879271 & 3.307297 \\ 1 & 1.447038 & 5.153408 & 1.697286 \\ 1 & 0.259209 & 6.321629 & 2.298569\end{array}$

$1_{\text {C10- }} \psi_{-1 \mathbf{a} 233 a 4 a}$

Number of imaginary frequencies : 0

The smallest frequencies are : $11.8943 \quad 17.5392 \quad 21.9039 \mathrm{~cm}(-1)$

Electronic energy : $\quad H F=-4125.4651604$ 
Zero-point correction $=$

Thermal correction to Energy=

Thermal correction to Enthalpy=

Thermal correction to Gibbs Free Energy=

Sum of electronic and zero-point Energies=

Sum of electronic and thermal Energies $=$

Sum of electronic and thermal Enthalpies=

Sum of electronic and thermal Free Energies=
1.300023 (Hartree/Particle)

1.378119

1.379063

1.188630

$-4124.165138$

$-4124.087042$

$-4124.086097$

$-4124.276530$

Cartesian Coordinates

$\begin{array}{cccc}6 & 1.665762 & -2.330930 & 5.122531 \\ 6 & 1.942949 & -3.557071 & 4.529962 \\ 6 & 2.177441 & -3.629590 & 3.159237 \\ 6 & 2.139584 & -2.483860 & 2.366533 \\ 6 & 1.857305 & -1.255119 & 2.969603 \\ 6 & 1.622809 & -1.180469 & 4.337044 \\ 6 & 2.466614 & -2.577132 & 0.898073 \\ 7 & 1.503344 & -1.897655 & 0.009629 \\ 6 & 0.431168 & -2.679883 & -0.545626 \\ 6 & -0.859819 & -2.616080 & -0.048483 \\ 6 & -1.912151 & -3.285263 & -0.753870 \\ 6 & -1.610013 & -4.050465 & -1.918688 \\ 6 & -0.264803 & -4.157461 & -2.341440 \\ 6 & 0.720894 & -3.476482 & -1.681028 \\ 6 & -3.267533 & -3.196472 & -0.341285 \\ 6 & -4.264481 & -3.814448 & -1.053975 \\ 6 & -3.959324 & -4.574898 & -2.203982 \\ 6 & -2.658463 & -4.690160 & -2.623877 \\ 6 & -1.178915 & -1.998312 & 1.269612 \\ 6 & -1.472458 & -0.653963 & 1.438585 \\ 6 & -1.844309 & -0.181825 & 2.728160 \\ 6 & -1.944020 & -1.028129 & 3.798123 \\ 6 & -1.675928 & -2.408349 & 3.654608 \\ 6 & -1.278235 & -2.902851 & 2.379645 \\ 6 & -1.797182 & -3.300827 & 4.746385 \\ 6 & -1.548651 & -4.640681 & 4.590534 \\ 6 & -1.148605 & -5.136915 & 3.332740 \\ 6 & -1.011062 & -4.292013 & 2.259364 \\ 15 & -1.530987 & 0.480732 & -0.031775 \\ 45 & 0.112746 & 0.629586 & -1.870114 \\ 6 & -0.350103 & -0.665471 & -3.261246 \\ 8 & -0.538681 & -1.331259 & -4.183647 \\ 15 & 1.871510 & -0.526278 & -0.914901 \\ 8 & 2.802072 & 0.222814 & 0.233188 \\ 6 & 3.628946 & 1.241466 & -0.201078 \\ 6 & 4.826499 & 0.928457 & -0.814683 \\ 6 & 5.630889 & 2.008117 & -1.305826 \\ 6 & 5.221913 & 3.353914 & -1.058771 \\ 6 & 4.011662 & 3.602500 & -0.368218 \\ 6 & 3.214512 & 2.566302 & 0.036510 \\ 6 & 6.021214 & 4.423225 & -1.531405 \\ 6 & 7.167129 & 4.186089 & -2.245472 \\ 6 & 7.553827 & 2.858852 & -2.525461 \\ 6 & 6.808111 & 1.800476 & -2.069129 \\ 6 & 5.203261 & -0.494538 & -0.988069\end{array}$




\begin{tabular}{|c|c|c|c|}
\hline & 4.319064 & -1.363245 & -1.605458 \\
\hline & 4.656514 & -2.702185 & -1.891922 \\
\hline & 5.865055 & -3.201460 & -1.491410 \\
\hline & 6.770311 & -2.393670 & -0.762385 \\
\hline & 6.438225 & -1.030976 & -0.495331 \\
\hline & 7.340269 & -0.270935 & 0.292833 \\
\hline & 8.511409 & -0.816658 & 0.756853 \\
\hline & 8.851940 & -2.152636 & 0.459130 \\
\hline & 7.992933 & -2.922403 & -0.281899 \\
\hline & 3.075568 & -0.933575 & -2.007522 \\
\hline & -3.293585 & 0.269372 & -0.526403 \\
\hline & -4.322382 & 0.324590 & 0.418576 \\
\hline & -5.646631 & 0.100877 & 0.048872 \\
\hline & -5.909179 & -0.174269 & -1.299327 \\
\hline & -4.908517 & -0.237776 & -2.269185 \\
\hline & -3.592461 & -0.005209 & -1.857062 \\
\hline & -6.785590 & 0.084077 & 1.066610 \\
\hline & -5.264873 & -0.541444 & -3.723416 \\
\hline & -1.503804 & 2.133830 & 0.763468 \\
\hline & -2.414038 & 3.120136 & 0.415804 \\
\hline & -2.327427 & 4.409556 & 0.962162 \\
\hline & -1.308718 & 4.664435 & 1.875334 \\
\hline & -0.370066 & 3.687377 & 2.249326 \\
\hline & -0.466961 & 2.433719 & 1.657695 \\
\hline & -3.363926 & 5.453235 & 0.552288 \\
\hline 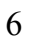 & -3.279632 & 5.686197 & -0.960444 \\
\hline & 0.696293 & 4.014789 & 3.294234 \\
\hline 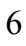 & 1.523869 & 2.787455 & 3.675037 \\
\hline & -0.223907 & 2.174578 & -2.934018 \\
\hline 3 & -0.439224 & 3.158540 & -3.502613 \\
\hline & 1.640970 & 5.091351 & 2.751113 \\
\hline & 0.019744 & 4.531810 & 4.569439 \\
\hline 5 & -3.152022 & 6.792498 & 1.253114 \\
\hline & -4.764429 & 4.941796 & 0.912651 \\
\hline | & 7.088836 & 0.756716 & 0.541932 \\
\hline & -2.065910 & 0.873660 & 2.867245 \\
\hline . & 8.229439 & -3.961457 & -0.507821 \\
\hline & -2.097885 & -2.897657 & 5.712743 \\
\hline & 5.694517 & 5.441437 & -1.324502 \\
\hline 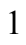 & -3.517492 & -2.608140 & 0.540044 \\
\hline & -5.300106 & -3.709500 & -0.734545 \\
\hline 1 & 3.711118 & 4.634054 & -0.189939 \\
\hline & -2.243325 & -0.648890 & 4.774682 \\
\hline | & 6.139363 & -4.231908 & -1.711297 \\
\hline & 8.448277 & 2.671030 & -3.115181 \\
\hline & 3.940433 & -3.309000 & -2.441334 \\
\hline 1 & -2.407533 & -5.271610 & -3.509856 \\
\hline & -0.033145 & -4.757996 & -3.219613 \\
\hline 1 & -0.942012 & -6.198201 & 3.212691 \\
\hline & 7.114158 & 0.784725 & -2.305323 \\
\hline & -0.686460 & -4.691979 & 1.300759 \\
\hline 1 & 1.752886 & -3.519357 & -2.025293 \\
\hline 1 & 7.769749 & 5.015237 & -2.608813 \\
\hline 1 & 9.180605 & -0.212976 & 1.365793 \\
\hline 1 & -4.758381 & -5.067194 & -2.754561 \\
\hline 1 & -1.652722 & -5.320367 & 5.433356 \\
\hline
\end{tabular}




\begin{tabular}{|c|c|c|c|}
\hline 1 & 9.786024 & -2.569990 & 0.827716 \\
\hline 1 & 2.260637 & 2.732649 & 0.535212 \\
\hline 1 & -4.076313 & 0.536298 & 1.456152 \\
\hline & -2.771133 & -0.053174 & -2.567903 \\
\hline 1 & -6.940360 & -0.357468 & -1.601379 \\
\hline 1 & -3.207497 & 2.885322 & -0.292902 \\
\hline 1 & 0.251517 & 1.652344 & 1.904744 \\
\hline 1 & -1.234192 & 5.652914 & 2.323493 \\
\hline 1 & 2.398049 & 5.350663 & 3.503484 \\
\hline 1 & 2.166776 & 4.738325 & 1.853915 \\
\hline 1 & 1.104840 & 6.010586 & 2.485340 \\
\hline 1 & 0.774285 & 4.746323 & 5.337906 \\
\hline 1 & -0.544713 & 5.455734 & 4.397713 \\
\hline 1 & -0.674710 & 3.785378 & 4.976752 \\
\hline 1 & 0.885401 & 1.978562 & 4.058509 \\
\hline 1 & 2.107517 & 2.394716 & 2.831029 \\
\hline 1 & 2.237623 & 3.052986 & 4.464875 \\
\hline 1 & -3.920191 & 7.504545 & 0.926149 \\
\hline 1 & -3.230464 & 6.703037 & 2.344449 \\
\hline 1 & -2.175024 & 7.230918 & 1.012231 \\
\hline 1 & -5.005553 & 4.001449 & 0.400516 \\
\hline 1 & -4.853252 & 4.767555 & 1.993252 \\
\hline 1 & -5.525454 & 5.679646 & 0.624900 \\
\hline 1 & -2.284190 & 6.048757 & -1.247446 \\
\hline 1 & -3.476480 & 4.770948 & -1.531267 \\
\hline 1 & -4.017978 & 6.437369 & -1.272093 \\
\hline 1 & 0.715315 & 1.790215 & -0.934747 \\
\hline 1 & 2.535821 & -3.633195 & 0.596548 \\
\hline 1 & 3.459671 & -2.141841 & 0.730106 \\
\hline 1 & 2.384568 & -4.593710 & 2.693753 \\
\hline 1 & 1.820380 & -0.354793 & 2.357178 \\
\hline 1 & 1.965270 & -4.463648 & 5.131608 \\
\hline 1 & 1.404509 & -0.216719 & 4.794789 \\
\hline 1 & 1.476148 & -2.270634 & 6.192367 \\
\hline 6 & -4.021205 & -0.785746 & -4.575611 \\
\hline 6 & -6.138023 & -1.797799 & -3.804648 \\
\hline 6 & -6.031340 & 0.651139 & -4.305286 \\
\hline 6 & -6.322440 & 0.510080 & 2.458631 \\
\hline 6 & -7.905111 & 1.033145 & 0.628447 \\
\hline 6 & -7.331862 & -1.345660 & 1.161687 \\
\hline 1 & -4.319882 & -1.038580 & -5.600765 \\
\hline 1 & -3.376284 & 0.100972 & -4.635504 \\
\hline 1 & -3.423012 & -1.621298 & -4.185788 \\
\hline 1 & -6.390394 & -2.013081 & -4.851480 \\
\hline 1 & -5.605340 & -2.668457 & -3.398605 \\
\hline 1 & -7.082215 & -1.691937 & -3.256949 \\
\hline 1 & -6.295216 & 0.462898 & -5.354864 \\
\hline 1 & -6.961044 & 0.845679 & -3.755653 \\
\hline 1 & -5.423300 & 1.564441 & -4.267921 \\
\hline 1 & -8.150877 & -1.397663 & 1.891882 \\
\hline 1 & -7.719398 & -1.701842 & 0.198775 \\
\hline 1 & -6.545138 & -2.041168 & 1.485167 \\
\hline 1 & -7.174819 & 0.497880 & 3.149539 \\
\hline 1 & -5.561405 & -0.168316 & 2.867126 \\
\hline 1 & -5.910429 & 1.528072 & 2.458084 \\
\hline 1 & -8.720850 & 1.021569 & 1.363473 \\
\hline
\end{tabular}


$1_{\text {C10- }-\psi_{-1 a 233 a 4 s}}$

Number of imaginary frequencies : 0

The smallest frequencies are : $10.6771 \quad 15.5280 \quad 18.3577 \mathrm{~cm}(-1)$

Electronic energy : $\quad H F=-4125.4653307$

Zero-point correction $=\quad 1.299402($ Hartree/Particle $)$

Thermal correction to Energy= $\quad 1.377724$

Thermal correction to Enthalpy= $\quad 1.378669$

Thermal correction to Gibbs Free Energy= 1.186584

Sum of electronic and zero-point Energies $=\quad-4124.165929$

Sum of electronic and thermal Energies $=\quad-4124.087606$

Sum of electronic and thermal Enthalpies $=\quad-4124.086662$

Sum of electronic and thermal Free Energies $=\quad-4124.278747$

\begin{tabular}{|c|c|c|c|}
\hline & \multicolumn{3}{|c|}{ Cartesian Coordinates } \\
\hline 6 & 6.021678 & 4.516792 & -1.390697 \\
\hline 6 & 5.233172 & 3.427254 & -0.947026 \\
\hline 6 & 5.647374 & 2.093257 & -1.243791 \\
\hline 6 & 6.818291 & 1.918545 & -2.024832 \\
\hline 6 & 7.553480 & 2.996275 & -2.452007 \\
\hline 6 & 7.161872 & 4.310894 & -2.123395 \\
\hline 6 & 4.853966 & 0.993188 & -0.781430 \\
\hline 6 & 3.660594 & 1.278541 & -0.146556 \\
\hline 6 & 3.241915 & 2.591695 & 0.142271 \\
\hline 6 & 4.029320 & 3.645348 & -0.235292 \\
\hline 6 & 5.237596 & -0.421621 & -1.000576 \\
\hline 6 & 4.352624 & -1.278244 & -1.633875 \\
\hline 6 & 4.695592 & -2.606738 & -1.959746 \\
\hline 6 & 5.910754 & -3.109420 & -1.584309 \\
\hline 6 & 6.818018 & -2.316544 & -0.841789 \\
\hline 6 & 6.480400 & -0.963828 & -0.534205 \\
\hline 6 & 8.048224 & -2.850953 & -0.387648 \\
\hline 6 & 8.909678 & -2.096828 & 0.366456 \\
\hline 6 & 8.564065 & -0.771750 & 0.704334 \\
\hline 6 & 7.385475 & -0.220507 & 0.266432 \\
\hline 8 & 3.103592 & -0.844481 & -2.014684 \\
\hline 15 & 1.906980 & -0.477822 & -0.89931 \\
\hline 8 & 2.845315 & 0.240387 & 0.260903 \\
\hline 7 & 1.552627 & -1.875572 & -0.010753 \\
\hline 6 & 0.491320 & -2.658367 & -0.585262 \\
\hline 6 & -0.795160 & -2.642826 & -0.073530 \\
\hline 6 & -1.838134 & -3.320103 & -0.784717 \\
\hline 6 & -1.531934 & -4.035709 & -1.979667 \\
\hline 6 & -0.190695 & -4.089651 & -2.423690 \\
\hline 6 & 0.786165 & -3.408537 & -1.750318 \\
\hline 6 & -2.571450 & -4.683065 & -2.691134 \\
\hline 6 & -3.867737 & -4.624496 & -2.246548 \\
\hline 6 & -4.176382 & -3.915919 & -1.064921 \\
\hline 6 & -3.188512 & -3.288561 & -0.347879 \\
\hline 6 & -1.118550 & -2.062359 & 1.259815 \\
\hline 6 & -1.461525 & -0.732572 & 1.451769 \\
\hline 6 & -1.846436 & -0.296650 & 2.749786 \\
\hline
\end{tabular}




\begin{tabular}{|c|c|c|c|}
\hline 6 & -1.904430 & -1.163849 & 3.806765 \\
\hline & -1.581933 & -2.529789 & 3.640002 \\
\hline & -1.175384 & -2.987775 & 2.354201 \\
\hline & -0.859199 & -4.363997 & 2.207301 \\
\hline & -0.958386 & -5.231727 & 3.266500 \\
\hline & -1.365726 & -4.771636 & 4.535837 \\
\hline & -1.661239 & -3.444620 & 4.717072 \\
\hline & -1.552173 & 0.418314 & -0.002966 \\
\hline & -1.624104 & 2.069465 & 0.795715 \\
\hline & -0.637349 & 2.421944 & 1.725925 \\
\hline & -0.604158 & 3.691526 & 2.290615 \\
\hline & -1.555465 & 4.629939 & 1.855703 \\
\hline & -2.525989 & 4.322442 & 0.906558 \\
\hline & -2.549576 & 3.019218 & 0.388870 \\
\hline & 0.416792 & 4.080600 & 3.359069 \\
\hline & 1.259800 & 2.888065 & 3.809706 \\
\hline & -3.565737 & 5.327047 & 0.415107 \\
\hline & -3.386685 & 5.540714 & -1.092438 \\
\hline & 2.506946 & -2.554524 & 0.886559 \\
\hline & 2.183733 & -2.438967 & 2.355183 \\
\hline & 1.867609 & -1.208699 & 2.937252 \\
\hline & 1.645593 & -1.113418 & 4.305620 \\
\hline & 1.733343 & -2.245129 & 5.113468 \\
\hline & 2.042844 & -3.473904 & 4.541675 \\
\hline ) & 2.266329 & -3.566566 & 3.170535 \\
\hline 45 & 0.126845 & 0.687548 & -1.798300 \\
\hline 5 & -0.243379 & 2.289034 & -2.766929 \\
\hline & -0.482796 & 3.303690 & -3.267428 \\
\hline & -0.288199 & -0.546750 & -3.255870 \\
\hline & -0.445058 & -1.182699 & -4.204888 \\
\hline & -3.298443 & 0.147620 & -0.526691 \\
\hline 5 & -4.334910 & 0.166865 & 0.402729 \\
\hline & -5.659958 & -0.048112 & 0.014972 \\
\hline & -5.909843 & -0.282388 & -1.338243 \\
\hline 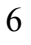 & -4.891557 & -0.317600 & -2.299294 \\
\hline & -3.583242 & -0.092062 & -1.870872 \\
\hline 5 & -6.753343 & -0.042147 & 1.081603 \\
\hline & -8.139931 & -0.299243 & 0.497784 \\
\hline 5 & -5.235175 & -0.570772 & -3.766029 \\
\hline ) & -3.985328 & -0.805332 & -4.611647 \\
\hline & -6.776645 & 1.319663 & 1.784886 \\
\hline & -6.456442 & -1.142879 & 2.107561 \\
\hline & -6.124620 & -1.810855 & -3.897756 \\
\hline 5 & -5.978112 & 0.650842 & -4.317902 \\
\hline & -3.440814 & 6.681520 & 1.107388 \\
\hline 6 & -4.971453 & 4.778076 & 0.687588 \\
\hline & 1.353576 & 5.158141 & 2.804392 \\
\hline & -0.311032 & 4.627718 & 4.592445 \\
\hline & 7.130571 & 0.798295 & 0.546348 \\
\hline & -2.109730 & 0.746761 & 2.906615 \\
\hline & 8.288642 & -3.881839 & -0.644607 \\
\hline & -1.969044 & -3.069612 & 5.692493 \\
\hline & 5.691504 & 5.525452 & -1.145902 \\
\hline & -3.442371 & -2.736405 & 0.555810 \\
\hline 1 & -5.208536 & -3.860793 & -0.724178 \\
\hline 1 & 3.727036 & 4.668432 & -0.017184 \\
\hline
\end{tabular}




\begin{tabular}{|c|c|c|c|}
\hline 1 & -2.212520 & -0.812641 & 4.791070 \\
\hline & 6.189163 & -4.131575 & -1.835515 \\
\hline & 8.443299 & 2.834045 & -3.056147 \\
\hline & 3.979982 & -3.202866 & -2.521063 \\
\hline & -2.317211 & -5.225172 & -3.600818 \\
\hline & 0.045380 & -4.653302 & -3.324839 \\
\hline & -0.716045 & -6.282926 & 3.126319 \\
\hline & 7.127823 & 0.913117 & -2.297977 \\
\hline & -0.530784 & -4.735739 & 1.238572 \\
\hline & 1.814798 & -3.417258 & -2.105630 \\
\hline & 7.756242 & 5.155538 & -2.463903 \\
\hline & 9.235341 & -0.181097 & 1.323668 \\
\hline & -4.660330 & -5.123112 & -2.800695 \\
\hline & -1.438894 & -5.469038 & 5.367372 \\
\hline & 9.849667 & -2.518467 & 0.714578 \\
\hline & 2.293084 & 2.735305 & 0.657455 \\
\hline & -4.106760 & 0.348352 & 1.452627 \\
\hline & -2.754972 & -0.111141 & -2.575432 \\
\hline & -6.934472 & -0.449563 & -1.663744 \\
\hline & -3.302373 & 2.746080 & -0.350017 \\
\hline & 0.095507 & 1.670879 & 2.019529 \\
\hline & -1.528077 & 5.630510 & 2.281899 \\
\hline & 2.088991 & 5.455530 & 3.564150 \\
\hline & 1.903608 & 4.787052 & 1.929195 \\
\hline & 0.806320 & 6.057713 & 2.497343 \\
\hline & 0.413950 & 4.890995 & 5.374108 \\
\hline 1 & -0.892663 & 5.529355 & 4.368148 \\
\hline & -0.998910 & 3.879734 & 5.007847 \\
\hline & 0.628651 & 2.076718 & 4.200005 \\
\hline - & 1.880393 & 2.480926 & 2.999457 \\
\hline & 1.940140 & 3.198055 & 4.612717 \\
\hline 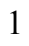 & -4.211085 & 7.363101 & 0.725050 \\
\hline & -3.581318 & 6.602468 & 2.193320 \\
\hline & -2.466446 & 7.150359 & 0.918876 \\
\hline & -5.147386 & 3.821639 & 0.178477 \\
\hline & -5.130788 & 4.619519 & 1.762511 \\
\hline 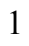 & -5.731927 & 5.486983 & 0.333293 \\
\hline & -2.385898 & 5.931028 & -1.317725 \\
\hline 1 & -3.514716 & 4.610605 & -1.659303 \\
\hline ] & -4.125491 & 6.262904 & -1.465516 \\
\hline 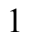 & 0.691859 & 1.802562 & -0.785939 \\
\hline 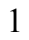 & 3.506654 & -2.136294 & 0.712372 \\
\hline[ & 2.499852 & -4.532950 & 2.722594 \\
\hline 1 & 1.794173 & -0.323120 & 2.307872 \\
\hline & 2.097439 & -4.367683 & 5.160252 \\
\hline 1 & 1.401788 & -0.147167 & 4.744932 \\
\hline 1 & 1.552898 & -2.169488 & 6.183916 \\
\hline 1 & -4.275669 & -1.028110 & -5.646113 \\
\hline 1 & -3.332561 & 0.076816 & -4.641989 \\
\hline 1 & -3.400104 & -1.656987 & -4.236856 \\
\hline 1 & -6.354970 & -1.998023 & -4.954941 \\
\hline 1 & -5.614054 & -2.697966 & -3.499889 \\
\hline 1 & -7.080051 & -1.703992 & -3.370638 \\
\hline 1 & -6.232427 & 0.500209 & -5.375911 \\
\hline 1 & -6.911278 & 0.840599 & -3.772556 \\
\hline 1 & -5.357657 & 1.553628 & -4.243980 \\
\hline
\end{tabular}




$\begin{array}{rrrr}1 & -7.225509 & -1.152445 & 2.891683 \\ 1 & -6.450420 & -2.131666 & 1.629632 \\ 1 & -5.483881 & -1.005164 & 2.597426 \\ 1 & -8.886705 & -0.285271 & 1.301658 \\ 1 & -8.427707 & 0.469677 & -0.230798 \\ 1 & -8.203759 & -1.279469 & 0.007743 \\ 1 & -7.538475 & 1.327127 & 2.576045 \\ 1 & -5.812951 & 1.563562 & 2.249241 \\ 1 & -7.019247 & 2.122923 & 1.076570 \\ 1 & 2.561434 & -3.614844 & 0.597207\end{array}$

$1_{\mathrm{C} 10-}-\psi_{-1225354 a}$

Number of imaginary frequencies : 0

The smallest frequencies are : $10.4242 \quad 13.1789 \quad 16.2948 \mathrm{~cm}(-1)$

Electronic energy : $\quad H F=-4125.4655762$

Zero-point correction $=\quad 1.299790($ Hartree/Particle $)$

Thermal correction to Energy= $\quad 1.378079$

Thermal correction to Enthalpy= $\quad 1.379023$

Thermal correction to Gibbs Free Energy= 1.186597

Sum of electronic and zero-point Energies $=\quad-4124.165786$

Sum of electronic and thermal Energies $=\quad-4124.087497$

Sum of electronic and thermal Enthalpies $=\quad-4124.086553$

Sum of electronic and thermal Free Energies $=\quad-4124.278979$

\section{Cartesian Coordinates}

$\begin{array}{ccccc}6 & 5.966180 & 4.481148 & -1.437287 \\ 6 & 5.176433 & 3.394249 & -0.989408 \\ 6 & 5.593361 & 2.058371 & -1.273942 \\ 6 & 6.768505 & 1.879052 & -2.047568 \\ 6 & 7.504826 & 2.954243 & -2.479223 \\ 6 & 7.110272 & 4.270900 & -2.162654 \\ 6 & 4.798050 & 0.960667 & -0.808680 \\ 6 & 3.600739 & 1.249549 & -0.182724 \\ 6 & 3.179267 & 2.564567 & 0.093746 \\ 6 & 3.968027 & 3.616321 & -0.286653 \\ 6 & 5.183430 & -0.454917 & -1.020107 \\ 6 & 4.302424 & -1.313581 & -1.655861 \\ 6 & 4.647000 & -2.642793 & -1.976885 \\ 6 & 5.860297 & -3.144178 & -1.593899 \\ 6 & 6.763596 & -2.348937 & -0.848926 \\ 6 & 6.424102 & -0.995525 & -0.546353 \\ 6 & 7.991865 & -2.881653 & -0.387662 \\ 6 & 8.849676 & -2.125122 & 0.368206 \\ 6 & 8.502192 & -0.799194 & 0.700750 \\ 6 & 7.325319 & -0.249607 & 0.256148 \\ 8 & 3.055385 & -0.881427 & -2.044216 \\ 15 & 1.851802 & -0.509016 & -0.939485 \\ 8 & 2.781248 & 0.214449 & 0.225218 \\ 7 & 1.490579 & -1.903063 & -0.047017 \\ 6 & 0.425260 & -2.681888 & -0.619507 \\ 6 & -0.865096 & -2.643513 & -0.117698 \\ 6 & -1.909312 & -3.320211 & -0.828682 \\ 6 & -1.594717 & -4.086801 & -1.989688 \\ 6 & -0.250610 & -4.159364 & -2.422892 \\ & & & \\ 6\end{array}$




\begin{tabular}{|c|c|c|c|}
\hline 5 & 0.723661 & -3.458483 & -1.766219 \\
\hline 5 & -2.629043 & -4.766020 & -2.678781 \\
\hline 6 & -3.928497 & -4.687502 & -2.248297 \\
\hline 6 & -4.248377 & -3.914849 & -1.110972 \\
\hline 6 & -3.266369 & -3.253704 & -0.416003 \\
\hline 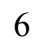 & -1.180642 & -2.071408 & 1.222323 \\
\hline 6 & -1.476391 & -0.735518 & 1.444572 \\
\hline 0 & -1.841699 & -0.310827 & 2.752181 \\
\hline 6 & -1.927812 & -1.196051 & 3.791550 \\
\hline 6 & -1.647974 & -2.567844 & 3.596898 \\
\hline 6 & -1.260153 & -3.014636 & 2.301326 \\
\hline 6 & -0.976791 & -4.395536 & 2.132042 \\
\hline 6 & -1.091152 & -5.277364 & 3.178136 \\
\hline 6 & -1.484128 & -4.828679 & 4.455901 \\
\hline 6 & -1.746943 & -3.497957 & 4.659034 \\
\hline 15 & -1.533442 & 0.449978 & 0.020405 \\
\hline 6 & -1.492053 & 2.080353 & 0.858762 \\
\hline 6 & -0.461226 & 2.356729 & 1.767340 \\
\hline 6 & -0.349231 & 3.607982 & 2.363116 \\
\hline 6 & -1.266153 & 4.602847 & 1.981984 \\
\hline 6 & -2.278759 & 4.369305 & 1.056447 \\
\hline 6 & -2.381039 & 3.083989 & 0.505058 \\
\hline 6 & 0.711060 & 3.917185 & 3.419639 \\
\hline 6 & 1.530736 & 2.681901 & 3.789567 \\
\hline 6 & -3.293403 & 5.430408 & 0.637367 \\
\hline 6 & -3.047189 & 6.774116 & \\
\hline 6 & 2.451543 & -2.593385 & 0.835371 \\
\hline 6 & 2.126957 & -2.513746 & 2.305724 \\
\hline 6 & 1.833207 & -1.293616 & 2.920342 \\
\hline 6 & 1.609880 & -1.232090 & 4.290329 \\
\hline 6 & 1.674356 & -2.386763 & 5.067446 \\
\hline 6 & 1.960901 & -3.605278 & 4.463101 \\
\hline 6 & 2.185438 & -3.664638 & 3.090190 \\
\hline 45 & 0.074288 & 0.659467 & -1.843678 \\
\hline 6 & -0.349227 & 2.231255 & -2.836623 \\
\hline 8 & -0.637394 & 3.230259 & -3.343445 \\
\hline 6 & -0.445625 & -0.606883 & -3.239512 \\
\hline 8 & -0.708006 & -1.252794 & -4.158367 \\
\hline 6 & -3.296328 & 0.279310 & -0.481884 \\
\hline 6 & -4.334044 & 0.274985 & 0.459641 \\
\hline 6 & -5.655575 & 0.106013 & 0.062833 \\
\hline 6 & -5.918956 & -0.048900 & -1.309661 \\
\hline 6 & -4.913976 & -0.031764 & -2.271055 \\
\hline 6 & -3.594843 & 0.130188 & -1.826855 \\
\hline 6 & -6.803182 & 0.034561 & 1.068435 \\
\hline 6 & -6.343446 & 0.344438 & 2.491838 \\
\hline 6 & -5.169912 & -0.241189 & -3.760912 \\
\hline 6 & -6.655483 & -0.183703 & -4.107850 \\
\hline 6 & -7.898735 & 1.038441 & 0.696163 \\
\hline 6 & -7.380507 & -1.385620 & 1.051730 \\
\hline 6 & -4.632195 & -1.622838 & -4.151718 \\
\hline 6 & -4.447545 & 0.835823 & -4.579028 \\
\hline 6 & -3.214104 & 5.640611 & -0.879145 \\
\hline 6 & -4.701779 & 4.953596 & 1.014230 \\
\hline 6 & 0.026130 & 4.419790 & 4.696193 \\
\hline 6 & 1.664897 & 4.996830 & 2.899031 \\
\hline
\end{tabular}




\begin{tabular}{|c|c|c|c|}
\hline & 7.068824 & 0.769993 & 0.531765 \\
\hline & -2.067041 & 0.738435 & 2.928866 \\
\hline 1 & 8.233829 & -3.913147 & -0.640734 \\
\hline 1 & -2.041107 & -3.130830 & 5.641662 \\
\hline & 5.633603 & 5.491288 & -1.202079 \\
\hline & -3.529660 & -2.653686 & 0.454153 \\
\hline & -5.283902 & -3.839431 & -0.784944 \\
\hline & 3.661868 & 4.640693 & -0.078999 \\
\hline & -2.221146 & -0.854755 & 4.783821 \\
\hline & 6.140301 & -4.166946 & -1.840809 \\
\hline & 8.397778 & 2.788247 & -3.077698 \\
\hline & 3.933148 & -3.240100 & -2.539362 \\
\hline & -2.367419 & -5.348974 & -3.560676 \\
\hline & -0.010336 & -4.755256 & -3.301913 \\
\hline & -0.872334 & -6.331220 & 3.020410 \\
\hline ] & 7.080483 & 0.871974 & -2.311654 \\
\hline & -0.657996 & -4.760313 & 1.157599 \\
\hline & 1.754644 & -3.479071 & -2.115136 \\
\hline & 7.705420 & 5.113546 & -2.506744 \\
\hline & 9.170642 & -0.206516 & 1.321203 \\
\hline & -4.716567 & -5.210494 & -2.785813 \\
\hline & -1.570736 & -5.537228 & 5.276657 \\
\hline 1 & 9.788250 & -2.545472 & 0.721663 \\
\hline & 2.226929 & 2.711252 & 0.601892 \\
\hline 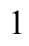 & -4.092477 & 0.398747 & 1.512377 \\
\hline & -2.776360 & 0.130767 & -2.543253 \\
\hline 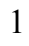 & -6.951095 & -0.189820 & -1.625001 \\
\hline 1 & -3.167817 & 2.866946 & -0.216780 \\
\hline & 0.242720 & 1.562294 & 2.016463 \\
\hline 1 & -1.177939 & 5.588969 & 2.433074 \\
\hline & 0.696873 & 1.806424 & -0.902759 \\
\hline 1 & 2.516039 & -3.646864 & 0.523939 \\
\hline & 3.446694 & -2.161143 & 0.671167 \\
\hline & 2.401424 & -4.622599 & 2.616157 \\
\hline 1 & 1.779642 & -0.388332 & 2.316493 \\
\hline | & 1.998518 & -4.516208 & 5.057361 \\
\hline 1 & 1.382991 & -0.274306 & 4.756372 \\
\hline & 1.493482 & -2.336377 & 6.139295 \\
\hline 1 & -6.786767 & -0.305209 & -5.190428 \\
\hline ] & -7.224567 & -0.986497 & -3.621904 \\
\hline 1 & -7.104425 & 0.777301 & -3.824249 \\
\hline 1 & -4.769263 & -1.798758 & -5.227512 \\
\hline 1 & -3.562451 & -1.724538 & -3.925414 \\
\hline 1 & -5.160921 & -2.414868 & -3.604637 \\
\hline & -4.655972 & 0.698239 & -5.648132 \\
\hline 1 & -4.783496 & 1.841810 & -4.295600 \\
\hline 1 & -3.357363 & 0.798982 & -4.455825 \\
\hline 1 & -7.201669 & 0.298226 & 3.173987 \\
\hline 1 & -5.599119 & -0.377519 & 2.853769 \\
\hline 1 & -5.911052 & 1.350900 & 2.570835 \\
\hline 1 & -8.207743 & -1.473686 & 1.769098 \\
\hline 1 & -7.765728 & -1.660335 & 0.061897 \\
\hline 1 & -6.611744 & -2.119813 & 1.329676 \\
\hline 1 & -8.720443 & 0.989055 & 1.422971 \\
\hline 1 & -7.509430 & 2.064878 & 0.697032 \\
\hline 1 & -8.326259 & 0.843615 & -0.294341 \\
\hline
\end{tabular}




$\begin{array}{rrrr}1 & -3.798556 & 7.500001 & 0.982446 \\ 1 & -3.125349 & 6.702836 & 2.410615 \\ 1 & -2.059983 & 7.184214 & 1.068712 \\ 1 & -3.931952 & 6.409758 & -1.194628 \\ 1 & -2.210456 & 5.968467 & -1.179203 \\ 1 & -3.443323 & 4.724949 & -1.437282 \\ 1 & -5.450775 & 5.696066 & 0.707237 \\ 1 & -4.959561 & 4.001706 & 0.532148 \\ 1 & -4.790392 & 4.812269 & 2.099622 \\ 1 & 2.244342 & 2.934679 & 4.583703 \\ 1 & 0.886224 & 1.873597 & 4.163644 \\ 1 & 2.113102 & 2.294709 & 2.942062 \\ 1 & 2.416204 & 5.242387 & 3.661691 \\ 1 & 2.197637 & 4.653191 & 2.002286 \\ 1 & 1.135898 & 5.922314 & 2.641060 \\ 1 & 0.775133 & 4.622930 & 5.473177 \\ 1 & -0.535216 & 5.346832 & 4.531028 \\ 1 & -0.673211 & 3.670034 & 5.088700\end{array}$

$1_{\mathrm{C} 10-} \psi_{-1 \mathrm{a} 23354 \mathrm{~s}}$

Number of imaginary frequencies : 0

The smallest frequencies are : $12.7398 \quad 15.9075 \quad 23.3557 \mathrm{~cm}(-1)$

Electronic energy : $\quad H F=-4125.4644672$

Zero-point correction $=\quad 1.300068($ Hartree/Particle $)$

Thermal correction to Energy $=\quad 1.378048$

Thermal correction to Enthalpy= $\quad 1.378992$

Thermal correction to Gibbs Free Energy $=\quad 1.188903$

Sum of electronic and zero-point Energies $=\quad-4124.164399$

Sum of electronic and thermal Energies $=\quad-4124.086419$

Sum of electronic and thermal Enthalpies $=\quad-4124.085475$

Sum of electronic and thermal Free Energies $=\quad-4124.275565$

Cartesian Coordinates

\begin{tabular}{|c|c|c|c|}
\hline 6 & 6.159665 & 4.586284 & -1.001558 \\
\hline 6 & 5.405018 & 3.456877 & -0.601056 \\
\hline 6 & 5.771935 & 2.162442 & -1.080614 \\
\hline o & 6.859129 & 2.069997 & -1.986280 \\
\hline 6 & 7.561841 & 3.186394 & -2.366322 \\
\hline 6 & 7.219397 & 4.458497 & -1.862048 \\
\hline 6 & 5.012797 & 1.020999 & -0.665816 \\
\hline 6 & 3.890842 & 1.238710 & 0.108731 \\
\hline 6 & 3.524475 & 2.510166 & 0.588276 \\
\hline 6 & 4.283933 & 3.597642 & 0.252631 \\
\hline 6 & 5.355458 & -0.369493 & -1.046691 \\
\hline 6 & 4.404006 & -1.164874 & -1.664362 \\
\hline 6 & 4.691747 & -2.476079 & -2.097529 \\
\hline 6 & 5.924754 & -3.019376 & -1.861798 \\
\hline 6 & 6.909790 & -2.289343 & -1.155774 \\
\hline 6 & 6.623519 & -0.957298 & -0.728772 \\
\hline 6 & 8.165770 & -2.867506 & -0.850257 \\
\hline 6 & 9.102939 & -2.176996 & -0.126139 \\
\hline 0 & 8.810331 & -0.875176 & 0.331541 \\
\hline 6 & 7.607400 & -0.282325 & 0.038925 \\
\hline 8 & 3.143269 & -0.677526 & -1.926865 \\
\hline
\end{tabular}




\begin{tabular}{|c|c|c|c|}
\hline 15 & 2.039879 & -0.353104 & -0.703392 \\
\hline 8 & 3.097353 & 0.163236 & 0.458196 \\
\hline 7 & 1.624773 & -1.807582 & 0.054396 \\
\hline 6 & 0.561653 & -2.516567 & -0.606732 \\
\hline 6 & -0.706314 & -2.636400 & -0.060866 \\
\hline 6 & -1.730629 & -3.303949 & -0.808743 \\
\hline 6 & -1.443487 & -3.810072 & -2.110659 \\
\hline 6 & -0.135268 & -3.675101 & -2.625546 \\
\hline 6 & 0.835440 & -3.052617 & -1.889682 \\
\hline 6 & -2.467638 & -4.440265 & -2.858686 \\
\hline 6 & -3.735349 & -4.561190 & -2.350598 \\
\hline 6 & -4.025890 & -4.061192 & -1.063193 \\
\hline 6 & -3.050060 & -3.460101 & -0.308563 \\
\hline 6 & -1.041135 & -2.143728 & 1.304955 \\
\hline 6 & -1.472013 & -0.842798 & 1.536286 \\
\hline 6 & -1.874599 & -0.468457 & 2.845726 \\
\hline 6 & -1.849450 & -1.363789 & 3.882111 \\
\hline 6 & -1.427691 & -2.696362 & 3.677531 \\
\hline 6 & -1.023666 & -3.097182 & 2.371671 \\
\hline 6 & -0.630439 & -4.447377 & 2.178249 \\
\hline 6 & -0.631955 & -5.341220 & 3.220083 \\
\hline 6 & -1.017461 & -4.933788 & 4.514851 \\
\hline 6 & -1.402243 & -3.636146 & 4.735865 \\
\hline 15 & -1.597998 & 0.351675 & 0.121749 \\
\hline 6 & -2.058098 & 1.951955 & 0.906092 \\
\hline 6 & -1.209856 & 2.531087 & 1.858671 \\
\hline 6 & -1.411336 & 3.831153 & 2.307951 \\
\hline 6 & -2.480725 & 4.559514 & 1.761385 \\
\hline 6 & -3.338846 & 4.016920 & 0.809639 \\
\hline 6 & -3.112606 & 2.697905 & 5511 \\
\hline 6 & -0.455932 & & 3.29 \\
\hline 6 & 0.427003 & 3.471031 & 4.016978 \\
\hline 6 & -4.483025 & 4.799383 & 0.168968 \\
\hline 6 & -4.671115 & 6.176841 & 0.799016 \\
\hline 6 & 2.500801 & -2.549289 & 0.977588 \\
\hline 6 & 2.216123 & -2.332290 & 2.445638 \\
\hline 6 & 1.775911 & -1.107614 & 2.950252 \\
\hline 6 & 1.581931 & -0.935041 & 4.316359 \\
\hline 6 & 1.822123 & -1.982991 & 5.200363 \\
\hline 6 & 2.257333 & -3.208417 & 4.706157 \\
\hline 6 & 2.452022 & -3.378144 & 3.339070 \\
\hline 45 & 0.300417 & 1.018202 & -1.297664 \\
\hline 6 & 0.086096 & 2.895623 & -1.663032 \\
\hline 8 & -0.057109 & 4.038920 & -1.751214 \\
\hline 6 & 0.018885 & 0.292775 & -3.080671 \\
\hline 8 & -0.072979 & -0.104887 & -4.160161 \\
\hline 6 & -3.200470 & -0.154766 & -0.622296 \\
\hline 6 & -4.301071 & -0.418389 & 0.192778 \\
\hline 6 & -5.540878 & -0.741940 & -0.352928 \\
\hline 6 & -5.652872 & -0.772018 & -1.749169 \\
\hline 6 & -4.574795 & -0.496628 & -2.593977 \\
\hline 6 & -3.341965 & -0.198526 & -2.003267 \\
\hline 6 & -6.717692 & -1.009209 & 0.585011 \\
\hline 6 & -7.947545 & -1.526417 & -0.156904 \\
\hline 6 & -4.694586 & -0.472509 & -4.116728 \\
\hline 6 & -6.101755 & -0.821450 & -4.594467 \\
\hline
\end{tabular}




\begin{tabular}{|c|c|c|c|}
\hline 6 & -6.326873 & -2.055144 & 1.636041 \\
\hline 6 & -7.091302 & 0.300602 & 1.289720 \\
\hline 6 & -3.720379 & -1.481769 & -4.731990 \\
\hline 6 & -4.351610 & 0.934873 & -4.620710 \\
\hline 6 & -4.174959 & 4.989142 & -1.321619 \\
\hline 6 & -5.794807 & 4.020302 & 0.320870 \\
\hline 6 & -1.227854 & 5.270198 & 4.370011 \\
\hline 6 & 0.446724 & 5.456805 & 2.520922 \\
\hline 1 & 7.396015 & 0.716614 & 0.410877 \\
\hline 1 & -2.221503 & 0.546032 & 3.028198 \\
\hline 1 & 8.365188 & -3.880739 & -1.196779 \\
\hline 1 & -1.701197 & -3.304195 & 5.729512 \\
\hline 1 & 5.868462 & 5.563178 & -0.617844 \\
\hline 1 & -3.290916 & -3.080111 & 0.682385 \\
\hline 1 & -5.035573 & -4.153627 & -0.665719 \\
\hline 1 & 4.022009 & 4.589177 & 0.618331 \\
\hline 1 & -2.164902 & -1.060017 & 4.879778 \\
\hline 1 & 6.158465 & -4.027801 & -2.199334 \\
\hline 1 & 8.386666 & 3.089299 & -3.068695 \\
\hline 1 & 3.922477 & -3.029097 & -2.630811 \\
\hline 1 & -2.225645 & -4.817806 & -3.851474 \\
\hline 1 & 0.087238 & -4.069013 & -3.615947 \\
\hline 1 & -0.330146 & -6.371940 & 3.047274 \\
\hline 1 & 7.127761 & 1.098310 & -2.393032 \\
\hline 1 & -0.324749 & -4.774834 & 1.186590 \\
\hline 1 & 1.841093 & -2.941199 & -2.286506 \\
\hline 1 & 7.787576 & 5.334028 & -2.167489 \\
\hline 1 & 9.543079 & -0.336665 & 0.928345 \\
\hline 1 & -4.518127 & -5.039178 & -2.935713 \\
\hline 1 & -1.007951 & -5.650212 & 5.333222 \\
\hline 1 & 10.062637 & -2.632051 & \\
\hline 1 & 2.642030 & 2.590818 & 1.219206 \\
\hline 1 & -4.184126 & -0.362314 & 1.274919 \\
\hline 1 & -2.473480 & 0.016418 & -2.624702 \\
\hline 1 & -6.614879 & -1.015102 & -2.190881 \\
\hline 1 & -3.758486 & 2.260427 & -0.363855 \\
\hline 1 & -0.372070 & 1.947702 & 2.238263 \\
\hline 1 & -2.631852 & 5.585668 & 2.087825 \\
\hline 1 & 0.725131 & 1.642458 & 0.126485 \\
\hline 1 & 2.419837 & -3.619157 & 0.736626 \\
\hline 1 & 3.544668 & -2.271130 & 0.775254 \\
\hline 1 & 2.779817 & -4.344533 & 2.954371 \\
\hline 1 & 1.575435 & -0.284040 & 2.268359 \\
\hline 1 & 2.433688 & -4.040833 & 5.384674 \\
\hline 1 & 1.230159 & 0.025584 & 4.689710 \\
\hline 1 & 1.662556 & -1.847993 & 6.268231 \\
\hline 1 & -6.135294 & -0.794500 & -5.690857 \\
\hline 1 & -6.401320 & -1.830308 & -4.280991 \\
\hline 1 & -6.851305 & -0.108979 & -4.226220 \\
\hline 1 & -3.792435 & -1.457275 & -5.827764 \\
\hline 1 & -2.677227 & -1.271308 & -4.465057 \\
\hline 1 & -3.950680 & -2.500463 & -4.395290 \\
\hline 1 & -4.446595 & 0.981670 & -5.713879 \\
\hline 1 & -5.027920 & 1.684380 & -4.188924 \\
\hline 1 & -3.323932 & 1.224503 & -4.366088 \\
\hline 1 & -8.750514 & -1.737223 & 0.560650 \\
\hline
\end{tabular}




$\begin{array}{lrrr}1 & -8.337508 & -0.792891 & -0.873757 \\ 1 & -7.734406 & -2.457254 & -0.699655 \\ 1 & -7.920014 & 0.137270 & 1.992273 \\ 1 & -6.246437 & 0.714302 & 1.855565 \\ 1 & -7.409587 & 1.059666 & 0.562588 \\ 1 & -7.169371 & -2.240294 & 2.315676 \\ 1 & -6.063393 & -3.011780 & 1.164606 \\ 1 & -5.474741 & -1.740099 & 2.251376 \\ 1 & -5.515855 & 6.688834 & 0.321140 \\ 1 & -4.889194 & 6.109776 & 1.872945 \\ 1 & -3.787275 & 6.813606 & 0.665842 \\ 1 & -4.976752 & 5.560471 & -1.809080 \\ 1 & -3.232703 & 5.534993 & -1.460907 \\ 1 & -4.081972 & 4.029239 & -1.845284 \\ 1 & -6.622764 & 4.579817 & -0.134794 \\ 1 & -5.753023 & 3.038507 & -0.168549 \\ 1 & -6.038133 & 3.857277 & 1.379219 \\ 1 & 1.048148 & 3.979177 & 4.765133 \\ 1 & -0.174066 & 2.713231 & 4.538475 \\ 1 & 1.110907 & 2.952365 & 3.332142 \\ 1 & 1.170183 & 5.939445 & 3.192553 \\ 1 & 1.005368 & 4.922557 & 1.739375 \\ 1 & -0.136042 & 6.244870 & 2.028005 \\ 1 & -0.527018 & 5.715933 & 5.087956 \\ 1 & -1.822305 & 6.089539 & 3.950125 \\ 1 & -1.907125 & 4.611616 & 4.926631\end{array}$

$1_{\text {C10- }} \psi_{\text {-1s2a3a4a }}$

Number of imaginary frequencies : 0

The smallest frequencies are : $13.9669 \quad 16.6829 \quad 22.2289 \mathrm{~cm}(-1)$

Electronic energy : $\quad \mathrm{HF}=-4125.464155$

Zero-point correction $=\quad 1.300916($ Hartree/Particle $)$

Thermal correction to Energy $=\quad 1.378451$

Thermal correction to Enthalpy $=\quad 1.379395$

Thermal correction to Gibbs Free Energy= 1.191467

Sum of electronic and zero-point Energies $=\quad-4124.163239$

Sum of electronic and thermal Energies $=\quad-4124.085704$

Sum of electronic and thermal Enthalpies $=\quad-4124.084760$

Sum of electronic and thermal Free Energies $=\quad-4124.272688$

Cartesian Coordinates

$\begin{array}{lrrr}6 & 1.772160 & -1.580194 & 5.240207 \\ 6 & 2.179475 & -2.844576 & 4.828186 \\ 6 & 2.400309 & -3.100704 & 3.477792 \\ 6 & 2.221053 & -2.101161 & 2.522566 \\ 6 & 1.811581 & -0.834956 & 2.944999 \\ 6 & 1.590229 & -0.576334 & 4.292434 \\ 6 & 2.539152 & -2.393198 & 1.076417 \\ 7 & 1.621175 & -1.775510 & 0.102531 \\ 6 & 0.576942 & -2.585374 & -0.465624 \\ 6 & -0.701851 & -2.625564 & 0.064977 \\ 6 & -1.725600 & -3.346483 & -0.631221 \\ 6 & -1.413778 & -4.022613 & -1.847660 \\ 6 & -0.083803 & -4.001886 & -2.323803\end{array}$




\begin{tabular}{|c|c|c|c|}
\hline 6 & 0.878274 & -3.300178 & -1.650651 \\
\hline 6 & -3.065489 & -3.395157 & -0.162630 \\
\hline & -4.036034 & -4.062992 & -0.866242 \\
\hline & -3.719567 & -4.735555 & -2.066775 \\
\hline & -2.435023 & -4.712832 & -2.545906 \\
\hline & -1.045115 & -2.020540 & 1.382085 \\
\hline & -1.465509 & -0.704644 & 1.512248 \\
\hline 6 & -1.895228 & -0.239445 & 2.784929 \\
\hline 6 & -1.891859 & -1.055844 & 3.883564 \\
\hline 6 & -1.468657 & -2.400658 & 3.784423 \\
\hline 6 & -1.046909 & -2.895797 & 2.517154 \\
\hline 6 & -1.470065 & -3.262491 & 4.906841 \\
\hline 6 & -1.089535 & -4.574936 & 4.786641 \\
\hline 6 & -0.675618 & -5.073038 & 3.533615 \\
\hline 6 & -0.650311 & -4.255986 & 2.430512 \\
\hline 15 & -1.565093 & 0.384607 & 0.008455 \\
\hline 45 & 0.197445 & 0.745103 & -1.707444 \\
\hline 6 & -0.104510 & -0.446881 & -3.224217 \\
\hline 8 & -0.176791 & -1.077222 & -4.187242 \\
\hline 15 & 1.987774 & -0.411789 & -0.831561 \\
\hline 8 & 2.962972 & 0.318172 & 0.284584 \\
\hline 6 & 3.781354 & 1.333126 & -0.172221 \\
\hline 6 & 4.958287 & 1.010779 & -0.818271 \\
\hline 6 & 5.759146 & 2.085218 & -1.324811 \\
\hline 6 & 5.368142 & 3.433367 & -1.059804 \\
\hline 6 & 4.178432 & 3.689570 & -0.336142 \\
\hline 6 & 3.383258 & 2.658137 & 0.083385 \\
\hline 6 & 6.166708 & 4.497397 & -1.545516 \\
\hline 6 & 7.293070 & 4.253403 & -2.287981 \\
\hline 6 & 7.660660 & 2.924329 & -2.584738 \\
\hline$b$ & 6.915720 & 1.870484 & -2.116886 \\
\hline 6 & 5.314183 & -0.417160 & -0.993494 \\
\hline 6 & 4.402206 & -1.281383 & -1.577453 \\
\hline 6 & 4.714969 & -2.629739 & -1.848548 \\
\hline 6 & 5.926243 & -3.139845 & -1.469831 \\
\hline 6 & 6.860544 & -2.334424 & -0.776539 \\
\hline 6 & 6.553273 & -0.963541 & -0.522539 \\
\hline 6 & 7.483123 & -0.205440 & 0.234826 \\
\hline 6 & 8.656840 & -0.760872 & 0.680213 \\
\hline 6 & 8.972790 & -2.105330 & 0.393401 \\
\hline 6 & 8.086785 & -2.873365 & -0.316852 \\
\hline 8 & 3.155346 & -0.837741 & -1.958774 \\
\hline 6 & -3.254104 & -0.037561 & -0.595701 \\
\hline 6 & -4.337048 & -0.063951 & 0.286871 \\
\hline 6 & -5.620554 & -0.365003 & -0.162140 \\
\hline 6 & -5.782469 & -0.646470 & -1.523993 \\
\hline 6 & -4.725631 & -0.625574 & -2.435282 \\
\hline 6 & -3.453210 & -0.314468 & -1.944435 \\
\hline 6 & -6.818745 & -0.434347 & 0.782959 \\
\hline 6 & -4.987301 & -0.899948 & -3.915091 \\
\hline 6 & -1.816118 & 2.055800 & 0.734167 \\
\hline 6 & -2.803997 & 2.902947 & 0.226607 \\
\hline 6 & -2.935232 & 4.210208 & 0.690427 \\
\hline 6 & -2.059763 & 4.645505 & 1.694303 \\
\hline 6 & -1.058271 & 3.828464 & 2.218751 \\
\hline 6 & -0.933990 & 2.535560 & 1.699390 \\
\hline
\end{tabular}




\begin{tabular}{|c|c|c|c|}
\hline 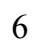 & -4.001919 & 5.160903 & 0.150404 \\
\hline & -0.150301 & 4.262200 & 3.369034 \\
\hline & -0.214344 & 2.382139 & -2.606902 \\
\hline & -0.486106 & 3.408805 & -3.063710 \\
\hline & 7.251172 & 0.828544 & 0.476100 \\
\hline & -2.240717 & 0.786069 & 2.891423 \\
\hline & 8.303711 & -3.918762 & -0.532898 \\
\hline & -1.788140 & -2.859646 & 5.867730 \\
\hline & 5.855731 & 5.517595 & -1.324581 \\
\hline & -3.325341 & -2.878426 & 0.759371 \\
\hline & -5.060099 & -4.073551 & -0.498123 \\
\hline & 3.893611 & 4.722042 & -0.139443 \\
\hline & -2.226725 & -0.678990 & 4.849609 \\
\hline & 6.179920 & -4.177590 & -1.679987 \\
\hline & 8.539507 & 2.731763 & -3.196011 \\
\hline & 3.982096 & -3.238883 & -2.372187 \\
\hline 1 & -2.174475 & -5.226415 & -3.470285 \\
\hline & 0.157469 & -4.533419 & -3.242934 \\
\hline & -0.371646 & -6.113420 & 3.441142 \\
\hline & 7.205976 & 0.852773 & -2.364877 \\
\hline & -0.321369 & -4.654444 & 1.472646 \\
\hline & 1.897750 & -3.257048 & -2.026713 \\
\hline & 7.895118 & 5.078989 & -2.660304 \\
\hline & 9.347313 & -0.158365 & 1.266112 \\
\hline 1 & -4.497076 & -5.271402 & -2.607381 \\
\hline 1 & -1.102542 & -5.231842 & 5.653486 \\
\hline & 9.909276 & -2.530382 & 0.746806 \\
\hline . & 2.447335 & 2.825618 & 0.611923 \\
\hline & -4.166709 & 0.161279 & 1.336842 \\
\hline 1 & -2.592114 & -0.287888 & -2.608370 \\
\hline 1 & -6.780528 & -0.888117 & -1.889359 \\
\hline 1 & -3.471755 & 2.527380 & -0.544763 \\
\hline 1 & -0.149423 & 1.876337 & 2.076861 \\
\hline ( & -2.169671 & 5.659239 & 2.073466 \\
\hline 1 & 0.690958 & 1.813681 & -0.607278 \\
\hline 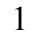 & 2.543226 & -3.479648 & 0.903570 \\
\hline 1 & 3.560389 & -2.048655 & 0.865190 \\
\hline 1 & 2.706112 & -4.097333 & 3.157871 \\
\hline 1 & 1.664997 & -0.048806 & 2.207712 \\
\hline 1 & 2.311391 & -3.641356 & 5.557673 \\
\hline 1 & 1.271425 & 0.418099 & 4.603965 \\
\hline 1 & 1.591867 & -1.379152 & 6.294274 \\
\hline U & -3.691595 & -1.001228 & -4.716439 \\
\hline 6 & -5.746960 & -2.219142 & -4.083944 \\
\hline 6 & -5.824198 & 0.248796 & -4.489444 \\
\hline 6 & -6.480132 & 0.085203 & 2.179222 \\
\hline 6 & -7.976759 & 0.405998 & 0.235552 \\
\hline 6 & -7.263201 & -1.896053 & 0.907644 \\
\hline 1 & -3.922614 & -1.231186 & -5.764205 \\
\hline 1 & -3.124680 & -0.061095 & -4.708361 \\
\hline 1 & -3.041619 & -1.800424 & -4.334859 \\
\hline 1 & -5.925884 & -2.417389 & -5.149160 \\
\hline 1 & -5.165668 & -3.056416 & -3.674988 \\
\hline 1 & -6.723583 & -2.209522 & -3.585425 \\
\hline 1 & -6.018193 & 0.084560 & -5.558140 \\
\hline & -6.793455 & 0.341326 & -3.983597 \\
\hline
\end{tabular}




$\begin{array}{rrrr}1 & -5.299376 & 1.207427 & -4.383789 \\ 1 & -7.374244 & 0.045839 & 2.814040 \\ 1 & -5.705233 & -0.518134 & 2.670607 \\ 1 & -6.136363 & 1.128236 & 2.152008 \\ 1 & -8.132237 & -1.979435 & 1.574466 \\ 1 & -7.544426 & -2.321392 & -0.064172 \\ 1 & -6.456786 & -2.513501 & 1.326865 \\ 1 & -8.828919 & 0.371092 & 0.927153 \\ 1 & -7.680962 & 1.456481 & 0.117245 \\ 1 & -8.332448 & 0.046480 & -0.737113 \\ 6 & -4.877993 & 4.503294 & -0.914039 \\ 6 & -4.912119 & 5.616278 & 1.296417 \\ 6 & -3.319484 & 6.379899 & -0.479246 \\ 6 & -0.347114 & 5.728575 & 3.746058 \\ 6 & -0.481167 & 3.403147 & 4.596196 \\ 6 & 1.321822 & 4.063702 & 2.994978 \\ 1 & -5.634114 & 5.218725 & -1.261148 \\ 1 & -4.295680 & 4.189922 & -1.790228 \\ 1 & -5.409564 & 3.624007 & -0.524440 \\ 1 & -5.685104 & 6.299647 & 0.919830 \\ 1 & -5.416475 & 4.758724 & 1.761289 \\ 1 & -4.358881 & 6.144974 & 2.081845 \\ 1 & -4.070410 & 7.071880 & -0.884026 \\ 1 & -2.714521 & 6.934891 & 0.247939 \\ 1 & -2.658840 & 6.075974 & -1.301674 \\ 1 & 0.334533 & 5.993101 & 4.564188 \\ 1 & -0.130588 & 6.399482 & 2.904655 \\ 1 & -1.367708 & 5.931461 & 4.094583 \\ 1 & 1.969926 & 4.394261 & 3.817503 \\ 1 & 1.565017 & 3.012119 & 2.796039 \\ 1 & 1.583437 & 4.649834 & 2.103441 \\ 1 & 0.156414 & 3.682893 & 5.445949 \\ 1 & -1.528128 & 3.539136 & 4.898407 \\ 1 & -0.328784 & 2.333308 & 4.399665\end{array}$

$1_{\mathrm{C} 10-}-\psi_{-1 \mathrm{~s} 2 \mathrm{a3a} a \mathrm{~s}}$

Number of imaginary frequencies : 0

The smallest frequencies are : $13.4373 \quad 14.0084 \quad 22.3170 \mathrm{~cm}(-1)$

Electronic energy : $\quad H F=-4125.4648234$

Zero-point correction $=\quad 1.299902($ Hartree/Particle $)$

Thermal correction to Energy= $\quad 1.377914$

Thermal correction to Enthalpy= $\quad 1.378859$

Thermal correction to Gibbs Free Energy $=\quad 1.188305$

Sum of electronic and zero-point Energies $=\quad-4124.164921$

Sum of electronic and thermal Energies $=\quad-4124.086909$

Sum of electronic and thermal Enthalpies $=\quad-4124.085965$

Sum of electronic and thermal Free Energies $=\quad-4124.276519$

Cartesian Coordinates

$\begin{array}{llll}6 & 1.778008 & -1.629324 & 5.239012 \\ 6 & 2.199712 & -2.885120 & 4.814997 \\ 6 & 2.428581 & -3.124096 & 3.462989 \\ 6 & 2.240255 & -2.116821 & 2.517315 \\ 6 & 1.813517 & -0.860066 & 2.951346\end{array}$




\begin{tabular}{|c|c|c|c|}
\hline 6 & 1.586603 & -0.618107 & 4.300979 \\
\hline 6 & 2.565726 & -2.394243 & 1.069757 \\
\hline & 1.651756 & -1.769921 & 0.097058 \\
\hline & 0.610915 & -2.574354 & -0.484564 \\
\hline & -0.665562 & -2.636549 & 0.050011 \\
\hline & -1.686355 & -3.351860 & -0.656367 \\
\hline & -1.376278 & -3.992364 & -1.892358 \\
\hline p & -0.049350 & -3.947596 & -2.375218 \\
\hline & 0.911575 & -3.257557 & -1.688334 \\
\hline 6 & -3.022921 & -3.425307 & -0.180791 \\
\hline 6 & -3.991326 & -4.083616 & -0.896116 \\
\hline 5 & -3.677344 & -4.718595 & -2.117588 \\
\hline 6 & -2.396111 & -4.671300 & -2.603782 \\
\hline 6 & -1.015177 & -2.046073 & 1.371965 \\
\hline 6 & -1.449115 & -0.734788 & 1.505846 \\
\hline 6 & -1.889408 & -0.278938 & 2.777865 \\
\hline 6 & -1.886632 & -1.101362 & 3.872288 \\
\hline 6 & -1.451205 & -2.441798 & 3.768896 \\
\hline 6 & -1.016285 & -2.926651 & 2.502023 \\
\hline 6 & -1.451256 & -3.309089 & 4.887226 \\
\hline 6 & -1.058474 & -4.617505 & 4.762341 \\
\hline 6 & -0.632984 & -5.105749 & 3.509215 \\
\hline 6 & -0.607850 & -4.282883 & 2.410548 \\
\hline & -1.554449 & 0.355654 & 0.003939 \\
\hline 45 & 0.226054 & 0.774733 & -1.671331 \\
\hline 6 & -0.053479 & -0.377667 & -3.222409 \\
\hline 8 & -0.115780 & -0.985993 & -4.200193 \\
\hline 15 & 2.017183 & -0.389663 & -0.811931 \\
\hline 8 & 2.990852 & 0.320296 & 0.318542 \\
\hline 6 & 3.797448 & 1.353214 & -0.118362 \\
\hline 6 & 4.972386 & 1.057900 & -0.781035 \\
\hline 6 & 5.756381 & 2.152337 & -1.270990 \\
\hline 6 & 5.353447 & 3.489812 & -0.972054 \\
\hline 6 & 4.169386 & 3.716808 & -0.229590 \\
\hline 6 & 3.389349 & 2.667615 & 0.173648 \\
\hline 6 & 6.135046 & 4.573199 & -1.442236 \\
\hline 6 & 7.256045 & 4.358299 & -2.201636 \\
\hline 6 & 7.635197 & 3.040331 & -2.531474 \\
\hline 6 & 6.906975 & 1.968089 & -2.079293 \\
\hline 6 & 5.344503 & -0.362287 & -0.984257 \\
\hline 6 & 4.438134 & -1.228563 & -1.573828 \\
\hline 6 & 4.761419 & -2.571018 & -1.861118 \\
\hline 6 & 5.980779 & -3.072897 & -1.497813 \\
\hline 6 & 6.912981 & -2.265361 & -0.804183 \\
\hline 6 & 6.593394 & -0.901112 & -0.530432 \\
\hline 6 & 7.522103 & -0.142677 & 0.228028 \\
\hline 6 & 8.706085 & -0.690680 & 0.655136 \\
\hline 6 & 9.033805 & -2.027903 & 0.348579 \\
\hline 6 & 8.149212 & -2.796640 & -0.362661 \\
\hline 8 & 3.186253 & -0.791776 & -1.945825 \\
\hline 6 & -3.222641 & -0.106609 & -0.625863 \\
\hline 6 & -4.306435 & -0.173494 & 0.244005 \\
\hline 6 & -5.589337 & -0.473880 & -0.218322 \\
\hline 6 & -5.747560 & -0.703693 & -1.585506 \\
\hline 6 & -4.679029 & -0.648598 & -2.490155 \\
\hline 6 & -3.413458 & -0.346400 & -1.986230 \\
\hline
\end{tabular}




\begin{tabular}{|c|c|c|c|}
\hline 6 & -6.743460 & -0.514220 & 0.780913 \\
\hline 6 & -4.933243 & -0.874378 & -3.979326 \\
\hline & -1.875285 & 2.015551 & 0.727432 \\
\hline & -2.903264 & 2.816526 & 0.225465 \\
\hline & -3.094366 & 4.115183 & 0.692023 \\
\hline & -2.237004 & 4.589002 & 1.693834 \\
\hline 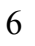 & -1.194536 & 3.819044 & 2.211300 \\
\hline & -1.013003 & 2.533587 & 1.690736 \\
\hline 6 & -4.213017 & 5.009147 & 0.160251 \\
\hline 6 & -0.302710 & 4.293720 & 3.357887 \\
\hline 6 & -0.195756 & 2.431946 & -2.529329 \\
\hline 8 & -0.477056 & 3.465575 & -2.963779 \\
\hline 1 & 7.281543 & 0.885618 & 0.484516 \\
\hline 1 & -2.245452 & 0.742715 & 2.886332 \\
\hline 1 & 8.375161 & -3.836907 & -0.593670 \\
\hline 1 & -1.778580 & -2.914067 & 5.848244 \\
\hline 1 & 5.815303 & 5.584756 & -1.195500 \\
\hline 1 & -3.281865 & -2.935826 & 0.756594 \\
\hline 1 & -5.013122 & -4.113885 & -0.522836 \\
\hline 1 & 3.876644 & 4.741373 & -0.005114 \\
\hline 1 & -2.230953 & -0.732660 & 4.838156 \\
\hline 1 & 6.243018 & -4.105822 & -1.720799 \\
\hline 1 & 8.509512 & 2.871284 & -3.156043 \\
\hline 1 & 4.029646 & -3.182325 & -2.383863 \\
\hline 1 & -2.137095 & -5.156476 & -3.543828 \\
\hline 1 & 0.190943 & -4.452450 & -3.309544 \\
\hline 1 & -0.319616 & -6.143051 & 3.413443 \\
\hline 1 & 7.205626 & 0.959303 & -2.352660 \\
\hline 1 & -0.270118 & -4.672970 & 1.452289 \\
\hline 1 & 1.928511 & -3.197077 & -2.069117 \\
\hline 1 & 7.844830 & 5.198671 & -2.561902 \\
\hline 1 & 9.395596 & -0.087865 & 1.241859 \\
\hline 1 & -4.453819 & -5.245653 & -2.668201 \\
\hline 1 & -1.070549 & -5.278985 & 5.625726 \\
\hline 1 & 9.978250 & -2.447012 & 0.687592 \\
\hline 1 & 2.458294 & 2.812703 & 0.717202 \\
\hline 1 & -4.150502 & 0.023662 & 1.304134 \\
\hline 1 & -2.549185 & -0.292523 & -2.644393 \\
\hline 1 & -6.739709 & -0.931301 & -1.970043 \\
\hline 1 & -3.555341 & 2.412507 & -0.544355 \\
\hline 1 & -0.198730 & 1.909443 & 2.065329 \\
\hline 1 & -2.394146 & 5.594952 & 2.077226 \\
\hline 1 & 0.700894 & 1.813441 & -0.534047 \\
\hline 1 & 2.572970 & -3.479008 & 0.886815 \\
\hline 1 & 3.587254 & -2.045673 & 0.866077 \\
\hline 1 & 2.746775 & -4.113715 & 3.133518 \\
\hline 1 & 1.656767 & -0.066664 & 2.223682 \\
\hline 1 & 2.337964 & -3.688030 & 5.536550 \\
\hline 1 & 1.253818 & 0.368948 & 4.620796 \\
\hline 1 & 1.592395 & -1.441294 & 6.294562 \\
\hline 6 & -3.633654 & -0.948732 & -4.777473 \\
\hline 6 & -5.690715 & -2.187818 & -4.194869 \\
\hline 6 & -5.768643 & 0.291829 & -4.519732 \\
\hline 6 & -8.054075 & -0.960110 & 0.138519 \\
\hline 6 & -6.413948 & -1.495247 & 1.912446 \\
\hline 6 & -6.943487 & 0.890574 & 1.362540 \\
\hline
\end{tabular}




$\begin{array}{lrrr}1 & -3.859686 & -1.144840 & -5.833198 \\ 1 & -3.067654 & -0.008950 & -4.736708 \\ 1 & -2.984778 & -1.759417 & -4.418682 \\ 1 & -5.857644 & -2.355718 & -5.267182 \\ 1 & -5.113045 & -3.035433 & -3.803254 \\ 1 & -6.672653 & -2.192956 & -3.707002 \\ 1 & -5.958620 & 0.161959 & -5.593875 \\ 1 & -6.739871 & 0.367278 & -4.014920 \\ 1 & -5.244644 & 1.246765 & -4.381305 \\ 1 & -8.846539 & -0.990281 & 0.896901 \\ 1 & -8.382629 & -0.270670 & -0.649547 \\ 1 & -7.973044 & -1.965570 & -0.295629 \\ 1 & -7.743015 & 0.885513 & 2.115810 \\ 1 & -6.032042 & 1.270513 & 1.842161 \\ 1 & -7.227331 & 1.601453 & 0.574951 \\ 1 & -7.232861 & -1.519825 & 2.643832 \\ 1 & -6.282649 & -2.513607 & 1.523185 \\ 1 & -5.498012 & -1.225406 & 2.453296 \\ 6 & -5.026805 & 4.324952 & -0.936737 \\ 6 & -5.165969 & 5.367545 & 1.305832 \\ 6 & -3.609358 & 6.290478 & -0.424303 \\ 6 & -0.519430 & 5.767561 & 3.692975 \\ 6 & -0.636871 & 3.462406 & 4.603014 \\ 6 & 1.175692 & 4.100303 & 3.005437 \\ 1 & -5.824681 & 4.997421 & -1.276248 \\ 1 & -4.409967 & 4.077498 & -1.810568 \\ 1 & -5.502552 & 3.399736 & -0.582467 \\ 1 & -5.974175 & 6.015894 & 0.941266 \\ 1 & -5.623596 & 4.464690 & 1.732103 \\ 1 & -4.655090 & 5.899458 & 2.117557 \\ 1 & -4.402137 & 6.938000 & -0.822715 \\ 1 & -3.057521 & 6.867951 & 0.327125 \\ 1 & -2.916535 & 6.058792 & -1.243675 \\ 1 & 0.157535 & 6.064627 & 4.503780 \\ 1 & -0.311997 & 6.416352 & 2.832135 \\ 1 & -1.542477 & 5.967308 & 4.035785 \\ 1 & 1.810843 & 4.475576 & 3.818731 \\ 1 & 1.435009 & 3.044029 & 2.857005 \\ 1 & 1.437762 & 4.650096 & 2.091134 \\ 1 & -0.009567 & 3.768183 & 5.451496 \\ 1 & -1.687722 & 3.595660 & 4.892503 \\ 1 & -0.472163 & 2.389988 & 4.432190\end{array}$

$1_{\mathrm{C} 10-} \psi_{-1 \mathrm{~s} 2 \mathrm{a3} 54 \mathrm{a}}$

Number of imaginary frequencies : 0

The smallest frequencies are : $12.6334 \quad 16.6126 \quad 20.6035 \mathrm{~cm}(-1)$

Electronic energy : $\quad \mathrm{HF}=-4125.464467$

Zero-point correction $=\quad 1.299832($ Hartree/Particle $)$

Thermal correction to Energy= $\quad 1.378003$

Thermal correction to Enthalpy= $\quad 1.378947$

Thermal correction to Gibbs Free Energy $=\quad 1.187792$

Sum of electronic and zero-point Energies $=\quad-4124.164635$

Sum of electronic and thermal Energies $=\quad-4124.086464$

Sum of electronic and thermal Enthalpies $=\quad-4124.085520$

Sum of electronic and thermal Free Energies $=\quad-4124.276675$ 
Cartesian Coordinates

$\begin{array}{crrr}\cdots & 1.651017 & -2.216684 & 5.103859 \\ 6 & 1.981408 & -3.446010 & 4.545155 \\ 6 & 2.233203 & -3.544524 & 3.179223 \\ 6 & 2.161073 & -2.421712 & 2.356280 \\ 6 & 1.826758 & -1.190126 & 2.925674 \\ 6 & 1.575206 & -1.089259 & 4.288757 \\ 6 & 2.507382 & -2.543223 & 0.892919 \\ 7 & 1.563868 & -1.867058 & -0.017156 \\ 6 & 0.514570 & -2.651072 & -0.610798 \\ 6 & -0.780941 & -2.638702 & -0.122097 \\ 6 & -1.809130 & -3.315669 & -0.856002 \\ 6 & -1.475212 & -4.048388 & -2.033306 \\ 6 & -0.125694 & -4.098964 & -2.451588 \\ 6 & 0.833502 & -3.403139 & -1.768131 \\ 6 & -3.170977 & -3.273580 & -0.456679 \\ 6 & -4.141163 & -3.912926 & -1.187452 \\ 6 & -3.802093 & -4.651678 & -2.341592 \\ 6 & -2.495965 & -4.715314 & -2.753898 \\ 6 & -1.124687 & -2.090818 & 1.221041 \\ 6 & -1.482263 & -0.770802 & 1.450099 \\ 6 & -1.905299 & -0.381460 & 2.751019 \\ 6 & -1.967845 & -1.279357 & 3.781282 \\ 6 & -1.614040 & -2.632993 & 3.581362 \\ 6 & -1.184422 & -3.048433 & 2.288472 \\ 6 & -1.688373 & -3.577385 & 4.632769 \\ 6 & -1.366380 & -4.893643 & 4.420204 \\ 6 & -0.937118 & -5.312142 & 3.143892 \\ 6 & -0.842328 & -4.414649 & 2.109065 \\ 15 & -1.545518 & 0.428458 & 0.037357 \\ 45 & 0.125044 & 0.717811 & -1.755292 \\ 6 & -0.352158 & -0.513494 & -3.195312 \\ 8 & -0.587503 & -1.150591 & -4.127394 \\ 15 & 1.917531 & -0.460249 & -0.891766 \\ 8 & 2.865244 & 0.239577 & 0.270221 \\ 6 & 3.674795 & 1.284573 & -0.131921 \\ 6 & 4.865180 & 1.009630 & -0.776186 \\ 6 & 5.652892 & 2.118166 & -1.228104 \\ 6 & 5.236605 & 3.447174 & -0.910955 \\ 6 & 4.034995 & 3.652636 & -0.191297 \\ 6 & 3.251974 & 2.591549 & 0.173576 \\ 6 & 6.021000 & 4.544301 & -1.343015 \\ 6 & 7.158267 & 4.351223 & -2.083809 \\ 6 & 7.551095 & 3.042288 & -2.432983 \\ 6 & 6.820198 & 1.957126 & -2.017359 \\ 6 & 5.248810 & -0.402210 & -1.012898 \\ 6 & 4.359386 & -1.253290 & -1.648169 \\ 6 & 4.701862 & -2.578297 & -1.988899 \\ 6 & 5.919866 & -3.083790 & -1.626479 \\ 6 & 6.831502 & -2.297623 & -0.882397 \\ 6 & 6.494997 & -0.948097 & -0.560031 \\ 6 & 7.404745 & -0.211856 & 0.241920 \\ 6 & 8.586504 & -0.766361 & 0.666905 \\ 6 & 8.930929 & -2.087868 & 0.314039\end{array}$




\begin{tabular}{|c|c|c|c|}
\hline & 8.065056 & -2.835430 & -0.441523 \\
\hline & 3.107966 & -0.816762 & -2.018130 \\
\hline & -3.283966 & 0.179085 & -0.521702 \\
\hline & -4.338641 & 0.094143 & 0.396275 \\
\hline & -5.642598 & -0.122997 & -0.032710 \\
\hline & -5.872303 & -0.236422 & -1.415018 \\
\hline & -4.850323 & -0.138916 & -2.353914 \\
\hline & -3.547890 & 0.063564 & -1.877103 \\
\hline & -6.806965 & -0.276634 & 0.943853 \\
\hline & -5.073557 & -0.292466 & -3.856117 \\
\hline & -1.626305 & 2.063003 & 0.872741 \\
\hline & -2.494634 & 3.041968 & 0.382288 \\
\hline & -2.490980 & 4.331188 & 0.908799 \\
\hline & -1.611703 & 4.609552 & 1.964728 \\
\hline & -0.729612 & 3.657735 & 2.475288 \\
\hline & -0.729573 & 2.387316 & 1.887114 \\
\hline & -3.418759 & 5.426978 & 0.387043 \\
\hline & 0.169169 & 3.922444 & 3.683043 \\
\hline & -0.263443 & 2.323400 & -2.712773 \\
\hline & -0.531997 & 3.336963 & -3.201202 \\
\hline & 7.150930 & 0.803955 & 0.533364 \\
\hline & -2.195644 & 0.650901 & 2.931573 \\
\hline & 8.304503 & -3.863624 & -0.709877 \\
\hline & -2.013954 & -3.233879 & 5.614003 \\
\hline & 5.689896 & 5.548769 & -1.082605 \\
\hline & -3.448557 & -2.706405 & 0.430531 \\
\hline & -5.181802 & -3.845706 & -0.875819 \\
\hline & 3.730264 & 4.671237 & 0.044416 \\
\hline & -2.301908 & -0.962093 & 4.768782 \\
\hline ] & 6.197413 & -4.103048 & -1.890133 \\
\hline & 8.438246 & 2.890580 & -3.043751 \\
\hline & 3.985156 & -3.169217 & -2.554255 \\
\hline 1 & -2.218849 & -5.273717 & -3.646915 \\
\hline & 0.131176 & -4.673017 & -3.340385 \\
\hline ] & -0.674178 & -6.354655 & 2.978422 \\
\hline & 7.130289 & 0.956296 & -2.306367 \\
\hline l & -0.496233 & -4.755800 & 1.135326 \\
\hline & 1.868611 & -3.408253 & -2.103075 \\
\hline . & 7.749223 & 5.201933 & -2.415079 \\
\hline 1 & 9.261325 & -0.181158 & 1.287562 \\
\hline & -4.580334 & -5.161389 & -2.905640 \\
\hline 1 & -1.435747 & -5.613994 & 5.232278 \\
\hline & 9.873511 & -2.512058 & 0.651904 \\
\hline l & 2.307665 & 2.723416 & 0.697461 \\
\hline & -4.123412 & 0.191276 & 1.457315 \\
\hline 1 & -2.716771 & 0.128538 & -2.575965 \\
\hline I & -6.890936 & -0.409234 & -1.757407 \\
\hline 1 & -3.172135 & 2.783655 & -0.428499 \\
\hline 1 & -0.036811 & 1.624591 & 2.248911 \\
\hline 1 & -1.622928 & 5.607214 & 2.398301 \\
\hline 1 & 0.694352 & 1.825379 & -0.736121 \\
\hline 1 & 2.564743 & -3.604491 & 0.607920 \\
\hline 1 & 3.509591 & -2.126023 & 0.729991 \\
\hline 1 & 2.481644 & -4.511864 & 2.741572 \\
\hline 1 & 1.762179 & -0.308249 & 2.289954 \\
\hline 1 & 2.028843 & -4.336272 & 5.169316 \\
\hline
\end{tabular}




$\begin{array}{rrrr}1 & 1.318388 & -0.122335 & 4.719713 \\ 1 & 1.446948 & -2.136775 & 6.169724 \\ 6 & -6.554419 & -0.269188 & -4.227127 \\ 6 & -4.375640 & 0.843697 & -4.613348 \\ 6 & -4.482699 & -1.635800 & -4.299077 \\ 6 & -6.380648 & -0.048109 & 2.392696 \\ 6 & -7.909682 & 0.732619 & 0.608533 \\ 6 & -7.361381 & -1.701480 & 0.832575 \\ 1 & -6.662944 & -0.344731 & -5.316370 \\ 1 & -7.103706 & -1.112375 & -3.789205 \\ 1 & -7.041008 & 0.661936 & -3.908143 \\ 1 & -4.569289 & 0.752217 & -5.690133 \\ 1 & -4.743689 & 1.824691 & -4.285262 \\ 1 & -3.286082 & 0.831641 & -4.480255 \\ 1 & -4.605909 & -1.771261 & -5.382431 \\ 1 & -3.411283 & -1.705966 & -4.069873 \\ 1 & -4.984020 & -2.469767 & -3.789628 \\ 1 & -7.248136 & -0.165925 & 3.054220 \\ 1 & -5.619820 & -0.770457 & 2.718373 \\ 1 & -5.981976 & 0.963095 & 2.547206 \\ 1 & -8.208436 & -1.839002 & 1.518273 \\ 1 & -7.712282 & -1.930112 & -0.181330 \\ 1 & -6.590633 & -2.438413 & 1.097612 \\ 1 & -8.746292 & 0.629122 & 1.312387 \\ 1 & -7.534307 & 1.761779 & 0.679155 \\ 1 & -8.311471 & 0.592240 & -0.401900 \\ 6 & -4.289654 & 4.945440 & -0.771630 \\ 6 & -4.344088 & 5.893308 & 1.516456 \\ 6 & -2.580095 & 6.609525 & -0.109478 \\ 6 & 0.108818 & 5.375521 & 4.148020 \\ 6 & -0.303138 & 3.030468 & 4.838363 \\ 6 & 1.628275 & 3.594001 & 3.353515 \\ 1 & -4.934283 & 5.765795 & -1.111622 \\ 1 & -3.687045 & 4.622685 & -1.630878 \\ 1 & -4.943454 & 4.112936 & -0.479235 \\ 1 & -5.020857 & 6.679098 & 1.154578 \\ 1 & -4.958629 & 5.062919 & 1.888395 \\ 1 & -3.785887 & 6.303759 & 2.366627 \\ 1 & -3.233141 & 7.402892 & -0.497499 \\ 1 & -1.967276 & 7.046365 & 0.688133 \\ 1 & -1.906457 & 6.298639 & -0.918587 \\ 1 & 0.779128 & 5.515750 & 5.005339 \\ 1 & 0.429114 & 6.070282 & 3.360852 \\ 1 & -0.899307 & 5.663621 & 4.471961 \\ 1 & 2.263917 & 3.773524 & 4.230834 \\ 1 & 1.769862 & 2.545760 & 3.060222 \\ 1 & 1.999190 & 4.227890 & 2.536753 \\ 1 & 0.320539 & 3.190167 & 5.728500 \\ 1 & -1.343220 & 3.257551 & 5.107764 \\ 1 & -0.253033 & 1.964584 & 4.579352\end{array}$

$\mathbf{1}_{\mathrm{C} 10-} \psi_{-1 \mathrm{~s} 2 \mathrm{a} 3 \mathrm{~s} 4 \mathrm{~s}}$

Number of imaginary frequencies : 0

The smallest frequencies are : $9.6177 \quad 13.8704 \quad 20.5918 \mathrm{~cm}(-1)$

Electronic energy : $\quad \mathrm{HF}=-4125.4648353$ 
Zero-point correction $=$

Thermal correction to Energy=

Thermal correction to Enthalpy=

Thermal correction to Gibbs Free Energy=

Sum of electronic and zero-point Energies=

Sum of electronic and thermal Energies $=$

Sum of electronic and thermal Enthalpies=

Sum of electronic and thermal Free Energies=
1.300114 (Hartree/Particle)

1.378090

1.379034

1.188234

$-4124.164722$

$-4124.086745$

$-4124.085801$

$-4124.276602$

Cartesian Coordinates

$\begin{array}{crrr}6 & 1.767964 & -1.756750 & 5.197682 \\ 6 & 2.190732 & -3.001726 & 4.744887 \\ 6 & 2.437962 & -3.204326 & 3.389835 \\ 6 & 2.266607 & -2.170477 & 2.470362 \\ 6 & 1.839370 & -0.924053 & 2.933659 \\ 6 & 1.593730 & -0.718514 & 4.285798 \\ 6 & 2.607385 & -2.401301 & 1.018254 \\ 7 & 1.660952 & -1.799993 & 0.060583 \\ 6 & 0.642425 & -2.640830 & -0.508807 \\ 6 & -0.648016 & -2.681098 & -0.008135 \\ 6 & -1.645496 & -3.435014 & -0.708526 \\ 6 & -1.288078 & -4.170842 & -1.877155 \\ 6 & 0.055600 & -4.153259 & -2.315497 \\ 6 & 0.986137 & -3.398943 & -1.654950 \\ 6 & -2.999687 & -3.470148 & -0.282657 \\ 6 & -3.939493 & -4.188944 & -0.978453 \\ 6 & -3.577739 & -4.923522 & -2.128318 \\ 6 & -2.278710 & -4.911827 & -2.566704 \\ 6 & -1.016673 & -2.091473 & 1.310026 \\ 6 & -1.455361 & -0.785086 & 1.467860 \\ 6 & -1.903089 & -0.354801 & 2.747118 \\ 6 & -1.904472 & -1.197138 & 3.825611 \\ 6 & -1.460562 & -2.533007 & 3.699815 \\ 6 & -1.014114 & -2.991619 & 2.427228 \\ 6 & -1.460989 & -3.419071 & 4.803483 \\ 6 & -1.052975 & -4.720812 & 4.660105 \\ 6 & -0.611493 & -5.182448 & 3.402905 \\ 6 & -0.587885 & -4.341467 & 2.317981 \\ 15 & -1.557129 & 0.342562 & -0.003902 \\ 45 & 0.152360 & 0.677911 & -1.755904 \\ 6 & -0.237484 & -0.590482 & -3.187209 \\ 8 & -0.419409 & -1.262631 & -4.106690 \\ 15 & 1.979233 & -0.416909 & -0.863146 \\ 8 & 2.911858 & 0.345074 & 0.269929 \\ 6 & 3.694682 & 1.395441 & -0.167807 \\ 6 & 4.889701 & 1.127441 & -0.805923 \\ 6 & 5.651273 & 2.238627 & -1.293919 \\ 6 & 5.204821 & 3.567049 & -1.017842 \\ 6 & 4.000596 & 3.767742 & -0.301013 \\ 6 & 3.243233 & 2.701044 & 0.099782 \\ 6 & 5.963705 & 4.667524 & -1.485484 \\ 6 & 7.104779 & 4.477003 & -2.221119 \\ 6 & 7.527760 & 3.166983 & -2.528751 \\ 6 & 6.821977 & 2.079101 & -2.078339 \\ 6 & 5.306164 & -0.282680 & -0.991934\end{array}$




\begin{tabular}{|c|c|c|c|}
\hline & 4.437109 & -1.178095 & -1.593798 \\
\hline & 4.810215 & -2.507858 & -1.880260 \\
\hline & 6.037794 & -2.971290 & -1.494612 \\
\hline & 6.929571 & -2.136047 & -0.780713 \\
\hline & 6.562864 & -0.782043 & -0.515137 \\
\hline & 7.453621 & 0.005862 & 0.258556 \\
\hline & 8.645026 & -0.505335 & 0.710107 \\
\hline & 9.018771 & -1.832705 & 0.413623 \\
\hline & 8.172251 & -2.628929 & -0.313599 \\
\hline & 3.175830 & -0.785032 & -1.980208 \\
\hline & -3.258322 & -0.028377 & -0.603566 \\
\hline & -4.335812 & -0.084108 & 0.280711 \\
\hline & -5.634491 & -0.300460 & -0.176094 \\
\hline & -5.827878 & -0.463277 & -1.554032 \\
\hline & -4.771488 & -0.413951 & -2.465840 \\
\hline & -3.485483 & -0.194967 & -1.963436 \\
\hline & -6.778308 & -0.321289 & 0.836536 \\
\hline & -4.949105 & -0.630914 & -3.966237 \\
\hline & -1.794343 & 1.995547 & 0.766713 \\
\hline & -2.796016 & 2.848609 & 0.297841 \\
\hline & -2.946088 & 4.132481 & 0.816284 \\
\hline & -2.074574 & 4.537755 & 1.836045 \\
\hline & -1.057422 & 3.715160 & 2.321010 \\
\hline & -0.913979 & 2.447130 & 1.746483 \\
\hline & -4.036547 & 5.081449 & 0.322848 \\
\hline & -0.160421 & 4.110613 & 3.493665 \\
\hline 6 & -0.277684 & 2.256137 & -2.744152 \\
\hline & -0.568645 & 3.247007 & -3.264331 \\
\hline & 7.177107 & 1.026961 & 0.507557 \\
\hline ] & -2.258122 & 0.665207 & 2.873891 \\
\hline & 8.434402 & -3.662076 & -0.538363 \\
\hline 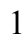 & -1.799331 & -3.043126 & 5.768328 \\
\hline & 5.610169 & 5.671973 & -1.256396 \\
\hline & -3.294997 & -2.905371 & 0.600389 \\
\hline & -4.974177 & -4.190910 & -0.642566 \\
\hline & 3.674242 & 4.786001 & -0.094955 \\
\hline 1 & -2.255287 & -0.847707 & 4.796283 \\
\hline & 6.337949 & -3.994194 & -1.715898 \\
\hline | & 8.418386 & 3.016467 & -3.134734 \\
\hline & 4.109287 & -3.137520 & -2.423203 \\
\hline & -1.984117 & -5.468488 & -3.455176 \\
\hline l & 0.331218 & -4.728086 & -3.198145 \\
\hline & -0.283597 & -6.213770 & 3.292348 \\
\hline 1 & 7.155007 & 1.076645 & -2.334601 \\
\hline & -0.235176 & -4.713810 & 1.358037 \\
\hline 1 & 2.016186 & -3.357609 & -2.002225 \\
\hline & 7.675993 & 5.330092 & -2.579777 \\
\hline 1 & 9.304545 & 0.119229 & 1.308467 \\
\hline 1 & -4.332764 & -5.492942 & -2.666099 \\
\hline & -1.064729 & -5.396882 & 5.512137 \\
\hline 1 & 9.968436 & -2.222571 & 0.772385 \\
\hline & 2.298014 & 2.826576 & 0.623504 \\
\hline 1 & -4.157509 & 0.059885 & 1.345748 \\
\hline & -2.636731 & -0.149255 & -2.642051 \\
\hline 1 & -6.834985 & -0.630166 & -1.925465 \\
\hline 1 & -3.463559 & 2.495331 & -0.483618 \\
\hline
\end{tabular}




\begin{tabular}{|c|c|c|c|}
\hline 1 & -0.122815 & 1.780866 & 2.097581 \\
\hline & -2.202232 & 5.530503 & 2.262396 \\
\hline & 0.648750 & 1.810063 & -0.723460 \\
\hline & 2.666385 & -3.480679 & 0.812735 \\
\hline & 3.609724 & -1.997660 & 0.822333 \\
\hline & 2.757568 & -4.185550 & 3.037500 \\
\hline & 1.695474 & -0.112359 & 2.223655 \\
\hline & 2.316873 & -3.824554 & 5.445911 \\
\hline & 1.262761 & 0.261163 & 4.629727 \\
\hline 1 & 1.568406 & -1.597385 & 6.255403 \\
\hline & -6.418505 & -0.674418 & -4.377970 \\
\hline 6 & -4.269985 & 0.501510 & -4.745943 \\
\hline & -4.300695 & -1.968899 & -4.341633 \\
\hline & -8.121676 & -0.658167 & 0.194728 \\
\hline & -6.492956 & -1.370698 & 1.917462 \\
\hline & -6.890051 & 1.064152 & 1.484669 \\
\hline & -6.492742 & -0.804947 & -5.464908 \\
\hline [ & -6.952742 & -1.513240 & -3.913735 \\
\hline 1 & -6.943853 & 0.254269 & -4.118972 \\
\hline 1 & -4.418600 & 0.358668 & -5.824391 \\
\hline 1 & -4.690455 & 1.478114 & -4.472197 \\
\hline & -3.187078 & 0.542393 & -4.571275 \\
\hline & -4.396233 & -2.152579 & -5.420655 \\
\hline & -3.232507 & -1.991688 & -4.088840 \\
\hline 1 & -4.782784 & -2.799262 & -3.808266 \\
\hline & -8.904534 & -0.672519 & 0.963505 \\
\hline 1 & -8.416774 & 0.084325 & -0.557585 \\
\hline . & -8.110391 & -1.646426 & -0.283313 \\
\hline & -7.680704 & 1.070034 & 2.247267 \\
\hline 1 & -5.953065 & 1.367595 & 1.969116 \\
\hline & -7.139732 & 1.826884 & 0.734796 \\
\hline 1 & -7.306235 & -1.386955 & 2.655351 \\
\hline & -6.417594 & -2.374637 & 1.479162 \\
\hline ] & -5.559653 & -1.174271 & 2.460227 \\
\hline 6 & -4.887733 & 4.457453 & -0.781674 \\
\hline 6 & -4.963536 & 5.445349 & 1.487820 \\
\hline 5 & -3.389764 & 6.354168 & -0.233700 \\
\hline & -0.369610 & 5.560172 & 3.925319 \\
\hline 6 & -0.497558 & 3.202864 & 4.683597 \\
\hline ) & 1.316216 & 3.936063 & 3.126748 \\
\hline 1 & -5.666139 & 5.166343 & -1.090971 \\
\hline ] & -4.292175 & 4.213186 & -1.671069 \\
\hline 1 & -5.390401 & 3.539361 & -0.445789 \\
\hline 1 & -5.751745 & 6.130440 & 1.147628 \\
\hline ] & -5.448805 & 4.549516 & 1.897821 \\
\hline 1 & -4.426436 & 5.939725 & 2.306237 \\
\hline ] & -4.160639 & 7.043979 & -0.603056 \\
\hline 1 & -2.805794 & 6.886541 & 0.526907 \\
\hline 1 & -2.717015 & 6.118539 & -1.068472 \\
\hline 1 & 0.307777 & 5.798550 & 4.754969 \\
\hline 1 & -0.155990 & 6.264011 & 3.110507 \\
\hline 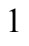 & -1.392464 & 5.742246 & 4.278540 \\
\hline 1 & 1.953402 & 4.230109 & 3.971328 \\
\hline & 1.567990 & 2.896748 & 2.879947 \\
\hline & 1.585067 & 4.565987 & 2.267941 \\
\hline & 0.130162 & 3.453140 & 5.549765 \\
\hline
\end{tabular}


$1_{\text {C10- }} \Psi$-1s2s3a4a

Number of imaginary frequencies : 0

The smallest frequencies are : $12.5686 \quad 16.3492 \quad 22.3959 \mathrm{~cm}(-1)$

Electronic energy : $\quad \mathrm{HF}=-4125.4647591$

Zero-point correction $=\quad 1.301137$ (Hartree/Particle)

Thermal correction to Energy= $\quad 1.378583$

Thermal correction to Enthalpy= $\quad 1.379527$

Thermal correction to Gibbs Free Energy= 1.191858

Sum of electronic and zero-point Energies $=\quad-4124.163622$

Sum of electronic and thermal Energies $=\quad-4124.086176$

Sum of electronic and thermal Enthalpies $=\quad-4124.085232$

Sum of electronic and thermal Free Energies $=\quad-4124.272901$

\begin{tabular}{crrr} 
& \multicolumn{3}{c}{ Cartesian Coordinates } \\
6 & 1.774022 & -1.567157 & 5.240999 \\
6 & 2.179251 & -2.832754 & 4.830769 \\
6 & 2.399607 & -3.091323 & 3.480858 \\
6 & 2.222135 & -2.092851 & 2.524053 \\
6 & 1.814807 & -0.825343 & 2.944687 \\
6 & 1.593737 & -0.564427 & 4.291750 \\
6 & 2.540744 & -2.387505 & 1.078412 \\
7 & 1.624882 & -1.769789 & 0.102468 \\
6 & 0.580964 & -2.579279 & -0.466668 \\
6 & -0.699295 & -2.615768 & 0.060516 \\
6 & -1.722040 & -3.338004 & -0.635720 \\
6 & -1.407603 & -4.019089 & -1.848762 \\
6 & -0.076510 & -4.001000 & -2.321893 \\
6 & 0.884538 & -3.297906 & -1.648774 \\
6 & -3.062920 & -3.384635 & -0.169789 \\
6 & -4.031858 & -4.055612 & -0.872674 \\
6 & -3.712788 & -4.733092 & -2.069751 \\
6 & -2.427321 & -4.712103 & -2.546393 \\
6 & -1.043335 & -2.007896 & 1.376095 \\
6 & -1.460631 & -0.690883 & 1.505236 \\
6 & -1.888618 & -0.223590 & 2.777761 \\
6 & -1.886821 & -1.039092 & 3.877105 \\
6 & -1.467231 & -2.385108 & 3.778903 \\
6 & -1.046947 & -2.882177 & 2.511885 \\
6 & -1.469973 & -3.245943 & 4.902316 \\
6 & -1.092634 & -4.559349 & 4.782913 \\
6 & -0.681551 & -5.059779 & 3.529856 \\
6 & -0.654647 & -4.243729 & 2.426043 \\
15 & -1.559786 & 0.397268 & 0.000638 \\
45 & 0.203750 & 0.749793 & -1.710117 \\
6 & -0.105747 & -0.444227 & -3.223565 \\
8 & -0.185708 & -1.077140 & -4.184277 \\
15 & 1.993428 & -0.407384 & -0.832850 \\
8 & 2.968145 & 0.322523 & 0.283827 \\
6 & 3.788233 & 1.336257 & -0.172355 \\
6 & 4.965524 & 1.012281 & -0.816952 \\
6 & 5.768282 & 2.085638 & -1.322797
\end{tabular}




\begin{tabular}{|c|c|c|c|}
\hline & 5.378762 & 3.434330 & -1.058344 \\
\hline & 4.188619 & 3.692175 & -0.335961 \\
\hline & 3.391607 & 2.661828 & 0.082745 \\
\hline & 6.179301 & 4.497255 & -1.543228 \\
\hline & 7.306176 & 4.251711 & -2.284408 \\
\hline & 7.672305 & 2.922126 & -2.580684 \\
\hline & 6.925435 & 1.869315 & -2.113589 \\
\hline & 5.320093 & -0.416117 & -0.991326 \\
\hline & 4.407934 & -1.279678 & -1.576001 \\
\hline & 4.719971 & -2.628252 & -1.846902 \\
\hline & 5.930472 & -3.139332 & -1.467065 \\
\hline & 6.864680 & -2.334684 & -0.772767 \\
\hline & 6.558249 & -0.963549 & -0.519074 \\
\hline & 7.487959 & -0.206247 & 0.239262 \\
\hline & 8.660763 & -0.762657 & 0.685837 \\
\hline & 8.975930 & -2.107355 & 0.399296 \\
\hline & 8.090016 & -2.874640 & -0.311876 \\
\hline & 3.161812 & -0.835172 & -1.958574 \\
\hline & -3.252159 & -0.021281 & -0.597371 \\
\hline & -4.330808 & -0.046049 & 0.290814 \\
\hline & -5.615787 & -0.352815 & -0.149980 \\
\hline & -5.784656 & -0.636104 & -1.510763 \\
\hline 0 & -4.732550 & -0.616663 & -2.427576 \\
\hline & -3.457604 & -0.303117 & -1.944120 \\
\hline 6 & -6.807663 & -0.429524 & 0.802665 \\
\hline 0 & -5.001009 & -0.900324 & -3.904572 \\
\hline & -1.807985 & 2.068551 & 0.726939 \\
\hline 6 & -2.797216 & 2.913113 & 0.232934 \\
\hline & -2.941208 & 4.218984 & 0.710982 \\
\hline 6 & -2.069749 & 4.652595 & 1.712221 \\
\hline 5 & -1.054761 & 3.833758 & 2.222493 \\
\hline 6 & -0.922549 & 2.549604 & 1.694391 \\
\hline 6 & -4.051426 & 5.095441 & 0.134197 \\
\hline & -0.145462 & 4.266639 & 3.372810 \\
\hline 6 & -0.199762 & 2.387898 & -2.612247 \\
\hline & -0.466757 & 3.415555 & -3.069224 \\
\hline ] & 7.256619 & 0.827918 & 0.480348 \\
\hline 1 & -2.231707 & 0.802823 & 2.883800 \\
\hline & 8.306299 & -3.920205 & -0.527755 \\
\hline 1 & -1.786237 & -2.841367 & 5.863067 \\
\hline & 5.869446 & 5.517880 & -1.322688 \\
\hline 1 & -3.324597 & -2.864151 & 0.749633 \\
\hline & -5.056790 & -4.064956 & -0.506939 \\
\hline 1 & 3.905014 & 4.725025 & -0.139489 \\
\hline | & -2.219946 & -0.660376 & 4.843029 \\
\hline & 6.183587 & -4.177231 & -1.677146 \\
\hline 1 & 8.551569 & 2.728336 & -3.190971 \\
\hline & 3.987271 & -3.236688 & -2.371599 \\
\hline 1 & -2.164794 & -5.229470 & -3.468091 \\
\hline & 0.166469 & -4.535955 & -3.238578 \\
\hline 1 & -0.380785 & -6.101146 & 3.437988 \\
\hline 1 & 7.214602 & 0.851202 & -2.361196 \\
\hline 1 & -0.327543 & -4.644087 & 1.468357 \\
\hline 1 & 1.905110 & -3.257246 & -2.022075 \\
\hline 1 & 7.909768 & 5.076462 & -2.656081 \\
\hline 1 & 9.351119 & -0.160736 & 1.272474 \\
\hline
\end{tabular}




\begin{tabular}{|c|c|c|c|}
\hline 1 & -4.489117 & -5.271358 & -2.609655 \\
\hline & -1.106468 & -5.215474 & 5.650343 \\
\hline & 9.911711 & -2.533185 & 0.753629 \\
\hline & 2.455449 & 2.830606 & 0.610504 \\
\hline & -4.155989 & 0.182955 & 1.339184 \\
\hline & -2.599621 & -0.279571 & -2.612334 \\
\hline & -6.784248 & -0.880585 & -1.870198 \\
\hline & -3.470629 & 2.545998 & -0.540688 \\
\hline & -0.133762 & 1.891563 & 2.064976 \\
\hline & -2.182100 & 5.657514 & 2.107646 \\
\hline & 0.698091 & 1.817980 & -0.609859 \\
\hline & 2.542829 & -3.474232 & 0.907309 \\
\hline & 3.562871 & -2.045341 & 0.867604 \\
\hline & 2.703804 & -4.088930 & 3.162455 \\
\hline & 1.669832 & -0.040017 & 2.206212 \\
\hline & 2.309971 & -3.628660 & 5.561434 \\
\hline & 1.276662 & 0.431006 & 4.601862 \\
\hline & 1.594192 & -1.364259 & 6.294789 \\
\hline ) & -3.709019 & -1.002361 & -4.711759 \\
\hline & -5.756933 & -2.223220 & -4.061658 \\
\hline 2 & -5.845028 & 0.241339 & -4.482401 \\
\hline 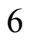 & -6.456261 & 0.063676 & 2.205278 \\
\hline 0 & -7.964082 & 0.426318 & 0.276459 \\
\hline 2 & -7.260859 & -1.890228 & 0.907641 \\
\hline 1 & -3.944586 & -1.238607 & -5.757101 \\
\hline 1 & -3.144673 & -0.060691 & -4.711430 \\
\hline 1 & -3.055066 & -1.797816 & -4.329136 \\
\hline 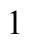 & -5.942469 & -2.427137 & -5.124675 \\
\hline & -5.169659 & -3.056404 & -3.652947 \\
\hline 1 & -6.730091 & -2.214875 & -3.556377 \\
\hline 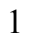 & -6.045278 & 0.067926 & -5.548473 \\
\hline 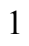 & -6.811442 & 0.335017 & -3.971342 \\
\hline 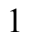 & -5.322413 & 1.202296 & -4.388420 \\
\hline & -7.345141 & 0.013456 & 2.846658 \\
\hline | & -5.677933 & -0.550250 & 2.678049 \\
\hline & -6.111377 & 1.106483 & 2.195635 \\
\hline 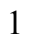 & -8.126583 & -1.977549 & 1.578211 \\
\hline & -7.550764 & -2.300092 & -0.068211 \\
\hline 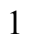 & -6.456398 & -2.518918 & 1.313587 \\
\hline ] & -8.814910 & 0.379170 & 0.969015 \\
\hline 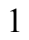 & -7.665589 & 1.478451 & 0.181030 \\
\hline 1 & -8.322380 & 0.089079 & -0.703280 \\
\hline 6 & -4.100575 & 6.476924 & 0.781458 \\
\hline 6 & -3.821954 & 5.280241 & -1.370266 \\
\hline 0 & -5.406151 & 4.413285 & 0.362239 \\
\hline 6 & -0.343073 & 5.731896 & 3.754479 \\
\hline 5 & -0.473284 & 3.404116 & 4.598411 \\
\hline 6 & 1.326656 & 4.071612 & 2.996757 \\
\hline 1 & -4.913976 & 7.063244 & 0.335715 \\
\hline 1 & -4.291181 & 6.419305 & 1.861030 \\
\hline 1 & -3.169624 & 7.036710 & 0.624782 \\
\hline 1 & -4.607807 & 5.915949 & -1.800292 \\
\hline 1 & -2.853591 & 5.758878 & -1.564275 \\
\hline 1 & -3.834655 & 4.325773 & -1.910233 \\
\hline 1 & -6.216646 & 5.027143 & -0.053405 \\
\hline 1 & -5.458499 & 3.427332 & -0.117540 \\
\hline
\end{tabular}




$\begin{array}{rrrr}1 & -5.601634 & 4.275604 & 1.434098 \\ 1 & 0.342285 & 5.995164 & 4.569935 \\ 1 & -0.131926 & 6.405486 & 2.913877 \\ 1 & -1.361925 & 5.932766 & 4.109012 \\ 1 & 1.975045 & 4.402605 & 3.818885 \\ 1 & 1.571943 & 3.020837 & 2.796335 \\ 1 & 1.586107 & 4.659179 & 2.105539 \\ 1 & 0.164579 & 3.682861 & 5.448333 \\ 1 & -1.520206 & 3.537385 & 4.902104 \\ 1 & -0.319361 & 2.335112 & 4.398552\end{array}$

$1_{\mathrm{C} 10-} \Psi_{-1 \mathrm{~s} 2 \mathrm{~s} 3 \mathrm{a} 4 \mathrm{~s}}$

Number of imaginary frequencies : 0

The smallest frequencies are : $11.1874 \quad 16.6703 \quad 19.8584 \mathrm{~cm}(-1)$

Electronic energy : $\quad H F=-4125.4651167$

Zero-point correction $=\quad 1.300249($ Hartree/Particle $)$

Thermal correction to Energy $=\quad 1.378129$

Thermal correction to Enthalpy= $\quad 1.379073$

Thermal correction to Gibbs Free Energy $=\quad 1.189005$

Sum of electronic and zero-point Energies $=\quad-4124.164868$

Sum of electronic and thermal Energies $=\quad-4124.086988$

Sum of electronic and thermal Enthalpies $=\quad-4124.086044$

Sum of electronic and thermal Free Energies $=\quad-4124.276112$

\section{Cartesian Coordinates}

$\begin{array}{cccc}6 & 1.746594 & -1.633746 & 5.235188 \\ 6 & 2.149370 & -2.895987 & 4.812314 \\ 6 & 2.375018 & -3.139764 & 3.460587 \\ 6 & 2.203826 & -2.130003 & 2.514026 \\ 6 & 1.797753 & -0.866368 & 2.947436 \\ 6 & 1.572311 & -0.619986 & 4.296512 \\ 6 & 2.528869 & -2.407966 & 1.066041 \\ 7 & 1.618105 & -1.779112 & 0.092108 \\ 6 & 0.575876 & -2.580811 & -0.491218 \\ 6 & -0.707888 & -2.616033 & 0.026933 \\ 6 & -1.729768 & -3.327536 & -0.680975 \\ 6 & -1.411147 & -3.997957 & -1.898851 \\ 6 & -0.076762 & -3.982262 & -2.363121 \\ 6 & 0.883817 & -3.290287 & -1.677665 \\ 6 & -3.072728 & -3.374632 & -0.220869 \\ 6 & -4.039743 & -4.036417 & -0.935026 \\ 6 & -3.717769 & -4.700541 & -2.138835 \\ 6 & -2.430048 & -4.678926 & -2.609383 \\ 6 & -1.055058 & -2.020806 & 1.346882 \\ 6 & -1.467721 & -0.703943 & 1.489696 \\ 6 & -1.897363 & -0.249727 & 2.766241 \\ 6 & -1.903626 & -1.078635 & 3.855655 \\ 6 & -1.489731 & -2.425179 & 3.742742 \\ 6 & -1.065670 & -2.908375 & 2.471701 \\ 6 & -1.498341 & -3.298903 & 4.856331 \\ 6 & -1.123874 & -4.611692 & 4.722608 \\ 6 & -0.711753 & -5.099178 & 3.464783 \\ 6 & -0.678680 & -4.270475 & 2.370676 \\ 15 & -1.561160 & 0.398914 & -0.003874\end{array}$




\begin{tabular}{|c|c|c|c|}
\hline 45 & 0.205850 & 0.757286 & -1.707111 \\
\hline 6 & -0.101682 & -0.425227 & -3.230527 \\
\hline 8 & -0.180919 & -1.045930 & -4.199180 \\
\hline 15 & 1.990989 & -0.408379 & -0.829213 \\
\hline 8 & 2.962004 & 0.312240 & 0.296981 \\
\hline 6 & 3.779842 & 1.332202 & -0.149944 \\
\hline 6 & 4.958255 & 1.017322 & -0.797141 \\
\hline 6 & 5.756658 & 2.097428 & -1.295639 \\
\hline 6 & 5.362287 & 3.442667 & -1.021166 \\
\hline 6 & 4.171878 & 3.690781 & -0.295888 \\
\hline 6 & 3.378816 & 2.654459 & 0.115409 \\
\hline 6 & 6.158068 & 4.512164 & -1.499363 \\
\hline 6 & 7.284912 & 4.276324 & -2.243717 \\
\hline 6 & 7.655861 & 2.950327 & -2.549854 \\
\hline 6 & 6.913748 & 1.891280 & -2.089282 \\
\hline 6 & 5.318419 & -0.408380 & -0.981752 \\
\hline 6 & 4.409430 & -1.271161 & -1.572394 \\
\hline 6 & 4.725537 & -2.617179 & -1.851115 \\
\hline 6 & 5.937979 & -3.126383 & -1.474968 \\
\hline 6 & 6.870092 & -2.322597 & -0.776851 \\
\hline 6 & 6.558934 & -0.954265 & -0.513997 \\
\hline 6 & 7.486954 & -0.198301 & 0.247729 \\
\hline 6 & 8.662657 & -0.753026 & 0.688749 \\
\hline 6 & 8.982390 & -2.094698 & 0.393252 \\
\hline 6 & 8.098148 & -2.860844 & -0.321227 \\
\hline 8 & 3.162948 & -0.827501 & -1.954256 \\
\hline 6 & -3.251412 & -0.017153 & -0.609695 \\
\hline 6 & -4.324182 & -0.074471 & 0.275348 \\
\hline 6 & -5.611595 & -0.389530 & -0.165610 \\
\hline 6 & -5.787594 & -0.633609 & -1.528283 \\
\hline 6 & -4.732289 & -0.583124 & -2.448487 \\
\hline 6 & -3.460780 & -0.272654 & -1.964866 \\
\hline 6 & -6.747588 & -0.455963 & 0.853539 \\
\hline 6 & -5.003824 & -0.832110 & -3.931079 \\
\hline 6 & -1.811855 & 2.063753 & 0.736756 \\
\hline 6 & -2.799029 & 2.914922 & 0.249010 \\
\hline 6 & -2.936546 & 4.218883 & 0.735246 \\
\hline 6 & -2.066620 & 4.639608 & 1.743407 \\
\hline 6 & -1.057448 & 3.812170 & 2.251093 \\
\hline 6 & -0.927153 & 2.533054 & 1.710658 \\
\hline 6 & -4.030527 & 5.113024 & 0.153763 \\
\hline 6 & -0.152705 & 4.230560 & 3.410323 \\
\hline 6 & -0.194997 & 2.397221 & -2.605929 \\
\hline 8 & -0.464510 & 3.424576 & -3.061943 \\
\hline 1 & 7.252192 & 0.833500 & 0.495532 \\
\hline 1 & -2.235362 & 0.777182 & 2.883249 \\
\hline 1 & 8.318017 & -3.904197 & -0.544057 \\
\hline 1 & -1.816054 & -2.904550 & 5.820844 \\
\hline 1 & 5.844455 & 5.529980 & -1.271266 \\
\hline 1 & -3.336835 & -2.862433 & 0.702990 \\
\hline 1 & -5.066576 & -4.048096 & -0.574694 \\
\hline 1 & 3.884636 & 4.721122 & -0.091688 \\
\hline 1 & -2.237788 & -0.709940 & 4.825106 \\
\hline 1 & 6.194483 & -4.162174 & -1.691245 \\
\hline 1 & 8.535030 & 2.764339 & -3.162698 \\
\hline 1 & 3.994415 & -3.225100 & -2.378658 \\
\hline
\end{tabular}




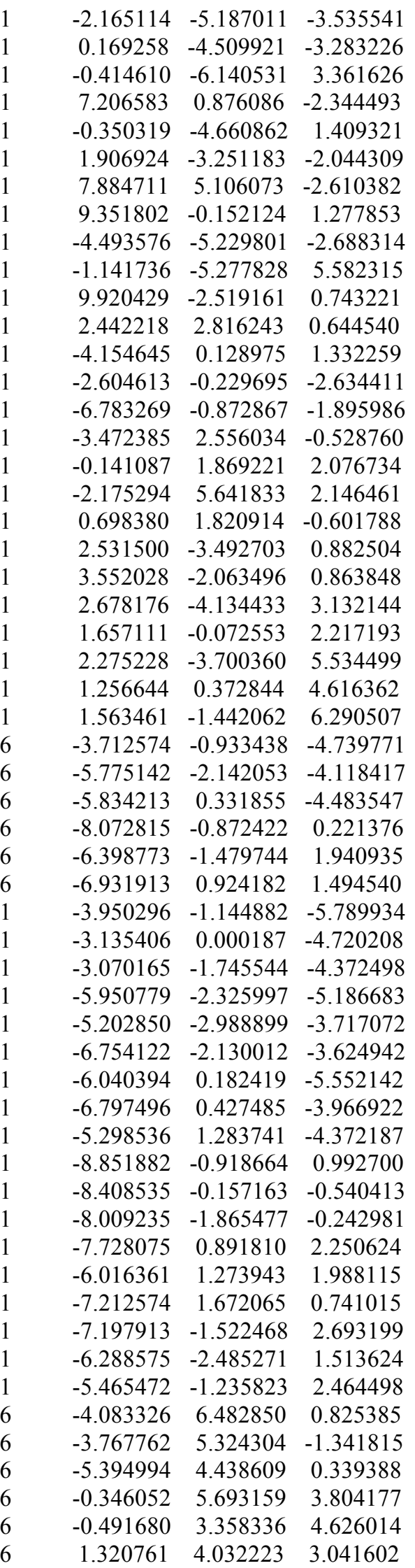




$\begin{array}{rrrr}1 & -4.887193 & 7.080824 & 0.377842 \\ 1 & -4.290744 & 6.406319 & 1.900697 \\ 1 & -3.147955 & 7.041569 & 0.693168 \\ 1 & -4.536724 & 5.978348 & -1.774966 \\ 1 & -2.789901 & 5.794482 & -1.506626 \\ 1 & -3.782148 & 4.380740 & -1.900483 \\ 1 & -6.189845 & 5.065258 & -0.087270 \\ 1 & -5.443704 & 3.459832 & -0.155146 \\ 1 & -5.617701 & 4.288444 & 1.404151 \\ 1 & 0.336078 & 5.946053 & 4.625603 \\ 1 & -0.127443 & 6.373395 & 2.970873 \\ 1 & -1.365914 & 5.895265 & 4.155076 \\ 1 & 1.965341 & 4.351350 & 3.871370 \\ 1 & 1.562332 & 2.982416 & 2.831744 \\ 1 & 1.588849 & 4.628080 & 2.158493 \\ 1 & 0.141094 & 3.627720 & 5.482731 \\ 1 & -1.540287 & 3.492115 & 4.923614 \\ 1 & -0.339369 & 2.290678 & 4.417790\end{array}$

$1_{\mathrm{C} 10-}-\psi_{-1223354 a}$

Number of imaginary frequencies : 0

The smallest frequencies are : $12.1367 \quad 14.0162 \quad 21.1607 \mathrm{~cm}(-1)$

Electronic energy : $\quad H F=-4125.4648926$

Zero-point correction $=\quad 1.299908($ Hartree/Particle)

Thermal correction to Energy= $\quad 1.377965$

Thermal correction to Enthalpy= $\quad 1.378909$

Thermal correction to Gibbs Free Energy= $\quad 1.188159$

Sum of electronic and zero-point Energies $=\quad-4124.164985$

Sum of electronic and thermal Energies $=\quad-4124.086928$

Sum of electronic and thermal Enthalpies $=\quad-4124.085983$

Sum of electronic and thermal Free Energies $=\quad-4124.276734$

Cartesian Coordinates

$\begin{array}{cccc}6 & 1.839608 & -0.918899 & 2.946179 \\ 6 & 2.255483 & -2.170768 & 2.487636 \\ 6 & 2.425013 & -3.200423 & 3.412714 \\ 6 & 2.188537 & -2.987853 & 4.768083 \\ 6 & 1.776755 & -1.737299 & 5.215814 \\ 6 & 1.603511 & -0.703733 & 4.298626 \\ 6 & 2.587963 & -2.411894 & 1.034705 \\ 7 & 1.648237 & -1.799918 & 0.076982 \\ 15 & 1.978456 & -0.419285 & -0.847028 \\ 45 & 0.161444 & 0.699665 & -1.729297 \\ 6 & -0.237757 & -0.560171 & -3.164888 \\ 8 & -0.423893 & -1.231856 & -4.083894 \\ 6 & 0.622629 & -2.629438 & -0.496518 \\ 6 & -0.672200 & -2.646850 & -0.006403 \\ 6 & -1.676627 & -3.383798 & -0.714377 \\ 6 & -1.321478 & -4.131415 & -1.876192 \\ 6 & 0.026761 & -4.141062 & -2.300810 \\ 6 & 0.963756 & -3.398331 & -1.636079 \\ 6 & -3.036572 & -3.385139 & -0.306775 \\ 6 & -3.985915 & -4.077282 & -1.016362 \\ 6 & -3.625492 & -4.829248 & -2.155436\end{array}$




\begin{tabular}{|c|c|c|c|}
\hline 6 & -2.320038 & -4.853775 & -2.574107 \\
\hline 6 & -1.034408 & -2.056014 & 1.313009 \\
\hline & -1.458329 & -0.745744 & 1.478645 \\
\hline & -1.891246 & -0.314419 & 2.762584 \\
\hline & -1.889466 & -1.159266 & 3.839096 \\
\hline & -1.461598 & -2.499612 & 3.705097 \\
\hline & -1.033345 & -2.959907 & 2.426833 \\
\hline & -1.460707 & -3.388751 & 4.806391 \\
\hline & -1.069895 & -4.694820 & 4.655412 \\
\hline & -0.649544 & -5.158982 & 3.391853 \\
\hline & -0.626952 & -4.315360 & 2.308935 \\
\hline 15 & -1.558049 & 0.384539 & 0.010251 \\
\hline & -3.263144 & 0.020699 & -0.583996 \\
\hline & -4.339993 & -0.050893 & 0.309082 \\
\hline & -5.629833 & -0.299008 & -0.145525 \\
\hline & -5.819339 & -0.472925 & -1.527783 \\
\hline & -4.773771 & -0.395231 & -2.442466 \\
\hline U & -3.488320 & -0.149521 & -1.940293 \\
\hline 6 & -6.816929 & -0.425581 & 0.807542 \\
\hline 6 & -7.968531 & 0.474546 & 0.348547 \\
\hline 6 & -4.953868 & -0.595390 & -3.944836 \\
\hline 6 & -4.293843 & -1.920187 & -4.342724 \\
\hline 6 & -1.775084 & 2.039388 & 0.780675 \\
\hline 6 & -0.887305 & 2.482406 & 1.763612 \\
\hline 6 & -1.015694 & 3.748420 & 2.335751 \\
\hline 6 & -2.028711 & 4.587348 & 1.854727 \\
\hline 6 & -2.901312 & 4.191920 & 0.838948 \\
\hline 6 & -2.760389 & 2.904096 & 0.314788 \\
\hline 6 & -4.016052 & 5.085888 & 0.299697 \\
\hline 6 & -3.805847 & 5.310996 & -1.202104 \\
\hline 6 & -0.111485 & 4.136814 & 3.505860 \\
\hline 6 & -0.455645 & 3.235865 & 4.698955 \\
\hline 6 & -7.282353 & -1.886148 & 0.818147 \\
\hline 6 & -6.452419 & -0.028290 & 2.237056 \\
\hline 6 & -4.289030 & 0.554437 & -4.711482 \\
\hline 6 & -6.424615 & -0.647841 & -4.350675 \\
\hline 6 & -5.367918 & 4.397097 & 0.526986 \\
\hline 6 & -4.054954 & 6.448896 & 0.985527 \\
\hline 6 & 1.362332 & 3.945464 & 3.135780 \\
\hline 6 & -0.302505 & 5.589710 & 3.935354 \\
\hline 8 & 3.160798 & -0.801711 & -1.974356 \\
\hline 6 & 4.420572 & -1.210179 & -1.599508 \\
\hline 6 & 5.305342 & -0.326279 & -1.003413 \\
\hline 6 & 6.561043 & -0.841045 & -0.540495 \\
\hline 6 & 6.910181 & -2.198040 & -0.814160 \\
\hline 6 & 6.002225 & -3.021249 & -1.521584 \\
\hline 6 & 4.776041 & -2.543105 & -1.893523 \\
\hline 6 & 8.152449 & -2.705729 & -0.362013 \\
\hline 6 & 9.015236 & -1.921006 & 0.358550 \\
\hline 6 & 8.658890 & -0.590776 & 0.663494 \\
\hline 6 & 7.468239 & -0.065374 & 0.226447 \\
\hline 6 & 4.907216 & 1.088429 & -0.811627 \\
\hline 6 & 3.720911 & 1.370005 & -0.163185 \\
\hline 6 & 3.286534 & 2.680536 & 0.108550 \\
\hline 6 & 4.052587 & 3.738661 & -0.298310 \\
\hline y & 5.248323 & 3.524475 & -1.025431 \\
\hline
\end{tabular}




\begin{tabular}{|c|c|c|c|}
\hline 6 & 5.677174 & 2.191147 & -1.305860 \\
\hline 6 & 6.838764 & 2.018764 & -2.101061 \\
\hline 6 & 7.552666 & 3.098773 & -2.557604 \\
\hline 6 & 7.147501 & 4.413346 & -2.245637 \\
\hline 6 & 6.015447 & 4.616452 & -1.499531 \\
\hline 8 & 2.929389 & 0.328354 & 0.279987 \\
\hline 6 & -0.261878 & 2.291310 & -2.701342 \\
\hline 8 & -0.557423 & 3.289909 & -3.203549 \\
\hline 1 & 7.205228 & 0.957776 & 0.481674 \\
\hline 1 & -2.237022 & 0.708162 & 2.894703 \\
\hline 1 & 8.401067 & -3.740855 & -0.592935 \\
\hline 1 & -1.783573 & -3.011179 & 5.775914 \\
\hline 1 & 5.675442 & 5.624759 & -1.266956 \\
\hline 1 & -3.330300 & -2.808760 & 0.568921 \\
\hline 1 & -5.026635 & -4.040750 & -0.700100 \\
\hline 1 & 3.739495 & 4.760483 & -0.089393 \\
\hline 1 & -2.226928 & -0.808845 & 4.814149 \\
\hline 1 & 6.289169 & -4.046533 & -1.749299 \\
\hline 1 & 8.435853 & 2.938393 & -3.171893 \\
\hline 1 & 4.063345 & -3.162897 & -2.432609 \\
\hline 1 & -2.026879 & -5.422682 & -3.455283 \\
\hline 1 & 0.300781 & -4.725711 & -3.177489 \\
\hline 1 & -0.337156 & -6.194376 & 3.274710 \\
\hline 1 & 7.157957 & 1.012746 & -2.360907 \\
\hline 1 & -0.290330 & -4.690192 & 1.344235 \\
\hline 1 & 1.997679 & -3.376273 & -1.973195 \\
\hline 1 & 7.725025 & 5.260062 & -2.609263 \\
\hline 1 & 9.331390 & 0.024679 & 1.256816 \\
\hline 1 & -4.386831 & -5.381453 & -2.702321 \\
\hline 1 & -1.080167 & -5.372814 & 5.505935 \\
\hline 1 & 9.964546 & -2.322116 & 0.705656 \\
\hline 1 & 2.346933 & 2.816641 & 0.639749 \\
\hline 1 & -4.154277 & 0.095959 & 1.370166 \\
\hline 1 & -2.641974 & -0.087439 & -2.620823 \\
\hline 1 & -6.825738 & -0.672637 & -1.890177 \\
\hline 1 & -3.436331 & 2.565707 & -0.469629 \\
\hline 1 & -0.104554 & 1.806218 & 2.114207 \\
\hline 1 & -2.139781 & 5.577162 & 2.286919 \\
\hline 1 & 0.669496 & 1.819084 & -0.688330 \\
\hline 1 & 2.629099 & -3.492839 & 0.833270 \\
\hline 1 & 3.596160 & -2.024997 & 0.834554 \\
\hline 1 & 2.735629 & -4.185956 & 3.064340 \\
\hline 1 & 1.697601 & -0.110480 & 2.232164 \\
\hline 1 & 2.313683 & -3.807304 & 5.473225 \\
\hline 1 & 1.281065 & 0.280089 & 4.638729 \\
\hline 1 & 1.585362 & -1.569880 & 6.273797 \\
\hline 1 & -6.502746 & -0.762846 & -5.439063 \\
\hline 1 & -6.947850 & -1.499375 & -3.897087 \\
\hline 1 & -6.958256 & 0.271146 & -4.074612 \\
\hline 1 & -4.385629 & -2.084866 & -5.425099 \\
\hline 1 & -3.225984 & -1.939142 & -4.088459 \\
\hline 1 & -4.771710 & -2.762883 & -3.825827 \\
\hline 1 & -4.445966 & 0.427320 & -5.790668 \\
\hline 1 & -4.713527 & 1.523964 & -4.419547 \\
\hline 1 & -3.204788 & 0.599189 & -4.545969 \\
\hline 1 & -7.338792 & -0.108986 & 2.878791 \\
\hline
\end{tabular}




$\begin{array}{lrrr}1 & -5.679833 & -0.680532 & 2.665734 \\ 1 & -6.095027 & 1.009006 & 2.291074 \\ 1 & -8.143051 & -2.011936 & 1.489074 \\ 1 & -7.583532 & -2.226778 & -0.180613 \\ 1 & -6.479173 & -2.546776 & 1.172909 \\ 1 & -8.813837 & 0.389772 & 1.044233 \\ 1 & -7.659873 & 1.527813 & 0.320902 \\ 1 & -8.339179 & 0.207583 & -0.647997 \\ 1 & -4.870527 & 7.049876 & 0.563929 \\ 1 & -4.236097 & 6.361801 & 2.064760 \\ 1 & -3.123388 & 7.009765 & 0.836168 \\ 1 & -4.591854 & 5.965293 & -1.602945 \\ 1 & -2.835707 & 5.785834 & -1.397427 \\ 1 & -3.835553 & 4.372458 & -1.768627 \\ 1 & -6.184680 & 5.022576 & 0.141956 \\ 1 & -5.426197 & 3.425709 & 0.019033 \\ 1 & -5.547486 & 4.226214 & 1.596974 \\ 1 & 0.381503 & 5.821810 & 4.761370 \\ 1 & -0.084644 & 6.289662 & 3.118350 \\ 1 & -1.321387 & 5.784535 & 4.293095 \\ 1 & 2.004867 & 4.232893 & 3.978611 \\ 1 & 1.601855 & 2.903348 & 2.888856 \\ 1 & 1.636070 & 4.571776 & 2.275843 \\ 1 & 0.175763 & 3.482209 & 5.563603 \\ 1 & -1.504668 & 3.365433 & 4.997038 \\ 1 & -0.306544 & 2.173272 & 4.464607\end{array}$

$1_{\mathrm{C} 10-} \psi_{-1225354 \mathrm{~s}}$

Number of imaginary frequencies : 0

The smallest frequencies are : $12.2545 \quad 16.0827 \quad 22.4819 \mathrm{~cm}(-1)$

Electronic energy : $\quad \mathrm{HF}=-4125.4648618$

Zero-point correction $=\quad 1.299757$ (Hartree/Particle)

Thermal correction to Energy= $\quad 1.377804$

Thermal correction to Enthalpy= $\quad 1.378748$

Thermal correction to Gibbs Free Energy $=\quad 1.187968$

Sum of electronic and zero-point Energies $=\quad-4124.165105$

Sum of electronic and thermal Energies $=\quad-4124.087058$

Sum of electronic and thermal Enthalpies $=\quad-4124.086114$

Sum of electronic and thermal Free Energies $=\quad-4124.276894$

\begin{tabular}{cccc}
\multicolumn{4}{c}{ Cartesian Coordinates } \\
$\ldots$ & 1.772474 & -1.091614 & 2.972849 \\
6 & 2.211035 & -2.321595 & 2.479799 \\
6 & 2.427400 & -3.364722 & 3.381163 \\
6 & 2.214035 & -3.187481 & 4.744802 \\
6 & 1.778442 & -1.957273 & 5.226977 \\
6 & 1.558808 & -0.911556 & 4.334831 \\
6 & 2.510371 & -2.544449 & 1.015369 \\
7 & 1.642280 & -1.804521 & 0.083458 \\
15 & 2.060015 & -0.349470 & -0.672373 \\
45 & 0.323503 & 1.033366 & -1.243648 \\
6 & 0.065949 & 0.353187 & -3.047364 \\
8 & -0.011152 & -0.033582 & -4.131975 \\
6 & 0.584430 & -2.512623 & -0.587407
\end{tabular}




\begin{tabular}{|c|c|c|c|}
\hline 6 & -0.689022 & -2.633045 & -0.054681 \\
\hline & -1.705388 & -3.301241 & -0.813372 \\
\hline & -1.406121 & -3.803295 & -2.114111 \\
\hline & -0.093649 & -3.664928 & -2.616879 \\
\hline & 0.869780 & -3.044954 & -1.869603 \\
\hline & -3.028905 & -3.461925 & -0.325343 \\
\hline & -3.996971 & -4.062328 & -1.090539 \\
\hline & -3.694570 & -4.557095 & -2.377175 \\
\hline & -2.422470 & -4.432726 & -2.873299 \\
\hline & -1.043125 & -2.141073 & 1.306743 \\
\hline & -1.498180 & -0.846788 & 1.529069 \\
\hline & -1.935237 & -0.479270 & 2.829562 \\
\hline & -1.912375 & -1.371985 & 3.868083 \\
\hline & -1.460573 & -2.696149 & 3.673652 \\
\hline & -1.028268 & -3.092316 & 2.375416 \\
\hline & -1.438631 & -3.634082 & 4.733365 \\
\hline & -1.028861 & -4.925510 & 4.521393 \\
\hline & -0.616389 & -5.329017 & 3.233808 \\
\hline & -0.611637 & -4.436635 & 2.190707 \\
\hline & -1.608024 & 0.348744 & 0.114424 \\
\hline & -3.193953 & -0.164092 & -0.659665 \\
\hline & -4.309106 & -0.427638 & 0.135297 \\
\hline & -5.537794 & -0.754700 & -0.432712 \\
\hline & -5.623452 & -0.787909 & -1.830753 \\
\hline & -4.530300 & -0.512101 & -2.655854 \\
\hline & -3.309107 & -0.210741 & -2.042904 \\
\hline & -6.732223 & -1.019434 & 0.483343 \\
\hline$)$ & -6.360088 & -2.056562 & 1.549726 \\
\hline & -4.623602 & -0.483990 & -4.180347 \\
\hline 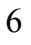 & -3.623821 & -1.474778 & -4.784427 \\
\hline ) & -2.094218 & 1.943269 & 0.895342 \\
\hline 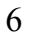 & -1.283612 & 2.517251 & 1.876389 \\
\hline f & -1.498879 & 3.818050 & 2.327606 \\
\hline 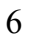 & -2.554386 & 4.544836 & 1.762881 \\
\hline 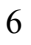 & -3.378946 & 4.004608 & 0.774405 \\
\hline 5 & -3.133566 & 2.694593 & 0.353760 \\
\hline 5 & -4.503694 & 4.791223 & 0.103853 \\
\hline ) & -4.144427 & 5.001106 & -1.372563 \\
\hline 5 & -0.585451 & 4.385501 & 3.412691 \\
\hline 6 & -0.855536 & 3.643133 & 4.726586 \\
\hline 5 & -7.122987 & 0.294388 & 1.171035 \\
\hline 5 & -7.945425 & -1.545477 & -0.279479 \\
\hline 6 & -4.293860 & 0.931710 & -4.669966 \\
\hline 6 & -6.016627 & -0.852364 & -4.684138 \\
\hline 6 & -5.817562 & 4.006775 & 0.200258 \\
\hline 6 & -4.719875 & 6.160292 & 0.743477 \\
\hline 5 & 0.881915 & 4.187462 & 3.010965 \\
\hline 6 & -0.815274 & 5.876677 & 3.646262 \\
\hline 8 & 3.155277 & -0.674808 & -1.903925 \\
\hline 6 & 4.421793 & -1.152730 & -1.652197 \\
\hline 6 & 5.373738 & -0.350061 & -1.044434 \\
\hline 6 & 6.649816 & -0.927380 & -0.740300 \\
\hline 6 & 6.942819 & -2.256807 & -1.170685 \\
\hline 6 & 5.956116 & -2.994768 & -1.865949 \\
\hline & 4.716086 & -2.461651 & -2.088082 \\
\hline 6 & 8.206987 & -2.824162 & -0.878959 \\
\hline
\end{tabular}




\begin{tabular}{|c|c|c|c|}
\hline & 9.146021 & -2.125606 & -0.165016 \\
\hline & 8.847267 & -0.826340 & 0.296057 \\
\hline & 7.636135 & -0.243933 & 0.016723 \\
\hline & 5.023921 & 1.037598 & -0.660448 \\
\hline & 3.907770 & 1.245423 & 0.124704 \\
\hline & 3.530719 & 2.513965 & 0.602759 \\
\hline & 4.274941 & 3.608735 & 0.257092 \\
\hline & 5.390586 & 3.477869 & -0.605419 \\
\hline & 5.767340 & 2.186018 & -1.084678 \\
\hline & 6.847107 & 2.102812 & -2.000050 \\
\hline & 7.534417 & 3.225591 & -2.389435 \\
\hline & 7.182886 & 4.495287 & -1.885435 \\
\hline & 6.129507 & 4.614064 & -1.015847 \\
\hline & 3.127875 & 0.161512 & 0.481919 \\
\hline & 0.153960 & 2.927231 & -1.550163 \\
\hline & 0.044630 & 4.076457 & -1.602277 \\
\hline & 7.420099 & 0.753204 & 0.390879 \\
\hline & -2.308664 & 0.527360 & 3.002998 \\
\hline & 8.411339 & -3.835628 & -1.227771 \\
\hline & -1.760215 & -3.305777 & 5.721135 \\
\hline & 5.831217 & 5.588889 & -0.632302 \\
\hline & -3.279295 & -3.086273 & 0.664917 \\
\hline & -5.009762 & -4.158428 & -0.701974 \\
\hline & 4.003717 & 4.598196 & 0.622263 \\
\hline & -2.254637 & -1.073233 & 4.858390 \\
\hline & 6.194436 & -4.001093 & -2.206529 \\
\hline & 8.353871 & 3.135354 & -3.098994 \\
\hline & 3.946658 & -3.021181 & -2.614191 \\
\hline & -2.170702 & -4.806989 & -3.864921 \\
\hline ] & 0.138508 & -4.055919 & -3.606211 \\
\hline & -0.295885 & -6.355196 & 3.067697 \\
\hline ] & 7.122433 & 1.132986 & -2.406762 \\
\hline & -0.284922 & -4.760480 & 1.204567 \\
\hline & 1.879003 & -2.932847 & -2.256406 \\
\hline I & 7.738841 & 5.376031 & -2.198332 \\
\hline ] & 9.581880 & -0.281545 & 0.884823 \\
\hline 1 & -4.471496 & -5.034067 & -2.970869 \\
\hline & -1.021837 & -5.640424 & 5.341122 \\
\hline 1 & 10.112098 & -2.572393 & 0.057889 \\
\hline l & 2.652015 & 2.588667 & 1.238869 \\
\hline 1 & -4.212647 & -0.368752 & 1.219264 \\
\hline 1 & -2.429796 & 0.005656 & -2.648566 \\
\hline 1 & -6.576767 & -1.033434 & -2.289657 \\
\hline 1 & -3.748914 & 2.262949 & -0.433880 \\
\hline & -0.454740 & 1.937564 & 2.284750 \\
\hline 1 & -2.727958 & 5.563937 & 2.095004 \\
\hline | & 0.720484 & 1.598198 & 0.214776 \\
\hline 1 & 2.431266 & -3.615025 & 0.777138 \\
\hline 1 & 3.555785 & -2.266481 & 0.821231 \\
\hline 1 & 2.754293 & -4.335031 & 3.005633 \\
\hline 1 & 1.590510 & -0.269132 & 2.284698 \\
\hline 1 & 2.374850 & -4.017948 & 5.429522 \\
\hline 1 & 1.208814 & 0.053805 & 4.698189 \\
\hline 1 & 1.603498 & -1.816575 & 6.291673 \\
\hline 1 & -6.030035 & -0.826613 & -5.780991 \\
\hline 1 & -6.307836 & -1.865079 & -4.375039 \\
\hline
\end{tabular}




\begin{tabular}{|c|c|c|c|}
\hline & -6.782777 & -0.150038 & -4.330931 \\
\hline & -3.675595 & -1.442523 & -5.881115 \\
\hline & .588575 & -1.253417 & -4.496468 \\
\hline & -3.847533 & -2.498907 & -4.460283 \\
\hline & -4.373053 & 0.984941 & -5.764116 \\
\hline & -4.987243 & 1.668388 & -4.242966 \\
\hline & -3.274543 & 1.234074 & -4.39724 \\
\hline & -8.761467 & -1.755318 & 0.423477 \\
\hline & -8.324115 & -0.817590 & \\
\hline & -7.718036 & -2.478517 & -0.812636 \\
\hline & -7.965950 & 0.134340 & 1.85 \\
\hline & -6.290614 & 0.713145 & 1.751479 \\
\hline & -7.426881 & 1.048080 & 0.432214 \\
\hline & -7.215983 & -2.239438 & 2.213018 \\
\hline & -6.084003 & -3.015 & 1.09( \\
\hline & -5.521845 & -1.734513 & 2.180301 \\
\hline & -5.550198 & & \\
\hline & -4.97 & 6.07 & \\
\hline & -3.835317 & 6.802834 & 0.648878 \\
\hline & -4.929165 & 5.578903 & -1.879839 \\
\hline & -3.198197 & 5.548938 & -1.471735 \\
\hline & -4.032694 & 4.048330 & -1.905564 \\
\hline & -6.629762 & 4.567205 & -0.281966 \\
\hline & -5.754727 & 3.028341 & -0.293761 \\
\hline & -6.099719 & & 1.247675 \\
\hline & -0.121768 & 6.240 & 4.414747 \\
\hline & -0.640154 & 6.463660 & 2.735445 \\
\hline & -1.831981 & 6.087259 & 4.001327 \\
\hline & 1.545627 & 4.663235 & 3.745212 \\
\hline & 1.157508 & 3.125505 & 2.967048 \\
\hline & 1.084066 & 4.631651 & 2.025948 \\
\hline & -0.194475 & 4.013370 & 5.522119 \\
\hline & -1.893504 & 3.789459 & 5.053181 \\
\hline & -0.686877 & 2.562753 & 4.62604 \\
\hline
\end{tabular}

$1_{\text {C11- }} \psi_{-1 \text {-1a2a3a4a }}$

Number of imaginary frequencies : 0

The smallest frequencies are : $12.5151 \quad 14.9655 \quad 19.3352 \mathrm{~cm}(-1)$

Electronic energy : $\quad H F=-4125.4634992$

Zero-point correction $=\quad 1.299040($ Hartree/Particle $)$

Thermal correction to Energy= $\quad 1.377837$

Thermal correction to Enthalpy= $\quad 1.378781$

Thermal correction to Gibbs Free Energy $=\quad 1.183930$

Sum of electronic and zero-point Energies $=\quad-4124.164459$

Sum of electronic and thermal Energies $=\quad-4124.085662$

Sum of electronic and thermal Enthalpies $=\quad-4124.084718$

Sum of electronic and thermal Free Energies $=\quad-4124.279569$

Cartesian Coordinates

$\begin{array}{lllll}6 & 3.994354 & -0.091012 & 4.176616 \\ 6 & 4.830116 & -0.851140 & 3.366008 \\ 6 & 4.289910 & -1.656045 & 2.365715 \\ 6 & 2.913010 & -1.703095 & 2.157980\end{array}$




\begin{tabular}{|c|c|c|c|}
\hline 6 & 2.081056 & -0.932671 & 2.972081 \\
\hline 6 & 2.615358 & -0.138263 & 3.977503 \\
\hline & 2.338825 & -2.603428 & 1.096089 \\
\hline & 1.372671 & -1.959979 & 0.174748 \\
\hline & 0.277333 & -2.782289 & -0.261751 \\
\hline & -0.970838 & -2.670017 & 0.326474 \\
\hline & -2.064142 & -3.423020 & -0.204517 \\
\hline 5 & -1.841514 & -4.310691 & -1.299544 \\
\hline 6 & -0.535058 & -4.446853 & -1.822901 \\
\hline 6 & 0.492007 & -3.690762 & -1.324747 \\
\hline 6 & -3.381531 & -3.304954 & 0.310585 \\
\hline 6 & -4.418954 & -4.015571 & -0.238936 \\
\hline 6 & -4.193241 & -4.898277 & -1.318115 \\
\hline & -2.930684 & -5.041823 & -1.834373 \\
\hline 6 & -1.168116 & -1.889875 & 1.581007 \\
\hline 6 & -1.478156 & -0.538323 & 1.600501 \\
\hline 6 & -1.706310 & 0.102337 & 2.850257 \\
\hline 6 & -1.611834 & -0.579537 & 4.032979 \\
\hline 6 & -1.282201 & -1.954176 & 4.049028 \\
\hline 6 & -1.066926 & -2.623806 & 2.809484 \\
\hline 6 & -1.154063 & -2.669299 & 5.263275 \\
\hline 6 & -0.824982 & -4.000609 & 5.263364 \\
\hline 6 & -0.614906 & -4.671637 & 4.040739 \\
\hline 6 & -0.735980 & -4.004298 & 2.846667 \\
\hline 15 & -1.658009 & 0.391641 & 0.002059 \\
\hline 45 & -0.099530 & 0.330061 & -1.925716 \\
\hline 6 & -0.629194 & -1.108051 & -3.142022 \\
\hline 8 & -0.865598 & -1.865770 & -3.978658 \\
\hline 15 & 1.711303 & -0.701653 & -0.911879 \\
\hline 8 & 2.653298 & 0.189048 & 0.103344 \\
\hline 6 & 3.490187 & 1.133386 & -0.447861 \\
\hline 6 & 4.682972 & 0.734110 & -1.019373 \\
\hline 6 & 5.508359 & 1.738439 & -1.623808 \\
\hline 6 & 5.121974 & 3.110056 & -1.531094 \\
\hline 6 & 3.912727 & 3.454126 & -0.880869 \\
\hline 6 & 3.097816 & 2.483844 & -0.364998 \\
\hline 6 & 5.940605 & 4.106734 & -2.115648 \\
\hline 6 & 7.085177 & 3.773331 & -2.792643 \\
\hline 6 & 7.450060 & 2.416999 & -2.920535 \\
\hline 6 & 6.684717 & 1.428515 & -2.352965 \\
\hline 6 & 5.033987 & -0.704944 & -1.045025 \\
\hline 6 & 4.138129 & -1.621787 & -1.566701 \\
\hline 6 & 4.474783 & -2.981071 & -1.745862 \\
\hline 6 & 5.685437 & -3.448777 & -1.315000 \\
\hline 6 & 6.592316 & -2.586053 & -0.651674 \\
\hline 6 & 6.264621 & -1.204624 & -0.504909 \\
\hline 6 & 7.149954 & -0.387264 & 0.242841 \\
\hline 6 & 8.300893 & -0.901454 & 0.788032 \\
\hline 6 & 8.638698 & -2.259127 & 0.608955 \\
\hline 6 & 7.797091 & -3.080984 & -0.096226 \\
\hline 8 & 2.886669 & -1.231702 & -1.984522 \\
\hline 6 & -3.439626 & 0.085867 & -0.347489 \\
\hline 6 & -4.402522 & 0.243167 & 0.653455 \\
\hline 6 & -5.744301 & -0.035266 & 0.405955 \\
\hline 6 & -6.091111 & -0.477019 & -0.877252 \\
\hline 6 & -5.157603 & -0.644382 & -1.900546 \\
\hline
\end{tabular}




\begin{tabular}{|c|c|c|c|}
\hline 6 & -3.821036 & -0.348927 & -1.612375 \\
\hline & -6.814923 & 0.077797 & 1.489602 \\
\hline & -5.606429 & -1.113281 & -3.283454 \\
\hline & -1.623660 & 2.141264 & 0.554207 \\
\hline & -2.557448 & 3.057769 & 0.077893 \\
\hline & -2.453374 & 4.415586 & 0.393574 \\
\hline & -1.400273 & 4.816107 & 1.218423 \\
\hline & -0.448185 & 3.920239 & 1.719098 \\
\hline 0 & -0.558201 & 2.581843 & 1.344481 \\
\hline б & -3.454923 & 5.449841 & -0.118079 \\
\hline 6 & 0.649580 & 4.416122 & 2.660341 \\
\hline 6 & -0.521458 & 1.749413 & -3.123433 \\
\hline 8 & -0.786983 & 2.668618 & -3.773609 \\
\hline 1 & 6.893693 & 0.656488 & 0.406160 \\
\hline 1 & -1.959898 & 1.159367 & 2.869384 \\
\hline 1 & 8.031180 & -4.136536 & -0.228870 \\
\hline 1 & -1.319859 & -2.135900 & 6.198251 \\
\hline 1 & 5.629720 & 5.146732 & -2.024898 \\
\hline 1 & -3.570294 & -2.619698 & 1.135509 \\
\hline 1 & -5.426969 & -3.891051 & 0.152961 \\
\hline 1 & 3.628410 & 4.503899 & -0.821527 \\
\hline 1 & -1.791173 & -0.069798 & 4.978905 \\
\hline 1 & 5.955994 & -4.494614 & -1.450593 \\
\hline 1 & 8.343228 & 2.149639 & -3.480878 \\
\hline 1 & 3.751222 & -3.630384 & -2.234240 \\
\hline 1 & -2.742002 & -5.717170 & -2.667717 \\
\hline 1 & -0.366861 & -5.138712 & -2.646693 \\
\hline 1 & -0.357446 & -5.728302 & 4.043737 \\
\hline 1 & 6.974882 & 0.388172 & -2.473599 \\
\hline 1 & -0.576597 & -4.539968 & 1.912986 \\
\hline 1 & 1.493775 & -3.763055 & -1.745472 \\
\hline 1 & 7.702908 & 4.546810 & -3.243000 \\
\hline 1 & 8.955388 & -0.255379 & 1.368955 \\
\hline 1 & -5.023822 & -5.462118 & -1.737840 \\
\hline 1 & -0.725453 & -4.541278 & 6.201658 \\
\hline 1 & 9.556302 & -2.651944 & 1.040575 \\
\hline 1 & 2.145616 & 2.720366 & 0.107367 \\
\hline 1 & -4.090661 & 0.583940 & 1.637726 \\
\hline 1 & -3.049621 & -0.468011 & -2.369825 \\
\hline 1 & -7.136949 & -0.704851 & -1.082966 \\
\hline 1 & -3.369694 & 2.703319 & -0.552563 \\
\hline 1 & 0.179885 & 1.854145 & 1.683172 \\
\hline 1 & -1.318491 & 5.869545 & 1.486903 \\
\hline 1 & 1.774890 & -3.411913 & 1.583460 \\
\hline 1 & 0.553685 & 1.589026 & -1.166866 \\
\hline 1 & 3.155841 & -3.086103 & 0.538159 \\
\hline 1 & 4.952007 & -2.242927 & 1.726766 \\
\hline 1 & 1.007809 & -0.941149 & 2.793374 \\
\hline 1 & 5.909251 & -0.817151 & 3.503011 \\
\hline 1 & 1.948770 & 0.448674 & 4.608255 \\
\hline 1 & 4.413434 & 0.538110 & 4.959193 \\
\hline 6 & -4.420269 & -1.473550 & -4.175608 \\
\hline 6 & -6.491617 & -2.357822 & -3.164174 \\
\hline 6 & -6.399592 & 0.014901 & -3.951975 \\
\hline 6 & -6.265128 & 0.675503 & 2.783447 \\
\hline 6 & -7.962765 & 0.969873 & 1.006598 \\
\hline
\end{tabular}




$\begin{array}{lrrr}6 & -7.349993 & -1.324713 & 1.800770 \\ 1 & -4.784467 & -1.847825 & -5.140670 \\ 1 & -3.777980 & -0.608346 & -4.386091 \\ 1 & -3.801238 & -2.260911 & -3.722863 \\ 1 & -6.800221 & -2.696057 & -4.162251 \\ 1 & -5.943896 & -3.178909 & -2.682369 \\ 1 & -7.405600 & -2.173748 & -2.587173 \\ 1 & -6.730975 & -0.289313 & -4.954197 \\ 1 & -7.290470 & 0.284403 & -3.370533 \\ 1 & -5.783944 & 0.917752 & -4.058091 \\ 1 & -7.069801 & 0.749429 & 3.525732 \\ 1 & -5.471898 & 0.055422 & 3.222298 \\ 1 & -5.863980 & 1.685966 & 2.627951 \\ 1 & -8.128926 & -1.276995 & 2.573870 \\ 1 & -7.786686 & -1.803229 & 0.915127 \\ 1 & -6.545195 & -1.974108 & 2.171862 \\ 1 & -8.726975 & 1.061640 & 1.789856 \\ 1 & -7.602826 & 1.978618 & 0.766239 \\ 1 & -8.456777 & 0.569557 & 0.113491 \\ 6 & -4.544454 & 4.821420 & -0.984386 \\ 6 & -4.132743 & 6.138147 & 1.071846 \\ 6 & -2.718180 & 6.494722 & -0.962637 \\ 6 & 1.500991 & 3.269005 & 3.202610 \\ 6 & 1.563847 & 5.397362 & 1.920377 \\ 6 & 0.009036 & 5.127593 & 3.858087 \\ 1 & -5.241412 & 5.599731 & -1.319959 \\ 1 & -4.130796 & 4.342165 & -1.881107 \\ 1 & -5.128314 & 4.071504 & -0.433607 \\ 1 & -4.858828 & 6.882538 & 0.718272 \\ 1 & -4.670511 & 5.409545 & 1.692725 \\ 1 & -3.412842 & 6.658418 & 1.715083 \\ 1 & -3.424792 & 7.243858 & -1.344789 \\ 1 & -1.951656 & 7.025741 & -0.385327 \\ 1 & -2.223532 & 6.024875 & -1.822560 \\ 1 & 2.243700 & 3.659301 & 3.910249 \\ 1 & 0.886416 & 2.530823 & 3.737312 \\ 1 & 2.052981 & 2.740820 & 2.413639 \\ 1 & 0.785811 & 5.470116 & 4.554776 \\ 1 & -0.574199 & 6.006494 & 3.558860 \\ 1 & -0.661066 & 4.451424 & 4.405033 \\ 1 & 2.345850 & 5.773080 & 2.593994 \\ 1 & 2.059163 & 4.910305 & 1.069860 \\ 1 & 1.010502 & 6.262341 & 1.534619\end{array}$

$1_{\mathrm{C} 11-}-\psi_{-12 a 2 a 3 a 4 s}$

Number of imaginary frequencies : 0

The smallest frequencies are : $12.2563 \quad 18.1381 \quad 19.3587 \mathrm{~cm}(-1)$

Electronic energy : $\quad \mathrm{HF}=-4125.4639105$

Zero-point correction $=\quad 1.298632($ Hartree/Particle)

Thermal correction to Energy= $\quad 1.377467$

Thermal correction to Enthalpy= $\quad 1.378411$

Thermal correction to Gibbs Free Energy $=\quad 1.183644$

Sum of electronic and zero-point Energies $=\quad-4124.165279$

Sum of electronic and thermal Energies $=\quad-4124.086443$

Sum of electronic and thermal Enthalpies $=\quad-4124.085499$ 
Cartesian Coordinates

\begin{tabular}{|c|c|c|c|}
\hline 6 & 4.002243 & 0.210322 & 4.164989 \\
\hline 6 & 4.852280 & -0.602193 & 3.423176 \\
\hline 6 & 4.331015 & -1.464585 & 2.461279 \\
\hline 6 & 2.959003 & -1.519425 & 2.225056 \\
\hline 6 & 2.112672 & -0.696964 & 2.970693 \\
\hline 6 & 2.628079 & 0.157467 & 3.935837 \\
\hline 6 & 2.406089 & -2.481770 & 1.207168 \\
\hline 7 & 1.444632 & -1.899641 & 0.241879 \\
\hline 6 & 0.363845 & -2.755752 & -0.165886 \\
\hline 6 & -0.882921 & -2.652091 & 0.426594 \\
\hline 6 & -1.963627 & -3.445270 & -0.068768 \\
\hline 6 & -1.734060 & -4.352574 & -1.145638 \\
\hline 6 & -0.430276 & -4.472588 & -1.679477 \\
\hline 6 & 0.587048 & -3.686480 & -1.207601 \\
\hline 6 & -3.276180 & -3.346017 & 0.462912 \\
\hline 6 & -4.303974 & -4.091622 & -0.056855 \\
\hline 6 & -4.072002 & -4.991814 & -1.120346 \\
\hline 6 & -2.813640 & -5.118205 & -1.650859 \\
\hline 6 & -1.094804 & -1.820262 & 1.643459 \\
\hline 6 & -1.445785 & -0.479682 & 1.591544 \\
\hline 6 & -1.692052 & 0.217250 & 2.806940 \\
\hline 6 & -1.578104 & -0.400445 & 4.023229 \\
\hline 6 & -1.208094 & -1.761892 & 4.110478 \\
\hline 6 & -0.972044 & -2.486948 & 2.906716 \\
\hline 6 & -1.060974 & -2.411028 & 5.359278 \\
\hline 6 & -0.694033 & -3.730759 & 5.425777 \\
\hline 6 & -0.464398 & -4.456836 & 4.238404 \\
\hline 6 & -0.603413 & -3.854286 & 3.012452 \\
\hline 15 & -1.651756 & 0.361807 & -0.055533 \\
\hline 45 & -0.052558 & 0.292325 & -1.948099 \\
\hline 6 & -0.555764 & -1.181532 & -3.131754 \\
\hline 8 & -0.783496 & -1.961658 & -3.949968 \\
\hline 15 & 1.768670 & -0.680382 & -0.892507 \\
\hline 8 & 2.693230 & 0.268921 & 0.086271 \\
\hline 6 & 3.531246 & 1.190119 & -0.500826 \\
\hline 6 & 4.734560 & 0.770493 & -1.034556 \\
\hline 6 & 5.566161 & 1.748694 & -1.671842 \\
\hline 6 & 5.173408 & 3.121395 & -1.649671 \\
\hline 6 & 3.951654 & 3.489944 & -1.037371 \\
\hline 6 & 3.131634 & 2.541166 & -0.490207 \\
\hline 6 & 5.998569 & 4.093329 & -2.265909 \\
\hline 6 & 7.155741 & 3.733583 & -2.907027 \\
\hline 6 & 7.527586 & 2.374328 & -2.965276 \\
\hline 6 & 6.756055 & 1.410057 & -2.365526 \\
\hline 6 & 5.090047 & -0.666866 & -0.990139 \\
\hline 6 & 4.204827 & -1.607825 & -1.486471 \\
\hline 6 & 4.548889 & -2.972104 & -1.603025 \\
\hline 6 & 5.753435 & -3.416644 & -1.132110 \\
\hline 6 & 6.646447 & -2.523498 & -0.490649 \\
\hline 6 & 6.313414 & -1.137988 & -0.410086 \\
\hline 6 & 7.184837 & -0.285269 & 0.314134 \\
\hline 6 & 8.326917 & -0.771668 & 0.901684 \\
\hline
\end{tabular}




\begin{tabular}{|c|c|c|c|}
\hline 6 & 8.669322 & -2.135500 & 0.790356 \\
\hline 6 & 7.842000 & -2.990168 & 0.107593 \\
\hline & 2.958078 & -1.238252 & -1.936693 \\
\hline & -3.404887 & -0.063672 & -0.420467 \\
\hline & -4.380738 & 0.052664 & 0.564634 \\
\hline & -5.715733 & -0.265141 & 0.303862 \\
\hline & -6.037689 & -0.705965 & -0.980156 \\
\hline & -5.081987 & -0.834997 & -1.996668 \\
\hline & -3.761256 & -0.503938 & -1.695102 \\
\hline & -6.740816 & -0.116237 & 1.425830 \\
\hline & -5.509080 & -1.306818 & -3.384999 \\
\hline y & -1.752870 & 2.127885 & 0.438705 \\
\hline & -2.817437 & 2.937224 & 0.052032 \\
\hline & -2.840567 & 4.295968 & 0.381009 \\
\hline & -1.779731 & 4.805087 & 1.132443 \\
\hline & -0.698902 & 4.016913 & 1.546178 \\
\hline 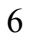 & -0.686555 & 2.679342 & 1.154840 \\
\hline & -4.000379 & 5.207408 & -0.017803 \\
\hline & 0.416774 & 4.624152 & 2.396712 \\
\hline & -0.410111 & 1.658711 & -3.226352 \\
\hline & -0.618661 & 2.538528 & -3.947628 \\
\hline & 6.923969 & 0.764303 & 0.424959 \\
\hline & -1.978705 & 1.265272 & 2.772963 \\
\hline & 8.080330 & -4.049989 & 0.026641 \\
\hline & -1.242867 & -1.836614 & 6.266549 \\
\hline & 5.682705 & 5.135121 & -2.229287 \\
\hline & -3.468127 & -2.648315 & 1.276699 \\
\hline & -5.309420 & -3.981922 & 0.346053 \\
\hline & 3.663275 & 4.540006 & -1.032210 \\
\hline I & -1.773592 & 0.151882 & 4.941674 \\
\hline & 6.029088 & -4.466418 & -1.218097 \\
\hline 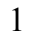 & 8.431360 & 2.085141 & -3.497050 \\
\hline & 3.836525 & -3.645210 & -2.075196 \\
\hline & -2.620772 & -5.807911 & -2.471396 \\
\hline L & -0.255583 & -5.179116 & -2.489394 \\
\hline & -0.177781 & -5.504460 & 4.294838 \\
\hline 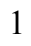 & 7.052353 & 0.366546 & -2.431920 \\
\hline & -0.429569 & -4.430685 & 2.105959 \\
\hline | & 1.586096 & -3.751077 & -1.635508 \\
\hline 1 & 7.778538 & 4.487945 & -3.382151 \\
\hline & 8.970571 & -0.098640 & 1.463810 \\
\hline 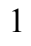 & -4.894897 & -5.583396 & -1.516287 \\
\hline & -0.579929 & -4.220558 & 6.389989 \\
\hline 1 & 9.579505 & -2.506496 & 1.255666 \\
\hline ] & 2.170050 & 2.795773 & -0.047330 \\
\hline 1 & -4.096883 & 0.400832 & 1.557448 \\
\hline 1 & -2.980496 & -0.587447 & -2.447187 \\
\hline 1 & -7.071397 & -0.959089 & -1.207160 \\
\hline 1 & -3.636298 & 2.497995 & -0.511803 \\
\hline ] & 0.146186 & 2.033378 & 1.435475 \\
\hline 1 & -1.797571 & 5.857702 & 1.414495 \\
\hline 1 & 1.844278 & -3.268048 & 1.732169 \\
\hline 1 & 0.550037 & 1.578414 & -1.194048 \\
\hline 1 & 3.234015 & -2.985354 & 0.684879 \\
\hline 1 & 5.004648 & -2.091351 & 1.874360 \\
\hline & 1.044148 & -0.709927 & 2.765953 \\
\hline
\end{tabular}




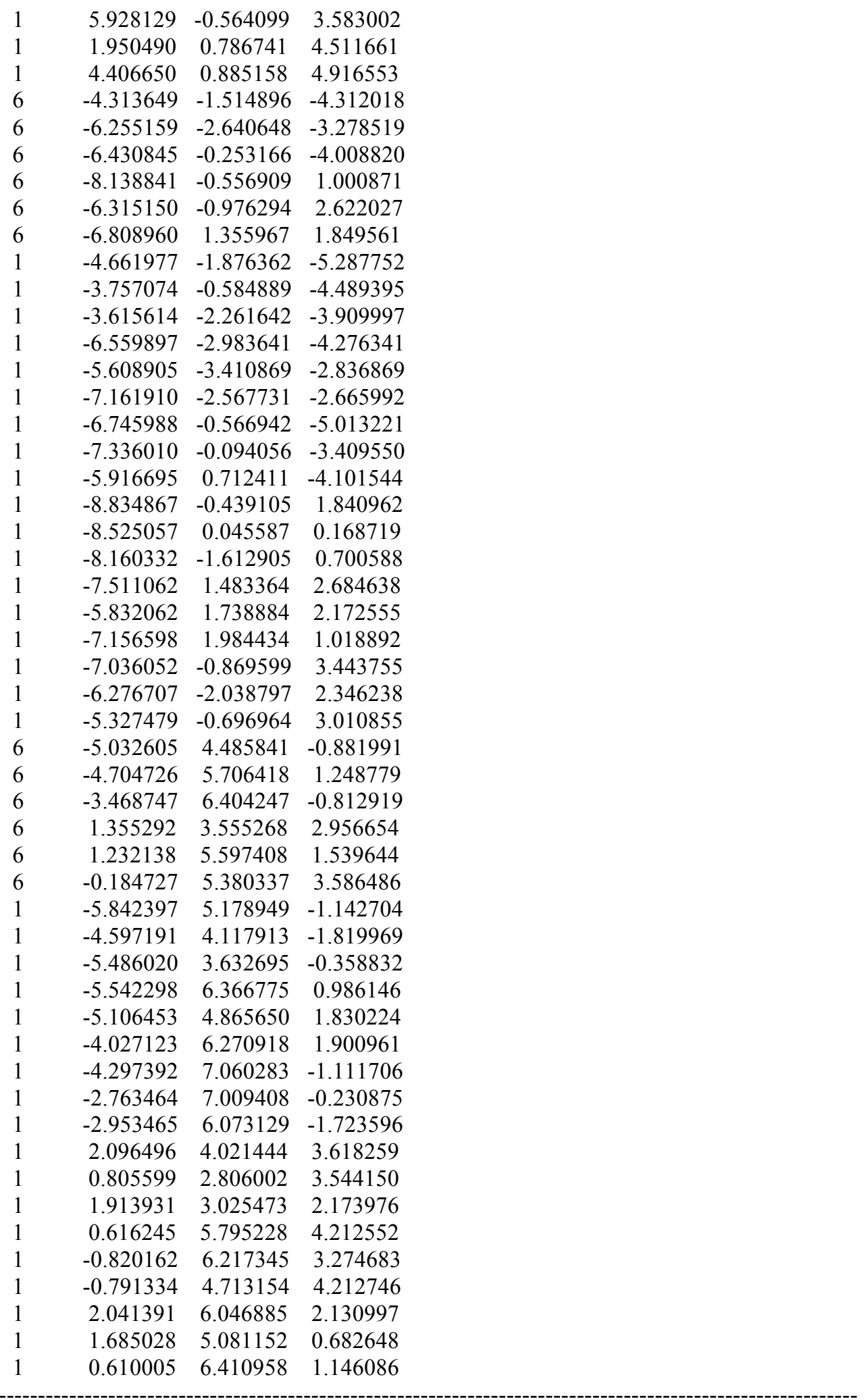

$1_{\text {C11- }} \psi_{\text {-1a2a3s4a }}$

Number of imaginary frequencies : 0

The smallest frequencies are : 8.8583 $16.7141 \quad 20.2316 \mathrm{~cm}(-1)$ 
Electronic energy : $\quad H F=-4125.4638595$

Zero-point correction $=$

1.298999 (Hartree/Particle)

Thermal correction to Energy= $\quad 1.377788$

Thermal correction to Enthalpy= $\quad 1.378732$

Thermal correction to Gibbs Free Energy= 1.183968

Sum of electronic and zero-point Energies $=\quad-4124.164860$

Sum of electronic and thermal Energies $=\quad-4124.086071$

Sum of electronic and thermal Enthalpies $=\quad-4124.085127$

Sum of electronic and thermal Free Energies $=\quad-4124.279892$

\begin{tabular}{|c|c|c|c|}
\hline & \multicolumn{3}{|c|}{ Cartesian Coordinates } \\
\hline 6 & 3.961532 & -0.155273 & 4.198907 \\
\hline 6 & 4.810003 & -0.871408 & 3.361723 \\
\hline 6 & 4.282590 & -1.661427 & 2.342991 \\
\hline 6 & 2.905805 & -1.735957 & 2.142495 \\
\hline 6 & 2.060975 & -1.008737 & 2.982345 \\
\hline 6 & 2.582735 & -0.231559 & 4.007720 \\
\hline 6 & 2.343921 & -2.623210 & 1.063380 \\
\hline 7 & 1.364175 & -1.975184 & 0.159544 \\
\hline 6 & 0.277004 & -2.803827 & -0.285054 \\
\hline 6 & -0.977591 & -2.697040 & 0.290630 \\
\hline 6 & -2.057262 & -3.470435 & -0.240879 \\
\hline 6 & -1.812251 & -4.378738 & -1.314127 \\
\hline 6 & -0.501193 & -4.499694 & -1.830278 \\
\hline 6 & 0.510546 & -3.719775 & -1.338045 \\
\hline 6 & -3.379192 & -3.368792 & 0.266420 \\
\hline 6 & -4.396976 & -4.121697 & -0.263970 \\
\hline 6 & -4.148466 & -5.024335 & -1.320826 \\
\hline 6 & -2.882927 & -5.146573 & -1.834109 \\
\hline 6 & -1.189481 & -1.927680 & 1.550458 \\
\hline 6 & -1.496913 & -0.575801 & 1.589780 \\
\hline 6 & -1.738428 & 0.044465 & 2.847644 \\
\hline 6 & -1.661392 & -0.656599 & 4.019995 \\
\hline 6 & -1.332346 & -2.031319 & 4.017285 \\
\hline 6 & -1.101301 & -2.680543 & 2.769740 \\
\hline 6 & -1.218922 & -2.764978 & 5.221899 \\
\hline 6 & -0.887654 & -4.095577 & 5.205833 \\
\hline 6 & -0.659805 & -4.746406 & 3.975608 \\
\hline 6 & -0.766921 & -4.060685 & 2.790627 \\
\hline 15 & -1.659151 & 0.393508 & 0.013715 \\
\hline 45 & -0.128406 & 0.320021 & -1.926896 \\
\hline 6 & -0.761996 & -1.129898 & -3.075801 \\
\hline 8 & -1.108645 & -1.890535 & -3.870354 \\
\hline 15 & 1.694020 & -0.709114 & -0.921453 \\
\hline 8 & 2.633217 & 0.180811 & 0.098864 \\
\hline 6 & 3.455869 & 1.138760 & -0.450519 \\
\hline 6 & 4.650865 & 0.756710 & -1.028910 \\
\hline 6 & 5.461648 & 1.773270 & -1.632711 \\
\hline 6 & 5.058188 & 3.139523 & -1.534290 \\
\hline 6 & 3.847194 & 3.466042 & -0.878284 \\
\hline 6 & 3.046826 & 2.484018 & -0.361505 \\
\hline 6 & 5.861826 & 4.148329 & -2.118840 \\
\hline 6 & 7.008149 & 3.831604 & -2.800895 \\
\hline 6 & 7.389899 & 2.480445 & -2.934140 \\
\hline 6 & 6.639204 & 1.480563 & -2.366946 \\
\hline
\end{tabular}




\begin{tabular}{|c|c|c|c|}
\hline & 5.018674 & -0.677867 & -1.062806 \\
\hline & 4.132252 & -1.602937 & -1.586114 \\
\hline & 4.486564 & -2.956611 & -1.775064 \\
\hline & 5.704531 & -3.411225 & -1.350940 \\
\hline & 6.601924 & -2.541166 & -0.684166 \\
\hline & 6.256751 & -1.165035 & -0.528424 \\
\hline & 7.132253 & -0.340999 & 0.223554 \\
\hline & 8.290396 & -0.843710 & 0.764198 \\
\hline & 8.645660 & -2.195654 & 0.575794 \\
\hline & 7.813834 & -3.023885 & -0.133596 \\
\hline & 2.874077 & -1.227824 & -1.996242 \\
\hline & -3.444023 & 0.132817 & -0.352000 \\
\hline & -4.414915 & 0.218728 & 0.654168 \\
\hline & -5.756028 & -0.014445 & 0.372962 \\
\hline & -6.106720 & -0.330011 & -0.951057 \\
\hline & -5.170042 & -0.407299 & -1.977101 \\
\hline & -3.827780 & -0.173066 & -1.648457 \\
\hline & -6.833619 & 0.021559 & 1.455080 \\
\hline & -5.526292 & -0.805760 & -3.406801 \\
\hline & -1.595020 & 2.128913 & 0.609802 \\
\hline & -2.523258 & 3.069946 & 0.172060 \\
\hline & -2.398912 & 4.416665 & 0.526894 \\
\hline & -1.331428 & 4.779592 & 1.350553 \\
\hline & -0.386939 & 3.857418 & 1.816464 \\
\hline & -0.518414 & 2.531792 & 1.405424 \\
\hline & -3.392533 & 5.478618 & 0.057807 \\
\hline & 0.720954 & 4.312537 & 2.766419 \\
\hline & -0.480968 & 1.695766 & -3.194803 \\
\hline & -0.693408 & 2.582892 & -3.906462 \\
\hline ] & 6.862559 & 0.698266 & 0.393790 \\
\hline & -1.989506 & 1.101728 & 2.880735 \\
\hline & 8.061346 & -4.075497 & -0.273030 \\
\hline & -1.397436 & -2.245899 & 6.162584 \\
\hline & 5.537933 & 5.183962 & -2.023836 \\
\hline 1 & -3.585113 & -2.669623 & 1.075828 \\
\hline & -5.406501 & -4.020229 & 0.129462 \\
\hline 1 & 3.549093 & 4.511749 & -0.815661 \\
\hline & -1.852605 & -0.162203 & 4.971675 \\
\hline & 5.988163 & -4.452617 & -1.493875 \\
\hline ] & 8.284447 & 2.226183 & -3.498358 \\
\hline & 3.769483 & -3.612278 & -2.264597 \\
\hline 1 & -2.676824 & -5.834438 & -2.652943 \\
\hline & -0.316919 & -5.203097 & -2.640726 \\
\hline 1 & -0.399220 & -5.802247 & 3.965094 \\
\hline & 6.942089 & 0.444401 & -2.491964 \\
\hline 1 & -0.592531 & -4.582423 & 1.851878 \\
\hline | & 1.516458 & -3.783093 & -1.750422 \\
\hline 1 & 7.614272 & 4.614254 & -3.251167 \\
\hline 1 & 8.937098 & -0.193010 & 1.348684 \\
\hline & -4.964692 & -5.616387 & -1.729058 \\
\hline 1 & -0.799563 & -4.650745 & 6.136768 \\
\hline 1 & 9.568789 & -2.579308 & 1.003860 \\
\hline 1 & 2.094130 & 2.707433 & 0.116549 \\
\hline 1 & -4.105417 & 0.465943 & 1.666560 \\
\hline 1 & -3.058246 & -0.239137 & -2.414696 \\
\hline 1 & -7.153872 & -0.523806 & -1.176145 \\
\hline
\end{tabular}




\begin{tabular}{|c|c|c|c|}
\hline & -3.347658 & 2.744348 & -0.458184 \\
\hline ] & 0.209177 & 1.784455 & 1.722864 \\
\hline & -1.232790 & 5.823494 & 1.649050 \\
\hline | & 1.795742 & -3.451147 & 1.535299 \\
\hline & 0.531523 & 1.590721 & -1.194301 \\
\hline | & 3.167208 & -3.080707 & 0.493967 \\
\hline & 4.954092 & -2.215465 & 1.684884 \\
\hline ] & 0.986909 & -1.041840 & 2.811455 \\
\hline & 5.888935 & -0.814905 & 3.492836 \\
\hline 1 & 1.906535 & 0.318631 & 4.660859 \\
\hline 1 & 4.370354 & 0.461345 & 4.996778 \\
\hline 6 & -7.031975 & -0.773126 & -3.659051 \\
\hline 6 & -4.856119 & 0.139954 & -4.409898 \\
\hline ) & -5.024142 & -2.235278 & -3.642826 \\
\hline & -6.279347 & 0.462068 & 2.808567 \\
\hline 0 & -7.945354 & 0.997899 & 1.057742 \\
\hline & -7.419179 & -1.385780 & 1.616629 \\
\hline 1 & -7.235887 & -1.027239 & -4.706781 \\
\hline 1 & -7.571328 & -1.501593 & -3.040470 \\
\hline 1 & -7.456371 & 0.221498 & -3.468922 \\
\hline ] & -5.144680 & -0.133745 & -5.433149 \\
\hline 1 & -5.160411 & 1.180857 & -4.239752 \\
\hline 1 & -3.760510 & 0.097709 & -4.363153 \\
\hline & -5.243714 & -2.555741 & -4.670587 \\
\hline 1 & -3.940222 & -2.317714 & -3.486964 \\
\hline 1 & -5.510987 & -2.937261 & -2.952658 \\
\hline 1 & -7.087637 & 0.474331 & 3.550519 \\
\hline 1 & -5.503695 & -0.220803 & 3.180822 \\
\hline & -5.854142 & 1.473502 & 2.766219 \\
\hline 1 & -8.204069 & -1.390019 & 2.385016 \\
\hline 1 & -7.862675 & -1.756320 & 0.684221 \\
\hline 1 & -6.640270 & -2.096496 & 1.924919 \\
\hline 1 & -8.716477 & 1.033295 & 1.838815 \\
\hline 1 & -7.549111 & 2.013409 & 0.928329 \\
\hline 1 & -8.439573 & 0.710114 & 0.122359 \\
\hline 6 & -4.506182 & 4.888839 & -0.805135 \\
\hline 6 & -4.040326 & 6.148739 & 1.274609 \\
\hline 6 & -2.653236 & 6.532778 & -0.772987 \\
\hline 6 & 1.578345 & 3.144205 & 3.249672 \\
\hline 6 & 1.627616 & 5.326916 & 2.062936 \\
\hline 6 & 0.088961 & 4.969380 & 3.999587 \\
\hline 1 & -5.196940 & 5.685827 & -1.108071 \\
\hline 1 & -4.115588 & 4.427296 & -1.721117 \\
\hline 1 & -5.091527 & 4.133316 & -0.263809 \\
\hline 1 & -4.760355 & 6.911991 & 0.950085 \\
\hline 1 & -4.579430 & 5.414365 & 1.887289 \\
\hline 1 & -3.302692 & 6.643439 & 1.917909 \\
\hline 1 & -3.353900 & 7.302708 & -1.123477 \\
\hline 1 & -1.868204 & 7.036270 & -0.195903 \\
\hline 1 & -2.181186 & 6.077701 & -1.653255 \\
\hline 1 & 2.331067 & 3.504678 & 3.962699 \\
\hline 1 & 0.970467 & 2.385539 & 3.762510 \\
\hline 1 & 2.118870 & 2.647366 & 2.432711 \\
\hline 1 & 0.870488 & 5.282900 & 4.704599 \\
\hline 1 & -0.498821 & 5.858738 & 3.742970 \\
\hline 1 & -0.575427 & 4.268042 & 4.521287 \\
\hline
\end{tabular}




$\begin{array}{llll}1 & 2.410636 & 5.678326 & 2.748322 \\ 1 & 2.122560 & 4.876490 & 1.192331 \\ 1 & 1.070017 & 6.204868 & 1.714538\end{array}$

$1_{\text {C11- }} \psi_{-1 \text { 1a2a3s4s }}$

Number of imaginary frequencies : 0

The smallest frequencies are : $10.3093 \quad 16.6025 \quad 17.2134 \mathrm{~cm}(-1)$

Electronic energy : $\quad \mathrm{HF}=-4125.4641763$

Zero-point correction $=\quad 1.298577($ Hartree/Particle $)$

Thermal correction to Energy= $\quad 1.377451$

Thermal correction to Enthalpy= $\quad 1.378396$

Thermal correction to Gibbs Free Energy= $\quad 1.183307$

Sum of electronic and zero-point Energies $=\quad-4124.165600$

Sum of electronic and thermal Energies $=\quad-4124.086725$

Sum of electronic and thermal Enthalpies $=\quad-4124.085781$

Sum of electronic and thermal Free Energies $=\quad-4124.280869$

Cartesian Coordinates

$\begin{array}{cccc}6 & -0.660310 & 2.611293 & 1.242523 \\ 6 & -1.725188 & 2.093947 & 0.499637 \\ 6 & -2.760945 & 2.933422 & 0.098428 \\ 6 & -2.757047 & 4.289213 & 0.438609 \\ 6 & -1.696426 & 4.766290 & 1.210922 \\ 6 & -0.644183 & 3.947737 & 1.639108 \\ 15 & -1.662636 & 0.336121 & -0.029331 \\ 6 & -3.426594 & -0.031779 & -0.399115 \\ 6 & -4.408164 & 0.087678 & 0.584845 \\ 6 & -5.749350 & -0.162817 & 0.300483 \\ 6 & -6.083873 & -0.543946 & -1.005212 \\ 6 & -5.126402 & -0.671206 & -2.014145 \\ 6 & -3.793503 & -0.407829 & -1.685126 \\ 6 & -6.780649 & 0.001632 & 1.415598 \\ 6 & -6.792458 & 1.465838 & 1.871939 \\ 6 & -5.460847 & -1.138683 & -3.428424 \\ 6 & -4.892466 & -2.550794 & -3.614620 \\ 6 & -3.887846 & 5.231302 & 0.028236 \\ 6 & -3.315493 & 6.415121 & -0.757955 \\ 6 & 0.468570 & 4.522942 & 2.515400 \\ 6 & -0.140869 & 5.258843 & 3.714020 \\ 6 & -1.461302 & -0.547745 & 1.593435 \\ 6 & -1.095119 & -1.885178 & 1.618352 \\ 6 & -0.962743 & -2.572600 & 2.870905 \\ 6 & -1.209709 & -1.872985 & 4.087614 \\ 6 & -1.600536 & -0.515977 & 4.026114 \\ 6 & -1.719957 & 0.122432 & 2.821627 \\ 6 & -0.570234 & -3.935060 & 2.954099 \\ 6 & -0.420106 & -4.555948 & 4.169616 \\ 6 & -0.662223 & -3.855021 & 5.369311 \\ 6 & -1.051702 & -2.540946 & 5.325036 \\ 6 & -0.868551 & -2.703046 & 0.393048 \\ 6 & 0.383629 & -2.795089 & -0.189469 \\ 6 & 0.632971 & -3.740321 & -1.212377 \\ 6 & -0.361955 & -4.563308 & -1.668434 \\ 6 & -1.671149 & -4.461224 & -1.143462\end{array}$




\begin{tabular}{|c|c|c|c|}
\hline 6 & -1.930332 & -3.525102 & -0.098034 \\
\hline 6 & -2.726298 & -5.274574 & -1.624994 \\
\hline 6 & -3.989826 & -5.167067 & -1.103155 \\
\hline 6 & -4.252282 & -4.235422 & -0.074937 \\
\hline & -3.248984 & -3.440734 & 0.420145 \\
\hline 7 & 1.449424 & -1.921158 & 0.218361 \\
\hline 15 & 1.745271 & -0.688709 & -0.909453 \\
\hline 8 & 2.938865 & -1.216694 & -1.964773 \\
\hline 6 & 4.197061 & -1.563643 & -1.529419 \\
\hline 6 & 5.066221 & -0.610743 & -1.027401 \\
\hline 6 & 6.301586 & -1.064546 & -0.458879 \\
\hline 6 & 6.662987 & -2.441589 & -0.559782 \\
\hline 6 & 5.785689 & -3.344310 & -1.209646 \\
\hline 6 & 4.569312 & -2.918641 & -1.667237 \\
\hline 6 & 7.157690 & -0.204123 & 0.274426 \\
\hline 6 & 8.311798 & -0.674800 & 0.851199 \\
\hline 6 & 8.682391 & -2.029340 & 0.719116 \\
\hline 6 & 7.870420 & -2.891461 & 0.027298 \\
\hline 6 & 4.682956 & 0.819978 & -1.050964 \\
\hline 6 & 3.476073 & 1.209284 & -0.502953 \\
\hline 6 & 3.051449 & 2.552192 & -0.468479 \\
\hline 6 & 3.849165 & 3.524020 & -1.008005 \\
\hline 6 & 5.072904 & 3.187411 & -1.634769 \\
\hline 6 & 5.491487 & 1.822852 & -1.679621 \\
\hline 6 & 5.875155 & 4.183553 & -2.242531 \\
\hline 6 & 7.034379 & 3.855168 & -2.896679 \\
\hline 6 & 7.431529 & 2.504241 & -2.977103 \\
\hline 6 & 6.682650 & & -2.386078 \\
\hline 8 & 2.659869 & 0.264025 & 0.075561 \\
\hline 6 & 2.434982 & -2.498983 & 1.161467 \\
\hline 6 & 2.972721 & -1.546079 & 2.196176 \\
\hline 6 & 4.344354 & -1.471700 & 2.430003 \\
\hline 6 & 4.852559 & -0.623719 & 3.411484 \\
\hline 6 & 3.989840 & 0.153395 & 4.176256 \\
\hline 6 & 2.616282 & 0.081090 & \\
\hline 6 & 2.113605 & -0.756902 & 2.962850 \\
\hline 45 & -0.102292 & 0.259956 & -1.944989 \\
\hline 6 & -0.482103 & 1.593699 & -3.251447 \\
\hline 8 & -0.710602 & 2.453323 & -3.990937 \\
\hline 6 & -0.675507 & -1.235056 & -3.068380 \\
\hline 8 & -0.986606 & -2.026007 & -3.848062 \\
\hline 6 & -8.191884 & -0.368990 & 0.967318 \\
\hline 6 & -6.404887 & -0.900789 & 2.597032 \\
\hline 6 & -6.965220 & -1.185383 & -3.684804 \\
\hline 6 & -4.831290 & -0.197932 & -4.462409 \\
\hline 6 & -4.928249 & 4.537969 & -0.849087 \\
\hline 6 & -4.593679 & 5.746663 & 1.287378 \\
\hline 6 & 1.387080 & 3.430753 & 3.062175 \\
\hline 6 & 1.306807 & 5.506066 & 1.692376 \\
\hline 1 & 6.875040 & 0.837870 & 0.401805 \\
\hline 1 & -2.018379 & 1.167625 & 2.805896 \\
\hline 1 & 8.130493 & -3.944837 & -0.069383 \\
\hline 1 & -1.243125 & -1.985076 & 6.241832 \\
\hline 1 & 5.539872 & 5.218505 & -2.188472 \\
\hline 1 & -3.463158 & -2.722135 & 1.209977 \\
\hline 1 & -5.260193 & -4.143541 & 0.324484 \\
\hline
\end{tabular}




\begin{tabular}{|c|c|c|c|}
\hline 1 & 3.540915 & 4.568412 & -0.985917 \\
\hline & -1.805185 & 0.015916 & 4.954538 \\
\hline & 6.083066 & -4.386658 & -1.312088 \\
\hline 1 & 8.336879 & 2.239971 & -3.519068 \\
\hline 1 & 3.867574 & -3.599123 & -2.144772 \\
\hline 1 & -2.510561 & -5.984739 & -2.422002 \\
\hline 1 & -0.165553 & -5.288220 & -2.456773 \\
\hline 1 & -0.114581 & -5.599030 & 4.207619 \\
\hline 1 & 6.998047 & 0.480245 & -2.469558 \\
\hline 1 & -0.385472 & -4.494009 & 2.038931 \\
\hline 1 & 1.636843 & -3.791791 & -1.631150 \\
\hline 1 & 7.639359 & 4.627993 & -3.365090 \\
\hline 1 & 8.943121 & 0.003470 & 1.420963 \\
\hline 1 & -4.793965 & -5.793829 & -1.482661 \\
\hline 1 & -0.539542 & -4.359233 & 6.324998 \\
\hline 1 & 9.601887 & -2.387756 & 1.175906 \\
\hline 1 & 2.089615 & 2.781630 & -0.012530 \\
\hline 1 & -4.119658 & 0.392639 & 1.590297 \\
\hline 1 & -3.016289 & -0.497506 & -2.441138 \\
\hline 1 & -7.124994 & -0.745379 & -1.240966 \\
\hline 1 & -3.578546 & 2.520209 & -0.486677 \\
\hline 1 & 0.147075 & 1.940512 & 1.538465 \\
\hline 1 & -1.691867 & 5.817458 & 1.499204 \\
\hline 1 & 1.899373 & -3.311452 & 1.673834 \\
\hline 1 & 0.509638 & 1.564626 & -1.230186 \\
\hline 1 & 3.270889 & -2.969609 & 0.621896 \\
\hline 1 & 5.027722 & -2.071336 & 1.826069 \\
\hline 1 & 1.044828 & -0.783825 & 2.759574 \\
\hline 1 & 5.928011 & -0.569465 & 3.569265 \\
\hline 1 & 1.929127 & 0.682111 & 4.543301 \\
\hline 1 & 4.383747 & 0.815962 & 4.944163 \\
\hline 1 & -7.153017 & -1.494028 & -4.720858 \\
\hline 1 & -7.473217 & -1.909241 & -3.034899 \\
\hline 1 & -7.435351 & -0.203709 & -3.540539 \\
\hline 1 & -5.092624 & -0.526000 & -5.477071 \\
\hline 1 & -5.193364 & 0.830622 & -4.335616 \\
\hline 1 & -3.735575 & -0.178066 & -4.402049 \\
\hline 1 & -5.105181 & -2.919972 & -4.627389 \\
\hline 1 & -3.804576 & -2.574917 & -3.468295 \\
\hline 1 & -5.337495 & -3.249996 & -2.893835 \\
\hline 1 & -8.890039 & -0.242250 & 1.804388 \\
\hline 1 & -8.544668 & 0.270206 & 0.147861 \\
\hline 1 & -8.256239 & -1.414961 & 0.640141 \\
\hline 1 & -7.499512 & 1.604352 & 2.701137 \\
\hline 1 & -5.804248 & 1.798895 & 2.215044 \\
\hline 1 & -7.102051 & 2.127237 & 1.051753 \\
\hline 1 & -7.133363 & -0.786136 & 3.410944 \\
\hline 1 & -6.399665 & -1.956991 & 2.296603 \\
\hline 1 & -5.413393 & -0.667045 & 3.005515 \\
\hline 1 & -5.719300 & 5.250796 & -1.114155 \\
\hline 1 & -4.492279 & 4.164649 & -1.784689 \\
\hline 1 & -5.405779 & 3.692696 & -0.334296 \\
\hline 1 & -5.409687 & 6.430030 & 1.015931 \\
\hline 1 & -5.025337 & 4.916159 & 1.861995 \\
\hline 1 & -3.909299 & 6.291437 & 1.949168 \\
\hline 1 & -4.122595 & 7.094221 & -1.064125 \\
\hline
\end{tabular}




$\begin{array}{lrrr}1 & -2.600460 & 6.999094 & -0.166141 \\ 1 & -2.799237 & 6.072300 & -1.663735 \\ 1 & 2.130330 & 3.873112 & 3.737824 \\ 1 & 0.820784 & 2.680334 & 3.632102 \\ 1 & 1.942719 & 2.906346 & 2.273531 \\ 1 & 0.655671 & 5.651418 & 4.359905 \\ 1 & -0.763940 & 6.108760 & 3.412106 \\ 1 & -0.762690 & 4.584254 & 4.316961 \\ 1 & 2.108265 & 5.936619 & 2.307932 \\ 1 & 1.773184 & 5.002952 & 0.834808 \\ 1 & 0.699479 & 6.333014 & 1.303838\end{array}$

$1_{\text {C11- }} \psi_{-1 \mathrm{a} 2 \mathrm{~s} 3 \mathrm{a} 4 \mathrm{a}}$

Number of imaginary frequencies : 0

The smallest frequencies are : $10.8474 \quad 18.6578 \quad 19.5769 \mathrm{~cm}(-1)$

Electronic energy : $\quad H F=-4125.4643117$

Zero-point correction $=\quad 1.298988$ (Hartree/Particle)

Thermal correction to Energy= $\quad 1.377663$

Thermal correction to Enthalpy= $\quad 1.378607$

Thermal correction to Gibbs Free Energy= $\quad 1.185056$

Sum of electronic and zero-point Energies $=\quad-4124.165323$

Sum of electronic and thermal Energies $=\quad-4124.086648$

Sum of electronic and thermal Enthalpies $=\quad-4124.085704$

Sum of electronic and thermal Free Energies $=\quad-4124.279256$

Cartesian Coordinates

$\begin{array}{cccc}6 & -0.567480 & 2.572659 & 1.316555 \\ 6 & -1.631466 & 2.116544 & 0.525925 \\ 6 & -2.573411 & 3.021037 & 0.058918 \\ 6 & -2.494681 & 4.382689 & 0.385029 \\ 6 & -1.449180 & 4.797609 & 1.204205 \\ 6 & -0.476878 & 3.908056 & 1.693168 \\ 15 & -1.660548 & 0.361451 & -0.011116 \\ 6 & -3.446035 & 0.059669 & -0.348631 \\ 6 & -4.408576 & 0.285272 & 0.639983 \\ 6 & -5.756315 & 0.031890 & 0.398195 \\ 6 & -6.109782 & -0.460023 & -0.865023 \\ 6 & -5.176403 & -0.702577 & -1.873223 \\ 6 & -3.833657 & -0.426104 & -1.592871 \\ 6 & -6.831054 & 0.238333 & 1.463863 \\ 6 & -7.450132 & -1.119750 & 1.813450 \\ 6 & -5.631952 & -1.225798 & -3.234545 \\ 6 & -6.398784 & -0.113794 & -3.958671 \\ 6 & -3.566106 & 5.329276 & -0.151211 \\ 6 & 0.618882 & 4.418187 & 2.629056 \\ 6 & -0.023568 & 5.119027 & 3.832139 \\ 6 & -1.470989 & -0.546419 & 1.600096 \\ 6 & -1.135861 & -1.892234 & 1.603744 \\ 6 & -1.021672 & -2.603112 & 2.844589 \\ 6 & -1.247086 & -1.916123 & 4.072749 \\ 6 & -1.602647 & -0.548478 & 4.033423 \\ 6 & -1.711946 & 0.110435 & 2.839177 \\ 6 & -0.667358 & -3.977009 & 2.904836 \\ 6 & -0.531046 & -4.620974 & 4.110038\end{array}$




\begin{tabular}{|c|c|c|c|}
\hline 6 & -0.749082 & -3.932146 & 5.321297 \\
\hline 6 & -1.102642 & -2.607316 & 5.298949 \\
\hline 6 & -0.925306 & -2.690368 & 0.362727 \\
\hline 6 & 0.325958 & -2.800309 & -0.218699 \\
\hline 6 & 0.551461 & -3.716644 & -1.272817 \\
\hline 6 & -0.470009 & -4.477902 & -1.774180 \\
\hline 6 & -1.779530 & -4.345648 & -1.257312 \\
\hline 6 & -2.010559 & -3.456538 & -0.165288 \\
\hline 6 & -2.864640 & -5.078441 & -1.797911 \\
\hline 6 & -4.129743 & -4.940017 & -1.285771 \\
\hline 6 & -4.361837 & -4.062539 & -0.203692 \\
\hline 6 & -3.329005 & -3.347022 & 0.348016 \\
\hline 7 & 1.410423 & -1.959029 & 0.207875 \\
\hline 15 & 1.726853 & -0.713133 & -0.900043 \\
\hline 8 & 2.910371 & -1.244517 & -1.964159 \\
\hline 6 & 4.170303 & -1.601686 & -1.542853 \\
\hline 6 & 5.049353 & -0.657569 & -1.041621 \\
\hline 6 & 6.290697 & -1.122092 & -0.494901 \\
\hline 6 & 6.645843 & -2.499177 & -0.616190 \\
\hline 6 & 5.754908 & -3.392257 & -1.260803 \\
\hline 6 & 4.534005 & -2.957243 & -1.696959 \\
\hline 6 & 7.160679 & -0.272950 & 0.235191 \\
\hline 6 & 8.322694 & -0.753419 & 0.787570 \\
\hline 6 & 8.687299 & -2.107259 & 0.633768 \\
\hline 6 & 7.861173 & -2.959207 & -0.053859 \\
\hline 6 & 4.670640 & 0.774590 & -1.042444 \\
\hline 6 & 3.472538 & 1.161314 & -0.473645 \\
\hline 6 & 3.054310 & 2.505312 & -0.414594 \\
\hline 6 & 3.848288 & 3.481078 & -0.952464 \\
\hline 6 & 5.061614 & 3.148190 & -1.601017 \\
\hline 6 & 5.474385 & 1.782838 & -1.668965 \\
\hline 6 & 5.858648 & 4.149072 & -2.207825 \\
\hline 6 & 7.007086 & 3.824875 & -2.882775 \\
\hline 6 & 7.397960 & 2.473677 & -2.985911 \\
\hline 6 & 6.654015 & 1.481677 & -2.396455 \\
\hline 8 & 2.656496 & 0.210720 & 0.097801 \\
\hline 6 & 2.389227 & -2.578071 & 1.131765 \\
\hline 6 & 2.953373 & -1.656975 & 2.181045 \\
\hline 6 & 4.329994 & -1.587665 & 2.384824 \\
\hline 6 & 4.860677 & -0.764157 & 3.375059 \\
\hline 6 & 4.015645 & -0.008369 & 4.180087 \\
\hline 6 & 2.637008 & -0.078165 & 3.985131 \\
\hline 6 & 2.112010 & -0.890268 & 2.988956 \\
\hline 45 & -0.102347 & 0.270343 & -1.933165 \\
\hline 6 & -0.484064 & 1.636785 & -3.203746 \\
\hline 8 & -0.712259 & 2.521799 & -3.912832 \\
\hline 6 & -0.640294 & -1.213643 & -3.090244 \\
\hline 8 & -0.889196 & -2.007035 & -3.889009 \\
\hline 6 & -6.264396 & 0.847832 & 2.744793 \\
\hline 6 & -7.920067 & 1.176762 & 0.934173 \\
\hline 6 & -4.451671 & -1.653153 & -4.104663 \\
\hline 6 & -6.545042 & -2.443275 & -3.060664 \\
\hline 6 & 1.489437 & 3.282550 & 3.164832 \\
\hline 6 & 1.515093 & 5.412973 & 1.884923 \\
\hline 1 & 6.883535 & 0.768301 & 0.379308 \\
\hline 1 & -1.984523 & 1.162862 & 2.841443 \\
\hline
\end{tabular}




\begin{tabular}{|c|c|c|c|}
\hline & 8.116149 & -4.012248 & -0.166515 \\
\hline & -1.275872 & -2.060887 & 6.225002 \\
\hline & 5.527883 & 5.184395 & -2.136005 \\
\hline & -3.520497 & -2.667212 & 1.176334 \\
\hline & -5.370510 & -3.944010 & 0.188009 \\
\hline & 3.543662 & 4.526016 & -0.912342 \\
\hline & -1.790273 & -0.025776 & 4.970637 \\
\hline & 6.046075 & -4.434899 & -1.377081 \\
\hline & 8.294247 & 2.212970 & -3.544406 \\
\hline & 3.822173 & -3.630543 & -2.169960 \\
\hline & -2.670794 & -5.752236 & -2.631324 \\
\hline & -0.295160 & -5.171110 & -2.595433 \\
\hline & -0.255303 & -5.672812 & 4.130826 \\
\hline & 6.964008 & 0.444999 & -2.497962 \\
\hline & -0.501955 & -4.526091 & 1.980035 \\
\hline & 1.555669 & -3.785440 & -1.688461 \\
\hline & 7.608003 & 4.601381 & -3.350332 \\
\hline & 8.965097 & -0.083455 & 1.354763 \\
\hline & -4.956789 & -5.505765 & -1.709921 \\
\hline & -0.636809 & -4.454234 & 6.268636 \\
\hline & 9.613268 & -2.473347 & 1.070985 \\
\hline & 2.099849 & 2.732636 & 0.057817 \\
\hline 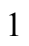 & -4.092811 & 0.666202 & 1.607994 \\
\hline & -3.063512 & -0.596498 & -2.341754 \\
\hline . & -7.161303 & -0.664733 & -1.066666 \\
\hline & -3.386760 & 2.664794 & -0.572310 \\
\hline & 0.178224 & 1.853458 & 1.656106 \\
\hline ] & -1.378929 & 5.846668 & 1.483924 \\
\hline & 1.839438 & -3.389315 & 1.630613 \\
\hline ] & 0.529185 & 1.558040 & -1.205418 \\
\hline & 3.211294 & -3.054797 & 0.576280 \\
\hline . & 4.999104 & -2.171540 & 1.750433 \\
\hline 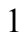 & 1.038503 & -0.916605 & 2.812989 \\
\hline & 5.939534 & -0.712527 & 3.508680 \\
\hline 1 & 1.963457 & 0.504906 & 4.611999 \\
\hline . & 4.427152 & 0.634968 & 4.955108 \\
\hline l & -4.821677 & -2.065563 & -5.051827 \\
\hline & -3.792823 & -0.811371 & -4.354593 \\
\hline 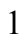 & -3.849591 & -2.430675 & -3.613040 \\
\hline 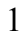 & -6.861035 & -2.818776 & -4.042948 \\
\hline ! & -6.015868 & -3.254043 & -2.542568 \\
\hline 1 & -7.454448 & -2.213470 & -2.492903 \\
\hline . & -6.734668 & -0.458768 & -4.946082 \\
\hline 1 & -7.284969 & 0.202625 & -3.393976 \\
\hline & -5.763356 & 0.769151 & -4.106651 \\
\hline 1 & -7.071692 & 0.985023 & 3.475161 \\
\hline 1 & -5.507531 & 0.202562 & 3.210668 \\
\hline L & -5.811707 & 1.831923 & 2.563470 \\
\hline l & -8.231519 & -1.001011 & 2.576278 \\
\hline 1 & -7.907035 & -1.600457 & 0.939451 \\
\hline 1 & -6.689119 & -1.803903 & 2.212337 \\
\hline 1 & -8.692194 & 1.332111 & 1.699498 \\
\hline 1 & -7.502009 & 2.157793 & 0.673032 \\
\hline 1 & -8.417896 & 0.777364 & 0.042682 \\
\hline 1 & 2.231893 & 3.682385 & 3.867454 \\
\hline 1 & 0.888102 & 2.536480 & 3.703568 \\
\hline
\end{tabular}




$\begin{array}{rrrr}1 & 2.042909 & 2.761326 & 2.372273 \\ 1 & 0.753101 & 5.466671 & 4.526384 \\ 1 & -0.616481 & 5.993434 & 3.539303 \\ 1 & -0.684125 & 4.434061 & 4.379820 \\ 1 & 2.293642 & 5.801207 & 2.555455 \\ 1 & 2.015051 & 4.932617 & 1.033279 \\ 1 & 0.947151 & 6.269093 & 1.500721 \\ 6 & -3.362712 & 6.766815 & 0.319287 \\ 6 & -3.531208 & 5.317314 & -1.683610 \\ 6 & -4.944128 & 4.858839 & 0.331414 \\ 1 & -5.729331 & 5.526457 & -0.048283 \\ 1 & -5.178719 & 3.843230 & -0.012762 \\ 1 & -4.998403 & 4.862031 & 1.428300 \\ 1 & -4.294420 & 5.995937 & -2.087999 \\ 1 & -2.552539 & 5.645726 & -2.056511 \\ 1 & -3.724285 & 4.318595 & -2.093105 \\ 1 & -4.157347 & 7.404081 & -0.088788 \\ 1 & -3.402755 & 6.851115 & 1.413118 \\ 1 & -2.404475 & 7.178056 & -0.023307\end{array}$

$1_{\text {C11- }} \psi_{-1 \text { 1a2s3a4s }}$

Number of imaginary frequencies : 0

The smallest frequencies are : $13.7275 \quad 19.566320 .6612 \mathrm{~cm}(-1)$

Electronic energy : $\quad \mathrm{HF}=-4125.4646479$

Zero-point correction $=\quad 1.300310($ Hartree/Particle $)$

Thermal correction to Energy= $\quad 1.378524$

Thermal correction to Enthalpy $=\quad 1.379468$

Thermal correction to Gibbs Free Energy $=1.187595$

Sum of electronic and zero-point Energies $=\quad-4124.164338$

Sum of electronic and thermal Energies $=\quad-4124.086124$

Sum of electronic and thermal Enthalpies $=\quad-4124.085180$

Sum of electronic and thermal Free Energies $=\quad-4124.277053$

Cartesian Coordinates

$\begin{array}{cccc}6 & -0.589059 & 2.566379 & 1.351188 \\ 6 & -1.650643 & 2.106645 & 0.559499 \\ 6 & -2.597699 & 3.006858 & 0.094225 \\ 6 & -2.522607 & 4.369227 & 0.418202 \\ 6 & -1.481227 & 4.787215 & 1.241141 \\ 6 & -0.506757 & 3.901066 & 1.732116 \\ 15 & -1.658368 & 0.357512 & 0.005114 \\ 6 & -3.437198 & 0.042637 & -0.350337 \\ 6 & -4.404269 & 0.220629 & 0.635184 \\ 6 & -5.753737 & -0.036925 & 0.380541 \\ 6 & -6.100335 & -0.475982 & -0.898271 \\ 6 & -5.153292 & -0.674539 & -1.911237 \\ 6 & -3.817305 & -0.402722 & -1.616145 \\ 6 & -6.766039 & 0.141552 & 1.510348 \\ 6 & -5.599832 & -1.157884 & -3.289777 \\ 6 & -3.586130 & 5.315179 & -0.134495 \\ 6 & 0.585975 & 4.414855 & 2.669400 \\ 6 & -0.059423 & 5.117839 & 3.869550 \\ 6 & -1.471442 & -0.568469 & 1.605082 \\ 6 & -1.137207 & -1.914046 & 1.586330\end{array}$




\begin{tabular}{|c|c|c|c|}
\hline 6 & -1.021311 & -2.645443 & 2.814408 \\
\hline 6 & -1.246466 & -1.978800 & 4.053736 \\
\hline 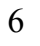 & -1.600970 & -0.610334 & 4.037338 \\
\hline & -1.709200 & 0.069366 & 2.854315 \\
\hline ) & -0.666394 & -4.019861 & 2.851161 \\
\hline & -0.530423 & -4.684113 & 4.045328 \\
\hline f & -0.749412 & -4.015883 & 5.268012 \\
\hline 5 & -1.102692 & -2.690774 & 5.268069 \\
\hline 6 & -0.934737 & -2.687898 & 0.329253 \\
\hline 6 & 0.310281 & -2.780875 & -0.268546 \\
\hline 6 & 0.526037 & -3.675757 & -1.342784 \\
\hline 6 & -0.497399 & -4.435954 & -1.842510 \\
\hline 6 & -1.801038 & -4.319903 & -1.307400 \\
\hline 6 & -2.023657 & -3.447441 & -0.200434 \\
\hline 6 & -2.887156 & -5.056876 & -1.840458 \\
\hline 6 & -4.145835 & -4.934861 & -1.309223 \\
\hline 6 & -4.370948 & -4.068347 & -0.216812 \\
\hline 6 & -3.336952 & -3.350054 & 0.328797 \\
\hline 7 & 1.398841 & -1.949355 & 0.167234 \\
\hline 15 & 1.723696 & -0.685786 & -0.917313 \\
\hline 8 & 2.903720 & -1.201484 & -1.991547 \\
\hline 6 & 4.158629 & -1.580281 & -1.573225 \\
\hline 6 & 5.045131 & -0.655120 & -1.050345 \\
\hline 6 & 6.279787 & -1.143116 & -0.508884 \\
\hline 6 & 6.620664 & -2.521386 & -0.655337 \\
\hline 6 & 5.722736 & -3.392579 & -1.319785 \\
\hline 6 & 4.508165 & -2.936360 & -1.752024 \\
\hline 6 & 7.156302 & -0.317433 & \\
\hline 6 & 8.311216 & -0.820781 & 0.786928 \\
\hline 6 & 8.661967 & -2.175250 & 0.608383 \\
\hline 6 & 7.829202 & -3.004988 & -0.098090 \\
\hline 6 & 4.679948 & 0.780407 & -1.023111 \\
\hline 6 & 3.483671 & 1.166931 & -0.450248 \\
\hline 6 & 3.078564 & 2.513383 & -0.363688 \\
\hline 6 & 3.883920 & 3.492516 & -0.877904 \\
\hline 6 & 5.095729 & 3.161429 & -1.530145 \\
\hline 6 & 5.495236 & 1.793792 & -1.625987 \\
\hline 6 & 5.904244 & 4.167016 & -2.113548 \\
\hline 6 & 7.051339 & 3.845891 & -2.792228 \\
\hline 6 & 7.429267 & 2.493390 & -2.922896 \\
\hline 6 & 6.673983 & 1.496537 & -2.356498 \\
\hline 8 & 2.656408 & 0.213052 & 0.099042 \\
\hline 6 & 2.370152 & -2.583353 & 1.089566 \\
\hline 6 & 2.935828 & -1.678625 & 2.152353 \\
\hline 6 & 4.311981 & -1.623653 & 2.363452 \\
\hline 6 & 4.845131 & -0.816700 & 3.365895 \\
\hline 6 & 4.003031 & -0.062222 & 4.175159 \\
\hline 6 & 2.624860 & -0.116693 & 3.972192 \\
\hline 6 & 2.097491 & -0.913260 & 2.964786 \\
\hline 45 & -0.100408 & 0.327904 & -1.925188 \\
\hline 6 & -0.557565 & 1.750784 & -3.106231 \\
\hline 8 & -0.845923 & 2.669340 & -3.747142 \\
\hline 6 & -0.609765 & -1.103384 & -3.158058 \\
\hline 8 & -0.836531 & -1.850931 & -4.006542 \\
\hline 6 & 1.457299 & 3.281724 & 3.209415 \\
\hline 6 & 1.482308 & 5.408682 & 1.924094 \\
\hline
\end{tabular}




\begin{tabular}{|c|c|c|c|}
\hline ] & 6.889877 & 0.723802 & 0.403129 \\
\hline & -1.979721 & 1.122230 & 2.873047 \\
\hline & 8.073395 & -4.058303 & -0.230297 \\
\hline 1 & -1.275968 & -2.160059 & 6.203206 \\
\hline 1 & 5.583434 & 5.203796 & -2.020570 \\
\hline & -3.522904 & -2.679449 & 1.166485 \\
\hline & -5.375082 & -3.964449 & 0.190043 \\
\hline & 3.589909 & 4.539454 & -0.815832 \\
\hline & -1.787342 & -0.103348 & 4.983382 \\
\hline L & 6.003252 & -4.435849 & -1.454930 \\
\hline & 8.324506 & 2.235713 & -3.484461 \\
\hline & 3.791196 & -3.592750 & -2.240705 \\
\hline & -2.699238 & -5.720268 & -2.683513 \\
\hline & -0.328319 & -5.116327 & -2.675642 \\
\hline & -0.254176 & -5.736012 & 4.048574 \\
\hline & 6.973967 & 0.459236 & -2.479296 \\
\hline ] & -0.500383 & -4.552643 & 1.916929 \\
\hline & 1.525281 & -3.733213 & -1.771735 \\
\hline ] & 7.661098 & 4.626119 & -3.241800 \\
\hline & 8.958784 & -0.168625 & 1.368816 \\
\hline ] & -4.973718 & -5.504383 & -1.726565 \\
\hline & -0.637740 & -4.553976 & 6.206417 \\
\hline & 9.582537 & -2.559464 & 1.041426 \\
\hline 1 & 2.124744 & 2.739965 & 0.110171 \\
\hline & -4.101488 & 0.562828 & 1.624436 \\
\hline$\frac{1}{1}$ & -3.041499 & -0.544329 & -2.365031 \\
\hline & -7.145844 & -0.679257 & -1.120348 \\
\hline ] & -3.408256 & 2.647738 & -0.539185 \\
\hline$\frac{1}{1}$ & 0.163356 & 1.850770 & 1.684049 \\
\hline & -1.414575 & 5.836745 & 1.520005 \\
\hline 1 & 1.813225 & -3.396941 & 1.576566 \\
\hline & 0.540226 & 1.589833 & -1.159744 \\
\hline 1 & 3.191714 & -3.058712 & 0.532074 \\
\hline 1 & 4.979086 & -2.205958 & 1.725468 \\
\hline ] & 1.024756 & -0.926920 & 2.782955 \\
\hline 1 & 5.923714 & -0.776617 & 3.505543 \\
\hline 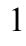 & 1.953446 & 0.466304 & 4.601463 \\
\hline 1 & 4.416552 & 0.568412 & 4.959469 \\
\hline & 2.195346 & 3.684029 & 3.915182 \\
\hline 1 & 0.855886 & 2.533686 & 3.745374 \\
\hline ] & 2.016036 & 2.762692 & 2.419186 \\
\hline 1 & 0.715692 & 5.469515 & 4.563493 \\
\hline 1 & -0.654110 & 5.989909 & 3.573460 \\
\hline 1 & -0.718903 & 4.433090 & 4.418762 \\
\hline 1 & 2.261546 & 5.797129 & 2.593714 \\
\hline l & 1.980909 & 4.927103 & 1.072294 \\
\hline 1 & 0.914427 & 6.264610 & 1.539392 \\
\hline 6 & -3.392675 & 6.752210 & 0.341530 \\
\hline 6 & -3.520907 & 5.304099 & -1.666167 \\
\hline 6 & -4.972812 & 4.842912 & 0.319377 \\
\hline 1 & -5.750721 & 5.507984 & -0.079386 \\
\hline 1 & -5.197156 & 3.825705 & -0.026698 \\
\hline 1 & -5.051528 & 4.848356 & 1.414750 \\
\hline 1 & -4.271339 & 5.988145 & -2.085154 \\
\hline 1 & -2.532579 & 5.624542 & -2.020132 \\
\hline 1 & -3.712855 & 4.306570 & -2.079214 \\
\hline
\end{tabular}


$1_{\text {C11- }-\psi_{-12} \text { 253s4a }}$

Number of imaginary frequencies : 0

The smallest frequencies are : $10.3326 \quad 17.2702 \quad 18.6978 \mathrm{~cm}(-1)$

Electronic energy : $\quad \mathrm{HF}=-4125.464607$

Zero-point correction $=\quad 1.299037$ (Hartree/Particle)

Thermal correction to Energy= $\quad 1.377672$

Thermal correction to Enthalpy= $\quad 1.378616$

Thermal correction to Gibbs Free Energy= 1.185322

Sum of electronic and zero-point Energies $=\quad-4124.165570$

Sum of electronic and thermal Energies $=\quad-4124.086935$

Sum of electronic and thermal Enthalpies $=\quad-4124.085991$

Sum of electronic and thermal Free Energies $=\quad-4124.279285$

Cartesian Coordinates

$\begin{array}{cccc}6 & -0.554389 & 2.541505 & 1.374404 \\ 6 & -1.622833 & 2.100583 & 0.581199 \\ 6 & -2.569646 & 3.012224 & 0.139274 \\ 6 & -2.490776 & 4.367059 & 0.491976 \\ 6 & -1.439438 & 4.767396 & 1.310938 \\ 6 & -0.462316 & 3.869746 & 1.775361 \\ 15 & -1.657118 & 0.356523 & 0.011910 \\ 6 & -3.439448 & 0.076996 & -0.351261 \\ 6 & -4.420289 & 0.242931 & 0.635869 \\ 6 & -5.762689 & 0.021249 & 0.351532 \\ 6 & -6.103205 & -0.378145 & -0.952999 \\ 6 & -5.156051 & -0.539637 & -1.958873\end{array}$




\begin{tabular}{|c|c|c|c|}
\hline 6 & -3.813954 & -0.304567 & -1.629590 \\
\hline 6 & -6.856084 & 0.171088 & 1.408122 \\
\hline & -5.498830 & -1.004451 & -3.371489 \\
\hline & -3.570660 & 5.320096 & -0.014713 \\
\hline & 0.639616 & 4.363730 & 2.712639 \\
\hline & 0.004117 & 5.032337 & 3.937581 \\
\hline o & -1.479682 & -0.582321 & 1.603665 \\
\hline 6 & -1.143644 & -1.927690 & 1.584987 \\
\hline 6 & -1.032984 & -2.658105 & 2.815842 \\
\hline 6 & -1.267358 & -1.991704 & 4.053756 \\
\hline 6 & -1.625574 & -0.624360 & 4.035525 \\
\hline 6 & -1.727475 & 0.054321 & 2.851995 \\
\hline 6 & -0.671202 & -4.030900 & 2.857676 \\
\hline 6 & -0.539519 & -4.693074 & 4.053493 \\
\hline 6 & -0.769336 & -4.025029 & 5.274076 \\
\hline 6 & -1.128186 & -2.701525 & 5.269900 \\
\hline 6 & -0.922982 & -2.713468 & 0.336842 \\
\hline 6 & 0.331623 & -2.812888 & -0.239830 \\
\hline 6 & 0.576363 & -3.746331 & -1.274783 \\
\hline 6 & -0.424176 & -4.553290 & -1.746530 \\
\hline 6 & -1.736220 & -4.439968 & -1.230926 \\
\hline 6 & -1.992750 & -3.510401 & -0.178940 \\
\hline 6 & -2.797398 & -5.234743 & -1.729976 \\
\hline 6 & -4.064914 & -5.113505 & -1.221100 \\
\hline 6 & -4.326060 & -4.184074 & -0.190612 \\
\hline 6 & -3.316629 & -3.408860 & 0.322649 \\
\hline 7 & 1.407170 & -1.960179 & 0.186852 \\
\hline 15 & 1.713449 & -0.705098 & -0.913527 \\
\hline 8 & 2.897469 & -1.221041 & -1.984617 \\
\hline 6 & 4.162736 & -1.570231 & -1.573035 \\
\hline 6 & 5.035508 & -0.623807 & -1.065245 \\
\hline 6 & 6.282391 & -1.083833 & -0.527187 \\
\hline 6 & 6.649488 & -2.456174 & -0.665330 \\
\hline 6 & 5.764536 & -3.349656 & -1.317640 \\
\hline 6 & 4.538176 & -2.920619 & -1.744153 \\
\hline 6 & 7.146112 & -0.235762 & 0.211561 \\
\hline 6 & 8.313550 & -0.712337 & 0.755816 \\
\hline 6 & 8.690380 & -2.060700 & 0.584416 \\
\hline 6 & 7.870397 & -2.911783 & -0.111590 \\
\hline 6 & 4.645924 & 0.805407 & -1.048994 \\
\hline 6 & 3.447532 & 1.177056 & -0.471014 \\
\hline 6 & 3.020613 & 2.517452 & -0.393462 \\
\hline 6 & 3.805911 & 3.505022 & -0.922569 \\
\hline 6 & 5.018693 & 3.188265 & -1.580172 \\
\hline 6 & 5.440349 & 1.826655 & -1.666404 \\
\hline 6 & 5.806694 & 4.201838 & -2.177641 \\
\hline 6 & 6.954793 & 3.893685 & -2.860658 \\
\hline 6 & 7.354303 & 2.546516 & -2.981794 \\
\hline 6 & 6.619181 & 1.542403 & -2.401852 \\
\hline 8 & 2.640438 & 0.213856 & 0.091597 \\
\hline 6 & 2.400605 & -2.579078 & 1.096093 \\
\hline 6 & 2.951911 & -1.668192 & 2.160913 \\
\hline 6 & 4.327842 & -1.576693 & 2.360829 \\
\hline 6 & 4.846804 & -0.764040 & 3.366043 \\
\hline 6 & 3.990745 & -0.042480 & 4.190701 \\
\hline 6 & 2.612891 & -0.135742 & 4.000276 \\
\hline
\end{tabular}




\begin{tabular}{|c|c|c|c|}
\hline 6 & 2.099341 & -0.935512 & 2.988207 \\
\hline 45 & -0.126124 & 0.282612 & -1.926193 \\
\hline 6 & -0.541092 & 1.652808 & -3.182684 \\
\hline 8 & -0.801547 & 2.540256 & -3.877490 \\
\hline 6 & -0.721614 & -1.189198 & -3.067993 \\
\hline 8 & -1.044982 & -1.965796 & -3.857173 \\
\hline 6 & 1.521379 & 3.220811 & 3.213044 \\
\hline 6 & 1.523424 & 5.380897 & 1.984097 \\
\hline 1 & 6.859747 & 0.801037 & 0.369060 \\
\hline 1 & -2.000810 & 1.106414 & 2.869128 \\
\hline 1 & 8.134648 & -3.961025 & -0.237518 \\
\hline 1 & -1.309347 & -2.169937 & 6.203049 \\
\hline 1 & 5.469291 & 5.233944 & -2.091790 \\
\hline 1 & -3.530114 & -2.692283 & 1.114068 \\
\hline 1 & -5.337693 & -4.077849 & 0.195802 \\
\hline 1 & 3.494438 & 4.547284 & -0.868326 \\
\hline 1 & -1.819887 & -0.117911 & 4.980228 \\
\hline 1 & 6.064647 & -4.388202 & -1.447078 \\
\hline 1 & 8.250230 & 2.298684 & -3.546678 \\
\hline 1 & 3.830026 & -3.594517 & -2.221813 \\
\hline 1 & -2.582998 & -5.941273 & -2.530572 \\
\hline 1 & -0.230562 & -5.272087 & -2.541130 \\
\hline 1 & -0.257832 & -5.743516 & 4.059197 \\
\hline 1 & 6.935582 & 0.509168 & -2.517406 \\
\hline 1 & -0.495303 & -4.565601 & 1.926467 \\
\hline 1 & 1.582315 & -3.802642 & -1.688403 \\
\hline 1 & 7.548748 & 4.679881 & -3.320847 \\
\hline 1 & 8.950908 & -0.043552 & 1.330052 \\
\hline 1 & -4.873689 & -5.725522 & -1.614564 \\
\hline 1 & -0.661177 & -4.561547 & 6.213792 \\
\hline 1 & 9.620698 & -2.423554 & 1.015039 \\
\hline 1 & 2.066994 & 2.732721 & 0.086455 \\
\hline 1 & -4.118152 & 0.554571 & 1.632762 \\
\hline 1 & -3.039230 & -0.426107 & -2.383680 \\
\hline 1 & -7.151613 & -0.563270 & -1.179203 \\
\hline 1 & -3.386689 & 2.667169 & -0.493271 \\
\hline 1 & 0.192387 & 1.815798 & 1.697347 \\
\hline 1 & -1.368316 & 5.810826 & 1.610872 \\
\hline 1 & 1.867636 & -3.409375 & 1.581260 \\
\hline 1 & 0.517783 & 1.570021 & -1.207566 \\
\hline 1 & 3.229168 & -3.030904 & 0.529893 \\
\hline 1 & 5.005281 & -2.134387 & 1.711883 \\
\hline 1 & 1.025699 & -0.980962 & 2.817068 \\
\hline 1 & 5.925050 & -0.694244 & 3.496367 \\
\hline 1 & 1.930913 & 0.418981 & 4.643462 \\
\hline 1 & 4.392931 & 0.591914 & 4.977922 \\
\hline 1 & 2.268152 & 3.607873 & 3.918306 \\
\hline 1 & 0.929368 & 2.458661 & 3.739213 \\
\hline 1 & 2.070247 & 2.721456 & 2.403241 \\
\hline 1 & 0.784990 & 5.369545 & 4.632264 \\
\hline 1 & -0.597658 & 5.908838 & 3.670128 \\
\hline 1 & -0.646418 & 4.330306 & 4.475568 \\
\hline 1 & 2.306747 & 5.756504 & 2.656241 \\
\hline 1 & 2.017356 & 4.923836 & 1.116266 \\
\hline 1 & 0.947905 & 6.243460 & 1.626522 \\
\hline 6 & -3.359136 & 6.751619 & 0.470153 \\
\hline
\end{tabular}




$\begin{array}{rrrr}6 & -3.562595 & 5.327187 & -1.547629 \\ 6 & -4.939773 & 4.842366 & 0.486401 \\ 1 & -5.732598 & 5.512089 & 0.126729 \\ 1 & -5.177775 & 3.829337 & 0.136587 \\ 1 & -4.975330 & 4.834441 & 1.584030 \\ 1 & -4.330048 & 6.013893 & -1.929693 \\ 1 & -2.589348 & 5.656433 & -1.933866 \\ 1 & -3.767071 & 4.334826 & -1.967069 \\ 1 & -4.159298 & 7.394423 & 0.082058 \\ 1 & -3.382896 & 6.822605 & 1.565379 \\ 1 & -2.405626 & 7.166184 & 0.118410 \\ 6 & -7.004054 & -1.029875 & -3.624443 \\ 6 & -4.857618 & -0.070089 & -4.404324 \\ 6 & -4.950425 & -2.423749 & -3.560739 \\ 6 & -6.311650 & 0.715123 & 2.727706 \\ 6 & -7.937635 & 1.136263 & 0.911558 \\ 6 & -7.481116 & -1.202508 & 1.676181 \\ 1 & -7.199110 & -1.339849 & -4.658737 \\ 1 & -7.520729 & -1.743579 & -2.970014 \\ 1 & -7.459174 & -0.040758 & -3.482835 \\ 1 & -5.160604 & -2.783841 & -4.577221 \\ 1 & -3.864265 & -2.467101 & -3.405455 \\ 1 & -5.413102 & -3.119418 & -2.847806 \\ 1 & -5.125265 & -0.391613 & -5.419419 \\ 1 & -5.204705 & 0.963241 & -4.274183 \\ 1 & -3.761611 & -0.066049 & -4.345314 \\ 1 & -7.131915 & 0.819566 & 3.448977 \\ 1 & -5.565530 & 0.045635 & 3.176210 \\ 1 & -5.853200 & 1.705330 & 2.601409 \\ 1 & -8.718727 & 1.254208 & 1.674439 \\ 1 & -7.515706 & 2.128601 & 0.705370 \\ 1 & -8.425701 & 0.783179 & -0.004369 \\ 1 & -8.277218 & -1.121969 & 2.428751 \\ 1 & -7.921363 & -1.636593 & 0.769934 \\ 1 & -6.728240 & -1.906948 & 2.054346\end{array}$

$1_{\mathrm{C} 11-}-\psi_{-1225354 s}$

Number of imaginary frequencies : 0

The smallest frequencies are : $12.5750 \quad 17.3646 \quad 19.3952 \mathrm{~cm}(-1)$

Electronic energy : $\quad H F=-4125.4647554$

Zero-point correction $=\quad 1.299060($ Hartree/Particle $)$

Thermal correction to Energy $=\quad 1.377628$

Thermal correction to Enthalpy= $\quad 1.378572$

Thermal correction to Gibbs Free Energy= 1.185431

Sum of electronic and zero-point Energies $=\quad-4124.165696$

Sum of electronic and thermal Energies $=\quad-4124.087127$

Sum of electronic and thermal Enthalpies $=\quad-4124.086183$

Sum of electronic and thermal Free Energies $=\quad-4124.279324$

Cartesian Coordinates

$\begin{array}{llll}6 & -0.590995 & 2.527441 & 1.421607 \\ 6 & -1.650085 & 2.073612 & 0.623589 \\ 6 & -2.607810 & 2.972914 & 0.179204 \\ 6 & -2.546544 & 4.328796 & 0.530639\end{array}$




\begin{tabular}{|c|c|c|c|}
\hline 5 & -1.507365 & 4.740907 & 1.359390 \\
\hline 6 & -0.521851 & 3.855172 & 1.829165 \\
\hline 1 & -1.648349 & 0.337248 & 0.032147 \\
\hline 6 & -3.424135 & 0.038035 & -0.344195 \\
\hline 6 & -4.398612 & 0.132437 & 0.649444 \\
\hline 6 & -5.744745 & -0.090053 & 0.363232 \\
\hline 6 & -6.092127 & -0.401668 & -0.957321 \\
\hline 6 & -5.143173 & -0.491005 & -1.978457 \\
\hline 6 & -3.803576 & -0.271441 & -1.644787 \\
\hline 6 & -6.766431 & 0.017204 & 1.494295 \\
\hline 6 & -5.496626 & -0.876655 & -3.412742 \\
\hline 6 & -3.619696 & 5.273509 & -0.005150 \\
\hline 6 & 0.569207 & 4.361646 & 2.772337 \\
\hline 6 & -0.078348 & 5.033885 & 3.988795 \\
\hline 6 & -1.464440 & -0.626634 & 1.606775 \\
\hline 6 & -1.130810 & -1.971839 & 1.556061 \\
\hline 6 & -1.017405 & -2.731292 & 2.767802 \\
\hline 6 & -1.248530 & -2.094227 & 4.021555 \\
\hline 6 & -1.602233 & -0.725561 & 4.036618 \\
\hline 6 & -1.704477 & -0.017578 & 2.869840 \\
\hline 6 & -0.659300 & -4.105446 & 2.774793 \\
\hline 6 & -0.529971 & -4.797721 & 3.953655 \\
\hline 6 & -0.758101 & -4.159340 & 5.190527 \\
\hline 6 & -1.111834 & -2.834676 & 5.219588 \\
\hline 6 & -0.921498 & -2.719944 & 0.283824 \\
\hline 6 & 0.323761 & -2.786606 & -0.317703 \\
\hline 6 & 0.553879 & -3.671894 & -1.397041 \\
\hline 6 & -0.452250 & -4.459494 & -1.889192 \\
\hline 6 & -1.754988 & -4.377716 & -1.345468 \\
\hline 6 & -1.996342 & -3.500412 & -0.246024 \\
\hline 6 & -2.821055 & -5.154279 & -1.862492 \\
\hline 6 & -4.079493 & -5.064524 & -1.325527 \\
\hline 6 & -4.325709 & -4.186970 & -0.247053 \\
\hline 6 & -3.311516 & -3.429896 & 0.283782 \\
\hline 7 & 1.402900 & -1.947908 & 0.128017 \\
\hline 15 & 1.717800 & -0.665070 & -0.936650 \\
\hline 8 & 2.898838 & -1.153121 & -2.021952 \\
\hline 6 & 4.157895 & -1.530090 & -1.614704 \\
\hline 6 & 5.038952 & -0.608838 & -1.076049 \\
\hline 6 & 6.278699 & -1.098788 & -0.547743 \\
\hline 6 & 6.629293 & -2.471230 & -0.723074 \\
\hline 6 & 5.736074 & -3.335426 & -1.402816 \\
\hline 6 & 4.516943 & -2.879571 & -1.822228 \\
\hline 6 & 7.151034 & -0.282326 & 0.216106 \\
\hline 6 & 8.311056 & -0.788269 & 0.749626 \\
\hline 6 & 8.671607 & -2.135979 & 0.541952 \\
\hline 6 & 7.843011 & -2.957137 & -0.179260 \\
\hline 6 & 4.664109 & 0.823392 & -1.018104 \\
\hline 6 & 3.467224 & 1.190456 & -0.433872 \\
\hline 6 & 3.056323 & 2.532561 & -0.313428 \\
\hline 6 & 3.855575 & 3.527646 & -0.806093 \\
\hline 6 & 5.066525 & 3.217954 & -1.470385 \\
\hline 6 & 5.472151 & 1.854845 & -1.599870 \\
\hline 6 & 5.868433 & 4.240872 & -2.032406 \\
\hline 6 & 7.014787 & 3.941352 & -2.722158 \\
\hline 6 & 7.398477 & 2.594119 & -2.886215 \\
\hline
\end{tabular}




\begin{tabular}{|c|c|c|c|}
\hline 6 & 6.649749 & 1.580629 & -2.341080 \\
\hline 8 & 2.646023 & 0.220455 & 0.095096 \\
\hline & 2.384086 & -2.587688 & 1.036578 \\
\hline 5 & 2.939613 & -1.697381 & 2.116659 \\
\hline 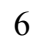 & 4.315481 & -1.625197 & 2.324247 \\
\hline 5 & 4.839619 & -0.831012 & 3.341466 \\
\hline 5 & 3.988753 & -0.106532 & 4.168833 \\
\hline 5 & 2.610841 & -0.179043 & 3.969790 \\
\hline 6 & 2.092254 & -0.962955 & 2.947950 \\
\hline 45 & -0.120089 & 0.355365 & -1.912131 \\
\hline 6 & -0.646086 & 1.797765 & -3.040172 \\
\hline 8 & -0.996694 & 2.729526 & -3.629070 \\
\hline 6 & -0.672016 & -1.061774 & -3.140127 \\
\hline 8 & -0.956174 & -1.801819 & -3.978228 \\
\hline 6 & 1.454336 & 3.226985 & 3.285787 \\
\hline 6 & 1.452139 & 5.379166 & 2.043127 \\
\hline 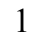 & 6.877567 & 0.753402 & 0.401105 \\
\hline 1 & -1.974892 & 1.034702 & 2.911541 \\
\hline 1 & 8.094593 & -4.005653 & -0.333880 \\
\hline 1 & -1.290628 & -2.325886 & 6.165808 \\
\hline 1 & 5.543153 & 5.273625 & -1.913748 \\
\hline 1 & -3.515938 & -2.749401 & 1.109518 \\
\hline 1 & -5.330290 & -4.106701 & 0.162862 \\
\hline 1 & 3.557247 & 4.571404 & -0.716962 \\
\hline 1 & -1.792474 & -0.241387 & 4.993745 \\
\hline 1 & 6.024071 & -4.373522 & -1.560191 \\
\hline 1 & 8.292902 & 2.354002 & -3.456773 \\
\hline 1 & 3.803358 & -3.530730 & -2.322703 \\
\hline 1 & -2.617672 & -5.820821 & -2.699458 \\
\hline 1 & -0.269718 & -5.139054 & -2.720140 \\
\hline 1 & -0.251544 & -5.848856 & 3.933473 \\
\hline 1 & 6.953758 & 0.547978 & -2.489992 \\
\hline 1 & -0.485155 & -4.616274 & 1.829911 \\
\hline 1 & 1.552492 & -3.705969 & -1.829846 \\
\hline 1 & 7.619390 & 4.734892 & -3.155078 \\
\hline 1 & 8.955257 & -0.143434 & 1.343296 \\
\hline 1 & -4.892366 & -5.662087 & -1.732463 \\
\hline 1 & -0.652346 & -4.719595 & 6.116561 \\
\hline 1 & 9.596266 & -2.522111 & 0.964447 \\
\hline 1 & 2.103456 & 2.743092 & 0.169595 \\
\hline 1 & -4.099788 & 0.386151 & 1.665988 \\
\hline 1 & -3.030200 & -0.347607 & -2.406556 \\
\hline 1 & -7.137300 & -0.577013 & -1.196069 \\
\hline 1 & -3.415372 & 2.618678 & -0.460672 \\
\hline 1 & 0.169413 & 1.812511 & 1.737497 \\
\hline 1 & -1.451192 & 5.785071 & 1.660043 \\
\hline 1 & 1.838804 & -3.418058 & 1.507758 \\
\hline 1 & 0.534306 & 1.611642 & -1.146662 \\
\hline 1 & 3.210826 & -3.041578 & 0.468953 \\
\hline 1 & 4.989231 & -2.183741 & 1.672164 \\
\hline 1 & 1.019078 & -0.991167 & 2.770373 \\
\hline 1 & 5.917960 & -0.776992 & 3.478339 \\
\hline 1 & 1.932793 & 0.379896 & 4.613588 \\
\hline 1 & 4.395111 & 0.514481 & 4.964495 \\
\hline 1 & 2.190519 & 3.622090 & 3.997557 \\
\hline 1 & 0.862674 & 2.461321 & 3.807423 \\
\hline
\end{tabular}




\begin{tabular}{|c|c|c|c|}
\hline & 2.015776 & 2.730749 & 2.482908 \\
\hline & 0.695994 & 5.379688 & 4.686542 \\
\hline & -0.683142 & 5.905459 & 3.712401 \\
\hline & -0.728473 & 4.331538 & 4.526807 \\
\hline & 230791 & 5.761749 & 2.716799 \\
\hline & .951203 & 4.919714 & 1.17938 \\
\hline & 1.874062 & 6.237009 & 1.6783 \\
\hline & .459274 & 6.696717 & 0.521989 \\
\hline & .529695 & 5.315139 & -1.535285 \\
\hline & -5.004780 & 4.763361 & 0.411605 \\
\hline & -5.787131 & 5.431111 & 0.026335 \\
\hline & -5.210332 & 3.756296 & 0.026313 \\
\hline & -5.098463 & 4.727994 & 1.505237 \\
\hline & -4.295912 & 5.987494 & -1.944639 \\
\hline & -2.547211 & 5.681 & -1 \\
\hline & -3.67 & 4.32 & -1.986006 \\
\hline & -4.256578 & 7.33 & 0.116409 \\
\hline & -3.528825 & 6.738970 & 1.616840 \\
\hline & -2.501973 & 7.140481 & 0.219954 \\
\hline & -7.001846 & -0.846620 & -3.66807 \\
\hline & -4.827898 & 0.084247 & $-4.4028 c$ \\
\hline & -4.98 & -2.3 & \\
\hline & -8.18 & -0.3 & 3830 \\
\hline & -6.39 & -0.9 & 1959 \\
\hline & -6.759339 & 1.447883 & 2.045570 \\
\hline & -7.202322 & -1.092996 & -4.718325 \\
\hline & -7.540299 & -1.581285 & -3.056043 \\
\hline & -7.430127 & 0.145136 & -3.471857 \\
\hline & -5.213065 & -2.612379 & -4.695840 \\
\hline & -3.904 & -2.378921 & -3.51 \\
\hline & -5.4 & -3.0 & -2.98045 \\
\hline & -5.10 & -0.1 & -5.4 \\
\hline & -5.140650 & 1.121056 & -4.222684 \\
\hline & -3.732195 & 0.050201 & -4.349480 \\
\hline & -8.875821 & -0.226618 & 1.882105 \\
\hline & -8.534939 & 0.383790 & 0.258069 \\
\hline & -8.263639 & -1.331890 & 0.642920 \\
\hline 1 & -7.115185 & -0.898001 & 3.435698 \\
\hline & -6.395133 & -1.998039 & 2.242779 \\
\hline & -5.396398 & -0.763827 & 3.029128 \\
\hline & -7.465523 & 1.539425 & 2.881977 \\
\hline & -5.767943 & 1.744814 & 2.410459 \\
\hline & -7.058 & 2.166957 & 1.2713 \\
\hline
\end{tabular}

$1_{C 11-} \psi_{-1 s 2 a 3 a 4 a}$

Number of imaginary frequencies : 0

The smallest frequencies are : $11.6799 \quad 18.1198 \quad 20.4621 \mathrm{~cm}(-1)$

Electronic energy : $\quad H F=-4125.4633022$

Zero-point correction $=\quad 1.299618($ Hartree/Particle $)$

Thermal correction to Energy= $\quad 1.378051$

Thermal correction to Enthalpy= $\quad 1.378995$

Thermal correction to Gibbs Free Energy $=\quad 1.186270$

Sum of electronic and zero-point Energies $=\quad-4124.163684$

Sum of electronic and thermal Energies $=\quad-4124.085251$

Sum of electronic and thermal Enthalpies $=\quad-4124.084307$ 
Cartesian Coordinates

\begin{tabular}{|c|c|c|c|}
\hline 6 & 3.899702 & 0.633761 & 4.003200 \\
\hline 6 & 4.764319 & -0.224948 & 3.333905 \\
\hline 6 & 4.255789 & -1.206230 & 2.486277 \\
\hline 6 & 2.882556 & -1.334032 & 2.290520 \\
\hline 6 & 2.019207 & -0.476169 & 2.975490 \\
\hline 6 & 2.523310 & 0.498751 & 3.826642 \\
\hline 6 & 2.344651 & -2.383915 & 1.354901 \\
\hline 7 & 1.422606 & -1.872987 & 0.313073 \\
\hline 6 & 0.365998 & -2.760602 & -0.091368 \\
\hline 6 & -0.889773 & -2.678805 & 0.485598 \\
\hline 6 & -1.951317 & -3.488390 & -0.025890 \\
\hline 6 & -1.692183 & -4.395938 & -1.095997 \\
\hline 6 & -0.379306 & -4.496974 & -1.609860 \\
\hline 6 & 0.617275 & -3.691604 & -1.126891 \\
\hline 6 & -3.275637 & -3.401182 & 0.478596 \\
\hline 6 & -4.285027 & -4.160051 & -0.058127 \\
\hline 6 & -4.022688 & -5.062242 & -1.112898 \\
\hline 6 & -2.752727 & -5.176969 & -1.617853 \\
\hline 6 & -1.143147 & -1.849846 & 1.696435 \\
\hline 6 & -1.515861 & -0.516114 & 1.633856 \\
\hline 6 & -1.836810 & 0.168284 & 2.839618 \\
\hline 6 & -1.762635 & -0.450992 & 4.057652 \\
\hline 6 & -1.363775 & -1.803790 & 4.157203 \\
\hline 6 & -1.059442 & -2.518497 & 2.962725 \\
\hline 6 & -1.265078 & -2.456880 & 5.408568 \\
\hline 6 & -0.879935 & -3.770972 & 5.486562 \\
\hline 6 & -0.581403 & -4.486511 & 4.308349 \\
\hline 6 & -0.672404 & -3.879656 & 3.079828 \\
\hline 15 & -1.652230 & 0.333211 & -0.016406 \\
\hline 45 & -0.064285 & 0.238472 & -1.936020 \\
\hline 6 & -0.512365 & -1.246295 & -3.128510 \\
\hline 8 & -0.687148 & -2.032865 & -3.953825 \\
\hline 15 & 1.765521 & -0.709099 & -0.874294 \\
\hline 8 & 2.705427 & 0.271200 & 0.052666 \\
\hline 6 & 3.544117 & 1.158827 & -0.583458 \\
\hline 6 & 4.740733 & 0.706745 & -1.104364 \\
\hline 6 & 5.573252 & 1.649574 & -1.791409 \\
\hline 6 & 5.188309 & 3.024443 & -1.829223 \\
\hline 6 & 3.972891 & 3.428129 & -1.225911 \\
\hline 6 & 3.150989 & 2.509641 & -0.632176 \\
\hline 6 & 6.016104 & 3.961738 & -2.493763 \\
\hline 6 & 7.167533 & 3.565891 & -3.124004 \\
\hline 6 & 7.531122 & 2.203150 & -3.122501 \\
\hline 6 & 6.756982 & 1.272165 & -2.475423 \\
\hline 6 & 5.085625 & -0.728600 & -0.985780 \\
\hline 6 & 4.187319 & -1.687924 & -1.420774 \\
\hline 6 & 4.514038 & -3.060925 & -1.447887 \\
\hline 6 & 5.715831 & -3.489088 & -0.954807 \\
\hline 6 & 6.624599 & -2.567913 & -0.379346 \\
\hline 6 & 6.307700 & -1.176357 & -0.384691 \\
\hline 6 & 7.194325 & -0.290330 & 0.279118 \\
\hline 6 & 8.337041 & -0.751868 & 0.885151 \\
\hline
\end{tabular}




\begin{tabular}{|c|c|c|c|}
\hline 6 & 8.664960 & -2.123467 & 0.854597 \\
\hline 6 & 7.821293 & -3.009766 & 0.235433 \\
\hline & 2.944757 & -1.330082 & -1.894721 \\
\hline & -3.420394 & -0.000618 & -0.410566 \\
\hline & -4.424838 & 0.263170 & 0.524531 \\
\hline & -5.760197 & -0.009079 & 0.238275 \\
\hline & -6.057470 & -0.561289 & -1.013766 \\
\hline ठ & -5.081134 & -0.840137 & -1.970888 \\
\hline & -3.752813 & -0.545138 & -1.646921 \\
\hline & -6.877621 & 0.241983 & 1.248664 \\
\hline & -5.480612 & -1.419470 & -3.326657 \\
\hline 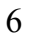 & -1.662634 & 2.104158 & 0.471709 \\
\hline & -2.587532 & 2.990851 & -0.083745 \\
\hline & -2.552268 & 4.348895 & 0.227136 \\
\hline & -1.574509 & 4.795116 & 1.127307 \\
\hline & -0.629752 & 3.937507 & 1.691231 \\
\hline & -0.669969 & 2.589379 & 1.319202 \\
\hline & -3.558385 & 5.341596 & -0.352512 \\
\hline & 0.370104 & 4.383684 & 2.758202 \\
\hline & -0.564716 & 1.619247 & -3.148435 \\
\hline & -0.882777 & 2.518065 & -3.803217 \\
\hline & 6.945990 & 0.766928 & 0.327443 \\
\hline & -2.144899 & 1.210304 & 2.798156 \\
\hline & 8.047357 & -4.075201 & 0.218825 \\
\hline & -1.500547 & -1.890467 & 6.308504 \\
\hline & 5.707050 & 5.006166 & -2.502860 \\
\hline & -3.491460 & -2.704997 & 1.287239 \\
\hline & -5.298083 & -4.063700 & 0.327919 \\
\hline & 3.690017 & 4.479134 & -1.263284 \\
\hline 1 & -2.013559 & 0.093631 & 4.967388 \\
\hline & 5.977651 & -4.545700 & -0.974163 \\
\hline ] & 8.430257 & 1.884519 & -3.645227 \\
\hline & 3.794114 & -3.756911 & -1.872586 \\
\hline & -2.536100 & -5.866793 & -2.432310 \\
\hline 1 & -0.180840 & -5.202613 & -2.415019 \\
\hline & -0.279858 & -5.529424 & 4.373382 \\
\hline 1 & 7.046387 & 0.224756 & -2.495318 \\
\hline & -0.445707 & -4.448296 & 2.180167 \\
\hline ] & 1.620874 & -3.737745 & -1.545140 \\
\hline & 7.792208 & 4.294092 & -3.636080 \\
\hline ] & 8.992969 & -0.052272 & 1.398529 \\
\hline 1 & -4.831095 & -5.664678 & -1.522126 \\
\hline & -0.804806 & -4.264261 & 6.452809 \\
\hline 1 & 9.576286 & -2.474666 & 1.332828 \\
\hline & 2.198004 & 2.788042 & -0.188385 \\
\hline 1 & -4.152299 & 0.691618 & 1.485479 \\
\hline & -2.951106 & -0.743451 & -2.354477 \\
\hline t & -7.098281 & -0.783239 & -1.250582 \\
\hline . & -3.341528 & 2.603499 & -0.764825 \\
\hline & 0.064495 & 1.894140 & 1.732111 \\
\hline 1 & -1.559738 & 5.849256 & 1.396546 \\
\hline & 1.753115 & -3.110802 & 1.930173 \\
\hline 1 & 0.547472 & 1.536667 & -1.208064 \\
\hline & 3.178234 & -2.944114 & 0.903303 \\
\hline & 4.940308 & -1.864290 & 1.948356 \\
\hline & 0.945762 & -0.555267 & 2.808336 \\
\hline
\end{tabular}




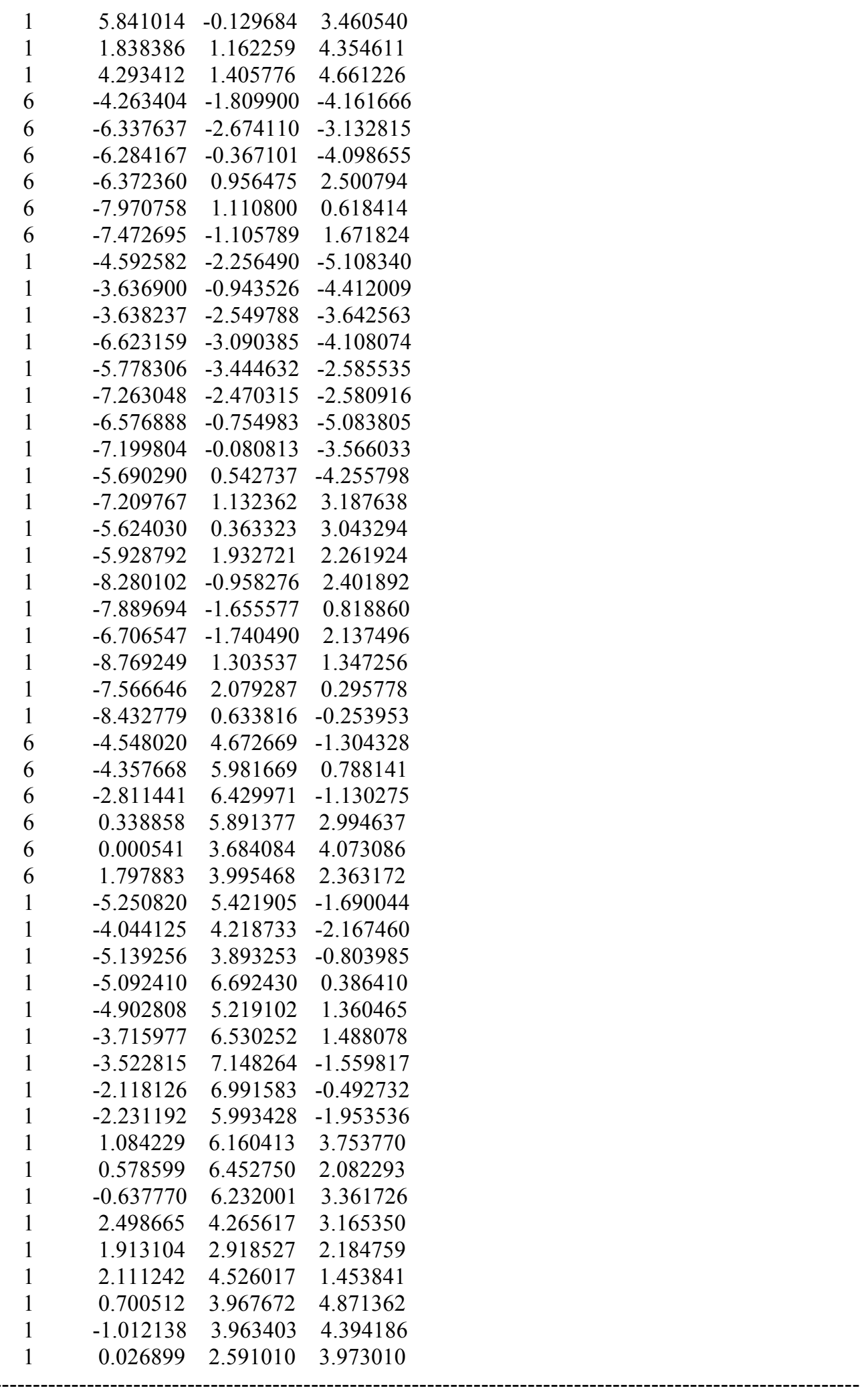

$1_{\mathrm{C} 11-} \psi_{-1 \text { 122a3a4s }}$

Number of imaginary frequencies : 0

The smallest frequencies are : $9.7893 \quad 16.2158 \quad 19.4861 \mathrm{~cm}(-1)$ 
Electronic energy : $\quad H F=-4125.4636512$

Zero-point correction $=$

1.298845 (Hartree/Particle)

Thermal correction to Energy $=\quad 1.377513$

Thermal correction to Enthalpy= $\quad 1.378457$

Thermal correction to Gibbs Free Energy= 1.184399

Sum of electronic and zero-point Energies $=\quad-4124.164807$

Sum of electronic and thermal Energies $=\quad-4124.086139$

Sum of electronic and thermal Enthalpies $=\quad-4124.085194$

Sum of electronic and thermal Free Energies $=\quad-4124.279252$

\begin{tabular}{crrr} 
& \multicolumn{3}{c}{ Cartesian Coordinates } \\
\cline { 1 - 1 }$\ldots \ldots \ldots \ldots \ldots \ldots \ldots \ldots \ldots \ldots \ldots \ldots$. \\
\hline 6 & 3.942370 & 0.712993 & 4.020744 \\
6 & 4.805772 & -0.176955 & 3.391968 \\
6 & 4.299928 & -1.159565 & 2.544150 \\
6 & 2.930251 & -1.260070 & 2.311518 \\
6 & 2.068239 & -0.369257 & 2.954442 \\
6 & 2.569294 & 0.609974 & 3.801573 \\
6 & 2.395910 & -2.315605 & 1.380193 \\
7 & 1.478362 & -1.812139 & 0.331328 \\
6 & 0.419771 & -2.699077 & -0.069426 \\
6 & -0.831117 & -2.623425 & 0.519588 \\
6 & -1.893610 & -3.439500 & 0.021765 \\
6 & -1.644881 & -4.334625 & -1.061041 \\
6 & -0.339402 & -4.422508 & -1.595251 \\
6 & 0.661686 & -3.620806 & -1.115153 \\
6 & -3.209191 & -3.370902 & 0.551876 \\
6 & -4.219772 & -4.136690 & 0.027918 \\
6 & -3.967604 & -5.026954 & -1.039457 \\
6 & -2.706938 & -5.121996 & -1.570653 \\
6 & -1.078617 & -1.774744 & 1.717405 \\
6 & -1.462138 & -0.445237 & 1.623159 \\
6 & -1.767843 & 0.270406 & 2.813813 \\
6 & -1.673404 & -0.316429 & 4.047171 \\
6 & -1.269723 & -1.664729 & 4.177401 \\
6 & -0.977781 & -2.409602 & 2.998310 \\
6 & -1.154228 & -2.285244 & 5.443986 \\
6 & -0.766061 & -3.596285 & 5.550884 \\
6 & -0.481580 & -4.342051 & 4.387658 \\
6 & -0.588180 & -3.767107 & 3.145251 \\
15 & -1.639826 & 0.345914 & -0.052490 \\
45 & -0.001394 & 0.301239 & -1.921106 \\
6 & -0.401332 & -1.172882 & -3.140791 \\
8 & -0.538359 & -1.959857 & -3.972763 \\
15 & 1.823019 & -0.648292 & -0.855160 \\
8 & 2.762357 & 0.332142 & 0.072248 \\
6 & 3.616103 & 1.206879 & -0.561402 \\
6 & 4.814548 & 0.740792 & -1.065117 \\
6 & 5.664921 & 1.672390 & -1.745680 \\
6 & 5.293045 & 3.050531 & -1.796231 \\
6 & 4.072646 & 3.468332 & -1.212974 \\
6 & 3.235335 & 2.560456 & -0.624753 \\
6 & 6.138021 & 3.976676 & -2.454769 \\
6 & 7.294191 & 3.567089 & -3.067272 \\
6 & 7.645468 & 2.201217 & -3.053173 \\
6 & 6.854351 & 1.280754 & -2.411568 \\
\hline & & & \\
6
\end{tabular}




\begin{tabular}{|c|c|c|c|}
\hline & 5.140934 & -0.698466 & -0.941037 \\
\hline & 4.237199 & -1.646110 & -1.390247 \\
\hline & 4.549266 & -3.022431 & -1.419198 \\
\hline & 5.739213 & -3.465764 & -0.910956 \\
\hline & 6.649491 & -2.556965 & -0.318417 \\
\hline & 6.349098 & -1.161774 & -0.324498 \\
\hline & 7.236180 & -0.287413 & 0.353827 \\
\hline & 8.363882 & -0.763769 & 0.976244 \\
\hline & 8.675617 & -2.139259 & 0.947871 \\
\hline & 7.831387 & -3.014233 & 0.313478 \\
\hline & 3.003154 & -1.272181 & -1.874145 \\
\hline & -3.370344 & -0.141153 & -0.453087 \\
\hline & -4.380037 & -0.007218 & 0.495160 \\
\hline & -5.698541 & -0.365445 & 0.205680 \\
\hline & -5.969655 & -0.860732 & -1.070309 \\
\hline & -4.978896 & -1.010975 & -2.049427 \\
\hline & -3.674526 & -0.643004 & -1.718388 \\
\hline & -6.762228 & -0.199018 & 1.288627 \\
\hline & -5.354347 & -1.529677 & -3.436256 \\
\hline & -1.814543 & 2.121610 & 0.389279 \\
\hline & -2.879674 & 2.884229 & -0.093419 \\
\hline & -2.985391 & 4.240076 & 0.212181 \\
\hline & -2.008036 & 4.807021 & 1.040402 \\
\hline & -0.926966 & 4.075119 & 1.533813 \\
\hline & -0.827087 & 2.730455 & 1.161360 \\
\hline & -4.141882 & 5.099752 & -0.295274 \\
\hline & 0.083175 & 4.654275 & 2.524054 \\
\hline & -0.426666 & 1.690960 & -3.155494 \\
\hline & -0.688202 & 2.585945 & -3.839491 \\
\hline & 6.999237 & 0.772577 & 0.400628 \\
\hline & -2.086001 & 1.307962 & 2.748152 \\
\hline & 8.045356 & -4.082173 & 0.297566 \\
\hline & -1.379572 & -1.696225 & 6.331934 \\
\hline & 5.838463 & 5.023740 & -2.473805 \\
\hline & -3.417486 & -2.682076 & 1.369171 \\
\hline & -5.227322 & -4.052623 & 0.431428 \\
\hline & 3.798808 & 4.521247 & -1.261856 \\
\hline & -1.913582 & 0.251515 & 4.945468 \\
\hline & 5.989630 & -4.525135 & -0.930765 \\
\hline & 8.548682 & 1.871602 & -3.561861 \\
\hline & 3.827774 & -3.708727 & -1.856715 \\
\hline & -2.498098 & -5.803299 & -2.394315 \\
\hline & -0.148941 & -5.118842 & -2.410438 \\
\hline & -0.178647 & -5.382858 & 4.476008 \\
\hline & 7.134694 & 0.230789 & -2.421972 \\
\hline & -0.372710 & -4.357778 & 2.257070 \\
\hline & 1.660229 & -3.660955 & -1.545797 \\
\hline & 7.932126 & 4.286770 & -3.575024 \\
\hline 1 & 9.020345 & -0.073180 & 1.501012 \\
\hline & -4.776589 & -5.636391 & -1.437036 \\
\hline & -0.677922 & -4.064347 & 6.528512 \\
\hline & 9.575254 & -2.502244 & 1.439267 \\
\hline 1 & 2.276361 & 2.848370 & -0.201388 \\
\hline & -4.135792 & 0.387091 & 1.481102 \\
\hline 1 & -2.865604 & -0.744367 & -2.438437 \\
\hline 1 & -6.990608 & -1.141532 & -1.320846 \\
\hline
\end{tabular}




\begin{tabular}{|c|c|c|c|}
\hline & -3.632606 & 2.403129 & -0.712211 \\
\hline & 0.013671 & 2.129772 & 1.518246 \\
\hline & -2.103226 & 5.857216 & 1.308077 \\
\hline & 1.803139 & -3.039077 & 1.958220 \\
\hline & 0.559089 & 1.586110 & -1.131781 \\
\hline & 3.231235 & -2.879082 & 0.936072 \\
\hline & 4.984942 & -1.840548 & 2.035963 \\
\hline ] & 0.999564 & -0.423660 & 2.752678 \\
\hline & 5.880276 & -0.104283 & 3.548862 \\
\hline 1 & 1.883906 & 1.300915 & 4.292229 \\
\hline 1 & 4.335350 & 1.485641 & 4.678228 \\
\hline 6 & -4.122705 & -1.812920 & -4.293023 \\
\hline & -6.150777 & -2.832741 & -3.319203 \\
\hline ) & -6.208606 & -0.472098 & -4.144174 \\
\hline & -8.133787 & -0.695611 & 0.839923 \\
\hline 0 & -6.352422 & -0.996796 & 2.532709 \\
\hline & -6.882245 & 1.286598 & 1.648738 \\
\hline & -4.433574 & -2.215345 & -5.265358 \\
\hline & -3.535037 & -0.906793 & -4.490206 \\
\hline & -3.465806 & -2.554540 & -3.818459 \\
\hline & -6.407842 & -3.207932 & -4.318718 \\
\hline & -5.558591 & -3.603819 & -2.808748 \\
\hline 1 & -7.089766 & -2.705286 & -2.767518 \\
\hline & -6.489676 & -0.815923 & -5.148930 \\
\hline 1 & -7.132761 & -0.258120 & -3.592640 \\
\hline 1 & -5.656573 & 0.470834 & -4.251066 \\
\hline 1 & -8.858456 & -0.565218 & 1.653562 \\
\hline ] & -8.510471 & -0.137130 & -0.026532 \\
\hline 1 & -8.116956 & -1.762630 & 0.581021 \\
\hline 1 & -7.615152 & 1.428708 & 2.454527 \\
\hline 1 & -5.926658 & 1.707305 & 1.987684 \\
\hline 1 & -7.217053 & 1.872064 & 0.782121 \\
\hline 1 & -7.099973 & -0.872982 & 3.327806 \\
\hline 1 & -6.280801 & -2.068874 & 2.304934 \\
\hline 1 & -5.383737 & -0.676851 & 2.937625 \\
\hline 6 & -5.099159 & 4.312145 & -1.187636 \\
\hline 6 & -4.938370 & 5.638298 & 0.898463 \\
\hline 6 & -3.587863 & 6.273394 & -1.109952 \\
\hline 6 & -0.103479 & 6.154376 & 2.737446 \\
\hline 6 & -0.113447 & 3.947533 & 3.871788 \\
\hline 6 & 1.515816 & 4.417555 & 2.038856 \\
\hline 1 & -5.916286 & 4.965990 & -1.517635 \\
\hline 1 & -4.599540 & 3.929380 & -2.086867 \\
\hline 1 & -5.550487 & 3.460451 & -0.659686 \\
\hline 1 & -5.774635 & 6.259248 & 0.549832 \\
\hline 1 & -5.354434 & 4.816341 & 1.496213 \\
\hline 1 & -4.321558 & 6.256352 & 1.562121 \\
\hline 1 & -4.409769 & 6.895758 & -1.489057 \\
\hline 1 & -2.933203 & 6.918657 & -0.511942 \\
\hline 1 & -3.009076 & 5.914969 & -1.970889 \\
\hline 1 & 0.652456 & 6.522896 & 3.442256 \\
\hline 1 & 0.013214 & 6.717513 & 1.802393 \\
\hline 1 & -1.086937 & 6.392320 & 3.162276 \\
\hline 1 & 2.232246 & 4.847940 & 2.751269 \\
\hline 1 & 1.755968 & 3.350833 & 1.947086 \\
\hline 1 & 1.685182 & 4.893041 & 1.063117 \\
\hline
\end{tabular}




$\begin{array}{rrrr}1 & 0.598041 & 4.331193 & 4.616011 \\ 1 & -1.129170 & 4.111354 & 4.256661 \\ 1 & 0.040008 & 2.863346 & 3.786734\end{array}$

$1_{\text {C11- }} \psi_{-1 \text { s2a3s4a }}$

Number of imaginary frequencies : 0

The smallest frequencies are : $7.7292 \quad 10.3899 \quad 15.5034 \mathrm{~cm}(-1)$

Electronic energy : $\quad H F=-4125.4635423$

Zero-point correction $=\quad 1.298648($ Hartree/Particle $)$

Thermal correction to Energy= $\quad 1.377499$

Thermal correction to Enthalpy= $\quad 1.378443$

Thermal correction to Gibbs Free Energy= $\quad 1.182259$

Sum of electronic and zero-point Energies $=\quad-4124.164894$

Sum of electronic and thermal Energies $=\quad-4124.086043$

Sum of electronic and thermal Enthalpies= $\quad-4124.085099$

Sum of electronic and thermal Free Energies $=\quad-4124.281283$

Cartesian Coordinates

$\begin{array}{cccc}\cdots & \ldots \ldots \ldots \ldots \ldots \ldots \ldots \ldots \ldots & \ldots \ldots \ldots \ldots \\ 6 & 3.909180 & 0.600720 & 4.009979 \\ 6 & 4.766776 & -0.254866 & 3.328011 \\ 6 & 4.249189 & -1.231324 & 2.480269 \\ 6 & 2.874060 & -1.356676 & 2.296572 \\ 6 & 2.017685 & -0.501722 & 2.994016 \\ 6 & 2.531044 & 0.467644 & 3.846039 \\ 6 & 2.325746 & -2.403435 & 1.363846 \\ 7 & 1.401644 & -1.886235 & 0.327066 \\ 6 & 0.349549 & -2.778446 & -0.079956 \\ 6 & -0.916540 & -2.678051 & 0.470636 \\ 6 & -1.968916 & -3.503129 & -0.036001 \\ 6 & -1.685289 & -4.457125 & -1.058742 \\ 6 & -0.362914 & -4.572641 & -1.545271 \\ 6 & 0.621619 & -3.743052 & -1.078899 \\ 6 & -3.303514 & -3.400160 & 0.437542 \\ 6 & -4.296850 & -4.194543 & -0.077932 \\ 6 & -4.009342 & -5.145266 & -1.081673 \\ 6 & -2.730714 & -5.270858 & -1.560488 \\ 6 & -1.176045 & -1.838931 & 1.673770 \\ 6 & -1.525393 & -0.499089 & 1.611673 \\ 6 & -1.840260 & 0.189566 & 2.816903 \\ 6 & -1.786259 & -0.432509 & 4.034431 \\ 6 & -1.409364 & -1.791438 & 4.134591 \\ 6 & -1.107709 & -2.509206 & 2.941238 \\ 6 & -1.326909 & -2.445945 & 5.386400 \\ 6 & -0.958297 & -3.764543 & 5.466689 \\ 6 & -0.659870 & -4.482694 & 4.290132 \\ 6 & -0.735923 & -3.874634 & 3.061171 \\ 15 & -1.644264 & 0.362671 & -0.032880 \\ 45 & -0.078961 & 0.206434 & -1.951833 \\ 6 & -0.636922 & -1.314365 & -3.048561 \\ 8 & -0.926454 & -2.120927 & -3.820538 \\ 15 & 1.747934 & -0.730160 & -0.867183 \\ 8 & 2.677619 & 0.265808 & 0.057159 \\ 6 & 3.508899 & 1.154382 & -0.588667 \\ 6 & 4.709572 & 0.709335 & -1.107159\end{array}$




\begin{tabular}{|c|c|c|c|}
\hline 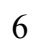 & 5.529431 & 1.652646 & -1.809223 \\
\hline & 5.130424 & 3.022858 & -1.862499 \\
\hline 6 & 3.912891 & 3.420896 & -1.260052 \\
\hline & 3.102295 & 2.500767 & -0.653552 \\
\hline & 5.945684 & 3.960601 & -2.541733 \\
\hline & 7.098236 & 3.568976 & -3.172498 \\
\hline & 7.475211 & 2.209993 & -3.156753 \\
\hline & 6.713570 & 1.279132 & -2.494822 \\
\hline & 5.072753 & -0.720140 & -0.974272 \\
\hline 6 & 4.184749 & -1.694716 & -1.395802 \\
\hline 6 & 4.529057 & -3.063743 & -1.411797 \\
\hline & 5.739808 & -3.471762 & -0.923695 \\
\hline O & 6.640992 & -2.533462 & -0.364040 \\
\hline & 6.304773 & -1.146501 & -0.377671 \\
\hline 6 & 7.185217 & -0.243242 & 0.270912 \\
\hline U & 8.340434 & -0.683946 & 0.868768 \\
\hline 6 & 8.687234 & -2.050971 & 0.845700 \\
\hline 6 & 7.849545 & -2.953805 & 0.242510 \\
\hline 8 & 2.937974 & -1.357341 & -1.871686 \\
\hline 6 & -3.412831 & 0.053584 & -0.438467 \\
\hline 6 & -4.421430 & 0.204831 & 0.521964 \\
\hline 6 & -5.750240 & -0.054195 & 0.208099 \\
\hline 6 & -6.049609 & -0.471641 & -1.100456 \\
\hline 6 & -5.074436 & -0.617891 & -2.081656 \\
\hline 6 & -3.746882 & -0.349649 & -1.721565 \\
\hline 6 & -6.867825 & 0.062787 & 1.242795 \\
\hline 6 & -5.372131 & -1.109907 & -3.495156 \\
\hline 6 & -1.633381 & 2.126435 & 0.478847 \\
\hline 6 & -2.594246 & 3.019845 & 0.002706 \\
\hline 6 & -2.557199 & 4.366590 & 0.361177 \\
\hline 6 & -1.543524 & 4.789610 & 1.231263 \\
\hline 6 & -0.566926 & 3.922058 & 1.723189 \\
\hline 6 & -0.607352 & 2.589294 & 1.299652 \\
\hline 6 & -3.593772 & 5.370352 & -0.141326 \\
\hline 6 & 0.452820 & 4.337871 & 2.783890 \\
\hline 6 & -0.484453 & 1.516178 & -3.273364 \\
\hline 8 & -0.735653 & 2.366406 & -4.016493 \\
\hline 1 & 6.922836 & 0.810837 & 0.313409 \\
\hline 1 & -2.129878 & 1.236852 & 2.774697 \\
\hline 1 & 8.090098 & -4.016142 & 0.232194 \\
\hline 1 & -1.560662 & -1.876452 & 6.284834 \\
\hline 1 & 5.625668 & 5.001563 & -2.562039 \\
\hline 1 & -3.540247 & -2.666460 & 1.206852 \\
\hline 1 & -5.316570 & -4.088399 & 0.286504 \\
\hline 1 & 3.618481 & 4.468247 & -1.309060 \\
\hline 1 & -2.033874 & 0.115244 & 4.943184 \\
\hline 1 & 6.015530 & -4.524938 & -0.935898 \\
\hline 1 & 8.374711 & 1.893945 & -3.680423 \\
\hline 1 & 3.815232 & -3.772533 & -1.825541 \\
\hline 1 & -2.495259 & -5.994453 & -2.339608 \\
\hline 1 & -0.147744 & -5.310557 & -2.316418 \\
\hline 1 & -0.369974 & -5.528821 & 4.356554 \\
\hline 1 & 7.013273 & 0.234546 & -2.504557 \\
\hline 1 & -0.508072 & -4.446438 & 2.163840 \\
\hline 1 & 1.632975 & -3.801190 & -1.476580 \\
\hline 1 & 7.713184 & 4.297215 & -3.696165 \\
\hline
\end{tabular}




\begin{tabular}{|c|c|c|c|}
\hline & 8.991614 & 0.028891 & 1.369830 \\
\hline & -4.806149 & -5.771502 & -1.477118 \\
\hline & -0.895324 & -4.258983 & 6.433209 \\
\hline & 9.608263 & -2.385641 & 1.317158 \\
\hline & 2.148004 & 2.775856 & -0.210680 \\
\hline & -4.151916 & 0.529184 & 1.523755 \\
\hline & -2.950121 & -0.463678 & -2.453543 \\
\hline & -7.086884 & -0.687098 & -1.349820 \\
\hline & -3.379233 & 2.647868 & -0.651207 \\
\hline 1 & 0.147890 & 1.883322 & 1.654432 \\
\hline 1 & -1.526106 & 5.833357 & 1.538066 \\
\hline 1 & 1.731936 & -3.125993 & 1.942459 \\
\hline 1 & 0.540879 & 1.527144 & -1.274845 \\
\hline 1 & 3.153722 & -2.969268 & 0.908877 \\
\hline & 4.927823 & -1.888339 & 1.933842 \\
\hline & 0.942295 & -0.579896 & 2.837623 \\
\hline 1 & 5.844591 & -0.161598 & 3.445982 \\
\hline 1 & 1.852323 & 1.128119 & 4.385271 \\
\hline 1 & 4.309475 & 1.368862 & 4.668617 \\
\hline 6 & -6.868858 & -1.143475 & -3.794613 \\
\hline & -4.700978 & -0.195179 & -4.526346 \\
\hline 6 & -4.816220 & -2.531979 & -3.636980 \\
\hline 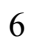 & -6.373907 & 0.655316 & 2.561444 \\
\hline & -7.987117 & 0.963927 & 0.711383 \\
\hline 6 & -7.424723 & -1.336766 & 1.526495 \\
\hline 1 & -7.030633 & -1.468053 & -4.830194 \\
\hline 1 & -7.404340 & -1.849969 & -3.147624 \\
\hline 1 & -7.330555 & -0.153883 & -3.680376 \\
\hline & -4.933587 & -0.539768 & -5.542457 \\
\hline l & -5.056605 & 0.838978 & -4.429884 \\
\hline & -3.607544 & -0.183971 & -4.431245 \\
\hline 1 & -4.995988 & -2.915532 & -4.650745 \\
\hline 1 & -3.735173 & -2.569313 & -3.448909 \\
\hline 1 & -5.298462 & -3.212114 & -2.922042 \\
\hline 1 & -7.212216 & 0.737964 & 3.264547 \\
\hline 1 & -5.609119 & 0.027752 & 3.038510 \\
\hline 1 & -5.955262 & 1.661449 & 2.424436 \\
\hline 1 & -8.237466 & -1.286370 & 2.263801 \\
\hline 1 & -7.823292 & -1.810849 & 0.620924 \\
\hline 1 & -6.640327 & -1.990759 & 1.931532 \\
\hline 1 & -8.782606 & 1.062488 & 1.461991 \\
\hline 1 & -7.608864 & 1.969662 & 0.486009 \\
\hline 1 & -8.446984 & 0.567410 & -0.201318 \\
\hline 6 & -4.655762 & 4.713157 & -1.020828 \\
\hline 6 & -4.305272 & 6.019452 & 1.051190 \\
\hline 6 & -2.889556 & 6.452058 & -0.967006 \\
\hline 6 & 0.446550 & 5.842094 & 3.044311 \\
\hline 6 & 0.079693 & 3.621842 & 4.089152 \\
\hline 6 & 1.872185 & 3.934793 & 2.375761 \\
\hline 1 & -5.383729 & 5.467936 & -1.344099 \\
\hline 1 & -4.223027 & 4.266564 & -1.925164 \\
\hline 1 & -5.208434 & 3.929780 & -0.483575 \\
\hline 1 & -5.060839 & 6.734400 & 0.698780 \\
\hline 1 & -4.815021 & 5.263969 & 1.663544 \\
\hline 1 & -3.613481 & 6.567061 & 1.702179 \\
\hline 1 & -3.619079 & 7.182559 & -1.342220 \\
\hline
\end{tabular}




$\begin{array}{rrrr}1 & -2.144465 & 6.999163 & -0.376640 \\ 1 & -2.374797 & 6.012034 & -1.830864 \\ 1 & 1.209764 & 6.088970 & 3.793146 \\ 1 & 0.676276 & 6.414145 & 2.135985 \\ 1 & -0.517537 & 6.190725 & 3.435829 \\ 1 & 2.579281 & 4.180998 & 3.180096 \\ 1 & 1.972184 & 2.859489 & 2.180277 \\ 1 & 2.191308 & 4.475787 & 1.474774 \\ 1 & 0.789488 & 3.878330 & 4.888100 \\ 1 & -0.926157 & 3.912658 & 4.421503 \\ 1 & 0.086029 & 2.530689 & 3.966591\end{array}$

\section{$\mathbf{1}_{\mathrm{C} 11-} \psi_{-1 \mathrm{1s2a3s4s}}$}

Number of imaginary frequencies : 0

The smallest frequencies are : $14.0276 \quad 16.2907 \quad 21.9407 \mathrm{~cm}(-1)$

Electronic energy : $\quad H F=-4125.4640179$

Zero-point correction $=\quad 1.299585($ Hartree/Particle $)$

Thermal correction to Energy= $\quad 1.377938$

Thermal correction to Enthalpy= $\quad 1.378882$

Thermal correction to Gibbs Free Energy= 1.186796

Sum of electronic and zero-point Energies $=\quad-4124.164433$

Sum of electronic and thermal Energies $=\quad-4124.086080$

Sum of electronic and thermal Enthalpies $=\quad-4124.085136$

Sum of electronic and thermal Free Energies $=\quad-4124.277222$

Cartesian Coordinates

$\begin{array}{rrrr}0 & 3.893201 & 0.651768 & 4.002550 \\ 6 & 4.765887 & -0.195201 & 3.328748 \\ 6 & 4.266328 & -1.179063 & 2.478863 \\ 6 & 2.894206 & -1.321183 & 2.285174 \\ 6 & 2.022764 & -0.474969 & 2.974458 \\ 6 & 2.518006 & 0.502504 & 3.827981 \\ 6 & 2.366661 & -2.373044 & 1.345808 \\ 7 & 1.437228 & -1.867215 & 0.307955 \\ 6 & 0.390180 & -2.765741 & -0.097628 \\ 6 & -0.866601 & -2.696320 & 0.478411 \\ 6 & -1.915892 & -3.527696 & -0.023383 \\ 6 & -1.641336 & -4.452644 & -1.074396 \\ 6 & -0.328882 & -4.533083 & -1.593560 \\ 6 & 0.653793 & -3.701660 & -1.125902 \\ 6 & -3.240856 & -3.453751 & 0.481178 \\ 6 & -4.234539 & -4.249624 & -0.030668 \\ 6 & -3.955237 & -5.174840 & -1.060493 \\ 6 & -2.685865 & -5.271426 & -1.570059 \\ 6 & -1.129645 & -1.872747 & 1.690907 \\ 6 & -1.518574 & -0.543410 & 1.634898 \\ 6 & -1.850344 & 0.130601 & 2.843498 \\ 6 & -1.772699 & -0.494615 & 4.058359 \\ 6 & -1.355526 & -1.842168 & 4.151774 \\ 6 & -1.038649 & -2.546388 & 2.954328 \\ 6 & -1.250159 & -2.499715 & 5.400321 \\ 6 & -0.845781 & -3.808217 & 5.472968 \\ 6 & -0.534201 & -4.513465 & 4.291936 \\ 6 & -0.631892 & -3.902371 & 3.066093\end{array}$




\begin{tabular}{|c|c|c|c|}
\hline 15 & -1.654196 & 0.318208 & -0.007813 \\
\hline & -0.072767 & 0.242583 & -1.927532 \\
\hline 6 & -0.571313 & -1.249932 & -3.089292 \\
\hline 8 & -0.815772 & -2.038274 & -3.895288 \\
\hline & 1.768336 & -0.695372 & -0.874491 \\
\hline 8 & 2.699837 & 0.289817 & 0.056400 \\
\hline 6 & 3.526552 & 1.190751 & -0.576376 \\
\hline 6 & 4.726638 & 0.755210 & -1.103471 \\
\hline 6 & 5.545654 & 1.710802 & -1.788985 \\
\hline 6 & 5.145111 & 3.081402 & -1.818168 \\
\hline 6 & 3.928047 & 3.468374 & -1.207188 \\
\hline 6 & 3.118646 & 2.537604 & -0.615360 \\
\hline 6 & 5.959257 & 4.031147 & -2.481862 \\
\hline 6 & 7.112008 & 3.651272 & -3.119504 \\
\hline 6 & 7.490989 & 2.292730 & -3.126268 \\
\hline 6 & 6.730495 & 1.350006 & -2.480009 \\
\hline 6 & 5.089445 & -0.676188 & -0.991342 \\
\hline 6 & 4.201720 & -1.645197 & -1.426550 \\
\hline 6 & 4.545366 & -3.014034 & -1.458356 \\
\hline 6 & 5.754029 & -3.428700 & -0.970567 \\
\hline 6 & 6.653381 & -2.497947 & -0.395696 \\
\hline 6 & 6.318958 & -1.110507 & -0.395514 \\
\hline 6 & 7.196944 & -0.215518 & 0.267817 \\
\hline 6 & 8.347839 & -0.664479 & 0.867783 \\
\hline 6 & 8.693010 & -2.031667 & 0.831428 \\
\hline 6 & 7.858017 & -2.926573 & \\
\hline 8 & 2.953991 & -1.301568 & -1.896856 \\
\hline 6 & -3.408755 & -0.036504 & -0.436095 \\
\hline 6 & -4.432662 & 0.112672 & 0.499631 \\
\hline 6 & -5.760145 & -0.149002 & 0.164380 \\
\hline 6 & -6.038819 & -0.564735 & -1.143993 \\
\hline 6 & -5.038914 & -0.716827 & -2.106549 \\
\hline 6 & -3.721321 & -0.448810 & -1.725522 \\
\hline 6 & -6.839265 & 0.044739 & 1.228345 \\
\hline 6 & -5.310654 & -1.202376 & -3.527902 \\
\hline 6 & -1.709507 & 2.081933 & 0.502839 \\
\hline 6 & -2.681143 & 2.947176 & -0.003945 \\
\hline 6 & -2.681553 & 4.298975 & 0.336740 \\
\hline 6 & -1.697094 & 4.756690 & 1.223347 \\
\hline 6 & -0.708905 & 3.919222 & 1.742226 \\
\hline 6 & -0.711913 & 2.580458 & 1.337022 \\
\hline 6 & -3.735370 & 5.269753 & -0.193465 \\
\hline 6 & 0.298263 & 4.374244 & 2.798395 \\
\hline 6 & -0.560580 & 1.602236 & -3.169202 \\
\hline 8 & -0.870965 & 2.482398 & -3.852326 \\
\hline 1 & 6.935652 & 0.838390 & 0.320453 \\
\hline 1 & -2.171329 & 1.168769 & 2.806181 \\
\hline 1 & 8.097417 & -3.989018 & 0.191971 \\
\hline 1 & -1.495810 & -1.940982 & 6.302330 \\
\hline 1 & 5.638477 & 5.072071 & -2.484506 \\
\hline 1 & -3.467849 & -2.742165 & 1.273727 \\
\hline 1 & -5.247277 & -4.163911 & 0.357676 \\
\hline 1 & 3.633560 & 4.516416 & -1.237335 \\
\hline 1 & -2.033391 & 0.041729 & 4.970244 \\
\hline 1 & 6.028902 & -4.481911 & -0.993910 \\
\hline 1 & 8.391163 & 1.986818 & -3.654782 \\
\hline
\end{tabular}




\begin{tabular}{|c|c|c|c|}
\hline & 3.832604 & -3.717650 & -1.882539 \\
\hline & -2.457768 & -5.974604 & -2.369830 \\
\hline 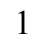 & -0.119075 & -5.247281 & -2.388216 \\
\hline & -0.217134 & -5.552021 & 4.352570 \\
\hline & 7.031515 & 0.305979 & -2.506071 \\
\hline & -0.395078 & -4.463909 & 2.164586 \\
\hline & 1.656910 & -3.734998 & -1.546586 \\
\hline & 7.725963 & 4.388903 & -3.631052 \\
\hline & 8.996937 & 0.041768 & 1.380736 \\
\hline 1 & -4.751407 & -5.804472 & -1.451864 \\
\hline 1 & -0.765306 & -4.304980 & 6.437001 \\
\hline & 9.610691 & -2.372834 & 1.304748 \\
\hline 1 & 2.164503 & 2.803308 & -0.166187 \\
\hline & -4.189746 & 0.446208 & 1.507960 \\
\hline & -2.912059 & -0.563026 & -2.443460 \\
\hline & -7.068988 & -0.772196 & -1.419351 \\
\hline & -3.442175 & 2.549350 & -0.671081 \\
\hline 1 & 0.053006 & 1.899217 & 1.717280 \\
\hline & -1.712326 & 5.803567 & 1.519746 \\
\hline 1 & 1.784365 & -3.109292 & 1.918655 \\
\hline & 0.531046 & 1.549304 & -1.207589 \\
\hline 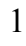 & 3.206001 & -2.921454 & 0.890334 \\
\hline 1 & 4.956824 & -1.827610 & 1.937044 \\
\hline & 0.949809 & -0.564901 & 2.808863 \\
\hline 1 & 5.841752 & -0.088568 & 3.453402 \\
\hline 1 & 1.827036 & 1.156626 & 4.359730 \\
\hline 1 & 4.279760 & 1.425637 & 4.662617 \\
\hline 0 & -6.802800 & -1.282115 & -3.840317 \\
\hline 6 & -4.661692 & -0.251801 & -4.541078 \\
\hline 6 & -4.706565 & -2.602759 & -3.685166 \\
\hline 0 & -8.227900 & -0.350278 & 0.732739 \\
\hline 6 & -6.510649 & -0.816501 & 2.453798 \\
\hline 6 & -6.878403 & 1.523240 & 1.634212 \\
\hline 1 & -6.945063 & -1.608718 & -4.878141 \\
\hline 1 & -7.320815 & -2.006549 & -3.198871 \\
\hline 1 & -7.297018 & -0.308049 & -3.728753 \\
\hline 1 & -4.870642 & -0.591487 & -5.564083 \\
\hline l & -5.054734 & 0.767833 & -4.435823 \\
\hline 1 & -3.570451 & -0.203066 & -4.432218 \\
\hline 1 & -4.869767 & -2.979410 & -4.704300 \\
\hline 1 & -3.625528 & -2.604621 & -3.494000 \\
\hline 1 & -5.167302 & -3.308052 & -2.980553 \\
\hline 1 & -8.961823 & -0.201469 & 1.534886 \\
\hline 1 & -8.549331 & 0.259312 & -0.121340 \\
\hline 1 & -8.271909 & -1.406778 & 0.437562 \\
\hline 1 & -7.616952 & 1.683820 & 2.431358 \\
\hline 1 & -5.905796 & 1.876222 & 2.000787 \\
\hline 1 & -7.160862 & 2.153655 & 0.780795 \\
\hline 1 & -7.274492 & -0.680478 & 3.231174 \\
\hline 1 & -6.487243 & -1.881772 & 2.187956 \\
\hline 1 & -5.539116 & -0.563815 & 2.897355 \\
\hline 6 & -4.699572 & 4.598671 & -1.169390 \\
\hline 6 & -4.553320 & 5.820303 & 0.980243 \\
\hline 6 & -3.047467 & 6.426658 & -0.925473 \\
\hline 6 & 0.242051 & 5.878774 & 3.050222 \\
\hline 6 & -0.039987 & 3.653639 & 4.110236 \\
\hline
\end{tabular}




$\begin{array}{lrrr}6 & 1.727605 & 4.016940 & 2.380602 \\ 1 & -5.437470 & 5.331153 & -1.520182 \\ 1 & -4.180245 & 4.203770 & -2.052262 \\ 1 & -5.252162 & 3.772944 & -0.700666 \\ 1 & -5.316964 & 6.521614 & 0.617552 \\ 1 & -5.065731 & 5.009322 & 1.514871 \\ 1 & -3.927775 & 6.356527 & 1.704150 \\ 1 & -3.796873 & 7.125610 & -1.320747 \\ 1 & -2.380492 & 6.995895 & -0.267121 \\ 1 & -2.450449 & 6.057311 & -1.769195 \\ 1 & 0.995220 & 6.155177 & 3.798920 \\ 1 & 0.454324 & 6.453076 & 2.139103 \\ 1 & -0.734040 & 6.197065 & 3.437901 \\ 1 & 2.434765 & 4.305652 & 3.170487 \\ 1 & 1.864556 & 2.942079 & 2.205076 \\ 1 & 2.014225 & 4.550935 & 1.464469 \\ 1 & 0.669101 & 3.936235 & 4.900837 \\ 1 & -1.051039 & 3.914768 & 4.451135 \\ 1 & -0.000819 & 2.562521 & 3.993984\end{array}$

$1_{\text {C11- }} \psi_{-13233 a 4 a}$

Number of imaginary frequencies : 0

The smallest frequencies are : $13.1870 \quad 19.5803 \quad 21.9060 \mathrm{~cm}(-1)$

Electronic energy : $\quad H F=-4125.4638963$

Zero-point correction $=\quad 1.299885$ (Hartree/Particle)

Thermal correction to Energy= $\quad 1.378214$

Thermal correction to Enthalpy= $\quad 1.379159$

Thermal correction to Gibbs Free Energy= $\quad 1.187296$

Sum of electronic and zero-point Energies $=\quad-4124.164011$

Sum of electronic and thermal Energies $=\quad-4124.085682$

Sum of electronic and thermal Enthalpies= $\quad-4124.084738$

Sum of electronic and thermal Free Energies= $\quad-4124.276601$

Cartesian Coordinates

$\begin{array}{lrrr}6 & 3.909481 & 0.631683 & 4.002979 \\ 6 & 4.763194 & -0.248183 & 3.347399 \\ 6 & 4.243014 & -1.228397 & 2.505577 \\ 6 & 2.868958 & -1.334669 & 2.302928 \\ 6 & 2.016315 & -0.455554 & 2.974354 \\ 6 & 2.532068 & 0.519025 & 3.818779 \\ 6 & 2.318903 & -2.383784 & 1.373490 \\ 7 & 1.407157 & -1.866585 & 0.326126 \\ 6 & 0.340573 & -2.742204 & -0.077872 \\ 6 & -0.918189 & -2.635096 & 0.487878 \\ 6 & -1.988501 & -3.432900 & -0.023665 \\ 6 & -1.734743 & -4.356833 & -1.080963 \\ 6 & -0.419242 & -4.484331 & -1.582454 \\ 6 & 0.585746 & -3.688453 & -1.101004 \\ 6 & -3.315807 & -3.316801 & 0.466711 \\ 6 & -4.333052 & -4.064331 & -0.071036 \\ 6 & -4.076306 & -4.983448 & -1.112500 \\ 6 & -2.803639 & -5.125787 & -1.603893 \\ 6 & -1.166398 & -1.792668 & 1.690148 \\ 6 & -1.521514 & -0.454850 & 1.615846\end{array}$




\begin{tabular}{|c|c|c|c|}
\hline 6 & -1.839553 & 0.241622 & 2.815644 \\
\hline 6 & -1.779055 & -0.369851 & 4.038387 \\
\hline 6 & -1.397938 & -1.726772 & 4.149567 \\
\hline 6 & -1.097324 & -2.453497 & 2.961583 \\
\hline 6 & -1.314081 & -2.372296 & 5.405883 \\
\hline 5 & -0.947330 & -3.690961 & 5.494853 \\
\hline 6 & -0.652880 & -4.418579 & 4.323034 \\
\hline 6 & -0.729390 & -3.818945 & 3.089945 \\
\hline 15 & -1.648038 & 0.384555 & -0.041541 \\
\hline 5 & -0.046993 & 0.254144 & -1.940675 \\
\hline 6 & -0.529673 & -1.237715 & -3.110865 \\
\hline 8 & -0.731231 & -2.033135 & -3.921288 \\
\hline 15 & 1.766856 & -0.711144 & -0.864968 \\
\hline 8 & 2.715741 & 0.262446 & 0.061027 \\
\hline 6 & 3.565963 & 1.138023 & -0.576108 \\
\hline 6 & 4.759644 & 0.670678 & -1.090437 \\
\hline 6 & 5.604703 & 1.601249 & -1.779020 \\
\hline 6 & 5.234640 & 2.979965 & -1.824894 \\
\hline 6 & 4.021222 & 3.399434 & -1.228473 \\
\hline 6 & 3.187361 & 2.492675 & -0.633453 \\
\hline 6 & 6.074914 & 3.905324 & -2.490518 \\
\hline 6 & 7.224483 & 3.494372 & -3.114423 \\
\hline 6 & 7.573360 & 2.127832 & -3.105290 \\
\hline 6 & 6.786852 & 1.208151 & -2.456927 \\
\hline 6 & 5.088009 & -0.768007 & -0.964601 \\
\hline 6 & 4.180378 & -1.719046 & -1.398574 \\
\hline 6 & 4.491956 & -3.095720 & -1.419074 \\
\hline 6 & 5.687395 & -3.535344 & -0.920798 \\
\hline 6 & 6.604714 & -2.622022 & -0.346423 \\
\hline 6 & 6.303209 & -1.227058 & -0.358007 \\
\hline 6 & 7.197998 & -0.348366 & 0.304541 \\
\hline 6 & 8.334164 & -0.820116 & 0.915050 \\
\hline 6 & 8.646927 & -2.195384 & 0.890725 \\
\hline 6 & 7.794896 & -3.074712 & 0.273058 \\
\hline 8 & 2.943490 & -1.350022 & -1.878066 \\
\hline 6 & -3.416655 & 0.045170 & -0.433001 \\
\hline 6 & -4.418079 & 0.279775 & 0.513900 \\
\hline 6 & -5.746576 & -0.039591 & 0.245752 \\
\hline 6 & -6.042302 & -0.599240 & -1.003923 \\
\hline 6 & -5.070484 & -0.844596 & -1.974401 \\
\hline 6 & -3.747953 & -0.506961 & -1.665919 \\
\hline 6 & -6.854056 & 0.146094 & 1.281286 \\
\hline 6 & -5.462485 & -1.431511 & -3.329567 \\
\hline 6 & -1.652114 & 2.156108 & 0.441524 \\
\hline 6 & -2.578079 & 3.043530 & -0.096056 \\
\hline 6 & -2.546569 & 4.403972 & 0.227219 \\
\hline 6 & -1.568235 & 4.845854 & 1.120891 \\
\hline 6 & -0.613556 & 3.980268 & 1.669555 \\
\hline 6 & -0.651341 & 2.639492 & 1.287453 \\
\hline 6 & -3.595909 & 5.330779 & -0.383643 \\
\hline 6 & 0.391717 & 4.421567 & 2.734178 \\
\hline 6 & -0.458612 & 1.619934 & -3.203494 \\
\hline 8 & -0.712163 & 2.505173 & -3.903272 \\
\hline 1 & 6.961291 & 0.711767 & 0.348074 \\
\hline 1 & -2.135890 & 1.286673 & 2.766242 \\
\hline 1 & 8.009189 & -4.142642 & 0.261080 \\
\hline
\end{tabular}




\begin{tabular}{|c|c|c|c|}
\hline & -1.546208 & -1.796441 & 6.300680 \\
\hline & 5.777055 & 4.952941 & -2.505518 \\
\hline & -3.529167 & -2.604395 & 1.262085 \\
\hline & -5.348650 & -3.939911 & 0.300564 \\
\hline & 3.749606 & 4.453159 & -1.272320 \\
\hline & -2.027471 & 0.184598 & 4.942879 \\
\hline & 5.937501 & -4.594874 & -0.935286 \\
\hline & 8.470959 & 1.797131 & -3.623133 \\
\hline & 3.765500 & -3.785181 & -1.843329 \\
\hline 1 & -2.591477 & -5.828356 & -2.408586 \\
\hline & -0.225386 & -5.202214 & -2.377841 \\
\hline & -0.366129 & -5.465097 & 4.396639 \\
\hline & 7.065064 & 0.157649 & -2.471242 \\
\hline & -0.505757 & -4.396933 & 2.195484 \\
\hline & 1.591962 & -3.754984 & -1.510143 \\
\hline 1 & 7.858881 & 4.213494 & -3.627377 \\
\hline 1 & 8.996646 & -0.125775 & 1.427149 \\
\hline 1 & -4.890996 & -5.576504 & -1.523094 \\
\hline & -0.883677 & -4.178544 & 6.464806 \\
\hline ] & 9.553230 & -2.554734 & 1.372439 \\
\hline ] & 2.235528 & 2.783632 & -0.195469 \\
\hline & -4.147728 & 0.714117 & 1.472890 \\
\hline 1 & -2.948282 & -0.683879 & -2.381516 \\
\hline & -7.077746 & -0.858169 & -1.224222 \\
\hline 1 & -3.341279 & 2.665907 & -0.776099 \\
\hline & 0.085390 & 1.941547 & 1.691642 \\
\hline & -1.549881 & 5.894076 & 1.403880 \\
\hline 1 & 1.715764 & -3.098132 & 1.952348 \\
\hline & 0.567144 & 1.549360 & -1.210508 \\
\hline l & 3.145618 & -2.958980 & 0.928125 \\
\hline & 4.919426 & -1.902454 & 1.977205 \\
\hline 1 & 0.942696 & -0.516715 & 2.800187 \\
\hline 1 & 5.840574 & -0.170030 & 3.479744 \\
\hline 1 & 1.855574 & 1.199932 & 4.335368 \\
\hline 1 & 4.312563 & 1.402844 & 4.656293 \\
\hline 6 & -4.242993 & -1.905391 & -4.118228 \\
\hline 5 & -6.394313 & -2.633447 & -3.148586 \\
\hline 6 & -6.182313 & -0.348956 & -4.141478 \\
\hline 6 & -6.360467 & 0.875941 & 2.529345 \\
\hline 6 & -8.009861 & 0.955547 & 0.685544 \\
\hline 6 & -7.361813 & -1.237154 & 1.705152 \\
\hline 1 & -4.568355 & -2.366271 & -5.059417 \\
\hline | & -3.569036 & -1.079899 & -4.380606 \\
\hline 1 & -3.667082 & -2.655545 & -3.557808 \\
\hline L & -6.647600 & -3.061371 & -4.127460 \\
\hline 1 & -5.907925 & -3.416842 & -2.552146 \\
\hline 1 & -7.338438 & -2.368672 & -2.658138 \\
\hline 1 & -6.474189 & -0.736924 & -5.126931 \\
\hline 1 & -7.090678 & 0.000063 & -3.634041 \\
\hline 1 & -5.531751 & 0.521239 & -4.299251 \\
\hline 1 & -7.191769 & 1.003811 & 3.233972 \\
\hline 1 & -5.572781 & 0.316819 & 3.052144 \\
\hline 1 & -5.971313 & 1.874845 & 2.290584 \\
\hline 1 & -8.159126 & -1.142115 & 2.454840 \\
\hline 1 & -7.766446 & -1.802691 & 0.856373 \\
\hline 1 & -6.549751 & -1.830045 & 2.148013 \\
\hline
\end{tabular}


$1_{\mathrm{C} 11-}-\psi_{-1 \mathbf{1} 233 a 4 \mathrm{~s}}$

Number of imaginary frequencies : 0

The smallest frequencies are : $12.5829 \quad 18.8150 \quad 22.6676 \mathrm{~cm}(-1)$

Electronic energy : $\quad H F=-4125.4639897$

Zero-point correction $=\quad 1.299958($ Hartree/Particle $)$

Thermal correction to Energy $=\quad 1.378062$

Thermal correction to Enthalpy $=\quad 1.379006$

Thermal correction to Gibbs Free Energy= 1.188003

Sum of electronic and zero-point Energies $=\quad-4124.164032$

Sum of electronic and thermal Energies $=\quad-4124.085928$

Sum of electronic and thermal Enthalpies $=\quad-4124.084984$

Sum of electronic and thermal Free Energies $=\quad-4124.275987$

Cartesian Coordinates

$\begin{array}{rrrr}6 & 3.900046 & 0.374950 & 4.195596 \\ 6 & 4.783368 & -0.451649 & 3.510422 \\ 6 & 4.308169 & -1.330656 & 2.539644 \\ 6 & 2.948570 & -1.391523 & 2.240977 \\ 6 & 2.068505 & -0.556681 & 2.931777 \\ 6 & 2.538293 & 0.317856 & 3.902323 \\ 6 & 2.446165 & -2.369312 & 1.210995 \\ 7 & 1.485055 & -1.813962 & 0.230563 \\ 6 & 0.423575 & -2.686319 & -0.192906 \\ 6 & -0.822241 & -2.633530 & 0.408796 \\ 6 & -1.886924 & -3.434489 & -0.109180 \\ 6 & -1.644147 & -4.291515 & -1.223887\end{array}$




\begin{tabular}{|c|c|c|c|}
\hline 6 & -0.342803 & -4.357778 & -1.771337 \\
\hline 6 & 0.659861 & -3.570639 & -1.271503 \\
\hline 6 & -3.197356 & -3.391561 & 0.435897 \\
\hline 6 & -4.208238 & -4.146850 & -0.102833 \\
\hline & -3.962520 & -4.997620 & -1.203215 \\
\hline ) & -2.707009 & -5.066374 & -1.750356 \\
\hline 6 & -1.057756 & -1.840206 & 1.646969 \\
\hline 0 & -1.456307 & -0.511831 & 1.623275 \\
\hline 6 & -1.747040 & 0.144061 & 2.851435 \\
\hline 6 & -1.617538 & -0.496671 & 4.054339 \\
\hline 6 & -1.192692 & -1.843670 & 4.113205 \\
\hline 6 & -0.921230 & -2.531478 & 2.895016 \\
\hline 6 & -1.033803 & -2.517245 & 5.347473 \\
\hline 6 & -0.623387 & -3.825238 & 5.386040 \\
\hline 6 & -0.360284 & -4.514999 & 4.184047 \\
\hline 6 & -0.509103 & -3.888132 & 2.971470 \\
\hline 15 & -1.658258 & 0.359335 & -0.006475 \\
\hline 45 & -0.042415 & 0.403681 & -1.887850 \\
\hline 6 & -0.450887 & -1.017870 & -3.165495 \\
\hline 8 & -0.597856 & -1.772833 & -4.025003 \\
\hline 15 & 1.798853 & -0.585307 & -0.897320 \\
\hline 8 & 2.749366 & 0.339779 & 0.074165 \\
\hline 6 & 3.605966 & 1.242084 & -0.516168 \\
\hline 6 & 4.798585 & 0.795055 & -1.049417 \\
\hline 6 & 5.656590 & 1.756417 & -1.676602 \\
\hline 6 & 5.294948 & 3.138017 & -1.649945 \\
\hline 6 & 4.077697 & 3.531895 & -1.043711 \\
\hline 6 & 3.234669 & & -0.503798 \\
\hline 6 & 6.147031 & 4.092979 & -2.256002 \\
\hline 6 & 7.300560 & 3.709466 & -2.890044 \\
\hline 6 & 7.642132 & 2.342421 & -2.951435 \\
\hline 6 & 6.843802 & 1.393497 & -2.362409 \\
\hline 6 & 5.109666 & -0.652809 & -1.018026 \\
\hline 6 & 4.198077 & -1.558672 & -1.532464 \\
\hline 6 & 4.502242 & -2.930363 & -1.669267 \\
\hline 6 & 5.687295 & -3.419155 & -1.192089 \\
\hline 6 & 6.599497 & -2.564521 & -0.526046 \\
\hline 6 & 6.311101 & -1.169813 & -0.432672 \\
\hline 6 & 7.201985 & -0.353387 & 0.309576 \\
\hline 6 & 8.320662 & -0.883374 & 0.903979 \\
\hline 6 & 8.619698 & -2.256477 & 0.780515 \\
\hline 6 & 7.772940 & -3.076354 & 0.079213 \\
\hline 8 & 2.961197 & -1.143623 & -1.973155 \\
\hline 6 & -3.399094 & -0.094591 & -0.402477 \\
\hline 6 & -4.395763 & 0.008842 & 0.563563 \\
\hline 6 & -5.716589 & -0.349170 & 0.282594 \\
\hline 6 & -6.004524 & -0.803566 & -1.005190 \\
\hline 6 & -5.028232 & -0.916695 & -2.003536 \\
\hline 6 & -3.720302 & -0.555832 & -1.678949 \\
\hline 6 & -6.762639 & -0.245105 & 1.390799 \\
\hline 6 & -5.419150 & -1.394036 & -3.400956 \\
\hline 6 & -1.806417 & 2.118699 & 0.501853 \\
\hline 6 & -2.816847 & 2.922091 & -0.016503 \\
\hline 6 & -2.886258 & 4.284560 & 0.289357 \\
\hline 6 & -1.926685 & 4.813066 & 1.155082 \\
\hline 6 & -0.892270 & 4.034022 & 1.689310 \\
\hline
\end{tabular}




\begin{tabular}{|c|c|c|c|}
\hline 6 & -0.827721 & 2.690315 & 1.319588 \\
\hline 6 & -4.009478 & 5.119995 & -0.321679 \\
\hline 6 & 0.105690 & 4.579758 & 2.711502 \\
\hline 6 & -0.529284 & 1.855044 & -3.026975 \\
\hline 8 & -0.840711 & 2.788222 & -3.634583 \\
\hline 1 & 6.973276 & 0.702654 & 0.429975 \\
\hline 1 & -2.078714 & 1.179443 & 2.838981 \\
\hline 1 & 7.978536 & -4.142131 & -0.012222 \\
\hline 1 & -1.243179 & -1.971497 & 6.266402 \\
\hline 1 & 5.855479 & 5.141757 & -2.216812 \\
\hline 1 & -3.402408 & -2.730756 & 1.276978 \\
\hline 1 & -5.211479 & -4.085119 & 0.314772 \\
\hline 1 & 3.811876 & 4.587983 & -1.034527 \\
\hline 1 & -1.843851 & 0.026258 & 4.983069 \\
\hline 1 & 5.931415 & -4.475448 & -1.292243 \\
\hline 1 & 8.543645 & 2.035151 & -3.476873 \\
\hline 1 & 3.777178 & -3.574818 & -2.161485 \\
\hline 1 & -2.502709 & -5.717251 & -2.599359 \\
\hline 1 & -0.157129 & -5.026249 & -2.610585 \\
\hline 1 & -0.039915 & -5.553745 & 4.218339 \\
\hline 1 & 7.116737 & 0.343668 & -2.430640 \\
\hline 1 & -0.309762 & -4.436364 & 2.052820 \\
\hline 1 & 1.656705 & -3.595381 & -1.707444 \\
\hline 1 & 7.944100 & 4.451535 & -3.356856 \\
\hline 1 & 8.979608 & -0.238212 & 1.480838 \\
\hline 1 & -4.772171 & -5.597850 & -1.613259 \\
\hline 1 & -0.501309 & -4.333928 & 6.339431 \\
\hline 1 & 9.512403 & -2.661887 & 1.250970 \\
\hline 1 & 2.279284 & 2.870552 & -0.061143 \\
\hline 1 & -4.138015 & 0.371904 & 1.558140 \\
\hline 1 & -2.921094 & -0.634659 & -2.412533 \\
\hline 1 & -7.027430 & -1.083086 & -1.248631 \\
\hline 1 & -3.562527 & 2.479044 & -0.676089 \\
\hline 1 & -0.020096 & 2.060051 & 1.700514 \\
\hline 1 & -1.983323 & 5.863591 & 1.423181 \\
\hline 1 & 1.900909 & -3.175661 & 1.722994 \\
\hline 1 & 0.533308 & 1.653733 & -1.05 \\
\hline 1 & 3.299958 & -2.844111 & 0.703326 \\
\hline 1 & 5.008533 & -1.966883 & 1.995457 \\
\hline 1 & 1.010852 & -0.574083 & 2.676689 \\
\hline 1 & 5.850025 & -0.410529 & 3.722599 \\
\hline 1 & 1.834751 & 0.958378 & 4.43 \\
\hline 1 & 4.269459 & 1.064269 & 4.951915 \\
\hline 6 & -4.196568 & -1.662431 & -4.275854 \\
\hline 6 & -6.225192 & -2.693625 & -3.314822 \\
\hline 6 & -6.270471 & -0.309952 & -4.071390 \\
\hline 6 & -8.144425 & -0.703635 & 0.932731 \\
\hline 6 & -6.335090 & -1.127730 & 2.570100 \\
\hline 6 & -6.870223 & 1.211676 & 1.855424 \\
\hline 1 & -4.518661 & -2.038350 & -5.255143 \\
\hline 1 & -3.606593 & -0.754338 & -4.456256 \\
\hline 1 & -3.538271 & -2.419132 & -3.827069 \\
\hline 1 & -6.486269 & -3.041573 & -4.323092 \\
\hline 1 & -5.638652 & -3.482090 & -2.824596 \\
\hline 1 & -7.163019 & -2.572892 & -2.759768 \\
\hline 1 & -6.563156 & -0.622492 & -5.083046 \\
\hline
\end{tabular}




$\begin{array}{rrrr}1 & -7.187868 & -0.104620 & -3.505389 \\ 1 & -5.711560 & 0.631294 & -4.156155 \\ 1 & -8.855862 & -0.619374 & 1.763901 \\ 1 & -8.528902 & -0.089163 & 0.108441 \\ 1 & -8.140834 & -1.752391 & 0.607692 \\ 1 & -7.599891 & 1.299348 & 2.671767 \\ 1 & -5.912072 & 1.600812 & 2.222142 \\ 1 & -7.202776 & 1.861479 & 1.035069 \\ 1 & -7.066625 & -1.055352 & 3.386241 \\ 1 & -6.273215 & -2.181935 & 2.268100 \\ 1 & -5.356643 & -0.839387 & 2.975689 \\ 6 & -3.962909 & 6.578379 & 0.126317 \\ 6 & -3.888980 & 5.083378 & -1.849672 \\ 6 & -5.364365 & 4.534749 & 0.094798 \\ 6 & -0.034621 & 6.086023 & 2.919077 \\ 6 & -0.155286 & 3.884138 & 4.053742 \\ 6 & 1.544253 & 4.295072 & 2.270656 \\ 1 & -4.788323 & 7.132516 & -0.338146 \\ 1 & -4.071914 & 6.678458 & 1.214128 \\ 1 & -3.028444 & 7.070043 & -0.173259 \\ 1 & -4.674601 & 5.698311 & -2.309335 \\ 1 & -2.916163 & 5.471623 & -2.178031 \\ 1 & -3.990168 & 4.065788 & -2.245771 \\ 1 & -6.183357 & 5.117987 & -0.347557 \\ 1 & -5.483078 & 3.494147 & -0.234073 \\ 1 & -5.484215 & 4.556718 & 1.186236 \\ 1 & 0.711505 & 6.427821 & 3.647466 \\ 1 & 0.131681 & 6.644444 & 1.988764 \\ 1 & -1.021700 & 6.359570 & 3.312833 \\ 1 & 2.251903 & 4.696778 & 3.008181 \\ 1 & 1.751574 & 3.221294 & 2.178277 \\ 1 & 1.760948 & 4.771024 & 1.304564 \\ 1 & 0.542429 & 4.249813 & 4.819606 \\ 1 & -1.177547 & 4.079226 & 4.404850 \\ 1 & -0.030138 & 2.796049 & 3.977072\end{array}$

$1_{\mathrm{C} 11-}-\psi_{-1223354}$

Number of imaginary frequencies : 0

The smallest frequencies are : $12.9120 \quad 18.402421 .9526 \mathrm{~cm}(-1)$

Electronic energy : $\quad H F=-4125.4641945$

Zero-point correction $=\quad 1.299707($ Hartree/Particle $)$

Thermal correction to Energy= $\quad 1.377965$

Thermal correction to Enthalpy= $\quad 1.378909$

Thermal correction to Gibbs Free Energy $=\quad 1.187860$

Sum of electronic and zero-point Energies $=\quad-4124.164488$

Sum of electronic and thermal Energies $=\quad-4124.086230$

Sum of electronic and thermal Enthalpies $=\quad-4124.085286$

Sum of electronic and thermal Free Energies $=\quad-4124.276335$

Cartesian Coordinates

$\begin{array}{llll}6 & -0.663292 & 2.572859 & 1.411750 \\ 6 & -1.660425 & 2.100934 & 0.555181 \\ 6 & -2.593805 & 2.992547 & 0.037706 \\ 6 & -2.573025 & 4.345467 & 0.389389\end{array}$




\begin{tabular}{|c|c|c|c|}
\hline 6 & -1.597517 & 4.775953 & 1.291586 \\
\hline & -0.635470 & 3.906239 & 1.821121 \\
\hline & -1.645452 & 0.345530 & 0.017577 \\
\hline 6 & -3.408997 & 0.031541 & -0.406020 \\
\hline 6 & -4.429709 & 0.182473 & 0.541619 \\
\hline 6 & -5.754914 & -0.074154 & 0.209254 \\
\hline & -6.037652 & -0.486596 & -1.104788 \\
\hline & -5.050263 & -0.628050 & -2.074277 \\
\hline & -3.727188 & -0.365210 & -1.695115 \\
\hline & -6.883356 & 0.030023 & 1.233846 \\
\hline & -7.383381 & -1.384045 & 1.550990 \\
\hline 0 & -5.328503 & -1.096867 & -3.499438 \\
\hline 0 & -4.723766 & -2.494322 & -3.677810 \\
\hline 0 & -3.631276 & 5.273887 & -0.202787 \\
\hline 6 & -3.499078 & 5.283401 & -1.730187 \\
\hline 0 & 0.367540 & 4.335021 & 2.893094 \\
\hline 6 & 1.794644 & 3.951604 & 2.490919 \\
\hline 6 & -1.516981 & -0.552083 & 1.639563 \\
\hline 6 & -1.147851 & -1.888174 & 1.664319 \\
\hline 6 & -1.061368 & -2.589516 & 2.913090 \\
\hline 6 & -1.361688 & -1.905561 & 4.126670 \\
\hline 6 & -1.758971 & -0.550149 & 4.064381 \\
\hline 6 & -1.833536 & 0.101470 & 2.863373 \\
\hline 6 & -0.672945 & -3.953087 & 2.994847 \\
\hline 6 & -0.577914 & -4.590778 & 4.207284 \\
\hline 6 & -0.873715 & -3.905874 & 5.404207 \\
\hline 6 & -1.259558 & -2.590559 & 5.360695 \\
\hline 6 & -0.894192 & -2.689184 & 0.434027 \\
\hline 6 & 0.362275 & -2.756880 & -0.143842 \\
\hline 6 & 0.620733 & -3.676904 & -1.187479 \\
\hline 6 & -0.365704 & -4.496464 & -1.667355 \\
\hline 6 & -1.677309 & -4.417785 & -1.146179 \\
\hline 6 & -1.948435 & -3.506073 & -0.082414 \\
\hline 6 & -2.723933 & -5.226922 & -1.653091 \\
\hline 6 & -3.992926 & -5.134278 & -1.142323 \\
\hline 6 & -4.270132 & -4.220315 & -0.102069 \\
\hline 6 & -3.274536 & -3.433256 & 0.420109 \\
\hline 7 & 1.416961 & -1.874300 & 0.276834 \\
\hline 15 & 1.757899 & -0.684643 & -0.885186 \\
\hline 8 & 2.934167 & -1.283289 & -1.922385 \\
\hline 6 & 4.180357 & -1.646983 & -1.463033 \\
\hline 6 & 5.078273 & -0.694241 & -1.013099 \\
\hline 6 & 6.305526 & -1.150579 & -0.429168 \\
\hline 6 & 6.626692 & -2.540950 & -0.454988 \\
\hline 6 & 5.717093 & -3.452732 & -1.043916 \\
\hline 6 & 4.511136 & -3.018112 & -1.520832 \\
\hline 6 & 7.194106 & -0.275775 & 0.246831 \\
\hline 6 & 8.342452 & -0.746240 & 0.835101 \\
\hline 6 & 8.674307 & -2.115858 & 0.773704 \\
\hline 6 & 7.828950 & -2.991730 & 0.142149 \\
\hline 6 & 4.728224 & 0.742273 & -1.096774 \\
\hline 6 & 3.534698 & 1.178779 & -0.555856 \\
\hline 6 & 3.140468 & 2.530239 & -0.564437 \\
\hline 6 & 3.956335 & 3.464706 & -1.141361 \\
\hline 6 & 5.166230 & 3.078086 & -1.766676 \\
\hline 6 & 5.553512 & 1.703351 & -1.766833 \\
\hline
\end{tabular}




\begin{tabular}{|c|c|c|c|}
\hline 5 & 5.986379 & 4.032903 & -2.415502 \\
\hline 6 & 7.132300 & 3.654638 & -3.066284 \\
\hline 6 & 7.498114 & 2.292930 & -3.101716 \\
\hline 6 & 6.731614 & 1.344905 & -2.470467 \\
\hline 8 & 2.701318 & 0.273322 & 0.061377 \\
\hline 6 & 2.345258 & -2.408622 & 1.301394 \\
\hline 6 & 2.881922 & -1.379250 & 2.260048 \\
\hline 6 & 4.255269 & -1.246537 & 2.450991 \\
\hline 6 & 4.762032 & -0.280043 & 3.316476 \\
\hline 6 & 3.895367 & 0.557836 & 4.009225 \\
\hline 6 & 2.518809 & 0.416494 & 3.838327 \\
\hline 6 & 2.016624 & -0.543067 & 2.968776 \\
\hline 45 & -0.077884 & 0.288982 & -1.912009 \\
\hline 6 & -0.597296 & 1.690944 & -3.093298 \\
\hline 8 & -0.937909 & 2.600521 & -3.721231 \\
\hline 6 & -0.586297 & -1.174258 & -3.105369 \\
\hline 8 & -0.834329 & -1.946489 & -3.925910 \\
\hline 6 & -6.420488 & 0.678601 & 2.537585 \\
\hline 6 & -8.038323 & 0.868037 & 0.676553 \\
\hline 6 & -6.822291 & -1.173731 & -3.803879 \\
\hline 6 & -4.685084 & -0.132743 & -4.503330 \\
\hline 6 & -3.493992 & 6.709897 & 0.296033 \\
\hline 6 & -5.024983 & 4.764740 & 0.186922 \\
\hline 6 & 0.339433 & 5.839028 & 3.153581 \\
\hline 6 & -0.000406 & 3.615090 & 4.197395 \\
\hline 1 & 6.943341 & 0.779622 & 0.318175 \\
\hline 1 & -2.140293 & 1.144619 & 2.848777 \\
\hline 1 & 8.058134 & -4.055868 & 0.101608 \\
\hline 1 & -1.492272 & -2.047128 & 6.275386 \\
\hline 1 & 5.675812 & 5.076746 & -2.395925 \\
\hline 1 & -3.502692 & -2.728692 & 1.218549 \\
\hline 1 & -5.283065 & -4.136671 & 0.286101 \\
\hline 1 & 3.672649 & 4.516134 & -1.148233 \\
\hline 1 & -2.006929 & -0.029723 & 4.988943 \\
\hline 1 & 5.981984 & -4.507887 & -1.086979 \\
\hline 1 & 8.392794 & 1.988974 & -3.640589 \\
\hline 1 & 3.790889 & -3.707346 & -1.955881 \\
\hline 1 & -2.497125 & -5.920017 & -2.461983 \\
\hline 1 & -0.159474 & -5.200117 & -2.472290 \\
\hline 1 & -0.275103 & -5.634663 & 4.244780 \\
\hline 1 & 7.022307 & 0.298708 & -2.518748 \\
\hline 1 & -0.448171 & -4.499118 & 2.080812 \\
\hline 1 & 1.623813 & -3.708759 & -1.608299 \\
\hline 1 & 7.750895 & 4.396319 & -3.566258 \\
\hline 1 & 8.999989 & -0.055387 & 1.358161 \\
\hline 1 & -4.790643 & -5.756949 & -1.541579 \\
\hline 1 & -0.795609 & -4.423578 & 6.357364 \\
\hline 1 & 9.590125 & -2.474016 & 1.237984 \\
\hline 1 & 2.191756 & 2.796181 & -0.103870 \\
\hline 1 & -4.172874 & 0.506337 & 1.547168 \\
\hline 1 & -2.921562 & -0.476834 & -2.417643 \\
\hline 1 & -7.071696 & -0.699741 & -1.368345 \\
\hline 1 & -3.354084 & 2.624566 & -0.650750 \\
\hline 1 & 0.079492 & 1.871932 & 1.799634 \\
\hline 1 & -1.586505 & 5.818337 & 1.595838 \\
\hline 1 & 1.758741 & -3.152251 & 1.860322 \\
\hline
\end{tabular}




$\begin{array}{lrrr}1 & 0.548095 & 1.573772 & -1.171036 \\ 1 & 3.179560 & -2.953367 & 0.832403 \\ 1 & 4.940894 & -1.887707 & 1.894435 \\ 1 & 0.942893 & -0.626338 & 2.806156 \\ 1 & 5.838835 & -0.179570 & 3.438208 \\ 1 & 1.832110 & 1.062570 & 4.385288 \\ 1 & 4.287520 & 1.318680 & 4.681064 \\ 1 & -6.971083 & -1.486131 & -4.845122 \\ 1 & -7.335270 & -1.907612 & -3.169091 \\ 1 & -7.316469 & -0.201746 & -3.675254 \\ 1 & -4.898998 & -0.459290 & -5.529546 \\ 1 & -5.077936 & 0.885334 & -4.383266 \\ 1 & -3.593226 & -0.085411 & -4.399306 \\ 1 & -4.892949 & -2.857932 & -4.700658 \\ 1 & -3.641382 & -2.497687 & -3.494328 \\ 1 & -5.179298 & -3.209064 & -2.979448 \\ 1 & -7.268745 & 0.761581 & 3.228643 \\ 1 & -5.646168 & 0.087575 & 3.044439 \\ 1 & -6.026125 & 1.690179 & 2.370865 \\ 1 & -8.200699 & -1.349766 & 2.284242 \\ 1 & -7.757526 & -1.894932 & 0.654833 \\ 1 & -6.574237 & -1.995176 & 1.973994 \\ 1 & -8.837577 & 0.952920 & 1.424673 \\ 1 & -7.704616 & 1.882821 & 0.423496 \\ 1 & -8.481758 & 0.427009 & -0.223693 \\ 1 & -4.273644 & 7.333709 & -0.159072 \\ 1 & -3.611693 & 6.780563 & 1.385256 \\ 1 & -2.524469 & 7.147114 & 0.024936 \\ 1 & -5.801145 & 5.418084 & -0.234029 \\ 1 & -5.212621 & 3.747758 & -0.180883 \\ 1 & -5.147745 & 4.753241 & 1.278258 \\ 1 & -4.253661 & 5.946785 & -2.174151 \\ 1 & -2.508168 & 5.643068 & -2.036001 \\ 1 & -3.635681 & 4.285736 & -2.164731 \\ 1 & 1.089243 & 6.094740 & 3.912955 \\ 1 & 0.574870 & 6.414595 & 2.249003 \\ 1 & -0.634263 & 6.175653 & 3.531613 \\ 1 & 2.497064 & 4.217037 & 3.293231 \\ 1 & 1.910933 & 2.876059 & 2.305644 \\ 1 & 2.105308 & 4.488695 & 1.584514 \\ 1 & 0.702061 & 3.883501 & 4.998760 \\ 1 & -1.011666 & 3.891249 & 4.525575 \\ 1 & 0.022932 & 2.523878 & 4.078077\end{array}$

$1_{\mathrm{C} 11-}-\psi_{-1223344 s}$

Number of imaginary frequencies : 0

The smallest frequencies are : $11.5755 \quad 18.8802 \quad 19.7735 \mathrm{~cm}(-1)$

Electronic energy : $\quad H F=-4125.4638539$

Zero-point correction $=\quad 1.299143($ Hartree/Particle)

Thermal correction to Energy= $\quad 1.377587$

Thermal correction to Enthalpy= $\quad 1.378531$

Thermal correction to Gibbs Free Energy $=\quad 1.185981$

Sum of electronic and zero-point Energies $=\quad-4124.164711$

Sum of electronic and thermal Energies $=\quad-4124.086267$

Sum of electronic and thermal Enthalpies $=\quad-4124.085322$ 
Cartesian Coordinates

\begin{tabular}{|c|c|c|c|}
\hline 6 & 3.905902 & 0.319851 & 4.222300 \\
\hline 6 & 4.788847 & -0.495022 & 3.522719 \\
\hline 6 & 4.313404 & -1.355114 & 2.535351 \\
\hline 6 & 2.953904 & -1.408771 & 2.234324 \\
\hline 6 & 2.074630 & -0.584225 & 2.938335 \\
\hline 6 & 2.544679 & 0.271223 & 3.925572 \\
\hline 6 & 2.451613 & -2.372093 & 1.190682 \\
\hline 7 & 1.486708 & -1.806535 & 0.219483 \\
\hline 6 & 0.427062 & -2.679943 & -0.206202 \\
\hline 6 & -0.825531 & -2.615221 & 0.380011 \\
\hline 6 & -1.879708 & -3.437389 & -0.128189 \\
\hline 6 & -1.614368 & -4.342108 & -1.199450 \\
\hline 6 & -0.307616 & -4.411832 & -1.734240 \\
\hline 6 & 0.680487 & -3.592964 & -1.257028 \\
\hline 6 & -3.198248 & -3.381944 & 0.394821 \\
\hline 6 & -4.194321 & -4.173172 & -0.119973 \\
\hline 6 & -3.923169 & -5.079320 & -1.168631 \\
\hline 6 & -2.660115 & -5.158597 & -1.696106 \\
\hline 6 & -1.063501 & -1.817167 & 1.616243 \\
\hline 6 & -1.446245 & -0.483682 & 1.602717 \\
\hline 6 & -1.727392 & 0.168539 & 2.834969 \\
\hline 6 & -1.606813 & -0.481031 & 4.033858 \\
\hline 6 & -1.197582 & -1.832964 & 4.084058 \\
\hline 6 & -0.932801 & -2.516295 & 2.861704 \\
\hline 6 & -1.044318 & -2.513908 & 5.315056 \\
\hline 6 & -0.643491 & -3.824867 & 5.347483 \\
\hline 6 & -0.384331 & -4.509772 & 4.141937 \\
\hline 6 & -0.528851 & -3.876068 & 2.932432 \\
\hline 15 & -1.649353 & 0.403821 & -0.016355 \\
\hline 45 & -0.041143 & 0.425537 & -1.891879 \\
\hline 6 & -0.543248 & -1.019127 & -3.107947 \\
\hline 8 & -0.791457 & -1.793720 & -3.925827 \\
\hline 15 & 1.799592 & -0.571162 & -0.900895 \\
\hline 8 & 2.748313 & 0.347138 & 0.080168 \\
\hline 6 & 3.607867 & 1.253552 & -0.498526 \\
\hline 6 & 4.802078 & 0.810664 & -1.032050 \\
\hline 6 & 5.663241 & 1.777370 & -1.646724 \\
\hline 6 & 5.303385 & 3.159101 & -1.607096 \\
\hline 6 & 4.084713 & 3.548374 & -1.000842 \\
\hline 6 & 3.238759 & 2.611598 & -0.473340 \\
\hline 6 & 6.158700 & 4.119224 & -2.200297 \\
\hline 6 & 7.313893 & 3.740837 & -2.834370 \\
\hline 6 & 7.653799 & 2.374024 & -2.908847 \\
\hline 6 & 6.852256 & 1.420084 & -2.332411 \\
\hline 6 & 5.112733 & -0.637572 & -1.014245 \\
\hline 6 & 4.202475 & -1.538208 & -1.540056 \\
\hline 6 & 4.508174 & -2.907968 & -1.692673 \\
\hline 6 & 5.692406 & -3.401680 & -1.218680 \\
\hline 6 & 6.602254 & -2.554277 & -0.540173 \\
\hline 6 & 6.312933 & -1.160821 & -0.431679 \\
\hline 6 & 7.201466 & -0.352715 & 0.322411 \\
\hline 6 & 8.318612 & -0.889083 & 0.913988 \\
\hline
\end{tabular}




\begin{tabular}{|c|c|c|c|}
\hline & 8.618558 & -2.260549 & 0.775742 \\
\hline & 7.774148 & -3.072587 & 0.062567 \\
\hline & 2.966046 & -1.120144 & -1.977656 \\
\hline & -3.383564 & -0.064237 & -0.420839 \\
\hline & -4.373911 & -0.079539 & 0.562050 \\
\hline & -5.678554 & -0.470986 & 0.265521 \\
\hline & -5.973120 & -0.819764 & -1.058721 \\
\hline & -5.011949 & -0.790150 & -2.071465 \\
\hline & -3.710601 & -0.416280 & -1.724453 \\
\hline & -6.714719 & -0.508371 & 1.388510 \\
\hline & -5.308329 & -1.203219 & -3.511367 \\
\hline & -1.823637 & 2.156722 & 0.505682 \\
\hline & -2.874203 & 2.932377 & 0.026853 \\
\hline & -2.985950 & 4.285053 & 0.360949 \\
\hline & -2.025756 & 4.831537 & 1.214291 \\
\hline & -0.950704 & 4.081047 & 1.708065 \\
\hline & -0.845861 & 2.747503 & 1.310813 \\
\hline & -4.154830 & 5.088325 & -0.205696 \\
\hline & 0.051506 & 4.645592 & 2.715575 \\
\hline & -0.455299 & 1.845507 & -3.098238 \\
\hline & -0.718360 & 2.755650 & -3.761362 \\
\hline & 6.971896 & 0.701704 & 0.454654 \\
\hline & -2.048766 & 1.207109 & 2.828203 \\
\hline & 7.980405 & -4.137181 & -0.040504 \\
\hline & -1.249296 & -1.970619 & 6.236425 \\
\hline & 5.868345 & 5.167921 & -2.151077 \\
\hline & -3.419810 & -2.686640 & 1.203121 \\
\hline 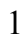 & -5.202905 & -4.104810 & 0.284497 \\
\hline & 3.820458 & 4.604665 & -0.981657 \\
\hline & -1.826998 & 0.038822 & 4.965755 \\
\hline 1 & 5.937655 & -4.456497 & -1.330897 \\
\hline & 8.556660 & 2.070999 & -3.434441 \\
\hline 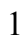 & 3.784179 & -3.546078 & -2.194780 \\
\hline & -2.437602 & -5.846834 & -2.510349 \\
\hline 1 & -0.105937 & -5.111547 & -2.543771 \\
\hline & -0.070039 & -5.550539 & 4.170749 \\
\hline 1 & 7.124057 & 0.370666 & -2.410691 \\
\hline 1 & -0.331381 & -4.422079 & 2.012101 \\
\hline & 1.682769 & -3.624186 & -1.680156 \\
\hline 1 & 7.960041 & 4.486843 & -3.291197 \\
\hline & 8.975527 & -0.250313 & 1.500217 \\
\hline 1 & -4.720324 & -5.708023 & -1.559630 \\
\hline & -0.525077 & -4.339185 & 6.298306 \\
\hline 1 & 9.510016 & -2.671025 & 1.244175 \\
\hline ] & 2.282156 & 2.879785 & -0.031504 \\
\hline & -4.116839 & 0.208896 & 1.581016 \\
\hline 1 & -2.925752 & -0.402350 & -2.478040 \\
\hline & -6.985650 & -1.124775 & -1.307035 \\
\hline 1 & -3.620329 & 2.474226 & -0.621190 \\
\hline & -0.010494 & 2.137713 & 1.664988 \\
\hline 1 & -2.114902 & 5.873639 & 1.505568 \\
\hline 1 & 1.909439 & -3.186988 & 1.692437 \\
\hline 1 & 0.543060 & 1.684805 & -1.076832 \\
\hline 1 & 3.305872 & -2.838083 & 0.675975 \\
\hline 1 & 5.013293 & -1.982753 & 1.980689 \\
\hline 1 & 1.017465 & -0.593967 & 2.680899 \\
\hline
\end{tabular}




\begin{tabular}{|c|c|c|c|}
\hline 1 & 5.855292 & -0.459673 & 3.737010 \\
\hline 1 & 1.841694 & 0.903846 & 4.466558 \\
\hline 1 & 4.275386 & 0.994093 & 4.992086 \\
\hline 6 & -6.805232 & -1.342585 & -3.779598 \\
\hline 6 & -4.746365 & -0.164307 & -4.488860 \\
\hline 6 & -4.640428 & -2.558296 & -3.770999 \\
\hline 6 & -8.055189 & -1.073486 & 0.926065 \\
\hline 6 & -6.201495 & -1.391618 & 2.532918 \\
\hline 6 & -6.944657 & 0.915008 & 1.908780 \\
\hline 1 & -6.967970 & -1.602764 & -4.833056 \\
\hline 1 & -7.261859 & -2.138042 & -3.177083 \\
\hline 1 & -7.344915 & -0.407261 & -3.580957 \\
\hline 1 & -4.991375 & -0.448501 & -5.520629 \\
\hline 1 & -5.173120 & 0.829704 & -4.301380 \\
\hline 1 & -3.653981 & -0.077754 & -4.429783 \\
\hline 1 & -4.811100 & -2.879244 & -4.807896 \\
\hline 1 & -3.556213 & -2.516587 & -3.603236 \\
\hline 1 & -5.047337 & -3.327774 & -3.101353 \\
\hline 1 & -8.755608 & -1.099721 & 1.770367 \\
\hline 1 & -8.513872 & -0.459405 & 0.140677 \\
\hline 1 & -7.956145 & -2.099481 & 0.546816 \\
\hline 1 & -7.660866 & 0.908315 & 2.741666 \\
\hline 1 & -6.015653 & 1.374782 & 2.268857 \\
\hline 1 & -7.352645 & 1.559255 & 1.118919 \\
\hline 1 & -6.936422 & -1.420037 & 3.348574 \\
\hline 1 & -6.040425 & -2.423891 & \\
\hline 1 & -5.256119 & -1.028368 & 2.955786 \\
\hline 6 & -4.164917 & 6.532523 & 0.287982 \\
\hline 6 & -4.057480 & 5.104987 & -1.735705 \\
\hline 6 & -5.475759 & 4.430508 & 0.211889 \\
\hline 6 & -0.149877 & 6.138121 & 2.967119 \\
\hline 6 & -0.135793 & 3.904958 & 4.046038 \\
\hline 6 & 1.486292 & 4.437299 & 2.223495 \\
\hline 1 & -5.021633 & 7.063690 & -0.145597 \\
\hline 1 & -4.259244 & 6.593296 & 1.380067 \\
\hline 1 & -3.258378 & 7.074630 & -0.010294 \\
\hline 1 & -4.886631 & 5.683845 & -2.164785 \\
\hline 1 & -3.116296 & 5.563861 & -2.064969 \\
\hline 1 & -4.100943 & 4.095173 & -2.161606 \\
\hline 1 & -6.326716 & 4.995870 & -0.191493 \\
\hline 1 & -5.558656 & 3.399875 & -0.156465 \\
\hline 1 & -5.574644 & 4.403831 & 1.305222 \\
\hline 1 & 0.601246 & 6.494474 & 3.683231 \\
\hline 1 & -0.035608 & 6.727152 & 2.047925 \\
\hline 1 & -1.136403 & 6.356227 & 3.395408 \\
\hline 1 & 2.199114 & 4.860760 & 2.943566 \\
\hline 1 & 1.737399 & 3.375735 & 2.108228 \\
\hline 1 & 1.647179 & 4.935527 & 1.257771 \\
\hline 1 & 0.562811 & 4.288535 & 4.802190 \\
\hline 1 & -1.156714 & 4.037091 & 4.429009 \\
\hline 1 & 0.045061 & 2.827241 & 3.938633 \\
\hline
\end{tabular}

$1_{\text {C12- }} \psi_{\text {-1a2a3a4a }}$

Number of imaginary frequencies : 0 
The smallest frequencies are : $11.3487 \quad 16.5139 \quad 18.9036 \mathrm{~cm}(-1)$ Electronic energy : $\quad H F=-4125.4610947$

Zero-point correction $=\quad 1.299973($ Hartree/Particle $)$

Thermal correction to Energy $=\quad 1.378266$

Thermal correction to Enthalpy= $\quad 1.379210$

Thermal correction to Gibbs Free Energy= $\quad 1.187077$

Sum of electronic and zero-point Energies $=\quad-4124.161122$

Sum of electronic and thermal Energies $=\quad-4124.082828$

Sum of electronic and thermal Enthalpies $=\quad-4124.081884$

Sum of electronic and thermal Free Energies $=\quad-4124.274017$

Cartesian Coordinates

$\begin{array}{ccccc}6 & 3.260863 & -6.128838 & 1.714638 \\ 6 & 3.060439 & -5.235713 & 2.763426 \\ 6 & 2.708397 & -3.918503 & 2.497018 \\ 6 & 2.569518 & -3.462447 & 1.183964 \\ 6 & 2.794573 & -4.358812 & 0.139325 \\ 6 & 3.127592 & -5.684610 & 0.403090 \\ 6 & 2.239341 & -2.007249 & 0.981067 \\ 7 & 1.585272 & -1.663300 & -0.291568 \\ 6 & 0.476603 & -2.411372 & -0.806154 \\ 6 & -0.727944 & -2.548769 & -0.132798 \\ 6 & -1.828664 & -3.193143 & -0.781667 \\ 6 & -1.677060 & -3.683140 & -2.112060 \\ 6 & -0.424678 & -3.551148 & -2.752317 \\ 6 & 0.615339 & -2.929000 & -2.118468 \\ 6 & -3.099149 & -3.321995 & -0.160460 \\ 6 & -4.153627 & -3.897922 & -0.823836 \\ 6 & -3.994597 & -4.391114 & -2.137426 \\ 6 & -2.780485 & -4.283724 & -2.766130 \\ 6 & -0.901118 & -2.058492 & 1.261995 \\ 6 & -1.195321 & -0.729882 & 1.537911 \\ 6 & -1.371536 & -0.327993 & 2.890160 \\ 6 & -1.262158 & -1.222601 & 3.920623 \\ 6 & -0.987305 & -2.586673 & 3.671018 \\ 6 & -0.811421 & -3.016357 & 2.323831 \\ 6 & -0.897936 & -3.530001 & 4.722803 \\ 6 & -0.661633 & -4.855361 & 4.457796 \\ 6 & -0.497301 & -5.288407 & 3.125241 \\ 6 & -0.559294 & -4.392946 & 2.086864 \\ 15 & -1.498810 & 0.444682 & 0.126989 \\ 45 & -0.100672 & 0.904129 & -1.904863 \\ 6 & -0.584601 & -0.275139 & -3.391066 \\ 8 & -0.804561 & -0.803698 & -4.392501 \\ 15 & 1.795461 & -0.164302 & -1.077936 \\ 8 & 2.556770 & 0.616812 & 0.171683 \\ 6 & 3.365854 & 1.692032 & -0.145818 \\ 6 & 4.631918 & 1.461341 & -0.650455 \\ 6 & 5.430360 & 2.592871 & -1.013783 \\ 6 & 4.937160 & 3.907963 & -0.757255 \\ 6 & 3.653413 & 4.072729 & -0.185368 \\ 6 & 2.868500 & 2.987086 & 0.097986 \\ 6 & 5.730177 & 5.029100 & -1.103648 \\ 6 & 6.951945 & 4.870476 & -1.704920 \\ 6 & 7.426007 & 3.573639 & -1.994513\end{array}$




\begin{tabular}{|c|c|c|c|}
\hline & 6.687051 & 2.466585 & -1.658984 \\
\hline & 5.081312 & 0.062766 & -0.847186 \\
\hline & 4.293993 & -0.794184 & -1.595902 \\
\hline & 4.673630 & -2.121321 & -1.873445 \\
\hline & 5.834391 & -2.618905 & -1.346509 \\
\hline & 6.643603 & -1.819235 & -0.504972 \\
\hline & 6.266504 & -0.466883 & -0.242274 \\
\hline & 7.067673 & 0.284699 & 0.655386 \\
\hline & 8.189097 & -0.259009 & 1.230573 \\
\hline & 8.575949 & -1.584840 & 0.942666 \\
\hline & 7.813173 & -2.346119 & 0.095411 \\
\hline & 3.092096 & -0.354412 & -2.110070 \\
\hline & -3.269739 & 0.071850 & -0.213717 \\
\hline & -4.181689 & -0.084772 & 0.833063 \\
\hline & -5.522979 & -0.358819 & 0.577643 \\
\hline & -5.923933 & -0.457900 & -0.760670 \\
\hline & -5.041354 & -0.303681 & -1.830430 \\
\hline & -3.701161 & -0.039862 & -1.531074 \\
\hline & -6.539532 & -0.579350 & 1.696405 \\
\hline & -5.549263 & -0.388928 & -3.268209 \\
\hline & -1.591660 & 2.083629 & 0.951708 \\
\hline & -2.611842 & 2.970686 & 0.614840 \\
\hline & -2.626591 & 4.273563 & 1.117432 \\
\hline & -1.604867 & 4.646997 & 1.993223 \\
\hline & -0.568294 & 3.780732 & 2.358548 \\
\hline & -0.558162 & 2.505097 & 1.793159 \\
\hline 6 & -3.721945 & 5.273368 & 0.750605 \\
\hline & 0.484916 & 4.238026 & 3.368084 \\
\hline & -0.779163 & 2.468696 & -2.738384 \\
\hline & -1.215749 & 3.459227 & -3.146614 \\
\hline & 6.777662 & 1.303869 & 0.897404 \\
\hline ] & -1.612467 & 0.708217 & 3.114023 \\
\hline & 8.085934 & -3.377323 & -0.124860 \\
\hline & -1.029884 & -3.179903 & 5.745794 \\
\hline & 5.338326 & 6.023073 & -0.891921 \\
\hline & -3.238555 & -2.936952 & 0.848272 \\
\hline 1 & -5.123978 & -3.972885 & -0.336287 \\
\hline & 3.286496 & 5.081374 & 0.000830 \\
\hline 1 & -1.405529 & -0.895148 & 4.949758 \\
\hline & 6.136410 & -3.645880 & -1.545829 \\
\hline & 8.383569 & 3.449227 & -2.495196 \\
\hline 1 & 4.026260 & -2.722706 & -2.507867 \\
\hline & -2.641895 & -4.655695 & -3.780390 \\
\hline 1 & -0.307608 & -3.929872 & -3.766408 \\
\hline & -0.298347 & -6.337646 & 2.918438 \\
\hline 1 & 7.060854 & 1.474925 & -1.900566 \\
\hline & -0.403326 & -4.739190 & 1.067487 \\
\hline 1 & 1.572744 & -2.802241 & -2.622316 \\
\hline 1 & 7.549651 & 5.738829 & -1.972091 \\
\hline & 8.782052 & 0.337375 & 1.920405 \\
\hline 1 & -4.836573 & -4.855662 & -2.646517 \\
\hline & -0.602209 & -5.574484 & 5.271753 \\
\hline 1 & 9.469325 & -2.001367 & 1.402007 \\
\hline & 1.864513 & 3.094030 & 0.505844 \\
\hline 1 & -3.828771 & 0.004557 & 1.857774 \\
\hline 1 & -2.966969 & 0.078282 & -2.324538 \\
\hline
\end{tabular}




\begin{tabular}{|c|c|c|c|}
\hline 1 & -6.972082 & -0.667813 & -0.974574 \\
\hline & -3.397000 & 2.637708 & -0.059658 \\
\hline & 0.253232 & 1.813621 & 2.017378 \\
\hline & -1.616362 & 5.653489 & 2.411744 \\
\hline & 3.171526 & -1.432245 & 1.025312 \\
\hline & 1.634165 & -1.657561 & 1.833248 \\
\hline & 2.523057 & -3.229731 & 3.321956 \\
\hline & 2.705118 & -4.019226 & -0.889489 \\
\hline 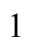 & 3.155986 & -5.570448 & 3.794503 \\
\hline & 3.285801 & -6.373459 & -0.424344 \\
\hline & 3.520630 & -7.165465 & 1.918208 \\
\hline & 0.507765 & 2.064174 & -0.971769 \\
\hline & -4.747854 & 4.680574 & -0.213666 \\
\hline 6 & -4.459344 & 5.712893 & 2.020159 \\
\hline 6 & -3.087249 & 6.494522 & 0.076592 \\
\hline 6 & 1.484574 & 3.133345 & 3.707372 \\
\hline 6 & 1.258040 & 5.435756 & 2.807964 \\
\hline 6 & -0.213490 & 4.650067 & 4.669673 \\
\hline 6 & -4.403643 & -0.428471 & -4.278036 \\
\hline 6 & -6.387381 & -1.655987 & -3.462546 \\
\hline 6 & -6.412790 & 0.844242 & -3.556323 \\
\hline 6 & -5.922604 & -0.393700 & 3.081254 \\
\hline 6 & -7.695659 & 0.415423 & 1.551408 \\
\hline 6 & -7.080898 & -2.010613 & 1.607150 \\
\hline I & -5.514959 & 5.431700 & -0.440425 \\
\hline 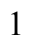 & -4.289735 & 4.380885 & -1.165405 \\
\hline 1 & -5.258574 & 3.805551 & 0.210762 \\
\hline 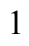 & 2.196488 & 3.501117 & 4.456991 \\
\hline 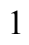 & 0.988772 & 2.248311 & 4.129201 \\
\hline ] & 2.069533 & 2.814412 & 2.834424 \\
\hline 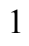 & 0.528529 & 4.965246 & 5.415513 \\
\hline 1 & -0.908758 & 5.485069 & 4.522719 \\
\hline 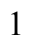 & -0.782410 & 3.811495 & 5.092286 \\
\hline 1 & 2.000479 & 5.786071 & 3.537628 \\
\hline 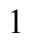 & 1.793767 & 5.163715 & 1.888730 \\
\hline 1 & 0.597387 & 6.279137 & 2.573449 \\
\hline 1 & -5.250082 & 6.433546 & 1.771664 \\
\hline 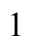 & -4.928696 & 4.854617 & 2.518698 \\
\hline 1 & -3.788928 & 6.193532 & 2.742975 \\
\hline 1 & -3.861987 & 7.220877 & -0.203952 \\
\hline 1 & -2.375888 & 7.006816 & 0.735667 \\
\hline 1 & -2.549352 & 6.202503 & -0.834927 \\
\hline 1 & -4.809541 & -0.527550 & -5.292641 \\
\hline 1 & -3.797926 & 0.487612 & -4.260276 \\
\hline 1 & -3.738064 & -1.284184 & -4.098649 \\
\hline 1 & -6.734332 & -1.722984 & -4.502211 \\
\hline 1 & -5.791524 & -2.552273 & -3.243827 \\
\hline 1 & -7.276550 & -1.675332 & -2.821136 \\
\hline 1 & -6.788352 & 0.818549 & -4.588342 \\
\hline 1 & -7.278819 & 0.899341 & -2.884627 \\
\hline 1 & -5.832285 & 1.767718 & -3.429608 \\
\hline 1 & -6.685553 & -0.567357 & 3.850521 \\
\hline 1 & -5.102473 & -1.101289 & 3.265696 \\
\hline 1 & -5.535240 & 0.623598 & 3.223899 \\
\hline 1 & -7.831269 & -2.185861 & 2.389972 \\
\hline 1 & -7.555549 & -2.213423 & 0.639017 \\
\hline
\end{tabular}


$1_{\text {C12- } \Psi_{-1 a 2 a 3 a 4 s}}$

Number of imaginary frequencies : 0

The smallest frequencies are : $8.9076 \quad 15.1932 \quad 17.7447 \mathrm{~cm}(-1)$

Electronic energy : $\quad H F=-4125.4617142$

Zero-point correction $=\quad 1.299730$ (Hartree/Particle)

Thermal correction to Energy= $\quad 1.378122$

Thermal correction to Enthalpy= $\quad 1.379066$

Thermal correction to Gibbs Free Energy= $\quad 1.186224$

Sum of electronic and zero-point Energies= $\quad-4124.161984$

Sum of electronic and thermal Energies $=\quad-4124.083592$

Sum of electronic and thermal Enthalpies = $\quad-4124.082648$

Sum of electronic and thermal Free Energies $=\quad-4124.275490$

Cartesian Coordinates

$\begin{array}{cccc}\cdots & 3.349749 & -6.066439 & 1.802212 \\ 6 & 3.093636 & -5.176431 & 2.841029 \\ 6 & 2.731771 & -3.864591 & 2.559766 \\ 6 & 2.641073 & -3.411117 & 1.242065 \\ 6 & 2.920003 & -4.304823 & 0.207949 \\ 6 & 3.261722 & -5.625155 & 0.485507 \\ 6 & 2.298908 & -1.962177 & 1.018740 \\ 7 & 1.640012 & -1.645901 & -0.259281 \\ 6 & 0.553301 & -2.427338 & -0.771363 \\ 6 & -0.656275 & -2.573803 & -0.108462 \\ 6 & -1.736507 & -3.254359 & -0.754447 \\ 6 & -1.558846 & -3.772713 & -2.070811 \\ 6 & -0.302000 & -3.629778 & -2.700025 \\ 6 & 0.717840 & -2.971668 & -2.069488 \\ 6 & -3.012086 & -3.392110 & -0.145420 \\ 6 & -4.046138 & -4.006179 & -0.806731 \\ 6 & -3.860978 & -4.528314 & -2.105564 \\ 6 & -2.642048 & -4.411011 & -2.722961 \\ 6 & -0.850641 & -2.057583 & 1.274031 \\ 6 & -1.160910 & -0.726601 & 1.521080 \\ 6 & -1.341027 & -0.298020 & 2.864324 \\ 6 & -1.227279 & -1.170360 & 3.913537 \\ 6 & -0.946998 & -2.538042 & 3.692820 \\ 6 & -0.761010 & -2.993364 & 2.355342 \\ 6 & -0.862200 & -3.460222 & 4.763773 \\ 6 & -0.622546 & -4.790165 & 4.526509 \\ 6 & -0.446861 & -5.248354 & 3.203844 \\ 6 & -0.501900 & -4.373491 & 2.147808 \\ 15 & -1.482778 & 0.411860 & 0.083443 \\ 45 & -0.075955 & 0.858923 & -1.934355 \\ 6 & -0.530204 & -0.359716 & -3.397338 \\ 8 & -0.738227 & -0.915668 & -4.386371 \\ 15 & 1.831897 & -0.158374 & -1.069529 \\ 8 & 2.562187 & 0.658564 & 0.176075 \\ 6 & 3.348545 & 1.748132 & -0.148894\end{array}$




\begin{tabular}{|c|c|c|c|}
\hline 6 & 4.624870 & 1.540140 & -0.636876 \\
\hline 6 & 5.400553 & 2.684792 & -1.008808 \\
\hline & 4.874492 & 3.991540 & -0.775909 \\
\hline & 3.581481 & 4.134301 & -0.219172 \\
\hline & 2.818936 & 3.034750 & 0.070905 \\
\hline & 5.644578 & 5.126029 & -1.130596 \\
\hline & 6.875596 & 4.987570 & -1.717770 \\
\hline U & 7.382422 & 3.698245 & -1.984230 \\
\hline & 6.666289 & 2.578982 & -1.639876 \\
\hline & 5.109731 & 0.150329 & -0.808821 \\
\hline & 4.353268 & -0.736887 & -1.554161 \\
\hline & 4.775665 & -2.054343 & -1.816712 \\
\hline & 5.945811 & -2.512519 & -1.274939 \\
\hline & 6.722425 & -1.682148 & -0.432306 \\
\hline & 6.302896 & -0.339288 & -0.186219 \\
\hline & 7.071214 & 0.443650 & 0.713246 \\
\hline & 8.201555 & -0.061509 & 1.305800 \\
\hline & 8.630900 & -1.377636 & 1.033699 \\
\hline & 7.900543 & -2.168681 & 0.185014 \\
\hline & 3.144182 & -0.338587 & -2.084436 \\
\hline & -3.239671 & -0.016440 & -0.264319 \\
\hline$b$ & -4.155454 & -0.132956 & 0.776294 \\
\hline & -5.503953 & -0.399006 & 0.527792 \\
\hline & -5.901369 & -0.544479 & -0.802169 \\
\hline & -5.005906 & -0.435714 & -1.874412 \\
\hline & -3.668192 & -0.170830 & -1.582068 \\
\hline & -6.463470 & -0.507265 & 1.710945 \\
\hline f & -5.512805 & -0.576479 & -3.308183 \\
\hline b & -1.659249 & 2.058708 & 0.881352 \\
\hline 6 & -2.744038 & 2.870986 & 0.558097 \\
\hline & -2.850633 & 4.166954 & 1.066214 \\
\hline & -1.847677 & 4.614116 & 1.928703 \\
\hline 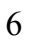 & -0.744513 & 3.826143 & 2.276540 \\
\hline & -0.648208 & 2.554205 & 1.709683 \\
\hline 6 & -4.037444 & 5.069151 & 0.733036 \\
\hline & 0.294396 & 4.364960 & 3.260445 \\
\hline 5 & -0.759842 & 2.393636 & -2.818083 \\
\hline & -1.199079 & 3.367315 & -3.261970 \\
\hline & 6.748004 & 1.455686 & 0.942984 \\
\hline ] & -1.588948 & 0.740915 & 3.066332 \\
\hline & 8.206142 & -3.193204 & -0.022988 \\
\hline 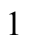 & -1.001657 & -3.090290 & 5.778750 \\
\hline & 5.227476 & 6.113407 & -0.937020 \\
\hline 1 & -3.173083 & -2.983336 & 0.850869 \\
\hline | & -5.021841 & -4.084866 & -0.331219 \\
\hline & 3.189442 & 5.136541 & -0.051297 \\
\hline 1 & -1.373415 & -0.822039 & 4.935417 \\
\hline & 6.280691 & -3.531309 & -1.463472 \\
\hline t & 8.347482 & 3.589217 & -2.473985 \\
\hline & 4.153480 & -2.679874 & -2.453128 \\
\hline 1 & -2.484051 & -4.803912 & -3.726410 \\
\hline 1 & -0.165610 & -4.029928 & -3.703408 \\
\hline 1 & -0.242860 & -6.300719 & 3.018979 \\
\hline 1 & 7.065601 & 1.593154 & -1.863747 \\
\hline & -0.334187 & -4.739500 & 1.137181 \\
\hline & 1.678362 & -2.836279 & -2.564969 \\
\hline
\end{tabular}




\begin{tabular}{|c|c|c|c|}
\hline & 7.455590 & 5.865781 & -1.991680 \\
\hline ] & 8.768592 & 0.557970 & 1.997064 \\
\hline 1 & -4.687435 & -5.021577 & -2.613090 \\
\hline 1 & -0.568320 & -5.493315 & 5.354655 \\
\hline ] & 9.531049 & -1.763411 & 1.506445 \\
\hline 1 & 1.808231 & 3.124445 & 0.465607 \\
\hline ] & -3.813711 & -0.003800 & 1.802989 \\
\hline 1 & -2.931146 & -0.078542 & -2.376254 \\
\hline & -6.947939 & -0.747670 & -1.020335 \\
\hline 1 & -3.513796 & 2.483267 & -0.103744 \\
\hline 1 & 0.210517 & 1.919850 & 1.928960 \\
\hline 1 & -1.929116 & 5.615452 & 2.351652 \\
\hline 1 & 3.225502 & -1.377198 & 1.051549 \\
\hline 1 & 1.690962 & -1.605158 & 1.865797 \\
\hline & 2.498330 & -3.179619 & 3.375567 \\
\hline ] & 2.863320 & -3.965688 & -0.823384 \\
\hline 1 & 3.150403 & -5.509827 & 3.875446 \\
\hline 1 & 3.462022 & -6.312297 & -0.334175 \\
\hline 1 & 3.616276 & -7.099073 & 2.017028 \\
\hline 1 & 0.498782 & 2.048137 & -1.016577 \\
\hline & -4.996824 & 4.417741 & -0.261803 \\
\hline 6 & -4.815511 & 5.371767 & 2.018575 \\
\hline 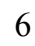 & -3.532693 & 6.378145 & 0.117742 \\
\hline & 1.340752 & 3.316000 & 3.634292 \\
\hline 0 & 1.011700 & 5.568142 & 2.640237 \\
\hline 6 & -0.404512 & 4.802917 & 4.553054 \\
\hline 6 & -4.369338 & -0.595103 & -4.320486 \\
\hline 6 & -6.297943 & -1.882611 & -3.464716 \\
\hline 6 & -6.427387 & 0.611051 & -3.629092 \\
\hline 6 & -7.883035 & -0.865756 & 1.280470 \\
\hline 6 & -5.969476 & -1.594993 & 2.672469 \\
\hline 6 & -6.509361 & 0.840034 & 2.441921 \\
\hline 1 & -5.835581 & 5.096485 & -0.461565 \\
\hline 1 & -4.507941 & 4.204969 & -1.221782 \\
\hline 1 & -5.417355 & 3.477665 & 0.121486 \\
\hline 1 & 2.033910 & 3.736875 & 4.373475 \\
\hline 1 & 0.882680 & 2.422899 & 4.081134 \\
\hline 1 & 1.942983 & 2.996931 & 2.773592 \\
\hline 1 & 0.334676 & 5.173034 & 5.275947 \\
\hline 1 & -1.129857 & 5.607603 & 4.385131 \\
\hline 1 & -0.938017 & 3.962464 & 5.016033 \\
\hline 1 & 1.750736 & 5.977419 & 3.342248 \\
\hline 1 & 1.542388 & 5.279721 & 1.722837 \\
\hline 1 & 0.312873 & 6.373193 & 2.382200 \\
\hline 1 & -5.672816 & 6.023579 & 1.802416 \\
\hline 1 & -5.198628 & 4.447225 & 2.470942 \\
\hline 1 & -4.193338 & 5.877818 & 2.767022 \\
\hline 1 & -4.378992 & 7.030053 & -0.137455 \\
\hline 1 & -2.882597 & 6.935322 & 0.802916 \\
\hline 1 & -2.964047 & 6.185193 & -0.801077 \\
\hline 1 & -4.774581 & -0.721731 & -5.332303 \\
\hline 1 & -3.792873 & 0.339881 & -4.316645 \\
\hline 1 & -3.677462 & -1.427335 & -4.131581 \\
\hline 1 & -6.650910 & -1.989706 & -4.499110 \\
\hline 1 & -5.662598 & -2.747371 & -3.230358 \\
\hline 1 & -7.179068 & -1.923365 & -2.813253 \\
\hline
\end{tabular}




$\begin{array}{lrrr}1 & -6.798924 & 0.541234 & -4.660481 \\ 1 & -7.297866 & 0.648731 & -2.962240 \\ 1 & -5.886649 & 1.561324 & -3.527413 \\ 1 & -8.530138 & -0.936255 & 2.163904 \\ 1 & -8.316731 & -0.106127 & 0.617582 \\ 1 & -7.921290 & -1.834692 & 0.765419 \\ 1 & -7.167883 & 0.779034 & 3.318931 \\ 1 & -5.517350 & 1.155194 & 2.789662 \\ 1 & -6.897757 & 1.628160 & 1.783208 \\ 1 & -6.643791 & -1.674554 & 3.535772 \\ 1 & -5.943234 & -2.574425 & 2.176683 \\ 1 & -4.962473 & -1.390658 & 3.058362\end{array}$

$1_{\text {C12- }-\psi_{-1 a 2 a 354 a}}$

Number of imaginary frequencies : 0

The smallest frequencies are : $10.3593 \quad 16.6852 \quad 17.7791 \mathrm{~cm}(-1)$

Electronic energy : $\quad H F=-4125.4611166$

Zero-point correction $=\quad 1.300143($ Hartree/Particle)

Thermal correction to Energy $=\quad 1.378372$

Thermal correction to Enthalpy= $\quad 1.379316$

Thermal correction to Gibbs Free Energy $=\quad 1.187511$

Sum of electronic and zero-point Energies $=\quad-4124.160974$

Sum of electronic and thermal Energies $=\quad-4124.082745$

Sum of electronic and thermal Enthalpies $=\quad-4124.081801$

Sum of electronic and thermal Free Energies $=\quad-4124.273606$

Cartesian Coordinates

$\begin{array}{lrrr}6 & 3.201782 & -6.169682 & 1.650534 \\ 6 & 2.976509 & -5.290296 & 2.705696 \\ 6 & 2.636085 & -3.968158 & 2.448004 \\ 6 & 2.535215 & -3.493825 & 1.138133 \\ 6 & 2.783154 & -4.377157 & 0.087449 \\ 6 & 3.103955 & -5.707623 & 0.341829 \\ 6 & 2.221200 & -2.034204 & 0.944010 \\ 7 & 1.574931 & -1.676500 & -0.329022 \\ 6 & 0.467149 & -2.416342 & -0.857137 \\ 6 & -0.743341 & -2.553092 & -0.194256 \\ 6 & -1.841772 & -3.186656 & -0.858111 \\ 6 & -1.676602 & -3.684175 & -2.184132 \\ 6 & -0.418172 & -3.552611 & -2.813163 \\ 6 & 0.615098 & -2.927522 & -2.171270 \\ 6 & -3.122121 & -3.299923 & -0.254933 \\ 6 & -4.172372 & -3.869628 & -0.930437 \\ 6 & -3.997075 & -4.380482 & -2.234669 \\ 6 & -2.773355 & -4.287461 & -2.846367 \\ 6 & -0.920719 & -2.089199 & 1.209522 \\ 6 & -1.199906 & -0.764530 & 1.517880 \\ 6 & -1.379177 & -0.393647 & 2.878530 \\ 6 & -1.289137 & -1.314472 & 3.887130 \\ 6 & -1.030995 & -2.675379 & 3.605015 \\ 6 & -0.850417 & -3.074168 & 2.248831 \\ 6 & -0.963077 & -3.644806 & 4.634514 \\ 6 & -0.742877 & -4.966408 & 4.339504 \\ 6 & -0.573145 & -5.369003 & 2.998163\end{array}$




\begin{tabular}{|c|c|c|c|}
\hline 6 & -0.614453 & -4.448206 & 1.981210 \\
\hline 15 & -1.497454 & 0.448162 & 0.140820 \\
\hline 4 & -0.121652 & 0.923577 & -1.885302 \\
\hline 6 & -0.715345 & -0.248914 & -3.336306 \\
\hline 8 & -1.051290 & -0.775680 & -4.305946 \\
\hline 15 & 1.782777 & -0.165327 & -1.092812 \\
\hline 8 & 2.547298 & 0.594466 & 0.169356 \\
\hline 6 & 3.356043 & 1.674846 & -0.128880 \\
\hline 6 & 4.621390 & 1.453355 & -0.639764 \\
\hline 6 & 5.419524 & 2.591176 & -0.983724 \\
\hline 6 & 4.927330 & 3.901466 & -0.701865 \\
\hline 6 & 3.645172 & 4.055913 & -0.123648 \\
\hline 6 & 2.860451 & 2.965496 & 0.141521 \\
\hline 6 & 5.719799 & 5.028726 & -1.029104 \\
\hline 6 & 6.940201 & 4.881053 & -1.635868 \\
\hline 6 & 7.413337 & 3.589671 & -1.950280 \\
\hline 6 & 6.674904 & 2.476673 & -1.633724 \\
\hline 6 & 5.070586 & 0.058384 & -0.861040 \\
\hline 6 & 4.282011 & -0.785894 & -1.622729 \\
\hline 6 & 4.661151 & -2.108409 & -1.922498 \\
\hline 6 & 5.822388 & -2.614960 & -1.405326 \\
\hline 6 & 6.633213 & -1.829206 & -0.552282 \\
\hline 6 & 6.256763 & -0.481298 & -0.266816 \\
\hline 6 & 7.059867 & 0.255281 & 0.641550 \\
\hline 6 & 8.182579 & -0.297774 & 1.205220 \\
\hline 6 & 8.568844 & -1.618612 & 0.894682 \\
\hline 6 & 7.804070 & -2.365881 & 0.036848 \\
\hline 8 & 3.079992 & -0.337727 & -2.128530 \\
\hline 6 & -3.274072 & 0.105050 & -0.196855 \\
\hline 6 & -4.170253 & -0.161278 & 0.845169 \\
\hline 6 & -5.511985 & -0.413223 & 0.585589 \\
\hline 6 & -5.946591 & -0.358024 & -0.749799 \\
\hline 6 & -5.086044 & -0.074530 & -1.806088 \\
\hline 6 & -3.734148 & 0.138085 & -1.503712 \\
\hline 6 & -6.505359 & -0.755431 & 1.694551 \\
\hline 6 & -5.533806 & -0.021157 & -3.264224 \\
\hline 6 & -1.564506 & 2.069058 & 1.002781 \\
\hline 6 & -2.566209 & 2.982121 & 0.680353 \\
\hline 6 & -2.553431 & 4.276433 & 1.204781 \\
\hline 6 & -1.528611 & 4.611231 & 2.092902 \\
\hline 6 & -0.512506 & 3.716563 & 2.446340 \\
\hline 6 & -0.525735 & 2.452653 & 1.855365 \\
\hline 6 & -3.619020 & 5.309897 & 0.843632 \\
\hline 6 & 0.542204 & 4.129970 & 3.472926 \\
\hline 6 & -0.750032 & 2.510305 & -2.717726 \\
\hline 8 & -1.156357 & 3.518205 & -3.115140 \\
\hline 1 & 6.770487 & 1.270346 & 0.900930 \\
\hline 1 & -1.610730 & 0.639294 & 3.125886 \\
\hline 1 & 8.076383 & -3.393306 & -0.201018 \\
\hline 1 & -1.098868 & -3.317330 & 5.664459 \\
\hline 1 & 5.328589 & 6.018619 & -0.797973 \\
\hline 1 & -3.272568 & -2.907646 & 0.749717 \\
\hline 1 & -5.150387 & -3.928735 & -0.456124 \\
\hline 1 & 3.279177 & 5.061013 & 0.082472 \\
\hline 1 & -1.436252 & -1.011153 & 4.923081 \\
\hline 1 & 6.124192 & -3.638437 & -1.622206 \\
\hline
\end{tabular}




\begin{tabular}{|c|c|c|c|}
\hline 1 & 8.369833 & 3.474362 & -2.455173 \\
\hline 1 & 4.013037 & -2.698623 & -2.566598 \\
\hline 1 & -2.623741 & -4.666454 & -3.856447 \\
\hline & -0.291944 & -3.932607 & -3.825709 \\
\hline 1 & -0.386189 & -6.415470 & 2.767523 \\
\hline & 7.048165 & 1.489664 & -1.894443 \\
\hline 1 & -0.453932 & -4.772584 & 0.955445 \\
\hline 1 & 1.576694 & -2.799268 & -2.666435 \\
\hline 1 & 7.537532 & 5.754068 & -1.888286 \\
\hline 1 & 8.777019 & 0.287247 & 1.903461 \\
\hline 1 & -4.835898 & -4.839627 & -2.753449 \\
\hline 1 & -0.700088 & -5.705819 & 5.136139 \\
\hline 1 & 9.463311 & -2.042560 & 1.345034 \\
\hline 1 & 1.858199 & 3.065698 & 0.555551 \\
\hline 1 & -3.799118 & -0.181993 & 1.866756 \\
\hline 1 & -3.020413 & 0.338058 & -2.300825 \\
\hline 1 & -6.998614 & -0.543999 & -0.958962 \\
\hline 1 & -3.356720 & 2.677714 & -0.001692 \\
\hline 1 & 0.271895 & 1.741604 & 2.066669 \\
\hline 1 & -1.520543 & 5.609934 & 2.529909 \\
\hline 1 & 3.159378 & -1.469349 & 0.993782 \\
\hline 1 & 1.617371 & -1.683497 & 1.796782 \\
\hline 1 & 2.428197 & -3.290273 & 3.276537 \\
\hline 1 & 2.720790 & -4.022349 & -0.938314 \\
\hline 1 & 3.042120 & -5.639593 & 3.734303 \\
\hline 1 & 3.280913 & -6.386235 & -0.490262 \\
\hline 1 & 3.452554 & -7.209950 & 1.846744 \\
\hline 1 & 0.506529 & 2.066560 & -0.945962 \\
\hline 6 & -4.649656 & 4.759477 & -0.140575 \\
\hline 6 & -4.357673 & 5.750301 & 2.112037 \\
\hline 6 & -2.944853 & 6.523728 & 0.194767 \\
\hline 6 & 1.533275 & 3.006866 & 3.774291 \\
\hline 6 & 1.326290 & 5.341362 & 2.959636 \\
\hline 6 & -0.159059 & 4.499109 & 4.785826 \\
\hline 6 & -7.053561 & -0.053419 & -3.405664 \\
\hline 6 & -5.021434 & 1.265961 & -3.922178 \\
\hline 6 & -4.951573 & -1.233391 & -3.998556 \\
\hline 6 & -5.849436 & -0.766875 & 3.074199 \\
\hline 6 & -7.636284 & 0.278238 & 1.710130 \\
\hline 6 & -7.089547 & -2.149525 & 1.440554 \\
\hline 1 & -5.390306 & 5.536366 & -0.368485 \\
\hline 1 & -4.188441 & 4.455776 & -1.089807 \\
\hline 1 & -5.192528 & 3.896501 & 0.267843 \\
\hline 1 & 2.249463 & 3.344246 & 4.534060 \\
\hline 1 & 1.031496 & 2.112516 & 4.168399 \\
\hline 1 & 2.113509 & 2.712353 & 2.889607 \\
\hline 1 & 0.581089 & 4.784744 & 5.545350 \\
\hline 1 & -0.850937 & 5.341147 & 4.664729 \\
\hline 1 & -0.733096 & 3.648403 & 5.176145 \\
\hline 1 & 2.068780 & 5.658332 & 3.704302 \\
\hline 1 & 1.863392 & 5.098957 & 2.032959 \\
\hline 1 & 0.673763 & 6.198567 & 2.754688 \\
\hline 1 & -5.126461 & 6.495231 & 1.866247 \\
\hline 1 & -4.854568 & 4.897880 & 2.593449 \\
\hline 1 & -3.682140 & 6.202762 & 2.848172 \\
\hline 1 & -3.696804 & 7.275646 & -0.080421 \\
\hline
\end{tabular}


$1_{\text {C12- }} \psi_{-1 \text { a2a3s4s }}$

Number of imaginary frequencies : 0

The smallest frequencies are : $12.7657 \quad 15.6138 \quad 17.3453 \mathrm{~cm}(-1)$

Electronic energy : $\quad \mathrm{HF}=-4125.4615124$

Zero-point correction $=\quad 1.299517($ Hartree/Particle)

Thermal correction to Energy $=\quad 1.377948$

Thermal correction to Enthalpy= $\quad 1.378892$

Thermal correction to Gibbs Free Energy= 1.186212

Sum of electronic and zero-point Energies $=\quad-4124.161995$

Sum of electronic and thermal Energies $=\quad-4124.083565$

Sum of electronic and thermal Enthalpies $=\quad-4124.082621$

Sum of electronic and thermal Free Energies $=\quad-4124.275301$

\begin{tabular}{rrrr} 
& \multicolumn{4}{c}{ Cartesian Coordinates } \\
\hline 6 & 3.298548 & -5.944456 & 2.084200 \\
6 & 2.998360 & -5.005897 & 3.067361 \\
6 & 2.625940 & -3.716864 & 2.707070 \\
6 & 2.566955 & -3.334254 & 1.365114 \\
6 & 2.886279 & -4.276512 & 0.387273 \\
6 & 3.240019 & -5.574532 & 0.744077 \\
6 & 2.223532 & -1.901109 & 1.059152 \\
7 & 1.603579 & -1.649631 & -0.251998 \\
6 & 0.534695 & -2.456280 & -0.763539 \\
6 & -0.694807 & -2.579500 & -0.133856 \\
6 & -1.750006 & -3.296959 & -0.781453 \\
6 & -1.519718 & -3.901807 & -2.051868 \\
6 & -0.245453 & -3.774175 & -2.649572 \\
6 & 0.743183 & -3.061204 & -2.028809 \\
6 & -3.046150 & -3.405110 & -0.211789 \\
6 & -4.046400 & -4.085466 & -0.860517 \\
6 & -3.806151 & -4.702990 & -2.107033 \\
6 & -2.568557 & -4.607760 & -2.689962 \\
6 & -0.922537 & -2.042250 & 1.235078
\end{tabular}




\begin{tabular}{|c|c|c|c|}
\hline 6 & -1.227335 & -0.708719 & 1.469986 \\
\hline 6 & -1.430790 & -0.274205 & 2.808997 \\
\hline 6 & -1.344553 & -1.142207 & 3.863959 \\
\hline 6 & -1.065037 & -2.511838 & 3.655161 \\
\hline 6 & -0.854341 & -2.973482 & 2.323925 \\
\hline 6 & -1.000821 & -3.427927 & 4.732810 \\
\hline 6 & -0.754246 & -4.758599 & 4.507994 \\
\hline 6 & -0.550299 & -5.223259 & 3.191701 \\
\hline 6 & -0.587218 & -4.354359 & 2.130052 \\
\hline 15 & -1.503109 & 0.447131 & 0.033865 \\
\hline 4 & -0.116179 & 0.798528 & -2.017043 \\
\hline 6 & -0.695005 & -0.506348 & -3.359074 \\
\hline 8 & -1.027489 & -1.121059 & -4.276351 \\
\hline 15 & 1.797063 & -0.191048 & -1.114465 \\
\hline 8 & 2.518699 & 0.675069 & 0.104750 \\
\hline 6 & 3.315337 & 1.746199 & -0.255849 \\
\hline 6 & 4.597241 & 1.512006 & -0.716700 \\
\hline 6 & 5.384460 & 2.636085 & -1.125301 \\
\hline 6 & 4.861927 & 3.954102 & -0.957140 \\
\hline 6 & 3.561558 & 4.126643 & -0.426786 \\
\hline 6 & 2.789528 & 3.043938 & -0.099684 \\
\hline 6 & 5.643471 & 5.068585 & -1.348166 \\
\hline 6 & 6.882889 & 4.899301 & -1.909075 \\
\hline 6 & 7.386577 & 3.597321 & -2.112449 \\
\hline 6 & 6.658856 & 2.497335 & -1.732055 \\
\hline 6 & 5.074692 & 0.113490 & -0.827236 \\
\hline 6 & 4.320309 & -0.795381 & -1.548311 \\
\hline 6 & 4.734697 & -2.125569 & -1.753238 \\
\hline 6 & 5.894053 & -2.571384 & -1.178891 \\
\hline 6 & 6.666800 & -1.715212 & -0.358885 \\
\hline 6 & 6.255461 & -0.360700 & -0.170121 \\
\hline 6 & 7.018433 & 0.450167 & 0.709035 \\
\hline 6 & 8.136725 & -0.040814 & 1.335515 \\
\hline 6 & 8.558632 & -1.369680 & 1.119509 \\
\hline 6 & 7.832752 & -2.186917 & 0.292066 \\
\hline 8 & 3.121497 & -0.407245 & -2.108554 \\
\hline 6 & -3.276392 & 0.099793 & -0.315060 \\
\hline 6 & -4.201331 & -0.041127 & 0.719250 \\
\hline 6 & -5.552464 & -0.258387 & 0.454254 \\
\hline 6 & -5.956446 & -0.329950 & -0.884979 \\
\hline 6 & -5.056705 & -0.189561 & -1.944068 \\
\hline 6 & -3.711089 & 0.022983 & -1.632439 \\
\hline 6 & -6.520960 & -0.389069 & 1.628536 \\
\hline 6 & -5.464694 & -0.321364 & -3.409201 \\
\hline 6 & -1.589370 & 2.079397 & 0.873783 \\
\hline 6 & -2.680490 & 2.924820 & 0.691076 \\
\hline 6 & -2.722842 & 4.183340 & 1.296477 \\
\hline 6 & -1.650428 & 4.552718 & 2.110417 \\
\hline 6 & -0.541089 & 3.725082 & 2.322435 \\
\hline 6 & -0.511310 & 2.498232 & 1.659441 \\
\hline 6 & -3.915589 & 5.122936 & 1.126102 \\
\hline 6 & 0.565455 & 4.166405 & 3.280962 \\
\hline 6 & -0.683638 & 2.284822 & -3.050416 \\
\hline 8 & -1.029123 & 3.235100 & -3.612679 \\
\hline 1 & 6.700477 & 1.472785 & 0.895532 \\
\hline 1 & -1.671954 & 0.767675 & 3.003572 \\
\hline
\end{tabular}




\begin{tabular}{|c|c|c|c|}
\hline 1 & 8.132407 & -3.221060 & 0.127447 \\
\hline & -1.160494 & -3.052272 & 5.742701 \\
\hline & 5.228840 & 6.065381 & -1.203830 \\
\hline & -3.248958 & -2.925203 & 0.744695 \\
\hline 1 & -5.036544 & -4.144384 & -0.413228 \\
\hline & 3.171924 & 5.136808 & -0.308236 \\
\hline 1 & -1.509097 & -0.788093 & 4.881039 \\
\hline & 6.222966 & -3.599361 & -1.323085 \\
\hline 1 & 8.358405 & 3.462769 & -2.582052 \\
\hline 1 & 4.115930 & -2.771432 & -2.372554 \\
\hline & -2.369989 & -5.067200 & -3.657285 \\
\hline 1 & -0.070241 & -4.232668 & -3.621500 \\
\hline 1 & -0.339985 & -6.276022 & 3.016668 \\
\hline 1 & 7.055775 & 1.501042 & -1.908530 \\
\hline 1 & -0.399773 & -4.727175 & 1.125726 \\
\hline 1 & 1.716689 & -2.938706 & -2.501368 \\
\hline 1 & 7.471894 & 5.762267 & -2.210783 \\
\hline 1 & 8.699805 & 0.600195 & 2.010198 \\
\hline 1 & -4.607231 & -5.243806 & -2.606266 \\
\hline 1 & -0.715149 & -5.457065 & 5.340964 \\
\hline 1 & 9.449331 & -1.744068 & 1.618589 \\
\hline 1 & 1.775229 & 3.156079 & 0.279604 \\
\hline 1 & -3.859767 & 0.030582 & 1.751445 \\
\hline 1 & -2.976332 & 0.132316 & -2.427026 \\
\hline 1 & -7.006376 & -0.498701 & -1.107189 \\
\hline 1 & -3.507374 & 2.591992 & 0.069564 \\
\hline 1 & 0.342307 & 1.830550 & 1.777510 \\
\hline 1 & -1.683376 & 5.520762 & 2.610499 \\
\hline 1 & 3.147112 & -1.311399 & 1.090881 \\
\hline 1 & 1.590934 & -1.505480 & 1.870257 \\
\hline 1 & 2.360802 & -2.993325 & 3.478599 \\
\hline 1 & 2.856555 & -3.992125 & -0.661786 \\
\hline 1 & 3.030931 & -5.283590 & 4.119128 \\
\hline 1 & 3.474278 & -6.300166 & -0.032293 \\
\hline 1 & 3.575939 & -6.959438 & 2.360635 \\
\hline 1 & 0.469137 & 2.028272 & -1.164942 \\
\hline 6 & -4.969171 & 4.552787 & 0.177903 \\
\hline 6 & -4.576587 & 5.351168 & 2.490090 \\
\hline 6 & -3.438685 & 6.463567 & 0.558452 \\
\hline 6 & 1.607980 & 3.072378 & 3.507846 \\
\hline 6 & 1.271141 & 5.404848 & 2.720159 \\
\hline 6 & -0.050239 & 4.507341 & 4.643498 \\
\hline 6 & -6.980233 & -0.302607 & -3.594396 \\
\hline 6 & -4.870806 & 0.825911 & -4.235024 \\
\hline 6 & -4.926156 & -1.656935 & -3.936049 \\
\hline 6 & -7.942707 & -0.717381 & 1.180445 \\
\hline 6 & -6.048845 & -1.509530 & 2.563114 \\
\hline 6 & -6.554329 & 0.938878 & 2.395107 \\
\hline 1 & -5.806800 & 5.256605 & 0.093483 \\
\hline 1 & -4.569196 & 4.392471 & -0.831782 \\
\hline 1 & -5.376223 & 3.597574 & 0.538408 \\
\hline 1 & 2.353356 & 3.421465 & 4.233465 \\
\hline 1 & 1.155596 & 2.156189 & 3.911999 \\
\hline 1 & 2.149918 & 2.810051 & 2.589828 \\
\hline 1 & 0.736711 & 4.799830 & 5.351386 \\
\hline 1 & -0.763806 & 5.337659 & 4.585190 \\
\hline
\end{tabular}




$\begin{array}{rrrr}1 & -0.577718 & 3.640928 & 5.063912 \\ 1 & 2.050209 & 5.748497 & 3.413975 \\ 1 & 1.752894 & 5.181845 & 1.758924 \\ 1 & 0.573985 & 6.236636 & 2.561071 \\ 1 & -5.434798 & 6.029351 & 2.390120 \\ 1 & -4.940865 & 4.405012 & 2.912272 \\ 1 & -3.883670 & 5.796317 & 3.214619 \\ 1 & -4.289988 & 7.144238 & 0.423269 \\ 1 & -2.720559 & 6.961386 & 1.221074 \\ 1 & -2.955494 & 6.327565 & -0.417513 \\ 1 & -7.221774 & -0.364678 & -4.662930 \\ 1 & -7.468051 & -1.154321 & -3.103542 \\ 1 & -7.427910 & 0.621439 & -3.205485 \\ 1 & -5.196595 & 0.743596 & -5.280230 \\ 1 & -5.199293 & 1.801711 & -3.853921 \\ 1 & -3.773089 & 0.819319 & -4.239174 \\ 1 & -5.182916 & -1.784655 & -4.996728 \\ 1 & -3.833991 & -1.719971 & -3.841407 \\ 1 & -5.354475 & -2.498284 & -3.374970 \\ 1 & -8.593443 & -0.813396 & 2.058787 \\ 1 & -8.367036 & 0.070086 & 0.544657 \\ 1 & -7.988799 & -1.666231 & 0.630047 \\ 1 & -7.211544 & 0.861006 & 3.271857 \\ 1 & -5.558021 & 1.236127 & 2.747434 \\ 1 & -6.937959 & 1.746877 & 1.757770 \\ 1 & -6.735861 & -1.609479 & 3.414127 \\ 1 & -6.023584 & -2.473305 & 2.037441 \\ 1 & -5.045985 & -1.326367 & 2.969516\end{array}$

$1_{\text {C12- }} \psi_{-122 s 3 a 4 a}$

Number of imaginary frequencies : 0

The smallest frequencies are : $9.4350 \quad 16.2779 \quad 17.3123 \mathrm{~cm}(-1)$

Electronic energy : $\quad \mathrm{HF}=-4125.4617875$

Zero-point correction $=\quad 1.299604$ (Hartree/Particle $)$

Thermal correction to Energy= $\quad 1.378148$

Thermal correction to Enthalpy= $\quad 1.379092$

Thermal correction to Gibbs Free Energy= $\quad 1.185304$

Sum of electronic and zero-point Energies $=\quad-4124.162184$

Sum of electronic and thermal Energies $=\quad-4124.083639$

Sum of electronic and thermal Enthalpies $=\quad-4124.082695$

Sum of electronic and thermal Free Energies $=\quad-4124.276484$

Cartesian Coordinates

$\begin{array}{rrrr}6 & 3.222118 & -6.063725 & 1.937447 \\ 6 & 2.959586 & -5.148355 & 2.952647 \\ 6 & 2.611096 & -3.840961 & 2.636863 \\ 6 & 2.538788 & -3.417464 & 1.307959 \\ 6 & 2.823024 & -4.336541 & 0.297787 \\ 6 & 3.152427 & -5.652326 & 0.610049 \\ 6 & 2.214828 & -1.970459 & 1.048342 \\ 7 & 1.584404 & -1.673606 & -0.247884 \\ 6 & 0.491474 & -2.444514 & -0.763459 \\ 6 & -0.732674 & -2.554688 & -0.121434 \\ 6 & -1.816584 & -3.217533 & -0.779075\end{array}$




\begin{tabular}{|c|c|c|c|}
\hline 6 & -1.625520 & -3.767934 & -2.080241 \\
\hline 6 & -0.353269 & -3.666021 & -2.686258 \\
\hline f & 0.667311 & -3.015112 & -2.049299 \\
\hline 6 & -3.107834 & -3.306228 & -0.195539 \\
\hline f & -4.145833 & -3.902831 & -0.866323 \\
\hline b & -3.946600 & -4.462111 & -2.147581 \\
\hline 6 & -2.711382 & -4.393668 & -2.740093 \\
\hline 6 & -0.941029 & -2.035164 & 1.257401 \\
\hline 6 & -1.243614 & -0.703715 & 1.506652 \\
\hline 6 & -1.448292 & -0.284105 & 2.850512 \\
\hline 6 & -1.357652 & -1.162834 & 3.895998 \\
\hline 6 & -1.078201 & -2.530426 & 3.671984 \\
\hline 6 & -0.874832 & -2.978690 & 2.335039 \\
\hline 6 & -1.013742 & -3.458850 & 4.738698 \\
\hline 6 & -0.777355 & -4.788764 & 4.497345 \\
\hline 6 & -0.585855 & -5.240483 & 3.174830 \\
\hline 6 & -0.621591 & -4.359196 & 2.123146 \\
\hline 5 & -1.513481 & 0.462230 & 0.077890 \\
\hline 45 & -0.095312 & 0.839435 & -1.951923 \\
\hline 6 & -0.589581 & -0.395086 & -3.390201 \\
\hline 8 & -0.818661 & -0.962841 & -4.367636 \\
\hline 15 & 1.798563 & -0.200595 & -1.080509 \\
\hline 8 & 2.554631 & 0.620543 & 0.146978 \\
\hline 6 & 3.354404 & 1.693153 & -0.201768 \\
\hline 6 & 4.622779 & 1.457795 & -0.697889 \\
\hline 6 & 5.410944 & 2.583922 & -1.098430 \\
\hline 6 & 4.905412 & 3.902173 & -0.885940 \\
\hline 6 & 3.620708 & & -0.318101 \\
\hline 6 & 2.845889 & 2.990985 & 0.001617 \\
\hline 6 & 5.687562 & 5.018331 & -1.270887 \\
\hline 6 & 6.910547 & 4.850998 & -1.867381 \\
\hline 6 & 7.396865 & 3.549684 & -2.113123 \\
\hline 6 & 6.668599 & 2.447704 & -1.739610 \\
\hline 6 & 5.084691 & 0.057519 & -0.846083 \\
\hline 6 & 4.306246 & -0.832042 & -1.565611 \\
\hline 6 & 4.700006 & -2.164267 & -1.795976 \\
\hline 6 & 5.864991 & -2.631720 & -1.251009 \\
\hline 6 & 6.665169 & -1.795602 & -0.436722 \\
\hline 6 & 6.273639 & -0.439175 & -0.220992 \\
\hline 6 & 7.064879 & 0.350729 & 0.652212 \\
\hline 6 & 8.190541 & -0.161422 & 1.247733 \\
\hline 6 & 8.591997 & -1.492051 & 1.005311 \\
\hline 6 & 7.838974 & -2.289557 & 0.182912 \\
\hline 8 & 3.102615 & -0.421379 & -2.098995 \\
\hline 6 & -3.295740 & 0.139516 & -0.260082 \\
\hline 6 & -4.224690 & 0.084009 & 0.782307 \\
\hline 6 & -5.572372 & -0.162232 & 0.528538 \\
\hline 6 & -5.959412 & -0.350098 & -0.804232 \\
\hline 6 & -5.060139 & -0.297136 & -1.870201 \\
\hline 6 & -3.716463 & -0.049205 & -1.572591 \\
\hline 6 & -6.601728 & -0.285630 & 1.650846 \\
\hline 6 & -5.555985 & -0.478485 & -3.303568 \\
\hline 6 & -1.552637 & 2.099671 & 0.909417 \\
\hline 6 & -2.557960 & 3.014479 & 0.631769 \\
\hline 6 & -2.547266 & 4.297350 & 1.197746 \\
\hline 6 & -1.505476 & 4.620414 & 2.062268 \\
\hline
\end{tabular}




\begin{tabular}{|c|c|c|c|}
\hline ) & -0.469822 & 3.719900 & 2.362744 \\
\hline & -0.492573 & 2.472897 & 1.747548 \\
\hline & -3.688540 & 5.255905 & 0.866376 \\
\hline & 0.608497 & 4.115972 & 3.371289 \\
\hline & -0.692165 & 2.381001 & -2.885070 \\
\hline & -1.060912 & 3.363664 & -3.371389 \\
\hline & 6.763569 & 1.374333 & 0.859287 \\
\hline & -1.690302 & 0.755445 & 3.057008 \\
\hline & 8.122847 & -3.324737 & -0.001812 \\
\hline & -1.166316 & -3.093956 & 5.753625 \\
\hline & 5.286323 & 6.015212 & -1.093088 \\
\hline & -3.276902 & -2.870859 & 0.787651 \\
\hline & -5.133977 & -3.936998 & -0.411229 \\
\hline & 3.244567 & 5.084554 & -0.164551 \\
\hline & -1.520736 & -0.819863 & 4.917139 \\
\hline & 6.177811 & -3.661707 & -1.415142 \\
\hline & 8.355329 & 3.417476 & -2.610064 \\
\hline & 4.060626 & -2.794477 & -2.410358 \\
\hline & -2.543186 & -4.814200 & -3.730588 \\
\hline & -0.205691 & -4.089098 & -3.678590 \\
\hline & -0.386164 & -6.293081 & 2.986723 \\
\hline & 7.051407 & 1.451985 & -1.948117 \\
\hline & -0.443560 & -4.721225 & 1.113013 \\
\hline & 1.638820 & -2.908594 & -2.529867 \\
\hline & 7.499750 & 5.715476 & -2.164359 \\
\hline & 8.775557 & 0.464070 & 1.918308 \\
\hline & -4.775290 & -4.944006 & -2.662394 \\
\hline & -0.738474 & -5.496746 & 5.322255 \\
\hline & 9.488663 & -1.883390 & 1.480109 \\
\hline & 1.842200 & 3.102306 & 0.408934 \\
\hline & -3.882815 & 0.237635 & 1.803082 \\
\hline & -2.970539 & -0.005813 & -2.362600 \\
\hline & -7.009709 & -0.548840 & -1.017594 \\
\hline & -3.369463 & 2.727118 & -0.036105 \\
\hline & 0.304640 & 1.754995 & 1.935458 \\
\hline & -1.489692 & 5.602263 & 2.530741 \\
\hline & 3.147019 & -1.394899 & 1.086971 \\
\hline & 1.595462 & -1.589661 & 1.876765 \\
\hline & 2.373628 & -3.135372 & 3.433663 \\
\hline & 2.783955 & -4.020603 & -0.741893 \\
\hline & 3.002221 & -5.458189 & 3.995026 \\
\hline & 3.358398 & -6.359322 & -0.191147 \\
\hline & 3.479685 & -7.092692 & 2.179145 \\
\hline & 0.500808 & 2.026433 & -1.047448 \\
\hline & -3.539582 & 6.604334 & 1.565721 \\
\hline & -3.723553 & 5.502231 & -0.646094 \\
\hline & -5.015767 & 4.628847 & 1.311033 \\
\hline & 1.604880 & 2.987232 & 3.631692 \\
\hline & 1.380848 & 5.335527 & 2.859295 \\
\hline & -0.060337 & 4.463515 & 4.706945 \\
\hline & -4.401542 & -0.573025 & -4.298917 \\
\hline & -6.381658 & -1.763572 & -3.419543 \\
\hline & -6.428629 & 0.723449 & -3.681530 \\
\hline & -6.032007 & 0.138477 & 3.003671 \\
\hline & -7.823645 & 0.590177 & 1.357337 \\
\hline & -7.039131 & -1.751755 & 1.750800 \\
\hline
\end{tabular}




$\begin{array}{rrrr}1 & -4.380192 & 7.255007 & 1.293670 \\ 1 & -3.543153 & 6.504037 & 2.658925 \\ 1 & -2.616686 & 7.119390 & 1.269401 \\ 1 & 2.340257 & 3.312717 & 4.378216 \\ 1 & 1.110597 & 2.088490 & 4.025547 \\ 1 & 2.162515 & 2.703296 & 2.729261 \\ 1 & 0.699254 & 4.729916 & 5.454118 \\ 1 & -0.749258 & 5.311973 & 4.619699 \\ 1 & -0.629782 & 3.608085 & 5.093997 \\ 1 & 2.138492 & 5.642543 & 3.592804 \\ 1 & 1.899158 & 5.107121 & 1.918525 \\ 1 & 0.723231 & 6.194746 & 2.680692 \\ 1 & -4.535289 & 6.197273 & -0.899991 \\ 1 & -2.779595 & 5.939470 & -0.995961 \\ 1 & -3.890338 & 4.577802 & -1.211732 \\ 1 & -5.852559 & 5.301556 & 1.079345 \\ 1 & -5.208501 & 3.673587 & 0.806034 \\ 1 & -5.019924 & 4.442879 & 2.393342 \\ 1 & -4.797142 & -0.734445 & -5.309577 \\ 1 & -3.799429 & 0.345133 & -4.328852 \\ 1 & -3.734549 & -1.413164 & -4.062464 \\ 1 & -6.725959 & -1.898685 & -4.453538 \\ 1 & -5.778212 & -2.639120 & -3.144955 \\ 1 & -7.271675 & -1.750614 & -2.778842 \\ 1 & -6.794960 & 0.622273 & -4.712188 \\ 1 & -7.301629 & 0.816768 & -3.023337 \\ 1 & -5.857999 & 1.659080 & -3.615658 \\ 1 & -6.812115 & 0.063105 & 3.771651 \\ 1 & -5.198798 & -0.501764 & 3.322741 \\ 1 & -5.678717 & 1.178364 & 2.988301 \\ 1 & -7.785477 & -1.878192 & 2.546973 \\ 1 & -7.484673 & -2.109151 & 0.813695 \\ 1 & -6.182728 & -2.398553 & 1.985231 \\ 1 & -8.553829 & 0.504091 & 2.172890 \\ 1 & -7.542267 & 1.647332 & 1.267760 \\ 1 & -8.334710 & 0.300424 & 0.431829\end{array}$

$1_{\mathrm{C} 12-} \psi_{-12253 a 4 s}$

Number of imaginary frequencies : 0

The smallest frequencies are : $14.0946 \quad 16.155720 .9921 \mathrm{~cm}(-1)$

Electronic energy : $\quad H F=-4125.4622275$

Zero-point correction $=\quad 1.303213$ (Hartree/Particle)

Thermal correction to Energy= $\quad 1.380757$

Thermal correction to Enthalpy= $\quad 1.381701$

Thermal correction to Gibbs Free Energy= 1.193039

Sum of electronic and zero-point Energies $=\quad-4124.159014$

Sum of electronic and thermal Energies $=\quad-4124.081470$

Sum of electronic and thermal Enthalpies $=\quad-4124.080526$

Sum of electronic and thermal Free Energies $=\quad-4124.269188$

\section{Cartesian Coordinates}

$\begin{array}{llll}6 & 3.396343 & -6.071062 & 1.742896 \\ 6 & 3.187052 & -5.178461 & 2.790525 \\ 6 & 2.808681 & -3.868916 & 2.522849\end{array}$




\begin{tabular}{|c|c|c|c|}
\hline 6 & 2.650855 & -3.419644 & 1.209486 \\
\hline 6 & 2.885490 & -4.314738 & 0.166096 \\
\hline & 3.245466 & -5.633296 & 0.431209 \\
\hline & 2.287834 & -1.972303 & 1.005619 \\
\hline & 1.633154 & -1.641160 & -0.269853 \\
\hline & 0.536721 & -2.406752 & -0.785464 \\
\hline & -0.669774 & -2.551902 & -0.117912 \\
\hline & -1.756429 & -3.223422 & -0.762343 \\
\hline & -1.591384 & -3.728372 & -2.085248 \\
\hline 6 & -0.339143 & -3.581308 & -2.722905 \\
\hline 6 & 0.688432 & -2.935579 & -2.091947 \\
\hline 6 & -3.025716 & -3.366478 & -0.142429 \\
\hline 6 & -4.067560 & -3.971631 & -0.799484 \\
\hline & -3.895341 & -4.480286 & -2.105515 \\
\hline 6 & -2.681844 & -4.358623 & -2.733184 \\
\hline 6 & -0.858933 & -2.043738 & 1.268045 \\
\hline 6 & -1.183673 & -0.717941 & 1.523381 \\
\hline 6 & -1.370992 & -0.301624 & 2.869845 \\
\hline 6 & -1.244308 & -1.178908 & 3.913358 \\
\hline 6 & -0.939989 & -2.540112 & 3.684114 \\
\hline 6 & -0.750932 & -2.984148 & 2.343490 \\
\hline 6 & -0.831498 & -3.466537 & 4.749044 \\
\hline 6 & -0.563412 & -4.789484 & 4.502813 \\
\hline 6 & -0.385474 & -5.236925 & 3.176773 \\
\hline 6 & -0.466115 & -4.357606 & 2.125953 \\
\hline & -1.509504 & 0.433895 & 0.096608 \\
\hline 45 & -0.082295 & 0.900107 & -1.899430 \\
\hline 6 & -0.550580 & -0.296479 & -3.376714 \\
\hline 8 & -0.762816 & -0.843198 & -4.369800 \\
\hline 15 & 1.822954 & -0.141586 & -1.060087 \\
\hline 8 & 2.569904 & 0.656034 & 0.188785 \\
\hline 6 & 3.362455 & 1.742336 & -0.131860 \\
\hline 6 & 4.633150 & 1.529402 & -0.632564 \\
\hline 6 & 5.414621 & 2.671639 & -0.999358 \\
\hline 6 & 4.900787 & 3.980055 & -0.749117 \\
\hline 6 & 3.613369 & 4.127257 & -0.180707 \\
\hline 6 & 2.844596 & 3.030780 & 0.104511 \\
\hline 6 & 5.676940 & 5.111953 & -1.098817 \\
\hline 6 & 6.901867 & 4.969718 & -1.697712 \\
\hline 6 & 7.396296 & 3.679177 & -1.981236 \\
\hline 6 & 6.674200 & 2.562173 & -1.642046 \\
\hline 6 & 5.104958 & 0.137257 & -0.822099 \\
\hline 6 & 4.333423 & -0.735296 & -1.569282 \\
\hline 6 & 4.736189 & -2.056853 & -1.841463 \\
\hline 6 & 5.904149 & -2.532904 & -1.310460 \\
\hline 6 & 6.697923 & -1.716883 & -0.469851 \\
\hline 6 & 6.297292 & -0.370281 & -0.212575 \\
\hline 6 & 7.083315 & 0.397933 & 0.684319 \\
\hline 6 & 8.212519 & -0.124517 & 1.263905 \\
\hline 6 & 8.622730 & -1.444482 & 0.981182 \\
\hline 6 & 7.875034 & -2.221579 & 0.134766 \\
\hline 8 & 3.126066 & -0.317054 & -2.087934 \\
\hline 6 & -3.265503 & 0.005832 & -0.257273 \\
\hline 6 & -4.180764 & -0.146711 & 0.779734 \\
\hline 6 & -5.522844 & -0.438572 & 0.523363 \\
\hline 6 & -5.915653 & -0.562542 & -0.810090 \\
\hline
\end{tabular}




\begin{tabular}{|c|c|c|c|}
\hline 6 & -5.021739 & -0.414187 & -1.878673 \\
\hline 6 & -3.689503 & -0.130673 & -1.578736 \\
\hline & -6.482850 & -0.600228 & 1.700593 \\
\hline & -5.525161 & -0.531236 & -3.315859 \\
\hline & -1.676912 & 2.071400 & 0.914838 \\
\hline & -2.748506 & 2.898344 & 0.609038 \\
\hline & -2.838103 & 4.195361 & 1.131367 \\
\hline & -1.831555 & 4.623430 & 1.991096 \\
\hline 6 & -0.734173 & 3.812259 & 2.326259 \\
\hline 0 & -0.654847 & 2.549242 & 1.747580 \\
\hline 6 & -4.032836 & 5.059588 & 0.736489 \\
\hline 6 & 0.314518 & 4.329268 & 3.311172 \\
\hline 6 & -0.679091 & 2.464995 & -2.794965 \\
\hline 8 & -1.046947 & 3.458679 & -3.258909 \\
\hline 1 & 6.775237 & 1.412796 & 0.922141 \\
\hline 1 & -1.633997 & 0.732147 & 3.079148 \\
\hline 1 & 8.165948 & -3.248659 & -0.081479 \\
\hline 1 & -0.973748 & -3.105637 & 5.766886 \\
\hline 1 & 5.269360 & 6.100593 & -0.891859 \\
\hline 1 & -3.173336 & -2.970445 & 0.860704 \\
\hline 1 & -5.037877 & -4.054643 & -0.313134 \\
\hline 1 & 3.230540 & 5.130724 & 0.000355 \\
\hline 1 & -1.395754 & -0.839447 & 4.937440 \\
\hline 1 & 6.223927 & -3.555263 & -1.505803 \\
\hline 1 & 8.356370 & 3.567544 & -2.480123 \\
\hline 1 & 4.100763 & -2.671236 & -2.475620 \\
\hline 1 & -2.534105 & -4.742218 & -3.741803 \\
\hline 1 & -0.211954 & -3.969120 & -3.732335 \\
\hline 1 & -0.161362 & -6.283924 & 2.984915 \\
\hline 1 & 7.063728 & 1.575445 & -1.878922 \\
\hline 1 & -0.300192 & -4.714189 & 1.111694 \\
\hline 1 & 1.645816 & -2.798939 & -2.593249 \\
\hline 1 & 7.486486 & 5.846063 & -1.967741 \\
\hline 1 & 8.793591 & 0.484194 & 1.953085 \\
\hline 1 & -4.726828 & -4.968030 & -2.610154 \\
\hline 1 & -0.489025 & -5.495730 & 5.326741 \\
\hline 1 & 9.522099 & -1.844098 & 1.443828 \\
\hline 1 & 1.837521 & 3.124428 & 0.507715 \\
\hline 1 & -3.842669 & -0.033075 & 1.809570 \\
\hline 1 & -2.952539 & -0.011080 & -2.369585 \\
\hline 1 & -6.957417 & -0.782375 & -1.034271 \\
\hline 1 & -3.532486 & 2.538262 & -0.056551 \\
\hline 1 & 0.197565 & 1.902877 & 1.955639 \\
\hline 1 & -1.891174 & 5.619820 & 2.423703 \\
\hline 1 & 3.205850 & -1.375447 & 1.056053 \\
\hline 1 & 1.669464 & -1.638093 & 1.854589 \\
\hline 1 & 2.617713 & -3.181121 & 3.347409 \\
\hline 1 & 2.782029 & -3.980233 & -0.862966 \\
\hline 1 & 3.297250 & -5.507957 & 3.821812 \\
\hline 1 & 3.410690 & -6.321441 & -0.395443 \\
\hline 1 & 3.676949 & -7.101999 & 1.947635 \\
\hline 1 & 0.489329 & 2.061152 & -0.946499 \\
\hline 6 & -4.015722 & 6.425107 & 1.417113 \\
\hline 6 & -4.010703 & 5.275640 & -0.781108 \\
\hline 6 & -5.332311 & 4.343890 & 1.126513 \\
\hline 6 & 1.351458 & 3.265436 & 3.668992 \\
\hline
\end{tabular}




$\begin{array}{rrrr}6 & 1.041637 & 5.532007 & 2.701375 \\ 6 & -0.373956 & 4.759919 & 4.611937 \\ 6 & -4.378657 & -0.542505 & -4.324785 \\ 6 & -6.319233 & -1.828816 & -3.495977 \\ 6 & -6.430516 & 0.667773 & -3.619984 \\ 6 & -7.889897 & -0.989643 & 1.255651 \\ 6 & -5.963881 & -1.696499 & 2.639117 \\ 6 & -6.569457 & 0.727062 & 2.463017 \\ 1 & -4.894931 & 7.003391 & 1.106350 \\ 1 & -4.048422 & 6.339061 & 2.511100 \\ 1 & -3.126788 & 7.007983 & 1.143591 \\ 1 & 2.052111 & 3.671120 & 4.409592 \\ 1 & 0.885558 & 2.372577 & 4.108170 \\ 1 & 1.946711 & 2.948880 & 2.802546 \\ 1 & 0.372683 & 5.113269 & 5.335562 \\ 1 & -1.090787 & 5.574588 & 4.456731 \\ 1 & -0.914452 & 3.919924 & 5.067613 \\ 1 & 1.789463 & 5.924092 & 3.403871 \\ 1 & 1.563344 & 5.248796 & 1.777233 \\ 1 & 0.350756 & 6.348300 & 2.457855 \\ 1 & -4.851091 & 5.912972 & -1.088098 \\ 1 & -3.079832 & 5.763803 & -1.097093 \\ 1 & -4.093765 & 4.329938 & -1.330897 \\ 1 & -6.201419 & 4.950602 & 0.838409 \\ 1 & -5.432637 & 3.367860 & 0.634207 \\ 1 & -5.379745 & 4.178336 & 2.211136 \\ 1 & -4.780839 & -0.658321 & -5.339092 \\ 1 & -3.799592 & 0.390537 & -4.310461 \\ 1 & -3.689721 & -1.378157 & -4.140862 \\ 1 & -6.661385 & -1.920460 & -4.535403 \\ 1 & -5.693743 & -2.701673 & -3.265020 \\ 1 & -7.208443 & -1.870082 & -2.855728 \\ 1 & -6.804228 & 0.614911 & -4.651610 \\ 1 & -7.299160 & 0.702964 & -2.950482 \\ 1 & -5.881883 & 1.612234 & -3.506558 \\ 1 & -8.535570 & -1.108675 & 2.134895 \\ 1 & -8.346700 & -0.223636 & 0.616026 \\ 1 & -7.896165 & -1.942555 & 0.709994 \\ 1 & -7.227367 & 0.625054 & 3.336707 \\ 1 & -5.587354 & 1.062339 & 2.819965 \\ 1 & -6.979462 & 1.519591 & 1.822823 \\ 1 & -6.635813 & -1.807830 & 3.500763 \\ 1 & -5.918911 & -2.665203 & 2.123659 \\ 1 & -4.961362 & -1.478528 & 3.029263\end{array}$

$\mathbf{1}_{\text {C12- } \psi_{-122 s 34 a}}$

Number of imaginary frequencies : 0

The smallest frequencies are : $14.0418 \quad 15.9654 \quad 18.7983 \mathrm{~cm}(-1)$

Electronic energy : $\quad H F=-4125.4619298$

Zero-point correction $=\quad 1.300255($ Hartree/Particle $)$

Thermal correction to Energy= $\quad 1.378457$

Thermal correction to Enthalpy= $\quad 1.379401$

Thermal correction to Gibbs Free Energy $=\quad 1.188243$

Sum of electronic and zero-point Energies $=\quad-4124.161675$

Sum of electronic and thermal Energies $=\quad-4124.083473$ 
Sum of electronic and thermal Enthalpies=

Sum of electronic and thermal Free Energies=
$-4124.082529$

$-4124.273687$

Cartesian Coordinates

$\begin{array}{crrrr}6 & 3.184003 & -6.050822 & 1.946427 \\ 6 & 2.907082 & -5.129581 & 2.952425 \\ 6 & 2.558975 & -3.825157 & 2.624085 \\ 6 & 2.501501 & -3.410478 & 1.291691 \\ 6 & 2.799147 & -4.335664 & 0.290900 \\ 6 & 3.128210 & -5.648427 & 0.615557 \\ 6 & 2.181073 & -1.965339 & 1.018152 \\ 7 & 1.553773 & -1.678656 & -0.282305 \\ 6 & 0.466004 & -2.457435 & -0.797162 \\ 6 & -0.760765 & -2.566535 & -0.159896 \\ 6 & -1.834634 & -3.251704 & -0.811964 \\ 6 & -1.624921 & -3.843703 & -2.091907 \\ 6 & -0.352609 & -3.732227 & -2.696863 \\ 6 & 0.653498 & -3.046961 & -2.073046 \\ 6 & -3.129467 & -3.339323 & -0.236129 \\ 6 & -4.147752 & -3.990742 & -0.886450 \\ 6 & -3.927535 & -4.597928 & -2.141763 \\ 6 & -2.692148 & -4.519811 & -2.731923 \\ 6 & -0.969672 & -2.051693 & 1.220948 \\ 6 & -1.252053 & -0.718911 & 1.485278 \\ 6 & -1.446814 & -0.309345 & 2.833827 \\ 6 & -1.368540 & -1.199521 & 3.870337 \\ 6 & -1.107527 & -2.568292 & 3.632061 \\ 6 & -0.911236 & -3.006123 & 2.290651 \\ 6 & -1.050913 & -3.506558 & 4.690644 \\ 6 & -0.826312 & -4.836244 & 4.437808 \\ 6 & -0.638468 & -5.277566 & 3.111275 \\ 6 & -0.668253 & -4.386898 & 2.067399 \\ 15 & -1.505532 & 0.474335 & 0.077640 \\ 45 & -0.139346 & 0.826731 & -1.990467 \\ 6 & -0.750009 & -0.443087 & -3.351026 \\ 8 & -1.096877 & -1.035405 & -4.277770 \\ 15 & 1.764370 & -0.209371 & -1.121615 \\ 8 & 2.510014 & 0.621855 & 0.106949 \\ 6 & 3.314896 & 1.691305 & -0.239684 \\ 6 & 4.587620 & 1.452216 & -0.722920 \\ 6 & 5.381631 & 2.575508 & -1.120107 \\ 6 & 4.876255 & 3.895422 & -0.918121 \\ 6 & 3.586354 & 4.071517 & -0.363622 \\ 6 & 2.806863 & 2.991112 & -0.046228 \\ 6 & 5.664142 & 5.008789 & -1.299381 \\ 6 & 6.893086 & 4.837164 & -1.882257 \\ 6 & 7.379596 & 3.534093 & -2.117994 \\ 6 & 6.645506 & 2.434783 & -1.747977 \\ 6 & 5.048866 & 0.050991 & -0.863316 \\ 6 & 4.276002 & -0.838539 & -1.588785 \\ 6 & 4.671716 & -2.170805 & -1.815917 \\ 6 & 5.831613 & -2.638517 & -1.260387 \\ 6 & 6.624199 & -1.802835 & -0.438226 \\ 6 & 6.231476 & -0.446118 & -0.226704 \\ 6 & 7.014443 & 0.343573 & 0.654099\end{array}$




\begin{tabular}{|c|c|c|c|}
\hline 6 & 8.133703 & -0.169228 & 1.261057 \\
\hline & 8.536706 & -1.500167 & 1.022884 \\
\hline & 7.791486 & -2.297380 & 0.193105 \\
\hline & 3.076428 & -0.427984 & -2.131064 \\
\hline & -3.298952 & 0.209044 & -0.246659 \\
\hline & -4.222062 & 0.086492 & 0.799857 \\
\hline & -5.572489 & -0.118708 & 0.539586 \\
\hline & -5.981899 & -0.189378 & -0.803258 \\
\hline & -5.093643 & -0.054270 & -1.865680 \\
\hline & -3.740302 & 0.139208 & -1.558774 \\
\hline & -6.595709 & -0.313032 & 1.657723 \\
\hline & -5.510052 & -0.173039 & -3.329044 \\
\hline & -1.486679 & 2.095669 & 0.939464 \\
\hline & -2.487291 & 3.030755 & 0.719251 \\
\hline & -2.440699 & 4.296854 & 1.320030 \\
\hline & -1.372917 & 4.577364 & 2.167276 \\
\hline & -0.343836 & 3.652771 & 2.413750 \\
\hline & -0.397915 & 2.427293 & 1.758111 \\
\hline & -3.574951 & 5.281863 & 1.047033 \\
\hline & 0.755979 & 3.995734 & 3.418591 \\
\hline & -0.714556 & 2.344694 & -2.971929 \\
\hline & -1.067284 & 3.315094 & -3.493867 \\
\hline & 6.711469 & 1.367360 & 0.857972 \\
\hline & -1.671993 & 0.731881 & 3.050684 \\
\hline & 8.076510 & -3.332796 & 0.011483 \\
\hline & -1.199195 & -3.148785 & 5.708731 \\
\hline & 5.262796 & 6.007009 & -1.129525 \\
\hline 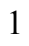 & -3.315225 & -2.865589 & 0.726609 \\
\hline & -5.136013 & -4.035790 & -0.433214 \\
\hline & 3.210170 & 5.083415 & -0.219226 \\
\hline & -1.525180 & -0.864426 & 4.895076 \\
\hline & 6.145819 & -3.668518 & -1.421826 \\
\hline 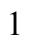 & 8.342951 & 3.398332 & -2.604409 \\
\hline & 4.037934 & -2.800781 & -2.436365 \\
\hline 1 & -2.509447 & -4.970261 & -3.706574 \\
\hline & -0.192905 & -4.179895 & -3.676460 \\
\hline 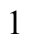 & -0.446567 & -6.329864 & 2.913715 \\
\hline 1 & 7.028748 & 1.437721 & -1.948984 \\
\hline & -0.493649 & -4.742467 & 1.054516 \\
\hline 1 & 1.625604 & -2.935948 & -2.551286 \\
\hline & 7.486919 & 5.699498 & -2.176220 \\
\hline 1 & 8.712317 & 0.455938 & 1.937462 \\
\hline & -4.742445 & -5.116257 & -2.642479 \\
\hline 1 & -0.792971 & -5.551712 & 5.256476 \\
\hline 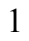 & 9.428296 & -1.891994 & 1.506752 \\
\hline 1 & 1.800524 & 3.105558 & 0.353459 \\
\hline 1 & -3.868087 & 0.151037 & 1.825863 \\
\hline & -3.007620 & 0.232756 & -2.357455 \\
\hline 1 & -7.036513 & -0.356244 & -1.014238 \\
\hline & -3.323802 & 2.772870 & 0.070563 \\
\hline 1 & 0.396108 & 1.693928 & 1.897692 \\
\hline 1 & -1.331065 & 5.543044 & 2.666840 \\
\hline 1 & 3.114745 & -1.391901 & 1.053023 \\
\hline 1 & 1.561659 & -1.575116 & 1.842296 \\
\hline 1 & 2.311269 & -3.114930 & 3.413564 \\
\hline & 2.771378 & -4.026255 & -0.751093 \\
\hline
\end{tabular}




\begin{tabular}{|c|c|c|c|}
\hline 1 & 2.938291 & -5.432473 & 3.997250 \\
\hline ] & 3.345147 & -6.360117 & -0.178558 \\
\hline & 3.441630 & -7.077429 & 2.197910 \\
\hline 1 & 0.484762 & 2.032615 & -1.131073 \\
\hline & -3.374808 & 6.614612 & 1.763377 \\
\hline & -3.661136 & 5.556625 & -0.458668 \\
\hline f & -4.896219 & 4.671092 & 1.530128 \\
\hline 6 & 1.753290 & 2.852989 & 3.602217 \\
\hline 6 & 1.523607 & 5.238459 & 2.958103 \\
\hline 6 & 0.110324 & 4.274177 & 4.781750 \\
\hline 6 & -7.026121 & -0.132785 & -3.505173 \\
\hline 6 & -4.904365 & 0.971503 & -4.150150 \\
\hline 6 & -4.991902 & -1.512065 & -3.866961 \\
\hline 6 & -5.996198 & -0.052674 & 3.038552 \\
\hline 6 & -7.779814 & 0.640361 & 1.468843 \\
\hline 6 & -7.097825 & -1.761133 & 1.619997 \\
\hline 1 & -4.211502 & 7.286021 & 1.532683 \\
\hline 1 & -3.342059 & 6.495109 & 2.854105 \\
\hline 1 & -2.452569 & 7.116625 & 1.443455 \\
\hline 1 & 2.504810 & 3.139085 & 4.348840 \\
\hline 1 & 1.264271 & 1.936746 & 3.960841 \\
\hline 1 & 2.290822 & 2.614331 & 2.674889 \\
\hline 1 & 0.882478 & 4.501674 & 5.528926 \\
\hline 1 & -0.579600 & 5.125772 & 4.748835 \\
\hline 1 & -0.453436 & 3.399740 & 5.133386 \\
\hline 1 & 2.293024 & 5.505004 & 3.695152 \\
\hline 1 & 2.027838 & 5.057591 & 1.999635 \\
\hline 1 & 0.866718 & 6.107814 & 2.834816 \\
\hline 1 & -4.463899 & 6.276425 & -0.668149 \\
\hline 1 & -2.720581 & 5.977454 & -0.836806 \\
\hline 1 & -3.873979 & 4.648183 & -1.034839 \\
\hline 1 & -5.730205 & 5.356951 & 1.328714 \\
\hline 1 & -5.118138 & 3.720211 & 1.028909 \\
\hline 1 & -4.868134 & 4.479972 & 2.611106 \\
\hline 1 & -7.275126 & -0.184455 & -4.572528 \\
\hline 1 & -7.522049 & -0.981413 & -3.017032 \\
\hline 1 & -7.458956 & 0.794352 & -3.106905 \\
\hline 1 & -5.238663 & 0.902527 & -5.193593 \\
\hline 1 & -5.214412 & 1.949567 & -3.759434 \\
\hline 1 & -3.806916 & 0.947189 & -4.162250 \\
\hline 1 & -5.254157 & -1.628497 & -4.927573 \\
\hline 1 & -3.900365 & -1.591380 & -3.777449 \\
\hline 1 & -5.429741 & -2.351358 & -3.310170 \\
\hline 1 & -6.769035 & -0.184113 & 3.806312 \\
\hline 1 & -5.179986 & -0.748636 & 3.274595 \\
\hline 1 & -5.609520 & 0.971441 & 3.128072 \\
\hline 1 & -7.846092 & -1.928855 & 2.406599 \\
\hline 1 & -7.562612 & -2.009415 & 0.657632 \\
\hline 1 & -6.270759 & -2.464668 & 1.785901 \\
\hline 1 & -8.506824 & 0.504980 & 2.280606 \\
\hline 1 & -7.451371 & 1.687505 & 1.482582 \\
\hline 1 & -8.309606 & 0.468171 & 0.524771 \\
\hline
\end{tabular}

$1_{\mathrm{C} 12-} \psi_{-12253545}$

Number of imaginary frequencies : 0 
The smallest frequencies are : $8.9063 \quad 14.9262 \quad 18.9082 \mathrm{~cm}(-1)$ Electronic energy : $\quad \mathrm{HF}=-4125.4621252$

Zero-point correction $=\quad 1.300070($ Hartree/Particle $)$

Thermal correction to Energy $=\quad 1.378291$

Thermal correction to Enthalpy= $\quad 1.379235$

Thermal correction to Gibbs Free Energy= $\quad 1.187431$

Sum of electronic and zero-point Energies $=\quad-4124.162055$

Sum of electronic and thermal Energies $=\quad-4124.083834$

Sum of electronic and thermal Enthalpies $=\quad-4124.082890$

Sum of electronic and thermal Free Energies $=\quad-4124.274694$

Cartesian Coordinates

$\begin{array}{ccccc}6 & 3.243087 & -6.038304 & 1.918009 \\ 6 & 2.965817 & -5.122920 & 2.929275 \\ 6 & 2.607735 & -3.819438 & 2.608116 \\ 6 & 2.540350 & -3.399728 & 1.277717 \\ 6 & 2.838180 & -4.318933 & 0.271561 \\ 6 & 3.177333 & -5.630939 & 0.589143 \\ 6 & 2.209355 & -1.955345 & 1.012963 \\ 7 & 1.578451 & -1.664768 & -0.284898 \\ 6 & 0.496512 & -2.449216 & -0.803243 \\ 6 & -0.726853 & -2.577329 & -0.162621 \\ 6 & -1.795134 & -3.270333 & -0.815645 \\ 6 & -1.583726 & -3.847319 & -2.102229 \\ 6 & -0.315533 & -3.714815 & -2.711564 \\ 6 & 0.685651 & -3.024762 & -2.085142 \\ 6 & -3.086266 & -3.379917 & -0.234820 \\ 6 & -4.098467 & -4.038776 & -0.887288 \\ 6 & -3.876099 & -4.631534 & -2.149034 \\ 6 & -2.644605 & -4.531746 & -2.743869 \\ 6 & -0.938916 & -2.069735 & 1.220361 \\ 6 & -1.234601 & -0.740092 & 1.486945 \\ 6 & -1.431473 & -0.334101 & 2.835876 \\ 6 & -1.344320 & -1.225562 & 3.870836 \\ 6 & -1.072248 & -2.591656 & 3.630081 \\ 6 & -0.871567 & -3.025324 & 2.287938 \\ 6 & -1.007342 & -3.531390 & 4.686963 \\ 6 & -0.770364 & -4.858385 & 4.431611 \\ 6 & -0.577831 & -5.295503 & 3.104270 \\ 6 & -0.615481 & -4.403322 & 2.062058 \\ 15 & -1.503723 & 0.446754 & 0.077572 \\ 45 & -0.134200 & 0.837634 & -1.973858 \\ 6 & -0.721189 & -0.421227 & -3.354653 \\ 8 & -1.057807 & -1.008721 & -4.288296 \\ 15 & 1.778067 & -0.187823 & -1.113695 \\ 8 & 2.516303 & 0.640395 & 0.121255 \\ 6 & 3.312283 & 1.718804 & -0.217552 \\ 6 & 4.587039 & 1.493452 & -0.701972 \\ 6 & 5.372168 & 2.625948 & -1.090489 \\ 6 & 4.856396 & 3.940300 & -0.878500 \\ 6 & 3.564867 & 4.101944 & -0.323377 \\ 6 & 2.793783 & 3.012998 & -0.014991 \\ 6 & 5.635733 & 5.062739 & -1.250716 \\ 6 & 6.866114 & 4.905176 & -1.834509 \\ 6 & 7.362854 & 3.607799 & -2.080158\end{array}$




\begin{tabular}{|c|c|c|c|}
\hline & 6.637333 & 2.499950 & -1.718896 \\
\hline & 5.059760 & 0.097020 & -0.851986 \\
\hline & 4.294693 & -0.793934 & -1.583945 \\
\hline & 4.701472 & -2.121276 & -1.820199 \\
\hline & 5.865038 & -2.583149 & -1.267445 \\
\hline & 6.650363 & -1.746551 & -0.439217 \\
\hline & 6.246227 & -0.394666 & -0.218369 \\
\hline & 7.022248 & 0.395412 & 0.668207 \\
\hline & 8.145662 & -0.112127 & 1.271872 \\
\hline & 8.559958 & -1.437907 & 1.024477 \\
\hline & 7.821673 & -2.235586 & 0.188957 \\
\hline & 3.091971 & -0.389658 & -2.124009 \\
\hline & -3.287275 & 0.141210 & -0.261135 \\
\hline & -4.209323 & 0.001401 & 0.776222 \\
\hline & -5.562441 & -0.210117 & 0.514526 \\
\hline & -5.972394 & -0.265364 & -0.823799 \\
\hline & -5.076672 & -0.118649 & -1.885359 \\
\hline & -3.728028 & 0.078188 & -1.577406 \\
\hline & -6.525891 & -0.365379 & 1.690415 \\
\hline & -5.491453 & -0.227749 & -3.350395 \\
\hline & -1.542199 & 2.070679 & 0.934260 \\
\hline & -2.567701 & 2.974628 & 0.698493 \\
\hline & -2.563514 & 4.245867 & 1.289685 \\
\hline & -1.511080 & 4.564317 & 2.143015 \\
\hline & -0.457315 & 3.672321 & 2.404929 \\
\hline & -0.471003 & 2.440256 & 1.759646 \\
\hline & -3.716473 & 5.199672 & 0.985592 \\
\hline & 0.630289 & 4.060093 & 3.406789 \\
\hline & -0.748172 & 2.364097 & -2.919843 \\
\hline & -1.134539 & 3.338377 & -3.409637 \\
\hline & 6.710604 & 1.415151 & 0.879134 \\
\hline & -1.666606 & 0.704549 & 3.054292 \\
\hline 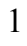 & 8.115507 & -3.267253 & 0.000174 \\
\hline & -1.159126 & -3.176964 & 5.705701 \\
\hline & 5.226539 & 6.056434 & -1.073213 \\
\hline & -3.275174 & -2.917837 & 0.733370 \\
\hline & -5.084419 & -4.099891 & -0.431316 \\
\hline ] & 3.180831 & 5.109643 & -0.170522 \\
\hline & -1.502802 & -0.893752 & 4.896362 \\
\hline ] & 6.187959 & -3.609308 & -1.435954 \\
\hline & 8.327402 & 3.483353 & -2.567235 \\
\hline 1 & 4.073204 & -2.751881 & -2.445587 \\
\hline ] & -2.460277 & -4.970815 & -3.723389 \\
\hline & -0.155004 & -4.151183 & -3.696113 \\
\hline 1 & -0.375790 & -6.345577 & 2.904947 \\
\hline & 7.028489 & 1.507461 & -1.927219 \\
\hline 1 & -0.436614 & -4.755067 & 1.048577 \\
\hline & 1.654832 & -2.898853 & -2.565748 \\
\hline 1 & 7.453298 & 5.774347 & -2.121625 \\
\hline 1 & 8.718818 & 0.513156 & 1.952800 \\
\hline & -4.686429 & -5.156039 & -2.650760 \\
\hline 1 & -0.730642 & -5.575077 & 5.248915 \\
\hline 1 & 9.454744 & -1.825555 & 1.505803 \\
\hline 1 & 1.785972 & 3.116493 & 0.384081 \\
\hline & -3.863314 & 0.059997 & 1.807833 \\
\hline 1 & -2.995025 & 0.182908 & -2.374299 \\
\hline
\end{tabular}




\begin{tabular}{|c|c|c|c|}
\hline 1 & -7.024033 & -0.426748 & -1.043306 \\
\hline & -3.388125 & 2.689855 & 0.040709 \\
\hline & 0.340953 & 1.730093 & 1.913502 \\
\hline & -1.500735 & 5.535447 & 2.633635 \\
\hline & 3.139129 & -1.375762 & 1.050508 \\
\hline & 1.588305 & -1.574582 & 1.840132 \\
\hline & 2.359644 & -3.114002 & 3.401771 \\
\hline & 2.801639 & -4.005963 & -0.769067 \\
\hline & 3.004593 & -5.429847 & 3.972661 \\
\hline & 3.394216 & -6.338073 & -0.209045 \\
\hline & 3.508602 & -7.064261 & 2.163871 \\
\hline 1 & 0.473855 & 2.038876 & -1.096099 \\
\hline 6 & -3.593560 & 6.521425 & 1.738796 \\
\hline & -3.735418 & 5.503701 & -0.516992 \\
\hline & -5.041433 & 4.540651 & 1.388708 \\
\hline & 1.646965 & 2.940472 & 3.624289 \\
\hline & 1.376843 & 5.303595 & 2.914441 \\
\hline & -0.024028 & 4.363778 & 4.760276 \\
\hline 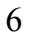 & -7.007703 & -0.202132 & -3.528216 \\
\hline & -4.897627 & 0.931197 & -4.160059 \\
\hline ) & -4.958565 & -1.555834 & -3.901404 \\
\hline & -7.954027 & -0.661973 & 1.240435 \\
\hline & -6.060323 & -1.522316 & 2.583073 \\
\hline & -6.540083 & 0.933833 & 2.504346 \\
\hline & -4.443989 & 7.168264 & 1.488940 \\
\hline & -3.599424 & 6.376574 & 2.827038 \\
\hline 1 & -2.678799 & 7.064108 & 1.467828 \\
\hline & 2.387446 & 3.257572 & 4.369378 \\
\hline & 1.171539 & 2.024227 & 4.000704 \\
\hline & 2.196409 & 2.689429 & 2.707235 \\
\hline & 0.742360 & 4.623278 & 5.502914 \\
\hline 1 & -0.727171 & 5.203178 & 4.704321 \\
\hline & -0.574545 & 3.490079 & 5.133764 \\
\hline ( & 2.140505 & 5.603606 & 3.644600 \\
\hline & 1.884859 & 5.106265 & 1.961140 \\
\hline I & 0.704177 & 6.157046 & 2.766943 \\
\hline 1 & -4.559068 & 6.189803 & -0.756726 \\
\hline & -2.796785 & 5.976264 & -0.833939 \\
\hline 1 & -3.870439 & 4.597820 & -1.120633 \\
\hline t & -5.882142 & 5.213366 & 1.171592 \\
\hline 1 & -5.219477 & 3.602748 & 0.846783 \\
\hline ] & -5.057521 & 4.314488 & 2.463222 \\
\hline ] & -7.254518 & -0.247271 & -4.596392 \\
\hline 1 & -7.495366 & -1.059934 & -3.047877 \\
\hline ] & -7.451169 & 0.717019 & -3.123179 \\
\hline 1 & -5.228131 & 0.867462 & -5.205089 \\
\hline & -5.220706 & 1.901870 & -3.761512 \\
\hline 1 & -3.799922 & 0.920670 & -4.168891 \\
\hline 1 & -5.222914 & -1.666457 & -4.962154 \\
\hline 1 & -3.865776 & -1.621919 & -3.815939 \\
\hline 1 & -5.383710 & -2.405657 & -3.350592 \\
\hline 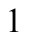 & -8.600972 & -0.777341 & 2.119233 \\
\hline 1 & -8.371383 & 0.150450 & 0.631890 \\
\hline & -8.015757 & -1.592576 & 0.661228 \\
\hline & -7.207382 & 0.835314 & 3.371327 \\
\hline & -5.542386 & 1.196713 & 2.877857 \\
\hline
\end{tabular}




$\begin{array}{lrrr}1 & -6.899959 & 1.773939 & 1.895331 \\ 1 & -6.739103 & -1.639975 & 3.438498 \\ 1 & -6.054799 & -2.468943 & 2.026725 \\ 1 & -5.050141 & -1.365589 & 2.982552\end{array}$

$1_{\mathrm{C} 12-} \psi_{-1 \mathrm{~s} 223 \mathrm{a} a \mathrm{a}}$

Number of imaginary frequencies : 0

The smallest frequencies are : $12.8646 \quad 15.5962 \quad 17.8599 \mathrm{~cm}(-1)$

Electronic energy : $\quad H F=-4125.4602146$

Zero-point correction $=\quad 1.300153$ (Hartree/Particle)

Thermal correction to Energy= $\quad 1.378360$

Thermal correction to Enthalpy= $\quad 1.379305$

Thermal correction to Gibbs Free Energy $=\quad 1.187506$

Sum of electronic and zero-point Energies $=\quad-4124.160062$

Sum of electronic and thermal Energies $=\quad-4124.081854$

Sum of electronic and thermal Enthalpies $=\quad-4124.080910$

Sum of electronic and thermal Free Energies $=\quad-4124.272708$

Cartesian Coordinates

$\begin{array}{cccc}6 & -1.023806 & 2.642597 & 1.811056 \\ 6 & -1.882499 & 2.034478 & 0.897654 \\ 6 & -3.009263 & 2.731492 & 0.451803 \\ 6 & -3.312106 & 4.000183 & 0.933173 \\ 6 & -2.465559 & 4.554543 & 1.902271 \\ 6 & -1.322260 & 3.898697 & 2.358388 \\ 15 & -1.504016 & 0.406328 & 0.121363 \\ 6 & -3.205171 & -0.165332 & -0.297682 \\ 6 & -4.148877 & -0.367618 & 0.712479 \\ 6 & -5.462487 & -0.711939 & 0.405605 \\ 6 & -5.799378 & -0.850122 & -0.947261 \\ 6 & -4.880149 & -0.664984 & -1.980832 \\ 6 & -3.572298 & -0.314928 & -1.629387 \\ 6 & -6.512751 & -0.968296 & 1.485202 \\ 6 & -7.739340 & -0.082197 & 1.245462 \\ 6 & -5.317614 & -0.795370 & -3.438413 \\ 6 & -6.180230 & 0.419975 & -3.796731 \\ 6 & -4.548593 & 4.764871 & 0.465480 \\ 6 & -4.131166 & 6.134673 & -0.079081 \\ 6 & -0.431437 & 4.469672 & 3.462406 \\ 6 & 1.016727 & 4.575976 & 2.976713 \\ 6 & -1.135616 & -0.715566 & 1.559898 \\ 6 & -0.735620 & -2.024499 & 1.315622 \\ 6 & -0.585648 & -2.948624 & 2.399705 \\ 6 & -0.800154 & -2.503434 & 3.736564 \\ 6 & -1.186906 & -1.161440 & 3.952751 \\ 6 & -1.362350 & -0.302908 & 2.900675 \\ 6 & -0.236535 & -4.309161 & 2.194182 \\ 6 & -0.114511 & -5.174501 & 3.252611 \\ 6 & -0.312012 & -4.724040 & 4.574930 \\ 6 & -0.645043 & -3.413820 & 4.809574 \\ 6 & -0.518484 & -2.532337 & -0.067880 \\ 6 & 0.679373 & -2.332676 & -0.737647 \\ 6 & 0.845814 & -2.835213 & -2.052957 \\ 6 & -0.157701 & -3.511338 & -2.690066\end{array}$




$\begin{array}{cccc}6 & -1.395551 & -3.729020 & -2.045115 \\ 6 & -1.574398 & -3.252234 & -0.713394 \\ 6 & -2.457673 & -4.402822 & -2.696477 \\ 6 & -3.657497 & -4.599508 & -2.061796 \\ 6 & -3.842919 & -4.124186 & -0.745481 \\ 6 & -2.829142 & -3.474303 & -0.086877 \\ 7 & 1.752513 & -1.541117 & -0.214030 \\ 15 & 1.910033 & -0.031656 & -0.993434 \\ 8 & 3.197909 & -0.179200 & -2.045789 \\ 6 & 4.421190 & -0.583484 & -1.553131 \\ 6 & 5.192653 & 0.291385 & -0.807968 \\ 6 & 6.402608 & -0.206030 & -0.225015 \\ 6 & 6.820661 & -1.542041 & -0.508189 \\ 6 & 6.026444 & -2.358292 & -1.347947 \\ 6 & 4.841927 & -1.893782 & -1.851667 \\ 6 & 7.190292 & 0.561346 & 0.671198 \\ 6 & 8.336561 & 0.048791 & 1.225499 \\ 6 & 8.763533 & -1.259943 & 0.916605 \\ 6 & 8.015154 & -2.036437 & 0.070265 \\ 6 & 4.704716 & 1.673902 & -0.592135 \\ 6 & 3.438126 & 1.861286 & -0.072088 \\ 6 & 2.903440 & 3.136260 & 0.190156 \\ 6 & 3.652422 & 4.249166 & -0.081600 \\ 6 & 4.935390 & 4.130215 & -0.666901 \\ 6 & 5.464488 & 2.834044 & -0.948906 \\ 6 & 5.691945 & 5.279438 & -1.003011 \\ 6 & 6.911293 & 5.166294 & -1.619171 \\ 6 & 7.419774 & 3.888693 & -1.934506 \\ 6 & 6.717376 & 2.755150 & -1.609041 \\ 6 & 2.667608 & 0.759748 & 0.249458 \\ 6 & -5.5 .502232 & 4.950797 & 1.650926 \\ 6 & -0.881182 & 5.856981 & 3.914617 \\ 1 & 6.869903 & 1.567473 & 0.928926 \\ 6 & -1.702122 & 0.710340 & 3.098588 \\ 1 & 8.318868 & -3.055425 & -0.165824 \\ 6 & -0.806596 & -3.051823 & 5.824148 \\ 6 & 2.416639 & -1.869637 & 1.056683 \\ 6 & -6.273391 & 6.257951 & -0.771233 \\ 6 & 2.833542 & -3.305189 & 1.240165 \\ 6 & 3.030449 & -3.758478 & 2.547013 \\ 6 & 3.460804 & -5.055191 & 2.797197 \\ 6 & 3.683009 & -5.931994 & 1.738721 \\ 6 & 3.490316 & -5.490926 & 0.433773 \\ 6 & 3.078201 & -4.184240 & 0.185870 \\ 45 & -0.026648 & 0.977311 & -1.794877 \\ 6 & -0.692105 & 2.558661 & -2.618585 \\ 6 & -1.127171 & 3.551692 & -3.021653 \\ 6 & -0.414010 & -0.183638 & -3.321367 \\ 6 & -5.983505 & -0.672094 & 2.887089 \\ 6 & -0.7259301 & -2.442657 & 1.436151 \\ 6 & -3.106600 & 0.924833\end{array}$




\begin{tabular}{|c|c|c|c|}
\hline 1 & -4.801502 & -4.271412 & -0.251368 \\
\hline 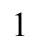 & 3.258083 & 5.242941 & 0.124937 \\
\hline 1 & -1.367448 & -0.823266 & 4.972649 \\
\hline & 6.359498 & -3.372133 & -1.564405 \\
\hline & 8.375082 & 3.800420 & -2.447064 \\
\hline & 4.206744 & -2.509218 & -2.484915 \\
\hline & -2.297917 & -4.760805 & -3.712750 \\
\hline & -0.020410 & -3.876166 & -3.706741 \\
\hline & 0.156625 & -6.211891 & 3.069522 \\
\hline 1 & 7.117738 & 1.778907 & -1.870160 \\
\hline 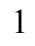 & -0.057084 & -4.666499 & 1.182454 \\
\hline & 1.794838 & -2.654188 & -2.556167 \\
\hline & 7.480688 & 6.055860 & -1.878327 \\
\hline & 8.918310 & 0.656525 & 1.914975 \\
\hline 1 & -4.466791 & -5.121876 & -2.567836 \\
\hline 1 & -0.203351 & -5.418599 & 5.404942 \\
\hline 1 & 9.676259 & -1.651768 & 1.359349 \\
\hline 1 & 1.899736 & 3.206776 & 0.602313 \\
\hline 1 & -3.845293 & -0.243264 & 1.749486 \\
\hline 1 & -2.817267 & -0.150169 & -2.394446 \\
\hline 1 & -6.824773 & -1.116070 & -1.203391 \\
\hline 1 & -3.654944 & 2.266494 & -0.287576 \\
\hline 1 & -0.120387 & 2.116598 & 2.127194 \\
\hline 1 & -2.714822 & 5.531992 & 2.309256 \\
\hline 1 & 3.312030 & -1.240805 & 1.118344 \\
\hline 1 & 1.785300 & -1.571840 & 1.909569 \\
\hline 1 & 2.826207 & -3.085182 & 3.380579 \\
\hline 1 & 2.940120 & -3.847136 & -0.838107 \\
\hline 1 & 3.601747 & -5.388203 & 3.823598 \\
\hline 1 & 3.663101 & -6.167045 & -0.401230 \\
\hline 1 & 4.004744 & -6.953424 & 1.930164 \\
\hline 1 & 0.470566 & 2.104989 & -0.761192 \\
\hline 1 & -6.178733 & 4.611785 & -0.941927 \\
\hline 1 & -4.676695 & 3.883367 & -1.532647 \\
\hline 1 & -5.659765 & 3.039289 & -0.315575 \\
\hline 1 & -0.209828 & 6.221574 & 4.702159 \\
\hline 1 & -0.852497 & 6.585178 & 3.093800 \\
\hline 1 & -1.896700 & 5.846421 & 4.330709 \\
\hline 1 & 1.646813 & 5.026111 & 3.755457 \\
\hline 1 & 1.446336 & 3.595065 & 2.741057 \\
\hline 1 & 1.085755 & 5.205600 & 2.079344 \\
\hline 1 & 0.128540 & 3.927554 & 5.493969 \\
\hline 1 & -1.515579 & 3.426591 & 5.043930 \\
\hline 1 & -0.110952 & 2.528609 & 4.434192 \\
\hline 1 & -6.398963 & 5.502760 & 1.338229 \\
\hline 1 & -5.824364 & 3.979211 & 2.049517 \\
\hline 1 & -5.035932 & 5.510921 & 2.470697 \\
\hline 1 & -5.011496 & 6.688841 & -0.431491 \\
\hline 1 & -3.637027 & 6.750724 & 0.681711 \\
\hline 1 & -3.438536 & 6.024790 & -0.923668 \\
\hline 1 & -4.480197 & -0.983827 & -5.420227 \\
\hline 1 & -3.537275 & 0.082552 & -4.376811 \\
\hline 1 & -3.450202 & -1.680429 & -4.154060 \\
\hline 1 & -6.406443 & -2.183623 & -4.699609 \\
\hline 1 & -5.544916 & -2.959020 & -3.351530 \\
\hline 1 & -7.062460 & -2.082856 & -3.063589 \\
\hline
\end{tabular}




$\begin{array}{rrrr}1 & -6.511070 & 0.364055 & -4.842832 \\ 1 & -7.074047 & 0.482436 & -3.162870 \\ 1 & -5.614409 & 1.352630 & -3.669253 \\ 1 & -6.771717 & -0.863813 & 3.626004 \\ 1 & -5.127615 & -1.308783 & 3.149227 \\ 1 & -5.676257 & 0.376607 & 2.994715 \\ 1 & -8.491160 & -0.255491 & 2.026958 \\ 1 & -7.467064 & 0.981164 & 1.269834 \\ 1 & -8.218611 & -0.283303 & 0.280100 \\ 1 & -7.704510 & -2.647874 & 2.186800 \\ 1 & -7.330667 & -2.725280 & 0.454933 \\ 1 & -6.072515 & -3.095945 & 1.652312\end{array}$

$1_{\text {C12- }} \psi_{-152 a 3 a 4 s}$

Number of imaginary frequencies : 0

The smallest frequencies are : $12.8324 \quad 15.9696 \quad 18.3391 \mathrm{~cm}(-1)$

Electronic energy : $\quad H F=-4125.4609966$

Zero-point correction $=\quad 1.300289($ Hartree/Particle $)$

Thermal correction to Energy= $\quad 1.378444$

Thermal correction to Enthalpy= $\quad 1.379389$

Thermal correction to Gibbs Free Energy= $\quad 1.187993$

Sum of electronic and zero-point Energies $=\quad-4124.160708$

Sum of electronic and thermal Energies $=\quad-4124.082552$

Sum of electronic and thermal Enthalpies $=\quad-4124.081608$

Sum of electronic and thermal Free Energies $=\quad-4124.273003$

Cartesian Coordinates

$\begin{array}{llll}6 & 3.711985 & -5.900958 & 1.769023 \\ 6 & 3.479619 & -5.022424 & 2.823837 \\ 6 & 3.042620 & -3.729005 & 2.567907 \\ 6 & 2.849447 & -3.280715 & 1.258809 \\ 6 & 3.103683 & -4.161563 & 0.208287 \\ 6 & 3.522391 & -5.465024 & 0.461870 \\ 6 & 2.425141 & -1.848253 & 1.068485 \\ 7 & 1.761643 & -1.529210 & -0.205089 \\ 6 & 0.694429 & -2.330557 & -0.725685 \\ 6 & -0.501911 & -2.535876 & -0.055180 \\ 6 & -1.550817 & -3.270176 & -0.695237 \\ 6 & -1.367206 & -3.757008 & -2.022508 \\ 6 & -0.132128 & -3.530559 & -2.669840 \\ 6 & 0.864978 & -2.840140 & -2.037738 \\ 6 & -2.803161 & -3.497400 & -0.066012 \\ 6 & -3.808665 & -4.168135 & -0.715960 \\ 6 & -3.617244 & -4.657711 & -2.026181 \\ 6 & -2.421416 & -4.451089 & -2.665340 \\ 6 & -0.728304 & -2.020045 & 1.323531 \\ 6 & -1.143700 & -0.713747 & 1.554544 \\ 6 & -1.385362 & -0.293766 & 2.890488 \\ 6 & -1.210440 & -1.143111 & 3.950133 \\ 6 & -0.808429 & -2.482645 & 3.746991 \\ 6 & -0.578233 & -2.935105 & 2.415242 \\ 6 & -0.653212 & -3.383634 & 4.827846 \\ 6 & -0.306067 & -4.692295 & 4.605411 \\ 6 & -0.094332 & -5.150450 & 3.287988\end{array}$




\begin{tabular}{|c|c|c|c|}
\hline 6 & -0.215647 & -4.293905 & 2.222314 \\
\hline 1 & -1.511832 & 0.393956 & 0.104173 \\
\hline 4 & -0.025917 & 0.969829 & -1.804007 \\
\hline 6 & -0.382321 & -0.197657 & -3.332599 \\
\hline 8 & -0.526696 & -0.745313 & -4.337433 \\
\hline 15 & 1.914008 & -0.023446 & -0.992160 \\
\hline 8 & 2.664809 & 0.778004 & 0.247922 \\
\hline 6 & 3.431938 & 1.880782 & -0.077471 \\
\hline 6 & 4.701135 & 1.695404 & -0.591602 \\
\hline 6 & 5.458201 & 2.856635 & -0.950716 \\
\hline 6 & 4.923123 & 4.152212 & -0.677187 \\
\hline 6 & 3.636904 & 4.269318 & -0.098624 \\
\hline 6 & 2.890990 & 3.154927 & 0.175288 \\
\hline 6 & 5.677150 & 5.302575 & -1.015062 \\
\hline 6 & 6.899804 & 5.190988 & -1.624922 \\
\hline 6 & 7.414255 & 3.913805 & -1.932100 \\
\hline 6 & 6.714369 & 2.779249 & -1.604761 \\
\hline 6 & 5.194455 & 0.313653 & -0.799725 \\
\hline 6 & 4.428784 & -0.567175 & -1.543931 \\
\hline 6 & 4.856638 & -1.876317 & -1.837397 \\
\hline 6 & 6.041784 & -2.333889 & -1.328801 \\
\hline 6 & 6.829387 & -1.511765 & -0.488549 \\
\hline 6 & 6.404409 & -0.176798 & -0.210913 \\
\hline 6 & 7.185126 & 0.596645 & 0.686155 \\
\hline 6 & 8.331329 & 0.090683 & 1.246604 \\
\hline 6 & 8.765305 & -1.217026 & 0.943091 \\
\hline 6 & 8.023788 & -1.999212 & 0.095932 \\
\hline 8 & 3.204719 & -0.170460 & -2.041085 \\
\hline 6 & -3.201535 & -0.202064 & -0.326443 \\
\hline 6 & -4.149460 & -0.389618 & 0.674045 \\
\hline 6 & -5.464755 & -0.744748 & 0.368501 \\
\hline 6 & -5.795704 & -0.909395 & -0.977428 \\
\hline 6 & -4.864044 & -0.739021 & -2.010074 \\
\hline 6 & -3.561947 & -0.379827 & -1.660795 \\
\hline 6 & -6.469442 & -0.900448 & 1.508040 \\
\hline 6 & -5.298393 & -0.897057 & -3.465962 \\
\hline 6 & -1.918106 & 2.019202 & 0.873610 \\
\hline 6 & -3.060696 & 2.697863 & 0.438978 \\
\hline 6 & -3.373363 & 3.965920 & 0.917464 \\
\hline 6 & -2.525033 & 4.534356 & 1.876581 \\
\hline 6 & -1.369783 & 3.894637 & 2.325243 \\
\hline 6 & -1.059343 & 2.641976 & 1.777345 \\
\hline 6 & -4.616517 & 4.720622 & 0.451053 \\
\hline 6 & -0.478915 & 4.479216 & 3.422060 \\
\hline 6 & -0.716117 & 2.540081 & -2.629721 \\
\hline 8 & -1.170787 & 3.523659 & -3.034031 \\
\hline 1 & 6.859176 & 1.602033 & 0.939789 \\
\hline 1 & -1.734939 & 0.718027 & 3.078584 \\
\hline 1 & 8.332867 & -3.017549 & -0.135969 \\
\hline 1 & -0.826452 & -3.015905 & 5.838415 \\
\hline 1 & 5.254027 & 6.280629 & -0.789726 \\
\hline 1 & -2.968499 & -3.118016 & 0.940702 \\
\hline 1 & -4.766224 & -4.318727 & -0.220965 \\
\hline 1 & 3.237690 & 5.262567 & 0.101071 \\
\hline 1 & -1.402175 & -0.799313 & 4.966112 \\
\hline 1 & 6.380202 & -3.346763 & -1.541442 \\
\hline
\end{tabular}




\begin{tabular}{|c|c|c|c|}
\hline & 8.372274 & 3.826590 & -2.439752 \\
\hline & 4.226311 & -2.496556 & -2.470810 \\
\hline & -2.258516 & -4.818205 & -3.677848 \\
\hline & 0.008894 & -3.901807 & -3.683680 \\
\hline & 0.187008 & -6.186767 & 3.114473 \\
\hline & 7.119498 & 1.803283 & -1.859461 \\
\hline & -0.025333 & -4.657140 & 1.214665 \\
\hline & 1.812186 & -2.653722 & -2.542483 \\
\hline & 7.467289 & 6.081387 & -1.885400 \\
\hline & 8.907557 & 0.702838 & 1.936801 \\
\hline & -4.419475 & -5.197755 & -2.524816 \\
\hline & -0.197678 & -5.379851 & 5.441255 \\
\hline & 9.677906 & -1.603609 & 1.390664 \\
\hline & 1.885005 & 3.223441 & 0.581998 \\
\hline & -3.862230 & -0.236939 & 1.714157 \\
\hline & -2.803419 & -0.226523 & -2.424898 \\
\hline & -6.816753 & -1.177812 & -1.240539 \\
\hline & -3.709220 & 2.221612 & -0.290852 \\
\hline & -0.146282 & 2.128622 & 2.086703 \\
\hline & -2.782002 & 5.510875 & 2.280952 \\
\hline & 3.316803 & -1.214095 & 1.129249 \\
\hline & 1.790213 & -1.550333 & 1.918628 \\
\hline & 2.829148 & -3.054969 & 3.398619 \\
\hline & 2.967569 & -3.828297 & -0.817203 \\
\hline ] & 3.617557 & -5.351695 & 3.851852 \\
\hline & 3.702644 & -6.142682 & -0.370302 \\
\hline & 4.038858 & -6.919890 & 1.965010 \\
\hline 1 & 0.457950 & 2.104519 & -0.771107 \\
\hline & -5.378443 & 3.962434 & -0.634887 \\
\hline 6 & -5.559378 & 4.927072 & 1.641691 \\
\hline 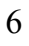 & -4.205209 & 6.081549 & -0.120249 \\
\hline 6 & -0.937875 & 5.865537 & 3.868062 \\
\hline 6 & -0.522888 & 3.547007 & 4.639437 \\
\hline 5 & 0.966883 & 4.593923 & 2.931254 \\
\hline 5 & -4.106495 & -0.902046 & -4.422030 \\
\hline & -6.058793 & -2.212371 & -3.658809 \\
\hline 6 & -6.211345 & 0.278719 & -3.832636 \\
\hline 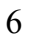 & -7.827371 & -1.400982 & 1.023396 \\
\hline 6 & -5.936474 & -1.903420 & 2.538164 \\
\hline 6 & -6.668224 & 0.465346 & 2.177073 \\
\hline l & -6.260757 & 4.540714 & -0.936917 \\
\hline 1 & -4.763665 & 3.803846 & -1.531006 \\
\hline 1 & -5.731065 & 2.981442 & -0.286316 \\
\hline 1 & -0.265699 & 6.240373 & 4.650067 \\
\hline ] & -0.919233 & 6.588832 & 3.042606 \\
\hline 1 & -1.951159 & 5.849343 & 4.289345 \\
\hline 1 & 1.594770 & 5.057039 & 3.704121 \\
\hline 1 & 1.404953 & 3.614695 & 2.704532 \\
\hline 1 & 1.028372 & 5.215370 & 2.027631 \\
\hline 1 & 0.092961 & 3.951458 & 5.454234 \\
\hline 1 & -1.549572 & 3.437131 & 5.013389 \\
\hline 1 & -0.141401 & 2.545553 & 4.402172 \\
\hline 1 & -6.459792 & 5.471535 & 1.326441 \\
\hline 1 & -5.876252 & 3.963143 & 2.062170 \\
\hline 1 & -5.087279 & 5.504384 & 2.446040 \\
\hline 1 & -5.089534 & 6.630066 & -0.471588 \\
\hline
\end{tabular}




$\begin{array}{rrrr}1 & -3.702507 & 6.709260 & 0.625276 \\ 1 & -3.521962 & 5.958613 & -0.970588 \\ 1 & -4.458846 & -1.062841 & -5.448769 \\ 1 & -3.564840 & 0.052835 & -4.413608 \\ 1 & -3.395150 & -1.705185 & -4.182127 \\ 1 & -6.347535 & -2.329310 & -4.711772 \\ 1 & -5.429836 & -3.068606 & -3.381053 \\ 1 & -6.977988 & -2.259515 & -3.063049 \\ 1 & -6.533753 & 0.204011 & -4.880179 \\ 1 & -7.111359 & 0.305608 & -3.205354 \\ 1 & -5.687182 & 1.235495 & -3.706290 \\ 1 & -8.505510 & -1.517372 & 1.878211 \\ 1 & -8.300621 & -0.698784 & 0.325290 \\ 1 & -7.747704 & -2.377956 & 0.528151 \\ 1 & -7.356023 & 0.381139 & 3.029475 \\ 1 & -5.722476 & 0.882710 & 2.546379 \\ 1 & -7.097037 & 1.186363 & 1.468131 \\ 1 & -6.646033 & -2.004726 & 3.370300 \\ 1 & -5.806386 & -2.896640 & 2.088141 \\ 1 & -4.971410 & -1.601342 & 2.964578\end{array}$

$1_{\mathrm{C} 12-} \psi_{-15223 s 4 a}$

Number of imaginary frequencies : 0

The smallest frequencies are : $10.6710 \quad 14.9037 \quad 15.9722 \mathrm{~cm}(-1)$

Electronic energy : $\quad H F=-4125.4602286$

Zero-point correction $=\quad 1.299784$ (Hartree/Particle)

Thermal correction to Energy $=\quad 1.378164$

Thermal correction to Enthalpy= $\quad 1.379109$

Thermal correction to Gibbs Free Energy $=\quad 1.186243$

Sum of electronic and zero-point Energies $=\quad-4124.160444$

Sum of electronic and thermal Energies $=\quad-4124.082064$

Sum of electronic and thermal Enthalpies $=\quad-4124.081120$

Sum of electronic and thermal Free Energies $=\quad-4124.273986$

\begin{tabular}{|c|c|c|c|}
\hline \multicolumn{4}{|c|}{ Cartesian Coordinates } \\
\hline 6 & 3.641009 & -5.959157 & 1.661155 \\
\hline 6 & 3.411557 & -5.096295 & 2.729558 \\
\hline 6 & 2.979086 & -3.797688 & 2.493725 \\
\hline 6 & 2.787275 & -3.328469 & 1.191683 \\
\hline 6 & 3.037906 & -4.193794 & 0.127545 \\
\hline 6 & 3.452288 & -5.502450 & 0.361027 \\
\hline 6 & 2.3712 & -1.8906 & 1.024748 \\
\hline 7 & 1.719176 & -1.543486 & -0.247054 \\
\hline 6 & 0.649664 & -2.325071 & -0.792644 \\
\hline 6 & -0.549936 & -2.546023 & -0.132275 \\
\hline 6 & -1.602123 & -3.251158 & -0.800316 \\
\hline 6 & -1.413749 & -3.702432 & -2.139666 \\
\hline 6 & -0.173864 & -3.465062 & -2.773851 \\
\hline 6 & 0.822761 & -2.795848 & -2.119024 \\
\hline 6 & & & -0.188 \\
\hline 6 & .869217 & -4.124227 & -0.867921 \\
\hline 6 & -3.671430 & -4.581050 & -2.188345 \\
\hline 6 & -2.466716 & -4.372075 & -2.808878 \\
\hline 6 & -0.771512 & -2.084216 & 1.266459 \\
\hline
\end{tabular}




\begin{tabular}{|c|c|c|c|}
\hline 6 & -1.165706 & -0.782779 & 1.554025 \\
\hline 6 & -1.401953 & -0.415834 & 2.906417 \\
\hline 6 & -1.238469 & -1.310994 & 3.929176 \\
\hline 6 & -0.853216 & -2.645790 & 3.669142 \\
\hline 6 & -0.631081 & -3.045737 & 2.319340 \\
\hline 6 & -0.705346 & -3.591906 & 4.711783 \\
\hline 6 & -0.371757 & -4.893704 & 4.435268 \\
\hline 6 & -0.167372 & -5.299739 & 3.099772 \\
\hline 6 & -0.282687 & -4.399281 & 2.070304 \\
\hline 15 & -1.515525 & 0.391318 & 0.154900 \\
\hline 4 & -0.059240 & 1.008791 & -1.768605 \\
\hline 6 & -0.485722 & -0.115619 & -3.310947 \\
\hline 8 & -0.703034 & -0.622937 & -4.324175 \\
\hline 15 & 1.881666 & -0.020823 & -1.000882 \\
\hline 8 & 2.649833 & 0.745809 & 0.250801 \\
\hline 6 & 3.422886 & 1.849490 & -0.058473 \\
\hline 6 & 4.685878 & 1.664499 & -0.588054 \\
\hline 6 & 5.448195 & 2.826345 & -0.933374 \\
\hline 6 & 4.925177 & 4.120529 & -0.631730 \\
\hline 6 & 3.646168 & 4.236261 & -0.037305 \\
\hline 6 & 2.894539 & 3.122675 & 0.224544 \\
\hline 6 & 5.683753 & 5.271418 & -0.957497 \\
\hline 6 & 6.899212 & 5.162036 & -1.581941 \\
\hline 6 & 7.401658 & 3.886853 & -1.916233 \\
\hline 6 & 6.697181 & 2.751613 & -1.601373 \\
\hline 6 & 5.166349 & 0.283134 & -0.826369 \\
\hline 6 & 4.386750 & -0.576477 & -1.580681 \\
\hline 6 & 4.799047 & -1.884449 & -1.900289 \\
\hline 6 & 5.983527 & -2.362386 & -1.409187 \\
\hline 6 & 6.786354 & -1.562524 & -0.561934 \\
\hline 6 & 6.376807 & -0.228675 & -0.257152 \\
\hline 6 & 7.173453 & 0.521712 & 0.645550 \\
\hline 6 & 8.320183 & -0.004643 & 1.185843 \\
\hline 6 & 8.738525 & -1.310979 & 0.855860 \\
\hline 6 & 7.981376 & -2.071387 & 0.002784 \\
\hline 8 & 3.163489 & -0.157724 & -2.061777 \\
\hline 6 & -3.222439 & -0.136484 & -0.288063 \\
\hline 6 & -4.158189 & -0.452488 & 0.704302 \\
\hline 6 & -5.468032 & -0.775034 & 0.372060 \\
\hline 6 & -5.828097 & -0.751185 & -0.986770 \\
\hline 6 & -4.927031 & -0.422705 & -1.995092 \\
\hline 6 & -3.608211 & -0.133735 & -1.618518 \\
\hline 6 & -6.507352 & -1.153364 & 1.426050 \\
\hline 6 & -5.299589 & -0.362477 & -3.473998 \\
\hline 6 & -1.853145 & 1.999756 & 0.988033 \\
\hline 6 & -2.952615 & 2.754838 & 0.568419 \\
\hline 6 & -3.195914 & 4.022521 & 1.087382 \\
\hline 6 & -2.327939 & 4.507002 & 2.075212 \\
\hline 6 & -1.216624 & 3.787106 & 2.512321 \\
\hline 6 & -0.969987 & 2.540902 & 1.920050 \\
\hline 6 & -4.384281 & 4.868622 & 0.634622 \\
\hline 6 & -0.307039 & 4.278819 & 3.638893 \\
\hline 6 & -0.747450 & 2.601130 & -2.550462 \\
\hline 8 & -1.196065 & 3.597905 & -2.928724 \\
\hline 1 & 6.859748 & 1.525681 & 0.919482 \\
\hline 1 & -1.739564 & 0.591366 & 3.136431 \\
\hline
\end{tabular}




\begin{tabular}{|c|c|c|c|}
\hline & 8.278490 & -3.088426 & -0.249585 \\
\hline & -0.872655 & -3.264158 & 5.736993 \\
\hline & 5.269860 & 6.248301 & -0.710957 \\
\hline & -3.034370 & -3.134889 & 0.827704 \\
\hline & -4.831971 & -4.279110 & -0.384442 \\
\hline & 3.256752 & 5.228698 & 0.184370 \\
\hline & -1.426357 & -1.008117 & 4.958792 \\
\hline & 6.310127 & -3.374733 & -1.641890 \\
\hline & 8.353816 & 3.801960 & -2.435185 \\
\hline & 4.157757 & -2.487583 & -2.539183 \\
\hline & -2.298783 & -4.709146 & -3.830949 \\
\hline & -0.029347 & -3.809253 & -3.796708 \\
\hline & 0.104109 & -6.330636 & 2.883584 \\
\hline & 7.092670 & 1.777467 & -1.877324 \\
\hline & -0.099003 & -4.722924 & 1.048200 \\
\hline & 1.773015 & -2.600462 & -2.614240 \\
\hline & 7.470131 & 6.052923 & -1.833084 \\
\hline & 8.908988 & 0.590171 & 1.880581 \\
\hline & -4.477089 & -5.091963 & -2.711218 \\
\hline & -0.267992 & -5.615610 & 5.242293 \\
\hline & 9.651650 & -1.713782 & 1.287816 \\
\hline & 1.894210 & 3.191396 & 0.645252 \\
\hline & -3.844908 & -0.441207 & 1.745648 \\
\hline & -2.866615 & 0.118335 & -2.374513 \\
\hline & -6.855570 & -0.991974 & -1.253017 \\
\hline & -3.618305 & 2.340556 & -0.184417 \\
\hline & -0.087803 & 1.969206 & 2.215728 \\
\hline ] & -2.534030 & 5.481528 & 2.512129 \\
\hline & 3.266209 & -1.263191 & 1.103209 \\
\hline & 1.732664 & -1.605131 & 1.876486 \\
\hline & 2.770170 & -3.135265 & 3.334752 \\
\hline & 2.902902 & -3.844207 & -0.892656 \\
\hline ] & 3.548648 & -5.441828 & 3.752339 \\
\hline & 3.630361 & -6.167693 & -0.481575 \\
\hline 1 & 3.964981 & -6.981944 & 1.841279 \\
\hline & 0.454914 & 2.119589 & -0.724844 \\
\hline 6 & -5.176150 & 4.200594 & -0.488407 \\
\hline 6 & -5.329043 & 5.090399 & 1.820774 \\
\hline & -3.882723 & 6.220992 & 0.117508 \\
\hline 5 & -0.712443 & 5.657634 & 4.154584 \\
\hline 6 & -0.391540 & 3.288553 & 4.807262 \\
\hline 6 & 1.142920 & 4.361169 & 3.154783 \\
\hline 5 & -6.795713 & -0.558486 & -3.703802 \\
\hline 6 & -4.903151 & 1.004464 & -4.046268 \\
\hline 6 & -4.546260 & -1.464620 & -4.225330 \\
\hline 5 & -5.948941 & -1.067169 & 2.845138 \\
\hline 6 & -7.710545 & -0.209073 & 1.330000 \\
\hline j & -6.968120 & -2.595199 & 1.187039 \\
\hline 1 & -6.010742 & 4.848501 & -0.784709 \\
\hline 1 & -4.556826 & 4.030821 & -1.379323 \\
\hline 1 & -5.602874 & 3.236244 & -0.180369 \\
\hline 1 & -0.032547 & 5.962894 & 4.959829 \\
\hline 1 & -0.656405 & 6.422101 & 3.368887 \\
\hline 1 & -1.729687 & 5.660982 & 4.566593 \\
\hline 1 & 1.791245 & 4.738047 & 3.957146 \\
\hline 1 & 1.534737 & 3.382177 & 2.853863 \\
\hline
\end{tabular}




$\begin{array}{rrrr}1 & 1.232956 & 5.043862 & 2.299053 \\ 1 & 0.235524 & 3.627948 & 5.642851 \\ 1 & -1.423215 & 3.199143 & 5.172853 \\ 1 & -0.047142 & 2.286401 & 4.521386 \\ 1 & -6.191298 & 5.698119 & 1.514619 \\ 1 & -5.708062 & 4.133561 & 2.203799 \\ 1 & -4.834067 & 5.610578 & 2.649931 \\ 1 & -4.727234 & 6.834519 & -0.224143 \\ 1 & -3.353104 & 6.790525 & 0.890501 \\ 1 & -3.197143 & 6.085811 & -0.729050 \\ 1 & -7.016955 & -0.483658 & -4.775910 \\ 1 & -7.137273 & -1.546585 & -3.369255 \\ 1 & -7.392786 & 0.205402 & -3.188601 \\ 1 & -5.186635 & 1.068012 & -5.105233 \\ 1 & -5.409489 & 1.818572 & -3.510702 \\ 1 & -3.821420 & 1.185655 & -3.987138 \\ 1 & -4.788022 & -1.431400 & -5.296458 \\ 1 & -3.458614 & -1.360043 & -4.123585 \\ 1 & -4.820582 & -2.455615 & -3.841051 \\ 1 & -6.725598 & -1.356686 & 3.564238 \\ 1 & -5.095249 & -1.742848 & 2.994611 \\ 1 & -5.627397 & -0.048489 & 3.098248 \\ 1 & -7.750738 & -2.871698 & 1.906537 \\ 1 & -7.375757 & -2.737990 & 0.178584 \\ 1 & -6.132052 & -3.296165 & 1.316947 \\ 1 & -8.452554 & -0.459052 & 2.100117 \\ 1 & -7.403998 & 0.834113 & 1.482042 \\ 1 & -8.212868 & -0.272156 & 0.357337\end{array}$

$1_{\mathrm{C} 12-}-\psi_{-152 a 354 s}$

Number of imaginary frequencies : 0

The smallest frequencies are : $10.9959 \quad 14.8201 \quad 15.4124 \mathrm{~cm}(-1)$

Electronic energy : $\quad H F=-4125.4606383$

Zero-point correction $=\quad 1.299804$ (Hartree/Particle)

Thermal correction to Energy= $\quad 1.378110$

Thermal correction to Enthalpy= $\quad 1.379054$

Thermal correction to Gibbs Free Energy= $\quad 1.186652$

Sum of electronic and zero-point Energies $=\quad-4124.160834$

Sum of electronic and thermal Energies $=\quad-4124.082528$

Sum of electronic and thermal Enthalpies $=\quad-4124.081584$

Sum of electronic and thermal Free Energies $=\quad-4124.273986$

\section{Cartesian Coordinates}

$\begin{array}{lrrrr}6 & 3.745158 & -5.870961 & 1.775351 \\ 6 & 3.484433 & -4.999522 & 2.829435 \\ 6 & 3.025330 & -3.714173 & 2.571656 \\ 6 & & 2.838366 & -3.266474 & 1.261446 \\ 6 & 3.119375 & -4.140257 & 0.211919 \\ 6 & 3.560253 & -5.436052 & 0.467180 \\ 6 & & 2.391729 & -1.841131 & 1.068563 \\ 7 & 1.739288 & -1.530978 & -0.212637 \\ 6 & 0.689111 & -2.347073 & -0.745143 \\ 6 & -0.509434 & -2.576779 & -0.086159 \\ 6 & -1.535711 & -3.335678 & -0.735234\end{array}$




\begin{tabular}{|c|c|c|c|}
\hline 6 & -1.322788 & -3.834400 & -2.053813 \\
\hline 6 & -0.088708 & -3.576465 & -2.691580 \\
\hline & 0.882495 & -2.854073 & -2.055353 \\
\hline 6 & -2.788857 & -3.591419 & -0.118721 \\
\hline & -3.761795 & -4.309350 & -0.768535 \\
\hline 0 & -3.539138 & -4.813948 & -2.067398 \\
\hline 6 & -2.343986 & -4.575925 & -2.696007 \\
\hline 0 & -0.756326 & -2.085396 & 1.297934 \\
\hline 6 & -1.189463 & -0.789255 & 1.550278 \\
\hline 6 & -1.457669 & -0.400866 & 2.891123 \\
\hline 6 & -1.285835 & -1.268559 & 3.935752 \\
\hline 6 & -0.858576 & -2.596915 & 3.711488 \\
\hline 6 & -0.605370 & -3.019665 & 2.374271 \\
\hline 6 & -0.699817 & -3.514887 & 4.777275 \\
\hline 6 & -0.326253 & -4.812692 & 4.534989 \\
\hline 6 & -0.091630 & -5.242162 & 3.211932 \\
\hline 6 & -0.216662 & -4.368205 & 2.160847 \\
\hline 15 & -1.528704 & 0.359774 & 0.126267 \\
\hline 45 & -0.069594 & 0.949508 & -1.807055 \\
\hline 6 & -0.473483 & -0.232483 & -3.312178 \\
\hline 8 & -0.685063 & -0.782844 & -4.303874 \\
\hline 15 & 1.884392 & -0.026180 & -1.002954 \\
\hline 8 & 2.633238 & 0.782254 & 0.233639 \\
\hline 6 & 3.385133 & 1.894228 & -0.097150 \\
\hline 6 & 4.654183 & 1.722589 & -0.616259 \\
\hline 6 & 5.395219 & 2.891581 & -0.983632 \\
\hline 6 & 4.844908 & 4.181368 & -0.713174 \\
\hline 6 & 3.560563 & & -0.128033 \\
\hline 6 & 2.829788 & 3.162453 & 0.154647 \\
\hline 6 & 5.582270 & 5.339857 & -1.059959 \\
\hline 6 & 6.803251 & 5.241314 & -1.675333 \\
\hline 6 & 7.332730 & 3.969464 & -1.979156 \\
\hline 6 & 6.649238 & 2.827429 & -1.643243 \\
\hline 6 & 5.163219 & 0.346216 & -0.820935 \\
\hline 6 & 4.405324 & -0.547052 & -1.558269 \\
\hline 6 & 4.849850 & -1.851134 & -1.849645 \\
\hline 6 & 6.044049 & -2.290762 & -1.346347 \\
\hline 6 & 6.824861 & -1.455448 & -0.512832 \\
\hline 6 & 6.382468 & -0.125957 & -0.236492 \\
\hline 6 & 7.156642 & 0.660909 & 0.654551 \\
\hline 6 & 8.312817 & 0.172853 & 1.210306 \\
\hline 6 & 8.763954 & -1.129249 & 0.907604 \\
\hline 6 & 8.029259 & -1.924203 & 0.066367 \\
\hline 8 & 3.174907 & -0.167364 & -2.053308 \\
\hline 6 & -3.230856 & -0.168946 & -0.334502 \\
\hline 6 & -4.198814 & -0.397036 & 0.643297 \\
\hline 6 & -5.518790 & -0.682303 & 0.298817 \\
\hline 6 & -5.847236 & -0.727432 & -1.062489 \\
\hline 6 & -4.901209 & -0.507104 & -2.066247 \\
\hline 6 & -3.587036 & -0.235061 & -1.675333 \\
\hline 6 & -6.543019 & -0.896160 & 1.412350 \\
\hline 6 & -5.228256 & -0.574856 & -3.555940 \\
\hline 6 & -1.883822 & 1.972380 & 0.945579 \\
\hline 6 & -3.006779 & 2.703649 & 0.547245 \\
\hline 6 & -3.271187 & 3.963377 & 1.075675 \\
\hline 6 & -2.397048 & 4.466046 & 2.048438 \\
\hline
\end{tabular}




\begin{tabular}{|c|c|c|c|}
\hline 6 & -1.263628 & 3.769016 & 2.466422 \\
\hline 6 & -0.999564 & 2.529900 & 1.867094 \\
\hline & -4.486592 & 4.781210 & 0.643688 \\
\hline & -0.352203 & 4.273485 & 3.585929 \\
\hline & -0.793769 & 2.500942 & -2.637418 \\
\hline & -1.269420 & 3.472083 & -3.047642 \\
\hline & 6.817627 & 1.662221 & 0.907228 \\
\hline & -1.820860 & 0.603056 & 3.094922 \\
\hline & 8.351647 & -2.938641 & -0.164575 \\
\hline & -0.891384 & -3.169159 & 5.792285 \\
\hline & 5.147500 & 6.313327 & -0.836892 \\
\hline & -2.981358 & -3.200478 & 0.878971 \\
\hline & -4.717550 & -4.487030 & -0.280078 \\
\hline & 3.150317 & 5.273863 & 0.069493 \\
\hline & -1.497156 & -0.948177 & 4.955517 \\
\hline & 6.395392 & -3.299353 & -1.558383 \\
\hline & 8.289365 & 3.892356 & -2.491036 \\
\hline & 4.225458 & -2.482288 & -2.478098 \\
\hline & -2.158778 & -4.947850 & -3.702862 \\
\hline & 0.073217 & -3.952363 & -3.700565 \\
\hline & 0.210564 & -6.269760 & 3.022448 \\
\hline & 7.065812 & 1.855633 & -1.895439 \\
\hline & -0.009408 & -4.709947 & 1.149173 \\
\hline & 1.829276 & -2.646395 & -2.552204 \\
\hline & 7.357928 & 6.137751 & -1.942625 \\
\hline & 8.883779 & 0.794911 & 1.896014 \\
\hline & -4.318847 & -5.383717 & -2.568243 \\
\hline & -0.214700 & -5.513594 & 5.359270 \\
\hline & 9.684374 & -1.501564 & 1.351248 \\
\hline 1 & 1.826278 & 3.220290 & 0.569235 \\
\hline & -3.918338 & -0.330494 & 1.694307 \\
\hline & -2.819287 & -0.056453 & -2.425182 \\
\hline & -6.873677 & -0.940652 & -1.346729 \\
\hline & -3.678025 & 2.275850 & -0.192262 \\
\hline 1 & -0.106291 & 1.971817 & 2.155516 \\
\hline & -2.617284 & 5.435160 & 2.490605 \\
\hline ] & 3.271407 & -1.191811 & 1.141324 \\
\hline & 1.740999 & -1.555993 & 1.911181 \\
\hline & 2.790084 & -3.046463 & 3.401560 \\
\hline & 2.986479 & -3.807213 & -0.814045 \\
\hline & 3.617590 & -5.328537 & 3.858162 \\
\hline 1 & 3.761883 & -6.108349 & -0.364448 \\
\hline & 4.089892 & -6.883722 & 1.972744 \\
\hline 1 & 0.420644 & 2.100349 & -0.795348 \\
\hline ) & -5.305641 & 4.071188 & -0.433234 \\
\hline 6 & -5.394835 & 5.025549 & 1.853659 \\
\hline 5 & -4.019444 & 6.125605 & 0.075830 \\
\hline 5 & -0.772012 & 5.647040 & 4.104096 \\
\hline 6 & -0.416931 & 3.282145 & 4.754846 \\
\hline ) & 1.093618 & 4.374203 & 3.093348 \\
\hline 6 & -6.726534 & -0.709789 & -3.816212 \\
\hline 5 & -4.736132 & 0.697689 & -4.256746 \\
\hline 6 & -4.520665 & -1.793532 & -4.159791 \\
\hline 6 & -7.905527 & -1.330063 & 0.877783 \\
\hline 6 & -6.046312 & -1.981114 & 2.375232 \\
\hline 6 & -6.723050 & 0.421320 & 2.176055 \\
\hline
\end{tabular}




$\begin{array}{rrrr}1 & -6.171878 & 4.688192 & -0.703435 \\ 1 & -4.720433 & 3.904652 & -1.347474 \\ 1 & -5.685824 & 3.098123 & -0.091496 \\ 1 & -0.087950 & 5.963364 & 4.901505 \\ 1 & -0.735754 & 6.410960 & 3.316724 \\ 1 & -1.784542 & 5.636245 & 4.527403 \\ 1 & 1.741975 & 4.756661 & 3.893020 \\ 1 & 1.495660 & 3.400897 & 2.787529 \\ 1 & 1.170915 & 5.060211 & 2.239046 \\ 1 & 0.209349 & 3.630380 & 5.587399 \\ 1 & -1.445523 & 3.178246 & 5.125448 \\ 1 & -0.059980 & 2.284948 & 4.467034 \\ 1 & -6.274825 & 5.612420 & 1.557694 \\ 1 & -5.748334 & 4.076682 & 2.278230 \\ 1 & -4.881902 & 5.577900 & 2.650332 \\ 1 & -4.882118 & 6.721459 & -0.251630 \\ 1 & -3.470165 & 6.718537 & 0.817085 \\ 1 & -3.361060 & 5.977327 & -0.789917 \\ 1 & -6.912627 & -0.725224 & -4.897438 \\ 1 & -7.134657 & -1.641271 & -3.403323 \\ 1 & -7.292637 & 0.131322 & -3.394913 \\ 1 & -4.990515 & 0.663257 & -5.324377 \\ 1 & -5.204400 & 1.592547 & -3.825856 \\ 1 & -3.647563 & 0.823061 & -4.187032 \\ 1 & -4.739636 & -1.869706 & -5.233761 \\ 1 & -3.430732 & -1.733745 & -4.043225 \\ 1 & -4.853863 & -2.720690 & -3.674188 \\ 1 & -8.595991 & -1.492643 & 1.715051 \\ 1 & -8.355760 & -0.568780 & 0.228219 \\ 1 & -7.842099 & -2.269861 & 0.313573 \\ 1 & -7.423849 & 0.289871 & 3.011820 \\ 1 & -5.774182 & 0.790642 & 2.586041 \\ 1 & -7.127734 & 1.200914 & 1.516891 \\ 1 & -6.774121 & -2.131889 & 3.183758 \\ 1 & -5.920603 & -2.940057 & 1.855328 \\ 1 & -5.086345 & -1.726029 & 2.841629\end{array}$

$1_{\mathrm{C} 12-} \psi_{-15233 a 4 a}$

Number of imaginary frequencies : 0

The smallest frequencies are : $11.0258 \quad 14.5999 \quad 16.2482 \mathrm{~cm}(-1)$

Electronic energy : $\quad H F=-4125.4605538$

Zero-point correction $=\quad 1.299898($ Hartree/Particle $)$

Thermal correction to Energy= $\quad 1.378159$

Thermal correction to Enthalpy= $\quad 1.379103$

Thermal correction to Gibbs Free Energy $=\quad 1.186703$

Sum of electronic and zero-point Energies $=\quad-4124.160655$

Sum of electronic and thermal Energies $=\quad-4124.082395$

Sum of electronic and thermal Enthalpies $=\quad-4124.081451$

Sum of electronic and thermal Free Energies $=\quad-4124.273851$

\section{Cartesian Coordinates}

\begin{tabular}{|c|c|c|c|}
\hline & 3.662325 & -5.898845 & 1.815930 \\
\hline & 3.437705 & -5.008141 & 2.8622 \\
\hline 6 & 3.008029 & -3.714863 & 2.5939 \\
\hline
\end{tabular}




\begin{tabular}{|c|c|c|c|}
\hline 6 & 2.814408 & -3.278596 & 1.280813 \\
\hline 6 & 3.060955 & -4.171564 & 0.238776 \\
\hline b & 3.472307 & -5.475003 & 0.504947 \\
\hline & 2.398608 & -1.845100 & 1.078784 \\
\hline 7 & 1.744743 & -1.528854 & -0.200118 \\
\hline 5 & 0.673932 & -2.323178 & -0.724468 \\
\hline 6 & -0.527948 & -2.518281 & -0.060678 \\
\hline f & -1.579877 & -3.242843 & -0.706859 \\
\hline 5 & -1.393637 & -3.731277 & -2.033140 \\
\hline 6 & -0.152671 & -3.516658 & -2.673071 \\
\hline 6 & 0.846910 & -2.834832 & -2.035576 \\
\hline 6 & -2.837342 & -3.460421 & -0.084372 \\
\hline 6 & -3.845696 & -4.121346 & -0.739967 \\
\hline 6 & -3.651322 & -4.612459 & -2.049182 \\
\hline 6 & -2.450040 & -4.416832 & -2.681405 \\
\hline 6 & -0.755307 & -2.003746 & 1.318317 \\
\hline 6 & -1.163056 & -0.695633 & 1.551990 \\
\hline 6 & -1.401849 & -0.277520 & 2.889282 \\
\hline 6 & -1.228579 & -1.128983 & 3.947328 \\
\hline 6 & -0.833864 & -2.470247 & 3.741318 \\
\hline 6 & -0.609508 & -2.921836 & 2.408301 \\
\hline 6 & -0.681218 & -3.373872 & 4.820331 \\
\hline 6 & -0.342422 & -4.684227 & 4.594902 \\
\hline 6 & -0.136928 & -5.141561 & 3.276213 \\
\hline 6 & -0.255558 & -4.282463 & 2.212278 \\
\hline 15 & -1.516795 & 0.423568 & 0.106301 \\
\hline 45 & -0.021706 & 0.980449 & -1.803578 \\
\hline 6 & -0.391381 & -0.184244 & -3.331085 \\
\hline 8 & -0.542774 & -0.729265 & -4.336356 \\
\hline 15 & 1.911946 & -0.028263 & -0.995248 \\
\hline 8 & 2.676619 & 0.773128 & 0.236275 \\
\hline 6 & 3.453663 & 1.865444 & -0.102221 \\
\hline 6 & 4.719807 & 1.661427 & -0.616603 \\
\hline 6 & 5.488391 & 2.810275 & -0.990103 \\
\hline 6 & 4.966944 & 4.114414 & -0.731594 \\
\hline 6 & 3.682628 & 4.251347 & -0.153136 \\
\hline 6 & 2.925781 & 3.147941 & 0.135514 \\
\hline 6 & 5.732360 & 5.252685 & -1.084462 \\
\hline 6 & 6.952980 & 5.121020 & -1.694475 \\
\hline 6 & 7.453925 & 3.834961 & -1.986731 \\
\hline 6 & 6.742751 & 2.711839 & -1.644710 \\
\hline 6 & 5.196515 & 0.272151 & -0.812101 \\
\hline 6 & 4.418531 & -0.605830 & -1.546905 \\
\hline 6 & 4.829147 & -1.923334 & -1.827104 \\
\hline 6 & 6.008789 & -2.391053 & -1.314886 \\
\hline 6 & 6.808218 & -1.570646 & -0.484250 \\
\hline 6 & 6.401095 & -0.227443 & -0.220160 \\
\hline 6 & 7.193424 & 0.545439 & 0.667210 \\
\hline 6 & 8.333827 & 0.030525 & 1.231361 \\
\hline 6 & 8.749912 & -1.286090 & 0.941617 \\
\hline 6 & 7.996902 & -2.067522 & 0.103964 \\
\hline 8 & 3.198891 & -0.198028 & -2.046284 \\
\hline 6 & -3.217049 & -0.138983 & -0.328260 \\
\hline 6 & -4.173856 & -0.336795 & 0.670163 \\
\hline 6 & -5.485028 & -0.679421 & 0.347564 \\
\hline 6 & -5.804638 & -0.820697 & -1.008648 \\
\hline
\end{tabular}




\begin{tabular}{|c|c|c|c|}
\hline 6 & -4.873597 & -0.633251 & -2.031729 \\
\hline & -3.568865 & -0.289033 & -1.664944 \\
\hline & -6.549543 & -0.924532 & 1.416084 \\
\hline & -5.300616 & -0.763073 & -3.492690 \\
\hline & -1.882438 & 2.048149 & 0.896725 \\
\hline & -3.022085 & 2.750919 & 0.514545 \\
\hline & -3.314481 & 4.008709 & 1.045535 \\
\hline & -2.434725 & 4.543927 & 1.987863 \\
\hline & -1.268802 & 3.876478 & 2.382613 \\
\hline & -0.990549 & 2.638893 & 1.796659 \\
\hline & -4.591246 & 4.715218 & 0.594640 \\
\hline & -0.344881 & 4.420993 & 3.473483 \\
\hline & -0.680401 & 2.559127 & -2.638885 \\
\hline & -1.108911 & 3.549911 & -3.053552 \\
\hline & 6.881166 & 1.557727 & 0.910455 \\
\hline & -1.745757 & 0.735639 & 3.080370 \\
\hline & 8.292245 & -3.092270 & -0.117302 \\
\hline & -0.849832 & -3.006743 & 5.831909 \\
\hline & 5.319773 & 6.237812 & -0.870708 \\
\hline & -3.003346 & -3.082509 & 0.922636 \\
\hline & -4.806030 & -4.265723 & -0.248478 \\
\hline & 3.293378 & 5.250879 & 0.034639 \\
\hline & -1.416920 & -0.785453 & 4.964063 \\
\hline & 6.333460 & -3.410563 & -1.517033 \\
\hline 1 & 8.410353 & 3.731707 & -2.494367 \\
\hline & 4.190341 & -2.542363 & -2.453158 \\
\hline & -2.284518 & -4.785797 & -3.692791 \\
\hline & -0.008910 & -3.889824 & -3.685818 \\
\hline & 0.137638 & -6.179303 & 3.100395 \\
\hline 1 & 7.137293 & 1.728619 & -1.887873 \\
\hline & -0.070309 & -4.645093 & 1.203519 \\
\hline I & 1.798421 & -2.657788 & -2.535398 \\
\hline & 7.529173 & 6.002334 & -1.966495 \\
\hline & 8.919256 & 0.642551 & 1.913894 \\
\hline 1 & -4.455846 & -5.144682 & -2.552426 \\
\hline & -0.235927 & -5.373737 & 5.429391 \\
\hline ] & 9.657990 & -1.679804 & 1.392172 \\
\hline & 1.921344 & 3.231844 & 0.543040 \\
\hline 1 & -3.884198 & -0.206831 & 1.710482 \\
\hline 1 & -2.803622 & -0.126720 & -2.420194 \\
\hline 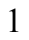 & -6.827583 & -1.085704 & -1.276387 \\
\hline 1 & -3.703463 & 2.305716 & -0.208837 \\
\hline & -0.081468 & 2.101319 & 2.074762 \\
\hline 1 & -2.663332 & 5.508905 & 2.429507 \\
\hline & 3.293617 & -1.215782 & 1.140883 \\
\hline 1 & 1.760672 & -1.538720 & 1.923731 \\
\hline & 2.801255 & -3.030850 & 3.418164 \\
\hline 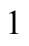 & 2.925515 & -3.848517 & -0.790078 \\
\hline 1 & 3.576198 & -5.327558 & 3.893299 \\
\hline & 3.646713 & -6.162099 & -0.320717 \\
\hline 1 & 3.983684 & -6.917633 & 2.021543 \\
\hline ] & 0.470935 & 2.109940 & -0.769831 \\
\hline 6 & -4.789971 & 6.060627 & 1.287257 \\
\hline 6 & -4.527986 & 4.958886 & -0.917474 \\
\hline 6 & -5.800275 & 3.826890 & 0.916021 \\
\hline 6 & -0.761721 & 5.810093 & 3.951721 \\
\hline
\end{tabular}




$\begin{array}{rrrr}6 & -0.392626 & 3.468035 & 4.674752 \\ 6 & 1.095416 & 4.509543 & 2.962216 \\ 6 & -4.111691 & -0.672211 & -4.447257 \\ 6 & -5.986409 & -2.112593 & -3.725641 \\ 6 & -6.276965 & 0.371050 & -3.824715 \\ 6 & -6.053858 & -0.559605 & 2.814067 \\ 6 & -7.796871 & -0.083644 & 1.125644 \\ 6 & -6.924888 & -2.410671 & 1.411158 \\ 1 & -5.718895 & 6.524148 & 0.931849 \\ 1 & -4.872270 & 5.954623 & 2.376795 \\ 1 & -3.971251 & 6.758506 & 1.069565 \\ 1 & -0.062897 & 6.157220 & 4.723089 \\ 1 & -0.746334 & 6.545868 & 3.137323 \\ 1 & -1.764505 & 5.810412 & 4.397441 \\ 1 & 1.747528 & 4.936219 & 3.735984 \\ 1 & 1.503612 & 3.525361 & 2.703607 \\ 1 & 1.159859 & 5.152121 & 2.073677 \\ 1 & 0.244386 & 3.842895 & 5.487472 \\ 1 & -1.416106 & 3.375107 & 5.062030 \\ 1 & -0.038771 & 2.462514 & 4.412958 \\ 1 & -5.432084 & 5.481810 & -1.257659 \\ 1 & -3.658883 & 5.575335 & -1.180728 \\ 1 & -4.454434 & 4.021975 & -1.482873 \\ 1 & -6.729084 & 4.312435 & 0.587068 \\ 1 & -5.739587 & 2.851814 & 0.414672 \\ 1 & -5.877887 & 3.644354 & 1.996357 \\ 1 & -4.458600 & -0.796833 & -5.480779 \\ 1 & -3.604425 & 0.300334 & -4.390290 \\ 1 & -3.370615 & -1.457976 & -4.247120 \\ 1 & -6.287878 & -2.206630 & -4.777458 \\ 1 & -5.302906 & -2.939586 & -3.491855 \\ 1 & -6.888585 & -2.236900 & -3.114723 \\ 1 & -6.591970 & 0.310521 & -4.875315 \\ 1 & -7.179482 & 0.329720 & -3.202101 \\ 1 & -5.807435 & 1.351275 & -3.668340 \\ 1 & -6.854620 & -0.728158 & 3.545064 \\ 1 & -5.195656 & -1.171281 & 3.123154 \\ 1 & -5.762155 & 0.497623 & 2.876738 \\ 1 & -7.705000 & -2.612958 & 2.157678 \\ 1 & -7.304873 & -2.733931 & 0.433659 \\ 1 & -6.054272 & -3.033527 & 1.658787 \\ 1 & -8.556001 & -0.254342 & 1.900519 \\ 1 & -7.558150 & 0.988330 & 1.119494 \\ 1 & -8.254924 & -0.331120 & 0.160854\end{array}$

$1_{\text {C12- } \Psi-152 s 3 a 4 s}$

Number of imaginary frequencies : 0

The smallest frequencies are : $3.2965 \quad 16.0320 \quad 16.4108 \mathrm{~cm}(-1)$

Electronic energy : $\quad H F=-4125.4609718$

Zero-point correction $=\quad 1.300069($ Hartree/Particle $)$

Thermal correction to Energy= $\quad 1.378300$

Thermal correction to Enthalpy= $\quad 1.379245$

Thermal correction to Gibbs Free Energy $=\quad 1.185870$

Sum of electronic and zero-point Energies $=\quad-4124.160903$

Sum of electronic and thermal Energies $=\quad-4124.082671$ 
Sum of electronic and thermal Enthalpies=

Sum of electronic and thermal Free Energies=
$-4124.081727$

$-4124.275102$

Cartesian Coordinates

\begin{tabular}{|c|c|c|c|}
\hline 6 & 3.617090 & -5.931997 & 1.782746 \\
\hline 6 & 3.381232 & -5.051025 & 2.834784 \\
\hline 6 & 2.956442 & -3.754608 & 2.573850 \\
\hline 6 & 2.778955 & -3.305697 & 1.262706 \\
\hline 6 & 3.036307 & -4.189122 & 0.215155 \\
\hline 6 & 3.442882 & -5.495549 & 0.473661 \\
\hline 6 & 2.368314 & -1.869675 & 1.068300 \\
\hline 7 & 1.724861 & -1.543410 & -0.213241 \\
\hline 6 & 0.655529 & -2.330917 & -0.750739 \\
\hline 6 & -0.551525 & -2.527757 & -0.096856 \\
\hline 6 & -1.602522 & -3.240750 & -0.757637 \\
\hline 6 & -1.408119 & -3.719602 & -2.086284 \\
\hline 6 & -0.161077 & -3.506036 & -2.714649 \\
\hline 6 & 0.836025 & -2.832444 & -2.064760 \\
\hline 6 & -2.866802 & -3.454742 & -0.147668 \\
\hline 6 & -3.872956 & -4.105098 & -0.817168 \\
\hline 6 & -3.670259 & -4.587926 & -2.128173 \\
\hline 6 & -2.462512 & -4.394554 & -2.748624 \\
\hline 6 & -0.784989 & -2.031958 & 1.287995 \\
\hline 6 & -1.185436 & -0.725206 & 1.540155 \\
\hline 6 & -1.435021 & -0.328436 & 2.882062 \\
\hline 6 & -1.280014 & -1.199334 & 3.926953 \\
\hline 6 & -0.891194 & -2.539307 & 3.701933 \\
\hline 6 & -0.654506 & -2.969091 & 2.363966 \\
\hline 6 & -0.753815 & -3.461426 & 4.767181 \\
\hline 6 & -0.417235 & -4.769051 & 4.523438 \\
\hline 6 & -0.199099 & -5.204824 & 3.199548 \\
\hline 6 & -0.303554 & -4.327643 & 2.148964 \\
\hline 15 & -1.525424 & 0.419522 & 0.111366 \\
\hline 45 & -0.034286 & 0.984257 & -1.797445 \\
\hline 6 & -0.420235 & -0.170754 & -3.328817 \\
\hline 8 & -0.584423 & -0.708709 & -4.335755 \\
\hline 15 & 1.897079 & -0.036714 & -0.996199 \\
\hline 8 & 2.663008 & 0.753075 & 0.242355 \\
\hline 6 & 3.439853 & 1.848120 & -0.088205 \\
\hline 6 & 4.705234 & 1.647868 & -0.606059 \\
\hline 6 & 5.472953 & 2.799230 & -0.973507 \\
\hline 6 & 4.951572 & 4.101470 & -0.705709 \\
\hline 6 & 3.668752 & 4.234243 & -0.123114 \\
\hline 6 & 2.912635 & 3.128927 & 0.160292 \\
\hline 6 & 5.715660 & 5.242325 & -1.053104 \\
\hline 6 & 6.934991 & 5.115041 & -1.666569 \\
\hline 6 & 7.435932 & 3.831043 & -1.967793 \\
\hline 6 & 6.726045 & 2.705447 & -1.631255 \\
\hline 6 & 5.182208 & 0.260074 & -0.810949 \\
\hline 6 & 4.403853 & -0.613354 & -1.550669 \\
\hline 6 & 4.814676 & -1.928945 & -1.839546 \\
\hline 6 & 5.995128 & -2.399368 & -1.331653 \\
\hline 6 & 6.795396 & -1.583784 & -0.497073 \\
\hline 6 & 6.387809 & -0.242586 & -0.223764 \\
\hline 6 & 7.181103 & 0.525227 & 0.667133 \\
\hline
\end{tabular}




\begin{tabular}{|c|c|c|c|}
\hline & 8.322873 & 0.007596 & 1.225995 \\
\hline & 8.739373 & -1.306854 & 0.927198 \\
\hline & 7.985428 & -2.083534 & 0.085972 \\
\hline & 3.184396 & -0.201982 & -2.047441 \\
\hline & -3.233367 & -0.119550 & -0.322093 \\
\hline & -4.179049 & -0.332294 & 0.675915 \\
\hline & -5.494605 & -0.683188 & 0.363817 \\
\hline & -5.830740 & -0.801622 & -0.985637 \\
\hline & -4.904104 & -0.595129 & -2.016456 \\
\hline & -3.599068 & -0.255555 & -1.660445 \\
\hline & -6.494282 & -0.890286 & 1.500301 \\
\hline & -5.346182 & -0.705644 & -3.474365 \\
\hline & -1.863323 & 2.040081 & 0.921242 \\
\hline & -2.970181 & 2.784734 & 0.520446 \\
\hline & -3.217709 & 4.057050 & 1.040329 \\
\hline & -2.340123 & 4.553231 & 2.006406 \\
\hline & -1.213754 & 3.837436 & 2.428856 \\
\hline & -0.968178 & 2.594052 & 1.840703 \\
\hline & -4.435091 & 4.832651 & 0.540502 \\
\hline & -0.296317 & 4.338851 & 3.545576 \\
\hline & -0.674657 & 2.573331 & -2.626165 \\
\hline & -1.088121 & 3.572485 & -3.036348 \\
\hline & 6.868609 & 1.535786 & 0.917173 \\
\hline & -1.772365 & 0.684252 & 3.087061 \\
\hline & 8.281174 & -3.106625 & -0.142326 \\
\hline & -0.931892 & -3.110640 & 5.782923 \\
\hline & 5.303057 & 6.225880 & -0.832254 \\
\hline 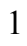 & -3.040336 & -3.080135 & 0.859529 \\
\hline & -4.839959 & -4.244933 & -0.337635 \\
\hline & 3.280090 & 5.232551 & 0.072083 \\
\hline & -1.476678 & -0.872096 & 4.947482 \\
\hline & 6.320128 & -3.417349 & -1.540837 \\
\hline 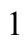 & 8.391335 & 3.731426 & -2.478083 \\
\hline & 4.175611 & -2.544315 & -2.468959 \\
\hline 1 & -2.290444 & -4.756509 & -3.761405 \\
\hline & -0.011041 & -3.872110 & -3.729066 \\
\hline 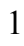 & 0.074160 & -6.240356 & 3.009272 \\
\hline 1 & 7.120571 & 1.723974 & -1.881404 \\
\hline & -0.108947 & -4.674305 & 1.136432 \\
\hline 1 & 1.791709 & -2.654849 & -2.556234 \\
\hline & 7.510183 & 5.998292 & -1.934395 \\
\hline 1 & 8.909105 & 0.615747 & 1.911299 \\
\hline & -4.473617 & -5.111489 & -2.642272 \\
\hline 1 & -0.321974 & -5.472779 & 5.347361 \\
\hline ] & 9.648560 & -1.702724 & 1.373612 \\
\hline 1 & 1.909735 & 3.210136 & 0.572393 \\
\hline 1 & -3.888301 & -0.214598 & 1.719524 \\
\hline & -2.839516 & -0.087280 & -2.420149 \\
\hline 1 & -6.852510 & -1.063407 & -1.252844 \\
\hline & -3.653542 & 2.366622 & -0.217738 \\
\hline 1 & -0.082179 & 2.025264 & 2.130515 \\
\hline 1 & -2.537876 & 5.526863 & 2.443687 \\
\hline 1 & 3.264256 & -1.242841 & 1.141190 \\
\hline 1 & 1.724778 & -1.567999 & 1.910731 \\
\hline 1 & 2.740996 & -3.078227 & 3.402076 \\
\hline & 2.913134 & -3.855689 & -0.811932 \\
\hline
\end{tabular}




\begin{tabular}{|c|c|c|c|}
\hline 1 & 3.507026 & -5.380536 & 3.864276 \\
\hline | & 3.626162 & -6.175042 & -0.356362 \\
\hline & 3.934814 & -6.953104 & 1.982421 \\
\hline & 0.464755 & 2.105642 & -0.758476 \\
\hline & -4.590717 & 6.184041 & 1.232943 \\
\hline & -4.288051 & 5.080511 & -0.965341 \\
\hline & -5.705771 & 4.014216 & 0.799260 \\
\hline & -0.688502 & 5.728141 & 4.043615 \\
\hline & -0.387279 & 3.365240 & 4.727625 \\
\hline & 1.153782 & 4.402703 & 3.059444 \\
\hline ) & -4.164778 & -0.622063 & -4.438955 \\
\hline & -6.052746 & -2.043075 & -3.715101 \\
\hline ) & -6.309937 & 0.445046 & -3.785882 \\
\hline 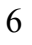 & -7.848907 & -1.385256 & 1.000539 \\
\hline & -5.949997 & -1.926066 & 2.491461 \\
\hline ) & -6.704315 & 0.445504 & 2.223233 \\
\hline & -5.475336 & 6.699802 & 0.838602 \\
\hline & -4.728815 & 6.077732 & 2.316859 \\
\hline 1 & -3.725870 & 6.836747 & 1.057830 \\
\hline & 0.000709 & 6.040908 & 4.838000 \\
\hline 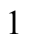 & -0.634874 & 6.480251 & 3.245938 \\
\hline | & -1.701561 & 5.744994 & 4.465286 \\
\hline & 1.805433 & 4.785154 & 3.856429 \\
\hline & 1.538107 & 3.416742 & 2.772151 \\
\hline & 1.249334 & 5.073038 & 2.194582 \\
\hline & 0.242757 & 3.710980 & 5.558426 \\
\hline 1 & -1.419446 & 3.288524 & 5.094886 \\
\hline & -0.050986 & 2.356822 & 4.454236 \\
\hline 1 & -5.153216 & 5.640127 & -1.346071 \\
\hline & -3.383721 & 5.663935 & -1.181353 \\
\hline 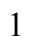 & -4.220593 & 4.144160 & -1.532911 \\
\hline I & -6.589118 & 4.560095 & 0.441160 \\
\hline & -5.685235 & 3.044601 & 0.284649 \\
\hline l & -5.838189 & 3.821993 & 1.872284 \\
\hline & -4.522607 & -0.732594 & -5.470353 \\
\hline 1 & -3.643786 & 0.342865 & -4.378318 \\
\hline [ & -3.432864 & -1.419750 & -4.252067 \\
\hline 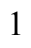 & -6.352053 & -2.127567 & -4.768303 \\
\hline 1 & -5.383091 & -2.881780 & -3.482732 \\
\hline ] & -6.959335 & -2.155622 & -3.108650 \\
\hline 1 & -6.638149 & 0.397870 & -4.833145 \\
\hline 1 & -7.205541 & 0.410866 & -3.152911 \\
\hline 1 & -5.824567 & 1.417137 & -3.626748 \\
\hline 1 & -8.522542 & -1.542547 & 1.852358 \\
\hline 1 & -8.332032 & -0.660917 & 0.332493 \\
\hline 1 & -7.761373 & -2.341069 & 0.466681 \\
\hline 1 & -7.394350 & 0.320586 & 3.068846 \\
\hline 1 & -5.762491 & 0.852075 & 2.613811 \\
\hline 1 & -7.134384 & 1.193804 & 1.544017 \\
\hline 1 & -6.655147 & -2.060266 & 3.322696 \\
\hline 1 & -5.816310 & -2.902641 & 2.007602 \\
\hline 1 & -4.984996 & -1.632818 & 2.924255 \\
\hline
\end{tabular}

$1_{\mathrm{C} 12-}-\psi_{-1523354 a}$

Number of imaginary frequencies : 0 
The smallest frequencies are : $10.4577 \quad 12.5701 \quad 14.7916 \mathrm{~cm}(-1)$ Electronic energy : $\quad H F=-4125.4605264$

Zero-point correction $=\quad 1.299756($ Hartree/Particle $)$

Thermal correction to Energy $=\quad 1.378084$

Thermal correction to Enthalpy= $\quad 1.379028$

Thermal correction to Gibbs Free Energy= $\quad 1.185486$

Sum of electronic and zero-point Energies $=\quad-4124.160770$

Sum of electronic and thermal Energies $=\quad-4124.082442$

Sum of electronic and thermal Enthalpies $=\quad-4124.081498$

Sum of electronic and thermal Free Energies $=\quad-4124.275040$

\section{Cartesian Coordinates}

$\begin{array}{ccccc}6 & 3.647308 & -5.912559 & 1.767186 \\ 6 & 3.421603 & -5.031097 & 2.821071 \\ 6 & 2.991514 & -3.735719 & 2.563808 \\ 6 & 2.798610 & -3.288154 & 1.254340 \\ 6 & 3.045959 & -4.172006 & 0.204749 \\ 6 & 3.457694 & -5.477507 & 0.459807 \\ 6 & 2.383511 & -1.852805 & 1.064269 \\ 7 & 1.729970 & -1.525427 & -0.212175 \\ 6 & 0.658266 & -2.316269 & -0.740354 \\ 6 & -0.541552 & -2.518111 & -0.074921 \\ 6 & -1.591380 & -3.246769 & -0.720255 \\ 6 & -1.400671 & -3.743579 & -2.042772 \\ 6 & -0.163289 & -3.517661 & -2.686354 \\ 6 & 0.830740 & -2.823534 & -2.053434 \\ 6 & -2.849943 & -3.463494 & -0.100098 \\ 6 & -3.853234 & -4.134461 & -0.753615 \\ 6 & -3.651386 & -4.641553 & -2.054966 \\ 6 & -2.448841 & -4.446767 & -2.684441 \\ 6 & -0.768176 & -2.016116 & 1.308705 \\ 6 & -1.175053 & -0.710478 & 1.556353 \\ 6 & -1.418287 & -0.307302 & 2.897433 \\ 6 & -1.248997 & -1.170768 & 3.946209 \\ 6 & -0.852953 & -2.509482 & 3.726287 \\ 6 & -0.625067 & -2.946348 & 2.389027 \\ 6 & -0.702413 & -3.424517 & 4.795889 \\ 6 & -0.362178 & -4.732160 & 4.557232 \\ 6 & -0.153371 & -5.175118 & 3.234211 \\ 6 & -0.270074 & -4.304696 & 2.179296 \\ 15 & -1.519738 & 0.427732 & 0.124549 \\ 45 & -0.034611 & 1.001296 & -1.788551 \\ 6 & -0.436388 & -0.148677 & -3.317806 \\ 8 & -0.630204 & -0.675986 & -4.325668 \\ 15 & 1.899042 & -0.018481 & -0.994447 \\ 8 & 2.668500 & 0.769553 & 0.242622 \\ 6 & 3.447538 & 1.863136 & -0.086720 \\ 6 & 4.711799 & 1.661291 & -0.606711 \\ 6 & 5.482265 & 2.812271 & -0.969825 \\ 6 & 4.965046 & 4.114829 & -0.695240 \\ 6 & 3.682752 & 4.248812 & -0.111654 \\ 6 & 2.923710 & 3.144307 & 0.166715 \\ 6 & 5.732569 & 5.254987 & -1.037288 \\ 6 & 6.951129 & 5.127006 & -1.652153 \\ 6 & 7.447733 & 3.843003 & -1.960399\end{array}$




\begin{tabular}{|c|c|c|c|}
\hline & 6.734484 & 2.717961 & -1.629150 \\
\hline & 5.184924 & 0.272964 & -0.817652 \\
\hline & 4.402801 & -0.596114 & -1.558621 \\
\hline & 4.809511 & -1.911639 & -1.853315 \\
\hline & 5.989745 & -2.387040 & -1.349705 \\
\hline & 6.793702 & -1.576601 & -0.513700 \\
\hline & 6.390432 & -0.235215 & -0.234775 \\
\hline & 7.187664 & 0.526920 & 0.657505 \\
\hline & 8.329007 & 0.003913 & 1.212261 \\
\hline & 8.741193 & -1.310581 & 0.907839 \\
\hline & 7.983394 & -2.081935 & 0.065220 \\
\hline & 3.182586 & -0.180894 & -2.050379 \\
\hline & -3.217628 & -0.121296 & -0.330461 \\
\hline & -4.176328 & -0.387756 & 0.655234 \\
\hline & -5.477714 & -0.732654 & 0.310009 \\
\hline & -5.802030 & -0.791258 & -1.056707 \\
\hline & -4.877845 & -0.515990 & -2.060090 \\
\hline & -3.571718 & -0.191233 & -1.668020 \\
\hline & -6.538180 & -1.065522 & 1.358892 \\
\hline & -5.214628 & -0.556690 & -3.548640 \\
\hline & -1.881089 & 2.042388 & 0.935180 \\
\hline 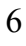 & -3.019951 & 2.752529 & 0.565331 \\
\hline & -3.305521 & 4.005490 & 1.111380 \\
\hline & -2.421275 & 4.526185 & 2.057674 \\
\hline 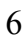 & -1.257223 & 3.849353 & 2.442228 \\
\hline & -0.984825 & 2.618379 & 1.840280 \\
\hline 6 & -4.579879 & 4.722552 & 0.670786 \\
\hline & -0.329006 & 4.376760 & 3.537876 \\
\hline 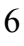 & -0.701670 & 2.583702 & -2.610418 \\
\hline 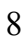 & -1.136291 & 3.574891 & -3.017500 \\
\hline & 6.878583 & 1.537411 & 0.911993 \\
\hline ] & -1.763181 & 0.703556 & 3.098549 \\
\hline & 8.275692 & -3.105038 & -0.167429 \\
\hline & -0.873765 & -3.068387 & 5.810928 \\
\hline l & 5.323244 & 6.238698 & -0.811127 \\
\hline & -3.022626 & -3.075552 & 0.902311 \\
\hline l & -4.815112 & -4.275190 & -0.264062 \\
\hline & 3.296860 & 5.247237 & 0.088532 \\
\hline 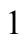 & -1.441257 & -0.839035 & 4.966106 \\
\hline 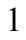 & 6.311572 & -3.405156 & -1.563100 \\
\hline | & 8.402435 & 3.742951 & -2.471916 \\
\hline 1 & 4.167132 & -2.522776 & -2.483469 \\
\hline & -2.280133 & -4.819982 & -3.693839 \\
\hline 1 & -0.017811 & -3.893295 & -3.697979 \\
\hline & 0.122186 & -6.210744 & 3.047804 \\
\hline 1 & 7.125727 & 1.736560 & -1.884662 \\
\hline & -0.082416 & -4.656684 & 1.167262 \\
\hline 1 & 1.779554 & -2.638815 & -2.555600 \\
\hline 1 & 7.528983 & 6.009790 & -1.915741 \\
\hline 1 & 8.918232 & 0.607824 & 1.898744 \\
\hline 1 & -4.453051 & -5.178663 & -2.557447 \\
\hline & -0.257135 & -5.430500 & 5.384542 \\
\hline 1 & 9.650076 & -1.710725 & 1.351055 \\
\hline 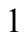 & 1.920689 & 3.226257 & 0.578185 \\
\hline 1 & -3.888262 & -0.316085 & 1.701643 \\
\hline 1 & -2.813092 & 0.025066 & -2.418081 \\
\hline
\end{tabular}




\begin{tabular}{|c|c|c|c|}
\hline 1 & -6.819755 & -1.057462 & -1.334945 \\
\hline & -3.704947 & 2.318165 & -0.161296 \\
\hline & -0.077200 & 2.073793 & 2.109416 \\
\hline & -2.644749 & 5.486961 & 2.510997 \\
\hline & 3.278900 & -1.224743 & 1.132050 \\
\hline & 1.745477 & -1.553086 & 1.911532 \\
\hline & 2.783410 & -3.059210 & 3.393847 \\
\hline & 2.910641 & -3.839975 & -0.821235 \\
\hline & 3.559478 & -5.359488 & 3.849373 \\
\hline & 3.632780 & -6.157383 & -0.371675 \\
\hline & 3.969078 & -6.932931 & 1.964135 \\
\hline & 0.467914 & 2.125996 & -0.753612 \\
\hline & -4.772708 & 6.059537 & 1.381033 \\
\hline & -4.516963 & 4.984736 & -0.838320 \\
\hline & -5.791650 & 3.834271 & 0.980812 \\
\hline & -0.741252 & 5.760181 & 4.036110 \\
\hline & -0.375836 & 3.407711 & 4.726253 \\
\hline & 1.110073 & 4.468688 & 3.023755 \\
\hline & -6.705151 & -0.771268 & -3.799885 \\
\hline & -4.807708 & 0.768164 & -4.205940 \\
\hline & -4.445880 & -1.708858 & -4.204205 \\
\hline ) & -6.039182 & -0.815437 & 2.780620 \\
\hline & -7.788046 & -0.206496 & 1.140098 \\
\hline ) & -6.912006 & -2.547236 & 1.238649 \\
\hline 1 & -5.699929 & 6.531554 & 1.032442 \\
\hline & -4.854715 & 5.939426 & 2.469156 \\
\hline & -3.951286 & 6.756940 & 1.172117 \\
\hline 1 & -0.040741 & 6.094332 & 4.811673 \\
\hline & -0.724411 & 6.507367 & 3.232206 \\
\hline 1 & -1.743681 & 5.757047 & 4.482626 \\
\hline & 1.765825 & 4.881541 & 3.801949 \\
\hline 1 & 1.514363 & 3.487358 & 2.748710 \\
\hline 1 & 1.173945 & 5.124529 & 2.144910 \\
\hline & 0.264317 & 3.770020 & 5.542179 \\
\hline 1 & -1.398471 & 3.312093 & 5.115119 \\
\hline & -0.025216 & 2.404929 & 4.450064 \\
\hline 1 & -5.418748 & 5.516452 & -1.170984 \\
\hline & -3.644992 & 5.599731 & -1.095358 \\
\hline ] & -4.449172 & 4.054052 & -1.415102 \\
\hline 1 & -6.719292 & 4.329973 & 0.664064 \\
\hline & -5.736349 & 2.868927 & 0.460827 \\
\hline 1 & -5.866115 & 3.631332 & 2.057610 \\
\hline & -6.900987 & -0.767226 & -4.879485 \\
\hline 1 & -7.053643 & -1.736385 & -3.410075 \\
\hline 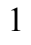 & -7.315132 & 0.023046 & -3.349995 \\
\hline 1 & -5.072125 & 0.758109 & -5.271615 \\
\hline 1 & -5.323024 & 1.616963 & -3.737172 \\
\hline 1 & -3.727832 & 0.955355 & -4.141258 \\
\hline 1 & -4.662028 & -1.748202 & -5.280653 \\
\hline 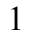 & -3.359763 & -1.603313 & -4.084435 \\
\hline 1 & -4.733012 & -2.670704 & -3.759381 \\
\hline 1 & -6.834866 & -1.053472 & 3.497725 \\
\hline 1 & -5.173678 & -1.443348 & 3.032647 \\
\hline 1 & -5.757076 & 0.234949 & 2.933506 \\
\hline 1 & -7.697626 & -2.804581 & 1.962079 \\
\hline 1 & -7.284330 & -2.798215 & 0.237389 \\
\hline
\end{tabular}




$\begin{array}{llrr}1 & -6.042722 & -3.186237 & 1.447234 \\ 1 & -8.544279 & -0.439346 & 1.901557 \\ 1 & -7.550682 & 0.862633 & 1.217823 \\ 1 & -8.248462 & -0.377598 & 0.159991\end{array}$

$1_{\mathrm{C} 12-} \psi_{-1 \mathrm{~s} 2 \mathrm{~s} 3 \mathrm{~s} 4 \mathrm{~s}}$

Number of imaginary frequencies : 0

The smallest frequencies are : $10.2085 \quad 15.6372 \quad 15.7969 \mathrm{~cm}(-1)$

Electronic energy : $\quad H F=-4125.4607624$

Zero-point correction $=\quad 1.299430($ Hartree/Particle $)$

Thermal correction to Energy= $\quad 1.377873$

Thermal correction to Enthalpy= $\quad 1.378817$

Thermal correction to Gibbs Free Energy= $\quad 1.185373$

Sum of electronic and zero-point Energies $=\quad-4124.161332$

Sum of electronic and thermal Energies $=\quad-4124.082889$

Sum of electronic and thermal Enthalpies = $\quad-4124.081945$

Sum of electronic and thermal Free Energies $=\quad-4124.275390$

Cartesian Coordinates

$\begin{array}{cccc}6 & 3.684648 & -5.939622 & 1.647735 \\ 6 & 3.449618 & -5.083248 & 2.720131 \\ 6 & 3.010008 & -3.785934 & 2.490332 \\ 6 & 2.816793 & -3.311519 & 1.190415 \\ 6 & 3.072648 & -4.170397 & 0.122306 \\ 6 & 3.494116 & -5.477815 & 0.349655 \\ 6 & 2.392954 & -1.875375 & 1.029440 \\ 7 & 1.738395 & -1.527085 & -0.240875 \\ 6 & 0.672025 & -2.313129 & -0.786269 \\ 6 & -0.524611 & -2.543137 & -0.123754 \\ 6 & -1.571126 & -3.259506 & -0.788591 \\ 6 & -1.383305 & -3.708689 & -2.128436 \\ 6 & -0.147770 & -3.458718 & -2.766401 \\ 6 & 0.845136 & -2.781876 & -2.113470 \\ 6 & -2.825116 & -3.507238 & -0.170895 \\ 6 & -3.827766 & -4.159283 & -0.844066 \\ 6 & -3.631802 & -4.612153 & -2.166177 \\ 6 & -2.432546 & -4.389117 & -2.792785 \\ 6 & -0.752568 & -2.077841 & 1.272806 \\ 6 & -1.162861 & -0.779600 & 1.552700 \\ 6 & -1.407178 & -0.408425 & 2.902440 \\ 6 & -1.238301 & -1.297213 & 3.930009 \\ 6 & -0.838889 & -2.629289 & 3.677590 \\ 6 & -0.607106 & -3.033062 & 2.330537 \\ 6 & -0.686419 & -3.569305 & 4.725050 \\ 6 & -0.340165 & -4.869293 & 4.455647 \\ 6 & -0.127202 & -5.279477 & 3.122756 \\ 6 & -0.246051 & -4.384588 & 2.088843 \\ 15 & -1.517130 & 0.387699 & 0.148835 \\ 45 & -0.049726 & 1.021004 & -1.756359 \\ 6 & -0.458054 & -0.100797 & -3.304513 \\ 8 & -0.663525 & -0.608209 & -4.320181 \\ 15 & 1.894476 & -0.001280 & -0.989527 \\ 8 & 2.659399 & 0.764353 & 0.264563 \\ 6 & 3.426990 & 1.872772 & -0.041374\end{array}$




\begin{tabular}{|c|c|c|c|}
\hline 6 & 4.691031 & 1.696093 & -0.571143 \\
\hline 6 & 5.447674 & 2.863378 & -0.911030 \\
\hline & 4.918119 & 4.153668 & -0.603868 \\
\hline & 3.637856 & 4.260596 & -0.010468 \\
\hline & 2.891728 & 3.142084 & 0.245496 \\
\hline & 5.671327 & 5.309749 & -0.923606 \\
\hline & 6.887787 & 5.209247 & -1.547569 \\
\hline & 7.396603 & 3.938114 & -1.887535 \\
\hline & 6.697432 & 2.797972 & -1.578578 \\
\hline & 5.178194 & 0.318081 & -0.815268 \\
\hline & 4.402536 & -0.542517 & -1.572638 \\
\hline y & 4.821801 & -1.846739 & -1.898534 \\
\hline & 6.009397 & -2.320318 & -1.410756 \\
\hline & 6.808439 & -1.519976 & -0.560389 \\
\hline & 6.391677 & -0.189877 & -0.249036 \\
\hline & 7.184563 & 0.560499 & 0.656967 \\
\hline & 8.334521 & 0.037966 & 1.194078 \\
\hline & 8.760091 & -1.264317 & 0.857370 \\
\hline & 8.006694 & -2.024820 & 0.001064 \\
\hline & 3.176678 & -0.128425 & -2.051193 \\
\hline & -3.209434 & -0.165992 & -0.316778 \\
\hline & -4.151545 & -0.487561 & 0.659860 \\
\hline & -5.459727 & -0.822854 & 0.316711 \\
\hline 5 & -5.809936 & -0.794739 & -1.039926 \\
\hline ) & -4.895707 & -0.456668 & -2.040321 \\
\hline & -3.584528 & -0.163913 & -1.653866 \\
\hline & -6.457403 & -1.154109 & 1.426087 \\
\hline & -5.257932 & -0.392018 & -3.522146 \\
\hline & -1.900466 & 1.985007 & 0.984288 \\
\hline$b$ & -3.032628 & 2.697701 & 0.597002 \\
\hline ) & -3.313312 & 3.959958 & 1.123315 \\
\hline 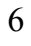 & -2.444433 & 4.477555 & 2.085623 \\
\hline f & -1.295381 & 3.792184 & 2.498540 \\
\hline & -1.016853 & 2.559208 & 1.902727 \\
\hline 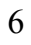 & -4.555023 & 4.700189 & 0.630329 \\
\hline & -0.384061 & 4.318209 & 3.608905 \\
\hline 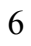 & -0.738298 & 2.611628 & -2.543415 \\
\hline & -1.187302 & 3.604661 & -2.930265 \\
\hline & 6.865313 & 1.561278 & 0.936096 \\
\hline & -1.754609 & 0.596820 & 3.126512 \\
\hline & 8.309340 & -3.038942 & -0.256436 \\
\hline ] & -0.860747 & -3.238580 & 5.748130 \\
\hline & 5.252427 & 6.283410 & -0.672796 \\
\hline 1 & -2.995186 & -3.158134 & 0.845864 \\
\hline & -4.786406 & -4.325941 & -0.355836 \\
\hline 1 & 3.243071 & 5.250040 & 0.215171 \\
\hline ] & -1.432153 & -0.991418 & 4.957645 \\
\hline & 6.341449 & -3.329714 & -1.648517 \\
\hline | & 8.349504 & 3.860304 & -2.406228 \\
\hline & 4.183259 & -2.450422 & -2.539656 \\
\hline 1 & -2.266422 & -4.723615 & -3.816015 \\
\hline & -0.002977 & -3.800499 & -3.790032 \\
\hline 1 & 0.153459 & -6.309063 & 2.912124 \\
\hline 1 & 7.097887 & 1.827106 & -1.858873 \\
\hline & -0.055712 & -4.711198 & 1.068858 \\
\hline & 1.792498 & -2.578497 & -2.611079 \\
\hline
\end{tabular}




\begin{tabular}{|c|c|c|c|}
\hline 1 & 7.454586 & 6.104063 & -1.794019 \\
\hline & 8.920303 & 0.632625 & 1.891496 \\
\hline 1 & -4.434119 & -5.131782 & -2.685557 \\
\hline & -0.233279 & -5.586713 & 5.266244 \\
\hline 1 & 9.675749 & -1.664068 & 1.286788 \\
\hline 1 & 1.890097 & 3.203869 & 0.663796 \\
\hline 1 & -3.857475 & -0.467011 & 1.708918 \\
\hline 1 & -2.837793 & 0.093824 & -2.402674 \\
\hline 1 & -6.830162 & -1.038144 & -1.322718 \\
\hline 1 & -3.708656 & 2.265107 & -0.139296 \\
\hline 1 & -0.111651 & 2.015430 & 2.181836 \\
\hline 1 & -2.666514 & 5.444349 & 2.526519 \\
\hline 1 & 3.284419 & -1.243306 & 1.110096 \\
\hline 1 & 1.753037 & -1.596504 & 1.882342 \\
\hline 1 & 2.795188 & -3.129297 & 3.334477 \\
\hline 1 & 2.935805 & -3.816472 & -0.896147 \\
\hline 1 & 3.587726 & -5.433092 & 3.741310 \\
\hline 1 & 3.676212 & -6.138048 & -0.496020 \\
\hline 1 & 4.014183 & -6.961447 & 1.823172 \\
\hline 1 & 0.449612 & 2.129754 & -0.702357 \\
\hline 6 & -4.740228 & 6.050533 & 1.317337 \\
\hline 6 & -4.427352 & 4.943587 & -0.878239 \\
\hline 6 & -5.800847 & 3.848992 & 0.904148 \\
\hline 6 & -0.811378 & 5.695489 & 4.111414 \\
\hline 6 & -0.437521 & 3.341718 & 4.790740 \\
\hline 6 & 1.059929 & 4.423512 & 3.111354 \\
\hline 6 & & -0.588573 & -3.764370 \\
\hline 6 & -4.859050 & 0.977634 & -4.086703 \\
\hline 6 & -4.498098 & -1.490490 & -4.272647 \\
\hline 6 & -7.773868 & -1.701036 & 0.879406 \\
\hline 6 & -5.868459 & -2.207515 & 2.371849 \\
\hline 6 & -6.754300 & 0.127270 & 2.214459 \\
\hline 1 & -5.640921 & 6.541521 & 0.927786 \\
\hline 1 & -4.866943 & 5.946105 & 2.402811 \\
\hline 1 & -3.893681 & 6.724165 & 1.132210 \\
\hline 1 & -0.125499 & 6.026472 & 4.901278 \\
\hline 1 & -0.784458 & 6.449714 & 3.314405 \\
\hline 1 & -1.821381 & 5.683593 & 4.540484 \\
\hline 1 & 1.704656 & 4.834679 & 3.899625 \\
\hline 1 & 1.473432 & 3.447051 & 2.832518 \\
\hline 1 & 1.127980 & 5.086959 & 2.238594 \\
\hline 1 & 0.189680 & 3.704475 & 5.616439 \\
\hline 1 & -1.463855 & 3.235388 & 5.166698 \\
\hline 1 & -0.074915 & 2.343608 & 4.513366 \\
\hline 1 & -5.307185 & 5.483953 & -1.253137 \\
\hline 1 & -3.536854 & 5.543566 & -1.105990 \\
\hline 1 & -4.346994 & 4.005977 & -1.442607 \\
\hline 1 & -6.701478 & 4.367721 & 0.548682 \\
\hline 1 & -5.756985 & 2.876303 & 0.396342 \\
\hline 1 & -5.920110 & 3.661807 & 1.979573 \\
\hline 1 & -6.964794 & -0.509377 & -4.837916 \\
\hline 1 & -7.095895 & -1.578235 & -3.436873 \\
\hline 1 & -7.354358 & 0.172599 & -3.251091 \\
\hline 1 & -5.138865 & 1.046731 & -5.146302 \\
\hline 1 & -5.366200 & 1.789689 & -3.548742 \\
\hline 1 & -3.777491 & 1.158218 & -4.022944 \\
\hline
\end{tabular}




$\begin{array}{llll}1 & -4.735034 & -1.455406 & -5.344817 \\ 1 & -3.411340 & -1.382410 & -4.165681 \\ 1 & -4.771229 & -2.483383 & -3.892219 \\ 1 & -8.439088 & -1.964024 & 1.711553 \\ 1 & -8.303620 & -0.965178 & 0.261566 \\ 1 & -7.618757 & -2.607194 & 0.278342 \\ 1 & -7.450365 & -0.079504 & 3.038980 \\ 1 & -5.842142 & 0.562767 & 2.642535 \\ 1 & -7.213024 & 0.886538 & 1.566792 \\ 1 & -6.582223 & -2.433135 & 3.175329 \\ 1 & -5.659947 & -3.145216 & 1.839447 \\ 1 & -4.935827 & -1.879001 & 2.847675\end{array}$

\section{Cartesian coordinates for intermediate 2}

$2_{\text {C1c- }}-\psi_{-122 a 3 a 4 a}$

Number of imaginary frequencies : 0

The smallest frequencies are : $13.0220 \quad 15.0431 \quad 20.4019 \mathrm{~cm}(-1)$

Electronic energy : $\quad H F=-4012.1866048$

Zero-point correction $=\quad 1.291065($ Hartree/Particle $)$

Thermal correction to Energy= $\quad 1.367019$

Thermal correction to Enthalpy= $\quad 1.367963$

Thermal correction to Gibbs Free Energy $=\quad 1.181587$

Sum of electronic and zero-point Energies $=\quad-4010.895540$

Sum of electronic and thermal Energies $=\quad-4010.819586$

Sum of electronic and thermal Enthalpies $=\quad-4010.818642$

Sum of electronic and thermal Free Energies $=\quad-4011.005018$

Cartesian Coordinates

$\begin{array}{cccc}6 & -2.311145 & 3.015174 & 0.314337 \\ 6 & -1.411690 & 2.017523 & 0.684310 \\ 6 & -0.314680 & 2.333014 & 1.490651 \\ 6 & -0.137780 & 3.624603 & 1.987037 \\ 6 & -1.059804 & 4.601987 & 1.597118 \\ 6 & -2.143835 & 4.328978 & 0.759402 \\ 15 & -1.552791 & 0.314563 & 0.019644 \\ 6 & -1.431656 & -0.728844 & 1.553788 \\ 6 & -1.232432 & -2.097294 & 1.442239 \\ 6 & -1.178242 & -2.916736 & 2.614910 \\ 6 & -1.337216 & -2.317820 & 3.898100 \\ 6 & -1.561550 & -0.924570 & 3.976743 \\ 6 & -1.608540 & -0.157648 & 2.843351 \\ 6 & -1.266353 & -3.124534 & 5.058915 \\ 6 & -1.045901 & -4.474739 & 4.963485 \\ 6 & -0.889736 & -5.074511 & 3.695803 \\ 6 & -0.957524 & -4.317786 & 2.552245 \\ 6 & -1.112257 & -2.765967 & 0.118096 \\ 6 & 0.100444 & -2.809121 & -0.546809 \\ 6 & 0.222926 & -3.489169 & -1.782160 \\ 6 & -0.859031 & -4.106998 & -2.350354 \\ 6 & -2.120129 & -4.075607 & -1.711299 \\ 6 & -2.253762 & -3.400660 & -0.461136 \\ 6 & -3.533425 & -3.360658 & 0.151639\end{array}$




\begin{tabular}{|c|c|c|c|}
\hline 5 & -4.616700 & -3.960848 & -0.439563 \\
\hline 6 & -4.476863 & -4.645113 & -1.667142 \\
\hline 6 & -3.255128 & -4.696827 & -2.288683 \\
\hline 7 & 1.245176 & -2.120706 & -0.010818 \\
\hline 1 & 1.652463 & -0.803242 & -0.986413 \\
\hline 8 & 2.858485 & -1.300333 & -2.030001 \\
\hline 6 & 4.135365 & -1.591398 & -1.607826 \\
\hline 6 & 4.973825 & -0.597104 & -1.133420 \\
\hline 6 & 6.257864 & -0.983667 & -0.628329 \\
\hline 6 & 6.685098 & -2.340088 & -0.749615 \\
\hline 6 & 5.820118 & -3.290560 & -1.345923 \\
\hline 6 & 4.563570 & -2.928558 & -1.748376 \\
\hline 6 & 7.112401 & -0.074864 & 0.046104 \\
\hline 6 & 8.328471 & -0.477623 & 0.540792 \\
\hline 6 & 8.760241 & -1.812128 & 0.390615 \\
\hline 6 & 7.949279 & -2.721968 & -0.238721 \\
\hline 6 & 4.501562 & 0.806267 & -1.125883 \\
\hline 6 & 3.291283 & 1.102284 & -0.531266 \\
\hline 6 & 2.751006 & 2.402134 & -0.498232 \\
\hline 6 & 3.447366 & 3.431286 & -1.072218 \\
\hline 6 & 4.676744 & 3.193079 & -1.732578 \\
\hline 6 & 5.204805 & 1.867453 & -1.785221 \\
\hline 6 & 5.373459 & 4.246984 & -2.372124 \\
\hline 6 & 6.532608 & 4.009594 & -3.064832 \\
\hline 6 & 7.034733 & 2.694889 & -3.155313 \\
\hline 6 & 6.389463 & 1.653911 & -2.534721 \\
\hline 8 & 2.564880 & 0.083146 & 0.061328 \\
\hline 6 & 0.991300 & 3.981152 & 2.953891 \\
\hline 6 & 1.829675 & 2.761000 & 3.329904 \\
\hline 6 & -3.122167 & 5.443740 & 0.392871 \\
\hline 6 & -4.216346 & 4.962339 & -0.557397 \\
\hline 6 & -3.335725 & 0.120283 & -0.379398 \\
\hline 6 & -3.680941 & -0.304442 & -1.659125 \\
\hline 6 & -5.018634 & -0.460815 & -2.031662 \\
\hline 6 & -5.991065 & -0.192555 & -1.067430 \\
\hline 6 & -5.679923 & 0.217073 & 0.236167 \\
\hline 6 & -4.334450 & 0.369135 & 0.565637 \\
\hline 6 & -6.799462 & 0.446314 & 1.250323 \\
\hline 6 & -6.278855 & 1.039453 & 2.558246 \\
\hline 6 & -5.414027 & -0.839218 & -3.457792 \\
\hline 6 & -4.228042 & -1.379256 & -4.257430 \\
\hline 45 & -0.135716 & 0.281998 & -1.930043 \\
\hline 6 & -1.211378 & 1.462831 & -2.920550 \\
\hline 8 & -1.807611 & 2.225407 & -3.555011 \\
\hline 6 & -7.461182 & -0.900949 & 1.562171 \\
\hline 6 & -7.846623 & 1.409129 & 0.681360 \\
\hline 6 & -6.506884 & -1.911294 & -3.454989 \\
\hline 6 & -5.937258 & 0.420766 & -4.157494 \\
\hline 6 & 2.143516 & -2.930629 & 0.837452 \\
\hline 6 & 2.876143 & -2.150235 & 1.893888 \\
\hline 6 & 2.179350 & -1.365064 & 2.814870 \\
\hline 6 & 2.862778 & -0.669087 & 3.802947 \\
\hline 6 & 4.253594 & -0.737341 & 3.877091 \\
\hline 6 & 4.953284 & -1.516031 & 2.962394 \\
\hline 6 & 4.263754 & -2.223340 & 1.980765 \\
\hline 6 & 1.911350 & 5.028983 & 2.320826 \\
\hline
\end{tabular}




\begin{tabular}{|c|c|c|c|}
\hline 6 & 0.386319 & 4.549298 & 4.243339 \\
\hline 6 & -3.793913 & 5.963396 & 1.669026 \\
\hline 6 & -2.367567 & 6.588660 & -0.290723 \\
\hline 1 & 0.794372 & 0.362463 & -3.245901 \\
\hline 1 & 6.785582 & 0.952288 & 0.186033 \\
\hline 1 & -1.781877 & 0.911533 & 2.936541 \\
\hline 1 & 8.258279 & -3.760795 & -0.348186 \\
\hline 1 & -1.389041 & -2.646949 & 6.029982 \\
\hline 1 & 4.956773 & 5.251484 & -2.311043 \\
\hline 1 & -3.655382 & -2.827394 & 1.092814 \\
\hline 1 & -5.593699 & -3.902340 & 0.036238 \\
\hline 1 & 3.046748 & 4.443954 & -1.055933 \\
\hline 1 & -1.699625 & -0.468289 & 4.956198 \\
\hline 1 & 6.163050 & -4.318035 & -1.456378 \\
\hline 1 & 7.938543 & 2.501166 & -3.728703 \\
\hline 1 & 3.872048 & -3.646982 & -2.184164 \\
\hline 1 & -3.135278 & -5.212874 & -3.240426 \\
\hline 1 & -0.764373 & -4.622884 & -3.304439 \\
\hline 1 & -0.717016 & -6.145929 & 3.624692 \\
\hline 1 & 6.783736 & 0.645527 & -2.627127 \\
\hline 1 & -0.842692 & -4.794404 & 1.580804 \\
\hline 1 & 1.192807 & -3.495433 & -2.277455 \\
\hline 1 & 7.056034 & 4.826040 & -3.556899 \\
\hline 1 & 8.960986 & 0.238305 & 1.060889 \\
\hline 1 & -5.341402 & -5.127085 & -2.119085 \\
\hline 1 & -0.991218 & -5.086084 & \\
\hline 1 & 9.727184 & -2.117577 & 0.783545 \\
\hline 1 & 1.783460 & 2.554959 & -0.022167 \\
\hline 1 & -4.049048 & 0.694173 & 1.563851 \\
\hline 1 & -2.878467 & -0.520468 & -2.361813 \\
\hline 1 & -7.040457 & -0.306305 & -1.338210 \\
\hline 1 & -3.149884 & 2.755727 & -0.327396 \\
\hline 1 & 0.390487 & 1.543070 & \\
\hline 1 & -0.931844 & 5.617791 & 1.971546 \\
\hline 1 & 1.494145 & -3.673583 & 1.323059 \\
\hline 1 & 2.861410 & -3.494087 & 0.220267 \\
\hline 1 & 4.814336 & -2.827868 & 1.259387 \\
\hline 1 & 1.095528 & -1.284259 & 2.735492 \\
\hline 1 & 6.039771 & -1.573314 & 3.002043 \\
\hline 1 & 2.306524 & -0.067722 & 4.520526 \\
\hline 1 & 4.787122 & -0.183694 & 4.646919 \\
\hline 1 & 2.603771 & 3.050350 & 4.052358 \\
\hline 1 & 1.214670 & 1.978661 & 3.797262 \\
\hline 1 & 2.342215 & 2.318101 & 2.465370 \\
\hline 1 & 1.181497 & 4.788122 & 4.962212 \\
\hline 1 & -0.184508 & 5.468669 & 4.066179 \\
\hline 1 & -0.288146 & 3.821925 & 4.714345 \\
\hline 1 & 2.704301 & 5.314538 & 3.025164 \\
\hline 1 & 2.394071 & 4.634870 & 1.416818 \\
\hline 1 & 1.367322 & 5.940337 & 2.044065 \\
\hline 1 & -4.891476 & 5.794601 & -0.792955 \\
\hline 1 & -3.804215 & 4.592480 & -1.505228 \\
\hline 1 & -4.824308 & 4.161881 & -0.113824 \\
\hline 1 & -4.505063 & 6.764807 & 1.427293 \\
\hline 1 & -4.347341 & 5.161190 & 2.175247 \\
\hline 1 & -3.066306 & 6.368988 & 2.382639 \\
\hline
\end{tabular}




\begin{tabular}{|c|c|c|c|}
\hline & -3.063884 & 7.392655 & -0.564597 \\
\hline & -1.599291 & 7.025255 & 0.358817 \\
\hline & -1.874040 & 6.241199 & -1.207330 \\
\hline & -8.641229 & 1.581511 & 1.419438 \\
\hline & -7.398538 & 2.380465 & 0.435162 \\
\hline & -8.324377 & 1.021909 & -0.226074 \\
\hline & -7.117474 & 1.212266 & 3.244380 \\
\hline & -5.575170 & 0.367656 & 3.066840 \\
\hline & -5.777319 & 2.003713 & 2.397344 \\
\hline & -8.271011 & -0.773005 & 2.293362 \\
\hline & -7.890273 & -1.362189 & 0.663791 \\
\hline & -6.731575 & -1.605383 & 1.984049 \\
\hline & -4.566142 & -1.677941 & -5.257870 \\
\hline & -3.438451 & -0.628779 & -4.395271 \\
\hline & -3.782350 & -2.260966 & -3.775446 \\
\hline & -6.224975 & 0.194920 & -5.193465 \\
\hline & -6.815967 & 0.832633 & -3.644742 \\
\hline & -5.165882 & 1.202169 & -4.181861 \\
\hline & -6.759602 & -2.192202 & -4.485886 \\
\hline & -6.166417 & -2.814339 & -2.930812 \\
\hline & -7.433473 & -1.569987 & -2.97824 \\
\hline
\end{tabular}

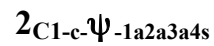

Number of imaginary frequencies : 0

The smallest frequencies are : $13.7459 \quad 15.4330 \quad 19.7352 \mathrm{~cm}(-1)$

Electronic energy : $\quad H F=-4012.1869948$

Zero-point correction $=\quad 1.290694($ Hartree/Particle $)$

Thermal correction to Energy $=\quad 1.366818$

Thermal correction to Enthalpy= $\quad 1.367763$

Thermal correction to Gibbs Free Energy $=\quad 1.180259$

Sum of electronic and zero-point Energies $=\quad-4010.896301$

Sum of electronic and thermal Energies $=\quad-4010.820176$

Sum of electronic and thermal Enthalpies $=\quad-4010.819232$

Sum of electronic and thermal Free Energies $=\quad-4011.006735$

Cartesian Coordinates

$\begin{array}{cccc}6 & -3.682595 & -0.295318 & -1.693468 \\ 6 & -3.325089 & 0.100058 & -0.403998 \\ 6 & -4.314085 & 0.314921 & 0.550650 \\ 6 & -5.666343 & 0.163354 & 0.233688 \\ 6 & -5.991862 & -0.201698 & -1.074247 \\ 6 & -5.020684 & -0.443431 & -2.054753 \\ 15 & -1.544390 & 0.305085 & -0.001897 \\ 45 & -0.121380 & 0.282226 & -1.944780 \\ 1 & 0.811151 & 0.365389 & -3.259052 \\ 6 & -6.708137 & 0.387019 & 1.327502 \\ 6 & -6.442506 & -0.595903 & 2.474656 \\ 6 & -5.429710 & -0.792447 & -3.484464 \\ 6 & -6.528657 & -1.858524 & -3.493188 \\ 6 & -1.429950 & -0.737486 & 1.533433 \\ 6 & -1.228070 & -2.105282 & 1.422138 \\ 6 & -1.187831 & -2.927215 & 2.593275 \\ 6 & -1.367967 & -2.331728 & 3.875198\end{array}$




\begin{tabular}{|c|c|c|c|}
\hline 6 & -1.595958 & -0.938977 & 3.953547 \\
\hline 6 & -1.625244 & -0.168859 & 2.821466 \\
\hline b & -0.963846 & -4.327600 & 2.530060 \\
\hline & -0.912640 & -5.087213 & 3.672565 \\
\hline y & -1.089831 & -4.490983 & 4.939189 \\
\hline & -1.314110 & -3.141397 & 5.034841 \\
\hline 6 & -1.102313 & -2.768810 & 0.096429 \\
\hline 6 & 0.110965 & -2.808422 & -0.567428 \\
\hline 6 & 0.232214 & -3.477449 & -1.808962 \\
\hline 6 & -0.852085 & -4.084014 & -2.385189 \\
\hline 6 & -2.114258 & -4.053125 & -1.748086 \\
\hline 6 & -2.245643 & -3.393148 & -0.489909 \\
\hline 6 & -3.252635 & -4.659487 & -2.334643 \\
\hline 6 & -4.474847 & -4.607584 & -1.713868 \\
\hline 6 & -4.611597 & -3.939066 & -0.477207 \\
\hline 6 & -3.525391 & -3.353320 & 0.122741 \\
\hline 7 & 1.254895 & -2.122954 & -0.026441 \\
\hline 6 & 2.146433 & -2.930125 & 0.831405 \\
\hline 6 & 2.869221 & -2.146214 & 1.892128 \\
\hline 6 & 2.163756 & -1.358359 & 2.804174 \\
\hline 6 & 2.837866 & -0.656898 & 3.794708 \\
\hline 6 & 4.228128 & -0.722703 & 3.880555 \\
\hline 6 & 4.936438 & -1.504539 & 2.975257 \\
\hline 6 & 4.256140 & -2.217033 & 1.990872 \\
\hline 15 & 1.664836 & -0.801041 & -0.994759 \\
\hline 8 & 2.567699 & 0.084957 & 0.061831 \\
\hline 6 & 3.297574 & 1.106257 & -0.522571 \\
\hline 6 & 4.513211 & 0.813396 & -1.107962 \\
\hline 6 & 5.220644 & 1.877464 & -1.758125 \\
\hline 6 & 4.690390 & 3.202257 & -1.706111 \\
\hline 6 & 3.454875 & 3.436952 & -1.056068 \\
\hline 6 & 2.755059 & 2.405260 & -0.490978 \\
\hline 6 & 6.412004 & 1.667818 & -2.498081 \\
\hline 6 & 7.061330 & 2.711573 & -3.109702 \\
\hline 6 & 6.556758 & 4.025357 & -3.019506 \\
\hline 6 & 5.391254 & 4.259046 & -2.336291 \\
\hline 6 & 4.986742 & -0.589615 & -1.116298 \\
\hline 6 & 4.152350 & -1.582782 & -1.600172 \\
\hline 6 & 4.581105 & -2.919727 & -1.740216 \\
\hline 6 & 5.834558 & -3.282749 & -1.329105 \\
\hline 6 & 6.695201 & -2.333547 & -0.724565 \\
\hline 6 & 6.267075 & -0.977419 & -0.602849 \\
\hline 6 & 7.116758 & -0.070358 & 0.080020 \\
\hline 6 & 8.329476 & -0.474270 & 0.581952 \\
\hline 6 & 8.762410 & -1.808344 & 0.431268 \\
\hline 6 & 7.955857 & -2.716636 & -0.205890 \\
\hline 8 & 2.879020 & -1.290278 & -2.032011 \\
\hline 6 & -1.427243 & 2.008007 & 0.667382 \\
\hline 6 & -0.334882 & 2.335094 & 1.475189 \\
\hline 6 & -0.178676 & 3.625705 & 1.980921 \\
\hline 6 & -1.115310 & 4.591477 & 1.596382 \\
\hline 6 & -2.193999 & 4.307540 & 0.755189 \\
\hline 6 & -2.342752 & 2.993397 & 0.304460 \\
\hline 6 & 0.945786 & 3.992743 & 2.949286 \\
\hline 6 & 1.859002 & 5.045464 & 2.314385 \\
\hline 6 & -3.189289 & 5.408523 & 0.392521 \\
\hline
\end{tabular}




\begin{tabular}{|c|c|c|c|}
\hline 6 & -3.896187 & 5.884766 & 1.666554 \\
\hline 6 & 0.335887 & 4.559620 & 4.236908 \\
\hline 6 & 1.792421 & 2.779419 & 3.329344 \\
\hline 6 & -2.449191 & 6.585035 & -0.252070 \\
\hline 6 & -4.253379 & 4.923467 & -0.589822 \\
\hline 6 & -6.604251 & 1.826140 & 1.845796 \\
\hline 6 & -8.134333 & 0.162433 & 0.832437 \\
\hline 6 & -5.950478 & 0.483627 & -4.156247 \\
\hline 6 & -4.253954 & -1.325583 & -4.303793 \\
\hline 1 & 6.788674 & 0.956283 & 0.220723 \\
\hline 1 & -1.799101 & 0.900305 & 2.914535 \\
\hline 1 & 8.265675 & -3.755166 & -0.315836 \\
\hline 1 & -1.452738 & -2.666624 & 6.005153 \\
\hline 1 & 4.972646 & 5.262781 & -2.275777 \\
\hline 1 & -3.645135 & -2.830858 & 1.070555 \\
\hline 1 & -5.588208 & -3.879857 & -0.000729 \\
\hline 1 & 3.052697 & 4.449022 & -1.040924 \\
\hline 1 & -1.749132 & -0.485228 & 4.931940 \\
\hline 1 & 6.178264 & -4.310010 & -1.439122 \\
\hline 1 & 7.970349 & 2.520784 & -3.675793 \\
\hline 1 & 3.892493 & -3.636967 & -2.182579 \\
\hline 1 & -3.135149 & -5.164183 & -3.292757 \\
\hline 1 & -0.758387 & -4.589648 & -3.344838 \\
\hline 1 & -0.736943 & -6.158148 & 3.601483 \\
\hline 1 & 6.808414 & 0.660260 & -2.590278 \\
\hline 1 & -0.833293 & -4.801101 & 1.559047 \\
\hline 1 & 1.202690 & -3.483093 & -2.303172 \\
\hline 1 & 7.083408 & 4.843999 & -3.504431 \\
\hline 1 & 8.958298 & 0.240327 & 1.108321 \\
\hline 1 & -5.341942 & -5.077902 & -2.173160 \\
\hline 1 & -1.048272 & -5.104650 & 5.836044 \\
\hline 1 & 9.726679 & -2.114718 & 0.829997 \\
\hline 1 & 1.782955 & 2.555326 & -0.023378 \\
\hline 1 & -4.026760 & 0.608759 & 1.560357 \\
\hline 1 & -2.886048 & -0.495457 & -2.407368 \\
\hline 1 & -7.040246 & -0.306996 & -1.344541 \\
\hline 1 & -3.178621 & 2.724561 & -0.337295 \\
\hline 1 & 0.381694 & 1.553800 & 1.730385 \\
\hline 1 & -1.003448 & 5.606528 & 1.977907 \\
\hline 1 & 1.493639 & -3.672715 & 1.313024 \\
\hline 6 & -1.194619 & 1.466995 & -2.933368 \\
\hline 1 & 2.870265 & -3.493958 & 0.221567 \\
\hline 1 & 4.813617 & -2.823455 & 1.276395 \\
\hline 1 & 1.080726 & -1.278551 & 2.714491 \\
\hline 1 & 6.022612 & -1.560227 & 3.024413 \\
\hline 1 & 2.274835 & -0.053132 & 4.504923 \\
\hline 1 & 4.754522 & -0.164830 & 4.652233 \\
\hline 1 & 2.560319 & 3.075115 & 4.055808 \\
\hline 1 & 1.181286 & 1.992010 & 3.793270 \\
\hline 1 & 2.313410 & 2.340873 & 2.467640 \\
\hline 1 & 1.128993 & 4.804773 & 4.955905 \\
\hline 1 & -0.240263 & 5.475248 & 4.057917 \\
\hline 1 & -0.334329 & 3.829006 & 4.708932 \\
\hline 1 & 2.649753 & 5.338091 & 3.018319 \\
\hline 1 & 2.344668 & 4.652464 & 1.411439 \\
\hline 1 & 1.308545 & 5.952228 & 2.035207 \\
\hline
\end{tabular}




$\begin{array}{rrrr}1 & -4.941426 & 5.746034 & -0.822185 \\ 1 & -3.813036 & 4.582807 & -1.535998 \\ 1 & -4.852619 & 4.100739 & -0.176772 \\ 1 & -4.616961 & 6.679100 & 1.429860 \\ 1 & -4.445172 & 5.060592 & 2.141497 \\ 1 & -3.189776 & 6.285082 & 2.404203 \\ 1 & -3.159955 & 7.376653 & -0.524751 \\ 1 & -1.705874 & 7.028542 & 0.421286 \\ 1 & -1.928478 & 6.268644 & -1.164881 \\ 1 & -7.318387 & 1.991195 & 2.663897 \\ 1 & -5.601815 & 2.060107 & 2.226580 \\ 1 & -6.835233 & 2.544584 & 1.048141 \\ 1 & -8.841013 & 0.331141 & 1.654851 \\ 1 & -8.402054 & 0.853871 & 0.023177 \\ 1 & -8.286667 & -0.863667 & 0.473235 \\ 1 & -7.176715 & -0.453701 & 3.279145 \\ 1 & -6.522991 & -1.633695 & 2.123567 \\ 1 & -5.442878 & -0.469893 & 2.910100 \\ 1 & -4.601995 & -1.600952 & -5.307518 \\ 1 & -3.459297 & -0.579051 & -4.432753 \\ 1 & -3.812202 & -2.220775 & -3.843216 \\ 1 & -6.250377 & 0.278894 & -5.193159 \\ 1 & -6.821026 & 0.892469 & -3.627392 \\ 1 & -5.173269 & 1.259529 & -4.173860 \\ 1 & -6.785391 & -2.124195 & -4.527113 \\ 1 & -6.191543 & -2.770458 & -2.982036 \\ 1 & -7.452177 & -1.518895 & -3.009504 \\ 8 & -1.788539 & 2.233558 & -3.565178\end{array}$

$2_{\text {C1-c- }-\psi-1 a 2 a 3 s 4 a}$

Number of imaginary frequencies : 0

The smallest frequencies are : $12.0511 \quad 14.5809 \quad 18.5516 \mathrm{~cm}(-1)$

Electronic energy : $\quad H F=-4012.1852253$

Zero-point correction $=\quad 1.290711($ Hartree/Particle $)$

Thermal correction to Energy= $\quad 1.367026$

Thermal correction to Enthalpy= $\quad 1.367971$

Thermal correction to Gibbs Free Energy $=\quad 1.178858$

Sum of electronic and zero-point Energies $=\quad-4010.894515$

Sum of electronic and thermal Energies $=\quad-4010.818199$

Sum of electronic and thermal Enthalpies $=\quad-4010.817255$

Sum of electronic and thermal Free Energies $=\quad-4011.006367$

Cartesian Coordinates

$\begin{array}{cccc}6 & -2.295546 & 3.013306 & 0.394665 \\ 6 & -1.410835 & 1.995598 & 0.744060 \\ 6 & -0.313871 & 2.276348 & 1.563101 \\ 6 & -0.118599 & 3.555488 & 2.084544 \\ 6 & -1.021677 & 4.555778 & 1.707955 \\ 6 & -2.107432 & 4.316121 & 0.862575 \\ 15 & -1.565385 & 0.318956 & 0.017534 \\ 6 & -1.469775 & -0.780125 & 1.511348 \\ 6 & -1.275667 & -2.144737 & 1.357507 \\ 6 & -1.244738 & -3.001770 & 2.505340 \\ 6 & -1.425395 & -2.443350 & 3.804096\end{array}$




\begin{tabular}{|c|c|c|c|}
\hline 6 & -1.644809 & -1.052171 & 3.924109 \\
\hline 6 & -1.665812 & -0.249330 & 2.815479 \\
\hline & -1.378042 & -3.286312 & 4.940113 \\
\hline & -1.158396 & -4.633260 & 4.806656 \\
\hline & -0.979011 & -5.192973 & 3.524001 \\
\hline & -1.024212 & -4.400811 & 2.403571 \\
\hline & -1.130768 & -2.781486 & 0.019560 \\
\hline & 0.095929 & -2.818918 & -0.619171 \\
\hline & 0.249748 & -3.503971 & -1.848422 \\
\hline 6 & -0.809638 & -4.150063 & -2.426954 \\
\hline 6 & -2.082648 & -4.133163 & -1.811179 \\
\hline & -2.253811 & -3.429503 & -0.581615 \\
\hline 6 & -3.547193 & -3.394534 & 0.001191 \\
\hline 6 & -4.602610 & -4.045847 & -0.586854 \\
\hline 6 & -4.420405 & -4.774182 & -1.782754 \\
\hline 6 & -3.188613 & -4.807075 & -2.384543 \\
\hline 7 & 1.228365 & -2.132160 & -0.055883 \\
\hline 5 & 1.636806 & -0.795335 & -1.005036 \\
\hline 8 & 2.846180 & -1.272095 & -2.056270 \\
\hline 6 & 4.122174 & -1.573063 & -1.638382 \\
\hline 6 & 4.959089 & -0.591508 & -1.135510 \\
\hline 6 & 6.240205 & -0.991339 & -0.633200 \\
\hline 6 & 6.668342 & -2.344203 & -0.786669 \\
\hline 6 & 5.806690 & -3.278474 & -1.412576 \\
\hline 6 & 4.552314 & -2.905974 & -1.812047 \\
\hline 6 & 7.090065 & -0.100581 & 0.070547 \\
\hline 6 & 8.302648 & -0.516396 & 0.563014 \\
\hline 6 & 8.735660 & -1.846493 & 0.380757 \\
\hline 6 & 7.929219 & -2.739431 & -0.277856 \\
\hline 6 & 4.489755 & 0.812191 & -1.095373 \\
\hline 6 & 3.278723 & 1.096855 & -0.497104 \\
\hline 6 & 2.743345 & 2.397578 & -0.431349 \\
\hline 6 & 3.444637 & 3.438451 & -0.977434 \\
\hline 6 & 4.674726 & 3.212818 & -1.641033 \\
\hline 6 & 5.198774 & 1.887308 & -1.725425 \\
\hline 6 & 5.376474 & 4.280172 & -2.252045 \\
\hline 6 & 6.537189 & 4.056656 & -2.946800 \\
\hline 6 & 7.035863 & 2.743170 & -3.068221 \\
\hline 6 & 6.385410 & 1.688973 & -2.475953 \\
\hline 8 & 2.547409 & 0.065201 & 0.066157 \\
\hline 6 & 1.012519 & 3.876038 & 3.061413 \\
\hline 6 & 1.842599 & 2.639530 & 3.399993 \\
\hline 6 & -3.052990 & 5.457946 & 0.493698 \\
\hline 6 & -4.178286 & 4.999308 & -0.431414 \\
\hline 6 & -3.348504 & 0.162924 & -0.396111 \\
\hline 6 & -3.705150 & -0.124589 & -1.706212 \\
\hline 6 & -5.050960 & -0.244326 & -2.081945 \\
\hline 6 & -6.012516 & -0.094370 & -1.087649 \\
\hline 6 & -5.686742 & 0.187301 & 0.250588 \\
\hline 6 & -4.342566 & 0.316330 & 0.579378 \\
\hline 6 & -6.801162 & 0.323533 & 1.286753 \\
\hline 6 & -6.264436 & 0.714064 & 2.662222 \\
\hline 6 & -5.392030 & -0.516178 & -3.544955 \\
\hline 6 & -6.897457 & -0.585412 & -3.786917 \\
\hline 45 & -0.126822 & 0.347370 & -1.915717 \\
\hline 6 & -1.073347 & 1.717424 & -2.788035 \\
\hline
\end{tabular}




\begin{tabular}{|c|c|c|c|}
\hline & -1.547785 & 2.627866 & -3.322967 \\
\hline & -7.527270 & -1.019611 & 1.419438 \\
\hline & -7.797850 & 1.400613 & 0.845549 \\
\hline & -4.816872 & 0.608959 & -4.413524 \\
\hline & -4.774797 & -1.850819 & -3.973370 \\
\hline & 2.129134 & -2.957037 & 0.776226 \\
\hline y & 2.836657 & -2.199949 & 1.866111 \\
\hline & 2.113045 & -1.472483 & 2.813115 \\
\hline & 2.770735 & -0.798473 & 3.833401 \\
\hline & 4.162572 & -0.829004 & 3.912760 \\
\hline & 4.889032 & -1.549685 & 2.971554 \\
\hline f & 4.225385 & -2.237138 & 1.958483 \\
\hline & 1.939773 & 4.936988 & 2.461381 \\
\hline b & 0.409357 & 4.409238 & 4.366527 \\
\hline & -3.686344 & 6.029147 & 1.767101 \\
\hline & -2.263938 & 6.557925 & -0.224330 \\
\hline 1 & 0.809010 & 0.457259 & -3.224677 \\
\hline & 6.762034 & 0.922392 & 0.235823 \\
\hline 1 & -1.833810 & 0.817577 & 2.939279 \\
\hline & 8.238891 & -3.775122 & -0.412267 \\
\hline & -1.517050 & -2.838794 & 5.923272 \\
\hline & 4.962403 & 5.284053 & -2.167433 \\
\hline ] & -3.697951 & -2.836260 & 0.923463 \\
\hline & -5.588401 & -3.999571 & -0.129041 \\
\hline 1 & 3.047630 & 4.451844 & -0.935990 \\
\hline & -1.797818 & -0.627021 & 4.915261 \\
\hline 1 & 6.150077 & -4.302759 & -1.548305 \\
\hline & 7.941109 & 2.561065 & -3.643161 \\
\hline & 3.864499 & -3.613411 & -2.270808 \\
\hline 1 & -3.038431 & -5.348179 & -3.317807 \\
\hline & -0.687062 & -4.681791 & -3.369118 \\
\hline 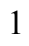 & -0.805963 & -6.261871 & 3.422348 \\
\hline ] & 6.777144 & 0.682044 & -2.592088 \\
\hline 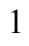 & -0.891168 & -4.847873 & 1.420502 \\
\hline 1 & 1.228744 & -3.500053 & -2.325095 \\
\hline & 7.064510 & 4.883473 & -3.416906 \\
\hline 1 & 8.931374 & 0.185445 & 1.106386 \\
\hline & -5.262954 & -5.295591 & -2.231909 \\
\hline 1 & -1.121430 & -5.272621 & 5.685599 \\
\hline 1 & 9.699852 & -2.162217 & 0.772314 \\
\hline 1 & 1.775387 & 2.541530 & 0.046642 \\
\hline 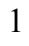 & -4.046735 & 0.541791 & 1.601645 \\
\hline d & -2.911289 & -0.258668 & -2.442642 \\
\hline 1 & -7.063654 & -0.193672 & -1.350911 \\
\hline 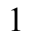 & -3.136670 & 2.780243 & -0.253898 \\
\hline 1 & 0.378259 & 1.470466 & 1.809278 \\
\hline 1 & -0.874653 & 5.563577 & 2.096872 \\
\hline 1 & 1.485102 & -3.724330 & 1.230056 \\
\hline 1 & 2.862657 & -3.490312 & 0.150911 \\
\hline 1 & 4.796822 & -2.798028 & 1.218248 \\
\hline 1 & 1.027753 & -1.422706 & 2.731885 \\
\hline 1 & 5.976436 & -1.577950 & 3.016646 \\
\hline 1 & 2.193381 & -0.244377 & 4.572018 \\
\hline ] & 4.676042 & -0.291947 & 4.707609 \\
\hline 1 & 2.622253 & 2.901619 & 4.126915 \\
\hline 1 & 1.222775 & 1.849775 & 3.847722 \\
\hline
\end{tabular}




$\begin{array}{rrrr}1 & 2.346805 & 2.216371 & 2.520580 \\ 1 & 1.204993 & 4.624992 & 5.092216 \\ 1 & -0.158703 & 5.334892 & 4.214446 \\ 1 & -0.267860 & 3.671256 & 4.816513 \\ 1 & 2.734678 & 5.193935 & 3.174453 \\ 1 & 2.419794 & 4.568921 & 1.545076 \\ 1 & 1.402882 & 5.861050 & 2.214499 \\ 1 & -4.832288 & 5.849077 & -0.664219 \\ 1 & -3.794454 & 4.608585 & -1.382780 \\ 1 & -4.800864 & 4.221529 & 0.031720 \\ 1 & -4.371387 & 6.850270 & 1.516562 \\ 1 & -4.261483 & 5.259663 & 2.298865 \\ 1 & -2.935897 & 6.425240 & 2.461927 \\ 1 & -2.930628 & 7.384921 & -0.503668 \\ 1 & -1.467323 & 6.972727 & 0.405290 \\ 1 & -1.800147 & 6.171396 & -1.141082 \\ 1 & -8.592555 & 1.511468 & 1.595407 \\ 1 & -7.302208 & 2.373446 & 0.732612 \\ 1 & -8.279615 & 1.158421 & -0.108977 \\ 1 & -7.097606 & 0.807567 & 3.370064 \\ 1 & -5.574490 & -0.038734 & 3.066225 \\ 1 & -5.742084 & 1.679909 & 2.636732 \\ 1 & -8.342428 & -0.944406 & 2.151890 \\ 1 & -7.963357 & -1.346529 & 0.467479 \\ 1 & -6.838330 & -1.803865 & 1.760253 \\ 1 & -7.090469 & -0.778118 & -4.849710 \\ 1 & -7.368616 & -1.396505 & -3.216374 \\ 1 & -7.399475 & 0.355922 & -3.527664 \\ 1 & -5.003274 & -2.053656 & -5.028609 \\ 1 & -3.682777 & -1.854958 & -3.863296 \\ 1 & -5.172235 & -2.677642 & -3.370786 \\ 1 & -5.078497 & 0.445630 & -5.467623 \\ 1 & -5.215855 & 1.586376 & -4.112227 \\ 1 & -3.723038 & 0.659439 & -4.350504\end{array}$

$2_{\text {C1-c- }-\psi_{-1 a 2 a 3 s 4 s}}$

Number of imaginary frequencies : 0

The smallest frequencies are : $12.5520 \quad 15.2408 \quad 17.2435 \mathrm{~cm}(-1)$

Electronic energy : $\quad H F=-4012.1855671$

Zero-point correction $=\quad 1.290400($ Hartree/Particle $)$

Thermal correction to Energy= $\quad 1.366749$

Thermal correction to Enthalpy= $\quad 1.367693$

Thermal correction to Gibbs Free Energy $=\quad 1.178340$

Sum of electronic and zero-point Energies $=\quad-4010.895167$

Sum of electronic and thermal Energies $=\quad-4010.818818$

Sum of electronic and thermal Enthalpies $=\quad-4010.817874$

Sum of electronic and thermal Free Energies $=\quad-4011.007227$

Cartesian Coordinates

$\begin{array}{rrrr}6 & -3.688537 & -0.165459 & -1.752608 \\ 6 & -3.331252 & 0.099560 & -0.434079 \\ 6 & -4.324148 & 0.224311 & 0.537199 \\ 6 & -5.672191 & 0.093588 & 0.209308 \\ 6 & -6.000144 & -0.150148 & -1.130219\end{array}$




\begin{tabular}{|c|c|c|c|}
\hline 6 & -5.031174 & -0.276031 & -2.128716 \\
\hline 1 & -1.555878 & 0.295803 & -0.007406 \\
\hline & -0.107043 & 0.348287 & -1.931017 \\
\hline 1 & 0.832941 & 0.475042 & -3.235967 \\
\hline 6 & -6.716568 & 0.226958 & 1.316161 \\
\hline 6 & -6.425697 & -0.805680 & 2.412179 \\
\hline 6 & -5.374208 & -0.536039 & -3.594072 \\
\hline 6 & -4.728431 & 0.542678 & -4.471782 \\
\hline 6 & -1.450273 & -0.783409 & 1.501810 \\
\hline 6 & -1.240744 & -2.147292 & 1.363319 \\
\hline 6 & -1.202670 & -2.992600 & 2.518800 \\
\hline 6 & -1.394874 & -2.423950 & 3.811262 \\
\hline 6 & -1.630538 & -1.034303 & 3.916518 \\
\hline 6 & -1.655455 & -0.241886 & 2.800133 \\
\hline 6 & -0.967234 & -4.389969 & 2.430451 \\
\hline 6 & -0.918426 & -5.171247 & 3.558331 \\
\hline 6 & -1.109069 & -4.601485 & 4.835045 \\
\hline 6 & -1.343375 & -3.255723 & 4.955276 \\
\hline 6 & -1.094787 & -2.788987 & 0.028723 \\
\hline 6 & 0.127161 & -2.809347 & -0.619887 \\
\hline 6 & 0.275713 & -3.480540 & -1.857347 \\
\hline 6 & -0.784952 & -4.125815 & -2.435049 \\
\hline 6 & -2.052406 & -4.127242 & -1.807679 \\
\hline 6 & -2.217146 & -3.442751 & -0.566498 \\
\hline 6 & -3.159216 & -4.800379 & -2.380646 \\
\hline 6 & -4.384215 & -4.788736 & -1.764482 \\
\hline 6 & -4.558799 & -4.084345 & -0.553121 \\
\hline 6 & -3.503931 & -3.430059 & 0.032084 \\
\hline 7 & 1.256568 & -2.115374 & -0.059854 \\
\hline 6 & 2.151678 & -2.926777 & 0.791142 \\
\hline 6 & 2.855294 & -2.153809 & 1.872509 \\
\hline 6 & 2.130761 & -1.398140 & 2.796569 \\
\hline 6 & 2.786075 & -0.709480 & 3.808520 \\
\hline 6 & 4.176555 & -0.754707 & 3.903275 \\
\hline 6 & 4.903944 & -1.503720 & 2.985357 \\
\hline 6 & 4.242496 & -2.204635 & 1.980016 \\
\hline 15 & 1.661951 & -0.779357 & -1.010992 \\
\hline 8 & 2.560354 & 0.091367 & 0.062207 \\
\hline 6 & 3.291896 & 1.122886 & -0.500723 \\
\hline 6 & 4.509342 & 0.840073 & -1.086972 \\
\hline 6 & 5.219584 & 1.915073 & -1.715671 \\
\hline 6 & 4.689304 & 3.238803 & -1.642420 \\
\hline 6 & 3.451919 & 3.462612 & -0.991883 \\
\hline 6 & 2.749958 & 2.421544 & -0.447090 \\
\hline 6 & 6.413816 & 1.718093 & -2.454431 \\
\hline 6 & 7.065480 & 2.772178 & -3.045535 \\
\hline 6 & 6.560417 & 4.084171 & -2.934823 \\
\hline 6 & 5.392392 & 4.306149 & -2.251929 \\
\hline 6 & 4.983278 & -0.562517 & -1.117453 \\
\hline 6 & 4.152536 & -1.548005 & -1.622923 \\
\hline 6 & 4.584793 & -2.881519 & -1.784811 \\
\hline 6 & 5.836317 & -3.250175 & -1.372927 \\
\hline 6 & 6.691948 & -2.310973 & -0.746170 \\
\hline 6 & 6.261045 & -0.957806 & -0.603246 \\
\hline 6 & 7.104680 & -0.062067 & 0.101681 \\
\hline 6 & 8.314386 & -0.473448 & 0.604835 \\
\hline
\end{tabular}




\begin{tabular}{|c|c|c|c|}
\hline 6 & 8.750319 & -1.804005 & 0.433136 \\
\hline 6 & 7.949615 & -2.701708 & -0.225988 \\
\hline 8 & 2.879983 & -1.250186 & -2.053845 \\
\hline 6 & -1.458264 & 1.981956 & 0.708264 \\
\hline 6 & -0.364295 & 2.303198 & 1.516376 \\
\hline 6 & -0.220630 & 3.583469 & 2.051190 \\
\hline 6 & -1.170624 & 4.547021 & 1.693968 \\
\hline 6 & -2.251475 & 4.269395 & 0.853870 \\
\hline 6 & -2.389845 & 2.963137 & 0.377503 \\
\hline 6 & 0.907103 & 3.942282 & 3.018830 \\
\hline 6 & 1.809298 & 5.011350 & 2.395457 \\
\hline 6 & -3.260993 & 5.366098 & 0.518798 \\
\hline 6 & -3.982507 & 5.790919 & 1.802675 \\
\hline 6 & 0.302545 & 4.484368 & 4.319550 \\
\hline 6 & 1.763193 & 2.727884 & 3.373312 \\
\hline 6 & -2.536287 & 6.573111 & -0.085519 \\
\hline 6 & -4.310837 & 4.895191 & -0.485629 \\
\hline 6 & -6.646964 & 1.639896 & 1.907726 \\
\hline 6 & -8.137724 & -0.008029 & 0.811239 \\
\hline 6 & -4.831150 & -1.909742 & -3.999811 \\
\hline 6 & -6.879066 & -0.520119 & -3.850779 \\
\hline 1 & 6.773906 & 0.961254 & 0.259209 \\
\hline 1 & -1.835639 & 0.824223 & 2.913247 \\
\hline 1 & 8.261558 & -3.737735 & -0.352320 \\
\hline 1 & -1.491323 & -2.800663 & 5.933644 \\
\hline 1 & 4.973522 & 5.308717 & -2.175867 \\
\hline 1 & -3.650320 & -2.885640 & 0.963908 \\
\hline 1 & -5.538680 & -4.057853 & -0.081260 \\
\hline 1 & 3.050377 & 4.474553 & -0.960082 \\
\hline 1 & -1.792053 & -0.601170 & 4.902860 \\
\hline 1 & 6.182021 & -4.274865 & -1.4 \\
\hline 1 & 7.976723 & 2.591102 & -3.611250 \\
\hline 1 & 3.900798 & -3.592233 & \\
\hline 1 & -3.015066 & -5.324370 & -3.3 \\
\hline 1 & -0.666931 & -4.644197 & -3.385201 \\
\hline 1 & -0.733956 & -6.239205 & 3.467479 \\
\hline 1 & 6.810638 & 0.712238 & -2.562277 \\
\hline 1 & -0.825677 & -4.844103 & \\
\hline 1 & 1.250652 & -3.465367 & -2.342184 \\
\hline 1 & 7.088745 & 4.910952 & -3.403854 \\
\hline 1 & 8.938380 & 0.232246 & 1.148670 \\
\hline 1 & -5.227123 & -5.310253 & -2.212864 \\
\hline 1 & -1.069233 & -5.232306 & 5.720006 \\
\hline 1 & 9.712154 & -2.116267 & 0.833174 \\
\hline 1 & 1.776160 & 2.563387 & 0.019780 \\
\hline 1 & -4.036596 & 0.432303 & 1.567650 \\
\hline 1 & -2.893595 & -0.287041 & -2.489846 \\
\hline 1 & -7.047646 & -0.241939 & -1.402376 \\
\hline 1 & -3.227825 & 2.699140 & -0.263429 \\
\hline 1 & 0.362831 & 1.524476 & 1.748910 \\
\hline 1 & -1.066984 & 5.554844 & 2.096230 \\
\hline 1 & 1.504187 & -3.685731 & 1.254118 \\
\hline 6 & -1.081644 & 1.696056 & -2.807271 \\
\hline 1 & 2.887537 & -3.471067 & 0.178166 \\
\hline 1 & 4.814945 & -2.786764 & 1.257178 \\
\hline 1 & 1.047250 & -1.334802 & 2.701182 \\
\hline
\end{tabular}




$\begin{array}{rrrr}1 & 5.990434 & -1.543363 & 3.042076 \\ 1 & 2.207995 & -0.132232 & 4.528604 \\ 1 & 4.688239 & -0.206722 & 4.691754 \\ 1 & 2.532756 & 3.014813 & 4.101532 \\ 1 & 1.158304 & 1.928801 & 3.825212 \\ 1 & 2.282741 & 2.307752 & 2.501551 \\ 1 & 1.099162 & 4.721291 & 5.037413 \\ 1 & -0.278833 & 5.400276 & 4.159914 \\ 1 & -0.361147 & 3.742884 & 4.783656 \\ 1 & 2.602600 & 5.297717 & 3.099109 \\ 1 & 2.291753 & 4.636713 & 1.483056 \\ 1 & 1.250841 & 5.918719 & 2.134694 \\ 1 & -5.005828 & 5.715905 & -0.703419 \\ 1 & -3.857203 & 4.583054 & -1.435446 \\ 1 & -4.905119 & 4.056066 & -0.099405 \\ 1 & -4.714378 & 6.581152 & 1.587068 \\ 1 & -4.520854 & 4.943291 & 2.247608 \\ 1 & -3.285986 & 6.178255 & 2.556559 \\ 1 & -3.258406 & 7.361086 & -0.338058 \\ 1 & -1.803681 & 7.008504 & 0.604545 \\ 1 & -2.006136 & 6.292750 & -1.004706 \\ 1 & -7.358076 & 1.744058 & 2.738468 \\ 1 & -5.646627 & 1.879557 & 2.290797 \\ 1 & -6.902445 & 2.391965 & 1.149678 \\ 1 & -8.845617 & 0.088172 & 1.644199 \\ 1 & -8.429955 & 0.724440 & 0.048006 \\ 1 & -8.260878 & -1.013574 & 0.388270 \\ 1 & -7.170922 & -0.730441 & 3.215484 \\ 1 & -6.466523 & -1.825854 & 2.007344 \\ 1 & -5.435572 & -0.668710 & 2.865470 \\ 1 & -7.071649 & -0.695587 & -4.916641 \\ 1 & -7.401494 & -1.307253 & -3.291800 \\ 1 & -7.329886 & 0.446165 & -3.589116 \\ 1 & -5.048428 & -2.108828 & -5.058274 \\ 1 & -3.744317 & -1.977952 & -3.861878 \\ 1 & -5.291813 & -2.704929 & -3.399273 \\ 1 & -5.004874 & 0.389436 & -5.523535 \\ 1 & -5.060245 & 1.546769 & -4.176586 \\ 1 & -3.633437 & 0.522185 & -4.415815 \\ 8 & -1.582057 & 2.590760 & -3.344705\end{array}$

$2_{\text {C1-c- }-\psi_{-12323 a 4 a}}$

Number of imaginary frequencies : 0

The smallest frequencies are : $14.4581 \quad 15.5372 \quad 20.2672 \mathrm{~cm}(-1)$

Electronic energy : $\quad \mathrm{HF}=-4012.187141$

Zero-point correction $=\quad 1.291200($ Hartree/Particle $)$

Thermal correction to Energy= $\quad 1.367139$

Thermal correction to Enthalpy $=\quad 1.368083$

Thermal correction to Gibbs Free Energy= 1.181596

Sum of electronic and zero-point Energies $=\quad-4010.895941$

Sum of electronic and thermal Energies $=\quad-4010.820002$

Sum of electronic and thermal Enthalpies $=\quad-4010.819058$

Sum of electronic and thermal Free Energies $=\quad-4011.005545$

Cartesian Coordinates 


\begin{tabular}{cccc}
\hline 6 & 2.166863 & -1.358939 & 2.813811 \\
6 & 2.873076 & -2.141168 & 1.897618 \\
6 & 4.260520 & -2.206862 & 1.992605 \\
6 & 4.940384 & -1.494620 & 2.977511 \\
6 & 4.231245 & -0.718789 & 3.887373 \\
6 & 2.840515 & -0.658546 & 3.805374 \\
6 & 2.149549 & -2.925089 & 0.837493 \\
7 & 1.255402 & -2.117461 & -0.017306 \\
15 & 1.664414 & -0.799595 & -0.991072 \\
45 & -0.127553 & 0.275018 & -1.945914 \\
1 & 0.764253 & 0.270980 & -3.289709 \\
6 & 0.113416 & -2.805381 & -0.559738 \\
6 & -1.103912 & -2.759598 & 0.096634 \\
6 & -2.242593 & -3.391540 & -0.491521 \\
6 & -2.101477 & -4.064544 & -1.742056 \\
6 & -0.835569 & -4.099724 & -2.371243 \\
6 & 0.243627 & -3.485677 & -1.794221 \\
6 & -3.526054 & -3.352164 & 0.113117 \\
6 & -4.606079 & -3.949643 & -0.486986 \\
6 & -4.459481 & -4.629615 & -1.716111 \\
6 & -3.233463 & -4.682047 & -2.329062 \\
6 & -1.229538 & -2.093427 & 1.421779 \\
6 & -1.421229 & -0.724175 & 1.537108 \\
6 & -1.599456 & -0.155593 & 2.827647 \\
6 & -1.562372 & -0.926256 & 3.958866 \\
6 & -1.347088 & -2.320732 & 3.876726 \\
6 & -1.185489 & -2.916668 & 2.592415 \\
6 & -1.287283 & -3.131368 & 5.035416 \\
6 & -1.074735 & -4.482603 & 4.937008 \\
6 & -0.915494 & -5.079380 & 3.668305 \\
6 & -0.972605 & -4.318819 & 2.526697 \\
15 & -1.540397 & 0.324819 & 0.006907 \\
6 & -3.326854 & 0.135580 & -0.379755 \\
6 & -4.312750 & 0.372688 & 0.582428 \\
6 & -5.662031 & 0.216662 & 0.272542 \\
6 & -5.991293 & -0.176205 & -1.032123 \\
6 & -5.032395 & -0.432516 & -2.012649 \\
6 & -3.689088 & -0.278117 & -1.657808 \\
6 & -6.768503 & 0.423852 & 1.305699 \\
6 & -6.225503 & 0.934188 & 2.639126 \\
6 & -5.446234 & -0.801803 & -3.435921 \\
6 & -5.955794 & 0.467749 & -4.128290 \\
6 & -1.402079 & 2.025777 & 0.677386 \\
6 & -0.304052 & 2.344286 & 1.488135 \\
6 & -0.136538 & 3.630281 & 1.991540 \\
6 & -1.070278 & 4.609751 & 1.615173 \\
6 & -2.150291 & 4.333712 & 0.781040 \\
6 & -2.302706 & 3.019083 & 0.318248 \\
6 & -3.187643 & 5.381530 & 0.383177 \\
6 & -3.212202 & 5.524381 & -1.142373 \\
6 & 0.995421 & 3.990074 & 2.953682 \\
6 & 1.910483 & 5.038750 & 2.314556 \\
6 & -7.791632 & 1.440980 & 0.789902 \\
6 & -7.465288 & -0.918592 & 1.556352 \\
6 & -6.555602 & -1.856815 & -3.426053 \\
& & & \\
6 & & &
\end{tabular}




\begin{tabular}{|c|c|c|c|}
\hline 6 & -4.275627 & -1.359117 & -4.246445 \\
\hline 6 & -4.569091 & 4.930227 & 0.873925 \\
\hline & -2.892669 & 6.752453 & 0.985957 \\
\hline & 0.397262 & 4.558859 & 4.246060 \\
\hline & 1.837483 & 2.771745 & 3.327094 \\
\hline & 2.877257 & -1.293433 & -2.028407 \\
\hline & 4.151509 & -1.584509 & -1.598405 \\
\hline & 4.986884 & -0.589852 & -1.119461 \\
\hline 6 & 6.268204 & -0.975762 & -0.607085 \\
\hline b & 6.696386 & -2.332142 & -0.725420 \\
\hline & 5.834913 & -3.283182 & -1.325873 \\
\hline 6 & 4.580625 & -2.921684 & -1.735756 \\
\hline & 7.957857 & -2.713486 & -0.207477 \\
\hline & 8.765123 & -1.803165 & 0.425880 \\
\hline & 8.332248 & -0.468691 & 0.573094 \\
\hline & 7.118761 & -0.066448 & 0.071649 \\
\hline & 4.513629 & 0.813137 & -1.116014 \\
\hline & 3.298955 & 1.108756 & -0.530026 \\
\hline & 2.757112 & 2.408109 & -0.502972 \\
\hline & 3.456234 & 3.437034 & -1.074078 \\
\hline 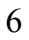 & 4.690241 & 3.199082 & -1.725740 \\
\hline & 5.220085 & 1.873947 & -1.772491 \\
\hline & 6.409810 & 1.660377 & -2.513960 \\
\hline & 7.057953 & 2.700956 & -3.132190 \\
\hline & 6.553849 & 4.015286 & -3.047312 \\
\hline & 5.389976 & 4.252632 & -2.362588 \\
\hline & 2.569725 & 0.090117 & 0.060159 \\
\hline 1 & 6.790733 & 0.960594 & 0.209580 \\
\hline & -1.766372 & 0.914385 & 2.923565 \\
\hline 1 & 8.267680 & -3.752296 & -0.314726 \\
\hline & -1.411929 & -2.655851 & 6.007255 \\
\hline 1 & 4.971777 & 5.256765 & -2.306049 \\
\hline & -3.653171 & -2.822399 & 1.055594 \\
\hline & -5.586063 & -3.892807 & -0.016995 \\
\hline 1 & 3.054570 & 4.449344 & -1.062028 \\
\hline & -1.701324 & -0.472088 & 4.939176 \\
\hline 1 & 6.178682 & -4.310652 & -1.433711 \\
\hline & 7.965632 & 2.507181 & -3.699414 \\
\hline ] & 3.891799 & -3.640514 & -2.175079 \\
\hline & -3.107786 & -5.195904 & -3.281208 \\
\hline ] & -0.735205 & -4.614964 & -3.325067 \\
\hline & -0.748799 & -6.151589 & 3.594784 \\
\hline & 6.805776 & 0.652286 & -2.602131 \\
\hline 1 & -0.855462 & -4.793321 & 1.554519 \\
\hline & 1.217093 & -3.493633 & -2.282362 \\
\hline 1 & 7.079569 & 4.831405 & -3.537465 \\
\hline & 8.961733 & 0.247584 & 1.096378 \\
\hline 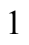 & -5.321894 & -5.108253 & -2.175580 \\
\hline 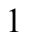 & -1.028526 & -5.097007 & 5.833129 \\
\hline 1 & 9.729974 & -2.108202 & 0.824227 \\
\hline 1 & 1.786784 & 2.561177 & -0.032662 \\
\hline & -4.013775 & 0.688614 & 1.579684 \\
\hline 1 & -2.896583 & -0.489795 & -2.372839 \\
\hline & -7.044339 & -0.289486 & -1.288730 \\
\hline & -3.142389 & 2.766310 & -0.328839 \\
\hline & 0.404770 & 1.555993 & 1.743204 \\
\hline
\end{tabular}




$\begin{array}{rrrr}1 & -0.943628 & 5.619186 & 2.000774 \\ 1 & 1.498306 & -3.668742 & 1.319586 \\ 6 & -1.197860 & 1.446089 & -2.951085 \\ 1 & -5.525343 & 0.224061 & 3.098782 \\ 1 & -5.713151 & 1.899593 & 2.530343 \\ 1 & -7.054556 & 1.077702 & 3.343438 \\ 1 & -8.275878 & 1.113353 & -0.137516 \\ 1 & -8.582850 & 1.593288 & 1.536104 \\ 1 & -7.320355 & 2.413340 & 0.595743 \\ 1 & -7.909166 & -1.327608 & 0.640249 \\ 1 & -6.754018 & -1.660449 & 1.943699 \\ 1 & -8.269591 & -0.802382 & 2.295473 \\ 1 & -5.171536 & 1.236044 & -4.159991 \\ 1 & -6.258580 & 0.248595 & -5.161396 \\ 1 & -6.821777 & 0.893216 & -3.604953 \\ 1 & -7.475426 & -1.499418 & -2.948052 \\ 1 & -6.815812 & -2.137124 & -4.455183 \\ 1 & -6.228034 & -2.763468 & -2.899678 \\ 1 & -3.476549 & -0.620459 & -4.392255 \\ 1 & -3.838566 & -2.247138 & -3.768105 \\ 1 & -4.627259 & -1.652863 & -5.243669 \\ 1 & 2.872824 & -3.487573 & 0.225793 \\ 1 & 4.818583 & -2.809415 & 1.275329 \\ 1 & 1.083288 & -1.283882 & 2.728096 \\ 1 & 6.026895 & -1.546108 & 3.023831 \\ 1 & 2.276555 & -0.060139 & 4.519376 \\ 1 & 4.757327 & -0.161473 & 4.659682 \\ 1 & 2.612819 & 3.062175 & 4.047788 \\ 1 & 1.224584 & 1.988372 & 3.795387 \\ 1 & 2.348800 & 2.329847 & 2.461383 \\ 1 & 1.196601 & 4.795369 & 4.961046 \\ 1 & -0.171715 & 5.479942 & 4.072889 \\ 1 & -0.276281 & 3.832621 & 4.720126 \\ 1 & 2.707223 & 5.325697 & 3.014064 \\ 1 & 2.388356 & 4.644498 & 1.408007 \\ 1 & 1.363590 & 5.949215 & 2.040638 \\ 1 & -3.665270 & 7.465822 & 0.672670 \\ 1 & -2.893388 & 6.729082 & 2.083529 \\ 1 & -1.925504 & 7.148185 & 0.650076 \\ 1 & -5.333014 & 5.669045 & 0.596523 \\ 1 & -4.865364 & 3.967695 & 0.437056 \\ 1 & -4.582416 & 4.823206 & 1.966808 \\ 1 & -3.947809 & 6.283237 & -1.441434 \\ 1 & -2.231036 & 5.833503 & -1.524786 \\ 1 & -3.483769 & 4.586617 & -1.640935 \\ 8 & -1.789131 & 2.202334 & -3.597780\end{array}$

$2_{\text {C1-c- }-\psi-1 a 2 s 3 a 4 s}$

Number of imaginary frequencies : 0

The smallest frequencies are : $14.0626 \quad 16.9330 \quad 18.9197 \mathrm{~cm}(-1)$

Electronic energy : $\quad \mathrm{HF}=-4012.1873103$

Zero-point correction $=\quad 1.290735$ (Hartree/Particle)

Thermal correction to Energy= $\quad 1.366841$

Thermal correction to Enthalpy $=\quad 1.367785$

Thermal correction to Gibbs Free Energy $=\quad 1.180433$ 
Sum of electronic and zero-point Energies= Sum of electronic and thermal Energies= Sum of electronic and thermal Enthalpies= Sum of electronic and thermal Free Energies=
$-4010.896576$

$-4010.820469$

$-4010.819525$

$-4011.006877$

Cartesian Coordinates

\begin{tabular}{|c|c|c|c|}
\hline 6 & -3.717737 & -0.218709 & -1.690576 \\
\hline 6 & -3.338479 & 0.148820 & -0.398694 \\
\hline 6 & -4.311511 & 0.338574 & 0.578498 \\
\hline 6 & -5.668641 & 0.178102 & 0.286174 \\
\hline 6 & -6.015702 & -0.157842 & -1.023846 \\
\hline 6 & -5.062424 & -0.364988 & -2.029367 \\
\hline 15 & -1.549577 & 0.328173 & -0.013203 \\
\hline 45 & -0.116597 & 0.306912 & -1.948474 \\
\hline 1 & 0.829193 & 0.395281 & -3.252669 \\
\hline 6 & -6.692240 & 0.343408 & 1.408250 \\
\hline 6 & -6.394083 & -0.688133 & 2.503729 \\
\hline 6 & -5.502608 & -0.686900 & -3.456220 \\
\hline 6 & -6.560797 & -1.793726 & -3.461871 \\
\hline 6 & -1.441421 & -0.736230 & 1.507441 \\
\hline 6 & -1.247746 & -2.103765 & 1.379003 \\
\hline 6 & -1.218561 & -2.940930 & 2.540008 \\
\hline 6 & -1.400114 & -2.360597 & 3.828760 \\
\hline 6 & -1.617645 & -0.967221 & 3.924399 \\
\hline 6 & -1.637192 & -0.182841 & 2.802094 \\
\hline 6 & -1.003917 & -4.341998 & 2.460196 \\
\hline 6 & -0.963063 & -5.116312 & 3.593231 \\
\hline 6 & -1.141529 & -4.534964 & 4.866529 \\
\hline 6 & -1.356803 & -3.185196 & 4.978251 \\
\hline 6 & -1.114313 & -2.752386 & 0.046311 \\
\hline 6 & 0.105500 & -2.791809 & -0.605262 \\
\hline 6 & 0.237021 & -3.448900 & -1.852141 \\
\hline 6 & -0.843662 & -4.043740 & -2.446593 \\
\hline 6 & -2.112093 & -4.013460 & -1.822386 \\
\hline 6 & -2.253621 & -3.366496 & -0.558369 \\
\hline 6 & -3.245876 & -4.611764 & -2.425608 \\
\hline 6 & -4.472621 & -4.567960 & -1.813380 \\
\hline 6 & -4.619089 & -3.913980 & -0.570071 \\
\hline 6 & -3.538063 & -3.333550 & 0.044201 \\
\hline 7 & 1.247133 & -2.116997 & -0.046168 \\
\hline 6 & 2.132738 & -2.939935 & 0.802869 \\
\hline 6 & 2.852444 & -2.174358 & 1.878846 \\
\hline 6 & 2.142946 & -1.409214 & 2.806859 \\
\hline 6 & 2.813199 & -0.727408 & 3.813684 \\
\hline 6 & 4.203675 & -0.788689 & 3.898977 \\
\hline 6 & 4.916076 & -1.546995 & 2.976960 \\
\hline 6 & 4.239607 & -2.241233 & 1.977074 \\
\hline 15 & 1.665747 & -0.787002 & -0.999444 \\
\hline 8 & 2.572771 & 0.082707 & 0.066659 \\
\hline 6 & 3.309741 & 1.105293 & -0.506695 \\
\hline 6 & 4.524287 & 0.810680 & -1.093269 \\
\hline 6 & 5.239461 & 1.876586 & -1.731709 \\
\hline 6 & 4.717357 & 3.204072 & -1.667460 \\
\hline 6 & 3.482483 & 3.440042 & -1.016646 \\
\hline 6 & 2.775397 & 2.407209 & -0.462734 \\
\hline
\end{tabular}




\begin{tabular}{|c|c|c|c|}
\hline 6 & 6.430727 & 1.666686 & -2.471740 \\
\hline 6 & 7.087448 & 2.712252 & -3.072227 \\
\hline & 6.590796 & 4.028190 & -2.970119 \\
\hline & 5.425739 & 4.262520 & -2.286361 \\
\hline & 4.988963 & -0.595003 & -1.115167 \\
\hline & 4.149454 & -1.577771 & -1.611227 \\
\hline & 4.571399 & -2.915430 & -1.765560 \\
\hline & 5.821758 & -3.290200 & -1.355524 \\
\hline & 6.686257 & -2.352990 & -0.737922 \\
\hline & 6.265601 & -0.995810 & -0.602644 \\
\hline & 7.118281 & -0.101260 & 0.092847 \\
\hline & 8.326812 & -0.517875 & 0.594477 \\
\hline & 8.752446 & -1.852753 & 0.430604 \\
\hline ) & 7.942881 & -2.749209 & -0.219368 \\
\hline & 2.878269 & -1.272999 & -2.041082 \\
\hline & -1.405069 & 2.020620 & 0.677898 \\
\hline b & -0.310209 & 2.319344 & 1.500213 \\
\hline & -0.135678 & 3.597226 & 2.021296 \\
\hline D & -1.056848 & 4.589696 & 1.648258 \\
\hline & -2.132870 & 4.334143 & 0.802374 \\
\hline & -2.296047 & 3.025394 & 0.325735 \\
\hline & 0.989653 & 3.934059 & 2.999305 \\
\hline 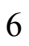 & 1.914689 & 4.991263 & 2.389171 \\
\hline 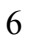 & -3.144577 & 5.403511 & 0.394843 \\
\hline 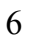 & -3.103725 & 5.586528 & -1.126220 \\
\hline b & 0.381463 & 4.480418 & 4.296715 \\
\hline ) & 1.823430 & 2.705278 & 3.356948 \\
\hline & -4.550680 & 4.958094 & 0.814116 \\
\hline & -2.859384 & 6.754280 & 1.045965 \\
\hline 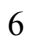 & -6.595291 & 1.755694 & 1.995171 \\
\hline 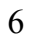 & -8.124972 & 0.125390 & 0.929087 \\
\hline 6 & -6.091683 & 0.585483 & -4.076391 \\
\hline 6 & -4.334608 & -1.152517 & -4.324859 \\
\hline & 6.795738 & 0.925648 & 0.243964 \\
\hline & -1.803764 & 0.886295 & 2.908337 \\
\hline & 8.246915 & -3.788303 & -0.339646 \\
\hline ] & -1.496070 & -2.721611 & 5.953893 \\
\hline & 5.013257 & 5.268202 & -2.216827 \\
\hline 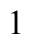 & -3.662971 & -2.823043 & 0.997883 \\
\hline 1 & -5.599216 & -3.862577 & -0.099919 \\
\hline & 3.086759 & 4.454451 & -0.991499 \\
\hline 1 & -1.770466 & -0.524905 & 4.908069 \\
\hline & 6.159695 & -4.318162 & -1.476340 \\
\hline 1 & 7.996187 & 2.521330 & -3.638724 \\
\hline & 3.880431 & -3.624123 & -2.217790 \\
\hline 1 & -3.120624 & -5.106115 & -3.388047 \\
\hline l & -0.742123 & -4.539825 & -3.410387 \\
\hline 1 & -0.794572 & -6.187474 & 3.509299 \\
\hline 1 & 6.821061 & 0.657624 & -2.572999 \\
\hline ] & -0.872579 & -4.804237 & 1.483886 \\
\hline 1 & 1.212579 & -3.453993 & -2.336039 \\
\hline ( & 7.123204 & 4.848159 & -3.446431 \\
\hline 1 & 8.957950 & 0.187095 & 1.130954 \\
\hline ] & -5.336018 & -5.033493 & -2.284371 \\
\hline 1 & -1.107997 & -5.160171 & 5.755733 \\
\hline 1 & 9.713441 & -2.169199 & 0.829370 \\
\hline
\end{tabular}




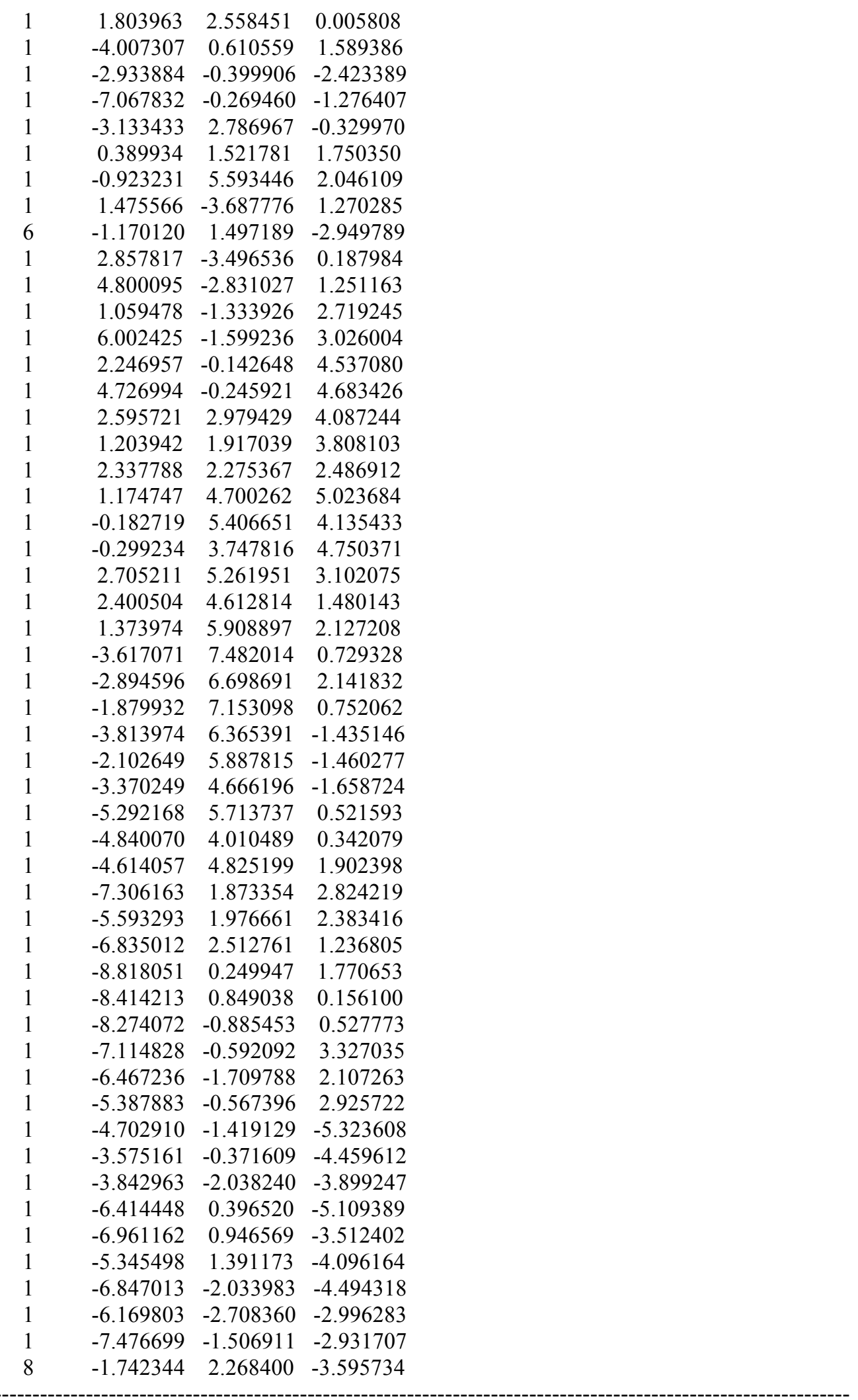

$\mathbf{2}_{\text {C1-c- }} \boldsymbol{\psi}_{\text {-1a2s3s4a }}$ (3s converted to 3a during optimization)

Number of imaginary frequencies : 0 
The smallest frequencies are : $14.9085 \quad 17.6921 \quad 19.2353 \mathrm{~cm}(-1)$

Electronic energy : $\quad H F=-4012.1871116$

Zero-point correction $=\quad 1.290629($ Hartree/Particle $)$

Thermal correction to Energy= $\quad 1.366767$

Thermal correction to Enthalpy= $\quad 1.367711$

Thermal correction to Gibbs Free Energy $=\quad 1.180501$

Sum of electronic and zero-point Energies $=\quad-4010.896483$

Sum of electronic and thermal Energies $=\quad-4010.820344$

Sum of electronic and thermal Enthalpies $=\quad-4010.819400$

Sum of electronic and thermal Free Energies $=\quad-4011.006611$

Cartesian Coordinates

\begin{tabular}{cccc}
\hline 6 & -2.269372 & 3.049134 & 0.323719 \\
6 & -1.382428 & 2.043974 & 0.683182 \\
6 & -0.284175 & 2.345278 & 1.500017 \\
6 & -0.102035 & 3.627695 & 2.007377 \\
6 & -1.019356 & 4.620989 & 1.626584 \\
6 & -2.099050 & 4.361442 & 0.786724 \\
15 & -1.538666 & 0.349828 & 0.002254 \\
6 & -1.434043 & -0.710018 & 1.525127 \\
6 & -1.258391 & -2.079986 & 1.397457 \\
6 & -1.235866 & -2.915805 & 2.559703 \\
6 & -1.400414 & -2.330423 & 3.848574 \\
6 & -1.596920 & -0.933806 & 3.943272 \\
6 & -1.614552 & -0.151374 & 2.819635 \\
6 & -1.362647 & -3.153660 & 4.999252 \\
6 & -1.169205 & -4.506843 & 4.888707 \\
6 & -1.008040 & -5.093141 & 3.615376 \\
6 & -1.043593 & -4.320247 & 2.481178 \\
6 & -1.131659 & -2.734544 & 0.066684 \\
6 & 0.090915 & -2.796083 & -0.578497 \\
6 & 0.221661 & -3.471936 & -1.815217 \\
6 & -0.862849 & -4.061529 & -2.407898 \\
6 & -2.135803 & -4.000401 & -1.795232 \\
6 & -2.276921 & -3.334732 & -0.540931 \\
6 & -3.568377 & -3.260041 & 0.043202 \\
6 & -4.657270 & -3.812632 & -0.582846 \\
6 & -4.510060 & -4.492071 & -1.812430 \\
6 & -3.275754 & -4.581317 & -2.403918 \\
7 & 1.236807 & -2.123907 & -0.024611 \\
15 & 1.661523 & -0.801848 & -0.986707 \\
8 & 2.868883 & -1.302348 & -2.027514 \\
6 & 4.140402 & -1.608489 & -1.599740 \\
6 & 4.985290 & -0.625129 & -1.114090 \\
6 & 6.262482 & -1.026530 & -0.603456 \\
6 & 6.677782 & -2.386198 & -0.729792 \\
6 & 5.807701 & -3.324878 & -1.337214 \\
6 & 4.557263 & -2.948703 & -1.745730 \\
6 & 7.121199 & -0.129604 & 0.081514 \\
6 & 8.330182 & -0.546653 & 0.581682 \\
6 & 8.750263 & -1.884357 & 0.426844 \\
6 & 7.934954 & -2.782919 & -0.212949 \\
6 & 4.525856 & 0.782320 & -1.100365 \\
6 & 3.315024 & 1.085563 & -0.510541 \\
6 & 2.786260 & 2.389987 & -0.473156 \\
& & & \\
6
\end{tabular}




\begin{tabular}{|c|c|c|c|}
\hline 6 & 3.494993 & 3.416135 & -1.037300 \\
\hline 6 & 4.725931 & 3.170876 & -1.692054 \\
\hline 6 & 5.242407 & 1.840899 & -1.749412 \\
\hline 6 & 5.435719 & 4.222180 & -2.321424 \\
\hline 6 & 6.596652 & 3.978392 & -3.008880 \\
\hline 6 & 7.087509 & 2.659760 & -3.104178 \\
\hline 6 & 6.429399 & 1.621041 & -2.493388 \\
\hline 8 & 2.576117 & 0.069370 & 0.071956 \\
\hline 6 & 1.027899 & 3.969142 & 2.978515 \\
\hline 6 & 1.855229 & 2.739589 & 3.348318 \\
\hline 6 & -3.120495 & 5.423782 & 0.386723 \\
\hline 6 & -2.781621 & 6.800673 & 0.951729 \\
\hline 6 & -3.327718 & 0.172478 & -0.381839 \\
\hline 6 & -3.695116 & -0.211854 & -1.667093 \\
\hline 6 & -5.038670 & -0.388606 & -2.011929 \\
\hline 6 & -5.991288 & -0.190086 & -1.012474 \\
\hline 6 & -5.656548 & 0.179052 & 0.298124 \\
\hline 6 & -4.308593 & 0.365051 & 0.595761 \\
\hline 6 & -6.752842 & 0.313320 & 1.353924 \\
\hline 6 & -6.210517 & 0.824947 & 2.687071 \\
\hline 6 & -5.456491 & -0.712905 & -3.445116 \\
\hline 6 & -6.585580 & -1.746079 & -3.471346 \\
\hline 45 & -0.112066 & 0.305678 & -1.937300 \\
\hline 6 & -1.165977 & 1.494932 & -2.939638 \\
\hline 8 & -1.746185 & 2.261164 & -3.584526 \\
\hline 6 & -7.836285 & 1.286240 & 0.878324 \\
\hline 6 & -7.374361 & -1.068260 & 1.590669 \\
\hline 6 & -5.942006 & 0.585699 & -4.100488 \\
\hline 6 & -4.292858 & -1.266213 & -4.268912 \\
\hline 6 & 2.120850 & -2.945699 & 0.827187 \\
\hline 6 & 2.845647 & -2.174072 & 1.895380 \\
\hline 6 & 2.139728 & -1.399485 & 2.818223 \\
\hline 6 & 2.813731 & -0.706265 & 3.814581 \\
\hline 6 & 4.204590 & -0.766213 & 3.894647 \\
\hline 6 & 4.913332 & -1.535278 & 2.978690 \\
\hline 6 & 4.233113 & -2.240489 & 1.989028 \\
\hline 6 & 0.426702 & 4.534440 & 4.271048 \\
\hline 6 & 1.957686 & 5.013280 & 2.353429 \\
\hline 6 & -3.177060 & 5.534661 & -1.140733 \\
\hline 6 & -4.499098 & 5.013039 & 0.918547 \\
\hline 1 & 0.830577 & 0.377942 & -3.244910 \\
\hline 1 & 6.803052 & 0.899675 & 0.225637 \\
\hline 1 & -1.768132 & 0.919839 & 2.924487 \\
\hline 1 & 8.234776 & -3.824017 & -0.326267 \\
\hline 1 & -1.488696 & -2.686289 & 5.974884 \\
\hline 1 & 5.027646 & 5.229992 & -2.256878 \\
\hline 1 & -3.693358 & -2.735829 & 0.989266 \\
\hline 1 & -5.643906 & -3.722236 & -0.132255 \\
\hline 1 & 3.103247 & 4.432206 & -1.017454 \\
\hline 1 & -1.737211 & -0.487857 & 4.927168 \\
\hline 1 & 6.141555 & -4.354899 & -1.451659 \\
\hline 1 & 7.992838 & 2.461269 & -3.673524 \\
\hline 1 & 3.862528 & -3.658323 & -2.190621 \\
\hline 1 & -3.149900 & -5.093852 & -3.356762 \\
\hline 1 & -0.761933 & -4.572822 & -3.363786 \\
\hline 1 & -0.857136 & -6.166991 & 3.532313 \\
\hline
\end{tabular}




\begin{tabular}{|c|c|c|c|}
\hline . & 6.815201 & 0.609722 & -2.589420 \\
\hline & -0.925600 & -4.786370 & 1.505035 \\
\hline & 1.199945 & -3.495432 & -2.292969 \\
\hline & 7.130185 & 4.792920 & -3.493204 \\
\hline & 8.966030 & 0.160273 & 1.109961 \\
\hline & -5.379163 & -4.939919 & -2.290178 \\
\hline & -1.139863 & -5.130938 & 5.778844 \\
\hline & 9.711643 & -2.201164 & 0.824393 \\
\hline & 1.817794 & 2.548994 & -0.001168 \\
\hline & -4.005084 & 0.660044 & 1.598049 \\
\hline & -2.906121 & -0.391258 & -2.394618 \\
\hline & -7.043550 & -0.330163 & -1.258355 \\
\hline 1 & -3.110981 & 2.808139 & -0.325487 \\
\hline 1 & 0.413464 & 1.547089 & 1.755308 \\
\hline & -0.879594 & 5.628336 & 2.013200 \\
\hline 1 & 1.461605 & -3.687626 & 1.301039 \\
\hline & 2.842423 & -3.509077 & 0.214254 \\
\hline & 4.790887 & -2.837687 & 1.267071 \\
\hline & 1.056007 & -1.325167 & 2.734230 \\
\hline & 5.999855 & -1.587183 & 3.024406 \\
\hline 1 & 2.250178 & -0.113609 & 4.533669 \\
\hline & 4.731098 & -0.214316 & 4.670561 \\
\hline 1 & 2.630450 & 3.017364 & 4.074117 \\
\hline 1 & 1.232048 & 1.959982 & 3.809238 \\
\hline 1 & 2.365484 & 2.297287 & 2.482122 \\
\hline & 2.751824 & 5.287593 & 3.060910 \\
\hline & 2.438650 & 4.620772 & 1.447793 \\
\hline 1 & 1.421884 & 5.930999 & 2.081841 \\
\hline 1 & 1.223420 & 4.758183 & 4.993051 \\
\hline 1 & -0.132361 & 5.462073 & 4.100403 \\
\hline & -0.257001 & 3.811387 & 4.735345 \\
\hline 1 & -3.540891 & 7.525968 & 0.633247 \\
\hline & -2.766212 & 6.803957 & 2.049379 \\
\hline & -1.809745 & 7.162768 & 0.592126 \\
\hline 1 & -3.895440 & 6.310295 & -1.438388 \\
\hline 1 & -2.196377 & 5.803852 & -1.553396 \\
\hline 1 & -3.491023 & 4.596690 & -1.613274 \\
\hline 1 & -5.256451 & 5.754809 & 0.631004 \\
\hline 1 & -4.818498 & 4.039852 & 0.523842 \\
\hline 1 & -4.492045 & 4.943387 & 2.014513 \\
\hline & -8.170287 & -1.008378 & 2.345457 \\
\hline 1 & -7.812818 & -1.482719 & 0.674198 \\
\hline 1 & -6.617722 & -1.777638 & 1.952861 \\
\hline 1 & -7.032776 & 0.919582 & 3.407402 \\
\hline 1 & -5.471289 & 0.139940 & 3.123277 \\
\hline 1 & -5.743539 & 1.813968 & 2.586303 \\
\hline 1 & -8.617987 & 1.385040 & 1.643253 \\
\hline 1 & -7.418305 & 2.284181 & 0.693537 \\
\hline 1 & -8.323962 & 0.951900 & -0.044828 \\
\hline 1 & -6.841271 & -1.994424 & -4.509655 \\
\hline 1 & -6.281584 & -2.672514 & -2.965872 \\
\hline 1 & -7.503197 & -1.382568 & -2.993901 \\
\hline 1 & -4.648481 & -1.534415 & -5.271891 \\
\hline 1 & -3.485854 & -0.533668 & -4.400507 \\
\hline 1 & -3.865246 & -2.168446 & -3.809369 \\
\hline 1 & -6.244995 & 0.403122 & -5.140651 \\
\hline
\end{tabular}




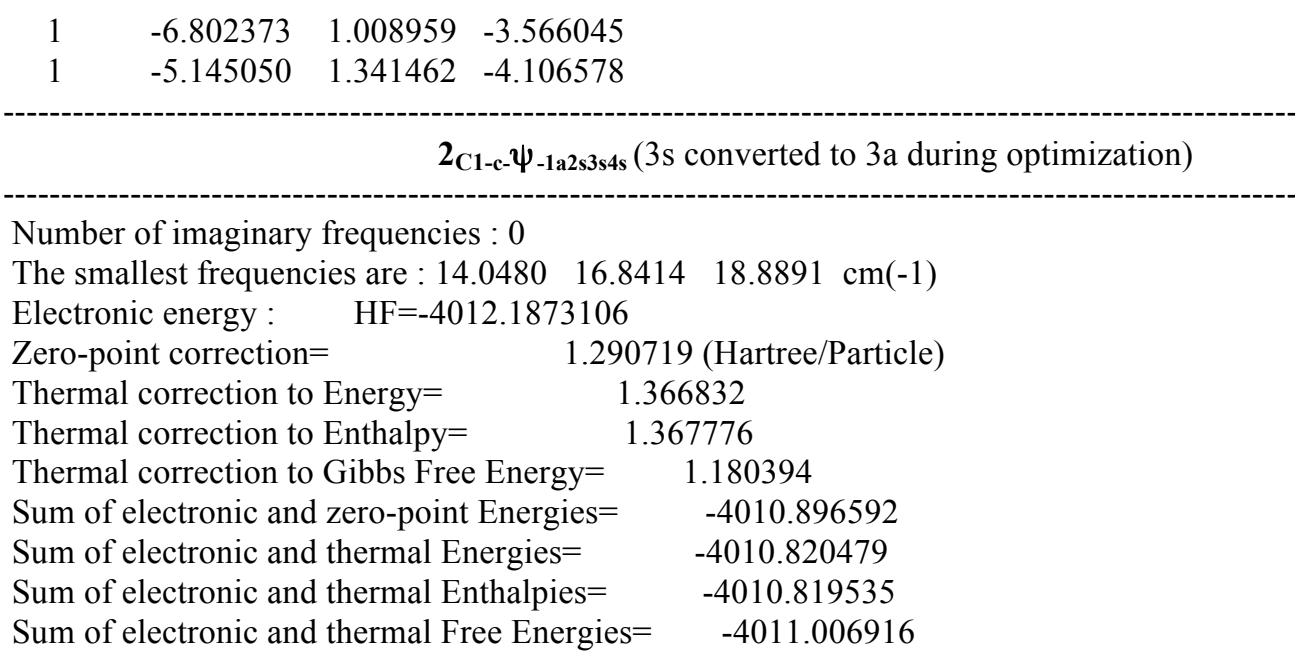

Cartesian Coordinates

$\begin{array}{cccc}6 & -3.718084 & -0.219075 & -1.690134 \\ 6 & -3.338726 & 0.149267 & -0.398512 \\ 6 & -4.311696 & 0.339831 & 0.578581 \\ 6 & -5.668862 & 0.179420 & 0.286386 \\ 6 & -6.016017 & -0.157301 & -1.023416 \\ 6 & -5.062802 & -0.365271 & -2.028824 \\ 15 & -1.549733 & 0.328226 & -0.013250 \\ 45 & -0.117365 & 0.305811 & -1.948794 \\ 6 & -1.170421 & 1.496233 & -2.950541 \\ 8 & -1.742162 & 2.267641 & -3.596695 \\ 6 & -6.692381 & 0.345216 & 1.408462 \\ 6 & -8.125258 & 0.128273 & 0.929238 \\ 6 & -5.503027 & -0.687979 & -3.455484 \\ 6 & -6.561595 & -1.794444 & -3.460481 \\ 6 & -1.441627 & -0.736039 & 1.507451 \\ 6 & -1.247465 & -2.103511 & 1.379189 \\ 6 & -1.217967 & -2.940505 & 2.540321 \\ 6 & -1.399743 & -2.360061 & 3.828993 \\ 6 & -1.617919 & -0.966767 & 3.924431 \\ 6 & -1.637739 & -0.182557 & 2.802019 \\ 6 & -1.002607 & -4.341468 & 2.460724 \\ 6 & -0.961248 & -5.115576 & 3.593885 \\ 6 & -1.139919 & -4.534117 & 4.867098 \\ 6 & -1.355913 & -3.184443 & 4.978613 \\ 6 & -1.114039 & -2.752430 & 0.046640 \\ 6 & 0.105771 & -2.792395 & -0.604891 \\ 6 & 0.237227 & -3.450328 & -1.851339 \\ 6 & -0.843491 & -4.045470 & -2.445413 \\ 6 & -2.111957 & -4.014555 & -1.821293 \\ 6 & -2.253431 & -3.366738 & -0.557709 \\ 6 & -3.245810 & -4.613074 & -2.424167 \\ 6 & -4.472578 & -4.568670 & -1.812019 \\ 6 & -4.618978 & -3.913898 & -0.569123 \\ 6 & -3.537886 & -3.333220 & 0.044804 \\ 7 & 1.247489 & -2.117253 & -0.046357 \\ 6 & 2.134027 & -2.939879 & 0.802004 \\ 6 & 2.852656 & -2.174312 & 1.878749\end{array}$




\begin{tabular}{|c|c|c|c|}
\hline & 2.142176 & -1.410085 & 2.806749 \\
\hline & 2.811393 & -0.728391 & 3.814337 \\
\hline & 4.201861 & -0.788751 & 3.900355 \\
\hline & 4.915271 & -1.546042 & 2.978279 \\
\hline & 4.239826 & -2.240244 & 1.977667 \\
\hline & 1.665324 & -0.787193 & -0.999937 \\
\hline & 2.572173 & 0.082763 & 0.066135 \\
\hline & 3.309182 & 1.105367 & -0.507089 \\
\hline & 4.523856 & 0.810829 & -1.093438 \\
\hline & 5.239163 & 1.876812 & -1.731610 \\
\hline & 4.717025 & 3.204285 & -1.667340 \\
\hline & 3.481993 & 3.440167 & -1.016793 \\
\hline & 2.774800 & 2.407270 & -0.463138 \\
\hline & 6.430602 & 1.667020 & -2.471395 \\
\hline & 7.087440 & 2.712664 & -3.071620 \\
\hline & 6.590743 & 4.028581 & -2.969486 \\
\hline & 5.425525 & 4.262813 & -2.285968 \\
\hline & 4.988540 & -0.594848 & -1.115544 \\
\hline & 4.149173 & -1.577526 & -1.612018 \\
\hline & 4.571368 & -2.915039 & -1.767027 \\
\hline & 5.821732 & -3.289830 & -1.357047 \\
\hline & 6.686004 & -2.352803 & -0.738841 \\
\hline & 6.265147 & -0.995744 & -0.603000 \\
\hline & 7.117532 & -0.101431 & 0.093141 \\
\hline & 8.325982 & -0.518148 & 0.594892 \\
\hline & 8.751830 & -1.852884 & 0.430451 \\
\hline & 7.942560 & -2.749114 & -0.220202 \\
\hline & 2.877917 & -1.272808 & -2.041674 \\
\hline & -1.404651 & 2.020707 & 0.677625 \\
\hline & -0.309980 & 2.319183 & 1.500270 \\
\hline & -0.135123 & 3.597143 & 2.021065 \\
\hline & -1.055831 & 4.589859 & 1.647547 \\
\hline & -2.131688 & 4.334514 & 0.801384 \\
\hline & -2.295128 & 3.025743 & 0.324919 \\
\hline & 0.990043 & 3.933820 & 2.999314 \\
\hline & 1.823685 & 2.704964 & 3.356992 \\
\hline & -3.142966 & 5.404115 & 0.393407 \\
\hline 6 & -2.857359 & 6.754987 & 1.044137 \\
\hline & 0.381626 & 4.480068 & 4.296661 \\
\hline & 1.915233 & 4.991035 & 2.389434 \\
\hline & -3.101913 & 5.586652 & -1.127710 \\
\hline & -4.549260 & 4.959317 & 0.812694 \\
\hline 6 & -6.394815 & -0.686836 & 2.503628 \\
\hline 6 & -6.594587 & 1.757256 & 1.995829 \\
\hline & -6.091637 & 0.584196 & -4.076520 \\
\hline & -4.335110 & -1.154577 & -4.323718 \\
\hline & 0.828088 & 0.392936 & -3.253339 \\
\hline & 6.794796 & 0.925351 & 0.244706 \\
\hline & -1.804680 & 0.886539 & 2.908108 \\
\hline & 8.246757 & -3.788109 & -0.340925 \\
\hline 1 & -1.495351 & -2.720772 & 5.954189 \\
\hline & 5.013003 & 5.268477 & -2.216423 \\
\hline 1 & -3.662782 & -2.822067 & 0.998142 \\
\hline & -5.599116 & -3.862004 & -0.099041 \\
\hline & 3.086259 & 4.454572 & -0.991627 \\
\hline & -1.770961 & -0.524376 & 4.908034 \\
\hline
\end{tabular}




\begin{tabular}{|c|c|c|c|}
\hline & 6.159838 & -4.317678 & -1.478353 \\
\hline & 7.996311 & 2.521817 & -3.637931 \\
\hline 1 & 3.880544 & -3.623598 & -2.219688 \\
\hline 1 & -3.120603 & -5.108061 & -3.386284 \\
\hline & -0.741981 & -4.542239 & -3.408858 \\
\hline & -0.792194 & -6.186661 & 3.510115 \\
\hline & 6.820984 & 0.657980 & -2.572681 \\
\hline & -0.871110 & -4.803809 & 1.484484 \\
\hline & 1.212803 & -3.455803 & -2.335202 \\
\hline & 7.123242 & 4.848611 & -3.445591 \\
\hline & 8.956880 & 0.186627 & 1.131906 \\
\hline & -5.336035 & -5.034344 & -2.282759 \\
\hline & -1.105973 & -5.159156 & 5.756404 \\
\hline & 9.712760 & -2.169409 & 0.829314 \\
\hline & 1.803269 & 2.558450 & 0.005229 \\
\hline & -4.007408 & 0.612289 & 1.589317 \\
\hline & -2.934275 & -0.401000 & -2.422816 \\
\hline & -7.068176 & -0.268856 & -1.275884 \\
\hline & -3.132366 & 2.787474 & -0.331036 \\
\hline & 0.389790 & 1.521423 & 1.750823 \\
\hline & -0.921976 & 5.593645 & 2.045229 \\
\hline & 1.477758 & -3.689032 & 1.268588 \\
\hline 1 & 2.859961 & -3.494979 & 0.186803 \\
\hline & 4.801148 & -2.829284 & 1.251784 \\
\hline ] & 1.058735 & -1.335451 & 2.718565 \\
\hline & 6.001631 & -1.597502 & 3.027861 \\
\hline 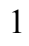 & 2.244308 & -0.144421 & 4.537717 \\
\hline 1 & 4.724391 & -0.246040 & 4.685368 \\
\hline & -6.847803 & -2.035302 & -4.492790 \\
\hline 1 & -6.170959 & -2.708900 & -2.994245 \\
\hline & -7.477452 & -1.506965 & -2.930600 \\
\hline 1 & -6.414395 & 0.394667 & -5.109418 \\
\hline ] & -6.961033 & 0.945927 & -3.512818 \\
\hline | & -5.345194 & 1.389634 & -4.096775 \\
\hline 1 & -4.703466 & -1.422059 & -5.322214 \\
\hline | & -3.575528 & -0.373916 & -4.459190 \\
\hline 1 & -3.843604 & -2.039988 & -3.897287 \\
\hline & -7.115777 & -0.590970 & 3.326766 \\
\hline 1 & -6.468039 & -1.708318 & 2.106730 \\
\hline ] & -5.388695 & -0.566433 & 2.925925 \\
\hline l & -8.818309 & 0.253818 & 1.770680 \\
\hline 1 & -8.413754 & 0.851845 & 0.155904 \\
\hline 1 & -8.275249 & -0.882610 & 0.528361 \\
\hline 1 & -7.305110 & 1.874909 & 2.825175 \\
\hline & -5.592386 & 1.977702 & 2.383825 \\
\hline 1 & -6.834276 & 2.514700 & 1.237829 \\
\hline 1 & -5.290485 & 5.715036 & 0.519698 \\
\hline 1 & -4.838857 & 4.011562 & 0.341084 \\
\hline 1 & -4.612828 & 4.826978 & 1.901033 \\
\hline 1 & -3.811598 & 6.365927 & -1.436892 \\
\hline 1 & -2.100612 & 5.887136 & -1.461816 \\
\hline 1 & -3.369073 & 4.666364 & -1.659973 \\
\hline 1 & -3.614809 & 7.482866 & 0.727268 \\
\hline 1 & -2.892615 & 6.699743 & 2.140019 \\
\hline 1 & -1.877769 & 7.153400 & 0.750139 \\
\hline 1 & 2.595832 & 2.978976 & 4.087489 \\
\hline
\end{tabular}




$\begin{array}{rrrr}1 & 1.204050 & 1.916721 & 3.807928 \\ 1 & 2.338167 & 2.275108 & 2.487004 \\ 1 & 2.706141 & 5.260999 & 3.102183 \\ 1 & 2.400538 & 4.612928 & 1.479990 \\ 1 & 1.374780 & 5.909042 & 2.128235 \\ 1 & 1.174817 & 4.700362 & 5.023599 \\ 1 & -0.182997 & 5.406012 & 4.135265 \\ 1 & -0.298731 & 3.747204 & 4.750408\end{array}$

$2_{\text {C1-c- }} \psi_{-1 \text { 1s2a3a4a }}$

Number of imaginary frequencies : 0

The smallest frequencies are : $13.7625 \quad 17.0293 \quad 17.8631 \mathrm{~cm}(-1)$

Electronic energy : $\quad H F=-4012.1864767$

Zero-point correction $=\quad 1.290638($ Hartree/Particle $)$

Thermal correction to Energy= $\quad 1.366677$

Thermal correction to Enthalpy= $\quad 1.367621$

Thermal correction to Gibbs Free Energy= $\quad 1.180990$

Sum of electronic and zero-point Energies $=\quad-4010.895838$

Sum of electronic and thermal Energies $=\quad-4010.819800$

Sum of electronic and thermal Enthalpies $=\quad-4010.818856$

Sum of electronic and thermal Free Energies $=\quad-4011.005487$

\begin{tabular}{|c|c|c|c|}
\hline & \multicolumn{3}{|c|}{ Cartesian Coordinates } \\
\hline 6 & 2.074620 & -1.247524 & 2.843564 \\
\hline 6 & 2.802845 & -2.060079 & 1.971280 \\
\hline 6 & 4.191964 & -2.074825 & 2.060916 \\
\hline 6 & 4.852996 & -1.277242 & 2.991948 \\
\hline 6 & 4.123418 & -0.462551 & 3.849759 \\
\hline 6 & 2.730581 & -0.456448 & 3.777752 \\
\hline 6 & 2.099641 & -2.910559 & 0.949476 \\
\hline 7 & 1.219309 & -2.150241 & 0.038370 \\
\hline 15 & 1.631237 & -0.868355 & -0.982676 \\
\hline 45 & -0.151064 & 0.188159 & -1.968471 \\
\hline 1 & 0.774088 & 0.208664 & -3.289592 \\
\hline 6 & 0.092138 & -2.864616 & -0.502243 \\
\hline 6 & -1.141560 & -2.792130 & 0.119381 \\
\hline 6 & -2.271425 & -3.425599 & -0.483709 \\
\hline 6 & -2.102230 & -4.146393 & -1.703410 \\
\hline 6 & -0.817774 & -4.219946 & -2.290576 \\
\hline 6 & 0.249574 & -3.590563 & -1.707569 \\
\hline 6 & -3.573530 & -3.336507 & 0.074304 \\
\hline 6 & -4.644665 & -3.937163 & -0.538086 \\
\hline 6 & -4.469866 & -4.670368 & -1.732646 \\
\hline 6 & -3.225512 & -4.768931 & -2.301869 \\
\hline 6 & -1.302100 & -2.109134 & 1.431081 \\
\hline 6 & -1.497550 & -0.740452 & 1.529839 \\
\hline 6 & -1.739417 & -0.166949 & 2.808673 \\
\hline 6 & -1.748770 & -0.930327 & 3.944884 \\
\hline 6 & -1.520913 & -2.324096 & 3.880986 \\
\hline 6 & -1.303871 & -2.926287 & 2.608163 \\
\hline 6 & -1.506940 & -3.127418 & 5.045944 \\
\hline 6 & -1.286481 & -4.478703 & 4.964514 \\
\hline 6 & -1.073186 & -5.081998 & 3.707169 \\
\hline 6 & -1.085129 & -4.328072 & 2.559507 \\
\hline
\end{tabular}




\begin{tabular}{|c|c|c|c|}
\hline & -1.544560 & 0.311153 & -0.003637 \\
\hline 6 & -3.338424 & 0.226085 & -0.395285 \\
\hline 6 & -4.318743 & 0.581274 & 0.534700 \\
\hline 6 & -5.671389 & 0.471454 & 0.219659 \\
\hline 6 & -6.009493 & -0.002217 & -1.055125 \\
\hline 6 & -5.056838 & -0.370582 & -2.005424 \\
\hline 6 & -3.710968 & -0.257884 & -1.646067 \\
\hline 6 & -6.774165 & 0.825735 & 1.215678 \\
\hline 6 & -6.214232 & 1.417887 & 2.507451 \\
\hline 6 & -5.479152 & -0.811699 & -3.405557 \\
\hline 6 & -5.969489 & 0.425290 & -4.167534 \\
\hline 6 & -1.288016 & 1.993299 & 0.682009 \\
\hline 6 & -0.228168 & 2.220076 & 1.556498 \\
\hline 6 & 0.008748 & 3.491770 & 2.092282 \\
\hline 6 & -0.809969 & 4.537245 & 1.666303 \\
\hline 6 & -1.854864 & 4.351437 & 0.749059 \\
\hline 6 & -2.086049 & 3.064066 & 0.270254 \\
\hline 6 & -2.720707 & 5.540381 & 0.336386 \\
\hline 6 & -3.394488 & 6.135262 & 1.578104 \\
\hline 6 & 1.073398 & 3.653071 & 3.177685 \\
\hline 6 & 0.618888 & 2.848276 & 4.403054 \\
\hline 6 & -7.729057 & 1.853848 & 0.600261 \\
\hline 6 & -7.551411 & -0.447898 & 1.567039 \\
\hline 6 & -6.605816 & -1.845959 & -3.335668 \\
\hline 6 & -4.319656 & -1.431622 & -4.185096 \\
\hline 6 & -1.842730 & 6.605265 & -0.328784 \\
\hline 6 & -3.816929 & 5.141536 & -0.649283 \\
\hline 6 & 2.428895 & 3.124380 & 2.698758 \\
\hline 6 & 1.256750 & 5.107399 & 3.603535 \\
\hline 8 & 2.848325 & -1.401767 & -1.995594 \\
\hline 6 & 4.123690 & -1.660218 & -1.548242 \\
\hline 6 & 4.950516 & -0.634709 & -1.121421 \\
\hline 6 & 6.235202 & -0.982228 & -0.590971 \\
\hline 6 & 6.672954 & -2.340110 & -0.634397 \\
\hline 6 & 5.818492 & -3.329168 & -1.180647 \\
\hline 6 & 4.562289 & -2.999624 & -1.611311 \\
\hline 6 & 7.938080 & -2.682968 & -0.098605 \\
\hline 6 & 8.739950 & -1.733087 & 0.481061 \\
\hline 6 & 8.297986 & -0.395437 & 0.554233 \\
\hline 6 & 7.080518 & -0.030403 & 0.034154 \\
\hline 6 & 4.461091 & 0.761107 & -1.189262 \\
\hline 6 & 3.242398 & 1.068680 & -0.618555 \\
\hline 6 & 2.673723 & 2.355441 & -0.668890 \\
\hline 6 & 3.355439 & 3.363603 & -1.294686 \\
\hline 6 & 4.595194 & 3.111871 & -1.930548 \\
\hline 6 & 5.149229 & 1.795868 & -1.903941 \\
\hline 6 & 6.342855 & 1.562355 & -2.632982 \\
\hline 6 & 6.973036 & 2.578222 & -3.308354 \\
\hline 6 & 6.445606 & 3.886060 & -3.295842 \\
\hline 6 & 5.277330 & 4.140482 & -2.624728 \\
\hline 8 & 2.531958 & 0.069823 & 0.029220 \\
\hline 1 & 6.745189 & 1.000380 & 0.114748 \\
\hline 1 & -1.913862 & 0.903030 & 2.892659 \\
\hline 1 & 8.255296 & -3.723864 & -0.148519 \\
\hline 1 & -1.673301 & -2.646335 & 6.008791 \\
\hline 1 & 4.841670 & 5.138794 & -2.624318 \\
\hline
\end{tabular}




\begin{tabular}{|c|c|c|c|}
\hline & -3.722232 & -2.765162 & 0.989021 \\
\hline & -5.638180 & -3.839960 & -0.104513 \\
\hline & 2.936823 & 4.367860 & -1.336790 \\
\hline & -1.933164 & -0.469839 & 4.914932 \\
\hline & 6.169588 & -4.358550 & -1.231195 \\
\hline & 7.884319 & 2.369467 & -3.864391 \\
\hline & 3.879982 & -3.746526 & -2.012155 \\
\hline & -3.078952 & -5.321546 & -3.228908 \\
\hline & -0.694219 & -4.772587 & -3.220423 \\
\hline & -0.900561 & -6.154080 & 3.646741 \\
\hline & 6.756495 & 0.557916 & -2.665156 \\
\hline & -0.925561 & -4.808491 & 1.596353 \\
\hline & 1.234995 & -3.621888 & -2.169697 \\
\hline & 6.957042 & 4.683128 & -3.830515 \\
\hline & 8.923609 & 0.353464 & 1.034736 \\
\hline & -5.325805 & -5.150530 & -2.202530 \\
\hline & -1.275728 & -5.087705 & 5.865448 \\
\hline & 9.707711 & -2.008558 & 0.893626 \\
\hline & 1.701160 & 2.515018 & -0.206619 \\
\hline & -4.012872 & 0.953299 & 1.509577 \\
\hline & -2.923783 & -0.553537 & -2.336734 \\
\hline & -7.065072 & -0.080836 & -1.314638 \\
\hline 1 & -2.900306 & 2.874719 & -0.425269 \\
\hline & 0.404163 & 1.381937 & 1.857390 \\
\hline 1 & -0.643471 & 5.535792 & 2.065387 \\
\hline & 1.441414 & -3.627632 & 1.461293 \\
\hline & -1.203170 & 1.355118 & -2.998864 \\
\hline & -5.561276 & 0.711556 & 3.037098 \\
\hline & -5.644127 & 2.338257 & 2.320046 \\
\hline I & -7.039289 & 1.671757 & 3.184764 \\
\hline & -8.222922 & 1.477399 & -0.303287 \\
\hline 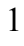 & -8.516793 & 2.117111 & 1.318767 \\
\hline 1 & -7.196080 & 2.774877 & 0.330623 \\
\hline & -8.018018 & -0.899450 & 0.682830 \\
\hline 1 & -6.887996 & -1.199503 & 2.015403 \\
\hline 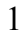 & -8.349052 & -0.225206 & 2.288702 \\
\hline 1 & -5.173610 & 1.178508 & -4.241537 \\
\hline 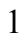 & -6.275715 & 0.153251 & -5.186970 \\
\hline 1 & -6.828842 & 0.892932 & -3.670021 \\
\hline l & -7.514147 & -1.451336 & -2.865091 \\
\hline I & -6.881610 & -2.169181 & -4.348121 \\
\hline 1 & -6.287825 & -2.733099 & -2.771932 \\
\hline 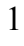 & -3.508092 & -0.715302 & -4.369088 \\
\hline 1 & -3.897781 & -2.300490 & -3.660481 \\
\hline l & -4.677112 & -1.771944 & -5.165300 \\
\hline 1 & 2.835001 & -3.502704 & 0.381267 \\
\hline 1 & 4.766044 & -2.702816 & 1.378990 \\
\hline 1 & 0.987670 & -1.219193 & 2.765087 \\
\hline 1 & 5.940821 & -1.290461 & 3.034222 \\
\hline ] & 2.153654 & 0.174886 & 4.452883 \\
\hline 1 & 4.634641 & 0.167759 & 4.574644 \\
\hline 1 & 2.040497 & 5.170619 & 4.368933 \\
\hline 1 & 1.564407 & 5.743021 & 2.763102 \\
\hline 1 & 0.341647 & 5.529942 & 4.038105 \\
\hline 1 & 3.163417 & 3.183187 & 3.514170 \\
\hline 1 & 2.389010 & 2.076320 & 2.375803 \\
\hline
\end{tabular}




$\begin{array}{rrrr}1 & 2.811494 & 3.719089 & 1.859056 \\ 1 & 1.357011 & 2.930404 & 5.213171 \\ 1 & -0.343165 & 3.219986 & 4.781378 \\ 1 & 0.494031 & 1.783201 & 4.166775 \\ 1 & -4.414556 & 6.023556 & -0.911655 \\ 1 & -3.404940 & 4.733964 & -1.581500 \\ 1 & -4.501402 & 4.395093 & -0.222609 \\ 1 & -2.452827 & 7.465820 & -0.634683 \\ 1 & -1.061880 & 6.976042 & 0.346184 \\ 1 & -1.350167 & 6.203996 & -1.223787 \\ 1 & -4.030148 & 6.985236 & 1.295689 \\ 1 & -4.028933 & 5.389474 & 2.075341 \\ 1 & -2.665499 & 6.498099 & 2.312551 \\ 8 & -1.777856 & 2.111083 & -3.660845\end{array}$

$2_{\text {C1-c- }-\psi_{-1 s 2 a 3 a 4 s}}$

Number of imaginary frequencies : 0

The smallest frequencies are : $11.8133 \quad 18.6389 \quad 20.9886 \mathrm{~cm}(-1)$

Electronic energy : $\quad \mathrm{HF}=-4012.1879461$

Zero-point correction $=\quad 1.291259($ Hartree/Particle $)$

Thermal correction to Energy= $\quad 1.367064$

Thermal correction to Enthalpy $=\quad 1.368008$

Thermal correction to Gibbs Free Energy $=\quad 1.182223$

Sum of electronic and zero-point Energies $=\quad-4010.896687$

Sum of electronic and thermal Energies $=\quad-4010.820882$

Sum of electronic and thermal Enthalpies $=\quad-4010.819938$

Sum of electronic and thermal Free Energies $=\quad-4011.005723$

\begin{tabular}{cccc}
\multicolumn{4}{c}{ Cartesian Coordinates } \\
\hline 6 & -4.044313 & -0.060458 & -1.313353 \\
6 & -3.440758 & 0.515753 & -0.192195 \\
6 & -4.211865 & 1.236589 & 0.712510 \\
6 & -5.585544 & 1.409084 & 0.512402 \\
6 & -6.155234 & 0.828857 & -0.620677 \\
6 & -5.408276 & 0.090565 & -1.549488 \\
15 & -1.635273 & 0.250474 & 0.029686 \\
45 & -0.405664 & 0.114817 & -2.038568 \\
1 & 0.453175 & 0.155986 & -3.402864 \\
6 & -6.382798 & 2.228084 & 1.525819 \\
6 & -6.260177 & 1.581547 & 2.910410 \\
6 & -6.086448 & -0.477764 & -2.794380 \\
6 & -7.224696 & -1.417110 & -2.383471 \\
6 & -1.643490 & -0.962577 & 1.434087 \\
6 & -1.447646 & -2.317364 & 1.219982 \\
6 & -1.473473 & -3.229828 & 2.327224 \\
6 & -1.725419 & -2.736472 & 3.640272 \\
6 & -1.963154 & -1.353151 & 3.816490 \\
6 & -1.924557 & -0.499068 & 2.749377 \\
6 & -1.226579 & -4.618808 & 2.171249 \\
6 & -1.222132 & -5.463083 & 3.254356 \\
6 & -1.473098 & -4.967999 & 4.550741 \\
6 & -1.720899 & -3.631704 & 4.736136 \\
6 & -1.259353 & -2.902967 & -0.135500 \\
6 & -0.020909 & -2.924967 & -0.750768
\end{tabular}




\begin{tabular}{|c|c|c|c|}
\hline 6 & 0.155290 & -3.582027 & -1.993404 \\
\hline 6 & -0.896985 & -4.190741 & -2.621824 \\
\hline b & -2.186848 & -4.167898 & -2.042144 \\
\hline & -2.373759 & -3.522058 & -0.783592 \\
\hline D & -3.294223 & -4.778648 & -2.680415 \\
\hline & -4.539213 & -4.754851 & -2.104760 \\
\hline 6 & -4.729202 & -4.109309 & -0.863521 \\
\hline 5 & -3.676580 & -3.505109 & -0.221649 \\
\hline 7 & 1.096487 & -2.224783 & -0.168694 \\
\hline 6 & 2.046271 & -3.040228 & 0.618402 \\
\hline 6 & 2.651142 & -2.337344 & 1.804291 \\
\hline 6 & 1.841410 & -1.906020 & 2.857186 \\
\hline 6 & 2.402720 & -1.330749 & 3.990646 \\
\hline 6 & 3.783635 & -1.160202 & 4.079033 \\
\hline 6 & 4.595468 & -1.571716 & 3.027406 \\
\hline 6 & 4.030061 & -2.165657 & 1.901551 \\
\hline 15 & 1.456147 & -0.871755 & -1.116207 \\
\hline 8 & 2.338530 & 0.021816 & -0.050640 \\
\hline 6 & 3.037599 & 1.071368 & -0.629057 \\
\hline 6 & 4.268903 & 0.820844 & -1.198821 \\
\hline 6 & 4.961205 & 1.915681 & -1.814808 \\
\hline 6 & 4.396854 & 3.225550 & -1.744468 \\
\hline 6 & 3.139804 & 3.413903 & -1.120422 \\
\hline 6 & 2.453502 & 2.352136 & -0.597463 \\
\hline 6 & 6.172152 & 1.752628 & -2.534298 \\
\hline 6 & 6.808964 & 2.826036 & -3.106764 \\
\hline 6 & 6.270945 & 4.124693 & -2.996035 \\
\hline 6 & 5.085438 & 4.313562 & -2.333435 \\
\hline 6 & 4.772182 & -0.570099 & -1.242659 \\
\hline 6 & 3.966360 & -1.565353 & -1.769438 \\
\hline 6 & 4.442157 & -2.877531 & -1.981051 \\
\hline 6 & 5.705614 & -3.220868 & -1.585010 \\
\hline 6 & 6.530702 & -2.279452 & -0.921490 \\
\hline 6 & 6.058691 & -0.945553 & -0.735085 \\
\hline 6 & 6.867335 & -0.051199 & 0.011203 \\
\hline 6 & 8.082961 & -0.445699 & 0.514126 \\
\hline 6 & 8.561764 & -1.754461 & 0.295875 \\
\hline 6 & 7.795988 & -2.650427 & -0.405683 \\
\hline 8 & 2.677976 & -1.301633 & -2.174400 \\
\hline 6 & -1.040096 & 1.790625 & 0.831344 \\
\hline 6 & -0.033722 & 1.761100 & 1.791180 \\
\hline 6 & 0.504560 & 2.946116 & 2.312889 \\
\hline 6 & 0.036645 & 4.153630 & 1.800480 \\
\hline 6 & -0.949679 & 4.218281 & 0.802324 \\
\hline 6 & -1.483318 & 3.022672 & 0.332940 \\
\hline 6 & 1.575878 & 2.858309 & 3.398762 \\
\hline 6 & 1.013723 & 2.079065 & 4.593786 \\
\hline 6 & -1.375989 & 5.574544 & 0.244231 \\
\hline 6 & -1.831770 & 6.494264 & 1.381549 \\
\hline 6 & 2.810534 & 2.136265 & 2.846376 \\
\hline 6 & 2.014670 & 4.233392 & 3.895295 \\
\hline 6 & -0.178913 & 6.204244 & -0.477655 \\
\hline 6 & -2.523487 & 5.454285 & -0.756423 \\
\hline 6 & -5.822494 & 3.654770 & 1.574300 \\
\hline 6 & -7.865086 & 2.312343 & 1.171504 \\
\hline 6 & -6.651667 & 0.679072 & -3.626095 \\
\hline
\end{tabular}




\begin{tabular}{|c|c|c|c|}
\hline 6 & -5.114842 & -1.263475 & -3.673085 \\
\hline & 6.503541 & 0.955509 & 0.201123 \\
\hline & -2.098932 & 0.561688 & 2.915707 \\
\hline & 8.140599 & -3.671116 & -0.566827 \\
\hline & -1.914584 & -3.233571 & 5.731210 \\
\hline & 4.640729 & 5.305126 & -2.259318 \\
\hline & -3.838253 & -3.002791 & 0.730041 \\
\hline & -5.718438 & -4.090911 & -0.410996 \\
\hline & 2.713342 & 4.415564 & -1.084419 \\
\hline & -2.175391 & -0.977856 & 4.816849 \\
\hline & 6.082741 & -4.229036 & -1.748439 \\
\hline 1 & 7.734088 & 2.670540 & -3.657343 \\
\hline & 3.780317 & -3.593286 & -2.464263 \\
\hline & -3.133831 & -5.266099 & -3.640986 \\
\hline & -0.758320 & -4.687098 & -3.580754 \\
\hline & -1.024945 & -6.522977 & 3.111205 \\
\hline & 6.595561 & 0.757760 & -2.642204 \\
\hline & -1.035795 & -5.016852 & 1.176773 \\
\hline & 1.144512 & -3.571967 & -2.448125 \\
\hline & 6.787662 & 4.967252 & -3.449734 \\
\hline & 8.679216 & 0.256849 & 1.092077 \\
\hline & -5.383363 & -5.225608 & -2.603819 \\
\hline & -1.468085 & -5.647704 & 5.399684 \\
\hline & 9.528649 & -2.052278 & 0.694667 \\
\hline & 1.473899 & 2.465786 & -0.141182 \\
\hline & -3.732343 & 1.694041 & 1.577092 \\
\hline & -3.417411 & -0.614071 & -2.010148 \\
\hline & -7.220077 & 0.960929 & -0.802674 \\
\hline & -2.253668 & 3.030659 & -0.435542 \\
\hline 1 & 0.349355 & 0.800852 & 2.139417 \\
\hline & 0.451258 & 5.085229 & 2.179779 \\
\hline 1 & 1.463071 & -3.900343 & 0.976327 \\
\hline & -1.555176 & 1.209684 & -3.039834 \\
\hline 1 & 2.843630 & -3.444913 & -0.023493 \\
\hline & 4.671323 & -2.501588 & 1.086628 \\
\hline 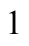 & 0.763237 & -2.039330 & 2.787262 \\
\hline 1 & 5.674547 & -1.437880 & 3.076851 \\
\hline & 1.757968 & -1.021286 & 4.812309 \\
\hline 1 & 4.221713 & -0.703616 & 4.964605 \\
\hline & 2.767931 & 4.115728 & 4.684604 \\
\hline 1 & 2.470666 & 4.831165 & 3.095423 \\
\hline & 1.178842 & 4.804788 & 4.319939 \\
\hline & 3.576030 & 2.046757 & 3.629561 \\
\hline 1 & 2.583782 & 1.123233 & 2.488053 \\
\hline & 3.247188 & 2.699042 & 2.010594 \\
\hline 1 & 1.783211 & 1.964949 & 5.370448 \\
\hline & 0.157979 & 2.605166 & 5.037982 \\
\hline 1 & 0.678782 & 1.073587 & 4.310271 \\
\hline l & -2.808612 & 6.452981 & -1.110315 \\
\hline 1 & -2.243226 & 4.863371 & -1.637967 \\
\hline 1 & -3.414467 & 4.995521 & -0.306092 \\
\hline & -2.143681 & 7.467254 & 0.979270 \\
\hline 1 & -2.686580 & 6.062758 & 1.919005 \\
\hline & -1.036411 & 6.682410 & 2.112453 \\
\hline & -0.454048 & 7.180877 & -0.898541 \\
\hline & 0.672366 & 6.359287 & 0.197162 \\
\hline
\end{tabular}




$\begin{array}{lrrr}1 & 0.154710 & 5.561511 & -1.303693 \\ 1 & -6.371195 & 4.256594 & 2.311421 \\ 1 & -4.760981 & 3.674029 & 1.854162 \\ 1 & -5.921408 & 4.145150 & 0.596939 \\ 1 & -8.392007 & 2.905514 & 1.929568 \\ 1 & -8.028543 & 2.799893 & 0.201862 \\ 1 & -8.336912 & 1.321632 & 1.142998 \\ 1 & -6.824729 & 2.160966 & 3.653350 \\ 1 & -6.660548 & 0.559550 & 2.902428 \\ 1 & -5.219565 & 1.527716 & 3.253793 \\ 1 & -5.647429 & -1.651265 & -4.550893 \\ 1 & -4.291096 & -0.636779 & -4.040664 \\ 1 & -4.682626 & -2.123287 & -3.142851 \\ 1 & -7.134777 & 0.294973 & -4.534700 \\ 1 & -7.399510 & 1.259959 & -3.072363 \\ 1 & -5.851818 & 1.366215 & -3.932038 \\ 1 & -7.719295 & -1.827308 & -3.274257 \\ 1 & -6.840518 & -2.258739 & -1.791857 \\ 1 & -7.990544 & -0.907334 & -1.786201 \\ 8 & -2.182619 & 1.937213 & -3.685732\end{array}$

$2_{\mathrm{C} 1-\mathrm{c}-} \psi_{-1222354 a}$

Number of imaginary frequencies : 0

The smallest frequencies are : $11.3738 \quad 16.2061 \quad 20.9569 \mathrm{~cm}(-1)$

Electronic energy : $\quad \mathrm{HF}=-4012.1851424$

Zero-point correction $=\quad 1.291082($ Hartree/Particle $)$

Thermal correction to Energy $=\quad 1.367098$

Thermal correction to Enthalpy= $\quad 1.368043$

Thermal correction to Gibbs Free Energy $=\quad 1.180703$

Sum of electronic and zero-point Energies $=\quad-4010.894061$

Sum of electronic and thermal Energies $=\quad-4010.818044$

Sum of electronic and thermal Enthalpies $=\quad-4010.817100$

Sum of electronic and thermal Free Energies $=\quad-4011.004440$

\begin{tabular}{cccc}
\multicolumn{4}{c}{ Cartesian Coordinates } \\
\hline $6 \ldots \ldots \ldots \ldots \ldots \ldots \ldots \ldots \ldots \ldots \ldots$ \\
6 & -3.693084 & -0.104980 & -1.732605 \\
6 & -3.331993 & 0.237386 & -0.436960 \\
6 & -4.324625 & 0.474547 & 0.522420 \\
6 & -5.670408 & 0.373955 & 0.192208 \\
6 & -6.000805 & 0.042727 & -1.133469 \\
6 & -5.040260 & -0.191341 & -2.112175 \\
45 & -1.545740 & 0.322178 & -0.014956 \\
4 & -0.130919 & 0.248143 & -1.966408 \\
1 & 0.763186 & 0.223828 & -3.307116 \\
6 & -6.781058 & 0.582465 & 1.219403 \\
6 & -7.751717 & 1.663396 & 0.732842 \\
6 & -5.382110 & -0.542307 & -3.558053 \\
6 & -4.687438 & 0.445573 & -4.502872 \\
6 & -1.515777 & -0.760601 & 1.495594 \\
6 & -1.322869 & -2.127765 & 1.375219 \\
6 & -1.336595 & -2.964007 & 2.539848 \\
6 & -1.566867 & -2.382465 & 3.819961 \\
6 & -1.793085 & -0.989669 & 3.904691 \\
6 & -1.768245 & -0.207713 & 2.781657
\end{tabular}




\begin{tabular}{|c|c|c|c|}
\hline 6 & -1.115541 & -4.364866 & 2.472368 \\
\hline 6 & -1.115920 & -5.136593 & 3.608178 \\
\hline & -1.344353 & -4.553612 & 4.872355 \\
\hline & -1.566106 & -3.203887 & 4.972288 \\
\hline & -1.144971 & -2.797320 & 0.058443 \\
\hline & 0.097883 & -2.864569 & -0.545325 \\
\hline & 0.280390 & -3.604631 & -1.738398 \\
\hline & -0.767102 & -4.270308 & -2.316720 \\
\hline & -2.058257 & -4.213594 & -1.742210 \\
\hline & -2.257828 & -3.456406 & -0.549452 \\
\hline & -3.155427 & -4.897841 & -2.321003 \\
\hline & -4.404040 & -4.824875 & -1.758901 \\
\hline & -4.613531 & -4.042790 & -0.601846 \\
\hline & -3.568885 & -3.377331 & -0.010701 \\
\hline & 1.213912 & -2.144844 & 0.010232 \\
\hline & 2.093628 & -2.909694 & 0.918483 \\
\hline & 2.783843 & -2.066387 & 1.954957 \\
\hline & 2.044746 & -1.264543 & 2.828012 \\
\hline & 2.689367 & -0.481400 & 3.776728 \\
\hline & 4.081420 & -0.484579 & 3.862512 \\
\hline & 4.821671 & -1.288368 & 3.003605 \\
\hline 6 & 4.172101 & -2.078095 & 2.057944 \\
\hline & 1.626654 & -0.854045 & -0.999614 \\
\hline & 2.522562 & 0.070761 & 0.029297 \\
\hline 6 & 3.243410 & 1.075978 & -0.595105 \\
\hline 6 & 4.464553 & 0.771886 & -1.162540 \\
\hline 6 & 5.163541 & 1.815698 & -1.853309 \\
\hline 0 & 4.618154 & 3.135544 & -1.858132 \\
\hline 6 & 3.376159 & 3.383178 & -1.224963 \\
\hline 6 & 2.683919 & 2.367480 & -0.623551 \\
\hline 6 & 6.360407 & 1.588619 & -2.579054 \\
\hline 6 & 7.001527 & 2.613209 & -3.230572 \\
\hline 6 & 6.482460 & 3.923974 & -3.196135 \\
\hline 6 & 5.311369 & 4.172903 & -2.527846 \\
\hline 6 & 4.948460 & -0.626691 & -1.113230 \\
\hline 6 & 4.121711 & -1.643521 & -1.560514 \\
\hline 6 & 4.557616 & -2.983077 & -1.640267 \\
\hline 6 & 5.809779 & -3.322537 & -1.205765 \\
\hline 6 & 6.662991 & -2.344060 & -0.639018 \\
\hline 6 & 6.228359 & -0.985844 & -0.578486 \\
\hline 6 & 7.071513 & -0.046056 & 0.067445 \\
\hline 6 & 8.284159 & -0.422230 & 0.590854 \\
\hline 6 & 8.723377 & -1.759696 & 0.500418 \\
\hline 6 & 7.923327 & -2.698449 & -0.099484 \\
\hline o & 2.849978 & -1.375701 & -2.012429 \\
\hline 6 & -1.320135 & 1.991138 & 0.712014 \\
\hline 6 & -0.257327 & 2.215931 & 1.583376 \\
\hline 6 & -0.042225 & 3.477362 & 2.150839 \\
\hline 6 & -0.884048 & 4.517576 & 1.756848 \\
\hline 6 & -1.933128 & 4.334303 & 0.844190 \\
\hline 6 & -2.145520 & 3.054102 & 0.337735 \\
\hline 6 & 1.022794 & 3.630157 & 3.236992 \\
\hline 6 & 0.573686 & 2.802323 & 4.448952 \\
\hline 6 & -2.821451 & 5.516692 & 0.461615 \\
\hline 6 & -3.495682 & 6.077600 & 1.718670 \\
\hline 6 & 2.382336 & 3.119266 & 2.750128 \\
\hline
\end{tabular}




\begin{tabular}{|c|c|c|c|}
\hline 6 & 1.195386 & 5.078923 & 3.685731 \\
\hline 6 & -1.964224 & 6.607295 & -0.188864 \\
\hline & -3.917385 & 5.119384 & -0.525398 \\
\hline & -7.534425 & -0.739456 & 1.405888 \\
\hline & -6.235523 & 1.016884 & 2.578380 \\
\hline & -4.889898 & -1.961465 & -3.859306 \\
\hline & -6.883201 & -0.486345 & -3.829114 \\
\hline & 6.738215 & 0.984172 & 0.161897 \\
\hline & -1.940382 & 0.861240 & 2.881409 \\
\hline & 8.238112 & -3.739357 & -0.162865 \\
\hline & -1.742952 & -2.737579 & 5.940538 \\
\hline & 4.882017 & 5.173808 & -2.510779 \\
\hline & -3.743271 & -2.772762 & 0.878134 \\
\hline & -5.612306 & -3.963793 & -0.177820 \\
\hline & 2.964576 & 4.390920 & -1.249796 \\
\hline & -1.987079 & -0.544845 & 4.880121 \\
\hline & 6.158460 & -4.352038 & -1.269150 \\
\hline & 7.915067 & 2.409246 & -3.784690 \\
\hline & 3.876749 & -3.722656 & -2.056813 \\
\hline & -2.984007 & -5.480477 & -3.225096 \\
\hline & -0.621783 & -4.844599 & -3.230217 \\
\hline & -0.941094 & -6.207363 & 3.532685 \\
\hline & 6.767754 & 0.582296 & -2.628143 \\
\hline & -0.943446 & -4.830799 & 1.504269 \\
\hline & 1.271232 & -3.625572 & -2.189271 \\
\hline & 7.002523 & 4.727900 & -3.711887 \\
\hline & 8.908000 & 0.317606 & 1.087452 \\
\hline & -5.239400 & -5.355026 & -2.211260 \\
\hline & -1.343428 & -5.176769 & 5.763635 \\
\hline & 9.687417 & -2.043968 & 0.915719 \\
\hline & 1.709605 & 2.524197 & -0.164072 \\
\hline ] & -4.027030 & 0.739920 & 1.534068 \\
\hline & -2.901876 & -0.309399 & -2.455293 \\
\hline & -7.053686 & -0.030124 & -1.398525 \\
\hline & -2.964144 & 2.865096 & -0.352622 \\
\hline | & 0.392007 & 1.381707 & 1.858469 \\
\hline $\mathbf{l}$ & -0.732017 & 5.509462 & 2.177879 \\
\hline & 1.437077 & -3.637302 & 1.417506 \\
\hline 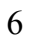 & -1.053249 & 1.601648 & -2.887880 \\
\hline & 2.837173 & -3.490143 & 0.348865 \\
\hline 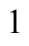 & 4.754807 & -2.697266 & 1.375199 \\
\hline & 0.958510 & -1.237808 & 2.738769 \\
\hline | & 5.909004 & -1.299129 & 3.056935 \\
\hline ] & 2.104285 & 0.141143 & 4.452973 \\
\hline & 4.583651 & 0.139447 & 4.599021 \\
\hline L & 1.981459 & 5.136587 & 4.449185 \\
\hline & 1.494018 & 5.730581 & 2.854362 \\
\hline l & 0.278627 & 5.486616 & 4.130787 \\
\hline & 3.116604 & 3.173541 & 3.566050 \\
\hline & 2.352263 & 2.075295 & 2.412868 \\
\hline & 2.759532 & 3.728893 & 1.918687 \\
\hline & 1.312912 & 2.872295 & 5.259237 \\
\hline & -0.389023 & 3.163897 & 4.835303 \\
\hline & 0.451528 & 1.741468 & 4.193054 \\
\hline 1 & -4.529026 & 5.997372 & -0.768451 \\
\hline 1 & -3.503633 & 4.735297 & -1.466808 \\
\hline
\end{tabular}




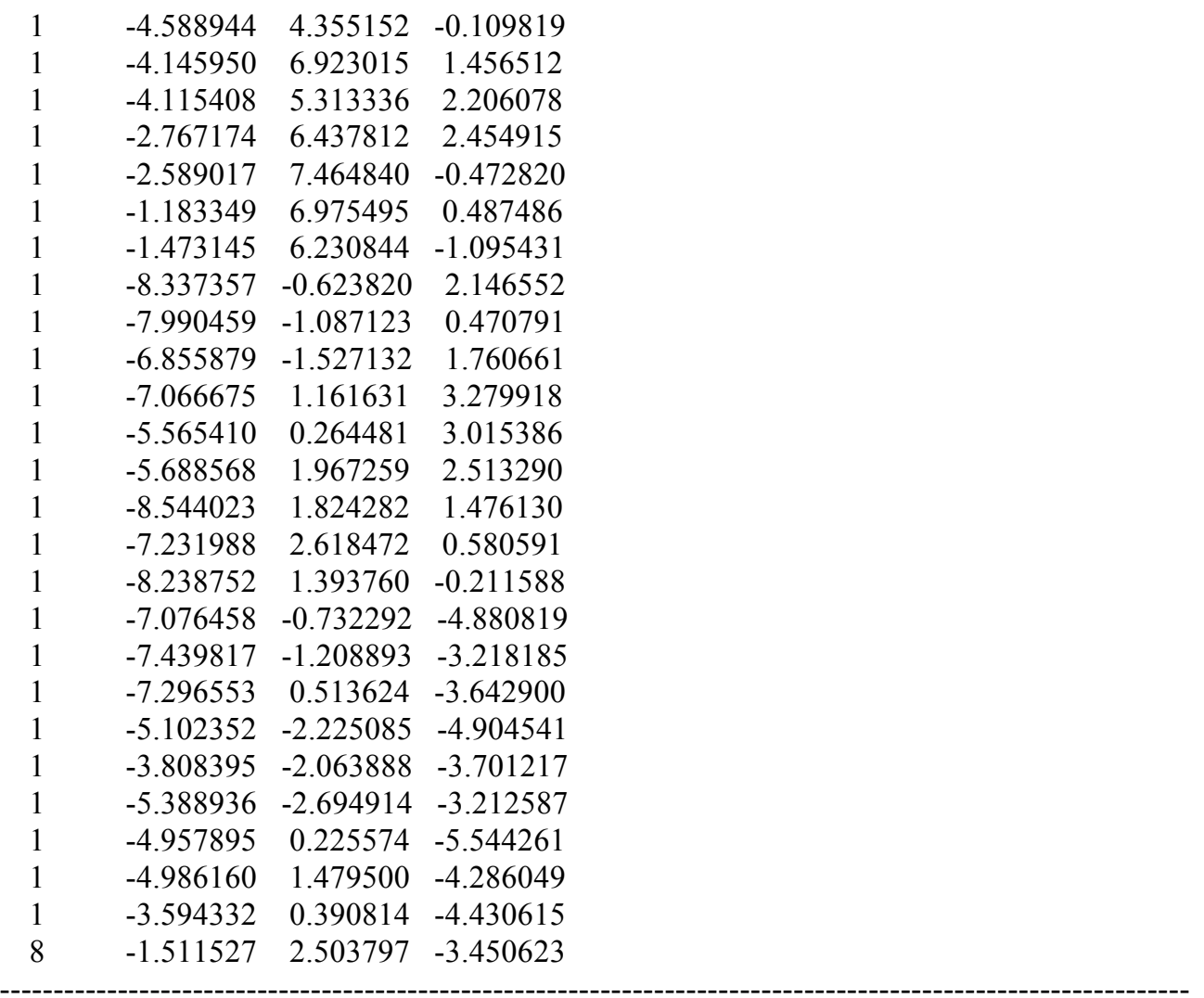

$2_{\mathrm{C} 1-\mathrm{c}-} \psi_{-1 \mathrm{~s} 2 \mathrm{2} 3 \mathrm{~s} 4 \mathrm{~s}}$

Number of imaginary frequencies : 0

The smallest frequencies are : $9.1842 \quad 15.737920 .7273 \mathrm{~cm}(-1)$

Electronic energy : $\quad \mathrm{HF}=-4012.1854578$

Zero-point correction $=\quad 1.291131$ (Hartree/Particle)

Thermal correction to Energy= $\quad 1.367112$

Thermal correction to Enthalpy $=\quad 1.368056$

Thermal correction to Gibbs Free Energy $=\quad 1.180003$

Sum of electronic and zero-point Energies $=\quad-4010.894327$

Sum of electronic and thermal Energies $=\quad-4010.818346$

Sum of electronic and thermal Enthalpies $=\quad-4010.817401$

Sum of electronic and thermal Free Energies $=\quad-4011.005455$

Cartesian Coordinates

$\begin{array}{cccc}6 & -3.688400 & -0.163343 & -1.782112 \\ 6 & -3.336808 & 0.191086 & -0.482498 \\ 6 & -4.335913 & 0.415540 & 0.464160 \\ 6 & -5.683505 & 0.295259 & 0.130381 \\ 6 & -6.004624 & -0.041980 & -1.190149 \\ 6 & -5.029611 & -0.269449 & -2.164358 \\ 15 & -1.556849 & 0.315702 & -0.043600 \\ 45 & -0.112964 & 0.244471 & -1.969286 \\ 1 & 0.828201 & 0.281266 & -3.278280 \\ 6 & -6.734536 & 0.529611 & 1.213467 \\ 6 & -6.498967 & -0.460950 & 2.360428 \\ 6 & -5.367335 & -0.648650 & -3.605002 \\ 6 & -4.640637 & 0.290593 & -4.574508\end{array}$




\begin{tabular}{|c|c|c|c|}
\hline 6 & -1.523023 & -0.737608 & 1.488360 \\
\hline 6 & -1.306643 & -2.103015 & 1.394475 \\
\hline 5 & -1.314375 & -2.918897 & 2.573338 \\
\hline & -1.564162 & -2.319432 & 3.841288 \\
\hline ) & -1.814496 & -0.929441 & 3.899428 \\
\hline & -1.793314 & -0.166707 & 2.762934 \\
\hline 6 & -1.069056 & -4.316659 & 2.531767 \\
\hline 6 & -1.064474 & -5.068484 & 3.680848 \\
\hline 6 & -1.312124 & -4.467757 & 4.933044 \\
\hline 6 & -1.557805 & -3.120541 & 5.007841 \\
\hline 6 & -1.115020 & -2.793872 & 0.091150 \\
\hline 6 & 0.131089 & -2.863466 & -0.505175 \\
\hline 6 & 0.324559 & -3.627331 & -1.681300 \\
\hline 6 & -0.715346 & -4.313382 & -2.250001 \\
\hline 6 & -2.010037 & -4.253264 & -1.683590 \\
\hline 6 & -2.220256 & -3.472572 & -0.508139 \\
\hline 6 & -3.100606 & -4.956332 & -2.252547 \\
\hline 6 & -4.352571 & -4.877263 & -1.698651 \\
\hline 6 & -4.572226 & -4.071649 & -0.559638 \\
\hline 6 & -3.534061 & -3.389306 & 0.023194 \\
\hline 7 & 1.238409 & -2.123650 & 0.040700 \\
\hline 6 & 2.119028 & -2.861843 & 0.970487 \\
\hline 6 & 2.793563 & -1.993185 & 1.996338 \\
\hline 6 & 2.041216 & -1.182971 & 2.850115 \\
\hline 6 & 2.672189 & -0.376733 & 3.788618 \\
\hline 6 & 4.063533 & -0.365469 & 3.883933 \\
\hline 6 & 4.816954 & -1.177627 & 4504 \\
\hline 6 & & -1.989914 & 2.108690 \\
\hline 15 & 1.642651 & -0.845206 & -0.988259 \\
\hline 8 & 2.536918 & 0.093696 & 0.028936 \\
\hline 6 & 3.264060 & 1.088469 & -0.603814 \\
\hline 6 & 4.486316 & 0.773984 & -1.163226 \\
\hline 6 & 5.192437 & 1.808347 & -1.860940 \\
\hline 6 & 4.652342 & 3.130273 & -1.880762 \\
\hline 6 & 3.408338 & 3.388801 & -1.255999 \\
\hline 6 & 2.709746 & 2.381606 & -0.647903 \\
\hline 6 & 6.391666 & 1.569759 & -2.579084 \\
\hline 6 & 7.039637 & 2.585599 & -3.237517 \\
\hline 6 & 6.525568 & 3.898658 & -3.217925 \\
\hline 6 & 5.352585 & 4.158496 & -2.557180 \\
\hline 6 & 4.964246 & -0.626056 & -1.098563 \\
\hline 6 & 4.134762 & -1.643652 & -1.539046 \\
\hline 6 & 4.564532 & -2.986026 & -1.602553 \\
\hline 6 & 5.812894 & -3.326804 & -1.158233 \\
\hline 6 & 6.668202 & -2.346377 & -0.598089 \\
\hline 6 & 6.240056 & -0.985461 & -0.554398 \\
\hline 6 & 7.084474 & -0.042891 & 0.085833 \\
\hline 6 & 8.292355 & -0.419461 & 0.619890 \\
\hline 6 & 8.725363 & -1.759982 & 0.546132 \\
\hline 6 & 7.923983 & -2.701117 & -0.048197 \\
\hline 8 & 2.865742 & -1.374076 & -1.997693 \\
\hline 6 & -1.371879 & 1.999042 & 0.662323 \\
\hline 6 & -0.309381 & 2.252022 & 1.526597 \\
\hline 6 & -0.126072 & 3.517524 & 2.095336 \\
\hline 6 & -0.997612 & 4.535076 & 1.706192 \\
\hline 6 & -2.043328 & 4.325790 & 0.795625 \\
\hline
\end{tabular}




\begin{tabular}{|c|c|c|c|}
\hline 6 & -2.227648 & 3.039291 & 0.292826 \\
\hline 6 & 0.935600 & 3.697392 & 3.180519 \\
\hline & 0.499719 & 2.869939 & 4.397578 \\
\hline & -2.964609 & 5.484906 & 0.419418 \\
\hline & -3.702100 & 5.971132 & 1.672147 \\
\hline & 2.304816 & 3.207808 & 2.698970 \\
\hline U & 1.081547 & 5.152215 & 3.619175 \\
\hline & -2.136143 & 6.633411 & -0.165452 \\
\hline o & -4.008453 & 5.078432 & -0.618520 \\
\hline & -6.612715 & 1.965496 & 1.737299 \\
\hline & -8.158734 & 0.330381 & 0.701656 \\
\hline 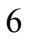 & -4.908055 & -2.087776 & -3.859794 \\
\hline 6 & -6.864236 & -0.562942 & -3.893104 \\
\hline & 6.755806 & 0.989942 & 0.167358 \\
\hline & -1.982280 & 0.901069 & 2.842915 \\
\hline & 8.233940 & -3.744166 & -0.098774 \\
\hline & -1.750022 & -2.640683 & 5.966470 \\
\hline & 4.927075 & 5.161169 & -2.551550 \\
\hline & -3.715291 & -2.766855 & 0.898510 \\
\hline & -5.573809 & -3.986865 & -0.143122 \\
\hline & 3.000513 & 4.397705 & -1.293084 \\
\hline & -2.023070 & -0.470634 & 4.865340 \\
\hline & 6.156740 & -4.358642 & -1.208490 \\
\hline & 7.954846 & 2.372849 & -3.785550 \\
\hline & 3.881961 & -3.726899 & -2.014125 \\
\hline & -2.921657 & -5.557710 & -3.142823 \\
\hline & -0.561159 & -4.906532 & -3.149916 \\
\hline & -0.870887 & -6.137241 & 3.625280 \\
\hline & 6.795487 & 0.561488 & -2.616533 \\
\hline 1 & -0.881682 & -4.796440 & 1.573311 \\
\hline & 1.318097 & -3.650881 & -2.126096 \\
\hline ] & 7.051054 & 4.695615 & -3.738972 \\
\hline & 8.917199 & 0.322558 & 1.111960 \\
\hline & -5.182923 & -5.421024 & -2.144036 \\
\hline & -1.307103 & -5.075256 & 5.835029 \\
\hline & 9.685673 & -2.044524 & 0.969802 \\
\hline 1 & 1.733162 & 2.545491 & -0.196186 \\
\hline & -4.054481 & 0.688680 & 1.480913 \\
\hline 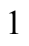 & -2.890054 & -0.362186 & -2.498773 \\
\hline & -7.051647 & -0.126947 & -1.466186 \\
\hline 1 & -3.045460 & 2.829217 & -0.392662 \\
\hline & 0.362307 & 1.434277 & 1.797982 \\
\hline & -0.871597 & 5.529590 & 2.129502 \\
\hline 1 & 1.465598 & -3.586130 & 1.478318 \\
\hline & -1.023478 & 1.606365 & -2.892247 \\
\hline 1 & 2.872703 & -3.445796 & 0.418213 \\
\hline & 4.774310 & -2.615585 & 1.440849 \\
\hline 1 & 0.955346 & -1.168098 & 2.753167 \\
\hline & 5.903871 & -1.177695 & 3.105820 \\
\hline 1 & 2.076791 & 0.252487 & 4.449420 \\
\hline 1 & 4.554951 & 0.276122 & 4.612601 \\
\hline & 1.869052 & 5.230155 & 4.379366 \\
\hline 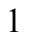 & 1.364182 & 5.804029 & 2.782352 \\
\hline & 0.158877 & 5.545064 & 4.065282 \\
\hline & 3.036655 & 3.281031 & 3.515559 \\
\hline & 2.295175 & 2.161063 & 2.368773 \\
\hline
\end{tabular}




$\begin{array}{rrrr}1 & 2.672217 & 3.819042 & 1.864305 \\ 1 & 1.235219 & 2.960391 & 5.209267 \\ 1 & -0.470973 & 3.215427 & 4.778763 \\ 1 & 0.398953 & 1.805144 & 4.148768 \\ 1 & -4.639692 & 5.941968 & -0.863184 \\ 1 & -3.545572 & 4.731387 & -1.551440 \\ 1 & -4.669581 & 4.283142 & -0.248348 \\ 1 & -4.371417 & 6.805210 & 1.421526 \\ 1 & -4.312241 & 5.166592 & 2.104362 \\ 1 & -3.010822 & 6.321858 & 2.448182 \\ 1 & -2.791331 & 7.468467 & -0.447628 \\ 1 & -1.399115 & 7.019730 & 0.548571 \\ 1 & -1.595540 & 6.309046 & -1.063893 \\ 1 & -7.331859 & 2.139866 & 2.549083 \\ 1 & -5.609720 & 2.181982 & 2.127111 \\ 1 & -6.823252 & 2.689809 & 0.939479 \\ 1 & -8.871231 & 0.506652 & 1.517440 \\ 1 & -8.406987 & 1.029809 & -0.106859 \\ 1 & -8.324126 & -0.691343 & 0.335904 \\ 1 & -7.246243 & -0.315531 & 3.152187 \\ 1 & -6.581816 & -1.496433 & 2.003320 \\ 1 & -5.506721 & -0.346142 & 2.815005 \\ 1 & -7.051396 & -0.822467 & -4.942642 \\ 1 & -7.444982 & -1.262125 & -3.277794 \\ 1 & -7.256223 & 0.449254 & -3.727974 \\ 1 & -5.115183 & -2.377291 & -4.899338 \\ 1 & -3.831166 & -2.209215 & -3.684689 \\ 1 & -5.431924 & -2.789602 & -3.197650 \\ 1 & -4.924210 & 0.056826 & -5.609324 \\ 1 & -4.897102 & 1.340348 & -4.381258 \\ 1 & -3.550465 & 0.192470 & -4.507702 \\ 8 & -1.467614 & 2.515450 & -3.454763\end{array}$

$2_{\text {C1-c- }-\psi-152 s 3 a 4 a}$

Number of imaginary frequencies : 0

The smallest frequencies are : $12.4226 \quad 17.4233 \quad 21.9093 \mathrm{~cm}(-1)$

Electronic energy : $\quad H F=-4012.1869352$

Zero-point correction $=\quad 1.291335($ Hartree/Particle $)$

Thermal correction to Energy= $\quad 1.367107$

Thermal correction to Enthalpy= $\quad 1.368051$

Thermal correction to Gibbs Free Energy= 1.182491

Sum of electronic and zero-point Energies $=\quad-4010.895600$

Sum of electronic and thermal Energies $=\quad-4010.819828$

Sum of electronic and thermal Enthalpies $=\quad-4010.818884$

Sum of electronic and thermal Free Energies $=\quad-4011.004445$

Cartesian Coordinates

$\begin{array}{llll}6 & 2.052270 & -1.278679 & 2.829295 \\ 6 & 2.776635 & -2.089941 & 1.952559 \\ 6 & 4.165107 & -2.118685 & 2.048068 \\ 6 & 4.829458 & -1.335626 & 2.988981 \\ 6 & 4.103847 & -0.521728 & 3.850921 \\ 6 & 2.711456 & -0.502313 & 3.773482 \\ 6 & 2.070165 & -2.922477 & 0.918336\end{array}$




\begin{tabular}{|c|c|c|c|}
\hline 7 & 1.206483 & -2.142483 & 0.008105 \\
\hline 15 & 1.639111 & -0.851822 & -0.992763 \\
\hline 4 & -0.127883 & 0.231416 & -1.978604 \\
\hline 1 & 0.810747 & 0.257762 & -3.290127 \\
\hline 6 & 0.072947 & -2.835070 & -0.547273 \\
\hline 6 & -1.161632 & -2.758197 & 0.072036 \\
\hline 6 & -2.297424 & -3.367497 & -0.544397 \\
\hline 6 & -2.134202 & -4.066188 & -1.777674 \\
\hline 6 & -0.849212 & -4.144798 & -2.362980 \\
\hline 6 & 0.224738 & -3.541052 & -1.765104 \\
\hline 6 & -3.599359 & -3.274625 & 0.013402 \\
\hline 6 & -4.676318 & -3.850355 & -0.612690 \\
\hline 6 & -4.507880 & -4.561244 & -1.821552 \\
\hline 6 & -3.263597 & -4.663257 & -2.390384 \\
\hline 6 & -1.318815 & -2.088732 & 1.390916 \\
\hline 6 & -1.496874 & -0.718703 & 1.502160 \\
\hline 6 & -1.738562 & -0.155129 & 2.785590 \\
\hline 6 & -1.765214 & -0.929925 & 3.913839 \\
\hline 6 & -1.555320 & -2.325921 & 3.837009 \\
\hline 6 & -1.338186 & -2.917837 & 2.559397 \\
\hline 6 & -1.559538 & -3.141358 & 4.993551 \\
\hline 6 & -1.356896 & -4.494613 & 4.899283 \\
\hline 6 & -1.144235 & -5.087802 & 3.637015 \\
\hline 6 & -1.138691 & -4.321918 & 2.497236 \\
\hline 15 & -1.533075 & 0.346138 & -0.023180 \\
\hline 6 & -3.329979 & 0.269383 & -0.406361 \\
\hline 6 & -4.300659 & 0.559768 & 0.556419 \\
\hline 6 & -5.655626 & 0.421525 & 0.265380 \\
\hline 6 & -6.008279 & 0.010738 & -1.027584 \\
\hline 6 & -5.066097 & -0.288131 & -2.011802 \\
\hline 6 & -3.715632 & -0.166381 & -1.670371 \\
\hline 6 & -6.744164 & 0.654900 & 1.311248 \\
\hline 6 & -6.173345 & 1.150543 & 2.638559 \\
\hline 6 & -5.502335 & -0.677110 & -3.423187 \\
\hline 6 & -5.973716 & 0.592495 & -4.142060 \\
\hline 6 & -1.269278 & 2.019867 & 0.679658 \\
\hline 6 & -0.209882 & 2.230225 & 1.565260 \\
\hline 6 & 0.024090 & 3.487545 & 2.124453 \\
\hline 6 & -0.794217 & 4.547539 & 1.716666 \\
\hline 6 & -1.828748 & 4.380128 & 0.791573 \\
\hline 6 & -2.057237 & 3.097505 & 0.284770 \\
\hline 6 & -2.737833 & 5.524797 & 0.347217 \\
\hline 6 & -2.630577 & 5.702067 & -1.171252 \\
\hline 6 & 1.082665 & 3.628860 & 3.219339 \\
\hline 6 & 0.615387 & 2.810275 & 4.430703 \\
\hline 6 & -7.750025 & 1.694728 & 0.807895 \\
\hline 6 & -7.466277 & -0.673169 & 1.568670 \\
\hline 6 & -6.646915 & -1.693111 & -3.383177 \\
\hline 6 & -4.356933 & -1.293027 & -4.226962 \\
\hline 6 & -4.187560 & 5.190109 & 0.719798 \\
\hline 6 & -2.372377 & 6.851784 & 1.007530 \\
\hline 6 & 2.438806 & 3.099140 & 2.743360 \\
\hline 6 & 1.272104 & 5.076165 & 3.666623 \\
\hline 8 & 2.855726 & -1.385026 & -2.006064 \\
\hline 6 & 4.124820 & -1.665524 & -1.553953 \\
\hline 6 & 4.961711 & -0.655868 & -1.109471 \\
\hline
\end{tabular}




\begin{tabular}{|c|c|c|c|}
\hline & 6.238580 & -1.025930 & -0.575497 \\
\hline & 6.659441 & -2.388656 & -0.632529 \\
\hline & 5.795876 & -3.360223 & -1.195596 \\
\hline & 4.546651 & -3.009650 & -1.630070 \\
\hline & 7.916697 & -2.753914 & -0.093017 \\
\hline & 8.726833 & -1.821252 & 0.502876 \\
\hline & 8.301358 & -0.479048 & 0.589284 \\
\hline & 7.091909 & -0.092430 & 0.066178 \\
\hline & 4.489705 & 0.746567 & -1.161567 \\
\hline & 3.271442 & 1.061598 & -0.593795 \\
\hline & 2.719731 & 2.356161 & -0.629137 \\
\hline & 3.418393 & 3.364349 & -1.235864 \\
\hline & 4.658620 & 3.105803 & -1.867936 \\
\hline & 5.195255 & 1.782397 & -1.857431 \\
\hline & 6.389923 & 1.543887 & -2.583160 \\
\hline & 7.037302 & 2.561169 & -3.239867 \\
\hline y & 6.527079 & 3.875582 & -3.210919 \\
\hline & 5.358300 & 4.135468 & -2.542822 \\
\hline & 2.544260 & 0.063340 & 0.036059 \\
\hline & 6.769072 & 0.941426 & 0.157449 \\
\hline & -1.900398 & 0.916021 & 2.879986 \\
\hline & 8.220962 & -3.798130 & -0.153283 \\
\hline & -1.725223 & -2.668033 & 5.960364 \\
\hline & 4.935713 & 5.139304 & -2.530040 \\
\hline & -3.743046 & -2.720018 & 0.939405 \\
\hline & -5.669502 & -3.749545 & -0.178798 \\
\hline & 3.013115 & 4.374485 & -1.265781 \\
\hline & -1.949291 & -0.476792 & 4.887425 \\
\hline & 6.134144 & -4.393373 & -1.255959 \\
\hline 1 & 7.948946 & 2.348592 & -3.793861 \\
\hline & 3.857388 & -3.743202 & -2.043484 \\
\hline ] & -3.121720 & -5.199347 & -3.327808 \\
\hline & -0.730185 & -4.680944 & -3.303013 \\
\hline & -0.986113 & -6.161500 & 3.566461 \\
\hline & 6.790484 & 0.534643 & -2.627978 \\
\hline & -0.980279 & -4.794177 & 1.529840 \\
\hline 1 & 1.211136 & -3.577205 & -2.224871 \\
\hline & 7.052111 & 4.673622 & -3.730758 \\
\hline 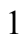 & 8.933348 & 0.256078 & 1.082557 \\
\hline ] & -5.368444 & -5.021884 & -2.302425 \\
\hline 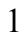 & -1.359835 & -5.113015 & 5.793864 \\
\hline 1 & 9.688413 & -2.113888 & 0.918066 \\
\hline & 1.746638 & 2.521864 & -0.170167 \\
\hline 1 & -3.984680 & 0.889395 & 1.543323 \\
\hline & -2.936250 & -0.425903 & -2.383865 \\
\hline 1 & -7.066525 & -0.084700 & -1.269683 \\
\hline l & -2.870255 & 2.929189 & -0.421683 \\
\hline 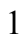 & 0.418020 & 1.384658 & 1.854384 \\
\hline 1 & -0.624565 & 5.532699 & 2.140656 \\
\hline 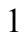 & 1.399314 & -3.635781 & 1.418769 \\
\hline 6 & -1.164237 & 1.405374 & -3.016231 \\
\hline ] & -5.478061 & 0.426837 & 3.084929 \\
\hline 1 & -5.647034 & 2.107785 & 2.525925 \\
\hline 1 & -6.989621 & 1.305358 & 3.355296 \\
\hline 1 & -8.248039 & 1.379954 & -0.116757 \\
\hline 1 & -8.531794 & 1.861698 & 1.560925 \\
\hline
\end{tabular}




$\begin{array}{rrrr}1 & -7.258830 & 2.656811 & 0.612849 \\ 1 & -7.941667 & -1.064263 & 0.660721 \\ 1 & -6.763399 & -1.434852 & 1.932832 \\ 1 & -8.250018 & -0.544153 & 2.327462 \\ 1 & -5.163957 & 1.332411 & -4.197799 \\ 1 & -6.291956 & 0.359447 & -5.167461 \\ 1 & -6.820442 & 1.059548 & -3.622619 \\ 1 & -7.550656 & -1.294489 & -2.907283 \\ 1 & -6.923752 & -1.985950 & -4.404416 \\ 1 & -6.347140 & -2.599518 & -2.840212 \\ 1 & -3.536221 & -0.584588 & -4.399179 \\ 1 & -3.944372 & -2.181068 & -3.727623 \\ 1 & -4.725793 & -1.602343 & -5.213176 \\ 1 & 2.801966 & -3.518634 & 0.349731 \\ 1 & 4.736294 & -2.745723 & 1.362858 \\ 1 & 0.966076 & -1.239101 & 2.746550 \\ 1 & 5.916902 & -1.359569 & 3.036020 \\ 1 & 2.137483 & 0.127976 & 4.452108 \\ 1 & 4.617854 & 0.097320 & 4.583501 \\ 1 & 2.053835 & 5.123912 & 4.435211 \\ 1 & 1.585672 & 5.722435 & 2.836591 \\ 1 & 0.358316 & 5.497596 & 4.104888 \\ 1 & 3.167102 & 3.141562 & 3.565368 \\ 1 & 2.395655 & 2.056250 & 2.404487 \\ 1 & 2.831738 & 3.704052 & 1.915793 \\ 1 & 1.346618 & 2.879756 & 5.248254 \\ 1 & -0.348855 & 3.180039 & 4.805484 \\ 1 & 0.489308 & 1.748839 & 4.179257 \\ 1 & -3.051747 & 7.637261 & 0.653284 \\ 1 & -2.463729 & 6.807766 & 2.100596 \\ 1 & -1.350360 & 7.165912 & 0.759437 \\ 1 & -4.858315 & 6.002808 & 0.410065 \\ 1 & -4.536446 & 4.269996 & 0.233896 \\ 1 & -4.294019 & 5.058256 & 1.804868 \\ 1 & -3.276483 & 6.525325 & -1.505097 \\ 1 & -1.600477 & 5.937691 & -1.467873 \\ 1 & -2.935524 & 4.800673 & -1.715434 \\ 8 & -1.727866 & 2.161378 & -3.687595\end{array}$

$2_{\text {C1-c- }} \psi_{-1 s 2 s 3 a 4 s}$

Number of imaginary frequencies : 0

The smallest frequencies are : $12.5807 \quad 17.9057 \quad 21.2230 \mathrm{~cm}(-1)$

Electronic energy : $\quad H F=-4012.186995$

Zero-point correction $=\quad 1.290980$ (Hartree/Particle)

Thermal correction to Energy= $\quad 1.366874$

Thermal correction to Enthalpy= $\quad 1.367819$

Thermal correction to Gibbs Free Energy $=\quad 1.181566$

Sum of electronic and zero-point Energies $=\quad-4010.896015$

Sum of electronic and thermal Energies $=\quad-4010.820121$

Sum of electronic and thermal Enthalpies = $\quad-4010.819176$

Sum of electronic and thermal Free Energies= $\quad-4011.005429$

Cartesian Coordinates

$6 \quad-3.706622-0.191339-1.695862$ 


\begin{tabular}{|c|c|c|c|}
\hline 6 & -3.328625 & 0.238016 & -0.423013 \\
\hline 6 & -4.305151 & 0.517361 & 0.528851 \\
\hline 6 & -5.663081 & 0.383015 & 0.227771 \\
\hline 6 & -6.007415 & -0.015363 & -1.065780 \\
\hline 6 & -5.051006 & -0.310416 & -2.045343 \\
\hline & -1.535533 & 0.334475 & -0.027025 \\
\hline & -0.123155 & 0.231827 & -1.977064 \\
\hline 1 & 0.816378 & 0.264077 & -3.287932 \\
\hline 6 & -6.693402 & 0.635501 & 1.326639 \\
\hline 6 & -6.453446 & -0.369555 & 2.460428 \\
\hline 6 & -5.479898 & -0.693114 & -3.460626 \\
\hline 6 & -6.614128 & -1.721293 & -3.430391 \\
\hline 6 & -1.497645 & -0.724700 & 1.502121 \\
\hline 6 & -1.313160 & -2.094074 & 1.394761 \\
\hline 6 & -1.331161 & -2.920735 & 2.564657 \\
\hline 6 & -1.553380 & -2.326999 & 3.840502 \\
\hline 6 & -1.768913 & -0.931737 & 3.913703 \\
\hline 6 & -1.743203 & -0.159107 & 2.783690 \\
\hline 6 & -1.125644 & -4.324062 & 2.505707 \\
\hline 6 & -1.130178 & -5.087514 & 3.647078 \\
\hline 6 & -1.347820 & -4.492532 & 4.907714 \\
\hline 6 & -1.556386 & -3.139986 & 4.998803 \\
\hline 6 & -1.152308 & -2.763574 & 0.076737 \\
\hline 6 & 0.082721 & -2.834588 & -0.542693 \\
\hline 6 & 0.236600 & -3.537402 & -1.762037 \\
\hline 6 & -0.835677 & -4.143057 & -2.361023 \\
\hline 6 & -2.120731 & -4.070379 & -1.775240 \\
\hline 6 & -2.286384 & -3.375366 & -0.540120 \\
\hline 6 & -3.247720 & -4.670015 & -2.389727 \\
\hline 6 & -4.491893 & -4.575324 & -1.819716 \\
\hline 6 & -4.662595 & -3.868703 & -0.608681 \\
\hline 6 & -3.588664 & -3.288660 & 0.018664 \\
\hline 7 & 1.213595 & -2.137805 & 0.012825 \\
\hline 6 & 2.075796 & -2.910989 & 0.930353 \\
\hline 6 & 2.777528 & -2.071023 & 1.961808 \\
\hline 6 & 2.048935 & -1.256602 & 2.832077 \\
\hline 6 & 2.703824 & -0.471916 & 3.772368 \\
\hline 6 & 4.096115 & -0.486465 & 3.852568 \\
\hline 6 & 4.825932 & -1.303749 & 2.997408 \\
\hline 6 & 4.165899 & -2.094817 & 2.060170 \\
\hline 15 & 1.644559 & -0.847960 & -0.989972 \\
\hline 8 & 2.546567 & 0.071381 & 0.037938 \\
\hline 6 & 3.272920 & 1.069277 & -0.593338 \\
\hline 6 & 4.492675 & 0.755244 & -1.158496 \\
\hline 6 & 5.197129 & 1.790807 & -1.855863 \\
\hline 6 & 4.657666 & 3.113040 & -1.870652 \\
\hline 6 & 3.415807 & 3.370591 & -1.241387 \\
\hline 6 & 2.718413 & 2.362465 & -0.633151 \\
\hline 6 & 6.393407 & 1.552987 & -2.579171 \\
\hline 6 & 7.039566 & 2.569940 & -3.237593 \\
\hline 6 & 6.526463 & 3.883319 & -3.212877 \\
\hline 6 & 5.356143 & 4.142436 & -2.547191 \\
\hline 6 & 4.966932 & -0.646307 & -1.102501 \\
\hline 6 & 4.131983 & -1.658147 & -1.545686 \\
\hline 6 & 4.554984 & -3.002141 & -1.616614 \\
\hline 6 & 5.803850 & -3.350133 & -1.179020 \\
\hline
\end{tabular}




\begin{tabular}{|c|c|c|c|}
\hline & 6.665742 & -2.375881 & -0.618058 \\
\hline & 6.243468 & -1.013391 & -0.565751 \\
\hline & 7.095081 & -0.077060 & 0.074084 \\
\hline & 8.304357 & -0.460820 & 0.599691 \\
\hline & 8.731332 & -1.802836 & 0.517807 \\
\hline & 7.922813 & -2.738158 & -0.076089 \\
\hline & 2.863515 & -1.379938 & -2.001023 \\
\hline & -1.296992 & 2.012925 & 0.673504 \\
\hline & -0.235650 & 2.237512 & 1.553337 \\
\hline & -0.017120 & 3.497208 & 2.113091 \\
\hline & -0.852063 & 4.546121 & 1.709758 \\
\hline & -1.889029 & 4.364955 & 0.790083 \\
\hline & -2.103683 & 3.078944 & 0.285210 \\
\hline & 1.042787 & 3.651945 & 3.204873 \\
\hline & 0.585491 & 2.832629 & 4.419579 \\
\hline & -2.811196 & 5.498988 & 0.344988 \\
\hline y & -2.699183 & 5.679938 & -1.172820 \\
\hline & 2.403048 & 3.133432 & 2.728221 \\
\hline & 1.219498 & 5.102392 & 3.647181 \\
\hline & -4.258431 & 5.145997 & 0.709667 \\
\hline & -2.464981 & 6.829224 & 1.009087 \\
\hline & -6.542991 & 2.062434 & 1.864806 \\
\hline & -8.127975 & 0.462649 & 0.834719 \\
\hline & -5.962389 & 0.576613 & -4.171917 \\
\hline & -4.327022 & -1.291796 & -4.266558 \\
\hline & 6.771060 & 0.956735 & 0.161799 \\
\hline & -1.908612 & 0.911713 & 2.875205 \\
\hline & 8.228259 & -3.782221 & -0.132870 \\
\hline & -1.726004 & -2.665370 & 5.964293 \\
\hline & 4.931348 & 5.145376 & -2.537717 \\
\hline & -3.736451 & -2.734783 & 0.944978 \\
\hline 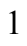 & -5.656139 & -3.775852 & -0.174339 \\
\hline & 3.008348 & 4.379740 & -1.274886 \\
\hline & -1.956063 & -0.477148 & 4.886021 \\
\hline & 6.143163 & -4.383161 & -1.235420 \\
\hline & 7.952487 & 2.357884 & -3.789680 \\
\hline 1 & 3.867002 & -3.737655 & -2.028681 \\
\hline & -3.103898 & -5.202725 & -3.328781 \\
\hline 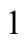 & -0.715173 & -4.676514 & -3.302397 \\
\hline & -0.967397 & -6.160687 & 3.579168 \\
\hline & 6.796222 & 0.544494 & -2.620713 \\
\hline 1 & -0.963309 & -4.797549 & 1.539560 \\
\hline & 1.222897 & -3.569434 & -2.222294 \\
\hline 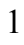 & 7.050526 & 4.681120 & -3.734060 \\
\hline & 8.935058 & 0.276470 & 1.091381 \\
\hline 1 & -5.350735 & -5.038843 & -2.300880 \\
\hline 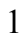 & -1.349881 & -5.109085 & 5.803568 \\
\hline & 9.692790 & -2.093177 & 0.934884 \\
\hline 1 & 1.743897 & 2.526827 & -0.176793 \\
\hline 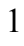 & -4.002758 & 0.834254 & 1.526945 \\
\hline 1 & -2.921499 & -0.444316 & -2.405415 \\
\hline & -7.060070 & -0.105039 & -1.324378 \\
\hline 1 & -2.918307 & 2.899309 & -0.416560 \\
\hline ] & 0.405141 & 1.400176 & 1.838376 \\
\hline 1 & -0.693334 & 5.533498 & 2.132871 \\
\hline 1 & 1.404699 & -3.622725 & 1.432643 \\
\hline
\end{tabular}




$\begin{array}{lrrr}6 & -1.160365 & 1.410216 & -3.009206 \\ 1 & 2.810517 & -3.508986 & 0.367485 \\ 1 & 4.740416 & -2.724121 & 1.379805 \\ 1 & 0.962841 & -1.220451 & 2.746625 \\ 1 & 5.913348 & -1.323950 & 3.046752 \\ 1 & 2.126570 & 0.161153 & 4.445625 \\ 1 & 4.606789 & 0.139035 & 4.581989 \\ 1 & 2.003547 & 5.160265 & 4.412714 \\ 1 & 1.523389 & 5.749182 & 2.813961 \\ 1 & 0.303266 & 5.516101 & 4.087656 \\ 1 & 3.132365 & 3.186306 & 3.548695 \\ 1 & 2.369912 & 2.088780 & 2.393449 \\ 1 & 2.788569 & 3.739047 & 1.897687 \\ 1 & 1.317765 & 2.910943 & 5.235406 \\ 1 & -0.381257 & 3.194749 & 4.795385 \\ 1 & 0.468468 & 1.769422 & 4.171136 \\ 1 & -3.154927 & 7.606008 & 0.656095 \\ 1 & -2.556880 & 6.780998 & 2.101939 \\ 1 & -1.447270 & 7.158487 & 0.762928 \\ 1 & -3.351637 & 6.497519 & -1.507936 \\ 1 & -1.670199 & 5.925915 & -1.464899 \\ 1 & -2.993453 & 4.776491 & -1.719490 \\ 1 & -4.938306 & 5.947091 & 0.389600 \\ 1 & -4.590398 & 4.217340 & 0.228041 \\ 1 & -4.370784 & 5.020611 & 1.794902 \\ 1 & -7.263989 & 2.242285 & 2.673647 \\ 1 & -5.540257 & 2.252139 & 2.267174 \\ 1 & -6.731568 & 2.801576 & 1.074977 \\ 1 & -8.825798 & 0.655588 & 1.659312 \\ 1 & -8.372114 & 1.164221 & 0.026611 \\ 1 & -8.319290 & -0.556763 & 0.475450 \\ 1 & -7.181946 & -0.218843 & 3.268644 \\ 1 & -6.560270 & -1.400231 & 2.095783 \\ 1 & -5.449447 & -0.274111 & 2.893494 \\ 1 & -4.689875 & -1.592903 & -5.257542 \\ 1 & -3.510753 & -0.575617 & -4.427506 \\ 1 & -3.910023 & -2.182445 & -3.775556 \\ 1 & -6.277848 & 0.346964 & -5.198947 \\ 1 & -6.813912 & 1.032961 & -3.650883 \\ 1 & -5.159098 & 1.323940 & -4.222409 \\ 1 & -6.884778 & -2.010652 & -4.454312 \\ 1 & -6.306078 & -2.627911 & -2.892366 \\ 1 & -724839 & -1.335457 & -2.954498 \\ 1 & 2.169664 & -3.675902\end{array}$

$2_{\text {C1-c- }} \psi_{-1225354 a}$

Number of imaginary frequencies : 0

The smallest frequencies are : $11.9826 \quad 16.1639 \quad 21.5615 \mathrm{~cm}(-1)$

Electronic energy : $\quad H F=-4012.1855317$

Zero-point correction $=\quad 1.291436$ (Hartree/Particle)

Thermal correction to Energy= $\quad 1.367334$

Thermal correction to Enthalpy= $\quad 1.368278$

Thermal correction to Gibbs Free Energy= $\quad 1.181472$

Sum of electronic and zero-point Energies $=\quad-4010.894095$

Sum of electronic and thermal Energies $=\quad-4010.818197$ 
Sum of electronic and thermal Enthalpies=

Sum of electronic and thermal Free Energies=
$-4010.817253$

$-4011.004060$

Cartesian Coordinates

\begin{tabular}{|c|c|c|c|}
\hline 6 & -3.724156 & -0.059000 & -1.736767 \\
\hline 6 & -3.341908 & 0.283631 & -0.447090 \\
\hline 6 & -4.320543 & 0.514434 & 0.529346 \\
\hline 6 & -5.671248 & 0.394378 & 0.226188 \\
\hline 6 & -6.022436 & 0.058105 & -1.092803 \\
\hline 6 & -5.077953 & -0.161799 & -2.090048 \\
\hline 15 & -1.548322 & 0.345675 & -0.043329 \\
\hline 45 & -0.124640 & 0.230458 & -1.985911 \\
\hline 1 & 0.779367 & 0.172008 & -3.318065 \\
\hline 6 & -6.765475 & 0.577602 & 1.276516 \\
\hline 6 & -7.765909 & 1.643831 & 0.818631 \\
\hline 6 & -5.444952 & -0.507414 & -3.531145 \\
\hline 6 & -4.851428 & 0.548612 & -4.470726 \\
\hline 6 & -1.524195 & -0.738019 & 1.468337 \\
\hline 6 & -1.332936 & -2.105711 & 1.351583 \\
\hline 6 & -1.350143 & -2.939510 & 2.518381 \\
\hline 6 & -1.583333 & -2.355497 & 3.796711 \\
\hline 6 & -1.808355 & -0.962279 & 3.877902 \\
\hline 6 & -1.778983 & -0.182861 & 2.753387 \\
\hline 6 & -1.129486 & -4.340623 & 2.454556 \\
\hline 6 & -1.132540 & -5.109961 & 3.592055 \\
\hline 6 & -1.363656 & -4.524319 & 4.854431 \\
\hline 6 & -1.585471 & -3.174321 & 4.950832 \\
\hline 6 & -1.150738 & -2.782017 & 0.038721 \\
\hline 6 & 0.096448 & -2.862135 & -0.553540 \\
\hline 6 & 0.285439 & -3.612514 & -1.739127 \\
\hline 6 & -0.760732 & -4.276771 & -2.321238 \\
\hline 6 & -2.056242 & -4.208737 & -1.757603 \\
\hline 6 & -2.261589 & -3.441048 & -0.572645 \\
\hline 6 & -3.151631 & -4.894495 & -2.337681 \\
\hline 6 & -4.403241 & -4.815237 & -1.782841 \\
\hline 6 & -4.617622 & -4.024731 & -0.632427 \\
\hline 6 & -3.574931 & -3.356215 & -0.041202 \\
\hline 7 & 1.212132 & -2.147204 & 0.009163 \\
\hline 6 & 2.090490 & -2.921933 & 0.909769 \\
\hline 6 & 2.785685 & -2.087762 & 1.950016 \\
\hline 6 & 2.050634 & -1.291823 & 2.831893 \\
\hline 6 & 2.700000 & -0.518389 & 3.785357 \\
\hline 6 & 4.092338 & -0.524771 & 3.866523 \\
\hline 6 & 4.828431 & -1.322266 & 2.998256 \\
\hline 6 & 4.174182 & -2.102912 & 2.048349 \\
\hline 15 & 1.630588 & -0.857208 & -0.998600 \\
\hline 8 & 2.521165 & 0.070733 & 0.032339 \\
\hline 6 & 3.239871 & 1.077706 & -0.592643 \\
\hline 6 & 4.462980 & 0.776845 & -1.157474 \\
\hline 6 & 5.159231 & 1.821310 & -1.850188 \\
\hline 6 & 4.609497 & 3.139240 & -1.858923 \\
\hline 6 & 3.366307 & 3.384178 & -1.227166 \\
\hline 6 & 2.676599 & 2.367622 & -0.624270 \\
\hline 6 & 6.357501 & 1.596412 & -2.574320 \\
\hline 6 & 6.995683 & 2.621477 & -3.227975 \\
\hline
\end{tabular}




\begin{tabular}{|c|c|c|c|}
\hline 6 & 6.472191 & 3.930568 & -3.197382 \\
\hline & 5.299715 & 4.177252 & -2.530705 \\
\hline & 4.953037 & -0.619229 & -1.103739 \\
\hline & 4.132067 & -1.641155 & -1.550038 \\
\hline & 4.576472 & -2.978002 & -1.629111 \\
\hline & 5.830397 & -3.309680 & -1.193720 \\
\hline & 6.677155 & -2.326017 & -0.626227 \\
\hline & 6.234485 & -0.970397 & -0.567265 \\
\hline & 7.071735 & -0.024999 & 0.078209 \\
\hline & 8.286183 & -0.393554 & 0.602900 \\
\hline & 8.733168 & -1.728572 & 0.514342 \\
\hline & 7.938984 & -2.672598 & -0.085137 \\
\hline & 2.859334 & -1.381842 & -2.003691 \\
\hline & -1.295297 & 2.008998 & 0.689654 \\
\hline & -0.229538 & 2.209110 & 1.570413 \\
\hline & -0.006729 & 3.453613 & 2.161485 \\
\hline & -0.843351 & 4.513134 & 1.790899 \\
\hline & -1.886018 & 4.356981 & 0.873513 \\
\hline & -2.103056 & 3.085319 & 0.333900 \\
\hline 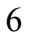 & 1.055786 & 3.580054 & 3.254279 \\
\hline & 0.590591 & 2.742907 & 4.453690 \\
\hline & -2.819273 & 5.499945 & 0.477421 \\
\hline & -2.751770 & 5.715987 & -1.038123 \\
\hline & 2.411400 & 3.059041 & 2.767732 \\
\hline & 1.245346 & 5.020714 & 3.722829 \\
\hline & -4.254888 & 5.134641 & 0.874766 \\
\hline & -2.456297 & 6.815244 & 1.161910 \\
\hline & -7.495353 & -0.757295 & 1.465865 \\
\hline & -6.201138 & 1.007002 & 2.629531 \\
\hline & -4.869077 & -1.881562 & -3.887278 \\
\hline & -6.954648 & -0.549472 & -3.751829 \\
\hline & 6.732570 & 1.003456 & 0.171068 \\
\hline & -1.949245 & 0.886559 & 2.851850 \\
\hline & 8.259870 & -3.711720 & -0.147233 \\
\hline & -1.764409 & -2.705762 & 5.917611 \\
\hline & 4.866910 & 5.176715 & -2.516619 \\
\hline & -3.750700 & -2.746949 & 0.843971 \\
\hline 1 & -5.618217 & -3.942186 & -0.213476 \\
\hline & 2.951635 & 4.390557 & -1.254313 \\
\hline ] & -2.003972 & -0.514823 & 4.851831 \\
\hline & 6.185395 & -4.337038 & -1.256842 \\
\hline ] & 7.910339 & 2.419139 & -3.780846 \\
\hline 1 & 3.900624 & -3.721667 & -2.046443 \\
\hline & -2.976255 & -5.484771 & -3.236029 \\
\hline ] & -0.611080 & -4.859069 & -3.228939 \\
\hline & -0.957765 & -6.180919 & 3.519154 \\
\hline ] & 6.768329 & 0.591382 & -2.620541 \\
\hline & -0.955397 & -4.809017 & 1.488001 \\
\hline 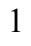 & 1.280178 & -3.642526 & -2.180789 \\
\hline 1 & 6.989954 & 4.734893 & -3.714825 \\
\hline ] & 8.905372 & 0.350461 & 1.099073 \\
\hline 1 & -5.236965 & -5.347583 & -2.235606 \\
\hline & -1.364746 & -5.145508 & 5.747081 \\
\hline 1 & 9.698509 & -2.006813 & 0.930710 \\
\hline & 1.702162 & 2.523534 & -0.164870 \\
\hline & -4.009012 & 0.784440 & 1.535374 \\
\hline
\end{tabular}




$\begin{array}{rrrr}1 & -2.945769 & -0.255536 & -2.475102 \\ 1 & -7.079025 & -0.033165 & -1.336406 \\ 1 & -2.923351 & 2.924244 & -0.365809 \\ 1 & 0.410481 & 1.363373 & 1.831803 \\ 1 & -0.681978 & 5.488629 & 2.239633 \\ 1 & 1.432027 & -3.649949 & 1.405712 \\ 6 & -1.017476 & 1.564297 & -2.961554 \\ 1 & 2.830365 & -3.501732 & 0.334608 \\ 1 & 4.753133 & -2.718242 & 1.359081 \\ 1 & 0.963895 & -1.263493 & 2.746930 \\ 1 & 5.915963 & -1.335470 & 3.047428 \\ 1 & 2.118556 & 0.099326 & 4.468966 \\ 1 & 4.597979 & 0.091954 & 4.606856 \\ 1 & 2.032590 & 5.058095 & 4.486334 \\ 1 & 1.551199 & 5.680510 & 2.900584 \\ 1 & 0.334333 & 5.433442 & 4.174852 \\ 1 & 3.142263 & 3.094280 & 3.587768 \\ 1 & 2.370369 & 2.019567 & 2.418601 \\ 1 & 2.799985 & 3.673907 & 1.945472 \\ 1 & 1.324089 & 2.797437 & 5.270415 \\ 1 & -0.371900 & 3.108623 & 4.836908 \\ 1 & 0.460942 & 1.686312 & 4.184334 \\ 1 & -3.152894 & 7.600140 & 0.841496 \\ 1 & -2.523675 & 6.743976 & 2.255226 \\ 1 & -1.443730 & 7.148219 & 0.899943 \\ 1 & -3.409289 & 6.544615 & -1.333776 \\ 1 & -1.730818 & 5.962956 & -1.356217 \\ 1 & -3.069203 & 4.827644 & -1.596178 \\ 1 & -4.945027 & 5.943843 & 0.600434 \\ 1 & -4.598938 & 4.221139 & 0.373284 \\ 1 & -4.334628 & 4.973671 & 1.958193 \\ 1 & -8.288955 & -0.657291 & 2.218760 \\ 1 & -7.960224 & -1.106591 & 0.535780 \\ 1 & -6.801035 & -1.536658 & 1.807341 \\ 1 & -7.020965 & 1.126953 & 3.348892 \\ 1 & -5.508361 & 0.262040 & 3.043759 \\ 1 & -5.673894 & 1.968347 & 2.566540 \\ 1 & -8.549057 & 1.778509 & 1.576693 \\ 1 & -7.271617 & 2.612335 & 0.669267 \\ 1 & -8.262755 & 1.373919 & -0.120669 \\ 1 & -7.166138 & -0.799143 & -4.799140 \\ 1 & -7.440234 & -1.312304 & -3.129194 \\ 1 & -7.427971 & 0.418942 & -3.543193 \\ 1 & -5.113478 & -2.138000 & -4.927173 \\ 1 & -3.776621 & -1.910333 & -3.786036 \\ 1 & -5.282995 & -2.661645 & -3.235377 \\ 1 & -5.129218 & 0.330036 & -5.510557 \\ 1 & -5.221867 & 1.552170 & -4.224356 \\ 1 & -3.756091 & 0.573836 & -4.421941 \\ 8 & -1.441214 & 2.450742 & -3.574275\end{array}$

$2_{\text {C1-c- }} \psi_{-1252354 s}$

Number of imaginary frequencies : 0

The smallest frequencies are : $10.9893 \quad 15.9613 \quad 21.3249 \mathrm{~cm}(-1)$

Electronic energy : $\quad H F=-4012.1855518$ 
Zero-point correction $=$

Thermal correction to Energy=

Thermal correction to Enthalpy=

Thermal correction to Gibbs Free Energy=

Sum of electronic and zero-point Energies=

Sum of electronic and thermal Energies=

Sum of electronic and thermal Enthalpies=

Sum of electronic and thermal Free Energies=
1.291190 (Hartree/Particle)

1.367157

1.368101

1.180542

$-4010.894362$

$-4010.818395$

$-4010.817450$

$-4011.005010$

Cartesian Coordinates

$\begin{array}{cccc}6 & 5.330984 & 4.187182 & -2.521256 \\ 6 & 4.635743 & 3.150811 & -1.852114 \\ 6 & 5.181692 & 1.831242 & -1.842493 \\ 6 & 6.381395 & 1.603284 & -2.563304 \\ 6 & 7.024475 & 2.626883 & -3.214433 \\ 6 & 6.504701 & 3.937488 & -3.184593 \\ 6 & 4.480604 & 0.788591 & -1.152062 \\ 6 & 3.257347 & 1.093374 & -0.589606 \\ 6 & 2.697160 & 2.384381 & -0.623419 \\ 6 & 3.391164 & 3.399092 & -1.224404 \\ 6 & 4.964842 & -0.609680 & -1.099094 \\ 6 & 4.140016 & -1.627272 & -1.548172 \\ 6 & 4.577475 & -2.966389 & -1.626771 \\ 6 & 5.828614 & -3.304707 & -1.188463 \\ 6 & 6.679168 & -2.325518 & -0.618949 \\ 6 & 6.243320 & -0.967642 & -0.560196 \\ 6 & 7.937962 & -2.678817 & -0.075119 \\ 6 & 8.735215 & -1.739297 & 0.527309 \\ 6 & 8.294719 & -0.402123 & 0.615861 \\ 6 & 7.083607 & -0.026970 & 0.088162 \\ 8 & 2.869121 & -1.360455 & -2.003186 \\ 15 & 1.643460 & -0.839621 & -0.993126 \\ 8 & 2.534933 & 0.089401 & 0.035346 \\ 7 & 1.231943 & -2.130243 & 0.016585 \\ 6 & 0.121068 & -2.854968 & -0.543025 \\ 6 & -1.126344 & -2.782852 & 0.049940 \\ 6 & -2.234321 & -3.445961 & -0.561390 \\ 6 & -2.025935 & -4.212114 & -1.746775 \\ 6 & -0.729973 & -4.274648 & -2.309878 \\ 6 & 0.313130 & -3.604531 & -1.728484 \\ 6 & -3.119578 & -4.899740 & -2.328426 \\ 6 & -4.372146 & -4.821126 & -1.776009 \\ 6 & -4.589372 & -4.031081 & -0.625702 \\ 6 & -3.548644 & -3.362431 & -0.031619 \\ 6 & -1.315042 & -2.104640 & 1.360468 \\ 6 & -1.522435 & -0.738869 & 1.469540 \\ 6 & -1.783982 & -0.179839 & 2.751281 \\ 6 & -1.806244 & -0.954229 & 3.879725 \\ 6 & -1.567157 & -2.345490 & 3.805715 \\ 6 & -1.325779 & -2.933161 & 2.530626 \\ 6 & -1.091673 & -4.332304 & 2.473317 \\ 6 & -1.089349 & -5.096431 & 3.614294 \\ 6 & -1.328062 & -4.507188 & 4.873631 \\ 6 & -1.563079 & -3.158980 & 4.963611 \\ 15 & -1.556568 & 0.331617 & -0.050935\end{array}$




\begin{tabular}{|c|c|c|c|}
\hline 6 & -1.334108 & 2.004148 & 0.671148 \\
\hline 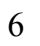 & -0.269836 & 2.226521 & 1.548334 \\
\hline 6 & -0.069397 & 3.476200 & 2.136225 \\
\hline 6 & -0.925699 & 4.519432 & 1.763524 \\
\hline 6 & -1.967343 & 4.341526 & 0.848987 \\
\hline 6 & -2.163868 & 3.063698 & 0.315759 \\
\hline & 0.990007 & 3.624208 & 3.229397 \\
\hline f & 0.535910 & 2.786177 & 4.432484 \\
\hline ) & -2.914873 & 5.469044 & 0.441978 \\
\hline 6 & -2.825079 & 5.688895 & -1.072028 \\
\hline 6 & 2.111917 & -2.891685 & 0.927363 \\
\hline 6 & 2.794986 & -2.045600 & 1.966170 \\
\hline 6 & 2.049450 & -1.246835 & 2.836548 \\
\hline 6 & 2.687598 & -0.461612 & 3.787926 \\
\hline 6 & 4.079302 & -0.459594 & 3.878962 \\
\hline 6 & 4.825905 & -1.260090 & 3.022468 \\
\hline 6 & 4.182907 & -2.051932 & 2.074140 \\
\hline 45 & -0.118817 & 0.240325 & -1.981264 \\
\hline 1 & 0.828763 & 0.268680 & -3.285932 \\
\hline 6 & -3.347363 & 0.242101 & -0.462343 \\
\hline 6 & -4.330730 & 0.460344 & 0.503690 \\
\hline 6 & -5.684383 & 0.339690 & 0.193151 \\
\hline 6 & -6.028638 & 0.021104 & -1.126557 \\
\hline 6 & -5.071148 & -0.192522 & -2.120428 \\
\hline 6 & -3.723469 & -0.096631 & -1.758965 \\
\hline 6 & -6.719318 & 0.531472 & 1.301135 \\
\hline 6 & -6.477324 & -0.521065 & 2.390321 \\
\hline 6 & -5.432141 & -0.549420 & -3.561021 \\
\hline 6 & -4.721545 & 0.406805 & -4.525955 \\
\hline 6 & -6.579300 & 1.933304 & 1.904619 \\
\hline 6 & -8.152082 & 0.373240 & 0.798549 \\
\hline 6 & -4.976811 & -1.984105 & -3.846872 \\
\hline 6 & -6.933535 & -0.460168 & -3.823295 \\
\hline 6 & -4.351101 & 5.080450 & 0.813839 \\
\hline 6 & -2.584985 & 6.788725 & 1.134742 \\
\hline 6 & 2.354215 & 3.121055 & 2.748022 \\
\hline 6 & 1.157075 & 5.069959 & 3.690749 \\
\hline 1 & 6.749237 & 1.003061 & 0.181069 \\
\hline 1 & -1.964822 & 0.888383 & 2.843763 \\
\hline 1 & 8.253773 & -3.719489 & -0.137192 \\
\hline 1 & -1.748372 & -2.687910 & 5.927957 \\
\hline 1 & 4.901051 & 5.187892 & -2.507849 \\
\hline 1 & -3.727266 & -2.752000 & 0.852676 \\
\hline 1 & -5.591358 & -3.947482 & -0.210079 \\
\hline 1 & 2.979209 & 4.406553 & -1.253234 \\
\hline 1 & -2.007021 & -0.504061 & 4.851357 \\
\hline 1 & 6.178413 & -4.333882 & -1.250873 \\
\hline 1 & 7.940194 & 2.422242 & -3.764690 \\
\hline 1 & 3.898783 & -3.706630 & -2.045647 \\
\hline 1 & -2.942232 & -5.489629 & -3.226650 \\
\hline 1 & -0.577549 & -4.856210 & -3.217599 \\
\hline 1 & -0.904533 & -6.166048 & 3.546643 \\
\hline 1 & 6.789490 & 0.597104 & -2.608849 \\
\hline 1 & -0.911668 & -4.803213 & 1.509057 \\
\hline 1 & 1.307499 & -3.628746 & -2.171246 \\
\hline 1 & 7.026354 & 4.740617 & -3.699977 \\
\hline
\end{tabular}




\begin{tabular}{|c|c|c|c|}
\hline 1 & 8.916330 & 0.338310 & 1.114359 \\
\hline | & -5.204814 & -5.353456 & -2.230803 \\
\hline & -1.324745 & -5.124314 & 5.769080 \\
\hline & 9.698030 & -2.022724 & 0.946011 \\
\hline & 1.721091 & 2.541752 & -0.168046 \\
\hline & -4.032410 & 0.718937 & 1.519448 \\
\hline & -2.940226 & -0.295509 & -2.491308 \\
\hline & -7.080059 & -0.061801 & -1.385774 \\
\hline & -2.983416 & 2.885432 & -0.380529 \\
\hline & 0.384806 & 1.392676 & 1.811696 \\
\hline & -0.780595 & 5.499303 & 2.208329 \\
\hline & 1.456542 & -3.621844 & 1.424200 \\
\hline & -1.062639 & 1.531206 & -2.967523 \\
\hline 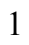 & 2.859748 & -3.469072 & 0.360329 \\
\hline 1 & 4.770472 & -2.668771 & 1.393426 \\
\hline 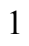 & 0.963331 & -1.224757 & 2.743251 \\
\hline 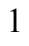 & 5.913050 & -1.266683 & 3.079931 \\
\hline [ & 2.097654 & 0.158645 & 4.461948 \\
\hline 1 & 4.576363 & 0.165968 & 4.617674 \\
\hline 1 & -7.137725 & -0.702647 & -4.873748 \\
\hline & -7.503656 & -1.169853 & -3.210036 \\
\hline 1 & -7.323429 & 0.548849 & -3.635274 \\
\hline 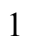 & -5.016270 & 0.185935 & -5.560483 \\
\hline ] & -4.981946 & 1.452251 & -4.315151 \\
\hline 1 & -3.630151 & 0.315500 & -4.472754 \\
\hline 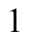 & -5.197664 & -2.255058 & -4.888565 \\
\hline 1 & -3.898012 & -2.110517 & -3.687447 \\
\hline 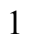 & -5.493133 & -2.697017 & -3.190624 \\
\hline 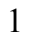 & -7.205588 & -0.405284 & 3.204477 \\
\hline 1 & -6.584297 & -1.535492 & 1.983187 \\
\hline 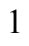 & -5.473314 & -0.442988 & 2.827034 \\
\hline 1 & -8.852132 & 0.519005 & 1.630911 \\
\hline D & -8.401015 & 1.113074 & 0.026925 \\
\hline D & -8.335682 & -0.627943 & 0.387703 \\
\hline 1 & -7.304722 & 2.072084 & 2.717638 \\
\hline 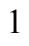 & -5.579192 & 2.109117 & 2.319531 \\
\hline 1 & -6.768646 & 2.706917 & 1.148701 \\
\hline 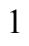 & -5.049220 & 5.876300 & 0.521094 \\
\hline 1 & -4.670742 & 4.157892 & 0.312508 \\
\hline l & -4.448978 & 4.925868 & 1.896697 \\
\hline 1 & -3.490011 & 6.507965 & -1.377682 \\
\hline 1 & -1.802731 & 5.951772 & -1.372449 \\
\hline 1 & -3.119445 & 4.796416 & -1.636184 \\
\hline 1 & -3.293233 & 7.561042 & 0.809292 \\
\hline ] & -2.662683 & 6.711993 & 2.227040 \\
\hline 1 & -1.576498 & 7.142681 & 0.884904 \\
\hline 1 & 1.943310 & 5.123395 & 4.454352 \\
\hline 1 & 1.452921 & 5.730206 & 2.865210 \\
\hline 1 & 0.239648 & 5.470892 & 4.140364 \\
\hline 1 & 3.082605 & 3.171080 & 3.569467 \\
\hline 1 & 2.329932 & 2.079415 & 2.403412 \\
\hline 1 & 2.735528 & 3.738222 & 1.924085 \\
\hline 1 & 1.266880 & 2.856840 & 5.250241 \\
\hline 1 & -0.433085 & 3.138371 & 4.811995 \\
\hline 1 & 0.423964 & 1.726191 & 4.168492 \\
\hline 8 & -1.533798 & 2.385445 & -3.590521 \\
\hline
\end{tabular}


$2_{\text {C1-t- }} \psi_{-1 \text {-12a3a4a }}$

Number of imaginary frequencies : 0

The smallest frequencies are : $12.5138 \quad 15.7012 \quad 21.2490 \mathrm{~cm}(-1)$

Electronic energy : $\quad H F=-4012.1882914$

Zero-point correction $=\quad 1.290638($ Hartree/Particle $)$

Thermal correction to Energy $=\quad 1.366789$

Thermal correction to Enthalpy= $\quad 1.367733$

Thermal correction to Gibbs Free Energy= $\quad 1.179943$

Sum of electronic and zero-point Energies $=\quad-4010.897654$

Sum of electronic and thermal Energies $=\quad-4010.821503$

Sum of electronic and thermal Enthalpies $=\quad-4010.820559$

Sum of electronic and thermal Free Energies $=\quad-4011.008349$

Cartesian Coordinates

$\begin{array}{cccc}6 & -3.689349 & -0.252302 & -1.823141 \\ 6 & -3.383461 & 0.170856 & -0.533378 \\ 6 & -4.404488 & 0.374743 & 0.398656 \\ 6 & -5.737217 & 0.171938 & 0.049949 \\ 6 & -6.011128 & -0.249415 & -1.258069 \\ 6 & -5.015342 & -0.469248 & -2.210760 \\ 15 & -1.626078 & 0.385097 & -0.058772 \\ 45 & -0.045690 & 0.325260 & -1.848383 \\ 6 & 0.980994 & 0.840386 & -3.320370 \\ 8 & 1.627454 & 1.231625 & -4.195558 \\ 6 & -6.878594 & 0.353700 & 1.048567 \\ 6 & -7.929358 & 1.312870 & 0.480305 \\ 6 & -5.379057 & -0.864101 & -3.641495 \\ 6 & -5.908942 & 0.381214 & -4.362104 \\ 6 & -1.582561 & -0.606911 & 1.515307 \\ 6 & -1.382881 & -1.978082 & 1.467737 \\ 6 & -1.390292 & -2.751634 & 2.672496 \\ 6 & -1.612445 & -2.103932 & 3.922090 \\ 6 & -1.840422 & -0.709185 & 3.934647 \\ 6 & -1.826845 & 0.012940 & 2.771498 \\ 6 & -1.170618 & -4.154292 & 2.674846 \\ 6 & -1.159160 & -4.865939 & 3.848888 \\ 6 & -1.374854 & -4.217540 & 5.083394 \\ 6 & -1.598872 & -2.865056 & 5.115592 \\ 6 & -1.195743 & -2.703237 & 0.182637 \\ 6 & 0.057831 & -2.808701 & -0.390693 \\ 6 & 0.248266 & -3.556328 & -1.577446 \\ 6 & -0.808358 & -4.175132 & -2.189948 \\ 6 & -2.111180 & -4.075326 & -1.648199 \\ 6 & -2.312342 & -3.336299 & -0.444502 \\ 6 & -3.220422 & -4.694746 & -2.274833 \\ 6 & -4.480729 & -4.578403 & -1.745878 \\ 6 & -4.686285 & -3.829538 & -0.566131 \\ 6 & -3.629194 & -3.233278 & 0.074325 \\ 7 & 1.177214 & -2.113477 & 0.189561 \\ 6 & 2.052517 & -2.926185 & 1.060934 \\ 6 & 2.777329 & -2.128192 & 2.108451 \\ 6 & 2.066215 & -1.346724 & 3.021142 \\ & & & \\ 6\end{array}$




\begin{tabular}{|c|c|c|c|}
\hline 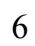 & 2.739802 & -0.598720 & 3.977715 \\
\hline & 4.133164 & -0.616630 & 4.029944 \\
\hline U & 4.846327 & -1.400399 & 3.129632 \\
\hline & 4.167572 & -2.156847 & 2.178038 \\
\hline & 1.632711 & -0.823631 & -0.804591 \\
\hline & 2.587210 & 0.046784 & 0.229153 \\
\hline & 3.294255 & 1.047885 & -0.415975 \\
\hline & 4.488556 & 0.731314 & -1.031676 \\
\hline & 5.163860 & 1.758903 & -1.767287 \\
\hline 6 & 4.620307 & 3.079273 & -1.780402 \\
\hline U & 3.408569 & 3.343794 & -1.097932 \\
\hline 6 & 2.743137 & 2.344125 & -0.440447 \\
\hline U & 6.333011 & 1.512627 & -2.530744 \\
\hline & 6.948368 & 2.520652 & -3.230902 \\
\hline O & 6.429763 & 3.832022 & -3.210065 \\
\hline U & 5.285863 & 4.099121 & -2.502929 \\
\hline 6 & 4.961059 & -0.670812 & -0.987215 \\
\hline 6 & 4.111884 & -1.678168 & -1.412361 \\
\hline 6 & 4.532740 & -3.022730 & -1.495794 \\
\hline 6 & 5.789134 & -3.374141 & -1.082909 \\
\hline 6 & 6.664314 & -2.403455 & -0.536289 \\
\hline 6 & 6.247015 & -1.039816 & -0.477007 \\
\hline 6 & 7.113843 & -0.104208 & 0.143000 \\
\hline 6 & 8.331125 & -0.491111 & 0.647407 \\
\hline 6 & 8.751819 & -1.834852 & 0.560822 \\
\hline 6 & 7.929994 & -2.768693 & -0.017169 \\
\hline 8 & 2.834340 & -1.395420 & -1.833754 \\
\hline 6 & -1.487148 & 2.099163 & 0.571655 \\
\hline 6 & -0.404349 & 2.411104 & 1.397444 \\
\hline 6 & -0.238378 & 3.698596 & 1.906198 \\
\hline 6 & -1.163487 & 4.673103 & 1.515887 \\
\hline 6 & -2.242647 & 4.399206 & 0.671527 \\
\hline 6 & -2.394128 & 3.090157 & 0.206751 \\
\hline 6 & 0.881924 & 4.049269 & 2.885586 \\
\hline 6 & 1.824017 & 5.078163 & 2.253490 \\
\hline 6 & -3.227960 & 5.510447 & 0.312235 \\
\hline 6 & -3.862772 & 6.062115 & 1.593804 \\
\hline 6 & 0.269778 & 4.639241 & 4.161703 \\
\hline 6 & 1.697515 & 2.820488 & 3.286100 \\
\hline 6 & -2.485719 & 6.636000 & -0.415807 \\
\hline 6 & -4.351636 & 5.014441 & -0.595453 \\
\hline 6 & -7.520862 & -1.012036 & 1.316734 \\
\hline 6 & -6.393455 & 0.922234 & 2.380813 \\
\hline 6 & -6.458181 & -1.950021 & -3.654411 \\
\hline 6 & -4.169837 & -1.395228 & -4.411310 \\
\hline 1 & 6.795627 & 0.931472 & 0.231868 \\
\hline 1 & -2.004653 & 1.084486 & 2.815385 \\
\hline 1 & 8.231534 & -3.813676 & -0.078184 \\
\hline 1 & -1.768103 & -2.350077 & 6.060235 \\
\hline 1 & 4.858074 & 5.100714 & -2.493678 \\
\hline 1 & -3.799621 & -2.652257 & 0.979376 \\
\hline 1 & -5.693114 & -3.718816 & -0.167536 \\
\hline 1 & 2.995832 & 4.351190 & -1.129660 \\
\hline 1 & -2.029224 & -0.214781 & 4.886740 \\
\hline 1 & 6.124731 & -4.407922 & -1.147938 \\
\hline 1 & 7.840898 & 2.303116 & -3.813341 \\
\hline
\end{tabular}




\begin{tabular}{|c|c|c|c|}
\hline . & 3.836955 & -3.757365 & -1.896284 \\
\hline 1 & -3.049381 & -5.262403 & -3.188556 \\
\hline 1 & -0.661918 & -4.743122 & -3.107246 \\
\hline 1 & -0.985235 & -5.939284 & 3.827777 \\
\hline & 6.738446 & 0.505009 & -2.568994 \\
\hline 1 & -1.009768 & -4.668652 & 1.729550 \\
\hline & 1.249335 & -3.610312 & -2.001863 \\
\hline & 6.929100 & 4.622151 & -3.765982 \\
\hline & 8.973786 & 0.245097 & 1.125060 \\
\hline 1 & -5.325500 & -5.059263 & -2.234978 \\
\hline 1 & -1.363523 & -4.793489 & 6.005763 \\
\hline 1 & 9.719652 & -2.127571 & 0.961167 \\
\hline 1 & 1.787696 & 2.517197 & 0.051650 \\
\hline 1 & -4.145470 & 0.697795 & 1.404357 \\
\hline 1 & -2.867432 & -0.410903 & -2.518274 \\
\hline 1 & -7.050530 & -0.410594 & -1.542916 \\
\hline 1 & -3.223393 & 2.829085 & -0.446092 \\
\hline 1 & 0.294871 & 1.620050 & 1.668219 \\
\hline 1 & -1.044558 & 5.687188 & 1.897792 \\
\hline 1 & 1.386570 & -3.652212 & 1.549469 \\
\hline 1 & -1.227867 & 1.215047 & -2.550768 \\
\hline 1 & 2.772801 & -3.506633 & 0.461923 \\
\hline 1 & 4.727337 & -2.760322 & 1.462741 \\
\hline 1 & 0.978180 & -1.312932 & 2.960513 \\
\hline 1 & 5.934722 & -1.419999 & 3.154327 \\
\hline 1 & 2.175166 & 0.005074 & 4.686462 \\
\hline 1 & 4.658701 & -0.020789 & 4.773365 \\
\hline 1 & 2.462540 & 3.106063 & 4.019677 \\
\hline 1 & 1.063950 & 2.050134 & 3.748794 \\
\hline 1 & 2.220476 & 2.361754 & 2.436121 \\
\hline 1 & 1.059732 & 4.870608 & 4.888620 \\
\hline 1 & -0.282051 & 5.567199 & 3.970011 \\
\hline 1 & -0.423168 & 3.927881 & 4.630309 \\
\hline 1 & 2.610802 & 5.362137 & 2.965365 \\
\hline 1 & 2.314248 & 4.668927 & 1.360330 \\
\hline 1 & 1.294367 & 5.992575 & 1.959111 \\
\hline 1 & -5.038209 & 5.841532 & -0.815827 \\
\hline 1 & -3.971076 & 4.638170 & -1.553437 \\
\hline 1 & -4.939631 & 4.214792 & -0.123684 \\
\hline 1 & -4.577941 & 6.859645 & 1.351433 \\
\hline 1 & -4.404884 & 5.274692 & 2.133936 \\
\hline 1 & -3.117009 & 6.483963 & 2.278380 \\
\hline 1 & -3.183264 & 7.440360 & -0.685792 \\
\hline 1 & -1.695614 & 7.077290 & 0.203868 \\
\hline 1 & -2.020646 & 6.266388 & -1.338563 \\
\hline 1 & -8.348581 & -0.915212 & 2.032461 \\
\hline 1 & -7.922018 & -1.462865 & 0.400357 \\
\hline 1 & -6.786190 & -1.709883 & 1.741459 \\
\hline 1 & -7.246608 & 1.053499 & 3.058142 \\
\hline 1 & -5.679296 & 0.254343 & 2.880758 \\
\hline 1 & -5.915455 & 1.903364 & 2.255826 \\
\hline 1 & -8.743212 & 1.455698 & 1.203669 \\
\hline 1 & -7.490248 & 2.296233 & 0.266904 \\
\hline 1 & -8.378648 & 0.939302 & -0.447293 \\
\hline 1 & -4.483415 & -1.714598 & -5.413395 \\
\hline 1 & -3.390279 & -0.634409 & -4.543989 \\
\hline
\end{tabular}


$2_{\text {C1-t- }} \psi_{-1 \text { 12a3a4s }}$

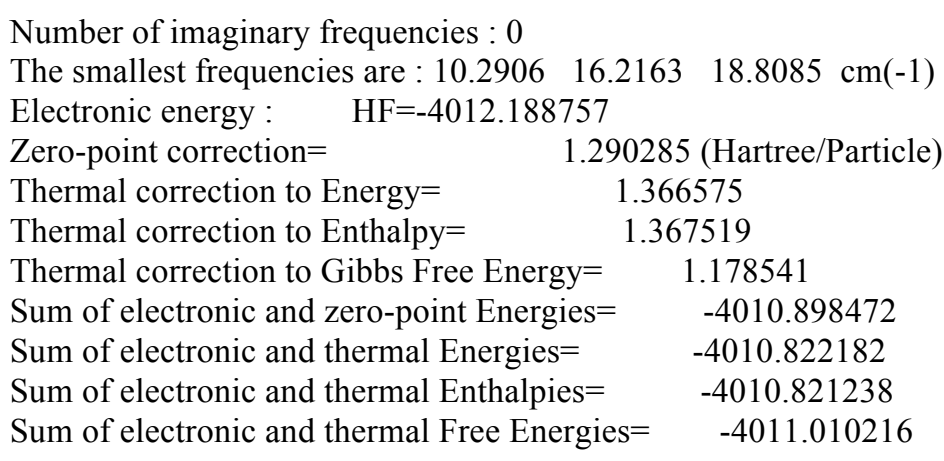

Cartesian Coordinates

$\begin{array}{cccc}6 & -2.376519 & 3.084846 & 0.229930 \\ 6 & -1.493271 & 2.073356 & 0.598352 \\ 6 & -0.413188 & 2.358823 & 1.437027 \\ 6 & -0.229266 & 3.637851 & 1.959968 \\ 6 & -1.129587 & 4.633537 & 1.565721 \\ 6 & -2.199596 & 4.388934 & 0.700845 \\ 15 & -1.641745 & 0.368311 & -0.055085 \\ 6 & -1.601451 & -0.643544 & 1.506745 \\ 6 & -1.388849 & -2.012017 & 1.444116 \\ 6 & -1.390049 & -2.799721 & 2.639278 \\ 6 & -1.623888 & -2.169694 & 3.895521 \\ 6 & -1.869471 & -0.778082 & 3.923486 \\ 6 & -1.858070 & -0.041563 & 2.769239 \\ 6 & -1.603122 & -2.944587 & 5.079920 \\ 6 & -1.360774 & -4.293546 & 5.032237 \\ 6 & -1.133872 & -4.924575 & 3.790705 \\ 6 & -1.152537 & -4.199232 & 2.625153 \\ 6 & -1.204010 & -2.716590 & 0.147895 \\ 6 & 0.044298 & -2.802465 & -0.439451 \\ 6 & 0.224767 & -3.516676 & -1.648282 \\ 6 & -0.836130 & -4.122721 & -2.266364 \\ 6 & -2.132511 & -4.048717 & -1.705284 \\ 6 & -2.323479 & -3.343154 & -0.480204 \\ 6 & -3.632298 & -3.271659 & 0.063688 \\ 6 & -4.690655 & -3.873078 & -0.569729 \\ 6 & -4.496046 & -4.583955 & -1.774559 \\ 6 & -3.244594 & -4.664043 & -2.330782 \\ 7 & 1.166803 & -2.120784 & 0.150676 \\ 15 & 1.624468 & -0.813431 & -0.819174 \\ 8 & 2.827572 & -1.367811 & -1.856087 \\ 6 & 4.106122 & -1.653568 & -1.439750 \\ 6 & 4.953014 & -0.650529 & -1.000150\end{array}$




\begin{tabular}{|c|c|c|c|}
\hline 6 & 6.241076 & -1.023382 & -0.498146 \\
\hline 6 & 6.662376 & -2.384669 & -0.579222 \\
\hline D & 5.788843 & -3.349431 & -1.138832 \\
\hline & 4.530598 & -2.995500 & -1.543991 \\
\hline$b$ & 7.106690 & -0.094626 & 0.133785 \\
\hline 5 & 8.326374 & -0.485401 & 0.629232 \\
\hline 6 & 8.750824 & -1.826436 & 0.521413 \\
\hline 5 & 7.930331 & -2.753939 & -0.068562 \\
\hline 6 & 4.476305 & 0.750599 & -1.021706 \\
\hline 6 & 3.281867 & 1.053878 & -0.399596 \\
\hline 6 & 2.727483 & 2.348893 & -0.402570 \\
\hline 6 & 3.390083 & 3.361126 & -1.043513 \\
\hline 6 & 4.602012 & 3.111114 & -1.731213 \\
\hline 6 & 5.148289 & 1.791892 & -1.740948 \\
\hline 6 & 5.264807 & 4.144503 & -2.436869 \\
\hline 6 & 6.408474 & 3.891761 & -3.149644 \\
\hline 6 & 6.929527 & 2.581937 & -3.193536 \\
\hline 6 & 6.316965 & 1.560953 & -2.509929 \\
\hline 8 & 2.577783 & 0.040295 & 0.229797 \\
\hline 6 & 0.883435 & 3.956372 & 2.958387 \\
\hline 6 & 1.692405 & 2.714119 & 3.329648 \\
\hline 6 & -3.148930 & 5.527057 & 0.328772 \\
\hline 6 & -4.225914 & 5.081580 & -0.657643 \\
\hline 6 & -3.398859 & 0.168933 & -0.535970 \\
\hline 6 & -3.710451 & -0.239573 & -1.833651 \\
\hline 6 & -5.035927 & -0.423565 & -2.225265 \\
\hline 6 & -6.037804 & -0.190635 & -1.273434 \\
\hline 6 & -5.757199 & 0.198897 & 0.037282 \\
\hline 6 & -4.416844 & 0.374937 & 0.389822 \\
\hline 6 & -6.833680 & 0.419848 & 1.097667 \\
\hline 6 & -8.240168 & 0.158871 & 0.565529 \\
\hline 6 & -5.407499 & -0.811187 & -3.655931 \\
\hline 6 & -4.198613 & -1.315590 & -4.443658 \\
\hline 45 & -0.059080 & 0.344009 & -1.844664 \\
\hline 6 & 0.963960 & 0.887302 & -3.308592 \\
\hline 8 & 1.607731 & 1.294955 & -4.178279 \\
\hline 6 & -6.771667 & 1.869101 & 1.593246 \\
\hline 6 & -6.583873 & -0.537097 & 2.270036 \\
\hline 6 & -5.968547 & 0.428355 & -4.362306 \\
\hline 6 & -6.465165 & -1.918663 & -3.663349 \\
\hline 6 & 2.040127 & -2.952214 & 1.006102 \\
\hline 6 & 2.769928 & -2.177059 & 2.067196 \\
\hline 6 & 2.064225 & -1.417021 & 3.001980 \\
\hline 6 & 2.743752 & -0.691414 & 3.971690 \\
\hline 6 & 4.137387 & -0.710465 & 4.015199 \\
\hline 6 & 4.845161 & -1.473149 & 3.092768 \\
\hline 6 & 4.160553 & -2.207506 & 2.128283 \\
\hline 6 & 1.834466 & 5.001512 & 2.367620 \\
\hline 6 & 0.258039 & 4.509210 & 4.244693 \\
\hline 6 & -3.844084 & 6.039090 & 1.595300 \\
\hline 6 & -2.356811 & 6.668880 & -0.316713 \\
\hline 1 & 6.785937 & 0.938775 & 0.238573 \\
\hline 1 & -2.045307 & 1.027854 & 2.825470 \\
\hline 1 & 8.234844 & -3.796962 & -0.146168 \\
\hline 1 & -1.781452 & -2.443194 & 6.030182 \\
\hline 1 & 4.835094 & 5.144961 & -2.409928 \\
\hline
\end{tabular}




\begin{tabular}{|c|c|c|c|}
\hline & -3.793650 & -2.715958 & 0.986448 \\
\hline & -5.689814 & -3.796492 & -0.145361 \\
\hline & 2.974766 & 4.367859 & -1.057698 \\
\hline & -2.067896 & -0.297357 & 4.880617 \\
\hline & 6.127220 & -4.381166 & -1.220103 \\
\hline & 7.821748 & 2.376083 & -3.780680 \\
\hline & 3.835895 & -3.726014 & -1.953726 \\
\hline & -3.082550 & -5.203034 & -3.263316 \\
\hline & -0.697287 & -4.663802 & -3.200945 \\
\hline & -0.945802 & -5.995221 & 3.757441 \\
\hline & 6.724262 & 0.554917 & -2.565755 \\
\hline & -0.983712 & -4.699817 & 1.673811 \\
\hline & 1.221194 & -3.554978 & -2.085193 \\
\hline & 6.905735 & 4.692261 & -3.692434 \\
\hline & 8.968132 & 0.245505 & 1.116145 \\
\hline & -5.342234 & -5.065260 & -2.260674 \\
\hline & -1.343744 & -4.880085 & 5.947823 \\
\hline & 9.720542 & -2.122242 & 0.914876 \\
\hline & 1.771805 & 2.511241 & 0.092588 \\
\hline & -4.162596 & 0.679686 & 1.404925 \\
\hline & -2.889185 & -0.407377 & -2.527273 \\
\hline & -7.075957 & -0.322366 & -1.570471 \\
\hline ] & -3.203231 & 2.845671 & -0.434964 \\
\hline & 0.271528 & 1.554099 & 1.703890 \\
\hline l & -0.996221 & 5.641705 & 1.958676 \\
\hline & 1.372073 & -3.684611 & 1.482067 \\
\hline 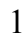 & -1.249021 & 1.232435 & -2.535627 \\
\hline 1 & 2.757120 & -3.524236 & 0.395146 \\
\hline & 4.715643 & -2.794921 & 1.396201 \\
\hline 1 & 0.975667 & -1.381906 & 2.949454 \\
\hline & 5.933691 & -1.493640 & 3.110570 \\
\hline 1 & 2.183601 & -0.103952 & 4.697487 \\
\hline ] & 4.667335 & -0.132049 & 4.769184 \\
\hline | & 2.458441 & 2.977978 & 4.070315 \\
\hline 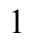 & 1.054792 & 1.936282 & 3.773945 \\
\hline & 2.212512 & 2.272295 & 2.468736 \\
\hline 1 & 1.039851 & 4.720480 & 4.986457 \\
\hline & -0.293408 & 5.441353 & 4.072594 \\
\hline 1 & -0.439054 & 3.783992 & 4.685233 \\
\hline ] & 2.614630 & 5.260667 & 3.096004 \\
\hline 1 & 2.332738 & 4.618993 & 1.467131 \\
\hline 1 & 1.311033 & 5.926667 & 2.096767 \\
\hline 1 & -4.880818 & 5.929435 & -0.894890 \\
\hline 1 & -3.794966 & 4.722042 & -1.600679 \\
\hline & -4.857983 & 4.283926 & -0.244999 \\
\hline 1 & -4.531677 & 6.859288 & 1.348484 \\
\hline 1 & -4.428138 & 5.240741 & 2.072298 \\
\hline 1 & -3.128005 & 6.417245 & 2.335207 \\
\hline 1 & -3.032330 & 7.488242 & -0.597040 \\
\hline 1 & -1.599424 & 7.083264 & 0.359513 \\
\hline 1 & -1.844282 & 6.326547 & -1.224743 \\
\hline 1 & -7.346803 & -0.397388 & 3.047775 \\
\hline 1 & -6.629027 & -1.582369 & 1.935778 \\
\hline 1 & -5.602281 & -0.380914 & 2.735764 \\
\hline 1 & -8.973736 & 0.326489 & 1.364258 \\
\hline 1 & -8.497627 & 0.831664 & -0.262552 \\
\hline
\end{tabular}


$2_{\text {C1-t- }} \psi_{-1 \text { 1a2a3s4a }}$

Number of imaginary frequencies : 0

The smallest frequencies are : $13.7156 \quad 17.0340 \quad 21.1760 \mathrm{~cm}(-1)$

Electronic energy : $\quad H F=-4012.1878123$

Zero-point correction $=\quad 1.291092($ Hartree/Particle)

Thermal correction to Energy $=\quad 1.367118$

Thermal correction to Enthalpy= $\quad 1.368062$

Thermal correction to Gibbs Free Energy $=\quad 1.180746$

Sum of electronic and zero-point Energies $=\quad-4010.896720$

Sum of electronic and thermal Energies $=\quad-4010.820695$

Sum of electronic and thermal Enthalpies= $\quad-4010.819751$

Sum of electronic and thermal Free Energies $=\quad-4011.007066$

Cartesian Coordinates

$\begin{array}{cccc}6 & 5.273148 & 4.145181 & -2.470109 \\ 6 & 4.608810 & 3.119130 & -1.755267 \\ 6 & 5.154053 & 1.799385 & -1.752009 \\ 6 & 6.323514 & 1.560293 & -2.517273 \\ 6 & 6.937624 & 2.574273 & -3.209862 \\ 6 & 6.417403 & 3.884798 & -3.179191 \\ 6 & 4.480368 & 0.765452 & -1.023850 \\ 6 & 3.285656 & 1.075965 & -0.405789 \\ 6 & 2.732626 & 2.371522 & -0.420857 \\ 6 & 3.396647 & 3.376960 & -1.070908 \\ 6 & 4.955295 & -0.636273 & -0.990201 \\ 6 & 4.107945 & -1.641753 & -1.423158 \\ 6 & 4.530781 & -2.985085 & -1.516794 \\ 6 & 5.787844 & -3.337591 & -1.106999 \\ 6 & 6.661733 & -2.369532 & -0.553638 \\ 6 & 6.242024 & -1.007122 & -0.483282 \\ 6 & 7.928284 & -2.736610 & -0.037949 \\ 6 & 8.748334 & -1.806041 & 0.547741 \\ 6 & 8.325056 & -0.463901 & 0.645675 \\ 6 & 7.107087 & -0.075045 & 0.144413 \\ 8 & 2.829646 & -1.357490 & -1.841169 \\ 15 & 1.628705 & -0.800527 & -0.803029 \\ 8 & 2.580435 & 0.069885 & 0.233145 \\ 7 & 1.189832 & -2.098070 & 0.188485 \\ 6 & 0.090811 & -2.817453 & -0.401501 \\ 6 & -1.173873 & -2.724310 & 0.148750\end{array}$




\begin{tabular}{|c|c|c|c|}
\hline 6 & -2.264721 & -3.401529 & -0.478751 \\
\hline & -2.022087 & -4.189734 & -1.643110 \\
\hline & -0.709364 & -4.267831 & -2.164934 \\
\hline & 0.318524 & -3.592674 & -1.563662 \\
\hline & -3.097263 & -4.888548 & -2.244034 \\
\hline & -4.366267 & -4.801306 & -1.730704 \\
\hline f & -4.616004 & -3.994822 & -0.599171 \\
\hline ) & -3.592069 & -3.318016 & 0.015556 \\
\hline 6 & -1.387266 & -2.005868 & 1.434226 \\
\hline 6 & -1.603027 & -0.638088 & 1.496278 \\
\hline 6 & -1.861227 & -0.033767 & 2.757404 \\
\hline 6 & -1.873860 & -0.767639 & 3.912864 \\
\hline 6 & -1.627889 & -2.159138 & 3.887030 \\
\hline 6 & -1.389976 & -2.791266 & 2.632373 \\
\hline 6 & -1.146554 & -4.190090 & 2.623900 \\
\hline 6 & -1.129899 & -4.912210 & 3.791541 \\
\hline 6 & -1.363487 & -4.279207 & 5.030665 \\
\hline 6 & -1.609138 & -2.930800 & 5.073598 \\
\hline 15 & -1.644241 & 0.374550 & -0.062301 \\
\hline 6 & -1.513641 & 2.080477 & 0.592414 \\
\hline 6 & -0.429492 & 2.387848 & 1.418000 \\
\hline 6 & -0.270895 & 3.668733 & 1.945489 \\
\hline 6 & -1.206029 & 4.641647 & 1.575332 \\
\hline 6 & -2.287988 & 4.371908 & 0.733345 \\
\hline 6 & -2.430984 & 3.069260 & 0.248317 \\
\hline 6 & 0.852009 & 4.013145 & 2.924087 \\
\hline 6 & 0.242664 & 4.580395 & 4.211847 \\
\hline 6 & -3.286414 & 5.479357 & 0.399605 \\
\hline 6 & -2.560294 & 6.625289 & -0.312598 \\
\hline 6 & 2.077576 & -2.901448 & 1.057366 \\
\hline 6 & 2.783067 & -2.099366 & 2.114575 \\
\hline 6 & 2.052515 & -1.346424 & 3.035566 \\
\hline 6 & 2.707069 & -0.595717 & 4.003219 \\
\hline 6 & 4.100297 & -0.581427 & 7600 \\
\hline 6 & 4.832940 & -1.337277 & 3.149028 \\
\hline 6 & 4.173597 & -2.097409 & 2.186845 \\
\hline 45 & -0.052472 & 0.348243 & -1.842000 \\
\hline 6 & -3.399210 & 0.169034 & -0.546493 \\
\hline 6 & -4.427153 & 0.329766 & 0.391440 \\
\hline 6 & -5.755888 & 0.147809 & 0.027617 \\
\hline 6 & -6.030069 & -0.204749 & -1.305278 \\
\hline 6 & -5.031471 & -0.368586 & -2.260636 \\
\hline 6 & -3.703235 & -0.182130 & -1.853335 \\
\hline 6 & -6.901116 & 0.285421 & 1.028957 \\
\hline 6 & -7.536668 & -1.092756 & 1.244017 \\
\hline 6 & -5.317408 & -0.688558 & -3.725615 \\
\hline 6 & -4.521589 & -1.923647 & -4.157500 \\
\hline 6 & -6.422384 & 0.807230 & 2.382565 \\
\hline 6 & -7.956983 & 1.259158 & 0.495488 \\
\hline 6 & -6.796619 & -0.964740 & -3.980754 \\
\hline 6 & -4.892854 & 0.512788 & -4.579280 \\
\hline 6 & -4.410900 & 4.987966 & -0.509556 \\
\hline 6 & -3.918529 & 6.001332 & 1.694881 \\
\hline 6 & 1.677136 & 2.783777 & 3.302413 \\
\hline 6 & 1.783636 & 5.058065 & 2.302784 \\
\hline 1 & 6.786810 & 0.959232 & 0.241913 \\
\hline
\end{tabular}




\begin{tabular}{|c|c|c|c|}
\hline & -2.050854 & 1.035356 & 2.810409 \\
\hline 1 & 8.231749 & -3.780496 & -0.107535 \\
\hline & -1.791329 & -2.426824 & 6.021767 \\
\hline & 4.844174 & 5.146170 & -2.453303 \\
\hline & -3.796220 & -2.697440 & 0.886968 \\
\hline & -5.628886 & -3.909331 & -0.211281 \\
\hline & 2.982664 & 4.384041 & -1.095090 \\
\hline & -2.073992 & -0.285475 & 4.868894 \\
\hline & 6.124978 & -4.370369 & -1.179501 \\
\hline & 7.830435 & 2.362195 & -3.793885 \\
\hline & 3.835440 & -3.718084 & -1.921406 \\
\hline & -2.892998 & -5.493757 & -3.126273 \\
\hline & -0.532336 & -4.868234 & -3.055858 \\
\hline & -0.937773 & -5.982233 & 3.761412 \\
\hline & 6.730192 & 0.553492 & -2.563013 \\
\hline & -0.970787 & -4.694038 & 1.675693 \\
\hline & 1.327101 & -3.630356 & -1.971879 \\
\hline & 6.915806 & 4.679695 & -3.729114 \\
\hline & 8.966336 & 0.269477 & 1.129495 \\
\hline & -5.185025 & -5.341983 & -2.200520 \\
\hline & -1.348101 & -4.863321 & 5.947827 \\
\hline & 9.716736 & -2.100243 & 0.945606 \\
\hline & 1.776761 & 2.539020 & 0.072478 \\
\hline & -4.171646 & 0.604487 & 1.412146 \\
\hline & -2.882969 & -0.300246 & -2.560786 \\
\hline & -7.067887 & -0.354631 & -1.595917 \\
\hline & -3.261834 & 2.811714 & -0.403943 \\
\hline & 0.276289 & 1.597629 & 1.673618 \\
\hline & -1.093112 & 5.650744 & 1.971976 \\
\hline & 1.422267 & -3.642686 & 1.537478 \\
\hline & -1.231620 & 1.244752 & -2.542563 \\
\hline & 2.810767 & -3.464515 & 0.457850 \\
\hline & 4.748598 & -2.680207 & 1.466427 \\
\hline & 0.963917 & -1.338823 & 2.974475 \\
\hline & 5.921375 & -1.332713 & 3.176860 \\
\hline & 2.127553 & -0.015080 & 4.719229 \\
\hline & 4.610545 & 0.016771 & 4.809747 \\
\hline & 2.445597 & 3.063171 & 4.034876 \\
\hline & 1.050561 & 2.003890 & 3.758560 \\
\hline & 2.196520 & 2.338179 & 2.443159 \\
\hline & 1.034885 & 4.805288 & 4.938343 \\
\hline & -0.315171 & 5.508092 & 4.036819 \\
\hline & -0.444007 & 3.858255 & 4.673079 \\
\hline & 2.571527 & 5.337275 & 3.015327 \\
\hline & 2.272939 & 4.666213 & 1.401413 \\
\hline & 1.245821 & 5.972793 & 2.024581 \\
\hline & -5.106673 & 5.812020 & -0.711759 \\
\hline & -4.032916 & 4.631300 & -1.476056 \\
\hline & -4.988131 & 4.175009 & -0.047363 \\
\hline & -4.643943 & 6.795128 & 1.471417 \\
\hline & -4.448318 & 5.198490 & 2.224458 \\
\hline & -3.172610 & 6.419166 & 2.381774 \\
\hline & -3.267294 & 7.427928 & -0.562373 \\
\hline & -1.769753 & 7.062382 & 0.309470 \\
\hline & -2.098663 & 6.277537 & -1.245523 \\
\hline & -8.368067 & -1.026830 & 1.958992 \\
\hline
\end{tabular}




$\begin{array}{lrrr}1 & -7.931242 & -1.511803 & 0.309945 \\ 1 & -6.799808 & -1.801398 & 1.646358 \\ 1 & -7.278353 & 0.912095 & 3.060953 \\ 1 & -5.708431 & 0.123977 & 2.861543 \\ 1 & -5.946422 & 1.793418 & 2.293606 \\ 1 & -8.769900 & 1.372824 & 1.225016 \\ 1 & -7.523100 & 2.251486 & 0.315971 \\ 1 & -8.407150 & 0.916350 & -0.443417 \\ 1 & -6.948761 & -1.209694 & -5.039454 \\ 1 & -7.159795 & -1.815568 & -3.388760 \\ 1 & -7.425011 & -0.093521 & -3.754773 \\ 1 & -4.720360 & -2.149942 & -5.213789 \\ 1 & -3.438926 & -1.783303 & -4.049269 \\ 1 & -4.804957 & -2.799634 & -3.560311 \\ 1 & -5.089072 & 0.314195 & -5.641737 \\ 1 & -5.450084 & 1.414741 & -4.293891 \\ 1 & -3.822978 & 0.732369 & -4.471890 \\ 6 & 0.978970 & 0.876118 & -3.306502 \\ 8 & 1.626769 & 1.272316 & -4.178313\end{array}$

$2_{\text {C1-t- }-\psi_{-1 a 2 a 3 s 4 s}}$

Number of imaginary frequencies : 0

The smallest frequencies are : $12.8642 \quad 16.3827 \quad 17.3807 \mathrm{~cm}(-1)$

Electronic energy : $\quad H F=-4012.1881059$

Zero-point correction $=\quad 1.291136$ (Hartree/Particle)

Thermal correction to Energy $=\quad 1.367139$

Thermal correction to Enthalpy= $\quad 1.368083$

Thermal correction to Gibbs Free Energy= $\quad 1.180344$

Sum of electronic and zero-point Energies $=\quad-4010.896970$

Sum of electronic and thermal Energies $=\quad-4010.820967$

Sum of electronic and thermal Enthalpies $=\quad-4010.820022$

Sum of electronic and thermal Free Energies $=\quad-4011.007762$

Cartesian Coordinates

$\begin{array}{llll}6 & 5.293355 & 4.154021 & -2.408504 \\ 6 & 4.621518 & 3.121755 & -1.709805 \\ 6 & 5.162083 & 1.800157 & -1.719481 \\ 6 & 6.334699 & 1.566099 & -2.481478 \\ 6 & 6.956163 & 2.586172 & -3.158387 \\ 6 & 6.440537 & 3.898141 & -3.114480 \\ 6 & 4.481254 & 0.760133 & -1.006807 \\ 6 & 3.284529 & 1.067378 & -0.391015 \\ 6 & 2.735634 & 2.364781 & -0.394348 \\ 6 & 3.406396 & 3.375499 & -1.029104 \\ 6 & 4.952059 & -0.643168 & -0.986212 \\ 6 & 4.104034 & -1.641602 & -1.433899 \\ 6 & 4.523856 & -2.984926 & -1.540200 \\ 6 & 5.777994 & -3.345251 & -1.128173 \\ 6 & 6.651732 & -2.385647 & -0.559985 \\ 6 & 6.235330 & -1.022927 & -0.477142 \\ 6 & 7.914670 & -2.761695 & -0.041886 \\ 6 & 8.734339 & -1.839630 & 0.557647 \\ 6 & 8.314325 & -0.497388 & 0.667506 \\ 6 & 7.099924 & -0.099891 & 0.164434\end{array}$




\begin{tabular}{|c|c|c|c|}
\hline & 2.828362 & -1.349516 & -1.854613 \\
\hline 15 & 1.624061 & -0.799954 & -0.816591 \\
\hline & 2.573335 & 0.056408 & 0.233528 \\
\hline & 1.175657 & -2.107343 & 0.157304 \\
\hline & 0.070929 & -2.811068 & -0.440668 \\
\hline & -1.191447 & -2.718413 & 0.114751 \\
\hline & -2.289102 & -3.377907 & -0.519121 \\
\hline & -2.056280 & -4.148301 & -1.697178 \\
\hline & -0.745996 & -4.225945 & -2.225300 \\
\hline 6 & 0.288978 & -3.568228 & -1.616576 \\
\hline & -3.138762 & -4.829713 & -2.304843 \\
\hline & -4.405105 & -4.741186 & -1.785073 \\
\hline 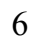 & -4.644771 & -3.950891 & -0.639955 \\
\hline 6 & -3.613657 & -3.292020 & -0.017910 \\
\hline 6 & -1.398997 & -2.013672 & 1.408259 \\
\hline 6 & -1.606170 & -0.645294 & 1.483040 \\
\hline 6 & -1.862817 & -0.052265 & 2.749762 \\
\hline 6 & -1.884783 & -0.798268 & 3.897493 \\
\hline 6 & -1.649119 & -2.191219 & 3.858070 \\
\hline 6 & -1.410710 & -2.811561 & 2.597789 \\
\hline 6 & -1.177141 & -4.211807 & 2.574941 \\
\hline 6 & -1.170058 & -4.946520 & 3.734791 \\
\hline 6 & -1.404045 & -4.325152 & 4.979765 \\
\hline 6 & -1.640401 & -2.975550 & 5.036344 \\
\hline 15 & -1.643595 & 0.379993 & -0.067930 \\
\hline 6 & -1.513414 & 2.081112 & 0.598971 \\
\hline 6 & -0.432139 & 2.377527 & 1.432231 \\
\hline 6 & -0.268194 & 3.654652 & 1.966734 \\
\hline 6 & -1.190886 & 4.636881 & 1.590330 \\
\hline 6 & -2.264459 & 4.380643 & 0.733370 \\
\hline 6 & -2.419862 & 3.078539 & 0.249837 \\
\hline 6 & 0.848610 & 3.985610 & 2.956687 \\
\hline 6 & 0.230530 & 4.540633 & 4.245557 \\
\hline 6 & -3.242991 & 5.501754 & 0.386162 \\
\hline 6 & -2.483783 & 6.672486 & -0.246821 \\
\hline 6 & 2.055985 & -2.924374 & 1.020669 \\
\hline 6 & 2.761598 & -2.136858 & 2.088887 \\
\hline 6 & 2.031716 & -1.388460 & 3.014112 \\
\hline 6 & 2.686389 & -0.650597 & 3.991501 \\
\hline 6 & 4.079471 & -0.644891 & 4.051708 \\
\hline 6 & 4.811492 & -1.396423 & 3.139042 \\
\hline 6 & 4.151780 & -2.143639 & 2.166981 \\
\hline 45 & -0.051614 & 0.364208 & -1.846848 \\
\hline 6 & -3.398768 & 0.175442 & -0.553191 \\
\hline 6 & -4.419134 & 0.306440 & 0.389003 \\
\hline 6 & -5.753306 & 0.113459 & 0.035755 \\
\hline 6 & -6.038219 & -0.206514 & -1.297704 \\
\hline 6 & -5.039594 & -0.339199 & -2.265742 \\
\hline 6 & -3.712457 & -0.153101 & -1.867551 \\
\hline 6 & -6.829289 & 0.261179 & 1.110204 \\
\hline 6 & -6.822787 & 1.702615 & 1.632817 \\
\hline 6 & -5.336460 & -0.632913 & -3.734756 \\
\hline 6 & -4.542475 & -1.859127 & -4.195032 \\
\hline 6 & -8.227924 & -0.048387 & 0.583224 \\
\hline 6 & -6.533331 & -0.705369 & 2.263545 \\
\hline 6 & -6.817031 & -0.907153 & -3.984858 \\
\hline
\end{tabular}




\begin{tabular}{|c|c|c|c|}
\hline 6 & -4.920523 & 0.583749 & -4.570766 \\
\hline 6 & -4.317356 & 5.046296 & -0.598763 \\
\hline 6 & -3.939795 & 5.976160 & 1.666396 \\
\hline 6 & 1.668139 & 2.750219 & 3.327504 \\
\hline 6 & 1.787829 & 5.034399 & 2.353529 \\
\hline 1 & 6.782178 & 0.934261 & 0.271085 \\
\hline 1 & -2.044300 & 1.017664 & 2.813604 \\
\hline 1 & 8.215614 & -3.805634 & -0.121020 \\
\hline 1 & -1.822673 & -2.480512 & 5.989190 \\
\hline 1 & 4.867883 & 5.156290 & -2.381662 \\
\hline 1 & -3.808905 & -2.682898 & 0.863928 \\
\hline 1 & -5.656071 & -3.863763 & -0.248095 \\
\hline 1 & 2.995686 & 4.384107 & -1.043855 \\
\hline 1 & -2.084015 & -0.324544 & 4.857933 \\
\hline 1 & 6.112747 & -4.378081 & -1.210386 \\
\hline 1 & 7.851327 & 2.377948 & -3.740189 \\
\hline 1 & 3.828605 & -3.711511 & -1.956349 \\
\hline 1 & -2.942687 & -5.421949 & -3.197678 \\
\hline 1 & -0.576292 & -4.812089 & -3.127075 \\
\hline 1 & -0.985293 & -6.017486 & 3.693958 \\
\hline 1 & 6.737995 & 0.558451 & -2.537282 \\
\hline 1 & -1.001466 & -4.706588 & 1.621889 \\
\hline 1 & 1.295534 & -3.605796 & -2.029781 \\
\hline 1 & 6.944821 & 4.697881 & -3.651882 \\
\hline 1 & 8.955272 & 0.229096 & 1.162051 \\
\hline 1 & -5.229698 & -5.268068 & -2.260374 \\
\hline 1 & -1.396251 & -4.919154 & 5.890656 \\
\hline 1 & 9.699943 & -2.140708 & 0.957168 \\
\hline 1 & 1.777654 & 2.529753 & 0.095673 \\
\hline 1 & -4.166086 & 0.563540 & 1.417304 \\
\hline 1 & -2.896700 & -0.252860 & -2.583008 \\
\hline 1 & -7.073894 & -0.357373 & -1.588375 \\
\hline 1 & -3.248502 & 2.829851 & -0.409169 \\
\hline 1 & 0.267453 & 1.581686 & 1.687419 \\
\hline 1 & -1.073308 & 5.643273 & 1.992544 \\
\hline 1 & 1.395523 & -3.667769 & 1.490307 \\
\hline 1 & -1.229265 & 1.269342 & -2.539256 \\
\hline 1 & 2.788682 & -3.484365 & 0.417680 \\
\hline 1 & 4.726433 & -2.722738 & 1.443358 \\
\hline 1 & 0.943596 & -1.373437 & 2.948241 \\
\hline 1 & 5.899812 & -1.398441 & 3.171382 \\
\hline 1 & 2.107009 & -0.073267 & 4.710320 \\
\hline 1 & 4.590070 & -0.056731 & 4.811492 \\
\hline 1 & 2.433769 & 3.020383 & 4.066337 \\
\hline 1 & 1.037163 & 1.967884 & 3.773284 \\
\hline 1 & 2.190137 & 2.311576 & 2.466166 \\
\hline 1 & 1.017438 & 4.757418 & 4.980258 \\
\hline 1 & -0.325207 & 5.470502 & 4.075272 \\
\hline 1 & -0.460338 & 3.814620 & 4.694347 \\
\hline 1 & 2.571476 & 5.303066 & 3.074734 \\
\hline 1 & 2.282270 & 4.650763 & 1.451412 \\
\hline 1 & 1.255240 & 5.954026 & 2.081574 \\
\hline 1 & -4.992342 & 5.882942 & -0.819020 \\
\hline 1 & -3.885665 & 4.710042 & -1.550065 \\
\hline 1 & -4.928870 & 4.229295 & -0.193203 \\
\hline 1 & -4.649458 & 6.782946 & 1.438515 \\
\hline
\end{tabular}




$\begin{array}{rrrr}1 & -4.499726 & 5.155823 & 2.135026 \\ 1 & -3.226679 & 6.359922 & 2.406296 \\ 1 & -3.180905 & 7.480051 & -0.507818 \\ 1 & -1.730450 & 7.093908 & 0.429575 \\ 1 & -1.971370 & 6.357538 & -1.164777 \\ 1 & -7.560378 & 1.823078 & 2.437875 \\ 1 & -5.841996 & 1.994079 & 2.030359 \\ 1 & -7.080859 & 2.407386 & 0.831515 \\ 1 & -8.959973 & 0.060154 & 1.393521 \\ 1 & -8.526159 & 0.636587 & -0.220717 \\ 1 & -8.304223 & -1.076414 & 0.205300 \\ 1 & -7.292688 & -0.604058 & 3.050666 \\ 1 & -6.548401 & -1.746580 & 1.914657 \\ 1 & -5.553761 & -0.523773 & 2.723884 \\ 1 & -6.975921 & -1.131001 & -5.047262 \\ 1 & -7.174485 & -1.770648 & -3.407973 \\ 1 & -7.445842 & -0.042253 & -3.736908 \\ 1 & -4.746661 & -2.064790 & -5.254558 \\ 1 & -3.459300 & -1.720836 & -4.089054 \\ 1 & -4.822343 & -2.746868 & -3.613656 \\ 1 & -5.125434 & 0.403705 & -5.634907 \\ 1 & -5.476327 & 1.479948 & -4.265112 \\ 1 & -3.850107 & 0.802775 & -4.468380 \\ 6 & 0.983054 & 0.905089 & -3.304408 \\ 8 & 1.633507 & 1.310783 & -4.169842 \\ --------------------------------------------------------------------- \\ -\end{array}$

Number of imaginary frequencies : 0

The smallest frequencies are : $13.9690 \quad 18.5049 \quad 21.3550 \mathrm{~cm}(-1)$

Electronic energy : $\quad \mathrm{HF}=-4012.1888359$

Zero-point correction $=\quad 1.291403$ (Hartree/Particle)

Thermal correction to Energy= $\quad 1.367434$

Thermal correction to Enthalpy= $\quad 1.368378$

Thermal correction to Gibbs Free Energy $=\quad 1.180759$

Sum of electronic and zero-point Energies $=\quad-4010.897433$

Sum of electronic and thermal Energies $=\quad-4010.821402$

Sum of electronic and thermal Enthalpies $=\quad-4010.820458$

Sum of electronic and thermal Free Energies $=\quad-4011.008077$

Cartesian Coordinates

$\begin{array}{cccc}6 & -2.351498 & 3.065304 & 0.197370 \\ 6 & -1.468565 & 2.062292 & 0.569239 \\ 6 & -0.391161 & 2.360584 & 1.413200 \\ 6 & -0.221866 & 3.639982 & 1.931055 \\ 6 & -1.135717 & 4.632217 & 1.539288 \\ 6 & -2.197707 & 4.373689 & 0.676178 \\ 15 & -1.633080 & 0.353413 & -0.069723 \\ 6 & -1.567378 & -0.652131 & 1.494143 \\ 6 & -1.349554 & -2.020010 & 1.431885 \\ 6 & -1.340353 & -2.804974 & 2.629154 \\ 6 & -1.566215 & -2.172551 & 3.885836 \\ 6 & -1.812891 & -0.781026 & 3.913365 \\ 6 & -1.814003 & -0.047446 & 2.757379 \\ 6 & -1.535175 & -2.944777 & 5.071706\end{array}$




\begin{tabular}{|c|c|c|c|}
\hline 6 & -1.290111 & -4.293318 & 5.025392 \\
\hline 6 & -1.069978 & -4.926453 & 3.783792 \\
\hline 6 & -1.098427 & -4.203725 & 2.616769 \\
\hline 6 & -1.160400 & -2.727512 & 0.137051 \\
\hline f & 0.089111 & -2.804010 & -0.449721 \\
\hline & 0.278212 & -3.524612 & -1.653400 \\
\hline 6 & -0.775376 & -4.145062 & -2.269246 \\
\hline f & -2.073502 & -4.077841 & -1.711574 \\
\hline 5 & -2.272835 & -3.367933 & -0.490103 \\
\hline 6 & -3.583478 & -3.305468 & 0.050109 \\
\hline 6 & -4.636256 & -3.915568 & -0.584535 \\
\hline 6 & -4.433262 & -4.629424 & -1.786201 \\
\hline 6 & -3.179123 & -4.704600 & -2.337082 \\
\hline 7 & 1.204481 & -2.104609 & 0.133059 \\
\hline 15 & 1.638858 & -0.794929 & -0.844638 \\
\hline 8 & 2.851248 & -1.330781 & -1.880351 \\
\hline 6 & 4.126527 & -1.619104 & -1.455385 \\
\hline 6 & 4.969757 & -0.619586 & -1.000465 \\
\hline 6 & 6.252373 & -0.997573 & -0.487816 \\
\hline 6 & 6.674293 & -2.358153 & -0.576334 \\
\hline 6 & 5.806148 & -3.318015 & -1.152558 \\
\hline 6 & 4.551913 & -2.960316 & -1.566496 \\
\hline 6 & 7.110757 & -0.075075 & 0.162843 \\
\hline 6 & 8.324836 & -0.470593 & 0.668247 \\
\hline 6 & 8.750512 & -1.810541 & 0.552436 \\
\hline 6 & 7.936566 & -2.732361 & -0.055246 \\
\hline 6 & 4.496195 & 0.782546 & -1.020909 \\
\hline 6 & 3.291302 & 1.085289 & -0.418719 \\
\hline 6 & 2.738555 & 2.380775 & -0.426809 \\
\hline 6 & 3.416176 & 3.395963 & -1.046881 \\
\hline 6 & 4.641635 & 3.148028 & -1.710828 \\
\hline 6 & 5.183722 & 1.827024 & -1.721009 \\
\hline 6 & 5.321392 & 4.185413 & -2.394162 \\
\hline 6 & 6.477589 & 3.935207 & -3.087264 \\
\hline 6 & 6.994210 & 2.623721 & -3.133764 \\
\hline 6 & 6.365279 & 1.598888 & -2.471089 \\
\hline 8 & 2.578849 & 0.073814 & 0.203352 \\
\hline 6 & 0.886636 & 3.972860 & 2.929435 \\
\hline 6 & 1.711015 & 2.741170 & 3.301166 \\
\hline 6 & -3.214363 & 5.434099 & 0.258875 \\
\hline 6 & -2.929726 & 6.795091 & 0.888331 \\
\hline 6 & -3.407307 & 0.175226 & -0.498841 \\
\hline 6 & -3.761897 & -0.253620 & -1.774109 \\
\hline 6 & -5.105132 & -0.419273 & -2.126424 \\
\hline 6 & -6.067965 & -0.144844 & -1.153876 \\
\hline 6 & -5.744593 & 0.271650 & 0.143852 \\
\hline 6 & -4.396221 & 0.426447 & 0.456134 \\
\hline 6 & -6.852589 & 0.501584 & 1.169953 \\
\hline 6 & -6.309605 & 1.030820 & 2.496091 \\
\hline 6 & -5.526608 & -0.838566 & -3.534130 \\
\hline 6 & -4.343482 & -1.344532 & -4.358520 \\
\hline 45 & -0.082486 & 0.298143 & -1.885737 \\
\hline 6 & 0.882313 & 0.800024 & -3.402088 \\
\hline 8 & 1.471917 & 1.179484 & -4.321526 \\
\hline 6 & -7.558487 & -0.832279 & 1.440331 \\
\hline 6 & -7.866750 & 1.517030 & 0.633831 \\
\hline
\end{tabular}




\begin{tabular}{|c|c|c|c|}
\hline & -6.569812 & -1.958461 & -3.473955 \\
\hline & -6.130315 & 0.378437 & -4.244300 \\
\hline 6 & 2.088592 & -2.917522 & 0.995248 \\
\hline & 2.798342 & -2.130190 & 2.061253 \\
\hline & 2.073503 & -1.379092 & 2.988319 \\
\hline & 2.732277 & -0.649638 & 3.969199 \\
\hline & 4.125233 & -0.654954 & 4.031605 \\
\hline & 4.852449 & -1.407624 & 3.116136 \\
\hline & 4.188365 & -2.146116 & 2.140270 \\
\hline & 0.255242 & 4.518196 & 4.216137 \\
\hline & 1.824617 & 5.029593 & 2.338379 \\
\hline 6 & -3.185798 & 5.595454 & -1.264911 \\
\hline & -4.614798 & 4.987848 & 0.698475 \\
\hline & 6.788333 & 0.957007 & 0.274947 \\
\hline & -2.002665 & 1.021894 & 2.812974 \\
\hline 1 & 8.241658 & -3.774751 & -0.138795 \\
\hline 1 & -1.707760 & -2.441634 & 6.022107 \\
\hline 1 & 4.894498 & 5.187064 & -2.366143 \\
\hline 1 & -3.751405 & -2.751060 & 0.971666 \\
\hline 1 & -5.637525 & -3.841151 & -0.164224 \\
\hline 1 & 3.003274 & 4.403669 & -1.063137 \\
\hline 1 & -2.002942 & -0.298499 & 4.871292 \\
\hline & 6.145082 & -4.349124 & -1.239154 \\
\hline 1 & 7.896260 & 2.419631 & -3.706321 \\
\hline 1 & 3.860925 & -3.687354 & -1.988627 \\
\hline 1 & -3.010149 & -5.246898 & -3.266425 \\
\hline 1 & -0.629932 & -4.690633 & -3.200185 \\
\hline 1 & -0.879166 & -5.996647 & 3.751559 \\
\hline 1 & 6.769997 & 0.592004 & -2.529205 \\
\hline 1 & -0.934361 & -4.706094 & 1.665572 \\
\hline 1 & 1.275710 & -3.554823 & -2.088518 \\
\hline 1 & 6.988050 & 4.738848 & -3.612889 \\
\hline 1 & 8.960792 & 0.255528 & 1.169724 \\
\hline 1 & -5.274816 & -5.117180 & -2.273964 \\
\hline 1 & -1.265442 & -4.877751 & 5.942152 \\
\hline 1 & 9.715720 & -2.110175 & 0.954011 \\
\hline 1 & 1.773380 & 2.541108 & 0.050313 \\
\hline 1 & -4.100858 & 0.752318 & 1.450706 \\
\hline 1 & -2.964865 & -0.454574 & -2.486805 \\
\hline 1 & -7.120176 & -0.263817 & -1.412378 \\
\hline 1 & -3.176352 & 2.824883 & -0.472291 \\
\hline 1 & 0.297756 & 1.561695 & 1.686002 \\
\hline 1 & -1.010039 & 5.636953 & 1.937687 \\
\hline 1 & 1.431114 & -3.661033 & 1.468831 \\
\hline 1 & -1.303962 & 1.118719 & -2.600513 \\
\hline 1 & 2.819276 & -3.478038 & 0.390242 \\
\hline 1 & 4.759684 & -2.727380 & 1.415835 \\
\hline 1 & 0.985883 & -1.355159 & 2.921578 \\
\hline 1 & 5.940662 & -1.418395 & 3.150047 \\
\hline 1 & 2.155863 & -0.071010 & 4.689413 \\
\hline 1 & 4.639381 & -0.074294 & 4.794746 \\
\hline 1 & 2.475495 & 3.015120 & 4.039817 \\
\hline 1 & 1.083497 & 1.956840 & 3.748251 \\
\hline 1 & 2.234170 & 2.303603 & 2.439969 \\
\hline 1 & 2.600855 & 5.299276 & 3.067159 \\
\hline 1 & 2.328445 & 4.652262 & 1.438796 \\
\hline
\end{tabular}




$\begin{array}{rrrr}1 & 1.289828 & 5.947868 & 2.066470 \\ 1 & 1.034790 & 4.734853 & 4.958739 \\ 1 & -0.303780 & 5.445965 & 4.045654 \\ 1 & -0.435641 & 3.785961 & 4.654972 \\ 1 & -3.686183 & 7.518330 & 0.558652 \\ 1 & -2.967006 & 6.757816 & 1.984887 \\ 1 & -1.949100 & 7.187546 & 0.589763 \\ 1 & -3.914537 & 6.354070 & -1.581027 \\ 1 & -2.193171 & 5.914911 & -1.607144 \\ 1 & -3.431712 & 4.661799 & -1.784306 \\ 1 & -5.363326 & 5.735766 & 0.404018 \\ 1 & -4.905286 & 4.031982 & 0.243382 \\ 1 & -4.664912 & 4.870382 & 1.789255 \\ 1 & -8.661062 & 1.685932 & 1.373163 \\ 1 & -7.387546 & 2.483073 & 0.427426 \\ 1 & -8.347671 & 1.178208 & -0.291356 \\ 1 & -7.139222 & 1.191117 & 3.196110 \\ 1 & -5.613884 & 0.324878 & 2.969269 \\ 1 & -5.791573 & 1.991458 & 2.371479 \\ 1 & -8.362828 & -0.699701 & 2.176640 \\ 1 & -8.004234 & -1.252322 & 0.530137 \\ 1 & -6.852537 & -1.573001 & 1.839698 \\ 1 & -4.696167 & -1.669016 & -5.345876 \\ 1 & -3.586340 & -0.567335 & -4.523564 \\ 1 & -3.854244 & -2.203372 & -3.877695 \\ 1 & -6.442620 & 0.112788 & -5.263564 \\ 1 & -7.009785 & 0.763317 & -3.712439 \\ 1 & -5.398348 & 1.193402 & -4.315674 \\ 1 & -6.842828 & -2.271886 & -4.490324 \\ 1 & -6.172320 & -2.834288 & -2.943925 \\ 1 & -7.494577 & -1.648888 & -2.972525\end{array}$

$2_{\text {C1-t- }} \Psi_{-12253 a 4 s}$

Number of imaginary frequencies : 0

The smallest frequencies are : $14.8586 \quad 20.2627 \quad 21.7310 \mathrm{~cm}(-1)$

Electronic energy : $\quad H F=-4012.1890197$

Zero-point correction $=\quad 1.292545($ Hartree/Particle $)$

Thermal correction to Energy= $\quad 1.368288$

Thermal correction to Enthalpy= $\quad 1.369232$

Thermal correction to Gibbs Free Energy $=\quad 1.182584$

Sum of electronic and zero-point Energies $=\quad-4010.896474$

Sum of electronic and thermal Energies $=\quad-4010.820732$

Sum of electronic and thermal Enthalpies $=\quad-4010.819788$

Sum of electronic and thermal Free Energies $=\quad-4011.006435$

Cartesian Coordinates

$\begin{array}{crrr}6 & -3.787726 & -0.193444 & -1.774225 \\ 6 & -3.417627 & 0.196516 & -0.486764 \\ 6 & -4.395721 & 0.418160 & 0.478621 \\ 6 & -5.752336 & 0.270107 & 0.177989 \\ 6 & -6.091199 & -0.095277 & -1.125618 \\ 6 & -5.131710 & -0.335120 & -2.118307 \\ 15 & -1.639142 & 0.355802 & -0.065608 \\ 45 & -0.092333 & 0.326668 & -1.886952\end{array}$




\begin{tabular}{|c|c|c|c|}
\hline 6 & 0.873110 & 0.847784 & -3.396111 \\
\hline & 1.463609 & 1.238252 & -4.310380 \\
\hline & -6.783671 & 0.488348 & 1.283480 \\
\hline & -6.519521 & -0.514773 & 2.413201 \\
\hline & -5.571393 & -0.689730 & -3.537964 \\
\hline & -6.595309 & -1.828529 & -3.517852 \\
\hline & -1.576884 & -0.686231 & 1.474795 \\
\hline & -1.371744 & -2.054167 & 1.380068 \\
\hline & -1.373513 & -2.868436 & 2.557480 \\
\hline & -1.596933 & -2.264985 & 3.828635 \\
\hline & -1.828250 & -0.871916 & 3.889788 \\
\hline & -1.818801 & -0.110178 & 2.752076 \\
\hline & -1.145559 & -4.268781 & 2.511048 \\
\hline & -1.128270 & -5.020379 & 3.659916 \\
\hline & -1.346027 & -4.415823 & 4.916157 \\
\hline & -1.577466 & -3.066334 & 4.995133 \\
\hline & -1.185069 & -2.729332 & 0.068070 \\
\hline & 0.064565 & -2.794729 & -0.519838 \\
\hline & 0.250770 & -3.480018 & -1.744391 \\
\hline & -0.806248 & -4.073842 & -2.380183 \\
\hline & -2.104246 & -4.016881 & -1.821229 \\
\hline & -2.300419 & -3.345523 & -0.577557 \\
\hline & -3.212480 & -4.617868 & -2.466860 \\
\hline & -4.465553 & -4.557534 & -1.911995 \\
\hline & -4.664959 & -3.882948 & -0.687390 \\
\hline & -3.610562 & -3.295358 & -0.034473 \\
\hline 1 & 1.182509 & -2.117037 & 0.083452 \\
\hline 5 & 2.059217 & -2.956279 & 0.927516 \\
\hline 6 & 2.770804 & -2.201136 & 2.015388 \\
\hline 6 & 2.048278 & -1.479681 & 2.967496 \\
\hline 6 & 2.709398 & -0.783428 & 3.970798 \\
\hline 6 & 4.102495 & -0.791874 & 4.030457 \\
\hline b & 4.827476 & -1.514110 & 3.089001 \\
\hline 6 & 4.160936 & -2.220261 & 2.091236 \\
\hline 15 & 1.626590 & -0.786852 & -0.861743 \\
\hline 8 & 2.565470 & 0.056830 & 0.207783 \\
\hline 6 & 3.280745 & 1.079781 & -0.392763 \\
\hline 5 & 4.486647 & 0.787718 & -0.998233 \\
\hline 6 & 5.177199 & 1.845229 & -1.675441 \\
\hline 6 & 4.636998 & 3.166518 & -1.639698 \\
\hline 6 & 3.410066 & 3.402497 & -0.974156 \\
\hline 6 & 2.729480 & 2.375731 & -0.376811 \\
\hline 6 & 6.360548 & 1.630851 & -2.426799 \\
\hline 6 & 6.993019 & 2.668217 & -3.066165 \\
\hline 6 & 6.478263 & 3.979274 & -2.993985 \\
\hline 6 & 5.320306 & 4.216802 & -2.299384 \\
\hline 6 & 4.958708 & -0.614945 & -1.004988 \\
\hline 6 & 4.115241 & -1.603906 & -1.481891 \\
\hline 6 & 4.539535 & -2.943037 & -1.619381 \\
\hline 6 & 5.792708 & -3.310320 & -1.210546 \\
\hline 6 & 6.660748 & -2.363438 & -0.613026 \\
\hline 6 & 6.239906 & -1.004524 & -0.497590 \\
\hline 6 & 7.097871 & -0.096300 & 0.173380 \\
\hline 6 & 8.310480 & -0.503295 & 0.673096 \\
\hline 6 & 8.735143 & -1.841037 & 0.530847 \\
\hline 6 & 7.921585 & -2.749492 & -0.097153 \\
\hline
\end{tabular}




\begin{tabular}{|c|c|c|c|}
\hline & 2.841406 & -1.305104 & -1.903753 \\
\hline & -1.456281 & 2.049648 & 0.606743 \\
\hline & -0.382605 & 2.316104 & 1.465782 \\
\hline & -0.191616 & 3.586248 & 1.998445 \\
\hline & -1.074591 & 4.603596 & 1.600618 \\
\hline & -2.129094 & 4.377901 & 0.719084 \\
\hline & -2.311596 & 3.075864 & 0.232625 \\
\hline & 0.907318 & 3.882243 & 3.018532 \\
\hline & 0.261544 & 4.400751 & 4.309170 \\
\hline & -3.095785 & 5.473326 & 0.274277 \\
\hline & -2.985417 & 5.654223 & -1.243861 \\
\hline & 1.864579 & 4.943719 & 2.468321 \\
\hline & 1.714933 & 2.633490 & 3.369767 \\
\hline & -4.529081 & 5.064657 & 0.635026 \\
\hline & -2.802623 & 6.815844 & 0.938517 \\
\hline & -6.662981 & 1.916132 & 1.827511 \\
\hline & -8.215592 & 0.285983 & 0.795095 \\
\hline & -6.207581 & 0.551399 & -4.174879 \\
\hline & -4.395339 & -1.131278 & -4.407413 \\
\hline & 6.776544 & 0.933755 & 0.305517 \\
\hline & -1.994946 & 0.959599 & 2.833794 \\
\hline & 8.225834 & -3.790263 & -0.201437 \\
\hline & -1.747805 & -2.585030 & 5.957181 \\
\hline & 4.894770 & 5.218295 & -2.251678 \\
\hline & -3.777541 & -2.768521 & 0.903882 \\
\hline & -5.665503 & -3.820542 & -0.264105 \\
\hline & 2.998357 & 4.410823 & -0.970714 \\
\hline & -2.014281 & -0.410980 & 4.859085 \\
\hline & 6.130700 & -4.339813 & -1.317662 \\
\hline & 7.896489 & 2.474617 & -3.640123 \\
\hline & 3.848673 & -3.660701 & -2.057413 \\
\hline & -3.046096 & -5.130043 & -3.413577 \\
\hline & -0.663524 & -4.590528 & -3.327866 \\
\hline & -0.948215 & -6.091357 & 3.601830 \\
\hline & 6.764009 & 0.624803 & -2.504494 \\
\hline & -0.983828 & -4.748984 & 1.548076 \\
\hline & 1.248369 & -3.503193 & -2.179688 \\
\hline & 6.991589 & 4.792831 & -3.501244 \\
\hline & 8.946162 & 0.211792 & 1.190512 \\
\hline & -5.309186 & -5.026817 & -2.414080 \\
\hline & -1.330185 & -5.022940 & 5.818276 \\
\hline & 9.699255 & -2.149748 & 0.928159 \\
\hline & 1.763277 & 2.527540 & 0.100777 \\
\hline & -4.097587 & 0.709034 & 1.485807 \\
\hline & -2.997973 & -0.373548 & -2.500240 \\
\hline & -7.142498 & -0.200122 & -1.385164 \\
\hline & -3.132010 & 2.860441 & -0.451061 \\
\hline & 0.288332 & 1.501006 & 1.734919 \\
\hline & -0.928753 & 5.602447 & 2.007115 \\
\hline & 1.396186 & -3.707863 & 1.380164 \\
\hline & -1.314333 & 1.152238 & -2.595679 \\
\hline & 2.788395 & -3.505737 & 0.310696 \\
\hline & 4.730026 & -2.778335 & 1.347078 \\
\hline & 0.960517 & -1.453682 & 2.904574 \\
\hline & 5.915761 & -1.526660 & 3.120156 \\
\hline & 2.134537 & -0.227986 & 4.710311 \\
\hline
\end{tabular}




$\begin{array}{rrrr}1 & 4.618497 & -0.237183 & 4.811455 \\ 1 & 2.474611 & 2.882271 & 4.122140 \\ 1 & 1.075447 & 1.845332 & 3.792502 \\ 1 & 2.241753 & 2.210820 & 2.503338 \\ 1 & 2.635510 & 5.182557 & 3.213244 \\ 1 & 2.374114 & 4.585756 & 1.563964 \\ 1 & 1.344991 & 5.876492 & 2.217300 \\ 1 & 1.031621 & 4.596820 & 5.067276 \\ 1 & -0.291962 & 5.334147 & 4.151418 \\ 1 & -0.438338 & 3.661559 & 4.721447 \\ 1 & -3.525822 & 7.563966 & 0.590208 \\ 1 & -2.886743 & 6.761099 & 2.031787 \\ 1 & -1.800961 & 7.188033 & 0.687830 \\ 1 & -3.676934 & 6.436100 & -1.586005 \\ 1 & -1.968443 & 5.951027 & -1.530671 \\ 1 & -3.227932 & 4.733183 & -1.787082 \\ 1 & -5.237623 & 5.840106 & 0.314187 \\ 1 & -4.824995 & 4.125361 & 0.150672 \\ 1 & -4.639839 & 4.933337 & 1.719703 \\ 1 & -7.381261 & 2.075504 & 2.643101 \\ 1 & -5.661085 & 2.127599 & 2.221496 \\ 1 & -6.875868 & 2.653712 & 1.042424 \\ 1 & -8.914676 & 0.451007 & 1.624684 \\ 1 & -8.480862 & 0.991461 & -0.002851 \\ 1 & -8.382385 & -0.733291 & 0.423227 \\ 1 & -7.243215 & -0.374363 & 3.227526 \\ 1 & -6.617180 & -1.546381 & 2.049308 \\ 1 & -5.513638 & -0.407063 & 2.839045 \\ 1 & -4.759447 & -1.408749 & -5.404848 \\ 1 & -3.653354 & -0.333691 & -4.541746 \\ 1 & -3.884560 & -2.005824 & -3.980908 \\ 1 & -6.529502 & 0.333111 & -5.202347 \\ 1 & -7.086838 & 0.891624 & -3.613201 \\ 1 & -5.491630 & 1.382630 & -4.213472 \\ 1 & -6.888034 & -2.088435 & -4.543855 \\ 1 & -6.170768 & -2.725885 & -3.048673 \\ 1 & -7.511775 & -1.563578 & -2.977173\end{array}$

\section{$2_{\mathrm{C} 1-\mathrm{t}-}-\psi_{-1225354 a}$}

Number of imaginary frequencies : 0

The smallest frequencies are : $13.8578 \quad 17.2388 \quad 20.4176 \mathrm{~cm}(-1)$

Electronic energy : $\quad H F=-4012.1884032$

Zero-point correction $=\quad 1.290583$ (Hartree/Particle)

Thermal correction to Energy= $\quad 1.366895$

Thermal correction to Enthalpy= $\quad 1.367839$

Thermal correction to Gibbs Free Energy $=\quad 1.178682$

Sum of electronic and zero-point Energies $=\quad-4010.897820$

Sum of electronic and thermal Energies $=\quad-4010.821508$

Sum of electronic and thermal Enthalpies $=\quad-4010.820564$

Sum of electronic and thermal Free Energies $=\quad-4011.009721$

Cartesian Coordinates

$\begin{array}{llll}6 & 5.351097 & 4.176183 & -2.348364 \\ 6 & 4.661481 & 3.139571 & -1.673814\end{array}$




\begin{tabular}{|c|c|c|c|}
\hline 6 & 5.189383 & 1.813013 & -1.697899 \\
\hline 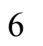 & 6.367135 & 1.579526 & -2.452240 \\
\hline & 7.005765 & 2.603933 & -3.106271 \\
\hline & 6.503238 & 3.920323 & -3.046173 \\
\hline & 4.491704 & 0.769070 & -1.006991 \\
\hline ) & 3.290908 & 1.078772 & -0.400172 \\
\hline & 2.752459 & 2.380347 & -0.393796 \\
\hline f & 3.440070 & 3.394243 & -1.004843 \\
\hline D & 4.952238 & -0.637682 & -0.999491 \\
\hline 6 & 4.100443 & -1.626130 & -1.462352 \\
\hline 6 & 4.514501 & -2.969991 & -1.585436 \\
\hline 6 & 5.765615 & -3.342005 & -1.174875 \\
\hline 6 & 6.641961 & -2.394575 & -0.590483 \\
\hline 6 & 6.231555 & -1.031248 & -0.490188 \\
\hline 6 & 7.900801 & -2.784121 & -0.072381 \\
\hline 6 & 8.722023 & -1.874781 & 0.544201 \\
\hline 6 & 8.307490 & -0.532462 & 0.672047 \\
\hline 6 & 7.097286 & -0.121996 & 0.169276 \\
\hline 8 & 2.826894 & -1.323545 & -1.882524 \\
\hline 15 & 1.621433 & -0.785702 & -0.839947 \\
\hline 8 & 2.568487 & 0.069209 & 0.212779 \\
\hline 7 & 1.183566 & -2.096667 & 0.134598 \\
\hline 6 & 0.078539 & -2.803390 & -0.459023 \\
\hline 6 & -1.183651 & -2.710489 & 0.097066 \\
\hline 6 & -2.280247 & -3.373429 & -0.535675 \\
\hline 6 & -2.045690 & -4.150557 & -1.709088 \\
\hline 6 & -0.735188 & -4.229317 & -2.236432 \\
\hline 6 & 0.297799 & -3.565438 & -1.631445 \\
\hline 6 & -3.126603 & -4.837765 & -2.313161 \\
\hline 6 & -4.393263 & -4.749237 & -1.794341 \\
\hline 6 & -4.635570 & -3.950711 & -0.655446 \\
\hline 6 & -3.605914 & -3.285315 & -0.037802 \\
\hline 6 & -1.389292 & -2.009341 & 1.393527 \\
\hline 6 & -1.593425 & -0.640987 & 1.477534 \\
\hline 6 & -1.843992 & -0.053818 & 2.748385 \\
\hline 6 & -1.862223 & -0.805498 & 3.892186 \\
\hline 6 & -1.629523 & -2.198819 & 3.844021 \\
\hline 6 & -1.397850 & -2.813304 & 2.579509 \\
\hline 6 & -1.165515 & -4.213594 & 2.549129 \\
\hline 6 & -1.153950 & -4.953956 & 3.705406 \\
\hline 6 & -1.381844 & -4.338475 & 4.954324 \\
\hline 6 & -1.616446 & -2.988841 & 5.018352 \\
\hline 15 & -1.639717 & 0.393471 & -0.065763 \\
\hline 6 & -1.472776 & 2.089217 & 0.605092 \\
\hline 6 & -0.391364 & 2.369931 & 1.450043 \\
\hline 6 & -0.215318 & 3.640716 & 1.986305 \\
\hline 6 & -1.127059 & 4.641956 & 1.612560 \\
\hline 6 & -2.193803 & 4.400501 & 0.750529 \\
\hline 6 & -2.354818 & 3.099774 & 0.253006 \\
\hline 6 & 0.900129 & 3.955841 & 2.982647 \\
\hline 6 & 0.279910 & 4.493042 & 4.278127 \\
\hline 6 & -3.204448 & 5.473047 & 0.350156 \\
\hline 6 & -4.609246 & 5.024116 & 0.771768 \\
\hline 6 & 2.070584 & -2.913181 & 0.991766 \\
\hline 6 & 2.769436 & -2.132767 & 2.069748 \\
\hline 6 & 2.033659 & -1.404312 & 3.006087 \\
\hline
\end{tabular}




\begin{tabular}{|c|c|c|c|}
\hline 6 & 2.681756 & -0.681765 & 3.999197 \\
\hline 6 & 4.074562 & -0.670440 & 4.063829 \\
\hline 6 & 4.812658 & -1.400905 & 3.139008 \\
\hline 6 & 4.159480 & -2.133533 & 2.151523 \\
\hline 45 & -0.075696 & 0.354221 & -1.868476 \\
\hline 6 & 0.900563 & 0.843382 & -3.382710 \\
\hline 8 & 1.495026 & 1.208015 & -4.304764 \\
\hline 6 & -3.408933 & 0.221650 & -0.517091 \\
\hline 6 & -4.413150 & 0.374378 & 0.448411 \\
\hline 6 & -5.750638 & 0.191147 & 0.118835 \\
\hline 6 & -6.060163 & -0.139686 & -1.212009 \\
\hline 6 & -5.087002 & -0.287910 & -2.195584 \\
\hline 6 & -3.747823 & -0.111880 & -1.819710 \\
\hline 6 & -6.868604 & 0.297014 & 1.154799 \\
\hline 6 & -7.508911 & -1.084773 & 1.330920 \\
\hline 6 & -5.409979 & -0.590056 & -3.656638 \\
\hline 6 & -4.645306 & -1.835729 & -4.114019 \\
\hline 6 & -6.351853 & 0.758630 & 2.516304 \\
\hline 6 & -7.932030 & 1.296419 & 0.688392 \\
\hline 6 & -6.898887 & -0.837858 & -3.882221 \\
\hline 6 & -4.982801 & 0.609958 & -4.510776 \\
\hline 6 & -2.918031 & 6.819724 & 1.008866 \\
\hline 6 & -3.165765 & 5.664847 & -1.169926 \\
\hline 6 & 1.717571 & 2.71 & 3.337360 \\
\hline 6 & 1.842414 & 5.011761 & 2.396827 \\
\hline 1 & 6.783540 & 0.911701 & 0.290730 \\
\hline 1 & -2.022835 & 1.016267 & 2.818625 \\
\hline 1 & 8.197026 & -3.828283 & -0.165195 \\
\hline 1 & -1.793685 & -2.498137 & 5.974390 \\
\hline 1 & 4.935053 & 5.182037 & -2.309787 \\
\hline 1 & -3.803397 & -2.669703 & 3741 \\
\hline 1 & -5.647165 & -3.862445 & -0.264524 \\
\hline 1 & 3.038409 & 4.406594 & -1.009975 \\
\hline 1 & -2.056029 & -0.336537 & 4.856063 \\
\hline 1 & 6.095546 & -4.375244 & -1.270284 \\
\hline 1 & 7.904513 & 2.395756 & -3.682539 \\
\hline 1 & 3.817049 & -3.687785 & -2.013022 \\
\hline 1 & -2.928556 & -5.435552 & -3.201831 \\
\hline 1 & -0.564218 & -4.820387 & -3.134740 \\
\hline 1 & -0.970180 & -6.024847 & 3.658494 \\
\hline 1 & 6.760972 & 0.568981 & -2.520751 \\
\hline 1 & -0.994241 & -4.704172 & 1.593121 \\
\hline 1 & 1.304586 & -3.602111 & -2.044211 \\
\hline 1 & 7.021244 & 4.723495 & -3.565088 \\
\hline 1 & 8.949147 & 0.183619 & 1.180639 \\
\hline 1 & -5.216312 & -5.281478 & -2.266344 \\
\hline 1 & -1.370573 & -4.936819 & 5.862338 \\
\hline 1 & 9.684373 & -2.186192 & 0.943653 \\
\hline 1 & 1.790046 & 2.546257 & 0.087194 \\
\hline 1 & -4.132477 & 0.637000 & 1.465558 \\
\hline 1 & -2.946518 & -0.226853 & -2.548860 \\
\hline 1 & -7.105319 & -0.287270 & -1.476726 \\
\hline 1 & -3.181977 & 2.872153 & -0.418448 \\
\hline 1 & 0.295099 & 1.563892 & 1.708027 \\
\hline 1 & -0.995128 & 5.640524 & 2.024122 \\
\hline 1 & 1.415937 & -3.666204 & 1.454121 \\
\hline
\end{tabular}




$\begin{array}{rrrr}1 & -1.266928 & 1.239382 & -2.560401 \\ 1 & 2.808205 & -3.462326 & 0.385096 \\ 1 & 4.739261 & -2.698227 & 1.420676 \\ 1 & 0.945765 & -1.394656 & 2.938652 \\ 1 & 5.900796 & -1.399301 & 3.175566 \\ 1 & 2.097064 & -0.121735 & 4.727368 \\ 1 & 4.580159 & -0.094850 & 4.836482 \\ 1 & 2.485971 & 2.974482 & 4.077040 \\ 1 & 1.086209 & 1.928830 & 3.776326 \\ 1 & 2.235498 & 2.284013 & 2.469463 \\ 1 & 1.065387 & 4.697169 & 5.018015 \\ 1 & -0.273529 & 5.426343 & 4.120058 \\ 1 & -0.413399 & 3.761534 & 4.714194 \\ 1 & 2.625121 & 5.267904 & 3.123567 \\ 1 & 2.337906 & 4.640219 & 1.490213 \\ 1 & 1.312833 & 5.936772 & 2.137843 \\ 1 & -3.671294 & 7.551844 & 0.691726 \\ 1 & -2.959209 & 6.759337 & 2.104264 \\ 1 & -1.935395 & 7.215813 & 0.721990 \\ 1 & -3.890371 & 6.431715 & -1.475544 \\ 1 & -2.170058 & 5.988097 & -1.499451 \\ 1 & -3.410719 & 4.742092 & -1.708950 \\ 1 & -5.351633 & 5.784104 & 0.493296 \\ 1 & -4.903517 & 4.082671 & 0.290552 \\ 1 & -4.665944 & 4.877640 & 1.858661 \\ 1 & -8.319226 & -1.041770 & 2.071323 \\ 1 & -7.933762 & -1.462607 & 0.392666 \\ 1 & -6.767596 & -1.815031 & 1.683216 \\ 1 & -7.188390 & 0.828867 & 3.222911 \\ 1 & -5.622573 & 0.056154 & 2.941946 \\ 1 & -5.881858 & 1.749697 & 2.460147 \\ 1 & -8.731950 & 1.374802 & 1.436729 \\ 1 & -7.500922 & 2.296438 & 0.550716 \\ 1 & -8.398190 & 1.000057 & -0.258583 \\ 1 & -7.078713 & -1.064974 & -4.940560 \\ 1 & -7.263459 & -1.691122 & -3.294653 \\ 1 & -7.506971 & 0.040434 & -3.629451 \\ 1 & -4.867933 & -2.049309 & -5.168223 \\ 1 & -3.558439 & -1.715727 & -4.024613 \\ 1 & -4.933106 & -2.711697 & -3.518878 \\ 1 & -5.204315 & 0.422078 & -5.570165 \\ 1 & -5.518847 & 1.519126 & -4.208210 \\ 1 & -3.907419 & 0.810972 & -4.424776\end{array}$

\section{$2_{\mathrm{C} 1-\mathrm{t}-} \boldsymbol{\psi}_{-1 \mathrm{a} 25354 \mathrm{~s}}$}

Number of imaginary frequencies : 0

The smallest frequencies are : $14.4258 \quad 16.4691 \quad 19.9889 \mathrm{~cm}(-1)$

Electronic energy : $\quad \mathrm{HF}=-4012.1884439$

Zero-point correction $=\quad 1.290953($ Hartree/Particle)

Thermal correction to Energy= $\quad 1.367023$

Thermal correction to Enthalpy= $\quad 1.367967$

Thermal correction to Gibbs Free Energy= $\quad 1.179938$

Sum of electronic and zero-point Energies $=\quad-4010.897491$

Sum of electronic and thermal Energies $=\quad-4010.821421$

Sum of electronic and thermal Enthalpies $=\quad-4010.820477$ 
Cartesian Coordinates

\begin{tabular}{|c|c|c|c|}
\hline 6 & 4.149708 & -2.159281 & 2.137811 \\
\hline 6 & 2.759249 & -2.166288 & 2.064584 \\
\hline 6 & 2.024406 & -1.447695 & 3.009286 \\
\hline 6 & 2.674303 & -0.725242 & 4.001367 \\
\hline 6 & 4.067463 & -0.704741 & 4.056478 \\
\hline 6 & 4.804538 & -1.426816 & 3.124199 \\
\hline 6 & 2.058392 & -2.938245 & 0.982057 \\
\hline 7 & 1.178712 & -2.109917 & 0.128728 \\
\hline 15 & 1.626028 & -0.787813 & -0.826025 \\
\hline 45 & -0.052821 & 0.381928 & -1.846646 \\
\hline 6 & 0.983726 & 0.941660 & -3.295975 \\
\hline 8 & 1.635680 & 1.359975 & -4.154202 \\
\hline 6 & 0.079198 & -2.807575 & -0.485997 \\
\hline 6 & -1.187853 & -2.721876 & 0.060180 \\
\hline 6 & -2.280624 & -3.369883 & -0.593525 \\
\hline 6 & -2.037370 & -4.126633 & -1.778452 \\
\hline 6 & -0.722301 & -4.199936 & -2.294975 \\
\hline 6 & 0.307262 & -3.549724 & -1.669416 \\
\hline 6 & -3.610784 & -3.284638 & -0.106750 \\
\hline 6 & -4.636145 & -3.935477 & -0.746590 \\
\hline 6 & -4.385691 & -4.715152 & -1.896781 \\
\hline 6 & -3.114617 & -4.799148 & -2.405128 \\
\hline 6 & -1.402174 & -2.040534 & 1.365257 \\
\hline 6 & -1.603450 & -0.673061 & 1.466050 \\
\hline 6 & -1.857490 & -0.102112 & 2.743351 \\
\hline 6 & -1.884363 & -0.869401 & 3.876737 \\
\hline 6 & -1.657609 & -2.262915 & 3.810854 \\
\hline 6 & -1.420575 & -2.860905 & 2.539501 \\
\hline 6 & -1.655087 & -3.069086 & 4.974233 \\
\hline 6 & -1.426071 & -4.418721 & 4.892819 \\
\hline 6 & -1.193312 & -5.018016 & 3.636835 \\
\hline 6 & -1.194266 & -4.261746 & 2.490883 \\
\hline 15 & -1.640129 & 0.380345 & -0.064803 \\
\hline 6 & -3.404247 & 0.208037 & -0.534359 \\
\hline 6 & -3.738667 & -0.089136 & -1.850808 \\
\hline 6 & -5.073089 & -0.252345 & -2.235120 \\
\hline 6 & -6.057775 & -0.128662 & -1.251926 \\
\hline 6 & -5.752119 & 0.158045 & 0.084744 \\
\hline 6 & -4.411454 & 0.330919 & 0.423819 \\
\hline 6 & -5.390157 & -0.524103 & -3.704079 \\
\hline 6 & -4.937535 & 0.681079 & -4.537482 \\
\hline 6 & -6.811191 & 0.267001 & 1.180949 \\
\hline 6 & -6.750576 & 1.659972 & 1.817925 \\
\hline 6 & -1.491324 & 2.068414 & 0.629524 \\
\hline 6 & -2.380403 & 3.076226 & 0.287539 \\
\hline 6 & -2.226902 & 4.373658 & 0.795901 \\
\hline 6 & -1.163700 & 4.613052 & 1.662982 \\
\hline 6 & -0.247131 & 3.613434 & 2.029652 \\
\hline 6 & -0.413546 & 2.347282 & 1.479581 \\
\hline 6 & 0.866338 & 3.925424 & 3.029189 \\
\hline 6 & 1.806892 & 4.986185 & 2.449151 \\
\hline 6 & -3.233493 & 5.447875 & 0.389574 \\
\hline
\end{tabular}




\begin{tabular}{|c|c|c|c|}
\hline 6 & -4.648140 & 4.986538 & 0.760953 \\
\hline 8 & 2.832473 & -1.321082 & -1.870109 \\
\hline & 4.108395 & -1.616248 & -1.452416 \\
\hline & 4.954059 & -0.622063 & -0.991056 \\
\hline & 6.238374 & -1.005541 & -0.487430 \\
\hline & 6.658103 & -2.366001 & -0.588899 \\
\hline & 5.786274 & -3.319986 & -1.169374 \\
\hline & 4.531157 & -2.957148 & -1.576247 \\
\hline & 7.922366 & -2.745862 & -0.076843 \\
\hline & 8.740120 & -1.829935 & 0.534567 \\
\hline & 8.316741 & -0.490394 & 0.663025 \\
\hline f & 7.101035 & -0.089141 & 0.166176 \\
\hline & 4.480165 & 0.780262 & -0.993076 \\
\hline 6 & 3.282239 & 1.076943 & -0.374349 \\
\hline & 2.730926 & 2.373174 & -0.361001 \\
\hline & 3.401097 & 3.393888 & -0.980164 \\
\hline$b$ & 4.617710 & 3.151833 & -1.662418 \\
\hline 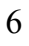 & 5.160004 & 1.831258 & -1.690512 \\
\hline 6 & 6.333681 & 1.609854 & -2.454704 \\
\hline & 6.954721 & 2.640506 & -3.115774 \\
\hline 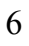 & 6.437589 & 3.951114 & -3.053062 \\
\hline ) & 5.289160 & 4.195124 & -2.344945 \\
\hline & 2.572237 & 0.056432 & 0.236438 \\
\hline ) & 1.686337 & 2.683880 & 3.377600 \\
\hline ) & 0.244575 & 4.454612 & 4.327131 \\
\hline 6 & -2.972114 & 6.783579 & 1.080199 \\
\hline 6 & -3.151400 & 5.665975 & -1.125457 \\
\hline f & -8.225602 & 0.047578 & 0.650894 \\
\hline 6 & -6.533177 & -0.795403 & 2.251748 \\
\hline 6 & -6.880926 & -0.744229 & -3.946643 \\
\hline ) & -4.641877 & -1.775494 & -4.173892 \\
\hline ] & 6.780933 & 0.942728 & 0.286922 \\
\hline . & -2.032507 & 0.967561 & 2.827069 \\
\hline . & 8.225873 & -3.787864 & -0.170392 \\
\hline ] & -1.835968 & -2.590869 & 5.935905 \\
\hline & 4.862364 & 5.196353 & -2.303741 \\
\hline 1 & -3.815745 & -2.680661 & 0.776736 \\
\hline & -5.651737 & -3.849615 & -0.366037 \\
\hline 1 & 2.988698 & 4.401912 & -0.981086 \\
\hline 1 & -2.080658 & -0.412892 & 4.846069 \\
\hline 1 & 6.123333 & -4.350875 & -1.265358 \\
\hline 1 & 7.850762 & 2.441888 & -3.699579 \\
\hline 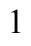 & 3.837394 & -3.679875 & -2.001552 \\
\hline 1 & -2.910150 & -5.381648 & -3.302427 \\
\hline 1 & -0.544874 & -4.775445 & -3.202088 \\
\hline 1 & -1.014288 & -6.089032 & 3.576290 \\
\hline 1 & 6.738240 & 0.603637 & -2.524969 \\
\hline 1 & -1.019617 & -4.739623 & 1.529049 \\
\hline 1 & 1.317188 & -3.581798 & -2.074723 \\
\hline 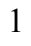 & 6.941670 & 4.759298 & -3.577873 \\
\hline 1 & 8.956215 & 0.230910 & 1.166963 \\
\hline ] & -5.206223 & -5.235806 & -2.385803 \\
\hline 1 & -1.422977 & -5.029666 & 5.792483 \\
\hline ] & 9.706796 & -2.133938 & 0.929251 \\
\hline 1 & 1.771943 & 2.529729 & 0.129666 \\
\hline 1 & -4.142809 & 0.558557 & 1.455058 \\
\hline
\end{tabular}




$\begin{array}{rrrr}1 & -2.933745 & -0.185144 & -2.578923 \\ 1 & -7.098645 & -0.261654 & -1.532423 \\ 1 & -3.204488 & 2.850371 & -0.388264 \\ 1 & 0.278040 & 1.543044 & 1.729723 \\ 1 & -1.037769 & 5.608849 & 2.083088 \\ 1 & 1.397866 & -3.689726 & 1.438465 \\ 1 & -1.233406 & 1.286471 & -2.534804 \\ 1 & 2.793955 & -3.487621 & 0.372937 \\ 1 & 4.727858 & -2.715654 & 1.399275 \\ 1 & 0.935839 & -1.445233 & 2.949171 \\ 1 & 5.892908 & -1.417701 & 3.153249 \\ 1 & 2.091033 & -0.171659 & 4.735561 \\ 1 & 4.574262 & -0.128577 & 4.827922 \\ 1 & 2.451336 & 2.940670 & 4.121865 \\ 1 & 1.055872 & 1.892790 & 3.808296 \\ 1 & 2.208827 & 2.261676 & 2.508322 \\ 1 & 2.590223 & 5.238476 & 3.176580 \\ 1 & 2.301573 & 4.621164 & 1.539393 \\ 1 & 1.275841 & 5.912220 & 2.196879 \\ 1 & 1.029475 & 4.657360 & 5.067997 \\ 1 & -0.311412 & 5.387157 & 4.173690 \\ 1 & -0.446689 & 3.719182 & 4.759783 \\ 1 & -3.724313 & 7.515979 & 0.761147 \\ 1 & -3.035942 & 6.700979 & 2.173100 \\ 1 & -1.987090 & 7.193313 & 0.822221 \\ 1 & -3.870625 & 6.434545 & -1.439537 \\ 1 & -2.148020 & 5.999017 & -1.420237 \\ 1 & -3.375352 & 4.750565 & -1.685899 \\ 1 & -5.384497 & 5.747665 & 0.469811 \\ 1 & -4.923808 & 4.050425 & 0.258677 \\ 1 & -4.738602 & 4.824935 & 1.843388 \\ 1 & -7.499200 & 1.746937 & 2.617002 \\ 1 & -5.768712 & 1.870621 & 2.259749 \\ 1 & -6.958350 & 2.441369 & 1.074996 \\ 1 & -8.945193 & 0.130827 & 1.475160 \\ 1 & -8.502282 & 0.795491 & -0.103333 \\ 1 & -8.347968 & -0.949415 & 0.208137 \\ 1 & -7.272975 & -0.726685 & 3.060835 \\ 1 & -6.594095 & -1.805685 & 1.825701 \\ 1 & -5.537776 & -0.683561 & 2.700983 \\ 1 & -7.054686 & -0.947011 & -5.010944 \\ 1 & -7.264372 & -1.603469 & -3.380391 \\ 1 & -7.477694 & 0.138054 & -3.681143 \\ 1 & -5.154531 & 0.512285 & -5.601016 \\ 1 & -5.461216 & 1.594298 & -4.225536 \\ 1 & -3.859885 & 0.863881 & -4.441306 \\ 1 & -4.859463 & -1.970735 & -5.232758 \\ 1 & -3.554108 & -1.673445 & -4.073755 \\ 1 & -4.947364 & -2.655558 & -3.593389\end{array}$

\section{$2_{\text {C1-t- }} \psi_{-1 \text { 1s2a3a4a }}$}

Number of imaginary frequencies : 0

The smallest frequencies are : $12.1664 \quad 19.9187 \quad 22.8473 \mathrm{~cm}(-1)$

Electronic energy :

$\mathrm{HF}=-4012.1880381$

Zero-point correction $=\quad 1.292512($ Hartree/Particle $)$ 
Thermal correction to Energy $=\quad 1.368223$

Thermal correction to Enthalpy= $\quad 1.369167$

Thermal correction to Gibbs Free Energy= $\quad 1.182614$

Sum of electronic and zero-point Energies $=\quad-4010.895526$

Sum of electronic and thermal Energies $=\quad-4010.819816$

Sum of electronic and thermal Enthalpies $=\quad-4010.818871$

Sum of electronic and thermal Free Energies $=\quad-4011.005425$

Cartesian Coordinates

$\begin{array}{cccc}0 & 1.963197 & -1.046297 & 3.034397 \\ 6 & 2.702072 & -1.902097 & 2.213997 \\ 6 & 4.091333 & -1.893005 & 2.300958 \\ 6 & 4.743147 & -1.025334 & 3.173246 \\ 6 & 4.003463 & -0.165826 & 3.977330 \\ 6 & 2.610443 & -0.185833 & 3.912004 \\ 6 & 2.009403 & -2.811765 & 1.238201 \\ 7 & 1.169818 & -2.099533 & 0.251970 \\ 15 & 1.623679 & -0.874477 & -0.821782 \\ 45 & -0.066341 & 0.203437 & -1.921321 \\ 6 & 0.902524 & 0.596329 & -3.466618 \\ 8 & 1.498936 & 0.902298 & -4.408683 \\ 6 & 0.074338 & -2.849459 & -0.303846 \\ 6 & -1.191335 & -2.734421 & 0.240050 \\ 6 & -2.290068 & -3.400451 & -0.384304 \\ 6 & -2.058551 & -4.190741 & -1.549113 \\ 6 & -0.742131 & -4.310274 & -2.052813 \\ 6 & 0.296063 & -3.654057 & -1.447402 \\ 6 & -3.618866 & -3.279207 & 0.098928 \\ 6 & -4.658643 & -3.910686 & -0.535957 \\ 6 & -4.424121 & -4.707079 & -1.678690 \\ 6 & -3.151567 & -4.840474 & -2.173747 \\ 6 & -1.417202 & -1.970041 & 1.495178 \\ 6 & -1.614021 & -0.598489 & 1.497507 \\ 6 & -1.920081 & 0.052831 & 2.724719 \\ 6 & -1.991067 & -0.638014 & 3.904083 \\ 6 & -1.762541 & -2.032570 & 3.939653 \\ 6 & -1.480316 & -2.712232 & 2.719650 \\ 6 & -1.811483 & -2.762184 & 5.151463 \\ 6 & -1.591124 & -4.115695 & 5.165165 \\ 6 & -1.314821 & -4.795838 & 3.960461 \\ 6 & -1.264274 & -4.114620 & 2.769372 \\ 15 & -1.601953 & 0.362734 & -0.097549 \\ 6 & -3.373735 & 0.229033 & -0.556397 \\ 6 & -4.381000 & 0.560286 & 0.353281 \\ 6 & -5.723801 & 0.408140 & 0.015933 \\ 6 & -6.024026 & -0.089806 & -1.258232 \\ 6 & -5.042856 & -0.440342 & -2.186808 \\ 6 & -3.706840 & -0.273998 & -1.810719 \\ 6 & -6.851783 & 0.728102 & 0.994512 \\ 6 & -6.330700 & 1.340437 & 2.293248 \\ 6 & -5.434284 & -0.937477 & -3.577550 \\ 6 & -5.999718 & 0.243964 & -4.374186 \\ 6 & -1.372641 & 2.075963 & 0.514411 \\ 6 & -0.335186 & 2.339953 & 1.405666 \\ 6 & -0.149626 & 3.621763 & 1.936952 \\ & & & \\ 6 & & \end{array}$




\begin{tabular}{|c|c|c|c|}
\hline 6 & -0.998757 & 4.635458 & 1.493283 \\
\hline & -2.024139 & 4.409961 & 0.563319 \\
\hline & -2.202046 & 3.113934 & 0.084837 \\
\hline & -2.929320 & 5.565694 & 0.140485 \\
\hline & -2.086733 & 6.657576 & -0.526601 \\
\hline & 0.880408 & 3.825695 & 3.048423 \\
\hline & 0.403510 & 3.036088 & 4.275433 \\
\hline & -7.832148 & 1.721605 & 0.363103 \\
\hline & -7.588728 & -0.571973 & 1.336967 \\
\hline & -6.496813 & -2.036027 & -3.479440 \\
\hline & -4.238120 & -1.508360 & -4.338226 \\
\hline & -3.629241 & 6.143169 & 1.376090 \\
\hline & -4.007149 & 5.122877 & -0.846569 \\
\hline b & 2.259782 & 3.314257 & 2.622904 \\
\hline & 1.022762 & 5.291680 & 3.449706 \\
\hline & 2.843223 & -1.492219 & -1.800801 \\
\hline b & 4.109447 & -1.749767 & -1.330984 \\
\hline & 4.954069 & -0.716697 & -0.960723 \\
\hline D & 6.228403 & -1.052645 & -0.401010 \\
\hline & 6.637365 & -2.419277 & -0.348691 \\
\hline & 5.765675 & -3.424720 & -0.834553 \\
\hline & 4.520806 & -3.099016 & -1.300462 \\
\hline 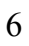 & 7.891192 & -2.750879 & 0.219785 \\
\hline 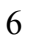 & 8.708935 & -1.780478 & 0.740226 \\
\hline 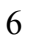 & 8.295588 & -0.431781 & 0.717662 \\
\hline$b$ & 7.089899 & -0.077695 & 0.163749 \\
\hline ) & 4.482254 & 0.678877 & -1.106647 \\
\hline & 3.271838 & 1.028880 & -0.541806 \\
\hline 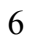 & 2.711602 & 2.314601 & -0.669002 \\
\hline 6 & 3.391918 & 3.275662 & -1.366730 \\
\hline & 4.624910 & 2.976561 & -1.995132 \\
\hline 6 & 5.172907 & 1.662050 & -1.887346 \\
\hline 6 & 6.362547 & 1.375942 & -2.604013 \\
\hline 6 & 6.993767 & 2.343160 & -3.346437 \\
\hline 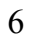 & 6.471033 & 3.651193 & -3.417318 \\
\hline & 5.307466 & 3.954478 & -2.758536 \\
\hline & 2.557831 & 0.071630 & 0.161039 \\
\hline & 6.776325 & 0.963134 & 0.170130 \\
\hline 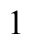 & -2.096256 & 1.125743 & 2.735079 \\
\hline | & 8.186568 & -3.799124 & 0.243458 \\
\hline | & -2.026575 & -2.222727 & 6.072798 \\
\hline 1 & 4.876334 & 4.952733 & -2.821194 \\
\hline & -3.811861 & -2.660023 & 0.973426 \\
\hline 1 & -5.673506 & -3.788229 & -0.161731 \\
\hline & 2.977867 & 4.277380 & -1.470151 \\
\hline 1 & -2.224777 & -0.117303 & 4.832146 \\
\hline l & 6.094858 & -4.462320 & -0.811507 \\
\hline 1 & 7.902250 & 2.095392 & -3.890935 \\
\hline 1 & 3.827264 & -3.857797 & -1.657895 \\
\hline 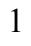 & -2.958124 & -5.445393 & -3.058584 \\
\hline 1 & -0.570359 & -4.921474 & -2.937343 \\
\hline (1) & -1.142949 & -5.869612 & 3.975535 \\
\hline 1 & 6.772277 & 0.369794 & -2.570461 \\
\hline 1 & -1.055659 & -4.653241 & 1.847259 \\
\hline 1 & 1.306884 & -3.721243 & -1.846237 \\
\hline 1 & 6.982992 & 4.409253 & -4.005543 \\
\hline
\end{tabular}




\begin{tabular}{|c|c|c|c|}
\hline 1 & 8.934328 & 0.334858 & 1.150637 \\
\hline 1 & -5.256221 & -5.210414 & -2.166802 \\
\hline 1 & -1.628810 & -4.667942 & 6.101241 \\
\hline 1 & 9.667504 & -2.047171 & 1.179212 \\
\hline 1 & 1.744477 & 2.510480 & -0.209849 \\
\hline 1 & -4.105625 & 0.944191 & 1.332301 \\
\hline 1 & -2.896720 & -0.533163 & -2.489043 \\
\hline 1 & -7.071886 & -0.208735 & -1.534028 \\
\hline 1 & -2.994979 & 2.891029 & -0.624730 \\
\hline 1 & 0.310235 & 1.520819 & 1.731567 \\
\hline 1 & -0.873981 & 5.640516 & 1.891028 \\
\hline 1 & 1.322396 & -3.477532 & 1.780437 \\
\hline 1 & -1.248403 & 1.077134 & -2.645251 \\
\hline 1 & -5.661417 & 0.657555 & 2.833673 \\
\hline 1 & -5.789728 & 2.279564 & 2.112634 \\
\hline 1 & -7.173546 & 1.567117 & 2.958229 \\
\hline 1 & -8.293781 & 1.329018 & -0.550576 \\
\hline 1 & -8.642943 & 1.954075 & 1.066469 \\
\hline 1 & -7.328402 & 2.662298 & 0.105198 \\
\hline 1 & -8.040778 & -1.031328 & 0.449122 \\
\hline 1 & -6.900996 & -1.305740 & 1.778928 \\
\hline 1 & -8.393289 & -0.380284 & 2.059843 \\
\hline 1 & -5.253553 & 1.043063 & -4.472844 \\
\hline 1 & -6.288917 & -0.076892 & -5.384375 \\
\hline 1 & -6.886988 & 0.671762 & -3.890039 \\
\hline 1 & -7.427713 & -1.684271 & -3.019087 \\
\hline 1 & -6.750909 & -2.403027 & -4.482682 \\
\hline 1 & -6.126370 & -2.885920 & -2.891105 \\
\hline 1 & -3.465527 & -0.752625 & -4.529686 \\
\hline 1 & -3.773581 & -2.342612 & -3.793725 \\
\hline 1 & -4.570118 & -1.887675 & -5.313124 \\
\hline 1 & 2.746886 & -3.456493 & 0.733404 \\
\hline 1 & 4.672170 & -2.555635 & 1.658395 \\
\hline 1 & 0.874767 & -1.041095 & 2.959590 \\
\hline 1 & 5.831117 & -1.020258 & 3.213730 \\
\hline 1 & 2.027691 & 0.479795 & 4.547720 \\
\hline 1 & 4.507178 & 0.519108 & 4.656508 \\
\hline 1 & 1.787182 & 5.386371 & 4.231233 \\
\hline 1 & 1.335502 & 5.917703 & 2.603943 \\
\hline 1 & 0.089669 & 5.702589 & 3.856029 \\
\hline 1 & 2.970194 & 3.416996 & 3.455119 \\
\hline 1 & 2.253170 & 2.254977 & 2.336340 \\
\hline 1 & 2.651892 & 3.890245 & 1.774516 \\
\hline 1 & 1.110190 & 3.155444 & 5.108724 \\
\hline 1 & -0.580630 & 3.390524 & 4.611587 \\
\hline 1 & 0.314362 & 1.963164 & 4.058504 \\
\hline 1 & -4.636693 & 5.981081 & -1.113320 \\
\hline 1 & -3.577716 & 4.727632 & -1.775788 \\
\hline 1 & -4.664075 & 4.352474 & -0.419060 \\
\hline 1 & -4.293975 & 6.967844 & 1.085791 \\
\hline 1 & -4.238585 & 5.377833 & 1.875141 \\
\hline 1 & -2.917445 & 6.536796 & 2.111533 \\
\hline 1 & -2.725325 & 7.495199 & -0.838055 \\
\hline 1 & -1.321455 & 7.057827 & 0.149472 \\
\hline 1 & -1.577359 & 6.269920 & -1.418082 \\
\hline
\end{tabular}


$2_{\mathrm{C} 1-\mathrm{t}-} \boldsymbol{\psi}_{-1 \mathrm{~s} 2 \mathrm{a3a4s}}$

Number of imaginary frequencies : 0

The smallest frequencies are : $9.6719 \quad 16.2880 \quad 20.2299 \mathrm{~cm}(-1)$

Electronic energy : $\quad H F=-4012.1884021$

Zero-point correction $=\quad 1.291656($ Hartree/Particle $)$

Thermal correction to Energy $=\quad 1.367626$

Thermal correction to Enthalpy= $\quad 1.368570$

Thermal correction to Gibbs Free Energy= 1.179866

Sum of electronic and zero-point Energies $=\quad-4010.896746$

Sum of electronic and thermal Energies $=\quad-4010.820776$

Sum of electronic and thermal Enthalpies $=\quad-4010.819832$

Sum of electronic and thermal Free Energies $=\quad-4011.008536$

Cartesian Coordinates

$\begin{array}{cccc}6 & -3.718300 & -0.252245 & -1.822262 \\ 6 & -3.380037 & 0.231928 & -0.557716 \\ 6 & -4.383297 & 0.538925 & 0.356042 \\ 6 & -5.731808 & 0.387448 & 0.023189 \\ 6 & -6.037755 & -0.077582 & -1.256535 \\ 6 & -5.052151 & -0.410149 & -2.195339 \\ 15 & -1.608471 & 0.366553 & -0.099047 \\ 45 & -0.073581 & 0.224573 & -1.924592 \\ 6 & 0.896511 & 0.646982 & -3.461261 \\ 8 & 1.492839 & 0.975604 & -4.395744 \\ 6 & -6.790791 & 0.707594 & 1.075438 \\ 6 & -6.574843 & -0.208562 & 2.286528 \\ 6 & -5.448909 & -0.882093 & -3.593296 \\ 6 & -6.512441 & -1.981252 & -3.512139 \\ 6 & -1.618217 & -0.610919 & 1.486832 \\ 6 & -1.423598 & -1.982578 & 1.468588 \\ 6 & -1.484949 & -2.739435 & 2.683912 \\ 6 & -1.763275 & -2.074343 & 3.912632 \\ 6 & -1.989688 & -0.679199 & 3.894011 \\ 6 & -1.920431 & 0.025726 & 2.722617 \\ 6 & -1.270986 & -4.142606 & 2.715921 \\ 6 & -1.319381 & -4.838123 & 3.898808 \\ 6 & -1.591510 & -4.172276 & 5.112428 \\ 6 & -1.810041 & -2.818434 & 5.115721 \\ 6 & -1.204719 & -2.731188 & 0.203316 \\ 6 & 0.058200 & -2.842371 & -0.347634 \\ 6 & 0.271240 & -3.628483 & -1.505548 \\ 6 & -0.772496 & -4.271626 & -2.115691 \\ 6 & -2.085668 & -4.157784 & -1.602119 \\ 6 & -2.308882 & -3.384473 & -0.424347 \\ 6 & -3.183384 & -4.797376 & -2.228909 \\ 6 & -4.452018 & -4.671938 & -1.721942 \\ 6 & -4.678167 & -3.891983 & -0.566221 \\ 6 & -3.634397 & -3.268055 & 0.069571 \\ 7 & 1.158683 & -2.106133 & 0.216362 \\ 6 & 1.994837 & -2.833216 & 1.194555 \\ 6 & 2.684981 & -1.938293 & 2.185875 \\ 6 & 1.944833 & -1.087286 & 3.010128 \\ 6 & 2.590054 & -0.240528 & 3.902366 \\ 6 & 3.982597 & -0.229652 & 3.979021\end{array}$




\begin{tabular}{|c|c|c|c|}
\hline & 4.723460 & -1.084418 & 3.171005 \\
\hline & 4.073550 & -1.938172 & 2.283600 \\
\hline & 1.616106 & -0.865964 & -0.837825 \\
\hline & 2.549818 & 0.062818 & 0.161508 \\
\hline & 3.271361 & 1.027392 & -0.522777 \\
\hline & 4.482710 & 0.680808 & -1.087720 \\
\hline & 5.181448 & 1.673320 & -1.849159 \\
\hline & 4.639971 & 2.991958 & -1.938113 \\
\hline & 3.405330 & 3.286495 & -1.310775 \\
\hline 6 & 2.717618 & 2.317534 & -0.631466 \\
\hline & 6.373166 & 1.393405 & -2.564836 \\
\hline & 7.012324 & 2.369642 & -3.288420 \\
\hline & 6.495976 & 3.681097 & -3.340367 \\
\hline O & 5.330669 & 3.979015 & -2.682210 \\
\hline 6 & 4.947002 & -0.719416 & -0.963367 \\
\hline U & 4.099090 & -1.741870 & -1.354979 \\
\hline 6 & 4.504060 & -3.093384 & -1.346515 \\
\hline 6 & 5.745521 & -3.433038 & -0.881488 \\
\hline & 6.619729 & -2.440407 & -0.374399 \\
\hline 6 & 6.217250 & -1.071186 & -0.404078 \\
\hline 6 & 7.080472 & -0.110475 & 0.181979 \\
\hline 6 & 8.282070 & -0.479952 & 0.734740 \\
\hline 6 & 8.689362 & -1.830688 & 0.734662 \\
\hline 6 & 7.869617 & -2.787844 & 0.193299 \\
\hline 8 & 2.835778 & -1.470037 & -1.824904 \\
\hline 6 & -1.380575 & 2.074245 & 0.527624 \\
\hline 6 & -0.347927 & 2.327390 & 1.427451 \\
\hline 6 & -0.157306 & 3.605686 & 1.965373 \\
\hline 6 & -0.990652 & 4.628322 & 1.512978 \\
\hline 6 & -2.005226 & 4.415606 & 0.567818 \\
\hline 6 & -2.196108 & 3.120718 & 0.090965 \\
\hline 6 & 0.864240 & 3.796770 & 3.086954 \\
\hline 6 & 0.377312 & 2.996994 & 4.303362 \\
\hline 6 & -2.882275 & 5.586238 & 0.126496 \\
\hline 6 & -2.004917 & 6.680393 & -0.490620 \\
\hline 6 & 2.245615 & 3.286002 & 2.666880 \\
\hline 6 & 1.006486 & 5.258626 & 3.502845 \\
\hline 6 & -3.624951 & 6.150859 & 1.342864 \\
\hline 6 & -3.922340 & 5.169844 & -0.911008 \\
\hline 6 & -6.661901 & 2.173200 & 1.506059 \\
\hline 6 & -8.211168 & 0.487231 & 0.561994 \\
\hline 6 & -6.015201 & 0.313904 & -4.367293 \\
\hline 6 & -4.256165 & -1.441489 & -4.367768 \\
\hline 1 & 6.771232 & 0.931411 & 0.205893 \\
\hline 1 & -2.094750 & 1.098654 & 2.746295 \\
\hline 1 & 8.160202 & -3.837674 & 0.199464 \\
\hline 1 & -2.021924 & -2.289805 & 6.044041 \\
\hline 1 & 4.904483 & 4.980195 & -2.730476 \\
\hline 1 & -3.822308 & -2.657940 & 0.952126 \\
\hline 1 & -5.690227 & -3.776473 & -0.182674 \\
\hline 1 & 2.996222 & 4.291612 & -1.400058 \\
\hline 1 & -2.220108 & -0.169030 & 4.828742 \\
\hline 1 & 6.069798 & -4.472419 & -0.875408 \\
\hline 1 & 7.922251 & 2.126547 & -3.832612 \\
\hline 1 & 3.808576 & -3.842651 & -1.719783 \\
\hline 1 & -2.996669 & -5.389010 & -3.124106 \\
\hline
\end{tabular}




\begin{tabular}{|c|c|c|c|}
\hline 1 & -0.607433 & -4.868936 & -3.010918 \\
\hline 1 & -1.149023 & -5.912239 & 3.900433 \\
\hline & 6.778119 & 0.384939 & -2.545876 \\
\hline & -1.065629 & -4.670425 & 1.786864 \\
\hline & 1.279660 & -3.692103 & -1.910985 \\
\hline & 7.014247 & 4.446287 & -3.913647 \\
\hline & 8.922230 & 0.275880 & 1.184300 \\
\hline & -5.287673 & -5.168766 & -2.210652 \\
\hline & -1.627417 & -4.735740 & 6.041858 \\
\hline 1 & 9.644834 & -2.109551 & 1.172830 \\
\hline 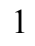 & 1.749332 & 2.510486 & -0.173367 \\
\hline & -4.112666 & 0.902323 & 1.346897 \\
\hline 1 & -2.909738 & -0.499902 & -2.506614 \\
\hline 1 & -7.082473 & -0.189735 & -1.538627 \\
\hline 1 & -2.985083 & 2.906169 & -0.625892 \\
\hline 1 & 0.291120 & 1.503106 & 1.752914 \\
\hline 1 & -0.860544 & 5.631591 & 1.913474 \\
\hline 1 & 1.306041 & -3.506666 & 1.724855 \\
\hline 1 & -1.258114 & 1.098735 & -2.641974 \\
\hline 1 & 2.733623 & -3.470882 & 0.682744 \\
\hline 1 & 4.655765 & -2.596631 & 1.637973 \\
\hline 1 & 0.857290 & -1.073647 & 2.925591 \\
\hline 1 & 5.811077 & -1.086054 & 3.219867 \\
\hline 1 & 2.006096 & 0.421864 & 4.540440 \\
\hline 1 & 4.484991 & 0.444613 & 4.669744 \\
\hline 1 & 1.761902 & 5.343983 & 4.294128 \\
\hline 1 & 1.331297 & 5.891136 & 2.666524 \\
\hline 1 & 0.069714 & 5.668753 & 3.901460 \\
\hline 1 & 2.949612 & 3.377569 & 3.505846 \\
\hline 1 & 2.238195 & 2.229886 & 2.368485 \\
\hline 1 & 2.646181 & 3.870132 & 1.828081 \\
\hline 1 & 1.077737 & 3.108779 & 5.142906 \\
\hline 1 & -0.609118 & 3.349122 & 4.635215 \\
\hline 1 & 0.289452 & 1.925916 & 4.077095 \\
\hline 1 & -4.526730 & 6.040462 & -1.195286 \\
\hline 1 & -3.457626 & 4.777907 & -1.824593 \\
\hline 1 & -4.608935 & 4.406120 & -0.521523 \\
\hline 1 & -2.625548 & 7.524665 & -0.819800 \\
\hline 1 & -1.267436 & 7.070557 & 0.221006 \\
\hline 1 & -1.460053 & 6.299765 & -1.363995 \\
\hline 1 & -4.265666 & 6.990649 & 1.041884 \\
\hline 1 & -4.265145 & 5.384863 & 1.800768 \\
\hline 1 & -2.937896 & 6.519175 & 2.114326 \\
\hline 1 & -7.389367 & 2.405308 & 2.295579 \\
\hline 1 & -5.662744 & 2.407138 & 1.895400 \\
\hline 1 & -6.856515 & 2.846185 & 0.660702 \\
\hline 1 & -8.931147 & 0.732344 & 1.353014 \\
\hline 1 & -8.440212 & 1.126847 & -0.300101 \\
\hline 1 & -8.385257 & -0.557076 & 0.271974 \\
\hline 1 & -7.329370 & -0.008607 & 3.059319 \\
\hline 1 & -6.659662 & -1.264416 & 1.995094 \\
\hline 1 & -5.586366 & -0.070096 & 2.742797 \\
\hline 1 & -4.591801 & -1.802705 & -5.348301 \\
\hline 1 & -3.482497 & -0.684141 & -4.548122 \\
\hline 1 & -3.792058 & -2.286287 & -3.839306 \\
\hline 1 & -6.309283 & 0.010862 & -5.381574 \\
\hline
\end{tabular}




$\begin{array}{lllll}1 & -6.899751 & 0.735171 & -3.872621 \\ 1 & -5.267663 & 1.112948 & -4.455384 \\ 1 & -6.768503 & -2.330427 & -4.521241 \\ 1 & -6.141341 & -2.841762 & -2.939738 \\ 1 & -7.442326 & -1.637094 & -3.044170\end{array}$

$2_{\text {C1-t- }} \psi_{-1 \text { 1s2a3s4a }}$

Number of imaginary frequencies : 0

The smallest frequencies are : $12.8309 \quad 16.6377 \quad 19.4631 \mathrm{~cm}(-1)$

Electronic energy : $\quad H F=-4012.1875646$

Zero-point correction $=\quad 1.291121$ (Hartree/Particle)

Thermal correction to Energy= $\quad 1.367077$

Thermal correction to Enthalpy= $\quad 1.368021$

Thermal correction to Gibbs Free Energy $=\quad 1.180762$

Sum of electronic and zero-point Energies $=\quad-4010.896444$

Sum of electronic and thermal Energies $=\quad-4010.820488$

Sum of electronic and thermal Enthalpies $=\quad-4010.819544$

Sum of electronic and thermal Free Energies $=\quad-4011.006803$

Cartesian Coordinates

$\begin{array}{cccc}0 & 5.223347 & 4.026860 & -2.706600 \\ 6 & 4.564759 & 3.032436 & -1.943425 \\ 6 & 5.127955 & 1.722522 & -1.862882 \\ 6 & 6.308619 & 1.458182 & -2.602256 \\ 6 & 6.916623 & 2.441274 & -3.343162 \\ 6 & 6.378317 & 3.744079 & -3.389282 \\ 6 & 4.460450 & 0.721250 & -1.085429 \\ 6 & 3.255640 & 1.050010 & -0.496698 \\ 6 & 2.683585 & 2.333542 & -0.590387 \\ 6 & 3.342196 & 3.311259 & -1.285952 \\ 6 & 4.949754 & -0.671041 & -0.966190 \\ 6 & 4.113274 & -1.707519 & -1.344381 \\ 6 & 4.538692 & -3.052719 & -1.336093 \\ 6 & 5.790886 & -3.371516 & -0.885244 \\ 6 & 6.656463 & -2.363623 & -0.393578 \\ 6 & 6.232726 & -1.000789 & -0.423141 \\ 6 & 7.919539 & -2.689404 & 0.157478 \\ 6 & 8.732274 & -1.717371 & 0.682695 \\ 6 & 8.304608 & -0.372957 & 0.682573 \\ 6 & 7.089710 & -0.024250 & 0.145641 \\ 8 & 2.841391 & -1.455238 & -1.800956 \\ 15 & 1.623873 & -0.858944 & -0.805852 \\ 8 & 2.557231 & 0.071520 & 0.192745 \\ 7 & 1.176366 & -2.108331 & 0.241607 \\ 6 & 0.099991 & -2.863502 & -0.345033 \\ 6 & -1.181336 & -2.749613 & 0.160873 \\ 6 & -2.256942 & -3.429929 & -0.488056 \\ 6 & -1.982617 & -4.255517 & -1.618568 \\ 6 & -0.651607 & -4.369308 & -2.084312 \\ 6 & 0.360942 & -3.682194 & -1.469901 \\ 6 & -3.047026 & -4.951200 & -2.242595 \\ 6 & -4.333274 & -4.824892 & -1.783624 \\ 6 & -4.613154 & -3.979503 & -0.687667 \\ 6 & -3.601434 & -3.304439 & -0.051830\end{array}$




\begin{tabular}{|c|c|c|c|}
\hline 6 & -1.434068 & -2.014269 & 1.428865 \\
\hline 6 & -1.637421 & -0.644706 & 1.473814 \\
\hline 6 & -1.950775 & -0.033132 & 2.719809 \\
\hline 6 & -2.025964 & -0.760672 & 3.876552 \\
\hline 6 & -1.791920 & -2.154676 & 3.869338 \\
\hline 6 & -1.499787 & -2.794479 & 2.630383 \\
\hline 6 & -1.273103 & -4.196216 & 2.639778 \\
\hline 6 & -1.325204 & -4.913557 & 3.809485 \\
\hline 6 & -1.613101 & -4.272699 & 5.032727 \\
\hline 6 & -1.842518 & -2.920926 & 5.058297 \\
\hline 15 & -1.617285 & 0.366236 & -0.088187 \\
\hline 6 & -1.401924 & 2.060968 & 0.576135 \\
\hline 6 & -0.368036 & 2.306454 & 1.476698 \\
\hline 6 & -0.191869 & 3.574266 & 2.043299 \\
\hline 6 & -1.046655 & 4.594361 & 1.625196 \\
\hline 6 & -2.069615 & 4.387750 & 0.688260 \\
\hline 6 & -2.238826 & 3.103901 & 0.174871 \\
\hline 6 & 0.834855 & 3.754582 & 3.161933 \\
\hline 6 & 2.217014 & 3.256939 & 2.728839 \\
\hline 6 & -2.979092 & 5.549654 & 0.292326 \\
\hline 6 & -2.139483 & 6.657133 & -0.352455 \\
\hline 6 & 2.023919 & -2.839053 & 1.207855 \\
\hline 6 & 2.711065 & -1.945536 & 2.201975 \\
\hline 6 & 1.966172 & -1.116383 & 3.043954 \\
\hline 6 & 2.607925 & -0.263948 & 3.933390 \\
\hline 6 & 4.001014 & -0.225803 & 3.989152 \\
\hline 6 & 4.746814 & -1.060596 & 3.164861 \\
\hline 6 & 4.100658 & -1.920411 & 2.280742 \\
\hline 45 & -0.052725 & 0.254211 & -1.889840 \\
\hline 6 & 0.966086 & 0.710081 & -3.386277 \\
\hline 8 & 1.605492 & 1.060659 & -4.283467 \\
\hline 6 & -3.384659 & 0.243275 & -0.566657 \\
\hline 6 & -4.403914 & 0.470382 & 0.367790 \\
\hline 6 & -5.739178 & 0.323028 & 0.013589 \\
\hline 6 & -6.031824 & -0.051407 & -1.309423 \\
\hline 6 & -5.043867 & -0.274615 & -2.262896 \\
\hline 6 & -3.707763 & -0.133470 & -1.861888 \\
\hline 6 & -6.877096 & 0.534887 & 1.010016 \\
\hline 6 & -7.626327 & -0.788476 & 1.201186 \\
\hline 6 & -5.343755 & -0.638650 & -3.714760 \\
\hline 6 & -4.695645 & -1.985111 & -4.050993 \\
\hline 6 & -6.369965 & 0.997148 & 2.374677 \\
\hline 6 & -7.843972 & 1.598561 & 0.479370 \\
\hline 6 & -6.841003 & -0.748839 & -3.989373 \\
\hline 6 & -4.765603 & 0.447808 & -4.629758 \\
\hline 6 & -4.057226 & 5.125314 & -0.702717 \\
\hline 6 & -3.678598 & 6.099781 & 1.540546 \\
\hline 6 & 0.972268 & 5.211136 & 3.597896 \\
\hline 6 & 0.356585 & 2.934924 & 4.368498 \\
\hline 1 & 6.765649 & 1.013182 & 0.168018 \\
\hline 1 & -2.130646 & 1.038408 & 2.762363 \\
\hline 1 & 8.226359 & -3.734597 & 0.163702 \\
\hline 1 & -2.065401 & -2.410408 & 5.994165 \\
\hline 1 & 4.780931 & 5.021232 & -2.749178 \\
\hline 1 & -3.827346 & -2.652869 & 0.791017 \\
\hline 1 & -5.639170 & -3.857297 & -0.346919 \\
\hline
\end{tabular}




\begin{tabular}{|c|c|c|c|}
\hline 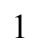 & 2.917757 & 4.310729 & -1.365904 \\
\hline 1 & -2.265966 & -0.270116 & 4.819280 \\
\hline 1 & 6.131094 & -4.405792 & -0.879336 \\
\hline 1 & 7.818494 & 2.210338 & -3.905730 \\
\hline & 3.849983 & -3.813891 & -1.697821 \\
\hline 1 & -2.819981 & -5.586254 & -3.097797 \\
\hline & -0.448165 & -5.002175 & -2.946676 \\
\hline 1 & -1.145136 & -5.985963 & 3.792709 \\
\hline & 6.729406 & 0.456111 & -2.587939 \\
\hline 1 & -1.054037 & -4.706779 & 1.704295 \\
\hline 1 & 1.382259 & -3.742502 & -1.842035 \\
\hline & 6.871714 & 4.514711 & -3.977018 \\
\hline 1 & 8.939941 & 0.394577 & 1.118948 \\
\hline 1 & -5.143234 & -5.363136 & -2.271302 \\
\hline 1 & -1.651716 & -4.853115 & 5.951554 \\
\hline 1 & 9.698152 & -1.979680 & 1.108071 \\
\hline 1 & 1.723739 & 2.514266 & -0.110470 \\
\hline 1 & -4.137910 & 0.765294 & 1.379623 \\
\hline 1 & -2.896117 & -0.309631 & -2.567226 \\
\hline 1 & -7.076227 & -0.164582 & -1.593499 \\
\hline 1 & -3.029696 & 2.894639 & -0.541279 \\
\hline 1 & 0.282104 & 1.482506 & 1.780184 \\
\hline 1 & -0.927771 & 5.589490 & 2.048940 \\
\hline 1 & 1.342510 & -3.522606 & 1.734944 \\
\hline 1 & -1.215218 & 1.159104 & -2.604619 \\
\hline 1 & 2.765622 & -3.465620 & 0.686505 \\
\hline 1 & 4.685549 & -2.563023 & 1.621732 \\
\hline 1 & 0.877012 & -1.125898 & 2.977706 \\
\hline 1 & 5.834901 & -1.041050 & 3.197618 \\
\hline 1 & 2.020984 & 0.381914 & 4.585368 \\
\hline 1 & 4.500083 & 0.453703 & 4.677169 \\
\hline 1 & 1.734974 & 5.289639 & 4.382856 \\
\hline 1 & 1.284684 & 5.857686 & 2.767599 \\
\hline 1 & 0.037320 & 5.609831 & 4.012046 \\
\hline 1 & 2.924725 & 3.343734 & 3.565153 \\
\hline 1 & 2.215328 & 2.203986 & 2.419415 \\
\hline 1 & 2.609694 & 3.852930 & 1.894633 \\
\hline 1 & 1.060466 & 3.036474 & 5.206500 \\
\hline 1 & -0.629461 & 3.278691 & 4.710003 \\
\hline 1 & 0.270978 & 1.867226 & 4.125879 \\
\hline 1 & -4.690045 & 5.987344 & -0.948705 \\
\hline 1 & -3.627696 & 4.753882 & -1.641701 \\
\hline 1 & -4.710671 & 4.342761 & -0.292608 \\
\hline 1 & -4.345746 & 6.928510 & 1.267887 \\
\hline 1 & -4.285355 & 5.323271 & 2.024992 \\
\hline 1 & -2.966868 & 6.480630 & 2.282721 \\
\hline 1 & -2.779824 & 7.500380 & -0.644562 \\
\hline 1 & -1.373583 & 7.043345 & 0.331023 \\
\hline 1 & -1.630976 & 6.289635 & -1.252943 \\
\hline 1 & -8.454870 & -0.661674 & 1.911134 \\
\hline 1 & -8.048570 & -1.161228 & 0.259914 \\
\hline 1 & -6.956053 & -1.562393 & 1.598853 \\
\hline 1 & -7.219090 & 1.137602 & 3.055268 \\
\hline 1 & -5.696958 & 0.262197 & 2.836693 \\
\hline 1 & -5.837725 & 1.955808 & 2.308813 \\
\hline 1 & -8.657213 & 1.765636 & 1.198351 \\
\hline
\end{tabular}




\begin{tabular}{|c|c|c|c|}
\hline 1 & -7.328505 & 2.555310 & 0.323506 \\
\hline & -8.302482 & 1.307155 & -0.472944 \\
\hline & -7.003555 & -1.012364 & -5.042083 \\
\hline & -7.313706 & -1.529648 & -3.378987 \\
\hline & -7.364462 & 0.198275 & -3.804318 \\
\hline & -4.879855 & -2.242689 & -5.102903 \\
\hline & -3.609333 & -1.974082 & -3.896387 \\
\hline & -5.110751 & -2.785550 & -3.425166 \\
\hline & -4.975468 & 0.209814 & -5.681328 \\
\hline 1 & -5.210958 & 1.426544 & -4.408334 \\
\hline 1 & -3.678050 & 0.543025 & -4.521116 \\
\hline
\end{tabular}

$2_{\text {C1-t- }-\psi_{-122 a 3 s 4 s}}$

Number of imaginary frequencies : 0

The smallest frequencies are : $11.8904 \quad 15.2979 \quad 18.1723 \mathrm{~cm}(-1)$

Electronic energy : $\quad H F=-4012.1877728$

Zero-point correction $=\quad 1.290964$ (Hartree/Particle)

Thermal correction to Energy= $\quad 1.367031$

Thermal correction to Enthalpy= $\quad 1.367975$

Thermal correction to Gibbs Free Energy $=\quad 1.179740$

Sum of electronic and zero-point Energies $=\quad-4010.896809$

Sum of electronic and thermal Energies $=\quad-4010.820742$

Sum of electronic and thermal Enthalpies $=\quad-4010.819798$

Sum of electronic and thermal Free Energies $=\quad-4011.008032$

Cartesian Coordinates

$\begin{array}{crrr}6 & -2.254412 & 3.109619 & 0.172221 \\ 6 & -1.418862 & 2.065218 & 0.573365 \\ 6 & -0.383305 & 2.309241 & 1.472618 \\ 6 & -0.205053 & 3.576056 & 2.040415 \\ 6 & -1.054919 & 4.598657 & 1.618885 \\ 6 & -2.076029 & 4.395140 & 0.679296 \\ 15 & -1.625177 & 0.369594 & -0.092292 \\ 6 & -1.649500 & -0.639222 & 1.471460 \\ 6 & -1.441522 & -2.008173 & 1.431110 \\ 6 & -1.505125 & -2.785134 & 2.634744 \\ 6 & -1.800902 & -2.142962 & 3.871517 \\ 6 & -2.040909 & -0.750031 & 3.874098 \\ 6 & -1.967147 & -0.025517 & 2.715245 \\ 6 & -1.848912 & -2.905766 & 5.062807 \\ 6 & -1.613674 & -4.256574 & 5.041384 \\ 6 & -1.322572 & -4.899921 & 3.820187 \\ 6 & -1.272992 & -4.185941 & 2.648344 \\ 6 & -1.191225 & -2.746082 & 0.164491 \\ 6 & 0.087570 & -2.858517 & -0.347817 \\ 6 & 0.343352 & -3.676838 & -1.474184 \\ 6 & -0.671373 & -4.367159 & -2.081462 \\ 6 & -1.999401 & -4.258667 & -1.605809 \\ 6 & -2.268971 & -3.431246 & -0.475583 \\ 6 & -3.610214 & -3.312199 & -0.027516 \\ 6 & -4.622533 & -3.998876 & -0.649828 \\ 6 & -4.347355 & -4.845289 & -1.746179 \\ 6 & -3.064950 & -4.962743 & -2.218242 \\ 7 & 1.166784 & -2.105364 & 0.235822\end{array}$




\begin{tabular}{|c|c|c|c|}
\hline & 1.617017 & -0.856045 & -0.810320 \\
\hline 8 & 2.833898 & -1.452453 & -1.805927 \\
\hline 6 & 4.104233 & -1.710527 & -1.348010 \\
\hline 6 & 4.943593 & -0.678196 & -0.964825 \\
\hline 6 & 6.224207 & -1.014301 & -0.420056 \\
\hline 6 & 6.643123 & -2.378699 & -0.394242 \\
\hline 6 & 5.775062 & -3.381921 & -0.891025 \\
\hline 6 & 4.524909 & -3.057237 & -1.343304 \\
\hline 6 & 7.083219 & -0.042760 & 0.154178 \\
\hline 6 & 8.295695 & -0.397555 & 0.692609 \\
\hline 6 & 8.718769 & -1.743418 & 0.688852 \\
\hline 6 & 7.903860 & -2.710773 & 0.158413 \\
\hline 6 & 4.459729 & 0.716277 & -1.080859 \\
\hline 6 & 3.254652 & 1.047666 & -0.494152 \\
\hline 6 & 2.687170 & 2.333372 & -0.585768 \\
\hline 6 & 3.351064 & 3.310798 & -1.276671 \\
\hline 6 & 4.574527 & 3.029645 & -1.931477 \\
\hline 6 & 5.132842 & 1.717477 & -1.853563 \\
\hline 6 & 5.238734 & 4.024132 & -2.689683 \\
\hline 6 & 6.394497 & 3.739398 & -3.370203 \\
\hline 6 & 6.927945 & 2.434501 & -3.326895 \\
\hline 6 & 6.314457 & 1.451245 & -2.590735 \\
\hline 8 & 2.551463 & 0.070016 & 0.191255 \\
\hline 6 & 0.819750 & 3.753381 & 3.161255 \\
\hline 6 & 0.957565 & 5.209036 & 3.600022 \\
\hline 6 & -2.971227 & 5.564538 & 0.272995 \\
\hline 6 & -4.027998 & 5.153880 & -0.749719 \\
\hline 6 & -3.387410 & 0.235673 & -0.585320 \\
\hline 6 & -3.700926 & -0.147815 & -1.885254 \\
\hline 6 & -5.029808 & -0.293602 & -2.293946 \\
\hline 6 & -6.032881 & -0.068542 & -1.348764 \\
\hline 6 & -5.750083 & 0.306101 & -0.029279 \\
\hline 6 & -4.413130 & 0.458785 & 0.333881 \\
\hline 6 & -6.832641 & 0.549749 & 1.020773 \\
\hline 6 & -8.239534 & 0.314223 & 0.477330 \\
\hline 6 & -5.318583 & -0.669955 & -3.745519 \\
\hline 6 & -6.813221 & -0.794328 & -4.029117 \\
\hline 45 & -0.055277 & 0.267005 & -1.889207 \\
\hline 6 & 0.963209 & 0.728448 & -3.384377 \\
\hline 8 & 1.601372 & 1.081810 & -4.281323 \\
\hline 6 & -6.614065 & -0.405133 & 2.200665 \\
\hline 6 & -6.747807 & 2.000666 & 1.508946 \\
\hline 6 & -4.658084 & -2.014122 & -4.067682 \\
\hline 6 & -4.743495 & 0.413659 & -4.665738 \\
\hline 6 & 2.013420 & -2.837521 & 1.202021 \\
\hline 6 & 2.699056 & -1.946117 & 2.199112 \\
\hline 6 & 1.952998 & -1.117110 & 3.040177 \\
\hline 6 & 2.593384 & -0.267140 & 3.932953 \\
\hline 6 & 3.986346 & -0.231549 & 3.993189 \\
\hline 6 & 4.733259 & -1.066227 & 3.169764 \\
\hline 6 & 4.088443 & -1.923420 & 2.282127 \\
\hline 6 & 0.338379 & 2.932056 & 4.365466 \\
\hline 6 & 2.202300 & 3.255114 & 2.730169 \\
\hline 6 & -2.113360 & 6.673515 & -0.345018 \\
\hline 6 & -3.694267 & 6.107761 & 1.510640 \\
\hline 1 & 6.762530 & 0.995647 & 0.179740 \\
\hline
\end{tabular}




\begin{tabular}{|c|c|c|c|}
\hline & -2.150672 & 1.045456 & 2.754525 \\
\hline & 8.207037 & -3.757042 & 0.161689 \\
\hline & -2.074650 & -2.393402 & 5.996974 \\
\hline & 4.800027 & 5.020231 & -2.730206 \\
\hline & -3.832782 & -2.658086 & 0.814668 \\
\hline & -5.645781 & -3.886819 & -0.297420 \\
\hline & 2.930269 & 4.311944 & -1.354987 \\
\hline & -2.283897 & -0.257627 & 4.815107 \\
\hline & 6.111634 & -4.417397 & -0.887820 \\
\hline & 7.830446 & 2.202102 & -3.887848 \\
\hline & 3.834183 & -3.814787 & -1.708747 \\
\hline & -2.841864 & -5.598310 & -3.074117 \\
\hline & -0.471687 & -5.000505 & -2.944355 \\
\hline & -1.138179 & -5.971636 & 3.806732 \\
\hline & 6.731598 & 0.447622 & -2.578562 \\
\hline & -1.051809 & -4.698400 & 1.714384 \\
\hline & 1.362658 & -3.735423 & -1.852167 \\
\hline & 6.892232 & 4.510145 & -3.954119 \\
\hline & 8.932631 & 0.366231 & 1.133204 \\
\hline & -5.157989 & -5.391542 & -2.223662 \\
\hline & -1.650317 & -4.834423 & 5.961904 \\
\hline & 9.682792 & -2.010543 & 1.115433 \\
\hline 1 & 1.726406 & 2.515873 & -0.108317 \\
\hline & -4.163944 & 0.755879 & 1.352096 \\
\hline & -2.882851 & -0.324079 & -2.583128 \\
\hline 1 & -7.070705 & -0.184415 & -1.647616 \\
\hline & -3.047733 & 2.901852 & -0.542020 \\
\hline 1 & 0.266674 & 1.484470 & 1.774294 \\
\hline & -0.932837 & 5.594179 & 2.040716 \\
\hline | & 1.331625 & -3.522257 & 1.726995 \\
\hline 1 & -1.214245 & 1.183150 & -2.595784 \\
\hline & 2.755844 & -3.462977 & 0.680417 \\
\hline 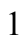 & 4.674398 & -2.565894 & 1.623907 \\
\hline & 0.864072 & -1.124798 & 2.970281 \\
\hline 1 & 5.821249 & -1.048665 & 3.206122 \\
\hline & 2.005432 & 0.378691 & 4.584065 \\
\hline 1 & 4.484463 & 0.445872 & 4.683942 \\
\hline I & 1.717549 & 5.285268 & 4.387834 \\
\hline & 1.273858 & 5.856522 & 2.771929 \\
\hline 1 & 0.021593 & 5.608252 & 4.011356 \\
\hline & 2.908310 & 3.339656 & 3.568150 \\
\hline 1 & 2.200248 & 2.202702 & 2.418804 \\
\hline 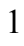 & 2.597367 & 3.852305 & 1.897961 \\
\hline 1 & 1.040319 & 3.032238 & 5.205246 \\
\hline 1 & -0.648387 & 3.275532 & 4.705229 \\
\hline 1 & 0.253174 & 1.864718 & 4.121116 \\
\hline 1 & -4.648377 & 6.021837 & -1.006304 \\
\hline 1 & -3.578090 & 4.783382 & -1.679516 \\
\hline 1 & -4.696680 & 4.374932 & -0.359138 \\
\hline 1 & -2.745182 & 7.519487 & -0.647508 \\
\hline 1 & -1.363176 & 7.055411 & 0.357844 \\
\hline 1 & -1.584653 & 6.310007 & -1.235437 \\
\hline 1 & -4.347788 & 6.945472 & 1.232390 \\
\hline 1 & -4.318875 & 5.331626 & 1.972590 \\
\hline 1 & -2.995388 & 6.473282 & 2.272665 \\
\hline & -7.492809 & 2.185043 & 2.294781 \\
\hline
\end{tabular}




$\begin{array}{lrrr}1 & -5.760098 & 2.243335 & 1.921288 \\ 1 & -6.946293 & 2.699827 & 0.686008 \\ 1 & -8.975880 & 0.497466 & 1.270078 \\ 1 & -8.477958 & 0.990084 & -0.353887 \\ 1 & -8.378596 & -0.718436 & 0.131897 \\ 1 & -7.377615 & -0.240797 & 2.972970 \\ 1 & -6.685388 & -1.451180 & 1.873556 \\ 1 & -5.631539 & -0.271330 & 2.671013 \\ 1 & -6.966294 & -1.066827 & -5.080980 \\ 1 & -7.283950 & -1.574311 & -3.416228 \\ 1 & -7.345851 & 0.149691 & -3.854984 \\ 1 & -4.942698 & 0.164689 & -5.716887 \\ 1 & -5.199658 & 1.390097 & -4.456469 \\ 1 & -3.657828 & 0.520073 & -4.548847 \\ 1 & -4.843271 & -2.286553 & -5.115710 \\ 1 & -3.571404 & -1.989656 & -3.917617 \\ 1 & -5.062101 & -2.811254 & -3.430245\end{array}$

$2_{\mathrm{C} 1-\mathrm{t}-} \psi-1 \mathrm{~s} 2 \mathrm{~s} 3 \mathrm{a} 4 \mathrm{a}$

Number of imaginary frequencies : 0

The smallest frequencies are : $14.9770 \quad 18.1859 \quad 21.3289 \mathrm{~cm}(-1)$

Electronic energy : $\quad H F=-4012.1884375$

Zero-point correction $=\quad 1.291526$ (Hartree/Particle)

Thermal correction to Energy= $\quad 1.367266$

Thermal correction to Enthalpy= $\quad 1.368210$

Thermal correction to Gibbs Free Energy= $\quad 1.182203$

Sum of electronic and zero-point Energies $=\quad-4010.896912$

Sum of electronic and thermal Energies $=\quad-4010.821171$

Sum of electronic and thermal Enthalpies $=\quad-4010.820227$

Sum of electronic and thermal Free Energies $=\quad-4011.006235$

Cartesian Coordinates

$\begin{array}{cccc}6 & -0.312704 & 2.309658 & 1.482552 \\ 6 & -1.347330 & 2.080954 & 0.572354 \\ 6 & -2.133563 & 3.144413 & 0.141020 \\ 6 & -1.925110 & 4.436349 & 0.630860 \\ 6 & -0.916584 & 4.625242 & 1.580105 \\ 6 & -0.102651 & 3.577629 & 2.027491 \\ 15 & -1.608605 & 0.388180 & -0.078852 \\ 6 & -3.386183 & 0.284537 & -0.523442 \\ 6 & -4.382657 & 0.591833 & 0.407044 \\ 6 & -5.728868 & 0.433687 & 0.086386 \\ 6 & -6.043509 & -0.027091 & -1.198576 \\ 6 & -5.073717 & -0.347769 & -2.149401 \\ 6 & -3.733018 & -0.191274 & -1.784587 \\ 6 & -6.846828 & 0.701417 & 1.092207 \\ 6 & -7.826592 & 1.735712 & 0.529292 \\ 6 & -5.480568 & -0.806255 & -3.549108 \\ 6 & -6.556874 & -1.893199 & -3.473404 \\ 6 & -2.824417 & 5.567480 & 0.135577 \\ 6 & -4.285197 & 5.228893 & 0.457544 \\ 6 & 0.920087 & 3.744775 & 3.152546 \\ 6 & 2.293459 & 3.214220 & 2.730653 \\ 6 & -1.617294 & -0.621148 & 1.485809\end{array}$




\begin{tabular}{|c|c|c|c|}
\hline & -1.427932 & -1.993257 & 1.440430 \\
\hline & -1.488209 & -2.771703 & 2.642309 \\
\hline 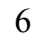 & -1.759979 & -2.128350 & 3.884037 \\
\hline & -1.981082 & -0.732142 & 3.892515 \\
\hline & -1.912887 & -0.006002 & 2.734197 \\
\hline & -1.278532 & -4.175828 & 2.648143 \\
\hline & -1.325375 & -4.892904 & 3.818214 \\
\hline & -1.591478 & -4.248676 & 5.044719 \\
\hline & -1.805387 & -2.894341 & 5.073335 \\
\hline & -1.210928 & -2.721530 & 0.162132 \\
\hline & 0.052220 & -2.826644 & -0.389681 \\
\hline & 0.265659 & -3.595350 & -1.559244 \\
\hline & -0.777698 & -4.227775 & -2.180719 \\
\hline & -2.091019 & -4.120665 & -1.666370 \\
\hline & -2.314473 & -3.365670 & -0.476703 \\
\hline ) & -3.188468 & -4.750022 & -2.303852 \\
\hline & -4.457739 & -4.631447 & -1.796816 \\
\hline & -4.684209 & -3.871278 & -0.628092 \\
\hline & -3.640070 & -3.259188 & 0.018631 \\
\hline 7 & 1.154290 & -2.101589 & 0.185574 \\
\hline & 1.610050 & -0.844590 & -0.849370 \\
\hline 8 & 2.822244 & -1.435286 & -1.853818 \\
\hline 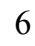 & 4.084179 & -1.727109 & -1.392068 \\
\hline 6 & 4.940664 & -0.719653 & -0.981107 \\
\hline 6 & 6.208224 & -1.092639 & -0.429760 \\
\hline ) & 6.599916 & -2.465292 & -0.428168 \\
\hline 6 & 5.717666 & -3.440476 & -0.954815 \\
\hline 6 & 4.478545 & -3.081704 & -1.411705 \\
\hline 6 & 7.079024 & -0.150679 & 0.175143 \\
\hline 5 & 8.277884 & -0.540636 & 0.719687 \\
\hline 6 & 8.674643 & -1.894217 & 0.691866 \\
\hline 6 & 7.847332 & -2.833832 & 0.131608 \\
\hline 6 & 4.487997 & 0.686392 & -1.079552 \\
\hline 6 & 3.279985 & 1.033148 & -0.507674 \\
\hline 6 & 2.736574 & 2.329404 & -0.594555 \\
\hline 6 & 3.431574 & 3.303835 & -1.258538 \\
\hline 6 & 4.663257 & 3.009870 & -1.891895 \\
\hline 6 & 5.194047 & 1.685634 & -1.825222 \\
\hline 6 & 5.361367 & 4.003456 & -2.620244 \\
\hline 6 & 6.523695 & 3.706996 & -3.284313 \\
\hline 6 & 7.029338 & 2.390685 & -3.254570 \\
\hline 6 & 6.382847 & 1.407869 & -2.546586 \\
\hline 8 & 2.551778 & 0.063090 & 0.161557 \\
\hline 6 & 1.992456 & -2.848249 & 1.147120 \\
\hline 6 & 2.682806 & -1.974392 & 2.156839 \\
\hline 6 & 4.071199 & -1.979508 & 2.257643 \\
\hline 6 & 4.720892 & -1.146608 & 3.164867 \\
\hline 6 & 3.980031 & -0.308067 & 3.989674 \\
\hline 6 & 2.587720 & -0.313912 & 3.909363 \\
\hline 6 & 1.942721 & -1.139288 & 2.997196 \\
\hline 45 & -0.082816 & 0.264729 & -1.912107 \\
\hline 6 & 0.882253 & 0.709633 & -3.445514 \\
\hline 8 & 1.474884 & 1.051861 & -4.377442 \\
\hline 6 & -7.589805 & -0.612294 & 1.362737 \\
\hline 6 & -6.312725 & 1.227530 & 2.422950 \\
\hline 6 & -4.296303 & -1.372928 & -4.331447 \\
\hline
\end{tabular}




\begin{tabular}{|c|c|c|c|}
\hline 6 & -6.035523 & 0.402287 & -4.311789 \\
\hline 6 & -2.492662 & 6.907213 & 0.787621 \\
\hline 6 & -2.659296 & 5.719179 & -1.380697 \\
\hline 6 & 1.087439 & 5.201117 & 3.578919 \\
\hline 6 & 0.418301 & 2.945743 & 4.363357 \\
\hline 1 & 6.777624 & 0.892820 & 0.220409 \\
\hline 1 & -2.083295 & 1.067103 & 2.777849 \\
\hline 1 & 8.129773 & -3.885791 & 0.116441 \\
\hline 1 & -2.012137 & -2.381937 & 6.011863 \\
\hline 1 & 4.943348 & 5.008757 & -2.651463 \\
\hline 1 & -3.826117 & -2.669342 & 0.914618 \\
\hline 1 & -5.696077 & -3.762474 & -0.242139 \\
\hline 1 & 3.030581 & 4.313553 & -1.330839 \\
\hline 1 & -2.206547 & -0.238743 & 4.837370 \\
\hline 1 & 6.033715 & -4.482295 & -0.970174 \\
\hline 1 & 7.936778 & 2.149198 & -3.803605 \\
\hline 1 & 3.776844 & -3.817842 & -1.799209 \\
\hline 1 & -3.001072 & -5.327552 & -3.208059 \\
\hline 1 & -0.612293 & -4.811267 & -3.084937 \\
\hline 1 & -1.158243 & -5.967367 & 3.799511 \\
\hline 1 & 6.779498 & 0.395931 & -2.544888 \\
\hline 1 & -1.077478 & -4.687567 & 1.709240 \\
\hline 1 & 1.274602 & -3.653670 & -1.964157 \\
\hline 1 & 7.047740 & 4.477329 & -3.845327 \\
\hline 1 & 8.924082 & 0.200918 & 1.184138 \\
\hline 1 & -5.293305 & -5.119162 & -2.294769 \\
\hline 1 & -1.626133 & -4.828792 & 5.963893 \\
\hline 1 & 9.628108 & -2.189346 & 1.123695 \\
\hline 1 & 1.770667 & 2.523065 & -0.131574 \\
\hline 1 & -4.095984 & 0.954482 & 1.391095 \\
\hline 1 & -2.930654 & -0.436828 & -2.476955 \\
\hline 1 & -7.094430 & -0.145271 & -1.462442 \\
\hline 1 & -2.923426 & 2.957488 & -0.585765 \\
\hline 1 & 0.310006 & 1.471429 & 1.803677 \\
\hline 1 & -0.764324 & 5.618286 & 1.992231 \\
\hline 1 & 1.304761 & -3.533256 & 1.663915 \\
\hline 1 & -1.269871 & 1.148584 & -2.611454 \\
\hline 1 & -5.632855 & 0.512755 & 2.906201 \\
\hline 1 & -5.779995 & 2.180177 & 2.302384 \\
\hline 1 & -7.148598 & 1.402688 & 3.111886 \\
\hline 1 & -8.292753 & 1.401643 & -0.405255 \\
\hline 1 & -8.633532 & 1.926212 & 1.249557 \\
\hline 1 & -7.321559 & 2.689455 & 0.328723 \\
\hline 1 & -8.045408 & -1.021106 & 0.452438 \\
\hline 1 & -6.905631 & -1.371650 & 1.765037 \\
\hline 1 & -8.392518 & -0.455780 & 2.096029 \\
\hline 1 & -5.279088 & 1.193302 & -4.396637 \\
\hline 1 & -6.336896 & 0.110787 & -5.327306 \\
\hline 1 & -6.912877 & 0.829774 & -3.809585 \\
\hline 1 & -7.482764 & -1.540421 & -3.003728 \\
\hline 1 & -6.817555 & -2.233193 & -4.484414 \\
\hline 1 & -6.196545 & -2.761711 & -2.906252 \\
\hline 1 & -3.516532 & -0.621892 & -4.511708 \\
\hline 1 & -3.837525 & -2.224494 & -3.809301 \\
\hline 1 & -4.639449 & -1.726081 & -5.312299 \\
\hline 1 & 2.731204 & -3.474596 & 0.621440 \\
\hline
\end{tabular}




$\begin{array}{rrrr}1 & 4.653522 & -2.625716 & 1.599859 \\ 1 & 0.855462 & -1.121145 & 2.910402 \\ 1 & 5.808352 & -1.151713 & 3.216140 \\ 1 & 2.003671 & 0.335921 & 4.560118 \\ 1 & 4.482284 & 0.349729 & 4.696219 \\ 1 & 1.843946 & 5.267194 & 4.371026 \\ 1 & 1.423960 & 5.834093 & 2.747617 \\ 1 & 0.158071 & 5.625100 & 3.980293 \\ 1 & 2.996873 & 3.283974 & 3.572240 \\ 1 & 2.268119 & 2.162038 & 2.419967 \\ 1 & 2.706423 & 3.800516 & 1.899407 \\ 1 & 1.119927 & 3.039174 & 5.204174 \\ 1 & -0.561847 & 3.313302 & 4.697012 \\ 1 & 0.310996 & 1.878062 & 4.129616 \\ 1 & -3.163340 & 7.682296 & 0.395833 \\ 1 & -2.624728 & 6.879484 & 1.877126 \\ 1 & -1.464191 & 7.224806 & 0.572961 \\ 1 & -4.948611 & 6.031501 & 0.108226 \\ 1 & -4.609122 & 4.298376 & -0.025513 \\ 1 & -4.433032 & 5.114831 & 1.539795 \\ 1 & -3.304165 & 6.525606 & -1.755363 \\ 1 & -1.621792 & 5.966497 & -1.639614 \\ 1 & -2.926211 & 4.802115 & -1.919060\end{array}$

$2_{\mathrm{C} 1-\mathrm{t}-} \boldsymbol{\psi}_{-1 \mathrm{~s} 2 \mathrm{~s} 3 \mathrm{a} 4 \mathrm{~s}}$

Number of imaginary frequencies : 0

The smallest frequencies are : $14.0298 \quad 19.3647 \quad 20.5392 \mathrm{~cm}(-1)$

Electronic energy : $\quad H F=-4012.1885147$

Zero-point correction $=\quad 1.291518($ Hartree/Particle)

Thermal correction to Energy= $\quad 1.367335$

Thermal correction to Enthalpy= $\quad 1.368279$

Thermal correction to Gibbs Free Energy $=\quad 1.181550$

Sum of electronic and zero-point Energies $=\quad-4010.896997$

Sum of electronic and thermal Energies $=\quad-4010.821180$

Sum of electronic and thermal Enthalpies $=\quad-4010.820236$

Sum of electronic and thermal Free Energies $=\quad-4011.006965$

\section{Cartesian Coordinates}

$\begin{array}{lrrr}6 & 5.358392 & 4.018100 & -2.610943 \\ 6 & 4.660798 & 3.022141 & -1.885341 \\ 6 & 5.195342 & 1.699466 & -1.817317 \\ 6 & 6.387334 & 1.425528 & -2.534880 \\ 6 & 7.033253 & 2.410547 & -3.240318 \\ 6 & 6.523818 & 3.725375 & -3.271214 \\ 6 & 4.489998 & 0.697921 & -1.074086 \\ 6 & 3.279136 & 1.040952 & -0.505942 \\ 6 & 2.731987 & 2.335451 & -0.594597 \\ 6 & 3.426065 & 3.312162 & -1.256149 \\ 6 & 4.946524 & -0.706768 & -0.973985 \\ 6 & 4.093916 & -1.716790 & -1.386778 \\ 6 & 4.491477 & -3.070442 & -1.403510 \\ 6 & 5.730087 & -3.425667 & -0.942448 \\ 6 & 6.608445 & -2.447723 & -0.414438 \\ 6 & 6.213439 & -1.076021 & -0.418647\end{array}$




\begin{tabular}{|c|c|c|c|}
\hline 6 & 7.855131 & -2.812646 & 0.149342 \\
\hline & 8.678572 & -1.870437 & 0.710937 \\
\hline 6 & 8.278484 & -0.517779 & 0.736111 \\
\hline 6 & 7.080241 & -0.131316 & 0.187734 \\
\hline 8 & 2.832767 & -1.428390 & -1.852931 \\
\hline 1 & 1.615888 & -0.842254 & -0.851712 \\
\hline 8 & 2.551887 & 0.068948 & 0.161406 \\
\hline 7 & 1.161254 & -2.101211 & 0.181141 \\
\hline 6 & 0.059712 & -2.826883 & -0.394384 \\
\hline 6 & -1.202448 & -2.727182 & 0.160903 \\
\hline 6 & -2.307109 & -3.368863 & -0.477882 \\
\hline 6 & -2.085779 & -4.116263 & -1.672715 \\
\hline 6 & -0.773750 & -4.218078 & -2.191403 \\
\hline 6 & 0.270824 & -3.588237 & -1.569118 \\
\hline 6 & -3.184109 & -4.743113 & -2.311085 \\
\hline 6 & -4.451776 & -4.630129 & -1.798965 \\
\hline 6 & -4.676068 & -3.876790 & -0.625364 \\
\hline 6 & -3.631590 & -3.265801 & 0.021802 \\
\hline 6 & -1.418953 & -2.001360 & 1.440078 \\
\hline 6 & -1.612242 & -0.629868 & 1.483660 \\
\hline 6 & -1.907078 & -0.014330 & 2.731817 \\
\hline 6 & -1.971708 & -0.739890 & 3.891002 \\
\hline 6 & -1.748350 & -2.135655 & 3.883516 \\
\hline 6 & -1.476664 & -2.779294 & 2.641999 \\
\hline 6 & -1.265091 & -4.183110 & 2.648040 \\
\hline 6 & -1.309778 & -4.899634 & 3.818483 \\
\hline 6 & -1.575444 & -4.255087 & 5.044994 \\
\hline 6 & -1.791300 & -2.901082 & 5.073297 \\
\hline 15 & -1.608646 & 0.374643 & -0.084471 \\
\hline 6 & -1.372119 & 2.071844 & 0.564675 \\
\hline 6 & -0.336048 & 2.314879 & 1.469545 \\
\hline 6 & -0.143024 & 3.584700 & 2.016244 \\
\hline 6 & -0.974399 & 4.620867 & 1.573815 \\
\hline 6 & -1.984861 & 4.418200 & 0.629504 \\
\hline 6 & -2.177982 & 3.123365 & 0.140611 \\
\hline 6 & 0.880014 & 3.764664 & 3.139115 \\
\hline 6 & 2.258376 & 3.247294 & 2.717056 \\
\hline 6 & -2.898460 & 5.537792 & 0.133848 \\
\hline 6 & -4.356023 & 5.179488 & 0.448698 \\
\hline 6 & 1.996847 & -2.843071 & 1.148564 \\
\hline 6 & 2.683449 & -1.964295 & 2.156734 \\
\hline 6 & 1.940746 & -1.124673 & 2.990286 \\
\hline 6 & 2.582814 & -0.293765 & 3.899494 \\
\hline 6 & 3.974887 & -0.287213 & 3.984035 \\
\hline 6 & 4.718328 & -1.130450 & 3.166351 \\
\hline 6 & 4.071523 & -1.968492 & 2.261802 \\
\hline 45 & -0.079314 & 0.260986 & -1.916539 \\
\hline 6 & 0.886115 & 0.711512 & -3.447895 \\
\hline 8 & 1.478996 & 1.057431 & -4.378291 \\
\hline 6 & -3.383809 & 0.252886 & -0.535105 \\
\hline 6 & -4.382883 & 0.547069 & 0.388344 \\
\hline 6 & -5.733059 & 0.396550 & 0.060372 \\
\hline 6 & -6.044276 & -0.046168 & -1.226253 \\
\hline 6 & -5.063401 & -0.361901 & -2.175488 \\
\hline 6 & -3.727755 & -0.212311 & -1.805210 \\
\hline 6 & -6.789656 & 0.679099 & 1.126319 \\
\hline
\end{tabular}




\begin{tabular}{|c|c|c|c|}
\hline & -6.652612 & 2.120998 & 1.626766 \\
\hline & -5.466815 & -0.809376 & -3.579626 \\
\hline & -6.033328 & 0.400939 & -4.330844 \\
\hline & -8.211649 & 0.491172 & 0.604339 \\
\hline & -6.577333 & -0.292604 & 2.294109 \\
\hline & -4.278417 & -1.358051 & -4.368367 \\
\hline & -6.532793 & -1.907249 & -3.513347 \\
\hline & -2.587236 & 6.880795 & 0.789209 \\
\hline & -2.728755 & 5.693648 & -1.381618 \\
\hline & 1.032816 & 5.223542 & 3.562307 \\
\hline & 0.388058 & 2.962762 & 4.352109 \\
\hline & 6.776215 & 0.911497 & 0.231034 \\
\hline & -2.079624 & 1.058391 & 2.774790 \\
\hline & 8.140158 & -3.863932 & 0.136120 \\
\hline & -1.997970 & -2.388602 & 6.011792 \\
\hline & 4.937494 & 5.022170 & -2.643133 \\
\hline & -3.817426 & -2.677968 & 0.919686 \\
\hline & -5.687266 & -3.772396 & -0.236602 \\
\hline & 3.022172 & 4.320646 & -1.329663 \\
\hline & -2.196305 & -0.246083 & 4.835859 \\
\hline & 6.048696 & -4.466736 & -0.955552 \\
\hline & 7.943246 & 2.171992 & -3.786402 \\
\hline & 3.792713 & -3.808747 & -1.792171 \\
\hline & -2.998637 & -5.314752 & -3.219423 \\
\hline 1 & -0.610163 & -4.795571 & -3.099793 \\
\hline & -1.141389 & -5.973908 & 3.800193 \\
\hline & 6.787016 & 0.414784 & -2.532212 \\
\hline & -1.064554 & -4.694809 & 1.708993 \\
\hline & 1.278502 & -3.642686 & -1.977674 \\
\hline 1 & 7.047478 & 4.497514 & -3.830101 \\
\hline & 8.921557 & 0.225840 & 1.201600 \\
\hline ] & -5.288030 & -5.116802 & -2.296785 \\
\hline & -1.608364 & -4.834858 & 5.964447 \\
\hline & 9.631498 & -2.162786 & 1.145841 \\
\hline ] & 1.763851 & 2.525869 & -0.135026 \\
\hline & -4.107531 & 0.893132 & 1.384342 \\
\hline 1 & -2.922272 & -0.451200 & -2.496216 \\
\hline & -7.090143 & -0.154908 & -1.505106 \\
\hline 1 & -2.969737 & 2.925480 & -0.581149 \\
\hline ] & 0.300133 & 1.485180 & 1.786600 \\
\hline l & -0.834316 & 5.615730 & 1.985919 \\
\hline 1 & 1.308324 & -3.526816 & 1.665896 \\
\hline & -1.268536 & 1.139566 & -2.619468 \\
\hline 1 & 2.737944 & -3.470606 & 0.627630 \\
\hline & 4.655986 & -2.617891 & 1.609059 \\
\hline 1 & 0.853869 & -1.106346 & 2.899510 \\
\hline l & 5.805633 & -1.135090 & 3.220964 \\
\hline ] & 1.996600 & 0.359859 & 4.544542 \\
\hline 1 & 4.474916 & 0.374803 & 4.688193 \\
\hline 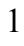 & 1.790496 & 5.299343 & 4.352426 \\
\hline 1 & 1.360282 & 5.858409 & 2.728837 \\
\hline ( & 0.100007 & 5.638319 & 3.965252 \\
\hline 1 & 2.961958 & 3.327757 & 3.557526 \\
\hline ] & 2.244384 & 2.193752 & 2.410005 \\
\hline 1 & 2.664059 & 3.835217 & 1.883387 \\
\hline 1 & 1.089761 & 3.064930 & 5.191845 \\
\hline
\end{tabular}




$\begin{array}{rrrr}1 & -0.595426 & 3.320738 & 4.686409 \\ 1 & 0.291661 & 1.893604 & 4.120275 \\ 1 & -3.268866 & 7.646785 & 0.398425 \\ 1 & -2.720087 & 6.848505 & 1.878500 \\ 1 & -1.563391 & 7.214236 & 0.576451 \\ 1 & -3.381073 & 6.493352 & -1.757824 \\ 1 & -1.693010 & 5.952878 & -1.635931 \\ 1 & -2.983147 & 4.774236 & -1.922076 \\ 1 & -5.028763 & 5.969527 & 0.088584 \\ 1 & -4.662896 & 4.240042 & -0.028561 \\ 1 & -4.509442 & 5.072454 & 1.530866 \\ 1 & -7.393401 & 2.323645 & 2.411974 \\ 1 & -5.660164 & 2.322538 & 2.048424 \\ 1 & -6.821134 & 2.837132 & 0.811584 \\ 1 & -8.929631 & 0.709146 & 1.405034 \\ 1 & -8.434868 & 1.167649 & -0.230801 \\ 1 & -8.394919 & -0.538752 & 0.271867 \\ 1 & -7.324576 & -0.118842 & 3.080297 \\ 1 & -6.676003 & -1.333441 & 1.957043 \\ 1 & -5.583674 & -0.184398 & 2.747766 \\ 1 & -4.618585 & -1.700402 & -5.354093 \\ 1 & -3.503139 & -0.599817 & -4.537790 \\ 1 & -3.815022 & -2.213709 & -3.857015 \\ 1 & -6.333904 & 0.115466 & -5.348304 \\ 1 & -6.913720 & 0.816936 & -3.824468 \\ 1 & -5.283531 & 1.198799 & -4.410157 \\ 1 & -6.791479 & -2.240092 & -4.527283 \\ 1 & -6.162933 & -2.777532 & -2.955085 \\ 1 & -7.461141 & -1.568094 & -3.038631\end{array}$

$2_{\text {C1-t- }} \psi_{-1 \mathrm{~s} 2 s 3 s 4 a}$

Number of imaginary frequencies : 0

The smallest frequencies are : $10.9892 \quad 14.6862 \quad 17.9996 \mathrm{~cm}(-1)$

Electronic energy : $\quad H F=-4012.1879643$

Zero-point correction $=\quad 1.290999($ Hartree/Particle $)$

Thermal correction to Energy= $\quad 1.367035$

Thermal correction to Enthalpy= $\quad 1.367979$

Thermal correction to Gibbs Free Energy $=\quad 1.179971$

Sum of electronic and zero-point Energies $=\quad-4010.896965$

Sum of electronic and thermal Energies $=\quad-4010.820929$

Sum of electronic and thermal Enthalpies $=\quad-4010.819985$

Sum of electronic and thermal Free Energies $=\quad-4011.007993$

\section{Cartesian Coordinates}

\begin{tabular}{|c|c|c|c|}
\hline 6 & 5.318868 & 4.054088 & -2.611403 \\
\hline 6 & 4.632347 & 3.053319 & -1.881868 \\
\hline 6 & 5.175290 & 1.733854 & -1.818864 \\
\hline 6 & 6.364156 & 1.467817 & -2.544492 \\
\hline 6 & 6.999220 & 2.457318 & -3.253509 \\
\hline 6 & 6.481489 & 3.768974 & -3.279939 \\
\hline 6 & 4.480466 & 0.726963 & -1.072865 \\
\hline 6 & & & $-0.4 \mathrm{C} \quad \mathrm{x}$ \\
\hline 6 & 2.716303 & 2.3535 & 79061 \\
\hline 6 & 3.400488 & 3.335353 & -1.243389 \\
\hline
\end{tabular}




\begin{tabular}{|c|c|c|c|}
\hline 6 & 4.947747 & -0.674460 & -0.975753 \\
\hline 6 & 4.101179 & -1.691119 & -1.384673 \\
\hline 6 & 4.509847 & -3.041545 & -1.403888 \\
\hline 6 & 5.753708 & -3.386889 & -0.949709 \\
\hline 6 & 6.626948 & -2.402098 & -0.426038 \\
\hline 6 & 6.220509 & -1.033756 & -0.427038 \\
\hline 6 & 7.879847 & -2.757127 & 0.130187 \\
\hline 6 & 8.698608 & -1.808578 & 0.687912 \\
\hline 6 & 8.287261 & -0.459398 & 0.716772 \\
\hline 6 & 7.082771 & -0.082443 & 0.175461 \\
\hline 8 & 2.835667 & -1.412796 & -1.845038 \\
\hline 15 & 1.618591 & -0.832959 & -0.840037 \\
\hline 8 & 2.552292 & 0.083635 & 0.170419 \\
\hline 7 & 1.177387 & -2.093518 & 0.196679 \\
\hline 6 & 0.095227 & -2.842807 & -0.385875 \\
\hline 6 & -1.180935 & -2.735053 & 0.134328 \\
\hline 6 & -2.261746 & -3.413287 & -0.508107 \\
\hline 6 & -1.997568 & -4.232085 & -1.645924 \\
\hline 6 & -0.671681 & -4.338010 & -2.127866 \\
\hline 6 & 0.345474 & -3.651809 & -1.520122 \\
\hline 6 & -3.066623 & -4.928916 & -2.260712 \\
\hline 6 & -4.347909 & -4.809525 & -1.786208 \\
\hline 6 & -4.618387 & -3.968763 & -0.684333 \\
\hline 6 & -3.601794 & -3.292751 & -0.057332 \\
\hline 6 & -1.423180 & -2.009517 & 1.410154 \\
\hline 6 & -1.625706 & -0.640243 & 1.467422 \\
\hline 6 & -1.929545 & -0.037949 & 2.720306 \\
\hline 6 & -1.996472 & -0.774368 & 3.871955 \\
\hline 6 & -1.763589 & -2.168494 & 3.852049 \\
\hline 6 & -1.480809 & -2.798843 & 2.606138 \\
\hline 6 & -1.254672 & -4.200600 & 2.603060 \\
\hline 6 & -1.298539 & -4.926959 & 3.767561 \\
\hline 6 & -1.577368 & -4.295481 & 4.997752 \\
\hline 6 & -1.805975 & -2.943814 & 5.035356 \\
\hline 15 & -1.617987 & 0.381870 & -0.086742 \\
\hline 6 & -1.386618 & 2.071181 & \\
\hline 6 & -0.349944 & 2.307976 & 1.490261 \\
\hline 6 & -0.168125 & 3.569527 & 2.059297 \\
\hline 6 & -1.016333 & 4.603315 & 1.643732 \\
\hline 6 & -2.030681 & 4.406681 & 0.702648 \\
\hline 6 & -2.206719 & 3.120770 & 0.184478 \\
\hline 6 & 0.858275 & 3.741291 & 3.180240 \\
\hline 6 & 2.237562 & 3.235169 & 2.747737 \\
\hline 6 & -2.973829 & 5.519862 & 0.250297 \\
\hline 6 & -4.416705 & 5.124845 & 0.589422 \\
\hline 6 & 2.027625 & -2.829890 & 1.156162 \\
\hline 6 & 2.705345 & -1.947525 & 2.166852 \\
\hline 6 & 1.953841 & -1.124863 & 3.009325 \\
\hline 6 & 2.587888 & -0.290764 & 3.921352 \\
\hline 6 & 3.980094 & -0.263798 & 3.999319 \\
\hline 6 & 4.732320 & -1.090437 & 3.172722 \\
\hline 6 & 4.093805 & -1.932111 & 2.265822 \\
\hline 45 & -0.073799 & 0.276944 & -1.905229 \\
\hline 6 & 0.899178 & 0.724276 & -3.433760 \\
\hline 8 & 1.493810 & 1.066825 & -4.364129 \\
\hline 6 & -3.392291 & 0.268780 & -0.544856 \\
\hline
\end{tabular}




\begin{tabular}{|c|c|c|c|}
\hline 6 & -4.400682 & 0.494938 & 0.402110 \\
\hline 6 & -5.740008 & 0.337802 & 0.066360 \\
\hline 6 & -6.048010 & -0.037886 & -1.252850 \\
\hline 6 & -5.071692 & -0.254300 & -2.219843 \\
\hline 6 & -3.731062 & -0.109607 & -1.835620 \\
\hline 6 & -6.865926 & 0.530640 & 1.080872 \\
\hline 6 & -7.579095 & -0.810035 & 1.290744 \\
\hline 6 & -5.388967 & -0.614262 & -3.669079 \\
\hline 6 & -4.739390 & -1.956067 & -4.020320 \\
\hline 6 & -6.346772 & 1.011218 & 2.434773 \\
\hline 6 & -7.870504 & 1.565872 & 0.564208 \\
\hline 6 & -6.889203 & -0.730094 & -3.924339 \\
\hline 6 & -4.827774 & 0.478849 & -4.586766 \\
\hline 6 & -2.674295 & 6.853381 & 0.930010 \\
\hline 6 & -2.841410 & 5.714240 & -1.264188 \\
\hline 6 & 1.006418 & 5.195946 & 3.619598 \\
\hline 6 & 0.373006 & 2.923293 & 4.385085 \\
\hline 1 & 6.770446 & 0.957794 & 0.221333 \\
\hline 1 & -2.108540 & 1.033367 & 2.772318 \\
\hline 1 & 8.173563 & -3.805977 & 0.114280 \\
\hline 1 & -2.021828 & -2.440352 & 5.976674 \\
\hline 1 & 4.891677 & 5.055607 & -2.639929 \\
\hline 1 & -3.820148 & -2.644017 & 0.789697 \\
\hline 1 & -5.641029 & -3.850666 & -0.332082 \\
\hline 1 & 2.990683 & 4.341767 & -1.312083 \\
\hline 1 & -2.228963 & -0.290996 & 4.820265 \\
\hline 1 & 6.080641 & -4.425343 & -0.964963 \\
\hline 1 & 7.906885 & 2.224732 & -3.806000 \\
\hline 1 & 3.814981 & -3.785802 & -1.788111 \\
\hline 1 & -2.847182 & -5.559335 & -3.121325 \\
\hline 1 & -0.475959 & -4.964504 & -2.996640 \\
\hline 1 & -1.118910 & -5.999246 & 3.741234 \\
\hline 1 & 6.769870 & 0.459483 & -2.545568 \\
\hline 1 & -1.042505 & -4.704079 & 1.662166 \\
\hline 1 & 1.362871 & -3.706231 & -1.903794 \\
\hline 1 & 6.996478 & 4.544689 & -3.841915 \\
\hline 1 & 8.926701 & 0.289162 & 1.179329 \\
\hline 1 & -5.161413 & -5.348908 & -2.266669 \\
\hline 1 & -1.609586 & -4.882891 & 5.912370 \\
\hline 1 & 9.656463 & -2.093308 & 1.116992 \\
\hline 1 & 1.750529 & 2.538001 & -0.112221 \\
\hline 1 & -4.123313 & 0.795044 & 1.409460 \\
\hline 1 & -2.927979 & -0.284237 & -2.551006 \\
\hline 1 & -7.095334 & -0.158371 & -1.522745 \\
\hline 1 & -2.999859 & 2.926972 & -0.536937 \\
\hline 1 & 0.293828 & 1.478288 & 1.791605 \\
\hline 1 & -0.887619 & 5.591010 & 2.076250 \\
\hline 1 & 1.349860 & -3.525779 & 1.671682 \\
\hline 1 & -1.250108 & 1.174771 & -2.604944 \\
\hline 1 & 2.775610 & -3.444219 & 0.629380 \\
\hline 1 & 4.684673 & -2.569091 & 1.606675 \\
\hline 1 & 0.866062 & -1.124066 & 2.925150 \\
\hline 1 & 5.819803 & -1.079295 & 3.222418 \\
\hline 1 & 1.995319 & 0.349327 & 4.573982 \\
\hline 1 & 4.473386 & 0.400898 & 4.705722 \\
\hline 1 & 1.769884 & 5.266501 & 4.404608 \\
\hline
\end{tabular}




$\begin{array}{lrrr}1 & 1.323738 & 5.842338 & 2.791068 \\ 1 & 0.074853 & 5.601046 & 4.035048 \\ 1 & 2.943710 & 3.309945 & 3.586554 \\ 1 & 2.228101 & 2.184840 & 2.429798 \\ 1 & 2.637384 & 3.834166 & 1.919096 \\ 1 & 1.076621 & 3.018146 & 5.224125 \\ 1 & -0.610890 & 3.273646 & 4.726168 \\ 1 & 0.279674 & 1.856798 & 4.140243 \\ 1 & -3.375777 & 7.615635 & 0.568160 \\ 1 & -2.787201 & 6.795104 & 2.020426 \\ 1 & -1.660179 & 7.209095 & 0.706964 \\ 1 & -3.518970 & 6.507827 & -1.607163 \\ 1 & -1.817484 & 6.002200 & -1.534833 \\ 1 & -3.088045 & 4.802512 & -1.820870 \\ 1 & -5.112567 & 5.913239 & 0.272362 \\ 1 & -4.718548 & 4.195887 & 0.089157 \\ 1 & -4.540436 & 4.978811 & 1.670872 \\ 1 & -8.399244 & -0.699640 & 2.013122 \\ 1 & -8.006457 & -1.198299 & 0.358041 \\ 1 & -6.884145 & -1.565042 & 1.682107 \\ 1 & -7.187987 & 1.138581 & 3.127678 \\ 1 & -5.652912 & 0.291980 & 2.890664 \\ 1 & -5.834715 & 1.979689 & 2.355304 \\ 1 & -8.675939 & 1.710403 & 1.296679 \\ 1 & -7.386994 & 2.537381 & 0.399036 \\ 1 & -8.337227 & 1.259959 & -0.379490 \\ 1 & -7.064521 & -0.990311 & -4.975820 \\ 1 & -7.350289 & -1.515365 & -3.310771 \\ 1 & -7.414450 & 0.213885 & -3.728635 \\ 1 & -4.936206 & -2.209192 & -5.071020 \\ 1 & -3.651192 & -1.941259 & -3.879953 \\ 1 & -5.143071 & -2.761268 & -3.393280 \\ 1 & -5.050465 & 0.244592 & -5.636532 \\ 1 & -5.274413 & 1.454661 & -4.355222 \\ 1 & -3.739264 & 0.578220 & -4.492072\end{array}$

$2_{\text {C1-t- }-1-1523354 s}$

Number of imaginary frequencies : 0

The smallest frequencies are : $14.3820 \quad 19.0798 \quad 22.1721 \mathrm{~cm}(-1)$

Electronic energy : $\quad H F=-4012.1879895$

Zero-point correction $=\quad 1.291610($ Hartree/Particle $)$

Thermal correction to Energy $=\quad 1.367340$

Thermal correction to Enthalpy= $\quad 1.368284$

Thermal correction to Gibbs Free Energy $=\quad 1.181919$

Sum of electronic and zero-point Energies $=\quad-4010.896380$

Sum of electronic and thermal Energies $=\quad-4010.820650$

Sum of electronic and thermal Enthalpies $=\quad-4010.819706$

Sum of electronic and thermal Free Energies $=\quad-4011.006071$

\section{Cartesian Coordinates}

\begin{tabular}{|c|c|c|c|}
\hline 6 & 1.936511 & -1.158100 & 3.008033 \\
\hline 6 & 2.680593 & -1.984661 & 2.162775 \\
\hline 6 & 4.069289 & -1.980514 & 2.260133 \\
\hline 6 & 4.715567 & -1.145101 & 3.167358 \\
\hline
\end{tabular}




\begin{tabular}{|c|c|c|c|}
\hline 6 & 3.970766 & -0.313532 & 3.995777 \\
\hline 6 & 2.578242 & -0.329862 & 3.920054 \\
\hline 6 & 1.994625 & -2.854227 & 1.146566 \\
\hline 7 & 1.157616 & -2.100889 & 0.188637 \\
\hline 15 & 1.616998 & -0.832989 & -0.830904 \\
\hline 45 & -0.046540 & 0.323229 & -1.886596 \\
\hline 6 & 0.073206 & -2.833696 & -0.410883 \\
\hline 6 & -1.204860 & -2.724294 & 0.104293 \\
\hline 6 & -2.288365 & -3.382092 & -0.554378 \\
\hline 6 & -2.025520 & -4.180384 & -1.707016 \\
\hline 6 & -0.697927 & -4.289682 & -2.183611 \\
\hline 6 & 0.322650 & -3.625345 & -1.557433 \\
\hline 6 & -3.629247 & -3.260437 & -0.106043 \\
\hline 6 & -4.647975 & -3.915393 & -0.751839 \\
\hline 6 & -4.379736 & -4.735028 & -1.870027 \\
\hline 6 & -3.097069 & -4.855869 & -2.340661 \\
\hline 6 & -1.447496 & -2.013601 & 1.387851 \\
\hline 6 & -1.636218 & -0.643075 & 1.458882 \\
\hline 6 & -1.940359 & -0.051816 & 2.716836 \\
\hline 6 & -2.022232 & -0.800965 & 3.859606 \\
\hline 6 & -1.805245 & -2.197264 & 3.825060 \\
\hline 6 & -1.520927 & -2.816052 & 2.573816 \\
\hline 6 & -1.864545 & -2.985895 & 4.998804 \\
\hline 6 & -1.650903 & -4.339518 & 4.946618 \\
\hline 6 & -1.370400 & -4.959563 & 3.710898 \\
\hline 6 & -1.310123 & -4.220052 & 2.555485 \\
\hline 15 & -1.612449 & 0.395290 & -0.084838 \\
\hline 6 & -3.379504 & 0.277318 & -0.568128 \\
\hline 6 & -4.399054 & 0.439308 & 0.371377 \\
\hline 6 & -5.736811 & 0.271433 & 0.017197 \\
\hline 6 & -6.028142 & -0.042142 & -1.316632 \\
\hline 6 & -5.031801 & -0.199814 & -2.282444 \\
\hline 6 & -3.701238 & -0.048736 & -1.881280 \\
\hline 6 & -6.809387 & 0.400007 & 1.098043 \\
\hline 6 & -6.746967 & 1.795840 & 1.727633 \\
\hline 6 & -5.329272 & -0.505462 & -3.748656 \\
\hline 6 & -4.609269 & -1.792199 & -4.164048 \\
\hline 6 & -1.396826 & 2.077304 & 0.608611 \\
\hline 6 & -0.360338 & 2.309743 & 1.515725 \\
\hline 6 & -0.186323 & 3.565145 & 2.100166 \\
\hline 6 & -1.041737 & 4.598547 & 1.697752 \\
\hline 6 & -2.056175 & 4.406491 & 0.755783 \\
\hline 6 & -2.225712 & 3.125232 & 0.223933 \\
\hline 6 & -2.999597 & 5.521103 & 0.307603 \\
\hline 6 & -4.446722 & 5.110357 & 0.606767 \\
\hline 6 & 0.840236 & 3.729242 & 3.222124 \\
\hline 6 & 2.220755 & 3.229846 & 2.785331 \\
\hline 6 & -8.218919 & 0.189059 & 0.551513 \\
\hline 6 & -6.552957 & -0.660055 & 2.176797 \\
\hline 6 & -6.821101 & -0.688840 & -4.013937 \\
\hline 6 & -4.825151 & 0.659109 & -4.609693 \\
\hline 6 & -2.723564 & 6.843064 & 1.018905 \\
\hline 6 & -2.835722 & 5.744428 & -1.199996 \\
\hline 6 & 0.985325 & 5.180370 & 3.674019 \\
\hline 6 & 0.357147 & 2.899398 & 4.419720 \\
\hline 8 & 2.830779 & -1.415388 & -1.838112 \\
\hline
\end{tabular}




\begin{tabular}{|c|c|c|c|}
\hline ) & 4.095455 & -1.699924 & -1.379567 \\
\hline & 4.944612 & -0.686909 & -0.967724 \\
\hline 6 & 6.217321 & -1.050799 & -0.422431 \\
\hline & 6.619745 & -2.420395 & -0.425408 \\
\hline & 5.743199 & -3.401386 & -0.950732 \\
\hline & 4.499970 & -3.051403 & -1.403291 \\
\hline & 7.872598 & -2.780242 & 0.127798 \\
\hline & 8.695205 & -1.835239 & 0.685889 \\
\hline & 8.288114 & -0.484823 & 0.718021 \\
\hline 6 & 7.083725 & -0.103140 & 0.179854 \\
\hline 6 & 4.478259 & 0.715576 & -1.055889 \\
\hline & 3.273516 & 1.048961 & -0.469589 \\
\hline 6 & 2.721739 & 2.342922 & -0.537618 \\
\hline v & 3.401492 & 3.326627 & -1.203772 \\
\hline 6 & 4.625657 & 3.044752 & -1.856841 \\
\hline U & 5.167840 & 1.724608 & -1.803363 \\
\hline 6 & 6.350551 & 1.459648 & -2.539249 \\
\hline 6 & 6.980221 & 2.450674 & -3.250899 \\
\hline 6 & 6.462882 & 3.762630 & -3.269451 \\
\hline 6 & 5.306494 & 4.046924 & -2.589797 \\
\hline 8 & 2.555113 & 0.066017 & 0.191509 \\
\hline 6 & 0.978055 & 0.813577 & -3.368470 \\
\hline 8 & 1.621151 & 1.184803 & -4.254578 \\
\hline 1 & 6.774856 & 0.938062 & 0.227754 \\
\hline 1 & -2.108947 & 1.020526 & 2.779670 \\
\hline 1 & 8.163279 & -3.829895 & 0.109074 \\
\hline 1 & -2.081227 & -2.491232 & 5.944574 \\
\hline 1 & 4.880142 & 5.048962 & -2.611600 \\
\hline 1 & -3.846857 & -2.626441 & 0.753098 \\
\hline 1 & -5.671557 & -3.799023 & -0.401560 \\
\hline 1 & 2.993058 & 4.334163 & -1.263563 \\
\hline 1 & -2.254963 & -0.325994 & 4.812082 \\
\hline 1 & 6.067359 & -4.440664 & -0.969162 \\
\hline 1 & 7.883126 & 2.219174 & -3.811576 \\
\hline 1 & 3.802878 & -3.792257 & -1.790065 \\
\hline 1 & -2.878726 & -5.470589 & -3.212816 \\
\hline 1 & -0.503343 & -4.901877 & -3.062765 \\
\hline 1 & -1.202504 & -6.033410 & 3.673157 \\
\hline 1 & 6.755293 & 0.450914 & -2.546320 \\
\hline 1 & -1.097059 & -4.714585 & 1.610054 \\
\hline 1 & 1.341909 & -3.683748 & -1.935501 \\
\hline 1 & 6.973403 & 4.539479 & -3.833923 \\
\hline 1 & 8.930962 & 0.260912 & 1.180409 \\
\hline 1 & -5.195472 & -5.257839 & -2.364705 \\
\hline 1 & -1.696158 & -4.937310 & 5.853928 \\
\hline 1 & 9.653034 & -2.123694 & 1.112527 \\
\hline 1 & 1.760483 & 2.527094 & -0.061682 \\
\hline 1 & -4.143908 & 0.685660 & 1.401566 \\
\hline 1 & -2.887783 & -0.178645 & -2.594145 \\
\hline 1 & -7.066575 & -0.169746 & -1.608423 \\
\hline 1 & -3.017614 & 2.934911 & -0.499704 \\
\hline 1 & 0.289234 & 1.480356 & 1.805450 \\
\hline 1 & -0.918101 & 5.582221 & 2.140909 \\
\hline 1 & 1.306058 & -3.543935 & 1.656048 \\
\hline 1 & -1.198743 & 1.263760 & -2.573232 \\
\hline 1 & 2.736103 & -3.475244 & 0.618291 \\
\hline
\end{tabular}




$\begin{array}{rrrr}1 & 4.654171 & -2.620153 & 1.598143 \\ 1 & 0.848627 & -1.147858 & 2.924871 \\ 1 & 5.803231 & -1.141858 & 3.214941 \\ 1 & 1.991653 & 0.314298 & 4.574128 \\ 1 & 4.470159 & 0.346914 & 4.701860 \\ 1 & -6.981276 & -0.912159 & -5.076257 \\ 1 & -7.240112 & -1.522847 & -3.435364 \\ 1 & -7.396373 & 0.216446 & -3.780197 \\ 1 & -5.027499 & 0.463141 & -5.671435 \\ 1 & -5.326812 & 1.596785 & -4.336612 \\ 1 & -3.744424 & 0.813062 & -4.498839 \\ 1 & -4.811749 & -2.015343 & -5.220414 \\ 1 & -3.521128 & -1.717865 & -4.044475 \\ 1 & -4.952113 & -2.643646 & -3.562304 \\ 1 & -7.304894 & -0.586212 & 2.974207 \\ 1 & -6.610765 & -1.671211 & 1.751791 \\ 1 & -5.563693 & -0.550834 & 2.640124 \\ 1 & -8.948244 & 0.289292 & 1.365285 \\ 1 & -8.477722 & 0.931037 & -0.214817 \\ 1 & -8.346361 & -0.811648 & 0.118775 \\ 1 & -7.501843 & 1.890596 & 2.519891 \\ 1 & -5.768266 & 2.005612 & 2.176430 \\ 1 & -6.945249 & 2.573663 & 0.978647 \\ 1 & -5.140428 & 5.898380 & 0.284123 \\ 1 & -4.729679 & 4.186264 & 0.086902 \\ 1 & -4.595675 & 4.947919 & 1.682584 \\ 1 & -3.509589 & 6.541206 & -1.542961 \\ 1 & -1.807550 & 6.041583 & -1.443331 \\ 1 & -3.065920 & 4.841401 & -1.777700 \\ 1 & -3.427877 & 7.605592 & 0.663163 \\ 1 & -2.850712 & 6.760365 & 2.106196 \\ 1 & -1.710411 & 7.214151 & 0.817579 \\ 1 & 1.749884 & 5.246213 & 4.458365 \\ 1 & 1.299409 & 5.834749 & 2.850545 \\ 1 & 0.053415 & 5.579369 & 4.094540 \\ 1 & 2.926565 & 3.299791 & 3.624830 \\ 1 & 2.214524 & 2.182056 & 2.458593 \\ 1 & 2.619231 & 3.836802 & 1.961831 \\ 1 & 1.060603 & 2.988453 & 5.259515 \\ 1 & -0.627577 & 3.244194 & 4.764032 \\ 1 & 0.266510 & 1.834890 & 4.165260\end{array}$

\section{$2_{\text {C2-c- }-\psi-122 a 3 a 4 a}$}

Number of imaginary frequencies : 0

The smallest frequencies are : $12.9863 \quad 17.2832 \quad 21.1947 \mathrm{~cm}(-1)$

Electronic energy : $\quad H F=-4012.1863263$

Zero-point correction $=\quad 1.290742($ Hartree/Particle)

Thermal correction to Energy= $\quad 1.366735$

Thermal correction to Enthalpy= $\quad 1.367679$

Thermal correction to Gibbs Free Energy $=\quad 1.181077$

Sum of electronic and zero-point Energies $=\quad-4010.895584$

Sum of electronic and thermal Energies $=\quad-4010.819591$

Sum of electronic and thermal Enthalpies $=\quad-4010.818647$ 
Cartesian Coordinates

$\begin{array}{cccc}\cdots & -2.151028 & 3.115133 & 0.567758 \\ 6 & -1.295950 & 2.053883 & 0.854444 \\ 6 & -0.223846 & 2.239572 & 1.731341 \\ 6 & -0.028206 & 3.460933 & 2.375270 \\ 6 & -0.901177 & 4.508347 & 2.060067 \\ 6 & -1.958019 & 4.367175 & 1.157449 \\ 15 & -1.441015 & 0.449603 & -0.018084 \\ 45 & 0.056429 & 0.650746 & -1.892276 \\ 1 & 1.038951 & 0.894997 & -3.148232 \\ 6 & 1.068254 & 3.668481 & 3.419392 \\ 6 & 0.422333 & 4.089748 & 4.744756 \\ 6 & -2.875583 & 5.553547 & 0.866656 \\ 6 & -2.045336 & 6.725304 & 0.332452 \\ 6 & -1.427980 & -0.773881 & 1.376915 \\ 6 & -1.242588 & -2.121594 & 1.111096 \\ 6 & -1.383228 & -3.088939 & 2.158028 \\ 6 & -1.720458 & -2.655343 & 3.471888 \\ 6 & -1.891146 & -1.272994 & 3.711959 \\ 6 & -1.748353 & -0.363009 & 2.699377 \\ 6 & -1.214785 & -4.480224 & 1.935781 \\ 6 & -1.388092 & -5.386324 & 2.952443 \\ 6 & -1.725256 & -4.950726 & 4.251084 \\ 6 & -1.878743 & -3.611232 & 4.503795 \\ 6 & -0.999759 & -2.637988 & -0.263200 \\ 6 & 0.264389 & -2.664725 & -0.826712 \\ 6 & 0.472060 & -3.270520 & -2.091085 \\ 6 & -0.568389 & -3.810320 & -2.796550 \\ 6 & -1.883432 & -3.760991 & -2.278876 \\ 6 & -2.104824 & -3.177090 & -0.996957 \\ 6 & -2.982856 & -4.277220 & -3.007981 \\ 6 & -4.256739 & -4.201550 & -2.504624 \\ 6 & -4.483612 & -3.603422 & -1.245833 \\ 6 & -3.435504 & -3.116563 & -0.505873 \\ 7 & 1.382048 & -1.997245 & -0.213427 \\ 6 & 2.317825 & -2.779673 & 0.614462 \\ 6 & 2.019549 & -2.773115 & 2.092044 \\ 6 & 1.858477 & -1.573300 & 2.791255 \\ 6 & 1.661423 & -1.582870 & 4.166605 \\ 6 & 1.624882 & -2.788725 & 4.864399 \\ 6 & 1.784975 & -3.985757 & 4.176458 \\ 6 & 1.979377 & -3.974316 & 2.797539 \\ 15 & 1.783854 & -0.587187 & -1.048114 \\ 8 & 2.708233 & 0.127621 & 0.124340 \\ 6 & 3.504694 & 1.173601 & -0.315553 \\ 6 & 4.718812 & 0.890507 & -0.909060 \\ 6 & 5.497909 & 1.989453 & -1.399205 \\ 6 & 5.033063 & 3.323363 & -1.188513 \\ 6 & 3.791421 & 3.540234 & -0.543964 \\ 6 & 3.025006 & 2.483882 & -0.131118\end{array}$




$\begin{array}{lrrr}6 & 6.699726 & 1.812444 & -2.130922 \\ 6 & 7.418806 & 2.889716 & -2.586366 \\ 6 & 6.977520 & 4.206239 & -2.339610 \\ 6 & 5.804824 & 4.412926 & -1.660181 \\ 6 & 5.113246 & -0.525370 & -1.099395 \\ 6 & 4.236319 & -1.385993 & -1.739187 \\ 6 & 4.580647 & -2.717254 & -2.051589 \\ 6 & 5.792195 & -3.216444 & -1.659525 \\ 6 & 6.692213 & -2.417466 & -0.914056 \\ 6 & 6.350812 & -1.063151 & -0.618038 \\ 6 & 7.246610 & -0.312332 & 0.185723 \\ 6 & 8.421478 & -0.858986 & 0.639034 \\ 6 & 8.771020 & -2.186446 & 0.314291 \\ 6 & 7.918259 & -2.947085 & -0.443339 \\ 8 & 2.993507 & -0.952717 & -2.139127 \\ 6 & -3.204369 & 0.331437 & -0.520460 \\ 6 & -4.244558 & 0.430400 & 0.407407 \\ 6 & -5.570442 & 0.280571 & 0.008192 \\ 6 & -5.821924 & 0.045700 & -1.351003 \\ 6 & -4.807579 & -0.062274 & -2.302462 \\ 6 & -3.489315 & 0.072993 & -1.857634 \\ 6 & -6.730179 & 0.315737 & 1.001560 \\ 6 & -7.371738 & -1.075970 & 1.051516 \\ 6 & -5.133241 & -0.249013 & -3.783328 \\ 6 & -5.591423 & 1.103797 & -4.341271 \\ 6 & -6.270799 & 0.677961 & 2.412576 \\ 6 & -7.773775 & 1.347781 & 0.563252 \\ 6 & -3.915477 & -0.715548 & -4.582154 \\ 6 & -6.247440 & -1.280713 & -3.977353 \\ 6 & 1.868572 & 2.392168 & 3.674723 \\ 6 & 2.034693 & 4.762274 & 2.955558 \\ 6 & -3.942965 & 5.216747 & -0.172521 \\ 6 & -3.582905 & 5.975189 & 2.159358 \\ 1 & 6.986913 & 0.708608 & 0.454124 \\ 1 & -1.891766 & 0.693937 & 2.911690 \\ 1 & 8.162776 & -3.979468 & -0.690268 \\ 1 & -2.130046 & -3.257207 & 5.502968 \\ 1 & 5.435130 & 5.421762 & -1.481591 \\ 1 & -3.625390 & -2.656310 & 0.462149 \\ 1 & -5.498866 & -3.524738 & -0.861074 \\ 1 & 3.440049 & 4.562319 & -0.409696 \\ 1 & -2.146729 & -0.941957 & 4.718000 \\ 1 & 6.072438 & -4.241283 & -1.897357 \\ 1 & 8.334532 & 2.724668 & -3.149661 \\ 1 & 3.864338 & -3.320405 & -2.605532 \\ 1 & -2.793569 & -4.728607 & -3.980995 \\ 1 & -0.401400 & -4.265533 & -3.771350 \\ 1 & -1.258761 & -6.448531 & 2.756632 \\ 1 & 7.048789 & 0.805201 & -2.342118 \\ 1 & -0.940794 & -4.830110 & 0.942440 \\ 1 & 1.482458 & -3.277013 & -2.496494 \\ 1 & 7.558943 & 5.050807 & -2.701984 \\ 1 & 9.086640 & -0.263288 & 1.260138\end{array}$




$\begin{array}{rrrr}1 & -5.094753 & -4.598461 & -3.074123 \\ 1 & -1.857435 & -5.678027 & 5.049063 \\ 1 & 9.707740 & -2.604753 & 0.675046 \\ 1 & 2.045561 & 2.622344 & 0.325280 \\ 1 & -4.004517 & 0.619984 & 1.451185 \\ 1 & -2.654659 & -0.040998 & -2.546686 \\ 1 & -6.856623 & -0.061788 & -1.675466 \\ 1 & -2.972642 & 2.956215 & -0.126915 \\ 1 & 0.447848 & 1.402176 & 1.923003 \\ 1 & -0.753695 & 5.473712 & 2.544820 \\ 6 & -0.943567 & 2.000496 & -2.740414 \\ 1 & 2.336688 & -3.813734 & 0.237912 \\ 1 & 3.327557 & -2.377269 & 0.462593 \\ 1 & 2.096802 & -4.914303 & 2.257513 \\ 1 & 1.889499 & -0.628305 & 2.248855 \\ 1 & 1.745220 & -4.933615 & 4.709712 \\ 1 & 1.538511 & -0.641069 & 4.699717 \\ 1 & 1.466662 & -2.793355 & 5.940994 \\ 1 & -4.205528 & -0.886822 & -5.626598 \\ 1 & -3.108792 & 0.029284 & -4.591465 \\ 1 & -3.507938 & -1.656505 & -4.186398 \\ 1 & -5.830055 & 1.019352 & -5.410405 \\ 1 & -6.486145 & 1.471042 & -3.822133 \\ 1 & -4.803011 & 1.860187 & -4.227899 \\ 1 & -6.438194 & -1.428772 & -5.048287 \\ 1 & -5.963859 & -2.250056 & -3.546104 \\ 1 & -7.195733 & -0.970130 & -3.522952 \\ 1 & -8.601274 & 1.380389 & 1.284463 \\ 1 & -7.334567 & 2.352319 & 0.507362 \\ 1 & -8.204510 & 1.116478 & -0.418015 \\ 1 & -8.209633 & -1.089127 & 1.761782 \\ 1 & -7.758638 & -1.384840 & 0.072342 \\ 1 & -6.640305 & -1.828650 & 1.376741 \\ 1 & -7.135816 & 0.698342 & 3.087392 \\ 1 & -5.557971 & -0.053534 & 2.816356 \\ 1 & -5.800876 & 1.669926 & 2.447062 \\ 1 & 2.807584 & 4.934901 & 3.716531 \\ 1 & 2.540768 & 4.472542 & 2.025110 \\ 1 & 1.522831 & 5.716014 & 2.778830 \\ 1 & 1.192136 & 4.228052 & 5.515744 \\ 1 & -0.129670 & 5.033078 & 4.655971 \\ 1 & -0.279255 & 3.323800 & 5.100677 \\ 1 & 2.614223 & 2.574110 & 4.458725 \\ 1 & 1.219934 & 1.572283 & 4.015609 \\ 1 & 2.411646 & 2.054961 & 2.781284 \\ 1 & -4.570358 & 6.098482 & -0.353976 \\ 1 & -3.501452 & 4.921979 & -1.133534 \\ 1 & -4.604725 & 4.406703 & 0.162867 \\ 1 & -2.695673 & 7.582536 & 0.112178 \\ 1 & -1.290119 & 7.060899 & 1.053276 \\ 1 & -1.525491 & 6.447855 & -0.593516 \\ 1 & -4.250638 & 6.826271 & 1.968963 \\ & -4.189500 & 5.152803 & 2.561119\end{array}$


$\begin{array}{llll}1 & -2.872776 & 6.278278 & 2.938489\end{array}$

$\begin{array}{llll}8 & -1.488502 & 2.867866 & -3.278266\end{array}$

$2_{\text {C2-c- }-\psi-12 a 3 a 4 s}$

Number of imaginary frequencies : 0

The smallest frequencies are : $11.1990 \quad 13.2772 \quad 20.4003 \mathrm{~cm}(-1)$

Electronic energy : $\quad H F=-4012.1868388$

Zero-point correction $=\quad 1.290861($ Hartree/Particle $)$

Thermal correction to Energy= $\quad 1.366851$

Thermal correction to Enthalpy= $\quad 1.367795$

Thermal correction to Gibbs Free Energy $=\quad 1.180009$

Sum of electronic and zero-point Energies $=\quad-4010.895978$

Sum of electronic and thermal Energies $=\quad-4010.819988$

Sum of electronic and thermal Enthalpies $=\quad-4010.819044$

Sum of electronic and thermal Free Energies $=\quad-4011.006829$

Cartesian Coordinates

$\begin{array}{cccc}6 & 5.751216 & 4.470505 & -1.675139 \\ 6 & 4.991953 & 3.373346 & -1.200824 \\ 6 & 5.474828 & 2.044582 & -1.403347 \\ 6 & 6.681312 & 1.879585 & -2.130128 \\ 6 & 7.387946 & 2.963841 & -2.588453 \\ 6 & 6.928841 & 4.275730 & -2.349561 \\ 6 & 4.708611 & 0.938002 & -0.910140 \\ 6 & 3.488866 & 1.208199 & -0.322225 \\ 6 & 2.991211 & 2.512930 & -0.146516 \\ 6 & 3.745240 & 3.577207 & -0.561806 \\ 6 & 5.122615 & -0.473595 & -1.090662 \\ 6 & 4.259603 & -1.350133 & -1.727783 \\ 6 & 4.622950 & -2.678562 & -2.030630 \\ 6 & 5.839310 & -3.159164 & -1.630457 \\ 6 & 6.725553 & -2.343607 & -0.886430 \\ 6 & 6.365325 & -0.991843 & -0.601128 \\ 6 & 7.956169 & -2.854187 & -0.406676 \\ 6 & 8.795721 & -2.077494 & 0.349416 \\ 6 & 8.427783 & -0.752393 & 0.663245 \\ 6 & 7.248005 & -0.224026 & 0.201066 \\ 8 & 3.012016 & -0.936815 & -2.133965 \\ 15 & 1.794356 & -0.581153 & -1.048856 \\ 8 & 2.705129 & 0.154243 & 0.121284 \\ 7 & 1.409612 & -1.989783 & -0.204133 \\ 6 & 0.303100 & -2.679955 & -0.812608 \\ 6 & -0.962054 & -2.669322 & -0.250629 \\ 6 & -2.055246 & -3.244471 & -0.974989 \\ 6 & -1.820524 & -3.845441 & -2.246737 \\ 6 & -0.505443 & -3.869772 & -2.766201 \\ 6 & 0.523126 & -3.297305 & -2.069282 \\ 6 & -2.905844 & -4.408212 & -2.962225 \\ 6 & -4.178164 & -4.370736 & -2.450942 \\ 6 & -4.418607 & -3.757441 & -1.202134 \\ 6 & -3.385834 & -3.213715 & -0.480350\end{array}$




$\begin{array}{cccc}6 & -1.218139 & -2.141152 & 1.116620 \\ 6 & -1.426311 & -0.793720 & 1.366594 \\ 6 & -1.758689 & -0.374149 & 2.683444 \\ 6 & -1.892988 & -1.275481 & 3.704953 \\ 6 & -1.701397 & -2.657649 & 3.480408 \\ 6 & -1.349555 & -3.099503 & 2.173216 \\ 6 & -1.158594 & -4.490353 & 1.966890 \\ 6 & -1.326135 & -5.388429 & 2.991536 \\ 6 & -1.679400 & -4.944732 & 4.283141 \\ 6 & -1.853000 & -3.605031 & 4.521157 \\ 15 & -1.447794 & 0.416756 & -0.040190 \\ 6 & -3.209892 & 0.289054 & -0.544710 \\ 6 & -3.501803 & 0.003932 & -1.879696 \\ 6 & -4.819834 & -0.107647 & -2.319164 \\ 6 & -5.839306 & 0.056705 & -1.372337 \\ 6 & -5.580797 & 0.313803 & -0.024557 \\ 6 & -4.246096 & 0.431915 & 0.372255 \\ 6 & -5.161511 & -0.355268 & -3.787278 \\ 6 & -5.725644 & 0.941904 & -4.378270 \\ 6 & -6.676815 & 0.452172 & 1.029658 \\ 6 & -6.469476 & -0.622610 & 2.104103 \\ 6 & 2.356596 & -2.752787 & 0.629538 \\ 6 & 2.052758 & -2.745043 & 2.105839 \\ 6 & 2.026055 & -3.943771 & 2.816039 \\ 6 & 1.823727 & -3.952290 & 4.193845 \\ 6 & 1.642720 & -2.754993 & 4.875920 \\ 6 & 1.666751 & -1.551494 & 4.173508 \\ 6 & 1.871687 & -1.544688 & 2.799318 \\ 45 & 0.050485 & 0.620004 & -1.910227 \\ 6 & -0.973528 & 1.932183 & -2.788618 \\ 8 & -1.529999 & 2.779249 & -3.346317 \\ 6 & -1.323530 & 2.029815 & 0.820887 \\ 6 & -2.191728 & 3.078732 & 0.527289 \\ 6 & -2.019974 & 4.334652 & 1.115413 \\ 6 & -0.972710 & 4.491238 & 2.026517 \\ 6 & -0.085110 & 3.457523 & 2.346504 \\ 6 & -0.258245 & 2.232932 & 1.702182 \\ 6 & -2.959697 & 5.503289 & 0.822659 \\ 6 & -2.149788 & 6.721071 & 0.365959 \\ 6 & 1.002741 & 3.682718 & 3.395978 \\ 6 & 0.344287 & 4.095307 & 4.717879 \\ 6 & -3.968526 & 5.170901 & -0.274841 \\ 6 & -3.735227 & 5.852308 & 2.097925 \\ 6 & 1.821102 & 2.419016 & 3.656625 \\ 6 & 1.954765 & 4.790475 & 2.935376 \\ 6 & -8.075727 & 0.275338 & 0.445520 \\ 6 & -6.599162 & 1.843455 & 1.668928 \\ 6 & -3.933475 & -0.763992 & -4.600115 \\ 6 & -6.203835 & -1.469704 & -3.918844 \\ 1 & 1.037105 & 0.870286 & -3.162008 \\ 1 & 6.974137 & 0.795298 & 0.461332 \\ 1 & -1.917153 & 0.682820 & 2.884550 \\ 1 & 8.215026 & -3.885005 & -0.645443\end{array}$




\begin{tabular}{|c|c|c|c|}
\hline & -2.116533 & -3.244400 & 5.514793 \\
\hline & 5.367888 & 5.475333 & -1.502766 \\
\hline & -3.589930 & -2.732044 & 0.474874 \\
\hline & -5.433515 & -3.708232 & -0.812502 \\
\hline & 3.379994 & 4.595223 & -0.433822 \\
\hline & -2.158389 & -0.937614 & 4.706176 \\
\hline & 6.133968 & -4.181715 & -1.860570 \\
\hline & 8.307651 & 2.807953 & -3.147860 \\
\hline & 3.916698 & -3.294206 & -2.583779 \\
\hline & -2.706253 & -4.869419 & -3.928502 \\
\hline & -0.328714 & -4.336253 & -3.733936 \\
\hline & -1.179634 & -6.450520 & 2.807563 \\
\hline & 7.044087 & 0.875911 & -2.335182 \\
\hline & -0.871476 & -4.846594 & 0.979557 \\
\hline & 1.533587 & -3.288667 & -2.474599 \\
\hline & 7.500544 & 5.126002 & -2.714050 \\
\hline & 9.082534 & -0.143869 & 1.282985 \\
\hline & -5.004946 & -4.807831 & -3.007143 \\
\hline & -1.807307 & -5.665812 & 5.087437 \\
\hline & 9.736074 & -2.481265 & 0.717212 \\
\hline & 2.008283 & 2.640620 & 0.305388 \\
\hline & -4.010024 & 0.637997 & 1.416100 \\
\hline & -2.669355 & -0.143825 & -2.565000 \\
\hline & -6.872400 & -0.025371 & -1.703530 \\
\hline & -3.006876 & 2.907648 & -0.172112 \\
\hline & 0.424447 & 1.405581 & 1.898614 \\
\hline & -0.844626 & 5.457413 & 2.514946 \\
\hline & 2.395017 & -3.787884 & 0.257339 \\
\hline & 3.359403 & -2.332992 & 0.479356 \\
\hline & 2.159464 & -4.884209 & 2.280523 \\
\hline & 1.893663 & -0.601483 & 2.253318 \\
\hline & 1.794034 & -4.898446 & 4.730785 \\
\hline & 1.528164 & -0.609439 & 4.702270 \\
\hline & 1.478084 & -2.757601 & 5.951554 \\
\hline & -4.229634 & -0.960857 & -5.638306 \\
\hline & -3.167721 & 0.022604 & -4.626040 \\
\hline & -3.471846 & -1.679547 & -4.203427 \\
\hline & -5.981183 & 0.802741 & -5.437636 \\
\hline & -6.632836 & 1.266537 & -3.852987 \\
\hline & -4.989786 & 1.754210 & -4.310574 \\
\hline & -6.422125 & -1.656647 & -4.978622 \\
\hline & -5.832790 & -2.405218 & -3.478890 \\
\hline & -7.153792 & -1.218195 & -3.432317 \\
\hline & -7.352689 & 1.943183 & 2.461871 \\
\hline 1 & -5.616701 & 2.041524 & 2.116572 \\
\hline & -6.790040 & 2.626005 & 0.922977 \\
\hline & -7.251426 & -0.552863 & 2.872325 \\
\hline 1 & -6.516466 & -1.627664 & 1.663386 \\
\hline 1 & -5.499125 & -0.527780 & 2.607866 \\
\hline 1 & -8.823785 & 0.381058 & 1.241400 \\
\hline 1 & -8.300633 & 1.030901 & -0.318160 \\
\hline & -8.210388 & -0.717839 & -0.002255 \\
\hline & 2.722341 & 4.974496 & 3.699054 \\
\hline
\end{tabular}




$\begin{array}{rrrr}1 & 2.468235 & 4.508043 & 2.006693 \\ 1 & 1.429548 & 5.736561 & 2.756674 \\ 1 & 1.108296 & 4.245956 & 5.492294 \\ 1 & -0.221591 & 5.030048 & 4.625914 \\ 1 & -0.347086 & 3.318924 & 5.071255 \\ 1 & 2.559753 & 2.612968 & 4.444368 \\ 1 & 1.183164 & 1.589731 & 3.994931 \\ 1 & 2.373900 & 2.089395 & 2.766339 \\ 1 & -4.609236 & 6.041912 & -0.461427 \\ 1 & -3.474472 & 4.913401 & -1.220864 \\ 1 & -4.624749 & 4.336179 & 0.005506 \\ 1 & -2.822114 & 7.560102 & 0.142126 \\ 1 & -1.442224 & 7.063176 & 1.130543 \\ 1 & -1.578122 & 6.494009 & -0.542935 \\ 1 & -4.419106 & 6.691716 & 1.912683 \\ 1 & -4.334229 & 4.996821 & 2.437964 \\ 1 & -3.067433 & 6.140811 & 2.919159\end{array}$

$2_{\text {C2-c- }- \text {-1a2a3s4a }}$

Number of imaginary frequencies : 0

The smallest frequencies are : $12.7273 \quad 17.719620 .3286 \mathrm{~cm}(-1)$

Electronic energy : $\quad H F=-4012.1855422$

Zero-point correction $=\quad 1.291372($ Hartree/Particle $)$

Thermal correction to Energy= $\quad 1.367283$

Thermal correction to Enthalpy= $\quad 1.368227$

Thermal correction to Gibbs Free Energy $=1.181438$

Sum of electronic and zero-point Energies $=\quad-4010.894170$

Sum of electronic and thermal Energies $=\quad-4010.818259$

Sum of electronic and thermal Enthalpies $=\quad-4010.817315$

Sum of electronic and thermal Free Energies $=\quad-4011.004104$

Cartesian Coordinates

$\begin{array}{crrr}6 & -3.491624 & 0.147011 & -1.904276 \\ 6 & -3.214519 & 0.322757 & -0.555654 \\ 6 & -4.266972 & 0.397354 & 0.365816 \\ 6 & -5.588633 & 0.299282 & -0.053297 \\ 6 & -5.832848 & 0.131712 & -1.427729 \\ 6 & -4.811465 & 0.064258 & -2.369966 \\ 15 & -1.456285 & 0.418739 & -0.029286 \\ 6 & -1.453224 & -0.828780 & 1.341990 \\ 6 & -1.238673 & -2.169883 & 1.066215 \\ 6 & -1.385707 & -3.149259 & 2.103604 \\ 6 & -1.760801 & -2.734022 & 3.413312 \\ 6 & -1.956837 & -1.356809 & 3.663045 \\ 6 & -1.805066 & -0.435858 & 2.662389 \\ 6 & -1.929339 & -3.701081 & 4.433094 \\ 6 & -1.746713 & -5.035616 & 4.173917 \\ 6 & -1.366521 & -5.453179 & 2.881514 \\ 6 & -1.183995 & -4.535686 & 1.876730 \\ 6 & -0.949399 & -2.685907 & -0.300513 \\ 6 & 0.331632 & -2.707682 & -0.825676\end{array}$




$\begin{array}{cccc}6 & 0.591409 & -3.368628 & -2.052398 \\ 6 & -0.410037 & -3.983613 & -2.752473 \\ 6 & -1.740967 & -3.945941 & -2.275537 \\ 6 & -2.018179 & -3.283895 & -1.043615 \\ 6 & -3.364717 & -3.231393 & -0.597541 \\ 6 & -4.372013 & -3.819244 & -1.321145 \\ 6 & -4.086780 & -4.505543 & -2.521383 \\ 6 & -2.799014 & -4.559201 & -2.990032 \\ 7 & 1.418722 & -1.997570 & -0.205591 \\ 15 & 1.787907 & -0.581520 & -1.046854 \\ 8 & 3.010442 & -0.923200 & -2.132603 \\ 6 & 4.260661 & -1.328099 & -1.726363 \\ 6 & 5.115578 & -0.447451 & -1.083941 \\ 6 & 6.361407 & -0.957263 & -0.593511 \\ 6 & 6.733340 & -2.305039 & -0.882528 \\ 6 & 5.855721 & -3.124481 & -1.632315 \\ 6 & 4.636652 & -2.652004 & -2.033915 \\ 6 & 7.236070 & -0.185052 & 0.213295 \\ 6 & 8.418766 & -0.705593 & 0.676854 \\ 6 & 8.797975 & -2.026768 & 0.359817 \\ 6 & 7.966647 & -2.807530 & -0.401151 \\ 6 & 4.690124 & 0.959749 & -0.896856 \\ 6 & 3.468746 & 1.216978 & -0.306683 \\ 6 & 2.963138 & 2.517347 & -0.120774 \\ 6 & 3.709273 & 3.589431 & -0.530392 \\ 6 & 4.956136 & 3.398523 & -1.173152 \\ 6 & 5.448178 & 2.074517 & -1.384025 \\ 6 & 5.706839 & 4.503737 & -1.642313 \\ 6 & 6.884910 & 4.321144 & -2.319431 \\ 6 & 7.353296 & 3.013945 & -2.566036 \\ 6 & 6.655078 & 1.922111 & -2.112805 \\ 8 & 2.692771 & 0.154195 & 0.128579 \\ 6 & -6.759960 & 0.342173 & 0.926536 \\ 6 & -7.769728 & 1.412232 & 0.498637 \\ 6 & -5.058514 & -0.079158 & -3.869553 \\ 6 & -4.429361 & -1.383678 & -4.367796 \\ 6 & -1.330859 & 2.006345 & 0.878905 \\ 6 & -2.211759 & 3.054031 & 0.623798 \\ 6 & -2.045491 & 4.295465 & 1.242526 \\ 6 & -0.981148 & 4.442968 & 2.134579 \\ 6 & -0.081694 & 3.408958 & 2.419252 \\ 6 & -0.258284 & 2.195241 & 1.755033 \\ 6 & 1.023811 & 3.625361 & 3.452161 \\ 6 & 1.955812 & 4.750924 & 2.993292 \\ 6 & -2.996415 & 5.463101 & 0.986693 \\ 6 & -3.661201 & 5.877193 & 2.304086 \\ 45 & 0.061687 & 0.655553 & -1.881796 \\ 1 & 1.047108 & 0.911781 & -3.132262 \\ 6 & 0.387648 & 4.008301 & 4.793788 \\ 6 & 1.860202 & 2.366366 & 3.674112 \\ 6 & -2.207670 & 6.646068 & 0.416153 \\ 6 & -4.098274 & 5.101528 & -0.007332 \\ 6 & 2.378812 & -2.750667 & 0.622517\end{array}$




\begin{tabular}{|c|c|c|c|}
\hline & 2.043531 & -2.786907 & 2.090821 \\
\hline & 1.836617 & -1.605132 & 2.808548 \\
\hline & 1.589654 & -1.647791 & 4.174937 \\
\hline & 1.550799 & -2.869380 & 4.845167 \\
\hline & 1.757340 & -4.048198 & 4.138717 \\
\hline & 2.000115 & -4.003717 & 2.767970 \\
\hline & -7.444778 & -1.029184 & 0.939797 \\
\hline & -6.307754 & 0.665182 & 2.349419 \\
\hline & -4.413693 & 1.104954 & -4.600304 \\
\hline & -6.545269 & -0.101787 & -4.213385 \\
\hline & 6.953665 & 0.831306 & 0.475981 \\
\hline & -1.967066 & 0.617080 & 2.881039 \\
\hline & 8.234345 & -3.835432 & -0.642745 \\
\hline & -2.212181 & -3.359358 & 5.428133 \\
\hline & 5.316582 & 5.504820 & -1.463744 \\
\hline & -3.596895 & -2.707175 & 0.327698 \\
\hline & -5.398657 & -3.752717 & -0.966935 \\
\hline & 3.337258 & 4.604052 & -0.395104 \\
\hline & -2.238791 & -1.039106 & 4.666338 \\
\hline & 6.159218 & -4.143605 & -1.866167 \\
\hline & 8.273465 & 2.867832 & -3.127329 \\
\hline & 3.938067 & -3.271306 & -2.592479 \\
\hline & -2.565965 & -5.070517 & -3.922978 \\
\hline & -0.199540 & -4.493874 & -3.690975 \\
\hline & -1.211079 & -6.510902 & 2.680521 \\
\hline & 7.024828 & 0.922138 & -2.323596 \\
\hline & -0.876273 & -4.874512 & 0.889513 \\
\hline & 1.613359 & -3.369270 & -2.427782 \\
\hline & 7.449958 & 5.177520 & -2.680002 \\
\hline & 9.067157 & -0.093879 & 1.300140 \\
\hline & -4.892468 & -4.978534 & -3.078787 \\
\hline & -1.887169 & -5.771960 & 4.962111 \\
\hline & 9.740495 & -2.424314 & 0.728854 \\
\hline & 1.980473 & 2.635469 & 0.334102 \\
\hline & -4.035334 & 0.536223 & 1.419194 \\
\hline & -2.655977 & 9999 & -2.601343 \\
\hline & -6.865451 & 281 & 62906 \\
\hline 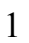 & -3.033176 & 2.893375 & -0.070202 \\
\hline & 0.429670 & 1.367034 & 1.928472 \\
\hline & -0.848294 & 5.402128 & 2.635578 \\
\hline & -0.849490 & 2.122495 & -2.630594 \\
\hline & 2.459353 & -3.774826 & 0.227536 \\
\hline & 3.368119 & -2.291923 & 0.501175 \\
\hline & 2.152312 & -4.929392 & 2.212161 \\
\hline & 1.871223 & -0.648571 & 2.286325 \\
\hline 1 & 1.715219 & -5.007829 & 4.650305 \\
\hline & 1.427865 & -0.720913 & 4.723440 \\
\hline & 1.353272 & -2.900246 & 5.914827 \\
\hline & -6.670496 & -0.200524 & -5.298981 \\
\hline & -7.062148 & -0.950207 & -3.746003 \\
\hline & -7.052196 & 0.822509 & -3.906651 \\
\hline & -4.611061 & 1.038089 & -5678622 \\
\hline & & & \\
\hline
\end{tabular}




$\begin{array}{rrrr}1 & -3.325083 & 1.129140 & -4.466760 \\ 1 & -4.577789 & -1.487731 & -5.451311 \\ 1 & -3.349576 & -1.421001 & -4.174697 \\ 1 & -4.885336 & -2.251891 & -3.874803 \\ 1 & -8.601278 & 1.455164 & 1.214600 \\ 1 & -7.302456 & 2.404914 & 0.464428 \\ 1 & -8.200090 & 1.211199 & -0.489352 \\ 1 & -8.298034 & -1.026388 & 1.631666 \\ 1 & -7.820258 & -1.309038 & -0.052321 \\ 1 & -6.746435 & -1.810769 & 1.267784 \\ 1 & -7.180094 & 0.701597 & 3.014028 \\ 1 & -5.623337 & -0.094642 & 2.749807 \\ 1 & -5.807029 & 1.641516 & 2.405339 \\ 1 & 2.735738 & 4.929435 & 3.745672 \\ 1 & 2.454517 & 4.490186 & 2.050399 \\ 1 & 1.418202 & 5.694674 & 2.841088 \\ 1 & 1.165465 & 4.152786 & 5.555540 \\ 1 & -0.188594 & 4.939027 & 4.729359 \\ 1 & -0.289373 & 3.219422 & 5.147054 \\ 1 & 2.614210 & 2.555490 & 4.448304 \\ 1 & 1.238936 & 1.525233 & 4.013537 \\ 1 & 2.396281 & 2.056682 & 2.766759 \\ 1 & -4.758544 & 5.965347 & -0.155070 \\ 1 & -3.691813 & 4.825355 & -0.989049 \\ 1 & -4.719347 & 4.269302 & 0.352052 \\ 1 & -2.878759 & 7.493460 & 0.221247 \\ 1 & -1.428117 & 6.992225 & 1.105623 \\ 1 & -1.722178 & 6.373121 & -0.529762 \\ 1 & -4.353338 & 6.713106 & 2.135247 \\ 1 & -4.234714 & 5.044712 & 2.732843 \\ 1 & -2.929274 & 6.200285 & 3.054311 \\ 8 & -1.304851 & 3.087604 & -3.077915\end{array}$

$2_{\text {C2-c- }-\psi_{-12 a 3 s 4 s}}$

Number of imaginary frequencies : 0

The smallest frequencies are : $12.9174 \quad 18.0642 \quad 21.2270 \mathrm{~cm}(-1)$

Electronic energy : $\quad H F=-4012.1857774$

Zero-point correction $=\quad 1.291078($ Hartree/Particle $)$

Thermal correction to Energy= $\quad 1.367099$

Thermal correction to Enthalpy= $\quad 1.368043$

Thermal correction to Gibbs Free Energy= 1.180476

Sum of electronic and zero-point Energies $=\quad-4010.894699$

Sum of electronic and thermal Energies $=\quad-4010.818679$

Sum of electronic and thermal Enthalpies $=\quad-4010.817734$

Sum of electronic and thermal Free Energies $=\quad-4011.005301$

Cartesian Coordinates

$\begin{array}{llll}6 & 2.012954 & -4.003848 & 2.747365 \\ 6 & 2.055737 & -2.786416 & 2.071156 \\ 6 & 1.861991 & -1.604584 & 2.792515 \\ 6 & 1.627367 & -1.648004 & 4.161202\end{array}$




$\begin{array}{cccc}6 & 1.588201 & -2.870236 & 4.830099 \\ 6 & 1.782860 & -4.049061 & 4.120248 \\ 6 & 2.381775 & -2.751116 & 0.600502 \\ 7 & 1.424193 & -1.990535 & -0.223896 \\ 15 & 1.793983 & -0.566531 & -1.050877 \\ 45 & 0.068420 & 0.675280 & -1.879911 \\ 1 & 1.057887 & 0.946072 & -3.124282 \\ 6 & 0.332917 & -2.692760 & -0.845546 \\ 6 & -0.943601 & -2.677995 & -0.309913 \\ 6 & -2.018981 & -3.267734 & -1.049137 \\ 6 & -1.753410 & -3.911510 & -2.293144 \\ 6 & -0.426742 & -3.941784 & -2.782754 \\ 6 & 0.581812 & -3.337521 & -2.083110 \\ 6 & -3.360672 & -3.222665 & -0.587860 \\ 6 & -4.374974 & -3.799344 & -1.310392 \\ 6 & -4.101816 & -4.466582 & -2.524149 \\ 6 & -2.818670 & -4.513752 & -3.006305 \\ 6 & -1.223943 & -2.168391 & 1.060196 \\ 6 & -1.443471 & -0.828357 & 1.336627 \\ 6 & -1.789367 & -0.438084 & 2.659215 \\ 6 & -1.931542 & -1.360898 & 3.659966 \\ 6 & -1.733383 & -2.737311 & 3.407916 \\ 6 & -1.363385 & -3.149660 & 2.096071 \\ 6 & -1.896334 & -3.706629 & 4.426516 \\ 6 & -1.715205 & -5.040548 & 4.163161 \\ 6 & -1.341398 & -5.455293 & 2.867903 \\ 6 & -1.162837 & -4.535441 & 1.864636 \\ 15 & -1.457542 & 0.415806 & -0.038745 \\ 6 & -1.364737 & 2.006000 & 0.868371 \\ 6 & -2.262496 & 3.040654 & 0.618077 \\ 6 & -2.112918 & 4.282666 & 1.241091 \\ 6 & -1.056449 & 4.438702 & 2.141365 \\ 6 & -0.141269 & 3.417181 & 2.421169 \\ 6 & -0.295009 & 2.207679 & 1.744686 \\ 6 & -3.079860 & 5.438625 & 0.990103 \\ 6 & -4.122399 & 5.098283 & -0.072818 \\ 6 & 0.958338 & 3.639795 & 3.458880 \\ 6 & 1.798144 & 2.383553 & 3.685803 \\ 6 & -3.210114 & 0.293945 & -0.577539 \\ 6 & -3.485507 & 0.150468 & -1.934279 \\ 6 & -4.800778 & 0.068079 & -2.401291 \\ 6 & -5.830612 & 0.097300 & -1.458267 \\ 6 & -5.586516 & 0.215660 & -0.084108 \\ 6 & -4.262261 & 0.323593 & 0.337652 \\ 6 & -6.697812 & 0.228687 & 0.963819 \\ 6 & -8.082877 & 0.039598 & 0.350731 \\ 6 & -5.046891 & -0.046343 & -3.904044 \\ 6 & -6.533273 & -0.054537 & -4.251040 \\ 8 & 3.018171 & -0.896689 & -2.137696 \\ 6 & 4.266659 & -1.308393 & -1.733226 \\ 6 & 5.121818 & -0.435992 & -1.080064 \\ 6 & 6.365830 & -0.953401 & -0.592874 \\ 6 & 6.735388 & -2.298897 & -0.895380\end{array}$




$\begin{array}{rrrr}6 & 5.857259 & -3.109071 & -1.654659 \\ 6 & 4.639837 & -2.630025 & -2.053402 \\ 6 & 7.967052 & -2.808838 & -0.417634 \\ 6 & 8.798910 & -2.037746 & 0.352546 \\ 6 & 8.421891 & -0.719231 & 0.682931 \\ 6 & 7.240884 & -0.191459 & 0.223213 \\ 6 & 4.698274 & 0.970084 & -0.879873 \\ 6 & 3.475684 & 1.224119 & -0.290625 \\ 6 & 2.972049 & 2.523656 & -0.093939 \\ 6 & 3.721155 & 3.598243 & -0.491174 \\ 6 & 4.969418 & 3.411038 & -1.132302 \\ 6 & 5.459503 & 2.088139 & -1.354528 \\ 6 & 6.667804 & 1.940434 & -2.081956 \\ 6 & 7.369111 & 3.035207 & -2.523170 \\ 6 & 6.902607 & 4.340896 & -2.265225 \\ 6 & 5.723300 & 4.519252 & -1.589140 \\ 8 & 2.696161 & 0.159292 & 0.133089 \\ 6 & -6.681792 & 1.572324 & 1.702128 \\ 6 & -6.461835 & -0.912357 & 1.961271 \\ 6 & -4.425025 & -1.347487 & -4.420365 \\ 6 & -4.394273 & 1.144046 & -4.617256 \\ 6 & 1.887276 & 4.768577 & 3.001800 \\ 6 & 0.315928 & 4.021397 & 4.797824 \\ 6 & -3.815738 & 5.771041 & 2.292803 \\ 6 & -2.301155 & 6.668570 & 0.512142 \\ 1 & 6.960178 & 0.822716 & 0.496014 \\ 1 & -1.955025 & 0.613818 & 2.879966 \\ 1 & 8.233051 & -3.834676 & -0.669635 \\ 1 & -2.174772 & -3.367358 & 5.423617 \\ 1 & 5.334444 & 5.519310 & -1.401980 \\ 1 & -3.584009 & -2.710838 & 0.347074 \\ 1 & -5.397759 & -3.740036 & -0.943641 \\ 1 & 3.350677 & 4.612273 & -0.347492 \\ 1 & -2.209006 & -1.045078 & 4.665098 \\ 1 & 6.159060 & -4.126476 & -1.898007 \\ 1 & 8.290280 & 2.892690 & -3.083745 \\ 1 & 3.940104 & -3.241987 & -2.618664 \\ 1 & -2.595349 & -5.010841 & -3.949276 \\ 1 & -0.224970 & -4.438563 & -3.730371 \\ 1 & -1.187720 & -6.512661 & 2.663692 \\ 1 & 7.036162 & 0.941837 & -2.301487 \\ 1 & -0.860251 & -4.871528 & 0.874862 \\ 1 & 1.600529 & -3.333924 & -2.467435 \\ 1 & 7.470086 & 5.199592 & -2.616328 \\ 1 & 9.070645 & -0.115380 & 1.313461 \\ 1 & -4.913064 & -4.931034 & -3.080681 \\ 1 & -1.852184 & -5.778738 & 4.950241 \\ 1 & 9.740158 & -2.440996 & 0.718625 \\ 1 & 1.988081 & 2.638991 & 0.358787 \\ 1 & -4.041483 & 0.431654 & 1.399446 \\ 1 & -2.647711 & 0.098790 & -2.631043 \\ 1 & -6.858464 & 0.025270 & -1.802001 \\ & -3.080898 & 2.871207 & -0.077837\end{array}$




$\begin{array}{rrrr}1 & 0.407119 & 1.390177 & 1.910823 \\ 1 & -0.942495 & 5.394588 & 2.653068 \\ 6 & -0.849850 & 2.139844 & -2.624957 \\ 1 & 2.451783 & -3.775471 & 0.204102 \\ 1 & 3.374439 & -2.301030 & 0.474350 \\ 1 & 2.156206 & -4.929547 & 2.189207 \\ 1 & 1.899430 & -0.647618 & 2.271513 \\ 1 & 1.741395 & -5.009192 & 4.630958 \\ 1 & 1.478377 & -0.720936 & 4.712870 \\ 1 & 1.401061 & -2.901548 & 5.901610 \\ 1 & -6.656457 & -0.129943 & -5.338746 \\ 1 & -7.055833 & -0.909836 & -3.803141 \\ 1 & -7.036329 & 0.865597 & -3.925996 \\ 1 & -4.594969 & 1.095961 & -5.695961 \\ 1 & -4.788180 & 2.097160 & -4.240620 \\ 1 & -3.305194 & 1.157369 & -4.487032 \\ 1 & -4.573861 & -1.436888 & -5.505192 \\ 1 & -3.345682 & -1.392373 & -4.226503 \\ 1 & -4.885380 & -2.219490 & -3.938026 \\ 1 & -7.441846 & 1.584083 & 2.495041 \\ 1 & -5.709343 & 1.779167 & 2.167378 \\ 1 & -6.902443 & 2.397233 & 1.012030 \\ 1 & -7.255974 & -0.927710 & 2.719810 \\ 1 & -6.464347 & -1.884181 & 1.449218 \\ 1 & -5.503279 & -0.817982 & 2.487292 \\ 1 & -8.841390 & 0.049263 & 1.143636 \\ 1 & -8.335202 & 0.843133 & -0.352863 \\ 1 & -8.169867 & -0.919436 & -0.176473 \\ 1 & 2.663096 & 4.952733 & 3.757087 \\ 1 & 2.391264 & 4.508035 & 2.061618 \\ 1 & 1.345220 & 5.709021 & 2.844608 \\ 1 & 1.090609 & 4.168684 & 5.562186 \\ 1 & -0.262795 & 4.950386 & 4.731602 \\ 1 & -0.359710 & 3.230448 & 5.149136 \\ 1 & 2.544808 & 2.574386 & 4.466688 \\ 1 & 1.176725 & 1.539461 & 4.017903 \\ 1 & 2.343350 & 2.076780 & 2.782792 \\ 1 & -4.787243 & 5.957966 & -0.224205 \\ 1 & -3.659782 & 4.862866 & -1.040251 \\ 1 & -4.749809 & 4.246567 & 0.222532 \\ 1 & -2.989896 & 7.502067 & 0.319429 \\ 1 & -1.568594 & 7.011536 & 1.252478 \\ 1 & -1.761530 & 6.453064 & -0.418982 \\ 1 & -4.517717 & 6.600922 & 2.134698 \\ 1 & -4.390238 & 4.905654 & 2.649586 \\ 1 & -3.125304 & 6.066738 & 3.092294 \\ 8 & -1.311718 & 3.102001 & -3.071698\end{array}$

$2_{\text {C2-c- }-\psi_{-12} 233 a 4 a}$

Number of imaginary frequencies : 0

The smallest frequencies are : $13.6091 \quad 16.736921 .0194 \mathrm{~cm}(-1)$

Electronic energy : $\quad H F=-4012.1870477$ 
Zero-point correction $=$

Thermal correction to Energy=

Thermal correction to Enthalpy=

Thermal correction to Gibbs Free Energy=

Sum of electronic and zero-point Energies=

Sum of electronic and thermal Energies=

Sum of electronic and thermal Enthalpies=

Sum of electronic and thermal Free Energies=
1.290801 (Hartree/Particle)

1.366729

1.367673

1.180960

$-4010.896246$

$-4010.820319$

$-4010.819375$

$-4011.006088$

\section{Cartesian Coordinates}

$\begin{array}{cccc}0 & -3.475367 & 0.046195 & -1.881244 \\ 6 & -3.192971 & 0.323748 & -0.547438 \\ 6 & -4.235212 & 0.431307 & 0.377542 \\ 6 & -5.559925 & 0.268234 & -0.020624 \\ 6 & -5.808647 & 0.013364 & -1.376750 \\ 6 & -4.792619 & -0.101783 & -2.325554 \\ 15 & -1.430957 & 0.455115 & -0.041605 \\ 6 & -1.417461 & -0.752440 & 1.367874 \\ 6 & -1.222158 & -2.102153 & 1.119812 \\ 6 & -1.368365 & -3.057897 & 2.176870 \\ 6 & -1.719474 & -2.610614 & 3.482516 \\ 6 & -1.895617 & -1.226139 & 3.705488 \\ 6 & -1.748592 & -0.327619 & 2.683389 \\ 6 & -1.887243 & -3.555387 & 4.523182 \\ 6 & -1.729611 & -4.897347 & 4.286722 \\ 6 & -1.377100 & -5.346412 & 2.996761 \\ 6 & -1.194222 & -4.451219 & 1.972153 \\ 6 & -0.964625 & -2.635828 & -0.245302 \\ 6 & 0.304743 & -2.667227 & -0.796915 \\ 6 & 0.525819 & -3.293747 & -2.048950 \\ 6 & -0.506203 & -3.848781 & -2.754874 \\ 6 & -1.826221 & -3.794990 & -2.250383 \\ 6 & -2.061011 & -3.191126 & -0.980176 \\ 6 & -3.396060 & -3.128538 & -0.501440 \\ 6 & -4.435908 & -3.631377 & -1.242357 \\ 6 & -4.195870 & -4.248342 & -2.489576 \\ 6 & -2.917306 & -4.327093 & -2.980567 \\ 7 & 1.414294 & -1.983492 & -0.186402 \\ 15 & 1.809258 & -0.581250 & -1.037394 \\ 8 & 3.024707 & -0.952420 & -2.120230 \\ 6 & 4.269415 & -1.371542 & -1.711215 \\ 6 & 5.138236 & -0.496797 & -1.079523 \\ 6 & 6.377511 & -1.019911 & -0.586599 \\ 6 & 6.729148 & -2.375656 & -0.863504 \\ 6 & 5.837708 & -3.190007 & -1.602612 \\ 6 & 4.624446 & -2.704073 & -2.005847 \\ 6 & 7.264940 & -0.252727 & 0.210952 \\ 6 & 8.441371 & -0.785634 & 0.676408 \\ 6 & 8.800958 & -2.114937 & 0.370730 \\ 6 & 7.956522 & -2.891009 & -0.380553 \\ 6 & 4.733640 & 0.918611 & -0.908295 \\ 6 & 3.515829 & 1.200611 & -0.322076\end{array}$




$\begin{array}{rrrr}6 & 3.026647 & 2.509730 & -0.155276 \\ 6 & 3.787426 & 3.566267 & -0.577828 \\ 6 & 5.032567 & 3.350019 & -1.215818 \\ 6 & 5.506855 & 2.016798 & -1.409233 \\ 6 & 5.798648 & 4.438958 & -1.698066 \\ 6 & 6.974751 & 4.231948 & -2.371485 \\ 6 & 7.425413 & 2.915536 & -2.601283 \\ 6 & 6.712053 & 1.839044 & -2.135152 \\ 8 & 2.725380 & 0.154506 & 0.128374 \\ 6 & -6.719373 & 0.301452 & 0.973277 \\ 6 & -7.785599 & 1.302735 & 0.518946 \\ 6 & -5.115825 & -0.311788 & -3.803877 \\ 6 & -6.222351 & -1.354239 & -3.983823 \\ 6 & -1.308208 & 2.065999 & 0.822036 \\ 6 & -2.192633 & 3.102826 & 0.562399 \\ 6 & -2.044004 & 4.350979 & 1.184162 \\ 6 & -0.985786 & 4.513696 & 2.073963 \\ 6 & -0.070042 & 3.485743 & 2.355454 \\ 6 & -0.232447 & 2.272204 & 1.696367 \\ 6 & 1.035797 & 3.715252 & 3.384793 \\ 6 & 1.973765 & 4.826420 & 2.903413 \\ 6 & -3.061955 & 5.449304 & 0.885052 \\ 6 & -4.460874 & 4.957099 & 1.277243 \\ 45 & 0.076400 & 0.632300 & -1.907307 \\ 6 & -0.930774 & 1.953151 & -2.790498 \\ 8 & -1.481041 & 2.799651 & -3.355125 \\ 6 & 0.404872 & 4.125808 & 4.720703 \\ 6 & 1.863856 & 2.454675 & 3.629592 \\ 6 & -3.037930 & 5.774097 & -0.612569 \\ 6 & -2.776490 & 6.734860 & 1.656605 \\ 6 & 2.354965 & -2.749229 & 0.651956 \\ 6 & 2.043189 & -2.736911 & 2.126504 \\ 6 & 1.878284 & -1.532609 & 2.817260 \\ 6 & 1.662969 & -1.534007 & 4.189767 \\ 6 & 1.613090 & -2.736178 & 4.893277 \\ 6 & 1.777937 & -3.937398 & 4.213987 \\ 6 & 1.989945 & -3.934194 & 2.837540 \\ 6 & -7.332526 & -1.102087 & 1.048990 \\ 6 & -6.265178 & 0.698579 & 2.376679 \\ 6 & -5.583455 & 1.029291 & -4.381884 \\ 6 & -3.894075 & -0.780935 & -4.594938 \\ 1 & 1.066243 & 0.862674 & -3.160425 \\ 1 & 6.997384 & 0.769829 & 0.465042 \\ 1 & -1.898259 & 0.731027 & 2.882492 \\ 1 & 8.208846 & -3.924920 & -0.612856 \\ 1 & -2.150024 & -3.190752 & 5.515563 \\ 1 & 5.421780 & 5.447390 & -1.532681 \\ 1 & -3.595978 & -2.654749 & 0.457972 \\ 1 & -5.454587 & -3.550992 & -0.866966 \\ 1 & 3.428935 & 4.587512 & -0.456625 \\ 1 & -2.160261 & -0.884279 & 4.705542 \\ & 6.125838 & -4.215791 & -1.826526 \\ 6.343894 & 2.749867 & -3.159888\end{array}$




$\begin{array}{rrrr}1 & 3.914684 & -3.318689 & -2.555605 \\ 1 & -2.717733 & -4.793453 & -3.944413 \\ 1 & -0.328611 & -4.319804 & -3.720251 \\ 1 & -1.243702 & -6.410437 & 2.813972 \\ 1 & 7.068287 & 0.831671 & -2.333349 \\ 1 & -0.908488 & -4.811700 & 0.985965 \\ 1 & 1.539990 & -3.303869 & -2.444762 \\ 1 & 7.551694 & 5.075969 & -2.742196 \\ 1 & 9.099917 & -0.177461 & 1.292459 \\ 1 & -5.027347 & -4.657489 & -3.059996 \\ 1 & -1.869776 & -5.616204 & 5.090964 \\ 1 & 9.738748 & -2.522343 & 0.741055 \\ 1 & 2.044879 & 2.646903 & 0.296270 \\ 1 & -3.997994 & 0.638844 & 1.418602 \\ 1 & -2.639671 & -0.073621 & -2.567942 \\ 1 & -6.842261 & -0.105534 & -1.700588 \\ 1 & -3.016932 & 2.937411 & -0.130985 \\ 1 & 0.461490 & 1.450026 & 1.873758 \\ 1 & -0.861238 & 5.468529 & 2.580455 \\ 1 & 2.391462 & -3.785052 & 0.281596 \\ 1 & 3.359673 & -2.332664 & 0.505589 \\ 1 & 2.110781 & -4.877468 & 2.304030 \\ 1 & 1.921814 & -0.590654 & 2.270290 \\ 1 & 1.728194 & -4.882215 & 4.751797 \\ 1 & 1.537353 & -0.589185 & 4.716771 \\ 1 & 1.440898 & -2.734561 & 5.967738 \\ 1 & -4.182328 & -0.971441 & -5.636531 \\ 1 & -3.093822 & -0.029524 & -4.616042 \\ 1 & -3.478645 & -1.711823 & -4.184046 \\ 1 & -5.820899 & 0.927159 & -5.449725 \\ 1 & -6.481150 & 1.397714 & -3.868737 \\ 1 & -4.800497 & 1.792875 & -4.279777 \\ 1 & -6.411999 & -1.518087 & -5.052646 \\ 1 & -5.931490 & -2.315574 & -3.539758 \\ 1 & -7.172879 & -1.044667 & -3.533449 \\ 1 & -8.614800 & 1.325312 & 1.238538 \\ 1 & -7.371337 & 2.317024 & 0.450563 \\ 1 & -8.209573 & 1.048299 & -0.459501 \\ 1 & -8.165429 & -1.121096 & 1.764982 \\ 1 & -7.719442 & -1.433011 & 0.076954 \\ 1 & -6.584088 & -1.835158 & 1.380272 \\ 1 & -7.130569 & 0.720982 & 3.050954 \\ 1 & -5.542900 & -0.015105 & 2.795315 \\ 1 & -5.808336 & 1.697423 & 2.390997 \\ 1 & 2.754736 & 5.016653 & 3.651879 \\ 1 & 2.470954 & 4.543461 & 1.966019 \\ 1 & 1.440158 & 5.769076 & 2.731453 \\ 1 & 1.185488 & 4.273396 & 5.478912 \\ 1 & -0.160807 & 5.061843 & 4.644375 \\ 1 & -0.279687 & 3.349382 & 5.086952 \\ 1 & 2.614269 & 2.650184 & 4.405709 \\ & 1.234476 & 1.622082 & 3.975796 \\ & 2.403987 & 2.128996 & 2.730291\end{array}$




$\begin{array}{lrrr}1 & -3.534698 & 7.488472 & 1.409396 \\ 1 & -2.810399 & 6.579260 & 2.742791 \\ 1 & -1.797114 & 7.158353 & 1.399092 \\ 1 & -3.758605 & 6.571047 & -0.840283 \\ 1 & -2.043188 & 6.116734 & -0.925048 \\ 1 & -3.299443 & 4.905926 & -1.228798 \\ 1 & -5.210543 & 5.731315 & 1.065429 \\ 1 & -4.751215 & 4.054784 & 0.723541 \\ 1 & -4.509069 & 4.723357 & 2.349164\end{array}$

$2_{\mathrm{C} 2-\mathrm{c}-} \psi_{-1 \mathrm{a} 2 \mathrm{~s} 3 \mathrm{a} 4 \mathrm{~s}}$

Number of imaginary frequencies : 0

The smallest frequencies are : $13.0046 \quad 16.9777 \quad 21.4520 \mathrm{~cm}(-1)$

Electronic energy : $\quad H F=-4012.1871835$

Zero-point correction $=\quad 1.291116($ Hartree/Particle $)$

Thermal correction to Energy=

1.366960

Thermal correction to Enthalpy=

1.367905

Thermal correction to Gibbs Free Energy=

Sum of electronic and zero-point Energies=

1.181780

Sum of electronic and thermal Energies=

$-4010.896067$

$-4010.820223$

Sum of electronic and thermal Enthalpies=

$-4010.819279$

Sum of electronic and thermal Free Energies=

$-4011.005404$

Cartesian Coordinates

$\begin{array}{cccc}6 & -0.238502 & 2.230254 & 1.713572 \\ 6 & -1.306125 & 2.045778 & 0.824320 \\ 6 & -2.160039 & 3.103952 & 0.545554 \\ 6 & -1.987019 & 4.352560 & 1.160451 \\ 6 & -0.940438 & 4.491909 & 2.068067 \\ 6 & -0.057031 & 3.442457 & 2.370565 \\ 15 & -1.449385 & 0.438718 & -0.045968 \\ 45 & 0.053943 & 0.630825 & -1.912803 \\ 6 & -0.980925 & 1.897625 & -2.842706 \\ 8 & -1.544530 & 2.705943 & -3.448540 \\ 6 & -2.957099 & 5.483885 & 0.825740 \\ 6 & -4.387791 & 5.037955 & 1.150593 \\ 6 & 1.031609 & 3.648738 & 3.423063 \\ 6 & 1.988791 & 4.758523 & 2.978028 \\ 6 & -1.429617 & -0.779111 & 1.354943 \\ 6 & -1.226179 & -2.126264 & 1.098908 \\ 6 & -1.364755 & -3.089086 & 2.150854 \\ 6 & -1.717793 & -2.651937 & 3.459251 \\ 6 & -1.901349 & -1.269817 & 3.690676 \\ 6 & -1.761251 & -0.364383 & 2.673658 \\ 6 & -1.181031 & -4.480031 & 1.938367 \\ 6 & -1.357364 & -5.382289 & 2.957872 \\ 6 & -1.712367 & -4.943118 & 4.250509 \\ 6 & -1.878629 & -3.603618 & 4.494682 \\ 6 & -0.965452 & -2.650882 & -0.269068 \\ 6 & 0.302987 & -2.665267 & -0.823375 \\ 6 & 0.528871 & -3.281282 & -2.079665\end{array}$




$\begin{array}{cccc}6 & -0.497098 & -3.848758 & -2.784174 \\ 6 & -1.815290 & -3.820698 & -2.272906 \\ 6 & -2.055847 & -3.221612 & -1.001252 \\ 6 & -2.897709 & -4.378895 & -2.996174 \\ 6 & -4.173069 & -4.339396 & -2.492473 \\ 6 & -4.418501 & -3.731366 & -1.242216 \\ 6 & -3.388785 & -3.190784 & -0.513471 \\ 7 & 1.408323 & -1.979924 & -0.207002 \\ 6 & 2.353710 & -2.750821 & 0.621305 \\ 6 & 2.044159 & -2.757870 & 2.096285 \\ 6 & 2.006076 & -3.963791 & 2.793454 \\ 6 & 1.797556 & -3.985464 & 4.170249 \\ 6 & 1.621315 & -2.794145 & 4.864000 \\ 6 & 1.655990 & -1.583423 & 4.174410 \\ 6 & 1.867316 & -1.563699 & 2.801341 \\ 15 & 1.797688 & -0.568013 & -1.043645 \\ 8 & 2.711528 & 0.157893 & 0.129589 \\ 6 & 3.500281 & 1.210454 & -0.308499 \\ 6 & 4.718907 & 0.937685 & -0.897568 \\ 6 & 5.490227 & 2.043275 & -1.384983 \\ 6 & 5.013497 & 3.373181 & -1.175512 \\ 6 & 3.767853 & 3.579425 & -0.535233 \\ 6 & 3.008810 & 2.516490 & -0.125604 \\ 6 & 6.695926 & 1.876493 & -2.112687 \\ 6 & 7.407533 & 2.959857 & -2.565370 \\ 6 & 6.954493 & 4.272595 & -2.319615 \\ 6 & 5.777818 & 4.469293 & -1.644118 \\ 6 & 5.126447 & -0.474737 & -1.085756 \\ 6 & 4.259357 & -1.343572 & -1.727868 \\ 6 & 4.617115 & -2.671485 & -2.039357 \\ 6 & 5.831539 & -3.159781 & -1.642645 \\ 6 & 6.721409 & -2.352768 & -0.893681 \\ 6 & 6.367078 & -1.001306 & -0.599785 \\ 6 & 7.253342 & -0.242272 & 0.206816 \\ 6 & 8.431048 & -0.778566 & 0.665152 \\ 6 & 8.793216 & -2.103217 & 0.342826 \\ 6 & 7.950053 & -2.871581 & -0.417736 \\ 8 & 3.013546 & -0.922230 & -2.131262 \\ 6 & -3.217964 & 0.325518 & -0.536141 \\ 6 & -3.518896 & 0.042639 & -1.869173 \\ 6 & -4.839608 & -0.072218 & -2.300617 \\ 6 & -5.852532 & 0.079454 & -1.345323 \\ 6 & -5.585715 & 0.329883 & 0.002797 \\ 6 & -4.249018 & 0.460537 & 0.389466 \\ 6 & -5.184667 & -0.304582 & -3.770526 \\ 6 & -6.279941 & -1.365083 & -3.913053 \\ 6 & -6.677092 & 0.427648 & 1.067483 \\ 6 & -6.596214 & 1.785203 & 1.773963 \\ 6 & -5.680023 & 1.020121 & -4.362461 \\ 6 & -3.972107 & -0.772796 & -4.575492 \\ 6 & -6.466074 & -0.695002 & 2.091231 \\ 6 & -8.079327 & 0.280588 & 0.482815 \\ 6 & -2.856179 & 5.812380 & -0.667906\end{array}$




$\begin{array}{rrrr}6 & -2.663822 & 6.756584 & 1.615580 \\ 6 & 0.375130 & 4.046194 & 4.750747 \\ 6 & 1.844357 & 2.378385 & 3.667448 \\ 1 & 1.054682 & 0.883721 & -3.152589 \\ 1 & 6.983942 & 0.776594 & 0.473500 \\ 1 & -1.914975 & 0.692340 & 2.879900 \\ 1 & 8.204427 & -3.901955 & -0.663123 \\ 1 & -2.143219 & -3.246340 & 5.489253 \\ 1 & 5.399170 & 5.474970 & -1.466453 \\ 1 & -3.596284 & -2.712357 & 0.442645 \\ 1 & -5.435151 & -3.683789 & -0.857284 \\ 1 & 3.407588 & 4.598453 & -0.401348 \\ 1 & -2.166119 & -0.935463 & 4.693243 \\ 1 & 6.121905 & -4.182011 & -1.879545 \\ 1 & 8.326480 & 2.802645 & -3.125647 \\ 1 & 3.908543 & -3.280576 & -2.596688 \\ 1 & -2.693535 & -4.837383 & -3.962790 \\ 1 & -0.315963 & -4.313928 & -3.751721 \\ 1 & -1.216596 & -6.444282 & 2.768902 \\ 1 & 7.054059 & 0.872250 & -2.323012 \\ 1 & -0.893150 & -4.833212 & 0.950180 \\ 1 & 1.541811 & -3.275185 & -2.478633 \\ 1 & 7.530121 & 5.122097 & -2.679690 \\ 1 & 9.088650 & -0.176745 & 1.288404 \\ 1 & -4.997846 & -4.771987 & -3.055082 \\ 1 & -1.847421 & -5.667460 & 5.050692 \\ 1 & 9.732018 & -2.513263 & 0.707627 \\ 1 & 2.027062 & 2.646496 & 0.328271 \\ 1 & -4.006801 & 0.661602 & 1.432994 \\ 1 & -2.691364 & -0.102306 & -2.560677 \\ 1 & -6.887442 & -0.008155 & -1.668558 \\ 1 & -2.975893 & 2.956238 & -0.162072 \\ 1 & 0.434685 & 1.393746 & 1.903089 \\ 1 & -0.799000 & 5.445769 & 2.571838 \\ 1 & 2.394410 & -3.782039 & 0.238787 \\ 1 & 3.356456 & -2.328291 & 0.478823 \\ 1 & 2.135836 & -4.899352 & 2.248574 \\ 1 & 1.897940 & -0.615269 & 2.264876 \\ 1 & 1.758952 & -4.936996 & 4.697024 \\ 1 & 1.521176 & -0.646100 & 4.712483 \\ 1 & 1.451879 & -2.806932 & 5.938816 \\ 1 & -4.273870 & -0.969902 & -5.612028 \\ 1 & -3.174935 & -0.018708 & -4.610694 \\ 1 & -3.548372 & -1.700791 & -4.165877 \\ 1 & -5.938239 & 0.895476 & -5.423039 \\ 1 & -6.571650 & 1.388177 & -3.838800 \\ 1 & -4.904446 & 1.794385 & -4.290823 \\ 1 & -6.487924 & -1.548873 & -4.975290 \\ 1 & -5.966214 & -2.314838 & -3.459595 \\ 1 & -7.225242 & -1.061830 & -3.447760 \\ & -6.760721 & 1.851246 & 2.559805 \\ 1 & 1.950816 & 2.247371 \\ 1 & & 2.606723 & 1.066417\end{array}$




$\begin{array}{rrrr}1 & -7.233995 & -0.648861 & 2.875298 \\ 1 & -6.533706 & -1.679837 & 1.609937 \\ 1 & -5.485361 & -0.631710 & 2.580026 \\ 1 & -8.823237 & 0.359710 & 1.285619 \\ 1 & -8.302660 & 1.066131 & -0.250604 \\ 1 & -8.222777 & -0.693949 & -0.001446 \\ 1 & 2.756461 & 4.928694 & 3.744823 \\ 1 & 2.502009 & 4.486423 & 2.046130 \\ 1 & 1.468283 & 5.709443 & 2.812027 \\ 1 & 1.139950 & 4.178841 & 5.527662 \\ 1 & -0.183104 & 4.986686 & 4.673371 \\ 1 & -0.322230 & 3.269578 & 5.091734 \\ 1 & 2.584169 & 2.559132 & 4.457246 \\ 1 & 1.202618 & 1.548057 & 3.995818 \\ 1 & 2.395482 & 2.057861 & 2.772963 \\ 1 & -3.390706 & 7.532736 & 1.345086 \\ 1 & -2.742239 & 6.596089 & 2.698790 \\ 1 & -1.664340 & 7.154171 & 1.397065 \\ 1 & -3.537809 & 6.634855 & -0.923541 \\ 1 & -1.837276 & 6.119715 & -0.936097 \\ 1 & -3.122241 & 4.954616 & -1.296578 \\ 1 & -5.099683 & 5.839357 & 0.911305 \\ 1 & -4.682826 & 4.151039 & 0.575559 \\ 1 & -4.493919 & 4.798271 & 2.217058\end{array}$

$2_{\text {C2-c- }-\psi_{-12} 25354 a}$

Number of imaginary frequencies : 0

The smallest frequencies are : $11.4856 \quad 16.2180 \quad 20.4232 \mathrm{~cm}(-1)$

Electronic energy : $\quad H F=-4012.1862369$

Zero-point correction $=\quad 1.291771($ Hartree/Particle $)$

Thermal correction to Energy= $\quad 1.367478$

Thermal correction to Enthalpy= $\quad 1.368422$

Thermal correction to Gibbs Free Energy= 1.182522

Sum of electronic and zero-point Energies $=\quad-4010.894465$

Sum of electronic and thermal Energies $=\quad-4010.818759$

Sum of electronic and thermal Enthalpies $=\quad-4010.817814$

Sum of electronic and thermal Free Energies $=\quad-4011.003715$

Cartesian Coordinates

$\begin{array}{cccc}6 & 1.948880 & -3.993043 & 2.781911 \\ 6 & 2.010686 & -2.778819 & 2.101655 \\ 6 & 1.814691 & -1.592565 & 2.814818 \\ 6 & 1.560745 & -1.627557 & 4.180007 \\ 6 & 1.503306 & -2.846717 & 4.853481 \\ 6 & 1.698533 & -4.030213 & 4.151538 \\ 6 & 2.353777 & -2.748550 & 0.635042 \\ 7 & 1.404721 & -1.987390 & -0.198579 \\ 15 & 1.790756 & -0.579275 & -1.045591 \\ 45 & 0.075332 & 0.658736 & -1.902273 \\ 6 & -0.817252 & 2.119135 & -2.683360 \\ 8 & -1.256690 & 3.078997 & -3.156754\end{array}$




$\begin{array}{cccc}6 & 0.314951 & -2.689076 & -0.824520 \\ 6 & -0.969644 & -2.653762 & -0.308813 \\ 6 & -2.038511 & -3.246441 & -1.056104 \\ 6 & -1.758354 & -3.913153 & -2.284982 \\ 6 & -0.424903 & -3.962145 & -2.753591 \\ 6 & 0.577317 & -3.355406 & -2.047691 \\ 6 & -3.387079 & -3.188264 & -0.615968 \\ 6 & -4.392493 & -3.776446 & -1.342110 \\ 6 & -4.104787 & -4.464663 & -2.540624 \\ 6 & -2.815443 & -4.522546 & -3.004054 \\ 6 & -1.262250 & -2.128696 & 1.053478 \\ 6 & -1.464515 & -0.784241 & 1.321701 \\ 6 & -1.816783 & -0.382045 & 2.639378 \\ 6 & -1.984505 & -1.296987 & 3.642938 \\ 6 & -1.802647 & -2.677352 & 3.399935 \\ 6 & -1.423668 & -3.102055 & 2.094527 \\ 6 & -1.987907 & -3.638092 & 4.422788 \\ 6 & -1.816674 & -4.975463 & 4.170755 \\ 6 & -1.431859 & -5.402512 & 2.882817 \\ 6 & -1.233991 & -4.491388 & 1.875124 \\ 15 & -1.453351 & 0.458706 & -0.055336 \\ 6 & -1.303784 & 2.041118 & 0.857905 \\ 6 & -2.180777 & 3.095259 & 0.648462 \\ 6 & -2.007318 & 4.320476 & 1.309317 \\ 6 & -0.930166 & 4.442993 & 2.182529 \\ 6 & -0.020071 & 3.397299 & 2.411884 \\ 6 & -0.211864 & 2.205910 & 1.721506 \\ 6 & -3.018831 & 5.438652 & 1.067137 \\ 6 & -2.701865 & 6.696344 & 1.871725 \\ 6 & 1.111596 & 3.585597 & 3.421425 \\ 6 & 1.964097 & 2.326941 & 3.573302 \\ 6 & -3.215672 & 0.380095 & -0.573100 \\ 6 & -3.502954 & 0.211599 & -1.920584 \\ 6 & -4.826057 & 0.119152 & -2.376119 \\ 6 & -5.839762 & 0.161815 & -1.424430 \\ 6 & -5.584806 & 0.312968 & -0.049973 \\ 6 & -4.261707 & 0.434122 & 0.357782 \\ 6 & -6.744539 & 0.288506 & 0.943784 \\ 6 & -6.285294 & 0.565194 & 2.374058 \\ 6 & -5.084525 & -0.025205 & -3.873752 \\ 6 & -6.573960 & -0.057189 & -4.205208 \\ 8 & 3.015425 & -0.939777 & -2.123120 \\ 6 & 4.259905 & -1.353869 & -1.708530 \\ 6 & 5.119886 & -0.477435 & -1.067203 \\ 6 & 6.358069 & -0.995759 & -0.566724 \\ 6 & 6.719119 & -2.348545 & -0.845814 \\ 6 & 5.838347 & -3.163897 & -1.596400 \\ 6 & 4.625835 & -2.682565 & -2.007346 \\ 6 & 7.945079 & -2.859415 & -0.354596 \\ 6 & 8.779283 & -2.081705 & 0.406372 \\ 6 & 8.410549 & -0.755206 & 0.713459 \\ 6 & 7.235247 & -0.226654 & 0.240289 \\ 6 & 4.707699 & 0.935450 & -0.895241\end{array}$




$\begin{array}{rrrr}6 & 3.484978 & 1.210723 & -0.316210 \\ 6 & 2.989826 & 2.517962 & -0.150751 \\ 6 & 3.748969 & 3.578243 & -0.567082 \\ 6 & 4.998769 & 3.368349 & -1.197927 \\ 6 & 5.479632 & 2.037401 & -1.389958 \\ 6 & 6.689917 & 1.865402 & -2.108760 \\ 6 & 7.401650 & 2.945343 & -2.569415 \\ 6 & 6.944183 & 4.259628 & -2.340876 \\ 6 & 5.763212 & 4.461014 & -1.674264 \\ 8 & 2.696432 & 0.160096 & 0.127584 \\ 6 & -7.791239 & 1.342143 & 0.568785 \\ 6 & -7.384557 & -1.104469 & 0.910660 \\ 6 & -4.452222 & -1.327371 & -4.374866 \\ 6 & -4.453423 & 1.161096 & -4.612254 \\ 6 & 2.022180 & 4.735272 & 2.978881 \\ 6 & 0.510109 & 3.914317 & 4.793101 \\ 6 & -4.414200 & 4.949195 & 1.474566 \\ 6 & -3.024863 & 5.810133 & -0.419692 \\ 1 & 1.071479 & 0.892969 & -3.148591 \\ 1 & 6.960546 & 0.793762 & 0.495397 \\ 1 & -1.968427 & 0.673399 & 2.853401 \\ 1 & 8.204715 & -3.891136 & -0.588582 \\ 1 & -2.273829 & -3.289035 & 5.414393 \\ 1 & 5.381159 & 5.467688 & -1.510046 \\ 1 & -3.623015 & -2.662134 & 0.307664 \\ 1 & -5.420385 & -3.710899 & -0.991259 \\ 1 & 3.385278 & 4.597718 & -0.446446 \\ 1 & -2.267634 & -0.971816 & 4.643504 \\ 1 & 6.134023 & -4.186835 & -1.823505 \\ 1 & 8.324143 & 2.784068 & -3.122682 \\ 1 & 3.925351 & -3.298263 & -2.567408 \\ 1 & -2.579847 & -5.036232 & -3.935051 \\ 1 & -0.212729 & -4.476044 & -3.689718 \\ 1 & -1.285251 & -6.462577 & 2.687581 \\ 1 & 7.051512 & 0.859748 & -2.305985 \\ 1 & -0.923223 & -4.837662 & 0.891468 \\ 1 & 1.601746 & -3.366490 & -2.415760 \\ 1 & 7.519840 & 5.106448 & -2.707190 \\ 1 & 3.347956 & -2.299252 & 0.517728 \\ 1 & 2.092765 & -4.922205 & 2.229709 \\ 1 & 1.864198 & -0.638948 & 2.288502 \\ 1 & -4.909694 & -0.145781 & 1.336719 \\ 1 & -1.969571 & -5.706877 & -3.100178 \\ 1 & 9.716057 & -2.485645 & 4.961218 \\ 1 & 2.005152 & 2.650954 & 0.783020 \\ 1 & -4.024638 & 0.565155 & 1.410909 \\ 1 & -2.673233 & 0.140967 & -2.625032 \\ 1 & -6.874151 & 0.071308 & -1.750244 \\ 1 & -3.018743 & 2.962693 & -0.035557 \\ & 0.474189 & 1.370264 & 1.862253 \\ 1 & 2.784399 & 5.379498 & 2.716794 \\ 1 & & & \\ 1 & & & \end{array}$




$\begin{array}{rrrr}1 & 1.642902 & -4.987585 & 4.666022 \\ 1 & 1.407855 & -0.697146 & 4.725196 \\ 1 & 1.300440 & -2.871990 & 5.922284 \\ 1 & -6.707576 & -0.152276 & -5.290125 \\ 1 & -7.080873 & -0.911354 & -3.737413 \\ 1 & -7.084908 & 0.862061 & -3.890069 \\ 1 & -4.653389 & 1.086605 & -5.689559 \\ 1 & -4.865125 & 2.114411 & -4.256025 \\ 1 & -3.364806 & 1.196765 & -4.482412 \\ 1 & -4.609176 & -1.434424 & -5.456888 \\ 1 & -3.370720 & -1.359170 & -4.190411 \\ 1 & -4.899641 & -2.196886 & -3.876310 \\ 1 & -8.621571 & 1.323710 & 1.287221 \\ 1 & -7.358260 & 2.350851 & 0.580575 \\ 1 & -8.217038 & 1.172909 & -0.427168 \\ 1 & -8.219901 & -1.161522 & 1.621703 \\ 1 & -7.774691 & -1.353269 & -0.084025 \\ 1 & -6.651227 & -1.874823 & 1.186013 \\ 1 & -7.150649 & 0.547152 & 3.048487 \\ 1 & -5.573968 & -0.190399 & 2.733776 \\ 1 & -5.813018 & 1.552253 & 2.468871 \\ 1 & 2.820200 & 4.893207 & 3.716744 \\ 1 & 2.498077 & 4.513038 & 2.014615 \\ 1 & 1.473932 & 5.679407 & 2.875233 \\ 1 & 1.307577 & 4.034482 & 5.538597 \\ 1 & -0.071585 & 4.843726 & 4.779362 \\ 1 & -0.153300 & 3.108656 & 5.134276 \\ 1 & 2.743075 & 2.497173 & 4.326905 \\ 1 & 1.361498 & 1.469734 & 3.906126 \\ 1 & 2.469610 & 2.050012 & 2.638009 \\ 1 & -3.455949 & 7.465802 & 1.663904 \\ 1 & -2.715343 & 6.507759 & 2.953105 \\ 1 & -1.723028 & 7.116441 & 1.606718 \\ 1 & -3.739658 & 6.623173 & -0.605232 \\ 1 & -2.033072 & 6.148988 & -0.745415 \\ 1 & -3.313478 & 4.965244 & -1.055677 \\ 1 & -5.160063 & 5.736446 & 1.300607 \\ 1 & -4.724920 & 4.065166 & 0.902830 \\ 1 & -4.442491 & 4.685728 & 2.540262\end{array}$

$2_{\mathrm{C} 2-\mathrm{c}-}-\psi_{-1 \mathrm{a} 2 \mathrm{~s} 3 \mathrm{~s} 4 \mathrm{~s}}$

Number of imaginary frequencies : 0

The smallest frequencies are : $12.6671 \quad 15.9488 \quad 17.1706 \mathrm{~cm}(-1)$

Electronic energy : $\quad H F=-4012.1861786$

Zero-point correction $=\quad 1.291022($ Hartree/Particle $)$

Thermal correction to Energy= $\quad 1.367052$

Thermal correction to Enthalpy= $\quad 1.367996$

Thermal correction to Gibbs Free Energy= 1.179939

Sum of electronic and zero-point Energies $=\quad-4010.895157$

Sum of electronic and thermal Energies $=\quad-4010.819126$

Sum of electronic and thermal Enthalpies $=\quad-4010.818182$

Sum of electronic and thermal Free Energies $=\quad-4011.006240$ 
Cartesian Coordinates

$\begin{array}{cccc}0 & & & \\ 6 & 2.003399 & -4.015450 & 2.727341 \\ 6 & 2.050431 & -2.795453 & 2.056101 \\ 6 & 1.857835 & -1.616056 & 2.781616 \\ 6 & 1.620848 & -1.664093 & 4.149687 \\ 6 & 1.577804 & -2.888779 & 4.813804 \\ 6 & 1.770665 & -4.065322 & 4.099646 \\ 6 & 2.378715 & -2.754262 & 0.586130 \\ 7 & 1.424039 & -1.986887 & -0.235185 \\ 15 & 1.795931 & -0.558284 & -1.052998 \\ 45 & 0.070726 & 0.685992 & -1.878777 \\ 6 & -0.835045 & 2.157862 & -2.623461 \\ 8 & -1.283007 & 3.125813 & -3.071810 \\ 6 & 0.333282 & -2.683092 & -0.864385 \\ 6 & -0.945498 & -2.666629 & -0.334089 \\ 6 & -2.019205 & -3.250272 & -1.080742 \\ 6 & -1.749552 & -3.889260 & -2.326476 \\ 6 & -0.420970 & -3.921059 & -2.810523 \\ 6 & 0.586019 & -3.322950 & -2.103642 \\ 6 & -3.362706 & -3.205995 & -0.624470 \\ 6 & -4.374252 & -3.780226 & -1.352994 \\ 6 & -4.097173 & -4.441757 & -2.568914 \\ 6 & -2.812477 & -4.486893 & -3.046815 \\ 6 & -1.227691 & -2.165737 & 1.039171 \\ 6 & -1.443592 & -0.827389 & 1.326659 \\ 6 & -1.787687 & -0.446695 & 2.652571 \\ 6 & -1.932301 & -1.377005 & 3.645980 \\ 6 & -1.738045 & -2.751959 & 3.382819 \\ 6 & -1.369037 & -3.154694 & 2.067645 \\ 6 & -1.902357 & -3.728954 & 4.393846 \\ 6 & -1.722958 & -5.061096 & 4.120228 \\ 6 & -1.349666 & -5.466223 & 2.821884 \\ 6 & -1.170255 & -4.538920 & 1.825660 \\ 15 & -1.456360 & 0.427984 & -0.038119 \\ 6 & -1.349765 & 2.010679 & 0.879873 \\ 6 & -2.235487 & 3.051113 & 0.639489 \\ 6 & -2.087920 & 4.288205 & 1.283095 \\ 6 & -1.034553 & 4.433260 & 2.181653 \\ 6 & -0.120652 & 3.399690 & 2.448203 \\ 6 & -0.278822 & 2.199496 & 1.764026 \\ 6 & -3.092594 & 5.398646 & 0.984046 \\ 6 & -2.819151 & 6.664831 & 1.791024 \\ 6 & 0.980296 & 3.610201 & 3.486886 \\ 6 & 1.821352 & 2.352051 & 3.697248 \\ 6 & -3.214717 & 0.322834 & -0.564263 \\ 6 & -3.498879 & 0.190785 & -1.920185 \\ 6 & -4.817111 & 0.109256 & -2.379365 \\ 6 & -5.840496 & 0.126571 & -1.429313 \\ 6 & -5.588205 & 0.234663 & -0.055443 \\ 6 & -4.261390 & 0.344951 & 0.358464 \\ 6 & -6.693454 & 0.213650 & 0.999542 \\ & & & \\ 6\end{array}$




\begin{tabular}{rrrr}
6 & -8.084542 & 0.075524 & 0.386429 \\
6 & -5.072514 & 0.002608 & -3.881135 \\
6 & -6.561061 & -0.012445 & -4.218493 \\
8 & 3.020473 & -0.882663 & -2.141195 \\
6 & 4.268052 & -1.298921 & -1.738541 \\
6 & 5.124139 & -0.432126 & -1.079177 \\
6 & 6.367182 & -0.954548 & -0.594772 \\
6 & 6.735032 & -2.298453 & -0.906232 \\
6 & 5.856176 & -3.102263 & -1.671383 \\
6 & 4.639598 & -2.618864 & -2.067429 \\
6 & 7.965826 & -2.813311 & -0.431526 \\
6 & 8.798406 & -2.048625 & 0.344232 \\
6 & 8.422977 & -0.731929 & 0.683545 \\
6 & 7.242891 & -0.199407 & 0.226929 \\
6 & 4.702668 & 0.973256 & -0.870104 \\
6 & 3.480114 & 1.225504 & -0.279994 \\
6 & 2.979113 & 2.524582 & -0.073790 \\
6 & 3.730543 & 3.600461 & -0.463138 \\
6 & 4.978460 & 3.415380 & -1.105539 \\
6 & 5.466130 & 2.093138 & -1.336787 \\
6 & 6.674305 & 1.948176 & -2.064973 \\
6 & 7.377666 & 3.044649 & -2.498623 \\
6 & 6.913501 & 4.349408 & -2.231844 \\
6 & 5.734450 & 4.525306 & -1.554676 \\
8 & 2.698233 & 0.159290 & 0.135748 \\
6 & -6.657256 & 1.513144 & 1.811539 \\
6 & -6.465016 & -0.980478 & 1.934786 \\
6 & -4.446683 & -1.292073 & -4.409307 \\
6 & -4.431941 & 1.201110 & -4.591485 \\
6 & 1.908180 & 4.745126 & 3.042696 \\
6 & 0.340116 & 3.975081 & 4.831660 \\
6 & -4.505270 & 4.908195 & 1.323945 \\
6 & -3.025273 & 5.753814 & -0.505607 \\
1 & 1.062907 & 0.956481 & -3.120617 \\
1 & 6.963425 & 0.813240 & 0.506592 \\
1 & -1.950166 & 0.603966 & 2.881773 \\
1 & 8.230558 & -3.837746 & -0.690461 \\
1 & -2.179938 & -3.397017 & 5.393650 \\
1 & 5.347434 & 5.524772 & -1.360661 \\
1 & -3.589165 & -2.697643 & 0.311756 \\
1 & -5.398528 & -3.723627 & -0.990398 \\
1 & 3.362263 & 4.614211 & -0.312023 \\
1 & -2.208012 & -1.068411 & 4.653827 \\
1 & 6.156761 & -4.118375 & -1.921553 \\
1 & 8.298651 & 2.904286 & -3.060043 \\
1 & 3.939591 & -3.226033 & -2.637445 \\
1 & -2.585745 & -4.979807 & -3.991152 \\
1 & -0.216489 & -4.414325 & -3.759386 \\
1 & -1.197177 & -6.522158 & 2.609524 \\
1 & 7.040851 & 0.950426 & -2.291275 \\
& 1.606460 & -3.867983 & 0.833327 \\
\hline & 7.482604 & 5.209439 & -2.483199 \\
6 & & -2.577006
\end{tabular}




$\begin{array}{rrrr}1 & 9.072242 & -0.133302 & 1.318511 \\ 1 & -4.906686 & -4.903421 & -3.130250 \\ 1 & -1.860726 & -5.805105 & 4.901663 \\ 1 & 9.738980 & -2.455611 & 0.707898 \\ 1 & 1.995678 & 2.638528 & 0.380533 \\ 1 & -4.034340 & 0.441935 & 1.420058 \\ 1 & -2.665567 & 0.144376 & -2.622575 \\ 1 & -6.870135 & 0.052365 & -1.767100 \\ 1 & -3.054505 & 2.901215 & -0.063829 \\ 1 & 0.416922 & 1.375734 & 1.925533 \\ 1 & -0.910884 & 5.378768 & 2.705550 \\ 1 & 2.446100 & -3.776884 & 0.184846 \\ 1 & 3.372866 & -2.306486 & 0.463243 \\ 1 & 2.145492 & -4.939319 & 2.165884 \\ 1 & 1.897836 & -0.657332 & 2.264131 \\ 1 & 1.726268 & -5.027296 & 4.606624 \\ 1 & 1.472514 & -0.738657 & 4.704356 \\ 1 & 1.388810 & -2.923838 & 5.884879 \\ 1 & -6.690916 & -0.081323 & -5.305852 \\ 1 & -7.075387 & -0.873953 & -3.772959 \\ 1 & -7.067705 & 0.902319 & -3.884007 \\ 1 & -4.634371 & 1.155016 & -5.669938 \\ 1 & -4.834243 & 2.149186 & -4.211071 \\ 1 & -3.342823 & 1.224054 & -4.463193 \\ 1 & -4.601401 & -1.375232 & -5.493790 \\ 1 & -3.366034 & -1.333579 & -4.221936 \\ 1 & -4.900054 & -2.169458 & -3.930098 \\ 1 & -7.429591 & 1.496537 & 2.592356 \\ 1 & -5.689705 & 1.667414 & 2.305276 \\ 1 & -6.847795 & 2.382651 & 1.168602 \\ 1 & -7.248205 & -1.020444 & 2.703878 \\ 1 & -6.492206 & -1.925196 & 1.375442 \\ 1 & -5.496523 & -0.927454 & 2.448697 \\ 1 & -8.838726 & 0.062245 & 1.183359 \\ 1 & -8.325736 & 0.914115 & -0.279362 \\ 1 & -8.191702 & -0.857239 & -0.182321 \\ 1 & 2.684422 & 4.920273 & 3.799686 \\ 1 & 2.411716 & 4.496466 & 2.099074 \\ 1 & 1.365873 & 5.687332 & 2.897900 \\ 1 & 1.116116 & 4.109531 & 5.597066 \\ 1 & -0.235737 & 4.906599 & 4.779124 \\ 1 & -0.337205 & 3.181057 & 5.172712 \\ 1 & 2.571827 & 2.535271 & 4.476299 \\ 1 & 1.201567 & 1.504835 & 4.024412 \\ 1 & 2.361931 & 2.054230 & 2.788487 \\ 1 & -3.568899 & 7.427220 & 1.544927 \\ 1 & -2.876951 & 6.484047 & 2.872352 \\ 1 & -1.833021 & 7.090395 & 1.564582 \\ 1 & -3.730856 & 6.563471 & -0.735968 \\ 1 & -2.018678 & 6.089597 & -0.786282 \\ 1 & -3.280186 & 4.900139 & -1.144712 \\ 1 & -5.242076 & 5.695202 & 1.114084 \\ 1 & -4.786611 & 4.025902 & 0.734954\end{array}$


$2_{\text {C2-t- }} \psi_{-1 \text {-2a3a4a }}$

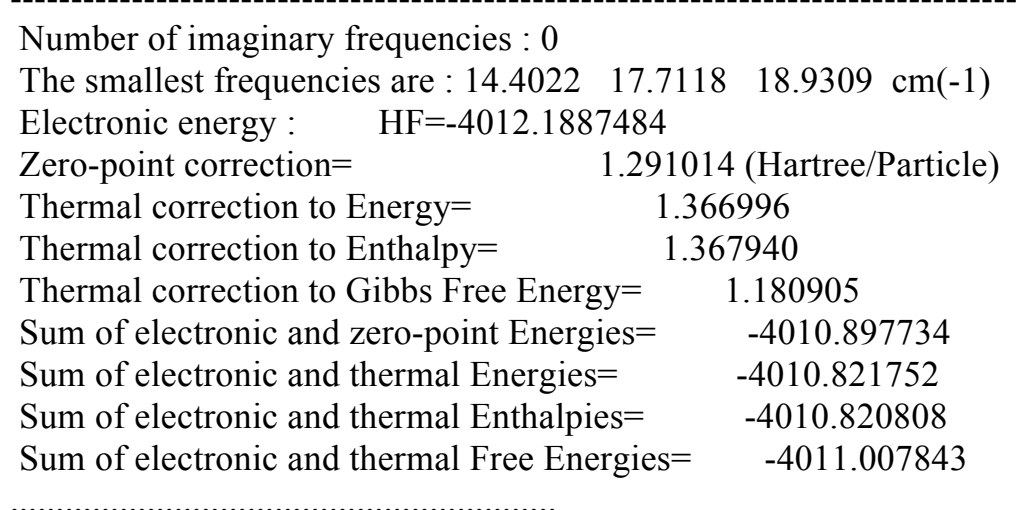

Cartesian Coordinates

$\begin{array}{cccc}0 & -3.488765 & 0.086297 & -2.005419 \\ 6 & -3.232073 & 0.398982 & -0.674373 \\ 6 & -4.290298 & 0.542696 & 0.227425 \\ 6 & -5.609113 & 0.388686 & -0.191878 \\ 6 & -5.832573 & 0.077721 & -1.540065 \\ 6 & -4.799291 & -0.082093 & -2.463736 \\ 15 & -1.489894 & 0.516808 & -0.111810 \\ 6 & -1.547853 & -0.657935 & 1.326591 \\ 6 & -1.374376 & -2.018578 & 1.127293 \\ 6 & -1.596589 & -2.938644 & 2.204472 \\ 6 & -1.999153 & -2.444176 & 3.477814 \\ 6 & -2.143875 & -1.049426 & 3.653523 \\ 6 & -1.924131 & -0.186596 & 2.614408 \\ 6 & -2.252490 & -3.352635 & 4.533411 \\ 6 & -2.126019 & -4.704972 & 4.342699 \\ 6 & -1.716900 & -5.201046 & 3.087319 \\ 6 & -1.451181 & -4.342087 & 2.049937 \\ 6 & -1.047993 & -2.610566 & -0.198775 \\ 6 & 0.255762 & -2.710655 & -0.652281 \\ 6 & 0.547601 & -3.425106 & -1.840717 \\ 6 & -0.446593 & -4.000705 & -2.582883 \\ 6 & -1.797944 & -3.876224 & -2.184317 \\ 6 & -2.105451 & -3.181351 & -0.977791 \\ 6 & -3.467900 & -3.059676 & -0.600193 \\ 6 & -4.465293 & -3.598786 & -1.373691 \\ 6 & -4.154839 & -4.302864 & -2.557550 \\ 6 & -2.847833 & -4.435181 & -2.953161 \\ 7 & 1.337439 & -2.016982 & -0.004638 \\ 15 & 1.776376 & -0.638112 & -0.872681 \\ 8 & 3.000843 & -1.073022 & -1.938174 \\ 6 & 4.243309 & -1.479530 & -1.513486 \\ 6 & 5.114182 & -0.583795 & -0.914666 \\ 6 & 6.355408 & -1.084955 & -0.406983 \\ 6 & 6.708010 & -2.449896 & -0.632200\end{array}$




$\begin{array}{rrrr}6 & 5.814941 & -3.291752 & -1.337538 \\ 6 & 4.600781 & -2.821947 & -1.757585 \\ 6 & 7.244979 & -0.284957 & 0.355066 \\ 6 & 8.424065 & -0.797303 & 0.836500 \\ 6 & 8.783869 & -2.137651 & 0.583089 \\ 6 & 7.937670 & -2.944207 & -0.133451 \\ 6 & 4.703936 & 0.834574 & -0.795010 \\ 6 & 3.490969 & 1.126989 & -0.203899 \\ 6 & 2.986092 & 2.437352 & -0.099415 \\ 6 & 3.725463 & 3.481096 & -0.587761 \\ 6 & 4.966526 & 3.250247 & -1.228494 \\ 6 & 5.458096 & 1.915508 & -1.357233 \\ 6 & 5.712069 & 4.322866 & -1.775214 \\ 6 & 6.884822 & 4.096752 & -2.448302 \\ 6 & 7.352824 & 2.776501 & -2.612664 \\ 6 & 6.659238 & 1.716134 & -2.084168 \\ 8 & 2.716611 & 0.088009 & 0.290075 \\ 6 & -6.791024 & 0.514476 & 0.767481 \\ 6 & -7.777158 & 1.566327 & 0.249324 \\ 6 & -5.102417 & -0.364995 & -3.934706 \\ 6 & -6.198898 & -1.424059 & -4.077558 \\ 6 & -1.339401 & 2.142639 & 0.718419 \\ 6 & -2.202335 & 3.194690 & 0.424073 \\ 6 & -2.039683 & 4.441065 & 1.034181 \\ 6 & -0.992640 & 4.590784 & 1.946865 \\ 6 & -0.111733 & 3.552395 & 2.268835 \\ 6 & -0.288727 & 2.330750 & 1.620089 \\ 6 & 0.971507 & 3.768874 & 3.325050 \\ 6 & 1.945783 & 4.854519 & 2.857906 \\ 6 & -2.980243 & 5.612780 & 0.757572 \\ 6 & -3.664152 & 6.034141 & 2.063177 \\ 45 & 0.141878 & 0.620518 & -1.843279 \\ 6 & 1.183783 & 1.208524 & -3.279464 \\ 8 & 1.823639 & 1.627654 & -4.145816 \\ 6 & 0.312950 & 4.207668 & 4.638438 \\ 6 & 1.765142 & 2.493595 & 3.605681 \\ 6 & -2.178223 & 6.792014 & 0.198005 \\ 6 & -4.068710 & 5.253172 & -0.251417 \\ 6 & 2.246118 & -2.777003 & 0.873247 \\ 6 & 1.858912 & -2.747702 & 2.328735 \\ 6 & 1.681364 & -1.530680 & 2.993136 \\ 6 & 1.373570 & -1.507272 & 4.347335 \\ 6 & 1.243043 & -2.698994 & 5.058499 \\ 6 & 1.419903 & -3.912836 & 4.405313 \\ 6 & 1.724522 & -3.934124 & 3.046305 \\ 6 & -7.495631 & -0.843039 & 0.867965 \\ 6 & -6.352455 & 0.928678 & 2.170749 \\ 6 & -5.575345 & 0.939871 & -4.586185 \\ 6 & -3.867368 & -0.865710 & -4.684044 \\ 1 & 6.976595 & 0.746661 & 0.568795 \\ 1 & -2.053352 & 0.880714 & 2.777442 \\ 1 & 8.190783 & -3.986189 & -0.325495 \\ & -2.556254 & -2.951296 & 5.499512 \\ 6\end{array}$




$\begin{array}{rrrr}1 & 5.322419 & 5.333283 & -1.658973 \\ 1 & -3.721954 & -2.511113 & 0.305233 \\ 1 & -5.504897 & -3.477689 & -1.074728 \\ 1 & 3.351980 & 4.501689 & -0.516304 \\ 1 & -2.444497 & -0.670468 & 4.629714 \\ 1 & 6.103109 & -4.325070 & -1.523821 \\ 1 & 8.268951 & 2.594840 & -3.170159 \\ 1 & 3.894096 & -3.457745 & -2.286434 \\ 1 & -2.592924 & -4.969160 & -3.867561 \\ 1 & -0.213853 & -4.543448 & -3.497619 \\ 1 & -1.605782 & -6.273273 & 2.941322 \\ 1 & 7.027797 & 0.704530 & -2.231913 \\ 1 & -1.123352 & -4.741079 & 1.092228 \\ 1 & 1.586846 & -3.489300 & -2.157282 \\ 1 & 7.446098 & 4.927883 & -2.868801 \\ 1 & 9.084780 & -0.164189 & 1.424493 \\ 1 & -4.954451 & -4.735471 & -3.155529 \\ 1 & -2.332959 & -5.395804 & 5.157018 \\ 1 & 9.723567 & -2.528922 & 0.965810 \\ 1 & 2.007631 & 2.587322 & 0.354386 \\ 1 & -4.072158 & 0.777903 & 1.266312 \\ 1 & -2.640332 & -0.028145 & -2.676732 \\ 1 & -6.860829 & -0.045045 & -1.879723 \\ 1 & -3.006063 & 3.031121 & -0.289604 \\ 1 & 0.377178 & 1.494045 & 1.831345 \\ 1 & -0.862736 & 5.555659 & 2.437512 \\ 1 & -1.008146 & 1.591771 & -2.486482 \\ 1 & 2.296706 & -3.816431 & 0.515267 \\ 1 & 3.255776 & -2.360004 & 0.765319 \\ 1 & 1.853301 & -4.887030 & 2.532187 \\ 1 & 1.790437 & -0.599297 & 2.437327 \\ 1 & 1.310793 & -4.848472 & 4.950297 \\ 1 & 1.237275 & -0.553293 & 4.854605 \\ 1 & 0.998259 & -2.679512 & 6.118625 \\ 1 & -4.139986 & -1.113018 & -5.717972 \\ 1 & -3.071774 & -0.111453 & -4.731919 \\ 1 & -3.452897 & -1.771195 & -4.218588 \\ 1 & -5.796440 & 0.780937 & -5.650613 \\ 1 & -6.484681 & 1.323286 & -4.105434 \\ 1 & -4.803569 & 1.717085 & -4.512223 \\ 1 & -5.844794 & 1.902824 & 2.168688 \\ 1 & 2.710081 & 5.036003 & 3.625467 \\ 1 & -6.378169 & -1.634446 & -5.140071 \\ 1 & -5.903337 & -2.363561 & -3.592061 \\ 1 & -7.155795 & -1.104159 & -3.648358 \\ 1 & -8.623301 & 1.667416 & 0.942072 \\ 1 & -7.294644 & 2.548337 & 0.159805 \\ 1 & -8.187335 & 1.305353 & -0.733404 \\ 1 & -8.355724 & -0.780935 & 1.548392 \\ 1 & -7.865155 & -1.186799 & -0.106150 \\ 1 & -6.811731 & -1.609286 & 1.257225 \\ & -7.231929 & 1.015874 & 2.821048 \\ 1 & & & \\ 1 & & \end{array}$




$\begin{array}{rrrr}1 & 2.462047 & 4.551849 & 1.937164 \\ 1 & 1.437205 & 5.806422 & 2.662437 \\ 1 & 1.075122 & 4.349302 & 5.416299 \\ 1 & -0.232312 & 5.153400 & 4.535291 \\ 1 & -0.396720 & 3.449275 & 4.994540 \\ 1 & 2.501931 & 2.683807 & 4.396014 \\ 1 & 1.109888 & 1.680595 & 3.950109 \\ 1 & 2.317824 & 2.142060 & 2.723863 \\ 1 & -4.724318 & 6.118733 & -0.409419 \\ 1 & -3.649897 & 4.974577 & -1.226677 \\ 1 & -4.697445 & 4.423274 & 0.099808 \\ 1 & -2.843364 & 7.641262 & -0.008791 \\ 1 & -1.409364 & 7.136951 & 0.899948 \\ 1 & -1.677634 & 6.517167 & -0.739151 \\ 1 & -4.350539 & 6.871763 & 1.880000 \\ 1 & -4.247361 & 5.205712 & 2.486385 \\ 1 & -2.943186 & 6.357893 & 2.823624\end{array}$

$2_{\mathrm{C} 2-\mathrm{t}-} \psi_{-1 \mathrm{a} 2 \mathrm{a} 3 \mathrm{a} 4 \mathrm{~s}}$

Number of imaginary frequencies : 0

The smallest frequencies are : $13.9125 \quad 17.0882 \quad 18.2370 \mathrm{~cm}(-1)$

Electronic energy : $\quad \mathrm{HF}=-4012.1891096$

Zero-point correction $=\quad 1.291061($ Hartree/Particle $)$

Thermal correction to Energy= $\quad 1.366896$

Thermal correction to Enthalpy= $\quad 1.367840$

Thermal correction to Gibbs Free Energy $=\quad 1.181618$

Sum of electronic and zero-point Energies $=\quad-4010.898048$

Sum of electronic and thermal Energies $=\quad-4010.822214$

Sum of electronic and thermal Enthalpies $=\quad-4010.821270$

Sum of electronic and thermal Free Energies $=\quad-4011.007491$

Cartesian Coordinates

$\begin{array}{cccc}6 & -2.257547 & 3.187274 & 0.427677 \\ 6 & -1.373645 & 2.149691 & 0.710746 \\ 6 & -0.321385 & 2.350932 & 1.607683 \\ 6 & -0.167616 & 3.567800 & 2.270280 \\ 6 & -1.066777 & 4.593326 & 1.957190 \\ 6 & -2.109339 & 4.434576 & 1.040723 \\ 15 & -1.489183 & 0.521757 & -0.120456 \\ 45 & 0.162055 & 0.665893 & -1.829704 \\ 6 & 1.215436 & 1.310190 & -3.233381 \\ 8 & 1.858607 & 1.771405 & -4.075634 \\ 6 & 0.912014 & 3.791247 & 3.328726 \\ 6 & 0.250151 & 4.232156 & 4.639694 \\ 6 & -3.065452 & 5.595375 & 0.769836 \\ 6 & -2.274387 & 6.817177 & 0.291254 \\ 6 & -1.547673 & -0.648604 & 1.323593 \\ 6 & -1.376391 & -2.009615 & 1.125434 \\ 6 & -1.597291 & -2.930006 & 2.201273 \\ 6 & -1.995643 & -2.435654 & 3.475789 \\ 6 & -2.136476 & -1.040681 & 3.652300\end{array}$




$\begin{array}{cccc}6 & -1.919262 & -0.177136 & 2.612584 \\ 6 & -1.457999 & -4.333471 & 2.043004 \\ 6 & -1.724078 & -5.193242 & 3.079525 \\ 6 & -2.127209 & -4.697448 & 4.337154 \\ 6 & -2.248379 & -3.345039 & 4.530840 \\ 6 & -1.061654 & -2.591405 & -0.206604 \\ 6 & 0.235945 & -2.672777 & -0.680517 \\ 6 & 0.512077 & -3.346345 & -1.896384 \\ 6 & -0.491607 & -3.903280 & -2.640376 \\ 6 & -1.836442 & -3.804388 & -2.213746 \\ 6 & -2.128327 & -3.148465 & -0.982017 \\ 6 & -2.895513 & -4.348975 & -2.980327 \\ 6 & -4.195983 & -4.239389 & -2.557598 \\ 6 & -4.491318 & -3.570827 & -1.349419 \\ 6 & -3.484962 & -3.047332 & -0.577187 \\ 7 & 1.324943 & -1.999379 & -0.023741 \\ 6 & 2.216736 & -2.776740 & 0.856366 \\ 6 & 1.837711 & -2.737082 & 2.314265 \\ 6 & 1.672858 & -1.517785 & 2.977958 \\ 6 & 1.378246 & -1.490377 & 4.335194 \\ 6 & 1.248178 & -2.679607 & 5.050348 \\ 6 & 1.412974 & -3.895731 & 4.398136 \\ 6 & 1.705036 & -3.921070 & 3.036599 \\ 15 & 1.785637 & -0.617021 & -0.874183 \\ 8 & 2.733092 & 0.082091 & 0.298813 \\ 6 & 3.521392 & 1.116752 & -0.181527 \\ 6 & 4.731357 & 0.816071 & -0.774760 \\ 6 & 5.499127 & 1.894186 & -1.323715 \\ 6 & 5.023723 & 3.233304 & -1.179925 \\ 6 & 3.785010 & 3.472104 & -0.537548 \\ 6 & 3.032834 & 2.431922 & -0.061702 \\ 6 & 6.698439 & 1.688607 & -2.051952 \\ 6 & 7.405216 & 2.746453 & -2.567861 \\ 6 & 6.953098 & 4.070321 & -2.388749 \\ 6 & 5.782662 & 4.302955 & -1.713879 \\ 6 & 5.124308 & -0.605976 & -0.908977 \\ 6 & 4.242428 & -1.484930 & -1.516610 \\ 6 & 4.581033 & -2.830637 & -1.769507 \\ 6 & 5.788814 & -3.319677 & -1.352942 \\ 6 & 6.693431 & -2.495011 & -0.642101 \\ 6 & 6.358740 & -1.127584 & -0.405148 \\ 6 & 7.258640 & -0.346032 & 0.363949 \\ 6 & 8.431256 & -0.877767 & 0.840174 \\ 6 & 8.773795 & -2.220244 & 0.574535 \\ 6 & 7.916885 & -3.009496 & -0.148472 \\ 8 & 3.006872 & -1.057264 & -1.941065 \\ 6 & -3.220720 & 0.373221 & -0.707273 \\ 6 & -4.284447 & 0.460302 & 0.186027 \\ 6 & -5.601840 & 0.283438 & -0.242413 \\ 6 & -5.814774 & 0.021225 & -1.596920 \\ 6 & -4.766989 & -0.082516 & -2.520884 \\ 6 & -3.465266 & 0.095958 & -2.052838 \\ & -6.727417 & 0.359065 & 0.786793\end{array}$




$\begin{array}{rrrr}6 & -6.489674 & -0.704997 & 1.865725 \\ 6 & -5.061187 & -0.321062 & -4.001610 \\ 6 & -5.563879 & 0.994182 & -4.608562 \\ 6 & -8.100640 & 0.107807 & 0.169935 \\ 6 & -6.738834 & 1.751204 & 1.428333 \\ 6 & -3.814286 & -0.766326 & -4.765997 \\ 6 & -6.132441 & -1.400035 & -4.184362 \\ 6 & 1.708267 & 2.518707 & 3.615854 \\ 6 & 1.884083 & 4.878018 & 2.859551 \\ 6 & -4.100351 & 5.253854 & -0.299671 \\ 6 & -3.812045 & 5.942979 & 2.062701 \\ 1 & 7.003726 & 0.687035 & 0.587013 \\ 1 & -2.047198 & 0.890091 & 2.776735 \\ 1 & 8.156363 & -4.052961 & -0.349726 \\ 1 & -2.548045 & -2.944546 & 5.498560 \\ 1 & 5.405168 & 5.316626 & -1.586412 \\ 1 & -3.726937 & -2.526139 & 0.348029 \\ 1 & -5.526966 & -3.467365 & -1.030058 \\ 1 & 3.424235 & 4.496394 & -0.454772 \\ 1 & -2.433200 & -0.661556 & 4.629616 \\ 1 & 6.062801 & -4.355597 & -1.546066 \\ 1 & 8.319540 & 2.560100 & -3.126761 \\ 1 & 3.865566 & -3.454655 & -2.300782 \\ 1 & -2.653019 & -4.854789 & -3.913946 \\ 1 & -0.271235 & -4.413150 & -3.576848 \\ 1 & -1.618098 & -6.265706 & 2.931483 \\ 1 & 7.054802 & 0.674338 & -2.211005 \\ 1 & -1.135103 & -4.730813 & 1.082881 \\ 1 & 1.545713 & -3.390007 & -2.234194 \\ 1 & 7.524717 & 4.899343 & -2.799368 \\ 1 & 9.100195 & -0.258447 & 1.433533 \\ 1 & -5.002451 & -4.663406 & -3.152512 \\ 1 & -2.334107 & -5.388932 & 5.150927 \\ 1 & 9.708614 & -2.626879 & 0.953166 \\ 1 & 2.055440 & 2.588031 & 0.392406 \\ 1 & -4.084654 & 0.661948 & 1.238126 \\ 1 & -2.610794 & 0.019247 & -2.721985 \\ 1 & -6.834204 & -0.112133 & -1.951820 \\ 1 & -3.063122 & 3.013604 & -0.281959 \\ 1 & 0.361856 & 1.525242 & 1.807002 \\ 1 & -0.954980 & 5.555019 & 2.458260 \\ 1 & -0.990526 & 1.642174 & -2.462247 \\ 1 & 2.241982 & -3.817696 & 0.500280 \\ 1 & 3.236009 & -2.384694 & 0.744296 \\ 1 & 1.824818 & -4.875838 & 2.523737 \\ 1 & 1.779842 & -0.587132 & 2.420733 \\ 1 & 1.304300 & -4.829780 & 4.945920 \\ 1 & 1.252357 & -0.534495 & 4.841664 \\ 1 & 1.013719 & -2.656470 & 6.112732 \\ 1 & -4.078817 & -0.976280 & -5.810247 \\ 1 & -3.033369 & 0.004401 & -4.779972 \\ 1 & -3.383959 & -1.681950 & -4.336371 \\ & -5.784830 & 0.865632 & -5.677121\end{array}$




$\begin{array}{rrrr}1 & -6.479917 & 1.341775 & -4.113619 \\ 1 & -4.808675 & 1.784892 & -4.510739 \\ 1 & -6.299860 & -1.581786 & -5.254085 \\ 1 & -5.818316 & -2.346862 & -3.725440 \\ 1 & -7.099355 & -1.115712 & -3.752566 \\ 1 & -7.516561 & 1.810746 & 2.201649 \\ 1 & -5.779470 & 1.999072 & 1.900612 \\ 1 & -6.950926 & 2.523543 & 0.677479 \\ 1 & -7.284313 & -0.666342 & 2.623061 \\ 1 & -6.491699 & -1.712089 & 1.426986 \\ 1 & -5.530816 & -0.571110 & 2.382853 \\ 1 & -8.871627 & 0.168322 & 0.948457 \\ 1 & -8.349750 & 0.852579 & -0.596843 \\ 1 & -8.169022 & -0.889749 & -0.283604 \\ 1 & 2.645375 & 5.066325 & 3.628489 \\ 1 & 2.404255 & 4.572528 & 1.941886 \\ 1 & 1.372135 & 5.826685 & 2.657068 \\ 1 & 1.010781 & 4.376400 & 5.418518 \\ 1 & -0.295793 & 5.177162 & 4.534371 \\ 1 & -0.459109 & 3.473581 & 4.996256 \\ 1 & 2.436536 & 2.711148 & 4.413526 \\ 1 & 1.053029 & 1.702188 & 3.952121 \\ 1 & 2.271956 & 2.170535 & 2.739741 \\ 1 & -4.758777 & 6.116364 & -0.462673 \\ 1 & -3.631129 & 5.009182 & -1.261092 \\ 1 & -4.735150 & 4.408091 & -0.004044 \\ 1 & -2.956315 & 7.653148 & 0.085458 \\ 1 & -1.548038 & 7.161517 & 1.037061 \\ 1 & -1.725789 & 6.592575 & -0.632283 \\ 1 & -4.507327 & 6.775684 & 1.890536 \\ 1 & -4.395429 & 5.084257 & 2.421015 \\ 1 & -3.128058 & 6.241359 & 2.866704\end{array}$

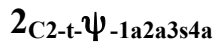

Number of imaginary frequencies : 1

The smallest frequencies are : $-1.7031 \quad 12.6933 \quad 16.0179 \mathrm{~cm}(-1)$

Electronic energy : $\quad H F=-4012.1883536$

Zero-point correction $=\quad 1.290763$ (Hartree/Particle)

Thermal correction to Energy= $\quad 1.365840$

Thermal correction to Enthalpy= $\quad 1.366784$

Thermal correction to Gibbs Free Energy $=\quad 1.182332$

Sum of electronic and zero-point Energies $=\quad-4010.897591$

Sum of electronic and thermal Energies $=\quad-4010.822514$

Sum of electronic and thermal Enthalpies $=\quad-4010.821569$

Sum of electronic and thermal Free Energies $=\quad-4011.006022$

Cartesian Coordinates

$\begin{array}{llll}6 & 1.729104 & -3.935222 & 3.021083 \\ 6 & 1.852868 & -2.742852 & 2.311170 \\ 6 & 1.663506 & -1.531990 & 2.983684 \\ 6 & 1.355610 & -1.520831 & 4.338088\end{array}$




$\begin{array}{cccc}6 & 1.236155 & -2.718373 & 5.041348 \\ 6 & 1.424145 & -3.926215 & 4.380057 \\ 6 & 2.243753 & -2.762206 & 0.856316 \\ 7 & 1.336248 & -1.999089 & -0.021050 \\ 15 & 1.780670 & -0.612555 & -0.873808 \\ 45 & 0.166083 & 0.696190 & -1.809293 \\ 6 & 1.239641 & 1.382985 & -3.177554 \\ 8 & 1.897396 & 1.876198 & -3.989817 \\ 6 & 0.261633 & -2.697440 & -0.675477 \\ 6 & -1.044663 & -2.609316 & -0.226598 \\ 6 & -2.091634 & -3.209634 & -0.998316 \\ 6 & -1.769676 & -3.939655 & -2.179806 \\ 6 & -0.416533 & -4.039436 & -2.579717 \\ 6 & 0.565528 & -3.422998 & -1.854154 \\ 6 & -3.457191 & -3.099150 & -0.628191 \\ 6 & -4.442235 & -3.695692 & -1.375460 \\ 6 & -4.114261 & -4.452130 & -2.521428 \\ 6 & -2.805606 & -4.562458 & -2.917567 \\ 6 & -1.379042 & -2.025641 & 1.101262 \\ 6 & -1.557031 & -0.667599 & 1.312810 \\ 6 & -1.937666 & -0.208981 & 2.603989 \\ 6 & -2.156599 & -1.081620 & 3.635034 \\ 6 & -2.003792 & -2.473758 & 3.448010 \\ 6 & -1.597046 & -2.955745 & 2.171135 \\ 6 & -2.252972 & -3.391737 & 4.496478 \\ 6 & -2.116449 & -4.741634 & 4.296429 \\ 6 & -1.700519 & -5.225336 & 3.038467 \\ 6 & -1.439743 & -4.357064 & 2.007694 \\ 15 & -1.493234 & 0.523428 & -0.111060 \\ 6 & -1.384519 & 2.138610 & 0.746166 \\ 6 & -2.290785 & 3.163811 & 0.492233 \\ 6 & -2.164103 & 4.400370 & 1.130272 \\ 6 & -1.108130 & 4.566843 & 2.029628 \\ 6 & -0.184796 & 3.553852 & 2.312871 \\ 6 & -0.327367 & 2.342883 & 1.636544 \\ 6 & -3.153792 & 5.540990 & 0.898332 \\ 6 & -4.245636 & 5.163913 & -0.100868 \\ 6 & 0.910359 & 3.784811 & 3.353724 \\ 6 & 1.725533 & 2.520079 & 3.621649 \\ 6 & -3.221013 & 0.391537 & -0.709161 \\ 6 & -3.449832 & 0.187334 & -2.061624 \\ 6 & -4.750406 & 0.031443 & -2.560326 \\ 6 & -5.801622 & 0.075604 & -1.649546 \\ 6 & -5.605000 & 0.282348 & -0.272901 \\ 6 & -4.301222 & 0.437586 & 0.181857 \\ 6 & -6.804623 & 0.296038 & 0.672609 \\ 6 & -6.404822 & 0.641460 & 2.105981 \\ 6 & -4.945503 & -0.153223 & -4.062936 \\ 6 & -6.406802 & -0.392952 & -4.432049 \\ 8 & 3.004436 & -1.039956 & -1.943439 \\ 6 & 4.244887 & -1.456309 & -1.521849 \\ 6 & 5.118270 & -0.571428 & -0.910465 \\ 6 & 6.356567 & -1.083514 & -0.406525\end{array}$




\begin{tabular}{rrrr}
6 & 6.704245 & -2.446987 & -0.647483 \\
6 & 5.809556 & -3.276464 & -1.365201 \\
6 & 4.598161 & -2.796769 & -1.782097 \\
6 & 7.930853 & -2.952250 & -0.152172 \\
6 & 8.778737 & -2.157782 & 0.575762 \\
6 & 8.423850 & -0.819088 & 0.844390 \\
6 & 7.247812 & -0.296233 & 0.366795 \\
6 & 4.713529 & 0.847059 & -0.774547 \\
6 & 3.500840 & 1.136665 & -0.181614 \\
6 & 2.999217 & 2.447333 & -0.064738 \\
6 & 3.741848 & 3.493805 & -0.542152 \\
6 & 4.983731 & 3.266166 & -1.182467 \\
6 & 5.472246 & 1.931542 & -1.323620 \\
6 & 6.674536 & 1.736400 & -2.049785 \\
6 & 7.371869 & 2.800276 & -2.566176 \\
6 & 6.906610 & 4.119941 & -2.389883 \\
6 & 5.732949 & 4.342283 & -1.717116 \\
8 & 2.723058 & 0.094265 & 0.299133 \\
6 & -7.830507 & 1.333647 & 0.205282 \\
6 & -7.447769 & -1.095392 & 0.678568 \\
6 & -4.129142 & -1.354967 & -4.549883 \\
6 & -4.463476 & 1.112935 & -4.781323 \\
6 & 1.861716 & 4.883570 & 2.869785 \\
6 & 0.269400 & 4.214734 & 4.678608 \\
6 & -3.830565 & 5.902919 & 2.225315 \\
6 & -2.408286 & 6.763760 & 0.354234 \\
1 & 6.983210 & 0.733910 & 0.592157 \\
1 & -2.073366 & 0.856108 & 2.775286 \\
1 & 8.180184 & -3.992866 & -0.356202 \\
1 & -2.560931 & -2.999281 & 5.464912 \\
1 & 5.345454 & 5.352452 & -1.591937 \\
1 & -3.722028 & -2.518756 & 0.254232 \\
1 & -5.484183 & -3.580839 & -1.082950 \\
1 & 3.370847 & 4.514644 & -0.461963 \\
1 & -2.461770 & -0.712339 & 4.613507 \\
1 & 6.094233 & -4.308446 & -1.563862 \\
1 & 8.288866 & 2.621916 & -3.123310 \\
1 & 3.891079 & -3.423234 & -2.321284 \\
1 & -2.538558 & -5.127257 & -3.809705 \\
1 & -0.172210 & -4.601361 & -3.479768 \\
1 & -2.591601 & 0.153404 & -2.731970 \\
1 & -6.819765 & -0.054526 & -2.011371 \\
1 & -1.579733 & -6.295495 & 2.885202 \\
1 & 7.041102 & 0.725441 & -2.206690 \\
1 & -1.105536 & -4.747527 & 1.048703 \\
1 & 1.605465 & -3.473167 & -2.170560 \\
1 & 7.470743 & 4.953817 & -2.801026 \\
1 & 9.085919 & -0.195705 & 1.441191 \\
1 & -4.902831 & -4.932845 & -3.096385 \\
1 & -2.319717 & -5.439784 & 5.105406 \\
1 & 9.716032 & -2.557395 & 0.955748 \\
& 2.019795 & 2.595515 & 0.387487 \\
\hline
\end{tabular}




$\begin{array}{rrrr}1 & -3.100186 & 2.987550 & -0.211811 \\ 1 & 0.369732 & 1.524837 & 1.818796 \\ 1 & -1.005698 & 5.523613 & 2.541966 \\ 1 & -0.966992 & 1.713660 & -2.414311 \\ 1 & 2.297053 & -3.799521 & 0.492844 \\ 1 & 3.253098 & -2.343201 & 0.753832 \\ 1 & 1.866776 & -4.883426 & 2.500615 \\ 1 & 1.763812 & -0.595185 & 2.435293 \\ 1 & 1.323311 & -4.866523 & 4.918546 \\ 1 & 1.209659 & -0.571516 & 4.851449 \\ 1 & 0.991120 & -2.708078 & 6.101547 \\ 1 & -6.494681 & -0.536338 & -5.516363 \\ 1 & -6.806420 & -1.293485 & -3.946390 \\ 1 & -7.046349 & 0.457564 & -4.162411 \\ 1 & -4.592931 & 1.007872 & -5.867083 \\ 1 & -5.034263 & 1.992205 & -4.455093 \\ 1 & -3.401672 & 1.313096 & -4.589459 \\ 1 & -4.254021 & -1.481098 & -5.633924 \\ 1 & -3.055764 & -1.240791 & -4.352879 \\ 1 & -4.459309 & -2.278990 & -4.058117 \\ 1 & -8.685789 & 1.358325 & 0.893677 \\ 1 & -7.389179 & 2.338548 & 0.178439 \\ 1 & -8.221813 & 1.114004 & -0.794969 \\ 1 & -8.323694 & -1.111624 & 1.341346 \\ 1 & -7.780630 & -1.398639 & -0.321938 \\ 1 & -6.736855 & -1.850509 & 1.040881 \\ 1 & -7.297025 & 0.652307 & 2.744650 \\ 1 & -5.707665 & -0.093653 & 2.530226 \\ 1 & -5.938325 & 1.633873 & 2.171501 \\ 1 & 2.635544 & 5.077767 & 3.624642 \\ 1 & 2.367418 & 4.586539 & 1.941328 \\ 1 & 1.335595 & 5.827172 & 2.680077 \\ 1 & 1.043742 & 4.364283 & 5.442750 \\ 1 & -0.287901 & 5.154565 & 4.587631 \\ 1 & -0.424970 & 3.448012 & 5.046634 \\ 1 & 2.464864 & 2.717312 & 4.407858 \\ 1 & 1.085321 & 1.694766 & 3.965249 \\ 1 & 2.278403 & 2.181796 & 2.734926 \\ 1 & -4.935928 & 6.007538 & -0.226539 \\ 1 & -3.833842 & 4.925103 & -1.089594 \\ 1 & -4.836814 & 4.302631 & 0.240078 \\ 1 & -3.109395 & 7.591142 & 0.180273 \\ 1 & -1.640018 & 7.121682 & 1.050305 \\ 1 & -1.915254 & 6.530989 & -0.598177 \\ 1 & -4.551493 & 6.717786 & 2.075196 \\ 1 & -4.374383 & 5.041882 & 2.635962 \\ 1 & -3.109333 & 6.234767 & 2.982040\end{array}$

$2_{\mathrm{C} 2-\mathrm{t}-\psi_{-1} \text { a2a3s4s }}$

Number of imaginary frequencies : 1

The smallest frequencies are : -12.9941 $14.8502 \quad 16.5879 \mathrm{~cm}(-1)$

Electronic energy : $\quad H F=-4012.1887861$ 
Zero-point correction $=$

Thermal correction to Energy=

Thermal correction to Enthalpy=

Thermal correction to Gibbs Free Energy=

Sum of electronic and zero-point Energies=

Sum of electronic and thermal Energies=

Sum of electronic and thermal Enthalpies=

Sum of electronic and thermal Free Energies=
1.291252 (Hartree/Particle)

1.366128

1.367072

1.183900

$-4010.897534$

$-4010.822659$

$-4010.821714$

$-4011.004887$

Cartesian Coordinates

\begin{tabular}{cccc}
0 & 5.776146 & 4.261579 & -1.769073 \\
6 & 5.014488 & 3.202085 & -1.219002 \\
6 & 5.487416 & 1.859916 & -1.340816 \\
6 & 6.686759 & 1.640332 & -2.064797 \\
6 & 7.396124 & 2.688377 & -2.596925 \\
6 & 6.946680 & 4.015861 & -2.439158 \\
6 & 4.717080 & 0.792149 & -0.775149 \\
6 & 3.506707 & 1.104437 & -0.188911 \\
6 & 3.020442 & 2.422399 & -0.090949 \\
6 & 3.775510 & 3.453440 & -0.581862 \\
6 & 5.109949 & -0.632160 & -0.884100 \\
6 & 4.230240 & -1.522280 & -1.478408 \\
6 & 4.572217 & -2.870688 & -1.711287 \\
6 & 5.779879 & -3.351578 & -1.285236 \\
6 & 6.681283 & -2.514899 & -0.584399 \\
6 & 6.343984 & -1.144421 & -0.369547 \\
6 & 7.904309 & -3.019821 & -0.080016 \\
6 & 8.758407 & -2.218163 & 0.632614 \\
6 & 8.413257 & -0.872237 & 0.876532 \\
6 & 7.241045 & -0.349711 & 0.389261 \\
8 & 2.994089 & -1.104990 & -1.910792 \\
15 & 1.771165 & -0.640409 & -0.856696 \\
8 & 2.715888 & 0.079098 & 0.306733 \\
7 & 1.304509 & -2.001768 & 0.023943 \\
6 & 0.218856 & -2.697822 & -0.614323 \\
6 & -1.086108 & -2.577972 & -0.169177 \\
6 & -2.141734 & -3.184131 & -0.924931 \\
6 & -1.829456 & -3.951863 & -2.084992 \\
6 & -0.477752 & -4.080772 & -2.482063 \\
6 & 0.512846 & -3.458533 & -1.773290 \\
6 & -2.873425 & -4.582350 & -2.804794 \\
6 & -4.181036 & -4.441742 & -2.413723 \\
6 & -4.497882 & -3.650646 & -1.288378 \\
6 & -3.505619 & -3.044451 & -0.558393 \\
6 & -1.414912 & -1.954789 & 1.142056 \\
6 & -1.571161 & -0.588992 & 1.318254 \\
6 & -1.955240 & -0.092725 & 2.594456 \\
6 & -2.198214 & -0.935577 & 3.644719 \\
6 & -2.068126 & -2.334334 & 3.493773 \\
6 & -1.656818 & -2.854136 & 2.233464 \\
6 & -1.518514 & -4.261365 & 2.107771 \\
6 & -1.799802 & -5.098618 & 3.158647 \\
\hline
\end{tabular}




$\begin{array}{cccc}6 & -2.220021 & -4.576969 & 4.399876 \\ 6 & -2.339728 & -3.220670 & 4.563512 \\ 15 & -1.482220 & 0.567728 & -0.134119 \\ 6 & -3.203457 & 0.418475 & -0.745703 \\ 6 & -3.416732 & 0.138765 & -2.091720 \\ 6 & -4.703582 & -0.081832 & -2.587902 \\ 6 & -5.770666 & -0.012564 & -1.687514 \\ 6 & -5.590045 & 0.271294 & -0.328541 \\ 6 & -4.289434 & 0.484850 & 0.126542 \\ 6 & -4.896218 & -0.338219 & -4.081257 \\ 6 & -4.723905 & 0.993555 & -4.821868 \\ 6 & -6.741863 & 0.342093 & 0.672745 \\ 6 & -6.509404 & -0.683427 & 1.789155 \\ 6 & 2.201490 & -2.761267 & 0.915018 \\ 6 & 1.807887 & -2.711033 & 2.368082 \\ 6 & 1.668609 & -3.887862 & 3.100395 \\ 6 & 1.358116 & -3.848735 & 4.457643 \\ 6 & 1.177669 & -2.626316 & 5.093640 \\ 6 & 1.312949 & -1.444096 & 4.367565 \\ 6 & 1.628750 & -1.485228 & 3.015587 \\ 45 & 0.177589 & 0.657453 & -1.838139 \\ 6 & 1.245923 & 1.231606 & -3.261325 \\ 8 & 1.900669 & 1.642671 & -4.120239 \\ 6 & -1.366494 & 2.200137 & 0.686350 \\ 6 & -2.256264 & 3.232834 & 0.405064 \\ 6 & -2.113533 & 4.480507 & 1.018966 \\ 6 & -1.069216 & 4.644382 & 1.932544 \\ 6 & -0.163761 & 3.623491 & 2.243252 \\ 6 & -0.313819 & 2.405805 & 1.581623 \\ 6 & -3.080107 & 5.634258 & 0.755209 \\ 6 & -2.301172 & 6.868125 & 0.287988 \\ 6 & 0.921458 & 3.853247 & 3.294572 \\ 6 & 0.270721 & 4.314855 & 4.603856 \\ 6 & -4.110102 & 5.291359 & -0.318718 \\ 6 & -3.831999 & 5.962633 & 2.050121 \\ 6 & 1.711693 & 2.579453 & 3.593016 \\ 6 & 1.897392 & 4.927806 & 2.805295 \\ 6 & -8.093579 & 0.041032 & 0.030940 \\ 6 & -6.800967 & 1.751569 & 1.273307 \\ 6 & -6.281443 & -0.899824 & -4.395409 \\ 6 & -3.856016 & -1.337720 & -4.597583 \\ 1 & 6.984076 & 0.686256 & 0.595788 \\ 1 & -2.074079 & 0.978161 & 2.739244 \\ 1 & 8.145798 & -4.065901 & -0.264607 \\ 1 & -2.650830 & -2.798830 & 5.518448 \\ 1 & 5.400555 & 5.277877 & -1.657918 \\ 1 & -3.764643 & -2.430136 & 0.303290 \\ 1 & -5.538710 & -3.517193 & -0.999150 \\ 1 & 3.416635 & 4.479599 & -0.515885 \\ 1 & -2.505536 & -0.536857 & 4.610871 \\ 1 & 6.056350 & -4.389536 & -1.463276 \\ 1 & 8.310372 & 2.491266 & -3.152251 \\ 1 & 3.859162 & -3.501784 & -2.237238\end{array}$




$\begin{array}{lrrr}1 & -2.613936 & -5.174783 & -3.681107 \\ 1 & -0.241551 & -4.670312 & -3.366480 \\ 1 & -1.692312 & -6.173951 & 3.034321 \\ 1 & 7.040922 & 0.622913 & -2.207634 \\ 1 & -1.182105 & -4.681463 & 1.162140 \\ 1 & 1.552406 & -3.531902 & -2.087146 \\ 1 & 7.520371 & 4.837058 & -2.862410 \\ 1 & 9.079869 & -0.242780 & 1.461785 \\ 1 & -4.976701 & -4.925314 & -2.976425 \\ 1 & -2.440117 & -5.251200 & 5.224581 \\ 1 & 9.692925 & -2.617502 & 1.019661 \\ 1 & 2.042989 & 2.587890 & 0.359506 \\ 1 & -4.115725 & 0.696088 & 1.181551 \\ 1 & -2.550278 & 0.090160 & -2.749293 \\ 1 & -6.777620 & -0.191575 & -2.053993 \\ 1 & -3.062961 & 3.054667 & -0.302269 \\ 1 & 0.371566 & 1.582067 & 1.781931 \\ 1 & -0.961381 & 5.606327 & 2.433904 \\ 1 & -0.938155 & 1.671790 & -2.479477 \\ 1 & 2.242408 & -3.805378 & 0.569836 \\ 1 & 3.215969 & -2.356398 & 0.807122 \\ 1 & 1.798194 & -4.847485 & 2.599106 \\ 1 & 1.741499 & -0.560887 & 2.448834 \\ 1 & 1.244741 & -4.777133 & 5.014015 \\ 1 & 1.174336 & -0.483431 & 4.861390 \\ 1 & 0.926900 & -2.592734 & 6.152004 \\ 1 & -6.357258 & -1.116087 & -5.468511 \\ 1 & -6.470252 & -1.836170 & -3.851996 \\ 1 & -7.084802 & -0.192662 & -4.153305 \\ 1 & -4.853286 & 0.851841 & -5.903711 \\ 1 & -5.463784 & 1.731469 & -4.484449 \\ 1 & -3.725533 & 1.418097 & -4.653935 \\ 1 & -4.031307 & -1.540583 & -5.662492 \\ 1 & -2.828168 & -0.966408 & -4.505006 \\ 1 & -3.918730 & -2.289186 & -4.053127 \\ 1 & -7.605060 & 1.817886 & 2.018650 \\ 1 & -5.862279 & 2.030189 & 1.769518 \\ 1 & -7.001555 & 2.498276 & 0.493830 \\ 1 & -7.316417 & -0.627779 & 2.532258 \\ 1 & -6.496299 & -1.704822 & 1.385625 \\ 1 & -5.560503 & -0.523282 & 2.316842 \\ 1 & -8.883573 & 0.098513 & 0.790415 \\ 1 & -8.344453 & 0.761155 & -0.758392 \\ 1 & -8.125411 & -0.968104 & -0.400745 \\ 1 & 2.664846 & 5.121335 & 3.566799 \\ 1 & 2.409523 & 4.606891 & 1.888323 \\ 1 & 1.389641 & 5.876762 & 2.593523 \\ 1 & 1.037448 & 4.463089 & 5.375874 \\ 1 & -0.268889 & 5.262596 & 4.491106 \\ 1 & -0.441487 & 3.565874 & 4.974413 \\ 1 & 2.442680 & 2.776249 & 4.387107 \\ 1 & 1.053157 & 1.769557 & 3.938818 \\ & 2.271918 & 2.219565 & 2.719524\end{array}$




$\begin{array}{lllr}1 & -4.774321 & 6.150116 & -0.478012 \\ 1 & -3.636797 & 5.055924 & -1.280446 \\ 1 & -4.739913 & 4.439565 & -0.029876 \\ 1 & -2.991623 & 7.698481 & 0.088131 \\ 1 & -1.579516 & 7.214371 & 1.037410 \\ 1 & -1.749074 & 6.656965 & -0.636620 \\ 1 & -4.535657 & 6.789646 & 1.884641 \\ 1 & -4.406882 & 5.094504 & 2.399593 \\ 1 & -3.151934 & 6.260468 & 2.857671\end{array}$

$2_{\text {C2-t- } \Psi-1 \text { a2s3a4a }}$

Number of imaginary frequencies : 0

The smallest frequencies are : $14.6279 \quad 16.2365 \quad 19.2948 \mathrm{~cm}(-1)$

Electronic energy : $\quad \mathrm{HF}=-4012.189355$

Zero-point correction $=\quad 1.291166($ Hartree/Particle $)$

Thermal correction to Energy= $\quad 1.367065$

Thermal correction to Enthalpy= $\quad 1.368009$

Thermal correction to Gibbs Free Energy= 1.181339

Sum of electronic and zero-point Energies $=\quad-4010.898189$

Sum of electronic and thermal Energies $=\quad-4010.822290$

Sum of electronic and thermal Enthalpies $=\quad-4010.821346$

Sum of electronic and thermal Free Energies $=\quad-4011.008015$

Cartesian Coordinates

$\begin{array}{cccc}6 & 1.718063 & -3.944007 & 3.024148 \\ 6 & 1.851746 & -2.754778 & 2.310951 \\ 6 & 1.675709 & -1.540454 & 2.980749 \\ 6 & 1.370615 & -1.522554 & 4.335686 \\ 6 & 1.240775 & -2.717000 & 5.042274 \\ 6 & 1.415724 & -3.928212 & 4.383704 \\ 6 & 2.238213 & -2.779812 & 0.854981 \\ 7 & 1.333690 & -2.012186 & -0.020502 \\ 15 & 1.776505 & -0.627712 & -0.877434 \\ 45 & 0.143850 & 0.639444 & -1.840109 \\ 6 & 1.186686 & 1.236711 & -3.271958 \\ 8 & 1.827258 & 1.661779 & -4.134838 \\ 6 & 0.250766 & -2.698488 & -0.673775 \\ 6 & -1.052701 & -2.598716 & -0.219461 \\ 6 & -2.111541 & -3.163482 & -1.000895 \\ 6 & -1.805203 & -3.853844 & -2.210368 \\ 6 & -0.454254 & -3.977268 & -2.610679 \\ 6 & 0.541113 & -3.406313 & -1.866469 \\ 6 & -3.473778 & -3.042265 & -0.621961 \\ 6 & -4.471635 & -3.580047 & -1.395874 \\ 6 & -4.162255 & -4.280508 & -2.582160 \\ 6 & -2.855740 & -4.410423 & -2.980045 \\ 6 & -1.376357 & -2.016053 & 1.111245 \\ 6 & -1.545962 & -0.656692 & 1.321808 \\ 6 & -1.920281 & -0.194978 & 2.613742 \\ 6 & -2.140454 & -1.065864 & 3.646064 \\ 6 & -1.998340 & -2.459479 & 3.459033\end{array}$




$\begin{array}{cccc}6 & -1.598367 & -2.944372 & 2.181277 \\ 6 & -2.251630 & -3.375969 & 4.507671 \\ 6 & -2.127236 & -4.726938 & 4.306070 \\ 6 & -1.720156 & -5.213550 & 3.046335 \\ 6 & -1.454857 & -4.346720 & 2.015425 \\ 15 & -1.485149 & 0.529159 & -0.107085 \\ 6 & -1.324708 & 2.146577 & 0.735931 \\ 6 & -2.172444 & 3.204650 & 0.444830 \\ 6 & -2.010805 & 4.449458 & 1.068780 \\ 6 & -0.976504 & 4.587195 & 1.990683 \\ 6 & -0.099664 & 3.535931 & 2.306305 \\ 6 & -0.276339 & 2.323312 & 1.648846 \\ 6 & -2.988221 & 5.573583 & 0.733194 \\ 6 & -2.694649 & 6.853648 & 1.510725 \\ 6 & 0.980026 & 3.741332 & 3.368201 \\ 6 & 1.771473 & 2.462842 & 3.639676 \\ 6 & -3.230163 & 0.425566 & -0.664394 \\ 6 & -3.491899 & 0.115353 & -1.994979 \\ 6 & -4.804071 & -0.054577 & -2.448291 \\ 6 & -5.833425 & 0.096918 & -1.518934 \\ 6 & -5.604985 & 0.405380 & -0.170820 \\ 6 & -4.284952 & 0.567040 & 0.242125 \\ 6 & -6.782005 & 0.508458 & 0.797485 \\ 6 & -6.341784 & 0.931739 & 2.197582 \\ 6 & -5.112288 & -0.332416 & -3.919232 \\ 6 & -3.880719 & -0.834172 & -4.673694 \\ 8 & 2.999726 & -1.056491 & -1.946418 \\ 6 & 4.240892 & -1.470397 & -1.525155 \\ 6 & 5.114454 & -0.582320 & -0.918945 \\ 6 & 6.354825 & -1.091287 & -0.416755 \\ 6 & 6.703449 & -2.455136 & -0.654372 \\ 6 & 5.807414 & -3.288461 & -1.366016 \\ 6 & 4.594240 & -2.811676 & -1.780985 \\ 6 & 7.932388 & -2.957171 & -0.161575 \\ 6 & 8.781703 & -2.159277 & 0.560925 \\ 6 & 8.425837 & -0.820278 & 0.826621 \\ 6 & 7.247503 & -0.300508 & 0.351292 \\ 6 & 4.708141 & 0.836023 & -0.785994 \\ 6 & 3.496366 & 1.126640 & -0.191431 \\ 6 & 2.996936 & 2.437781 & -0.071599 \\ 6 & 3.739849 & 3.483885 & -0.549380 \\ 6 & 4.978990 & 3.255153 & -1.194583 \\ 6 & 5.465508 & 1.920056 & -1.337881 \\ 6 & 6.665084 & 1.723935 & -2.068316 \\ 6 & 7.361674 & 2.787208 & -2.586941 \\ 6 & 6.898578 & 4.107342 & -2.408390 \\ 6 & 5.727612 & 4.330676 & -1.731279 \\ 8 & 2.718184 & 0.085567 & 0.291810 \\ 6 & -7.797510 & 1.536278 & 0.288343 \\ 6 & -7.453681 & -0.865474 & 0.903446 \\ 6 & -6.212344 & -1.387593 & -4.063233 \\ 6 & -5.583253 & 0.976039 & -4.564960 \\ 6 & 1.957486 & 4.829641 & 2.913695\end{array}$




$\begin{array}{rrrr}6 & 0.318804 & 4.169137 & 4.683902 \\ 6 & -4.411829 & 5.119359 & 1.079840 \\ 6 & -2.904917 & 5.891982 & -0.763618 \\ 1 & 6.982303 & 0.729955 & 0.574382 \\ 1 & -2.047329 & 0.871218 & 2.785747 \\ 1 & 8.182401 & -3.998102 & -0.363155 \\ 1 & -2.553497 & -2.981869 & 5.477351 \\ 1 & 5.341759 & 5.341241 & -1.604139 \\ 1 & -3.727754 & -2.496174 & 0.285156 \\ 1 & -5.511048 & -3.461416 & -1.095251 \\ 1 & 3.370660 & 4.505130 & -0.465912 \\ 1 & -2.439115 & -0.694227 & 4.625677 \\ 1 & 6.092623 & -4.320911 & -1.561437 \\ 1 & 8.276386 & 2.608032 & -3.147553 \\ 1 & 3.885536 & -3.440888 & -2.314955 \\ 1 & -2.601662 & -4.941100 & -3.896593 \\ 1 & -0.222732 & -4.516008 & -3.528087 \\ 1 & -1.610369 & -6.284707 & 2.891748 \\ 1 & 7.029919 & 0.712608 & -2.226917 \\ 1 & -1.128509 & -4.738645 & 1.054311 \\ 1 & 1.580093 & -3.470171 & -2.183945 \\ 1 & 7.462185 & 4.940768 & -2.821166 \\ 1 & 9.089007 & -0.194126 & 1.419286 \\ 1 & -4.962399 & -4.711822 & -3.180352 \\ 1 & -2.333947 & -5.423887 & 5.115211 \\ 1 & 9.720859 & -2.556414 & 0.938907 \\ 1 & 2.020053 & 2.586695 & 0.386047 \\ 1 & -4.063234 & 0.803723 & 1.280065 \\ 1 & -2.645984 & 0.002643 & -2.669761 \\ 1 & -6.862543 & -0.031629 & -1.853568 \\ 1 & -2.974055 & 3.058924 & -0.278438 \\ 1 & 0.383784 & 1.481263 & 1.857044 \\ 1 & -0.841559 & 5.540991 & 2.496558 \\ 1 & -1.005956 & 1.614370 & -2.478528 \\ 1 & 2.283333 & -3.818094 & 0.492982 \\ 1 & 3.250137 & -2.367864 & 0.748452 \\ 1 & 1.845669 & -4.894914 & 2.506051 \\ 1 & 1.783371 & -0.606630 & 2.428753 \\ 1 & 1.307040 & -4.866075 & 4.924934 \\ 1 & 1.235734 & -0.570516 & 4.846996 \\ 1 & 0.998055 & -2.701694 & 6.102942 \\ 1 & -4.157002 & -1.077284 & -5.707639 \\ 1 & -3.083119 & -0.082073 & -4.721538 \\ 1 & -3.467465 & -1.742365 & -4.212471 \\ 1 & -5.808612 & 0.821181 & -5.629087 \\ 1 & -6.489695 & 1.360599 & -4.079642 \\ 1 & -4.808719 & 1.750556 & -4.491420 \\ 1 & -6.393774 & -1.594241 & -5.126094 \\ 1 & -5.919118 & -2.329519 & -3.581060 \\ 1 & -7.167699 & -1.065917 & -3.631965 \\ 1 & -8.640249 & 1.615140 & 0.988043 \\ & -8.209625 & 1.266565 & -0.691195 \\ & & & \\ 1 & & \end{array}$




$\begin{array}{rrrr}1 & -8.306098 & -0.825064 & 1.595117 \\ 1 & -7.827511 & -1.215455 & -0.066847 \\ 1 & -6.746600 & -1.616132 & 1.281948 \\ 1 & -7.218517 & 1.001618 & 2.853674 \\ 1 & -5.651169 & 0.207533 & 2.650303 \\ 1 & -5.853111 & 1.915509 & 2.192502 \\ 1 & 2.720154 & 5.002348 & 3.684909 \\ 1 & 2.475521 & 4.534337 & 1.991501 \\ 1 & 1.451510 & 5.784417 & 2.726030 \\ 1 & 1.079186 & 4.299400 & 5.465498 \\ 1 & -0.221746 & 5.118296 & 4.589302 \\ 1 & -0.394919 & 3.409825 & 5.029867 \\ 1 & 2.506678 & 2.645287 & 4.433314 \\ 1 & 1.114371 & 1.647801 & 3.975548 \\ 1 & 2.325840 & 2.118432 & 2.756119 \\ 1 & -3.423701 & 7.626377 & 1.236230 \\ 1 & -2.769140 & 6.702793 & 2.595562 \\ 1 & -1.696276 & 7.250186 & 1.285285 \\ 1 & -3.605126 & 6.698378 & -1.020183 \\ 1 & -1.894404 & 6.219226 & -1.039733 \\ 1 & -3.154869 & 5.024564 & -1.385772 \\ 1 & -5.133674 & 5.912204 & 0.841994 \\ 1 & -4.707112 & 4.223207 & 0.518865 \\ 1 & -4.501212 & 4.890060 & 2.150105\end{array}$

$2_{\mathrm{C} 3-\mathrm{c}-} \psi_{-1 \mathrm{a} 2 \mathrm{a} 3 \mathrm{a} 4 \mathrm{a}}$

Number of imaginary frequencies : 0

The smallest frequencies are : $14.3517 \quad 17.026922 .0422 \mathrm{~cm}(-1)$

Electronic energy : $\quad H F=-4012.1854084$

Zero-point correction $=\quad 1.291878$ (Hartree/Particle)

Thermal correction to Energy= $\quad 1.367556$

Thermal correction to Enthalpy $=\quad 1.368501$

Thermal correction to Gibbs Free Energy $=\quad 1.183146$

Sum of electronic and zero-point Energies $=\quad-4010.893530$

Sum of electronic and thermal Energies $=\quad-4010.817852$

Sum of electronic and thermal Enthalpies $=\quad-4010.816908$

Sum of electronic and thermal Free Energies $=\quad-4011.002262$

Cartesian Coordinates

$\begin{array}{cccc}6 & 2.604151 & -4.468104 & 0.059016 \\ 6 & 2.479629 & -3.551408 & 1.102439 \\ 6 & 2.685539 & -3.993751 & 2.411430 \\ 6 & 3.008045 & -5.318730 & 2.674933 \\ 6 & 3.112624 & -6.231648 & 1.628647 \\ 6 & 2.909677 & -5.801102 & 0.321655 \\ 6 & 2.179067 & -2.090724 & 0.897351 \\ 7 & 1.519071 & -1.740373 & -0.366693 \\ 15 & 1.747601 & -0.259009 & -1.164836 \\ 45 & -0.132207 & 0.745638 & -1.982148 \\ 1 & 0.695189 & 0.996993 & -3.341335\end{array}$




$\begin{array}{cccc}6 & 0.370343 & -2.427474 & -0.878969 \\ 6 & -0.810373 & -2.575832 & -0.168751 \\ 6 & -1.933780 & -3.209084 & -0.788517 \\ 6 & -1.837023 & -3.651175 & -2.140625 \\ 6 & -0.627286 & -3.451336 & -2.843502 \\ 6 & 0.443483 & -2.862975 & -2.228220 \\ 6 & -3.172080 & -3.376817 & -0.115119 \\ 6 & -4.242633 & -3.965523 & -0.741069 \\ 6 & -4.132496 & -4.428087 & -2.070548 \\ 6 & -2.954594 & -4.267019 & -2.755003 \\ 6 & -0.934685 & -2.064980 & 1.221496 \\ 6 & -1.164356 & -0.717598 & 1.468601 \\ 6 & -1.290606 & -0.277199 & 2.814014 \\ 6 & -1.179738 & -1.149697 & 3.864052 \\ 6 & -0.956949 & -2.527787 & 3.643642 \\ 6 & -0.843700 & -2.997424 & 2.303482 \\ 6 & -0.853562 & -3.446970 & 4.715739 \\ 6 & -0.660029 & -4.784032 & 4.476312 \\ 6 & -0.557700 & -5.255738 & 3.150247 \\ 6 & -0.638410 & -4.385772 & 2.092112 \\ 15 & -1.440965 & 0.450051 & 0.041479 \\ 6 & -3.212162 & 0.120024 & -0.317808 \\ 6 & -4.178164 & 0.135518 & 0.690951 \\ 6 & -5.521242 & -0.087613 & 0.393674 \\ 6 & -5.862510 & -0.328232 & -0.944066 \\ 6 & -4.921151 & -0.361916 & -1.973967 \\ 6 & -3.583814 & -0.142505 & -1.632920 \\ 6 & -6.597206 & -0.127416 & 1.477643 \\ 6 & -7.794259 & 0.742978 & 1.083300 \\ 6 & -5.354038 & -0.550687 & -3.426688 \\ 6 & -6.381139 & -1.678683 & -3.549709 \\ 6 & -1.456621 & 2.094653 & 0.856283 \\ 6 & -0.362849 & 2.476332 & 1.638401 \\ 6 & -0.320518 & 3.729704 & 2.249760 \\ 6 & -1.374763 & 4.613283 & 1.994751 \\ 6 & -2.458536 & 4.281168 & 1.178665 \\ 6 & -2.489186 & 3.000355 & 0.622441 \\ 6 & -3.579631 & 5.292758 & 0.948162 \\ 6 & -4.244148 & 5.623598 & 2.289039 \\ 6 & 0.802404 & 4.139667 & 3.202449 \\ 6 & 1.528477 & 5.372334 & 2.655424 \\ 6 & -7.057260 & -1.580040 & 1.648927 \\ 6 & -6.075775 & 0.374015 & 2.823502 \\ 6 & -5.980119 & 0.762744 & -3.910480 \\ 6 & -4.172761 & -0.887935 & -4.337260 \\ 6 & -3.000195 & 6.571164 & 0.333824 \\ 6 & -4.653456 & 4.757730 & 0.002977 \\ 6 & 0.196726 & 4.471138 & 4.571671 \\ 6 & 1.829163 & 3.026476 & 3.402965 \\ 8 & 3.057822 & -0.469191 & -2.166426 \\ 6 & 4.259087 & -0.857335 & -1.609054 \\ 6 & 5.021062 & 0.057012 & -0.901454 \\ 6 & 6.207479 & -0.412074 & -0.253855\end{array}$




\begin{tabular}{|c|c|c|c|}
\hline & 6.611838 & -1.770574 & -0.429898 \\
\hline & 5.825887 & -2.635439 & -1.228118 \\
\hline & 4.663552 & -2.192282 & -1.799177 \\
\hline & 7.784735 & -2.236361 & 0.213112 \\
\hline & 8.523452 & -1.409416 & 1.019280 \\
\hline & 8.108729 & -0.075964 & 1.222076 \\
\hline & 6.983517 & 0.409287 & 0.603834 \\
\hline & 4.535241 & 1.453686 & -0.806124 \\
\hline ) & 3.248600 & 1.673890 & -0.354558 \\
\hline ) & 2.668448 & 2.954785 & -0.290324 \\
\hline 6 & 3.408220 & 4.042958 & -0.670940 \\
\hline 6 & 4.726094 & 3.887171 & -1.163178 \\
\hline 6 & 5.294138 & 2.580432 & -1.258121 \\
\hline 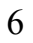 & 6.579831 & 2.453590 & -1.842830 \\
\hline 6 & 7.277921 & 3.557116 & -2.266434 \\
\hline 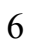 & 6.730137 & 4.850192 & -2.133323 \\
\hline 6 & 5.477436 & 5.005955 & -1.598389 \\
\hline 3 & 2.484923 & 0.589993 & 0.053455 \\
\hline [ & 6.670280 & 1.435674 & 0.778317 \\
\hline 1 & -1.483714 & 0.772447 & 3.020368 \\
\hline 1 & 8.079953 & -3.273382 & 0.058919 \\
\hline 1 & -0.937777 & -3.068591 & 5.733649 \\
\hline 1 & 5.028921 & 5.994491 & -1.509186 \\
\hline 1 & -3.273271 & -3.018922 & 0.907645 \\
\hline 1 & -5.185730 & -4.074515 & -0.209169 \\
\hline 1 & 2.977916 & 5.042610 & -0.629226 \\
\hline 1 & -1.277803 & -0.790085 & 4.887698 \\
\hline 1 & 6.146816 & -3.667624 & -1.358836 \\
\hline | & 8.260491 & 3.431755 & -2.715792 \\
\hline 1 & 4.033636 & -2.844557 & -2.399522 \\
\hline | & -2.856961 & -4.606854 & -3.785466 \\
\hline | & -0.557503 & -3.779192 & -3.879329 \\
\hline 1 & -0.394419 & -6.314947 & 2.964329 \\
\hline | & 7.011230 & 1.463517 & -1.964782 \\
\hline | & -0.536408 & -4.760441 & 1.076170 \\
\hline 1 & 1.378822 & -2.718438 & -2.766878 \\
\hline 1 & 7.295745 & 5.716331 & -2.468825 \\
\hline 1 & 8.683309 & 0.572751 & 1.879463 \\
\hline 1 & -4.984015 & -4.906622 & -2.549953 \\
\hline 1 & -0.587118 & -5.483551 & 5.306091 \\
\hline 1 & 9.419780 & -1.779331 & 1.511588 \\
\hline 1 & 1.638853 & 3.047166 & 0.051820 \\
\hline 1 & -3.868741 & 0.335923 & 1.714694 \\
\hline 1 & -2.801993 & -0.178862 & -2.389171 \\
\hline 1 & -6.910722 & -0.496917 & -1.188992 \\
\hline 1 & -3.325074 & 2.696384 & -0.002302 \\
\hline 1 & 0.449617 & 1.765961 & 1.787308 \\
\hline 1 & -1.352682 & 5.599673 & 2.458605 \\
\hline 1 & 3.124337 & -1.533799 & 0.922009 \\
\hline & 1.596043 & -1.721322 & 1.756570 \\
\hline & -1.308185 & 2.005559 & -2.735367 \\
\hline & 2.577979 & -3.287633 & 3.235550 \\
\hline & & -4.140313 & -0.966 \\
\hline
\end{tabular}




$\begin{array}{rrrr}1 & 3.158389 & -5.643434 & 3.702627 \\ 1 & 2.990178 & -6.506189 & -0.503239 \\ 1 & 3.350675 & -7.273647 & 1.831263 \\ 1 & 2.597887 & 3.360110 & 4.111290 \\ 1 & 1.372178 & 2.116800 & 3.816364 \\ 1 & 2.341166 & 2.760130 & 2.468596 \\ 1 & 0.988404 & 4.749197 & 5.280284 \\ 1 & -0.511192 & 5.307193 & 4.522128 \\ 1 & -0.337221 & 3.604640 & 4.984026 \\ 1 & 2.316480 & 5.690013 & 3.351398 \\ 1 & 2.003585 & 5.151724 & 1.690377 \\ 1 & 0.849904 & 6.221791 & 2.511369 \\ 1 & -5.055582 & 6.349675 & 2.144813 \\ 1 & -4.673679 & 4.722312 & 2.746442 \\ 1 & -3.534737 & 6.056037 & 3.005226 \\ 1 & -5.433954 & 5.516581 & -0.134623 \\ 1 & -4.245402 & 4.518808 & -0.987882 \\ 1 & -5.138971 & 3.854944 & 0.398660 \\ 1 & -3.799281 & 7.303241 & 0.155546 \\ 1 & -2.257886 & 7.045710 & 0.986752 \\ 1 & -2.514556 & 6.359005 & -0.627263 \\ 1 & -4.534983 & -1.063040 & -5.358278 \\ 1 & -3.438855 & -0.072948 & -4.391937 \\ 1 & -3.651786 & -1.796326 & -4.003232 \\ 1 & -6.654007 & -1.825074 & -4.602994 \\ 1 & -5.969784 & -2.623159 & -3.170041 \\ 1 & -7.308004 & -1.467509 & -3.003455 \\ 1 & -6.299092 & 0.674894 & -4.958155 \\ 1 & -6.858110 & 1.036104 & -3.311159 \\ 1 & -5.257656 & 1.587252 & -3.843261 \\ 1 & -6.889633 & 0.368819 & 3.559399 \\ 1 & -5.274515 & -0.262047 & 3.221622 \\ 1 & -5.694872 & 1.402366 & 2.754535 \\ 1 & -8.554652 & 0.718546 & 1.874984 \\ 1 & -7.493272 & 1.788331 & 0.937183 \\ 1 & -8.276438 & 0.401523 & 0.160063 \\ 1 & -7.829553 & -1.652318 & 2.426934 \\ 1 & -7.477087 & -1.985364 & 0.719484 \\ 1 & -6.216031 & -2.221379 & 1.945589 \\ 8 & -1.966140 & 2.833607 & -3.205356\end{array}$

$2_{\text {C3-c- }-\psi-1 \text { 2a3a4s }}$

Number of imaginary frequencies : 0

The smallest frequencies are : $14.3350 \quad 17.2821 \quad 21.0351 \mathrm{~cm}(-1)$

Electronic energy : $\quad H F=-4012.1859234$

Zero-point correction $=\quad 1.291318$ (Hartree/Particle)

Thermal correction to Energy= $\quad 1.367142$

Thermal correction to Enthalpy $=\quad 1.368086$

Thermal correction to Gibbs Free Energy= 1.182051

Sum of electronic and zero-point Energies $=\quad-4010.894605$

Sum of electronic and thermal Energies $=\quad-4010.818781$

Sum of electronic and thermal Enthalpies $=\quad-4010.817837$ 
Cartesian Coordinates

$\begin{array}{cccc}\cdots & & & \\ 6 & 5.431712 & 5.080419 & -1.537689 \\ 6 & 4.690806 & 3.949041 & -1.117308 \\ 6 & 5.276305 & 2.650298 & -1.215876 \\ 6 & 6.568982 & 2.544077 & -1.789276 \\ 6 & 7.256818 & 3.659402 & -2.198420 \\ 6 & 6.691330 & 4.944436 & -2.061622 \\ 6 & 4.527664 & 1.510938 & -0.778702 \\ 6 & 3.234382 & 1.711571 & -0.337019 \\ 6 & 2.637305 & 2.984505 & -0.269884 \\ 6 & 3.366492 & 4.084516 & -0.636540 \\ 6 & 5.031737 & 0.121317 & -0.881357 \\ 6 & 4.287497 & -0.795966 & -1.603834 \\ 6 & 4.711389 & -2.123395 & -1.804079 \\ 6 & 5.875107 & -2.556639 & -1.228198 \\ 6 & 6.642730 & -1.689569 & -0.414637 \\ 6 & 6.218747 & -0.338472 & -0.228269 \\ 6 & 7.816386 & -2.146149 & 0.233576 \\ 6 & 8.536962 & -1.317737 & 1.054526 \\ 6 & 8.102531 & 0.007823 & 1.267517 \\ 6 & 6.976198 & 0.484336 & 0.644538 \\ 8 & 3.085262 & -0.418230 & -2.166378 \\ 15 & 1.765892 & -0.237305 & -1.171033 \\ 8 & 2.481494 & 0.615345 & 0.057793 \\ 7 & 1.559500 & -1.729623 & -0.386411 \\ 6 & 0.424025 & -2.428593 & -0.911690 \\ 6 & -0.759375 & -2.597401 & -0.210813 \\ 6 & -1.874537 & -3.228006 & -0.847091 \\ 6 & -1.768047 & -3.646456 & -2.205917 \\ 6 & -0.553546 & -3.432888 & -2.896382 \\ 6 & 0.509885 & -2.847658 & -2.265332 \\ 6 & -2.880498 & -4.253609 & -2.838099 \\ 6 & -4.061510 & -4.430680 & -2.162953 \\ 6 & -4.180135 & -3.993470 & -0.825616 \\ 6 & -3.115508 & -3.412311 & -0.183265 \\ 6 & -0.898414 & -2.102451 & 1.183727 \\ 6 & -1.147103 & -0.760444 & 1.439859 \\ 6 & -1.287891 & -0.331510 & 2.787340 \\ 6 & -1.176871 & -1.211681 & 3.831146 \\ 6 & -0.936400 & -2.585311 & 3.601207 \\ 6 & -0.804513 & -3.042457 & 2.258404 \\ 6 & -0.580137 & -4.426204 & 2.037182 \\ 6 & -0.499042 & -5.304065 & 3.088766 \\ 6 & -0.619644 & -4.844670 & 4.417697 \\ 6 & -0.831715 & -3.512158 & 4.666512 \\ 15 & -1.441563 & 0.407602 & 0.017869 \\ 6 & -1.495848 & 2.049138 & 0.839160 \\ 6 & -0.413893 & 2.457184 & 1.624090 \\ 6 & -0.407191 & 3.708794 & 2.241020 \\ 6 & -1.483780 & 4.565412 & 1.986292\end{array}$




$\begin{array}{rrrr}6 & -2.556806 & 4.207289 & 1.166926 \\ 6 & -2.553015 & 2.927248 & 0.608990 \\ 6 & 0.702525 & 4.145268 & 3.197515 \\ 6 & 1.751198 & 3.054280 & 3.407029 \\ 6 & -3.702807 & 5.189465 & 0.931249 \\ 6 & -4.754295 & 4.629423 & -0.024995 \\ 6 & 2.214944 & -2.078271 & 0.880324 \\ 6 & 2.531854 & -3.536349 & 1.078946 \\ 6 & 2.675876 & -4.444686 & 0.030726 \\ 6 & 2.996441 & -5.775325 & 0.287313 \\ 6 & 3.195101 & -6.211545 & 1.593094 \\ 6 & 3.070874 & -5.306765 & 2.644284 \\ 6 & 2.733390 & -3.984351 & 2.386686 \\ 45 & -0.128482 & 0.737359 & -1.993719 \\ 1 & 0.717170 & 1.035037 & -3.333003 \\ 6 & -3.211714 & 0.055796 & -0.328241 \\ 6 & -4.152382 & 0.036755 & 0.696135 \\ 6 & -5.507543 & -0.172053 & 0.427234 \\ 6 & -5.885467 & -0.351964 & -0.904629 \\ 6 & -4.961542 & -0.356262 & -1.958176 \\ 6 & -3.617021 & -0.161425 & -1.645760 \\ 6 & -6.495254 & -0.192371 & 1.591705 \\ 6 & -7.923968 & -0.483513 & 1.140957 \\ 6 & -5.439323 & -0.500422 & -3.401585 \\ 6 & -4.278744 & -0.718199 & -4.371703 \\ 6 & -6.482002 & 1.171905 & 2.291059 \\ 6 & -6.078473 & -1.283902 & 2.585161 \\ 6 & -6.395853 & -1.687927 & -3.537407 \\ 6 & -6.165473 & 0.790372 & -3.798704 \\ 6 & -4.386778 & 5.498577 & 2.267394 \\ 6 & -3.152365 & 6.484863 & 0.325849 \\ 6 & 1.404744 & 5.391037 & 2.648944 \\ 6 & 0.084790 & 4.469162 & 4.563191 \\ 1 & 6.647455 & 1.504480 & 0.827001 \\ 1 & -1.493507 & 0.714649 & 2.999751 \\ 1 & 8.126914 & -3.177471 & 0.071421 \\ 1 & -0.929882 & -3.143400 & 5.686703 \\ 1 & 4.969550 & 6.062412 & -1.445909 \\ 1 & -3.222988 & -3.071599 & 0.844983 \\ 1 & -5.125654 & -4.114920 & -0.300919 \\ 1 & 2.922983 & 5.078283 & -0.592368 \\ 1 & -1.286912 & -0.861839 & 4.856961 \\ 1 & 6.211223 & -3.582944 & -1.366808 \\ 1 & 8.245154 & 3.549701 & -2.639145 \\ 1 & 4.095174 & -2.777921 & -2.416008 \\ 1 & -2.776081 & -4.574868 & -3.873752 \\ 1 & -0.474444 & -3.745816 & -3.936106 \\ 1 & -0.320952 & -6.359617 & 2.895796 \\ 1 & 7.014289 & 1.560573 & -1.914142 \\ 1 & -0.463430 & -4.790346 & 1.018924 \\ 1 & 1.447781 & -2.690072 & -2.795766 \\ 1 & 7.249007 & 5.820005 & -2.385735 \\ 1 & 8.662594 & 0.657254 & 1.936618\end{array}$




\begin{tabular}{rrrr}
1 & -4.908975 & -4.903051 & -2.655465 \\
1 & -0.545942 & -5.550210 & 5.242295 \\
1 & 9.433979 & -1.680682 & 1.550756 \\
1 & 1.603551 & 3.061501 & 0.063364 \\
1 & -3.824828 & 0.199956 & 1.723058 \\
1 & -2.854463 & -0.176143 & -2.422100 \\
1 & -6.938335 & -0.496181 & -1.138276 \\
1 & -3.380973 & 2.603413 & -0.016134 \\
1 & 0.417490 & 1.768627 & 1.771300 \\
1 & -1.488250 & 5.550943 & 2.452459 \\
1 & 3.153097 & -1.509913 & 0.914637 \\
1 & 1.621660 & -1.720939 & 1.737558 \\
6 & -1.316471 & 1.990788 & -2.738374 \\
1 & 2.610433 & -3.284921 & 3.214351 \\
1 & 2.525245 & -4.112248 & -0.993758 \\
1 & 3.217494 & -5.636123 & 3.671036 \\
1 & 3.092132 & -6.474112 & -0.541303 \\
1 & 3.445007 & -7.251672 & 1.790979 \\
1 & 2.508549 & 3.405809 & 4.118935 \\
1 & 1.310543 & 2.136472 & 3.820201 \\
1 & 2.274578 & 2.795799 & 2.476802 \\
1 & 0.868168 & 4.764490 & 5.274012 \\
1 & -0.638519 & 5.291574 & 4.508527 \\
1 & -0.434086 & 3.593924 & 4.976264 \\
1 & 2.181926 & 5.728941 & 3.347569 \\
1 & 1.889550 & 5.176887 & 1.687265 \\
1 & 0.708186 & 6.224558 & 2.497513 \\
1 & -5.211020 & 6.209323 & 2.119489 \\
1 & -4.803351 & 4.586280 & 2.714911 \\
1 & -3.692797 & 5.940392 & 2.992989 \\
1 & -5.556787 & 5.365289 & -0.161104 \\
1 & -4.333351 & 4.411562 & -1.015412 \\
1 & -5.213716 & 3.708224 & 0.359575 \\
1 & -3.969411 & 7.195056 & 0.140577 \\
1 & -2.430504 & 6.978206 & 0.987728 \\
1 & -2.650866 & 6.288490 & -0.630443 \\
1 & -4.670222 & -0.861904 & -5.386742 \\
1 & -3.596459 & 0.141173 & -4.407550 \\
1 & -3.692383 & -1.608908 & -4.107409 \\
1 & -6.779362 & -1.319461 & 3.430031 \\
1 & -6.079839 & -2.270592 & 2.102884 \\
1 & -6.719136 & -1.794508 & -4.581345 \\
1 & -5.900369 & -2.620791 & -3.237516 \\
1 & -7.299942 & -1.572939 & -2.927858 \\
1 & -6.516821 & 0.730225 & -4.837887 \\
1 & -7.037040 & 0.979804 & -3.159278 \\
1 & -5.494416 & 1.656200 & -3.716672 \\
1 & -8.590647 & -0.495065 & 2.012541 \\
1 & -8.301798 & 0.281370 & 0.450465 \\
1 & -8.006646 & -1.461816 & 0.649742 \\
& -7.152268 & 1.163660 & 3.161315 \\
\hline & & & \\
1 & & &
\end{tabular}


$\begin{array}{lllll}1 & -5.073958 & -1.116577 & 2.994463\end{array}$

$\begin{array}{lllll}8 & -1.977754 & 2.821778 & -3.198386\end{array}$

$2_{\text {C3-c- }-\psi_{-1 a 2 a 3 s 4 a}}$

$\begin{array}{lcc}\text { Number of imaginary frequencies : } 0 & & \\ \text { The smallest frequencies are }: 9.8002 & 13.0349 & 17.5307 \mathrm{~cm}(-1) \\ \text { Electronic energy : HF=-4012.18447 } & \\ \text { Zero-point correction= } & 1.290941 \text { (Hartree/Particle) } \\ \text { Thermal correction to Energy= } & 1.367076 \\ \text { Thermal correction to Enthalpy= } & 1.368020 \\ \text { Thermal correction to Gibbs Free Energy= } & 1.179048 \\ \text { Sum of electronic and zero-point Energies= } & -4010.893529 \\ \text { Sum of electronic and thermal Energies }= & -4010.817394 \\ \text { Sum of electronic and thermal Enthalpies }= & -4010.816450 \\ \text { Sum of electronic and thermal Free Energies }= & -4011.005422\end{array}$

Cartesian Coordinates

$\begin{array}{cccc}\cdots & -3.640461 & 0.048724 & -1.611647 \\ 6 & -3.206639 & 0.136520 & -0.296036 \\ 6 & -4.119907 & -0.026785 & 0.751623 \\ 6 & -5.461164 & -0.283015 & 0.491244 \\ 6 & -5.868525 & -0.353693 & -0.851040 \\ 6 & -4.987405 & -0.187382 & -1.916834 \\ 15 & -1.428729 & 0.464803 & 0.036472 \\ 45 & -0.107030 & 0.802976 & -1.971440 \\ 1 & 0.747243 & 1.088825 & -3.306486 \\ 6 & -6.482681 & -0.502963 & 1.605575 \\ 6 & -7.071629 & -1.912695 & 1.483167 \\ 6 & -5.448255 & -0.174772 & -3.372928 \\ 6 & -5.554905 & 1.289153 & -3.818304 \\ 6 & -1.144020 & -0.740274 & 1.428190 \\ 6 & -0.941764 & -2.084375 & 1.141821 \\ 6 & -0.871384 & -3.048827 & 2.197025 \\ 6 & -0.978619 & -2.614871 & 3.549953 \\ 6 & -1.169643 & -1.239004 & 3.809986 \\ 6 & -1.258273 & -0.334595 & 2.785136 \\ 6 & -0.694153 & -4.434787 & 1.947329 \\ 6 & -0.634583 & -5.335465 & 2.980928 \\ 6 & -0.731777 & -4.899201 & 4.319593 \\ 6 & -0.898432 & -3.565804 & 4.596018 \\ 6 & -0.821663 & -2.556392 & -0.263069 \\ 6 & 0.367194 & -2.410452 & -0.960017 \\ 6 & 0.445034 & -2.811126 & -2.319543 \\ 6 & -0.631753 & -3.359587 & -2.960479 \\ 6 & -1.853725 & -3.547952 & -2.275979 \\ 6 & -1.953156 & -3.144478 & -0.911819 \\ 6 & -2.979146 & -4.118192 & -2.918494 \\ 6 & -4.170770 & -4.260661 & -2.254117 \\ 6 & -4.282216 & -3.836676 & -0.912506 \\ 6 & -3.201853 & -3.301562 & -0.255777 \\ 7 & 1.520524 & -1.750313 & -0.422503\end{array}$




$\begin{array}{cccc}6 & 2.171494 & -2.141896 & 0.834031 \\ 6 & 2.450598 & -3.611423 & 1.001941 \\ 6 & 2.576173 & -4.500696 & -0.064997 \\ 6 & 2.861202 & -5.844253 & 0.164437 \\ 6 & 3.042717 & -6.313018 & 1.461476 \\ 6 & 2.937973 & -5.427701 & 2.531181 \\ 6 & 2.635906 & -4.091891 & 2.300504 \\ 15 & 1.757691 & -0.246404 & -1.174012 \\ 8 & 2.489813 & 0.565114 & 0.073238 \\ 6 & 3.264850 & 1.654068 & -0.297800 \\ 6 & 4.554129 & 1.436770 & -0.743232 \\ 6 & 5.326651 & 2.569695 & -1.154866 \\ 6 & 4.768008 & 3.877769 & -1.028071 \\ 6 & 3.446017 & 4.029815 & -0.545993 \\ 6 & 2.693734 & 2.937286 & -0.204476 \\ 6 & 6.617625 & 2.449389 & -1.729251 \\ 6 & 7.329094 & 3.559012 & -2.112761 \\ 6 & 6.790178 & 4.852147 & -1.947847 \\ 6 & 5.532828 & 5.002557 & -1.422406 \\ 6 & 5.029534 & 0.039574 & -0.875281 \\ 6 & 4.267588 & -0.846487 & -1.618017 \\ 6 & 4.664350 & -2.177980 & -1.845592 \\ 6 & 5.817380 & -2.647532 & -1.276606 \\ 6 & 6.600800 & -1.814360 & -0.442973 \\ 6 & 6.204936 & -0.458713 & -0.229629 \\ 6 & 6.976858 & 0.329502 & 0.662111 \\ 6 & 8.090582 & -0.183823 & 1.278477 \\ 6 & 8.497286 & -1.513763 & 1.039452 \\ 6 & 7.762130 & -2.309380 & 0.199186 \\ 8 & 3.073985 & -0.431970 & -2.173045 \\ 6 & -1.452936 & 2.092785 & 0.883890 \\ 6 & -0.368874 & 2.475810 & 1.678182 \\ 6 & -0.340719 & 3.726257 & 2.296954 \\ 6 & -1.396708 & 4.605758 & 2.034529 \\ 6 & -2.472290 & 4.270816 & 1.208349 \\ 6 & -2.491086 & 2.991781 & 0.648781 \\ 6 & 0.771636 & 4.138873 & 3.260980 \\ 6 & 0.152040 & 4.473267 & 4.623271 \\ 6 & -3.588477 & 5.282819 & 0.956128 \\ 6 & -2.997736 & 6.533106 & 0.296115 \\ 6 & 1.796755 & 3.026553 & 3.475060 \\ 6 & 1.502457 & 5.370967 & 2.718952 \\ 6 & -4.674850 & 4.728532 & 0.035750 \\ 6 & -4.242043 & 5.666405 & 2.288148 \\ 6 & -5.858810 & -0.372285 & 2.993441 \\ 6 & -7.605884 & 0.532587 & 1.486841 \\ 6 & -6.810794 & -0.843343 & -3.549305 \\ 6 & -4.447473 & -0.900425 & -4.277270 \\ 1 & 6.669123 & 1.352252 & 0.865204 \\ 1 & -1.431351 & 0.712503 & 3.020110 \\ 1 & 8.051085 & -3.343555 & 0.016716 \\ 1 & -0.977707 & -3.214139 & 5.623846 \\ 1 & 5.091025 & 5.991683 & -1.309434\end{array}$




$\begin{array}{rrrr}1 & -3.301682 & -2.977731 & 0.778830 \\ 1 & -5.234385 & -3.935010 & -0.394542 \\ 1 & 3.022858 & 5.031307 & -0.480511 \\ 1 & -1.260636 & -0.906935 & 4.843515 \\ 1 & 6.132058 & -3.677539 & -1.436384 \\ 1 & 8.315606 & 3.438698 & -2.554806 \\ 1 & 4.036026 & -2.806593 & -2.472264 \\ 1 & -2.879235 & -4.429362 & -3.957729 \\ 1 & -0.558622 & -3.661015 & -4.004007 \\ 1 & -0.492056 & -6.392151 & 2.765829 \\ 1 & 7.042513 & 1.459830 & -1.875646 \\ 1 & -0.596843 & -4.782864 & 0.921501 \\ 1 & 1.388193 & -2.670709 & -2.845408 \\ 1 & 7.366386 & 5.722979 & -2.251683 \\ 1 & 8.662033 & 0.439453 & 1.962652 \\ 1 & -5.032346 & -4.691728 & -2.759521 \\ 1 & -0.675915 & -5.623161 & 5.129498 \\ 1 & 9.384567 & -1.906035 & 1.530846 \\ 1 & 1.661145 & 3.028755 & 0.128668 \\ 1 & -3.763423 & 0.041433 & 1.777008 \\ 1 & -2.905219 & 0.161521 & -2.406852 \\ 1 & -6.918585 & -0.550996 & -1.059243 \\ 1 & -3.318982 & 2.685617 & 0.014202 \\ 1 & 0.448307 & 1.770962 & 1.827509 \\ 1 & -1.381244 & 5.592299 & 2.498423 \\ 1 & 3.124330 & -1.598886 & 0.875945 \\ 1 & 1.590632 & -1.787112 & 1.700692 \\ 6 & -1.210326 & 2.152106 & -2.674767 \\ 1 & 2.529228 & -3.406835 & 3.142253 \\ 1 & 2.439788 & -4.142366 & -1.082810 \\ 1 & 3.072070 & -5.782452 & 3.551173 \\ 1 & 2.942693 & -6.527569 & -0.678472 \\ 1 & 3.264692 & -7.363255 & 1.638138 \\ 1 & 2.556687 & 3.361510 & 4.192193 \\ 1 & 1.335181 & 2.117257 & 3.884123 \\ 1 & 2.320554 & 2.758837 & 2.547633 \\ 1 & 0.936631 & 4.752899 & 5.339103 \\ 1 & -0.555208 & 5.309272 & 4.564956 \\ 1 & -0.386049 & 3.607868 & 5.032340 \\ 1 & 2.282729 & 5.691045 & 3.422518 \\ 1 & 1.987885 & 5.148942 & 1.759358 \\ 1 & 0.824358 & 6.219232 & 2.565797 \\ 1 & -5.047528 & 6.394290 & 2.121829 \\ 1 & -4.677646 & 4.786685 & 2.779813 \\ 1 & -7.075485 & -0.868722 & -4.613850 \\ 1 & -3.526394 & 6.118840 & 2.985349 \\ 1 & -5.452402 & 5.488499 & -0.112456 \\ 1 & -4.280087 & 4.464373 & -0.954224 \\ 1 & -5.160992 & 3.838465 & 0.458287 \\ 1 & -3.787856 & 7.270698 & 0.101067 \\ & -2.242355 & 7.015647 & 0.928070 \\ 1 & -2.523336 & 6.282729 & -0.661600 \\ 1 & & -1.878297 & -3.180545\end{array}$




$\begin{array}{rrrr}1 & -7.612957 & -0.300936 & -3.033519 \\ 1 & -4.820182 & -0.909990 & -5.309919 \\ 1 & -3.462816 & -0.416574 & -4.297162 \\ 1 & -4.304838 & -1.940194 & -3.954218 \\ 1 & -5.891665 & 1.351624 & -4.862226 \\ 1 & -6.271556 & 1.840300 & -3.194884 \\ 1 & -4.585696 & 1.799749 & -3.745239 \\ 1 & -6.623790 & -0.550110 & 3.759722 \\ 1 & -5.054958 & -1.104105 & 3.154046 \\ 1 & -5.447115 & 0.630918 & 3.164889 \\ 1 & -8.338854 & 0.394879 & 2.293105 \\ 1 & -7.208085 & 1.553119 & 1.561838 \\ 1 & -8.143974 & 0.452860 & 0.534734 \\ 1 & -7.831940 & -2.077787 & 2.258546 \\ 1 & -7.547965 & -2.080155 & 0.509317 \\ 1 & -6.290565 & -2.674265 & 1.613152 \\ 8 & -1.797950 & 3.051571 & -3.106374\end{array}$

$2_{\text {C3-c- }-} \Psi-1$ a2a3s4s

Number of imaginary frequencies : 0

The smallest frequencies are : $13.7994 \quad 16.9471 \quad 17.9102 \mathrm{~cm}(-1)$

Electronic energy : $\quad \mathrm{HF}=-4012.1844245$

Zero-point correction $=\quad 1.291104$ (Hartree/Particle)

Thermal correction to Energy= $\quad 1.367106$

Thermal correction to Enthalpy= $\quad 1.368051$

Thermal correction to Gibbs Free Energy $=\quad 1.180613$

Sum of electronic and zero-point Energies $=\quad-4010.893320$

Sum of electronic and thermal Energies $=\quad-4010.817318$

Sum of electronic and thermal Enthalpies $=\quad-4010.816374$

Sum of electronic and thermal Free Energies $=\quad-4011.003812$

Cartesian Coordinates

$\begin{array}{crrr}6 & -3.640949 & -0.094470 & -1.677258 \\ 6 & -3.217356 & 0.028737 & -0.357248 \\ 6 & -4.148820 & -0.055574 & 0.676827 \\ 6 & -5.503528 & -0.245006 & 0.412557 \\ 6 & -5.902126 & -0.338477 & -0.926679 \\ 6 & -4.994828 & -0.264345 & -1.986660 \\ 15 & -1.451745 & 0.390212 & 0.001086 \\ 45 & -0.123088 & 0.762509 & -1.991226 \\ 6 & -1.255111 & 2.100350 & -2.676069 \\ 8 & -1.852368 & 3.005663 & -3.080256 \\ 6 & -6.480443 & -0.321405 & 1.584603 \\ 6 & -6.048373 & -1.444109 & 2.535699 \\ 6 & -5.418176 & -0.364624 & -3.451000 \\ 6 & -4.912973 & 0.863792 & -4.217131 \\ 6 & -1.153668 & -0.780952 & 1.419396 \\ 6 & -0.887580 & -2.119113 & 1.162153 \\ 6 & -0.790034 & -3.060609 & 2.236145 \\ 6 & -0.944753 & -2.609156 & 3.578472 \\ 6 & -1.203223 & -1.239000 & 3.809442\end{array}$




$\begin{array}{cccc}6 & -1.310608 & -0.357143 & 2.766969 \\ 6 & -0.540234 & -4.440167 & 2.015289 \\ 6 & -0.462061 & -5.319587 & 3.065901 \\ 6 & -0.610133 & -4.866187 & 4.393947 \\ 6 & -0.843301 & -3.537241 & 4.642929 \\ 6 & -0.733321 & -2.613479 & -0.230752 \\ 6 & 0.450687 & -2.428028 & -0.926463 \\ 6 & 0.553561 & -2.861183 & -2.274269 \\ 6 & -0.485961 & -3.496114 & -2.897038 \\ 6 & -1.694957 & -3.740393 & -2.206358 \\ 6 & -1.827669 & -3.284264 & -0.862173 \\ 6 & -2.772240 & -4.423614 & -2.820393 \\ 6 & -3.946588 & -4.634947 & -2.144298 \\ 6 & -4.096620 & -4.151594 & -0.826727 \\ 6 & -3.066174 & -3.494458 & -0.201606 \\ 7 & 1.571858 & -1.711261 & -0.394490 \\ 6 & 2.229872 & -2.065844 & 0.869576 \\ 6 & 2.573267 & -3.520894 & 1.047105 \\ 6 & 2.764493 & -4.403407 & -0.015904 \\ 6 & 3.103754 & -5.732816 & 0.221061 \\ 6 & 3.275127 & -6.193576 & 1.522452 \\ 6 & 3.106606 & -5.314103 & 2.588557 \\ 6 & 2.749816 & -3.993073 & 2.349760 \\ 15 & 1.766545 & -0.215003 & -1.172621 \\ 8 & 2.468399 & 0.636998 & 0.065017 \\ 6 & 3.216727 & 1.740829 & -0.315503 \\ 6 & 4.514602 & 1.552231 & -0.748871 \\ 6 & 5.260011 & 2.700290 & -1.168855 \\ 6 & 4.666075 & 3.994527 & -1.061969 \\ 6 & 3.337134 & 4.117431 & -0.590701 \\ 6 & 2.611648 & 3.009638 & -0.240820 \\ 6 & 6.558007 & 2.607782 & -1.732594 \\ 6 & 7.242562 & 3.731354 & -2.124331 \\ 6 & 6.668378 & 5.011551 & -1.979053 \\ 6 & 5.403566 & 5.134571 & -1.464540 \\ 6 & 5.028396 & 0.167085 & -0.862398 \\ 6 & 4.296214 & -0.747449 & -1.600615 \\ 6 & 4.735608 & -2.066987 & -1.818581 \\ 6 & 5.900151 & -2.497345 & -1.242294 \\ 6 & 6.653189 & -1.635386 & -0.409996 \\ 6 & 6.215034 & -0.291173 & -0.207397 \\ 6 & 6.958151 & 0.525842 & 0.683000 \\ 6 & 8.084089 & 0.050733 & 1.307790 \\ 6 & 8.532671 & -1.267439 & 1.078879 \\ 6 & 7.826232 & -2.090424 & 0.240379 \\ 8 & 3.092712 & -0.375381 & -2.163626 \\ 6 & -1.537026 & 2.024485 & 0.835327 \\ 6 & -0.463738 & 2.449787 & 1.622900 \\ 6 & -0.485574 & 3.695331 & 2.251672 \\ 6 & -1.581549 & 4.529881 & 2.004732 \\ 6 & -2.647130 & 4.154164 & 1.183683 \\ 6 & -2.615007 & 2.879189 & 0.615803 \\ 6 & 0.615684 & 4.148642 & 3.210233\end{array}$




$\begin{array}{rrrr}6 & -0.007748 & 4.465877 & 4.574889 \\ 6 & -3.814921 & 5.111510 & 0.953529 \\ 6 & -3.292529 & 6.419659 & 0.350765 \\ 6 & 1.679196 & 3.072667 & 3.423256 \\ 6 & 1.300645 & 5.403607 & 2.660777 \\ 6 & -4.855367 & 4.531699 & -0.003346 \\ 6 & -4.503882 & 5.401519 & 2.291292 \\ 6 & -7.911465 & -0.606801 & 1.136652 \\ 6 & -6.471230 & 1.017167 & 2.332856 \\ 6 & -6.934431 & -0.435472 & -3.614578 \\ 6 & -4.808478 & -1.628702 & -4.065626 \\ 1 & 0.731568 & 1.084746 & -3.318634 \\ 1 & 6.618164 & 1.539947 & 0.878076 \\ 1 & -1.530095 & 0.685951 & 2.979965 \\ 1 & 8.147608 & -3.116370 & 0.065724 \\ 1 & -0.960309 & -3.172207 & 5.662462 \\ 1 & 4.934629 & 6.112744 & -1.366535 \\ 1 & -3.195284 & -3.124350 & 0.813933 \\ 1 & -5.037575 & -4.301845 & -0.301876 \\ 1 & 2.887587 & 5.108185 & -0.540086 \\ 1 & -1.329191 & -0.893377 & 4.834838 \\ 1 & 6.247732 & -3.517883 & -1.394724 \\ 1 & 8.235224 & 3.631956 & -2.557730 \\ 1 & 4.130806 & -2.717503 & -2.445977 \\ 1 & -2.648045 & -4.772212 & -3.844942 \\ 1 & -0.389736 & -3.831076 & -3.928451 \\ 1 & -0.263667 & -6.371478 & 2.872677 \\ 1 & 7.010401 & 1.628410 & -1.864032 \\ 1 & -0.398505 & -4.800060 & 0.998572 \\ 1 & 1.487864 & -2.682373 & -2.804218 \\ 1 & 7.223599 & 5.893678 & -2.289314 \\ 1 & 8.632716 & 0.695389 & 1.990832 \\ 1 & -4.768019 & -5.162496 & -2.624408 \\ 1 & -0.539302 & -5.573050 & 5.217656 \\ 1 & 9.429201 & -1.629273 & 1.576800 \\ 1 & 1.574893 & 3.077327 & 0.085002 \\ 1 & -3.808042 & 0.045582 & 1.707166 \\ 1 & -2.891963 & -0.050675 & -2.469283 \\ 1 & -6.956851 & -0.472890 & -1.148466 \\ 1 & -3.435245 & 2.542345 & -0.012514 \\ 1 & 0.382826 & 1.778561 & 1.763842 \\ 1 & -1.607479 & 5.511623 & 2.478148 \\ 1 & 3.155737 & -1.478917 & 0.917366 \\ 1 & 1.624979 & -1.733569 & 1.728773 \\ 1 & 2.590804 & -3.314390 & 3.188496 \\ 1 & 2.210655 & 2.822234 & 2.495447 \\ 1 & 2.639346 & -4.051039 & -1.037235 \\ 1 & 3.232285 & -5.662643 & 3.611762 \\ 1 & 3.235465 & -6.411642 & -0.619117 \\ 1 & 3.539094 & -7.233033 & 1.705003 \\ 1 & 2.428383 & 3.434446 & 4.138652 \\ & 0.771311 & 4.771282 & 5.286164\end{array}$




$\begin{array}{rrrr}1 & -0.740632 & 5.279663 & 4.519312 \\ 1 & -0.516275 & 3.584786 & 4.988293 \\ 1 & 2.071343 & 5.754491 & 3.360217 \\ 1 & 1.790311 & 5.194959 & 1.700360 \\ 1 & 0.591557 & 6.225965 & 2.506227 \\ 1 & -5.341767 & 6.096864 & 2.146880 \\ 1 & -4.902526 & 4.479276 & 2.734787 \\ 1 & -3.818234 & 5.853593 & 3.018488 \\ 1 & -5.675909 & 5.248694 & -0.132502 \\ 1 & -4.431746 & 4.330985 & -0.996393 \\ 1 & -5.290873 & 3.596755 & 0.376376 \\ 1 & -4.124181 & 7.113183 & 0.167241 \\ 1 & -2.580693 & 6.926184 & 1.013596 \\ 1 & -2.787253 & 6.235267 & -0.606067 \\ 1 & -7.186210 & -0.491693 & -4.681059 \\ 1 & -7.360703 & -1.324313 & -3.131756 \\ 1 & -7.433430 & 0.452431 & -3.204712 \\ 1 & -5.090811 & -1.711846 & -5.124220 \\ 1 & -3.712226 & -1.622705 & -4.010466 \\ 1 & -5.160089 & -2.528949 & -3.544057 \\ 1 & -5.254399 & 0.825722 & -5.260233 \\ 1 & -5.289493 & 1.793557 & -3.770557 \\ 1 & -3.818077 & 0.919993 & -4.234564 \\ 1 & -8.567988 & -0.662912 & 2.014185 \\ 1 & -8.305007 & 0.183715 & 0.484986 \\ 1 & -7.990578 & -1.563334 & 0.603774 \\ 1 & -7.133097 & 0.972851 & 3.208480 \\ 1 & -5.467029 & 1.286213 & 2.685080 \\ 1 & -6.826190 & 1.829166 & 1.684060 \\ 1 & -6.743393 & -1.514153 & 3.383210 \\ 1 & -6.046540 & -2.413401 & 2.019686 \\ 1 & -5.042734 & -1.284662 & 2.945422\end{array}$

$2_{C 3-c-}-1$-1a2s3a4a

Number of imaginary frequencies : 0

The smallest frequencies are : $14.9974 \quad 15.092121 .7346 \mathrm{~cm}(-1)$

Electronic energy : $\quad H F=-4012.1860296$

Zero-point correction $=\quad 1.291585$ (Hartree/Particle)

Thermal correction to Energy= $\quad 1.367403$

Thermal correction to Enthalpy= $\quad 1.368347$

Thermal correction to Gibbs Free Energy $=\quad 1.182284$

Sum of electronic and zero-point Energies $=\quad-4010.894445$

Sum of electronic and thermal Energies $=\quad-4010.818626$

Sum of electronic and thermal Enthalpies $=\quad-4010.817682$

Sum of electronic and thermal Free Energies $=\quad-4011.003745$

Cartesian Coordinates

$\begin{array}{llll}6 & 5.517342 & 4.988585 & -1.596983 \\ 6 & 4.759373 & 3.874545 & -1.161121 \\ 6 & 5.321032 & 2.564753 & -1.252072 \\ 6 & 6.607310 & 2.430162 & -1.833746\end{array}$




$\begin{array}{cccc}6 & 7.311875 & 3.529257 & -2.258182 \\ 6 & 6.770360 & 4.825365 & -2.128918 \\ 6 & 4.555414 & 1.442839 & -0.799345 \\ 6 & 3.269018 & 1.670632 & -0.350995 \\ 6 & 2.695038 & 2.954495 & -0.291192 \\ 6 & 3.441202 & 4.038104 & -0.672294 \\ 6 & 5.033788 & 0.043411 & -0.891227 \\ 6 & 4.268052 & -0.867862 & -1.598773 \\ 6 & 4.666025 & -2.205114 & -1.786473 \\ 6 & 5.824911 & -2.653621 & -1.212582 \\ 6 & 6.613914 & -1.791901 & -0.414019 \\ 6 & 6.216543 & -0.430993 & -0.240845 \\ 6 & 7.783054 & -2.263031 & 0.231918 \\ 6 & 8.524746 & -1.438785 & 1.038119 \\ 6 & 8.116927 & -0.102748 & 1.237927 \\ 6 & 6.995493 & 0.387593 & 0.616839 \\ 8 & 3.069923 & -0.473991 & -2.158946 \\ 15 & 1.758816 & -0.254805 & -1.160309 \\ 8 & 2.498929 & 0.591426 & 0.057926 \\ 7 & 1.518386 & -1.733646 & -0.361840 \\ 6 & 0.369474 & -2.415292 & -0.881384 \\ 6 & -0.817039 & -2.555666 & -0.179337 \\ 6 & -1.939906 & -3.183014 & -0.806119 \\ 6 & -1.836423 & -3.626370 & -2.157367 \\ 6 & -0.620746 & -3.434295 & -2.852048 \\ 6 & 0.449418 & -2.852371 & -2.229703 \\ 6 & -2.952643 & -4.237572 & -2.778761 \\ 6 & -4.135094 & -4.394623 & -2.101353 \\ 6 & -4.251383 & -3.931947 & -0.772465 \\ 6 & -3.183111 & -3.345636 & -0.140286 \\ 6 & -0.947418 & -2.042724 & 1.209560 \\ 6 & -1.171705 & -0.693969 & 1.453963 \\ 6 & -1.305833 & -0.252271 & 2.798327 \\ 6 & -1.208305 & -1.124748 & 3.849687 \\ 6 & -0.990941 & -2.504114 & 3.631796 \\ 6 & -0.869061 & -2.974991 & 2.292841 \\ 6 & -0.669131 & -4.364512 & 2.083973 \\ 6 & -0.602354 & -5.234372 & 3.143210 \\ 6 & -0.713430 & -4.761465 & 4.468108 \\ 6 & -0.901482 & -3.423207 & 4.705201 \\ 15 & -1.438137 & 0.473927 & 0.024267 \\ 6 & -1.445242 & 2.116385 & 0.843728 \\ 6 & -0.344143 & 2.486598 & 1.628427 \\ 6 & -0.298886 & 3.725850 & 2.258585 \\ 6 & -1.359877 & 4.617320 & 2.031122 \\ 6 & -2.446428 & 4.297062 & 1.221957 \\ 6 & -2.474575 & 3.024217 & 0.634567 \\ 6 & 0.831879 & 4.122565 & 3.207450 \\ 6 & 1.854824 & 3.003063 & 3.391576 \\ 6 & -3.616572 & 5.248585 & 0.981253 \\ 6 & -3.436420 & 6.585523 & 1.695289 \\ 6 & 2.170410 & -2.087378 & 0.905353 \\ 6 & 2.460566 & -3.549872 & 1.112398\end{array}$




$\begin{array}{cccc}6 & 2.588316 & -4.466554 & 0.069325 \\ 6 & 2.883433 & -5.801571 & 0.333399 \\ 6 & 3.072832 & -6.234248 & 1.641742 \\ 6 & 2.965253 & -5.321454 & 2.687795 \\ 6 & 2.652866 & -3.994336 & 2.422713 \\ 45 & -0.114283 & 0.753131 & -1.991101 \\ 1 & 0.737725 & 1.009189 & -3.334322 \\ 6 & -3.212306 & 0.152022 & -0.329205 \\ 6 & -4.171514 & 0.153811 & 0.686992 \\ 6 & -5.513847 & -0.082879 & 0.398469 \\ 6 & -5.863587 & -0.312973 & -0.939323 \\ 6 & -4.930178 & -0.330513 & -1.976318 \\ 6 & -3.591490 & -0.104667 & -1.643006 \\ 6 & -6.584021 & -0.144482 & 1.487210 \\ 6 & -6.039416 & 0.261171 & 2.855455 \\ 6 & -5.370872 & -0.514505 & -3.427366 \\ 6 & -4.195142 & -0.854565 & -4.344222 \\ 6 & -7.748496 & 0.790079 & 1.145211 \\ 6 & -7.097175 & -1.585963 & 1.585407 \\ 6 & -6.402993 & -1.638163 & -3.548978 \\ 6 & -5.994030 & 0.802590 & -3.904888 \\ 6 & -4.905981 & 4.598873 & 1.498598 \\ 6 & -3.754522 & 5.521996 & -0.520291 \\ 6 & 1.559555 & 5.356477 & 2.665092 \\ 6 & 0.239181 & 4.445265 & 4.584526 \\ 1 & 6.687508 & 1.415964 & 0.788988 \\ 1 & -1.494992 & 0.798434 & 3.003092 \\ 1 & 8.072927 & -3.301880 & 0.079908 \\ 1 & -0.992050 & -3.043757 & 5.722165 \\ 1 & 5.073711 & 5.979582 & -1.510778 \\ 1 & -3.289737 & -2.986345 & 0.881511 \\ 1 & -5.197968 & -4.039952 & -0.246390 \\ 1 & 3.015975 & 5.040037 & -0.633484 \\ 1 & -1.312676 & -0.763976 & 4.872298 \\ 1 & 6.140666 & -3.687623 & -1.341506 \\ 1 & 8.294748 & 3.398007 & -2.705192 \\ 1 & 4.034267 & -2.854978 & -2.387466 \\ 1 & -2.849922 & -4.577942 & -3.808535 \\ 1 & -0.545811 & -3.763386 & -3.887104 \\ 1 & -0.443050 & -6.294518 & 2.959177 \\ 1 & 7.033980 & 1.437665 & -1.952639 \\ 1 & -0.559357 & -4.740276 & 1.069221 \\ 1 & 1.389146 & -2.713850 & -2.762172 \\ 1 & 7.341063 & 5.687935 & -2.464984 \\ 1 & 8.693857 & 0.543930 & 1.895260 \\ 1 & -4.985375 & -4.870418 & -2.585659 \\ 1 & -0.651426 & -5.460936 & 5.298816 \\ 1 & 9.418154 & -1.812736 & 1.532680 \\ 1 & 1.665213 & 3.052886 & 0.048451 \\ 1 & -3.856824 & 0.349148 & 1.710167 \\ 1 & -2.814708 & -0.135208 & -2.404511 \\ 1 & -6.912367 & -0.488675 & -1.177483 \\ 1 & -3.318756 & 2.736759 & 0.008578\end{array}$




$\begin{array}{rrrr}1 & 0.466646 & 1.771503 & 1.763180 \\ 1 & -1.328306 & 5.591237 & 2.514967 \\ 1 & 3.119057 & -1.536328 & 0.933949 \\ 1 & 1.585701 & -1.713834 & 1.761636 \\ 6 & -1.283237 & 1.997924 & -2.779094 \\ 1 & 2.542325 & -3.288296 & 3.246509 \\ 1 & 2.445046 & -4.136871 & -0.957103 \\ 1 & 3.104985 & -5.647886 & 3.716439 \\ 1 & 2.966473 & -6.506557 & -0.491327 \\ 1 & 3.302756 & -7.277838 & 1.845563 \\ 1 & 2.628308 & 3.326157 & 4.099610 \\ 1 & 1.395646 & 2.091178 & 3.797658 \\ 1 & 2.361541 & 2.744195 & 2.452398 \\ 1 & 1.039051 & 4.708893 & 5.289403 \\ 1 & -0.461106 & 5.288283 & 4.550259 \\ 1 & -0.298751 & 3.579018 & 4.992268 \\ 1 & 2.354051 & 5.664967 & 3.357840 \\ 1 & 2.026673 & 5.141465 & 1.694870 \\ 1 & 0.883632 & 6.209947 & 2.533001 \\ 1 & -5.762534 & 5.266342 & 1.333618 \\ 1 & -5.120656 & 3.651788 & 0.986794 \\ 1 & -4.838916 & 4.393175 & 2.575222 \\ 1 & -4.300046 & 7.230815 & 1.491082 \\ 1 & -3.367551 & 6.465095 & 2.784249 \\ 1 & -2.540451 & 7.117028 & 1.349517 \\ 1 & -4.579400 & 6.223435 & -0.704388 \\ 1 & -2.835861 & 5.963354 & -0.927487 \\ 1 & -3.965868 & 4.608817 & -1.088632 \\ 1 & -4.563036 & -1.026981 & -5.363666 \\ 1 & -3.458963 & -0.041980 & -4.401843 \\ 1 & -3.675602 & -1.765207 & -4.013982 \\ 1 & -6.677701 & -1.782960 & -4.601979 \\ 1 & -5.995418 & -2.584767 & -3.170287 \\ 1 & -7.328600 & -1.423240 & -3.002040 \\ 1 & -6.319754 & 0.718451 & -4.950781 \\ 1 & -6.867222 & 1.078178 & -3.299517 \\ 1 & -5.267564 & 1.623859 & -3.840459 \\ 1 & -6.844616 & 0.218222 & 3.599652 \\ 1 & -5.241598 & -0.410468 & 3.198954 \\ 1 & -5.646089 & 1.286597 & 2.851279 \\ 1 & -8.512534 & 0.745846 & 1.932699 \\ 1 & -7.409541 & 1.831026 & 1.063426 \\ 1 & -8.237291 & 0.522469 & 0.201139 \\ 1 & -7.871703 & -1.667582 & 2.360162 \\ 1 & -7.531770 & -1.931556 & 0.639032 \\ 1 & -6.280334 & -2.271124 & 1.850019 \\ 8 & -1.933605 & 2.812836 & -3.281097\end{array}$

$2_{\mathrm{C} 3-\mathrm{c}-} \psi_{-1 \mathrm{a} 2 \mathrm{~s} 3 \mathrm{a} 4 \mathrm{~s}}$

Number of imaginary frequencies : 0

The smallest frequencies are : $13.8016 \quad 14.6255 \quad 18.7019 \mathrm{~cm}(-1)$

Electronic energy : $\quad \mathrm{HF}=-4012.1861545$ 
Zero-point correction $=$

Thermal correction to Energy=

Thermal correction to Enthalpy=

Thermal correction to Gibbs Free Energy=

Sum of electronic and zero-point Energies=

Sum of electronic and thermal Energies=

Sum of electronic and thermal Enthalpies=

Sum of electronic and thermal Free Energies=
1.291491 (Hartree/Particle)

1.367405

1.368349

1.181228

$-4010.894664$

$-4010.818749$

$-4010.817805$

$-4011.004926$

Cartesian Coordinates

$\begin{array}{cccc}0 & -3.642961 & -0.043945 & -1.632748 \\ 6 & -3.219365 & 0.153964 & -0.318149 \\ 6 & -4.144457 & 0.107677 & 0.720392 \\ 6 & -5.498892 & -0.127145 & 0.470252 \\ 6 & -5.894791 & -0.290418 & -0.858958 \\ 6 & -4.989540 & -0.253966 & -1.927600 \\ 15 & -1.437578 & 0.463672 & 0.012747 \\ 45 & -0.114385 & 0.762614 & -1.996597 \\ 1 & 0.743645 & 1.034833 & -3.333001 \\ 6 & -6.465271 & -0.209099 & 1.650777 \\ 6 & -6.006804 & -1.323378 & 2.600088 \\ 6 & -5.486401 & -0.379623 & -3.366581 \\ 6 & -6.200782 & 0.924137 & -3.742015 \\ 6 & -1.170580 & -0.726567 & 1.422169 \\ 6 & -0.956608 & -2.072239 & 1.153632 \\ 6 & -0.893691 & -3.024979 & 2.219976 \\ 6 & -1.021758 & -2.577666 & 3.566519 \\ 6 & -1.224937 & -1.200252 & 3.809054 \\ 6 & -1.305729 & -0.307382 & 2.773465 \\ 6 & -0.703809 & -4.412110 & 1.987352 \\ 6 & -0.652781 & -5.301661 & 3.031038 \\ 6 & -0.770650 & -4.852119 & 4.363538 \\ 6 & -0.949074 & -3.516897 & 4.623582 \\ 6 & -0.819474 & -2.559452 & -0.244169 \\ 6 & 0.374973 & -2.416355 & -0.932299 \\ 6 & 0.466503 & -2.834442 & -2.285693 \\ 6 & -0.601983 & -3.396030 & -2.929028 \\ 6 & -1.828339 & -3.583142 & -2.252317 \\ 6 & -1.942018 & -3.162677 & -0.894415 \\ 6 & -2.944468 & -4.168854 & -2.897848 \\ 6 & -4.136021 & -4.323697 & -2.236424 \\ 6 & -4.262387 & -3.881924 & -0.901360 \\ 6 & -3.195308 & -3.319545 & -0.246054 \\ 7 & 1.520348 & -1.742864 & -0.394427 \\ 6 & 2.162897 & -2.112071 & 0.873048 \\ 6 & 2.444021 & -3.578092 & 1.066540 \\ 6 & 2.582956 & -4.482424 & 0.014071 \\ 6 & 2.869879 & -5.821554 & 0.265851 \\ 6 & 3.039986 & -6.270707 & 1.571348 \\ 6 & 2.921522 & -5.370312 & 2.626889 \\ 6 & 2.617239 & -4.038979 & 2.373798 \\ 15 & 1.759194 & -0.251606 & -1.170339\end{array}$




$\begin{array}{rrrr}8 & 2.490191 & 0.579878 & 0.063570 \\ 6 & 3.262579 & 1.664901 & -0.324991 \\ 6 & 4.551865 & 1.443721 & -0.768342 \\ 6 & 5.320358 & 2.571833 & -1.200191 \\ 6 & 4.758157 & 3.880228 & -1.094212 \\ 6 & 3.436997 & 4.036731 & -0.611233 \\ 6 & 2.688431 & 2.947896 & -0.250110 \\ 6 & 6.610363 & 2.445422 & -1.775427 \\ 6 & 7.317596 & 3.550377 & -2.179683 \\ 6 & 6.775222 & 4.844566 & -2.035569 \\ 6 & 5.518795 & 5.000315 & -1.509471 \\ 6 & 5.031244 & 0.045945 & -0.877230 \\ 6 & 4.270980 & -0.854823 & -1.603771 \\ 6 & 4.671625 & -2.188734 & -1.809188 \\ 6 & 5.826756 & -2.645062 & -1.233815 \\ 6 & 6.609040 & -1.795336 & -0.416018 \\ 6 & 6.209383 & -0.437486 & -0.225222 \\ 6 & 6.980910 & 0.368433 & 0.650934 \\ 6 & 8.097700 & -0.130706 & 1.273388 \\ 6 & 8.508008 & -1.463315 & 1.056453 \\ 6 & 7.773358 & -2.275621 & 0.231866 \\ 8 & 3.076092 & -0.452998 & -2.165358 \\ 6 & -1.437702 & 2.097689 & 0.849665 \\ 6 & -0.344106 & 2.456879 & 1.649370 \\ 6 & -0.294723 & 3.697023 & 2.278042 \\ 6 & -1.338602 & 4.602517 & 2.028190 \\ 6 & -2.415302 & 4.293915 & 1.201227 \\ 6 & -2.453796 & 3.016898 & 0.624331 \\ 6 & 0.822877 & 4.080304 & 3.247797 \\ 6 & 0.207618 & 4.390434 & 4.617856 \\ 6 & -3.554083 & 5.269775 & 0.912338 \\ 6 & -3.600491 & 5.559619 & -0.592199 \\ 6 & 1.839170 & 2.955544 & 3.436535 \\ 6 & 1.564101 & 5.317197 & 2.731354 \\ 6 & -3.385782 & 6.595852 & 1.649164 \\ 6 & -4.883928 & 4.644353 & 1.349481 \\ 6 & -7.894498 & -0.518750 & 1.214065 \\ 6 & -6.472190 & 1.127663 & 2.400614 \\ 6 & -4.339421 & -0.603744 & -4.351654 \\ 6 & -6.460821 & -1.552142 & -3.505714 \\ 1 & 6.670657 & 1.393746 & 0.836649 \\ 1 & -1.483916 & 0.741720 & 2.995951 \\ 1 & 8.065154 & -3.311873 & 0.066439 \\ 1 & -1.044129 & -3.155010 & 5.646518 \\ 1 & 5.074514 & 5.989989 & -1.412114 \\ 1 & -3.310899 & -2.971354 & 0.779147 \\ 1 & -5.218243 & -3.983777 & -0.391368 \\ 1 & 3.011470 & 5.038036 & -0.560815 \\ 1 & -1.330543 & -0.857469 & 4.837715 \\ 1 & 6.144499 & -3.676616 & -1.376648 \\ 1 & 8.303341 & 3.425415 & -2.622145 \\ 1 & 4.045262 & -2.829972 & -2.424850 \\ 1 & -2.833735 & -4.492509 & -3.932053 \\ & & & \end{array}$




$\begin{array}{rrrr}1 & -0.518268 & -3.710196 & -3.967991 \\ 1 & -0.500665 & -6.359576 & 2.828967 \\ 1 & 7.037786 & 1.454701 & -1.905793 \\ 1 & -0.589214 & -4.770220 & 0.966782 \\ 1 & 1.413136 & -2.696047 & -2.805665 \\ 1 & 7.348066 & 5.711753 & -2.355740 \\ 1 & 8.668894 & 0.506151 & 1.945158 \\ 1 & -4.986423 & -4.780868 & -2.738167 \\ 1 & -0.721202 & -5.567151 & 5.181744 \\ 1 & 9.397707 & -1.844247 & 1.552373 \\ 1 & 1.656839 & 3.041845 & 0.085308 \\ 1 & -3.803269 & 0.255502 & 1.745172 \\ 1 & -2.892771 & -0.039902 & -2.420933 \\ 1 & -6.947721 & -0.454886 & -1.077853 \\ 1 & -3.292889 & 2.737749 & -0.012241 \\ 1 & 0.458698 & 1.734890 & 1.794317 \\ 1 & -1.300762 & 5.578677 & 2.507202 \\ 1 & 3.114201 & -1.566170 & 0.912746 \\ 1 & 1.575025 & -1.744419 & 1.729659 \\ 6 & -1.267622 & 2.035727 & -2.760396 \\ 1 & 2.499038 & -3.342311 & 3.204420 \\ 1 & 2.455411 & -4.139281 & -1.010066 \\ 1 & 3.046272 & -5.709767 & 3.653249 \\ 1 & 2.961892 & -6.516823 & -0.566129 \\ 1 & 3.263590 & -7.317499 & 1.765551 \\ 1 & 2.604465 & 3.270208 & 4.157162 \\ 1 & 1.371760 & 2.042222 & 3.829689 \\ 1 & 2.356877 & 2.702140 & 2.501783 \\ 1 & 0.995272 & 4.648398 & 5.338460 \\ 1 & -0.493195 & 5.232839 & 4.578361 \\ 1 & -0.336475 & 3.520239 & 5.008716 \\ 1 & 2.349699 & 5.612932 & 3.439614 \\ 1 & 2.044669 & 5.111891 & 1.765611 \\ 1 & 0.894984 & 6.176062 & 2.600295 \\ 1 & -5.714401 & 5.332277 & 1.141049 \\ 1 & -5.091009 & 3.705615 & 0.819850 \\ 1 & -4.883818 & 4.430257 & 2.426533 \\ 1 & -4.228683 & 7.257391 & 1.412823 \\ 1 & -3.368598 & 6.462073 & 2.738703 \\ 1 & -2.467082 & 7.117151 & 1.350876 \\ 1 & -4.406202 & 6.271276 & -0.817769 \\ 1 & -2.654888 & 5.996094 & -0.938588 \\ 1 & -3.783770 & 4.653599 & -1.181825 \\ 1 & -4.744043 & -0.735935 & -5.363091 \\ 1 & -3.647594 & 0.247738 & -4.390349 \\ 1 & -3.760844 & -1.503130 & -4.099412 \\ 1 & -6.790317 & -1.645146 & -4.548943 \\ 1 & -5.978367 & -2.495012 & -3.216256 \\ 1 & -7.361188 & -1.428741 & -2.892447 \\ 1 & -6.569201 & 0.877668 & -4.775979 \\ 1 & -7.059270 & 1.118898 & -3.086520 \\ 1 & -5.516992 & 1.780110 & -3.663676 \\ 1 & -8.544806 & -0.578979 & 2.095911\end{array}$




$\begin{array}{rrrr}1 & -8.302905 & 0.261469 & 0.558921 \\ 1 & -7.962424 & -1.480203 & 0.688369 \\ 1 & -7.140407 & 1.074033 & 3.270757 \\ 1 & -5.474076 & 1.402820 & 2.763814 \\ 1 & -6.828537 & 1.939159 & 1.752333 \\ 1 & -6.680261 & -1.387842 & 3.465398 \\ 1 & -6.016950 & -2.297961 & 2.094323 \\ 1 & -4.991115 & -1.157699 & 2.981927 \\ 8 & -1.902169 & 2.876595 & -3.239701\end{array}$

$2_{\text {C3-c- }-\psi-122 s 354 a}$

Number of imaginary frequencies : 0

The smallest frequencies are : $15.0703 \quad 15.3089 \quad 21.6036 \mathrm{~cm}(-1)$

Electronic energy : $\quad \mathrm{HF}=-4012.1860094$

Zero-point correction $=\quad 1.291719($ Hartree/Particle $)$

Thermal correction to Energy=

1.367490

Thermal correction to Enthalpy=

1.368434

Thermal correction to Gibbs Free Energy=

Sum of electronic and zero-point Energies=

1.182540

Sum of electronic and thermal Energies=

$-4010.894291$

Sum of electronic and thermal Enthalpies=

$-4010.818520$

Sum of electronic and thermal Free Energies=

$-4010.817576$

$-4011.003469$

\section{Cartesian Coordinates}

$\begin{array}{cccc}6 & -3.584463 & -0.102618 & -1.645971 \\ 6 & -3.209025 & 0.152622 & -0.330818 \\ 6 & -4.170654 & 0.150870 & 0.683109 \\ 6 & -5.511812 & -0.087932 & 0.390878 \\ 6 & -5.857660 & -0.317304 & -0.948064 \\ 6 & -4.921783 & -0.331036 & -1.982881 \\ 15 & -1.436427 & 0.476694 & 0.027810 \\ 45 & -0.113589 & 0.752507 & -1.989208 \\ 1 & 0.719530 & 0.974335 & -3.349911 \\ 6 & -6.585008 & -0.151452 & 1.476568 \\ 6 & -7.098838 & -1.592897 & 1.571309 \\ 6 & -5.358345 & -0.513683 & -3.435347 \\ 6 & -5.980022 & 0.803836 & -3.913530 \\ 6 & -1.171354 & -0.692701 & 1.456439 \\ 6 & -0.948420 & -2.041606 & 1.211506 \\ 6 & -0.870502 & -2.974174 & 2.294605 \\ 6 & -0.991574 & -2.503516 & 3.633720 \\ 6 & -1.207653 & -1.124039 & 3.852034 \\ 6 & -1.304614 & -0.251206 & 2.800925 \\ 6 & -0.671546 & -4.363811 & 2.085455 \\ 6 & -0.605007 & -5.233912 & 3.144504 \\ 6 & -0.715417 & -4.761218 & 4.469538 \\ 6 & -0.902493 & -3.422888 & 4.706927 \\ 6 & -0.818576 & -2.554769 & -0.177393 \\ 6 & 0.368347 & -2.416307 & -0.879142 \\ 6 & 0.448524 & -2.855177 & -2.226907 \\ 6 & -0.621654 & -3.437351 & -2.848880\end{array}$




$\begin{array}{cccc}6 & -1.837696 & -3.627827 & -2.154371 \\ 6 & -1.941576 & -3.182277 & -0.803887 \\ 6 & -2.953798 & -4.239716 & -2.775277 \\ 6 & -4.136469 & -4.395689 & -2.098002 \\ 6 & -4.253160 & -3.930828 & -0.769927 \\ 6 & -3.185117 & -3.343395 & -0.138369 \\ 7 & 1.517869 & -1.735317 & -0.360048 \\ 6 & 2.169800 & -2.088502 & 0.907308 \\ 6 & 2.459519 & -3.550971 & 1.115126 \\ 6 & 2.587159 & -4.468132 & 0.072461 \\ 6 & 2.881839 & -5.803123 & 0.337145 \\ 6 & 3.070928 & -6.235293 & 1.645701 \\ 6 & 2.963501 & -5.322013 & 2.691347 \\ 6 & 2.651545 & -3.994914 & 2.425656 \\ 15 & 1.758622 & -0.257186 & -1.159707 \\ 8 & 2.498798 & 0.589965 & 0.057948 \\ 6 & 3.268130 & 1.669233 & -0.352148 \\ 6 & 4.554470 & 1.441729 & -0.800806 \\ 6 & 5.319259 & 2.563626 & -1.254968 \\ 6 & 4.756900 & 3.873193 & -1.165091 \\ 6 & 3.438872 & 4.036513 & -0.675780 \\ 6 & 2.693492 & 2.952858 & -0.293274 \\ 6 & 6.605359 & 2.429165 & -1.837058 \\ 6 & 7.309111 & 3.528223 & -2.262938 \\ 6 & 6.766910 & 4.824153 & -2.134779 \\ 6 & 5.514040 & 4.987213 & -1.602442 \\ 6 & 5.033629 & 0.042467 & -0.891242 \\ 6 & 4.268183 & -0.870119 & -1.597418 \\ 6 & 4.666671 & -2.207478 & -1.783287 \\ 6 & 5.825898 & -2.654675 & -1.209069 \\ 6 & 6.614780 & -1.791443 & -0.412011 \\ 6 & 6.216806 & -0.430491 & -0.240566 \\ 6 & 6.995656 & 0.389638 & 0.615740 \\ 6 & 8.117571 & -0.099291 & 1.237074 \\ 6 & 8.525989 & -1.435390 & 1.038929 \\ 6 & 7.784396 & -2.261102 & 0.234138 \\ 8 & 3.069822 & -0.477671 & -2.158019 \\ 6 & -1.447596 & 2.118357 & 0.848702 \\ 6 & -0.345947 & 2.490940 & 1.631566 \\ 6 & -0.301785 & 3.730677 & 2.260787 \\ 6 & -1.364695 & 4.620187 & 2.034411 \\ 6 & -2.452044 & 4.297433 & 1.227337 \\ 6 & -2.478916 & 3.024161 & 0.640776 \\ 6 & 0.830453 & 4.130423 & 3.206592 \\ 6 & 0.240428 & 4.456685 & 4.583958 \\ 6 & -3.623817 & 5.247045 & 0.987023 \\ 6 & -3.761295 & 5.521534 & -0.514391 \\ 6 & 1.854282 & 3.011883 & 3.391675 \\ 6 & 1.556408 & 5.363202 & 2.659332 \\ 6 & -3.446350 & 6.583626 & 1.702396 \\ 6 & -4.912493 & 4.594822 & 1.503006 \\ 6 & -6.044176 & 0.252088 & 2.846928 \\ 6 & -7.748414 & 0.783760 & 1.132593\end{array}$




$\begin{array}{rrrr}6 & -6.389889 & -1.637373 & -3.561118 \\ 6 & -4.179828 & -0.852826 & -4.348975 \\ 1 & 6.687234 & 1.418095 & 0.786588 \\ 1 & -1.492841 & 0.799599 & 3.005946 \\ 1 & 8.074735 & -3.300011 & 0.083431 \\ 1 & -0.992491 & -3.043546 & 5.723982 \\ 1 & 5.069885 & 5.978047 & -1.517062 \\ 1 & -3.292076 & -2.982309 & 0.882770 \\ 1 & -5.199887 & -4.038273 & -0.243976 \\ 1 & 3.013119 & 5.038252 & -0.637737 \\ 1 & -1.311461 & -0.763438 & 4.874759 \\ 1 & 6.142079 & -3.688721 & -1.336587 \\ 1 & 8.291862 & 3.397074 & -2.710245 \\ 1 & 4.034954 & -2.858446 & -2.383139 \\ 1 & -2.850743 & -4.581658 & -3.804497 \\ 1 & -0.546509 & -3.767926 & -3.883450 \\ 1 & -0.446403 & -6.294117 & 2.960206 \\ 1 & 7.032535 & 1.436783 & -1.955103 \\ 1 & -0.562354 & -4.739530 & 1.070634 \\ 1 & 1.388531 & -2.717738 & -2.759172 \\ 1 & 7.336966 & 5.686701 & -2.472001 \\ 1 & 8.694421 & 0.548575 & 1.893308 \\ 1 & -4.986617 & -4.872260 & -2.581782 \\ 1 & -0.653630 & -5.460894 & 5.300089 \\ 1 & 9.419774 & -1.808219 & 1.533655 \\ 1 & 1.663792 & 3.051024 & 0.046842 \\ 1 & -3.858746 & 0.345229 & 1.707341 \\ 1 & -2.805624 & -0.129879 & -2.405554 \\ 1 & -6.905507 & -0.494676 & -1.189041 \\ 1 & -3.323433 & 2.735009 & 0.016071 \\ 1 & 0.466329 & 1.777318 & 1.765294 \\ 1 & -1.333923 & 5.594581 & 2.517349 \\ 1 & 3.118622 & -1.537712 & 0.935485 \\ 1 & 1.585299 & -1.714300 & 1.763438 \\ 6 & -1.275897 & 2.005969 & -2.772811 \\ 1 & 2.541208 & -3.288459 & 3.249120 \\ 1 & 2.444165 & -4.138804 & -0.954123 \\ 1 & 3.103034 & -5.648040 & 3.720148 \\ 1 & 2.964807 & -6.508487 & -0.487265 \\ 1 & -5.125864 & 3.648233 & 0.989763 \\ 1 & -4.311340 & 4.387652 & 2.579353 \\ 1 & 3.300521 & -7.278861 & 1.850004 \\ 1 & 2.628683 & 3.336953 & 4.097796 \\ 1 & 1.396137 & 2.100671 & 3.800436 \\ 1 & 2.359741 & 2.751165 & 2.452320 \\ 1 & 1.041685 & 4.722343 & 5.286488 \\ 1 & -0.459986 & 5.299562 & 4.548888 \\ 1 & -0.296545 & 3.591504 & 4.995170 \\ 1 & 2.351969 & 5.674228 & 3.349720 \\ 1 & 2.021869 & 5.145616 & 1.688885 \\ 1 & 0.879637 & 6.215808 & 2.525936 \\ & -5.769857 & 5.261399 & 1.338684 \\ 1 & & & \\ 1 & & & \end{array}$




$\begin{array}{rrrr}1 & -3.377032 & 6.462171 & 2.791218 \\ 1 & -2.551574 & 7.117396 & 1.357034 \\ 1 & -4.588100 & 6.220698 & -0.698527 \\ 1 & -2.843583 & 5.966026 & -0.920322 \\ 1 & -3.969340 & 4.608252 & -1.083831 \\ 1 & -6.661955 & -1.780667 & -4.615007 \\ 1 & -5.982787 & -2.584369 & -3.182882 \\ 1 & -7.316905 & -1.423610 & -3.016106 \\ 1 & -4.544479 & -1.023691 & -5.369846 \\ 1 & -3.443055 & -0.040514 & -4.403083 \\ 1 & -3.661703 & -1.764037 & -4.018231 \\ 1 & -6.304222 & 0.720076 & -4.959926 \\ 1 & -6.853944 & 1.079839 & -3.309391 \\ 1 & -5.253130 & 1.624622 & -3.847841 \\ 1 & -6.851347 & 0.207731 & 3.588905 \\ 1 & -5.247113 & -0.419966 & 3.191370 \\ 1 & -5.651040 & 1.277600 & 2.845545 \\ 1 & -8.514805 & 0.738343 & 1.917727 \\ 1 & -7.409214 & 1.824821 & 1.053397 \\ 1 & -8.234344 & 0.517631 & 0.186624 \\ 1 & -7.876020 & -1.675125 & 2.343336 \\ 1 & -7.530352 & -1.937360 & 0.623113 \\ 1 & -6.283186 & -2.278615 & 1.838078 \\ 8 & -1.922108 & 2.826923 & -3.270445\end{array}$

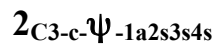

Number of imaginary frequencies : 0

The smallest frequencies are : $14.3340 \quad 16.1016 \quad 21.1108 \mathrm{~cm}(-1)$

Electronic energy : $\quad H F=-4012.1861324$

Zero-point correction $=\quad 1.291472($ Hartree/Particle $)$

Thermal correction to Energy=

1.367369

Thermal correction to Enthalpy=

1.368313

Thermal correction to Gibbs Free Energy=

Sum of electronic and zero-point Energies $=$

Sum of electronic and thermal Energies $=$

1.181583

$-4010.894660$

$-4010.818763$

Sum of electronic and thermal Enthalpies=

$-4010.817819$

Sum of electronic and thermal Free Energies=

$-4011.004550$

\section{Cartesian Coordinates}

$\begin{array}{cccc}6 & -3.597843 & -0.048613 & -1.663535 \\ 6 & -3.205658 & 0.177010 & -0.344245 \\ 6 & -4.151631 & 0.128871 & 0.675656 \\ 6 & -5.494650 & -0.138401 & 0.399152 \\ 6 & -5.859249 & -0.329324 & -0.935988 \\ 6 & -4.932788 & -0.290465 & -1.985711 \\ 15 & -1.431466 & 0.498550 & 0.011847 \\ 45 & -0.098452 & 0.770419 & -1.996560 \\ 1 & 0.757878 & 1.013623 & -3.339518 \\ 6 & -6.483465 & -0.235032 & 1.560031 \\ 6 & -6.020831 & -1.337350 & 2.521430 \\ 6 & -5.385068 & -0.441975 & -3.437188\end{array}$




$\begin{array}{cccc}6 & -5.998525 & 0.890810 & -3.883025 \\ 6 & -1.177774 & -0.676681 & 1.438225 \\ 6 & -0.968385 & -2.026938 & 1.189372 \\ 6 & -0.911899 & -2.965465 & 2.268639 \\ 6 & -1.043128 & -2.499960 & 3.608567 \\ 6 & -1.243918 & -1.118875 & 3.831315 \\ 6 & -1.317550 & -0.239646 & 2.783531 \\ 6 & -0.727087 & -4.356357 & 2.054931 \\ 6 & -0.683971 & -5.232278 & 3.110420 \\ 6 & -0.804519 & -4.764759 & 4.436387 \\ 6 & -0.977877 & -3.425406 & 4.678222 \\ 6 & -0.839221 & -2.535537 & -0.201112 \\ 6 & 0.350419 & -2.407560 & -0.899920 \\ 6 & 0.428043 & -2.840916 & -2.249489 \\ 6 & -0.648406 & -3.406061 & -2.876596 \\ 6 & -1.868782 & -3.583105 & -2.186073 \\ 6 & -1.969211 & -3.144019 & -0.833320 \\ 6 & -2.992636 & -4.174961 & -2.812461 \\ 6 & -4.179431 & -4.315184 & -2.138879 \\ 6 & -4.292689 & -3.853605 & -0.809288 \\ 6 & -3.217010 & -3.287376 & -0.171750 \\ 7 & 1.505884 & -1.741246 & -0.374783 \\ 6 & 2.150060 & -2.109429 & 0.892417 \\ 6 & 2.419687 & -3.576805 & 1.092041 \\ 6 & 2.548370 & -4.486844 & 0.043174 \\ 6 & 2.823262 & -5.827506 & 0.299964 \\ 6 & 2.991482 & -6.272718 & 1.607078 \\ 6 & 2.883230 & -5.366879 & 2.658999 \\ 6 & 2.590587 & -4.033856 & 2.400931 \\ 15 & 1.761988 & -0.259373 & -1.162134 \\ 8 & 2.502794 & 0.573320 & 0.065152 \\ 6 & 3.283480 & 1.648722 & -0.333460 \\ 6 & 4.570355 & 1.413400 & -0.776568 \\ 6 & 5.347325 & 2.531971 & -1.218177 \\ 6 & 4.795507 & 3.845589 & -1.122088 \\ 6 & 3.475885 & 4.016622 & -0.639864 \\ 6 & 2.719104 & 2.936668 & -0.269566 \\ 6 & 6.635704 & 2.390879 & -1.793623 \\ 6 & 7.351268 & 3.487038 & -2.207163 \\ 6 & 6.819305 & 4.786561 & -2.072613 \\ 6 & 5.564655 & 4.956318 & -1.546638 \\ 6 & 5.037670 & 0.010679 & -0.875385 \\ 6 & 4.268100 & -0.889372 & -1.593060 \\ 6 & 4.656031 & -2.228496 & -1.788271 \\ 6 & 5.808170 & -2.690566 & -1.211543 \\ 6 & 6.599922 & -1.841063 & -0.402672 \\ 6 & 6.212987 & -0.478084 & -0.222192 \\ 6 & 6.994012 & 0.328019 & 0.645323 \\ 6 & 8.107791 & -0.176045 & 1.269214 \\ 6 & 8.505442 & -1.514098 & 1.062475 \\ 6 & 7.761402 & -2.326503 & 0.246461 \\ 8 & 3.075875 & -0.481939 & -2.156085 \\ 6 & -1.441001 & 2.137658 & 0.837465\end{array}$




\begin{tabular}{|c|c|c|c|}
\hline & -0.342588 & 2.503952 & 1.627634 \\
\hline & -0.297636 & 3.741873 & 2.260308 \\
\hline & -1.354617 & 4.636754 & 2.027499 \\
\hline 6 & -2.438354 & 4.320518 & 1.212806 \\
\hline & -2.468459 & 3.047227 & 0.626079 \\
\hline & 0.829923 & 4.134482 & 3.214680 \\
\hline & 0.231940 & 4.465544 & 4.587450 \\
\hline & -3.597523 & 5.281671 & 0.956864 \\
\hline & -3.690554 & 5.578509 & -0.543851 \\
\hline & 1.844715 & 3.009092 & 3.408250 \\
\hline & 1.568298 & 5.361948 & 2.672161 \\
\hline & -3.429525 & 6.606370 & 1.696289 \\
\hline & -4.905543 & 4.633259 & 1.425635 \\
\hline & -7.896408 & -0.577160 & 1.095517 \\
\hline & -6.534467 & 1.103063 & 2.305436 \\
\hline & -6.429124 & -1.552513 & -3.578492 \\
\hline & -4.218204 & -0.775164 & -4.368557 \\
\hline & 6.693632 & 1.357696 & 0.822982 \\
\hline & -1.494298 & 0.812539 & 2.991774 \\
\hline & 8.043372 & -3.366695 & 0.088916 \\
\hline & -1.074992 & -3.049805 & 5.696000 \\
\hline & 5.128384 & 5.950234 & -1.456593 \\
\hline & -3.320653 & -2.927141 & 0.850338 \\
\hline & -5.244013 & -3.943107 & -0.287842 \\
\hline & 3.058354 & 5.021670 & -0.597704 \\
\hline 1 & -1.353642 & -0.761856 & 4.854684 \\
\hline 1 & 6.116105 & -3.726178 & -1.346254 \\
\hline & 8.335559 & 3.350851 & -2.649557 \\
\hline 1 & 4.021882 & -2.868759 & -2.397066 \\
\hline 1 & -2.892498 & -4.513880 & -3.842968 \\
\hline 1 & -0.575315 & -3.732311 & -3.912682 \\
\hline & -0.535657 & -6.293380 & 2.922702 \\
\hline & 7.055207 & 1.395810 & -1.916485 \\
\hline & -0.608247 & -4.728412 & 1.039785 \\
\hline & 1.370916 & -2.712733 & -2.779037 \\
\hline & 7.398697 & 5.646691 & -2.399947 \\
\hline & 8.686466 & 0.461201 & 1.934178 \\
\hline & -5.035671 & -4.776064 & -2.627133 \\
\hline & -0.761212 & -5.469177 & 5.264096 \\
\hline 1 & 9.392888 & -1.898961 & 1.559391 \\
\hline 1 & 1.688061 & 3.041221 & 0.064565 \\
\hline 1 & -3.834711 & 0.295002 & 1.705553 \\
\hline 1 & -2.829566 & -0.047308 & -2.433887 \\
\hline 1 & -6.903403 & -0.520372 & -1.172505 \\
\hline & -3.310339 & 2.762149 & -0.004232 \\
\hline 1 & 0.465921 & 1.786682 & 1.764679 \\
\hline & -1.321830 & 5.610702 & 2.511175 \\
\hline & 3.105798 & -1.570999 & 0.926274 \\
\hline & 1.567689 & -1.732797 & 1.748934 \\
\hline & -1.250467 & 2.026549 & -2.790786 \\
\hline & 2.478223 & -3.333200 & 3.229079 \\
\hline 1 & 2.421738 & -4.146889 & -0.982133 \\
\hline & & & \\
\hline
\end{tabular}




$\begin{array}{rrrr}1 & 2.907095 & -6.527091 & -0.529257 \\ 1 & 3.205565 & -7.320747 & 1.805304 \\ 1 & 2.615302 & 3.328994 & 4.120873 \\ 1 & 1.377060 & 2.100998 & 3.813145 \\ 1 & 2.356513 & 2.744832 & 2.473316 \\ 1 & 1.029808 & 4.726391 & 5.295607 \\ 1 & -0.462678 & 5.312931 & 4.546917 \\ 1 & -0.313615 & 3.604162 & 4.995320 \\ 1 & 2.361020 & 5.667833 & 3.368097 \\ 1 & 2.039154 & 5.140681 & 1.705153 \\ 1 & 0.898290 & 6.219048 & 2.533517 \\ 1 & -5.751723 & 5.310133 & 1.246019 \\ 1 & -5.112440 & 3.696096 & 0.893249 \\ 1 & -4.872714 & 4.409690 & 2.500315 \\ 1 & -4.287425 & 7.256857 & 1.484559 \\ 1 & -3.383397 & 6.467661 & 2.784335 \\ 1 & -2.525919 & 7.142076 & 1.378012 \\ 1 & -4.504614 & 6.289068 & -0.740928 \\ 1 & -2.757347 & 6.018154 & -0.918507 \\ 1 & -3.892662 & 4.674668 & -1.130407 \\ 1 & -6.701995 & -1.676780 & -4.634550 \\ 1 & -6.033509 & -2.510248 & -3.214818 \\ 1 & -7.354754 & -1.336250 & -3.032140 \\ 1 & -4.594571 & -0.923880 & -5.388674 \\ 1 & -3.474357 & 0.030940 & -4.416368 \\ 1 & -3.705555 & -1.697327 & -4.059529 \\ 1 & -6.335654 & 0.830962 & -4.926997 \\ 1 & -6.862795 & 1.162910 & -3.263275 \\ 1 & -5.262723 & 1.703035 & -3.809620 \\ 1 & -8.563516 & -0.647573 & 1.963946 \\ 1 & -8.307636 & 0.190948 & 0.427953 \\ 1 & -7.932316 & -1.542317 & 0.573276 \\ 1 & -7.219048 & 1.037275 & 3.161901 \\ 1 & -5.550379 & 1.401061 & 2.688266 \\ 1 & -6.895216 & 1.904784 & 1.647523 \\ 1 & -6.710310 & -1.414781 & 3.372978 \\ 1 & -6.000164 & -2.312725 & 2.016963 \\ 1 & -5.016806 & -1.148897 & 2.923417 \\ 8 & -1.889712 & 2.846594 & -3.298737\end{array}$

$2_{\text {C3-t- } \psi-1 \text { a2a3a4a }}$

Number of imaginary frequencies : 0

The smallest frequencies are : $12.5715 \quad 15.9462 \quad 19.2470 \mathrm{~cm}(-1)$

Electronic energy : $\quad H F=-4012.1864072$

Zero-point correction $=\quad 1.291088$ (Hartree/Particle)

Thermal correction to Energy= $\quad 1.367130$

Thermal correction to Enthalpy= $\quad 1.368074$

Thermal correction to Gibbs Free Energy= 1.180088

Sum of electronic and zero-point Energies $=\quad-4010.895319$

Sum of electronic and thermal Energies $=\quad-4010.819277$

Sum of electronic and thermal Enthalpies $=\quad-4010.818333$ 
Cartesian Coordinates

$\begin{array}{cccc}\cdots & -0.380165 & 2.504087 & 1.603455 \\ 6 & -1.459258 & 2.177269 & 0.778146 \\ 6 & -2.455709 & 3.118970 & 0.531018 \\ 6 & -2.401597 & 4.385812 & 1.116252 \\ 6 & -1.329285 & 4.667651 & 1.966323 \\ 6 & -0.313915 & 3.744959 & 2.238010 \\ 15 & -1.500054 & 0.530710 & -0.030102 \\ 6 & -3.274154 & 0.245055 & -0.405765 \\ 6 & -4.219874 & 0.241420 & 0.622717 \\ 6 & -5.568407 & 0.023136 & 0.351238 \\ 6 & -5.936129 & -0.192907 & -0.982620 \\ 6 & -5.016924 & -0.200386 & -2.032834 \\ 6 & -3.671366 & 0.015294 & -1.719685 \\ 6 & -6.625046 & -0.022388 & 1.453578 \\ 6 & -7.171774 & -1.450969 & 1.553887 \\ 6 & -5.493640 & -0.381029 & -3.473212 \\ 6 & -6.255825 & 0.881638 & -3.892677 \\ 6 & -3.481948 & 5.439943 & 0.879969 \\ 6 & -2.847839 & 6.691892 & 0.264882 \\ 6 & 0.788549 & 4.097799 & 3.236462 \\ 6 & 0.146494 & 4.434107 & 4.587892 \\ 6 & -1.291227 & -0.638447 & 1.409892 \\ 6 & -1.093722 & -1.993586 & 1.180211 \\ 6 & -1.086234 & -2.922658 & 2.269803 \\ 6 & -1.258361 & -2.443233 & 3.600249 \\ 6 & -1.445263 & -1.057295 & 3.803896 \\ 6 & -1.469489 & -0.187287 & 2.746578 \\ 6 & -0.912752 & -4.317907 & 2.075518 \\ 6 & -0.923230 & -5.184733 & 3.139487 \\ 6 & -1.087210 & -4.703687 & 4.455714 \\ 6 & -1.247771 & -3.359582 & 4.679509 \\ 6 & -0.919465 & -2.520240 & -0.198201 \\ 6 & 0.301155 & -2.423920 & -0.845877 \\ 6 & 0.431083 & -2.886839 & -2.181105 \\ 6 & -0.625172 & -3.449264 & -2.843257 \\ 6 & -1.878519 & -3.591119 & -2.205011 \\ 6 & -2.031409 & -3.121922 & -0.867669 \\ 6 & -2.981614 & -4.182830 & -2.866966 \\ 6 & -4.196901 & -4.296694 & -2.240978 \\ 6 & -4.362464 & -3.803343 & -0.928554 \\ 6 & -3.308145 & -3.235535 & -0.257656 \\ 7 & 1.439895 & -1.761248 & -0.281065 \\ 15 & 1.733864 & -0.284103 & -1.065433 \\ 8 & 3.057820 & -0.539658 & -2.053458 \\ 6 & 4.242250 & -0.959760 & -1.485765 \\ 6 & 5.021735 & -0.068717 & -0.766633 \\ 6 & 6.187632 & -0.569489 & -0.106633 \\ 6 & 6.557786 & -1.937959 & -0.279260 \\ 6 & 5.759167 & -2.779370 & -1.089635\end{array}$




$\begin{array}{cccc}6 & 4.616515 & -2.304145 & -1.674643 \\ 6 & 6.975250 & 0.230702 & 0.760430 \\ 6 & 8.079260 & -0.284922 & 1.392221 \\ 6 & 8.459915 & -1.629271 & 1.194200 \\ 6 & 7.709326 & -2.435676 & 0.378144 \\ 6 & 4.570769 & 1.339508 & -0.675511 \\ 6 & 3.285124 & 1.586493 & -0.234795 \\ 6 & 2.727292 & 2.878763 & -0.193456 \\ 6 & 3.490565 & 3.949435 & -0.578106 \\ 6 & 4.811683 & 3.765161 & -1.051192 \\ 6 & 5.356760 & 2.447323 & -1.127156 \\ 6 & 5.588967 & 4.865948 & -1.486654 \\ 6 & 6.845283 & 4.682261 & -2.003811 \\ 6 & 7.370892 & 3.378154 & -2.118224 \\ 6 & 6.647213 & 2.291393 & -1.694154 \\ 8 & 2.501337 & 0.518070 & 0.173024 \\ 6 & 2.040467 & -2.135063 & 1.005918 \\ 6 & 2.285198 & -3.605161 & 1.217029 \\ 6 & 2.390807 & -4.065540 & 2.531611 \\ 6 & 2.657554 & -5.401930 & 2.800848 \\ 6 & 2.803721 & -6.307865 & 1.753769 \\ 6 & 2.701636 & -5.859288 & 0.440925 \\ 6 & 2.453755 & -4.515465 & 0.174065 \\ 45 & -0.071199 & 0.800145 & -1.945533 \\ 6 & -6.053237 & 0.366975 & 2.815464 \\ 6 & -7.770439 & 0.941879 & 1.129357 \\ 6 & -4.328580 & -0.586372 & -4.440110 \\ 6 & -6.422253 & -1.593242 & -3.587782 \\ 6 & -4.574629 & 4.945240 & -0.065240 \\ 6 & -4.138648 & 5.803336 & 2.216511 \\ 6 & 1.762995 & 2.942402 & 3.458597 \\ 6 & 1.581357 & 5.307925 & 2.733967 \\ 1 & 6.687472 & 1.265192 & 0.931291 \\ 1 & -1.637544 & 0.868981 & 2.940694 \\ 1 & 7.978419 & -3.480230 & 0.226914 \\ 1 & -1.376275 & -2.973147 & 5.689718 \\ 1 & 5.157889 & 5.863390 & -1.411968 \\ 1 & -3.451442 & -2.851464 & 0.750730 \\ 1 & -5.336347 & -3.870432 & -0.447385 \\ 1 & 3.077445 & 4.956907 & -0.552866 \\ 1 & -1.584757 & -0.688923 & 4.819560 \\ 1 & 6.054546 & -3.819200 & -1.220159 \\ 1 & 8.356883 & 3.230947 & -2.553265 \\ 1 & 3.980638 & -2.939376 & -2.286815 \\ 1 & -2.841782 & -4.546151 & -3.884309 \\ 1 & -0.511593 & -3.800131 & -3.867615 \\ 1 & -0.782944 & -6.249386 & 2.966408 \\ 1 & 7.061913 & 1.292435 & -1.801017 \\ 1 & -0.758783 & -4.701331 & 1.069277 \\ 1 & 1.396865 & -2.780095 & -2.672095 \\ 1 & 7.431177 & 5.534742 & -2.339473 \\ 1 & 8.663292 & 0.347898 & 2.056758 \\ 1 & -5.036447 & -4.760650 & -2.754637 \\ & & & \\ 6\end{array}$




$\begin{array}{rrrr}1 & -1.086537 & -5.401229 & 5.290369 \\ 1 & 9.339587 & -2.023480 & 1.697598 \\ 1 & 1.695792 & 2.995818 & 0.134963 \\ 1 & -3.891395 & 0.417717 & 1.644332 \\ 1 & -2.907290 & 0.014561 & -2.493730 \\ 1 & -6.988839 & -0.361185 & -1.210157 \\ 1 & -3.278659 & 2.852586 & -0.127466 \\ 1 & 0.398641 & 1.761007 & 1.770901 \\ 1 & -1.287045 & 5.645196 & 2.447120 \\ 1 & 3.001253 & -1.608501 & 1.067624 \\ 1 & 1.437133 & -1.749252 & 1.843799 \\ 1 & -1.367619 & 1.655525 & -2.456544 \\ 1 & 2.247990 & -3.364575 & 3.354822 \\ 1 & 2.380708 & -4.172116 & -0.855175 \\ 1 & 2.729397 & -5.740668 & 3.832506 \\ 1 & 2.816608 & -6.558804 & -0.384615 \\ 1 & 2.996758 & -7.358424 & 1.960073 \\ 1 & 2.516001 & 3.235974 & 4.200747 \\ 1 & 1.255753 & 2.045740 & 3.840659 \\ 1 & 2.300506 & 2.668737 & 2.541039 \\ 1 & 0.921923 & 4.672590 & 5.328136 \\ 1 & -0.527839 & 5.296646 & 4.525834 \\ 1 & -0.433666 & 3.582740 & 4.968284 \\ 1 & 2.354945 & 5.585588 & 3.462495 \\ 1 & 2.082771 & 5.081485 & 1.783660 \\ 1 & 0.941327 & 6.184750 & 2.577806 \\ 1 & -4.922136 & 6.557625 & 2.063266 \\ 1 & -4.603455 & 4.922208 & 2.678187 \\ 1 & -3.418689 & 6.215717 & 2.933796 \\ 1 & -5.328923 & 5.730972 & -0.198280 \\ 1 & -4.178272 & 4.697201 & -1.058049 \\ 1 & -5.089043 & 4.057822 & 0.328337 \\ 1 & -3.614110 & 7.458084 & 0.085583 \\ 1 & -2.085847 & 7.132846 & 0.918977 \\ 1 & -2.370588 & 6.458410 & -0.695382 \\ 1 & -4.716201 & -0.749950 & -5.453714 \\ 1 & -3.663212 & 0.285639 & -4.482807 \\ 1 & -3.725475 & -1.462664 & -4.165198 \\ 1 & -6.748756 & -1.719320 & -4.628643 \\ 1 & -5.905249 & -2.511879 & -3.280173 \\ 1 & -7.325934 & -1.490835 & -2.975018 \\ 1 & -6.606323 & 0.791913 & -4.930029 \\ 1 & -7.132253 & 1.057395 & -3.255900 \\ 1 & -5.611568 & 1.768179 & -3.827958 \\ 1 & -6.847848 & 0.335882 & 3.571496 \\ 1 & -5.261647 & -0.319344 & 3.144938 \\ 1 & -5.642238 & 1.385607 & 2.808032 \\ 1 & -8.523534 & 0.917815 & 1.928221 \\ 1 & -7.404500 & 1.973075 & 1.041571 \\ 1 & -8.279915 & 0.687074 & 0.192655 \\ 1 & -7.942270 & -1.514971 & 2.334276 \\ 1 & -7.622300 & -1.784427 & 0.610641 \\ 1 & -6.370484 & -2.156568 & 1.812262\end{array}$


$\begin{array}{llll}6 & 0.724205 & 1.461989 & -3.496819\end{array}$

$8 \quad 1.191448 \quad 1.916576 \quad-4.451688$

$2_{\mathrm{C} 3-\mathrm{t}-}-\psi_{-1 \mathrm{a} 2 \mathrm{a} 3 \mathrm{a} 4 \mathrm{~s}}$

Number of imaginary frequencies : 0

The smallest frequencies are : $13.0819 \quad 14.0688 \quad 19.2342 \mathrm{~cm}(-1)$

Electronic energy : $\quad H F=-4012.1870456$

Zero-point correction $=\quad 1.291258($ Hartree/Particle $)$

Thermal correction to Energy= $\quad 1.367228$

Thermal correction to Enthalpy= $\quad 1.368172$

Thermal correction to Gibbs Free Energy $=\quad 1.180397$

Sum of electronic and zero-point Energies $=\quad-4010.895788$

Sum of electronic and thermal Energies $=\quad-4010.819818$

Sum of electronic and thermal Enthalpies $=\quad-4010.818873$

Sum of electronic and thermal Free Energies $=\quad-4011.006649$

\section{Cartesian Coordinates}

$\begin{array}{cccc}0 & 2.501556 & -4.494378 & 0.184799 \\ 6 & 2.325096 & -3.582398 & 1.225054 \\ 6 & 2.431636 & -4.038553 & 2.540991 \\ 6 & 2.707101 & -5.372443 & 2.814152 \\ 6 & 2.861254 & -6.280085 & 1.769768 \\ 6 & 2.758302 & -5.835694 & 0.455540 \\ 6 & 2.070040 & -2.114798 & 1.008800 \\ 7 & 1.463809 & -1.750779 & -0.278543 \\ 15 & 1.746968 & -0.275960 & -1.070674 \\ 45 & -0.063944 & 0.787788 & -1.962525 \\ 6 & 0.723375 & 1.440116 & -3.522569 \\ 8 & 1.183802 & 1.886914 & -4.484329 \\ 6 & 0.329940 & -2.426782 & -0.837330 \\ 6 & -0.888422 & -2.529132 & -0.186459 \\ 6 & -1.996270 & -3.146628 & -0.847782 \\ 6 & -1.841477 & -3.627387 & -2.180645 \\ 6 & -0.591029 & -3.478528 & -2.823225 \\ 6 & 0.461273 & -2.899521 & -2.168920 \\ 6 & -3.270759 & -3.264178 & -0.233682 \\ 6 & -4.320243 & -3.850664 & -0.895961 \\ 6 & -4.152640 & -4.356322 & -2.203443 \\ 6 & -2.940239 & -4.236437 & -2.833937 \\ 6 & -1.066222 & -1.991497 & 1.186845 \\ 6 & -1.275775 & -0.635932 & 1.402195 \\ 6 & -1.456309 & -0.172548 & 2.734228 \\ 6 & -1.425477 & -1.031818 & 3.800258 \\ 6 & -1.229442 & -2.418369 & 3.610749 \\ 6 & -1.052302 & -2.909745 & 2.285322 \\ 6 & -1.213437 & -3.323778 & 4.699196 \\ 6 & -1.042915 & -4.668763 & 4.488912 \\ 6 & -0.873665 & -5.161665 & 3.177727 \\ 6 & -0.868185 & -4.305563 & 2.105116 \\ 15 & -1.495639 & 0.514802 & -0.051760 \\ 6 & -3.261377 & 0.194807 & -0.438884\end{array}$




$\begin{array}{lrrr}6 & -4.207610 & 0.185681 & 0.580942 \\ 6 & -5.559458 & -0.037912 & 0.309395 \\ 6 & -5.926975 & -0.250177 & -1.019815 \\ 6 & -4.998304 & -0.258704 & -2.069336 \\ 6 & -3.657084 & -0.040012 & -1.756449 \\ 6 & -6.552044 & -0.043571 & 1.469755 \\ 6 & -6.546431 & 1.332123 & 2.146643 \\ 6 & -5.473615 & -0.449795 & -3.508817 \\ 6 & -6.380414 & -1.679131 & -3.618608 \\ 6 & -1.502694 & 2.166245 & 0.747691 \\ 6 & -0.426535 & 2.529799 & 1.561437 \\ 6 & -0.399945 & 3.768547 & 2.203017 \\ 6 & -1.453035 & 4.653356 & 1.948585 \\ 6 & -2.523307 & 4.335789 & 1.108606 \\ 6 & -2.537117 & 3.070282 & 0.518318 \\ 6 & -3.649705 & 5.345256 & 0.893646 \\ 6 & -4.330468 & 5.634853 & 2.236060 \\ 6 & 0.703014 & 4.159172 & 3.186959 \\ 6 & 1.465653 & 5.376699 & 2.656066 \\ 6 & -6.137245 & -1.117413 & 2.483159 \\ 6 & -7.977483 & -0.346830 & 1.016793 \\ 6 & -6.258739 & 0.797619 & -3.931586 \\ 6 & -4.307044 & -0.637367 & -4.477366 \\ 6 & -3.074286 & 6.644691 & 0.321009 \\ 6 & -4.710768 & 4.827651 & -0.075261 \\ 6 & 0.069227 & 4.504967 & 4.539851 \\ 6 & 1.702234 & 3.026835 & 3.418623 \\ 8 & 3.079958 & -0.523110 & -2.048418 \\ 6 & 4.264609 & -0.928631 & -1.470668 \\ 6 & 5.030545 & -0.026657 & -0.750482 \\ 6 & 6.196295 & -0.513408 & -0.079785 \\ 6 & 6.580675 & -1.879041 & -0.243671 \\ 6 & 5.796032 & -2.731731 & -1.055952 \\ 6 & 4.652938 & -2.270163 & -1.650913 \\ 6 & 7.732192 & -2.362814 & 0.424112 \\ 6 & 8.469102 & -1.545735 & 1.242002 \\ 6 & 8.074181 & -0.204264 & 1.431444 \\ 6 & 6.969915 & 0.298042 & 0.789466 \\ 6 & 4.566182 & 1.377955 & -0.670872 \\ 6 & 3.274539 & 1.615737 & -0.242827 \\ 6 & 2.703692 & 2.902798 & -0.217089 \\ 6 & 3.460120 & 3.978033 & -0.602359 \\ 6 & 4.787575 & 3.803230 & -1.061084 \\ 6 & 5.345766 & 2.490143 & -1.122989 \\ 6 & 6.642874 & 2.342799 & -1.676966 \\ 6 & 7.360334 & 3.433604 & -2.101216 \\ 6 & 6.821481 & 4.733405 & -2.000272 \\ 6 & 5.558576 & 4.908503 & -1.496354 \\ 8 & 2.497992 & 0.543006 & 0.167261 \\ 1 & 6.670986 & 1.330496 & 0.953520 \\ 1 & -1.632219 & 0.884153 & 2.918014 \\ 1 & 8.012346 & -3.405379 & 0.279323 \\ & -1.345894 & -2.928291 & 5.705378\end{array}$




$\begin{array}{rrrr}1 & 5.117382 & 5.902254 & -1.432275 \\ 1 & -3.416938 & -2.866080 & 0.769311 \\ 1 & -5.292890 & -3.921379 & -0.412868 \\ 1 & 3.037026 & 4.981577 & -0.589125 \\ 1 & -1.567041 & -0.654216 & 4.812241 \\ 1 & 6.102456 & -3.769194 & -1.179673 \\ 1 & 8.351770 & 3.292918 & -2.525909 \\ 1 & 4.027035 & -2.913979 & -2.264385 \\ 1 & -2.799612 & -4.608211 & -3.848107 \\ 1 & -0.476414 & -3.837632 & -3.844617 \\ 1 & -0.725445 & -6.226951 & 3.015484 \\ 1 & 7.067999 & 1.347188 & -1.773452 \\ 1 & -0.710128 & -4.697791 & 1.102879 \\ 1 & 1.424892 & -2.787469 & -2.663141 \\ 1 & 7.402553 & 5.589222 & -2.335825 \\ 1 & 8.647290 & 0.436948 & 2.097441 \\ 1 & -4.988805 & -4.833805 & -2.710164 \\ 1 & -1.038319 & -5.358025 & 5.330406 \\ 1 & 9.348867 & -1.929294 & 1.753405 \\ 1 & 1.667735 & 3.012148 & 0.099807 \\ 1 & -3.888307 & 0.363288 & 1.607728 \\ 1 & -2.891082 & -0.040025 & -2.528571 \\ 1 & -6.976347 & -0.415240 & -1.255634 \\ 1 & -3.358766 & 2.776006 & -0.129489 \\ 1 & 0.379093 & 1.813463 & 1.718357 \\ 1 & -1.443889 & 5.628465 & 2.435921 \\ 1 & 3.027180 & -1.581076 & 1.066285 \\ 1 & 1.465628 & -1.729853 & 1.846197 \\ 1 & -1.366403 & 1.632606 & -2.476034 \\ 1 & 2.282086 & -3.336576 & 3.362162 \\ 1 & 2.427246 & -4.154373 & -0.845450 \\ 1 & 2.779404 & -5.707986 & 3.846823 \\ 1 & 2.879295 & -6.536600 & -0.367954 \\ 1 & 3.061030 & -7.328768 & 1.979181 \\ 1 & 2.452720 & 3.345176 & 4.153037 \\ 1 & 1.214662 & 2.125167 & 3.814353 \\ 1 & 2.241495 & 2.751778 & 2.502579 \\ 1 & 0.848971 & 4.764014 & 5.268494 \\ 1 & -0.616757 & 5.357922 & 4.474732 \\ 1 & -0.495044 & 3.651097 & 4.937987 \\ 1 & 2.238743 & 5.684801 & 3.372821 \\ 1 & 1.964192 & 5.143691 & 1.705827 \\ 1 & 0.804622 & 6.235798 & 2.488605 \\ 1 & -5.142210 & 6.362747 & 2.103296 \\ 1 & -4.763555 & 4.719640 & 2.661452 \\ 1 & -3.631032 & 6.048003 & 2.973138 \\ 1 & -5.501169 & 5.579917 & -0.191097 \\ 1 & -4.294853 & 4.628829 & -1.071276 \\ 1 & -5.184562 & 3.904600 & 0.286337 \\ 1 & -3.877762 & 7.374292 & 0.152521 \\ 1 & -2.344583 & 7.108172 & 0.995872 \\ 1 & -2.574732 & 6.463131 & -0.639101 \\ 1 & -4.693779 & -0.802585 & -5.491052 \\ & & & \end{array}$




$\begin{array}{rrrr}1 & -3.652688 & 0.243061 & -4.517749 \\ 1 & -3.692832 & -1.507261 & -4.206589 \\ 1 & -6.704254 & -1.815566 & -4.659036 \\ 1 & -5.846712 & -2.586854 & -3.306925 \\ 1 & -7.285740 & -1.590527 & -3.006122 \\ 1 & -6.609313 & 0.697356 & -4.967936 \\ 1 & -7.137628 & 0.960826 & -3.294973 \\ 1 & -5.630001 & 1.695552 & -3.871752 \\ 1 & -8.648815 & -0.346505 & 1.884875 \\ 1 & -8.353895 & 0.405139 & 0.311501 \\ 1 & -8.054108 & -1.333511 & 0.541440 \\ 1 & -7.224449 & 1.337591 & 3.010877 \\ 1 & -5.547524 & 1.613439 & 2.504473 \\ 1 & -6.883825 & 2.110787 & 1.449653 \\ 1 & -6.835158 & -1.132616 & 3.331163 \\ 1 & -6.147188 & -2.113905 & 2.021565 \\ 1 & -5.130189 & -0.948525 & 2.885704\end{array}$

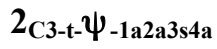

\begin{tabular}{|c|c|c|c|}
\hline \multicolumn{4}{|c|}{ Number of imaginary frequencies : 0} \\
\hline \multicolumn{4}{|c|}{ The smallest frequencies are : 8.1794} \\
\hline \multicolumn{2}{|c|}{ Electronic energy : } & \multicolumn{2}{|c|}{$\mathrm{HF}=-4012.1859666$} \\
\hline \multicolumn{3}{|c|}{ Zero-point correction $=$} & \\
\hline \multicolumn{4}{|c|}{ Thermal correction to Energy= } \\
\hline \multicolumn{4}{|c|}{ Thermal correction to Enthalpy= } \\
\hline \multirow{5}{*}{\multicolumn{4}{|c|}{$\begin{array}{l}\text { Thermal correction to Gibbs Free Energy= } \\
\text { Sum of electronic and zero-point Energies= } \\
\text { Sum of electronic and thermal Energies= } \\
\text { Sum of electronic and thermal Enthalpies= } \\
\text { Sum of electronic and thermal Free Energies= }\end{array}$}} \\
\hline & & & \\
\hline & & & \\
\hline & & & \\
\hline & & & \\
\hline \multicolumn{4}{|c|}{ Cartesian Coordinates } \\
\hline 6 & 5.645933 & 4.818596 & -1.457013 \\
\hline 6 & 4.857909 & 3.724496 & -1.024083 \\
\hline 6 & 5.389470 & 2.401392 & -1.104314 \\
\hline 6 & 6.677843 & 2.234005 & -1.672721 \\
\hline 6 & 7.412192 & 3.314643 & -2.094197 \\
\hline 6 & 6.899925 & 4.623665 & -1.975673 \\
\hline 6 & 4.592153 & 1.300422 & -0.655685 \\
\hline 6 & 3.309899 & 1.559375 & -0.212108 \\
\hline 6 & 2.765163 & 2.857286 & -0.167418 \\
\hline 6 & 3.538963 & 3.921018 & -0.549889 \\
\hline 6 & 5.026651 & -0.112518 & -0.753953 \\
\hline 6 & 4.236132 & -0.990744 & -1.476853 \\
\hline 6 & 4.593455 & -2.338892 & -1.671410 \\
\hline 6 & 5.730277 & -2.830737 & -1.088670 \\
\hline 6 & 6.539778 & -2.002574 & -0.275424 \\
\hline 6 & 6.186600 & -0.630362 & -0.096891 \\
\hline 6 & 7.685515 & -2.516999 & 0.379312 \\
\hline 6 & 8.446478 & -1.723131 & 1.198055 \\
\hline 6 & 8.082546 & -0.374994 & 1.401720 \\
\hline
\end{tabular}




$\begin{array}{cccc}6 & 6.984508 & 0.156630 & 0.772816 \\ 8 & 3.057160 & -0.553359 & -2.042806 \\ 15 & 1.735990 & -0.283181 & -1.054966 \\ 8 & 2.515201 & 0.496954 & 0.190947 \\ 7 & 1.414955 & -1.760513 & -0.282849 \\ 6 & 0.265383 & -2.404404 & -0.847823 \\ 6 & -0.952944 & -2.489719 & -0.194624 \\ 6 & -2.072174 & -3.087129 & -0.856729 \\ 6 & -1.926968 & -3.568500 & -2.190520 \\ 6 & -0.676293 & -3.433402 & -2.836116 \\ 6 & 0.385481 & -2.870515 & -2.182964 \\ 6 & -3.034345 & -4.164008 & -2.841102 \\ 6 & -4.252474 & -4.253474 & -2.216064 \\ 6 & -4.410037 & -3.746254 & -0.908687 \\ 6 & -3.347739 & -3.187115 & -0.242413 \\ 6 & -1.115872 & -1.961367 & 1.184428 \\ 6 & -1.289971 & -0.603290 & 1.417420 \\ 6 & -1.460898 & -0.152907 & 2.755039 \\ 6 & -1.454555 & -1.025978 & 3.810170 \\ 6 & -1.289850 & -2.414031 & 3.603584 \\ 6 & -1.122872 & -2.892758 & 2.272191 \\ 6 & -0.968427 & -4.290119 & 2.075700 \\ 6 & -0.992893 & -5.158744 & 3.137922 \\ 6 & -1.153262 & -4.678377 & 4.454879 \\ 6 & -1.295086 & -3.332669 & 4.681042 \\ 15 & -1.482479 & 0.577052 & -0.016325 \\ 6 & -1.477884 & 2.214060 & 0.811103 \\ 6 & -0.392521 & 2.563537 & 1.619005 \\ 6 & -0.352428 & 3.797250 & 2.269442 \\ 6 & -1.403016 & 4.689966 & 2.031941 \\ 6 & -2.485275 & 4.383936 & 1.203305 \\ 6 & -2.510889 & 3.123953 & 0.601259 \\ 6 & 0.767039 & 4.177846 & 3.238590 \\ 6 & 1.745695 & 3.028345 & 3.473939 \\ 6 & -3.606190 & 5.403492 & 1.007248 \\ 6 & -4.704870 & 4.883816 & 0.082315 \\ 6 & 2.013351 & -2.155600 & 0.999174 \\ 6 & 2.239048 & -3.630707 & 1.196073 \\ 6 & 2.398118 & -4.533652 & 0.145190 \\ 6 & 2.628855 & -5.882812 & 0.400320 \\ 6 & 2.722996 & -6.344476 & 1.709222 \\ 6 & 2.585992 & -5.446327 & 2.764168 \\ 6 & 2.336477 & -4.104377 & 2.506550 \\ 45 & -0.032212 & 0.871604 & -1.913208 \\ 6 & 0.805713 & 1.577761 & -3.422185 \\ 8 & 1.306165 & 2.067813 & -4.342010 \\ 6 & -3.241860 & 0.269939 & -0.435619 \\ 6 & -4.202671 & 0.142206 & 0.574598 \\ 6 & -5.530325 & -0.124809 & 0.262691 \\ 6 & -5.873397 & -0.255909 & -1.093101 \\ 6 & -4.942929 & -0.128541 & -2.121519 \\ 6 & -3.612772 & 0.130917 & -1.765469 \\ 6 & -6.599652 & -0.307538 & 1.338193\end{array}$




\begin{tabular}{|c|c|c|c|}
\hline & -6.045307 & -0.086355 & 2.744129 \\
\hline & -5.326350 & -0.189368 & -3.598674 \\
\hline & -6.712833 & -0.794884 & -3.807849 \\
\hline & -7.740161 & 0.690435 & 1.113091 \\
\hline 6 & -7.148592 & -1.736841 & 1.264860 \\
\hline 6 & -4.322918 & -1.032384 & -4.391145 \\
\hline & -5.323137 & 1.242592 & -4.148131 \\
\hline & -4.241854 & 5.727207 & 2.363950 \\
\hline & -3.030206 & 6.683818 & 0.393554 \\
\hline & 1.547242 & 5.373257 & 2.683271 \\
\hline & 0.156988 & 4.554219 & 4.594115 \\
\hline & 6.709490 & 1.193839 & 0.948097 \\
\hline & -1.614932 & 0.904835 & 2.951167 \\
\hline & 7.941740 & -3.564153 & 0.223825 \\
\hline & -1.419776 & -2.946244 & 5.691725 \\
\hline & 5.224970 & 5.820110 & -1.379267 \\
\hline & -3.484333 & -2.796271 & 0.764779 \\
\hline & -5.383447 & -3.798563 & -0.424289 \\
\hline & 3.136250 & 4.932655 & -0.522629 \\
\hline & -1.590835 & -0.657822 & 4.826317 \\
\hline & 6.012403 & -3.873718 & -1.223318 \\
\hline & 8.396346 & 3.158724 & -2.530371 \\
\hline & 3.948773 & -2.963456 & -2.285448 \\
\hline & -2.899736 & -4.536937 & -3.855836 \\
\hline & -0.569091 & -3.790779 & -3.858938 \\
\hline & -0.866259 & -6.224783 & 2.962797 \\
\hline & 7.082350 & 1.231202 & -1.782722 \\
\hline & -0.816553 & -4.674077 & 1.069351 \\
\hline & 1.349294 & -2.770672 & -2.679343 \\
\hline & 7.494179 & 5.471109 & -2.309388 \\
\hline & 8.674769 & 0.247950 & 2.068340 \\
\hline & -5.099900 & -4.704280 & -2.728273 \\
\hline & -1.164085 & -5.377612 & 5.288040 \\
\hline & 9.321544 & -2.130152 & 1.699286 \\
\hline & 1.734199 & 2.983971 & 0.159325 \\
\hline & -3.895303 & 0.249233 & 1.612350 \\
\hline & -2.843315 & 0.235547 & -2.528478 \\
\hline & -6.910867 & -0.470611 & -1.342871 \\
\hline 1 & -3.339393 & 2.839006 & -0.042215 \\
\hline 1 & 0.410586 & 1.841019 & 1.761750 \\
\hline & -1.380972 & 5.662764 & 2.523401 \\
\hline 1 & 2.981136 & -1.642557 & 1.064432 \\
\hline 1 & 1.416371 & -1.770198 & 1.841612 \\
\hline & -1.293458 & 1.796549 & -2.396278 \\
\hline & 2.200214 & -3.409298 & 3.335875 \\
\hline & 2.330957 & -4.180395 & -0.881119 \\
\hline & 2.651628 & -5.795187 & 3.792862 \\
\hline & 2.736560 & -6.576219 & -0.431320 \\
\hline & 2.902652 & -7.399152 & 1.906363 \\
\hline & 2.505408 & 3.337366 & 4.202828 \\
\hline & 1.242311 & 2.138987 & 3.877648 \\
\hline & 2.276348 & 2.736543 & 2.558085 \\
\hline & & 4804207 & 53090 \\
\hline
\end{tabular}




$\begin{array}{rrrr}1 & -0.509294 & 5.422416 & 4.527110 \\ 1 & -0.421844 & 3.718921 & 5.009971 \\ 1 & 2.335051 & 5.675002 & 3.386619 \\ 1 & 2.029028 & 5.118846 & 1.729938 \\ 1 & 0.900515 & 6.242661 & 2.512717 \\ 1 & -5.053317 & 6.456885 & 2.239826 \\ 1 & -4.665885 & 4.824872 & 2.823872 \\ 1 & -3.518702 & 6.153794 & 3.069611 \\ 1 & -5.487660 & 5.645661 & -0.022207 \\ 1 & -4.325229 & 4.661477 & -0.923113 \\ 1 & -5.180293 & 3.975218 & 0.476602 \\ 1 & -3.826312 & 7.425193 & 0.241982 \\ 1 & -2.268457 & 7.142943 & 1.035223 \\ 1 & -2.568063 & 6.478499 & -0.580437 \\ 1 & -6.924955 & -0.870959 & -4.881753 \\ 1 & -6.778604 & -1.805603 & -3.381325 \\ 1 & -7.508184 & -0.181404 & -3.366055 \\ 1 & -4.621749 & -1.070474 & -5.447117 \\ 1 & -3.304888 & -0.625505 & -4.357094 \\ 1 & -4.285918 & -2.060129 & -4.007870 \\ 1 & -5.599521 & 1.247571 & -5.211464 \\ 1 & -6.039970 & 1.874294 & -3.606895 \\ 1 & -4.331462 & 1.704503 & -4.054896 \\ 1 & -6.843832 & -0.231396 & 3.482603 \\ 1 & -5.240351 & -0.794286 & 2.986270 \\ 1 & -5.655719 & 0.932159 & 2.874191 \\ 1 & -8.508512 & 0.572433 & 1.888967 \\ 1 & -7.372158 & 1.723790 & 1.156182 \\ 1 & -8.229194 & 0.550792 & 0.141727 \\ 1 & -7.933619 & -1.884837 & 2.018852 \\ 1 & -7.583391 & -1.962573 & 0.283210 \\ 1 & -6.353558 & -2.469907 & 1.460043\end{array}$

$2_{\mathrm{C} 3-\mathrm{t}-{ }_{-1}-1 \mathrm{a} 2 \mathrm{a} 34 \mathrm{~s}}$

Number of imaginary frequencies : 0

The smallest frequencies are : $11.7986 \quad 12.9455 \quad 16.1111 \mathrm{~cm}(-1)$

Electronic energy : $\quad H F=-4012.1863384$

Zero-point correction $=\quad 1.290575($ Hartree/Particle $)$

Thermal correction to Energy= $\quad 1.366843$

Thermal correction to Enthalpy= $\quad 1.367787$

Thermal correction to Gibbs Free Energy $=\quad 1.178230$

Sum of electronic and zero-point Energies $=\quad-4010.895763$

Sum of electronic and thermal Energies $=\quad-4010.819496$

Sum of electronic and thermal Enthalpies $=\quad-4010.818552$

Sum of electronic and thermal Free Energies $=\quad-4011.008109$

Cartesian Coordinates

$\begin{array}{llll}6 & 2.394326 & -4.536872 & 0.129991 \\ 6 & 2.237960 & -3.635008 & 1.182222 \\ 6 & 2.336268 & -4.110567 & 2.491950 \\ 6 & 2.583355 & -5.453329 & 2.747595\end{array}$




$\begin{array}{cccc}6 & 2.717337 & -6.350387 & 1.691338 \\ 6 & 2.622772 & -5.886800 & 0.383149 \\ 6 & 2.014281 & -2.159256 & 0.987942 \\ 7 & 1.414126 & -1.761046 & -0.292401 \\ 15 & 1.734526 & -0.281527 & -1.060411 \\ 45 & -0.031979 & 0.875202 & -1.919361 \\ 6 & 0.806554 & 1.580431 & -3.428738 \\ 8 & 1.307072 & 2.069952 & -4.348769 \\ 6 & 0.262551 & -2.402951 & -0.855661 \\ 6 & -0.955005 & -2.484395 & -0.200745 \\ 6 & -2.076784 & -3.078365 & -0.861134 \\ 6 & -1.935081 & -3.562501 & -2.194125 \\ 6 & -0.684537 & -3.432707 & -2.841196 \\ 6 & 0.379517 & -2.871197 & -2.190327 \\ 6 & -3.352298 & -3.168037 & -0.245933 \\ 6 & -4.419039 & -3.720496 & -0.910332 \\ 6 & -4.265072 & -4.232944 & -2.216176 \\ 6 & -3.046571 & -4.153205 & -2.842124 \\ 6 & -1.114538 & -1.955153 & 1.178380 \\ 6 & -1.285921 & -0.596679 & 1.411216 \\ 6 & -1.448911 & -0.145339 & 2.749425 \\ 6 & -1.438095 & -1.017841 & 3.805138 \\ 6 & -1.280323 & -2.406616 & 3.598314 \\ 6 & -1.120640 & -2.886210 & 2.266354 \\ 6 & -1.284599 & -3.324931 & 4.676070 \\ 6 & -1.148642 & -4.671173 & 4.449662 \\ 6 & -0.995004 & -5.152360 & 3.132189 \\ 6 & -0.971643 & -4.284141 & 2.069643 \\ 15 & -1.482918 & 0.581243 & -0.024184 \\ 6 & -3.239748 & 0.261089 & -0.446427 \\ 6 & -4.195548 & 0.112436 & 0.558003 \\ 6 & -5.523609 & -0.177007 & 0.248828 \\ 6 & -5.870062 & -0.297898 & -1.102054 \\ 6 & -4.938363 & -0.143081 & -2.132834 \\ 6 & -3.613993 & 0.128634 & -1.779946 \\ 6 & -6.525897 & -0.353759 & 1.388745 \\ 6 & -6.655627 & 0.968351 & 2.153886 \\ 6 & -5.326599 & -0.191418 & -3.609759 \\ 6 & -4.324784 & -1.026739 & -4.412496 \\ 6 & -1.491556 & 2.219587 & 0.800191 \\ 6 & -0.409648 & 2.575882 & 1.609822 \\ 6 & -0.378754 & 3.809283 & 2.261097 \\ 6 & -1.432766 & 4.696542 & 2.018757 \\ 6 & -2.507511 & 4.387190 & 1.181630 \\ 6 & -2.527856 & 3.124576 & 0.584549 \\ 6 & -3.633866 & 5.400353 & 0.983123 \\ 6 & -4.340165 & 5.630737 & 2.323966 \\ 6 & 0.735606 & 4.196035 & 3.233616 \\ 6 & 1.513534 & 5.392819 & 2.677925 \\ 6 & -6.031309 & -1.449184 & 2.341778 \\ 6 & -7.911063 & -0.756506 & 0.889809 \\ 6 & -5.326501 & 1.245279 & -4.147067 \\ 6 & -6.713437 & -0.795090 & -3.822330\end{array}$




$\begin{array}{rrrr}6 & -3.057157 & 6.725449 & 0.474202 \\ 6 & -4.673401 & 4.918799 & -0.026750 \\ 6 & 0.119539 & 4.573497 & 4.586053 \\ 6 & 1.717571 & 3.050648 & 3.475188 \\ 8 & 3.058684 & -0.547515 & -2.045039 \\ 6 & 4.235706 & -0.986704 & -1.476399 \\ 6 & 5.023711 & -0.110670 & -0.748136 \\ 6 & 6.180927 & -0.630798 & -0.088050 \\ 6 & 6.534255 & -2.002664 & -0.269041 \\ 6 & 5.727589 & -2.828317 & -1.087669 \\ 6 & 4.593127 & -2.334469 & -1.673330 \\ 6 & 7.677219 & -2.519351 & 0.388768 \\ 6 & 8.435224 & -1.728082 & 1.212754 \\ 6 & 8.070974 & -0.380394 & 1.418820 \\ 6 & 6.975635 & 0.153417 & 0.787071 \\ 6 & 4.589178 & 1.302112 & -0.647321 \\ 6 & 3.305368 & 1.560200 & -0.207654 \\ 6 & 2.761156 & 2.858279 & -0.160934 \\ 6 & 3.536981 & 3.922891 & -0.536722 \\ 6 & 4.857595 & 3.727271 & -1.006605 \\ 6 & 5.388673 & 2.404140 & -1.089435 \\ 6 & 6.679018 & 2.237965 & -1.653717 \\ 6 & 7.415524 & 3.319619 & -2.068778 \\ 6 & 6.903602 & 4.628534 & -1.947577 \\ 6 & 5.647830 & 4.822406 & -1.432842 \\ 8 & 2.508866 & 0.497113 & 0.189655 \\ 1 & 6.700216 & 1.190188 & 0.964278 \\ 1 & -1.599809 & 0.912695 & 2.946044 \\ 1 & 7.933660 & -3.566168 & 0.231382 \\ 1 & -1.403921 & -2.937801 & 5.687126 \\ 1 & 5.227140 & 5.823886 & -1.353171 \\ 1 & -3.485562 & -2.771811 & 0.759428 \\ 1 & -5.393525 & -3.762511 & -0.426345 \\ 1 & 3.134722 & 4.934663 & -0.507675 \\ 1 & -1.567288 & -0.648676 & 4.821859 \\ 1 & 6.009816 & -3.871055 & -1.223974 \\ 1 & 8.401169 & 3.164627 & -2.501901 \\ 1 & 3.950145 & -2.957009 & -2.291228 \\ 1 & -2.915303 & -4.529580 & -3.856028 \\ 1 & -2.847398 & 0.249646 & -2.543478 \\ 1 & -6.901025 & -0.524503 & -1.358476 \\ 1 & -0.579528 & -3.791854 & -3.863629 \\ 1 & -0.872790 & -6.218879 & 2.956854 \\ 1 & 7.083322 & 1.235303 & -1.765748 \\ 1 & -0.825235 & -4.668998 & 1.062838 \\ 1 & 1.342917 & -2.774078 & -2.688141 \\ 1 & 7.499578 & 5.476772 & -2.276163 \\ 1 & 8.660741 & 0.240418 & 2.089591 \\ 1 & -5.115629 & -4.679166 & -2.727237 \\ 1 & -1.158839 & -5.370204 & 5.282994 \\ & 9.308141 & -2.136859 & 1.716297 \\ 1 & & & \\ 6 & & & \end{array}$




$\begin{array}{rrrr}1 & -3.353032 & 2.835379 & -0.061571 \\ 1 & 0.397303 & 1.858027 & 1.754093 \\ 1 & -1.419456 & 5.668216 & 2.512414 \\ 1 & 2.982961 & -1.647806 & 1.052513 \\ 1 & 1.419258 & -1.774421 & 1.831986 \\ 1 & -1.292293 & 1.802177 & -2.401360 \\ 1 & 2.202824 & -3.416190 & 3.322305 \\ 1 & 2.326855 & -4.182244 & -0.895823 \\ 1 & 2.649597 & -5.803594 & 3.775775 \\ 1 & 2.728324 & -6.579311 & -0.449511 \\ 1 & 2.895085 & -7.405677 & 1.886906 \\ 1 & 2.473409 & 3.364082 & 4.206210 \\ 1 & 1.216039 & 2.160303 & 3.878992 \\ 1 & 2.252842 & 2.758743 & 2.562073 \\ 1 & 0.910590 & 4.827893 & 5.303988 \\ 1 & -0.549364 & 5.439312 & 4.514819 \\ 1 & -0.457810 & 3.737169 & 5.001930 \\ 1 & 2.298228 & 5.698839 & 3.382907 \\ 1 & 1.998952 & 5.137835 & 1.726547 \\ 1 & 0.864356 & 6.259580 & 2.503283 \\ 1 & -5.155721 & 6.357197 & 2.206962 \\ 1 & -4.772003 & 4.695499 & 2.704835 \\ 1 & -3.655079 & 6.018949 & 3.087780 \\ 1 & -5.453236 & 5.681939 & -0.143110 \\ 1 & -4.232531 & 4.741633 & -1.016182 \\ 1 & -5.167260 & 3.993255 & 0.297587 \\ 1 & -3.863589 & 7.454434 & 0.318114 \\ 1 & -2.346189 & 7.169491 & 1.181192 \\ 1 & -2.536972 & 6.586141 & -0.482000 \\ 1 & -6.927201 & -0.859628 & -4.896682 \\ 1 & -6.779212 & -1.810414 & -3.407167 \\ 1 & -7.508110 & -0.186553 & -3.372526 \\ 1 & -4.621902 & -1.049408 & -5.469441 \\ 1 & -3.305420 & -0.623979 & -4.370572 \\ 1 & -4.291989 & -2.059774 & -4.043293 \\ 1 & -5.610396 & 1.259385 & -5.208356 \\ 1 & -6.039467 & 1.872641 & -3.595697 \\ 1 & -4.334218 & 1.706296 & -4.056810 \\ 1 & -8.588010 & -0.886340 & 1.743617 \\ 1 & -8.352133 & 0.006672 & 0.236004 \\ 1 & -7.886641 & -1.707281 & 0.340521 \\ 1 & -7.350601 & 0.857747 & 2.997339 \\ 1 & -5.692307 & 1.307011 & 2.556060 \\ 1 & -7.041458 & 1.761433 & 1.500178 \\ 1 & -6.740489 & -1.581286 & 3.170023 \\ 1 & -5.941752 & -2.412702 & 1.821466 \\ 1 & -5.052661 & -1.215994 & 2.780847\end{array}$

$2_{C 3-t-} \psi_{-12 a 23 a 4 a}$

Number of imaginary frequencies : 0

The smallest frequencies are : $11.5416 \quad 15.0272 \quad 17.1133 \mathrm{~cm}(-1)$

Electronic energy : $\quad H F=-4012.1870479$ 
Zero-point correction $=$

Thermal correction to Energy=

Thermal correction to Enthalpy=

Thermal correction to Gibbs Free Energy=

Sum of electronic and zero-point Energies=

Sum of electronic and thermal Energies=

Sum of electronic and thermal Enthalpies=

Sum of electronic and thermal Free Energies=
1.290582 (Hartree/Particle)

1.366855

1.367799

1.178222

$-4010.896466$

$-4010.820193$

$-4010.819249$

$-4011.008826$

Cartesian Coordinates

\begin{tabular}{cccc}
\hline 6 & 2.470403 & -4.510444 & 0.145907 \\
6 & 2.300869 & -3.605199 & 1.193136 \\
6 & 2.407121 & -4.071515 & 2.505558 \\
6 & 2.675240 & -5.408833 & 2.768642 \\
6 & 2.822252 & -6.309759 & 1.717370 \\
6 & 2.719673 & -5.855235 & 0.406605 \\
6 & 2.054750 & -2.134360 & 0.989239 \\
7 & 1.452129 & -1.754992 & -0.295162 \\
15 & 1.740246 & -0.271715 & -1.070271 \\
45 & -0.070455 & 0.806083 & -1.947473 \\
6 & 0.716281 & 1.465432 & -3.504455 \\
8 & 1.177162 & 1.916364 & -4.464083 \\
6 & 0.313890 & -2.416695 & -0.862362 \\
6 & -0.906439 & -2.516806 & -0.214625 \\
6 & -2.019432 & -3.112148 & -0.887918 \\
6 & -1.867480 & -3.572066 & -2.228652 \\
6 & -0.613499 & -3.429561 & -2.865472 \\
6 & 0.443489 & -2.872501 & -2.199979 \\
6 & -3.296882 & -3.225844 & -0.279323 \\
6 & -4.353225 & -3.783244 & -0.955690 \\
6 & -4.188794 & -4.267448 & -2.271684 \\
6 & -2.972365 & -4.154765 & -2.895623 \\
6 & -1.079095 & -1.998472 & 1.167182 \\
6 & -1.276433 & -0.644732 & 1.404980 \\
6 & -1.451139 & -0.200809 & 2.744527 \\
6 & -1.424533 & -1.076850 & 3.796846 \\
6 & -1.238870 & -2.461725 & 3.584832 \\
6 & -1.069615 & -2.933689 & 2.251357 \\
6 & -1.226562 & -3.384256 & 4.658780 \\
6 & -1.067033 & -4.727135 & 4.426968 \\
6 & -0.905879 & -5.200753 & 3.107670 \\
6 & -0.897158 & -4.327845 & 2.048670 \\
15 & -1.492210 & 0.530226 & -0.028627 \\
6 & -3.269237 & 0.246898 & -0.393356 \\
6 & -4.205680 & 0.237684 & 0.643789 \\
6 & -5.556261 & 0.018789 & 0.383949 \\
6 & -5.936608 & -0.187506 & -0.948140 \\
6 & -5.027163 & -0.187748 & -2.006778 \\
6 & -3.678390 & 0.025425 & -1.704773 \\
6 & -6.602019 & -0.038916 & 1.496087 \\
6 & -7.738944 & 0.945598 & 1.204724 \\
6 & -5.516709 & -0.357348 & -3.444248
\end{tabular}




$\begin{array}{rrrr}6 & -6.456177 & -1.561178 & -3.558220 \\ 6 & -1.455256 & 2.173046 & 0.786153 \\ 6 & -0.377918 & 2.504449 & 1.618518 \\ 6 & -0.323615 & 3.739015 & 2.258117 \\ 6 & -1.348009 & 4.661984 & 1.992332 \\ 6 & -2.410254 & 4.376663 & 1.138357 \\ 6 & -2.450765 & 3.108855 & 0.543661 \\ 6 & -3.540133 & 5.362114 & 0.848288 \\ 6 & -4.872883 & 4.743750 & 1.288657 \\ 6 & 0.775727 & 4.095753 & 3.258508 \\ 6 & 1.561061 & 5.312542 & 2.759984 \\ 6 & -7.164056 & -1.463193 & 1.570550 \\ 6 & -6.012306 & 0.311589 & 2.860955 \\ 6 & -6.271626 & 0.913882 & -3.850876 \\ 6 & -4.360970 & -0.567366 & -4.421354 \\ 6 & -3.586502 & 5.654599 & -0.655576 \\ 6 & -3.362326 & 6.686246 & 1.586318 \\ 6 & 0.132175 & 4.423709 & 4.611312 \\ 6 & 1.757819 & 2.946182 & 3.477268 \\ 8 & 3.066563 & -0.515536 & -2.057911 \\ 6 & 4.250955 & -0.936453 & -1.490648 \\ 6 & 5.026358 & -0.048318 & -0.763614 \\ 6 & 6.191703 & -0.550631 & -0.103806 \\ 6 & 6.565818 & -1.916941 & -0.284789 \\ 6 & 5.771524 & -2.754810 & -1.103066 \\ 6 & 4.629081 & -2.278586 & -1.687704 \\ 6 & 7.716782 & -2.416237 & 0.372434 \\ 6 & 8.462934 & -1.613581 & 1.196219 \\ 6 & 8.078205 & -0.271638 & 1.402518 \\ 6 & 6.974702 & 0.245532 & 0.771106 \\ 6 & 4.571799 & 1.358165 & -0.664189 \\ 6 & 3.284599 & 1.599398 & -0.224785 \\ 6 & 2.724436 & 2.890364 & -0.175143 \\ 6 & 3.486653 & 3.965223 & -0.550098 \\ 6 & 4.809097 & 3.786926 & -1.021717 \\ 6 & 5.356661 & 2.470670 & -1.106132 \\ 6 & 6.648670 & 2.321199 & -1.671333 \\ 6 & 7.371412 & 3.412310 & -2.085689 \\ 6 & 6.843261 & 4.714626 & -1.962819 \\ 6 & 5.585417 & 4.892276 & -1.447253 \\ 8 & 2.502086 & 0.526741 & 0.174137 \\ 1 & 6.683627 & 1.277974 & 0.948569 \\ 1 & -1.618833 & 0.854340 & 2.945096 \\ 1 & 7.988993 & -3.459036 & 0.214817 \\ 1 & -1.352907 & -3.003540 & 5.671428 \\ 1 & 5.152387 & 5.888377 & -1.366153 \\ 1 & -3.439485 & -2.849018 & 0.731944 \\ 1 & -5.328062 & -3.848947 & -0.476238 \\ 1 & 3.071714 & 4.971755 & -0.518107 \\ 1 & -1.561370 & -0.714189 & 4.814921 \\ 1 & 6.069972 & -3.792944 & -1.239928 \\ 1 & 8.358658 & 3.270040 & -2.519518 \\ 1 & 3.996287 & -2.911284 & -2.305665 \\ & & & \end{array}$




$\begin{array}{rrrr}1 & -2.833136 & -4.511384 & -3.915413 \\ 1 & -0.500417 & -3.774308 & -3.891953 \\ 1 & -0.766417 & -6.264469 & 2.928276 \\ 1 & 7.065327 & 1.323776 & -1.784672 \\ 1 & -0.745546 & -4.705507 & 1.039891 \\ 1 & 1.409369 & -2.763762 & -2.690350 \\ 1 & 7.428436 & 5.570596 & -2.290767 \\ 1 & 8.658602 & 0.358010 & 2.073221 \\ 1 & -5.030000 & -4.723684 & -2.789554 \\ 1 & -1.064995 & -5.429422 & 5.257629 \\ 1 & 9.342166 & -2.009017 & 1.699423 \\ 1 & 1.692339 & 3.003405 & 0.152771 \\ 1 & -3.868389 & 0.409148 & 1.663338 \\ 1 & -2.921559 & 0.029013 & -2.485902 \\ 1 & -6.991447 & -0.354120 & -1.167026 \\ 1 & -3.274989 & 2.847686 & -0.118764 \\ 1 & 0.404808 & 1.765148 & 1.783803 \\ 1 & -1.308419 & 5.632458 & 2.482730 \\ 1 & 3.015223 & -1.607346 & 1.052249 \\ 1 & 1.452278 & -1.753084 & 1.829759 \\ 1 & -1.372118 & 1.654901 & -2.456496 \\ 1 & 2.264026 & -3.374383 & 3.331945 \\ 1 & 2.397031 & -4.162365 & -0.881753 \\ 1 & 2.747583 & -5.752239 & 3.798722 \\ 1 & 2.835393 & -6.550832 & -0.422132 \\ 1 & 3.016396 & -7.361059 & 1.918802 \\ 1 & 2.508799 & 3.242530 & 4.220369 \\ 1 & 1.256404 & 2.045084 & 3.856563 \\ 1 & 2.297307 & 2.678772 & 2.559025 \\ 1 & 0.906917 & 4.661704 & 5.352422 \\ 1 & -0.544853 & 5.284242 & 4.553572 \\ 1 & -0.444547 & 3.568384 & 4.988069 \\ 1 & 2.332948 & 5.592439 & 3.489475 \\ 1 & 2.063903 & 5.092191 & 1.808960 \\ 1 & 0.915809 & 6.185998 & 2.606913 \\ 1 & -5.700640 & 5.435525 & 1.082201 \\ 1 & -5.086486 & 3.804629 & 0.761885 \\ 1 & -7.140917 & 1.093704 & -3.205443 \\ 1 & -5.619121 & 1.794502 & -3.787379 \\ 1 & -4.870268 & 4.530961 & 2.366010 \\ 1 & -4.196776 & 7.356780 & 1.345355 \\ 1 & -3.353069 & 6.552529 & 2.675923 \\ 1 & -2.436098 & 7.197286 & 1.293460 \\ 1 & -4.394737 & 6.363727 & -0.879963 \\ 1 & -2.642394 & 6.096387 & -0.999004 \\ 1 & -3.764983 & 4.749630 & -1.248441 \\ 1 & -4.757984 & -0.724470 & -5.432343 \\ 1 & -3.689847 & 0.300051 & -4.466641 \\ 1 & -3.762014 & -1.448912 & -4.154262 \\ 1 & -6.791005 & -1.679186 & -4.597356 \\ 1 & -754720 & -2.485640 & -3.259161 \\ 1 & & & \\ 1 & & & \\ 1 & & & \\ 1 & & & \end{array}$




$\begin{array}{rrrr}1 & -6.798770 & 0.265301 & 3.624715 \\ 1 & -5.221369 & -0.388254 & 3.162713 \\ 1 & -5.594992 & 1.327427 & 2.876665 \\ 1 & -8.487927 & 0.907864 & 2.006982 \\ 1 & -7.362749 & 1.975186 & 1.143346 \\ 1 & -8.255638 & 0.721604 & 0.264036 \\ 1 & -7.928057 & -1.535159 & 2.356575 \\ 1 & -7.626816 & -1.772181 & 0.624957 \\ 1 & -6.368283 & -2.182653 & 1.806817\end{array}$

$2_{\mathrm{C} 3-\mathrm{t}-\mathrm{-}-1 \mathrm{a} 2 \mathrm{s3a4s}}$

Number of imaginary frequencies : 0

The smallest frequencies are : $12.4198 \quad 16.4650 \quad 18.8714 \mathrm{~cm}(-1)$

Electronic energy : $\quad H F=-4012.1873662$

Zero-point correction $=\quad 1.291463($ Hartree/Particle $)$

Thermal correction to Energy=

1.367427

Thermal correction to Enthalpy=

1.368371

Thermal correction to Gibbs Free Energy=

Sum of electronic and zero-point Energies=

Sum of electronic and thermal Energies $=$

1.180311

$-4010.895904$

Sum of electronic and thermal Enthalpies=

$-4010.819940$

Sum of electronic and thermal Free Energies=

$-4010.818995$

$-4011.007055$

Cartesian Coordinates

$\begin{array}{cccc}6 & 5.588600 & 4.887775 & -1.409708 \\ 6 & 4.810624 & 3.780897 & -0.991221 \\ 6 & 5.356323 & 2.464430 & -1.083826 \\ 6 & 6.648252 & 2.316625 & -1.649626 \\ 6 & 7.372654 & 3.409270 & -2.056976 \\ 6 & 6.846308 & 4.711539 & -1.926102 \\ 6 & 4.569630 & 1.350306 & -0.649379 \\ 6 & 3.282798 & 1.590588 & -0.208424 \\ 6 & 2.724420 & 2.882003 & -0.150747 \\ 6 & 3.488295 & 3.958078 & -0.518845 \\ 6 & 5.021410 & -0.056341 & -0.758603 \\ 6 & 4.244446 & -0.937471 & -1.492433 \\ 6 & 4.618957 & -2.279337 & -1.697924 \\ 6 & 5.759637 & -2.762444 & -1.115418 \\ 6 & 6.555610 & -1.932062 & -0.291138 \\ 6 & 6.184927 & -0.566001 & -0.101359 \\ 6 & 7.704791 & -2.438627 & 0.363665 \\ 6 & 8.452405 & -1.643321 & 1.193239 \\ 6 & 8.071011 & -0.301766 & 1.408103 \\ 6 & 6.969285 & 0.222371 & 0.779350 \\ 8 & 3.061784 & -0.509343 & -2.057924 \\ 15 & 1.735205 & -0.268784 & -1.069970 \\ 8 & 2.498410 & 0.516094 & 0.182237 \\ 7 & 1.440223 & -1.758110 & -0.308908 \\ 6 & 0.300048 & -2.409950 & -0.883452 \\ 6 & -0.920381 & -2.515399 & -0.236488 \\ 6 & -2.034610 & -3.101136 & -0.916254\end{array}$




$\begin{array}{cccc}6 & -1.884847 & -3.541901 & -2.263711 \\ 6 & -0.630944 & -3.393621 & -2.899307 \\ 6 & 0.428118 & -2.849182 & -2.226726 \\ 6 & -2.991252 & -4.112729 & -2.938316 \\ 6 & -4.206252 & -4.236088 & -2.313682 \\ 6 & -4.367079 & -3.774885 & -0.989100 \\ 6 & -3.310816 & -3.224431 & -0.306767 \\ 6 & -1.090992 & -2.009955 & 1.150368 \\ 6 & -1.279729 & -0.657001 & 1.400104 \\ 6 & -1.446502 & -0.223029 & 2.743696 \\ 6 & -1.423231 & -1.108266 & 3.788568 \\ 6 & -1.250120 & -2.492709 & 3.564094 \\ 6 & -1.086137 & -2.954346 & 2.226339 \\ 6 & -0.922490 & -4.347788 & 2.011574 \\ 6 & -0.935237 & -5.229562 & 3.063095 \\ 6 & -1.091996 & -4.766235 & 4.386673 \\ 6 & -1.242542 & -3.424457 & 4.630150 \\ 15 & -1.493860 & 0.530147 & -0.023462 \\ 6 & -1.461788 & 2.167912 & 0.802062 \\ 6 & -0.385512 & 2.497089 & 1.636743 \\ 6 & -0.329687 & 3.731972 & 2.275664 \\ 6 & -1.348610 & 4.658936 & 2.003179 \\ 6 & -2.408430 & 4.376780 & 1.145111 \\ 6 & -2.454474 & 3.106198 & 0.556436 \\ 6 & 0.766964 & 4.086206 & 3.279857 \\ 6 & 1.745468 & 2.934319 & 3.502399 \\ 6 & -3.521532 & 5.374769 & 0.833219 \\ 6 & -3.350046 & 6.692765 & 1.583660 \\ 6 & 2.039581 & -2.150696 & 0.972974 \\ 6 & 2.280013 & -3.624035 & 1.164985 \\ 6 & 2.447601 & -4.521756 & 0.110968 \\ 6 & 2.692025 & -5.869355 & 0.361778 \\ 6 & 2.791629 & -6.334198 & 1.669148 \\ 6 & 2.646503 & -5.440843 & 2.727137 \\ 6 & 2.383298 & -4.100705 & 2.473908 \\ 45 & -0.069368 & 0.826233 & -1.937019 \\ 6 & 0.728236 & 1.513358 & -3.476345 \\ 8 & 1.198060 & 1.984913 & -4.421623 \\ 6 & -3.269728 & 0.245521 & -0.393913 \\ 6 & -4.202526 & 0.202524 & 0.638294 \\ 6 & -5.556823 & -0.020896 & 0.377991 \\ 6 & -5.941908 & -0.188547 & -0.953201 \\ 6 & -5.028195 & -0.156882 & -2.014917 \\ 6 & -3.683120 & 0.053967 & -1.712648 \\ 6 & -6.534175 & -0.086769 & 1.550330 \\ 6 & -7.965477 & -0.367284 & 1.100935 \\ 6 & -5.519182 & -0.290257 & -3.455715 \\ 6 & -4.366355 & -0.505868 & -4.435446 \\ 6 & -6.521341 & 1.248752 & 2.302453 \\ 6 & -6.105693 & -1.211458 & 2.501063 \\ 6 & -6.481495 & -1.472880 & -3.594979 \\ 6 & -6.248730 & 1.002834 & -3.838880 \\ 6 & -4.873083 & 4.769750 & 1.231246\end{array}$




$\begin{array}{rrrr}6 & -3.521399 & 5.675639 & -0.669914 \\ 6 & 1.557295 & 5.301016 & 2.784383 \\ 6 & 0.118891 & 4.416109 & 4.630009 \\ 1 & 6.680651 & 1.254307 & 0.963556 \\ 1 & -1.605906 & 0.831574 & 2.953423 \\ 1 & 7.974458 & -3.481067 & 0.199472 \\ 1 & -1.364942 & -3.051479 & 5.646152 \\ 1 & 5.156941 & 5.883960 & -1.322556 \\ 1 & -3.451464 & -2.862339 & 0.710499 \\ 1 & -5.340644 & -3.852543 & -0.509086 \\ 1 & 3.074729 & 4.964973 & -0.480891 \\ 1 & -1.553804 & -0.753287 & 4.810170 \\ 1 & 6.055334 & -3.800493 & -1.258726 \\ 1 & 8.359805 & 3.268321 & -2.491450 \\ 1 & 3.984679 & -2.906257 & -2.320277 \\ 1 & -2.853933 & -4.452447 & -3.964120 \\ 1 & -0.519281 & -3.724583 & -3.930463 \\ 1 & -0.802375 & -6.292539 & 2.874543 \\ 1 & 7.063449 & 1.319311 & -1.769135 \\ 1 & -0.774585 & -4.717709 & 0.999391 \\ 1 & 1.394401 & -2.737862 & -2.715724 \\ 1 & 7.432753 & 5.568702 & -2.248622 \\ 1 & 8.652542 & 0.321948 & 2.083353 \\ 1 & -5.048821 & -4.683470 & -2.837011 \\ 1 & -1.093387 & -5.475593 & 5.211295 \\ 1 & 9.330238 & -2.044337 & 1.694468 \\ 1 & 1.692242 & 2.994475 & 0.177173 \\ 1 & -3.869496 & 0.344699 & 1.666388 \\ 1 & -2.927827 & 0.081018 & -2.494668 \\ 1 & -6.993668 & -0.349913 & -1.180113 \\ 1 & -3.276616 & 2.847300 & -0.109571 \\ 1 & 0.396043 & 1.756703 & 1.802897 \\ 1 & -1.305695 & 5.630976 & 2.490161 \\ 1 & 3.001996 & -1.627975 & 1.041801 \\ 1 & 1.437540 & -1.774279 & 1.815914 \\ 1 & -1.366970 & 1.686981 & -2.437208 \\ 1 & 2.242186 & -3.409223 & 3.305343 \\ 1 & 2.376637 & -4.165658 & -0.914174 \\ 1 & 2.716659 & -5.792296 & 3.754653 \\ 1 & 2.806345 & -6.559031 & -0.472078 \\ 1 & -5.081745 & 3.835522 & 0.694217 \\ 1 & -4.905363 & 4.552798 & 2.307245 \\ 1 & 2.982061 & -7.387641 & 1.862758 \\ 1 & 2.494460 & 3.228977 & 4.248173 \\ 1 & 1.240616 & 2.034448 & 3.880012 \\ 1 & 2.287659 & 2.665466 & 2.586151 \\ 1 & 0.891165 & 4.653407 & 5.373916 \\ 1 & -0.556545 & 5.277657 & 4.568811 \\ 1 & -0.460703 & 3.562067 & 5.005228 \\ 1 & 2.327355 & 5.578570 & 3.516688 \\ & 2.062893 & 5.079596 & 1.835080 \\ & 0.915060 & 6.176332 & 2.629372 \\ 1 & -5.686133 & 5.472047 & 1.003084 \\ 1 & & & \\ 1 & & \end{array}$




$\begin{array}{rrrr}1 & -4.173934 & 7.371066 & 1.328509 \\ 1 & -3.365884 & 6.551733 & 2.672309 \\ 1 & -2.414144 & 7.199260 & 1.314755 \\ 1 & -4.316726 & 6.392684 & -0.914535 \\ 1 & -2.563787 & 6.110853 & -0.983111 \\ 1 & -3.689105 & 4.774362 & -1.271593 \\ 1 & -4.765313 & -0.637663 & -5.449301 \\ 1 & -3.678092 & 0.348553 & -4.466183 \\ 1 & -3.785618 & -1.403913 & -4.182528 \\ 1 & -6.807669 & -1.570485 & -4.638902 \\ 1 & -5.990454 & -2.410427 & -3.303500 \\ 1 & -7.384375 & -1.357705 & -2.983717 \\ 1 & -6.613638 & 0.946385 & -4.873629 \\ 1 & -7.112143 & 1.189093 & -3.187377 \\ 1 & -5.577866 & 1.868483 & -3.762777 \\ 1 & -8.624833 & -0.413408 & 1.976907 \\ 1 & -8.351388 & 0.420628 & 0.441358 \\ 1 & -8.048810 & -1.327670 & 0.575511 \\ 1 & -7.203075 & 1.209258 & 3.162806 \\ 1 & -5.522660 & 1.500135 & 2.680934 \\ 1 & -6.848899 & 2.069464 & 1.650576 \\ 1 & -6.783546 & -1.260580 & 3.364001 \\ 1 & -6.136991 & -2.185487 & 1.995035 \\ 1 & -5.087730 & -1.069162 & 2.886365\end{array}$

$2_{\mathrm{C} 3-\mathrm{t}-} \psi_{-1 \mathrm{a} 2 \mathrm{~s} 3 \mathrm{~s} 4 \mathrm{a}}$

Number of imaginary frequencies : 0

The smallest frequencies are : $11.7662 \quad 12.9003 \quad 14.8972 \mathrm{~cm}(-1)$

Electronic energy : $\quad H F=-4012.1865878$

Zero-point correction $=\quad 1.290344($ Hartree/Particle $)$

Thermal correction to Energy= $\quad 1.366725$

Thermal correction to Enthalpy= $\quad 1.367669$

Thermal correction to Gibbs Free Energy= 1.177228

Sum of electronic and zero-point Energies $=\quad-4010.896243$

Sum of electronic and thermal Energies $=\quad-4010.819863$

Sum of electronic and thermal Enthalpies $=\quad-4010.818919$

Sum of electronic and thermal Free Energies $=\quad-4011.009360$

Cartesian Coordinates

$\begin{array}{llll}6 & 5.651214 & 4.841430 & -1.420850 \\ 6 & 4.862685 & 3.743922 & -0.997557 \\ 6 & 5.395940 & 2.421896 & -1.083736 \\ 6 & 6.686587 & 2.259248 & -1.648347 \\ 6 & 7.421434 & 3.343154 & -2.060444 \\ 6 & 6.907390 & 4.650923 & -1.935870 \\ 6 & 4.598278 & 1.317480 & -0.644354 \\ 6 & 3.314250 & 1.572326 & -0.203578 \\ 6 & 2.767730 & 2.869309 & -0.153876 \\ 6 & 3.541732 & 3.936119 & -0.527227 \\ 6 & 5.034626 & -0.094376 & -0.749526 \\ 6 & 4.247486 & -0.969041 & -1.480389\end{array}$




$\begin{array}{cccc}6 & 4.606918 & -2.315601 & -1.681937 \\ 6 & 5.742214 & -2.809777 & -1.098138 \\ 6 & 6.547808 & -1.985807 & -0.276779 \\ 6 & 6.192629 & -0.615034 & -0.091301 \\ 6 & 7.691441 & -2.503106 & 0.379390 \\ 6 & 8.448305 & -1.713517 & 1.206031 \\ 6 & 8.082236 & -0.366998 & 1.416490 \\ 6 & 6.986234 & 0.167411 & 0.786404 \\ 8 & 3.069405 & -0.529603 & -2.046792 \\ 15 & 1.745662 & -0.269449 & -1.059790 \\ 8 & 2.519914 & 0.506748 & 0.191711 \\ 7 & 1.429118 & -1.752688 & -0.296994 \\ 6 & 0.279955 & -2.394613 & -0.865163 \\ 6 & -0.938587 & -2.482681 & -0.212741 \\ 6 & -2.059029 & -3.071853 & -0.879887 \\ 6 & -1.914737 & -3.543313 & -2.217353 \\ 6 & -0.662694 & -3.409014 & -2.860459 \\ 6 & 0.400096 & -2.853213 & -2.202843 \\ 6 & -3.024715 & -4.127115 & -2.874097 \\ 6 & -4.244326 & -4.213896 & -2.251495 \\ 6 & -4.400747 & -3.716132 & -0.940341 \\ 6 & -3.335827 & -3.169480 & -0.267997 \\ 6 & -1.100214 & -1.962916 & 1.169778 \\ 6 & -1.272929 & -0.606117 & 1.410853 \\ 6 & -1.439464 & -0.162598 & 2.751231 \\ 6 & -1.431779 & -1.041716 & 3.801400 \\ 6 & -1.270485 & -2.428922 & 3.586441 \\ 6 & -1.106669 & -2.900433 & 2.252059 \\ 6 & -0.955156 & -4.296895 & 2.047183 \\ 6 & -0.979443 & -5.171575 & 3.104411 \\ 6 & -1.136750 & -4.698429 & 4.424406 \\ 6 & -1.275690 & -3.353788 & 4.658568 \\ 15 & -1.473032 & 0.579488 & -0.016755 \\ 6 & -1.478294 & 2.211730 & 0.819012 \\ 6 & -0.392923 & 2.570573 & 1.629692 \\ 6 & -0.369241 & 3.795859 & 2.288683 \\ 6 & -1.436659 & 4.681406 & 2.066507 \\ 6 & -2.511715 & 4.366660 & 1.239663 \\ 6 & -2.517640 & 3.108864 & 0.622577 \\ 6 & 0.750304 & 4.185898 & 3.253854 \\ 6 & 1.741133 & 3.045677 & 3.483520 \\ 6 & -3.693280 & 5.305329 & 1.006049 \\ 6 & -3.544176 & 6.626962 & 1.754713 \\ 6 & 2.027945 & -2.153058 & 0.983202 \\ 6 & 2.253640 & -3.628919 & 1.174051 \\ 6 & 2.413102 & -4.527838 & 0.119763 \\ 6 & 2.643802 & -5.877953 & 0.369948 \\ 6 & 2.737584 & -6.344546 & 1.677126 \\ 6 & 2.600323 & -5.450381 & 2.735425 \\ 6 & 2.350843 & -4.107515 & 2.482750 \\ 6 & -0.026019 & 0.884528 & -1.913244 \\ 8 & 0.810455 & 1.599357 & -3.419255 \\ & 1.310066 & 2.094762 & -4.336613\end{array}$




$\begin{array}{rrrr}6 & -3.234274 & 0.270349 & -0.428689 \\ 6 & -4.186894 & 0.126567 & 0.587397 \\ 6 & -5.515533 & -0.144148 & 0.282856 \\ 6 & -5.869308 & -0.255897 & -1.072105 \\ 6 & -4.947930 & -0.108815 & -2.106021 \\ 6 & -3.615301 & 0.148650 & -1.757178 \\ 6 & -6.573467 & -0.354065 & 1.364754 \\ 6 & -6.005671 & -0.162320 & 2.769672 \\ 6 & -5.342926 & -0.148169 & -3.580780 \\ 6 & -6.732750 & -0.746650 & -3.787418 \\ 6 & -7.720974 & 0.642766 & 1.172895 \\ 6 & -7.117818 & -1.783743 & 1.265574 \\ 6 & -4.348429 & -0.983669 & -4.392203 \\ 6 & -5.339085 & 1.291215 & -4.110648 \\ 6 & -4.978936 & 4.622910 & 1.490272 \\ 6 & -3.812876 & 5.613458 & -0.490623 \\ 6 & 1.516367 & 5.389251 & 2.695551 \\ 6 & 0.144552 & 4.555361 & 4.613226 \\ 1 & 6.709333 & 1.203180 & 0.967111 \\ 1 & -1.592118 & 0.894173 & 2.953590 \\ 1 & 7.949295 & -3.549065 & 0.218638 \\ 1 & -1.397980 & -2.972953 & 5.671662 \\ 1 & 5.228915 & 5.842026 & -1.338603 \\ 1 & -3.471936 & -2.784609 & 0.741620 \\ 1 & -5.375559 & -3.765057 & -0.458173 \\ 1 & 3.137806 & 4.947141 & -0.495609 \\ 1 & -1.564868 & -0.679130 & 4.819965 \\ 1 & 6.025928 & -3.851632 & -1.238066 \\ 1 & 8.407408 & 3.190884 & -2.493782 \\ 1 & 3.964983 & -2.937110 & -2.301930 \\ 1 & -2.891049 & -4.492548 & -3.891671 \\ 1 & -0.555639 & -3.759876 & -3.885548 \\ 1 & -0.855100 & -6.236836 & 2.923028 \\ 1 & 7.092492 & 1.257524 & -1.762928 \\ 1 & -0.805976 & -4.675222 & 1.038298 \\ 1 & 1.364516 & -2.752455 & -2.697870 \\ 1 & 7.502064 & 5.500986 & -2.262092 \\ 1 & 8.671084 & 0.252380 & 2.089390 \\ 1 & -5.093953 & -4.654802 & -2.768718 \\ 1 & -1.147493 & -5.402403 & 5.253565 \\ 1 & 9.321706 & -2.122778 & 1.708343 \\ 1 & 1.735535 & 2.993090 & 0.170027 \\ 1 & -3.871974 & 0.221561 & 1.624061 \\ 1 & -2.852297 & 0.265532 & -2.524895 \\ 1 & -6.907858 & -0.471638 & -1.316440 \\ 1 & -3.349403 & 2.825827 & -0.020958 \\ 1 & 0.419019 & 1.856564 & 1.764567 \\ 1 & -1.421151 & 5.645584 & 2.570292 \\ 1 & 2.995778 & -1.640321 & 1.050366 \\ 1 & 1.431284 & -1.771292 & 1.827461 \\ & 2.289837 & 1.808369 & -2.391965 \\ & 2.346319 & -3.415435 & 3.314591 \\ & & -4.170748 & -0.905268\end{array}$




$\begin{array}{rrrr}1 & 2.665797 & -5.803069 & 3.762826 \\ 1 & 2.751817 & -6.568237 & -0.464244 \\ 1 & 2.917231 & -7.399951 & 1.870335 \\ 1 & 2.500121 & 3.360686 & 4.210599 \\ 1 & 1.247632 & 2.150615 & 3.886833 \\ 1 & 2.271977 & 2.760910 & 2.565602 \\ 1 & 0.941267 & 4.808707 & 5.325230 \\ 1 & -0.527022 & 5.419718 & 4.552253 \\ 1 & -0.426479 & 3.715357 & 5.030397 \\ 1 & 2.305061 & 5.697743 & 3.394992 \\ 1 & 1.995335 & 5.140104 & 1.739361 \\ 1 & 0.860569 & 6.252648 & 2.529362 \\ 1 & -5.844209 & 5.278640 & 1.323784 \\ 1 & -5.170366 & 3.679810 & 0.962050 \\ 1 & -4.924796 & 4.399735 & 2.564091 \\ 1 & -4.414845 & 7.263924 & 1.554088 \\ 1 & -3.488297 & 6.480727 & 2.841292 \\ 1 & -2.651946 & 7.180476 & 1.434736 \\ 1 & -4.657708 & 6.291463 & -0.672310 \\ 1 & -2.902450 & 6.097950 & -0.865912 \\ 1 & -3.979100 & 4.709418 & -1.088546 \\ 1 & -6.953380 & -0.807488 & -4.860570 \\ 1 & -6.797806 & -1.763007 & -3.374259 \\ 1 & -7.522849 & -0.137180 & -3.330866 \\ 1 & -4.652966 & -1.003037 & -5.447050 \\ 1 & -3.327877 & -0.583344 & -4.356753 \\ 1 & -4.315539 & -2.017585 & -4.025689 \\ 1 & -5.627006 & 1.312002 & -5.170704 \\ 1 & -6.046981 & 1.918853 & -3.553114 \\ 1 & -4.344257 & 1.747340 & -4.022414 \\ 1 & -6.796076 & -0.327569 & 3.512605 \\ 1 & -5.195359 & -0.872311 & 2.987227 \\ 1 & -5.618793 & 0.854561 & 2.919661 \\ 1 & -8.483166 & 0.500695 & 1.950791 \\ 1 & -7.359117 & 1.677030 & 1.239918 \\ 1 & -8.215797 & 0.524290 & 0.201598 \\ 1 & -7.892350 & -1.951806 & 2.026147 \\ 1 & -7.565153 & -1.988455 & 0.284917 \\ 1 & -6.318078 & -2.518544 & 1.433329\end{array}$

$2_{\mathrm{C} 3-\mathrm{t}-\mathrm{\psi}-1 \mathrm{a} 2 \mathrm{~s} 3 \mathrm{~s} 4 \mathrm{~s}}$

Number of imaginary frequencies : 0

The smallest frequencies are : $10.9845 \quad 14.0377 \quad 15.2193 \mathrm{~cm}(-1)$

Electronic energy : $\quad \mathrm{HF}=-4012.1867705$

Zero-point correction $=\quad 1.290582($ Hartree/Particle $)$

Thermal correction to Energy= $\quad 1.366823$

Thermal correction to Enthalpy= $\quad 1.367767$

Thermal correction to Gibbs Free Energy $=\quad 1.178017$

Sum of electronic and zero-point Energies $=\quad-4010.896189$

Sum of electronic and thermal Energies $=\quad-4010.819948$

Sum of electronic and thermal Enthalpies $=\quad-4010.819003$

Sum of electronic and thermal Free Energies $=\quad-4011.008753$ 


\begin{tabular}{|c|c|c|c|}
\hline & \multicolumn{3}{|c|}{ Cartesian Coordinates } \\
\hline 6 & -3.645844 & 0.119070 & -1.740133 \\
\hline 6 & -3.247490 & 0.250265 & -0.413354 \\
\hline 6 & -4.189381 & 0.129052 & 0.607642 \\
\hline 6 & -5.529704 & -0.127817 & 0.321773 \\
\hline 6 & -5.900861 & -0.244030 & -1.022586 \\
\hline 6 & -4.982370 & -0.119907 & -2.069607 \\
\hline 15 & -1.481292 & 0.554333 & -0.018199 \\
\hline 45 & -0.047153 & 0.867700 & -1.922434 \\
\hline 6 & 0.772094 & 1.578398 & -3.439781 \\
\hline 8 & 1.258723 & 2.068413 & -4.366941 \\
\hline 6 & -6.517426 & -0.276673 & 1.478340 \\
\hline 6 & -6.048218 & -1.401798 & 2.409249 \\
\hline 6 & -5.404570 & -0.158477 & -3.537495 \\
\hline 6 & -5.481114 & 1.287220 & -4.044320 \\
\hline 6 & -1.272124 & -0.639496 & 1.400528 \\
\hline 6 & -1.097435 & -1.993952 & 1.147551 \\
\hline 6 & -1.099027 & -2.940103 & 2.222287 \\
\hline 6 & -1.257464 & -2.479389 & 3.561105 \\
\hline 6 & -1.416359 & -1.093872 & 3.788333 \\
\hline 6 & -1.431542 & -0.206500 & 2.745091 \\
\hline 6 & -0.946278 & -4.334693 & 2.005837 \\
\hline 6 & -0.965612 & -5.217786 & 3.056088 \\
\hline 6 & -1.118683 & -4.755365 & 4.380450 \\
\hline 6 & -1.257794 & -3.412795 & 4.625839 \\
\hline 6 & -0.931388 & -2.501086 & -0.239413 \\
\hline 6 & 0.288993 & -2.400768 & -0.887165 \\
\hline 6 & 0.416904 & -2.849231 & -2.227468 \\
\hline 6 & -0.639985 & -3.406476 & -2.893151 \\
\hline 6 & -1.893177 & -3.553629 & -2.255357 \\
\hline 6 & -2.045362 & -3.093201 & -0.914869 \\
\hline 6 & -2.995663 & -4.143457 & -2.919462 \\
\hline 6 & -4.214316 & -4.252045 & -2.298618 \\
\hline 6 & -4.378339 & -3.767646 & -0.983312 \\
\hline 6 & -3.322397 & -3.208985 & -0.306516 \\
\hline 7 & 1.432038 & -1.753987 & -0.312069 \\
\hline 6 & 2.030616 & -2.154886 & 0.967995 \\
\hline 6 & 2.263235 & -3.630062 & 1.155324 \\
\hline 6 & 2.429964 & -4.525324 & 0.099051 \\
\hline 6 & 2.667177 & -5.874833 & 0.346465 \\
\hline 6 & 2.760404 & -6.344228 & 1.652688 \\
\hline 6 & 2.616188 & -5.453490 & 2.712970 \\
\hline 6 & 2.360157 & -4.111357 & 2.463036 \\
\hline 15 & 1.738407 & -0.264940 & -1.067788 \\
\hline 8 & 2.503040 & 0.512432 & 0.188721 \\
\hline 6 & 3.291638 & 1.584813 & -0.199517 \\
\hline 6 & 4.578747 & 1.340363 & -0.637165 \\
\hline 6 & 5.370616 & 2.451940 & -1.068802 \\
\hline 6 & 4.828646 & 3.770102 & -0.978187 \\
\hline 6 & 3.504957 & 3.951499 & -0.511233 \\
\hline 6 & 2.736618 & 2.877932 & -0.145363 \\
\hline
\end{tabular}




$\begin{array}{rrrr}6 & 6.664104 & 2.300140 & -1.629924 \\ 6 & 7.393411 & 3.390586 & -2.034476 \\ 6 & 6.870644 & 4.694468 & -1.905445 \\ 6 & 5.611575 & 4.874576 & -1.393731 \\ 6 & 5.024774 & -0.068064 & -0.747315 \\ 6 & 4.245865 & -0.944526 & -1.484788 \\ 6 & 4.614995 & -2.287679 & -1.691528 \\ 6 & 5.751783 & -2.776898 & -1.106444 \\ 6 & 6.549058 & -1.951480 & -0.278443 \\ 6 & 6.184023 & -0.584046 & -0.087610 \\ 6 & 6.969232 & 0.199453 & 0.796678 \\ 6 & 8.066632 & -0.330625 & 1.427995 \\ 6 & 8.442552 & -1.673591 & 1.212236 \\ 6 & 7.693917 & -2.464239 & 0.379134 \\ 8 & 3.066453 & -0.510340 & -2.052595 \\ 6 & -1.481142 & 2.185601 & 0.819959 \\ 6 & -0.401633 & 2.536897 & 1.641431 \\ 6 & -0.371480 & 3.766171 & 2.292807 \\ 6 & -1.423247 & 4.664330 & 2.048836 \\ 6 & -2.490228 & 4.358583 & 1.207951 \\ 6 & -2.506386 & 3.094900 & 0.603276 \\ 6 & 0.737466 & 4.146991 & 3.273708 \\ 6 & 0.113831 & 4.500406 & 4.629320 \\ 6 & -3.642443 & 5.321902 & 0.931198 \\ 6 & -3.674010 & 5.652110 & -0.565466 \\ 6 & 1.727249 & 3.005703 & 3.502007 \\ 6 & 1.509469 & 5.357001 & 2.738703 \\ 6 & -3.507621 & 6.629471 & 1.706790 \\ 6 & -4.966435 & 4.660918 & 1.333800 \\ 6 & -7.926499 & -0.617491 & 1.000933 \\ 6 & -6.579782 & 1.038647 & 2.263112 \\ 6 & -6.769216 & -0.819550 & -3.726261 \\ 6 & -4.388406 & -0.930417 & -4.383915 \\ 1 & 6.684606 & 1.232384 & 0.981574 \\ 1 & -1.582556 & 0.848770 & 2.956632 \\ 1 & 7.959376 & -3.507644 & 0.214220 \\ 1 & -1.376416 & -3.040080 & 5.642384 \\ 1 & 5.182658 & 5.872078 & -1.308155 \\ 1 & -3.463517 & -2.835321 & 0.706465 \\ 1 & -5.352391 & -3.837046 & -0.502682 \\ 1 & 3.094245 & 4.959667 & -0.476088 \\ 1 & -1.542696 & -0.739468 & 4.810651 \\ 1 & 6.043128 & -3.816076 & -1.250501 \\ 1 & 8.381752 & 3.246618 & -2.465243 \\ 1 & 3.979448 & -2.910641 & -2.316589 \\ 1 & -2.855872 & -4.499472 & -3.939496 \\ 1 & -0.526916 & -3.749534 & -3.920208 \\ 1 & -0.840724 & -6.281432 & 2.865851 \\ 1 & 7.076679 & 1.301553 & -1.747919 \\ 1 & -0.801102 & -4.704846 & 0.993385 \\ 1 & 1.382599 & -2.739313 & -2.718018 \\ 1 & 7.460962 & 5.549824 & -2.225687 \\ & 8.648904 & 0.289389 & 2.106009\end{array}$




$\begin{array}{rrrr}1 & -5.057145 & -4.701062 & -2.819752 \\ 1 & -1.125760 & -5.465944 & 5.203988 \\ 1 & 9.316975 & -2.079379 & 1.715583 \\ 1 & 1.702864 & 2.993446 & 0.176534 \\ 1 & -3.869673 & 0.231278 & 1.644667 \\ 1 & -2.888760 & 0.218968 & -2.515648 \\ 1 & -6.942035 & -0.441724 & -1.261542 \\ 1 & -3.331568 & 2.818525 & -0.051606 \\ 1 & 0.401673 & 1.815798 & 1.788680 \\ 1 & -1.401080 & 5.632265 & 2.545195 \\ 1 & 2.995858 & -1.637617 & 1.038084 \\ 1 & 1.430988 & -1.778298 & 1.812443 \\ 1 & -1.324918 & 1.770471 & -2.403537 \\ 1 & 2.219760 & -3.421736 & 3.296119 \\ 1 & 2.363834 & -4.165892 & -0.925244 \\ 1 & 2.681402 & -5.808389 & 3.739629 \\ 1 & 2.780837 & -6.562464 & -0.489166 \\ 1 & 2.945188 & -7.399164 & 1.843607 \\ 1 & 2.481558 & 3.316226 & 4.235858 \\ 1 & 1.232278 & 2.107614 & 3.896640 \\ 1 & 2.263195 & 2.727638 & 2.584904 \\ 1 & 0.900402 & 4.749575 & 5.354027 \\ 1 & -0.560958 & 5.362312 & 4.567910 \\ 1 & -0.459099 & 3.653850 & 5.030469 \\ 1 & 2.288922 & 5.657749 & 3.451711 \\ 1 & 2.001130 & 5.118727 & 1.786174 \\ 1 & 0.856011 & 6.222384 & 2.574195 \\ 1 & -5.805798 & 5.341397 & 1.136890 \\ 1 & -5.153345 & 3.734997 & 0.774895 \\ 1 & -4.974651 & 4.414081 & 2.403780 \\ 1 & -4.359621 & 7.282179 & 1.478579 \\ 1 & -3.500843 & 6.464979 & 2.792251 \\ 1 & -2.595290 & 7.175741 & 1.434846 \\ 1 & -4.496755 & 6.346045 & -0.784891 \\ 1 & -2.736367 & 6.126066 & -0.882842 \\ 1 & -3.819628 & 4.757272 & -1.182559 \\ 1 & -7.003760 & -0.884152 & -4.796268 \\ 1 & -6.782948 & -1.839527 & -3.317853 \\ 1 & -7.579995 & -0.249914 & -3.255229 \\ 1 & -4.731064 & -0.975615 & -5.426066 \\ 1 & -3.397479 & -0.460537 & -4.391122 \\ 1 & -4.270897 & -1.957996 & -4.016233 \\ 1 & -5.786596 & 1.310406 & -5.099494 \\ 1 & -6.210159 & 1.870342 & -3.466004 \\ 1 & -4.507993 & 1.789364 & -3.962580 \\ 1 & -8.592664 & -0.728389 & 1.865808 \\ 1 & -8.347184 & 0.170024 & 0.362579 \\ 1 & -7.950861 & -1.562733 & 0.442506 \\ 1 & -7.276180 & 0.948050 & 3.107774 \\ 1 & -5.599939 & 1.321440 & 2.667853 \\ 1 & -6.928414 & 1.861335 & 1.624628 \\ 1 & -6.741936 & -1.511193 & 3.253795 \\ 1 & -6.013484 & -2.362596 & 1.878347\end{array}$


4. Cartesian coordinates for intermediate 3

\begin{tabular}{|c|c|}
\hline & $3_{\text {IA-si- }}-\psi_{-1 \mathbf{a} 2 \mathbf{a} 3 \mathbf{a} 4 \mathbf{a}}$ \\
\hline \multicolumn{2}{|l|}{ Number of imaginary frequencies : 0} \\
\hline \multicolumn{2}{|l|}{ The smallest frequencies are $: 17.3155 \quad 19.1151$} \\
\hline & \\
\hline Zero-point correction $=\quad 1.454953$ & 1.454953 (Hartree/Particle) \\
\hline Thermal correction to Energy= & 1.539368 \\
\hline Thermal correction to Enthalpy $=$ & 1.540312 \\
\hline Thermal correction to Gibbs Free Energy= & \\
\hline Sum of electronic and zero-point Energies $=$ & $\begin{array}{l}1.338446 \\
-4359.437540\end{array}$ \\
\hline Sum of electronic and thermal Energies= & -4359.353125 \\
\hline Sum of electronic and thermal Enthalpies= & -4359.352181 \\
\hline Sum of electronic and thermal Free Energies= & -4359.554047 \\
\hline
\end{tabular}

Cartesian Coordinates

$\begin{array}{cccc}6 & 2.821079 & 0.833469 & -3.802335 \\ 6 & 1.956964 & -0.264910 & -3.898614 \\ 6 & 2.509370 & -1.505267 & -4.232993 \\ 6 & 3.879545 & -1.652245 & -4.429608 \\ 6 & 4.726421 & -0.555822 & -4.308998 \\ 6 & 4.188060 & 0.692213 & -4.000362 \\ 6 & 0.481706 & -0.100652 & -3.718768 \\ 6 & -0.127090 & 0.841621 & -4.738704 \\ 45 & -0.118153 & 0.398542 & -1.651412 \\ 6 & 0.373400 & 2.266816 & -1.560931 \\ 8 & 0.478256 & 3.398139 & -1.785276 \\ 15 & 1.567338 & -0.675555 & -0.371020 \\ 8 & 2.837736 & -1.320846 & -1.240151 \\ 6 & 4.038736 & -1.699611 & -0.700710 \\ 6 & 4.898797 & -0.769121 & -0.146432 \\ 6 & 6.075608 & -1.250717 & 0.513942 \\ 6 & 6.401658 & -2.638908 & 0.453631 \\ 6 & 5.545805 & -3.522674 & -0.249526 \\ 6 & 4.380593 & -3.066588 & -0.801000 \\ 6 & 6.907351 & -0.404599 & 1.290506 \\ 6 & 8.009319 & -0.899364 & 1.944399 \\ 6 & 8.348351 & -2.265380 & 1.851975 \\ 6 & 7.555629 & -3.114133 & 1.122002 \\ 6 & 4.569125 & 0.671030 & -0.233729 \\ 6 & 3.339828 & 1.122371 & 0.206840 \\ 6 & 2.976035 & 2.481830 & 0.174057 \\ 6 & 3.837095 & 3.403036 & -0.356445 \\ 6 & 5.079124 & 2.995548 & -0.899250 \\ 6 & 5.451858 & 1.617969 & -0.848970 \\ 6 & 5.941541 & 3.929429 & -1.524279 \\ 6 & 7.116874 & 3.527289 & -2.105009 \\ 6 & 7.472841 & 2.162069 & -2.088280 \\ 6 & 6.664988 & 1.234935 & -1.477311 \\ 8 & 2.424062 & 0.230489 & 0.718074 \\ 7 & 1.057385 & -1.879457 & 0.715034\end{array}$




\begin{tabular}{|c|c|c|c|}
\hline 6 & 0.047024 & -2.737294 & 0.158817 \\
\hline 6 & -1.255656 & -2.672910 & 0.619204 \\
\hline & -2.265948 & -3.455869 & -0.017795 \\
\hline & -1.908358 & -4.327659 & -1.088929 \\
\hline & -0.551605 & -4.423442 & -1.475004 \\
\hline & 0.397345 & -3.640983 & -0.871987 \\
\hline & -2.918282 & -5.084321 & -1.733472 \\
\hline & -4.229687 & -4.979078 & -1.345098 \\
\hline & -4.587938 & -4.110848 & -0.289609 \\
\hline & -3.630336 & -3.377511 & 0.364211 \\
\hline & -1.597495 & -1.871125 & 1.824631 \\
\hline f & -1.864341 & -0.512769 & 1.764198 \\
\hline & -2.227965 & 0.173697 & 2.955662 \\
\hline ) & -2.311273 & -0.475442 & 4.158202 \\
\hline & -2.033180 & -1.858118 & 4.254762 \\
\hline 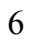 & -1.674242 & -2.569999 & 3.073583 \\
\hline$b$ & -1.391757 & -3.956754 & 3.189016 \\
\hline & -1.458579 & -4.593551 & 4.403659 \\
\hline$b$ & -1.816250 & -3.882598 & 5.568555 \\
\hline b & -2.097457 & -2.542463 & 5.491746 \\
\hline & -1.843691 & 0.353746 & 0.114700 \\
\hline y & -1.925209 & 2.116050 & 0.626487 \\
\hline 6 & -0.930472 & 2.607208 & 1.477358 \\
\hline 5 & -0.888858 & 3.954897 & 1.830724 \\
\hline 6 & -1.837960 & 4.807413 & 1.254576 \\
\hline 6 & -2.829029 & 4.353922 & 0.382267 \\
\hline 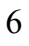 & -2.863057 & 2.988629 & 0.082638 \\
\hline & 0.145193 & 4.509936 & 2.809666 \\
\hline 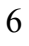 & -3.841291 & 5.339507 & -0.198989 \\
\hline 6 & 1.900653 & -2.499025 & 1.766012 \\
\hline 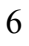 & 2.373722 & -1.559250 & 2.842309 \\
\hline 6 & 3.727814 & -1.475673 & 3.159232 \\
\hline 6 & 4.171194 & -0.608678 & 4.154753 \\
\hline 6 & 3.259200 & 0.174586 & 4.854219 \\
\hline 6 & 1.901295 & 0.082547 & 4.553311 \\
\hline ) & 1.464043 & -0.773461 & 3.551218 \\
\hline 6 & -3.569725 & -0.014491 & -0.420968 \\
\hline 6 & -3.806405 & -0.527586 & -1.692774 \\
\hline 6 & -5.104685 & -0.842442 & -2.110651 \\
\hline 6 & -6.146636 & -0.623392 & -1.209170 \\
\hline 6 & -5.944911 & -0.110357 & 0.077833 \\
\hline 6 & -4.638628 & 0.187491 & 0.456862 \\
\hline 6 & -5.407896 & -1.365728 & -3.513983 \\
\hline 6 & -7.128920 & 0.060209 & 1.027433 \\
\hline ) & -0.308833 & -1.154169 & -3.193516 \\
\hline 1 & 6.648329 & 0.647325 & 1.384309 \\
\hline 1 & -2.449465 & 1.237078 & 2.914915 \\
\hline 1 & 7.790683 & -4.175710 & 1.054788 \\
\hline 1 & -2.372663 & -1.978691 & 6.382071 \\
\hline 1 & 5.639867 & 4.975853 & -1.544542 \\
\hline 1 & -3.915524 & -2.704864 & 1.171409 \\
\hline 1 & -5.632945 & -4.015869 & 0.000723 \\
\hline 1 & 3.563580 & 4.456757 & -0.396695 \\
\hline 1 & -2.596405 & 0.068167 & 5.058254 \\
\hline 1 & 5.819879 & -4.573864 & -0.323170 \\
\hline 1 & 8.392436 & 1.838788 & -2.571098 \\
\hline
\end{tabular}




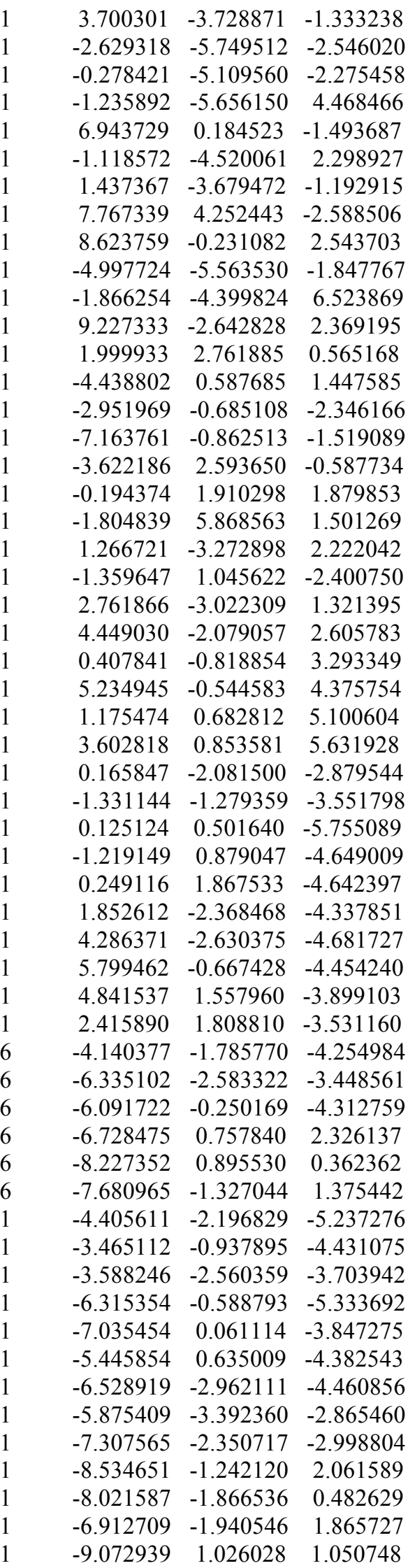




$\begin{array}{rrrr}1 & -7.855752 & 1.892347 & 0.091592 \\ 1 & -8.617353 & 0.425512 & -0.548084 \\ 1 & -7.610806 & 0.879338 & 2.966996 \\ 1 & -5.987811 & 0.180840 & 2.895923 \\ 1 & -6.313426 & 1.757863 & 2.139001 \\ 6 & -4.876519 & 4.648958 & -1.084821 \\ 6 & -4.588566 & 6.040733 & 0.940741 \\ 6 & -3.102915 & 6.381376 & -1.045724 \\ 6 & 0.964103 & 3.399537 & 3.466415 \\ 6 & 1.095215 & 5.452624 & 2.064168 \\ 6 & -0.562922 & 5.285073 & 3.926760 \\ 1 & -5.591623 & 5.390821 & -1.462144 \\ 1 & -4.416470 & 4.163884 & -1.955180 \\ 1 & -5.448023 & 3.889440 & -0.533135 \\ 1 & -3.815485 & 7.093107 & -1.484407 \\ 1 & -2.381394 & 6.956158 & -0.452482 \\ 1 & -2.552730 & 5.900793 & -1.865105 \\ 1 & -5.324606 & 6.747646 & 0.534728 \\ 1 & -5.126261 & 5.313521 & 1.563485 \\ 1 & -3.914172 & 6.606643 & 1.594548 \\ 1 & 0.173890 & 5.674729 & 4.641764 \\ 1 & -1.134165 & 6.140336 & 3.547212 \\ 1 & -1.256958 & 4.636546 & 4.477247 \\ 1 & 1.857319 & 5.850434 & 2.747926 \\ 1 & 1.611693 & 4.928337 & 1.248874 \\ 1 & 0.561725 & 6.303794 & 1.623375 \\ 1 & 1.650799 & 3.830094 & 4.206588 \\ 1 & 0.316650 & 2.681055 & 3.988816 \\ 1 & 1.576530 & 2.837262 & 2.749175\end{array}$

$3_{\text {IA-si }-\Psi-12 a 2 a 3 a 4 s}$

Number of imaginary frequencies : 0

The smallest frequencies are : $15.4400 \quad 21.761924 .1488 \mathrm{~cm}(-1)$

Electronic energy : $\quad H F=-4360.8930464$

Zero-point correction $=\quad 1.454524($ Hartree/Particle $)$

Thermal correction to Energy= $\quad 1.539100$

Thermal correction to Enthalpy= $\quad 1.540045$

Thermal correction to Gibbs Free Energy $=\quad 1.337602$

Sum of electronic and zero-point Energies $=\quad-4359.438522$

Sum of electronic and thermal Energies $=\quad-4359.353946$

Sum of electronic and thermal Enthalpies $=\quad-4359.353002$

Sum of electronic and thermal Free Energies $=\quad-4359.555445$

\section{Cartesian Coordinates}

$\begin{array}{cccc}6 & 2.825412 & 0.926536 & -3.778157 \\ 6 & 1.969948 & -0.175881 & -3.901271 \\ 6 & 2.532594 & -1.404540 & -4.260448 \\ 6 & 3.904500 & -1.536842 & -4.455664 \\ 6 & 4.742560 & -0.436795 & -4.309122 \\ 6 & 4.193960 & 0.800216 & -3.974951 \\ 6 & 0.492987 & -0.025721 & -3.722952 \\ 6 & -0.119047 & 0.933735 & -4.724831 \\ 45 & -0.115514 & 0.426878 & -1.647450 \\ 6 & 0.371976 & 2.294676 & -1.517911\end{array}$




\begin{tabular}{|c|c|c|c|}
\hline 3 & 0.476100 & 3.430406 & -1.718696 \\
\hline 15 & 1.567977 & -0.666395 & -0.384806 \\
\hline 8 & 2.839313 & -1.294617 & -1.264461 \\
\hline 6 & 4.037204 & -1.687298 & -0.728068 \\
\hline 6 & 4.897348 & -0.769811 & -0.152701 \\
\hline 6 & 6.069916 & -1.267267 & 0.503480 \\
\hline 6 & 6.391752 & -2.655222 & 0.419055 \\
\hline 6 & 5.536579 & -3.523033 & -0.304436 \\
\hline 6 & 4.375277 & -3.053145 & -0.852631 \\
\hline 6 & 6.900702 & -0.438500 & 1.299553 \\
\hline 6 & 7.997420 & -0.949147 & 1.950047 \\
\hline 6 & 8.332136 & -2.314477 & 1.834445 \\
\hline 6 & 7.540510 & -3.146791 & 1.084626 \\
\hline 6 & 4.571878 & 0.672768 & -0.213069 \\
\hline 6 & 3.342298 & 1.119594 & 0.231769 \\
\hline 6 & 2.984094 & 2.481058 & 0.228156 \\
\hline 6 & 3.851323 & 3.410075 & -0.278179 \\
\hline 6 & 5.093836 & 3.009341 & -0.825038 \\
\hline 6 & 5.460408 & 1.629332 & -0.804514 \\
\hline 6 & 5.963125 & 3.953306 & -1.424909 \\
\hline 6 & 7.139106 & 3.559176 & -2.009859 \\
\hline 6 & 7.488812 & 2.192285 & -2.023073 \\
\hline 6 & 6.674345 & 1.255217 & -1.436585 \\
\hline 8 & 2.421510 & 0.221099 & 0.721058 \\
\hline 7 & 1.058513 & -1.887794 & 0.681477 \\
\hline 6 & 0.047609 & -2.737098 & 0.113864 \\
\hline 6 & -1.253603 & -2.682833 & 0.579840 \\
\hline 6 & -2.265071 & -3.457830 & -0.064643 \\
\hline 6 & -1.909958 & -4.309814 & -1.152496 \\
\hline 6 & -0.555086 & -4.393903 & -1.547776 \\
\hline 6 & 0.395220 & -3.620925 & -0.934756 \\
\hline 6 & -2.919976 & -5.060801 & -1.803398 \\
\hline 6 & -4.228856 & -4.970577 & -1.403348 \\
\hline 6 & -4.584463 & -4.121683 & -0.331401 \\
\hline 6 & -3.627609 & -3.391592 & 0.327090 \\
\hline 6 & -1.593500 & -1.897591 & 1.796270 \\
\hline 6 & -1.862470 & -0.539089 & 1.751562 \\
\hline 6 & -2.226559 & 0.132290 & 2.951301 \\
\hline 6 & -2.307685 & -0.531291 & 4.146276 \\
\hline 6 & -2.027623 & -1.914514 & 4.226400 \\
\hline 6 & -1.668341 & -2.611584 & 3.036544 \\
\hline 6 & -1.384255 & -3.999383 & 3.135008 \\
\hline 6 & -1.449378 & -4.650665 & 4.341964 \\
\hline 6 & -1.806992 & -3.954188 & 5.515632 \\
\hline 6 & -2.090040 & -2.613690 & 5.455232 \\
\hline 15 & -1.843021 & 0.345035 & 0.110791 \\
\hline 6 & -1.949485 & 2.101301 & 0.639143 \\
\hline 6 & -0.965866 & 2.594624 & 1.501548 \\
\hline 6 & -0.945813 & 3.937678 & 1.874243 \\
\hline 6 & -1.897343 & 4.786246 & 1.296744 \\
\hline 6 & -2.870114 & 4.333203 & 0.403583 \\
\hline 6 & -2.892184 & 2.969438 & 0.095655 \\
\hline 6 & 0.070646 & 4.492289 & 2.871671 \\
\hline 6 & -3.880348 & 5.317167 & -0.183962 \\
\hline 6 & 1.896614 & -2.519049 & 1.729505 \\
\hline 6 & 2.370772 & -1.591293 & 2.815829 \\
\hline
\end{tabular}




\begin{tabular}{|c|c|c|c|}
\hline 6 & 3.723907 & -1.521600 & 3.140147 \\
\hline 6 & 4.169762 & -0.665906 & 4.144397 \\
\hline & 3.261195 & 0.120723 & 4.844367 \\
\hline & 1.904148 & 0.043245 & 4.535408 \\
\hline & 1.464326 & -0.802571 & 3.525873 \\
\hline & -3.562579 & -0.037105 & -0.434990 \\
\hline & -3.799190 & -0.524763 & -1.720860 \\
\hline & -5.092734 & -0.838923 & -2.141163 \\
\hline & -6.141835 & -0.650762 & -1.232251 \\
\hline & -5.937864 & -0.174439 & 0.063048 \\
\hline & -4.629157 & 0.130074 & 0.443435 \\
\hline & -5.394407 & -1.334403 & -3.554823 \\
\hline & -7.062342 & 0.013130 & 1.078700 \\
\hline & -0.292938 & -1.094859 & -3.222905 \\
\hline & 6.644638 & 0.612333 & 1.411849 \\
\hline & -2.450171 & 1.195540 & 2.923474 \\
\hline & 7.772254 & -4.207769 & 0.999091 \\
\hline & -2.365383 & -2.060915 & 6.352378 \\
\hline & 5.666321 & 5.001328 & -1.422433 \\
\hline & -3.912672 & -2.730790 & 1.144775 \\
\hline & -5.628129 & -4.039984 & -0.032707 \\
\hline & 3.583273 & 4.465733 & -0.294797 \\
\hline & -2.592906 & 0.001250 & 5.052896 \\
\hline & 5.807674 & -4.573550 & -0.396368 \\
\hline & 8.408914 & 1.875911 & -2.509509 \\
\hline & 3.695389 & -3.703355 & -1.400072 \\
\hline & -2.632849 & -5.710581 & -2.628969 \\
\hline & -0.284228 & -5.064967 & -2.361671 \\
\hline & -1.225501 & -5.713722 & 4.393874 \\
\hline & 6.948345 & 0.204140 & -1.475791 \\
\hline & -1.111411 & -4.551549 & 2.237858 \\
\hline & 1.434185 & -3.651994 & -1.259941 \\
\hline & 7.794915 & 4.292235 & -2.473834 \\
\hline & 8.610941 & -0.294185 & 2.564816 \\
\hline & -4.997107 & -5.551827 & -1.909361 \\
\hline & -1.855526 & -4.482993 & 6.464664 \\
\hline ] & 9.206935 & -2.704521 & 2.349407 \\
\hline & 2.007844 & 2.756602 & 0.622416 \\
\hline & -4.438984 & 0.506025 & 1.448162 \\
\hline & -2.944527 & -0.659859 & -2.378873 \\
\hline & -7.154161 & -0.887894 & -1.552450 \\
\hline & -3.642304 & 2.573230 & -0.584482 \\
\hline & -0.221914 & 1.902458 & 1.898159 \\
\hline . & -1.880204 & 5.844291 & 1.558117 \\
\hline & 1.258681 & -3.294774 & 2.176790 \\
\hline & -1.358414 & 1.083513 & -2.387419 \\
\hline & 2.757296 & -3.041352 & 1.282651 \\
\hline & 4.442853 & -2.126799 & 2.585611 \\
\hline ] & 0.409184 & -0.835577 & 3.261626 \\
\hline & 5.232840 & -0.612941 & 4.371473 \\
\hline & 1.181094 & 0.647510 & 5.082032 \\
\hline & 3.606737 & 0.791375 & 5.628413 \\
\hline 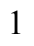 & 0.185796 & -2.026197 & -2.927082 \\
\hline & -1.313349 & -1.217761 & -3.587401 \\
\hline & 0.138769 & 0.616885 & -5.747273 \\
\hline & -1.211642 & 0.961647 & -4.637992 \\
\hline
\end{tabular}




\begin{tabular}{lrrr}
1 & 0.249734 & 1.959950 & -4.605775 \\
1 & 1.882730 & -2.270266 & -4.385515 \\
1 & 4.319624 & -2.506252 & -4.727169 \\
1 & 5.816789 & -0.537118 & -4.453868 \\
1 & 4.840489 & 1.668544 & -3.853433 \\
1 & 2.411471 & 1.892617 & -3.488230 \\
6 & -4.124724 & -1.728808 & -4.306306 \\
6 & -6.311222 & -2.561089 & -3.514083 \\
6 & -6.088936 & -0.208346 & -4.329268 \\
6 & -8.422166 & -0.407857 & 0.528381 \\
6 & -6.767066 & -0.841307 & 2.317816 \\
6 & -7.143437 & 1.491333 & 1.477336 \\
1 & -4.388088 & -2.119687 & -5.297314 \\
1 & -3.455247 & -0.872757 & -4.464040 \\
1 & -3.567192 & -2.511741 & -3.772638 \\
1 & -6.314316 & -0.528808 & -5.355680 \\
1 & -7.033110 & 0.087382 & -3.854682 \\
1 & -5.449237 & 0.682321 & -4.384426 \\
1 & -6.498885 & -2.923021 & -4.533685 \\
1 & -5.846070 & -3.376604 & -2.944354 \\
1 & -7.286851 & -2.345027 & -3.063111 \\
1 & -7.914678 & 1.639908 & 2.245244 \\
1 & -6.193584 & 1.866134 & 1.879986 \\
1 & -7.406080 & 2.113362 & 0.611627 \\
1 & -7.559956 & -0.714866 & 3.067371 \\
1 & -6.720299 & -1.906785 & 2.054344 \\
1 & -5.814633 & -0.575400 & 2.794133 \\
1 & -9.192988 & -0.260577 & 1.295419 \\
1 & -8.713345 & 0.186288 & -0.347320 \\
1 & -8.437575 & -1.468615 & 0.245244 \\
6 & -4.843874 & 4.643868 & -1.158931 \\
6 & -4.706255 & 5.934609 & 0.949907 \\
6 & -3.136139 & 6.423533 & -0.938960 \\
6 & 0.884498 & 3.382152 & 3.535096 \\
6 & 1.027971 & 5.441924 & 2.144624 \\
6 & -0.656804 & 5.259135 & 3.981960 \\
1 & -5.551287 & 5.386646 & -1.548756 \\
1 & -4.319416 & 4.204717 & -2.017300 \\
1 & -5.431483 & 3.851446 & -0.676046 \\
1 & -3.851373 & 7.127897 & -1.384905 \\
1 & -2.471975 & 6.998555 & -0.282756 \\
1 & -2.523819 & 6.002396 & -1.746899 \\
1 & -5.437238 & 6.647915 & 0.545671 \\
1 & -5.258145 & 5.159880 & 1.498976 \\
1 & -4.080186 & 6.473746 & 1.671252 \\
1 & 0.068077 & 5.647625 & 4.709631 \\
1 & -1.225174 & 6.114580 & 3.598680 \\
1 & -1.356581 & 4.605433 & 4.518907 \\
1 & 1.778374 & 5.839973 & 2.841083 \\
1 & 1.558246 & 4.922587 & 1.334934 \\
1 & 0.497442 & 6.292482 & 1.699086 \\
1 & 1.553457 & 3.811413 & 4.292025 \\
& 0.232356 & 2.654102 & 4.038281 \\
\hline & 1.515284 & 2.831779 & 2.824765
\end{tabular}

$3_{\text {IA-si }-\Psi-1 \text { a2a3s4a }}$ 
Number of imaginary frequencies : 0

The smallest frequencies are : $12.8357 \quad 16.3703 \quad 22.6895 \mathrm{~cm}(-1)$

Electronic energy : $\quad H F=-4360.8925217$

Zero-point correction $=\quad 1.453931($ Hartree/Particle $)$

Thermal correction to Energy= $\quad 1.538732$

Thermal correction to Enthalpy= $\quad 1.539676$

Thermal correction to Gibbs Free Energy= 1.335869

Sum of electronic and zero-point Energies $=\quad-4359.438591$

Sum of electronic and thermal Energies $=\quad-4359.353790$

Sum of electronic and thermal Enthalpies $=\quad-4359.352846$

Sum of electronic and thermal Free Energies $=\quad-4359.556653$

Cartesian Coordinates

\begin{tabular}{cccc}
\hline 6 & 2.810870 & 0.775109 & -3.795270 \\
6 & 1.906397 & -0.289257 & -3.904086 \\
6 & 2.412563 & -1.548582 & -4.240234 \\
6 & 3.777791 & -1.747247 & -4.425510 \\
6 & 4.665445 & -0.685570 & -4.287683 \\
6 & 4.173003 & 0.581437 & -3.978719 \\
6 & 0.438555 & -0.069345 & -3.729738 \\
6 & -0.127300 & 0.908755 & -4.740297 \\
45 & -0.138648 & 0.431771 & -1.657659 \\
6 & 0.370214 & 2.292690 & -1.553919 \\
8 & 0.472832 & 3.426546 & -1.766385 \\
15 & 1.559469 & -0.650890 & -0.391041 \\
8 & 2.838190 & -1.284430 & -1.257479 \\
6 & 4.033063 & -1.669977 & -0.708859 \\
6 & 4.889552 & -0.743294 & -0.143520 \\
6 & 6.061281 & -1.227372 & 0.523509 \\
6 & 6.386229 & -2.615660 & 0.461485 \\
6 & 5.534429 & -3.496115 & -0.250695 \\
6 & 4.374161 & -3.037242 & -0.810370 \\
6 & 6.887972 & -0.384176 & 1.308559 \\
6 & 7.984260 & -0.882098 & 1.969590 \\
6 & 8.322230 & -2.248334 & 1.876135 \\
6 & 7.534352 & -3.094158 & 1.137518 \\
6 & 4.560334 & 0.696748 & -0.229103 \\
6 & 3.328036 & 1.147378 & 0.204260 \\
6 & 2.965190 & 2.507162 & 0.171410 \\
6 & 3.827559 & 3.427859 & -0.357981 \\
6 & 5.070819 & 3.019965 & -0.897704 \\
6 & 5.445599 & 1.643191 & -0.841069 \\
6 & 5.932922 & 3.952474 & -1.525046 \\
6 & 7.110650 & 3.549995 & -2.100556 \\
6 & 7.470214 & 2.185906 & -2.074960 \\
6 & 6.662270 & 1.259782 & -1.462546 \\
8 & 2.407487 & 0.254907 & 0.706115 \\
7 & 1.051726 & -1.859536 & 0.688853 \\
6 & 0.056202 & -2.726710 & 0.120755 \\
6 & -1.249210 & -2.686298 & 0.576558 \\
6 & -2.235666 & -3.509310 & -0.049378 \\
6 & -1.846871 & -4.408908 & -1.086544 \\
6 & -0.490522 & -4.458672 & -1.483050 \\
6 & 0.431375 & -3.627909 & -0.903722 \\
& & & \\
\hline
\end{tabular}




\begin{tabular}{|c|c|c|c|}
\hline & -2.823490 & -5.243630 & -1.683926 \\
\hline & -4.134509 & -5.186600 & -1.285886 \\
\hline & -4.527845 & -4.277774 & -0.278941 \\
\hline & -3.602972 & -3.465363 & 0.327903 \\
\hline & -1.603838 & -1.897134 & 1.788150 \\
\hline & -1.867727 & -0.536981 & 1.750810 \\
\hline & -2.231310 & 0.131763 & 2.952189 \\
\hline & -2.320032 & -0.535641 & 4.144007 \\
\hline & -2.042556 & -1.919638 & 4.219382 \\
\hline & -1.680169 & -2.613677 & 3.028370 \\
\hline & -1.390223 & -4.000505 & 3.127278 \\
\hline & -1.456837 & -4.653693 & 4.333127 \\
\hline & -1.821681 & -3.960843 & 5.506529 \\
\hline & -2.107803 & -2.621065 & 5.446839 \\
\hline & -1.849187 & 0.362961 & 0.121178 \\
\hline & -1.925943 & 2.117904 & 0.659011 \\
\hline & -0.926328 & 2.600931 & 1.508867 \\
\hline & -0.880943 & 3.946429 & 1.870841 \\
\hline & -1.831828 & 4.804258 & 1.305632 \\
\hline & -2.827361 & 4.358915 & 0.434371 \\
\hline & -2.865366 & 2.995793 & 0.125999 \\
\hline & 0.158660 & 4.495342 & 2.847333 \\
\hline & -3.840009 & 5.350935 & -0.135181 \\
\hline & 1.892597 & -2.482031 & 1.740780 \\
\hline 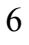 & 2.357266 & -1.548980 & 2.826239 \\
\hline & 3.709763 & -1.461372 & 3.148853 \\
\hline & 4.144858 & -0.604214 & 4.156455 \\
\hline & 3.225916 & 0.163787 & 4.863656 \\
\hline & 1.869488 & 0.066463 & 4.557840 \\
\hline & 1.440696 & -0.778190 & 3.542526 \\
\hline & -3.577572 & 0.010152 & -0.413551 \\
\hline & -3.819175 & -0.429528 & -1.705488 \\
\hline 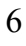 & -5.119413 & -0.723012 & -2.143181 \\
\hline & -6.159316 & -0.569532 & -1.232647 \\
\hline 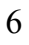 & -5.951223 & -0.125921 & 0.084997 \\
\hline & -4.649410 & 0.160219 & 0.477147 \\
\hline 5 & -5.325662 & -1.199663 & -3.578496 \\
\hline & -7.135580 & -0.006956 & 1.042366 \\
\hline & -0.395964 & -1.094830 & -3.215822 \\
\hline . & 6.629322 & 0.667801 & 1.403166 \\
\hline & -2.449606 & 1.196293 & 2.926486 \\
\hline . & 7.768722 & -4.155819 & 1.069212 \\
\hline & -2.386655 & -2.069801 & 6.343837 \\
\hline . & 5.629119 & 4.998149 & -1.551277 \\
\hline & -3.916037 & -2.764326 & 1.100429 \\
\hline & -5.573784 & -4.218822 & 0.015513 \\
\hline . & 3.553663 & 4.481380 & -0.400364 \\
\hline 1 & -2.606418 & -0.005824 & 5.051819 \\
\hline 1 & 5.807503 & -4.547527 & -0.324973 \\
\hline & 8.392532 & 1.862549 & -2.552480 \\
\hline 1 & 3.698468 & -3.698683 & -1.349473 \\
\hline & -2.508476 & -5.930927 & -2.467849 \\
\hline 1 & -0.195649 & -5.154900 & -2.266875 \\
\hline & -1.227939 & -5.715730 & 4.384015 \\
\hline 1 & 6.944185 & 0.210127 & -1.471977 \\
\hline 1 & -1.110158 & -4.551411 & 2.231679 \\
\hline
\end{tabular}




\begin{tabular}{|c|c|c|c|}
\hline 1 & 1.470472 & -3.635599 & -1.229123 \\
\hline & 7.760686 & 4.274104 & -2.586174 \\
\hline & 8.594948 & -0.216244 & 2.575402 \\
\hline & -4.876531 & -5.831865 & -1.751274 \\
\hline & -1.871906 & -4.491224 & 6.454590 \\
\hline & 9.196650 & -2.628257 & 2.399249 \\
\hline & 1.988168 & 2.787755 & 0.560059 \\
\hline & -4.449665 & 0.509343 & 1.487027 \\
\hline & -2.970926 & -0.547555 & -2.376801 \\
\hline & -7.175417 & -0.801132 & -1.545930 \\
\hline & -3.627729 & 2.607185 & -0.544552 \\
\hline & -0.187993 & 1.900438 & 1.901015 \\
\hline & -1.796090 & 5.863518 & 1.560136 \\
\hline & 1.258731 & -3.260591 & 2.188906 \\
\hline & -1.375997 & 1.105943 & -2.388669 \\
\hline & 2.757377 & -3.000173 & 1.297496 \\
\hline & 4.436197 & -2.054864 & 2.591740 \\
\hline & 0.385473 & -0.828027 & 3.281913 \\
\hline & 5.207532 & -0.536761 & 4.381680 \\
\hline & 1.138069 & 0.652977 & 5.112462 \\
\hline & 3.562741 & 0.834182 & 5.651744 \\
\hline & 0.031843 & -2.048151 & -2.910175 \\
\hline & -1.422058 & -1.165687 & -3.580391 \\
\hline & 0.108068 & 0.567130 & -5.760158 \\
\hline ] & -1.215991 & 0.997170 & -4.649662 \\
\hline & 0.296543 & 1.914931 & -4.634897 \\
\hline & 1.723430 & -2.384705 & -4.356667 \\
\hline & 4.149112 & -2.738636 & -4.680706 \\
\hline & 5.734702 & -0.838743 & -4.422377 \\
\hline 1 & 4.858153 & 1.421190 & -3.866528 \\
\hline & 2.440926 & 1.764235 & -3.522938 \\
\hline 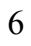 & -6.799078 & -1.410560 & -3.916743 \\
\hline 6 & -4.758781 & -0.156807 & -4.548551 \\
\hline 3 & -4.594162 & -2.531658 & -3.774436 \\
\hline 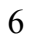 & -6.731019 & 0.590228 & 2.388845 \\
\hline & -8.217643 & 0.890879 & 0.433715 \\
\hline & -7.711480 & -1.405473 & 1.292361 \\
\hline & -6.893973 & -1.743454 & -4.958053 \\
\hline 1 & -7.259354 & -2.180908 & -3.284452 \\
\hline 1 & -7.380896 & -0.485417 & -3.812339 \\
\hline l & -4.706022 & -2.880988 & -4.810055 \\
\hline 1 & -3.520157 & -2.449727 & -3.562034 \\
\hline d & -5.001322 & -3.301919 & -3.106702 \\
\hline 1 & -4.901548 & -0.485691 & -5.586618 \\
\hline & -5.263255 & 0.810591 & -4.426764 \\
\hline 1 & -3.684061 & 0.006683 & -4.398820 \\
\hline 1 & -8.566080 & -1.352925 & 1.980529 \\
\hline 1 & -8.058447 & -1.877888 & 0.364945 \\
\hline 1 & -6.954964 & -2.063181 & 1.741774 \\
\hline 1 & -9.064512 & 0.986783 & 1.126290 \\
\hline 1 & -7.828152 & 1.897957 & 0.235410 \\
\hline 1 & -8.609813 & 0.492445 & -0.509334 \\
\hline 1 & -7.613121 & 0.668975 & 3.036713 \\
\hline 1 & -5.994062 & -0.032253 & 2.913948 \\
\hline 1 & -6.310528 & 1.598789 & 2.277147 \\
\hline 6 & -4.869213 & 4.672778 & -1.037398 \\
\hline
\end{tabular}




$\begin{array}{rrrr}6 & -4.593485 & 6.030224 & 1.013723 \\ 6 & -3.102037 & 6.409208 & -0.961678 \\ 6 & 0.990799 & 3.382321 & 3.482238 \\ 6 & 1.095566 & 5.454430 & 2.106245 \\ 6 & -0.545214 & 5.250723 & 3.980634 \\ 1 & -5.582194 & 5.419940 & -1.408296 \\ 1 & -4.402761 & 4.201065 & -1.911710 \\ 1 & -5.444420 & 3.905081 & -0.501419 \\ 1 & -3.815821 & 7.124743 & -1.392070 \\ 1 & -2.385665 & 6.977972 & -0.356618 \\ 1 & -2.546385 & 5.944004 & -1.786224 \\ 1 & -5.328870 & 6.743470 & 0.617646 \\ 1 & -5.132627 & 5.290888 & 1.620713 \\ 1 & -3.922194 & 6.584510 & 1.680624 \\ 1 & 0.195070 & 5.638654 & 4.693020 \\ 1 & -1.128195 & 6.104943 & 3.616805 \\ 1 & -1.227787 & 4.589282 & 4.530095 \\ 1 & 1.862941 & 5.845525 & 2.787974 \\ 1 & 1.605873 & 4.945324 & 1.277562 \\ 1 & 0.553467 & 6.309724 & 1.684405 \\ 1 & 1.678423 & 3.807548 & 4.224681 \\ 1 & 0.351971 & 2.651668 & 3.998025 \\ 1 & 1.603587 & 2.835419 & 2.753216\end{array}$

$3_{\text {IA-si- }} \boldsymbol{\psi}_{-1 \mathrm{a} 2 \mathrm{a} 3 \mathrm{~s} 4 \mathrm{~s}}$

Number of imaginary frequencies : 0

The smallest frequencies are : $11.8250 \quad 18.9534 \quad 19.7009 \mathrm{~cm}(-1)$

Electronic energy : $\quad H F=-4360.8928418$

Zero-point correction $=\quad 1.454338($ Hartree/Particle $)$

Thermal correction to Energy $=\quad 1.539047$

Thermal correction to Enthalpy= $\quad 1.539991$

Thermal correction to Gibbs Free Energy= $\quad 1.336425$

Sum of electronic and zero-point Energies $=\quad-4359.438504$

Sum of electronic and thermal Energies $=\quad-4359.353795$

Sum of electronic and thermal Enthalpies $=\quad-4359.352851$

Sum of electronic and thermal Free Energies $=\quad-4359.556417$

\section{Cartesian Coordinates}

$\begin{array}{cccc}6 & 2.825766 & 0.861502 & -3.784074 \\ 6 & 1.944140 & -0.221349 & -3.898970 \\ 6 & 2.477180 & -1.466558 & -4.245785 \\ 6 & 3.845878 & -1.633341 & -4.437417 \\ 6 & 4.710203 & -0.552802 & -4.298056 \\ 6 & 4.191050 & 0.700344 & -3.976756 \\ 6 & 0.471558 & -0.031995 & -3.725578 \\ 6 & -0.108291 & 0.944375 & -4.730150 \\ 45 & -0.126912 & 0.439934 & -1.651909 \\ 6 & 0.369031 & 2.303877 & -1.525402 \\ 8 & 0.468758 & 3.440336 & -1.724394 \\ 15 & 1.561314 & -0.650128 & -0.385413 \\ 8 & 2.839035 & -1.278772 & -1.255967 \\ 6 & 4.036767 & -1.664446 & -0.714263 \\ 6 & 4.891010 & -0.740946 & -0.140144 \\ 6 & 6.064105 & -1.229397 & 0.521591\end{array}$




\begin{tabular}{|c|c|c|c|}
\hline 6 & 6.393106 & -2.615990 & 0.444082 \\
\hline 6 & 5.544290 & -3.490876 & -0.278435 \\
\hline D & 4.382366 & -3.029178 & -0.832208 \\
\hline & 6.888167 & -0.392888 & 1.316530 \\
\hline 6 & 7.985671 & -0.895159 & 1.972245 \\
\hline 5 & 8.327778 & -2.259196 & 1.863242 \\
\hline 6 & 7.542519 & -3.098805 & 1.114827 \\
\hline 6 & 4.559524 & 0.699570 & -0.210071 \\
\hline 6 & 3.327174 & 1.144591 & 0.228763 \\
\hline 6 & 2.964212 & 2.504700 & 0.213514 \\
\hline 6 & 3.827337 & 3.432611 & -0.301784 \\
\hline 6 & 5.071239 & 3.032074 & -0.845507 \\
\hline 6 & 5.444734 & 1.654287 & -0.809441 \\
\hline 6 & 5.935207 & 3.973462 & -1.456879 \\
\hline 6 & 7.113203 & 3.578763 & -2.037295 \\
\hline 6 & 7.470625 & 2.213852 & -2.033745 \\
\hline 6 & 6.660995 & 1.279236 & -1.436674 \\
\hline 8 & 2.407484 & 0.245830 & 0.720554 \\
\hline 7 & 1.052320 & -1.868482 & 0.683074 \\
\hline 6 & 0.058240 & -2.732756 & 0.107913 \\
\hline 6 & -1.248216 & -2.693613 & 0.560271 \\
\hline 6 & -2.235080 & -3.509776 & -0.073060 \\
\hline 6 & -1.846336 & -4.402303 & -1.116068 \\
\hline 6 & -0.488655 & -4.453404 & -1.508152 \\
\hline 6 & 0.434090 & -3.629257 & -0.920442 \\
\hline 6 & -2.824505 & -5.228534 & -1.722650 \\
\hline 6 & -4.136632 & -5.168516 & -1.328367 \\
\hline 6 & -4.529282 & -4.266165 & -0.315292 \\
\hline 6 & -3.602915 & -3.463284 & 0.301595 \\
\hline 6 & -1.606921 & -1.910735 & 1.773967 \\
\hline 6 & -1.876219 & -0.551904 & 1.738621 \\
\hline 6 & -2.247324 & 0.111640 & 2.940549 \\
\hline 6 & -2.337868 & -0.559895 & 4.130098 \\
\hline 6 & -2.056313 & -1.943156 & 4.202554 \\
\hline 6 & -1.686303 & -2.631928 & 3.010960 \\
\hline 6 & -1.392754 & -4.018233 & 3.106020 \\
\hline 6 & -1.462563 & -4.675730 & 4.309312 \\
\hline 6 & -1.834399 & -3.987965 & 5.483560 \\
\hline 6 & -2.124480 & -2.648915 & 5.427387 \\
\hline 15 & -1.851383 & 0.349813 & 0.109489 \\
\hline 6 & -1.954441 & 2.101405 & 0.653650 \\
\hline 6 & -0.963212 & 2.588304 & 1.510900 \\
\hline 6 & -0.938590 & 3.928960 & 1.891381 \\
\hline 6 & -1.897770 & 4.780839 & 1.331299 \\
\hline 6 & -2.880410 & 4.333751 & 0.446124 \\
\hline 6 & -2.903800 & 2.972466 & 0.126854 \\
\hline 6 & 0.089863 & 4.477904 & 2.879532 \\
\hline 6 & -3.904060 & 5.319478 & -0.115014 \\
\hline 6 & 1.892653 & -2.495592 & 1.732566 \\
\hline 6 & 2.353179 & -1.567291 & 2.823960 \\
\hline 6 & 3.705232 & -1.477638 & 3.147951 \\
\hline 6 & 4.137545 & -0.622549 & 4.158464 \\
\hline 6 & 3.216275 & 0.141748 & 4.866710 \\
\hline 6 & 1.860323 & 0.042648 & 4.559277 \\
\hline 6 & 1.434150 & -0.800586 & 3.541619 \\
\hline 6 & -3.570389 & -0.019022 & -0.443552 \\
\hline
\end{tabular}




\begin{tabular}{|c|c|c|c|}
\hline ) & -3.805886 & -0.437417 & -1.747802 \\
\hline & -5.099629 & -0.729799 & -2.193063 \\
\hline & -6.151055 & -0.598658 & -1.283769 \\
\hline & -5.947397 & -0.185639 & 0.038776 \\
\hline & -4.644310 & 0.102503 & 0.439445 \\
\hline & -5.299251 & -1.189523 & -3.635464 \\
\hline & -7.079000 & -0.042330 & 1.054872 \\
\hline & -0.346425 & -1.077380 & -3.224968 \\
\hline & 6.626432 & 0.657142 & 1.423385 \\
\hline 1 & -2.469977 & 1.175232 & 2.917584 \\
\hline 1 & 7.779953 & -4.158934 & 1.034451 \\
\hline 1 & -2.409000 & -2.101670 & 6.325062 \\
\hline 1 & 5.632698 & 5.019795 & -1.466821 \\
\hline 1 & -3.915373 & -2.766981 & 1.078990 \\
\hline 1 & -5.575975 & -4.203604 & -0.024137 \\
\hline 1 & 3.554023 & 4.486713 & -0.328984 \\
\hline 1 & -2.629740 & -0.033824 & 5.038348 \\
\hline 1 & 5.820933 & -4.540375 & -0.365359 \\
\hline 1 & 8.392673 & 1.896820 & -2.516020 \\
\hline 1 & 3.708379 & -3.685515 & -1.379598 \\
\hline 1 & -2.510254 & -5.911017 & -2.511078 \\
\hline 1 & -0.193146 & -5.145561 & -2.295321 \\
\hline 1 & -1.230927 & -5.737293 & 4.357573 \\
\hline 1 & 6.941024 & 0.229365 & -1.463342 \\
\hline 1 & -1.107599 & -4.565057 & 2.209498 \\
\hline 1 & 1.474469 & -3.639176 & -1.241703 \\
\hline 1 & 7.764852 & 4.309755 & -2.510285 \\
\hline 1 & 8.594123 & -0.234492 & 2.585938 \\
\hline 1 & -4.880013 & -5.806636 & -1.801415 \\
\hline 1 & -1.887025 & -4.521816 & 6.429541 \\
\hline 1 & 9.203175 & -2.642509 & 2.382222 \\
\hline 1 & 1.986953 & 2.780181 & 0.605074 \\
\hline 1 & -4.458783 & 0.430530 & 1.461675 \\
\hline 1 & -2.953888 & -0.537459 & -2.417520 \\
\hline 1 & -7.161280 & -0.825832 & -1.611939 \\
\hline 1 & -3.660715 & 2.580836 & -0.548453 \\
\hline 1 & -0.216733 & 1.892905 & 1.896756 \\
\hline 1 & -1.879547 & 5.836579 & 1.601825 \\
\hline 1 & 1.259959 & -3.278531 & 2.174648 \\
\hline 1 & -1.363459 & 1.110976 & -2.388536 \\
\hline 1 & 2.759827 & -3.008602 & 1.287774 \\
\hline 1 & 4.433475 & -2.067044 & 2.588757 \\
\hline 1 & 0.379259 & -0.851365 & 3.279421 \\
\hline 1 & 5.199874 & -0.553314 & 4.384801 \\
\hline 1 & 1.127351 & 0.627102 & 5.114063 \\
\hline 1 & 3.551006 & 0.810949 & 5.656690 \\
\hline 1 & 0.099249 & -2.024675 & -2.925907 \\
\hline 1 & -1.369675 & -1.165658 & -3.594238 \\
\hline 1 & 0.139281 & 0.616871 & -5.751739 \\
\hline 1 & -1.198875 & 1.010782 & -4.644764 \\
\hline 1 & 0.294933 & 1.957696 & -4.613228 \\
\hline 1 & 1.806632 & -2.317180 & -4.365493 \\
\hline 1 & 4.238052 & -2.614598 & -4.700395 \\
\hline 1 & 5.781918 & -0.680777 & -4.439355 \\
\hline 1 & 4.857977 & 1.554054 & -3.861105 \\
\hline 1 & 2.435101 & 1.839818 & -3.502172 \\
\hline
\end{tabular}




\begin{tabular}{|c|c|c|c|}
\hline 6 & -6.771234 & -1.391257 & -3.986132 \\
\hline 6 & -4.722183 & -0.140724 & -4.592808 \\
\hline & -4.572062 & -2.522899 & -3.839106 \\
\hline & -8.436420 & -0.435727 & 0.478332 \\
\hline & -6.794695 & -0.949275 & 2.258593 \\
\hline & -7.160884 & 1.417413 & 1.516981 \\
\hline & -6.859773 & -1.710406 & -5.03230 \\
\hline & -7.238756 & -2.168111 & -3.36741 \\
\hline & -7.350928 & -0.465696 & -3.87393 \\
\hline 1 & -4.678637 & -2.862804 & -4.87847 \\
\hline & -3.499263 & -2.446587 & -3.61834 \\
\hline 1 & -4.986468 & -3.297301 & -3.18051 \\
\hline & -4.863694 & -0.457905 & -5.63468 \\
\hline 1 & -5.220274 & 0.828904 & -4.462815 \\
\hline & -3.646950 & 0.013490 & -4.438120 \\
\hline 1 & -7.943956 & 1.535732 & 2.278194 \\
\hline 1 & -6.216271 & 1.768770 & \\
\hline 1 & -7.406458 & 2.078743 & 0.675627 \\
\hline 1 & -7.592100 & -0.851690 & \\
\hline 1 & -6.749952 & -2.002748 & 1.950761 \\
\hline 1 & -5.844686 & -0.706831 & 2.751975 \\
\hline 1 & -9.211028 & -0.321547 & 1.247235 \\
\hline 1 & -8.722100 & 0.197863 & -0.371087 \\
\hline 1 & -8.452826 & -1.482430 & 0.147518 \\
\hline 6 & -4.864609 & 4.657897 & -1.100936 \\
\hline 6 & -4.730659 & 5.899166 & \\
\hline 6 & -3.179039 & 6.453245 & -0.847460 \\
\hline 6 & 0.923865 & 3.365 & 3.513191 \\
\hline 6 & 1.026772 & 5.446464 & 2.150884 \\
\hline & -0.626040 & 5.222230 & 4.012452 \\
\hline 1 & -5.578319 & 5.402902 & -1.474773 \\
\hline 1 & -4.338226 & 4.239632 & -1.968508 \\
\hline 1 & -5.445831 & 3.852077 & -0.632929 \\
\hline 1 & -3.906654 & 7.159145 & -1.270230 \\
\hline 1 & -2.517294 & 7.021029 & -0.182673 \\
\hline 1 & -2.568213 & 6.060454 & -1.670559 \\
\hline 1 & -5.472300 & 6.613697 & 0.656171 \\
\hline 1 & -5.270029 & 5.104526 & 1.571151 \\
\hline 1 & -4.105629 & 6.427130 & 1.768854 \\
\hline 1 & 0.107134 & 5.608426 & 4.733041 \\
\hline & -1.209739 & 6.076641 & 3.650311 \\
\hline 1 & -1.309855 & 4.554078 & 4.552171 \\
\hline & 1.786429 & 5.838924 & 2.840427 \\
\hline & 1.546428 & 4.944439 & 1.323622 \\
\hline & 0.482446 & 6.300198 & 1.728744 \\
\hline & 1.604027 & 3.789567 & 4.263042 \\
\hline & 0.286009 & 2.627136 & 4.019377 \\
\hline & 1.544697 & 2.827297 & 2.78457 \\
\hline
\end{tabular}

$3_{\text {IA-si- }} \Psi-1 \mathbf{a 2 s 3 a 4 a}$

Number of imaginary frequencies : 0

The smallest frequencies are : $14.5151 \quad 22.2732 \quad 23.9589 \mathrm{~cm}(-1)$ Electronic energy :

Zero-point correction $=$ $\mathrm{HF}=-4360.8933265$

Thermal correction to Energy=

1.454469 (Hartree/Particle) 1.539043 
Thermal correction to Enthalpy= $\quad 1.539988$

Thermal correction to Gibbs Free Energy $=\quad 1.337682$

Sum of electronic and zero-point Energies $=\quad-4359.438858$

Sum of electronic and thermal Energies $=\quad-4359.354283$

Sum of electronic and thermal Enthalpies $=\quad-4359.353339$

Sum of electronic and thermal Free Energies $=\quad-4359.555645$

Cartesian Coordinates

$\begin{array}{cccc}6 & 2.803214 & 0.983051 & -3.759765 \\ 6 & 1.933942 & -0.105796 & -3.904514 \\ 6 & 2.479680 & -1.331943 & -4.296684 \\ 6 & 3.848893 & -1.475847 & -4.502381 \\ 6 & 4.701093 & -0.389984 & -4.332876 \\ 6 & 4.169073 & 0.845157 & -3.966670 \\ 6 & 0.460006 & 0.057147 & -3.712981 \\ 6 & -0.147038 & 1.046231 & -4.688794 \\ 45 & -0.128591 & 0.471504 & -1.623505 \\ 6 & 0.387853 & 2.328371 & -1.457365 \\ 8 & 0.509665 & 3.466004 & -1.636052 \\ 15 & 1.553879 & -0.664809 & -0.397794 \\ 8 & 2.815096 & -1.277268 & -1.302871 \\ 6 & 4.013970 & -1.691712 & -0.785556 \\ 6 & 4.883640 & -0.795009 & -0.192200 \\ 6 & 6.056647 & -1.316536 & 0.444182 \\ 6 & 6.368659 & -2.704006 & 0.322913 \\ 6 & 5.503505 & -3.547424 & -0.417367 \\ 6 & 4.342174 & -3.056222 & -0.946536 \\ 6 & 6.896652 & -0.513923 & 1.257249 \\ 6 & 7.992770 & -1.048376 & 1.889374 \\ 6 & 8.317843 & -2.412445 & 1.737191 \\ 6 & 7.517263 & -3.220011 & 0.970016 \\ 6 & 4.566741 & 0.650638 & -0.211027 \\ 6 & 3.343776 & 1.093483 & 0.255799 \\ 6 & 2.996585 & 2.457179 & 0.295834 \\ 6 & 3.867763 & 3.393883 & -0.189155 \\ 6 & 5.102653 & 2.999875 & -0.757929 \\ 6 & 5.458540 & 1.617114 & -0.780721 \\ 6 & 5.974898 & 3.954133 & -1.336797 \\ 6 & 7.143319 & 3.568136 & -1.942005 \\ 6 & 7.482439 & 2.199610 & -1.997351 \\ 6 & 6.664902 & 1.252114 & -1.432310 \\ 8 & 2.419310 & 0.188188 & 0.725271 \\ 7 & 1.046488 & -1.910305 & 0.641370 \\ 6 & 0.031098 & -2.741302 & 0.055515 \\ 6 & -1.271842 & -2.683086 & 0.516196 \\ 6 & -2.287266 & -3.433217 & -0.151563 \\ 6 & -1.933584 & -4.271328 & -1.250750 \\ 6 & -0.577045 & -4.363122 & -1.638529 \\ 6 & 0.376360 & -3.608694 & -1.007625 \\ 6 & -2.946967 & -5.000155 & -1.921219 \\ 6 & -4.258132 & -4.899895 & -1.531086 \\ 6 & -4.612898 & -4.062231 & -0.450060 \\ 6 & -3.652288 & -3.355889 & 0.228596 \\ 6 & -1.606664 & -1.930127 & 1.755150 \\ 6 & -1.863754 & -0.568679 & 1.755652\end{array}$




\begin{tabular}{|c|c|c|c|}
\hline & -2.219864 & 0.067651 & 2.976733 \\
\hline & -2.303717 & -0.632313 & 4.150294 \\
\hline & -2.033933 & -2.019551 & 4.185658 \\
\hline & -1.684410 & -2.682139 & 2.973137 \\
\hline & -1.410324 & -4.074446 & 3.028072 \\
\hline & -1.475666 & -4.762061 & 4.214692 \\
\hline & -1.823292 & -4.099633 & 5.410701 \\
\hline & -2.096671 & -2.755977 & 5.392675 \\
\hline & -1.842951 & 0.368554 & 0.146966 \\
\hline & -1.900322 & 2.109928 & 0.725951 \\
\hline & -0.913030 & 2.561714 & 1.613422 \\
\hline & -0.855059 & 3.896537 & 1.998430 \\
\hline & -1.772493 & 4.789402 & 1.418628 \\
\hline & -2.738962 & 4.378193 & 0.506328 \\
\hline & -2.797424 & 3.015407 & 0.180720 \\
\hline & 0.168078 & 4.411036 & 3.010256 \\
\hline & 1.141302 & 5.363523 & 2.307850 \\
\hline & -3.729448 & 5.333966 & -0.153316 \\
\hline & -3.516837 & 5.301374 & -1.671676 \\
\hline & 1.889236 & -2.568839 & 1.668648 \\
\hline & 2.366028 & -1.669330 & 2.777318 \\
\hline & 3.721388 & -1.596853 & 3.091545 \\
\hline & 4.168878 & -0.766258 & 4.115896 \\
\hline & 3.259761 & -0.008952 & 4.846997 \\
\hline & 1.900553 & -0.090747 & 4.548751 \\
\hline & 1.459270 & -0.910011 & 3.518181 \\
\hline & -3.577622 & 0.040545 & -0.390782 \\
\hline ) & -3.824524 & -0.422596 & -1.679558 \\
\hline & -5.125579 & -0.724703 & -2.098874 \\
\hline 6 & -6.159414 & -0.548277 & -1.179205 \\
\hline & -5.947655 & -0.087655 & 0.126302 \\
\hline 6 & -4.639845 & 0.207239 & 0.503307 \\
\hline 6 & -5.438181 & -1.189642 & -3.520734 \\
\hline 6 & -7.125047 & 0.031150 & 1.092583 \\
\hline 6 & -0.335373 & -1.013444 & -3.231556 \\
\hline 6 & -0.550213 & 5.162363 & 4.137091 \\
\hline 6 & 0.964723 & 3.273942 & 3.648130 \\
\hline 0 & -5.160462 & 4.891150 & 0.173979 \\
\hline 6 & -3.556334 & 6.774040 & 0.321538 \\
\hline 1 & 6.648161 & 0.535324 & 1.397745 \\
\hline & -2.434738 & 1.133166 & 2.982244 \\
\hline 1 & 7.741554 & -4.279932 & 0.856334 \\
\hline & -2.363759 & -2.228712 & 6.307519 \\
\hline 1 & 5.686506 & 5.003931 & -1.301587 \\
\hline & -3.936189 & -2.703552 & 1.052889 \\
\hline 1 & -5.658065 & -3.971112 & -0.159240 \\
\hline 1 & 3.608300 & 4.451786 & -0.172128 \\
\hline 1 & -2.582173 & -0.126486 & 5.074131 \\
\hline 1 & 5.766620 & -4.597151 & -0.537223 \\
\hline 1 & 8.396581 & 1.890642 & -2.499537 \\
\hline 1 & 3.654266 & -3.688392 & -1.505047 \\
\hline 1 & -2.660590 & -5.640364 & -2.754494 \\
\hline 1 & -0.307571 & -5.023472 & -2.461591 \\
\hline 1 & -1.259279 & -5.827771 & 4.232833 \\
\hline 1 & 6.930340 & 0.200420 & -1.503474 \\
\hline 1 & -1.144409 & -4.601093 & 2.113699 \\
\hline
\end{tabular}




\begin{tabular}{|c|c|c|c|}
\hline & 1.416841 & -3.643502 & -1.327456 \\
\hline 1 & 7.801402 & 4.309344 & -2.389526 \\
\hline & 8.613383 & -0.413497 & 2.517947 \\
\hline | & -5.028961 & -5.463529 & -2.052906 \\
\hline & -1.871508 & -4.656967 & 6.343288 \\
\hline 1 & 9.192257 & -2.821387 & 2.237949 \\
\hline ] & 2.025708 & 2.728550 & 0.706428 \\
\hline l & -4.433262 & 0.570448 & 1.507005 \\
\hline & -2.975122 & -0.553550 & -2.345260 \\
\hline 1 & -7.178402 & -0.780889 & -1.488326 \\
\hline 1 & -3.546659 & 2.660163 & -0.525850 \\
\hline & -0.197665 & 1.841100 & 2.011691 \\
\hline & -1.718875 & 5.839839 & 1.696370 \\
\hline 1 & 1.253571 & -3.356941 & 2.097056 \\
\hline 1 & -1.366715 & 1.162277 & -2.341032 \\
\hline ] & 2.749385 & -3.077549 & 1.205664 \\
\hline & 4.440223 & -2.180126 & 2.513947 \\
\hline 1 & 0.402006 & -0.946177 & 3.263625 \\
\hline & 5.233519 & -0.710251 & 4.334887 \\
\hline 1 & 1.176816 & 0.489091 & 5.120338 \\
\hline & 3.606413 & 0.641654 & 5.647320 \\
\hline l & 1.643822 & 3.676479 & 4.410781 \\
\hline$\frac{1}{1}$ & 0.301761 & 2.548230 & 4.140168 \\
\hline & 1.582805 & 2.726225 & 2.924452 \\
\hline 1 & 0.178775 & 5.523811 & 4.874621 \\
\hline & -1.105947 & 6.034021 & 3.772318 \\
\hline 1 & -1.259844 & 4.506154 & 4.657848 \\
\hline 1 & 1.899798 & 5.727838 & 3.013939 \\
\hline | & 1.661065 & 4.858158 & 1.482527 \\
\hline 1 & 0.626607 & 6.236632 & 1.888397 \\
\hline 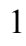 & -4.292685 & 7.417267 & -0.176660 \\
\hline 1 & -3.713638 & 6.871776 & 1.403653 \\
\hline 1 & -2.560660 & 7.167925 & 0.080450 \\
\hline 1 & -4.206115 & 5.997129 & -2.169299 \\
\hline 1 & -2.490579 & 5.594684 & -1.929215 \\
\hline 1 & -3.691089 & 4.303180 & -2.091450 \\
\hline 1 & -5.886521 & 5.556646 & -0.312189 \\
\hline 1 & -5.366362 & 3.868907 & -0.167868 \\
\hline 1 & -5.343472 & 4.923998 & 1.256245 \\
\hline 1 & 0.133559 & -1.956964 & -2.958938 \\
\hline 1 & -1.359718 & -1.114992 & -3.591289 \\
\hline 1 & 0.101163 & 0.750259 & -5.719838 \\
\hline 1 & -1.238715 & 1.084365 & -4.594567 \\
\hline 1 & 0.233883 & 2.065275 & -4.548281 \\
\hline 1 & 1.818628 & -2.186511 & -4.438853 \\
\hline 1 & 4.250953 & -2.443256 & -4.799480 \\
\hline 1 & 5.773342 & -0.499791 & -4.485177 \\
\hline 1 & 4.826634 & 1.702576 & -3.827585 \\
\hline 1 & 2.402672 & 1.946610 & -3.443956 \\
\hline 6 & -4.175390 & -1.580421 & -4.285582 \\
\hline 6 & -6.366945 & -2.407587 & -3.501961 \\
\hline 6 & -6.124682 & -0.040900 & -4.268638 \\
\hline 6 & -6.709564 & 0.619760 & 2.439234 \\
\hline 6 & -8.210441 & 0.932215 & 0.495147 \\
\hline 6 & -7.702099 & -1.367522 & 1.340644 \\
\hline 1 & -4.446747 & -1.952635 & -5.281593 \\
\hline
\end{tabular}




$\begin{array}{lrrr}1 & -3.500832 & -0.726461 & -4.432499 \\ 1 & -3.620474 & -2.376008 & -3.768248 \\ 1 & -6.356850 & -0.337471 & -5.300698 \\ 1 & -7.063920 & 0.253843 & -3.783672 \\ 1 & -5.476555 & 0.844495 & -4.307963 \\ 1 & -6.569327 & -2.740213 & -4.528671 \\ 1 & -5.904064 & -3.242771 & -2.959787 \\ 1 & -7.335505 & -2.194167 & -3.034739 \\ 1 & -8.555457 & -1.314900 & 2.030341 \\ 1 & -8.051486 & -1.837925 & 0.412987 \\ 1 & -6.946606 & -2.027127 & 1.788907 \\ 1 & -9.055240 & 1.020826 & 1.191215 \\ 1 & -7.824237 & 1.941758 & 0.304049 \\ 1 & -8.604622 & 0.540435 & -0.449972 \\ 1 & -7.585912 & 0.692446 & 3.095557 \\ 1 & -5.966735 & -0.005616 & 2.952607 \\ 1 & -6.291969 & 1.629809 & 2.332103\end{array}$

$3_{\text {IA-si- }} \psi_{-1 \mathbf{a} 2 s 3 a 4 s}$

Number of imaginary frequencies : 0

The smallest frequencies are : $15.676320 .364023 .4112 \mathrm{~cm}(-1)$

Electronic energy :

Zero-point correction=

$\mathrm{HF}=-4360.8936905$

Thermal correction to Energy=

Thermal correction to Enthalpy=

1.454658 (Hartree/Particle)

1.539167

1.540111

Thermal correction to Gibbs Free Energy=

Sum of electronic and zero-point Energies $=$

Sum of electronic and thermal Energies $=$

1.337713

$-4359.439033$

Sum of electronic and thermal Enthalpies=

$-4359.354524$

Sum of electronic and thermal Free Energies=

$-4359.353579$

$-4359.555978$

\section{Cartesian Coordinates}

$\begin{array}{cccc}6 & -4.626728 & 0.144870 & 0.478373 \\ 6 & -3.565993 & -0.005535 & -0.410595 \\ 6 & -3.812481 & -0.459170 & -1.706909 \\ 6 & -5.109763 & -0.757745 & -2.127178 \\ 6 & -6.152276 & -0.590026 & -1.206871 \\ 6 & -5.938809 & -0.147219 & 0.099005 \\ 15 & -1.839430 & 0.349878 & 0.133372 \\ 6 & -1.933384 & 2.092530 & 0.704145 \\ 6 & -0.951200 & 2.566594 & 1.585311 \\ 6 & -0.924777 & 3.900918 & 1.975217 \\ 6 & -1.867523 & 4.772351 & 1.403497 \\ 6 & -2.830559 & 4.339472 & 0.497324 \\ 6 & -2.857763 & 2.976352 & 0.168800 \\ 6 & 0.091100 & 4.435712 & 2.983821 \\ 6 & 0.902990 & 3.313033 & 3.628147 \\ 6 & -3.848098 & 5.272396 & -0.154267 \\ 6 & -3.729757 & 6.708241 & 0.349356 \\ 6 & -5.421656 & -1.215797 & -3.551223 \\ 6 & -6.333399 & -2.446805 & -3.536101 \\ 6 & -7.055771 & 0.004615 & 1.129258 \\ 6 & -7.113214 & 1.458338 & 1.611958 \\ 45 & -0.122542 & 0.471960 & -1.631900\end{array}$




\begin{tabular}{|c|c|c|c|}
\hline & -0.317163 & -1.006458 & -3.246613 \\
\hline & 0.477504 & 0.068574 & -3.719612 \\
\hline & -0.127309 & 1.059994 & -4.694300 \\
\hline & 1.952804 & -0.090473 & -3.903679 \\
\hline & 2.819536 & 0.998769 & -3.747385 \\
\hline & 4.187026 & 0.863506 & -3.945190 \\
\hline & 4.723165 & -0.369171 & -4.313628 \\
\hline & 3.873570 & -1.455098 & -4.495196 \\
\hline & 2.502778 & -1.313868 & -4.298553 \\
\hline & 0.377355 & 2.332829 & -1.457245 \\
\hline & 0.490545 & 3.471804 & -1.632832 \\
\hline & 1.563903 & -0.655137 & -0.403377 \\
\hline & 1.058466 & -1.901828 & 0.635244 \\
\hline & 1.896727 & -2.554042 & 1.670112 \\
\hline & 2.370172 & -1.648744 & 2.775562 \\
\hline & 3.723647 & -1.581924 & 3.099015 \\
\hline & 4.168768 & -0.746808 & 4.120803 \\
\hline & 3.259143 & 0.021604 & 4.839404 \\
\hline & 1.901723 & -0.053673 & 4.531393 \\
\hline & 1.462736 & -0.878477 & 3.504338 \\
\hline & 2.831811 & -1.261349 & -1.303081 \\
\hline & 4.028285 & -1.673114 & -0.777891 \\
\hline & 4.891934 & -0.774038 & -0.179329 \\
\hline & 6.062379 & -1.292374 & 0.464282 \\
\hline & 6.378111 & -2.679269 & 0.346023 \\
\hline & 5.519439 & -3.525200 & -0.398883 \\
\hline & 4.360404 & -3.036979 & -0.935838 \\
\hline ) & 7.523836 & -3.192314 & 1.000565 \\
\hline 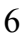 & 8.318209 & -2.382344 & 1.771640 \\
\hline ) & 7.989650 & -1.018702 & 1.920239 \\
\hline 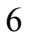 & 6.896149 & -0.487157 & 1.281176 \\
\hline 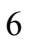 & 4.571814 & 0.670936 & -0.201686 \\
\hline & 3.344798 & 1.111481 & 0.256652 \\
\hline 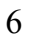 & 2.994415 & 2.474580 & 0.293419 \\
\hline ) & 3.866359 & 3.412585 & -0.187745 \\
\hline 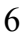 & 5.105464 & 3.020641 & -0.748677 \\
\hline f & 5.464809 & 1.638751 & -0.767158 \\
\hline & 6.676161 & 1.275789 & -1.410608 \\
\hline 5 & 7.494693 & 2.224385 & -1.972331 \\
\hline ) & 7.151785 & 3.592141 & -1.921405 \\
\hline 5 & 5.978794 & 3.976160 & -1.323837 \\
\hline & 2.420016 & 0.204528 & 0.721892 \\
\hline 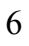 & 0.046349 & -2.736930 & 0.049520 \\
\hline 5 & -1.255205 & -2.690392 & 0.515855 \\
\hline 6 & -2.268482 & -3.445166 & -0.149523 \\
\hline 6 & -1.914001 & -4.273684 & -1.255728 \\
\hline 6 & -0.558878 & -4.352664 & -1.651071 \\
\hline 6 & 0.392605 & -3.595964 & -1.019887 \\
\hline 6 & -2.924958 & -5.006695 & -1.925305 \\
\hline ) & -4.234350 & -4.920787 & -1.526250 \\
\hline 6 & -4.589689 & -4.093204 & -0.437661 \\
\hline 6 & -3.632076 & -3.381143 & 0.239205 \\
\hline 6 & -1.592213 & -1.937877 & 1.753916 \\
\hline 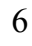 & -1.855935 & -0.577758 & 1.748423 \\
\hline U & -2.214230 & 0.061707 & 2.967099 \\
\hline j & -2.295166 & -0.634622 & 4.143319 \\
\hline
\end{tabular}




\begin{tabular}{|c|c|c|c|}
\hline 6 & -2.020711 & -2.020705 & 4.183855 \\
\hline 6 & -1.667338 & -2.685961 & 2.974015 \\
\hline & -1.388255 & -4.077059 & 3.033423 \\
\hline & -1.452489 & -4.761078 & 4.222126 \\
\hline & -1.803951 & -4.096037 & 5.415660 \\
\hline & -2.082136 & -2.753468 & 5.393195 \\
\hline 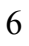 & 1.051102 & 5.396297 & 2.274556 \\
\hline & -0.635910 & 5.184235 & 4.106851 \\
\hline 6 & -3.618348 & 5.274666 & -1.670394 \\
\hline & -5.267179 & 4.775332 & 0.146514 \\
\hline & -4.156675 & -1.584619 & -4.323195 \\
\hline 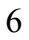 & -6.126912 & -0.072281 & -4.289583 \\
\hline & -8.424630 & -0.365105 & 0.564780 \\
\hline & -6.764989 & -0.919986 & 2.318211 \\
\hline & 6.644743 & 0.561747 & 1.419067 \\
\hline & -2.433166 & 1.126347 & 2.968984 \\
\hline & 7.750905 & -4.251903 & 0.889307 \\
\hline & -2.352326 & -2.224367 & 6.306057 \\
\hline & 5.687569 & 5.025285 & -1.292042 \\
\hline & -3.917874 & -2.734813 & 1.068266 \\
\hline & -5.634092 & -4.013881 & -0.141122 \\
\hline & 3.604916 & 4.469924 & -0.173204 \\
\hline & -2.575267 & -0.126453 & 5.065378 \\
\hline & 5.785644 & -4.574431 & -0.516234 \\
\hline & 8.412674 & 1.916881 & -2.468373 \\
\hline & 3.677703 & -3.671195 & -1.498357 \\
\hline & -2.638013 & -5.639075 & -2.764343 \\
\hline 1 & -0.288844 & -5.005806 & -2.479695 \\
\hline & -1.232438 & -5.825967 & 4.243995 \\
\hline 1 & 6.944778 & 0.224690 & -1.478228 \\
\hline & -1.119802 & -4.605340 & 2.120734 \\
\hline ] & 1.431785 & -3.621686 & -1.344770 \\
\hline & 7.810668 & 4.334250 & -2.366241 \\
\hline 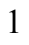 & 8.605401 & -0.381855 & 2.551599 \\
\hline 1 & -5.003490 & -5.488136 & -2.046498 \\
\hline 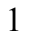 & -1.851354 & -4.650653 & 6.349906 \\
\hline 1 & 9.190439 & -2.789004 & 2.278035 \\
\hline & 2.020929 & 2.744651 & 0.698601 \\
\hline 1 & -4.428874 & 0.492651 & 1.491786 \\
\hline & -2.962273 & -0.581059 & -2.373143 \\
\hline 1 & -7.167246 & -0.816338 & -1.526619 \\
\hline 1 & -3.604674 & 2.604084 & -0.531323 \\
\hline & -0.216031 & 1.862354 & 1.976978 \\
\hline ] & -1.837991 & 5.823074 & 1.683831 \\
\hline & 1.258988 & -3.339221 & 2.100862 \\
\hline 1 & -1.365130 & 1.154565 & -2.350062 \\
\hline & 2.758127 & -3.066281 & 1.213294 \\
\hline 1 & 4.443340 & -2.173156 & 2.530572 \\
\hline ] & 0.407273 & -0.908817 & 3.241515 \\
\hline 1 & 5.232083 & -0.695740 & 4.347264 \\
\hline 1 & 1.177624 & 0.535781 & 5.092622 \\
\hline & 3.603997 & 0.676080 & 5.637314 \\
\hline 1 & 1.574553 & 3.728564 & 4.390434 \\
\hline & 0.249491 & 2.579511 & 4.121405 \\
\hline & 1.530581 & 2.771911 & 2.907836 \\
\hline & 0.089066 & 5.557820 & 4.842231 \\
\hline
\end{tabular}




$\begin{array}{lrrr}1 & -1.201724 & 6.047717 & 3.738292 \\ 1 & -1.337679 & 4.522474 & 4.631227 \\ 1 & 1.803740 & 5.777572 & 2.977963 \\ 1 & 1.578327 & 4.891001 & 1.453968 \\ 1 & 0.523782 & 6.257893 & 1.846959 \\ 1 & -4.484972 & 7.334582 & -0.142062 \\ 1 & -3.897625 & 6.778740 & 1.432048 \\ 1 & -2.747464 & 7.142485 & 0.123360 \\ 1 & -4.331643 & 5.948217 & -2.164777 \\ 1 & -2.603175 & 5.616177 & -1.911195 \\ 1 & -3.744324 & 4.276289 & -2.106853 \\ 1 & -6.008810 & 5.432796 & -0.326903 \\ 1 & -5.438490 & 3.758549 & -0.229596 \\ 1 & -5.461665 & 4.769057 & 1.227332 \\ 1 & 0.153084 & -1.949849 & -2.975875 \\ 1 & -1.339364 & -1.109100 & -3.612164 \\ 1 & 0.126255 & 0.768684 & -5.725372 \\ 1 & -1.219505 & 1.095120 & -4.604830 \\ 1 & 0.250709 & 2.079310 & -4.547986 \\ 1 & 1.843779 & -2.168408 & -4.450100 \\ 1 & 4.278962 & -2.420460 & -4.794412 \\ 1 & 5.796648 & -0.476924 & -4.458584 \\ 1 & 4.842613 & 1.720912 & -3.797059 \\ 1 & 2.415364 & 1.960086 & -3.429243 \\ 1 & -4.426314 & -1.949856 & -5.322261 \\ 1 & -3.491564 & -0.721940 & -4.462691 \\ 1 & -3.592367 & -2.379276 & -3.814689 \\ 1 & -6.533658 & -2.777727 & -4.563818 \\ 1 & -5.857587 & -3.277803 & -2.998491 \\ 1 & -7.303409 & -2.248574 & -3.065251 \\ 1 & -6.359461 & -0.366399 & -5.322260 \\ 1 & -7.068321 & 0.207191 & -3.799921 \\ 1 & -5.490796 & 0.821860 & -4.326750 \\ 1 & -7.895917 & 1.577391 & 2.373368 \\ 1 & -6.164822 & 1.785445 & 2.056418 \\ 1 & -7.344672 & 2.138394 & 0.781363 \\ 1 & -7.548387 & -0.820084 & 3.081733 \\ 1 & -6.738955 & -1.970579 & 1.998529 \\ 1 & -5.803120 & -0.694608 & 2.796852 \\ 1 & -9.189708 & -0.243868 & 1.342031 \\ 1 & -8.708406 & 0.277756 & -0.278441 \\ 1 & -8.460457 & -1.409648 & 0.228572\end{array}$

$3_{\text {IA-si }-} \psi-1 \mathbf{a} 253 s 4 a$

Number of imaginary frequencies : 0

The smallest frequencies are : $15.4734 \quad 21.603024 .3468 \mathrm{~cm}(-1)$

Electronic energy : $\quad H F=-4360.8933413$

Zero-point correction $=\quad 1.454311($ Hartree/Particle $)$

Thermal correction to Energy $=\quad 1.538952$

Thermal correction to Enthalpy= $\quad 1.539896$

Thermal correction to Gibbs Free Energy= $\quad 1.337314$

Sum of electronic and zero-point Energies $=\quad-4359.439030$

Sum of electronic and thermal Energies $=\quad-4359.354389$

Sum of electronic and thermal Enthalpies $=\quad-4359.353445$

Sum of electronic and thermal Free Energies $=\quad-4359.556027$ 
Cartesian Coordinates

$\begin{array}{cccc}\cdots & 2.801222 & 0.943108 & -3.737034 \\ 6 & 1.900171 & -0.115595 & -3.908013 \\ 6 & 2.409939 & -1.352166 & -4.315189 \\ 6 & 3.776181 & -1.537038 & -4.507393 \\ 6 & 4.660851 & -0.483173 & -4.305455 \\ 6 & 4.164330 & 0.763010 & -3.926630 \\ 6 & 0.431146 & 0.088211 & -3.721542 \\ 6 & -0.141270 & 1.103599 & -4.690721 \\ 45 & -0.146181 & 0.500794 & -1.630602 \\ 6 & 0.373987 & 2.353650 & -1.451723 \\ 8 & 0.494157 & 3.492641 & -1.622623 \\ 15 & 1.551932 & -0.633885 & -0.417180 \\ 8 & 2.820508 & -1.234989 & -1.320446 \\ 6 & 4.013556 & -1.655187 & -0.793474 \\ 6 & 4.879539 & -0.761605 & -0.190597 \\ 6 & 6.047117 & -1.285270 & 0.453514 \\ 6 & 6.357966 & -2.672949 & 0.332468 \\ 6 & 5.497232 & -3.513715 & -0.415915 \\ 6 & 4.341013 & -3.019993 & -0.953977 \\ 6 & 6.881968 & -0.484851 & 1.273978 \\ 6 & 7.972233 & -1.021882 & 1.913981 \\ 6 & 8.296166 & -2.386357 & 1.762701 \\ 6 & 7.500535 & -3.191675 & 0.988023 \\ 6 & 4.563914 & 0.684129 & -0.210087 \\ 6 & 3.337883 & 1.127603 & 0.248031 \\ 6 & 2.992412 & 2.491835 & 0.287611 \\ 6 & 3.865594 & 3.427366 & -0.196170 \\ 6 & 5.102032 & 3.031834 & -0.760596 \\ 6 & 5.459249 & 1.649383 & -0.775976 \\ 6 & 5.975034 & 3.984105 & -1.341517 \\ 6 & 7.146202 & 3.596517 & -1.940277 \\ 6 & 7.488236 & 2.228375 & -1.985764 \\ 6 & 6.669647 & 1.282641 & -1.419320 \\ 8 & 2.409213 & 0.222976 & 0.709955 \\ 7 & 1.050500 & -1.881538 & 0.620119 \\ 6 & 0.047765 & -2.725504 & 0.030971 \\ 6 & -1.254675 & -2.693850 & 0.495914 \\ 6 & -2.248852 & -3.491817 & -0.149743 \\ 6 & -1.870498 & -4.360762 & -1.216485 \\ 6 & -0.517371 & -4.401780 & -1.624872 \\ 6 & 0.411831 & -3.593400 & -1.025838 \\ 6 & -2.853676 & -5.175793 & -1.830021 \\ 6 & -4.161253 & -5.127934 & -1.419626 \\ 6 & -4.545032 & -4.245970 & -0.385496 \\ 6 & -3.613646 & -3.453093 & 0.237057 \\ 6 & -1.599319 & -1.946088 & 1.736480 \\ 6 & -1.865506 & -0.586025 & 1.748006 \\ 6 & -2.224738 & 0.039684 & 2.973680 \\ 6 & -2.302412 & -0.668249 & 4.142728 \\ 6 & -2.019113 & -2.052855 & 4.168873 \\ 6 & -1.665648 & -2.705028 & 2.951786 \\ 6 & -1.372330 & -4.093815 & 3.000766 \\ 6 & -1.425278 & -4.787439 & 4.184479 \\ & & & \\ 6 & & & \end{array}$




\begin{tabular}{|c|c|c|c|}
\hline 6 & -1.779534 & -4.135595 & 5.384253 \\
\hline 6 & -2.070141 & -2.795614 & 5.372653 \\
\hline & -1.850822 & 0.369101 & 0.151178 \\
\hline & -1.921853 & 2.105757 & 0.743371 \\
\hline & -0.933804 & 2.563710 & 1.626632 \\
\hline & -0.887198 & 3.897547 & 2.016946 \\
\hline & -1.819576 & 4.782817 & 1.449498 \\
\hline & -2.789810 & 4.365054 & 0.544299 \\
\hline & -2.833702 & 3.003836 & 0.210252 \\
\hline & 0.138614 & 4.419122 & 3.022297 \\
\hline & 1.095262 & 5.384822 & 2.315355 \\
\hline & -3.803965 & 5.310558 & -0.093866 \\
\hline & -3.615394 & 5.292991 & -1.615448 \\
\hline ) & 1.893828 & -2.535639 & 1.650386 \\
\hline & 2.361201 & -1.637826 & 2.764298 \\
\hline & 3.713993 & -1.567403 & 3.089992 \\
\hline ) & 4.153160 & -0.745733 & 4.125100 \\
\hline & 3.238034 & 0.004225 & 4.856177 \\
\hline ) & 1.881381 & -0.075010 & 4.545883 \\
\hline & 1.448595 & -0.884369 & 3.503897 \\
\hline 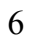 & -3.581954 & 0.038739 & -0.390701 \\
\hline ) & -3.822508 & -0.370309 & -1.692834 \\
\hline f & -5.122293 & -0.653702 & -2.138395 \\
\hline ) & -6.161951 & -0.529094 & -1.223178 \\
\hline ) & -5.954908 & -0.120111 & 0.105991 \\
\hline b & -4.654348 & 0.166161 & 0.503454 \\
\hline 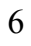 & -5.328051 & -1.084720 & -3.588163 \\
\hline f & -7.139029 & -0.041982 & 1.068501 \\
\hline ) & -0.396030 & -0.963397 & -3.249776 \\
\hline 6 & -0.577782 & 5.158006 & 4.158522 \\
\hline 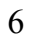 & 0.952512 & 3.287914 & 3.648339 \\
\hline 5 & -5.222566 & 4.842496 & 0.252107 \\
\hline 6 & -3.645953 & 6.749452 & 0.389657 \\
\hline 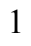 & 6.633872 & 0.564601 & 1.413837 \\
\hline 1 & -2.447365 & 1.103564 & 2.985517 \\
\hline . & 7.724118 & -4.251786 & 0.874703 \\
\hline 1 & -2.341337 & -2.275514 & 6.290381 \\
\hline & 5.685039 & 5.033660 & -1.313189 \\
\hline 1 & -3.919680 & -2.770309 & 1.028585 \\
\hline 1 & -5.588643 & -4.192842 & -0.081968 \\
\hline ] & 3.606806 & 4.485371 & -0.181382 \\
\hline 1 & -2.584024 & -0.170794 & 5.070126 \\
\hline ] & 5.759419 & -4.563756 & -0.535058 \\
\hline 1 & 8.405220 & 1.918222 & -2.481970 \\
\hline . & 3.657288 & -3.651292 & -1.518474 \\
\hline 1 & -2.546513 & -5.840617 & -2.636144 \\
\hline 1 & -0.230709 & -5.074205 & -2.432153 \\
\hline 1 & -1.193627 & -5.850011 & 4.197123 \\
\hline 1 & 6.937062 & 0.231006 & -1.483648 \\
\hline 1 & -1.100560 & -4.613709 & 2.084350 \\
\hline 1 & 1.448986 & -3.595569 & -1.357591 \\
\hline 1 & 7.804579 & 4.336197 & -2.389862 \\
\hline 1 & 8.589024 & -0.388825 & 2.548127 \\
\hline 1 & -4.908275 & -5.758593 & -1.896909 \\
\hline 1 & -1.818239 & -4.697702 & 6.314403 \\
\hline 1 & 9.165899 & -2.797365 & 2.269878 \\
\hline
\end{tabular}




\begin{tabular}{|c|c|c|c|}
\hline & 2.021040 & 2.764566 & 0.696142 \\
\hline & -4.456096 & 0.493253 & 1.521142 \\
\hline & -2.973425 & -0.471588 & -2.366065 \\
\hline & -7.177195 & -0.757433 & -1.541731 \\
\hline & -3.584663 & 2.644139 & -0.492149 \\
\hline & -0.208574 & 1.848701 & 2.017006 \\
\hline & -1.775824 & 5.832287 & 1.732593 \\
\hline & 1.261398 & -3.328421 & 2.075000 \\
\hline & -1.384192 & 1.205292 & -2.333935 \\
\hline & 2.758456 & -3.038746 & 1.190006 \\
\hline & 4.437458 & -2.146837 & 2.514414 \\
\hline & 0.393510 & -0.918105 & 3.240345 \\
\hline & 5.216076 & -0.691572 & 4.352868 \\
\hline & 1.152761 & 0.498605 & 5.117521 \\
\hline & 3.577886 & 0.646994 & 5.665684 \\
\hline & 1.633760 & 3.693861 & 4.407225 \\
\hline & 0.300760 & 2.553475 & 4.142244 \\
\hline & 1.569876 & 2.749774 & 2.916888 \\
\hline & 0.153836 & 5.524340 & 4.891019 \\
\hline & -1.146177 & 6.025079 & 3.802434 \\
\hline & -1.275553 & 4.492011 & 4.682848 \\
\hline & 1.856933 & 5.753359 & 3.015796 \\
\hline & 1.611608 & 4.888468 & 1.482501 \\
\hline & 0.567804 & 6.254616 & 1.904960 \\
\hline ] & -4.398336 & 7.385307 & -0.093840 \\
\hline & -3.790356 & 6.836746 & 1.474466 \\
\hline 1 & -2.659523 & 7.159984 & 0.138550 \\
\hline 1 & -4.327679 & 5.976909 & -2.096797 \\
\hline & -2.600419 & 5.611247 & -1.886828 \\
\hline l & -3.774198 & 4.294186 & -2.039970 \\
\hline & -5.966559 & 5.501228 & -0.215815 \\
\hline l & -5.417533 & 3.820464 & -0.096624 \\
\hline ] & -5.388594 & 4.861849 & 1.337437 \\
\hline | & 0.040144 & -1.924898 & -2.982877 \\
\hline l & -1.422287 & -1.026573 & -3.615491 \\
\hline & 0.094927 & 0.805072 & -5.723848 \\
\hline 1 & -1.230291 & 1.182983 & -4.595496 \\
\hline & 0.278436 & 2.106432 & -4.544257 \\
\hline 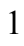 & 1.722757 & -2.181648 & -4.480366 \\
\hline ] & 4.150678 & -2.511538 & -4.817120 \\
\hline l & 5.731106 & -0.625926 & -4.443923 \\
\hline 1 & 4.847343 & 1.596492 & -3.765655 \\
\hline . & 2.428148 & 1.913962 & -3.409289 \\
\hline 6 & -6.800733 & -1.293469 & -3.930745 \\
\hline f & -4.769532 & -0.005434 & -4.522871 \\
\hline 6 & -4.588564 & -2.404347 & -3.831346 \\
\hline 6 & -6.736667 & 0.508438 & 2.435228 \\
\hline 6 & -8.230211 & 0.867184 & 0.494042 \\
\hline 6 & -7.705761 & -1.452171 & 1.270302 \\
\hline 1 & -6.895520 & -1.592857 & -4.982183 \\
\hline 1 & -7.255107 & -2.086727 & -3.322987 \\
\hline 1 & -7.387994 & -0.375796 & -3.795434 \\
\hline 1 & -4.696268 & -2.715371 & -4.879535 \\
\hline 1 & -3.515480 & -2.324829 & -3.613375 \\
\hline 1 & -4.993477 & -3.201011 & -3.194025 \\
\hline 1 & -4.914614 & -0.297914 & -5.571463 \\
\hline
\end{tabular}




$\begin{array}{rrrr}1 & -5.277932 & 0.954712 & -4.365112 \\ 1 & -3.694984 & 0.157676 & -4.371062 \\ 1 & -8.564177 & -1.426980 & 1.955258 \\ 1 & -8.044811 & -1.897413 & 0.326693 \\ 1 & -6.947716 & -2.118296 & 1.704145 \\ 1 & -9.078733 & 0.925653 & 1.188762 \\ 1 & -7.853406 & 1.885971 & 0.336808 \\ 1 & -8.616798 & 0.501795 & -0.464587 \\ 1 & -7.617617 & 0.553715 & 3.087890 \\ 1 & -5.991681 & -0.125972 & 2.934171 \\ 1 & -6.326910 & 1.524685 & 2.361320\end{array}$

$3_{\text {IA-si- }} \psi_{\text {-1a2s334s }}$

Number of imaginary frequencies : 0

The smallest frequencies are : $17.6747 \quad 21.0527 \quad 24.1054 \mathrm{~cm}(-1)$

Electronic energy : $\quad H F=-4360.8935158$

Zero-point correction $=\quad 1.454682($ Hartree/Particle)

Thermal correction to Energy $=\quad 1.539145$

Thermal correction to Enthalpy= $\quad 1.540089$

Thermal correction to Gibbs Free Energy= $\quad 1.338173$

Sum of electronic and zero-point Energies $=\quad-4359.438834$

Sum of electronic and thermal Energies $=\quad-4359.354371$

Sum of electronic and thermal Enthalpies $=\quad-4359.353427$

Sum of electronic and thermal Free Energies $=\quad-4359.555342$

\section{Cartesian Coordinates}

$\begin{array}{cccc}6 & 2.805481 & 0.990606 & -3.723043 \\ 6 & 1.914504 & -0.074391 & -3.906990 \\ 6 & 2.436796 & -1.302918 & -4.322411 \\ 6 & 3.805476 & -1.474197 & -4.509926 \\ 6 & 4.679961 & -0.414447 & -4.294949 \\ 6 & 4.170927 & 0.824091 & -3.907891 \\ 6 & 0.443161 & 0.115282 & -3.723934 \\ 6 & -0.134649 & 1.134154 & -4.686149 \\ 45 & -0.142311 & 0.505486 & -1.631282 \\ 6 & 0.360283 & 2.362254 & -1.435428 \\ 8 & 0.463962 & 3.504565 & -1.594541 \\ 15 & 1.559403 & -0.629015 & -0.423740 \\ 8 & 2.833403 & -1.216241 & -1.328389 \\ 6 & 4.026135 & -1.635018 & -0.799365 \\ 6 & 4.885774 & -0.741701 & -0.186990 \\ 6 & 6.053080 & -1.264769 & 0.458125 \\ 6 & 6.369896 & -2.650441 & 0.329489 \\ 6 & 5.515673 & -3.489883 & -0.427765 \\ 6 & 4.359710 & -2.997344 & -0.967495 \\ 6 & 6.881889 & -0.466258 & 1.286544 \\ 6 & 7.971728 & -1.003098 & 1.927429 \\ 6 & 8.301321 & -2.365433 & 1.769194 \\ 6 & 7.511817 & -3.168916 & 0.986402 \\ 6 & 4.564101 & 0.702913 & -0.198198 \\ 6 & 3.334359 & 1.138225 & 0.257884 \\ 6 & 2.983125 & 2.500854 & 0.305634 \\ 6 & 3.854737 & 3.443270 & -0.167553 \\ 6 & 5.095217 & 3.056669 & -0.729322\end{array}$




\begin{tabular}{|c|c|c|c|}
\hline 6 & 5.457868 & 1.675764 & -0.753408 \\
\hline 6 & 5.967119 & 4.016611 & -1.299170 \\
\hline f & 7.142368 & 3.638012 & -1.895705 \\
\hline 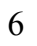 & 7.489821 & 2.271545 & -1.949996 \\
\hline$b$ & 6.672484 & 1.318507 & -1.394100 \\
\hline & 2.408231 & 0.226621 & 0.710716 \\
\hline 1 & 1.063276 & -1.886826 & 0.603947 \\
\hline 6 & 0.062120 & -2.730166 & 0.011187 \\
\hline b & -1.239528 & -2.705874 & 0.479035 \\
\hline 6 & -2.234493 & -3.497567 & -0.172262 \\
\hline 6 & -1.857589 & -4.355122 & -1.248592 \\
\hline 6 & -0.504722 & -4.392039 & -1.658497 \\
\hline 6 & 0.425163 & -3.588525 & -1.053588 \\
\hline 6 & -2.842398 & -5.161747 & -1.870634 \\
\hline 6 & -4.150112 & -5.114268 & -1.460567 \\
\hline 6 & -4.532080 & -4.242485 & -0.417065 \\
\hline 6 & -3.599085 & -3.459988 & 0.215799 \\
\hline 6 & -1.584703 & -1.967770 & 1.724561 \\
\hline 6 & -1.856168 & -0.609050 & 1.741681 \\
\hline 6 & -2.214875 & 0.010210 & 2.970670 \\
\hline 6 & -2.288972 & -0.703797 & 4.136547 \\
\hline 6 & -2.003368 & -2.087966 & 4.155807 \\
\hline 6 & -1.648697 & -2.733127 & 2.935464 \\
\hline 6 & -1.353102 & -4.121626 & 2.976679 \\
\hline 6 & -1.404883 & -4.821624 & 4.156650 \\
\hline 6 & -1.760034 & -4.176689 & 5.359974 \\
\hline 6 & -2.052885 & -2.837181 & 5.355681 \\
\hline 15 & -1.846467 & 0.347995 & 0.145788 \\
\hline 6 & -1.950726 & 2.081131 & 0.742649 \\
\hline 6 & -0.967760 & 2.554404 & 1.623197 \\
\hline 6 & -0.949320 & 3.885674 & 2.024394 \\
\hline 6 & -1.902767 & 4.754628 & 1.466705 \\
\hline 6 & -2.868585 & 4.321931 & 0.563528 \\
\hline 6 & -2.886384 & 2.962045 & 0.222382 \\
\hline 6 & 0.069956 & 4.420601 & 3.029431 \\
\hline 6 & 1.014648 & 5.396991 & 2.321188 \\
\hline 6 & -3.897197 & 5.251384 & -0.075013 \\
\hline 6 & -3.669801 & 5.272007 & -1.591502 \\
\hline 6 & 1.904923 & -2.540682 & 1.635565 \\
\hline 6 & 2.368111 & -1.643632 & 2.751950 \\
\hline 6 & 3.719360 & -1.576049 & 3.084435 \\
\hline 6 & 4.155286 & -0.754321 & 4.120949 \\
\hline 6 & 3.238445 & -0.000738 & 4.845992 \\
\hline 6 & 1.883235 & -0.076625 & 4.528507 \\
\hline 6 & 1.453632 & -0.886963 & 3.486062 \\
\hline 6 & -3.570439 & -0.002098 & -0.404944 \\
\hline 6 & -3.812805 & -0.387131 & -1.717973 \\
\hline 6 & -5.109425 & -0.662316 & -2.165528 \\
\hline 6 & -6.156089 & -0.553233 & -1.247992 \\
\hline 6 & -5.945494 & -0.175939 & 0.084428 \\
\hline 6 & -4.640073 & 0.101224 & 0.485937 \\
\hline 6 & -5.316542 & -1.073855 & -3.621368 \\
\hline 6 & -7.071835 & -0.068396 & 1.111163 \\
\hline 6 & -0.376517 & -0.947022 & -3.262936 \\
\hline 6 & -0.654335 & 5.151357 & 4.165818 \\
\hline 6 & 0.898027 & 3.299651 & 3.655612 \\
\hline
\end{tabular}




\begin{tabular}{|c|c|c|c|}
\hline 6 & -5.310485 & 4.736370 & 0.223098 \\
\hline 6 & -3.792073 & 6.682675 & 0.444223 \\
\hline 1 & 6.629353 & 0.581385 & 1.431897 \\
\hline 1 & -2.440111 & 1.073413 & 2.987982 \\
\hline 1 & 7.739861 & -4.227451 & 0.867381 \\
\hline 1 & -2.324941 & -2.322520 & 6.276209 \\
\hline 1 & 5.672966 & 5.064813 & -1.264175 \\
\hline 1 & -3.904759 & -2.784324 & 1.014060 \\
\hline 1 & -5.576095 & -4.187994 & -0.115111 \\
\hline 1 & 3.591947 & 4.500121 & -0.145702 \\
\hline 1 & -2.570051 & -0.211381 & 5.066798 \\
\hline 1 & 5.782666 & -4.538052 & -0.552575 \\
\hline 1 & 8.410173 & 1.968631 & -2.444444 \\
\hline 1 & 3.680983 & -3.627523 & -1.539210 \\
\hline 1 & -2.536833 & -5.818585 & -2.683884 \\
\hline 1 & -0.218829 & -5.056690 & -2.472469 \\
\hline 1 & -1.171753 & -5.883922 & 4.163661 \\
\hline 1 & 6.944337 & 0.268408 & -1.464608 \\
\hline 1 & -1.080969 & -4.636006 & 2.057259 \\
\hline 1 & 1.461925 & -3.586908 & -1.386732 \\
\hline 1 & 7.799882 & 4.383529 & -2.336828 \\
\hline 1 & 8.583741 & -0.371589 & 2.567718 \\
\hline 1 & -4.898751 & -5.737290 & -1.945320 \\
\hline 1 & -1.797767 & -4.743847 & 6.287089 \\
\hline 1 & 9.170580 & -2.776299 & 2.277298 \\
\hline 1 & 2.009187 & 2.767176 & 0.712249 \\
\hline 1 & -4.449218 & 0.402948 & 1.515368 \\
\hline 1 & -2.963922 & -0.473147 & -2.393463 \\
\hline 1 & -7.168325 & -0.768964 & -1.577653 \\
\hline 1 & -3.634096 & 2.590820 & -0.477330 \\
\hline 1 & -0.225083 & 1.852282 & 2.004380 \\
\hline 1 & -1.879355 & 5.803163 & 1.755726 \\
\hline 1 & 1.272747 & -3.335179 & 2.057406 \\
\hline 1 & -1.384998 & 1.205788 & -2.330988 \\
\hline 1 & 2.771556 & -3.041759 & 1.176659 \\
\hline 1 & 4.444554 & -2.157366 & 2.512886 \\
\hline 1 & 0.399988 & -0.917101 & 3.216376 \\
\hline 1 & 5.217127 & -0.702617 & 4.354209 \\
\hline 1 & 1.153250 & 0.500599 & 5.094795 \\
\hline 1 & 3.575828 & 0.642520 & 5.656120 \\
\hline 1 & 1.572909 & 3.713989 & 4.415594 \\
\hline 1 & 0.255572 & 2.555843 & 4.147800 \\
\hline 1 & 1.523478 & 2.771093 & 2.924137 \\
\hline 1 & 0.073438 & 5.525148 & 4.898334 \\
\hline 1 & -1.231839 & 6.012630 & 3.810405 \\
\hline 1 & -1.344945 & 4.477833 & 4.690008 \\
\hline 1 & 1.769674 & 5.778629 & 3.021828 \\
\hline 1 & 1.539524 & 4.904395 & 1.491389 \\
\hline 1 & 0.475763 & 6.257593 & 1.906231 \\
\hline 1 & -4.555506 & 7.306631 & -0.037433 \\
\hline 1 & -3.956547 & 6.739162 & 1.528274 \\
\hline 1 & -2.815214 & 7.129701 & 0.219619 \\
\hline 1 & -4.389014 & 5.945184 & -2.077763 \\
\hline 1 & -2.657402 & 5.623476 & -1.829942 \\
\hline 1 & -3.788387 & 4.277192 & -2.038318 \\
\hline 1 & -6.059842 & 5.392056 & -0.240499 \\
\hline
\end{tabular}




\begin{tabular}{|c|c|c|c|}
\hline & -5.472682 & 3.722485 & -0.1648 \\
\hline & -5.502310 & 4.714651 & 1.304213 \\
\hline & 0.067342 & -1.906828 & -3.002722 \\
\hline & -1.401373 & -1.016352 & -3.631533 \\
\hline & 0.106932 & 0.846362 & -5.721072 \\
\hline & -1.224493 & 1.204121 & -4.5934 \\
\hline & 0.276537 & 2.139044 & \\
\hline & 1.757619 & -2.136987 & -4.497392 \\
\hline & 4.189917 & -2.442779 & -4.825 \\
\hline & 5.752073 & -0.546734 & -4.429 \\
\hline & 4.846077 & 1.661865 & -3.736417 \\
\hline & 2.422349 & 1.955119 & -3.388274 \\
\hline & -6.789872 & -1.270203 & -3.96918 \\
\hline & -4.750582 & 0.011680 & \\
\hline & -4.584567 & -2.39 & \\
\hline & -8.434524 & -0.42 & \\
\hline & -6.786420 & -1.029342 & 2.2 \\
\hline & -7.143118 & 1.368760 & 1.64 \\
\hline & -6.883901 & -1.555586 & -5.02459 \\
\hline & -7.250245 & -2.068562 & -3.37284 \\
\hline & -7.372593 & -0.35 & -3.82 \\
\hline & & -2.6 & \\
\hline & -3.51 & -2.32 & -3.6 \\
\hline & -4.994421 & -3.196478 & -3.2 \\
\hline & -4.897948 & -0.268034 & -5.595990 \\
\hline & -5.252014 & 0.973660 & -4.37572 \\
\hline & -3.674912 & 0.165376 & $-4.39107 t$ \\
\hline & -7.930378 & 1.457821 & 2.40112 \\
\hline & -6.199021 & 1.689086 & 2.098630 \\
\hline & -7.375864 & 2.072963 & \\
\hline & -7.579642 & -0.961468 & 3.02890 \\
\hline & -6.747323 & -2.068484 & 1.918 \\
\hline & -5.832782 & -0.811889 & $2.77017^{\prime}$ \\
\hline & -9.205147 & -0.337941 & 1.300667 \\
\hline & -8.717910 & 0.242681 & -0.29735 \\
\hline & -8.4610 & -1.4596 & 0.152 \\
\hline
\end{tabular}

$3_{\text {IA-si }-\Psi-1 \text { s2a3a4a }}$

Number of imaginary frequencies : 0

The smallest frequencies are : $5.0087 \quad 16.0855 \quad 22.4735 \mathrm{~cm}(-1)$

Electronic energy : $\quad H F=-4360.8917771$

Zero-point correction $=\quad 1.453438($ Hartree/Particle $)$

Thermal correction to Energy= $\quad 1.538308$

Thermal correction to Enthalpy= $\quad 1.539253$

Thermal correction to Gibbs Free Energy= $\quad 1.334385$

Sum of electronic and zero-point Energies $=\quad-4359.438339$

Sum of electronic and thermal Energies $=\quad-4359.353469$

Sum of electronic and thermal Enthalpies $=\quad-4359.352525$

Sum of electronic and thermal Free Energies $=\quad-4359.557392$

Cartesian Coordinates

$\begin{array}{llll}6 & -3.824138 & -0.797187 & -1.611446 \\ 6 & -3.585118 & -0.046109 & -0.464007 \\ 6 & -4.658006 & 0.360477 & 0.334021\end{array}$




\begin{tabular}{|c|c|c|c|}
\hline 6 & -5.968896 & 0.032384 & -0.001944 \\
\hline 6 & -6.172067 & -0.726834 & -1.160449 \\
\hline 6 & -5.126608 & -1.153225 & -1.980049 \\
\hline 15 & -1.850040 & 0.341314 & 0.031434 \\
\hline 6 & -1.902946 & -0.313600 & 1.777074 \\
\hline 6 & -1.579130 & -1.635935 & 2.031335 \\
\hline 6 & -1.661182 & -2.158948 & 3.364955 \\
\hline 6 & -2.092380 & -1.312027 & 4.426398 \\
\hline 6 & -2.440180 & 0.025955 & 4.130329 \\
\hline 6 & -2.347770 & 0.506045 & 2.852257 \\
\hline 6 & -2.163055 & -1.821263 & 5.744593 \\
\hline 6 & -1.819300 & -3.121273 & 6.014785 \\
\hline 6 & -1.390306 & -3.965711 & 4.969676 \\
\hline 6 & -1.316039 & -3.499809 & 3.679953 \\
\hline 6 & -1.181659 & -2.591391 & 0.962590 \\
\hline 6 & 0.132939 & -2.686487 & 0.544223 \\
\hline 6 & 0.535554 & -3.737715 & -0.313453 \\
\hline 6 & -0.372954 & -4.644581 & -0.791533 \\
\hline 6 & -1.742012 & -4.529241 & -0.457654 \\
\hline 6 & -2.153060 & -3.499338 & 0.440217 \\
\hline 6 & -3.529730 & -3.399434 & 0.770245 \\
\hline 6 & -4.446970 & -4.266830 & 0.232580 \\
\hline 6 & -4.036122 & -5.290218 & -0.650359 \\
\hline 6 & -2.711960 & -5.417066 & -0.985559 \\
\hline 7 & 1.101295 & -1.710376 & 0.963370 \\
\hline 6 & 1.927923 & -2.100271 & 2.130744 \\
\hline 6 & 2.364570 & -0.962513 & 3.014111 \\
\hline 6 & 3.712929 & -0.794444 & 3.321502 \\
\hline 6 & 4.132359 & 0.259875 & 4.128637 \\
\hline 6 & 3.200577 & 1.151305 & \\
\hline 6 & 1.847278 & 0.976517 & 4.362733 \\
\hline 6 & 1.432760 & -0.070456 & 3.548916 \\
\hline 6 & -7.158108 & 0.439076 & 0.865833 \\
\hline 6 & -8.208687 & 1.164188 & 0.018815 \\
\hline 6 & -5.429966 & -1.951036 & -3.247540 \\
\hline 6 & -6.130201 & -1.029709 & -4.252773 \\
\hline 45 & -0.141351 & 0.115611 & -1.734750 \\
\hline 6 & -0.336911 & -1.671085 & -2.997864 \\
\hline 6 & 0.447753 & -0.717927 & -3.694685 \\
\hline 6 & 1.923741 & -0.904213 & -3.839654 \\
\hline 6 & 2.479828 & -2.181196 & -3.963025 \\
\hline 6 & 3.852771 & -2.355126 & -4.111809 \\
\hline 6 & 4.698399 & -1.251945 & -4.151040 \\
\hline 6 & 4.155909 & 0.028486 & -4.056481 \\
\hline 6 & 2.786018 & 0.197486 & -3.908941 \\
\hline 6 & 0.323311 & 1.978984 & -1.941916 \\
\hline 8 & 0.406870 & 3.066390 & -2.331708 \\
\hline 15 & 1.583569 & -0.694439 & -0.311822 \\
\hline 8 & 2.415435 & 0.404724 & 0.602368 \\
\hline 6 & 3.333412 & 1.200558 & -0.046704 \\
\hline 6 & 4.573233 & 0.690812 & -0.382508 \\
\hline 6 & 5.460003 & 1.524065 & -1.139739 \\
\hline 6 & 5.080545 & 2.869394 & -1.431959 \\
\hline 6 & 3.827106 & 3.353564 & -0.987057 \\
\hline 6 & 2.961028 & 2.529345 & -0.322196 \\
\hline 6 & 6.684471 & 1.047805 & -1.675592 \\
\hline
\end{tabular}




\begin{tabular}{|c|c|c|c|}
\hline 6 & 7.496553 & 1.861715 & -2.426280 \\
\hline 6 & 7.133948 & 3.200991 & -2.682377 \\
\hline & 5.948225 & 3.688269 & -2.195501 \\
\hline & 4.908638 & -0.710141 & -0.044482 \\
\hline & 4.061426 & -1.725013 & -0.449962 \\
\hline & 4.406313 & -3.087320 & -0.308789 \\
\hline & 5.560413 & -3.437022 & 0.336457 \\
\hline & 6.401841 & -2.442386 & 0.893637 \\
\hline & 6.074477 & -1.065596 & 0.707885 \\
\hline & 7.542830 & -2.791192 & 1.655931 \\
\hline & 8.321068 & -1.826344 & 2.243235 \\
\hline & 7.980863 & -0.465842 & 2.091227 \\
\hline & 6.891807 & -0.094952 & 1.340916 \\
\hline 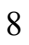 & 2.871219 & -1.442770 & -1.067993 \\
\hline & -1.888809 & 2.153536 & 0.336237 \\
\hline & -0.954990 & 2.715412 & 1.204628 \\
\hline b & -0.934377 & 4.092555 & 1.452551 \\
\hline & -1.822633 & 4.894048 & 0.734410 \\
\hline D & -2.737764 & 4.365882 & -0.185946 \\
\hline & -2.768417 & 2.983372 & -0.361451 \\
\hline & -0.049532 & 4.637416 & 2.574651 \\
\hline y & 1.403635 & 4.186359 & 2.408705 \\
\hline 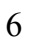 & -3.676854 & 5.303350 & -0.942616 \\
\hline ) & -2.845209 & 6.251286 & -1.813257 \\
\hline ) & -0.063952 & 6.162470 & 2.643946 \\
\hline 6 & -0.598984 & 4.091222 & 3.899737 \\
\hline 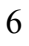 & -4.642750 & 4.544440 & -1.849993 \\
\hline & -4.508674 & 6.118321 & 0.053947 \\
\hline 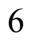 & -6.746382 & 1.371385 & 2.003946 \\
\hline 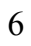 & -7.775816 & -0.824273 & 1.475917 \\
\hline & -4.161705 & -2.501091 & -3.895861 \\
\hline 6 & -6.342202 & -3.139506 & -2.927758 \\
\hline & 6.630911 & 0.956495 & 1.246494 \\
\hline & -2.619658 & 1.541149 & 2.660782 \\
\hline 1 & 7.779436 & -3.847582 & 1.777614 \\
\hline & -2.494528 & -1.155161 & 6.539993 \\
\hline ] & 5.641962 & 4.713432 & -2.399733 \\
\hline & -3.859258 & -2.607113 & 1.440599 \\
\hline 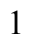 & -5.501059 & -4.158476 & 0.481580 \\
\hline 1 & 3.548278 & 4.382854 & -1.208866 \\
\hline . & -2.784089 & 0.672718 & 4.937008 \\
\hline 1 & 5.836536 & -4.484096 & 0.450110 \\
\hline & 8.424944 & 1.467205 & -2.833402 \\
\hline 1 & 3.738312 & -3.834646 & -0.732801 \\
\hline & -2.381934 & -6.200648 & -1.666184 \\
\hline 1 & -0.057994 & -5.448155 & -1.455759 \\
\hline l & -1.118266 & -4.996079 & 5.186937 \\
\hline 1 & 6.969246 & 0.012781 & -1.504833 \\
\hline 1 & -0.986999 & -4.166393 & 2.885293 \\
\hline 1 & 1.583772 & -3.794801 & -0.602220 \\
\hline 1 & 7.787888 & 3.837051 & -3.274450 \\
\hline I & 8.584219 & 0.297874 & 2.577027 \\
\hline 1 & -4.773556 & -5.975137 & -1.063978 \\
\hline 1 & -1.875318 & -3.503711 & 7.031281 \\
\hline 1 & 9.189726 & -2.105844 & 2.834795 \\
\hline 1 & 1.980108 & 2.866690 & 0.002995 \\
\hline
\end{tabular}




\begin{tabular}{|c|c|c|c|}
\hline 1 & -4.459044 & 0.947598 & 1.226314 \\
\hline & -2.969401 & -1.106504 & -2.207475 \\
\hline & -7.192641 & -0.993976 & -1.434738 \\
\hline & -3.479016 & 2.533029 & -1.050194 \\
\hline & -0.260444 & 2.060572 & 1.736336 \\
\hline & -1.816282 & 5.969483 & 0.899024 \\
\hline & 1.293561 & -2.783583 & 2.713857 \\
\hline & -1.397036 & 0.615848 & -2.567099 \\
\hline & 2.807426 & -2.682394 & 1.813221 \\
\hline & 4.449513 & -1.483086 & 2.904753 \\
\hline & 0.378858 & -0.185912 & 3.298415 \\
\hline 1 & 5.192359 & 0.384483 & 4.341596 \\
\hline 1 & 1.110002 & 1.665199 & 4.774703 \\
\hline & 3.523863 & 1.979740 & 5.275673 \\
\hline 1 & 0.141445 & -2.531783 & -2.535066 \\
\hline & -1.360297 & -1.854718 & -3.326058 \\
\hline & 1.823986 & -3.051166 & -3.937267 \\
\hline & 4.263148 & -3.360116 & -4.198139 \\
\hline & 5.773828 & -1.383177 & -4.256822 \\
\hline & 4.808764 & 0.900160 & -4.085242 \\
\hline & 2.376606 & 1.202625 & -3.803261 \\
\hline & -4.426481 & -3.094269 & -4.780337 \\
\hline & -3.489483 & -1.700160 & -4.231877 \\
\hline & -3.606930 & -3.154603 & -3.207348 \\
\hline & -6.355903 & -1.573289 & -5.180394 \\
\hline & -7.074879 & -0.637820 & -3.855203 \\
\hline & -5.494022 & -0.172238 & -4.508454 \\
\hline 1 & -6.546629 & -3.712871 & -3.841787 \\
\hline & -5.864014 & -3.813532 & -2.204587 \\
\hline 1 & -7.309833 & -2.830013 & -2.515393 \\
\hline & -9.059200 & 1.464057 & 0.645441 \\
\hline ] & -7.790197 & 2.070272 & -0.438202 \\
\hline & -8.603321 & 0.535050 & -0.787690 \\
\hline & -8.630991 & -0.565727 & 2.115138 \\
\hline 1 & -8.133111 & -1.517308 & 0.704019 \\
\hline & -7.040841 & -1.359245 & 2.092314 \\
\hline 1 & -7.632217 & 1.659145 & 2.584036 \\
\hline & -6.042961 & 0.892153 & 2.698070 \\
\hline 1 & -6.281821 & 2.293256 & 1.626998 \\
\hline & -5.307258 & 5.255505 & -2.356869 \\
\hline 1 & -4.116712 & 3.974956 & -2.626694 \\
\hline & -5.275512 & 3.846636 & -1.283852 \\
\hline & -3.501440 & 6.925136 & -2.380737 \\
\hline 1 & -2.168652 & 6.872366 & -1.213768 \\
\hline & -2.233527 & 5.687662 & -2.529829 \\
\hline 1 & -5.191131 & 6.791568 & -0.482049 \\
\hline & -5.114802 & 5.459848 & 0.690430 \\
\hline 1 & -3.884902 & 6.736868 & 0.710166 \\
\hline & 2.001243 & 4.518384 & 3.269132 \\
\hline 1 & 1.509382 & 3.095445 & 2.348457 \\
\hline 1 & 1.850192 & 4.623710 & 1.505738 \\
\hline & 0.001757 & 4.453087 & 4.745977 \\
\hline 1 & -1.637045 & 4.414693 & 4.056339 \\
\hline & -0.586315 & 2.993457 & 3.919763 \\
\hline & 0.603673 & 6.500457 & 3.446631 \\
\hline 1 & 0.286963 & 6.616631 & 1.708189 \\
\hline
\end{tabular}




$\begin{array}{rrrr}1 & -1.063653 & 6.557655 & 2.864857 \\ 6 & -0.165088 & 0.042539 & -4.853708 \\ 1 & 0.212126 & 1.069903 & -4.929672 \\ 1 & 0.082813 & -0.460822 & -5.801064 \\ 1 & -1.256784 & 0.095132 & -4.766824\end{array}$

$3_{\text {IA-si }-} \psi_{-1 \text {-1s2a3a4s }}$

Number of imaginary frequencies : 0

The smallest frequencies are : $9.8447 \quad 19.7332 \quad 23.6765 \mathrm{~cm}(-1)$

Electronic energy : $\quad H F=-4360.8922326$

Zero-point correction $=\quad 1.453917$ (Hartree/Particle)

Thermal correction to Energy= $\quad 1.538668$

Thermal correction to Enthalpy= $\quad 1.539612$

Thermal correction to Gibbs Free Energy $=1.335540$

Sum of electronic and zero-point Energies $=\quad-4359.438315$

Sum of electronic and thermal Energies $=\quad-4359.353565$

Sum of electronic and thermal Enthalpies $=\quad-4359.352621$

Sum of electronic and thermal Free Energies $=\quad-4359.556692$

Cartesian Coordinates

$\begin{array}{cccc}6 & 2.817483 & 0.663812 & -3.842265 \\ 6 & 1.982412 & -0.460340 & -3.881147 \\ 6 & 2.567120 & -1.702202 & -4.147014 \\ 6 & 3.941039 & -1.824447 & -4.333279 \\ 6 & 4.759033 & -0.701782 & -4.270076 \\ 6 & 4.188614 & 0.546954 & -4.028346 \\ 6 & 0.502452 & -0.326745 & -3.713334 \\ 6 & -0.129918 & 0.528613 & -4.793671 \\ 45 & -0.111295 & 0.286942 & -1.687276 \\ 6 & 0.334690 & 2.167172 & -1.689720 \\ 8 & 0.420291 & 3.289326 & -1.962597 \\ 15 & 1.605776 & -0.658882 & -0.347564 \\ 8 & 2.878353 & -1.370201 & -1.165676 \\ 6 & 4.061651 & -1.718352 & -0.565347 \\ 6 & 4.927663 & -0.752797 & -0.083570 \\ 6 & 6.080689 & -1.186407 & 0.647797 \\ 6 & 6.378605 & -2.579376 & 0.736449 \\ 6 & 5.520695 & -3.513409 & 0.105389 \\ 6 & 4.378277 & -3.093978 & -0.518947 \\ 6 & 6.914090 & -0.281381 & 1.353527 \\ 6 & 7.990161 & -0.727865 & 2.080828 \\ 6 & 8.301500 & -2.102560 & 2.136283 \\ 6 & 7.507251 & -3.005977 & 1.477364 \\ 6 & 4.622063 & 0.677688 & -0.308002 \\ 6 & 3.391200 & 1.179100 & 0.068267 \\ 6 & 3.044364 & 2.534325 & -0.080924 \\ 6 & 3.928911 & 3.399638 & -0.664326 \\ 6 & 5.175146 & 2.934009 & -1.148163 \\ 6 & 5.528219 & 1.560089 & -0.980995 \\ 6 & 6.062604 & 3.803470 & -1.828609 \\ 6 & 7.242725 & 3.341191 & -2.352259 \\ 6 & 7.580031 & 1.977656 & -2.218241 \\ 6 & 6.748583 & 1.113175 & -1.549869 \\ 8 & 2.461376 & 0.345731 & 0.645939\end{array}$




\begin{tabular}{|c|c|c|c|}
\hline 7 & 1.103894 & -1.760989 & 0.845849 \\
\hline 6 & 0.130451 & -2.695722 & 0.352463 \\
\hline f & -1.177530 & -2.647390 & 0.799931 \\
\hline f & -2.154572 & -3.509910 & 0.215468 \\
\hline 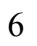 & -1.758324 & -4.440155 & -0.791108 \\
\hline 6 & -0.396947 & -4.510936 & -1.164480 \\
\hline 6 & 0.518853 & -3.655134 & -0.612164 \\
\hline 6 & -2.734053 & -5.279927 & -1.383330 \\
\hline 6 & -4.050098 & -5.201638 & -1.004876 \\
\hline 6 & -4.446567 & -4.277261 & -0.012876 \\
\hline 6 & -3.523368 & -3.458796 & 0.587420 \\
\hline 6 & -1.563880 & -1.776613 & 1.941783 \\
\hline 6 & -1.893008 & -0.440703 & 1.779303 \\
\hline 6 & -2.329568 & 0.302814 & 2.910966 \\
\hline 6 & -2.406047 & -0.263392 & 4.155233 \\
\hline 6 & -2.053076 & -1.617163 & 4.356860 \\
\hline 6 & -1.634035 & -2.389964 & 3.235723 \\
\hline 6 & -1.290273 & -3.750400 & 3.453014 \\
\hline 6 & -1.351792 & -4.303605 & 4.708304 \\
\hline 6 & -1.765903 & -3.531595 & 5.814033 \\
\hline 6 & -2.109693 & -2.215714 & 5.638005 \\
\hline 15 & -1.851986 & 0.316904 & 0.075607 \\
\hline 6 & -1.983003 & 2.107350 & 0.474637 \\
\hline 6 & -1.077280 & 2.670157 & \\
\hline 6 & -1.115308 & 4.034237 & 1.680194 \\
\hline 6 & -2.034224 & 4.828792 & 0.994084 \\
\hline 6 & -2.918996 & 4.304398 & 0.042094 \\
\hline 6 & -2.893636 & 2.930315 & -0.191426 \\
\hline 6 & -0.236709 & 4.568258 & \\
\hline 6 & -3.891010 & 5.235012 & -0.680954 \\
\hline 6 & 1.903839 & -2.224894 & 2.004418 \\
\hline 6 & 2.351831 & -1.136927 & 2.942963 \\
\hline 6 & 3.699568 & -1.009614 & 3.271633 \\
\hline 6 & 4.132588 & 0.011960 & 4.112883 \\
\hline 6 & 3.215590 & 0.913196 & 4.642490 \\
\hline 6 & 1.862399 & 0.778007 & 4.335319 \\
\hline 6 & 1.433917 & -0.238972 & 3.491663 \\
\hline 6 & -3.563934 & -0.111633 & -0.463170 \\
\hline 6 & -3.776059 & -0.744684 & -1.688881 \\
\hline 6 & -5.063922 & -1.086322 & -2.105391 \\
\hline 6 & -6.133457 & -0.772038 & -1.257087 \\
\hline 6 & -5.954223 & -0.151858 & -0.020424 \\
\hline 6 & -4.649786 & 0.170943 & 0.360215 \\
\hline 6 & -5.338890 & -1.751164 & -3.453421 \\
\hline 6 & -7.101879 & 0.175289 & 0.932089 \\
\hline 6 & -0.258679 & -1.367271 & -3.120742 \\
\hline 1 & 6.676401 & 0.779473 & 1.333323 \\
\hline 1 & -2.609067 & 1.346493 & 2.791681 \\
\hline 1 & 7.721244 & -4.073119 & 1.523418 \\
\hline 1 & -2.430749 & -1.605511 & 6.481112 \\
\hline 1 & 5.776686 & 4.848796 & -1.938408 \\
\hline 1 & -3.841859 & -2.743529 & 1.344220 \\
\hline 1 & -5.495136 & -4.206851 & 0.270595 \\
\hline 1 & 3.672363 & 4.450913 & -0.788701 \\
\hline 1 & -2.743103 & 0.326371 & 5.007205 \\
\hline 1 & 5.773896 & -4.571630 & 0.145067 \\
\hline
\end{tabular}




\begin{tabular}{|c|c|c|c|}
\hline & 8.504700 & 1.605684 & -2.654054 \\
\hline ] & 3.699001 & -3.797329 & -0.996414 \\
\hline & -2.414739 & -5.987747 & -2.146963 \\
\hline | & -0.092651 & -5.240476 & -1.913627 \\
\hline & -1.081383 & -5.347279 & 4.851500 \\
\hline & 7.014624 & 0.062052 & -1.471821 \\
\hline & -0.973703 & -4.360723 & 2.609561 \\
\hline ] & 1.561172 & -3.679193 & -0.924620 \\
\hline & 7.912007 & 4.016862 & -2.879788 \\
\hline & 8.605859 & -0.013706 & 2.623228 \\
\hline 1 & -4.791936 & -5.850505 & -1.466032 \\
\hline & -1.811068 & -3.982855 & 6.802451 \\
\hline & 9.160551 & -2.441904 & 2.710303 \\
\hline & 2.068566 & 2.856350 & 0.273366 \\
\hline & -4.479737 & 0.658991 & 1.319009 \\
\hline 1 & -2.907751 & -0.967123 & -2.303862 \\
\hline & -7.141593 & -1.025211 & -1.578530 \\
\hline 1 & -3.580910 & 2.483160 & -0.905531 \\
\hline 1 & -0.353400 & 2.023548 & 1.872484 \\
\hline 1 & -2.074195 & 5.895146 & 1.206311 \\
\hline & 1.246945 & -2.921285 & 2.545513 \\
\hline & -1.367792 & 0.854613 & -2.474996 \\
\hline 1 & 2.775399 & -2.809993 & 1.670073 \\
\hline & 4.426207 & -1.700315 & 2.840691 \\
\hline 1 & 0.381507 & -0.318583 & 3.221940 \\
\hline 1 & 5.192341 & 0.105998 & 4.342291 \\
\hline 1 & 1.136074 & 1.475091 & 4.753261 \\
\hline ] & 3.550660 & 1.717784 & 5.293881 \\
\hline & 0.243259 & -2.257708 & -2.747301 \\
\hline 1 & -1.275250 & -1.546410 & -3.471854 \\
\hline & 0.135576 & 0.130625 & -5.785336 \\
\hline 1 & -1.222945 & 0.540424 & -4.708031 \\
\hline 1 & 0.216977 & 1.568951 & -4.763251 \\
\hline 1 & 1.932646 & -2.586149 & -4.205654 \\
\hline 1 & 4.373447 & -2.803976 & -4.531464 \\
\hline 1 & 5.834771 & -0.793513 & -4.408875 \\
\hline 1 & 4.819802 & 1.433170 & -3.974047 \\
\hline 1 & 2.385327 & 1.641758 & -3.628212 \\
\hline 6 & -4.054699 & -2.219911 & -4.133312 \\
\hline 5 & -6.242900 & -2.974841 & -3.273509 \\
\hline 6 & -6.033313 & -0.737562 & -4.369908 \\
\hline 5 & -8.458256 & -0.260721 & 0.384571 \\
\hline 6 & -6.870025 & -0.551643 & 2.262485 \\
\hline 6 & -7.148186 & 1.689042 & 1.171355 \\
\hline 1 & -4.298887 & -2.726841 & -5.075505 \\
\hline 1 & -3.388495 & -1.382104 & -4.379222 \\
\hline 1 & -3.501345 & -2.930539 & -3.502588 \\
\hline 1 & -6.239881 & -1.185177 & -5.351728 \\
\hline 1 & -6.987727 & -0.396263 & -3.949571 \\
\hline 1 & -5.401563 & 0.146613 & -4.526383 \\
\hline 1 & -6.422488 & -3.455550 & -4.244406 \\
\hline 1 & -5.770743 & -3.712984 & -2.611600 \\
\hline 1 & -7.222011 & -2.718422 & -2.852251 \\
\hline 1 & -7.936267 & 1.938834 & 1.894610 \\
\hline 1 & -6.199536 & 2.078269 & 1.562968 \\
\hline 1 & -7.366880 & 2.223781 & 0.237704 \\
\hline
\end{tabular}




$\begin{array}{lrrr}1 & -7.686188 & -0.334406 & 2.964735 \\ 1 & -6.834912 & -1.639117 & 2.111164 \\ 1 & -5.929786 & -0.253842 & 2.743891 \\ 1 & -9.246390 & -0.003974 & 1.103694 \\ 1 & -8.700694 & 0.242065 & -0.560511 \\ 1 & -8.504457 & -1.345072 & 0.219504 \\ 6 & -4.752432 & 4.492239 & -1.699757 \\ 6 & -4.824942 & 5.892161 & 0.341306 \\ 6 & -3.101741 & 6.317119 & -1.425483 \\ 6 & -0.322852 & 6.085909 & 2.952289 \\ 6 & -0.722798 & 3.935579 & 4.122536 \\ 6 & 1.231204 & 4.195929 & 2.588165 \\ 1 & -5.434502 & 5.198600 & -2.189643 \\ 1 & -4.145466 & 4.020828 & -2.483336 \\ 1 & -5.367367 & 3.713188 & -1.229071 \\ 1 & -3.786504 & 6.981949 & -1.969213 \\ 1 & -2.507848 & 6.939295 & -0.745367 \\ 1 & -2.413705 & 5.868047 & -2.153498 \\ 1 & -5.528690 & 6.567670 & -0.163651 \\ 1 & -5.410944 & 5.135187 & 0.879717 \\ 1 & -4.275443 & 6.482048 & 1.084884 \\ 1 & 1.843421 & 4.551880 & 3.428282 \\ 1 & 1.387673 & 3.111891 & 2.515748 \\ 1 & 1.618633 & 4.661693 & 1.671758 \\ 1 & -0.113714 & 4.283641 & 4.968488 \\ 1 & -1.768659 & 4.204493 & 4.323657 \\ 1 & -0.661995 & 2.839479 & 4.090853 \\ 1 & 0.337301 & 6.418554 & 3.763287 \\ 1 & -0.005512 & 6.599226 & 2.035328 \\ 1 & -1.337767 & 6.421372 & 3.200877\end{array}$

$3_{\text {IA-si }-} \psi_{-1 \text { 1s2a3s4a }}$

Number of imaginary frequencies : 0

The smallest frequencies are : $16.004620 .423625 .9078 \mathrm{~cm}(-1)$

Electronic energy : $\quad \mathrm{HF}=-4360.8917396$

Zero-point correction $=\quad 1.454422($ Hartree/Particle)

Thermal correction to Energy= $\quad 1.538922$

Thermal correction to Enthalpy= $\quad 1.539866$

Thermal correction to Gibbs Free Energy $=\quad 1.337948$

Sum of electronic and zero-point Energies $=\quad-4359.437318$

Sum of electronic and thermal Energies $=\quad-4359.352818$

Sum of electronic and thermal Enthalpies $=\quad-4359.351873$

Sum of electronic and thermal Free Energies $=\quad-4359.553792$

Cartesian Coordinates

$\begin{array}{cccc}6 & -4.668978 & 0.247508 & 0.402603 \\ 6 & -3.588837 & -0.032449 & -0.446081 \\ 6 & -3.822179 & -0.638183 & -1.670944 \\ 6 & -5.122026 & -0.964894 & -2.086301 \\ 6 & -6.168903 & -0.683302 & -1.215913 \\ 6 & -5.969319 & -0.075733 & 0.035983 \\ 15 & -1.857700 & 0.348833 & 0.063453 \\ 6 & -1.915781 & 2.143069 & 0.453842 \\ 6 & -0.977531 & 2.675361 & 1.335868\end{array}$




\begin{tabular}{|c|c|c|c|}
\hline 6 & -0.963500 & 4.041333 & 1.638632 \\
\hline 5 & -1.863183 & 4.864777 & 0.960423 \\
\hline p & -2.784216 & 4.367838 & 0.028553 \\
\hline & -2.808745 & 2.993055 & -0.200766 \\
\hline f & -0.068170 & 4.547866 & 2.770173 \\
\hline 5 & 1.384336 & 4.107813 & 2.570986 \\
\hline 6 & -3.729840 & 5.329327 & -0.688721 \\
\hline 5 & -2.904028 & 6.295898 & -1.544348 \\
\hline 6 & -5.321506 & -1.608718 & -3.455906 \\
\hline 6 & -4.606545 & -2.963283 & -3.485181 \\
\hline 6 & -7.165492 & 0.186899 & 0.948561 \\
\hline 6 & -8.194627 & 1.059617 & 0.222603 \\
\hline 45 & -0.151132 & 0.240646 & -1.728068 \\
\hline 6 & -0.383542 & -1.452606 & -3.104295 \\
\hline 6 & 0.439139 & -0.479205 & -3.728290 \\
\hline 6 & -0.133958 & 0.371808 & -4.844019 \\
\hline 6 & 1.910669 & -0.697764 & -3.870851 \\
\hline 6 & 2.801189 & 0.383892 & -3.879273 \\
\hline 6 & 4.166793 & 0.188296 & -4.033196 \\
\hline 6 & 4.676797 & -1.099335 & -4.191523 \\
\hline 6 & 3.803483 & -2.181362 & -4.210147 \\
\hline 6 & 2.434675 & -1.980567 & -4.056835 \\
\hline 6 & 0.319838 & 2.111118 & -1.814317 \\
\hline 8 & 0.403817 & 3.220787 & -2.135024 \\
\hline 15 & 1.576821 & -0.656887 & -0.354581 \\
\hline 7 & 1.084681 & -1.732128 & 0.865871 \\
\hline 6 & 1.898583 & -2.180457 & 2.021191 \\
\hline 6 & 2.342414 & -1.087561 & 2.955880 \\
\hline 6 & 3.690152 & -0.952305 & 3.281584 \\
\hline 6 & 4.117078 & 0.059802 & 4.137360 \\
\hline 6 & 3.193660 & 0.941902 & 4.687194 \\
\hline 6 & 1.840718 & 0.798960 & 4.382716 \\
\hline 6 & 1.418672 & -0.206338 & 3.521706 \\
\hline 8 & 2.864465 & -1.371789 & -1.142675 \\
\hline 6 & 4.046858 & -1.698572 & -0.530819 \\
\hline 6 & 4.899788 & -0.715605 & -0.063379 \\
\hline 6 & 6.055763 & -1.122147 & 0.678008 \\
\hline 6 & 6.368417 & -2.509970 & 0.792863 \\
\hline 6 & 5.523732 & -3.464738 & 0.174453 \\
\hline 6 & 4.379184 & -3.069650 & -0.461606 \\
\hline 6 & 7.499311 & -2.910209 & 1.544865 \\
\hline 6 & 8.282255 & -1.985670 & 2.188005 \\
\hline 6 & 7.957366 & -0.615502 & 2.104518 \\
\hline 6 & 6.878079 & -0.194505 & 1.366716 \\
\hline 6 & 4.582285 & 0.703904 & -0.333425 \\
\hline 6 & 3.342998 & 1.208537 & 0.011691 \\
\hline 6 & 2.984699 & 2.551649 & -0.206159 \\
\hline 6 & 3.862837 & 3.396777 & -0.827419 \\
\hline 6 & 5.116708 & 2.922210 & -1.281192 \\
\hline 6 & 5.483938 & 1.562834 & -1.042640 \\
\hline 6 & 6.713072 & 1.102368 & -1.581631 \\
\hline 6 & 7.538683 & 1.942101 & -2.287887 \\
\hline 6 & 7.186186 & 3.292718 & -2.493417 \\
\hline 6 & 5.997753 & 3.766659 & -2.000160 \\
\hline 8 & 2.411445 & 0.392608 & 0.614425 \\
\hline 6 & 0.121134 & -2.687535 & 0.391343 \\
\hline
\end{tabular}




\begin{tabular}{|c|c|c|c|}
\hline 6 & -1.191927 & -2.627827 & 0.821186 \\
\hline & -2.154331 & -3.526311 & 0.266541 \\
\hline & -1.732448 & -4.525628 & -0.660168 \\
\hline & -0.367203 & -4.596079 & -1.020897 \\
\hline & 0.529676 & -3.688901 & -0.521453 \\
\hline & -2.686678 & -5.432969 & -1.183835 \\
\hline & -4.007049 & -5.349132 & -0.823208 \\
\hline & -4.433302 & -4.341081 & 0.069817 \\
\hline & -3.530680 & -3.457591 & 0.606110 \\
\hline & -1.585610 & -1.734869 & 1.944376 \\
\hline & -1.898650 & -0.397062 & 1.769974 \\
\hline & -2.328058 & 0.363779 & 2.893161 \\
\hline & -2.416502 & -0.188689 & 4.142251 \\
\hline & -2.078136 & -1.544216 & 4.358151 \\
\hline & -1.662196 & -2.332697 & 3.246575 \\
\hline & -1.323657 & -3.691582 & 3.482661 \\
\hline & -1.391268 & -4.229116 & 4.744440 \\
\hline & -1.806289 & -3.442625 & 5.839254 \\
\hline & -2.142458 & -2.127229 & 5.646071 \\
\hline & -0.086016 & 6.069363 & 2.895099 \\
\hline & -0.601012 & 3.952116 & 4.080501 \\
\hline & -4.711371 & 4.599407 & -1.603444 \\
\hline & -4.544714 & 6.122272 & 0.339068 \\
\hline & -6.794389 & -1.840379 & -3.782947 \\
\hline & -4.732326 & -0.694136 & -4.536085 \\
\hline & -6.762587 & 0.904390 & 2.235420 \\
\hline & -7.806532 & -1.152646 & 1.328031 \\
\hline & 6.629033 & 0.863220 & 1.324559 \\
\hline & -2.592640 & 1.410112 & 2.762088 \\
\hline 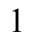 & 7.724581 & -3.973904 & 1.612092 \\
\hline & -2.462093 & -1.504832 & 6.480752 \\
\hline & 5.699964 & 4.801433 & -2.164587 \\
\hline ] & -3.869135 & -2.680881 & 1.290160 \\
\hline & -5.486573 & -4.258677 & 0.330502 \\
\hline 1 & 3.594394 & 4.437177 & -1.006016 \\
\hline & -2.749232 & 0.413340 & 4.987277 \\
\hline 1 & 5.790014 & -4.518912 & 0.232131 \\
\hline & 8.470345 & 1.559571 & -2.698939 \\
\hline 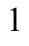 & 3.711446 & -3.786926 & -0.934931 \\
\hline 1 & -2.346678 & -6.197021 & -1.881570 \\
\hline & -0.044678 & -5.369396 & -1.716660 \\
\hline 1 & -1.124369 & -5.271780 & 4.900944 \\
\hline & 6.991728 & 0.060152 & -1.449383 \\
\hline 1 & -1.004531 & -4.314164 & 2.649244 \\
\hline & 1.574110 & -3.713854 & -0.826918 \\
\hline 1 & 7.850537 & 3.948642 & -3.051211 \\
\hline 1 & 8.564963 & 0.115433 & 2.633550 \\
\hline 1 & -4.731610 & -6.049767 & -1.232746 \\
\hline 1 & -1.856941 & -3.881672 & 6.832894 \\
\hline 1 & 9.143285 & -2.304724 & 2.770630 \\
\hline 1 & 2.004122 & 2.882191 & 0.126097 \\
\hline 1 & -4.477735 & 0.725446 & 1.359631 \\
\hline 1 & -2.967871 & -0.859446 & -2.307706 \\
\hline 1 & -7.185346 & -0.937663 & -1.509643 \\
\hline 1 & -3.522597 & 2.565278 & -0.900656 \\
\hline & -0.270703 & 2.003985 & 1.829532 \\
\hline
\end{tabular}




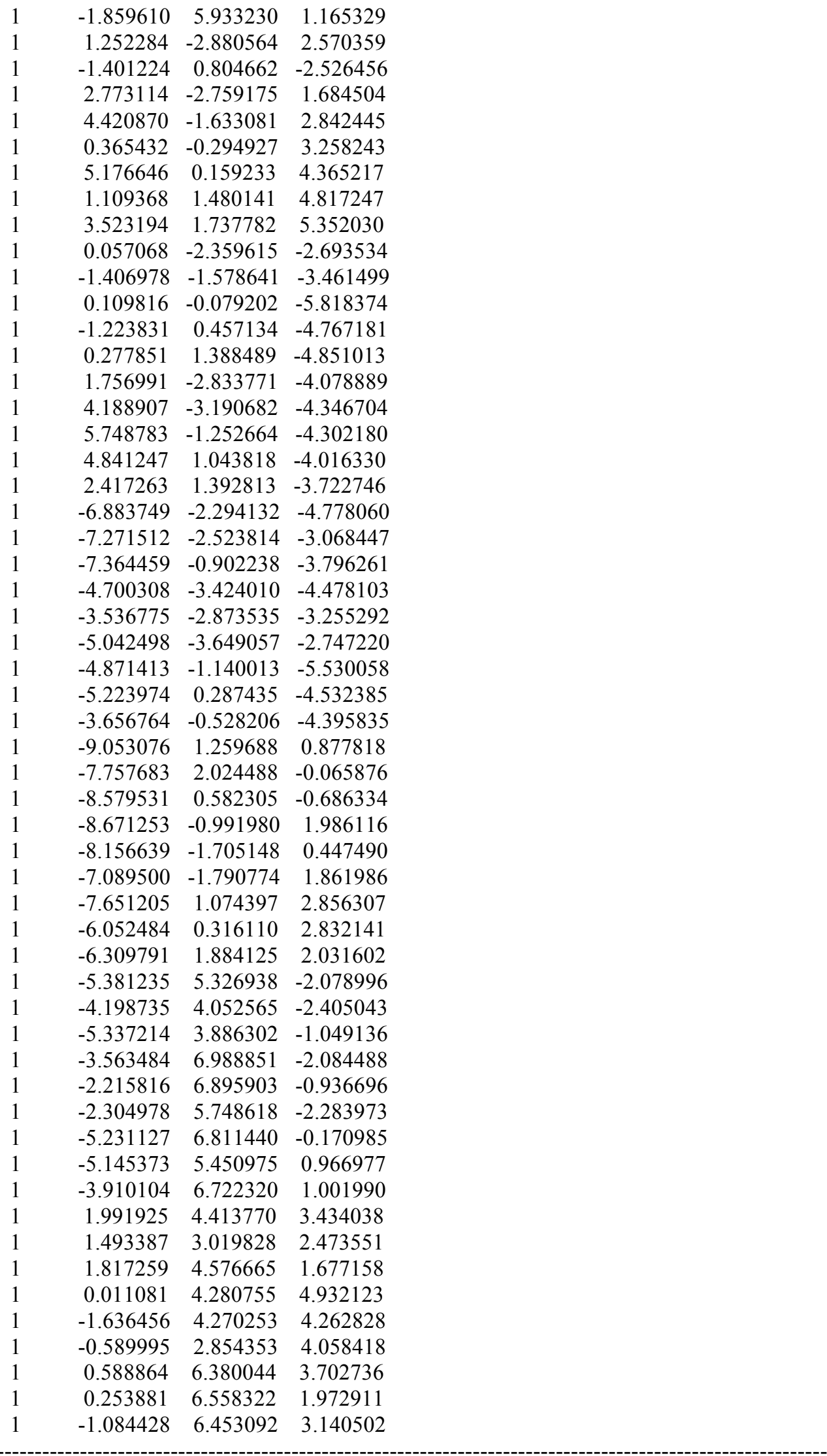


$3_{\text {IA-si- }} \psi_{-1 \mathrm{~s} 2 \mathrm{a} 3 \mathrm{~s} 4 \mathrm{~s}}$

Number of imaginary frequencies : 0

The smallest frequencies are : $11.0711 \quad 13.1220 \quad 21.8658 \mathrm{~cm}(-1)$

Electronic energy : $\quad H F=-4360.8920345$

Zero-point correction $=\quad 1.453980($ Hartree/Particle $)$

Thermal correction to Energy= $\quad 1.538736$

Thermal correction to Enthalpy= $\quad 1.539680$

Thermal correction to Gibbs Free Energy $=\quad 1.334972$

Sum of electronic and zero-point Energies $=\quad-4359.438054$

Sum of electronic and thermal Energies $=\quad-4359.353299$

Sum of electronic and thermal Enthalpies $=\quad-4359.352355$

Sum of electronic and thermal Free Energies $=\quad-4359.557063$

Cartesian Coordinates

$\begin{array}{cccc}6 & 2.813116 & 0.415724 & -3.872696 \\ 6 & 1.931147 & -0.672894 & -3.872898 \\ 6 & 2.466236 & -1.950694 & -4.062058 \\ 6 & 3.837354 & -2.140203 & -4.208954 \\ 6 & 4.702041 & -1.051525 & -4.180758 \\ 6 & 4.181020 & 0.231347 & -4.020031 \\ 6 & 0.457417 & -0.466638 & -3.734568 \\ 6 & -0.119444 & 0.384922 & -4.847852 \\ 45 & -0.144598 & 0.239468 & -1.733058 \\ 6 & 0.306734 & 2.115341 & -1.809817 \\ 8 & 0.377943 & 3.227688 & -2.124223 \\ 15 & 1.587850 & -0.647276 & -0.359682 \\ 8 & 2.881551 & -1.352367 & -1.146422 \\ 6 & 4.062391 & -1.675128 & -0.529243 \\ 6 & 4.909485 & -0.688939 & -0.057698 \\ 6 & 6.063786 & -1.091588 & 0.688410 \\ 6 & 6.380580 & -2.478411 & 0.804641 \\ 6 & 5.541770 & -3.436192 & 0.182975 \\ 6 & 4.398723 & -3.045076 & -0.458210 \\ 6 & 6.880383 & -0.161263 & 1.380300 \\ 6 & 7.957967 & -0.578702 & 2.122592 \\ 6 & 8.286821 & -1.947828 & 2.207786 \\ 6 & 7.509567 & -2.874934 & 1.561494 \\ 6 & 4.586781 & 0.729609 & -0.327797 \\ 6 & 3.343565 & 1.227890 & 0.012418 \\ 6 & 2.979348 & 2.569407 & -0.205391 \\ 6 & 3.855822 & 3.419617 & -0.822034 \\ 6 & 5.114066 & 2.951768 & -1.270707 \\ 6 & 5.487100 & 1.593910 & -1.032152 \\ 6 & 5.994311 & 3.801542 & -1.984382 \\ 6 & 7.187717 & 3.334368 & -2.472059 \\ 6 & 7.546485 & 1.985506 & -2.265756 \\ 6 & 6.721793 & 1.140712 & -1.564488 \\ 8 & 2.413613 & 0.407368 & 0.611017 \\ 7 & 1.100948 & -1.725817 & 0.859820 \\ 6 & 0.143017 & -2.687082 & 0.385990 \\ 6 & -1.167930 & -2.641283 & 0.824087 \\ 6 & -2.126292 & -3.545491 & 0.272414 \\ 6 & -1.703039 & -4.532751 & -0.666458 \\ 6 & -0.339951 & -4.588367 & -1.037734 \\ & & & \\ 6\end{array}$




\begin{tabular}{|c|c|c|c|}
\hline 6 & 0.553481 & -3.678721 & -0.536370 \\
\hline 6 & -2.653510 & -5.443498 & -1.191035 \\
\hline 6 & -3.971393 & -5.375147 & -0.818266 \\
\hline 6 & -4.398487 & -4.381136 & 0.090014 \\
\hline 6 & -3.499884 & -3.493616 & 0.626244 \\
\hline 6 & -1.565228 & -1.749715 & 1.946445 \\
\hline 6 & -1.891100 & -0.415917 & 1.765257 \\
\hline 6 & -2.324729 & 0.346383 & 2.885704 \\
\hline 6 & -2.404791 & -0.200830 & 4.137923 \\
\hline 6 & -2.054776 & -1.552331 & 4.359737 \\
\hline 6 & -1.634540 & -2.342371 & 3.250977 \\
\hline 6 & -1.285579 & -3.697730 & 3.491893 \\
\hline 6 & -1.346515 & -4.230242 & 4.756096 \\
\hline 6 & -1.764955 & -3.441989 & 5.848437 \\
\hline 6 & -2.111750 & -2.130106 & 5.650379 \\
\hline 15 & -1.856489 & 0.320242 & 0.053676 \\
\hline 6 & -1.952927 & 2.114880 & 0.436687 \\
\hline 6 & -1.021305 & 2.669503 & 1.311730 \\
\hline 6 & -1.040402 & 4.034571 & 1.617991 \\
\hline 6 & -1.965430 & 4.836315 & 0.947863 \\
\hline 6 & -2.876553 & 4.318433 & 0.017542 \\
\hline 6 & -2.870665 & 2.943413 & -0.211350 \\
\hline 6 & -0.148413 & 4.560996 & 2.742881 \\
\hline 6 & -3.859096 & 5.254986 & -0.683014 \\
\hline 6 & 1.911568 & -2.164645 & 2.021127 \\
\hline 6 & 2.349005 & -1.065641 & \\
\hline 6 & 3.694761 & -0.928627 & 3.284996 \\
\hline 6 & 4.116845 & 0.088758 & 4.136942 \\
\hline 6 & 3.190573 & 0.975213 & 4.674753 \\
\hline 6 & 1.839550 & 0.831075 & 4.362441 \\
\hline 6 & 1.422221 & -0.180184 & 3.506157 \\
\hline 6 & -3.580199 & -0.086285 & -0.460662 \\
\hline 6 & -3.814013 & -0.678876 & -1.696179 \\
\hline 6 & -5.110925 & -0.996070 & -2.114891 \\
\hline 6 & -6.166120 & -0.719323 & -1.244069 \\
\hline 6 & -5.964719 & -0.132633 & 0.011432 \\
\hline 6 & -4.659058 & 0.180152 & 0.383650 \\
\hline 6 & -5.311039 & -1.636586 & -3.486590 \\
\hline 6 & -7.101557 & 0.174000 & 0.984675 \\
\hline 6 & -0.358634 & -1.449646 & -3.117082 \\
\hline 1 & 6.628107 & 0.895649 & 1.337003 \\
\hline 1 & -2.598431 & 1.389723 & 2.750467 \\
\hline 1 & 7.737909 & -3.937903 & 1.629828 \\
\hline 1 & -2.434593 & -1.506652 & 6.483023 \\
\hline 1 & 5.692024 & 4.834954 & -2.149168 \\
\hline 1 & -3.839204 & -2.728180 & 1.322771 \\
\hline 1 & -5.449615 & -4.314429 & 0.363503 \\
\hline 1 & 3.583208 & 4.458988 & -1.000337 \\
\hline 1 & -2.740160 & 0.402616 & 4.980907 \\
\hline 1 & 5.811258 & -4.489460 & 0.242297 \\
\hline 1 & 8.482473 & 1.608457 & -2.672023 \\
\hline 1 & 3.735170 & -3.764768 & -0.933869 \\
\hline 1 & -2.312696 & -6.197590 & -1.899114 \\
\hline 1 & -0.016174 & -5.352790 & -1.742691 \\
\hline 1 & -1.071808 & -5.270266 & 4.916586 \\
\hline 1 & 7.005517 & 0.099971 & -1.431403 \\
\hline
\end{tabular}




\begin{tabular}{|c|c|c|c|}
\hline & -0.964220 & -4.321333 & 2.660090 \\
\hline & 1.596004 & -3.692819 & -0.849165 \\
\hline 1 & 7.851431 & 3.994327 & -3.025829 \\
\hline & 8.561075 & 0.154256 & 2.653957 \\
\hline & -4.693229 & -6.078287 & -1.228278 \\
\hline & -1.810106 & -3.877103 & 6.844069 \\
\hline & 9.146384 & -2.264048 & 2.794110 \\
\hline & 1.995468 & 2.894123 & 0.122805 \\
\hline & -4.476720 & 0.645092 & 1.351460 \\
\hline 1 & -2.958804 & -0.893873 & -2.333894 \\
\hline 1 & -7.178945 & -0.963309 & -1.551206 \\
\hline 1 & -3.579388 & 2.499788 & -0.906549 \\
\hline 1 & -0.293934 & 2.014924 & 1.798169 \\
\hline L & -1.990375 & 5.903552 & 1.158020 \\
\hline & 1.265404 & -2.863759 & 2.571693 \\
\hline & -1.399074 & 0.794431 & -2.531404 \\
\hline & 2.789004 & -2.742684 & 1.690831 \\
\hline 1 & 4.428033 & -1.612288 & 2.854670 \\
\hline & 0.370643 & -0.269164 & 3.236189 \\
\hline 1 & 5.174965 & 0.189033 & 4.371039 \\
\hline & 1.105987 & 1.515859 & 4.787533 \\
\hline L & 3.516459 & 1.775042 & 5.336598 \\
\hline ] & 0.088761 & -2.354599 & -2.709441 \\
\hline & -1.379986 & -1.582686 & -3.477188 \\
\hline 1 & 0.130538 & -0.059529 & -5.823644 \\
\hline & -1.210165 & 0.461180 & -4.773491 \\
\hline 1 & 0.284283 & 1.404866 & -4.848912 \\
\hline 1 & 1.795423 & -2.809077 & -4.091439 \\
\hline 1 & 4.231306 & -3.145911 & -4.347772 \\
\hline 1 & 5.775834 & -1.195842 & -4.285940 \\
\hline & 4.848658 & 1.092011 & -3.996225 \\
\hline 1 & 2.420582 & 1.421077 & -3.714479 \\
\hline 5 & -6.784786 & -1.848779 & -3.823862 \\
\hline 6 & -4.702805 & -0.733425 & -4.565703 \\
\hline 5 & -4.614559 & -3.001259 & -3.507649 \\
\hline 6 & -8.465477 & -0.246986 & 0.443747 \\
\hline 5 & -6.857038 & -0.581782 & 2.296528 \\
\hline & -7.142549 & 1.682457 & 1.257563 \\
\hline 1 & -6.872929 & -2.297537 & -4.821365 \\
\hline 1 & -7.275644 & -2.528923 & -3.115679 \\
\hline 1 & -7.343196 & -0.903670 & -3.837434 \\
\hline 1 & -4.718089 & -3.469203 & -4.496288 \\
\hline 1 & -3.542903 & -2.922352 & -3.282858 \\
\hline 1 & -5.055834 & -3.675407 & -2.761779 \\
\hline & -4.841135 & -1.180710 & -5.559166 \\
\hline 1 & -5.181810 & 0.254397 & -4.568510 \\
\hline 1 & -3.626190 & -0.580595 & -4.419395 \\
\hline 1 & -7.928101 & 1.918709 & 1.988101 \\
\hline 1 & -6.191656 & 2.059059 & 1.655805 \\
\hline 1 & -7.360885 & 2.238949 & 0.336572 \\
\hline 1 & -7.663039 & -0.375166 & 3.013603 \\
\hline 1 & -6.830094 & -1.665994 & 2.122115 \\
\hline 1 & -5.908886 & -0.299280 & 2.771694 \\
\hline 1 & -9.243791 & -0.006357 & 1.178986 \\
\hline 1 & -8.719456 & 0.278561 & -0.485750 \\
\hline 1 & -8.516072 & -1.326964 & 0.253504 \\
\hline
\end{tabular}




$\begin{array}{rrrr}6 & -4.752665 & 4.516128 & -1.676887 \\ 6 & -4.761485 & 5.920793 & 0.361984 \\ 6 & -3.081008 & 6.329148 & -1.450215 \\ 6 & -0.201515 & 6.081472 & 2.869908 \\ 6 & -0.654689 & 3.950905 & 4.057088 \\ 6 & 1.312212 & 4.155608 & 2.529695 \\ 1 & -5.442003 & 5.226532 & -2.150504 \\ 1 & -4.170402 & 4.037730 & -2.474902 \\ 1 & -5.360920 & 3.743152 & -1.187819 \\ 1 & -3.774227 & 6.997521 & -1.978683 \\ 1 & -2.466182 & 6.948778 & -0.786549 \\ 1 & -2.414387 & 5.873051 & -2.193679 \\ 1 & -5.474162 & 6.599517 & -0.125878 \\ 1 & -5.337318 & 5.168427 & 0.917594 \\ 1 & -4.188755 & 6.509082 & 1.089144 \\ 1 & 1.921936 & 4.482111 & 3.383574 \\ 1 & 1.446352 & 3.069891 & 2.438320 \\ 1 & 1.722975 & 4.628799 & 1.627646 \\ 1 & -0.043033 & 4.293293 & 4.903603 \\ 1 & -1.695853 & 4.243614 & 4.249363 \\ 1 & -0.617312 & 2.853702 & 4.033457 \\ 1 & 0.471816 & 6.407421 & 3.672796 \\ 1 & 0.119420 & 6.579301 & 1.945673 \\ 1 & -1.206904 & 6.441053 & 3.123321\end{array}$

$3_{\text {IA-si }-\Psi-1 s 2 s 3 a 4 a}$

Number of imaginary frequencies : 0

The smallest frequencies are : $10.9230 \quad 18.9665 \quad 24.3858 \mathrm{~cm}(-1)$

Electronic energy : $\quad \mathrm{HF}=-4360.8924061$

Zero-point correction $=\quad 1.454507($ Hartree/Particle $)$

Thermal correction to Energy= $\quad 1.538886$

Thermal correction to Enthalpy $=\quad 1.539830$

Thermal correction to Gibbs Free Energy $=1.337478$

Sum of electronic and zero-point Energies $=\quad-4359.437899$

Sum of electronic and thermal Energies $=\quad-4359.353520$

Sum of electronic and thermal Enthalpies $=\quad-4359.352576$

Sum of electronic and thermal Free Energies $=\quad-4359.554928$

Cartesian Coordinates

$\begin{array}{lrrr}6 & -2.163317 & -1.951020 & 5.696473 \\ 6 & -2.092648 & -1.406852 & 4.392147 \\ 6 & -1.673575 & -2.229033 & 3.306595 \\ 6 & -1.341676 & -3.581725 & 3.583640 \\ 6 & -1.416042 & -4.081542 & 4.860490 \\ 6 & -1.831828 & -3.261232 & 5.929826 \\ 6 & -1.589695 & -1.670707 & 1.987625 \\ 6 & -1.901441 & -0.339038 & 1.770047 \\ 6 & -2.335603 & 0.455337 & 2.868168 \\ 6 & -2.428542 & -0.058059 & 4.133318 \\ 6 & -1.200528 & -2.598629 & 0.892013 \\ 6 & 0.113376 & -2.690530 & 0.471211 \\ 6 & 0.508740 & -3.718036 & -0.417867 \\ 6 & -0.406663 & -4.601532 & -0.925755 \\ 6 & -1.775143 & -4.485347 & -0.589901\end{array}$




\begin{tabular}{|c|c|c|c|}
\hline 6 & -2.178252 & -3.482397 & 0.341359 \\
\hline 6 & -2.752388 & -5.346721 & -1.147420 \\
\hline 6 & -4.075582 & -5.220308 & -0.808011 \\
\hline 6 & -4.478245 & -4.224385 & 0.109513 \\
\hline 6 & -3.553730 & -3.383131 & 0.675567 \\
\hline 7 & 1.087698 & -1.733648 & 0.919280 \\
\hline 6 & 1.909534 & -2.161566 & 2.076386 \\
\hline 6 & 2.355373 & -1.052141 & 2.990542 \\
\hline 6 & 3.704552 & -0.907094 & 3.305892 \\
\hline 6 & 4.132807 & 0.119668 & 4.143332 \\
\hline 6 & 3.209293 & 1.006807 & 4.684905 \\
\hline 6 & 1.855127 & 0.854952 & 4.390242 \\
\hline 6 & 1.431742 & -0.164828 & 3.547045 \\
\hline 15 & -1.848257 & 0.359818 & 0.042059 \\
\hline 45 & -0.138621 & 0.173946 & -1.730658 \\
\hline 15 & 1.577553 & -0.685744 & -0.326652 \\
\hline 8 & 2.417127 & 0.381496 & 0.617025 \\
\hline 6 & 3.340380 & 1.187160 & -0.011790 \\
\hline 6 & 4.577762 & 0.677982 & -0.356908 \\
\hline 6 & 5.472806 & 1.525824 & -1.087625 \\
\hline 6 & 5.100201 & 2.879286 & -1.350449 \\
\hline 6 & 3.846721 & 3.358540 & -0.900769 \\
\hline 6 & 2.975427 & 2.523944 & -0.256127 \\
\hline 6 & 6.704898 & 1.061755 & -1.616095 \\
\hline 6 & 7.526932 & 1.891655 & -2.337961 \\
\hline 6 & 7.168013 & 3.236311 & -2.569730 \\
\hline 6 & 5.977193 & 3.713895 & -2.085774 \\
\hline 6 & 4.901700 & -0.734461 & -0.057147 \\
\hline 6 & 4.047877 & -1.730739 & -0.494269 \\
\hline 6 & 4.383613 & -3.099046 & -0.395348 \\
\hline 6 & 5.534024 & -3.476404 & 0.240832 \\
\hline 6 & 6.380121 & -2.505342 & 0.831206 \\
\hline 6 & 6.062653 & -1.121215 & 0.687293 \\
\hline 6 & 7.516360 & -2.885675 & 1.585503 \\
\hline 6 & 8.299471 & -1.945014 & 2.204541 \\
\hline 6 & 7.969145 & -0.578068 & 2.093744 \\
\hline 6 & 6.884932 & -0.176438 & 1.352342 \\
\hline 8 & 2.859644 & -1.422090 & -1.103382 \\
\hline 6 & -3.587633 & -0.005428 & -0.458269 \\
\hline 6 & -4.657117 & 0.353592 & 0.368090 \\
\hline 6 & -5.967523 & 0.024185 & 0.030562 \\
\hline 6 & -6.175213 & -0.672364 & -1.166211 \\
\hline 6 & -5.133851 & -1.051675 & -2.013126 \\
\hline 6 & -3.830915 & -0.704781 & -1.637037 \\
\hline 6 & -5.439607 & -1.796012 & -3.312193 \\
\hline 6 & -6.361186 & -2.989737 & -3.042849 \\
\hline 6 & -7.154218 & 0.354549 & 0.933975 \\
\hline 6 & -7.787744 & -0.956815 & 1.413801 \\
\hline 6 & -1.872839 & 2.163438 & 0.392451 \\
\hline 6 & -0.934797 & 2.699026 & 1.279452 \\
\hline 6 & -0.907296 & 4.065188 & 1.561967 \\
\hline 6 & -1.790838 & 4.897160 & 0.862270 \\
\hline 6 & -2.699288 & 4.399008 & -0.073918 \\
\hline 6 & -2.737760 & 3.016688 & -0.283611 \\
\hline 6 & -0.019828 & 4.577106 & 2.698226 \\
\hline 6 & -0.574645 & 4.005242 & 4.010170 \\
\hline
\end{tabular}




\begin{tabular}{|c|c|c|c|}
\hline ) & -3.663647 & 5.290016 & -0.853982 \\
\hline & -3.368059 & 5.158308 & -2.352352 \\
\hline & 1.430059 & 4.118537 & 2.523160 \\
\hline & -0.021101 & 6.100365 & 2.803447 \\
\hline & -5.105001 & 4.846048 & -0.575945 \\
\hline & -3.539649 & 6.762654 & -0.471883 \\
\hline & -6.130294 & -0.830068 & -4.281287 \\
\hline v & -4.173277 & -2.330732 & -3.977127 \\
\hline U & -8.195369 & 1.170681 & 0.161439 \\
\hline 6 & -6.737212 & 1.159587 & 2.163119 \\
\hline 6 & -0.329682 & -1.576361 & -3.044921 \\
\hline 6 & 0.455287 & -0.602034 & -3.711906 \\
\hline 6 & 1.931788 & -0.782099 & -3.864304 \\
\hline 6 & 2.792289 & 0.322650 & -3.905576 \\
\hline 6 & 4.161330 & 0.161556 & -4.070685 \\
\hline 6 & 4.705207 & -1.114297 & -4.208858 \\
\hline 6 & 3.861876 & -2.219898 & -4.194423 \\
\hline 6 & 2.489658 & -2.053483 & -4.029908 \\
\hline 6 & 0.337479 & 2.038936 & -1.888913 \\
\hline 8 & 0.432186 & 3.134538 & -2.251956 \\
\hline 6 & -0.156432 & 0.191018 & -4.849820 \\
\hline 1 & 6.631756 & 0.879268 & 1.289522 \\
\hline 1 & -2.599161 & 1.497467 & 2.705253 \\
\hline 1 & 7.745210 & -3.946996 & 1.674730 \\
\hline 1 & -2.484647 & -1.303486 & 6.511121 \\
\hline 1 & 5.674964 & 4.744219 & -2.268958 \\
\hline 1 & -3.875131 & -2.611394 & 1.373004 \\
\hline 1 & -5.531559 & -4.117363 & 0.363208 \\
\hline 1 & 3.573625 & 4.394056 & -1.099518 \\
\hline 1 & -2.763656 & 0.570151 & 4.958139 \\
\hline 1 & 5.803279 & -4.528299 & 0.321641 \\
\hline 1 & 8.461178 & 1.506007 & -2.740157 \\
\hline 1 & 3.712139 & -3.828762 & -0.843814 \\
\hline 1 & -2.428786 & -6.109786 & -1.853904 \\
\hline 1 & -0.097469 & -5.386088 & -1.614939 \\
\hline 1 & -1.154309 & -5.120266 & 5.048722 \\
\hline 1 & 6.989047 & 0.023952 & -1.462854 \\
\hline 1 & -1.023035 & -4.229788 & 2.769657 \\
\hline 1 & 1.556911 & -3.774509 & -0.706900 \\
\hline 1 & 7.829686 & 3.884775 & -3.139313 \\
\hline 1 & 8.576454 & 0.166002 & 2.604468 \\
\hline 1 & -4.818484 & -5.884942 & -1.244308 \\
\hline 1 & -1.887686 & -3.670362 & 6.935886 \\
\hline 1 & 9.164495 & -2.248662 & 2.789479 \\
\hline 1 & 1.996196 & 2.858800 & 0.076599 \\
\hline 1 & -4.455658 & 0.895822 & 1.288135 \\
\hline 1 & -2.978253 & -0.982040 & -2.251755 \\
\hline 1 & -7.196389 & -0.934023 & -1.443571 \\
\hline 1 & -3.451157 & 2.595632 & -0.991383 \\
\hline 1 & -0.246388 & 2.025375 & 1.795342 \\
\hline 1 & -1.774416 & 5.964470 & 1.061063 \\
\hline 1 & 1.268482 & -2.854669 & 2.640394 \\
\hline 1 & -1.390303 & 0.701736 & -2.552656 \\
\hline 1 & 2.784127 & -2.742868 & 1.744190 \\
\hline 1 & 4.435086 & -1.592010 & 2.872727 \\
\hline 1 & 0.377403 & -0.261396 & 3.290497 \\
\hline
\end{tabular}




$\begin{array}{rrrr}1 & 5.193380 & 0.226519 & 4.363023 \\ 1 & 1.124034 & 1.540511 & 4.818184 \\ 1 & 3.539735 & 1.814007 & 5.335512 \\ 1 & 0.149663 & -2.449396 & -2.606935 \\ 1 & -1.353059 & -1.752148 & -3.377958 \\ 1 & 0.093619 & -0.284995 & -5.810623 \\ 1 & -1.248238 & 0.240601 & -4.763643 \\ 1 & 0.220072 & 1.220388 & -4.895862 \\ 1 & 1.835791 & -2.925315 & -4.025617 \\ 1 & 4.273346 & -3.221072 & -4.313453 \\ 1 & 5.779802 & -1.240159 & -4.328411 \\ 1 & 4.811575 & 1.035575 & -4.079864 \\ 1 & 2.382720 & 1.323749 & -3.766639 \\ 1 & -4.439477 & -2.888255 & -4.884094 \\ 1 & -3.493400 & -1.523502 & -4.280782 \\ 1 & -3.626671 & -3.013897 & -3.311067 \\ 1 & -6.359887 & -1.334699 & -5.229727 \\ 1 & -7.071767 & -0.445848 & -3.868694 \\ 1 & -5.486256 & 0.031072 & -4.502770 \\ 1 & -6.564982 & -3.525671 & -3.979390 \\ 1 & -5.890855 & -3.694588 & -2.344273 \\ 1 & -7.328827 & -2.690422 & -2.623275 \\ 1 & -9.052558 & 1.401335 & 0.808163 \\ 1 & -7.772309 & 2.121137 & -0.188487 \\ 1 & -8.579892 & 0.633673 & -0.713835 \\ 1 & -8.635720 & -0.752092 & 2.081371 \\ 1 & -8.160402 & -1.562672 & 0.578561 \\ 1 & -7.058583 & -1.563260 & 1.967767 \\ 1 & -7.620142 & 1.378981 & 2.776586 \\ 1 & -6.026736 & 0.610003 & 2.795291 \\ 1 & -6.279770 & 2.119159 & 1.887278 \\ 1 & -4.251480 & 7.356259 & -1.059377 \\ 1 & -3.766357 & 6.933183 & 0.588658 \\ 1 & -2.536288 & 7.156865 & -0.677684 \\ 1 & -5.811956 & 5.473656 & -1.135120 \\ 1 & -5.281475 & 3.804341 & -0.872401 \\ 1 & -5.344359 & 4.936292 & 0.492080 \\ 1 & -4.060802 & 5.780705 & -2.935096 \\ 1 & -2.345354 & 5.485978 & -2.578688 \\ 1 & -3.470888 & 4.124460 & -2.703939 \\ 1 & 2.029570 & 4.426452 & 3.391227 \\ 1 & 1.527606 & 3.028504 & 2.438307 \\ 1 & 1.880680 & 4.572707 & 1.630544 \\ 1 & 0.027306 & 4.343885 & 4.865141 \\ 1 & -1.610691 & 4.332155 & 4.173021 \\ 1 & -0.569579 & 2.907230 & 4.005366 \\ 1 & 0.649600 & 6.413137 & 3.613774 \\ 1 & 0.333706 & 6.573746 & 1.878777 \\ 1 & -1.017016 & 6.499314 & 3.034137\end{array}$

$3_{\text {IA-si- }} \psi_{-122 s 3 a 4 s}$

Number of imaginary frequencies : 0

The smallest frequencies are : $14.2112 \quad 23.1412 \quad 25.6460 \mathrm{~cm}(-1)$

Electronic energy :

Zero-point correction $=$

$\mathrm{HF}=-4360.8926339$

1.454450 (Hartree/Particle) 


$\begin{array}{lc}\text { Thermal correction to Energy= } & 1.538916 \\ \text { Thermal correction to Enthalpy= } & 1.539860 \\ \text { Thermal correction to Gibbs Free Energy }= & 1.337675 \\ \text { Sum of electronic and zero-point Energies }= & -4359.438184 \\ \text { Sum of electronic and thermal Energies }= & -4359.353718 \\ \text { Sum of electronic and thermal Enthalpies }= & -4359.352774 \\ \text { Sum of electronic and thermal Free Energies }= & -4359.554959\end{array}$

Cartesian Coordinates

\begin{tabular}{cccc}
\hline 6 & 2.472687 & -2.006641 & -4.044844 \\
6 & 1.927936 & -0.731076 & -3.867658 \\
6 & 2.800532 & 0.364683 & -3.891368 \\
6 & 4.168512 & 0.190159 & -4.051047 \\
6 & 4.699180 & -1.089983 & -4.200531 \\
6 & 3.843792 & -2.186376 & -4.203756 \\
6 & 0.453227 & -0.536919 & -3.718475 \\
6 & -0.145186 & 0.284527 & -4.843045 \\
45 & -0.140471 & 0.205732 & -1.724971 \\
6 & 0.326999 & 2.075668 & -1.845312 \\
8 & 0.415003 & 3.178600 & -2.187283 \\
15 & -1.851891 & 0.342792 & 0.051328 \\
6 & -1.906513 & 2.142230 & 0.419026 \\
6 & -0.968482 & 2.684955 & 1.301546 \\
6 & -0.956354 & 4.049765 & 1.591125 \\
6 & -1.853360 & 4.874431 & 0.899661 \\
6 & -2.762359 & 4.369668 & -0.032434 \\
6 & -2.789018 & 2.987406 & -0.244436 \\
6 & -0.066650 & 4.567043 & 2.723055 \\
6 & -0.083159 & 6.089810 & 2.833630 \\
6 & -3.733677 & 5.253664 & -0.812006 \\
6 & -3.617019 & 6.727728 & -0.433101 \\
6 & -1.895323 & -0.376214 & 1.770795 \\
6 & -1.577693 & -1.709618 & 1.968294 \\
6 & -1.657356 & -2.288285 & 3.278226 \\
6 & -2.075852 & -1.483737 & 4.377134 \\
6 & -2.414207 & -0.131702 & 4.139804 \\
6 & -2.326754 & 0.401081 & 2.881899 \\
6 & -1.322542 & -3.644590 & 3.533187 \\
6 & -1.393620 & -4.164314 & 4.802183 \\
6 & -1.808525 & -3.361379 & 5.885089 \\
6 & -2.142710 & -2.048319 & 5.673064 \\
6 & -1.185769 & -2.616114 & 0.856346 \\
6 & 0.127710 & -2.690629 & 0.430381 \\
6 & 0.526029 & -3.695427 & -0.482859 \\
6 & -0.386603 & -4.570328 & -1.010449 \\
6 & -1.755080 & -4.467538 & -0.670668 \\
6 & -2.160552 & -3.490956 & 0.287199 \\
6 & -3.536087 & -3.404377 & 0.625025 \\
6 & -4.458721 & -4.230432 & 0.034125 \\
6 & -4.054113 & -5.198739 & -0.911664 \\
6 & -2.730477 & -5.314419 & -1.252917 \\
7 & 1.096833 & -1.735882 & 0.894044 \\
15 & 1.584012 & -0.670363 & -0.338076 \\
8 & 2.869262 & -1.391316 & -1.123940 \\
6 & 4.054293 & -1.710123 & -0.513455 \\
& & & \\
6 & &
\end{tabular}




\begin{tabular}{|c|c|c|c|}
\hline 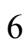 & 4.907070 & -0.720929 & -0.058780 \\
\hline & 6.064891 & -1.118554 & 0.684549 \\
\hline & 6.379531 & -2.504857 & 0.812665 \\
\hline & 5.534954 & -3.467064 & 0.205790 \\
\hline & 4.388244 & -3.079982 & -0.431309 \\
\hline & 6.886822 & -0.183143 & 1.363133 \\
\hline & 7.967618 & -0.595512 & 2.103600 \\
\hline & 8.294626 & -1.964360 & 2.199862 \\
\hline & 7.512124 & -2.896185 & 1.566822 \\
\hline & 4.585751 & 0.695389 & -0.341910 \\
\hline & 3.346764 & 1.200650 & 0.003320 \\
\hline & 2.983477 & 2.540260 & -0.227443 \\
\hline & 3.857574 & 3.381815 & -0.859119 \\
\hline & 5.112150 & 2.906853 & -1.310331 \\
\hline & 5.483405 & 1.550518 & -1.060814 \\
\hline & 5.990523 & 3.748057 & -2.036394 \\
\hline & 7.180587 & 3.273889 & -2.525508 \\
\hline & 7.537353 & 1.926180 & -2.308567 \\
\hline & 6.714222 & 1.089833 & -1.595451 \\
\hline & 2.420207 & 0.388427 & 0.618693 \\
\hline & 1.912974 & -2.171075 & 2.052303 \\
\hline & 2.354938 & -1.067216 & 2.975070 \\
\hline & 3.702740 & -0.924390 & 3.297178 \\
\hline & 4.127889 & 0.098697 & 4.140706 \\
\hline & 3.202637 & 0.984471 & 4.681541 \\
\hline & 1.849690 & 0.834478 & 4.380410 \\
\hline 6 & 1.429433 & -0.181835 & 3.531550 \\
\hline ) & -3.586486 & -0.038166 & -0.454317 \\
\hline 6 & -4.652850 & 0.274781 & 0.384520 \\
\hline 6 & -5.968009 & -0.046467 & 0.039468 \\
\hline & -6.179595 & -0.686343 & -1.182211 \\
\hline 6 & -5.131754 & -1.024192 & -2.048027 \\
\hline o & -3.832231 & -0.690348 & -1.663724 \\
\hline 6 & -7.090554 & 0.278184 & 1.022965 \\
\hline 6 & -8.466671 & -0.107782 & 0.487352 \\
\hline 6 & -5.440233 & -1.713692 & -3.376226 \\
\hline 6 & -4.172920 & -2.190835 & -4.081462 \\
\hline 5 & -0.344082 & -1.515638 & -3.072589 \\
\hline 6 & -6.332979 & -2.937679 & -3.148422 \\
\hline 5 & -6.163429 & -0.720280 & -4.292345 \\
\hline 6 & -6.846853 & -0.501395 & 2.321366 \\
\hline 6 & -7.099951 & 1.781203 & 1.322043 \\
\hline 6 & -0.606245 & 3.984700 & 4.036674 \\
\hline 6 & 1.386711 & 4.124177 & 2.536341 \\
\hline 6 & -3.437748 & 5.120650 & -2.310363 \\
\hline 6 & -5.172509 & 4.802911 & -0.532618 \\
\hline 1 & 6.636130 & 0.873762 & 1.311197 \\
\hline 1 & -2.591566 & 1.445240 & 2.735060 \\
\hline 1 & 7.738874 & -3.958883 & 1.644164 \\
\hline 1 & -2.463402 & -1.414184 & 6.498432 \\
\hline 1 & 5.689563 & 4.780558 & -2.209131 \\
\hline 1 & -3.858825 & -2.653793 & 1.345103 \\
\hline 1 & -5.512365 & -4.132827 & 0.289424 \\
\hline 1 & 3.585583 & 4.419600 & -1.047311 \\
\hline 1 & -2.746926 & 0.483521 & 4.975291 \\
\hline 1 & 5.802798 & -4.520228 & 0.273766 \\
\hline
\end{tabular}




\begin{tabular}{|c|c|c|c|}
\hline & 8.470546 & 1.543260 & -2.715775 \\
\hline & 3.719433 & -3.802685 & -0.894905 \\
\hline 1 & -2.405283 & -6.056779 & -1.980384 \\
\hline & -0.075234 & -5.335609 & -1.720053 \\
\hline & -1.129968 & -5.205423 & 4.973822 \\
\hline & 6.996463 & 0.049813 & -1.453854 \\
\hline & -1.004649 & -4.278995 & 2.708208 \\
\hline & 1.573339 & -3.739520 & -0.776692 \\
\hline & 7.843058 & 3.927337 & -3.088427 \\
\hline & 8.574814 & 0.141342 & 2.624819 \\
\hline & -4.795952 & -5.851491 & -1.367281 \\
\hline & -1.861574 & -3.786290 & 6.884735 \\
\hline & 9.156872 & -2.276542 & 2.784411 \\
\hline & 2.002714 & 2.871265 & 0.104010 \\
\hline & -4.458397 & 0.774189 & 1.332669 \\
\hline & -2.980318 & -0.937083 & -2.291870 \\
\hline & -7.196420 & -0.938118 & -1.476447 \\
\hline & -3.503947 & 2.561149 & -0.947564 \\
\hline & -0.265601 & 2.017249 & 1.805616 \\
\hline & -1.846070 & 5.941453 & 1.100688 \\
\hline & 1.269168 & -2.867541 & 2.609030 \\
\hline & -1.393765 & 0.747609 & -2.534919 \\
\hline 1 & 2.788899 & -2.750541 & 1.720345 \\
\hline & 4.434862 & -1.607756 & 2.864286 \\
\hline ] & 0.376267 & -0.275818 & 3.269520 \\
\hline & 5.187533 & 0.203963 & 4.365589 \\
\hline 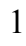 & 1.117051 & 1.518903 & 4.807658 \\
\hline ] & 3.530796 & 1.788946 & 5.336649 \\
\hline & 0.124119 & -2.401891 & -2.649380 \\
\hline 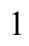 & -1.368035 & -1.673789 & -3.412745 \\
\hline & 0.101353 & -0.176558 & -5.811994 \\
\hline 1 & -1.236361 & 0.346866 & -4.758731 \\
\hline ] & 0.244340 & 1.309727 & -4.868951 \\
\hline & 1.809348 & -2.871265 & -4.054557 \\
\hline 1 & 4.244977 & -3.190550 & -4.332485 \\
\hline & 5.772996 & -1.226290 & -4.315580 \\
\hline 1 & 4.828566 & 1.056902 & -4.046308 \\
\hline & 2.401170 & 1.368609 & -3.742270 \\
\hline 1 & -4.439831 & -2.709899 & -5.010773 \\
\hline ] & -3.512366 & -1.356726 & -4.353446 \\
\hline ] & -3.604774 & -2.893606 & -3.455466 \\
\hline l & -6.395594 & -1.187580 & -5.259095 \\
\hline 1 & -7.107052 & -0.373555 & -3.852602 \\
\hline 1 & -5.539364 & 0.162471 & -4.483757 \\
\hline & -6.541925 & -3.434442 & -4.105247 \\
\hline 1 & -5.835918 & -3.663474 & -2.491072 \\
\hline l & -7.298735 & -2.678354 & -2.698992 \\
\hline 1 & -7.889093 & 2.019990 & 2.047734 \\
\hline 1 & -6.147731 & 2.127315 & 1.742829 \\
\hline 1 & -7.293675 & 2.361881 & 0.410408 \\
\hline 1 & -7.636116 & -0.283827 & 3.053754 \\
\hline 1 & -6.849889 & -1.583539 & 2.132708 \\
\hline 1 & -5.883403 & -0.248229 & 2.782736 \\
\hline 1 & -9.235609 & 0.150275 & 1.226475 \\
\hline 1 & -8.710138 & 0.425922 & -0.440466 \\
\hline 1 & -8.545932 & -1.185721 & 0.295793 \\
\hline
\end{tabular}




$\begin{array}{lrrr}1 & -4.333698 & 7.316146 & -1.019948 \\ 1 & -3.842147 & 6.899067 & 0.627645 \\ 1 & -2.616409 & 7.127206 & -0.642170 \\ 1 & -5.881762 & 5.419955 & -1.100698 \\ 1 & -5.342234 & 3.756320 & -0.816359 \\ 1 & -5.414802 & 4.904781 & 0.533637 \\ 1 & -4.131756 & 5.740935 & -2.893861 \\ 1 & -2.415586 & 5.449820 & -2.537074 \\ 1 & -3.538486 & 4.086096 & -2.660604 \\ 1 & 1.989374 & 4.436631 & 3.400560 \\ 1 & 1.495350 & 3.035382 & 2.448232 \\ 1 & 1.825563 & 4.585268 & 1.641373 \\ 1 & -0.000922 & 4.325050 & 4.888567 \\ 1 & -1.643918 & 4.301463 & 4.208805 \\ 1 & -0.591315 & 2.886792 & 4.026898 \\ 1 & 0.589004 & 6.406920 & 3.641060 \\ 1 & 0.261106 & 6.569961 & 1.908470 \\ 1 & -1.081841 & 6.477467 & 3.071479\end{array}$

$3_{\text {IA-si- }} \psi_{-1 \mathbf{1 s 2} 2354 a}$

Number of imaginary frequencies : 0

The smallest frequencies are : $18.6782 \quad 20.641326 .8005 \mathrm{~cm}(-1)$

Electronic energy : $\quad H F=-4360.8924402$

Zero-point correction $=\quad 1.454635($ Hartree/Particle)

Thermal correction to Energy= $\quad 1.538999$

Thermal correction to Enthalpy= $\quad 1.539943$

Thermal correction to Gibbs Free Energy $=\quad 1.338852$

Sum of electronic and zero-point Energies $=\quad-4359.437805$

Sum of electronic and thermal Energies $=\quad-4359.353441$

Sum of electronic and thermal Enthalpies $=\quad-4359.352497$

Sum of electronic and thermal Free Energies $=\quad-4359.553588$

Cartesian Coordinates

$\begin{array}{cccc}6 & 2.435732 & -1.979136 & -4.057085 \\ 6 & 1.916028 & -0.694685 & -3.870479 \\ 6 & 2.810103 & 0.383996 & -3.877563 \\ 6 & 4.175096 & 0.183838 & -4.031041 \\ 6 & 4.680843 & -1.105407 & -4.189909 \\ 6 & 3.803923 & -2.184496 & -4.209710 \\ 6 & 0.445106 & -0.471640 & -3.728854 \\ 6 & -0.124693 & 0.379915 & -4.845826 \\ 45 & -0.144383 & 0.251982 & -1.729524 \\ 6 & 0.330297 & 2.121855 & -1.816258 \\ 8 & 0.414098 & 3.231430 & -2.137182 \\ 15 & -1.849479 & 0.364692 & 0.059622 \\ 6 & -1.902004 & 2.157022 & 0.454394 \\ 6 & -0.962688 & 2.688825 & 1.342028 \\ 6 & -0.953696 & 4.049211 & 1.651575 \\ 6 & -1.859517 & 4.880426 & 0.979576 \\ 6 & -2.771585 & 4.386246 & 0.044935 \\ 6 & -2.789796 & 3.008429 & -0.192755 \\ 6 & -0.057964 & 4.553707 & 2.784408 \\ 6 & -0.072492 & 6.075278 & 2.911303 \\ 6 & -3.758945 & 5.274816 & -0.707904\end{array}$




\begin{tabular}{|c|c|c|c|}
\hline 6 & -3.646844 & 6.743620 & -0.308029 \\
\hline & -1.897148 & -0.381818 & 1.765417 \\
\hline & -1.591479 & -1.721190 & 1.938997 \\
\hline & -1.672801 & -2.319943 & 3.240492 \\
\hline & -2.085839 & -1.530184 & 4.352249 \\
\hline & -2.416709 & -0.172656 & 4.137155 \\
\hline & -2.323987 & 0.380440 & 2.888656 \\
\hline & -1.341268 & -3.680668 & 3.475889 \\
\hline & -1.413106 & -4.218838 & 4.737167 \\
\hline & -1.825506 & -3.431158 & 5.832105 \\
\hline & -2.154670 & -2.113892 & 5.639617 \\
\hline b & -1.199977 & -2.614796 & 0.815763 \\
\hline 6 & 0.113947 & -2.680623 & 0.389259 \\
\hline 6 & 0.520934 & -3.686233 & -0.519484 \\
\hline 6 & -0.378092 & -4.592406 & -1.016946 \\
\hline 6 & -1.744094 & -4.515242 & -0.660224 \\
\hline 6 & -2.165186 & -3.509633 & 0.260118 \\
\hline 6 & -3.543010 & -3.431893 & 0.592797 \\
\hline 6 & -4.446669 & -4.315386 & 0.058206 \\
\hline 6 & -4.020984 & -5.331329 & -0.826100 \\
\hline 6 & -2.699841 & -5.422105 & -1.181932 \\
\hline 7 & 1.080390 & -1.728628 & 0.864856 \\
\hline 15 & 1.577311 & -0.653274 & -0.353705 \\
\hline 8 & 2.863380 & -1.371267 & -1.141255 \\
\hline 6 & 4.044976 & -1.700935 & -0.529405 \\
\hline 6 & 4.899885 & -0.720129 & -0.061044 \\
\hline 6 & 6.055209 & -1.129820 & 0.679676 \\
\hline 6 & 6.365008 & -2.518404 & 0.793138 \\
\hline 6 & 5.518132 & -3.470872 & 0.174150 \\
\hline 6 & 4.374346 & -3.072779 & -0.461393 \\
\hline 6 & 6.879813 & -0.204567 & 1.368867 \\
\hline 6 & 7.958626 & -0.628488 & 2.105687 \\
\hline 6 & 8.280703 & -1.999396 & 2.187730 \\
\hline 6 & 7.495441 & -2.921692 & 1.544197 \\
\hline 6 & 4.585334 & 0.700386 & -0.329446 \\
\hline 6 & 3.347254 & 1.207415 & 0.016520 \\
\hline 6 & 2.992193 & 2.551850 & -0.198714 \\
\hline 6 & 3.872481 & 3.395864 & -0.818491 \\
\hline 6 & 5.125058 & 2.918952 & -1.273456 \\
\hline 6 & 5.489074 & 1.558324 & -1.037217 \\
\hline 6 & 6.007941 & 3.762402 & -1.991346 \\
\hline 6 & 7.195007 & 3.286334 & -2.485848 \\
\hline 6 & 7.544293 & 1.934539 & -2.282614 \\
\hline 6 & 6.716906 & 1.095704 & -1.577377 \\
\hline 8 & 2.413755 & 0.392535 & 0.617669 \\
\hline 6 & 1.892482 & -2.181149 & 2.019923 \\
\hline 6 & 2.339430 & -1.090826 & 2.956099 \\
\hline 6 & 3.687631 & -0.959223 & 3.281366 \\
\hline 6 & 4.117415 & 0.051228 & 4.137659 \\
\hline 6 & 3.196419 & 0.935284 & 4.688423 \\
\hline 6 & 1.842977 & 0.795821 & 4.384548 \\
\hline 6 & 1.418085 & -0.207851 & 3.523061 \\
\hline 6 & -3.581702 & -0.008691 & -0.452556 \\
\hline 6 & -4.660788 & 0.269819 & 0.397790 \\
\hline 6 & -5.960769 & -0.061005 & 0.036004 \\
\hline 6 & -6.161287 & -0.669469 & -1.215176 \\
\hline
\end{tabular}




\begin{tabular}{|c|c|c|c|}
\hline ) & -5.115994 & -0.944671 & -2.089686 \\
\hline & -3.816139 & -0.614869 & -1.677122 \\
\hline & -7.150153 & 0.172907 & 0.965213 \\
\hline & -6.760784 & 0.964793 & 2.212246 \\
\hline & -5.317779 & -1.589632 & -3.458541 \\
\hline & -6.791592 & -1.814620 & -3.786239 \\
\hline & -0.380444 & -1.442016 & -3.103972 \\
\hline U & -4.609226 & -2.947573 & -3.484146 \\
\hline U & -4.723624 & -0.681092 & -4.541003 \\
\hline 6 & -7.695083 & -1.188829 & 1.411882 \\
\hline 6 & -8.251082 & 0.950433 & 0.236941 \\
\hline 6 & -0.591649 & 3.957830 & 4.094322 \\
\hline 6 & 1.394147 & 4.111993 & 2.584962 \\
\hline 6 & -3.483064 & 5.165808 & -2.212104 \\
\hline 6 & -5.190283 & 4.808568 & -0.413455 \\
\hline 1 & 6.633048 & 0.853728 & 1.327770 \\
\hline 1 & -2.583514 & 1.428148 & 2.758334 \\
\hline 1 & 7.718535 & -3.985916 & 1.610303 \\
\hline 1 & -2.472033 & -1.490532 & 6.474445 \\
\hline 1 & 5.712674 & 4.798185 & -2.153957 \\
\hline 1 & -3.883107 & -2.647421 & 1.267783 \\
\hline 1 & -5.500932 & -4.227323 & 0.313120 \\
\hline 1 & 3.606800 & 4.437335 & -0.994957 \\
\hline 1 & -2.747063 & 0.430476 & 4.982336 \\
\hline 1 & 5.782204 & -4.525655 & 0.230848 \\
\hline 1 & 8.474789 & 1.550355 & -2.694765 \\
\hline 1 & 3.705027 & -3.788063 & -0.935521 \\
\hline 1 & -2.360366 & -6.191433 & -1.874101 \\
\hline 1 & -0.056741 & -5.370300 & -1.708123 \\
\hline 1 & -1.151542 & -5.262926 & 4.893184 \\
\hline 1 & 6.993015 & 0.052571 & -1.446991 \\
\hline 1 & -1.023986 & -4.304175 & 2.642448 \\
\hline 1 & 1.566146 & -3.716199 & -0.821986 \\
\hline 1 & 7.860746 & 3.941525 & -3.042847 \\
\hline 1 & 8.568046 & 0.100701 & 2.635029 \\
\hline 1 & -4.746801 & -6.031864 & -1.233562 \\
\hline 1 & -1.879581 & -3.870737 & 6.825328 \\
\hline 1 & 9.141404 & -2.320793 & 2.769554 \\
\hline 1 & 2.012612 & 2.884376 & 0.134701 \\
\hline 1 & -4.469287 & 0.750338 & 1.353503 \\
\hline 1 & -2.962426 & -0.836041 & -2.314605 \\
\hline 1 & -7.177024 & -0.930211 & -1.505202 \\
\hline 1 & -3.504236 & 2.590128 & -0.900974 \\
\hline 1 & -0.256292 & 2.015330 & 1.833302 \\
\hline 1 & -1.856683 & 5.943948 & 1.198492 \\
\hline 1 & 1.243630 & -2.879870 & 2.567851 \\
\hline 1 & -1.391088 & 0.821410 & -2.530270 \\
\hline 1 & 2.765281 & -2.762244 & 1.682838 \\
\hline 1 & 4.416482 & -1.641438 & 2.841359 \\
\hline 1 & 0.364534 & -0.293622 & 3.259964 \\
\hline 1 & 5.177309 & 0.147889 & 4.365186 \\
\hline 1 & 1.113522 & 1.478534 & 4.819858 \\
\hline 1 & 3.528212 & 1.730016 & 5.353509 \\
\hline 1 & 0.058190 & -2.349555 & -2.692092 \\
\hline 1 & -1.404075 & -1.566332 & -3.461201 \\
\hline 1 & 0.119103 & -0.072407 & -5.819562 \\
\hline
\end{tabular}




$\begin{array}{rrrr}1 & -1.214385 & 0.468210 & -4.770307 \\ 1 & 0.289644 & 1.395564 & -4.853010 \\ 1 & 1.755134 & -2.829981 & -4.080099 \\ 1 & 4.186026 & -3.195024 & -4.346678 \\ 1 & 5.752362 & -1.262290 & -4.300112 \\ 1 & 4.852460 & 1.037049 & -4.013124 \\ 1 & 2.429378 & 1.394044 & -3.720406 \\ 1 & -6.882712 & -2.264474 & -4.782961 \\ 1 & -7.271300 & -2.499014 & -3.074388 \\ 1 & -7.358142 & -0.874316 & -3.795861 \\ 1 & -4.863136 & -1.130273 & -5.533395 \\ 1 & -5.211847 & 0.302154 & -4.542089 \\ 1 & -3.647675 & -0.518588 & -4.400375 \\ 1 & -4.701739 & -3.409049 & -4.476841 \\ 1 & -3.539922 & -2.861998 & -3.250280 \\ 1 & -5.050853 & -3.630393 & -2.746954 \\ 1 & -9.100277 & 1.124764 & 0.911070 \\ 1 & -7.884837 & 1.927858 & -0.103338 \\ 1 & -8.634619 & 0.413270 & -0.638387 \\ 1 & -8.550967 & -1.058699 & 2.088143 \\ 1 & -8.030318 & -1.794577 & 0.560736 \\ 1 & -6.924065 & -1.759937 & 1.947143 \\ 1 & -7.647394 & 1.125127 & 2.838537 \\ 1 & -6.019708 & 0.434530 & 2.825267 \\ 1 & -6.351740 & 1.951688 & 1.956574 \\ 1 & -4.374787 & 7.336390 & -0.876306 \\ 1 & -3.859188 & 6.897258 & 0.758036 \\ 1 & -2.651454 & 7.152497 & -0.523817 \\ 1 & -5.913867 & 5.435437 & -0.951889 \\ 1 & -5.360280 & 3.768879 & -0.721024 \\ 1 & -5.413414 & 4.880190 & 0.659570 \\ 1 & -4.180145 & 5.800362 & -2.776236 \\ 1 & -2.460888 & 5.490488 & -2.446458 \\ 1 & -3.595923 & 4.138209 & -2.578542 \\ 1 & 2.001957 & 4.416139 & 3.448521 \\ 1 & 1.502261 & 3.024103 & 2.485804 \\ 1 & 1.827748 & 4.581592 & 1.691812 \\ 1 & 0.019833 & 4.285971 & 4.946614 \\ 1 & -1.627161 & 4.276147 & 4.276194 \\ 1 & -0.581045 & 2.860084 & 4.071458 \\ 1 & 0.605466 & 6.383238 & 3.717432 \\ 1 & 0.265695 & 6.564937 & 1.988881 \\ 1 & -1.069048 & 6.461299 & 3.160357\end{array}$

\section{$3_{\text {IA-si- }} \psi_{-1}-1253344 \mathrm{~s}$}

Number of imaginary frequencies : 0

The smallest frequencies are : $12.8161 \quad 18.990524 .0963 \mathrm{~cm}(-1)$

Electronic energy : $\quad \mathrm{HF}=-4360.8925286$

Zero-point correction $=\quad 1.454573($ Hartree/Particle)

Thermal correction to Energy= $\quad 1.538971$

Thermal correction to Enthalpy= $\quad 1.539915$

Thermal correction to Gibbs Free Energy $=\quad 1.337903$

Sum of electronic and zero-point Energies $=\quad-4359.437955$

Sum of electronic and thermal Energies $=\quad-4359.353558$

Sum of electronic and thermal Enthalpies $=\quad-4359.352614$ 
Cartesian Coordinates

\begin{tabular}{|c|c|c|c|}
\hline 6 & -3.827362 & -0.599811 & -1.696299 \\
\hline 6 & -3.587088 & -0.030479 & -0.451103 \\
\hline 6 & -4.662474 & 0.225188 & 0.401669 \\
\hline 6 & -5.969890 & -0.083050 & 0.030598 \\
\hline 6 & -6.177126 & -0.646279 & -1.234892 \\
\hline 6 & -5.126443 & -0.906896 & -2.115833 \\
\hline 15 & -1.855837 & 0.340158 & 0.068039 \\
\hline 6 & -1.897836 & -0.433762 & 1.761936 \\
\hline 6 & -1.582643 & -1.773691 & 1.915768 \\
\hline 6 & -1.659255 & -2.392192 & 3.207977 \\
\hline 6 & -2.076762 & -1.622000 & 4.331580 \\
\hline 6 & -2.415059 & -0.263331 & 4.137361 \\
\hline 6 & -2.327200 & 0.309160 & 2.897000 \\
\hline 6 & -2.141884 & -2.225617 & 5.609912 \\
\hline 6 & -1.805349 & -3.543825 & 5.782096 \\
\hline 6 & -1.388784 & -4.312375 & 4.675070 \\
\hline 6 & -1.320137 & -3.754429 & 3.422221 \\
\hline 6 & -1.186812 & -2.645573 & 0.777145 \\
\hline 6 & 0.125227 & -2.689016 & 0.342052 \\
\hline 6 & 0.535203 & -3.666027 & -0.596012 \\
\hline 6 & -0.359823 & -4.563253 & -1.116371 \\
\hline 6 & -1.723872 & -4.508681 & -0.748567 \\
\hline 6 & -2.146549 & -3.536518 & 0.206350 \\
\hline 6 & -3.520700 & -3.487553 & 0.558157 \\
\hline 6 & -4.420549 & -4.363042 & 0.004443 \\
\hline 6 & -3.994321 & -5.341429 & -0.920967 \\
\hline 6 & -2.675810 & -5.406805 & -1.291890 \\
\hline 7 & 1.085607 & -1.740176 & 0.835440 \\
\hline 6 & 1.893581 & -2.204765 & 1.988637 \\
\hline 6 & 2.336178 & -1.124092 & 2.937907 \\
\hline 6 & 3.683699 & -0.990310 & 3.265080 \\
\hline 6 & 4.109069 & 0.013682 & 4.131143 \\
\hline 6 & 3.184304 & 0.888649 & 4.690077 \\
\hline 6 & 1.831468 & 0.746030 & 4.385015 \\
\hline 6 & 1.410984 & -0.251136 & 3.513936 \\
\hline 6 & -7.102029 & 0.186111 & 1.020785 \\
\hline 6 & -7.130498 & 1.677040 & 1.375798 \\
\hline 6 & -5.332470 & -1.527372 & -3.495872 \\
\hline 6 & -4.718743 & -0.615805 & -4.564634 \\
\hline 45 & -0.148497 & 0.276441 & -1.724559 \\
\hline 6 & -0.390970 & -1.387693 & -3.134211 \\
\hline 6 & 0.440495 & -0.408936 & -3.737418 \\
\hline 6 & -0.123337 & 0.468812 & -4.836857 \\
\hline 6 & 1.910459 & -0.637367 & -3.879628 \\
\hline 6 & 2.423856 & -1.921486 & -4.085540 \\
\hline 6 & 3.791380 & -2.131961 & -4.236978 \\
\hline 6 & 4.674044 & -1.058147 & -4.197042 \\
\hline 6 & 4.174645 & 0.231113 & -4.019353 \\
\hline 6 & 2.810395 & 0.436383 & -3.866498 \\
\hline 6 & 0.324775 & 2.147219 & -1.777986 \\
\hline 8 & 0.409931 & 3.261449 & -2.082089 \\
\hline 15 & 1.580185 & -0.645048 & -0.366393 \\
\hline
\end{tabular}




\begin{tabular}{|c|c|c|c|}
\hline 8 & 2.411024 & 0.390004 & 0.621549 \\
\hline 6 & 3.343219 & 1.217168 & 0.035374 \\
\hline & 4.583583 & 0.719934 & -0.316706 \\
\hline & 5.484564 & 1.591488 & -1.011099 \\
\hline & 5.115959 & 2.954274 & -1.227039 \\
\hline & 3.862132 & 3.420280 & -0.764365 \\
\hline D & 2.984435 & 2.563838 & -0.158142 \\
\hline & 6.713431 & 1.140656 & -1.558857 \\
\hline$b$ & 7.537594 & 1.992409 & -2.252278 \\
\hline f & 7.183758 & 3.345906 & -2.435439 \\
\hline & 5.995594 & 3.810919 & -1.933159 \\
\hline b & 4.902642 & -0.703271 & -0.068462 \\
\hline & 4.051366 & -1.680422 & -0.550941 \\
\hline$b$ & 4.383563 & -3.052280 & -0.497481 \\
\hline f & 5.527243 & -3.455037 & 0.135361 \\
\hline$b$ & 6.371337 & -2.507872 & 0.766118 \\
\hline 6 & 6.058070 & -1.118861 & 0.668855 \\
\hline$b$ & 7.501917 & -2.917243 & 1.513667 \\
\hline$b$ & 8.284109 & -2.000735 & 2.169095 \\
\hline 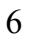 & 7.958569 & -0.629794 & 2.103111 \\
\hline 5 & 6.879481 & -0.199875 & 1.370192 \\
\hline 5 & 2.870657 & -1.345994 & -1.162427 \\
\hline$b$ & -1.923527 & 2.127075 & 0.487993 \\
\hline 6 & -0.986654 & 2.655129 & 1.380349 \\
\hline 6 & -0.984348 & 4.012737 & 1.702457 \\
\hline 6 & -1.890419 & 4.846697 & 1.034292 \\
\hline 6 & -2.798675 & 4.357279 & 0.093287 \\
\hline 6 & -2.815385 & 2.980628 & -0.151411 \\
\hline 6 & -0.091934 & 4.510756 & 2.840731 \\
\hline 6 & 1.363244 & 4.081864 & 2.635415 \\
\hline 6 & -3.776430 & 5.251245 & -0.666249 \\
\hline 6 & -5.212009 & 4.781156 & -0.402178 \\
\hline 6 & -0.117280 & 6.030649 & 2.984805 \\
\hline 6 & -0.620650 & 3.896705 & 4.144268 \\
\hline 6 & -3.673378 & 6.716488 & -0.251089 \\
\hline 6 & -3.474537 & 5.156817 & -2.166550 \\
\hline 6 & -8.470892 & -0.194919 & 0.463087 \\
\hline 5 & -6.858638 & -0.638599 & 2.290821 \\
\hline 6 & -6.808059 & -1.724394 & -3.834323 \\
\hline 6 & -4.646153 & -2.896948 & -3.534129 \\
\hline 1 & 6.630036 & 0.858189 & 1.341649 \\
\hline 1 & -2.591134 & 1.357619 & 2.782683 \\
\hline t & 7.727592 & -3.981616 & 1.567376 \\
\hline 1 & -2.462683 & -1.616952 & 6.454219 \\
\hline 1 & 5.696841 & 4.847973 & -2.080596 \\
\hline 1 & -3.858674 & -2.734960 & 1.269118 \\
\hline 1 & -5.471960 & -4.299431 & 0.277498 \\
\hline 1 & 3.593121 & 4.463504 & -0.924673 \\
\hline 1 & -2.747092 & 0.325145 & 4.992158 \\
\hline 1 & 5.793284 & -4.509869 & 0.180823 \\
\hline 1 & 8.468975 & 1.617316 & -2.670749 \\
\hline 1 & 3.716387 & -3.764387 & -0.979370 \\
\hline 1 & -2.335409 & -6.149251 & -2.012339 \\
\hline 1 & -0.036505 & -5.316431 & -1.833539 \\
\hline 1 & -1.121585 & -5.357303 & 4.815061 \\
\hline 1 & 6.992635 & 0.096431 & -1.444249 \\
\hline
\end{tabular}




\begin{tabular}{|c|c|c|c|}
\hline 1 & -0.999967 & -4.363110 & 2.579016 \\
\hline & 1.578744 & -3.678606 & -0.905259 \\
\hline & 7.846887 & 4.011229 & -2.983464 \\
\hline & 8.565449 & 0.094565 & 2.641917 \\
\hline & -4.717207 & -6.035046 & -1.345081 \\
\hline & -1.856808 & -3.998968 & 6.768413 \\
\hline & 9.144948 & -2.326893 & 2.748059 \\
\hline & 2.003561 & 2.887530 & 0.179856 \\
\hline & -4.475565 & 0.670395 & 1.377897 \\
\hline & -2.975082 & -0.807148 & -2.340243 \\
\hline & -7.191238 & -0.885644 & -1.541258 \\
\hline 1 & -3.528644 & 2.565866 & -0.862944 \\
\hline & -0.275641 & 1.980672 & 1.863658 \\
\hline & -1.889875 & 5.908771 & 1.260144 \\
\hline & 1.243317 & -2.909801 & 2.526593 \\
\hline & -1.397314 & 0.860402 & -2.511168 \\
\hline & 2.767949 & -2.781493 & 1.648150 \\
\hline & 4.415471 & -1.665107 & 2.818616 \\
\hline & 0.357810 & -0.339108 & 3.250319 \\
\hline & 5.168452 & 0.112534 & 4.360084 \\
\hline & 1.098982 & 1.421195 & 4.827041 \\
\hline & 3.512684 & 1.678538 & 5.362587 \\
\hline & 0.041073 & -2.305913 & -2.740061 \\
\hline & -1.414602 & -1.498012 & -3.495370 \\
\hline & 0.119125 & 0.035766 & -5.819645 \\
\hline & -1.212716 & 0.560915 & -4.760403 \\
\hline & 0.296134 & 1.482241 & -4.822226 \\
\hline & 1.738726 & -2.768068 & -4.125305 \\
\hline & 4.168352 & -3.142242 & -4.389187 \\
\hline 1 & 5.745031 & -1.219135 & -4.306526 \\
\hline & 4.856507 & 1.080309 & -3.985406 \\
\hline 1 & 2.434887 & 1.445831 & -3.693592 \\
\hline & -6.900493 & -2.157544 & -4.838317 \\
\hline & -7.302856 & -2.411758 & -3.135987 \\
\hline & -7.359935 & -0.775352 & -3.832780 \\
\hline & -4.859230 & -1.051564 & -5.562876 \\
\hline 1 & -5.192634 & 0.374420 & -4.557239 \\
\hline & -3.641356 & -0.470221 & -4.416806 \\
\hline ] & -4.751497 & -3.351017 & -4.529021 \\
\hline & -3.574378 & -2.829093 & -3.306131 \\
\hline 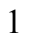 & -5.093901 & -3.577449 & -2.797923 \\
\hline & -7.927196 & 1.880649 & 2.104055 \\
\hline & -6.184347 & 2.017174 & 1.814931 \\
\hline l & -7.323908 & 2.288891 & 0.484620 \\
\hline & -7.654285 & -0.455208 & 3.025650 \\
\hline 1 & -6.851285 & -1.713007 & 2.062615 \\
\hline & -5.900711 & -0.394444 & 2.767996 \\
\hline 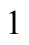 & -9.245397 & 0.018024 & 1.210730 \\
\hline & -8.720406 & 0.376904 & -0.440005 \\
\hline 1 & -8.533641 & -1.263992 & 0.222068 \\
\hline 1 & -4.394951 & 7.312896 & -0.823701 \\
\hline t & -3.900526 & 6.859351 & 0.813442 \\
\hline 1 & -2.676286 & 7.130009 & -0.449430 \\
\hline & -5.925041 & 5.405874 & -0.956991 \\
\hline & -5.372492 & 3.740324 & -0.711620 \\
\hline & -5.458166 & 4.854416 & 0.665560 \\
\hline
\end{tabular}




$\begin{array}{lrrr}1 & -4.168381 & 5.789251 & -2.737077 \\ 1 & -2.452286 & 5.494083 & -2.381246 \\ 1 & -3.570707 & 4.130932 & -2.542755 \\ 1 & 1.969460 & 4.380542 & 3.502021 \\ 1 & 1.479375 & 2.996038 & 2.523420 \\ 1 & 1.792606 & 4.565145 & 1.747509 \\ 1 & -0.010610 & 4.219327 & 4.999690 \\ 1 & -1.657985 & 4.206408 & 4.330534 \\ 1 & -0.603132 & 2.799333 & 4.109520 \\ 1 & 0.557267 & 6.334143 & 3.795477 \\ 1 & 0.219022 & 6.533136 & 2.068619 \\ 1 & -1.116966 & 6.406788 & 3.236452\end{array}$

$3_{\text {IB-re- }-\Psi-122 a 3 a 4 a}$

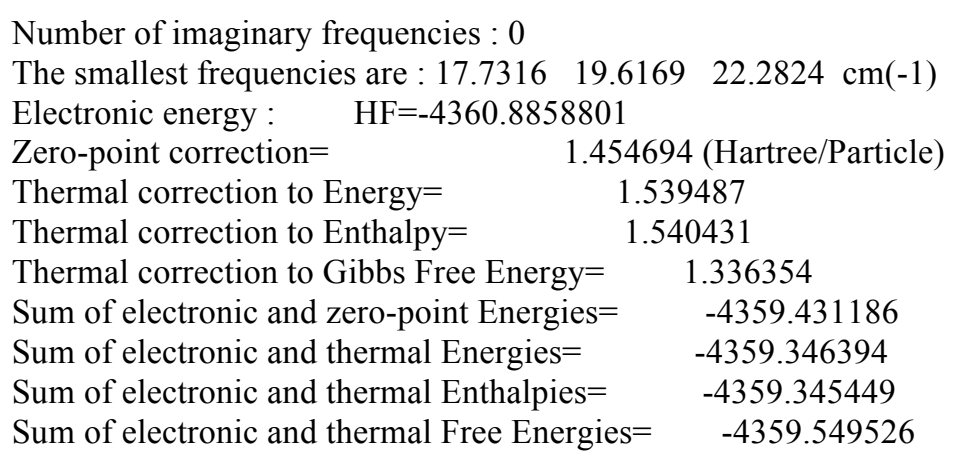

Cartesian Coordinates

$\begin{array}{cccc}6 & -1.927108 & 3.035459 & -4.307908 \\ 6 & -0.697479 & 2.995277 & -3.642013 \\ 6 & -0.114398 & 4.216878 & -3.279905 \\ 6 & -0.754978 & 5.422979 & -3.531599 \\ 6 & -1.996688 & 5.443343 & -4.164101 \\ 6 & -2.576224 & 4.241886 & -4.555622 \\ 6 & 0.011097 & 1.699968 & -3.394530 \\ 6 & 1.425718 & 1.688572 & -3.928979 \\ 45 & -0.148799 & 1.066865 & -1.264351 \\ 15 & 1.733955 & -0.370238 & -0.947637 \\ 7 & 1.404449 & -2.004941 & -0.628976 \\ 6 & 2.359655 & -3.022686 & -0.128388 \\ 6 & 2.965772 & -2.746947 & 1.221746 \\ 6 & 2.160558 & -2.449501 & 2.322268 \\ 6 & 2.723635 & -2.234563 & 3.572703 \\ 6 & 4.105663 & -2.304322 & 3.741910 \\ 6 & 4.914602 & -2.601407 & 2.650896 \\ 6 & 4.345161 & -2.826416 & 1.399530 \\ 15 & -1.566381 & -0.016791 & 0.441773 \\ 6 & -1.312646 & -1.623610 & 1.345512 \\ 6 & -1.062429 & -2.777618 & 0.619809 \\ 6 & -0.960171 & -4.042926 & 1.286412 \\ 6 & -1.123227 & -4.107170 & 2.700849 \\ 6 & -1.382693 & -2.912840 & 3.411289 \\ 6 & -1.470960 & -1.713668 & 2.756339 \\ 6 & -0.689050 & -5.250946 & 0.590440\end{array}$




\begin{tabular}{|c|c|c|c|}
\hline & -0.586458 & -6.445460 & 1.259448 \\
\hline & -0.752880 & -6.501598 & 2.659230 \\
\hline & -1.015591 & -5.353781 & 3.362156 \\
\hline & -0.902276 & -2.785877 & -0.860541 \\
\hline & 0.329236 & -2.518147 & -1.431368 \\
\hline & 0.542583 & -2.732738 & -2.812901 \\
\hline & -0.479594 & -3.146526 & -3.625703 \\
\hline & -1.777417 & -3.338575 & -3.099081 \\
\hline & -1.991538 & -3.177086 & -1.697147 \\
\hline & -2.868431 & -3.690499 & -3.931303 \\
\hline & -4.122238 & -3.870545 & -3.405870 \\
\hline & -4.337371 & -3.712442 & -2.018565 \\
\hline & -3.298008 & -3.385902 & -1.184243 \\
\hline & -1.568991 & 1.215544 & 1.806606 \\
\hline & -2.564930 & 2.187581 & 1.866511 \\
\hline & -2.502545 & 3.223020 & 2.801988 \\
\hline & -1.427544 & 3.233760 & 3.692783 \\
\hline & -0.417834 & 2.265571 & 3.671003 \\
\hline & -0.488223 & 1.274819 & 2.691043 \\
\hline & 0.718145 & 2.326577 & 4.691520 \\
\hline & -3.565159 & 4.317850 & 2.877830 \\
\hline & -3.372096 & -0.198676 & 0.085878 \\
\hline & -4.226146 & -0.702620 & 1.072083 \\
\hline & -5.588824 & -0.861314 & 0.834694 \\
\hline & -6.073538 & -0.490115 & -0.424013 \\
\hline & -5.255813 & 0.027645 & -1.428515 \\
\hline & -3.889785 & 0.164266 & -1.154161 \\
\hline & -6.542341 & -1.437914 & 1.879738 \\
\hline & -5.876064 & 0.434517 & -2.763916 \\
\hline & 0.401486 & 2.635826 & -0.277544 \\
\hline & 0.494228 & 3.700340 & 0.166316 \\
\hline & -0.708328 & 0.483092 & -3.307371 \\
\hline & 2.681365 & -0.101590 & 0.382065 \\
\hline & 3.535786 & 0.975822 & 0.376064 \\
\hline & 4.726822 & 0.913067 & -0.323739 \\
\hline & 5.553111 & 2.084701 & -0.350481 \\
\hline & 5.180553 & 3.223939 & 0.425923 \\
\hline & 3.983481 & 3.193579 & 1.180126 \\
\hline & 3.165631 & 2.098117 & 1.141315 \\
\hline & 6.001406 & 4.377695 & 0.411561 \\
\hline & 7.135528 & 4.428220 & -0.357085 \\
\hline & 7.486729 & 3.320111 & -1.156070 \\
\hline & 6.718908 & 2.182002 & -1.152742 \\
\hline & 5.083528 & -0.327329 & -1.048684 \\
\hline & 4.183762 & -0.884499 & -1.939030 \\
\hline & 4.517546 & -1.995035 & -2.744927 \\
\hline & 5.732662 & -2.605611 & -2.600582 \\
\hline & 6.649019 & -2.156851 & -1.617774 \\
\hline & 6.321341 & -1.016467 & -0.825011 \\
\hline & 7.215441 & -0.650083 & 0.212796 \\
\hline & 8.375126 & -1.352574 & 0.431976 \\
\hline & 8.712434 & -2.456596 & -0.378248 \\
\hline & 7.861747 & -2.848165 & -1.380440 \\
\hline & 2.938002 & -0.334287 & -2.120559 \\
\hline & 6.960397 & 0.185960 & 0.858867 \\
\hline & -1.677466 & -0.813130 & 3.329227 \\
\hline
\end{tabular}




\begin{tabular}{|c|c|c|c|}
\hline ] & 8.095178 & -3.710510 & -2.003712 \\
\hline & -1.143220 & -5.378900 & 4.443489 \\
\hline & 5.701283 & 5.230848 & 1.018550 \\
\hline$\frac{1}{1}$ & -3.474837 & -3.253343 & -0.117889 \\
\hline & -5.338662 & -3.841352 & -1.611333 \\
\hline & 3.709321 & 4.067567 & 1.769888 \\
\hline & -1.514235 & -2.960889 & 4.491671 \\
\hline & 6.000214 & -3.460858 & -3.218910 \\
\hline & 8.371244 & 3.369253 & -1.787266 \\
\hline & 3.788034 & -2.343376 & -3.473031 \\
\hline & -2.688486 & -3.810509 & -4.998777 \\
\hline & -0.314850 & -3.299986 & -4.691182 \\
\hline & -0.376772 & -7.357439 & 0.705168 \\
\hline & 6.998390 & 1.343504 & -1.785072 \\
\hline & -0.561604 & -5.226657 & -0.489892 \\
\hline & 1.534490 & -2.537690 & -3.218954 \\
\hline & 7.755440 & 5.321725 & -0.364709 \\
\hline & 9.036432 & -1.057744 & 1.243721 \\
\hline & -4.955003 & -4.135192 & -4.054188 \\
\hline & -0.670087 & -7.454788 & 3.176160 \\
\hline & 9.636542 & -3.000695 & -0.197793 \\
\hline & 2.222218 & 2.062506 & 1.682717 \\
\hline & -3.813184 & -0.976243 & 2.039124 \\
\hline ] & -3.205857 & 0.559722 & -1.900772 \\
\hline & -7.138162 & -0.607748 & -0.627419 \\
\hline ] & -3.391264 & 2.138773 & 1.161640 \\
\hline & 0.296570 & 0.521325 & 2.615526 \\
\hline & -1.371818 & 4.030246 & 4.434769 \\
\hline ] & 1.775463 & -3.952448 & -0.067794 \\
\hline & -1.477984 & 1.923551 & -1.361725 \\
\hline 1 & 3.158967 & -3.205258 & -0.863077 \\
\hline & 4.987124 & -3.050854 & 0.546413 \\
\hline 1 & 1.085728 & -2.356767 & 2.184478 \\
\hline & 5.995595 & -2.654077 & 2.765784 \\
\hline 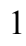 & 2.077324 & -2.010145 & 4.420509 \\
\hline ] & 4.547575 & -2.128059 & 4.720414 \\
\hline & -0.212862 & -0.434377 & -3.619898 \\
\hline ] & -1.791515 & 0.461818 & -3.424269 \\
\hline & 2.092724 & 2.366785 & -3.381774 \\
\hline 1 & 1.863811 & 0.688970 & -3.875078 \\
\hline & 1.427298 & 2.011332 & -4.981832 \\
\hline 1 & 0.848359 & 4.217058 & -2.770313 \\
\hline l & -0.282880 & 6.355234 & -3.227341 \\
\hline | & -2.500223 & 6.388017 & -4.357762 \\
\hline 1 & -3.536702 & 4.237039 & -5.069290 \\
\hline & -2.378406 & 2.105805 & -4.649862 \\
\hline 6 & -4.826393 & 0.937561 & -3.749400 \\
\hline 6 & -6.585220 & -0.766782 & -3.397058 \\
\hline 6 & -6.887327 & 1.561467 & -2.524630 \\
\hline 6 & -5.839486 & -1.730171 & 3.203825 \\
\hline 6 & -7.676667 & -0.444334 & 2.151580 \\
\hline 6 & -7.129111 & -2.752586 & 1.353381 \\
\hline 1 & -7.335328 & 1.881300 & -3.475188 \\
\hline 1 & -7.703306 & 1.249144 & -1.861323 \\
\hline 1 & -6.400833 & 2.433675 & -2.068572 \\
\hline 1 & -5.307482 & 1.222265 & -4.694424 \\
\hline
\end{tabular}




$\begin{array}{rrrr}1 & -4.300448 & 1.821823 & -3.366480 \\ 1 & -4.081318 & 0.161242 & -3.976331 \\ 1 & -7.039949 & -0.478068 & -4.354350 \\ 1 & -5.871764 & -1.578749 & -3.590615 \\ 1 & -7.383948 & -1.165515 & -2.759424 \\ 1 & -6.561650 & -2.138731 & 3.921901 \\ 1 & -5.035966 & -2.470319 & 3.088922 \\ 1 & -5.408851 & -0.823097 & 3.647986 \\ 1 & -8.359607 & -0.845157 & 2.912559 \\ 1 & -7.282046 & 0.510971 & 2.521216 \\ 1 & -8.270954 & -0.235248 & 1.254047 \\ 1 & -7.839074 & -3.172940 & 2.078575 \\ 1 & -7.663546 & -2.616248 & 0.404912 \\ 1 & -6.336956 & -3.495775 & 1.190077 \\ 6 & -4.663845 & 4.132658 & 1.832908 \\ 6 & -4.219646 & 4.302245 & 4.263629 \\ 6 & -2.903271 & 5.679054 & 2.638312 \\ 6 & 1.625727 & 1.099681 & 4.614384 \\ 6 & 1.558347 & 3.582103 & 4.435422 \\ 6 & 0.139830 & 2.384808 & 6.110089 \\ 1 & -5.405166 & 4.935479 & 1.934059 \\ 1 & -4.269138 & 4.176298 & 0.809643 \\ 1 & -5.193183 & 3.177878 & 1.954586 \\ 1 & -4.984609 & 5.087443 & 4.332762 \\ 1 & -4.707181 & 3.337637 & 4.456975 \\ 1 & -3.493680 & 4.476447 & 5.067049 \\ 1 & -3.653825 & 6.480388 & 2.673227 \\ 1 & -2.140999 & 5.905104 & 3.393776 \\ 1 & -2.417351 & 5.709319 & 1.654460 \\ 1 & 2.387548 & 1.150684 & 5.402820 \\ 1 & 1.055626 & 0.171282 & 4.760622 \\ 1 & 2.157910 & 1.020942 & 3.657282 \\ 1 & 0.952383 & 2.419521 & 6.848086 \\ 1 & -0.486977 & 3.269486 & 6.272026 \\ 1 & -0.471283 & 1.497906 & 6.322837 \\ 1 & 2.403227 & 3.629869 & 5.135846 \\ 1 & 1.961773 & 3.584135 & 3.413729 \\ 1 & 0.967958 & 4.498547 & 4.557630\end{array}$

$3_{\text {IB-re }-} \psi_{-1 \text {-1a2a3a4s }}$

Number of imaginary frequencies : 0

The smallest frequencies are : $16.7743 \quad 20.040922 .8853 \mathrm{~cm}(-1)$

Electronic energy : $\quad H F=-4360.8864693$

Zero-point correction $=\quad 1.457833($ Hartree/Particle)

Thermal correction to Energy $=\quad 1.541753$

Thermal correction to Enthalpy= $\quad 1.542697$

Thermal correction to Gibbs Free Energy $=\quad 1.342162$

Sum of electronic and zero-point Energies $=\quad-4359.428636$

Sum of electronic and thermal Energies $=\quad-4359.344716$

Sum of electronic and thermal Enthalpies $=\quad-4359.343772$

Sum of electronic and thermal Free Energies $=\quad-4359.544308$

Cartesian Coordinates

$6 \quad-1.940790 \quad 2.935302 \quad-4.395051$ 


\begin{tabular}{|c|c|c|c|}
\hline 6 & -0.716554 & 2.918511 & -3.718607 \\
\hline 6 & -0.147744 & 4.151471 & -3.372587 \\
\hline 6 & -0.797080 & 5.346942 & -3.650449 \\
\hline 6 & -2.033666 & 5.344630 & -4.293485 \\
\hline 6 & -2.598951 & 4.131338 & -4.668738 \\
\hline 6 & 0.002555 & 1.634535 & -3.443919 \\
\hline 6 & 1.414827 & 1.623051 & -3.984149 \\
\hline 45 & -0.153114 & 1.052067 & -1.295938 \\
\hline 15 & 1.742395 & -0.356983 & -0.947927 \\
\hline 7 & 1.446607 & -1.996229 & -0.614235 \\
\hline 6 & 2.433821 & -2.979435 & -0.106935 \\
\hline 6 & 3.004764 & -2.695856 & 1.257677 \\
\hline 6 & 2.173867 & -2.396280 & 2.338414 \\
\hline 6 & 2.707070 & -2.181893 & 3.602016 \\
\hline 6 & 4.084259 & -2.254815 & 3.805358 \\
\hline 6 & 4.918923 & -2.552109 & 2.734016 \\
\hline 6 & 4.379790 & -2.775141 & 1.468982 \\
\hline 15 & -1.571611 & -0.006108 & 0.412453 \\
\hline 6 & -1.305987 & -1.596734 & 1.340219 \\
\hline 6 & -1.029936 & -2.756098 & 0.633752 \\
\hline 6 & -0.914535 & -4.009896 & 1.319210 \\
\hline 6 & -1.090468 & -4.058510 & 2.732489 \\
\hline 6 & -1.374806 & -2.858674 & 3.423757 \\
\hline 6 & -1.475366 & -1.670386 & 2.751072 \\
\hline 6 & -0.616172 & -5.221037 & 0.641557 \\
\hline 6 & -0.499197 & -6.404971 & 1.327222 \\
\hline 6 & -0.677909 & -6.446036 & 2.725874 \\
\hline 6 & -0.967450 & -5.294008 & 3.411306 \\
\hline 6 & -0.855788 & -2.791454 & -0.844444 \\
\hline 6 & 0.381620 & -2.541590 & -1.409636 \\
\hline 6 & 0.609535 & -2.797726 & -2.781873 \\
\hline 6 & -0.404676 & -3.234087 & -3.593068 \\
\hline 6 & -1.708760 & -3.408609 & -3.074692 \\
\hline 6 & -1.936348 & -3.207580 & -1.680153 \\
\hline 6 & -2.792554 & -3.783327 & -3.906270 \\
\hline 6 & -4.050573 & -3.951331 & -3.386368 \\
\hline 6 & -4.277260 & -3.758935 & -2.005321 \\
\hline 6 & -3.245810 & -3.406427 & -1.171879 \\
\hline 6 & -1.601583 & 1.235807 & 1.768078 \\
\hline 6 & -2.626682 & 2.175973 & 1.839870 \\
\hline 6 & -2.595966 & 3.200378 & 2.788911 \\
\hline 6 & -1.521322 & 3.233094 & 3.679631 \\
\hline 6 & -0.481147 & 2.297978 & 3.644216 \\
\hline 6 & -0.521562 & 1.318547 & 2.651451 \\
\hline 6 & 0.653316 & 2.379372 & 4.665207 \\
\hline 6 & -3.708059 & 4.242301 & 2.896336 \\
\hline 6 & -3.373424 & -0.208461 & 0.047180 \\
\hline 6 & -4.223239 & -0.704682 & 1.032221 \\
\hline 6 & -5.590122 & -0.873514 & 0.802050 \\
\hline 6 & -6.081512 & -0.518990 & -0.453444 \\
\hline 6 & -5.260336 & -0.008759 & -1.466969 \\
\hline 6 & -3.898189 & 0.139584 & -1.198504 \\
\hline 6 & -6.463693 & -1.435076 & 1.921700 \\
\hline 6 & -5.884761 & 0.380009 & -2.805803 \\
\hline 6 & 0.382069 & 2.640664 & -0.333006 \\
\hline 8 & 0.459707 & 3.707500 & 0.108241 \\
\hline
\end{tabular}




\begin{tabular}{|c|c|c|c|}
\hline 6 & -0.706743 & 0.414869 & -3.325935 \\
\hline 8 & 2.675785 & -0.063192 & 0.385960 \\
\hline 6 & 3.524871 & 1.017810 & 0.381400 \\
\hline 6 & 4.724048 & 0.955358 & -0.304000 \\
\hline 6 & 5.548375 & 2.128781 & -0.323924 \\
\hline 6 & 5.163598 & 3.269783 & 0.443978 \\
\hline 6 & 3.956596 & 3.239943 & 1.182275 \\
\hline 6 & 3.142063 & 2.142345 & 1.137172 \\
\hline 6 & 5.982359 & 4.425035 & 0.436935 \\
\hline 6 & 7.126513 & 4.475508 & -0.316732 \\
\hline 6 & 7.490029 & 3.365768 & -1.107796 \\
\hline 6 & 6.724241 & 2.226233 & -1.111242 \\
\hline 6 & 5.090267 & -0.282984 & -1.027981 \\
\hline 6 & 4.202401 & -0.837535 & -1.931760 \\
\hline 6 & 4.553405 & -1.931979 & -2.752224 \\
\hline 6 & 5.769541 & -2.538622 & -2.600828 \\
\hline 6 & 6.669157 & -2.100877 & -1.597578 \\
\hline 6 & 6.327818 & -0.970031 & -0.796973 \\
\hline 6 & 7.206487 & -0.612046 & 0.256731 \\
\hline 6 & 8.363077 & -1.316183 & 0.486724 \\
\hline 6 & 8.713556 & -2.411677 & -0.329532 \\
\hline 6 & 7.879234 & -2.792982 & -1.349365 \\
\hline 8 & 2.951276 & -0.300755 & -2.114134 \\
\hline 1 & 6.940941 & 0.217916 & 0.906527 \\
\hline 1 & -1.698856 & -0.765192 & 3.309907 \\
\hline 1 & 8.123746 & -3.647931 & -1.978538 \\
\hline 1 & -1.104865 & -5.307306 & 4.491641 \\
\hline 1 & 5.672593 & 5.279398 & 1.037353 \\
\hline 1 & -3.431172 & -3.245347 & -0.110782 \\
\hline 1 & -5.280463 & -3.882726 & \\
\hline 1 & 3.672918 & 4.115325 & 1.765424 \\
\hline 1 & -1.514454 & -2.893424 & 4.503626 \\
\hline 1 & 6.050469 & -3.382481 & -3.228749 \\
\hline 1 & 8.382621 & 3.414630 & -1.727536 \\
\hline 1 & 3.835305 & -2.268159 & -3.497351 \\
\hline 1 & -2.603993 & -3.931431 & -4.968706 \\
\hline 1 & -0.228810 & -3.420267 & -4.651512 \\
\hline 1 & -0.268671 & -7.320247 & 0.786814 \\
\hline 1 & 7.013582 & 1.386786 & -1.737772 \\
\hline 1 & -0.478936 & -5.210028 & -0.437711 \\
\hline 1 & 1.608101 & -2.621285 & -3.180090 \\
\hline 1 & 7.744825 & 5.370145 & -0.318805 \\
\hline 1 & 9.011907 & -1.029011 & 1.311190 \\
\hline 1 & -4.877587 & -4.233745 & -4.034524 \\
\hline 1 & -0.583290 & -7.390883 & 3.255926 \\
\hline 1 & 9.635208 & -2.957012 & -0.140450 \\
\hline 1 & 2.192355 & 2.105970 & 1.667336 \\
\hline 1 & -3.814946 & -0.968027 & 2.006423 \\
\hline 1 & -3.218964 & 0.533127 & -1.950184 \\
\hline 1 & -7.143734 & -0.637293 & -0.658158 \\
\hline 1 & -3.453324 & 2.108789 & 1.137152 \\
\hline 1 & 0.283866 & 0.587742 & 2.568593 \\
\hline 1 & -1.494043 & 4.016637 & 4.436673 \\
\hline 1 & 1.891175 & -3.935077 & -0.067681 \\
\hline 1 & -1.487787 & 1.898518 & -1.414953 \\
\hline 1 & 3.253415 & -3.120588 & -0.827788 \\
\hline
\end{tabular}




\begin{tabular}{|c|c|c|c|}
\hline 1 & 5.042801 & -2.998631 & 0.631666 \\
\hline & 1.102898 & -2.300831 & 2.174862 \\
\hline & 5.996813 & -2.605302 & 2.874575 \\
\hline & 2.041101 & -1.955270 & 4.433822 \\
\hline & 4.502391 & -2.079603 & 4.794426 \\
\hline & -0.204699 & -0.506798 & -3.615821 \\
\hline 1 & -1.789813 & 0.382369 & -3.439789 \\
\hline & 2.078192 & 2.319381 & -3.455954 \\
\hline 1 & 1.862021 & 0.628786 & -3.910096 \\
\hline & 1.407990 & 1.922080 & -5.043970 \\
\hline 1 & 0.810514 & 4.168776 & -2.854771 \\
\hline 1 & -0.336366 & 6.288723 & -3.358339 \\
\hline 1 & -2.544371 & 6.281062 & -4.507357 \\
\hline 1 & -3.555217 & 4.108794 & -5.189759 \\
\hline 1 & -2.380498 & 1.995455 & -4.724114 \\
\hline 6 & -4.836736 & 0.860255 & -3.804284 \\
\hline 6 & -6.605166 & -0.825842 & -3.417439 \\
\hline 6 & -6.887724 & 1.517158 & -2.579952 \\
\hline 6 & -7.923034 & -1.586568 & 1.501301 \\
\hline 6 & -5.939611 & -2.818409 & 2.326535 \\
\hline 0 & -6.408419 & -0.490838 & 3.128411 \\
\hline 1 & -7.338400 & 1.824357 & -3.533440 \\
\hline 1 & -7.702353 & 1.220483 & -1.907993 \\
\hline 1 & -6.393426 & 2.393484 & -2.140433 \\
\hline 1 & -5.319822 & 1.127437 & -4.753406 \\
\hline 1 & -4.307330 & 1.750605 & -3.441039 \\
\hline 1 & -4.094421 & 0.077366 & -4.017327 \\
\hline 1 & -7.054374 & -0.550095 & -4.381103 \\
\hline 1 & -5.900519 & -1.649194 & -3.594549 \\
\hline 1 & -7.410352 & -1.203255 & -2.775393 \\
\hline 1 & -8.506911 & -1.997205 & 2.334724 \\
\hline 1 & -8.373843 & -0.623961 & 1.227677 \\
\hline 1 & -8.034783 & -2.273019 & 0.651727 \\
\hline 1 & -6.547822 & -3.233712 & 3.141478 \\
\hline 1 & -5.988834 & -3.517983 & 1.481080 \\
\hline 1 & -4.898371 & -2.787728 & 2.673179 \\
\hline 1 & -7.014000 & -0.888770 & 3.953912 \\
\hline 1 & -5.385725 & -0.353380 & 3.501352 \\
\hline 1 & -6.803093 & 0.499840 & 2.867138 \\
\hline 6 & -4.770043 & 4.070076 & 1.811950 \\
\hline 6 & -4.393396 & 4.106054 & 4.260696 \\
\hline 6 & -3.112353 & 5.647146 & 2.759266 \\
\hline 6 & 1.572091 & 1.159815 & 4.600748 \\
\hline 6 & 1.480450 & 3.640766 & 4.396070 \\
\hline 6 & 0.076231 & 2.445979 & 6.083855 \\
\hline 1 & -5.543629 & 4.839366 & 1.930510 \\
\hline 1 & -4.348641 & 4.177349 & 0.804095 \\
\hline 1 & -5.267341 & 3.092456 & 1.871729 \\
\hline 1 & -5.198446 & 4.846766 & 4.360342 \\
\hline 1 & -4.835484 & 3.107613 & 4.378665 \\
\hline 1 & -3.693172 & 4.262063 & 5.090504 \\
\hline 1 & -3.905151 & 6.404556 & 2.824981 \\
\hline 1 & -2.381037 & 5.866236 & 3.546455 \\
\hline & -2.607977 & 5.766123 & 1.791743 \\
\hline 1 & 2.330916 & 1.224090 & 5.391098 \\
\hline & 1.009315 & 0.227861 & 4.752838 \\
\hline
\end{tabular}


$3_{\text {IB-re }-} \psi_{-1 \text { a2a3s4a }}$

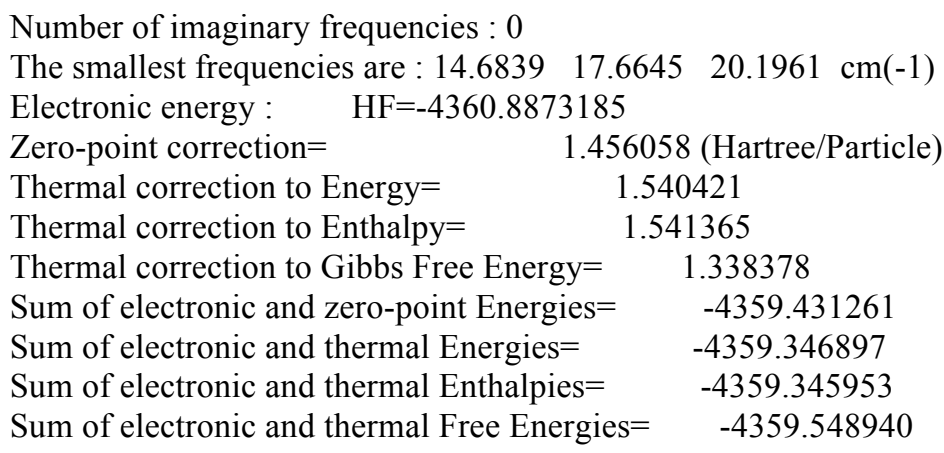

Cartesian Coordinates

$\begin{array}{cccc}6 & -2.058927 & 2.869477 & -4.296805 \\ 6 & -0.780065 & 2.840111 & -3.728517 \\ 6 & -0.150283 & 4.067995 & -3.483172 \\ 6 & -0.782717 & 5.272267 & -3.761211 \\ 6 & -2.065795 & 5.284423 & -4.304109 \\ 6 & -2.697585 & 4.075592 & -4.572940 \\ 6 & -0.068559 & 1.552945 & -3.449975 \\ 6 & 1.330902 & 1.507079 & -4.021064 \\ 45 & -0.176946 & 1.033168 & -1.280928 \\ 15 & 1.704060 & -0.389859 & -0.935411 \\ 7 & 1.387557 & -2.017430 & -0.567558 \\ 6 & 2.365079 & -3.008729 & -0.055906 \\ 6 & 2.960467 & -2.707279 & 1.293811 \\ 6 & 2.146588 & -2.394201 & 2.383373 \\ 6 & 2.700044 & -2.158464 & 3.634424 \\ 6 & 4.080861 & -2.222369 & 3.814991 \\ 6 & 4.898419 & -2.535105 & 2.734794 \\ 6 & 4.338834 & -2.780898 & 1.482928 \\ 15 & -1.573146 & 0.023522 & 0.477323 \\ 6 & -1.327361 & -1.562903 & 1.414160 \\ 6 & -1.085474 & -2.734046 & 0.713932 \\ 6 & -0.983730 & -3.983734 & 1.410916 \\ 6 & -1.143522 & -4.013686 & 2.826889 \\ 6 & -1.399043 & -2.802406 & 3.509656 \\ 6 & -1.483342 & -1.618771 & 2.827047 \\ 6 & -0.711781 & -5.208338 & 0.744641 \\ 6 & -0.606895 & -6.385868 & 1.443057 \\ 6 & -0.771731 & -6.408130 & 2.843797 \\ 6 & -1.034341 & -5.243505 & 3.518497 \\ 6 & -0.926741 & -2.790649 & -0.766121 \\ 6 & 0.309152 & -2.563347 & -1.344515 \\ 6 & 0.530115 & -2.862419 & -2.709227\end{array}$




\begin{tabular}{|c|c|c|c|}
\hline & -0.484979 & -3.333341 & -3.499417 \\
\hline & -1.786155 & -3.494469 & -2.969575 \\
\hline & -2.012553 & -3.228655 & -1.585414 \\
\hline & -2.864912 & -3.929105 & -3.778371 \\
\hline & -4.119955 & -4.087012 & -3.248950 \\
\hline & -4.350725 & -3.810806 & -1.883126 \\
\hline & -3.323179 & -3.402357 & -1.070152 \\
\hline & -1.547246 & 1.292806 & 1.805944 \\
\hline & -2.518424 & 2.291333 & 1.831356 \\
\hline & -2.422687 & 3.362335 & 2.723152 \\
\hline & -1.344496 & 3.378244 & 3.610007 \\
\hline & -0.361736 & 2.382677 & 3.624672 \\
\hline & -0.461852 & 1.356788 & 2.684397 \\
\hline & 0.772870 & 2.449005 & 4.646367 \\
\hline & -3.444682 & 4.497445 & 2.748559 \\
\hline & -3.379612 & -0.154714 & 0.134145 \\
\hline & -4.244815 & -0.636125 & 1.127651 \\
\hline & -5.598494 & -0.817045 & 0.874892 \\
\hline & -6.076395 & -0.496282 & -0.407169 \\
\hline & -5.250362 & -0.002106 & -1.409967 \\
\hline & -3.888080 & 0.162754 & -1.115099 \\
\hline & -6.562912 & -1.359124 & 1.928230 \\
\hline & -5.753141 & 0.359018 & -2.804749 \\
\hline & 0.403487 & 2.644914 & -0.386030 \\
\hline & 0.513667 & 3.726692 & 0.009368 \\
\hline & -0.784458 & 0.344187 & -3.279934 \\
\hline 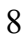 & 2.660206 & -0.084588 & 0.379481 \\
\hline 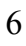 & 3.522375 & 0.985620 & 0.341149 \\
\hline 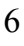 & 4.709269 & 0.895527 & -0.362442 \\
\hline f & 5.546408 & 2.058596 & -0.420090 \\
\hline & 5.186590 & 3.219635 & 0.329730 \\
\hline 5 & 3.992044 & 3.218308 & 1.088497 \\
\hline f & 3.164898 & 2.129254 & 1.080434 \\
\hline 6 & 6.017230 & 4.365626 & 0.284721 \\
\hline ( & 7.149148 & 4.387664 & -0.488368 \\
\hline 6 & 7.487989 & 3.257554 & -1.261253 \\
\hline 5 & 6.710355 & 2.126659 & -1.228253 \\
\hline 5 & 5.050121 & -0.361096 & -1.066867 \\
\hline 5 & 4.142169 & -0.923179 & -1.945573 \\
\hline 5 & 4.467514 & -2.039375 & -2.747232 \\
\hline 6 & 5.678599 & -2.657426 & -2.600984 \\
\hline 6 & 6.598985 & -2.209143 & -1.621550 \\
\hline 6 & 6.283104 & -1.057549 & -0.840263 \\
\hline 6 & 7.181970 & -0.687531 & 0.192042 \\
\hline 6 & 8.334159 & -1.399778 & 0.418981 \\
\hline 6 & 8.659457 & -2.516358 & -0.378955 \\
\hline 6 & 7.804933 & -2.909804 & -1.377199 \\
\hline 8 & 2.895751 & -0.373126 & -2.120583 \\
\hline 1 & 6.935624 & 0.158957 & 0.827994 \\
\hline 1 & -1.683801 & -0.703495 & 3.378514 \\
\hline 1 & 8.029828 & -3.780917 & -1.991356 \\
\hline 1 & -1.159948 & -5.241892 & 4.600356 \\
\hline 1 & 5.726290 & 5.235753 & 0.871760 \\
\hline 1 & -3.512779 & -3.183787 & -0.019968 \\
\hline 1 & -5.353526 & -3.916812 & -1.473703 \\
\hline 1 & 3.727645 & 4.109332 & 1.656756 \\
\hline
\end{tabular}




\begin{tabular}{|c|c|c|c|}
\hline 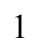 & -1.528771 & -2.824909 & 4.591070 \\
\hline [ & 5.939781 & -3.517956 & -3.214688 \\
\hline 1 & 8.370841 & 3.283651 & -1.896178 \\
\hline & 3.734939 & -2.383357 & -3.474550 \\
\hline & -2.674132 & -4.132146 & -4.831278 \\
\hline & -0.311151 & -3.563268 & -4.549537 \\
\hline & -0.396386 & -7.310770 & 0.910939 \\
\hline & 6.980682 & 1.271264 & -1.841565 \\
\hline & -0.584414 & -5.212278 & -0.335898 \\
\hline 1 & 1.527100 & -2.700305 & -3.116986 \\
\hline & 7.776795 & 5.275236 & -0.519508 \\
\hline & 8.999079 & -1.102727 & 1.226958 \\
\hline 1 & -4.942615 & -4.417232 & -3.879687 \\
\hline & -0.687405 & -7.348289 & 3.383822 \\
\hline 1 & 9.577804 & -3.068128 & -0.192496 \\
\hline 1 & 2.224359 & 2.114092 & 1.628086 \\
\hline 1 & -3.841198 & -0.875781 & 2.107725 \\
\hline 1 & -3.205532 & 0.543022 & -1.872252 \\
\hline 1 & -7.134812 & -0.640126 & -0.615353 \\
\hline 1 & -3.348155 & 2.236651 & 1.130556 \\
\hline 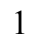 & 0.303404 & 0.581468 & 2.634674 \\
\hline 1 & -1.263333 & 4.201994 & 4.319068 \\
\hline 1 & 1.804165 & -3.952002 & 0.011420 \\
\hline 1 & -1.501976 & 1.895875 & -1.389097 \\
\hline 1 & 3.171393 & -3.179675 & -0.785402 \\
\hline 1 & 4.987821 & -3.017704 & 0.638421 \\
\hline 1 & 1.072588 & -2.308000 & 2.235675 \\
\hline 1 & 5.978679 & -2.583214 & 2.858253 \\
\hline 1 & 2.047431 & -1.921862 & 4.473988 \\
\hline 1 & 4.515071 & -2.029206 & 4.793758 \\
\hline 1 & -0.300026 & -0.590303 & -3.558399 \\
\hline 1 & -1.869489 & 0.319404 & -3.367558 \\
\hline 1 & 2.019935 & 2.200129 & -3.522832 \\
\hline 1 & 1.761450 & 0.506313 & -3.934852 \\
\hline 1 & 1.306126 & 1.783264 & -5.086721 \\
\hline 1 & 0.845190 & 4.075341 & -3.041133 \\
\hline 1 & -0.271027 & 6.209039 & -3.548756 \\
\hline 1 & -2.563196 & 6.227058 & -4.521821 \\
\hline 1 & -3.693996 & 4.065775 & -5.012149 \\
\hline 1 & -2.561584 & 1.933423 & -4.539854 \\
\hline 6 & -7.253506 & 0.128428 & -2.963878 \\
\hline 6 & -5.466608 & 1.841125 & -3.070920 \\
\hline 6 & -5.029517 & -0.505676 & -3.843384 \\
\hline 6 & -5.866714 & -1.642592 & 3.257664 \\
\hline 6 & -7.675590 & -0.336083 & 2.180698 \\
\hline 6 & -7.176594 & -2.671336 & 1.427652 \\
\hline 1 & -5.333205 & -0.217551 & -4.859227 \\
\hline 1 & -3.937336 & -0.413494 & -3.779526 \\
\hline 1 & -5.270631 & -1.567887 & -3.702654 \\
\hline 1 & -7.561796 & 0.401488 & -3.981101 \\
\hline 1 & -7.525159 & -0.924331 & -2.810418 \\
\hline 1 & -7.840213 & 0.741597 & -2.267492 \\
\hline 1 & -5.796030 & 2.117046 & -4.082563 \\
\hline 1 & -6.002220 & 2.478278 & -2.354738 \\
\hline 1 & -4.398987 & 2.080001 & -2.989399 \\
\hline 1 & -6.595034 & -2.035141 & 3.978441 \\
\hline
\end{tabular}




$\begin{array}{rrrr}1 & -5.070365 & -2.392027 & 3.152771 \\ 1 & -5.428317 & -0.735161 & 3.693066 \\ 1 & -8.369739 & -0.708249 & 2.946181 \\ 1 & -7.259603 & 0.616126 & 2.535108 \\ 1 & -8.261201 & -0.128793 & 1.277074 \\ 1 & -7.885795 & -3.068080 & 2.166657 \\ 1 & -7.719892 & -2.542443 & 0.483516 \\ 1 & -6.398240 & -3.429976 & 1.268557 \\ 6 & -4.562241 & 4.294404 & 1.727444 \\ 6 & -4.082228 & 4.588731 & 4.139145 \\ 6 & -2.733598 & 5.815504 & 2.422726 \\ 6 & 1.665004 & 1.209665 & 4.595665 \\ 6 & 1.630780 & 3.688453 & 4.372732 \\ 6 & 0.187541 & 2.540672 & 6.060456 \\ 1 & -5.271321 & 5.129671 & 1.789198 \\ 1 & -4.179777 & 4.263531 & 0.699019 \\ 1 & -5.125735 & 3.369652 & 1.911204 \\ 1 & -4.818173 & 5.403563 & 4.167520 \\ 1 & -4.601012 & 3.655893 & 4.396105 \\ 1 & -3.341592 & 4.786252 & 4.923526 \\ 1 & -3.452098 & 6.646457 & 2.416864 \\ 1 & -1.954264 & 6.054931 & 3.156444 \\ 1 & -2.258079 & 5.768327 & 1.434359 \\ 1 & 2.431484 & 1.270997 & 5.378872 \\ 1 & 1.084454 & 0.292439 & 4.768684 \\ 1 & 2.190732 & 1.100193 & 3.637897 \\ 1 & 0.995917 & 2.578687 & 6.802914 \\ 1 & -0.429352 & 3.436029 & 6.200906 \\ 1 & -0.436369 & 1.665770 & 6.285450 \\ 1 & 2.468014 & 3.740991 & 5.081853 \\ 1 & 2.047012 & 3.662578 & 3.356621 \\ 1 & 1.051419 & 4.614981 & 4.468163\end{array}$

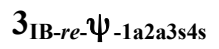

Number of imaginary frequencies : 0

The smallest frequencies are : $14.3362 \quad 18.0812 \quad 23.4769 \mathrm{~cm}(-1)$

Electronic energy : $\quad \mathrm{HF}=-4360.8876202$

Zero-point correction $=\quad 1.456317$ (Hartree/Particle)

Thermal correction to Energy $=\quad 1.540556$

Thermal correction to Enthalpy $=\quad 1.541500$

Thermal correction to Gibbs Free Energy $=\quad 1.339704$

Sum of electronic and zero-point Energies $=\quad-4359.431303$

Sum of electronic and thermal Energies $=\quad-4359.347064$

Sum of electronic and thermal Enthalpies $=\quad-4359.346120$

Sum of electronic and thermal Free Energies $=\quad-4359.547917$

Cartesian Coordinates

$\begin{array}{llll}6 & -2.038089 & 2.842532 & -4.344917 \\ 6 & -0.767099 & 2.826414 & -3.758877 \\ 6 & -0.151383 & 4.060546 & -3.509194 \\ 6 & -0.790064 & 5.258218 & -3.800876 \\ 6 & -2.065469 & 5.257214 & -4.361816 \\ 6 & -2.683231 & 4.042053 & -4.634481 \\ 6 & -0.047787 & 1.546383 & -3.467830\end{array}$




\begin{tabular}{|c|c|c|c|}
\hline 6 & 1.357022 & 1.510369 & -4.026621 \\
\hline 45 & -0.171395 & 1.033307 & -1.298225 \\
\hline 15 & 1.713547 & -0.379742 & -0.935224 \\
\hline 7 & 1.401864 & -2.007039 & -0.561915 \\
\hline 6 & 2.381840 & -2.990850 & -0.040306 \\
\hline 6 & 2.966297 & -2.682745 & 1.312793 \\
\hline 6 & 2.143856 & -2.365395 & 2.394661 \\
\hline 6 & 2.687278 & -2.126535 & 3.649491 \\
\hline 6 & 4.066474 & -2.191241 & 3.841655 \\
\hline 6 & 4.892631 & -2.507255 & 2.768994 \\
\hline 6 & 4.343137 & -2.756162 & 1.513289 \\
\hline 15 & -1.573275 & 0.024264 & 0.453319 \\
\hline 6 & -1.329782 & -1.555560 & 1.403405 \\
\hline 6 & -1.074619 & -2.730329 & 0.714467 \\
\hline 6 & -0.972207 & -3.974515 & 1.420799 \\
\hline 6 & -1.144348 & -3.995515 & 2.835340 \\
\hline 6 & -1.411200 & -2.780609 & 3.507235 \\
\hline 6 & -1.496404 & -1.602237 & 2.815448 \\
\hline 6 & -0.687924 & -5.201972 & 0.765113 \\
\hline 6 & -0.582717 & -6.374043 & 1.472660 \\
\hline 6 & -0.759591 & -6.387553 & 2.872057 \\
\hline 6 & -1.034354 & -5.219844 & 3.536472 \\
\hline 6 & -0.906939 & -2.795916 & -0.763707 \\
\hline 6 & 0.328847 & -2.562632 & -1.339494 \\
\hline 6 & 0.554649 & -2.866857 & -2.702420 \\
\hline 6 & -0.455156 & -3.349592 & -3.492527 \\
\hline 6 & -1.756090 & -3.519569 & -2.964573 \\
\hline 6 & -1.986988 & -3.247567 & -1.582645 \\
\hline 6 & -2.830052 & -3.970107 & -3.771144 \\
\hline 6 & -4.083738 & -4.138050 & -3.241141 \\
\hline 6 & -4.318545 & -3.855577 & -1.877182 \\
\hline 6 & -3.295902 & -3.430475 & -1.066849 \\
\hline 6 & -1.571369 & 1.293822 & 1.781893 \\
\hline 6 & -2.568049 & 2.265907 & 1.822535 \\
\hline 6 & -2.500044 & 3.325112 & 2.730674 \\
\hline 6 & -1.419298 & 3.359626 & 3.613773 \\
\hline 6 & -0.410117 & 2.390618 & 3.612470 \\
\hline 6 & -0.486365 & 1.374481 & 2.659517 \\
\hline 6 & 0.726875 & 2.474026 & 4.630387 \\
\hline 6 & -3.566018 & 4.417489 & 2.788533 \\
\hline 6 & -3.375364 & -0.162132 & 0.092158 \\
\hline 6 & -4.246904 & -0.625683 & 1.080012 \\
\hline 6 & -5.604410 & -0.806372 & 0.824967 \\
\hline 6 & -6.075680 & -0.504275 & -0.458588 \\
\hline 6 & -5.235753 & -0.026768 & -1.465520 \\
\hline 6 & -3.878527 & 0.140141 & -1.167201 \\
\hline 6 & -6.503537 & -1.328391 & 1.944439 \\
\hline 6 & -5.731557 & 0.314119 & -2.868685 \\
\hline 6 & 0.386301 & 2.650344 & -0.398014 \\
\hline 8 & 0.478611 & 3.731397 & 0.003880 \\
\hline 6 & -0.755438 & 0.332342 & -3.300488 \\
\hline 8 & 2.660673 & -0.065273 & 0.383825 \\
\hline 6 & 3.519784 & 1.007309 & 0.345704 \\
\hline 6 & 4.712218 & 0.916652 & -0.348291 \\
\hline 6 & 5.546948 & 2.081568 & -0.404646 \\
\hline 6 & 5.178018 & 3.245464 & 0.336401 \\
\hline
\end{tabular}




\begin{tabular}{|c|c|c|c|}
\hline 6 & 3.977151 & 3.245182 & 1.085233 \\
\hline & 3.153150 & 2.153808 & 1.075987 \\
\hline & 6.006315 & 4.393167 & 0.292785 \\
\hline & 7.144812 & 4.414114 & -0.470639 \\
\hline & 7.492825 & 3.281079 & -1.235097 \\
\hline & 6.717503 & 2.148538 & -1.203246 \\
\hline & 5.060268 & -0.342042 & -1.045677 \\
\hline & 4.159129 & -0.908647 & -1.928584 \\
\hline & 4.491701 & -2.026067 & -2.725471 \\
\hline & 5.702912 & -2.641433 & -2.569745 \\
\hline & 6.615691 & -2.189053 & -1.585097 \\
\hline & 6.292890 & -1.035878 & -0.808999 \\
\hline & 7.184166 & -0.661912 & 0.228455 \\
\hline & 8.335449 & -1.372406 & 0.465539 \\
\hline & 8.667599 & -2.490753 & -0.327104 \\
\hline & 7.820704 & -2.887617 & -1.330484 \\
\hline & 2.912311 & -0.362611 & -2.112985 \\
\hline & 6.932425 & 0.186138 & 0.860239 \\
\hline & -1.703974 & -0.684073 & 3.359259 \\
\hline & 8.050950 & -3.759996 & -1.940833 \\
\hline & -1.169389 & -5.211544 & 4.617164 \\
\hline & 5.708380 & 5.265462 & 0.873063 \\
\hline & -3.487061 & -3.206655 & -0.018043 \\
\hline & -5.320127 & -3.969428 & -1.466684 \\
\hline & 3.705988 & 4.138097 & 1.647302 \\
\hline & -1.548412 & -2.795908 & 4.587857 \\
\hline & 5.969805 & -3.503054 & -3.179430 \\
\hline 1 & 8.381033 & 3.306122 & -1.862546 \\
\hline & 3.764146 & -2.373116 & -3.456383 \\
\hline & -2.636956 & -4.177832 & -4.822712 \\
\hline & -0.276924 & -3.583515 & -4.541031 \\
\hline & -0.362669 & -7.301469 & 0.948854 \\
\hline 1 & 6.995020 & 1.290855 & -1.810112 \\
\hline & -0.551233 & -5.212494 & -0.314237 \\
\hline 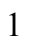 & 1.551287 & -2.699223 & -3.108961 \\
\hline & 7.770647 & 5.302998 & -0.500716 \\
\hline 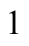 & 8.994297 & -1.072482 & 1.277421 \\
\hline 1 & -4.902285 & -4.481184 & -3.870338 \\
\hline & -0.674892 & -7.323532 & 3.419236 \\
\hline ] & 9.585150 & -3.041070 & -0.132603 \\
\hline & 2.208587 & 2.138387 & 1.616516 \\
\hline ] & -3.856418 & -0.854588 & 2.070497 \\
\hline & -3.190915 & 0.510278 & -1.924653 \\
\hline . & -7.130433 & -0.644416 & -0.676652 \\
\hline ] & -3.398403 & 2.198435 & 1.123971 \\
\hline ] & 0.295981 & 0.616951 & 2.601367 \\
\hline 1 & -1.360041 & 4.174722 & 4.334937 \\
\hline & 1.826634 & -3.937650 & 0.025786 \\
\hline 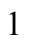 & -1.501952 & 1.886055 & -1.420187 \\
\hline & 3.194100 & -3.158995 & -0.763767 \\
\hline & 4.998909 & -2.995536 & 0.674742 \\
\hline 1 & 1.071234 & -2.278354 & 2.237584 \\
\hline 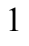 & 5.971816 & -2.555559 & 2.901377 \\
\hline 1 & 2.028056 & -1.887053 & 4.483021 \\
\hline & 4.492756 & -1.995898 & 4.823462 \\
\hline & -0.260893 & -0.599033 & -3.571521 \\
\hline
\end{tabular}




\begin{tabular}{|c|c|c|c|}
\hline 1 & -1.839317 & 0.298408 & -3.398898 \\
\hline & 2.036137 & 2.210126 & -3.524122 \\
\hline & 1.794831 & 0.513314 & -3.934704 \\
\hline & 1.339151 & 1.784092 & -5.093047 \\
\hline & 0.837673 & 4.078019 & -3.053186 \\
\hline & -0.289527 & 6.200198 & -3.584909 \\
\hline & -2.567797 & 6.194721 & -4.590097 \\
\hline & -3.673419 & 4.021904 & -5.087219 \\
\hline & -2.528998 & 1.901179 & -4.591465 \\
\hline 6 & -7.230166 & 0.076356 & -3.035184 \\
\hline & -5.448374 & 1.793216 & -3.154137 \\
\hline & -4.998656 & -0.561684 & -3.891298 \\
\hline & -7.954943 & -1.490635 & 1.500526 \\
\hline b & -5.992885 & -2.698474 & 2.407423 \\
\hline & -6.470744 & -0.344092 & 3.119526 \\
\hline & -5.301390 & -0.292255 & -4.912557 \\
\hline 1 & -3.907506 & -0.459797 & -3.825660 \\
\hline & -5.232487 & -1.623137 & -3.734091 \\
\hline 1 & -7.531514 & 0.333711 & -4.058585 \\
\hline & -7.499998 & -0.974801 & -2.868365 \\
\hline 1 & -7.824297 & 0.697774 & -2.352586 \\
\hline 1 & -5.769823 & 2.053252 & -4.172586 \\
\hline 1 & -5.992551 & 2.438719 & -2.452149 \\
\hline & -4.382341 & 2.036782 & -3.066466 \\
\hline 1 & -8.555494 & -1.872107 & 2.336007 \\
\hline | & -8.398182 & -0.536905 & 1.186579 \\
\hline 1 & -8.053181 & -2.204990 & 0.672616 \\
\hline 1 & -6.615407 & -3.081177 & 3.227602 \\
\hline & -6.033004 & -3.428173 & 1.587311 \\
\hline l & -4.956571 & -2.659606 & 2.767709 \\
\hline & -7.109230 & -0.703366 & 3.937971 \\
\hline ] & -5.458461 & -0.212287 & 3.521118 \\
\hline 1 & -6.838506 & 0.643870 & 2.812528 \\
\hline 5 & -4.663861 & 4.210654 & 1.746937 \\
\hline 6 & -4.220397 & 4.419596 & 4.174467 \\
\hline ? & -2.909606 & 5.777276 & 2.527321 \\
\hline 6 & 1.630386 & 1.242651 & 4.584320 \\
\hline 6 & 1.570794 & 3.720354 & 4.344507 \\
\hline 6 & 0.148003 & 2.569015 & 6.046878 \\
\hline 1 & -5.407379 & 5.013154 & 1.833452 \\
\hline 1 & -4.268796 & 4.236480 & 0.723207 \\
\hline 1 & -5.190450 & 3.256674 & 1.886529 \\
\hline 1 & -4.986751 & 5.204303 & 4.233310 \\
\hline 1 & -4.706294 & 3.456499 & 4.380079 \\
\hline 1 & -3.494243 & 4.605318 & 4.975207 \\
\hline 1 & -3.664243 & 6.575297 & 2.543963 \\
\hline 1 & -2.152335 & 6.021562 & 3.282147 \\
\hline 1 & -2.418952 & 5.791711 & 1.545423 \\
\hline 1 & 2.395614 & 1.313441 & 5.367950 \\
\hline 1 & 1.057570 & 0.320996 & 4.759622 \\
\hline 1 & 2.158506 & 1.134150 & 3.627902 \\
\hline 1 & 0.960243 & 2.615928 & 6.784523 \\
\hline 1 & -0.473344 & 3.461213 & 6.187341 \\
\hline 1 & -0.468899 & 1.691232 & 6.279768 \\
\hline 1 & 2.410079 & 3.787213 & 5.050039 \\
\hline 1 & 1.983258 & 3.691810 & 3.326875 \\
\hline
\end{tabular}


$3_{\text {IB-re- }} \psi_{-12 a 2 s 3 a 4 a}$

Number of imaginary frequencies : 0

The smallest frequencies are : $12.9155 \quad 17.3317 \quad 19.0374 \mathrm{~cm}(-1)$

Electronic energy : $\quad H F=-4360.8866538$

Zero-point correction $=\quad 1.454587$ (Hartree/Particle)

Thermal correction to Energy= $\quad 1.539314$

Thermal correction to Enthalpy $=\quad 1.540258$

Thermal correction to Gibbs Free Energy= $\quad 1.335895$

Sum of electronic and zero-point Energies $=\quad-4359.432066$

Sum of electronic and thermal Energies $=\quad-4359.347340$

Sum of electronic and thermal Enthalpies $=\quad-4359.346396$

Sum of electronic and thermal Free Energies $=\quad-4359.550759$

Cartesian Coordinates

$\begin{array}{cccc}6 & -1.938597 & 3.008738 & -4.341953 \\ 6 & -0.722657 & 2.984498 & -3.650899 \\ 6 & -0.164071 & 4.212809 & -3.273712 \\ 6 & -0.816844 & 5.410277 & -3.534576 \\ 6 & -2.046226 & 5.414614 & -4.191238 \\ 6 & -2.600519 & 4.206532 & -4.598315 \\ 6 & -0.001633 & 1.697542 & -3.395241 \\ 6 & 1.411547 & 1.697850 & -3.933146 \\ 45 & -0.154836 & 1.074037 & -1.260964 \\ 15 & 1.726248 & -0.364214 & -0.947677 \\ 7 & 1.403385 & -2.002420 & -0.636934 \\ 6 & 2.376092 & -3.014056 & -0.157503 \\ 6 & 2.970991 & -2.759636 & 1.202087 \\ 6 & 2.156326 & -2.497088 & 2.304493 \\ 6 & 2.710178 & -2.303537 & 3.562667 \\ 6 & 4.092005 & -2.359022 & 3.737937 \\ 6 & 4.910380 & -2.621597 & 2.645041 \\ 6 & 4.350419 & -2.825967 & 1.386011 \\ 15 & -1.563800 & -0.003310 & 0.453863 \\ 6 & -1.321459 & -1.616737 & 1.347975 \\ 6 & -1.070351 & -2.766779 & 0.617247 \\ 6 & -0.973588 & -4.036320 & 1.277249 \\ 6 & -1.143029 & -4.107909 & 2.690543 \\ 6 & -1.403744 & -2.916940 & 3.406389 \\ 6 & -1.486641 & -1.714157 & 2.757724 \\ 6 & -0.700511 & -5.240798 & 0.576280 \\ 6 & -0.602017 & -6.438972 & 1.239463 \\ 6 & -0.774725 & -6.502362 & 2.638062 \\ 6 & -1.039457 & -5.357928 & 3.345829 \\ 6 & -0.908445 & -2.771615 & -0.862734 \\ 6 & 0.325233 & -2.517490 & -1.434591 \\ 6 & 0.539077 & -2.750589 & -2.813166 \\ 6 & -0.485056 & -3.165364 & -3.623183 \\ 6 & -1.786133 & -3.338295 & -3.097087 \\ 6 & -2.000419 & -3.159849 & -1.697345 \\ 6 & -2.879869 & -3.689273 & -3.926206 \\ 6 & -4.135856 & -3.852132 & -3.399664 \\ 6 & -4.350850 & -3.676657 & -2.014389 \\ & & & \\ 6\end{array}$




\begin{tabular}{|c|c|c|c|}
\hline 6 & -3.309110 & -3.350055 & -1.183227 \\
\hline 6 & -1.536230 & 1.217052 & 1.827356 \\
\hline & -2.507789 & 2.204506 & 1.898288 \\
\hline & -2.428030 & 3.235080 & 2.844522 \\
\hline & -1.357808 & 3.221328 & 3.733499 \\
\hline & -0.362911 & 2.229960 & 3.698385 \\
\hline & -0.450981 & 1.250256 & 2.714164 \\
\hline & 0.769465 & 2.257988 & 4.724188 \\
\hline & 0.183014 & 2.278571 & 6.140724 \\
\hline & -3.511970 & 4.309536 & 2.852972 \\
\hline & -4.880186 & 3.651529 & 3.068785 \\
\hline y & -3.374812 & -0.159994 & 0.112443 \\
\hline & -4.234425 & -0.618003 & 1.116159 \\
\hline ) & -5.597612 & -0.777105 & 0.880994 \\
\hline & -6.076999 & -0.452753 & -0.392873 \\
\hline b & -5.253173 & 0.015643 & -1.416236 \\
\hline & -3.886962 & 0.155322 & -1.142725 \\
\hline & -6.554336 & -1.323042 & 1.939401 \\
\hline y & -5.864756 & 0.365478 & -2.771843 \\
\hline 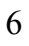 & 1.671466 & 1.029381 & 4.617259 \\
\hline$b$ & 1.618005 & 3.515599 & 4.510012 \\
\hline & -3.300963 & 5.342018 & 3.957047 \\
\hline 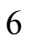 & -3.500913 & 5.039831 & 1.504731 \\
\hline & 0.399978 & 2.646104 & -0.281821 \\
\hline 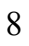 & 0.499324 & 3.710914 & 0.160007 \\
\hline ) & -0.711758 & 0.475987 & -3.301640 \\
\hline 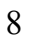 & 2.676272 & -0.103541 & 0.381768 \\
\hline 6 & 3.528431 & 0.975999 & 0.380615 \\
\hline 6 & 4.718935 & 0.918629 & -0.320275 \\
\hline f & 5.544456 & 2.091089 & -0.340534 \\
\hline ) & 5.170140 & 3.226109 & 0.441096 \\
\hline 6 & 3.971984 & 3.191266 & 1.193287 \\
\hline ) & 3.155702 & 2.094769 & 1.149797 \\
\hline 6 & 5.989791 & 4.380781 & 0.433235 \\
\hline 5 & 7.124616 & 4.436293 & -0.334019 \\
\hline 6 & 7.477489 & 3.332581 & -1.138294 \\
\hline 6 & 6.710720 & 2.193744 & -1.141439 \\
\hline 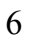 & 5.074929 & -0.314090 & -1.058449 \\
\hline 6 & 4.175779 & -0.860575 & -1.955938 \\
\hline f & 4.514653 & -1.952960 & -2.784313 \\
\hline 6 & 5.731427 & -2.562588 & -2.650178 \\
\hline 5 & 6.644760 & -2.130568 & -1.656894 \\
\hline 6 & 6.314129 & -1.003936 & -0.845893 \\
\hline 6 & 7.205969 & -0.652757 & 0.199037 \\
\hline 5 & 8.365854 & -1.357545 & 0.409320 \\
\hline 6 & 8.705958 & -2.447945 & -0.417997 \\
\hline j & 7.857986 & -2.824020 & -1.428440 \\
\hline 8 & 2.926036 & -0.315819 & -2.124227 \\
\hline 1 & 6.948793 & 0.172812 & 0.857643 \\
\hline 1 & -1.691201 & -0.816147 & 3.335367 \\
\hline 1 & 8.094044 & -3.675615 & -2.065358 \\
\hline 1 & -1.171918 & -5.388524 & 4.426444 \\
\hline 1 & 5.688199 & 5.230570 & 1.044198 \\
\hline & -3.486525 & -3.199667 & -0.119291 \\
\hline 1 & -5.353497 & -3.789474 & -1.605087 \\
\hline & 3.695053 & 4.063371 & 1.784533 \\
\hline
\end{tabular}




\begin{tabular}{|c|c|c|c|}
\hline & -1.539707 & -2.970601 & 4.485966 \\
\hline & 6.002797 & -3.404308 & -3.285160 \\
\hline & 8.362474 & 3.385706 & -1.768506 \\
\hline 1 & 3.787686 & -2.285704 & -3.522352 \\
\hline & -2.700641 & -3.822634 & -4.992207 \\
\hline & -0.319172 & -3.334764 & -4.686046 \\
\hline & -0.390530 & -7.348161 & 0.681307 \\
\hline & 6.991685 & 1.358961 & -1.777950 \\
\hline & -0.567481 & -5.211318 & -0.503270 \\
\hline & 1.535210 & -2.574138 & -3.217539 \\
\hline & 7.743700 & 5.330398 & -0.336557 \\
\hline & 9.025327 & -1.075105 & 1.226939 \\
\hline & -4.970352 & -4.116575 & -4.045948 \\
\hline & -0.695088 & -7.458307 & 3.150375 \\
\hline & 9.630252 & -2.993950 & -0.244409 \\
\hline & 2.211536 & 2.056157 & 1.689768 \\
\hline & -3.824942 & -0.857883 & 2.093697 \\
\hline & -3.197253 & 0.510642 & -1.903837 \\
\hline & -7.141732 & -0.572507 & -0.594351 \\
\hline & -3.337753 & 2.185052 & 1.193035 \\
\hline & 0.317698 & 0.481075 & 2.633306 \\
\hline & -1.283055 & 4.004085 & 4.485412 \\
\hline$\frac{1}{1}$ & 1.811052 & -3.956711 & -0.123327 \\
\hline & -1.484249 & 1.931363 & -1.359976 \\
\hline l & 3.182023 & -3.162365 & -0.892368 \\
\hline & 4.999799 & -3.023345 & 0.531804 \\
\hline 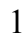 & 1.080966 & -2.417320 & 2.163182 \\
\hline 1 & 5.991360 & -2.663005 & 2.764489 \\
\hline & 2.056972 & -2.106967 & 4.412005 \\
\hline 1 & 4.526307 & -2.198788 & 4.722589 \\
\hline & 2.436147 & 1.059499 & 5.404019 \\
\hline 1 & 1.097356 & 0.100839 & 4.745475 \\
\hline ] & 2.199533 & 0.968821 & 3.656434 \\
\hline 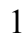 & 0.991277 & 2.285090 & 6.884236 \\
\hline 1 & -0.438282 & 3.162748 & 6.324776 \\
\hline 1 & -0.435842 & 1.390234 & 6.323178 \\
\hline 1 & 2.453734 & 3.541515 & 5.222396 \\
\hline & 2.036350 & 3.540279 & 3.494640 \\
\hline 1 & 1.031374 & 4.432106 & 4.647747 \\
\hline 1 & -4.105314 & 6.087652 & 3.922651 \\
\hline 1 & -3.318218 & 4.885640 & 4.955471 \\
\hline 1 & -2.350984 & 5.879088 & 3.839463 \\
\hline 1 & -4.266315 & 5.827681 & 1.490369 \\
\hline 1 & -2.525183 & 5.508562 & 1.321877 \\
\hline & -3.704108 & 4.362585 & 0.666310 \\
\hline 1 & -5.672688 & 4.412008 & 3.068274 \\
\hline 1 & -5.119431 & 2.923683 & 2.283193 \\
\hline 1 & -4.915853 & 3.126752 & 4.032790 \\
\hline 1 & -0.209693 & -0.439840 & -3.609010 \\
\hline 1 & -1.794531 & 0.447875 & -3.420310 \\
\hline 1 & 2.071687 & 2.388128 & -3.392726 \\
\hline 1 & 1.861086 & 0.703816 & -3.873735 \\
\hline 1 & 1.406013 & 2.013340 & -4.988211 \\
\hline 1 & 0.787606 & 4.224167 & -2.743882 \\
\hline 1 & -0.364770 & 6.348283 & -3.217909 \\
\hline 1 & -2.559733 & 6.352576 & -4.391234 \\
\hline
\end{tabular}




$\begin{array}{rrrr}1 & -3.550341 & 4.189973 & -5.130999 \\ 1 & -2.367226 & 2.073394 & -4.697602 \\ 6 & -4.800969 & 0.769582 & -3.787515 \\ 6 & -6.622374 & -0.840902 & -3.335511 \\ 6 & -6.831963 & 1.541428 & -2.595112 \\ 6 & -5.859944 & -1.555695 & 3.279592 \\ 6 & -7.706454 & -0.338940 & 2.165526 \\ 6 & -7.116053 & -2.664193 & 1.453022 \\ 1 & -7.275440 & 1.820524 & -3.560565 \\ 1 & -7.652872 & 1.298382 & -1.909124 \\ 1 & -6.310391 & 2.420681 & -2.195119 \\ 1 & -5.273895 & 0.999522 & -4.751268 \\ 1 & -4.254141 & 1.665577 & -3.466209 \\ 1 & -4.076053 & -0.040188 & -3.956028 \\ 1 & -7.070513 & -0.586071 & -4.305376 \\ 1 & -5.941751 & -1.689089 & -3.488525 \\ 1 & -7.433494 & -1.173389 & -2.676198 \\ 1 & -6.585602 & -1.936048 & 4.009490 \\ 1 & -5.052642 & -2.296443 & 3.202594 \\ 1 & -5.436421 & -0.627815 & 3.686463 \\ 1 & -8.397180 & -0.731138 & 2.923935 \\ 1 & -7.332690 & 0.630581 & 2.519397 \\ 1 & -8.287962 & -0.160532 & 1.253235 \\ 1 & -7.807176 & -3.083771 & 2.196618 \\ 1 & -7.665130 & -2.561641 & 0.508588 \\ 1 & -6.308215 & -3.391610 & 1.294569\end{array}$

$3_{\text {IB-re- }} \psi_{-12 a 23 a 4 s}$

Number of imaginary frequencies : 0

The smallest frequencies are : $15.3323 \quad 16.6759 \quad 21.1646 \mathrm{~cm}(-1)$

Electronic energy : $\quad H F=-4360.8871603$

Zero-point correction $=\quad 1.454526($ Hartree/Particle $)$

Thermal correction to Energy $=\quad 1.539257$

Thermal correction to Enthalpy $=\quad 1.540201$

Thermal correction to Gibbs Free Energy= $\quad 1.336105$

Sum of electronic and zero-point Energies $=\quad-4359.432635$

Sum of electronic and thermal Energies $=\quad-4359.347904$

Sum of electronic and thermal Enthalpies $=\quad-4359.346959$

Sum of electronic and thermal Free Energies $=\quad-4359.551056$

Cartesian Coordinates

$\begin{array}{cccc}6 & 1.851164 & -3.195545 & -4.183215 \\ 6 & 0.644475 & -3.168642 & -3.475442 \\ 6 & 0.134890 & -4.389922 & -3.014971 \\ 6 & 0.826551 & -5.578335 & -3.209565 \\ 6 & 2.045985 & -5.582153 & -3.883829 \\ 6 & 2.550551 & -4.383535 & -4.375564 \\ 6 & -0.115031 & -1.893272 & -3.276620 \\ 6 & -1.560264 & -1.987819 & -3.714237 \\ 45 & 0.121586 & -1.104439 & -1.213467 \\ 15 & -1.739628 & 0.362765 & -0.934972 \\ 7 & -1.379485 & 1.998671 & -0.657690 \\ 6 & -2.325949 & 3.040389 & -0.189798 \\ 6 & -2.942245 & 2.802006 & 1.162611\end{array}$




\begin{tabular}{|c|c|c|c|}
\hline 6 & -2.143409 & 2.547264 & 2.278361 \\
\hline 6 & -2.716176 & 2.351352 & 3.527734 \\
\hline 6 & -4.101061 & 2.399655 & 3.680150 \\
\hline 6 & -4.903381 & 2.657758 & 2.574213 \\
\hline 6 & -4.324525 & 2.863292 & 1.324043 \\
\hline 15 & 1.556706 & 0.048340 & 0.434105 \\
\hline 5 & 1.332049 & 1.673497 & 1.314477 \\
\hline 5 & 1.084847 & 2.816018 & 0.570599 \\
\hline 6 & 0.998183 & 4.094048 & 1.212865 \\
\hline 6 & 1.173874 & 4.183105 & 2.624344 \\
\hline 6 & 1.429849 & 3.000040 & 3.354626 \\
\hline 6 & 1.504121 & 1.787844 & 2.721579 \\
\hline 6 & 0.731302 & 5.290275 & 0.495672 \\
\hline 6 & 0.643860 & 6.498067 & 1.142545 \\
\hline 6 & 0.822352 & 6.579163 & 2.539767 \\
\hline 6 & 1.081657 & 5.443004 & 3.262578 \\
\hline 6 & 0.923901 & 2.784352 & -0.908085 \\
\hline 6 & -0.300657 & 2.479773 & -1.475476 \\
\hline 6 & -0.501200 & 2.625495 & -2.867750 \\
\hline 6 & 0.526781 & 3.004757 & -3.690044 \\
\hline 6 & 1.816045 & 3.240868 & -3.160648 \\
\hline 6 & 2.016622 & 3.150053 & -1.750794 \\
\hline 6 & 2.910606 & 3.573334 & -3.996545 \\
\hline 6 & 4.154296 & 3.802263 & -3.466037 \\
\hline 6 & 4.356796 & 3.709561 & -2.070707 \\
\hline 6 & 3.314445 & 3.400680 & -1.233946 \\
\hline 6 & 1.578971 & -1.146849 & 1.829140 \\
\hline 6 & 2.572493 & -2.111023 & 1.906131 \\
\hline 6 & 2.524406 & -3.127385 & 2.869270 \\
\hline 6 & 1.464939 & -3.120751 & 3.771194 \\
\hline 6 & 0.448223 & -2.151721 & 3.730417 \\
\hline 6 & 0.503854 & -1.188539 & 2.727958 \\
\hline 6 & -0.680870 & -2.193088 & 4.759341 \\
\hline 6 & -0.094748 & -2.215608 & 6.175679 \\
\hline 6 & 3.621946 & -4.187653 & 2.868909 \\
\hline 6 & 4.991738 & -3.510023 & 2.995484 \\
\hline 6 & 3.353855 & 0.207785 & 0.034544 \\
\hline 6 & 4.237215 & 0.671340 & 1.005645 \\
\hline 6 & 5.601218 & 0.814432 & 0.742494 \\
\hline 6 & 6.053828 & 0.459093 & -0.527576 \\
\hline 6 & 5.197729 & -0.020850 & -1.527108 \\
\hline 6 & 3.838135 & -0.132740 & -1.229550 \\
\hline 6 & 6.513258 & 1.357124 & 1.840577 \\
\hline 6 & 5.781545 & -0.415935 & -2.882393 \\
\hline 6 & -1.594975 & -0.972653 & 4.658113 \\
\hline 6 & -1.518482 & -3.457113 & 4.537795 \\
\hline 6 & 3.473830 & -5.180620 & 4.018412 \\
\hline 6 & 3.554352 & -4.965226 & 1.548549 \\
\hline 6 & -0.389693 & -2.639176 & -0.147144 \\
\hline 8 & -0.480235 & -3.695873 & 0.315167 \\
\hline 6 & 0.551324 & -0.642583 & -3.318507 \\
\hline 8 & -2.697778 & 0.142942 & 0.395421 \\
\hline 6 & -3.542029 & -0.943005 & 0.407911 \\
\hline 6 & -4.725329 & -0.906275 & -0.306161 \\
\hline 6 & -5.538435 & -2.087001 & -0.319791 \\
\hline 6 & -5.161570 & -3.207669 & 0.481255 \\
\hline
\end{tabular}




\begin{tabular}{|c|c|c|c|}
\hline 6 & -3.973546 & -3.149967 & 1.248347 \\
\hline 6 & -3.167317 & -2.046141 & 1.198272 \\
\hline & -5.969546 & -4.370638 & 0.478478 \\
\hline & -7.094305 & -4.447510 & -0.301719 \\
\hline & -7.449420 & -3.357598 & -1.123764 \\
\hline & -6.694515 & -2.210910 & -1.132152 \\
\hline & -5.087574 & 0.319020 & -1.054117 \\
\hline & -4.186949 & 0.872073 & -1.946478 \\
\hline & -4.524767 & 1.970325 & -2.767424 \\
\hline & -5.746365 & 2.571551 & -2.639089 \\
\hline & -6.666234 & 2.126182 & -1.658008 \\
\hline y & -6.332907 & 0.999920 & -0.847547 \\
\hline & -7.231349 & 0.638438 & 0.188297 \\
\hline & -8.401169 & 1.330003 & 0.387321 \\
\hline & -8.744342 & 2.418326 & -0.441366 \\
\hline & -7.888858 & 2.806574 & -1.440738 \\
\hline & -2.936375 & 0.328962 & -2.116630 \\
\hline & -6.972575 & -0.185238 & 0.848508 \\
\hline & 1.707214 & 0.896860 & 3.310174 \\
\hline & -8.126438 & 3.657707 & -2.077700 \\
\hline & 1.218637 & 5.487886 & 4.342135 \\
\hline & -5.667036 & -5.209519 & 1.103885 \\
\hline & 3.482133 & 3.318330 & -0.160966 \\
\hline & 5.351187 & 3.872175 & -1.658487 \\
\hline & -3.695756 & -4.009938 & 1.856804 \\
\hline & 1.570521 & 3.067288 & 4.432837 \\
\hline & -6.017026 & 3.416638 & -3.269902 \\
\hline & -8.326537 & -3.427902 & -1.763191 \\
\hline & -3.793056 & 2.316617 & -3.494272 \\
\hline & 2.741087 & 3.641315 & -5.070288 \\
\hline & 0.371423 & 3.103208 & -4.763461 \\
\hline ] & 0.436929 & 7.401219 & 0.572994 \\
\hline & -6.976558 & -1.386030 & -1.781146 \\
\hline & 0.595564 & 5.244932 & -0.583014 \\
\hline & -1.487370 & 2.403195 & -3.273412 \\
\hline & -7.703979 & -5.348047 & -0.300354 \\
\hline 1 & -9.066101 & 1.038710 & 1.197368 \\
\hline & 4.988936 & 4.055251 & -4.116697 \\
\hline ] & 0.751585 & 7.542676 & 3.039031 \\
\hline & -9.676543 & 2.953615 & -0.276954 \\
\hline ] & -2.229628 & -1.990206 & 1.748036 \\
\hline & 3.856735 & 0.930878 & 1.992542 \\
\hline & 3.127162 & -0.488733 & -1.971244 \\
\hline ] & 7.113688 & 0.553641 & -0.755872 \\
\hline & 3.391813 & -2.088310 & 1.188984 \\
\hline 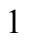 & -0.283859 & -0.439269 & 2.639735 \\
\hline & 1.414775 & -3.893194 & 4.535695 \\
\hline & -1.732427 & 3.965139 & -0.149914 \\
\hline & 1.463280 & -1.942403 & -1.309119 \\
\hline & -3.118622 & 3.210323 & -0.934904 \\
\hline 1 & -4.960513 & 3.054920 & 0.458635 \\
\hline & -1.065304 & 2.473067 & 2.154519 \\
\hline & -5.986238 & 2.694886 & 2.677049 \\
\hline & -2.075205 & 2.158165 & 4.387278 \\
\hline & -4.550560 & 2.237725 & 4.657673 \\
\hline & -2.352354 & -1.008766 & 5.451595 \\
\hline
\end{tabular}




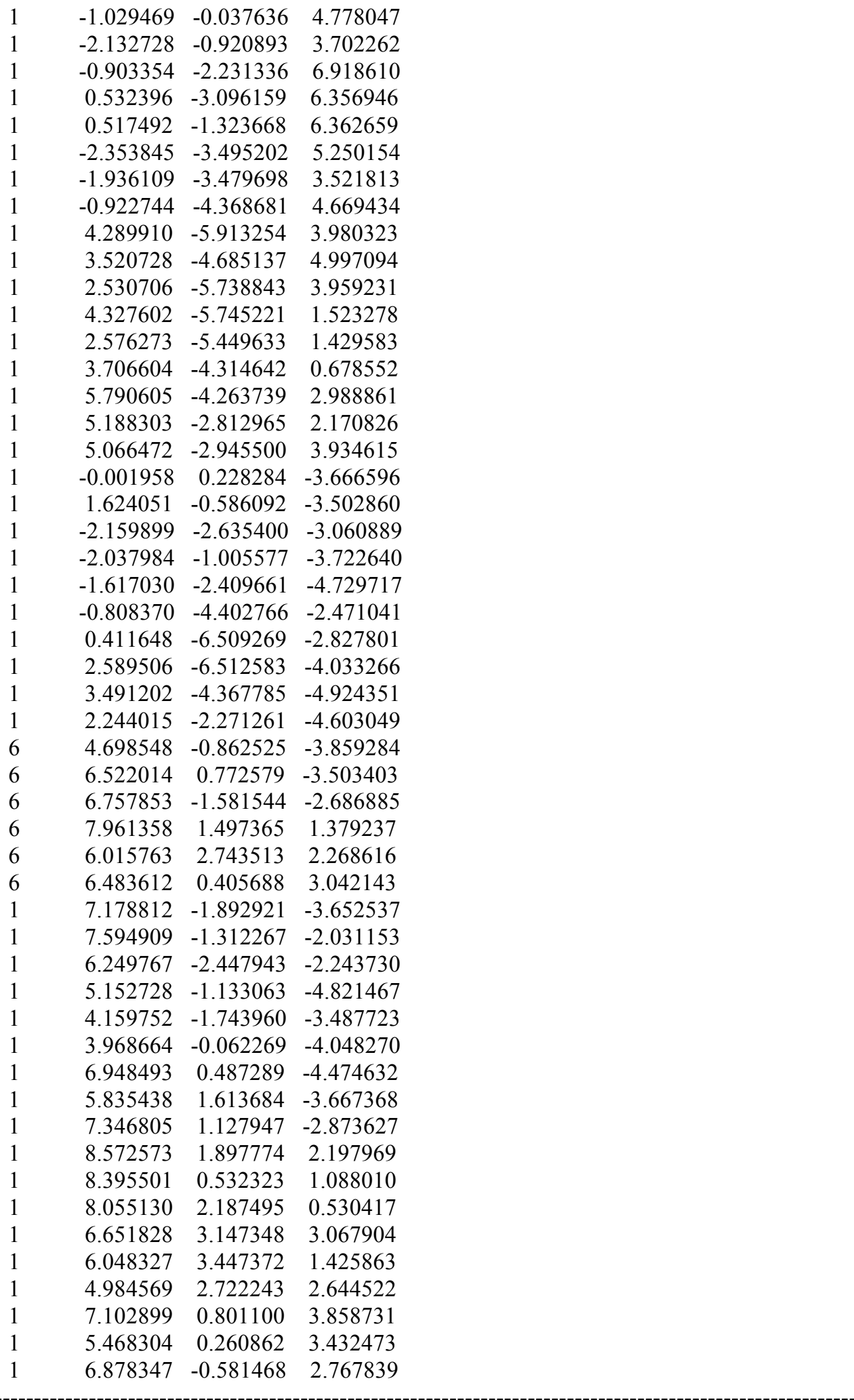

$3_{\text {IB-re- }} \psi_{-12 a 2334 a}$

Number of imaginary frequencies : 0

The smallest frequencies are : $18.9496 \quad 20.6717 \quad 25.7445 \mathrm{~cm}(-1)$ 
Electronic energy : $\quad \mathrm{HF}=-4360.8880581$

Zero-point correction $=$

1.457380 (Hartree/Particle)

Thermal correction to Energy= $\quad 1.541219$

Thermal correction to Enthalpy= $\quad 1.542163$

Thermal correction to Gibbs Free Energy $=\quad 1.342526$

Sum of electronic and zero-point Energies $=\quad-4359.430679$

Sum of electronic and thermal Energies $=\quad-4359.346840$

Sum of electronic and thermal Enthalpies $=\quad-4359.345895$

Sum of electronic and thermal Free Energies $=\quad-4359.545532$

Cartesian Coordinates

$\begin{array}{cccc}0 & -2.048707 & 2.917156 & -4.267973 \\ 6 & -0.774595 & 2.890138 & -3.689135 \\ 6 & -0.157715 & 4.119511 & -3.419603 \\ 6 & -0.798287 & 5.322353 & -3.684783 \\ 6 & -2.076630 & 5.331739 & -4.238732 \\ 6 & -2.695466 & 4.121785 & -4.531408 \\ 6 & -0.053726 & 1.605022 & -3.425265 \\ 6 & 1.350771 & 1.581967 & -3.985601 \\ 45 & -0.172366 & 1.048050 & -1.266988 \\ 15 & 1.712951 & -0.373537 & -0.936185 \\ 7 & 1.402619 & -2.006856 & -0.588190 \\ 6 & 2.385483 & -2.997975 & -0.086712 \\ 6 & 2.975464 & -2.710074 & 1.268520 \\ 6 & 2.157773 & -2.414686 & 2.360372 \\ 6 & 2.707497 & -2.194276 & 3.615902 \\ 6 & 4.088124 & -2.255001 & 3.798866 \\ 6 & 4.909500 & -2.548810 & 2.716255 \\ 6 & 4.353757 & -2.779912 & 1.459933 \\ 15 & -1.567284 & 0.011026 & 0.474207 \\ 6 & -1.322917 & -1.585231 & 1.393350 \\ 6 & -1.069469 & -2.746832 & 0.682081 \\ 6 & -0.961529 & -4.002804 & 1.366149 \\ 6 & -1.126831 & -4.049258 & 2.780994 \\ 6 & -1.394826 & -2.847287 & 3.475395 \\ 6 & -1.485093 & -1.657162 & 2.804940 \\ 6 & -0.677886 & -5.217531 & 0.687494 \\ 6 & -0.566549 & -6.401919 & 1.373227 \\ 6 & -0.736590 & -6.440770 & 2.772985 \\ 6 & -1.010845 & -5.285687 & 3.459483 \\ 6 & -0.907513 & -2.786866 & -0.797899 \\ 6 & 0.327952 & -2.549673 & -1.372612 \\ 6 & 0.550472 & -2.833102 & -2.740477 \\ 6 & -0.462230 & -3.301197 & -3.535684 \\ 6 & -1.762927 & -3.474060 & -3.008072 \\ 6 & -1.991106 & -3.220401 & -1.622132 \\ 6 & -2.839684 & -3.908838 & -3.819601 \\ 6 & -4.094280 & -4.075735 & -3.291471 \\ 6 & -4.327610 & -3.807203 & -1.924448 \\ 6 & -3.301516 & -3.400404 & -1.108976 \\ 6 & -1.546821 & 1.258399 & 1.821912 \\ 6 & -2.523972 & 2.241488 & 1.868747 \\ 6 & -2.447754 & 3.297709 & 2.786069 \\ 6 & -1.375257 & 3.314215 & 3.672267 \\ 6 & -0.375746 & 2.326842 & 3.662588\end{array}$




\begin{tabular}{|c|c|c|c|}
\hline 6 & -0.460820 & 1.319707 & 2.706221 \\
\hline 6 & 0.759401 & 2.390385 & 4.683751 \\
\hline 6 & 0.177275 & 2.453780 & 6.100763 \\
\hline 6 & -3.538526 & 4.364968 & 2.765776 \\
\hline 6 & -4.901137 & 3.704901 & 3.009970 \\
\hline 6 & -3.373689 & -0.156718 & 0.125594 \\
\hline 6 & -4.247515 & -0.618493 & 1.120873 \\
\hline 6 & -5.602362 & -0.787196 & 0.864691 \\
\hline 6 & -6.071172 & -0.478308 & -0.423888 \\
\hline 6 & -5.236058 & -0.003283 & -1.428533 \\
\hline 6 & -3.873707 & 0.152494 & -1.129025 \\
\hline 6 & -6.573561 & -1.320377 & 1.916447 \\
\hline 6 & -5.730640 & 0.354392 & -2.827186 \\
\hline 6 & 1.665229 & 1.162055 & 4.612582 \\
\hline 6 & 1.602995 & 3.643326 & 4.427048 \\
\hline 6 & -3.328651 & 5.433334 & 3.835268 \\
\hline 6 & -3.538608 & 5.052017 & 1.394661 \\
\hline 6 & 0.390034 & 2.651070 & -0.344280 \\
\hline 8 & 0.486213 & 3.727857 & 0.067870 \\
\hline 6 & -0.759904 & 0.386992 & -3.280552 \\
\hline 8 & 2.666736 & -0.080357 & 0.383040 \\
\hline 6 & 3.520949 & 0.996682 & 0.357951 \\
\hline 6 & 4.709246 & 0.922414 & -0.344994 \\
\hline 6 & 5.538805 & 2.091471 & -0.388069 \\
\hline 6 & 5.169842 & 3.241694 & 0.373878 \\
\hline 6 & 3.973625 & 3.224378 & 1.129868 \\
\hline 6 & 3.153825 & 2.129945 & 1.108407 \\
\hline 6 & 5.993233 & 4.393324 & 0.343597 \\
\hline 6 & 7.126768 & 4.431236 & -0.426578 \\
\hline 6 & 7.474553 & 3.312027 & -1.211327 \\
\hline 6 & 6.704027 & 2.175899 & \\
\hline 6 & 5.058799 & -0.324371 & -1.062667 \\
\hline 6 & 4.155733 & -0.882709 & -1.948895 \\
\hline 6 & 4.489541 & -1.988060 & -2.762019 \\
\hline 6 & 5.704302 & -2.599937 & -2.620583 \\
\hline 6 & 6.620157 & -2.156682 & -1.634636 \\
\hline 6 & 6.295559 & -1.015830 & -0.841255 \\
\hline 6 & 7.189992 & -0.651951 & 0.197086 \\
\hline 6 & 8.346157 & -1.359507 & 0.418399 \\
\hline 6 & 8.680182 & -2.464804 & -0.391522 \\
\hline 6 & 7.830087 & -2.852364 & -1.395826 \\
\hline 8 & 2.905729 & -0.339559 & -2.120059 \\
\hline 1 & 6.937172 & 0.185680 & 0.842125 \\
\hline 1 & -1.693482 & -0.749252 & 3.365561 \\
\hline 1 & 8.061688 & -3.715038 & -2.019329 \\
\hline 1 & -1.140816 & -5.297046 & 4.540766 \\
\hline 1 & 5.695582 & 5.254957 & 0.939724 \\
\hline 1 & -3.491191 & -3.187948 & -0.057789 \\
\hline 1 & -5.330368 & -3.915257 & -1.515129 \\
\hline 1 & 3.701944 & 4.107466 & 1.707020 \\
\hline 1 & -1.528830 & -2.882151 & 4.555960 \\
\hline 1 & 5.971872 & -3.452047 & -3.243208 \\
\hline 1 & 8.358673 & 3.350785 & -1.843818 \\
\hline 1 & 3.759997 & -2.328873 & -3.493864 \\
\hline 1 & -2.648072 & -4.104419 & -4.873773 \\
\hline 1 & -0.286636 & -3.520343 & -4.587822 \\
\hline
\end{tabular}




\begin{tabular}{|c|c|c|c|}
\hline & -0.346999 & -7.319309 & 0.831841 \\
\hline & 6.981204 & 1.328988 & -1.814699 \\
\hline & -0.546681 & -5.208691 & -0.392570 \\
\hline & 1.546728 & -2.661883 & -3.146454 \\
\hline & 7.748816 & 5.323067 & -0.446270 \\
\hline & 9.007512 & -1.067487 & 1.231119 \\
\hline & -4.914959 & -4.405948 & -3.924818 \\
\hline & -0.647038 & -7.386128 & 3.303003 \\
\hline & 9.601677 & -3.012794 & -0.209506 \\
\hline & 2.212084 & 2.102840 & 1.653402 \\
\hline & -3.850090 & -0.849851 & 2.105595 \\
\hline & -3.184784 & 0.521776 & -1.885797 \\
\hline & -7.129450 & -0.616986 & -0.636156 \\
\hline & -3.354835 & 2.199052 & 1.165658 \\
\hline & 0.311699 & 0.552587 & 2.644348 \\
\hline & -1.302106 & 4.118281 & 4.401510 \\
\hline & 1.831506 & -3.946341 & -0.032822 \\
\hline & -1.502313 & 1.904889 & -1.368898 \\
\hline & 3.194736 & -3.153861 & -0.816255 \\
\hline & 5.005684 & -3.001891 & 0.613640 \\
\hline & 1.083816 & -2.329236 & 2.211656 \\
\hline & 5.989721 & -2.593438 & 2.841289 \\
\hline 1 & 2.051982 & -1.972342 & 4.457175 \\
\hline & 4.519200 & -2.073884 & 4.781309 \\
\hline ] & 2.432580 & 1.219896 & 5.395189 \\
\hline & 1.094702 & 0.236237 & 4.772557 \\
\hline 1 & 2.190170 & 1.072697 & 3.652321 \\
\hline & 0.987805 & 2.485362 & 6.841154 \\
\hline & -0.445478 & 3.341940 & 6.258959 \\
\hline ] & -0.438935 & 1.570216 & 6.312845 \\
\hline & 2.440320 & 3.695066 & 5.136147 \\
\hline ] & 2.018542 & 3.636415 & 3.410248 \\
\hline ] & 1.013396 & 4.561747 & 4.536436 \\
\hline 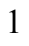 & -4.138191 & 6.172236 & 3.781913 \\
\hline 1 & -3.337355 & 5.008893 & 4.847789 \\
\hline 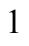 & -2.382913 & 5.972504 & 3.695237 \\
\hline 1 & -4.307973 & 5.835355 & 1.359795 \\
\hline & -2.566181 & 5.519313 & 1.191623 \\
\hline 1 & -3.742707 & 4.348096 & 0.578395 \\
\hline 1 & -5.699439 & 4.458867 & 2.984196 \\
\hline ] & -5.137372 & 2.946307 & 2.252856 \\
\hline 1 & -4.929671 & 3.215772 & 3.992778 \\
\hline 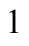 & -0.264564 & -0.538169 & -3.571290 \\
\hline 1 & -1.843957 & 0.353359 & -3.377951 \\
\hline & 2.030516 & 2.268593 & -3.466024 \\
\hline 1 & 1.787813 & 0.582682 & -3.917983 \\
\hline 1 & 1.332943 & 1.881816 & -5.044943 \\
\hline 1 & 0.834173 & 4.128924 & -2.969605 \\
\hline 1 & -0.296731 & 6.260169 & -3.453602 \\
\hline 1 & -2.580391 & 6.273262 & -4.446352 \\
\hline 1 & -3.687908 & 4.110133 & -4.979466 \\
\hline 1 & -2.541006 & 1.980575 & -4.529573 \\
\hline 6 & -7.228196 & 0.113732 & -2.997343 \\
\hline 6 & -5.451752 & 1.838879 & -3.088441 \\
\hline 6 & -4.994920 & -0.502802 & -3.863167 \\
\hline 6 & -5.900845 & -1.523509 & 3.272393 \\
\hline
\end{tabular}




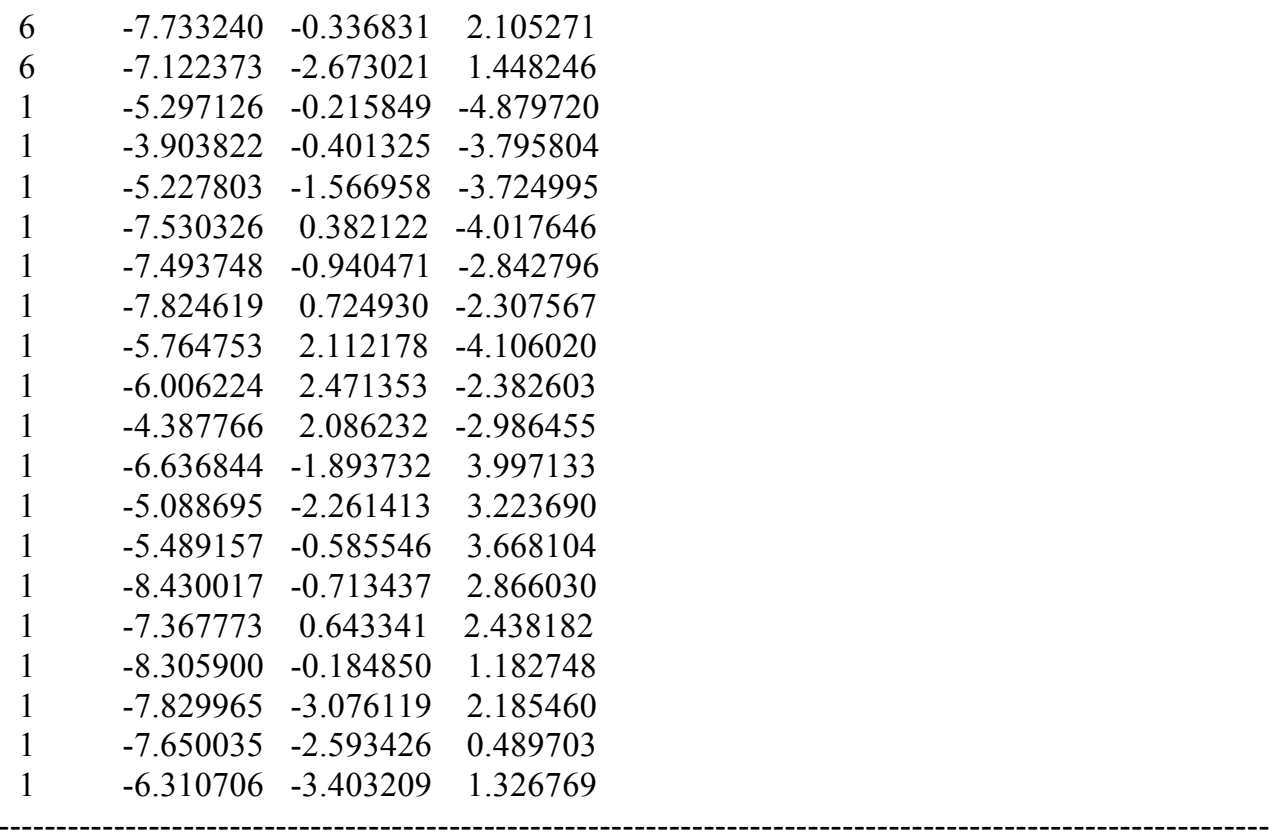

$3_{\text {IB-re- }} \psi_{-1 \mathrm{a} 2 \mathrm{~s} 3 \mathrm{~s} 4 \mathrm{~s}}$

Number of imaginary frequencies : 0

The smallest frequencies are : $16.2488 \quad 19.449120 .8502 \mathrm{~cm}(-1)$

Electronic energy : $\quad H F=-4360.8881471$

Zero-point correction $=\quad 1.458169$ (Hartree/Particle)

Thermal correction to Energy $=\quad 1.541902$

Thermal correction to Enthalpy= $\quad 1.542846$

Thermal correction to Gibbs Free Energy $=\quad 1.342584$

Sum of electronic and zero-point Energies $=\quad-4359.429978$

Sum of electronic and thermal Energies $=\quad-4359.346245$

Sum of electronic and thermal Enthalpies $=\quad-4359.345301$

Sum of electronic and thermal Free Energies $=\quad-4359.545563$

\begin{tabular}{cccc}
\multicolumn{4}{c}{ Cartesian Coordinates } \\
\hline $6 \ldots \ldots \ldots \ldots \ldots \ldots \ldots \ldots \ldots \ldots \ldots \ldots \ldots \ldots \ldots \ldots$ \\
6 & -2.518517 & 2.256135 & 1.882834 \\
6 & -1.548521 & 1.265659 & 1.830412 \\
6 & -0.459731 & 1.315805 & 2.712084 \\
6 & -0.365279 & 2.317559 & 3.672984 \\
6 & -1.355352 & 3.314076 & 3.686378 \\
6 & -2.428822 & 3.311153 & 2.801105 \\
15 & -1.574011 & 0.025902 & 0.475373 \\
6 & -3.380813 & -0.144797 & 0.127250 \\
6 & -4.244268 & -0.646992 & 1.103805 \\
6 & -5.599306 & -0.842400 & 0.844707 \\
6 & -6.078070 & -0.503095 & -0.426848 \\
6 & -5.248294 & 0.020907 & -1.418902 \\
6 & -3.891742 & 0.191711 & -1.120275 \\
6 & -6.488197 & -1.423799 & 1.943736 \\
6 & -7.931875 & -1.615407 & 1.486212 \\
6 & -5.752773 & 0.402632 & -2.808339 \\
6 & -5.020605 & -0.436467 & -3.862116 \\
6 & 0.770568 & 2.365954 & 4.694091 \\
6 & 1.668444 & 1.132403 & 4.613243
\end{tabular}




\begin{tabular}{|c|c|c|c|}
\hline 6 & -3.500909 & 4.397566 & 2.781008 \\
\hline 6 & -3.284338 & 5.450028 & 3.865045 \\
\hline 45 & -0.179371 & 1.066462 & -1.260427 \\
\hline 6 & 0.400703 & 2.661154 & -0.334810 \\
\hline 8 & 0.508856 & 3.735012 & 0.081939 \\
\hline 6 & -0.070536 & 1.631111 & -3.418196 \\
\hline 6 & -0.785495 & 0.418565 & -3.272985 \\
\hline 6 & 1.329895 & 1.598019 & -3.987831 \\
\hline 15 & 1.700625 & -0.365158 & -0.945133 \\
\hline 8 & 2.656611 & -0.085816 & 0.375510 \\
\hline 6 & 3.518419 & 0.985259 & 0.358154 \\
\hline 6 & 4.706131 & 0.907843 & -0.345398 \\
\hline 6 & 5.544730 & 2.070785 & -0.378590 \\
\hline 6 & 5.184918 & 3.217115 & 0.393498 \\
\hline 6 & 3.988290 & 3.202809 & 1.148873 \\
\hline 6 & 3.159821 & 2.115183 & 1.117732 \\
\hline 6 & 6.017652 & 4.362233 & 0.373760 \\
\hline 6 & 7.151695 & 4.397824 & -0.395774 \\
\hline 6 & 7.490480 & 3.282971 & -1.190595 \\
\hline 6 & 6.710695 & 2.153052 & -1.182392 \\
\hline 6 & 5.046246 & -0.335301 & -1.073825 \\
\hline 6 & 4.139127 & -0.879084 & -1.964965 \\
\hline 6 & 4.465506 & -1.978888 & -2.788623 \\
\hline 6 & 5.675764 & -2.600911 & -2.652394 \\
\hline 6 & 6.594420 & -2.173687 & -1.661956 \\
\hline 6 & 6.277967 & -1.037473 & -0.858613 \\
\hline 6 & 7.798977 & -2.880565 & -1.428693 \\
\hline 6 & 8.651551 & -2.508051 & -0.420801 \\
\hline 6 & 8.325687 & -1.407086 & 0.398272 \\
\hline 6 & 7.174957 & -0.689093 & 0.182804 \\
\hline 8 & 2.892639 & -0.325595 & -2.129792 \\
\hline 7 & 1.385258 & -1.999735 & -0.607504 \\
\hline 6 & 0.307642 & -2.533955 & -1.394207 \\
\hline 6 & -0.928727 & -2.767089 & -0.819498 \\
\hline 6 & -2.014947 & -3.191187 & -1.645118 \\
\hline 6 & -1.789324 & -3.435624 & -3.033263 \\
\hline 6 & -0.488477 & -3.265612 & -3.561421 \\
\hline 6 & 0.527720 & -2.809051 & -2.764073 \\
\hline 6 & -2.868505 & -3.859897 & -3.846977 \\
\hline 6 & -4.122296 & -4.029186 & -3.317889 \\
\hline 6 & -4.352102 & -3.773164 & -1.947915 \\
\hline 6 & -3.324606 & -3.373040 & -1.130809 \\
\hline 6 & -1.086051 & -2.735238 & 0.661408 \\
\hline 6 & -1.329730 & -1.577402 & 1.381928 \\
\hline 6 & -1.484320 & -1.658349 & 2.793917 \\
\hline 6 & -1.397525 & -2.853551 & 3.455952 \\
\hline 6 & -1.141142 & -4.052287 & 2.751651 \\
\hline 6 & -0.980988 & -3.996156 & 1.336624 \\
\hline 6 & -0.704021 & -5.206704 & 0.648256 \\
\hline 6 & -0.596177 & -6.396742 & 1.324676 \\
\hline 6 & -0.762791 & -6.445486 & 2.724507 \\
\hline 6 & -1.029192 & -5.294308 & 3.420540 \\
\hline 6 & 2.366559 & -2.995872 & -0.113830 \\
\hline 6 & 2.959694 & -2.722412 & 1.243239 \\
\hline 6 & 2.147705 & -2.422929 & 2.338274 \\
\hline 6 & 2.701846 & -2.222465 & 3.595258 \\
\hline
\end{tabular}




\begin{tabular}{|c|c|c|c|}
\hline & 4.081211 & -2.308632 & 3.777358 \\
\hline & 4.897033 & -2.605771 & 2.691553 \\
\hline & 4.336872 & -2.815632 & 1.433450 \\
\hline & -5.941556 & -2.792160 & 2.369489 \\
\hline & -6.490778 & -0.476999 & 3.149156 \\
\hline & -7.250849 & 0.161793 & -2.975174 \\
\hline & -5.479762 & 1.891946 & -3.047857 \\
\hline & 1.622398 & 3.615334 & 4.447304 \\
\hline & 0.188692 & 2.421839 & 6.111532 \\
\hline & -3.471887 & 5.099227 & 1.417725 \\
\hline & -4.879114 & 3.762799 & 3.000302 \\
\hline & 6.928432 & 0.144736 & 0.835082 \\
\hline & -1.684441 & -0.753038 & 3.361594 \\
\hline & 8.024390 & -3.739527 & -2.059569 \\
\hline & -1.155532 & -5.313090 & 4.502137 \\
\hline & 5.726862 & 5.220811 & 0.977631 \\
\hline 1 & -3.511798 & -3.167541 & -0.077457 \\
\hline & -5.354122 & -3.888552 & -1.538934 \\
\hline & 3.723476 & 4.083033 & 1.733530 \\
\hline & -1.525343 & -2.895081 & 4.537026 \\
\hline & 5.937398 & -3.449002 & -3.282991 \\
\hline & 8.375047 & 3.320213 & -1.822554 \\
\hline 1 & 3.734127 & -2.307424 & -3.524225 \\
\hline & -2.679091 & -4.046750 & -4.903119 \\
\hline ] & -0.315527 & -3.478066 & -4.615362 \\
\hline & -0.381983 & -7.310943 & 0.775798 \\
\hline 1 & 6.981141 & 1.309543 & -1.811914 \\
\hline & -0.575768 & -5.190621 & -0.432031 \\
\hline & 1.524647 & -2.640764 & -3.169614 \\
\hline 1 & 7.781076 & 5.284648 & -0.407245 \\
\hline & 8.989046 & -1.126892 & 1.213514 \\
\hline ] & -4.944921 & -4.352479 & -3.952251 \\
\hline & -0.676330 & -7.395266 & 3.247067 \\
\hline ] & 9.568809 & -3.064531 & -0.243195 \\
\hline 1 & 2.217404 & 2.091280 & 1.661691 \\
\hline & -3.848188 & -0.901433 & 2.085866 \\
\hline 1 & -3.210100 & 0.589930 & -1.869010 \\
\hline & -7.131019 & -0.652052 & -0.647754 \\
\hline 1 & -3.351405 & 2.222499 & 1.181317 \\
\hline ] & 0.307876 & 0.544372 & 2.644305 \\
\hline 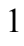 & -1.273484 & 4.115296 & 4.417773 \\
\hline 1 & 1.811437 & -3.943567 & -0.066772 \\
\hline & -1.504270 & 1.932941 & -1.349731 \\
\hline ] & 3.174655 & -3.147279 & -0.845536 \\
\hline & 4.984984 & -3.040373 & 0.584938 \\
\hline 1 & 1.074944 & -2.318877 & 2.192543 \\
\hline l & 5.976392 & -2.669801 & 2.815399 \\
\hline ] & 2.050414 & -1.996689 & 4.438693 \\
\hline 1 & 4.515547 & -2.144257 & 4.761288 \\
\hline 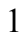 & 2.435636 & 1.178865 & 5.396735 \\
\hline 1 & 1.092037 & 0.208891 & 4.765346 \\
\hline ( & 2.193349 & 1.047653 & 3.652520 \\
\hline 1 & 0.999286 & 2.445482 & 6.852167 \\
\hline 1 & -0.431092 & 3.310999 & 6.275612 \\
\hline 1 & -0.430615 & 1.538994 & 6.317578 \\
\hline 1 & 2.463367 & 3.652864 & 5.152967 \\
\hline
\end{tabular}




$\begin{array}{rrrr}1 & 2.033038 & 3.616747 & 3.428492 \\ 1 & 1.040414 & 4.536941 & 4.569835 \\ 1 & -4.078517 & 6.205186 & 3.808944 \\ 1 & -3.314987 & 5.014585 & 4.872511 \\ 1 & -2.326825 & 5.972620 & 3.743860 \\ 1 & -4.235986 & 5.887504 & 1.377208 \\ 1 & -2.493575 & 5.563399 & 1.237642 \\ 1 & -3.664838 & 4.404310 & 0.591061 \\ 1 & -5.658798 & 4.536359 & 2.994565 \\ 1 & -5.130155 & 3.035907 & 2.217817 \\ 1 & -4.924807 & 3.243288 & 3.966664 \\ 1 & -7.108258 & -0.889982 & 3.958338 \\ 1 & -5.482404 & -0.314150 & 3.549120 \\ 1 & -6.903330 & 0.503031 & 2.876391 \\ 1 & -8.524354 & -2.043181 & 2.304851 \\ 1 & -8.402939 & -0.665714 & 1.201897 \\ 1 & -8.003621 & -2.303960 & 0.633960 \\ 1 & -6.556370 & -3.213973 & 3.176131 \\ 1 & -5.960000 & -3.500415 & 1.530295 \\ 1 & -4.908013 & -2.736523 & 2.735256 \\ 1 & -5.322765 & -0.129298 & -4.872851 \\ 1 & -3.929209 & -0.339775 & -3.792838 \\ 1 & -5.256071 & -1.502765 & -3.744213 \\ 1 & -5.807774 & 2.182496 & -4.055891 \\ 1 & -6.024942 & 2.511280 & -2.323373 \\ 1 & -4.414944 & 2.139930 & -2.957250 \\ 1 & -7.558513 & 0.448876 & -3.988753 \\ 1 & -7.514224 & -0.895502 & -2.839990 \\ 1 & -7.844951 & 0.758379 & -2.270735 \\ 1 & -0.299857 & -0.509579 & -3.570329 \\ 1 & -1.870456 & 0.393775 & -3.361049 \\ 1 & 2.018873 & 2.277207 & -3.470791 \\ 1 & 1.758915 & 0.594815 & -3.925838 \\ 1 & 1.307698 & 1.901038 & -5.046190 \\ 6 & -0.782440 & 2.923713 & -3.669129 \\ 6 & -0.151374 & 4.146354 & -3.401908 \\ 6 & -0.784892 & 5.356008 & -3.652963 \\ 6 & -2.070402 & 5.379242 & -4.189568 \\ 6 & -2.703600 & 4.176079 & -4.479825 \\ 6 & -2.063811 & 2.964688 & -4.231016 \\ 1 & 0.846016 & 4.144948 & -2.964111 \\ 1 & -0.272176 & 6.288288 & -3.423852 \\ 1 & -2.568690 & 6.326120 & -4.385737 \\ 1 & -3.702093 & 4.175071 & -4.914447 \\ 1 & -2.567565 & 2.033741 & -4.490890\end{array}$

$3_{\text {IB-re }-}-\psi_{-122 a 3 a 4 a}$

Number of imaginary frequencies : 0

The smallest frequencies are : $3.7897 \quad 12.4226 \quad 18.9238 \mathrm{~cm}(-1)$

Electronic energy : $\quad H F=-4360.8848724$

Zero-point correction $=\quad 1.454172($ Hartree/Particle)

Thermal correction to Energy= $\quad 1.539235$

Thermal correction to Enthalpy $=\quad 1.540180$

Thermal correction to Gibbs Free Energy $=\quad 1.332254$

Sum of electronic and zero-point Energies $=\quad-4359.430700$ 
Sum of electronic and thermal Energies= Sum of electronic and thermal Enthalpies= Sum of electronic and thermal Free Energies=
$-4359.345637$

$-4359.344693$

$-4359.552618$

Cartesian Coordinates

$\begin{array}{cccc}6 & -1.719356 & 3.444859 & -4.033864 \\ 6 & -0.529816 & 3.357968 & -3.302953 \\ 6 & -0.009212 & 4.543319 & -2.766583 \\ 6 & -0.673621 & 5.753707 & -2.914788 \\ 6 & -1.876385 & 5.816533 & -3.615711 \\ 6 & -2.391910 & 4.655003 & -4.179582 \\ 6 & 0.206051 & 2.061170 & -3.160876 \\ 6 & 1.657332 & 2.158051 & -3.577899 \\ 45 & -0.070843 & 1.172390 & -1.147188 \\ 15 & 1.782983 & -0.313994 & -0.922002 \\ 7 & 1.405547 & -1.950682 & -0.669382 \\ 6 & 2.318331 & -3.000563 & -0.156106 \\ 6 & 2.905836 & -2.731027 & 1.203650 \\ 6 & 2.085745 & -2.418961 & 2.289622 \\ 6 & 2.636329 & -2.150049 & 3.535840 \\ 6 & 4.018346 & -2.189116 & 3.715846 \\ 6 & 4.840396 & -2.514446 & 2.642679 \\ 6 & 4.284500 & -2.787601 & 1.395128 \\ 15 & -1.565108 & -0.049398 & 0.415109 \\ 6 & -1.375328 & -1.710381 & 1.239626 \\ 6 & -1.092002 & -2.823897 & 0.465199 \\ 6 & -1.019340 & -4.124406 & 1.063601 \\ 6 & -1.244727 & -4.266475 & 2.463286 \\ 6 & -1.548601 & -3.114388 & 3.223525 \\ 6 & -1.613459 & -1.881518 & 2.631439 \\ 6 & -0.721373 & -5.292380 & 0.312940 \\ 6 & -0.647070 & -6.522543 & 0.918589 \\ 6 & -0.871103 & -6.655371 & 2.305128 \\ 6 & -1.164248 & -5.547831 & 3.058870 \\ 6 & -0.889324 & -2.737059 & -1.005739 \\ 6 & 0.349046 & -2.409080 & -1.529194 \\ 6 & 0.578683 & -2.488805 & -2.922531 \\ 6 & -0.430490 & -2.826194 & -3.785092 \\ 6 & -1.731174 & -3.085596 & -3.297289 \\ 6 & -1.962889 & -3.062363 & -1.889583 \\ 6 & -2.806205 & -3.374452 & -4.173867 \\ 6 & -4.062700 & -3.623008 & -3.684609 \\ 6 & -4.298143 & -3.593107 & -2.291915 \\ 6 & -3.274554 & -3.330928 & -1.416712 \\ 6 & -1.633981 & 1.083498 & 1.863341 \\ 6 & -2.625802 & 2.063519 & 1.940109 \\ 6 & -2.632819 & 2.994709 & 2.976261 \\ 6 & -1.643250 & 2.890115 & 3.963179 \\ 6 & -0.641138 & 1.920556 & 3.920104 \\ 6 & -0.629039 & 1.045635 & 2.827596 \\ 6 & 0.369423 & 1.720306 & 5.049534 \\ 6 & -3.684566 & 4.097939 & 3.074377 \\ 6 & -3.348570 & -0.192875 & -0.052783 \\ 6 & -4.261247 & -0.754876 & 0.844847 \\ 6 & -5.606334 & -0.893524 & 0.514165\end{array}$




\begin{tabular}{|c|c|c|c|}
\hline 6 & -6.014059 & -0.435405 & -0.742851 \\
\hline & -5.136820 & 0.144318 & -1.659706 \\
\hline & -3.788984 & 0.250913 & -1.296696 \\
\hline & -6.622794 & -1.529110 & 1.460636 \\
\hline & -5.674731 & 0.637321 & -3.001770 \\
\hline & 0.426543 & 2.628107 & 0.026985 \\
\hline & 0.505186 & 3.646350 & 0.570714 \\
\hline & -0.477162 & 0.823628 & -3.277153 \\
\hline & 2.751870 & -0.118553 & 0.401234 \\
\hline & 3.612625 & 0.954237 & 0.419383 \\
\hline & 4.790580 & 0.905852 & -0.301815 \\
\hline & 5.622841 & 2.072683 & -0.307339 \\
\hline & 5.267849 & 3.190810 & 0.507392 \\
\hline & 4.084785 & 3.142533 & 1.283196 \\
\hline & 3.261694 & 2.051591 & 1.226830 \\
\hline & 6.094998 & 4.340142 & 0.512239 \\
\hline & 7.217588 & 4.406178 & -0.272203 \\
\hline & 7.551837 & 3.318708 & -1.106236 \\
\hline & 6.777801 & 2.184927 & -1.122932 \\
\hline & 5.127733 & -0.321585 & -1.057073 \\
\hline & 4.212791 & -0.852489 & -1.948657 \\
\hline & 4.525223 & -1.958026 & -2.769912 \\
\hline & 5.732102 & -2.588654 & -2.640624 \\
\hline 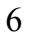 & 6.662814 & -2.165392 & -1.660374 \\
\hline & 6.357076 & -1.030225 & -0.851283 \\
\hline & 7.265627 & -0.687597 & 0.182342 \\
\hline & 8.419150 & -1.405941 & 0.380680 \\
\hline & 8.735789 & -2.503211 & -0.446925 \\
\hline & 7.869962 & -2.873370 & -1.444129 \\
\hline & 2.974445 & -0.278561 & -2.112165 \\
\hline & 7.028037 & 0.144054 & 0.840570 \\
\hline & -1.863486 & -1.018357 & 3.242828 \\
\hline & 8.087122 & -3.730465 & -2.080429 \\
\hline & -1.338985 & -5.632648 & 4.130548 \\
\hline & 5.809620 & 5.177509 & 1.147675 \\
\hline & -3.468520 & -3.296642 & -0.345507 \\
\hline ] & -5.303393 & -3.770852 & -1.913632 \\
\hline & 3.826233 & 3.997614 & 1.906530 \\
\hline & -1.738011 & -3.221596 & 4.291190 \\
\hline ] & 5.982480 & -3.439641 & -3.271933 \\
\hline & 8.427919 & 3.380744 & -1.747944 \\
\hline ] & 3.789326 & -2.287247 & -3.500179 \\
\hline & -2.610916 & -3.392205 & -5.245214 \\
\hline 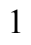 & -0.250687 & -2.871956 & -4.858249 \\
\hline & -0.415510 & -7.403219 & 0.323850 \\
\hline 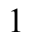 & 7.043453 & 1.361132 & -1.780389 \\
\hline ] & -0.550601 & -5.207167 & -0.758370 \\
\hline ] & 1.571387 & -2.246502 & -3.296989 \\
\hline 1 & 7.842139 & 5.296465 & -0.264404 \\
\hline & 9.092417 & -1.128472 & 1.188696 \\
\hline 1 & -4.882700 & -3.840838 & -4.365820 \\
\hline & -0.809967 & -7.635951 & 2.771332 \\
\hline 1 & 9.655880 & -3.059296 & -0.283333 \\
\hline 1 & 2.334163 & 1.998319 & 1.790768 \\
\hline & -3.908575 & -1.093537 & 1.815153 \\
\hline & -3.057709 & 0.683500 & -1.975091 \\
\hline
\end{tabular}




\begin{tabular}{|c|c|c|c|}
\hline 1 & -7.064881 & -0.534905 & -1.016725 \\
\hline & -3.391495 & 2.096847 & 1.168876 \\
\hline & 0.156243 & 0.289981 & 2.748800 \\
\hline & -1.664292 & 3.593203 & 4.793095 \\
\hline & 1.707439 & -3.913598 & -0.109682 \\
\hline & -1.400386 & 2.030924 & -1.216186 \\
\hline & 3.126726 & -3.201978 & -0.876471 \\
\hline & 4.936736 & -3.021369 & 0.552217 \\
\hline & 1.009709 & -2.347900 & 2.141879 \\
\hline & 5.921048 & -2.546646 & 2.767746 \\
\hline & 1.982372 & -1.904241 & 4.372690 \\
\hline 1 & 4.450178 & -1.968081 & 4.689701 \\
\hline & 0.070551 & -0.036177 & -3.660863 \\
\hline & -1.547508 & 0.790345 & -3.479378 \\
\hline & 2.256384 & 2.769813 & -2.890144 \\
\hline & 2.124762 & 1.172219 & -3.627231 \\
\hline & 1.729310 & 2.625081 & -4.572468 \\
\hline & 0.921584 & 4.508860 & -2.202146 \\
\hline & -0.250638 & 6.655413 & -2.475932 \\
\hline & -2.398514 & 6.764163 & -3.729163 \\
\hline & -3.320115 & 4.685843 & -4.748689 \\
\hline & -2.120598 & 2.551074 & -4.508013 \\
\hline & -4.575133 & 1.242321 & -3.868367 \\
\hline & -6.298175 & -0.532699 & -3.769538 \\
\hline ) & -6.735417 & 1.716457 & -2.756791 \\
\hline & -5.986238 & -1.979333 & 2.773786 \\
\hline & -7.724669 & -0.514541 & 1.783739 \\
\hline & -7.242049 & -2.760853 & 0.791004 \\
\hline & -7.123217 & 2.093458 & -3.712789 \\
\hline 1 & -7.588125 & 1.336253 & -2.181018 \\
\hline & -6.310660 & 2.565214 & -2.205254 \\
\hline 1 & -4.999263 & 1.594116 & -4.818112 \\
\hline & -4.099749 & 2.102249 & -3.378393 \\
\hline 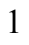 & -3.795705 & 0.504355 & -4.106487 \\
\hline 1 & -6.706411 & -0.184449 & -4.728107 \\
\hline ] & -5.543919 & -1.302920 & -3.979669 \\
\hline 1 & -7.116386 & -1.007277 & -3.213315 \\
\hline & -6.749106 & -2.439281 & 3.414561 \\
\hline 1 & -5.197070 & -2.726559 & 2.611279 \\
\hline & -5.552265 & -1.138457 & 3.330361 \\
\hline ] & -8.462073 & -0.955384 & 2.468038 \\
\hline 1 & -7.306223 & 0.378234 & 2.266586 \\
\hline ] & -8.261004 & -0.187156 & 0.884843 \\
\hline 1 & -7.996976 & -3.213452 & 1.448040 \\
\hline & -7.733054 & -2.515128 & -0.158723 \\
\hline 1 & -6.476533 & -3.522189 & 0.588729 \\
\hline j & -4.673606 & 4.061306 & 1.911225 \\
\hline 6 & -4.476225 & 3.938880 & 4.377019 \\
\hline 6 & -2.985650 & 5.461767 & 3.061606 \\
\hline 5 & 0.283376 & 2.813400 & 6.111747 \\
\hline 6 & 0.070224 & 0.372894 & 5.720060 \\
\hline 5 & 1.800126 & 1.701593 & 4.505524 \\
\hline 1 & -5.406300 & 4.869988 & 2.027348 \\
\hline & -4.174711 & 4.204578 & 0.944067 \\
\hline & -5.231038 & 3.115718 & 1.872520 \\
\hline & -5.234066 & 4.729505 & 4.461324 \\
\hline
\end{tabular}




$\begin{array}{rrrr}1 & -4.993412 & 2.970675 & 4.406103 \\ 1 & -3.833492 & 4.001738 & 5.263356 \\ 1 & -3.726806 & 6.270937 & 3.112010 \\ 1 & -2.303064 & 5.583905 & 3.911182 \\ 1 & -2.400760 & 5.591711 & 2.141720 \\ 1 & 1.042874 & 2.636852 & 6.883834 \\ 1 & 0.466955 & 3.809394 & 5.688400 \\ 1 & -0.692844 & 2.826696 & 6.613006 \\ 1 & 2.513886 & 1.542450 & 5.325163 \\ 1 & 1.965149 & 0.898368 & 3.775814 \\ 1 & 2.049167 & 2.658113 & 4.025725 \\ 1 & 0.775540 & 0.185621 & 6.541613 \\ 1 & -0.946666 & 0.357654 & 6.134927 \\ 1 & 0.152589 & -0.460134 & 5.009087\end{array}$

$3_{\text {IB-re- }} \psi_{-1 \text { s2a3a4s }}$

Number of imaginary frequencies : 0

The smallest frequencies are : $8.7186 \quad 17.241620 .6122 \mathrm{~cm}(-1)$

Electronic energy : $\quad \mathrm{HF}=-4360.8857835$

Zero-point correction $=\quad 1.454959($ Hartree/Particle $)$

Thermal correction to Energy= $\quad 1.539709$

Thermal correction to Enthalpy= $\quad 1.540653$

Thermal correction to Gibbs Free Energy= $\quad 1.335398$

Sum of electronic and zero-point Energies $=\quad-4359.430825$

Sum of electronic and thermal Energies $=\quad-4359.346075$

Sum of electronic and thermal Enthalpies $=\quad-4359.345131$

Sum of electronic and thermal Free Energies $=\quad-4359.550386$

Cartesian Coordinates

$\begin{array}{cccc}6 & -1.716337 & 3.359259 & -4.107477 \\ 6 & -0.543207 & 3.307544 & -3.347124 \\ 6 & -0.065882 & 4.509046 & -2.806739 \\ 6 & -0.757640 & 5.700810 & -2.978207 \\ 6 & -1.945544 & 5.727869 & -3.706362 \\ 6 & -2.417196 & 4.550306 & -4.275319 \\ 6 & 0.220737 & 2.030401 & -3.177526 \\ 6 & 1.677405 & 2.158551 & -3.567058 \\ 45 & -0.072111 & 1.157068 & -1.160685 \\ 15 & 1.794494 & -0.309737 & -0.904501 \\ 7 & 1.426880 & -1.946887 & -0.639674 \\ 6 & 2.353960 & -2.987373 & -0.132656 \\ 6 & 2.938100 & -2.718519 & 1.228514 \\ 6 & 2.114358 & -2.419764 & 2.315409 \\ 6 & 2.660965 & -2.164277 & 3.566092 \\ 6 & 4.042432 & -2.203895 & 3.749669 \\ 6 & 4.868339 & -2.513097 & 2.674728 \\ 6 & 4.316551 & -2.772558 & 1.422521 \\ 15 & -1.566685 & -0.057541 & 0.401519 \\ 6 & -1.365922 & -1.703940 & 1.253244 \\ 6 & -1.066324 & -2.825179 & 0.496212 \\ 6 & -0.981033 & -4.116085 & 1.113057 \\ 6 & -1.210420 & -4.240932 & 2.513831 \\ 6 & -1.528193 & -3.081212 & 3.256652 \\ 6 & -1.605014 & -1.857509 & 2.646821\end{array}$




\begin{tabular}{|c|c|c|c|}
\hline 6 & -0.665373 & -5.290835 & 0.380258 \\
\hline & -0.578037 & -6.511067 & 1.003780 \\
\hline & -0.806247 & -6.626895 & 2.391199 \\
\hline & -1.116407 & -5.512630 & 3.127901 \\
\hline & -0.859072 & -2.756291 & -0.974984 \\
\hline & 0.377161 & -2.421913 & -1.498709 \\
\hline & 0.613558 & -2.521137 & -2.889618 \\
\hline & -0.388216 & -2.884250 & -3.750362 \\
\hline & -1.687181 & -3.153843 & -3.263202 \\
\hline & -1.924728 & -3.110586 & -1.856964 \\
\hline & -2.754163 & -3.474992 & -4.138322 \\
\hline & -4.007761 & -3.737424 & -3.648679 \\
\hline & -4.247939 & -3.690347 & -2.257257 \\
\hline b & -3.232861 & -3.393535 & -1.383345 \\
\hline & -1.678347 & 1.096740 & 1.830444 \\
\hline & -2.692662 & 2.055673 & 1.882410 \\
\hline b & -2.729752 & 3.003205 & 2.902725 \\
\hline & -1.749030 & 2.935497 & 3.901452 \\
\hline D & -0.725785 & 1.988096 & 3.883348 \\
\hline & -0.681970 & 1.097512 & 2.804137 \\
\hline & 0.289973 & 1.842366 & 5.016225 \\
\hline ) & -3.804463 & 4.086493 & 2.970484 \\
\hline ) & -3.341207 & -0.232522 & -0.085755 \\
\hline ) & -4.260904 & -0.736463 & 0.829832 \\
\hline$b$ & -5.610883 & -0.881504 & 0.503698 \\
\hline 6 & -6.011200 & -0.490834 & -0.773451 \\
\hline 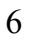 & -5.117384 & 0.028028 & -1.719109 \\
\hline & -3.773354 & 0.143242 & -1.358653 \\
\hline 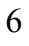 & -6.567497 & -1.448761 & 1.550401 \\
\hline 6 & -5.647127 & 0.467503 & -3.082773 \\
\hline 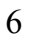 & 0.387433 & 2.634866 & 0.002697 \\
\hline$x$ & 0.446695 & 3.658594 & 0.538210 \\
\hline 6 & -0.433050 & 0.776908 & -3.293958 \\
\hline 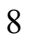 & 2.756337 & -0.096868 & 0.421554 \\
\hline 6 & 3.604809 & 0.986463 & 0.430077 \\
\hline ) & 4.788460 & 0.939967 & -0.281345 \\
\hline 6 & 5.608658 & 2.115403 & -0.297671 \\
\hline & 5.233616 & 3.243001 & 0.494803 \\
\hline 6 & 4.042641 & 3.195561 & 1.258402 \\
\hline 6 & 3.232808 & 2.094189 & 1.213330 \\
\hline 6 & 6.048426 & 4.401128 & 0.488881 \\
\hline 6 & 7.178175 & 4.466407 & -0.285236 \\
\hline 5 & 7.532076 & 3.369172 & -1.098044 \\
\hline 6 & 6.770261 & 2.227041 & -1.103843 \\
\hline 5 & 5.139714 & -0.291175 & -1.023805 \\
\hline 6 & 4.235246 & -0.835355 & -1.918119 \\
\hline 5 & 4.565964 & -1.938375 & -2.735731 \\
\hline 6 & 5.778824 & -2.555445 & -2.597269 \\
\hline 6 & 6.697786 & -2.120896 & -1.610751 \\
\hline 5 & 6.374618 & -0.986750 & -0.807435 \\
\hline 6 & 7.271314 & -0.631481 & 0.232180 \\
\hline 5 & 8.430077 & -1.338020 & 0.442339 \\
\hline 6 & 8.763752 & -2.434911 & -0.379100 \\
\hline 6 & 7.909909 & -2.816414 & -1.382458 \\
\hline 8 & 2.990543 & -0.278234 & -2.090447 \\
\hline 1 & 7.019553 & 0.199795 & 0.885694 \\
\hline
\end{tabular}




\begin{tabular}{|c|c|c|c|}
\hline & -1.861294 & -0.987757 & 3.246112 \\
\hline . & 8.140680 & -3.673322 & -2.014170 \\
\hline 1 & -1.294233 & -5.584429 & 4.200020 \\
\hline & 5.747757 & 5.245626 & 1.107618 \\
\hline 1 & -3.431454 & -3.345523 & -0.313486 \\
\hline & -5.250876 & -3.879203 & -1.878757 \\
\hline 1 & 3.767175 & 4.059150 & 1.862448 \\
\hline & -1.717054 & -3.174827 & 4.325697 \\
\hline & 6.042821 & -3.404564 & -3.225507 \\
\hline & 8.413814 & 3.430113 & -1.732058 \\
\hline 1 & 3.838673 & -2.276208 & -3.470664 \\
\hline 1 & -2.555140 & -3.507095 & -5.208644 \\
\hline 1 & -0.203482 & -2.945885 & -4.821881 \\
\hline 1 & -0.332952 & -7.397153 & 0.422657 \\
\hline 1 & 7.051192 & 1.395983 & -1.745519 \\
\hline 1 & -0.491522 & -5.218863 & -0.691545 \\
\hline 1 & 1.605488 & -2.274625 & -3.263656 \\
\hline 1 & 7.793204 & 5.363323 & -0.285753 \\
\hline 1 & 9.093973 & -1.051692 & 1.255004 \\
\hline 1 & -4.821265 & -3.981768 & -4.328729 \\
\hline 1 & -0.734578 & -7.599831 & 2.871683 \\
\hline 1 & 9.687627 & -2.981792 & -0.206056 \\
\hline 1 & 2.299114 & 2.041073 & 1.766497 \\
\hline 1 & -3.921808 & -1.023298 & 1.824237 \\
\hline 1 & -3.034420 & 0.530963 & -2.055742 \\
\hline 1 & -7.059292 & -0.586472 & -1.050456 \\
\hline 1 & -3.451258 & 2.060748 & 1.103609 \\
\hline 1 & 0.124299 & 0.362541 & 2.741557 \\
\hline 1 & -1.794498 & 3.650369 & 4.720155 \\
\hline 1 & 1.753641 & -3.907665 & -0.089636 \\
\hline 1 & -1.416215 & 1.989713 & -1.260715 \\
\hline 1 & 3.164141 & -3.176188 & -0.854357 \\
\hline 1 & 4.971376 & -2.995995 & 0.578825 \\
\hline 1 & 1.038505 & -2.350278 & 2.165061 \\
\hline 1 & 5.948712 & -2.544077 & 2.802467 \\
\hline 1 & 2.003926 & -1.928991 & 4.403259 \\
\hline 1 & 4.471124 & -1.995041 & 4.727564 \\
\hline 1 & 0.139367 & -0.074426 & -3.660115 \\
\hline 1 & -1.498429 & 0.720024 & -3.516155 \\
\hline 1 & 2.249572 & 2.783446 & -2.868166 \\
\hline 1 & 2.166811 & 1.183021 & -3.606621 \\
\hline 1 & 1.758605 & 2.626730 & -4.560322 \\
\hline 1 & 0.851313 & 4.501659 & -2.219558 \\
\hline 1 & -0.368506 & 6.615793 & -2.535551 \\
\hline 1 & -2.489728 & 6.660707 & -3.837308 \\
\hline 1 & -3.332157 & 4.554100 & -4.866066 \\
\hline 1 & -2.079751 & 2.452849 & -4.588406 \\
\hline 6 & -4.526039 & 0.938535 & -4.003234 \\
\hline 6 & -6.368005 & -0.696651 & -3.769089 \\
\hline 6 & -6.625649 & 1.631340 & -2.887268 \\
\hline 6 & -7.993169 & -1.586432 & 1.023353 \\
\hline 6 & -6.084622 & -2.839299 & 1.980904 \\
\hline 6 & -6.594500 & -0.515977 & 2.766806 \\
\hline 1 & -7.008154 & 1.975685 & -3.857776 \\
\hline 1 & -7.488133 & 1.345045 & -2.272978 \\
\hline 1 & -6.131662 & 2.480282 & -2.396944 \\
\hline
\end{tabular}




$\begin{array}{lrrr}1 & -4.942049 & 1.240685 & -4.973198 \\ 1 & -3.999334 & 1.805955 & -3.584306 \\ 1 & -3.792295 & 0.140973 & -4.188310 \\ 1 & -6.756922 & -0.378180 & -4.745745 \\ 1 & -5.678238 & -1.534816 & -3.934405 \\ 1 & -7.217033 & -1.068503 & -3.182703 \\ 1 & -8.637423 & -2.007001 & 1.805821 \\ 1 & -8.419900 & -0.617696 & 0.733518 \\ 1 & -8.045380 & -2.258745 & 0.156774 \\ 1 & -6.748413 & -3.251087 & 2.753119 \\ 1 & -6.087875 & -3.535339 & 1.131383 \\ 1 & -5.067544 & -2.822156 & 2.393427 \\ 1 & -7.258496 & -0.918697 & 3.543652 \\ 1 & -5.599935 & -0.386205 & 3.211770 \\ 1 & -6.966553 & 0.478363 & 2.487370 \\ 6 & -4.773372 & 4.015161 & 1.792158 \\ 6 & -4.612091 & 3.928441 & 4.263216 \\ 6 & -3.133487 & 5.464258 & 2.949838 \\ 6 & 0.130509 & 2.920169 & 6.085455 \\ 6 & 0.081299 & 0.474490 & 5.678806 \\ 6 & 1.717524 & 1.923264 & 4.469633 \\ 1 & -5.526732 & 4.807387 & 1.887589 \\ 1 & -4.261801 & 4.160211 & 0.831841 \\ 1 & -5.306982 & 3.055963 & 1.753262 \\ 1 & -5.387064 & 4.704328 & 4.326070 \\ 1 & -5.109728 & 2.950143 & 4.297989 \\ 1 & -3.983622 & 4.016084 & 5.157726 \\ 1 & -3.892142 & 6.258263 & 2.973818 \\ 1 & -2.470065 & 5.613911 & 3.810126 \\ 1 & -2.534157 & 5.592885 & 2.039147 \\ 1 & 0.883008 & 2.773356 & 6.870518 \\ 1 & 0.274107 & 3.927990 & 5.674921 \\ 1 & -0.854984 & 2.881310 & 6.566940 \\ 1 & 2.444189 & 1.837059 & 5.288664 \\ 1 & 1.937637 & 1.117844 & 3.757704 \\ 1 & 1.889963 & 2.884221 & 3.965241 \\ 1 & 0.799341 & 0.329762 & 6.497791 \\ 1 & -0.931183 & 0.390591 & 6.096365 \\ 1 & 0.216930 & -0.348590 & 4.964513\end{array}$

$3_{\text {IB-re- }} \Psi-1$ s2a3s4a

Number of imaginary frequencies : 0

The smallest frequencies are : $12.5474 \quad 18.139620 .4856 \mathrm{~cm}(-1)$

Electronic energy : $\quad H F=-4360.8864898$

Zero-point correction $=\quad 1.454793($ Hartree/Particle $)$

Thermal correction to Energy $=\quad 1.539465$

Thermal correction to Enthalpy $=\quad 1.540410$

Thermal correction to Gibbs Free Energy $=\quad 1.335902$

Sum of electronic and zero-point Energies $=\quad-4359.431697$

Sum of electronic and thermal Energies $=\quad-4359.347024$

Sum of electronic and thermal Enthalpies $=\quad-4359.346080$

Sum of electronic and thermal Free Energies $=\quad-4359.550588$

Cartesian Coordinates 


\begin{tabular}{|c|c|c|c|}
\hline 6 & 7.821483 & -2.856186 & -1.438607 \\
\hline 6 & 6.603441 & -2.170279 & -1.664392 \\
\hline 6 & 6.278578 & -1.030687 & -0.869308 \\
\hline 6 & 7.179886 & -0.661915 & 0.161610 \\
\hline 6 & 8.344876 & -1.359021 & 0.369475 \\
\hline & 8.680212 & -2.460508 & -0.445008 \\
\hline 6 & 5.038378 & -0.343292 & -1.085065 \\
\hline 6 & 4.133872 & -0.900136 & -1.971159 \\
\hline 6 & 4.464001 & -2.011588 & -2.777381 \\
\hline 6 & 5.680494 & -2.621244 & -2.639884 \\
\hline 6 & 4.684022 & 0.893789 & -0.353946 \\
\hline 6 & 3.497414 & 0.950250 & 0.353676 \\
\hline 6 & 3.128126 & 2.066958 & 1.126052 \\
\hline 6 & 3.944314 & 3.163631 & 1.167408 \\
\hline 6 & 5.136950 & 3.201150 & 0.406500 \\
\hline 6 & 5.507634 & 2.067300 & -0.378831 \\
\hline 6 & 5.955656 & 4.356511 & 0.394013 \\
\hline 6 & 7.085089 & 4.413886 & -0.381059 \\
\hline 6 & 7.433902 & 3.311341 & -1.188823 \\
\hline 6 & 6.668353 & 2.171696 & -1.187575 \\
\hline 8 & 2.643149 & -0.129129 & 0.354951 \\
\hline 15 & 1.687268 & -0.364994 & -0.973734 \\
\hline 8 & 2.888814 & -0.347606 & -2.150384 \\
\hline 45 & -0.162932 & 1.116154 & -1.261385 \\
\hline 6 & -0.755416 & 0.549013 & -3.303047 \\
\hline 6 & -0.016913 & 1.752924 & -3.393819 \\
\hline 6 & 1.394323 & 1.714192 & -3.936467 \\
\hline 6 & -0.702678 & 3.064547 & -3.617547 \\
\hline 6 & -1.974873 & 3.143165 & -4.196147 \\
\hline 6 & -2.589349 & 4.371545 & -4.423773 \\
\hline 6 & -1.939010 & 5.555461 & -4.094986 \\
\hline 6 & -0.662156 & 5.495467 & -3.540959 \\
\hline 6 & -0.053950 & 4.268587 & -3.311086 \\
\hline 15 & -1.571645 & 0.039176 & 0.456657 \\
\hline 6 & -3.389479 & 0.002755 & 0.122371 \\
\hline 6 & -4.286979 & -0.438496 & 1.105834 \\
\hline 6 & -5.643220 & -0.565094 & 0.835532 \\
\hline 6 & -6.092142 & -0.226051 & -0.452339 \\
\hline 6 & -5.234361 & 0.231460 & -1.445679 \\
\hline 6 & -3.869840 & 0.337883 & -1.134125 \\
\hline 6 & -6.637553 & -1.089851 & 1.869224 \\
\hline 6 & -7.242649 & -2.401540 & 1.355891 \\
\hline 6 & -5.707896 & 0.621067 & -2.843450 \\
\hline 6 & -4.980119 & -0.230047 & -3.890235 \\
\hline 6 & 0.425403 & 2.678030 & -0.289319 \\
\hline 8 & 0.532942 & 3.742659 & 0.151107 \\
\hline 7 & 1.316436 & -1.994939 & -0.673862 \\
\hline 6 & 0.250348 & -2.476179 & -1.509064 \\
\hline 6 & -1.001153 & -2.719785 & -0.973395 \\
\hline 6 & -2.075800 & -3.086876 & -1.840264 \\
\hline 6 & -1.820757 & -3.285614 & -3.230343 \\
\hline 6 & -0.506113 & -3.111199 & -3.721466 \\
\hline 6 & 0.496513 & -2.696316 & -2.884748 \\
\hline 6 & -3.402157 & -3.257513 & -1.364630 \\
\hline 6 & -4.414430 & -3.617991 & -2.218349 \\
\hline 6 & -4.153562 & -3.838915 & -3.589005 \\
\hline
\end{tabular}




\begin{tabular}{|c|c|c|c|}
\hline & -2.885582 & -3.668969 & -4.082671 \\
\hline & -1.199529 & -2.759084 & 0.501211 \\
\hline & -1.433879 & -1.631989 & 1.270721 \\
\hline & -1.664522 & -1.785932 & 2.666756 \\
\hline & -1.638649 & -3.014518 & 3.268510 \\
\hline & -1.377284 & -4.181823 & 2.515204 \\
\hline & -1.158230 & -4.058057 & 1.112803 \\
\hline & -1.326688 & -5.458103 & 3.124098 \\
\hline & -1.064787 & -6.580754 & 2.381299 \\
\hline & -0.841892 & -6.466789 & 0.993395 \\
\hline & -0.889529 & -5.241508 & 0.374872 \\
\hline & 2.246301 & -3.038227 & -0.176062 \\
\hline & 2.848049 & -2.773366 & 1.177865 \\
\hline & 2.035591 & -2.520113 & 2.284702 \\
\hline & 2.597860 & -2.277874 & 3.531465 \\
\hline & 3.983038 & -2.280187 & 3.689824 \\
\hline & 4.797646 & -2.545364 & 2.594915 \\
\hline 6 & 4.230437 & -2.796552 & 1.347883 \\
\hline 6 & -1.427765 & 1.168516 & 1.900409 \\
\hline 6 & -2.297085 & 2.253616 & 2.037773 \\
\hline 6 & -2.130795 & 3.174813 & 3.070366 \\
\hline 6 & -1.101828 & 2.950752 & 3.995423 \\
\hline 6 & -0.226978 & 1.869180 & 3.896758 \\
\hline 6 & -0.377266 & 1.009012 & 2.802153 \\
\hline 6 & -3.032278 & 4.397819 & 3.228529 \\
\hline 6 & -2.176821 & 5.664802 & 3.119523 \\
\hline 6 & 0.788556 & 1.523625 & 4.987425 \\
\hline 6 & 2.189345 & 1.324356 & 4.404037 \\
\hline 6 & 0.877443 & 2.599750 & 6.066684 \\
\hline 6 & 0.327472 & 0.218537 & 5.651283 \\
\hline 6 & -4.115496 & 4.461451 & 2.154119 \\
\hline 6 & -3.722387 & 4.361502 & 4.596478 \\
\hline 6 & -5.976446 & -1.369473 & 3.217398 \\
\hline 6 & -7.751900 & -0.061765 & 2.089866 \\
\hline 6 & -7.208161 & 0.411687 & -3.031663 \\
\hline 6 & -5.398691 & 2.104404 & -3.074752 \\
\hline 1 & 6.927329 & 0.172963 & 0.810162 \\
\hline 1 & -1.868920 & -0.909015 & 3.275808 \\
\hline 1 & 8.052892 & -3.717300 & -2.064325 \\
\hline 1 & -1.497881 & -5.526403 & 4.197531 \\
\hline 1 & 5.657515 & 5.205519 & 1.007772 \\
\hline 1 & -3.615363 & -3.078132 & -0.311367 \\
\hline 1 & -5.428030 & -3.728545 & -1.837460 \\
\hline 1 & 3.669991 & 4.032669 & 1.764007 \\
\hline 1 & -1.820428 & -3.106122 & 4.339019 \\
\hline 1 & 5.945052 & -3.476198 & -3.259909 \\
\hline 1 & 8.314556 & 3.366130 & -1.824929 \\
\hline 1 & 3.731867 & -2.359957 & -3.502787 \\
\hline 1 & -2.673343 & -3.824173 & -5.139599 \\
\hline 1 & -0.311014 & -3.285153 & -4.778605 \\
\hline 1 & -0.631605 & -7.358161 & 0.406768 \\
\hline 1 & 6.945750 & 1.337314 & -1.826328 \\
\hline 1 & -0.716399 & -5.175186 & -0.697354 \\
\hline 1 & 1.501693 & -2.518082 & -3.264358 \\
\hline 1 & 7.703134 & 5.308719 & -0.386958 \\
\hline 1 & 9.012301 & -1.061517 & 1.175215 \\
\hline
\end{tabular}




\begin{tabular}{|c|c|c|c|}
\hline 1 & -4.964390 & -4.131914 & -4.252509 \\
\hline . & -1.026014 & -7.557620 & 2.857557 \\
\hline 1 & 9.608865 & -2.999866 & -0.273955 \\
\hline & 2.192712 & 2.022763 & 1.678826 \\
\hline & -3.908460 & -0.693721 & 2.091395 \\
\hline & -3.161480 & 0.683798 & -1.883803 \\
\hline & -7.152356 & -0.330533 & -0.674160 \\
\hline & -3.101434 & 2.376238 & 1.315458 \\
\hline & 0.310069 & 0.168646 & 2.679775 \\
\hline 1 & -0.992733 & 3.645341 & 4.825687 \\
\hline 1 & 1.642087 & -3.955434 & -0.124519 \\
\hline 1 & -1.472712 & 2.004273 & -1.332616 \\
\hline 1 & 3.047325 & -3.231623 & -0.906507 \\
\hline 1 & 4.875297 & -2.991286 & 0.489844 \\
\hline 1 & 0.955102 & -2.486709 & 2.155910 \\
\hline 1 & 5.880434 & -2.551916 & 2.704184 \\
\hline 1 & 1.951289 & -2.081829 & 4.386693 \\
\hline 1 & 4.422311 & -2.077886 & 4.664645 \\
\hline 1 & -0.281788 & -0.376372 & -3.626165 \\
\hline 1 & -1.839009 & 0.549016 & -3.410454 \\
\hline 1 & 2.085319 & 2.359046 & -3.379133 \\
\hline 1 & 1.802480 & 0.700906 & -3.905114 \\
\hline 1 & 1.400605 & 2.057992 & -4.982450 \\
\hline 1 & 0.937692 & 4.238703 & -2.861470 \\
\hline 1 & -0.135834 & 6.412299 & -3.282255 \\
\hline 1 & -2.417111 & 6.515841 & -4.275110 \\
\hline 1 & -3.581105 & 4.399364 & -4.872613 \\
\hline 1 & -2.491234 & 2.228533 & -4.486991 \\
\hline 1 & -5.274444 & 0.078734 & -4.902695 \\
\hline 1 & -3.887939 & -0.145486 & -3.819484 \\
\hline 1 & -5.228130 & -1.293154 & -3.771738 \\
\hline 1 & -7.494750 & 0.700127 & -4.050984 \\
\hline 1 & -7.495415 & -0.639314 & -2.894617 \\
\hline 1 & -7.799972 & 1.024666 & -2.339543 \\
\hline 1 & -5.696810 & 2.402443 & -4.089950 \\
\hline 1 & -5.947188 & 2.734217 & -2.362052 \\
\hline 1 & -4.331002 & 2.328219 & -2.958012 \\
\hline 1 & -6.727281 & -1.742430 & 3.925348 \\
\hline 1 & -5.189723 & -2.132189 & 3.140488 \\
\hline 1 & -5.534311 & -0.463303 & 3.652063 \\
\hline 1 & -8.466059 & -0.428975 & 2.839099 \\
\hline 1 & -7.341528 & 0.890054 & 2.451603 \\
\hline 1 & -8.315372 & 0.144657 & 1.172198 \\
\hline 1 & -7.960241 & -2.804068 & 2.083637 \\
\hline 1 & -7.773544 & -2.267075 & 0.405452 \\
\hline 1 & -6.459924 & -3.156491 & 1.199658 \\
\hline 1 & -4.735320 & 5.353437 & 2.310402 \\
\hline 1 & -3.689486 & 4.529845 & 1.145009 \\
\hline 1 & -4.780608 & 3.588183 & 2.187572 \\
\hline 1 & -4.376160 & 5.236011 & 4.715380 \\
\hline 1 & -4.341434 & 3.460774 & 4.700877 \\
\hline 1 & -3.004667 & 4.372751 & 5.425497 \\
\hline 1 & -2.806400 & 6.560145 & 3.212856 \\
\hline 1 & -1.411496 & 5.713539 & 3.903593 \\
\hline 1 & -1.664727 & 5.707744 & 2.149462 \\
\hline 1 & 1.632540 & 2.313228 & 6.809696 \\
\hline
\end{tabular}


$3_{\text {IB-re- }} \psi_{-1 \mathrm{~s} 2 \mathrm{a3s} 4 \mathrm{~s}}$

Number of imaginary frequencies : 0

The smallest frequencies are : $14.1593 \quad 18.6166 \quad 24.9584 \mathrm{~cm}(-1)$

Electronic energy : $\quad H F=-4360.8866614$

Zero-point correction $=\quad 1.455757($ Hartree/Particle $)$

Thermal correction to Energy= $\quad 1.540079$

Thermal correction to Enthalpy= $\quad 1.541024$

Thermal correction to Gibbs Free Energy= 1.338462

Sum of electronic and zero-point Energies $=\quad-4359.430905$

Sum of electronic and thermal Energies $=\quad-4359.346582$

Sum of electronic and thermal Enthalpies $=\quad-4359.345638$

Sum of electronic and thermal Free Energies $=\quad-4359.548199$

\begin{tabular}{cccc}
\multicolumn{4}{c}{ Cartesian Coordinates } \\
\hline 6 & 7.880858 & -2.765984 & -1.445719 \\
6 & 6.660644 & -2.080381 & -1.660556 \\
6 & 6.322657 & -0.967708 & -0.833638 \\
6 & 7.213885 & -0.624671 & 0.214772 \\
6 & 8.381734 & -1.320472 & 0.410227 \\
6 & 8.729678 & -2.395203 & -0.434131 \\
6 & 5.081126 & -0.280370 & -1.039736 \\
6 & 4.186829 & -0.812259 & -1.951243 \\
6 & 4.530776 & -1.895894 & -2.789135 \\
6 & 5.748864 & -2.504221 & -2.658445 \\
6 & 4.714934 & 0.931443 & -0.273446 \\
6 & 3.525236 & 0.957303 & 0.429905 \\
6 & 3.145628 & 2.046750 & 1.235313 \\
6 & 3.952878 & 3.148342 & 1.312676 \\
6 & 5.147725 & 3.218118 & 0.557430 \\
6 & 5.529915 & 2.110826 & -0.259421 \\
6 & 5.957941 & 4.379354 & 0.581735 \\
6 & 7.089544 & 4.467532 & -0.187333 \\
6 & 7.449804 & 3.391473 & -1.025316 \\
6 & 6.692939 & 2.246565 & -1.059884 \\
8 & 2.677279 & -0.127104 & 0.388484 \\
15 & 1.730730 & -0.305617 & -0.955463 \\
8 & 2.941388 & -0.257735 & -2.124391 \\
45 & -0.118955 & 1.189620 & -1.182636 \\
6 & -0.588306 & 0.806195 & -3.294387 \\
6 & 0.127049 & 2.027924 & -3.224455 \\
6 & 1.569589 & 2.069182 & -3.679733 \\
6 & -0.576046 & 3.341940 & -3.368884 \\
6 & -1.815762 & 3.449885 & -4.009879 \\
6 & -2.443953 & 4.682750 & -4.163448 \\
6 & -1.839884 & 5.843710 & -3.694463
\end{tabular}




\begin{tabular}{|c|c|c|c|}
\hline & -0.594862 & 5.756706 & -3.075588 \\
\hline & 0.027491 & 4.525093 & -2.921052 \\
\hline & -1.575109 & -0.022542 & 0.417906 \\
\hline & -3.371589 & -0.074947 & -0.007951 \\
\hline & -4.304140 & -0.582679 & 0.898824 \\
\hline & -5.656695 & -0.670041 & 0.577016 \\
\hline & -6.060212 & -0.225296 & -0.687896 \\
\hline & -5.156501 & 0.293084 & -1.616374 \\
\hline & -3.805916 & 0.357400 & -1.255134 \\
\hline & -6.621053 & -1.263726 & 1.602198 \\
\hline & -6.575027 & -0.432939 & 2.889862 \\
\hline & -5.579139 & 0.802987 & -2.991862 \\
\hline 0 & -4.813407 & 0.040740 & -4.078377 \\
\hline & 0.393415 & 2.670062 & -0.048253 \\
\hline & 0.464529 & 3.698335 & 0.477379 \\
\hline 1 & 1.343502 & -1.944277 & -0.734111 \\
\hline 6 & 0.291394 & -2.382173 & -1.611079 \\
\hline 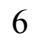 & -0.957577 & -2.689830 & -1.103077 \\
\hline & -2.022318 & -3.010614 & -1.998921 \\
\hline 6 & -1.763877 & -3.085897 & -3.399969 \\
\hline 6 & -0.452063 & -2.846336 & -3.870397 \\
\hline 6 & 0.542987 & -2.487603 & -2.999205 \\
\hline 6 & -3.344999 & -3.245250 & -1.540434 \\
\hline 6 & -4.351841 & -3.545317 & -2.423035 \\
\hline 6 & -4.087873 & -3.642912 & -3.807373 \\
\hline 6 & -2.822255 & -3.413177 & -4.283225 \\
\hline 6 & -1.172561 & -2.810721 & 0.363347 \\
\hline 6 & -1.435909 & -1.720558 & 1.175030 \\
\hline 6 & -1.687615 & -1.936531 & 2.558928 \\
\hline 6 & -1.657255 & -3.190960 & 3.106088 \\
\hline 6 & -1.370620 & -4.321557 & 2.307302 \\
\hline 6 & -1.127681 & -4.133872 & 0.916202 \\
\hline 6 & -1.317846 & -5.623702 & 2.858721 \\
\hline 6 & -1.031848 & -6.709043 & 2.070619 \\
\hline 6 & -0.786704 & -6.531100 & 0.693069 \\
\hline 6 & -0.835785 & -5.279668 & 0.129642 \\
\hline 6 & 2.261013 & -3.012858 & -0.269188 \\
\hline 6 & 2.859886 & -2.798719 & 1.095052 \\
\hline 6 & 2.046575 & -2.569941 & 2.206881 \\
\hline 6 & 2.608376 & -2.369248 & 3.461371 \\
\hline 6 & 3.992954 & -2.390657 & 3.623059 \\
\hline 6 & 4.807957 & -2.633117 & 2.523238 \\
\hline 6 & 4.241536 & -2.841173 & 1.267989 \\
\hline 6 & -1.537343 & 1.037558 & 1.918275 \\
\hline 6 & -2.490309 & 2.038239 & 2.116214 \\
\hline 6 & -2.416135 & 2.885047 & 3.220974 \\
\hline 6 & -1.385467 & 2.673514 & 4.146757 \\
\hline 6 & -0.421835 & 1.677991 & 3.982206 \\
\hline 6 & -0.489965 & 0.892575 & 2.825958 \\
\hline 6 & -3.434953 & 3.996878 & 3.463571 \\
\hline 6 & -2.707405 & 5.342613 & 3.550287 \\
\hline 6 & 0.605138 & 1.335134 & 5.062565 \\
\hline 6 & 2.018903 & 1.248455 & 4.482613 \\
\hline 6 & 0.624240 & 2.356505 & 6.197206 \\
\hline 6 & 0.217053 & -0.028517 & 5.650780 \\
\hline 6 & -4.469355 & 4.088280 & 2.343640 \\
\hline
\end{tabular}




\begin{tabular}{|c|c|c|c|}
\hline 6 & -4.179272 & 3.727554 & 4.776192 \\
\hline 6 & -8.063680 & -1.286384 & 1.103922 \\
\hline 6 & -6.198848 & -2.707016 & 1.904478 \\
\hline 6 & -7.071722 & 0.618097 & -3.252836 \\
\hline 6 & -5.258239 & 2.299008 & -3.084287 \\
\hline 1 & 6.951913 & 0.189039 & 0.885993 \\
\hline 1 & -1.912352 & -1.089305 & 3.201916 \\
\hline 1 & 8.122445 & -3.606305 & -2.095396 \\
\hline 1 & -1.506935 & -5.742904 & 3.924672 \\
\hline 1 & 5.651580 & 5.207869 & 1.219006 \\
\hline 1 & -3.559882 & -3.161100 & -0.475373 \\
\hline 1 & -5.363125 & -3.704572 & -2.053756 \\
\hline 1 & 3.669561 & 3.996350 & 1.934825 \\
\hline 1 & -1.855310 & -3.331805 & 4.168295 \\
\hline 1 & 6.023352 & -3.337653 & -3.302957 \\
\hline 1 & 8.332430 & 3.471304 & -1.655984 \\
\hline 1 & 3.809396 & -2.224707 & -3.533960 \\
\hline 1 & -2.607813 & -3.474773 & -5.349274 \\
\hline 1 & -0.252219 & -2.926280 & -4.937963 \\
\hline 1 & -0.558410 & -7.393563 & 0.071020 \\
\hline 1 & 6.978817 & 1.432368 & -1.720628 \\
\hline 1 & -0.647584 & -5.161342 & -0.935565 \\
\hline 1 & 1.544115 & -2.259495 & -3.361235 \\
\hline 1 & 7.700768 & 5.366766 & -0.165108 \\
\hline 1 & 9.041590 & -1.042975 & 1.229230 \\
\hline 1 & -4.893950 & -3.890894 & -4.494619 \\
\hline 1 & -0.991512 & -7.706052 & 2.503036 \\
\hline 1 & 9.660393 & -2.933846 & -0.272279 \\
\hline 1 & 2.208670 & 1.979584 & 1.781975 \\
\hline 1 & -3.966806 & -0.925281 & 1.875776 \\
\hline 1 & -3.066814 & 0.750025 & -1.950617 \\
\hline 1 & -7.111111 & -0.287805 & -0.955171 \\
\hline 1 & -3.291405 & 2.151724 & 1.389446 \\
\hline 1 & 0.258736 & 0.115223 & 2.655425 \\
\hline 1 & -1.346414 & 3.306169 & 5.031158 \\
\hline 1 & 1.648726 & -3.925952 & -0.251600 \\
\hline 1 & -1.441760 & 2.058760 & -1.237479 \\
\hline 1 & 3.062388 & -3.186281 & -1.004515 \\
\hline 1 & 4.886899 & -3.015370 & 0.405969 \\
\hline 1 & 0.966482 & -2.519219 & 2.076522 \\
\hline 1 & 5.890313 & -2.654205 & 2.634933 \\
\hline 1 & 1.962241 & -2.191233 & 4.320832 \\
\hline 1 & 4.431372 & -2.221606 & 4.604517 \\
\hline 1 & -0.075455 & -0.073900 & -3.679345 \\
\hline 1 & -1.663717 & 0.798271 & -3.468950 \\
\hline 1 & 2.212859 & 2.655141 & -3.010248 \\
\hline 1 & 1.995077 & 1.064591 & -3.736964 \\
\hline 1 & 1.633887 & 2.529605 & -4.677725 \\
\hline 1 & 0.994177 & 4.473478 & -2.421947 \\
\hline 1 & -0.104390 & 6.655784 & -2.707374 \\
\hline 1 & -2.328613 & 6.807875 & -3.816452 \\
\hline 1 & -3.409293 & 4.733251 & -4.664726 \\
\hline 1 & -2.294626 & 2.556292 & -4.409599 \\
\hline 1 & -5.069829 & 0.432235 & -5.072356 \\
\hline 1 & -3.725154 & 0.117701 & -3.958238 \\
\hline 1 & -5.065626 & -1.027488 & -4.055714 \\
\hline
\end{tabular}




$\begin{array}{rrrr}1 & -7.318337 & 0.989990 & -4.255455 \\ 1 & -7.366873 & -0.438770 & -3.212026 \\ 1 & -7.688154 & 1.176437 & -2.536492 \\ 1 & -5.519930 & 2.684908 & -4.079802 \\ 1 & -5.829053 & 2.868758 & -2.339158 \\ 1 & -4.194572 & 2.505120 & -2.911520 \\ 1 & -8.713550 & -1.718867 & 1.875169 \\ 1 & -8.438677 & -0.278123 & 0.885907 \\ 1 & -8.176313 & -1.898400 & 0.199610 \\ 1 & -6.872234 & -3.156874 & 2.646720 \\ 1 & -6.239355 & -3.322941 & 0.995633 \\ 1 & -5.177009 & -2.767994 & 2.301253 \\ 1 & -7.249913 & -0.859364 & 3.644302 \\ 1 & -5.569160 & -0.397169 & 3.326268 \\ 1 & -6.891748 & 0.600941 & 2.699629 \\ 1 & -5.176890 & 4.897969 & 2.562845 \\ 1 & -4.005549 & 4.308473 & 1.373583 \\ 1 & -5.050707 & 3.161919 & 2.242391 \\ 1 & -4.915993 & 4.519710 & 4.966617 \\ 1 & -4.715895 & 2.770215 & 4.732133 \\ 1 & -3.500880 & 3.692269 & 5.637269 \\ 1 & -3.428429 & 6.156555 & 3.705779 \\ 1 & -1.990588 & 5.372789 & 4.379639 \\ 1 & -2.155837 & 5.548452 & 2.623757 \\ 1 & 1.393685 & 2.079024 & 6.928797 \\ 1 & 0.860644 & 3.365097 & 5.834115 \\ 1 & -0.332643 & 2.399034 & 6.732769 \\ 1 & 2.724962 & 0.925700 & 5.260442 \\ 1 & 2.100933 & 0.528235 & 3.658404 \\ 1 & 2.354003 & 2.227680 & 4.115320 \\ 1 & 0.924085 & -0.325501 & 6.438096 \\ 1 & -0.788270 & 0.006990 & 6.092175 \\ 1 & 0.215728 & -0.813768 & 4.883171\end{array}$

$3_{\text {IB-re- }}-\psi_{-1 \mathrm{~s} 233 a 4 a}$

Number of imaginary frequencies : 0

The smallest frequencies are : $10.9685 \quad 16.5297 \quad 19.0063 \mathrm{~cm}(-1)$

Electronic energy : $\quad \mathrm{HF}=-4360.8864983$

Zero-point correction $=\quad 1.455090($ Hartree/Particle $)$

Thermal correction to Energy $=\quad 1.539589$

Thermal correction to Enthalpy= $\quad 1.540533$

Thermal correction to Gibbs Free Energy= $\quad 1.337149$

Sum of electronic and zero-point Energies $=\quad-4359.431409$

Sum of electronic and thermal Energies $=\quad-4359.346909$

Sum of electronic and thermal Enthalpies $=\quad-4359.345965$

Sum of electronic and thermal Free Energies $=\quad-4359.549349$

Cartesian Coordinates

$\begin{array}{lllll}6 & 1.819476 & -3.138581 & -4.145880 \\ 6 & & 0.634879 & -3.201658 & -3.402977 \\ 6 & & 0.245886 & -4.454746 & -2.911131 \\ 6 & 1.030788 & -5.583237 & -3.110685 \\ 6 & & 2.226189 & -5.494867 & -3.820479 \\ 6 & & 2.611695 & -4.265632 & -4.343837\end{array}$




\begin{tabular}{|c|c|c|c|}
\hline 6 & -0.221140 & -1.990280 & -3.187208 \\
\hline 6 & -1.681285 & -2.218735 & -3.515245 \\
\hline 45 & 0.068764 & -1.138921 & -1.163243 \\
\hline & -1.776338 & 0.332916 & -0.881288 \\
\hline 7 & -1.383129 & 1.953272 & -0.551621 \\
\hline 6 & -2.292812 & 2.985691 & 0.003868 \\
\hline 6 & -2.890498 & 2.656601 & 1.345729 \\
\hline 6 & -2.077092 & 2.312614 & 2.427163 \\
\hline 6 & -2.638307 & 1.983765 & 3.654670 \\
\hline 6 & -4.022715 & 1.993696 & 3.819163 \\
\hline 6 & -4.837916 & 2.352988 & 2.751359 \\
\hline 6 & -4.271866 & 2.687617 & 1.523859 \\
\hline 15 & 1.558468 & 0.011015 & 0.448847 \\
\hline 6 & 1.410678 & 1.640522 & 1.344670 \\
\hline 6 & 1.116716 & 2.786675 & 0.624978 \\
\hline 6 & 1.049897 & 4.059506 & 1.280374 \\
\hline 6 & 1.299009 & 4.141452 & 2.680889 \\
\hline 6 & 1.621998 & 2.958020 & 3.383705 \\
\hline 6 & 1.676346 & 1.751913 & 2.737933 \\
\hline 6 & 0.733422 & 5.256983 & 0.586210 \\
\hline 6 & 0.662719 & 6.459131 & 1.245798 \\
\hline 6 & 0.910454 & 6.532778 & 2.632726 \\
\hline 6 & 1.222703 & 5.395319 & 3.332635 \\
\hline 6 & 0.909084 & 2.766283 & -0.847018 \\
\hline 6 & -0.324472 & 2.450971 & -1.388132 \\
\hline 6 & -0.549872 & 2.599141 & -2.777102 \\
\hline 6 & 0.455108 & 2.998452 & -3.617818 \\
\hline 6 & 1.750164 & 3.255950 & -3.113792 \\
\hline 6 & 1.980854 & 3.150286 & -1.709974 \\
\hline 6 & 2.820626 & 3.620395 & -3.967873 \\
\hline 6 & 4.075257 & 3.850039 & -3.462644 \\
\hline 6 & 4.310966 & 3.729238 & -2.074870 \\
\hline 6 & 3.288834 & 3.401729 & -1.219955 \\
\hline 6 & 1.591742 & -1.169037 & 1.856107 \\
\hline 6 & 2.553623 & -2.170574 & 1.918760 \\
\hline 6 & 2.530656 & -3.132013 & 2.933160 \\
\hline 6 & 1.533171 & -3.038900 & 3.906018 \\
\hline 6 & 0.549999 & -2.041575 & 3.873303 \\
\hline 6 & 0.573678 & -1.135427 & 2.812652 \\
\hline 6 & -0.472035 & -1.849874 & 4.994699 \\
\hline 6 & 3.601188 & -4.220465 & 2.937599 \\
\hline 6 & 3.335447 & 0.136732 & -0.035962 \\
\hline 6 & 4.299868 & 0.504447 & 0.908992 \\
\hline 6 & 5.632589 & 0.669936 & 0.546353 \\
\hline 6 & 5.973062 & 0.457160 & -0.796267 \\
\hline 6 & 5.040792 & 0.090524 & -1.765488 \\
\hline 6 & 3.709162 & -0.071112 & -1.358836 \\
\hline 6 & 6.701259 & 1.120170 & 1.540712 \\
\hline 6 & 5.476893 & -0.185832 & -3.204032 \\
\hline 6 & -0.392871 & -2.663426 & -0.060092 \\
\hline 8 & -0.485604 & -3.714816 & 0.413570 \\
\hline 6 & 0.332577 & -0.688675 & -3.299961 \\
\hline 8 & -2.775557 & 0.090230 & 0.410231 \\
\hline 6 & -3.629742 & -0.987328 & 0.354294 \\
\hline 6 & -4.793723 & -0.899679 & -0.384457 \\
\hline 6 & -5.622169 & -2.065455 & -0.473563 \\
\hline
\end{tabular}




\begin{tabular}{|c|c|c|c|}
\hline & -5.272736 & -3.231426 & 0.274452 \\
\hline & -4.100563 & -3.227993 & 1.068458 \\
\hline & -3.282947 & -2.131607 & 1.095231 \\
\hline & -6.094562 & -4.382041 & 0.194864 \\
\hline & -7.205809 & -4.402506 & -0.607845 \\
\hline & -7.534393 & -3.265707 & -1.375516 \\
\hline & -6.765797 & -2.130160 & -1.310201 \\
\hline & -5.112373 & 0.367402 & -1.080083 \\
\hline & -4.180166 & 0.936284 & -1.929662 \\
\hline & -4.474375 & 2.082620 & -2.699935 \\
\hline & -5.679202 & 2.714332 & -2.556791 \\
\hline & -6.626405 & 2.249871 & -1.611722 \\
\hline & -6.340007 & 1.073075 & -0.857062 \\
\hline & -7.264976 & 0.684330 & 0.145168 \\
\hline & -8.416137 & 1.400468 & 0.364403 \\
\hline & -8.713412 & 2.541004 & -0.410501 \\
\hline & -7.831637 & 2.954837 & -1.376107 \\
\hline & -2.940879 & 0.365692 & -2.100153 \\
\hline & -7.041197 & -0.182158 & 0.762308 \\
\hline & 1.932182 & 0.862537 & 3.308262 \\
\hline & -8.034258 & 3.844792 & -1.970684 \\
\hline & 1.415853 & 5.434942 & 4.403788 \\
\hline & -5.813871 & -5.256854 & 0.779966 \\
\hline & 3.482496 & 3.305533 & -0.152933 \\
\hline 1 & 5.312249 & 3.892898 & -1.679868 \\
\hline & -3.846050 & -4.120012 & 1.639397 \\
\hline & 1.830796 & 3.018861 & 4.451412 \\
\hline & -5.915246 & 3.597073 & -3.148802 \\
\hline & -8.401824 & -3.290545 & -2.031388 \\
\hline 1 & -3.726619 & 2.442387 & -3.403081 \\
\hline & 2.625786 & 3.704466 & -5.036253 \\
\hline 1 & 0.274520 & 3.102316 & -4.686769 \\
\hline & 0.416087 & 7.363385 & 0.694061 \\
\hline & -7.026520 & -1.267733 & -1.918234 \\
\hline 1 & 0.544595 & 5.217197 & -0.484713 \\
\hline & -1.538402 & 2.366012 & -3.167394 \\
\hline 1 & -7.826098 & -5.293950 & -0.665016 \\
\hline & -9.102715 & 1.087381 & 1.147822 \\
\hline 1 & 4.892159 & 4.121449 & -4.128261 \\
\hline ] & 0.852606 & 7.491734 & 3.142348 \\
\hline l & -9.631705 & 3.095144 & -0.230985 \\
\hline 1 & -2.364328 & -2.108732 & 1.675868 \\
\hline & 3.995510 & 0.663518 & 1.940173 \\
\hline 1 & 2.935160 & -0.350423 & -2.070836 \\
\hline 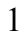 & 7.012889 & 0.588839 & -1.091727 \\
\hline 1 & 3.329493 & -2.208427 & 1.155019 \\
\hline l & -0.190849 & -0.357665 & 2.748483 \\
\hline 1 & 1.521543 & -3.761169 & 4.716772 \\
\hline 1 & -1.675248 & 3.890529 & 0.096840 \\
\hline ] & 1.423511 & -1.951668 & -1.291981 \\
\hline 1 & -3.094728 & 3.225533 & -0.711911 \\
\hline ] & -4.917563 & 2.948074 & 0.683663 \\
\hline 1 & -0.997707 & 2.267762 & 2.289706 \\
\hline 1 & -5.920328 & 2.363844 & 2.864573 \\
\hline 1 & -1.991817 & 1.713460 & 4.489685 \\
\hline 1 & -4.461507 & 1.724242 & 4.777675 \\
\hline
\end{tabular}




\begin{tabular}{|c|c|c|c|}
\hline 1 & -0.319121 & 0.120707 & -3.626584 \\
\hline 1 & 1.379293 & -0.538002 & -3.561805 \\
\hline 1 & -2.170687 & -2.906352 & -2.812509 \\
\hline 1 & -2.243217 & -1.282077 & -3.497764 \\
\hline 1 & -1.777380 & -2.660473 & -4.519342 \\
\hline 1 & -0.676258 & -4.540760 & -2.338610 \\
\hline 1 & 0.707476 & -6.539777 & -2.704425 \\
\hline 1 & 2.842346 & -6.378156 & -3.973853 \\
\hline 1 & 3.529905 & -4.179400 & -4.923092 \\
\hline 1 & 2.115325 & -2.194475 & -4.598853 \\
\hline 6 & 4.317034 & -0.025461 & -4.186066 \\
\hline 6 & 6.592770 & 0.764788 & -3.645172 \\
\hline 6 & 5.982908 & -1.631405 & -3.278805 \\
\hline 6 & 6.161962 & 1.204421 & 2.967328 \\
\hline 6 & 7.877692 & 0.138876 & 1.536641 \\
\hline 6 & 7.196527 & 2.512488 & 1.131845 \\
\hline 1 & 6.294003 & -1.880173 & -4.303222 \\
\hline 1 & 6.842874 & -1.789091 & -2.614793 \\
\hline 1 & 5.194868 & -2.336873 & -2.981575 \\
\hline 1 & 4.681441 & -0.146662 & -5.214300 \\
\hline 1 & 3.543248 & -0.785292 & -4.027590 \\
\hline 1 & 3.850027 & 0.966061 & -4.103153 \\
\hline 1 & 6.835898 & 0.586514 & -4.700616 \\
\hline 1 & 6.281344 & 1.813448 & -3.542289 \\
\hline 1 & 7.520951 & 0.625679 & -3.078622 \\
\hline 1 & 6.965442 & 1.514024 & 3.647503 \\
\hline 1 & 5.352425 & 1.940874 & 3.060720 \\
\hline 1 & 5.785392 & 0.234067 & 3.318199 \\
\hline 1 & 8.643614 & 0.463049 & 2.253839 \\
\hline 1 & 7.552331 & -0.869209 & 1.824470 \\
\hline 1 & 8.359142 & & \\
\hline 1 & 7.966082 & 2.868288 & 1.830408 \\
\hline 1 & 7.633353 & 2.512604 & 0.125251 \\
\hline 1 & 6.371392 & 3.237711 & 1.139923 \\
\hline 6 & 3.464764 & -5.170092 & 4.124821 \\
\hline 6 & 3.482804 & -5.043695 & 1.649405 \\
\hline 6 & 4.988074 & -3.569172 & 3.004259 \\
\hline 6 & -0.434906 & -2.979691 & 6.020852 \\
\hline 6 & -0.140774 & -0.535804 & 5.714990 \\
\hline 6 & -1.893570 & -1.769089 & 4.431627 \\
\hline 1 & 4.254033 & -5.931170 & 4.079868 \\
\hline 1 & 3.567554 & -4.646875 & 5.084559 \\
\hline 1 & 2.501592 & -5.696283 & 4.120435 \\
\hline 1 & 4.243899 & -5.835659 & 1.630375 \\
\hline 1 & 2.495653 & -5.518503 & 1.578160 \\
\hline 1 & 3.618394 & -4.428776 & 0.751402 \\
\hline 1 & 5.771451 & -4.339056 & 3.004794 \\
\hline 1 & 5.175729 & -2.906923 & 2.149710 \\
\hline 1 & 5.098758 & -2.973216 & 3.920252 \\
\hline 1 & -1.197636 & -2.803495 & 6.789921 \\
\hline 1 & -0.646019 & -3.954534 & 5.562478 \\
\hline 1 & 0.533903 & -3.042706 & 6.532460 \\
\hline 1 & -2.612407 & -1.595086 & 5.244247 \\
\hline 1 & -2.019279 & -0.949665 & 3.712053 \\
\hline 1 & -2.172142 & -2.708483 & 3.934685 \\
\hline 1 & -0.855572 & -0.350278 & 6.528902 \\
\hline
\end{tabular}


$3_{\text {IB-re- }} \psi-1$-1s2s3a4s

Number of imaginary frequencies : 0

The smallest frequencies are : $13.2671 \quad 16.0035 \quad 19.2395 \mathrm{~cm}(-1)$

Electronic energy : $\quad H F=-4360.8862345$

Zero-point correction $=\quad 1.455581$ (Hartree/Particle)

Thermal correction to Energy= $\quad 1.539925$

Thermal correction to Enthalpy= $\quad 1.540869$

Thermal correction to Gibbs Free Energy= $\quad 1.337181$

Sum of electronic and zero-point Energies $=\quad-4359.430653$

Sum of electronic and thermal Energies $=\quad-4359.346310$

Sum of electronic and thermal Enthalpies $=\quad-4359.345366$

Sum of electronic and thermal Free Energies $=\quad-4359.549053$

\begin{tabular}{cccc}
\hline & \multicolumn{3}{c}{ Cartesian Coordinates } \\
\hline 6 & -1.553710 & 3.597125 & -3.888322 \\
6 & -0.417306 & 3.520676 & -3.075551 \\
6 & -0.008968 & 4.690122 & -2.420463 \\
6 & -0.734320 & 5.869071 & -2.531586 \\
6 & -1.886931 & 5.917003 & -3.313026 \\
6 & -2.287820 & 4.774731 & -3.996792 \\
6 & 0.377331 & 2.255411 & -2.959045 \\
6 & 1.854776 & 2.457657 & -3.219827 \\
45 & -0.023238 & 1.210761 & -1.051046 \\
15 & 1.808684 & -0.292676 & -0.849492 \\
7 & 1.398900 & -1.925095 & -0.613204 \\
6 & 2.292904 & -2.993728 & -0.103088 \\
6 & 2.893929 & -2.732309 & 1.251605 \\
6 & 2.084903 & -2.425332 & 2.347293 \\
6 & 2.650292 & -2.158086 & 3.587526 \\
6 & 4.034273 & -2.194831 & 3.750881 \\
6 & 4.845042 & -2.516220 & 2.667797 \\
6 & 4.274885 & -2.787786 & 1.426803 \\
15 & -1.577148 & -0.079038 & 0.403482 \\
6 & -1.409573 & -1.736279 & 1.244196 \\
6 & -1.098025 & -2.851072 & 0.482628 \\
6 & -1.025430 & -4.147590 & 1.086688 \\
6 & -1.278480 & -4.285329 & 2.482089 \\
6 & -1.610445 & -3.132854 & 3.229780 \\
6 & -1.675955 & -1.902694 & 2.631084 \\
6 & -0.702309 & -5.315442 & 0.346543 \\
6 & -0.627477 & -6.542063 & 0.958906 \\
6 & -0.876951 & -6.670854 & 2.341717 \\
6 & -1.196602 & -5.563448 & 3.084739 \\
6 & -0.878624 & -2.750377 & -0.984007 \\
6 & 0.350356 & -2.367162 & -1.492800 \\
6 & 0.576476 & -2.381711 & -2.889462 \\
6 & -0.423844 & -2.716275 & -3.763143 \\
6 & -1.710520 & -3.051876 & -3.285458 \\
6 & -1.940478 & -3.086204 & -1.878106 \\
6 & -2.771092 & -3.365595 & -4.171202 \\
6 & -4.013839 & -3.689012 & -3.690468 \\
& & & \\
\hline
\end{tabular}




\begin{tabular}{|c|c|c|c|}
\hline 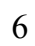 & -4.250807 & -3.704926 & -2.297893 \\
\hline & -3.240624 & -3.419779 & -1.414570 \\
\hline 0 & -1.741421 & 1.040767 & 1.852332 \\
\hline & -2.774653 & 1.967798 & 1.924604 \\
\hline & -2.841807 & 2.894582 & 2.968768 \\
\hline & -1.866354 & 2.834076 & 3.966070 \\
\hline & -0.814459 & 1.910114 & 3.925454 \\
\hline & -0.746693 & 1.044885 & 2.833212 \\
\hline & 0.216191 & 1.780890 & 5.045751 \\
\hline 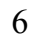 & -3.968745 & 3.924695 & 2.962956 \\
\hline & -3.325190 & -0.235491 & -0.168381 \\
\hline & -4.289517 & -0.764194 & 0.684587 \\
\hline & -5.620880 & -0.898211 & 0.284958 \\
\hline & -5.955345 & -0.469087 & -0.998840 \\
\hline & -5.014546 & 0.076630 & -1.882144 \\
\hline 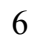 & -3.690832 & 0.178488 & -1.450275 \\
\hline 6 & -6.628151 & -1.500019 & 1.262543 \\
\hline 6 & -5.473421 & 0.559796 & -3.256887 \\
\hline 6 & 0.386957 & 2.605453 & 0.232428 \\
\hline 8 & 0.449768 & 3.599483 & 0.821113 \\
\hline 6 & -0.227653 & 1.000010 & -3.229514 \\
\hline 8 & 2.805719 & -0.121534 & 0.455961 \\
\hline 6 & 3.665300 & 0.954466 & 0.448322 \\
\hline 6 & 4.829401 & 0.897888 & -0.293444 \\
\hline 6 & 5.658722 & 2.065808 & -0.333276 \\
\hline 6 & 5.310314 & 3.200095 & 0.462197 \\
\hline 6 & 4.139719 & 3.162933 & 1.257514 \\
\hline 6 & 3.323178 & 2.065702 & 1.239653 \\
\hline 6 & 6.132238 & 4.352761 & 0.429613 \\
\hline 6 & 7.243256 & 4.405801 & -0.372034 \\
\hline 6 & 7.571425 & 3.301112 & -1.185535 \\
\hline 6 & 6.802340 & 2.164077 & -1.166523 \\
\hline 6 & 5.146779 & -0.338316 & -1.042411 \\
\hline 6 & 4.213041 & -0.867907 & -1.915100 \\
\hline 6 & 4.501345 & -1.986684 & -2.726948 \\
\hline 6 & 5.704285 & -2.627402 & -2.609182 \\
\hline 6 & 6.655090 & -2.201753 & -1.649596 \\
\hline 6 & 6.372190 & -1.055527 & -0.847727 \\
\hline 6 & 7.299480 & -0.709549 & 0.167987 \\
\hline 6 & 8.450553 & -1.434778 & 0.354731 \\
\hline 6 & 8.745009 & -2.542894 & -0.466775 \\
\hline 6 & 7.860074 & -2.916972 & -1.445526 \\
\hline 8 & 2.978505 & -0.281438 & -2.064570 \\
\hline 1 & 7.078484 & 0.130761 & 0.821111 \\
\hline 1 & -1.944656 & -1.039340 & 3.234094 \\
\hline 1 & 8.060137 & -3.782582 & -2.075869 \\
\hline 1 & -1.391863 & -5.645920 & 4.153070 \\
\hline 1 & 5.852331 & 5.202814 & 1.050464 \\
\hline 1 & -3.437316 & -3.422403 & -0.343360 \\
\hline 1 & -5.247133 & -3.935479 & -1.924757 \\
\hline 1 & 3.886272 & 4.028868 & 1.867909 \\
\hline 1 & -1.820056 & -3.237176 & 4.293911 \\
\hline 1 & 5.935879 & -3.488722 & -3.233648 \\
\hline 1 & 8.438862 & 3.352315 & -1.839829 \\
\hline 1 & 3.749811 & -2.320604 & -3.438793 \\
\hline 1 & -2.575610 & -3.343389 & -5.242485 \\
\hline
\end{tabular}




\begin{tabular}{|c|c|c|c|}
\hline . & -0.244105 & -2.713309 & -4.837302 \\
\hline & -0.376040 & -7.423167 & 0.372934 \\
\hline & 7.062915 & 1.326685 & -1.808708 \\
\hline & -0.512899 & -5.231665 & -0.721787 \\
\hline & 1.558572 & -2.094505 & -3.258714 \\
\hline & 7.863776 & 5.298686 & -0.392910 \\
\hline & 9.139237 & -1.154548 & 1.148673 \\
\hline & -4.821994 & -3.928970 & -4.378328 \\
\hline & -0.814805 & -7.648709 & 2.813495 \\
\hline & 9.663462 & -3.104320 & -0.312470 \\
\hline & 2.407117 & 2.019767 & 1.820831 \\
\hline 1 & -3.999757 & -1.081153 & 1.685660 \\
\hline & -2.916732 & 0.585016 & -2.097518 \\
\hline & -6.987816 & -0.555435 & -1.331886 \\
\hline & -3.532701 & 1.980016 & 1.142556 \\
\hline & 0.080358 & 0.334659 & 2.751161 \\
\hline & -1.922902 & 3.528940 & 4.798574 \\
\hline 1 & 1.663521 & -3.893436 & -0.050597 \\
\hline 1 & -1.368017 & 2.045189 & -1.154118 \\
\hline 1 & 3.092183 & -3.212752 & -0.828400 \\
\hline 1 & 4.916941 & -3.018738 & 0.575371 \\
\hline 1 & 1.006157 & -2.358322 & 2.212555 \\
\hline & 5.927183 & -2.546324 & 2.780092 \\
\hline & 2.006732 & -1.915893 & 4.432972 \\
\hline 1 & 4.476776 & -1.975496 & 4.720308 \\
\hline 1 & 0.395799 & 0.197272 & -3.622260 \\
\hline 1 & -1.272220 & 0.935201 & -3.533404 \\
\hline 1 & 2.345278 & 3.046726 & -2.433255 \\
\hline 1 & 2.382731 & 1.504192 & -3.291039 \\
\hline 1 & 1.997215 & 3.002044 & -4.166339 \\
\hline & 0.879727 & 4.667356 & -1.791684 \\
\hline 1 & -0.399035 & 6.757359 & -1.999519 \\
\hline 1 & -2.457742 & 6.839321 & -3.396449 \\
\hline 1 & -3.172899 & 4.796963 & -4.631088 \\
\hline 1 & -1.862384 & 2.722019 & -4.457319 \\
\hline 6 & -4.306096 & 1.048832 & -4.108929 \\
\hline 6 & -6.167823 & -0.577682 & -4.011201 \\
\hline U & -6.452143 & 1.725700 & -3.074685 \\
\hline 6 & -8.017880 & -1.646875 & 0.649230 \\
\hline 6 & -6.150636 & -2.892146 & 1.694239 \\
\hline 6 & -6.737863 & -0.593049 & 2.493694 \\
\hline 1 & -6.788061 & 2.098710 & -4.051851 \\
\hline 1 & -7.343221 & 1.429700 & -2.507551 \\
\hline 1 & -5.975153 & 2.557674 & -2.540369 \\
\hline 1 & -4.674380 & 1.380663 & -5.088437 \\
\hline 1 & -3.795419 & 1.901064 & -3.641713 \\
\hline 1 & -3.568552 & 0.252227 & -4.282584 \\
\hline 1 & -6.502111 & -0.229476 & -4.997899 \\
\hline 1 & -5.477690 & -1.417995 & -4.161949 \\
\hline 1 & -7.049599 & -0.955927 & -3.479967 \\
\hline 1 & -8.700187 & -2.095405 & 1.382359 \\
\hline 1 & -8.443120 & -0.678322 & 0.356695 \\
\hline 1 & -8.007267 & -2.299337 & -0.234144 \\
\hline 1 & -6.860533 & -3.334352 & 2.406261 \\
\hline 1 & -6.077193 & -3.567312 & 0.830994 \\
\hline 1 & -5.167373 & -2.866822 & 2.181505 \\
\hline
\end{tabular}




$\begin{array}{rrrr}1 & -7.438289 & -1.020595 & 3.224029 \\ 1 & -5.770693 & -0.460976 & 2.995235 \\ 1 & -7.106859 & 0.402943 & 2.214702 \\ 6 & -3.938953 & 4.831013 & 4.190676 \\ 6 & -3.825948 & 4.803383 & 1.713923 \\ 6 & -5.323367 & 3.206740 & 2.932112 \\ 6 & 0.045610 & 2.846612 & 6.125356 \\ 6 & 0.050995 & 0.403345 & 5.700046 \\ 6 & 1.631888 & 1.902969 & 4.476674 \\ 1 & -4.769354 & 5.546679 & 4.140290 \\ 1 & -4.050217 & 4.263313 & 5.123844 \\ 1 & -3.009499 & 5.411730 & 4.249174 \\ 1 & -4.617303 & 5.565028 & 1.688766 \\ 1 & -2.856193 & 5.318050 & 1.706424 \\ 1 & -3.894671 & 4.218708 & 0.788396 \\ 1 & -6.141636 & 3.939132 & 2.908609 \\ 1 & -5.431420 & 2.561108 & 2.050874 \\ 1 & -5.455975 & 2.580239 & 3.824343 \\ 1 & 0.810163 & 2.710114 & 6.900653 \\ 1 & 0.162366 & 3.860419 & 5.721119 \\ 1 & -0.933067 & 2.782715 & 6.617817 \\ 1 & 2.373779 & 1.843307 & 5.284597 \\ 1 & 1.865836 & 1.099985 & 3.766243 \\ 1 & 1.766604 & 2.866481 & 3.965475 \\ 1 & 0.791906 & 0.264114 & 6.499539 \\ 1 & -0.948978 & 0.296154 & 6.141740 \\ 1 & 0.182776 & -0.409576 & 4.973513\end{array}$

$3_{\text {IB-re- }}-\psi_{-1 s 23354 a}$

Number of imaginary frequencies : 0

The smallest frequencies are : $16.5604 \quad 17.5473 \quad 23.1792 \mathrm{~cm}(-1)$

Electronic energy : $\quad H F=-4360.8869258$

Zero-point correction $=\quad 1.455884($ Hartree/Particle $)$

Thermal correction to Energy $=\quad 1.540224$

Thermal correction to Enthalpy= $\quad 1.541168$

Thermal correction to Gibbs Free Energy= $\quad 1.338982$

Sum of electronic and zero-point Energies $=\quad-4359.431042$

Sum of electronic and thermal Energies $=\quad-4359.346702$

Sum of electronic and thermal Enthalpies $=\quad-4359.345757$

Sum of electronic and thermal Free Energies $=\quad-4359.547944$

Cartesian Coordinates

$\begin{array}{rrrr}6 & -7.839422 & 2.926936 & -1.352831 \\ 6 & -6.633811 & 2.224530 & -1.594021 \\ 6 & -6.331510 & 1.060147 & -0.826545 \\ 6 & -7.241673 & 0.681219 & 0.192865 \\ 6 & -8.393520 & 1.394828 & 0.416944 \\ 6 & -8.706575 & 2.522736 & -0.370041 \\ 6 & -5.102874 & 0.357436 & -1.055061 \\ 6 & -4.184135 & 0.920333 & -1.922953 \\ 6 & -4.495101 & 2.052969 & -2.706901 \\ 6 & -5.701598 & 2.679970 & -2.558255 \\ 6 & -4.772086 & -0.901676 & -0.350910 \\ 6 & -3.595869 & -0.983778 & 0.369534\end{array}$




\begin{tabular}{|c|c|c|c|}
\hline 6 & -3.240559 & -2.121347 & 1.117118 \\
\hline 6 & -4.060746 & -3.215925 & 1.115986 \\
\hline 6 & -5.244989 & -3.225843 & 0.340480 \\
\hline 6 & -5.603124 & -2.067729 & -0.415391 \\
\hline 6 & -6.069690 & -4.375798 & 0.286620 \\
\hline 6 & -7.192821 & -4.403699 & -0.499154 \\
\hline 6 & -7.530165 & -3.275481 & -1.275638 \\
\hline 6 & -6.758673 & -2.140681 & -1.234864 \\
\hline 8 & -2.739049 & 0.092136 & 0.404781 \\
\hline 15 & -1.760726 & 0.340362 & -0.902589 \\
\hline 8 & -2.943657 & 0.355767 & -2.101871 \\
\hline 45 & 0.090844 & -1.130351 & -1.185231 \\
\hline 6 & 0.486623 & -0.642314 & -3.291727 \\
\hline 6 & -0.156593 & -1.905124 & -3.252167 \\
\hline 6 & -1.606346 & -2.013448 & -3.673296 \\
\hline 6 & 0.618875 & -3.171562 & -3.448043 \\
\hline 6 & 1.880066 & -3.185830 & -4.056926 \\
\hline 6 & 2.577280 & -4.375591 & -4.245288 \\
\hline 6 & 2.025977 & -5.587098 & -3.842778 \\
\hline 6 & 0.762580 & -5.593298 & -3.256921 \\
\hline 6 & 0.069911 & -4.404327 & -3.069344 \\
\hline 15 & 1.561554 & -0.002443 & 0.450982 \\
\hline 6 & 3.347918 & 0.114987 & -0.003692 \\
\hline 6 & 4.293248 & 0.554909 & 0.933806 \\
\hline 6 & 5.628059 & 0.715414 & 0.584142 \\
\hline 6 & 6.000410 & 0.421816 & -0.738526 \\
\hline 6 & 5.093306 & -0.034244 & -1.688929 \\
\hline 6 & 3.754649 & -0.182815 & -1.294984 \\
\hline 6 & 6.676811 & 1.227058 & 1.569924 \\
\hline 6 & 7.200716 & 2.582727 & 1.082145 \\
\hline 6 & 5.500614 & -0.406453 & -3.112291 \\
\hline 6 & 4.575905 & 0.274492 & -4.125943 \\
\hline 6 & -0.406773 & -2.666672 & -0.116816 \\
\hline 8 & -0.480644 & -3.716430 & 0.363439 \\
\hline 7 & -1.378295 & 1.963836 & -0.579821 \\
\hline 6 & -0.324939 & 2.463540 & -1.421819 \\
\hline 6 & 0.920597 & 2.749021 & -0.891863 \\
\hline 6 & 1.982471 & 3.143069 & -1.763032 \\
\hline 6 & 1.722744 & 3.318483 & -3.154911 \\
\hline 6 & 0.416195 & 3.088641 & -3.643849 \\
\hline 6 & -0.573980 & 2.656980 & -2.801148 \\
\hline 6 & 3.303671 & 3.356899 & -1.290675 \\
\hline 6 & 4.305920 & 3.736975 & -2.147906 \\
\hline 6 & 4.036045 & 3.947632 & -3.518474 \\
\hline 6 & 2.772815 & 3.736165 & -4.009523 \\
\hline 6 & 1.132531 & 2.775320 & 0.580525 \\
\hline 6 & 1.408282 & 1.637530 & 1.320085 \\
\hline 6 & 1.659152 & 1.764088 & 2.714699 \\
\hline 6 & 1.611116 & 2.978861 & 3.343898 \\
\hline 6 & 1.308120 & 4.155664 & 2.621513 \\
\hline 6 & 1.070967 & 4.057946 & 1.219774 \\
\hline 6 & 1.237460 & 5.417852 & 3.257655 \\
\hline 6 & 0.940182 & 6.549392 & 2.542088 \\
\hline 6 & 0.701454 & 6.460362 & 1.154640 \\
\hline 6 & 0.767508 & 5.249890 & 0.509817 \\
\hline 6 & -2.294094 & 2.993964 & -0.030575 \\
\hline
\end{tabular}




\begin{tabular}{|c|c|c|c|}
\hline 6 & -2.881638 & 2.672816 & 1.317729 \\
\hline 6 & -2.059114 & 2.337812 & 2.394994 \\
\hline 6 & -2.608876 & 2.022980 & 3.631049 \\
\hline 6 & -3.991685 & 2.037424 & 3.808592 \\
\hline 6 & -4.816044 & 2.386162 & 2.744465 \\
\hline 6 & -4.261184 & 2.707084 & 1.508017 \\
\hline 6 & 1.579130 & -1.172753 & 1.866098 \\
\hline 6 & 2.538767 & -2.176281 & 1.933002 \\
\hline 6 & 2.512343 & -3.135385 & 2.948964 \\
\hline 6 & 1.514206 & -3.036616 & 3.920661 \\
\hline 6 & 0.535217 & -2.035234 & 3.885941 \\
\hline 6 & 0.560810 & -1.132421 & 2.821919 \\
\hline 6 & 3.579803 & -4.226924 & 2.955220 \\
\hline 6 & 4.969160 & -3.580474 & 3.020364 \\
\hline 6 & -0.483184 & -1.835253 & 5.009305 \\
\hline 6 & -1.906274 & -1.751148 & 4.451004 \\
\hline 6 & -0.446868 & -2.960599 & 6.040484 \\
\hline 6 & -0.145027 & -0.519566 & 5.723420 \\
\hline 6 & 3.440781 & -5.173515 & 4.144573 \\
\hline 6 & 3.457388 & -5.052769 & 1.668985 \\
\hline 6 & 6.105911 & 1.414810 & 2.974049 \\
\hline 6 & 7.839426 & 0.233074 & 1.660885 \\
\hline 6 & 6.931949 & 0.015579 & -3.434810 \\
\hline 6 & 5.392875 & -1.928487 & -3.262419 \\
\hline 1 & -7.005431 & -0.174901 & 0.819743 \\
\hline 1 & 1.899980 & 0.879307 & 3.298515 \\
\hline 1 & -8.054199 & 3.807266 & -1.957387 \\
\hline 1 & 1.422209 & 5.468226 & 4.329831 \\
\hline 1 & -5.781749 & -5.244171 & 0.877747 \\
\hline 1 & 3.522530 & 3.187301 & -0.237317 \\
\hline 1 & 5.317342 & 3.870970 & -1.768461 \\
\hline 1 & -3.798629 & -4.102218 & 1.692421 \\
\hline 1 & 1.808963 & 3.052093 & 4.412897 \\
\hline 1 & -5.950461 & 3.551971 & -3.160817 \\
\hline 1 & -8.406821 & -3.306332 & -1.918862 \\
\hline 1 & -3.758674 & 2.404268 & -3.426103 \\
\hline 1 & 2.554661 & 3.879341 & -5.066978 \\
\hline 1 & 0.215089 & 3.241693 & -4.703135 \\
\hline 1 & 0.464902 & 7.359430 & 0.590173 \\
\hline 1 & -7.026734 & -1.285497 & -1.849780 \\
\hline 1 & 0.583917 & 5.200440 & -0.561612 \\
\hline 1 & -1.571985 & 2.444844 & -3.179809 \\
\hline 1 & -7.815506 & -5.294530 & -0.536595 \\
\hline 1 & -9.068158 & 1.089681 & 1.213760 \\
\hline 1 & 4.836270 & 4.263575 & -4.184344 \\
\hline 1 & 0.886269 & 7.514883 & 3.039624 \\
\hline 1 & -9.625202 & 3.074981 & -0.186433 \\
\hline 1 & -2.312503 & -2.095888 & 1.682214 \\
\hline 1 & 3.968402 & 0.776937 & 1.946851 \\
\hline 1 & 3.006689 & -0.532478 & -2.003552 \\
\hline 1 & 7.041767 & 0.559126 & -1.022773 \\
\hline 1 & 3.315701 & -2.217898 & 1.170526 \\
\hline 1 & -0.199933 & -0.350966 & 2.756531 \\
\hline 1 & 1.500068 & -3.756520 & 4.733475 \\
\hline 1 & -1.683329 & 3.904700 & 0.050864 \\
\hline 1 & 1.428885 & -1.973397 & -1.288763 \\
\hline
\end{tabular}




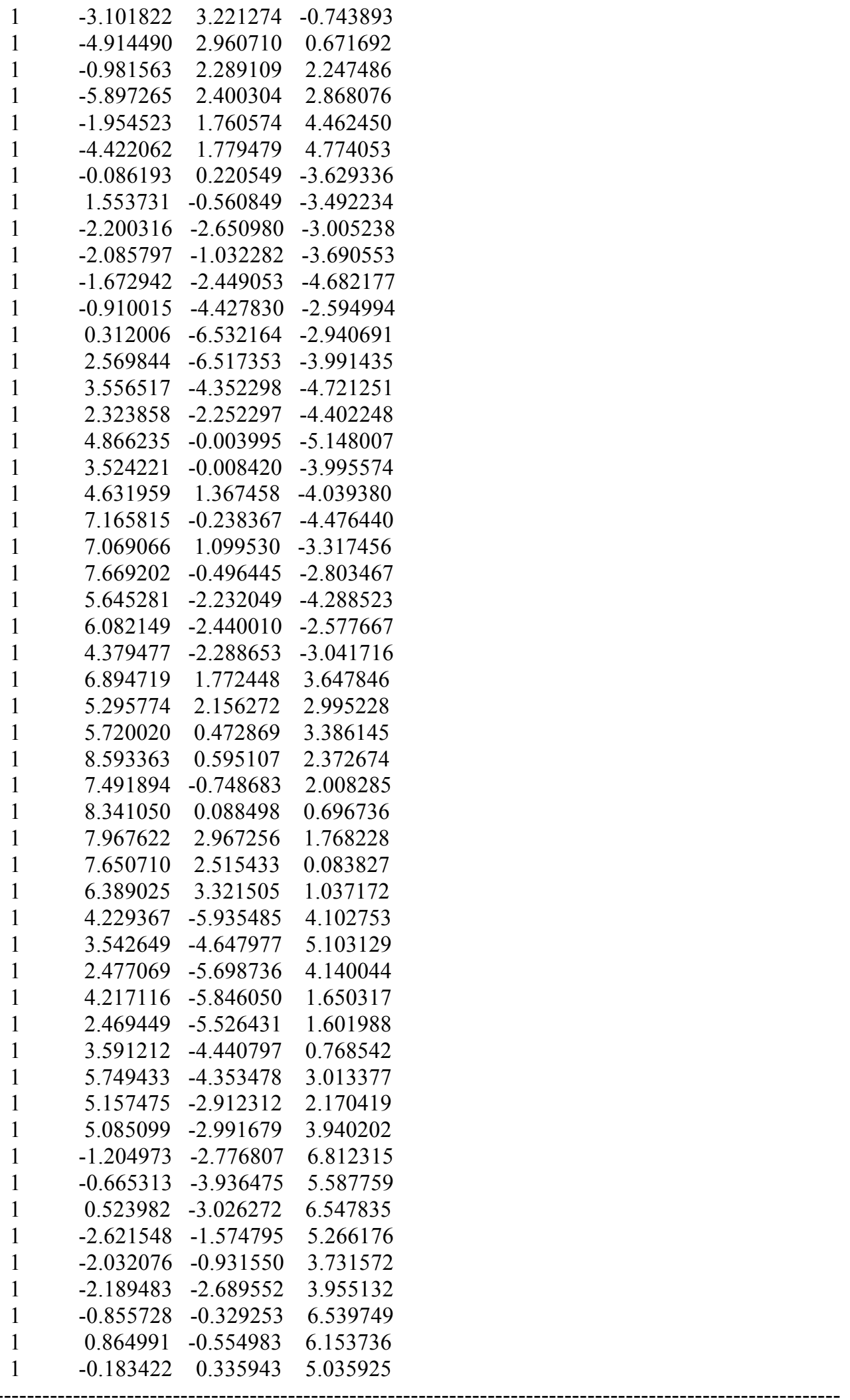

$3_{\text {IB-re- }}-\psi_{-1 \mathrm{~s} 25334 \mathrm{~s}}$

Number of imaginary frequencies : 0 
The smallest frequencies are : $16.3390 \quad 17.3676 \quad 21.1919 \mathrm{~cm}(-1)$ Electronic energy : $\quad H F=-4360.8869395$

Zero-point correction $=\quad 1.456344$ (Hartree/Particle)

Thermal correction to Energy $=\quad 1.540691$

Thermal correction to Enthalpy= $\quad 1.541635$

Thermal correction to Gibbs Free Energy= $\quad 1.338367$

Sum of electronic and zero-point Energies $=\quad-4359.430596$

Sum of electronic and thermal Energies $=\quad-4359.346249$

Sum of electronic and thermal Enthalpies $=\quad-4359.345305$

Sum of electronic and thermal Free Energies $=\quad-4359.548572$

\section{Cartesian Coordinates}

$\begin{array}{ccccc}6 & 1.855077 & -3.352089 & -3.915631 \\ 6 & 0.593292 & -3.300567 & -3.310154 \\ 6 & 0.030073 & -4.511711 & -2.885283 \\ 6 & 0.710126 & -5.714470 & -3.025487 \\ 6 & 1.974390 & -5.744709 & -3.608737 \\ 6 & 2.539512 & -4.555759 & -4.056715 \\ 6 & -0.167703 & -2.018742 & -3.165053 \\ 6 & -1.617142 & -2.125818 & -3.587652 \\ 45 & 0.080947 & -1.168686 & -1.128871 \\ 15 & -1.763734 & 0.322037 & -0.899565 \\ 7 & -1.375265 & 1.953081 & -0.629321 \\ 6 & -2.292578 & 3.007045 & -0.130635 \\ 6 & -2.898268 & 2.743398 & 1.221835 \\ 6 & -2.091211 & 2.437344 & 2.319015 \\ 6 & -2.656178 & 2.182081 & 3.561511 \\ 6 & -4.039789 & 2.229954 & 3.726284 \\ 6 & -4.849034 & 2.548091 & 2.641256 \\ 6 & -4.278707 & 2.807082 & 1.397223 \\ 15 & 1.557484 & 0.019174 & 0.457077 \\ 6 & 1.381118 & 1.671448 & 1.297588 \\ 6 & 1.118465 & 2.797501 & 0.533612 \\ 6 & 1.052994 & 4.090487 & 1.149732 \\ 6 & 1.269060 & 4.211515 & 2.553166 \\ 6 & 1.551161 & 3.046148 & 3.301484 \\ 6 & 1.606309 & 1.820320 & 2.693803 \\ 6 & 0.767585 & 5.271468 & 0.414353 \\ 6 & 0.699723 & 6.493505 & 1.036595 \\ 6 & 0.917911 & 6.605707 & 2.425924 \\ 6 & 1.196598 & 5.485369 & 3.165846 \\ 6 & 0.924317 & 2.736773 & -0.940714 \\ 6 & -0.311426 & 2.419192 & -1.476424 \\ 6 & -0.540317 & 2.542190 & -2.867065 \\ 6 & 0.463071 & 2.928013 & -3.715789 \\ 6 & 1.759265 & 3.192604 & -3.217809 \\ 6 & 1.994864 & 3.104187 & -1.813141 \\ 6 & 2.822229 & 3.559996 & -4.079382 \\ 6 & 4.074586 & 3.813658 & -3.581063 \\ 6 & 4.316704 & 3.706757 & -2.193688 \\ 6 & 3.302722 & 3.372352 & -1.331323 \\ 6 & 1.632990 & -1.138010 & 1.883050 \\ 6 & 2.617731 & -2.119246 & 1.927895 \\ 6 & 2.626114 & -3.090212 & 2.932423 \\ 6 & 1.646805 & -3.016795 & 3.925236\end{array}$




\begin{tabular}{|c|c|c|c|}
\hline 6 & 0.647220 & -2.036211 & 3.916445 \\
\hline 6 & 0.629821 & -1.128735 & 2.855624 \\
\hline b & -0.371889 & -1.886514 & 5.046698 \\
\hline & 3.695483 & -4.179426 & 2.894483 \\
\hline & 3.333792 & 0.166511 & -0.028628 \\
\hline & 4.262504 & 0.724047 & 0.852317 \\
\hline & 5.595007 & 0.901334 & 0.486264 \\
\hline 0 & 5.982565 & 0.485961 & -0.793078 \\
\hline & 5.085899 & -0.094792 & -1.691594 \\
\hline 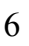 & 3.754256 & -0.244265 & -1.288143 \\
\hline & 6.557145 & 1.546276 & 1.483114 \\
\hline & 5.502474 & -0.582667 & -3.076850 \\
\hline & -0.423583 & -2.662815 & -0.004326 \\
\hline & -0.506141 & -3.698218 & 0.504772 \\
\hline ) & 0.490175 & -0.765219 & -3.248451 \\
\hline & -2.744679 & 0.121899 & 0.414145 \\
\hline 7 & -3.604833 & -0.952215 & 0.412159 \\
\hline & -4.778276 & -0.893083 & -0.315210 \\
\hline & -5.610993 & -2.059451 & -0.342401 \\
\hline ) & -5.258397 & -3.191003 & 0.454931 \\
\hline & -4.077165 & -3.156295 & 1.234194 \\
\hline b & -3.254931 & -2.063788 & 1.200060 \\
\hline & -6.085333 & -4.340443 & 0.438240 \\
\hline & -7.205459 & -4.393671 & -0.350567 \\
\hline & -7.537215 & -3.292698 & -1.167633 \\
\hline 6 & -6.763326 & -2.158755 & -1.163537 \\
\hline ? & -5.106181 & 0.341972 & -1.061969 \\
\hline f & -4.184114 & 0.876593 & -1.944010 \\
\hline 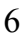 & -4.490865 & 1.985125 & -2.763401 \\
\hline 6 & -5.698126 & 2.616138 & -2.639849 \\
\hline 6 & -6.635301 & 2.190295 & -1.666869 \\
\hline & -6.335809 & 1.051279 & -0.860942 \\
\hline 6 & -7.250659 & 0.705003 & 0.165836 \\
\hline 6 & -8.404467 & 1.423861 & 0.360620 \\
\hline 6 & -8.714774 & 2.525282 & -0.463913 \\
\hline 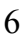 & -7.842779 & 2.898835 & -1.454477 \\
\hline 8 & -2.944010 & 0.304574 & -2.101201 \\
\hline I & -7.017344 & -0.129636 & 0.821879 \\
\hline & 1.837385 & 0.945378 & 3.295783 \\
\hline D & -8.055196 & 3.759130 & -2.088047 \\
\hline & 1.365023 & 5.553579 & 4.239716 \\
\hline 1 & -5.801711 & -5.188049 & 1.060748 \\
\hline & 3.502221 & 3.286267 & -0.264011 \\
\hline 1 & 5.317489 & 3.888404 & -1.805665 \\
\hline 1 & -3.818829 & -4.023141 & 1.841149 \\
\hline ] & 1.730024 & 3.137260 & 4.372414 \\
\hline 1 & -5.943839 & 3.469445 & -3.269861 \\
\hline ] & -8.411379 & -3.344234 & -1.812889 \\
\hline 1 & -3.749946 & 2.315669 & -3.487950 \\
\hline 1 & 2.622327 & 3.630562 & -5.147745 \\
\hline 1 & 0.280608 & 3.017802 & -4.785679 \\
\hline 1 & 0.477522 & 7.383922 & 0.452906 \\
\hline 1 & -7.027291 & -1.324729 & -1.808576 \\
\hline 1 & 0.600640 & 5.203289 & -0.658722 \\
\hline 1 & -1.531083 & 2.304415 & -3.249833 \\
\hline 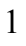 & -7.829992 & -5.283961 & -0.359185 \\
\hline
\end{tabular}




\begin{tabular}{|c|c|c|c|}
\hline 1 & -9.082751 & 1.143815 & 1.163532 \\
\hline & 4.885398 & 4.090070 & -4.251637 \\
\hline & 0.862660 & 7.580268 & 2.905270 \\
\hline & -9.635033 & 3.081894 & -0.303077 \\
\hline 1 & -2.327871 & -2.021407 & 1.765357 \\
\hline & 3.935950 & 1.038646 & 1.842662 \\
\hline 1 & 3.020093 & -0.685557 & -1.959703 \\
\hline & 7.016195 & 0.622684 & -1.097190 \\
\hline 1 & 3.382151 & -2.139307 & 1.152031 \\
\hline 1 & -0.158051 & -0.373502 & 2.799993 \\
\hline 1 & 1.661282 & -3.744101 & 4.731211 \\
\hline 1 & -1.678110 & 3.917343 & -0.077093 \\
\hline 1 & 1.414197 & -2.022563 & -1.198848 \\
\hline 1 & -3.089989 & 3.210767 & -0.862419 \\
\hline 1 & -4.920550 & 3.037395 & 0.545465 \\
\hline 1 & -1.013925 & 2.362795 & 2.182702 \\
\hline 1 & -5.930802 & 2.586235 & 2.754519 \\
\hline 1 & -2.012085 & 1.940630 & 4.406929 \\
\hline 1 & -4.483109 & 2.021109 & 4.697646 \\
\hline 1 & -0.072223 & 0.090116 & -3.620528 \\
\hline 1 & 1.559149 & -0.701311 & -3.446358 \\
\hline 1 & -2.221055 & -2.729667 & -2.897534 \\
\hline 1 & -2.084726 & -1.140417 & -3.645273 \\
\hline 1 & -1.685582 & -2.599888 & -4.578922 \\
\hline 1 & -0.950975 & -4.506051 & -2.412716 \\
\hline 1 & 0.248774 & -6.635465 & -2.674170 \\
\hline 1 & 2.508173 & -6.685939 & -3.720283 \\
\hline 1 & 3.519320 & -4.561911 & -4.531897 \\
\hline 1 & 2.309174 & -2.437480 & -4.296512 \\
\hline 6 & 6.944938 & -0.215353 & -3.415338 \\
\hline 6 & 5.366436 & -2.109040 & -3.120071 \\
\hline 6 & 4.598341 & 0.045117 & -4.142316 \\
\hline 6 & 7.956833 & 1.735089 & 0.904178 \\
\hline 6 & 6.023211 & 2.925994 & 1.887880 \\
\hline 6 & 6.668596 & 0.656440 & 2.726207 \\
\hline 1 & 4.882112 & -0.312966 & -5.141310 \\
\hline 1 & 3.540166 & -0.201048 & -3.990800 \\
\hline 1 & 4.681937 & 1.139995 & -4.132384 \\
\hline 1 & 7.186660 & -0.565301 & -4.426944 \\
\hline 1 & 7.101830 & 0.871772 & -3.394243 \\
\hline 1 & 7.663862 & -0.681527 & -2.729437 \\
\hline 1 & 5.625357 & -2.488792 & -4.118729 \\
\hline 1 & 6.038397 & -2.583223 & -2.392477 \\
\hline 1 & 4.344592 & -2.436252 & -2.888136 \\
\hline 1 & 8.604035 & 2.211781 & 1.651231 \\
\hline 1 & 8.421825 & 0.779313 & 0.630597 \\
\hline 1 & 7.948523 & 2.380572 & 0.015744 \\
\hline 1 & 6.693731 & 3.393859 & 2.621421 \\
\hline 1 & 5.962086 & 3.594098 & 1.018017 \\
\hline 1 & 5.023957 & 2.874487 & 2.339357 \\
\hline 1 & 7.340461 & 1.113168 & 3.465591 \\
\hline 1 & 5.696534 & 0.499355 & 3.209833 \\
\hline 1 & 7.073854 & -0.330025 & 2.465725 \\
\hline 6 & 3.603650 & -5.129629 & 4.085642 \\
\hline 6 & 3.517499 & -5.000296 & 1.611041 \\
\hline 6 & 5.087920 & -3.537949 & 2.903250 \\
\hline
\end{tabular}




$\begin{array}{rrrr}6 & -0.234264 & -2.976767 & 6.106601 \\ 6 & -0.147205 & -0.529145 & 5.725625 \\ 6 & -1.797830 & -1.941359 & 4.492477 \\ 1 & 4.390766 & -5.890595 & 4.010818 \\ 1 & 3.742203 & -4.606376 & 5.040898 \\ 1 & 2.641432 & -5.656523 & 4.117441 \\ 1 & 4.273649 & -5.795503 & 1.557284 \\ 1 & 2.525920 & -5.470447 & 1.582002 \\ 1 & 3.615648 & -4.383624 & 0.708851 \\ 1 & 5.863884 & -4.314888 & 2.878800 \\ 1 & 5.250066 & -2.883431 & 2.037809 \\ 1 & 5.239396 & -2.936804 & 3.809701 \\ 1 & -0.984200 & -2.821646 & 6.892530 \\ 1 & -0.398045 & -3.978140 & 5.687921 \\ 1 & 0.751630 & -2.962061 & 6.588544 \\ 1 & -2.527179 & -1.851224 & 5.308786 \\ 1 & -2.002393 & -1.127703 & 3.785234 \\ 1 & -1.982029 & -2.895741 & 3.979895 \\ 1 & -0.863529 & -0.386117 & 6.546359 \\ 1 & 0.866065 & -0.462046 & 6.144246 \\ 1 & -0.273468 & 0.304384 & 5.021912\end{array}$

$3_{\text {IC-re- }} \psi_{-1 \mathbf{a} 2 \mathrm{a} 3 \mathrm{a} 4 \mathrm{a}}$

Number of imaginary frequencies : 0

The smallest frequencies are : $15.8564 \quad 19.3356 \quad 22.9202 \mathrm{~cm}(-1)$

Electronic energy : $\quad H F=-4360.8789581$

Zero-point correction $=\quad 1.455689($ Hartree/Particle $)$

Thermal correction to Energy= $\quad 1.540002$

Thermal correction to Enthalpy= $\quad 1.540946$

Thermal correction to Gibbs Free Energy= $\quad 1.340330$

Sum of electronic and zero-point Energies= $\quad-4359.423269$

Sum of electronic and thermal Energies $=\quad-4359.338956$

Sum of electronic and thermal Enthalpies $=\quad-4359.338012$

Sum of electronic and thermal Free Energies $=\quad-4359.538628$

Cartesian Coordinates

$\begin{array}{cccc}6 & 1.388429 & -3.258249 & -3.320578 \\ 6 & 0.067290 & -2.779348 & -3.293267 \\ 6 & -0.948433 & -3.739289 & -3.167191 \\ 6 & -0.672510 & -5.098871 & -3.098209 \\ 6 & 0.641708 & -5.553715 & -3.141189 \\ 6 & 1.668996 & -4.617908 & -3.241092 \\ 6 & -0.282689 & -1.353749 & -3.542699 \\ 6 & -1.587099 & -1.157573 & -4.295543 \\ 45 & -0.091496 & 0.217493 & -1.826968 \\ 6 & 0.481100 & 2.061911 & -2.068757 \\ 8 & 0.612981 & 3.081736 & -2.599345 \\ 15 & -1.747534 & 0.552001 & -0.048254 \\ 6 & -1.869882 & 2.391205 & -0.065768 \\ 6 & -0.867398 & 3.138877 & 0.558036 \\ 6 & -0.859499 & 4.531353 & 0.493669 \\ 6 & -1.850619 & 5.151004 & -0.274494 \\ 6 & -2.847883 & 4.434017 & -0.939140\end{array}$




\begin{tabular}{|c|c|c|c|}
\hline 6 & -2.848391 & 3.041590 & -0.813315 \\
\hline 6 & 0.194766 & 5.371799 & 1.213847 \\
\hline y & -3.898939 & 5.179411 & -1.759964 \\
\hline & -3.512459 & 0.025717 & -0.198195 \\
\hline D & -3.889446 & -1.008409 & -1.052029 \\
\hline & -5.222523 & -1.447019 & -1.096871 \\
\hline y & -6.143543 & -0.810854 & -0.264756 \\
\hline f & -5.795975 & 0.224132 & 0.610545 \\
\hline 0 & -4.466053 & 0.628152 & 0.629194 \\
\hline 6 & -6.849559 & 0.837086 & 1.529836 \\
\hline 6 & -5.708121 & -2.537814 & -2.053524 \\
\hline 6 & -1.522258 & 0.219125 & 1.774195 \\
\hline 6 & -1.130444 & -1.035011 & 2.211320 \\
\hline 6 & -0.984384 & -1.301162 & 3.615064 \\
\hline 6 & -1.254870 & -0.269652 & 4.558788 \\
\hline 6 & -1.696225 & 0.985901 & 4.083106 \\
\hline 6 & -1.825927 & 1.219228 & 2.741580 \\
\hline 6 & -1.069541 & -0.514219 & 5.939868 \\
\hline 6 & -0.631917 & -1.735866 & 6.383702 \\
\hline 6 & -0.369938 & -2.765795 & 5.456810 \\
\hline 6 & -0.546714 & -2.557869 & 4.110896 \\
\hline 6 & -0.891415 & -2.191716 & 1.306631 \\
\hline 6 & 0.359911 & -2.444608 & 0.775842 \\
\hline 6 & 0.643973 & -3.689582 & 0.168615 \\
\hline 6 & -0.315921 & -4.660883 & 0.078433 \\
\hline 6 & -1.630990 & -4.415204 & 0.536456 \\
\hline 6 & -1.926412 & -3.167659 & 1.159657 \\
\hline 6 & -3.243788 & -2.946893 & 1.637413 \\
\hline 6 & -4.215558 & -3.905534 & 1.493976 \\
\hline 6 & -3.920354 & -5.140229 & 0.875011 \\
\hline 6 & -2.653928 & -5.386765 & 0.407258 \\
\hline 7 & 1.415539 & -1.476893 & 0.893279 \\
\hline 15 & 1.702125 & -0.562123 & -0.503838 \\
\hline 8 & 2.900759 & -1.298636 & -1.413849 \\
\hline 6 & 4.110193 & -1.659024 & -0.870116 \\
\hline 6 & 5.030177 & -0.696439 & -0.497883 \\
\hline 6 & 6.196391 & -1.129943 & 0.214060 \\
\hline 6 & 6.441821 & -2.526354 & 0.382304 \\
\hline 6 & 5.527200 & -3.465109 & -0.156328 \\
\hline 6 & 4.372988 & -3.040956 & -0.755516 \\
\hline 6 & 7.090339 & -0.217331 & 0.830164 \\
\hline 6 & 8.171696 & -0.661090 & 1.550766 \\
\hline 6 & 8.429149 & -2.041450 & 1.686469 \\
\hline 6 & 7.578213 & -2.951293 & 1.112848 \\
\hline 6 & 4.766741 & 0.727975 & -0.808378 \\
\hline 6 & 3.570346 & 1.306888 & -0.425823 \\
\hline 6 & 3.287706 & 2.670083 & -0.641137 \\
\hline 6 & 4.197210 & 3.460692 & -1.286989 \\
\hline 6 & 5.407879 & 2.911967 & -1.773316 \\
\hline 6 & 5.694230 & 1.530381 & -1.552139 \\
\hline 6 & 6.328815 & 3.709887 & -2.494564 \\
\hline 6 & 7.480555 & 3.171245 & -3.007360 \\
\hline 6 & 7.749252 & 1.798843 & -2.825388 \\
\hline 6 & 6.882670 & 1.002142 & -2.118763 \\
\hline 8 & 2.632317 & 0.575962 & 0.256130 \\
\hline 6 & 0.718508 & -0.372629 & -3.737073 \\
\hline
\end{tabular}




\begin{tabular}{|c|c|c|c|}
\hline 5 & 2.407626 & -1.755033 & 1.960804 \\
\hline & 2.890291 & -0.570869 & 2.758251 \\
\hline & 2.017368 & 0.420229 & 3.210205 \\
\hline & 2.481403 & 1.463393 & 4.000429 \\
\hline & 3.829750 & 1.538339 & 4.346547 \\
\hline & 4.707437 & 0.560412 & 3.892838 \\
\hline & 4.237865 & -0.487644 & 3.104761 \\
\hline & 6.891237 & 0.848759 & 0.753856 \\
\hline & -2.165591 & 2.197682 & 2.413705 \\
\hline & 7.751601 & -4.021198 & 1.221676 \\
\hline & -1.277977 & 0.292392 & 6.641437 \\
\hline & 6.092174 & 4.762782 & -2.642420 \\
\hline & -3.486141 & -1.995687 & 2.109005 \\
\hline & -5.223206 & -3.707658 & 1.855861 \\
\hline & 3.992642 & 4.517308 & -1.454178 \\
\hline & -1.930277 & 1.769933 & 4.802462 \\
\hline & 5.744321 & -4.527552 & -0.058737 \\
\hline & 8.649163 & 1.364172 & -3.254895 \\
\hline & 3.637927 & -3.736774 & -1.157524 \\
\hline & -2.410532 & -6.335398 & -0.070185 \\
\hline & -0.085490 & -5.623056 & -0.374127 \\
\hline & -0.026655 & -3.734955 & 5.811452 \\
\hline & 7.101486 & -0.055812 & -2.000867 \\
\hline & -0.343055 & -3.367177 & 3.412313 \\
\hline & 1.650532 & -3.863829 & -0.207738 \\
\hline & 8.177987 & 3.792457 & -3.564441 \\
\hline & 8.832633 & 0.059787 & 2.026936 \\
\hline & -4.699998 & -5.892018 & 0.769123 \\
\hline & -0.486531 & -1.914232 & 7.446590 \\
\hline 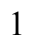 & 9.292799 & -2.379185 & 2.254496 \\
\hline & 2.338023 & 3.059302 & -0.279796 \\
\hline 1 & -4.154919 & 1.427725 & 1.295284 \\
\hline & -3.119130 & -1.481996 & -1.661064 \\
\hline & -7.182689 & -1.135706 & -0.292030 \\
\hline & -3.612790 & 2.448383 & -1.309426 \\
\hline & -0.090753 & 2.614285 & 1.114369 \\
\hline 1 & -1.845006 & 6.237493 & -0.358282 \\
\hline & 1.900881 & -2.449635 & 2.645719 \\
\hline 1 & -1.326617 & 0.793533 & -2.643729 \\
\hline & 3.274027 & -2.301415 & 1.559455 \\
\hline | & 4.934578 & -1.246011 & 2.743111 \\
\hline ] & 0.972703 & 0.384878 & 2.907940 \\
\hline & 5.765384 & 0.612019 & 4.143697 \\
\hline 1 & 1.783349 & 2.224618 & 4.347013 \\
\hline & 4.192932 & 2.359136 & 4.961471 \\
\hline 1 & 1.774206 & -0.614990 & -3.624968 \\
\hline & 0.527049 & 0.427286 & -4.453318 \\
\hline 1 & -1.983792 & -3.415145 & -3.131556 \\
\hline 1 & -1.498382 & -5.805054 & -3.014554 \\
\hline 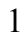 & 0.863475 & -6.618423 & -3.104696 \\
\hline 1 & 2.705508 & -4.949995 & -3.292999 \\
\hline 1 & 2.214604 & -2.561110 & -3.428837 \\
\hline 1 & -1.642540 & -1.845953 & -5.153460 \\
\hline & -2.483055 & -1.326784 & -3.686821 \\
\hline 1 & -1.658856 & -0.132686 & -4.675685 \\
\hline 6 & -4.558733 & -3.379792 & -2.599649 \\
\hline
\end{tabular}




$\begin{array}{rrrr}6 & -6.689821 & -3.489414 & -1.362155 \\ 6 & -6.411581 & -1.857589 & -3.234238 \\ 6 & -6.295345 & 2.011951 & 2.333488 \\ 6 & -8.038814 & 1.343256 & 0.707456 \\ 6 & -7.327765 & -0.236805 & 2.513838 \\ 1 & -7.088773 & 2.437872 & 2.960348 \\ 1 & -5.478612 & 1.706679 & 3.001385 \\ 1 & -5.921690 & 2.812663 & 1.680157 \\ 1 & -8.081617 & 0.175838 & 3.198026 \\ 1 & -7.778754 & -1.093307 & 1.997307 \\ 1 & -6.491046 & -0.612797 & 3.117888 \\ 1 & -8.793759 & 1.789571 & 1.368394 \\ 1 & -7.723927 & 2.110603 & -0.011536 \\ 1 & -8.530045 & 0.540157 & 0.145595 \\ 1 & -4.956774 & -4.198395 & -3.212896 \\ 1 & -3.902075 & -2.788376 & -3.248096 \\ 1 & -3.953981 & -3.822986 & -1.795092 \\ 1 & -6.986573 & -4.284559 & -2.058433 \\ 1 & -6.227570 & -3.963168 & -0.486093 \\ 1 & -7.610394 & -2.992306 & -1.035719 \\ 1 & -6.767285 & -2.606195 & -3.955546 \\ 1 & -7.276335 & -1.268413 & -2.903325 \\ 1 & -5.725462 & -1.179059 & -3.759242 \\ 6 & -4.925010 & 4.233896 & -2.380483 \\ 6 & -4.649620 & 6.167225 & -0.859814 \\ 6 & -3.206485 & 5.945029 & -2.892434 \\ 6 & 1.028124 & 4.535732 & 2.185123 \\ 6 & 1.129388 & 6.004059 & 0.177173 \\ 6 & -0.483420 & 6.481524 & 2.025215 \\ 1 & -3.947715 & 6.480164 & -3.501487 \\ 1 & -2.492335 & 6.685131 & -2.511702 \\ 1 & -2.656549 & 5.259097 & -3.549691 \\ 1 & -5.667410 & 4.813804 & -2.943187 \\ 1 & -4.462272 & 3.525685 & -3.079522 \\ 1 & -5.465764 & 3.656724 & -1.617619 \\ 1 & -5.412660 & 6.704934 & -1.438648 \\ 1 & -5.155978 & 5.644269 & -0.037795 \\ 1 & -3.982395 & 6.916980 & -0.417972 \\ 1 & 1.724879 & 5.184871 & 2.730481 \\ 1 & 0.393273 & 4.028109 & 2.925365 \\ 1 & 1.632341 & 3.771237 & 1.679740 \\ 1 & 0.271644 & 7.071303 & 2.561724 \\ 1 & -1.051023 & 7.176588 & 1.395592 \\ 1 & -1.173969 & 6.061626 & 2.768215 \\ 1 & 1.902658 & 6.606983 & 0.672683 \\ 1 & 1.632060 & 5.234437 & -0.424464 \\ 1 & 0.584048 & 6.657421 & -0.515146\end{array}$

$3_{\text {IC-re- }} \psi_{-1 \text { aza3a4s }}$

Number of imaginary frequencies : 0

The smallest frequencies are : $18.0256 \quad 20.633023 .9571 \mathrm{~cm}(-1)$

Electronic energy : $\quad H F=-4360.8794546$

Zero-point correction $=\quad 1.455952$ (Hartree/Particle)

Thermal correction to Energy= $\quad 1.540175$

Thermal correction to Enthalpy= $\quad 1.541119$ 
Thermal correction to Gibbs Free Energy= Sum of electronic and zero-point Energies= Sum of electronic and thermal Energies= Sum of electronic and thermal Enthalpies= Sum of electronic and thermal Free Energies=
1.340330
$-4359.423503$
$-4359.339280$
$-4359.338336$
$-4359.539125$

Cartesian Coordinates

\begin{tabular}{|c|c|c|c|}
\hline 6 & 1.409381 & -3.226791 & -3.342270 \\
\hline 6 & 0.087816 & -2.749360 & -3.314756 \\
\hline 6 & -0.927239 & -3.711050 & -3.198407 \\
\hline 6 & -0.650524 & -5.070750 & -3.136385 \\
\hline 6 & 0.664276 & -5.524077 & -3.177840 \\
\hline 6 & 1.690871 & -4.586722 & -3.270208 \\
\hline 6 & -0.263618 & -1.322208 & -3.553316 \\
\hline 6 & -1.565045 & -1.121822 & -4.310300 \\
\hline 45 & -0.082669 & 0.232790 & -1.822701 \\
\hline 6 & 0.487938 & 2.081354 & -2.039704 \\
\hline 8 & 0.620741 & 3.109445 & -2.554014 \\
\hline 15 & -1.747095 & 0.544833 & -0.047655 \\
\hline 6 & -1.907771 & 2.380741 & -0.072900 \\
\hline 6 & -0.912777 & 3.149056 & 0.536639 \\
\hline 6 & -0.929411 & 4.541077 & 0.465820 \\
\hline 6 & -1.936670 & 5.139408 & -0.297848 \\
\hline 6 & -2.925500 & 4.401099 & -0.952422 \\
\hline 6 & -2.904222 & 3.010002 & -0.815215 \\
\hline 6 & 0.128684 & 5.397778 & 1.160284 \\
\hline 6 & -3.992928 & 5.123423 & -1.772987 \\
\hline 6 & -3.503073 & -0.012483 & -0.192918 \\
\hline 6 & -3.879478 & -1.031659 & -1.070738 \\
\hline 6 & -5.206090 & -1.477033 & -1.116603 \\
\hline 6 & -6.133352 & -0.868051 & -0.262349 \\
\hline 6 & -5.784787 & 0.138959 & 0.636707 \\
\hline 6 & -4.453814 & 0.553999 & 0.653198 \\
\hline 6 & -6.769658 & 0.769739 & 1.617532 \\
\hline 6 & -5.689580 & -2.552658 & -2.091352 \\
\hline 6 & -1.513745 & 0.222938 & 1.776284 \\
\hline 6 & -1.121476 & -1.030264 & 2.215622 \\
\hline 6 & -0.972575 & -1.294802 & 3.618786 \\
\hline 6 & -1.238639 & -0.261353 & 4.561506 \\
\hline 6 & -1.678125 & 0.994220 & 4.084460 \\
\hline 6 & -1.811719 & 1.225569 & 2.742540 \\
\hline 6 & -1.051303 & -0.504725 & 5.942559 \\
\hline 6 & -0.616775 & -1.727265 & 6.386884 \\
\hline 6 & -0.360170 & -2.759452 & 5.460821 \\
\hline 6 & -0.538604 & -2.552590 & 4.115016 \\
\hline 6 & -0.890747 & -2.186544 & 1.309304 \\
\hline 6 & 0.356189 & -2.442447 & 0.769556 \\
\hline 6 & 0.627281 & -3.682891 & 0.147548 \\
\hline 6 & -0.341781 & -4.644668 & 0.049974 \\
\hline 6 & -1.652106 & -4.394738 & 0.519222 \\
\hline 6 & -1.933356 & -3.153309 & 1.160844 \\
\hline 6 & -3.244678 & -2.928505 & 1.653352 \\
\hline 6 & -4.224997 & -3.877412 & 1.505324 \\
\hline 6 & -3.944313 & -5.106128 & 0.867526 \\
\hline 6 & -2.683901 & -5.356379 & 0.385495 \\
\hline
\end{tabular}




\begin{tabular}{|c|c|c|c|}
\hline 7 & 1.417314 & -1.481139 & 0.889431 \\
\hline 15 & 1.707445 & -0.559136 & -0.501872 \\
\hline & 2.907159 & -1.290247 & -1.413682 \\
\hline & 4.115023 & -1.654149 & -0.868714 \\
\hline & 5.035974 & -0.693951 & -0.492805 \\
\hline & 6.201774 & -1.131762 & 0.217440 \\
\hline & 6.444770 & -2.529157 & 0.381301 \\
\hline & 5.527841 & -3.464817 & -0.158794 \\
\hline & 4.374437 & -3.036861 & -0.756788 \\
\hline 6 & 7.097819 & -0.222889 & 0.836016 \\
\hline & 8.179063 & -0.670879 & 1.554177 \\
\hline & 8.434224 & -2.052076 & 1.685200 \\
\hline 0 & 7.581003 & -2.958473 & 1.109535 \\
\hline 6 & 4.773049 & 0.732780 & -0.793670 \\
\hline 6 & 3.577318 & 1.309353 & -0.405474 \\
\hline 6 & 3.298033 & 2.675899 & -0.602529 \\
\hline 6 & 4.208558 & 3.472937 & -1.238653 \\
\hline 6 & 5.417331 & 2.927208 & -1.733335 \\
\hline 6 & 5.701236 & 1.542416 & -1.528719 \\
\hline 6 & 6.339319 & 3.731635 & -2.445890 \\
\hline 6 & 7.489603 & 3.196789 & -2.965946 \\
\hline 6 & 7.755893 & 1.821872 & -2.800178 \\
\hline 6 & 6.888359 & 1.018638 & -2.102129 \\
\hline 8 & 2.636844 & 0.573444 & 0.266518 \\
\hline 6 & 0.736571 & -0.337510 & -3.734794 \\
\hline 6 & 2.403480 & -1.759796 & 1.962359 \\
\hline 6 & 2.889572 & -0.573579 & 2.755199 \\
\hline 6 & 2.020834 & 0.426449 & 3.195666 \\
\hline 6 & 2.488849 & 1.477082 & 3.973282 \\
\hline 6 & 3.837117 & 1.549419 & 4.320505 \\
\hline 6 & 4.710349 & 0.561501 & 3.880219 \\
\hline 6 & 4.236857 & -0.493000 & 3.102984 \\
\hline 1 & 6.900786 & 0.843805 & 0.763416 \\
\hline 1 & -2.150044 & 2.204176 & 2.414195 \\
\hline 1 & 7.752453 & -4.029041 & 1.214858 \\
\hline 1 & -1.256016 & 0.303252 & 6.643636 \\
\hline 1 & 6.104717 & 4.786684 & -2.581150 \\
\hline 1 & -3.475676 & -1.981038 & 2.138767 \\
\hline 1 & -5.227897 & -3.676537 & 1.878544 \\
\hline 1 & 4.005998 & 4.532256 & -1.390921 \\
\hline 1 & -1.907883 & 1.780341 & 4.802929 \\
\hline 1 & 5.742698 & -4.527920 & -0.063401 \\
\hline 1 & 8.654683 & 1.390583 & -3.235403 \\
\hline 1 & 3.637403 & -3.729957 & -1.159943 \\
\hline 1 & -2.452120 & -6.300518 & -0.106510 \\
\hline 1 & -0.121506 & -5.603043 & -0.415498 \\
\hline 1 & -0.019968 & -3.729415 & 5.816236 \\
\hline 1 & 7.105216 & -0.041064 & -1.996500 \\
\hline 1 & -0.340022 & -3.363336 & 3.416576 \\
\hline 1 & 1.630377 & -3.861167 & -0.236348 \\
\hline 1 & 8.187768 & 3.823145 & -3.516310 \\
\hline 1 & 8.841662 & 0.047338 & 2.032060 \\
\hline 1 & -4.730014 & -5.851324 & 0.759555 \\
\hline 1 & -0.469981 & -1.904858 & 7.449704 \\
\hline 1 & 9.297809 & -2.393196 & 2.251300 \\
\hline 1 & 2.350085 & 3.062062 & -0.233932 \\
\hline
\end{tabular}




\begin{tabular}{|c|c|c|c|}
\hline & -4.149840 & 1.335605 & 1.347640 \\
\hline & -3.108790 & -1.485062 & -1.694390 \\
\hline ] & -7.167646 & -1.200417 & -0.299076 \\
\hline & -3.664304 & 2.401837 & -1.300083 \\
\hline & -0.120761 & 2.641098 & 1.086734 \\
\hline & -1.950753 & 6.225263 & -0.388238 \\
\hline & 1.890494 & -2.448847 & 2.648235 \\
\hline & -1.315369 & 0.814806 & -2.638939 \\
\hline & 3.268495 & -2.312239 & 1.565965 \\
\hline 1 & 4.930815 & -1.257881 & 2.749767 \\
\hline | & 0.976788 & 0.393908 & 2.891484 \\
\hline 1 & 5.768043 & 0.610693 & 4.132548 \\
\hline 1 & 1.794184 & 2.246757 & 4.307751 \\
\hline 1 & 4.203771 & 2.376022 & 4.925521 \\
\hline 1 & 1.792259 & -0.578982 & -3.620651 \\
\hline ] & 0.546480 & 0.468604 & -4.444401 \\
\hline & -1.962786 & -3.387838 & -3.163543 \\
\hline 1 & -1.476039 & -5.778109 & -3.058293 \\
\hline 1 & 0.887079 & -6.588719 & -3.146458 \\
\hline 1 & 2.727807 & -4.917535 & -3.321528 \\
\hline 1 & 2.235283 & -2.528435 & -3.444374 \\
\hline 1 & -1.615002 & -1.802472 & -5.174695 \\
\hline 1 & -2.463348 & -1.298529 & -3.707256 \\
\hline 1 & -1.637316 & -0.093644 & -4.681330 \\
\hline 6 & -4.537562 & -3.380801 & -2.652935 \\
\hline & -6.664883 & -3.521781 & -1.414970 \\
\hline 6 & -6.398791 & -1.855486 & -3.258527 \\
\hline 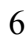 & -8.184390 & 0.218865 & 1.460825 \\
\hline 6 & -6.295764 & 0.471418 & 3.045569 \\
\hline 6 & -6.820669 & 2.285530 & 1.395318 \\
\hline ] & -8.851985 & 0.706429 & 2.182430 \\
\hline 1 & -8.589677 & 0.407740 & 0.458309 \\
\hline 1 & -8.228047 & -0.861201 & 1.652072 \\
\hline 1 & -7.493372 & 2.756031 & 2.124987 \\
\hline 1 & -5.834941 & 2.756284 & 1.501145 \\
\hline 1 & -7.197257 & 2.520167 & 0.390953 \\
\hline 1 & -6.987781 & 0.906286 & 3.779437 \\
\hline 1 & -6.251815 & -0.612219 & 3.221556 \\
\hline 1 & -5.296610 & 0.879249 & 3.245664 \\
\hline 1 & -4.932981 & -4.190639 & -3.279404 \\
\hline 1 & -3.883264 & -2.776736 & -3.292045 \\
\hline 1 & -3.931469 & -3.834859 & -1.855097 \\
\hline 1 & -6.956181 & -4.307492 & -2.124159 \\
\hline 1 & -6.198571 & -4.006807 & -0.547093 \\
\hline 1 & -7.588993 & -3.037036 & -1.080247 \\
\hline 1 & -6.755175 & -2.593233 & -3.990617 \\
\hline 1 & -7.264119 & -1.274229 & -2.915272 \\
\hline 1 & -5.716181 & -1.166680 & -3.774652 \\
\hline 6 & -4.978764 & 4.153979 & -2.421021 \\
\hline 6 & -4.785053 & 6.068726 & -0.862896 \\
\hline 6 & -3.318205 & 5.931891 & -2.886303 \\
\hline 6 & 0.952146 & 4.588224 & 2.162878 \\
\hline 6 & 1.069559 & 5.977300 & 0.098508 \\
\hline 6 & -0.535121 & 6.545084 & 1.929052 \\
\hline 1 & -4.073769 & 6.446308 & -3.495501 \\
\hline 1 & -2.637716 & 6.694712 & -2.489333 \\
\hline
\end{tabular}




$\begin{array}{lrrr}1 & -2.735748 & 5.277465 & -3.547641 \\ 1 & -5.730428 & 4.717569 & -2.988061 \\ 1 & -4.481912 & 3.470157 & -3.121109 \\ 1 & -5.512857 & 3.550200 & -1.674828 \\ 1 & -5.555028 & 6.597732 & -1.440647 \\ 1 & -5.288116 & 5.512476 & -0.060778 \\ 1 & -4.144823 & 6.825615 & -0.393952 \\ 1 & 1.646546 & 5.251530 & 2.693941 \\ 1 & 0.310024 & 4.104364 & 2.912854 \\ 1 & 1.558818 & 3.807489 & 1.686074 \\ 1 & 0.228128 & 7.145092 & 2.441944 \\ 1 & -1.093803 & 7.223737 & 1.274217 \\ 1 & -1.229980 & 6.162763 & 2.688149 \\ 1 & 1.866142 & 6.570219 & 0.568704 \\ 1 & 1.537895 & 5.177142 & -0.490301 \\ 1 & 0.532798 & 6.627551 & -0.603597\end{array}$

$3_{\text {IC-re- }} \Psi_{-12 a 2 a 3 s 4 a}$

Number of imaginary frequencies : 0

The smallest frequencies are : $17.1052 \quad 21.223923 .3114 \mathrm{~cm}(-1)$

Electronic energy : $\quad H F=-4360.8784373$

Zero-point correction $=\quad 1.455166($ Hartree/Particle $)$

Thermal correction to Energy $=\quad 1.539646$

Thermal correction to Enthalpy= $\quad 1.540590$

Thermal correction to Gibbs Free Energy $=\quad 1.338863$

Sum of electronic and zero-point Energies $=\quad-4359.423271$

Sum of electronic and thermal Energies $=\quad-4359.338791$

Sum of electronic and thermal Enthalpies $=\quad-4359.337847$

Sum of electronic and thermal Free Energies $=\quad-4359.539574$

Cartesian Coordinates

$\begin{array}{cccc}6 & 1.133440 & -3.272729 & -3.315108 \\ 6 & -0.126989 & -2.651587 & -3.346989 \\ 6 & -1.250039 & -3.491451 & -3.272214 \\ 6 & -1.129600 & -4.872069 & -3.176079 \\ 6 & 0.127792 & -5.468565 & -3.166387 \\ 6 & 1.256594 & -4.654909 & -3.231085 \\ 6 & -0.304917 & -1.197165 & -3.599727 \\ 6 & -1.545686 & -0.853188 & -4.402359 \\ 45 & -0.053286 & 0.288017 & -1.823807 \\ 6 & 0.552346 & 2.127616 & -2.011940 \\ 8 & 0.676200 & 3.164310 & -2.511785 \\ 15 & -1.725781 & 0.575724 & -0.052147 \\ 6 & -1.872839 & 2.412937 & -0.043781 \\ 6 & -0.860857 & 3.162852 & 0.560912 \\ 6 & -0.874152 & 4.556135 & 0.525302 \\ 6 & -1.899438 & 5.176093 & -0.196597 \\ 6 & -2.911351 & 4.457422 & -0.837182 \\ 6 & -2.886943 & 3.062892 & -0.741654 \\ 6 & 0.200792 & 5.391448 & 1.220560 \\ 6 & -4.013108 & 5.202618 & -1.589052 \\ 6 & -3.484287 & 0.029747 & -0.213951 \\ 6 & -3.864346 & -0.935401 & -1.137730 \\ 6 & -5.198045 & -1.382659 & -1.206137\end{array}$




\begin{tabular}{|c|c|c|c|}
\hline 6 & -6.111629 & -0.833902 & -0.312094 \\
\hline 5 & -5.759970 & 0.141753 & 0.633870 \\
\hline f & -4.438133 & 0.561120 & 0.665669 \\
\hline & -6.807218 & 0.669593 & 1.611214 \\
\hline$b$ & -5.605179 & -2.442162 & -2.231172 \\
\hline 5 & -1.499027 & 0.226903 & 1.769063 \\
\hline 6 & -1.116226 & -1.031739 & 2.202644 \\
\hline 5 & -0.959043 & -1.299081 & 3.605444 \\
\hline 6 & -1.213935 & -0.266718 & 4.552420 \\
\hline 6 & -1.651398 & 0.991562 & 4.081348 \\
\hline 6 & -1.788023 & 1.227300 & 2.740915 \\
\hline 6 & -1.016303 & -0.512336 & 5.931690 \\
\hline 6 & -0.580214 & -1.735977 & 6.371117 \\
\hline 6 & -0.332705 & -2.766865 & 5.441262 \\
\hline 6 & -0.522669 & -2.557996 & 4.097363 \\
\hline 6 & -0.892920 & -2.191063 & 1.297024 \\
\hline 6 & 0.349569 & -2.444331 & 0.745951 \\
\hline 6 & 0.620410 & -3.685908 & 0.125910 \\
\hline 6 & -0.334588 & -4.664383 & 0.066387 \\
\hline 6 & -1.630937 & -4.433827 & 0.581475 \\
\hline 6 & -1.922960 & -3.177824 & 1.188852 \\
\hline 6 & -3.226217 & -2.967537 & 1.709856 \\
\hline 6 & -4.175739 & -3.957041 & 1.653876 \\
\hline 6 & -3.874355 & -5.209161 & 1.074373 \\
\hline 6 & -2.632697 & -5.434416 & 0.537011 \\
\hline 7 & 1.415755 & -1.488621 & 0.869114 \\
\hline 15 & 1.718996 & -0.565848 & -0.517336 \\
\hline 8 & 2.900064 & -1.322687 & -1.438905 \\
\hline 6 & 4.109163 & -1.699443 & -0.904792 \\
\hline 6 & 5.045340 & -0.750957 & -0.536090 \\
\hline 6 & 6.211194 & -1.202796 & 0.165506 \\
\hline 6 & 6.439891 & -2.602781 & 0.326704 \\
\hline 6 & 5.508384 & -3.527107 & -0.207565 \\
\hline 6 & 4.355603 & -3.084901 & -0.796025 \\
\hline 6 & 7.121483 & -0.304537 & 0.778840 \\
\hline 6 & 8.202476 & -0.765214 & 1.489323 \\
\hline 6 & 8.443416 & -2.149245 & 1.617135 \\
\hline 6 & 7.576297 & -3.045554 & 1.046460 \\
\hline 6 & 4.799295 & 0.680196 & -0.829576 \\
\hline 6 & 3.618102 & 1.271691 & -0.421271 \\
\hline 6 & 3.359362 & 2.644906 & -0.598762 \\
\hline 6 & 4.273777 & 3.432659 & -1.240953 \\
\hline 6 & 5.465927 & 2.871850 & -1.758748 \\
\hline 6 & 5.730879 & 1.481095 & -1.569399 \\
\hline 6 & 6.390866 & 3.667420 & -2.477385 \\
\hline 6 & 7.525727 & 3.118793 & -3.016679 \\
\hline 6 & 7.773359 & 1.738822 & -2.864300 \\
\hline 6 & 6.902661 & 0.943543 & -2.161105 \\
\hline 8 & 2.675352 & 0.541458 & 0.253428 \\
\hline 6 & 0.792827 & -0.315653 & -3.722549 \\
\hline 6 & 2.403346 & -1.787695 & 1.936076 \\
\hline 6 & 2.907224 & -0.612640 & 2.733894 \\
\hline 6 & 2.050016 & 0.389694 & 3.191019 \\
\hline 6 & 2.533061 & 1.430343 & 3.972913 \\
\hline 6 & 3.885386 & 1.490795 & 4.306318 \\
\hline 6 & 4.747314 & 0.500807 & 3.848592 \\
\hline
\end{tabular}




\begin{tabular}{|c|c|c|c|}
\hline & 4.258555 & -0.544344 & 3.068200 \\
\hline & 6.935785 & 0.764338 & 0.708953 \\
\hline & -2.123880 & 2.208409 & 2.417781 \\
\hline & 7.736537 & -4.118063 & 1.149607 \\
\hline & -1.213398 & 0.295501 & 6.635125 \\
\hline & 6.171144 & 4.727037 & -2.601416 \\
\hline & -3.472276 & -2.002838 & 2.152048 \\
\hline & -5.171884 & -3.770250 & 2.050916 \\
\hline & 4.086774 & 4.496728 & -1.379513 \\
\hline & -1.875646 & 1.776512 & 4.802821 \\
\hline & 5.711653 & -4.592758 & -0.115533 \\
\hline & 8.660026 & 1.297441 & -3.314030 \\
\hline & 3.609384 & -3.771114 & -1.192817 \\
\hline & -2.389700 & -6.388894 & 0.070840 \\
\hline & -0.111379 & -5.626944 & -0.389495 \\
\hline & 0.009775 & -3.737721 & 5.792024 \\
\hline & 7.104527 & -0.120153 & -2.065608 \\
\hline & -0.330411 & -3.368668 & 3.397235 \\
\hline & 1.617802 & -3.855488 & -0.275104 \\
\hline & 8.226201 & 3.738561 & -3.571555 \\
\hline & 8.876092 & -0.054835 & 1.963491 \\
\hline & -4.634761 & -5.986701 & 1.041511 \\
\hline & -0.424697 & -1.915166 & 7.432425 \\
\hline & 9.307016 & -2.500435 & 2.177014 \\
\hline 1 & 2.425058 & 3.044313 & -0.210178 \\
\hline & -4.127886 & 1.315656 & 1.382750 \\
\hline & -3.101249 & -1.355680 & -1.795417 \\
\hline & -7.144665 & -1.172740 & -0.339230 \\
\hline & -3.661368 & 2.468428 & -1.220247 \\
\hline l & -0.060015 & 2.638120 & 1.081949 \\
\hline & -1.913450 & 6.263853 & -0.258626 \\
\hline 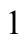 & 1.884231 & -2.474198 & 2.619782 \\
\hline 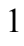 & -1.259923 & 0.932341 & -2.627599 \\
\hline | & 3.259542 & -2.348683 & 1.532303 \\
\hline l & 4.943491 & -1.311068 & 2.701509 \\
\hline & 1.002844 & 0.366833 & 2.896800 \\
\hline l & 5.807997 & 0.540956 & 4.089772 \\
\hline & 1.846878 & 2.201287 & 4.321620 \\
\hline 1 & 4.263958 & 2.309760 & 4.914372 \\
\hline ] & 1.814136 & -0.657814 & -3.564419 \\
\hline l & 0.710879 & 0.517100 & -4.421769 \\
\hline 1 & -2.246257 & -3.055007 & -3.310909 \\
\hline 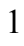 & -2.029615 & -5.484021 & -3.120592 \\
\hline 1 & 0.227779 & -6.551060 & -3.115966 \\
\hline & 2.250042 & -5.102284 & -3.241095 \\
\hline 1 & 2.034064 & -2.667401 & -3.381753 \\
\hline 1 & -1.637859 & -1.519071 & -5.274727 \\
\hline 1 & -2.473968 & -0.937199 & -3.828780 \\
\hline 1 & -1.494830 & 0.179299 & -4.764465 \\
\hline 0 & -7.096613 & -2.768250 & -2.176184 \\
\hline 6 & -5.294486 & -1.937528 & -3.643539 \\
\hline 5 & -4.834734 & -3.738423 & -1.961429 \\
\hline 6 & -6.266638 & 1.811477 & 2.469895 \\
\hline 6 & -8.031002 & 1.188732 & 0.849776 \\
\hline 6 & -7.229291 & -0.474587 & 2.540307 \\
\hline 1 & -7.057318 & 2.178881 & 3.136067 \\
\hline
\end{tabular}




$\begin{array}{rrrr}1 & -5.428181 & 1.491715 & 3.103137 \\ 1 & -5.928855 & 2.657801 & 1.855520 \\ 1 & -7.975825 & -0.126027 & 3.266886 \\ 1 & -7.669793 & -1.311562 & 1.984181 \\ 1 & -6.366026 & -0.860618 & 3.099658 \\ 1 & -8.779670 & 1.574031 & 1.554714 \\ 1 & -7.756007 & 2.005758 & 0.170064 \\ 1 & -8.515875 & 0.406828 & 0.253694 \\ 1 & -7.327660 & -3.540224 & -2.921065 \\ 1 & -7.397311 & -3.161000 & -1.196307 \\ 1 & -7.719614 & -1.893679 & -2.404974 \\ 1 & -5.628362 & -2.670230 & -4.390371 \\ 1 & -5.807490 & -0.988464 & -3.846340 \\ 1 & -4.222389 & -1.776710 & -3.800459 \\ 1 & -5.012853 & -4.464124 & -2.767862 \\ 1 & -3.752878 & -3.576462 & -1.882044 \\ 1 & -5.160192 & -4.192012 & -1.017249 \\ 6 & -5.044148 & 4.251844 & -2.193403 \\ 6 & -4.745903 & 6.141437 & -0.623883 \\ 6 & -3.391029 & 6.021083 & -2.725007 \\ 6 & 1.023338 & 4.557783 & 2.204041 \\ 6 & 1.139318 & 5.975166 & 0.159400 \\ 6 & -0.441130 & 6.535836 & 2.012128 \\ 1 & -4.171680 & 6.556606 & -3.282258 \\ 1 & -2.678403 & 6.766819 & -2.352555 \\ 1 & -2.855457 & 5.370929 & -3.428671 \\ 1 & -5.822529 & 4.830373 & -2.706727 \\ 1 & -4.596617 & 3.574135 & -2.931531 \\ 1 & -5.538932 & 3.641162 & -1.425189 \\ 1 & -5.545855 & 6.679632 & -1.150100 \\ 1 & -5.203391 & 5.578399 & 0.200432 \\ 1 & -4.076757 & 6.891255 & -0.185174 \\ 1 & 1.730308 & 5.204819 & 2.738596 \\ 1 & 0.381692 & 4.070230 & 2.951962 \\ 1 & 1.616473 & 3.777198 & 1.710398 \\ 1 & 0.334059 & 7.112057 & 2.534250 \\ 1 & -0.987997 & 7.237438 & 1.371676 \\ 1 & -1.141426 & 6.152248 & 2.765551 \\ 1 & 1.935861 & 6.567328 & 0.630673 \\ 1 & 1.608930 & 5.177331 & -0.431374 \\ 1 & 0.600715 & 6.626811 & -0.540024 \\ & & \end{array}$

$3_{\text {IC-re- }} \psi_{-12 a 2 a 3 s 4 s}$

Number of imaginary frequencies : 0

The smallest frequencies are : $16.976920 .548121 .4904 \mathrm{~cm}(-1)$

Electronic energy : $\quad H F=-4360.878744$

Zero-point correction $=\quad 1.455123($ Hartree/Particle $)$

Thermal correction to Energy $=\quad 1.539626$

Thermal correction to Enthalpy= $\quad 1.540570$

Thermal correction to Gibbs Free Energy $=\quad 1.338050$

Sum of electronic and zero-point Energies $=\quad-4359.423621$

Sum of electronic and thermal Energies $=\quad-4359.339118$

Sum of electronic and thermal Enthalpies $=\quad-4359.338174$

Sum of electronic and thermal Free Energies $=\quad-4359.540694$ 
Cartesian Coordinates

\begin{tabular}{|c|c|c|c|}
\hline 6 & 1.123467 & -3.261205 & -3.329818 \\
\hline 6 & -0.137159 & -2.640241 & -3.357219 \\
\hline 6 & -1.260076 & -3.480815 & -3.288114 \\
\hline 6 & -1.139242 & -4.862128 & -3.202976 \\
\hline 6 & 0.118330 & -5.458335 & -3.197904 \\
\hline 6 & 1.246962 & -4.643908 & -3.256039 \\
\hline 6 & -0.315345 & -1.184254 & -3.600463 \\
\hline 6 & -1.556936 & -0.835218 & -4.399621 \\
\hline 45 & -0.061862 & 0.289035 & -1.815317 \\
\hline 6 & 0.539604 & 2.131218 & -1.994035 \\
\hline 8 & 0.660927 & 3.170040 & -2.490067 \\
\hline 15 & -1.731280 & 0.560736 & -0.037893 \\
\hline 6 & -1.892312 & 2.396930 & -0.028104 \\
\hline 6 & -0.886477 & 3.153126 & 0.578858 \\
\hline 6 & -0.904780 & 4.546136 & 0.537122 \\
\hline 6 & -1.924190 & 5.158768 & -0.198975 \\
\hline 6 & -2.924712 & 4.433315 & -0.850008 \\
\hline 6 & -2.902737 & 3.039769 & -0.738533 \\
\hline 6 & 0.161956 & 5.388619 & 1.236085 \\
\hline 6 & -4.009346 & 5.171653 & -1.632991 \\
\hline 6 & -3.487857 & 0.006188 & -0.195671 \\
\hline 6 & -3.876493 & -0.944279 & -1.136627 \\
\hline 6 & -5.208534 & -1.382987 & -1.207571 \\
\hline 6 & -6.124835 & -0.844096 & -0.301948 \\
\hline 6 & -5.763914 & 0.105949 & 0.659040 \\
\hline 6 & -4.435619 & 0.518856 & 0.692386 \\
\hline 6 & -6.746157 & 0.690207 & 1.672045 \\
\hline 6 & -5.622395 & -2.428180 & -2.245334 \\
\hline 6 & -1.495655 & 0.208531 & 1.781386 \\
\hline 6 & -1.105613 & -1.049868 & 2.209051 \\
\hline 6 & -0.944038 & -1.322886 & 3.609986 \\
\hline 6 & -1.200689 & -0.295800 & 4.562063 \\
\hline 6 & -1.643321 & 0.963043 & 4.097377 \\
\hline 6 & -1.784715 & 1.204072 & 2.758113 \\
\hline 6 & -0.999398 & -0.547055 & 5.939792 \\
\hline 6 & -0.558605 & -1.771349 & 6.372632 \\
\hline 6 & -0.309814 & -2.797266 & 5.437585 \\
\hline 6 & -0.502929 & -2.582790 & 4.095036 \\
\hline 6 & -0.882564 & -2.203770 & 1.297027 \\
\hline 6 & 0.358029 & -2.452114 & 0.739699 \\
\hline 6 & 0.625966 & -3.688201 & 0.107666 \\
\hline 6 & -0.330759 & -4.664676 & 0.040122 \\
\hline 6 & -1.625636 & -4.437738 & 0.560457 \\
\hline 6 & -1.913346 & -3.188572 & 1.183530 \\
\hline 6 & -3.213547 & -2.981633 & 1.713033 \\
\hline 6 & -4.166311 & -3.967126 & 1.646147 \\
\hline 6 & -3.870748 & -5.211711 & 1.047573 \\
\hline 6 & -2.630730 & -5.434434 & 0.505263 \\
\hline 7 & 1.422630 & -1.494981 & 0.863841 \\
\hline 15 & 1.717983 & -0.565534 & -0.519556 \\
\hline 8 & 2.899078 & -1.313941 & -1.448004 \\
\hline 6 & 4.109258 & -1.691038 & -0.916384 \\
\hline 6 & 5.043853 & -0.742434 & -0.543998 \\
\hline 6 & 6.211330 & -1.195163 & 0.154275 \\
\hline
\end{tabular}




\begin{tabular}{|c|c|c|c|}
\hline 6 & 6.442593 & -2.595430 & 0.309308 \\
\hline 6 & 5.512190 & -3.519118 & -0.227938 \\
\hline & 4.358139 & -3.076479 & -0.813612 \\
\hline & 7.121001 & -0.297960 & 0.770111 \\
\hline & 8.203711 & -0.759789 & 1.477205 \\
\hline & 8.447131 & -2.143950 & 1.598905 \\
\hline & 7.580723 & -3.039299 & 1.025677 \\
\hline & 4.794905 & 0.689835 & -0.830486 \\
\hline & 3.613289 & 1.277414 & -0.417620 \\
\hline & 3.352950 & 2.651657 & -0.585019 \\
\hline & 4.265672 & 3.444475 & -1.223318 \\
\hline & 5.457476 & 2.888134 & -1.746792 \\
\hline & 5.724407 & 1.496506 & -1.566649 \\
\hline & 6.380384 & 3.689344 & -2.461731 \\
\hline & 7.515098 & 3.145547 & -3.006215 \\
\hline & 7.764682 & 1.764960 & -2.862864 \\
\hline & 6.896034 & 0.964176 & -2.163358 \\
\hline & 2.672073 & 0.542076 & 0.253014 \\
\hline & 0.782012 & -0.301686 & -3.719040 \\
\hline & 2.411272 & -1.789741 & 1.930827 \\
\hline & 2.910392 & -0.612192 & 2.728402 \\
\hline & 2.049295 & 0.386203 & 3.186996 \\
\hline & 2.529041 & 1.429943 & 3.966812 \\
\hline 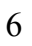 & 3.881845 & 1.497728 & 4.296825 \\
\hline & 4.747580 & 0.511687 & 3.837886 \\
\hline & 4.262061 & -0.536484 & 3.059591 \\
\hline & 6.933589 & 0.770894 & 0.704757 \\
\hline & -2.123357 & 2.185733 & 2.439764 \\
\hline & 7.742939 & -4.111961 & 1.124016 \\
\hline & -1.197720 & 0.256838 & 6.647360 \\
\hline & 6.159236 & 4.749479 & -2.578657 \\
\hline & -3.452742 & -2.022474 & 2.171078 \\
\hline & -5.160240 & -3.783563 & 2.050004 \\
\hline & 4.077821 & 4.509355 & -1.354266 \\
\hline & -1.867422 & 1.744383 & 4.822790 \\
\hline & 5.717434 & -4.584788 & -0.140663 \\
\hline 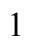 & 8.651222 & 1.327540 & -3.316696 \\
\hline & 3.613048 & -3.762389 & -1.213024 \\
\hline & -2.391989 & -6.383710 & 0.026461 \\
\hline ] & -0.110200 & -5.622280 & -0.427333 \\
\hline & 0.036081 & -3.768730 & 5.783279 \\
\hline 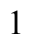 & 7.099353 & -0.099867 & -2.074879 \\
\hline & -0.310090 & -3.389653 & 3.390669 \\
\hline ] & 1.622052 & -3.854975 & -0.297695 \\
\hline & 8.213972 & 3.769680 & -3.558207 \\
\hline 1 & 8.876850 & -0.050269 & 1.953332 \\
\hline 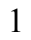 & -4.633909 & -5.986129 & 1.005775 \\
\hline ] & -0.400485 & -1.954965 & 7.432796 \\
\hline 1 & 9.312110 & -2.496037 & 2.156083 \\
\hline & 2.419236 & 3.047756 & -0.191458 \\
\hline 1 & -4.126563 & 1.256549 & 1.431005 \\
\hline & -3.116911 & -1.355805 & -1.803772 \\
\hline 1 & -7.157729 & -1.175629 & -0.342261 \\
\hline 1 & -3.672929 & 2.440593 & -1.218840 \\
\hline 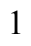 & -0.085557 & 2.633711 & 1.105109 \\
\hline & -1.940304 & 6.246143 & -0.267559 \\
\hline
\end{tabular}




\begin{tabular}{|c|c|c|c|}
\hline 1 & 1.895089 & -2.478441 & 2.614541 \\
\hline 1 & -1.271784 & 0.935272 & -2.612267 \\
\hline 1 & 3.269786 & -2.346974 & 1.526762 \\
\hline & 4.950087 & -1.299872 & 2.691718 \\
\hline 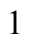 & 1.001594 & 0.358342 & 2.895293 \\
\hline & 5.808668 & 0.557275 & 4.076328 \\
\hline 1 & 1.839905 & 2.197797 & 4.316502 \\
\hline 1 & 4.257692 & 2.319211 & 4.903167 \\
\hline 1 & 1.803635 & -0.644451 & -3.564359 \\
\hline 1 & 0.698905 & 0.535491 & -4.412824 \\
\hline 1 & -2.256499 & -3.044398 & -3.322703 \\
\hline 1 & -2.039143 & -5.474660 & -3.152599 \\
\hline 1 & 0.218612 & -6.541164 & -3.156025 \\
\hline 1 & 2.240509 & -5.090958 & -3.269120 \\
\hline 1 & 2.023973 & -2.655137 & -3.391918 \\
\hline 1 & -1.649946 & -1.495570 & -5.276096 \\
\hline 1 & -2.484540 & -0.922854 & -3.825605 \\
\hline 1 & -1.506443 & 0.199551 & -4.755232 \\
\hline 6 & -7.116526 & -2.743657 & -2.198277 \\
\hline 6 & -5.304234 & -1.914070 & -3.652570 \\
\hline 6 & -4.862235 & -3.732622 & -1.985184 \\
\hline 6 & -8.156229 & 0.128390 & 1.511188 \\
\hline 6 & -6.260026 & 0.357444 & 3.088058 \\
\hline 6 & -6.815575 & 2.211596 & 1.494656 \\
\hline 1 & -8.819865 & 0.578316 & 2.260412 \\
\hline 1 & -8.577928 & 0.352283 & 0.522864 \\
\hline 1 & -8.184026 & -0.959017 & 1.659183 \\
\hline 1 & -7.487473 & 2.653938 & 2.242549 \\
\hline 1 & -5.833211 & 2.688517 & 1.605627 \\
\hline 1 & -7.201986 & 2.470833 & 0.500157 \\
\hline 1 & -6.948026 & 0.772615 & 3.836917 \\
\hline 1 & -6.213453 & -0.729681 & 3.238224 \\
\hline 1 & -5.260801 & 0.762557 & 3.292792 \\
\hline 1 & -7.350702 & -3.505732 & -2.952349 \\
\hline 1 & -7.424058 & -3.144687 & -1.224020 \\
\hline 1 & -7.732311 & -1.862149 & -2.419796 \\
\hline 1 & -5.644738 & -2.636411 & -4.406484 \\
\hline 1 & -5.806837 & -0.957839 & -3.847663 \\
\hline 1 & -4.230344 & -1.763448 & -3.806762 \\
\hline 1 & -5.044178 & -4.450803 & -2.797501 \\
\hline 1 & -3.779488 & -3.578101 & -1.902458 \\
\hline 1 & -5.192676 & -4.191092 & -1.045041 \\
\hline 6 & -4.996289 & 4.214365 & -2.297344 \\
\hline 6 & -4.795334 & 6.080464 & -0.681273 \\
\hline 6 & -3.358944 & 6.021040 & -2.730056 \\
\hline 6 & 0.987349 & 4.560578 & 2.221772 \\
\hline 6 & 1.100595 & 5.978411 & 0.178366 \\
\hline 6 & -0.491384 & 6.528330 & 2.024957 \\
\hline 1 & -4.128649 & 6.545741 & -3.312159 \\
\hline 1 & -2.680923 & 6.779083 & -2.320011 \\
\hline 1 & -2.779513 & 5.393584 & -3.419461 \\
\hline 1 & -5.757816 & 4.788784 & -2.839834 \\
\hline 1 & -4.502570 & 3.553766 & -3.021584 \\
\hline 1 & -5.518582 & 3.586121 & -1.563185 \\
\hline 1 & -5.578330 & 6.621423 & -1.229813 \\
\hline 1 & -5.281413 & 5.494258 & 0.109921 \\
\hline
\end{tabular}




$\begin{array}{rrrr}1 & -4.153405 & 6.826723 & -0.197734 \\ 1 & 1.689427 & 5.212081 & 2.757352 \\ 1 & 0.347342 & 4.069511 & 2.968805 \\ 1 & 1.585833 & 3.783443 & 1.728903 \\ 1 & 0.277431 & 7.111714 & 2.548582 \\ 1 & -1.042734 & 7.224322 & 1.382192 \\ 1 & -1.190376 & 6.139550 & 2.776942 \\ 1 & 1.890510 & 6.577008 & 0.652644 \\ 1 & 1.578938 & 5.183274 & -0.409119 \\ 1 & 0.560586 & 6.625277 & -0.524351\end{array}$

$3_{\text {IC-re- }} \psi_{-12253 a 4 a}$

Number of imaginary frequencies : 0

The smallest frequencies are : $17.7223 \quad 21.738924 .2891 \mathrm{~cm}(-1)$

Electronic energy : $\quad H F=-4360.8798638$

Zero-point correction $=\quad 1.456046($ Hartree/Particle $)$

Thermal correction to Energy= $\quad 1.540197$

Thermal correction to Enthalpy= $\quad 1.541141$

Thermal correction to Gibbs Free Energy $=\quad 1.340824$

Sum of electronic and zero-point Energies $=\quad-4359.423818$

Sum of electronic and thermal Energies $=\quad-4359.339667$

Sum of electronic and thermal Enthalpies $=\quad-4359.338723$

Sum of electronic and thermal Free Energies $=\quad-4359.539039$

\section{Cartesian Coordinates}

$\begin{array}{cccc}6 & 1.375549 & -3.162976 & -3.405013 \\ 6 & 0.058592 & -2.675274 & -3.354710 \\ 6 & -0.963239 & -3.629955 & -3.240399 \\ 6 & -0.697207 & -4.992678 & -3.202616 \\ 6 & 0.613225 & -5.456109 & -3.266059 \\ 6 & 1.646578 & -4.526079 & -3.356213 \\ 6 & -0.282649 & -1.241719 & -3.569006 \\ 6 & -1.584241 & -1.020415 & -4.319935 \\ 45 & -0.083981 & 0.279459 & -1.816293 \\ 6 & 0.513307 & 2.123805 & -2.001172 \\ 8 & 0.668366 & 3.154666 & -2.503520 \\ 15 & -1.738797 & 0.581417 & -0.028981 \\ 6 & -1.864000 & 2.418415 & -0.026186 \\ 6 & -0.865654 & 3.172367 & 0.604885 \\ 6 & -0.860491 & 4.560671 & 0.534005 \\ 6 & -1.846810 & 5.182539 & -0.248546 \\ 6 & -2.828340 & 4.460340 & -0.921322 \\ 6 & -2.829408 & 3.065170 & -0.782967 \\ 6 & 0.200166 & 5.402294 & 1.242285 \\ 6 & 1.015284 & 4.577288 & 2.238779 \\ 6 & -3.894175 & 5.111992 & -1.798544 \\ 6 & -3.776029 & 6.633355 & -1.828334 \\ 6 & -3.505805 & 0.059295 & -0.176697 \\ 6 & -3.892196 & -0.939446 & -1.066994 \\ 6 & -5.223873 & -1.383375 & -1.108614 \\ 6 & -6.131023 & -0.794695 & -0.228504 \\ 6 & -5.772442 & 0.199923 & 0.688657 \\ 6 & -4.447706 & 0.621073 & 0.692719 \\ 6 & -6.807722 & 0.737481 & 1.673762\end{array}$




\begin{tabular}{|c|c|c|c|}
\hline 6 & -5.717974 & -2.433862 & -2.105171 \\
\hline 6 & -1.504925 & 0.220900 & 1.786290 \\
\hline & -1.131993 & -1.046691 & 2.200696 \\
\hline & -0.983855 & -1.338980 & 3.598621 \\
\hline & -1.230417 & -0.318403 & 4.560608 \\
\hline & -1.651169 & 0.952699 & 4.108178 \\
\hline & -1.784951 & 1.210375 & 2.771303 \\
\hline & -1.043926 & -0.589852 & 5.936549 \\
\hline & -0.628970 & -1.827248 & 6.357804 \\
\hline & -0.391641 & -2.846587 & 5.412628 \\
\hline & -0.569409 & -2.612459 & 4.071173 \\
\hline & -0.917076 & -2.189835 & 1.273218 \\
\hline & 0.327353 & -2.451387 & 0.730172 \\
\hline & 0.587238 & -3.687162 & 0.094002 \\
\hline & -0.390626 & -4.638504 & -0.015549 \\
\hline & -1.699414 & -4.380482 & 0.453656 \\
\hline & -1.969654 & -3.143588 & 1.108987 \\
\hline & -3.280867 & -2.910780 & 1.597942 \\
\hline & -4.271311 & -3.846626 & 1.433402 \\
\hline & -4.000672 & -5.071241 & 0.783663 \\
\hline & -2.740619 & -5.329685 & 0.305128 \\
\hline & 1.398680 & -1.503383 & 0.864732 \\
\hline 1 & 1.697948 & -0.559592 & -0.510491 \\
\hline & 2.890320 & -1.286106 & -1.435133 \\
\hline 6 & 4.093756 & -1.672395 & -0.895441 \\
\hline & 5.024484 & -0.728900 & -0.501354 \\
\hline 6 & 6.184266 & -1.192029 & 0.202765 \\
\hline 0 & 6.412040 & -2.594705 & 0.341568 \\
\hline 6 & 5.485864 & -3.510645 & -0.216334 \\
\hline 6 & 4.337992 & -3.059593 & -0.807924 \\
\hline f & 7.088851 & -0.304203 & 0.839098 \\
\hline 6 & 8.164282 & -0.776631 & 1.550327 \\
\hline 6 & 8.404695 & -2.162615 & 1.656302 \\
\hline 6 & 7.542680 & -3.049246 & 1.063148 \\
\hline 6 & 4.777478 & 0.705692 & -0.776728 \\
\hline 6 & 3.587623 & 1.288218 & -0.379391 \\
\hline 6 & 3.324461 & 2.661524 & -0.550160 \\
\hline 6 & 4.245310 & 3.459960 & -1.169525 \\
\hline 5 & 5.448345 & 2.909989 & -1.673266 \\
\hline 6 & 5.716038 & 1.518392 & -1.494984 \\
\hline 6 & 6.380564 & 3.717373 & -2.368956 \\
\hline 6 & 7.525488 & 3.179477 & -2.897591 \\
\hline 6 & 7.775884 & 1.798689 & -2.757733 \\
\hline 6 & 6.898124 & 0.992135 & -2.076480 \\
\hline & 2.638006 & 0.550540 & 0.277509 \\
\hline 6 & 0.725383 & -0.262471 & -3.738944 \\
\hline 6 & 2.380301 & -1.812020 & 1.933605 \\
\hline 6 & 2.881464 & -0.646193 & 2.746655 \\
\hline 6 & 2.027621 & 0.362799 & 3.195770 \\
\hline 6 & 2.508717 & 1.393103 & 3.992273 \\
\hline 6 & 3.855447 & 1.435613 & 4.350342 \\
\hline 6 & 4.713864 & 0.438477 & 3.901885 \\
\hline 6 & 4.227315 & -0.595710 & 3.105470 \\
\hline 6 & 1.149306 & 5.986636 & 0.190318 \\
\hline U & -0.459637 & 6.545456 & 2.020785 \\
\hline 6 & -3.744579 & 4.592154 & -3.233362 \\
\hline
\end{tabular}




\begin{tabular}{|c|c|c|c|}
\hline 5 & -5.285646 & 4.751428 & -1.264746 \\
\hline & 6.903292 & 0.765629 & 0.785615 \\
\hline & -2.111401 & 2.199253 & 2.461840 \\
\hline & 7.702725 & -4.123309 & 1.148999 \\
\hline & -1.233299 & 0.208770 & 6.652485 \\
\hline & 6.158214 & 4.777447 & -2.484184 \\
\hline & -3.504508 & -1.966343 & 2.091968 \\
\hline & -5.274233 & -3.638541 & 1.802918 \\
\hline & 4.055672 & 4.524359 & -1.301538 \\
\hline & -1.866821 & 1.728836 & 4.841671 \\
\hline & 5.689298 & -4.577521 & -0.139942 \\
\hline & 8.670467 & 1.365621 & -3.199806 \\
\hline & 3.593955 & -3.737026 & -1.224529 \\
\hline & -2.516589 & -6.270610 & -0.196504 \\
\hline & -0.178894 & -5.594242 & -0.490360 \\
\hline & -0.066842 & -3.828384 & 5.749536 \\
\hline & 7.102841 & -0.071802 & -1.990968 \\
\hline & -0.385606 & -3.413857 & 3.358048 \\
\hline & 1.589373 & -3.870511 & -0.290181 \\
\hline & 8.231691 & 3.808228 & -3.434812 \\
\hline & 8.833694 & -0.074314 & 2.042132 \\
\hline & -4.793954 & -5.806393 & 0.663030 \\
\hline ] & -0.482798 & -2.026299 & 7.416904 \\
\hline & 9.263904 & -2.523171 & 2.216992 \\
\hline D & 2.380529 & 3.051712 & -0.175277 \\
\hline & -4.129724 & 1.395828 & 1.385051 \\
\hline - & -3.129263 & -1.384585 & -1.706044 \\
\hline 1 & -7.167181 & -1.129029 & -0.246151 \\
\hline & -3.588867 & 2.473613 & -1.293179 \\
\hline 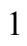 & -0.091099 & 2.650493 & 1.166603 \\
\hline & -1.834554 & 6.266702 & -0.333674 \\
\hline 1 & 1.857489 & -2.505126 & 2.607945 \\
\hline ] & -1.308608 & 0.893278 & -2.621871 \\
\hline 1 & 3.238282 & -2.369737 & 1.529359 \\
\hline 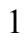 & 4.909732 & -1.368267 & 2.746527 \\
\hline | & 0.985230 & 0.353827 & 2.883880 \\
\hline 1 & 5.770326 & 0.464618 & 4.162610 \\
\hline & 1.825578 & 2.170403 & 4.332800 \\
\hline 1 & 4.232550 & 2.246374 & 4.970179 \\
\hline ] & 1.715093 & 5.230149 & 2.775634 \\
\hline 1 & 0.368460 & 4.093338 & 2.984633 \\
\hline 1 & 1.614998 & 3.795055 & 1.755543 \\
\hline l & 0.305247 & 7.136181 & 2.542066 \\
\hline 1 & -1.013119 & 7.233379 & 1.371297 \\
\hline & -1.158521 & 6.158904 & 2.774043 \\
\hline 1 & 1.950268 & 6.565743 & 0.670178 \\
\hline & 1.611144 & 5.189111 & -0.407317 \\
\hline 1 & 0.621608 & 6.651503 & -0.504822 \\
\hline 1 & -4.564182 & 7.051633 & -2.467101 \\
\hline l & -3.893649 & 7.074282 & -0.829874 \\
\hline 1 & -2.812672 & 6.961546 & -2.239213 \\
\hline | & -4.493682 & 5.056940 & -3.888774 \\
\hline 1 & -2.749092 & 4.827860 & -3.631915 \\
\hline & -3.877208 & 3.505168 & -3.294584 \\
\hline 1 & -6.064504 & 5.199208 & -1.896615 \\
\hline & -5.455212 & 3.667195 & -1.248876 \\
\hline
\end{tabular}




$\begin{array}{lrrr}1 & -5.422813 & 5.127437 & -0.242096 \\ 1 & 1.779509 & -0.514069 & -3.632374 \\ 1 & 0.539396 & 0.556439 & -4.434900 \\ 1 & -1.995525 & -3.298500 & -3.186508 \\ 1 & -1.527630 & -5.694366 & -3.125408 \\ 1 & 0.827381 & -6.522905 & -3.253205 \\ 1 & 2.680002 & -4.864612 & -3.424626 \\ 1 & 2.205853 & -2.469979 & -3.507621 \\ 1 & -1.640678 & -1.687077 & -5.194764 \\ 1 & -2.482297 & -1.201178 & -3.717713 \\ 1 & -1.650701 & 0.014060 & -4.674271 \\ 6 & -4.574812 & -3.269188 & -2.675055 \\ 6 & -6.714045 & -3.398932 & -1.454200 \\ 6 & -6.407813 & -1.703083 & -3.263404 \\ 6 & -6.246477 & 1.861010 & 2.542766 \\ 6 & -8.027094 & 1.279394 & 0.921660 \\ 6 & -7.242615 & -0.408568 & 2.595133 \\ 1 & -7.026023 & 2.225175 & 3.223691 \\ 1 & -5.404022 & 1.522023 & 3.160612 \\ 1 & -5.907800 & 2.714379 & 1.939903 \\ 1 & -7.981714 & -0.055001 & 3.326819 \\ 1 & -7.696640 & -1.235629 & 2.034964 \\ 1 & -6.383226 & -0.810511 & 3.148981 \\ 1 & -8.771926 & 1.660804 & 1.632794 \\ 1 & -7.745934 & 2.104162 & 0.254230 \\ 1 & -8.518542 & 0.510202 & 0.314166 \\ 1 & -4.978791 & -4.063136 & -3.316183 \\ 1 & -3.909835 & -2.664297 & -3.302109 \\ 1 & -3.977656 & -3.744466 & -1.883002 \\ 1 & -7.007015 & -4.170631 & -2.177841 \\ 1 & -6.265895 & -3.902194 & -0.587046 \\ 1 & -7.636175 & -2.906393 & -1.125296 \\ 1 & -6.770568 & -2.420578 & -4.012289 \\ 1 & -7.265942 & -1.115083 & -2.913443 \\ 1 & -5.711199 & -1.015042 & -3.761634\end{array}$

$3_{\text {IC-re- }} \Psi_{-122 s 3 a 4 s}$

Number of imaginary frequencies : 0

The smallest frequencies are : $18.027021 .785423 .5853 \mathrm{~cm}(-1)$

Electronic energy : $\quad H F=-4360.8800107$

Zero-point correction $=\quad 1.455716($ Hartree/Particle $)$

Thermal correction to Energy $=\quad 1.540011$

Thermal correction to Enthalpy= $\quad 1.540955$

Thermal correction to Gibbs Free Energy $=\quad 1.339861$

Sum of electronic and zero-point Energies $=\quad-4359.424295$

Sum of electronic and thermal Energies $=\quad-4359.340000$

Sum of electronic and thermal Enthalpies $=\quad-4359.339056$

Sum of electronic and thermal Free Energies $=\quad-4359.540150$

Cartesian Coordinates

$\begin{array}{cccc}6 & -3.892868 & -0.984596 & -1.060297 \\ 6 & -3.506977 & 0.024067 & -0.174448 \\ 6 & -4.451701 & 0.590771 & 0.678581 \\ 6 & -5.784427 & 0.180640 & 0.666026\end{array}$




\begin{tabular}{|c|c|c|c|}
\hline 5 & -6.142380 & -0.815775 & -0.241380 \\
\hline 6 & -5.222557 & -1.420709 & -1.106484 \\
\hline 5 & -1.744213 & 0.560025 & -0.025853 \\
\hline & -0.091072 & 0.264695 & -1.814363 \\
\hline 6 & 0.715038 & -0.279684 & -3.738147 \\
\hline 6 & -0.288927 & -1.262124 & -3.563328 \\
\hline 6 & -6.759313 & 0.797121 & 1.666029 \\
\hline 6 & -6.281042 & 0.456334 & 3.083210 \\
\hline 6 & -5.716202 & -2.482535 & -2.091266 \\
\hline 6 & -6.692397 & -3.455150 & -1.421214 \\
\hline 6 & -1.507021 & 0.211685 & 1.791620 \\
\hline 6 & -1.119854 & -1.049273 & 2.212549 \\
\hline 6 & -0.965374 & -1.332809 & 3.611280 \\
\hline 6 & -1.219402 & -0.309643 & 4.568388 \\
\hline 6 & -1.654675 & 0.954427 & 4.110052 \\
\hline 6 & -1.795090 & 1.203441 & 2.771984 \\
\hline 6 & -0.537983 & -2.599958 & 4.089078 \\
\hline 6 & -0.353521 & -2.825091 & 5.431142 \\
\hline 6 & -0.597232 & -1.802774 & 6.371518 \\
\hline 6 & -1.025720 & -0.571744 & 5.945135 \\
\hline 6 & -0.902174 & -2.194893 & 1.289678 \\
\hline 6 & 0.339859 & -2.453051 & 0.739909 \\
\hline 6 & 0.598848 & -3.688239 & 0.102357 \\
\hline 6 & -0.378018 & -4.641420 & -0.002151 \\
\hline 6 & -1.684442 & -4.386956 & 0.475610 \\
\hline 6 & -1.953025 & -3.151325 & 1.133713 \\
\hline 6 & -2.724728 & -5.338286 & 0.334276 \\
\hline 6 & -3.981167 & -5.083319 & 0.824183 \\
\hline 6 & -4.249329 & -3.860140 & 1.477883 \\
\hline 6 & -3.260483 & -2.921506 & 1.634062 \\
\hline 7 & 1.408500 & -1.501096 & 0.867398 \\
\hline 6 & 2.393731 & -1.800397 & 1.935529 \\
\hline 6 & 2.890917 & -0.629072 & 2.743265 \\
\hline 6 & 2.033395 & 0.377724 & 3.190439 \\
\hline 6 & 2.511350 & 1.413049 & 3.982305 \\
\hline 6 & 3.858511 & 1.462806 & 4.337816 \\
\hline 6 & 4.720547 & 0.467910 & 3.891353 \\
\hline 6 & 4.237184 & -0.571248 & 3.099489 \\
\hline 15 & 1.698784 & -0.559781 & -0.511241 \\
\hline 8 & 2.633719 & 0.558584 & 0.271099 \\
\hline 6 & 3.577458 & 1.300036 & -0.389918 \\
\hline 6 & 4.769921 & 0.723508 & -0.788243 \\
\hline 6 & 5.702031 & 1.539903 & -1.510683 \\
\hline 6 & 5.425679 & 2.929432 & -1.691969 \\
\hline 6 & 4.220409 & 3.473566 & -1.187197 \\
\hline 6 & 3.305652 & 2.671267 & -0.563830 \\
\hline 6 & 6.885990 & 1.019179 & -2.093346 \\
\hline 6 & 7.757541 & 1.829182 & -2.778472 \\
\hline 6 & 7.498698 & 3.208117 & -2.921232 \\
\hline 6 & 6.351677 & 3.740549 & -2.391602 \\
\hline 6 & 5.026122 & -0.708754 & -0.508970 \\
\hline 6 & 4.100127 & -1.659083 & -0.897696 \\
\hline 6 & 4.352185 & -3.044534 & -0.805120 \\
\hline 6 & 5.503911 & -3.486795 & -0.214364 \\
\hline 6 & 6.426154 & -2.563470 & 0.337866 \\
\hline 6 & 6.190059 & -1.162634 & 0.194354 \\
\hline
\end{tabular}




\begin{tabular}{|c|c|c|c|}
\hline 6 & 7.090879 & -0.267308 & 0.825527 \\
\hline 6 & 8.170473 & -0.730952 & 1.536211 \\
\hline & 8.419016 & -2.115126 & 1.646829 \\
\hline & 7.560865 & -3.008869 & 1.058776 \\
\hline & 2.893468 & -1.281639 & -1.436459 \\
\hline & -1.886433 & 2.396244 & -0.029420 \\
\hline & -0.890607 & 3.158426 & 0.595740 \\
\hline & -0.895102 & 4.546542 & 0.521988 \\
\hline & -1.886883 & 5.159766 & -0.260448 \\
\hline & -2.865668 & 4.429449 & -0.928595 \\
\hline & -2.859605 & 3.034809 & -0.783546 \\
\hline & 0.161331 & 5.396727 & 1.226382 \\
\hline & 1.105053 & 5.984987 & 0.171726 \\
\hline & -3.930032 & 5.071546 & -1.814814 \\
\hline & -3.756266 & 4.556891 & -3.248886 \\
\hline & -0.504920 & 6.537246 & 2.003264 \\
\hline & 0.983473 & 4.579422 & 2.223498 \\
\hline & -5.324103 & 4.693661 & -1.300482 \\
\hline & -3.828784 & 6.594240 & -1.838941 \\
\hline & 0.494660 & 2.111921 & -2.006488 \\
\hline & 0.640281 & 3.142847 & -2.511515 \\
\hline ) & -6.796637 & 2.318529 & 1.485674 \\
\hline & -8.180118 & 0.264346 & 1.501910 \\
\hline & -6.428472 & -1.768198 & -3.246142 \\
\hline & -4.571211 & -3.309250 & -2.669290 \\
\hline & 6.899126 & 0.801249 & 0.768571 \\
\hline & -2.130878 & 2.187643 & 2.457871 \\
\hline & 7.727193 & -4.081676 & 1.148323 \\
\hline & -1.220800 & 0.228686 & 6.657502 \\
\hline & 6.122855 & 4.799002 & -2.509036 \\
\hline & -3.481262 & -1.977944 & 2.131718 \\
\hline & -5.249274 & -3.655648 & 1.857047 \\
\hline & 4.024065 & 4.536459 & -1.321559 \\
\hline & -1.875984 & 1.732421 & 4.839884 \\
\hline 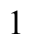 & 5.713401 & -4.552209 & -0.134050 \\
\hline & 8.653754 & 1.400310 & -3.221328 \\
\hline & 3.610739 & -3.727802 & -1.216881 \\
\hline & -2.502835 & -6.278178 & -0.170261 \\
\hline & -0.167049 & -5.596286 & -0.478993 \\
\hline ] & -0.018614 & -3.801966 & 5.772399 \\
\hline & 7.097171 & -0.043312 & -2.005496 \\
\hline ] & -0.349783 & -3.403422 & 3.379400 \\
\hline & 1.599021 & -3.869336 & -0.287992 \\
\hline 1 & 8.200052 & 3.839692 & -3.461481 \\
\hline 1 & 8.836859 & -0.022994 & 2.024024 \\
\hline ] & -4.773476 & -5.820579 & 0.710148 \\
\hline ] & -0.445604 & -1.994693 & 7.431163 \\
\hline & 9.281413 & -2.468683 & 2.207083 \\
\hline 1 & 2.360112 & 3.056756 & -0.188136 \\
\hline & -4.141018 & 1.365378 & 1.377958 \\
\hline 1 & -3.127114 & -1.437458 & -1.690364 \\
\hline 1 & -7.178381 & -1.143067 & -0.275720 \\
\hline 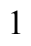 & -3.617767 & 2.436971 & -1.288463 \\
\hline 1 & -0.110379 & 2.643093 & 1.155667 \\
\hline & -1.881021 & 6.243657 & -0.349777 \\
\hline & 1.875888 & -2.493511 & 2.613654 \\
\hline
\end{tabular}




\begin{tabular}{|c|c|c|c|}
\hline & -1.321419 & 0.869793 & -2.618074 \\
\hline & 3.253567 & -2.355420 & 1.531552 \\
\hline & 4.922477 & -1.341914 & 2.741963 \\
\hline | & 0.990485 & 0.363042 & 2.880541 \\
\hline & 5.777328 & 0.499679 & 4.150140 \\
\hline & 1.825374 & 2.188587 & 4.321167 \\
\hline & 4.233105 & 2.277455 & 4.954066 \\
\hline & 1.679668 & 5.238171 & 2.757868 \\
\hline & 0.341059 & 4.092757 & 2.971405 \\
\hline & 1.587775 & 3.800173 & 1.741132 \\
\hline 1 & 0.256741 & 7.134577 & 2.521712 \\
\hline & -1.064375 & 7.219581 & 1.353008 \\
\hline & -1.199782 & 6.147774 & 2.758750 \\
\hline 1 & 1.902258 & 6.571209 & 0.649191 \\
\hline & 1.572186 & 5.189233 & -0.424178 \\
\hline 1 & 0.571851 & 6.644131 & -0.524656 \\
\hline & -4.616710 & 7.005643 & -2.482435 \\
\hline l & -3.958771 & 7.030324 & -0.839852 \\
\hline & -2.866310 & 6.934947 & -2.241595 \\
\hline & -4.503576 & 5.012778 & -3.912594 \\
\hline ] & -2.759237 & 4.806575 & -3.634919 \\
\hline & -3.873235 & 3.468181 & -3.312731 \\
\hline$\frac{1}{1}$ & -6.098953 & 5.137089 & -1.940364 \\
\hline & -5.482857 & 3.607664 & -1.292820 \\
\hline ] & -5.478835 & 5.062328 & -0.277598 \\
\hline & -7.461111 & 2.774318 & 2.232032 \\
\hline & -5.806137 & 2.777233 & 1.598634 \\
\hline 1 & -7.176019 & 2.585178 & 0.490316 \\
\hline & -8.840223 & 0.742655 & 2.236483 \\
\hline$\frac{1}{1}$ & -8.586308 & 0.479826 & 0.505126 \\
\hline & -8.234918 & -0.819114 & 1.669538 \\
\hline 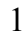 & -6.963044 & 0.881132 & 3.832229 \\
\hline$\frac{1}{1}$ & -6.249978 & -0.631894 & 3.231421 \\
\hline & -5.275322 & 0.846170 & 3.286621 \\
\hline 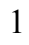 & -4.974108 & -4.108322 & -3.304737 \\
\hline & -3.916348 & -2.700331 & -3.303261 \\
\hline 1 & -3.963970 & -3.777672 & -1.880681 \\
\hline 1 & -6.792893 & -2.495583 & -3.984593 \\
\hline 1 & -7.288784 & -1.186542 & -2.891171 \\
\hline 1 & -5.745219 & -1.077138 & -3.758418 \\
\hline 1 & -6.991243 & -4.230899 & -2.138198 \\
\hline 1 & -6.223599 & -3.952734 & -0.561844 \\
\hline 1 & -7.612417 & -2.970197 & -1.075716 \\
\hline 1 & 1.770345 & -0.527252 & -3.633860 \\
\hline 1 & 0.524209 & 0.536954 & -4.435446 \\
\hline 6 & 0.057733 & -2.693838 & -3.346007 \\
\hline 6 & -1.593903 & -1.046545 & -4.310029 \\
\hline 6 & -0.960685 & -3.652191 & -3.232148 \\
\hline 6 & -0.689532 & -5.013787 & -3.190117 \\
\hline 6 & 0.622803 & -5.472345 & -3.249270 \\
\hline 6 & 1.652805 & -4.538521 & -3.339224 \\
\hline 6 & 1.376735 & -3.176590 & -3.391739 \\
\hline 1 & -1.994373 & -3.324718 & -3.182415 \\
\hline 1 & -1.517458 & -5.718450 & -3.112950 \\
\hline 1 & 0.841033 & -6.538270 & -3.233254 \\
\hline 1 & 2.687683 & -4.873277 & -3.404260 \\
\hline
\end{tabular}




$\begin{array}{llll}1 & 2.204713 & -2.480631 & -3.493191 \\ 1 & -1.652111 & -1.716132 & -5.182506 \\ 1 & -2.489072 & -1.227191 & -3.703491 \\ 1 & -1.664079 & -0.013409 & -4.667553\end{array}$

$3_{\text {IC-re- }} \psi_{-12 a 2 s 34 a}$

Number of imaginary frequencies : 0

The smallest frequencies are : $14.2102 \quad 17.6522 \quad 23.4981 \mathrm{~cm}(-1)$

Electronic energy : $\quad H F=-4360.8792338$

Zero-point correction $=\quad 1.454836($ Hartree/Particle $)$

Thermal correction to Energy= $\quad 1.539432$

Thermal correction to Enthalpy= $\quad 1.540376$

Thermal correction to Gibbs Free Energy $=\quad 1.337360$

Sum of electronic and zero-point Energies $=\quad-4359.424397$

Sum of electronic and thermal Energies $=\quad-4359.339802$

Sum of electronic and thermal Enthalpies $=\quad-4359.338857$

Sum of electronic and thermal Free Energies $=\quad-4359.541873$

Cartesian Coordinates

$\begin{array}{cccc}6 & 1.107574 & -3.252717 & -3.333428 \\ 6 & -0.151276 & -2.627913 & -3.355742 \\ 6 & -1.276338 & -3.465190 & -3.281815 \\ 6 & -1.159368 & -4.846806 & -3.195938 \\ 6 & 0.096396 & -5.446765 & -3.195651 \\ 6 & 1.227165 & -4.635760 & -3.259473 \\ 6 & -0.326187 & -1.171342 & -3.598283 \\ 6 & -1.571270 & -0.818268 & -4.390275 \\ 45 & -0.058974 & 0.302025 & -1.815307 \\ 6 & 0.551789 & 2.140985 & -1.992500 \\ 8 & 0.678620 & 3.181314 & -2.483842 \\ 15 & -1.721233 & 0.578745 & -0.033975 \\ 6 & -1.860619 & 2.415327 & -0.016438 \\ 6 & -0.851482 & 3.167994 & 0.598900 \\ 6 & -0.865045 & 4.557365 & 0.560280 \\ 6 & -1.885419 & 5.183857 & -0.174028 \\ 6 & -2.882913 & 4.464392 & -0.825870 \\ 6 & -2.859159 & 3.066139 & -0.724390 \\ 6 & 0.205854 & 5.394177 & 1.259605 \\ 6 & 1.032045 & 4.561069 & 2.240424 \\ 6 & -4.006008 & 5.121860 & -1.624040 \\ 6 & -3.907340 & 6.644906 & -1.625471 \\ 6 & -3.484330 & 0.047230 & -0.194815 \\ 6 & -3.871960 & -0.914642 & -1.118801 \\ 6 & -5.209127 & -1.351733 & -1.187141 \\ 6 & -6.117653 & -0.797457 & -0.291437 \\ 6 & -5.758194 & 0.174885 & 0.655301 \\ 6 & -4.433895 & 0.586679 & 0.684857 \\ 6 & -6.802087 & 0.708663 & 1.633040 \\ 6 & -5.625271 & -2.405102 & -2.214969 \\ 6 & -1.488204 & 0.218863 & 1.783395 \\ 6 & -1.102336 & -1.041863 & 2.207810 \\ 6 & -0.936274 & -1.316670 & 3.607976 \\ 6 & -1.183936 & -0.288903 & 4.561813 \\ 6 & -1.625764 & 0.971499 & 4.100390\end{array}$




\begin{tabular}{|c|c|c|c|}
\hline 6 & -1.772544 & 1.213805 & 2.762201 \\
\hline 5 & -0.975976 & -0.541468 & 5.938297 \\
\hline p & -0.537445 & -1.767575 & 6.368365 \\
\hline & -0.298275 & -2.794291 & 5.431733 \\
\hline & -0.498120 & -2.578627 & 4.090342 \\
\hline & -0.887798 & -2.196334 & 1.294098 \\
\hline 6 & 0.350009 & -2.450972 & 0.733407 \\
\hline 6 & 0.611680 & -3.689721 & 0.103846 \\
\hline 6 & -0.348151 & -4.663590 & 0.043891 \\
\hline 6 & -1.640567 & -4.430636 & 0.567966 \\
\hline 6 & -1.922974 & -3.177460 & 1.185509 \\
\hline 6 & -3.222323 & -2.963539 & 1.714606 \\
\hline 6 & -4.178097 & -3.946741 & 1.655536 \\
\hline 6 & -3.886315 & -5.196436 & 1.065866 \\
\hline 6 & -2.648194 & -5.425265 & 0.521711 \\
\hline 7 & 1.420559 & -1.500219 & 0.856467 \\
\hline 15 & 1.717096 & -0.564600 & -0.523005 \\
\hline 8 & 2.894170 & -1.311720 & -1.457059 \\
\hline 6 & 4.105201 & -1.693340 & -0.930758 \\
\hline 6 & 5.042981 & -0.748117 & -0.557603 \\
\hline 6 & 6.211265 & -1.206560 & 0.135821 \\
\hline 6 & 6.440004 & -2.608032 & 0.283884 \\
\hline 6 & 5.505781 & -3.527485 & -0.254073 \\
\hline 6 & 4.350844 & -3.079793 & -0.834052 \\
\hline 6 & 7.124161 & -0.314156 & 0.753741 \\
\hline 6 & 8.207712 & -0.781484 & 1.455958 \\
\hline 6 & 8.448692 & -2.166654 & 1.570525 \\
\hline 6 & 7.579033 & -3.057564 & 0.995255 \\
\hline 6 & 4.795759 & 0.685848 & -0.835991 \\
\hline 6 & 3.616433 & 1.273312 & -0.416440 \\
\hline 6 & 3.357907 & 2.648707 & -0.576789 \\
\hline 6 & 4.270497 & 3.443157 & -1.213312 \\
\hline 6 & 5.460013 & 2.887543 & -1.742605 \\
\hline 6 & 5.724766 & 1.494516 & -1.570524 \\
\hline 6 & 6.382540 & 3.690771 & -2.455781 \\
\hline 6 & 7.514704 & 3.147650 & -3.006201 \\
\hline 6 & 7.762054 & 1.765850 & -2.870753 \\
\hline 6 & 6.893766 & 0.963047 & -2.173126 \\
\hline 8 & 2.676071 & 0.536173 & 0.253857 \\
\hline 6 & 0.773663 & -0.292597 & -3.722625 \\
\hline 6 & 2.411585 & -1.810138 & 1.916975 \\
\hline 6 & 2.922794 & -0.643398 & 2.722165 \\
\hline 6 & 2.072657 & 0.363030 & 3.183662 \\
\hline 6 & 2.562545 & 1.395527 & 3.971980 \\
\hline 6 & 3.914759 & 1.443229 & 4.308074 \\
\hline 6 & 4.769584 & 0.448976 & 3.846391 \\
\hline 6 & 4.274069 & -0.587946 & 3.059127 \\
\hline 6 & 1.142905 & 5.984497 & 0.200513 \\
\hline 6 & -0.440787 & 6.533586 & 2.054621 \\
\hline 6 & -3.943654 & 4.637399 & -3.077281 \\
\hline 6 & -5.356910 & 4.731428 & -1.010798 \\
\hline 1 & 6.938826 & 0.755354 & 0.693888 \\
\hline 1 & -2.112200 & 2.196048 & 2.446431 \\
\hline 1 & 7.739261 & -4.131000 & 1.088244 \\
\hline 1 & -1.167248 & 0.262961 & 6.647209 \\
\hline 1 & 6.163116 & 4.751923 & -2.566545 \\
\hline
\end{tabular}




\begin{tabular}{|c|c|c|c|}
\hline & -3.459940 & -2.001060 & 2.165840 \\
\hline & -5.171540 & -3.756284 & 2.057710 \\
\hline & 4.083836 & 4.508928 & -1.338609 \\
\hline & -1.845617 & 1.752449 & 4.827505 \\
\hline & 5.708735 & -4.593960 & -0.171425 \\
\hline & 8.646538 & 1.329146 & -3.329258 \\
\hline & 3.601896 & -3.762071 & -1.232590 \\
\hline & -2.412938 & -6.377976 & 0.048010 \\
\hline & -0.132104 & -5.624011 & -0.419911 \\
\hline & 0.045481 & -3.767290 & 5.775236 \\
\hline & 7.095312 & -0.101833 & -2.090809 \\
\hline & -0.312664 & -3.386176 & 3.384761 \\
\hline & 1.605844 & -3.860984 & -0.304646 \\
\hline & 8.213300 & 3.773321 & -3.556801 \\
\hline & 8.883364 & -0.075543 & 1.933839 \\
\hline & -4.651300 & -5.969380 & 1.030754 \\
\hline & -0.373970 & -1.952014 & 7.427574 \\
\hline & 9.314335 & -2.523091 & 2.123895 \\
\hline & 2.425354 & 3.044420 & -0.180049 \\
\hline & -4.118687 & 1.341346 & 1.399821 \\
\hline & -3.112546 & -1.339746 & -1.777681 \\
\hline & -7.153313 & -1.128383 & -0.317802 \\
\hline & -3.632100 & 2.477212 & -1.216970 \\
\hline & -0.056144 & 2.642993 & 1.127903 \\
\hline ] & -1.892166 & 6.269796 & -0.232063 \\
\hline & 1.893256 & -2.500306 & 2.597628 \\
\hline . & -1.266114 & 0.957061 & -2.610172 \\
\hline & 3.264197 & -2.370937 & 1.505480 \\
\hline & 4.953574 & -1.358019 & 2.689363 \\
\hline . & 1.025731 & 0.350302 & 2.887592 \\
\hline & 5.830114 & 0.479453 & 4.089579 \\
\hline ] & 1.881889 & 2.170264 & 4.323138 \\
\hline & 4.298871 & 2.255820 & 4.921177 \\
\hline 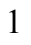 & 1.738303 & 5.209258 & 2.774522 \\
\hline & 0.393084 & 4.071070 & 2.989056 \\
\hline 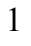 & 1.626171 & 3.782588 & 1.744501 \\
\hline ] & 0.331922 & 7.110998 & 2.579147 \\
\hline & -0.990081 & 7.235120 & 1.416278 \\
\hline . & -1.139790 & 6.144700 & 2.806567 \\
\hline & 1.937893 & 6.576948 & 0.674049 \\
\hline . & 1.614315 & 5.189649 & -0.393090 \\
\hline & 0.603188 & 6.637678 & -0.496547 \\
\hline . & -4.734865 & 7.067226 & -2.209431 \\
\hline . & -3.974223 & 7.062257 & -0.612334 \\
\hline & -2.972146 & 6.994171 & -2.081639 \\
\hline . & -4.738866 & 5.108101 & -3.671192 \\
\hline & -2.979240 & 4.895036 & -3.533960 \\
\hline 1 & -4.068718 & 3.550860 & -3.156749 \\
\hline 1 & -6.179933 & 5.183298 & -1.580854 \\
\hline 1 & -5.512834 & 3.644641 & -1.008197 \\
\hline . & -5.431867 & 5.083181 & 0.026962 \\
\hline 1 & 1.794788 & -0.638951 & -3.572690 \\
\hline & 0.690047 & 0.544732 & -4.416144 \\
\hline & -2.271523 & -3.025841 & -3.313477 \\
\hline 1 & -2.060894 & -5.456591 & -3.141098 \\
\hline 1 & 0.193627 & -6.529852 & -3.153263 \\
\hline
\end{tabular}




$\begin{array}{lrrr}1 & 2.219271 & -5.085867 & -3.276773 \\ 1 & 2.009716 & -2.649519 & -3.399688 \\ 1 & -1.672591 & -1.479245 & -5.265358 \\ 1 & -2.495595 & -0.901369 & -3.810149 \\ 1 & -1.518834 & 0.215891 & -4.747412 \\ 6 & -7.117785 & -2.725157 & -2.154528 \\ 6 & -5.318755 & -1.895116 & -3.626363 \\ 6 & -4.859148 & -3.705996 & -1.955686 \\ 6 & -6.244315 & 1.822603 & 2.516782 \\ 6 & -8.005916 & 1.268052 & 0.868379 \\ 6 & -7.259323 & -0.440778 & 2.538678 \\ 1 & -7.030891 & 2.190271 & 3.187677 \\ 1 & -5.413861 & 1.474167 & 3.145560 \\ 1 & -5.889503 & 2.675720 & 1.921865 \\ 1 & -8.004477 & -0.086443 & 3.263820 \\ 1 & -7.714092 & -1.258259 & 1.965545 \\ 1 & -6.411058 & -0.855947 & 3.099972 \\ 1 & -8.756238 & 1.652708 & 1.571963 \\ 1 & -7.705982 & 2.094477 & 0.210803 \\ 1 & -8.497124 & 0.508648 & 0.248589 \\ 1 & -7.355267 & -3.493891 & -2.900733 \\ 1 & -7.415858 & -3.119472 & -1.174472 \\ 1 & -7.738211 & -1.847423 & -2.378129 \\ 1 & -5.659290 & -2.622810 & -4.375085 \\ 1 & -5.828524 & -0.942908 & -3.822520 \\ 1 & -4.246610 & -1.738442 & -3.787306 \\ 1 & -5.045449 & -4.427001 & -2.764455 \\ 1 & -3.776060 & -3.549531 & -1.882359 \\ 1 & -5.181099 & -4.162618 & -1.011816\end{array}$

$3_{\mathrm{IC}-\mathrm{re}-} \psi_{-1 \mathrm{a} 2 \mathrm{2} 33 \mathrm{~s} 4 \mathrm{~s}}$

Number of imaginary frequencies : 0

The smallest frequencies are : $17.241620 .1353 \quad 22.6246 \mathrm{~cm}(-1)$

Electronic energy : $\quad H F=-4360.879299$

Zero-point correction $=\quad 1.454863($ Hartree/Particle)

Thermal correction to Energy= $\quad 1.539454$

Thermal correction to Enthalpy $=\quad 1.540398$

Thermal correction to Gibbs Free Energy $=\quad 1.337568$

Sum of electronic and zero-point Energies $=\quad-4359.424436$

Sum of electronic and thermal Energies $=\quad-4359.339845$

Sum of electronic and thermal Enthalpies $=\quad-4359.338901$

Sum of electronic and thermal Free Energies $=\quad-4359.541731$

\section{Cartesian Coordinates}

$\begin{array}{cccc}6 & 1.070638 & -3.216403 & -3.370616 \\ 6 & -0.185409 & -2.585710 & -3.388217 \\ 6 & -1.314476 & -3.419205 & -3.333607 \\ 6 & -1.203775 & -4.802652 & -3.270128 \\ 6 & 0.049355 & -5.407991 & -3.273567 \\ 6 & 1.183886 & -4.600872 & -3.318821 \\ 6 & -0.352546 & -1.124599 & -3.607396 \\ 6 & -1.597473 & -0.751304 & -4.390240 \\ 45 & -0.072358 & 0.319732 & -1.802037 \\ 6 & 0.548916 & 2.157331 & -1.957234\end{array}$




\begin{tabular}{|c|c|c|c|}
\hline & 0.686749 & 3.200096 & -2.440332 \\
\hline & -1.727499 & 0.575441 & -0.010172 \\
\hline & -1.864662 & 2.412175 & 0.023347 \\
\hline & -0.858412 & 3.159650 & 0.649682 \\
\hline & -0.861039 & 4.548888 & 0.604375 \\
\hline & -1.861295 & 5.179431 & -0.153515 \\
\hline & -2.851486 & 4.464571 & -0.821443 \\
\hline & -2.847390 & 3.067319 & -0.703088 \\
\hline & 0.204422 & 5.382089 & 1.315812 \\
\hline & 1.025364 & 4.544419 & 2.296908 \\
\hline & -3.928178 & 5.125558 & -1.678415 \\
\hline & -3.826825 & 6.648409 & -1.671824 \\
\hline & -3.492288 & 0.047778 & -0.172018 \\
\hline & -3.891526 & -0.892436 & -1.119017 \\
\hline & -5.227676 & -1.318302 & -1.190469 \\
\hline U & -6.136264 & -0.779788 & -0.276855 \\
\hline 6 & -5.765032 & 0.160562 & 0.689950 \\
\hline 6 & -4.433889 & 0.565131 & 0.720205 \\
\hline 6 & -6.737777 & 0.734652 & 1.718174 \\
\hline 6 & -5.654497 & -2.347983 & -2.238572 \\
\hline 6 & -1.487751 & 0.193494 & 1.801393 \\
\hline 6 & -1.101263 & -1.072570 & 2.209029 \\
\hline 6 & -0.931753 & -1.365403 & 3.604903 \\
\hline 6 & -1.175695 & -0.349633 & 4.572355 \\
\hline 6 & -1.616584 & 0.917257 & 4.128160 \\
\hline 6 & -1.767205 & 1.176610 & 2.793381 \\
\hline 6 & -0.965255 & -0.620263 & 5.945029 \\
\hline 6 & -0.528378 & -1.852684 & 6.358291 \\
\hline 6 & -0.293317 & -2.867778 & 5.408001 \\
\hline 6 & -0.495294 & -2.634422 & 4.069893 \\
\hline 6 & -0.893876 & -2.215062 & 1.279208 \\
\hline 6 & 0.339709 & -2.465656 & 0.707629 \\
\hline 6 & 0.591635 & -3.694183 & 0.054758 \\
\hline 6 & -0.374280 & -4.661182 & -0.019041 \\
\hline 6 & -1.662721 & -4.431130 & 0.515953 \\
\hline 6 & -1.934397 & -3.188755 & 1.159595 \\
\hline 6 & -3.228839 & -2.977460 & 1.701463 \\
\hline 6 & -4.191054 & -3.953226 & 1.627252 \\
\hline 6 & -3.911150 & -5.191672 & 1.008762 \\
\hline 6 & -2.677195 & -5.417845 & 0.454185 \\
\hline 7 & 1.414022 & -1.520532 & 0.838955 \\
\hline 15 & 1.706883 & -0.567042 & -0.528826 \\
\hline 8 & 2.880537 & -1.301256 & -1.477160 \\
\hline 6 & 4.091099 & -1.693601 & -0.957528 \\
\hline 6 & 5.031946 & -0.756296 & -0.572089 \\
\hline 6 & 6.199839 & -1.228134 & 0.113104 \\
\hline 6 & 6.424350 & -2.632286 & 0.241142 \\
\hline 6 & 5.486493 & -3.541334 & -0.307994 \\
\hline 6 & 4.332287 & -3.082003 & -0.880212 \\
\hline 6 & 7.116680 & -0.347530 & 0.741991 \\
\hline 6 & 8.199953 & -0.828138 & 1.435644 \\
\hline 6 & 8.436767 & -2.215545 & 1.530214 \\
\hline 6 & 7.563216 & -3.095426 & 0.944003 \\
\hline 6 & 4.788248 & 0.682434 & -0.828611 \\
\hline 6 & 3.610996 & 1.266568 & -0.398550 \\
\hline 6 & 3.356890 & 2.645412 & -0.534466 \\
\hline
\end{tabular}




\begin{tabular}{|c|c|c|c|}
\hline & 4.271762 & 3.447727 & -1.157646 \\
\hline & 5.458799 & 2.897264 & -1.697802 \\
\hline & 5.719074 & 1.500567 & -1.550247 \\
\hline & 6.383417 & 3.709710 & -2.397674 \\
\hline & 7.513283 & 3.172517 & -2.958545 \\
\hline & 7.756198 & 1.787764 & -2.847319 \\
\hline & 6.885835 & 0.975806 & -2.162958 \\
\hline & 2.668984 & 0.521857 & 0.260333 \\
\hline & 0.751889 & -0.250039 & -3.720330 \\
\hline & 2.404625 & -1.842380 & 1.896153 \\
\hline & 2.919432 & -0.684245 & 2.711587 \\
\hline & 2.072435 & 0.320714 & 3.182137 \\
\hline & 2.565784 & 1.345827 & 3.977866 \\
\hline & 3.918505 & 1.387372 & 4.312844 \\
\hline & 4.770128 & 0.394422 & 3.842615 \\
\hline & 4.271162 & -0.634972 & 3.047578 \\
\hline ) & 1.148663 & 5.979506 & 0.267079 \\
\hline & -0.450526 & 6.515640 & 2.112365 \\
\hline D & -3.775843 & 4.641292 & -3.125478 \\
\hline & -5.314774 & 4.736843 & -1.151954 \\
\hline & 6.934972 & 0.723304 & 0.697134 \\
\hline & -2.104834 & 2.163553 & 2.490511 \\
\hline & 7.720194 & -4.170570 & 1.021415 \\
\hline & -1.153536 & 0.175311 & 6.664614 \\
\hline & 6.167471 & 4.773375 & -2.489642 \\
\hline & -3.455958 & -2.022866 & 2.175052 \\
\hline & -5.180437 & -3.766873 & 2.040714 \\
\hline & 4.088866 & 4.516199 & -1.263992 \\
\hline & -1.832340 & 1.689406 & 4.865794 \\
\hline 1 & 5.686257 & -4.609463 & -0.240414 \\
\hline & 8.638846 & 1.356197 & -3.314142 \\
\hline ] & 3.580646 & -3.756223 & -1.287336 \\
\hline & -2.450852 & -6.362157 & -0.040170 \\
\hline & -0.166470 & -5.613268 & -0.503396 \\
\hline ] & 0.048822 & -3.845924 & 5.738268 \\
\hline & 7.083941 & -0.091009 & -2.099421 \\
\hline ] & -0.313621 & -3.433295 & 3.353517 \\
\hline & 1.582698 & -3.863263 & -0.362151 \\
\hline 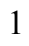 & 8.213524 & 3.805371 & -3.498745 \\
\hline 1 & 8.878717 & -0.131129 & 1.922148 \\
\hline ( & -4.681665 & -5.958453 & 0.961467 \\
\hline 1 & -0.363228 & -2.051128 & 7.414705 \\
\hline & 9.302289 & -2.582495 & 2.076856 \\
\hline 1 & 2.425789 & 3.036948 & -0.130000 \\
\hline & -4.117108 & 1.297088 & 1.461336 \\
\hline 1 & -3.137579 & -1.304943 & -1.792019 \\
\hline l & -7.171707 & -1.103461 & -0.315600 \\
\hline 1 & -3.615143 & 2.481470 & -1.207699 \\
\hline 1 & -0.072304 & 2.631275 & 1.189059 \\
\hline 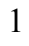 & -1.853982 & 6.264808 & -0.221841 \\
\hline 1 & 1.884869 & -2.537292 & 2.570853 \\
\hline ] & -1.279207 & 0.991770 & -2.583439 \\
\hline 1 & 3.255524 & -2.401625 & 1.479019 \\
\hline 1 & 4.948318 & -1.403796 & 2.670984 \\
\hline 1 & 1.025144 & 0.313263 & 2.887145 \\
\hline 1 & 5.830971 & 0.420156 & 4.084982 \\
\hline
\end{tabular}




$\begin{array}{rrrr}1 & 1.887527 & 2.119795 & 4.335377 \\ 1 & 4.305477 & 2.194160 & 4.931780 \\ 1 & 1.728901 & 5.190103 & 2.837586 \\ 1 & 0.383129 & 4.050732 & 3.040317 \\ 1 & 1.621674 & 3.768544 & 1.799317 \\ 1 & 0.317067 & 7.095348 & 2.641945 \\ 1 & -1.001504 & 7.215858 & 1.473966 \\ 1 & -1.150419 & 6.120522 & 2.860229 \\ 1 & 1.941473 & 6.567030 & 0.750277 \\ 1 & 1.622556 & 5.188431 & -0.329667 \\ 1 & 0.614923 & 6.639194 & -0.428365 \\ 1 & -4.623965 & 7.073378 & -2.294838 \\ 1 & -3.942141 & 7.063251 & -0.661947 \\ 1 & -2.870270 & 6.997654 & -2.081214 \\ 1 & -4.532619 & 5.111358 & -3.768262 \\ 1 & -2.784545 & 4.898815 & -3.520951 \\ 1 & -3.894437 & 3.554193 & -3.210604 \\ 1 & -6.098715 & 5.193138 & -1.771382 \\ 1 & -5.473326 & 3.650837 & -1.164368 \\ 1 & -5.454851 & 5.084337 & -0.119608 \\ 1 & 1.771441 & -0.604392 & -3.578603 \\ 1 & 0.671274 & 0.598405 & -4.400597 \\ 1 & -2.307733 & -2.975363 & -3.362899 \\ 1 & -2.108280 & -5.409188 & -3.230913 \\ 1 & 0.141791 & -6.492034 & -3.248731 \\ 1 & 2.174073 & -5.055065 & -3.338909 \\ 1 & 1.975933 & -2.616428 & -3.422426 \\ 1 & -1.705699 & -1.398054 & -5.275087 \\ 1 & -2.520563 & -0.837063 & -3.808584 \\ 1 & -1.539138 & 0.287872 & -4.731628 \\ 6 & -7.149776 & -2.656653 & -2.184167 \\ 6 & -5.344281 & -1.816579 & -3.641193 \\ 6 & -4.899013 & -3.659724 & -2.003106 \\ 6 & -8.155631 & 0.195966 & 1.546914 \\ 6 & -6.252124 & 0.359581 & 3.123893 \\ 6 & -6.787717 & 2.260644 & 1.581389 \\ 1 & -8.812211 & 0.638990 & 2.306381 \\ 1 & -8.574576 & 0.446958 & 0.563914 \\ 1 & -8.199058 & -0.893793 & 1.671754 \\ 1 & -7.457544 & 2.689861 & 2.338743 \\ 1 & -5.800705 & 2.722921 & 1.709609 \\ 1 & -7.166514 & 2.552551 & 0.592873 \\ 1 & -6.930330 & 0.767716 & 3.885505 \\ 1 & -6.222964 & -0.731445 & 3.247652 \\ 1 & -5.245293 & 0.743052 & 3.333374 \\ 1 & -7.393155 & -3.409213 & -2.944856 \\ 1 & -7.452190 & -3.066750 & -1.212081 \\ 1 & -7.762855 & -1.769860 & -2.391630 \\ 1 & -5.695667 & -2.526318 & -4.402065 \\ 1 & -5.842056 & -0.854531 & -3.819388 \\ 1 & -4.270565 & -1.671285 & -3.801849 \\ 1 & -5.091312 & -4.364949 & -2.824356 \\ 1 & -3.814824 & -3.512104 & -1.927342 \\ & -5.224542 & -4.130321 & -1.067365\end{array}$

$3_{\text {IC-re-- }} \psi_{-1 \text { s2a3a4a }}$ 
Number of imaginary frequencies : 0

The smallest frequencies are : $16.5314 \quad 20.2752 \quad 23.7539 \mathrm{~cm}(-1)$

Electronic energy : $\quad \mathrm{HF}=-4360.8778683$

Zero-point correction $=\quad 1.459806($ Hartree/Particle $)$

Thermal correction to Energy= $\quad 1.542641$

Thermal correction to Enthalpy= $\quad 1.543585$

Thermal correction to Gibbs Free Energy= 1.347323

Sum of electronic and zero-point Energies $=\quad-4359.418063$

Sum of electronic and thermal Energies $=\quad-4359.335227$

Sum of electronic and thermal Enthalpies $=\quad-4359.334283$

Sum of electronic and thermal Free Energies $=\quad-4359.530545$

Cartesian Coordinates

$\begin{array}{ccccc}6 & 4.217156 & -0.237281 & 3.115242 \\ 6 & 2.874868 & -0.381551 & 2.769967 \\ 6 & 1.970149 & 0.607676 & 3.158715 \\ 6 & 2.398499 & 1.715554 & 3.877103 \\ 6 & 3.743068 & 1.854223 & 4.218970 \\ 6 & 4.650995 & 0.873866 & 3.834844 \\ 6 & 2.428696 & -1.609861 & 2.019220 \\ 7 & 1.460961 & -1.384614 & 0.916992 \\ 15 & 1.760733 & -0.495200 & -0.493395 \\ 8 & 2.685653 & 0.657694 & 0.247547 \\ 6 & 3.635713 & 1.368971 & -0.438710 \\ 6 & & 4.835214 & 0.778513 & -0.790895 \\ 6 & 5.775622 & 1.561018 & -1.539296 \\ 6 & 5.496255 & 2.937755 & -1.797369 \\ 6 & 4.281058 & 3.500727 & -1.338941 \\ 6 & 3.361215 & 2.727359 & -0.686868 \\ 6 & 6.970926 & 1.016258 & -2.074916 \\ 6 & 7.850026 & 1.793297 & -2.787921 \\ 6 & 7.587239 & 3.161296 & -3.007741 \\ 6 & 6.429765 & 3.715228 & -2.524765 \\ 6 & 5.087418 & -0.638594 & -0.443533 \\ 6 & 4.167721 & -1.604428 & -0.808773 \\ 6 & 4.424768 & -2.984383 & -0.661382 \\ 6 & 5.566438 & -3.399584 & -0.032332 \\ 6 & 6.474978 & -2.453242 & 0.502867 \\ 6 & 6.239794 & -1.060248 & 0.296977 \\ 6 & 7.596120 & -2.865855 & 1.263461 \\ 6 & 8.441412 & -1.946502 & 1.830100 \\ 6 & 8.193937 & -0.568461 & 1.656395 \\ 6 & 7.128010 & -0.137059 & 0.905940 \\ 8 & 2.968001 & -1.249437 & -1.377483 \\ 6 & 0.427096 & -2.376244 & 0.800059 \\ 6 & -0.828160 & -2.153891 & 1.337025 \\ 6 & -1.838493 & -3.157166 & 1.199699 \\ 6 & -1.513360 & -4.398584 & 0.579315 \\ 6 & -0.196877 & -4.608724 & 0.109895 \\ 6 & 0.737057 & -3.612251 & 0.186749 \\ 6 & -3.160515 & -2.969343 & 1.679129 \\ 6 & -4.108336 & -3.952355 & 1.538659 \\ 6 & -3.782236 & -5.182143 & 0.925290 \\ 6 & -2.510489 & -5.397701 & 0.457014\end{array}$




\begin{tabular}{|c|c|c|c|}
\hline 5 & -1.097774 & -0.991386 & 2.225870 \\
\hline 6 & -1.511978 & 0.248325 & 1.766072 \\
\hline & -1.848406 & 1.254079 & 2.715903 \\
\hline & -1.728837 & 1.042588 & 4.062409 \\
\hline & -1.267024 & -0.196275 & 4.560992 \\
\hline & -0.962319 & -1.234235 & 3.634572 \\
\hline f & -1.100036 & -0.420458 & 5.947950 \\
\hline 5 & -0.646709 & -1.627872 & 6.414017 \\
\hline 6 & -0.348039 & -2.663265 & 5.504190 \\
\hline 6 & -0.506229 & -2.475192 & 4.153110 \\
\hline 15 & -1.734036 & 0.538465 & -0.064801 \\
\hline 6 & -3.465103 & -0.096728 & -0.210035 \\
\hline 6 & -3.793457 & -1.129643 & -1.083864 \\
\hline 6 & -5.103160 & -1.635218 & -1.132252 \\
\hline 6 & -6.050297 & -1.065090 & -0.282679 \\
\hline 6 & -5.750152 & -0.035031 & 0.616331 \\
\hline 6 & -4.443633 & 0.437401 & 0.635869 \\
\hline 6 & -5.532773 & -2.731292 & -2.109702 \\
\hline 6 & -6.212040 & -2.060148 & -3.309590 \\
\hline 6 & -6.828365 & 0.496366 & 1.557651 \\
\hline 6 & -8.032949 & 0.992958 & 0.751860 \\
\hline 45 & -0.041705 & 0.259592 & -1.819903 \\
\hline 6 & 0.797441 & -0.282031 & -3.725623 \\
\hline 6 & -0.158367 & -1.308948 & -3.527258 \\
\hline 6 & -1.464667 & -1.182412 & -4.291848 \\
\hline 6 & 0.262853 & -2.716327 & -3.275653 \\
\hline 6 & 1.600516 & -3.129073 & -3.358781 \\
\hline 6 & 1.952937 & -4.470631 & -3.266374 \\
\hline 6 & 0.978111 & -5.452842 & -3.106687 \\
\hline 6 & -0.357658 & -5.067068 & -3.045509 \\
\hline 6 & -0.699985 & -3.722239 & -3.110177 \\
\hline 6 & 0.468319 & 2.128973 & -2.018392 \\
\hline 8 & 0.569261 & 3.163118 & -2.527602 \\
\hline 6 & -1.993488 & 2.364027 & -0.131040 \\
\hline 6 & -1.091476 & 3.222576 & 0.493401 \\
\hline 6 & -1.234334 & 4.612456 & 0.408571 \\
\hline 6 & -2.265527 & 5.111598 & -0.385506 \\
\hline 6 & -3.154554 & 4.276769 & -1.075514 \\
\hline 6 & -3.011554 & 2.899316 & -0.924047 \\
\hline 6 & -0.306140 & 5.513566 & 1.221816 \\
\hline 6 & 1.157604 & 5.184900 & 0.915447 \\
\hline 6 & -4.249867 & 4.893719 & -1.943562 \\
\hline 6 & -3.604863 & 5.735296 & -3.049952 \\
\hline 6 & -0.531529 & 6.995302 & 0.931314 \\
\hline 6 & -0.573403 & 5.268307 & 2.712304 \\
\hline 6 & -5.130473 & 3.834437 & -2.602474 \\
\hline 6 & -5.148281 & 5.786328 & -1.080208 \\
\hline 6 & -6.323400 & 1.653969 & 2.416484 \\
\hline 6 & -7.267377 & -0.636986 & 2.492038 \\
\hline 6 & -4.346204 & -3.545112 & -2.619539 \\
\hline 6 & -6.515961 & -3.709335 & -1.458384 \\
\hline 1 & 6.935952 & 0.927864 & 0.799392 \\
\hline 1 & -2.211929 & 2.218381 & 2.372293 \\
\hline 1 & 7.761924 & -3.933642 & 1.401128 \\
\hline 1 & -1.336150 & 0.390326 & 6.635773 \\
\hline 1 & 6.198515 & 4.764876 & -2.701558 \\
\hline
\end{tabular}




\begin{tabular}{|c|c|c|c|}
\hline & -3.428031 & -2.022138 & 2.145075 \\
\hline & -5.121124 & -3.778117 & 1.898517 \\
\hline & 4.081367 & 4.554280 & -1.530003 \\
\hline & -1.989370 & 1.832081 & 4.766852 \\
\hline & 5.777829 & -4.460560 & 0.090709 \\
\hline & 8.755158 & 1.346232 & -3.193074 \\
\hline & 3.698665 & -3.686693 & -1.067446 \\
\hline & -2.243227 & -6.340981 & -0.018394 \\
\hline & 0.055706 & -5.564911 & -0.343244 \\
\hline & 0.008746 & -3.620869 & 5.876423 \\
\hline & 7.185018 & -0.038978 & -1.927405 \\
\hline & -0.275205 & -3.288336 & 3.467629 \\
\hline & 1.741242 & -3.759627 & -0.205135 \\
\hline & 8.294273 & 3.767038 & -3.569769 \\
\hline & 8.850512 & 0.160394 & 2.126431 \\
\hline & -4.542154 & -5.954479 & 0.823834 \\
\hline & -0.516585 & -1.790909 & 7.481337 \\
\hline & 9.292938 & -2.274538 & 2.421646 \\
\hline & 2.413629 & 3.128602 & -0.339062 \\
\hline & -4.169715 & 1.236430 & 1.318853 \\
\hline & -3.003958 & -1.550849 & -1.706633 \\
\hline & -7.071516 & -1.441875 & -0.311143 \\
\hline & -3.692055 & 2.220674 & -1.432142 \\
\hline & -0.269880 & 2.800301 & 1.076625 \\
\hline & -2.388744 & 6.188295 & -0.479374 \\
\hline & 1.911653 & -2.280633 & 2.720059 \\
\hline & -1.285572 & 0.801693 & -2.647129 \\
\hline & 3.310592 & -2.162423 & 1.661075 \\
\hline & 4.938291 & -0.994323 & 2.802148 \\
\hline & 0.929308 & 0.521777 & 2.855250 \\
\hline & 5.705837 & 0.973677 & 4.084600 \\
\hline & 1.675992 & 2.477296 & 4.168938 \\
\hline & 4.080206 & 2.725500 & 4.776613 \\
\hline & 1.863474 & -0.474295 & -3.613724 \\
\hline & 0.569225 & 0.506543 & -4.443683 \\
\hline & -1.747756 & -3.444815 & -3.040676 \\
\hline & -1.144373 & -5.812435 & -2.928970 \\
\hline & 1.255072 & -6.504296 & -3.059870 \\
\hline & 3.002133 & -4.752042 & -3.350736 \\
\hline & 2.385782 & -2.395436 & -3.515417 \\
\hline 1 & -1.473542 & -1.871578 & -5.150935 \\
\hline 1 & -2.355414 & -1.403024 & -3.692698 \\
\hline 1 & -1.589670 & -0.162149 & -4.670934 \\
\hline 1 & -7.131740 & 2.010469 & 3.067235 \\
\hline 1 & -5.488742 & 1.353643 & 3.064181 \\
\hline 1 & -5.992797 & 2.503521 & 1.803502 \\
\hline & -8.809850 & 1.375044 & 1.427554 \\
\hline 1 & -7.745079 & 1.807544 & 0.074434 \\
\hline 1 & -8.486155 & 0.199002 & 0.146622 \\
\hline 1 & -8.040578 & -0.284417 & 3.188092 \\
\hline 1 & -7.680635 & -1.489234 & 1.938233 \\
\hline 1 & -6.418655 & -1.002733 & 3.086034 \\
\hline 1 & -4.705288 & -4.377599 & -3.238117 \\
\hline 1 & -3.687277 & -2.942323 & -3.254563 \\
\hline 1 & -3.752645 & -3.966817 & -1.795251 \\
\hline 1 & -6.757883 & -4.514233 & -2.164436 \\
\hline
\end{tabular}




$\begin{array}{lrrr}1 & -6.080384 & -4.167664 & -0.560355 \\ 1 & -7.465194 & -3.239283 & -1.176841 \\ 1 & -6.528726 & -2.812141 & -4.045496 \\ 1 & -7.099058 & -1.491255 & -3.002545 \\ 1 & -5.524626 & -1.364214 & -3.809533 \\ 1 & -4.377498 & 6.176374 & -3.694284 \\ 1 & -3.002087 & 6.556882 & -2.644600 \\ 1 & -2.948647 & 5.119040 & -3.678087 \\ 1 & -5.907363 & 4.323485 & -3.203632 \\ 1 & -4.556376 & 3.182487 & -3.273228 \\ 1 & -5.636455 & 3.200901 & -1.861112 \\ 1 & -5.939781 & 6.236907 & -1.694134 \\ 1 & -5.629584 & 5.205409 & -0.282275 \\ 1 & -4.590770 & 6.604403 & -0.608702 \\ 1 & 0.162219 & 7.597647 & 1.531203 \\ 1 & -0.351832 & 7.236349 & -0.124413 \\ 1 & -1.548428 & 7.316860 & 1.189954 \\ 1 & 1.823303 & 5.873158 & 1.453240 \\ 1 & 1.425299 & 4.169144 & 1.232002 \\ 1 & 1.366255 & 5.281282 & -0.159221 \\ 1 & 0.076827 & 5.903260 & 3.329635 \\ 1 & -1.615952 & 5.500628 & 2.967900 \\ 1 & -0.383483 & 4.223575 & 2.992594\end{array}$

$3_{\text {IC-re }-} \psi_{-1 \mathrm{~s} 2 \mathrm{a} 3 \mathrm{a} 4 \mathrm{~s}}$

Number of imaginary frequencies : 0

The smallest frequencies are : $16.661520 .6737 \quad 21.6418 \mathrm{~cm}(-1)$

Electronic energy : $\quad \mathrm{HF}=-4360.8785183$

Zero-point correction $=\quad 1.459674$ (Hartree/Particle)

Thermal correction to Energy= $\quad 1.542579$

Thermal correction to Enthalpy= $\quad 1.543523$

Thermal correction to Gibbs Free Energy $=\quad 1.346653$

Sum of electronic and zero-point Energies $=\quad-4359.418844$

Sum of electronic and thermal Energies $=\quad-4359.335940$

Sum of electronic and thermal Enthalpies $=\quad-4359.334996$

Sum of electronic and thermal Free Energies $=\quad-4359.531865$

\section{Cartesian Coordinates}

$\begin{array}{cccc}6 & 1.620903 & -3.134619 & -3.341849 \\ 6 & 0.281172 & -2.727579 & -3.262260 \\ 6 & -0.677969 & -3.736359 & -3.093742 \\ 6 & -0.331641 & -5.079714 & -3.023902 \\ 6 & 1.005477 & -5.460455 & -3.084089 \\ 6 & 1.977033 & -4.475270 & -3.246666 \\ 6 & -0.147419 & -1.323755 & -3.519189 \\ 6 & -1.456635 & -1.206092 & -4.280394 \\ 45 & -0.036384 & 0.243540 & -1.815277 \\ 6 & 0.459783 & 2.116413 & -2.022073 \\ 8 & 0.548131 & 3.146297 & -2.542263 \\ 15 & -1.729825 & 0.509586 & -0.062647 \\ 6 & -2.026569 & 2.329793 & -0.144654 \\ 6 & -1.131968 & 3.211950 & 0.457271 \\ 6 & -1.304329 & 4.597934 & 0.363442 \\ 6 & -2.359948 & 5.069487 & -0.415778\end{array}$




\begin{tabular}{|c|c|c|c|}
\hline 6 & -3.245089 & 4.210955 & -1.081135 \\
\hline 5 & -3.070006 & 2.838085 & -0.922023 \\
\hline p & -0.384596 & 5.524682 & 1.157712 \\
\hline & -4.373802 & 4.795509 & -1.928742 \\
\hline f & -3.450795 & -0.154200 & -0.204883 \\
\hline & -3.773199 & -1.199356 & -1.073173 \\
\hline 6 & -5.080242 & -1.699160 & -1.130422 \\
\hline 6 & -6.045670 & -1.111502 & -0.304154 \\
\hline 6 & -5.751211 & -0.078156 & 0.584453 \\
\hline 6 & -4.436451 & 0.383359 & 0.620144 \\
\hline 6 & -6.777972 & 0.539576 & 1.530063 \\
\hline 6 & -5.504229 & -2.811203 & -2.092138 \\
\hline 6 & -1.502671 & 0.241829 & 1.771252 \\
\hline 6 & -1.075529 & -0.988682 & 2.243987 \\
\hline 6 & -0.936024 & -1.215447 & 3.654629 \\
\hline 6 & -1.249348 & -0.170664 & 4.570455 \\
\hline 6 & -1.722032 & 1.058815 & 4.059321 \\
\hline 6 & -1.845400 & 1.255045 & 2.710598 \\
\hline 6 & -1.080121 & -0.379271 & 5.959580 \\
\hline 6 & -0.616702 & -1.578042 & 6.437693 \\
\hline 6 & -0.309740 & -2.620278 & 5.538352 \\
\hline 6 & -0.469777 & -2.447340 & 4.185492 \\
\hline 6 & -0.802171 & -2.157239 & 1.364845 \\
\hline 6 & 0.450177 & -2.376245 & 0.819399 \\
\hline 6 & 0.758822 & -3.612413 & 0.205023 \\
\hline 6 & -0.173934 & -4.610632 & 0.134800 \\
\hline 6 & -1.486738 & -4.404543 & 0.616161 \\
\hline 6 & -1.809836 & -3.164141 & \\
\hline 6 & -3.127364 & -2.979459 & 1.732431 \\
\hline 6 & -4.072948 & -3.965811 & 1.603209 \\
\hline 6 & -3.749247 & -5.194919 & 0.986861 \\
\hline 6 & -2.482122 & -5.406693 & 0.504429 \\
\hline 7 & 1.479703 & -1.378792 & 0.926318 \\
\hline 15 & 1.772569 & -0.496760 & -0.490050 \\
\hline 8 & 2.984965 & -1.248850 & -1.368623 \\
\hline 6 & 4.187433 & -1.588080 & -0.795917 \\
\hline 6 & 5.100523 & -0.610873 & -0.444570 \\
\hline 6 & 6.258246 & -1.014569 & 0.297732 \\
\hline 6 & 6.504033 & -2.403080 & 0.520897 \\
\hline 6 & 5.599997 & -3.362746 & 0.002313 \\
\hline 6 & 4.453797 & -2.964003 & -0.629131 \\
\hline 6 & 7.141897 & -0.077506 & 0.891980 \\
\hline 6 & 8.213504 & -0.491794 & 1.644008 \\
\hline 6 & 8.471541 & -1.865680 & 1.834337 \\
\hline 6 & 7.630735 & -2.798080 & 1.282648 \\
\hline 6 & 4.834731 & 0.800812 & -0.803922 \\
\hline 6 & 3.631820 & 1.383689 & -0.450565 \\
\hline 6 & 3.346462 & 2.739113 & -0.702765 \\
\hline 6 & 4.257431 & 3.516056 & -1.363034 \\
\hline 6 & 5.474271 & 2.959481 & -1.825169 \\
\hline 6 & 5.765521 & 1.586391 & -1.560789 \\
\hline 6 & 6.398198 & 3.739701 & -2.561815 \\
\hline 6 & 7.557473 & 3.191735 & -3.047347 \\
\hline 6 & 7.832133 & 1.827198 & -2.820539 \\
\hline 6 & 6.962544 & 1.047683 & -2.098642 \\
\hline 8 & 2.689445 & 0.668320 & 0.241387 \\
\hline
\end{tabular}




\begin{tabular}{|c|c|c|c|}
\hline 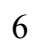 & 0.803031 & -0.292824 & -3.722277 \\
\hline 6 & 2.442904 & -1.581105 & 2.037192 \\
\hline 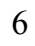 & 2.882703 & -0.337030 & 2.765689 \\
\hline & 1.971510 & 0.652897 & 3.137276 \\
\hline & 2.393412 & 1.780021 & 3.828794 \\
\hline & 3.738230 & 1.937116 & 4.161763 \\
\hline & 4.652345 & 0.955486 & 3.796178 \\
\hline & 4.224919 & -0.174907 & 3.102924 \\
\hline & 6.942122 & 0.984608 & 0.772586 \\
\hline 1 & -2.215730 & 2.213005 & 2.356983 \\
\hline & 7.804375 & -3.862836 & 1.433465 \\
\hline & -1.322999 & 0.436397 & 6.639211 \\
\hline & 6.158109 & 4.786501 & -2.743645 \\
\hline 1 & -3.391787 & -2.032557 & 2.201453 \\
\hline 1 & -5.082381 & -3.794647 & 1.973512 \\
\hline & 4.049194 & 4.567301 & -1.557727 \\
\hline 1 & -1.987748 & 1.853671 & 4.755734 \\
\hline 1 & 5.818480 & -4.420461 & 0.140114 \\
\hline 1 & 8.738776 & 1.384804 & -3.227437 \\
\hline 1 & 3.731031 & -3.677158 & -1.021761 \\
\hline 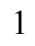 & -2.217106 & -6.349153 & 0.026048 \\
\hline 1 & 0.077292 & -5.565951 & -0.320922 \\
\hline 1 & 0.054718 & -3.571129 & 5.920319 \\
\hline 1 & 7.185304 & -0.005027 & -1.945758 \\
\hline 1 & -0.233118 & -3.265535 & 3.507973 \\
\hline 1 & 1.759908 & -3.757284 & -0.195812 \\
\hline 1 & 8.256937 & 3.799519 & -3.616596 \\
\hline 1 & 8.866509 & 0.247648 & 2.102342 \\
\hline 1 & -4.507354 & -5.970234 & 0.894905 \\
\hline 1 & -0.485079 & -1.729168 & 7.506575 \\
\hline 1 & 9.327492 & -2.180050 & 2.426913 \\
\hline 1 & 2.397624 & 3.134377 & -0.351334 \\
\hline 1 & -4.174697 & 1.184753 & 1.309003 \\
\hline 1 & -2.978755 & -1.627412 & -1.684693 \\
\hline 1 & -7.066501 & -1.481513 & -0.355735 \\
\hline 1 & -3.747351 & 2.142221 & -1.410105 \\
\hline 1 & -0.293306 & 2.811150 & 1.031203 \\
\hline 1 & -2.507656 & 6.142619 & -0.515530 \\
\hline 1 & 1.923944 & -2.239483 & 2.748294 \\
\hline 1 & -1.285188 & 0.777035 & -2.640137 \\
\hline 1 & 3.327677 & -2.138125 & 1.693159 \\
\hline 1 & 4.951080 & -0.932481 & 2.802980 \\
\hline 1 & 0.930385 & 0.552415 & 2.839704 \\
\hline 1 & 5.707147 & 1.069189 & 4.040064 \\
\hline 1 & 1.665587 & 2.541929 & 4.106819 \\
\hline 1 & 4.070714 & 2.823294 & 4.698317 \\
\hline 1 & 1.870303 & -0.479513 & -3.612534 \\
\hline 1 & 0.569093 & 0.493600 & -4.440840 \\
\hline 1 & -1.726469 & -3.462112 & -3.026954 \\
\hline 1 & -1.115915 & -5.827249 & -2.904056 \\
\hline 1 & 1.286606 & -6.510670 & -3.034103 \\
\hline 1 & 3.027117 & -4.753753 & -3.329557 \\
\hline 1 & 2.403729 & -2.398727 & -3.499590 \\
\hline 1 & -1.465465 & -1.899274 & -5.136238 \\
\hline 1 & -2.344566 & -1.426954 & -3.676994 \\
\hline 1 & -1.586546 & -0.188103 & -4.663935 \\
\hline
\end{tabular}




$\begin{array}{lrrr}6 & -4.312319 & -3.611875 & -2.608784 \\ 6 & -6.461144 & -3.798083 & -1.415203 \\ 6 & -6.210575 & -2.165524 & -3.290238 \\ 6 & -8.170637 & -0.058663 & 1.351869 \\ 6 & -6.329589 & 0.287215 & 2.974971 \\ 6 & -6.869868 & 2.048468 & 1.274353 \\ 1 & -8.870066 & 0.420482 & 2.048566 \\ 1 & -8.557856 & 0.098664 & 0.336826 \\ 1 & -8.184692 & -1.135932 & 1.562364 \\ 1 & -7.578257 & 2.512371 & 1.973904 \\ 1 & -5.902094 & 2.550670 & 1.400037 \\ 1 & -7.223215 & 2.250867 & 0.254384 \\ 1 & -7.049300 & 0.720999 & 3.682378 \\ 1 & -6.262455 & -0.790337 & 3.178272 \\ 1 & -5.346053 & 0.725697 & 3.187621 \\ 1 & -4.666167 & -4.453051 & -3.218593 \\ 1 & -3.667367 & -3.003936 & -3.253318 \\ 1 & -3.706091 & -4.020562 & -1.787002 \\ 1 & -6.708879 & -4.609498 & -2.111855 \\ 1 & -5.998741 & -4.246110 & -0.525700 \\ 1 & -7.408655 & -3.337951 & -1.112755 \\ 1 & -6.525054 & -2.931553 & -4.012463 \\ 1 & -7.102773 & -1.607114 & -2.979246 \\ 1 & -5.540822 & -1.465299 & -3.807794 \\ 6 & -5.234934 & 3.709971 & -2.571050 \\ 6 & -5.281192 & 5.661911 & -1.048172 \\ 6 & -3.776683 & 5.655692 & -3.047532 \\ 6 & -0.634618 & 6.998527 & 0.847643 \\ 6 & -0.640858 & 5.296024 & 2.652894 \\ 6 & 1.083231 & 5.215648 & 0.850303 \\ 1 & -4.575413 & 6.072012 & -3.676326 \\ 1 & -3.192113 & 6.496314 & -2.654758 \\ 1 & -3.114580 & 5.059873 & -3.688889 \\ 1 & -6.039524 & 4.175403 & -3.154283 \\ 1 & -4.654680 & 3.077726 & -3.255333 \\ 1 & -5.704448 & 3.058399 & -1.820885 \\ 1 & -6.092607 & 6.095319 & -1.648359 \\ 1 & -5.736628 & 5.066123 & -0.245956 \\ 1 & -4.736433 & 6.491290 & -0.581332 \\ 1 & 0.056634 & 7.619763 & 1.430902 \\ 1 & -0.469872 & 7.225965 & -0.213520 \\ 1 & -1.653070 & 7.309638 & 1.112724 \\ 1 & 1.738729 & 5.918467 & 1.381737 \\ 1 & 1.367694 & 4.206837 & 1.174553 \\ 1 & 1.289033 & 5.307854 & -0.225094 \\ 1 & -0.000066 & 5.951810 & 3.258207 \\ 1 & -1.686500 & 5.512274 & 2.909956 \\ 1 & -0.429771 & 4.259109 & 2.946917\end{array}$

$3_{\text {IC-re- }} \psi_{-1 \text { s2a3s4a }}$

Number of imaginary frequencies : 0

The smallest frequencies are : $14.7823 \quad 18.2003 \quad 20.3743 \mathrm{~cm}(-1)$ Electronic energy :

Zero-point correction $=$ $\mathrm{HF}=-4360.8780256$

Thermal correction to Energy=

1.455737 (Hartree/Particle) 1.540033 
$\begin{array}{lc}\text { Thermal correction to Enthalpy }= & 1.540977 \\ \text { Thermal correction to Gibbs Free Energy= } & 1.339565 \\ \text { Sum of electronic and zero-point Energies }= & -4359.422289 \\ \text { Sum of electronic and thermal Energies }= & -4359.337993 \\ \text { Sum of electronic and thermal Enthalpies }= & -4359.337049 \\ \text { Sum of electronic and thermal Free Energies }= & -4359.538461\end{array}$

Cartesian Coordinates

$\begin{array}{cccc}6 & 1.326606 & -3.252182 & -3.249091 \\ 6 & 0.048318 & -2.669392 & -3.275391 \\ 6 & -1.047565 & -3.539619 & -3.157369 \\ 6 & -0.884621 & -4.912562 & -3.023348 \\ 6 & 0.389995 & -5.471281 & -3.017250 \\ 6 & 1.492581 & -4.627115 & -3.125214 \\ 6 & -0.176899 & -1.227356 & -3.562443 \\ 6 & -1.423621 & -0.944919 & -4.380854 \\ 45 & 0.011780 & 0.290138 & -1.820595 \\ 6 & 0.567127 & 2.145380 & -2.008931 \\ 8 & 0.674679 & 3.179197 & -2.518172 \\ 15 & -1.704711 & 0.545083 & -0.084973 \\ 6 & -1.995873 & 2.366909 & -0.145684 \\ 6 & -1.085465 & 3.237888 & 0.449095 \\ 6 & -1.265685 & 4.624577 & 0.394677 \\ 6 & -2.351837 & 5.110159 & -0.333113 \\ 6 & -3.258232 & 4.264325 & -0.985174 \\ 6 & -3.066581 & 2.888683 & -0.874224 \\ 6 & -0.330748 & 5.536339 & 1.188795 \\ 6 & -4.434117 & 4.861520 & -1.756070 \\ 6 & -3.428054 & -0.111879 & -0.241689 \\ 6 & -3.759472 & -1.101764 & -1.157625 \\ 6 & -5.073760 & -1.604062 & -1.232140 \\ 6 & -6.020525 & -1.075851 & -0.360596 \\ 6 & -5.718080 & -0.075827 & 0.577239 \\ 6 & -4.412283 & 0.388881 & 0.622696 \\ 6 & -6.797597 & 0.433316 & 1.529428 \\ 6 & -5.424048 & -2.704897 & -2.234575 \\ 6 & -1.486760 & 0.263419 & 1.750135 \\ 6 & -1.076532 & -0.971943 & 2.226447 \\ 6 & -0.934197 & -1.193706 & 3.638860 \\ 6 & -1.234082 & -0.142520 & 4.551833 \\ 6 & -1.695741 & 1.089410 & 4.037335 \\ 6 & -1.817119 & 1.282366 & 2.688346 \\ 6 & -1.061045 & -0.345696 & 5.941356 \\ 6 & -0.604081 & -1.545120 & 6.423662 \\ 6 & -0.307631 & -2.593112 & 5.527644 \\ 6 & -0.473099 & -2.425763 & 4.174754 \\ 6 & -0.806746 & -2.150242 & 1.357939 \\ 6 & 0.447375 & -2.373566 & 0.819662 \\ 6 & 0.764561 & -3.618935 & 0.230067 \\ 6 & -0.153457 & -4.632748 & 0.192349 \\ 6 & -1.458222 & -4.437723 & 0.699734 \\ 6 & -1.799444 & -3.177574 & 1.271948 \\ 6 & -3.112465 & -3.003487 & 1.781363 \\ 6 & -4.023026 & -4.030193 & 1.749365 \\ 6 & -3.671618 & -5.285731 & 1.207051\end{array}$




\begin{tabular}{|c|c|c|c|}
\hline & -2.420117 & -5.477420 & 0.680111 \\
\hline & 1.481406 & -1.381378 & 0.927004 \\
\hline & 1.790935 & -0.509468 & -0.490627 \\
\hline & 2.981746 & -1.296219 & -1.374847 \\
\hline & 4.190757 & -1.641491 & -0.819169 \\
\hline & 5.119477 & -0.672210 & -0.486957 \\
\hline & 6.284816 & -1.084866 & 0.239198 \\
\hline & 6.522496 & -2.474753 & 0.461948 \\
\hline & 5.601710 & -3.427370 & -0.039418 \\
\hline 6 & 4.449231 & -3.019103 & -0.652688 \\
\hline & 7.185355 & -0.154333 & 0.818367 \\
\hline & 8.265205 & -0.576342 & 1.554140 \\
\hline O & 8.515514 & -1.951890 & 1.742059 \\
\hline 6 & 7.658127 & -2.878075 & 1.205729 \\
\hline 6 & 4.865195 & 0.743504 & -0.838793 \\
\hline 6 & 3.679378 & 1.341731 & -0.455919 \\
\hline 6 & 3.415379 & 2.706777 & -0.676920 \\
\hline 6 & 4.326791 & 3.476917 & -1.344322 \\
\hline 6 & 5.522242 & 2.904845 & -1.842230 \\
\hline 6 & 5.794532 & 1.523127 & -1.603305 \\
\hline 6 & 6.444824 & 3.679109 & -2.586735 \\
\hline 6 & 7.584290 & 3.117841 & -3.103011 \\
\hline 6 & 7.839580 & 1.745733 & -2.900423 \\
\hline 6 & 6.971160 & 0.971301 & -2.171644 \\
\hline 8 & 2.737521 & 0.630229 & 0.239822 \\
\hline 6 & 0.892691 & -0.312443 & -3.700956 \\
\hline 6 & 2.447105 & -1.599959 & 2.033392 \\
\hline 6 & 2.910007 & -0.360494 & 2.754245 \\
\hline 6 & 2.011907 & 0.636693 & 3.137964 \\
\hline 6 & 2.453799 & 1.764322 & 3.815923 \\
\hline 6 & 3.805831 & 1.913916 & 4.122636 \\
\hline 6 & 4.706741 & 0.924397 & 3.745123 \\
\hline 6 & 4.259225 & -0.206515 & 3.065736 \\
\hline 1 & 6.992854 & 0.909266 & 0.700856 \\
\hline 1 & -2.179974 & 2.242634 & 2.333685 \\
\hline 1 & 7.825333 & -3.943997 & 1.355467 \\
\hline 1 & -1.294859 & 0.475384 & 6.617650 \\
\hline 1 & 6.219888 & 4.732430 & -2.749138 \\
\hline 1 & -3.397963 & -2.036805 & 2.194260 \\
\hline 1 & -5.027023 & -3.873022 & 2.139041 \\
\hline 1 & 4.134848 & 4.535192 & -1.516428 \\
\hline 1 & -1.953752 & 1.889256 & 4.730941 \\
\hline 1 & 5.813035 & -4.486772 & 0.096260 \\
\hline 1 & 8.730206 & 1.293718 & -3.331392 \\
\hline 1 & 3.715610 & -3.728408 & -1.031057 \\
\hline 1 & -2.138382 & -6.434111 & 0.241064 \\
\hline 1 & 0.106912 & -5.597850 & -0.237650 \\
\hline 1 & 0.053229 & -3.544381 & 5.911983 \\
\hline 1 & 7.178325 & -0.087162 & -2.037064 \\
\hline 1 & -0.243317 & -3.249425 & 3.501691 \\
\hline 1 & 1.767440 & -3.761138 & -0.166352 \\
\hline 1 & 8.282809 & 3.721346 & -3.677938 \\
\hline 1 & 8.931192 & 0.158489 & 2.001029 \\
\hline 1 & -4.401467 & -6.092564 & 1.194054 \\
\hline 1 & -0.468721 & -1.692040 & 7.492653 \\
\hline 1 & 9.378481 & -2.272375 & 2.321001 \\
\hline
\end{tabular}




\begin{tabular}{|c|c|c|c|}
\hline & 2.483383 & 3.114781 & -0.296029 \\
\hline & -4.140176 & 1.161440 & 1.335789 \\
\hline & -2.976127 & -1.496744 & -1.807103 \\
\hline & -7.040017 & -1.452627 & -0.398180 \\
\hline & -3.756764 & 2.201867 & -1.356601 \\
\hline & -0.232548 & 2.825402 & 0.993255 \\
\hline & -2.509288 & 6.184709 & -0.396558 \\
\hline & 1.920264 & -2.247812 & 2.748258 \\
\hline & -1.198758 & 0.893322 & -2.650757 \\
\hline 1 & 3.321073 & -2.172161 & 1.685899 \\
\hline 1 & 4.974464 & -0.970352 & 2.755635 \\
\hline 1 & 0.965035 & 0.541477 & 2.859009 \\
\hline 1 & 5.766667 & 1.032381 & 3.968558 \\
\hline 1 & 1.735932 & 2.531917 & 4.103998 \\
\hline 1 & 4.154182 & 2.800498 & 4.648316 \\
\hline 1 & 1.924355 & -0.616894 & -3.532626 \\
\hline 1 & 0.786037 & 0.502810 & -4.417234 \\
\hline 1 & -2.056343 & -3.132818 & -3.191574 \\
\hline 1 & -1.765098 & -5.548424 & -2.933076 \\
\hline 1 & 0.523187 & -6.548339 & -2.936066 \\
\hline ] & 2.498782 & -5.044808 & -3.140624 \\
\hline 1 & 2.207527 & -2.624027 & -3.351572 \\
\hline 1 & -1.490007 & -1.637875 & -5.234345 \\
\hline & -2.352716 & -1.039928 & -3.810405 \\
\hline 1 & -1.401763 & 0.077985 & -4.772209 \\
\hline ? & -6.906802 & -3.071958 & -2.207714 \\
\hline 6 & -5.089687 & -2.240761 & -3.655432 \\
\hline 0 & -4.624624 & -3.968451 & -1.900001 \\
\hline 6 & -6.303478 & 1.597273 & 2.386636 \\
\hline 6 & -8.017549 & 0.914777 & 0.737817 \\
\hline & -7.211221 & -0.710325 & 2.462599 \\
\hline 1 & -7.116381 & 1.950642 & 3.033362 \\
\hline 1 & -5.469417 & 1.305820 & 3.039076 \\
\hline 1 & -5.975291 & 2.445710 & 1.769950 \\
\hline 1 & -7.984713 & -0.373041 & 3.165904 \\
\hline 1 & -7.615041 & -1.566057 & 1.907199 \\
\hline 1 & -6.352103 & -1.065112 & 3.048183 \\
\hline 1 & -8.791176 & 1.285438 & 1.423450 \\
\hline 1 & -7.748273 & 1.734513 & 0.059051 \\
\hline 1 & -8.468765 & 0.116935 & 0.136526 \\
\hline 1 & -7.096025 & -3.872955 & -2.933493 \\
\hline 1 & -7.222947 & -3.443244 & -1.224431 \\
\hline 1 & -7.547491 & -2.223001 & -2.480105 \\
\hline 1 & -5.396226 & -3.002559 & -4.384595 \\
\hline 1 & -5.612162 & -1.307024 & -3.900718 \\
\hline 1 & -4.016925 & -2.070294 & -3.796169 \\
\hline 1 & -4.759820 & -4.725928 & -2.685317 \\
\hline 1 & -3.550487 & -3.770331 & -1.797911 \\
\hline 1 & -4.961875 & -4.399343 & -0.949023 \\
\hline 6 & -5.328179 & 3.785389 & -2.368652 \\
\hline 6 & -5.292496 & 5.702755 & -0.804471 \\
\hline 6 & -3.907400 & 5.749707 & -2.887844 \\
\hline 6 & -0.572571 & 7.015294 & 0.896288 \\
\hline 6 & -0.578179 & 5.292507 & 2.683295 \\
\hline 6 & 1.133102 & 5.220505 & 0.869739 \\
\hline 1 & -4.743321 & 6.181532 & -3.454779 \\
\hline
\end{tabular}




$\begin{array}{rrrr}1 & -3.298448 & 6.580077 & -2.510517 \\ 1 & -3.287332 & 5.170755 & -3.584197 \\ 1 & -6.168437 & 4.258698 & -2.892158 \\ 1 & -4.788712 & 3.170370 & -3.100225 \\ 1 & -5.748368 & 3.115923 & -1.604650 \\ 1 & -6.146333 & 6.135097 & -1.343378 \\ 1 & -5.687058 & 5.088157 & 0.015672 \\ 1 & -4.728753 & 6.531568 & -0.359963 \\ 1 & 0.135182 & 7.624915 & 1.471976 \\ 1 & -0.425783 & 7.250116 & -0.165904 \\ 1 & -1.582557 & 7.334265 & 1.183382 \\ 1 & 1.795347 & 5.913626 & 1.405420 \\ 1 & 1.412166 & 4.207056 & 1.183894 \\ 1 & 1.336051 & 5.321605 & -0.205250 \\ 1 & 0.070342 & 5.937678 & 3.291869 \\ 1 & -1.620947 & 5.511371 & 2.949741 \\ 1 & -0.371155 & 4.250749 & 2.963060\end{array}$

$3_{\mathrm{IC}-\mathrm{re}-} \psi_{-1 \mathrm{~s} 2 \mathrm{a} \mathbf{3} 4 \mathrm{~s}}$

Number of imaginary frequencies : 0

The smallest frequencies are : $14.0387 \quad 16.3124 \quad 19.6567 \mathrm{~cm}(-1)$

Electronic energy :

Zero-point correction $=$

$\mathrm{HF}=-4360.8785273$

Thermal correction to Energy=

Thermal correction to Enthalpy=

1.455274 (Hartree/Particle)

$\quad 1.540728$

Thermal correction to Gibbs Free Energy=

Sum of electronic and zero-point Energies=

Sum of electronic and thermal Energies=

Sum of electronic and thermal Enthalpies=

Sum of electronic and thermal Free Energies=

1.337521

$-4359.423253$

$-4359.338744$

$-4359.337800$

$-4359.541007$

\section{Cartesian Coordinates}

$\begin{array}{cccc}6 & 1.371238 & -3.162756 & -3.292065 \\ 6 & 0.082223 & -2.603905 & -3.292536 \\ 6 & -0.994954 & -3.496390 & -3.167993 \\ 6 & -0.803699 & -4.867264 & -3.050108 \\ 6 & 0.481356 & -5.401295 & -3.064727 \\ 6 & 1.565809 & -4.535030 & -3.180809 \\ 6 & -0.171846 & -1.162147 & -3.559351 \\ 6 & -1.430491 & -0.891986 & -4.363679 \\ 45 & 0.003463 & 0.336083 & -1.801448 \\ 6 & 0.545937 & 2.197721 & -1.934531 \\ 8 & 0.663603 & 3.250897 & -2.400036 \\ 15 & -1.729869 & 0.525427 & -0.066660 \\ 6 & -2.072933 & 2.338841 & -0.117674 \\ 6 & -1.174969 & 3.229646 & 0.466125 \\ 6 & -1.374214 & 4.612665 & 0.396229 \\ 6 & -2.473349 & 5.074373 & -0.327621 \\ 6 & -3.371949 & 4.208438 & -0.963963 \\ 6 & -3.158384 & 2.836742 & -0.841567 \\ 6 & -0.439593 & 5.547493 & 1.163178 \\ 6 & -4.557315 & 4.781397 & -1.739173 \\ 6 & -3.437259 & -0.171860 & -0.223248 \\ 6 & -3.756576 & -1.151778 & -1.159636\end{array}$




\begin{tabular}{|c|c|c|c|}
\hline 6 & -5.060421 & -1.667368 & -1.245003 \\
\hline 6 & -6.022595 & -1.166131 & -0.365924 \\
\hline & -5.731504 & -0.187118 & 0.589999 \\
\hline & -4.427006 & 0.292951 & 0.645613 \\
\hline & -6.766912 & 0.371084 & 1.564217 \\
\hline & -5.394019 & -2.760625 & -2.262095 \\
\hline & -1.492494 & 0.237002 & 1.763980 \\
\hline & -1.053351 & -0.993280 & 2.227451 \\
\hline 0 & -0.891919 & -1.221668 & 3.636452 \\
\hline & -1.197908 & -0.180969 & 4.559299 \\
\hline 6 & -1.687825 & 1.045536 & 4.058222 \\
\hline 6 & -1.830020 & 1.244164 & 2.711926 \\
\hline 6 & -1.001738 & -0.388373 & 5.945115 \\
\hline 6 & -0.518244 & -1.582555 & 6.414358 \\
\hline 6 & -0.218579 & -2.621343 & 5.508700 \\
\hline 6 & -0.405389 & -2.449573 & 4.159173 \\
\hline 6 & -0.776195 & -2.161896 & 1.348190 \\
\hline 6 & 0.476957 & -2.371724 & 0.802294 \\
\hline 6 & 0.800758 & -3.610991 & 0.203309 \\
\hline 6 & -0.110033 & -4.631180 & 0.162065 \\
\hline 6 & -1.414077 & -4.449490 & 0.676403 \\
\hline 6 & -1.761159 & -3.196412 & 1.260362 \\
\hline 6 & -3.071435 & -3.036117 & 1.780908 \\
\hline 6 & -3.975501 & -4.068313 & 1.744287 \\
\hline 6 & -3.619150 & -5.316278 & 1.187990 \\
\hline 6 & -2.369047 & -5.495465 & 0.653237 \\
\hline 7 & 1.504880 & -1.373585 & 0.912483 \\
\hline 15 & 1.794831 & -0.475241 & -0.492827 \\
\hline 8 & 2.981796 & -1.237224 & -1.402326 \\
\hline 6 & 4.192744 & -1.596931 & -0.860712 \\
\hline 6 & 5.122712 & -0.636029 & -0.508815 \\
\hline 6 & 6.288983 & -1.065924 & 0.205547 \\
\hline 6 & 6.525490 & -2.460797 & 0.396468 \\
\hline 6 & 5.604260 & -3.401180 & -0.127176 \\
\hline 6 & 4.451033 & -2.978344 & -0.729259 \\
\hline 6 & 7.191230 & -0.149304 & 0.803982 \\
\hline 6 & 8.271088 & -0.588643 & 1.529492 \\
\hline 6 & 8.519776 & -1.968354 & 1.686826 \\
\hline 6 & 7.661220 & -2.881567 & 1.130380 \\
\hline 6 & 4.868936 & 0.786876 & -0.830448 \\
\hline 6 & 3.684266 & 1.378612 & -0.433964 \\
\hline 6 & 3.422246 & 2.748274 & -0.626367 \\
\hline 6 & 4.333004 & 3.530726 & -1.279921 \\
\hline 6 & 5.526638 & 2.967273 & -1.791840 \\
\hline 6 & 5.797594 & 1.580657 & -1.581292 \\
\hline 6 & 6.448461 & 3.755140 & -2.522821 \\
\hline 6 & 7.585910 & 3.202919 & -3.053142 \\
\hline 6 & 7.839810 & 1.826698 & -2.878794 \\
\hline 6 & 6.972195 & 1.038919 & -2.163451 \\
\hline 8 & 2.742102 & 0.656246 & 0.249773 \\
\hline 6 & 0.881159 & -0.227207 & -3.694637 \\
\hline 6 & 2.484422 & -1.600860 & 2.004614 \\
\hline 6 & 2.936983 & -0.369437 & 2.745639 \\
\hline 6 & 2.032405 & 0.616187 & 3.143939 \\
\hline 6 & 2.467681 & 1.736406 & 3.838313 \\
\hline 6 & 3.818849 & 1.890438 & 4.146707 \\
\hline
\end{tabular}




\begin{tabular}{|c|c|c|c|}
\hline & 4.725973 & 0.912326 & 3.754797 \\
\hline & 4.285174 & -0.211478 & 3.059519 \\
\hline & 6.999933 & 0.916854 & 0.709871 \\
\hline & -2.213470 & 2.199713 & 2.366275 \\
\hline & 7.827744 & -3.950717 & 1.256009 \\
\hline & -1.239518 & 0.425138 & 6.629114 \\
\hline & 6.224548 & 4.811808 & -2.663475 \\
\hline & -3.359086 & -2.075011 & 2.205814 \\
\hline & -4.977993 & -3.921032 & 2.141209 \\
\hline & 4.141561 & 4.592474 & -1.429865 \\
\hline & -1.950836 & 1.836413 & 4.760123 \\
\hline & 5.816071 & -4.463523 & -0.017690 \\
\hline & 8.728713 & 1.382228 & -3.321029 \\
\hline & 3.716666 & -3.677180 & -1.125930 \\
\hline & -2.083382 & -6.446769 & 0.205113 \\
\hline & 0.155780 & -5.591657 & -0.275045 \\
\hline & 0.161794 & -3.569016 & 5.882983 \\
\hline & 7.178395 & -0.022314 & -2.050825 \\
\hline & -0.173153 & -3.266309 & 3.478531 \\
\hline & 1.803765 & -3.744346 & -0.195809 \\
\hline & 8.283814 & 3.816921 & -3.617609 \\
\hline & 8.938348 & 0.135349 & 1.991899 \\
\hline & -4.343894 & -6.127652 & 1.171471 \\
\hline & -0.364965 & -1.732629 & 7.480482 \\
\hline & 9.382700 & -2.302587 & 2.258008 \\
\hline & 2.491494 & 3.148991 & -0.235328 \\
\hline & -4.173408 & 1.056626 & 1.379139 \\
\hline & -2.966766 & -1.524268 & -1.814109 \\
\hline & -7.035658 & -1.551792 & -0.423378 \\
\hline 1 & -3.841120 & 2.135313 & -1.313923 \\
\hline & -0.310779 & 2.834710 & 1.005286 \\
\hline & -2.645898 & 6.145852 & -0.403412 \\
\hline ] & 1.974630 & -2.270842 & 2.711223 \\
\hline & -1.206239 & 0.947933 & -2.625958 \\
\hline 1 & 3.362730 & -2.153326 & 1.636817 \\
\hline & 5.005141 & -0.966126 & 2.738073 \\
\hline 1 & 0.985857 & 0.518599 & 2.864127 \\
\hline ] & 5.785503 & 1.023856 & 3.978499 \\
\hline 1 & 1.745128 & 2.494544 & 4.138473 \\
\hline 1 & 4.161525 & 2.771821 & 4.684727 \\
\hline 1 & 1.918943 & -0.516189 & -3.537512 \\
\hline 1 & 0.755524 & 0.595152 & -4.399530 \\
\hline | & -2.011825 & -3.109287 & -3.184203 \\
\hline 1 & -1.670715 & -5.520713 & -2.954635 \\
\hline 1 & 0.636292 & -6.476216 & -2.994537 \\
\hline 1 & 2.579609 & -4.932932 & -3.215705 \\
\hline 1 & 2.238441 & -2.518008 & -3.405188 \\
\hline 1 & -1.496627 & -1.580888 & -5.220494 \\
\hline 1 & -2.352585 & -1.001604 & -3.784614 \\
\hline l & -1.425817 & 0.133267 & -4.749643 \\
\hline 6 & -6.874791 & -3.137212 & -2.255148 \\
\hline 6 & -5.047061 & -2.285591 & -3.676204 \\
\hline 6 & -4.589956 & -4.021302 & -1.927266 \\
\hline 6 & -8.136881 & -0.281259 & 1.399497 \\
\hline 6 & -6.292791 & 0.125885 & 3.001743 \\
\hline & -6.922263 & 1.877895 & 1.324655 \\
\hline
\end{tabular}




$\begin{array}{rrrr}1 & -8.840186 & 0.150158 & 2.122998 \\ 1 & -8.554277 & -0.115290 & 0.398036 \\ 1 & -8.100082 & -1.363226 & 1.581855 \\ 1 & -7.632133 & 2.309781 & 2.043133 \\ 1 & -5.970111 & 2.413553 & 1.431752 \\ 1 & -7.305542 & 2.074512 & 0.314169 \\ 1 & -7.020365 & 0.530191 & 3.718367 \\ 1 & -6.186697 & -0.949067 & 3.199824 \\ 1 & -5.324275 & 0.598248 & 3.209597 \\ 1 & -7.051599 & -3.928913 & -2.994143 \\ 1 & -7.199255 & -3.524409 & -1.280879 \\ 1 & -7.518062 & -2.288518 & -2.522157 \\ 1 & -5.344055 & -3.043408 & -4.413430 \\ 1 & -5.570172 & -1.351946 & -3.920559 \\ 1 & -3.973712 & -2.111195 & -3.805878 \\ 1 & -4.717608 & -4.778027 & -2.714582 \\ 1 & -3.517405 & -3.816503 & -1.820187 \\ 1 & -4.928698 & -4.455679 & -0.978283 \\ 6 & -5.429811 & 3.687451 & -2.351158 \\ 6 & -5.433142 & 5.611291 & -0.793724 \\ 6 & -4.044190 & 5.674292 & -2.873756 \\ 6 & -0.667198 & 7.015719 & 0.810613 \\ 6 & -0.702347 & 5.362921 & 2.663169 \\ 6 & 1.024938 & 5.209281 & 0.868620 \\ 1 & -4.886825 & 6.085148 & -3.446309 \\ 1 & -3.454369 & 6.519751 & -2.499669 \\ 1 & -3.409711 & 5.104455 & -3.564601 \\ 1 & -6.276856 & 4.144170 & -2.878523 \\ 1 & -4.876730 & 3.080208 & -3.079145 \\ 1 & -5.840503 & 3.013057 & -1.586410 \\ 1 & -6.287772 & 6.033813 & -1.339167 \\ 1 & -5.827808 & 4.991703 & 0.022829 \\ 1 & -4.882402 & 6.446293 & -0.344363 \\ 1 & 0.045133 & 7.641732 & 1.362596 \\ 1 & -0.517143 & 7.205312 & -0.260122 \\ 1 & -1.674194 & 7.356050 & 1.082923 \\ 1 & 1.687011 & 5.922648 & 1.377152 \\ 1 & 1.297721 & 4.209417 & 1.229436 \\ 1 & 1.234073 & 5.260085 & -0.208717 \\ 1 & -0.049469 & 6.020345 & 3.253712 \\ 1 & -1.743948 & 5.605903 & 2.912350 \\ 1 & -0.513132 & 4.328962 & 2.981404\end{array}$

$3_{\text {IC-re }-} \psi_{-1 \mathbf{1 s} 233 a 4 a}$

Number of imaginary frequencies : 0

The smallest frequencies are : $16.2171 \quad 19.5018 \quad 21.1717 \mathrm{~cm}(-1)$

Electronic energy : $\quad \mathrm{HF}=-4360.878948$

Zero-point correction $=\quad 1.455758($ Hartree/Particle $)$

Thermal correction to Energy= $\quad 1.540002$

Thermal correction to Enthalpy= $\quad 1.540946$

Thermal correction to Gibbs Free Energy= 1.339496

Sum of electronic and zero-point Energies $=\quad-4359.423190$

Sum of electronic and thermal Energies $=\quad-4359.338946$

Sum of electronic and thermal Enthalpies $=\quad-4359.338002$

Sum of electronic and thermal Free Energies $=\quad-4359.539452$ 
Cartesian Coordinates

\begin{tabular}{cccc}
\hline 6 & -2.997253 & 2.901340 & -0.930020 \\
6 & -1.976664 & 2.369242 & -0.148986 \\
6 & -1.069771 & 3.235205 & 0.467632 \\
6 & -1.218981 & 4.620239 & 0.380317 \\
6 & -2.261371 & 5.120201 & -0.408773 \\
6 & -3.148796 & 4.281703 & -1.085703 \\
15 & -1.723406 & 0.543726 & -0.073068 \\
45 & -0.022609 & 0.248924 & -1.817186 \\
6 & 0.494045 & 2.115339 & -2.028042 \\
8 & 0.591830 & 3.146455 & -2.543987 \\
6 & -0.291036 & 5.528865 & 1.186663 \\
6 & -0.559529 & 5.295253 & 2.678906 \\
6 & -4.287430 & 4.802680 & -1.959871 \\
6 & -5.625097 & 4.324192 & -1.381688 \\
6 & -1.507739 & 0.267672 & 1.760429 \\
6 & -1.100461 & -0.969543 & 2.232007 \\
6 & -0.970924 & -1.201529 & 3.643177 \\
6 & -1.274673 & -0.154470 & 4.559726 \\
6 & -1.728334 & 1.082531 & 4.049117 \\
6 & -1.842184 & 1.283287 & 2.700503 \\
6 & -0.521510 & -2.439831 & 4.173830 \\
6 & -0.369207 & -2.617140 & 5.527052 \\
6 & -0.667392 & -1.573061 & 6.427040 \\
6 & -1.114013 & -0.367802 & 5.949127 \\
6 & -0.828475 & -2.140215 & 1.354813 \\
6 & 0.432623 & -2.372331 & 0.836672 \\
6 & 0.748056 & -3.619044 & 0.249170 \\
6 & -0.189442 & -4.612352 & 0.164788 \\
6 & -1.512466 & -4.390444 & 0.610795 \\
6 & -1.840126 & -3.142037 & 1.215996 \\
6 & -2.511889 & -5.386932 & 0.486641 \\
6 & -3.787538 & -5.163162 & 0.940270 \\
6 & -4.115480 & -3.927470 & 1.540477 \\
6 & -3.166054 & -2.946050 & 1.681214 \\
7 & 1.465868 & -1.379648 & 0.946461 \\
15 & 1.768180 & -0.505788 & -0.474004 \\
8 & 2.970195 & -1.277305 & -1.350766 \\
6 & 4.175154 & -1.621179 & -0.786139 \\
6 & 5.095843 & -0.648650 & -0.441688 \\
6 & 6.255531 & -1.058090 & 0.294422 \\
6 & 6.496427 & -2.447741 & 0.515555 \\
6 & 5.585428 & -3.402981 & 0.000969 \\
6 & 4.437121 & -2.998474 & -0.622923 \\
6 & 7.146433 & -0.125098 & 0.884237 \\
6 & 8.220435 & -0.544457 & 1.629991 \\
6 & 8.473834 & -1.919554 & 1.817706 \\
6 & 7.625896 & -2.848052 & 1.270356 \\
6 & 4.838425 & 0.764051 & -0.803056 \\
6 & 3.642293 & 1.357233 & -0.444497 \\
6 & 3.365644 & 2.713911 & -0.699014 \\
6 & 4.278799 & 3.481875 & -1.366716 \\
6 & 5.488780 & 2.914867 & -1.833891 \\
6 & 5.771203 & 1.540381 & -1.567215 \\
& & & \\
6 & & &
\end{tabular}




\begin{tabular}{|c|c|c|c|}
\hline & 6.414334 & 3.686271 & -2.577731 \\
\hline & 7.566887 & 3.128448 & -3.067904 \\
\hline & 7.832639 & 1.762547 & -2.838745 \\
\hline & 6.961280 & 0.991386 & -2.110004 \\
\hline & 2.698383 & 0.649964 & 0.254523 \\
\hline & 2.435494 & -1.596169 & 2.049110 \\
\hline & 2.885279 & -0.359376 & 2.783254 \\
\hline & 4.230499 & -0.204985 & 3.112092 \\
\hline & 4.666754 & 0.917641 & 3.812122 \\
\hline & 3.758347 & 1.898800 & 4.193265 \\
\hline & 2.410698 & 1.749525 & 3.868311 \\
\hline & 1.980029 & 0.630421 & 3.169021 \\
\hline & -3.459334 & -0.080568 & -0.217925 \\
\hline & -3.795811 & -1.114491 & -1.087590 \\
\hline & -5.108730 & -1.612736 & -1.130536 \\
\hline & -6.050467 & -1.035377 & -0.279852 \\
\hline & -5.743037 & -0.001964 & 0.612659 \\
\hline & -4.433958 & 0.464004 & 0.626009 \\
\hline & -5.547872 & -2.709733 & -2.102596 \\
\hline & -6.530276 & -3.683202 & -1.443358 \\
\hline & -6.811700 & 0.533095 & 1.562793 \\
\hline & -7.208353 & -0.588737 & 2.529727 \\
\hline & -6.231425 & -2.039403 & -3.300462 \\
\hline & -4.366459 & -3.527719 & -2.616550 \\
\hline & -8.043896 & 0.990411 & 0.776116 \\
\hline & -6.308716 & 1.718325 & 2.384510 \\
\hline 6 & 0.839114 & -0.316452 & -3.710639 \\
\hline 5 & -0.139006 & -1.323286 & -3.527037 \\
\hline & 0.244302 & -2.738085 & -3.261951 \\
\hline 6 & 1.572701 & -3.192968 & -3.310003 \\
\hline & 1.879022 & -4.546048 & -3.209833 \\
\hline 6 & 0.871067 & -5.497496 & -3.070833 \\
\hline 6 & -0.450472 & -5.065759 & -3.010513 \\
\hline 6 & -0.750970 & -3.712839 & -3.097298 \\
\hline 6 & -1.433526 & -1.171399 & -4.307333 \\
\hline 6 & 1.173459 & 5.199505 & 0.884770 \\
\hline 6 & -0.516266 & 7.008662 & 0.885234 \\
\hline 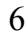 & -4.121801 & 4.259846 & -3.384066 \\
\hline 6 & -4.318633 & 6.326980 & -2.033249 \\
\hline 1 & 6.950460 & 0.937936 & 0.766651 \\
\hline & -2.199696 & 2.246534 & 2.347766 \\
\hline 1 & 7.796059 & -3.913615 & 1.419344 \\
\hline & -1.349433 & 0.449635 & 6.629259 \\
\hline 1 & 6.180909 & 4.734294 & -2.761150 \\
\hline & -3.434909 & -1.994419 & 2.137364 \\
\hline 1 & -5.130658 & -3.748010 & 1.890940 \\
\hline 1 & 4.077627 & 4.534049 & -1.563716 \\
\hline & -1.987215 & 1.879234 & 4.745997 \\
\hline 1 & 5.800634 & -4.461796 & 0.135321 \\
\hline 1 & 8.733809 & 1.312395 & -3.249252 \\
\hline 1 & 3.709690 & -3.708228 & -1.013187 \\
\hline 1 & -2.242865 & -6.336018 & 0.024122 \\
\hline 1 & 0.065969 & -5.575181 & -0.272997 \\
\hline 1 & -0.017464 & -3.572932 & 5.908611 \\
\hline 1 & 7.177297 & -0.062479 & -1.955503 \\
\hline 1 & -0.290505 & -3.259606 & 3.496291 \\
\hline
\end{tabular}




\begin{tabular}{|c|c|c|c|}
\hline & 1.760921 & -3.778129 & -0.115215 \\
\hline & 8.267736 & 3.729483 & -3.642577 \\
\hline ] & 8.879148 & 0.191875 & 2.085127 \\
\hline 1 & -4.548767 & -5.934134 & 0.838447 \\
\hline & -0.542037 & -1.727699 & 7.496169 \\
\hline & 9.331980 & -2.237941 & 2.404919 \\
\hline & 2.421999 & 3.118048 & -0.343962 \\
\hline & -4.155378 & 1.266497 & 1.302992 \\
\hline & -3.010416 & -1.543146 & -1.710581 \\
\hline & -7.072733 & -1.409572 & -0.302057 \\
\hline & -3.687621 & 2.225191 & -1.432435 \\
\hline & -0.244454 & 2.815848 & 1.047815 \\
\hline & -2.382564 & 6.195268 & -0.498899 \\
\hline & 1.917901 & -2.257660 & 2.758378 \\
\hline & -1.255668 & 0.796563 & -2.657302 \\
\hline ] & 3.315810 & -2.154347 & 1.695572 \\
\hline & 4.951605 & -0.962774 & 2.800757 \\
\hline & 0.936638 & 0.535869 & 2.877186 \\
\hline & 5.723736 & 1.025702 & 4.049032 \\
\hline & 1.687654 & 2.511483 & 4.158207 \\
\hline & 4.097538 & 2.778854 & 4.735652 \\
\hline & 1.898848 & -0.530526 & -3.579322 \\
\hline$\frac{1}{1}$ & 0.638553 & 0.475886 & -4.432725 \\
\hline & -1.789860 & -3.403639 & -3.041936 \\
\hline ] & -1.261757 & -5.784809 & -2.897108 \\
\hline & 1.113008 & -6.557171 & -3.018815 \\
\hline & 2.919989 & -4.860762 & -3.276740 \\
\hline 1 & 2.383425 & -2.483675 & -3.450672 \\
\hline & -1.447325 & -1.863177 & -5.164170 \\
\hline 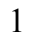 & -2.335712 & -1.370868 & -3.717282 \\
\hline & -1.532108 & -0.150039 & -4.691121 \\
\hline 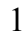 & -7.113064 & 2.084119 & 3.035048 \\
\hline$\frac{1}{1}$ & -5.464276 & 1.443794 & 3.030922 \\
\hline & -5.993058 & 2.553561 & 1.744162 \\
\hline 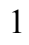 & -8.810781 & 1.372036 & 1.463292 \\
\hline & -7.789165 & 1.796538 & 0.075713 \\
\hline 1 & -8.498317 & 0.176642 & 0.198944 \\
\hline & -7.973564 & -0.237285 & 3.235146 \\
\hline 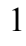 & -7.615271 & -1.460057 & 2.001170 \\
\hline 1 & -6.339832 & -0.925732 & 3.112045 \\
\hline & -4.729915 & -4.359811 & -3.233073 \\
\hline 1 & -3.709067 & -2.926230 & -3.254598 \\
\hline & -3.771126 & -3.949925 & -1.793569 \\
\hline 1 & -6.778185 & -4.489321 & -2.145919 \\
\hline & -6.091147 & -4.140395 & -0.546494 \\
\hline 1 & -7.476656 & -3.209806 & -1.157933 \\
\hline | & -6.554665 & -2.792195 & -4.032659 \\
\hline 1 & -7.114838 & -1.466734 & -2.990143 \\
\hline 1 & -5.544152 & -1.347276 & -3.805938 \\
\hline 1 & -4.925110 & 4.636307 & -4.031834 \\
\hline 1 & -3.162070 & 4.575199 & -3.813530 \\
\hline 1 & -4.156153 & 3.164270 & -3.416081 \\
\hline 1 & -5.150661 & 6.647674 & -2.672837 \\
\hline 1 & -4.469176 & 6.784139 & -1.046628 \\
\hline 1 & -3.395724 & 6.735124 & -2.464987 \\
\hline 1 & -6.458233 & 4.662772 & -2.012494 \\
\hline
\end{tabular}




$\begin{array}{rrrr}1 & -5.678490 & 3.229642 & -1.317721 \\ 1 & -5.780897 & 4.727576 & -0.372035 \\ 1 & 0.179848 & 7.614698 & 1.478648 \\ 1 & -0.339512 & 7.241765 & -0.172745 \\ 1 & -1.531797 & 7.333754 & 1.144548 \\ 1 & 1.837557 & 5.893879 & 1.416631 \\ 1 & 1.442584 & 4.187376 & 1.211413 \\ 1 & 1.383026 & 5.285987 & -0.190562 \\ 1 & 0.088915 & 5.936029 & 3.292157 \\ 1 & -1.602755 & 5.527977 & 2.931490 \\ 1 & -0.368636 & 4.252872 & 2.967157\end{array}$

$3_{\text {IC-re- }} \psi_{-1 \mathrm{~s} 2 \mathrm{~s} 3 \mathrm{a} 4 \mathrm{~s}}$

Number of imaginary frequencies : 0

The smallest frequencies are : $16.381120 .031422 .4955 \mathrm{~cm}(-1)$

Electronic energy : $\quad H F=-4360.8786788$

Zero-point correction $=\quad 1.459458($ Hartree/Particle $)$

Thermal correction to Energy= $\quad 1.542375$

Thermal correction to Enthalpy= $\quad 1.543319$

Thermal correction to Gibbs Free Energy= $\quad 1.346249$

Sum of electronic and zero-point Energies $=\quad-4359.419221$

Sum of electronic and thermal Energies $=\quad-4359.336304$

Sum of electronic and thermal Enthalpies $=\quad-4359.335360$

Sum of electronic and thermal Free Energies $=\quad-4359.532430$

Cartesian Coordinates

$\begin{array}{cccc}6 & -0.730541 & -3.703258 & -3.138985 \\ 6 & 0.235018 & -2.698540 & -3.296262 \\ 6 & 1.571357 & -3.115417 & -3.384054 \\ 6 & 1.920730 & -4.457809 & -3.296511 \\ 6 & 0.943544 & -5.438347 & -3.141447 \\ 6 & -0.391237 & -5.049161 & -3.080305 \\ 6 & -0.182433 & -1.286844 & -3.529899 \\ 6 & -1.494365 & -1.146116 & -4.282237 \\ 45 & -0.054047 & 0.271282 & -1.805998 \\ 6 & 0.774902 & -0.260271 & -3.720199 \\ 15 & -1.738536 & 0.536615 & -0.037535 \\ 6 & -3.472410 & -0.089131 & -0.192492 \\ 6 & -3.804964 & -1.125365 & -1.067748 \\ 6 & -5.116364 & -1.612803 & -1.127676 \\ 6 & -6.073131 & -1.028788 & -0.288851 \\ 6 & -5.768243 & -0.006006 & 0.608573 \\ 6 & -4.451751 & 0.452348 & 0.637664 \\ 6 & -5.553036 & -2.707817 & -2.103118 \\ 6 & -4.370180 & -3.511842 & -2.635375 \\ 6 & -6.785030 & 0.597271 & 1.574501 \\ 6 & -8.178141 & -0.003340 & 1.407245 \\ 6 & -1.990234 & 2.362805 & -0.093985 \\ 6 & -2.983246 & 2.904773 & -0.904160 \\ 6 & -3.103768 & 4.285963 & -1.078820 \\ 6 & -2.229381 & 5.116230 & -0.374375 \\ 6 & -1.220970 & 4.606135 & 0.450840 \\ 6 & -1.089860 & 3.219631 & 0.543860 \\ 6 & -0.297607 & 5.503492 & 1.274525\end{array}$




\begin{tabular}{|c|c|c|c|}
\hline 6 & -0.519809 & 6.987022 & 0.990436 \\
\hline 5 & -4.169243 & 4.817984 & -2.034743 \\
\hline b & -4.191697 & 6.343037 & -2.096307 \\
\hline & -1.518615 & 0.237803 & 1.791784 \\
\hline$b$ & -1.099734 & -1.002379 & 2.244897 \\
\hline 6 & -0.960937 & -1.253147 & 3.651327 \\
\hline 6 & -1.266526 & -0.221200 & 4.583879 \\
\hline 5 & -1.733814 & 1.018714 & 4.092710 \\
\hline 6 & -1.857638 & 1.237139 & 2.747401 \\
\hline 6 & -1.095614 & -0.452951 & 5.969119 \\
\hline 6 & -0.638366 & -1.662114 & 6.426940 \\
\hline 6 & -0.339800 & -2.691887 & 5.510664 \\
\hline 6 & -0.501572 & -2.496357 & 4.161102 \\
\hline 6 & -0.837128 & -2.157741 & 1.346279 \\
\hline 6 & 0.411716 & -2.377999 & 0.794241 \\
\hline 6 & 0.707877 & -3.605705 & 0.159110 \\
\hline 6 & -0.232476 & -4.595914 & 0.076187 \\
\hline 6 & -1.542729 & -4.386575 & 0.562772 \\
\hline 6 & -1.854072 & -3.153141 & 1.205402 \\
\hline 6 & -3.169700 & -2.963727 & 1.701546 \\
\hline 6 & -4.124117 & -3.939604 & 1.558400 \\
\hline 6 & -3.811746 & -5.162383 & 0.923863 \\
\hline 6 & -2.546970 & -5.378157 & 0.437061 \\
\hline 7 & 1.451396 & -1.393202 & 0.913588 \\
\hline 6 & 2.422897 & -1.630982 & 2.009739 \\
\hline 6 & 2.876711 & -0.410348 & 2.768184 \\
\hline 6 & 4.221626 & -0.271447 & 3.105179 \\
\hline 6 & 4.662414 & 0.832464 & 3.831603 \\
\hline 6 & 3.758819 & 1.810734 & 4.231083 \\
\hline 6 & 2.411592 & 1.677402 & 3.897586 \\
\hline 6 & 1.976409 & 0.576885 & 3.172060 \\
\hline 6 & 0.459706 & 2.140507 & -1.992842 \\
\hline 8 & 0.566169 & 3.178501 & -2.492915 \\
\hline 15 & 1.750670 & -0.496113 & -0.491947 \\
\hline 8 & 2.682483 & 0.646989 & 0.255066 \\
\hline 6 & 3.630135 & 1.363086 & -0.429377 \\
\hline 6 & 4.826012 & 0.773223 & -0.794729 \\
\hline 6 & 5.763978 & 1.561429 & -1.540245 \\
\hline 6 & 5.486860 & 2.941758 & -1.781062 \\
\hline 6 & 4.275893 & 3.502894 & -1.309369 \\
\hline 6 & 3.357622 & 2.724813 & -0.660768 \\
\hline 6 & 6.954671 & 1.019669 & -2.088983 \\
\hline 6 & 7.831961 & 1.802600 & -2.797786 \\
\hline 6 & 7.571937 & 3.173853 & -2.999926 \\
\hline 6 & 6.418559 & 3.725174 & -2.504364 \\
\hline 6 & 5.077689 & -0.647190 & -0.460506 \\
\hline 6 & 4.153886 & -1.609084 & -0.825923 \\
\hline 6 & 4.408698 & -2.990411 & -0.687981 \\
\hline 6 & 5.553717 & -3.411862 & -0.069340 \\
\hline 6 & 6.467896 & -2.470672 & 0.465328 \\
\hline 6 & 6.233846 & -1.075930 & 0.270225 \\
\hline 6 & 7.593376 & -2.890390 & 1.215534 \\
\hline 6 & 8.443956 & -1.976416 & 1.782952 \\
\hline 6 & 8.197382 & -0.596842 & 1.620753 \\
\hline 6 & 7.127359 & -0.158514 & 0.880207 \\
\hline 8 & 2.951435 & -1.248900 & -1.385676 \\
\hline
\end{tabular}




\begin{tabular}{|c|c|c|c|}
\hline 6 & -0.571316 & 5.252756 & 2.762910 \\
\hline 6 & 1.167170 & 5.174480 & 0.972497 \\
\hline 6 & -5.554084 & 4.339061 & -1.584742 \\
\hline 6 & -3.875042 & 4.287304 & -3.443493 \\
\hline 6 & -6.517316 & -3.695715 & -1.438234 \\
\hline 6 & -6.256508 & -2.037745 & -3.289571 \\
\hline 6 & -6.317452 & 0.326495 & 3.010095 \\
\hline 6 & -6.885472 & 2.108556 & 1.340468 \\
\hline 1 & 6.936423 & 0.907442 & 0.782683 \\
\hline 1 & -2.224119 & 2.202353 & 2.409514 \\
\hline 1 & 7.758300 & -3.959387 & 1.344612 \\
\hline 1 & -1.332207 & 0.353031 & 6.662371 \\
\hline 1 & 6.189278 & 4.777459 & -2.667527 \\
\hline 1 & -3.425325 & -2.021000 & 2.183722 \\
\hline 1 & -5.131732 & -3.764820 & 1.931956 \\
\hline 1 & 4.078343 & 4.559150 & -1.487272 \\
\hline 1 & -1.995467 & 1.803416 & 4.802044 \\
\hline 1 & 5.763553 & -4.474011 & 0.045975 \\
\hline 1 & 8.733586 & 1.357865 & -3.213189 \\
\hline 1 & 3.677917 & -3.688849 & -1.092287 \\
\hline 1 & -2.290842 & -6.315745 & -0.055500 \\
\hline 1 & 0.010597 & -5.546279 & -0.393876 \\
\hline 1 & 0.019562 & -3.650900 & 5.876738 \\
\hline 1 & 7.166586 & -0.037824 & -1.955031 \\
\hline 1 & -0.271340 & -3.304903 & 3.469884 \\
\hline 1 & 1.707101 & -3.752824 & -0.245670 \\
\hline 1 & 8.277692 & 3.784274 & -3.558489 \\
\hline 1 & 8.858019 & 0.127700 & 2.091757 \\
\hline 1 & -4.576814 & -5.929444 & 0.821016 \\
\hline 1 & -0.505404 & -1.831043 & 7.492993 \\
\hline 1 & 9.298897 & -2.309963 & 2.366431 \\
\hline 1 & 2.413355 & 3.124748 & -0.302303 \\
\hline 1 & -4.182176 & 1.248858 & 1.329422 \\
\hline 1 & -3.013465 & -1.556919 & -1.680732 \\
\hline 1 & -7.095829 & -1.393870 & -0.336754 \\
\hline 1 & -3.663315 & 2.234325 & -1.428395 \\
\hline 1 & -0.281388 & 2.793152 & 1.141957 \\
\hline 1 & -2.330506 & 6.192305 & -0.476258 \\
\hline 1 & 1.906553 & -2.305638 & 2.707277 \\
\hline 1 & -1.299604 & 0.824156 & -2.625552 \\
\hline 1 & 3.301138 & -2.183854 & 1.643099 \\
\hline 1 & 4.939216 & -1.026885 & 2.780253 \\
\hline 1 & 0.933277 & 0.495242 & 2.875280 \\
\hline 1 & 5.719163 & 0.928309 & 4.074743 \\
\hline 1 & 1.692132 & 2.437373 & 4.201376 \\
\hline 1 & 4.101351 & 2.676284 & 4.794324 \\
\hline 1 & 1.840722 & -0.456937 & -3.613600 \\
\hline 1 & 0.546574 & 0.536384 & -4.429245 \\
\hline 1 & -1.777949 & -3.424302 & -3.070371 \\
\hline 1 & -1.180039 & -5.793090 & -2.968452 \\
\hline 1 & 1.217737 & -6.490661 & -3.098406 \\
\hline 1 & 2.969268 & -4.741217 & -3.382174 \\
\hline 1 & 2.358549 & -2.382707 & -3.536162 \\
\hline 1 & -1.515927 & -1.828968 & -5.146117 \\
\hline 1 & -2.380974 & -1.364392 & -3.676046 \\
\hline 1 & -1.615723 & -0.122207 & -4.652781 \\
\hline
\end{tabular}




$\begin{array}{rrrr}1 & -8.869551 & 0.465395 & 2.118889 \\ 1 & -8.579548 & 0.165255 & 0.399561 \\ 1 & -8.186449 & -1.083131 & 1.604989 \\ 1 & -7.573869 & 2.562253 & 2.066215 \\ 1 & -5.915217 & 2.611407 & 1.441009 \\ 1 & -7.268431 & 2.322910 & 0.333858 \\ 1 & -7.031220 & 0.745746 & 3.732122 \\ 1 & -6.241604 & -0.753399 & 3.197126 \\ 1 & -5.334122 & 0.767864 & 3.217455 \\ 1 & -4.734011 & -4.338978 & -3.258381 \\ 1 & -3.720109 & -2.900036 & -3.271162 \\ 1 & -3.766678 & -3.940799 & -1.821981 \\ 1 & -6.773655 & -4.494709 & -2.146037 \\ 1 & -6.057120 & -4.160849 & -0.556302 \\ 1 & -7.460250 & -3.231779 & -1.127349 \\ 1 & -6.580389 & -2.789839 & -4.022195 \\ 1 & -7.142370 & -1.475430 & -2.967660 \\ 1 & -5.581333 & -1.336405 & -3.798606 \\ 1 & -4.616789 & 4.667432 & -4.159130 \\ 1 & -2.880247 & 4.607117 & -3.780729 \\ 1 & -3.901785 & 3.191479 & -3.484893 \\ 1 & -4.973057 & 6.671526 & -2.793287 \\ 1 & -4.414307 & 6.791164 & -1.119127 \\ 1 & -3.239015 & 6.753387 & -2.454849 \\ 1 & -6.324403 & 4.690876 & -2.284309 \\ 1 & -5.621112 & 3.244397 & -1.540637 \\ 1 & -5.799096 & 4.730749 & -0.588418 \\ 1 & 0.166132 & 7.585481 & 1.603103 \\ 1 & -0.326307 & 7.235636 & -0.061039 \\ 1 & -1.540215 & 7.306406 & 1.237685 \\ 1 & 1.832249 & 5.859897 & 1.514782 \\ 1 & 1.431719 & 4.156883 & 1.285847 \\ 1 & 1.379088 & 5.273618 & -0.101399 \\ 1 & 0.075399 & 5.886369 & 3.385290 \\ 1 & -1.615159 & 5.483300 & 3.014886 \\ 1 & -0.381463 & 4.207444 & 3.041010\end{array}$

$3_{\text {IC-re- }-} \psi_{-1 \mathrm{~s} 25334 a}$

Number of imaginary frequencies : 0

The smallest frequencies are : $18.0688 \quad 19.348120 .3822 \mathrm{~cm}(-1)$

Electronic energy :

Zero-point correction $=$ $\mathrm{HF}=-4360.8785507$

Thermal correction to Energy=

Thermal correction to Enthalpy=

1.455458 (Hartree/Particle)

Thermal correction to Gibbs Free Energy $=\quad 1.338950$

Sum of electronic and zero-point Energies $=\quad-4359.423093$

Sum of electronic and thermal Energies $=\quad-4359.338794$

Sum of electronic and thermal Enthalpies $=\quad-4359.337850$

Sum of electronic and thermal Free Energies $=\quad-4359.539600$

\section{Cartesian Coordinates}

$\begin{array}{rrrr}6 & 1.246584 & -3.180311 & -3.331414 \\ 6 & -0.024317 & -2.580836 & -3.334243 \\ 6 & -1.130865 & -3.439836 & -3.235152\end{array}$




\begin{tabular}{|c|c|c|c|}
\hline 6 & -0.985209 & -4.818122 & -3.143321 \\
\hline 6 & 0.282196 & -5.393022 & -3.160026 \\
\hline 6 & 1.395155 & -4.560314 & -3.248534 \\
\hline 6 & -0.233007 & -1.128588 & -3.580038 \\
\hline 6 & -1.481746 & -0.809817 & -4.381790 \\
\hline 45 & -0.015453 & 0.342809 & -1.801819 \\
\hline 6 & 0.535132 & 2.203893 & -1.928999 \\
\hline 8 & 0.631554 & 3.263345 & -2.385070 \\
\hline 15 & -1.714186 & 0.566112 & -0.038969 \\
\hline 6 & -1.958752 & 2.394234 & -0.052907 \\
\hline 6 & -1.047381 & 3.235639 & 0.590193 \\
\hline 6 & -1.182394 & 4.623619 & 0.537488 \\
\hline 6 & -2.213681 & 5.153474 & -0.246965 \\
\hline 6 & -3.103929 & 4.340412 & -0.951362 \\
\hline 6 & -2.969959 & 2.955217 & -0.825557 \\
\hline 6 & -0.251125 & 5.500324 & 1.375410 \\
\hline 6 & -4.217014 & 4.893516 & -1.838506 \\
\hline 6 & -3.452507 & -0.046800 & -0.205721 \\
\hline 6 & -3.801603 & -1.012024 & -1.141247 \\
\hline 6 & -5.122299 & -1.496176 & -1.219630 \\
\hline 6 & -6.054540 & -0.982800 & -0.324154 \\
\hline 6 & -5.733279 & -0.010636 & 0.636625 \\
\hline 6 & -4.424446 & 0.446664 & 0.676850 \\
\hline 6 & -6.800003 & 0.475098 & 1.615105 \\
\hline 6 & -5.493711 & -2.563322 & -2.250360 \\
\hline 6 & -1.490406 & 0.228804 & \\
\hline 6 & -1.078506 & -1.020125 & 2.220545 \\
\hline 6 & -0.923991 & -1.282960 & 3.624419 \\
\hline 6 & -1.211561 & -0.257436 & 4.569685 \\
\hline 6 & -1.679551 & 0.988474 & 4.095446 \\
\hline 6 & -1.814985 & 1.219764 & \\
\hline 6 & -1.021039 & -0.499324 & 5.950654 \\
\hline 6 & -0.560412 & -1.712641 & \\
\hline 6 & -0.278948 & -2.736363 & 5.465299 \\
\hline 6 & -0.460962 & -2.531022 & 4.119770 \\
\hline 6 & -0.823971 & -2.173907 & 1.315625 \\
\hline 6 & 0.424213 & -2.393794 & 0.762823 \\
\hline 6 & 0.725707 & -3.625624 & 7644 \\
\hline 6 & -0.202529 & -4.628959 & 0.077018 \\
\hline 6 & -1.502854 & -4.434763 & 0.596299 \\
\hline 6 & -1.827919 & -3.187952 & 1.206003 \\
\hline 6 & -3.136735 & -3.014156 & 1.726761 \\
\hline 6 & -4.058106 & -4.030112 & 1.670435 \\
\hline 6 & -3.722882 & -5.273144 & 1.090345 \\
\hline 6 & -2.476125 & -5.463026 & 0.551607 \\
\hline 7 & 1.467938 & -1.413900 & 0.888315 \\
\hline 15 & 1.771284 & -0.506000 & -0.508065 \\
\hline 8 & 2.951325 & -1.278563 & -1.420697 \\
\hline 6 & 4.159954 & -1.648881 & -0.879712 \\
\hline 6 & 5.096803 & -0.696959 & -0.520289 \\
\hline 6 & 6.257148 & -1.138902 & 0.195914 \\
\hline 6 & 6.483717 & -2.536166 & 0.380172 \\
\hline 6 & 5.557495 & -3.467022 & -0.151587 \\
\hline 6 & 4.408966 & -3.032632 & -0.754379 \\
\hline 6 & 7.162728 & -0.231226 & 0.802673 \\
\hline 6 & 8.236760 & -0.681276 & 1.530228 \\
\hline
\end{tabular}




\begin{tabular}{|c|c|c|c|}
\hline & 8.475614 & -2.063423 & 1.681500 \\
\hline & 7.613539 & -2.968226 & 1.116649 \\
\hline & 4.856073 & 0.728059 & -0.841247 \\
\hline & 3.672559 & 1.325792 & -0.451812 \\
\hline & 3.407035 & 2.690683 & -0.669888 \\
\hline & 4.321427 & 3.465609 & -1.327638 \\
\hline & 5.521124 & 2.898025 & -1.820145 \\
\hline & 5.790346 & 1.513545 & -1.593841 \\
\hline & 6.448831 & 3.678998 & -2.551197 \\
\hline & 7.590501 & 3.121858 & -3.066936 \\
\hline & 7.842389 & 1.747162 & -2.878150 \\
\hline 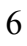 & 6.968797 & 0.966090 & -2.162760 \\
\hline & 2.731717 & 0.609494 & 0.242139 \\
\hline b & 0.846538 & -0.222980 & -3.701732 \\
\hline & 2.449122 & -1.679784 & 1.969793 \\
\hline & 2.922663 & -0.474998 & 2.740423 \\
\hline$b$ & 2.036162 & 0.516549 & 3.163712 \\
\hline & 2.489695 & 1.604169 & 3.897366 \\
\hline D & 3.841398 & 1.719141 & 4.219631 \\
\hline & 4.731008 & 0.735799 & 3.801150 \\
\hline & 4.272099 & -0.355137 & 3.066496 \\
\hline & 6.977929 & 0.836545 & 0.714009 \\
\hline & -2.181753 & 2.189511 & 2.429883 \\
\hline & 7.772592 & -4.039054 & 1.237552 \\
\hline 1 & -1.244389 & 0.303010 & 6.652491 \\
\hline & 6.225962 & 4.734243 & -2.703692 \\
\hline 1 & -3.410832 & -2.056091 & 2.167057 \\
\hline . & -5.058776 & -3.873523 & 2.068928 \\
\hline & 4.128094 & 4.523980 & -1.497438 \\
\hline 1 & -1.930903 & 1.767841 & 4.814323 \\
\hline & 5.761031 & -4.531457 & -0.046904 \\
\hline 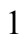 & 8.734674 & 1.298449 & -3.309137 \\
\hline 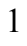 & 3.670546 & -3.725358 & -1.153616 \\
\hline | & -2.206838 & -6.409869 & 0.084319 \\
\hline ] & 0.045632 & -5.584250 & -0.381223 \\
\hline 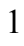 & 0.083580 & -3.698934 & 5.818688 \\
\hline 1 & 7.174004 & -0.094010 & -2.038969 \\
\hline & -0.242782 & -3.336325 & 3.421078 \\
\hline 1 & 1.725765 & -3.766966 & -0.266072 \\
\hline ] & 8.293197 & 3.730574 & -3.631184 \\
\hline 1 & 8.906616 & 0.035934 & 1.999416 \\
\hline 1 & -4.461479 & -6.071422 & 1.057953 \\
\hline 1 & -0.411368 & -1.889177 & 7.456168 \\
\hline 1 & 9.333832 & -2.406224 & 2.254702 \\
\hline 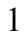 & 2.467244 & 3.092284 & -0.302419 \\
\hline 1 & -4.139343 & 1.201343 & 1.404198 \\
\hline 1 & -3.025678 & -1.403322 & -1.801684 \\
\hline 1 & -7.078044 & -1.348800 & -0.360370 \\
\hline 1 & -3.661721 & 2.297947 & -1.351128 \\
\hline 1 & -0.228359 & 2.794373 & 1.162750 \\
\hline 1 & -2.323138 & 6.231586 & -0.312531 \\
\hline 1 & 1.933164 & -2.359478 & 2.662585 \\
\hline 1 & -1.225543 & 0.977666 & -2.607731 \\
\hline 1 & 3.317975 & -2.235391 & 1.585360 \\
\hline 1 & 4.978568 & -1.113897 & 2.725276 \\
\hline 1 & 0.989469 & 0.448685 & 2.875484 \\
\hline
\end{tabular}




\begin{tabular}{|c|c|c|c|}
\hline 1 & 5.790909 & 0.818166 & 4.035326 \\
\hline 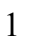 & 1.781134 & 2.367984 & 4.216585 \\
\hline & 4.198386 & 2.574798 & 4.788996 \\
\hline & 1.875186 & -0.544463 & -3.546949 \\
\hline 1 & 0.746040 & 0.611374 & -4.396505 \\
\hline & -2.134613 & -3.019507 & -3.250301 \\
\hline 1 & -1.873348 & -5.445142 & -3.067816 \\
\hline 1 & 0.401800 & -6.473638 & -3.111592 \\
\hline 1 & 2.395748 & -4.990340 & -3.280679 \\
\hline 1 & 2.135017 & -2.560522 & -3.420388 \\
\hline 1 & -1.561393 & -1.477579 & -5.253992 \\
\hline 1 & -2.407433 & -0.912245 & -3.807108 \\
\hline 1 & -1.452247 & 0.223660 & -4.743934 \\
\hline 6 & -6.980332 & -2.914163 & -2.221112 \\
\hline 6 & -5.166720 & -2.060657 & -3.659700 \\
\hline 6 & -4.706085 & -3.844914 & -1.959870 \\
\hline 6 & -6.280520 & 1.582097 & 2.530360 \\
\hline 6 & -8.008778 & 1.021086 & 0.848556 \\
\hline 6 & -7.237975 & -0.703770 & 2.491793 \\
\hline 1 & -7.082156 & 1.909680 & 3.204198 \\
\hline 1 & -5.444622 & 1.241780 & 3.156378 \\
\hline 1 & -5.946783 & 2.459960 & 1.960628 \\
\hline 1 & -8.002137 & -0.383628 & 3.212993 \\
\hline 1 & -7.662529 & -1.522333 & 1.897476 \\
\hline 1 & -6.386009 & -1.106084 & 3.056668 \\
\hline 1 & -8.776882 & 1.371129 & 1.551078 \\
\hline 1 & -7.721765 & 1.869190 & 0.213219 \\
\hline 1 & -8.472895 & 0.263495 & 0.206061 \\
\hline 1 & -7.185991 & -3.688133 & -2.971317 \\
\hline 1 & -7.291474 & -3.314384 & -1.247655 \\
\hline 1 & -7.613478 & -2.049177 & -2.458472 \\
\hline 1 & -5.480449 & -2.800518 & -4.408132 \\
\hline 1 & -5.687894 & -1.118817 & -3.875053 \\
\hline 1 & -4.094531 & -1.888573 & -3.802553 \\
\hline 1 & -4.858109 & -4.578575 & -2.764448 \\
\hline 1 & -3.628703 & -3.662685 & -1.863034 \\
\hline 1 & -5.039655 & -4.297792 & -1.018043 \\
\hline 6 & -4.251250 & 6.419495 & -1.851186 \\
\hline 6 & -3.996862 & 4.408645 & -3.276543 \\
\hline 6 & -5.573410 & 4.390095 & -1.329853 \\
\hline 6 & -0.468538 & 6.990283 & 1.121981 \\
\hline 6 & -0.526510 & 5.219474 & 2.858333 \\
\hline 6 & 1.214132 & 5.175567 & 1.070253 \\
\hline 1 & -6.383725 & 4.761937 & -1.971477 \\
\hline 1 & -5.632554 & 3.294082 & -1.322729 \\
\hline 1 & -5.763761 & 4.742509 & -0.307279 \\
\hline 1 & -5.066515 & 6.764040 & -2.499931 \\
\hline 1 & -4.429611 & 6.835556 & -0.851031 \\
\hline 1 & -3.318682 & 6.847132 & -2.241127 \\
\hline 1 & -4.780486 & 4.803049 & -3.937720 \\
\hline 1 & -3.025490 & 4.748703 & -3.658777 \\
\hline 1 & -4.017659 & 3.314567 & -3.351163 \\
\hline 1 & 0.228408 & 7.573627 & 1.736812 \\
\hline 1 & -0.287319 & 7.257047 & 0.072767 \\
\hline 1 & -1.483698 & 7.311013 & 1.388345 \\
\hline 1 & 1.876209 & 5.832100 & 1.650593 \\
\hline
\end{tabular}




$\begin{array}{cccc}1 & 1.472843 & 4.143301 & 1.337784 \\ 1 & 1.438649 & 5.325725 & 0.005282 \\ 1 & 0.116522 & 5.843239 & 3.494344 \\ 1 & -1.571841 & 5.440562 & 3.112675 \\ 1 & -0.333055 & 4.169396 & 3.115399\end{array}$

$3_{\text {IC-re- }} \psi_{-1 \mathrm{~s} 2 \mathrm{~s} 334 \mathrm{~s}}$

Number of imaginary frequencies : 0

The smallest frequencies are : $11.0943 \quad 16.5589 \quad 17.7235 \mathrm{~cm}(-1)$

Electronic energy : $\quad \mathrm{HF}=-4360.8785685$

Zero-point correction $=\quad 1.455245($ Hartree/Particle $)$

Thermal correction to Energy= $\quad 1.539586$

Thermal correction to Enthalpy= $\quad 1.540530$

Thermal correction to Gibbs Free Energy $=\quad 1.337872$

Sum of electronic and zero-point Energies $=\quad-4359.423323$

Sum of electronic and thermal Energies $=\quad-4359.338983$

Sum of electronic and thermal Enthalpies $=\quad-4359.338038$

Sum of electronic and thermal Free Energies $=\quad-4359.540696$

Cartesian Coordinates

$\begin{array}{cccc}6 & 1.249025 & -3.162201 & -3.342984 \\ 6 & -0.024294 & -2.567960 & -3.337945 \\ 6 & -1.126752 & -3.432006 & -3.237250 \\ 6 & -0.975059 & -4.810058 & -3.151930 \\ 6 & 0.294628 & -5.379605 & -3.176016 \\ 6 & 1.403704 & -4.541870 & -3.265751 \\ 6 & -0.239609 & -1.115484 & -3.577246 \\ 6 & -1.491520 & -0.797822 & -4.374742 \\ 45 & -0.024367 & 0.347580 & -1.793604 \\ 6 & 0.521869 & 2.210629 & -1.911648 \\ 8 & 0.618822 & 3.270810 & -2.365902 \\ 15 & -1.725737 & 0.554129 & -0.030150 \\ 6 & -1.981627 & 2.381206 & -0.039422 \\ 6 & -1.072604 & 3.223819 & 0.605487 \\ 6 & -1.206738 & 4.611705 & 0.549403 \\ 6 & -2.230545 & 5.140223 & -0.245481 \\ 6 & -3.116728 & 4.326031 & -0.953866 \\ 6 & -2.990012 & 2.940908 & -0.817248 \\ 6 & -0.279596 & 5.489563 & 1.390687 \\ 6 & -4.208334 & 4.878784 & -1.867799 \\ 6 & -3.460140 & -0.069675 & -0.201744 \\ 6 & -3.808258 & -1.032025 & -1.146588 \\ 6 & -5.124430 & -1.515539 & -1.229244 \\ 6 & -6.066396 & -1.007894 & -0.332130 \\ 6 & -5.746375 & -0.047029 & 0.632805 \\ 6 & -4.433148 & 0.410787 & 0.677449 \\ 6 & -6.757315 & 0.502525 & 1.637299 \\ 6 & -5.493317 & -2.581418 & -2.263214 \\ 6 & -1.499189 & 0.213136 & 1.791801 \\ 6 & -1.075857 & -1.033072 & 2.224940 \\ 6 & -0.917405 & -1.298336 & 3.627780 \\ 6 & -1.211559 & -0.277571 & 4.576015 \\ 6 & -1.690182 & 0.965688 & 4.105514 \\ 6 & -1.830028 & 1.199076 & 2.764609\end{array}$




\begin{tabular}{|c|c|c|c|}
\hline 6 & -1.016313 & -0.521164 & 5.956010 \\
\hline 6 & -0.545409 & -1.731933 & 6.394970 \\
\hline b & -0.258336 & -2.751407 & 5.463812 \\
\hline & -0.444541 & -2.544252 & 4.119144 \\
\hline f & -0.817787 & -2.183136 & 1.316774 \\
\hline o & 0.429342 & -2.398389 & 0.760135 \\
\hline 6 & 0.730868 & -3.627315 & 0.129165 \\
\hline 6 & -0.196454 & -4.631472 & 0.065865 \\
\hline 6 & -1.495823 & -4.441491 & 0.589212 \\
\hline 6 & -1.820180 & -3.198210 & 1.206061 \\
\hline 6 & -3.126788 & -3.027569 & 1.732895 \\
\hline 6 & -4.048343 & -4.043039 & 1.673253 \\
\hline 6 & -3.714534 & -5.282515 & 1.084668 \\
\hline 6 & -2.469017 & -5.469697 & 0.541907 \\
\hline 7 & 1.470900 & -1.416361 & 0.886014 \\
\hline 15 & 1.767805 & -0.502438 & -0.507731 \\
\hline 8 & 2.947312 & -1.268455 & -1.426423 \\
\hline 6 & 4.157163 & -1.639692 & -0.888785 \\
\hline 6 & 5.093335 & -0.688048 & -0.526893 \\
\hline 6 & 6.255288 & -1.131291 & 0.185832 \\
\hline 6 & 6.483731 & -2.528994 & 0.364405 \\
\hline 6 & 5.557913 & -3.458897 & -0.169732 \\
\hline 6 & 4.407998 & -3.023609 & -0.769273 \\
\hline 6 & 7.160687 & -0.224854 & 0.794756 \\
\hline 6 & 8.236264 & -0.676433 & 1.519062 \\
\hline 6 & 8.476985 & -2.058884 & 1.664619 \\
\hline 6 & 7.615166 & -2.962528 & 1.097539 \\
\hline 6 & 4.850219 & 0.737954 & -0.841819 \\
\hline 6 & 3.666575 & 1.332678 & -0.448076 \\
\hline 6 & 3.399376 & 2.698310 & -0.659400 \\
\hline 6 & 4.311892 & 3.477098 & -1.315154 \\
\hline 6 & 5.511270 & 2.912904 & -1.812330 \\
\hline 6 & 5.782338 & 1.527720 & -1.592606 \\
\hline 6 & 6.436834 & 3.698055 & -2.541607 \\
\hline 6 & 7.578112 & 3.144372 & -3.061924 \\
\hline 6 & 7.831772 & 1.769124 & -2.879646 \\
\hline 6 & 6.960339 & 0.984008 & -2.166044 \\
\hline 8 & 2.727524 & 0.612504 & 0.244154 \\
\hline 6 & 0.836379 & -0.205276 & -3.697501 \\
\hline 6 & 2.454334 & -1.680315 & 1.965865 \\
\hline 6 & 2.922864 & -0.475015 & 2.738856 \\
\hline 6 & 2.031836 & 0.510298 & 3.167150 \\
\hline 6 & 2.481162 & 1.598675 & 3.902296 \\
\hline 6 & 3.833142 & 1.721100 & 4.220561 \\
\hline 6 & 4.727279 & 0.744069 & 3.797007 \\
\hline 6 & 4.272461 & -0.347912 & 3.061430 \\
\hline 1 & 6.974587 & 0.843037 & 0.710386 \\
\hline 1 & -2.202980 & 2.167240 & 2.443104 \\
\hline 1 & 7.775705 & -4.033624 & 1.214022 \\
\hline 1 & -1.244532 & 0.277699 & 6.660216 \\
\hline 1 & 6.212609 & 4.753733 & -2.689038 \\
\hline 1 & -3.396816 & -2.072174 & 2.181911 \\
\hline 1 & -5.047518 & -3.888360 & 2.075819 \\
\hline 1 & 4.117220 & 4.536052 & -1.479722 \\
\hline 1 & -1.945792 & 1.741491 & 4.826723 \\
\hline 1 & 5.762928 & -4.523481 & -0.069558 \\
\hline
\end{tabular}




\begin{tabular}{|c|c|c|c|}
\hline & 8.723703 & 1.323239 & -3.314287 \\
\hline & 3.669981 & -3.715635 & -1.170486 \\
\hline & -2.201026 & -6.414192 & 0.069153 \\
\hline & 0.051824 & -5.584205 & -0.397639 \\
\hline & 0.111785 & -3.712166 & 5.814240 \\
\hline & 7.166881 & -0.076422 & -2.047304 \\
\hline & -0.222370 & -3.346412 & 3.418096 \\
\hline & 1.730229 & -3.765886 & -0.277194 \\
\hline & 8.279121 & 3.756285 & -3.624810 \\
\hline & 8.905986 & 0.039744 & 1.990010 \\
\hline 1 & -4.453151 & -6.080677 & 1.049683 \\
\hline & -0.392802 & -1.909830 & 7.456907 \\
\hline & 9.336462 & -2.402876 & 2.235210 \\
\hline L & 2.459851 & 3.097278 & -0.288365 \\
\hline & -4.156677 & 1.159218 & 1.418325 \\
\hline 1 & -3.030401 & -1.417685 & -1.807946 \\
\hline & -7.086961 & -1.374182 & -0.382031 \\
\hline 1 & -3.679867 & 2.283080 & -1.344864 \\
\hline & -0.254966 & 2.783638 & 1.180782 \\
\hline ] & -2.336079 & 6.218281 & -0.317807 \\
\hline & 1.942414 & -2.364199 & 2.657459 \\
\hline 1 & -1.236321 & 0.983962 & -2.595588 \\
\hline 1 & 3.325383 & -2.230815 & 1.579106 \\
\hline & 4.982291 & -1.101862 & 2.716555 \\
\hline 1 & 0.984693 & 0.437186 & 2.881904 \\
\hline 1 & 5.787465 & 0.832102 & 4.027845 \\
\hline 1 & 1.768987 & 2.357290 & 4.225761 \\
\hline ] & 4.186744 & 2.577554 & 4.790833 \\
\hline & 1.866561 & -0.523591 & -3.546455 \\
\hline 1 & 0.731257 & 0.631765 & -4.388349 \\
\hline & -2.132197 & -3.015783 & -3.245984 \\
\hline 1 & -1.860354 & -5.440967 & -3.075411 \\
\hline 1 & 0.418958 & -6.459891 & -3.132424 \\
\hline 1 & 2.405953 & -4.967534 & -3.303878 \\
\hline 1 & 2.134547 & -2.538607 & -3.433838 \\
\hline 1 & -1.573269 & -1.464930 & -5.247261 \\
\hline 1 & -2.415419 & -0.900865 & -3.797359 \\
\hline 1 & -1.463997 & 0.235961 & -4.736353 \\
\hline 6 & -6.981055 & -2.929113 & -2.243294 \\
\hline 5 & -5.157273 & -2.082123 & -3.671636 \\
\hline 6 & -4.710512 & -3.864791 & -1.967200 \\
\hline 6 & -8.143735 & -0.113207 & 1.468650 \\
\hline 6 & -6.269697 & 0.194465 & 3.058343 \\
\hline 6 & -6.882328 & 2.019368 & 1.454037 \\
\hline 1 & -8.829090 & 0.312718 & 2.212329 \\
\hline 1 & -8.567292 & 0.092187 & 0.477097 \\
\hline 1 & -8.130890 & -1.200598 & 1.619011 \\
\hline 1 & -7.573247 & 2.438941 & 2.197779 \\
\hline 1 & -5.918088 & 2.531533 & 1.566168 \\
\hline 1 & -7.273386 & 2.261172 & 0.456746 \\
\hline 1 & -6.978471 & 0.587677 & 3.799624 \\
\hline 1 & -6.184251 & -0.889180 & 3.215228 \\
\hline 1 & -5.288028 & 0.637805 & 3.268753 \\
\hline 1 & -7.183399 & -3.701746 & -2.995788 \\
\hline 1 & -7.299790 & -3.329935 & -1.272651 \\
\hline 1 & -7.611039 & -2.062718 & -2.483830 \\
\hline
\end{tabular}




$\begin{array}{lrrr}1 & -5.474464 & -2.819959 & -4.420629 \\ 1 & -5.669953 & -1.136242 & -3.889667 \\ 1 & -4.083316 & -1.919537 & -3.811426 \\ 1 & -4.862835 & -4.600599 & -2.769793 \\ 1 & -3.632902 & -3.684624 & -1.868483 \\ 1 & -5.047505 & -4.313683 & -1.024608 \\ 6 & -4.240559 & 6.404805 & -1.884442 \\ 6 & -3.950242 & 4.390836 & -3.298738 \\ 6 & -5.578327 & 4.378296 & -1.395520 \\ 6 & -0.498989 & 6.979251 & 1.137573 \\ 6 & -0.558741 & 5.207563 & 2.872679 \\ 6 & 1.187138 & 5.167613 & 1.089649 \\ 1 & -6.369488 & 4.749760 & -2.060861 \\ 1 & -5.639572 & 3.282366 & -1.387121 \\ 1 & -5.796745 & 4.734057 & -0.379749 \\ 1 & -5.040448 & 6.748593 & -2.552443 \\ 1 & -4.441468 & 6.823048 & -0.889469 \\ 1 & -3.299168 & 6.831070 & -2.254054 \\ 1 & -4.716744 & 4.782820 & -3.981130 \\ 1 & -2.969586 & 4.731140 & -3.656406 \\ 1 & -3.967542 & 3.296408 & -3.370594 \\ 1 & 0.193834 & 7.563551 & 1.756150 \\ 1 & -0.313332 & 7.247484 & 0.089524 \\ 1 & -1.516188 & 7.297320 & 1.399352 \\ 1 & 1.846347 & 5.821313 & 1.676447 \\ 1 & 1.445468 & 4.133891 & 1.351967 \\ 1 & 1.415943 & 5.324306 & 0.026556 \\ 1 & 0.080281 & 5.833355 & 3.510708 \\ 1 & -1.605539 & 5.425286 & 3.123849 \\ 1 & -0.362526 & 4.158185 & 3.130407\end{array}$

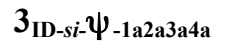

Number of imaginary frequencies : 0

The smallest frequencies are : $13.8379 \quad 17.2062 \quad 19.8242 \mathrm{~cm}(-1)$

Electronic energy : $\quad H F=-4360.8793799$

Zero-point correction $=\quad 1.454917$ (Hartree/Particle)

Thermal correction to Energy= $\quad 1.539632$

Thermal correction to Enthalpy= $\quad 1.540576$

Thermal correction to Gibbs Free Energy $=\quad 1.336483$

Sum of electronic and zero-point Energies $=\quad-4359.424463$

Sum of electronic and thermal Energies $=\quad-4359.339748$

Sum of electronic and thermal Enthalpies $=\quad-4359.338804$

Sum of electronic and thermal Free Energies $=\quad-4359.542897$

Cartesian Coordinates

$\begin{array}{lrrr}6 & 2.361092 & -1.077019 & 5.118506 \\ 6 & 1.624808 & -0.092885 & 4.442406 \\ 6 & 2.015096 & 1.243211 & 4.653669 \\ 6 & 3.059192 & 1.571790 & 5.504442 \\ 6 & 3.763747 & 0.576734 & 6.182704 \\ 6 & 3.408733 & -0.748654 & 5.977392 \\ 6 & 0.407597 & -0.459756 & 3.658475 \\ 6 & 0.089809 & -1.933007 & 3.595961\end{array}$




\begin{tabular}{|c|c|c|c|}
\hline 45 & 0.064769 & 0.588386 & 1.581163 \\
\hline 6 & -0.558006 & 2.420093 & 1.363172 \\
\hline 8 & -0.643132 & 3.552605 & 1.588984 \\
\hline 15 & 1.553659 & 0.433739 & -0.336962 \\
\hline 6 & 1.642552 & 2.190917 & -0.875354 \\
\hline 6 & 0.558580 & 2.743297 & -1.561495 \\
\hline 6 & 0.535817 & 4.093798 & -1.906137 \\
\hline 6 & 1.608359 & 4.888443 & -1.487254 \\
\hline 6 & 2.695653 & 4.373912 & -0.777147 \\
\hline 6 & 2.699615 & 3.007340 & -0.483631 \\
\hline 6 & -0.631951 & 4.708305 & -2.677046 \\
\hline 6 & 3.844843 & 5.295617 & -0.372276 \\
\hline 6 & 3.325551 & -0.035426 & -0.158838 \\
\hline 6 & 3.780449 & -0.621077 & 1.013440 \\
\hline 6 & 5.101502 & -1.091472 & 1.112872 \\
\hline 6 & 5.934826 & -0.925149 & 0.011493 \\
\hline 6 & 5.512948 & -0.309657 & -1.177891 \\
\hline 6 & 4.196293 & 0.123990 & -1.246150 \\
\hline 6 & 6.485380 & -0.168202 & -2.346547 \\
\hline 6 & 5.595962 & -1.718480 & 2.413548 \\
\hline 6 & 1.211854 & -0.439120 & -1.950815 \\
\hline 6 & 0.885343 & -1.785775 & -1.967944 \\
\hline 6 & 0.674645 & -2.464222 & -3.216931 \\
\hline 6 & 0.817560 & -1.748162 & -4.440060 \\
\hline 6 & 1.186164 & -0.385237 & -4.389631 \\
\hline 6 & 1.373972 & 0.243880 & -3.189490 \\
\hline 6 & 0.579842 & -2.403413 & -5.671460 \\
\hline 6 & 0.210042 & -3.723277 & -5.704766 \\
\hline 6 & 0.068136 & -4.441116 & -4.499261 \\
\hline 6 & 0.297610 & -3.832124 & -3.290041 \\
\hline 6 & 0.752776 & -2.632648 & -0.749746 \\
\hline 6 & -0.462517 & -2.744886 & -0.099184 \\
\hline 6 & -0.674226 & -3.768872 & \\
\hline 6 & 0.323239 & -4.650411 & 1.174259 \\
\hline 6 & 1.601375 & -4.533148 & 0.578638 \\
\hline 6 & 1.823639 & -3.510597 & -0.390959 \\
\hline 6 & 3.102445 & -3.423224 & -0.998595 \\
\hline 6 & 4.101873 & -4.304485 & -0.668924 \\
\hline 6 & 3.873185 & -5.326811 & 0.278078 \\
\hline 6 & 2.650377 & -5.432686 & 0.891419 \\
\hline 7 & -1.550896 & -1.865904 & -0.427048 \\
\hline 15 & -1.779465 & -0.595639 & 0.671689 \\
\hline 8 & -2.860860 & -1.107923 & 1.853894 \\
\hline 6 & -4.104404 & -1.601912 & 1.539616 \\
\hline 6 & -5.085429 & -0.774662 & 1.024032 \\
\hline 6 & -6.300127 & -1.383531 & 0.566599 \\
\hline 6 & -6.519558 & -2.776333 & 0.789426 \\
\hline 6 & -5.530429 & -3.538336 & 1.458546 \\
\hline 6 & -4.338699 & -2.967301 & 1.810933 \\
\hline 6 & -7.274552 & -0.666231 & -0.173288 \\
\hline 6 & -8.405684 & -1.286434 & -0.643999 \\
\hline 6 & -8.634658 & -2.655307 & -0.391740 \\
\hline 6 & -7.706943 & -3.381257 & 0.310508 \\
\hline 6 & -4.841805 & 0.682669 & 0.920068 \\
\hline 6 & -3.714967 & 1.144054 & 0.266291 \\
\hline 6 & -3.464957 & 2.515253 & 0.064178 \\
\hline
\end{tabular}




\begin{tabular}{|c|c|c|c|}
\hline & -4.332230 & 3.445272 & 0.567079 \\
\hline & -5.461833 & 3.041490 & 1.318561 \\
\hline & -5.717717 & 1.649943 & 1.514125 \\
\hline & -6.332655 & 3.998727 & 1.893337 \\
\hline & -7.404302 & 3.611168 & 2.655649 \\
\hline & -7.640038 & 2.239186 & 2.881648 \\
\hline & -6.821803 & 1.286453 & 2.326987 \\
\hline & -2.825937 & 0.255068 & -0.282644 \\
\hline & -0.664912 & 0.461600 & 3.618192 \\
\hline & -2.591715 & -2.454726 & -1.306742 \\
\hline & -3.189011 & -1.533596 & -2.338553 \\
\hline & -2.390133 & -0.706764 & -3.130223 \\
\hline & -2.958390 & 0.100076 & -4.106781 \\
\hline & -4.338173 & 0.096132 & -4.306422 \\
\hline & -5.141117 & -0.722183 & -3.519851 \\
\hline & -4.567645 & -1.532626 & -2.542799 \\
\hline & -7.101351 & 0.384032 & -0.394404 \\
\hline & 1.658683 & 1.292020 & -3.189328 \\
\hline & -7.858182 & -4.443175 & 0.499998 \\
\hline & 0.692618 & -1.832223 & -6.591888 \\
\hline & -6.121956 & 5.053850 & 1.723800 \\
\hline & 3.288842 & -2.634260 & -1.725876 \\
\hline & 5.079384 & -4.210897 & -1.138748 \\
\hline & -4.153657 & 4.508589 & 0.412612 \\
\hline & 1.317229 & 0.160560 & -5.323353 \\
\hline & -5.723846 & -4.590145 & 1.662524 \\
\hline & -8.474811 & 1.931772 & 3.507607 \\
\hline & -3.555325 & -3.534994 & 2.309293 \\
\hline & 2.462346 & -6.212143 & 1.628586 \\
\hline 1 & 0.149771 & -5.446424 & 1.896986 \\
\hline & -0.224381 & -5.488259 & -4.527165 \\
\hline 1 & -7.011941 & 0.234565 & 2.523342 \\
\hline & 0.185389 & -4.407012 & -2.373054 \\
\hline & -1.659080 & -3.849444 & 1.310694 \\
\hline & -8.063213 & 4.355389 & 3.096803 \\
\hline & -9.129473 & -0.716815 & -1.222687 \\
\hline ] & 4.671872 & -6.022890 & 0.525185 \\
\hline & 0.024907 & -4.218416 & -6.655130 \\
\hline 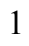 & -9.537833 & -3.132468 & -0.764754 \\
\hline . & -2.578065 & 2.798251 & -0.499100 \\
\hline . & 3.824871 & 0.591588 & -2.154222 \\
\hline 1 & 3.084337 & -0.720868 & 1.848696 \\
\hline & 6.959004 & -1.288377 & 0.066291 \\
\hline 1 & 3.529389 & 2.566830 & 0.063683 \\
\hline & -0.271030 & 2.093162 & -1.840182 \\
\hline 1 & 1.598201 & 5.951588 & -1.726638 \\
\hline l & -2.087555 & -3.281876 & -1.827042 \\
\hline 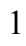 & 1.329580 & 1.382225 & 2.099855 \\
\hline 1 & -3.398334 & -2.910358 & -0.712684 \\
\hline 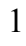 & -5.204914 & -2.162407 & -1.919435 \\
\hline 1 & -1.317502 & -0.674232 & -2.951757 \\
\hline . & -6.220780 & -0.728330 & -3.657883 \\
\hline 1 & -2.317358 & 0.737025 & -4.715122 \\
\hline 1 & -4.783574 & 0.732219 & -5.068459 \\
\hline 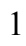 & -1.685113 & 0.081529 & 3.574587 \\
\hline & -0.572799 & 1.418511 & 4.131592 \\
\hline
\end{tabular}




\begin{tabular}{|c|c|c|c|}
\hline$\frac{1}{1}$ & 1.488643 & 2.043886 & 4.136847 \\
\hline & 3.324744 & 2.618371 & 5.642116 \\
\hline ] & 4.582470 & 0.835593 & 6.850565 \\
\hline 1 & 3.949625 & -1.546234 & 6.484946 \\
\hline & 2.106541 & -2.126752 & 4.995967 \\
\hline 1 & -0.025065 & -2.355465 & 4.607900 \\
\hline & -0.851633 & -2.104476 & 3.066539 \\
\hline & 0.871278 & -2.511386 & 3.084793 \\
\hline & 4.580219 & -2.740291 & 2.931462 \\
\hline & 6.931063 & -2.441540 & 2.242525 \\
\hline & 5.782554 & -0.593128 & 3.436719 \\
\hline & 5.862207 & 0.572539 & -3.527806 \\
\hline & 7.723855 & 0.613834 & -1.896083 \\
\hline & 6.905274 & -1.563096 & -2.823791 \\
\hline & 4.928260 & -3.169829 & 3.881415 \\
\hline & 3.595632 & -2.297491 & 3.117950 \\
\hline 1 & 4.450685 & -3.559920 & 2.212426 \\
\hline ] & 7.209921 & -2.921848 & 3.189055 \\
\hline$\frac{1}{1}$ & 6.874728 & -3.226482 & 1.475513 \\
\hline & 7.747408 & -1.757765 & 1.977481 \\
\hline l & 6.140828 & -0.995381 & 4.394807 \\
\hline & 6.520682 & 0.136659 & 3.077474 \\
\hline 1 & 4.844078 & -0.059018 & 3.628786 \\
\hline ] & 6.596660 & 0.658026 & -4.338565 \\
\hline & 4.987600 & 0.044475 & -3.931162 \\
\hline 1 & 5.552263 & 1.590002 & -3.253407 \\
\hline | & 7.611841 & -1.485601 & -3.661278 \\
\hline 1 & 7.394268 & -2.140590 & -2.029401 \\
\hline ] & 6.034416 & -2.136524 & -3.169754 \\
\hline 1 & 8.424863 & 0.732743 & -2.733147 \\
\hline 1 & 7.447667 & 1.615527 & -1.541193 \\
\hline 1 & 8.262014 & 0.109055 & -1.085072 \\
\hline 6 & -1.519461 & 3.639432 & -3.316762 \\
\hline 6 & -1.475950 & 5.541138 & -1.705882 \\
\hline 6 & -0.117711 & 5.611550 & -3.802523 \\
\hline 6 & 4.947456 & 4.550776 & 0.377563 \\
\hline 6 & 4.464236 & 5.916721 & -1.629428 \\
\hline 6 & 3.312283 & 6.406558 & 0.538371 \\
\hline 1 & -2.294768 & 4.118480 & -3.928011 \\
\hline 1 & -0.937415 & 2.975233 & -3.971562 \\
\hline 1 & -2.037753 & 3.013269 & -2.579068 \\
\hline 1 & -0.963428 & 6.030591 & -4.363459 \\
\hline 1 & 0.472212 & 6.456257 & -3.429003 \\
\hline 1 & 0.508726 & 5.048971 & -4.506960 \\
\hline 1 & -2.351762 & 5.964180 & -2.217085 \\
\hline 1 & -1.829877 & 4.927326 & -0.866488 \\
\hline 1 & -0.897460 & 6.371050 & -1.280932 \\
\hline 1 & 4.129171 & 7.074326 & 0.843961 \\
\hline 1 & 2.552055 & 7.019504 & 0.039418 \\
\hline 1 & 2.858834 & 5.986140 & 1.445238 \\
\hline 1 & 5.752744 & 5.250013 & 0.635983 \\
\hline 1 & 4.582663 & 4.108490 & 1.313418 \\
\hline 1 & 5.389699 & 3.748682 & -0.229564 \\
\hline 1 & 5.297060 & 6.578953 & -1.356870 \\
\hline 1 & 4.855715 & 5.139218 & -2.298894 \\
\hline 1 & 3.739495 & 6.512635 & -2.197114 \\
\hline
\end{tabular}


$3_{\text {ID-si- }} \psi_{-1 \text { a2a3a4s }}$

Number of imaginary frequencies : 0

The smallest frequencies are : $15.8684 \quad 17.8517 \quad 19.8321 \mathrm{~cm}(-1)$

Electronic energy : $\quad \mathrm{HF}=-4360.879696$

Zero-point correction $=\quad 1.454808($ Hartree/Particle $)$

Thermal correction to Energy $=\quad 1.539538$

Thermal correction to Enthalpy= $\quad 1.540482$

Thermal correction to Gibbs Free Energy $=1.336774$

Sum of electronic and zero-point Energies $=\quad-4359.424888$

Sum of electronic and thermal Energies $=\quad-4359.340158$

Sum of electronic and thermal Enthalpies $=\quad-4359.339214$

Sum of electronic and thermal Free Energies $=\quad-4359.542922$

Cartesian Coordinates

$\begin{array}{ccccc}6 & 2.355619 & -0.933213 & 5.140999 \\ 6 & 1.619979 & 0.029535 & 4.434213 \\ 6 & 2.010051 & 1.371462 & 4.605242 \\ 6 & 3.054001 & 1.726069 & 5.445664 \\ 6 & 3.758428 & 0.752251 & 6.154190 \\ 6 & 3.402956 & -0.578672 & 5.989910 \\ 6 & 0.403783 & -0.361344 & 3.659237 \\ 6 & 0.089610 & -1.836694 & 3.636850 \\ 45 & 0.057523 & 0.631085 & 1.561326 \\ 6 & -0.574881 & 2.452175 & 1.286469 \\ 8 & -0.667786 & 3.590264 & 1.478506 \\ 15 & 1.556058 & 0.425766 & -0.346589 \\ 6 & 1.660219 & 2.171534 & -0.918855 \\ 6 & 0.588028 & 2.716502 & -1.628919 \\ 6 & 0.574600 & 4.061627 & -1.995250 \\ 6 & 1.637827 & 4.861279 & -1.563633 \\ 6 & 2.707491 & 4.357192 & -0.819615 \\ 6 & 2.710863 & 2.992542 & -0.517386 \\ 6 & -0.578921 & 4.665838 & -2.795349 \\ 6 & 3.839658 & 5.289457 & -0.391416 \\ 6 & 3.325003 & -0.051482 & -0.154336 \\ 6 & 3.786174 & -0.599552 & 1.037925 \\ 6 & 5.102181 & -1.070886 & 1.144274 \\ 6 & 5.935262 & -0.953397 & 0.029563 \\ 6 & 5.505680 & -0.387196 & -1.175512 \\ 6 & 4.188758 & 0.058864 & -1.245758 \\ 6 & 6.398009 & -0.263524 & -2.409046 \\ 6 & 5.602032 & -1.652794 & 2.464315 \\ 6 & 1.215200 & -0.484246 & -1.940075 \\ 6 & 0.895817 & -1.832921 & -1.924748 \\ 6 & 0.693382 & -2.543431 & -3.157083 \\ 6 & 0.837561 & -1.857623 & -4.397362 \\ 6 & 1.194670 & -0.490991 & -4.379601 \\ 6 & 1.375173 & 0.168988 & -3.194728 \\ 6 & 0.611154 & -2.545450 & -5.613078 \\ 6 & 0.249842 & -3.867986 & -5.614869 \\ 6 & 0.104656 & -4.555533 & -4.392090 \\ 6 & 0.324001 & -3.914769 & -3.197527 \\ 6 & 0.765583 & -2.649917 & -0.686010 \\ 6 & & & \\ & & \end{array}$




\begin{tabular}{|c|c|c|c|}
\hline 6 & -0.448858 & -2.749153 & -0.031647 \\
\hline & -0.656420 & -3.748199 & 0.947855 \\
\hline & 0.344264 & -4.617522 & 1.291176 \\
\hline & 1.621367 & -4.512004 & 0.691160 \\
\hline & 1.839501 & -3.514663 & -0.305374 \\
\hline & 3.117315 & -3.438798 & -0.916583 \\
\hline & 4.119596 & -4.308041 & -0.564464 \\
\hline & 3.895235 & -5.305965 & 0.409182 \\
\hline & 2.673417 & -5.399760 & 1.026356 \\
\hline & -1.541058 & -1.884450 & -0.382795 \\
\hline & -1.779123 & -0.587834 & 0.682256 \\
\hline & -2.860736 & -1.072372 & 1.875509 \\
\hline & -4.102939 & -1.576380 & 1.571495 \\
\hline & -5.086338 & -0.763025 & 1.038483 \\
\hline & -6.301835 & -1.384266 & 0.599619 \\
\hline & -6.518294 & -2.771933 & 0.854905 \\
\hline & -5.524949 & -3.517392 & 1.536209 \\
\hline & -4.333439 & -2.936253 & 1.872365 \\
\hline & -7.280061 & -0.685998 & -0.153423 \\
\hline & -8.412181 & -1.318444 & -0.605140 \\
\hline & -8.638436 & -2.681284 & -0.319991 \\
\hline & -7.706653 & -3.389423 & 0.394890 \\
\hline & -4.844525 & 0.691028 & 0.891944 \\
\hline & -3.720362 & 1.133747 & 0.220518 \\
\hline & -3.477022 & 2.497835 & -0.031639 \\
\hline & -4.346599 & 3.441494 & 0.440780 \\
\hline & -5.471830 & 3.059990 & 1.210298 \\
\hline & -5.721999 & 1.675214 & 1.454963 \\
\hline & -6.344080 & 4.033479 & 1.754742 \\
\hline & -7.411686 & 3.668803 & 2.533854 \\
\hline & -7.641969 & 2.304609 & 2.807802 \\
\hline & -6.822236 & 1.336106 & 2.283406 \\
\hline & -2.827362 & 0.230594 & -0.296447 \\
\hline & -0.671790 & 0.556426 & 3.598866 \\
\hline 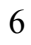 & -2.570979 & -2.497983 & -1.259492 \\
\hline & -3.177464 & -1.592167 & -2.299271 \\
\hline 6 & -2.381824 & -0.782349 & -3.111473 \\
\hline & -2.956107 & 0.026352 & -4.082726 \\
\hline 6 & -4.339357 & 0.039853 & -4.257213 \\
\hline o & -5.138682 & -0.765199 & -3.453392 \\
\hline & -4.558825 & -1.577526 & -2.481629 \\
\hline 1 & -7.109313 & 0.359008 & -0.399837 \\
\hline & 1.652829 & 1.218669 & -3.219630 \\
\hline 1 & -7.855324 & -4.446990 & 0.609119 \\
\hline & 0.725739 & -1.996953 & -6.546970 \\
\hline 1 & -6.137778 & 5.082867 & 1.548177 \\
\hline ] & 3.300660 & -2.666907 & -1.663383 \\
\hline 1 & 5.096807 & -4.224356 & -1.036695 \\
\hline 1 & -4.173012 & 4.499385 & 0.248091 \\
\hline & 1.324168 & 0.032631 & -5.326129 \\
\hline 1 & -5.715161 & -4.565095 & 1.763101 \\
\hline & -8.473635 & 2.016116 & 3.446757 \\
\hline 1 & -3.547416 & -3.491672 & 2.380330 \\
\hline 1 & 2.488722 & -6.160085 & 1.784050 \\
\hline 1 & 0.174194 & -5.394655 & 2.034934 \\
\hline & -0.182137 & -5.604621 & -4.394912 \\
\hline
\end{tabular}




\begin{tabular}{|c|c|c|c|}
\hline 1 & -7.008043 & 0.290760 & 2.515913 \\
\hline 1 & 0.209551 & -4.466787 & -2.266878 \\
\hline 1 & -1.640813 & -3.819193 & 1.407773 \\
\hline 1 & -8.071710 & 4.425499 & 2.951507 \\
\hline & -9.138996 & -0.763377 & -1.194100 \\
\hline & 4.696548 & -5.992620 & 0.673570 \\
\hline & 0.073342 & -4.388323 & -6.553318 \\
\hline 1 & -9.542427 & -3.168210 & -0.678092 \\
\hline 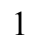 & -2.592815 & 2.764234 & -0.606964 \\
\hline 1 & 3.820440 & 0.491744 & -2.175122 \\
\hline 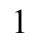 & 3.094545 & -0.667313 & 1.880166 \\
\hline & 6.954540 & -1.321653 & 0.098595 \\
\hline 1 & 3.530870 & 2.558470 & 0.050037 \\
\hline 1 & -0.241135 & 2.065509 & -1.907369 \\
\hline 1 & 1.633501 & 5.920999 & -1.817254 \\
\hline 1 & -2.052651 & -3.322150 & -1.770448 \\
\hline 1 & 1.316023 & 1.446618 & 2.060338 \\
\hline 1 & -3.372178 & -2.959012 & -0.662128 \\
\hline 1 & -5.193271 & -2.195707 & -1.844027 \\
\hline 1 & -1.306195 & -0.762477 & -2.951299 \\
\hline 1 & -6.220499 & -0.759355 & -3.573320 \\
\hline 1 & -2.317213 & 0.651898 & -4.705081 \\
\hline 1 & -4.790111 & 0.678192 & -5.014215 \\
\hline 1 & -1.691618 & 0.173954 & 3.566375 \\
\hline 1 & -0.580318 & 1.525729 & 4.088597 \\
\hline 1 & 1.483728 & 2.156002 & 4.064172 \\
\hline 1 & 3.319580 & 2.776414 & 5.550997 \\
\hline 1 & 4.577388 & 1.031286 & 6.813582 \\
\hline 1 & 3.943625 & -1.360446 & 6.521747 \\
\hline 1 & 2.101224 & -1.986263 & 5.050769 \\
\hline 1 & -0.020590 & -2.232901 & 4.659804 \\
\hline 1 & -0.853626 & -2.024589 & 3.116300 \\
\hline 1 & 0.870016 & -2.426729 & 3.137317 \\
\hline 6 & 4.588678 & -2.658353 & 3.017402 \\
\hline 6 & 6.938360 & -2.378617 & 2.316118 \\
\hline 6 & 5.787641 & -0.494842 & 3.450517 \\
\hline 6 & 7.802060 & -0.813077 & -2.171968 \\
\hline 6 & 5.770572 & -1.050049 & -3.566422 \\
\hline 6 & 6.522155 & 1.213894 & -2.799158 \\
\hline 1 & 4.942311 & -3.062323 & 3.976547 \\
\hline 1 & 3.607128 & -2.206893 & 3.198304 \\
\hline 1 & 4.451290 & -3.496708 & 2.321621 \\
\hline 1 & 7.221019 & -2.822156 & 3.279306 \\
\hline 1 & 6.882082 & -3.192377 & 1.579839 \\
\hline 1 & 7.752309 & -1.703572 & 2.022823 \\
\hline 1 & 6.154472 & -0.865101 & 4.418299 \\
\hline 1 & 6.518931 & 0.228245 & 3.064658 \\
\hline 1 & 4.846636 & 0.038452 & 3.631915 \\
\hline 1 & 8.400003 & -0.703975 & -3.085668 \\
\hline 1 & 8.324776 & -0.274994 & -1.370739 \\
\hline 1 & 7.785845 & -1.880725 & -1.915992 \\
\hline 1 & 7.144135 & 1.321656 & -3.698052 \\
\hline 1 & 5.547101 & 1.670017 & -3.012286 \\
\hline 1 & 6.991289 & 1.792501 & -1.992785 \\
\hline 1 & 6.380992 & -0.948104 & -4.474002 \\
\hline 1 & 5.708033 & -2.119433 & -3.323502 \\
\hline
\end{tabular}




$\begin{array}{lrrr}1 & 4.756770 & -0.704619 & -3.806429 \\ 6 & -1.439550 & 3.588542 & -3.457627 \\ 6 & -1.455346 & 5.488540 & -1.844479 \\ 6 & -0.047300 & 5.576169 & -3.906819 \\ 6 & 4.890920 & 4.573513 & 0.453681 \\ 6 & 4.531236 & 5.849500 & -1.639359 \\ 6 & 3.268771 & 6.443601 & 0.439264 \\ 1 & -2.205972 & 4.060214 & -4.085567 \\ 1 & -0.836214 & 2.931216 & -4.100016 \\ 1 & -1.966841 & 2.956112 & -2.732225 \\ 1 & -0.883744 & 5.982790 & -4.490313 \\ 1 & 0.519459 & 6.429834 & -3.518243 \\ 1 & 0.605861 & 5.022895 & -4.594142 \\ 1 & -2.318908 & 5.908859 & -2.378316 \\ 1 & -1.829652 & 4.868469 & -1.018670 \\ 1 & -0.893854 & 6.319496 & -1.399238 \\ 1 & 4.075961 & 7.113555 & 0.764948 \\ 1 & 2.548440 & 7.046350 & -0.126573 \\ 1 & 2.757667 & 6.066417 & 1.334439 \\ 1 & 5.677359 & 5.283124 & 0.740354 \\ 1 & 4.464367 & 4.161822 & 1.377458 \\ 1 & 5.372804 & 3.753142 & -0.094672 \\ 1 & 5.347674 & 6.526204 & -1.352815 \\ 1 & 4.959825 & 5.040825 & -2.246351 \\ 1 & 3.838901 & 6.414286 & -2.275483\end{array}$

$3_{\text {ID-si- }} \psi_{-1 \text {-12a3s4a }}$

Number of imaginary frequencies : 0

The smallest frequencies are : $13.7227 \quad 16.1401 \quad 19.6370 \mathrm{~cm}(-1)$

Electronic energy : $\quad H F=-4360.8793094$

Zero-point correction $=\quad 1.454671($ Hartree/Particle $)$

Thermal correction to Energy= $\quad 1.539456$

Thermal correction to Enthalpy $=\quad 1.540400$

Thermal correction to Gibbs Free Energy $=1.335799$

Sum of electronic and zero-point Energies $=\quad-4359.424638$

Sum of electronic and thermal Energies $=\quad-4359.339854$

Sum of electronic and thermal Enthalpies $=\quad-4359.338910$

Sum of electronic and thermal Free Energies $=\quad-4359.543511$

Cartesian Coordinates

$\begin{array}{cccc}6 & 2.396253 & -0.986759 & 5.126612 \\ 6 & 1.649346 & -0.018269 & 4.439794 \\ 6 & 2.028766 & 1.323921 & 4.631764 \\ 6 & 3.072657 & 1.673119 & 5.474468 \\ 6 & 3.787847 & 0.693424 & 6.163877 \\ 6 & 3.443636 & -0.637583 & 5.977662 \\ 6 & 0.432924 & -0.405679 & 3.664309 \\ 6 & 0.125509 & -1.881892 & 3.624397 \\ 45 & 0.076846 & 0.610490 & 1.577249 \\ 6 & -0.556918 & 2.436362 & 1.338536 \\ 8 & -0.652018 & 3.569830 & 1.554811 \\ 15 & 1.554244 & 0.441298 & -0.350445 \\ 6 & 1.602276 & 2.189880 & -0.921473 \\ 6 & 0.516139 & 2.699669 & -1.637745\end{array}$




\begin{tabular}{|c|c|c|c|}
\hline 6 & 0.463787 & 4.042648 & -2.006836 \\
\hline 6 & 1.499719 & 4.876014 & -1.570975 \\
\hline f & 2.580991 & 4.407597 & -0.821396 \\
\hline & 2.624278 & 3.043794 & -0.515562 \\
\hline f & -0.684078 & 4.611626 & -2.840237 \\
\hline & 3.676497 & 5.378067 & -0.382616 \\
\hline 6 & 3.334886 & 0.002074 & -0.174794 \\
\hline 5 & 3.801219 & -0.556639 & 1.006341 \\
\hline 6 & 5.124659 & -1.020193 & 1.106026 \\
\hline 6 & 5.947624 & -0.879111 & -0.006464 \\
\hline 6 & 5.513408 & -0.295019 & -1.207186 \\
\hline 6 & 4.196437 & 0.138933 & -1.272818 \\
\hline 6 & 6.474183 & -0.188916 & -2.389478 \\
\hline 6 & 5.630010 & -1.617911 & 2.416358 \\
\hline 6 & 1.220694 & -0.472149 & -1.943255 \\
\hline 6 & 0.903902 & -1.821112 & -1.930410 \\
\hline 6 & 0.700040 & -2.528989 & -3.164520 \\
\hline 6 & 0.842793 & -1.840355 & -4.403340 \\
\hline 6 & 1.203774 & -0.474642 & -4.383063 \\
\hline 6 & 1.383397 & 0.182829 & -3.197075 \\
\hline 6 & 0.612479 & -2.524511 & -5.620269 \\
\hline 6 & 0.249083 & -3.846554 & -5.624804 \\
\hline 6 & 0.106497 & -4.537251 & -4.403658 \\
\hline 6 & 0.329652 & -3.899917 & -3.207871 \\
\hline 6 & 0.775860 & -2.643579 & -0.695029 \\
\hline 6 & -0.439998 & -2.752139 & -0.045248 \\
\hline 6 & -0.648703 & -3.762506 & 0.922391 \\
\hline 6 & 0.352679 & -4.633725 & 1.258784 \\
\hline 6 & 1.631992 & -4.517741 & 0.665370 \\
\hline 6 & 1.851401 & -3.508435 & -0.318727 \\
\hline 6 & 3.131877 & -3.421669 & -0.922902 \\
\hline 6 & 4.134561 & -4.293751 & -0.578775 \\
\hline 6 & 3.908616 & -5.303571 & 0.382176 \\
\hline 6 & 2.685200 & -5.406325 & 0.994725 \\
\hline 7 & -1.531343 & -1.883045 & -0.388586 \\
\hline 15 & -1.764103 & -0.597146 & 0.690610 \\
\hline 8 & -2.843151 & -1.096371 & 1.881091 \\
\hline 6 & -4.084716 & -1.599602 & 1.573020 \\
\hline 6 & -5.069163 & -0.783774 & 1.045398 \\
\hline 6 & -6.280715 & -1.403884 & 0.595306 \\
\hline 6 & -6.494192 & -2.794591 & 0.836016 \\
\hline 6 & -5.502569 & -3.543123 & 1.516559 \\
\hline 6 & -4.313782 & -2.962147 & 1.862731 \\
\hline 6 & -7.257469 & -0.700343 & -0.154632 \\
\hline 6 & -8.385213 & -1.331594 & -0.618720 \\
\hline 6 & -8.608164 & -2.698249 & -0.349414 \\
\hline 6 & -7.678145 & -3.410969 & 0.363301 \\
\hline 6 & -4.831085 & 0.672999 & 0.923244 \\
\hline 6 & -3.703699 & 1.129051 & 0.266910 \\
\hline 6 & -3.449453 & 2.498382 & 0.059079 \\
\hline 6 & -4.317333 & 3.432738 & 0.552848 \\
\hline 6 & -5.451712 & 3.034990 & 1.300287 \\
\hline 6 & -5.709103 & 1.645109 & 1.505982 \\
\hline 6 & -6.324727 & 3.997149 & 1.863475 \\
\hline 6 & -7.399897 & 3.616194 & 2.624104 \\
\hline 6 & -7.636957 & 2.246190 & 2.860556 \\
\hline
\end{tabular}




\begin{tabular}{|c|c|c|c|}
\hline 6 & -6.816513 & 1.288701 & 2.317488 \\
\hline 8 & -2.815205 & 0.234540 & -0.274674 \\
\hline & -0.646792 & 0.507413 & 3.616315 \\
\hline & -2.568534 & -2.489267 & -1.260781 \\
\hline 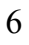 & -3.168710 & -1.587545 & -2.308066 \\
\hline & -2.372301 & -0.777253 & -3.119160 \\
\hline 0 & -2.943502 & 0.006996 & -4.112388 \\
\hline 6 & -4.323656 & -0.002063 & -4.308765 \\
\hline & -5.124227 & -0.803368 & -3.502481 \\
\hline & -4.547874 & -1.591548 & -2.509117 \\
\hline & -7.088659 & 0.347905 & -0.388539 \\
\hline & 1.661510 & 1.232546 & -3.220037 \\
\hline & -7.824886 & -4.471064 & 0.566076 \\
\hline & 0.725656 & -1.973683 & -6.552973 \\
\hline & -6.112871 & 5.050799 & 1.686363 \\
\hline & 3.316952 & -2.639410 & -1.657985 \\
\hline & 5.112709 & -4.203170 & -1.047727 \\
\hline & -4.134758 & 4.494903 & 0.395691 \\
\hline & 1.335451 & 0.050305 & -5.328554 \\
\hline & -5.691633 & -4.592935 & 1.734482 \\
\hline & -8.474531 & 1.944184 & 3.485402 \\
\hline & -3.528776 & -3.519678 & 2.369954 \\
\hline & 2.499545 & -6.175613 & 1.743083 \\
\hline & 0.181668 & -5.420051 & 1.992607 \\
\hline & -0.181334 & -5.586045 & -4.408509 \\
\hline & -7.007791 & 0.238541 & 2.521775 \\
\hline & 0.216987 & -4.454683 & -2.278659 \\
\hline & -1.634351 & -3.841376 & 1.378290 \\
\hline & -8.060601 & 4.364190 & 3.056098 \\
\hline 1 & -9.110974 & -0.772728 & -1.205371 \\
\hline & 4.710115 & -5.992126 & 0.640915 \\
\hline & 0.069341 & -4.364019 & -6.564233 \\
\hline & -9.508612 & -3.184284 & -0.717530 \\
\hline & -2.555441 & 2.774930 & -0.495816 \\
\hline & 3.816697 & 0.584024 & -2.188772 \\
\hline & 3.111410 & -0.643004 & 1.848370 \\
\hline & 6.972867 & -1.239432 & 0.047577 \\
\hline & 3.454831 & 2.635713 & 0.056192 \\
\hline 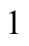 & -0.289245 & 2.022451 & -1.922627 \\
\hline & 1.462257 & 5.934529 & -1.828087 \\
\hline 1 & -2.059907 & -3.322112 & -1.767577 \\
\hline & 1.339585 & 1.417582 & 2.079973 \\
\hline & -3.374010 & -2.939353 & -0.661053 \\
\hline 1 & -5.183125 & -2.208973 & -1.871523 \\
\hline & -1.299007 & -0.740602 & -2.945330 \\
\hline 1 & -6.204190 & -0.813460 & -3.637878 \\
\hline & -2.304332 & 0.630405 & -4.736664 \\
\hline 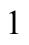 & -4.771108 & 0.616305 & -5.084061 \\
\hline & -1.664523 & 0.119725 & 3.581855 \\
\hline & -0.559769 & 1.471446 & 4.117128 \\
\hline 1 & 1.493873 & 2.112932 & 4.105699 \\
\hline & 3.329665 & 2.723748 & 5.596870 \\
\hline 1 & 4.606320 & 0.968303 & 6.825614 \\
\hline & 3.992960 & -1.423450 & 6.494354 \\
\hline & 2.150720 & -2.040280 & 5.018783 \\
\hline & 0.017639 & -2.290851 & 4.642645 \\
\hline
\end{tabular}




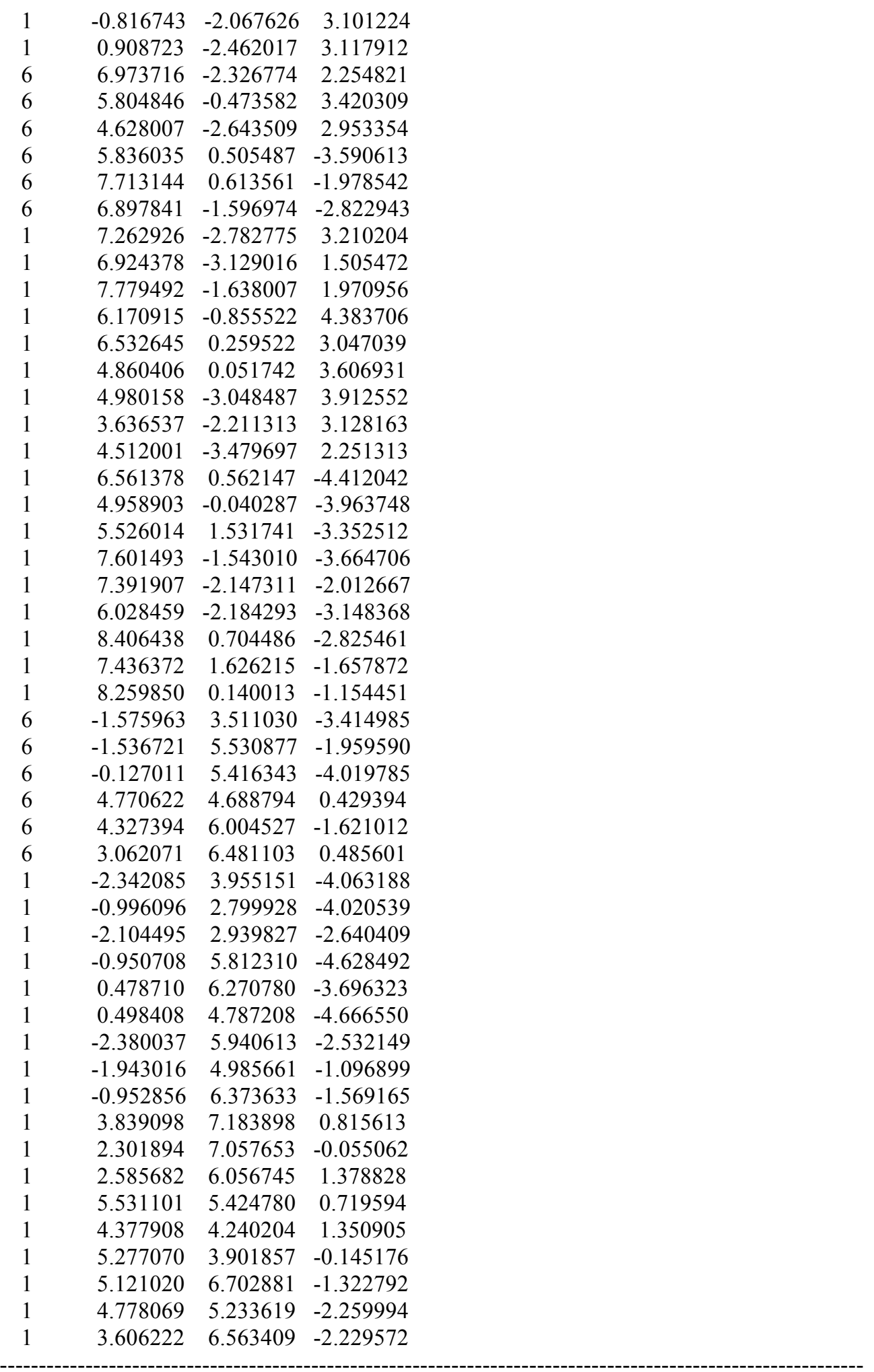

$3_{\text {ID-si }-\psi_{-12 a 2 a 3 s 4 s}}$

Number of imaginary frequencies : 0

The smallest frequencies are : $15.7962 \quad 17.1010 \quad 18.9866 \mathrm{~cm}(-1)$

Electronic energy : $\quad H F=-4360.8796972$ 
Zero-point correction $=$

Thermal correction to Energy=

Thermal correction to Enthalpy=

Thermal correction to Gibbs Free Energy=

Sum of electronic and zero-point Energies=

Sum of electronic and thermal Energies $=$

Sum of electronic and thermal Enthalpies=

Sum of electronic and thermal Free Energies=
1.454862 (Hartree/Particle) 1.539616

1.540560

1.336494

$-4359.424835$

$-4359.340081$

$-4359.339137$

$-4359.543203$

Cartesian Coordinates

$\begin{array}{llcc}6 & -4.563874 & -1.537598 & -2.514174 \\ 6 & -3.183430 & -1.548542 & -2.324256 \\ 6 & -2.387083 & -0.723181 & -3.120006 \\ 6 & -2.959875 & 0.095940 & -4.083404 \\ 6 & -4.342049 & 0.104748 & -4.266347 \\ 6 & -5.142096 & -0.714881 & -3.478163 \\ 6 & -2.579795 & -2.468623 & -1.295382 \\ 7 & -1.547581 & -1.870402 & -0.411254 \\ 15 & -1.783647 & -0.590460 & 0.674188 \\ 8 & -2.828943 & 0.247028 & -0.292160 \\ 6 & -3.720611 & 1.142339 & 0.240949 \\ 6 & -4.846844 & 0.689539 & 0.901871 \\ 6 & -5.723536 & 1.664845 & 1.481397 \\ 6 & -5.469761 & 3.053540 & 1.263851 \\ 6 & -4.341836 & 3.447072 & 0.504358 \\ 6 & -3.473655 & 2.510456 & 0.015738 \\ 6 & -6.826432 & 1.312469 & 2.300730 \\ 6 & -7.645341 & 2.272579 & 2.841528 \\ 6 & -7.411466 & 3.641220 & 2.594263 \\ 6 & -6.341153 & 4.018368 & 1.824862 \\ 6 & -5.091136 & -0.766396 & 1.022732 \\ 6 & -4.110230 & -1.589522 & 1.545149 \\ 6 & -4.344081 & -2.953131 & 1.825767 \\ 6 & -5.535935 & -3.526892 & 1.478369 \\ 6 & -6.525976 & -2.769706 & 0.805206 \\ 6 & -6.306436 & -1.378779 & 0.571219 \\ 6 & -7.714054 & -3.378213 & 0.332623 \\ 6 & -8.642491 & -2.657818 & -0.374342 \\ 6 & -8.413181 & -1.291230 & -0.638294 \\ 6 & -7.281317 & -0.667539 & -0.173923 \\ 8 & -2.867322 & -1.092842 & 1.858171 \\ 6 & -0.457394 & -2.743214 & -0.073734 \\ 6 & 0.756160 & -2.638317 & -0.728742 \\ 6 & 1.827912 & -3.512142 & -0.363554 \\ 6 & 1.609725 & -4.521456 & 0.620656 \\ 6 & 0.333838 & -4.630966 & 1.222565 \\ 6 & -0.665513 & -3.754810 & 0.892711 \\ 6 & 3.103648 & -3.432827 & -0.978494 \\ 6 & 4.105103 & -4.308190 & -0.639746 \\ 6 & 3.881235 & -5.316929 & 0.322856 \\ 6 & 2.660589 & -5.415883 & 0.941739 \\ 6 & 0.888062 & -1.801307 & -1.953526 \\ 6 & 1.212146 & -0.453809 & -1.944655 \\ 6 & 1.373296 & 0.221087 & -3.187683 \\ 6 & 1.189005 & -0.416992 & -4.383951\end{array}$




\begin{tabular}{|c|c|c|c|}
\hline 6 & 0.826934 & -1.781806 & -4.425835 \\
\hline 6 & 0.682247 & -2.489203 & -3.197891 \\
\hline 6 & 0.596671 & -2.447210 & -5.653262 \\
\hline 6 & 0.231873 & -3.768567 & -5.677991 \\
\hline 6 & 0.086984 & -4.477590 & -4.467518 \\
\hline 6 & 0.309435 & -3.858733 & -3.262052 \\
\hline 15 & 1.554232 & 0.427251 & -0.334527 \\
\hline 6 & 3.318355 & -0.065522 & -0.141696 \\
\hline 6 & 3.771182 & -0.634453 & 1.044021 \\
\hline 6 & 5.084601 & -1.112991 & 1.149556 \\
\hline 6 & 5.924670 & -0.978957 & 0.041850 \\
\hline 6 & 5.503818 & -0.390388 & -1.155376 \\
\hline 6 & 4.188314 & 0.059357 & -1.226179 \\
\hline 6 & 5.575797 & -1.719249 & 2.461913 \\
\hline 6 & 4.552653 & -2.724091 & 2.998070 \\
\hline 6 & 6.404656 & -0.239672 & -2.379249 \\
\hline 6 & 6.554744 & 1.249325 & -2.712901 \\
\hline 45 & 0.054849 & 0.610565 & 1.572722 \\
\hline 6 & -0.678640 & 0.507428 & 3.608969 \\
\hline 6 & 0.396579 & -0.410609 & 3.660908 \\
\hline 6 & 0.082599 & -1.885517 & 3.617148 \\
\hline 6 & 1.610733 & -0.030083 & 4.443872 \\
\hline 6 & 2.340663 & -1.001688 & 5.144458 \\
\hline 6 & 3.385699 & -0.658354 & 6.000700 \\
\hline 6 & 3.744596 & 0.669913 & 6.178609 \\
\hline 6 & 3.045721 & 1.652471 & 5.476679 \\
\hline 6 & 2.003822 & 1.309033 & 4.629078 \\
\hline 6 & -0.573378 & 2.437739 & 1.331003 \\
\hline 8 & -0.664928 & 3.571564 & 1.547960 \\
\hline 6 & 1.673149 & 2.179325 & -0.884830 \\
\hline 6 & 0.597673 & 2.741813 & -1.575992 \\
\hline 6 & 0.595862 & 4.088807 & -1.935611 \\
\hline 6 & 1.677555 & 4.871637 & -1.518990 \\
\hline 6 & 2.754094 & 4.348995 & -0.798062 \\
\hline 6 & 2.741682 & 2.983811 & -0.497983 \\
\hline 6 & -0.561661 & 4.710910 & -2.716250 \\
\hline 6 & -0.035881 & 5.634331 & -3.819744 \\
\hline 6 & 3.906823 & 5.263178 & -0.385859 \\
\hline 6 & 3.371561 & 6.394096 & 0.498541 \\
\hline 6 & -1.433865 & 3.647453 & -3.385622 \\
\hline 6 & -1.425050 & 5.525707 & -1.746873 \\
\hline 6 & 4.985563 & 4.517659 & 0.396749 \\
\hline 6 & 4.556467 & 5.857734 & -1.640395 \\
\hline 6 & 7.798067 & -0.820557 & -2.154734 \\
\hline 6 & 5.771783 & -0.974683 & -3.567188 \\
\hline 6 & 6.905959 & -2.454857 & 2.305958 \\
\hline 6 & 5.769667 & -0.577575 & 3.465409 \\
\hline 1 & -7.107927 & 0.380675 & -0.404388 \\
\hline 1 & 1.654738 & 1.269966 & -3.194552 \\
\hline 1 & -7.865192 & -4.438567 & 0.530747 \\
\hline 1 & 0.711362 & -1.882603 & -6.577492 \\
\hline 1 & -6.132014 & 5.071048 & 1.638966 \\
\hline 1 & 3.285890 & -2.653908 & -1.718100 \\
\hline 1 & 5.081168 & -4.220082 & -1.113812 \\
\hline 1 & -4.164991 & 4.508000 & 0.332349 \\
\hline 1 & 1.319232 & 0.123045 & -5.321141 \\
\hline
\end{tabular}




\begin{tabular}{|c|c|c|c|}
\hline & -5.728902 & -4.577320 & 1.689781 \\
\hline & -8.479151 & 1.973778 & 3.472912 \\
\hline & -3.560526 & -3.517354 & 2.327768 \\
\hline & 2.476183 & -6.185235 & 1.690346 \\
\hline & 0.163388 & -5.417096 & 1.956735 \\
\hline & -0.202171 & -5.525821 & -4.488677 \\
\hline & -7.015014 & 0.263387 & 2.513135 \\
\hline & 0.195258 & -4.426839 & -2.341092 \\
\hline & -1.649056 & -3.829725 & 1.353762 \\
\hline & -8.070803 & 4.391292 & 3.024744 \\
\hline & -9.137286 & -0.726174 & -1.221060 \\
\hline & 4.681825 & -6.008232 & 0.577171 \\
\hline & 0.052674 & -4.271682 & -6.625289 \\
\hline & -9.546232 & -3.137752 & -0.742383 \\
\hline & -2.587540 & 2.785477 & -0.552297 \\
\hline & 3.826974 & 0.510514 & -2.149492 \\
\hline & 3.075079 & -0.711157 & 1.881700 \\
\hline & 6.942426 & -1.351604 & 0.110231 \\
\hline & 3.565855 & 2.535769 & 0.052044 \\
\hline & -0.241790 & 2.101466 & -1.848340 \\
\hline & 1.683403 & 5.932610 & -1.767311 \\
\hline & -2.064760 & -3.288317 & -1.816765 \\
\hline 1 & 1.315924 & 1.415629 & 2.082520 \\
\hline & -3.382692 & -2.934274 & -0.703891 \\
\hline 1 & -5.198894 & -2.167405 & -1.888563 \\
\hline & -1.312435 & -0.699814 & -2.953109 \\
\hline & -6.223203 & -0.712404 & -3.604463 \\
\hline 1 & -2.320496 & 0.733120 & -4.693295 \\
\hline & -4.791431 & 0.751171 & -5.017276 \\
\hline I & -1.697984 & 0.124520 & 3.568365 \\
\hline & -0.589334 & 1.470372 & 4.111552 \\
\hline l & 1.481838 & 2.100209 & 4.093452 \\
\hline 1 & 3.314050 & 2.700950 & 5.592931 \\
\hline & 4.561773 & 0.940337 & 6.843768 \\
\hline ] & 3.921860 & -1.446695 & 6.527369 \\
\hline & 2.083219 & -2.053005 & 5.043564 \\
\hline 1 & -0.037664 & -2.294069 & 4.634136 \\
\hline & -0.855305 & -2.066896 & 3.084822 \\
\hline 1 & 0.868235 & -2.469447 & 3.118685 \\
\hline 1 & 7.180955 & -2.917334 & 3.262465 \\
\hline & 6.844770 & -3.255355 & 1.555683 \\
\hline 1 & 7.727328 & -1.782670 & 2.027251 \\
\hline & 6.127538 & -0.965396 & 4.429642 \\
\hline 1 & 6.511015 & 0.142348 & 3.093056 \\
\hline & 4.833980 & -0.035905 & 3.649521 \\
\hline 1 & 4.897380 & -3.139716 & 3.955453 \\
\hline 1 & 3.572382 & -2.269087 & 3.176898 \\
\hline 1 & 4.415395 & -3.554597 & 2.292885 \\
\hline 1 & 8.402296 & -0.691043 & -3.061615 \\
\hline 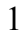 & 8.326241 & -0.318420 & -1.334086 \\
\hline 1 & 7.762143 & -1.895686 & -1.934204 \\
\hline ( & 7.162709 & 1.380950 & -3.618236 \\
\hline 1 & 5.586261 & 1.736048 & -2.885812 \\
\hline 1 & 7.052871 & 1.782566 & -1.892474 \\
\hline & 6.394971 & -0.859440 & -4.464363 \\
\hline 1 & 5.681386 & -2.049249 & -3.357601 \\
\hline
\end{tabular}




$\begin{array}{lrrr}1 & 4.769339 & -0.598552 & -3.808346 \\ 1 & -2.209471 & 4.131901 & -3.992189 \\ 1 & -0.840958 & 3.002496 & -4.049935 \\ 1 & -1.950714 & 3.001133 & -2.665110 \\ 1 & -0.875306 & 6.042993 & -4.397429 \\ 1 & 0.527048 & 6.487099 & -3.423636 \\ 1 & 0.618599 & 5.091233 & -4.513858 \\ 1 & -2.284368 & 5.967504 & -2.270097 \\ 1 & -1.805723 & 4.894727 & -0.932329 \\ 1 & -0.851560 & 6.340441 & -1.287131 \\ 1 & 4.191611 & 7.055743 & 0.808890 \\ 1 & 2.628400 & 7.009933 & -0.021942 \\ 1 & 2.897356 & 5.992843 & 1.403424 \\ 1 & 5.792030 & 5.212419 & 0.663489 \\ 1 & 4.596004 & 4.090756 & 1.329874 \\ 1 & 5.432591 & 3.704515 & -0.190925 \\ 1 & 5.390064 & 6.516843 & -1.362660 \\ 1 & 4.953882 & 5.065381 & -2.288774 \\ 1 & 3.848155 & 6.450217 & -2.231963\end{array}$

$3_{\text {ID-si- }} \psi_{-1 \text {-1a2s3ata }}$

Number of imaginary frequencies : 0

The smallest frequencies are : $15.8379 \quad 18.3683 \quad 18.9300 \mathrm{~cm}(-1)$

Electronic energy : $\quad H F=-4360.8802007$

Zero-point correction $=\quad 1.454742$ (Hartree/Particle)

Thermal correction to Energy $=\quad 1.539433$

Thermal correction to Enthalpy= $\quad 1.540378$

Thermal correction to Gibbs Free Energy= $\quad 1.336566$

Sum of electronic and zero-point Energies $=\quad-4359.425459$

Sum of electronic and thermal Energies $=\quad-4359.340767$

Sum of electronic and thermal Enthalpies= $\quad-4359.339823$

Sum of electronic and thermal Free Energies $=\quad-4359.543635$

Cartesian Coordinates

$\begin{array}{cccc}6 & 2.426558 & -0.969783 & 5.121799 \\ 6 & 1.685483 & -0.007879 & 4.419910 \\ 6 & 2.084174 & 1.333351 & 4.577448 \\ 6 & 3.144779 & 1.687250 & 5.397091 \\ 6 & 3.857653 & 0.713661 & 6.097695 \\ 6 & 3.491475 & -0.616163 & 5.949047 \\ 6 & 0.458869 & -0.399620 & 3.662119 \\ 6 & 0.149039 & -1.875606 & 3.632038 \\ 45 & 0.080182 & 0.606231 & 1.572108 \\ 6 & -0.555177 & 2.436198 & 1.357298 \\ 8 & -0.641596 & 3.565278 & 1.599245 \\ 15 & 1.535806 & 0.431350 & -0.363846 \\ 6 & 1.559043 & 2.174853 & -0.946724 \\ 6 & 0.478716 & 2.673707 & -1.687243 \\ 6 & 0.419340 & 4.013335 & -2.054520 \\ 6 & 1.437323 & 4.866610 & -1.595626 \\ 6 & 2.501458 & 4.408928 & -0.824332 \\ 6 & 2.553925 & 3.041150 & -0.521708 \\ 6 & -0.708190 & 4.568729 & -2.924346 \\ 6 & -1.601517 & 3.461627 & -3.483544\end{array}$




\begin{tabular}{|c|c|c|c|}
\hline 6 & 3.610851 & 5.315670 & -0.299256 \\
\hline 6 & 3.424156 & 6.770304 & -0.721397 \\
\hline ) & 3.323358 & 0.024231 & -0.186515 \\
\hline & 3.788730 & -0.576360 & 0.974670 \\
\hline$b$ & 5.117436 & -1.025206 & 1.065649 \\
\hline 6 & 5.946984 & -0.823392 & -0.033553 \\
\hline 6 & 5.515852 & -0.187316 & -1.208428 \\
\hline f & 4.191831 & 0.225959 & -1.267863 \\
\hline 6 & 6.482272 & -0.009817 & -2.377333 \\
\hline 6 & 5.633285 & -1.656669 & 2.356653 \\
\hline 6 & 1.216197 & -0.497012 & -1.949857 \\
\hline 6 & 0.902342 & -1.846248 & -1.927747 \\
\hline 6 & 0.705819 & -2.564256 & -3.157292 \\
\hline 6 & 0.856576 & -1.885600 & -4.400659 \\
\hline 6 & 1.216905 & -0.519483 & -4.389337 \\
\hline 6 & 1.387394 & 0.148040 & -3.207759 \\
\hline 6 & 0.635190 & -2.579957 & -5.613418 \\
\hline 6 & 0.271775 & -3.902051 & -5.609542 \\
\hline 6 & 0.119694 & -4.582368 & -4.383770 \\
\hline 6 & 0.334695 & -3.935149 & -3.191776 \\
\hline 6 & 0.773007 & -2.659502 & -0.686607 \\
\hline 6 & -0.441579 & -2.762761 & -0.033440 \\
\hline 6 & -0.650227 & -3.770420 & 0.937199 \\
\hline 6 & 0.349047 & -4.644971 & 1.271360 \\
\hline 6 & 1.628009 & -4.532289 & 0.676276 \\
\hline 6 & 1.848926 & -3.522995 & -0.307518 \\
\hline 6 & 3.131980 & -3.433015 & -0.906278 \\
\hline 6 & 4.134752 & -4.303478 & -0.557951 \\
\hline 6 & 3.906013 & -5.316244 & 0.399212 \\
\hline 6 & 2.680253 & -5.421689 & 1.006653 \\
\hline 7 & -1.532131 & -1.892764 & -0.377049 \\
\hline 15 & -1.762442 & -0.601899 & 0.697759 \\
\hline 8 & -2.840450 & -1.093713 & 1.891881 \\
\hline 6 & -4.086045 & -1.589995 & 1.588879 \\
\hline 6 & -5.066981 & -0.769234 & 1.062441 \\
\hline 6 & -6.284638 & -1.382589 & 0.619487 \\
\hline 6 & -6.506424 & -2.771132 & 0.865367 \\
\hline 6 & -5.516393 & -3.524590 & 1.542765 \\
\hline 6 & -4.322342 & -2.950514 & 1.882248 \\
\hline 6 & -7.260068 & -0.674827 & -0.128232 \\
\hline 6 & -8.394286 & -1.299791 & -0.584975 \\
\hline 6 & -8.625419 & -2.663965 & -0.310126 \\
\hline 6 & -7.696867 & -3.380813 & 0.400329 \\
\hline 6 & -4.819692 & 0.685472 & 0.932119 \\
\hline 6 & -3.693538 & 1.130682 & 0.266012 \\
\hline 6 & -3.433172 & 2.497271 & 0.045883 \\
\hline 6 & -4.292856 & 3.439134 & 0.540204 \\
\hline 6 & -5.423987 & 3.052882 & 1.298456 \\
\hline 6 & -5.688504 & 1.665992 & 1.514268 \\
\hline 6 & -6.287129 & 4.023797 & 1.861836 \\
\hline 6 & -7.359469 & 3.654019 & 2.631915 \\
\hline 6 & -7.603649 & 2.286889 & 2.877785 \\
\hline 6 & -6.792870 & 1.321109 & 2.334843 \\
\hline 8 & -2.812864 & 0.226289 & -0.271512 \\
\hline 6 & -0.620406 & 0.513522 & 3.621192 \\
\hline 6 & -2.573313 & -2.501503 & -1.242963 \\
\hline
\end{tabular}




\begin{tabular}{|c|c|c|c|}
\hline & -3.172109 & -1.603573 & -2.294167 \\
\hline & -2.372724 & -0.806337 & -3.115146 \\
\hline & -2.942014 & -0.024012 & -4.110942 \\
\hline & -4.323294 & -0.021326 & -4.299444 \\
\hline & -5.126769 & -0.809729 & -3.483335 \\
\hline & -4.552280 & -1.596665 & -2.487879 \\
\hline & -1.571259 & 5.523271 & -2.093299 \\
\hline & -0.116195 & 5.330946 & -4.115504 \\
\hline & 3.617575 & 5.258077 & 1.233352 \\
\hline & 4.962133 & 4.830017 & -0.838549 \\
\hline & -7.085496 & 0.371594 & -0.365995 \\
\hline & 1.662887 & 1.198366 & -3.237625 \\
\hline & -7.849864 & -4.439248 & 0.607109 \\
\hline & 0.755146 & -2.036970 & -6.549863 \\
\hline & -6.069964 & 5.075058 & 1.677111 \\
\hline & 3.320902 & -2.650509 & -1.640362 \\
\hline & 5.115558 & -4.207687 & -1.019912 \\
\hline & -4.106363 & 4.499407 & 0.375417 \\
\hline & 1.354525 & -0.002354 & -5.338266 \\
\hline & -5.711040 & -4.572792 & 1.763518 \\
\hline & -8.439019 & 1.993784 & 3.509777 \\
\hline & -3.538245 & -3.512261 & 2.386247 \\
\hline & 2.492874 & -6.191833 & 1.753682 \\
\hline & 0.177231 & -5.430383 & 2.005954 \\
\hline & -0.169113 & -5.630900 & -4.381938 \\
\hline & -6.989422 & 0.273287 & 2.546159 \\
\hline & 0.214442 & -4.481877 & -2.258749 \\
\hline & -1.634783 & -3.845285 & 1.396230 \\
\hline & -8.012502 & 4.408630 & 3.064075 \\
\hline & -9.118987 & -0.737777 & -1.169922 \\
\hline & 4.707918 & -6.003322 & 0.660651 \\
\hline & 0.098866 & -4.427289 & -6.545933 \\
\hline & -9.530968 & -3.144936 & -0.672329 \\
\hline & -2.542758 & 2.766857 & -0.518975 \\
\hline & 3.813600 & 0.709206 & -2.164664 \\
\hline & 3.092490 & -0.706400 & 1.805171 \\
\hline & 6.976383 & -1.172816 & 0.013372 \\
\hline & 3.379046 & 2.651410 & 0.073595 \\
\hline & -0.310915 & 1.986521 & -1.991069 \\
\hline & 1.386571 & 5.921911 & -1.855391 \\
\hline & -2.068581 & -3.339335 & -1.745406 \\
\hline & 1.350155 & 1.411855 & 2.057661 \\
\hline & -3.379130 & -2.944923 & -0.638744 \\
\hline & -5.189638 & -2.204092 & -1.842805 \\
\hline & -1.298218 & -0.778586 & -2.947195 \\
\hline & -6.207497 & -0.810800 & -3.612815 \\
\hline & -2.300234 & 0.588697 & -4.743092 \\
\hline & -4.769312 & 0.595896 & -5.076482 \\
\hline & -2.360072 & 3.896758 & -4.146644 \\
\hline & -1.021703 & 2.735152 & -4.070668 \\
\hline & -2.138377 & 2.909914 & -2.700445 \\
\hline & -0.921193 & 5.711275 & -4.758351 \\
\hline & 0.486962 & 6.191602 & -3.804081 \\
\hline & 0.521580 & 4.676967 & -4.724485 \\
\hline 1 & -2.383396 & 5.938617 & -2.705453 \\
\hline & -2.024081 & 5.002713 & -1.238522 \\
\hline
\end{tabular}




$\begin{array}{lrrr}1 & -0.986308 & 6.362090 & -1.696670 \\ 1 & 4.245137 & 7.378861 & -0.321653 \\ 1 & 3.431182 & 6.885560 & -1.813153 \\ 1 & 2.486240 & 7.190802 & -0.336403 \\ 1 & 4.398587 & 5.916396 & 1.637523 \\ 1 & 2.651319 & 5.584881 & 1.639809 \\ 1 & 3.808986 & 4.245211 & 1.608623 \\ 1 & 5.774485 & 5.465422 & -0.460686 \\ 1 & 5.182752 & 3.797623 & -0.538103 \\ 1 & 4.983811 & 4.870496 & -1.935882 \\ 1 & -1.638822 & 0.126500 & 3.598163 \\ 1 & -0.526758 & 1.480430 & 4.115545 \\ 1 & 1.553113 & 2.118169 & 4.041032 \\ 1 & 3.417507 & 2.736845 & 5.491208 \\ 1 & 4.690576 & 0.992188 & 6.739582 \\ 1 & 4.037466 & -1.397714 & 6.475743 \\ 1 & 2.164992 & -2.021969 & 5.042184 \\ 1 & 0.045368 & -2.279198 & 4.652828 \\ 1 & -0.796871 & -2.061824 & 3.115367 \\ 1 & 0.928281 & -2.460280 & 3.124411 \\ 6 & 4.605546 & -2.635014 & 2.929260 \\ 6 & 6.936478 & -2.427169 & 2.147222 \\ 6 & 5.888890 & -0.526258 & 3.359394 \\ 6 & 5.856349 & 0.776486 & -3.527655 \\ 6 & 7.731604 & 0.746093 & -1.913006 \\ 6 & 6.884810 & -1.392061 & -2.903421 \\ 1 & 4.984714 & -3.072788 & 3.863356 \\ 1 & 3.654223 & -2.148161 & 3.167024 \\ 1 & 4.404528 & -3.452409 & 2.224479 \\ 1 & 7.227792 & -2.914033 & 3.086637 \\ 1 & 6.825435 & -3.211588 & 1.385664 \\ 1 & 7.769384 & -1.775733 & 1.854290 \\ 1 & 6.264054 & -0.929747 & 4.310605 \\ 1 & 6.637427 & 0.176612 & 2.969298 \\ 1 & 4.972238 & 0.037910 & 3.572771 \\ 1 & 6.589608 & 0.893637 & -4.335573 \\ 1 & 4.982035 & 0.263799 & -3.950880 \\ 1 & 5.546151 & 1.782190 & -3.212723 \\ 1 & 7.578842 & -1.294799 & -3.749309 \\ 1 & 7.381122 & -1.995906 & -2.133475 \\ 1 & 6.003699 & -1.948660 & -3.250749 \\ 1 & 8.426814 & 0.882566 & -2.752176 \\ 1 & 7.468783 & 1.739810 & -1.527444 \\ 1 & 8.272384 & 0.212859 & -1.122278\end{array}$

$3_{\text {ID-si- }} \psi_{-1 \mathbf{a} 2 s 3 a 4 s}$

Number of imaginary frequencies : 0

The smallest frequencies are : $14.6971 \quad 17.898519 .3011 \mathrm{~cm}(-1)$

Electronic energy : $\quad H F=-4360.8801827$

Zero-point correction $=\quad 1.454429($ Hartree/Particle $)$

Thermal correction to Energy= $\quad 1.539017$

Thermal correction to Enthalpy $=\quad 1.539961$

Thermal correction to Gibbs Free Energy $=\quad 1.336817$

Sum of electronic and zero-point Energies $=\quad-4359.425753$

Sum of electronic and thermal Energies $=\quad-4359.341166$ 
Sum of electronic and thermal Enthalpies=

Sum of electronic and thermal Free Energies=
$-4359.340222$

$-4359.543365$

Cartesian Coordinates

\begin{tabular}{|c|c|c|c|}
\hline 6 & 2.449587 & -0.655731 & 5.153859 \\
\hline 6 & 1.696583 & 0.262917 & 4.407975 \\
\hline 6 & 2.074197 & 1.615880 & 4.506856 \\
\hline 6 & 3.122085 & 2.023035 & 5.318024 \\
\hline 6 & 3.842905 & 1.093213 & 6.068057 \\
\hline 6 & 3.500594 & -0.247971 & 5.973984 \\
\hline 6 & 0.475895 & -0.178185 & 3.667841 \\
\hline 6 & 0.175324 & -1.655306 & 3.721789 \\
\hline 45 & 0.098784 & 0.704065 & 1.527783 \\
\hline 6 & -0.541115 & 2.517058 & 1.202264 \\
\hline 8 & -0.636954 & 3.656366 & 1.385538 \\
\hline 15 & 1.552272 & 0.415214 & -0.401913 \\
\hline 6 & 1.580500 & 2.125173 & -1.076668 \\
\hline 6 & 0.504497 & 2.583122 & -1.848971 \\
\hline 6 & 0.440982 & 3.903727 & -2.278561 \\
\hline 6 & 1.442064 & 4.786146 & -1.839250 \\
\hline 6 & 2.496875 & 4.372958 & -1.030614 \\
\hline 6 & 2.563813 & 3.017512 & -0.677874 \\
\hline 6 & -0.674959 & 4.407647 & -3.192988 \\
\hline 6 & -1.560264 & 3.269733 & -3.700814 \\
\hline 6 & 3.566322 & 5.323044 & -0.498640 \\
\hline 6 & 3.379456 & 6.752132 & -1.000613 \\
\hline 6 & 3.338324 & 0.004563 & -0.210209 \\
\hline 6 & 3.821982 & -0.477057 & 1.002072 \\
\hline 6 & 5.146253 & -0.922167 & 1.116119 \\
\hline 6 & 5.964242 & -0.846806 & -0.013081 \\
\hline 6 & 5.512904 & -0.345906 & -1.238843 \\
\hline 6 & 4.188345 & 0.077429 & -1.315613 \\
\hline 6 & 6.388073 & -0.283781 & -2.489394 \\
\hline 6 & 5.668261 & -1.437571 & 2.455109 \\
\hline 6 & 1.210899 & -0.600743 & -1.927982 \\
\hline 6 & 0.893393 & -1.944823 & -1.821515 \\
\hline 6 & 0.675427 & -2.733768 & -3.002697 \\
\hline 6 & 0.806404 & -2.130464 & -4.286232 \\
\hline 6 & 1.170655 & -0.767371 & -4.361514 \\
\hline 6 & 1.363906 & -0.031899 & -3.224387 \\
\hline 6 & 0.559733 & -2.893594 & -5.451820 \\
\hline 6 & 0.191497 & -4.211468 & -5.363283 \\
\hline 6 & 0.060991 & -4.818101 & -4.097043 \\
\hline 6 & 0.300782 & -4.103056 & -2.949375 \\
\hline 6 & 0.785381 & -2.683340 & -0.532919 \\
\hline 6 & -0.421691 & -2.761273 & 0.136981 \\
\hline 6 & -0.608916 & -3.711949 & 1.167711 \\
\hline 6 & 0.404215 & -4.553777 & 1.542245 \\
\hline 6 & 1.675467 & -4.463680 & 0.926841 \\
\hline 6 & 1.874470 & -3.512146 & -0.117159 \\
\hline 6 & 3.146934 & -3.449046 & -0.741254 \\
\hline 6 & 4.160163 & -4.293346 & -0.360939 \\
\hline 6 & 3.954100 & -5.248527 & 0.658610 \\
\hline 6 & 2.739796 & -5.324132 & 1.292956 \\
\hline 7 & -1.524100 & -1.924220 & -0.247450 \\
\hline
\end{tabular}




\begin{tabular}{|c|c|c|c|}
\hline & -1.745146 & -0.559253 & 0.734556 \\
\hline 8 & -2.818122 & -0.964228 & 1.963943 \\
\hline 6 & -4.063099 & -1.484136 & 1.701272 \\
\hline 6 & -5.047682 & -0.704957 & 1.121415 \\
\hline 6 & -6.265082 & -1.351768 & 0.727585 \\
\hline 6 & -6.480598 & -2.720869 & 1.069839 \\
\hline 6 & -5.485768 & -3.422231 & 1.794609 \\
\hline 6 & -4.293038 & -2.821674 & 2.089882 \\
\hline 6 & -7.246741 & -0.700806 & -0.062279 \\
\hline 6 & -8.379986 & -1.360175 & -0.470627 \\
\hline 6 & -8.603736 & -2.703569 & -0.103132 \\
\hline 6 & -7.669658 & -3.366062 & 0.651490 \\
\hline 6 & -4.803840 & 0.737525 & 0.886610 \\
\hline 6 & -3.680743 & 1.137219 & 0.186832 \\
\hline 6 & -3.425173 & 2.485426 & -0.131446 \\
\hline 6 & -4.286847 & 3.457512 & 0.296614 \\
\hline 6 & -5.414141 & 3.123391 & 1.084739 \\
\hline 6 & -5.673240 & 1.754956 & 1.400578 \\
\hline 6 & -6.278298 & 4.129587 & 1.580584 \\
\hline 6 & -7.346260 & 3.812848 & 2.379931 \\
\hline 6 & -7.584792 & 2.466117 & 2.724451 \\
\hline 6 & -6.772889 & 1.466432 & 2.248599 \\
\hline 8 & -2.798060 & 0.199753 & -0.286662 \\
\hline 6 & -0.608080 & 0.726511 & 3.57 \\
\hline 6 & -2.579133 & -2.595518 & -1.047921 \\
\hline 6 & -3.188480 & -1.780854 & -2.159563 \\
\hline 6 & -2.400536 & -1.020860 & -3.025326 \\
\hline 6 & -2.978586 & -0.316446 & -4.072718 \\
\hline 6 & -4.358118 & -0.355782 & -4.269393 \\
\hline 6 & -5.150650 & -1.106423 & -3.408557 \\
\hline 6 & -4.567151 & -1.815207 & -2.360824 \\
\hline 6 & -1.550068 & 5.406698 & -2.429537 \\
\hline 6 & -0.065439 & 5.101106 & -4.416951 \\
\hline 6 & 3.489362 & 5.338466 & 1.033 \\
\hline 6 & 4.952984 & 4.839364 & -0.938130 \\
\hline 1 & -7.078551 & 0.327837 & -0.371205 \\
\hline 1 & 1.642781 & 1.013512 & -3.320881 \\
\hline 1 & -7.817222 & -4.408557 & 0.930556 \\
\hline 1 & 0.664133 & -2.406964 & -6.420 \\
\hline 1 & -6.065435 & 5.165517 & 1.319934 \\
\hline 1 & 3.318680 & -2.706738 & -1.520583 \\
\hline 1 & 5.131909 & -4.222724 & -0.846361 \\
\hline 1 & -4.104708 & 4.503817 & 0.055542 \\
\hline 1 & 1.293361 & -0.307541 & -5.341469 \\
\hline 1 & -5.675322 & -4.453543 & 2.087446 \\
\hline 1 & -8.416489 & 2.216423 & 3.379513 \\
\hline 1 & -3.504229 & -3.344363 & 2.627531 \\
\hline 1 & 2.570105 & -6.050230 & 2.086884 \\
\hline 1 & 0.249051 & -5.295905 & 2.324063 \\
\hline 1 & -0.230453 & -5.863667 & -4.028323 \\
\hline 1 & -6.964943 & 0.435772 & 2.535110 \\
\hline 1 & 0.197111 & -4.593720 & -1.983691 \\
\hline 1 & -1.587228 & -3.769982 & 1.642665 \\
\hline 1 & -8.000061 & 4.594544 & 2.759609 \\
\hline 1 & -9.109612 & -0.842231 & -1.089156 \\
\hline 1 & 4.764087 & -5.915714 & 0.945369 \\
\hline
\end{tabular}




$\begin{array}{lrrr}1 & -0.001476 & -4.789809 & -6.263766 \\ 1 & -9.508174 & -3.212182 & -0.428496 \\ 1 & -2.536946 & 2.717488 & -0.716658 \\ 1 & 3.802112 & 0.455699 & -2.261434 \\ 1 & 3.140027 & -0.516492 & 1.853944 \\ 1 & 6.989211 & -1.197701 & 0.060850 \\ 1 & 3.384361 & 2.661094 & -0.055563 \\ 1 & -0.279558 & 1.879332 & -2.126879 \\ 1 & 1.383225 & 5.829630 & -2.141364 \\ 1 & -2.085196 & -3.474022 & -1.487913 \\ 1 & 1.367455 & 1.538263 & 1.968682 \\ 1 & -3.379142 & -2.983704 & -0.399400 \\ 1 & -5.197064 & -2.391810 & -1.681005 \\ 1 & -1.329147 & -0.957380 & -2.848969 \\ 1 & -6.230188 & -1.138205 & -3.543624 \\ 1 & -2.345906 & 0.268598 & -4.739249 \\ 1 & -4.811505 & 0.200675 & -5.086972 \\ 1 & -2.306718 & 3.665614 & -4.401105 \\ 1 & -0.972241 & 2.508852 & -4.233818 \\ 1 & -2.111094 & 2.766065 & -2.895265 \\ 1 & -0.860788 & 5.449598 & -5.089224 \\ 1 & 0.539150 & 5.974266 & -4.145669 \\ 6 & 0.575797 & 4.411710 & -4.981612 \\ 6 & 5.841769 & -0.235512 & 3.389184 \\ 6 & 7.809466 & -0.778120 & -2.233806 \\ 1 & 5.766520 & -1.168383 & -3.577249 \\ 1 & -2.360795 & 5.776807 & -3.071818 \\ 1 & -2.004511 & 4.937528 & -1.546309 \\ 1 & 5.043412 & -2.782684 & 4.038761 \\ 1 & -0.973997 & 6.273276 & -2.082873 \\ 1 & 4.686827 & -2.001016 & 3.220188 \\ 1 & 4.561316 & -3.313757 & 2.409981 \\ 1 & 3.435968 & 7.392189 & -0.593937 \\ 1 & 2.419485 & 7.176947 & -0.680408 \\ 1 & 4.315509 & -2.541235 & 3.303942 \\ 1 & 6.967367 & -2.991075 & 1.625628\end{array}$




$\begin{array}{llrl}1 & 7.814927 & -1.469085 & 1.994820 \\ 1 & 6.222044 & -0.557872 & 4.368740 \\ 1 & 6.558089 & 0.482075 & 2.966663 \\ 1 & 4.893509 & 0.289817 & 3.555460 \\ 1 & 8.394802 & -0.711191 & -3.159635 \\ 1 & 8.324104 & -0.175096 & -1.474676 \\ 1 & 7.827259 & -1.827102 & -1.910214 \\ 1 & 7.077927 & 1.220253 & -3.899332 \\ 1 & 5.481191 & 1.579274 & -3.220031 \\ 1 & 6.932112 & 1.811918 & -2.231384 \\ 1 & 6.364982 & -1.122831 & -4.497306 \\ 1 & 5.729115 & -2.217260 & -3.252757 \\ 1 & 4.743019 & -0.862263 & -3.829201\end{array}$

$3_{\text {ID-si }-\Psi-1 \text { a2s3s4a }}$

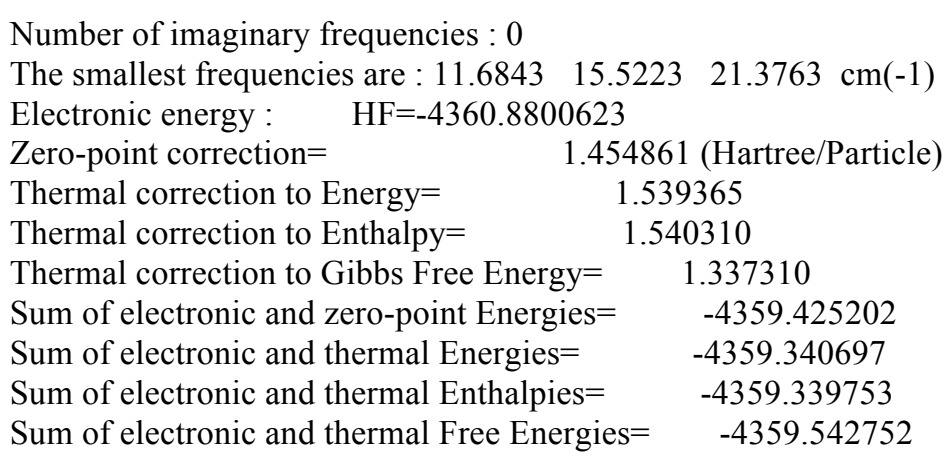

Cartesian Coordinates

$\begin{array}{cccc}6 & 2.463830 & -0.674653 & 5.135744 \\ 6 & 1.705084 & 0.246747 & 4.399123 \\ 6 & 2.079920 & 1.600018 & 4.504597 \\ 6 & 3.130323 & 2.004825 & 5.313643 \\ 6 & 3.856567 & 1.072219 & 6.054940 \\ 6 & 3.517295 & -0.269241 & 5.953902 \\ 6 & 0.482113 & -0.192620 & 3.661734 \\ 6 & 0.183342 & -1.670290 & 3.710894 \\ 45 & 0.097166 & 0.699040 & 1.528912 \\ 6 & -0.550456 & 2.510394 & 1.212627 \\ 8 & -0.654523 & 3.647812 & 1.403034 \\ 15 & 1.548180 & 0.420514 & -0.405897 \\ 6 & 1.562173 & 2.132326 & -1.076514 \\ 6 & 0.483878 & 2.587055 & -1.847590 \\ 6 & 0.410297 & 3.910683 & -2.266559 \\ 6 & 1.404332 & 4.797624 & -1.820191 \\ 6 & 2.461628 & 4.386790 & -1.013655 \\ 6 & 2.537784 & 3.029630 & -0.670146 \\ 6 & -0.709068 & 4.414382 & -3.176948 \\ 6 & -1.593405 & 3.276368 & -3.686008 \\ 6 & 3.528727 & 5.338885 & -0.480400 \\ 6 & 3.310212 & 6.777290 & -0.941479 \\ 6 & 3.337801 & 0.021825 & -0.224016 \\ 6 & 3.823782 & -0.467311 & 0.979892 \\ 6 & 5.154240 & -0.908365 & 1.089270 \\ 6 & 5.962926 & -0.818184 & -0.038852\end{array}$




\begin{tabular}{|c|c|c|c|}
\hline 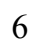 & 5.508328 & -0.304013 & -1.263822 \\
\hline & 4.186255 & 0.112993 & -1.336735 \\
\hline & 6.452656 & -0.257167 & -2.462934 \\
\hline & 5.680501 & -1.430047 & 2.423648 \\
\hline & 1.208013 & -0.596486 & -1.931007 \\
\hline & 0.893499 & -1.941436 & -1.825240 \\
\hline & 0.678505 & -2.729749 & -3.007725 \\
\hline & 0.811363 & -2.125358 & -4.290641 \\
\hline & 1.174052 & -0.761727 & -4.364579 \\
\hline 6 & 1.362509 & -0.026511 & -3.226758 \\
\hline v & 0.569515 & -2.888377 & -5.457303 \\
\hline 6 & 0.203638 & -4.207061 & -5.370665 \\
\hline 6 & 0.070543 & -4.814558 & -4.105161 \\
\hline 6 & 0.305813 & -4.099677 & -2.956415 \\
\hline 6 & 0.783335 & -2.682519 & -0.537807 \\
\hline 6 & -0.425826 & -2.762958 & 0.128099 \\
\hline 6 & -0.617436 & -3.718511 & 1.153529 \\
\hline 6 & 0.393505 & -4.563131 & 1.527475 \\
\hline 6 & 1.667129 & -4.470342 & 0.917449 \\
\hline 6 & 1.870599 & -3.513557 & -0.120949 \\
\hline 6 & 3.146149 & -3.447602 & -0.738165 \\
\hline 6 & 4.157817 & -4.294046 & -0.358350 \\
\hline 6 & 3.946632 & -5.255072 & 0.654640 \\
\hline 6 & 2.729632 & -5.333135 & 1.283490 \\
\hline 7 & -1.526486 & -1.923164 & -0.255081 \\
\hline & -1.747075 & -0.562692 & 0.733239 \\
\hline 8 & -2.818454 & -0.973334 & 1.962361 \\
\hline 6 & -4.061693 & -1.496423 & 1.697702 \\
\hline 6 & -5.048645 & -0.718654 & 1.119904 \\
\hline 6 & -6.263452 & -1.368314 & 0.722851 \\
\hline 6 & -6.474490 & -2.739316 & 1.060203 \\
\hline 6 & -5.477924 & -3.439606 & 1.783622 \\
\hline 6 & -4.287466 & -2.835952 & 2.081838 \\
\hline 6 & -7.246577 & -0.718034 & -0.065798 \\
\hline 6 & -8.377131 & -1.379915 & -0.477500 \\
\hline 6 & -8.596650 & -2.725277 & -0.114615 \\
\hline 6 & -7.660999 & -3.387129 & 0.638614 \\
\hline 6 & -4.809902 & 0.725606 & 0.891099 \\
\hline 6 & -3.687577 & 1.132132 & 0.194033 \\
\hline 6 & -3.436257 & 2.482505 & -0.118190 \\
\hline 6 & -4.301581 & 3.449796 & 0.313284 \\
\hline 6 & -5.428532 & 3.108466 & 1.098761 \\
\hline 6 & -5.683344 & 1.737829 & 1.408515 \\
\hline 6 & -6.296555 & 4.109698 & 1.597870 \\
\hline 6 & -7.364362 & 3.786050 & 2.394630 \\
\hline 6 & -7.598802 & 2.437077 & 2.733138 \\
\hline 6 & -6.783031 & 1.442100 & 2.254034 \\
\hline 8 & -2.801652 & 0.199854 & -0.283430 \\
\hline 6 & -0.603848 & 0.710823 & 3.577014 \\
\hline 6 & -2.580439 & -2.589659 & -1.060884 \\
\hline 6 & -3.187101 & -1.768113 & -2.168863 \\
\hline 6 & -2.396697 & -1.004287 & -3.028980 \\
\hline 6 & -2.972311 & -0.291703 & -4.072159 \\
\hline 6 & -4.351770 & -0.326701 & -4.270276 \\
\hline 6 & -5.146633 & -1.081729 & -3.415385 \\
\hline 6 & -4.565592 & -1.798685 & -2.371861 \\
\hline
\end{tabular}




\begin{tabular}{|c|c|c|c|}
\hline 6 & -1.584460 & 5.409488 & -2.408617 \\
\hline 6 & -0.103641 & 5.112672 & -4.400225 \\
\hline & 3.488964 & 5.316753 & 1.052994 \\
\hline & 4.910508 & 4.885769 & -0.966088 \\
\hline & -7.081362 & 0.312107 & -0.371371 \\
\hline & 1.639937 & 1.019517 & -3.321904 \\
\hline & -7.805280 & -4.431053 & 0.914034 \\
\hline & 0.675574 & -2.400913 & -6.425425 \\
\hline & -6.086796 & 5.147417 & 1.341844 \\
\hline & 3.321555 & -2.700807 & -1.511710 \\
\hline & 5.131479 & -4.219851 & -0.839388 \\
\hline 1 & -4.122927 & 4.497795 & 0.077052 \\
\hline & 1.298933 & -0.301374 & -5.344006 \\
\hline & -5.664257 & -4.472467 & 2.073044 \\
\hline & -8.430444 & 2.181856 & 3.386134 \\
\hline & -3.497356 & -3.357587 & 2.618659 \\
\hline & 2.556119 & -6.063313 & 2.072837 \\
\hline & 0.234900 & -5.309291 & 2.304742 \\
\hline & -0.219321 & -5.860650 & -4.037754 \\
\hline & -6.972063 & 0.409625 & 2.535973 \\
\hline & 0.200113 & -4.591252 & -1.991440 \\
\hline & -1.597775 & -3.778209 & 1.624078 \\
\hline & -8.021200 & 4.563939 & 2.776878 \\
\hline & -9.107894 & -0.862450 & -1.095087 \\
\hline & 4.754889 & -5.924402 & 0.941261 \\
\hline & 0.014393 & -4.785233 & -6.272051 \\
\hline & -9.499057 & -3.235868 & -0.442509 \\
\hline & -2.548250 & 2.719877 & -0.701569 \\
\hline & 3.792292 & 0.506155 & -2.270259 \\
\hline 1 & 3.143851 & -0.518427 & 1.832778 \\
\hline & 6.992582 & -1.164634 & 0.022025 \\
\hline & 3.360312 & 2.674844 & -0.049580 \\
\hline & -0.294687 & 1.879363 & -2.131111 \\
\hline & 1.337471 & 5.842855 & -2.114530 \\
\hline & -2.086025 & -3.465872 & -1.504904 \\
\hline & 1.363889 & 1.535285 & 1.970658 \\
\hline 1 & -3.381824 & -2.981016 & -0.415938 \\
\hline & -5.197272 & -2.378588 & -1.696512 \\
\hline l & -1.325296 & -0.944211 & -2.851399 \\
\hline & -6.226136 & -1.110412 & -3.551571 \\
\hline & -2.337702 & 0.296919 & -4.733772 \\
\hline & -4.803259 & 0.236390 & -5.084355 \\
\hline & -2.344604 & 3.673541 & -4.380494 \\
\hline 1 & -1.006039 & 2.520181 & -4.226294 \\
\hline & -2.138454 & 2.766789 & -2.880234 \\
\hline 1 & -0.901095 & 5.462560 & -5.069307 \\
\hline & 0.500804 & 5.985460 & -4.127354 \\
\hline 1 & 0.536901 & 4.425874 & -4.968812 \\
\hline & -2.399254 & 5.777361 & -3.047002 \\
\hline 1 & -2.033177 & 4.937843 & -1.523806 \\
\hline 1 & -1.010195 & 6.277873 & -2.063543 \\
\hline & 4.100489 & 7.419468 & -0.532563 \\
\hline 1 & 3.346071 & 6.868431 & -2.034950 \\
\hline & 2.349265 & 7.177335 & -0.593296 \\
\hline & 4.235247 & 6.009565 & 1.465100 \\
\hline & 2.500192 & 5.620835 & 1.421468 \\
\hline
\end{tabular}




\begin{tabular}{|c|c|c|c|}
\hline & 3.700838 & 4.319533 & 1.458210 \\
\hline & 5.689898 & 5.551983 & -0.572257 \\
\hline & 5.152124 & 3.865863 & -0.641859 \\
\hline & 4.967736 & 4.910158 & -2.062400 \\
\hline & -1.620087 & 0.317616 & 3.576705 \\
\hline & -0.515204 & 1.701893 & 4.021797 \\
\hline & 1.536298 & 2.353684 & 3.937036 \\
\hline & & 3.062098 & 5.368187 \\
\hline & 4.680009 & 1.390501 & 6.690562 \\
\hline & 4.075709 & -1.019444 & 6.512198 \\
\hline & 2.222676 & -1.733957 & 5.094468 \\
\hline & 0.088271 & -2.020659 & 4.752002 \\
\hline & -0.763088 & -1.891529 & 3.209260 \\
\hline & 0.964319 & -2.274633 & 3.229880 \\
\hline & 7.032085 & -2.128686 & 85674 \\
\hline & 19140 & -0.2 & \\
\hline & 4.697688 & -2.438356 & 3.025437 \\
\hline & 5.790449 & 0.356590 & -3.694567 \\
\hline & 7.687362 & 0.581469 & -2.117607 \\
\hline & 6.885521 & -1.684512 & -2.817358 \\
\hline & 7.336431 & -2.53 & 3.260569 \\
\hline & 6.986802 & -2.9 & 79597 \\
\hline & 7.825644 & -1.4 & 8328 \\
\hline & 6.231938 & -0.559144 & 4.341764 \\
\hline & 6.561425 & 0.490858 & 2.945498 \\
\hline & 4.898597 & 0.287021 & 3.535452 \\
\hline & 5.065702 & -2.786905 & 4.000715 \\
\hline & 3.703151 & -2.008774 & 3.188957 \\
\hline & 4.583714 & -3.312409 & 2.370489 \\
\hline & 6.504366 & 0.372789 & -4.527719 \\
\hline & 4.915531 & -0.221026 & -4.022353 \\
\hline & 5.470472 & 1.391337 & -3.513838 \\
\hline & 7.568071 & -1.675141 & -3.677974 \\
\hline & 7.406257 & -2.177299 & -1.987058 \\
\hline & 6.016523 & -2.301515 & -3.083599 \\
\hline & 8.372066 & 0.622855 & -2.975366 \\
\hline & 7.403898 & 1.610605 & -1.861473 \\
\hline & 8.245683 & 0.167767 & -1.269412 \\
\hline
\end{tabular}

$3_{\text {ID-si }-} \psi_{-1 \text { a2s3s4s }}$

Number of imaginary frequencies : 0

The smallest frequencies are : $11.0058 \quad 14.754120 .0558 \mathrm{~cm}(-1)$

Electronic energy : $\quad H F=-4360.8801695$

Zero-point correction $=\quad 1.454411$ (Hartree/Particle)

Thermal correction to Energy= $\quad 1.539144$

Thermal correction to Enthalpy= $\quad 1.540088$

Thermal correction to Gibbs Free Energy $=\quad 1.335881$

Sum of electronic and zero-point Energies $=\quad-4359.425758$

Sum of electronic and thermal Energies $=\quad-4359.341025$

Sum of electronic and thermal Enthalpies= $\quad-4359.340081$

Sum of electronic and thermal Free Energies= $\quad-4359.544289$

Cartesian Coordinates

$6 \quad-3.826746-0.565959-0.972581$ 


\begin{tabular}{|c|c|c|c|}
\hline 6 & -3.339335 & -0.012660 & 0.207563 \\
\hline 6 & -4.192346 & 0.150580 & 1.300292 \\
\hline 6 & -5.525562 & -0.247017 & 1.243741 \\
\hline 6 & -5.981278 & -0.821594 & 0.052743 \\
\hline 6 & -5.159570 & -0.990751 & -1.064297 \\
\hline & -1.551643 & 0.399053 & 0.380777 \\
\hline & -0.105409 & 0.637126 & -1.562488 \\
\hline 6 & 0.586847 & 0.599789 & -3.613324 \\
\hline 6 & -0.481735 & -0.326344 & -3.666443 \\
\hline 6 & -6.406134 & -0.067461 & 2.478530 \\
\hline 6 & -5.810546 & -0.875373 & 3.637972 \\
\hline 6 & -5.695908 & -1.572450 & -2.370669 \\
\hline 6 & -7.028038 & -2.298194 & -2.184359 \\
\hline 6 & -1.205189 & -0.560541 & 1.942404 \\
\hline 6 & -0.866178 & -1.902400 & 1.885607 \\
\hline 6 & -0.638618 & -2.644592 & 3.094893 \\
\hline 6 & -0.781740 & -1.997175 & 4.355398 \\
\hline 6 & -1.169124 & -0.638658 & 4.380599 \\
\hline 6 & -1.371616 & 0.051874 & 3.217264 \\
\hline 6 & -0.243797 & -4.009092 & 3.091824 \\
\hline 6 & 0.004401 & -4.678433 & 4.265039 \\
\hline 6 & -0.137460 & -4.028076 & 5.508180 \\
\hline 6 & -0.525726 & -2.713593 & 5.548233 \\
\hline 6 & -0.748486 & -2.685006 & 0.624477 \\
\hline 6 & 0.457047 & -2.769019 & -0.047175 \\
\hline 6 & 0.653301 & -3.750944 & -1.046317 \\
\hline 6 & -0.350587 & -4.616642 & -1.390282 \\
\hline 6 & -1.621386 & -4.521493 & -0.774654 \\
\hline 6 & -1.828224 & -3.540473 & 0.240024 \\
\hline 6 & -2.678184 & -5.402457 & -1.112841 \\
\hline 6 & -3.892647 & -5.318291 & -0.479755 \\
\hline 6 & -4.105894 & -4.335603 & 0.511794 \\
\hline 6 & -3.099785 & -3.471433 & 0.865290 \\
\hline 7 & 1.549361 & -1.904418 & 0.303931 \\
\hline 6 & 2.611377 & -2.531926 & 1.129659 \\
\hline 6 & 3.208317 & -1.673821 & 2.215198 \\
\hline 6 & 2.412751 & -0.885831 & 3.048564 \\
\hline 6 & 2.981564 & -0.149761 & 4.079249 \\
\hline 6 & 4.359086 & -0.183728 & 4.290559 \\
\hline 6 & 5.159413 & -0.960248 & 3.460379 \\
\hline 6 & 4.585262 & -1.701059 & 2.429852 \\
\hline 15 & 1.754638 & -0.576400 & -0.730248 \\
\hline 8 & 2.798963 & 0.232720 & 0.261078 \\
\hline 6 & 3.673832 & 1.158879 & -0.247480 \\
\hline 6 & 4.802933 & 0.741709 & -0.926687 \\
\hline 6 & 5.666919 & 1.745335 & -1.475593 \\
\hline 6 & 5.394620 & 3.122873 & -1.214807 \\
\hline 6 & 4.260475 & 3.478078 & -0.445961 \\
\hline 6 & 3.405315 & 2.516102 & 0.016562 \\
\hline 6 & 6.774151 & 1.433378 & -2.305331 \\
\hline 6 & 7.580409 & 2.420726 & -2.815299 \\
\hline 6 & 7.328425 & 3.777785 & -2.525237 \\
\hline 6 & 6.253164 & 4.116337 & -1.744893 \\
\hline 6 & 5.057810 & -0.706710 & -1.105222 \\
\hline 6 & 4.081769 & -1.514387 & -1.660091 \\
\hline 6 & 4.325140 & -2.862831 & -2.000117 \\
\hline
\end{tabular}




\begin{tabular}{|c|c|c|c|}
\hline 6 & 5.521786 & -3.442010 & -1.678808 \\
\hline & 6.506808 & -2.707167 & -0.974022 \\
\hline & 6.277863 & -1.328791 & -0.681558 \\
\hline & 7.248571 & -0.641804 & 0.091129 \\
\hline & 8.384501 & -1.276624 & 0.529658 \\
\hline & 8.622098 & -2.630017 & 0.210508 \\
\hline & 7.698738 & -3.326789 & -0.526311 \\
\hline & 2.832856 & -1.015000 & -1.944072 \\
\hline & -1.585428 & 2.129901 & 1.001254 \\
\hline & -0.510600 & 2.617717 & 1.756747 \\
\hline & -0.454896 & 3.951095 & 2.146562 \\
\hline & -1.465659 & 4.812752 & 1.688101 \\
\hline & -2.521352 & 4.367726 & 0.897926 \\
\hline 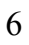 & -2.577007 & 3.003081 & 0.581641 \\
\hline & 0.662220 & 4.490685 & 3.039126 \\
\hline & 1.526650 & 5.470858 & 2.239931 \\
\hline y & -3.606357 & 5.289962 & 0.349407 \\
\hline & -3.541883 & 5.264905 & -1.183109 \\
\hline ) & 0.054791 & 5.218680 & 4.243881 \\
\hline & 1.557146 & 3.374856 & 3.578127 \\
\hline & -4.982673 & 4.799499 & 0.814150 \\
\hline & -3.433649 & 6.734097 & 0.811534 \\
\hline & 0.514410 & 2.466039 & -1.298035 \\
\hline & 0.598039 & 3.600528 & -1.514134 \\
\hline & -6.456300 & 1.417058 & 2.857421 \\
\hline ) & -7.836859 & -0.548292 & 2.251452 \\
\hline & -5.908970 & -0.412352 & -3.348915 \\
\hline & -4.701762 & -2.576263 & -2.959504 \\
\hline & 7.069003 & 0.395479 & 0.362760 \\
\hline & -1.666213 & 1.095802 & 3.275296 \\
\hline & 7.857108 & -4.377059 & -0.767761 \\
\hline & -0.639580 & -2.193905 & 6.498555 \\
\hline & 6.030122 & 5.159802 & -1.526111 \\
\hline & -3.276740 & -2.709425 & 1.623952 \\
\hline & -5.077479 & -4.256873 & 0.995797 \\
\hline & 4.068483 & 4.531464 & -0.247010 \\
\hline 1 & -1.301850 & -0.145811 & 5.343049 \\
\hline & 5.721867 & -4.481256 & -1.934328 \\
\hline 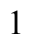 & 8.418248 & 2.152842 & -3.455167 \\
\hline . & 3.543556 & -3.411605 & -2.522137 \\
\hline & -2.502968 & -6.150446 & -1.884926 \\
\hline 1 & -0.188757 & -5.381369 & -2.148611 \\
\hline & 0.311517 & -5.721309 & 4.234801 \\
\hline 1 & 6.976827 & 0.393987 & -2.549957 \\
\hline & -0.131374 & -4.532764 & 2.144633 \\
\hline 1 & 1.631088 & -3.811916 & -1.521911 \\
\hline 1 & 7.977922 & 4.549594 & -2.931587 \\
\hline t & 9.105431 & -0.731223 & 1.134640 \\
\hline 1 & -4.697175 & -6.000422 & -0.745930 \\
\hline ] & 0.062234 & -4.570423 & 6.429362 \\
\hline 1 & 9.528712 & -3.118977 & 0.559114 \\
\hline ( & 2.512902 & 2.762391 & 0.589163 \\
\hline 1 & -3.804243 & 0.588929 & 2.218616 \\
\hline ] & -3.142116 & -0.672407 & -1.816324 \\
\hline 1 & -7.014314 & -1.151611 & -0.004440 \\
\hline 1 & -3.397353 & 2.623240 & -0.026796 \\
\hline
\end{tabular}




\begin{tabular}{|c|c|c|c|}
\hline$\frac{1}{1}$ & 0.277986 & 1.927046 & 2.054489 \\
\hline & -1.414667 & 5.864824 & 1.960665 \\
\hline & 2.128474 & -3.401194 & 1.599152 \\
\hline & -1.383215 & 1.443788 & -2.026581 \\
\hline & 3.417763 & -2.932406 & 0.496805 \\
\hline ] & 5.221108 & -2.299011 & 1.774397 \\
\hline & 1.342701 & -0.827751 & 2.861170 \\
\hline & 6.237823 & -0.987348 & 3.605285 \\
\hline & 2.342792 & 0.456350 & 4.720771 \\
\hline & 4.804878 & 0.397384 & 5.095056 \\
\hline & 2.305736 & 3.797220 & 4.260471 \\
\hline & 0.976092 & 2.629242 & 4.139623 \\
\hline & 2.105782 & 2.847561 & 2.786295 \\
\hline & 0.851491 & 5.592273 & 4.900912 \\
\hline & -0.555870 & 6.079708 & 3.948539 \\
\hline & -0.579876 & 4.543891 & 4.833088 \\
\hline & 2.336941 & 5.867877 & 2.866506 \\
\hline & 1.981448 & 4.976792 & 1.370614 \\
\hline L & 0.942348 & 6.321170 & 1.867654 \\
\hline & -4.238693 & 7.353082 & 0.395702 \\
\hline ] & -3.480657 & 6.823762 & 1.904750 \\
\hline & -2.482171 & 7.162281 & 0.470992 \\
\hline 1 & -4.301556 & 5.933860 & -1.610008 \\
\hline ] & -2.556420 & 5.596230 & -1.536736 \\
\hline | & -3.716965 & 4.259709 & -1.586526 \\
\hline$\frac{1}{1}$ & -5.773242 & 5.447467 & 0.412280 \\
\hline & -5.193017 & 3.775532 & 0.480680 \\
\hline 1 & -5.056461 & 4.815586 & 1.909777 \\
\hline ] & -7.065804 & 1.560707 & 3.759864 \\
\hline 1 & -5.459652 & 1.828846 & 3.060131 \\
\hline 1 & -6.903301 & 2.012178 & 2.050180 \\
\hline & -8.426955 & -0.393183 & 3.163573 \\
\hline 1 & -8.332020 & 0.003110 & 1.441809 \\
\hline 1 & -7.877048 & -1.619198 & 2.013398 \\
\hline 1 & -6.421280 & -0.755760 & 4.543171 \\
\hline 1 & -5.778784 & -1.945367 & 3.391527 \\
\hline 1 & -4.787455 & -0.561891 & 3.882018 \\
\hline 1 & -5.092227 & -2.982629 & -3.903090 \\
\hline | & -3.731496 & -2.120425 & -3.183059 \\
\hline 1 & -4.533439 & -3.413801 & -2.269327 \\
\hline 1 & -6.297199 & -0.781229 & -4.308897 \\
\hline 1 & -6.633230 & 0.307423 & -2.943991 \\
\hline 1 & -4.973472 & 0.124231 & -3.549572 \\
\hline 1 & -7.334711 & -2.747123 & -3.137652 \\
\hline 1 & -6.951929 & -3.107546 & -1.445189 \\
\hline 1 & -7.835864 & -1.623206 & -1.874923 \\
\hline 1 & 1.610038 & 0.225033 & -3.606496 \\
\hline l & 0.478322 & 1.575625 & -4.086475 \\
\hline 6 & -0.151973 & -1.798252 & -3.673740 \\
\hline 6 & -1.716895 & 0.065631 & -4.410008 \\
\hline 6 & -2.120421 & 1.406941 & -4.554405 \\
\hline 6 & -3.184459 & 1.764783 & -5.367751 \\
\hline 6 & -3.896952 & 0.794905 & -6.073843 \\
\hline 6 & -3.528824 & -0.535387 & -5.934625 \\
\hline 6 & -2.460396 & -0.893155 & -5.113598 \\
\hline 1 & -1.588778 & 2.188802 & -4.014316 \\
\hline
\end{tabular}


$3_{\text {ID-si- }} \psi_{-1 \text { s2a3a4a }}$

Number of imaginary frequencies : 0

The smallest frequencies are : $16.6679 \quad 19.808622 .1806 \mathrm{~cm}(-1)$

Electronic energy : $\quad \mathrm{HF}=-4360.8788286$

Zero-point correction $=\quad 1.455081($ Hartree/Particle)

Thermal correction to Energy= $\quad 1.539464$

Thermal correction to Enthalpy= $\quad 1.540409$

Thermal correction to Gibbs Free Energy $=\quad 1.338540$

Sum of electronic and zero-point Energies=

$-4359.423747$

Sum of electronic and thermal Energies $=$

$-4359.339364$

Sum of electronic and thermal Enthalpies=

$-4359.338420$

Sum of electronic and thermal Free Energies=

$-4359.540289$

Cartesian Coordinates

$\begin{array}{cccc}6 & 2.192922 & -1.438574 & 5.062682 \\ 6 & 1.517784 & -0.390108 & 4.420188 \\ 6 & 1.968745 & 0.913668 & 4.699915 \\ 6 & 3.014947 & 1.151000 & 5.578247 \\ 6 & 3.660631 & 0.092583 & 6.218060 \\ 6 & 3.242759 & -1.202651 & 5.948678 \\ 6 & 0.298643 & -0.663800 & 3.599494 \\ 6 & -0.063571 & -2.120088 & 3.438598 \\ 45 & 0.007401 & 0.531103 & 1.604291 \\ 6 & -0.579036 & 2.381520 & 1.465204 \\ 8 & -0.658881 & 3.503041 & 1.741532 \\ 15 & 1.551654 & 0.453065 & -0.275928 \\ 6 & 1.772291 & 2.238941 & -0.672956 \\ 6 & 0.775570 & 2.927567 & -1.361438 \\ 6 & 0.896428 & 4.294774 & -1.634115 \\ 6 & 2.017259 & 4.959112 & -1.135904 \\ 6 & 3.015426 & 4.306233 & -0.402003 \\ 6 & 2.879574 & 2.936168 & -0.186314 \\ 6 & -0.144516 & 4.984253 & -2.515473 \\ 6 & 4.219797 & 5.095567 & 0.107760 \\ 6 & 3.290953 & -0.129719 & -0.092491 \\ 6 & 3.678594 & -0.850083 & 1.028096 \\ 6 & 4.971849 & -1.394279 & 1.116399 \\ 6 & 5.850820 & -1.156033 & 0.064170 \\ 6 & 5.499428 & -0.403629 & -1.067402 \\ 6 & 4.204678 & 0.092986 & -1.132002 \\ 6 & 6.514223 & -0.191277 & -2.188899 \\ 6 & 5.403686 & -2.164521 & 2.362029 \\ 6 & 1.218978 & -0.277879 & -1.961765 \\ 6 & 0.842987 & -1.605885 & -2.091477 \\ 6 & 0.631373 & -2.174516 & -3.394187 \\ 6 & 0.828472 & -1.371597 & -4.554357\end{array}$




\begin{tabular}{|c|c|c|c|}
\hline 6 & 1.251471 & -0.034037 & -4.389264 \\
\hline 6 & 1.438601 & 0.490510 & -3.139690 \\
\hline & 0.593951 & -1.918744 & -5.838018 \\
\hline & 0.173551 & -3.215768 & -5.982340 \\
\hline & -0.024099 & -4.018669 & -4.839851 \\
\hline & 0.202554 & -3.515890 & -3.582157 \\
\hline & 0.663221 & -2.544631 & -0.948577 \\
\hline O & -0.564686 & -2.672307 & -0.325352 \\
\hline & -0.820645 & -3.762175 & 0.539457 \\
\hline 6 & 0.142773 & -4.699902 & 0.797304 \\
\hline & 1.430990 & -4.577556 & 0.225680 \\
\hline & 1.700101 & -3.485190 & -0.651570 \\
\hline 0 & 2.990292 & -3.390269 & -1.232691 \\
\hline 6 & 3.956740 & -4.327015 & -0.962557 \\
\hline 6 & 3.680362 & -5.418483 & -0.110409 \\
\hline 6 & 2.444506 & -5.535903 & 0.473754 \\
\hline 7 & -1.624148 & -1.740233 & -0.595746 \\
\hline 15 & -1.849481 & -0.567861 & 0.608370 \\
\hline 8 & -2.934510 & -1.178034 & 1.741838 \\
\hline 6 & -4.175152 & -1.648633 & 1.381975 \\
\hline 6 & -5.156377 & -0.786038 & 0.927989 \\
\hline 6 & -6.363142 & -1.359645 & 0.408882 \\
\hline 6 & -6.578668 & -2.766707 & 0.514512 \\
\hline 6 & -5.595164 & -3.575891 & 1.134508 \\
\hline 6 & -4.409460 & -3.031128 & 1.544722 \\
\hline 6 & -7.332377 & -0.587301 & -0.281206 \\
\hline 6 & -8.454898 & -1.171122 & -0.814743 \\
\hline 6 & -8.680918 & -2.556828 & -0.677079 \\
\hline 6 & -7.758248 & -3.334507 & -0.025521 \\
\hline 6 & -4.920428 & 0.675298 & 0.952593 \\
\hline 6 & -3.790591 & 1.196388 & 0.351967 \\
\hline 6 & -3.545346 & 2.579805 & 0.271370 \\
\hline 6 & -4.419782 & 3.459449 & 0.847049 \\
\hline 6 & -5.553720 & 2.987794 & 1.551008 \\
\hline 6 & -5.805863 & 1.583612 & 1.621068 \\
\hline 6 & -6.433110 & 3.887745 & 2.200434 \\
\hline 6 & -7.509530 & 3.431195 & 2.916450 \\
\hline 6 & -7.742337 & 2.043908 & 3.017841 \\
\hline 6 & -6.915807 & 1.146355 & 2.388255 \\
\hline 8 & -2.892545 & 0.364012 & -0.266974 \\
\hline 6 & -0.746000 & 0.291973 & 3.618853 \\
\hline 6 & -2.662351 & -2.220986 & -1.541821 \\
\hline 6 & -3.212050 & -1.183795 & -2.485707 \\
\hline 6 & -2.370495 & -0.301569 & -3.164842 \\
\hline 6 & -2.893043 & 0.641621 & -4.039099 \\
\hline 6 & -4.269375 & 0.717933 & -4.248942 \\
\hline 6 & -5.113952 & -0.159991 & -3.578595 \\
\hline 6 & -4.586066 & -1.106508 & -2.703502 \\
\hline 1 & -7.161127 & 0.478143 & -0.413912 \\
\hline 1 & 1.770569 & 1.521108 & -3.052884 \\
\hline 1 & -7.907407 & -4.408757 & 0.075800 \\
\hline 1 & 0.750666 & -1.282620 & -6.708050 \\
\hline 1 & -6.225070 & 4.954392 & 2.126462 \\
\hline 1 & 3.214761 & -2.549110 & -1.886824 \\
\hline 1 & 4.943795 & -4.228144 & -1.410694 \\
\hline 1 & -4.243554 & 4.532597 & 0.786813 \\
\hline
\end{tabular}




\begin{tabular}{|c|c|c|c|}
\hline 1 & 1.427282 & 0.577802 & -5.273564 \\
\hline 1 & -5.787885 & -4.640588 & 1.255216 \\
\hline 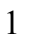 & -8.581509 & 1.679860 & 3.606475 \\
\hline & -3.633155 & -3.633438 & 2.011779 \\
\hline 1 & 2.219115 & -6.367839 & 1.139663 \\
\hline 1 & -0.066589 & -5.546498 & 1.449581 \\
\hline 1 & -0.357103 & -5.047539 & -4.955748 \\
\hline 1 & -7.104268 & 0.080605 & 2.488337 \\
\hline 1 & 0.048603 & -4.155849 & -2.715639 \\
\hline 1 & -1.813518 & -3.848342 & 0.976588 \\
\hline 1 & -8.174774 & 4.131571 & 3.416036 \\
\hline 1 & -9.174666 & -0.558791 & -1.353424 \\
\hline 1 & 4.452855 & -6.158416 & 0.087859 \\
\hline 1 & -0.008640 & -3.627686 & -6.972147 \\
\hline 1 & -9.577993 & -3.004914 & -1.097847 \\
\hline 1 & -2.659703 & 2.911845 & -0.262569 \\
\hline 1 & 3.886120 & 0.666445 & -1.998335 \\
\hline 1 & 2.950488 & -0.996367 & 1.828186 \\
\hline 1 & 6.855453 & -1.571062 & 0.113611 \\
\hline 1 & 3.639180 & 2.392047 & 0.369054 \\
\hline 1 & -0.102247 & 2.379503 & -1.713004 \\
\hline 1 & 2.127402 & 6.024958 & -1.323277 \\
\hline 1 & -2.169272 & -3.009358 & -2.128644 \\
\hline 1 & 1.273734 & 1.273120 & 2.187702 \\
\hline 1 & -3.492169 & -2.706130 & -1.005266 \\
\hline 1 & -5.255617 & -1.779661 & -2.165026 \\
\hline 1 & -1.300854 & -0.333257 & -2.969644 \\
\hline 1 & -6.191244 & -0.106016 & -3.725217 \\
\hline 1 & -2.219236 & 1.322250 & -4.558491 \\
\hline 1 & -4.679855 & 1.461644 & -4.928696 \\
\hline 1 & -1.777290 & -0.052608 & 3.546048 \\
\hline 1 & -0.626481 & 1.207182 & 4.198576 \\
\hline 1 & 1.489324 & 1.762214 & 4.214945 \\
\hline 1 & 3.328903 & 2.175925 & 5.767491 \\
\hline 1 & 4.481767 & 0.279859 & 6.906546 \\
\hline 1 & 3.735708 & -2.048625 & 6.425860 \\
\hline 1 & 1.887833 & -2.467570 & 4.890564 \\
\hline 1 & -0.209418 & -2.601392 & 4.419631 \\
\hline 1 & -1.000631 & -2.230488 & 2.886240 \\
\hline 1 & 0.709495 & -2.689491 & 2.904731 \\
\hline 6 & 4.300105 & -3.122934 & 2.814412 \\
\hline 6 & 6.663773 & -2.996570 & 2.125131 \\
\hline 6 & 5.694274 & -1.140840 & 3.464859 \\
\hline 6 & 5.987758 & 0.755121 & -3.266147 \\
\hline 6 & 7.806095 & 0.408005 & -1.623385 \\
\hline 6 & 6.825201 & -1.541831 & -2.843871 \\
\hline 1 & 4.613538 & -3.652925 & 3.724883 \\
\hline 1 & 3.365818 & -2.603815 & 3.051372 \\
\hline 1 & 4.088459 & -3.870240 & 2.038111 \\
\hline 1 & 6.889366 & -3.581441 & 3.025925 \\
\hline 1 & 6.532508 & -3.702475 & 1.293146 \\
\hline 1 & 7.545732 & -2.377591 & 1.918388 \\
\hline 1 & 6.009270 & -1.645255 & 4.389401 \\
\hline 1 & 6.499502 & -0.459744 & 3.157849 \\
\hline 1 & 4.809291 & -0.535816 & 3.697882 \\
\hline 1 & 6.755559 & 0.902466 & -4.036080 \\
\hline
\end{tabular}




$\begin{array}{lrrr}1 & 5.094864 & 0.356909 & -3.766669 \\ 1 & 5.737258 & 1.741752 & -2.852282 \\ 1 & 7.559918 & -1.417432 & -3.651091 \\ 1 & 7.239356 & -2.259865 & -2.124750 \\ 1 & 5.918770 & -1.983942 & -3.279072 \\ 1 & 8.531218 & 0.574439 & -2.431182 \\ 1 & 7.614973 & 1.373578 & -1.137631 \\ 1 & 8.283075 & -0.248347 & -0.886183 \\ 6 & 0.039234 & 6.499530 & -2.558326 \\ 6 & -0.001867 & 4.438883 & -3.942180 \\ 6 & -1.559638 & 4.691263 & -2.009754 \\ 6 & 5.217533 & 4.208865 & 0.849505 \\ 6 & 4.949556 & 5.738847 & -1.077129 \\ 6 & 3.742480 & 6.190309 & 1.067395 \\ 1 & -0.743909 & 6.947579 & -3.182739 \\ 1 & -0.035443 & 6.947472 & -1.558956 \\ 1 & 1.004202 & 6.787908 & -2.993869 \\ 1 & -2.298049 & 5.240146 & -2.609143 \\ 1 & -1.812504 & 3.626882 & -2.090504 \\ 1 & -1.677342 & 4.999555 & -0.961535 \\ 1 & -0.735131 & 4.910647 & -4.610705 \\ 1 & 1.001037 & 4.640739 & -4.341523 \\ 1 & -0.165598 & 3.353371 & -3.977755 \\ 1 & 4.597820 & 6.766418 & 1.445788 \\ 1 & 3.057404 & 6.894341 & 0.579637 \\ 1 & 3.217602 & 5.755848 & 1.927850 \\ 1 & 6.071650 & 4.812512 & 1.181420 \\ 1 & 4.774804 & 3.745909 & 1.740757 \\ 1 & 5.608600 & 3.406963 & 0.207685 \\ 1 & 5.823343 & 6.303205 & -0.724486 \\ 1 & 5.303148 & 4.975284 & -1.782588 \\ 1 & 4.309216 & 6.435551 & -1.631261\end{array}$

$3_{\text {ID-si- }} \psi_{-1 \text { s2a3a4s }}$

Number of imaginary frequencies : 0

The smallest frequencies are : $14.0168 \quad 17.5967 \quad 19.2820 \mathrm{~cm}(-1)$

Electronic energy : $\quad H F=-4360.8792979$

Zero-point correction $=\quad 1.454917$ (Hartree/Particle)

Thermal correction to Energy $=\quad 1.539520$

Thermal correction to Enthalpy $=\quad 1.540464$

Thermal correction to Gibbs Free Energy= $\quad 1.337037$

Sum of electronic and zero-point Energies $=\quad-4359.424381$

Sum of electronic and thermal Energies $=\quad-4359.339778$

Sum of electronic and thermal Enthalpies $=\quad-4359.338834$

Sum of electronic and thermal Free Energies $=\quad-4359.542261$

Cartesian Coordinates

$\begin{array}{lrrr}6 & 2.175251 & -1.121660 & 5.139578 \\ 6 & 1.488951 & -0.118613 & 4.439268 \\ 6 & 1.923933 & 1.204219 & 4.645624 \\ 6 & 2.965436 & 1.502798 & 5.510577 \\ 6 & 3.621336 & 0.489727 & 6.210497 \\ 6 & 3.219490 & -0.823450 & 6.013513 \\ 6 & 0.274760 & -0.451069 & 3.634055\end{array}$




\begin{tabular}{|c|c|c|c|}
\hline 6 & -0.076603 & -1.916773 & 3.558145 \\
\hline 45 & -0.012391 & 0.616397 & 1.563954 \\
\hline 6 & -0.603767 & 2.453181 & 1.321963 \\
\hline 8 & -0.696253 & 3.585631 & 1.545470 \\
\hline 15 & 1.544219 & 0.415384 & -0.299023 \\
\hline 6 & 1.833035 & 2.174274 & -0.770668 \\
\hline 6 & 0.850318 & 2.881893 & -1.460238 \\
\hline 6 & 1.018446 & 4.234642 & -1.775644 \\
\hline 6 & 2.180396 & 4.865670 & -1.330039 \\
\hline 6 & 3.168181 & 4.194761 & -0.598746 \\
\hline 6 & 2.980212 & 2.839530 & -0.334369 \\
\hline 6 & -0.019148 & 4.945808 & -2.643805 \\
\hline 6 & 4.416342 & 4.946494 & -0.139609 \\
\hline 6 & 3.268631 & -0.204183 & -0.092087 \\
\hline 6 & 3.678327 & -0.812037 & 1.089396 \\
\hline 6 & 4.969131 & -1.348394 & 1.204570 \\
\hline 6 & 5.831937 & -1.230251 & 0.112683 \\
\hline 6 & 5.455019 & -0.603222 & -1.079810 \\
\hline 6 & 4.160286 & -0.099574 & -1.161423 \\
\hline 6 & 6.384473 & -0.454442 & -2.282743 \\
\hline 6 & 5.419034 & -1.987928 & 2.515992 \\
\hline 6 & 1.189386 & -0.384247 & -1.948090 \\
\hline 6 & 0.820032 & -1.719382 & -2.012027 \\
\hline 6 & 0.610457 & -2.351969 & -3.284291 \\
\hline 6 & 0.792896 & -1.603322 & -4.482548 \\
\hline 6 & 1.195977 & -0.253013 & -4.383796 \\
\hline 6 & 1.387692 & 0.331937 & -3.161393 \\
\hline 6 & 0.564322 & -2.216519 & -5.737386 \\
\hline 6 & 0.166241 & -3.526092 & -5.816855 \\
\hline 6 & -0.015854 & -4.275623 & -4.636054 \\
\hline 6 & & -3.707910 & -3.405173 \\
\hline 6 & 0.643216 & -2.596370 & -0.820471 \\
\hline 6 & -0.582969 & -2.683424 & -0.186129 \\
\hline 6 & -0.834464 & -3.712526 & 0.750882 \\
\hline 6 & 0.133109 & -4.625737 & 1.073264 \\
\hline 6 & 1.419203 & -4.540426 & 0.490592 \\
\hline 6 & 1.681155 & -3.515079 & -0.466413 \\
\hline 6 & 2.967280 & -3.462233 & -1.061890 \\
\hline 6 & 3.938265 & -4.371604 & -0.724468 \\
\hline 6 & 3.670837 & -5.394938 & 0.210983 \\
\hline 6 & 2.437495 & -5.473357 & 0.806549 \\
\hline 7 & -1.642259 & -1.768306 & -0.509402 \\
\hline 15 & -1.869167 & -0.532853 & 0.628485 \\
\hline 8 & -2.959332 & -1.075597 & 1.790977 \\
\hline 6 & -4.200076 & -1.561266 & 1.451605 \\
\hline 6 & -5.177813 & -0.721291 & 0.950058 \\
\hline 6 & -6.385305 & -1.318522 & 0.459420 \\
\hline 6 & -6.604319 & -2.717712 & 0.637665 \\
\hline 6 & -5.623789 & -3.495917 & 1.300454 \\
\hline 6 & -4.437886 & -2.933083 & 1.684571 \\
\hline 6 & -7.351918 & -0.581357 & -0.271553 \\
\hline 6 & -8.474969 & -1.189712 & -0.775812 \\
\hline 6 & -8.704108 & -2.565926 & -0.567010 \\
\hline 6 & -7.784101 & -3.310457 & 0.125718 \\
\hline 6 & -4.937624 & 0.738521 & 0.892948 \\
\hline 6 & -3.805733 & 1.220628 & 0.264468 \\
\hline
\end{tabular}




\begin{tabular}{|c|c|c|c|}
\hline 6 & -3.560051 & 2.596087 & 0.096462 \\
\hline 6 & -4.434169 & 3.510742 & 0.615021 \\
\hline 6 & -5.568902 & 3.084514 & 1.346433 \\
\hline 6 & -5.822488 & 1.687438 & 1.503032 \\
\hline 6 & -6.448301 & 4.023478 & 1.938024 \\
\hline 6 & -7.526099 & 3.612824 & 2.679387 \\
\hline 6 & -7.760172 & 2.234690 & 2.866308 \\
\hline 6 & -6.933635 & 1.299342 & 2.294551 \\
\hline 8 & -2.907253 & 0.351946 & -0.299479 \\
\hline 6 & -0.775370 & 0.496939 & 3.587227 \\
\hline 6 & -2.669521 & -2.290702 & -1.444855 \\
\hline 6 & -3.219873 & -1.290814 & -2.428153 \\
\hline 6 & -2.379149 & -0.427080 & -3.131909 \\
\hline 6 & -2.902271 & 0.490605 & -4.032349 \\
\hline 6 & -4.278311 & 0.556855 & -4.247479 \\
\hline 6 & -5.121713 & -0.305381 & -3.555802 \\
\hline 6 & -4.593361 & -1.224284 & -2.651891 \\
\hline 1 & -7.178578 & 0.475431 & -0.458926 \\
\hline 1 & 1.710853 & 1.368196 & -3.123746 \\
\hline 1 & -7.935579 & -4.377687 & \\
\hline 1 & 0.708480 & -1.621603 & -6.638181 \\
\hline 1 & -6.239380 & 5.083361 & 1.798460 \\
\hline 1 & 3.184931 & -2.673149 & -1.780505 \\
\hline 1 & 4.925090 & -4.301479 & -1.178394 \\
\hline 1 & -4.257246 & 4.577899 & 0.487237 \\
\hline 1 & 1.355501 & 0.318870 & -5.297405 \\
\hline 1 & -5.818978 & -4.552558 & \\
\hline 1 & -8.600229 & 1.908515 & 3.475507 \\
\hline 1 & -3.664061 & -3.512221 & 2.183998 \\
\hline 1 & 2.217933 & -6.253921 & 1.533753 \\
\hline 1 & -0.071218 & -5.423819 & 1.785526 \\
\hline 1 & -0.330902 & -5.314574 & -4.700876 \\
\hline 1 & -7.122430 & 0.241953 & 2.460729 \\
\hline 1 & 0.060949 & -4.306572 & \\
\hline 1 & -1.826258 & -3.769976 & 1.194780 \\
\hline 1 & -8.191431 & 4.343324 & \\
\hline 1 & -9.192477 & -0.604597 & -1.346804 \\
\hline 1 & 4.448000 & -6.113444 & 0.462738 \\
\hline 1 & -0.010275 & -3.989028 & -6.784926 \\
\hline 1 & -9.601344 & -3.033499 & -0.965641 \\
\hline 1 & -2.672788 & 2.892677 & -0.455488 \\
\hline 1 & 3.834340 & 0.385226 & -2.080637 \\
\hline 1 & 2.969015 & -0.870015 & 1.917405 \\
\hline 1 & 6.832765 & -1.644562 & 0.190700 \\
\hline 1 & 3.729401 & 2.284375 & 0.223837 \\
\hline 1 & -0.057310 & 2.359812 & -1.772846 \\
\hline 1 & 2.329824 & 5.919754 & -1.554125 \\
\hline 1 & -2.166555 & -3.095913 & -1.999533 \\
\hline 1 & 1.250809 & 1.392852 & 2.107812 \\
\hline 1 & -3.499129 & -2.762468 & -0.895836 \\
\hline 1 & -5.262477 & -1.882197 & -2.094363 \\
\hline 1 & -1.310029 & -0.448338 & -2.932944 \\
\hline 1 & -6.198577 & -0.259395 & -3.708015 \\
\hline 1 & -2.229419 & 1.159675 & -4.567656 \\
\hline 1 & -4.689453 & 1.280521 & -4.948156 \\
\hline 1 & -1.804092 & 0.142017 & 3.530014 \\
\hline
\end{tabular}




\begin{tabular}{|c|c|c|c|}
\hline & -0.665329 & 1.447582 & 4.108880 \\
\hline & 1.436026 & 2.018535 & 4.112752 \\
\hline & 3.267285 & 2.540290 & 5.642212 \\
\hline & 4.438244 & 0.725061 & 6.889185 \\
\hline & 3.721059 & -1.635318 & 6.538532 \\
\hline & 1.882709 & -2.162236 & 5.024675 \\
\hline & -0.222054 & -2.340461 & 4.565511 \\
\hline & -1.010949 & -2.065067 & 3.009993 \\
\hline & 0.701236 & -2.511831 & 3.060217 \\
\hline & 4.346213 & -2.948319 & 3.035483 \\
\hline & 6.716034 & -2.782143 & 2.367386 \\
\hline & 5.654633 & -0.863252 & 3.530028 \\
\hline & 7.744538 & -1.108191 & -2.054528 \\
\hline & 5.743145 & -1.111319 & -3.511147 \\
\hline & 6.608751 & 1.037177 & -2.558824 \\
\hline & 4.664212 & -3.388872 & 3.990960 \\
\hline & 3.388484 & -2.447905 & 3.213475 \\
\hline 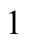 & 4.173982 & -3.764300 & 2.320982 \\
\hline & 6.958434 & -3.265818 & 3.322223 \\
\hline . & 6.627735 & -3.571921 & 1.608451 \\
\hline & 7.570751 & -2.145878 & 2.105068 \\
\hline . & 5.984616 & -1.273848 & 4.494805 \\
\hline ] & 6.431690 & -0.175299 & 3.170132 \\
\hline . & 4.742825 & -0.280379 & 3.708728 \\
\hline ] & 8.369518 & -0.981772 & -2.947664 \\
\hline & 8.282482 & -0.656140 & -1.211415 \\
\hline ] & 7.653441 & -2.186251 & -1.866375 \\
\hline 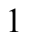 & 7.248341 & 1.172856 & -3.441540 \\
\hline | & 5.666244 & 1.568518 & -2.743628 \\
\hline L & 7.104266 & 1.521265 & -1.706710 \\
\hline & 6.387086 & -0.982772 & -4.391664 \\
\hline 1 & 5.605544 & -2.189323 & -3.353126 \\
\hline l & 4.761775 & -0.683539 & -3.753021 \\
\hline 5 & 0.170581 & 6.461379 & -2.652673 \\
\hline 5 & 0.125313 & 4.430199 & -4.081038 \\
\hline 6 & -1.437446 & 4.648934 & -2.147489 \\
\hline 5 & 5.374486 & 4.050087 & 0.642330 \\
\hline 5 & 5.163808 & 5.487591 & -1.363613 \\
\hline 5 & 4.007207 & 6.113514 & 0.765017 \\
\hline 1 & -0.618705 & 6.927680 & -3.255639 \\
\hline 1 & 0.112229 & 6.884377 & -1.641442 \\
\hline 1 & 1.129860 & 6.757647 & -3.095034 \\
\hline 1 & -2.169244 & 5.228237 & -2.725776 \\
\hline 1 & -1.704770 & 3.591334 & -2.267433 \\
\hline 1 & -1.549985 & 4.918658 & -1.088190 \\
\hline 1 & -0.609696 & 4.911772 & -4.740645 \\
\hline 1 & 1.127513 & 4.643397 & -4.475977 \\
\hline 1 & -0.033651 & 3.344857 & -4.136343 \\
\hline 1 & 4.896485 & 6.657420 & 1.111407 \\
\hline 1 & 3.361085 & 6.831722 & 0.245987 \\
\hline 1 & 3.464183 & 5.753127 & 1.648083 \\
\hline 1 & 6.262165 & 4.625790 & 0.933489 \\
\hline 1 & 4.916690 & 3.662080 & 1.561489 \\
\hline 1 & 5.716838 & 3.193381 & 0.045448 \\
\hline 1 & 6.063895 & 6.033480 & -1.050076 \\
\hline 1 & 5.478718 & 4.669651 & -2.025308 \\
\hline
\end{tabular}


$3_{\text {ID-si- }} \psi_{-1 \mathrm{~s} 2 \mathrm{a} 3 \mathrm{~s} 4 \mathrm{a}}$

Number of imaginary frequencies : 0

The smallest frequencies are : $16.8117 \quad 22.661924 .4499 \mathrm{~cm}(-1)$

Electronic energy : $\quad H F=-4360.8788247$

Zero-point correction $=\quad 1.456181$ (Hartree/Particle)

Thermal correction to Energy= $\quad 1.540326$

Thermal correction to Enthalpy $=\quad 1.541270$

Thermal correction to Gibbs Free Energy $=\quad 1.340512$

Sum of electronic and zero-point Energies $=\quad-4359.422644$

Sum of electronic and thermal Energies $=\quad-4359.338498$

Sum of electronic and thermal Enthalpies $=\quad-4359.337554$

Sum of electronic and thermal Free Energies $=\quad-4359.538313$

Cartesian Coordinates

$\begin{array}{cccc}6 & -2.280822 & -0.963553 & -5.146942 \\ 6 & -1.576894 & 0.012367 & -4.426013 \\ 6 & -2.001731 & 1.344756 & -4.588840 \\ 6 & -3.048205 & 1.678341 & -5.434749 \\ 6 & -3.720057 & 0.692281 & -6.157828 \\ 6 & -3.330014 & -0.629936 & -6.001988 \\ 6 & -0.355888 & -0.353397 & -3.645481 \\ 6 & -0.011150 & -1.822264 & -3.623665 \\ 45 & -0.036062 & 0.648132 & -1.551347 \\ 6 & 0.543057 & 2.484264 & -1.262103 \\ 8 & 0.606216 & 3.625145 & -1.449770 \\ 15 & -1.548617 & 0.417455 & 0.348779 \\ 6 & -1.713923 & 2.166884 & 0.901919 \\ 6 & -0.721029 & 2.757769 & 1.680289 \\ 6 & -0.800436 & 4.104240 & 2.054890 \\ 6 & -1.861070 & 4.855982 & 1.550980 \\ 6 & -2.842412 & 4.307834 & 0.714209 \\ 6 & -2.764030 & 2.949230 & 0.415933 \\ 6 & 0.223475 & 4.673675 & 3.036197 \\ 6 & -3.962262 & 5.198206 & 0.178898 \\ 6 & -3.305558 & -0.101311 & 0.141500 \\ 6 & -3.732569 & -0.677941 & -1.045589 \\ 6 & -5.039787 & -1.181124 & -1.167429 \\ 6 & -5.888020 & -1.058799 & -0.071727 \\ 6 & -5.494239 & -0.456534 & 1.133917 \\ 6 & -4.191514 & 0.014473 & 1.222280 \\ 6 & -6.481004 & -0.368514 & 2.296293 \\ 6 & -5.506110 & -1.793871 & -2.485266 \\ 6 & -1.209242 & -0.477990 & 1.950381 \\ 6 & -0.844271 & -1.815350 & 1.943634 \\ 6 & -0.629141 & -2.513185 & 3.181307 \\ 6 & -0.808266 & -1.827908 & 4.417330 \\ 6 & -1.221342 & -0.477212 & 4.390387 \\ 6 & -1.414964 & 0.170412 & 3.200962 \\ 6 & -0.567747 & -2.502194 & 5.637836 \\ 6 & -0.160819 & -3.811450 & 5.648449 \\ 6 & 0.016717 & -4.499149 & 4.430121 \\ 6 & -0.214919 & -3.871333 & 3.230954 \\ & & & \\ 6\end{array}$




\begin{tabular}{|c|c|c|c|}
\hline & -0.683310 & -2.636154 & 0.710532 \\
\hline & 0.537085 & -2.709685 & 0.064358 \\
\hline & 0.774711 & -3.709161 & -0.907919 \\
\hline & -0.201033 & -4.605907 & -1.251433 \\
\hline & -1.483353 & -4.529652 & -0.658716 \\
\hline & -1.732708 & -3.531280 & 0.329625 \\
\hline & -3.015591 & -3.486725 & 0.933603 \\
\hline & -3.991465 & -4.386459 & 0.583731 \\
\hline & -3.735509 & -5.384300 & -0.382021 \\
\hline 6 & -2.508836 & -5.448185 & -0.993026 \\
\hline & 1.606030 & -1.815388 & 0.414180 \\
\hline & 1.827330 & -0.535878 & -0.675287 \\
\hline & 2.908213 & -1.038130 & -1.865291 \\
\hline & 4.151393 & -1.535268 & -1.551918 \\
\hline 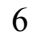 & 5.131817 & -0.713603 & -1.025676 \\
\hline 6 & 6.340450 & -1.327246 & -0.560010 \\
\hline 6 & 6.558356 & -2.719546 & -0.786429 \\
\hline 6 & 5.575525 & -3.474023 & -1.473031 \\
\hline & 4.388353 & -2.898157 & -1.833554 \\
\hline 6 & 7.308859 & -0.614792 & 0.192580 \\
\hline 6 & 8.432343 & -1.240210 & 0.674423 \\
\hline 6 & 8.659760 & -2.609064 & 0.419854 \\
\hline 6 & 7.738491 & -3.329686 & -0.296285 \\
\hline & 4.891385 & 0.743487 & -0.921599 \\
\hline 6 & 3.760380 & 1.203723 & -0.275757 \\
\hline 0 & 3.500198 & 2.573549 & -0.085482 \\
\hline 6 & 4.366137 & 3.504954 & -0.588263 \\
\hline 6 & 5.505174 & 3.102368 & -1.326172 \\
\hline 6 & 5.768997 & 1.710878 & -1.512456 \\
\hline 6 & 6.377484 & 4.060193 & -1.897744 \\
\hline & 7.458041 & 3.673313 & -2.647755 \\
\hline 6 & 7.701848 & 2.301222 & -2.864504 \\
\hline 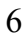 & 6.882159 & 1.347865 & -2.313053 \\
\hline & 2.874860 & 0.312534 & 0.276975 \\
\hline 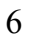 & 0.700841 & 0.587090 & -3.584496 \\
\hline & 2.645201 & -2.391020 & 1.303897 \\
\hline 5 & 3.208028 & -1.458497 & 2.344319 \\
\hline ) & 2.378724 & -0.642871 & 3.115718 \\
\hline 5 & 2.913484 & 0.188627 & 4.090298 \\
\hline 5 & 4.290011 & 0.218414 & 4.309814 \\
\hline & 5.122820 & -0.591267 & 3.545402 \\
\hline 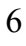 & 4.582765 & -1.425411 & 2.569303 \\
\hline & 7.136324 & 0.435550 & 0.414179 \\
\hline . & -1.740171 & 1.206698 & 3.219822 \\
\hline & 7.889284 & -4.391187 & -0.488470 \\
\hline 1 & -0.709585 & -1.954341 & 6.568344 \\
\hline 1 & 6.160945 & 5.115312 & -1.735580 \\
\hline 1 & -3.224506 & -2.715935 & 1.674262 \\
\hline 1 & -4.971950 & -4.329324 & 1.052630 \\
\hline & 4.179424 & 4.568167 & -0.443542 \\
\hline 1 & -1.384517 & 0.044742 & 5.332854 \\
\hline & 5.769499 & -4.524288 & -1.684295 \\
\hline 1 & 8.544087 & 1.994274 & -3.480629 \\
\hline 1 & 3.611665 & -3.460433 & -2.347788 \\
\hline 1 & -2.299173 & -6.208208 & -1.744485 \\
\hline 1 & -0.006513 & -5.383446 & -1.988694 \\
\hline
\end{tabular}




\begin{tabular}{|c|c|c|c|}
\hline & 0.338387 & -5.538015 & 4.439986 \\
\hline & 7.078602 & 0.295778 & -2.502115 \\
\hline & -0.075443 & -4.423394 & 2.303747 \\
\hline & 1.763007 & -3.758675 & -1.360859 \\
\hline & 8.118102 & 4.418118 & -3.086231 \\
\hline & 9.151477 & -0.674974 & 1.263116 \\
\hline & -4.516268 & -6.094870 & -0.644682 \\
\hline & 0.025938 & -4.321372 & 6.590631 \\
\hline & 9.557059 & -3.090342 & 0.801674 \\
\hline & 2.608300 & 2.852966 & 0.468275 \\
\hline & -3.843068 & 0.476717 & 2.142182 \\
\hline & -3.026284 & -0.743281 & -1.875634 \\
\hline & -6.901379 & -1.448411 & -0.144084 \\
\hline & -3.518124 & 2.482505 & -0.213519 \\
\hline & 0.118909 & 2.149375 & 2.024321 \\
\hline & -1.934861 & 5.909391 & 1.812152 \\
\hline & 2.149506 & -3.229562 & 1.814097 \\
\hline & -1.309524 & 1.434892 & -2.054157 \\
\hline & 3.468433 & -2.828783 & 0.719087 \\
\hline & 5.243175 & -2.045977 & 1.961047 \\
\hline & 1.309059 & -0.636293 & 2.918240 \\
\hline & 6.200359 & -0.570751 & 3.698127 \\
\hline & 2.248968 & 0.819027 & 4.680454 \\
\hline & 4.709969 & 0.874338 & 5.069465 \\
\hline & 1.728270 & 0.225142 & -3.552067 \\
\hline & 0.589813 & 1.553304 & -4.076372 \\
\hline & -1.501363 & 2.138653 & -4.036780 \\
\hline & -3.341665 & 2.721985 & -5.532623 \\
\hline & -4.540743 & 0.955002 & -6.821744 \\
\hline l & -3.844889 & -1.421394 & -6.544978 \\
\hline & -1.999152 & -2.010147 & -5.063449 \\
\hline 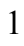 & 0.115032 & -2.213455 & -4.646659 \\
\hline & 0.931867 & -1.992197 & -3.096975 \\
\hline & -0.783052 & -2.429822 & -3.131719 \\
\hline 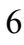 & -6.817986 & -2.563728 & -2.340426 \\
\hline & -5.723167 & -0.651548 & -3.483182 \\
\hline 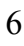 & -4.454572 & -2.768927 & -3.021243 \\
\hline & -5.886872 & 0.354159 & 3.503207 \\
\hline 6 & -7.734081 & 0.394381 & 1.853756 \\
\hline 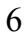 & -6.873492 & -1.783848 & 2.734822 \\
\hline & -7.074374 & -3.031339 & -3.299588 \\
\hline 1 & -6.741718 & -3.363564 & -1.590757 \\
\hline & -7.658264 & -1.913195 & -2.066679 \\
\hline 1 & -6.061983 & -1.043074 & -4.452734 \\
\hline & -6.487234 & 0.045044 & -3.112397 \\
\hline 1 & -4.801511 & -0.082707 & -3.656007 \\
\hline 1 & -4.782329 & -3.185005 & -3.984337 \\
\hline 1 & -3.484794 & -2.288530 & -3.190127 \\
\hline 1 & -4.301484 & -3.601632 & -2.321887 \\
\hline 1 & -6.629558 & 0.397293 & 4.309788 \\
\hline 1 & -5.002110 & -0.163097 & 3.898423 \\
\hline 1 & -5.601250 & 1.386521 & 3.261452 \\
\hline 1 & -7.593236 & -1.741812 & 3.563590 \\
\hline 1 & -7.338115 & -2.354376 & 1.921150 \\
\hline 1 & -5.994445 & -2.343730 & 3.081901 \\
\hline 1 & -8.446899 & 0.471555 & 2.685737 \\
\hline
\end{tabular}




$\begin{array}{rrrc}1 & -7.480345 & 1.412262 & 1.530408 \\ 1 & -8.249830 & -0.101374 & 1.022790 \\ 6 & 0.047162 & 6.174007 & 3.256843 \\ 6 & 0.045900 & 3.964788 & 4.385078 \\ 6 & 1.647171 & 4.432441 & 2.527432 \\ 6 & -4.936890 & 4.429967 & -0.711181 \\ 6 & -4.753733 & 5.788492 & 1.351324 \\ 6 & -3.353415 & 6.334336 & -0.650020 \\ 1 & 0.810036 & 6.534907 & 3.958098 \\ 1 & 0.161226 & 6.740323 & 2.323459 \\ 1 & -0.932209 & 6.415485 & 3.689092 \\ 1 & 2.377537 & 4.873864 & 3.218645 \\ 1 & 1.883634 & 3.363890 & 2.450949 \\ 1 & 1.795266 & 4.893075 & 1.540894 \\ 1 & 0.762049 & 4.353924 & 5.121713 \\ 1 & -0.966551 & 4.121337 & 4.780760 \\ 1 & 0.209193 & 2.882229 & 4.299814 \\ 1 & -4.145887 & 6.980102 & -1.052079 \\ 1 & -2.681462 & 6.965118 & -0.055774 \\ 1 & -2.777009 & 5.936744 & -1.495429 \\ 1 & -5.720262 & 5.109510 & -1.070012 \\ 1 & -4.440598 & 4.004161 & -1.593036 \\ 1 & -5.432599 & 3.612618 & -0.170329 \\ 1 & -5.564535 & 6.429305 & 0.979245 \\ 1 & -5.203986 & 4.994126 & 1.961100 \\ 1 & -4.125154 & 6.400308 & 2.009562\end{array}$

$3_{\text {ID-si- }} \psi_{-1 \text { s22a3s4s }}$

Number of imaginary frequencies : 0

The smallest frequencies are : $14.8709 \quad 18.1773 \quad 23.0431 \mathrm{~cm}(-1)$

Electronic energy : $\quad H F=-4360.8792632$

Zero-point correction $=\quad 1.455591$ (Hartree/Particle)

Thermal correction to Energy $=\quad 1.539948$

Thermal correction to Enthalpy= $\quad 1.540893$

Thermal correction to Gibbs Free Energy= $\quad 1.338684$

Sum of electronic and zero-point Energies= $\quad-4359.423672$

Sum of electronic and thermal Energies $=\quad-4359.339315$

Sum of electronic and thermal Enthalpies $=\quad-4359.338371$

Sum of electronic and thermal Free Energies= $\quad-4359.540579$

\begin{tabular}{cccc}
\multicolumn{4}{c}{ Cartesian Coordinates } \\
$\ldots \ldots \ldots \ldots \ldots \ldots \ldots \ldots \ldots \ldots \ldots \ldots$ \\
6 & 2.238866 & -0.973319 & 5.163053 \\
6 & 1.550291 & 0.006381 & 4.432530 \\
6 & 1.990186 & 1.334490 & 4.589824 \\
6 & 3.037164 & 1.660382 & 5.438194 \\
6 & 3.694814 & 0.670601 & 6.169162 \\
6 & 3.289109 & -0.647601 & 6.019841 \\
6 & 0.329371 & -0.350540 & 3.647411 \\
6 & -0.027655 & -1.816710 & 3.627515 \\
45 & 0.023550 & 0.651575 & 1.551334 \\
6 & -0.544095 & 2.487447 & 1.243096 \\
8 & -0.603354 & 3.631928 & 1.408611 \\
15 & 1.547517 & 0.402621 & -0.337844 \\
6 & 1.763813 & 2.150669 & -0.878613
\end{tabular}




\begin{tabular}{|c|c|c|c|}
\hline ) & 0.778954 & 2.778788 & -1.637577 \\
\hline & 0.892298 & 4.126284 & -1.998650 \\
\hline & 1.986557 & 4.839141 & -1.509635 \\
\hline & 2.964977 & 4.252343 & -0.696338 \\
\hline & 2.846679 & 2.895071 & -0.405379 \\
\hline & -0.138701 & 4.740504 & -2.944627 \\
\hline & 4.132649 & 5.095824 & -0.187882 \\
\hline & 3.291269 & -0.154253 & -0.119461 \\
\hline & 3.711935 & -0.731047 & 1.074149 \\
\hline & 5.011464 & -1.241984 & 1.200176 \\
\hline & 5.871527 & -1.130719 & 0.105332 \\
\hline & 5.483382 & -0.535658 & -1.099711 \\
\hline & 4.180120 & -0.055110 & -1.191362 \\
\hline & 6.406599 & -0.409763 & -2.310024 \\
\hline & 5.471079 & -1.853710 & 2.521472 \\
\hline 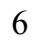 & 1.202517 & -0.471013 & -1.950816 \\
\hline 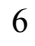 & 0.828066 & -1.805668 & -1.958269 \\
\hline 6 & 0.610926 & -2.490467 & -3.202222 \\
\hline 6 & 0.795082 & -1.794266 & -4.431280 \\
\hline 6 & 1.212825 & -0.445386 & -4.390511 \\
\hline & 1.410321 & 0.189479 & -3.194465 \\
\hline 6 & 0.554994 & -2.456656 & -5.658451 \\
\hline 6 & 0.144387 & -3.764566 & -5.681736 \\
\hline 6 & -0.038066 & -4.462953 & -4.470133 \\
\hline 6 & 0.192432 & -3.846775 & -3.264813 \\
\hline 6 & 0.662723 & -2.634044 & -0.731579 \\
\hline 6 & -0.555731 & -2.700956 & -0.080996 \\
\hline 6 & -0.793843 & -3.698992 & 0.892545 \\
\hline 6 & 0.179939 & -4.598869 & 1.234029 \\
\hline 6 & 1.460368 & -4.528776 & 0.636500 \\
\hline 6 & 1.709033 & -3.534246 & -0.355677 \\
\hline 6 & 2.989584 & -3.494241 & -0.964752 \\
\hline 6 & 3.966552 & -4.391016 & -0.611007 \\
\hline 6 & 3.712062 & -5.384655 & 0.359573 \\
\hline 6 & 2.485661 & -5.447346 & 0.971420 \\
\hline 7 & -1.620620 & -1.799784 & -0.425693 \\
\hline 15 & -1.841532 & -0.526935 & 0.671615 \\
\hline 8 & -2.926772 & -1.033121 & 1.856049 \\
\hline 6 & -4.169403 & -1.526993 & 1.534584 \\
\hline 6 & -5.146991 & -0.701205 & 1.009319 \\
\hline 6 & -6.353565 & -1.311104 & 0.533466 \\
\hline 6 & -6.573418 & -2.704698 & 0.749781 \\
\hline 6 & -5.594591 & -3.464109 & 1.436611 \\
\hline 6 & -4.408745 & -2.891398 & 1.806464 \\
\hline 6 & -7.317632 & -0.593277 & -0.219662 \\
\hline 6 & -8.438968 & -1.214928 & -0.711271 \\
\hline 6 & -8.668612 & -2.585189 & -0.466397 \\
\hline 6 & -7.751491 & -3.310928 & 0.249877 \\
\hline 6 & -4.905267 & 0.756316 & 0.915155 \\
\hline 6 & -3.771313 & 1.218753 & 0.276402 \\
\hline 6 & -3.509494 & 2.589106 & 0.093203 \\
\hline 6 & -4.376193 & 3.519038 & 0.597207 \\
\hline 6 & -5.518544 & 3.113854 & 1.328650 \\
\hline 6 & -5.784493 & 1.721628 & 1.506920 \\
\hline 6 & -6.392441 & 4.069576 & 1.901283 \\
\hline 6 & -7.476635 & 3.679935 & 2.644607 \\
\hline
\end{tabular}




\begin{tabular}{|c|c|c|c|}
\hline & -7.722787 & 2.307007 & 2.853258 \\
\hline & -6.901580 & 1.355641 & 2.300615 \\
\hline & -2.884674 & 0.329824 & -0.277300 \\
\hline & -0.719835 & 0.598352 & 3.581883 \\
\hline & -2.656224 & -2.359179 & -1.329540 \\
\hline & -3.208125 & -1.409074 & -2.360125 \\
\hline & -2.371580 & -0.577131 & -3.105926 \\
\hline & -2.897126 & 0.276241 & -4.066469 \\
\hline & -4.271590 & 0.311416 & -4.297992 \\
\hline & -5.111433 & -0.515321 & -3.560017 \\
\hline & -4.580567 & -1.370976 & -2.597522 \\
\hline & -7.143312 & 0.458303 & -0.433846 \\
\hline & 1.740568 & 1.224186 & -3.202628 \\
\hline & -7.903973 & -4.373493 & 0.434724 \\
\hline & 0.700621 & -1.900994 & -6.583714 \\
\hline & -6.174285 & 5.125301 & 1.745319 \\
\hline & 3.196600 & -2.727652 & -1.710641 \\
\hline & 4.947465 & -4.333620 & -1.079302 \\
\hline & -4.187662 & 4.582682 & 0.457731 \\
\hline & 1.377735 & 0.085283 & -5.327772 \\
\hline & -5.790373 & -4.515479 & 1.640548 \\
\hline & -8.568115 & 1.997812 & 3.464004 \\
\hline & -3.635265 & -3.457465 & 2.321330 \\
\hline & 2.277175 & -6.204685 & 1.725917 \\
\hline 1 & -0.014782 & -5.373941 & 1.973839 \\
\hline & -0.362570 & -5.500791 & -4.490349 \\
\hline & -7.099850 & 0.302759 & 2.483318 \\
\hline & 0.049671 & -4.406507 & -2.342685 \\
\hline & -1.780595 & -3.743778 & 1.349104 \\
\hline 1 & -8.137912 & 4.423171 & 3.083913 \\
\hline & -9.154664 & -0.645528 & -1.300138 \\
\hline ] & 4.493814 & -6.093064 & 0.625222 \\
\hline & -0.041471 & -4.265463 & -6.628925 \\
\hline & -9.564314 & -3.063416 & -0.855736 \\
\hline & -2.615158 & 2.869874 & -0.455527 \\
\hline & 3.844658 & 0.402361 & -2.121245 \\
\hline 1 & 3.003021 & -0.785690 & 1.902637 \\
\hline & 6.879153 & -1.526625 & 0.190869 \\
\hline 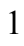 & 3.595187 & 2.400369 & 0.208684 \\
\hline & -0.084732 & 2.199458 & -1.973084 \\
\hline 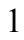 & 2.089940 & 5.891664 & -1.764209 \\
\hline l & -2.161323 & -3.193412 & -1.847498 \\
\hline & 1.297284 & 1.435873 & 2.057127 \\
\hline ] & -3.485006 & -2.800505 & -0.755161 \\
\hline & -5.246785 & -2.003890 & -2.008618 \\
\hline 1 & -1.304048 & -0.573656 & -2.897220 \\
\hline & -6.187577 & -0.490970 & -3.721827 \\
\hline 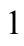 & -2.227101 & 0.920313 & -4.635335 \\
\hline 1 & -4.684507 & 0.984764 & -5.046172 \\
\hline 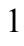 & -1.750016 & 0.244321 & 3.546936 \\
\hline 1 & -0.602581 & 1.564215 & 4.073016 \\
\hline ( & 1.501724 & 2.131068 & 4.031110 \\
\hline 1 & 3.342705 & 2.701013 & 5.531121 \\
\hline ] & 4.516459 & 0.927312 & 6.834242 \\
\hline 1 & 3.792320 & -1.442142 & 6.569233 \\
\hline 1 & 1.944474 & -2.016811 & 5.085187 \\
\hline
\end{tabular}




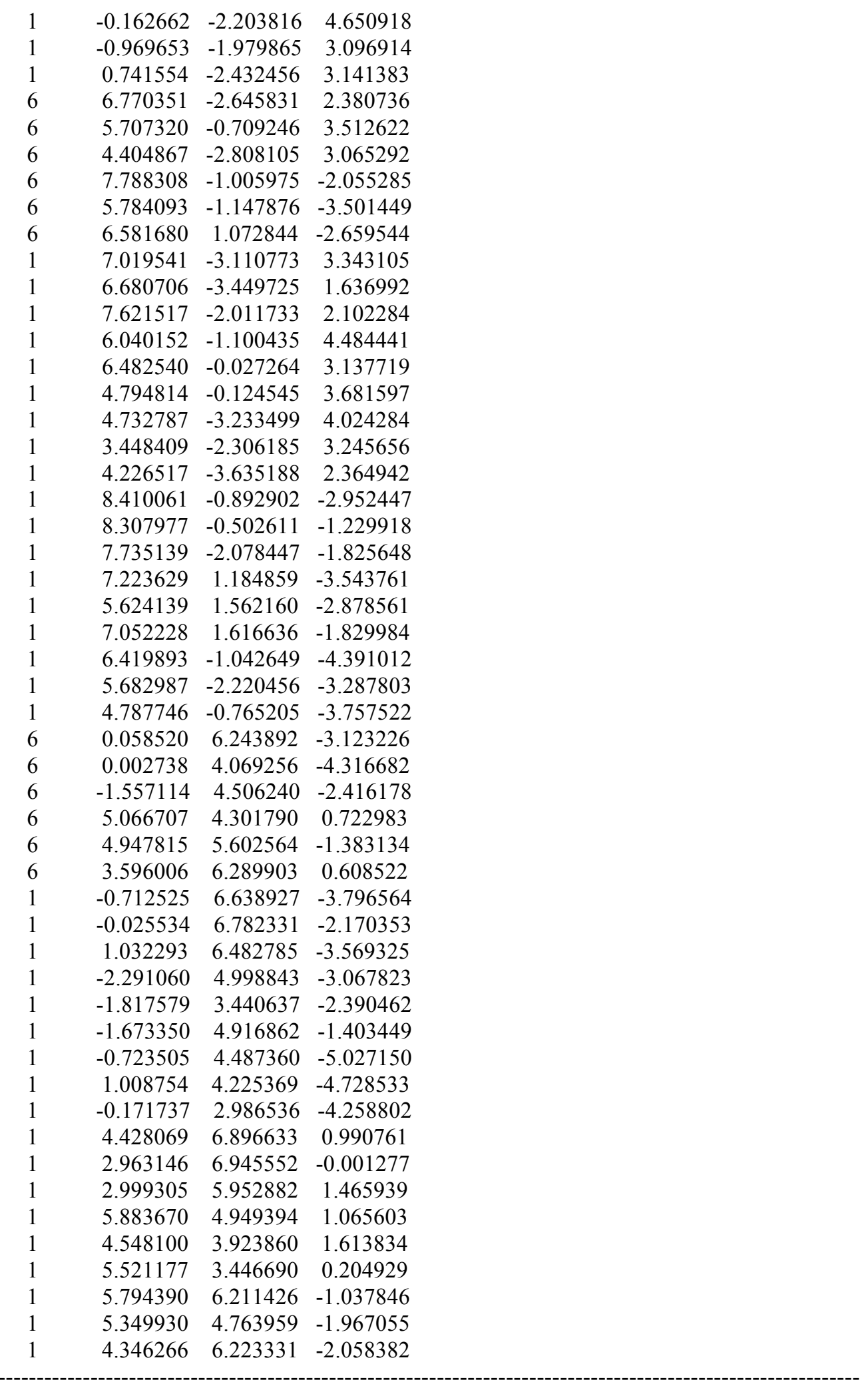

$3_{\text {ID-si }-\psi_{-122 s 3 a 4 a}}$

Number of imaginary frequencies : 0

The smallest frequencies are : $15.718920 .4458 \quad 22.7813 \mathrm{~cm}(-1)$ 
Electronic energy : $\quad H F=-4360.8796466$

Zero-point correction $=\quad 1.456446($ Hartree/Particle $)$

Thermal correction to Energy= $\quad 1.540505$

Thermal correction to Enthalpy= $\quad 1.541449$

Thermal correction to Gibbs Free Energy $=\quad 1.340380$

Sum of electronic and zero-point Energies $=\quad-4359.423201$

Sum of electronic and thermal Energies $=\quad-4359.339142$

Sum of electronic and thermal Enthalpies $=\quad-4359.338198$

Sum of electronic and thermal Free Energies $=\quad-4359.539267$

Cartesian Coordinates

$\begin{array}{crrr}0 & 2.264087 & -0.919574 & 5.144642 \\ 6 & 1.569853 & 0.054362 & 4.411686 \\ 6 & 2.008066 & 1.384279 & 4.558551 \\ 6 & 3.057730 & 1.717519 & 5.400792 \\ 6 & 3.719997 & 0.733661 & 6.135561 \\ 6 & 3.316759 & -0.586343 & 5.995527 \\ 6 & 0.344956 & -0.309576 & 3.635874 \\ 6 & -0.010915 & -1.776202 & 3.629107 \\ 45 & 0.025341 & 0.675258 & 1.537019 \\ 6 & -0.552706 & 2.504442 & 1.210467 \\ 8 & -0.619271 & 3.650311 & 1.362814 \\ 15 & 1.543482 & 0.411868 & -0.355828 \\ 6 & 1.729866 & 2.154787 & -0.920070 \\ 6 & 0.739828 & 2.765014 & -1.694216 \\ 6 & 0.837911 & 4.107712 & -2.062817 \\ 6 & 1.921970 & 4.845981 & -1.573057 \\ 6 & 2.898408 & 4.278184 & -0.752514 \\ 6 & 2.791782 & 2.918777 & -0.448601 \\ 6 & -0.198167 & 4.703824 & -3.015660 \\ 6 & 4.080450 & 5.064955 & -0.191411 \\ 6 & 3.297447 & -0.114694 & -0.140106 \\ 6 & 3.717270 & -0.694989 & 1.047774 \\ 6 & 5.023944 & -1.198144 & 1.175812 \\ 6 & 5.879174 & -1.071640 & 0.085787 \\ 6 & 5.494325 & -0.461092 & -1.118502 \\ 6 & 4.191127 & 0.008119 & -1.213644 \\ 6 & 6.484230 & -0.372731 & -2.278440 \\ 6 & 5.485936 & -1.809759 & 2.495824 \\ 6 & 1.200510 & -0.488373 & -1.952976 \\ 6 & 0.828356 & -1.823559 & -1.939752 \\ 6 & 0.609698 & -2.526196 & -3.173773 \\ 6 & 0.791261 & -1.847017 & -4.412885 \\ 6 & 1.209561 & -0.497760 & -4.392403 \\ 6 & 1.407362 & 0.154434 & -3.206099 \\ 6 & 0.548558 & -2.526310 & -5.630212 \\ 6 & 0.137365 & -3.834271 & -5.634682 \\ 6 & -0.042635 & -4.515774 & -4.413179 \\ 6 & 0.190908 & -3.883116 & -3.216952 \\ 6 & 0.666311 & -2.636574 & -0.701985 \\ 6 & -0.552819 & -2.701851 & -0.052555 \\ 6 & -0.790628 & -3.694700 & 0.926478 \\ 6 & 0.183354 & -4.592329 & 1.273090 \\ 6 & 1.464483 & -4.523737 & 0.676827 \\ 6 & 1.714062 & -3.531987 & -0.317933\end{array}$




\begin{tabular}{|c|c|c|c|}
\hline 6 & 2.995744 & -3.493301 & -0.924663 \\
\hline 6 & 3.970425 & -4.393099 & -0.572141 \\
\hline 6 & 3.714086 & -5.385214 & 0.399507 \\
\hline 6 & 2.488580 & -5.442995 & 1.013613 \\
\hline 7 & -1.619523 & -1.806253 & -0.405928 \\
\hline 15 & -1.839396 & -0.520131 & 0.676239 \\
\hline 8 & -2.918693 & -1.014733 & 1.870988 \\
\hline 6 & -4.160700 & -1.517005 & 1.560098 \\
\hline 6 & -5.142926 & -0.701045 & 1.028262 \\
\hline 6 & -6.349361 & -1.320472 & 0.564658 \\
\hline 6 & -6.563768 & -2.712060 & 0.798418 \\
\hline 6 & -5.579916 & -3.459838 & 1.490818 \\
\hline 6 & -4.394716 & -2.878741 & 1.849586 \\
\hline 6 & -7.318728 & -0.614683 & -0.193023 \\
\hline 6 & -8.439891 & -1.245744 & -0.672874 \\
\hline 6 & -8.663960 & -2.613800 & -0.411009 \\
\hline 6 & -7.741667 & -3.328026 & 0.310194 \\
\hline 6 & -4.906410 & 0.755905 & 0.915007 \\
\hline 6 & -3.776501 & 1.214369 & 0.266245 \\
\hline 6 & -3.520443 & 2.583289 & 0.065199 \\
\hline 6 & -4.388833 & 3.516221 & 0.560683 \\
\hline 6 & -5.526934 & 3.116098 & 1.301431 \\
\hline 6 & -5.786974 & 1.725282 & 1.498107 \\
\hline 6 & -6.402185 & 4.075716 & 1.865410 \\
\hline 6 & -7.482086 & 3.691434 & 2.617714 \\
\hline 6 & -7.722302 & 2.320330 & 2.844497 \\
\hline 6 & -6.899656 & 1.365145 & 2.300642 \\
\hline 8 & -2.888219 & 0.321886 & -0.279484 \\
\hline 6 & -0.706465 & 0.637363 & 3.571148 \\
\hline 6 & -2.656711 & -2.378927 & -1.300049 \\
\hline 6 & -3.213434 & -1.443320 & -2.341142 \\
\hline 6 & -2.380001 & -0.626162 & -3.106582 \\
\hline 6 & -2.910319 & 0.212541 & -4.077374 \\
\hline 6 & -4.286373 & 0.248003 & -4.299229 \\
\hline 6 & -5.123132 & -0.563709 & -3.541305 \\
\hline 6 & -4.587514 & -1.405079 & -2.568964 \\
\hline 1 & -7.148690 & 0.434896 & -0.420155 \\
\hline 1 & 1.737041 & 1.189257 & -3.229317 \\
\hline 1 & -7.889906 & -4.388846 & 0.508030 \\
\hline 1 & 0.692306 & -1.983378 & -6.563304 \\
\hline 1 & -6.188543 & 5.130198 & 1.695423 \\
\hline 1 & 3.205007 & -2.726533 & -1.669234 \\
\hline 1 & 4.949808 & -4.339979 & -1.043865 \\
\hline 1 & -4.204878 & 4.578753 & 0.407368 \\
\hline 1 & 1.373674 & 0.019079 & -5.337465 \\
\hline 1 & -5.771446 & -4.509313 & 1.708158 \\
\hline 1 & -8.564123 & 2.015681 & 3.462327 \\
\hline 1 & -3.617745 & -3.435937 & 2.368814 \\
\hline 1 & 2.279078 & -6.198259 & 1.769903 \\
\hline 1 & -0.011962 & -5.364931 & 2.015304 \\
\hline 1 & -0.367649 & -5.553631 & -4.418309 \\
\hline 1 & -7.093346 & 0.313915 & 2.497254 \\
\hline 1 & 0.049651 & -4.430195 & -2.287029 \\
\hline 1 & -1.778010 & -3.738901 & 1.381630 \\
\hline 1 & -8.144468 & 4.437654 & 3.050243 \\
\hline 1 & -9.159828 & -0.685710 & -1.265543 \\
\hline
\end{tabular}




\begin{tabular}{|c|c|c|c|}
\hline 1 & 4.493819 & -6.096041 & 0.664569 \\
\hline & -0.050893 & -4.347998 & -6.574492 \\
\hline & -9.559509 & -3.099564 & -0.791248 \\
\hline & -2.629290 & 2.860638 & -0.490353 \\
\hline & 3.849293 & 0.477417 & -2.132465 \\
\hline & 3.006282 & -0.762055 & 1.873556 \\
\hline & 6.891087 & -1.463977 & 0.162899 \\
\hline & 3.545666 & 2.446464 & 0.179992 \\
\hline & -0.112775 & 2.170567 & -2.031347 \\
\hline & 2.004161 & 5.895858 & -1.837032 \\
\hline & -2.161364 & -3.218626 & -1.808683 \\
\hline & 1.296137 & 1.472347 & 2.030894 \\
\hline & -3.483101 & -2.814692 & -0.718087 \\
\hline & -5.251033 & -2.026504 & -1.964983 \\
\hline & -1.310909 & -0.622822 & -2.906310 \\
\hline & -6.200365 & -0.538899 & -3.695662 \\
\hline & -2.242636 & 0.844613 & -4.662184 \\
\hline & -4.702865 & 0.909948 & -5.055556 \\
\hline & -1.736288 & 0.281447 & 3.545575 \\
\hline & -0.587566 & 1.606876 & 4.054619 \\
\hline & 1.515808 & 2.176732 & 3.997164 \\
\hline & 3.361493 & 2.759380 & 5.485696 \\
\hline & 4.543422 & 0.996179 & 6.796164 \\
\hline & 3.823814 & -1.376462 & 6.547777 \\
\hline ] & 1.971989 & -1.964239 & 5.074058 \\
\hline & -0.136127 & -2.156916 & 4.656113 \\
\hline 1 & -0.957971 & -1.943143 & 3.108768 \\
\hline 1 & 0.753964 & -2.394592 & 3.139492 \\
\hline & 4.424428 & -2.767466 & 3.042677 \\
\hline f & 6.787885 & -2.596861 & 2.352536 \\
\hline & 5.720069 & -0.663137 & 3.485034 \\
\hline 5 & 5.914251 & 0.407527 & -3.461236 \\
\hline 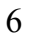 & 7.768025 & 0.328926 & -1.823343 \\
\hline 3 & 6.822411 & -1.789964 & -2.755220 \\
\hline ] & 4.752643 & -3.183415 & 4.005646 \\
\hline 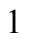 & 3.463200 & -2.272227 & 3.216527 \\
\hline 1 & 4.254730 & -3.601209 & 2.348313 \\
\hline & 7.039853 & -3.062886 & 3.313641 \\
\hline 1 & 6.700531 & -3.399365 & 1.606980 \\
\hline 1 & 7.636249 & -1.958747 & 2.074712 \\
\hline ] & 6.052256 & -1.052141 & 4.457892 \\
\hline 1 & 6.494992 & 0.018631 & 3.109238 \\
\hline & 4.806978 & -0.078776 & 3.651991 \\
\hline 1 & 6.663066 & 0.464362 & -4.261282 \\
\hline 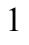 & 5.021220 & -0.073969 & -3.882074 \\
\hline 1 & 5.649689 & 1.435996 & -3.179979 \\
\hline 1 & 7.534378 & -1.753889 & -3.591042 \\
\hline 1 & 7.275907 & -2.393235 & -1.958811 \\
\hline 1 & 5.921047 & -2.312191 & -3.103834 \\
\hline 1 & 8.479780 & 0.394352 & -2.657153 \\
\hline 1 & 7.559193 & 1.349714 & -1.478352 \\
\hline 1 & 8.268410 & -0.204291 & -1.006472 \\
\hline 6 & -0.012558 & 6.206407 & -3.213495 \\
\hline 6 & -0.055795 & 4.018025 & -4.380324 \\
\hline 6 & -1.613356 & 4.466611 & -2.480075 \\
\hline 6 & 4.066680 & 6.528602 & -0.624043 \\
\hline
\end{tabular}




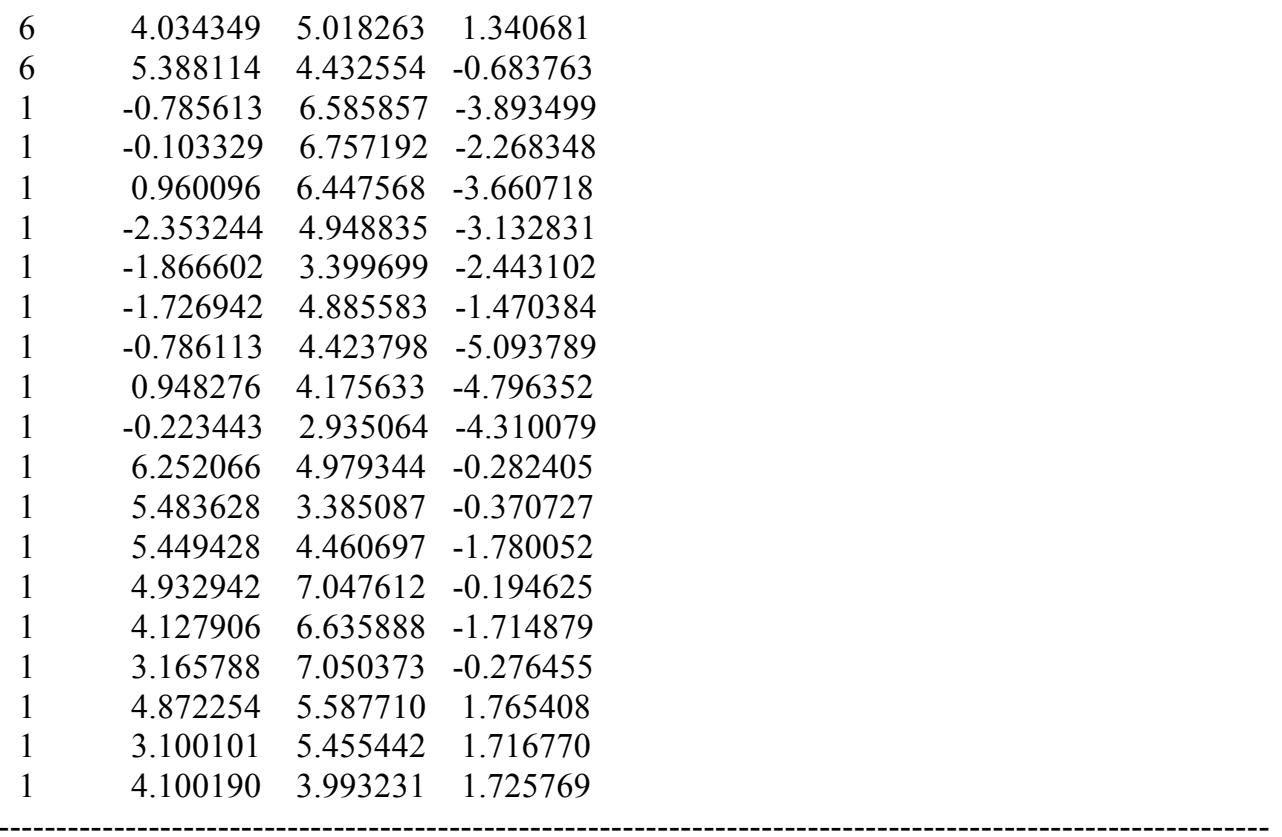

$3_{\text {ID-si- }-1 \text {-1s233a4s }}$

Number of imaginary frequencies : 0

The smallest frequencies are : $13.1820 \quad 17.8222 \quad 23.3447 \mathrm{~cm}(-1)$

Electronic energy : $\quad H F=-4360.8797149$

Zero-point correction $=\quad 1.455330($ Hartree/Particle $)$

Thermal correction to Energy $=\quad 1.539603$

Thermal correction to Enthalpy $=\quad 1.540547$

Thermal correction to Gibbs Free Energy= $\quad 1.338841$

Sum of electronic and zero-point Energies $=\quad-4359.424385$

Sum of electronic and thermal Energies $=\quad-4359.340112$

Sum of electronic and thermal Enthalpies $=\quad-4359.339168$

Sum of electronic and thermal Free Energies $=\quad-4359.540874$

\begin{tabular}{|c|c|c|c|}
\hline & \multicolumn{3}{|c|}{ Cartesian Coordinates } \\
\hline 6 & 2.244150 & -0.343035 & 5.201787 \\
\hline 6 & 1.561278 & 0.536312 & 4.348786 \\
\hline 6 & 2.017153 & 1.868210 & 4.322805 \\
\hline 6 & 3.078153 & 2.292027 & 5.108397 \\
\hline 6 & 3.732790 & 1.401571 & 5.959933 \\
\hline 6 & 3.308080 & 0.081438 & 5.996341 \\
\hline 6 & 0.333309 & 0.086831 & 3.622849 \\
\hline 6 & -0.019041 & -1.373307 & 3.774383 \\
\hline 45 & 0.011407 & 0.833580 & 1.429390 \\
\hline 6 & -0.585710 & 2.615681 & 0.918313 \\
\hline 8 & -0.669102 & 3.769646 & 0.963756 \\
\hline 15 & 1.539039 & 0.371664 & -0.404270 \\
\hline 6 & 1.797949 & 2.060289 & -1.089564 \\
\hline 6 & 0.831247 & 2.659622 & -1.899969 \\
\hline 6 & 0.966593 & 3.980959 & -2.328035 \\
\hline 6 & 2.068796 & 4.711628 & -1.867500 \\
\hline 6 & 3.025571 & 4.154895 & -1.016350 \\
\hline 6 & 2.880254 & 2.814731 & -0.651400 \\
\hline 6 & -0.057290 & 4.562305 & -3.302724 \\
\hline
\end{tabular}




\begin{tabular}{|c|c|c|c|}
\hline & 4.213621 & 4.936502 & -0.461487 \\
\hline & 3.270325 & -0.195076 & -0.122359 \\
\hline & 3.675144 & -0.662356 & 1.123795 \\
\hline & 4.962766 & -1.187033 & 1.303629 \\
\hline & 5.824270 & -1.205788 & 0.203906 \\
\hline & 5.452234 & -0.721932 & -1.054382 \\
\hline & 4.163043 & -0.216060 & -1.195129 \\
\hline & 6.373699 & -0.754158 & -2.271924 \\
\hline & 5.419653 & -1.668903 & 2.679033 \\
\hline & 1.181790 & -0.637178 & -1.931740 \\
\hline & 0.807749 & -1.967111 & -1.818042 \\
\hline & 0.578984 & -2.758008 & -2.994358 \\
\hline & 0.750892 & -2.172332 & -4.281520 \\
\hline & 1.169296 & -0.825527 & -4.363388 \\
\hline & 1.377991 & -0.087971 & -3.229690 \\
\hline & 0.498173 & -2.939641 & -5.443353 \\
\hline & 0.086441 & -4.243912 & -5.347382 \\
\hline & -0.083800 & -4.833120 & -4.077287 \\
\hline & 0.159823 & -4.114010 & -2.933193 \\
\hline & 0.663195 & -2.681358 & -0.519367 \\
\hline & -0.542731 & -2.689924 & 0.157577 \\
\hline & -0.754039 & -3.585312 & 1.232240 \\
\hline & 0.233386 & -4.439978 & 1.643658 \\
\hline & 1.501758 & -4.422936 & 1.017118 \\
\hline & 1.723926 & -3.531940 & -0.074595 \\
\hline & 2.996385 & -3.537325 & -0.701439 \\
\hline & 3.992784 & -4.374976 & -0.266699 \\
\hline & 3.763241 & -5.271154 & 0.800149 \\
\hline & 2.543109 & -5.291517 & 1.427529 \\
\hline & -1.621345 & -1.836854 & -0.257675 \\
\hline & -1.844057 & -0.456621 & 0.701823 \\
\hline & -2.911366 & -0.841308 & 1.945303 \\
\hline & -4.151052 & -1.379759 & 1.691397 \\
\hline & -5.143746 & -0.623412 & 1.094956 \\
\hline & -6.349832 & -1.292365 & 0.703608 \\
\hline & -6.549734 & -2.658335 & 1.066541 \\
\hline & -5.552458 & -3.332466 & 1.813190 \\
\hline & -4.369433 & -2.711356 & 2.105784 \\
\hline & -7.333274 & -0.667609 & -0.105446 \\
\hline & -8.453971 & -1.348855 & -0.512336 \\
\hline & -8.663426 & -2.688567 & -0.123265 \\
\hline & -7.727095 & -3.325917 & 0.649866 \\
\hline & -4.917912 & 0.816735 & 0.834298 \\
\hline & -3.800458 & 1.213481 & 0.125658 \\
\hline & -3.567153 & 2.553194 & -0.236619 \\
\hline & -4.439709 & 3.526055 & 0.166174 \\
\hline & -5.559365 & 3.198922 & 0.968762 \\
\hline & -5.800704 & 1.835747 & 1.320842 \\
\hline & -6.436401 & 4.205761 & 1.439854 \\
\hline & -7.500141 & 3.895527 & 2.247579 \\
\hline & -7.722298 & 2.554920 & 2.625342 \\
\hline & -6.897421 & 1.554110 & 2.174710 \\
\hline & -2.904448 & 0.278311 & -0.324641 \\
\hline & -0.719594 & 1.019463 & 3.457309 \\
\hline & -2.660171 & -2.488752 & -1.094219 \\
\hline & -3.231560 & -1.640559 & -2.200802 \\
\hline
\end{tabular}




\begin{tabular}{|c|c|c|c|}
\hline & -2.410513 & -0.865920 & -3.021878 \\
\hline & -2.954312 & -0.090296 & -4.036325 \\
\hline & -4.332212 & -0.080209 & -4.249957 \\
\hline & -5.156340 & -0.853736 & -3.440020 \\
\hline & -4.607101 & -1.629286 & -2.421521 \\
\hline & -7.174922 & 0.357275 & -0.431711 \\
\hline & 1.711047 & 0.940898 & -3.330887 \\
\hline & -7.863456 & -4.365375 & 0.945520 \\
\hline & 0.634304 & -2.467166 & -6.415134 \\
\hline & -6.237521 & 5.237475 & 1.152721 \\
\hline & 3.187947 & -2.844555 & -1.520110 \\
\hline & 4.969806 & -4.345364 & -0.746731 \\
\hline & -4.273566 & 4.566308 & -0.110584 \\
\hline & 1.325922 & -0.379808 & -5.345179 \\
\hline & -5.731886 & -4.359548 & 2.126462 \\
\hline & -8.551459 & 2.311253 & 3.285880 \\
\hline & -3.581669 & -3.212391 & 2.664762 \\
\hline & 2.353796 & -5.972543 & 2.256271 \\
\hline & 0.058857 & -5.137475 & 2.461629 \\
\hline & -0.409338 & -5.868211 & -4.003310 \\
\hline & -7.076188 & 0.528090 & 2.485935 \\
\hline & 0.026220 & -4.589786 & -1.963629 \\
\hline & -1.732187 & -3.590850 & 1.708993 \\
\hline & -8.163707 & 4.678256 & 2.607725 \\
\hline & -9.184842 & -0.851354 & -1.145996 \\
\hline & 4.558738 & -5.937260 & 1.127533 \\
\hline & -0.110164 & -4.825422 & -6.245031 \\
\hline & -9.558516 & -3.214387 & -0.447133 \\
\hline & -2.691519 & 2.773358 & -0.841309 \\
\hline & 3.838769 & 0.158304 & -2.165478 \\
\hline & 2.960740 & -0.623285 & 1.948397 \\
\hline & 6.821182 & -1.617877 & 0.329213 \\
\hline & 3.614006 & 2.354406 & 0.009005 \\
\hline & -0.040808 & 2.077320 & -2.206437 \\
\hline . & 2.179024 & 5.747069 & -2.175399 \\
\hline & -2.162379 & -3.363732 & -1.536718 \\
\hline [ & 1.279411 & 1.683758 & 1.831479 \\
\hline & -3.479184 & -2.879851 & -0.470669 \\
\hline . & -5.261805 & -2.216467 & -1.774977 \\
\hline ] & -1.340793 & -0.838797 & -2.826886 \\
\hline & -6.234644 & -0.847947 & -3.588719 \\
\hline 1 & -2.295947 & 0.511999 & -4.661729 \\
\hline & -4.760117 & 0.532480 & -5.040653 \\
\hline | & -1.749128 & 0.661940 & 3.471110 \\
\hline & -0.599876 & 2.036351 & 3.831128 \\
\hline 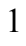 & 1.531310 & 2.589321 & 3.667449 \\
\hline I & 3.396719 & 3.331646 & 5.055682 \\
\hline 1 & 4.565865 & 1.734523 & 6.575114 \\
\hline 1 & 3.807058 & -0.638021 & 6.644262 \\
\hline 1 & 1.935257 & -1.382950 & 5.270722 \\
\hline 1 & -0.148628 & -1.640926 & 4.835893 \\
\hline . & -0.963816 & -1.598645 & 3.272048 \\
\hline 1 & 0.750094 & -2.038462 & 3.358028 \\
\hline 6 & 4.318174 & -2.485635 & 3.357565 \\
\hline 6 & 6.665214 & -2.552006 & 2.603938 \\
\hline 6 & 5.747580 & -0.431635 & 3.521942 \\
\hline
\end{tabular}




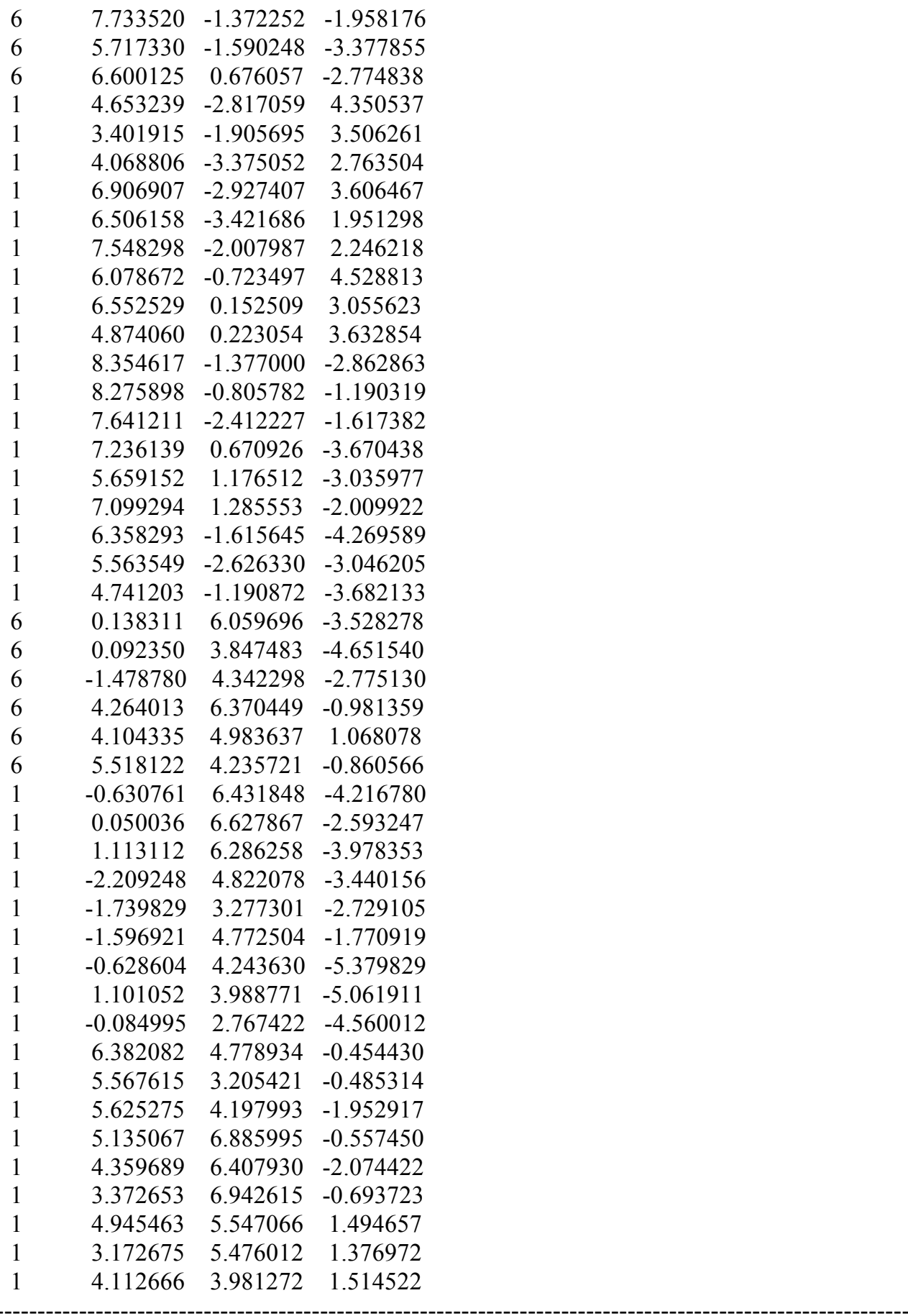

$3_{\text {ID-si }-} \psi_{-1 \text { s2s3s4a }}$

Number of imaginary frequencies : 0

The smallest frequencies are : $15.1284 \quad 22.6704 \quad 23.3770 \mathrm{~cm}(-1)$

Electronic energy : $\quad H F=-4360.8796069$

Zero-point correction $=\quad 1.455976$ (Hartree/Particle)

Thermal correction to Energy $=\quad 1.540163$

Thermal correction to Enthalpy= $\quad 1.541107$

Thermal correction to Gibbs Free Energy $=\quad 1.340143$

Sum of electronic and zero-point Energies $=\quad-4359.423631$ 
Sum of electronic and thermal Energies= Sum of electronic and thermal Enthalpies= Sum of electronic and thermal Free Energies=
$-4359.339444$

$-4359.338500$

$-4359.539464$

Cartesian Coordinates

$\begin{array}{cccc}6 & -2.286094 & -0.856385 & -5.153185 \\ 6 & -1.580244 & 0.104101 & -4.413679 \\ 6 & -2.002351 & 1.440259 & -4.551015 \\ 6 & -3.047603 & 1.792154 & -5.391055 \\ 6 & -3.721275 & 0.821510 & -6.133027 \\ 6 & -3.334262 & -0.504275 & -6.002103 \\ 6 & -0.359614 & -0.278893 & -3.640482 \\ 6 & -0.017324 & -1.748488 & -3.647239 \\ 45 & -0.035747 & 0.680008 & -1.530002 \\ 6 & 0.545732 & 2.510313 & -1.205219 \\ 8 & 0.608722 & 3.654222 & -1.373459 \\ 15 & -1.541639 & 0.412264 & 0.369126 \\ 6 & -1.696662 & 2.149162 & 0.959305 \\ 6 & -0.703267 & 2.726562 & 1.754112 \\ 6 & -0.781508 & 4.063666 & 2.147523 \\ 6 & -1.839147 & 4.833777 & 1.649429 \\ 6 & -2.812261 & 4.301207 & 0.801313 \\ 6 & -2.735226 & 2.942716 & 0.484550 \\ 6 & 0.241885 & 4.616734 & 3.139550 \\ 6 & -3.953412 & 5.128241 & 0.214416 \\ 6 & -3.303244 & -0.087178 & 0.153143 \\ 6 & -3.732845 & -0.642645 & -1.042941 \\ 6 & -5.044218 & -1.132481 & -1.174713 \\ 6 & -5.892594 & -1.021425 & -0.077999 \\ 6 & -5.495783 & -0.440930 & 1.137436 \\ 6 & -4.190288 & 0.021009 & 1.233986 \\ 6 & -6.480908 & -0.370944 & 2.302441 \\ 6 & -5.514860 & -1.716927 & -2.503823 \\ 6 & -1.205144 & -0.518585 & 1.949748 \\ 6 & -0.844542 & -1.856380 & 1.913027 \\ 6 & -0.632610 & -2.582522 & 3.134808 \\ 6 & -0.811225 & -1.924488 & 4.385654 \\ 6 & -1.219181 & -0.571896 & 4.388747 \\ 6 & -1.409242 & 0.103074 & 3.214039 \\ 6 & -0.574621 & -2.626891 & 5.590955 \\ 6 & -0.171207 & -3.937183 & 5.572612 \\ 6 & 0.006817 & -4.597607 & 4.339318 \\ 6 & -0.221582 & -3.942313 & 3.154303 \\ 6 & -0.687100 & -2.649855 & 0.661852 \\ 6 & 0.533083 & -2.715759 & 0.014445 \\ 6 & 0.766973 & -3.698851 & -0.975409 \\ 6 & -0.212374 & -4.585382 & -1.335176 \\ 6 & -1.495415 & -4.513176 & -0.743269 \\ 6 & -1.741162 & -3.531068 & 0.262073 \\ 6 & -3.025525 & -3.488236 & 0.863026 \\ 6 & -4.005872 & -4.376120 & 0.495870 \\ 6 & -3.753038 & -5.359519 & -0.485456 \\ 6 & -2.525499 & -5.419940 & -1.095179 \\ 7 & 1.604631 & -1.831094 & 0.380256 \\ 15 & 1.825667 & -0.528033 & -0.681391\end{array}$




\begin{tabular}{|c|c|c|c|}
\hline 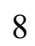 & 2.903674 & -1.005621 & -1.883625 \\
\hline & 4.148123 & -1.507928 & -1.583545 \\
\hline 6 & 5.129787 & -0.695833 & -1.044826 \\
\hline & 6.341632 & -1.317162 & -0.597933 \\
\hline & 6.560335 & -2.704244 & -0.853640 \\
\hline & 5.574498 & -3.446433 & -1.549250 \\
\hline 6 & 4.384902 & -2.864972 & -1.892387 \\
\hline 6 & 7.312965 & -0.618751 & 0.163977 \\
\hline U & 8.440242 & -1.251889 & 0.626472 \\
\hline 6 & 8.668557 & -2.614778 & 0.342461 \\
\hline 6 & 7.744192 & -3.322231 & -0.382745 \\
\hline 6 & 4.887981 & 0.758323 & -0.907183 \\
\hline 6 & 3.759120 & 1.202398 & -0.246189 \\
\hline 6 & 3.498933 & 2.567178 & -0.022261 \\
\hline 6 & 4.362970 & 3.510711 & -0.505250 \\
\hline 6 & 5.499422 & 3.126553 & -1.256768 \\
\hline 6 & 5.762931 & 1.740153 & -1.477910 \\
\hline 6 & 6.369292 & 4.098435 & -1.807915 \\
\hline 6 & 7.447187 & 3.730465 & -2.571138 \\
\hline 6 & 7.690572 & 2.364163 & -2.822324 \\
\hline 6 & 6.873202 & 1.397273 & -2.291293 \\
\hline 8 & 2.875453 & 0.297781 & 0.287343 \\
\hline 6 & 0.699018 & 0.658743 & -3.563891 \\
\hline 6 & 2.643831 & -2.426230 & 1.257810 \\
\hline 6 & 3.211448 & -1.512498 & 2.312280 \\
\hline 6 & 2.383266 & -0.718814 & 3.107340 \\
\hline 6 & 2.920721 & 0.101359 & 4.089932 \\
\hline 6 & 4.299330 & 0.140475 & 4.294537 \\
\hline 6 & 5.131011 & -0.648814 & 3.507821 \\
\hline 6 & 4.588128 & -1.471175 & 2.523281 \\
\hline 1 & 7.139775 & 0.426397 & 0.408486 \\
\hline 1 & -1.730220 & 1.140105 & 3.255587 \\
\hline 1 & 7.895449 & -4.379408 & -0.597090 \\
\hline 1 & -0.716287 & -2.099682 & 6.533341 \\
\hline 1 & 6.153030 & 5.149189 & -1.619173 \\
\hline 1 & -3.232703 & -2.727169 & 1.614222 \\
\hline 1 & -4.987244 & -4.320217 & 0.963168 \\
\hline 1 & 4.176587 & 4.570097 & -0.334414 \\
\hline 1 & -1.381058 & -0.070706 & 5.342596 \\
\hline 1 & 5.768326 & -4.492256 & -1.781668 \\
\hline 1 & 8.530637 & 2.072667 & -3.448819 \\
\hline 1 & 3.605972 & -3.418176 & -2.413115 \\
\hline 1 & -2.318800 & -6.168107 & -1.859251 \\
\hline 1 & -0.020390 & -5.350969 & -2.085483 \\
\hline 1 & 0.326257 & -5.637123 & 4.326247 \\
\hline 1 & 7.069360 & 0.350125 & -2.506416 \\
\hline 1 & -0.081551 & -4.473443 & 2.215009 \\
\hline 1 & 1.755511 & -3.744759 & -1.428328 \\
\hline 1 & 8.105453 & 4.485986 & -2.993693 \\
\hline 1 & 9.161704 & -0.697626 & 1.222707 \\
\hline 1 & -4.537155 & -6.061167 & -0.761767 \\
\hline 1 & 0.012794 & -4.468650 & 6.503350 \\
\hline 1 & 9.568812 & -3.102216 & 0.709195 \\
\hline 1 & 2.608671 & 2.833279 & 0.540673 \\
\hline 1 & -3.840362 & 0.468493 & 2.160627 \\
\hline 1 & -3.025152 & -0.702465 & -1.872244 \\
\hline
\end{tabular}




\begin{tabular}{|c|c|c|c|}
\hline . & -6.908638 & -1.402601 & -0.157477 \\
\hline & -3.490267 & 2.495624 & -0.161262 \\
\hline & 0.132802 & 2.109783 & 2.092261 \\
\hline & -1.902807 & 5.880908 & 1.929007 \\
\hline & 2.145797 & -3.271673 & 1.754169 \\
\hline & -1.308773 & 1.479624 & -2.015123 \\
\hline & 3.464372 & -2.856399 & 0.663630 \\
\hline & 5.247613 & -2.075020 & 1.897431 \\
\hline & 1.311610 & -0.719734 & 2.921821 \\
\hline & 6.209814 & -0.621324 & 3.650203 \\
\hline & 2.256759 & 0.715430 & 4.697765 \\
\hline & 4.721789 & 0.787467 & 5.060437 \\
\hline & 1.726102 & 0.295105 & -3.539647 \\
\hline & 0.588461 & 1.634188 & -4.037297 \\
\hline & -1.501315 & 2.222715 & -3.983307 \\
\hline & -3.338916 & 2.838156 & -5.468416 \\
\hline & -4.541052 & 1.098605 & -6.792212 \\
\hline 1 & -3.850611 & -1.284130 & -6.560276 \\
\hline 1 & -2.006867 & -1.905043 & -5.089532 \\
\hline 1 & 0.104483 & -2.121070 & -4.677651 \\
\hline 1 & 0.927633 & -1.929519 & -3.127769 \\
\hline & -0.788413 & -2.363587 & -3.163412 \\
\hline U & -6.832192 & -2.480036 & -2.373721 \\
\hline 6 & -5.724463 & -0.553597 & -3.478908 \\
\hline 6 & -4.470319 & -2.688334 & -3.059546 \\
\hline 6 & -5.884238 & 0.331374 & 3.520073 \\
\hline 6 & -7.736069 & 0.398027 & 1.876867 \\
\hline 6 & -6.870887 & -1.794319 & 2.717099 \\
\hline 1 & -7.092160 & -2.926710 & -3.341840 \\
\hline & -6.761736 & -3.294961 & -1.639988 \\
\hline & -7.667667 & -1.828989 & -2.086903 \\
\hline 1 & -6.063121 & -0.923813 & -4.456822 \\
\hline 1 & -6.486232 & 0.138697 & -3.095546 \\
\hline 1 & -4.799942 & 0.014606 & -3.638198 \\
\hline 1 & -4.801734 & -3.083267 & -4.030247 \\
\hline 1 & -3.497486 & -2.211270 & -3.220001 \\
\hline 1 & -4.322071 & -3.535639 & -2.376876 \\
\hline 1 & -6.626144 & 0.363337 & 4.327893 \\
\hline 1 & -5.000336 & -0.194033 & 3.906363 \\
\hline 1 & -5.596678 & 1.366702 & 3.293908 \\
\hline 1 & -7.586249 & -1.767886 & 3.550292 \\
\hline 1 & -7.339509 & -2.349851 & 1.895335 \\
\hline 1 & -5.989789 & -2.360280 & 3.048820 \\
\hline 1 & -8.448386 & 0.454698 & 2.710914 \\
\hline 1 & -7.486264 & 1.423976 & 1.576397 \\
\hline 1 & -8.251319 & -0.080786 & 1.035730 \\
\hline 6 & 0.067232 & 6.113469 & 3.385495 \\
\hline 6 & 0.064633 & 3.886346 & 4.476959 \\
\hline 6 & 1.665493 & 4.383386 & 2.626606 \\
\hline 6 & -3.906471 & 6.587574 & 0.659120 \\
\hline 6 & -3.859290 & 5.090520 & -1.316003 \\
\hline 6 & -5.295613 & 4.535425 & 0.659879 \\
\hline 1 & 0.829077 & 6.460645 & 4.094781 \\
\hline 1 & 0.184792 & 6.696046 & 2.462651 \\
\hline 1 & -0.912687 & 6.349460 & 3.819432 \\
\hline 1 & 2.396413 & 4.816943 & 3.322242 \\
\hline
\end{tabular}


$3_{\text {ID-si- }-\psi-122 s 3 s 4 s}$

Number of imaginary frequencies : 0

The smallest frequencies are : $15.1731 \quad 20.2175 \quad 23.6350 \mathrm{~cm}(-1)$

Electronic energy :

$\mathrm{HF}=-4360.8797544$

Zero-point correction $=$

1.455531 (Hartree/Particle)

Thermal correction to Energy= $\quad 1.539852$

Thermal correction to Enthalpy= $\quad 1.540796$

Thermal correction to Gibbs Free Energy $=\quad 1.339084$

Sum of electronic and zero-point Energies $=\quad-4359.424223$

Sum of electronic and thermal Energies $=\quad-4359.339902$

Sum of electronic and thermal Enthalpies $=\quad-4359.338958$

Sum of electronic and thermal Free Energies $=\quad-4359.540671$

\begin{tabular}{cccc}
\multicolumn{4}{c}{ Cartesian Coordinates } \\
\hline 6 & 2.274689 & -0.859005 & 5.169890 \\
6 & 1.573589 & 0.099929 & 4.423951 \\
6 & 1.996990 & 1.435936 & 4.558513 \\
6 & 3.039707 & 1.789032 & 5.401224 \\
6 & 3.709354 & 0.819828 & 6.148705 \\
6 & 3.320495 & -0.505740 & 6.021228 \\
6 & 0.355859 & -0.284293 & 3.646764 \\
6 & 0.015388 & -1.754282 & 3.650550 \\
45 & 0.036081 & 0.677131 & 1.534065 \\
6 & -0.537596 & 2.510689 & 1.212967 \\
8 & -0.588411 & 3.655684 & 1.377906 \\
15 & 1.541206 & 0.405428 & -0.361752 \\
6 & 1.712726 & 2.141262 & -0.950637 \\
6 & 0.720379 & 2.728264 & -1.739842 \\
6 & 0.810650 & 4.063626 & -2.136308 \\
6 & 1.879455 & 4.823280 & -1.645569 \\
6 & 2.852348 & 4.281264 & -0.803352 \\
6 & 2.763699 & 2.923737 & -0.485238 \\
6 & -0.210580 & 4.624901 & -3.125998 \\
6 & 4.003253 & 5.097253 & -0.220169 \\
6 & 3.298280 & -0.106401 & -0.137936 \\
6 & 3.728001 & -0.658574 & 1.064047 \\
6 & 5.034427 & -1.149634 & 1.196884 \\
6 & 5.890051 & -1.048381 & 0.097853 \\
6 & 5.492726 & -0.478277 & -1.116370
\end{tabular}




\begin{tabular}{|c|c|c|c|}
\hline ) & 4.184721 & -0.010719 & -1.212509 \\
\hline & 6.408955 & -0.377743 & -2.334499 \\
\hline & 5.504053 & -1.731506 & 2.528049 \\
\hline & 1.205260 & -0.518348 & -1.947190 \\
\hline & 0.842259 & -1.855440 & -1.915843 \\
\hline & 0.630710 & -2.577671 & -3.139675 \\
\hline & 0.810902 & -1.915953 & -4.388240 \\
\hline & 1.218831 & -0.563386 & -4.386461 \\
\hline & 1.409797 & 0.107535 & -3.209297 \\
\hline & 0.575063 & -2.614608 & -5.595882 \\
\hline & 0.171242 & -3.924800 & -5.581696 \\
\hline & -0.008209 & -4.588893 & -4.350524 \\
\hline & 0.219192 & -3.937219 & -3.163364 \\
\hline & 0.684764 & -2.651119 & -0.666727 \\
\hline & -0.533964 & -2.715662 & -0.016523 \\
\hline & -0.765585 & -3.698226 & 0.974351 \\
\hline 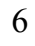 & 0.215100 & -4.584003 & 1.332855 \\
\hline 6 & 1.496948 & -4.512187 & 0.738162 \\
\hline 6 & 1.739729 & -3.531944 & -0.269583 \\
\hline 6 & 3.022356 & -3.488656 & -0.874302 \\
\hline & 4.004995 & -4.373382 & -0.505944 \\
\hline & 3.755809 & -5.354604 & 0.478556 \\
\hline 6 & 2.529154 & -5.416562 & 1.090041 \\
\hline 7 & -1.605274 & -1.829714 & -0.380140 \\
\hline 5 & -1.825400 & -0.526986 & 0.682142 \\
\hline 8 & -2.906407 & -1.003217 & 1.881933 \\
\hline 6 & -4.151372 & -1.502329 & 1.578713 \\
\hline 6 & -5.130295 & -0.686870 & 1.040076 \\
\hline 6 & -6.343084 & -1.304504 & 0.590649 \\
\hline 6 & -6.565342 & -2.691547 & 0.843636 \\
\hline 6 & -5.581852 & -3.437551 & 1.538498 \\
\hline 6 & -4.391278 & -2.859561 & 1.884095 \\
\hline 6 & -7.312032 & -0.602406 & -0.170894 \\
\hline 6 & -8.440443 & -1.231980 & -0.635480 \\
\hline 6 & -8.672316 & -2.594809 & -0.354103 \\
\hline 6 & -7.750294 & -3.305811 & 0.370609 \\
\hline 6 & -4.884380 & 0.766914 & 0.905022 \\
\hline 6 & -3.753339 & 1.208763 & 0.246230 \\
\hline 6 & -3.488590 & 2.573148 & 0.024981 \\
\hline 6 & -4.350370 & 3.518466 & 0.508537 \\
\hline 6 & -5.489013 & 3.136416 & 1.257828 \\
\hline 6 & -5.757107 & 1.750438 & 1.476202 \\
\hline 6 & -6.356656 & 4.110011 & 1.809456 \\
\hline 6 & -7.436703 & 3.744050 & 2.570599 \\
\hline 6 & -7.684612 & 2.378072 & 2.819124 \\
\hline 6 & -6.869511 & 1.409581 & 2.287510 \\
\hline 8 & -2.871636 & 0.302359 & -0.287435 \\
\hline 6 & -0.703097 & 0.652224 & 3.567643 \\
\hline 6 & -2.643757 & -2.421283 & -1.260878 \\
\hline 6 & -3.209237 & -1.504164 & -2.313635 \\
\hline 6 & -2.379896 & -0.705891 & -3.102898 \\
\hline 6 & -2.915627 & 0.117867 & -4.083399 \\
\hline 6 & -4.293683 & 0.155833 & -4.291972 \\
\hline 6 & -5.126510 & -0.638035 & -3.511130 \\
\hline 6 & -4.585347 & -1.463793 & -2.528455 \\
\hline 1 & -7.136176 & 0.442794 & -0.413261 \\
\hline
\end{tabular}




\begin{tabular}{|c|c|c|c|}
\hline . & 1.730720 & 1.144624 & -3.247345 \\
\hline 1 & -7.904300 & -4.362997 & 0.582948 \\
\hline 1 & 0.717857 & -2.084752 & -6.536599 \\
\hline 1 & -6.136930 & 5.160418 & 1.622792 \\
\hline & 3.226598 & -2.729384 & -1.628668 \\
\hline & 4.986130 & -4.315785 & -0.973717 \\
\hline & -4.160599 & 4.577581 & 0.339788 \\
\hline & 1.380428 & -0.058829 & -5.338591 \\
\hline & -5.778280 & -4.483464 & 1.768310 \\
\hline 1 & -8.526400 & 2.088084 & 3.444002 \\
\hline 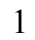 & -3.613874 & -3.415709 & 2.404033 \\
\hline & 2.325340 & -6.163441 & 1.856145 \\
\hline 1 & 0.025114 & -5.348533 & 2.084760 \\
\hline 1 & -0.327931 & -5.628360 & -4.340932 \\
\hline 1 & -7.069145 & 0.362667 & 2.500565 \\
\hline 1 & 0.078311 & -4.470900 & -2.225629 \\
\hline 1 & -1.753093 & -3.743941 & 1.429671 \\
\hline 1 & -8.093218 & 4.500862 & 2.993565 \\
\hline 1 & -9.160055 & -0.674885 & -1.231310 \\
\hline 1 & 4.542047 & -6.053531 & 0.755771 \\
\hline 1 & -0.011968 & -4.453509 & -6.514158 \\
\hline 1 & -9.573457 & -3.079387 & -0.722448 \\
\hline 1 & -2.596800 & 2.837483 & -0.536430 \\
\hline 1 & 3.841938 & 0.427278 & -2.149207 \\
\hline 1 & 3.019855 & -0.711144 & 1.893394 \\
\hline 1 & 6.901939 & -1.432276 & 0.187312 \\
\hline 1 & 3.518810 & 2.469484 & 0.155315 \\
\hline 1 & -0.123856 & 2.119577 & -2.072394 \\
\hline 1 & 1.952452 & 5.869457 & -1.926585 \\
\hline 1 & -2.146026 & -3.265984 & -1.758806 \\
\hline 1 & 1.311624 & 1.472111 & 2.020562 \\
\hline 1 & -3.465481 & -2.852147 & -0.668845 \\
\hline 1 & -5.245860 & -2.070941 & -1.906890 \\
\hline 1 & -1.308862 & -0.705643 & -2.913837 \\
\hline 1 & -6.204927 & -0.611396 & -3.656504 \\
\hline 1 & -2.250810 & 0.735872 & -4.686323 \\
\hline 1 & -4.714839 & 0.805596 & -5.056241 \\
\hline 1 & -1.729795 & 0.287904 & 3.539329 \\
\hline 1 & -0.594679 & 1.627430 & 4.042016 \\
\hline 1 & 1.499240 & 2.217142 & 3.986193 \\
\hline 1 & 3.332388 & 2.834827 & 5.476113 \\
\hline 1 & 4.527392 & 1.097870 & 6.809646 \\
\hline 1 & 3.833537 & -1.284508 & 6.583938 \\
\hline 1 & 1.993743 & -1.907365 & 5.109044 \\
\hline 1 & -0.112339 & -2.127567 & 4.679992 \\
\hline 1 & -0.926047 & -1.936359 & 3.125109 \\
\hline 1 & 0.790020 & -2.368275 & 3.171039 \\
\hline 6 & 6.818051 & -2.501200 & 2.401114 \\
\hline 6 & 5.718931 & -0.566605 & 3.500120 \\
\hline 6 & 4.455548 & -2.697006 & 3.086651 \\
\hline 6 & 7.800733 & -0.944605 & -2.067411 \\
\hline 6 & 5.790235 & -1.166884 & -3.494924 \\
\hline 6 & 6.560938 & 1.093296 & -2.737627 \\
\hline 1 & 7.075412 & -2.944929 & 3.371305 \\
\hline 1 & 6.744056 & -3.319099 & 1.671151 \\
\hline 1 & 7.656990 & -1.855724 & 2.111932 \\
\hline
\end{tabular}




$\begin{array}{lrrr}1 & 6.057523 & -0.935492 & 4.478602 \\ 1 & 6.482788 & 0.122094 & 3.114327 \\ 1 & 4.796513 & 0.005225 & 3.658632 \\ 1 & 4.787004 & -3.094129 & 4.056491 \\ 1 & 3.486524 & -2.213503 & 3.249866 \\ 1 & 4.299954 & -3.543039 & 2.403770 \\ 1 & 8.417485 & -0.848168 & -2.969956 \\ 1 & 8.314918 & -0.408968 & -1.259069 \\ 1 & 7.765846 & -2.010592 & -1.806474 \\ 1 & 7.193393 & 1.181237 & -3.631396 \\ 1 & 5.595671 & 1.562868 & -2.964749 \\ 1 & 7.032109 & 1.672926 & -1.932902 \\ 1 & 6.420370 & -1.087214 & -4.391181 \\ 1 & 5.702802 & -2.231881 & -3.240749 \\ 1 & 4.788060 & -0.805412 & -3.758962 \\ 6 & -0.027539 & 6.121232 & -3.368302 \\ 6 & -0.037140 & 3.896433 & -4.465037 \\ 6 & -1.635785 & 4.398153 & -2.614433 \\ 6 & 3.979994 & 6.552441 & -0.679795 \\ 6 & 3.900143 & 5.075652 & 1.310069 \\ 6 & 5.338816 & 4.479805 & -0.651671 \\ 1 & -0.789235 & 6.475107 & -4.074425 \\ 1 & -0.138697 & 6.701763 & -2.443374 \\ 1 & 0.952488 & 6.352499 & -3.804493 \\ 1 & -2.363502 & 4.836764 & -3.310244 \\ 1 & -1.879071 & 3.331993 & -2.526591 \\ 1 & -1.780777 & 4.870129 & -1.632771 \\ 1 & -0.750377 & 4.280019 & -5.207434 \\ 1 & 0.976412 & 4.040841 & -4.862554 \\ 1 & -0.207695 & 2.816238 & -4.364572 \\ 1 & 6.176686 & 5.045334 & -0.221990 \\ 1 & 5.438206 & 3.437662 & -0.322233 \\ 1 & 5.444561 & 4.496667 & -1.744651 \\ 1 & 4.824614 & 7.092897 & -0.234041 \\ 1 & 4.072667 & 6.640696 & -1.770163 \\ 1 & 3.061135 & 7.066164 & -0.369227 \\ 1 & 4.706917 & 5.672746 & 1.756603 \\ 1 & 2.941110 & 5.494122 & 1.642922 \\ 1 & 3.976288 & 4.058319 & 1.713603\end{array}$

$3_{\text {IIA-si- }} \psi_{-1 \mathbf{a} 2 a 3 a 4 a}$

Number of imaginary frequencies : 0

The smallest frequencies are : $20.4469 \quad 22.1782 \quad 24.6060 \mathrm{~cm}(-1)$

Electronic energy : $\quad H F=-4360.8808171$

Zero-point correction $=\quad 1.455406($ Hartree/Particle $)$

Thermal correction to Energy $=\quad 1.539711$

Thermal correction to Enthalpy= $\quad 1.540655$

Thermal correction to Gibbs Free Energy= $\quad 1.339989$

Sum of electronic and zero-point Energies $=\quad-4359.425411$

Sum of electronic and thermal Energies $=\quad-4359.341106$

Sum of electronic and thermal Enthalpies $=\quad-4359.340162$

Sum of electronic and thermal Free Energies $=\quad-4359.540829$

Cartesian Coordinates 


$\begin{array}{cccc}0 & 4.096830 & -2.221440 & 2.425531 \\ 6 & 2.757188 & -2.066324 & 2.073088 \\ 6 & 1.947475 & -1.247011 & 2.862176 \\ 6 & 2.466642 & -0.604349 & 3.978215 \\ 6 & 3.808153 & -0.762956 & 4.323182 \\ 6 & 4.622266 & -1.573077 & 3.541160 \\ 6 & 2.220279 & -2.849612 & 0.900943 \\ 7 & 1.211768 & -2.185209 & 0.040443 \\ 6 & 0.101986 & -3.013563 & -0.332880 \\ 6 & -1.120929 & -2.880405 & 0.295753 \\ 6 & -2.225897 & -3.671874 & -0.150631 \\ 6 & -2.031924 & -4.626383 & -1.193151 \\ 6 & -0.742410 & -4.781215 & -1.755495 \\ 6 & 0.290846 & -3.982415 & -1.346132 \\ 6 & -3.129864 & -5.404290 & -1.635459 \\ 6 & -4.371820 & -5.251369 & -1.072402 \\ 6 & -4.566602 & -4.309257 & -0.038888 \\ 6 & -3.521731 & -3.541866 & 0.412649 \\ 6 & -1.280546 & -2.077865 & 1.541226 \\ 6 & -1.601647 & -0.728859 & 1.573784 \\ 6 & -1.858733 & -0.115455 & 2.833713 \\ 6 & -1.738683 & -0.801937 & 4.009891 \\ 6 & -1.353321 & -2.161493 & 4.014167 \\ 6 & -1.141239 & -2.815867 & 2.767311 \\ 6 & -0.763575 & -4.184585 & 2.793415 \\ 6 & -0.586033 & -4.849290 & 3.982088 \\ 6 & -0.788636 & -4.189879 & 5.211622 \\ 6 & -1.169550 & -2.872500 & 5.223054 \\ 15 & -1.803362 & 0.259680 & 0.004018 \\ 45 & -0.491211 & -0.010185 & -2.001749 \\ 15 & 1.458799 & -0.801466 & -0.918938 \\ 8 & 2.638831 & -1.104771 & -2.061181 \\ 6 & 3.813383 & -1.737702 & -1.728627 \\ 6 & 4.774027 & -1.103280 & -0.963168 \\ 6 & 5.896351 & -1.890568 & -0.528678 \\ 6 & 6.052805 & -3.226062 & -1.009437 \\ 6 & 5.094259 & -3.764958 & -1.902456 \\ 6 & 3.984755 & -3.041507 & -2.240028 \\ 6 & 6.832077 & -1.421264 & 0.429507 \\ 6 & 7.868839 & -2.212190 & 0.860167 \\ 6 & 8.040177 & -3.516485 & 0.351845 \\ 6 & 7.145193 & -4.009122 & -0.562533 \\ 6 & 4.617618 & 0.316713 & -0.568536 \\ 6 & 3.451067 & 0.778485 & 0.018313 \\ 6 & 3.345565 & 2.068867 & 0.575504 \\ 6 & 4.404776 & 2.926829 & 0.518170 \\ 6 & 5.576994 & 2.568514 & -0.189741 \\ 6 & 5.671804 & 1.271555 & -0.776183 \\ 6 & 6.619867 & 3.505066 & -0.384524 \\ 6 & 7.710906 & 3.197701 & -1.157584 \\ 6 & 7.792986 & 1.930499 & -1.770271 \\ 6 & 6.806212 & 0.993619 & -1.580078 \\ 8 & 2.367002 & -0.027649 & 0.219050 \\ 6 & -1.651318 & 1.988408 & 0.610814 \\ 6 & -2.485139 & 2.981785 & 0.094831 \\ & & & \end{array}$




\begin{tabular}{|c|c|c|c|}
\hline & -2.328297 & 4.318134 & 0.470472 \\
\hline & -1.347515 & 4.619602 & 1.420239 \\
\hline & -0.513822 & 3.646343 & 1.979410 \\
\hline & -0.641631 & 2.336958 & 1.512602 \\
\hline & 0.457673 & 4.025388 & 3.098688 \\
\hline & -0.316018 & 4.734410 & 4.217603 \\
\hline & -3.189384 & 5.437641 & -0.111799 \\
\hline & -2.284908 & 6.421726 & -0.862777 \\
\hline & -3.622704 & 0.048443 & -0.231578 \\
\hline & -4.520297 & 0.463066 & 0.756804 \\
\hline & -5.889606 & 0.250856 & 0.617994 \\
\hline & -6.336139 & -0.397381 & -0.540672 \\
\hline & -5.469947 & -0.834461 & -1.542537 \\
\hline & -4.102450 & -0.599774 & -1.365281 \\
\hline & -6.896351 & 0.693516 & 1.677959 \\
\hline & -7.898954 & 1.671886 & 1.057151 \\
\hline 6 & -6.016397 & -1.500771 & -2.804764 \\
\hline 6 & -6.723363 & -0.435078 & -3.650158 \\
\hline 6 & -6.222224 & 1.391082 & 2.857658 \\
\hline 6 & -7.642383 & -0.534452 & 2.211221 \\
\hline 6 & -4.907669 & -2.131412 & -3.646626 \\
\hline 6 & -7.015649 & -2.603817 & -2.443848 \\
\hline 6 & 1.130019 & 2.800854 & 3.718944 \\
\hline 6 & 1.539155 & 4.966602 & 2.561043 \\
\hline 6 & -4.241011 & 4.911838 & -1.086413 \\
\hline 6 & -3.919005 & 6.171980 & 1.018376 \\
\hline 6 & -0.951068 & -1.605757 & -3.013100 \\
\hline 8 & -1.277839 & -2.252717 & -3.916621 \\
\hline 6 & 0.119248 & 2.005216 & -2.959995 \\
\hline 6 & -1.118973 & 2.805279 & -3.327196 \\
\hline 6 & 0.436993 & 0.871582 & -3.742975 \\
\hline 6 & 1.183178 & 2.861839 & -2.365350 \\
\hline 6 & 2.504795 & 2.831695 & -2.838551 \\
\hline 6 & 3.425814 & 3.806055 & -2.472502 \\
\hline 6 & 3.056025 & 4.840220 & -1.617414 \\
\hline 6 & 1.763009 & 4.861499 & -1.101474 \\
\hline 6 & 0.845282 & 3.882156 & -1.461791 \\
\hline 1 & 6.702525 & -0.429401 & 0.853710 \\
\hline 1 & -2.148177 & 0.931290 & 2.870333 \\
\hline 1 & 7.247693 & -5.020811 & -0.953046 \\
\hline 1 & -1.333078 & -2.347001 & 6.162881 \\
\hline 1 & 6.521533 & 4.487807 & 0.076441 \\
\hline 1 & -3.688987 & -2.808393 & 1.200049 \\
\hline 1 & -5.554974 & -4.182921 & 0.399398 \\
\hline 1 & 4.339526 & 3.918781 & 0.963748 \\
\hline 1 & -1.934477 & -0.305306 & 4.959685 \\
\hline 1 & 5.241716 & -4.771135 & -2.291099 \\
\hline 1 & 8.642532 & 1.693298 & -2.406945 \\
\hline 1 & 3.218359 & -3.434280 & -2.904933 \\
\hline 1 & -2.965408 & -6.125555 & -2.434763 \\
\hline 1 & -0.595312 & -5.523896 & -2.538089 \\
\hline 1 & -0.287802 & -5.895127 & 3.974424 \\
\hline 1 & 6.882418 & 0.027486 & -2.072411 \\
\hline 1 & -0.605325 & -4.714247 & 1.856285 \\
\hline 1 & 1.281250 & -4.071562 & -1.791877 \\
\hline 1 & 8.500972 & 3.928975 & -1.311867 \\
\hline
\end{tabular}




\begin{tabular}{|c|c|c|c|}
\hline & 8.560495 & -1.829373 & 1.607451 \\
\hline & -5.209529 & -5.851468 & -1.421689 \\
\hline 1 & -0.643209 & -4.727808 & 6.145490 \\
\hline & 8.869864 & -4.129798 & 0.695457 \\
\hline & 2.395868 & 2.348600 & 1.027305 \\
\hline & -4.138555 & 0.966784 & 1.640450 \\
\hline & -3.388194 & -0.928704 & -2.113541 \\
\hline & -7.406777 & -0.561227 & -0.664507 \\
\hline & -3.265290 & 2.698801 & -0.608986 \\
\hline & 0.019582 & 1.558642 & 1.892259 \\
\hline & -1.237126 & 5.653503 & 1.747431 \\
\hline & 1.712814 & -3.742806 & 1.293242 \\
\hline & -1.840631 & 0.471151 & -2.672588 \\
\hline & 3.063579 & -3.222775 & 0.301532 \\
\hline & 4.744746 & -2.850225 & 1.812993 \\
\hline & 0.909607 & -1.091710 & 2.575097 \\
\hline & 5.674281 & -1.700501 & 3.790463 \\
\hline & 1.816266 & 0.025971 & 4.583659 \\
\hline & 4.214204 & -0.253470 & 5.194474 \\
\hline & -0.164426 & 3.924368 & -1.053695 \\
\hline & 1.453527 & 5.653414 & -0.419469 \\
\hline & 3.773814 & 5.613754 & -1.348190 \\
\hline$\frac{1}{1}$ & 4.438995 & 3.761735 & -2.870634 \\
\hline & 2.805583 & 2.062878 & -3.548002 \\
\hline 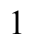 & -0.178229 & 0.646912 & -4.615400 \\
\hline & 1.464107 & 0.517503 & -3.823855 \\
\hline 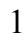 & -1.705586 & 3.108410 & -2.450858 \\
\hline ] & -0.832458 & 3.728031 & -3.856380 \\
\hline & -1.782160 & 2.226673 & -3.980192 \\
\hline 1 & -6.980769 & 1.686243 & 3.593513 \\
\hline & -5.504699 & 0.734986 & 3.368842 \\
\hline 1 & -5.692924 & 2.302186 & 2.546689 \\
\hline ] & -8.625735 & 2.003382 & 1.810984 \\
\hline | & -7.388179 & 2.561300 & 0.665471 \\
\hline 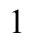 & -8.461849 & 1.219701 & 0.231890 \\
\hline | & -8.368399 & -0.237321 & 2.979973 \\
\hline 1 & -8.192956 & -1.057613 & 1.419979 \\
\hline & -6.944486 & -1.251618 & 2.662970 \\
\hline 1 & -7.388610 & -3.085824 & -3.357206 \\
\hline 1 & -6.538996 & -3.375304 & -1.824966 \\
\hline 1 & -7.887870 & -2.222430 & -1.899670 \\
\hline 1 & -7.125532 & -0.878994 & -4.571122 \\
\hline 1 & -7.557787 & 0.026404 & -3.106798 \\
\hline 1 & -6.026989 & 0.364309 & -3.935524 \\
\hline & -5.348155 & -2.628547 & -4.520283 \\
\hline 1 & -4.194848 & -1.386842 & -4.024582 \\
\hline 1 & -4.345364 & -2.887740 & -3.080458 \\
\hline 1 & -2.880724 & 7.239763 & -1.289879 \\
\hline 1 & -1.529530 & 6.868842 & -0.204343 \\
\hline 1 & -1.755250 & 5.924468 & -1.687008 \\
\hline 1 & -4.545298 & 6.975159 & 0.607576 \\
\hline 1 & -4.571679 & 5.485997 & 1.574480 \\
\hline 1 & -3.226015 & 6.628833 & 1.734919 \\
\hline 1 & -4.840201 & 5.748371 & -1.467399 \\
\hline 1 & -3.788368 & 4.412396 & -1.953001 \\
\hline 1 & -4.929679 & 4.204021 & -0.604836 \\
\hline
\end{tabular}




$\begin{array}{rrrr}1 & 1.764818 & 3.114344 & 4.557668 \\ 1 & 0.387111 & 2.091481 & 4.110772 \\ 1 & 1.774287 & 2.258911 & 3.015176 \\ 1 & 2.251673 & 5.224543 & 3.356398 \\ 1 & 2.097027 & 4.501092 & 1.737517 \\ 1 & 1.109774 & 5.903608 & 2.183070 \\ 1 & 0.363207 & 4.994091 & 5.040536 \\ 1 & -0.789083 & 5.662896 & 3.877165 \\ 1 & -1.104378 & 4.085922 & 4.622052\end{array}$

$3_{\text {IIA-si- }-\psi \text {-1a2a3a4s }}$

Number of imaginary frequencies : 0

The smallest frequencies are : $17.5613 \quad 20.002722 .7511 \mathrm{~cm}(-1)$

Electronic energy : $\quad H F=-4360.8813938$

Zero-point correction $=\quad 1.455270($ Hartree/Particle $)$

Thermal correction to Energy $=\quad 1.539661$

Thermal correction to Enthalpy= $\quad 1.540605$

Thermal correction to Gibbs Free Energy= $\quad 1.339119$

Sum of electronic and zero-point Energies $=\quad-4359.426124$

Sum of electronic and thermal Energies $=\quad-4359.341733$

Sum of electronic and thermal Enthalpies $=\quad-4359.340789$

Sum of electronic and thermal Free Energies $=\quad-4359.542275$

Cartesian Coordinates

$\begin{array}{cccc}6 & -2.502572 & 2.979733 & 0.090183 \\ 6 & -1.668345 & 1.986888 & 0.607840 \\ 6 & -0.658145 & 2.337277 & 1.508524 \\ 6 & -0.530930 & 3.646951 & 1.974433 \\ 6 & -1.361894 & 4.619873 & 1.410859 \\ 6 & -2.339915 & 4.317902 & 0.458348 \\ 15 & -1.809570 & 0.256761 & 0.000375 \\ 6 & -3.627374 & 0.035847 & -0.238360 \\ 6 & -4.524920 & 0.444050 & 0.744548 \\ 6 & -5.899036 & 0.232087 & 0.606788 \\ 6 & -6.345876 & -0.412408 & -0.547635 \\ 6 & -5.472162 & -0.847694 & -1.551595 \\ 6 & -4.108569 & -0.613107 & -1.376004 \\ 6 & -6.833542 & 0.712518 & 1.715247 \\ 6 & -6.020386 & -1.514096 & -2.812923 \\ 6 & 0.439334 & 4.027083 & 3.094363 \\ 6 & -3.187203 & 5.441059 & -0.137752 \\ 45 & -0.494720 & -0.009839 & -2.002778 \\ 6 & -0.955887 & -1.604809 & -3.014670 \\ 8 & -1.286640 & -2.251171 & -3.917125 \\ 6 & 0.117861 & 2.005188 & -2.960413 \\ 6 & 0.434643 & 0.871765 & -3.743903 \\ 6 & 1.182345 & 2.860351 & -2.364726 \\ 6 & 2.504114 & 2.829045 & -2.837409 \\ 6 & 3.426103 & 3.802196 & -2.470605 \\ 6 & 3.056937 & 4.836548 & -1.615533 \\ 6 & 1.763759 & 4.858957 & -1.100078 \\ 6 & 0.845118 & 3.880538 & -1.460722 \\ 6 & -1.119753 & 2.806414 & -3.326804 \\ 15 & 1.455162 & -0.801063 & -0.919950\end{array}$




$\begin{array}{lrrr}8 & 2.361320 & -0.026820 & 0.219263 \\ 6 & 3.446457 & 0.778434 & 0.021269 \\ 6 & 4.613545 & 0.316871 & -0.564683 \\ 6 & 5.668225 & 1.271663 & -0.770032 \\ 6 & 5.573270 & 2.567974 & -0.182156 \\ 6 & 4.400620 & 2.925671 & 0.525392 \\ 6 & 3.341181 & 2.067888 & 0.580652 \\ 6 & 6.616595 & 3.504428 & -0.374967 \\ 6 & 7.708171 & 3.197671 & -1.147515 \\ 6 & 7.790406 & 1.931169 & -1.761615 \\ 6 & 6.803234 & 0.994334 & -1.573283 \\ 6 & 4.770256 & -1.102803 & -0.960352 \\ 6 & 3.810488 & -1.736480 & -1.727493 \\ 6 & 3.982207 & -3.039957 & -2.239578 \\ 6 & 5.091036 & -3.763952 & -1.900964 \\ 6 & 6.048542 & -3.225898 & -1.006326 \\ 6 & 5.891903 & -1.890623 & -0.525008 \\ 6 & 7.140060 & -4.009607 & -0.558434 \\ 6 & 8.034040 & -3.517810 & 0.357372 \\ 6 & 7.862556 & -2.213707 & 0.866130 \\ 6 & 6.826634 & -1.422170 & 0.434570 \\ 8 & 2.636515 & -1.102972 & -2.061095 \\ 6 & 1.208000 & -2.185387 & 0.038364 \\ 6 & & & \end{array}$




\begin{tabular}{|c|c|c|c|}
\hline 1 & -5.555731 & -4.193247 & 0.395231 \\
\hline 1 & 4.335241 & 3.916951 & 0.972470 \\
\hline 1 & -1.942950 & -0.310497 & 4.955887 \\
\hline & 5.238732 & -4.769907 & -2.290088 \\
\hline 1 & 8.640402 & 1.694470 & -2.397876 \\
\hline & 3.216731 & -3.432012 & -2.905954 \\
\hline 1 & -2.963557 & -6.128963 & -2.440942 \\
\hline 1 & -0.594732 & -5.523553 & -2.543967 \\
\hline 1 & -0.294629 & -5.899485 & 3.969623 \\
\hline 1 & 6.879562 & 0.028747 & -2.066672 \\
\hline 1 & -0.608846 & -4.717276 & 1.851820 \\
\hline 1 & 1.280113 & -4.069761 & -1.796192 \\
\hline 1 & 8.498572 & 3.928901 & -1.300285 \\
\hline 1 & 8.553426 & -1.831519 & 1.614464 \\
\hline 1 & -5.207872 & -5.859640 & -1.426932 \\
\hline 1 & -0.654063 & -4.733822 & 6.140936 \\
\hline 1 & 8.863035 & -4.131632 & 0.701746 \\
\hline 1 & 2.391328 & 2.346895 & 1.032561 \\
\hline 1 & -4.151016 & 0.947662 & 1.634694 \\
\hline 1 & -3.393668 & -0.939684 & -2.124615 \\
\hline 1 & -7.412818 & -0.579917 & -0.679079 \\
\hline 1 & -3.284614 & 2.696446 & -0.611823 \\
\hline 1 & 0.004427 & 1.559953 & 1.887907 \\
\hline 1 & -1.249998 & 5.654730 & 1.734637 \\
\hline 1 & 1.706262 & -3.741589 & 1.293932 \\
\hline 1 & -1.844373 & 0.472286 & -2.673084 \\
\hline 1 & 3.058823 & -3.223609 & 0.303534 \\
\hline 1 & 4.738541 & -2.848000 & 1.815975 \\
\hline 1 & 0.901990 & -1.090118 & 2.572144 \\
\hline 1 & 5.665965 & -1.694471 & 3.792186 \\
\hline 1 & 1.806401 & 0.031395 & 4.579502 \\
\hline 1 & 4.204190 & -0.245552 & 5.192465 \\
\hline 1 & -0.164573 & 3.923301 & -1.052414 \\
\hline 1 & 1.455301 & 5.651160 & -0.417996 \\
\hline 1 & 3.775278 & 5.609408 & -1.345876 \\
\hline 1 & 4.439408 & 3.756920 & -2.868321 \\
\hline 1 & 2.804259 & 2.060425 & -3.547323 \\
\hline 1 & -0.180830 & 0.647923 & -4.616348 \\
\hline 1 & 1.461376 & 0.516576 & -3.824675 \\
\hline 1 & -1.705621 & 3.108863 & -2.449815 \\
\hline 1 & -0.832744 & 3.729465 & -3.855219 \\
\hline 1 & -1.783575 & 2.228856 & -3.980073 \\
\hline 6 & -4.911733 & -2.133072 & -3.663293 \\
\hline 6 & -7.008763 & -2.626516 & -2.450041 \\
\hline 6 & -6.740973 & -0.451948 & -3.651369 \\
\hline 6 & -8.297703 & 0.394996 & 1.423881 \\
\hline 6 & -6.449976 & 0.026577 & 3.031775 \\
\hline 6 & -6.695858 & 2.231934 & 1.868533 \\
\hline 1 & -8.924888 & 0.759001 & 2.247481 \\
\hline 1 & -8.648686 & 0.881700 & 0.504845 \\
\hline 1 & -8.472648 & -0.684612 & 1.330166 \\
\hline 1 & -7.336418 & 2.594370 & 2.683945 \\
\hline 1 & -5.665144 & 2.533966 & 2.094876 \\
\hline 1 & -7.000290 & 2.745941 & 0.947225 \\
\hline 1 & -7.114423 & 0.355965 & 3.841958 \\
\hline 1 & -6.537314 & -1.064417 & 2.944290 \\
\hline
\end{tabular}




$\begin{array}{rrrr}1 & -5.420077 & 0.252963 & 3.334841 \\ 1 & -7.382325 & -3.108874 & -3.363000 \\ 1 & -6.522464 & -3.395962 & -1.836138 \\ 1 & -7.881228 & -2.253951 & -1.900254 \\ 1 & -7.145031 & -0.897041 & -4.570938 \\ 1 & -7.576164 & 0.001493 & -3.102588 \\ 1 & -6.052576 & 0.353496 & -3.939000 \\ 1 & -5.353120 & -2.628524 & -4.537465 \\ 1 & -4.205542 & -1.381822 & -4.040462 \\ 1 & -4.341930 & -2.888691 & -3.103738 \\ 6 & 1.110195 & 2.803124 & 3.717368 \\ 6 & 1.522461 & 4.966431 & 2.556764 \\ 6 & -0.335513 & 4.738599 & 4.210846 \\ 6 & -4.219715 & 4.921595 & -1.135707 \\ 6 & -3.935708 & 6.176210 & 0.979348 \\ 6 & -2.266000 & 6.424427 & -0.869483 \\ 1 & -2.852652 & 7.242812 & -1.308351 \\ 1 & -1.523831 & 6.871532 & -0.196363 \\ 1 & -1.720570 & 5.926995 & -1.683252 \\ 1 & -4.546266 & 6.986317 & 0.558536 \\ 1 & -4.606425 & 5.494096 & 1.518486 \\ 1 & -3.254031 & 6.623932 & 1.712413 \\ 1 & -4.804872 & 5.761970 & -1.529871 \\ 1 & -3.750090 & 4.419693 & -1.991833 \\ 1 & -4.923738 & 4.218638 & -0.670774 \\ 1 & 1.743915 & 3.117556 & 4.556554 \\ 1 & 0.366657 & 2.094366 & 4.109084 \\ 1 & 1.755363 & 2.260157 & 3.015182 \\ 1 & 2.233675 & 5.225464 & 3.352932 \\ 1 & 2.081488 & 4.498662 & 1.735300 \\ 1 & 1.094548 & 5.902981 & 2.175986 \\ 1 & 0.342697 & 4.999638 & 5.034181 \\ 1 & -0.807712 & 5.666579 & 3.867842 \\ 1 & -1.124687 & 4.091279 & 4.615581\end{array}$

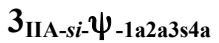

Number of imaginary frequencies : 0

The smallest frequencies are : $19.5486 \quad 21.5722 \quad 25.4904 \mathrm{~cm}(-1)$

Electronic energy : $\quad H F=-4360.8806543$

Zero-point correction $=\quad 1.458706($ Hartree/Particle $)$

Thermal correction to Energy= $\quad 1.542337$

Thermal correction to Enthalpy= $\quad 1.543281$

Thermal correction to Gibbs Free Energy $=\quad 1.344550$

Sum of electronic and zero-point Energies $=\quad-4359.421948$

Sum of electronic and thermal Energies $=\quad-4359.338317$

Sum of electronic and thermal Enthalpies $=\quad-4359.337373$

Sum of electronic and thermal Free Energies $=\quad-4359.536105$

Cartesian Coordinates

$\begin{array}{llll}6 & 4.085457 & -2.314594 & 2.362173 \\ 6 & 2.747344 & -2.139545 & 2.013167 \\ 6 & 1.943577 & -1.335165 & 2.823376 \\ 6 & 2.467195 & -0.724783 & 3.955340 \\ 6 & 3.807108 & -0.903226 & 4.296764\end{array}$




\begin{tabular}{|c|c|c|c|}
\hline 6 & 4.615103 & -1.699760 & 3.494675 \\
\hline 6 & 2.205310 & -2.885409 & 0.819057 \\
\hline & 1.199926 & -2.190713 & -0.021377 \\
\hline & 0.088212 & -3.005023 & -0.419642 \\
\hline & -1.133918 & -2.890276 & 0.214783 \\
\hline & -2.237011 & -3.676416 & -0.245650 \\
\hline & -2.036766 & -4.623094 & -1.293960 \\
\hline 5 & -0.750164 & -4.753750 & -1.868993 \\
\hline & 0.277577 & -3.949738 & -1.455294 \\
\hline 6 & -3.123705 & -5.423305 & -1.723776 \\
\hline 6 & -4.361556 & -5.295392 & -1.147288 \\
\hline 6 & -4.567904 & -4.347002 & -0.121996 \\
\hline 6 & -3.533770 & -3.559684 & 0.319501 \\
\hline 6 & -1.287182 & -2.127502 & 1.485984 \\
\hline 6 & -1.594856 & -0.777404 & 1.563112 \\
\hline 6 & -1.835201 & -0.199666 & 2.843020 \\
\hline 6 & -1.720670 & -0.925219 & 3.996113 \\
\hline 6 & -1.352964 & -2.289051 & 3.955319 \\
\hline 6 & -1.149806 & -2.905220 & 2.687653 \\
\hline 6 & -0.783451 & -4.277156 & 2.669693 \\
\hline 6 & -0.612929 & -4.981829 & 3.836162 \\
\hline 6 & -0.809994 & -4.360991 & 5.086517 \\
\hline 6 & -1.176609 & -3.040593 & 5.140564 \\
\hline & -1.801996 & 0.252719 & 0.023493 \\
\hline 45 & -0.492415 & 0.058787 & -1.990145 \\
\hline 5 & 1.453970 & -0.779874 & -0.937895 \\
\hline 8 & 2.633350 & -1.053466 & -2.088480 \\
\hline 6 & 3.806857 & -1.696898 & -1.772865 \\
\hline 6 & 4.767829 & -1.084175 & -0.990295 \\
\hline 6 & 5.889377 & -1.883530 & -0.576997 \\
\hline 6 & 6.044929 & -3.205783 & -1.093148 \\
\hline 6 & 5.085938 & -3.720040 & -2.000134 \\
\hline 6 & 3.977054 & -2.986933 & -2.318567 \\
\hline 6 & 6.825350 & -1.439880 & 0.392811 \\
\hline 6 & 7.861884 & -2.242346 & 0.802306 \\
\hline 6 & 8.032242 & -3.532994 & 0.259804 \\
\hline 6 & 7.136851 & -4.000931 & -0.667208 \\
\hline 6 & 4.612118 & 0.325012 & -0.558456 \\
\hline 6 & 3.445737 & 0.770294 & 0.040846 \\
\hline 6 & 3.337363 & 2.047422 & 0.627286 \\
\hline 6 & 4.395267 & 2.908245 & 0.590250 \\
\hline 6 & 5.568130 & 2.568365 & -0.125888 \\
\hline 6 & 5.664722 & 1.286010 & -0.743544 \\
\hline 6 & 6.609768 & 3.510704 & -0.298798 \\
\hline 6 & 7.700635 & 3.223563 & -1.079808 \\
\hline 6 & 7.784131 & 1.971683 & -1.722998 \\
\hline 6 & 6.798891 & 1.029094 & -1.554742 \\
\hline 8 & 2.364818 & -0.044367 & 0.223259 \\
\hline 6 & -1.652499 & 1.965660 & 0.670503 \\
\hline 6 & -2.486955 & 2.968212 & 0.174840 \\
\hline 6 & -2.329207 & 4.296788 & 0.578427 \\
\hline 6 & -1.349199 & 4.577631 & 1.535277 \\
\hline 6 & -0.513021 & 3.593888 & 2.071711 \\
\hline 6 & -0.640185 & 2.295105 & 1.576526 \\
\hline 6 & 0.462939 & 3.951017 & 3.194134 \\
\hline 6 & -0.306265 & 4.633852 & 4.332130 \\
\hline
\end{tabular}




\begin{tabular}{|c|c|c|c|}
\hline & -3.186633 & 5.431810 & 0.020456 \\
\hline & -2.277527 & 6.440612 & -0.691322 \\
\hline & -3.621618 & 0.046680 & -0.216695 \\
\hline & -4.514253 & 0.330101 & 0.826639 \\
\hline & -5.880780 & 0.130727 & 0.674447 \\
\hline & -6.344935 & -0.342815 & -0.565161 \\
\hline & -5.489614 & -0.623118 & -1.624692 \\
\hline & -4.114849 & -0.439351 & -1.417805 \\
\hline & -6.877158 & 0.401479 & 1.800113 \\
\hline & -7.902337 & 1.445443 & 1.344746 \\
\hline & -5.968491 & -1.116917 & -2.987795 \\
\hline & -5.427492 & -2.529028 & -3.232459 \\
\hline & -6.195122 & 0.926877 & 3.061657 \\
\hline & -7.598345 & -0.903335 & 2.155830 \\
\hline & -7.491115 & -1.162582 & -3.085306 \\
\hline & -5.448214 & -0.177552 & -4.083222 \\
\hline & 1.142071 & 2.715137 & 3.783734 \\
\hline & 1.539219 & 4.906262 & 2.670687 \\
\hline & -4.227215 & 4.935664 & -0.981143 \\
\hline & -3.928684 & 6.130117 & 1.165345 \\
\hline & -0.985807 & -1.500216 & -3.039308 \\
\hline & -1.364131 & -2.109027 & -3.949759 \\
\hline & 0.106573 & 2.107888 & -2.886444 \\
\hline & -1.139317 & 2.910715 & -3.219950 \\
\hline & 0.424780 & 0.999075 & -3.704872 \\
\hline & 1.170502 & 2.952487 & -2.274886 \\
\hline & 2.494552 & 2.919303 & -2.742156 \\
\hline & 3.421513 & 3.880980 & -2.359407 \\
\hline & 3.056621 & 4.904773 & -1.489848 \\
\hline 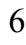 & 1.760777 & 4.930164 & -0.981657 \\
\hline & 0.834630 & 3.965829 & -1.361657 \\
\hline & 6.696263 & -0.459095 & 0.842188 \\
\hline ] & -2.109746 & 0.849367 & 2.913855 \\
\hline & 7.238876 & -5.002111 & -1.084060 \\
\hline 1 & -1.333636 & -2.543546 & 6.096839 \\
\hline & 6.510349 & 4.481990 & 0.185606 \\
\hline 1 & -3.707612 & -2.825748 & 1.105208 \\
\hline & -5.557186 & -4.231703 & 0.316521 \\
\hline & 4.328524 & 3.889378 & 1.058917 \\
\hline ] & -1.905808 & -0.456525 & 4.962102 \\
\hline & 5.232671 & -4.715749 & -2.415129 \\
\hline 1 & 8.633480 & 1.751185 & -2.365926 \\
\hline & 3.210399 & -3.361456 & -2.993628 \\
\hline 1 & -2.952793 & -6.143100 & -2.523086 \\
\hline & -0.599505 & -5.486578 & -2.660194 \\
\hline 1 & -0.324343 & -6.029579 & 3.794485 \\
\hline ] & 6.875780 & 0.075343 & -2.070552 \\
\hline & -0.628907 & -4.777837 & 1.716158 \\
\hline 1 & 1.266317 & -4.023134 & -1.907628 \\
\hline 1 & 8.489531 & 3.959491 & -1.217087 \\
\hline 1 & 8.554040 & -1.879630 & 1.559079 \\
\hline 1 & -5.189594 & -5.914847 & -1.485183 \\
\hline 1 & -0.670551 & -4.930400 & 6.002460 \\
\hline 1 & 8.861598 & -4.155682 & 0.586965 \\
\hline 1 & 2.386779 & 2.313624 & 1.084900 \\
\hline 1 & -4.124292 & 0.713809 & 1.765291 \\
\hline
\end{tabular}




\begin{tabular}{|c|c|c|c|}
\hline 1 & -3.413991 & -0.677685 & -2.213537 \\
\hline & -7.415753 & -0.487968 & -0.694671 \\
\hline & -3.268741 & 2.699719 & -0.533264 \\
\hline & 0.024527 & 1.509818 & 1.934851 \\
\hline & -1.239443 & 5.604198 & 1.885112 \\
\hline & 1.692668 & -3.786480 & 1.185690 \\
\hline & -1.836339 & 0.573191 & -2.640637 \\
\hline & 3.045912 & -3.245946 & 0.208105 \\
\hline & 4.729220 & -2.931368 & 1.733196 \\
\hline & 0.907604 & -1.163395 & 2.538990 \\
\hline & 5.665875 & -1.841944 & 3.741175 \\
\hline & 1.822268 & -0.103299 & 4.575618 \\
\hline & 4.216938 & -0.419062 & 5.180646 \\
\hline & -0.178184 & 4.019074 & -0.960093 \\
\hline & 1.455638 & 5.716312 & -0.291092 \\
\hline & 3.779290 & 5.668232 & -1.205396 \\
\hline & 4.435805 & 3.834178 & -2.754438 \\
\hline & 2.793893 & 2.157337 & -3.459466 \\
\hline & -0.193474 & 0.799391 & -4.581288 \\
\hline & 1.453174 & 0.653235 & -3.802608 \\
\hline & -1.719948 & 3.180025 & -2.329165 \\
\hline 1 & -0.863223 & 3.851836 & -3.721611 \\
\hline & -1.804344 & 2.348746 & -3.886265 \\
\hline ] & -6.946678 & 1.096921 & 3.842847 \\
\hline & -5.460786 & 0.214143 & 3.460952 \\
\hline & -5.684321 & 1.882392 & 2.882741 \\
\hline I & -8.619789 & 1.651565 & 2.150407 \\
\hline & -7.409557 & 2.390425 & 1.080920 \\
\hline 1 & -8.474928 & 1.111920 & 0.471111 \\
\hline ] & -8.318046 & -0.734033 & 2.968123 \\
\hline I & -8.151809 & -1.314399 & 1.302881 \\
\hline 1 & -6.883115 & -1.666315 & 2.490761 \\
\hline & -5.805279 & -0.510242 & -5.067036 \\
\hline 1 & -5.802171 & 0.849483 & -3.923959 \\
\hline & -4.351433 & -0.152599 & -4.124818 \\
\hline 1 & -5.736956 & -2.889811 & -4.222887 \\
\hline 1 & -4.331116 & -2.564405 & -3.191694 \\
\hline 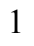 & -5.810006 & -3.229086 & -2.478577 \\
\hline 1 & -7.786299 & -1.508302 & -4.083978 \\
\hline . & -7.929161 & -1.857574 & -2.357148 \\
\hline 1 & -7.941940 & -0.173292 & -2.931804 \\
\hline 1 & -2.871968 & 7.269040 & -1.099891 \\
\hline 1 & -1.530489 & 6.870811 & -0.012574 \\
\hline 1 & -1.737892 & 5.970005 & -1.524672 \\
\hline 1 & -4.552648 & 6.944496 & 0.773382 \\
\hline 1 & -4.584915 & 5.426257 & 1.694144 \\
\hline 1 & -3.242813 & 6.565695 & 1.901806 \\
\hline 1 & -4.817255 & 5.784842 & -1.348311 \\
\hline 1 & -3.765211 & 4.456624 & -1.854320 \\
\hline 1 & -4.926264 & 4.220351 & -0.527572 \\
\hline 1 & 1.778740 & 3.011544 & 4.627209 \\
\hline 1 & 0.403727 & 1.993433 & 4.161515 \\
\hline 1 & 1.785867 & 2.193450 & 3.064530 \\
\hline 1 & 2.258665 & 5.144878 & 3.465885 \\
\hline 1 & 2.089469 & 4.462390 & 1.830247 \\
\hline 1 & 1.105903 & 5.852049 & 2.320111 \\
\hline
\end{tabular}




$\begin{array}{rrrr}1 & 0.377230 & 4.879237 & 5.155947 \\ 1 & -0.784873 & 5.567359 & 4.013773 \\ 1 & -1.089637 & 3.974219 & 4.728150\end{array}$

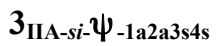

Number of imaginary frequencies : 0

The smallest frequencies are : $20.311022 .815926 .6286 \mathrm{~cm}(-1)$

Electronic energy : $\quad H F=-4360.8809188$

Zero-point correction $=\quad 1.458054($ Hartree/Particle $)$

Thermal correction to Energy= $\quad 1.541609$

Thermal correction to Enthalpy= $\quad 1.542553$

Thermal correction to Gibbs Free Energy= $\quad 1.344781$

Sum of electronic and zero-point Energies $=\quad-4359.422865$

Sum of electronic and thermal Energies $=\quad-4359.339310$

Sum of electronic and thermal Enthalpies $=\quad-4359.338366$

Sum of electronic and thermal Free Energies $=\quad-4359.536138$

Cartesian Coordinates

$\begin{array}{cccc}6 & -2.535959 & 2.923973 & 0.159564 \\ 6 & -1.683008 & 1.938090 & 0.657684 \\ 6 & -0.675537 & 2.289057 & 1.560819 \\ 6 & -0.571250 & 3.591938 & 2.051119 \\ 6 & -1.424495 & 4.558919 & 1.510876 \\ 6 & -2.399464 & 4.256909 & 0.555167 \\ 15 & -1.805047 & 0.219572 & 0.018938 \\ 6 & -3.622077 & -0.002901 & -0.224436 \\ 6 & -4.516323 & 0.304741 & 0.802386 \\ 6 & -5.888871 & 0.119439 & 0.648435 \\ 6 & -6.352581 & -0.372862 & -0.578164 \\ 6 & -5.487832 & -0.684985 & -1.628310 \\ 6 & -4.116069 & -0.509465 & -1.421079 \\ 6 & -6.814830 & 0.467545 & 1.812762 \\ 6 & -5.966211 & -1.200174 & -2.984303 \\ 6 & 0.399770 & 3.970250 & 3.171012 \\ 6 & -3.277107 & 5.372769 & -0.009360 \\ 45 & -0.496859 & 0.025408 & -1.993610 \\ 6 & -0.977767 & -1.547223 & -3.028460 \\ 8 & -1.356614 & -2.167626 & -3.930812 \\ 6 & 0.076955 & 2.073948 & -2.904511 \\ 6 & 0.408253 & 0.964280 & -3.716311 \\ 6 & 1.130309 & 2.933531 & -2.295569 \\ 6 & 2.455136 & 2.917741 & -2.761387 \\ 6 & 3.367737 & 3.893715 & -2.379656 \\ 6 & 2.986940 & 4.915209 & -1.514193 \\ 6 & 1.690326 & 4.923428 & -1.007321 \\ 6 & 0.779658 & 3.943656 & -1.384772 \\ 6 & -1.178205 & 2.860708 & -3.242416 \\ 15 & 1.460817 & -0.779828 & -0.938272 \\ 8 & 2.362084 & -0.026340 & 0.218677 \\ 6 & 3.433554 & 0.800068 & 0.034661 \\ 6 & 4.605197 & 0.367634 & -0.563942 \\ 6 & 5.645505 & 1.341485 & -0.751874 \\ 6 & 5.532748 & 2.624332 & -0.137884 \\ 6 & 4.356715 & 2.950811 & 0.579278 \\ & & & \\ 6 & & & \end{array}$




\begin{tabular}{|c|c|c|c|}
\hline ) & 3.310231 & 2.076377 & 0.619865 \\
\hline & 6.561939 & 3.579633 & -0.314311 \\
\hline & 7.656053 & 3.304297 & -1.094973 \\
\hline & 7.755528 & 2.051623 & -1.734254 \\
\hline & 6.782651 & 1.096849 & -1.562768 \\
\hline U & 4.778595 & -1.041192 & -0.990581 \\
\hline & 3.824768 & -1.669643 & -1.769435 \\
\hline & 4.009446 & -2.960633 & -2.307912 \\
\hline & 5.127273 & -3.678706 & -1.986755 \\
\hline & 6.081059 & -3.147947 & -1.083825 \\
\hline & 5.910190 & -1.824945 & -0.574275 \\
\hline & 7.182891 & -3.927889 & -0.655149 \\
\hline & 8.073637 & -3.444682 & 0.268414 \\
\hline & 7.888360 & -2.153532 & 0.804668 \\
\hline & 6.841903 & -1.365502 & 0.392411 \\
\hline & 2.643243 & -1.042440 & -2.087869 \\
\hline & 1.226564 & -2.189022 & -0.013797 \\
\hline & 0.123120 & -3.018880 & -0.403074 \\
\hline & -1.098495 & -2.913102 & 0.233777 \\
\hline & -2.194777 & -3.713463 & -0.217779 \\
\hline & -1.987825 & -4.664695 & -1.260598 \\
\hline & -0.701412 & -4.785731 & -1.838320 \\
\hline & 0.319260 & -3.968346 & -1.433101 \\
\hline & -3.068142 & -5.477859 & -1.682665 \\
\hline & -4.305717 & -5.357917 & -1.103776 \\
\hline 6 & -4.518297 & -4.405807 & -0.083183 \\
\hline & -3.490836 & -3.605846 & 0.350941 \\
\hline 6 & -1.258644 & -2.142974 & 1.499274 \\
\hline & -1.587157 & -0.797353 & 1.565979 \\
\hline & -1.835317 & -0.213828 & 2.841679 \\
\hline 6 & -1.707017 & -0.928390 & 4.000301 \\
\hline & -1.317223 & -2.286265 & 3.969657 \\
\hline 6 & -1.107528 & -2.909245 & 2.706477 \\
\hline & -0.720030 & -4.275436 & 2.698244 \\
\hline 6 & -0.534001 & -4.967561 & 3.869819 \\
\hline 6 & -0.736633 & -4.339609 & 5.115761 \\
\hline & -1.124859 & -3.025083 & 5.160401 \\
\hline 6 & 2.241518 & -2.864681 & 0.830546 \\
\hline & 2.774053 & -2.104205 & 2.019824 \\
\hline 6 & 1.961084 & -1.302782 & 2.823808 \\
\hline & 2.477402 & -0.678257 & 3.951391 \\
\hline 6 & 3.819037 & -0.839395 & 4.294700 \\
\hline & 4.636088 & -1.633100 & 3.498989 \\
\hline & 4.113807 & -2.261988 & 2.370791 \\
\hline 1 & 6.701680 & -0.384184 & 0.837256 \\
\hline 1 & -2.125016 & 0.831528 & 2.904670 \\
\hline 1 & 7.296209 & -4.929845 & -1.067185 \\
\hline 1 & -1.287176 & -2.522969 & 6.113136 \\
\hline & 6.450205 & 4.550949 & 0.167340 \\
\hline 1 & -3.669947 & -2.869224 & 1.133211 \\
\hline 1 & -5.507384 & -4.297389 & 0.357492 \\
\hline 1 & 4.278055 & 3.931847 & 1.046337 \\
\hline 1 & -1.897484 & -0.455079 & 4.963008 \\
\hline 1 & 5.285172 & -4.675011 & -2.396185 \\
\hline & 8.607469 & 1.840138 & -2.376782 \\
\hline & 3.246168 & -3.348072 & -2.979511 \\
\hline
\end{tabular}




\begin{tabular}{|c|c|c|c|}
\hline . & -2.892597 & -6.200742 & -2.478167 \\
\hline & -0.545516 & -5.521773 & -2.625510 \\
\hline 1 & -0.228783 & -6.010853 & 3.835756 \\
\hline & 6.871724 & 0.142583 & -2.075679 \\
\hline & -0.561070 & -4.781304 & 1.748158 \\
\hline & 1.307140 & -4.033922 & -1.888605 \\
\hline & 8.435288 & 4.049956 & -1.234882 \\
\hline & 8.576910 & -1.778849 & 1.558906 \\
\hline & -5.128806 & -5.986957 & -1.435974 \\
\hline 1 & -0.584677 & -4.899116 & 6.035803 \\
\hline 1 & 8.910771 & -4.055719 & 0.597732 \\
\hline 1 & 2.357749 & 2.329996 & 1.080616 \\
\hline 1 & -4.134028 & 0.703959 & 1.740499 \\
\hline 1 & -3.413653 & -0.766636 & -2.209578 \\
\hline 1 & -7.421119 & -0.508969 & -0.718027 \\
\hline 1 & -3.313977 & 2.639250 & -0.546154 \\
\hline 1 & 0.002991 & 1.516851 & 1.921909 \\
\hline 1 & -1.333596 & 5.588633 & 1.856888 \\
\hline 1 & 1.741432 & -3.770399 & 1.202957 \\
\hline 1 & -1.847887 & 0.518426 & -2.646464 \\
\hline 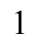 & 3.086790 & -3.217403 & 0.221530 \\
\hline 1 & 4.764665 & -2.876234 & 1.746642 \\
\hline 1 & 0.923454 & -1.144375 & 2.537802 \\
\hline 1 & 5.688229 & -1.761949 & 3.746995 \\
\hline 1 & 1.825087 & -0.059592 & 4.566805 \\
\hline 1 & 4.223084 & -0.344231 & 5.175140 \\
\hline 1 & -0.233397 & 3.980134 & -0.983014 \\
\hline 1 & 1.371510 & 5.707108 & -0.320222 \\
\hline 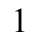 & 3.698334 & 5.689853 & -1.231528 \\
\hline 1 & 4.383272 & 3.860177 & -2.772879 \\
\hline 1 & 2.765940 & 2.158362 & -3.476628 \\
\hline 1 & -0.208043 & 0.751287 & -4.590937 \\
\hline 1 & 1.440457 & 0.629229 & -3.811437 \\
\hline 1 & -1.762400 & 3.128338 & -2.353242 \\
\hline 1 & -0.912873 & 3.802444 & -3.748725 \\
\hline 1 & -1.836534 & 2.287599 & -3.905898 \\
\hline 6 & -7.488730 & -1.250401 & -3.082795 \\
\hline 6 & -5.447443 & -0.275533 & -4.093027 \\
\hline 6 & -5.422981 & -2.614774 & -3.209040 \\
\hline 6 & -8.281431 & 0.183334 & 1.499010 \\
\hline 6 & -6.419834 & -0.366911 & 3.037103 \\
\hline 6 & -6.678029 & 1.959195 & 2.141000 \\
\hline 1 & -8.900792 & 0.444085 & 2.366519 \\
\hline 1 & -8.642693 & 0.776009 & 0.648815 \\
\hline 1 & -8.455713 & -0.877631 & 1.277782 \\
\hline 1 & -7.317778 & 2.224023 & 2.993753 \\
\hline 1 & -5.647411 & 2.234007 & 2.399671 \\
\hline 1 & -6.982890 & 2.576690 & 1.285870 \\
\hline 1 & -7.077600 & -0.135985 & 3.885918 \\
\hline 1 & -6.507449 & -1.440444 & 2.822868 \\
\hline 1 & -5.387227 & -0.176846 & 3.355807 \\
\hline 1 & -5.802015 & -0.623463 & -5.072526 \\
\hline 1 & -5.804855 & 0.752580 & -3.949048 \\
\hline 1 & -4.350704 & -0.247376 & -4.132850 \\
\hline 1 & -5.732207 & -2.990171 & -4.194111 \\
\hline 1 & -4.326568 & -2.648058 & -3.167909 \\
\hline
\end{tabular}




$\begin{array}{rrrr}1 & -5.804805 & -3.304578 & -2.445411 \\ 1 & -7.781590 & -1.614422 & -4.075655 \\ 1 & -7.926696 & -1.932886 & -2.342887 \\ 1 & -7.942051 & -0.259556 & -2.948131 \\ 6 & 1.094568 & 2.747799 & 3.770466 \\ 6 & 1.464407 & 4.933984 & 2.639528 \\ 6 & -0.377398 & 4.652749 & 4.303700 \\ 6 & -4.291868 & 4.854678 & -1.026358 \\ 6 & -4.049296 & 6.046972 & 1.129979 \\ 6 & -2.385047 & 6.405980 & -0.707491 \\ 1 & -2.995343 & 7.219943 & -1.121698 \\ 1 & -1.657337 & 6.854035 & -0.019379 \\ 1 & -1.824235 & 5.950697 & -1.535425 \\ 1 & -4.684835 & 6.851247 & 0.735675 \\ 1 & -4.697582 & 5.325350 & 1.644670 \\ 1 & -3.381607 & 6.490615 & 1.878375 \\ 1 & -4.902602 & 5.689032 & -1.393650 \\ 1 & -3.805628 & 4.396321 & -1.897568 \\ 1 & -4.973931 & 4.114384 & -0.587350 \\ 1 & 1.725769 & 3.058933 & 4.612744 \\ 1 & 0.365688 & 2.018646 & 4.152191 \\ 1 & 1.746820 & 2.230019 & 3.056060 \\ 1 & 2.179831 & 5.189420 & 3.433153 \\ 1 & 2.021163 & 4.488395 & 1.804236 \\ 1 & 1.019408 & 5.870621 & 2.279093 \\ 1 & 0.303233 & 4.912653 & 5.125401 \\ 1 & -0.867040 & 5.578048 & 3.978365 \\ 1 & -1.152814 & 3.987003 & 4.705118\end{array}$

$3_{\text {IIA-si- }} \psi_{-12 a 233 a 4 a}$

Number of imaginary frequencies : 0

The smallest frequencies are : $18.427520 .559623 .7123 \mathrm{~cm}(-1)$

Electronic energy : $\quad H F=-4360.8804627$

Zero-point correction $=\quad 1.454932($ Hartree/Particle $)$

Thermal correction to Energy= $\quad 1.539443$

Thermal correction to Enthalpy= $\quad 1.540387$

Thermal correction to Gibbs Free Energy= $\quad 1.338409$

Sum of electronic and zero-point Energies $=\quad-4359.425531$

Sum of electronic and thermal Energies $=\quad-4359.341020$

Sum of electronic and thermal Enthalpies $=\quad-4359.340076$

Sum of electronic and thermal Free Energies $=\quad-4359.542053$

Cartesian Coordinates

$\begin{array}{cccc}6 & 2.562214 & 2.841035 & -2.842015 \\ 6 & 1.237809 & 2.883856 & -2.377005 \\ 6 & 0.901289 & 3.911488 & -1.480721 \\ 6 & 1.825709 & 4.885052 & -1.120434 \\ 6 & 3.121217 & 4.851377 & -1.629270 \\ 6 & 3.488352 & 3.810269 & -2.476986 \\ 6 & 0.174089 & 2.029347 & -2.974150 \\ 6 & -1.065437 & 2.828012 & -3.338689 \\ 45 & -0.456637 & 0.016088 & -2.019105 \\ 6 & 0.492256 & 0.893744 & -3.753339 \\ 15 & 1.472455 & -0.800261 & -0.918941\end{array}$




\begin{tabular}{|c|c|c|c|}
\hline 7 & 1.197316 & -2.178840 & 0.039859 \\
\hline 6 & 2.187019 & -2.857791 & 0.910992 \\
\hline & 2.733183 & -2.077906 & 2.081222 \\
\hline & 1.933426 & -1.246525 & 2.868132 \\
\hline & 2.461011 & -0.605821 & 3.981330 \\
\hline & 3.800833 & -0.778585 & 4.326074 \\
\hline & 4.605036 & -1.600501 & 3.546248 \\
\hline & 4.071311 & -2.246547 & 2.433172 \\
\hline & 2.656454 & -1.123642 & -2.052150 \\
\hline & 3.822856 & -1.765209 & -1.707531 \\
\hline & 4.785614 & -1.134601 & -0.941332 \\
\hline & 5.898315 & -1.929143 & -0.495672 \\
\hline & 6.045011 & -3.269271 & -0.966462 \\
\hline & 5.085897 & -3.805579 & -1.860466 \\
\hline & 3.984885 & -3.074285 & -2.208598 \\
\hline & 6.832944 & -1.461934 & 0.464546 \\
\hline & 7.859975 & -2.259396 & 0.906363 \\
\hline & 8.021988 & -3.568688 & 0.407944 \\
\hline & 7.127579 & -4.059288 & -0.508122 \\
\hline & 4.639356 & 0.289309 & -0.557198 \\
\hline & 5.701212 & 1.235296 & -0.765481 \\
\hline & 5.610787 & 2.537497 & -0.189864 \\
\hline & 4.435327 & 2.909951 & 0.505376 \\
\hline & 3.370134 & 2.059313 & 0.562439 \\
\hline & 3.472757 & 0.762542 & 0.019999 \\
\hline & 6.839864 & 0.943036 & -1.558228 \\
\hline 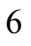 & 7.834683 & 1.871443 & -1.747869 \\
\hline & 7.756650 & 3.143980 & -1.145707 \\
\hline 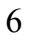 & 6.661822 & 3.465084 & -0.383657 \\
\hline K & 2.386230 & -0.039954 & 0.223803 \\
\hline ) & 0.078126 & -2.990307 & -0.341784 \\
\hline 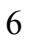 & -1.147506 & -2.837220 & 0.276978 \\
\hline f & -2.261753 & -3.609519 & -0.178714 \\
\hline 5 & -2.075449 & -4.566178 & -1.220560 \\
\hline 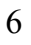 & -0.783934 & -4.743345 & -1.771497 \\
\hline 5 & 0.259626 & -3.963078 & -1.352470 \\
\hline 5 & -3.183396 & -5.323846 & -1.672920 \\
\hline f & -4.426895 & -5.149820 & -1.119645 \\
\hline 5 & -4.614102 & -4.205620 & -0.086388 \\
\hline 5 & -3.559320 & -3.458311 & 0.375032 \\
\hline 5 & -1.301025 & -2.030155 & 1.520080 \\
\hline 5 & -1.596967 & -0.675306 & 1.548126 \\
\hline 6 & -1.840859 & -0.053740 & 2.806714 \\
\hline 6 & -1.738992 & -0.740205 & 3.984785 \\
\hline 6 & -1.383130 & -2.107633 & 3.993142 \\
\hline 6 & -1.179664 & -2.768196 & 2.748106 \\
\hline 6 & -0.828744 & -4.143947 & 2.778125 \\
\hline 6 & -0.668944 & -4.809903 & 3.968624 \\
\hline 6 & -0.863222 & -4.144544 & 5.196296 \\
\hline 6 & -1.218031 & -2.819903 & 5.203992 \\
\hline 15 & -1.787687 & 0.307549 & -0.027945 \\
\hline 6 & -3.607273 & 0.090185 & -0.268914 \\
\hline 6 & -4.502457 & 0.416797 & 0.754928 \\
\hline 6 & -5.865499 & 0.160264 & 0.626362 \\
\hline 6 & -6.311456 & -0.419251 & -0.568514 \\
\hline 6 & -5.449600 & -0.756701 & -1.611280 \\
\hline
\end{tabular}




\begin{tabular}{|c|c|c|c|}
\hline & -4.086586 & -0.496722 & -1.435633 \\
\hline & -6.860365 & 0.442904 & 1.750673 \\
\hline & -5.995611 & -1.362359 & -2.903676 \\
\hline & -1.639678 & 2.035344 & 0.582520 \\
\hline & -0.612159 & 2.378349 & 1.473069 \\
\hline & -0.499415 & 3.671710 & 1.974237 \\
\hline & -1.366717 & 4.651204 & 1.464473 \\
\hline & -2.359789 & 4.358667 & 0.531786 \\
\hline & -2.496184 & 3.026131 & 0.115668 \\
\hline & 0.489691 & 4.035134 & 3.083622 \\
\hline & 1.575325 & 4.965100 & 2.534875 \\
\hline & -3.332658 & 5.408281 & -0.003263 \\
\hline & -3.254639 & 5.456113 & -1.533001 \\
\hline & -0.256883 & 4.749301 & 4.217607 \\
\hline & 1.153086 & 2.798981 & 3.691396 \\
\hline & -4.756481 & 5.031032 & 0.423708 \\
\hline ) & -3.029424 & 6.807106 & 0.527319 \\
\hline & -0.922541 & -1.578711 & -3.026049 \\
\hline & -1.258453 & -2.236098 & -3.918424 \\
\hline & 6.710268 & -0.466015 & 0.881228 \\
\hline & -2.108382 & 0.998831 & 2.841300 \\
\hline & 7.222858 & -5.074579 & -0.891047 \\
\hline & -1.374388 & -2.289443 & 6.142256 \\
\hline & 6.566829 & 4.452146 & 0.068700 \\
\hline 1 & -3.718825 & -2.725049 & 1.164277 \\
\hline & -5.604436 & -4.060346 & 0.342161 \\
\hline & 4.372812 & 3.906352 & 0.941249 \\
\hline & -1.925652 & -0.236760 & 4.932864 \\
\hline & 5.225834 & -4.815846 & -2.241202 \\
\hline 1 & 8.687612 & 1.623356 & -2.375805 \\
\hline & 3.217918 & -3.464809 & -2.874194 \\
\hline ] & -3.025185 & -6.047660 & -2.471185 \\
\hline & -0.643075 & -5.487883 & -2.553481 \\
\hline & -0.391473 & -5.861464 & 3.963836 \\
\hline 1 & 6.913295 & -0.027746 & -2.041809 \\
\hline & -0.677578 & -4.678478 & 1.842601 \\
\hline ] & 1.252151 & -4.068564 & -1.789878 \\
\hline & 8.553039 & 3.868487 & -1.299440 \\
\hline 1 & 8.551055 & -1.877817 & 1.654818 \\
\hline ] & -5.271666 & -5.735931 & -1.475750 \\
\hline l & -0.731842 & -4.683551 & 6.131622 \\
\hline 1 & 8.843972 & -4.187311 & 0.760445 \\
\hline . & 2.419215 & 2.349515 & 1.004658 \\
\hline 1 & -4.122428 & 0.873513 & 1.664660 \\
\hline & -3.374430 & -0.762357 & -2.210129 \\
\hline 1 & -7.376888 & -0.618337 & -0.684151 \\
\hline l & -3.295780 & 2.754878 & -0.574368 \\
\hline 1 & 0.067179 & 1.599423 & 1.818932 \\
\hline 1 & -1.266471 & 5.670544 & 1.830439 \\
\hline ] & 1.659160 & -3.738387 & 1.305198 \\
\hline 1 & -1.792569 & 0.507248 & -2.708027 \\
\hline 1 & 3.026099 & -3.251110 & 0.318379 \\
\hline 1 & 4.711896 & -2.884223 & 1.822083 \\
\hline 1 & 0.897028 & -1.079382 & 2.582258 \\
\hline 1 & 5.655834 & -1.738888 & 3.794671 \\
\hline 1 & 1.818669 & 0.034551 & 4.584779 \\
\hline
\end{tabular}




$\begin{array}{lrrr}1 & 4.213309 & -0.270564 & 5.195205 \\ 1 & 1.795469 & 3.099983 & 4.528957 \\ 1 & 0.404234 & 2.094862 & 4.081403 \\ 1 & 1.788776 & 2.253898 & 2.982644 \\ 1 & 0.437812 & 4.986500 & 5.034255 \\ 1 & -0.713259 & 5.691640 & 3.893627 \\ 1 & -1.053560 & 4.113515 & 4.626002 \\ 1 & 2.302172 & 5.211466 & 3.320977 \\ 1 & 2.115448 & 4.496595 & 1.701323 \\ 1 & 1.150541 & 5.908193 & 2.166918 \\ 1 & -3.744896 & 7.523878 & 0.105170 \\ 1 & -3.117629 & 6.862816 & 1.619980 \\ 1 & -2.022584 & 7.141296 & 0.244241 \\ 1 & -3.946879 & 6.214060 & -1.923571 \\ 1 & -2.242484 & 5.719295 & -1.869284 \\ 1 & -3.523979 & 4.497709 & -1.993387 \\ 1 & -5.478398 & 5.763348 & 0.037778 \\ 1 & -5.051365 & 4.042673 & 0.047927 \\ 1 & -4.844391 & 5.013553 & 1.518153 \\ 1 & -0.110754 & 3.960694 & -1.077707 \\ 1 & 1.521834 & 5.684674 & -0.445403 \\ 1 & 3.842792 & 5.621361 & -1.360029 \\ 1 & 4.503147 & 3.756469 & -2.869658 \\ 1 & 2.861225 & 2.065507 & -3.544785 \\ 1 & -0.117410 & 0.669174 & -4.629622 \\ 1 & 1.518081 & 0.534174 & -3.825204 \\ 1 & -1.662358 & 3.107300 & -2.462241 \\ 1 & -0.783141 & 3.764684 & -3.845226 \\ 1 & -1.717104 & 2.257260 & -4.010094 \\ 6 & -4.878322 & -1.869647 & -3.814310 \\ 6 & -6.921333 & -2.543447 & -2.596688 \\ 6 & -6.784727 & -0.283262 & -3.653811 \\ 6 & -6.198956 & 1.115382 & 2.952092 \\ 6 & -7.980408 & 1.359335 & 1.248657 \\ 6 & -7.460031 & -0.888003 & 2.220040 \\ 1 & -5.314354 & -2.332899 & -4.708526 \\ 1 & -4.221046 & -1.059774 & -4.157443 \\ 1 & -4.257764 & -2.627156 & -3.314659 \\ 1 & -7.303446 & -2.973464 & -3.532096 \\ 1 & -6.380695 & -3.332790 & -2.057866 \\ 1 & -7.790605 & -2.254074 & -1.994144 \\ 1 & -7.185853 & -0.684498 & -4.594587 \\ 1 & -7.629509 & 0.088807 & -3.060210 \\ 1 & -6.143631 & 0.574054 & -3.897520 \\ 1 & -6.951223 & 1.304822 & 3.728117 \\ 1 & -5.418487 & 0.484759 & 3.399056 \\ 1 & -5.749127 & 2.081203 & 2.685296 \\ 1 & -8.168990 & -0.721384 & 3.042416 \\ 1 & -7.999246 & -1.402189 & 1.414852 \\ 1 & -6.673691 & -1.564691 & 2.580886 \\ 1 & -8.697656 & 1.559373 & 2.055940 \\ 1 & -7.579625 & 2.322744 & 0.907764 \\ 1 & -8.539441 & 0.916364 & 0.415884\end{array}$

$3_{\text {IIA-si- }} \psi_{-1 \mathrm{a} 2 s 334 s}$ 
Number of imaginary frequencies : 0

The smallest frequencies are : $16.2537 \quad 18.2541 \quad 19.9029 \mathrm{~cm}(-1)$

Electronic energy :

$\mathrm{HF}=-4360.8805471$

Zero-point correction $=$

1.454229 (Hartree/Particle)

Thermal correction to Energy=

1.539048

Thermal correction to Enthalpy= $\quad 1.539993$

Thermal correction to Gibbs Free Energy=

Sum of electronic and zero-point Energies=

Sum of electronic and thermal Energies $=$

1.336029

$-4359.426319$

Sum of electronic and thermal Enthalpies=

$-4359.341499$

Sum of electronic and thermal Free Energies=

$-4359.340554$

$-4359.544518$

Cartesian Coordinates

$\begin{array}{cccc}6 & 2.483316 & 2.927804 & -2.857892 \\ 6 & 1.162517 & 2.906630 & -2.382990 \\ 6 & 0.787972 & 3.905574 & -1.470346 \\ 6 & 1.673533 & 4.907327 & -1.091968 \\ 6 & 2.968118 & 4.933872 & -1.604338 \\ 6 & 3.371205 & 3.927103 & -2.476756 \\ 6 & 0.130215 & 2.019645 & -2.987154 \\ 45 & -0.460592 & -0.000790 & -2.016734 \\ 6 & 0.485990 & 0.886968 & -3.752083 \\ 15 & 1.473758 & -0.802255 & -0.914896 \\ 7 & 1.207584 & -2.183860 & 0.042718 \\ 6 & 2.207779 & -2.861364 & 0.902974 \\ 6 & 2.748360 & -2.089469 & 2.080836 \\ 6 & 1.942027 & -1.274618 & 2.877928 \\ 6 & 2.463445 & -0.647308 & 4.001720 \\ 6 & 3.803791 & -0.815904 & 4.345994 \\ 6 & 4.615159 & -1.619765 & 3.554640 \\ 6 & 4.087499 & -2.253299 & 2.431669 \\ 8 & 2.657831 & -1.120097 & -2.050561 \\ 6 & 3.830888 & -1.749709 & -1.707495 \\ 6 & 4.789333 & -1.107147 & -0.945824 \\ 6 & 5.910171 & -1.888746 & -0.499075 \\ 6 & 6.068841 & -3.229356 & -0.964605 \\ 6 & 5.113495 & -3.778141 & -1.855133 \\ 6 & 4.004833 & -3.058871 & -2.204353 \\ 6 & 6.842023 & -1.407682 & 0.456921 \\ 6 & 7.877479 & -2.193205 & 0.900339 \\ 6 & 8.050785 & -3.503347 & 0.407890 \\ 6 & 7.159672 & -4.006892 & -0.504361 \\ 6 & 4.629270 & 0.317926 & -0.571690 \\ 6 & 5.677323 & 1.275372 & -0.796151 \\ 6 & 5.565917 & 2.586296 & -0.244533 \\ 6 & 4.385940 & 2.952353 & 0.446023 \\ 6 & 3.335223 & 2.085151 & 0.523997 \\ 6 & 3.458683 & 0.780435 & 0.006142 \\ 6 & 6.823111 & 0.985654 & -1.579771 \\ 6 & 7.805041 & 1.924844 & -1.782388 \\ 6 & 7.705088 & 3.207703 & -1.205792 \\ 6 & 6.602545 & 3.526734 & -0.454260 \\ 8 & 2.387781 & -0.039586 & 0.227447 \\ 6 & 0.092388 & -3.001745 & -0.337740 \\ 6 & -1.133516 & -2.855440 & 0.281752\end{array}$




\begin{tabular}{|c|c|c|c|}
\hline 6 & -2.244085 & -3.634892 & -0.170816 \\
\hline 6 & -2.053643 & -4.592004 & -1.211425 \\
\hline 6 & -0.761625 & -4.761998 & -1.763821 \\
\hline 6 & 0.277857 & -3.974552 & -1.347844 \\
\hline 6 & -3.157608 & -5.357413 & -1.660518 \\
\hline 6 & -4.401313 & -5.190260 & -1.105159 \\
\hline 6 & -4.592104 & -4.246414 & -0.072368 \\
\hline 6 & -3.541455 & -3.490988 & 0.385663 \\
\hline 6 & -1.294099 & -2.049073 & 1.523845 \\
\hline 6 & -1.606821 & -0.698358 & 1.549670 \\
\hline 6 & -1.865734 & -0.079969 & 2.806948 \\
\hline 6 & -1.758133 & -0.764290 & 3.985833 \\
\hline 6 & -1.382010 & -2.126314 & 3.996406 \\
\hline 6 & -1.166785 & -2.784953 & 2.752509 \\
\hline 6 & -0.797132 & -4.155638 & 2.784125 \\
\hline 6 & -0.628575 & -4.817872 & 3.975508 \\
\hline 6 & -0.833276 & -4.153931 & 5.202231 \\
\hline 6 & -1.207865 & -2.834702 & 5.208201 \\
\hline 15 & -1.793797 & 0.286409 & -0.026277 \\
\hline 6 & -3.610841 & 0.066567 & -0.277978 \\
\hline 6 & -4.514258 & 0.421828 & 0.720959 \\
\hline 6 & -5.883009 & 0.178259 & 0.581699 \\
\hline 6 & -6.321074 & -0.426277 & -0.598078 \\
\hline 6 & -5.442339 & -0.804017 & -1.620084 \\
\hline 6 & -4.083017 & -0.551036 & -1.436206 \\
\hline 6 & -6.818849 & 0.540678 & 1.733183 \\
\hline 6 & -6.425277 & -0.289040 & 2.961798 \\
\hline 6 & -5.974445 & -1.437602 & -2.905124 \\
\hline 6 & -6.981553 & -2.547243 & -2.588218 \\
\hline 6 & -1.650785 & 2.010822 & 0.596111 \\
\hline 6 & -0.617982 & 2.342781 & 1.485050 \\
\hline 6 & -0.500700 & 3.630549 & 1.998905 \\
\hline 6 & -1.364632 & 4.617760 & 1.498695 \\
\hline 6 & -2.359203 & 4.3380 & 0.563958 \\
\hline 6 & -2.506327 & 3.008256 & 0.141169 \\
\hline 6 & 0.488115 & 3.981250 & 3.112327 \\
\hline 6 & 1.558660 & 4.940703 & 2.584966 \\
\hline 6 & -3.306901 & 5.404381 & 0.017006 \\
\hline 6 & -3.177989 & 5.468586 & -1.508876 \\
\hline 6 & -0.267434 & 4.656523 & 4.264245 \\
\hline 6 & 1.169961 & 2.740473 & 3.688677 \\
\hline 6 & -4.748140 & 5.040521 & 0.392248 \\
\hline 6 & -3.002160 & 6.792906 & 0.572972 \\
\hline 6 & -0.905984 & -1.595711 & -3.034561 \\
\hline 8 & -1.228972 & -2.241785 & -3.940138 \\
\hline 6 & -6.691044 & 2.032783 & 2.058433 \\
\hline 6 & -8.282152 & 0.249605 & 1.410910 \\
\hline 6 & -6.666760 & -0.348857 & -3.733036 \\
\hline 6 & -4.855692 & -2.052660 & -3.745865 \\
\hline 1 & 6.710357 & -0.410642 & 0.868410 \\
\hline 1 & -2.145706 & 0.969375 & 2.840118 \\
\hline 1 & 7.264095 & -5.022957 & -0.882839 \\
\hline 1 & -1.373248 & -2.305713 & 6.145746 \\
\hline 1 & 6.490288 & 4.520890 & -0.021747 \\
\hline 1 & -3.704777 & -2.757021 & 1.173896 \\
\hline 1 & -5.581606 & -4.108922 & 0.360176 \\
\hline
\end{tabular}




\begin{tabular}{|c|c|c|c|}
\hline & 4.308751 & 3.955515 & 0.863272 \\
\hline & -1.954988 & -0.263049 & 4.933010 \\
\hline & 5.262436 & -4.788530 & -2.232162 \\
\hline & 8.664493 & 1.677479 & -2.401661 \\
\hline & 3.240396 & -3.459472 & -2.866882 \\
\hline & -2.996467 & -6.081049 & -2.458361 \\
\hline & -0.617246 & -5.506967 & -2.544759 \\
\hline & -0.335984 & -5.865325 & 3.972182 \\
\hline & 6.913103 & 0.007463 & -2.045133 \\
\hline & -0.637771 & -4.688843 & 1.849179 \\
\hline & 1.270198 & -4.074945 & -1.786964 \\
\hline & 8.490878 & 3.941210 & -1.370923 \\
\hline & 8.566525 & -1.801663 & 1.645511 \\
\hline & -5.243256 & -5.781509 & -1.459360 \\
\hline & -0.694713 & -4.689906 & 6.138263 \\
\hline & 8.879244 & -4.112416 & 0.761873 \\
\hline & 2.381728 & 2.366976 & 0.966232 \\
\hline & -4.146904 & 0.892340 & 1.631795 \\
\hline & -3.362773 & -0.845424 & -2.192608 \\
\hline & -7.384395 & -0.613488 & -0.730227 \\
\hline & -3.308421 & 2.746685 & -0.549745 \\
\hline & 0.062668 & 1.559221 & 1.817733 \\
\hline 1 & -1.258280 & 5.633716 & 1.872310 \\
\hline & 1.691243 & -3.752071 & 1.289057 \\
\hline I & -1.802683 & 0.468832 & -2.706961 \\
\hline & 3.049455 & -3.238865 & 0.304134 \\
\hline 1 & 4.733172 & -2.877786 & 1.812356 \\
\hline 1 & 0.904653 & -1.111495 & 2.593153 \\
\hline & 5.666595 & -1.754060 & 3.802593 \\
\hline 1 & 1.815298 & -0.021723 & 4.614331 \\
\hline & 4.211268 & -0.318613 & 5.223651 \\
\hline 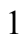 & 1.814556 & 3.030472 & 4.528418 \\
\hline ] & 0.431169 & 2.020281 & 4.068374 \\
\hline | & 1.806296 & 2.218110 & 2.963631 \\
\hline ] & 0.425012 & 4.887199 & 5.084731 \\
\hline | & -0.741454 & 5.597512 & 3.962125 \\
\hline l & -1.051735 & 3.997401 & 4.659586 \\
\hline & 2.285005 & 5.174795 & 3.375153 \\
\hline 1 & 2.102701 & 4.503470 & 1.737075 \\
\hline ] & 1.120672 & 5.888269 & 2.245359 \\
\hline l & -3.700561 & 7.522393 & 0.144110 \\
\hline 1 & -3.114042 & 6.835034 & 1.664121 \\
\hline 1 & -1.985730 & 7.120154 & 0.316424 \\
\hline 1 & -3.839934 & 6.246271 & -1.912978 \\
\hline 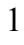 & -2.149018 & 5.711597 & -1.808132 \\
\hline 1 & -3.452978 & 4.521018 & -1.988102 \\
\hline 1 & -5.447336 & 5.785682 & -0.010617 \\
\hline 1 & -5.041936 & 4.059920 & -0.004128 \\
\hline 1 & -4.874549 & 5.013007 & 1.482758 \\
\hline 1 & -7.342221 & 2.295536 & 2.903006 \\
\hline 1 & -5.665982 & 2.312982 & 2.331595 \\
\hline 1 & -6.988461 & 2.648587 & 1.199267 \\
\hline 1 & -8.910695 & 0.532660 & 2.264665 \\
\hline 1 & -8.631851 & 0.820560 & 0.541044 \\
\hline 1 & -8.456342 & -0.816368 & 1.215785 \\
\hline 1 & -7.084337 & -0.057370 & 3.809534 \\
\hline
\end{tabular}




$\begin{array}{lrrr}1 & -6.510041 & -1.363350 & 2.749225 \\ 1 & -5.392252 & -0.095619 & 3.278146 \\ 1 & -5.285442 & -2.529696 & -4.635910 \\ 1 & -4.134457 & -1.303927 & -4.098658 \\ 1 & -4.304278 & -2.822470 & -3.187038 \\ 1 & -7.061868 & -0.768910 & -4.668164 \\ 1 & -7.504236 & 0.103931 & -3.186958 \\ 1 & -5.963193 & 0.453101 & -3.992429 \\ 1 & -7.332290 & -3.011255 & -3.519451 \\ 1 & -6.518643 & -3.330860 & -1.974061 \\ 1 & -7.867663 & -2.178177 & -2.058427 \\ 1 & -0.225503 & 3.910611 & -1.070175 \\ 1 & 1.336897 & 5.680171 & -0.401046 \\ 1 & 3.660449 & 5.725647 & -1.321710 \\ 1 & 4.384260 & 3.923949 & -2.876841 \\ 1 & 2.807815 & 2.181998 & -3.581223 \\ 1 & -0.108867 & 0.637240 & -4.631705 \\ 1 & 1.521959 & 0.554349 & -3.808268 \\ 6 & -1.125814 & 2.783856 & -3.369242 \\ 1 & -1.737778 & 3.053148 & -2.500000 \\ 1 & -0.862107 & 3.724282 & -3.878839 \\ 1 & -1.756194 & 2.192544 & -4.043231\end{array}$

$3_{\text {IIA-si- }} \psi_{-1}$ a2s3s4a

Number of imaginary frequencies :0

The smallest frequencies are :20.1023 $21.504923 .1581 \mathrm{~cm}(-1)$

Electronic energy : $\quad H F=-4360.8804629$

Zero-point correction $=\quad 1.455591$ (Hartree/Particle)

Thermal correction to Energy= $\quad 1.539825$

Thermal correction to Enthalpy= $\quad 1.540769$

Thermal correction to Gibbs Free Energy $=\quad 1.340025$

Sum of electronic and zero-point Energies $=\quad-4359.424872$

Sum of electronic and thermal Energies $=\quad-4359.340638$

Sum of electronic and thermal Enthalpies $=\quad-4359.339694$

Sum of electronic and thermal Free Energies $=\quad-4359.540438$

Cartesian Coordinates

$\begin{array}{cccc}6 & 2.507819 & 2.895521 & -2.809993 \\ 6 & 1.184941 & 2.918433 & -2.339214 \\ 6 & 0.840358 & 3.933834 & -1.432002 \\ 6 & 1.755530 & 4.913456 & -1.064618 \\ 6 & 3.049570 & 4.898864 & -1.577984 \\ 6 & 3.424736 & 3.870972 & -2.438149 \\ 6 & 0.128021 & 2.058366 & -2.941185 \\ 6 & -1.123481 & 2.846892 & -3.288857 \\ 45 & -0.467830 & 0.031254 & -2.007666 \\ 6 & 0.456046 & 0.937244 & -3.738411 \\ 15 & 1.474724 & -0.778012 & -0.928047 \\ 7 & 1.221900 & -2.172252 & 0.014028 \\ 6 & 2.228825 & -2.850292 & 0.866250 \\ 6 & 2.768327 & -2.084029 & 2.048456 \\ 6 & 1.961388 & -1.272728 & 2.848554 \\ 6 & 2.482457 & -0.647392 & 3.973553 \\ 6 & 3.823081 & -0.815977 & 4.317016\end{array}$




\begin{tabular}{|c|c|c|c|}
\hline 6 & 4.634638 & -1.617853 & 3.523895 \\
\hline 6 & 4.107391 & -2.248582 & 2.399078 \\
\hline 3 & 2.657808 & -1.070020 & -2.070313 \\
\hline & 3.832916 & -1.703224 & -1.740370 \\
\hline$b$ & 4.790929 & -1.072447 & -0.968531 \\
\hline 5 & 5.914726 & -1.859974 & -0.538850 \\
\hline 6 & 6.075225 & -3.191318 & -1.029632 \\
\hline 5 & 5.118774 & -3.726109 & -1.927406 \\
\hline 6 & 4.007829 & -3.002953 & -2.260890 \\
\hline 6 & 6.848138 & -1.395117 & 0.423612 \\
\hline 6 & 7.886821 & -2.186293 & 0.849199 \\
\hline 6 & 8.062095 & -3.486334 & 0.331374 \\
\hline 6 & 7.169302 & -3.974690 & -0.587488 \\
\hline 6 & 4.629387 & 0.344283 & -0.564416 \\
\hline 6 & 5.678944 & 1.305513 & -0.765962 \\
\hline 6 & 5.575421 & 2.599978 & -0.175300 \\
\hline 6 & 4.398508 & 2.950471 & 0.528748 \\
\hline 6 & 3.344009 & 2.086308 & 0.580162 \\
\hline 6 & 3.459670 & 0.797023 & 0.022838 \\
\hline 6 & 6.817280 & 1.036347 & -1.567317 \\
\hline 6 & 7.799823 & 1.978922 & -1.750933 \\
\hline 6 & 7.709198 & 3.243519 & -1.134090 \\
\hline 6 & 6.614109 & 3.542594 & -0.363565 \\
\hline 8 & 2.381680 & -0.018575 & 0.220994 \\
\hline 6 & 0.111453 & -2.995417 & -0.369819 \\
\hline 6 & -1.113821 & -2.864508 & 0.255141 \\
\hline 6 & -2.215856 & -3.659165 & -0.192686 \\
\hline 6 & -2.012195 & -4.629081 & -1.218757 \\
\hline 6 & -0.722473 & -4.775635 & -1.783051 \\
\hline 6 & 0.304692 & -3.964257 & -1.382239 \\
\hline 6 & -3.099328 & -5.434764 & -1.637722 \\
\hline 6 & -4.340692 & -5.289089 & -1.072912 \\
\hline 6 & -4.549861 & -4.318877 & -0.068819 \\
\hline 6 & -3.515039 & -3.527470 & 0.363503 \\
\hline 6 & -1.273470 & -2.072542 & 1.507277 \\
\hline 6 & -1.590678 & -0.723277 & 1.552450 \\
\hline 6 & -1.837171 & -0.118726 & 2.818815 \\
\hline 6 & -1.718179 & -0.816485 & 3.988561 \\
\hline 6 & -1.339258 & -2.177717 & 3.979734 \\
\hline 6 & -1.132368 & -2.821684 & 2.726594 \\
\hline 6 & -0.756370 & -4.191069 & 2.740254 \\
\hline 6 & -0.576398 & -4.866043 & 3.922751 \\
\hline 6 & -0.774688 & -4.216985 & 5.158505 \\
\hline 6 & -1.153553 & -2.899221 & 5.182111 \\
\hline 15 & -1.794889 & 0.274335 & -0.010425 \\
\hline 6 & -3.611666 & 0.048534 & -0.261350 \\
\hline 6 & -4.514809 & 0.318330 & 0.776843 \\
\hline 6 & -5.876034 & 0.084396 & 0.622063 \\
\hline 6 & -6.325772 & -0.401784 & -0.617973 \\
\hline 6 & -5.461070 & -0.661829 & -1.675059 \\
\hline 6 & -4.091290 & -0.450188 & -1.462934 \\
\hline 6 & -6.874589 & 0.299772 & 1.757848 \\
\hline 6 & -5.924317 & -1.171736 & -3.037708 \\
\hline 6 & -1.666192 & 1.995459 & 0.621618 \\
\hline 6 & -0.634931 & 2.340386 & 1.507142 \\
\hline 6 & -0.540108 & 3.626591 & 2.030243 \\
\hline
\end{tabular}




\begin{tabular}{|c|c|c|c|}
\hline & -1.429874 & 4.599354 & 1.546596 \\
\hline & -2.427115 & 4.305647 & 0.618965 \\
\hline & -2.544394 & 2.978571 & 0.180588 \\
\hline & 0.452565 & 3.988494 & 3.136999 \\
\hline & 1.519003 & 4.943844 & 2.594255 \\
\hline & -3.425862 & 5.345210 & 0.113516 \\
\hline & -3.361662 & 5.424351 & -1.415635 \\
\hline & -0.295493 & 4.673221 & 4.288094 \\
\hline & 1.139556 & 2.753529 & 3.720439 \\
\hline & -4.837635 & 4.928548 & 0.544384 \\
\hline & -3.147373 & 6.739029 & 0.669933 \\
\hline & -0.949721 & -1.553577 & -3.023352 \\
\hline & -1.317314 & -2.184873 & -3.922754 \\
\hline & 6.715331 & -0.406635 & 0.854693 \\
\hline & -2.119480 & 0.929544 & 2.865729 \\
\hline & 7.274985 & -4.983199 & -0.985308 \\
\hline & -1.313035 & -2.381087 & 6.126719 \\
\hline & 6.509065 & 4.523298 & 0.100254 \\
\hline & -3.689456 & -2.780013 & 1.136279 \\
\hline & -5.541474 & -4.189602 & 0.360797 \\
\hline & 4.325464 & 3.941178 & 0.975775 \\
\hline & -1.907353 & -0.326673 & 4.943245 \\
\hline & 5.268976 & -4.729196 & -2.322932 \\
\hline & 8.652635 & 1.748349 & -2.385678 \\
\hline 1 & 3.242605 & -3.393036 & -2.928743 \\
\hline & -2.926099 & -6.172550 & -2.419937 \\
\hline & -0.569075 & -5.526090 & -2.557011 \\
\hline & -0.279513 & -5.912144 & 3.905302 \\
\hline & 6.900022 & 0.072403 & -2.062848 \\
\hline 1 & -0.601216 & -4.713368 & 1.798488 \\
\hline & 1.295486 & -4.049200 & -1.828118 \\
\hline ] & 8.495934 & 3.979411 & -1.283372 \\
\hline & 8.576891 & -1.807113 & 1.599794 \\
\hline & -5.168956 & -5.912255 & -1.403362 \\
\hline 1 & -0.627195 & -4.762983 & 6.087349 \\
\hline & 8.893171 & -4.099899 & 0.671166 \\
\hline 1 & 2.390491 & 2.359918 & 1.027087 \\
\hline & -4.137300 & 0.713513 & 1.715864 \\
\hline 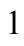 & -3.382678 & -0.678954 & -2.254286 \\
\hline ] & -7.391770 & -0.576737 & -0.749576 \\
\hline l & -3.346485 & 2.705433 & -0.505765 \\
\hline 1 & 0.062157 & 1.567531 & 1.830839 \\
\hline & -1.344405 & 5.613502 & 1.930246 \\
\hline 1 & 1.718682 & -3.746796 & 1.247297 \\
\hline & -1.806802 & 0.524509 & -2.683068 \\
\hline 1 & 3.070849 & -3.218446 & 0.262044 \\
\hline l & 4.753528 & -2.870717 & 1.777902 \\
\hline 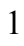 & 0.924283 & -1.109389 & 2.563341 \\
\hline 1 & 5.686107 & -1.752393 & 3.771673 \\
\hline 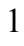 & 1.834292 & -0.022859 & 4.587306 \\
\hline 1 & 4.230617 & -0.320173 & 5.195486 \\
\hline ( & 1.783243 & 3.051191 & 4.558179 \\
\hline 1 & 0.404362 & 2.031867 & 4.104267 \\
\hline . & 1.778450 & 2.229977 & 2.998660 \\
\hline 1 & 0.402296 & 4.908098 & 5.102769 \\
\hline 1 & -0.768907 & 5.613486 & 3.983107 \\
\hline
\end{tabular}




$\begin{array}{rrrr}1 & -1.078886 & 4.018795 & 4.692737 \\ 1 & 2.248793 & 5.188705 & 3.378108 \\ 1 & 2.059075 & 4.497605 & 1.748537 \\ 1 & 1.076539 & 5.886069 & 2.245370 \\ 1 & -3.881611 & 7.448916 & 0.268910 \\ 1 & -3.226513 & 6.770356 & 1.764277 \\ 1 & -2.150467 & 7.100226 & 0.384961 \\ 1 & -4.070602 & 6.176979 & -1.786008 \\ 1 & -2.357096 & 5.711924 & -1.754867 \\ 1 & -3.617483 & 4.470106 & -1.892277 \\ 1 & -5.578150 & 5.654568 & 0.182512 \\ 1 & -5.115280 & 3.943498 & 0.147884 \\ 1 & -4.915187 & 4.882487 & 1.638898 \\ 1 & -0.171384 & 3.969291 & -1.026969 \\ 1 & 1.445254 & 5.703106 & -0.380864 \\ 1 & 3.763781 & 5.673679 & -1.303054 \\ 1 & 4.438625 & 3.832213 & -2.834943 \\ 1 & 2.812910 & 2.130578 & -3.521692 \\ 1 & -0.157321 & 0.717086 & -4.613287 \\ 1 & 1.486017 & 0.592737 & -3.823058 \\ 1 & -1.717766 & 3.109829 & -2.405445 \\ 1 & -0.855492 & 3.791990 & -3.787369 \\ 1 & -1.774020 & 2.275854 & -3.961889 \\ 6 & -7.445840 & -1.219723 & -3.151455 \\ 6 & -5.392878 & -0.247069 & -4.140140 \\ 6 & -5.379767 & -2.586629 & -3.256987 \\ 6 & -6.218417 & 0.909598 & 2.995126 \\ 6 & -7.999848 & 1.237087 & 1.308610 \\ 6 & -7.467222 & -1.058845 & 2.150438 \\ 1 & -7.729831 & -1.570915 & -4.151450 \\ 1 & -7.891289 & -1.911874 & -2.425169 \\ 1 & -7.899131 & -0.230173 & -3.007122 \\ 1 & -5.742449 & -0.591707 & -5.122468 \\ 1 & -5.746108 & 0.782613 & -3.997240 \\ 1 & -4.295789 & -0.224950 & -4.173821 \\ 1 & -5.673538 & -2.959886 & -4.247570 \\ 1 & -4.284207 & -2.621568 & -3.197242 \\ 1 & -5.775036 & -3.276479 & -2.500384 \\ 1 & -6.972797 & 1.053400 & 3.778862 \\ 1 & -5.435151 & 0.260680 & 3.409690 \\ 1 & -5.773962 & 1.890679 & 2.779311 \\ 1 & -8.178352 & -0.943353 & 2.979655 \\ 1 & -8.001991 & -1.529204 & 1.316065 \\ 1 & -6.677211 & -1.750064 & 2.473649 \\ 1 & -8.715539 & 1.388929 & 2.127623 \\ 1 & -7.604184 & 2.219817 & 1.021244 \\ 1 & -8.560252 & 0.839264 & 0.454466\end{array}$

$3_{\text {IIA-si- }} \Psi_{-1 \mathrm{a} 2 \mathrm{~s} 354 \mathrm{~s}}$

Number of imaginary frequencies : 0

The smallest frequencies are : $20.0447 \quad 21.8854 \quad 23.1823 \mathrm{~cm}(-1)$

Electronic energy : $\quad \mathrm{HF}=-4360.88042$

Zero-point correction $=\quad 1.455602($ Hartree/Particle $)$

Thermal correction to Energy= $\quad 1.539833$

Thermal correction to Enthalpy= $\quad 1.540778$ 
Thermal correction to Gibbs Free Energy= Sum of electronic and zero-point Energies= Sum of electronic and thermal Energies= Sum of electronic and thermal Enthalpies= Sum of electronic and thermal Free Energies=

\author{
1.339856 \\ $-4359.424818$ \\ $-4359.340587$ \\ $-4359.339642$ \\ $-4359.540564$
}

Cartesian Coordinates

\begin{tabular}{|c|c|c|c|}
\hline 6 & 2.516976 & 2.902257 & -2.796090 \\
\hline 6 & 1.193943 & 2.925565 & -2.325848 \\
\hline 6 & 0.850458 & 3.938185 & -1.415108 \\
\hline 6 & 1.766669 & 4.915576 & -1.044545 \\
\hline 6 & 3.060943 & 4.900840 & -1.557352 \\
\hline 6 & 3.435167 & 3.875098 & -2.420482 \\
\hline 6 & 0.135750 & 2.070290 & -2.932293 \\
\hline 6 & -1.113092 & 2.863671 & -3.278587 \\
\hline 45 & -0.467666 & 0.041855 & -2.008154 \\
\hline 6 & 0.461912 & 0.951606 & -3.733690 \\
\hline 15 & 1.471416 & -0.778350 & -0.931264 \\
\hline 7 & 1.214374 & -2.175938 & 0.004431 \\
\hline 6 & 2.216679 & -2.858906 & 0.858151 \\
\hline 6 & 2.757845 & -2.094858 & 2.041128 \\
\hline 6 & 1.952802 & -1.280995 & 2.840638 \\
\hline 6 & 2.475387 & -0.655210 & 3.964611 \\
\hline 6 & 3.815689 & -0.826540 & 4.308147 \\
\hline 6 & 4.625180 & -1.631559 & 3.516147 \\
\hline 6 & 4.096439 & -2.262305 & 2.391983 \\
\hline 8 & 2.655573 & -1.067891 & -2.072876 \\
\hline 6 & 3.828980 & -1.704287 & -1.742862 \\
\hline 6 & 4.786979 & -1.077370 & -0.967868 \\
\hline 6 & 5.909136 & -1.867958 & -0.539438 \\
\hline 6 & 6.068068 & -3.198058 & -1.034093 \\
\hline 6 & 5.111599 & -3.728793 & -1.934250 \\
\hline 6 & 4.002178 & -3.002876 & -2.266764 \\
\hline 6 & 6.842312 & -1.407501 & 0.425335 \\
\hline 6 & 7.879408 & -2.201545 & 0.849490 \\
\hline 6 & 8.053213 & -3.500258 & 0.327855 \\
\hline 6 & 7.160527 & -3.984432 & -0.593333 \\
\hline 6 & 4.626875 & 0.338121 & -0.558710 \\
\hline 6 & 5.677826 & 1.298866 & -0.755011 \\
\hline 6 & 5.575542 & 2.590773 & -0.158514 \\
\hline 6 & 4.398618 & 2.939554 & 0.546487 \\
\hline 6 & 3.342900 & 2.076597 & 0.593065 \\
\hline 6 & 3.457165 & 0.789947 & 0.029204 \\
\hline 6 & 6.816258 & 1.031821 & -1.556913 \\
\hline 6 & 7.800070 & 1.973980 & -1.735783 \\
\hline 6 & 7.710717 & 3.235851 & -1.113209 \\
\hline 6 & 6.615622 & 3.532826 & -0.341868 \\
\hline 8 & 2.377712 & -0.024778 & 0.221790 \\
\hline 6 & 0.101566 & -2.993802 & -0.383757 \\
\hline 6 & -1.123190 & -2.861731 & 0.241838 \\
\hline 6 & -2.228678 & -3.648768 & -0.210173 \\
\hline 6 & -2.029377 & -4.613090 & -1.242283 \\
\hline 6 & -0.740100 & -4.762194 & -1.807048 \\
\hline 6 & 0.290826 & -3.957857 & -1.401419 \\
\hline 6 & -3.120457 & -5.410544 & -1.666621 \\
\hline
\end{tabular}




\begin{tabular}{|c|c|c|c|}
\hline 6 & -4.361047 & -5.262185 & -1.100820 \\
\hline 6 & -4.565329 & -4.298019 & -0.089856 \\
\hline & -3.526844 & -3.514453 & 0.347878 \\
\hline & -1.280385 & -2.075481 & 1.497528 \\
\hline & -1.595094 & -0.725907 & 1.547978 \\
\hline & -1.840081 & -0.126185 & 2.816842 \\
\hline b & -1.723516 & -0.829556 & 3.983731 \\
\hline o & -1.348814 & -2.191863 & 3.969203 \\
\hline 6 & -1.142116 & -2.830489 & 2.713386 \\
\hline 6 & -0.770389 & -4.201072 & 2.721028 \\
\hline 6 & -0.594412 & -4.882206 & 3.900579 \\
\hline 6 & -0.792466 & -4.238375 & 5.139132 \\
\hline 6 & -1.167056 & -2.919515 & 5.168465 \\
\hline 15 & -1.798023 & 0.276906 & -0.011697 \\
\hline 6 & -3.614270 & 0.047577 & -0.267628 \\
\hline 6 & -4.516680 & 0.286294 & 0.771370 \\
\hline 6 & -5.881596 & 0.048814 & 0.614651 \\
\hline 6 & -6.332280 & -0.404117 & -0.631844 \\
\hline 6 & -5.461302 & -0.637103 & -1.696838 \\
\hline 6 & -4.095928 & -0.425875 & -1.482895 \\
\hline 6 & -6.810548 & 0.255154 & \\
\hline 6 & -5.924535 & -1.132474 & -3.065349 \\
\hline 6 & -1.676114 & 1.996719 & 0.625540 \\
\hline 6 & -0.646310 & 2.342045 & 1.512510 \\
\hline 6 & -0.552519 & 3.628117 & 2.035935 \\
\hline 6 & -1.440953 & 4.600997 & 1.549873 \\
\hline 6 & -2.437081 & 4.306975 & 0.620972 \\
\hline 6 & -2.555077 & & \\
\hline 6 & 0.438780 & 3.989273 & 3.144234 \\
\hline 6 & 1.508292 & 4.941827 & 2.602748 \\
\hline 6 & -3.428954 & 5.348673 & 0.105974 \\
\hline 6 & -3.338433 & 5.435725 & -1.421526 \\
\hline 6 & -0.310100 & 4.676360 & 4.293320 \\
\hline 6 & 1.123209 & 2.753700 & 3.729575 \\
\hline 6 & -4.848011 & 4.929543 & 0.509008 \\
\hline 6 & -3.160768 & 6.739448 & 0.674751 \\
\hline 6 & -0.954619 & -1.536636 & -3.031800 \\
\hline 8 & -1.322508 & -2.162158 & -3.935051 \\
\hline 1 & 6.710697 & -0.420110 & 0.859234 \\
\hline 1 & -2.118219 & 0.922923 & 2.867881 \\
\hline 1 & 7.265059 & -4.991891 & -0.994106 \\
\hline 1 & -1.326292 & -2.405339 & 6.115264 \\
\hline 1 & 6.511633 & 4.511546 & 0.126352 \\
\hline 1 & -3.696972 & -2.771234 & 1.126259 \\
\hline 1 & -5.556062 & -4.168553 & 0.341526 \\
\hline 1 & 4.326678 & 3.928126 & 0.998415 \\
\hline 1 & -1.911176 & -0.343300 & 4.940517 \\
\hline 1 & 5.260577 & -4.731002 & -2.332451 \\
\hline 1 & 8.652903 & 1.745232 & -2.371161 \\
\hline 1 & 3.236979 & -3.389768 & -2.936502 \\
\hline 1 & -2.951008 & -6.144077 & -2.453646 \\
\hline 1 & -0.590018 & -5.508696 & -2.585475 \\
\hline 1 & -0.300989 & -5.929199 & 3.878637 \\
\hline 1 & 6.897948 & 0.070022 & -2.056784 \\
\hline 1 & -0.615727 & -4.719433 & 1.777001 \\
\hline 1 & 1.281274 & -4.044527 & -1.847767 \\
\hline
\end{tabular}




\begin{tabular}{|c|c|c|c|}
\hline 1 & 8.498471 & 3.971409 & -1.258735 \\
\hline 1 & 8.569369 & -1.825666 & 1.601844 \\
\hline & -5.192484 & -5.878944 & -1.435305 \\
\hline & -0.648187 & -4.789271 & 6.065579 \\
\hline 1 & 8.883040 & -4.116129 & 0.666522 \\
\hline | & 2.389545 & 2.349178 & 1.041073 \\
\hline 1 & -4.145836 & 0.652388 & 1.727716 \\
\hline 1 & -3.386717 & -0.634693 & -2.278985 \\
\hline 1 & -7.395046 & -0.578239 & -0.773307 \\
\hline 1 & -3.355920 & 2.705668 & -0.503311 \\
\hline 1 & 0.050419 & 1.569486 & 1.837463 \\
\hline 1 & -1.355288 & 5.615569 & 1.932402 \\
\hline 1 & 1.701668 & -3.753150 & 1.238066 \\
\hline 1 & -1.803628 & 0.543928 & -2.682574 \\
\hline 1 & 3.057948 & -3.230641 & 0.254993 \\
\hline 1 & 4.741261 & -2.886279 & 1.771269 \\
\hline 1 & 0.916282 & -1.115025 & 2.554903 \\
\hline 1 & 5.676336 & -1.768325 & 3.763993 \\
\hline 1 & 1.828802 & -0.027898 & 4.577238 \\
\hline 1 & 4.224581 & -0.330384 & 5.185787 \\
\hline 1 & 1.765695 & 3.051127 & 4.568310 \\
\hline 1 & 0.386952 & 2.032579 & 4.112387 \\
\hline 1 & 1.763042 & 2.229557 & 3.008963 \\
\hline 1 & 0.386564 & 4.910589 & 5.109134 \\
\hline 1 & -0.781058 & 5.617280 & 3.986550 \\
\hline 1 & -1.095592 & 4.023899 & 4.697050 \\
\hline 1 & 2.235142 & 5.188364 & 3.388809 \\
\hline 1 & 2.051343 & 4.491930 & 1.760843 \\
\hline 1 & 1.068320 & 5.883397 & 2.248971 \\
\hline 1 & -3.892285 & 7.449948 & 0.269844 \\
\hline 1 & -3.252689 & 6.762888 & 1.768315 \\
\hline 1 & -2.161807 & 7.105665 & 0.403799 \\
\hline 1 & -4.040789 & 6.190462 & -1.800131 \\
\hline 1 & -2.328194 & 5.724481 & -1.742415 \\
\hline 1 & -3.586405 & 4.483623 & -1.906625 \\
\hline 1 & -5.581929 & 5.654683 & 0.132220 \\
\hline 1 & -5.115896 & 3.943962 & 0.106955 \\
\hline 1 & -4.947415 & 4.883671 & 1.601634 \\
\hline 1 & -0.161390 & 3.973564 & -1.010246 \\
\hline 1 & 1.457031 & 5.703549 & -0.358606 \\
\hline 1 & 3.775976 & 5.673942 & -1.279787 \\
\hline 1 & 4.449241 & 3.836098 & -2.816794 \\
\hline 1 & 2.821195 & 2.139327 & -3.510331 \\
\hline 1 & -0.150558 & 0.736894 & -4.610554 \\
\hline 1 & 1.491130 & 0.604776 & -3.818090 \\
\hline 1 & -1.708012 & 3.123738 & -2.394817 \\
\hline 1 & -0.841893 & 3.810477 & -3.772110 \\
\hline 1 & -1.763928 & 2.297471 & -3.955449 \\
\hline 6 & -7.446142 & -1.166547 & -3.185736 \\
\hline 6 & -5.381614 & -0.207996 & -4.162116 \\
\hline 6 & -5.391467 & -2.551529 & -3.288544 \\
\hline 6 & -8.271782 & -0.030011 & 1.472797 \\
\hline 6 & -6.385122 & -0.701548 & 2.931372 \\
\hline 6 & -6.711463 & 1.702234 & 2.304385 \\
\hline 1 & -7.728639 & -1.504539 & -4.190712 \\
\hline 1 & -7.901086 & -1.862713 & -2.469356 \\
\hline
\end{tabular}




$\begin{array}{lrrr}1 & -7.891859 & -0.174890 & -3.032531 \\ 1 & -5.730749 & -0.545716 & -5.146989 \\ 1 & -5.726832 & 0.824005 & -4.016327 \\ 1 & -4.284380 & -0.194559 & -4.192615 \\ 1 & -5.687182 & -2.920001 & -4.280407 \\ 1 & -4.296227 & -2.594656 & -3.226921 \\ 1 & -5.792489 & -3.240507 & -2.533841 \\ 1 & -8.893468 & 0.135532 & 2.361776 \\ 1 & -8.647481 & 0.631313 & 0.681376 \\ 1 & -8.425778 & -1.069302 & 1.155081 \\ 1 & -7.351553 & 1.848311 & 3.184935 \\ 1 & -5.688307 & 1.976273 & 2.589603 \\ 1 & -7.042042 & 2.404950 & 1.528265 \\ 1 & -7.036069 & -0.580540 & 3.807886 \\ 1 & -6.455529 & -1.746310 & 2.599336 \\ 1 & -5.351164 & -0.527046 & 3.255760\end{array}$

$3_{\text {IIA-si- }} \psi_{-1 \mathbf{1 s 2 a 3 a 4 a}}$

Number of imaginary frequencies : 0

The smallest frequencies are : $19.6880 \quad 21.451922 .7391 \mathrm{~cm}(-1)$

Electronic energy : $\quad H F=-4360.8795815$

Zero-point correction $=\quad 1.455534($ Hartree/Particle $)$

Thermal correction to Energy= $\quad 1.539833$

Thermal correction to Enthalpy= $\quad 1.540777$

Thermal correction to Gibbs Free Energy= 1.339999

Sum of electronic and zero-point Energies $=\quad-4359.424048$

Sum of electronic and thermal Energies $=\quad-4359.339748$

Sum of electronic and thermal Enthalpies $=\quad-4359.338804$

Sum of electronic and thermal Free Energies $=\quad-4359.539583$

Cartesian Coordinates

$\begin{array}{cccc}6 & 2.501702 & 3.057729 & -2.643786 \\ 6 & 1.163026 & 3.014553 & -2.222595 \\ 6 & 0.745429 & 3.986186 & -1.299564 \\ 6 & 1.610905 & 4.975948 & -0.848869 \\ 6 & 2.924915 & 5.024834 & -1.307011 \\ 6 & 3.365775 & 4.051274 & -2.199001 \\ 6 & 0.163012 & 2.131165 & -2.884066 \\ 6 & -1.089210 & 2.888206 & -3.291361 \\ 45 & -0.399915 & 0.075902 & -1.976426 \\ 6 & 0.558190 & 1.025269 & -3.669652 \\ 15 & 1.546852 & -0.723878 & -0.891609 \\ 7 & 1.286040 & -2.096872 & 0.080001 \\ 6 & 2.275932 & -2.739612 & 0.977977 \\ 6 & 2.794129 & -1.914701 & 2.130112 \\ 6 & 1.972531 & -1.057950 & 2.865375 \\ 6 & 2.478311 & -0.344249 & 3.943024 \\ 6 & 3.817608 & -0.476352 & 4.308819 \\ 6 & 4.641072 & -1.331780 & 3.586772 \\ 6 & 4.129613 & -2.046366 & 2.505312 \\ 8 & 2.708665 & -1.057980 & -2.046911 \\ 6 & 3.864213 & -1.727687 & -1.718315 \\ 6 & 4.848497 & -1.120205 & -0.961371 \\ 6 & 5.942420 & -1.941304 & -0.517789\end{array}$




\begin{tabular}{|c|c|c|c|}
\hline & 6.052677 & -3.285802 & -0.985497 \\
\hline & 5.078729 & -3.797150 & -1.877987 \\
\hline & 3.994498 & -3.039070 & -2.222651 \\
\hline & 6.891930 & -1.495577 & 0.438227 \\
\hline & 7.898085 & -2.318849 & 0.880680 \\
\hline & 8.024491 & -3.633241 & 0.384983 \\
\hline & 7.115717 & -4.102404 & -0.528225 \\
\hline & 4.740102 & 0.305701 & -0.574640 \\
\hline & 5.824108 & 1.225322 & -0.785837 \\
\hline & 5.775302 & 2.524377 & -0.196583 \\
\hline & 4.627257 & 2.912271 & 0.535346 \\
\hline & 3.545129 & 2.084799 & 0.604620 \\
\hline & 3.596075 & 0.802521 & 0.026760 \\
\hline & 6.949794 & 0.906844 & -1.587460 \\
\hline & 7.969268 & 1.807973 & -1.776214 \\
\hline & 7.928350 & 3.079507 & -1.168147 \\
\hline & 6.847799 & 3.425833 & -0.396884 \\
\hline & 2.480112 & 0.042514 & 0.227399 \\
\hline & 0.182881 & -2.933550 & -0.294965 \\
\hline & -1.043470 & -2.807125 & 0.328847 \\
\hline & -2.140758 & -3.608634 & -0.117588 \\
\hline & -1.936898 & -4.565844 & -1.155442 \\
\hline & -0.644295 & -4.715134 & -1.711056 \\
\hline & 0.382061 & -3.907924 & -1.300556 \\
\hline & -3.027106 & -5.357260 & -1.593640 \\
\hline 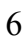 & -4.271242 & -5.210776 & -1.033917 \\
\hline & -4.477724 & -4.260249 & -0.009945 \\
\hline & -3.439063 & -3.484823 & 0.440985 \\
\hline & -1.212769 & -1.997559 & 1.568539 \\
\hline & -1.553702 & -0.652903 & 1.586890 \\
\hline ) & -1.822477 & -0.031124 & 2.839366 \\
\hline & -1.694849 & -0.704594 & 4.022880 \\
\hline 5 & -1.290834 & -2.058449 & 4.041451 \\
\hline & -1.067420 & -2.722006 & 2.801170 \\
\hline 5 & -0.678209 & -4.087347 & 2.840617 \\
\hline 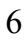 & -0.499933 & -4.739939 & 4.035757 \\
\hline 5 & -0.712880 & -4.071283 & 5.258756 \\
\hline 6 & -1.105271 & -2.757333 & 5.257283 \\
\hline 15 & -1.782296 & 0.294572 & -0.003263 \\
\hline 6 & -3.574842 & -0.075242 & -0.270528 \\
\hline 6 & -4.506754 & 0.188260 & 0.738792 \\
\hline 6 & -5.845936 & -0.161761 & 0.588127 \\
\hline 6 & -6.229879 & -0.778347 & -0.610462 \\
\hline 6 & -5.329101 & -1.060291 & -1.636219 \\
\hline 6 & -3.991233 & -0.699219 & -1.441084 \\
\hline 6 & -6.880922 & 0.071811 & 1.686940 \\
\hline 6 & -5.807359 & -1.687791 & -2.945470 \\
\hline 6 & -1.806699 & 2.045542 & 0.563283 \\
\hline 6 & -0.867362 & 2.532853 & 1.472226 \\
\hline 6 & -0.901328 & 3.867394 & 1.900414 \\
\hline 6 & -1.822614 & 4.719523 & 1.293508 \\
\hline 6 & -2.727185 & 4.281700 & 0.315693 \\
\hline 0 & -2.724907 & 2.929263 & -0.016211 \\
\hline 6 & -0.017922 & 4.321123 & 3.064383 \\
\hline 6 & -3.662331 & 5.288690 & -0.350960 \\
\hline 6 & -0.765561 & -1.519506 & -3.020523 \\
\hline
\end{tabular}




\begin{tabular}{|c|c|c|c|}
\hline 8 & -1.024007 & -2.177270 & -3.938242 \\
\hline ] & 6.797445 & -0.494932 & 0.850808 \\
\hline & -2.138198 & 1.008295 & 2.863584 \\
\hline & 7.183984 & -5.120667 & -0.909016 \\
\hline & -1.277550 & -2.225359 & 6.191868 \\
\hline & 6.783458 & 4.411792 & 0.063013 \\
\hline & -3.612532 & -2.746998 & 1.222758 \\
\hline & -5.469858 & -4.134402 & 0.420627 \\
\hline & 4.598835 & 3.899452 & 0.995006 \\
\hline & -1.902673 & -0.201562 & 4.966951 \\
\hline & 5.193966 & -4.809474 & -2.261515 \\
\hline & 8.812205 & 1.539899 & -2.409395 \\
\hline & 3.219928 & -3.409463 & -2.890860 \\
\hline & -2.854431 & -6.084636 & -2.385658 \\
\hline & -0.487878 & -5.462316 & -2.487536 \\
\hline & -0.193491 & -5.783427 & 4.038631 \\
\hline & 6.993385 & -0.062535 & -2.077558 \\
\hline & -0.513180 & -4.623732 & 1.908410 \\
\hline & 1.375507 & -3.996498 & -1.738288 \\
\hline & 8.742639 & 3.783449 & -1.323697 \\
\hline & 8.600659 & -1.953585 & 1.626523 \\
\hline & -5.101735 & -5.823888 & -1.378204 \\
\hline & -0.566742 & -4.599886 & 6.197822 \\
\hline & 8.830693 & -4.272435 & 0.737219 \\
\hline & 2.619830 & 2.376018 & 1.091711 \\
\hline & -4.173866 & 0.673504 & 1.652498 \\
\hline & -3.248172 & -0.915057 & -2.202176 \\
\hline & -7.277142 & -1.049508 & -0.741944 \\
\hline & -3.440818 & 2.541739 & -0.738403 \\
\hline & -0.124502 & 1.850061 & 1.890199 \\
\hline & -1.846551 & 5.766490 & 1.586039 \\
\hline & 1.757368 & -3.617688 & 1.389448 \\
\hline & -1.747189 & 0.526607 & -2.674366 \\
\hline & 3.128001 & -3.133339 & 0.403798 \\
\hline & 4.786235 & -2.704544 & 1.934215 \\
\hline & 0.938202 & -0.923613 & 2.557604 \\
\hline & 5.691178 & -1.440314 & 3.852948 \\
\hline & 1.819579 & 0.320969 & 4.500899 \\
\hline & 4.215036 & 0.088664 & 5.149308 \\
\hline & -0.285240 & 3.983323 & -0.948533 \\
\hline . & 1.239639 & 5.727817 & -0.151791 \\
\hline & 3.600367 & 5.810929 & -0.973271 \\
\hline & 4.391932 & 4.069689 & -2.562978 \\
\hline 1 & 2.860644 & 2.334969 & -3.374400 \\
\hline & -0.012734 & 0.788268 & -4.568465 \\
\hline 1 & 1.601318 & 0.714025 & -3.714250 \\
\hline & -1.716904 & 3.164185 & -2.434815 \\
\hline 1 & -0.815292 & 3.823897 & -3.804291 \\
\hline 1 & -1.706644 & 2.291878 & -3.972692 \\
\hline 5 & -4.644974 & -2.234897 & -3.774055 \\
\hline 6 & -6.778132 & -2.842500 & -2.681925 \\
\hline 5 & -6.523961 & -0.606158 & -3.762575 \\
\hline 6 & -6.289358 & 0.798274 & 2.893143 \\
\hline & -8.038736 & 0.916046 & 1.144692 \\
\hline 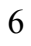 & -7.413618 & -1.285372 & 2.161093 \\
\hline & -5.034592 & -2.724970 & -4.675398 \\
\hline
\end{tabular}




\begin{tabular}{lrrr}
1 & -3.961784 & -1.443971 & -4.109192 \\
1 & -4.060600 & -2.978493 & -3.213437 \\
1 & -7.084857 & -3.295741 & -3.633718 \\
1 & -6.303987 & -3.623705 & -2.073326 \\
1 & -7.693096 & -2.519968 & -2.171282 \\
1 & -6.876451 & -1.015697 & -4.719225 \\
1 & -7.393368 & -0.205292 & -3.225692 \\
1 & -5.849308 & 0.232206 & -3.980667 \\
1 & -7.067536 & 0.944514 & 3.652786 \\
1 & -5.476769 & 0.226590 & 3.361571 \\
1 & -5.900822 & 1.789359 & 2.623220 \\
1 & -8.158344 & -1.149549 & 2.957083 \\
1 & -7.893079 & -1.845545 & 1.348788 \\
1 & -6.599983 & -1.905572 & 2.561103 \\
1 & -8.782262 & 1.093158 & 1.933346 \\
1 & -7.681783 & 1.892236 & 0.791383 \\
1 & -8.555410 & 0.427028 & 0.310360 \\
6 & -4.664081 & 4.617550 & -1.288503 \\
6 & -4.455836 & 6.057151 & 0.711436 \\
6 & -2.816846 & 6.272191 & -1.169460 \\
6 & -0.089184 & 5.828748 & 3.299340 \\
6 & -0.510049 & 3.616303 & 4.335627 \\
6 & 1.445538 & 3.946155 & 2.826404 \\
1 & 0.569850 & 6.100759 & 4.133243 \\
1 & 0.239220 & 6.398777 & 2.420190 \\
1 & -1.100889 & 6.159910 & 3.565311 \\
1 & 0.083268 & 3.932492 & 5.204588 \\
1 & -1.562532 & 3.856230 & 4.537605 \\
1 & -0.422424 & 2.524886 & 4.251565 \\
1 & 2.072509 & 4.332761 & 3.640940 \\
1 & 1.590604 & 2.859314 & 2.801335 \\
1 & 1.814207 & 4.367262 & 1.880429 \\
1 & -3.458006 & 7.029133 & -1.641419 \\
1 & -2.080633 & 6.796176 & -0.546665 \\
1 & -2.266706 & 5.752652 & -1.965687 \\
1 & -5.321501 & 5.377694 & -1.728915 \\
1 & -4.171313 & 4.094239 & -2.117950 \\
1 & -5.300131 & 3.895398 & -0.758800 \\
1 & -5.142225 & 6.766676 & 0.230549 \\
1 & -5.054715 & 5.373026 & 1.326908 \\
\hline & -3.809399 & 6.634044 & 1.383181
\end{tabular}

$3_{\text {IIA-si- }} \psi_{-1 s 223 a 4 s}$

Number of imaginary frequencies : 0

The smallest frequencies are : $18.3367 \quad 21.1850 \quad 22.9943 \mathrm{~cm}(-1)$

Electronic energy : $\quad H F=-4360.880077$

Zero-point correction $=\quad 1.455460($ Hartree/Particle $)$

Thermal correction to Energy $=\quad 1.539805$

Thermal correction to Enthalpy= $\quad 1.540750$

Thermal correction to Gibbs Free Energy $=\quad 1.339828$

Sum of electronic and zero-point Energies $=\quad-4359.424617$

Sum of electronic and thermal Energies $=\quad-4359.340272$

Sum of electronic and thermal Enthalpies $=\quad-4359.339327$

Sum of electronic and thermal Free Energies $=\quad-4359.540249$ 
Cartesian Coordinates

\begin{tabular}{|c|c|c|c|}
\hline 6 & -2.742399 & 2.877962 & -0.020136 \\
\hline 6 & -1.824793 & 1.998836 & 0.568163 \\
\hline 6 & -0.896620 & 2.492263 & 1.484960 \\
\hline 6 & -0.944965 & 3.825998 & 1.915590 \\
\hline 6 & -1.866979 & 4.672163 & 1.302236 \\
\hline 6 & -2.754193 & 4.230328 & 0.310403 \\
\hline 15 & -1.785517 & 0.247620 & 0.000380 \\
\hline 6 & -3.576247 & -0.121226 & -0.275224 \\
\hline 6 & -4.515550 & 0.190774 & 0.704023 \\
\hline 6 & -5.867277 & -0.120741 & 0.539217 \\
\hline 6 & -6.249489 & -0.759106 & -0.642044 \\
\hline 6 & -5.331200 & -1.101903 & -1.641643 \\
\hline 6 & -3.990941 & -0.774015 & -1.435763 \\
\hline 6 & -6.845972 & 0.233593 & 1.656609 \\
\hline 6 & -5.807660 & -1.768023 & -2.932049 \\
\hline 6 & -0.071340 & 4.285621 & 3.084347 \\
\hline 6 & 1.397386 & 3.932214 & 2.846151 \\
\hline 6 & -3.684036 & 5.233832 & -0.368118 \\
\hline 6 & -2.831441 & 6.292008 & -1.079224 \\
\hline 45 & -0.407004 & 0.036558 & -1.974150 \\
\hline 6 & -0.755695 & -1.570169 & -3.006736 \\
\hline 8 & -1.008053 & -2.234826 & -3.921203 \\
\hline 6 & 0.114204 & 2.094146 & -2.903196 \\
\hline 6 & 0.529585 & 0.988639 & -3.678618 \\
\hline 6 & 1.097051 & 3.001747 & -2.249389 \\
\hline 6 & 2.434020 & 3.067536 & -2.672533 \\
\hline 6 & 3.280160 & 4.078773 & -2.233142 \\
\hline 6 & 2.823211 & 5.046785 & -1.343166 \\
\hline 6 & 1.511285 & 4.974805 & -0.881830 \\
\hline 6 & 0.662990 & 3.968816 & -1.329082 \\
\hline 6 & -1.153805 & 2.822270 & -3.314149 \\
\hline 15 & 1.554133 & -0.725926 & -0.885981 \\
\hline 8 & 2.480599 & 0.054517 & 0.229880 \\
\hline 6 & 3.577552 & 0.839109 & 0.017292 \\
\hline 6 & 4.729234 & 0.360643 & -0.584246 \\
\hline 6 & 5.794237 & 1.299608 & -0.805627 \\
\hline 6 & 5.720307 & 2.602502 & -0.227566 \\
\hline 6 & 4.566260 & 2.973814 & 0.503531 \\
\hline 6 & 3.501342 & 2.125125 & 0.583756 \\
\hline 6 & 6.774159 & 3.523517 & -0.437887 \\
\hline 6 & 7.860261 & 3.192016 & -1.207863 \\
\hline 6 & 7.925830 & 1.916114 & -1.804580 \\
\hline 6 & 6.924803 & 0.996521 & -1.606261 \\
\hline 6 & 4.862787 & -1.065634 & -0.961613 \\
\hline 6 & 3.887564 & -1.695265 & -1.712262 \\
\hline 6 & 4.040721 & -3.006779 & -2.209705 \\
\hline 6 & 5.139202 & -3.743183 & -1.863352 \\
\hline 6 & 6.105663 & -3.209343 & -0.975899 \\
\hline 6 & 5.972297 & -1.864233 & -0.515790 \\
\hline 6 & 7.184215 & -4.004065 & -0.516319 \\
\hline 6 & 8.086393 & -3.513271 & 0.391965 \\
\hline 6 & 7.937512 & -2.198508 & 0.880300 \\
\hline 6 & 6.915831 & -1.395927 & 0.435488 \\
\hline 8 & 2.719044 & -1.049110 & -2.041763 \\
\hline
\end{tabular}




\begin{tabular}{|c|c|c|c|}
\hline 7 & 1.313050 & -2.098201 & 0.091869 \\
\hline 6 & 0.223583 & -2.954128 & -0.279872 \\
\hline & -1.004422 & -2.846872 & 0.344248 \\
\hline & -2.087809 & -3.670051 & -0.097053 \\
\hline & -1.868460 & -4.626004 & -1.133076 \\
\hline & -0.574264 & -4.753501 & -1.690357 \\
\hline & 0.438411 & -3.927801 & -1.282958 \\
\hline & -2.944386 & -5.438512 & -1.567730 \\
\hline & -4.189031 & -5.316604 & -1.003448 \\
\hline & -4.409722 & -4.370860 & 0.021804 \\
\hline & -3.386453 & -3.571643 & 0.466511 \\
\hline & -1.190205 & -2.035423 & 1.580109 \\
\hline & -1.555421 & -0.697078 & 1.592095 \\
\hline f & -1.843820 & -0.077074 & 2.841051 \\
\hline & -1.710486 & -0.744422 & 4.027461 \\
\hline & -1.281102 & -2.090299 & 4.052745 \\
\hline$b$ & -1.038238 & -2.753242 & 2.815900 \\
\hline & -0.624042 & -4.110999 & 2.861804 \\
\hline D & -0.440344 & -4.756511 & 4.059944 \\
\hline & -0.672803 & -4.088280 & 5.279611 \\
\hline & -1.089539 & -2.781871 & 5.271825 \\
\hline & 2.315608 & -2.722937 & 0.988642 \\
\hline f & 2.820203 & -1.887077 & 2.138732 \\
\hline & 1.982228 & -1.048604 & 2.876519 \\
\hline 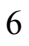 & 2.475634 & -0.323760 & 3.952432 \\
\hline$b$ & 3.819010 & -0.425268 & 4.313078 \\
\hline 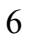 & 4.658973 & -1.261855 & 3.587947 \\
\hline f & 4.159680 & -1.988512 & 2.508864 \\
\hline & -0.162211 & 5.791241 & 3.325170 \\
\hline 6 & -0.556696 & 3.568913 & 4.351318 \\
\hline 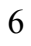 & -4.590894 & 4.574513 & -1.404863 \\
\hline 5 & -4.574554 & 5.911269 & 0.679069 \\
\hline & 6.804261 & -0.394774 & 0.842708 \\
\hline & -2.176436 & 0.957106 & 2.860467 \\
\hline 1 & 7.269861 & -5.023095 & -0.891511 \\
\hline & -1.277361 & -2.250483 & 6.203737 \\
\hline I & 6.690718 & 4.512087 & 0.013301 \\
\hline & -3.573053 & -2.837701 & 1.249428 \\
\hline 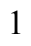 & -5.402002 & -4.266612 & 0.457060 \\
\hline | & 4.519296 & 3.964347 & 0.954274 \\
\hline ( & -1.933221 & -0.242557 & 4.968741 \\
\hline 1 & 5.271626 & -4.755429 & -2.241509 \\
\hline & 8.773178 & 1.659240 & -2.436516 \\
\hline 1 & 3.271811 & -3.394732 & -2.874424 \\
\hline & -2.759995 & -6.162627 & -2.360081 \\
\hline 1 & -0.405838 & -5.499526 & -2.465414 \\
\hline 1 & -0.114552 & -5.794090 & 4.067786 \\
\hline 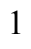 & 6.987007 & 0.023834 & -2.087704 \\
\hline 1 & -0.443853 & -4.646978 & 1.932185 \\
\hline 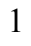 & 1.432650 & -4.000501 & -1.721669 \\
\hline 1 & 8.660090 & 3.910657 & -1.371012 \\
\hline ( & 8.635115 & -1.816515 & 1.622428 \\
\hline 1 & -5.008920 & -5.945442 & -1.344617 \\
\hline ] & -0.522474 & -4.611341 & 6.221114 \\
\hline 1 & 8.904769 & -4.135753 & 0.746051 \\
\hline ( & 2.571496 & 2.400704 & 1.071396 \\
\hline
\end{tabular}




\begin{tabular}{|c|c|c|c|}
\hline & -4.191463 & 0.691807 & 1.615253 \\
\hline ] & -3.241539 & -1.031196 & -2.177523 \\
\hline & -7.299377 & -0.999940 & -0.794540 \\
\hline & -3.447370 & 2.488215 & -0.751611 \\
\hline & -0.151686 & 1.814695 & 1.908004 \\
\hline & -1.904413 & 5.717991 & 1.597829 \\
\hline & 1.813288 & -3.609513 & 1.401741 \\
\hline ] & -1.763504 & 0.458093 & -2.670943 \\
\hline & 3.173752 & -3.101882 & 0.413595 \\
\hline 1 & 4.828748 & -2.631795 & 1.935205 \\
\hline 1 & 0.943943 & -0.938020 & 2.572526 \\
\hline & 5.712281 & -1.346364 & 3.850181 \\
\hline & 1.803614 & 0.324928 & 4.513780 \\
\hline ] & 4.206573 & 0.148502 & 5.152244 \\
\hline 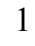 & -0.367620 & 3.949647 & -0.977974 \\
\hline 1 & 1.127712 & 5.721115 & -0.185239 \\
\hline & 3.484827 & 5.846056 & -1.013005 \\
\hline 1 & 4.305092 & 4.114941 & -2.599180 \\
\hline 1 & 2.805213 & 2.348024 & -3.400232 \\
\hline 1 & -0.038396 & 0.732033 & -4.573906 \\
\hline 1 & 1.578199 & 0.696219 & -3.721869 \\
\hline 1 & -1.787400 & 3.087068 & -2.458671 \\
\hline 1 & -0.900801 & 3.761824 & -3.830654 \\
\hline & -1.758128 & 2.209669 & -3.992832 \\
\hline 1 & 0.489644 & 6.067864 & 4.163199 \\
\hline 1 & 0.163680 & 6.368126 & 2.449457 \\
\hline 1 & -1.179122 & 6.109031 & 3.587594 \\
\hline 1 & 0.031876 & 3.887282 & 5.222668 \\
\hline & -1.612038 & 3.796215 & 4.553104 \\
\hline$\frac{1}{1}$ & -0.457318 & 2.478993 & 4.261924 \\
\hline 1 & 2.017600 & 4.318245 & 3.666098 \\
\hline 1 & 1.557529 & 2.847910 & 2.808510 \\
\hline 1 & 1.761702 & 4.369813 & 1.905884 \\
\hline 1 & -3.474598 & 7.026898 & -1.582040 \\
\hline 1 & -2.185284 & 6.838006 & -0.380572 \\
\hline 1 & -2.184309 & 5.833074 & -1.839073 \\
\hline 1 & -5.242949 & 5.331978 & -1.857671 \\
\hline 1 & -4.018340 & 4.107447 & -2.217020 \\
\hline 1 & -5.236735 & 3.807007 & -0.957603 \\
\hline 1 & -5.243962 & 6.636139 & 0.196818 \\
\hline 1 & -5.197091 & 5.172938 & 1.201674 \\
\hline 1 & -3.992189 & 6.453216 & 1.433856 \\
\hline 6 & -4.643403 & -2.315356 & -3.757173 \\
\hline 6 & -6.752872 & -2.933917 & -2.626448 \\
\hline 6 & -6.551499 & -0.722645 & -3.771424 \\
\hline 6 & -8.280025 & -0.172951 & 1.328358 \\
\hline 6 & -6.423748 & -0.497874 & 2.936759 \\
\hline 6 & -6.822515 & 1.747665 & 1.896512 \\
\hline 1 & -8.940898 & 0.102837 & 2.159815 \\
\hline 1 & -8.654807 & 0.333302 & 0.429455 \\
\hline 1 & -8.375580 & -1.256170 & 1.178477 \\
\hline 1 & -7.495686 & 2.014267 & 2.722482 \\
\hline 1 & -5.819967 & 2.113077 & 2.153040 \\
\hline 1 & -7.155737 & 2.289772 & 1.001807 \\
\hline 1 & -7.117085 & -0.267457 & 3.756933 \\
\hline 1 & -6.428844 & -1.585479 & 2.783684 \\
\hline
\end{tabular}




$\begin{array}{llll}1 & -5.415159 & -0.215143 & 3.263877 \\ 1 & -7.066717 & -3.415800 & -3.561862 \\ 1 & -6.253130 & -3.690797 & -2.007606 \\ 1 & -7.663906 & -2.615455 & -2.106225 \\ 1 & -6.905178 & -1.165284 & -4.712713 \\ 1 & -7.423788 & -0.322005 & -3.239387 \\ 1 & -5.893926 & 0.120906 & -4.019498 \\ 1 & -5.031974 & -2.821327 & -4.650159 \\ 1 & -3.968103 & -1.522576 & -4.104452 \\ 1 & -4.050447 & -3.046681 & -3.189296\end{array}$

$3_{\text {IIA-si- }} \psi_{-1 \text { s2a3s4a }}$

Number of imaginary frequencies : 0

The smallest frequencies are : $15.9151 \quad 21.4770 \quad 23.1974 \mathrm{~cm}(-1)$

Electronic energy : $\quad H F=-4360.8795246$

Zero-point correction $=\quad 1.455414($ Hartree/Particle $)$

Thermal correction to Energy $=\quad 1.539805$

Thermal correction to Enthalpy= $\quad 1.540749$

Thermal correction to Gibbs Free Energy $=\quad 1.338991$

Sum of electronic and zero-point Energies $=\quad-4359.424111$

Sum of electronic and thermal Energies $=\quad-4359.339720$

Sum of electronic and thermal Enthalpies $=\quad-4359.338776$

Sum of electronic and thermal Free Energies $=\quad-4359.540534$

Cartesian Coordinates

$\begin{array}{cccc}6 & -2.760650 & 2.866875 & 0.049436 \\ 6 & -1.827417 & 1.988429 & 0.612034 \\ 6 & -0.893577 & 2.475068 & 1.526782 \\ 6 & -0.946568 & 3.802721 & 1.974773 \\ 6 & -1.883656 & 4.649923 & 1.384873 \\ 6 & -2.784661 & 4.212998 & 0.403423 \\ 15 & -1.785848 & 0.247493 & 0.015064 \\ 6 & -3.577956 & -0.113419 & -0.266240 \\ 6 & -4.509507 & 0.090067 & 0.761640 \\ 6 & -5.854737 & -0.207006 & 0.580667 \\ 6 & -6.258514 & -0.700958 & -0.672053 \\ 6 & -5.363772 & -0.906375 & -1.716230 \\ 6 & -4.011153 & -0.619626 & -1.481748 \\ 6 & -6.885081 & -0.036827 & 1.695549 \\ 6 & -5.777724 & -1.430911 & -3.089167 \\ 6 & -0.067158 & 4.252364 & 3.143244 \\ 6 & 1.399996 & 3.896291 & 2.900134 \\ 6 & -3.744604 & 5.211465 & -0.239844 \\ 6 & -2.925245 & 6.242693 & -1.025004 \\ 45 & -0.407868 & 0.087755 & -1.963444 \\ 6 & -0.808085 & -1.504184 & -2.998134 \\ 8 & -1.125269 & -2.157557 & -3.900767 \\ 6 & 0.129225 & 2.155139 & -2.844036 \\ 6 & 0.529320 & 1.064331 & -3.649712 \\ 6 & 1.126796 & 3.039584 & -2.179604 \\ 6 & 2.456609 & 3.112261 & -2.623813 \\ 6 & 3.313107 & 4.110814 & -2.175442 \\ 6 & 2.872981 & 5.059814 & -1.257108 \\ 6 & 1.568865 & 4.980100 & -0.775317\end{array}$




\begin{tabular}{|c|c|c|c|}
\hline 6 & 0.710600 & 3.985876 & -1.230019 \\
\hline & -1.130548 & 2.910034 & -3.233194 \\
\hline 1 & 1.552707 & -0.706483 & -0.900374 \\
\hline & 2.479081 & 0.058931 & 0.224861 \\
\hline & 3.581079 & 0.840153 & 0.028417 \\
\hline & 4.729967 & 0.369003 & -0.584110 \\
\hline & 5.797562 & 1.308524 & -0.790631 \\
\hline & 5.730536 & 2.599928 & -0.186754 \\
\hline 6 & 4.580943 & 2.959849 & 0.556930 \\
\hline 6 & 3.512754 & 2.114137 & 0.622819 \\
\hline 6 & 6.786039 & 3.521832 & -0.384180 \\
\hline 0 & 7.867259 & 3.202394 & -1.166109 \\
\hline 6 & 7.926013 & 1.938225 & -1.787941 \\
\hline 6 & 6.923282 & 1.017766 & -1.602463 \\
\hline 6 & 4.859988 & -1.050536 & -0.986817 \\
\hline 6 & 3.882980 & -1.665805 & -1.746819 \\
\hline 6 & 4.034169 & -2.968507 & -2.267536 \\
\hline 6 & 5.132353 & -3.712068 & -1.935633 \\
\hline 6 & 6.100441 & -3.195036 & -1.040065 \\
\hline 6 & 5.968959 & -1.858210 & -0.556137 \\
\hline & 7.178744 & -3.998746 & -0.595898 \\
\hline 6 & 8.082336 & -3.525030 & 0.320081 \\
\hline 6 & 7.935056 & -2.219085 & 0.832068 \\
\hline 0 & 6.913696 & -1.407796 & 0.402497 \\
\hline 8 & 2.714794 & -1.012256 & -2.063126 \\
\hline 7 & 1.311896 & -2.093467 & 0.056130 \\
\hline 6 & 0.225343 & -2.947369 & -0.329356 \\
\hline 6 & -1.003907 & -2.853174 & 0.294821 \\
\hline 6 & -2.079665 & -3.684176 & -0.151376 \\
\hline 6 & -1.845322 & -4.651448 & -1.173374 \\
\hline 6 & -0.551773 & -4.758973 & -1.736419 \\
\hline 6 & 0.448699 & -3.914426 & -1.337108 \\
\hline 6 & -2.904446 & -5.497365 & -1.585097 \\
\hline 6 & -4.149054 & -5.391002 & -1.019011 \\
\hline 6 & -4.390883 & -4.419606 & -0.023237 \\
\hline 6 & -3.382759 & -3.591738 & 0.403303 \\
\hline 6 & -1.188642 & -2.064021 & 1.545839 \\
\hline 6 & -1.548565 & -0.724811 & 1.586808 \\
\hline 6 & -1.822659 & -0.126109 & 2.849488 \\
\hline 6 & -1.685790 & -0.817055 & 4.021763 \\
\hline 6 & -1.262884 & -2.165175 & 4.018005 \\
\hline 6 & -1.030182 & -2.805448 & 2.767190 \\
\hline 6 & -0.616475 & -4.164116 & 2.785601 \\
\hline 6 & -0.426394 & -4.832080 & 3.970369 \\
\hline 6 & -0.651019 & -4.186772 & 5.203807 \\
\hline 6 & -1.065410 & -2.879779 & 5.222798 \\
\hline 6 & 2.314595 & -2.731229 & 0.943832 \\
\hline 6 & 2.820380 & -1.912297 & 2.105522 \\
\hline 6 & 1.983083 & -1.086096 & 2.857799 \\
\hline 6 & 2.477650 & -0.378521 & 3.944727 \\
\hline 6 & 3.821523 & -0.485239 & 4.301940 \\
\hline 6 & 4.660840 & -1.309682 & 3.562272 \\
\hline 6 & 4.160328 & -2.019306 & 2.472511 \\
\hline 6 & -0.153578 & 5.756441 & 3.395462 \\
\hline 6 & -0.551506 & 3.527566 & 4.406166 \\
\hline 6 & -4.722103 & 4.538809 & -1.201829 \\
\hline
\end{tabular}




\begin{tabular}{|c|c|c|c|}
\hline 6 & -4.564929 & 5.923013 & 0.841537 \\
\hline 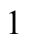 & 6.803344 & -0.414023 & 0.827647 \\
\hline & -2.149912 & 0.909212 & 2.890845 \\
\hline & 7.263123 & -5.011073 & -0.989079 \\
\hline & -1.245801 & -2.364978 & 6.165444 \\
\hline & 6.707588 & 4.501706 & 0.086527 \\
\hline & -3.582568 & -2.841324 & 1.166833 \\
\hline & -5.386321 & -4.321642 & 0.405684 \\
\hline & 4.539613 & 3.940571 & 1.029172 \\
\hline & -1.899170 & -0.332198 & 4.974033 \\
\hline & 5.263361 & -4.717591 & -2.331773 \\
\hline 1 & 8.769259 & 1.691386 & -2.429308 \\
\hline & 3.264039 & -3.343890 & -2.938002 \\
\hline & -2.706613 & -6.234222 & -2.362324 \\
\hline & -0.372842 & -5.508875 & -2.505370 \\
\hline & -0.101265 & -5.869804 & 3.956771 \\
\hline & 6.979779 & 0.054854 & -2.103818 \\
\hline & -0.441366 & -4.683199 & 1.845511 \\
\hline & 1.442835 & -3.974429 & -1.777751 \\
\hline & 8.668291 & 3.921866 & -1.319441 \\
\hline & 8.633594 & -1.850969 & 1.580299 \\
\hline & -4.955532 & -6.045410 & -1.342993 \\
\hline & -0.495625 & -4.727452 & 6.134470 \\
\hline & 8.900495 & -4.154471 & 0.662160 \\
\hline & 2.586731 & 2.383615 & 1.120727 \\
\hline & -4.167452 & 0.489758 & 1.712311 \\
\hline & -3.280185 & -0.793043 & -2.266875 \\
\hline & -7.312243 & -0.926785 & -0.823718 \\
\hline & -3.472009 & 2.480046 & -0.677312 \\
\hline & -0.139811 & 1.796749 & 1.932818 \\
\hline & -1.923921 & 5.691391 & 1.695038 \\
\hline ] & 1.811421 & -3.622747 & 1.345162 \\
\hline & -1.756379 & 0.547041 & -2.647940 \\
\hline & 3.172214 & -3.103201 & 0.363580 \\
\hline & 4.828904 & -2.652995 & 1.887709 \\
\hline 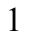 & 0.944394 & -0.970510 & 2.557002 \\
\hline & 5.714639 & -1.397803 & 3.821402 \\
\hline & 1.806492 & 0.261889 & 4.516646 \\
\hline ] & 4.210034 & 0.075380 & 5.149516 \\
\hline & -0.313493 & 3.959387 & -0.860971 \\
\hline ] & 1.199081 & 5.711875 & -0.056383 \\
\hline & 3.542020 & 5.850067 & -0.920145 \\
\hline & 4.332186 & 4.152528 & -2.556955 \\
\hline ] & 2.812809 & 2.408498 & -3.374117 \\
\hline & -0.045227 & 0.835871 & -4.548469 \\
\hline 1 & 1.575365 & 0.764711 & -3.705892 \\
\hline & -1.750200 & 3.175888 & -2.367629 \\
\hline 1 & -0.865741 & 3.851544 & -3.740278 \\
\hline & -1.753170 & 2.317238 & -3.913573 \\
\hline 1 & 0.505710 & 6.026112 & 4.229940 \\
\hline 1 & 0.165462 & 6.339445 & 2.521305 \\
\hline & -1.167598 & 6.073983 & 3.668999 \\
\hline 1 & 0.038658 & 3.839460 & 5.278811 \\
\hline & -1.606401 & 3.754178 & 4.610824 \\
\hline & -0.452856 & 2.438196 & 4.308699 \\
\hline & 2.022110 & 4.273084 & 3.722920 \\
\hline
\end{tabular}




$\begin{array}{rrrr}1 & 1.556632 & 2.811818 & 2.852847 \\ 1 & 1.76511 & 4.340796 & 1.963531 \\ 1 & -3.587328 & 6.986557 & -1.488747 \\ 1 & -2.216880 & 6.779319 & -0.381248 \\ 1 & -2.346719 & 5.760507 & -1.824659 \\ 1 & -5.400569 & 5.291876 & -1.622018 \\ 1 & -4.210376 & 4.055668 & -2.044238 \\ 1 & -5.337839 & 3.781793 & -0.697338 \\ 1 & -5.266518 & 6.630740 & 0.380266 \\ 1 & -5.149421 & 5.202409 & 1.428745 \\ 1 & -3.937324 & 6.492079 & 1.537456 \\ 6 & -7.292106 & -1.564555 & -3.226528 \\ 6 & -5.283857 & -0.468999 & -4.177052 \\ 6 & -5.150343 & -2.811079 & -3.310274 \\ 6 & -6.276028 & 0.574092 & 2.956123 \\ 6 & -8.020026 & 0.879497 & 1.227078 \\ 6 & -7.455127 & -1.412790 & 2.058789 \\ 1 & -7.050891 & 0.678622 & 3.725990 \\ 1 & -5.478786 & -0.053969 & 3.376194 \\ 1 & -5.862086 & 1.573560 & 2.766089 \\ 1 & -8.198375 & -1.320373 & 2.862338 \\ 1 & -7.947286 & -1.892752 & 1.203969 \\ 1 & -6.660017 & -2.084743 & 2.409152 \\ 1 & -8.758979 & 1.008960 & 2.029167 \\ 1 & -7.637571 & 1.872384 & 0.957249 \\ 1 & -8.549317 & 0.476670 & 0.355571 \\ 1 & -5.600804 & -0.823817 & -5.166795 \\ 1 & -5.695171 & 0.538452 & -4.030602 \\ 1 & -4.189417 & -0.386030 & -4.195501 \\ 1 & -5.405209 & -3.190559 & -4.309271 \\ 1 & -4.055902 & -2.783697 & -3.231304 \\ 1 & -5.516366 & -3.531200 & -2.566955 \\ 1 & -7.540440 & -1.924579 & -4.232854 \\ 1 & -7.708077 & -2.285878 & -2.511234 \\ 1 & -7.803196 & -0.603378 & -3.083519\end{array}$

$3_{\text {IIA-si- }} \psi_{-1 \mathrm{~s} 2 \mathrm{a3s} 4 \mathrm{~s}}$

Number of imaginary frequencies : 0

The smallest frequencies are : $17.526522 .616124 .6734 \mathrm{~cm}(-1)$

Electronic energy : $\quad \mathrm{HF}=-4360.8798776$

Zero-point correction $=\quad 1.455509($ Hartree/Particle $)$

Thermal correction to Energy= $\quad 1.539773$

Thermal correction to Enthalpy= $\quad 1.540717$

Thermal correction to Gibbs Free Energy $=\quad 1.340121$

Sum of electronic and zero-point Energies $=\quad-4359.424369$

Sum of electronic and thermal Energies $=\quad-4359.340104$

Sum of electronic and thermal Enthalpies $=\quad-4359.339160$

Sum of electronic and thermal Free Energies $=\quad-4359.539757$

Cartesian Coordinates

$\begin{array}{llll}6 & -2.795828 & 2.843489 & 0.050632 \\ 6 & -1.850778 & 1.974430 & 0.608166 \\ 6 & -0.916884 & 2.470257 & 1.517936 \\ 6 & -0.980893 & 3.796945 & 1.967052\end{array}$




\begin{tabular}{|c|c|c|c|}
\hline 6 & -1.927893 & 4.635474 & 1.380435 \\
\hline 6 & -2.828264 & 4.190230 & 0.402084 \\
\hline 1 & -1.788476 & 0.233694 & 0.011971 \\
\hline 6 & -3.576043 & -0.142438 & -0.278176 \\
\hline 6 & -4.510069 & 0.052162 & 0.741044 \\
\hline 6 & -5.859457 & -0.243207 & 0.555352 \\
\hline 6 & -6.260618 & -0.727090 & -0.696288 \\
\hline 6 & -5.355147 & -0.930018 & -1.738771 \\
\hline 6 & -4.007093 & -0.645203 & -1.500231 \\
\hline 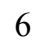 & -6.827438 & -0.032970 & 1.718494 \\
\hline 6 & -5.765000 & -1.454862 & -3.113357 \\
\hline & -0.100433 & 4.254281 & 3.131713 \\
\hline & 1.368017 & 3.905698 & 2.884944 \\
\hline 6 & -3.796020 & 5.180975 & -0.241424 \\
\hline 6 & -2.984021 & 6.230291 & -1.010308 \\
\hline 45 & -0.407378 & 0.082846 & -1.962838 \\
\hline 6 & -0.797108 & -1.510443 & -2.999374 \\
\hline 8 & -1.108299 & -2.165817 & -3.902600 \\
\hline 6 & 0.116392 & 2.154783 & -2.842150 \\
\hline 6 & 0.520198 & 1.067106 & -3.650167 \\
\hline & 1.111293 & 3.043043 & -2.179055 \\
\hline 6 & 2.440948 & 3.118817 & -2.623409 \\
\hline 6 & 3.294573 & 4.120607 & -2.177054 \\
\hline & 2.851702 & 5.070181 & -1.260635 \\
\hline 6 & 1.547922 & 4.987445 & -0.778549 \\
\hline 0 & 0.692506 & 3.989587 & -1.230801 \\
\hline$b$ & -1.149200 & 2.902839 & -3.225576 \\
\hline 15 & 1.555657 & -0.703024 & -0.899635 \\
\hline 8 & 2.479121 & 0.065754 & 0.225828 \\
\hline 6 & 3.577774 & 0.851588 & 0.028862 \\
\hline 6 & 4.728427 & 0.384635 & -0.583568 \\
\hline 6 & 5.792019 & 1.328320 & -0.791439 \\
\hline 6 & 5.719513 & 2.620346 & -0.189529 \\
\hline 6 & 4.568708 & 2.976291 & 0.554196 \\
\hline 6 & 3.504278 & 2.125967 & 0.621917 \\
\hline 6 & 6.770820 & 3.546632 & -0.388952 \\
\hline 6 & 7.853186 & 3.230678 & -1.170707 \\
\hline 6 & 7.917461 & 1.965664 & -1.790273 \\
\hline 6 & 6.918861 & 1.041092 & -1.602968 \\
\hline 6 & 4.863839 & -1.034762 & -0.985119 \\
\hline 6 & 3.889203 & -1.654013 & -1.744891 \\
\hline 6 & 4.044608 & -2.956840 & -2.263935 \\
\hline 6 & 5.145106 & -3.696447 & -1.930891 \\
\hline 6 & 6.111391 & -3.175159 & -1.035837 \\
\hline 6 & 5.975450 & -1.838210 & -0.553407 \\
\hline 6 & 7.192327 & -3.974786 & -0.590688 \\
\hline 6 & 8.094304 & -3.497006 & 0.324756 \\
\hline 6 & 7.942715 & -2.190959 & 0.835211 \\
\hline 6 & 6.918685 & -1.383540 & 0.404717 \\
\hline & 2.719014 & -1.004482 & -2.062173 \\
\hline 7 & 1.319632 & -2.091174 & 0.056724 \\
\hline 6 & 0.235313 & -2.948921 & -0.327023 \\
\hline 6 & -0.993356 & -2.858937 & 0.298909 \\
\hline 6 & -2.068031 & -3.691739 & -0.145883 \\
\hline 6 & -1.833252 & -4.657160 & -1.169445 \\
\hline 6 & -0.539987 & -4.761599 & -1.733835 \\
\hline
\end{tabular}




\begin{tabular}{|c|c|c|c|}
\hline 6 & 0.459372 & -3.915184 & -1.335377 \\
\hline & -2.891688 & -5.503829 & -1.581349 \\
\hline & -4.136038 & -5.399121 & -1.014354 \\
\hline & -4.378004 & -4.429654 & -0.016685 \\
\hline & -3.370443 & -3.601825 & 0.411110 \\
\hline & -1.180927 & -2.069871 & 1.549031 \\
\hline & -1.548954 & -0.732996 & 1.586834 \\
\hline & -1.827055 & -0.133421 & 2.848107 \\
\hline & -1.687548 & -0.821875 & 4.021767 \\
\hline & -1.257808 & -2.167798 & 4.020826 \\
\hline & -1.019925 & -2.808663 & 2.771373 \\
\hline & -0.599531 & -4.165195 & 2.792057 \\
\hline & -0.407550 & -4.830430 & 3.978034 \\
\hline & -0.636963 & -4.184412 & 5.210262 \\
\hline & -1.058126 & -2.879565 & 5.226934 \\
\hline & 2.323814 & -2.723752 & 0.946485 \\
\hline & 2.825215 & -1.901500 & 2.107777 \\
\hline & 1.984122 & -1.077320 & 2.858049 \\
\hline & 2.475058 & -0.365674 & 3.943947 \\
\hline & 3.819043 & -0.466591 & 4.302444 \\
\hline & 4.662133 & -1.289130 & 3.564912 \\
\hline & 4.165300 & -2.002557 & 2.475944 \\
\hline & -0.194780 & 5.758365 & 3.380919 \\
\hline & -0.576532 & 3.529119 & 4.397574 \\
\hline & -4.753943 & 4.503688 & -1.219530 \\
\hline & -4.635781 & 5.871583 & 0.838524 \\
\hline & 6.805103 & -0.389586 & 0.828624 \\
\hline & -2.158126 & 0.900699 & 2.887265 \\
\hline & 7.280073 & -4.987263 & -0.982746 \\
\hline & -1.242470 & -2.364431 & 6.168625 \\
\hline & 6.688135 & 4.526968 & 0.080070 \\
\hline I & -3.570714 & -2.853456 & 1.177136 \\
\hline & -5.373230 & -4.332772 & 0.412818 \\
\hline & 4.523147 & 3.957525 & 1.024980 \\
\hline & -1.903672 & -0.336403 & 4.973103 \\
\hline & 5.279351 & -4.702064 & -2.325708 \\
\hline 1 & 8.761672 & 1.721429 & -2.431370 \\
\hline & 3.275757 & -3.335509 & -2.934011 \\
\hline 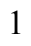 & -2.693807 & -6.239453 & -2.359726 \\
\hline . & -0.360463 & -5.510404 & -2.503721 \\
\hline & -0.077303 & -5.866558 & 3.966432 \\
\hline ] & 6.979504 & 0.077544 & -2.102616 \\
\hline & -0.420974 & -4.684613 & 1.852780 \\
\hline 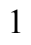 & 1.452881 & -3.972682 & -1.777766 \\
\hline & 8.650923 & 3.953464 & -1.325605 \\
\hline 1 & 8.640087 & -1.819676 & 1.582965 \\
\hline ] & -4.942238 & -6.053615 & -1.338863 \\
\hline 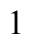 & -0.480008 & -4.722984 & 6.141882 \\
\hline 1 & 8.914554 & -4.123321 & 0.667564 \\
\hline 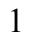 & 2.577270 & 2.392326 & 1.119749 \\
\hline 1 & -4.177811 & 0.444121 & 1.701327 \\
\hline & -3.273332 & -0.815163 & -2.283349 \\
\hline 1 & -7.311184 & -0.948649 & -0.860608 \\
\hline ] & -3.507361 & 2.449991 & -0.672302 \\
\hline 1 & -0.154040 & 1.799305 & 1.919381 \\
\hline 1 & -1.976421 & 5.676799 & 1.690063 \\
\hline
\end{tabular}




\begin{tabular}{|c|c|c|c|}
\hline . & 1.823877 & -3.616826 & 1.348322 \\
\hline 1 & -1.758374 & 0.534925 & -2.646941 \\
\hline 1 & 3.183490 & -3.092936 & 0.367520 \\
\hline 1 & 4.836888 & -2.634373 & 1.892546 \\
\hline & 0.945313 & -0.966038 & 2.556095 \\
\hline 1 & 5.716083 & -1.372608 & 3.824972 \\
\hline & 1.800906 & 0.273482 & 4.513809 \\
\hline 1 & 4.204772 & 0.097154 & 5.149218 \\
\hline & -0.331256 & 3.960517 & -0.860846 \\
\hline 1 & 1.176320 & 5.720045 & -0.061434 \\
\hline 1 & 3.518275 & 5.863258 & -0.925462 \\
\hline 1 & 4.313451 & 4.164565 & -2.558838 \\
\hline 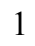 & 2.799262 & 2.414880 & -3.372503 \\
\hline 1 & -0.055630 & 0.836554 & -4.547554 \\
\hline 1 & 1.567607 & 0.772941 & -3.709534 \\
\hline 1 & -1.768522 & 3.161294 & -2.357516 \\
\hline 1 & -0.892013 & 3.847848 & -3.730001 \\
\hline 1 & -1.769658 & 2.308261 & -3.906418 \\
\hline 1 & 0.464604 & 6.033568 & 4.213504 \\
\hline 1 & 0.119212 & 6.341189 & 2.504814 \\
\hline 1 & -1.209972 & 6.070984 & 3.655763 \\
\hline 1 & 0.014869 & 3.845773 & 5.267668 \\
\hline 1 & -1.631953 & 3.750275 & 4.605535 \\
\hline 1 & -0.471995 & 2.440141 & 4.301528 \\
\hline 1 & 1.991172 & 4.292398 & 3.702318 \\
\hline 1 & 1.531083 & 2.821833 & 2.845471 \\
\hline 1 & 1.726597 & 4.344995 & 1.943430 \\
\hline 1 & -3.652828 & 6.965124 & -1.478745 \\
\hline 1 & -2.293604 & 6.776090 & -0.355015 \\
\hline 1 & -2.387104 & 5.762111 & -1.804766 \\
\hline 1 & -5.439439 & 5.250717 & -1.639070 \\
\hline 1 & -4.226308 & 4.036231 & -2.061043 \\
\hline 1 & -5.363288 & 3.732577 & -0.729047 \\
\hline 1 & -5.338084 & 6.578967 & 0.377731 \\
\hline 1 & -5.221034 & 5.138573 & 1.409433 \\
\hline 1 & -4.021081 & 6.436851 & 1.549015 \\
\hline 6 & -7.279082 & -1.586258 & -3.257885 \\
\hline 6 & -5.265195 & -0.496190 & -4.201368 \\
\hline 6 & -5.139409 & -2.836881 & -3.328862 \\
\hline 6 & -8.261539 & -0.416721 & 1.363270 \\
\hline 6 & -6.382537 & -0.900842 & 2.902091 \\
\hline 6 & -6.817769 & 1.443761 & 2.130372 \\
\hline 1 & -8.912602 & -0.251504 & 2.231042 \\
\hline 1 & -8.656771 & 0.188215 & 0.537104 \\
\hline 1 & -8.346571 & -1.475497 & 1.086117 \\
\hline 1 & -7.490653 & 1.607390 & 2.983010 \\
\hline 1 & -5.818039 & 1.787139 & 2.424394 \\
\hline 1 & -7.158895 & 2.081585 & 1.304399 \\
\hline 1 & -7.061886 & -0.764378 & 3.754384 \\
\hline 1 & -6.391357 & -1.965073 & 2.630462 \\
\hline 1 & -5.368445 & -0.654850 & 3.241962 \\
\hline 1 & -5.577736 & -0.853061 & -5.191800 \\
\hline 1 & -5.676425 & 0.511923 & -4.059262 \\
\hline 1 & -4.170686 & -0.413787 & -4.214835 \\
\hline 1 & -5.392038 & -3.219252 & -4.327370 \\
\hline 1 & -4.045177 & -2.810892 & -3.246161 \\
\hline
\end{tabular}




$\begin{array}{llll}1 & -5.508880 & -3.554003 & -2.584222 \\ 1 & -7.522764 & -1.946584 & -4.265265 \\ 1 & -7.700127 & -2.306372 & -2.544421 \\ 1 & -7.789641 & -0.624298 & -3.118340\end{array}$

$3_{\text {IIA-si- }} \psi_{-1 \mathrm{~s} 2 \mathrm{~s} 3 \mathrm{a} 4 \mathrm{a}}$

Number of imaginary frequencies : 0

The smallest frequencies are : $18.1844 \quad 18.9646 \quad 23.5161 \mathrm{~cm}(-1)$

Electronic energy : $\quad H F=-4360.8795009$

Zero-point correction $=\quad 1.455441$ (Hartree/Particle)

Thermal correction to Energy= $\quad 1.539708$

Thermal correction to Enthalpy= $\quad 1.540652$

Thermal correction to Gibbs Free Energy= 1.339829

Sum of electronic and zero-point Energies $=\quad-4359.424060$

Sum of electronic and thermal Energies $=\quad-4359.339793$

Sum of electronic and thermal Enthalpies $=\quad-4359.338849$

Sum of electronic and thermal Free Energies $=\quad-4359.539672$

Cartesian Coordinates

$\begin{array}{cccc}6 & -2.843480 & 2.874051 & -0.028786 \\ 6 & -1.856309 & 2.043698 & 0.499316 \\ 6 & -0.908802 & 2.582683 & 1.376496 \\ 6 & -1.004997 & 3.905428 & 1.816252 \\ 6 & -2.016380 & 4.706579 & 1.271541 \\ 6 & -2.932029 & 4.220587 & 0.335414 \\ 15 & -1.771478 & 0.283703 & -0.034119 \\ 6 & -3.553802 & -0.132934 & -0.303056 \\ 6 & -4.497080 & 0.142492 & 0.692510 \\ 6 & -5.832104 & -0.221450 & 0.538006 \\ 6 & -6.199755 & -0.868608 & -0.649614 \\ 6 & -5.286264 & -1.167693 & -1.659376 \\ 6 & -3.953564 & -0.788779 & -1.461681 \\ 6 & -6.877104 & 0.029571 & 1.623598 \\ 6 & -5.746166 & -1.830915 & -2.957546 \\ 6 & -0.101709 & 4.407522 & 2.945089 \\ 6 & -4.035842 & 5.083420 & -0.273870 \\ 45 & -0.370624 & 0.038236 & -1.988837 \\ 6 & -0.698025 & -1.586422 & -2.997727 \\ 8 & -0.942087 & -2.272402 & -3.898613 \\ 6 & 0.149691 & 2.091357 & -2.934193 \\ 6 & 0.552368 & 0.979931 & -3.707686 \\ 6 & 1.145172 & 2.997229 & -2.297534 \\ 6 & 2.486154 & 3.027088 & -2.714022 \\ 6 & 3.350927 & 4.029997 & -2.294176 \\ 6 & 2.908782 & 5.028406 & -1.431144 \\ 6 & 1.593025 & 4.995118 & -0.977773 \\ 6 & 0.725491 & 3.995389 & -1.403668 \\ 6 & -1.128630 & 2.813838 & -3.320697 \\ 15 & 1.588030 & -0.702800 & -0.879731 \\ 8 & 2.515695 & 0.094958 & 0.222320 \\ 6 & 3.625297 & 0.860310 & 0.007665 \\ 6 & 4.774061 & 0.358758 & -0.580573 \\ 6 & 5.852252 & 1.280694 & -0.809816 \\ 6 & 5.792658 & 2.592504 & -0.250474\end{array}$




\begin{tabular}{|c|c|c|c|}
\hline 6 & 4.640331 & 2.988469 & 0.470337 \\
\hline 6 & 3.564144 & 2.154842 & 0.557354 \\
\hline & 6.859454 & 3.496665 & -0.468306 \\
\hline & 7.944887 & 3.140206 & -1.227984 \\
\hline & 7.996651 & 1.855340 & -1.806454 \\
\hline & 6.982776 & 0.951700 & -1.600382 \\
\hline & 4.892761 & -1.074786 & -0.934722 \\
\hline & 3.914797 & -1.705768 & -1.680630 \\
\hline & 4.056263 & -3.027092 & -2.155180 \\
\hline & 5.143514 & -3.770408 & -1.788707 \\
\hline & 6.110072 & -3.233082 & -0.903439 \\
\hline & 5.989979 & -1.878552 & -0.467950 \\
\hline & 7.175208 & -4.032854 & -0.421756 \\
\hline & 8.076759 & -3.537236 & 0.484510 \\
\hline & 7.941143 & -2.212081 & 0.947962 \\
\hline & 6.932869 & -1.404951 & 0.481401 \\
\hline & 2.754580 & -1.052873 & -2.026388 \\
\hline & 1.339370 & -2.054733 & 0.124640 \\
\hline 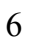 & 0.249574 & -2.916056 & -0.233597 \\
\hline & -0.981359 & -2.793430 & 0.382204 \\
\hline & -2.063755 & -3.623327 & -0.048590 \\
\hline$b$ & -1.841101 & -4.600902 & -1.063413 \\
\hline & -0.543971 & -4.743830 & -1.609795 \\
\hline & 0.468022 & -3.911694 & -1.214023 \\
\hline & -2.917135 & -5.418643 & -1.488037 \\
\hline 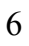 & -4.165664 & -5.278952 & -0.936492 \\
\hline 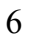 & -4.390477 & -4.309913 & 0.065952 \\
\hline 6 & -3.365732 & -3.508528 & 0.503199 \\
\hline b & -1.168070 & -1.954895 & 1.600149 \\
\hline 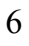 & -1.529929 & -0.615644 & 1.582435 \\
\hline 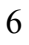 & -1.807214 & 0.036254 & 2.817472 \\
\hline & -1.671418 & -0.603652 & 4.018632 \\
\hline 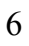 & -1.250042 & -1.951197 & 4.073633 \\
\hline & -1.015107 & -2.644280 & 2.851710 \\
\hline 6 & -0.608578 & -4.003082 & 2.927897 \\
\hline ) & -0.424601 & -4.621567 & 4.140109 \\
\hline 6 & -0.648468 & -3.923592 & 5.344624 \\
\hline 6 & -1.057669 & -2.615315 & 5.307838 \\
\hline & 2.336082 & -2.663859 & 1.038331 \\
\hline 6 & 2.837486 & -1.807800 & 2.175020 \\
\hline & 2.002959 & -0.941363 & 2.883820 \\
\hline 6 & 2.494021 & -0.199612 & 3.949323 \\
\hline 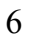 & 3.831322 & -0.312258 & 4.328651 \\
\hline 6 & 4.667875 & -1.176312 & 3.632260 \\
\hline 6 & 4.171086 & -1.919245 & 2.563185 \\
\hline ] & 6.831445 & -0.395151 & 0.869441 \\
\hline 1 & -2.135987 & 1.071955 & 2.814073 \\
\hline & 7.250667 & -5.059432 & -0.778087 \\
\hline 1 & -1.238777 & -2.061401 & 6.227902 \\
\hline & 6.787132 & 4.492301 & -0.031052 \\
\hline 1 & -3.552969 & -2.757883 & 1.269302 \\
\hline 1 & -5.386048 & -4.190599 & 0.490090 \\
\hline 1 & 4.603897 & 3.985708 & 0.907167 \\
\hline 1 & -1.886343 & -0.078242 & 4.948835 \\
\hline 1 & 5.266432 & -4.790405 & -2.148832 \\
\hline . & 8.843659 & 1.578616 & -2.430413 \\
\hline
\end{tabular}




\begin{tabular}{|c|c|c|c|}
\hline . & 3.287239 & -3.417515 & -2.818424 \\
\hline & -2.729877 & -6.160776 & -2.262851 \\
\hline 1 & -0.372696 & -5.506647 & -2.367693 \\
\hline & -0.105202 & -5.660710 & 4.171085 \\
\hline & 7.034839 & -0.028421 & -2.067784 \\
\hline & -0.435049 & -4.561656 & 2.010382 \\
\hline & 1.464766 & -3.995533 & -1.644940 \\
\hline & 8.754903 & 3.845967 & -1.397009 \\
\hline & 8.638290 & -1.825495 & 1.688139 \\
\hline 1 & -4.985341 & -5.912172 & -1.270143 \\
\hline 1 & -0.497690 & -4.425417 & 6.297552 \\
\hline & 8.884464 & -4.163538 & 0.855949 \\
\hline 1 & 2.636557 & 2.450640 & 1.037877 \\
\hline 1 & -4.177458 & 0.653201 & 1.596957 \\
\hline 1 & -3.202058 & -1.014706 & -2.211523 \\
\hline 1 & -7.243795 & -1.150259 & -0.785400 \\
\hline 1 & -3.568302 & 2.456415 & -0.727741 \\
\hline 1 & -0.117158 & 1.938441 & 1.765264 \\
\hline 1 & -2.096701 & 5.739885 & 1.594385 \\
\hline 1 & 1.829942 & -3.541327 & 1.466068 \\
\hline 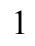 & -1.725147 & 0.439822 & -2.701331 \\
\hline 1 & 3.195943 & -3.056179 & 0.474959 \\
\hline 1 & 4.837498 & -2.584668 & 2.012196 \\
\hline 1 & 0.970074 & -0.823058 & 2.564656 \\
\hline 1 & 5.716868 & -1.269471 & 3.908494 \\
\hline 1 & 1.825138 & 0.472431 & 4.486709 \\
\hline 1 & 4.217152 & 0.274700 & 5.159464 \\
\hline 1 & -0.307268 & 4.004523 & -1.057128 \\
\hline 1 & 1.225865 & 5.769892 & -0.304774 \\
\hline 1 & 3.584052 & 5.822354 & -1.116302 \\
\hline 1 & 4.378258 & 4.035946 & -2.655169 \\
\hline 1 & 2.847938 & 2.284131 & -3.422312 \\
\hline 1 & -0.027472 & 0.718864 & -4.593927 \\
\hline 1 & 1.598914 & 0.682387 & -3.761733 \\
\hline 1 & -1.757997 & 3.053274 & -2.455515 \\
\hline 1 & -0.893949 & 3.767366 & -3.819796 \\
\hline 1 & -1.731254 & 2.206863 & -4.006023 \\
\hline 6 & -4.570056 & -2.377755 & -3.766717 \\
\hline 6 & -6.699389 & -2.995043 & -2.673130 \\
\hline 6 & -6.474697 & -0.779533 & -3.802931 \\
\hline 6 & -6.299928 & 0.792741 & 2.814163 \\
\hline 6 & -8.041207 & 0.847001 & 1.054660 \\
\hline 6 & -7.399198 & -1.320423 & 2.128966 \\
\hline 1 & -7.085802 & 0.957772 & 3.561933 \\
\hline 1 & -5.489682 & 0.237928 & 3.306345 \\
\hline 1 & -5.911566 & 1.776801 & 2.518760 \\
\hline 1 & -8.152409 & -1.171631 & 2.914581 \\
\hline 1 & -7.865188 & -1.907214 & 1.327678 \\
\hline 1 & -6.582674 & -1.920232 & 2.553034 \\
\hline 1 & -8.796038 & 1.024670 & 1.832334 \\
\hline 1 & -7.696801 & 1.824052 & 0.690792 \\
\hline 1 & -8.541083 & 0.337260 & 0.222511 \\
\hline 1 & -6.996034 & -3.473007 & -3.615992 \\
\hline 1 & -6.214141 & -3.755277 & -2.047166 \\
\hline 1 & -7.620470 & -2.676549 & -2.171024 \\
\hline 1 & -6.814830 & -1.216055 & -4.752057 \\
\hline
\end{tabular}




$\begin{array}{rrrr}1 & -7.353636 & -0.380463 & -3.280480 \\ 1 & -5.811854 & 0.064164 & -4.036210 \\ 1 & -4.946203 & -2.894074 & -4.659065 \\ 1 & -3.896794 & -1.583968 & -4.115162 \\ 1 & -3.977724 & -3.099224 & -3.185963 \\ 6 & -0.178885 & 5.922600 & 3.126997 \\ 6 & -0.565734 & 3.743164 & 4.248385 \\ 6 & 1.360297 & 4.033863 & 2.696661 \\ 6 & -4.016375 & 6.515592 & 0.253908 \\ 6 & -3.857209 & 5.133842 & -1.795408 \\ 6 & -5.401727 & 4.470360 & 0.059084 \\ 1 & -6.207293 & 5.069743 & -0.386122 \\ 1 & -5.500400 & 3.446405 & -0.323357 \\ 1 & -5.563638 & 4.441513 & 1.145059 \\ 1 & -4.633607 & 5.764212 & -2.249526 \\ 1 & -2.878931 & 5.556155 & -2.063377 \\ 1 & -3.929907 & 4.139100 & -2.252119 \\ 1 & -4.825930 & 7.090165 & -0.213466 \\ 1 & -4.170180 & 6.556744 & 1.340027 \\ 1 & -3.073854 & 7.026623 & 0.019015 \\ 1 & 0.513441 & 6.232119 & 3.919849 \\ 1 & 0.102000 & 6.461217 & 2.212444 \\ 1 & -1.180151 & 6.255972 & 3.426724 \\ 1 & 0.037595 & 4.095255 & 5.096523 \\ 1 & -1.617666 & 3.978296 & 4.458818 \\ 1 & -0.466619 & 2.650362 & 4.197852 \\ 1 & 1.996656 & 4.452911 & 3.487351 \\ 1 & 1.513596 & 2.948042 & 2.707872 \\ 1 & 1.712273 & 4.422858 & 1.730927\end{array}$

$3_{\text {IIA-si- }-\Psi \text {-1s2s3a4s }}$

Number of imaginary frequencies : 0

The smallest frequencies are : $18.234120 .0192 \quad 23.4494 \mathrm{~cm}(-1)$

Electronic energy : $\quad H F=-4360.879554$

Zero-point correction $=\quad 1.455597$ (Hartree/Particle)

Thermal correction to Energy= $\quad 1.539891$

Thermal correction to Enthalpy= $\quad 1.540835$

Thermal correction to Gibbs Free Energy $=\quad 1.339745$

Sum of electronic and zero-point Energies $=\quad-4359.423957$

Sum of electronic and thermal Energies $=\quad-4359.339664$

Sum of electronic and thermal Enthalpies $=\quad-4359.338719$

Sum of electronic and thermal Free Energies $=\quad-4359.539809$

\section{Cartesian Coordinates}

$\begin{array}{cccc}6 & -2.851569 & 2.873037 & -0.016851 \\ 6 & -1.871558 & 2.034112 & 0.512555 \\ 6 & -0.922928 & 2.564472 & 1.393975 \\ 6 & -1.010965 & 3.886694 & 1.836893 \\ 6 & -2.008521 & 4.698707 & 1.283566 \\ 6 & -2.923624 & 4.222633 & 0.341661 \\ 15 & -1.783562 & 0.277159 & -0.034057 \\ 6 & -3.566006 & -0.140511 & -0.306669 \\ 6 & -4.500695 & 0.095733 & 0.699073 \\ 6 & -5.841789 & -0.264869 & 0.545734\end{array}$




\begin{tabular}{|c|c|c|c|}
\hline 5 & -6.220853 & -0.862967 & -0.657381 \\
\hline 6 & -5.309080 & -1.123007 & -1.687107 \\
\hline & -3.977576 & -0.755887 & -1.488488 \\
\hline & -6.810711 & -0.017537 & 1.700518 \\
\hline ) & -5.787462 & -1.743375 & -2.999306 \\
\hline & -0.111107 & 4.377673 & 2.973362 \\
\hline 6 & -4.006482 & 5.103533 & -0.280492 \\
\hline 45 & -0.375701 & 0.047804 & -1.986538 \\
\hline b & -0.682479 & -1.571069 & -3.012424 \\
\hline 8 & -0.894982 & -2.250025 & -3.926369 \\
\hline 6 & 0.148251 & 2.104603 & -2.916827 \\
\hline 6 & 0.551491 & 0.998190 & -3.697310 \\
\hline 6 & 1.143919 & 3.005957 & -2.273767 \\
\hline 6 & 2.483925 & 3.040759 & -2.692953 \\
\hline 6 & 3.348588 & 4.041336 & -2.267240 \\
\hline 6 & 2.906931 & 5.033082 & -1.396345 \\
\hline 6 & 1.591917 & 4.995238 & -0.941126 \\
\hline 6 & 0.724784 & 3.997421 & -1.372282 \\
\hline 6 & -1.128100 & 2.831489 & -3.301154 \\
\hline 15 & 1.582469 & -0.698384 & -0.879749 \\
\hline 8 & 2.509146 & 0.092371 & 0.228125 \\
\hline 6 & 3.618790 & 0.859338 & 0.018745 \\
\hline 6 & 4.767638 & 0.363006 & -0.573702 \\
\hline 6 & 5.844990 & 1.287501 & -0.796698 \\
\hline 6 & 5.784584 & 2.595272 & -0.228122 \\
\hline 6 & 4.632512 & 2.984882 & 0.496505 \\
\hline 6 & 3.557273 & 2.149546 & 0.578356 \\
\hline 6 & 6.850173 & & -0.440671 \\
\hline 6 & 7.935236 & 3.152136 & -1.203914 \\
\hline 6 & 7.987859 & 1.871339 & -1.791274 \\
\hline 6 & 6.975152 & 0.965186 & -1.590479 \\
\hline 6 & 4.887400 & -1.067528 & -0.939171 \\
\hline 6 & 3.909699 & -1.693249 & -1.689759 \\
\hline 6 & 4.052437 & -3.010320 & -2.175589 \\
\hline 6 & 5.140792 & -3.755421 & -1.816009 \\
\hline 6 & 6.107135 & -3.224536 & -0.926621 \\
\hline 6 & 5.985594 & -1.873970 & -0.479480 \\
\hline 6 & 7.173559 & -4.026991 & -0.452372 \\
\hline 6 & 8.074802 & -3.538054 & 0.457851 \\
\hline 6 & 7.937541 & -2.217193 & 0.932987 \\
\hline 6 & 6.928067 & -1.407361 & 0.473715 \\
\hline 8 & 2.748860 & -1.038368 & -2.029575 \\
\hline 7 & 1.335544 & -2.058956 & 0.113394 \\
\hline 6 & 0.248247 & -2.919468 & -0.254265 \\
\hline 6 & -0.985183 & -2.801913 & 0.357451 \\
\hline 6 & -2.065711 & -3.628338 & -0.083901 \\
\hline 6 & -1.838529 & -4.598554 & -1.104799 \\
\hline 6 & -0.538914 & -4.737948 & -1.645997 \\
\hline 6 & 0.471298 & -3.908481 & -1.240186 \\
\hline 6 & -2.912315 & -5.413505 & -1.539943 \\
\hline 6 & -4.162817 & -5.278254 & -0.992219 \\
\hline 6 & -4.391992 & -4.316469 & 0.016256 \\
\hline 6 & -3.369850 & -3.517687 & 0.463962 \\
\hline 6 & -1.175841 & -1.973934 & 1.581789 \\
\hline 6 & -1.539801 & -0.635136 & 1.574990 \\
\hline 6 & -1.816278 & 0.006078 & 2.815769 \\
\hline
\end{tabular}




\begin{tabular}{|c|c|c|c|}
\hline 6 & -1.681093 & -0.644304 & 4.011566 \\
\hline 6 & -1.260131 & -1.992268 & 4.055143 \\
\hline & -1.023187 & -2.674151 & 2.827397 \\
\hline & -0.615273 & -4.033162 & 2.892135 \\
\hline & -0.432308 & -4.662107 & 4.099114 \\
\hline & -0.658532 & -3.975117 & 5.309523 \\
\hline & -1.068836 & -2.666974 & 5.283742 \\
\hline & 2.331972 & -2.673575 & 1.023609 \\
\hline & 2.831093 & -1.824686 & 2.166571 \\
\hline & 1.993778 & -0.966400 & 2.881955 \\
\hline & 2.482849 & -0.230248 & 3.952241 \\
\hline & 3.820854 & -0.340510 & 4.329829 \\
\hline & 4.660107 & -1.196681 & 3.626977 \\
\hline & 4.165277 & -1.934154 & 2.553231 \\
\hline & 6.825415 & -0.401057 & 0.870421 \\
\hline & -2.143113 & 1.042295 & 2.821245 \\
\hline & 7.250325 & -5.050334 & -0.817612 \\
\hline & -1.251644 & -2.121232 & 6.208329 \\
\hline & 6.777171 & 4.494582 & 0.003587 \\
\hline & -3.560234 & -2.771760 & 1.234493 \\
\hline & -5.389705 & -4.202620 & 0.436516 \\
\hline & 4.595460 & 3.978826 & 0.940748 \\
\hline & -1.895323 & -0.126636 & 4.946259 \\
\hline & 5.264833 & -4.772107 & -2.184986 \\
\hline & 8.834570 & 1.599847 & -2.417928 \\
\hline & 3.283557 & -3.395867 & -2.841853 \\
\hline & -2.721528 & -6.150177 & -2.319081 \\
\hline & -0.364226 & -5.495601 & -2.408277 \\
\hline & -0.111931 & -5.701170 & 4.121309 \\
\hline 1 & 7.027828 & -0.011651 & -2.064637 \\
\hline & -0.439932 & -4.583546 & 1.970050 \\
\hline & 1.469824 & -3.989253 & -1.667434 \\
\hline & 8.744300 & 3.859953 & -1.368843 \\
\hline & 8.634395 & -1.836140 & 1.676300 \\
\hline 1 & -4.980974 & -5.909668 & -1.332886 \\
\hline & -0.508557 & -4.485240 & 6.258155 \\
\hline 1 & 8.883532 & -4.166462 & 0.823455 \\
\hline & 2.629887 & 2.440682 & 1.061826 \\
\hline 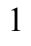 & -4.180209 & 0.568328 & 1.626598 \\
\hline 1 & -3.231934 & -0.954640 & -2.251898 \\
\hline & -7.262785 & -1.140117 & -0.802747 \\
\hline 1 & -3.578743 & 2.462355 & -0.717769 \\
\hline & -0.136084 & 1.914310 & 1.782692 \\
\hline ] & -2.077979 & 5.733606 & 1.603427 \\
\hline & 1.826217 & -3.554480 & 1.444738 \\
\hline & -1.727902 & 0.451911 & -2.702266 \\
\hline 1 & 3.192903 & -3.060882 & 0.458409 \\
\hline t & 4.833757 & -2.593195 & 1.997093 \\
\hline 1 & 0.960309 & -0.849536 & 2.564078 \\
\hline & 5.709688 & -1.287787 & 3.901671 \\
\hline 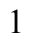 & 1.811936 & 0.435566 & 4.494801 \\
\hline & 4.205094 & 0.242218 & 5.164348 \\
\hline 1 & -0.307358 & 4.003301 & -1.023946 \\
\hline 1 & 1.224865 & 5.765039 & -0.262333 \\
\hline & 3.581984 & 5.825380 & -1.076898 \\
\hline & 4.375278 & 4.050923 & -2.629988 \\
\hline
\end{tabular}




$\begin{array}{rrrr}1 & 2.844838 & 2.303928 & -3.408045 \\ 1 & -0.026822 & 0.743711 & -4.586483 \\ 1 & 1.598022 & 0.700601 & -3.751525 \\ 1 & -1.757695 & 3.067176 & -2.435224 \\ 1 & -0.890907 & 3.787283 & -3.794780 \\ 1 & -1.731134 & 2.229489 & -3.990526 \\ 6 & -4.620559 & -2.195465 & -3.875832 \\ 6 & -6.672791 & -2.964661 & -2.734339 \\ 6 & -6.596392 & -0.691148 & -3.766914 \\ 6 & -8.232161 & -0.469277 & 1.376724 \\ 6 & -6.330035 & -0.800498 & 2.928718 \\ 6 & -6.849510 & 1.478901 & 2.029128 \\ 1 & -8.886759 & -0.269883 & 2.234578 \\ 1 & -8.647607 & 0.068363 & 0.514671 \\ 1 & -8.283193 & -1.545882 & 1.168482 \\ 1 & -7.520629 & 1.665699 & 2.878382 \\ 1 & -5.860028 & 1.872044 & 2.294583 \\ 1 & -7.220491 & 2.057766 & 1.172936 \\ 1 & -7.007318 & -0.634862 & 3.777506 \\ 1 & -6.307733 & -1.878688 & 2.720479 \\ 1 & -5.321579 & -0.503242 & 3.243965 \\ 1 & -6.995920 & -3.405852 & -3.686485 \\ 1 & -6.121789 & -3.732603 & -2.175703 \\ 1 & -7.578941 & -2.716515 & -2.169263 \\ 1 & -6.950774 & -1.100475 & -4.722878 \\ 1 & -7.473831 & -0.359469 & -3.197232 \\ 1 & -5.983457 & 0.193652 & -3.983578 \\ 1 & -5.006057 & -2.676243 & -4.783848 \\ 1 & -3.990538 & -1.356111 & -4.197771 \\ 1 & -3.983390 & -2.924343 & -3.355434 \\ 6 & -0.188922 & 5.890699 & 3.170522 \\ 6 & -0.578558 & 3.700762 & 4.268958 \\ 6 & 1.351336 & 4.006114 & 2.724920 \\ 6 & -3.951647 & 6.542471 & 0.226482 \\ 6 & -3.827618 & 5.129568 & -1.802580 \\ 6 & -5.386864 & 4.530424 & 0.060934 \\ 1 & -6.176559 & 5.139955 & -0.398775 \\ 1 & -5.508677 & 3.501169 & -0.300396 \\ 1 & -5.551461 & 4.527286 & 1.146639 \\ 1 & -4.591486 & 5.769743 & -2.264136 \\ 1 & -2.841481 & 5.528828 & -2.076887 \\ 1 & -3.921260 & 4.130884 & -2.246688 \\ 1 & -4.743677 & 7.130844 & -0.253595 \\ 1 & -4.110354 & 6.604152 & 1.310891 \\ 1 & -2.994848 & 7.025105 & -0.010801 \\ 1 & 0.495153 & 6.190847 & 3.974052 \\ 1 & 0.102858 & 6.438814 & 2.265014 \\ 1 & -1.193172 & 6.221798 & 3.462665 \\ 1 & 0.023658 & 4.043596 & 5.121639 \\ 1 & -1.630520 & 3.935367 & 4.479873 \\ 1 & -0.480848 & 2.608428 & 4.208103 \\ 1 & 1.986839 & 4.421679 & 3.518161 \\ 1 & 1.504383 & 2.920144 & 2.731663 \\ & 1.703987 & 4.399456 & 1.761253\end{array}$

$3_{\text {IIA-si- }} \psi_{-1522354 a}$ 
Number of imaginary frequencies : 0

The smallest frequencies are : $18.670920 .5548 \quad 25.0304 \mathrm{~cm}(-1)$

Electronic energy : $\quad \mathrm{HF}=-4360.8794556$

Zero-point correction $=\quad 1.456147($ Hartree/Particle $)$

Thermal correction to Energy $=\quad 1.540185$

Thermal correction to Enthalpy= $\quad 1.541129$

Thermal correction to Gibbs Free Energy= $\quad 1.341376$

Sum of electronic and zero-point Energies $=\quad-4359.423309$

Sum of electronic and thermal Energies $=\quad-4359.339271$

Sum of electronic and thermal Enthalpies $=\quad-4359.338326$

Sum of electronic and thermal Free Energies $=\quad-4359.538080$

Cartesian Coordinates

$\begin{array}{cccc}6 & -2.879701 & 2.815853 & 0.045942 \\ 6 & -1.877586 & 1.991799 & 0.554638 \\ 6 & -0.933393 & 2.530028 & 1.435746 \\ 6 & -1.045959 & 3.844422 & 1.896311 \\ 6 & -2.073552 & 4.638792 & 1.372051 \\ 6 & -2.987493 & 4.153999 & 0.433953 \\ 15 & -1.774091 & 0.242438 & -0.011754 \\ 6 & -3.554956 & -0.167161 & -0.298645 \\ 6 & -4.498108 & 0.039213 & 0.718413 \\ 6 & -5.838567 & -0.273376 & 0.529175 \\ 6 & -6.226034 & -0.788854 & -0.720230 \\ 6 & -5.318648 & -1.003569 & -1.751462 \\ 6 & -3.971342 & -0.697558 & -1.509302 \\ 6 & -6.882751 & -0.086864 & 1.628446 \\ 6 & -5.712119 & -1.554182 & -3.120089 \\ 6 & -0.141750 & 4.344600 & 3.025164 \\ 6 & -4.111180 & 5.006192 & -0.152742 \\ 45 & -0.376049 & 0.055653 & -1.971015 \\ 6 & -0.738412 & -1.563897 & -2.974451 \\ 8 & -1.029312 & -2.245499 & -3.864971 \\ 6 & 0.123607 & 2.119389 & -2.889360 \\ 6 & 0.531022 & 1.021494 & -3.681292 \\ 6 & 1.116930 & 3.027162 & -2.251525 \\ 6 & 2.449533 & 3.084046 & -2.690860 \\ 6 & 3.307397 & 4.091169 & -2.266512 \\ 6 & 2.867093 & 5.065087 & -1.375080 \\ 6 & 1.560523 & 5.003319 & -0.898337 \\ 6 & 0.699347 & 4.000647 & -1.330072 \\ 6 & -1.161528 & 2.839705 & -3.259100 \\ 15 & 1.592694 & -0.690588 & -0.881681 \\ 8 & 2.517320 & 0.099253 & 0.229123 \\ 6 & 3.609800 & 0.889275 & 0.014620 \\ 6 & 4.762299 & 0.415322 & -0.588656 \\ 6 & 5.822451 & 1.358436 & -0.814739 \\ 6 & 5.743793 & 2.662348 & -0.239729 \\ 6 & 4.591395 & 3.028351 & 0.496631 \\ 6 & 3.530874 & 2.174714 & 0.582948 \\ 6 & 6.791271 & 3.588816 & -0.457487 \\ 6 & 7.876218 & 3.260892 & -1.230698 \\ 6 & 7.947252 & 1.983076 & -1.822683 \\ 6 & 6.952177 & 1.058467 & -1.617602 \\ & & & \\ 6\end{array}$




\begin{tabular}{|c|c|c|c|}
\hline 6 & 4.902441 & -1.011848 & -0.959721 \\
\hline & 3.930798 & -1.650227 & -1.707568 \\
\hline & 4.092715 & -2.962712 & -2.199761 \\
\hline & 5.194477 & -3.691776 & -1.848120 \\
\hline & 6.156056 & -3.149320 & -0.960613 \\
\hline & 6.014962 & -1.802611 & -0.507525 \\
\hline & 7.237531 & -3.936323 & -0.494592 \\
\hline & 8.135028 & -3.436454 & 0.413352 \\
\hline & 7.978534 & -2.120019 & 0.894737 \\
\hline & 6.954075 & -1.324655 & 0.443447 \\
\hline & 2.757623 & -1.012992 & -2.038471 \\
\hline & 1.360095 & -2.057684 & 0.105037 \\
\hline & 0.285707 & -2.932758 & -0.266896 \\
\hline 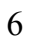 & -0.949592 & -2.838500 & 0.345828 \\
\hline & -2.011160 & -3.693795 & -0.088799 \\
\hline 6 & -1.756923 & -4.680918 & -1.087076 \\
\hline G & -0.457935 & -4.785818 & -1.637505 \\
\hline 6 & 0.528828 & -3.920278 & -1.249829 \\
\hline b & -2.801801 & -5.549338 & -1.488026 \\
\hline 6 & -4.051882 & -5.446941 & -0.933667 \\
\hline 6 & -4.313141 & -4.457940 & 0.039510 \\
\hline & -3.319167 & -3.607599 & 0.455102 \\
\hline 6 & -1.150784 & -2.021688 & 1.576993 \\
\hline 6 & -1.525617 & -0.685612 & 1.585183 \\
\hline 6 & -1.805222 & -0.058015 & 2.832315 \\
\hline 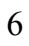 & -1.663387 & -0.718992 & 4.021264 \\
\hline 6 & -1.228924 & -2.063004 & 4.050808 \\
\hline 6 & -0.987934 & -2.731531 & 2.816302 \\
\hline 6 & -0.562169 & -4.085720 & 2.868760 \\
\hline 6 & -0.367640 & -4.722438 & 4.069818 \\
\hline & -0.599567 & -4.048961 & 5.286746 \\
\hline 6 & -1.026248 & -2.745951 & 5.273087 \\
\hline 6 & 2.368790 & -2.665277 & 1.007080 \\
\hline 6 & 2.857091 & -1.818831 & 2.156356 \\
\hline 6 & 2.007138 & -0.983005 & 2.883037 \\
\hline 6 & 2.487489 & -0.245616 & 3.956461 \\
\hline 6 & 3.829542 & -0.332139 & 4.325666 \\
\hline & 4.681657 & -1.165809 & 3.611181 \\
\hline 6 & 4.195505 & -1.904643 & 2.534478 \\
\hline & 6.836818 & -0.321865 & 0.845088 \\
\hline 1 & -2.141003 & 0.975359 & 2.848007 \\
\hline & 7.329134 & -4.956767 & -0.864499 \\
\hline 1 & -1.212755 & -2.210017 & 6.202663 \\
\hline 1 & 6.703834 & 4.578503 & -0.009438 \\
\hline ] & -3.534890 & -2.843374 & 1.200485 \\
\hline 1 & -5.312702 & -4.364720 & 0.459696 \\
\hline & 4.541617 & 4.018663 & 0.947580 \\
\hline 1 & -1.881691 & -0.212932 & 4.961309 \\
\hline 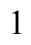 & 5.333041 & -4.704778 & -2.222040 \\
\hline 1 & 8.794006 & 1.728729 & -2.456441 \\
\hline 1 & 3.327581 & -3.357658 & -2.864754 \\
\hline 1 & -2.588433 & -6.300269 & -2.247457 \\
\hline 1 & -0.263943 & -5.550971 & -2.387521 \\
\hline & -0.033477 & -5.757311 & 4.082131 \\
\hline 1 & 7.018129 & 0.084287 & -2.095581 \\
\hline 1 & -0.381519 & -4.626305 & 1.941946 \\
\hline
\end{tabular}




\begin{tabular}{|c|c|c|c|}
\hline . & 1.527212 & -3.978154 & -1.680879 \\
\hline 1 & 8.671115 & 3.983631 & -1.399724 \\
\hline 1 & 8.672540 & -1.730928 & 1.636546 \\
\hline & -4.847508 & -6.118561 & -1.249107 \\
\hline & -0.440302 & -4.565149 & 6.230572 \\
\hline 1 & 8.955650 & -4.053041 & 0.772521 \\
\hline & 2.604017 & 2.446856 & 1.078182 \\
\hline 1 & -4.169457 & 0.457070 & 1.665991 \\
\hline & -3.232000 & -0.873475 & -2.285894 \\
\hline 1 & -7.276723 & -1.024189 & -0.878815 \\
\hline 1 & -3.601707 & 2.400148 & -0.656835 \\
\hline & -0.129388 & 1.891240 & 1.808011 \\
\hline 1 & -2.168737 & 5.664896 & 1.713435 \\
\hline 1 & 1.878779 & -3.558616 & 1.420241 \\
\hline 1 & -1.729446 & 0.472440 & -2.670808 \\
\hline 1 & 3.234804 & -3.032160 & 0.436106 \\
\hline 1 & 4.874054 & -2.544673 & 1.968308 \\
\hline 1 & 0.970048 & -0.883510 & 2.571374 \\
\hline 1 & 5.734461 & -1.237820 & 3.879173 \\
\hline 1 & 1.806694 & 0.403339 & 4.507144 \\
\hline 1 & 4.207021 & 0.251759 & 5.162452 \\
\hline 1 & -0.327839 & 3.988763 & -0.967398 \\
\hline 1 & 1.194471 & 5.757811 & -0.201936 \\
\hline 1 & 3.537393 & 5.861390 & -1.055396 \\
\hline 1 & 4.327859 & 4.119435 & -2.645507 \\
\hline 1 & 2.807955 & 2.360922 & -3.421011 \\
\hline 1 & -0.050500 & 0.768494 & -4.568857 \\
\hline 1 & 1.580158 & 0.734989 & -3.744994 \\
\hline 1 & -1.783461 & 3.068544 & -2.385597 \\
\hline 1 & -0.935411 & 3.798536 & -3.751962 \\
\hline 1 & -1.768888 & 2.236272 & -3.944077 \\
\hline 6 & -7.223127 & -1.709097 & -3.270998 \\
\hline 6 & -5.218349 & -0.603807 & -4.218234 \\
\hline 6 & -5.065592 & -2.929772 & -3.312106 \\
\hline 6 & -6.283063 & 0.515323 & 2.897566 \\
\hline 6 & -7.994975 & 0.847162 & 1.140274 \\
\hline 6 & -7.481043 & -1.452347 & 1.985269 \\
\hline 1 & -7.066461 & 0.628696 & 3.657467 \\
\hline 1 & -5.497940 & -0.122009 & 3.326468 \\
\hline 1 & -5.854786 & 1.510105 & 2.714250 \\
\hline 1 & -8.233789 & -1.345832 & 2.778098 \\
\hline 1 & -7.969712 & -1.926171 & 1.125120 \\
\hline 1 & -6.702343 & -2.136623 & 2.348163 \\
\hline 1 & -8.749414 & 0.983027 & 1.926831 \\
\hline 1 & -7.594040 & 1.836819 & 0.883658 \\
\hline 1 & -8.509777 & 0.454710 & 0.255305 \\
\hline 1 & -5.524456 & -0.975845 & -5.205057 \\
\hline 1 & -5.639448 & 0.402073 & -4.090079 \\
\hline 1 & -4.124416 & -0.511774 & -4.229836 \\
\hline 1 & -5.302416 & -3.327428 & -4.308465 \\
\hline 1 & -3.972684 & -2.888902 & -3.218550 \\
\hline 1 & -5.434200 & -3.642293 & -2.562808 \\
\hline 1 & -7.455860 & -2.095052 & -4.271431 \\
\hline 1 & -7.638807 & -2.418777 & -2.544086 \\
\hline 1 & -7.747238 & -0.751130 & -3.155980 \\
\hline 6 & -0.224744 & 5.858279 & 3.215472 \\
\hline
\end{tabular}




$\begin{array}{rrrr}6 & -0.598871 & 3.668692 & 4.324935 \\ 6 & 1.321213 & 3.980146 & 2.770334 \\ 6 & -4.112185 & 6.430419 & 0.396232 \\ 6 & -3.946740 & 5.081486 & -1.674898 \\ 6 & -5.462383 & 4.362717 & 0.183861 \\ 1 & -6.283793 & 4.952761 & -0.244612 \\ 1 & -5.544136 & 3.342340 & -0.212591 \\ 1 & -5.612727 & 4.313317 & 1.270715 \\ 1 & -4.733867 & 5.709987 & -2.112868 \\ 1 & -2.975302 & 5.518381 & -1.944634 \\ 1 & -4.012446 & 4.093077 & -2.146302 \\ 1 & -4.933985 & 6.998573 & -0.057418 \\ 1 & -4.259696 & 6.452775 & 1.483790 \\ 1 & -3.179638 & 6.960458 & 0.163577 \\ 1 & 0.470202 & 6.166868 & 4.006415 \\ 1 & 0.049021 & 6.401945 & 2.301603 \\ 1 & -1.225517 & 6.186480 & 3.522232 \\ 1 & 0.003785 & 4.018219 & 5.174623 \\ 1 & -1.651872 & 3.895585 & 4.538990 \\ 1 & -0.493479 & 2.576823 & 4.265661 \\ 1 & 1.956776 & 4.390642 & 3.566118 \\ 1 & 1.479387 & 2.894733 & 2.766568 \\ 1 & 1.670016 & 4.384418 & 1.809701\end{array}$

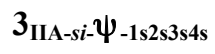

Number of imaginary frequencies : 0

The smallest frequencies are : $17.2351 \quad 21.046524 .6069 \mathrm{~cm}(-1)$

Electronic energy : $\quad \mathrm{HF}=-4360.8795274$

Zero-point correction $=\quad 1.455937$ (Hartree/Particle)

Thermal correction to Energy= $\quad 1.540056$

Thermal correction to Enthalpy= $\quad 1.541000$

Thermal correction to Gibbs Free Energy $=\quad 1.340731$

Sum of electronic and zero-point Energies $=\quad-4359.423590$

Sum of electronic and thermal Energies $=\quad-4359.339471$

Sum of electronic and thermal Enthalpies $=\quad-4359.338527$

Sum of electronic and thermal Free Energies $=\quad-4359.538796$

Cartesian Coordinates

$\begin{array}{cccc}6 & -2.888483 & 2.841190 & 0.056502 \\ 6 & -1.885293 & 2.011551 & 0.554899 \\ 6 & -0.932334 & 2.542746 & 1.431201 \\ 6 & -1.035359 & 3.856038 & 1.896704 \\ 6 & -2.056036 & 4.659687 & 1.373325 \\ 6 & -2.978632 & 4.182714 & 0.439535 \\ 15 & -1.779610 & 0.264870 & -0.020685 \\ 6 & -3.558907 & -0.151472 & -0.311404 \\ 6 & -4.491873 & 0.000893 & 0.716250 \\ 6 & -5.833109 & -0.332286 & 0.535499 \\ 6 & -6.230421 & -0.802591 & -0.722472 \\ 6 & -5.326349 & -0.962614 & -1.773718 \\ 6 & -3.984665 & -0.646476 & -1.538356 \\ 6 & -6.791737 & -0.193986 & 1.717019 \\ 6 & -5.728884 & -1.489697 & -3.149526 \\ 6 & -0.128679 & 4.344529 & 3.028781\end{array}$




\begin{tabular}{|c|c|c|c|}
\hline 6 & -4.086893 & 5.052298 & -0.152076 \\
\hline 45 & -0.379210 & 0.081533 & -1.978220 \\
\hline 6 & -0.754414 & -1.529721 & -2.990777 \\
\hline 8 & -1.061570 & -2.201462 & -3.883307 \\
\hline 6 & 0.129901 & 2.146253 & -2.884952 \\
\hline 6 & 0.529706 & 1.052030 & -3.685698 \\
\hline 6 & 1.129396 & 3.040691 & -2.238461 \\
\hline 6 & 2.467091 & 3.081225 & -2.664539 \\
\hline 6 & 3.333347 & 4.077367 & -2.231599 \\
\hline 6 & 2.896134 & 5.057991 & -1.345892 \\
\hline 6 & 1.583836 & 5.013490 & -0.883401 \\
\hline 6 & 0.715012 & 4.020604 & -1.322280 \\
\hline 6 & -1.152069 & 2.875913 & -3.247152 \\
\hline 15 & 1.584400 & -0.681615 & -0.892340 \\
\hline 8 & 2.508973 & 0.098623 & 0.225057 \\
\hline 6 & 3.614168 & 0.873990 & 0.023781 \\
\hline 6 & 4.764419 & 0.390560 & -0.576547 \\
\hline 6 & 5.836581 & 1.323012 & -0.790770 \\
\hline 6 & 5.770803 & 2.623560 & -0.206506 \\
\hline 6 & 4.618647 & 2.998246 & 0.525779 \\
\hline 6 & 3.547574 & 2.156771 & 0.599726 \\
\hline 6 & 6.830772 & 3.538674 & -0.411515 \\
\hline 6 & 7.915496 & 3.203582 & -1.181915 \\
\hline 6 & 7.973517 & 1.929907 & -1.784121 \\
\hline 6 & 6.966311 & 1.015952 & -1.591071 \\
\hline 6 & & -1.034999 & -0.958901 \\
\hline 6 & 3.915692 & -1.656914 & -1.715949 \\
\hline 6 & 4.065451 & -2.967028 & -2.218235 \\
\hline 6 & 5.158275 & -3.710453 & -1.868887 \\
\hline 6 & 6.122453 & -3.185249 & -0.973839 \\
\hline 6 & 5.993812 & -1.841014 & -0.509987 \\
\hline 6 & 7.193905 & -3.987455 & -0.510719 \\
\hline 6 & 8.093459 & -3.504708 & 0.404482 \\
\hline 6 & 7.949234 & -2.190635 & 0.896106 \\
\hline 6 & 6.934679 & -1.380898 & 0.447930 \\
\hline 8 & 2.750992 & -1.004250 & -2.046758 \\
\hline 7 & 1.340240 & -2.053838 & 0.084895 \\
\hline 6 & 0.259412 & -2.918632 & -0.292868 \\
\hline 6 & -0.974173 & -2.820939 & 0.322530 \\
\hline 6 & -2.042688 & -3.665343 & -0.115687 \\
\hline 6 & -1.797253 & -4.646264 & -1.122195 \\
\hline 6 & -0.499624 & -4.756724 & -1.674902 \\
\hline 6 & 0.494186 & -3.901255 & -1.282646 \\
\hline 6 & -2.849396 & -5.502821 & -1.529383 \\
\hline 6 & -4.098137 & -5.394261 & -0.973240 \\
\hline 6 & -4.350207 & -4.411651 & 0.008846 \\
\hline 6 & -3.349073 & -3.573257 & 0.431595 \\
\hline 6 & -1.168758 & -2.009963 & 1.557952 \\
\hline 6 & -1.533948 & -0.671511 & 1.572375 \\
\hline 6 & -1.806111 & -0.047820 & 2.823025 \\
\hline 6 & -1.669592 & -0.716225 & 4.008709 \\
\hline 6 & -1.247803 & -2.064337 & 4.031293 \\
\hline 6 & -1.011096 & -2.727633 & 2.793281 \\
\hline 6 & -0.596612 & -4.085574 & 2.838245 \\
\hline 6 & -0.410121 & -4.731221 & 4.035796 \\
\hline 6 & -0.638774 & -4.063186 & 5.256377 \\
\hline
\end{tabular}




\begin{tabular}{|c|c|c|c|}
\hline 6 & -1.053502 & -2.756290 & 5.249847 \\
\hline & 2.338622 & -2.674736 & 0.989280 \\
\hline & 2.835534 & -1.836129 & 2.140649 \\
\hline & 1.994291 & -0.992474 & 2.868719 \\
\hline & 2.481380 & -0.265471 & 3.946142 \\
\hline & 3.821307 & -0.370572 & 4.318412 \\
\hline & 4.664405 & -1.212504 & 3.603073 \\
\hline & 4.171548 & -1.940880 & 2.522241 \\
\hline & 6.826753 & -0.380214 & 0.857240 \\
\hline & -2.132366 & 0.988405 & 2.843917 \\
\hline & 7.276041 & -5.005765 & -0.888636 \\
\hline & -1.236945 & -2.224265 & 6.182266 \\
\hline & 6.753510 & 4.525582 & 0.044355 \\
\hline & -3.557888 & -2.813912 & 1.184931 \\
\hline & -5.348926 & -4.314731 & 0.430204 \\
\hline & 4.577697 & 3.986445 & 0.982316 \\
\hline & -1.882080 & -0.212720 & 4.951465 \\
\hline & 5.287673 & -4.721744 & -2.250613 \\
\hline & 8.819963 & 1.670304 & -2.416152 \\
\hline & 3.298405 & -3.348448 & -2.888898 \\
\hline & -2.642940 & -6.249181 & -2.295199 \\
\hline & -0.312347 & -5.517785 & -2.430777 \\
\hline & -0.084882 & -5.768981 & 4.042502 \\
\hline & 7.022779 & 0.045027 & -2.076778 \\
\hline & -0.418755 & -4.622010 & 1.908483 \\
\hline & 1.491629 & -3.963665 & -1.715229 \\
\hline & 8.720143 & 3.917736 & -1.341023 \\
\hline & 8.644763 & -1.814843 & 1.643325 \\
\hline & -4.899806 & -6.056266 & -1.293702 \\
\hline & -0.486275 & -4.586568 & 6.197348 \\
\hline & 8.906166 & -4.132986 & 0.761382 \\
\hline & 2.620014 & 2.438163 & 1.088576 \\
\hline & -4.165150 & 0.383761 & 1.682046 \\
\hline & -3.251373 & -0.789521 & -2.327069 \\
\hline 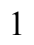 & -7.275236 & -1.052114 & -0.883461 \\
\hline & -3.619062 & 2.430055 & -0.640383 \\
\hline 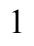 & -0.129784 & 1.898898 & 1.797758 \\
\hline & -2.138401 & 5.687735 & 1.711990 \\
\hline & 1.834912 & -3.560781 & 1.401948 \\
\hline & -1.729750 & 0.508893 & -2.676651 \\
\hline & 3.200438 & -3.054435 & 0.420263 \\
\hline 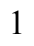 & 4.843061 & -2.588331 & 1.956198 \\
\hline & 0.959057 & -0.878967 & 2.555399 \\
\hline 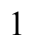 & 5.715417 & -1.299482 & 3.873640 \\
\hline & 1.807575 & 0.389573 & 4.498182 \\
\hline & 4.203984 & 0.205112 & 5.158517 \\
\hline 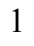 & -0.315364 & 4.021740 & -0.968643 \\
\hline & 1.219692 & 5.773783 & -0.192390 \\
\hline 1 & 3.572776 & 5.846476 & -1.020371 \\
\hline & 4.358038 & 4.092156 & -2.599829 \\
\hline 1 & 2.824383 & 2.353605 & -3.390753 \\
\hline & -0.055807 & 0.808619 & -4.573249 \\
\hline 1 & 1.576926 & 0.759762 & -3.754068 \\
\hline 1 & -1.774830 & 3.095864 & -2.371960 \\
\hline & -0.922212 & 3.839822 & -3.728195 \\
\hline & -1.760289 & 2.282805 & -3.940377 \\
\hline
\end{tabular}




$\begin{array}{rrrr}6 & -7.242355 & -1.621737 & -3.301254 \\ 6 & -5.224198 & -0.537683 & -4.240946 \\ 6 & -5.101154 & -2.872763 & -3.352945 \\ 6 & -8.219662 & -0.600579 & 1.362682 \\ 6 & -6.306589 & -1.100542 & 2.855471 \\ 6 & -6.815441 & 1.262187 & 2.194728 \\ 1 & -8.864599 & -0.485821 & 2.243042 \\ 1 & -8.639194 & 0.025396 & 0.564566 \\ 1 & -8.281185 & -1.649756 & 1.045637 \\ 1 & -7.480955 & 1.368482 & 3.062067 \\ 1 & -5.821044 & 1.617567 & 2.492883 \\ 1 & -7.184702 & 1.927604 & 1.403020 \\ 1 & -6.975007 & -1.018063 & 3.723247 \\ 1 & -6.293390 & -2.152165 & 2.538700 \\ 1 & -5.292973 & -0.843269 & 3.189310 \\ 1 & -5.534680 & -0.899858 & -5.230008 \\ 1 & -5.634434 & 0.471856 & -4.106227 \\ 1 & -4.129618 & -0.457006 & -4.253414 \\ 1 & -5.347126 & -3.262048 & -4.350485 \\ 1 & -4.007456 & -2.844339 & -3.262702 \\ 1 & -5.474382 & -3.585238 & -2.605575 \\ 1 & -7.481528 & -1.976243 & -4.311766 \\ 1 & -7.666276 & -2.346805 & -2.594656 \\ 1 & -7.754054 & -0.660995 & -3.157363 \\ 6 & -0.208873 & 5.856654 & 3.232628 \\ 6 & -0.587149 & 3.659972 & 4.323628 \\ 6 & 1.333384 & 3.978345 & 2.770889 \\ 6 & -4.060134 & 6.479787 & 0.388239 \\ 6 & -3.919988 & 5.116686 & -1.674506 \\ 6 & -5.451220 & 4.439951 & 0.186785 \\ 1 & -6.258332 & 5.045303 & -0.247493 \\ 1 & -5.556211 & 3.418955 & -0.202463 \\ 1 & -5.603928 & 4.401906 & 1.273503 \\ 1 & -4.699159 & 5.752277 & -2.116472 \\ 1 & -2.943347 & 5.540695 & -1.945871 \\ 1 & -3.997257 & 4.126435 & -2.140289 \\ 1 & -4.871697 & 7.060282 & -0.068219 \\ 1 & -4.206247 & 6.511792 & 1.475725 \\ 1 & -3.118112 & 6.990747 & 0.151158 \\ 1 & 0.483630 & 6.156112 & 4.029183 \\ 1 & 0.070168 & 6.408601 & 2.325413 \\ 1 & -1.210382 & 6.183868 & 3.538087 \\ 1 & 0.018954 & 3.999564 & 5.174874 \\ 1 & -1.638533 & 3.890751 & 4.541452 \\ 1 & -0.487346 & 2.568169 & 4.255542 \\ 1 & 1.970935 & 4.388780 & 3.565128 \\ 1 & 1.490051 & 2.892843 & 2.767552 \\ 1 & 1.680957 & 4.381548 & 1.809457\end{array}$

$3_{\text {IIB-re- }} \psi_{-1 \mathbf{a} 2 a 3 a 4 a}$

Number of imaginary frequencies : 0

The smallest frequencies are : $19.927522 .510322 .8766 \mathrm{~cm}(-1)$ Electronic energy : $\quad \mathrm{HF}=-4360.8843153$

Zero-point correction $=\quad 1.457681$ (Hartree/Particle) Thermal correction to Energy $=\quad 1.541059$ 
Thermal correction to Enthalpy= $\quad 1.542003$

Thermal correction to Gibbs Free Energy $=\quad 1.344380$

Sum of electronic and zero-point Energies $=\quad-4359.426634$

Sum of electronic and thermal Energies $=\quad-4359.343256$

Sum of electronic and thermal Enthalpies $=\quad-4359.342312$

Sum of electronic and thermal Free Energies $=\quad-4359.539935$

Cartesian Coordinates

$\begin{array}{cccc}0 & -1.218466 & 4.377817 & -1.563423 \\ 6 & -0.973447 & 3.311000 & -2.437277 \\ 6 & -1.925456 & 3.090541 & -3.449878 \\ 6 & -3.050908 & 3.890243 & -3.575603 \\ 6 & -3.271931 & 4.948890 & -2.694173 \\ 6 & -2.348237 & 5.180834 & -1.683723 \\ 6 & 0.268918 & 2.497622 & -2.305137 \\ 6 & 1.341422 & 3.076677 & -1.414215 \\ 45 & -0.155631 & 0.282219 & -1.844964 \\ 6 & 0.686919 & 1.623531 & -3.335394 \\ 15 & -1.583327 & 0.111454 & 0.081313 \\ 6 & -3.226184 & -0.678439 & -0.209344 \\ 6 & -3.620520 & -1.053518 & -1.487828 \\ 6 & -4.887442 & -1.599123 & -1.721820 \\ 6 & -5.743462 & -1.744818 & -0.629823 \\ 6 & -5.377472 & -1.385781 & 0.673886 \\ 6 & -4.103613 & -0.857932 & 0.864491 \\ 6 & -5.352519 & -1.983505 & -3.125168 \\ 6 & -4.201834 & -1.973029 & -4.130525 \\ 6 & -6.358615 & -1.584737 & 1.828182 \\ 6 & -5.780443 & -1.111590 & 3.159964 \\ 6 & -2.092385 & 1.763622 & 0.726910 \\ 6 & -3.395551 & 2.221669 & 0.548410 \\ 6 & -3.811517 & 3.458437 & 1.045688 \\ 6 & -2.870564 & 4.226683 & 1.729835 \\ 6 & -1.537828 & 3.827586 & 1.886331 \\ 6 & -1.157889 & 2.590650 & 1.361199 \\ 6 & -0.575987 & 4.741820 & 2.646023 \\ 6 & 0.854965 & 4.212217 & 2.640762 \\ 6 & -5.264672 & 3.920492 & 0.935403 \\ 6 & -6.070540 & 3.053809 & -0.031277 \\ 6 & -1.090021 & -0.811453 & 1.624528 \\ 6 & -0.618795 & -2.117815 & 1.544058 \\ 6 & -0.304293 & -2.840456 & 2.741965 \\ 6 & -0.474931 & -2.215475 & 4.011431 \\ 6 & -0.973925 & -0.895533 & 4.057362 \\ 6 & -1.266528 & -0.218619 & 2.904007 \\ 6 & -0.116201 & -2.910897 & 5.190974 \\ 6 & 0.397453 & -4.180314 & 5.128841 \\ 6 & 0.559933 & -4.810510 & 3.876943 \\ 6 & 0.212514 & -4.163403 & 2.717269 \\ 6 & -0.434049 & -2.858559 & 0.261136 \\ 6 & 0.761120 & -2.796233 & -0.431961 \\ 6 & 0.994335 & -3.621479 & -1.556042 \\ 6 & 0.025437 & -4.470284 & -2.015228 \\ 6 & -1.228914 & -4.537741 & -1.365778 \\ 6 & -1.454921 & -3.749981 & -0.197235\end{array}$




\begin{tabular}{|c|c|c|c|}
\hline 6 & -2.699094 & -3.884031 & 0.471179 \\
\hline & -3.674582 & -4.718774 & -0.013546 \\
\hline & -3.452780 & -5.483017 & -1.179766 \\
\hline & -2.253733 & -5.393199 & -1.839020 \\
\hline & 1.808693 & -1.907344 & -0.016862 \\
\hline & 2.927649 & -2.570850 & 0.699107 \\
\hline & 3.493803 & -1.863800 & 1.905038 \\
\hline & 4.865819 & -1.920861 & 2.142540 \\
\hline & 5.418490 & -1.325592 & 3.275019 \\
\hline & 4.601400 & -0.665938 & 4.185145 \\
\hline & 3.227645 & -0.605645 & 3.954276 \\
\hline & 2.681434 & -1.200509 & 2.825798 \\
\hline & -0.309688 & -1.190403 & -3.084569 \\
\hline & -0.473741 & -1.793127 & -4.060548 \\
\hline 15 & 1.881151 & -0.419857 & -0.830958 \\
\hline & 2.778995 & 0.312411 & 0.353068 \\
\hline 6 & 3.763160 & 1.246233 & 0.205118 \\
\hline & 4.911816 & 1.013068 & -0.531801 \\
\hline 6 & 5.805497 & 2.121291 & -0.729854 \\
\hline & 5.583197 & 3.346913 & -0.032564 \\
\hline 6 & 4.469367 & 3.460121 & 0.835522 \\
\hline & 3.572468 & 2.436572 & 0.938146 \\
\hline & 6.900565 & 2.062760 & -1.628975 \\
\hline & 7.737024 & 3.138057 & -1.804365 \\
\hline & 7.532738 & 4.333784 & -1.086303 \\
\hline & 6.472191 & 4.431306 & -0.221871 \\
\hline & 5.210271 & -0.330512 & -1.083667 \\
\hline & 4.278566 & -1.003932 & -1.848904 \\
\hline & 4.562803 & -2.224428 & -2.497763 \\
\hline 5 & 5.770231 & -2.833379 & -2.299191 \\
\hline & 6.720764 & -2.264226 & -1.416260 \\
\hline & 6.445360 & -1.007541 & -0.797196 \\
\hline 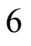 & 7.925108 & -2.945337 & -1.113930 \\
\hline & 8.819202 & -2.431569 & -0.210185 \\
\hline 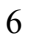 & 8.535491 & -1.208203 & 0.431399 \\
\hline & 7.386188 & -0.513683 & 0.142778 \\
\hline 3 & 3.022277 & -0.484263 & -2.048702 \\
\hline & -1.040153 & 4.851658 & 4.102766 \\
\hline & -0.560723 & 6.135422 & 2.007924 \\
\hline 6 & -5.907845 & 3.821237 & 2.323787 \\
\hline & -5.345957 & 5.368962 & 0.444911 \\
\hline 6 & -6.411958 & -0.974871 & -3.583120 \\
\hline & -5.956042 & -3.391027 & -3.121718 \\
\hline & -6.698573 & -3.073174 & 1.960561 \\
\hline & -7.641186 & -0.790999 & 1.558270 \\
\hline & 7.173336 & 0.414777 & 0.665945 \\
\hline & -1.654704 & 0.794246 & 2.973151 \\
\hline & 8.116029 & -3.897707 & -1.606923 \\
\hline & -0.249668 & -2.408837 & 6.148338 \\
\hline & 6.285576 & 5.352360 & 0.328965 \\
\hline & -2.884390 & -3.295363 & 1.368195 \\
\hline & -4.630006 & -4.791855 & 0.502937 \\
\hline & 4.326682 & 4.381488 & 1.398980 \\
\hline & -1.119243 & -0.420186 & 5.026789 \\
\hline & 6.006896 & -3.774098 & -2.793400 \\
\hline 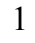 & 8.561507 & 3.066922 & -2.510304 \\
\hline
\end{tabular}




\begin{tabular}{|c|c|c|c|}
\hline & 3.801965 & -2.646335 & -3.151368 \\
\hline & -2.063355 & -5.982909 & -2.734721 \\
\hline & 0.197957 & -5.089873 & -2.893878 \\
\hline & 0.961886 & -5.820067 & 3.830704 \\
\hline & 7.067487 & 1.154251 & -2.201792 \\
\hline & 0.339885 & -4.667709 & 1.761617 \\
\hline & 1.960666 & -3.546964 & -2.053915 \\
\hline & 8.206026 & 5.175758 & -1.228909 \\
\hline & 9.230553 & -0.812214 & 1.168578 \\
\hline & -4.232870 & -6.146089 & -1.548141 \\
\hline & 0.679198 & -4.704377 & 6.039132 \\
\hline & 9.736120 & -2.967927 & 0.022488 \\
\hline & 2.691235 & 2.498367 & 1.571863 \\
\hline & -3.781697 & -0.563510 & 1.860582 \\
\hline & -2.920095 & -0.914771 & -2.305540 \\
\hline & -6.739265 & -2.156473 & -0.796316 \\
\hline & -4.103478 & 1.595757 & 0.015139 \\
\hline & -0.130832 & 2.236835 & 1.465984 \\
\hline & -3.182036 & 5.179946 & 2.156958 \\
\hline & 2.517562 & -3.535895 & 1.029419 \\
\hline & -1.532534 & 0.668968 & -2.510920 \\
\hline & 3.746331 & -2.812643 & 0.005209 \\
\hline & 5.516633 & -2.425561 & 1.426805 \\
\hline & 1.616760 & -1.119242 & 2.625144 \\
\hline & 6.494218 & -1.372537 & 3.434699 \\
\hline & 2.574664 & -0.088576 & 4.656232 \\
\hline & 5.030774 & -0.195139 & 5.067042 \\
\hline & 0.126008 & 1.544680 & -4.265653 \\
\hline & 1.751286 & 1.416961 & -3.447038 \\
\hline 1 & -0.520726 & 4.583222 & -0.754602 \\
\hline & -2.497524 & 5.997670 & -0.977473 \\
\hline l & -4.150541 & 5.582627 & -2.801971 \\
\hline & -3.760506 & 3.690709 & -4.376697 \\
\hline & -1.788881 & 2.271196 & -4.153420 \\
\hline & 1.573268 & 4.117192 & -1.693871 \\
\hline & 1.061253 & 3.083589 & -0.353116 \\
\hline 1 & 2.271519 & 2.506062 & -1.511467 \\
\hline & -4.565952 & -2.305222 & -5.111052 \\
\hline 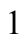 & -3.775055 & -0.970149 & -4.266466 \\
\hline 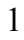 & -3.392989 & -2.651311 & -3.825070 \\
\hline 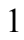 & -6.759350 & -1.215130 & -4.597326 \\
\hline 1 & -7.286158 & -0.974555 & -2.919647 \\
\hline & -6.002735 & 0.043989 & -3.596524 \\
\hline 1 & -6.285549 & -3.662266 & -4.133562 \\
\hline & -5.213005 & -4.131087 & -2.796121 \\
\hline 1 & -6.828161 & -3.474286 & -2.462175 \\
\hline l & -6.516849 & -1.264973 & 3.958792 \\
\hline 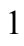 & -4.873087 & -1.666518 & 3.436510 \\
\hline 1 & -5.531994 & -0.041784 & 3.138284 \\
\hline ] & -8.359079 & -0.936575 & 2.376684 \\
\hline 1 & -7.430237 & 0.284820 & 1.484336 \\
\hline ] & -8.133034 & -1.099210 & 0.627927 \\
\hline 1 & -7.425502 & -3.229122 & 2.769149 \\
\hline 1 & -7.132900 & -3.482718 & 1.039984 \\
\hline 1 & -5.800679 & -3.658589 & 2.202304 \\
\hline 1 & 1.506325 & 4.895361 & 3.201250 \\
\hline
\end{tabular}




$\begin{array}{rrrr}1 & 0.932004 & 3.223824 & 3.114793 \\ 1 & 1.252335 & 4.142835 & 1.618702 \\ 1 & -0.363274 & 5.501372 & 4.674044 \\ 1 & -2.050406 & 5.271303 & 4.181562 \\ 1 & -1.049733 & 3.864877 & 4.584920 \\ 1 & 0.126774 & 6.795079 & 2.554333 \\ 1 & -0.220986 & 6.089687 & 0.963961 \\ 1 & -1.548626 & 6.611023 & 2.017766 \\ 1 & -6.394698 & 5.692124 & 0.402640 \\ 1 & -4.817151 & 6.067985 & 1.104322 \\ 1 & -4.920300 & 5.464958 & -0.562015 \\ 1 & -6.959982 & 4.135145 & 2.284597 \\ 1 & -5.876292 & 2.788171 & 2.695924 \\ 1 & -5.394426 & 4.456057 & 3.057095 \\ 1 & -7.096239 & 3.437460 & -0.103436 \\ 1 & -5.636513 & 3.060690 & -1.040000 \\ 1 & -6.135822 & 2.008623 & 0.302828\end{array}$

$3_{\text {IIB-re- }} \psi_{-1 \mathrm{a} 2 \mathrm{aza} a \mathrm{~s}}$

Number of imaginary frequencies : 0

The smallest frequencies are : $20.187922 .2662 \quad 25.2236 \mathrm{~cm}(-1)$

Electronic energy : $\quad H F=-4360.8852456$

Zero-point correction $=\quad 1.457434($ Hartree/Particle $)$

Thermal correction to Energy= $\quad 1.540891$

Thermal correction to Enthalpy= $\quad 1.541835$

Thermal correction to Gibbs Free Energy= 1.343943

Sum of electronic and zero-point Energies $=\quad-4359.427812$

Sum of electronic and thermal Energies $=\quad-4359.344355$

Sum of electronic and thermal Enthalpies $=\quad-4359.343410$

Sum of electronic and thermal Free Energies $=\quad-4359.541302$

\section{Cartesian Coordinates}

$\begin{array}{cccc}6 & -3.413549 & 2.223427 & 0.555079 \\ 6 & -2.107721 & 1.768303 & 0.723472 \\ 6 & -1.171069 & 2.595875 & 1.354132 \\ 6 & -1.550258 & 3.829858 & 1.886085 \\ 6 & -2.883647 & 4.228352 & 1.734993 \\ 6 & -3.827522 & 3.461036 & 1.053808 \\ 15 & -1.589256 & 0.121013 & 0.071649 \\ 45 & -0.158073 & 0.293081 & -1.852058 \\ 6 & -0.303652 & -1.175720 & -3.096378 \\ 8 & -0.458355 & -1.776283 & -4.075229 \\ 6 & -0.587114 & 4.741838 & 2.646691 \\ 6 & -1.048211 & 4.845302 & 4.104926 \\ 6 & -5.278753 & 3.930624 & 0.945737 \\ 6 & -5.919933 & 3.845516 & 2.336267 \\ 6 & -1.100249 & -0.804226 & 1.615921 \\ 6 & -0.625599 & -2.109421 & 1.537161 \\ 6 & -0.315003 & -2.832350 & 2.735992 \\ 6 & -0.495169 & -2.209819 & 4.005196 \\ 6 & -0.997847 & -0.891230 & 4.049628 \\ 6 & -1.284729 & -0.213371 & 2.895306 \\ 6 & 0.206162 & -4.153592 & 2.712515 \\ 6 & 0.548517 & -4.801304 & 3.873322\end{array}$




\begin{tabular}{|c|c|c|c|}
\hline 6 & 0.376168 & -4.173578 & 5.125142 \\
\hline 6 & -0.141687 & -2.905827 & 5.185976 \\
\hline & -0.439988 & -2.851387 & 0.255350 \\
\hline & 0.752358 & -2.787986 & -0.442106 \\
\hline & 0.980991 & -3.614157 & -1.566719 \\
\hline & 0.011838 & -4.466312 & -2.019876 \\
\hline & -1.238282 & -4.538538 & -1.362372 \\
\hline & -1.459850 & -3.747654 & -0.195594 \\
\hline & -2.262007 & -5.402818 & -1.822167 \\
\hline & -3.455063 & -5.497513 & -1.152220 \\
\hline & -3.672048 & -4.728609 & 0.011907 \\
\hline & -2.697970 & -3.884706 & 0.482595 \\
\hline & 1.801929 & -1.900155 & -0.029137 \\
\hline & 1.875768 & -0.410370 & -0.838566 \\
\hline & 3.019570 & -0.470614 & -2.053859 \\
\hline D & 4.273060 & -0.996374 & -1.852321 \\
\hline & 5.204782 & -0.329139 & -1.081713 \\
\hline 6 & 6.436170 & -1.011944 & -0.793349 \\
\hline ) & 6.708275 & -2.268040 & -1.415117 \\
\hline 6 & 5.758229 & -2.830807 & -2.302695 \\
\hline & 4.553986 & -2.216243 & -2.503610 \\
\hline & 7.376240 & -0.524366 & 0.150648 \\
\hline & 8.521928 & -1.224219 & 0.440644 \\
\hline & 8.802533 & -2.447026 & -0.203420 \\
\hline 6 & 7.909000 & -2.954799 & -1.111110 \\
\hline & 4.909397 & 1.014308 & -0.527762 \\
\hline 6 & 3.758476 & 1.250724 & 0.204673 \\
\hline f & 3.568581 & 2.441421 & 0.937437 \\
\hline 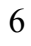 & 4.469912 & 3.461581 & 0.840514 \\
\hline 5 & 5.587701 & 3.344899 & -0.021955 \\
\hline & 5.808176 & 2.119487 & -0.720194 \\
\hline 6 & 6.482065 & 4.425916 & -0.205146 \\
\hline f & 7.546250 & 4.325526 & -1.064752 \\
\hline 6 & 7.748736 & 3.130298 & -1.784134 \\
\hline 6 & 6.906954 & 2.058207 & -1.614614 \\
\hline & 2.770151 & 0.320612 & 0.348799 \\
\hline 6 & 2.918656 & -2.563864 & 0.690025 \\
\hline 6 & 3.479942 & -1.859564 & 1.899993 \\
\hline 6 & 4.850377 & -1.923080 & 2.145180 \\
\hline 5 & 5.399394 & -1.332159 & 3.281672 \\
\hline 6 & 4.580160 & -0.670451 & 4.188358 \\
\hline 5 & 3.208066 & -0.603458 & 3.949745 \\
\hline 6 & 2.665438 & -1.193836 & 2.817141 \\
\hline 6 & -3.224636 & -0.679131 & -0.230886 \\
\hline 6 & -3.616087 & -1.050890 & -1.515671 \\
\hline 6 & -4.870702 & -1.613091 & -1.751885 \\
\hline 6 & -5.725905 & -1.794549 & -0.657400 \\
\hline 5 & -5.361466 & -1.444527 & 0.643676 \\
\hline 6 & -4.096857 & -0.884594 & 0.834555 \\
\hline 6 & -5.335249 & -1.985161 & -3.158633 \\
\hline 6 & -6.421472 & -0.994198 & -3.592242 \\
\hline 6 & -6.270578 & -1.637880 & 1.856033 \\
\hline 6 & -5.549851 & -2.487660 & 2.910190 \\
\hline 6 & -5.904543 & -3.406951 & -3.176572 \\
\hline 6 & -4.192998 & -1.929889 & -4.172086 \\
\hline 6 & -6.603924 & -0.265581 & 2.453409 \\
\hline
\end{tabular}




\begin{tabular}{|c|c|c|c|}
\hline 6 & -7.578906 & -2.337043 & 1.497734 \\
\hline 6 & 0.683102 & 1.640135 & -3.339104 \\
\hline & 0.262680 & 2.510058 & -2.306649 \\
\hline & -0.983604 & 3.317886 & -2.435407 \\
\hline & -1.934723 & 3.096368 & -3.448689 \\
\hline & -3.063565 & 3.891578 & -3.572149 \\
\hline & -3.288823 & 4.946821 & -2.687770 \\
\hline & -2.366475 & 5.179087 & -1.676196 \\
\hline & -1.233866 & 4.380002 & -1.557542 \\
\hline & 1.333665 & 3.089770 & -1.414351 \\
\hline & -0.574229 & 6.137991 & 2.014195 \\
\hline f & 0.844111 & 4.213088 & 2.636487 \\
\hline & -5.351677 & 5.377427 & 0.448434 \\
\hline 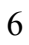 & -6.092175 & 3.066348 & -0.016937 \\
\hline & 7.165506 & 0.403523 & 0.675681 \\
\hline & -1.674644 & 0.798853 & 2.963671 \\
\hline 1 & 8.097566 & -3.906580 & -1.606139 \\
\hline & -0.282507 & -2.405554 & 6.143215 \\
\hline D & 6.296616 & 5.346797 & 0.346378 \\
\hline & -2.877683 & -3.293461 & 1.378402 \\
\hline & -4.623107 & -4.804109 & 0.537427 \\
\hline & 4.327876 & 4.382906 & 1.404204 \\
\hline & -1.149766 & -0.417219 & 5.018719 \\
\hline 1 & 5.992607 & -3.771210 & -2.798597 \\
\hline 1 & 8.576077 & 3.057017 & -2.486495 \\
\hline | & 3.793336 & -2.633121 & -3.160698 \\
\hline 1 & -2.076043 & -5.995617 & -2.716774 \\
\hline & 0.182142 & -5.086925 & -2.898246 \\
\hline & 0.954076 & -5.809456 & 3.828041 \\
\hline 1 & 7.072684 & 1.150185 & -2.188535 \\
\hline & 0.341007 & -4.656087 & 1.756934 \\
\hline 1 & 1.944750 & -3.538359 & -2.069533 \\
\hline | & 8.223771 & 5.164893 & -1.202598 \\
\hline 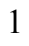 & 9.216492 & -0.832984 & 1.180825 \\
\hline I & -4.233818 & -6.167098 & -1.511427 \\
\hline | & 0.653793 & -4.698194 & 6.036377 \\
\hline 1 & 9.716638 & -2.987658 & 0.030420 \\
\hline & 2.684219 & 2.506278 & 1.566430 \\
\hline 1 & -3.789396 & -0.591457 & 1.838167 \\
\hline 1 & -2.918061 & -0.894261 & -2.332006 \\
\hline 1 & -6.709103 & -2.226616 & -0.833080 \\
\hline 1 & -4.125337 & 1.594676 & 0.029386 \\
\hline 1 & -0.142802 & 2.243542 & 1.452166 \\
\hline 1 & -3.193919 & 5.180703 & 2.164909 \\
\hline & 2.508897 & -3.530455 & 1.016203 \\
\hline 1 & -1.534264 & 0.677753 & -2.520646 \\
\hline 1 & 3.740136 & -2.802912 & -0.001386 \\
\hline 1 & 5.502935 & -2.429465 & 1.432214 \\
\hline 1 & 1.602215 & -1.107311 & 2.610827 \\
\hline$\frac{1}{1}$ & 6.473958 & -1.384317 & 3.447493 \\
\hline 1 & 2.553541 & -0.084448 & 4.648799 \\
\hline 1 & 5.006615 & -0.203170 & 5.073536 \\
\hline 1 & 0.122380 & 1.562073 & -4.269521 \\
\hline 1 & 1.747788 & 1.435416 & -3.450924 \\
\hline 1 & -0.536567 & 4.586368 & -0.748600 \\
\hline 1 & -2.518832 & 5.993591 & -0.967930 \\
\hline
\end{tabular}




$\begin{array}{rrrr}1 & -4.169584 & 5.577784 & -2.794241 \\ 1 & -3.772168 & 3.691426 & -4.373967 \\ 1 & -1.794551 & 2.280090 & -4.155080 \\ 1 & 1.562489 & 4.131624 & -1.691442 \\ 1 & 1.053616 & 3.093178 & -0.353311 \\ 1 & 2.265294 & 2.521768 & -1.512857 \\ 1 & -4.559701 & -2.243253 & -5.157826 \\ 1 & -3.781823 & -0.917691 & -4.285750 \\ 1 & -3.371573 & -2.603393 & -3.890977 \\ 1 & -6.772028 & -1.226918 & -4.607127 \\ 1 & -7.289950 & -1.024119 & -2.922053 \\ 1 & -6.035643 & 0.033878 & -3.593063 \\ 1 & -6.225933 & -3.671097 & -4.192916 \\ 1 & -5.144234 & -4.133740 & -2.860643 \\ 1 & -6.775441 & -3.520898 & -2.520140 \\ 1 & -8.191874 & -2.460177 & 2.399557 \\ 1 & -8.170445 & -1.758528 & 0.776756 \\ 1 & -7.406261 & -3.336700 & 1.076771 \\ 1 & -7.220266 & -0.376442 & 3.356011 \\ 1 & -5.697143 & 0.287504 & 2.732279 \\ 1 & -7.165277 & 0.348196 & 1.735523 \\ 1 & -6.198398 & -2.639752 & 3.783420 \\ 1 & -5.290486 & -3.477775 & 2.510692 \\ 1 & -4.623474 & -2.019154 & 3.267694 \\ 1 & 1.496305 & 4.894793 & 3.197761 \\ 1 & 0.922582 & 3.223208 & 3.107217 \\ 1 & 1.239509 & 4.147233 & 1.613461 \\ 1 & -0.369702 & 5.491906 & 4.677783 \\ 1 & -2.058017 & 5.265257 & 4.187739 \\ 1 & -1.057440 & 3.856288 & 4.582522 \\ 1 & 0.111442 & 6.796880 & 2.563807 \\ 1 & -0.233451 & 6.097092 & 0.970350 \\ 1 & -1.563113 & 6.611537 & 2.025018 \\ 1 & -6.398215 & 5.708422 & 0.412189 \\ 1 & -4.812637 & 6.075750 & 1.100252 \\ 1 & -4.932909 & 5.465002 & -0.562009 \\ 1 & -6.971806 & 4.160042 & 2.294386 \\ 1 & -5.889573 & 2.817608 & 2.722069 \\ 1 & -5.405661 & 4.489771 & 3.060795 \\ 1 & -7.113738 & 3.460488 & -0.091923 \\ 1 & -5.657416 & 3.065134 & -1.025446 \\ 1 & -6.168980 & 2.023755 & 0.321373\end{array}$

$3_{\text {IIB-re- }} \psi_{-1 \text {-1a2a3s4a }}$

Number of imaginary frequencies : 0

The smallest frequencies are : $19.171122 .3718 \quad 23.7174 \mathrm{~cm}(-1)$

Electronic energy :

Zero-point correction=

$\mathrm{HF}=-4360.8847697$

Thermal correction to Energy=

Thermal correction to Enthalpy=

1.456912 (Hartree/Particle)

1.540541

1.541485

Thermal correction to Gibbs Free Energy $=\quad 1.342920$

Sum of electronic and zero-point Energies $=\quad-4359.427857$

Sum of electronic and thermal Energies $=\quad-4359.344229$

Sum of electronic and thermal Enthalpies $=\quad-4359.343285$

Sum of electronic and thermal Free Energies $=\quad-4359.541849$ 


\section{Cartesian Coordinates}

$\begin{array}{cccc}\cdots & 4.876692 & -1.902771 & 2.128700 \\ 6 & 3.503023 & -1.862839 & 1.897517 \\ 6 & 2.685799 & -1.214959 & 2.824845 \\ 6 & 3.228725 & -0.618010 & 3.953754 \\ 6 & 4.604220 & -0.660960 & 4.178356 \\ 6 & 5.426157 & -1.305492 & 3.261745 \\ 6 & 2.939700 & -2.569422 & 0.690162 \\ 7 & 1.814033 & -1.909837 & -0.019447 \\ 15 & 1.878790 & -0.419597 & -0.828449 \\ 8 & 2.773530 & 0.312900 & 0.357960 \\ 6 & 3.747398 & 1.257843 & 0.213130 \\ 6 & 4.894638 & 1.042324 & -0.531162 \\ 6 & 5.773489 & 2.162339 & -0.728877 \\ 6 & 5.540772 & 3.381422 & -0.023680 \\ 6 & 4.432374 & 3.475525 & 0.853584 \\ 6 & 3.548409 & 2.440678 & 0.956076 \\ 6 & 6.862932 & 2.121883 & -1.635699 \\ 6 & 7.684154 & 3.208733 & -1.811996 \\ 6 & 7.469718 & 4.398321 & -1.086699 \\ 6 & 6.414490 & 4.477996 & -0.213955 \\ 6 & 5.207554 & -0.295362 & -1.089060 \\ 6 & 4.281635 & -0.978900 & -1.852261 \\ 6 & 4.579605 & -2.193912 & -2.505451 \\ 6 & 5.795881 & -2.787120 & -2.313523 \\ 6 & 6.742380 & -2.207757 & -1.432961 \\ 6 & 6.452475 & -0.956841 & -0.808899 \\ 6 & 7.957229 & -2.873200 & -1.137948 \\ 6 & 8.848004 & -2.350139 & -0.236291 \\ 6 & 8.550016 & -1.133129 & 0.410790 \\ 6 & 7.390142 & -0.453419 & 0.129153 \\ 8 & 3.018646 & -0.474103 & -2.047488 \\ 6 & 0.776023 & -2.806745 & -0.441444 \\ 6 & -0.425219 & -2.876134 & 0.240690 \\ 6 & -1.428230 & -3.789506 & -0.216677 \\ 6 & -1.168289 & -4.609624 & -1.355977 \\ 6 & 0.087658 & -4.523730 & -2.000413 \\ 6 & 1.031702 & -3.640930 & -1.554326 \\ 6 & -2.680147 & -3.929152 & 0.435752 \\ 6 & -3.623267 & -4.813494 & -0.026076 \\ 6 & -3.363398 & -5.617785 & -1.156410 \\ 6 & -2.160193 & -5.514727 & -1.805627 \\ 6 & -0.618674 & -2.143622 & 1.528171 \\ 6 & -1.092610 & -0.839213 & 1.623886 \\ 6 & -1.268767 & -0.259098 & 2.909134 \\ 6 & -0.972528 & -0.946181 & 4.055290 \\ 6 & -0.468850 & -2.263787 & 3.995151 \\ 6 & -0.298570 & -2.876216 & 2.719382 \\ 6 & -0.103733 & -2.967746 & 5.167707 \\ 6 & 0.417135 & -4.233489 & 5.093368 \\ 6 & 0.580765 & -4.851014 & 3.835444 \\ 6 & 0.226656 & -4.195824 & 2.682346 \\ 15 & -1.585564 & 0.101677 & 0.094027 \\ 6 & -3.227660 & -0.679662 & -0.210308 \\ & & & \\ 6\end{array}$




$\begin{array}{cccc}6 & -3.633874 & -0.994205 & -1.495583 \\ 6 & -4.915752 & -1.500908 & -1.752967 \\ 6 & -5.768992 & -1.686894 & -0.670515 \\ 6 & -5.387249 & -1.389890 & 0.649927 \\ 6 & -4.108666 & -0.889404 & 0.859671 \\ 6 & -5.292680 & -1.838989 & -3.192535 \\ 6 & -4.439772 & -3.025575 & -3.655032 \\ 6 & -6.367312 & -1.625578 & 1.798069 \\ 6 & -6.699744 & -3.119122 & 1.883983 \\ 45 & -0.163397 & 0.280783 & -1.831583 \\ 6 & 0.672432 & 1.621167 & -3.326425 \\ 6 & 0.256201 & 2.491144 & -2.291371 \\ 6 & 1.330438 & 3.073809 & -1.404846 \\ 6 & -0.991164 & 3.298430 & -2.413723 \\ 6 & -1.948180 & 3.076093 & -3.421391 \\ 6 & -3.078912 & 3.869594 & -3.537396 \\ 6 & -3.299237 & 4.924913 & -2.651700 \\ 6 & -2.370757 & 5.158215 & -1.646004 \\ 6 & -1.236833 & 4.359375 & -1.533425 \\ 6 & -0.382660 & -1.208430 & -3.039066 \\ 8 & -0.646179 & -1.829004 & -3.982033 \\ 6 & -2.101697 & 1.746996 & 0.752741 \\ 6 & -1.169447 & 2.575967 & 1.387775 \\ 6 & -1.553318 & 3.810465 & 1.915960 \\ 6 & -2.887863 & 4.204855 & 1.762487 \\ 1 & -3.827010 & 3.434137 & 1.078753 \\ 1 & -1.117411 & -0.480971 & 5.029667 \\ 1 & 6.043061 & -3.723042 & -2.811654 \\ 6 & -3.407886 & 2.199525 & 0.579166 \\ 6 & -0.593178 & 4.727971 & 2.673819 \\ 1 & -1.942691 & -6.128067 & -2.679033 \\ 6 & -0.585420 & 6.122168 & 2.036844 \\ 1 & -2.828342 & -5.160221 & -2.862162 \\ 1 & -0.989225 & -5.857434 & 3.778900 \\ 6 & -5.283304 & 3.887265 & 0.973793 \\ 1 & -1.037309 & 1.218116 & -2.213795 \\ 6 & -5.375294 & 5.337814 & 0.491739 \\ 6 & 0.839819 & 4.204135 & 2.663289 \\ 6 & -1.053375 & 4.834934 & 4.132014 \\ 6 & -6.085964 & 3.020222 & 0.004451 \\ 6 & -5.922279 & 3.774345 & 2.363177 \\ 6 & -5.790556 & -1.190156 & 3.143278 \\ 6 & -7.654021 & -0.829607 & 1.555492 \\ 6 & -6.764455 & -2.218359 & -3.332804 \\ 1 & -5.021503 & -0.632782 & -4.100336 \\ 1 & 7.166918 & 0.470248 & 0.656444 \\ 1 & -356354 & -4.691530 & 1.722558\end{array}$




\begin{tabular}{|c|c|c|c|}
\hline & 1.999721 & -3.554454 & -2.046758 \\
\hline & 8.130940 & 5.249655 & -1.230186 \\
\hline & 9.242529 & -0.730138 & 1.146569 \\
\hline | & -4.119064 & -6.316323 & -1.509367 \\
\hline & 0.704167 & -4.763945 & 5.998282 \\
\hline & 9.773264 & -2.874503 & -0.009384 \\
\hline & 2.671490 & 2.487643 & 1.597086 \\
\hline & -3.781386 & -0.636063 & 1.865281 \\
\hline & -2.939732 & -0.836892 & -2.316684 \\
\hline & -6.769627 & -2.078305 & -0.844785 \\
\hline & -4.114904 & 1.572311 & 0.046422 \\
\hline & -0.140413 & 2.226523 & 1.488393 \\
\hline & -3.202063 & 5.156312 & 2.191629 \\
\hline & 2.536873 & -3.538753 & 1.017016 \\
\hline & -1.538960 & 0.677440 & -2.490643 \\
\hline & 3.758625 & -2.801882 & -0.006667 \\
\hline & 5.531180 & -2.395022 & 1.407620 \\
\hline & 1.619347 & -1.147244 & 2.628698 \\
\hline & 6.503081 & -1.338733 & 3.416690 \\
\hline & 2.571867 & -0.113105 & 4.660942 \\
\hline & 5.031123 & -0.188520 & 5.060575 \\
\hline & 0.107301 & 1.542743 & -4.254083 \\
\hline 1 & 1.736907 & 1.418163 & -3.443024 \\
\hline & -0.535700 & 4.565566 & -0.727795 \\
\hline ] & -2.520286 & 5.972464 & -0.936829 \\
\hline & -4.181292 & 5.555035 & -2.752471 \\
\hline & -3.792534 & 3.668375 & -4.334508 \\
\hline ] & -1.811325 & 2.260191 & -4.128991 \\
\hline & 1.555731 & 4.115919 & -1.683876 \\
\hline 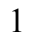 & 1.055584 & 3.077587 & -0.342182 \\
\hline & 2.262818 & 2.508002 & -1.507931 \\
\hline 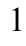 & -6.992122 & -2.431378 & -4.384881 \\
\hline$\frac{1}{1}$ & -7.013793 & -3.119427 & -2.757660 \\
\hline & -7.428938 & -1.407023 & -3.007490 \\
\hline 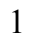 & -5.320426 & -0.862889 & -5.131672 \\
\hline & -5.589292 & 0.247079 & -3.769987 \\
\hline 1 & -3.959114 & -0.356645 & -4.123211 \\
\hline & -4.676105 & -3.284917 & -4.696276 \\
\hline 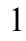 & -3.365606 & -2.805631 & -3.598583 \\
\hline 1 & -4.628417 & -3.907883 & -3.028924 \\
\hline | & -6.527421 & -1.367226 & 3.936775 \\
\hline 1 & -4.883085 & -1.751680 & 3.405354 \\
\hline & -5.543477 & -0.119707 & 3.151569 \\
\hline 1 & -7.427982 & -3.304516 & 2.685183 \\
\hline & -7.127907 & -3.502057 & 0.949217 \\
\hline 1 & -5.798456 & -3.705742 & 2.109677 \\
\hline 1 & -8.366508 & -1.000026 & 2.373804 \\
\hline 1 & -7.447434 & 0.248677 & 1.509808 \\
\hline 1 & -8.151303 & -1.114112 & 0.620710 \\
\hline 1 & 1.490881 & 4.890696 & 3.219955 \\
\hline 1 & 0.922665 & 3.216808 & 3.138494 \\
\hline 1 & 1.232945 & 4.134544 & 1.639576 \\
\hline 1 & -0.376566 & 5.485872 & 4.701981 \\
\hline 1 & -2.064471 & 5.251931 & 4.214095 \\
\hline 1 & -1.059048 & 3.847740 & 4.613260 \\
\hline 1 & 0.099400 & 6.784675 & 2.583164 \\
\hline
\end{tabular}




$\begin{array}{lrrr}1 & -0.246545 & 6.078896 & 0.992505 \\ 1 & -1.575592 & 6.592974 & 2.048076 \\ 1 & -6.426128 & 5.654704 & 0.454597 \\ 1 & -4.848772 & 6.036574 & 1.153239 \\ 1 & -4.953462 & 5.441996 & -0.516063 \\ 1 & -6.976935 & 4.080329 & 2.328838 \\ 1 & -5.881612 & 2.738863 & 2.728088 \\ 1 & -5.411672 & 4.408153 & 3.099337 \\ 1 & -7.113302 & 3.399894 & -0.065726 \\ 1 & -5.652807 & 3.030886 & -1.004685 \\ 1 & -6.147040 & 1.973966 & 0.335950\end{array}$

$3_{\text {IIB-re- }}-\psi_{-122 a 354 s}$

Number of imaginary frequencies : 0

The smallest frequencies are : $19.1910 \quad 21.4223 \quad 25.2253 \mathrm{~cm}(-1)$

Electronic energy : $\quad H F=-4360.8854314$

Zero-point correction $=\quad 1.456567($ Hartree/Particle $)$

Thermal correction to Energy= $\quad 1.540313$

Thermal correction to Enthalpy= $\quad 1.541257$

Thermal correction to Gibbs Free Energy= $\quad 1.342215$

Sum of electronic and zero-point Energies $=\quad-4359.428864$

Sum of electronic and thermal Energies $=\quad-4359.345119$

Sum of electronic and thermal Enthalpies $=\quad-4359.344174$

Sum of electronic and thermal Free Energies $=\quad-4359.543217$

Cartesian Coordinates

$\begin{array}{cccc}6 & 4.857207 & -1.906677 & 2.132812 \\ 6 & 3.484251 & -1.867081 & 1.897498 \\ 6 & 2.663449 & -1.224041 & 2.825104 \\ 6 & 3.202254 & -0.631618 & 3.958359 \\ 6 & 4.577101 & -0.674177 & 4.187061 \\ 6 & 5.402540 & -1.313749 & 3.270146 \\ 6 & 2.925034 & -2.569827 & 0.685994 \\ 7 & 1.804669 & -1.906039 & -0.028225 \\ 15 & 1.875307 & -0.413166 & -0.831865 \\ 8 & 2.770377 & 0.312556 & 0.358548 \\ 6 & 3.745633 & 1.256632 & 0.215978 \\ 6 & 4.894544 & 1.038719 & -0.525074 \\ 6 & 5.777351 & 2.156175 & -0.718972 \\ 6 & 5.545571 & 3.375489 & -0.013874 \\ 6 & 4.433958 & 3.472498 & 0.858957 \\ 6 & 3.546790 & 2.439993 & 0.958029 \\ 6 & 6.870133 & 2.112952 & -1.621647 \\ 6 & 7.695512 & 3.197289 & -1.793845 \\ 6 & 7.481910 & 4.387045 & -1.068546 \\ 6 & 6.423449 & 4.469469 & -0.199991 \\ 6 & 5.204494 & -0.299237 & -1.083901 \\ 6 & 4.278099 & -0.977648 & -1.851089 \\ 6 & 4.573122 & -2.192740 & -2.505429 \\ 6 & 5.786305 & -2.791345 & -2.310581 \\ 6 & 6.732450 & -2.217341 & -1.426123 \\ 6 & 6.445894 & -0.966109 & -0.801151 \\ 6 & 7.943510 & -2.888363 & -1.128183 \\ 6 & 8.833595 & -2.370378 & -0.222912\end{array}$




\begin{tabular}{|c|c|c|c|}
\hline 6 & 8.538818 & -1.152925 & 0.424844 \\
\hline & 7.382787 & -0.467857 & 0.140432 \\
\hline 8 & 3.017676 & -0.467465 & -2.048813 \\
\hline 6 & 0.765829 & -2.800106 & -0.454267 \\
\hline 6 & -0.435524 & -2.870886 & 0.227105 \\
\hline 6 & -1.437947 & -3.783708 & -0.232404 \\
\hline o & -1.179349 & -4.601271 & -1.373419 \\
\hline 6 & 0.076744 & -4.513865 & -2.017591 \\
\hline 6 & 1.020990 & -3.632319 & -1.568987 \\
\hline 6 & -2.689020 & -3.923016 & 0.420600 \\
\hline 6 & -3.635079 & -4.802950 & -0.042886 \\
\hline 6 & -3.377087 & -5.605468 & -1.174956 \\
\hline 6 & -2.173245 & -5.503892 & -1.823889 \\
\hline 6 & -0.632534 & -2.141352 & 1.515651 \\
\hline 6 & -1.103645 & -0.836036 & 1.613397 \\
\hline 6 & -1.286224 & -0.260718 & 2.899946 \\
\hline 6 & -1.002105 & -0.954131 & 4.045420 \\
\hline 6 & -0.502851 & -2.273317 & 3.983383 \\
\hline 6 & -0.323160 & -2.879877 & 2.706177 \\
\hline 6 & -0.149921 & -2.983826 & 5.155730 \\
\hline 6 & 0.368379 & -4.250491 & 5.079830 \\
\hline 6 & 0.542082 & -4.862008 & 3.820332 \\
\hline 6 & 0.199833 & -4.200345 & 2.667378 \\
\hline 15 & -1.589856 & 0.114140 & 0.086354 \\
\hline 6 & -3.226767 & -0.672713 & -0.230385 \\
\hline 6 & -3.635369 & -0.976634 & -1.521831 \\
\hline 6 & -4.905326 & -1.501660 & -1.779124 \\
\hline 6 & -5.752529 & -1.730743 & -0.693091 \\
\hline 6 & -5.367005 & -1.450186 & 0.624215 \\
\hline 6 & -4.097954 & -0.915174 & 0.832559 \\
\hline 6 & -5.284562 & -1.829585 & -3.221176 \\
\hline 6 & -4.434788 & -3.015795 & -3.690657 \\
\hline 6 & -6.273661 & -1.684717 & 1.831640 \\
\hline 6 & -6.621440 & -0.330078 & 2.460609 \\
\hline 45 & -0.163394 & 0.295754 & -1.834715 \\
\hline 6 & 0.674275 & 1.638123 & -3.327048 \\
\hline 6 & 0.256126 & 2.505650 & -2.290780 \\
\hline 6 & 1.328925 & 3.088116 & -1.402376 \\
\hline 6 & -0.993751 & 3.309759 & -2.410775 \\
\hline 6 & -1.946682 & 3.091305 & -3.423104 \\
\hline 6 & -3.079465 & 3.882096 & -3.537531 \\
\hline 6 & -3.305446 & 4.931145 & -2.645984 \\
\hline 6 & -2.381177 & 5.160229 & -1.635402 \\
\hline 6 & -1.246226 & 4.363152 & -1.523229 \\
\hline 6 & -0.383650 & -1.190832 & -3.045431 \\
\hline 8 & -0.641724 & -1.808057 & -3.992050 \\
\hline 6 & -2.111844 & 1.753389 & 0.756255 \\
\hline 6 & -1.175479 & 2.584013 & 1.383332 \\
\hline 6 & -1.557302 & 3.815878 & 1.918697 \\
\hline 6 & -2.892766 & 4.209639 & 1.773291 \\
\hline 6 & -3.836113 & 3.439857 & 1.094301 \\
\hline 6 & -3.420410 & 2.203893 & 0.593676 \\
\hline 6 & -0.593978 & 4.733126 & 2.672864 \\
\hline 6 & -0.586783 & 6.126709 & 2.034418 \\
\hline 6 & -5.289522 & 3.903594 & 0.990579 \\
\hline 6 & -5.369416 & 5.349832 & 0.492707 \\
\hline
\end{tabular}




\begin{tabular}{|c|c|c|c|}
\hline & 0.838379 & 4.207770 & 2.659379 \\
\hline & -1.050084 & 4.842278 & 4.132198 \\
\hline & -6.102181 & 3.035061 & 0.030881 \\
\hline & -5.926448 & 3.816498 & 2.382899 \\
\hline & -7.573954 & -2.390211 & 1.456001 \\
\hline & -5.545725 & -2.552017 & 2.866220 \\
\hline & -6.757738 & -2.204185 & -3.363383 \\
\hline & -5.011799 & -0.620121 & -4.124148 \\
\hline & 7.161811 & 0.456136 & 0.668123 \\
\hline & -1.673237 & 0.751836 & 2.980036 \\
\hline & 8.143031 & -3.836519 & -1.625861 \\
\hline & -0.290970 & -2.494933 & 6.118803 \\
\hline & 6.229819 & 5.385505 & 0.356783 \\
\hline & -2.900399 & -3.308899 & 1.293997 \\
\hline & -4.595297 & -4.880255 & 0.465281 \\
\hline & 4.284681 & 4.388763 & 1.429002 \\
\hline & -1.152334 & -0.492446 & 5.020679 \\
\hline & 6.031147 & -3.727567 & -2.809314 \\
\hline & 8.518383 & 3.138110 & -2.502763 \\
\hline & 3.815904 & -2.618917 & -3.160391 \\
\hline & -1.957626 & -6.116119 & -2.698546 \\
\hline & 0.271718 & -5.148164 & -2.880892 \\
\hline & 0.949041 & -5.868952 & 3.762401 \\
\hline & 7.043931 & 1.209036 & -2.199690 \\
\hline 1 & 0.337383 & -4.691553 & 1.706393 \\
\hline & 1.989276 & -3.545371 & -2.060783 \\
\hline & 8.146421 & 5.236361 & -1.208744 \\
\hline & 9.230748 & -0.753890 & 1.163322 \\
\hline & -4.134269 & -6.301287 & -1.530027 \\
\hline 1 & 0.645953 & -4.786126 & 5.984640 \\
\hline & 9.755835 & -2.899048 & 0.006293 \\
\hline ] & 2.667539 & 2.489572 & 1.595569 \\
\hline & -3.781138 & -0.668368 & 1.845665 \\
\hline & -2.946598 & -0.797330 & -2.342765 \\
\hline & -6.741326 & -2.142729 & -0.874434 \\
\hline & -4.132474 & 1.573716 & 0.070341 \\
\hline 1 & -0.144890 & 2.236332 & 1.474348 \\
\hline & -3.204635 & 5.160965 & 2.204376 \\
\hline 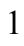 & 2.517393 & -3.538312 & 1.009469 \\
\hline & -1.537236 & 0.695963 & -2.494528 \\
\hline & 3.746628 & -2.803829 & -0.007180 \\
\hline & 5.514349 & -2.395492 & 1.411833 \\
\hline & 1.597423 & -1.156838 & 2.626252 \\
\hline ] & 6.478982 & -1.346768 & 3.428493 \\
\hline & 2.542696 & -0.130691 & 4.665855 \\
\hline 1 & 5.000730 & -0.205376 & 5.072793 \\
\hline & 0.110301 & 1.560062 & -4.255415 \\
\hline & 1.739064 & 1.436147 & -3.442566 \\
\hline 1 & -0.548072 & 4.566003 & -0.714232 \\
\hline 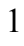 & -2.534768 & 5.970192 & -0.922245 \\
\hline 1 & -4.188318 & 5.560164 & -2.746389 \\
\hline & -3.789660 & 3.684327 & -4.338539 \\
\hline 1 & -1.804780 & 2.281069 & -4.136188 \\
\hline . & 1.551794 & 4.131588 & -1.678282 \\
\hline 1 & 1.053813 & 3.088153 & -0.339949 \\
\hline 1 & 2.262503 & 2.524301 & -1.506569 \\
\hline
\end{tabular}




$\begin{array}{rrrr}1 & -6.988036 & -2.401085 & -4.418043 \\ 1 & -7.008439 & -3.113418 & -2.802017 \\ 1 & -7.419858 & -1.396558 & -3.024308 \\ 1 & -5.318979 & -0.842996 & -5.154619 \\ 1 & -5.571856 & 0.261517 & -3.785489 \\ 1 & -3.947987 & -0.350408 & -4.153405 \\ 1 & -4.671886 & -3.269187 & -4.733273 \\ 1 & -3.360304 & -2.797389 & -3.632710 \\ 1 & -4.624809 & -3.901145 & -3.069172 \\ 1 & -8.180181 & -2.553132 & 2.356100 \\ 1 & -8.178609 & -1.796971 & 0.758432 \\ 1 & -7.388207 & -3.372081 & 1.000097 \\ 1 & -7.237008 & -0.467356 & 3.360183 \\ 1 & -5.719523 & 0.225201 & 2.751076 \\ 1 & -7.188439 & 0.294321 & 1.756389 \\ 1 & -6.193021 & -2.728945 & 3.735664 \\ 1 & -5.278588 & -3.530759 & 2.444299 \\ 1 & -4.623156 & -2.084950 & 3.234855 \\ 1 & 1.490879 & 4.892387 & 3.216763 \\ 1 & 0.920530 & 3.219266 & 3.132235 \\ 1 & 1.230390 & 4.140142 & 1.635177 \\ 1 & -0.371686 & 5.494245 & 4.699070 \\ 1 & -2.060954 & 5.259291 & 4.216663 \\ 1 & -1.054134 & 3.855905 & 4.615088 \\ 1 & 0.100891 & 6.788901 & 2.577534 \\ 1 & -0.251937 & 6.082121 & 0.988853 \\ 1 & -1.576397 & 6.598681 & 2.048947 \\ 1 & -6.417505 & 5.675857 & 0.456331 \\ 1 & -4.833771 & 6.050827 & 1.144517 \\ 1 & -4.951006 & 5.439207 & -0.517721 \\ 1 & -6.978303 & 4.131577 & 2.344794 \\ 1 & -5.895412 & 2.787821 & 2.766599 \\ 1 & -5.409671 & 4.459138 & 3.107055 \\ 1 & -7.124961 & 3.426229 & -0.043233 \\ 1 & -5.668768 & 3.032507 & -0.978331 \\ 1 & -6.175661 & 1.993010 & 0.371833\end{array}$

$3_{\text {IIB-re- }} \psi_{-122 s 3 a 4 a}$

Number of imaginary frequencies : 0

The smallest frequencies are : $14.8401 \quad 18.8683 \quad 23.2454 \mathrm{~cm}(-1)$ Electronic energy :

Zero-point correction $=$ $\mathrm{HF}=-4360.8847859$

Thermal correction to Energy=

Thermal correction to Enthalpy $=\quad 1.540700$

1.455490 (Hartree/Particle)

Thermal correction to Gibbs Free Energy $=\quad 1.339406$

Sum of electronic and zero-point Energies $=\quad-4359.429295$

Sum of electronic and thermal Energies $=\quad-4359.345030$

Sum of electronic and thermal Enthalpies $=\quad-4359.344086$

Sum of electronic and thermal Free Energies $=\quad-4359.545380$

Cartesian Coordinates

$\begin{array}{llll}6 & -1.923712 & 3.162976 & -3.351146 \\ 6 & -0.947410 & 3.363957 & -2.357686 \\ 6 & -1.147886 & 4.442540 & -1.486494\end{array}$




\begin{tabular}{|c|c|c|c|}
\hline 6 & -2.254107 & 5.281560 & -1.599223 \\
\hline 6 & -3.198616 & 5.071513 & -2.594776 \\
\hline 6 & -3.024884 & 3.996735 & -3.468260 \\
\hline 6 & 0.279397 & 2.523265 & -2.252071 \\
\hline 6 & 1.371971 & 3.068809 & -1.365178 \\
\hline 45 & -0.160281 & 0.299888 & -1.834378 \\
\hline 6 & -0.312579 & -1.160150 & -3.088898 \\
\hline 8 & -0.470234 & -1.762127 & -4.066207 \\
\hline 15 & -1.584929 & 0.098728 & 0.088529 \\
\hline 6 & -2.091915 & 1.748803 & 0.732764 \\
\hline 6 & -1.158122 & 2.569729 & 1.379885 \\
\hline 6 & -1.532574 & 3.810028 & 1.894926 \\
\hline 6 & -2.859868 & 4.226332 & 1.712446 \\
\hline 6 & -3.794423 & 3.462928 & 1.018058 \\
\hline 6 & -3.384889 & 2.217881 & 0.532384 \\
\hline 6 & -0.575092 & 4.705212 & 2.683565 \\
\hline 6 & -5.257593 & 3.881201 & 0.879240 \\
\hline 6 & -3.229275 & -0.683982 & -0.208971 \\
\hline 6 & -3.615176 & -1.055966 & -1.490801 \\
\hline 6 & -4.886032 & -1.586215 & -1.737224 \\
\hline 6 & -5.754281 & -1.718881 & -0.653559 \\
\hline 6 & -5.397588 & -1.362456 & 0.653580 \\
\hline 6 & -4.118975 & -0.850820 & 0.857445 \\
\hline 6 & -6.396557 & -1.552738 & 1.794049 \\
\hline 6 & -5.342273 & -1.969002 & -3.143804 \\
\hline 6 & -1.091977 & -0.822959 & \\
\hline 6 & -0.621255 & -2.129103 & 1.548527 \\
\hline 6 & -0.300890 & -2.852337 & 2.744168 \\
\hline 6 & -0.463766 & -2.227097 & 4.014540 \\
\hline 6 & -0.962661 & -0.907193 & 4.063378 \\
\hline 6 & -1.262227 & -0.230147 & 2.911878 \\
\hline 6 & -0.096841 & -2.922097 & 5.191795 \\
\hline 6 & 0.417240 & -4.191191 & \\
\hline 6 & 0.571285 & -4.821823 & 3.873599 \\
\hline 6 & 0.215681 & -4.175199 & 2.716132 \\
\hline 6 & -0.443395 & -2.865475 & 0.262422 \\
\hline 6 & 0.745707 & -2.798016 & -0.440950 \\
\hline 6 & 0.967711 & -3.614779 & -1.573582 \\
\hline 6 & -0.005140 & -4.461000 & -2.029223 \\
\hline 6 & -1.252498 & -4.535336 & -1.367098 \\
\hline 6 & -1.468307 & -3.754303 & -0.192183 \\
\hline 6 & -2.706414 & -3.892553 & 0.486730 \\
\hline 6 & -3.684426 & -4.728223 & 0.008536 \\
\hline 6 & -3.472306 & -5.486823 & -1.163094 \\
\hline 6 & -2.280186 & -5.390841 & -1.834073 \\
\hline 7 & 1.799827 & -1.915738 & -0.027517 \\
\hline 15 & 1.874913 & -0.421601 & -0.829431 \\
\hline 8 & 3.010814 & -0.479676 & -2.051476 \\
\hline 6 & 4.267899 & -1.001286 & -1.860864 \\
\hline 6 & 5.203335 & -0.331903 & -1.097227 \\
\hline 6 & 6.441034 & -1.009188 & -0.822857 \\
\hline 6 & 6.713580 & -2.261977 & -1.451027 \\
\hline 6 & 5.757305 & -2.827559 & -2.330138 \\
\hline 6 & 4.548149 & -2.218458 & -2.517580 \\
\hline 6 & 7.387449 & -0.519757 & 0.113692 \\
\hline 6 & 8.539408 & -1.214641 & 0.390628 \\
\hline
\end{tabular}




\begin{tabular}{|c|c|c|c|}
\hline & 8.820218 & -2.433898 & -0.259964 \\
\hline & 7.920673 & -2.943450 & -1.160734 \\
\hline & 4.906079 & 1.006316 & -0.532027 \\
\hline & 3.764138 & 1.230992 & 0.218048 \\
\hline & 3.583987 & 2.410905 & 0.971009 \\
\hline & 4.479086 & 3.436201 & 0.869171 \\
\hline & 5.581246 & 3.335085 & -0.015198 \\
\hline & 5.796887 & 2.117179 & -0.727625 \\
\hline & 6.465880 & 4.422982 & -0.204787 \\
\hline & 7.515927 & 4.335840 & -1.083090 \\
\hline & 7.714069 & 3.147330 & -1.814734 \\
\hline & 6.881698 & 2.068824 & -1.639666 \\
\hline & 2.777823 & 0.297870 & 0.359067 \\
\hline ) & 0.674345 & 1.664571 & -3.305255 \\
\hline & 2.920860 & -2.588758 & 0.676875 \\
\hline & 3.498607 & -1.891326 & 1.883025 \\
\hline b & 2.694981 & -1.232131 & 2.814403 \\
\hline & 3.251289 & -0.643798 & 3.941297 \\
\hline ) & 4.626871 & -0.707244 & 4.160366 \\
\hline & 5.435222 & -1.363918 & 3.240363 \\
\hline & 4.872318 & -1.952218 & 2.109189 \\
\hline & 7.177206 & 0.405412 & 0.643644 \\
\hline & -1.650011 & 0.782682 & 2.983659 \\
\hline & 8.109393 & -3.892753 & -1.660440 \\
\hline & -0.224093 & -2.419927 & 6.149953 \\
\hline & 6.284457 & 5.338319 & 0.357191 \\
\hline & -2.884664 & -3.307804 & 1.387813 \\
\hline & -4.634604 & -4.805427 & 0.533920 \\
\hline & 4.343765 & 4.350251 & 1.446181 \\
\hline 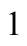 & -1.102356 & -0.431910 & 5.033673 \\
\hline & 5.991257 & -3.765735 & -2.830436 \\
\hline 1 & 8.530326 & 3.084485 & -2.530958 \\
\hline & 3.782958 & -2.637867 & -3.167745 \\
\hline & -2.097679 & -5.975681 & -2.734589 \\
\hline & 0.159212 & -5.074242 & -2.913859 \\
\hline & 0.973334 & -5.831223 & 3.825061 \\
\hline ] & 7.043372 & 1.165812 & -2.222668 \\
\hline & 0.336506 & -4.679427 & 1.759562 \\
\hline 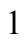 & 1.929001 & -3.535810 & -2.080599 \\
\hline ] & 8.185700 & 5.180560 & -1.226002 \\
\hline 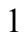 & 9.238944 & -0.822074 & 1.125395 \\
\hline 1 & -4.254479 & -6.150023 & -1.526758 \\
\hline & 0.705713 & -4.714744 & 6.034845 \\
\hline 1 & 9.739285 & -2.970511 & -0.036567 \\
\hline & 2.711862 & 2.464620 & 1.618175 \\
\hline 1 & -3.805102 & -0.554755 & 1.856184 \\
\hline l & -2.905277 & -0.925623 & -2.301643 \\
\hline 1 & -6.753475 & -2.117963 & -0.829527 \\
\hline 1 & -4.098621 & 1.594638 & 0.002809 \\
\hline ] & -0.136412 & 2.205632 & 1.499294 \\
\hline 1 & -3.162665 & 5.187739 & 2.123765 \\
\hline ] & 2.509034 & -3.554011 & 1.004133 \\
\hline 1 & -1.537180 & 0.700942 & -2.493598 \\
\hline ] & 3.733345 & -2.829854 & -0.024462 \\
\hline 1 & 5.516604 & -2.453883 & 1.385456 \\
\hline 1 & 1.628911 & -1.147760 & 2.622844 \\
\hline
\end{tabular}




\begin{tabular}{|c|c|c|c|}
\hline$\frac{1}{1}$ & 6.512174 & -1.413302 & 3.390668 \\
\hline & 2.604753 & -0.128644 & 4.650634 \\
\hline & 5.064424 & -0.241242 & 5.040790 \\
\hline ] & 0.100792 & 1.613462 & -4.229838 \\
\hline & 1.734909 & 1.449068 & -3.435386 \\
\hline & -0.430863 & 4.634172 & -0.691470 \\
\hline & -2.367804 & 6.109369 & -0.899426 \\
\hline & -4.058780 & 5.731363 & -2.693866 \\
\hline & -3.753802 & 3.811039 & -4.255279 \\
\hline & -1.822933 & 2.333208 & -4.048460 \\
\hline & 1.618531 & 4.109973 & -1.629511 \\
\hline & 1.104027 & 3.061242 & -0.301053 \\
\hline & 2.291268 & 2.485493 & -1.484278 \\
\hline ) & -4.184213 & -1.964391 & -4.140776 \\
\hline & -5.952580 & -3.373810 & -3.143019 \\
\hline & -6.392665 & -0.954505 & -3.610182 \\
\hline כ & -5.834176 & -1.093010 & 3.137540 \\
\hline & -7.665824 & -0.744146 & 1.504151 \\
\hline ) & -6.754647 & -3.037996 & 1.917340 \\
\hline & -4.543838 & -2.291209 & -5.124752 \\
\hline 1 & -3.749771 & -0.964022 & -4.270469 \\
\hline & -3.382063 & -2.649158 & -3.832169 \\
\hline & -6.273824 & -3.645731 & -4.157357 \\
\hline 1 & -5.216243 & -4.116881 & -2.808622 \\
\hline & -6.831208 & -3.451269 & -2.491416 \\
\hline$\frac{1}{1}$ & -6.732191 & -1.191789 & -4.627724 \\
\hline & -7.272973 & -0.950669 & -2.954840 \\
\hline ] & -5.978060 & 0.062384 & -3.618549 \\
\hline$\frac{1}{1}$ & -6.579894 & -1.257892 & 3.925405 \\
\hline & -4.929989 & -1.650956 & 3.417914 \\
\hline 1 & -5.587436 & -0.022618 & 3.136259 \\
\hline & -7.501883 & -3.186790 & 2.708598 \\
\hline 1 & -7.170427 & -3.443499 & 0.986563 \\
\hline ] & -5.868316 & -3.632038 & 2.179892 \\
\hline 1 & -8.388055 & -0.860381 & 2.323387 \\
\hline 1 & -7.437856 & 0.326126 & 1.404828 \\
\hline 1 & -8.160086 & -1.065733 & 0.579576 \\
\hline 6 & 0.859599 & 4.184481 & 2.662545 \\
\hline 5 & -0.564428 & 6.123135 & 2.101848 \\
\hline 6 & -1.043223 & 4.756733 & 4.142777 \\
\hline 5 & -5.428383 & 5.398889 & 0.942099 \\
\hline 6 & -5.858254 & 3.389805 & -0.442146 \\
\hline 6 & -6.032690 & 3.243455 & 2.039062 \\
\hline 1 & 1.505336 & 4.854828 & 3.244724 \\
\hline 1 & 0.942971 & 3.184160 & 3.109638 \\
\hline l & 1.259929 & 4.146575 & 1.639977 \\
\hline 1 & 0.120821 & 6.760945 & 2.676119 \\
\hline 1 & -0.222359 & 6.122029 & 1.057873 \\
\hline 1 & -1.552577 & 6.596826 & 2.131121 \\
\hline 1 & -0.372706 & 5.389079 & 4.740564 \\
\hline 1 & -2.057076 & 5.165325 & 4.233139 \\
\hline 1 & -1.047227 & 3.751914 & 4.586628 \\
\hline 1 & -7.104448 & 3.476826 & 1.968710 \\
\hline 1 & -5.919850 & 2.149575 & 2.025373 \\
\hline 1 & -5.666389 & 3.604896 & 3.009126 \\
\hline 1 & -6.874343 & 3.789095 & -0.557388 \\
\hline
\end{tabular}




$\begin{array}{llll}1 & -5.258394 & 3.719909 & -1.300865 \\ 1 & -5.939704 & 2.295627 & -0.487931 \\ 1 & -6.482940 & 5.661511 & 0.789338 \\ 1 & -5.134050 & 5.815100 & 1.913185 \\ 1 & -4.839881 & 5.899675 & 0.161849\end{array}$

$3_{\text {IIB-re- }} \psi_{-1 \mathrm{a} 233 \mathrm{a} 4 \mathrm{~s}}$

Number of imaginary frequencies : 0

The smallest frequencies are : $17.682720 .9057 \quad 21.6629 \mathrm{~cm}(-1)$

Electronic energy : $\quad \mathrm{HF}=-4360.8837907$

Zero-point correction $=\quad 1.455034$ (Hartree/Particle)

Thermal correction to Energy $=\quad 1.539443$

Thermal correction to Enthalpy= $\quad 1.540387$

Thermal correction to Gibbs Free Energy $=\quad 1.338671$

Sum of electronic and zero-point Energies $=\quad-4359.428757$

Sum of electronic and thermal Energies $=\quad-4359.344348$

Sum of electronic and thermal Enthalpies $=\quad-4359.343403$

Sum of electronic and thermal Free Energies $=\quad-4359.545120$

Cartesian Coordinates

$\begin{array}{cccc}6 & -1.738622 & 3.419823 & -3.362852 \\ 6 & -0.821805 & 3.516906 & -2.300669 \\ 6 & -1.025104 & 4.556381 & -1.382926 \\ 6 & -2.070483 & 5.465039 & -1.525342 \\ 6 & -2.942469 & 5.370179 & -2.601967 \\ 6 & -2.775583 & 4.327152 & -3.512902 \\ 6 & 0.360463 & 2.617698 & -2.178279 \\ 6 & 1.452536 & 3.105052 & -1.257820 \\ 45 & -0.171755 & 0.409333 & -1.815112 \\ 6 & -0.401569 & -1.014920 & -3.101457 \\ 8 & -0.614516 & -1.577981 & -4.091340 \\ 15 & -1.616287 & 0.150916 & 0.090127 \\ 6 & -2.098950 & 1.788560 & 0.784974 \\ 6 & -1.140553 & 2.573197 & 1.445671 \\ 6 & -1.479924 & 3.810274 & 1.987276 \\ 6 & -2.795653 & 4.268677 & 1.811262 \\ 6 & -3.755654 & 3.539973 & 1.118126 \\ 6 & -3.383326 & 2.288620 & 0.608812 \\ 6 & -0.503887 & 4.659530 & 2.802696 \\ 6 & -5.201113 & 4.006973 & 0.953350 \\ 6 & -3.265510 & -0.614517 & -0.227351 \\ 6 & -3.676678 & -0.909394 & -1.526518 \\ 6 & -4.928461 & -1.472758 & -1.772734 \\ 6 & -5.751323 & -1.751898 & -0.673697 \\ 6 & -5.366862 & -1.483030 & 0.640645 \\ 6 & -4.115469 & -0.894422 & 0.840427 \\ 6 & -6.228535 & -1.828637 & 1.855997 \\ 6 & -5.420618 & -1.773981 & -3.187039 \\ 6 & -1.136311 & -0.839941 & 1.594262 \\ 6 & -0.696591 & -2.154070 & 1.465308 \\ 6 & -0.398423 & -2.925573 & 2.636872 \\ 6 & -0.554286 & -2.342983 & 3.928371 \\ 6 & -1.019283 & -1.013191 & 4.022348 \\ 6 & -1.295405 & -0.288424 & 2.894191 \\ & & & \\ 6\end{array}$




\begin{tabular}{|c|c|c|c|}
\hline 6 & -0.210812 & -3.087904 & 5.082131 \\
\hline 6 & 0.275211 & -4.365027 & 4.974265 \\
\hline & 0.424742 & -4.952729 & 3.700341 \\
\hline & 0.091297 & -4.257309 & 2.564933 \\
\hline & -0.520636 & -2.850306 & 0.155357 \\
\hline & 0.673276 & -2.767228 & -0.537460 \\
\hline & 0.899517 & -3.544194 & -1.697080 \\
\hline & -0.070207 & -4.375738 & -2.184499 \\
\hline & -1.317705 & -4.476680 & -1.526183 \\
\hline & -1.540541 & -3.728981 & -0.330932 \\
\hline & -2.775428 & -3.900305 & 0.344483 \\
\hline & -3.742446 & -4.738647 & -0.150981 \\
\hline 0 & -3.524212 & -5.464027 & -1.341844 \\
\hline & -2.335550 & -5.332631 & -2.013128 \\
\hline & 1.733911 & -1.916180 & -0.079243 \\
\hline & 1.847180 & -0.402559 & -0.834659 \\
\hline 8 & 2.982538 & -0.453429 & -2.058358 \\
\hline 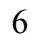 & 4.228629 & -1.006452 & -1.883424 \\
\hline & 5.176943 & -0.380214 & -1.099263 \\
\hline 4 & 6.399201 & -1.091374 & -0.842882 \\
\hline 6 & 6.647837 & -2.330451 & -1.507017 \\
\hline 6 & 5.682596 & -2.847771 & -2.405928 \\
\hline 6 & 4.486923 & -2.207707 & -2.577622 \\
\hline 6 & 7.352148 & -0.649139 & 0.110250 \\
\hline 6 & 8.488116 & -1.375718 & 0.370603 \\
\hline 6 & 8.745816 & -2.581150 & -0.314599 \\
\hline 6 & 7.839431 & -3.045177 & -1.233106 \\
\hline 6 & 4.909259 & 0.945649 & -0.492188 \\
\hline 6 & 3.773755 & 1.169371 & 0.267134 \\
\hline 6 & 3.620240 & 2.325701 & 1.060848 \\
\hline o & 4.537811 & 3.333800 & 0.993578 \\
\hline 6 & 5.636079 & 3.239491 & 0.103450 \\
\hline 6 & 5.823639 & 2.042907 & -0.651830 \\
\hline & 6.544243 & 4.313516 & -0.050255 \\
\hline 5 & 7.590796 & 4.233862 & -0.933485 \\
\hline & 7.761437 & 3.067632 & -1.706723 \\
\hline 5 & 6.905474 & 2.002388 & -1.567642 \\
\hline & 2.767420 & 0.253895 & 0.376898 \\
\hline 5 & 0.742753 & 1.762883 & -3.240609 \\
\hline 5 & 2.835228 & -2.633413 & 0.613003 \\
\hline & 3.419326 & -1.983115 & 1.841955 \\
\hline 6 & 2.621288 & -1.344752 & 2.792453 \\
\hline & 3.181458 & -0.802824 & 3.940496 \\
\hline 6 & 4.555420 & -0.892017 & 4.161188 \\
\hline 6 & 5.358211 & -1.527357 & 3.221595 \\
\hline & 4.791353 & -2.069495 & 2.069457 \\
\hline 1 & 7.159384 & 0.264714 & 0.665754 \\
\hline & -1.657929 & 0.731000 & 2.999750 \\
\hline 1 & 8.010546 & -3.983189 & -1.759753 \\
\hline & -0.332688 & -2.617303 & 6.056849 \\
\hline 1 & 6.383970 & 5.212609 & 0.543471 \\
\hline & -2.957546 & -3.344902 & 1.262307 \\
\hline 1 & -4.689732 & -4.843131 & 0.376543 \\
\hline 1 & 4.423302 & 4.229721 & 1.602618 \\
\hline 1 & -1.150631 & -0.568890 & 5.008338 \\
\hline 1 & 5.898368 & -3.774597 & -2.934794 \\
\hline
\end{tabular}




\begin{tabular}{|c|c|c|c|}
\hline & 8.574899 & 3.011963 & -2.426704 \\
\hline & 3.716424 & -2.590762 & -3.243717 \\
\hline ] & -2.147570 & -5.890862 & -2.929273 \\
\hline & 0.098882 & -4.961165 & -3.086865 \\
\hline & 0.806457 & -5.967780 & 3.617461 \\
\hline & 7.045557 & 1.117296 & -2.182842 \\
\hline & 0.209851 & -4.729551 & 1.592003 \\
\hline & 1.864331 & -3.450227 & -2.194113 \\
\hline & 8.278857 & 5.068093 & -1.048376 \\
\hline 1 & 9.192939 & -1.019379 & 1.118636 \\
\hline 1 & -4.299394 & -6.125754 & -1.722519 \\
\hline 1 & 0.545951 & -4.927147 & 5.864955 \\
\hline 1 & 9.652591 & -3.143237 & -0.104007 \\
\hline 1 & 2.749019 & 2.375906 & 1.709451 \\
\hline 1 & -3.792522 & -0.659390 & 1.854787 \\
\hline 1 & -2.996768 & -0.691553 & -2.344595 \\
\hline & -6.723622 & -2.205168 & -0.857429 \\
\hline 1 & -4.121586 & 1.689220 & 0.080221 \\
\hline & -0.127531 & 2.180749 & 1.550403 \\
\hline 1 & -3.067393 & 5.232321 & 2.236999 \\
\hline & 2.399140 & -3.597589 & 0.910609 \\
\hline & -1.530948 & 0.891866 & -2.461287 \\
\hline ] & 3.646902 & -2.872841 & -0.089999 \\
\hline & 5.431145 & -2.555750 & 1.331362 \\
\hline 1 & 1.556904 & -1.240533 & 2.600736 \\
\hline & 6.433836 & -1.596512 & 3.373655 \\
\hline 1 & 2.539490 & -0.304526 & 4.665844 \\
\hline 1 & 4.996021 & -0.462740 & 5.058599 \\
\hline 1 & 0.188009 & 1.752233 & -4.177696 \\
\hline 1 & 1.796938 & 1.511104 & -3.355590 \\
\hline & -0.357687 & 4.659548 & -0.530441 \\
\hline 1 & -2.192411 & 6.258821 & -0.788193 \\
\hline 1 & -3.747476 & 6.091489 & -2.729459 \\
\hline 1 & -3.455336 & 4.226998 & -4.357182 \\
\hline 1 & -1.637915 & 2.618900 & -4.092964 \\
\hline 1 & 1.729656 & 4.147460 & -1.484133 \\
\hline 1 & 1.168246 & 3.070185 & -0.198551 \\
\hline 1 & 2.357534 & 2.501407 & -1.382521 \\
\hline 6 & -4.351340 & -1.490063 & -4.240205 \\
\hline 6 & -5.810199 & -3.251100 & -3.302820 \\
\hline 6 & -6.641610 & -0.897436 & -3.487112 \\
\hline 5 & -7.512526 & -2.557954 & 1.469233 \\
\hline 6 & -5.434957 & -2.735965 & 2.805057 \\
\hline 6 & -6.611833 & -0.544912 & 2.599207 \\
\hline & -4.743486 & -1.729767 & -5.236742 \\
\hline 1 & -4.048249 & -0.434215 & -4.252670 \\
\hline 1 & -3.452779 & -2.101182 & -4.081327 \\
\hline 1 & -6.184360 & -3.465983 & -4.312948 \\
\hline 1 & -4.941280 & -3.895821 & -3.116859 \\
\hline 1 & -6.597302 & -3.534099 & -2.592790 \\
\hline 1 & -7.009168 & -1.088659 & -4.504456 \\
\hline 1 & -7.467824 & -1.093029 & -2.792296 \\
\hline 1 & -6.387326 & 0.168043 & -3.413841 \\
\hline 1 & -8.088074 & -2.796463 & 2.372549 \\
\hline 1 & -8.155366 & -1.945268 & 0.824302 \\
\hline 1 & -7.307286 & -3.503889 & 0.950320 \\
\hline
\end{tabular}




$\begin{array}{lrrr}1 & -7.202547 & -0.784116 & 3.493987 \\ 1 & -5.728849 & 0.020186 & 2.923894 \\ 1 & -7.217935 & 0.112868 & 1.962229 \\ 1 & -6.043142 & -2.989242 & 3.683811 \\ 1 & -5.153032 & -3.676872 & 2.313075 \\ 1 & -4.514916 & -2.260033 & 3.169517 \\ 6 & 0.911389 & 4.088930 & 2.795264 \\ 6 & -0.434828 & 6.085171 & 2.244565 \\ 6 & -0.991921 & 4.704104 & 4.255664 \\ 6 & -5.431516 & 5.400243 & 1.531938 \\ 6 & -5.581886 & 4.038078 & -0.529367 \\ 6 & -6.119749 & 3.019438 & 1.681988 \\ 1 & 1.571339 & 4.725228 & 3.399247 \\ 1 & 0.952199 & 3.077403 & 3.222675 \\ 1 & 1.325488 & 4.056889 & 1.778188 \\ 1 & 0.258336 & 6.691024 & 2.843428 \\ 1 & -0.070584 & 6.089679 & 1.208076 \\ 1 & -1.407462 & 6.590667 & 2.260954 \\ 1 & -0.307391 & 5.301386 & 4.873250 \\ 1 & -1.991342 & 5.147823 & 4.339647 \\ 1 & -1.038606 & 3.692867 & 4.682013 \\ 1 & -7.168101 & 3.337984 & 1.599861 \\ 1 & -6.042707 & 2.011681 & 1.254532 \\ 1 & -5.867257 & 2.953015 & 2.748984 \\ 1 & -6.633802 & 4.334071 & -0.642151 \\ 1 & -4.960897 & 4.755720 & -1.078247 \\ 1 & -5.457785 & 3.061194 & -1.012293 \\ 1 & -6.475364 & 5.698162 & 1.371222 \\ 1 & -5.244369 & 5.435529 & 2.613099 \\ 1 & -4.795485 & 6.152917 & 1.046828\end{array}$

$3_{\text {IIB-re- }} \psi_{-1 \mathbf{a} 25354 a}$

Number of imaginary frequencies : 0

The smallest frequencies are : $18.1682 \quad 20.392922 .4385 \mathrm{~cm}(-1)$

Electronic energy : $\quad H F=-4360.884219$

Zero-point correction $=\quad 1.457198($ Hartree/Particle $)$

Thermal correction to Energy= $\quad 1.540867$

Thermal correction to Enthalpy= $\quad 1.541811$

Thermal correction to Gibbs Free Energy= $\quad 1.342677$

Sum of electronic and zero-point Energies $=\quad-4359.427021$

Sum of electronic and thermal Energies $=\quad-4359.343352$

Sum of electronic and thermal Enthalpies $=\quad-4359.342408$

Sum of electronic and thermal Free Energies $=\quad-4359.541542$

\section{Cartesian Coordinates}

$\begin{array}{cccc}6 & 4.844868 & -1.930986 & 2.133686 \\ 6 & 3.473320 & -1.877835 & 1.892703 \\ 6 & 2.654822 & -1.228444 & 2.817876 \\ 6 & 3.194756 & -0.642610 & 3.954068 \\ 6 & 4.568141 & -0.698678 & 4.188406 \\ 6 & 5.391193 & -1.345072 & 3.274132 \\ 6 & 2.913515 & -2.573703 & 0.677534 \\ 7 & 1.796283 & -1.903842 & -0.035515 \\ 15 & 1.871038 & -0.408063 & -0.832682\end{array}$




\begin{tabular}{|c|c|c|c|}
\hline 3 & 2.768702 & 0.310671 & 0.359802 \\
\hline 6 & 3.756801 & 1.242259 & 0.223788 \\
\hline 6 & 4.904109 & 1.015652 & -0.517170 \\
\hline 6 & 5.800251 & 2.124166 & -0.702426 \\
\hline 6 & 5.582689 & 3.341025 & 0.011323 \\
\hline 6 & 4.471385 & 3.445073 & 0.883701 \\
\hline 6 & 3.571756 & 2.422728 & 0.974069 \\
\hline 6 & 6.893073 & 2.074661 & -1.604770 \\
\hline 6 & 7.731763 & 3.150137 & -1.768147 \\
\hline 6 & 7.532067 & 4.336923 & -1.034144 \\
\hline 6 & 6.473804 & 4.425693 & -0.165967 \\
\hline 6 & 5.200319 & -0.321433 & -1.085298 \\
\hline 6 & 4.267645 & -0.984097 & -1.858575 \\
\hline 6 & 4.551370 & -2.195854 & -2.523803 \\
\hline 6 & 5.758332 & -2.808402 & -2.333058 \\
\hline 6 & 6.709194 & -2.251970 & -1.442420 \\
\hline 6 & 6.434623 & -1.003217 & -0.807236 \\
\hline 6 & 7.913317 & -2.937440 & -1.149209 \\
\hline 6 & 8.807711 & -2.435833 & -0.238925 \\
\hline 6 & 8.524537 & -1.220844 & 0.418687 \\
\hline 6 & 7.375689 & -0.521988 & 0.138996 \\
\hline 8 & 3.012080 & -0.459838 & -2.051056 \\
\hline 6 & 0.756562 & -2.792889 & -0.469613 \\
\hline 6 & -0.446470 & -2.867338 & 0.209057 \\
\hline 6 & -1.449571 & -3.774166 & -0.261987 \\
\hline 6 & -1.186937 & -4.582462 & -1.409314 \\
\hline 6 & 0.070734 & -4.491571 & -2.049381 \\
\hline 6 & 1.014372 & -3.615069 & -1.590796 \\
\hline 6 & -2.703677 & -3.920804 & 0.384711 \\
\hline 6 & -3.644463 & -4.801989 & -0.088253 \\
\hline 6 & -3.381541 & -5.594402 & -1.226122 \\
\hline 6 & -2.177248 & -5.482814 & -1.871657 \\
\hline 6 & -0.637758 & -2.149180 & 1.505420 \\
\hline 6 & -1.097124 & -0.840686 & 1.617027 \\
\hline 6 & -1.266655 & -0.272490 & 2.908253 \\
\hline 6 & -0.980349 & -0.976565 & 4.046649 \\
\hline 6 & -0.492605 & -2.299458 & 3.971241 \\
\hline 6 & -0.326668 & -2.898617 & 2.688497 \\
\hline 6 & -0.138276 & -3.021631 & 5.136079 \\
\hline 6 & 0.368163 & -4.292300 & 5.047786 \\
\hline 6 & 0.528568 & -4.896162 & 3.782865 \\
\hline 6 & 0.184992 & -4.223142 & 2.636935 \\
\hline 15 & -1.586325 & 0.123064 & 0.101984 \\
\hline 6 & -3.235711 & -0.643972 & -0.203418 \\
\hline 6 & -3.646620 & -0.935093 & -1.492825 \\
\hline 6 & -4.927507 & -1.441057 & -1.755124 \\
\hline 6 & -5.774017 & -1.655130 & -0.672514 \\
\hline 6 & -5.388570 & -1.382654 & 0.652078 \\
\hline 6 & -4.112397 & -0.875870 & 0.866285 \\
\hline 6 & -5.306953 & -1.757910 & -3.198790 \\
\hline 6 & -4.467268 & -2.950542 & -3.670655 \\
\hline 6 & -6.354474 & -1.673606 & 1.800763 \\
\hline 6 & -6.600267 & -3.184863 & 1.878399 \\
\hline 45 & -0.168586 & 0.315458 & -1.827594 \\
\hline 6 & 0.680680 & 1.656101 & -3.311403 \\
\hline 6 & 0.270067 & 2.521155 & -2.269072 \\
\hline
\end{tabular}




\begin{tabular}{|c|c|c|c|}
\hline 6 & 1.349687 & 3.087891 & -1.378370 \\
\hline 6 & -0.965635 & 3.346954 & -2.391573 \\
\hline & -1.906622 & 3.162050 & -3.421293 \\
\hline & -3.011974 & 3.988424 & -3.552002 \\
\hline & -3.222353 & 5.038747 & -2.658292 \\
\hline & -2.320683 & 5.223268 & -1.618894 \\
\hline & -1.210934 & 4.392717 & -1.493047 \\
\hline & -0.405192 & -1.165401 & -3.043597 \\
\hline & -0.672682 & -1.774516 & -3.992839 \\
\hline & -2.084512 & 1.766274 & 0.777918 \\
\hline & -1.141035 & 2.597996 & 1.401385 \\
\hline O & -1.511953 & 3.838492 & 1.916091 \\
\hline & -2.848684 & 4.245769 & 1.769352 \\
\hline ס & -3.799846 & 3.464448 & 1.123255 \\
\hline & -3.385992 & 2.223712 & 0.624762 \\
\hline ר & -0.542955 & 4.755254 & 2.664386 \\
\hline f & 0.885926 & 4.220397 & 2.658637 \\
\hline & -5.278641 & 3.834938 & 1.032792 \\
\hline f & -6.067740 & 2.847771 & 1.903930 \\
\hline & -1.000702 & 4.877198 & 4.122540 \\
\hline & -0.523551 & 6.145660 & 2.019461 \\
\hline כ & -5.554842 & 5.250359 & 1.531778 \\
\hline ? & -5.776184 & 3.731126 & -0.411484 \\
\hline ) & -5.802260 & -1.216718 & 3.149912 \\
\hline & -7.687769 & -0.956119 & 1.565373 \\
\hline 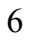 & -6.783305 & -2.117280 & -3.344542 \\
\hline ס & -5.019336 & -0.546859 & -4.095188 \\
\hline & 7.163369 & 0.399764 & 0.674068 \\
\hline & -1.645266 & 0.742695 & 2.996992 \\
\hline - & 8.103909 & -3.883318 & -1.654661 \\
\hline 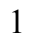 & -0.268712 & -2.538502 & 6.103541 \\
\hline ] & 6.290548 & 5.339946 & 0.397203 \\
\hline t & -2.922061 & -3.312609 & 1.261194 \\
\hline 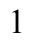 & -4.603529 & -4.891326 & 0.418774 \\
\hline I & 4.332474 & 4.358708 & 1.460528 \\
\hline D & -1.119977 & -0.521154 & 5.026384 \\
\hline 1 & 5.994447 & -3.742417 & -2.840085 \\
\hline & 8.554374 & 3.086252 & -2.476962 \\
\hline 1 & 3.791074 & -2.608261 & -3.183935 \\
\hline 1 & -1.956935 & -6.086323 & -2.751161 \\
\hline 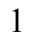 & 0.267121 & -5.119375 & -2.917078 \\
\hline 1 & 0.926424 & -5.906134 & 3.715085 \\
\hline ] & 7.056363 & 1.173209 & -2.189682 \\
\hline 1 & 0.312527 & -4.708458 & 1.671593 \\
\hline 1 & 1.984428 & -3.525278 & -2.078170 \\
\hline 1 & 8.206973 & 5.179125 & -1.167434 \\
\hline 1 & 9.219780 & -0.834819 & 1.160957 \\
\hline 1 & -4.135764 & -6.290011 & -1.587800 \\
\hline 1 & 0.646706 & -4.836858 & 5.946955 \\
\hline 1 & 9.724504 & -2.975466 & -0.013459 \\
\hline 1 & 2.692560 & 2.478334 & 1.611136 \\
\hline 1 & -3.784253 & -0.637337 & 1.875718 \\
\hline 1 & -2.955602 & -0.762038 & -2.313233 \\
\hline 1 & -6.771892 & -2.051777 & -0.850264 \\
\hline 1 & -4.116472 & 1.592039 & 0.125696 \\
\hline 1 & -0.113757 & 2.242350 & 1.498396 \\
\hline
\end{tabular}




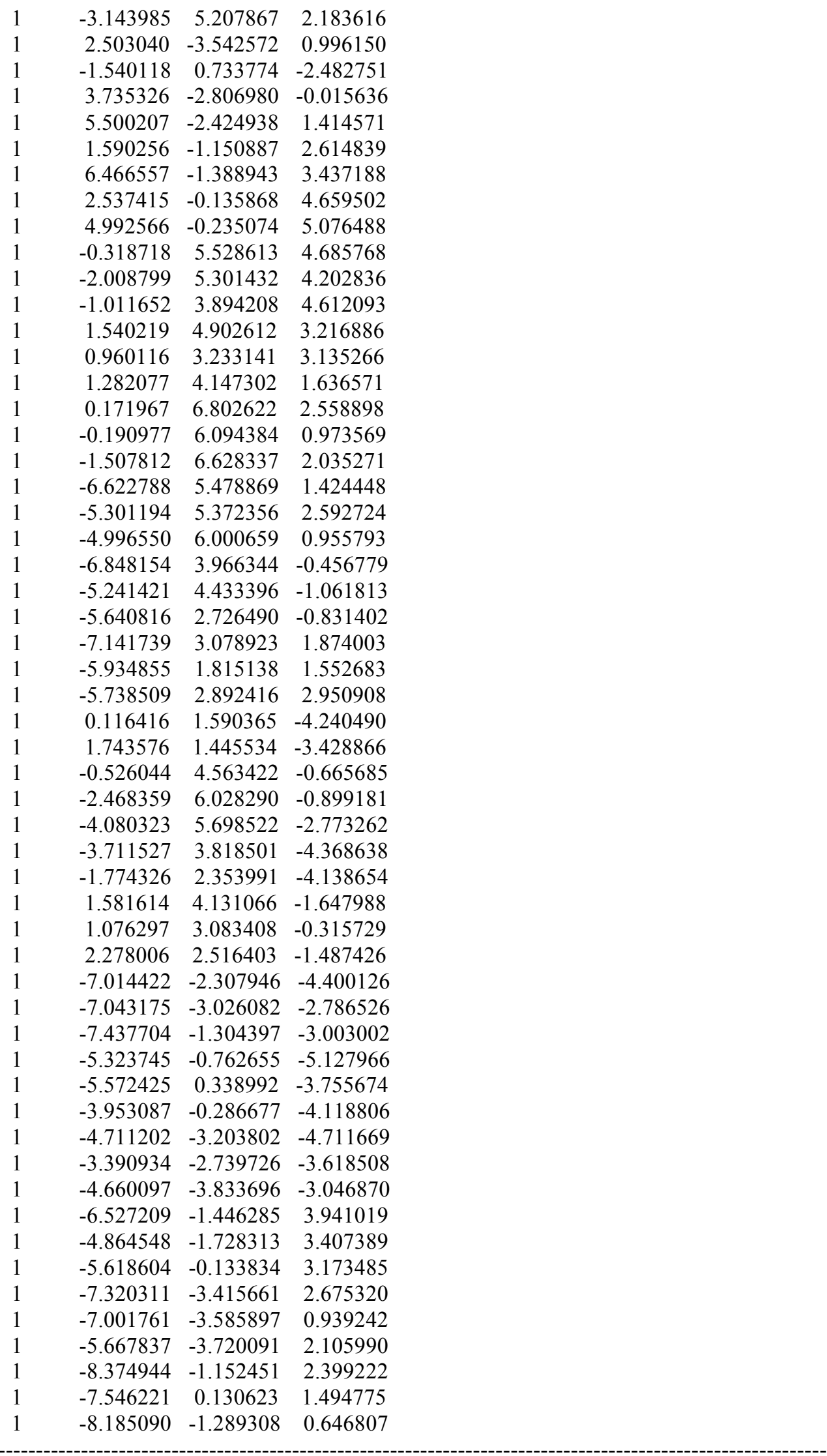


$3_{\text {IIB-re- }} \Psi-1 \mathrm{a} 2 \mathrm{~s} 3 \mathrm{~s} 4 \mathrm{~s}$

Number of imaginary frequencies : 0

The smallest frequencies are : $17.7522 \quad 19.8455 \quad 21.1222 \mathrm{~cm}(-1)$

Electronic energy : $\quad H F=-4360.8840523$

Zero-point correction $=\quad 1.454860$ (Hartree/Particle)

Thermal correction to Energy $=\quad 1.539422$

Thermal correction to Enthalpy= $\quad 1.540366$

Thermal correction to Gibbs Free Energy= $\quad 1.337655$

Sum of electronic and zero-point Energies $=\quad-4359.429192$

Sum of electronic and thermal Energies $=\quad-4359.344631$

Sum of electronic and thermal Enthalpies $=\quad-4359.343686$

Sum of electronic and thermal Free Energies= $\quad-4359.546397$

Cartesian Coordinates

$\begin{array}{cccc}6 & -1.777402 & 3.375172 & -3.369501 \\ 6 & -0.855301 & 3.488963 & -2.313371 \\ 6 & -1.066705 & 4.529296 & -1.398694 \\ 6 & -2.124195 & 5.424209 & -1.538760 \\ 6 & -3.001348 & 5.313366 & -2.609632 \\ 6 & -2.827425 & 4.267964 & -3.516516 \\ 6 & 0.337599 & 2.603241 & -2.191187 \\ 6 & 1.427919 & 3.106414 & -1.276850 \\ 45 & -0.178774 & 0.399352 & -1.808503 \\ 6 & -0.446235 & -1.049294 & -3.059266 \\ 8 & -0.720219 & -1.632133 & -4.022672 \\ 15 & -1.618718 & 0.153247 & 0.099879 \\ 6 & -2.100571 & 1.788980 & 0.800348 \\ 6 & -1.141338 & 2.578123 & 1.454708 \\ 6 & -1.481908 & 3.816488 & 1.993075 \\ 6 & -2.800988 & 4.268643 & 1.825520 \\ 6 & -3.762787 & 3.533831 & 1.141705 \\ 6 & -3.388036 & 2.283705 & 0.631529 \\ 6 & -0.502652 & 4.677597 & 2.792010 \\ 6 & -5.212259 & 3.992131 & 0.987876 \\ 6 & -3.269117 & -0.604020 & -0.224627 \\ 6 & -3.683500 & -0.868265 & -1.523497 \\ 6 & -4.941035 & -1.419367 & -1.787153 \\ 6 & -5.762288 & -1.726636 & -0.700134 \\ 6 & -5.372465 & -1.485826 & 0.623790 \\ 6 & -4.123976 & -0.904368 & 0.838476 \\ 6 & -6.239525 & -1.852388 & 1.829980 \\ 6 & -5.330144 & -1.710260 & -3.234805 \\ 6 & -1.143277 & -0.847255 & 1.597103 \\ 6 & -0.701539 & -2.160166 & 1.462714 \\ 6 & -0.405720 & -2.935772 & 2.632988 \\ 6 & -0.568403 & -2.359028 & 3.926386 \\ 6 & -1.036925 & -1.030781 & 4.024714 \\ 6 & -1.308831 & -0.301565 & 2.898634 \\ 6 & -0.227335 & -3.107243 & 5.078730 \\ 6 & 0.263604 & -4.382202 & 4.968215 \\ 6 & 0.421016 & -4.963960 & 3.692620 \\ 6 & 0.089882 & -4.265358 & 2.558471 \\ 6 & -0.514190 & -2.856340 & 0.153447 \\ 6 & 0.686819 & -2.773643 & -0.527088\end{array}$




\begin{tabular}{|c|c|c|c|}
\hline 6 & 0.934238 & -3.567881 & -1.670937 \\
\hline 6 & -0.015457 & -4.428415 & -2.146961 \\
\hline & -1.268082 & -4.534372 & -1.499077 \\
\hline & -1.520590 & -3.751991 & -0.332053 \\
\hline & -2.767453 & -3.914021 & 0.322880 \\
\hline & -3.714605 & -4.781460 & -0.161600 \\
\hline & -3.462325 & -5.549380 & -1.318400 \\
\hline & -2.262954 & -5.424396 & -1.971449 \\
\hline & 1.737992 & -1.911541 & -0.067531 \\
\hline & 1.845336 & -0.400807 & -0.829743 \\
\hline$\gamma$ & 2.980727 & -0.452562 & -2.053480 \\
\hline & 4.229637 & -0.998394 & -1.876817 \\
\hline$b$ & 5.175157 & -0.364389 & -1.095467 \\
\hline & 6.400846 & -1.068632 & -0.836340 \\
\hline & 6.655443 & -2.308919 & -1.495895 \\
\hline & 5.692958 & -2.834050 & -2.393154 \\
\hline & 4.494156 & -2.200545 & -2.567081 \\
\hline & 7.351848 & -0.618201 & 0.114947 \\
\hline 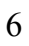 & 8.491390 & -1.338326 & 0.377732 \\
\hline & 8.754941 & -2.544887 & -0.303227 \\
\hline & 7.850635 & -3.016715 & -1.219766 \\
\hline & 4.901248 & 0.963184 & -0.495121 \\
\hline & 3.764489 & 1.185360 & 0.262757 \\
\hline & 3.604636 & 2.345664 & 1.049267 \\
\hline & 4.516962 & 3.358152 & 0.976143 \\
\hline ) & 5.616094 & 3.264169 & 0.087098 \\
\hline & 5.810148 & 2.064055 & -0.660913 \\
\hline 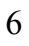 & 6.518793 & 4.341930 & -0.072641 \\
\hline ) & 7.566121 & 4.262391 & -0.954946 \\
\hline 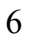 & 7.743110 & 3.092433 & -1.721109 \\
\hline ) & 6.892603 & 2.023632 & -1.576006 \\
\hline & 2.763630 & 0.264498 & 0.378444 \\
\hline 6 & 0.724579 & 1.745102 & -3.249365 \\
\hline & 2.842889 & -2.618414 & 0.630013 \\
\hline 6 & 3.416182 & -1.959520 & 1.859365 \\
\hline & 2.607592 & -1.328862 & 2.806060 \\
\hline 6 & 3.157890 & -0.777585 & 3.954445 \\
\hline 5 & 4.532187 & -0.849595 & 4.179235 \\
\hline & 5.345375 & -1.477344 & 3.243448 \\
\hline 6 & 4.788529 & -2.028909 & 2.090906 \\
\hline & 7.154676 & 0.296741 & 0.667155 \\
\hline ] & -1.673263 & 0.716979 & 3.006201 \\
\hline & 8.026244 & -3.955714 & -1.743159 \\
\hline 1 & -0.354853 & -2.640615 & 6.054628 \\
\hline | & 6.353599 & 5.243686 & 0.515677 \\
\hline ] & -2.975417 & -3.328743 & 1.216272 \\
\hline 1 & -4.670449 & -4.878132 & 0.351443 \\
\hline & 4.397624 & 4.257138 & 1.579734 \\
\hline t & -1.173190 & -0.591308 & 5.012178 \\
\hline & 5.913505 & -3.761387 & -2.919136 \\
\hline 1 & 8.557181 & 3.036657 & -2.440393 \\
\hline 1 & 3.725960 & -2.589367 & -3.232382 \\
\hline 1 & -2.050968 & -6.009231 & -2.865501 \\
\hline 1 & 0.173831 & -5.034587 & -3.031439 \\
\hline 1 & 0.807207 & -5.977100 & 3.607135 \\
\hline 1 & 7.037594 & 1.135585 & -2.185783 \\
\hline
\end{tabular}




\begin{tabular}{|c|c|c|c|}
\hline . & 0.215494 & -4.733970 & 1.584714 \\
\hline & 1.902527 & -3.470363 & -2.160198 \\
\hline & 8.249934 & 5.099446 & -1.074535 \\
\hline & 9.194591 & -0.975871 & 1.124348 \\
\hline & -4.219898 & -6.236528 & -1.689143 \\
\hline ] & 0.532522 & -4.946825 & 5.857869 \\
\hline & 9.664566 & -3.101674 & -0.090863 \\
\hline & 2.733330 & 2.395052 & 1.697719 \\
\hline & -3.801883 & -0.689258 & 1.857481 \\
\hline & -3.005929 & -0.642099 & -2.342668 \\
\hline & -6.734374 & -2.174295 & -0.886760 \\
\hline & -4.127203 & 1.681413 & 0.107775 \\
\hline . & -0.126339 & 2.189430 & 1.555016 \\
\hline 1 & -3.073852 & 5.232516 & 2.250018 \\
\hline 1 & 2.413384 & -3.585343 & 0.928488 \\
\hline 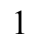 & -1.537476 & 0.877884 & -2.454724 \\
\hline & 3.659398 & -2.852678 & -0.069141 \\
\hline & 5.436169 & -2.509065 & 1.355647 \\
\hline 1 & 1.542397 & -1.238534 & 2.611426 \\
\hline & 6.421224 & -1.533464 & 3.399359 \\
\hline 1 & 2.507971 & -0.285645 & 4.677032 \\
\hline 1 & 4.964932 & -0.413067 & 5.076969 \\
\hline 1 & 0.167639 & 1.723297 & -4.184897 \\
\hline 1 & 1.780480 & 1.500868 & -3.364567 \\
\hline 1 & -0.396293 & 4.644091 & -0.550013 \\
\hline 1 & -2.251589 & 6.219540 & -0.804119 \\
\hline 1 & -3.816081 & 6.023909 & -2.735678 \\
\hline 1 & -3.511749 & 4.154307 & -4.355404 \\
\hline 1 & -1.671295 & 2.572505 & -4.097105 \\
\hline 1 & 1.695919 & 4.149354 & -1.511565 \\
\hline 1 & 1.145989 & 3.077180 & -0.216750 \\
\hline 1 & 2.337655 & 2.509092 & -1.398733 \\
\hline 6 & -6.802378 & -2.090604 & -3.375049 \\
\hline 6 & -5.072452 & -0.478300 & -4.110983 \\
\hline 6 & -4.476797 & -2.879424 & -3.739643 \\
\hline 6 & -7.517407 & -2.584406 & 1.427661 \\
\hline 6 & -5.447603 & -2.766498 & 2.773794 \\
\hline 6 & -6.635148 & -0.579746 & 2.585960 \\
\hline 1 & -7.042354 & -2.250727 & -4.433747 \\
\hline 1 & -7.041303 & -3.021924 & -2.845850 \\
\hline 1 & -7.466591 & -1.301036 & -2.999434 \\
\hline 1 & -5.386194 & -0.680022 & -5.143742 \\
\hline 1 & -5.635713 & 0.392068 & -3.749265 \\
\hline 1 & -4.011064 & -0.200593 & -4.143786 \\
\hline 1 & -4.723977 & -3.112725 & -4.784629 \\
\hline 1 & -3.403597 & -2.653136 & -3.688899 \\
\hline 1 & -4.651930 & -3.779020 & -3.134443 \\
\hline 1 & -8.094275 & -2.839736 & 2.325556 \\
\hline 1 & -8.162511 & -1.966916 & 0.789713 \\
\hline 1 & -7.304358 & -3.521305 & 0.895811 \\
\hline 1 & -7.233850 & -0.832404 & 3.471737 \\
\hline 1 & -5.757277 & -0.015988 & 2.926429 \\
\hline 1 & -7.237332 & 0.084017 & 1.951416 \\
\hline 1 & -6.059677 & -3.031440 & 3.646402 \\
\hline 1 & -5.159527 & -3.701109 & 2.273480 \\
\hline 1 & -4.531229 & -2.291488 & 3.148452 \\
\hline
\end{tabular}




$\begin{array}{rrrr}6 & 0.914698 & 4.112802 & 2.777470 \\ 6 & -0.444504 & 6.097006 & 2.216739 \\ 6 & -0.977589 & 4.737181 & 4.248673 \\ 6 & -5.447368 & 5.382616 & 1.571201 \\ 6 & -5.604390 & 4.024061 & -0.491908 \\ 6 & -6.119131 & 2.997185 & 1.721245 \\ 1 & 1.577504 & 4.757441 & 3.369355 \\ 1 & 0.963527 & 3.105570 & 3.213890 \\ 1 & 1.319361 & 4.072984 & 1.756923 \\ 1 & 0.253598 & 6.711910 & 2.800458 \\ 1 & -0.092477 & 6.090446 & 1.176028 \\ 1 & -1.418563 & 6.599528 & 2.238579 \\ 1 & -0.290119 & 5.344944 & 4.852597 \\ 1 & -1.978063 & 5.177682 & 4.336741 \\ 1 & -1.015740 & 3.731147 & 4.687752 \\ 1 & -7.169783 & 3.310717 & 1.650054 \\ 1 & -6.041113 & 1.991502 & 1.289273 \\ 1 & -5.856427 & 2.928022 & 2.785586 \\ 1 & -6.661974 & 4.302873 & -0.595278 \\ 1 & -5.000102 & 4.755337 & -1.041223 \\ 1 & -5.466666 & 3.052376 & -0.981982 \\ 1 & -6.494049 & 5.674533 & 1.418021 \\ 1 & -5.253186 & 5.416477 & 2.651179 \\ 1 & -4.819228 & 6.140323 & 1.083651\end{array}$

$3_{\text {IIB-re- }} \psi_{-1 \text { s2aza4a }}$

Number of imaginary frequencies : 0

The smallest frequencies are : $18.3016 \quad 20.030922 .7503 \mathrm{~cm}(-1)$

Electronic energy : $\quad \mathrm{HF}=-4360.8854876$

Zero-point correction $=\quad 1.455534$ (Hartree/Particle)

Thermal correction to Energy $=\quad 1.539663$

Thermal correction to Enthalpy= $\quad 1.540607$

Thermal correction to Gibbs Free Energy $=1.340346$

Sum of electronic and zero-point Energies $=\quad-4359.429954$

Sum of electronic and thermal Energies $=\quad-4359.345824$

Sum of electronic and thermal Enthalpies $=\quad-4359.344880$

Sum of electronic and thermal Free Energies $=\quad-4359.545141$

Cartesian Coordinates

$\begin{array}{cccc}6 & -1.923682 & 2.959511 & -3.569920 \\ 6 & -0.903299 & 3.178466 & -2.626700 \\ 6 & -1.047425 & 4.289211 & -1.785091 \\ 6 & -2.149200 & 5.135852 & -1.872905 \\ 6 & -3.145187 & 4.901227 & -2.811841 \\ 6 & -3.022443 & 3.799989 & -3.659855 \\ 6 & 0.305437 & 2.312815 & -2.530633 \\ 6 & 1.451468 & 2.874696 & -1.725113 \\ 45 & -0.197812 & 0.156796 & -1.939964 \\ 6 & -0.379613 & -1.406233 & -3.061768 \\ 8 & -0.537882 & -2.080635 & -3.990453 \\ 15 & -1.628088 & 0.171682 & -0.000215 \\ 6 & -1.991004 & 1.875569 & 0.597977 \\ 6 & -0.962998 & 2.596581 & 1.204201 \\ 6 & -1.205847 & 3.831568 & 1.816195\end{array}$




\begin{tabular}{|c|c|c|c|}
\hline 6 & -2.497234 & 4.353925 & 1.731619 \\
\hline & -3.534213 & 3.693142 & 1.061605 \\
\hline & -3.261719 & 2.446080 & 0.500842 \\
\hline & -0.075819 & 4.556530 & 2.548597 \\
\hline & -4.937441 & 4.300092 & 1.045380 \\
\hline & -3.314624 & -0.537005 & -0.252960 \\
\hline & -3.698825 & -1.020172 & -1.498559 \\
\hline & -4.965793 & -1.577606 & -1.700451 \\
\hline 6 & -5.833456 & -1.627919 & -0.609373 \\
\hline & -5.480251 & -1.158048 & 0.661924 \\
\hline 6 & -4.207755 & -0.615460 & 0.820917 \\
\hline 6 & -6.461415 & -1.288623 & 1.825991 \\
\hline 6 & -5.417081 & -2.078344 & -3.071706 \\
\hline 6 & -1.192375 & -0.692143 & 1.598046 \\
\hline 6 & -0.730155 & -2.002412 & 1.589634 \\
\hline 6 & -0.443631 & -2.672414 & 2.825844 \\
\hline 6 & -0.631218 & -1.987641 & 4.061215 \\
\hline 6 & -1.128956 & -0.666290 & 4.035892 \\
\hline 6 & -1.397893 & -0.043320 & 2.847422 \\
\hline 6 & -0.295785 & -2.627827 & 5.277982 \\
\hline 6 & 0.207716 & -3.902958 & 5.284762 \\
\hline 6 & 0.382524 & -4.594020 & 4.067486 \\
\hline 6 & 0.059707 & -3.999901 & 2.872624 \\
\hline 6 & -0.532165 & -2.810680 & 0.351729 \\
\hline 6 & 0.681460 & -2.818683 & -0.310675 \\
\hline 6 & 0.929104 & -3.736670 & -1.357284 \\
\hline 6 & -0.040515 & -4.609929 & -1.768646 \\
\hline 6 & -1.316135 & -4.599628 & -1.158049 \\
\hline 6 & -1.561825 & -3.707695 & -0.071655 \\
\hline 6 & -2.833695 & -3.745893 & 0.555822 \\
\hline 6 & -3.814621 & -4.595415 & 0.110147 \\
\hline 6 & -3.569216 & -5.472660 & -0.968891 \\
\hline 6 & -2.345213 & -5.473036 & -1.587517 \\
\hline 7 & 1.727590 & -1.908089 & 0.060790 \\
\hline 15 & 1.827158 & -0.489287 & -0.865505 \\
\hline 8 & 2.982915 & -0.664902 & -2.057939 \\
\hline 6 & 4.216782 & -1.210542 & -1.793365 \\
\hline 6 & 5.152232 & -0.513764 & -1.054996 \\
\hline 6 & 6.354907 & -1.207342 & -0.684409 \\
\hline 6 & 6.599851 & -2.517025 & -1.197596 \\
\hline 6 & 5.652476 & -3.118397 & -2.062351 \\
\hline 6 & 4.473545 & -2.485049 & -2.341358 \\
\hline 6 & 7.290864 & -0.672781 & 0.238232 \\
\hline 6 & 8.407895 & -1.380462 & 0.608954 \\
\hline 6 & 8.662581 & -2.659304 & 0.071728 \\
\hline 6 & 7.771960 & -3.212383 & -0.811897 \\
\hline 6 & 4.891416 & 0.878830 & -0.618331 \\
\hline 6 & 3.739378 & 1.213491 & 0.072870 \\
\hline 6 & 3.588142 & 2.466537 & 0.705435 \\
\hline 6 & 4.525408 & 3.443889 & 0.534337 \\
\hline 6 & 5.646243 & 3.219223 & -0.301970 \\
\hline 6 & 5.831040 & 1.932180 & -0.890365 \\
\hline 6 & 6.578462 & 4.250809 & -0.565675 \\
\hline 6 & 7.646292 & 4.041842 & -1.400652 \\
\hline 6 & 7.815066 & 2.783136 & -2.012144 \\
\hline 6 & 6.936475 & 1.757622 & -1.761627 \\
\hline
\end{tabular}




\begin{tabular}{|c|c|c|c|}
\hline & 2.711684 & 0.336357 & 0.268264 \\
\hline & 0.628331 & 1.372528 & -3.537933 \\
\hline & 2.817428 & -2.509423 & 0.867958 \\
\hline & 3.337967 & -1.707698 & 2.035006 \\
\hline & 2.499976 & -0.955468 & 2.860251 \\
\hline & 3.011012 & -0.279109 & 3.959589 \\
\hline$y$ & 4.372248 & -0.342173 & 4.254527 \\
\hline & 5.214082 & -1.088560 & 3.438888 \\
\hline ) & 4.697421 & -1.767092 & 2.337048 \\
\hline & 7.098653 & 0.299959 & 0.683031 \\
\hline & -1.784540 & 0.972172 & 2.865193 \\
\hline 1 & 7.940406 & -4.206227 & -1.224623 \\
\hline & -0.440140 & -2.079802 & 6.208131 \\
\hline 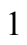 & 6.419536 & 5.221023 & -0.096585 \\
\hline & -3.036770 & -3.068877 & 1.384099 \\
\hline 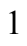 & -4.792461 & -4.591054 & 0.589514 \\
\hline I & 4.408902 & 4.413030 & 1.017513 \\
\hline & -1.291746 & -0.145251 & 4.978888 \\
\hline & 5.867743 & -4.101142 & -2.478328 \\
\hline & 8.645940 & 2.622639 & -2.695576 \\
\hline & 3.716966 & -2.927408 & -2.985924 \\
\hline 1 & -2.139745 & -6.147151 & -2.417932 \\
\hline 1 & 0.147884 & -5.307049 & -2.583639 \\
\hline & 0.774250 & -5.608611 & 4.075959 \\
\hline 1 & 7.077061 & 0.798734 & -2.253602 \\
\hline & 0.197109 & -4.551469 & 1.944821 \\
\hline 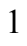 & 1.909303 & -3.721364 & -1.832565 \\
\hline 1 & 8.352782 & 4.843638 & -1.602071 \\
\hline 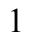 & 9.099544 & -0.951552 & 1.330734 \\
\hline ] & -4.352659 & -6.149187 & -1.304328 \\
\hline & 0.470711 & -4.384878 & 6.223512 \\
\hline 1 & 9.554424 & -3.206111 & 0.368656 \\
\hline & 2.703093 & 2.619524 & 1.319466 \\
\hline 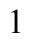 & -3.895719 & -0.243973 & 1.793852 \\
\hline 1 & -2.984836 & -0.966713 & -2.314279 \\
\hline D & -6.825646 & -2.057810 & -0.748074 \\
\hline 1 & -4.049721 & 1.891599 & 0.000110 \\
\hline & 0.032778 & 2.149753 & 1.252227 \\
\hline 1 & -2.714387 & 5.306390 & 2.210720 \\
\hline . & 2.396130 & -3.446174 & 1.258970 \\
\hline . & -1.565770 & 0.525188 & -2.633422 \\
\hline 1 & 3.662307 & -2.804070 & 0.227992 \\
\hline & 5.366823 & -2.342249 & 1.695400 \\
\hline 1 & 1.445439 & -0.871567 & 2.608722 \\
\hline & 6.280725 & -1.140243 & 3.650193 \\
\hline 1 & 2.339950 & 0.303167 & 4.589883 \\
\hline l & 4.772635 & 0.192865 & 5.113095 \\
\hline 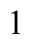 & 0.014981 & 1.275202 & -4.432797 \\
\hline 1 & 1.675486 & 1.112705 & -3.693583 \\
\hline 1 & -0.289107 & 4.499693 & -1.035551 \\
\hline 1 & -2.220011 & 5.986192 & -1.194447 \\
\hline 1 & -4.004225 & 5.565552 & -2.890179 \\
\hline 1 & -3.788502 & 3.599178 & -4.406791 \\
\hline 1 & -1.861644 & 2.108918 & -4.246371 \\
\hline 1 & 1.700340 & 3.901507 & -2.039004 \\
\hline 1 & 1.237940 & 2.910896 & -0.650685 \\
\hline
\end{tabular}




\begin{tabular}{|c|c|c|c|}
\hline & 2.354939 & 2.271150 & -1.866733 \\
\hline 6 & -4.250265 & -2.186037 & -4.052518 \\
\hline & -6.057396 & -3.464722 & \\
\hline & -6.443702 & -1.090450 & -3.63735 \\
\hline 6 & -5.923062 & -0.650503 & 3.10482 \\
\hline & -7.788002 & -0.607354 & 1.47538 \\
\hline & -6.712216 & -2.775165 & 2.10460 \\
\hline & -4.606926 & -2.595504 & -5.00615 \\
\hline & -3.795527 & -1.210319 & -4.2696 \\
\hline & -3.464233 & -2.854351 & -3.673 \\
\hline & -6.369730 & -3.818245 & -3.94 \\
\hline 1 & -5.342777 & -4.190705 & -2.546 \\
\hline 1 & -6.947949 & -3.466839 & -2.315 \\
\hline 1 & -6.785303 & -1.416186 & -4.629 \\
\hline 1 & -7.324968 & -1.006247 & -2.98869 \\
\hline 1 & -6.007479 & -0.088136 & -3.74058 \\
\hline 1 & -6.662972 & -0.751531 & $3.908^{\prime}$ \\
\hline 1 & -4.996510 & -1.131070 & 3.4481 \\
\hline 1 & -5.721585 & 0.421294 & 2.9715 \\
\hline 1 & -7.420907 & -2.895915 & 2.935 \\
\hline 1 & -7.132170 & -3.291341 & \\
\hline 1 & -5.779011 & -3.284055 & 2.383 \\
\hline 1 & -8.496216 & -0.703074 & 2.309 \\
\hline 1 & -7.642928 & 0.462944 & 1.27837 \\
\hline 1 & -8.261691 & -1.047933 & 0.590 \\
\hline 6 & -0.587179 & 5.715937 & 3.402 \\
\hline 6 & 0.654342 & 3.581817 & 3.4799 \\
\hline 6 & 0.907465 & 5.125333 & 1.519867 \\
\hline 6 & -5.905041 & 3.487393 & 0.187694 \\
\hline 6 & -5.475321 & 4.331077 & 2.48102 \\
\hline 6 & -4.902860 & 5.727192 & 0.49034 \\
\hline 1 & 0.250366 & 6.165742 & 3.950279 \\
\hline 1 & -1.038918 & 6.510370 & 2.795000 \\
\hline 1 & -1.328099 & 5.383771 & 4.14096 \\
\hline 1 & 1.479069 & 4.092816 & 3.994442 \\
\hline 1 & -0.027261 & 3.185539 & 4.245246 \\
\hline 1 & 1.081400 & 2.722674 & 2.947977 \\
\hline 1 & 1.738464 & 5.638494 & 2.024329 \\
\hline 1 & 1.334775 & 4.343090 & 0.879781 \\
\hline 1 & 0.408745 & 5.854815 & 0.866281 \\
\hline 1 & -5.909076 & 6.166667 & 0.517008 \\
\hline 1 & -4.242041 & 6.385704 & 1.067248 \\
\hline & -4.558236 & 5.730240 & -0.551225 \\
\hline 1 & -6.493212 & 4.743823 & 2.499905 \\
\hline & -5.511805 & 3.320466 & 2.908892 \\
\hline 1 & -4.852539 & 4.949675 & $3.13903^{\prime}$ \\
\hline & -6.892459 & 3.966297 & 0.19046 \\
\hline & -5.567062 & 3.422185 & -0.85493 \\
\hline 1 & -6.035363 & 2.464475 & 0.56789 \\
\hline
\end{tabular}

$3_{\text {IIB-re- }} \Psi-1 \mathrm{~s} 2 \mathrm{a} 3 \mathrm{a} 4 \mathrm{~s}$

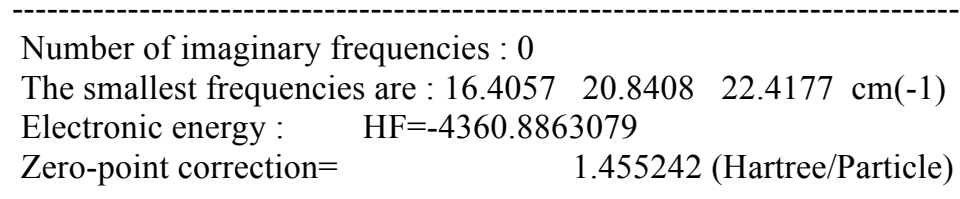




$\begin{array}{lc}\text { Thermal correction to Energy= } & 1.539521 \\ \text { Thermal correction to Enthalpy= } & 1.540465 \\ \text { Thermal correction to Gibbs Free Energy= } & 1.339119 \\ \text { Sum of electronic and zero-point Energies }= & -4359.431066 \\ \text { Sum of electronic and thermal Energies }= & -4359.346787 \\ \text { Sum of electronic and thermal Enthalpies }= & -4359.345843 \\ \text { Sum of electronic and thermal Free Energies }= & -4359.547189\end{array}$

Cartesian Coordinates

$\begin{array}{ccccc}6 & -1.969981 & 2.948952 & -3.533369 \\ 6 & -0.937130 & 3.184251 & -2.607601 \\ 6 & -1.075176 & 4.305140 & -1.778622 \\ 6 & -2.182142 & 5.145675 & -1.861085 \\ 6 & -3.190183 & 4.894941 & -2.782683 \\ 6 & -3.073575 & 3.783587 & -3.618647 \\ 6 & 0.278686 & 2.327441 & -2.518450 \\ 6 & 1.419394 & 2.891574 & -1.707730 \\ 45 & -0.201219 & 0.157051 & -1.946277 \\ 6 & -0.380159 & -1.395802 & -3.081041 \\ 8 & -0.549086 & -2.067878 & -4.009534 \\ 15 & -1.619878 & 0.153618 & -0.001906 \\ 6 & -1.997098 & 1.851893 & 0.602370 \\ 6 & -0.979679 & 2.574266 & 1.224891 \\ 6 & -1.236289 & 3.803968 & 1.841257 \\ 6 & -2.528224 & 4.323090 & 1.739547 \\ 6 & -3.551160 & 3.665625 & 1.045214 \\ 6 & -3.267232 & 2.419747 & 0.486715 \\ 6 & -0.118630 & 4.529375 & 2.592131 \\ 6 & -4.956141 & 4.268369 & 1.000951 \\ 6 & -3.302649 & -0.562936 & -0.251999 \\ 6 & -3.696462 & -1.043895 & -1.499591 \\ 6 & -4.962653 & -1.596118 & -1.693696 \\ 6 & -5.830266 & -1.649810 & -0.595584 \\ 6 & -5.465039 & -1.189115 & 0.670562 \\ 6 & -4.187217 & -0.646726 & 0.820711 \\ 6 & -6.381492 & -1.262042 & 1.890637 \\ 6 & -5.424519 & -2.088477 & -3.064526 \\ 6 & -1.176445 & -0.716515 & 1.591061 \\ 6 & -0.713925 & -2.026491 & 1.574050 \\ 6 & -0.425518 & -2.704214 & 2.805476 \\ 6 & -0.611683 & -2.027488 & 4.045437 \\ 6 & -1.107797 & -0.705481 & 4.028936 \\ 6 & -1.378542 & -0.075122 & 2.844677 \\ 6 & -0.274899 & -2.675434 & 5.257702 \\ 6 & 0.228959 & -3.950397 & 5.255571 \\ 6 & 0.403155 & -4.633282 & 4.033541 \\ 6 & 0.078871 & -4.031547 & 2.842922 \\ 6 & -0.518341 & -2.826031 & 0.330441 \\ 6 & 0.692059 & -2.824631 & -0.337767 \\ 6 & 0.936859 & -3.732970 & -1.393458 \\ 6 & -0.032819 & -4.605000 & -1.807790 \\ 6 & -1.305648 & -4.603312 & -1.190988 \\ 6 & -1.547575 & -3.722293 & -0.095146 \\ 6 & -2.815670 & -3.768372 & 0.539113 \\ 6 & -3.797707 & -4.615314 & 0.091122\end{array}$




\begin{tabular}{|c|c|c|c|}
\hline & -3.556328 & -5.482306 & -0.997178 \\
\hline & -2.335575 & -5.474822 & -1.622475 \\
\hline 7 & 1.736439 & -1.913062 & 0.036206 \\
\hline & 1.828317 & -0.486477 & -0.878915 \\
\hline & 2.988434 & -0.645242 & -2.069249 \\
\hline & 4.226672 & -1.180916 & -1.804295 \\
\hline & 5.153981 & -0.479394 & -1.059994 \\
\hline & 6.362660 & -1.163335 & -0.691203 \\
\hline & 6.621302 & -2.467646 & -1.211332 \\
\hline 6 & 5.680900 & -3.073746 & -2.080400 \\
\hline & 4.496302 & -2.450416 & -2.357919 \\
\hline & 7.291679 & -0.624922 & 0.236150 \\
\hline & 8.415069 & -1.323617 & 0.604699 \\
\hline & 8.683196 & -2.596848 & 0.060770 \\
\hline 0 & 7.799498 & -3.153651 & -0.827470 \\
\hline & 4.879057 & 0.907999 & -0.615239 \\
\hline 6 & 3.722240 & 1.226307 & 0.075654 \\
\hline 6 & 3.555586 & 2.474035 & 0.714776 \\
\hline 6 & 4.483087 & 3.461998 & 0.552021 \\
\hline 6 & 5.608564 & 3.253957 & -0.282454 \\
\hline 6 & 5.808366 & 1.972617 & -0.878496 \\
\hline 6 & 6.530509 & 4.296888 & -0.537325 \\
\hline 6 & 7.602514 & 4.104365 & -1.370914 \\
\hline 6 & 7.786146 & 2.851341 & -1.989776 \\
\hline 6 & 6.917817 & 1.815078 & -1.747939 \\
\hline 8 & 2.704639 & 0.335999 & 0.263495 \\
\hline 6 & 0.608793 & 1.399412 & -3.534239 \\
\hline 6 & 2.829881 & -2.515357 & 0.838055 \\
\hline 6 & 3.348114 & -1.720885 & 2.011316 \\
\hline 6 & 2.508356 & -0.976958 & 2.842263 \\
\hline 6 & 3.017528 & -0.307397 & 3.946622 \\
\hline 6 & 4.378755 & -0.369656 & 4.241569 \\
\hline 6 & 5.222444 & -1.107848 & 3.420422 \\
\hline 6 & 4.707752 & -1.778873 & 2.313060 \\
\hline 1 & 7.089191 & 0.343286 & 0.686200 \\
\hline 1 & -1.764075 & 0.940506 & 2.869287 \\
\hline 1 & 7.978388 & -4.143496 & -1.245374 \\
\hline 1 & -0.418313 & -2.133437 & 6.191519 \\
\hline 1 & 6.360263 & 5.262466 & -0.062689 \\
\hline 1 & -3.013728 & -3.100354 & 1.375532 \\
\hline 1 & -4.774309 & -4.615947 & 0.573405 \\
\hline 1 & 4.355012 & 4.427218 & 1.040120 \\
\hline 1 & -1.267786 & -0.189698 & 4.975318 \\
\hline 1 & 5.906048 & -4.052433 & -2.500702 \\
\hline 1 & 8.620433 & 2.703906 & -2.671981 \\
\hline 1 & 3.744460 & -2.897424 & -3.004893 \\
\hline 1 & -2.133571 & -6.141414 & -2.459781 \\
\hline 1 & 0.153254 & -5.294179 & -2.630047 \\
\hline 1 & 0.795661 & -5.647603 & 4.034999 \\
\hline 1 & 7.069828 & 0.860677 & -2.245267 \\
\hline 1 & 0.215845 & -4.576706 & 1.911294 \\
\hline 1 & 1.914558 & -3.710244 & -1.873787 \\
\hline 1 & 8.301061 & 4.914751 & -1.565594 \\
\hline 1 & 9.101243 & -0.892006 & 1.330087 \\
\hline 1 & -4.340103 & -6.157501 & -1.334550 \\
\hline 1 & 0.493021 & -4.438427 & 6.190857 \\
\hline
\end{tabular}




\begin{tabular}{|c|c|c|c|}
\hline 1 & 9.579826 & -3.136601 & 0.356187 \\
\hline & 2.666488 & 2.615274 & 1.325953 \\
\hline & -3.875898 & -0.280317 & 1.798480 \\
\hline & -2.986730 & -0.989199 & -2.318950 \\
\hline & -6.823054 & -2.071782 & -0.737367 \\
\hline & -4.045480 & 1.867212 & -0.031825 \\
\hline & 0.017477 & 2.131446 & 1.281563 \\
\hline & -2.755599 & 5.271881 & 2.220855 \\
\hline & 2.413507 & -3.457461 & 1.221548 \\
\hline & -1.575234 & 0.519729 & -2.629969 \\
\hline & 3.675564 & -2.800462 & 0.194810 \\
\hline & 5.378956 & -2.347262 & 1.667299 \\
\hline & 1.453800 & -0.893323 & 2.591172 \\
\hline 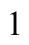 & 6.289109 & -1.158727 & 3.631682 \\
\hline & 2.345081 & 0.269293 & 4.580576 \\
\hline & 4.777749 & 0.159795 & 5.104239 \\
\hline & -0.005563 & 1.305684 & -4.428842 \\
\hline & 1.657947 & 1.150262 & -3.693719 \\
\hline & -0.303844 & 4.533404 & -1.048096 \\
\hline & -2.245756 & 6.004291 & -1.192471 \\
\hline & -4.054011 & 5.553626 & -2.856581 \\
\hline & -3.848958 & 3.569835 & -4.352246 \\
\hline & -1.913503 & 2.090329 & -4.200166 \\
\hline & 1.670408 & 3.917504 & -2.022939 \\
\hline & 1.197188 & 2.931188 & -0.634863 \\
\hline & 2.323856 & 2.288171 & -1.842325 \\
\hline 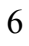 & -4.263455 & -2.201355 & -4.051780 \\
\hline 0 & -6.077164 & -3.469409 & -2.952809 \\
\hline 6 & -6.445057 & -1.089575 & -3.622175 \\
\hline 6 & -7.721795 & -1.919168 & 1.573708 \\
\hline 6 & -5.699480 & -2.082381 & 2.992833 \\
\hline 6 & -6.648393 & 0.156951 & 2.406491 \\
\hline l & -4.628004 & -2.606176 & -5.004444 \\
\hline 1 & -3.804818 & -1.227585 & -4.269530 \\
\hline 1 & -3.478608 & -2.874597 & -3.679202 \\
\hline 1 & -6.394476 & -3.815546 & -3.945449 \\
\hline 1 & -5.368335 & -4.203837 & -2.548171 \\
\hline 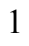 & -6.966669 & -3.466384 & -2.311606 \\
\hline 1 & -6.793478 & -1.408609 & -4.614119 \\
\hline 1 & -7.323159 & -1.000648 & -2.969874 \\
\hline 1 & -6.000681 & -0.090687 & -3.723285 \\
\hline 1 & -8.339888 & -1.955011 & 2.479706 \\
\hline 1 & -8.284420 & -1.361143 & 0.814303 \\
\hline 1 & -7.596219 & -2.951197 & 1.219385 \\
\hline l & -7.276821 & 0.127913 & 3.306973 \\
\hline 1 & -5.717408 & 0.678394 & 2.665653 \\
\hline 1 & -7.171052 & 0.758375 & 1.650211 \\
\hline 1 & -6.341352 & -2.131526 & 3.882837 \\
\hline 1 & -5.512610 & -3.112330 & 2.659691 \\
\hline$\frac{1}{1}$ & -4.738370 & -1.652286 & 3.303453 \\
\hline 6 & -0.648144 & 5.671129 & 3.458521 \\
\hline 6 & 0.616660 & 3.550382 & 3.515103 \\
\hline 6 & 0.864993 & 5.122804 & 1.577580 \\
\hline 6 & -5.875067 & 3.514894 & 0.040684 \\
\hline 6 & -5.561342 & 4.195789 & 2.408183 \\
\hline 6 & -4.909456 & 5.731677 & 0.551036 \\
\hline
\end{tabular}




$\begin{array}{rrrr}1 & 0.180536 & 6.121689 & 4.019288 \\ 1 & -1.103719 & 6.469680 & 2.859775 \\ 1 & -1.391045 & 5.320444 & 4.186646 \\ 1 & 1.429567 & 4.065323 & 4.044157 \\ 1 & -0.065591 & 3.133801 & 4.268929 \\ 1 & 1.061316 & 2.705272 & 2.974852 \\ 1 & 1.691902 & 5.630494 & 2.094141 \\ 1 & 1.297298 & 4.355250 & 0.922984 \\ 1 & 0.363975 & 5.862390 & 0.937349 \\ 1 & -5.921682 & 6.157637 & 0.555219 \\ 1 & -4.290999 & 6.355457 & 1.207643 \\ 1 & -4.511771 & 5.813873 & -0.468153 \\ 1 & -6.578277 & 4.611621 & 2.410569 \\ 1 & -5.620976 & 3.156174 & 2.757920 \\ 1 & -4.966670 & 4.760555 & 3.137218 \\ 1 & -6.858009 & 4.002412 & 0.013410 \\ 1 & -5.473953 & 3.508429 & -0.981690 \\ 1 & -6.036832 & 2.473308 & 0.350732\end{array}$

$3_{\text {IIB-re- }} \psi_{-1 \mathrm{~s} 2 \mathrm{a} 3 \mathrm{~s} 4 \mathrm{a}}$

Number of imaginary frequencies : 0

The smallest frequencies are : $16.7409 \quad 19.8985 \quad 20.2727 \mathrm{~cm}(-1)$

Electronic energy : $\quad H F=-4360.8860336$

Zero-point correction $=\quad 1.454886($ Hartree/Particle $)$

Thermal correction to Energy= $\quad 1.539312$

Thermal correction to Enthalpy= $\quad 1.540256$

Thermal correction to Gibbs Free Energy= $\quad 1.338279$

Sum of electronic and zero-point Energies $=\quad-4359.431147$

Sum of electronic and thermal Energies $=\quad-4359.346721$

Sum of electronic and thermal Enthalpies $=\quad-4359.345777$

Sum of electronic and thermal Free Energies $=\quad-4359.547754$

Cartesian Coordinates

$\begin{array}{cccc}6 & -1.982434 & 2.915102 & -3.553278 \\ 6 & -0.935709 & 3.152900 & -2.643675 \\ 6 & -1.062174 & 4.274810 & -1.814134 \\ 6 & -2.172316 & 5.112823 & -1.879221 \\ 6 & -3.194857 & 4.858406 & -2.783714 \\ 6 & -3.089634 & 3.746597 & -3.620556 \\ 6 & 0.279197 & 2.293350 & -2.566889 \\ 6 & 1.435520 & 2.865422 & -1.784233 \\ 45 & -0.205912 & 0.142936 & -1.956616 \\ 6 & -0.453091 & -1.451854 & -3.014571 \\ 8 & -0.706349 & -2.163413 & -3.893483 \\ 15 & -1.630283 & 0.175499 & -0.010033 \\ 6 & -1.966718 & 1.879216 & 0.598283 \\ 6 & -0.920021 & 2.574538 & 1.201633 \\ 6 & -1.135860 & 3.800033 & 1.840494 \\ 6 & -2.421684 & 4.341039 & 1.784111 \\ 6 & -3.477982 & 3.705895 & 1.119312 \\ 6 & -3.231416 & 2.465516 & 0.531883 \\ 6 & 0.014897 & 4.496997 & 2.567807 \\ 6 & -4.872613 & 4.332232 & 1.134725 \\ 6 & -3.325323 & -0.502980 & -0.276031\end{array}$




\begin{tabular}{|c|c|c|c|}
\hline 6 & -3.718591 & -0.938725 & -1.530824 \\
\hline 6 & -4.999434 & -1.460972 & -1.757462 \\
\hline p & -5.864968 & -1.546974 & -0.672179 \\
\hline & -5.501376 & -1.120477 & 0.616944 \\
\hline & -4.226056 & -0.598140 & 0.794532 \\
\hline & -6.485520 & -1.273348 & 1.775702 \\
\hline b & -5.355094 & -1.951260 & -3.158516 \\
\hline b & -1.218763 & -0.695413 & 1.590359 \\
\hline 6 & -0.758244 & -2.005519 & 1.585500 \\
\hline 6 & -0.484783 & -2.677101 & 2.824998 \\
\hline 6 & -0.690653 & -1.995067 & 4.058824 \\
\hline 6 & -1.188650 & -0.673819 & 4.029170 \\
\hline 6 & -1.440266 & -0.048356 & 2.838469 \\
\hline 6 & -0.377631 & -2.638751 & 5.279660 \\
\hline 6 & 0.122488 & -3.915154 & 5.292420 \\
\hline 6 & 0.319649 & -4.602266 & 4.076501 \\
\hline 6 & 0.019366 & -4.004342 & 2.877561 \\
\hline 6 & -0.540856 & -2.817613 & 0.353448 \\
\hline 6 & 0.682555 & -2.819312 & -0.290703 \\
\hline 6 & 0.964253 & -3.763476 & -1.305285 \\
\hline 6 & 0.025523 & -4.680932 & -1.691621 \\
\hline 6 & -1.257266 & -4.684808 & -1.095769 \\
\hline 6 & -1.547085 & -3.746375 & -0.060206 \\
\hline 6 & -2.831523 & -3.785114 & 0.541433 \\
\hline 6 & -3.772781 & -4.698440 & 0.136488 \\
\hline 6 & -3.478355 & -5.631375 & -0.881393 \\
\hline 6 & -2.247639 & -5.619037 & -1.486022 \\
\hline 7 & 1.710426 & -1.887105 & 0.079722 \\
\hline 15 & 1.822987 & -0.493378 & -0.883019 \\
\hline 8 & 2.983431 & -0.710492 & -2.065425 \\
\hline 6 & 4.212906 & -1.254276 & -1.775945 \\
\hline 6 & 5.146089 & -0.541179 & -1.050428 \\
\hline 6 & 6.339005 & -1.230435 & -0.643421 \\
\hline 6 & 6.581498 & -2.557226 & -1.111600 \\
\hline 6 & 5.641567 & -3.177403 & -1.971197 \\
\hline 6 & 4.470285 & -2.545207 & -2.284086 \\
\hline 6 & 7.266056 & -0.672409 & 0.274268 \\
\hline 6 & 8.372253 & -1.375557 & 0.683717 \\
\hline 6 & 8.624808 & -2.672865 & 0.191408 \\
\hline 6 & 7.743039 & -3.247783 & -0.687219 \\
\hline 6 & 4.891999 & 0.865619 & -0.659309 \\
\hline 6 & 3.738734 & 1.226173 & 0.015633 \\
\hline 6 & 3.590793 & 2.498992 & 0.608017 \\
\hline 6 & 4.534793 & 3.465417 & 0.412625 \\
\hline 6 & 5.659803 & 3.209080 & -0.408737 \\
\hline 6 & 5.840204 & 1.903877 & -0.956756 \\
\hline 6 & 6.600511 & 4.226213 & -0.697202 \\
\hline 6 & 7.672888 & 3.984896 & -1.517599 \\
\hline 6 & 7.838005 & 2.706891 & -2.088890 \\
\hline 6 & 6.950699 & 1.695243 & -1.813715 \\
\hline 8 & 2.707611 & 0.357603 & 0.231497 \\
\hline 6 & 0.590737 & 1.349481 & -3.575486 \\
\hline 6 & 2.786053 & -2.457007 & 0.927416 \\
\hline 6 & 3.281956 & -1.611216 & 2.073759 \\
\hline 6 & 2.427008 & -0.831794 & 2.855713 \\
\hline 6 & 2.918187 & -0.108702 & 3.934290 \\
\hline
\end{tabular}




\begin{tabular}{|c|c|c|c|}
\hline 6 & 4.275140 & -0.152195 & 4.252361 \\
\hline & 5.133152 & -0.926011 & 3.480307 \\
\hline & 4.636773 & -1.650638 & 2.398680 \\
\hline & 7.074712 & 0.315638 & 0.684407 \\
\hline & -1.827217 & 0.967069 & 2.853420 \\
\hline & 7.910414 & -4.255388 & -1.065577 \\
\hline & -0.537664 & -2.092729 & 6.208380 \\
\hline & 6.444855 & 5.211269 & -0.258956 \\
\hline & -3.073661 & -3.065890 & 1.322885 \\
\hline & -4.756059 & -4.703018 & 0.603914 \\
\hline & 4.420064 & 4.449743 & 0.864622 \\
\hline & -1.365846 & -0.155208 & 4.970875 \\
\hline & 5.855577 & -4.173139 & -2.355772 \\
\hline & 8.673002 & 2.520120 & -2.760524 \\
\hline & 3.720545 & -3.001748 & -2.926597 \\
\hline & -2.007442 & -6.328285 & -2.276892 \\
\hline & 0.246203 & -5.408223 & -2.471325 \\
\hline & 0.710859 & -5.617018 & 4.088777 \\
\hline & 7.088412 & 0.720587 & -2.274553 \\
\hline & 0.174761 & -4.554285 & 1.951640 \\
\hline & 1.951237 & -3.742972 & -1.765172 \\
\hline & 8.386013 & 4.775691 & -1.738163 \\
\hline & 9.056901 & -0.928556 & 1.401175 \\
\hline & -4.232064 & -6.353658 & -1.187719 \\
\hline & 0.366738 & -4.400400 & 6.234516 \\
\hline & 9.508191 & -3.216114 & 0.518592 \\
\hline & 2.701735 & 2.678581 & 1.208743 \\
\hline & -3.912148 & -0.256694 & 1.777658 \\
\hline & -3.007072 & -0.881011 & -2.349557 \\
\hline & -6.860520 & -1.962183 & -0.818851 \\
\hline & -4.034101 & 1.929695 & 0.034012 \\
\hline & 0.067941 & 2.110246 & 1.228239 \\
\hline & -2.618673 & 5.287906 & 2.282692 \\
\hline & 2.359179 & -3.380248 & 1.344044 \\
\hline & -1.572954 & 0.507655 & -2.647104 \\
\hline & 3.643490 & -2.771490 & 0.313840 \\
\hline 1 & 5.319035 & -2.245307 & 1.789032 \\
\hline & 1.375622 & -0.761147 & 2.585188 \\
\hline & 6.196954 & -0.962606 & 3.708660 \\
\hline ] & 2.234386 & 0.496369 & 4.528915 \\
\hline & 4.659504 & 0.419636 & 5.094374 \\
\hline 1 & -0.036674 & 1.245776 & -4.459857 \\
\hline & 1.636976 & 1.096734 & -3.747818 \\
\hline 1 & -0.282079 & 4.503268 & -1.092765 \\
\hline & -2.227563 & 5.971895 & -1.210525 \\
\hline 1 & -4.061565 & 5.514678 & -2.843525 \\
\hline 1 & -3.876421 & 3.530124 & -4.341141 \\
\hline & -1.934516 & 2.056938 & -4.221580 \\
\hline 1 & 1.683013 & 3.886430 & -2.117643 \\
\hline & 1.233217 & 2.919566 & -0.708070 \\
\hline 1 & 2.335972 & 2.257696 & -1.926622 \\
\hline 6 & -6.837043 & -2.292095 & -3.293766 \\
\hline 6 & -5.018075 & -0.873461 & -4.196203 \\
\hline 6 & -4.537103 & -3.213713 & -3.453775 \\
\hline 6 & -5.941006 & -0.682187 & 3.074631 \\
\hline 6 & -7.802918 & -0.564339 & 1.445839 \\
\hline
\end{tabular}




$\begin{array}{lrrr}6 & -6.755742 & -2.764302 & 2.008415 \\ 1 & -7.051428 & -2.606103 & -4.323073 \\ 1 & -7.131625 & -3.119523 & -2.635634 \\ 1 & -7.477079 & -1.428598 & -3.069250 \\ 1 & -5.302074 & -1.216624 & -5.199854 \\ 1 & -5.560072 & 0.058850 & -3.990330 \\ 1 & -3.945963 & -0.639086 & -4.225297 \\ 1 & -4.749741 & -3.580415 & -4.467525 \\ 1 & -3.457122 & -3.028005 & -3.382349 \\ 1 & -4.780743 & -4.012797 & -2.740817 \\ 1 & -6.681470 & -0.804175 & 3.875133 \\ 1 & -5.018332 & -1.181457 & 3.401400 \\ 1 & -5.731235 & 0.392146 & 2.977436 \\ 1 & -7.470172 & -2.901204 & 2.831556 \\ 1 & -7.176512 & -3.249282 & 1.118681 \\ 1 & -5.829734 & -3.291623 & 2.277211 \\ 1 & -8.511899 & -0.674609 & 2.277284 \\ 1 & -7.643416 & 0.509113 & 1.280120 \\ 1 & -8.282877 & -0.972326 & 0.548535 \\ 6 & -0.476654 & 5.602013 & 3.502280 \\ 6 & 0.792912 & 3.486454 & 3.419756 \\ 6 & 0.948535 & 5.133410 & 1.531939 \\ 6 & -5.867357 & 3.543572 & 0.285622 \\ 6 & -5.384843 & 4.352097 & 2.579982 \\ 6 & -4.826516 & 5.765024 & 0.595054 \\ 1 & 0.374996 & 6.028397 & 4.047302 \\ 1 & -0.954300 & 6.425818 & 2.957616 \\ 1 & -1.190478 & 5.220819 & 4.244094 \\ 1 & 1.620763 & 3.987196 & 3.939267 \\ 1 & 0.142887 & 3.031654 & 4.180262 \\ 1 & 1.226700 & 2.668112 & 2.831872 \\ 1 & 1.798451 & 5.623452 & 2.028005 \\ 1 & 1.350047 & 4.394277 & 0.826679 \\ 1 & 0.415716 & 5.895346 & 0.945887 \\ 1 & -5.825203 & 6.219380 & 0.645031 \\ 1 & -4.145205 & 6.406723 & 1.167063 \\ 1 & -4.500895 & 5.775401 & -0.452815 \\ 1 & -6.396035 & 4.779237 & 2.622434 \\ 1 & -5.428799 & 3.335790 & 2.994027 \\ 1 & -4.741679 & 4.952242 & 3.235428 \\ 1 & -6.847999 & 4.035346 & 0.314312 \\ 1 & -5.551483 & 3.487178 & -0.764335 \\ 1 & -6.003583 & 2.517591 & 0.654948\end{array}$

$3_{\text {IIB-re- }} \psi_{-1 \text { s2a3s4s }}$

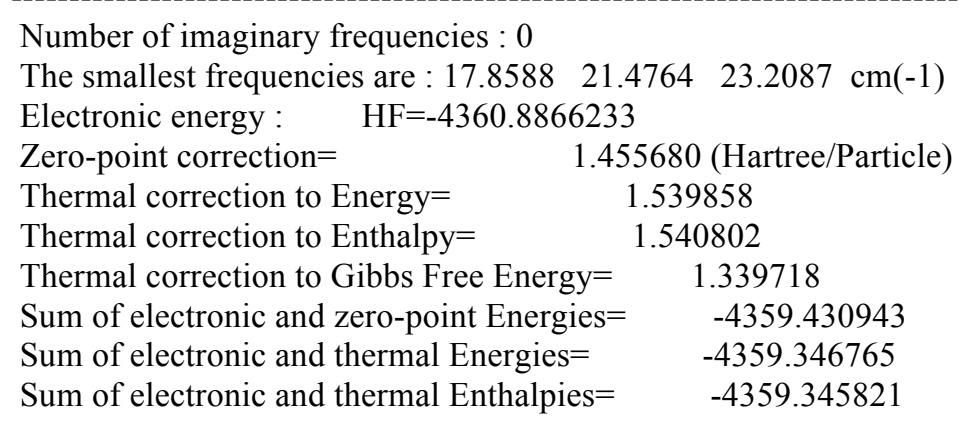


Cartesian Coordinates

\begin{tabular}{|c|c|c|c|}
\hline 6 & -2.035503 & 2.913709 & -3.489352 \\
\hline 6 & -0.996408 & 3.172667 & -2.576376 \\
\hline 6 & -1.150851 & 4.289036 & -1.744365 \\
\hline 6 & -2.279731 & 5.101494 & -1.810022 \\
\hline 6 & -3.294148 & 4.826575 & -2.717561 \\
\hline 6 & -3.161573 & 3.719370 & -3.556876 \\
\hline 6 & 0.238049 & 2.340553 & -2.498570 \\
\hline 6 & 1.368751 & 2.921240 & -1.685994 \\
\hline 45 & -0.205846 & 0.163027 & -1.937588 \\
\hline 6 & -0.445045 & -1.395086 & -3.050112 \\
\hline 8 & -0.708479 & -2.074287 & -3.951521 \\
\hline 15 & -1.611252 & 0.134690 & 0.014721 \\
\hline 6 & -1.999424 & 1.820465 & 0.645415 \\
\hline 6 & -0.985980 & 2.547019 & 1.268924 \\
\hline 6 & -1.252185 & 3.767478 & 1.899713 \\
\hline 6 & -2.550721 & 4.272869 & 1.812598 \\
\hline 6 & -3.571495 & 3.610020 & 1.120166 \\
\hline 6 & -3.277566 & 2.373574 & 0.546819 \\
\hline 6 & -0.137469 & 4.497857 & 2.649910 \\
\hline 6 & -4.983870 & 4.196528 & 1.090762 \\
\hline 6 & -3.294800 & -0.570209 & -0.246245 \\
\hline 6 & -3.718670 & -0.945820 & -1.513935 \\
\hline 6 & -5.005294 & -1.444989 & -1.734697 \\
\hline 6 & -5.855382 & -1.575656 & -0.634787 \\
\hline 6 & -5.457008 & -1.219314 & 0.660216 \\
\hline 6 & -4.170390 & -0.713267 & 0.831603 \\
\hline 6 & -6.363900 & -1.348953 & 1.883398 \\
\hline 6 & -5.397535 & -1.857127 & -3.151270 \\
\hline 6 & -1.163017 & -0.763135 & 1.588082 \\
\hline 6 & -0.698987 & -2.071953 & 1.546084 \\
\hline 6 & -0.405757 & -2.769103 & 2.766255 \\
\hline 6 & -0.593597 & -2.114252 & 4.017847 \\
\hline 6 & -1.092632 & -0.793285 & 4.025228 \\
\hline 6 & -1.363967 & -0.142763 & 2.852296 \\
\hline 6 & -0.253903 & -2.780971 & 5.219042 \\
\hline 6 & 0.256491 & -4.053044 & 5.195617 \\
\hline 6 & 0.435355 & -4.713502 & 3.962033 \\
\hline 6 & 0.107550 & -4.093628 & 2.781696 \\
\hline 6 & -0.493753 & -2.852258 & 0.290790 \\
\hline 6 & 0.721810 & -2.832194 & -0.368217 \\
\hline 6 & 0.990732 & -3.737872 & -1.420585 \\
\hline 6 & 0.045410 & -4.637534 & -1.831514 \\
\hline 6 & -1.230093 & -4.661929 & -1.220644 \\
\hline 6 & -1.503857 & -3.767897 & -0.142379 \\
\hline 6 & -2.778279 & -3.833268 & 0.477170 \\
\hline 6 & -3.729168 & -4.723183 & 0.043705 \\
\hline 6 & -3.453694 & -5.606741 & -1.022364 \\
\hline 6 & -2.229830 & -5.573172 & -1.640167 \\
\hline 7 & 1.753340 & -1.910828 & 0.017707 \\
\hline 15 & 1.834111 & -0.476945 & -0.887000 \\
\hline 8 & 2.994867 & -0.618201 & -2.079373 \\
\hline 6 & 4.240822 & -1.137814 & -1.818450 \\
\hline
\end{tabular}




\begin{tabular}{|c|c|c|c|}
\hline ) & 5.159745 & -0.426362 & -1.073099 \\
\hline & 6.378246 & -1.094391 & -0.708175 \\
\hline 6 & 6.655040 & -2.392829 & -1.233532 \\
\hline & 5.722437 & -3.008345 & -2.104390 \\
\hline & 4.528661 & -2.400889 & -2.378041 \\
\hline & 7.299907 & -0.546279 & 0.220760 \\
\hline & 8.433103 & -1.230470 & 0.586427 \\
\hline & 8.718835 & -2.497805 & 0.037695 \\
\hline & 7.842865 & -3.063594 & -0.852572 \\
\hline 6 & 4.866532 & 0.954980 & -0.621873 \\
\hline 6 & 3.707346 & 1.252051 & 0.074252 \\
\hline & 3.523610 & 2.494014 & 0.719668 \\
\hline 6 & 4.435951 & 3.496369 & 0.559188 \\
\hline & 5.562095 & 3.309347 & -0.279336 \\
\hline 6 & 5.779449 & 2.034160 & -0.882300 \\
\hline U & 6.467692 & 4.367125 & -0.531262 \\
\hline 6 & 7.540436 & 4.194574 & -1.368310 \\
\hline 6 & 7.741521 & 2.947281 & -1.993432 \\
\hline 6 & 6.889305 & 1.897019 & -1.754646 \\
\hline 8 & 2.705704 & 0.343435 & 0.260750 \\
\hline 6 & 0.582939 & 1.425110 & -3.521383 \\
\hline 6 & 2.853060 & -2.510770 & 0.813181 \\
\hline 6 & 3.365471 & -1.720206 & 1.991456 \\
\hline 6 & 2.517735 & -0.997716 & 2.833121 \\
\hline 6 & 3.020837 & -0.332052 & 3.942577 \\
\hline 6 & 4.384120 & -0.376150 & 4.231423 \\
\hline 6 & 5.235870 & -1.092073 & 3.398965 \\
\hline 6 & 4.727170 & -1.759641 & 2.286725 \\
\hline 1 & 7.084071 & 0.417439 & 0.674248 \\
\hline 1 & -1.751122 & 0.871773 & 2.893641 \\
\hline 1 & 8.035715 & -4.049228 & -1.274193 \\
\hline 1 & -0.399841 & -2.255144 & 6.161666 \\
\hline 1 & 6.284229 & 5.327779 & -0.051590 \\
\hline 1 & -3.002566 & -3.154762 & 1.298502 \\
\hline 1 & -4.706377 & -4.746894 & 0.523422 \\
\hline 1 & 4.295177 & 4.457229 & 1.052359 \\
\hline 1 & -1.252902 & -0.294622 & 4.980646 \\
\hline 1 & 5.960992 & -3.981880 & -2.529254 \\
\hline 1 & 8.576567 & 2.815540 & -2.677906 \\
\hline 1 & 3.782955 & -2.856326 & -3.026194 \\
\hline 1 & -2.002634 & -6.247297 & -2.464881 \\
\hline 1 & 0.253780 & -5.332018 & -2.643869 \\
\hline 1 & 0.834459 & -5.725118 & 3.946002 \\
\hline 1 & 7.054587 & 0.947090 & -2.256359 \\
\hline 1 & 0.249021 & -4.622538 & 1.841455 \\
\hline 1 & 1.971203 & -3.697041 & -1.893689 \\
\hline 1 & 8.226377 & 5.016183 & -1.560789 \\
\hline 1 & 9.113397 & -0.791976 & 1.313220 \\
\hline 1 & -4.215032 & -6.310121 & -1.352852 \\
\hline 1 & 0.522952 & -4.555555 & 6.122515 \\
\hline 1 & 9.622936 & -3.026141 & 0.330974 \\
\hline 1 & 2.634369 & 2.619653 & 1.333842 \\
\hline 1 & -3.841754 & -0.416634 & 1.827176 \\
\hline 1 & -3.025248 & -0.850290 & -2.344721 \\
\hline 1 & -6.857244 & -1.967191 & -0.787539 \\
\hline 1 & -4.053472 & 1.818444 & 0.027695 \\
\hline
\end{tabular}




\begin{tabular}{|c|c|c|c|}
\hline 1 & 0.016289 & 2.114342 & 1.313406 \\
\hline 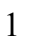 & -2.785148 & 5.214516 & 2.304513 \\
\hline & 2.444104 & -3.458966 & 1.190193 \\
\hline & -1.579904 & 0.527093 & -2.612285 \\
\hline 1 & 3.700437 & -2.783938 & 0.166856 \\
\hline 1 & 5.404498 & -2.310675 & 1.632395 \\
\hline 1 & 1.461096 & -0.927496 & 2.586577 \\
\hline 1 & 6.304095 & -1.128201 & 3.605253 \\
\hline 1 & 2.342147 & 0.227478 & 4.585177 \\
\hline 1 & 4.778347 & 0.150246 & 5.098147 \\
\hline 1 & -0.030964 & 1.327264 & -4.415876 \\
\hline 1 & 1.636126 & 1.195454 & -3.683188 \\
\hline 1 & -0.377547 & 4.533970 & -1.021093 \\
\hline 1 & -2.354807 & 5.957903 & -1.139953 \\
\hline 1 & -4.175887 & 5.462597 & -2.777173 \\
\hline 1 & -3.942165 & 3.485040 & -4.278745 \\
\hline 1 & -1.966865 & 2.058330 & -4.159514 \\
\hline 1 & 1.607824 & 3.949428 & -2.003369 \\
\hline 1 & 1.140781 & 2.961664 & -0.614140 \\
\hline 1 & 2.280934 & 2.328462 & -1.815397 \\
\hline 6 & -6.882075 & -2.193242 & -3.268029 \\
\hline 6 & -5.086709 & -0.721474 & -4.134338 \\
\hline 6 & -4.586499 & -3.098873 & -3.538547 \\
\hline 6 & -7.706591 & -1.992329 & 1.548612 \\
\hline 6 & -5.676476 & -2.213759 & 2.947535 \\
\hline 6 & -6.626305 & 0.047936 & 2.459365 \\
\hline 1 & -7.120937 & -2.453564 & -4.306903 \\
\hline 1 & -7.159988 & -3.054033 & -2.646454 \\
\hline 1 & -7.517465 & -1.343988 & -2.984074 \\
\hline 1 & -5.397734 & -1.006070 & -5.148306 \\
\hline 1 & -5.620353 & 0.198321 & -3.860480 \\
\hline 1 & -4.015014 & -0.486828 & -4.177143 \\
\hline 1 & -4.831753 & -3.414311 & -4.562116 \\
\hline 1 & -3.505514 & -2.911141 & -3.493319 \\
\hline 1 & -4.804386 & -3.934218 & -2.859630 \\
\hline 1 & -8.314240 & -2.075132 & 2.458627 \\
\hline 1 & -8.279472 & -1.398186 & 0.825352 \\
\hline 1 & -7.582650 & -3.004562 & 1.140878 \\
\hline 1 & -7.244291 & -0.017174 & 3.365243 \\
\hline 1 & -5.691574 & 0.558887 & 2.726493 \\
\hline 1 & -7.157984 & 0.678250 & 1.733451 \\
\hline 1 & -6.315546 & -2.300464 & 3.836609 \\
\hline 1 & -5.489571 & -3.228928 & 2.571916 \\
\hline 1 & -4.715187 & -1.796099 & 3.273978 \\
\hline 6 & -0.673951 & 5.621875 & 3.535058 \\
\hline 6 & 0.617526 & 3.516850 & 3.554764 \\
\hline 6 & 0.829244 & 5.116669 & 1.634270 \\
\hline 6 & -5.903607 & 3.434611 & 0.137660 \\
\hline 6 & -5.574721 & 4.112638 & 2.503513 \\
\hline 6 & -4.959189 & 5.661699 & 0.644736 \\
\hline 1 & 0.153694 & 6.075497 & 4.094872 \\
\hline 1 & -1.144089 & 6.422321 & 2.950325 \\
\hline 1 & -1.406527 & 5.252766 & 4.264503 \\
\hline 1 & 1.427485 & 4.035559 & 4.084577 \\
\hline 1 & -0.053839 & 3.081559 & 4.307788 \\
\hline 1 & 1.069183 & 2.684343 & 3.000981 \\
\hline
\end{tabular}




$\begin{array}{rrrr}1 & 1.654778 & 5.627477 & 2.149892 \\ 1 & 1.263902 & 4.363626 & 0.964634 \\ 1 & 0.312989 & 5.858603 & 1.009148 \\ 1 & -5.975583 & 6.077068 & 0.666929 \\ 1 & -4.336662 & 6.289536 & 1.293641 \\ 1 & -4.579395 & 5.752436 & -0.380667 \\ 1 & -6.596691 & 4.515668 & 2.516674 \\ 1 & -5.618031 & 3.071397 & 2.850654 \\ 1 & -4.980469 & 4.682825 & 3.228621 \\ 1 & -6.892656 & 3.910199 & 0.122664 \\ 1 & -5.514071 & 3.435690 & -0.889269 \\ 1 & -6.048957 & 2.390065 & 0.445981\end{array}$

$3_{\text {IIB-re- }} \psi_{-1 \mathbf{s} 2 \mathbf{s} 3 \mathrm{a} a \mathrm{a}}$

Number of imaginary frequencies : 0

The smallest frequencies are : $14.2480 \quad 18.3114 \quad 20.1652 \mathrm{~cm}(-1)$

Electronic energy : $\quad H F=-4360.8847151$

Zero-point correction $=\quad 1.455100($ Hartree/Particle $)$

Thermal correction to Energy= $\quad 1.539544$

Thermal correction to Enthalpy= $\quad 1.540488$

Thermal correction to Gibbs Free Energy $=\quad 1.337507$

Sum of electronic and zero-point Energies $=\quad-4359.429615$

Sum of electronic and thermal Energies $=\quad-4359.345171$

Sum of electronic and thermal Enthalpies $=\quad-4359.344227$

Sum of electronic and thermal Free Energies $=\quad-4359.547208$

Cartesian Coordinates

$\begin{array}{cccc}6 & -1.847131 & 3.016276 & -3.657238 \\ 6 & -0.877477 & 3.208427 & -2.657188 \\ 6 & -1.065107 & 4.295429 & -1.793044 \\ 6 & -2.159549 & 5.147152 & -1.915628 \\ 6 & -3.098911 & 4.945407 & -2.918327 \\ 6 & -2.935181 & 3.865955 & -3.785967 \\ 6 & 0.327628 & 2.341339 & -2.527581 \\ 6 & 1.451185 & 2.906795 & -1.693635 \\ 45 & -0.188595 & 0.186021 & -1.946937 \\ 6 & -0.383000 & -1.371528 & -3.075011 \\ 8 & -0.552457 & -2.040327 & -4.005700 \\ 15 & -1.623874 & 0.196190 & -0.011402 \\ 6 & -1.977493 & 1.896674 & 0.600295 \\ 6 & -0.941507 & 2.611886 & 1.209216 \\ 6 & -1.177654 & 3.835354 & 1.838012 \\ 6 & -2.475957 & 4.358511 & 1.783179 \\ 6 & -3.518294 & 3.700692 & 1.128184 \\ 6 & -3.245459 & 2.465003 & 0.532514 \\ 6 & -0.042924 & 4.549878 & 2.575071 \\ 6 & 0.685617 & 3.560917 & 3.492862 \\ 6 & -4.958253 & 4.214118 & 1.138058 \\ 6 & -5.089354 & 5.571923 & 1.822699 \\ 6 & -3.314891 & -0.501687 & -0.262785 \\ 6 & -3.705809 & -0.970190 & -1.511493 \\ 6 & -4.972524 & -1.529646 & -1.711851 \\ 6 & -5.831955 & -1.597407 & -0.615285 \\ 6 & -5.469891 & -1.146589 & 0.660955\end{array}$




\begin{tabular}{|c|c|c|c|}
\hline 6 & -4.200563 & -0.596273 & 0.816653 \\
\hline 5 & -6.441282 & -1.302366 & 1.830705 \\
\hline b & -5.428593 & -2.012469 & -3.088025 \\
\hline 6 & -1.195388 & -0.679394 & 1.583024 \\
\hline f & -0.747515 & -1.994685 & 1.570001 \\
\hline p & -0.471782 & -2.672968 & 2.804266 \\
\hline 6 & -0.657290 & -1.992233 & 4.042203 \\
\hline 6 & -1.140868 & -0.665659 & 4.021322 \\
\hline 6 & -1.398285 & -0.034225 & 2.834798 \\
\hline 6 & -0.333090 & -2.641330 & 5.257288 \\
\hline 6 & 0.158151 & -3.921209 & 5.260057 \\
\hline 6 & 0.331646 & -4.607975 & 4.040132 \\
\hline 6 & 0.019283 & -4.005212 & 2.846819 \\
\hline 6 & -0.551051 & -2.798750 & 0.328977 \\
\hline 6 & 0.664692 & -2.808158 & -0.329542 \\
\hline 6 & 0.912246 & -3.720492 & -1.381037 \\
\hline 6 & -0.059656 & -4.586661 & -1.801345 \\
\hline 6 & -1.337083 & -4.575036 & -1.194631 \\
\hline 6 & -1.582937 & -3.689655 & -0.102678 \\
\hline 6 & -2.856542 & -3.729547 & 0.521364 \\
\hline 6 & -3.838552 & -4.572940 & 0.066059 \\
\hline 6 & -3.593381 & -5.442121 & -1.019395 \\
\hline 6 & -2.367484 & -5.441784 & -1.634057 \\
\hline 7 & 1.714188 & -1.905636 & 0.051927 \\
\hline 15 & 1.827491 & -0.482739 & -0.866201 \\
\hline 8 & 2.985853 & -0.660577 & -2.056021 \\
\hline 6 & 4.215317 & -1.215516 & -1.790328 \\
\hline 6 & 5.153311 & -0.528775 & -1.045712 \\
\hline 6 & 6.350049 & -1.232344 & -0.674715 \\
\hline 6 & 6.587850 & -2.540988 & -1.193801 \\
\hline 6 & 5.639290 & -3.131425 & -2.064775 \\
\hline 6 & 4.465519 & -2.488760 & -2.344230 \\
\hline 6 & 7.286364 & -0.708925 & 0.253971 \\
\hline 6 & 8.397301 & -1.426081 & 0.624807 \\
\hline 6 & 8.645215 & -2.703786 & 0.081718 \\
\hline 6 & 7.753935 & -3.246252 & -0.807817 \\
\hline 6 & 4.900450 & 0.863537 & -0.603712 \\
\hline 6 & 3.748015 & 1.201947 & 0.084892 \\
\hline 6 & 3.601010 & 2.453725 & 0.720789 \\
\hline 6 & 4.544617 & 3.426257 & 0.557023 \\
\hline 6 & 5.667879 & 3.197962 & -0.275018 \\
\hline 6 & 5.847523 & 1.912154 & -0.867749 \\
\hline 6 & 6.607535 & 4.224890 & -0.530513 \\
\hline 6 & 7.677875 & 4.012616 & -1.361407 \\
\hline 6 & 7.841730 & 2.755336 & -1.977179 \\
\hline 6 & 6.955676 & 1.734297 & -1.734872 \\
\hline 8 & 2.716586 & 0.327850 & 0.274828 \\
\hline 6 & 0.676349 & 1.400083 & -3.525200 \\
\hline 6 & 2.797026 & -2.519504 & 0.859486 \\
\hline 6 & 3.320195 & -1.728048 & 2.032368 \\
\hline 6 & 2.484780 & -0.975958 & 2.860311 \\
\hline 6 & 2.997107 & -0.309571 & 3.965109 \\
\hline 6 & 4.357174 & -0.382761 & 4.263054 \\
\hline 6 & 5.196453 & -1.129219 & 3.444861 \\
\hline 6 & 4.678500 & -1.797595 & 2.337400 \\
\hline 6 & 0.940610 & 5.131402 & 1.553733 \\
\hline
\end{tabular}




\begin{tabular}{|c|c|c|c|}
\hline 6 & -0.547849 & 5.699720 & 3.445729 \\
\hline 6 & -5.488851 & 4.353934 & -0.290563 \\
\hline 6 & -5.824595 & 3.203161 & 1.901655 \\
\hline 1 & 7.099015 & 0.262681 & 0.703326 \\
\hline 1 & -1.775839 & 0.984651 & 2.856496 \\
\hline 1 & 7.917090 & -4.239074 & -1.225101 \\
\hline 1 & -0.475843 & -2.096183 & 6.189372 \\
\hline 1 & 6.452351 & 5.194237 & -0.058408 \\
\hline 1 & -3.060378 & -3.059366 & 1.355231 \\
\hline 1 & -4.817756 & -4.570250 & 0.542465 \\
\hline 1 & 4.431386 & 4.394445 & 1.042902 \\
\hline 1 & -1.301873 & -0.147342 & 4.966106 \\
\hline 1 & 5.849262 & -4.113448 & -2.485147 \\
\hline 1 & 8.674740 & 2.592456 & -2.657438 \\
\hline 1 & 3.708237 & -2.922742 & -2.993636 \\
\hline 1 & -2.161273 & -6.110245 & -2.468842 \\
\hline 1 & 0.128360 & -5.279347 & -2.620191 \\
\hline 1 & 0.714145 & -5.626096 & 4.045300 \\
\hline 1 & 7.092522 & 0.776546 & -2.230098 \\
\hline 1 & 0.155602 & -4.553550 & 1.916965 \\
\hline 1 & 1.894278 & -3.706660 & -1.852339 \\
\hline 1 & 8.390184 & 4.810839 & -1.556407 \\
\hline 1 & 9.089286 & -1.005671 & 1.351258 \\
\hline 1 & -4.378233 & -6.113233 & -1.362361 \\
\hline 1 & 0.412699 & -4.410074 & 6.197542 \\
\hline 1 & 9.532348 & -3.258099 & 0.378848 \\
\hline 1 & 2.714374 & 2.610028 & 1.331496 \\
\hline 1 & -3.883798 & -0.235736 & 1.792438 \\
\hline 1 & -2.997096 & -0.903931 & -2.331119 \\
\hline 1 & -6.824510 & -2.026956 & -0.752144 \\
\hline 1 & -4.049102 & 1.916488 & 0.044986 \\
\hline 1 & 0.053262 & 2.161857 & 1.244107 \\
\hline 1 & -2.681455 & 5.301666 & 2.280142 \\
\hline 1 & 2.366890 & -3.454850 & 1.244309 \\
\hline 1 & -1.547797 & 0.571114 & -2.650493 \\
\hline 1 & 3.641465 & -2.817441 & 0.220422 \\
\hline 1 & 5.346063 & -2.372925 & 1.694010 \\
\hline 1 & 1.431304 & -0.884560 & 2.607040 \\
\hline 1 & 6.262189 & -1.188892 & 3.658621 \\
\hline 1 & 2.327994 & 0.272880 & 4.597332 \\
\hline 1 & 4.758624 & 0.144401 & 5.125986 \\
\hline 1 & 1.516191 & 4.061148 & 4.008577 \\
\hline 1 & 0.004863 & 3.161507 & 4.257308 \\
\hline 1 & 1.103767 & 2.703890 & 2.950584 \\
\hline 1 & 0.294104 & 6.141443 & 3.993625 \\
\hline 1 & -1.002204 & 6.502188 & 2.851214 \\
\hline 1 & -1.284151 & 5.360206 & 4.185672 \\
\hline 1 & 1.773931 & 5.633247 & 2.065671 \\
\hline 1 & 1.364692 & 4.359558 & 0.899456 \\
\hline 1 & 0.444275 & 5.874317 & 0.913851 \\
\hline 1 & -6.134695 & 5.903795 & 1.786088 \\
\hline 1 & -4.795107 & 5.534060 & 2.879470 \\
\hline 1 & -4.482635 & 6.339075 & 1.323023 \\
\hline 1 & -6.546537 & 4.649986 & -0.272497 \\
\hline 1 & -4.929920 & 5.121876 & -0.837905 \\
\hline 1 & -5.411848 & 3.421470 & -0.862374 \\
\hline
\end{tabular}




$\begin{array}{rrrr}1 & -6.868574 & 3.543310 & 1.942820 \\ 1 & -5.811897 & 2.216790 & 1.418624 \\ 1 & -5.466547 & 3.079009 & 2.932755 \\ 1 & 0.086489 & 1.299938 & -4.435230 \\ 1 & 1.727137 & 1.140288 & -3.653874 \\ 1 & -0.348099 & 4.480866 & -0.997607 \\ 1 & -2.271011 & 5.974728 & -1.214374 \\ 1 & -3.949488 & 5.615601 & -3.026689 \\ 1 & -3.659539 & 3.689699 & -4.579247 \\ 1 & -1.751293 & 2.183134 & -4.351154 \\ 1 & 1.701421 & 3.935658 & -1.999903 \\ 1 & 1.209847 & 2.941247 & -0.625183 \\ 1 & 2.360799 & 2.307882 & -1.813596 \\ 6 & -4.258539 & -2.143150 & -4.062733 \\ 6 & -6.104213 & -3.382874 & -2.985974 \\ 6 & -6.426453 & -0.995223 & -3.653246 \\ 6 & -5.865396 & -0.757274 & 3.136406 \\ 6 & -7.743665 & -0.553351 & 1.530571 \\ 6 & -6.746509 & -2.790416 & 2.038163 \\ 1 & -4.617611 & -2.548591 & -5.017192 \\ 1 & -3.786411 & -1.176149 & -4.280813 \\ 1 & -3.485553 & -2.823318 & -3.678103 \\ 1 & -6.413571 & -3.723265 & -3.983028 \\ 1 & -5.412235 & -4.128460 & -2.572595 \\ 1 & -7.002377 & -3.367231 & -2.357119 \\ 1 & -6.770170 & -1.305286 & -4.649669 \\ 1 & -7.308933 & -0.893984 & -3.008516 \\ 1 & -5.964473 & -0.003536 & -3.746699 \\ 1 & -6.590515 & -0.901966 & 3.947169 \\ 1 & -4.939670 & -1.274273 & 3.426066 \\ 1 & -5.650865 & 0.318156 & 3.074647 \\ 1 & -7.455596 & -2.923271 & 2.866448 \\ 1 & -7.189070 & -3.251366 & 1.146258 \\ 1 & -5.831710 & -3.345271 & 2.289404 \\ 1 & -8.450488 & -0.669657 & 2.363108 \\ 1 & -7.560069 & 0.520711 & 1.393110 \\ 1 & -8.236233 & -0.926209 & 0.624510\end{array}$

$3_{\text {IIB-re- }} \Psi-1 \mathrm{~s} 2 s 334 \mathrm{~s}$

Number of imaginary frequencies : 0

The smallest frequencies are : $16.8819 \quad 21.1137 \quad 22.9999 \mathrm{~cm}(-1)$

Electronic energy : $\quad H F=-4360.8843394$

Zero-point correction $=\quad 1.456059($ Hartree/Particle $)$

Thermal correction to Energy= $\quad 1.540247$

Thermal correction to Enthalpy $=\quad 1.541191$

Thermal correction to Gibbs Free Energy= 1.339905

Sum of electronic and zero-point Energies $=\quad-4359.428280$

Sum of electronic and thermal Energies $=\quad-4359.344092$

Sum of electronic and thermal Enthalpies $=\quad-4359.343148$

Sum of electronic and thermal Free Energies $=\quad-4359.544434$

Cartesian Coordinates

$\begin{array}{llll}6 & -1.788144 & 3.175459 & -3.636915 \\ 6 & -0.862092 & 3.311765 & -2.587917\end{array}$




\begin{tabular}{|c|c|c|c|}
\hline 5 & -1.064344 & 4.374300 & -1.696736 \\
\hline & -2.123806 & 5.263912 & -1.849665 \\
\hline & -3.012967 & 5.124584 & -2.907601 \\
\hline & -2.841415 & 4.063132 & -3.795172 \\
\hline & 0.324020 & 2.421244 & -2.444409 \\
\hline & 1.433110 & 2.956650 & -1.572604 \\
\hline 4 & -0.217555 & 0.257789 & -1.927248 \\
\hline 6 & -0.446474 & -1.261975 & -3.100819 \\
\hline 8 & -0.634369 & -1.895931 & -4.051955 \\
\hline 15 & -1.637520 & 0.207257 & 0.022381 \\
\hline b & -1.958851 & 1.892056 & 0.689521 \\
\hline 6 & -0.912669 & 2.568624 & 1.326224 \\
\hline 6 & -1.120973 & 3.798570 & 1.951107 \\
\hline 6 & -2.396347 & 4.371908 & 1.854460 \\
\hline 6 & -3.446204 & 3.752226 & 1.175458 \\
\hline 6 & -3.206430 & 2.500694 & 0.596442 \\
\hline 6 & 0.016333 & 4.482834 & 2.713788 \\
\hline 6 & 0.770022 & 3.462077 & 3.574176 \\
\hline 6 & -4.861161 & 4.331502 & 1.135253 \\
\hline 6 & -4.952076 & 5.698296 & 1.808564 \\
\hline 6 & -3.340230 & -0.464290 & -0.225148 \\
\hline 6 & -3.762492 & -0.863596 & -1.491511 \\
\hline 6 & -5.021267 & -1.433145 & -1.686500 \\
\hline 6 & -5.845862 & -1.598680 & -0.567616 \\
\hline 6 & -5.451735 & -1.222926 & 0.718330 \\
\hline 6 & -4.191682 & -0.638682 & 609 \\
\hline 6 & -6.298985 & -1.486107 & 1.964055 \\
\hline 6 & -5.509535 & -1.836352 & -3.077075 \\
\hline 6 & -1.198765 & -0.744387 & 1.568887 \\
\hline 6 & -0.777776 & -2.066954 & 1.493674 \\
\hline 6 & -0.507318 & -2.802872 & 2.696195 \\
\hline 6 & -0.671752 & -2.173381 & 3.963960 \\
\hline 6 & -1.123207 & -0.836018 & 4.004792 \\
\hline 6 & -1.375001 & -0.148720 & 2.848474 \\
\hline 6 & -0.355395 & -2.881206 & 894 \\
\hline 6 & 0.110161 & -4.169385 & 5.092512 \\
\hline 6 & 0.266539 & -4.804376 & 3.842624 \\
\hline 6 & -0.039041 & -4.144003 & 2.678389 \\
\hline 6 & -0.588511 & -2.817490 & 0.217450 \\
\hline 6 & 0.629660 & -2.803246 & -0.436679 \\
\hline 6 & 0.879970 & -3.669833 & -1.525324 \\
\hline 6 & -0.091941 & -4.513333 & -1.988585 \\
\hline 6 & -1.371648 & -4.524136 & -1.387172 \\
\hline 6 & -1.619510 & -3.687930 & -0.257275 \\
\hline 6 & -2.892949 & -3.758750 & 0.362718 \\
\hline 6 & -3.873293 & -4.583100 & -0.129858 \\
\hline 6 & -3.628506 & -5.398953 & -1.255854 \\
\hline 6 & -2.401581 & -5.369195 & -1.867954 \\
\hline 7 & 1.680551 & -1.920947 & -0.014851 \\
\hline 15 & 1.799441 & -0.465892 & -0.878595 \\
\hline 8 & 2.956540 & -0.603848 & -2.075379 \\
\hline 6 & 4.183565 & -1.174046 & -1.831679 \\
\hline 6 & 5.125243 & -0.522184 & -1.060533 \\
\hline 6 & 6.318951 & -1.245493 & -0.719231 \\
\hline 6 & 6.551346 & -2.532554 & -1.291684 \\
\hline 6 & 5.600093 & -3.082652 & -2.185801 \\
\hline
\end{tabular}




\begin{tabular}{|c|c|c|c|}
\hline 6 & 4.428791 & -2.424302 & -2.437702 \\
\hline 6 & 7.257957 & -0.763791 & 0.229022 \\
\hline & 8.366672 & -1.499502 & 0.568947 \\
\hline & 8.609393 & -2.754788 & -0.026485 \\
\hline & 7.715225 & -3.257207 & -0.936505 \\
\hline & 4.880769 & 0.854491 & -0.567960 \\
\hline & 3.728080 & 1.175117 & 0.128483 \\
\hline & 3.582268 & 2.407439 & 0.801455 \\
\hline & 4.531028 & 3.380142 & 0.673402 \\
\hline & 5.657332 & 3.173301 & -0.160221 \\
\hline & 5.833514 & 1.906938 & -0.794575 \\
\hline & 6.601656 & 4.204344 & -0.379070 \\
\hline & 7.673493 & 4.015390 & -1.213638 \\
\hline ) & 7.834179 & 2.778495 & -1.870110 \\
\hline & 6.943414 & 1.753561 & -1.664267 \\
\hline & 2.694357 & 0.297726 & 0.289430 \\
\hline ) & 0.683928 & 1.500910 & -3.458308 \\
\hline & 2.757630 & -2.571432 & 0.771309 \\
\hline D & 3.288655 & -1.821860 & 1.967962 \\
\hline & 2.459023 & -1.091494 & 2.820889 \\
\hline & 2.976972 & -0.463130 & 3.945068 \\
\hline & 4.337233 & -0.552934 & 4.237777 \\
\hline & 5.170762 & -1.277906 & 3.394812 \\
\hline 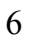 & 4.647080 & -1.908379 & 2.267863 \\
\hline & 0.980906 & 5.129123 & 1.713091 \\
\hline b & -0.493926 & 5.579197 & 3.649622 \\
\hline & -5.344213 & 4.483977 & -0.309585 \\
\hline & -5.797415 & 3.366188 & 1.872671 \\
\hline & 7.074492 & 0.189705 & 0.717207 \\
\hline & -1.727435 & 0.877092 & 2.915275 \\
\hline & 7.874654 & -4.232533 & -1.394535 \\
\hline 1 & -0.482644 & -2.373965 & 6.103330 \\
\hline & 6.448516 & 5.158444 & 0.123685 \\
\hline & -3.095370 & -3.131524 & 1.228996 \\
\hline 1 & -4.851925 & -4.605637 & 0.348326 \\
\hline & 4.419390 & 4.333505 & 1.188204 \\
\hline 1 & -1.263746 & -0.355493 & 4.972506 \\
\hline & 5.806135 & -4.047066 & -2.646970 \\
\hline 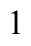 & 8.668325 & 2.634933 & -2.553310 \\
\hline . & 3.670107 & -2.828140 & -3.104682 \\
\hline & -2.193921 & -5.997892 & -2.732713 \\
\hline 1 & 0.097910 & -5.169839 & -2.836274 \\
\hline & 0.631021 & -5.828249 & 3.800916 \\
\hline 1 & 7.078013 & 0.812342 & -2.190830 \\
\hline & 0.085059 & -4.653395 & 1.725080 \\
\hline 1 & 1.864825 & -3.638128 & -1.989168 \\
\hline l & 8.389328 & 4.816835 & -1.380524 \\
\hline 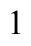 & 9.061125 & -1.111494 & 1.310902 \\
\hline 1 & -4.412669 & -6.053646 & -1.630643 \\
\hline 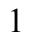 & 0.358904 & -4.703788 & 6.006390 \\
\hline 1 & 9.494995 & -3.323801 & 0.246430 \\
\hline ] & 2.690504 & 2.550675 & 1.408127 \\
\hline 1 & -3.857031 & -0.338052 & 1.858479 \\
\hline ] & -3.077388 & -0.737513 & -2.324418 \\
\hline 1 & -6.824494 & -2.052179 & -0.706999 \\
\hline 1 & -4.017249 & 1.977483 & 0.092292 \\
\hline
\end{tabular}




\begin{tabular}{|c|c|c|c|}
\hline & 0.064691 & 2.084236 & 1.378661 \\
\hline ] & -2.577076 & 5.326887 & 2.338421 \\
\hline 1 & 2.317395 & -3.514171 & 1.126395 \\
\hline 1 & -1.572341 & 0.687993 & -2.616188 \\
\hline ] & 3.598449 & -2.856961 & 0.121455 \\
\hline 1 & 5.310366 & -2.466989 & 1.605645 \\
\hline ] & 1.405647 & -0.985326 & 2.572968 \\
\hline 1 & 6.236574 & -1.350152 & 3.604310 \\
\hline & 2.312111 & 0.102916 & 4.596330 \\
\hline 1 & 4.743281 & -0.055235 & 5.115919 \\
\hline 1 & 1.586442 & 3.955860 & 4.117634 \\
\hline 1 & 0.101573 & 3.001850 & 4.314925 \\
\hline 1 & 1.216125 & 2.649075 & 2.988486 \\
\hline 1 & 0.344575 & 5.986051 & 4.228921 \\
\hline 1 & -0.942749 & 6.418165 & 3.103813 \\
\hline l & -1.236217 & 5.195627 & 4.361549 \\
\hline & 1.807562 & 5.624753 & 2.241702 \\
\hline 1 & 1.415421 & 4.395224 & 1.022602 \\
\hline & 0.465170 & 5.889082 & 1.109652 \\
\hline 1 & -5.979454 & 6.076825 & 1.734648 \\
\hline & -4.696183 & 5.653097 & 2.875078 \\
\hline 1 & -4.295004 & 6.434927 & 1.326969 \\
\hline$\frac{1}{1}$ & -6.393273 & 4.809815 & -0.321113 \\
\hline & -4.749662 & 5.232010 & -0.847095 \\
\hline 1 & -5.278809 & 3.546318 & -0.874824 \\
\hline 1 & -6.821631 & 3.763326 & 1.892246 \\
\hline 1 & -5.829131 & 2.384923 & 1.382553 \\
\hline 1 & -5.472127 & 3.213712 & 2.910724 \\
\hline 1 & 0.111671 & 1.429879 & -4.382095 \\
\hline 1 & 1.734888 & 1.236336 & -3.574618 \\
\hline & -0.385539 & 4.511025 & -0.858729 \\
\hline 1 & -2.246258 & 6.073803 & -1.129630 \\
\hline 1 & -3.834391 & 5.826269 & -3.039335 \\
\hline 1 & -3.530326 & 3.932088 & -4.627779 \\
\hline 1 & -1.682914 & 2.359088 & -4.349041 \\
\hline 1 & 1.695880 & 3.991618 & -1.846511 \\
\hline 1 & 1.168587 & 2.965152 & -0.508638 \\
\hline 1 & 2.341060 & 2.355240 & -1.689977 \\
\hline 6 & -4.355926 & -1.937012 & -4.075071 \\
\hline 6 & -6.210318 & -3.197461 & -3.039172 \\
\hline 6 & -6.497209 & -0.772620 & -3.569891 \\
\hline 6 & -7.628330 & -2.159557 & 1.633086 \\
\hline 6 & -5.519357 & -2.409568 & 2.909905 \\
\hline 6 & -6.598795 & -0.168449 & 2.684865 \\
\hline 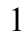 & -4.733149 & -2.296212 & -5.040972 \\
\hline 1 & -3.875084 & -0.967306 & -4.258552 \\
\hline 1 & -3.586055 & -2.641285 & -3.729646 \\
\hline 1 & -6.520253 & -3.486140 & -4.052092 \\
\hline 1 & -5.534715 & -3.974416 & -2.656965 \\
\hline 1 & -7.112890 & -3.193244 & -2.416606 \\
\hline 1 & -6.866263 & -1.023929 & -4.573811 \\
\hline 1 & -7.364608 & -0.689403 & -2.902288 \\
\hline 1 & -6.017721 & 0.213897 & -3.620937 \\
\hline 1 & -8.195340 & -2.330009 & 2.556957 \\
\hline 1 & -8.250576 & -1.538815 & 0.975558 \\
\hline 1 & -7.486831 & -3.135837 & 1.150820 \\
\hline
\end{tabular}




$\begin{array}{llcc}1 & -7.183692 & -0.357556 & 3.595182 \\ 1 & -5.682878 & 0.356487 & 2.983552 \\ 1 & -7.181932 & 0.507112 & 2.045113 \\ 1 & -6.108156 & -2.613796 & 3.814451 \\ 1 & -5.298834 & -3.373698 & 2.430813 \\ 1 & -4.565067 & -1.969438 & 3.229386\end{array}$

$3_{\text {IIB-re- }} \Psi-15233 s 4 a$

Number of imaginary frequencies : 0

The smallest frequencies are : $17.9717 \quad 20.0903 \quad 21.8954 \mathrm{~cm}(-1)$

Electronic energy : $\quad \mathrm{HF}=-4360.885221$

Zero-point correction $=\quad 1.455437$ (Hartree/Particle)

Thermal correction to Energy $=\quad 1.539788$

Thermal correction to Enthalpy= $\quad 1.540732$

Thermal correction to Gibbs Free Energy $=\quad 1.338549$

Sum of electronic and zero-point Energies $=\quad-4359.429784$

Sum of electronic and thermal Energies $=\quad-4359.345433$

Sum of electronic and thermal Enthalpies $=\quad-4359.344489$

Sum of electronic and thermal Free Energies $=\quad-4359.546672$

Cartesian Coordinates

$\begin{array}{cccc}6 & -1.903513 & 2.986114 & -3.612692 \\ 6 & -0.916089 & 3.196767 & -2.633568 \\ 6 & -1.102388 & 4.283949 & -1.769527 \\ 6 & -2.213291 & 5.117359 & -1.870929 \\ 6 & -3.171264 & 4.895831 & -2.851473 \\ 6 & -3.008494 & 3.816500 & -3.719657 \\ 6 & 0.301701 & 2.344494 & -2.521586 \\ 6 & 1.426988 & 2.919153 & -1.696404 \\ 45 & -0.194901 & 0.187736 & -1.943213 \\ 6 & -0.450356 & -1.382905 & -3.036435 \\ 8 & -0.714121 & -2.068610 & -3.932707 \\ 15 & -1.621858 & 0.186031 & -0.003334 \\ 6 & -1.985320 & 1.877821 & 0.626052 \\ 6 & -0.951622 & 2.598213 & 1.232030 \\ 6 & -1.195403 & 3.812353 & 1.875875 \\ 6 & -2.500008 & 4.321168 & 1.839817 \\ 6 & -3.541229 & 3.658206 & 1.188145 \\ 6 & -3.260372 & 2.432253 & 0.576814 \\ 6 & -0.060706 & 4.530873 & 2.608604 \\ 6 & 0.682265 & 3.540874 & 3.513828 \\ 6 & -4.986681 & 4.155152 & 1.214755 \\ 6 & -5.127714 & 5.502889 & 1.916962 \\ 6 & -3.312852 & -0.503836 & -0.264149 \\ 6 & -3.726225 & -0.889835 & -1.528261 \\ 6 & -5.011478 & -1.402635 & -1.753293 \\ 6 & -5.858098 & -1.533561 & -0.657639 \\ 6 & -5.470921 & -1.163780 & 0.642443 \\ 6 & -4.194546 & -0.643448 & 0.817844 \\ 6 & -6.434630 & -1.369166 & 1.810801 \\ 6 & -5.393206 & -1.827882 & -3.168408 \\ 6 & -1.190149 & -0.708106 & 1.577547 \\ 6 & -0.738450 & -2.021802 & 1.548064 \\ 6 & -0.456817 & -2.711376 & 2.775570\end{array}$




\begin{tabular}{|c|c|c|c|}
\hline 6 & -0.644639 & -2.044733 & 4.020958 \\
\hline & -1.133757 & -0.720078 & 4.015458 \\
\hline & -1.393354 & -0.076529 & 2.836172 \\
\hline & -0.315493 & -2.704115 & 5.229152 \\
\hline & 0.184876 & -3.980333 & 5.218671 \\
\hline & 0.363303 & -4.652560 & 3.991448 \\
\hline & 0.045293 & -4.040039 & 2.804596 \\
\hline & -0.532357 & -2.816051 & 0.301191 \\
\hline & 0.688498 & -2.813678 & -0.348342 \\
\hline & 0.959192 & -3.732960 & -1.388373 \\
\hline & 0.011689 & -4.632097 & -1.794452 \\
\hline & -1.267464 & -4.642307 & -1.191133 \\
\hline & -1.545206 & -3.731124 & -0.127885 \\
\hline & -2.824359 & -3.784519 & 0.483546 \\
\hline & -3.773298 & -4.680482 & 0.057838 \\
\hline & -3.492988 & -5.581329 & -0.992127 \\
\hline & -2.265793 & -5.558222 & -1.603336 \\
\hline & 1.726908 & -1.900566 & 0.038569 \\
\hline & 1.829335 & -0.473634 & -0.874310 \\
\hline & 2.988568 & -0.637246 & -2.065403 \\
\hline & 4.223858 & -1.180605 & -1.802514 \\
\hline & 5.155363 & -0.487173 & -1.055865 \\
\hline & 6.359070 & -1.179905 & -0.687321 \\
\hline & 6.610152 & -2.484162 & -1.211081 \\
\hline 6 & 5.667715 & -3.080696 & -2.084538 \\
\hline & 4.487400 & -2.449016 & -2.361578 \\
\hline 6 & 7.289765 & -0.650371 & 0.243535 \\
\hline 0 & 8.407656 & -1.357711 & 0.612305 \\
\hline & 8.668507 & -2.630942 & 0.064789 \\
\hline 6 & 7.783087 & -3.179084 & -0.827121 \\
\hline f & 4.889397 & 0.901119 & -0.609278 \\
\hline 6 & 3.734217 & 1.225595 & 0.081289 \\
\hline 6 & 3.574899 & 2.473989 & 0.720742 \\
\hline 6 & 4.508695 & 3.456306 & 0.559511 \\
\hline 6 & 5.633800 & 3.241852 & -0.273715 \\
\hline 6 & 5.825951 & 1.959790 & -0.870636 \\
\hline 6 & 6.562998 & 4.278925 & -0.526296 \\
\hline 6 & 7.635007 & 4.079971 & -1.358333 \\
\hline 6 & 7.811225 & 2.826305 & -1.978070 \\
\hline 6 & 6.935571 & 1.795671 & -1.738644 \\
\hline 3 & 2.712473 & 0.339786 & 0.269465 \\
\hline 6 & 0.651099 & 1.410113 & -3.526028 \\
\hline 6 & 2.817086 & -2.509375 & 0.840500 \\
\hline 6 & 3.333003 & -1.720549 & 2.018215 \\
\hline 6 & 2.490194 & -0.983701 & 2.852233 \\
\hline 6 & 2.996145 & -0.320601 & 3.961986 \\
\hline 6 & 4.357121 & -0.381544 & 4.258499 \\
\hline 6 & 5.203832 & -1.112367 & 3.433836 \\
\hline 6 & 4.692275 & -1.777691 & 2.321615 \\
\hline 6 & 0.909384 & 5.126851 & 1.582795 \\
\hline 6 & -0.567246 & 5.670813 & 3.491142 \\
\hline 6 & -5.528563 & 4.306542 & -0.208683 \\
\hline 6 & -5.836245 & 3.124867 & 1.971424 \\
\hline 1 & 7.092514 & 0.317693 & 0.696282 \\
\hline 1 & -1.774264 & 0.940873 & 2.868504 \\
\hline & 7.956372 & -4.168724 & -1.247864 \\
\hline
\end{tabular}




\begin{tabular}{|c|c|c|c|}
\hline & -0.461248 & -2.169212 & 6.166690 \\
\hline & 6.398394 & 5.245185 & -0.051068 \\
\hline 1 & -3.055959 & -3.092025 & 1.291778 \\
\hline 1 & -4.752673 & -4.695562 & 0.532987 \\
\hline & 4.386189 & 4.421865 & 1.048338 \\
\hline & -1.295941 & -0.213019 & 4.966096 \\
\hline & 5.887757 & -4.058809 & -2.508869 \\
\hline & 8.645606 & 2.673922 & -2.659074 \\
\hline & 3.734753 & -2.888423 & -3.012658 \\
\hline & -2.034194 & -6.244800 & -2.416468 \\
\hline & 0.221725 & -5.338811 & -2.595732 \\
\hline & 0.754219 & -5.667479 & 3.985507 \\
\hline & 7.081938 & 0.840794 & -2.236725 \\
\hline & 0.186427 & -4.578286 & 1.869623 \\
\hline & 1.943622 & -3.705105 & -1.853945 \\
\hline & 8.339251 & 4.885856 & -1.551148 \\
\hline & 9.095106 & -0.933021 & 1.340568 \\
\hline & -4.253414 & -6.288898 & -1.315635 \\
\hline & 0.443505 & -4.477062 & 6.150888 \\
\hline ] & 9.560944 & -3.177498 & 0.360398 \\
\hline & 2.686961 & 2.620373 & 1.331952 \\
\hline & -3.865144 & -0.337786 & 1.808115 \\
\hline$\frac{1}{1}$ & -3.029461 & -0.795359 & -2.356708 \\
\hline & -6.856739 & -1.941261 & -0.804293 \\
\hline l & -4.063245 & 1.880455 & 0.091825 \\
\hline & 0.048419 & 2.159041 & 1.252079 \\
\hline 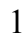 & -2.711088 & 5.256861 & 2.348468 \\
\hline 1 & 2.396722 & -3.451704 & 1.219311 \\
\hline & -1.553638 & 0.574315 & -2.641065 \\
\hline 1 & 3.664009 & -2.794099 & 0.198680 \\
\hline & 5.365436 & -2.341056 & 1.673515 \\
\hline 1 & 1.435608 & -0.901753 & 2.600261 \\
\hline$\frac{1}{1}$ & 6.270312 & -1.162177 & 3.646413 \\
\hline 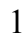 & 2.321368 & 0.249764 & 4.599157 \\
\hline 1 & 4.753469 & 0.143054 & 5.125344 \\
\hline 1 & 1.513610 & 4.043203 & 4.026266 \\
\hline 1 & 0.010176 & 3.131735 & 4.280806 \\
\hline & 1.101448 & 2.689909 & 2.962814 \\
\hline 1 & 0.275942 & 6.116359 & 4.034033 \\
\hline 1 & -1.034095 & 6.472903 & 2.905869 \\
\hline 1 & -1.293626 & 5.320332 & 4.235765 \\
\hline 1 & 1.744695 & 5.629469 & 2.090704 \\
\hline 1 & 1.331070 & 4.363302 & 0.917267 \\
\hline 1 & 0.401942 & 5.872066 & 0.954435 \\
\hline ] & -6.177051 & 5.823100 & 1.893116 \\
\hline 1 & -4.824106 & 5.455665 & 2.970708 \\
\hline 1 & -4.533993 & 6.282885 & 1.421582 \\
\hline 1 & -6.587927 & 4.595704 & -0.179681 \\
\hline 1 & -4.978390 & 5.083574 & -0.752412 \\
\hline 1 & -5.450203 & 3.380294 & -0.790503 \\
\hline 1 & -6.884324 & 3.451107 & 2.021139 \\
\hline 1 & -5.812952 & 2.143838 & 1.477838 \\
\hline 1 & -5.471742 & 2.994087 & 2.999426 \\
\hline 1 & 0.055253 & 1.308603 & -4.431997 \\
\hline 1 & 1.703219 & 1.160150 & -3.662765 \\
\hline 1 & -0.370560 & 4.484688 & -0.991574 \\
\hline
\end{tabular}




$\begin{array}{rrrr}1 & -2.322747 & 5.946088 & -1.170764 \\ 1 & -4.035467 & 5.550946 & -2.942832 \\ 1 & -3.747427 & 3.624419 & -4.495683 \\ 1 & -1.809583 & 2.152176 & -4.306163 \\ 1 & 1.669419 & 3.948374 & -2.007729 \\ 1 & 1.191802 & 2.955974 & -0.626567 \\ 1 & 2.339306 & 2.325033 & -1.819863 \\ 6 & -6.872106 & -2.186865 & -3.286731 \\ 6 & -5.096472 & -0.692658 & -4.155900 \\ 6 & -4.562951 & -3.059556 & -3.546576 \\ 6 & -5.850618 & -0.880343 & 3.135066 \\ 6 & -7.739393 & -0.607934 & 1.554306 \\ 6 & -6.738101 & -2.864894 & 1.954956 \\ 1 & -7.104162 & -2.458486 & -4.324238 \\ 1 & -7.138585 & -3.047202 & -2.659402 \\ 1 & -7.520854 & -1.345039 & -3.010890 \\ 1 & -5.396631 & -0.988382 & -5.169948 \\ 1 & -5.647932 & 0.218944 & -3.890601 \\ 1 & -4.028852 & -0.439457 & -4.193756 \\ 1 & -4.802360 & -3.385066 & -4.568335 \\ 1 & -3.485109 & -2.855264 & -3.502562 \\ 1 & -4.769005 & -3.893604 & -2.862526 \\ 1 & -6.571811 & -1.057528 & 3.942888 \\ 1 & -4.924648 & -1.410394 & 3.398948 \\ 1 & -5.634308 & 0.196406 & 3.116398 \\ 1 & -7.448221 & -3.033930 & 2.775796 \\ 1 & -7.177574 & -3.288080 & 1.043115 \\ 1 & -5.822288 & -3.427536 & 2.184338 \\ 1 & -8.436356 & -0.756342 & 2.390032 \\ 1 & -7.555918 & 0.470600 & 1.457474 \\ 1 & -8.244318 & -0.944022 & 0.640867\end{array}$

$3_{\text {IIB-re- }-} \psi_{-1522354 s}$

Number of imaginary frequencies : 0

The smallest frequencies are : $19.5913 \quad 21.019922 .3347 \mathrm{~cm}(-1)$

Electronic energy : $\quad H F=-4360.8846465$

Zero-point correction $=\quad 1.455690($ Hartree/Particle $)$

Thermal correction to Energy= $\quad 1.539934$

Thermal correction to Enthalpy= $\quad 1.540878$

Thermal correction to Gibbs Free Energy $=\quad 1.339678$

Sum of electronic and zero-point Energies $=\quad-4359.428957$

Sum of electronic and thermal Energies $=\quad-4359.344713$

Sum of electronic and thermal Enthalpies $=\quad-4359.343769$

Sum of electronic and thermal Free Energies $=\quad-4359.544968$

Cartesian Coordinates

$\begin{array}{lrrr}6 & -1.852166 & 3.144934 & -3.585456 \\ 6 & -0.907143 & 3.300902 & -2.555865 \\ 6 & -1.111120 & 4.361120 & -1.662511 \\ 6 & -2.190602 & 5.230117 & -1.794965 \\ 6 & -3.099030 & 5.071139 & -2.833448 \\ 6 & -2.926134 & 4.010913 & -3.722400 \\ 6 & 0.294095 & 2.427312 & -2.430529 \\ 6 & 1.404746 & 2.972607 & -1.566979\end{array}$




\begin{tabular}{|c|c|c|c|}
\hline 45 & -0.224508 & 0.262103 & -1.917085 \\
\hline 6 & -0.531556 & -1.270178 & -3.052972 \\
\hline 8 & -0.838207 & -1.916180 & -3.964892 \\
\hline 15 & -1.636163 & 0.194393 & 0.035550 \\
\hline 6 & -1.969156 & 1.871539 & 0.715591 \\
\hline 6 & -0.924858 & 2.554688 & 1.347978 \\
\hline 6 & -1.141227 & 3.777391 & 1.984207 \\
\hline 6 & -2.424278 & 4.336084 & 1.905223 \\
\hline 6 & -3.473965 & 3.708800 & 1.232877 \\
\hline 6 & -3.225093 & 2.465439 & 0.640625 \\
\hline 6 & -0.004379 & 4.468158 & 2.741515 \\
\hline 6 & 0.763039 & 3.449546 & 3.592216 \\
\hline 6 & -4.896763 & 4.269635 & 1.214638 \\
\hline 6 & -4.997478 & 5.628613 & 1.902181 \\
\hline 6 & -3.336435 & -0.472659 & -0.229654 \\
\hline 6 & -3.774605 & -0.783497 & -1.510852 \\
\hline 6 & -5.050209 & -1.306983 & -1.738039 \\
\hline 6 & -5.866596 & -1.544929 & -0.630504 \\
\hline 6 & -5.453445 & -1.260043 & 0.677213 \\
\hline 6 & -4.188254 & -0.700818 & 0.854172 \\
\hline 6 & -6.305821 & -1.573742 & 1.908238 \\
\hline 6 & -5.460266 & -1.661156 & -3.165559 \\
\hline 6 & -1.193829 & -0.767817 & 1.572387 \\
\hline 6 & -0.759081 & -2.085495 & 1.488465 \\
\hline 6 & -0.477363 & -2.824010 & 2.687448 \\
\hline 6 & -0.646945 & -2.202862 & 3.958693 \\
\hline 6 & -1.114727 & -0.871438 & 4.007592 \\
\hline 6 & -1.375105 & -0.180878 & 2.855389 \\
\hline 6 & -0.319436 & -2.912055 & 5.138790 \\
\hline 6 & 0.162909 & -4.193672 & 5.076696 \\
\hline 6 & 0.325572 & -4.8203 & \\
\hline 6 & 0.008896 & -4.158761 & 2.662936 \\
\hline 6 & -0.563805 & -2.832921 & \\
\hline 6 & 0.653253 & -2.806397 & -0.445474 \\
\hline 6 & 0.916344 & -3.679725 & -1.526106 \\
\hline 6 & -0.035468 & -4.556872 & -1.967937 \\
\hline 6 & -1.310879 & -4.590798 & -1.357980 \\
\hline 6 & -1.578934 & -3.729576 & -0.251511 \\
\hline 6 & -2.852027 & -3.812510 & 0.367669 \\
\hline 6 & -3.807186 & -4.683640 & -0.093817 \\
\hline 6 & -3.538739 & -5.531402 & -1.189856 \\
\hline 6 & -2.315326 & -5.482262 & -1.807254 \\
\hline 7 & 1.696253 & -1.913615 & -0.026282 \\
\hline 15 & 1.802200 & -0.456312 & -0.887479 \\
\hline 8 & 2.956360 & -0.582313 & -2.088324 \\
\hline 6 & 4.189270 & -1.142023 & -1.850161 \\
\hline 6 & 5.127965 & -0.483870 & -1.080572 \\
\hline 6 & 6.328711 & -1.197960 & -0.744439 \\
\hline 6 & 6.570298 & -2.481598 & -1.320694 \\
\hline 6 & 5.621219 & -3.037462 & -2.213511 \\
\hline 6 & 4.443580 & -2.388560 & -2.460249 \\
\hline 6 & 7.266207 & -0.710857 & 0.202574 \\
\hline 6 & 8.381825 & -1.438252 & 0.537826 \\
\hline 6 & 8.633395 & -2.689978 & -0.061379 \\
\hline 6 & 7.741069 & -3.197466 & -0.970373 \\
\hline 6 & 4.873627 & 0.889314 & -0.583269 \\
\hline
\end{tabular}




\begin{tabular}{|c|c|c|c|}
\hline & 3.720608 & 1.197807 & 0.118076 \\
\hline & 3.566631 & 2.426471 & 0.795857 \\
\hline & 4.506905 & 3.407400 & 0.668512 \\
\hline & 5.632259 & 3.212916 & -0.169370 \\
\hline & 5.816906 & 1.950431 & -0.809061 \\
\hline & 6.567343 & 4.252518 & -0.387069 \\
\hline & 7.638218 & 4.075577 & -1.225531 \\
\hline & 7.807157 & 2.842494 & -1.887123 \\
\hline & 6.925467 & 1.809461 & -1.682545 \\
\hline & 2.695148 & 0.311101 & 0.279658 \\
\hline & 0.656091 & 1.515879 & -3.452409 \\
\hline & 2.783447 & -2.556998 & 0.752229 \\
\hline & 3.310255 & -1.808325 & 1.951227 \\
\hline b & 2.475717 & -1.089854 & 2.809307 \\
\hline & 2.990172 & -0.462444 & 3.935657 \\
\hline & 4.351819 & -0.541209 & 4.225081 \\
\hline b & 5.190326 & -1.253969 & 3.376610 \\
\hline & 4.670141 & -1.883637 & 2.247626 \\
\hline D & 0.948235 & 5.126057 & 1.736907 \\
\hline & -0.516930 & 5.556602 & 3.685210 \\
\hline & -5.398782 & 4.430095 & -0.222820 \\
\hline & -5.811506 & 3.284706 & 1.953199 \\
\hline & 7.076197 & 0.239873 & 0.693646 \\
\hline & -1.739671 & 0.840343 & 2.927741 \\
\hline & 7.907312 & -4.170304 & -1.431270 \\
\hline & -0.451592 & -2.410784 & 6.096707 \\
\hline & 6.407856 & 5.203428 & 0.119738 \\
\hline & -3.073071 & -3.163466 & 1.213188 \\
\hline & -4.783269 & -4.719193 & 0.388073 \\
\hline 1 & 4.389062 & 4.357951 & 1.187114 \\
\hline & -1.260218 & -0.398193 & 4.978137 \\
\hline ] & 5.834151 & -3.998908 & -2.677737 \\
\hline & 8.640477 & 2.708370 & -2.573240 \\
\hline & 3.686336 & -2.797482 & -3.125719 \\
\hline 1 & -2.091327 & -6.129351 & -2.654191 \\
\hline & 0.168101 & -5.226714 & -2.801889 \\
\hline 1 & 0.703799 & -5.838974 & 3.776060 \\
\hline & 7.066325 & 0.871344 & -2.213014 \\
\hline 1 & 0.138662 & -4.662603 & 1.707468 \\
\hline ] & 1.898754 & -3.633241 & -1.993857 \\
\hline 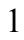 & 8.346969 & 4.883494 & -1.391469 \\
\hline 1 & 9.074916 & -1.046395 & 1.279033 \\
\hline & -4.303876 & -6.219552 & -1.542765 \\
\hline 1 & 0.420330 & -4.729062 & 5.987587 \\
\hline & 9.524330 & -3.252364 & 0.207892 \\
\hline 1 & 2.675435 & 2.560304 & 1.405424 \\
\hline l & -3.848173 & -0.457778 & 1.860868 \\
\hline 1 & -3.096915 & -0.623268 & -2.344829 \\
\hline 1 & -6.851606 & -1.975349 & -0.787731 \\
\hline 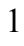 & -4.034993 & 1.937474 & 0.139994 \\
\hline 1 & 0.058199 & 2.080653 & 1.387651 \\
\hline ] & -2.611463 & 5.284950 & 2.398612 \\
\hline 1 & 2.355047 & -3.506648 & 1.103358 \\
\hline 1 & -1.579345 & 0.698083 & -2.595050 \\
\hline 1 & 3.625117 & -2.829311 & 0.097943 \\
\hline 1 & 5.337127 & -2.432664 & 1.581122 \\
\hline
\end{tabular}




\begin{tabular}{|c|c|c|c|}
\hline 1 & 1.421010 & -0.992476 & 2.563319 \\
\hline 1 & 6.257240 & -1.317168 & 3.583447 \\
\hline 1 & 2.321617 & 0.094297 & 4.591124 \\
\hline & 4.755078 & -0.044238 & 5.104920 \\
\hline 1 & 1.576994 & 3.947634 & 4.135432 \\
\hline 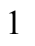 & 0.102237 & 2.977581 & 4.332442 \\
\hline 1 & 1.214429 & 2.645158 & 2.998828 \\
\hline 1 & 0.322570 & 5.969588 & 4.258671 \\
\hline 1 & -0.978485 & 6.392866 & 3.145864 \\
\hline 1 & -1.249414 & 5.163734 & 4.402212 \\
\hline 1 & 1.774434 & 5.626219 & 2.261942 \\
\hline 1 & 1.384138 & 4.398385 & 1.040597 \\
\hline 1 & 0.422410 & 5.884202 & 1.139923 \\
\hline 1 & -6.030352 & 5.994662 & 1.843596 \\
\hline 1 & -4.728693 & 5.576608 & 2.965200 \\
\hline 1 & -4.355296 & 6.378018 & 1.420135 \\
\hline 1 & -6.452620 & 4.740358 & -0.218764 \\
\hline 1 & -4.821897 & 5.193243 & -0.758292 \\
\hline 1 & -5.325673 & 3.500100 & -0.799690 \\
\hline 1 & -6.840435 & 3.668270 & 1.989195 \\
\hline 1 & -5.836556 & 2.308241 & 1.453230 \\
\hline 1 & -5.471724 & 3.125822 & 2.985619 \\
\hline 1 & 0.077670 & 1.443602 & -4.372239 \\
\hline 1 & 1.708998 & 1.263292 & -3.577124 \\
\hline 1 & -0.420025 & 4.510773 & -0.836701 \\
\hline 1 & -2.314399 & 6.038317 & -1.073245 \\
\hline 1 & -3.936871 & 5.756074 & -2.948784 \\
\hline 1 & -3.630939 & 3.862727 & -4.538738 \\
\hline 1 & -1.747210 & 2.328882 & -4.298256 \\
\hline 1 & 1.655172 & 4.010493 & -1.841387 \\
\hline 1 & 1.148532 & 2.976744 & -0.500963 \\
\hline 1 & 2.317641 & 2.380240 & -1.692296 \\
\hline 6 & -6.954656 & -1.953193 & -3.284035 \\
\hline 6 & -5.125082 & -0.508871 & -4.120411 \\
\hline 6 & -4.683989 & -2.913144 & -3.590201 \\
\hline 6 & -7.621106 & -2.259206 & 1.546605 \\
\hline 6 & -5.524493 & -2.509062 & 2.840593 \\
\hline 6 & -6.632088 & -0.278884 & 2.659004 \\
\hline 1 & -7.208035 & -2.159583 & -4.331552 \\
\hline 1 & -7.252553 & -2.834432 & -2.701914 \\
\hline 1 & -7.564603 & -1.101397 & -2.955284 \\
\hline 1 & -5.464432 & -0.753298 & -5.135621 \\
\hline 1 & -5.619628 & 0.421601 & -3.811783 \\
\hline 1 & -4.046974 & -0.311523 & -4.180117 \\
\hline 1 & -4.936763 & -3.191803 & -4.622683 \\
\hline 1 & -3.598548 & -2.755289 & -3.537490 \\
\hline 1 & -4.925508 & -3.761584 & -2.935817 \\
\hline 1 & -8.188025 & -2.474760 & 2.461071 \\
\hline 1 & -8.253754 & -1.626908 & 0.910558 \\
\hline 1 & -7.458296 & -3.213587 & 1.028409 \\
\hline 1 & -7.226216 & -0.497964 & 3.556654 \\
\hline 1 & -5.725129 & 0.247891 & 2.981372 \\
\hline 1 & -7.214768 & 0.406086 & 2.028810 \\
\hline 1 & -6.123495 & -2.745982 & 3.730276 \\
\hline 1 & -5.280894 & -3.456734 & 2.340587 \\
\hline 1 & -4.582747 & -2.063432 & 3.187602 \\
\hline
\end{tabular}


$3_{\text {IIC-re- }} \Psi-1$-1azazata

Number of imaginary frequencies : 0

The smallest frequencies are : $9.3365 \quad 14.5356 \quad 18.1103 \mathrm{~cm}(-1)$

Electronic energy : $\quad H F=-4360.8929345$

Zero-point correction $=\quad 1.454414$ (Hartree/Particle)

Thermal correction to Energy $=\quad 1.539194$

Thermal correction to Enthalpy= $\quad 1.540138$

Thermal correction to Gibbs Free Energy= 1.334843

Sum of electronic and zero-point Energies $=\quad-4359.438520$

Sum of electronic and thermal Energies $=\quad-4359.353741$

Sum of electronic and thermal Enthalpies $=\quad-4359.352797$

Sum of electronic and thermal Free Energies $=\quad-4359.558091$

\begin{tabular}{|c|c|c|c|}
\hline & \multicolumn{3}{|c|}{ Cartesian Coordinates } \\
\hline 6 & 2.260060 & 1.395661 & -4.151365 \\
\hline 6 & 2.028793 & 2.212883 & -3.037379 \\
\hline 6 & 3.125182 & 2.860659 & -2.460052 \\
\hline 6 & 4.413463 & 2.666271 & -2.951437 \\
\hline 6 & 4.630983 & 1.820723 & -4.032897 \\
\hline 6 & 3.543521 & 1.193012 & -4.638476 \\
\hline 6 & 0.644056 & 2.460791 & -2.535499 \\
\hline 6 & -0.244662 & 3.122090 & -3.570659 \\
\hline 45 & -0.331943 & 0.670049 & -1.62635 \\
\hline 6 & -0.660151 & -0.450748 & -3.151351 \\
\hline 8 & -0.857502 & -0.917356 & -4.194356 \\
\hline 15 & -1.862872 & 0.325405 & 0.242076 \\
\hline 6 & -2.008109 & 1.972843 & 1.039947 \\
\hline 6 & -1.021486 & 2.413110 & 1.924177 \\
\hline 6 & -1.022842 & 3.721851 & 2.411702 \\
\hline 6 & -2.002575 & 4.594967 & 1.929195 \\
\hline 6 & -2.981999 & 4.197335 & 1.014904 \\
\hline 6 & -2.981264 & 2.867011 & 0.593996 \\
\hline 6 & 0.046514 & 4.220080 & 3.383905 \\
\hline 6 & -3.991819 & 5.215623 & 0.489098 \\
\hline 6 & -3.610809 & -0.044444 & -0.221501 \\
\hline 6 & -3.957151 & -0.248288 & -1.552956 \\
\hline 6 & -5.280154 & -0.511076 & -1.921776 \\
\hline 6 & -6.234577 & -0.579714 & -0.906126 \\
\hline 6 & -5.920637 & -0.392530 & 0.445581 \\
\hline 6 & -4.594081 & -0.117705 & 0.769440 \\
\hline 6 & -7.013925 & -0.514713 & 1.506037 \\
\hline 6 & -5.697480 & -0.676428 & -3.382002 \\
\hline 6 & -1.682001 & -0.852737 & 1.668265 \\
\hline 6 & -1.387778 & -2.186444 & 1.417506 \\
\hline 6 & -1.292720 & -3.117625 & 2.501751 \\
\hline 6 & -1.505218 & -2.667953 & 3.837265 \\
\hline 6 & -1.827506 & -1.309438 & 4.055640 \\
\hline 6 & -1.910246 & -0.432592 & 3.007151 \\
\hline 6 & -1.380705 & -3.581085 & 4.911355 \\
\hline 6 & -1.058925 & -4.894352 & 4.682950 \\
\hline 6 & -0.853858 & -5.348302 & 3.362896 \\
\hline 6 & -0.972041 & -4.486084 & 2.301072 \\
\hline
\end{tabular}




\begin{tabular}{|c|c|c|c|}
\hline & -1.205252 & -2.723788 & 0.039418 \\
\hline & 0.023072 & -2.669577 & -0.594893 \\
\hline & 0.214628 & -3.291992 & -1.849863 \\
\hline & -0.818179 & -3.929135 & -2.482705 \\
\hline & -2.101172 & -3.973539 & -1.890056 \\
\hline & -2.298013 & -3.379468 & -0.607959 \\
\hline & -3.589848 & -3.448451 & -0.023969 \\
\hline & -4.627518 & -4.061885 & -0.679434 \\
\hline & -4.426387 & -4.656651 & -1.944412 \\
\hline 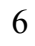 & -3.190145 & -4.609048 & -2.536163 \\
\hline & 1.126720 & -1.977615 & 0.008014 \\
\hline & 1.512702 & -0.489992 & -0.713804 \\
\hline & 2.737626 & -0.720006 & -1.821201 \\
\hline & 3.891193 & -1.397210 & -1.506827 \\
\hline & 4.818722 & -0.869253 & -0.626718 \\
\hline & 5.909145 & -1.712927 & -0.225201 \\
\hline 6 & 6.086873 & -2.985189 & -0.846851 \\
\hline . & 5.178249 & -3.402192 & -1.850364 \\
\hline & 4.091522 & -2.632737 & -2.160089 \\
\hline 6 & 6.789642 & -1.363257 & 0.830546 \\
\hline 6 & 7.795672 & -2.209515 & 1.227823 \\
\hline 6 & 7.988751 & -3.450557 & 0.585857 \\
\hline 6 & 7.147146 & -3.826312 & -0.429702 \\
\hline & 4.661009 & 0.505252 & -0.097024 \\
\hline 6 & 3.471429 & 0.908503 & 0.480369 \\
\hline & 3.323096 & 2.165673 & 1.101689 \\
\hline & 4.354848 & 3.061471 & 1.095183 \\
\hline ) & 5.562238 & 2.753149 & 0.422315 \\
\hline & 5.714123 & 1.476420 & -0.197982 \\
\hline 6 & 6.602432 & 3.708889 & 0.322054 \\
\hline & 7.744852 & 3.438528 & -0.387264 \\
\hline 6 & 7.883067 & 2.193869 & -1.036173 \\
\hline 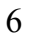 & 6.898745 & 1.240856 & -0.942101 \\
\hline & 2.395776 & 0.067933 & 0.566174 \\
\hline 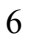 & 0.394040 & 2.689444 & -1.162501 \\
\hline & 2.035844 & -2.829175 & 0.811737 \\
\hline 5 & 2.564386 & -2.244400 & 2.097279 \\
\hline & 1.757414 & -1.502904 & 2.962411 \\
\hline 5 & 2.260284 & -1.025759 & 4.165010 \\
\hline 5 & 3.582603 & -1.280992 & 4.525827 \\
\hline & 4.393107 & -2.018509 & 3.671273 \\
\hline 6 & 3.884254 & -2.497544 & 2.465796 \\
\hline & 6.640289 & -0.421696 & 1.352473 \\
\hline 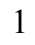 & -2.163958 & 0.605943 & 3.204758 \\
\hline & 7.268205 & -4.788449 & -0.925897 \\
\hline 1 & -1.541996 & -3.214454 & 5.924193 \\
\hline 1 & 6.465644 & 4.672540 & 0.811128 \\
\hline 1 & -3.756996 & -2.986847 & 0.947903 \\
\hline 1 & -5.616311 & -4.087196 & -0.225062 \\
\hline & 4.251744 & 4.029841 & 1.583447 \\
\hline 1 & -2.008343 & -0.968438 & 5.074411 \\
\hline & 5.343846 & -4.356021 & -2.348500 \\
\hline 1 & 8.774593 & 1.988603 & -1.624620 \\
\hline 1 & 3.365569 & -2.938623 & -2.910057 \\
\hline 1 & -3.021436 & -5.061028 & -3.512667 \\
\hline 1 & -0.671000 & -4.395365 & -3.455689 \\
\hline
\end{tabular}




\begin{tabular}{|c|c|c|c|}
\hline & -0.602468 & -6.391443 & 3.185299 \\
\hline & 7.012718 & 0.297638 & -1.469901 \\
\hline & -0.817848 & -4.853035 & 1.288268 \\
\hline & 1.202145 & -3.237460 & -2.305742 \\
\hline & 8.534299 & 4.182559 & -0.463907 \\
\hline & 8.445387 & -1.921609 & 2.051441 \\
\hline & -5.255497 & -5.150371 & -2.447154 \\
\hline & -0.961047 & -5.588040 & 5.514733 \\
\hline & 8.793270 & -4.108728 & 0.905427 \\
\hline 1 & 2.370996 & 2.384600 & 1.581810 \\
\hline 1 & -4.311443 & 0.044402 & 1.807005 \\
\hline & -3.172764 & -0.200225 & -2.302432 \\
\hline & -7.270737 & -0.787030 & -1.174950 \\
\hline ] & -3.730650 & 2.517962 & -0.111891 \\
\hline & -0.241634 & 1.715556 & 2.231526 \\
\hline 1 & -1.996129 & 5.630014 & 2.268533 \\
\hline 1 & 1.443043 & -3.721577 & 1.059200 \\
\hline 1 & -1.627589 & 1.437298 & -2.133728 \\
\hline & 2.878686 & -3.184812 & 0.199462 \\
\hline ] & 4.530969 & -3.066705 & 1.796263 \\
\hline ] & 0.737108 & -1.269777 & 2.666786 \\
\hline 1 & 5.430986 & -2.217903 & 3.931841 \\
\hline 1 & 1.613109 & -0.450015 & 4.825361 \\
\hline & 3.977665 & -0.901781 & 5.465977 \\
\hline 1 & 1.216154 & 2.656356 & -0.446202 \\
\hline 1 & -0.436394 & 3.334883 & -0.869886 \\
\hline 1 & 2.965908 & 3.529897 & -1.614996 \\
\hline ] & 5.251306 & 3.180900 & -2.483439 \\
\hline & 5.640534 & 1.659737 & -4.406813 \\
\hline 1 & 3.698615 & 0.535082 & -5.491730 \\
\hline & 1.418925 & 0.882462 & -4.616448 \\
\hline 1 & 0.242981 & 4.034710 & -3.947006 \\
\hline 1 & -0.433328 & 2.478143 & -4.438771 \\
\hline 1 & -1.215627 & 3.400833 & -3.143357 \\
\hline 6 & -4.492322 & -0.732404 & -4.318915 \\
\hline 6 & -6.497159 & -1.969538 & -3.565764 \\
\hline 6 & -6.567133 & 0.521357 & -3.781356 \\
\hline 5 & -6.487761 & -0.235021 & 2.912357 \\
\hline 5 & -8.138562 & 0.483078 & 1.211105 \\
\hline 5 & -7.577484 & -1.939995 & 1.485593 \\
\hline 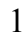 & -4.834195 & -0.884908 & -5.350497 \\
\hline 1 & -3.909223 & 0.198043 & -4.302955 \\
\hline 1 & -3.817997 & -1.561337 & -4.064354 \\
\hline 1 & -6.871832 & 0.440570 & -4.833731 \\
\hline 1 & -7.478192 & 0.585471 & -3.173027 \\
\hline 1 & -6.016724 & 1.463645 & -3.659369 \\
\hline 1 & -6.799357 & -2.080568 & -4.615777 \\
\hline 1 & -5.890733 & -2.842982 & -3.292060 \\
\hline 1 & -7.410711 & -1.988558 & -2.959280 \\
\hline 1 & -8.925079 & 0.406706 & 1.973883 \\
\hline 1 & -7.761167 & 1.513730 & 1.217460 \\
\hline 1 & -8.607490 & 0.305199 & 0.235989 \\
\hline 1 & -7.303087 & -0.342168 & 3.638925 \\
\hline 1 & -5.694046 & -0.936974 & 3.202802 \\
\hline 1 & -6.093497 & 0.785318 & 3.006445 \\
\hline 1 & -8.385578 & -2.043157 & 2.222501 \\
\hline
\end{tabular}




$\begin{array}{lrrr}1 & -7.985829 & -2.208747 & 0.503391 \\ 1 & -6.797774 & -2.671149 & 1.739109 \\ 6 & 0.766043 & 3.066374 & 4.083822 \\ 6 & 1.070893 & 5.045603 & 2.595626 \\ 6 & -0.573626 & 5.098787 & 4.474919 \\ 6 & -4.987651 & 4.592961 & -0.487471 \\ 6 & -4.781020 & 5.817392 & 1.656247 \\ 6 & -3.235545 & 6.326198 & -0.248742 \\ 1 & 1.466870 & 3.464651 & 4.828230 \\ 1 & 0.058368 & 2.407941 & 4.606701 \\ 1 & 1.352717 & 2.446176 & 3.395311 \\ 1 & 1.890701 & 5.374897 & 3.249651 \\ 1 & 1.501665 & 4.461924 & 1.769884 \\ 1 & 0.609783 & 5.939201 & 2.155862 \\ 1 & 0.202154 & 5.418314 & 5.182993 \\ 1 & -1.035937 & 6.008017 & 4.074429 \\ 1 & -1.339660 & 4.550706 & 5.038576 \\ 1 & -3.940098 & 7.060907 & -0.661513 \\ 1 & -2.543713 & 6.863429 & 0.411148 \\ 1 & -2.649785 & 5.911674 & -1.080671 \\ 1 & -5.505797 & 6.554793 & 1.285857 \\ 1 & -5.336463 & 5.039817 & 2.196780 \\ 1 & -4.131471 & 6.327017 & 2.377999 \\ 1 & -5.697020 & 5.358402 & -0.826556 \\ 1 & -4.490387 & 4.188403 & -1.379040 \\ 1 & -5.569551 & 3.784392 & -0.025474\end{array}$

$3_{\text {IIC-re- }} \psi_{-1 \mathrm{a} 2 \mathrm{2a3a4s}}$

Number of imaginary frequencies : 0

The smallest frequencies are : $14.6054 \quad 18.8388 \quad 22.4950 \mathrm{~cm}(-1)$

Electronic energy : $\quad H F=-4360.8938695$

Zero-point correction $=\quad 1.454464($ Hartree/Particle $)$

Thermal correction to Energy $=\quad 1.539056$

Thermal correction to Enthalpy= $\quad 1.540000$

Thermal correction to Gibbs Free Energy= $\quad 1.336933$

Sum of electronic and zero-point Energies $=\quad-4359.439406$

Sum of electronic and thermal Energies $=\quad-4359.354814$

Sum of electronic and thermal Enthalpies $=\quad-4359.353870$

Sum of electronic and thermal Free Energies $=\quad-4359.556936$

Cartesian Coordinates

$\begin{array}{cccc}6 & 2.336718 & 0.988257 & -4.274020 \\ 6 & 2.074261 & 1.919554 & -3.260864 \\ 6 & 3.153764 & 2.631894 & -2.729669 \\ 6 & 4.455120 & 2.391153 & -3.162727 \\ 6 & 4.702965 & 1.434065 & -4.139776 \\ 6 & 3.632976 & 0.739796 & -4.702103 \\ 6 & 0.675580 & 2.209388 & -2.823153 \\ 6 & -0.190709 & 2.738570 & -3.948753 \\ 45 & -0.304135 & 0.523943 & -1.740821 \\ 6 & -0.597034 & -0.762173 & -3.137351 \\ 8 & -0.775925 & -1.343286 & -4.124579 \\ 15 & -1.863718 & 0.338964 & 0.125962 \\ 6 & -2.075968 & 2.044537 & 0.774127\end{array}$




\begin{tabular}{|c|c|c|c|}
\hline 6 & -1.061311 & 2.596104 & 1.557282 \\
\hline & -1.131762 & 3.916145 & 2.007263 \\
\hline & -2.221239 & 4.686493 & 1.590336 \\
\hline & -3.235477 & 4.177451 & 0.773420 \\
\hline & -3.152230 & 2.840149 & 0.381817 \\
\hline & -0.021504 & 4.517474 & 2.869351 \\
\hline & -4.401809 & 5.074050 & 0.360580 \\
\hline 0 & -3.590085 & -0.128140 & -0.324502 \\
\hline 6 & -3.909851 & -0.523669 & -1.622752 \\
\hline 6 & -5.220746 & -0.851006 & -1.971709 \\
\hline 6 & -6.203795 & -0.771045 & -0.976948 \\
\hline 6 & -5.915817 & -0.390374 & 0.334487 \\
\hline 6 & -4.590747 & -0.074315 & 0.641711 \\
\hline 6 & -6.969242 & -0.296810 & 1.436253 \\
\hline 6 & -5.605232 & -1.236195 & -3.399490 \\
\hline 6 & -1.687365 & -0.672506 & 1.679257 \\
\hline 6 & -1.371308 & -2.021535 & 1.586726 \\
\hline 6 & -1.280488 & -2.822213 & 2.771090 \\
\hline 6 & -1.518753 & -2.226785 & 4.043653 \\
\hline 6 & -1.856380 & -0.856028 & 4.101599 \\
\hline 6 & -1.935017 & -0.105281 & 2.959144 \\
\hline 6 & -1.405680 & -3.011226 & 5.216109 \\
\hline 6 & -1.070627 & -4.338941 & 5.143403 \\
\hline 6 & -0.838275 & -4.936886 & 3.886718 \\
\hline 6 & -0.943703 & -4.201158 & 2.732399 \\
\hline 6 & -1.150615 & -2.703661 & 0.280501 \\
\hline 6 & 0.087516 & -2.678007 & -0.336898 \\
\hline 6 & 0.320803 & -3.423898 & -1.514959 \\
\hline 6 & -0.679399 & -4.157913 & -2.092307 \\
\hline 6 & -1.970049 & -4.183765 & -1.515990 \\
\hline 6 & -2.209755 & -3.462241 & -0.308326 \\
\hline 6 & -3.509237 & -3.513814 & 0.260460 \\
\hline 6 & -4.511821 & -4.234962 & -0.337783 \\
\hline 6 & -4.268610 & -4.953563 & -1.529059 \\
\hline 6 & -3.024423 & -4.924401 & -2.105028 \\
\hline 7 & 1.161521 & -1.895433 & 0.206355 \\
\hline 15 & 1.546169 & -0.499185 & -0.681914 \\
\hline 8 & 2.787925 & -0.846869 & -1.737257 \\
\hline 6 & 3.924651 & -1.502126 & -1.328406 \\
\hline 6 & 4.844050 & -0.892261 & -0.494320 \\
\hline 6 & 5.914569 & -1.700909 & 0.018530 \\
\hline 6 & 6.079515 & -3.038341 & -0.452516 \\
\hline 6 & 5.182055 & -3.551699 & -1.420751 \\
\hline 6 & 4.115159 & -2.805136 & -1.836470 \\
\hline 6 & 6.785830 & -1.248451 & 1.043055 \\
\hline 6 & 7.769597 & -2.060928 & 1.550782 \\
\hline 6 & 7.949126 & -3.370098 & 1.057567 \\
\hline 6 & 7.116907 & -3.844481 & 0.076494 \\
\hline 6 & 4.696010 & 0.534528 & -0.123947 \\
\hline 6 & 3.502440 & 1.019302 & 0.378846 \\
\hline 6 & 3.368418 & 2.338785 & 0.857724 \\
\hline 6 & 4.415236 & 3.212293 & 0.773762 \\
\hline 6 & 5.627584 & 2.813289 & 0.160544 \\
\hline 6 & 5.766767 & 1.473411 & -0.312219 \\
\hline 6 & 6.684489 & 3.736547 & -0.027977 \\
\hline 6 & 7.831003 & 3.373648 & -0.687821 \\
\hline
\end{tabular}




\begin{tabular}{|c|c|c|c|}
\hline & 7.956798 & 2.063404 & -1.194671 \\
\hline & 6.956637 & 1.140583 & -1.009200 \\
\hline & 2.403682 & 0.219220 & 0.532821 \\
\hline & 0.391033 & 2.588823 & -1.489927 \\
\hline & 2.051445 & -2.624714 & 1.141490 \\
\hline & 2.549428 & -1.866386 & 2.345962 \\
\hline & 1.722697 & -1.015775 & 3.082990 \\
\hline & 2.199601 & -0.372273 & 4.216517 \\
\hline & 3.515182 & -0.567497 & 4.635824 \\
\hline & 4.344370 & -1.414090 & 3.910179 \\
\hline & 3.861634 & -2.059202 & 2.773210 \\
\hline & 6.645833 & -0.251910 & 1.453508 \\
\hline & -2.198382 & 0.946591 & 3.035439 \\
\hline & 7.227191 & -4.858603 & -0.305490 \\
\hline & -1.587508 & -2.533806 & 6.177971 \\
\hline & 6.557198 & 4.749833 & 0.351109 \\
\hline & -3.711062 & -2.956454 & 1.173915 \\
\hline & -5.506831 & -4.248123 & 0.102818 \\
\hline & 4.319576 & 4.229811 & 1.150725 \\
\hline & -2.052303 & -0.402652 & 5.072674 \\
\hline & 5.339621 & -4.557699 & -1.805919 \\
\hline & 8.851447 & 1.782058 & -1.745763 \\
\hline & 3.399319 & -3.181776 & -2.563796 \\
\hline & -2.822163 & -5.471412 & -3.024928 \\
\hline ] & -0.499166 & -4.720134 & -3.007230 \\
\hline & -0.575555 & -5.990892 & 3.832351 \\
\hline & 7.062738 & 0.143753 & -1.428831 \\
\hline & -0.767867 & -4.678675 & 1.770544 \\
\hline & 1.315192 & -3.387513 & -1.956685 \\
\hline 1 & 8.632999 & 4.093291 & -0.834602 \\
\hline & 8.412182 & -1.692069 & 2.347320 \\
\hline ] & -5.070923 & -5.528633 & -1.986806 \\
\hline & -0.982940 & -4.933225 & 6.049929 \\
\hline 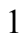 & 8.735738 & -4.001231 & 1.464378 \\
\hline & 2.413945 & 2.623516 & 1.294876 \\
\hline & -4.335463 & 0.234326 & 1.655146 \\
\hline 1 & -3.110501 & -0.573130 & -2.356675 \\
\hline & -7.230885 & -1.013630 & -1.241620 \\
\hline 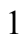 & -3.928832 & 2.405049 & -0.241715 \\
\hline 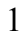 & -0.209244 & 1.969470 & 1.825398 \\
\hline 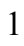 & -2.285784 & 5.724632 & 1.912345 \\
\hline 1 & 1.452600 & -3.476630 & 1.495336 \\
\hline & -1.606981 & 1.212000 & -2.340298 \\
\hline 1 & 2.908854 & -3.059326 & 0.604647 \\
\hline & 4.523066 & -2.713390 & 2.203297 \\
\hline 1 & 0.707721 & -0.831501 & 2.737787 \\
\hline 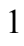 & 5.376823 & -1.570752 & 4.217538 \\
\hline 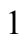 & 1.537185 & 0.287177 & 4.776035 \\
\hline 1 & 3.889957 & -0.058110 & 5.521144 \\
\hline 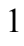 & 1.197699 & 2.643407 & -0.756834 \\
\hline 1 & -0.449778 & 3.255522 & -1.287979 \\
\hline & 2.970868 & 3.389618 & -1.968274 \\
\hline 1 & 5.279130 & 2.959097 & -2.733508 \\
\hline 1 & 5.722538 & 1.238089 & -4.466859 \\
\hline 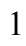 & 3.811890 & -0.005970 & -5.474611 \\
\hline & 1.509520 & 0.422882 & -4.701984 \\
\hline
\end{tabular}




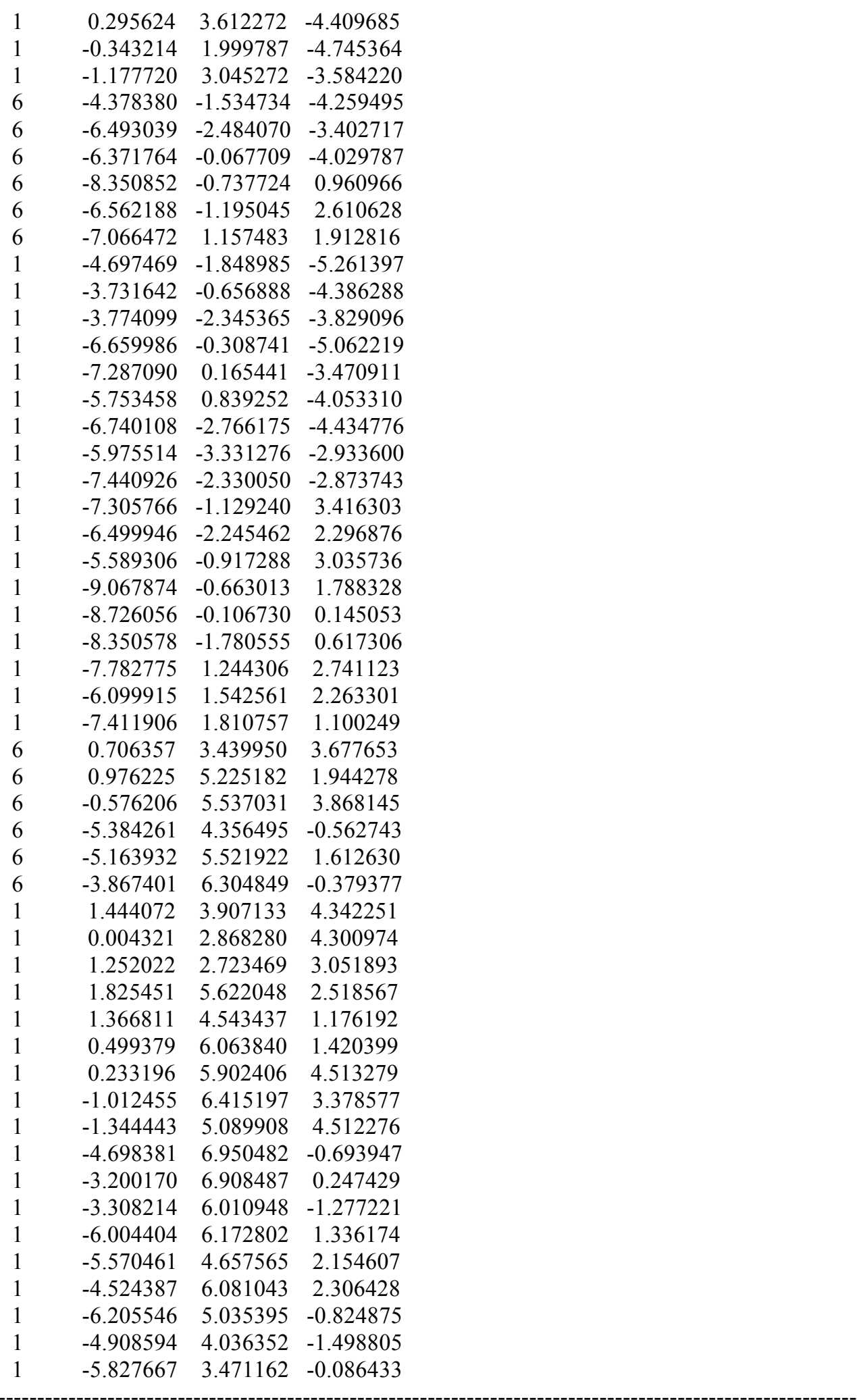

$3_{\text {IIC-re- }} \psi_{-1 \text { a2a3s4a }}$

Number of imaginary frequencies : 0

The smallest frequencies are : $11.5537 \quad 12.7202 \quad 15.8420 \mathrm{~cm}(-1)$ 
Electronic energy : $\quad H F=-4360.8929964$

Zero-point correction $=\quad 1.453770($ Hartree/Particle $)$

Thermal correction to Energy= $\quad 1.538803$

Thermal correction to Enthalpy= $\quad 1.539747$

Thermal correction to Gibbs Free Energy= $\quad 1.333224$

Sum of electronic and zero-point Energies $=\quad-4359.439226$

Sum of electronic and thermal Energies $=\quad-4359.354194$

Sum of electronic and thermal Enthalpies $=\quad-4359.353250$

Sum of electronic and thermal Free Energies $=\quad-4359.559773$

\begin{tabular}{|c|c|c|c|}
\hline & \multicolumn{3}{|c|}{ Cartesian Coordinates } \\
\hline 6 & 2.277381 & 1.324432 & -4.167986 \\
\hline 6 & 2.054901 & 2.169106 & -3.072779 \\
\hline 6 & 3.158680 & 2.814578 & -2.507085 \\
\hline 6 & 4.445562 & 2.590703 & -2.989776 \\
\hline 6 & 4.653749 & 1.718644 & -4.051863 \\
\hline 6 & 3.558997 & 1.093486 & -4.646989 \\
\hline 6 & 0.672210 & 2.443008 & -2.578767 \\
\hline 6 & -0.206771 & 3.093093 & -3.629357 \\
\hline 45 & -0.321361 & 0.681851 & -1.640568 \\
\hline 6 & -0.697028 & -0.472672 & -3.127947 \\
\hline 8 & -0.956007 & -0.964149 & -4.146108 \\
\hline 15 & -1.862160 & 0.359643 & 0.222575 \\
\hline 6 & -2.025181 & 2.011165 & 1.005919 \\
\hline 6 & -1.021899 & 2.467834 & 1.861759 \\
\hline 6 & -1.036366 & 3.772533 & 2.358574 \\
\hline 6 & -2.053170 & 4.624894 & 1.916658 \\
\hline 6 & -3.054968 & 4.209149 & 1.034735 \\
\hline 6 & -3.034286 & 2.883275 & 0.599143 \\
\hline 6 & 0.059911 & 4.282249 & 3.294101 \\
\hline 6 & -4.118154 & 5.201343 & 0.566529 \\
\hline 6 & -3.599605 & -0.028619 & -0.258366 \\
\hline 6 & -3.939810 & -0.186541 & -1.592117 \\
\hline 6 & -5.259584 & -0.456145 & -1.981107 \\
\hline 6 & -6.212877 & -0.600764 & -0.978504 \\
\hline 6 & -5.901090 & -0.465084 & 0.385343 \\
\hline 6 & -4.588374 & -0.163629 & 0.725602 \\
\hline 6 & -6.987475 & -0.679447 & 1.437810 \\
\hline 6 & -5.575270 & -0.587340 & -3.468754 \\
\hline 6 & -1.696721 & -0.797893 & 1.667037 \\
\hline 6 & -1.412421 & -2.137322 & 1.437833 \\
\hline 6 & -1.336139 & -3.053662 & 2.536837 \\
\hline 6 & -1.561251 & -2.583215 & 3.863237 \\
\hline 6 & -1.869365 & -1.218037 & 4.059131 \\
\hline 6 & -1.930661 & -0.355184 & 2.997678 \\
\hline 6 & -1.465149 & -3.483388 & 4.951277 \\
\hline 6 & -1.157256 & -4.803606 & 4.745646 \\
\hline 6 & -0.935967 & -5.277350 & 3.435250 \\
\hline 6 & -1.026628 & -4.428265 & 2.360259 \\
\hline 6 & -1.213074 & -2.697970 & 0.071270 \\
\hline 6 & 0.023871 & -2.648412 & -0.546998 \\
\hline 6 & 0.239648 & -3.305496 & -1.780331 \\
\hline 6 & -0.772192 & -3.986693 & -2.400797 \\
\hline 6 & -2.060810 & -4.037569 & -1.820641 \\
\hline 6 & -2.288693 & -3.387674 & -0.570926 \\
\hline
\end{tabular}




\begin{tabular}{|c|c|c|c|}
\hline 6 & -3.590255 & -3.450229 & -0.008154 \\
\hline 6 & -4.602254 & -4.123807 & -0.645348 \\
\hline & -4.366164 & -4.785396 & -1.870029 \\
\hline & -3.122559 & -4.736429 & -2.445633 \\
\hline & 1.115865 & -1.936354 & 0.054811 \\
\hline 15 & 1.516146 & -0.472654 & -0.707769 \\
\hline & 2.740684 & -0.745295 & -1.805971 \\
\hline 6 & 3.876656 & -1.441540 & -1.469700 \\
\hline & 4.809997 & -0.913045 & -0.596505 \\
\hline & 5.877660 & -1.770894 & -0.164985 \\
\hline & 6.029677 & -3.062406 & -0.752788 \\
\hline & 5.119706 & -3.482952 & -1.753524 \\
\hline & 4.053271 & -2.696899 & -2.091160 \\
\hline & 6.757885 & -1.415706 & 0.889362 \\
\hline & 7.739969 & -2.275468 & 1.316391 \\
\hline & 7.908574 & -3.536456 & 0.707099 \\
\hline & 7.066237 & -3.917655 & -0.305722 \\
\hline & 4.680692 & 0.477834 & -0.103483 \\
\hline & 3.499318 & 0.925889 & 0.457848 \\
\hline 6 & 3.384324 & 2.199788 & 1.051997 \\
\hline 6 & 4.439383 & 3.067628 & 1.029392 \\
\hline 6 & 5.639117 & 2.713565 & 0.365431 \\
\hline 6 & 5.758286 & 1.419207 & -0.224554 \\
\hline 6 & 6.703551 & 3.639533 & 0.243629 \\
\hline 6 & 7.838493 & 3.323504 & -0.458993 \\
\hline 6 & 7.945015 & 2.060427 & -1.077748 \\
\hline 6 & 6.936841 & 1.135201 & -0.960977 \\
\hline 8 & 2.397726 & 0.121375 & 0.556334 \\
\hline 6 & 0.422045 & 2.699243 & -1.209602 \\
\hline 6 & 2.004572 & -2.761072 & 0.908633 \\
\hline 6 & 2.520918 & -2.119423 & 2.171613 \\
\hline 6 & 1.704810 & -1.335008 & 2.988831 \\
\hline 6 & 2.196296 & -0.789267 & 4.166441 \\
\hline 6 & 3.516346 & -1.020965 & 4.551675 \\
\hline 6 & 4.334580 & -1.804913 & 3.747226 \\
\hline 6 & 3.837389 & -2.350506 & 2.565274 \\
\hline 1 & 6.625765 & -0.459223 & 1.388273 \\
\hline 1 & -2.174573 & 0.688866 & 3.177634 \\
\hline 1 & 7.167998 & -4.894696 & -0.776437 \\
\hline 1 & -1.637023 & -3.100885 & 5.956456 \\
\hline 1 & 6.591457 & 4.617563 & 0.709986 \\
\hline 1 & -3.785780 & -2.941715 & 0.935101 \\
\hline 1 & -5.596391 & -4.149412 & -0.202642 \\
\hline 1 & 4.360427 & 4.048853 & 1.495845 \\
\hline 1 & -2.057121 & -0.860647 & 5.070986 \\
\hline 1 & 5.267248 & -4.452291 & -2.226757 \\
\hline 1 & 8.831136 & 1.818444 & -1.660377 \\
\hline 1 & 3.327956 & -3.004989 & -2.840989 \\
\hline 1 & -2.927908 & -5.231344 & -3.396212 \\
\hline 1 & -0.602614 & -4.488876 & -3.351977 \\
\hline 1 & -0.693823 & -6.325545 & 3.275401 \\
\hline 1 & 7.027339 & 0.175945 & -1.463917 \\
\hline 1 & -0.859849 & -4.811156 & 1.355451 \\
\hline 1 & 1.232371 & -3.250632 & -2.223906 \\
\hline 1 & 8.646502 & 4.045273 & -0.553092 \\
\hline 1 & 8.389413 & -1.982698 & 2.138518 \\
\hline
\end{tabular}




\begin{tabular}{|c|c|c|}
\hline-5.175274 & -5.324205 & -2.358515 \\
\hline-1.081992 & -5.487329 & 5.587967 \\
\hline 8.694378 & -4.205357 & 1.050111 \\
\hline 2.437713 & 2.453904 & 1.524793 \\
\hline-4.312598 & -0.036321 & 1.769606 \\
\hline-3.159682 & -0.094191 & -2.343514 \\
\hline-7.240983 & -0.828558 & -1.253839 \\
\hline-3.799028 & 2.520547 & -0.082989 \\
\hline-0.219414 & 1.783505 & 2.139719 \\
\hline-2.061747 & 5.656750 & 2.265413 \\
\hline 1.397966 & -3.634457 & 1.188396 \\
\hline-1.608210 & 1.459709 & -2.154269 \\
\hline 2.851667 & -3.154019 & 0.325610 \\
\hline 4.490844 & -2.953374 & 1.932983 \\
\hline 0.687169 & -1.120864 & 2.670945 \\
\hline 5.370130 & -1.988556 & 4.028099 \\
\hline 1.542875 & -0.175682 & 4.785772 \\
\hline 3.903509 & -0.587944 & 5.471643 \\
\hline 1.242701 & 2.668220 & -0.490927 \\
\hline-0.401069 & 3.359333 & -0.928919 \\
\hline 3.007213 & 3.502753 & -1.675855 \\
\hline 5.289477 & 3.103132 & -2.530303 \\
\hline 5.661921 & 1.534751 & -4.418833 \\
\hline 3.707198 & 0.414618 & -5.484935 \\
\hline 1.430061 & 0.811308 & -4.622162 \\
\hline 0.290217 & 3.993832 & -4.021816 \\
\hline-0.397969 & 2.433624 & -4.485278 \\
\hline-1.176976 & 3.388226 & -3.212135 \\
\hline-7.067011 & -0.777452 & -3.730147 \\
\hline-5.118058 & 0.678824 & -4.204054 \\
\hline-4.828688 & -1.799753 & -4.033920 \\
\hline-6.468203 & -0.458718 & 2.857278 \\
\hline-8.147449 & 0.291908 & 1.197358 \\
\hline-7.498918 & -2.121425 & 1.342649 \\
\hline-7.245967 & -0.849022 & -4.810369 \\
\hline-7.449071 & -1.700304 & -3.274598 \\
\hline-7.660731 & 0.065218 & -3.352043 \\
\hline-5.034232 & -1.908358 & -5.107728 \\
\hline-3.742074 & -1.708775 & -3.909519 \\
\hline-5.143106 & -2.721758 & -3.527764 \\
\hline-5.361938 & 0.604294 & -5.272288 \\
\hline-5.617704 & 1.571001 & -3.803945 \\
\hline-4.034311 & 0.837623 & -4.126931 \\
\hline-8.929411 & 0.146820 & 1.954822 \\
\hline-7.806439 & 1.333390 & 1.259268 \\
\hline-8.611442 & 0.150401 & 0.214031 \\
\hline-7.277528 & -0.634924 & 3.577049 \\
\hline-5.649073 & -1.147179 & 3.108105 \\
\hline-6.110099 & 0.568082 & 3.007912 \\
\hline-8.297441 & -2.294167 & 2.076945 \\
\hline-7.903903 & -2.352387 & 0.349604 \\
\hline-6.689856 & -2.834830 & 1.552827 \\
\hline 0.777942 & 3.133726 & 4.005991 \\
\hline 1.076003 & 5.073441 & 2.460763 \\
\hline-0.519211 & 5.197956 & 4.376600 \\
\hline-5.138216 & 4.557406 & -0.370624 \\
\hline
\end{tabular}




$\begin{array}{lrrr}6 & -4.872057 & 5.763667 & 1.776207 \\ 6 & -3.434013 & 6.345836 & -0.189212 \\ 1 & 1.497853 & 3.537777 & 4.728845 \\ 1 & 0.069799 & 2.497459 & 4.555426 \\ 1 & 1.342890 & 2.488897 & 3.321841 \\ 1 & 1.917192 & 5.405506 & 3.085704 \\ 1 & 1.476699 & 4.465908 & 1.637211 \\ 1 & 0.615121 & 5.963418 & 2.013098 \\ 1 & 0.278237 & 5.517476 & 5.060019 \\ 1 & -0.970425 & 6.107771 & 3.964912 \\ 1 & -1.283882 & 4.679603 & 4.969517 \\ 1 & -4.180603 & 7.063966 & -0.554522 \\ 1 & -2.727596 & 6.894769 & 0.445102 \\ 1 & -2.877704 & 5.963817 & -1.055793 \\ 1 & -5.637280 & 6.480078 & 1.448374 \\ 1 & -5.375811 & 4.962408 & 2.332405 \\ 1 & -4.207049 & 6.287408 & 2.473293 \\ 1 & -5.886123 & 5.303388 & -0.667821 \\ 1 & -4.670947 & 4.177188 & -1.288569 \\ 1 & -5.672547 & 3.726460 & 0.109047\end{array}$

$3_{\text {IIC-re- }} \Psi-1$ a2a3s4s

Number of imaginary frequencies : 0

The smallest frequencies are : $13.7323 \quad 17.1238 \quad 22.5290 \mathrm{~cm}(-1)$

Electronic energy : $\quad H F=-4360.893585$

Zero-point correction $=\quad 1.454802$ (Hartree/Particle)

Thermal correction to Energy $=\quad 1.539169$

Thermal correction to Enthalpy= $\quad 1.540113$

Thermal correction to Gibbs Free Energy $=\quad 1.337818$

Sum of electronic and zero-point Energies $=\quad-4359.438783$

Sum of electronic and thermal Energies = $\quad-4359.354416$

Sum of electronic and thermal Enthalpies= $\quad-4359.353472$

Sum of electronic and thermal Free Energies $=\quad-4359.555767$

Cartesian Coordinates

$\begin{array}{cccc}6 & 2.330092 & 1.045211 & -4.256601 \\ 6 & 2.075668 & 1.967759 & -3.233346 \\ 6 & 3.160370 & 2.668987 & -2.697928 \\ 6 & 4.459116 & 2.426039 & -3.137636 \\ 6 & 4.698917 & 1.478413 & -4.125872 \\ 6 & 3.623587 & 0.795270 & -4.691984 \\ 6 & 0.679740 & 2.257507 & -2.787558 \\ 6 & -0.189627 & 2.801247 & -3.904189 \\ 45 & -0.299166 & 0.565564 & -1.720623 \\ 6 & -0.648797 & -0.709325 & -3.114789 \\ 8 & -0.900307 & -1.278809 & -4.093325 \\ 15 & -1.853570 & 0.339901 & 0.140686 \\ 6 & -2.086662 & 2.027951 & 0.823955 \\ 6 & -1.074212 & 2.582681 & 1.606809 \\ 6 & -1.167616 & 3.889926 & 2.089063 \\ 6 & -2.280516 & 4.645073 & 1.706739 \\ 6 & -3.293977 & 4.132545 & 0.890363 \\ 6 & -3.185214 & 2.807955 & 0.464909 \\ 6 & -0.054614 & 4.491106 & 2.947824\end{array}$




\begin{tabular}{|c|c|c|c|}
\hline 6 & -4.493366 & 5.004145 & 0.520925 \\
\hline & -3.573778 & -0.124616 & -0.332801 \\
\hline & -3.899850 & -0.392231 & -1.656335 \\
\hline & -5.212684 & -0.688748 & -2.035635 \\
\hline & -6.187252 & -0.739241 & -1.037037 \\
\hline & -5.890745 & -0.490273 & 0.309185 \\
\hline & -4.574000 & -0.176957 & 0.639322 \\
\hline & -6.940864 & -0.525401 & 1.418672 \\
\hline & -5.513552 & -0.938768 & -3.511898 \\
\hline & -1.680516 & -0.704117 & 1.669067 \\
\hline & -1.371849 & -2.051310 & 1.541239 \\
\hline & -1.290924 & -2.883758 & 2.704509 \\
\hline & -1.532697 & -2.320147 & 3.991083 \\
\hline & -1.859153 & -0.948430 & 4.084214 \\
\hline & -1.926690 & -0.167008 & 2.961894 \\
\hline & -1.433492 & -3.136284 & 5.143041 \\
\hline & -1.107431 & -4.463957 & 5.037434 \\
\hline & -0.869415 & -5.029789 & 3.767085 \\
\hline & -0.961608 & -4.263275 & 2.631870 \\
\hline & -1.147018 & -2.701775 & 0.219621 \\
\hline & 0.095206 & -2.667973 & -0.388936 \\
\hline & 0.339476 & -3.408574 & -1.568271 \\
\hline & -0.650751 & -4.154280 & -2.148367 \\
\hline & -1.946267 & -4.186707 & -1.582399 \\
\hline & -2.201799 & -3.453823 & -0.385228 \\
\hline 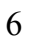 & -3.510438 & -3.493628 & 0.162366 \\
\hline & -4.504700 & -4.222954 & -0.440135 \\
\hline & -4.240875 & -4.968212 & -1.610112 \\
\hline & -2.989052 & -4.944727 & -2.169308 \\
\hline 7 & 1.165902 & -1.894889 & 0.175207 \\
\hline & 1.551114 & -0.478705 & -0.679727 \\
\hline & 2.794827 & -0.803414 & -1.740031 \\
\hline 5 & 3.933221 & -1.462945 & -1.344229 \\
\hline 6 & 4.849812 & -0.866908 & -0.497254 \\
\hline 6 & 5.922995 & -1.681717 & -0.000285 \\
\hline 6 & 6.093388 & -3.008406 & -0.498904 \\
\hline 6 & 5.198879 & -3.504914 & -1.478619 \\
\hline 6 & 4.129400 & -2.754101 & -1.879732 \\
\hline 6 & 6.791873 & -1.247060 & 1.033824 \\
\hline 6 & 7.778360 & -2.066196 & 1.525506 \\
\hline 6 & 7.963134 & -3.364245 & 1.005453 \\
\hline 6 & 7.133403 & -3.821349 & 0.014050 \\
\hline 6 & 4.696190 & 0.552328 & -0.100681 \\
\hline 6 & 3.500789 & 1.023226 & 0.410982 \\
\hline 6 & 3.360709 & 2.334232 & 0.911043 \\
\hline 6 & 4.402940 & 3.214366 & 0.838443 \\
\hline 6 & 5.616738 & 2.830930 & 0.218107 \\
\hline 6 & 5.762550 & 1.498916 & -0.274432 \\
\hline 6 & 6.669286 & 3.761917 & 0.043560 \\
\hline 6 & 7.818078 & 3.414002 & -0.620365 \\
\hline 6 & 7.950565 & 2.111866 & -1.146027 \\
\hline 6 & 6.954444 & 1.181859 & -0.975327 \\
\hline 8 & 2.405717 & 0.215286 & 0.550931 \\
\hline 6 & 0.402005 & 2.623565 & -1.448352 \\
\hline 6 & 2.055564 & -2.644488 & 1.094812 \\
\hline 6 & 2.551609 & -1.910438 & 2.315012 \\
\hline
\end{tabular}




\begin{tabular}{|c|c|c|c|}
\hline 6 & 1.721107 & -1.077396 & 3.067384 \\
\hline 6 & 2.195120 & -0.450573 & 4.211346 \\
\hline & 3.511573 & -0.647522 & 4.627177 \\
\hline & 4.344128 & -1.478429 & 3.887306 \\
\hline & 3.864515 & -2.105487 & 2.738939 \\
\hline & 6.647602 & -0.259718 & 1.464622 \\
\hline & -2.183184 & 0.884488 & 3.063607 \\
\hline & 7.247836 & -4.826980 & -0.388605 \\
\hline & -1.618496 & -2.683168 & 6.115978 \\
\hline & 6.537015 & 4.768935 & 0.437334 \\
\hline & -3.724986 & -2.920510 & 1.063375 \\
\hline & -5.506587 & -4.225020 & -0.014822 \\
\hline & 4.302422 & 4.225499 & 1.230954 \\
\hline & -2.055747 & -0.519760 & 5.066284 \\
\hline & 5.361058 & -4.501939 & -1.884659 \\
\hline & 8.847148 & 1.842700 & -1.700052 \\
\hline & 3.416119 & -3.118010 & -2.615978 \\
\hline & -2.774013 & -5.503667 & -3.079124 \\
\hline & -0.458929 & -4.720868 & -3.058234 \\
\hline & -0.612850 & -6.083650 & 3.686653 \\
\hline & 7.065492 & 0.191742 & -1.409312 \\
\hline & -0.781075 & -4.716619 & 1.659223 \\
\hline ] & 1.336589 & -3.366020 & -2.003146 \\
\hline & 8.616865 & 4.139419 & -0.755836 \\
\hline 1 & 8.418984 & -1.711532 & 2.330026 \\
\hline & -5.035639 & -5.551069 & -2.070870 \\
\hline 1 & -1.030705 & -5.082792 & 5.928393 \\
\hline & 8.751798 & -4.000761 & 1.399731 \\
\hline & 2.405952 & 2.607220 & 1.355328 \\
\hline 1 & -4.319087 & 0.042645 & 1.675802 \\
\hline & -3.111512 & -0.361057 & -2.404198 \\
\hline ] & -7.210946 & -0.975126 & -1.313284 \\
\hline & -3.959269 & 2.371011 & -0.160012 \\
\hline & -0.205001 & 1.969118 & 1.848743 \\
\hline & -2.364944 & 5.672610 & 2.056864 \\
\hline & 1.456529 & -3.503600 & 1.430174 \\
\hline ] & -1.596758 & 1.275558 & -2.302424 \\
\hline & 2.913513 & -3.067363 & 0.549544 \\
\hline 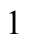 & 4.528980 & -2.746174 & 2.157365 \\
\hline & 0.705855 & -0.891830 & 2.724383 \\
\hline & 5.377149 & -1.636213 & 4.192181 \\
\hline 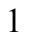 & 1.530336 & 0.197951 & 4.780864 \\
\hline & 3.884610 & -0.151475 & 5.520772 \\
\hline ] & 1.212643 & 2.669390 & -0.718968 \\
\hline & -0.436207 & 3.290186 & -1.234951 \\
\hline 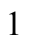 & 2.983920 & 3.419173 & -1.927549 \\
\hline & 5.287456 & 2.984789 & -2.704660 \\
\hline t & 5.716343 & 1.280781 & -4.458587 \\
\hline 1 & 3.796492 & 0.056566 & -5.472641 \\
\hline 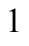 & 1.497993 & 0.486949 & -4.685006 \\
\hline 1 & 0.298026 & 3.676870 & -4.360103 \\
\hline ] & -0.350174 & 2.070090 & -4.706405 \\
\hline 1 & -1.173522 & 3.109089 & -3.532080 \\
\hline 6 & -7.003477 & -1.141188 & -3.775705 \\
\hline 6 & -5.042740 & 0.263494 & -4.340251 \\
\hline 6 & -4.769225 & -2.196607 & -3.971266 \\
\hline
\end{tabular}




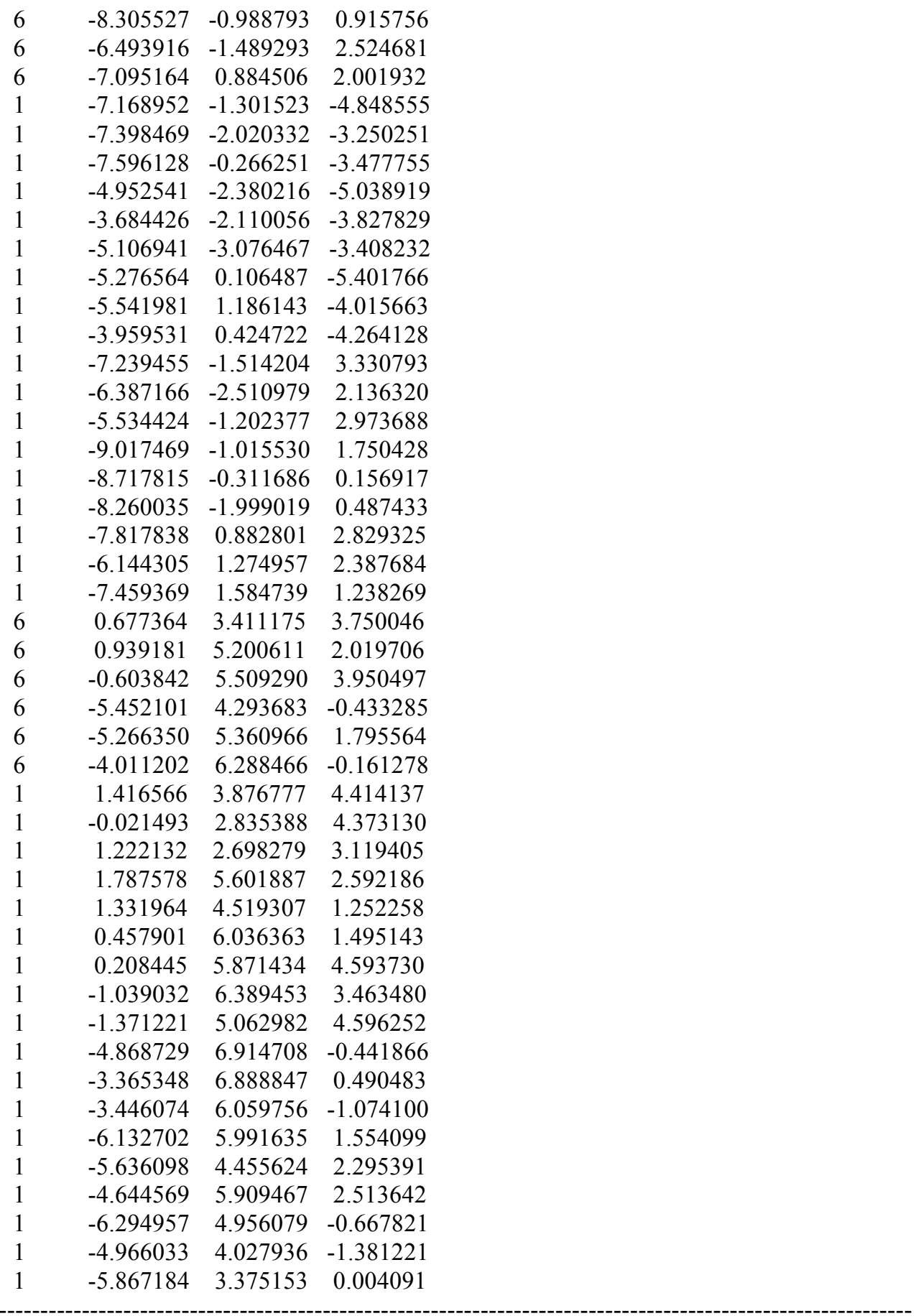

$3_{\text {IIC-re- }} \psi_{\text {-1a2s3ata }}$

Number of imaginary frequencies : 0

The smallest frequencies are : $12.1421 \quad 15.8806 \quad 22.4932 \mathrm{~cm}(-1)$

Electronic energy : $\quad H F=-4360.8940715$

Zero-point correction $=\quad 1.453733$ (Hartree/Particle)

Thermal correction to Energy $=\quad 1.538602$

Thermal correction to Enthalpy $=\quad 1.539546$

Thermal correction to Gibbs Free Energy= $\quad 1.335019$

Sum of electronic and zero-point Energies $=\quad-4359.440339$ 
Sum of electronic and thermal Energies= Sum of electronic and thermal Enthalpies= Sum of electronic and thermal Free Energies=
$-4359.355469$

$-4359.354525$

$-4359.559052$

Cartesian Coordinates

\begin{tabular}{|c|c|c|c|}
\hline 6 & 2.253305 & 1.325259 & -4.182587 \\
\hline 6 & 2.015277 & 2.162438 & -3.084840 \\
\hline 6 & 3.107393 & 2.825568 & -2.516890 \\
\hline 6 & 4.397973 & 2.626801 & -3.000633 \\
\hline 6 & 4.622063 & 1.762249 & -4.065603 \\
\hline 6 & 3.538897 & 1.118813 & -4.662239 \\
\hline 6 & 0.627212 & 2.412894 & -2.593211 \\
\hline 6 & -0.261438 & 3.042956 & -3.647586 \\
\hline 45 & -0.338104 & 0.636828 & -1.647799 \\
\hline 6 & -0.662377 & -0.520800 & -3.146023 \\
\hline 8 & -0.859121 & -1.013984 & -4.176808 \\
\hline 15 & -1.860749 & 0.314389 & 0.234432 \\
\hline 6 & -2.004810 & 1.972441 & 1.006088 \\
\hline 6 & -1.013488 & 2.428387 & 1.883402 \\
\hline 6 & -1.023923 & 3.735998 & 2.361714 \\
\hline 6 & -2.019061 & 4.604289 & 1.884572 \\
\hline 6 & -2.996610 & 4.190568 & 0.983179 \\
\hline 6 & -2.981905 & 2.854671 & 0.565759 \\
\hline 6 & 0.046110 & 4.246929 & 3.326361 \\
\hline 6 & 0.784720 & 3.100731 & 4.019333 \\
\hline 6 & -4.064171 & 5.120274 & 0.412955 \\
\hline 6 & -3.975526 & 6.532353 & 0.984948 \\
\hline 6 & -3.607918 & -0.058980 & -0.228599 \\
\hline 6 & -3.938669 & -0.331507 & -1.551593 \\
\hline 6 & -5.260129 & -0.594923 & -1.925778 \\
\hline 6 & -6.230810 & -0.586062 & -0.923561 \\
\hline 6 & -5.934380 & -0.321944 & 0.419672 \\
\hline 6 & -4.606604 & -0.059057 & 0.749652 \\
\hline 6 & -7.043326 & -0.365653 & 1.469963 \\
\hline 6 & -5.655985 & -0.827790 & -3.382847 \\
\hline 6 & -1.682254 & -0.828493 & 1.689806 \\
\hline 6 & -1.372007 & -2.164303 & 1.474050 \\
\hline 6 & -1.269756 & -3.067244 & 2.581159 \\
\hline 6 & -1.489428 & -2.586024 & 3.904561 \\
\hline 6 & -1.827442 & -1.226150 & 4.087742 \\
\hline 6 & -1.919802 & -0.377453 & 3.017192 \\
\hline 6 & -1.359832 & -3.471116 & 5.001361 \\
\hline 6 & -1.027110 & -4.786995 & 4.806441 \\
\hline 6 & -0.815585 & -5.272222 & 3.498605 \\
\hline 6 & -0.937908 & -4.437741 & 2.415311 \\
\hline 6 & -1.181639 & -2.730830 & 0.109286 \\
\hline 6 & 0.045673 & -2.674631 & -0.527214 \\
\hline 6 & 0.241745 & -3.320919 & -1.769424 \\
\hline 6 & -0.784992 & -3.981057 & -2.388765 \\
\hline 6 & -2.067319 & -4.026007 & -1.794855 \\
\hline 6 & -2.268656 & -3.408984 & -0.524364 \\
\hline 6 & -3.560541 & -3.474309 & 0.059953 \\
\hline 6 & -4.594068 & -4.107204 & -0.583372 \\
\hline 6 & -4.389013 & -4.723392 & -1.837589 \\
\hline 6 & -3.152454 & -4.679774 & -2.429443 \\
\hline
\end{tabular}




\begin{tabular}{|c|c|c|c|}
\hline 7 & 1.140766 & -1.953242 & 0.056716 \\
\hline 15 & 1.516395 & -0.485799 & -0.712564 \\
\hline & 2.739902 & -0.742168 & -1.815220 \\
\hline & 3.887269 & -1.421262 & -1.482245 \\
\hline & 4.817598 & -0.877734 & -0.614909 \\
\hline & 5.900651 & -1.719524 & -0.189498 \\
\hline & 6.067798 & -3.009402 & -0.776960 \\
\hline & 5.156950 & -3.445417 & -1.770254 \\
\hline & 4.077181 & -2.675414 & -2.101953 \\
\hline 6 & 6.783053 & -1.350362 & 0.858202 \\
\hline & 7.781264 & -2.194711 & 1.278655 \\
\hline & 7.964509 & -3.453555 & 0.669231 \\
\hline 0 & 7.120415 & -3.848411 & -0.336869 \\
\hline 6 & 4.670017 & 0.511489 & -0.121842 \\
\hline 6 & 3.483828 & 0.942565 & 0.442905 \\
\hline 6 & 3.350698 & 2.215198 & 1.036061 \\
\hline 6 & 4.393399 & 3.097912 & 1.011388 \\
\hline 6 & 5.596433 & 2.761135 & 0.344467 \\
\hline 6 & 5.732980 & 1.468990 & -0.246588 \\
\hline 6 & 6.646670 & 3.702928 & 0.219982 \\
\hline 6 & 7.783802 & 3.404436 & -0.486645 \\
\hline 6 & 7.906805 & 2.143755 & -1.107164 \\
\hline 6 & 6.912994 & 1.203487 & -0.987719 \\
\hline 8 & 2.395775 & 0.120870 & 0.546687 \\
\hline 6 & 0.369422 & 2.670764 & -1.226383 \\
\hline 6 & 2.045477 & -2.763827 & 0.906826 \\
\hline 6 & 2.565284 & -2.114487 & 2.164678 \\
\hline 6 & 1.750011 & -1.335173 & 2.987826 \\
\hline 6 & 2.246278 & -0.787714 & 4.162707 \\
\hline 6 & 3.570296 & -1.011276 & 4.538846 \\
\hline 6 & 4.388164 & -1.788657 & 3.727802 \\
\hline 6 & 3.886105 & -2.336626 & 2.549042 \\
\hline 6 & 1.053913 & 5.086835 & 2.532157 \\
\hline 6 & -0.577041 & 5.115696 & 4.423765 \\
\hline 6 & -3.879780 & 5.204997 & -1.107730 \\
\hline 6 & -5.455821 & 4.560415 & 0.730567 \\
\hline 1 & 6.640607 & -0.395302 & 1.356965 \\
\hline 1 & -2.185563 & 0.662603 & 3.188918 \\
\hline 1 & 7.233337 & -4.824281 & -0.807480 \\
\hline 1 & -1.526561 & -3.080797 & 6.004405 \\
\hline 1 & 6.521577 & 4.678859 & 0.687422 \\
\hline 1 & -3.731665 & -2.995968 & 1.022766 \\
\hline 1 & -5.582527 & -4.128308 & -0.128233 \\
\hline 1 & 4.302454 & 4.077826 & 1.478444 \\
\hline 1 & -2.014083 & -0.861509 & 5.097241 \\
\hline 1 & 5.315192 & -4.413487 & -2.242613 \\
\hline 1 & 8.794202 & 1.915604 & -1.693423 \\
\hline 1 & 3.350964 & -2.995027 & -2.845942 \\
\hline 1 & -2.980652 & -5.148552 & -3.397490 \\
\hline 1 & -0.633368 & -4.464682 & -3.352533 \\
\hline 1 & -0.556040 & -6.317555 & 3.347630 \\
\hline 1 & 7.015777 & 0.246575 & -1.492720 \\
\hline 1 & -0.778975 & -4.828427 & 1.412156 \\
\hline 1 & 1.228233 & -3.265824 & -2.227075 \\
\hline 1 & 8.580713 & 4.138176 & -0.582819 \\
\hline 1 & 8.432337 & -1.891193 & 2.095579 \\
\hline
\end{tabular}




\begin{tabular}{|c|c|c|}
\hline-5.214658 & -5.231562 & -2.331629 \\
\hline-0.925997 & -5.458992 & 5.655450 \\
\hline 8.763028 & -4.110068 & 1.006775 \\
\hline 2.400671 & 2.455707 & 1.509919 \\
\hline-4.335571 & 0.158053 & 1.780286 \\
\hline-3.143693 & -0.334598 & -2.291311 \\
\hline-7.266000 & -0.790105 & -1.197457 \\
\hline-3.734800 & 2.503203 & -0.138131 \\
\hline-0.228836 & 1.737021 & 2.192060 \\
\hline-2.018042 & 5.636364 & 2.226699 \\
\hline 1.451860 & -3.643662 & 1.193949 \\
\hline-1.641465 & 1.385874 & -2.166053 \\
\hline 2.892559 & -3.148254 & 0.318191 \\
\hline 4.539100 & -2.934957 & 1.911976 \\
\hline 0.728736 & -1.126691 & 2.677460 \\
\hline 5.426918 & -1.965535 & 4.001028 \\
\hline 1.593305 & -0.179783 & 4.787929 \\
\hline 3.960716 & -0.576816 & 5.456749 \\
\hline 1.487127 & 3.506057 & 4.758490 \\
\hline 0.086584 & 2.436178 & 4.547619 \\
\hline 1.372065 & 2.485340 & 3.326661 \\
\hline 0.199744 & 5.443181 & 5.127113 \\
\hline-1.053442 & 6.019931 & 4.028623 \\
\hline-1.332110 & 4.556861 & 4.991733 \\
\hline 1.876008 & 5.423127 & 3.179679 \\
\hline 1.483374 & 4.510522 & 1.700764 \\
\hline 0.579111 & 5.976777 & 2.099707 \\
\hline-4.766050 & 7.156984 & 0.550163 \\
\hline-4.109423 & 6.542744 & 2.074514 \\
\hline-3.015429 & 7.010077 & 0.751059 \\
\hline-4.624885 & 5.881562 & -1.547881 \\
\hline-2.881548 & 5.588460 & -1.359106 \\
\hline-3.990459 & 4.226411 & -1.592013 \\
\hline-6.233680 & 5.208409 & 0.304790 \\
\hline-5.600912 & 3.553196 & 0.319262 \\
\hline-5.618170 & 4.504810 & 1.815154 \\
\hline 1.187953 & 2.660068 & -0.504957 \\
\hline-0.468363 & 3.315749 & -0.952816 \\
\hline 2.943966 & 3.508790 & -1.683900 \\
\hline 5.232299 & 3.153915 & -2.540323 \\
\hline 5.633362 & 1.598480 & -4.433496 \\
\hline 3.699090 & 0.445568 & -5.502507 \\
\hline 1.415816 & 0.799463 & -4.640134 \\
\hline 0.220920 & 3.950474 & -4.042625 \\
\hline-0.439618 & 2.377278 & -4.501344 \\
\hline-1.236818 & 3.323243 & -3.232541 \\
\hline-4.436595 & -1.034312 & -4.279822 \\
\hline-6.548823 & -2.065189 & -3.508174 \\
\hline-6.422765 & 0.403241 & -3.880207 \\
\hline-6.550716 & 0.078622 & 2.845811 \\
\hline-8.197187 & 0.556059 & 1.062368 \\
\hline-7.557046 & -1.805326 & 1.585930 \\
\hline-4.764695 & -1.242221 & -5.306189 \\
\hline-3.792995 & -0.145697 & -4.318999 \\
\hline-3.825412 & -1.883207 & -3.943666 \\
\hline-6.713124 & 0.276092 & -4.932103 \\
\hline
\end{tabular}




$\begin{array}{lrrr}1 & -7.336665 & 0.575162 & -3.297444 \\ 1 & -5.803531 & 1.307077 & -3.806410 \\ 1 & -6.816852 & -2.231175 & -4.560018 \\ 1 & -6.026060 & -2.959780 & -3.145291 \\ 1 & -7.485455 & -1.967598 & -2.946201 \\ 1 & -8.990235 & 0.527803 & 1.821436 \\ 1 & -7.857501 & 1.595603 & 0.968660 \\ 1 & -8.649014 & 0.263924 & 0.107176 \\ 1 & -7.381583 & 0.049799 & 3.561963 \\ 1 & -5.760709 & -0.577457 & 3.234991 \\ 1 & -6.164416 & 1.106653 & 2.826344 \\ 1 & -8.361138 & -1.868471 & 2.331750 \\ 1 & -7.955122 & -2.176270 & 0.633038 \\ 1 & -6.751137 & -2.482121 & 1.901031\end{array}$

$3_{\text {IIC-re- }} \Psi_{-12 \text {-1233a4s }}$

Number of imaginary frequencies : 0

The smallest frequencies are : $14.6403 \quad 16.6083 \quad 22.1412 \mathrm{~cm}(-1)$

Electronic energy : $\quad \mathrm{HF}=-4360.894598$

Zero-point correction $=\quad 1.454017$ (Hartree/Particle)

Thermal correction to Energy= $\quad 1.538745$

Thermal correction to Enthalpy= $\quad 1.539689$

Thermal correction to Gibbs Free Energy= $\quad 1.336249$

Sum of electronic and zero-point Energies $=\quad-4359.440581$

Sum of electronic and thermal Energies $=\quad-4359.355853$

Sum of electronic and thermal Enthalpies $=\quad-4359.354909$

Sum of electronic and thermal Free Energies $=\quad-4359.558349$

\begin{tabular}{cccc}
\multicolumn{4}{c}{ Cartesian Coordinates } \\
\hline 6 & -3.940495 & -0.356736 & -1.570738 \\
6 & -3.605308 & -0.072903 & -0.247248 \\
6 & -4.600308 & -0.067583 & 0.726161 \\
6 & -5.933376 & -0.333201 & 0.403354 \\
6 & -6.236657 & -0.597166 & -0.933235 \\
6 & -5.260677 & -0.616814 & -1.937969 \\
15 & -1.861600 & 0.308962 & 0.219682 \\
45 & -0.337000 & 0.628589 & -1.658207 \\
6 & 0.627733 & 2.400853 & -2.611514 \\
6 & 0.363077 & 2.667380 & -1.247607 \\
6 & -6.979613 & -0.317796 & 1.516109 \\
6 & -6.582061 & -1.323510 & 2.603503 \\
6 & -5.661496 & -0.863917 & -3.391372 \\
6 & -6.545516 & -2.109252 & -3.501834 \\
6 & -1.688384 & -0.830011 & 1.679265 \\
6 & -1.377079 & -2.166271 & 1.468720 \\
6 & -1.281999 & -3.066767 & 2.578537 \\
6 & -1.513335 & -2.583298 & 3.899084 \\
6 & -1.852124 & -1.222939 & 4.076857 \\
6 & -1.934398 & -0.375976 & 3.004033 \\
6 & -0.946471 & -4.437056 & 2.418346 \\
6 & -0.833011 & -5.269359 & 3.504254 \\
6 & -1.057507 & -4.782182 & 4.809184 \\
6 & -1.393177 & -3.466291 & 4.998652 \\
6 & -1.179136 & -2.736483 & 0.106753
\end{tabular}




\begin{tabular}{|c|c|c|c|}
\hline 6 & 0.050716 & -2.678012 & -0.524687 \\
\hline 6 & 0.255244 & -3.329878 & -1.762561 \\
\hline 6 & -0.765376 & -3.999280 & -2.381930 \\
\hline 6 & -2.049622 & -4.048581 & -1.792500 \\
\hline 6 & -2.260254 & -3.424040 & -0.527100 \\
\hline 6 & -3.126681 & -4.716409 & -2.426022 \\
\hline 6 & -4.363756 & -4.769306 & -1.836264 \\
\hline 6 & -4.577843 & -4.146548 & -0.586871 \\
\hline 6 & -3.553714 & -3.495550 & 0.053624 \\
\hline 7 & 1.140826 & -1.951020 & 0.061654 \\
\hline 6 & 2.045302 & -2.756846 & 0.916699 \\
\hline 6 & 2.557731 & -2.102788 & 2.175097 \\
\hline 6 & 1.735651 & -1.325593 & 2.993393 \\
\hline 6 & 2.224809 & -0.773300 & 4.168987 \\
\hline 6 & 3.548417 & -0.989890 & 4.550643 \\
\hline 6 & 4.373027 & -1.765186 & 3.744410 \\
\hline 6 & 3.878074 & -2.318056 & 2.564946 \\
\hline 15 & 1.516957 & -0.485674 & -0.711476 \\
\hline 8 & 2.390950 & 0.126109 & 0.548945 \\
\hline 6 & 3.476271 & 0.951542 & 0.445153 \\
\hline 6 & 4.666085 & 0.521559 & -0.112881 \\
\hline 6 & 5.726671 & 1.481740 & -0.237401 \\
\hline 6 & 5.583098 & 2.776474 & 0.346354 \\
\hline 6 & 4.375910 & 3.112925 & 1.005830 \\
\hline 6 & 3.336118 & 2.226770 & 1.030884 \\
\hline 6 & 6.911274 & 1.216278 & -0.971261 \\
\hline 6 & 7.902647 & 2.159122 & -1.090565 \\
\hline 6 & 7.772484 & 3.422601 & -0.477252 \\
\hline 6 & 6.630892 & 3.721039 & 0.222133 \\
\hline 6 & 4.819232 & -0.869030 & -0.600473 \\
\hline 6 & 3.893418 & -1.417916 & -1.469333 \\
\hline 6 & 4.089166 & -2.673148 & -2.084983 \\
\hline 6 & 5.169722 & -3.439272 & -1.747178 \\
\hline 6 & 6.075566 & -2.997966 & -0.751642 \\
\hline 6 & 5.902954 & -1.706768 & -0.168619 \\
\hline 6 & 6.780584 & -1.332357 & 0.881254 \\
\hline 6 & 7.779275 & -2.173037 & 1.307936 \\
\hline 6 & 7.967873 & -3.433225 & 0.702948 \\
\hline 6 & 7.128542 & -3.833075 & -0.305151 \\
\hline 8 & 2.745060 & -0.743773 & -1.808527 \\
\hline 6 & -2.018490 & 1.968071 & 0.987657 \\
\hline 6 & -1.029117 & 2.429485 & 1.864311 \\
\hline 6 & -1.046127 & 3.737119 & 2.342278 \\
\hline 6 & -2.043748 & 4.600842 & 1.862301 \\
\hline 6 & -3.019067 & 4.182392 & 0.960545 \\
\hline 6 & -3.000938 & 2.844970 & 0.547384 \\
\hline 6 & 0.019712 & 4.253097 & 3.308890 \\
\hline 6 & 1.026415 & 5.095892 & 2.516424 \\
\hline 6 & -4.080118 & 5.112471 & 0.378287 \\
\hline 6 & -3.861043 & 5.212891 & -1.136854 \\
\hline 6 & -0.609466 & 5.120651 & 4.403814 \\
\hline 6 & 0.760690 & 3.110276 & 4.004860 \\
\hline 6 & -5.476823 & 4.545031 & 0.656822 \\
\hline 6 & -4.010491 & 6.519087 & 0.966347 \\
\hline 6 & -0.658019 & -0.538167 & -3.149993 \\
\hline 8 & -0.857050 & -1.037579 & -4.177291 \\
\hline
\end{tabular}




\begin{tabular}{|c|c|c|c|}
\hline 6 & -7.050099 & 1.088952 & 2.121606 \\
\hline 6 & -8.370940 & -0.692457 & 1.012923 \\
\hline 6 & -6.439807 & 0.356264 & -3.897260 \\
\hline 6 & -4.444955 & -1.070568 & -4.292108 \\
\hline 1 & 6.633892 & -0.376215 & 1.376729 \\
\hline 1 & -2.199013 & 0.665003 & 3.171889 \\
\hline 1 & 7.245524 & -4.810067 & -0.772425 \\
\hline 1 & -1.569359 & -3.074274 & 5.999410 \\
\hline 1 & 6.500409 & 4.698933 & 0.683954 \\
\hline 1 & -3.733678 & -3.008400 & 1.010969 \\
\hline 1 & -5.567097 & -4.178281 & -0.134530 \\
\hline 1 & 4.279673 & 4.095071 & 1.467092 \\
\hline 1 & -2.046229 & -0.856175 & 5.084178 \\
\hline 1 & 5.332380 & -4.408203 & -2.216254 \\
\hline 1 & 8.793737 & 1.930783 & -1.671118 \\
\hline 1 & 3.366608 & -2.996659 & -2.830826 \\
\hline 1 & -2.947692 & -5.189733 & -3.390533 \\
\hline 1 & -0.607161 & -4.488417 & -3.341842 \\
\hline 1 & -0.570457 & -6.314552 & 3.357568 \\
\hline 1 & 7.019762 & 0.257102 & -1.470733 \\
\hline 1 & -0.777531 & -4.829400 & 1.417487 \\
\hline 1 & 1.243355 & -3.272475 & -2.216336 \\
\hline 1 & 8.567509 & 4.158405 & -0.573259 \\
\hline 1 & 8.426531 & -1.865519 & 2.126398 \\
\hline 1 & -5.183093 & -5.289599 & -2.328142 \\
\hline 1 & -0.963677 & -5.452594 & 5.660280 \\
\hline 1 & 8.766673 & -4.086816 & 1.045467 \\
\hline 1 & 2.383209 & 2.466440 & 1.499360 \\
\hline 1 & -4.334312 & 0.157033 & 1.758812 \\
\hline 1 & -3.146462 & -0.368729 & -2.311426 \\
\hline 1 & -7.270303 & -0.793916 & -1.210595 \\
\hline 1 & -3.753438 & 2.489209 & -0.154953 \\
\hline 1 & -0.241238 & 1.741841 & 2.173298 \\
\hline 1 & -2.046274 & 5.633595 & 2.202312 \\
\hline 1 & 1.453791 & -3.638242 & 1.203326 \\
\hline 1 & -1.641709 & 1.370894 & -2.183623 \\
\hline 1 & 2.896055 & -3.139413 & 0.332117 \\
\hline 1 & 4.536240 & -2.914737 & 1.931673 \\
\hline 1 & 0.714620 & -1.122938 & 2.678470 \\
\hline 1 & 5.411517 & -1.936684 & 4.022038 \\
\hline 1 & 1.566600 & -0.167281 & 4.790544 \\
\hline 1 & 3.933284 & -0.551699 & 5.469115 \\
\hline 1 & 1.460287 & 3.518843 & 4.744893 \\
\hline 1 & 0.063523 & 2.444299 & 4.532616 \\
\hline 1 & 1.351420 & 2.495807 & 3.314258 \\
\hline 1 & 0.164323 & 5.451906 & 5.108699 \\
\hline 1 & -1.088412 & 6.022598 & 4.006523 \\
\hline 1 & -1.363758 & 4.559548 & 4.970575 \\
\hline 1 & 1.845211 & 5.436448 & 3.165881 \\
\hline 1 & 1.460651 & 4.519953 & 1.687290 \\
\hline 1 & 0.549324 & 5.983248 & 2.081188 \\
\hline 1 & -4.796134 & 7.143694 & 0.522789 \\
\hline 1 & -4.164939 & 6.517176 & 2.053270 \\
\hline 1 & -3.049243 & 7.005098 & 0.755648 \\
\hline 1 & -4.601321 & 5.887150 & -1.588637 \\
\hline 1 & -2.860433 & 5.606507 & -1.361546 \\
\hline
\end{tabular}




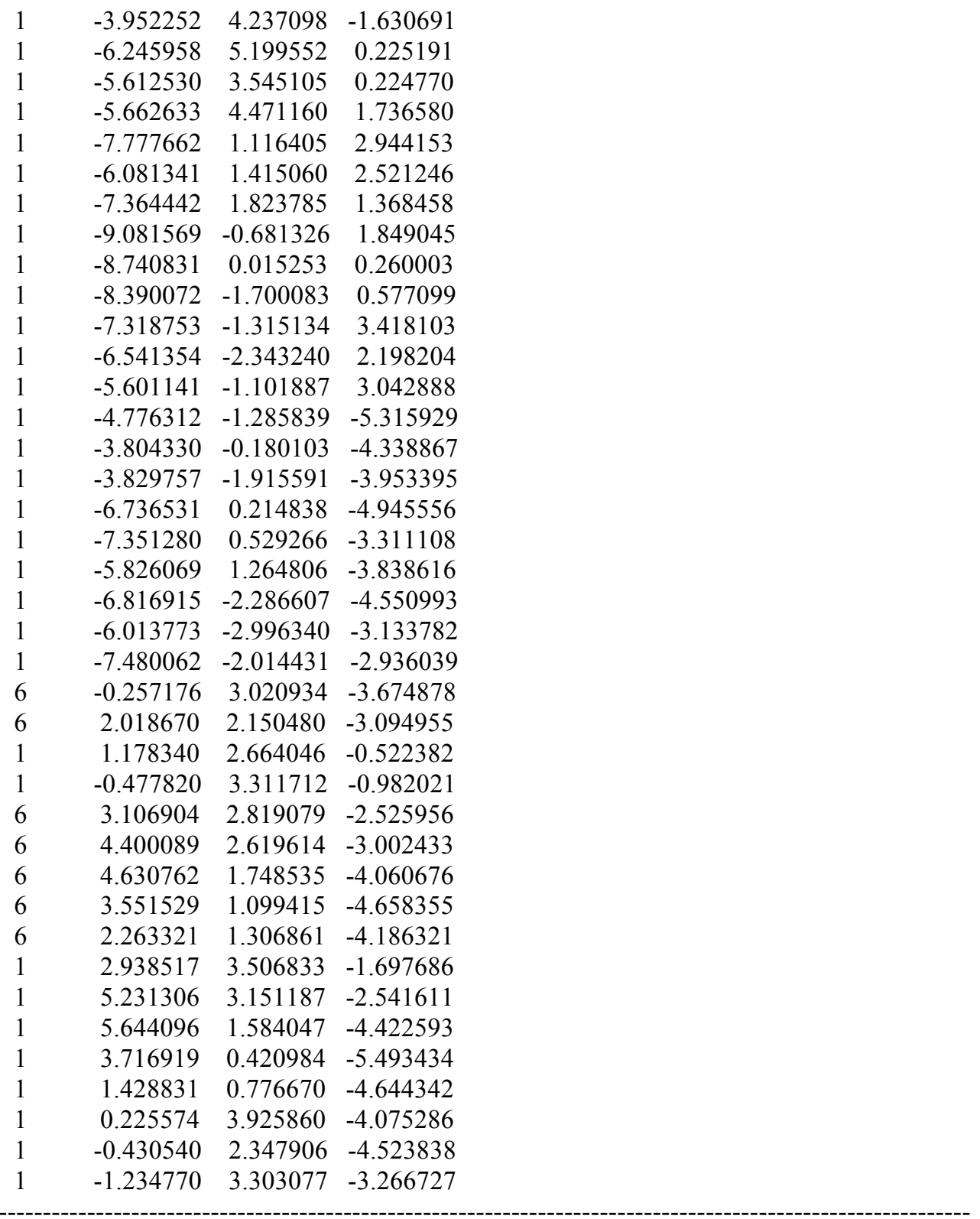

$3_{\text {IIC-re- }} \psi_{-12 \text { 2as3s4a }}$

Number of imaginary frequencies : 0

The smallest frequencies are : $14.3719 \quad 19.5122 \quad 22.7884 \mathrm{~cm}(-1)$

Electronic energy : $\quad H F=-4360.8940128$

Zero-point correction $=\quad 1.453798($ Hartree/Particle $)$

Thermal correction to Energy= $\quad 1.538697$

Thermal correction to Enthalpy= $\quad 1.539641$

Thermal correction to Gibbs Free Energy $=\quad 1.335325$

Sum of electronic and zero-point Energies $=\quad-4359.440215$

Sum of electronic and thermal Energies $=\quad-4359.355316$

Sum of electronic and thermal Enthalpies $=\quad-4359.354372$

Sum of electronic and thermal Free Energies $=\quad-4359.558688$

Cartesian Coordinates 


\begin{tabular}{|c|c|c|c|}
\hline 6 & 2.226028 & 1.264789 & -4.211260 \\
\hline 6 & 1.987444 & 2.118348 & -3.126235 \\
\hline 6 & 3.078511 & 2.793619 & -2.570693 \\
\hline 6 & 4.368683 & 2.591777 & -3.054112 \\
\hline & 4.593630 & 1.711356 & -4.105875 \\
\hline & 3.511393 & 1.054764 & -4.689918 \\
\hline & 0.599384 & 2.369421 & -2.635222 \\
\hline 6 & -0.297990 & 2.972039 & -3.698852 \\
\hline 4 & -0.350017 & 0.605931 & -1.653498 \\
\hline 6 & -0.692496 & -0.583909 & -3.121696 \\
\hline 8 & -0.921352 & -1.099138 & -4.135146 \\
\hline 15 & -1.865979 & 0.299161 & 0.231874 \\
\hline 6 & -1.988982 & 1.953937 & 1.014488 \\
\hline 6 & -1.004814 & 2.387401 & 1.911437 \\
\hline 6 & -1.008220 & 3.689472 & 2.405373 \\
\hline 6 & -1.982740 & 4.576347 & 1.919488 \\
\hline 6 & -2.950527 & 4.185642 & 0.997706 \\
\hline 6 & -2.947853 & 2.853416 & 0.568509 \\
\hline & 0.038067 & 4.174299 & 3.409161 \\
\hline 6 & 0.764219 & 3.010333 & 4.085501 \\
\hline & -3.999587 & 5.134340 & 0.424104 \\
\hline & -3.890898 & 6.543019 & 1.001044 \\
\hline & -3.618991 & -0.042447 & -0.231124 \\
\hline 6 & -3.959021 & -0.276376 & -1.553857 \\
\hline 6 & -5.291671 & -0.479690 & -1.939562 \\
\hline & -6.261709 & -0.473955 & -0.942781 \\
\hline 6 & -5.952473 & -0.257585 & 0.411398 \\
\hline & -4.622047 & -0.037087 & 0.747959 \\
\hline 6 & -7.060029 & -0.310345 & 1.462545 \\
\hline 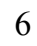 & -5.606384 & -0.680627 & -3.419738 \\
\hline 6 & -1.701407 & -0.857226 & 1.675428 \\
\hline 6 & -1.386356 & -2.189842 & 1.449950 \\
\hline 6 & -1.293320 & -3.102189 & 2.551567 \\
\hline 6 & -1.533459 & -2.633833 & 3.876049 \\
\hline 5 & -1.878966 & -1.276858 & 4.067420 \\
\hline 6 & -1.957763 & -0.418698 & 3.003760 \\
\hline 6 & -1.414713 & -3.527302 & 4.967177 \\
\hline 6 & -1.070103 & -4.839252 & 4.766493 \\
\hline 6 & -0.834509 & -5.311167 & 3.458102 \\
\hline 6 & -0.947287 & -4.468560 & 2.379967 \\
\hline 6 & -1.173498 & -2.754583 & 0.087098 \\
\hline 6 & 0.065928 & -2.703887 & -0.525477 \\
\hline 6 & 0.296379 & -3.386697 & -1.742124 \\
\hline 6 & -0.703419 & -4.095072 & -2.351933 \\
\hline 6 & -1.996366 & -4.142719 & -1.781086 \\
\hline 6 & -2.239463 & -3.463294 & -0.550217 \\
\hline 6 & -3.545374 & -3.518650 & 0.002581 \\
\hline 6 & -4.547083 & -4.217671 & -0.623195 \\
\hline 6 & -4.295480 & -4.910254 & -1.827617 \\
\hline 6 & -3.048008 & -4.866326 & -2.395240 \\
\hline 7 & 1.145746 & -1.965292 & 0.065624 \\
\hline 15 & 1.510468 & -0.496328 & -0.705035 \\
\hline 8 & 2.741295 & -0.741534 & -1.802338 \\
\hline 6 & 3.901073 & -1.396334 & -1.465322 \\
\hline 6 & 4.820108 & -0.832622 & -0.598495 \\
\hline 6 & 5.918566 & -1.652611 & -0.170129 \\
\hline
\end{tabular}




\begin{tabular}{|c|c|c|c|}
\hline ) & 6.110222 & -2.940885 & -0.753838 \\
\hline & 5.209647 & -3.396209 & -1.747871 \\
\hline & 4.116430 & -2.647265 & -2.083145 \\
\hline & 6.793580 & -1.263780 & 0.876678 \\
\hline & 7.806596 & -2.088568 & 1.300694 \\
\hline & 8.012981 & -3.345978 & 0.695736 \\
\hline & 7.177383 & -3.759171 & -0.310082 \\
\hline & 4.644673 & 0.556086 & -0.112970 \\
\hline & 3.447612 & 0.966112 & 0.444237 \\
\hline & 3.280340 & 2.243303 & 1.017530 \\
\hline & 4.302065 & 3.150010 & 0.985689 \\
\hline & 5.517049 & 2.831520 & 0.331458 \\
\hline & 5.686845 & 1.535596 & -0.243059 \\
\hline & 6.547206 & 3.794845 & 0.203573 \\
\hline & 7.696269 & 3.513298 & -0.490443 \\
\hline & 7.851389 & 2.249018 & -1.096164 \\
\hline & 6.877368 & 1.288647 & -0.974073 \\
\hline & 2.381171 & 0.116931 & 0.557047 \\
\hline & 0.345040 & 2.651119 & -1.272071 \\
\hline & 2.063645 & -2.766790 & 0.910509 \\
\hline & 2.566535 & -2.116767 & 2.174621 \\
\hline & 1.730250 & -1.366158 & 3.003235 \\
\hline & 2.209960 & -0.820972 & 4.186095 \\
\hline & 3.538189 & -1.015942 & 4.563230 \\
\hline & 4.377306 & -1.763147 & 3.745509 \\
\hline & 3.891561 & -2.310620 & 2.559776 \\
\hline & 1.060172 & 5.046069 & 2.670005 \\
\hline & -0.618877 & 5.004130 & 4.517462 \\
\hline & -3.808713 & 5.221667 & -1.095600 \\
\hline & -5.401146 & 4.595292 & 0.734125 \\
\hline & 6.633853 & -0.309082 & 1.371068 \\
\hline & -2.227461 & 0.619466 & 3.180966 \\
\hline & 7.308453 & -4.734278 & -0.777570 \\
\hline & -1.598827 & -3.146317 & 5.970772 \\
\hline 1 & 6.397222 & 4.773056 & 0.658736 \\
\hline & -3.750673 & -2.987893 & 0.930891 \\
\hline & -5.543969 & -4.238971 & -0.187701 \\
\hline & 4.184679 & 4.134138 & 1.437909 \\
\hline & -2.081045 & -0.922380 & 5.077557 \\
\hline & 5.387216 & -4.361870 & -2.218272 \\
\hline & 8.747854 & 2.034087 & -1.673552 \\
\hline & 3.397968 & -2.980943 & -2.828431 \\
\hline & -2.842228 & -5.384959 & -3.330654 \\
\hline & -0.521351 & -4.619547 & -3.288639 \\
\hline & -0.563655 & -6.352879 & 3.302089 \\
\hline & 7.004617 & 0.329542 & -1.469139 \\
\hline & -0.768468 & -4.850546 & 1.376844 \\
\hline & 1.291201 & -3.331097 & -2.181249 \\
\hline & 8.477839 & 4.263097 & -0.588599 \\
\hline & 8.451679 & -1.770480 & 2.116826 \\
\hline & -5.096192 & -5.468877 & -2.307624 \\
\hline & -0.977215 & -5.517760 & 5.611255 \\
\hline & 8.822841 & -3.986885 & 1.036253 \\
\hline & 2.320977 & 2.467632 & 1.480389 \\
\hline & -4.346867 & 0.152330 & 1.782786 \\
\hline & -3.168683 & -0.289490 & -2.300647 \\
\hline
\end{tabular}




\begin{tabular}{|c|c|c|c|}
\hline 1 & -7.301745 & -0.641864 & -1.214912 \\
\hline & -3.696362 & 2.518537 & -0.148232 \\
\hline & -0.235450 & 1.681899 & 2.226961 \\
\hline & -1.974570 & 5.604231 & 2.274122 \\
\hline & 1.485638 & -3.659652 & 1.189079 \\
\hline & -1.654992 & 1.344719 & -2.178917 \\
\hline & 2.919769 & -3.130563 & 0.321794 \\
\hline & 4.560087 & -2.886899 & 1.918513 \\
\hline & 0.704790 & -1.180252 & 2.692113 \\
\hline & 5.419399 & -1.917464 & 4.019650 \\
\hline & 1.540463 & -0.239289 & 4.818518 \\
\hline & 3.915086 & -0.583199 & 5.487564 \\
\hline & 1.448883 & 3.396276 & 4.851208 \\
\hline & 0.054802 & 2.331846 & 4.580047 \\
\hline & 1.368118 & 2.413527 & 3.390703 \\
\hline & 0.137253 & 5.314371 & 5.250580 \\
\hline & -1.092161 & 5.916798 & 4.138286 \\
\hline & -1.384018 & 4.422601 & 5.047874 \\
\hline & 1.862120 & 5.366114 & 3.350036 \\
\hline & 1.516224 & 4.501072 & 1.832162 \\
\hline & 0.590665 & 5.946795 & 2.254622 \\
\hline 1 & -4.666905 & 7.182618 & 0.561907 \\
\hline & -4.033554 & 6.552882 & 2.089499 \\
\hline 1 & -2.920591 & 7.004306 & 0.776280 \\
\hline & -4.542701 & 5.910654 & -1.535133 \\
\hline ] & -2.804617 & 5.591886 & -1.343129 \\
\hline 1 & -3.932954 & 4.246884 & -1.584408 \\
\hline & -6.167113 & 5.256943 & 0.307711 \\
\hline 1 & -5.560185 & 3.591910 & 0.318698 \\
\hline 1 & -5.568635 & 4.538273 & 1.817852 \\
\hline 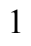 & 1.166790 & 2.661916 & -0.554553 \\
\hline 1 & -0.496648 & 3.294196 & -1.005886 \\
\hline & 2.915068 & 3.488499 & -1.747403 \\
\hline 1 & 5.201992 & 3.129482 & -2.604459 \\
\hline & 5.604650 & 1.545219 & -4.473470 \\
\hline 1 & 3.672324 & 0.368632 & -5.519581 \\
\hline t & 1.389301 & 0.728405 & -4.658035 \\
\hline ] & 0.177738 & 3.872541 & -4.117214 \\
\hline 1 & -0.478993 & 2.286491 & -4.536321 \\
\hline 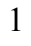 & -1.272405 & 3.257303 & -3.284775 \\
\hline 5 & -7.104759 & -0.804197 & -3.683351 \\
\hline 5 & -5.078336 & 0.518043 & -4.218585 \\
\hline 6 & -4.924378 & -1.960516 & -3.912147 \\
\hline 6 & -6.566721 & 0.125540 & 2.841003 \\
\hline 6 & -8.220424 & 0.607942 & 1.066415 \\
\hline 6 & -7.564954 & -1.754195 & 1.567206 \\
\hline 1 & -7.280225 & -0.933735 & -4.758745 \\
\hline 1 & -7.540922 & -1.673633 & -3.174401 \\
\hline 1 & -7.653254 & 0.092285 & -3.365641 \\
\hline 1 & -5.123474 & -2.112202 & -4.981836 \\
\hline 1 & -3.835853 & -1.925906 & -3.777038 \\
\hline 1 & -5.297741 & -2.836089 & -3.365535 \\
\hline 1 & -5.312721 & 0.395530 & -5.284528 \\
\hline 1 & -5.538937 & 1.454089 & -3.875885 \\
\hline 1 & -3.989455 & 0.629800 & -4.132995 \\
\hline 1 & -9.008548 & 0.570966 & 1.830134 \\
\hline
\end{tabular}


$3_{\text {IIC-re- }} \psi_{-1225354 s}$

Number of imaginary frequencies : 0

The smallest frequencies are : $11.4727 \quad 17.7491 \quad 21.1621 \mathrm{~cm}(-1)$

Electronic energy :

$\mathrm{HF}=-4360.8943601$

Zero-point correction $=$

1.454204 (Hartree/Particle)

Thermal correction to Energy= $\quad 1.538893$

Thermal correction to Enthalpy $=\quad 1.539837$

Thermal correction to Gibbs Free Energy= $\quad 1.336299$

Sum of electronic and zero-point Energies $=\quad-4359.440156$

Sum of electronic and thermal Energies $=\quad-4359.355467$

Sum of electronic and thermal Enthalpies $=\quad-4359.354523$

Sum of electronic and thermal Free Energies $=\quad-4359.558061$

Cartesian Coordinates

$\begin{array}{cccc}6 & 2.240826 & 1.307570 & -4.189795 \\ 6 & 1.995307 & 2.151568 & -3.098842 \\ 6 & 3.082035 & 2.825693 & -2.533461 \\ 6 & 4.374677 & 2.632172 & -3.013751 \\ 6 & 4.606448 & 1.761337 & -4.071983 \\ 6 & 3.528635 & 1.105910 & -4.665456 \\ 6 & 0.604521 & 2.394732 & -2.611683 \\ 6 & -0.290269 & 3.002466 & -3.674539 \\ 45 & -0.343656 & 0.619766 & -1.648123 \\ 6 & -0.677617 & -0.560009 & -3.125853 \\ 8 & -0.904676 & -1.071553 & -4.141523 \\ 15 & -1.866254 & 0.290142 & 0.224308 \\ 6 & -2.024904 & 1.940438 & 1.010168 \\ 6 & -1.040385 & 2.393490 & 1.896593 \\ 6 & -1.063369 & 3.695466 & 2.389922 \\ 6 & -2.060718 & 4.562566 & 1.915047 \\ 6 & -3.031012 & 4.152229 & 1.004310 \\ 6 & -3.007820 & 2.819832 & 0.576171 \\ 6 & -0.006644 & 4.202423 & 3.371265 \\ 6 & 0.737195 & 3.053193 & 4.053496 \\ 6 & -4.094177 & 5.084299 & 0.429350 \\ 6 & -4.029145 & 6.485688 & 1.030188 \\ 6 & -3.610684 & -0.076063 & -0.249909 \\ 6 & -3.952884 & -0.294657 & -1.578410 \\ 6 & -5.281839 & -0.501510 & -1.961844 \\ 6 & -6.256514 & -0.518636 & -0.962170 \\ 6 & -5.942994 & -0.328027 & 0.389789 \\ 6 & -4.609971 & -0.097378 & 0.724252 \\ 6 & -6.990252 & -0.346899 & 1.502122 \\ 6 & -5.599882 & -0.688195 & -3.443806 \\ 6 & -1.696907 & -0.866886 & 1.666895\end{array}$




\begin{tabular}{|c|c|c|c|}
\hline 6 & -1.379391 & -2.198180 & 1.437907 \\
\hline 6 & -1.288884 & -3.114546 & 2.536042 \\
\hline & -1.534812 & -2.651601 & 3.861301 \\
\hline & -1.879411 & -1.295011 & 4.056737 \\
\hline & -1.954053 & -0.432336 & 2.996177 \\
\hline & -1.421524 & -3.549795 & 4.949161 \\
\hline & -1.076255 & -4.860932 & 4.744435 \\
\hline & -0.834107 & -5.327242 & 3.435170 \\
\hline & -0.941638 & -4.480043 & 2.360151 \\
\hline & -1.163541 & -2.756076 & 0.073071 \\
\hline & 0.075958 & -2.697661 & -0.538815 \\
\hline & 0.308253 & -3.372222 & -1.759661 \\
\hline & -0.689540 & -4.080090 & -2.373467 \\
\hline & -1.982314 & -4.135422 & -1.802841 \\
\hline & -2.227564 & -3.463788 & -0.568208 \\
\hline & -3.533680 & -3.524208 & -0.016234 \\
\hline & -4.533025 & -4.222439 & -0.646516 \\
\hline & -4.279118 & -4.908940 & -1.853917 \\
\hline & -3.031677 & -4.858813 & -2.421088 \\
\hline & 1.153376 & -1.959627 & 0.057538 \\
\hline & 1.516928 & -0.485547 & -0.704002 \\
\hline 8 & 2.748614 & -0.723342 & -1.801771 \\
\hline 6 & 3.907219 & -1.381775 & -1.467505 \\
\hline & 4.825383 & -0.824762 & -0.595529 \\
\hline 6 & 5.921928 & -1.648917 & -0.170193 \\
\hline & 6.113221 & -2.933596 & -0.761856 \\
\hline 6 & 5.214035 & -3.381327 & -1.760561 \\
\hline 0 & 4.122240 & -2.628975 & -2.092949 \\
\hline 6 & 6.795248 & -1.268011 & 0.880926 \\
\hline 6 & 7.806520 & -2.096691 & 1.301490 \\
\hline f & 8.012861 & -3.350235 & 0.688540 \\
\hline 6 & 7.178721 & -3.755906 & -0.321537 \\
\hline 6 & 4.651295 & 0.560779 & -0.100566 \\
\hline 6 & 3.454719 & 0.968570 & 0.459383 \\
\hline 6 & 3.291524 & 2.239802 & 1.047181 \\
\hline 6 & 4.315540 & 3.144195 & 1.024348 \\
\hline 6 & 5.529185 & 2.829702 & 0.365770 \\
\hline 6 & 5.695707 & 1.539070 & -0.221354 \\
\hline 6 & 6.561503 & 3.791851 & 0.246862 \\
\hline 6 & 7.709660 & 3.514371 & -0.450288 \\
\hline 6 & 7.861743 & 2.255558 & -1.068040 \\
\hline 6 & 6.885637 & 1.296234 & -0.954690 \\
\hline & 2.385231 & 0.122288 & 0.562338 \\
\hline 6 & 0.343421 & 2.664985 & -1.247570 \\
\hline 6 & 2.069171 & -2.762300 & 0.903756 \\
\hline 6 & 2.566437 & -2.114429 & 2.171288 \\
\hline 6 & 1.726829 & -1.363628 & 2.996318 \\
\hline 6 & 2.202243 & -0.815342 & 4.179418 \\
\hline 6 & 3.529100 & -1.009676 & 4.561751 \\
\hline 6 & 4.371111 & -1.758672 & 3.748589 \\
\hline 6 & 3.890076 & -2.307541 & 2.561551 \\
\hline 6 & 0.998854 & 5.065937 & 2.600027 \\
\hline 6 & -0.649167 & 5.047086 & 4.476403 \\
\hline 6 & -3.875058 & 5.199054 & -1.084846 \\
\hline 6 & -5.489046 & 4.509477 & 0.702574 \\
\hline 1 & 6.635537 & -0.316566 & 1.381512 \\
\hline
\end{tabular}




\begin{tabular}{|c|c|c|c|}
\hline & -2.221982 & 0.605785 & 3.176469 \\
\hline & 7.309684 & -4.728015 & -0.795254 \\
\hline & -1.610335 & -3.173137 & 5.953511 \\
\hline & 6.413929 & 4.765962 & 0.711498 \\
\hline & -3.742450 & -2.994504 & 0.912461 \\
\hline & -5.530840 & -4.246642 & -0.213379 \\
\hline & 4.201604 & 4.123546 & 1.487596 \\
\hline & -2.083270 & -0.944141 & 5.067770 \\
\hline & 5.391452 & -4.344051 & -2.237004 \\
\hline & 8.757569 & 2.044081 & -1.647690 \\
\hline & 3.405006 & -2.956926 & -2.841935 \\
\hline & -2.824398 & -5.371847 & -3.359255 \\
\hline & -0.505922 & -4.598486 & -3.313258 \\
\hline & -0.562427 & -6.368274 & 3.276068 \\
\hline & 7.010478 & 0.341587 & -1.458945 \\
\hline & -0.758055 & -4.857577 & 1.356197 \\
\hline & 1.302754 & -3.310922 & -2.198611 \\
\hline & 8.492899 & 4.263295 & -0.541571 \\
\hline & 8.450243 & -1.784764 & 2.121060 \\
\hline & -5.078342 & -5.467089 & -2.336960 \\
\hline & -0.987677 & -5.543130 & 5.586686 \\
\hline & 8.821441 & -3.994202 & 1.026303 \\
\hline & 2.334202 & 2.461144 & 1.515660 \\
\hline & -4.341842 & 0.080872 & 1.765276 \\
\hline 1 & -3.163480 & -0.291798 & -2.326320 \\
\hline & -7.293006 & -0.684232 & -1.241545 \\
\hline 1 & -3.757017 & 2.470887 & -0.133041 \\
\hline & -0.253171 & 1.703286 & 2.201984 \\
\hline & -2.067625 & 5.591171 & 2.267458 \\
\hline 1 & 1.491330 & -3.656760 & 1.177521 \\
\hline & -1.650309 & 1.357650 & -2.172103 \\
\hline 1 & 2.927985 & -3.123129 & 0.317085 \\
\hline ] & 4.561390 & -2.883633 & 1.922998 \\
\hline | & 0.702591 & -1.178023 & 2.681198 \\
\hline l & 5.412120 & -1.912606 & 4.027060 \\
\hline 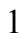 & 1.530659 & -0.230443 & 4.806779 \\
\hline 1 & 3.902921 & -0.574756 & 5.486314 \\
\hline & 1.430594 & 3.454343 & 4.803345 \\
\hline 1 & 0.040234 & 2.376427 & 4.567606 \\
\hline ] & 1.334773 & 2.451448 & 3.357539 \\
\hline 1 & 0.117470 & 5.371438 & 5.192251 \\
\hline 1 & -1.131383 & 5.952395 & 4.090907 \\
\hline 1 & -1.403563 & 4.471462 & 5.028239 \\
\hline 1 & 1.812130 & 5.399289 & 3.260027 \\
\hline 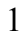 & 1.440690 & 4.508673 & 1.762374 \\
\hline 1 & 0.518501 & 5.958370 & 2.179064 \\
\hline 1 & -4.815495 & 7.112478 & 0.590995 \\
\hline 1 & -4.185501 & 6.473507 & 2.116770 \\
\hline 1 & -3.068615 & 6.975784 & 0.825687 \\
\hline 1 & -4.618102 & 5.874231 & -1.530672 \\
\hline 1 & -2.876264 & 5.599310 & -1.305687 \\
\hline 1 & -3.961941 & 4.227442 & -1.588036 \\
\hline 1 & -6.260542 & 5.166100 & 0.278380 \\
\hline 1 & -5.621858 & 3.513663 & 0.260008 \\
\hline 1 & -5.673829 & 4.423387 & 1.781624 \\
\hline & 1.162049 & 2.672028 & -0.526190 \\
\hline
\end{tabular}




$\begin{array}{lrrr}1 & -0.501070 & 3.304159 & -0.980664 \\ 1 & 2.913162 & 3.513209 & -1.705064 \\ 1 & 5.204608 & 3.168513 & -2.556236 \\ 1 & 5.619383 & 1.601704 & -4.437183 \\ 1 & 3.694974 & 0.427171 & -5.500113 \\ 1 & 1.407487 & 0.771999 & -4.643860 \\ 1 & 0.184000 & 3.908108 & -4.083370 \\ 1 & -0.464352 & 2.322922 & -4.518306 \\ 1 & -1.267784 & 3.280628 & -3.263041 \\ 6 & -7.098408 & -0.814427 & -3.706212 \\ 6 & -5.078017 & 0.519046 & -4.233632 \\ 6 & -4.914762 & -1.961314 & -3.949766 \\ 6 & -8.384586 & -0.692662 & 0.986093 \\ 6 & -6.600661 & -1.395485 & 2.551280 \\ 6 & -7.051657 & 1.037404 & 2.158557 \\ 1 & -7.274834 & -0.932768 & -4.782755 \\ 1 & -7.530989 & -1.690828 & -3.206341 \\ 1 & -7.649860 & 0.076502 & -3.378210 \\ 1 & -5.119441 & -2.106298 & -5.019378 \\ 1 & -3.825723 & -1.921519 & -3.820573 \\ 1 & -5.280027 & -2.842954 & -3.407209 \\ 1 & -5.313870 & 0.404535 & -5.300168 \\ 1 & -5.541687 & 1.450386 & -3.882434 \\ 1 & -3.989478 & 0.634437 & -4.149051 \\ 1 & -7.342621 & -1.418942 & 3.360789 \\ 1 & -6.557897 & -2.398183 & 2.105411 \\ 1 & -5.622514 & -1.192909 & 3.005788 \\ 1 & -9.094187 & -0.708617 & 1.823036 \\ 1 & -8.751045 & 0.045840 & 0.261710 \\ 1 & -8.411282 & -1.682537 & 0.511893 \\ 1 & -7.774215 & 1.038341 & 2.986031 \\ 1 & -6.079075 & 1.346404 & 2.562519 \\ 1 & -7.367862 & 1.799652 & 1.433859\end{array}$

$3_{\text {IIC-re- }} \Psi$-1s2a3a4a

Number of imaginary frequencies : 0

The smallest frequencies are : $10.8214 \quad 16.8509 \quad 19.9157 \mathrm{~cm}(-1)$

Electronic energy :

Zero-point correction $=$ $\mathrm{HF}=-4360.8916419$

Thermal correction to Energy $=\quad 1.539531$

Thermal correction to Enthalpy= $\quad 1.540475$

Thermal correction to Gibbs Free Energy $=\quad 1.337099$

Sum of electronic and zero-point Energies $=\quad-4359.436611$

Sum of electronic and thermal Energies $=\quad-4359.352111$

Sum of electronic and thermal Enthalpies $=\quad-4359.351167$

Sum of electronic and thermal Free Energies $=\quad-4359.554543$

Cartesian Coordinates

$\begin{array}{llll}6 & 2.321205 & 1.356494 & -4.182232 \\ 6 & 2.065977 & 2.194713 & -3.089066 \\ 6 & 3.147330 & 2.868533 & -2.513546 \\ 6 & 4.444161 & 2.678276 & -2.984141 \\ 6 & 4.684912 & 1.814397 & -4.045914 \\ 6 & 3.612500 & 1.161130 & -4.651001\end{array}$




\begin{tabular}{|c|c|c|c|}
\hline 6 & 0.671137 & 2.435071 & -2.611402 \\
\hline 6 & -0.214752 & 3.049178 & -3.677545 \\
\hline 4 & -0.277195 & 0.648483 & -1.670576 \\
\hline & -0.527891 & -0.542646 & -3.153351 \\
\hline & -0.665663 & -1.073970 & -4.174685 \\
\hline 1 & -1.845092 & 0.327224 & 0.176438 \\
\hline & -2.111065 & 1.999332 & 0.892373 \\
\hline 6 & -1.197179 & 2.532478 & 1.799345 \\
\hline 6 & -1.344679 & 3.835124 & 2.292704 \\
\hline 6 & -2.390832 & 4.607581 & 1.787635 \\
\hline 6 & -3.291109 & 4.121499 & 0.830344 \\
\hline 6 & -3.146054 & 2.803107 & 0.406736 \\
\hline 6 & -0.419618 & 4.337073 & 3.401249 \\
\hline 6 & -4.395188 & 5.031060 & 0.294892 \\
\hline 6 & -3.557109 & -0.150346 & -0.325724 \\
\hline 6 & -3.851603 & -0.424029 & -1.657135 \\
\hline 6 & -5.151752 & -0.750378 & -2.056138 \\
\hline 6 & -6.136927 & -0.810478 & -1.069711 \\
\hline 6 & -5.873404 & -0.559843 & 0.282425 \\
\hline 6 & -4.569107 & -0.223258 & 0.635580 \\
\hline 6 & -6.994400 & -0.679378 & 1.313678 \\
\hline 6 & -5.515966 & -0.985234 & -3.521105 \\
\hline 6 & -1.676700 & -0.756130 & 1.681321 \\
\hline 6 & -1.326581 & -2.090396 & 1.524610 \\
\hline 6 & -1.224896 & -2.948232 & 2.666950 \\
\hline 6 & -1.488746 & -2.425078 & 3.966100 \\
\hline 6 & -1.875041 & -1.071306 & 4.088545 \\
\hline 6 & -1.966121 & -0.266755 & 2.984306 \\
\hline 6 & -1.360905 & -3.264954 & 5.098055 \\
\hline 6 & -0.988021 & -4.577280 & 4.960237 \\
\hline 6 & -0.733994 & -5.104673 & 3.676533 \\
\hline 6 & -0.853764 & -4.314652 & 2.560200 \\
\hline 6 & -1.102965 & -2.698928 & 0.183884 \\
\hline 6 & 0.126496 & -2.623360 & -0.445958 \\
\hline 6 & 0.346176 & -3.292270 & -1.672200 \\
\hline 6 & -0.658110 & -3.993513 & -2.282533 \\
\hline 6 & -1.940279 & -4.066575 & -1.691739 \\
\hline 6 & -2.165180 & -3.427440 & -0.436549 \\
\hline 6 & -3.456210 & -3.523373 & 0.145922 \\
\hline 6 & -4.464107 & -4.210329 & -0.482511 \\
\hline 6 & -4.234342 & -4.850068 & -1.720331 \\
\hline 6 & -2.999442 & -4.774273 & -2.312097 \\
\hline 7 & 1.201725 & -1.865261 & 0.130067 \\
\hline 15 & 1.581096 & -0.427600 & -0.692825 \\
\hline 8 & 2.803469 & -0.730693 & -1.788205 \\
\hline 6 & 3.933126 & -1.426620 & -1.427986 \\
\hline 6 & 4.870110 & -0.878644 & -0.570827 \\
\hline 6 & 5.927804 & -1.731680 & -0.106701 \\
\hline 6 & 6.064879 & -3.045982 & -0.645564 \\
\hline 6 & 5.153519 & -3.491018 & -1.634267 \\
\hline 6 & 4.098333 & -2.704559 & -2.004615 \\
\hline 6 & 6.813648 & -1.346149 & 0.932337 \\
\hline 6 & 7.785190 & -2.200725 & 1.392382 \\
\hline 6 & 7.937533 & -3.486547 & 0.832518 \\
\hline 6 & 7.090730 & -3.896074 & -0.165363 \\
\hline 6 & 4.754319 & 0.530142 & -0.129409 \\
\hline
\end{tabular}




\begin{tabular}{|c|c|c|c|}
\hline & 3.576117 & 1.014515 & 0.407536 \\
\hline & 3.473563 & 2.311620 & 0.949023 \\
\hline & 4.540278 & 3.163602 & 0.903772 \\
\hline & 5.739117 & 2.770183 & 0.261022 \\
\hline & 5.843201 & 1.454158 & -0.282362 \\
\hline & 6.814294 & 3.678772 & 0.107414 \\
\hline & 7.944118 & 3.326182 & -0.586094 \\
\hline & 8.035483 & 2.041152 & -1.160466 \\
\hline & 7.018142 & 1.131191 & -1.008480 \\
\hline & 2.461632 & 0.231083 & 0.536811 \\
\hline & 0.393488 & 2.696710 & -1.249347 \\
\hline & 2.095365 & -2.624667 & 1.036858 \\
\hline & 2.572077 & -1.909271 & 2.275900 \\
\hline ) & 1.727648 & -1.093035 & 3.030852 \\
\hline & 2.185293 & -0.475916 & 4.186604 \\
\hline & 3.499139 & -0.667581 & 4.612759 \\
\hline ) & 4.345428 & -1.482993 & 3.870774 \\
\hline & 3.882569 & -2.098919 & 2.709600 \\
\hline & 6.694933 & -0.368137 & 1.391317 \\
\hline & -2.277177 & 0.767039 & 3.110059 \\
\hline & 7.181006 & -4.891378 & -0.598638 \\
\hline & -1.562364 & -2.842779 & 6.081602 \\
\hline & 6.713617 & 4.673771 & 0.539301 \\
\hline & -3.647572 & -3.026706 & 1.095805 \\
\hline ] & -5.451657 & -4.258718 & -0.027368 \\
\hline & 4.469032 & 4.162818 & 1.331795 \\
\hline & -2.102661 & -0.675166 & 5.077730 \\
\hline & 5.291227 & -4.478016 & -2.072618 \\
\hline & 8.917621 & 1.769313 & -1.735963 \\
\hline 1 & 3.374480 & -3.030030 & -2.748300 \\
\hline & -2.808097 & -5.259529 & -3.268321 \\
\hline ] & -0.486901 & -4.493662 & -3.234539 \\
\hline & -0.443474 & -6.147404 & 3.570757 \\
\hline & 7.098904 & 0.153158 & -1.475482 \\
\hline ] & -0.662310 & -4.737701 & 1.576003 \\
\hline & 1.332794 & -3.222047 & -2.125791 \\
\hline ] & 8.759650 & 4.035420 & -0.706847 \\
\hline & 8.439375 & -1.884006 & 2.201745 \\
\hline 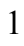 & -5.040103 & -5.399854 & -2.202315 \\
\hline ] & -0.888550 & -5.214286 & 5.835997 \\
\hline 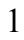 & 8.715148 & -4.151366 & 1.201221 \\
\hline 1 & 2.526370 & 2.595256 & 1.399388 \\
\hline ] & -4.327248 & -0.009012 & 1.673746 \\
\hline 1 & -3.045458 & -0.376565 & -2.383225 \\
\hline & -7.156078 & -1.063942 & -1.362842 \\
\hline 1 & -3.836319 & 2.385634 & -0.321703 \\
\hline l & -0.362961 & 1.912966 & 2.138572 \\
\hline 1 & -2.515440 & 5.627936 & 2.143586 \\
\hline 1 & 1.508440 & -3.500863 & 1.347419 \\
\hline 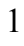 & -1.585416 & 1.358540 & -2.230587 \\
\hline 1 & 2.963269 & -3.020675 & 0.487716 \\
\hline ( & 4.557848 & -2.725980 & 2.125527 \\
\hline 1 & 0.714460 & -0.912505 & 2.679559 \\
\hline ] & 5.376137 & -1.636784 & 4.185694 \\
\hline 1 & 1.509590 & 0.160551 & 4.757519 \\
\hline 1 & 3.859974 & -0.179532 & 5.515706 \\
\hline
\end{tabular}




\begin{tabular}{|c|c|c|c|}
\hline 1 & 1.199941 & 2.697237 & -0.513877 \\
\hline & -0.456252 & 3.330693 & -0.989355 \\
\hline & 2.969845 & 3.554980 & -1.686627 \\
\hline & 5.269695 & 3.213294 & -2.516761 \\
\hline & 5.700882 & 1.658345 & -4.403986 \\
\hline & 3.785430 & 0.488691 & -5.489358 \\
\hline & 1.492603 & 0.823023 & -4.646650 \\
\hline & 0.260098 & 3.960915 & -4.071957 \\
\hline & -0.376439 & 2.377324 & -4.529612 \\
\hline & -1.197571 & 3.319007 & -3.273078 \\
\hline & -4.279444 & -1.048059 & -4.415572 \\
\hline & -6.276162 & -2.304844 & -3.678143 \\
\hline & -6.401790 & 0.172230 & -3.996579 \\
\hline & -6.521909 & -0.327209 & 2.722809 \\
\hline & -8.142216 & 0.266439 & 0.945702 \\
\hline & -7.508226 & -2.123320 & 1.333006 \\
\hline & -4.583076 & -1.251213 & -5.450398 \\
\hline & -3.720503 & -0.102868 & -4.419801 \\
\hline & -3.594773 & -1.848988 & -4.104677 \\
\hline & -6.668163 & 0.042182 & -5.054425 \\
\hline & -7.334400 & 0.234748 & -3.421582 \\
\hline & -5.880501 & 1.133151 & -3.893957 \\
\hline 1 & -6.541980 & -2.464840 & -4.731641 \\
\hline & -5.655140 & -3.148861 & -3.351775 \\
\hline & -7.207342 & -2.326111 & -3.099395 \\
\hline & -8.948540 & 0.194054 & 1.687913 \\
\hline & -7.799394 & 1.309034 & 0.920301 \\
\hline 1 & -8.574948 & 0.032941 & -0.034420 \\
\hline & -7.354483 & -0.436776 & 3.429171 \\
\hline I & -5.712423 & -0.987717 & 3.063349 \\
\hline & -6.167294 & 0.710008 & 2.787895 \\
\hline 1 & -8.338713 & -2.225567 & 2.044722 \\
\hline 1 & -7.870118 & -2.446210 & 0.348842 \\
\hline & -6.713548 & -2.815106 & 1.644667 \\
\hline 5 & -0.578597 & 5.833578 & 3.660671 \\
\hline & -0.764266 & 3.582878 & 4.691908 \\
\hline 6 & 1.045218 & 4.068846 & 3.046695 \\
\hline f & -5.269769 & 4.330858 & -0.743489 \\
\hline 5 & -5.295787 & 5.480539 & 1.450443 \\
\hline 6 & -3.760437 & 6.257694 & -0.369238 \\
\hline & 0.122787 & 6.147780 & 4.443745 \\
\hline 1 & -0.363908 & 6.428025 & 2.763095 \\
\hline & -1.586836 & 6.089166 & 4.009593 \\
\hline 1 & -0.121150 & 3.917199 & 5.517604 \\
\hline & -1.808546 & 3.757923 & 4.982478 \\
\hline 1 & -0.624180 & 2.499654 & 4.575804 \\
\hline 1 & 1.706679 & 4.502970 & 3.808217 \\
\hline 1 & 1.265346 & 2.994824 & 3.009452 \\
\hline 1 & 1.306471 & 4.513225 & 2.075876 \\
\hline 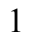 & -4.538430 & 6.917585 & -0.776363 \\
\hline 1 & -3.161917 & 6.846852 & 0.335809 \\
\hline 1 & -3.103182 & 5.957473 & -1.196197 \\
\hline 1 & -6.093538 & 6.137870 & 1.079089 \\
\hline 1 & -5.768352 & 4.617282 & 1.937618 \\
\hline 1 & -4.740492 & 6.034847 & 2.216666 \\
\hline 1 & -6.046509 & 5.021403 & -1.095501 \\
\hline
\end{tabular}


$3_{\text {IIC-re- }} \Psi$-1s2a3a4s

Number of imaginary frequencies : 0

The smallest frequencies are : $9.1498 \quad 19.069923 .4549 \mathrm{~cm}(-1)$

Electronic energy : $\quad \mathrm{HF}=-4360.8924035$

Zero-point correction $=\quad 1.454700($ Hartree/Particle $)$

Thermal correction to Energy= $\quad 1.539261$

Thermal correction to Enthalpy= $\quad 1.540206$

Thermal correction to Gibbs Free Energy $=\quad 1.336780$

Sum of electronic and zero-point Energies $=\quad-4359.437704$

Sum of electronic and thermal Energies $=\quad-4359.353142$

Sum of electronic and thermal Enthalpies $=\quad-4359.352198$

Sum of electronic and thermal Free Energies $=\quad-4359.555623$

\section{Cartesian Coordinates}

$\begin{array}{cccc}6 & 2.350944 & 1.357066 & -4.170802 \\ 6 & 2.077099 & 2.197933 & -3.084212 \\ 6 & 3.145542 & 2.888414 & -2.504329 \\ 6 & 4.447961 & 2.712619 & -2.964831 \\ 6 & 4.707532 & 1.845814 & -4.019769 \\ 6 & 3.648097 & 1.175153 & -4.628671 \\ 6 & 0.675707 & 2.424377 & -2.619581 \\ 6 & -0.209386 & 3.015670 & -3.699090 \\ 45 & -0.261221 & 0.633680 & -1.669029 \\ 6 & -0.491468 & -0.566392 & -3.147265 \\ 8 & -0.622295 & -1.105561 & -4.165457 \\ 15 & -1.831794 & 0.306061 & 0.165371 \\ 6 & -2.171239 & 1.976889 & 0.857674 \\ 6 & -1.268168 & 2.581686 & 1.730382 \\ 6 & -1.475190 & 3.887529 & 2.194158 \\ 6 & -2.581304 & 4.584878 & 1.705919 \\ 6 & -3.480086 & 4.023553 & 0.790255 \\ 6 & -3.264581 & 2.708916 & 0.386452 \\ 6 & -0.547269 & 4.477472 & 3.256456 \\ 6 & -4.664454 & 4.843300 & 0.281422 \\ 6 & -3.526205 & -0.223241 & -0.343982 \\ 6 & -3.817097 & -0.508481 & -1.677331 \\ 6 & -5.112693 & -0.838611 & -2.074931 \\ 6 & -6.109450 & -0.887127 & -1.091560 \\ 6 & -5.848967 & -0.624801 & 0.254019 \\ 6 & -4.539753 & -0.290475 & 0.607403 \\ 6 & -6.921375 & -0.657516 & 1.341385 \\ 6 & -5.472846 & -1.090300 & -3.537953 \\ 6 & -1.646062 & -0.759462 & 1.680024 \\ 6 & -1.292703 & -2.094660 & 1.534410 \\ 6 & -1.182518 & -2.939675 & 2.685150 \\ 6 & -1.440759 & -2.403274 & 3.980141 \\ 6 & -1.827078 & -1.048698 & 4.091015 \\ 6 & -1.925111 & -0.255383 & 2.979018 \\ 6 & -1.303093 & -3.230258 & 5.120495 \\ 6 & -0.925938 & -4.542513 & 4.994978 \\ 6 & -0.677479 & -5.083039 & 3.715541\end{array}$




\begin{tabular}{|c|c|c|c|}
\hline 6 & -0.806778 & -4.305881 & 2.591388 \\
\hline 6 & -1.068627 & -2.714943 & 0.198593 \\
\hline & 0.158347 & -2.632560 & -0.434999 \\
\hline & 0.383011 & -3.306097 & -1.657716 \\
\hline & -0.615000 & -4.022345 & -2.260677 \\
\hline & -1.893740 & -4.108176 & -1.663858 \\
\hline & -2.123111 & -3.462216 & -0.412928 \\
\hline & -3.410347 & -3.571371 & 0.175129 \\
\hline & -4.410364 & -4.278777 & -0.443191 \\
\hline & -4.176355 & -4.925046 & -1.676737 \\
\hline & -2.945104 & -4.835807 & -2.274294 \\
\hline & 1.230585 & -1.868141 & 0.138273 \\
\hline & 1.599406 & -0.425190 & -0.678280 \\
\hline & 2.829233 & -0.708226 & -1.769575 \\
\hline & 3.970833 & -1.383736 & -1.409105 \\
\hline & 4.897809 & -0.820355 & -0.550792 \\
\hline & 5.971930 & -1.655304 & -0.090925 \\
\hline & 6.134392 & -2.964059 & -0.636524 \\
\hline & 5.229102 & -3.424052 & -1.624024 \\
\hline & 4.157898 & -2.657155 & -1.988905 \\
\hline & 6.850474 & -1.258396 & 0.950045 \\
\hline & 7.840285 & -2.095428 & 1.403499 \\
\hline & 8.018632 & -3.374270 & 0.835565 \\
\hline & 7.178339 & -3.795517 & -0.162927 \\
\hline & 4.754343 & 0.584104 & -0.102806 \\
\hline & 3.565746 & 1.041181 & 0.435264 \\
\hline & 3.434000 & 2.332887 & 0.983277 \\
\hline & 4.481074 & 3.209034 & 0.940742 \\
\hline & 5.688885 & 2.843862 & 0.297944 \\
\hline 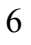 & 5.823571 & 1.531911 & -0.248763 \\
\hline & 6.744244 & 3.776349 & 0.149508 \\
\hline & 7.884014 & 3.450267 & -0.540640 \\
\hline 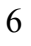 & 8.005664 & 2.168886 & -1.117509 \\
\hline & 7.007633 & 1.236739 & -0.971822 \\
\hline 3 & 2.468987 & 0.233050 & 0.559102 \\
\hline & 0.384709 & 2.694622 & -1.262429 \\
\hline 5 & 2.134093 & -2.627740 & 1.035557 \\
\hline & 2.616025 & -1.919135 & 2.276526 \\
\hline 5 & 1.774389 & -1.111236 & 3.043627 \\
\hline 6 & 2.238350 & -0.502797 & 4.201466 \\
\hline & 3.555098 & -0.695427 & 4.618035 \\
\hline 6 & 4.398770 & -1.501956 & 3.863605 \\
\hline & 3.929943 & -2.108842 & 2.700125 \\
\hline 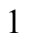 & 6.711599 & -0.286489 & 1.416269 \\
\hline & -2.235207 & 0.779962 & 3.094572 \\
\hline & 7.287854 & -4.786453 & -0.601749 \\
\hline & -1.499879 & -2.797877 & 6.100547 \\
\hline & 6.620513 & 4.767808 & 0.583525 \\
\hline 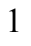 & -3.603849 & -3.069618 & 1.121835 \\
\hline & -5.395608 & -4.337605 & 0.015441 \\
\hline & 4.387756 & 4.204788 & 1.372597 \\
\hline & -2.047449 & -0.642584 & 5.077737 \\
\hline 1 & 5.384588 & -4.406997 & -2.065506 \\
\hline & 8.895670 & 1.917720 & -1.690271 \\
\hline 1 & 3.436936 & -2.994858 & -2.730089 \\
\hline 1 & -2.750659 & -5.325830 & -3.227461 \\
\hline
\end{tabular}




\begin{tabular}{|c|c|c|c|}
\hline & -0.441265 & -4.526652 & -3.210023 \\
\hline & -0.383305 & -6.125695 & 3.619547 \\
\hline & 7.110897 & 0.262007 & -1.441341 \\
\hline & -0.619429 & -4.739070 & 1.610843 \\
\hline & 1.368213 & -3.226939 & -2.113691 \\
\hline & 8.684267 & 4.177467 & -0.656800 \\
\hline & 8.488679 & -1.770180 & 2.214156 \\
\hline . & -4.975944 & -5.490220 & -2.151119 \\
\hline & -0.818437 & -5.169341 & 5.877107 \\
\hline 1 & 8.810692 & -4.024861 & 1.198858 \\
\hline 1 & 2.481020 & 2.590968 & 1.437566 \\
\hline & -4.309732 & -0.059093 & 1.647110 \\
\hline & -3.009144 & -0.462443 & -2.401488 \\
\hline ] & -7.124432 & -1.137381 & -1.394097 \\
\hline & -3.948485 & 2.238059 & -0.314039 \\
\hline 1 & -0.393682 & 2.015906 & 2.062467 \\
\hline 1 & -2.755697 & 5.604426 & 2.042809 \\
\hline 1 & 1.553672 & -3.508729 & 1.344664 \\
\hline 1 & -1.574953 & 1.325529 & -2.240331 \\
\hline 1 & 3.000303 & -3.016039 & 0.478129 \\
\hline . & 4.603412 & -2.728175 & 2.105738 \\
\hline & 0.758943 & -0.928062 & 2.699980 \\
\hline 1 & 5.432005 & -1.655499 & 4.170118 \\
\hline & 1.565891 & 0.129015 & 4.780880 \\
\hline 1 & 3.920480 & -0.214069 & 5.522756 \\
\hline 1 & 1.186291 & 2.711155 & -0.521784 \\
\hline 1 & -0.475104 & 3.319501 & -1.013738 \\
\hline ] & 2.953600 & 3.576258 & -1.681675 \\
\hline & 5.262960 & 3.261181 & -2.494890 \\
\hline ] & 5.728063 & 1.700914 & -4.369528 \\
\hline 1 & 3.835871 & 0.499831 & -5.461509 \\
\hline 1 & 1.532619 & 0.810174 & -4.637898 \\
\hline 1 & 0.255473 & 3.930912 & -4.097251 \\
\hline 1 & -0.352485 & 2.333999 & -4.546619 \\
\hline 1 & -1.199964 & 3.273854 & -3.306871 \\
\hline 6 & -4.234847 & -1.148687 & -4.430595 \\
\hline 6 & -6.219019 & -2.419482 & -3.682576 \\
\hline 5 & -6.369867 & 0.053080 & -4.026421 \\
\hline 5 & -8.268464 & -1.146161 & 0.815974 \\
\hline 5 & -6.487679 & -1.597535 & 2.472748 \\
\hline 6 & -7.104303 & 0.759711 & 1.897903 \\
\hline 1 & -4.535494 & -1.362220 & -5.464205 \\
\hline 1 & -3.684751 & -0.198340 & -4.441041 \\
\hline l & -3.543079 & -1.940913 & -4.113062 \\
\hline l & -6.634619 & -0.091589 & -5.082777 \\
\hline 1 & -7.303515 & 0.113390 & -3.453069 \\
\hline 1 & -5.857555 & 1.019927 & -3.934578 \\
\hline 1 & -6.474311 & -2.596985 & -4.735901 \\
\hline 1 & -5.592655 & -3.252780 & -3.338895 \\
\hline 1 & -7.154752 & -2.441177 & -3.111316 \\
\hline 1 & -7.246528 & -1.610413 & 3.266593 \\
\hline 1 & -6.370735 & -2.626045 & 2.106452 \\
\hline 1 & -5.537265 & -1.297556 & 2.932256 \\
\hline 1 & -8.998049 & -1.171654 & 1.635310 \\
\hline 1 & -8.673103 & -0.484816 & 0.039292 \\
\hline 1 & -8.199422 & -2.161164 & 0.402247 \\
\hline
\end{tabular}




$\begin{array}{rrrr}1 & -7.841350 & 0.762465 & 2.712512 \\ 1 & -6.165270 & 1.169149 & 2.292473 \\ 1 & -7.463675 & 1.442482 & 1.116196 \\ 6 & -0.751618 & 5.980148 & 3.439444 \\ 6 & -0.843659 & 3.781819 & 4.591102 \\ 6 & 0.919999 & 4.239930 & 2.889650 \\ 6 & -5.517906 & 4.066207 & -0.719571 \\ 6 & -5.556505 & 5.238662 & 1.463181 \\ 6 & -4.148244 & 6.107101 & -0.414542 \\ 1 & -0.039502 & 6.360194 & 4.182625 \\ 1 & -0.584045 & 6.530841 & 2.504733 \\ 1 & -1.757142 & 6.221718 & 3.805517 \\ 1 & -0.198282 & 4.180198 & 5.386058 \\ 1 & -1.888206 & 3.937017 & 4.891657 \\ 1 & -0.668830 & 2.699300 & 4.528278 \\ 1 & 1.577851 & 4.736080 & 3.615527 \\ 1 & 1.175038 & 3.173693 & 2.907386 \\ 1 & 1.152637 & 4.637779 & 1.891863 \\ 1 & -4.988395 & 6.702446 & -0.796882 \\ 1 & -3.571147 & 6.746825 & 0.263863 \\ 1 & -3.501051 & 5.850135 & -1.263402 \\ 1 & -6.412165 & 5.832254 & 1.114031 \\ 1 & -5.948707 & 4.349374 & 1.974207 \\ 1 & -5.017033 & 5.839933 & 2.204909 \\ 1 & -6.361811 & 4.688008 & -1.044054 \\ 1 & -4.948193 & 3.789225 & -1.616308 \\ 1 & -5.933195 & 3.146536 & -0.284174\end{array}$

$3_{\text {IIC-re- }} \psi_{-1 \text { s2a3s4a }}$

Number of imaginary frequencies : 0

The smallest frequencies are : $16.0420 \quad 16.7794 \quad 23.7031 \mathrm{~cm}(-1)$

Electronic energy : $\quad H F=-4360.8916882$

Zero-point correction $=\quad 1.455141$ (Hartree/Particle)

Thermal correction to Energy= $\quad 1.539522$

Thermal correction to Enthalpy= $\quad 1.540466$

Thermal correction to Gibbs Free Energy $=\quad 1.338267$

Sum of electronic and zero-point Energies $=\quad-4359.436547$

Sum of electronic and thermal Energies $=\quad-4359.352166$

Sum of electronic and thermal Enthalpies $=\quad-4359.351222$

Sum of electronic and thermal Free Energies $=\quad-4359.553421$

Cartesian Coordinates

$\begin{array}{cccc}6 & 2.315067 & 1.341670 & -4.173554 \\ 6 & 2.064591 & 2.191421 & -3.088220 \\ 6 & 3.149328 & 2.865487 & -2.519292 \\ 6 & 4.445024 & 2.664567 & -2.988550 \\ 6 & 4.681035 & 1.789311 & -4.042095 \\ 6 & 3.605243 & 1.135510 & -4.640707 \\ 6 & 0.671231 & 2.440607 & -2.611070 \\ 6 & -0.213198 & 3.050476 & -3.681221 \\ 45 & -0.283134 & 0.666588 & -1.658667 \\ 6 & -0.587824 & -0.527949 & -3.127750 \\ 8 & -0.794191 & -1.056442 & -4.139456 \\ 15 & -1.849747 & 0.341213 & 0.185390\end{array}$




\begin{tabular}{|c|c|c|c|}
\hline 6 & -2.114311 & 2.006529 & 0.915858 \\
\hline 6 & -1.195279 & 2.533054 & 1.821772 \\
\hline b & -1.339031 & 3.833236 & 2.322503 \\
\hline & -2.387339 & 4.609295 & 1.826735 \\
\hline D & -3.295396 & 4.128194 & 0.874544 \\
\hline 5 & -3.153559 & 2.811924 & 0.443012 \\
\hline 6 & -0.410641 & 4.329635 & 3.430833 \\
\hline 5 & -4.397377 & 5.043066 & 0.343816 \\
\hline 6 & -3.558268 & -0.130018 & -0.329504 \\
\hline 6 & -3.854445 & -0.353420 & -1.664374 \\
\hline 6 & -5.156152 & -0.667466 & -2.080367 \\
\hline 6 & -6.137037 & -0.788267 & -1.101731 \\
\hline 6 & -5.869060 & -0.589856 & 0.263804 \\
\hline 6 & -4.573257 & -0.248359 & 0.629596 \\
\hline 6 & -6.987056 & -0.765708 & 1.290040 \\
\hline 6 & -5.425708 & -0.854016 & -3.571270 \\
\hline 6 & -1.690001 & -0.759656 & 1.676131 \\
\hline 6 & -1.345281 & -2.093205 & 1.504378 \\
\hline 6 & -1.253470 & -2.964718 & 2.637890 \\
\hline 6 & -1.526372 & -2.456445 & 3.941136 \\
\hline 6 & -1.907027 & -1.102368 & 4.077624 \\
\hline 6 & -1.984674 & -0.283733 & 2.982894 \\
\hline 6 & -1.411842 & -3.310439 & 5.063974 \\
\hline 6 & -1.041168 & -4.621995 & 4.913919 \\
\hline 6 & -0.775121 & -5.133940 & 3.626482 \\
\hline 6 & -0.882802 & -4.330323 & 2.518644 \\
\hline 6 & -1.113115 & -2.693136 & 0.160766 \\
\hline 6 & 0.122671 & -2.620140 & -0.456861 \\
\hline 6 & 0.358398 & -3.301803 & -1.673262 \\
\hline 6 & -0.631374 & -4.027720 & -2.278174 \\
\hline 6 & -1.917758 & -4.105874 & -1.696803 \\
\hline 6 & -2.165554 & -3.433937 & -0.463071 \\
\hline 6 & -3.464823 & -3.523185 & 0.101435 \\
\hline 6 & -4.455768 & -4.241396 & -0.519630 \\
\hline 6 & -4.199258 & -4.924195 & -1.728370 \\
\hline 6 & -2.956994 & -4.852321 & -2.304311 \\
\hline 7 & 1.192643 & -1.860018 & 0.126526 \\
\hline 15 & 1.575265 & -0.421112 & -0.692169 \\
\hline 8 & 2.798455 & -0.723807 & -1.786414 \\
\hline 6 & 3.928359 & -1.419899 & -1.427281 \\
\hline 6 & 4.863669 & -0.874464 & -0.566693 \\
\hline 6 & 5.919970 & -1.729070 & -0.102357 \\
\hline 6 & 6.058934 & -3.041400 & -0.645445 \\
\hline 6 & 5.151173 & -3.482456 & -1.639213 \\
\hline 6 & 4.096624 & -2.695010 & -2.009395 \\
\hline 6 & 6.802309 & -1.347103 & 0.940976 \\
\hline 6 & 7.772112 & -2.203411 & 1.401476 \\
\hline 6 & 7.926332 & -3.487286 & 0.837624 \\
\hline 6 & 7.083093 & -3.893234 & -0.164735 \\
\hline 6 & 4.747928 & 0.533335 & -0.122552 \\
\hline 6 & 3.569146 & 1.017043 & 0.413573 \\
\hline 6 & 3.465791 & 2.313623 & 0.956134 \\
\hline 6 & 4.532658 & 3.165576 & 0.913013 \\
\hline 6 & 5.732703 & 2.772453 & 0.272293 \\
\hline 6 & 5.837569 & 1.456962 & -0.272225 \\
\hline 6 & 6.808921 & 3.680480 & 0.122766 \\
\hline
\end{tabular}




\begin{tabular}{|c|c|c|c|}
\hline 6 & 7.940742 & 3.327736 & -0.567380 \\
\hline 6 & 8.033008 & 2.043259 & -1.142837 \\
\hline & 7.014450 & 1.133944 & -0.995220 \\
\hline & 2.454747 & 0.233078 & 0.540784 \\
\hline & 0.397063 & 2.712165 & -1.249531 \\
\hline & 2.086899 & -2.623172 & 1.029638 \\
\hline & 2.557888 & -1.914219 & 2.274370 \\
\hline & 1.707904 & -1.106496 & 3.032110 \\
\hline & 2.159735 & -0.495494 & 4.193450 \\
\hline & 3.473028 & -0.685171 & 4.622261 \\
\hline & 4.324627 & -1.492533 & 3.877528 \\
\hline & 3.867613 & -2.102579 & 2.710987 \\
\hline & 6.681776 & -0.370790 & 1.403143 \\
\hline & -2.290921 & 0.750374 & 3.118033 \\
\hline & 7.174845 & -4.887004 & -0.601208 \\
\hline & -1.621403 & -2.899475 & 6.050576 \\
\hline & 6.707677 & 4.675046 & 0.555511 \\
\hline & -3.675275 & -3.000310 & 1.033351 \\
\hline & -5.449080 & -4.286215 & -0.076783 \\
\hline & 4.461176 & 4.164289 & 1.342183 \\
\hline & -2.140414 & -0.717613 & 5.069918 \\
\hline & 5.290934 & -4.467347 & -2.081637 \\
\hline ] & 8.916855 & 1.771332 & -1.715665 \\
\hline & 3.375837 & -3.017654 & -2.757246 \\
\hline & -2.746806 & -5.363246 & -3.243099 \\
\hline & -0.444773 & -4.546704 & -3.217082 \\
\hline & -0.484883 & -6.175689 & 3.510725 \\
\hline & 7.095921 & 0.156355 & -1.463031 \\
\hline & -0.681491 & -4.742232 & 1.531716 \\
\hline 1 & 1.348558 & -3.229243 & -2.118420 \\
\hline & 8.757259 & 4.036432 & -0.684602 \\
\hline 1 & 8.423342 & -1.889704 & 2.214384 \\
\hline ] & -4.991499 & -5.498394 & -2.204018 \\
\hline & -0.952015 & -5.269964 & 5.782717 \\
\hline 1 & 8.702501 & -4.153510 & 1.206838 \\
\hline & 2.518231 & 2.596251 & 1.406286 \\
\hline 1 & -4.333032 & -0.069994 & 1.674782 \\
\hline & -3.055300 & -0.272219 & -2.396844 \\
\hline 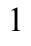 & -7.152843 & -1.044060 & -1.397289 \\
\hline 1 & -3.848914 & 2.398285 & -0.282743 \\
\hline & -0.360394 & 1.909945 & 2.152798 \\
\hline 1 & -2.505784 & 5.629491 & 2.185311 \\
\hline & 1.501853 & -3.503000 & 1.333352 \\
\hline ] & -1.587337 & 1.392397 & -2.205843 \\
\hline & 2.957300 & -3.013138 & 0.480135 \\
\hline . & 4.546761 & -2.723424 & 2.124778 \\
\hline 1 & 0.694655 & -0.928935 & 2.679374 \\
\hline t & 5.354772 & -1.644900 & 4.195007 \\
\hline 1 & 1.479752 & 0.133713 & 4.767240 \\
\hline & 3.829259 & -0.202058 & 5.529676 \\
\hline 1 & 1.205354 & 2.715465 & -0.516005 \\
\hline & -0.449523 & 3.351533 & -0.991869 \\
\hline 1 & 2.975624 & 3.559431 & -1.697770 \\
\hline 1 & 5.273441 & 3.199749 & -2.526511 \\
\hline & 5.696103 & 1.624596 & -4.398859 \\
\hline & 3.774930 & 0.453625 & -5.472078 \\
\hline
\end{tabular}




\begin{tabular}{|c|c|c|c|}
\hline & 1.483446 & 0.806371 & -4.630909 \\
\hline & 0.265066 & 3.956677 & -4.084156 \\
\hline 1 & -0.379494 & 2.372015 & -4.527327 \\
\hline & -1.194535 & 3.328040 & -3.278271 \\
\hline & -6.904192 & -1.089618 & -3.868403 \\
\hline & -4.980271 & 0.402196 & -4.330787 \\
\hline & -4.634428 & -2.063427 & -4.078809 \\
\hline & -6.511805 & -0.489657 & 2.715199 \\
\hline & -8.135582 & 0.198068 & 0.974293 \\
\hline & -7.500403 & -2.208940 & 1.236559 \\
\hline & -7.049322 & -1.204070 & -4.950039 \\
\hline & -7.278114 & -2.004457 & -3.390532 \\
\hline & -7.528313 & -0.248574 & -3.538775 \\
\hline L & -4.792503 & -2.197800 & -5.157725 \\
\hline & -3.555697 & -1.952019 & -3.909561 \\
\hline & -4.955721 & -2.979759 & -3.566964 \\
\hline & -5.192734 & 0.291591 & -5.402603 \\
\hline 1 & -5.513367 & 1.291660 & -3.969450 \\
\hline & -3.903735 & 0.591993 & -4.228450 \\
\hline & -8.941108 & 0.086966 & 1.712638 \\
\hline & -7.791842 & 1.240308 & 1.003228 \\
\hline 1 & -8.568915 & 0.016773 & -0.016565 \\
\hline 1 & -7.340986 & -0.645378 & 3.416894 \\
\hline 1 & -5.695838 & -1.162286 & 3.014765 \\
\hline 1 & -6.164798 & 0.544543 & 2.838906 \\
\hline 1 & -8.328019 & -2.347427 & 1.945442 \\
\hline 1 & -7.866745 & -2.482358 & 0.239377 \\
\hline 1 & -6.704086 & -2.915032 & 1.509707 \\
\hline 6 & -0.552307 & 5.829390 & 3.682203 \\
\hline 6 & -0.769189 & 3.585962 & 4.723817 \\
\hline 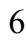 & 1.052054 & 4.042802 & 3.083149 \\
\hline 6 & -5.304137 & 4.334657 & -0.661155 \\
\hline 6 & -5.268218 & 5.534771 & 1.504908 \\
\hline 6 & -3.754202 & 6.243659 & -0.358873 \\
\hline 1 & 0.155083 & 6.140243 & 4.461211 \\
\hline 1 & -0.334190 & 6.416220 & 2.780460 \\
\hline 1 & -1.556283 & 6.098326 & 4.033265 \\
\hline & -0.126034 & 3.917186 & 5.550733 \\
\hline 1 & -1.812757 & 3.773874 & 5.008765 \\
\hline 1 & -0.640728 & 2.500714 & 4.613167 \\
\hline 1 & 1.715309 & 4.469903 & 3.847021 \\
\hline 1 & 1.260245 & 2.966351 & 3.047581 \\
\hline 1 & 1.322404 & 4.483708 & 2.113196 \\
\hline 1 & -4.527746 & 6.909566 & -0.764808 \\
\hline & -3.131065 & 6.834894 & 0.322917 \\
\hline 1 & -3.118566 & 5.914113 & -1.191640 \\
\hline 1 & -6.062193 & 6.195548 & 1.131744 \\
\hline 1 & -5.745388 & 4.692238 & 2.022367 \\
\hline 1 & -4.691175 & 6.099732 & 2.246713 \\
\hline 1 & -6.083064 & 5.027012 & -1.004717 \\
\hline 1 & -4.750982 & 3.996458 & -1.547276 \\
\hline 1 & -5.807768 & 3.463150 & -0.221067 \\
\hline
\end{tabular}

$3_{\text {IIC-re- }}-\psi_{-15223 s 4 s}$

Number of imaginary frequencies : 0 
The smallest frequencies are : $10.746920 .086224 .4145 \mathrm{~cm}(-1)$ Electronic energy : $\quad H F=-4360.8922328$

Zero-point correction $=\quad 1.454670($ Hartree/Particle $)$

Thermal correction to Energy $=\quad 1.539187$

Thermal correction to Enthalpy= $\quad 1.540132$

Thermal correction to Gibbs Free Energy= $\quad 1.337443$

Sum of electronic and zero-point Energies $=\quad-4359.437563$

Sum of electronic and thermal Energies $=\quad-4359.353045$

Sum of electronic and thermal Enthalpies $=\quad-4359.352101$

Sum of electronic and thermal Free Energies $=\quad-4359.554790$

Cartesian Coordinates

$\begin{array}{cccc}6 & 2.322705 & 1.339234 & -4.179127 \\ 6 & 2.052650 & 2.189198 & -3.098701 \\ 6 & 3.122744 & 2.885274 & -2.528527 \\ 6 & 4.423737 & 2.706000 & -2.991920 \\ 6 & 4.679553 & 1.830958 & -4.041036 \\ 6 & 3.618219 & 1.154756 & -4.640583 \\ 6 & 0.652891 & 2.416718 & -2.629771 \\ 6 & -0.238518 & 2.999816 & -3.708743 \\ 45 & -0.273984 & 0.629593 & -1.666675 \\ 6 & -0.538407 & -0.586004 & -3.125722 \\ 8 & -0.722589 & -1.136487 & -4.130036 \\ 15 & -1.845700 & 0.302906 & 0.169817 \\ 6 & -2.186355 & 1.970809 & 0.864101 \\ 6 & -1.285665 & 2.568014 & 1.743702 \\ 6 & -1.494998 & 3.869049 & 2.219209 \\ 6 & -2.598291 & 4.571441 & 1.732176 \\ 6 & -3.493809 & 4.017859 & 0.808114 \\ 6 & -3.278400 & 2.706041 & 0.395344 \\ 6 & -0.565629 & 4.445058 & 3.287375 \\ 6 & -4.675265 & 4.842194 & 0.300277 \\ 6 & -3.534115 & -0.225637 & -0.353855 \\ 6 & -3.814799 & -0.494334 & -1.687238 \\ 6 & -5.109642 & -0.814351 & -2.107262 \\ 6 & -6.112586 & -0.887327 & -1.138624 \\ 6 & -5.860834 & -0.640693 & 0.217210 \\ 6 & -4.560679 & -0.304923 & 0.588117 \\ 6 & -6.947254 & -0.683620 & 1.291068 \\ 6 & -5.364066 & -1.041576 & -3.596036 \\ 6 & -1.664941 & -0.762221 & 1.683421 \\ 6 & -1.294673 & -2.092371 & 1.538536 \\ 6 & -1.184899 & -2.938042 & 2.689527 \\ 6 & -1.461273 & -2.406597 & 3.982757 \\ 6 & -1.863768 & -1.056485 & 4.092254 \\ 6 & -1.961309 & -0.262858 & 2.980658 \\ 6 & -1.326898 & -3.233969 & 5.123161 \\ 6 & -0.934757 & -4.542034 & 4.999567 \\ 6 & -0.666520 & -5.077328 & 3.722058 \\ 6 & -0.792423 & -4.299712 & 2.597773 \\ 6 & -1.052456 & -2.714733 & 0.206746 \\ 6 & 0.182029 & -2.634118 & -0.412959 \\ 6 & 0.427657 & -3.333799 & -1.617074 \\ 6 & -0.551013 & -4.085958 & -2.208042 \\ 6 & -1.835703 & -4.173717 & -1.624111 \\ & & & \\ 6\end{array}$




\begin{tabular}{|c|c|c|c|}
\hline & -2.093256 & -3.483178 & -0.402931 \\
\hline & -3.391587 & -3.578154 & 0.162600 \\
\hline & -4.373245 & -4.319600 & -0.445677 \\
\hline & -4.106503 & -5.022270 & -1.640770 \\
\hline & -2.864671 & -4.945465 & -2.217248 \\
\hline & 1.240628 & -1.847762 & 0.155977 \\
\hline & 1.599049 & -0.412424 & -0.680317 \\
\hline & 2.828173 & -0.703717 & -1.770312 \\
\hline & 3.968043 & -1.379326 & -1.403387 \\
\hline & 4.893536 & -0.812404 & -0.546040 \\
\hline & 5.961818 & -1.647526 & -0.073176 \\
\hline & 6.120651 & -2.962274 & -0.605261 \\
\hline & 5.220377 & -3.425621 & -1.595706 \\
\hline & 4.154942 & -2.656986 & -1.973762 \\
\hline & 6.837618 & -1.244294 & 0.967790 \\
\hline & 7.819725 & -2.082390 & 1.435673 \\
\hline 6 & 7.993437 & -3.368313 & 0.882358 \\
\hline 6 & 7.156920 & -3.794868 & -0.117009 \\
\hline 6 & 4.755614 & 0.597349 & -0.113904 \\
\hline 6 & 3.568643 & 1.067736 & 0.416203 \\
\hline 6 & 3.445054 & 2.366459 & 0.949826 \\
\hline 6 & 4.498400 & 3.234647 & 0.900372 \\
\hline 6 & 5.704788 & 2.854881 & 0.263543 \\
\hline 6 & 5.831178 & 1.536476 & -0.269225 \\
\hline 6 & 6.766531 & 3.778657 & 0.106913 \\
\hline 6 & 7.904610 & 3.437909 & -0.578930 \\
\hline 6 & 8.018156 & 2.149875 & -1.142439 \\
\hline 6 & 7.014052 & 1.225723 & -0.987750 \\
\hline 8 & 2.465804 & 0.268717 & 0.546953 \\
\hline 6 & 0.367084 & 2.693311 & -1.272466 \\
\hline 6 & 2.148550 & -2.584456 & 1.067363 \\
\hline 6 & 2.606410 & -1.856579 & 2.306115 \\
\hline 6 & 1.746727 & -1.048809 & 3.052903 \\
\hline 6 & 2.189003 & -0.424029 & 4.210671 \\
\hline 6 & 3.501804 & -0.599490 & 4.646766 \\
\hline 6 & 4.363346 & -1.405791 & 3.912356 \\
\hline 6 & 3.916122 & -2.029399 & 2.749273 \\
\hline 1 & 6.702294 & -0.266395 & 1.422426 \\
\hline 1 & -2.283361 & 0.769014 & 3.095061 \\
\hline 1 & 7.263434 & -4.790730 & -0.545273 \\
\hline 1 & -1.538827 & -2.805360 & 6.101699 \\
\hline 1 & 6.649086 & 4.775271 & 0.530749 \\
\hline 1 & -3.608708 & -3.039877 & 1.083885 \\
\hline 1 & -5.366929 & -4.366779 & -0.004320 \\
\hline 1 & 4.411129 & 4.235466 & 1.321495 \\
\hline 1 & -2.097675 & -0.654474 & 5.077532 \\
\hline 1 & 5.374784 & -4.412284 & -2.029180 \\
\hline 1 & 8.906960 & 1.886900 & -1.711765 \\
\hline 1 & 3.439206 & -2.997079 & -2.718771 \\
\hline 1 & -2.647714 & -5.470936 & -3.146434 \\
\hline 1 & -0.356911 & -4.618445 & -3.137814 \\
\hline 1 & -0.359754 & -6.116470 & 3.627405 \\
\hline 1 & 7.111735 & 0.245452 & -1.446715 \\
\hline 1 & -0.589293 & -4.729631 & 1.618933 \\
\hline 1 & 1.416427 & -3.253215 & -2.064178 \\
\hline 1 & 8.709845 & 4.158463 & -0.701776 \\
\hline
\end{tabular}




\begin{tabular}{|c|c|c|c|}
\hline 1 & 8.465679 & -1.752443 & 2.246366 \\
\hline & -4.890850 & -5.615214 & -2.106427 \\
\hline & -0.830439 & -5.169360 & 5.881721 \\
\hline & 8.779253 & -4.019864 & 1.257305 \\
\hline & 2.493145 & 2.637306 & 1.398802 \\
\hline & -4.341545 & -0.081592 & 1.631927 \\
\hline & -3.005935 & -0.439448 & -2.411429 \\
\hline & -7.123750 & -1.137938 & -1.446351 \\
\hline 1 & -3.960631 & 2.239859 & -0.310047 \\
\hline & -0.412462 & 1.999427 & 2.074533 \\
\hline 1 & -2.773596 & 5.588238 & 2.077012 \\
\hline 1 & 1.580083 & -3.472180 & 1.379460 \\
\hline 1 & -1.590391 & 1.320281 & -2.231565 \\
\hline 1 & 3.026298 & -2.963448 & 0.521899 \\
\hline 1 & 4.602805 & -2.649457 & 2.171019 \\
\hline 1 & 0.733558 & -0.881564 & 2.694644 \\
\hline 1 & 5.393534 & -1.546507 & 4.235051 \\
\hline 1 & 1.502296 & 0.206712 & 4.774581 \\
\hline 1 & 3.850253 & -0.105492 & 5.551352 \\
\hline 1 & 1.171445 & 2.714676 & -0.534913 \\
\hline 1 & -0.492873 & 3.317754 & -1.022985 \\
\hline 1 & 2.933096 & 3.579778 & -1.711010 \\
\hline 1 & 5.240369 & 3.258659 & -2.529589 \\
\hline 1 & 5.698747 & 1.683579 & -4.393580 \\
\hline 1 & 3.803512 & 0.472667 & -5.468444 \\
\hline 1 & 1.502660 & 0.786898 & -4.637169 \\
\hline 1 & 0.223226 & 3.912580 & -4.116083 \\
\hline 1 & -0.385961 & 2.311793 & -4.550500 \\
\hline 1 & -1.227365 & 3.259915 & -3.313105 \\
\hline 6 & -6.834529 & -1.316432 & -3.899542 \\
\hline 6 & -4.940978 & 0.209304 & -4.377089 \\
\hline 6 & -4.543127 & -2.242121 & -4.076879 \\
\hline 6 & -8.284077 & -1.182874 & 0.749012 \\
\hline 6 & -6.522003 & -1.619479 & 2.428940 \\
\hline 6 & -7.148973 & 0.732634 & 1.844169 \\
\hline 1 & -6.966078 & -1.466513 & -4.978569 \\
\hline 1 & -7.194706 & -2.223527 & -3.396863 \\
\hline 1 & -7.479427 & -0.478828 & -3.603057 \\
\hline 1 & -4.697043 & -2.402007 & -5.152893 \\
\hline 1 & -3.467182 & -2.101445 & -3.911151 \\
\hline 1 & -4.842979 & -3.155588 & -3.547297 \\
\hline 1 & -5.135923 & 0.071049 & -5.449112 \\
\hline 1 & -5.501539 & 1.091889 & -4.041454 \\
\hline 1 & -3.871215 & 0.428551 & -4.262868 \\
\hline 1 & -7.291335 & -1.637054 & 3.212544 \\
\hline 1 & -6.393052 & -2.647548 & 2.065379 \\
\hline 1 & -5.580110 & -1.312512 & 2.901087 \\
\hline 1 & -9.022113 & -1.217760 & 1.560404 \\
\hline 1 & -8.686900 & -0.522823 & -0.029638 \\
\hline 1 & -8.201586 & -2.195789 & 0.332707 \\
\hline 1 & -7.899019 & 0.730838 & 2.646880 \\
\hline 1 & -6.218876 & 1.148437 & 2.253167 \\
\hline 1 & -7.500337 & 1.412637 & 1.056392 \\
\hline 6 & -0.811713 & 5.931658 & 3.535049 \\
\hline 6 & -0.811868 & 3.688652 & 4.599159 \\
\hline 6 & 0.899374 & 4.265861 & 2.880258 \\
\hline
\end{tabular}




$\begin{array}{rrrr}6 & -5.523617 & 4.073196 & -0.711278 \\ 6 & -5.573113 & 5.228377 & 1.480638 \\ 6 & -4.154666 & 6.111044 & -0.383138 \\ 1 & -0.103516 & 6.301166 & 4.287214 \\ 1 & -0.669030 & 6.526220 & 2.623385 \\ 1 & -1.820931 & 6.126431 & 3.918874 \\ 1 & -0.164200 & 4.078601 & 5.396377 \\ 1 & -1.854647 & 3.796756 & 4.925605 \\ 1 & -0.601874 & 2.615681 & 4.492855 \\ 1 & 1.559724 & 4.748392 & 3.613127 \\ 1 & 1.181063 & 3.206911 & 2.846281 \\ 1 & 1.099489 & 4.710890 & 1.895557 \\ 1 & -4.992370 & 6.709965 & -0.765246 \\ 1 & -3.580701 & 6.745077 & 0.303213 \\ 1 & -3.503189 & 5.860104 & -1.230583 \\ 1 & -6.426569 & 5.825449 & 1.132014 \\ 1 & -5.968490 & 4.335062 & 1.982127 \\ 1 & -5.037023 & 5.823137 & 2.230052 \\ 1 & -6.364656 & 4.698409 & -1.036660 \\ 1 & -4.948304 & 3.801801 & -1.606262 \\ 1 & -5.942657 & 3.150958 & -0.284771\end{array}$

$3_{\text {IIC-re- }} \Psi-1$ s2s3a4a

Number of imaginary frequencies : 0

The smallest frequencies are : $12.8480 \quad 16.499922 .4265 \mathrm{~cm}(-1)$

Electronic energy : $\quad H F=-4360.8924674$

Zero-point correction $=\quad 1.455322($ Hartree/Particle $)$

Thermal correction to Energy= $\quad 1.539630$

Thermal correction to Enthalpy= $\quad 1.540574$

Thermal correction to Gibbs Free Energy $=\quad 1.338355$

Sum of electronic and zero-point Energies $=\quad-4359.437145$

Sum of electronic and thermal Energies $=\quad-4359.352837$

Sum of electronic and thermal Enthalpies $=\quad-4359.351893$

Sum of electronic and thermal Free Energies $=\quad-4359.554112$

Cartesian Coordinates

$\begin{array}{cccc}6 & 2.316627 & 1.367293 & -4.174651 \\ 6 & 2.053976 & 2.205852 & -3.083490 \\ 6 & 3.130172 & 2.886401 & -2.506230 \\ 6 & 4.429147 & 2.702619 & -2.973420 \\ 6 & 4.677393 & 1.838127 & -4.032990 \\ 6 & 3.610172 & 1.177956 & -4.639669 \\ 6 & 0.656432 & 2.438909 & -2.610275 \\ 6 & -0.230908 & 3.041813 & -3.681696 \\ 45 & -0.282756 & 0.650038 & -1.665146 \\ 6 & -0.531719 & -0.539843 & -3.149306 \\ 8 & -0.670118 & -1.068289 & -4.172061 \\ 15 & -1.841678 & 0.322276 & 0.184256 \\ 6 & -2.094004 & 1.989131 & 0.914929 \\ 6 & -1.177102 & 2.514116 & 1.830076 \\ 6 & -1.324823 & 3.808143 & 2.334938 \\ 6 & -2.374076 & 4.592643 & 1.839163 \\ 6 & -3.268629 & 4.116888 & 0.878853 \\ 6 & -3.120740 & 2.799279 & 0.440551\end{array}$




\begin{tabular}{|c|c|c|c|}
\hline 6 & -0.403138 & 4.300011 & 3.451599 \\
\hline 5 & -4.386645 & 4.966995 & 0.280462 \\
\hline b & -3.559249 & -0.138190 & -0.316175 \\
\hline & -3.854689 & -0.418058 & -1.646212 \\
\hline$b$ & -5.157859 & -0.731927 & -2.045174 \\
\hline f & -6.145605 & -0.772173 & -1.060294 \\
\hline 6 & -5.883049 & -0.509069 & 0.289712 \\
\hline 6 & -4.574439 & -0.188769 & 0.643357 \\
\hline 6 & -7.007695 & -0.603274 & 1.319854 \\
\hline 6 & -5.523344 & -0.971935 & -3.509004 \\
\hline 6 & -1.674769 & -0.770016 & 1.682265 \\
\hline 6 & -1.322517 & -2.102431 & 1.517894 \\
\hline 6 & -1.218120 & -2.966751 & 2.655051 \\
\hline 6 & -1.481148 & -2.451277 & 3.957420 \\
\hline 6 & -1.870608 & -1.099092 & 4.087820 \\
\hline 6 & -1.964790 & -0.288592 & 2.988205 \\
\hline 6 & -1.348706 & -3.296884 & 5.084557 \\
\hline 6 & -0.972089 & -4.607308 & 4.938900 \\
\hline 6 & -0.719131 & -5.127119 & 3.651914 \\
\hline 6 & -0.843516 & -4.331492 & 2.540053 \\
\hline 6 & -1.099767 & -2.703186 & 0.173835 \\
\hline 6 & 0.129738 & -2.627264 & -0.455454 \\
\hline 6 & 0.349108 & -3.294464 & -1.682714 \\
\hline 6 & -0.655476 & -3.994623 & -2.294021 \\
\hline 6 & -1.938599 & -4.065442 & -1.704837 \\
\hline 6 & -2.163536 & -3.426408 & -0.449711 \\
\hline 6 & -3.456287 & -3.515904 & 0.129812 \\
\hline 6 & -4.465962 & -4.198258 & -0.500634 \\
\hline 6 & -4.235693 & -4.840159 & -1.737349 \\
\hline 6 & -2.999277 & -4.769619 & -2.326686 \\
\hline 7 & 1.205092 & -1.870142 & 0.121620 \\
\hline 15 & 1.579231 & -0.426812 & -0.693599 \\
\hline 8 & 2.800611 & -0.720260 & -1.792474 \\
\hline 6 & 3.934273 & -1.412561 & -1.437986 \\
\hline 6 & 4.870526 & -0.864204 & -0.580235 \\
\hline 6 & 5.933446 & -1.714347 & -0.122883 \\
\hline 6 & 6.076469 & -3.024792 & -0.669531 \\
\hline 6 & 5.164804 & -3.469484 & -1.658137 \\
\hline 6 & 4.104372 & -2.686767 & -2.021442 \\
\hline 6 & 6.819201 & -1.329989 & 0.916650 \\
\hline 6 & 7.796784 & -2.181622 & 1.369304 \\
\hline 6 & 7.955401 & -3.463089 & 0.801247 \\
\hline 6 & 7.108425 & -3.871707 & -0.196871 \\
\hline 6 & 4.748835 & 0.541877 & -0.131994 \\
\hline 6 & 3.569051 & 1.017561 & 0.409196 \\
\hline 6 & 3.459441 & 2.312472 & 0.954368 \\
\hline 6 & 4.521329 & 3.170597 & 0.910270 \\
\hline 6 & 5.721621 & 2.785857 & 0.265018 \\
\hline 6 & 5.832470 & 1.472314 & -0.282942 \\
\hline 6 & 6.791473 & 3.701034 & 0.113023 \\
\hline 6 & 7.922363 & 3.357159 & -0.583108 \\
\hline 6 & 8.020295 & 2.074545 & -1.161810 \\
\hline 6 & 7.008297 & 1.158353 & -1.011544 \\
\hline 8 & 2.459998 & 0.226357 & 0.538762 \\
\hline 6 & 0.373142 & 2.704462 & -1.250007 \\
\hline 6 & 2.104356 & -2.632758 & 1.020128 \\
\hline
\end{tabular}




\begin{tabular}{|c|c|c|c|}
\hline 6 & 2.581655 & -1.925143 & 2.263363 \\
\hline 6 & 1.735545 & -1.119693 & 3.027923 \\
\hline & 2.193687 & -0.510811 & 4.187891 \\
\hline & 3.509774 & -0.699855 & 4.608336 \\
\hline & 4.357810 & -1.504355 & 3.856515 \\
\hline & 3.894310 & -2.112595 & 2.691587 \\
\hline & 6.695382 & -0.355755 & 1.382285 \\
\hline & -2.277289 & 0.743987 & 3.120442 \\
\hline & 7.203339 & -4.863947 & -0.636144 \\
\hline & -1.549429 & -2.880651 & 6.070791 \\
\hline & 6.685744 & 4.694019 & 0.548322 \\
\hline & -3.647172 & -3.017341 & 1.078593 \\
\hline & -5.455126 & -4.240714 & -0.048373 \\
\hline & 4.445336 & 4.168145 & 1.341397 \\
\hline & -2.097705 & -0.708922 & 5.079510 \\
\hline & 5.306583 & -4.453422 & -2.102041 \\
\hline & 8.903242 & 1.809587 & -1.739271 \\
\hline & 3.379894 & -3.012434 & -2.764492 \\
\hline & -2.808190 & -5.255868 & -3.282447 \\
\hline & -0.484131 & -4.494403 & -3.246196 \\
\hline & -0.425712 & -6.168394 & 3.540006 \\
\hline & 7.093906 & 0.182304 & -1.481845 \\
\hline ] & -0.652637 & -4.748586 & 1.553191 \\
\hline & 1.336005 & -3.224072 & -2.135908 \\
\hline 1 & 8.733708 & 4.071402 & -0.702599 \\
\hline & 8.450851 & -1.866040 & 2.179204 \\
\hline 1 & -5.042505 & -5.387137 & -2.220792 \\
\hline & -0.868862 & -5.248680 & 5.811030 \\
\hline & 8.737868 & -4.125473 & 1.164012 \\
\hline 1 & 2.511210 & 2.588977 & 1.406983 \\
\hline & -4.332391 & 0.033561 & 1.679819 \\
\hline I & -3.047175 & -0.384171 & -2.371549 \\
\hline & -7.166759 & -1.016445 & -1.354122 \\
\hline & -3.812528 & 2.398172 & -0.298595 \\
\hline & -0.345158 & 1.888801 & 2.164093 \\
\hline & -2.492262 & 5.606244 & 2.209916 \\
\hline ] & 1.521810 & -3.513502 & 1.325906 \\
\hline & -1.594631 & 1.357351 & -2.221011 \\
\hline 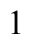 & 2.972266 & -3.021132 & 0.465637 \\
\hline & 4.570804 & -2.731002 & 2.099725 \\
\hline . & 0.720424 & -0.941183 & 2.681212 \\
\hline 1 & 5.390199 & -1.655823 & 4.167024 \\
\hline & 1.516518 & 0.116594 & 4.766992 \\
\hline ] & 3.870990 & -0.218160 & 5.514532 \\
\hline & 1.176868 & 2.715825 & -0.511549 \\
\hline 1 & -0.483029 & 3.333187 & -0.996710 \\
\hline & 2.946956 & 3.573050 & -1.680715 \\
\hline t & 5.250563 & 3.242904 & -2.504854 \\
\hline 1 & 5.695204 & 1.686947 & -4.387935 \\
\hline 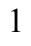 & 3.788934 & 0.504739 & -5.476182 \\
\hline 1 & 1.492241 & 0.828306 & -4.640208 \\
\hline 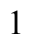 & 0.238483 & 3.955190 & -4.078831 \\
\hline 1 & -0.384903 & 2.365028 & -4.531306 \\
\hline 1 & -1.216925 & 3.306176 & -3.281642 \\
\hline 6 & -4.287390 & -1.047766 & -4.403120 \\
\hline . & -6.293764 & -2.286452 & -3.658682 \\
\hline
\end{tabular}




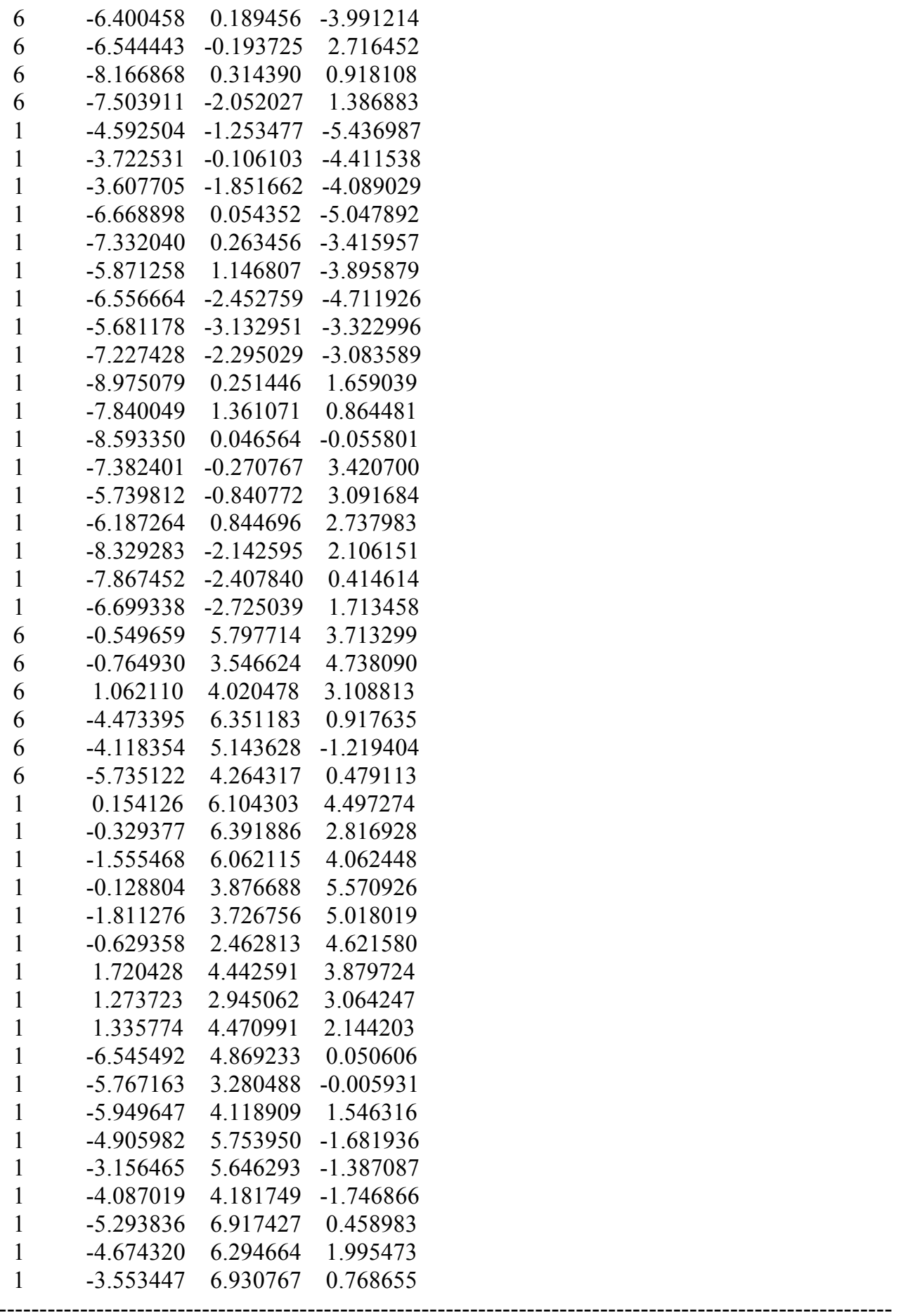

$3_{\text {IIC-re- }}-\psi_{-122 s 3 a 4 s}$

Number of imaginary frequencies : 0

The smallest frequencies are : $17.1812 \quad 23.5257 \quad 24.8176 \mathrm{~cm}(-1)$

Electronic energy : $\quad H F=-4360.8930761$

Zero-point correction $=\quad 1.455743($ Hartree/Particle $)$

Thermal correction to Energy $=\quad 1.539832$

Thermal correction to Enthalpy $=\quad 1.540776$

Thermal correction to Gibbs Free Energy $=\quad 1.340534$ 
Sum of electronic and zero-point Energies= Sum of electronic and thermal Energies= Sum of electronic and thermal Enthalpies= Sum of electronic and thermal Free Energies=
$-4359.437333$

$-4359.353245$

$-4359.352300$

$-4359.552542$

Cartesian Coordinates

\begin{tabular}{|c|c|c|c|}
\hline 6 & 2.305549 & 1.412557 & -4.154141 \\
\hline 6 & 2.044506 & 2.238872 & -3.053317 \\
\hline 6 & 3.121393 & 2.913702 & -2.470720 \\
\hline 6 & 4.419537 & 2.735968 & -2.942538 \\
\hline 6 & 4.666271 & 1.883340 & -4.012000 \\
\hline 6 & 3.598262 & 1.229311 & -4.623923 \\
\hline 6 & 0.647872 & 2.465610 & -2.574289 \\
\hline 6 & -0.242179 & 3.081854 & -3.635862 \\
\hline 45 & -0.287518 & 0.663440 & -1.649908 \\
\hline 6 & -0.536254 & -0.509980 & -3.146558 \\
\hline 8 & -0.675633 & -1.029503 & -4.173715 \\
\hline 15 & -1.846976 & 0.311617 & 0.190698 \\
\hline 6 & -2.105089 & 1.969268 & 0.939432 \\
\hline 6 & -1.192150 & 2.485999 & 1.862931 \\
\hline 6 & -1.333835 & 3.780696 & 2.367986 \\
\hline 6 & -2.367144 & 4.576941 & 1.857766 \\
\hline 6 & -3.256653 & 4.109179 & 0.888640 \\
\hline 6 & -3.123078 & 2.787256 & 0.459094 \\
\hline 6 & -0.418187 & 4.262058 & 3.494106 \\
\hline 6 & -4.349496 & 4.973689 & 0.265062 \\
\hline 6 & -3.562290 & -0.144891 & -0.320155 \\
\hline 6 & -3.859539 & -0.411798 & -1.656406 \\
\hline 6 & -5.160244 & -0.717028 & -2.056287 \\
\hline 6 & -6.154547 & -0.770257 & -1.070743 \\
\hline 6 & -5.888570 & -0.527058 & 0.277373 \\
\hline 6 & -4.575857 & -0.205920 & 0.631995 \\
\hline 6 & -6.956324 & -0.583148 & 1.368610 \\
\hline 6 & -5.527105 & -0.938199 & -3.522613 \\
\hline 6 & -1.682762 & -0.801547 & 1.673052 \\
\hline 6 & -1.328410 & -2.130964 & 1.490509 \\
\hline 6 & -1.228404 & -3.011947 & 2.615196 \\
\hline 6 & -1.499646 & -2.516757 & 3.923674 \\
\hline 6 & -1.889582 & -1.166649 & 4.072535 \\
\hline 6 & -1.977720 & -0.339399 & 2.984789 \\
\hline 6 & -1.372860 & -3.379367 & 5.038569 \\
\hline 6 & -0.992918 & -4.686707 & 4.875198 \\
\hline 6 & -0.730569 & -5.186130 & 3.582018 \\
\hline 6 & -0.849942 & -4.373890 & 2.481694 \\
\hline 6 & -1.101516 & -2.713876 & 0.139345 \\
\hline 6 & 0.130018 & -2.632290 & -0.485321 \\
\hline 6 & 0.352773 & -3.287630 & -1.718325 \\
\hline 6 & -0.649874 & -3.982751 & -2.338669 \\
\hline 6 & -1.934974 & -4.058440 & -1.754347 \\
\hline 6 & -2.163906 & -3.429843 & -0.494740 \\
\hline 6 & -3.458983 & -3.521685 & 0.079235 \\
\hline 6 & -4.466553 & -4.198810 & -0.560058 \\
\hline 6 & -4.232226 & -4.831724 & -1.800611 \\
\hline 6 & -2.993816 & -4.757230 & -2.385303 \\
\hline 7 & 1.203265 & -1.879881 & 0.101818 \\
\hline
\end{tabular}




\begin{tabular}{|c|c|c|c|}
\hline & 1.574840 & -0.425770 & -0.695300 \\
\hline 8 & 2.796787 & -0.703332 & -1.797729 \\
\hline 6 & 3.932762 & -1.395931 & -1.450884 \\
\hline 6 & 4.867272 & -0.853885 & -0.587217 \\
\hline 6 & 5.932831 & -1.705406 & -0.138804 \\
\hline 6 & 6.080739 & -3.009119 & -0.699949 \\
\hline 6 & 5.170676 & -3.446003 & -1.693487 \\
\hline 6 & 4.107326 & -2.663178 & -2.048109 \\
\hline 6 & 6.816271 & -1.329689 & 0.905840 \\
\hline 6 & 7.796641 & -2.182848 & 1.349508 \\
\hline 6 & 7.960528 & -3.457067 & 0.766821 \\
\hline 6 & 7.115644 & -3.857487 & -0.236382 \\
\hline 6 & 4.741067 & 0.546512 & -0.122907 \\
\hline 6 & 3.560031 & 1.010872 & 0.425128 \\
\hline 6 & 3.446035 & 2.298516 & 0.986381 \\
\hline 6 & 4.504496 & 3.161357 & 0.951053 \\
\hline 6 & 5.705541 & 2.789161 & 0.299838 \\
\hline 6 & 5.821124 & 1.482561 & -0.263432 \\
\hline 6 & 6.771817 & 3.710116 & 0.157730 \\
\hline 6 & 7.903792 & 3.378387 & -0.542521 \\
\hline 6 & 8.006511 & 2.102750 & -1.135652 \\
\hline 6 & 6.997922 & 1.181177 & -0.995744 \\
\hline 8 & 2.454558 & 0.213350 & 0.545405 \\
\hline 6 & 0.367609 & 2.713335 & -1.210130 \\
\hline 6 & 2.103155 & -2.650166 & 0.993186 \\
\hline 6 & 2.575620 & -1.954388 & 2.24 \\
\hline 6 & 1.723594 & -1.164021 & 3.018549 \\
\hline 6 & 2.177757 & -0.563240 & 4.184315 \\
\hline 6 & 3.495531 & -0.746528 & 4.602071 \\
\hline 6 & 4.349272 & -1.536748 & 3.841532 \\
\hline 6 & 3.889904 & -2.136224 & 2.670450 \\
\hline 1 & 6.688223 & -0.361522 & 1.38 \\
\hline 1 & -2.289235 & 0.691661 & 3.130823 \\
\hline 1 & 7.214487 & -4.844336 & -0.686795 \\
\hline 1 & -1.580368 & -2.978625 & 6.029806 \\
\hline 1 & 6.662535 & 4.697671 & 0.604345 \\
\hline 1 & -3.653148 & -3.028124 & 1.030205 \\
\hline 1 & -5.458328 & -4.241682 & -0.113692 \\
\hline 1 & 4.425266 & 4.153320 & 1.394328 \\
\hline 1 & -2.121047 & -0.791441 & 5.068972 \\
\hline 1 & 5.315952 & -4.424472 & -2.148238 \\
\hline 1 & 8.890431 & 1.847614 & -1.716033 \\
\hline 1 & 3.384003 & -2.983506 & -2.794611 \\
\hline 1 & -2.799878 & -5.235965 & -3.344265 \\
\hline 1 & -0.475564 & -4.474269 & -3.294601 \\
\hline 1 & -0.433940 & -6.224881 & 3.456018 \\
\hline 1 & 7.086947 & 0.210715 & -1.476908 \\
\hline 1 & -0.651741 & -4.775387 & 1.489825 \\
\hline 1 & 1.340884 & -3.212649 & -2.168099 \\
\hline 1 & 8.712420 & 4.096985 & -0.654116 \\
\hline 1 & 8.448803 & -1.874256 & 2.163624 \\
\hline 1 & -5.037732 & -5.374359 & -2.291084 \\
\hline 1 & -0.893992 & -5.341261 & 5.737978 \\
\hline 1 & 8.745276 & -4.120588 & 1.122500 \\
\hline 1 & 2.497452 & 2.565738 & 1.443858 \\
\hline 1 & -4.341968 & 0.008950 & 1.674408 \\
\hline
\end{tabular}




\begin{tabular}{|c|c|c|c|}
\hline 1 & -3.051734 & -0.371557 & -2.380956 \\
\hline & -7.172502 & -1.007475 & -1.373695 \\
\hline & -3.814101 & 2.391955 & -0.283994 \\
\hline & -0.366562 & 1.854880 & 2.201602 \\
\hline & -2.475642 & 5.593757 & 2.222613 \\
\hline & 1.522667 & -3.535978 & 1.287836 \\
\hline & -1.600378 & 1.377100 & -2.195798 \\
\hline & 2.973430 & -3.029464 & 0.436088 \\
\hline & 4.570886 & -2.742658 & 2.071397 \\
\hline & 0.706969 & -0.989625 & 2.674240 \\
\hline & 5.382980 & -1.683479 & 4.149942 \\
\hline 1 & 1.496250 & 0.053743 & 4.769602 \\
\hline 1 & 3.853718 & -0.271297 & 5.512872 \\
\hline & 1.173390 & 2.716438 & -0.473930 \\
\hline & -0.488924 & 3.337374 & -0.946500 \\
\hline & 2.939343 & 3.591293 & -1.637506 \\
\hline & 5.241563 & 3.271298 & -2.469350 \\
\hline & 5.683476 & 1.736741 & -4.370594 \\
\hline & 3.775744 & 0.565506 & -5.468192 \\
\hline & 1.480515 & 0.878238 & -4.623926 \\
\hline & 0.227113 & 3.999334 & -4.023564 \\
\hline & -0.399560 & 2.415410 & -4.493001 \\
\hline & -1.226599 & 3.342930 & -3.229558 \\
\hline & -4.292354 & -0.996252 & -4.419791 \\
\hline D & -6.292404 & -2.253684 & -3.690118 \\
\hline & -6.409214 & 0.226534 & -3.987608 \\
\hline f & -8.313796 & -1.029569 & 0.832418 \\
\hline 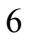 & -6.529415 & -1.575904 & 2.456807 \\
\hline & -7.116272 & 0.811690 & 1.984360 \\
\hline 1 & -4.598639 & -1.185943 & -5.456385 \\
\hline & -3.729724 & -0.053200 & -4.413737 \\
\hline 1 & -3.610006 & -1.803315 & -4.119983 \\
\hline & -6.678923 & 0.104710 & -5.045580 \\
\hline 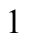 & -7.340352 & 0.289720 & -3.410494 \\
\hline 1 & -5.882796 & 1.184244 & -3.880177 \\
\hline ] & -6.554114 & -2.406851 & -4.745667 \\
\hline 1 & -5.676723 & -3.102414 & -3.365654 \\
\hline & -7.226258 & -2.273411 & -3.115724 \\
\hline ] & -7.281079 & -1.608693 & 3.256987 \\
\hline & -6.433390 & -2.590418 & 2.047567 \\
\hline ] & -5.569404 & -1.310289 & 2.917622 \\
\hline 1 & -9.040128 & -1.071420 & 1.653953 \\
\hline t & -8.709693 & -0.333965 & 0.081517 \\
\hline 1 & -8.265118 & -2.030480 & 0.383094 \\
\hline & -7.857925 & 0.791392 & 2.794486 \\
\hline 1 & -6.173553 & 1.187627 & 2.402161 \\
\hline & -7.458454 & 1.534002 & 1.231287 \\
\hline 6 & -0.565674 & 5.757226 & 3.768784 \\
\hline 6 & -0.786419 & 3.497472 & 4.772151 \\
\hline 5 & 1.048642 & 3.984812 & 3.155803 \\
\hline 6 & -4.394531 & 6.378303 & 0.860235 \\
\hline 5 & -4.076332 & 5.097056 & -1.239637 \\
\hline 6 & -5.717652 & 4.317268 & 0.483855 \\
\hline & 0.130367 & 6.055276 & 4.562884 \\
\hline & -0.335266 & 6.359972 & 2.880715 \\
\hline & -1.574978 & 6.019186 & 4.109726 \\
\hline
\end{tabular}




$\begin{array}{rrrr}1 & -0.154942 & 3.821104 & 5.610998 \\ 1 & -1.834307 & 3.674600 & 5.048154 \\ 1 & -0.649229 & 2.414736 & 4.647862 \\ 1 & 1.703553 & 4.399120 & 3.933868 \\ 1 & 1.259708 & 2.909643 & 3.101744 \\ 1 & 1.327014 & 4.444483 & 2.196871 \\ 1 & -6.507886 & 4.920916 & 0.017403 \\ 1 & -5.770324 & 3.310313 & 0.049611 \\ 1 & -5.944815 & 4.232712 & 1.554894 \\ 1 & -4.831212 & 5.737362 & -1.715935 \\ 1 & -3.088420 & 5.540843 & -1.422903 \\ 1 & -4.100710 & 4.122095 & -1.742824 \\ 1 & -5.197302 & 6.955062 & 0.383763 \\ 1 & -4.598227 & 6.360718 & 1.938838 \\ 1 & -3.456904 & 6.924437 & 0.695385\end{array}$

$3_{\text {IIC-re- }} \psi_{-1}-1223344 a$

Number of imaginary frequencies : 0

The smallest frequencies are : $16.1224 \quad 20.7513 \quad 23.3846 \mathrm{~cm}(-1)$

Electronic energy : $\quad H F=-4360.8924746$

Zero-point correction $=\quad 1.455539($ Hartree/Particle $)$

Thermal correction to Energy= $\quad 1.539732$

Thermal correction to Enthalpy= $\quad 1.540676$

Thermal correction to Gibbs Free Energy $=\quad 1.339468$

Sum of electronic and zero-point Energies $=\quad-4359.436936$

Sum of electronic and thermal Energies $=\quad-4359.352743$

Sum of electronic and thermal Enthalpies $=\quad-4359.351799$

Sum of electronic and thermal Free Energies $=\quad-4359.553007$

Cartesian Coordinates

$\begin{array}{cccc}6 & 3.888896 & -2.110200 & 2.689271 \\ 6 & 2.574389 & -1.931258 & 2.263170 \\ 6 & 1.722197 & -1.137009 & 3.032559 \\ 6 & 2.176375 & -0.529820 & 4.194988 \\ 6 & 3.494357 & -0.710131 & 4.613341 \\ 6 & 4.348312 & -1.504021 & 3.856867 \\ 6 & 2.101447 & -2.634071 & 1.015658 \\ 7 & 1.199872 & -1.868749 & 0.121300 \\ 15 & 1.574916 & -0.422520 & -0.687927 \\ 8 & 2.456555 & 0.223445 & 0.547700 \\ 6 & 3.559491 & 1.023083 & 0.417405 \\ 6 & 4.740774 & 0.555036 & -0.126912 \\ 6 & 5.819505 & 1.491066 & -0.277242 \\ 6 & 5.701502 & 2.803544 & 0.271813 \\ 6 & 4.499769 & 3.180957 & 0.918787 \\ 6 & 3.442952 & 2.316534 & 0.964469 \\ 6 & 6.997463 & 1.183406 & -1.005095 \\ 6 & 8.005052 & 2.104727 & -1.153637 \\ 6 & 7.899879 & 3.386599 & -0.574539 \\ 6 & 6.766617 & 3.724404 & 0.120695 \\ 6 & 4.867821 & -0.849537 & -0.578284 \\ 6 & 3.933085 & -1.399699 & -1.436636 \\ 6 & 4.108791 & -2.671447 & -2.023885 \\ 6 & 5.172670 & -3.450624 & -1.663013\end{array}$




\begin{tabular}{|c|c|c|c|}
\hline 6 & 6.082454 & -3.004993 & -0.673116 \\
\hline 6 & 5.934049 & -1.696594 & -0.123045 \\
\hline 6 & 7.117631 & -3.849026 & -0.202391 \\
\hline 6 & 7.962458 & -3.439775 & 0.797287 \\
\hline & 7.798378 & -2.160572 & 1.368890 \\
\hline b & 6.817706 & -1.311534 & 0.918030 \\
\hline & 2.796012 & -0.711591 & -1.788356 \\
\hline 0 & 0.129127 & -2.628798 & -0.460949 \\
\hline 6 & -1.104155 & -2.705987 & 0.160711 \\
\hline 0 & -2.158520 & -3.443954 & -0.462553 \\
\hline 0 & -1.913508 & -4.116079 & -1.696466 \\
\hline 6 & -0.628917 & -4.035255 & -2.281801 \\
\hline 6 & 0.361495 & -3.307401 & -1.679788 \\
\hline 6 & -3.457247 & -3.528628 & 0.103591 \\
\hline 6 & -4.450773 & -4.245776 & -0.514387 \\
\hline 6 & -4.195794 & -4.933305 & -1.720952 \\
\hline 6 & -2.954504 & -4.863772 & -2.299586 \\
\hline 6 & -1.333756 & -2.113155 & 1.507481 \\
\hline 6 & -1.684932 & -0.782337 & 1.684449 \\
\hline 6 & -1.979686 & -0.312615 & 2.993524 \\
\hline 6 & -1.891966 & -1.134116 & 4.085389 \\
\hline 6 & -1.502941 & -2.485286 & 3.943268 \\
\hline 6 & -1.233710 & -2.988296 & 2.637239 \\
\hline 6 & -1.376133 & -3.341546 & 5.063029 \\
\hline 6 & -0.997488 & -4.650169 & 4.907109 \\
\hline 6 & -0.736212 & -5.157117 & 3.616714 \\
\hline 6 & -0.855780 & -4.351242 & 2.511714 \\
\hline 15 & -1.844253 & 0.322862 & 0.197423 \\
\hline 6 & -3.555847 & -0.135738 & -0.319568 \\
\hline 6 & -3.842378 & -0.385549 & -1.651819 \\
\hline 6 & -5.145640 & -0.682027 & -2.075302 \\
\hline 6 & -6.139810 & -0.755276 & -1.105448 \\
\hline 6 & -5.884303 & -0.522337 & 0.257174 \\
\hline 6 & -4.583720 & -0.206658 & 0.630735 \\
\hline 6 & -5.405278 & -0.881488 & -3.566415 \\
\hline 6 & -4.605840 & -2.088140 & -4.066842 \\
\hline 6 & -7.019786 & -0.638585 & 1.272921 \\
\hline 6 & -8.179136 & 0.280532 & 0.874344 \\
\hline 45 & -0.287650 & 0.662406 & -1.648025 \\
\hline 6 & 0.371000 & 2.716251 & -1.237951 \\
\hline 6 & 0.646800 & 2.447851 & -2.599636 \\
\hline 6 & -0.246233 & 3.048333 & -3.668035 \\
\hline 6 & 2.041765 & 2.211965 & -3.078791 \\
\hline 6 & 2.297981 & 1.368639 & -4.167838 \\
\hline 6 & 3.589111 & 1.175306 & -4.637876 \\
\hline 6 & 4.660223 & 1.835721 & -4.038224 \\
\hline 6 & 4.418348 & 2.704238 & -2.980444 \\
\hline 6 & 3.121779 & 2.892204 & -2.508303 \\
\hline 6 & -0.576848 & -0.527810 & -3.123389 \\
\hline 8 & -0.772196 & -1.055188 & -4.137903 \\
\hline 6 & -2.103295 & 1.983225 & 0.938335 \\
\hline 6 & -1.187807 & 2.505514 & 1.856320 \\
\hline 6 & -1.338205 & 3.796975 & 2.366754 \\
\hline 6 & -2.389641 & 4.581193 & 1.874771 \\
\hline 6 & -3.283646 & 4.107468 & 0.912944 \\
\hline 6 & -3.132634 & 2.792376 & 0.468420 \\
\hline
\end{tabular}




\begin{tabular}{|c|c|c|c|}
\hline 6 & -0.415858 & 4.286699 & 3.483786 \\
\hline 6 & 1.049360 & 4.010004 & 3.138132 \\
\hline 6 & -4.404318 & 4.956498 & 0.318244 \\
\hline 6 & -5.750569 & 4.249186 & 0.515976 \\
\hline 6 & -0.563867 & 5.783524 & 3.749713 \\
\hline 6 & -0.775048 & 3.529514 & 4.768766 \\
\hline 6 & -4.493997 & 6.338404 & 0.959851 \\
\hline 6 & -4.137733 & 5.138365 & -1.181429 \\
\hline 6 & -6.573701 & -0.249453 & 2.680872 \\
\hline 6 & -7.510698 & -2.090141 & 1.310793 \\
\hline 6 & -6.880816 & -1.125549 & -3.870756 \\
\hline 6 & -4.960894 & 0.374440 & -4.327534 \\
\hline 1 & 6.689528 & -0.339214 & 1.386517 \\
\hline 1 & -2.291340 & 0.719208 & 3.133687 \\
\hline 1 & 7.216670 & -4.839701 & -0.644279 \\
\hline 1 & -1.582509 & -2.934739 & 6.052029 \\
\hline 1 & 6.655622 & 4.716551 & 0.556585 \\
\hline 1 & -3.664060 & -3.003322 & 1.034479 \\
\hline 1 & -5.443444 & -4.287231 & -0.069999 \\
\hline 1 & 4.418972 & 4.177616 & 1.351095 \\
\hline 1 & -2.123425 & -0.753780 & 5.079860 \\
\hline 1 & 5.318562 & -4.432650 & -2.109812 \\
\hline 1 & 8.890073 & 1.844483 & -1.730062 \\
\hline 1 & 3.385695 & -2.998381 & -2.767720 \\
\hline 1 & -2.746992 & -5.376871 & -3.237803 \\
\hline 1 & -0.444290 & -4.553167 & -3.221698 \\
\hline 1 & -0.440115 & -6.196703 & \\
\hline 1 & 7.088433 & 0.207966 & -1.475660 \\
\hline 1 & -0.657933 & -4.759014 & 1.522336 \\
\hline 1 & 1.349820 & -3.231209 & -2.128813 \\
\hline 1 & 8.707721 & 4.105000 & -0.692858 \\
\hline 1 & 8.450641 & -1.844750 & 2.180149 \\
\hline 1 & -4.989056 & -5.508319 & $-2.1 \mathrm{~S}$ \\
\hline 1 & -0.898552 & -5.299769 & 5.773626 \\
\hline 1 & 8.747357 & -4.100040 & 1.158659 \\
\hline 1 & 2.494216 & 2.586529 & 1.420146 \\
\hline 1 & -4.351393 & -0.003641 & 1.673392 \\
\hline 1 & -3.034174 & -0.337442 & -2.377142 \\
\hline 1 & -7.157776 & -0.995193 & -1.406519 \\
\hline 1 & -3.823306 & 2.393065 & -0.272726 \\
\hline 1 & -0.354714 & 1.880024 & 2.187165 \\
\hline 1 & -2.509803 & 5.593092 & 2.249600 \\
\hline 1 & 1.521748 & -3.518612 & 1.315666 \\
\hline 1 & -1.598994 & 1.379258 & -2.190832 \\
\hline 1 & 2.971394 & -3.015879 & 0.459751 \\
\hline 1 & 4.569965 & -2.719732 & 2.093448 \\
\hline 1 & 0.705334 & -0.965365 & 2.687523 \\
\hline 1 & 5.382138 & -1.648613 & 4.165878 \\
\hline 1 & 1.494643 & 0.089442 & 4.777522 \\
\hline 1 & 3.852474 & -0.229895 & 5.521537 \\
\hline 1 & 1.179317 & 2.730096 & -0.504611 \\
\hline 1 & -0.483669 & 3.345675 & -0.981044 \\
\hline 1 & 2.943688 & 3.581328 & -1.683676 \\
\hline 1 & 5.242793 & 3.244664 & -2.517472 \\
\hline 1 & 5.676109 & 1.681359 & -4.397279 \\
\hline 1 & 3.763131 & 0.498432 & -5.472440 \\
\hline
\end{tabular}




$\begin{array}{rrrr}1 & 1.470313 & 0.828584 & -4.626678 \\ 1 & 0.221840 & 3.959470 & -4.071790 \\ 1 & -0.407021 & 2.368361 & -4.514014 \\ 1 & -1.229618 & 3.315571 & -3.263125 \\ 1 & -7.017861 & -1.255586 & -4.951680 \\ 1 & -7.255160 & -2.034523 & -3.381969 \\ 1 & -7.510077 & -0.281875 & -3.558169 \\ 1 & -4.759628 & -2.225867 & -5.145901 \\ 1 & -3.528029 & -1.971155 & -3.895582 \\ 1 & -4.924631 & -3.004751 & -3.554362 \\ 1 & -5.160843 & 0.257522 & -5.401134 \\ 1 & -5.504140 & 1.261719 & -3.976083 \\ 1 & -3.886868 & 0.572025 & -4.212953 \\ 1 & -8.345297 & -2.195018 & 2.017380 \\ 1 & -7.859822 & -2.432307 & 0.328472 \\ 1 & -6.707639 & -2.764670 & 1.638026 \\ 1 & -7.419307 & -0.339752 & 3.374326 \\ 1 & -5.771492 & -0.900012 & 3.055205 \\ 1 & -6.219771 & 0.789606 & 2.721965 \\ 1 & -8.993031 & 0.203735 & 1.607702 \\ 1 & -7.856039 & 1.329161 & 0.838861 \\ 1 & -8.596992 & 0.025394 & -0.106606 \\ 1 & 0.140816 & 6.088925 & 4.533343 \\ 1 & -0.345835 & 6.380362 & 2.854563 \\ 1 & -1.569420 & 6.045710 & 4.101253 \\ 1 & -0.138197 & 3.858233 & 5.601573 \\ 1 & -1.821208 & 3.707736 & 5.050573 \\ 1 & -0.638399 & 2.446158 & 4.649490 \\ 1 & 1.708148 & 4.431421 & 3.909017 \\ 1 & 1.262851 & 2.935056 & 3.090644 \\ 1 & 1.320768 & 4.463228 & 2.174132 \\ 1 & -6.563332 & 4.853561 & 0.091246 \\ 1 & -5.780418 & 3.267302 & 0.026877 \\ 1 & -5.963183 & 4.098188 & 1.582811 \\ 1 & -4.927319 & 5.748104 & -1.641393 \\ 1 & -3.177308 & 5.644075 & -1.348350 \\ 1 & -4.104197 & 4.178268 & -1.712269 \\ 1 & -5.316006 & 6.904269 & 0.503553 \\ 1 & -4.694113 & 6.277912 & 2.037629 \\ 1 & -3.575428 & 6.920527 & 0.812204 \\ & & & \\ & & & \\ 1 & & \end{array}$

$3_{\text {IIC-re- }} \psi_{-1 \mathrm{~s} 22354 \mathrm{~s}}$

Number of imaginary frequencies : 0

The smallest frequencies are : $17.525920 .262524 .2435 \mathrm{~cm}(-1)$

Electronic energy : $\quad H F=-4360.8928248$

Zero-point correction $=\quad 1.455421$ (Hartree/Particle)

Thermal correction to Energy= $\quad 1.539599$

Thermal correction to Enthalpy= $\quad 1.540543$

Thermal correction to Gibbs Free Energy $=\quad 1.339627$

Sum of electronic and zero-point Energies $=\quad-4359.437404$

Sum of electronic and thermal Energies $=\quad-4359.353226$

Sum of electronic and thermal Enthalpies $=\quad-4359.352281$

Sum of electronic and thermal Free Energies $=\quad-4359.553197$

Cartesian Coordinates 


$\begin{array}{cccc}0 & 2.307453 & 1.385646 & -4.157466 \\ 6 & 2.050852 & 2.226178 & -3.066363 \\ 6 & 3.131525 & 2.900418 & -2.490010 \\ 6 & 4.428992 & 2.708684 & -2.958195 \\ 6 & 4.671117 & 1.842348 & -4.017688 \\ 6 & 3.599453 & 1.188465 & -4.623461 \\ 6 & 0.655283 & 2.464982 & -2.590346 \\ 6 & -0.232345 & 3.072482 & -3.659195 \\ 45 & -0.287994 & 0.678779 & -1.648582 \\ 6 & -0.580226 & -0.504440 & -3.129696 \\ 8 & -0.775315 & -1.027051 & -4.146643 \\ 15 & -1.849453 & 0.335329 & 0.193324 \\ 6 & -2.111515 & 1.989921 & 0.945578 \\ 6 & -1.192419 & 2.506514 & 1.863321 \\ 6 & -1.338999 & 3.795889 & 2.379837 \\ 6 & -2.388149 & 4.585251 & 1.891194 \\ 6 & -3.285749 & 4.117549 & 0.929810 \\ 6 & -3.142410 & 2.801863 & 0.483588 \\ 6 & -0.414302 & 4.278802 & 3.497863 \\ 6 & -4.396776 & 4.977026 & 0.331833 \\ 6 & -3.557855 & -0.122362 & -0.335457 \\ 6 & -3.846491 & -0.349260 & -1.675378 \\ 6 & -5.143878 & -0.651829 & -2.099379 \\ 6 & -6.139411 & -0.766901 & -1.127430 \\ 6 & -5.879173 & -0.569098 & 0.234643 \\ 6 & -4.580292 & -0.229484 & 0.608629 \\ 6 & -6.949453 & -0.702508 & 1.317406 \\ 6 & -5.405170 & -0.833088 & -3.593112 \\ 6 & -1.695796 & -0.781636 & 1.672130 \\ 6 & -1.347102 & -2.112051 & 1.487208 \\ 6 & -1.255234 & -2.995661 & 2.611126 \\ 6 & -1.531271 & -2.502101 & 3.919273 \\ 6 & -1.914879 & -1.150335 & 4.069631 \\ 6 & -1.993312 & -0.320182 & 2.983504 \\ 6 & -1.415350 & -3.367641 & 5.033096 \\ 6 & -1.040231 & -4.676292 & 4.869401 \\ 6 & -0.771080 & -5.173651 & 3.576885 \\ 6 & -0.880240 & -4.358653 & 2.477541 \\ 6 & -1.115254 & -2.697734 & 0.137714 \\ 6 & 0.119831 & -2.621943 & -0.480829 \\ 6 & 0.352014 & -3.295915 & -1.702285 \\ 6 & -0.639805 & -4.017701 & -2.309462 \\ 6 & -1.925876 & -4.096996 & -1.727075 \\ 6 & -2.170719 & -3.429929 & -0.490430 \\ 6 & -3.470470 & -3.513132 & 0.073562 \\ 6 & -4.464912 & -4.224702 & -0.549200 \\ 6 & -4.210942 & -4.906527 & -1.759157 \\ 6 & -2.968490 & -4.838069 & -2.335627 \\ 7 & 1.191129 & -1.866917 & 0.106692 \\ 15 & 1.571007 & -0.417343 & -0.694940 \\ 6 & 2.793976 & -0.704881 & -1.793383 \\ 6 & 3.927103 & -1.399669 & -1.441718 \\ 6 & 4.861273 & -0.858065 & -0.577352 \\ 6 & 5.921749 & -1.712384 & -0.122223 \\ & 6.065949 & -3.018882 & -0.677847 \\ 6 & & & \\ 6\end{array}$




\begin{tabular}{|c|c|c|c|}
\hline 6 & 5.158184 & -3.455116 & -1.673756 \\
\hline 6 & 4.099378 & -2.669138 & -2.034916 \\
\hline & 6.802953 & -1.336605 & 0.924296 \\
\hline & 7.777205 & -2.192802 & 1.375535 \\
\hline & 7.937105 & -3.470256 & 0.798838 \\
\hline & 7.094677 & -3.870470 & -0.206511 \\
\hline & 4.739221 & 0.544594 & -0.118721 \\
\hline & 3.558458 & 1.015308 & 0.424392 \\
\hline & 3.446836 & 2.305983 & 0.979065 \\
\hline & 4.508195 & 3.165232 & 0.942303 \\
\hline & 5.710128 & 2.785686 & 0.296925 \\
\hline & 5.823095 & 1.476058 & -0.259941 \\
\hline & 6.780559 & 3.701833 & 0.155121 \\
\hline & 7.914306 & 3.362454 & -0.538557 \\
\hline & 8.014621 & 2.083753 & -1.125472 \\
\hline & 7.001779 & 1.166820 & -0.985936 \\
\hline & 2.450732 & 0.221129 & 0.546071 \\
\hline & 0.376854 & 2.728297 & -1.228143 \\
\hline & 2.088125 & -2.638103 & 1.000548 \\
\hline & 2.557793 & -1.942681 & 2.253423 \\
\hline & 1.704737 & -1.150083 & 3.023536 \\
\hline ) & 2.155777 & -0.550910 & 4.191355 \\
\hline & 3.471323 & -0.737765 & 4.614447 \\
\hline & 4.326069 & -1.530124 & 3.857265 \\
\hline & 3.869839 & -2.128228 & 2.684284 \\
\hline & 6.677713 & -0.365826 & 1.396752 \\
\hline & -2.300562 & 0.712118 & 3.129785 \\
\hline & 7.190549 & -4.859592 & -0.652553 \\
\hline & -1.627305 & -2.967958 & 6.023816 \\
\hline & 6.673319 & 4.691768 & 0.596930 \\
\hline & -3.678956 & -2.990310 & 1.005808 \\
\hline & -5.459400 & -4.262007 & -0.107717 \\
\hline t & 4.431030 & 4.159520 & 1.380688 \\
\hline & -2.149428 & -0.776450 & 5.065821 \\
\hline 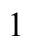 & 5.301244 & -4.435599 & -2.124851 \\
\hline & 8.900157 & 1.822475 & -1.700636 \\
\hline 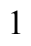 & 3.377792 & -2.988844 & -2.783339 \\
\hline 1 & -2.761398 & -5.346963 & -3.276216 \\
\hline & -0.455066 & -4.531829 & -3.251417 \\
\hline ] & -0.477309 & -6.213156 & 3.450417 \\
\hline & 7.089150 & 0.193784 & -1.462174 \\
\hline ] & -0.676488 & -4.759259 & 1.486451 \\
\hline & 1.341394 & -3.221112 & -2.149191 \\
\hline 1 & 8.726318 & 4.077295 & -0.649644 \\
\hline ] & 8.427460 & -1.884160 & 2.191158 \\
\hline ] & -5.005501 & -5.476166 & -2.236438 \\
\hline 1 & -0.949920 & -5.333225 & 5.731316 \\
\hline & 8.716823 & -4.136334 & 1.160756 \\
\hline 1 & 2.498016 & 2.577963 & 1.433364 \\
\hline . & -4.356432 & -0.043956 & 1.658865 \\
\hline 1 & -3.040343 & -0.277818 & -2.400919 \\
\hline 1 & -7.150246 & -1.016250 & -1.437105 \\
\hline 1 & -3.836826 & 2.406493 & -0.256245 \\
\hline 1 & -0.359516 & 1.877890 & 2.188633 \\
\hline & -2.503049 & 5.597078 & 2.267847 \\
\hline & 1.505770 & -3.523105 & 1.294029 \\
\hline
\end{tabular}




\begin{tabular}{|c|c|c|c|}
\hline 1 & -1.595798 & 1.404627 & -2.188147 \\
\hline & 2.959399 & -3.018944 & 0.446116 \\
\hline & 4.551407 & -2.736912 & 2.088207 \\
\hline & 0.689685 & -0.973890 & 2.675424 \\
\hline & 5.358015 & -1.679893 & 4.170076 \\
\hline & 1.473483 & 0.067034 & 4.774597 \\
\hline & 3.826911 & -0.263902 & 5.526974 \\
\hline & 1.183509 & 2.736164 & -0.492860 \\
\hline 1 & -0.476383 & 3.359356 & -0.970402 \\
\hline & 2.953269 & 3.587648 & -1.663844 \\
\hline 1 & 5.254112 & 3.243749 & -2.490135 \\
\hline 1 & 5.687711 & 1.684670 & -4.373296 \\
\hline 1 & 3.773726 & 0.513438 & -5.459463 \\
\hline 1 & 1.479263 & 0.850251 & -4.620822 \\
\hline 1 & 0.239863 & 3.983717 & -4.057867 \\
\hline 1 & -0.392424 & 2.396284 & -4.508331 \\
\hline 1 & -1.216282 & 3.341135 & -3.256703 \\
\hline 6 & -6.882437 & -1.063214 & -3.901150 \\
\hline 6 & -4.951595 & 0.424707 & -4.345479 \\
\hline 6 & -4.614691 & -2.042431 & -4.101910 \\
\hline 6 & -8.285129 & -1.188021 & 0.760027 \\
\hline 6 & -6.486211 & -1.712766 & 2.374538 \\
\hline 6 & -7.170202 & 0.663238 & 1.977463 \\
\hline 1 & -7.020020 & -1.174060 & -4.984166 \\
\hline 1 & -7.262933 & -1.978084 & -3.428618 \\
\hline 1 & -7.506551 & -0.221232 & -3.573993 \\
\hline 1 & -4.767433 & -2.171367 & -5.182272 \\
\hline 1 & -3.536534 & -1.934946 & -3.926549 \\
\hline 1 & -4.941596 & -2.960121 & -3.596285 \\
\hline 1 & -5.154956 & 0.317468 & -5.419437 \\
\hline 1 & -5.486686 & 1.313911 & -3.986538 \\
\hline 1 & -3.875790 & 0.613017 & -4.232810 \\
\hline 1 & -7.245361 & -1.814212 & 3.161836 \\
\hline 1 & -6.332516 & -2.705018 & 1.928788 \\
\hline 1 & -5.547385 & -1.416346 & 2.859540 \\
\hline 1 & -9.010996 & -1.290369 & 1.576666 \\
\hline 1 & -8.709697 & -0.484314 & 0.032769 \\
\hline 1 & -8.191825 & -2.169249 & 0.275552 \\
\hline 1 & -7.906218 & 0.583681 & 2.789183 \\
\hline 1 & -6.243137 & 1.068787 & 2.402297 \\
\hline 1 & -7.549316 & 1.392094 & 1.248914 \\
\hline 6 & -0.555852 & 5.775536 & 3.767817 \\
\hline 6 & -0.776866 & 3.519691 & 4.780773 \\
\hline 6 & 1.049724 & 3.996859 & 3.151536 \\
\hline 6 & -4.478029 & 6.358504 & 0.975566 \\
\hline 6 & -4.119302 & 5.159773 & -1.165840 \\
\hline 6 & -5.750303 & 4.281961 & 0.519941 \\
\hline 1 & 0.150264 & 6.075723 & 4.552169 \\
\hline 1 & -0.335327 & 6.373822 & 2.874248 \\
\hline 1 & -1.560182 & 6.041163 & 4.120248 \\
\hline 1 & -0.138632 & 3.843313 & 5.614503 \\
\hline 1 & -1.822242 & 3.701750 & 5.063034 \\
\hline 1 & -0.644981 & 2.436081 & 4.658475 \\
\hline 1 & 1.710118 & 4.412567 & 3.924140 \\
\hline 1 & 1.258580 & 2.921202 & 3.100122 \\
\hline 1 & 1.323410 & 4.452456 & 2.189305 \\
\hline
\end{tabular}




$\begin{array}{lrrr}1 & -6.553316 & 4.890228 & 0.082391 \\ 1 & -5.782706 & 3.296606 & 0.037749 \\ 1 & -5.975087 & 4.141264 & 1.585530 \\ 1 & -4.900008 & 5.778483 & -1.628978 \\ 1 & -3.152936 & 5.656399 & -1.325705 \\ 1 & -4.091874 & 4.200589 & -1.698664 \\ 1 & -5.294209 & 6.931274 & 0.517405 \\ 1 & -4.682344 & 6.297706 & 2.052531 \\ 1 & -3.554720 & 6.934101 & 0.831986\end{array}$

$3_{\text {IID-si- }} \psi_{-1 \text { a2a3a4a }}$

Number of imaginary frequencies : 0

The smallest frequencies are : $13.5028 \quad 19.7548 \quad 20.6772 \mathrm{~cm}(-1)$

Electronic energy : $\quad H F=-4360.8915146$

Zero-point correction $=\quad 1.454597$ (Hartree/Particle)

Thermal correction to Energy= $\quad 1.539416$

Thermal correction to Enthalpy= $\quad 1.540360$

Thermal correction to Gibbs Free Energy $=\quad 1.335979$

Sum of electronic and zero-point Energies $=\quad-4359.436918$

Sum of electronic and thermal Energies $=\quad-4359.352099$

Sum of electronic and thermal Enthalpies $=\quad-4359.351155$

Sum of electronic and thermal Free Energies $=\quad-4359.555536$

Cartesian Coordinates

$\begin{array}{cccc}6 & -1.585208 & 4.314637 & -2.157166 \\ 6 & -0.469214 & 3.652893 & -2.692391 \\ 6 & -0.304568 & 3.694191 & -4.082463 \\ 6 & -1.223590 & 4.340666 & -4.903323 \\ 6 & -2.331564 & 4.977166 & -4.355837 \\ 6 & -2.502597 & 4.962089 & -2.973075 \\ 6 & 0.527383 & 2.945199 & -1.828050 \\ 6 & 1.944682 & 2.944883 & -2.354054 \\ 45 & -0.226546 & 0.859229 & -1.365869 \\ 6 & 0.313870 & 2.790883 & -0.442975 \\ 15 & 1.742446 & -0.306320 & -0.825195 \\ 7 & 1.510581 & -1.924246 & -0.366772 \\ 6 & 2.516423 & -2.821939 & 0.249829 \\ 6 & 3.084927 & -2.380463 & 1.573986 \\ 6 & 2.268894 & -1.880926 & 2.590772 \\ 6 & 2.810296 & -1.502368 & 3.811233 \\ 6 & 4.181224 & -1.618600 & 4.038129 \\ 6 & 5.000596 & -2.118376 & 3.032731 \\ 6 & 4.453423 & -2.497049 & 1.808347 \\ 8 & 2.863979 & -0.267645 & -2.072423 \\ 6 & 4.087557 & -0.885124 & -1.957101 \\ 6 & 5.053578 & -0.385216 & -1.103957 \\ 6 & 6.245249 & -1.160232 & -0.912200 \\ 6 & 6.457790 & -2.332431 & -1.698709 \\ 6 & 5.487838 & -2.711603 & -2.658604 \\ 6 & 4.318475 & -2.012418 & -2.774702 \\ 6 & 7.201634 & -0.849297 & 0.088110 \\ 6 & 8.307399 & -1.639897 & 0.282978 \\ 6 & 8.528815 & -2.779631 & -0.517893\end{array}$




\begin{tabular}{|c|c|c|c|}
\hline 6 & 7.618890 & -3.115109 & -1.487336 \\
\hline & 4.824615 & 0.888040 & -0.382216 \\
\hline & 5.742791 & 1.986908 & -0.473826 \\
\hline & 5.534310 & 3.149415 & 0.328257 \\
\hline & 4.411804 & 3.208165 & 1.189805 \\
\hline & 3.499688 & 2.190433 & 1.207500 \\
\hline & 3.689279 & 1.058542 & 0.387316 \\
\hline & 6.845411 & 1.985174 & -1.365273 \\
\hline & 7.703769 & 3.054733 & -1.435938 \\
\hline & 7.512973 & 4.186521 & -0.616892 \\
\hline & 6.445490 & 4.229360 & 0.243075 \\
\hline & 2.724150 & 0.088733 & 0.446836 \\
\hline & 0.428419 & -2.580538 & -1.047488 \\
\hline & -0.746862 & -2.882488 & -0.382002 \\
\hline & -1.827590 & -3.479764 & -1.101792 \\
\hline & -1.679116 & -3.754401 & -2.493501 \\
\hline & -0.450259 & -3.459843 & -3.126598 \\
\hline & 0.574505 & -2.892252 & -2.418768 \\
\hline & -2.760285 & -4.326088 & -3.209016 \\
\hline & -3.944129 & -4.614489 & -2.579523 \\
\hline & -4.099069 & -4.334165 & -1.204016 \\
\hline & -3.066958 & -3.789307 & -0.482687 \\
\hline & -0.889686 & -2.649060 & 1.081758 \\
\hline & -1.281547 & -1.421637 & 1.598774 \\
\hline & -1.468606 & -1.291103 & 3.002209 \\
\hline & -1.250059 & -2.340858 & 3.853989 \\
\hline 6 & -0.830126 & -3.596843 & 3.360932 \\
\hline & -0.658141 & -3.759645 & 1.955650 \\
\hline 6 & -0.245914 & -5.030764 & 1.475653 \\
\hline 5 & -0.004498 & -6.069293 & 2.340548 \\
\hline f & -0.169942 & -5.898701 & 3.731332 \\
\hline 6 & -0.577088 & -4.686905 & 4.227509 \\
\hline & -1.622201 & -0.006262 & 0.438398 \\
\hline 6 & -3.350891 & -0.417341 & -0.051732 \\
\hline 6 & -4.301258 & -0.733863 & 0.923092 \\
\hline 6 & -5.619277 & -1.017692 & 0.573096 \\
\hline 6 & -5.952030 & -0.990549 & -0.787381 \\
\hline 5 & -5.027998 & -0.683740 & -1.787164 \\
\hline 6 & -3.717060 & -0.398950 & -1.393084 \\
\hline 6 & -6.678551 & -1.379559 & 1.613434 \\
\hline 6 & -5.463260 & -0.601312 & -3.249264 \\
\hline 6 & -1.849389 & 1.411774 & 1.580420 \\
\hline 6 & -0.821411 & 1.733996 & 2.467866 \\
\hline 6 & -0.883916 & 2.879997 & 3.260226 \\
\hline 6 & -1.985049 & 3.726650 & 3.092672 \\
\hline 6 & -3.012872 & 3.454146 & 2.184964 \\
\hline 6 & -2.933561 & 2.275676 & 1.439128 \\
\hline 6 & 0.239375 & 3.241778 & 4.231713 \\
\hline 6 & -4.185472 & 4.423857 & 2.044948 \\
\hline 6 & -0.407080 & 0.130848 & -3.134506 \\
\hline 8 & -0.442096 & -0.108973 & -4.267560 \\
\hline 1 & 7.035222 & 0.012884 & 0.728852 \\
\hline 1 & -1.797463 & -0.339567 & 3.411681 \\
\hline 1 & 7.763827 & -4.000709 & -2.104762 \\
\hline 1 & -0.710913 & -4.538952 & 5.298232 \\
\hline 1 & 6.269915 & 5.102306 & 0.870472 \\
\hline
\end{tabular}




\begin{tabular}{|c|c|c|c|}
\hline & -3.199635 & -3.570106 & 0.575278 \\
\hline . & -5.046658 & -4.549790 & -0.714156 \\
\hline 1 & 4.276247 & 4.083251 & 1.823855 \\
\hline & -1.401487 & -2.219917 & 4.926054 \\
\hline 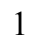 & 5.677241 & -3.583081 & -3.283084 \\
\hline 1 & 8.535728 & 3.030350 & -2.136225 \\
\hline 1 & 3.552859 & -2.295145 & -3.493327 \\
\hline . & -2.627884 & -4.532480 & -4.270290 \\
\hline & -0.337117 & -3.678978 & -4.187225 \\
\hline 1 & 0.314145 & -7.033224 & 1.950474 \\
\hline 1 & 7.000989 & 1.126481 & -2.013131 \\
\hline & -0.122084 & -5.179416 & 0.404779 \\
\hline 1 & 1.516500 & -2.647496 & -2.904905 \\
\hline & 8.203210 & 5.024547 & -0.678772 \\
\hline ] & 9.016554 & -1.387022 & 1.068084 \\
\hline 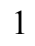 & -4.766681 & -5.058267 & -3.136691 \\
\hline & 0.025343 & -6.729651 & 4.405184 \\
\hline 1 & 9.411838 & -3.393462 & -0.356528 \\
\hline & 2.616765 & 2.217580 & 1.843976 \\
\hline 1 & -4.001307 & -0.742784 & 1.968573 \\
\hline . & -2.956384 & -0.157673 & -2.130181 \\
\hline 1 & -6.979042 & -1.214139 & -1.076034 \\
\hline ] & -3.712561 & 2.028590 & 0.722153 \\
\hline & 0.039910 & 1.068575 & 2.534450 \\
\hline 1 & -2.043551 & 4.636944 & 3.688328 \\
\hline 1 & 1.992872 & -3.777743 & 0.395708 \\
\hline 1 & -1.618743 & 1.564068 & -1.651454 \\
\hline 1 & 3.339062 & -3.026451 & -0.452677 \\
\hline 1 & 5.105641 & -2.872712 & 1.018016 \\
\hline 1 & 1.205538 & -1.750595 & 2.401935 \\
\hline . & 6.073578 & -2.207633 & 3.192424 \\
\hline l & 2.155023 & -1.111517 & 4.589079 \\
\hline 1 & 4.606631 & -1.316687 & 4.992899 \\
\hline 1 & 1.184110 & 2.697291 & 0.205850 \\
\hline 1 & -0.563206 & 3.206886 & 0.049835 \\
\hline 1 & 0.549149 & 3.196506 & -4.538145 \\
\hline 1 & -1.067692 & 4.345057 & -5.980466 \\
\hline 1 & -3.049669 & 5.485268 & -4.995686 \\
\hline 1 & -3.356373 & 5.466100 & -2.522290 \\
\hline 1 & -1.745735 & 4.316190 & -1.079946 \\
\hline 1 & 2.640667 & 2.540082 & -1.611539 \\
\hline 1 & 2.068126 & 2.351523 & -3.267377 \\
\hline 1 & 2.263256 & 3.972745 & -2.585737 \\
\hline 6 & 1.138659 & 2.042404 & 4.537079 \\
\hline 6 & 1.088920 & 4.349934 & 3.597447 \\
\hline 6 & -0.329772 & 3.739998 & 5.563374 \\
\hline 6 & -5.179786 & 3.979853 & 0.973426 \\
\hline 6 & -4.932088 & 4.509057 & 3.380905 \\
\hline 6 & -3.663033 & 5.812729 & 1.661925 \\
\hline 6 & -6.172767 & -1.183636 & 3.041681 \\
\hline 6 & -7.921731 & -0.502329 & 1.432327 \\
\hline 6 & -7.065074 & -2.852431 & 1.437900 \\
\hline 6 & -4.268441 & -0.521752 & -4.199197 \\
\hline 6 & -6.294934 & -1.826435 & -3.637674 \\
\hline 6 & -6.308901 & 0.665169 & -3.430530 \\
\hline 1 & 1.882933 & 2.320180 & 5.293959 \\
\hline
\end{tabular}




$\begin{array}{rrrr}1 & 0.559414 & 1.194617 & 4.929922 \\ 1 & 1.694429 & 1.688730 & 3.659448 \\ 1 & 1.953091 & 4.589844 & 4.232874 \\ 1 & 1.461959 & 4.044402 & 2.609441 \\ 1 & 0.507631 & 5.269932 & 3.456058 \\ 1 & 0.488515 & 3.962066 & 6.260882 \\ 1 & -0.916244 & 4.659447 & 5.454444 \\ 1 & -0.973309 & 2.982226 & 6.028842 \\ 1 & -4.498920 & 6.516289 & 1.549498 \\ 1 & -2.984866 & 6.225301 & 2.418434 \\ 1 & -3.118714 & 5.780899 & 0.707859 \\ 1 & -5.782171 & 5.200114 & 3.301392 \\ 1 & -5.321941 & 3.525436 & 3.674752 \\ 1 & -4.286336 & 4.868196 & 4.191483 \\ 1 & -5.998754 & 4.707301 & 0.908319 \\ 1 & -4.715693 & 3.914841 & -0.019935 \\ 1 & -5.626151 & 3.002648 & 1.203682 \\ 1 & -6.972547 & -1.426731 & 3.752576 \\ 1 & -5.321210 & -1.836797 & 3.274119 \\ 1 & -5.868173 & -0.144242 & 3.225822 \\ 1 & -7.840927 & -3.136395 & 2.162133 \\ 1 & -7.455487 & -3.055208 & 0.432588 \\ 1 & -6.197149 & -3.505741 & 1.601964 \\ 1 & -8.671566 & -0.747249 & 2.196350 \\ 1 & -7.671915 & 0.561861 & 1.534189 \\ 1 & -8.396439 & -0.642338 & 0.454242 \\ 1 & -6.583511 & -1.765016 & -4.695397 \\ 1 & -5.717970 & -2.750027 & -3.496058 \\ 1 & -7.219822 & -1.911256 & -3.054585 \\ 1 & -4.624096 & -0.515420 & -5.237425 \\ 1 & -3.681938 & 0.395004 & -4.053969 \\ 1 & -3.597865 & -1.384535 & -4.080580 \\ 1 & -6.631192 & 0.766239 & -4.476032 \\ 1 & -7.208129 & 0.645891 & -2.801580 \\ 1 & -5.732547 & 1.561602 & -3.165469\end{array}$

$3_{\text {IID-si- }} \psi_{-1 \mathrm{a} 2 \mathrm{a3a} 4 \mathrm{~s}}$

Number of imaginary frequencies : 0

The smallest frequencies are : $9.8472 \quad 20.058921 .1583 \mathrm{~cm}(-1)$

Electronic energy : $\quad H F=-4360.8922775$

Zero-point correction $=\quad 1.454137($ Hartree/Particle $)$

Thermal correction to Energy $=\quad 1.538968$

Thermal correction to Enthalpy= $\quad 1.539912$

Thermal correction to Gibbs Free Energy $=\quad 1.335428$

Sum of electronic and zero-point Energies $=\quad-4359.438140$

Sum of electronic and thermal Energies $=\quad-4359.353309$

Sum of electronic and thermal Enthalpies $=\quad-4359.352365$

Sum of electronic and thermal Free Energies $=\quad-4359.556850$

Cartesian Coordinates

$\begin{array}{lllll}6 & -1.606093 & 4.302278 & -2.151583 \\ 6 & -0.483180 & 3.651739 & -2.685789 \\ 6 & -0.321089 & 3.688973 & -4.076357 \\ 6 & -1.249418 & 4.319759 & -4.898755\end{array}$




\begin{tabular}{|c|c|c|c|}
\hline 6 & -2.364491 & 4.944751 & -4.352286 \\
\hline 6 & -2.532895 & 4.934149 & -2.969232 \\
\hline & 0.521346 & 2.956136 & -1.820862 \\
\hline & 1.938421 & 2.968156 & -2.347192 \\
\hline & -0.214282 & 0.863172 & -1.362577 \\
\hline & 0.310779 & 2.798198 & -0.435673 \\
\hline & 1.758509 & -0.300426 & -0.825901 \\
\hline & 1.530938 & -1.926107 & -0.393483 \\
\hline & 2.539528 & -2.833006 & 0.205653 \\
\hline & 3.111366 & -2.410420 & 1.534374 \\
\hline & 2.293370 & -1.951944 & 2.568900 \\
\hline & 2.837415 & -1.590483 & 3.793398 \\
\hline & 4.212685 & -1.681129 & 4.005833 \\
\hline & 5.033870 & -2.139897 & 2.982557 \\
\hline & 4.483778 & -2.503990 & 1.755160 \\
\hline & 2.887290 & -0.237729 & -2.065920 \\
\hline 6 & 4.118764 & -0.838802 & -1.950174 \\
\hline & 5.073193 & -0.334802 & -1.086442 \\
\hline & 6.275569 & -1.093896 & -0.898199 \\
\hline 6 & 6.511108 & -2.251837 & -1.699002 \\
\hline 0 & 5.550438 & -2.635363 & -2.666589 \\
\hline 6 & 4.370180 & -1.953938 & -2.778574 \\
\hline 0 & 7.219771 & -0.782797 & 0.113577 \\
\hline 5 & 8.337087 & -1.558068 & 0.304112 \\
\hline 6 & 8.582423 & -2.681820 & -0.512253 \\
\hline 6 & 7.683779 & -3.018260 & -1.491888 \\
\hline 6 & 4.821851 & 0.924619 & -0.348329 \\
\hline 6 & 5.722535 & 2.039229 & -0.419818 \\
\hline 6 & 5.488413 & 3.188153 & 0.394922 \\
\hline 6 & 4.360645 & 3.216166 & 1.251208 \\
\hline 6 & 3.466981 & 2.181861 & 1.252535 \\
\hline 6 & 3.680314 & 1.065129 & 0.417944 \\
\hline 6 & 6.831699 & 2.067113 & -1.302888 \\
\hline 6 & 7.671128 & 3.152501 & -1.355125 \\
\hline 6 & 7.454044 & 4.271538 & -0.525266 \\
\hline 6 & 6.380541 & 4.285369 & 0.327952 \\
\hline 8 & 2.733502 & 0.076325 & 0.456932 \\
\hline 6 & 0.451856 & -2.574913 & -1.086055 \\
\hline 6 & -0.726182 & -2.883452 & -0.428872 \\
\hline 6 & -1.806304 & -3.467391 & -1.160181 \\
\hline 6 & -1.653105 & -3.725072 & -2.554592 \\
\hline 6 & -0.419526 & -3.429508 & -3.178182 \\
\hline 6 & 0.603646 & -2.872096 & -2.459915 \\
\hline 6 & -2.733989 & -4.282458 & -3.281569 \\
\hline 6 & -3.921749 & -4.572931 & -2.660448 \\
\hline 6 & -4.081090 & -4.309289 & -1.282162 \\
\hline 6 & -3.049900 & -3.777666 & -0.549741 \\
\hline 6 & -0.874142 & -2.670154 & 1.037186 \\
\hline 6 & -1.265130 & -1.449322 & 1.569760 \\
\hline 6 & -1.458432 & -1.338377 & 2.973945 \\
\hline 6 & -1.253383 & -2.403055 & 3.810777 \\
\hline 6 & -0.837090 & -3.653753 & 3.301456 \\
\hline 6 & -0.652701 & -3.794724 & 1.895422 \\
\hline 6 & -0.239799 & -5.059155 & 1.398777 \\
\hline 6 & -0.011218 & -6.112618 & 2.248909 \\
\hline 6 & -0.190708 & -5.964271 & 3.640570 \\
\hline
\end{tabular}




\begin{tabular}{|c|c|c|c|}
\hline & -0.597661 & -4.758986 & 4.152519 \\
\hline & -1.606751 & -0.021196 & 0.425658 \\
\hline & -3.329197 & -0.439288 & -0.078840 \\
\hline & -4.267254 & -0.803415 & 0.881973 \\
\hline & -5.591494 & -1.080925 & 0.533886 \\
\hline & -5.945133 & -0.975148 & -0.812113 \\
\hline & -5.025151 & -0.615646 & -1.806097 \\
\hline & -3.711710 & -0.358482 & -1.417170 \\
\hline & -6.579882 & -1.455267 & 1.636433 \\
\hline 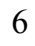 & -5.479744 & -0.468592 & -3.256761 \\
\hline 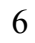 & -1.866982 & 1.377757 & 1.585870 \\
\hline & -0.845188 & 1.714584 & 2.474791 \\
\hline 6 & -0.937650 & 2.844714 & 3.287528 \\
\hline 0 & -2.063424 & 3.661830 & 3.138534 \\
\hline 6 & -3.086813 & 3.375483 & 2.230020 \\
\hline 0 & -2.977157 & 2.211888 & 1.464987 \\
\hline 6 & 0.183869 & 3.224399 & 4.254397 \\
\hline 6 & -4.294859 & 4.304860 & 2.117971 \\
\hline 6 & -0.406503 & 0.143810 & -3.134319 \\
\hline 8 & -0.470091 & -0.087207 & -4.267880 \\
\hline 1 & 7.034573 & 0.065998 & 0.766885 \\
\hline 1 & -1.783385 & -0.390728 & 3.395544 \\
\hline 1 & 7.846692 & -3.892725 & -2.120616 \\
\hline 1 & -0.741575 & -4.628051 & 5.224151 \\
\hline 1 & 6.185229 & 5.147602 & 0.964272 \\
\hline 1 & -3.186704 & -3.568969 & 0.510154 \\
\hline 1 & -5.033158 & -4.523412 & -0.799909 \\
\hline 1 & 4.206533 & 4.081287 & 1.894606 \\
\hline 1 & -1.411566 & -2.297766 & 4.883518 \\
\hline 1 & 5.755601 & -3.496818 & -3.299943 \\
\hline 1 & 8.508596 & 3.150775 & -2.049257 \\
\hline 1 & 3.610553 & -2.242653 & -3.501273 \\
\hline 1 & -2.598365 & -4.476335 & -4.344747 \\
\hline 1 & -0.302288 & -3.637704 & -4.240566 \\
\hline 1 & 0.308137 & -7.071205 & 1.846462 \\
\hline 1 & 7.007703 & 1.218475 & -1.958745 \\
\hline 1 & -0.105212 & -5.190445 & 0.326938 \\
\hline 1 & 1.548392 & -2.623722 & -2.939290 \\
\hline 1 & 8.129378 & 5.122539 & -0.573092 \\
\hline 1 & 9.036724 & -1.305644 & 1.097869 \\
\hline 1 & -4.744588 & -5.004520 & -3.226585 \\
\hline 1 & -0.005911 & -6.807071 & 4.302553 \\
\hline 1 & 9.474506 & -3.283314 & -0.354348 \\
\hline 1 & 2.581057 & 2.184880 & 1.885537 \\
\hline 1 & -3.964741 & -0.860416 & 1.927529 \\
\hline 1 & -2.958994 & -0.084330 & -2.150199 \\
\hline 1 & -6.974124 & -1.175024 & -1.105058 \\
\hline 1 & -3.754735 & 1.952617 & 0.750767 \\
\hline 1 & 0.034826 & 1.072297 & 2.526591 \\
\hline 1 & -2.145195 & 4.559359 & 3.750213 \\
\hline 1 & 2.016207 & -3.790716 & 0.338832 \\
\hline 1 & -1.608606 & 1.563023 & -1.644734 \\
\hline 1 & 3.359988 & -3.026531 & -0.502648 \\
\hline 1 & 5.136848 & -2.848647 & 0.951615 \\
\hline 1 & 1.225601 & -1.841376 & 2.392639 \\
\hline 1 & 6.110297 & -2.207841 & 3.129124 \\
\hline
\end{tabular}




\begin{tabular}{|c|c|c|c|}
\hline ] & 2.181063 & -1.235476 & 4.586953 \\
\hline & 4.639705 & -1.391733 & 4.963765 \\
\hline & 1.184452 & 2.712279 & 0.209663 \\
\hline 1 & -0.569177 & 3.203694 & 0.061187 \\
\hline 1 & 0.537544 & 3.199037 & -4.531234 \\
\hline & -1.095409 & 4.320871 & -5.976174 \\
\hline & -3.090256 & 5.440444 & -4.993231 \\
\hline & -3.392134 & 5.429647 & -2.519666 \\
\hline & -1.764813 & 4.306087 & -1.074277 \\
\hline & 2.637087 & 2.566414 & -1.605249 \\
\hline & 2.066256 & 2.377509 & -3.261592 \\
\hline & 2.249356 & 3.998845 & -2.576760 \\
\hline & 1.078155 & 2.028038 & 4.586114 \\
\hline & 1.035292 & 4.317356 & 3.596715 \\
\hline & -0.381726 & 3.754051 & 5.575306 \\
\hline & -5.234939 & 3.896105 & 0.985066 \\
\hline & -5.081233 & 4.259663 & 3.433042 \\
\hline & -3.830522 & 5.740378 & 1.850694 \\
\hline f & -7.951380 & -1.835229 & 1.085083 \\
\hline 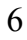 & -6.041905 & -2.652402 & 2.429341 \\
\hline 0 & -6.752734 & -0.254310 & 2.574518 \\
\hline כ & -4.306124 & -0.218930 & -4.203131 \\
\hline 6 & -6.198593 & -1.738682 & -3.720888 \\
\hline f & -6.438185 & 0.724285 & -3.351976 \\
\hline & 1.817441 & 2.316527 & 5.343864 \\
\hline l & 0.492448 & 1.189728 & 4.989036 \\
\hline ] & 1.639867 & 1.657482 & 3.719280 \\
\hline 1 & 1.897614 & 4.572622 & 4.228755 \\
\hline 1 & 1.410741 & 3.990321 & 2.616775 \\
\hline & 0.452341 & 5.232709 & 3.432850 \\
\hline 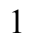 & 0.438600 & 3.978728 & 6.269374 \\
\hline & -0.955249 & 4.679322 & 5.449046 \\
\hline 1 & -1.035201 & 3.013331 & 6.054180 \\
\hline ] & -4.697803 & 6.407828 & 1.759195 \\
\hline 1 & -3.199267 & 6.132908 & 2.656588 \\
\hline 1 & -3.256935 & 5.803253 & 0.916203 \\
\hline 1 & -5.956971 & 4.920699 & 3.379969 \\
\hline 1 & -5.437577 & 3.241814 & 3.640377 \\
\hline | & -4.471176 & 4.580897 & 4.286284 \\
\hline 1 & -6.078124 & 4.596640 & 0.936150 \\
\hline ] & -4.732196 & 3.912251 & 0.008442 \\
\hline 1 & -5.652183 & 2.891052 & 1.134049 \\
\hline 1 & -8.616031 & -2.120459 & 1.910430 \\
\hline 1 & -8.428716 & -1.001022 & 0.555334 \\
\hline 1 & -7.890983 & -2.689752 & 0.397870 \\
\hline 1 & -7.430338 & -0.505386 & 3.401943 \\
\hline 1 & -5.797652 & 0.070093 & 3.007980 \\
\hline 1 & -7.181132 & 0.601143 & 2.035704 \\
\hline 1 & -6.731526 & -2.909095 & 3.244721 \\
\hline 1 & -5.942993 & -3.536488 & 1.785272 \\
\hline 1 & -5.059931 & -2.455799 & 2.878164 \\
\hline 1 & -6.528084 & -1.627296 & -4.762664 \\
\hline 1 & -5.527270 & -2.606115 & -3.667331 \\
\hline 1 & -7.087664 & -1.961829 & -3.118706 \\
\hline 1 & -4.673491 & -0.155181 & -5.235304 \\
\hline 1 & -3.786188 & 0.724180 & -3.984357 \\
\hline
\end{tabular}




$\begin{array}{lrrr}1 & -3.571246 & -1.034797 & -4.160975 \\ 1 & -6.770232 & 0.867442 & -4.389330 \\ 1 & -7.331961 & 0.581767 & -2.731516 \\ 1 & -5.945265 & 1.648851 & -3.023476\end{array}$

$3_{\text {IID-si- }} \psi-1$ a2a3s4a

Number of imaginary frequencies : 0

The smallest frequencies are : $16.2653 \quad 20.2462 \quad 21.2390 \mathrm{~cm}(-1)$

Electronic energy : $\quad H F=-4360.8917322$

Zero-point correction $=\quad 1.454628$ (Hartree/Particle)

Thermal correction to Energy= $\quad 1.539425$

Thermal correction to Enthalpy= $\quad 1.540370$

Thermal correction to Gibbs Free Energy= $\quad 1.336365$

Sum of electronic and zero-point Energies $=\quad-4359.437104$

Sum of electronic and thermal Energies $=\quad-4359.352307$

Sum of electronic and thermal Enthalpies $=\quad-4359.351363$

Sum of electronic and thermal Free Energies $=\quad-4359.555367$

Cartesian Coordinates

$\begin{array}{cccc}6 & -1.577825 & 4.368126 & -2.102324 \\ 6 & -0.499407 & 3.656459 & -2.648481 \\ 6 & -0.381327 & 3.638549 & -4.044100 \\ 6 & -1.309837 & 4.278453 & -4.858436 \\ 6 & -2.379867 & 4.968899 & -4.299332 \\ 6 & -2.505021 & 5.010145 & -2.912718 \\ 6 & 0.509513 & 2.956646 & -1.791921 \\ 6 & 1.921355 & 2.968243 & -2.332935 \\ 45 & -0.239605 & 0.880598 & -1.320600 \\ 6 & 0.311645 & 2.805934 & -0.402617 \\ 15 & 1.736693 & -0.289357 & -0.812278 \\ 7 & 1.522599 & -1.918916 & -0.388145 \\ 6 & 2.551477 & -2.819834 & 0.184150 \\ 6 & 3.113984 & -2.420032 & 1.524232 \\ 6 & 2.290387 & -1.970177 & 2.557953 \\ 6 & 2.825712 & -1.633369 & 3.793341 \\ 6 & 4.197621 & -1.742275 & 4.018009 \\ 6 & 5.024457 & -2.192133 & 2.995258 \\ 6 & 4.483575 & -2.529245 & 1.756106 \\ 8 & 2.849374 & -0.204362 & -2.064492 \\ 6 & 4.079554 & -0.811988 & -1.982328 \\ 6 & 5.047999 & -0.340684 & -1.115552 \\ 6 & 6.246162 & -1.115601 & -0.966008 \\ 6 & 6.462667 & -2.248014 & -1.807715 \\ 6 & 5.490007 & -2.590939 & -2.778412 \\ 6 & 4.313833 & -1.898098 & -2.853114 \\ 6 & 7.206776 & -0.844199 & 0.041825 \\ 6 & 8.320652 & -1.633621 & 0.191604 \\ 6 & 8.546492 & -2.731593 & -0.664552 \\ 6 & 7.632193 & -3.028935 & -1.642237 \\ 6 & 4.819561 & 0.907019 & -0.349876 \\ 6 & 5.733350 & 2.011927 & -0.414202 \\ 6 & 5.517628 & 3.154861 & 0.413995 \\ 6 & 4.393698 & 3.188633 & 1.274988 \\ 6 & 3.487692 & 2.165238 & 1.270234\end{array}$




\begin{tabular}{|c|c|c|c|}
\hline 6 & 3.684112 & 1.053577 & 0.424751 \\
\hline 6 & 6.837271 & 2.037502 & -1.303953 \\
\hline & 7.689179 & 3.113480 & -1.349214 \\
\hline & 7.490827 & 4.225361 & -0.505277 \\
\hline & 6.422407 & 4.242061 & 0.354231 \\
\hline & 2.726385 & 0.075743 & 0.462690 \\
\hline & 0.446688 & -2.572689 & -1.080917 \\
\hline & -0.738669 & -2.868236 & -0.431215 \\
\hline & -1.811049 & -3.462284 & -1.167266 \\
\hline & -1.632361 & -3.765750 & -2.549797 \\
\hline & -0.390310 & -3.483201 & -3.162972 \\
\hline & 0.618241 & -2.899229 & -2.445749 \\
\hline & -2.694932 & -4.357434 & -3.275902 \\
\hline & -3.894340 & -4.628284 & -2.669266 \\
\hline & -4.082437 & -4.311559 & -1.306340 \\
\hline & -3.066192 & -3.753657 & -0.571870 \\
\hline & -0.886964 & -2.664366 & 1.037168 \\
\hline & -1.272741 & -1.450628 & 1.588878 \\
\hline & -1.458002 & -1.355433 & 2.995306 \\
\hline & -1.248389 & -2.429820 & 3.817983 \\
\hline & -0.834035 & -3.673813 & 3.290601 \\
\hline & -0.658816 & -3.798776 & 1.881706 \\
\hline & -0.244225 & -5.056409 & 1.369280 \\
\hline & -0.007130 & -6.118320 & 2.206565 \\
\hline & -0.179110 & -5.986077 & 3.600684 \\
\hline & -0.586516 & -4.787570 & 4.127972 \\
\hline & -1.620169 & -0.011141 & 0.467339 \\
\hline 6 & -3.347873 & -0.417225 & -0.026979 \\
\hline & -4.279622 & -0.846492 & 0.927445 \\
\hline 5 & -5.591868 & -1.129935 & 0.567629 \\
\hline ) & -5.961672 & -0.945196 & -0.776327 \\
\hline 5 & -5.063676 & -0.509260 & -1.745244 \\
\hline 5 & -3.741716 & -0.271471 & -1.347016 \\
\hline & -6.620236 & -1.648263 & 1.571817 \\
\hline 5 & -5.438622 & -0.279214 & -3.206830 \\
\hline 5 & -1.854805 & 1.388796 & 1.630512 \\
\hline 5 & -0.831857 & 1.709780 & 2.524072 \\
\hline 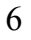 & -0.898648 & 2.857294 & 3.315018 \\
\hline 5 & -1.997321 & 3.705282 & 3.139314 \\
\hline 6 & -3.021756 & 3.432127 & 2.227981 \\
\hline & -2.939604 & 2.252006 & 1.485510 \\
\hline 6 & 0.222608 & 3.224895 & 4.286652 \\
\hline & -4.191541 & 4.404219 & 2.078136 \\
\hline 6 & -0.543136 & 0.135038 & -3.065866 \\
\hline & -0.751474 & -0.117575 & -4.177365 \\
\hline 1 & 7.036763 & -0.015098 & 0.724205 \\
\hline & -1.781672 & -0.412299 & 3.428275 \\
\hline & 7.780292 & -3.882646 & -2.302315 \\
\hline 1 & -0.723960 & -4.668533 & 5.201841 \\
\hline & 6.240783 & 5.099293 & 1.001277 \\
\hline 1 & -3.223405 & -3.512865 & 0.478498 \\
\hline & -5.042224 & -4.513386 & -0.834090 \\
\hline 1 & 4.252253 & 4.049692 & 1.926669 \\
\hline & -1.400592 & -2.338096 & 4.892794 \\
\hline 1 & 5.683515 & -3.429687 & -3.445000 \\
\hline 1 & 8.522052 & 3.109763 & -2.048849 \\
\hline
\end{tabular}




\begin{tabular}{|c|c|c|c|}
\hline 1 & 3.544693 & -2.151765 & -3.579063 \\
\hline . & -2.538345 & -4.585921 & -4.329285 \\
\hline & -0.255318 & -3.724505 & -4.216145 \\
\hline & 0.313642 & -7.071132 & 1.791716 \\
\hline 1 & 6.999432 & 1.195003 & -1.971025 \\
\hline & -0.113822 & -5.176004 & 0.295561 \\
\hline 1 & 1.571949 & -2.663930 & -2.913594 \\
\hline & 8.175924 & 5.068826 & -0.547496 \\
\hline 1 & 9.032991 & -1.412219 & 0.983315 \\
\hline 1 & -4.705438 & -5.080118 & -3.236372 \\
\hline 1 & 0.012410 & -6.835407 & 4.252335 \\
\hline 1 & 9.436238 & -3.344062 & -0.538985 \\
\hline 1 & 2.603517 & 2.173599 & 1.905737 \\
\hline 1 & -3.961003 & -0.957816 & 1.961429 \\
\hline 1 & -3.003757 & 0.048331 & -2.078016 \\
\hline 1 & -6.991730 & -1.150854 & -1.062064 \\
\hline 1 & -3.716134 & 2.004114 & 0.766066 \\
\hline 1 & 0.031229 & 1.046318 & 2.591320 \\
\hline 1 & -2.054609 & 4.619030 & 3.729674 \\
\hline 1 & 2.049854 & -3.792395 & 0.291149 \\
\hline 1 & -1.624757 & 1.607372 & -1.575659 \\
\hline 1 & 3.375913 & -2.976958 & -0.528688 \\
\hline 1 & 5.141103 & -2.866450 & 0.953007 \\
\hline 1 & 1.225685 & -1.846549 & 2.372072 \\
\hline 1 & 6.098214 & -2.274860 & 3.153720 \\
\hline 1 & 2.165498 & -1.282217 & 4.585477 \\
\hline 1 & 4.618031 & -1.473120 & 4.984727 \\
\hline 1 & 1.191420 & 2.719136 & 0.234805 \\
\hline 1 & -0.561643 & 3.218154 & 0.100725 \\
\hline 1 & 0.440192 & 3.093430 & -4.505668 \\
\hline 1 & -1.192360 & 4.235859 & -5.939648 \\
\hline 1 & -3.105133 & 5.472580 & -4.934584 \\
\hline 1 & -3.329241 & 5.555030 & -2.454589 \\
\hline 1 & -1.698642 & 4.414609 & -1.020907 \\
\hline 1 & 2.629237 & 2.571550 & -1.596789 \\
\hline 1 & 2.040138 & 2.373580 & -3.245652 \\
\hline 1 & 2.227428 & 3.998597 & -2.570423 \\
\hline 6 & 1.105816 & 2.021205 & 4.620255 \\
\hline 6 & 1.085399 & 4.312844 & 3.635973 \\
\hline 6 & -0.346847 & 3.755270 & 5.605831 \\
\hline 6 & -5.172655 & 3.965422 & 0.992198 \\
\hline 6 & -4.952541 & 4.486662 & 3.406050 \\
\hline 6 & -3.665170 & 5.794647 & 1.706691 \\
\hline 6 & -6.053879 & -1.731787 & 2.987781 \\
\hline 6 & -7.838843 & -0.719942 & 1.600017 \\
\hline 6 & -7.061202 & -3.056503 & 1.156630 \\
\hline 6 & -6.937991 & -0.429569 & -3.449379 \\
\hline 6 & -5.023058 & 1.137074 & -3.627173 \\
\hline 6 & -4.701998 & -1.301228 & -4.078420 \\
\hline 1 & 1.845326 & 2.303628 & 5.380090 \\
\hline 1 & 0.512508 & 1.187472 & 5.021587 \\
\hline 1 & 1.666635 & 1.646503 & 3.754416 \\
\hline 1 & 1.941313 & 4.566653 & 4.277182 \\
\hline 1 & 1.470769 & 3.981528 & 2.661426 \\
\hline 1 & 0.508070 & 5.229972 & 3.462771 \\
\hline 1 & 0.471078 & 3.980507 & 6.302627 \\
\hline
\end{tabular}




$\begin{array}{rrrr}1 & -0.921179 & 4.679692 & 5.477183 \\ 1 & -1.001532 & 3.014256 & 6.082518 \\ 1 & -4.500164 & 6.499163 & 1.593887 \\ 1 & -2.991136 & 6.202445 & 2.469369 \\ 1 & -3.115973 & 5.768981 & 0.755640 \\ 1 & -5.799761 & 5.180357 & 3.319338 \\ 1 & -5.348208 & 3.503532 & 3.692796 \\ 1 & -4.314242 & 4.841579 & 4.224361 \\ 1 & -5.988231 & 4.695778 & 0.917343 \\ 1 & -4.693642 & 3.902185 & 0.005560 \\ 1 & -5.624899 & 2.989165 & 1.212118 \\ 1 & -6.822788 & -2.115311 & 3.670319 \\ 1 & -5.192950 & -2.411961 & 3.047686 \\ 1 & -5.740034 & -0.748708 & 3.362390 \\ 1 & -7.823861 & -3.439102 & 1.848515 \\ 1 & -7.487983 & -3.075020 & 0.146144 \\ 1 & -6.210836 & -3.752118 & 1.177628 \\ 1 & -8.576938 & -1.083111 & 2.327722 \\ 1 & -7.550703 & 0.298473 & 1.891136 \\ 1 & -8.339359 & -0.660366 & 0.626277 \\ 1 & -5.321455 & 1.324849 & -4.667228 \\ 1 & -5.503066 & 1.895650 & -2.994700 \\ 1 & -3.937268 & 1.291710 & -3.568126 \\ 1 & -7.162935 & -0.233837 & -4.505375 \\ 1 & -7.291197 & -1.444338 & -3.224139 \\ 1 & -7.522928 & 0.279791 & -2.849135 \\ 1 & -4.944670 & -1.145141 & -5.138547 \\ 1 & -3.612622 & -1.221124 & -3.969571 \\ 1 & -4.987273 & -2.326079 & -3.806235\end{array}$

$3_{\text {IID-si- }-1 \text {-1a2a3s4s }}$

Number of imaginary frequencies : 0

The smallest frequencies are : $17.3521 \quad 18.8912 \quad 20.0967 \mathrm{~cm}(-1)$

Electronic energy : $\quad H F=-4360.8923688$

Zero-point correction $=\quad 1.454541$ (Hartree/Particle)

Thermal correction to Energy= $\quad 1.539352$

Thermal correction to Enthalpy $=\quad 1.540296$

Thermal correction to Gibbs Free Energy $=\quad 1.336389$

Sum of electronic and zero-point Energies $=\quad-4359.437828$

Sum of electronic and thermal Energies $=\quad-4359.353017$

Sum of electronic and thermal Enthalpies $=\quad-4359.352073$

Sum of electronic and thermal Free Energies $=\quad-4359.555980$

Cartesian Coordinates

$\begin{array}{cccc}6 & -1.735117 & 4.205500 & -2.132527 \\ 6 & -0.601811 & 3.584932 & -2.680453 \\ 6 & -0.469171 & 3.607046 & -4.074437 \\ 6 & -1.436502 & 4.191178 & -4.886344 \\ 6 & -2.562647 & 4.783521 & -4.326176 \\ 6 & -2.701409 & 4.790011 & -2.939782 \\ 6 & 0.438000 & 2.928274 & -1.826424 \\ 6 & 1.846754 & 2.981332 & -2.371840 \\ 45 & -0.232664 & 0.821222 & -1.354974 \\ 6 & 0.252460 & 2.768872 & -0.436889\end{array}$




$\begin{array}{cccc}15 & 1.772211 & -0.308252 & -0.852976 \\ 7 & 1.580542 & -1.947720 & -0.456494 \\ 6 & 2.615802 & -2.844593 & 0.111595 \\ 6 & 3.179553 & -2.438988 & 1.448914 \\ 6 & 2.346819 & -2.049273 & 2.499659 \\ 6 & 2.880038 & -1.693545 & 3.730480 \\ 6 & 4.259566 & -1.722701 & 3.932885 \\ 6 & 5.095509 & -2.114557 & 2.893732 \\ 6 & 4.556223 & -2.471988 & 1.659527 \\ 8 & 2.909574 & -0.190063 & -2.081921 \\ 6 & 4.168981 & -0.729633 & -1.962726 \\ 6 & 5.094005 & -0.186547 & -1.090142 \\ 6 & 6.333422 & -0.885060 & -0.905453 \\ 6 & 6.632379 & -2.020593 & -1.716461 \\ 6 & 5.694005 & -2.445384 & -2.688815 \\ 6 & 4.480385 & -1.824575 & -2.798274 \\ 6 & 7.255218 & -0.536297 & 0.114485 \\ 6 & 8.410847 & -1.253722 & 0.302785 \\ 6 & 8.718530 & -2.353990 & -0.524248 \\ 6 & 7.842870 & -2.726559 & -1.511869 \\ 6 & 4.773883 & 1.042103 & -0.327308 \\ 6 & 5.614561 & 2.204611 & -0.359938 \\ 6 & 5.313525 & 3.314913 & 0.486225 \\ 6 & 4.179256 & 3.259508 & 1.332676 \\ 6 & 3.342805 & 2.179400 & 1.297641 \\ 6 & 3.623025 & 1.099644 & 0.435684 \\ 6 & 6.728039 & 2.317405 & -1.230811 \\ 6 & 7.509739 & 3.446396 & -1.242035 \\ 6 & 7.226987 & 4.526926 & -0.381376 \\ 6 & 6.146936 & 4.458716 & 0.460873 \\ 8 & 2.733478 & 0.058254 & 0.443008 \\ 6 & 0.518947 & -2.602644 & -1.169521 \\ 6 & -0.661549 & -2.939084 & -0.530225 \\ 6 & -1.727172 & -3.519244 & -1.286466 \\ 6 & -1.549776 & -3.759618 & -2.681660 \\ 6 & -0.311260 & -3.441509 & -3.284192 \\ 6 & 0.692511 & -2.875770 & -2.545827 \\ 6 & -2.611813 & -4.321666 & -3.431845 \\ 6 & -3.807375 & -4.628512 & -2.834950 \\ 6 & -3.990132 & -4.384611 & -1.456395 \\ 6 & -2.976620 & -3.851145 & -0.700448 \\ 6 & -0.816884 & -2.776360 & 0.942501 \\ 6 & -1.216478 & -1.579599 & 1.521220 \\ 6 & -1.404325 & -1.518356 & 2.929041 \\ 6 & -1.188875 & -2.610481 & 3.726799 \\ 6 & -0.766057 & -3.839211 & 3.171116 \\ 6 & -0.583630 & -3.928821 & 1.760387 \\ 6 & -0.159927 & -5.171149 & 1.219006 \\ 6 & 0.076983 & -6.252650 & 2.030805 \\ 6 & -0.103130 & -6.155843 & 3.426888 \\ 6 & -0.517813 & -4.972414 & 3.981818 \\ 6 & -1.576950 & -0.118921 & 0.431250 \\ 6 & -3.312871 & -0.504122 & -0.051227 \\ 6 & -4.240849 & -0.880999 & 0.919759 \\ 6 & -5.589742 & -1.040432 & 0.608379 \\ & -5.992382 & -0.795990 & -0.711252\end{array}$




\begin{tabular}{|c|c|c|c|}
\hline 6 & -5.086962 & -0.426466 & -1.708305 \\
\hline 6 & -3.739273 & -0.307990 & -1.358134 \\
\hline & -6.563414 & -1.419116 & 1.723236 \\
\hline & -5.492414 & -0.142509 & -3.152302 \\
\hline & -1.838473 & 1.251073 & 1.627491 \\
\hline & -0.832081 & 1.576822 & 2.537783 \\
\hline 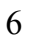 & -0.945724 & 2.692609 & 3.369634 \\
\hline & -2.071881 & 3.508279 & 3.215485 \\
\hline & -3.080938 & 3.230256 & 2.288706 \\
\hline & -2.952244 & 2.081277 & 1.507390 \\
\hline & 0.149032 & 3.058850 & 4.372102 \\
\hline y & -4.286881 & 4.159585 & 2.160001 \\
\hline & -0.508639 & 0.108311 & -3.118626 \\
\hline & -0.693643 & -0.108146 & -4.241758 \\
\hline & 7.023208 & 0.295064 & 0.775033 \\
\hline & -1.733022 & -0.587187 & 3.383716 \\
\hline & 8.054188 & -3.584659 & -2.148727 \\
\hline & -0.661275 & -4.881040 & 5.057586 \\
\hline & 5.901593 & 5.289866 & 1.120701 \\
\hline & -3.131915 & -3.661311 & 0.360479 \\
\hline & -4.945729 & -4.616410 & -0.990859 \\
\hline & 3.974124 & 4.097669 & 1.997490 \\
\hline & -1.343262 & -2.545073 & 4.803225 \\
\hline & 5.943559 & -3.290863 & -3.327739 \\
\hline & 8.352145 & 3.509323 & -1.927254 \\
\hline & 3.737720 & -2.150367 & -3.522929 \\
\hline & -2.457540 & -4.498533 & -4.495407 \\
\hline & -0.176558 & -3.636463 & -4.346981 \\
\hline & 0.403817 & -7.193584 & 1.594148 \\
\hline & 6.954594 & 1.499094 & -1.909440 \\
\hline & -0.023491 & -5.262965 & 0.143334 \\
\hline 1 & 1.639227 & -2.604554 & -3.008984 \\
\hline & 7.857081 & 5.413132 & -0.396940 \\
\hline 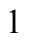 & 9.092628 & -0.973457 & 1.102700 \\
\hline & -4.618391 & -5.055943 & -3.420680 \\
\hline & 0.087992 & -7.020617 & 4.058018 \\
\hline 1 & 9.640199 & -2.909673 & -0.368428 \\
\hline & 2.450959 & 2.118135 & 1.918928 \\
\hline ] & -3.906808 & -1.024967 & 1.946994 \\
\hline & -3.004074 & -0.031712 & -2.109702 \\
\hline 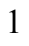 & -7.044245 & -0.893362 & -0.965352 \\
\hline & -3.719397 & 1.831517 & 0.779300 \\
\hline & 0.050113 & 0.937735 & 2.593850 \\
\hline ] & -2.165711 & 4.397461 & 3.837845 \\
\hline & 2.119021 & -3.819544 & 0.219656 \\
\hline 1 & -1.641512 & 1.501103 & -1.592732 \\
\hline & 3.438760 & -2.995087 & -0.604370 \\
\hline 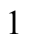 & 5.220106 & -2.760517 & 0.842655 \\
\hline & 1.274100 & -1.986959 & 2.331040 \\
\hline 1 & 6.174787 & -2.133809 & 3.033221 \\
\hline 1 & 2.212364 & -1.391015 & 4.536321 \\
\hline & 4.678507 & -1.437953 & 4.895776 \\
\hline 1 & 1.138093 & 2.713480 & 0.194466 \\
\hline & -0.632259 & 3.148732 & 0.072187 \\
\hline & 0.396279 & 3.138917 & -4.539584 \\
\hline & -1.305009 & 4.180439 & -5.966718 \\
\hline
\end{tabular}




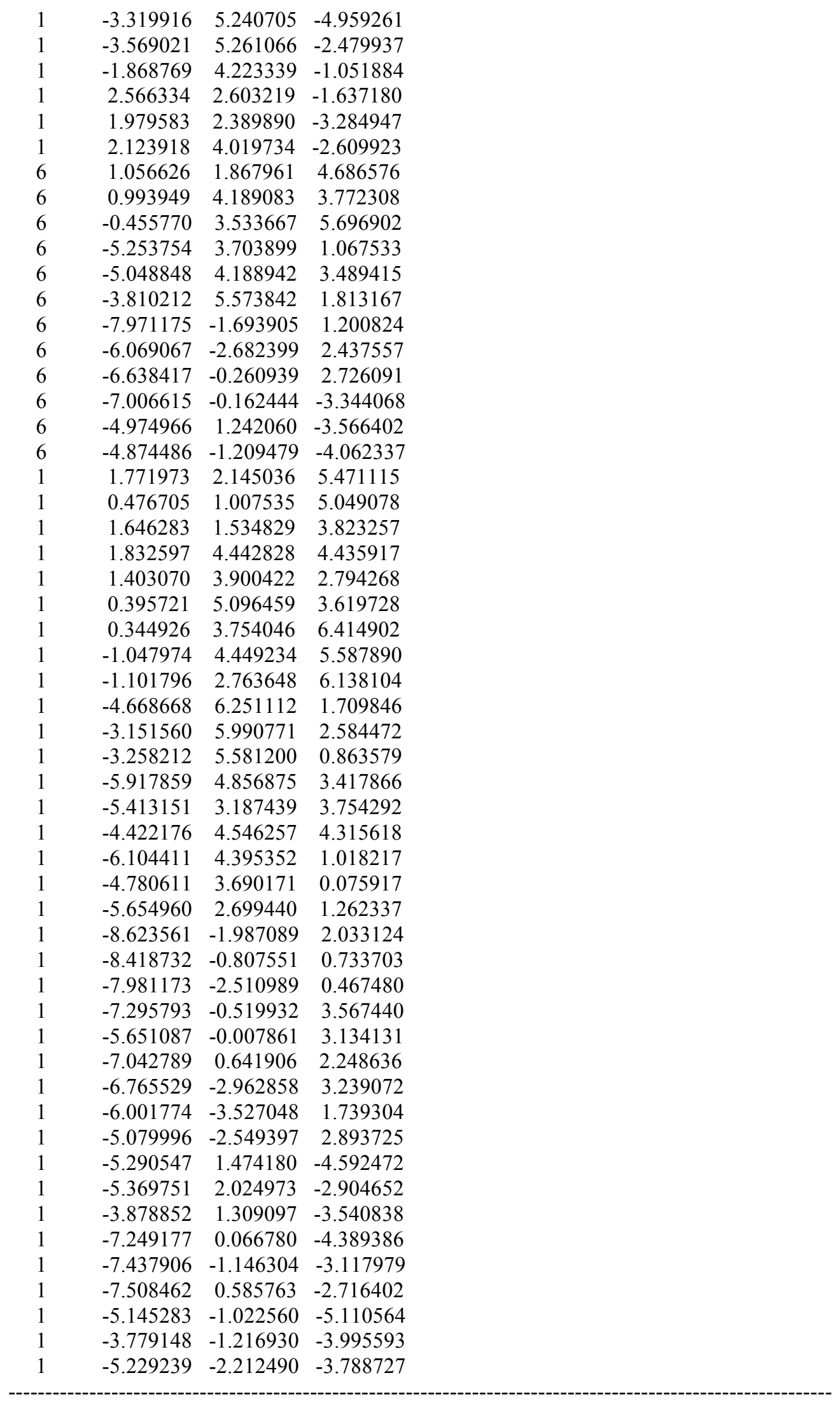

$3_{\text {IID-si- }} \psi_{-1 \mathrm{a} 2 \mathrm{~s} 3 \mathrm{a} 4 \mathrm{a}}$ 
Number of imaginary frequencies : 0

The smallest frequencies are : $10.5371 \quad 19.1620 \quad 21.1205 \mathrm{~cm}(-1)$

Electronic energy : $\quad H F=-4360.8925027$

Zero-point correction $=\quad 1.454423($ Hartree/Particle $)$

Thermal correction to Energy= $\quad 1.539324$

Thermal correction to Enthalpy= $\quad 1.540268$

Thermal correction to Gibbs Free Energy= $\quad 1.335395$

Sum of electronic and zero-point Energies $=\quad-4359.438080$

Sum of electronic and thermal Energies $=\quad-4359.353179$

Sum of electronic and thermal Enthalpies $=\quad-4359.352235$

Sum of electronic and thermal Free Energies $=\quad-4359.557108$

Cartesian Coordinates

$\begin{array}{cccc}6 & -1.503750 & 4.340933 & -2.273742 \\ 6 & -0.404662 & 3.625284 & -2.771262 \\ 6 & -0.224998 & 3.606428 & -4.160326 \\ 6 & -1.113242 & 4.250851 & -5.015220 \\ 6 & -2.204672 & 4.944611 & -4.504242 \\ 6 & -2.391637 & 4.985662 & -3.124342 \\ 6 & 0.565018 & 2.926528 & -1.870576 \\ 6 & 1.991405 & 2.901903 & -2.371971 \\ 45 & -0.221599 & 0.864990 & -1.373211 \\ 6 & 0.326799 & 2.808338 & -0.484968 \\ 15 & 1.738967 & -0.312304 & -0.815459 \\ 7 & 1.496727 & -1.927449 & -0.353401 \\ 6 & 2.503230 & -2.829733 & 0.255783 \\ 6 & 3.068527 & -2.398719 & 1.584794 \\ 6 & 2.248958 & -1.910346 & 2.604143 \\ 6 & 2.785629 & -1.548739 & 3.831866 \\ 6 & 4.155409 & -1.670047 & 4.062906 \\ 6 & 4.978530 & -2.156820 & 3.054153 \\ 6 & 4.436108 & -2.519059 & 1.822739 \\ 8 & 2.864503 & -0.280730 & -2.059001 \\ 6 & 4.087769 & -0.897751 & -1.942641 \\ 6 & 5.052934 & -0.402448 & -1.085760 \\ 6 & 6.243868 & -1.179694 & -0.897094 \\ 6 & 6.457735 & -2.346418 & -1.691343 \\ 6 & 5.488584 & -2.720212 & -2.654131 \\ 6 & 4.319222 & -2.020650 & -2.766315 \\ 6 & 7.198917 & -0.875839 & 0.106701 \\ 6 & 8.305366 & -1.666795 & 0.296429 \\ 6 & 8.528742 & -2.800040 & -0.513032 \\ 6 & 7.619503 & -3.129418 & -1.485164 \\ 6 & 4.826042 & 0.869682 & -0.361149 \\ 6 & 5.746904 & 1.966644 & -0.450332 \\ 6 & 5.534984 & 3.131996 & 0.346724 \\ 6 & 4.405784 & 3.196486 & 1.198842 \\ 6 & 3.491916 & 2.180129 & 1.214703 \\ 6 & 3.686853 & 1.043860 & 0.402191 \\ 6 & 6.854963 & 1.961370 & -1.335128 \\ 6 & 7.715266 & 3.029497 & -1.403234 \\ 6 & 7.521191 & 4.163758 & -0.588394 \\ 6 & 6.448372 & 4.210392 & 0.264584 \\ 8 & 2.722060 & 0.073284 & 0.458959 \\ & & & \\ 6\end{array}$




\begin{tabular}{|c|c|c|c|}
\hline & 0.414660 & -2.581942 & -1.035825 \\
\hline & -0.770406 & -2.859526 & -0.378100 \\
\hline & -1.851356 & -3.453707 & -1.100098 \\
\hline & -1.686950 & -3.764399 & -2.482409 \\
\hline & -0.445874 & -3.499874 & -3.105426 \\
\hline & 0.574749 & -2.923821 & -2.398327 \\
\hline & -2.765363 & -4.340361 & -3.198798 \\
\hline & -3.963948 & -4.590858 & -2.580927 \\
\hline & -4.136369 & -4.268325 & -1.216695 \\
\hline & -3.105064 & -3.725696 & -0.492532 \\
\hline & -0.919436 & -2.618060 & 1.083490 \\
\hline & -1.300387 & -1.386713 & 1.598090 \\
\hline & -1.497117 & -1.255196 & 3.000327 \\
\hline & -1.295650 & -2.307107 & 3.853363 \\
\hline & -0.883635 & -3.566869 & 3.363060 \\
\hline & -0.703738 & -3.731080 & 1.959012 \\
\hline & -0.296740 & -5.005063 & 1.482140 \\
\hline & -0.068567 & -6.045139 & 2.348851 \\
\hline & -0.242816 & -5.873363 & 3.738312 \\
\hline & -0.644738 & -4.658558 & 4.231466 \\
\hline & -1.623501 & 0.032965 & 0.437915 \\
\hline & -3.360566 & -0.358659 & -0.040601 \\
\hline 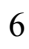 & -4.315848 & -0.636706 & 0.942218 \\
\hline & -5.630708 & -0.938336 & 0.597147 \\
\hline 6 & -5.959276 & -0.958051 & -0.765160 \\
\hline 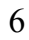 & -5.030975 & -0.692162 & -1.771860 \\
\hline 6 & -3.721290 & -0.392120 & -1.382479 \\
\hline f & -6.692576 & -1.277046 & 1.642314 \\
\hline 6 & -5.454792 & -0.661151 & -3.239714 \\
\hline 6 & -1.826386 & 1.451755 & 1.582281 \\
\hline 0 & -0.796236 & 1.757128 & 2.479806 \\
\hline 5 & -0.851606 & 2.894440 & 3.278902 \\
\hline 6 & -1.948273 & 3.757412 & 3.119654 \\
\hline 6 & -2.973677 & 3.498582 & 2.213076 \\
\hline 5 & -2.895897 & 2.326462 & 1.448885 \\
\hline 6 & 0.267114 & 3.237524 & 4.262306 \\
\hline 6 & -4.174883 & 4.421682 & 2.019933 \\
\hline 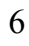 & -0.451771 & 0.099596 & -3.120395 \\
\hline 3 & -0.563162 & -0.163536 & -4.243151 \\
\hline ] & 7.030869 & -0.018900 & 0.754068 \\
\hline 1 & -1.818655 & -0.300427 & 3.408111 \\
\hline 1 & 7.765547 & -4.010335 & -2.108988 \\
\hline 1 & -0.784796 & -4.509443 & 5.301229 \\
\hline 1 & 6.269829 & 5.085322 & 0.888373 \\
\hline & -3.250901 & -3.475699 & 0.556974 \\
\hline 1 & -5.097060 & -4.449844 & -0.738119 \\
\hline 1 & 4.266177 & 4.075842 & 1.826012 \\
\hline 1 & -1.453689 & -2.184851 & 4.924327 \\
\hline 1 & 5.678718 & -3.587736 & -3.283860 \\
\hline 1 & 8.551355 & 3.002071 & -2.098473 \\
\hline 1 & 3.553278 & -2.298906 & -3.486454 \\
\hline 1 & -2.619992 & -4.577858 & -4.251822 \\
\hline 1 & -0.320959 & -3.747142 & -4.158490 \\
\hline 1 & 0.246698 & -7.011112 & 1.961084 \\
\hline 1 & 7.013448 & 1.101203 & -1.980206 \\
\hline 1 & -0.165246 & -5.154875 & 0.412328 \\
\hline
\end{tabular}




\begin{tabular}{|c|c|c|c|}
\hline$\frac{1}{1}$ & 1.526206 & -2.700243 & -2.876493 \\
\hline & 8.213050 & 5.000600 & -0.648166 \\
\hline & 9.013567 & -1.419197 & 1.084069 \\
\hline 1 & -4.784904 & -5.036397 & -3.139110 \\
\hline 1 & -0.058112 & -6.705563 & 4.413602 \\
\hline & 9.412505 & -3.413906 & -0.355948 \\
\hline & 2.602129 & 2.214237 & 1.841205 \\
\hline & -4.020666 & -0.608131 & 1.988605 \\
\hline & -2.956860 & -0.184082 & -2.125769 \\
\hline & -6.985939 & -1.190168 & -1.047504 \\
\hline & -3.680665 & 2.099532 & 0.728247 \\
\hline & 0.055029 & 1.079081 & 2.547880 \\
\hline & -1.992814 & 4.659768 & 3.724783 \\
\hline & 1.979904 & -3.786949 & 0.392915 \\
\hline & -1.605499 & 1.583348 & -1.669226 \\
\hline & 3.327312 & -3.028120 & -0.446785 \\
\hline ] & 5.090980 & -2.885839 & 1.030437 \\
\hline & 1.186657 & -1.776533 & 2.412089 \\
\hline 1 & 6.050677 & -2.249508 & 3.217567 \\
\hline & 2.127946 & -1.166820 & 4.612087 \\
\hline ] & 4.577198 & -1.381757 & 5.023475 \\
\hline & 1.187083 & 2.723366 & 0.178330 \\
\hline & -0.554381 & 3.242367 & -0.014518 \\
\hline 1 & 0.613813 & 3.058584 & -4.585754 \\
\hline & -0.947208 & 4.208592 & -6.090049 \\
\hline$\frac{1}{1}$ & -2.898719 & 5.451692 & -5.170925 \\
\hline & -3.234001 & 5.532231 & -2.702609 \\
\hline ] & -1.674301 & 4.389311 & -1.199023 \\
\hline ] & 2.672715 & 2.517719 & -1.605293 \\
\hline & 2.126746 & 2.278811 & -3.263229 \\
\hline 1 & 2.319212 & 3.920310 & -2.630672 \\
\hline 6 & 1.154028 & 2.027792 & 4.562154 \\
\hline 6 & 1.128979 & 4.346723 & 3.647754 \\
\hline 0 & -0.308178 & 3.725916 & 5.595348 \\
\hline 6 & -4.146827 & 5.621084 & 2.963351 \\
\hline 5 & -4.180124 & 4.945497 & 0.578548 \\
\hline 6 & -5.466504 & 3.637106 & 2.282369 \\
\hline 6 & -6.173592 & -1.105649 & 3.068743 \\
\hline 6 & -7.911613 & -0.365065 & 1.470347 \\
\hline 6 & -7.118546 & -2.738489 & 1.460937 \\
\hline 6 & -4.251826 & -0.718428 & -4.181837 \\
\hline 6 & -6.370428 & -1.841959 & -3.572537 \\
\hline 6 & -6.209647 & 0.649331 & -3.492991 \\
\hline 1 & 1.892962 & 2.290800 & 5.329512 \\
\hline 1 & 0.564040 & 1.180243 & 4.939137 \\
\hline 1 & 1.715389 & 1.680540 & 3.685383 \\
\hline 1 & 1.977354 & 4.588580 & 4.303278 \\
\hline 1 & 1.525911 & 4.041293 & 2.669473 \\
\hline 1 & 0.548830 & 5.265171 & 3.492342 \\
\hline 1 & 0.506967 & 3.933851 & 6.300775 \\
\hline 1 & -0.887254 & 4.650618 & 5.492864 \\
\hline 1 & -0.960301 & 2.967626 & 6.047852 \\
\hline 1 & -6.341228 & 4.287761 & 2.149166 \\
\hline 1 & -5.580533 & 2.785913 & 1.598779 \\
\hline 1 & -5.487538 & 3.247773 & 3.308999 \\
\hline 1 & -5.043034 & 5.605312 & 0.414434 \\
\hline
\end{tabular}




$\begin{array}{rrrr}1 & -3.269105 & 5.524254 & 0.372037 \\ 1 & -4.238438 & 4.132034 & -0.155697 \\ 1 & -5.031821 & 6.246545 & 2.791030 \\ 1 & -4.160258 & 5.314448 & 4.017351 \\ 1 & -3.263706 & 6.251831 & 2.799161 \\ 1 & -6.971788 & -1.346248 & 3.782257 \\ 1 & -5.329309 & -1.773980 & 3.284851 \\ 1 & -5.853345 & -0.073506 & 3.265011 \\ 1 & -7.888529 & -3.008965 & 2.196345 \\ 1 & -7.531488 & -2.924560 & 0.461534 \\ 1 & -6.264004 & -3.414030 & 1.604365 \\ 1 & -8.672512 & -0.602549 & 2.225815 \\ 1 & -7.634656 & 0.690440 & 1.591530 \\ 1 & -8.381736 & -0.476595 & 0.486197 \\ 1 & -6.632415 & -1.823041 & -4.638544 \\ 1 & -5.870064 & -2.796510 & -3.362796 \\ 1 & -7.311632 & -1.819785 & -3.010624 \\ 1 & -4.600467 & -0.758390 & -5.221708 \\ 1 & -3.609788 & 0.167168 & -4.093204 \\ 1 & -3.635045 & -1.610145 & -3.998338 \\ 1 & -6.527244 & 0.714181 & -4.542817 \\ 1 & -7.105495 & 0.725933 & -2.863127 \\ 1 & -5.571693 & 1.516822 & -3.277868\end{array}$

$3_{\text {IID-si- }} \psi_{-122 s 3 a 4 s}$

Number of imaginary frequencies : 0

The smallest frequencies are : $14.7257 \quad 18.0550 \quad 20.9996 \mathrm{~cm}(-1)$

Electronic energy : $\quad H F=-4360.8928692$

Zero-point correction $=\quad 1.454151($ Hartree/Particle $)$

Thermal correction to Energy= $\quad 1.539099$

Thermal correction to Enthalpy= $\quad 1.540044$

Thermal correction to Gibbs Free Energy= $\quad 1.334978$

Sum of electronic and zero-point Energies $=\quad-4359.438718$

Sum of electronic and thermal Energies $=\quad-4359.353770$

Sum of electronic and thermal Enthalpies $=\quad-4359.352826$

Sum of electronic and thermal Free Energies $=\quad-4359.557891$

\section{Cartesian Coordinates}

$\begin{array}{llll}6 & 6.439486 & 4.235027 & 0.400517 \\ 6 & 5.528768 & 3.152251 & 0.450701 \\ 6 & 5.748073 & 2.007751 & -0.374625 \\ 6 & 6.861251 & 2.028301 & -1.252999 \\ 6 & 7.718790 & 3.100103 & -1.289189 \\ 6 & 7.517013 & 4.212958 & -0.447338 \\ 6 & 4.828790 & 0.906942 & -0.319225 \\ 6 & 3.687522 & 1.058180 & 0.445912 \\ 6 & 3.484689 & 2.173347 & 1.284964 \\ 6 & 4.394669 & 3.193148 & 1.297857 \\ 6 & 5.054469 & -0.338880 & -1.089110 \\ 6 & 4.088209 & -0.798121 & -1.964916 \\ 6 & 4.319834 & -1.880509 & -2.840923 \\ 6 & 5.490808 & -2.581780 & -2.763676 \\ 6 & 6.459889 & -2.251580 & -1.784989 \\ 6 & 6.246343 & -1.122911 & -0.937503\end{array}$




\begin{tabular}{|c|c|c|c|}
\hline 6 & 7.622566 & -3.042104 & -1.616941 \\
\hline 6 & 8.532781 & -2.757462 & -0.631661 \\
\hline 6 & 8.309837 & -1.662845 & 0.229594 \\
\hline 6 & 7.202710 & -0.864343 & 0.077687 \\
\hline 8 & 2.862291 & -0.182221 & -2.050305 \\
\hline 1 & 1.742479 & -0.271780 & -0.804458 \\
\hline 8 & 2.726905 & 0.082606 & 0.477234 \\
\hline 7 & 1.521757 & -1.903429 & -0.392108 \\
\hline 6 & 0.443705 & -2.549308 & -1.088754 \\
\hline 6 & -0.737567 & -2.856097 & -0.437248 \\
\hline 6 & -1.812980 & -3.442666 & -1.173818 \\
\hline 6 & -1.650205 & -3.705873 & -2.566211 \\
\hline 6 & -0.413091 & -3.411540 & -3.183448 \\
\hline 6 & 0.604689 & -2.850818 & -2.460520 \\
\hline 6 & -2.723637 & -4.271982 & -3.297255 \\
\hline 6 & -3.913012 & -4.567250 & -2.681690 \\
\hline 6 & -4.081969 & -4.298296 & -1.305634 \\
\hline 6 & -3.058512 & -3.756829 & -0.569429 \\
\hline 6 & -0.890334 & -2.651271 & 1.029651 \\
\hline 6 & -1.287174 & -1.436442 & 1.571129 \\
\hline 6 & -1.487744 & -1.338147 & 2.975422 \\
\hline 6 & -1.281111 & -2.408499 & 3.804339 \\
\hline 6 & -0.854446 & -3.652237 & 3.286511 \\
\hline 6 & -0.665075 & -3.781267 & 1.880033 \\
\hline 6 & -0.240015 & -5.038389 & 1.375418 \\
\hline 6 & -0.003801 & -6.095809 & 2.218568 \\
\hline 6 & -0.188041 & -5.959139 & 3.610743 \\
\hline 6 & -0.607421 & -4.761317 & 4.130196 \\
\hline 15 & -1.626664 & 0.002935 & 0.442035 \\
\hline 6 & -1.867086 & 1.394760 & 1.613700 \\
\hline 6 & -0.841312 & 1.710858 & 2.512237 \\
\hline 6 & -0.916381 & 2.838934 & 3.322878 \\
\hline 6 & -2.028445 & 3.683340 & 3.172620 \\
\hline 6 & -3.051408 & 3.413838 & 2.266096 \\
\hline 6 & -2.956204 & 2.247812 & 1.494358 \\
\hline 6 & 0.203769 & 3.197148 & 4.299358 \\
\hline 6 & -0.366128 & 3.704887 & 5.627382 \\
\hline 6 & -4.257349 & 4.330391 & 2.068682 \\
\hline 6 & -5.550171 & 3.533940 & 2.281539 \\
\hline 6 & 2.544963 & -2.811097 & 0.179492 \\
\hline 6 & 3.102587 & -2.426143 & 1.526002 \\
\hline 6 & 2.278076 & -1.978265 & 2.559901 \\
\hline 6 & 2.809764 & -1.663398 & 3.802710 \\
\hline 6 & 4.178814 & -1.791102 & 4.034369 \\
\hline 6 & 5.006829 & -2.237028 & 3.010888 \\
\hline 6 & 4.469610 & -2.553008 & 1.764675 \\
\hline 45 & -0.227970 & 0.895292 & -1.335343 \\
\hline 6 & -0.423328 & 0.174334 & -3.107522 \\
\hline 8 & -0.492765 & -0.054274 & -4.241047 \\
\hline 6 & -3.353704 & -0.402915 & -0.059060 \\
\hline 6 & -4.293028 & -0.769434 & 0.900489 \\
\hline 6 & -5.612104 & -1.066423 & 0.547337 \\
\hline 6 & -5.960687 & -0.969145 & -0.800854 \\
\hline 6 & -5.041047 & -0.602985 & -1.792645 \\
\hline 6 & -3.731357 & -0.332382 & -1.399608 \\
\hline 6 & -6.597396 & -1.471202 & 1.642811 \\
\hline
\end{tabular}




\begin{tabular}{|c|c|c|c|}
\hline 6 & -6.769154 & -0.303639 & 2.621400 \\
\hline 6 & -5.491683 & -0.467326 & -3.245874 \\
\hline & -6.453640 & 0.721653 & -3.352228 \\
\hline & -7.970547 & -1.834969 & 1.084667 \\
\hline & -6.055073 & -2.691607 & 2.396645 \\
\hline & -4.315751 & -0.220521 & -4.189914 \\
\hline U & -6.205290 & -1.742416 & -3.704400 \\
\hline & -4.259659 & 5.509228 & 3.038043 \\
\hline 6 & -4.232165 & 4.884223 & 0.638606 \\
\hline & 1.092175 & 1.992133 & 4.614305 \\
\hline & 1.061576 & 4.298827 & 3.665434 \\
\hline & 7.034283 & -0.037947 & 0.763800 \\
\hline & -1.818090 & -0.395095 & 3.403308 \\
\hline & 7.768572 & -3.892854 & -2.281296 \\
\hline & -0.755134 & -4.639398 & 5.202379 \\
\hline & 6.254989 & 5.093309 & 1.045356 \\
\hline & -3.202206 & -3.544268 & 0.488777 \\
\hline & -5.035647 & -4.516721 & -0.828729 \\
\hline & 4.248248 & 4.056929 & 1.944802 \\
\hline & -1.444169 & -2.312984 & 4.877260 \\
\hline & 5.682447 & -3.417904 & -3.434085 \\
\hline & 8.558938 & 3.092312 & -1.980036 \\
\hline & 3.552642 & -2.124888 & -3.572156 \\
\hline & -2.580492 & -4.469826 & -4.358691 \\
\hline & -0.288519 & -3.625559 & -4.243822 \\
\hline & 0.325586 & -7.048435 & 1.810101 \\
\hline & 7.026367 & 1.185218 & -1.918566 \\
\hline & -0.101018 & -5.160781 & 0.303075 \\
\hline & 1.554684 & -2.607383 & -2.931972 \\
\hline & 8.206729 & 5.052992 & -0.482163 \\
\hline & 9.018950 & -1.451421 & 1.026913 \\
\hline 1 & -4.729855 & -5.006646 & -3.250497 \\
\hline & 0.003131 & -6.804858 & 4.267172 \\
\hline & 9.417102 & -3.377349 & -0.504200 \\
\hline & 2.591867 & 2.187865 & 1.907948 \\
\hline & -3.993583 & -0.821282 & 1.947272 \\
\hline & -2.977285 & -0.057914 & -2.131206 \\
\hline & -6.985700 & -1.182549 & -1.097840 \\
\hline ] & -3.738942 & 2.012272 & 0.774125 \\
\hline & 0.025096 & 1.050906 & 2.567826 \\
\hline ( & -2.086481 & 4.581082 & 3.783181 \\
\hline & 2.040560 & -3.783300 & 0.275729 \\
\hline & -1.617848 & 1.605316 & -1.621633 \\
\hline ] & 3.372404 & -2.964614 & -0.530465 \\
\hline & 5.128141 & -2.888718 & 0.961800 \\
\hline 1 & 1.215862 & -1.840387 & 2.369555 \\
\hline & 6.078639 & -2.333725 & 3.174510 \\
\hline 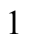 & 2.148844 & -1.314736 & 4.595270 \\
\hline & 4.596197 & -1.539222 & 5.007033 \\
\hline 1 & 1.831950 & 2.266290 & 5.376916 \\
\hline 1 & 0.502067 & 1.150902 & 5.004571 \\
\hline & 1.653002 & 1.631304 & 3.742691 \\
\hline 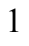 & 0.451903 & 3.917504 & 6.327983 \\
\hline & -0.939898 & 4.631749 & 5.515057 \\
\hline & -1.021036 & 2.955937 & 6.091264 \\
\hline & 1.913552 & 4.549970 & 4.312889 \\
\hline
\end{tabular}




$\begin{array}{rrrr}1 & 1.452702 & 3.982235 & 2.688284 \\ 1 & 0.479018 & 5.214468 & 3.502066 \\ 1 & -5.149667 & 6.127232 & 2.864571 \\ 1 & -4.285974 & 5.179080 & 4.084692 \\ 1 & -3.382653 & 6.154985 & 2.902266 \\ 1 & -5.094267 & 5.543546 & 0.467880 \\ 1 & -3.319174 & 5.470412 & 0.463955 \\ 1 & -4.269729 & 4.084214 & -0.111676 \\ 1 & -6.424150 & 4.183460 & 2.138250 \\ 1 & -5.641767 & 2.696679 & 1.577961 \\ 1 & -5.596778 & 3.123786 & 3.299080 \\ 1 & -7.456829 & -0.580491 & 3.432077 \\ 1 & -5.816522 & -0.006276 & 3.078091 \\ 1 & -7.184841 & 0.575959 & 2.112140 \\ 1 & -8.635008 & -2.136065 & 1.904463 \\ 1 & -8.445155 & -0.987688 & 0.573357 \\ 1 & -7.914312 & -2.674852 & 0.379386 \\ 1 & -6.741388 & -2.973880 & 3.206429 \\ 1 & -5.957348 & -3.555812 & 1.726288 \\ 1 & -5.071639 & -2.505916 & 2.847351 \\ 1 & -4.679691 & -0.164685 & -5.223756 \\ 1 & -3.799433 & 0.725435 & -3.975789 \\ 1 & -3.578348 & -1.033696 & -4.139491 \\ 1 & -6.789486 & 0.851299 & -4.390141 \\ 1 & -7.344752 & 0.583933 & -2.726838 \\ 1 & -5.962022 & 1.651525 & -3.037126 \\ 1 & -6.528868 & -1.639173 & -4.748837 \\ 1 & -5.532711 & -2.608126 & -3.640646 \\ 1 & -7.097732 & -1.962680 & -3.106254 \\ 6 & 0.293100 & 2.829317 & -0.410024 \\ 6 & 0.535430 & 2.972762 & -1.792716 \\ 6 & -0.443394 & 3.664349 & -2.689296 \\ 6 & 1.964594 & 2.978136 & -2.286616 \\ 1 & 1.152109 & 2.745088 & 0.255797 \\ 1 & -0.596264 & 3.243000 & 0.063578 \\ 6 & -0.261568 & 3.658034 & -4.078137 \\ 6 & -1.164329 & 4.284980 & -4.930732 \\ 6 & -2.272900 & 4.949112 & -4.417353 \\ 6 & -2.460641 & 4.980583 & -3.037305 \\ 6 & -1.558213 & 4.353143 & -2.188960 \\ 1 & 0.590959 & 3.133552 & -4.505501 \\ 1 & -0.996338 & 4.252021 & -6.005575 \\ 1 & -2.978916 & 5.441990 & -5.082116 \\ 1 & -3.315287 & 5.506746 & -2.614398 \\ 1 & -1.730423 & 4.391846 & -1.114126 \\ 1 & 2.648198 & 2.599240 & -1.519114 \\ 1 & 2.116305 & 2.365523 & -3.182449 \\ 1 & 2.275620 & 4.004556 & -2.533976\end{array}$

$3_{\text {IID-si- }} \psi_{-1 \mathrm{a} 233 s 4 a}$

Number of imaginary frequencies : 0

The smallest frequencies are : $10.767120 .383322 .0557 \mathrm{~cm}(-1)$ Electronic energy :

Zero-point correction= $\mathrm{HF}=-4360.8926034$

Thermal correction to Energy=

1.454356 (Hartree/Particle) 1.539165 
$\begin{array}{lc}\text { Thermal correction to Enthalpy }= & 1.540110 \\ \text { Thermal correction to Gibbs Free Energy }= & 1.335620 \\ \text { Sum of electronic and zero-point Energies }= & -4359.438248 \\ \text { Sum of electronic and thermal Energies }= & -4359.353438 \\ \text { Sum of electronic and thermal Enthalpies }= & -4359.352494 \\ \text { Sum of electronic and thermal Free Energies }= & -4359.556983\end{array}$

Cartesian Coordinates

$\begin{array}{cccc}6 & -1.647688 & 4.283543 & -2.139760 \\ 6 & -0.513980 & 3.634062 & -2.651385 \\ 6 & -0.347545 & 3.632014 & -4.042088 \\ 6 & -1.282273 & 4.223340 & -4.885883 \\ 6 & -2.409325 & 4.846396 & -4.361618 \\ 6 & -2.582295 & 4.874453 & -2.979449 \\ 6 & 0.493205 & 2.967242 & -1.767363 \\ 6 & 1.915135 & 2.996938 & -2.280386 \\ 45 & -0.235921 & 0.881507 & -1.303447 \\ 6 & 0.273758 & 2.818687 & -0.380833 \\ 15 & 1.752933 & -0.273978 & -0.810825 \\ 7 & 1.555797 & -1.913796 & -0.417538 \\ 6 & 2.592046 & -2.815686 & 0.139672 \\ 6 & 3.153457 & -2.429624 & 1.484210 \\ 6 & 2.325596 & -2.009732 & 2.527100 \\ 6 & 2.859045 & -1.680901 & 3.765387 \\ 6 & 4.233556 & -1.768312 & 3.983761 \\ 6 & 5.064629 & -2.189066 & 2.952074 \\ 6 & 4.525499 & -2.518076 & 1.709946 \\ 8 & 2.867465 & -0.153116 & -2.058813 \\ 6 & 4.106211 & -0.743896 & -1.981788 \\ 6 & 5.066141 & -0.271097 & -1.105921 \\ 6 & 6.275324 & -1.030445 & -0.965682 \\ 6 & 6.510676 & -2.146309 & -1.824405 \\ 6 & 5.544052 & -2.490517 & -2.800630 \\ 6 & 4.357365 & -1.814910 & -2.866554 \\ 6 & 7.228953 & -0.761462 & 0.049444 \\ 6 & 8.354527 & -1.536005 & 0.189114 \\ 6 & 8.599556 & -2.615893 & -0.684607 \\ 6 & 7.691931 & -2.911504 & -1.668991 \\ 6 & 4.816462 & 0.960394 & -0.320592 \\ 6 & 5.711539 & 2.081745 & -0.361993 \\ 6 & 5.468970 & 3.208592 & 0.481244 \\ 6 & 4.337872 & 3.211149 & 1.333484 \\ 6 & 3.451212 & 2.171398 & 1.308804 \\ 6 & 3.675302 & 1.074916 & 0.450983 \\ 6 & 6.822387 & 2.140407 & -1.241810 \\ 6 & 7.655427 & 3.231761 & -1.263438 \\ 6 & 7.430250 & 4.327409 & -0.405207 \\ 6 & 6.354587 & 4.312503 & 0.445043 \\ 8 & 2.737692 & 0.077312 & 0.471098 \\ 6 & 0.484234 & -2.561654 & -1.122200 \\ 6 & -0.701197 & -2.872352 & -0.479594 \\ 6 & -1.776017 & -3.441845 & -1.230656 \\ 6 & -1.601059 & -3.704076 & -2.622041 \\ 6 & -0.355721 & -3.416985 & -3.226451 \\ 6 & 0.655892 & -2.857464 & -2.494003\end{array}$




\begin{tabular}{|c|c|c|c|}
\hline 6 & -2.672592 & -4.256328 & -3.365900 \\
\hline & -3.876051 & -4.527984 & -2.767580 \\
\hline & -4.058309 & -4.256414 & -1.394070 \\
\hline & -3.033349 & -3.738465 & -0.642918 \\
\hline & -0.851130 & -2.699832 & 0.992641 \\
\hline & -1.247100 & -1.499916 & 1.566742 \\
\hline & -1.429051 & -1.430280 & 2.974996 \\
\hline & -1.207857 & -2.517341 & 3.777922 \\
\hline & -0.787091 & -3.749035 & 3.227080 \\
\hline & -0.614733 & -3.847874 & 1.815664 \\
\hline & -0.195488 & -5.093788 & 1.279156 \\
\hline & 0.049048 & -6.169515 & 2.096405 \\
\hline & -0.119075 & -6.063054 & 3.493252 \\
\hline & -0.530889 & -4.876357 & 4.043400 \\
\hline & -1.604831 & -0.044517 & 0.470902 \\
\hline & -3.333705 & -0.439980 & -0.028869 \\
\hline & -4.273528 & -0.862467 & 0.920800 \\
\hline & -5.595467 & -1.100176 & 0.561130 \\
\hline & -5.963429 & -0.883013 & -0.778639 \\
\hline & -5.057146 & -0.450784 & -1.741441 \\
\hline & -3.727945 & -0.258641 & -1.344080 \\
\hline & -6.634781 & -1.604301 & 1.561691 \\
\hline & -5.432335 & -0.157479 & -3.191311 \\
\hline & -1.854339 & 1.329416 & 1.661069 \\
\hline 6 & -0.831518 & 1.655303 & 2.559282 \\
\hline & -0.924328 & 2.775943 & 3.379126 \\
\hline 6 & -2.052253 & 3.601409 & 3.240303 \\
\hline & -3.073406 & 3.320011 & 2.335488 \\
\hline 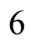 & -2.958462 & 2.164019 & 1.553108 \\
\hline 5 & 0.193128 & 3.146827 & 4.354084 \\
\hline & -4.309315 & 4.199874 & 2.160837 \\
\hline 6 & -0.531775 & 0.159215 & -3.061581 \\
\hline & -0.742793 & -0.069409 & -4.177687 \\
\hline & 7.044171 & 0.052648 & 0.745875 \\
\hline 1 & -1.757866 & -0.497094 & 3.425507 \\
\hline & 7.854499 & -3.752150 & -2.342320 \\
\hline 1 & -0.665732 & -4.777790 & 5.119653 \\
\hline & 6.152298 & 5.156599 & 1.103126 \\
\hline 1 & -3.185608 & -3.530520 & 0.414864 \\
\hline 1 & -5.020524 & -4.459105 & -0.927417 \\
\hline 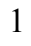 & 4.176184 & 4.061572 & 1.994433 \\
\hline 1 & -1.356535 & -2.445795 & 4.854748 \\
\hline 1 & 5.750626 & -3.317178 & -3.478306 \\
\hline 1 & 8.494281 & 3.252959 & -1.955585 \\
\hline & 3.591876 & -2.071629 & -3.595485 \\
\hline 1 & -2.520002 & -4.452455 & -4.426370 \\
\hline 1 & -0.222034 & -3.630839 & -4.285725 \\
\hline 1 & 0.372779 & -7.113284 & 1.663569 \\
\hline 1 & 7.005620 & 1.311191 & -1.920011 \\
\hline 1 & -0.068042 & -5.192963 & 0.202994 \\
\hline 1 & 1.610774 & -2.613679 & -2.955480 \\
\hline 1 & 8.100669 & 5.183311 & -0.429135 \\
\hline 1 & 9.061223 & -1.317026 & 0.986548 \\
\hline 1 & -4.694381 & -4.947340 & -3.349090 \\
\hline 1 & 0.078722 & -6.923060 & 4.128832 \\
\hline & 9.498502 & -3.216390 & -0.566960 \\
\hline
\end{tabular}




\begin{tabular}{|c|c|c|c|}
\hline 1 & 2.561584 & 2.155187 & 1.936411 \\
\hline & -3.955610 & -0.995287 & 1.952619 \\
\hline & -2.985794 & 0.059424 & -2.071300 \\
\hline & -6.999488 & -1.053735 & -1.065321 \\
\hline & -3.740255 & 1.921140 & 0.834593 \\
\hline & 0.047050 & 1.010706 & 2.606060 \\
\hline & -2.124822 & 4.492774 & 3.858575 \\
\hline & 2.097441 & -3.793256 & 0.233452 \\
\hline & -1.629631 & 1.592935 & -1.549282 \\
\hline & 3.416617 & -2.956359 & -0.576504 \\
\hline & 5.186153 & -2.830823 & 0.899580 \\
\hline 1 & 1.258348 & -1.902162 & 2.346081 \\
\hline & 6.140302 & -2.254532 & 3.105480 \\
\hline & 2.195546 & -1.352488 & 4.564493 \\
\hline & 4.652625 & -1.505165 & 4.952718 \\
\hline & 1.144518 & 2.747907 & 0.270983 \\
\hline & -0.614332 & 3.218783 & 0.106888 \\
\hline & 0.516177 & 3.133528 & -4.478264 \\
\hline & -1.125458 & 4.193147 & -5.962517 \\
\hline & -3.141606 & 5.309471 & -5.019357 \\
\hline & -3.452528 & 5.367311 & -2.548346 \\
\hline & -1.810832 & 4.316650 & -1.063070 \\
\hline & 2.614610 & 2.631125 & -1.520691 \\
\hline & 2.065464 & 2.384512 & -3.176458 \\
\hline 1 & 2.205631 & 4.027980 & -2.533618 \\
\hline & 1.096572 & 1.952310 & 4.666228 \\
\hline 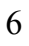 & 1.036604 & 4.258938 & 3.719132 \\
\hline ) & -0.379390 & 3.647358 & 5.683699 \\
\hline 6 & -4.307514 & 5.402150 & 3.100717 \\
\hline ) & -4.359541 & 4.718611 & 0.718194 \\
\hline ) & -5.566430 & 3.369132 & 2.446760 \\
\hline b & -6.092460 & -1.634678 & 2.989206 \\
\hline ) & -7.871456 & -0.699811 & 1.546771 \\
\hline 6 & -7.044603 & -3.030099 & 1.175212 \\
\hline 6 & -6.936430 & -0.257658 & -3.430584 \\
\hline 6 & -4.981711 & 1.264339 & -3.556319 \\
\hline 5 & -4.728578 & -1.164478 & -4.106092 \\
\hline & 1.833305 & 2.234244 & 5.428941 \\
\hline 1 & 0.517478 & 1.102770 & 5.054848 \\
\hline . & 1.661545 & 1.600900 & 3.793562 \\
\hline 1 & 1.886109 & 4.520681 & 4.365652 \\
\hline 1 & 1.430467 & 3.946816 & 2.741720 \\
\hline 1 & 0.442487 & 5.167242 & 3.556311 \\
\hline 1 & 0.437892 & 3.866367 & 6.383170 \\
\hline ] & -0.961275 & 4.569417 & 5.573668 \\
\hline 1 & -1.026838 & 2.892041 & 6.147707 \\
\hline 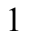 & -6.467088 & 3.985563 & 2.323558 \\
\hline 1 & -5.656891 & 2.510365 & 1.769327 \\
\hline 1 & -5.557327 & 2.984333 & 3.475162 \\
\hline 1 & -5.248712 & 5.346282 & 0.568849 \\
\hline 1 & -3.474304 & 5.329794 & 0.493918 \\
\hline 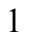 & -4.401816 & 3.901853 & -0.014278 \\
\hline 1 & -5.214555 & 5.998227 & 2.939154 \\
\hline & -4.297147 & 5.098278 & 4.155521 \\
\hline & -3.447179 & 6.060259 & 2.923159 \\
\hline & -6.874724 & -1.986104 & 3.673790 \\
\hline
\end{tabular}




$\begin{array}{lrrr}1 & -5.237479 & -2.316319 & 3.092877 \\ 1 & -5.778884 & -0.637359 & 3.326264 \\ 1 & -7.811207 & -3.410195 & 1.864242 \\ 1 & -7.455294 & -3.076998 & 0.158729 \\ 1 & -6.183133 & -3.710386 & 1.224317 \\ 1 & -8.610544 & -1.058501 & 2.275491 \\ 1 & -7.609333 & 0.332191 & 1.813739 \\ 1 & -8.363394 & -0.677747 & 0.567379 \\ 1 & -5.285181 & 1.504680 & -4.584094 \\ 1 & -5.435097 & 2.007770 & -2.886458 \\ 1 & -3.891909 & 1.391590 & -3.502048 \\ 1 & -7.160720 & -0.018473 & -4.477733 \\ 1 & -7.316932 & -1.269529 & -3.239440 \\ 1 & -7.498628 & 0.445875 & -2.802412 \\ 1 & -4.965472 & -0.956239 & -5.158472 \\ 1 & -3.637288 & -1.126890 & -3.994764 \\ 1 & -5.049644 & -2.189158 & -3.876802\end{array}$

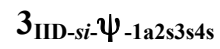

Number of imaginary frequencies : 0

The smallest frequencies are : $12.2711 \quad 19.4857 \quad 21.4692 \mathrm{~cm}(-1)$

Electronic energy :

$\mathrm{HF}=-4360.8928346$

Zero-point correction $=$

Thermal correction to Energy=

Thermal correction to Enthalpy=

1.454571 (Hartree/Particle)

Thermal correction to Gibbs Free Energy $=\quad 1.336130$

Sum of electronic and zero-point Energies=

Sum of electronic and thermal Energies=

$-4359.438263$

Sum of electronic and thermal Enthalpies=

$-4359.353530$

Sum of electronic and thermal Free Energies=

$-4359.352586$

$-4359.556705$

Cartesian Coordinates

$\begin{array}{cccc}6 & -1.557583 & 4.368897 & -2.171296 \\ 6 & -0.471477 & 3.644104 & -2.682877 \\ 6 & -0.325685 & 3.600187 & -4.075470 \\ 6 & -1.236767 & 4.225651 & -4.919727 \\ 6 & -2.316590 & 4.927816 & -4.394605 \\ 6 & -2.468074 & 4.996286 & -3.011872 \\ 6 & 0.517692 & 2.956562 & -1.794335 \\ 6 & 1.942122 & 2.970666 & -2.301483 \\ 45 & -0.234442 & 0.881238 & -1.323781 \\ 6 & 0.288204 & 2.816090 & -0.408443 \\ 15 & 1.745291 & -0.281681 & -0.807471 \\ 7 & 1.536742 & -1.915153 & -0.395418 \\ 6 & 2.568251 & -2.814464 & 0.174980 \\ 6 & 3.119050 & -2.422538 & 1.522105 \\ 6 & 2.284015 & -1.994839 & 2.556027 \\ 6 & 2.808250 & -1.665425 & 3.798168 \\ 6 & 4.180214 & -1.759166 & 4.029169 \\ 6 & 5.018385 & -2.186797 & 3.006070 \\ 6 & 4.488652 & -2.516935 & 1.760293 \\ 8 & 2.866481 & -0.183823 & -2.051365 \\ 6 & 4.102066 & -0.779568 & -1.962217 \\ 6 & 5.059454 & -0.302831 & -1.085885\end{array}$




\begin{tabular}{|c|c|c|c|}
\hline ) & 6.264081 & -1.066474 & -0.931075 \\
\hline & 6.498864 & -2.192198 & -1.776973 \\
\hline & 5.536669 & -2.540418 & -2.756148 \\
\hline & 4.353922 & -1.859391 & -2.836078 \\
\hline & 7.213681 & -0.790968 & 0.086050 \\
\hline & 8.334733 & -1.569270 & 0.239955 \\
\hline & 8.579087 & -2.659722 & -0.620718 \\
\hline & 7.675428 & -2.961500 & -1.606870 \\
\hline & 4.813286 & 0.938696 & -0.315609 \\
\hline 6 & 5.715582 & 2.053746 & -0.367440 \\
\hline U & 5.478583 & 3.191478 & 0.462372 \\
\hline & 4.345668 & 3.210396 & 1.311950 \\
\hline 0 & 3.451752 & 2.176600 & 1.295984 \\
\hline 6 & 3.670139 & 1.069409 & 0.450315 \\
\hline 6 & 6.828247 & 2.095114 & -1.245829 \\
\hline 6 & 7.668177 & 3.180880 & -1.278623 \\
\hline 6 & 7.448478 & 4.287634 & -0.433300 \\
\hline 6 & 6.371353 & 4.289230 & 0.415289 \\
\hline 8 & 2.724599 & 0.079156 & 0.476359 \\
\hline 6 & 0.471286 & -2.570462 & -1.102556 \\
\hline 6 & -0.721953 & -2.868483 & -0.468855 \\
\hline 6 & -1.784819 & -3.459252 & -1.220992 \\
\hline 6 & -1.585556 & -3.766495 & -2.599877 \\
\hline 6 & -0.334022 & -3.486483 & -3.194795 \\
\hline 6 & 0.662775 & -2.898589 & -2.464358 \\
\hline 6 & -2.638422 & -4.358769 & -3.339615 \\
\hline 6 & -3.847714 & -4.624182 & -2.750223 \\
\hline 6 & -4.056869 & -4.300024 & -1.391967 \\
\hline 6 & -3.050023 & -3.742996 & -0.644178 \\
\hline 6 & -0.885738 & -2.678857 & 0.999786 \\
\hline 6 & -1.274718 & -1.471196 & 1.561777 \\
\hline 6 & -1.470703 & -1.390735 & 2.967681 \\
\hline 6 & -1.272589 & -2.475385 & 3.779729 \\
\hline 6 & -0.858682 & -3.714961 & 3.241630 \\
\hline 6 & -0.668602 & -3.823749 & 1.833317 \\
\hline 6 & -0.251755 & -5.076433 & 1.310679 \\
\hline 6 & -0.028355 & -6.149250 & 2.137750 \\
\hline 6 & -0.216382 & -6.033383 & 3.531233 \\
\hline 6 & -0.625000 & -4.839639 & 4.068260 \\
\hline 15 & -1.615897 & -0.020571 & 0.453279 \\
\hline 6 & -3.343731 & -0.419272 & -0.047078 \\
\hline 6 & -4.280728 & -0.820877 & 0.906084 \\
\hline 6 & -5.602820 & -1.086359 & 0.552967 \\
\hline 6 & -5.971615 & -0.912914 & -0.787787 \\
\hline 6 & -5.060838 & -0.502441 & -1.763511 \\
\hline 6 & -3.738019 & -0.281256 & -1.371515 \\
\hline 6 & -6.585065 & -1.524647 & 1.639100 \\
\hline 6 & -5.436676 & -0.278529 & -3.226486 \\
\hline 6 & -1.863424 & 1.362432 & 1.633354 \\
\hline 6 & -0.841962 & 1.683973 & 2.534751 \\
\hline 6 & -0.927757 & 2.810844 & 3.346648 \\
\hline 6 & -2.045288 & 3.647762 & 3.194357 \\
\hline 6 & -3.064182 & 3.371816 & 2.285206 \\
\hline 6 & -2.958897 & 2.207280 & 1.513533 \\
\hline 6 & 0.186441 & 3.177128 & 4.326965 \\
\hline 6 & -4.276714 & 4.278358 & 2.083026 \\
\hline
\end{tabular}




\begin{tabular}{|c|c|c|c|}
\hline 6 & -0.532487 & 0.123795 & -3.065393 \\
\hline & -0.747893 & -0.139787 & -4.172874 \\
\hline & 7.029337 & 0.031792 & 0.772348 \\
\hline & -1.792341 & -0.450701 & 3.408979 \\
\hline & 7.837638 & -3.809958 & -2.270416 \\
\hline & -0.774185 & -4.732995 & 5.141860 \\
\hline & 6.173258 & 5.142124 & 1.063228 \\
\hline & -3.222051 & -3.495299 & 0.402043 \\
\hline & -5.025660 & -4.492627 & -0.934852 \\
\hline & 4.187906 & 4.068714 & 1.963517 \\
\hline & -1.432999 & -2.395345 & 4.854287 \\
\hline & 5.743447 & -3.374024 & -3.425195 \\
\hline & 8.508203 & 3.188970 & -1.969623 \\
\hline & 3.592556 & -2.118244 & -3.568477 \\
\hline & -2.466539 & -4.592077 & -4.389527 \\
\hline & -0.182984 & -3.730925 & -4.245046 \\
\hline & 0.294040 & -7.098084 & 1.715113 \\
\hline & 7.007141 & 1.256945 & -1.914088 \\
\hline & -0.108565 & -5.183682 & 0.237290 \\
\hline & 1.623075 & -2.662493 & -2.918242 \\
\hline & 8.124286 & 5.138992 & -0.465858 \\
\hline & 9.038421 & -1.344965 & 1.038559 \\
\hline & -4.651094 & -5.076041 & -3.328224 \\
\hline & -0.035961 & -6.891388 & 4.174624 \\
\hline & 9.474470 & -3.263210 & -0.491771 \\
\hline & 2.560346 & 2.173961 & 1.921280 \\
\hline & -3.971837 & -0.920550 & 1.946594 \\
\hline & -2.995863 & 0.020230 & -2.106132 \\
\hline & -7.002627 & -1.098020 & -1.075840 \\
\hline 1 & -3.738442 & 1.967792 & 0.791275 \\
\hline & 0.029422 & 1.030389 & 2.590758 \\
\hline & -2.110833 & 4.544722 & 3.805356 \\
\hline & 2.072415 & -3.791283 & 0.269847 \\
\hline & -1.622846 & 1.601092 & -1.587235 \\
\hline 1 & 3.398389 & -2.959661 & -0.533756 \\
\hline & 5.154738 & -2.836667 & 0.957143 \\
\hline ] & 1.218704 & -1.883475 & 2.365721 \\
\hline & 6.092213 & -2.257650 & 3.169673 \\
\hline & 2.139286 & -1.332444 & 4.590732 \\
\hline 1 & 4.591829 & -1.495775 & 5.001239 \\
\hline & 1.153533 & 2.739581 & 0.249796 \\
\hline & -0.599552 & 3.226513 & 0.071426 \\
\hline & 0.503941 & 3.045483 & -4.510358 \\
\hline 1 & -1.098243 & 4.162508 & -5.997446 \\
\hline & -3.028859 & 5.419821 & -5.053313 \\
\hline 1 & -3.300250 & 5.550505 & -2.580138 \\
\hline l & -1.698935 & 4.438224 & -1.093426 \\
\hline 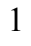 & 2.634859 & 2.589752 & -1.543151 \\
\hline 1 & 2.086682 & 2.363978 & -3.202332 \\
\hline ] & 2.246460 & 3.999848 & -2.545987 \\
\hline 6 & 1.084324 & 1.979532 & 4.642538 \\
\hline 5 & 1.036807 & 4.286916 & 3.697250 \\
\hline 6 & -0.391603 & 3.677955 & 5.654115 \\
\hline 6 & -4.291139 & 5.458312 & 3.050790 \\
\hline 6 & -4.251641 & 4.830030 & 0.651923 \\
\hline 6 & -5.563517 & 3.471285 & 2.293032 \\
\hline
\end{tabular}




$\begin{array}{rrrr}6 & -7.958935 & -1.877019 & 1.074701 \\ 6 & -6.041042 & -2.763293 & 2.361173 \\ 6 & -6.757087 & -0.382431 & 2.647428 \\ 6 & -6.937398 & -0.418997 & -3.467426 \\ 6 & -5.011701 & 1.132716 & -3.654798 \\ 6 & -4.709046 & -1.309778 & -4.094696 \\ 1 & 1.819462 & 2.258851 & 5.407744 \\ 1 & 0.500407 & 1.132573 & 5.029573 \\ 1 & 1.650727 & 1.625714 & 3.771742 \\ 1 & 1.883836 & 4.545229 & 4.348343 \\ 1 & 1.434691 & 3.974033 & 2.721702 \\ 1 & 0.446851 & 5.197456 & 3.531969 \\ 1 & 0.422640 & 3.895981 & 6.357464 \\ 1 & -0.972496 & 4.600342 & 5.541724 \\ 1 & -1.041793 & 2.922963 & 6.114807 \\ 1 & -6.442481 & 4.113756 & 2.148390 \\ 1 & -5.646911 & 2.633613 & 1.588740 \\ 1 & -5.608738 & 3.059877 & 3.310141 \\ 1 & -5.119289 & 5.480955 & 0.477035 \\ 1 & -3.343541 & 5.424590 & 0.480217 \\ 1 & -4.278196 & 4.028871 & -0.098011 \\ 1 & -5.184890 & 6.069805 & 2.873523 \\ 1 & -4.318734 & 5.129112 & 4.097711 \\ 1 & -3.418226 & 6.110098 & 2.917386 \\ 1 & -8.618438 & -2.207293 & 1.887263 \\ 1 & -8.440547 & -1.016709 & 0.592810 \\ 1 & -7.901535 & -2.693670 & 0.342697 \\ 1 & -7.439658 & -0.681625 & 3.454560 \\ 1 & -5.802773 & -0.092134 & 3.105327 \\ 1 & -7.178669 & 0.507756 & 2.161786 \\ 1 & -6.729108 & -3.068906 & 3.160897 \\ 1 & -5.938908 & -3.609100 & 1.668353 \\ 1 & -5.059857 & -2.587725 & 2.820579 \\ 1 & -5.309158 & 1.317091 & -4.695771 \\ 1 & -5.485964 & 1.898116 & -3.026141 \\ 1 & -3.924940 & 1.280169 & -3.596419 \\ 1 & -7.160515 & -0.230355 & -4.525111 \\ 1 & -7.299061 & -1.428782 & -3.233452 \\ 1 & -7.517042 & 0.300329 & -2.873970 \\ 1 & -4.952128 & -1.156488 & -5.155141 \\ 1 & -3.618982 & -1.237326 & -3.987748 \\ 1 & -5.001675 & -2.331364 & -3.818189\end{array}$

$3_{\text {IID-si- }} \psi_{-1 \mathbf{s} 2 a 3 a 4 a}$

Number of imaginary frequencies : 0

The smallest frequencies are : $14.0163 \quad 20.3276 \quad 21.7347 \mathrm{~cm}(-1)$

Electronic energy : $\quad H F=-4360.8905218$

Zero-point correction $=\quad 1.454160($ Hartree/Particle $)$

Thermal correction to Energy $=\quad 1.539111$

Thermal correction to Enthalpy= $\quad 1.540055$

Thermal correction to Gibbs Free Energy= 1.335651

Sum of electronic and zero-point Energies $=\quad-4359.436362$

Sum of electronic and thermal Energies $=\quad-4359.351411$

Sum of electronic and thermal Enthalpies $=\quad-4359.350466$

Sum of electronic and thermal Free Energies $=\quad-4359.554871$ 
Cartesian Coordinates

$\begin{array}{cccc}\cdots & -1.487173 & 4.506213 & -2.017830 \\ 6 & -0.410425 & 3.770466 & -2.532264 \\ 6 & -0.251866 & 3.747075 & -3.924077 \\ 6 & -1.143332 & 4.402074 & -4.765968 \\ 6 & -2.216333 & 5.113599 & -4.238581 \\ 6 & -2.379008 & 5.163157 & -2.856761 \\ 6 & 0.561300 & 3.055367 & -1.645485 \\ 6 & 1.997524 & 3.108694 & -2.114946 \\ 45 & -0.175338 & 0.948566 & -1.280996 \\ 6 & 0.290921 & 2.856793 & -0.275506 \\ 15 & 1.807776 & -0.196425 & -0.754751 \\ 7 & 1.601433 & -1.828924 & -0.336732 \\ 6 & 2.638398 & -2.720435 & 0.235288 \\ 6 & 3.179641 & -2.332928 & 1.587287 \\ 6 & 2.344570 & -1.876067 & 2.608293 \\ 6 & 2.861922 & -1.557844 & 3.856224 \\ 6 & 4.227260 & -1.691887 & 4.105595 \\ 6 & 5.066021 & -2.146111 & 3.094266 \\ 6 & 4.542967 & -2.465003 & 1.842959 \\ 8 & 2.917290 & -0.102610 & -2.010835 \\ 6 & 4.141463 & -0.724467 & -1.938939 \\ 6 & 5.115220 & -0.277163 & -1.065554 \\ 6 & 6.300194 & -1.072304 & -0.919939 \\ 6 & 6.501421 & -2.199272 & -1.772344 \\ 6 & 5.529450 & -2.512528 & -2.753761 \\ 6 & 4.364485 & -1.800467 & -2.824945 \\ 6 & 7.260727 & -0.825782 & 0.094381 \\ 6 & 8.359216 & -1.636730 & 0.243029 \\ 6 & 8.569271 & -2.731239 & -0.621839 \\ 6 & 7.655788 & -3.002615 & -1.607939 \\ 6 & 4.904891 & 0.970735 & -0.295774 \\ 6 & 5.833371 & 2.063237 & -0.361690 \\ 6 & 5.629674 & 3.213369 & 0.459640 \\ 6 & 4.500895 & 3.267673 & 1.312966 \\ 6 & 3.582511 & 2.255674 & 1.309793 \\ 6 & 3.769739 & 1.135170 & 0.475191 \\ 6 & 6.941026 & 2.069611 & -1.247224 \\ 6 & 7.808163 & 3.133280 & -1.293898 \\ 6 & 7.621949 & 4.251669 & -0.455717 \\ 6 & 6.549940 & 4.287413 & 0.398759 \\ 8 & 2.798373 & 0.169832 & 0.518273 \\ 6 & 0.537016 & -2.497483 & -1.033513 \\ 6 & -0.629991 & -2.849655 & -0.378805 \\ 6 & -1.678270 & -3.490962 & -1.111091 \\ 6 & -1.509380 & -3.744498 & -2.504691 \\ 6 & -0.292563 & -3.387134 & -3.128214 \\ 6 & 0.703366 & -2.786958 & -2.407483 \\ 6 & -2.555870 & -4.361249 & -3.233504 \\ 6 & -3.726230 & -4.718204 & -2.614368 \\ 6 & -3.900706 & -4.463642 & -1.236756 \\ 6 & -2.903146 & -3.872020 & -0.503086 \\ 6 & -0.798756 & -2.626609 & 1.084435 \\ 6 & -1.250300 & -1.418037 & 1.599525 \\ & & & \\ 6\end{array}$




\begin{tabular}{|c|c|c|c|}
\hline 6 & -1.478062 & -1.305777 & 2.997893 \\
\hline 6 & -1.229460 & -2.348578 & 3.850284 \\
\hline & -0.736673 & -3.579320 & 3.361704 \\
\hline & -0.533936 & -3.728709 & 1.958941 \\
\hline & -0.051746 & -4.976408 & 1.482746 \\
\hline & 0.232174 & -6.002536 & 2.349310 \\
\hline & 0.039210 & -5.843768 & 3.738062 \\
\hline & -0.439412 & -4.656756 & 4.230072 \\
\hline & -1.612594 & -0.008468 & 0.439926 \\
\hline & -3.285196 & -0.519772 & -0.145452 \\
\hline & -4.252818 & -0.932500 & 0.774228 \\
\hline & -5.538720 & -1.270653 & 0.358562 \\
\hline & -5.826876 & -1.175746 & -1.008892 \\
\hline & -4.882539 & -0.774268 & -1.954933 \\
\hline & -3.600873 & -0.454928 & -1.497323 \\
\hline & -6.610394 & -1.757284 & 1.332984 \\
\hline & -5.269201 & -0.641627 & -3.426674 \\
\hline & -2.025211 & 1.385772 & 1.565371 \\
\hline & -1.126590 & 1.799111 & 2.545964 \\
\hline & -1.377784 & 2.935074 & 3.325764 \\
\hline & -2.532234 & 3.670435 & 3.055150 \\
\hline & -3.430725 & 3.310940 & 2.041545 \\
\hline & -3.163328 & 2.155904 & 1.311281 \\
\hline & -0.409820 & 3.302512 & 4.449580 \\
\hline & -4.684174 & 4.149326 & 1.794502 \\
\hline & -0.333757 & 0.246913 & -3.060592 \\
\hline & -0.402723 & 0.008484 & -4.192301 \\
\hline & 7.101704 & 0.001286 & 0.782039 \\
\hline & -1.868407 & -0.375460 & 3.402383 \\
\hline 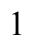 & 7.792705 & -3.852230 & -2.275675 \\
\hline & -0.596873 & -4.518880 & 5.298934 \\
\hline 1 & 6.377549 & 5.150248 & 1.040875 \\
\hline$\frac{1}{1}$ & -3.053734 & -3.674489 & 0.557080 \\
\hline | & -4.837305 & -4.737164 & -0.754370 \\
\hline 1 & 4.363462 & 4.135233 & 1.956726 \\
\hline | & -1.412134 & -2.240786 & 4.918980 \\
\hline 1 & 5.713539 & -3.344485 & -3.431437 \\
\hline & 8.643709 & 3.114658 & -1.990105 \\
\hline 1 & 3.596850 & -2.031183 & -3.559807 \\
\hline 1 & -2.407148 & -4.547628 & -4.296373 \\
\hline 1 & -0.163972 & -3.590724 & -4.190181 \\
\hline 1 & 0.606400 & -6.947586 & 1.962404 \\
\hline 1 & 7.094095 & 1.222090 & -1.910023 \\
\hline 1 & 0.094172 & -5.115659 & 0.413324 \\
\hline & 1.640163 & -2.504745 & -2.882387 \\
\hline 1 & 8.319136 & 5.085146 & -0.498617 \\
\hline 1 & 9.071693 & -1.435261 & 1.039913 \\
\hline 1 & -4.523119 & -5.195252 & -3.181046 \\
\hline 1 & 0.269539 & -6.664541 & 4.413292 \\
\hline 1 & 9.446837 & -3.361184 & -0.497017 \\
\hline 1 & 2.695326 & 2.279591 & 1.937430 \\
\hline 1 & -3.991473 & -0.978854 & 1.829170 \\
\hline 1 & -2.825902 & -0.144352 & -2.191669 \\
\hline 1 & -6.832231 & -1.427253 & -1.348117 \\
\hline 1 & -3.838838 & 1.846838 & 0.518522 \\
\hline & -0.211662 & 1.222248 & 2.704711 \\
\hline
\end{tabular}




\begin{tabular}{|c|c|c|c|}
\hline 1 & -2.745245 & 4.558226 & 3.646407 \\
\hline & 2.153381 & -3.702966 & 0.323090 \\
\hline & -1.570627 & 1.643659 & -1.586782 \\
\hline & 3.472504 & -2.854621 & -0.470576 \\
\hline & 5.209449 & -2.806537 & 1.049111 \\
\hline & 1.286393 & -1.731858 & 2.401079 \\
\hline & 6.135790 & -2.245021 & 3.270047 \\
\hline & 2.193021 & -1.197784 & 4.637174 \\
\hline & 4.634105 & -1.436655 & 5.081858 \\
\hline & 1.130458 & 2.759996 & 0.413543 \\
\hline & -0.620650 & 3.232966 & 0.186056 \\
\hline 1 & 0.572197 & 3.185070 & -4.359889 \\
\hline & -0.995731 & 4.354862 & -5.843264 \\
\hline & -2.913314 & 5.629555 & -4.895310 \\
\hline & -3.203662 & 5.728185 & -2.425087 \\
\hline & -1.631160 & 4.564764 & -0.939967 \\
\hline & 2.676873 & 2.690862 & -1.364322 \\
\hline & 2.169013 & 2.559170 & -3.047213 \\
\hline & 2.297012 & 4.153015 & -2.292118 \\
\hline & -0.754020 & 4.638139 & 5.103916 \\
\hline 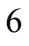 & -0.467440 & 2.210073 & 5.524438 \\
\hline & 1.018959 & 3.392044 & 3.903843 \\
\hline & -5.450558 & 3.689268 & 0.554994 \\
\hline ) & -5.611802 & 4.021270 & 3.008405 \\
\hline D & -4.307074 & 5.621155 & 1.597866 \\
\hline ) & -6.130566 & -1.725079 & 2.782573 \\
\hline 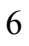 & -7.856954 & -0.873097 & 1.226540 \\
\hline ) & -6.978614 & -3.204688 & 0.988462 \\
\hline & -4.060166 & -0.342571 & -4.312415 \\
\hline ) & -5.914129 & -1.936913 & -3.927563 \\
\hline & -6.267697 & 0.513405 & -3.566545 \\
\hline 1 & -0.020557 & 4.863211 & 5.888309 \\
\hline & -0.731780 & 5.464680 & 4.381941 \\
\hline 1 & -1.742863 & 4.622473 & 5.579592 \\
\hline 1 & 0.217928 & 2.445878 & 6.350009 \\
\hline I & -1.480006 & 2.120797 & 5.939493 \\
\hline 1 & -0.180713 & 1.228629 & 5.123927 \\
\hline & 1.710244 & 3.714449 & 4.693981 \\
\hline 1 & 1.372799 & 2.418559 & 3.543902 \\
\hline . & 1.086571 & 4.111285 & 3.075729 \\
\hline 1 & -5.207977 & 6.220432 & 1.410230 \\
\hline 1 & -3.808897 & 6.046972 & 2.476727 \\
\hline 1 & -3.634387 & 5.746682 & 0.739198 \\
\hline 1 & -6.526112 & 4.611127 & 2.857118 \\
\hline ] & -5.906037 & 2.975216 & 3.167140 \\
\hline 1 & -5.132575 & 4.377577 & 3.928710 \\
\hline 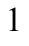 & -6.325577 & 4.333898 & 0.403384 \\
\hline 1 & -4.833460 & 3.745528 & -0.351888 \\
\hline 1 & -5.817278 & 2.658375 & 0.650777 \\
\hline 1 & -6.929965 & -2.083338 & 3.443474 \\
\hline 1 & -5.257664 & -2.372652 & 2.942756 \\
\hline 1 & -5.866613 & -0.708726 & 3.103504 \\
\hline 1 & -7.768761 & -3.570430 & 1.658310 \\
\hline & -7.340858 & -3.303810 & -0.042499 \\
\hline 1 & -6.107597 & -3.864295 & 1.105289 \\
\hline & -8.627853 & -1.216647 & 1.929336 \\
\hline
\end{tabular}




$\begin{array}{lrrr}1 & -7.620094 & 0.170976 & 1.469850 \\ 1 & -8.296732 & -0.891106 & 0.222334 \\ 1 & -6.193888 & -1.835676 & -4.984783 \\ 1 & -5.214301 & -2.778305 & -3.838922 \\ 1 & -6.823866 & -2.195104 & -3.371881 \\ 1 & -4.376751 & -0.294220 & -5.362119 \\ 1 & -3.593649 & 0.621656 & -4.069140 \\ 1 & -3.291506 & -1.124043 & -4.231568 \\ 1 & -6.554252 & 0.648053 & -4.618524 \\ 1 & -7.183888 & 0.332955 & -2.990077 \\ 1 & -5.828490 & 1.455156 & -3.211576\end{array}$

$3_{\text {IID-si- }} \Psi_{-1 \text { s2a3a4s }}$

Number of imaginary frequencies : 0

The smallest frequencies are : $13.8082 \quad 20.781322 .5058 \mathrm{~cm}(-1)$

Electronic energy : $\quad H F=-4360.8914717$

Zero-point correction $=\quad 1.454514$ (Hartree/Particle)

Thermal correction to Energy $=\quad 1.539208$

Thermal correction to Enthalpy= $\quad 1.540153$

Thermal correction to Gibbs Free Energy= $\quad 1.337025$

Sum of electronic and zero-point Energies $=\quad-4359.436958$

Sum of electronic and thermal Energies $=\quad-4359.352263$

Sum of electronic and thermal Enthalpies $=\quad-4359.351319$

Sum of electronic and thermal Free Energies $=\quad-4359.554447$

\section{Cartesian Coordinates}

$\begin{array}{cccc}6 & -1.622811 & 4.339022 & -2.058306 \\ 6 & -0.456465 & 3.732259 & -2.548673 \\ 6 & -0.226387 & 3.804679 & -3.928435 \\ 6 & -1.130562 & 4.426565 & -4.783549 \\ 6 & -2.288720 & 5.008682 & -4.281034 \\ 6 & -2.525345 & 4.963034 & -2.908823 \\ 6 & 0.526012 & 3.046757 & -1.650937 \\ 6 & 1.965487 & 3.116379 & -2.108467 \\ 45 & -0.171334 & 0.919923 & -1.286999 \\ 6 & 0.251873 & 2.843220 & -0.282958 \\ 15 & 1.824303 & -0.199327 & -0.755550 \\ 7 & 1.636764 & -1.838941 & -0.355588 \\ 6 & 2.678574 & -2.723700 & 0.217960 \\ 6 & 3.207627 & -2.336109 & 1.574800 \\ 6 & 2.358085 & -1.909048 & 2.596877 \\ 6 & 2.863393 & -1.587611 & 3.848882 \\ 6 & 4.230977 & -1.688724 & 4.101465 \\ 6 & 5.083997 & -2.113549 & 3.089297 \\ 6 & 4.573100 & -2.435549 & 1.833716 \\ 8 & 2.942442 & -0.078110 & -2.001837 \\ 6 & 4.179161 & -0.673643 & -1.921337 \\ 6 & 5.135970 & -0.207416 & -1.038741 \\ 6 & 6.338132 & -0.975071 & -0.887652 \\ 6 & 6.572976 & -2.092543 & -1.744298 \\ 6 & 5.614440 & -2.426213 & -2.732071 \\ 6 & 4.433004 & -1.742308 & -2.808115 \\ 6 & 7.284512 & -0.711749 & 0.135723 \\ 6 & 8.402286 & -1.495325 & 0.286765\end{array}$




\begin{tabular}{|c|c|c|c|}
\hline ) & 8.646541 & -2.578151 & -0.583729 \\
\hline & 7.746464 & -2.867101 & -1.577011 \\
\hline & 4.890131 & 1.030394 & -0.263137 \\
\hline & 5.791150 & 2.146631 & -0.312606 \\
\hline & 5.548086 & 3.286443 & 0.512966 \\
\hline & 4.409720 & 3.306456 & 1.355059 \\
\hline & 3.519279 & 2.270028 & 1.337924 \\
\hline & 3.744969 & 1.160069 & 0.499290 \\
\hline & 6.908692 & 2.187425 & -1.184996 \\
\hline & 7.747556 & 3.274037 & -1.215779 \\
\hline & 7.521687 & 4.382752 & -0.374639 \\
\hline & 6.439741 & 4.385227 & 0.467743 \\
\hline & 2.800807 & 0.167553 & 0.527665 \\
\hline & 0.585494 & -2.510326 & -1.069441 \\
\hline & -0.589085 & -2.869925 & -0.432504 \\
\hline 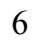 & -1.633442 & -3.492137 & -1.186128 \\
\hline 6 & -1.449536 & -3.727067 & -2.581099 \\
\hline 6 & -0.220798 & -3.374373 & -3.183700 \\
\hline 6 & 0.768768 & -2.786781 & -2.443738 \\
\hline & -2.495009 & -4.318669 & -3.332134 \\
\hline & -3.679409 & -4.664812 & -2.733949 \\
\hline 6 & -3.868832 & -4.427213 & -1.354996 \\
\hline 6 & -2.871740 & -3.863574 & -0.599326 \\
\hline 6 & -0.769358 & -2.673139 & 1.033030 \\
\hline 6 & -1.226778 & -1.475204 & 1.566558 \\
\hline 6 & -1.461510 & -1.386530 & 2.965364 \\
\hline 6 & -1.219292 & -2.445164 & 3.800197 \\
\hline 6 & -0.724651 & -3.667498 & 3.292491 \\
\hline 6 & -0.509658 & -3.790735 & 1.888974 \\
\hline 6 & -0.022979 & -5.029037 & 1.393415 \\
\hline 6 & 0.250940 & -6.072177 & 2.242750 \\
\hline 6 & 0.044113 & -5.940112 & 3.632347 \\
\hline 6 & -0.437189 & -4.761919 & 4.142675 \\
\hline 15 & -1.593720 & -0.053513 & 0.424988 \\
\hline 6 & -3.263434 & -0.564845 & -0.166602 \\
\hline 6 & -4.227410 & -0.964209 & 0.753037 \\
\hline 6 & -5.532800 & -1.260448 & 0.353263 \\
\hline 6 & -5.838448 & -1.141846 & -1.003308 \\
\hline 6 & -4.888316 & -0.754721 & -1.957989 \\
\hline 6 & -3.595354 & -0.476628 & -1.516966 \\
\hline 6 & -6.557296 & -1.650497 & 1.416679 \\
\hline 6 & -5.293709 & -0.591333 & -3.421323 \\
\hline 6 & -2.031949 & 1.320448 & 1.567479 \\
\hline 6 & -1.142687 & 1.741102 & 2.553467 \\
\hline 6 & -1.420215 & 2.860668 & 3.348697 \\
\hline 6 & -2.590699 & 3.573987 & 3.087408 \\
\hline 6 & -3.482221 & 3.206005 & 2.070795 \\
\hline 6 & -3.189218 & 2.066483 & 1.326801 \\
\hline 6 & -0.464916 & 3.232459 & 4.482114 \\
\hline 6 & -4.749446 & 4.023664 & 1.824902 \\
\hline 6 & -0.291256 & 0.242913 & -3.080231 \\
\hline 8 & -0.325482 & 0.030217 & -4.218538 \\
\hline 1 & 7.099497 & 0.105310 & 0.828762 \\
\hline 1 & -1.852218 & -0.462440 & 3.383826 \\
\hline 1 & 7.909199 & -3.709118 & -2.248596 \\
\hline 1 & -0.604438 & -4.644482 & 5.212494 \\
\hline
\end{tabular}




\begin{tabular}{|c|c|c|c|}
\hline ] & 6.236955 & 5.239595 & 1.112293 \\
\hline & -3.033983 & -3.676437 & 0.460902 \\
\hline & -4.818155 & -4.687554 & -0.890792 \\
\hline 1 & 4.242830 & 4.167091 & 2.001215 \\
\hline 1 & -1.408050 & -2.356758 & 4.869604 \\
\hline & 5.822424 & -3.251514 & -3.410961 \\
\hline & 8.591519 & 3.281271 & -1.901986 \\
\hline & 3.675246 & -1.990421 & -3.547752 \\
\hline & -2.334736 & -4.492629 & -4.395378 \\
\hline & -0.079509 & -3.566864 & -4.246113 \\
\hline & 0.628203 & -7.009981 & 1.841431 \\
\hline & 7.092535 & 1.347922 & -1.850223 \\
\hline & 0.134199 & -5.147396 & 0.323081 \\
\hline & 1.712708 & -2.502544 & -2.903395 \\
\hline & 8.196704 & 5.234815 & -0.405532 \\
\hline & 9.103616 & -1.281200 & 1.090218 \\
\hline ] & -4.475788 & -5.121226 & -3.318122 \\
\hline & 0.266475 & -6.774230 & 4.293763 \\
\hline ] & 9.539392 & -3.185855 & -0.456989 \\
\hline & 2.625443 & 2.266480 & 1.956499 \\
\hline ] & -3.964516 & -1.022015 & 1.809191 \\
\hline & -2.822345 & -0.173412 & -2.216732 \\
\hline & -6.853245 & -1.351225 & -1.336280 \\
\hline$\frac{1}{1}$ & -3.860649 & 1.751482 & 0.533231 \\
\hline & -0.216060 & 1.181529 & 2.706565 \\
\hline 1 & -2.822394 & 4.450264 & 3.688869 \\
\hline & 2.201450 & -3.710904 & 0.298545 \\
\hline ] & -1.579221 & 1.583439 & -1.597036 \\
\hline$\frac{1}{1}$ & 3.518329 & -2.846625 & -0.483425 \\
\hline | & 5.250579 & -2.753117 & 1.039257 \\
\hline 1 & 1.297037 & -1.790450 & 2.388004 \\
\hline ] & 6.155207 & -2.187324 & 3.268437 \\
\hline 1 & 2.183054 & -1.251443 & 4.630599 \\
\hline ] & 4.628312 & -1.431275 & 5.081053 \\
\hline 1 & 1.090423 & 2.760875 & 0.408823 \\
\hline 1 & -0.666739 & 3.204396 & 0.176714 \\
\hline | & 0.667290 & 3.347914 & -4.349153 \\
\hline 1 & -0.923568 & 4.455079 & -5.851674 \\
\hline ] & -2.995721 & 5.498062 & -4.947307 \\
\hline 1 & -3.420030 & 5.424678 & -2.493817 \\
\hline ] & -1.835530 & 4.313507 & -0.990580 \\
\hline 1 & 2.643165 & 2.744036 & -1.332778 \\
\hline 1 & 2.162177 & 2.532851 & -3.015017 \\
\hline 1 & 2.245029 & 4.159263 & -2.322159 \\
\hline 6 & -0.833498 & 4.556810 & 5.146221 \\
\hline 6 & -0.514874 & 2.129168 & 5.546305 \\
\hline 6 & 0.966873 & 3.348088 & 3.949480 \\
\hline 6 & -5.528882 & 3.524843 & 0.608612 \\
\hline 6 & -5.659576 & 3.920647 & 3.054171 \\
\hline 6 & -4.387010 & 5.492939 & 1.584952 \\
\hline 6 & -7.901847 & -2.046494 & 0.812984 \\
\hline 6 & -6.035837 & -2.839630 & 2.231910 \\
\hline 6 & -6.778246 & -0.451707 & 2.347864 \\
\hline 6 & -4.093207 & -0.299478 & -4.320654 \\
\hline 6 & -5.966913 & -1.868525 & -3.932144 \\
\hline 6 & -6.273985 & 0.582640 & -3.529042 \\
\hline
\end{tabular}




$\begin{array}{rrrr}1 & -0.107806 & 4.786195 & 5.936551 \\ 1 & -0.820311 & 5.389913 & 4.431589 \\ 1 & -1.824323 & 4.521793 & 5.616625 \\ 1 & 0.161732 & 2.366287 & 6.378707 \\ 1 & -1.528893 & 2.022289 & 5.953583 \\ 1 & -0.212208 & 1.155687 & 5.138130 \\ 1 & 1.645339 & 3.677869 & 4.747601 \\ 1 & 1.340792 & 2.382516 & 3.588791 \\ 1 & 1.030329 & 4.073032 & 3.125982 \\ 1 & -5.295335 & 6.081942 & 1.400142 \\ 1 & -3.874299 & 5.942019 & 2.443736 \\ 1 & -3.731728 & 5.601745 & 0.710627 \\ 1 & -6.578370 & 4.502678 & 2.900154 \\ 1 & -5.947788 & 2.878046 & 3.243492 \\ 1 & -5.171114 & 4.302798 & 3.958981 \\ 1 & -6.420940 & 4.146929 & 0.462462 \\ 1 & -4.930856 & 3.578788 & -0.311553 \\ 1 & -5.868417 & 2.486421 & 0.729015 \\ 1 & -8.593908 & -2.341936 & 1.611765 \\ 1 & -8.369076 & -1.217124 & 0.266925 \\ 1 & -7.804030 & -2.898416 & 0.126858 \\ 1 & -7.489099 & -0.707572 & 3.145446 \\ 1 & -5.843351 & -0.125253 & 2.821955 \\ 1 & -7.187594 & 0.403101 & 1.792399 \\ 1 & -6.755828 & -3.106300 & 3.017299 \\ 1 & -5.896139 & -3.722651 & 1.594326 \\ 1 & -5.077588 & -2.627576 & 2.722866 \\ 1 & -6.256826 & -1.748568 & -4.984678 \\ 1 & -5.280455 & -2.723050 & -3.863071 \\ 1 & -6.874345 & -2.118346 & -3.369084 \\ 1 & -4.426907 & -0.215784 & -5.362822 \\ 1 & -3.594090 & 0.644630 & -4.061977 \\ 1 & -3.347995 & -1.105483 & -4.273670 \\ 1 & -6.573555 & 0.737779 & -4.574516 \\ 1 & -7.184928 & 0.410386 & -2.942051 \\ 1 & -5.812393 & 1.511007 & -3.167033\end{array}$

$3_{\text {IID-si- }}-$-1s2a3s4a

Number of imaginary frequencies : 0

The smallest frequencies are : $9.7831 \quad 17.9625 \quad 20.8800 \mathrm{~cm}(-1)$

Electronic energy : $\quad H F=-4360.8908996$

Zero-point correction $=\quad 1.456191$ (Hartree/Particle)

Thermal correction to Energy $=\quad 1.540561$

Thermal correction to Enthalpy= $\quad 1.541506$

Thermal correction to Gibbs Free Energy= 1.338523

Sum of electronic and zero-point Energies $=\quad-4359.434709$

Sum of electronic and thermal Energies $=\quad-4359.350338$

Sum of electronic and thermal Enthalpies $=\quad-4359.349394$

Sum of electronic and thermal Free Energies $=\quad-4359.552376$

Cartesian Coordinates

\begin{tabular}{|c|c|c|c|}
\hline 6 & -1.649783 & 4.344596 & -1.999249 \\
\hline 6 & -0.498512 & 3.719621 & -2.501369 \\
\hline 6 & -0.305493 & 3.744880 & -3.888323 \\
\hline
\end{tabular}




$\begin{array}{cccc}6 & -1.233248 & 4.336550 & -4.739096 \\ 6 & -2.379310 & 4.933536 & -4.225149 \\ 6 & -2.577716 & 4.936311 & -2.846348 \\ 6 & 0.501600 & 3.050269 & -1.611001 \\ 6 & 1.936147 & 3.139639 & -2.080551 \\ 45 & -0.190315 & 0.929203 & -1.250772 \\ 6 & 0.243432 & 2.842350 & -0.239491 \\ 15 & 1.813191 & -0.205068 & -0.780285 \\ 7 & 1.632105 & -1.854125 & -0.416881 \\ 6 & 2.674034 & -2.749732 & 0.139192 \\ 6 & 3.211513 & -2.376820 & 1.497186 \\ 6 & 2.365430 & -1.970633 & 2.530842 \\ 6 & 2.877726 & -1.648345 & 3.779698 \\ 6 & 4.249077 & -1.731124 & 4.018681 \\ 6 & 5.098179 & -2.139276 & 2.996479 \\ 6 & 4.580364 & -2.459885 & 1.743201 \\ 8 & 2.924134 & -0.048289 & -2.028934 \\ 6 & 4.165633 & -0.636162 & -1.966466 \\ 6 & 5.125517 & -0.181108 & -1.080787 \\ 6 & 6.334044 & -0.943796 & -0.954336 \\ 6 & 6.570743 & -2.041358 & -1.835805 \\ 6 & 5.606964 & -2.362559 & -2.822482 \\ 6 & 4.420180 & -1.685918 & -2.875450 \\ 6 & 7.285176 & -0.696794 & 0.068780 \\ 6 & 8.409341 & -1.475629 & 0.195618 \\ 6 & -0.768851 & -2.722008 & 0.963349 \\ 6 & -1.211219 & -1.541875 & 1.546588 \\ 6 & -1.429342 & -1.500614 & 2.950158 \\ 6 & -1.188141 & -2.591060 & 3.743001 \\ 6 & -3.655619 & -2.537265 & -0.700008 \\ 6 & 7.750726 & -2.810910 & -1.693266 \\ 6 & 4.874363 & 1.034673 & -0.272268 \\ 6 & -0.712419 & -3.799015 & 3.184718 \\ 6 & -0.511673 & -3.872792 & 1.775420 \\ 6 & -0.766420 & 2.159182 & -0.289998 \\ 6 & 5.515818 & 3.271141 & 0.571105 \\ 6 & -0.044932 & -5.097685 & 1.229823 \\ 6 & 4.378775 & 3.257558 & 1.415360 \\ 6 & 3.496750 & 2.215045 & 1.368981 \\ 6 & 3.731143 & 1.132768 & 0.497806 \\ 6 & 6.882448 & 2.236003 & -1.162003 \\ 6 & 7.713188 & 3.329360 & -1.159360 \\ 6 & 7.480149 & 4.409382 & -0.283568 \\ 6 & 6.399092 & 4.377394 & 0.559299 \\ 6 & 2.798222 & 0.130287 & 0.503515 \\ 6 & 0.576430 & -2.506156 & -1.141233 \\ 6 & -0.600942 & -2.867844 & -0.510243 \\ 6 & -1.478631 & -3.448069 & -1.279430 \\ 6 & -3.651983 & -2.680163 \\ 6 & -3.308487 & -3.274497 \\ 6 & -2.751386 & -2.522225 \\ 6 & -4.175931 & 2.037079\end{array}$




\begin{tabular}{|c|c|c|c|}
\hline 6 & 0.025867 & -6.093649 & 3.432246 \\
\hline 6 & -0.433930 & -4.928672 & 3.990618 \\
\hline 15 & -1.581866 & -0.081197 & 0.462744 \\
\hline 6 & -3.273674 & -0.539148 & -0.109847 \\
\hline 6 & -4.222082 & -1.023705 & 0.800143 \\
\hline 6 & -5.530251 & -1.275547 & 0.403934 \\
\hline 6 & -5.877844 & -1.003739 & -0.931411 \\
\hline 6 & -4.961218 & -0.514028 & -1.856440 \\
\hline 6 & -3.643950 & -0.312563 & -1.424361 \\
\hline 6 & -6.575935 & -1.847550 & 1.360508 \\
\hline 6 & -5.312500 & -0.167710 & -3.300584 \\
\hline 6 & -1.971432 & 1.269981 & 1.647101 \\
\hline 6 & -1.070135 & 1.644758 & 2.640209 \\
\hline 6 & -1.313775 & 2.758271 & 3.455267 \\
\hline 6 & -2.461271 & 3.512255 & 3.205195 \\
\hline 6 & -3.364337 & 3.187632 & 2.183679 \\
\hline 6 & -3.106028 & 2.053379 & 1.420677 \\
\hline 6 & -0.355887 & 3.073558 & 4.603710 \\
\hline 6 & -4.603277 & 4.049381 & 1.947760 \\
\hline 6 & -0.426986 & 0.271792 & -3.040557 \\
\hline 8 & -0.598216 & 0.080917 & -4.170562 \\
\hline 1 & 7.098955 & 0.102758 & \\
\hline 1 & -1.809343 & -0.588868 & 3.404247 \\
\hline 1 & 7.914476 & -3.637235 & -2.383832 \\
\hline 1 & -0.591074 & -4.849591 & 5.065440 \\
\hline 1 & 6.190916 & 5.209563 & 1.230600 \\
\hline 1 & -3.064045 & -3.645940 & 0.361652 \\
\hline 1 & -4.872641 & -4.567763 & -1.017946 \\
\hline 1 & 4.207562 & 4.097304 & \\
\hline 1 & -1.365690 & -2.541024 & 4.816743 \\
\hline 1 & 5.815215 & -3.173088 & -3.518865 \\
\hline 1 & 8.556287 & 3.364351 & -1.845772 \\
\hline 1 & 3.658025 & -1.925705 & -3.613339 \\
\hline 1 & -2.378694 & -4.349763 & -4.513640 \\
\hline 1 & -0.105438 & -3.478815 & -4.341118 \\
\hline 1 & 0.581267 & -7.103057 & 1.597657 \\
\hline 1 & 7.071950 & 1.419286 & -1.853584 \\
\hline 1 & 0.102697 & -5.177831 & 0.154619 \\
\hline 1 & 1.701864 & -2.468048 & -2.975746 \\
\hline 1 & 8.148875 & 5.266951 & -0.288327 \\
\hline 1 & 9.114064 & -1.274403 & 0.999443 \\
\hline 1 & -4.539201 & -4.944886 & -3.457049 \\
\hline 1 & 0.240690 & -6.955463 & 4.059791 \\
\hline 1 & 9.553457 & -3.141300 & -0.592406 \\
\hline 1 & 2.603746 & 2.184131 & 1.988151 \\
\hline 1 & -3.922141 & -1.193827 & 1.831729 \\
\hline 1 & -2.895411 & 0.055328 & -2.120955 \\
\hline 1 & -6.904952 & -1.180502 & -1.245746 \\
\hline 1 & -3.786066 & 1.771992 & 0.621780 \\
\hline 1 & -0.161128 & 1.055014 & 2.782933 \\
\hline 1 & -2.665313 & 4.386937 & 3.819019 \\
\hline 1 & 2.193523 & -3.736207 & 0.210358 \\
\hline 1 & -1.596382 & 1.614854 & -1.503267 \\
\hline 1 & 3.509897 & -2.866029 & -0.568296 \\
\hline 1 & 5.255097 & -2.761155 & 0.940102 \\
\hline 1 & 1.300899 & -1.865881 & 2.332811 \\
\hline
\end{tabular}




\begin{tabular}{|c|c|c|c|}
\hline 1 & 6.171701 & -2.200420 & 3.166157 \\
\hline 1 & 2.200027 & -1.327864 & 4.570351 \\
\hline 1 & 4.652103 & -1.473522 & 4.995896 \\
\hline & 1.090632 & 2.768074 & 0.442476 \\
\hline 1 & -0.673396 & 3.195044 & 0.230783 \\
\hline 1 & 0.574387 & 3.267092 & -4.315543 \\
\hline 1 & -1.056405 & 4.327539 & -5.812978 \\
\hline 1 & -3.106192 & 5.397179 & -4.888432 \\
\hline 1 & -3.461483 & 5.411697 & -2.423600 \\
\hline 1 & -1.830505 & 4.358075 & -0.925045 \\
\hline 1 & 2.625348 & 2.783260 & -1.307037 \\
\hline 1 & 2.134866 & 2.554689 & -2.985562 \\
\hline 1 & 2.197142 & 4.185745 & -2.301678 \\
\hline 6 & -0.655973 & 4.418577 & 5.261403 \\
\hline 6 & -0.492534 & 1.975381 & 5.665806 \\
\hline 6 & 1.090896 & 3.101888 & 4.100504 \\
\hline 6 & -5.426270 & 3.562450 & 0.755335 \\
\hline 6 & -5.494450 & 4.001450 & 3.193953 \\
\hline 6 & -4.181984 & 5.497209 & 1.676303 \\
\hline 6 & -6.041736 & -1.986854 & 2.784732 \\
\hline 6 & -7.806877 & -0.936249 & 1.404099 \\
\hline 6 & -6.989522 & -3.240749 & 0.872827 \\
\hline 6 & -6.807351 & -0.299476 & -3.579114 \\
\hline 6 & -4.895718 & 1.281384 & -3.591527 \\
\hline 6 & -4.559339 & -1.112420 & -4.242739 \\
\hline 1 & 0.071834 & 4.607888 & 6.060380 \\
\hline 1 & -0.586592 & 5.247630 & 4.545255 \\
\hline 1 & -1.652364 & 4.442024 & 5.720343 \\
\hline 1 & 0.184462 & 2.168753 & 6.509212 \\
\hline 1 & -1.517700 & 1.931918 & 6.056394 \\
\hline 1 & -0.245479 & 0.985597 & 5.259162 \\
\hline 1 & 1.769127 & 3.401341 & 4.910612 \\
\hline 1 & 1.421570 & 2.114156 & 3.756224 \\
\hline 1 & 1.210953 & 3.817050 & 3.274441 \\
\hline 1 & -5.065799 & 6.123842 & 1.495928 \\
\hline 1 & -3.633552 & 5.935518 & 2.518633 \\
\hline 1 & -3.536974 & 5.560131 & 0.789336 \\
\hline 1 & -6.393591 & 4.614761 & 3.045876 \\
\hline 1 & -5.816973 & 2.973071 & 3.404568 \\
\hline 1 & -4.977232 & 4.379169 & 4.084363 \\
\hline 1 & -6.302344 & 4.210097 & 0.624229 \\
\hline 1 & -4.851340 & 3.589315 & -0.180579 \\
\hline 1 & -5.792202 & 2.535813 & 0.893924 \\
\hline 1 & -6.824705 & -2.400086 & 3.433127 \\
\hline 1 & -5.180148 & -2.666529 & 2.838019 \\
\hline 1 & -5.740626 & -1.018377 & 3.205313 \\
\hline 1 & -7.767868 & -3.660478 & 1.524622 \\
\hline 1 & -7.385314 & -3.219214 & -0.150231 \\
\hline 1 & -6.132299 & -3.928258 & 0.889977 \\
\hline 1 & -8.556625 & -1.344298 & 2.095144 \\
\hline 1 & -7.539348 & 0.069367 & 1.753670 \\
\hline 1 & -8.286475 & -0.833616 & 0.423589 \\
\hline 1 & -5.178015 & 1.557851 & -4.616318 \\
\hline 1 & -5.390757 & 1.980316 & -2.903537 \\
\hline 1 & -3.812277 & 1.438888 & -3.499592 \\
\hline 1 & -7.015662 & -0.019391 & -4.619397 \\
\hline
\end{tabular}




$\begin{array}{rrrrr}1 & -7.161947 & -1.329354 & -3.441698 \\ 1 & -7.403501 & 0.358891 & -2.933531 \\ 1 & -4.787925 & -0.870091 & -5.289659 \\ 1 & -3.471854 & -1.040803 & -4.113056 \\ 1 & -4.845820 & -2.156815 & -4.060217\end{array}$

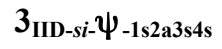

Number of imaginary frequencies : 0

The smallest frequencies are : $13.5710 \quad 19.3903 \quad 20.7645 \mathrm{~cm}(-1)$

Electronic energy : $\quad \mathrm{HF}=-4360.891525$

Zero-point correction $=\quad 1.456235($ Hartree/Particle $)$

Thermal correction to Energy= $\quad 1.540567$

Thermal correction to Enthalpy= $\quad 1.541511$

Thermal correction to Gibbs Free Energy $=\quad 1.339501$

Sum of electronic and zero-point Energies $=\quad-4359.435290$

Sum of electronic and thermal Energies $=\quad-4359.350959$

Sum of electronic and thermal Enthalpies $=\quad-4359.350014$

Sum of electronic and thermal Free Energies $=\quad-4359.552024$

Cartesian Coordinates

$\begin{array}{cccc}6 & -3.170688 & 2.035206 & 1.387717 \\ 6 & -2.021866 & 1.272781 & 1.613124 \\ 6 & -1.122427 & 1.668175 & 2.600210 \\ 6 & -1.378939 & 2.781789 & 3.409849 \\ 6 & -2.540070 & 3.515227 & 3.161281 \\ 6 & -3.441584 & 3.171626 & 2.144885 \\ 15 & -1.597275 & -0.080009 & 0.442788 \\ 6 & -3.269372 & -0.565736 & -0.162777 \\ 6 & -4.234677 & -1.025722 & 0.733193 \\ 6 & -5.535523 & -1.301745 & 0.316140 \\ 6 & -5.853629 & -1.080853 & -1.030483 \\ 6 & -4.911287 & -0.618903 & -1.951794 \\ 6 & -3.609831 & -0.389270 & -1.497225 \\ 6 & -6.556237 & -1.785292 & 1.345718 \\ 6 & -6.833664 & -0.648036 & 2.336155 \\ 6 & -5.235909 & -0.328728 & -3.415513 \\ 6 & -4.439580 & -1.282761 & -4.311065 \\ 6 & -0.417048 & 3.122402 & 4.547573 \\ 6 & 1.026924 & 3.152994 & 4.035718 \\ 6 & -4.690441 & 4.018372 & 1.906387 \\ 6 & -4.289489 & 5.477093 & 1.663749 \\ 45 & -0.188074 & 0.934882 & -1.259571 \\ 6 & -0.382948 & 0.264687 & -3.047491 \\ 8 & -0.514184 & 0.047309 & -4.178176 \\ 6 & 0.495720 & 3.065807 & -1.592422 \\ 6 & 0.231452 & 2.839845 & -0.225706 \\ 6 & -0.498641 & 3.755722 & -2.474119 \\ 6 & -1.620175 & 4.422763 & -1.960068 \\ 6 & -2.531787 & 5.052488 & -2.798406 \\ 6 & -2.346576 & 5.043812 & -4.178493 \\ 6 & -1.231865 & 4.399251 & -4.704714 \\ 6 & -0.320462 & 3.770818 & -3.863429 \\ 6 & 1.931301 & 3.166526 & -2.056473 \\ 15 & 1.812916 & -0.194287 & -0.771027\end{array}$




\begin{tabular}{|c|c|c|c|}
\hline 8 & 2.795699 & 0.157182 & 0.510448 \\
\hline 6 & 3.737141 & 1.151785 & 0.493779 \\
\hline & 4.878201 & 1.037204 & -0.277077 \\
\hline & 5.776893 & 2.155767 & -0.311847 \\
\hline & 5.536359 & 3.279995 & 0.535779 \\
\hline & 4.403253 & 3.282421 & 1.385368 \\
\hline 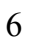 & 3.514340 & 2.244996 & 1.354670 \\
\hline & 6.425740 & 4.381096 & 0.505353 \\
\hline 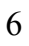 & 7.503076 & 4.395813 & -0.342797 \\
\hline b & 7.726460 & 3.302831 & -1.204923 \\
\hline & 6.889762 & 2.214221 & -1.189226 \\
\hline b & 5.122838 & -0.189535 & -1.070544 \\
\hline ) & 4.162309 & -0.648293 & -1.953216 \\
\hline$b$ & 4.413625 & -1.707557 & -2.851921 \\
\hline b & 5.596266 & -2.390634 & -2.788539 \\
\hline$b$ & 6.559006 & -2.065666 & -1.802040 \\
\hline 6 & 6.326917 & -0.957116 & -0.933238 \\
\hline$b$ & 7.733905 & -2.840779 & -1.647930 \\
\hline$b$ & 8.638153 & -2.561275 & -0.655728 \\
\hline b & 8.396806 & -1.487568 & 0.226800 \\
\hline 5 & 7.277743 & -0.703399 & 0.088533 \\
\hline 5 & 2.923758 & -0.054719 & -2.021827 \\
\hline 1 & 1.632196 & -1.839223 & -0.388829 \\
\hline 6 & 0.582341 & -2.508992 & -1.105883 \\
\hline 6 & -0.589755 & -2.877344 & -0.469579 \\
\hline 6 & -1.635566 & -3.492614 & -1.226509 \\
\hline 6 & -1.449729 & -3.730213 & -2.620449 \\
\hline 6 & -0.221847 & -3.372286 & -3.222054 \\
\hline 6 & 0.763988 & -2.777791 & -2.482217 \\
\hline 5 & -2.493723 & -4.324742 & -3.370612 \\
\hline 6 & -3.682930 & -4.659722 & -2.775861 \\
\hline 6 & -3.876581 & -4.412402 & -1.399459 \\
\hline 6 & -2.877372 & -3.854527 & -0.642355 \\
\hline 6 & -0.767024 & -2.706666 & 0.999441 \\
\hline 5 & -1.231509 & -1.522292 & 1.555482 \\
\hline 6 & -1.468995 & -1.461308 & 2.955398 \\
\hline 6 & -1.222164 & -2.534411 & 3.769994 \\
\hline 5 & -0.720753 & -3.744321 & 3.239275 \\
\hline 6 & -0.503024 & -3.839572 & 1.834007 \\
\hline 6 & -0.011944 & -5.066512 & 1.315218 \\
\hline 6 & 0.262525 & -6.125631 & 2.144456 \\
\hline 6 & 0.052265 & -6.021530 & 3.535888 \\
\hline 6 & -0.432596 & -4.854588 & 4.068325 \\
\hline 6 & 2.675108 & -2.723912 & 0.183271 \\
\hline 6 & 3.202098 & -2.336954 & 1.541359 \\
\hline 6 & 2.349970 & -1.918150 & 2.564910 \\
\hline 6 & 2.854581 & -1.586101 & 3.814437 \\
\hline 6 & 4.224001 & -1.670126 & 4.063793 \\
\hline 6 & 5.079107 & -2.089927 & 3.051320 \\
\hline 6 & 4.568925 & -2.421520 & 1.797873 \\
\hline 6 & -7.878163 & -2.200230 & 0.704752 \\
\hline 6 & -6.002075 & -2.996807 & 2.104819 \\
\hline 6 & -6.720025 & -0.505305 & -3.725765 \\
\hline 6 & -4.845694 & 1.118588 & -3.745341 \\
\hline 6 & -0.723146 & 4.476252 & 5.184039 \\
\hline 6 & -0.540186 & 2.040560 & 5.627780 \\
\hline
\end{tabular}




\begin{tabular}{|c|c|c|c|}
\hline 6 & -5.486251 & 3.539072 & 0.693096 \\
\hline 6 & -5.597334 & 3.937922 & 3.139241 \\
\hline 1 & 7.095241 & 0.106349 & 0.790736 \\
\hline 1 & -1.866206 & -0.547537 & 3.389988 \\
\hline 1 & 7.894263 & -3.675836 & -2.328714 \\
\hline 1 & -0.602535 & -4.758807 & 5.139866 \\
\hline 1 & 6.225167 & 5.223276 & 1.166419 \\
\hline 1 & -3.039137 & -3.665927 & 0.417556 \\
\hline 1 & -4.829517 & -4.662492 & -0.936190 \\
\hline 1 & 4.239664 & 4.130464 & 2.048861 \\
\hline 1 & -1.413821 & -2.468270 & 4.840477 \\
\hline 1 & 5.801952 & -3.208870 & -3.476627 \\
\hline 1 & 8.566761 & 3.323977 & -1.895330 \\
\hline 1 & 3.652980 & -1.949255 & -3.590642 \\
\hline 1 & -2.332817 & -4.499885 & -4.433637 \\
\hline 1 & -0.079203 & -3.563784 & -4.284447 \\
\hline 1 & 0.642757 & -7.054494 & 1.725545 \\
\hline 1 & 7.071550 & 1.387179 & -1.870506 \\
\hline 1 & 0.147758 & -5.163569 & 0.243101 \\
\hline 1 & 1.705607 & -2.486669 & -2.942163 \\
\hline 1 & 8.176362 & 5.249594 & -0.362070 \\
\hline 1 & 9.101422 & -1.281026 & 1.029365 \\
\hline 1 & -4.481194 & -5.109719 & -3.362269 \\
\hline 1 & 0.274720 & -6.868177 & 4.181164 \\
\hline 1 & 9.531969 & -3.169574 & -0.539056 \\
\hline 1 & 2.624718 & 2.226961 & 1.979211 \\
\hline 1 & -3.968240 & -1.151936 & 1.782514 \\
\hline 1 & -2.846329 & -0.039399 & -2.187270 \\
\hline 1 & -6.869117 & -1.269458 & -1.367410 \\
\hline 1 & -3.849437 & 1.738262 & 0.593144 \\
\hline 1 & -0.202799 & 1.094642 & 2.740126 \\
\hline 1 & -2.754924 & 4.390059 & 3.771141 \\
\hline 1 & 2.199592 & -3.712180 & 0.260641 \\
\hline 1 & -1.595067 & 1.615210 & -1.532888 \\
\hline 1 & 3.515964 & -2.842916 & -0.517661 \\
\hline 1 & 5.248133 & -2.733108 & 1.002524 \\
\hline 1 & 1.287035 & -1.812481 & 2.358598 \\
\hline 1 & 6.151455 & -2.151553 & 3.228288 \\
\hline 1 & 2.172053 & -1.256676 & 4.597212 \\
\hline 1 & 4.620851 & -1.404258 & 5.041322 \\
\hline 1 & 1.073447 & 2.758712 & 0.461909 \\
\hline 1 & -0.690604 & 3.182613 & 0.241445 \\
\hline 1 & 0.536050 & 3.258381 & -4.298246 \\
\hline 1 & -1.067012 & 4.382983 & -5.780413 \\
\hline 1 & -3.059935 & 5.538150 & -4.834251 \\
\hline 1 & -3.391837 & 5.562898 & -2.367683 \\
\hline 1 & -1.787499 & 4.445708 & -0.883804 \\
\hline 1 & 2.619617 & 2.792577 & -1.290658 \\
\hline 1 & 2.131681 & 2.605743 & -2.976225 \\
\hline 1 & 2.191425 & 4.218064 & -2.251513 \\
\hline 1 & 0.009546 & 4.686003 & 5.973372 \\
\hline 1 & -0.667183 & 5.292773 & 4.452476 \\
\hline 1 & -1.715796 & 4.499611 & 5.650987 \\
\hline 1 & 0.141414 & 2.250699 & 6.463432 \\
\hline 1 & -1.562450 & 1.997676 & 6.026011 \\
\hline 1 & -0.290601 & 1.045886 & 5.234570 \\
\hline
\end{tabular}




$\begin{array}{rrrr}1 & 1.707160 & 3.470160 & 4.837331 \\ 1 & 1.363240 & 2.162470 & 3.704948 \\ 1 & 1.137583 & 3.855433 & 3.197538 \\ 1 & -5.180627 & 6.089549 & 1.471515 \\ 1 & -3.768832 & 5.915254 & 2.523456 \\ 1 & -3.626045 & 5.563237 & 0.792810 \\ 1 & -6.502319 & 4.542227 & 2.989445 \\ 1 & -5.909636 & 2.902487 & 3.328992 \\ 1 & -5.095926 & 4.307334 & 4.042212 \\ 1 & -6.367012 & 4.178672 & 0.554110 \\ 1 & -4.891805 & 3.585369 & -0.229755 \\ 1 & -5.843931 & 2.506839 & 0.811999 \\ 1 & -8.564270 & -2.569412 & 1.477561 \\ 1 & -8.376014 & -1.361519 & 0.202118 \\ 1 & -7.738611 & -3.006339 & -0.028102 \\ 1 & -7.539951 & -0.974266 & 3.111934 \\ 1 & -5.916688 & -0.309362 & 2.835658 \\ 1 & -7.272928 & 0.217733 & 1.822760 \\ 1 & -6.731506 & -3.343149 & 2.849228 \\ 1 & -5.800565 & -3.832043 & 1.420740 \\ 1 & -5.070907 & -2.771741 & 2.639912 \\ 1 & -5.104263 & 1.352577 & -4.786810 \\ 1 & -5.375559 & 1.828761 & -3.096572 \\ 1 & -3.768690 & 1.299081 & -3.628222 \\ 1 & -6.907122 & -0.268680 & -4.780791 \\ 1 & -7.055251 & -1.537236 & -3.558490 \\ 1 & -7.347627 & 0.162870 & -3.121442 \\ 1 & -4.653375 & -1.079983 & -5.369481 \\ 1 & -3.356982 & -1.177985 & -4.163469 \\ 1 & -4.704241 & -2.327028 & -4.100101\end{array}$

$3_{\text {IID-si- }} \psi_{-1 \mathbf{s} 2 \mathbf{s} 3 a 4 a}$

Number of imaginary frequencies : 0

The smallest frequencies are : $15.7860 \quad 19.0668 \quad 21.8260 \mathrm{~cm}(-1)$

Electronic energy : $\quad H F=-4360.8912216$

Zero-point correction $=\quad 1.454539($ Hartree/Particle $)$

Thermal correction to Energy= $\quad 1.539315$

Thermal correction to Enthalpy= $\quad 1.540260$

Thermal correction to Gibbs Free Energy $=\quad 1.336801$

Sum of electronic and zero-point Energies $=\quad-4359.436682$

Sum of electronic and thermal Energies $=\quad-4359.351906$

Sum of electronic and thermal Enthalpies $=\quad-4359.350962$

Sum of electronic and thermal Free Energies $=\quad-4359.554421$

\section{Cartesian Coordinates}

$\begin{array}{cccc}6 & -3.126686 & 2.142211 & 1.382502 \\ 6 & -1.992497 & 1.364002 & 1.594079 \\ 6 & -1.072973 & 1.752847 & 2.571496 \\ 6 & -1.302252 & 2.871321 & 3.375355 \\ 6 & -2.462708 & 3.621464 & 3.144594 \\ 6 & -3.380685 & 3.282176 & 2.149000 \\ 15 & -1.608832 & -0.017696 & 0.443758 \\ 6 & -3.293426 & -0.497989 & -0.134725 \\ 6 & -4.268732 & -0.883176 & 0.788826\end{array}$




\begin{tabular}{|c|c|c|c|}
\hline 6 & -5.564232 & -1.190369 & 0.377283 \\
\hline 6 & -5.851329 & -1.099241 & -0.990655 \\
\hline 6 & -4.900489 & -0.721664 & -1.940149 \\
\hline 6 & -3.610855 & -0.430315 & -1.486298 \\
\hline 6 & -6.647532 & -1.635322 & 1.359028 \\
\hline 6 & -7.017638 & -3.093001 & 1.063383 \\
\hline 6 & -5.289912 & -0.580005 & -3.410525 \\
\hline 6 & -6.261451 & 0.598150 & -3.547025 \\
\hline 6 & -0.324465 & 3.205491 & 4.502022 \\
\hline 6 & 1.115122 & 3.206378 & 3.978555 \\
\hline 6 & -4.646413 & 4.088682 & 1.865270 \\
\hline 6 & -4.579365 & 4.643028 & 0.436717 \\
\hline 45 & -0.179221 & 0.931826 & -1.282592 \\
\hline 6 & -0.301531 & 0.246799 & -3.072982 \\
\hline 8 & -0.324152 & 0.028966 & -4.210625 \\
\hline 6 & 0.538003 & 3.049335 & -1.659336 \\
\hline 6 & 0.269790 & 2.854784 & -0.288867 \\
\hline 6 & -0.442862 & 3.740448 & -2.554566 \\
\hline 6 & -1.601558 & 4.358859 & -2.060573 \\
\hline 6 & -2.505811 & 4.982424 & -2.909470 \\
\hline 6 & -2.277601 & 5.018190 & -4.283458 \\
\hline 6 & -1.125831 & 4.426396 & -4.789363 \\
\hline 6 & -0.220537 & 3.803577 & -3.936035 \\
\hline 6 & 1.975226 & 3.101212 & -2.126056 \\
\hline 15 & 1.808935 & -0.203396 & -0.756882 \\
\hline 8 & 2.799837 & 0.161432 & 0.515583 \\
\hline 6 & 3.759067 & 1.138975 & 0.473057 \\
\hline 6 & 4.896556 & 0.986593 & -0.296499 \\
\hline 6 & 5.814473 & 2.088103 & -0.359622 \\
\hline 6 & 5.596743 & 3.236103 & 0.461396 \\
\hline 6 & 4.464690 & 3.279446 & \\
\hline 6 & 3.556867 & 2.257893 & 1.305709 \\
\hline 6 & 6.506353 & 4.319427 & 0.403225 \\
\hline 6 & 7.581255 & 4.294488 & -0.447908 \\
\hline 6 & 7.781452 & 3.177997 & -1.285364 \\
\hline 6 & 6.924872 & 2.105715 & -1.241635 \\
\hline 6 & 5.117571 & -0.258739 & -1.067202 \\
\hline 6 & 4.147193 & -0.713492 & -1.940823 \\
\hline 6 & 4.379317 & -1.787575 & -2.826930 \\
\hline 6 & 5.550230 & -2.489901 & -2.756107 \\
\hline 6 & 6.519727 & -2.168925 & -1.774807 \\
\hline 6 & 6.308877 & -1.044221 & -0.921684 \\
\hline 6 & 7.681092 & -2.962272 & -1.611163 \\
\hline 6 & 8.592199 & -2.683795 & -0.624890 \\
\hline 6 & 8.372371 & -1.592217 & 0.241234 \\
\hline 6 & 7.266902 & -0.790704 & 0.093317 \\
\hline 8 & 2.918242 & -0.101061 & -2.013247 \\
\hline 7 & 1.607464 & -1.838664 & -0.345624 \\
\hline 6 & 0.546954 & -2.505375 & -1.050337 \\
\hline 6 & -0.627886 & -2.850841 & -0.406215 \\
\hline 6 & -1.679080 & -3.472579 & -1.150624 \\
\hline 6 & -1.501585 & -3.722918 & -2.543644 \\
\hline 6 & -0.273525 & -3.382097 & -3.154304 \\
\hline 6 & 0.722339 & -2.794162 & -2.423210 \\
\hline 6 & -2.552048 & -4.318684 & -3.284306 \\
\hline 6 & -3.735008 & -4.655254 & -2.677819 \\
\hline
\end{tabular}




\begin{tabular}{|c|c|c|c|}
\hline & -3.918804 & -4.401370 & -1.301055 \\
\hline & -2.916767 & -3.832614 & -0.555736 \\
\hline & -0.802024 & -2.643899 & 1.058586 \\
\hline & -1.253701 & -1.441579 & 1.586768 \\
\hline & -1.485496 & -1.345523 & 2.985847 \\
\hline & -1.240684 & -2.398836 & 3.826266 \\
\hline & -0.749687 & -3.624907 & 3.323955 \\
\hline & -0.542630 & -3.757554 & 1.920127 \\
\hline & -0.062611 & -5.000572 & 1.429889 \\
\hline & 0.213939 & -6.038795 & 2.284448 \\
\hline & 0.016061 & -5.896958 & 3.674320 \\
\hline & -0.459671 & -4.714323 & 4.179640 \\
\hline & 2.644467 & -2.728646 & 0.228342 \\
\hline & 3.181400 & -2.336879 & 1.580878 \\
\hline & 2.339942 & -1.891062 & 2.601631 \\
\hline & 2.853067 & -1.563294 & 3.848869 \\
\hline & 4.220365 & -1.676957 & 4.097815 \\
\hline U & 5.065196 & -2.121064 & 3.087048 \\
\hline 6 & 4.546423 & -2.449488 & 1.836404 \\
\hline 6 & -6.180280 & -1.546637 & 2.810521 \\
\hline 6 & -7.891269 & -0.752800 & 1.211107 \\
\hline 6 & -4.078487 & -0.310024 & -4.302113 \\
\hline 6 & -5.967394 & -1.859782 & -3.908491 \\
\hline 6 & -0.600305 & 4.570181 & 5.129725 \\
\hline 6 & -0.457962 & 2.135611 & 5.592979 \\
\hline 6 & -4.819549 & 5.262066 & 2.825818 \\
\hline 6 & -5.871483 & 3.175280 & 1.999173 \\
\hline 1 & 7.100571 & 0.034005 & 0.782079 \\
\hline 1 & -1.874140 & -0.418989 & 3.400534 \\
\hline 1 & 7.825424 & -3.810071 & -2.279650 \\
\hline 1 & -0.620618 & -4.589478 & 5.249585 \\
\hline 1 & 6.323197 & 5.180483 & 1.044758 \\
\hline 1 & -3.073396 & -3.635852 & 0.503475 \\
\hline 1 & -4.865657 & -4.656311 & -0.828835 \\
\hline 1 & 4.316097 & 4.146071 & 1.953697 \\
\hline 1 & -1.425792 & -2.303635 & 4.895750 \\
\hline 1 & 5.741220 & -3.319927 & -3.434241 \\
\hline 1 & 8.619483 & 3.167758 & -1.978758 \\
\hline 1 & 3.613986 & -2.024385 & -3.562283 \\
\hline 1 & -2.396480 & -4.504570 & -4.346242 \\
\hline 1 & -0.137367 & -3.584928 & -4.215466 \\
\hline 1 & 0.586171 & -6.980257 & 1.887019 \\
\hline 1 & 7.088719 & 1.259758 & -1.903877 \\
\hline 1 & 0.087512 & -5.126681 & 0.359419 \\
\hline 1 & 1.666117 & -2.520361 & -2.889099 \\
\hline 1 & 8.270129 & 5.134958 & -0.488674 \\
\hline 1 & 9.082880 & -1.385473 & 1.038525 \\
\hline 1 & -4.534701 & -5.116132 & -3.253896 \\
\hline 1 & 0.240485 & -6.727167 & 4.339946 \\
\hline 1 & 9.475322 & -3.306054 & -0.500677 \\
\hline 1 & 2.666973 & 2.272677 & 1.929460 \\
\hline 1 & -4.007732 & -0.927297 & 1.843900 \\
\hline 1 & -2.830754 & -0.137951 & -2.182875 \\
\hline 1 & -6.862466 & -1.329236 & -1.327552 \\
\hline 1 & -3.824615 & 1.859250 & 0.596056 \\
\hline 1 & -0.163786 & 1.161768 & 2.706735 \\
\hline
\end{tabular}




\begin{tabular}{|c|c|c|c|}
\hline & -2.652150 & 4.497759 & 3.756951 \\
\hline & 2.160308 & -3.711708 & 0.316176 \\
\hline & -1.581180 & 1.608521 & -1.591042 \\
\hline & 3.480564 & -2.861790 & -0.475572 \\
\hline | & 5.217489 & -2.782252 & 1.042700 \\
\hline & 1.279546 & -1.762942 & 2.394761 \\
\hline & 6.136240 & -2.204659 & 3.262954 \\
\hline & 2.179234 & -1.213650 & 4.630287 \\
\hline & 4.623803 & -1.414358 & 5.073531 \\
\hline & 1.111714 & 2.766455 & 0.398329 \\
\hline & -0.642407 & 3.227897 & 0.174104 \\
\hline & 0.667503 & 3.338583 & -4.359630 \\
\hline & -0.924964 & 4.447438 & -5.858838 \\
\hline & -2.986022 & 5.507228 & -4.948487 \\
\hline & -3.396116 & 5.449928 & -2.490823 \\
\hline & -1.809678 & 4.342762 & -0.991250 \\
\hline & 2.653854 & 2.725739 & -1.352676 \\
\hline & 2.159741 & 2.510667 & -3.030627 \\
\hline & 2.264559 & 4.139866 & -2.347150 \\
\hline & 0.146401 & 4.774703 & 5.907240 \\
\hline & -0.540648 & 5.379091 & 4.390073 \\
\hline 1 & -1.585798 & 4.614800 & 5.609892 \\
\hline & 0.230990 & 2.342876 & 6.423336 \\
\hline 1 & -1.478722 & 2.111841 & 5.996783 \\
\hline ] & -0.225828 & 1.133816 & 5.207273 \\
\hline & 1.808621 & 3.512116 & 4.773215 \\
\hline 1 & 1.428285 & 2.208512 & 3.647887 \\
\hline & 1.232059 & 3.904044 & 3.137214 \\
\hline 1 & -5.484319 & 5.222640 & 0.208430 \\
\hline ] & -3.713170 & 5.309007 & 0.318357 \\
\hline 1 & -4.496995 & 3.844409 & -0.311517 \\
\hline 1 & -6.791584 & 3.738029 & 1.791627 \\
\hline 1 & -5.834335 & 2.330547 & 1.299175 \\
\hline 1 & -5.946966 & 2.766041 & 3.015531 \\
\hline | & -5.744465 & 5.801586 & 2.585937 \\
\hline 1 & -4.893474 & 4.930215 & 3.869665 \\
\hline 1 & -3.993003 & 5.980283 & 2.750527 \\
\hline 1 & -6.992441 & -1.858880 & 3.479107 \\
\hline 1 & -5.322106 & -2.202364 & 3.010085 \\
\hline 1 & -5.899421 & -0.520701 & 3.084892 \\
\hline 1 & -7.812444 & -3.434060 & 1.740758 \\
\hline 1 & -7.374836 & -3.224771 & 0.034205 \\
\hline 1 & -6.149358 & -3.750926 & 1.206817 \\
\hline 1 & -8.666090 & -1.068824 & 1.922371 \\
\hline 1 & -7.654949 & 0.299709 & 1.417010 \\
\hline 1 & -8.327817 & -0.807909 & 0.207005 \\
\hline 1 & -6.245330 & -1.753566 & -4.965686 \\
\hline 1 & -5.288633 & -2.718314 & -3.819192 \\
\hline 1 & -6.882968 & -2.093889 & -3.351816 \\
\hline 1 & -4.401058 & -0.241818 & -5.348892 \\
\hline 1 & -3.576898 & 0.635564 & -4.054201 \\
\hline 1 & -3.338191 & -1.119121 & -4.234462 \\
\hline 1 & -6.554130 & 0.734463 & -4.597068 \\
\hline 1 & -7.176443 & 0.443279 & -2.961286 \\
\hline 1 & -5.795779 & 1.530745 & -3.201746 \\
\hline
\end{tabular}


$3_{\text {IID-si- }- \text {-1s2s3a4s }}$

Number of imaginary frequencies : 0

The smallest frequencies are : $13.0324 \quad 16.423120 .0825 \mathrm{~cm}(-1)$

Electronic energy : $\quad H F=-4360.8917752$

Zero-point correction $=\quad 1.454286($ Hartree/Particle $)$

Thermal correction to Energy $=\quad 1.539225$

Thermal correction to Enthalpy= $\quad 1.540169$

Thermal correction to Gibbs Free Energy $=\quad 1.335104$

Sum of electronic and zero-point Energies $=\quad-4359.437490$

Sum of electronic and thermal Energies $=\quad-4359.352551$

Sum of electronic and thermal Enthalpies $=\quad-4359.351606$

Sum of electronic and thermal Free Energies $=\quad-4359.556671$

Cartesian Coordinates

$\begin{array}{cccc}6 & 4.442136 & -2.443601 & 1.877884 \\ 6 & 3.078454 & -2.339622 & 1.612016 \\ 6 & 2.229346 & -1.880273 & 2.620554 \\ 6 & 2.734356 & -1.527766 & 3.864445 \\ 6 & 4.100746 & -1.630538 & 4.122842 \\ 6 & 4.952647 & -2.090662 & 3.125266 \\ 6 & 2.550996 & -2.751538 & 0.261690 \\ 7 & 1.544221 & -1.850655 & -0.348999 \\ 6 & 0.481501 & -2.507268 & -1.059370 \\ 6 & -0.700955 & -2.838028 & -0.421795 \\ 6 & -1.751044 & -3.459304 & -1.168163 \\ 6 & -1.563092 & -3.728638 & -2.556218 \\ 6 & -0.330807 & -3.395111 & -3.162244 \\ 6 & 0.662482 & -2.804359 & -2.429892 \\ 6 & -2.607634 & -4.335596 & -3.296172 \\ 6 & -3.794648 & -4.664779 & -2.693504 \\ 6 & -3.990394 & -4.387238 & -1.322808 \\ 6 & -2.994934 & -3.805941 & -0.578363 \\ 6 & -0.885362 & -2.623469 & 1.040098 \\ 6 & -1.315228 & -1.410760 & 1.561920 \\ 6 & -1.570069 & -1.313440 & 2.957414 \\ 6 & -1.366748 & -2.373621 & 3.800016 \\ 6 & -0.895434 & -3.610160 & 3.304512 \\ 6 & -0.665182 & -3.744146 & 1.904637 \\ 6 & -0.203680 & -4.996678 & 1.420911 \\ 6 & 0.030923 & -6.043365 & 2.277827 \\ 6 & -0.192347 & -5.900830 & 3.663658 \\ 6 & -0.649336 & -4.708184 & 4.162792 \\ 15 & -1.625592 & 0.027198 & 0.418121 \\ 45 & -0.183679 & 0.943586 & -1.321791 \\ 15 & 1.784408 & -0.226979 & -0.782011 \\ 8 & 2.908673 & -0.169171 & -2.028130 \\ 6 & 4.126551 & -0.800754 & -1.928994 \\ 6 & 5.096899 & -0.341152 & -1.057788 \\ 6 & 6.275906 & -1.139799 & -0.885053 \\ 6 & 6.475617 & -2.288453 & -1.708381 \\ 6 & 5.507833 & -2.619197 & -2.687966 \\ 6 & 4.348919 & -1.900422 & -2.785736 \\ 6 & 7.231491 & -0.875292 & 0.129629 \\ 6 & 8.323778 & -1.689157 & 0.304906\end{array}$




\begin{tabular}{|c|c|c|c|}
\hline 6 & 8.532599 & -2.805398 & -0.531893 \\
\hline 6 & 7.623721 & -3.094866 & -1.516950 \\
\hline 6 & 4.886709 & 0.919353 & -0.309523 \\
\hline & 3.746417 & 1.096994 & 0.450343 \\
\hline & 3.556473 & 2.227167 & 1.270485 \\
\hline & 4.480251 & 3.234366 & 1.271668 \\
\hline & 5.615892 & 3.165908 & 0.428490 \\
\hline & 5.820508 & 2.006731 & -0.379811 \\
\hline & 6.541783 & 4.234852 & 0.363986 \\
\hline & 7.619921 & 4.185505 & -0.482095 \\
\hline & 7.807173 & 3.057947 & -1.307778 \\
\hline ס & 6.934641 & 1.998847 & -1.257035 \\
\hline & 2.770798 & 0.135746 & 0.494654 \\
\hline 0 & -1.942506 & 1.414847 & 1.580249 \\
\hline & -3.032429 & 2.258925 & 1.385490 \\
\hline & -3.216899 & 3.398866 & 2.173376 \\
\hline 0 & -2.287501 & 3.656259 & 3.183441 \\
\hline & -1.176251 & 2.832782 & 3.402983 \\
\hline 0 & -1.003240 & 1.729671 & 2.565353 \\
\hline & -0.203251 & 3.065426 & 4.559012 \\
\hline 6 & -0.410988 & 1.952331 & 5.593934 \\
\hline 6 & -4.415416 & 4.303718 & 1.892556 \\
\hline 6 & -5.708827 & 3.483300 & 1.962218 \\
\hline 6 & -3.327310 & -0.410897 & -0.144631 \\
\hline 6 & -4.302199 & -0.757992 & 0.786631 \\
\hline 6 & -5.598679 & -1.092970 & 0.388354 \\
\hline 6 & -5.888591 & -1.056505 & -0.976635 \\
\hline 6 & -4.931869 & -0.715982 & -1.941632 \\
\hline 6 & -3.646046 & -0.401656 & -1.501742 \\
\hline 6 & -6.622696 & -1.478722 & 1.454644 \\
\hline 6 & -6.852087 & -0.285330 & 2.389064 \\
\hline 6 & -5.317781 & -0.640092 & -3.418135 \\
\hline 6 & -6.105254 & -1.884150 & -3.838682 \\
\hline 6 & -6.092910 & -2.667196 & 2.266381 \\
\hline 6 & -7.966273 & -1.881535 & 0.853065 \\
\hline 6 & -6.188322 & 0.604646 & -3.627657 \\
\hline 6 & -4.092245 & -0.531814 & -4.324573 \\
\hline 6 & 1.244486 & 3.020645 & 4.062447 \\
\hline 6 & -0.425041 & 4.410081 & 5.248394 \\
\hline 6 & -4.266560 & 4.902101 & 0.488377 \\
\hline 6 & -4.528370 & 5.452546 & 2.891197 \\
\hline 6 & -0.378492 & 0.223925 & -3.092000 \\
\hline 8 & -0.481233 & -0.017365 & -4.220307 \\
\hline 6 & 0.598275 & 3.025808 & -1.720920 \\
\hline 6 & 2.043194 & 3.034229 & -2.167042 \\
\hline 6 & 0.304673 & 2.861420 & -0.350197 \\
\hline 6 & -0.341288 & 3.740704 & -2.640727 \\
\hline 6 & -1.450761 & 4.456036 & -2.167776 \\
\hline 6 & -2.308758 & 5.116308 & -3.037754 \\
\hline 6 & -2.079750 & 5.091075 & -4.411367 \\
\hline 6 & -0.976145 & 4.398217 & -4.897455 \\
\hline 6 & -0.118223 & 3.738848 & -4.023820 \\
\hline 1 & 7.074375 & -0.030534 & 0.795784 \\
\hline 1 & -1.941304 & -0.378648 & 3.369121 \\
\hline 1 & 7.759356 & -3.961373 & -2.162866 \\
\hline 1 & -0.828788 & -4.581864 & 5.229618 \\
\hline
\end{tabular}




\begin{tabular}{|c|c|c|c|}
\hline 1 & 6.368663 & 5.105036 & 0.995913 \\
\hline 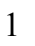 & -3.161429 & -3.587803 & 0.475442 \\
\hline & -4.942681 & -4.631727 & -0.855901 \\
\hline & 4.341645 & 4.109505 & 1.904916 \\
\hline 1 & -1.569493 & -2.275042 & 4.866063 \\
\hline & 5.690159 & -3.469355 & -3.343134 \\
\hline 1 & 8.647758 & 3.028639 & -1.997505 \\
\hline 1 & 3.586106 & -2.144855 & -3.521005 \\
\hline 1 & -2.443799 & -4.537056 & -4.354002 \\
\hline 1 & -0.188394 & -3.607752 & -4.220644 \\
\hline 1 & 0.389437 & -6.992090 & 1.885115 \\
\hline 1 & 7.088235 & 1.143941 & -1.910226 \\
\hline 1 & -0.034237 & -5.123975 & 0.353507 \\
\hline 1 & 1.608735 & -2.535866 & -2.893367 \\
\hline 1 & 8.321296 & 5.015296 & -0.528113 \\
\hline 1 & 9.032439 & -1.472661 & 1.101256 \\
\hline 1 & -4.588821 & -5.136776 & -3.268280 \\
\hline 1 & -0.002006 & -6.737999 & 4.331186 \\
\hline 1 & 9.405397 & -3.437446 & -0.386034 \\
\hline 1 & 2.662393 & 2.262403 & 1.887167 \\
\hline 1 & -4.049181 & -0.764834 & 1.846711 \\
\hline 1 & -2.864968 & -0.142990 & -2.210660 \\
\hline 1 & -6.895659 & -1.301183 & -1.307474 \\
\hline 1 & -3.745079 & 2.029442 & 0.593947 \\
\hline 1 & -0.129004 & 1.085721 & 2.687866 \\
\hline 1 & -2.429247 & 4.525710 & 3.817796 \\
\hline 1 & 2.041349 & -3.720040 & 0.365174 \\
\hline 1 & -1.569825 & 1.656489 & -1.626683 \\
\hline 1 & 3.393513 & -2.920260 & -0.426746 \\
\hline 1 & 5.118796 & -2.788369 & 1.094147 \\
\hline 1 & 1.168892 & -1.762660 & 2.408219 \\
\hline 1 & 6.022784 & -2.168102 & 3.309228 \\
\hline 1 & 2.054242 & -1.169239 & 4.636536 \\
\hline 1 & 4.497658 & -1.348622 & 5.095826 \\
\hline 1 & 1.134460 & 2.765491 & 0.350336 \\
\hline 1 & -0.603717 & 3.267558 & 0.092923 \\
\hline 1 & 0.731225 & 3.192326 & -4.429490 \\
\hline 1 & -0.777230 & 4.368563 & -5.967106 \\
\hline 1 & -2.750383 & 5.610131 & -5.092632 \\
\hline 1 & -3.160528 & 5.664014 & -2.636879 \\
\hline 1 & -1.652500 & 4.493054 & -1.097960 \\
\hline 1 & 2.701289 & 2.645945 & -1.382841 \\
\hline 1 & 2.222587 & 2.432398 & -3.064937 \\
\hline 1 & 2.363449 & 4.062972 & -2.391812 \\
\hline 1 & 0.314393 & 4.539052 & 6.048752 \\
\hline 1 & -0.310043 & 5.250194 & 4.551282 \\
\hline 1 & -1.417249 & 4.480221 & 5.711385 \\
\hline 1 & 0.268919 & 2.086202 & 6.446487 \\
\hline 1 & -1.440287 & 1.958964 & 5.976384 \\
\hline 1 & -0.218691 & 0.960006 & 5.164354 \\
\hline 1 & 1.935707 & 3.244181 & 4.885910 \\
\hline 1 & 1.515868 & 2.029465 & 3.678202 \\
\hline 1 & 1.412731 & 3.761320 & 3.267838 \\
\hline 1 & -5.123317 & 5.548783 & 0.254013 \\
\hline 1 & -3.355142 & 5.512358 & 0.421446 \\
\hline 1 & -4.211373 & 4.125953 & -0.285601 \\
\hline
\end{tabular}




$\begin{array}{rrrr}1 & -6.576519 & 4.123772 & 1.754245 \\ 1 & -5.720767 & 2.663598 & 1.232581 \\ 1 & -5.842896 & 3.045701 & 2.960386 \\ 1 & -5.406828 & 6.064447 & 2.650368 \\ 1 & -4.651131 & 5.090910 & 3.920480 \\ 1 & -3.652238 & 6.112816 & 2.860163 \\ 1 & -8.659708 & -2.166081 & 1.654599 \\ 1 & -8.431106 & -1.058653 & 0.295077 \\ 1 & -7.869172 & -2.742963 & 0.178943 \\ 1 & -6.807424 & -2.937758 & 3.055575 \\ 1 & -5.953320 & -3.548519 & 1.626300 \\ 1 & -5.131909 & -2.451350 & 2.750676 \\ 1 & -7.565826 & -0.549895 & 3.181127 \\ 1 & -5.923168 & 0.043674 & 2.872184 \\ 1 & -7.262784 & 0.570474 & 1.836910 \\ 1 & -6.353429 & -1.827846 & -4.906878 \\ 1 & -5.512776 & -2.793901 & -3.674772 \\ 1 & -7.050078 & -1.990949 & -3.292557 \\ 1 & -4.410424 & -0.528050 & -5.374874 \\ 1 & -3.526938 & 0.393793 & -4.155357 \\ 1 & -3.408636 & -1.380749 & -4.183587 \\ 1 & -6.482519 & 0.694869 & -4.682390 \\ 1 & -7.104615 & 0.564280 & -3.024841 \\ 1 & -5.642066 & 1.515585 & -3.349381\end{array}$

$3_{\text {IID-si- }-\Psi \text {-1s2s334a }}$

Number of imaginary frequencies : 0

The smallest frequencies are : $9.9137 \quad 18.349121 .5599 \mathrm{~cm}(-1)$

Electronic energy : $\quad H F=-4360.8914109$

Zero-point correction $=\quad 1.454917$ (Hartree/Particle)

Thermal correction to Energy= $\quad 1.539670$

Thermal correction to Enthalpy $=\quad 1.540614$

Thermal correction to Gibbs Free Energy $=\quad 1.336107$

Sum of electronic and zero-point Energies $=\quad-4359.436494$

Sum of electronic and thermal Energies $=\quad-4359.351741$

Sum of electronic and thermal Enthalpies $=\quad-4359.350797$

Sum of electronic and thermal Free Energies $=\quad-4359.555304$

\section{Cartesian Coordinates}

$\begin{array}{cccc}6 & -1.680316 & 4.339053 & -1.970138 \\ 6 & -0.507998 & 3.756969 & -2.476302 \\ 6 & -0.299994 & 3.830531 & -3.859125 \\ 6 & -1.232850 & 4.425636 & -4.702805 \\ 6 & -2.399232 & 4.977582 & -4.185514 \\ 6 & -2.612924 & 4.933221 & -2.809269 \\ 6 & 0.495602 & 3.081960 & -1.593835 \\ 6 & 1.928619 & 3.169448 & -2.068187 \\ 45 & -0.193939 & 0.956912 & -1.242227 \\ 6 & 0.242245 & 2.865921 & -0.222719 \\ 15 & 1.806499 & -0.184487 & -0.773326 \\ 7 & 1.614552 & -1.831467 & -0.404759 \\ 6 & 2.653978 & -2.730633 & 0.150074 \\ 6 & 3.185656 & -2.367010 & 1.512668 \\ 6 & 2.336789 & -1.962000 & 2.544317\end{array}$




$\begin{array}{cccc}6 & 2.844289 & -1.655583 & 3.799321 \\ 6 & 4.213532 & -1.750261 & 4.045337 \\ 6 & 5.065679 & -2.154834 & 3.024089 \\ 6 & 4.552508 & -2.462049 & 1.765763 \\ 8 & 2.912356 & -0.044193 & -2.030117 \\ 6 & 4.147135 & -0.646810 & -1.971944 \\ 6 & 5.114623 & -0.202845 & -1.089231 \\ 6 & 6.311984 & -0.981710 & -0.959924 \\ 6 & 6.532636 & -2.085757 & -1.837072 \\ 6 & 5.565985 & -2.393076 & -2.825479 \\ 6 & 4.388977 & -1.699461 & -2.881068 \\ 6 & 7.266147 & -0.743407 & 0.062401 \\ 6 & 8.377717 & -1.539250 & 0.194376 \\ 6 & 8.607635 & -2.609345 & -0.695670 \\ 6 & 7.700130 & -2.873188 & -1.689324 \\ 6 & 4.883026 & 1.023042 & -0.291166 \\ 6 & 5.789167 & 2.135373 & -0.329013 \\ 6 & 5.556752 & 3.262962 & 0.516306 \\ 6 & 4.423022 & 3.275446 & 1.364759 \\ 6 & 3.527678 & 2.243327 & 1.337314 \\ 6 & 3.744591 & 1.144650 & 0.482117 \\ 6 & 6.901764 & 2.184510 & -1.207297 \\ 6 & 7.746067 & 3.267177 & -1.225450 \\ 6 & 7.530840 & 4.363321 & -0.365212 \\ 6 & -3.288435 & -0.500315 & -0.126429 \\ 6 & -4.254959 & -0.976811 & 0.768963 \\ 6 & -5.551987 & -1.244945 & 0.345296 \\ 6 & -5.868285 & -1.002183 & -1.003182 \\ 6 & -4.934069 & -0.518291 & -1.913630 \\ 6 & -3.630101 & -0.297164 & -1.452835 \\ 6 & -6.615030 & -1.810029 & 1.286956 \\ 6 & 2.800653 & 0.151619 & 0.504296 \\ 6 & 0.559234 & -2.482094 & -1.131867 \\ 6 & -0.619284 & -2.844709 & -0.503984 \\ 6 & -1.671110 & -3.433855 & -1.273589 \\ 6 & -1.484739 & -3.649910 & -2.671317 \\ 6 & -0.255138 & -0.188652 & -3.368873 \\ 6 & -0.250287 & -3.300091 & -3.263814 \\ 6 & 0.741545 & -2.732389 & -2.511543 \\ 6 & -2.533035 & -4.218936 & -3.435001 \\ 6 & -3.726612 & -4.553405 & -2.849173 \\ 6 & -3.920253 & -4.330358 & -1.468720 \\ 6 & -2.918040 & -3.794487 & -0.699430 \\ 6 & -0.793816 & -2.697561 & 0.968240 \\ 6 & -1.243472 & -1.518595 & 1.547501 \\ 6 & -1.472684 & -1.479165 & 2.949689 \\ 6 & -1.229910 & -2.567501 & 3.744789 \\ 6 & -0.743177 & -3.772916 & 3.190539 \\ 6 & -0.53775 & -3.846919 & 1.782355 \\ 6 & -5.069417 & 1.240316 \\ 6 & & 1.287309 & 1.664936\end{array}$




\begin{tabular}{|c|c|c|c|}
\hline 6 & -1.043786 & 1.631311 & 2.649804 \\
\hline 6 & -1.262947 & 2.714722 & 3.502648 \\
\hline & -2.414374 & 3.487131 & 3.302780 \\
\hline & -3.338165 & 3.197478 & 2.296385 \\
\hline & -3.103336 & 2.080127 & 1.491154 \\
\hline & -0.283221 & 2.985491 & 4.644439 \\
\hline & -4.592555 & 4.031726 & 2.043592 \\
\hline & -0.398874 & 0.314126 & -3.041970 \\
\hline & -0.527227 & 0.133857 & -4.179367 \\
\hline & 7.091514 & 0.063346 & 0.770124 \\
\hline & -1.858929 & -0.569508 & 3.402505 \\
\hline & 7.852118 & -3.704964 & -2.376007 \\
\hline & -0.621436 & -4.819526 & 5.073381 \\
\hline & 6.259271 & 5.202708 & 1.142816 \\
\hline & -3.081798 & -3.624191 & 0.363478 \\
\hline & -4.874818 & -4.583639 & -1.011070 \\
\hline & 4.263682 & 4.126940 & 2.024844 \\
\hline & -1.414129 & -2.517248 & 4.817445 \\
\hline & 5.763781 & -3.206593 & -3.521440 \\
\hline & 8.586154 & 3.281173 & -1.916297 \\
\hline & 3.626119 & -1.927703 & -3.621651 \\
\hline & -2.370526 & -4.376545 & -4.500510 \\
\hline & -0.107158 & -3.476713 & -4.328707 \\
\hline & 0.580703 & -7.069603 & 1.613590 \\
\hline & 7.077428 & 1.355006 & -1.887144 \\
\hline & 0.093387 & -5.150231 & 0.165787 \\
\hline & 1.688371 & -2.447485 & -2.964187 \\
\hline & 8.210096 & 5.212316 & -0.386370 \\
\hline & 9.085085 & -1.345067 & 0.997608 \\
\hline 1 & -4.527772 & -4.985490 & -3.445016 \\
\hline & 0.230470 & -6.920575 & 4.074287 \\
\hline 1 & 9.495457 & -3.227246 & -0.583797 \\
\hline & 2.636938 & 2.234799 & 1.959939 \\
\hline & -3.978738 & -1.126598 & 1.810376 \\
\hline 1 & -2.868034 & 0.065052 & -2.137552 \\
\hline & -6.885290 & -1.194922 & -1.339717 \\
\hline ] & -3.810943 & 1.829196 & 0.701973 \\
\hline & -0.137570 & 1.029872 & 2.756044 \\
\hline 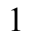 & -2.591984 & 4.341615 & 3.948590 \\
\hline & 2.173153 & -3.717448 & 0.213482 \\
\hline & -1.600036 & 1.641105 & -1.495998 \\
\hline & 3.492555 & -2.843304 & -0.554593 \\
\hline & 5.229239 & -2.763055 & 0.964274 \\
\hline 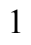 & 1.274030 & -1.848570 & 2.341283 \\
\hline & 6.138042 & -2.223893 & 3.198269 \\
\hline . & 2.164271 & -1.337407 & 4.589006 \\
\hline ] & 4.612708 & -1.504142 & 5.027086 \\
\hline ] & 1.092044 & 2.784587 & 0.455019 \\
\hline 1 & -0.671330 & 3.218809 & 0.253784 \\
\hline & 0.597516 & 3.392545 & -4.291626 \\
\hline 1 & -1.042828 & 4.454577 & -5.774095 \\
\hline & -3.130146 & 5.442686 & -4.843331 \\
\hline 1 & -3.514075 & 5.370713 & -2.381436 \\
\hline ] & -1.877132 & 4.314324 & -0.898887 \\
\hline & 2.619426 & 2.807533 & -1.298746 \\
\hline & 2.122595 & 2.588311 & -2.976826 \\
\hline
\end{tabular}




$\begin{array}{rrrr}1 & 2.192560 & 4.215747 & -2.284980 \\ 6 & -0.582430 & 4.292800 & 5.374627 \\ 6 & -0.383970 & 1.835024 & 5.654175 \\ 6 & 1.151189 & 3.055409 & 4.112372 \\ 6 & -4.724670 & 5.198785 & 3.018094 \\ 6 & -4.543239 & 4.599057 & 0.619134 \\ 6 & -5.831988 & 3.140875 & 2.190769 \\ 6 & -6.139332 & -1.843686 & 2.738050 \\ 6 & -7.888808 & -0.960715 & 1.229039 \\ 6 & -6.942455 & -3.244932 & 0.858143 \\ 6 & -6.739938 & -0.349099 & -3.683370 \\ 6 & -4.856101 & 1.265822 & -3.658100 \\ 6 & -4.462823 & -1.123857 & -4.287640 \\ 1 & 0.162039 & 4.450817 & 6.165028 \\ 1 & -0.538216 & 5.157469 & 4.699882 \\ 1 & -1.568268 & 4.281750 & 5.856345 \\ 1 & 0.301891 & 2.000826 & 6.496289 \\ 1 & -1.402155 & 1.753546 & 6.057011 \\ 1 & -0.124551 & 0.870359 & 5.197504 \\ 1 & 1.846767 & 3.306741 & 4.924157 \\ 1 & 1.476805 & 2.093551 & 3.698047 \\ 1 & 1.249237 & 3.822066 & 3.330876 \\ 1 & -6.744934 & 3.724558 & 2.011157 \\ 1 & -5.825958 & 2.306779 & 1.477639 \\ 1 & -5.894233 & 2.716988 & 3.201826 \\ 1 & -5.441446 & 5.198115 & 0.415729 \\ 1 & -3.667328 & 5.249701 & 0.488160 \\ 1 & -4.492693 & 3.807046 & -0.139588 \\ 1 & -5.637824 & 5.764522 & 2.794140 \\ 1 & -4.796676 & 4.858232 & 4.059226 \\ 1 & -3.880132 & 5.895609 & 2.941300 \\ 1 & -6.938142 & -2.237064 & 3.379371 \\ 1 & -5.263835 & -2.493210 & 2.872361 \\ 1 & -5.881728 & -0.840776 & 3.104468 \\ 1 & -7.721377 & -3.672009 & 1.504562 \\ 1 & -7.303844 & -3.290339 & -0.177071 \\ 1 & -6.053325 & -3.886166 & 0.935412 \\ 1 & -8.644186 & -1.366273 & 1.915235 \\ 1 & -7.686872 & 0.076363 & 1.526843 \\ 1 & -8.335320 & -0.941155 & 0.228087 \\ 1 & -5.121771 & 1.532952 & -4.689787 \\ 1 & -5.375065 & 1.960594 & -2.983637 \\ 1 & -3.777092 & 1.439537 & -3.545116 \\ 1 & -6.926265 & -0.086016 & -4.732223 \\ 1 & -7.080443 & -1.383090 & -3.541213 \\ 1 & -7.363535 & 0.307170 & -3.062017 \\ 1 & -4.666309 & -0.886759 & -5.340884 \\ 1 & -3.380310 & -1.036551 & -4.129028 \\ 1 & -4.739828 & -2.171443 & -4.110433\end{array}$

$3_{\text {IID-si- }} \Psi_{-1522354 s}$

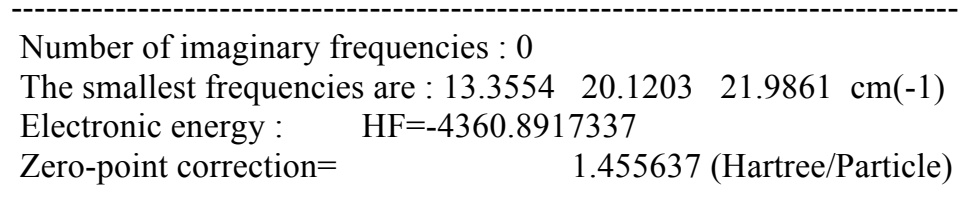




$\begin{array}{lc}\text { Thermal correction to Energy= } & 1.540018 \\ \text { Thermal correction to Enthalpy= } & 1.540962 \\ \text { Thermal correction to Gibbs Free Energy= } & 1.338584 \\ \text { Sum of electronic and zero-point Energies }= & -4359.436097 \\ \text { Sum of electronic and thermal Energies }= & -4359.351716 \\ \text { Sum of electronic and thermal Enthalpies }= & -4359.350772 \\ \text { Sum of electronic and thermal Free Energies }= & -4359.553150\end{array}$

Cartesian Coordinates

$\begin{array}{ccccc}6 & -1.618449 & 4.385285 & -1.981469 \\ 6 & -0.470119 & 3.753672 & -2.481402 \\ 6 & -0.270766 & 3.784038 & -3.867404 \\ 6 & -1.189042 & 4.389416 & -4.718877 \\ 6 & -2.331921 & 4.994322 & -4.206858 \\ 6 & -2.537450 & 4.989669 & -2.829090 \\ 6 & 0.519738 & 3.071962 & -1.589210 \\ 6 & 1.956419 & 3.150810 & -2.054131 \\ 45 & -0.192699 & 0.957424 & -1.235719 \\ 6 & 0.255227 & 2.862550 & -0.218522 \\ 15 & 1.806309 & -0.190847 & -0.774328 \\ 7 & 1.614670 & -1.839647 & -0.416396 \\ 6 & 2.653861 & -2.742662 & 0.132981 \\ 6 & 3.189431 & -2.384202 & 1.495505 \\ 6 & 2.343060 & -1.981251 & 2.530107 \\ 6 & 2.853657 & -1.676535 & 3.784142 \\ 6 & 4.223480 & -1.772262 & 4.026793 \\ 6 & 5.072985 & -2.175842 & 3.003064 \\ 6 & 4.556829 & -2.480261 & 1.745175 \\ 8 & 2.915814 & -0.038336 & -2.025189 \\ 6 & 4.152574 & -0.636977 & -1.968366 \\ 6 & 5.117128 & -0.196151 & -1.080737 \\ 6 & 6.317833 & -0.971360 & -0.957637 \\ 6 & 6.543909 & -2.066744 & -1.844293 \\ 6 & 5.578475 & -2.371159 & -2.834737 \\ 6 & 4.398512 & -1.682300 & -2.884840 \\ 6 & 7.270461 & -0.738416 & 0.067357 \\ 6 & 8.385880 & -1.530023 & 0.192301 \\ 6 & 8.621394 & -2.590630 & -0.707521 \\ 6 & 7.715159 & -2.849845 & -1.703534 \\ 6 & 4.879323 & 1.019606 & -0.268578 \\ 6 & 5.782159 & 2.135394 & -0.287195 \\ 6 & 5.544280 & 3.248941 & 0.575374 \\ 6 & 4.409090 & 3.245046 & 1.422146 \\ 6 & 3.516937 & 2.211080 & 1.376490 \\ 6 & 3.738658 & 1.127532 & 0.503661 \\ 6 & 6.896707 & 2.202207 & -1.161912 \\ 6 & 7.737606 & 3.287737 & -1.160731 \\ 6 & 7.516974 & 4.369314 & -0.283658 \\ 6 & 6.437904 & 4.346898 & 0.562050 \\ 8 & 2.795573 & 0.134274 & 0.509027 \\ 6 & 0.559071 & -2.485830 & -1.146251 \\ 6 & -0.620250 & -2.848277 & -0.519790 \\ 6 & -1.671124 & -3.435647 & -1.292401 \\ 6 & -1.483765 & -3.646590 & -2.690903 \\ 6 & -0.248189 & -3.296949 & -3.281348\end{array}$




\begin{tabular}{|c|c|c|c|}
\hline 6 & 0.743284 & -2.732416 & -2.526379 \\
\hline 6 & -2.532334 & -4.211068 & -3.457483 \\
\hline & -3.726825 & -4.546589 & -2.873783 \\
\hline & -3.920271 & -4.330425 & -1.492257 \\
\hline & -2.917980 & -3.798818 & -0.720100 \\
\hline & -0.794236 & -2.704804 & 0.953422 \\
\hline & -1.236540 & -1.525781 & 1.539064 \\
\hline & -1.462309 & -1.490011 & 2.941699 \\
\hline & -1.229740 & -2.584660 & 3.731298 \\
\hline & -0.753156 & -3.791240 & 3.170916 \\
\hline & -0.543405 & -3.859235 & 1.762720 \\
\hline & -0.073624 & -5.082261 & 1.215482 \\
\hline & 0.186220 & -6.163754 & 2.020185 \\
\hline & -0.017618 & -6.087128 & 3.414313 \\
\hline & -0.480700 & -4.924214 & 3.974187 \\
\hline & -1.596718 & -0.055768 & 0.463141 \\
\hline & -3.281788 & -0.513430 & -0.128202 \\
\hline & -4.230207 & -1.016451 & 0.763148 \\
\hline & -5.530932 & -1.300972 & 0.350831 \\
\hline & -5.870317 & -1.032790 & -0.982308 \\
\hline & -4.947790 & -0.517978 & -1.895421 \\
\hline & -3.643723 & -0.289120 & -1.449327 \\
\hline & -6.528164 & -1.851576 & 1.370174 \\
\hline & -5.291146 & -0.172801 & -3.342408 \\
\hline 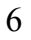 & -1.989298 & 1.283127 & 1.660362 \\
\hline & -1.069703 & 1.650827 & 2.645609 \\
\hline & -1.299993 & 2.752016 & 3.473190 \\
\hline 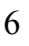 & -2.454800 & 3.513150 & 3.250377 \\
\hline 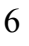 & -3.371818 & 3.196360 & 2.246222 \\
\hline 5 & -3.125936 & 2.063522 & 1.466918 \\
\hline ) & -0.327138 & 3.059242 & 4.611707 \\
\hline 5 & -4.620826 & 4.027408 & 1.958049 \\
\hline 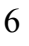 & -0.457702 & 0.292443 & -3.018497 \\
\hline 3 & -0.666789 & 0.090222 & -4.140147 \\
\hline 1 & 7.091847 & 0.060473 & 0.782893 \\
\hline & -1.840516 & -0.578634 & 3.398118 \\
\hline 1 & 7.871065 & -3.674713 & -2.397640 \\
\hline 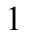 & -0.644787 & -4.849205 & 5.048265 \\
\hline . & 6.239241 & 5.180509 & 1.234456 \\
\hline 1 & -3.082086 & -3.632607 & 0.343298 \\
\hline | & -4.876273 & -4.584423 & -1.037546 \\
\hline 1 & 4.247031 & 4.085488 & 2.095709 \\
\hline & -1.413201 & -2.538479 & 4.804225 \\
\hline L & 5.779615 & -3.178790 & -3.536560 \\
\hline & 8.579213 & 3.315340 & -1.849312 \\
\hline 1 & 3.635896 & -1.908939 & -3.626270 \\
\hline 1 & -2.369432 & -4.364593 & -4.523489 \\
\hline 1 & -0.104587 & -3.470337 & -4.346681 \\
\hline 1 & 0.549924 & -7.089111 & 1.579413 \\
\hline 1 & 7.076736 & 1.384363 & -1.854666 \\
\hline 1 & 0.081269 & -5.158422 & 0.141013 \\
\hline 1 & 1.691363 & -2.447100 & -2.976500 \\
\hline 1 & 8.193646 & 5.220617 & -0.289652 \\
\hline 1 & 9.091936 & -1.339896 & 0.997655 \\
\hline 1 & -4.528332 & -4.974564 & -3.472074 \\
\hline & 0.192857 & -6.951494 & 4.039811 \\
\hline
\end{tabular}




\begin{tabular}{|c|c|c|c|}
\hline$\frac{1}{1}$ & 9.512253 & -3.205134 & -0.601242 \\
\hline & 2.625215 & 2.188289 & 1.997530 \\
\hline & -3.947941 & -1.179719 & 1.803028 \\
\hline | & -2.895590 & 0.096196 & -2.136482 \\
\hline & -6.885930 & -1.228243 & -1.315121 \\
\hline 1 & -3.826296 & 1.795202 & 0.677210 \\
\hline 1 & -0.159333 & 1.058231 & 2.766754 \\
\hline & -2.639389 & 4.381957 & 3.874722 \\
\hline & 2.171076 & -3.728715 & 0.194062 \\
\hline & -1.594219 & 1.656373 & -1.481635 \\
\hline & 3.490576 & -2.854269 & -0.574160 \\
\hline & 5.231797 & -2.779445 & 0.941514 \\
\hline & 1.280002 & -1.866269 & 2.329424 \\
\hline & 6.145578 & -2.246288 & 3.175128 \\
\hline & 2.175642 & -1.359624 & 4.575883 \\
\hline & 4.625079 & -1.527959 & 5.008004 \\
\hline & 1.099842 & 2.778936 & 0.465803 \\
\hline & -0.659668 & 3.223004 & 0.250062 \\
\hline L & 0.605291 & 3.298145 & -4.293492 \\
\hline & -1.007603 & 4.384483 & -5.792035 \\
\hline ] & -3.051489 & 5.467989 & -4.871047 \\
\hline & -3.420876 & 5.466493 & -2.406365 \\
\hline 1 & -1.806839 & 4.392223 & -0.908371 \\
\hline ] & 2.640865 & 2.787506 & -1.279808 \\
\hline | & 2.152705 & 2.566357 & -2.959910 \\
\hline 1 & 2.226283 & 4.195316 & -2.272202 \\
\hline 5 & -0.605720 & 4.408263 & 5.271220 \\
\hline 6 & -0.465818 & 1.963308 & 5.675834 \\
\hline 6 & 1.114836 & 3.072556 & 4.095291 \\
\hline 6 & -4.778895 & 5.198473 & 2.924081 \\
\hline 6 & -4.529961 & 4.588769 & 0.533229 \\
\hline 6 & -5.865528 & 3.139585 & 2.073932 \\
\hline 6 & -7.846002 & -2.273363 & 0.725033 \\
\hline 5 & -5.936565 & -3.078957 & 2.074072 \\
\hline 6 & -6.824163 & -0.764814 & 2.410457 \\
\hline 5 & -6.769702 & -0.387720 & -3.654184 \\
\hline 6 & -4.953303 & 1.301931 & -3.604066 \\
\hline 6 & -4.465945 & -1.055240 & -4.284617 \\
\hline 1 & 0.135935 & 4.592291 & 6.058636 \\
\hline 1 & -0.539829 & 5.235400 & 4.552559 \\
\hline 1 & -1.594469 & 4.442067 & 5.745550 \\
\hline 1 & 0.220783 & 2.149287 & 6.513141 \\
\hline 1 & -1.487879 & 1.931296 & 6.075666 \\
\hline 1 & -0.233981 & 0.970975 & 5.266378 \\
\hline 1 & 1.804507 & 3.359762 & 4.900125 \\
\hline 1 & 1.429900 & 2.082800 & 3.742933 \\
\hline 1 & 1.235313 & 3.790006 & 3.271198 \\
\hline 1 & -6.771139 & 3.725545 & 1.866750 \\
\hline 1 & -5.843548 & 2.302132 & 1.364434 \\
\hline 1 & -5.957076 & 2.720911 & 3.084904 \\
\hline 1 & -5.422460 & 5.185433 & 0.299631 \\
\hline 1 & -3.651150 & 5.240076 & 0.426564 \\
\hline 1 & -4.453646 & 3.792957 & -0.219448 \\
\hline 1 & -5.689706 & 5.758881 & 2.678224 \\
\hline 1 & -4.870715 & 4.861935 & 3.965023 \\
\hline 1 & -3.936674 & 5.899487 & 2.861802 \\
\hline
\end{tabular}




$\begin{array}{rrrr}1 & -8.512721 & -2.695531 & 1.487596 \\ 1 & -8.372265 & -1.426776 & 0.266384 \\ 1 & -7.693883 & -3.041435 & -0.045277 \\ 1 & -7.520082 & -1.140542 & 3.173124 \\ 1 & -5.912233 & -0.430326 & 2.921745 \\ 1 & -7.283279 & 0.113974 & 1.937997 \\ 1 & -6.647487 & -3.468599 & 2.814998 \\ 1 & -5.728823 & -3.884597 & 1.357031 \\ 1 & -5.002371 & -2.854884 & 2.604468 \\ 1 & -5.214493 & 1.572933 & -4.635956 \\ 1 & -5.515452 & 1.960922 & -2.928692 \\ 1 & -3.884859 & 1.520702 & -3.469438 \\ 1 & -6.970845 & -0.113142 & -4.697359 \\ 1 & -7.067109 & -1.437146 & -3.529232 \\ 1 & -7.417466 & 0.231493 & -3.019696 \\ 1 & -4.701105 & -0.818626 & -5.331367 \\ 1 & -3.387423 & -0.907846 & -4.145172 \\ 1 & -4.681811 & -2.118521 & -4.115007\end{array}$

\section{Cartesian coordinates for TS1}

$\operatorname{TS1}_{I L-s i}-\psi_{-1 \mathbf{a} 2 \mathrm{aza} a \mathrm{a}}$

Number of imaginary frequencies : 1

The smallest frequencies are : $-650.6146 \quad 12.5551 \quad 17.0224 \mathrm{~cm}(-1)$

Electronic energy : $\quad \mathrm{HF}=-4360.8595556$

Zero-point correction $=\quad 1.452199$ (Hartree/Particle)

Thermal correction to Energy= $\quad 1.536998$

Thermal correction to Enthalpy= $\quad 1.537943$

Thermal correction to Gibbs Free Energy $=\quad 1.334077$

Sum of electronic and zero-point Energies $=\quad-4359.407357$

Sum of electronic and thermal Energies $=\quad-4359.322557$

Sum of electronic and thermal Enthalpies $=\quad-4359.321613$

Sum of electronic and thermal Free Energies $=\quad-4359.525478$

Cartesian Coordinates

$\begin{array}{cccc}6 & -0.559913 & 1.398632 & 2.715488 \\ 6 & -1.615573 & 1.197340 & 1.822465 \\ 6 & -2.672002 & 2.103869 & 1.786003 \\ 6 & -2.688530 & 3.222916 & 2.621732 \\ 6 & -1.629893 & 3.382120 & 3.518060 \\ 6 & -0.566562 & 2.475345 & 3.601466 \\ 15 & -1.466325 & -0.080715 & 0.508123 \\ 45 & -0.040420 & 1.005501 & -1.094604 \\ 15 & 1.823495 & -0.340468 & -0.867561 \\ 8 & 2.947543 & -0.225318 & -2.129279 \\ 6 & 4.224577 & -0.717011 & -2.027114 \\ 6 & 5.152241 & -0.130874 & -1.182241 \\ 6 & 6.425041 & -0.771738 & -1.030327 \\ 6 & 6.754054 & -1.895504 & -1.846234 \\ 6 & 5.804815 & -2.373047 & -2.782954 \\ 6 & 4.561207 & -1.808555 & -2.858316 \\ 6 & 7.358266 & -0.374529 & -0.038895 \\ 6 & 8.553816 & -1.032468 & 0.115244\end{array}$




\begin{tabular}{|c|c|c|c|}
\hline & 8.889948 & -2.119765 & -0.718055 \\
\hline & 8.003427 & -2.540108 & -1.676621 \\
\hline & 4.782558 & 1.086357 & -0.424561 \\
\hline & 3.634359 & 1.071139 & 0.344605 \\
\hline & 3.223614 & 2.176242 & 1.116180 \\
\hline & 3.968600 & 3.323740 & 1.104088 \\
\hline & 5.125591 & 3.423289 & 0.294323 \\
\hline & 5.533702 & 2.304276 & -0.493971 \\
\hline & 5.871355 & 4.625681 & 0.236389 \\
\hline & 6.964277 & 4.740995 & -0.583546 \\
\hline & 7.348735 & 3.650662 & -1.391686 \\
\hline & 6.653982 & 2.467205 & -1.348433 \\
\hline & 2.870581 & -0.067572 & 0.393157 \\
\hline & -3.799521 & 4.269023 & 2.549582 \\
\hline & 0.555200 & 2.704078 & 4.613388 \\
\hline & -3.244306 & -0.402522 & 0.108041 \\
\hline & -4.097327 & -0.834134 & 1.129469 \\
\hline & -5.450275 & -1.056797 & 0.893908 \\
\hline & -5.926421 & -0.832779 & -0.403170 \\
\hline & -5.107516 & -0.411696 & -1.450892 \\
\hline & -3.749515 & -0.201225 & -1.172493 \\
\hline & -5.716200 & -0.133630 & -2.825758 \\
\hline & -4.653692 & -0.051887 & -3.919068 \\
\hline & -6.398859 & -1.560247 & 1.980614 \\
\hline & -5.722839 & -1.629691 & 3.348649 \\
\hline & -1.116768 & -1.641382 & 1.464193 \\
\hline & -0.814822 & -2.813814 & 0.786006 \\
\hline 6 & -0.635165 & -4.038966 & 1.506205 \\
\hline & -0.767797 & -4.047493 & 2.925167 \\
\hline 6 & -1.085262 & -2.839388 & 3.585562 \\
\hline ) & -1.253933 & -1.678338 & 2.879004 \\
\hline & -0.575655 & -5.254772 & 3.638705 \\
\hline 6 & -0.263743 & -6.416915 & 2.981229 \\
\hline & -0.133265 & -6.416671 & 1.576219 \\
\hline 6 & -0.316214 & -5.261303 & 0.857622 \\
\hline & -0.695455 & -2.867239 & -0.696815 \\
\hline & -1.803408 & -3.303972 & -1.486145 \\
\hline 6 & -1.660148 & -3.401631 & -2.902388 \\
\hline & -0.407940 & -3.110644 & -3.491064 \\
\hline 6 & 0.639573 & -2.687703 & -2.717744 \\
\hline 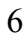 & 0.495385 & -2.536981 & -1.318358 \\
\hline 6 & -2.773934 & -3.785668 & -3.688899 \\
\hline ) & -3.983538 & -4.064133 & -3.104380 \\
\hline 6 & -4.127198 & -3.975224 & -1.702081 \\
\hline 6 & -3.064025 & -3.613731 & -0.913113 \\
\hline 7 & 1.587494 & -1.997157 & -0.560147 \\
\hline 6 & 2.603740 & -2.974158 & -0.105850 \\
\hline 6 & 3.245703 & -2.663709 & 1.220156 \\
\hline 6 & 2.466710 & -2.386938 & 2.344573 \\
\hline 5 & 3.062461 & -2.105136 & 3.566190 \\
\hline 6 & 4.451820 & -2.093338 & 3.682005 \\
\hline 6 & 5.234976 & -2.372434 & 2.567337 \\
\hline 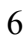 & 4.633096 & -2.660013 & 1.344523 \\
\hline 6 & -6.704427 & -1.235234 & -3.221457 \\
\hline 6 & -6.453509 & 1.209268 & -2.762392 \\
\hline 6 & -7.609935 & -0.629190 & 2.097514 \\
\hline
\end{tabular}




\begin{tabular}{|c|c|c|c|}
\hline 6 & -6.874760 & -2.969423 & 1.609463 \\
\hline 6 & 0.228550 & 2.750971 & -0.121792 \\
\hline 8 & 0.225706 & 3.900677 & 0.011658 \\
\hline 6 & -0.661909 & 1.625053 & -3.235306 \\
\hline 6 & 0.689457 & 1.925070 & -2.913333 \\
\hline 6 & -0.941912 & 0.416849 & -4.100344 \\
\hline 6 & -1.570171 & 2.802260 & -3.517927 \\
\hline 6 & -2.676346 & 3.188467 & -2.756507 \\
\hline 6 & -3.468192 & 4.271412 & -3.133323 \\
\hline 6 & -3.170984 & 4.991851 & -4.283700 \\
\hline 6 & -2.072053 & 4.622071 & -5.054509 \\
\hline 6 & -1.284766 & 3.542117 & -4.674422 \\
\hline 1 & 7.104772 & 0.448666 & 0.624220 \\
\hline 1 & -1.507807 & -0.768262 & 3.416261 \\
\hline 1 & 8.236879 & -3.390508 & -2.316205 \\
\hline 1 & -0.678504 & -5.237697 & 4.722837 \\
\hline 1 & 5.546584 & 5.463411 & 0.852254 \\
\hline 1 & -3.187428 & -3.536627 & 0.165825 \\
\hline 1 & -5.091378 & -4.192595 & -1.246007 \\
\hline 1 & 3.666940 & 4.185782 & 1.698262 \\
\hline 1 & -1.198233 & -2.845081 & 4.669035 \\
\hline 1 & 6.071719 & -3.213736 & -3.421349 \\
\hline 1 & 8.200371 & 3.749669 & -2.061174 \\
\hline 1 & 3.808802 & -2.180163 & -3.55 \\
\hline 1 & -2.647325 & -3.854209 & -4.768610 \\
\hline 1 & -0.298979 & -3.205797 & -4.570760 \\
\hline 1 & 0.112691 & -7.340814 & 1.058176 \\
\hline 1 & 6.957248 & 1.641521 & -1.986953 \\
\hline 1 & -0.217146 & -5.277511 & -0.225801 \\
\hline 1 & 1.598214 & -2.430926 & -3.165827 \\
\hline 1 & 7.526108 & 5.671332 & -0.623303 \\
\hline 1 & 9.245413 & -0.715182 & 0.892626 \\
\hline 1 & -4.833261 & -4.357834 & -3.717513 \\
\hline 1 & -0.115375 & -7.339337 & 3.537821 \\
\hline 1 & 9.842406 & -2.628610 & -0.589265 \\
\hline 1 & 2.310196 & 2.084542 & 1.701203 \\
\hline 1 & -3.690672 & -0.988339 & 2.125604 \\
\hline 1 & -3.063324 & 0.121489 & -1.954120 \\
\hline 1 & -6.984956 & -0.997025 & -0.600894 \\
\hline 1 & -3.481335 & 1.940527 & 1.078893 \\
\hline 1 & 0.277024 & 0.699706 & 2.707945 \\
\hline 1 & -1.628123 & 4.250160 & 4.176884 \\
\hline 1 & 2.069130 & -3.931943 & -0.026678 \\
\hline 1 & 3.383716 & -3.114141 & -0.870813 \\
\hline 1 & 5.254085 & -2.860950 & 0.469723 \\
\hline 1 & 1.384101 & -2.357323 & 2.244694 \\
\hline 1 & 6.320792 & -2.358231 & 2.640831 \\
\hline 1 & 2.436297 & -1.890504 & 4.431411 \\
\hline 1 & 4.920207 & -1.864632 & 4.637083 \\
\hline 1 & -1.460696 & 1.504249 & -1.622911 \\
\hline 1 & 0.976603 & 2.967162 & -2.764637 \\
\hline 1 & 1.477122 & 1.287073 & -3.313175 \\
\hline 1 & -2.918498 & 2.644336 & -1.842912 \\
\hline 1 & -4.316999 & 4.554281 & -2.513051 \\
\hline 1 & -3.786375 & 5.839977 & -4.575518 \\
\hline 1 & -1.821701 & 5.181561 & -5.953567 \\
\hline
\end{tabular}




$\begin{array}{rrrr}1 & -0.421431 & 3.261109 & -5.278138 \\ 1 & -1.881632 & -0.082849 & -3.839525 \\ 1 & -1.019597 & 0.720372 & -5.157317 \\ 1 & -0.137868 & -0.320523 & -4.002791 \\ 1 & -5.137745 & 0.086119 & -4.894262 \\ 1 & -3.975373 & 0.799524 & -3.776424 \\ 1 & -4.057539 & -0.974631 & -3.965392 \\ 1 & -7.085100 & -1.048227 & -4.234010 \\ 1 & -6.215873 & -2.218970 & -3.219398 \\ 1 & -7.575056 & -1.286285 & -2.557427 \\ 1 & -6.908575 & 1.445702 & -3.734023 \\ 1 & -7.250747 & 1.196813 & -2.008119 \\ 1 & -5.760979 & 2.023456 & -2.507878 \\ 1 & -7.572180 & -3.351373 & 2.367394 \\ 1 & -7.391478 & -2.986093 & 0.641630 \\ 1 & -6.026995 & -3.665415 & 1.551883 \\ 1 & -8.287054 & -0.986035 & 2.884974 \\ 1 & -7.300134 & 0.391339 & 2.356584 \\ 1 & -8.188785 & -0.577499 & 1.167813 \\ 1 & -6.443012 & -1.981707 & 4.097948 \\ 1 & -4.875145 & -2.328122 & 3.356847 \\ 1 & -5.359413 & -0.645746 & 3.674094 \\ 6 & 1.505063 & 1.509802 & 4.702304 \\ 6 & 1.356540 & 3.941420 & 4.194407 \\ 6 & -0.035032 & 2.930677 & 6.009478 \\ 6 & -4.938650 & 3.841382 & 1.625038 \\ 6 & -4.389615 & 4.511075 & 3.942650 \\ 6 & -3.207962 & 5.575191 & 2.007470 \\ 1 & -5.729573 & 4.601825 & 1.640382 \\ 1 & -4.608681 & 3.736754 & 0.582973 \\ 1 & -5.386792 & 2.888344 & 1.937619 \\ 1 & -3.984240 & 6.349785 & 1.939293 \\ 1 & -2.406780 & 5.958497 & 2.651267 \\ 1 & -2.785758 & 5.425420 & 1.004397 \\ 1 & -5.193159 & 5.257695 & 3.889218 \\ 1 & -4.813874 & 3.587354 & 4.357423 \\ 1 & -3.643423 & 4.885536 & 4.653281 \\ 1 & 2.192536 & 4.112954 & 4.886217 \\ 1 & 1.768615 & 3.817712 & 3.183511 \\ 1 & 0.734206 & 4.844865 & 4.186847 \\ 1 & 0.770691 & 3.087762 & 6.738824 \\ 1 & -0.688576 & 3.809696 & 6.051005 \\ 1 & -0.620898 & 2.061539 & 6.335885 \\ 1 & 2.250254 & 1.689222 & 5.487533 \\ 1 & 0.968257 & 0.584972 & 4.956306 \\ 1 & 2.057810 & 1.335069 & 3.770346\end{array}$

$\mathrm{TS1}_{\text {IL-si- }} \psi_{-1 \mathrm{a} 2 \mathrm{a} 3 \mathrm{a} 4 \mathrm{~s}}$

Number of imaginary frequencies : 1

The smallest frequencies are : -664.8906 $16.1349 \quad 17.7113 \mathrm{~cm}(-1)$

Electronic energy : $\quad H F=-4360.8603249$

Zero-point correction $=\quad 1.452282($ Hartree/Particle $)$

Thermal correction to Energy $=\quad 1.537098$

Thermal correction to Enthalpy $=\quad 1.538042$

Thermal correction to Gibbs Free Energy $=\quad 1.334504$ 
Sum of electronic and zero-point Energies= Sum of electronic and thermal Energies= Sum of electronic and thermal Enthalpies= Sum of electronic and thermal Free Energies=
$-4359.408043$

$-4359.323227$

$-4359.322283$

$-4359.525821$

Cartesian Coordinates

\begin{tabular}{|c|c|c|c|}
\hline 6 & 0.666196 & -1.419615 & 2.701953 \\
\hline 6 & 1.689493 & -1.166398 & 1.784923 \\
\hline 6 & 2.805108 & -1.998890 & 1.749404 \\
\hline 6 & 2.918075 & -3.088147 & 2.615444 \\
\hline 6 & 1.893193 & -3.294742 & 3.540896 \\
\hline 6 & 0.767469 & -2.466217 & 3.618607 \\
\hline 15 & 1.447625 & 0.100111 & 0.471466 \\
\hline 45 & 0.016242 & -1.024834 & -1.091984 \\
\hline 15 & -1.857864 & 0.304460 & -0.852896 \\
\hline 8 & -2.990532 & 0.174766 & -2.104947 \\
\hline 6 & -4.270206 & 0.657024 & -1.991962 \\
\hline 6 & -5.184901 & 0.064738 & -1.137545 \\
\hline 6 & -6.461242 & 0.695806 & -0.974645 \\
\hline 6 & -6.805999 & 1.816370 & -1.788470 \\
\hline 6 & -5.869340 & 2.300231 & -2.734585 \\
\hline 6 & -4.622327 & 1.745026 & -2.821177 \\
\hline 6 & -7.382749 & 0.291933 & 0.024987 \\
\hline 6 & -8.581521 & 0.941462 & 0.189777 \\
\hline 6 & -8.932903 & 2.026093 & -0.640712 \\
\hline 6 & -8.058371 & 2.452069 & -1.607763 \\
\hline 6 & -4.798433 & -1.149664 & -0.383238 \\
\hline 6 & -3.644121 & -1.125623 & 0.376729 \\
\hline 6 & -3.219788 & -2.227130 & 1.146942 \\
\hline 6 & -3.955709 & -3.380763 & 1.138601 \\
\hline 6 & -5.117038 & -3.489505 & 0.336166 \\
\hline 6 & -5.540256 & -2.373377 & -0.447990 \\
\hline 6 & -5.853281 & -4.697991 & 0.282543 \\
\hline 6 & -6.951209 & -4.821587 & -0.529474 \\
\hline 6 & -7.350713 & -3.733695 & -1.333587 \\
\hline 6 & -6.665533 & -2.544556 & -1.294218 \\
\hline 8 & -2.889236 & 0.019073 & 0.417665 \\
\hline 6 & 4.122089 & -4.027268 & 2.578474 \\
\hline 6 & -0.318832 & -2.741307 & 4.657879 \\
\hline 6 & 3.202876 & 0.466457 & 0.017898 \\
\hline 6 & 4.068735 & 0.902483 & 1.017086 \\
\hline 6 & 5.425293 & 1.111015 & 0.768228 \\
\hline 6 & 5.887146 & 0.877279 & -0.526675 \\
\hline 6 & 5.044215 & 0.462677 & -1.565679 \\
\hline 6 & 3.692572 & 0.258583 & -1.271771 \\
\hline 6 & 5.636516 & 0.188939 & -2.948510 \\
\hline 6 & 4.559801 & 0.073635 & -4.024822 \\
\hline 6 & 6.323158 & 1.563894 & 1.916637 \\
\hline 6 & 7.759977 & 1.817542 & 1.469148 \\
\hline 6 & 1.084175 & 1.647741 & 1.446430 \\
\hline 6 & 0.755773 & 2.819692 & 0.780305 \\
\hline 6 & 0.561124 & 4.036618 & 1.509378 \\
\hline 6 & 0.704914 & 4.037921 & 2.927119 \\
\hline 6 & 1.048544 & 2.830950 & 3.576061 \\
\hline 6 & 1.232448 & 1.677636 & 2.860159 \\
\hline
\end{tabular}




\begin{tabular}{|c|c|c|c|}
\hline 6 & 0.497906 & 5.237093 & 3.650219 \\
\hline 6 & 0.161383 & 6.397919 & 3.002739 \\
\hline & 0.020250 & 6.404810 & 1.598665 \\
\hline & 0.216976 & 5.257486 & 0.870982 \\
\hline & 0.625977 & 2.876271 & -0.700523 \\
\hline & 1.722513 & 3.333465 & -1.493582 \\
\hline & 1.572648 & 3.424353 & -2.909381 \\
\hline & 0.324521 & 3.105817 & -3.492675 \\
\hline & -0.712333 & 2.664377 & -2.714972 \\
\hline & -0.560865 & 2.520602 & -1.315700 \\
\hline & 2.676471 & 3.827820 & -3.700324 \\
\hline & 3.883045 & 4.128056 & -3.120327 \\
\hline & 4.033842 & 4.041994 & -1.718522 \\
\hline & 2.979837 & 3.664962 & -0.924764 \\
\hline & -1.639526 & 1.964381 & -0.549819 \\
\hline & -2.660380 & 2.928857 & -0.079277 \\
\hline b & -3.288103 & 2.605595 & 1.250544 \\
\hline & -2.498414 & 2.316341 & 2.364398 \\
\hline D & -3.082437 & 2.022359 & 3.588749 \\
\hline & -4.470628 & 2.012589 & 3.718483 \\
\hline & -5.264420 & 2.304243 & 2.614680 \\
\hline y & -4.674197 & 2.602239 & 1.388633 \\
\hline 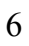 & 6.588315 & 1.314566 & -3.365931 \\
\hline 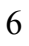 & 6.409966 & -1.133666 & -2.890063 \\
\hline 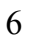 & 6.340480 & 0.473968 & 2.995467 \\
\hline b & 5.773160 & 2.867426 & 2.508088 \\
\hline & -0.185180 & -2.764072 & -0.100044 \\
\hline & -0.116415 & -3.907024 & 0.069076 \\
\hline 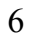 & 0.608704 & -1.637654 & -3.244547 \\
\hline f & -0.727620 & -1.970264 & -2.892083 \\
\hline ) & 0.839817 & -0.425167 & -4.118203 \\
\hline 6 & 1.537695 & -2.792936 & -3.548520 \\
\hline 6 & 2.675751 & -3.146263 & -2.819073 \\
\hline 6 & 3.483780 & -4.210129 & -3.215559 \\
\hline 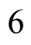 & 3.171167 & -4.943615 & -4.353514 \\
\hline & 2.040614 & -4.606071 & -5.092641 \\
\hline 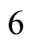 & 1.237401 & -3.544966 & -4.693348 \\
\hline & -7.117508 & -0.529497 & 0.685736 \\
\hline 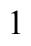 & 1.508037 & 0.769395 & 3.389343 \\
\hline | & -8.303811 & 3.300296 & -2.245727 \\
\hline & 0.609600 & 5.214836 & 4.733373 \\
\hline 1 & -5.517246 & -5.533579 & 0.895286 \\
\hline & 3.110485 & 3.589859 & 0.153863 \\
\hline 1 & 4.997010 & 4.271160 & -1.266013 \\
\hline & -3.645986 & -4.240528 & 1.731295 \\
\hline 1 & 1.170324 & 2.831096 & 4.658605 \\
\hline ] & -6.148606 & 3.138270 & -3.371151 \\
\hline 1 & -8.206451 & -3.839115 & -1.996832 \\
\hline 1 & -3.879023 & 2.121217 & -3.521159 \\
\hline 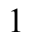 & 2.545016 & 3.892793 & -4.779690 \\
\hline 1 & 0.209981 & 3.195065 & -4.572313 \\
\hline t & -0.244897 & 7.328186 & 1.088783 \\
\hline 1 & -6.980287 & -1.720727 & -1.929567 \\
\hline 1 & 0.109981 & 5.278787 & -0.211623 \\
\hline 1 & -1.667093 & 2.387892 & -3.159534 \\
\hline 1 & -7.505611 & -5.756493 & -0.566025 \\
\hline
\end{tabular}




\begin{tabular}{|c|c|c|c|}
\hline 1 & -9.263879 & 0.619470 & 0.973362 \\
\hline 1 & 4.725143 & 4.436263 & -3.736858 \\
\hline 1 & 0.001833 & 7.314104 & 3.566490 \\
\hline & -9.887704 & 2.528274 & -0.503394 \\
\hline & -2.305054 & -2.127791 & 1.729080 \\
\hline 1 & 3.682730 & 1.065095 & 2.022479 \\
\hline 1 & 2.995832 & -0.067732 & -2.042344 \\
\hline 1 & 6.943201 & 1.022437 & -0.741945 \\
\hline 1 & 3.588614 & -1.800708 & 1.023174 \\
\hline 1 & -0.216370 & -0.778664 & 2.694744 \\
\hline 1 & 1.973506 & -4.134828 & 4.230467 \\
\hline 1 & -2.133786 & 3.891036 & 0.000954 \\
\hline 1 & -3.448325 & 3.066326 & -0.836518 \\
\hline 1 & -5.303536 & 2.811676 & 0.521798 \\
\hline 1 & -1.416942 & 2.283487 & 2.252926 \\
\hline 1 & -6.349467 & 2.290922 & 2.698797 \\
\hline 1 & -2.448162 & 1.796123 & 4.444972 \\
\hline 1 & -4.929890 & 1.774911 & 4.675809 \\
\hline 1 & 1.433872 & -1.501651 & -1.649196 \\
\hline 1 & -0.984403 & -3.018756 & -2.733419 \\
\hline 1 & -1.539967 & -1.354329 & -3.276732 \\
\hline 1 & 2.931470 & -2.591719 & -1.915327 \\
\hline 1 & 4.357964 & -4.468121 & -2.620318 \\
\hline 1 & 3.799454 & -5.776965 & -4.660048 \\
\hline 1 & 1.778176 & -5.175992 & -5.981643 \\
\hline 1 & 0.349603 & -3.288803 & -5.272137 \\
\hline 1 & 1.772784 & 0.097829 & -3.879282 \\
\hline 1 & 0.901922 & -0.729896 & -5.175845 \\
\hline 1 & 0.020720 & 0.293183 & -4.005075 \\
\hline 1 & 5.032819 & -0.068078 & -5.004919 \\
\hline 1 & 3.898644 & -0.787755 & -3.862036 \\
\hline 1 & 3.947153 & 0.985418 & -4.075437 \\
\hline 1 & 6.963133 & 1.129407 & -4.381088 \\
\hline 1 & 6.070262 & 2.283025 & -3.367021 \\
\hline 1 & 7.463392 & 1.397856 & -2.711143 \\
\hline 1 & 6.854677 & -1.363785 & -3.867949 \\
\hline 1 & 7.219726 & -1.096972 & -2.150175 \\
\hline 1 & 5.743742 & -1.964189 & -2.619109 \\
\hline 1 & 6.406206 & 3.206430 & 3.339106 \\
\hline 1 & 5.757027 & 3.663200 & 1.751178 \\
\hline 1 & 4.752147 & 2.756245 & 2.894951 \\
\hline 1 & 6.940358 & 0.795183 & 3.857815 \\
\hline 1 & 5.332516 & 0.233775 & 3.358025 \\
\hline 1 & 6.782493 & -0.452774 & 2.605006 \\
\hline 1 & 8.360200 & 2.150982 & 2.325130 \\
\hline 1 & 8.233683 & 0.910873 & 1.071635 \\
\hline 1 & 7.816233 & 2.600433 & 0.701313 \\
\hline 6 & 5.120923 & -3.644241 & 1.487243 \\
\hline 6 & 4.849105 & -3.977275 & 3.926694 \\
\hline 6 & 3.642855 & -5.456573 & 2.304386 \\
\hline 6 & -1.318598 & -1.589760 & 4.763845 \\
\hline 6 & -1.074675 & -4.013210 & 4.259452 \\
\hline 6 & 0.308553 & -2.936997 & 6.042292 \\
\hline 1 & -2.034179 & -1.795607 & 5.569814 \\
\hline 1 & -0.816961 & -0.639748 & 4.995996 \\
\hline 1 & -1.903482 & -1.448026 & 3.845941 \\
\hline
\end{tabular}




$\begin{array}{llll}1 & -1.875348 & -4.229111 & 4.980052 \\ 1 & -1.530284 & -3.902141 & 3.266013 \\ 1 & -0.409668 & -4.884890 & 4.220760 \\ 1 & -0.476510 & -3.113534 & 6.789360 \\ 1 & 0.989355 & -3.795232 & 6.078561 \\ 1 & 0.873015 & -2.047115 & 6.349981 \\ 1 & 5.974505 & -4.333421 & 1.511982 \\ 1 & 4.676398 & -3.706226 & 0.484724 \\ 1 & 5.513296 & -2.626410 & 1.622897 \\ 1 & 5.714501 & -4.653738 & 3.919905 \\ 1 & 5.214202 & -2.963179 & 4.137922 \\ 1 & 4.199442 & -4.278423 & 4.757383 \\ 1 & 4.498342 & -6.143929 & 2.255656 \\ 1 & 2.967872 & -5.823270 & 3.087046 \\ 1 & 3.105349 & -5.511752 & 1.348607\end{array}$

TS1 $_{I L-s i-} \Psi_{-1 \mathbf{a 2 a 3 s 4 a}}$

Number of imaginary frequencies : 1

The smallest frequencies are : $-632.5736 \quad 16.0480 \quad 18.5728 \mathrm{~cm}(-1)$

Electronic energy : $\quad H F=-4360.8592946$

Zero-point correction $=\quad 1.451500($ Hartree/Particle $)$

Thermal correction to Energy $=\quad 1.536625$

Thermal correction to Enthalpy= $\quad 1.537569$

Thermal correction to Gibbs Free Energy= $\quad 1.332431$

Sum of electronic and zero-point Energies= $\quad-4359.407795$

Sum of electronic and thermal Energies $=\quad-4359.322670$

Sum of electronic and thermal Enthalpies $=\quad-4359.321726$

Sum of electronic and thermal Free Energies $=\quad-4359.526864$

Cartesian Coordinates

$\begin{array}{cccc}6 & -0.229002 & 4.212829 & -4.033149 \\ 6 & -1.025478 & 3.305667 & -3.329320 \\ 6 & -2.177238 & 3.790166 & -2.697360 \\ 6 & -2.517056 & 5.135052 & -2.760622 \\ 6 & -1.709860 & 6.028988 & -3.460550 \\ 6 & -0.565585 & 5.562770 & -4.096076 \\ 6 & -0.662955 & 1.846225 & -3.329529 \\ 6 & -1.569897 & 0.980027 & -4.171583 \\ 45 & -0.038930 & 0.946925 & -1.195185 \\ 6 & 0.381861 & 2.728286 & -0.314138 \\ 8 & 0.361647 & 3.828847 & 0.038227 \\ 15 & -1.517076 & -0.049303 & 0.383860 \\ 6 & -1.591317 & 1.212214 & 1.723042 \\ 6 & -2.589344 & 2.183836 & 1.708630 \\ 6 & -2.558390 & 3.258364 & 2.600480 \\ 6 & -1.519438 & 3.303457 & 3.532919 \\ 6 & -0.511784 & 2.333933 & 3.588171 \\ 6 & -0.543418 & 1.309830 & 2.642073 \\ 6 & -3.605096 & 4.370644 & 2.567851 \\ 6 & 0.580664 & 2.425930 & 4.653162 \\ 6 & -3.319335 & -0.288058 & 0.016773 \\ 6 & -4.168644 & -0.750384 & 1.032891 \\ 6 & -5.521852 & -0.958706 & 0.804331 \\ 6 & -6.020377 & -0.672549 & -0.476595\end{array}$




\begin{tabular}{|c|c|c|c|}
\hline 6 & -5.214481 & -0.198201 & -1.505540 \\
\hline & -3.845121 & -0.019611 & -1.238140 \\
\hline & -5.766164 & 0.132896 & -2.890866 \\
\hline & -6.461017 & -1.498572 & 1.881176 \\
\hline & -1.249467 & -1.636472 & 1.332837 \\
\hline & -0.989477 & -2.817339 & 0.652513 \\
\hline & -0.883709 & -4.056801 & 1.365788 \\
\hline & -1.052799 & -4.072454 & 2.780523 \\
\hline & -1.327830 & -2.855753 & 3.444187 \\
\hline & -1.421493 & -1.682438 & 2.744577 \\
\hline & -0.935374 & -5.292957 & 3.487458 \\
\hline & -0.658530 & -6.462690 & 2.827702 \\
\hline & -0.488598 & -6.455572 & 1.427148 \\
\hline & -0.600375 & -5.287009 & 0.715105 \\
\hline & -0.826452 & -2.874174 & -0.825706 \\
\hline & 0.396870 & -2.590030 & -1.403295 \\
\hline & 0.588590 & -2.755012 & -2.795344 \\
\hline & -0.432570 & -3.185456 & -3.599999 \\
\hline & -1.708411 & -3.459174 & -3.053154 \\
\hline & -1.913008 & -3.297490 & -1.650201 \\
\hline & -3.205198 & -3.553885 & -1.122991 \\
\hline & -4.230228 & -3.964872 & -1.937802 \\
\hline & -4.014539 & -4.157390 & -3.320255 \\
\hline & -2.782065 & -3.900913 & -3.864572 \\
\hline & 1.477170 & -2.086509 & -0.603799 \\
\hline & 2.442748 & -3.097223 & -0.113352 \\
\hline & 3.055965 & -2.800137 & 1.229143 \\
\hline 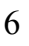 & 2.252017 & -2.518078 & 2.334623 \\
\hline & 2.819838 & -2.242382 & 3.570874 \\
\hline 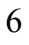 & 4.205922 & -2.242759 & 3.720963 \\
\hline & 5.013697 & -2.531773 & 2.626597 \\
\hline 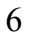 & 4.439614 & -2.813315 & 1.389027 \\
\hline f & 0.690902 & 1.481179 & -3.204226 \\
\hline & 1.754859 & -0.433489 & -0.881315 \\
\hline 3 & 2.922916 & -0.328980 & -2.099100 \\
\hline 5 & 4.180036 & -0.860239 & -1.959407 \\
\hline 5 & 5.097148 & -0.303163 & -1.084457 \\
\hline 6 & 6.350490 & -0.973803 & -0.902595 \\
\hline 6 & 6.675234 & -2.100261 & -1.716448 \\
\hline 6 & 5.739577 & -2.551663 & -2.679445 \\
\hline 6 & 4.512424 & -1.956778 & -2.785747 \\
\hline 6 & 7.266817 & -0.602740 & 0.114366 \\
\hline 6 & 8.443892 & -1.286969 & 0.293975 \\
\hline 6 & 8.777208 & -2.376759 & -0.537205 \\
\hline 6 & 7.905725 & -2.772927 & -1.519535 \\
\hline 6 & 4.734396 & 0.918436 & -0.331247 \\
\hline 5 & 3.560660 & 0.932308 & 0.399315 \\
\hline 6 & 3.156685 & 2.046911 & 1.160499 \\
\hline 6 & 3.933155 & 3.173219 & 1.177512 \\
\hline 6 & 5.118167 & 3.243535 & 0.406820 \\
\hline 6 & 5.519844 & 2.116167 & -0.372418 \\
\hline 6 & 5.896973 & 4.425850 & 0.376354 \\
\hline 6 & 7.017113 & 4.514538 & -0.409398 \\
\hline 6 & 7.396354 & 3.416833 & -1.210051 \\
\hline 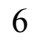 & 6.669339 & 2.252247 & -1.192309 \\
\hline & 2.759044 & -0.181273 & 0.417640 \\
\hline
\end{tabular}




\begin{tabular}{|c|c|c|c|}
\hline 1 & 7.014920 & 0.222173 & 0.775933 \\
\hline 1 & -1.644402 & -0.767249 & 3.286397 \\
\hline & 8.136609 & -3.624834 & -2.158042 \\
\hline & -1.066660 & -5.279781 & 4.568599 \\
\hline 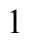 & 5.575538 & 5.270060 & 0.985081 \\
\hline & -3.381981 & -3.400259 & -0.059342 \\
\hline 1 & -5.218683 & -4.136160 & -1.515586 \\
\hline 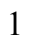 & 3.633614 & 4.042715 & 1.761696 \\
\hline 1 & -1.468248 & -2.864242 & 4.524517 \\
\hline 1 & 6.003173 & -3.396081 & -3.314313 \\
\hline 1 & 8.269922 & 3.495087 & -1.853545 \\
\hline 1 & 3.770749 & -2.307948 & -3.500228 \\
\hline 1 & -2.605905 & -4.031687 & -4.931513 \\
\hline 1 & -0.278889 & -3.317548 & -4.670137 \\
\hline 1 & -0.268551 & -7.384889 & 0.906686 \\
\hline 1 & 6.969578 & 1.421131 & -1.825074 \\
\hline 1 & -0.469869 & -5.299727 & -0.364908 \\
\hline 1 & 1.567596 & -2.521961 & -3.210667 \\
\hline 1 & 7.604389 & 5.429700 & -0.428608 \\
\hline 1 & 9.122619 & -0.988916 & 1.090086 \\
\hline 1 & -4.832360 & -4.496492 & -3.952782 \\
\hline 1 & -0.567552 & -7.395617 & 3.379144 \\
\hline 1 & 9.715127 & -2.906617 & -0.388078 \\
\hline 1 & 2.220528 & 1.981917 & 1.712183 \\
\hline 1 & -3.752792 & -0.951888 & 2.015931 \\
\hline 1 & -3.168933 & 0.324635 & -2.021350 \\
\hline 1 & -7.080261 & -0.830275 & -0.664261 \\
\hline 1 & -3.389196 & & 0.975522 \\
\hline 1 & 0.250571 & 0.563192 & 2.616368 \\
\hline 1 & -1.489815 & 4.129289 & 4.243627 \\
\hline 1 & 1.869185 & -4.032840 & -0.042647 \\
\hline 1 & 3.239088 & -3.272908 & -0.853659 \\
\hline 1 & 5.080086 & -3.022439 & 0.530529 \\
\hline 1 & 1.172406 & -2.479906 & 2.209713 \\
\hline 1 & 6.097309 & -2.531263 & \\
\hline 1 & 2.174612 & -2.023886 & 4.421110 \\
\hline 1 & 4.652534 & -2.018293 & 4.687427 \\
\hline 1 & -1.407132 & 1.617964 & -1.750362 \\
\hline 1 & 1.457085 & 2.245122 & -3.081433 \\
\hline 1 & 1.023415 & 0.560320 & -3.682392 \\
\hline 1 & -2.796659 & 3.105542 & -2.115182 \\
\hline 1 & -3.410630 & 5.489134 & -2.250294 \\
\hline 1 & -1.971262 & 7.083928 & -3.505180 \\
\hline 1 & 0.072685 & 6.250478 & -4.646880 \\
\hline 1 & 0.661574 & 3.853476 & -4.546042 \\
\hline 1 & -2.627285 & 1.127825 & -3.928218 \\
\hline 1 & -1.449695 & 1.257360 & -5.229788 \\
\hline 1 & -1.328302 & -0.082875 & -4.054347 \\
\hline 6 & 1.474844 & 1.186183 & 4.669686 \\
\hline 6 & 1.448988 & 3.658434 & 4.381211 \\
\hline 6 & -0.058113 & 2.553268 & 6.040949 \\
\hline 6 & -4.692856 & 4.113105 & 1.526372 \\
\hline 6 & -4.281218 & 4.491932 & 3.937456 \\
\hline 6 & -2.912783 & 5.691990 & 2.215718 \\
\hline 1 & -3.645408 & 6.509724 & 2.174006 \\
\hline 1 & -2.149386 & 5.965096 & 2.954376 \\
\hline
\end{tabular}




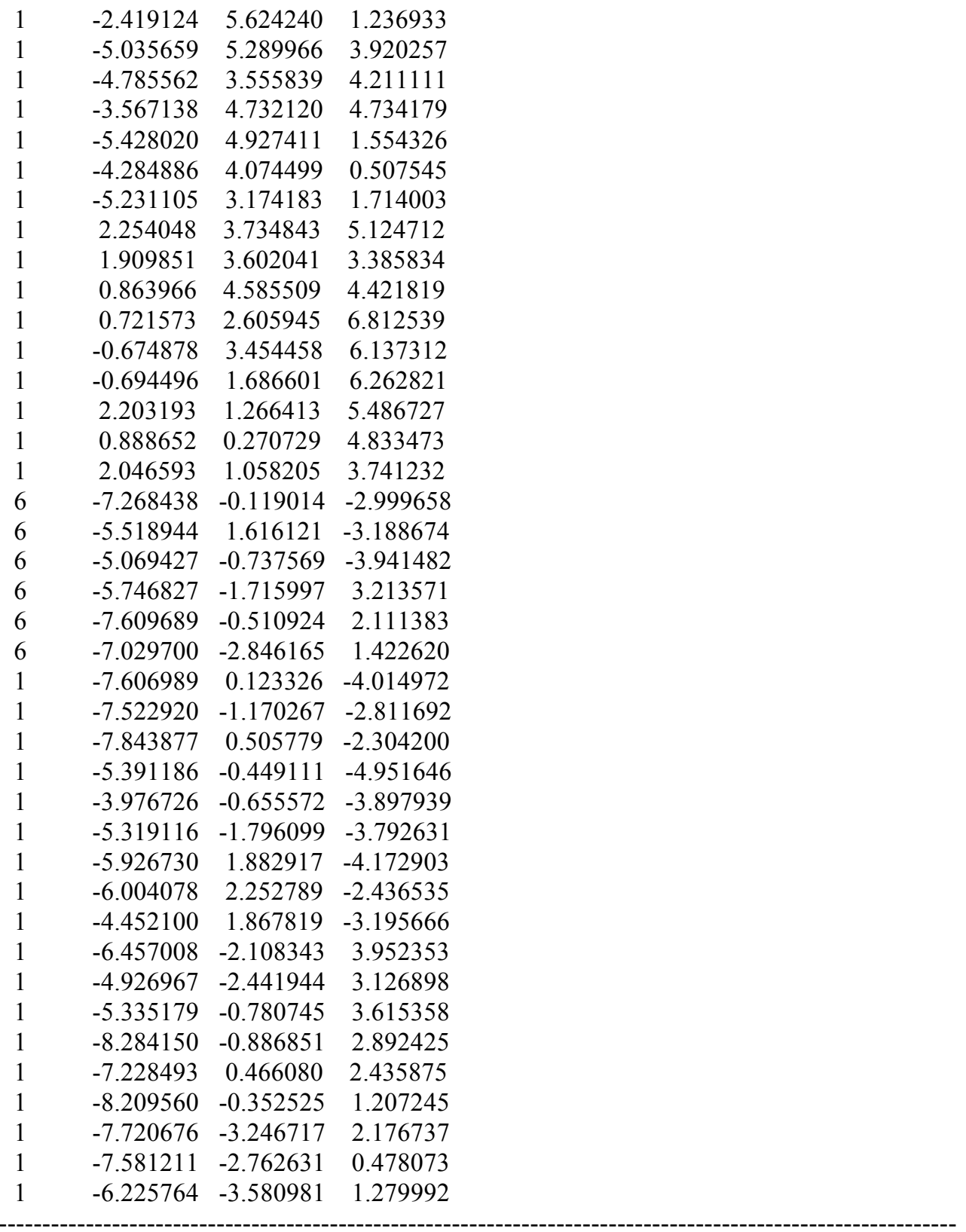

$\operatorname{TS1}_{I L-s i-} \psi_{-1 \mathrm{a} 2 \mathrm{a} 3 \mathrm{ss} 4 \mathrm{~s}}$

Number of imaginary frequencies : 1

The smallest frequencies are : $-637.0414 \quad 11.2362 \quad 16.5103 \mathrm{~cm}(-1)$

Electronic energy : $\quad H F=-4360.859777$

Zero-point correction $=\quad 1.451750$ (Hartree/Particle)

Thermal correction to Energy $=\quad 1.536814$

Thermal correction to Enthalpy= $\quad 1.537758$

Thermal correction to Gibbs Free Energy= $\quad 1.332991$

Sum of electronic and zero-point Energies= $\quad-4359.408027$

Sum of electronic and thermal Energies $=\quad-4359.322963$

Sum of electronic and thermal Enthalpies $=\quad-4359.322019$

Sum of electronic and thermal Free Energies $=\quad-4359.526786$

Cartesian Coordinates 


\begin{tabular}{cccc}
\hline 6 & -3.841425 & 0.033864 & -1.184814 \\
6 & -3.289149 & -0.323645 & 0.040222 \\
6 & -4.121989 & -0.836246 & 1.036405 \\
6 & -5.489886 & -0.997590 & 0.833439 \\
6 & -6.015775 & -0.611706 & -0.403898 \\
6 & -5.216822 & -0.092582 & -1.424634 \\
15 & -1.487258 & -0.082470 & 0.403642 \\
6 & -1.582430 & 1.120114 & 1.794750 \\
6 & -2.622562 & 2.045006 & 1.847948 \\
6 & -2.623510 & 3.071428 & 2.794698 \\
6 & -1.573291 & 3.113012 & 3.714150 \\
6 & -0.522065 & 2.189205 & 3.701182 \\
6 & -0.523607 & 1.216906 & 2.701105 \\
6 & -3.733739 & 4.118818 & 2.852655 \\
6 & -4.447821 & 4.030976 & 4.205746 \\
6 & 0.584963 & 2.274670 & 4.751494 \\
6 & 1.399139 & 3.551756 & 4.520251 \\
6 & -6.337885 & -1.589900 & 1.957277 \\
6 & -6.233711 & -0.699054 & 3.200679 \\
6 & -5.793275 & 0.305112 & -2.782824 \\
6 & -5.335916 & 1.719691 & -3.155248 \\
45 & -0.046001 & 0.988812 & -1.165000 \\
6 & 0.311943 & 2.744628 & -0.231557 \\
8 & 0.223310 & 3.827372 & 0.165161 \\
15 & 1.788553 & -0.361437 & -0.883000 \\
7 & 1.540812 & -2.028179 & -0.661188 \\
6 & 2.525564 & -3.037188 & -0.206556 \\
6 & 3.134633 & -2.771061 & 1.144026 \\
6 & 2.325133 & -2.559353 & 2.260873 \\
6 & 2.887141 & -2.308475 & 3.504983 \\
6 & 4.272899 & -2.264246 & 3.651548 \\
6 & 5.086591 & -2.483689 & 2.545438 \\
6 & 4.518423 & -2.740543 & 1.299849 \\
8 & 2.956442 & -0.195788 & -2.093569 \\
6 & 4.226677 & -0.698288 & -1.968661 \\
6 & 5.127872 & -0.146023 & -1.074423 \\
6 & 6.399993 & -0.786435 & -0.916055 \\
6 & 6.755691 & -1.874350 & -1.768530 \\
6 & 5.831999 & -2.319493 & -2.745950 \\
6 & 4.588447 & -1.756245 & -2.831830 \\
6 & 7.307645 & -0.423537 & 0.111603 \\
6 & 8.505397 & -1.077621 & 0.264951 \\
6 & 8.868862 & -2.127469 & -0.604120 \\
6 & 8.006424 & -2.516060 & -1.597345 \\
6 & 4.732328 & 1.042502 & -0.285615 \\
6 & 5.486045 & 2.261359 & -0.294007 \\
6 & 5.053253 & 3.357676 & 0.512263 \\
6 & 3.867756 & 3.237142 & 1.275870 \\
6 & 3.120555 & 2.091959 & 1.226913 \\
6 & 3.556572 & 1.007068 & 0.440791 \\
6 & 5.800731 & 4.560594 & 0.513761 \\
6 & 6.918915 & 4.698930 & -0.267467 \\
6 & 7.327641 & 3.632671 & -1.095479 \\
6 & 6.632094 & 2.449009 & -1.108489 \\
8 & 2.783474 & -0.127807 & 0.425874 \\
& & & \\
\hline & & &
\end{tabular}




\begin{tabular}{|c|c|c|c|}
\hline 6 & 0.676902 & 1.581978 & -3.155062 \\
\hline 6 & -0.712265 & 1.748456 & -3.316242 \\
\hline b & -1.243642 & 3.151770 & -3.452261 \\
\hline & -0.712887 & 3.941280 & -4.479630 \\
\hline & -1.203825 & 5.217683 & -4.732689 \\
\hline ) & -2.243926 & 5.730271 & -3.964362 \\
\hline & -2.783286 & 4.957415 & -2.940914 \\
\hline 6 & -2.286161 & 3.683227 & -2.686653 \\
\hline b & -1.475060 & 0.731109 & -4.133314 \\
\hline 6 & -1.188540 & -1.700170 & 1.288179 \\
\hline 6 & -0.916834 & -2.848680 & 0.559531 \\
\hline 6 & -0.799932 & -4.115805 & 1.220008 \\
\hline 6 & -0.969411 & -4.192078 & 2.632749 \\
\hline 6 & -1.247275 & -3.005266 & 3.347710 \\
\hline 6 & -1.351237 & -1.804312 & 2.697785 \\
\hline 6 & -0.846795 & -5.441284 & 3.286869 \\
\hline 6 & -0.563838 & -6.580270 & 2.577747 \\
\hline 6 & -0.391204 & -6.512430 & 1.179038 \\
\hline 6 & -0.507840 & -5.315143 & 0.517608 \\
\hline 6 & -0.751130 & -2.840935 & -0.919113 \\
\hline 6 & 0.470755 & -2.521183 & -1.481466 \\
\hline 6 & 0.668363 & -2.633619 & -2.877914 \\
\hline 6 & -0.349701 & -3.033062 & -3.702602 \\
\hline 6 & -1.627608 & -3.328104 & -3.171826 \\
\hline 6 & -1.833316 & -3.235750 & -1.762927 \\
\hline 6 & -3.122414 & -3.530198 & -1.247962 \\
\hline 6 & -4.146126 & -3.901387 & -2.083002 \\
\hline 6 & -3.934356 & -4.008730 & -3.475239 \\
\hline 6 & -2.702355 & -3.723038 & -4.005849 \\
\hline 6 & -3.123337 & 5.513842 & 2.681627 \\
\hline 6 & -4.774890 & 3.920693 & 1.752170 \\
\hline 6 & -0.030412 & 2.313355 & 6.154984 \\
\hline 6 & 1.525975 & 1.071188 & 4.694980 \\
\hline 6 & -5.818444 & -2.995122 & 2.286066 \\
\hline 6 & -7.811871 & -1.705858 & 1.577985 \\
\hline 6 & -5.302626 & -0.693600 & -3.837056 \\
\hline 6 & -7.320964 & 0.294653 & -2.795773 \\
\hline 1 & 7.033386 & 0.370816 & 0.801145 \\
\hline 1 & -1.575958 & -0.912881 & 3.277207 \\
\hline 1 & 8.260673 & -3.338157 & -2.265331 \\
\hline 1 & -0.978979 & -5.475195 & 4.367426 \\
\hline 1 & 5.456053 & 5.380185 & 1.143076 \\
\hline 1 & -3.296499 & -3.443375 & -0.176309 \\
\hline 1 & -5.130413 & -4.111811 & -1.668902 \\
\hline 1 & 3.542924 & 4.083675 & 1.879848 \\
\hline 1 & -1.381533 & -3.059575 & 4.427470 \\
\hline 1 & 6.119008 & -3.133418 & -3.409768 \\
\hline 1 & 8.199015 & 3.750775 & -1.735848 \\
\hline 1 & 3.856240 & -2.101235 & -3.559012 \\
\hline 1 & -2.526971 & -3.794969 & -5.078497 \\
\hline 1 & -0.193142 & -3.118634 & -4.777128 \\
\hline 1 & -0.165053 & -7.417494 & 0.619911 \\
\hline 1 & 6.954733 & 1.642955 & -1.762243 \\
\hline 1 & -0.375129 & -5.280716 & -0.561660 \\
\hline 1 & 1.648175 & -2.382100 & -3.280395 \\
\hline 1 & 7.481677 & 5.629530 & -0.262520 \\
\hline
\end{tabular}




\begin{tabular}{|c|c|c|c|}
\hline ] & 9.177488 & -0.786597 & 1.069236 \\
\hline & -4.753371 & -4.310631 & -4.124702 \\
\hline & -0.469204 & -7.535543 & 3.088809 \\
\hline 1 & 9.822951 & -2.633289 & -0.475587 \\
\hline 1 & 2.183935 & 1.990672 & 1.772114 \\
\hline & -3.694010 & -1.114616 & 1.997572 \\
\hline & -3.175059 & 0.410677 & -1.959884 \\
\hline & -7.082359 & -0.720072 & -0.575847 \\
\hline & -3.433440 & 1.968491 & 1.127673 \\
\hline & 0.300085 & 0.506283 & 2.626117 \\
\hline & -1.572100 & 3.896981 & 4.471416 \\
\hline & 1.969309 & -3.984987 & -0.166355 \\
\hline & 3.323863 & -3.173211 & -0.953077 \\
\hline & 5.162305 & -2.894585 & 0.432429 \\
\hline & 1.244449 & -2.557533 & 2.140105 \\
\hline & 6.169832 & -2.448622 & 2.645057 \\
\hline & 2.237825 & -2.145910 & 4.364498 \\
\hline & 4.714721 & -2.059581 & 4.624587 \\
\hline & -1.438848 & 1.616668 & -1.677366 \\
\hline & 1.319059 & 2.456491 & -3.055596 \\
\hline ] & 1.150384 & 0.703158 & -3.591431 \\
\hline & -2.704533 & 3.098844 & -1.865269 \\
\hline & -3.591242 & 5.349341 & -2.326023 \\
\hline ] & -2.628925 & 6.729023 & -4.158237 \\
\hline & -0.770322 & 5.812951 & -5.533658 \\
\hline$\frac{1}{1}$ & 0.095096 & 3.538433 & -5.090048 \\
\hline & -2.546321 & 0.733072 & -3.905715 \\
\hline ] & -1.382813 & 0.986710 & -5.200863 \\
\hline ] & -1.088106 & -0.282455 & -3.976256 \\
\hline & -3.909356 & 6.280793 & 2.708356 \\
\hline 1 & -2.403240 & 5.749816 & 3.474248 \\
\hline & -2.599201 & 5.595637 & 1.720118 \\
\hline 1 & -5.249358 & 4.779723 & 4.265285 \\
\hline & -4.899198 & 3.039786 & 4.346916 \\
\hline 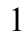 & -3.764178 & 4.209376 & 5.044647 \\
\hline ] & -5.548435 & 4.694750 & 1.834466 \\
\hline & -4.331798 & 4.001389 & 0.750273 \\
\hline 1 & -5.275371 & 2.945529 & 1.825170 \\
\hline & 2.219846 & 3.623462 & 5.246914 \\
\hline 1 & 1.834942 & 3.562330 & 3.511990 \\
\hline & 0.780245 & 4.451927 & 4.620874 \\
\hline 1 & 0.762357 & 2.361588 & 6.913386 \\
\hline ] & -0.679329 & 3.183977 & 6.305517 \\
\hline & -0.627739 & 1.412729 & 6.348320 \\
\hline 1 & 2.261808 & 1.138435 & 5.506452 \\
\hline & 0.977506 & 0.126457 & 4.818498 \\
\hline 1 & 2.089671 & 1.012766 & 3.754857 \\
\hline l & -7.680264 & 0.608858 & -3.783834 \\
\hline 1 & -7.731965 & -0.704911 & -2.606077 \\
\hline 1 & -7.741691 & 0.986909 & -2.054683 \\
\hline 1 & -5.639257 & -0.393842 & -4.839209 \\
\hline 1 & -4.208059 & -0.776180 & -3.853384 \\
\hline 1 & -5.698190 & -1.696804 & -3.629176 \\
\hline 1 & -5.802177 & 2.033438 & -4.098530 \\
\hline & -5.618825 & 2.445470 & -2.379789 \\
\hline 1 & -4.251395 & 1.790116 & -3.293185 \\
\hline
\end{tabular}




$\begin{array}{rrrr}1 & -8.375162 & -2.138335 & 2.414525 \\ 1 & -8.256508 & -0.727723 & 1.353449 \\ 1 & -7.961696 & -2.359485 & 0.708804 \\ 1 & -6.827896 & -1.119892 & 4.023087 \\ 1 & -5.200355 & -0.598965 & 3.555134 \\ 1 & -6.612784 & 0.310194 & 2.992340 \\ 1 & -6.409538 & -3.442708 & 3.096558 \\ 1 & -5.894704 & -3.653752 & 1.410140 \\ 1 & -4.768188 & -2.987411 & 2.606038\end{array}$

$\operatorname{TS1}_{I L-s i-} \psi-1 \mathbf{a} 2 s 3 a 4 a$

Number of imaginary frequencies : 1

The smallest frequencies are : $-672.0422 \quad 15.3939 \quad 18.6794 \mathrm{~cm}(-1)$

Electronic energy : $\quad H F=-4360.8605269$

Zero-point correction $=\quad 1.451265($ Hartree/Particle)

Thermal correction to Energy= $\quad 1.536468$

Thermal correction to Enthalpy= $\quad 1.537412$

Thermal correction to Gibbs Free Energy $=\quad 1.332163$

Sum of electronic and zero-point Energies $=\quad-4359.409262$

Sum of electronic and thermal Energies $=\quad-4359.324059$

Sum of electronic and thermal Enthalpies $=\quad-4359.323115$

Sum of electronic and thermal Free Energies $=\quad-4359.528364$

Cartesian Coordinates

$\begin{array}{cccc}6 & 0.476042 & -1.390789 & 2.687386 \\ 6 & 1.561360 & -1.239178 & 1.813535 \\ 6 & 2.599032 & -2.158398 & 1.845196 \\ 6 & 2.584956 & -3.236495 & 2.741650 \\ 6 & 1.508657 & -3.341557 & 3.617187 \\ 6 & 0.447848 & -2.419884 & 3.621061 \\ 15 & 1.467341 & 0.034116 & 0.491059 \\ 45 & 0.044071 & -1.013631 & -1.136169 \\ 15 & -1.810189 & 0.340094 & -0.881554 \\ 8 & -2.939080 & 0.249099 & -2.140606 \\ 6 & -4.214489 & 0.742285 & -2.026871 \\ 6 & -5.140795 & 0.146525 & -1.187289 \\ 6 & -6.411138 & 0.788594 & -1.021577 \\ 6 & -6.739901 & 1.924813 & -1.820085 \\ 6 & -5.792873 & 2.412862 & -2.753706 \\ 6 & -4.551061 & 1.846214 & -2.841535 \\ 6 & -7.341550 & 0.379589 & -0.032319 \\ 6 & -8.534512 & 1.038798 & 0.136098 \\ 6 & -8.870552 & 2.139053 & -0.680107 \\ 6 & -7.986672 & 2.570518 & -1.636197 \\ 6 & -4.771929 & -1.084828 & -0.452443 \\ 6 & -3.619207 & -1.087843 & 0.310223 \\ 6 & -3.203265 & -2.211834 & 1.051164 \\ 6 & -3.949185 & -3.358282 & 1.016779 \\ 6 & -5.113220 & -3.437232 & 0.215132 \\ 6 & -5.526049 & -2.299644 & -0.543569 \\ 6 & -5.860931 & -4.637160 & 0.135515 \\ 6 & -6.960874 & -4.732155 & -0.677542 \\ 6 & -7.350589 & -3.622843 & -1.456801 \\ 6 & -6.653689 & -2.441620 & -1.392228\end{array}$




\begin{tabular}{|c|c|c|c|}
\hline 8 & -2.853140 & 0.048933 & 0.379084 \\
\hline 6 & 3.744857 & -4.228506 & 2.714431 \\
\hline 6 & -0.696335 & -2.580475 & 4.620885 \\
\hline b & 3.253305 & 0.318386 & 0.102308 \\
\hline 6 & 4.122281 & 0.674638 & 1.138730 \\
\hline 6 & 5.476430 & 0.890947 & 0.902607 \\
\hline 6 & 5.937265 & 0.736331 & -0.409713 \\
\hline 6 & 5.102180 & 0.393018 & -1.472955 \\
\hline 6 & 3.743411 & 0.189573 & -1.194161 \\
\hline 6 & 5.698589 & 0.199438 & -2.867831 \\
\hline 6 & 4.623811 & 0.121227 & -3.948538 \\
\hline 6 & 6.438233 & 1.330285 & 2.004481 \\
\hline 6 & 5.778428 & 1.322765 & 3.382278 \\
\hline 6 & 1.145532 & 1.590770 & 1.467954 \\
\hline 6 & 0.841952 & 2.773520 & 0.810601 \\
\hline 6 & 0.670129 & 3.989438 & 1.549331 \\
\hline 6 & 0.814578 & 3.977919 & 2.966810 \\
\hline 6 & 1.139416 & 2.760595 & 3.606413 \\
\hline 6 & 1.299757 & 1.609762 & 2.882078 \\
\hline 6 & 0.626826 & 5.174114 & 3.699635 \\
\hline 6 & 0.307847 & 6.345312 & 3.061801 \\
\hline 6 & 0.166107 & 6.365257 & 1.658191 \\
\hline 6 & 0.344905 & 5.220675 & 0.921246 \\
\hline 6 & 0.718121 & 2.852985 & -0.669651 \\
\hline 6 & 1.826361 & 3.307936 & -1.447493 \\
\hline 6 & 1.677609 & 3.460081 & -2.858083 \\
\hline 6 & 0.423385 & 3.187961 & -3.452071 \\
\hline 6 & -0.621614 & 2.737075 & -2.690577 \\
\hline 6 & -0.475113 & 2.543511 & -1.296651 \\
\hline 6 & 2.787727 & 3.880096 & -3.631581 \\
\hline 6 & 3.999413 & 4.135158 & -3.040340 \\
\hline 6 & 4.151519 & 3.979963 & -1.644518 \\
\hline 6 & 3.090928 & 3.587331 & -0.867474 \\
\hline 7 & -1.568343 & 1.991274 & -0.548556 \\
\hline 6 & -2.583603 & 2.962861 & -0.079570 \\
\hline 6 & -3.220804 & 2.639299 & 1.245787 \\
\hline 6 & -2.438673 & 2.358627 & 2.367116 \\
\hline 6 & -3.031495 & 2.067876 & 3.588307 \\
\hline 6 & -4.420466 & 2.050002 & 3.706786 \\
\hline 6 & -5.206689 & 2.333778 & 2.595435 \\
\hline 6 & -4.608049 & 2.631019 & 1.373454 \\
\hline 6 & 6.632279 & 1.360372 & -3.225423 \\
\hline 6 & 6.492921 & -1.111849 & -2.879402 \\
\hline 6 & 7.651143 & 0.396183 & 2.055859 \\
\hline 6 & 6.905065 & 2.759085 & 1.701974 \\
\hline 6 & -0.210416 & -2.778027 & -0.202008 \\
\hline 8 & -0.193640 & -3.928104 & -0.072994 \\
\hline 6 & 0.653851 & -1.576398 & -3.297571 \\
\hline 6 & -0.690977 & -1.898876 & -2.969639 \\
\hline 6 & 0.911624 & -0.345681 & -4.137677 \\
\hline 6 & 1.573977 & -2.734502 & -3.617113 \\
\hline 6 & 2.706053 & -3.107117 & -2.887627 \\
\hline 6 & 3.510806 & -4.168004 & -3.297732 \\
\hline 6 & 3.200864 & -4.879762 & -4.450201 \\
\hline 6 & 2.076458 & -4.523128 & -5.189653 \\
\hline 6 & 1.276506 & -3.464515 & -4.776710 \\
\hline
\end{tabular}




\begin{tabular}{|c|c|c|c|}
\hline & -7.087533 & -0.453839 & 0.617792 \\
\hline & 1.555988 & 0.693142 & 3.406383 \\
\hline & -8.220190 & 3.430750 & -2.262455 \\
\hline & 0.738807 & 5.141252 & 4.782500 \\
\hline & -5.531948 & -5.489347 & 0.728901 \\
\hline & 3.220480 & 3.460832 & 0.206163 \\
\hline & 5.119488 & 4.166971 & -1.182364 \\
\hline & -3.642946 & -4.234778 & 1.586871 \\
\hline & 1.264343 & 2.750710 & 4.688564 \\
\hline & -6.059921 & 3.263119 & -3.379235 \\
\hline & -8.208090 & -3.705172 & -2.121042 \\
\hline & -3.799771 & 2.225049 & -3.531597 \\
\hline & 2.656874 & 3.995552 & -4.706772 \\
\hline & 0.310091 & 3.321746 & -4.527209 \\
\hline & -0.085397 & 7.296417 & 1.155612 \\
\hline & -6.961548 & -1.601076 & -2.008758 \\
\hline & 0.236555 & 5.252842 & -0.160960 \\
\hline & -1.581780 & 2.496668 & -3.144668 \\
\hline & -7.524286 & -5.660670 & -0.734157 \\
\hline & -9.224038 & 0.712389 & 0.911544 \\
\hline & 4.845522 & 4.457636 & -3.643989 \\
\hline & 0.162658 & 7.259283 & 3.632988 \\
\hline 1 & -9.820911 & 2.648859 & -0.540112 \\
\hline & -2.284880 & -2.136273 & 1.630769 \\
\hline ] & 3.727497 & 0.776442 & 2.146145 \\
\hline & 3.043862 & -0.069867 & -1.987396 \\
\hline 1 & 6.996677 & 0.894792 & -0.608065 \\
\hline 1 & 3.430145 & -2.046906 & 1.149716 \\
\hline & -0.347477 & -0.678827 & 2.628876 \\
\hline ] & 1.481776 & -4.164052 & 4.328347 \\
\hline & -2.049205 & 3.920031 & 0.007427 \\
\hline 1 & -3.366318 & 3.110337 & -0.840205 \\
\hline ] & -5.231572 & 2.836985 & 0.501678 \\
\hline | & -1.355808 & 2.334461 & 2.266572 \\
\hline 1 & -6.292295 & 2.316316 & 2.671108 \\
\hline 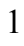 & -2.402995 & 1.852164 & 4.451647 \\
\hline l & -4.885976 & 1.814050 & 4.661517 \\
\hline & 1.460300 & -1.499244 & -1.684983 \\
\hline 1 & -0.965001 & -2.947225 & -2.841822 \\
\hline ] & -1.489807 & -1.261133 & -3.347027 \\
\hline l & 2.960375 & -2.570403 & -1.972905 \\
\hline 1 & 4.379731 & -4.441230 & -2.701541 \\
\hline 1 & 3.826322 & -5.711120 & -4.767755 \\
\hline 1 & 1.816225 & -5.075950 & -6.090043 \\
\hline 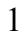 & 0.394036 & -3.192672 & -5.356535 \\
\hline 1 & 1.850608 & 0.155970 & -3.877814 \\
\hline 1 & 0.978234 & -0.624694 & -5.202221 \\
\hline 1 & 0.102782 & 0.382584 & -4.013385 \\
\hline 1 & 5.098041 & 0.036474 & -4.934579 \\
\hline 1 & 3.976389 & -0.757471 & -3.827646 \\
\hline 1 & 3.997051 & 1.024209 & -3.953384 \\
\hline 1 & 7.025254 & 1.224134 & -4.241540 \\
\hline 1 & 6.093327 & 2.316681 & -3.195892 \\
\hline 1 & 7.495740 & 1.435796 & -2.554229 \\
\hline 1 & 6.941173 & -1.280441 & -3.868112 \\
\hline 1 & 7.302311 & -1.103412 & -2.138325 \\
\hline
\end{tabular}




$\begin{array}{lrrr}1 & 5.839854 & -1.966566 & -2.656448 \\ 1 & 7.596068 & 3.112098 & 2.479541 \\ 1 & 7.425023 & 2.823767 & 0.737774 \\ 1 & 6.050902 & 3.449276 & 1.669391 \\ 1 & 8.338856 & 0.712077 & 2.851534 \\ 1 & 7.345050 & -0.637003 & 2.266074 \\ 1 & 8.217590 & 0.393456 & 1.117123 \\ 1 & 6.509544 & 1.625546 & 4.142411 \\ 1 & 4.935692 & 2.024600 & 3.442161 \\ 1 & 5.412272 & 0.323055 & 3.652919 \\ 6 & -1.634542 & -1.373600 & 4.619807 \\ 6 & -1.500987 & -3.834232 & 4.262288 \\ 6 & -0.135311 & -2.727968 & 6.039772 \\ 6 & 3.604587 & -5.318202 & 3.773787 \\ 6 & 3.796927 & -4.902590 & 1.338021 \\ 6 & 5.059741 & -3.480513 & 2.967534 \\ 1 & 4.462284 & -6.000326 & 3.717058 \\ 1 & 3.581157 & -4.902933 & 4.789789 \\ 1 & 2.698069 & -5.918833 & 3.625377 \\ 1 & 5.906075 & -4.180466 & 2.950822 \\ 1 & 5.251528 & -2.712177 & 2.207309 \\ 1 & 5.048945 & -2.985854 & 3.948054 \\ 1 & 4.616479 & -5.633237 & 1.300966 \\ 1 & 2.858660 & -5.430363 & 1.124089 \\ 1 & 3.960525 & -4.178404 & 0.530414 \\ 1 & -2.346484 & -3.960687 & 4.952131 \\ 1 & -1.901650 & -3.764958 & 3.241675 \\ 1 & -0.885239 & -4.740682 & 4.314142 \\ 1 & -0.956838 & -2.828256 & 6.761467 \\ 1 & 0.504213 & -3.611486 & 6.149064 \\ 1 & 0.456862 & -1.847979 & 6.323068 \\ 1 & -2.401010 & -1.499880 & 5.395023 \\ 1 & -1.090818 & -0.442795 & 4.835221 \\ 1 & -2.162050 & -1.242332 & 3.666117\end{array}$

$\operatorname{TS1}_{I L-s i-} \psi_{-1 \mathrm{a} 2 s 3 a 4 s}$

Number of imaginary frequencies : 1

The smallest frequencies are : $-653.5971 \quad 16.1934 \quad 17.8963 \mathrm{~cm}(-1)$

Electronic energy : $\quad H F=-4360.8609581$

Zero-point correction $=\quad 1.451804$ (Hartree/Particle)

Thermal correction to Energy= $\quad 1.536732$

Thermal correction to Enthalpy= $\quad 1.537676$

Thermal correction to Gibbs Free Energy= $\quad 1.333929$

Sum of electronic and zero-point Energies $=\quad-4359.409154$

Sum of electronic and thermal Energies $=\quad-4359.324226$

Sum of electronic and thermal Enthalpies $=\quad-4359.323282$

Sum of electronic and thermal Free Energies $=\quad-4359.527029$

Cartesian Coordinates

$\begin{array}{llll}6 & 0.506297 & -1.338990 & 2.708199 \\ 6 & 1.588563 & -1.200826 & 1.828438 \\ 6 & 2.630045 & -2.115598 & 1.875374 \\ 6 & 2.618992 & -3.178934 & 2.789847 \\ 6 & 1.546019 & -3.269274 & 3.671189\end{array}$




\begin{tabular}{|c|c|c|c|}
\hline 6 & 0.483410 & -2.350148 & 3.661278 \\
\hline 15 & 1.477878 & 0.040335 & 0.476634 \\
\hline & 0.030399 & -1.042535 & -1.107368 \\
\hline & -1.809788 & 0.333631 & -0.876161 \\
\hline & -2.945310 & 0.234473 & -2.129101 \\
\hline & -4.214446 & 0.744148 & -2.015764 \\
\hline & -5.143830 & 0.166718 & -1.166872 \\
\hline & -6.408984 & 0.820330 & -1.006673 \\
\hline 6 & -6.729976 & 1.950246 & -1.817301 \\
\hline 6 & -5.779108 & 2.422354 & -2.755050 \\
\hline 6 & -4.542175 & 1.844401 & -2.838926 \\
\hline 6 & -7.342264 & 0.428945 & -0.012957 \\
\hline 6 & -8.531326 & 1.097135 & 0.147058 \\
\hline 6 & -8.860246 & 2.190088 & -0.681738 \\
\hline 6 & -7.972758 & 2.605827 & -1.641377 \\
\hline 6 & -4.781779 & -1.055310 & -0.413418 \\
\hline 6 & -3.628613 & -1.052342 & 0.348707 \\
\hline 6 & -3.222544 & -2.164247 & 1.112789 \\
\hline 6 & -3.977449 & -3.305300 & 1.101419 \\
\hline 6 & -5.140967 & -3.391633 & 0.299733 \\
\hline 6 & -5.544388 & -2.266123 & -0.481681 \\
\hline 6 & -5.897570 & -4.587273 & 0.242354 \\
\hline 6 & -6.996781 & -4.689988 & -0.570787 \\
\hline 6 & -7.376957 & -3.593330 & -1.372422 \\
\hline 6 & -6.671638 & -2.416151 & -1.329403 \\
\hline 8 & -2.851941 & 0.078388 & 0.393205 \\
\hline 6 & 3.772187 & -4.179333 & 2.770350 \\
\hline 6 & -0.666769 & -2.503505 & 4.654961 \\
\hline 6 & 3.260547 & 0.319321 & 0.066701 \\
\hline 6 & 4.124448 & 0.731327 & 1.078717 \\
\hline 6 & 5.479311 & 0.959729 & 0.834567 \\
\hline 6 & 5.945004 & 0.750274 & -0.463148 \\
\hline 6 & 5.108214 & 0.345848 & -1.510933 \\
\hline 6 & 3.755351 & 0.136334 & -1.225580 \\
\hline 6 & 5.708269 & 0.100224 & -2.896074 \\
\hline 6 & 4.635424 & -0.023953 & -3.974736 \\
\hline 6 & 6.364586 & 1.446008 & 1.979921 \\
\hline 6 & 7.811233 & 1.666674 & 1.546445 \\
\hline 6 & 1.169907 & 1.622427 & 1.418747 \\
\hline 6 & 0.878409 & 2.792350 & 0.733386 \\
\hline 6 & 0.729294 & 4.029338 & 1.440508 \\
\hline 6 & 0.885341 & 4.053672 & 2.856544 \\
\hline 6 & 1.191971 & 2.847742 & 3.526152 \\
\hline 6 & 1.329381 & 1.675404 & 2.831396 \\
\hline 6 & 0.725297 & 5.272788 & 3.557590 \\
\hline 6 & 0.421228 & 6.431458 & 2.890255 \\
\hline 6 & 0.266194 & 6.415312 & 1.487854 \\
\hline 6 & 0.418508 & 5.248075 & 0.781369 \\
\hline 6 & 0.744850 & 2.831583 & -0.747184 \\
\hline 6 & 1.850286 & 3.253798 & -1.546582 \\
\hline 6 & 1.695185 & 3.350180 & -2.961431 \\
\hline 6 & 0.437626 & 3.058583 & -3.538596 \\
\hline 6 & -0.606336 & 2.644759 & -2.755047 \\
\hline 6 & -0.453945 & 2.506498 & -1.355212 \\
\hline 6 & 2.801982 & 3.737373 & -3.756244 \\
\hline 6 & 4.015541 & 4.017196 & -3.180653 \\
\hline
\end{tabular}




\begin{tabular}{|c|c|c|c|}
\hline & 4.173514 & 3.918089 & -1.780327 \\
\hline & 3.117777 & 3.553765 & -0.983183 \\
\hline & -1.544857 & 1.987609 & -0.580391 \\
\hline & -2.535232 & 2.985049 & -0.112143 \\
\hline & -3.171746 & 2.681842 & 1.218073 \\
\hline & -2.387595 & 2.413526 & 2.341021 \\
\hline & -2.978304 & 2.131661 & 3.565312 \\
\hline & -4.367110 & 2.111323 & 3.685264 \\
\hline & -5.155276 & 2.385429 & 2.572790 \\
\hline & -4.558738 & 2.673949 & 1.347730 \\
\hline & 6.639196 & 1.247929 & -3.300427 \\
\hline & 6.509185 & -1.206210 & -2.852619 \\
\hline & 6.356765 & 0.410401 & 3.109840 \\
\hline & 5.815473 & 2.780163 & 2.501142 \\
\hline & -0.235541 & -2.772130 & -0.110158 \\
\hline & -0.225975 & -3.914707 & 0.072994 \\
\hline ) & 0.607207 & -1.694857 & -3.257949 \\
\hline & -0.743692 & -1.961548 & -2.910334 \\
\hline D & 0.903083 & -0.503563 & -4.140103 \\
\hline & 1.484573 & -2.894471 & -3.538865 \\
\hline & 2.619889 & -3.266245 & -2.814607 \\
\hline & 3.381527 & -4.371816 & -3.187207 \\
\hline & 3.025186 & -5.128649 & -4.296601 \\
\hline & 1.898280 & -4.771618 & -5.031961 \\
\hline & 1.140405 & -3.669378 & -4.655744 \\
\hline & -7.094182 & -0.398658 & 0.646714 \\
\hline & 1.572877 & 0.768368 & 3.377961 \\
\hline & -8.200318 & 3.460639 & -2.277188 \\
\hline & 0.846481 & 5.267846 & 4.639948 \\
\hline & -5.576035 & -5.430105 & 0.852926 \\
\hline & 3.252492 & 3.468184 & 0.094250 \\
\hline ] & 5.143268 & 4.127197 & -1.331939 \\
\hline & -3.678455 & -4.172054 & 1.690027 \\
\hline & 1.321795 & 2.863860 & 4.607648 \\
\hline ] & -6.039223 & 3.269418 & -3.387809 \\
\hline & -8.233750 & -3.682447 & -2.036698 \\
\hline ] & -3.788765 & 2.212681 & -3.532228 \\
\hline & 2.666441 & 3.809461 & -4.834624 \\
\hline 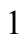 & 0.320222 & 3.148636 & -4.617834 \\
\hline 1 & 0.025778 & 7.336561 & 0.962125 \\
\hline & -6.971869 & -1.585507 & -1.962905 \\
\hline 1 & 0.300979 & 5.252257 & -0.300307 \\
\hline & -1.568590 & 2.387955 & -3.195284 \\
\hline 1 & -7.566939 & -5.615256 & -0.610313 \\
\hline & -9.223310 & 0.783641 & 0.925628 \\
\hline 1 & 4.858598 & 4.317139 & -3.799956 \\
\hline l & 0.297701 & 7.363286 & 3.437166 \\
\hline 1 & -9.807715 & 2.706996 & -0.548389 \\
\hline 1 & -2.303875 & -2.084273 & 1.691236 \\
\hline 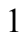 & 3.733914 & 0.880838 & 2.084414 \\
\hline 1 & 3.058257 & -0.165377 & -2.006235 \\
\hline 1 & 6.999567 & 0.910740 & -0.674796 \\
\hline 1 & 3.459454 & -2.014616 & 1.175997 \\
\hline 1 & -0.320530 & -0.631927 & 2.637050 \\
\hline 1 & 1.521085 & -4.080516 & 4.395071 \\
\hline 1 & -1.978373 & 3.930054 & -0.033062 \\
\hline
\end{tabular}




\begin{tabular}{|c|c|c|c|}
\hline 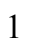 & -3.318381 & 3.145003 & -0.869891 \\
\hline | & -5.183512 & 2.871363 & 0.474921 \\
\hline & -1.304743 & 2.391536 & 2.239994 \\
\hline & -6.240738 & 2.367529 & 2.650429 \\
\hline & -2.348464 & 1.924936 & 4.429856 \\
\hline & -4.831116 & 1.881938 & 4.642324 \\
\hline & 1.438069 & -1.563858 & -1.652321 \\
\hline & -1.050666 & -2.995567 & -2.745798 \\
\hline & -1.524412 & -1.309936 & -3.301574 \\
\hline 1 & 2.909836 & -2.692032 & -1.933714 \\
\hline 1 & 4.253454 & -4.644674 & -2.595709 \\
\hline & 3.617197 & -5.994703 & -4.584190 \\
\hline & 1.602788 & -5.358211 & -5.899495 \\
\hline & 0.255452 & -3.396932 & -5.231444 \\
\hline 1 & 1.862016 & -0.029461 & -3.902415 \\
\hline & 0.951518 & -0.821224 & -5.194698 \\
\hline & 0.122459 & 0.257779 & -4.035988 \\
\hline & 5.111617 & -0.154119 & -4.954876 \\
\hline & 3.985606 & -0.894969 & -3.816446 \\
\hline & 4.011109 & 0.880292 & -4.021855 \\
\hline & 7.027096 & 1.074023 & -4.312739 \\
\hline & 6.100301 & 2.204578 & -3.303765 \\
\hline 1 & 7.506210 & 1.347463 & -2.637226 \\
\hline & 6.954686 & -1.417748 & -3.834266 \\
\hline & 7.321401 & -1.159315 & -2.116102 \\
\hline & 5.862890 & -2.053046 & -2.586065 \\
\hline & 6.428152 & 3.145876 & 3.336275 \\
\hline & 5.832768 & 3.544108 & 1.712064 \\
\hline & 4.781247 & 2.695629 & 2.859528 \\
\hline 1 & 6.959835 & 0.764757 & 3.956753 \\
\hline & 5.344619 & 0.209456 & 3.483310 \\
\hline ] & 6.782418 & -0.542936 & 2.769597 \\
\hline 1 & 8.402697 & 2.018941 & 2.400980 \\
\hline & 8.277526 & 0.741387 & 1.184243 \\
\hline 1 & 7.891277 & 2.425064 & 0.756594 \\
\hline & 3.635082 & -5.248300 & 3.851079 \\
\hline 6 & 3.803449 & -4.878959 & 1.406130 \\
\hline f & 5.096032 & -3.438847 & 2.994069 \\
\hline 6 & -1.596387 & -1.289790 & 4.649200 \\
\hline 6 & -1.475370 & -3.751579 & 4.284084 \\
\hline & -0.119532 & -2.658749 & 6.078060 \\
\hline 1 & -2.363794 & -1.407322 & 5.424860 \\
\hline d & -1.045219 & -0.362448 & 4.860723 \\
\hline 1 & -2.123507 & -1.157541 & 3.695492 \\
\hline 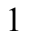 & -2.339138 & -3.866328 & 4.953080 \\
\hline 1 & -1.847045 & -3.685749 & 3.252307 \\
\hline 1 & -0.868699 & -4.662834 & 4.356618 \\
\hline 1 & -0.948313 & -2.758217 & 6.791485 \\
\hline 1 & 0.514659 & -3.545640 & 6.190625 \\
\hline 1 & 0.473631 & -1.782459 & 6.370582 \\
\hline 1 & 4.489197 & -5.935368 & 3.800152 \\
\hline 1 & 3.621877 & -4.814002 & 4.859277 \\
\hline 1 & 2.724824 & -5.847577 & 3.721291 \\
\hline 1 & 4.612159 & -5.621905 & 1.375802 \\
\hline 1 & 2.855885 & -5.396326 & 1.208178 \\
\hline & 3.971948 & -4.170308 & 0.586062 \\
\hline
\end{tabular}




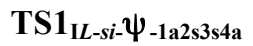

Number of imaginary frequencies : 1

The smallest frequencies are : $-630.6337 \quad 12.2062 \quad 18.5966 \mathrm{~cm}(-1)$

Electronic energy : $\quad H F=-4360.8601377$

Zero-point correction $=\quad 1.451794$ (Hartree/Particle)

Thermal correction to Energy= $\quad 1.536950$

Thermal correction to Enthalpy= $\quad 1.537895$

Thermal correction to Gibbs Free Energy= 1.332402

Sum of electronic and zero-point Energies= $\quad-4359.408344$

Sum of electronic and thermal Energies = $\quad-4359.323187$

Sum of electronic and thermal Enthalpies $=\quad-4359.322243$

Sum of electronic and thermal Free Energies $=\quad-4359.527735$

\section{Cartesian Coordinates}

\begin{tabular}{cccc}
$6 \ldots \ldots \ldots \ldots \ldots \ldots \ldots$ \\
6 & 0.777331 & -3.751600 & -4.696957 \\
6 & 1.338897 & -3.010616 & -3.651066 \\
6 & 2.374026 & -3.592805 & -2.912236 \\
6 & 2.832904 & -4.871403 & -3.208558 \\
6 & 2.262914 & -5.597155 & -4.250338 \\
6 & 1.231450 & -5.032915 & -4.993180 \\
6 & 0.861355 & -1.596133 & -3.460109 \\
45 & 0.134818 & -0.955240 & -1.269529 \\
6 & -0.191406 & -2.770442 & -0.436570 \\
8 & 0.005842 & -3.792858 & 0.067567 \\
15 & 1.496605 & 0.034002 & 0.398018 \\
6 & 3.330678 & 0.169759 & 0.158083 \\
6 & 3.918979 & -0.059558 & -1.077114 \\
6 & 5.309642 & 0.053068 & -1.254673 \\
6 & 6.074417 & 0.426818 & -0.155249 \\
6 & 5.513309 & 0.671541 & 1.108538 \\
6 & 4.139322 & 0.531297 & 1.245329 \\
6 & 5.923613 & -0.213005 & -2.628300 \\
6 & 7.450129 & -0.166219 & -2.605524 \\
6 & 6.408542 & 1.104947 & 2.267306 \\
6 & 7.524011 & 0.077553 & 2.485438 \\
6 & 1.402310 & -1.126476 & 1.824203 \\
6 & 2.346452 & -2.129415 & 1.985927 \\
6 & 2.224770 & -3.084975 & 3.004204 \\
6 & 1.138094 & -2.982201 & 3.867256 \\
6 & 0.165789 & -1.976268 & 3.736461 \\
6 & 0.294319 & -1.078424 & 2.681597 \\
6 & -0.950324 & -1.865865 & 4.775835 \\
6 & -0.319801 & -1.691776 & 6.163566 \\
6 & 3.296992 & -4.164697 & 3.127372 \\
6 & 4.663488 & -3.498725 & 3.332635 \\
6 & 1.224336 & 1.679091 & 1.247516 \\
6 & 0.946778 & 2.819850 & 0.510873 \\
6 & 0.810913 & 4.091380 & 1.163193 \\
6 & 0.977111 & 4.180252 & 2.575132 \\
6 & 1.278005 & 3.003522 & 3.297438 \\
\hline & & & \\
6 & & &
\end{tabular}




\begin{tabular}{|c|c|c|c|}
\hline 6 & 1.394020 & 1.800222 & 2.656384 \\
\hline 6 & 0.825572 & 5.429155 & 3.222832 \\
\hline b & 0.516585 & 6.557884 & 2.507807 \\
\hline & 0.347334 & 6.478301 & 1.109827 \\
\hline$b$ & 0.493047 & 5.280289 & 0.454697 \\
\hline f & 0.784664 & 2.815988 & -0.967723 \\
\hline 6 & -0.438665 & 2.515379 & -1.535114 \\
\hline 5 & -0.642647 & 2.672966 & -2.926248 \\
\hline 6 & 0.370856 & 3.105054 & -3.740492 \\
\hline 6 & 1.650785 & 3.384175 & -3.204835 \\
\hline 6 & 1.865095 & 3.234507 & -1.802097 \\
\hline 6 & 3.155780 & 3.512524 & -1.281528 \\
\hline 6 & 4.170599 & 3.932458 & -2.104343 \\
\hline 6 & 3.950560 & 4.096713 & -3.489738 \\
\hline 6 & 2.719234 & 3.819815 & -4.026756 \\
\hline 7 & -1.509428 & 2.010222 & -0.722082 \\
\hline 15 & -1.727109 & 0.337961 & -0.934729 \\
\hline 8 & -2.702615 & 0.086631 & 0.385089 \\
\hline 6 & -3.446532 & -1.071109 & 0.397949 \\
\hline 6 & -4.633267 & -1.123729 & -0.307846 \\
\hline 6 & -5.361108 & -2.358647 & -0.319746 \\
\hline 6 & -4.883615 & -3.459096 & 0.455021 \\
\hline 6 & -3.683032 & -3.324552 & 1.192043 \\
\hline 6 & -2.966287 & -2.159246 & 1.153065 \\
\hline 6 & -6.522311 & -2.558468 & -1.109556 \\
\hline 6 & -7.190018 & -3.758121 & -1.101367 \\
\hline 6 & -6.736035 & -4.829663 & -0.304576 \\
\hline 6 & -5.602114 & -4.679495 & 0.451277 \\
\hline 6 & -5.060554 & 0.057005 & -1.090985 \\
\hline 6 & -4.183933 & 0.616113 & -2.004664 \\
\hline 6 & -4.584775 & 1.644789 & -2.886285 \\
\hline 6 & -5.838316 & 2.183314 & -2.789340 \\
\hline 6 & -6.732651 & 1.742623 & -1.782464 \\
\hline 6 & -6.341081 & 0.676134 & -0.919093 \\
\hline 6 & -7.987702 & 2.369835 & -1.591684 \\
\hline 6 & -8.819860 & 1.986767 & -0.570570 \\
\hline 6 & -8.422508 & 0.954819 & 0.305114 \\
\hline 6 & -7.219693 & 0.314500 & 0.133463 \\
\hline 8 & -2.900891 & 0.148535 & -2.134603 \\
\hline 6 & -2.545495 & 2.997363 & -0.344325 \\
\hline 6 & -3.155473 & 2.811730 & 1.019797 \\
\hline 6 & -4.540089 & 2.834937 & 1.174864 \\
\hline 6 & -5.116465 & 2.715083 & 2.437224 \\
\hline 6 & -4.309930 & 2.581120 & 3.561754 \\
\hline 6 & -2.923869 & 2.563498 & 3.414028 \\
\hline 6 & -2.352856 & 2.669522 & 2.152827 \\
\hline 6 & -1.853137 & -0.659612 & 4.525296 \\
\hline 6 & -1.809115 & -3.133584 & 4.768911 \\
\hline 6 & 3.321076 & -4.999886 & 1.842044 \\
\hline 6 & 3.045972 & -5.106231 & 4.301968 \\
\hline 6 & -0.520625 & -1.367525 & -3.339804 \\
\hline 6 & 5.433153 & 0.855501 & -3.611592 \\
\hline 6 & 5.508633 & -1.603403 & -3.121952 \\
\hline 6 & 7.026112 & 2.467090 & 1.930550 \\
\hline 6 & 5.631389 & 1.241377 & 3.575287 \\
\hline 1 & -6.917980 & -0.466022 & 0.827452 \\
\hline
\end{tabular}




\begin{tabular}{|c|c|c|c|}
\hline$\frac{1}{1}$ & 1.619748 & 0.916533 & 3.247397 \\
\hline & -8.269763 & 3.177114 & -2.266517 \\
\hline & 0.955726 & 5.471260 & 4.303376 \\
\hline & -5.222624 & -5.502139 & 1.056082 \\
\hline & 3.337569 & 3.380201 & -0.215922 \\
\hline & 5.155604 & 4.130855 & -1.686054 \\
\hline & -3.320115 & -4.175423 & 1.767624 \\
\hline & 1.414589 & 3.066838 & 4.376497 \\
\hline & -6.155107 & 2.976411 & -3.464712 \\
\hline & -8.074529 & -3.884698 & -1.721815 \\
\hline & -3.871143 & 1.988838 & -3.632389 \\
\hline & 2.538847 & 3.933557 & -5.094942 \\
\hline & 0.208804 & 3.231593 & -4.810112 \\
\hline & 0.100152 & 7.374502 & 0.545341 \\
\hline & -6.880259 & -1.749006 & -1.740189 \\
\hline & 0.360832 & 5.238893 & -0.624434 \\
\hline & -1.625128 & 2.437190 & -3.332975 \\
\hline & -7.276886 & -5.773178 & -0.303603 \\
\hline L & -9.071760 & 0.667355 & 1.129179 \\
\hline & 4.764077 & 4.431701 & -4.129796 \\
\hline & 0.398664 & 7.513214 & 3.013921 \\
\hline & -9.777010 & 2.482569 & -0.426550 \\
\hline - & -2.023627 & -2.045828 & 1.685797 \\
\hline ] & 3.677098 & 0.700501 & 2.212953 \\
\hline & 3.275397 & -0.320216 & -1.916699 \\
\hline$\frac{1}{1}$ & 7.151021 & 0.533022 & -0.270129 \\
\hline & 3.193370 & -2.177063 & 1.301219 \\
\hline 1 & -0.453224 & -0.299611 & 2.529880 \\
\hline & 1.036737 & -3.696343 & 4.681790 \\
\hline 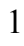 & -2.037287 & 3.972288 & -0.369762 \\
\hline 1 & -2.587665 & -3.070008 & 5.541141 \\
\hline & -2.309653 & -3.267091 & 3.801131 \\
\hline 1 & -1.215324 & -4.034038 & 4.967915 \\
\hline & -1.103259 & -1.579037 & 6.925090 \\
\hline 1 & 0.298681 & -2.550254 & 6.451292 \\
\hline 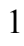 & 0.314519 & -0.795812 & 6.195984 \\
\hline 1 & -1.280634 & 0.278474 & 4.538451 \\
\hline 1 & -2.384338 & -0.715960 & 3.565867 \\
\hline & -2.614577 & -0.594207 & 5.313409 \\
\hline 1 & 3.841168 & -5.861231 & 4.346179 \\
\hline | & 3.044699 & -4.573939 & 5.262117 \\
\hline 1 & 2.092036 & -5.639775 & 4.202021 \\
\hline l & 4.927128 & -2.829371 & 2.503579 \\
\hline 1 & 4.675321 & -2.905943 & 4.257195 \\
\hline 1 & 5.452044 & -4.259641 & 3.408958 \\
\hline 1 & 2.355253 & -5.494736 & 1.679808 \\
\hline 1 & 3.532366 & -4.388713 & 0.955753 \\
\hline 1 & 4.095979 & -5.776082 & 1.905619 \\
\hline 1 & 4.846766 & 2.007418 & 3.512083 \\
\hline 1 & 5.161681 & 0.292972 & 3.870305 \\
\hline 1 & 6.314927 & 1.537466 & 4.380977 \\
\hline 1 & 8.159195 & -0.044162 & 1.600148 \\
\hline 1 & 8.172322 & 0.392108 & 3.314214 \\
\hline 1 & 7.109134 & -0.907118 & 2.738102 \\
\hline 1 & 7.646691 & 2.423835 & 1.027036 \\
\hline 1 & 6.243869 & 3.219751 & 1.763693 \\
\hline
\end{tabular}




$\begin{array}{rrrr}1 & 7.662344 & 2.815275 & 2.755553 \\ 1 & 4.426124 & -1.693347 & -3.264119 \\ 1 & 5.982646 & -1.820690 & -4.088352 \\ 1 & 5.814971 & -2.383815 & -2.411864 \\ 1 & 7.873773 & -0.916326 & -1.924907 \\ 1 & 7.837241 & -0.376415 & -3.610561 \\ 1 & 7.830493 & 0.820448 & -2.311737 \\ 1 & 4.338174 & 0.922782 & -3.646919 \\ 1 & 5.808247 & 1.845670 & -3.321216 \\ 1 & 5.794250 & 0.638564 & -4.626428 \\ 1 & -3.342622 & 3.042737 & -1.102298 \\ 1 & -5.179851 & 2.933411 & 0.296407 \\ 1 & -1.272213 & 2.620364 & 2.035264 \\ 1 & -6.200364 & 2.721505 & 2.534931 \\ 1 & -2.280355 & 2.465446 & 4.287466 \\ 1 & -4.756521 & 2.489310 & 4.549706 \\ 1 & -1.212580 & -2.207686 & -3.310176 \\ 1 & -0.927952 & -0.436287 & -3.731934 \\ 1 & 1.537647 & -1.543488 & -1.815058 \\ 1 & 2.814180 & -3.044271 & -2.077792 \\ 1 & 3.633811 & -5.304621 & -2.612659 \\ 1 & 2.618172 & -6.599695 & -4.478354 \\ 1 & 0.775852 & -5.590813 & -5.808625 \\ 1 & -0.022377 & -3.307267 & -5.288783 \\ 6 & 1.704109 & -0.567561 & -4.178976 \\ 1 & 2.765485 & -0.645426 & -3.921312 \\ 1 & 1.632994 & -0.743851 & -5.263645 \\ 1 & 1.365435 & 0.452859 & -3.963096\end{array}$

$\operatorname{TS1}_{I L-s i-} \Psi-1 \mathbf{a 2 s 3 3 4 s}$

Number of imaginary frequencies : 1

The smallest frequencies are : -629.5536 $9.6011 \quad 12.2708 \mathrm{~cm}(-1)$

Electronic energy : $\quad H F=-4360.8602403$

Zero-point correction $=\quad 1.451456($ Hartree/Particle $)$

Thermal correction to Energy $=\quad 1.536724$

Thermal correction to Enthalpy= $\quad 1.537669$

Thermal correction to Gibbs Free Energy $=\quad 1.330210$

Sum of electronic and zero-point Energies $=\quad-4359.408784$

Sum of electronic and thermal Energies $=\quad-4359.323516$

Sum of electronic and thermal Enthalpies $=\quad-4359.322572$

Sum of electronic and thermal Free Energies $=\quad-4359.530031$

Cartesian Coordinates

$\begin{array}{cccc}6 & -0.581618 & 3.988672 & -4.465529 \\ 6 & -1.220907 & 3.192997 & -3.508829 \\ 6 & -2.262625 & 3.760857 & -2.767462 \\ 6 & -2.650299 & 5.079649 & -2.973021 \\ 6 & -2.002640 & 5.860340 & -3.926450 \\ 6 & -0.965770 & 5.310244 & -4.671750 \\ 6 & -0.816918 & 1.747729 & -3.406223 \\ 6 & -1.711385 & 0.806957 & -4.180182 \\ 45 & -0.125256 & 0.975710 & -1.239959 \\ 6 & 0.216580 & 2.766963 & -0.348525 \\ 8 & 0.021218 & 3.767165 & 0.198794\end{array}$




$\begin{array}{lrrr}15 & -1.506947 & -0.050685 & 0.386156 \\ 6 & -3.336708 & -0.190421 & 0.115194 \\ 6 & -3.915384 & 0.105402 & -1.114458 \\ 6 & -5.299732 & -0.001164 & -1.310604 \\ 6 & -6.081138 & -0.439268 & -0.240580 \\ 6 & -5.531131 & -0.757091 & 1.006009 \\ 6 & -4.154917 & -0.615533 & 1.164383 \\ 6 & -5.901028 & 0.342238 & -2.672981 \\ 6 & -7.426495 & 0.263257 & -2.678606 \\ 6 & -6.363445 & -1.264984 & 2.182063 \\ 6 & -6.206743 & -0.311621 & 3.372115 \\ 6 & -1.439787 & 1.083323 & 1.834514 \\ 6 & -2.391970 & 2.077278 & 2.003820 \\ 6 & -2.275440 & 3.027879 & 3.027599 \\ 6 & -1.194810 & 2.918875 & 3.897523 \\ 6 & -0.220073 & 1.915405 & 3.764880 \\ 6 & -0.336488 & 1.031070 & 2.697705 \\ 6 & 0.890288 & 1.796728 & 4.809369 \\ 6 & 0.253393 & 1.617043 & 6.193357 \\ 6 & -3.341813 & 4.114369 & 3.143329 \\ 6 & -4.713560 & 3.459040 & 3.345301 \\ 6 & -1.239757 & -1.710881 & 1.207636 \\ 6 & -0.973258 & -2.839573 & 0.448555 \\ 6 & -0.845618 & -4.124356 & 1.075084 \\ 6 & -1.001988 & -4.238086 & 2.486301 \\ 6 & -1.287104 & -3.072749 & 3.232683 \\ 6 & -1.401321 & -1.856884 & 2.614656 \\ 6 & -0.857931 & -5.500455 & 3.109349 \\ 6 & -0.567259 & -6.618661 & 2.370707 \\ 6 & -0.409340 & -6.514730 & 0.972894 \\ 6 & -0.546921 & -5.303066 & 0.341549 \\ 6 & -0.814810 & -2.802471 & -1.029603 \\ 6 & 0.409344 & -2.494155 & -1.590930 \\ 6 & 0.608360 & -2.610889 & -2.986653 \\ 6 & -0.412293 & -3.005987 & -3.810874 \\ 6 & -1.693708 & -3.288231 & -3.280389 \\ 6 & -1.901691 & -3.183322 & -1.872750 \\ 6 & -3.194351 & -3.459886 & -1.356440 \\ 6 & -4.218562 & -3.834268 & -2.189430 \\ 6 & -4.004562 & -3.957179 & -3.580152 \\ 6 & -2.770544 & -3.682548 & -4.112194 \\ 7 & 1.484421 & -2.020228 & -0.764822 \\ 15 & 1.719322 & -0.345674 & -0.935314 \\ 8 & 2.692972 & -0.131718 & 0.393173 \\ 6 & 3.441361 & 1.022522 & 0.433137 \\ 6 & 4.629239 & 1.087501 & -0.269697 \\ 6 & 5.359389 & 2.321005 & -0.255218 \\ 6 & 4.883266 & 3.406007 & 0.541912 \\ 6 & 3.681859 & 3.258217 & 1.274972 \\ 6 & 2.962872 & 2.095589 & 1.210303 \\ 6 & 6.521582 & 2.535093 & -1.039952 \\ 6 & 7.191165 & 3.733224 & -1.006635 \\ 6 & 6.738425 & 4.788815 & -0.188124 \\ 6 & 5.603872 & 4.624988 & 0.563885 \\ 6 & 5.055749 & -0.078639 & -1.074447 \\ 6 & 4.179226 & -0.618919 & -1.999504 \\ & & & \\ 6\end{array}$




\begin{tabular}{|c|c|c|c|}
\hline & 4.578201 & -1.635361 & -2.895906 \\
\hline & 5.830021 & -2.179225 & -2.805671 \\
\hline & 6.724834 & -1.756446 & -1.791585 \\
\hline & 6.334607 & -0.703426 & -0.911238 \\
\hline & 7.979255 & -2.388061 & -1.611163 \\
\hline & 8.812512 & -2.021685 & -0.584841 \\
\hline & 8.416501 & -1.003433 & 0.307280 \\
\hline & 7.213921 & -0.359868 & 0.146791 \\
\hline & 2.899282 & -0.141848 & -2.126460 \\
\hline & 2.505396 & -3.029318 & -0.404998 \\
\hline & 3.125926 & -2.867676 & 0.957291 \\
\hline & 4.510882 & -2.908231 & 1.104024 \\
\hline & 5.095638 & -2.801936 & 2.363831 \\
\hline & 4.297099 & -2.663076 & 3.493393 \\
\hline & 2.910432 & -2.628203 & 3.353916 \\
\hline & 2.331264 & -2.723259 & 2.095635 \\
\hline y & 1.792021 & 0.589668 & 4.556722 \\
\hline & 1.751471 & 3.062875 & 4.812392 \\
\hline D & -3.355104 & 4.945269 & 1.854571 \\
\hline & -3.088678 & 5.058684 & 4.315256 \\
\hline & 0.549443 & 1.439043 & -3.295967 \\
\hline & -5.375193 & -0.649456 & -3.716535 \\
\hline & -5.510261 & 1.771695 & -3.064817 \\
\hline & -5.868616 & -2.663027 & 2.572772 \\
\hline & -7.849571 & -1.364752 & 1.847692 \\
\hline & 6.913197 & 0.409274 & 0.853720 \\
\hline & -1.620397 & -0.983388 & 3.222635 \\
\hline & 8.260162 & -3.184907 & -2.298772 \\
\hline & -0.979680 & -5.561500 & 4.189959 \\
\hline & 5.225402 & 5.435545 & 1.185417 \\
\hline & -3.369371 & -3.360835 & -0.285732 \\
\hline ] & -5.205814 & -4.029118 & -1.774896 \\
\hline & 3.320150 & 4.097322 & 1.868451 \\
\hline & -1.415515 & -3.155414 & 4.311419 \\
\hline & 6.145306 & -2.962498 & -3.493119 \\
\hline & 8.076329 & 3.871151 & -1.623732 \\
\hline 1 & 3.865113 & -1.965267 & -3.648805 \\
\hline & -2.595062 & -3.764373 & -5.184124 \\
\hline 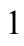 & -0.254794 & -3.099905 & -4.884541 \\
\hline & -0.177392 & -7.402977 & 0.389676 \\
\hline & 6.878696 & 1.737949 & -1.686617 \\
\hline l & -0.423958 & -5.242281 & -0.737786 \\
\hline & 1.591973 & -2.372416 & -3.389019 \\
\hline 1 & 7.280801 & 5.731234 & -0.167469 \\
\hline & 9.066502 & -0.729453 & 1.135335 \\
\hline 1 & -4.824728 & -4.259386 & -4.228008 \\
\hline 1 & -0.455487 & -7.584576 & 2.857756 \\
\hline ] & 9.769429 & -2.520346 & -0.449399 \\
\hline 1 & 2.018834 & 1.973150 & 1.738182 \\
\hline 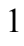 & -3.707002 & -0.846675 & 2.129035 \\
\hline 1 & -3.263455 & 0.412776 & -1.931638 \\
\hline ] & -7.153878 & -0.535239 & -0.378029 \\
\hline 1 & -3.236771 & 2.126618 & 1.316639 \\
\hline 1 & 0.417751 & 0.260086 & 2.539034 \\
\hline 1 & -1.098692 & 3.627676 & 4.717361 \\
\hline & 1.980026 & -3.994901 & -0.438189 \\
\hline
\end{tabular}




$\begin{array}{rrrr}1 & 2.529015 & 2.992932 & 5.585106 \\ 1 & 2.252945 & 3.202554 & 3.845894 \\ 1 & 1.158525 & 3.962713 & 5.016646 \\ 1 & 1.033309 & 1.500197 & 6.957876 \\ 1 & -0.365465 & 2.474852 & 6.482168 \\ 1 & -0.381974 & 0.721639 & 6.219158 \\ 1 & 1.217197 & -0.347283 & 4.558041 \\ 1 & 2.331245 & 0.652220 & 3.602198 \\ 1 & 2.546956 & 0.516723 & 5.350336 \\ 1 & -3.878490 & 5.819650 & 4.353302 \\ 1 & -3.095484 & 4.530252 & 5.277506 \\ 1 & -2.130321 & 5.584615 & 4.217155 \\ 1 & -4.976168 & 2.783549 & 2.520579 \\ 1 & -4.734333 & 2.874983 & 4.275113 \\ 1 & -5.497352 & 4.225976 & 3.410135 \\ 1 & -2.383717 & 5.428611 & 1.690473 \\ 1 & -3.573065 & 4.333471 & 0.970263 \\ 1 & -4.121290 & 5.730338 & 1.915141 \\ 1 & -8.275598 & -0.390902 & 1.574060 \\ 1 & -8.039966 & -2.067048 & 1.025771 \\ 1 & -8.400443 & -1.729146 & 2.724036 \\ 1 & -6.588440 & 0.688072 & 3.125256 \\ 1 & -6.772314 & -0.684225 & 4.236916 \\ 1 & -5.159964 & -0.200404 & 3.681853 \\ 1 & -5.975451 & -3.363075 & 1.733078 \\ 1 & -4.812333 & -2.661915 & 2.870562 \\ 1 & -6.452630 & -3.055072 & 3.416485 \\ 1 & -4.427119 & 1.898948 & -3.170588 \\ 1 & -5.965922 & 2.040837 & -4.027057 \\ 1 & -5.855807 & 2.494327 & -2.313136 \\ 1 & -7.877498 & 0.964053 & -1.963924 \\ 1 & -7.801864 & 0.523453 & -3.676426 \\ 1 & -7.789744 & -0.746401 & -2.448143 \\ 1 & -4.279146 & -0.701950 & -3.730363 \\ 1 & -5.743430 & -1.661451 & -3.502818 \\ 1 & -5.717070 & -0.367688 & -4.722082 \\ 1 & 3.296874 & -3.079447 & -1.168836 \\ 1 & 5.144388 & -3.007828 & 0.221184 \\ 1 & 1.250736 & -2.659500 & 1.983827 \\ 1 & 6.179884 & -2.821958 & 2.455618 \\ 1 & 2.273132 & -2.524650 & 4.231373 \\ 1 & 4.750506 & -2.580397 & 4.479025 \\ 1 & 1.285924 & 2.237456 & -3.222575 \\ 1 & 0.906988 & 0.506294 & -3.730937 \\ 1 & -1.516906 & 1.604154 & -1.779942 \\ 1 & -2.759444 & 3.167712 & -1.997552 \\ 1 & -3.455432 & 5.501498 & -2.374739 \\ 1 & -2.302316 & 6.894208 & -4.083314 \\ 1 & -0.450920 & 5.910474 & -5.419027 \\ 1 & 0.222607 & 3.557060 & -5.060273 \\ 1 & -1.615439 & 1.028227 & -5.254367 \\ 1 & -1.438846 & -0.241298 & -4.009133 \\ 1 & -2.768783 & 0.939177 & -3.929019\end{array}$

TS1 $_{I L-s i-} \Psi-1$ s2a3a4a 
Number of imaginary frequencies : 1

The smallest frequencies are : -654.3044 $9.7838 \quad 15.8018 \mathrm{~cm}(-1)$

Electronic energy : $\quad H F=-4360.8588707$

Zero-point correction $=\quad 1.451680($ Hartree/Particle $)$

Thermal correction to Energy= $\quad 1.536713$

Thermal correction to Enthalpy= $\quad 1.537657$

Thermal correction to Gibbs Free Energy= $\quad 1.332554$

Sum of electronic and zero-point Energies $=\quad-4359.407190$

Sum of electronic and thermal Energies $=\quad-4359.322158$

Sum of electronic and thermal Enthalpies $=\quad-4359.321213$

Sum of electronic and thermal Free Energies $=\quad-4359.526317$

Cartesian Coordinates

$\begin{array}{cccc}0 & 4.052948 & 1.110278 & 0.846329 \\ 6 & 3.184715 & 0.501720 & -0.064941 \\ 6 & 3.652362 & 0.131152 & -1.321520 \\ 6 & 4.993502 & 0.336591 & -1.676112 \\ 6 & 5.829836 & 0.937944 & -0.734872 \\ 6 & 5.384743 & 1.349019 & 0.526650 \\ 15 & 1.455055 & 0.129565 & 0.479445 \\ 6 & 1.102527 & 1.735738 & 1.358831 \\ 6 & 0.763537 & 2.863726 & 0.622816 \\ 6 & 0.587535 & 4.125563 & 1.275524 \\ 6 & 0.766041 & 4.220310 & 2.686365 \\ 6 & 1.123331 & 3.057831 & 3.405304 \\ 6 & 1.285836 & 1.858813 & 2.762694 \\ 6 & 0.583155 & 5.466908 & 3.332078 \\ 6 & 0.235568 & 6.583737 & 2.616693 \\ 6 & 0.058662 & 6.497538 & 1.219108 \\ 6 & 0.232691 & 5.303121 & 0.565516 \\ 6 & 0.617248 & 2.818975 & -0.857819 \\ 6 & 1.705539 & 3.219255 & -1.693506 \\ 6 & 1.558466 & 3.162006 & -3.111365 \\ 6 & 0.318151 & 2.763659 & -3.660321 \\ 6 & -0.716983 & 2.398451 & -2.842610 \\ 6 & -0.568565 & 2.401448 & -1.435179 \\ 6 & 2.654695 & 3.506338 & -3.939801 \\ 6 & 3.851852 & 3.894492 & -3.394317 \\ 6 & 3.999644 & 3.959093 & -1.991336 \\ 6 & 2.953291 & 3.638972 & -1.162994 \\ 7 & -1.647736 & 1.923466 & -0.619127 \\ 6 & -2.638687 & 2.940295 & -0.195496 \\ 6 & -3.266799 & 2.696302 & 1.150397 \\ 6 & -2.474289 & 2.478678 & 2.278116 \\ 6 & -3.055200 & 2.233419 & 3.514663 \\ 6 & -4.443336 & 2.205849 & 3.642583 \\ 6 & -5.240200 & 2.431385 & 2.525244 \\ 6 & -4.652849 & 2.678755 & 1.286826 \\ 6 & 5.571113 & -0.130212 & -3.012154 \\ 6 & 6.488320 & 0.938542 & -3.614783 \\ 6 & 6.345190 & 2.045276 & 1.488869 \\ 6 & 7.519600 & 1.114676 & 1.807978 \\ 45 & -0.043433 & -1.119782 & -0.928731 \\ 6 & -0.254363 & -2.752107 & 0.240134 \\ 8 & -0.220161 & -3.884161 & 0.479869\end{array}$




\begin{tabular}{|c|c|c|c|}
\hline 6 & 0.471124 & -1.999108 & -3.005794 \\
\hline 6 & 0.736969 & -0.906984 & -4.017334 \\
\hline & 1.343532 & -3.220484 & -3.188577 \\
\hline & 2.516185 & -3.506288 & -2.484342 \\
\hline & 3.269755 & -4.642112 & -2.773549 \\
\hline & 2.866636 & -5.516360 & -3.775910 \\
\hline & 1.701348 & -5.246753 & -4.488116 \\
\hline & 0.952644 & -4.113384 & -4.196185 \\
\hline & -0.869271 & -2.228534 & -2.594830 \\
\hline & -1.899118 & 0.252103 & -0.803186 \\
\hline & -2.953538 & 0.073067 & 0.466913 \\
\hline y & -3.724225 & -1.062122 & 0.488197 \\
\hline & -4.870355 & -1.120565 & -0.281332 \\
\hline & -5.626031 & -2.337471 & -0.274866 \\
\hline & -5.224229 & -3.406283 & 0.583688 \\
\hline & -4.068436 & -3.260091 & 1.388668 \\
\hline & -3.319885 & -2.116469 & 1.328942 \\
\hline & -6.744108 & -2.549215 & -1.121348 \\
\hline & -7.443321 & -3.730449 & -1.093171 \\
\hline & -7.065869 & -4.769172 & -0.216724 \\
\hline & -5.975338 & -4.606808 & 0.598546 \\
\hline & -5.230003 & 0.048305 & -1.115452 \\
\hline & -4.293419 & 0.573376 & -1.990469 \\
\hline 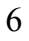 & -4.615588 & 1.617358 & -2.885791 \\
\hline & -5.855234 & 2.194342 & -2.852469 \\
\hline & -6.814474 & 1.779619 & -1.896757 \\
\hline & -6.498737 & 0.706252 & -1.010237 \\
\hline & -8.062465 & 2.438480 & -1.780383 \\
\hline & -8.960242 & 2.082366 & -0.806756 \\
\hline f & -8.636044 & 1.049360 & 0.097475 \\
\hline 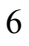 & -7.442348 & 0.377772 & -0.003357 \\
\hline 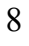 & -3.022268 & 0.060693 & -2.058461 \\
\hline f & 1.796509 & -1.059342 & 1.846468 \\
\hline & 2.908209 & -1.898932 & 1.743358 \\
\hline b & 3.096220 & -2.956892 & 2.627934 \\
\hline & 2.162896 & -3.124720 & 3.659160 \\
\hline 6 & 1.052355 & -2.292645 & 3.805724 \\
\hline f & 0.864377 & -1.279518 & 2.857883 \\
\hline 6 & 4.264809 & -3.930285 & 2.484320 \\
\hline 6 & 3.709086 & -5.320653 & 2.156602 \\
\hline & 0.065677 & -2.427985 & 4.965031 \\
\hline 6 & 0.163939 & -1.173300 & 5.841592 \\
\hline & 5.065540 & -3.988800 & 3.789330 \\
\hline 5 & 5.221797 & -3.528106 & 1.362436 \\
\hline ) & -1.364681 & -2.555244 & 4.431704 \\
\hline 6 & 0.356995 & -3.645839 & 5.838403 \\
\hline 6 & 6.377540 & -1.410427 & -2.763690 \\
\hline 6 & 4.481004 & -0.434039 & -4.036784 \\
\hline 6 & 6.872077 & 3.329577 & 0.838662 \\
\hline f & 5.667706 & 2.429704 & 2.802881 \\
\hline 1 & -7.199662 & -0.403664 & 0.712410 \\
\hline & 1.584736 & 0.988760 & 3.341223 \\
\hline 1 & -8.285668 & 3.247742 & -2.474652 \\
\hline 1 & 0.722371 & 5.516690 & 4.411116 \\
\hline 1 & -5.656260 & -5.405597 & 1.266930 \\
\hline & 3.080702 & 3.683883 & -0.082367 \\
\hline
\end{tabular}




\begin{tabular}{|c|c|c|c|}
\hline & 4.954796 & 4.262581 & -1.566102 \\
\hline & -3.770519 & -4.081995 & 2.038593 \\
\hline ] & 1.276664 & 3.129472 & 4.481501 \\
\hline 1 & -6.111478 & 2.997461 & -3.541587 \\
\hline & -8.293016 & -3.868207 & -1.758274 \\
\hline & -3.857181 & 1.943042 & -3.594510 \\
\hline & 2.523457 & 3.457023 & -5.020056 \\
\hline & 0.205521 & 2.736757 & -4.743459 \\
\hline & -0.215793 & 7.386241 & 0.655390 \\
\hline & -7.041426 & -1.763305 & -1.810942 \\
\hline & 0.099575 & 5.252137 & -0.513218 \\
\hline & -1.666526 & 2.068443 & -3.259501 \\
\hline & -7.631092 & -5.698168 & -0.200299 \\
\hline I & -9.336085 & 0.784893 & 0.886971 \\
\hline & 4.687506 & 4.159759 & -4.038738 \\
\hline 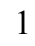 & 0.094698 & 7.536567 & 3.121603 \\
\hline & -9.912051 & 2.601221 & -0.720007 \\
\hline I & -2.409614 & -1.987709 & 1.908700 \\
\hline & 3.674091 & 1.399073 & 1.822871 \\
\hline & 2.950210 & -0.322084 & -2.020079 \\
\hline & 6.876139 & 1.098968 & -0.992456 \\
\hline & 3.624660 & -1.727466 & 0.944698 \\
\hline$\frac{1}{1}$ & -0.027541 & -0.650721 & 2.906437 \\
\hline & 2.310570 & -3.938868 & 4.365169 \\
\hline ] & -2.084653 & 3.889587 & -0.165235 \\
\hline & -3.426611 & 3.061211 & -0.955667 \\
\hline & -5.282931 & 2.831241 & 0.408736 \\
\hline 1 & -1.392332 & 2.461827 & 2.169573 \\
\hline & -6.325027 & 2.404323 & 2.609134 \\
\hline$\frac{1}{1}$ & -2.418643 & 2.059559 & 4.381453 \\
\hline & -4.900620 & 2.006045 & 4.609471 \\
\hline ] & 1.355571 & -1.683358 & -1.460610 \\
\hline$\frac{1}{1}$ & -1.168114 & -3.239078 & -2.311629 \\
\hline & -1.661964 & -1.624721 & -3.035119 \\
\hline 1 & 2.839635 & -2.838880 & -1.684098 \\
\hline & 4.173444 & -4.845500 & -2.201565 \\
\hline 1 & 3.452752 & -6.405481 & -3.997545 \\
\hline & 1.369555 & -5.925312 & -5.271318 \\
\hline | & 0.038759 & -3.908035 & -4.754086 \\
\hline 1 & 1.710426 & -0.425475 & -3.870817 \\
\hline | & 0.736073 & -1.331435 & -5.034757 \\
\hline 1 & -0.031097 & -0.128067 & -3.958409 \\
\hline & -0.379961 & -3.701424 & 6.649407 \\
\hline 1 & 0.293172 & -4.581961 & 5.268719 \\
\hline | & 1.349558 & -3.592152 & 6.303653 \\
\hline 1 & -2.065899 & -2.724846 & 5.259723 \\
\hline 1 & -1.693666 & -1.645471 & 3.914715 \\
\hline 1 & -1.451317 & -3.400331 & 3.734641 \\
\hline 1 & -0.532154 & -1.241096 & 6.688774 \\
\hline 1 & 1.178213 & -1.053647 & 6.244884 \\
\hline 1 & -0.084674 & -0.263388 & 5.279537 \\
\hline 1 & 6.053198 & -4.242796 & 1.315646 \\
\hline 1 & 4.730126 & -3.534134 & 0.380079 \\
\hline 1 & 5.651769 & -2.529641 & 1.520858 \\
\hline 1 & 5.907695 & -4.686244 & 3.687018 \\
\hline 1 & 5.473459 & -3.002219 & 4.045592 \\
\hline
\end{tabular}




$\begin{array}{lrrr}1 & 4.458897 & -4.330745 & 4.636189 \\ 1 & 4.527896 & -6.044029 & 2.040809 \\ 1 & 3.042808 & -5.692881 & 2.944195 \\ 1 & 3.137310 & -5.298652 & 1.218749 \\ 1 & 4.940916 & -0.707919 & -4.994777 \\ 1 & 3.848090 & -1.278821 & -3.734354 \\ 1 & 3.840640 & 0.442720 & -4.209316 \\ 1 & 6.849734 & 0.610092 & -4.598028 \\ 1 & 5.948448 & 1.885329 & -3.750095 \\ 1 & 7.372834 & 1.137626 & -2.998730 \\ 1 & 6.807147 & -1.785676 & -3.702618 \\ 1 & 7.200695 & -1.238869 & -2.058188 \\ 1 & 5.737560 & -2.199665 & -2.345530 \\ 1 & 7.586316 & 3.830278 & 1.506201 \\ 1 & 7.385386 & 3.135283 & -0.111179 \\ 1 & 6.050687 & 4.031992 & 0.641098 \\ 1 & 8.218167 & 1.603963 & 2.500019 \\ 1 & 7.168707 & 0.188187 & 2.280928 \\ 1 & 8.083738 & 0.838560 & 0.908940 \\ 1 & 6.388613 & 2.941098 & 3.453170 \\ 1 & 4.822721 & 3.114757 & 2.644697 \\ 1 & 5.297781 & 1.551477 & 3.347788\end{array}$

$\operatorname{TS1}_{I L-s i-}-\psi_{-1 s 2 a 3 a 4 s}$

Number of imaginary frequencies : 1

The smallest frequencies are : $-649.8387 \quad 15.6805 \quad 20.2153 \mathrm{~cm}(-1)$

Electronic energy : $\quad \mathrm{HF}=-4360.859892$

Zero-point correction $=\quad 1.452727($ Hartree/Particle $)$

Thermal correction to Energy= $\quad 1.537296$

Thermal correction to Enthalpy= $\quad 1.538240$

Thermal correction to Gibbs Free Energy $=\quad 1.336440$

Sum of electronic and zero-point Energies $=\quad-4359.407165$

Sum of electronic and thermal Energies $=\quad-4359.322596$

Sum of electronic and thermal Enthalpies $=\quad-4359.321652$

Sum of electronic and thermal Free Energies $=\quad-4359.523452$

Cartesian Coordinates

$\begin{array}{cccc}6 & -2.538239 & 3.409535 & -2.641732 \\ 6 & -1.330531 & 3.151709 & -3.295582 \\ 6 & -0.916557 & 4.055021 & -4.284323 \\ 6 & -1.676108 & 5.173666 & -4.604728 \\ 6 & -2.875293 & 5.416589 & -3.941181 \\ 6 & -3.302397 & 4.530328 & -2.959296 \\ 6 & -0.448075 & 1.943544 & -3.080339 \\ 6 & 0.883274 & 2.193273 & -2.652884 \\ 45 & 0.049181 & 1.106923 & -0.975911 \\ 15 & 1.910391 & -0.254078 & -0.815283 \\ 8 & 3.052097 & -0.078579 & -2.057522 \\ 6 & 4.327035 & -0.577079 & -1.955680 \\ 6 & 5.242105 & -0.022905 & -1.076002 \\ 6 & 6.513443 & -0.666664 & -0.928344 \\ 6 & 6.856163 & -1.760013 & -1.779068 \\ 6 & 5.920336 & -2.205477 & -2.744391 \\ 6 & 4.677084 & -1.639486 & -2.818436\end{array}$




\begin{tabular}{|c|c|c|c|}
\hline 6 & 7.431861 & -0.303247 & 0.089662 \\
\hline & 8.627257 & -0.963129 & 0.235809 \\
\hline & 8.978094 & -2.018322 & -0.632108 \\
\hline & 8.105482 & -2.406683 & -1.616495 \\
\hline & 4.855759 & 1.160498 & -0.275409 \\
\hline & 3.698845 & 1.103779 & 0.477369 \\
\hline & 3.267374 & 2.172052 & 1.286461 \\
\hline & 3.999459 & 3.327106 & 1.330132 \\
\hline & 5.166555 & 3.469801 & 0.541025 \\
\hline & 5.596593 & 2.386236 & -0.284735 \\
\hline & 5.902300 & 4.679902 & 0.539846 \\
\hline & 7.005283 & 4.836357 & -0.259661 \\
\hline & 7.411645 & 3.781587 & -1.103490 \\
\hline & 6.727487 & 2.591315 & -1.115687 \\
\hline & 2.944017 & -0.042678 & 0.467222 \\
\hline & -1.445795 & -0.132413 & 0.440514 \\
\hline 6 & -1.789307 & 1.046539 & 1.816039 \\
\hline 6 & -2.925126 & 1.856721 & 1.751182 \\
\hline & -3.138339 & 2.873033 & 2.677724 \\
\hline 6 & -2.196743 & 3.037430 & 3.701381 \\
\hline & -1.055036 & 2.241463 & 3.802858 \\
\hline & -0.849441 & 1.264110 & 2.821342 \\
\hline & -4.354365 & 3.793476 & 2.599555 \\
\hline 6 & -5.155220 & 3.705272 & 3.903002 \\
\hline & -0.058530 & 2.372221 & 4.954235 \\
\hline & 1.361934 & 2.546495 & 4.408369 \\
\hline & -1.105879 & -1.737057 & 1.330547 \\
\hline 6 & -0.757007 & -2.866683 & 0.603191 \\
\hline o & -0.587449 & -4.127056 & 1.260957 \\
\hline 6 & -0.780050 & -4.217862 & 2.670011 \\
\hline 0 & -1.144763 & -3.053046 & 3.381417 \\
\hline 6 & -1.303301 & -1.856464 & 2.733205 \\
\hline 6 & -0.225259 & -5.306567 & 0.557964 \\
\hline b & -0.058174 & -6.499151 & 1.216772 \\
\hline 6 & -0.249205 & -6.581376 & 2.612658 \\
\hline b & -0.603663 & -5.462363 & 3.321310 \\
\hline 6 & -0.589384 & -2.829145 & -0.874584 \\
\hline 5 & -1.665153 & -3.235982 & -1.722318 \\
\hline b & -1.488754 & -3.214355 & -3.137710 \\
\hline 6 & -0.235599 & -2.834158 & -3.670259 \\
\hline 6 & 0.783107 & -2.449551 & -2.840422 \\
\hline 6 & 0.607578 & -2.422773 & -1.436487 \\
\hline 6 & -2.929105 & -3.623590 & -1.205947 \\
\hline 6 & -3.962245 & -3.952880 & -2.046999 \\
\hline 6 & -3.782867 & -3.933089 & -3.447908 \\
\hline 5 & -2.570660 & -3.572624 & -3.979102 \\
\hline 7 & 1.668906 & -1.924690 & -0.608791 \\
\hline 6 & 2.660447 & -2.925329 & -0.150391 \\
\hline 6 & 3.258104 & -2.651533 & 1.203629 \\
\hline 6 & 2.439525 & -2.413411 & 2.308448 \\
\hline 6 & 2.991257 & -2.137336 & 3.551713 \\
\hline 6 & 4.376060 & -2.097804 & 3.709501 \\
\hline 6 & 5.198787 & -2.344463 & 2.615494 \\
\hline 6 & 4.640630 & -2.623499 & 1.370256 \\
\hline 6 & -3.175801 & -0.497452 & -0.106242 \\
\hline 6 & -3.631570 & -0.183625 & -1.386871 \\
\hline
\end{tabular}




\begin{tabular}{|c|c|c|c|}
\hline 6 & -4.973693 & -0.370245 & -1.731337 \\
\hline & -5.838610 & -0.884718 & -0.756755 \\
\hline & -5.409167 & -1.228640 & 0.524456 \\
\hline & -4.063224 & -1.024721 & 0.827770 \\
\hline & -5.540129 & 0.023117 & -3.095181 \\
\hline & -6.396646 & 1.282532 & -2.917469 \\
\hline & -6.336463 & -1.776567 & 1.606686 \\
\hline & -5.769291 & -3.089497 & 2.159447 \\
\hline 6 & 0.219277 & 2.749819 & 0.176342 \\
\hline & 0.147831 & 3.873041 & 0.446926 \\
\hline & -0.689580 & 0.834136 & -4.078660 \\
\hline y & -7.742239 & -2.056020 & 1.082001 \\
\hline & -6.439074 & -0.745271 & 2.737385 \\
\hline & -4.443043 & 0.324331 & -4.112208 \\
\hline & -6.405103 & -1.105300 & -3.666105 \\
\hline & -5.288218 & 3.423674 & 1.447722 \\
\hline & -3.876452 & 5.233341 & 2.382807 \\
\hline & -0.368231 & 3.561448 & 5.860214 \\
\hline & -0.117398 & 1.094579 & 5.800692 \\
\hline & 7.167702 & 0.496002 & 0.777516 \\
\hline & -1.604054 & -0.984478 & 3.307660 \\
\hline & 8.350118 & -3.232671 & -2.283299 \\
\hline & -0.753877 & -5.508968 & 4.399011 \\
\hline & 5.561717 & 5.490290 & 1.183198 \\
\hline & -3.080457 & -3.631991 & -0.127578 \\
\hline & -4.931636 & -4.226275 & -1.634224 \\
\hline & 3.679349 & 4.160087 & 1.954973 \\
\hline 1 & -1.306298 & -3.120521 & 4.456737 \\
\hline & 6.196805 & -3.023554 & -3.407612 \\
\hline & 8.271991 & 3.913935 & -1.755878 \\
\hline & 3.936837 & -1.990273 & -3.534315 \\
\hline 1 & -2.416974 & -3.554464 & -5.057356 \\
\hline & -0.100304 & -2.833927 & -4.751125 \\
\hline & 0.221644 & -7.389507 & 0.658311 \\
\hline 1 & 7.047258 & 1.792403 & -1.779915 \\
\hline & -0.080618 & -5.259117 & -0.519410 \\
\hline 1 & 1.740491 & -2.127897 & -3.245626 \\
\hline & 7.558813 & 5.772503 & -0.255617 \\
\hline ] & 9.307732 & -0.672247 & 1.033069 \\
\hline & -4.607652 & -4.208119 & -4.102334 \\
\hline . & -0.113814 & -7.532838 & 3.121631 \\
\hline & 9.930735 & -2.528354 & -0.509526 \\
\hline & 2.350285 & 2.046022 & 1.855567 \\
\hline ] & -3.703172 & -1.262800 & 1.827495 \\
\hline & -2.916237 & 0.212762 & -2.106273 \\
\hline 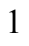 & -6.886974 & -1.017885 & -1.014000 \\
\hline & -3.644519 & 1.692674 & 0.953965 \\
\hline & 0.056526 & 0.653042 & 2.843718 \\
\hline & -2.365720 & 3.818311 & 4.439542 \\
\hline 1 & 2.114937 & -3.879482 & -0.115975 \\
\hline 1 & 3.464731 & -3.050128 & -0.892545 \\
\hline & 5.291057 & -2.792720 & 0.510245 \\
\hline & 1.360197 & -2.404689 & 2.175676 \\
\hline & 6.281321 & -2.308789 & 2.722633 \\
\hline & 2.334229 & -1.949748 & 4.400272 \\
\hline & 4.810731 & -1.873240 & 4.681356 \\
\hline
\end{tabular}




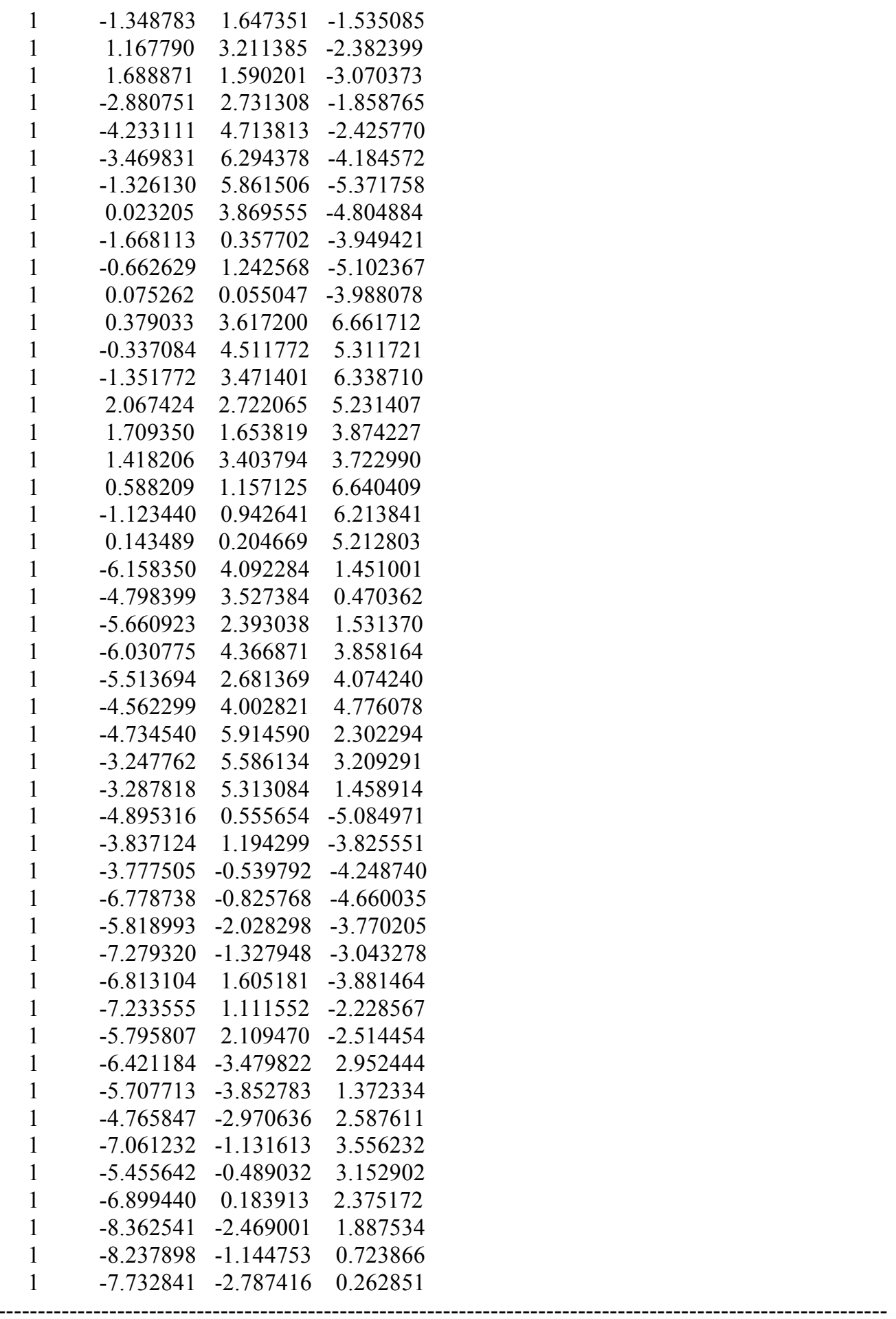

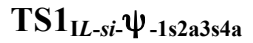

Number of imaginary frequencies : 1

The smallest frequencies are : -629.4129 $10.2022 \quad 18.4142 \mathrm{~cm}(-1)$

Electronic energy : $\quad H F=-4360.8581808$

Zero-point correction $=\quad 1.452294($ Hartree/Particle $)$

Thermal correction to Energy $=\quad 1.537208$

Thermal correction to Enthalpy= $\quad 1.538152$ 
Thermal correction to Gibbs Free Energy= Sum of electronic and zero-point Energies= Sum of electronic and thermal Energies= Sum of electronic and thermal Enthalpies= Sum of electronic and thermal Free Energies=

\author{
1.333564 \\ $-4359.405887$ \\ $-4359.320973$ \\ $-4359.320029$ \\ $-4359.524617$
}

Cartesian Coordinates

\begin{tabular}{|c|c|c|c|}
\hline 6 & 6.690701 & 2.361323 & -1.112680 \\
\hline 6 & 5.527825 & 2.211572 & -0.314504 \\
\hline 6 & 5.097802 & 3.333554 & 0.457727 \\
\hline 6 & 5.863235 & 4.524939 & 0.441602 \\
\hline 6 & 6.996789 & 4.627124 & -0.323092 \\
\hline 6 & 7.404217 & 3.534369 & -1.116603 \\
\hline 6 & 4.755906 & 1.004864 & -0.288808 \\
\hline 6 & 3.571456 & 1.003227 & 0.423022 \\
\hline 6 & 3.138607 & 2.111526 & 1.175733 \\
\hline 6 & 3.899727 & 3.248123 & 1.206786 \\
\hline 6 & 5.138535 & -0.209322 & -1.043370 \\
\hline 6 & 4.237147 & -0.764632 & -1.935949 \\
\hline 6 & 4.590280 & -1.850970 & -2.767133 \\
\hline 6 & 5.819254 & -2.439596 & -2.646232 \\
\hline 6 & 6.736406 & -1.992113 & -1.663950 \\
\hline 6 & 6.392510 & -0.873844 & -0.846975 \\
\hline 6 & 7.967176 & -2.659681 & -1.452000 \\
\hline 6 & 8.819914 & -2.266659 & -0.452105 \\
\hline 6 & 8.467250 & -1.184927 & 0.381782 \\
\hline 6 & 7.289950 & -0.505061 & 0.187566 \\
\hline 8 & 2.977620 & -0.241051 & -2.086842 \\
\hline 15 & 1.797434 & -0.371731 & -0.881444 \\
\hline 8 & 2.785760 & -0.123092 & 0.427660 \\
\hline 45 & -0.017230 & 0.983975 & -1.192799 \\
\hline 6 & 0.731611 & 1.538539 & -3.193483 \\
\hline 6 & -0.623189 & 1.889986 & -3.335923 \\
\hline 6 & -1.508095 & 1.017827 & -4.195198 \\
\hline 6 & -0.999254 & 3.345396 & -3.341263 \\
\hline 6 & -0.215110 & 4.251440 & -4.060771 \\
\hline 6 & -0.567683 & 5.596113 & -4.142471 \\
\hline 6 & -1.715980 & 6.057692 & -3.510495 \\
\hline 6 & -2.510294 & 5.164863 & -2.794852 \\
\hline 6 & -2.154397 & 3.825007 & -2.712425 \\
\hline 6 & 0.321445 & 2.752457 & -0.253818 \\
\hline 8 & 0.169086 & 3.784912 & 0.243704 \\
\hline 15 & -1.491692 & -0.051106 & 0.356512 \\
\hline 6 & -1.250079 & -1.677052 & 1.252658 \\
\hline 6 & -0.952878 & -2.830928 & 0.542528 \\
\hline 6 & -0.846899 & -4.092966 & 1.216399 \\
\hline 6 & -1.059683 & -4.161922 & 2.623320 \\
\hline 6 & -1.384181 & -2.974498 & 3.316890 \\
\hline 6 & -1.477076 & -1.780058 & 2.654237 \\
\hline 6 & -0.941140 & -5.404014 & 3.291119 \\
\hline 6 & -0.621177 & -6.544570 & 2.600272 \\
\hline 6 & -0.407709 & -6.484923 & 1.207016 \\
\hline 6 & -0.520224 & -5.294235 & 0.532723 \\
\hline 6 & -0.754891 & -2.841882 & -0.931557 \\
\hline 6 & 0.477835 & -2.530673 & -1.473205 \\
\hline
\end{tabular}




\begin{tabular}{|c|c|c|c|}
\hline & 0.701578 & -2.660483 & -2.864239 \\
\hline & -0.296656 & -3.082653 & -3.701572 \\
\hline & -1.581016 & -3.384231 & -3.190633 \\
\hline & -1.818017 & -3.258879 & -1.789183 \\
\hline & -3.116863 & -3.549328 & -1.295934 \\
\hline & -4.116298 & -3.959417 & -2.142406 \\
\hline & -3.869187 & -4.111569 & -3.524610 \\
\hline & -2.630398 & -3.820741 & -4.036128 \\
\hline & 1.532142 & -2.031515 & -0.636509 \\
\hline & 2.492408 & -3.037852 & -0.127972 \\
\hline & 3.063613 & -2.737895 & 1.232287 \\
\hline & 2.224164 & -2.467730 & 2.314358 \\
\hline & 2.752576 & -2.173727 & 3.563910 \\
\hline & 4.133951 & -2.145726 & 3.751229 \\
\hline & 4.976754 & -2.425604 & 2.681020 \\
\hline & 4.442258 & -2.724192 & 1.429868 \\
\hline & -1.590123 & 1.152273 & 1.749639 \\
\hline & -2.580849 & 2.136983 & 1.730917 \\
\hline & -2.627038 & 3.128657 & 2.706365 \\
\hline & -1.670285 & 3.089830 & 3.730075 \\
\hline & -0.666067 & 2.123034 & 3.779374 \\
\hline & -0.616023 & 1.180070 & 2.744742 \\
\hline & -3.688144 & 4.227302 & 2.697657 \\
\hline & -4.502009 & 4.167877 & 3.994968 \\
\hline & 0.306233 & 2.001334 & 4.953641 \\
\hline 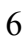 & 1.756127 & 1.996441 & 4.464011 \\
\hline & -3.293348 & -0.266342 & -0.036409 \\
\hline & -4.160244 & -0.712282 & 0.971985 \\
\hline & -5.513557 & -0.902187 & 0.730748 \\
\hline & -5.994266 & -0.618496 & -0.557450 \\
\hline & -5.169980 & -0.165045 & -1.581150 \\
\hline & -3.801558 & -0.000443 & -1.299122 \\
\hline 5 & -6.467494 & -1.434122 & 1.798124 \\
\hline & -7.641163 & -0.467049 & 1.983464 \\
\hline 5 & -5.703644 & 0.164462 & -2.974103 \\
\hline ) & -4.996474 & -0.708293 & -4.015780 \\
\hline 5 & -4.657951 & 4.085737 & 1.525739 \\
\hline 6 & -3.000269 & 5.591723 & 2.583293 \\
\hline & 0.151506 & 3.140159 & 5.958866 \\
\hline 6 & 0.018607 & 0.680194 & 5.679193 \\
\hline 6 & -7.204964 & -0.085322 & -3.101093 \\
\hline 6 & -5.451504 & 1.647306 & -3.270194 \\
\hline 6 & -5.780676 & -1.609101 & 3.151180 \\
\hline & -6.995400 & -2.802144 & 1.350674 \\
\hline 1 & 7.023032 & 0.314617 & 0.849889 \\
\hline & -1.739773 & -0.891424 & 3.221465 \\
\hline 1 & 8.213419 & -3.505121 & -2.093340 \\
\hline & -1.107095 & -5.431284 & 4.367200 \\
\hline 1 & 5.520796 & 5.365134 & 1.044462 \\
\hline & -3.318448 & -3.431695 & -0.232061 \\
\hline 1 & -5.108422 & -4.167760 & -1.746212 \\
\hline 1 & 3.577781 & 4.112129 & 1.786809 \\
\hline 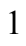 & -1.563923 & -3.020982 & 4.390690 \\
\hline 1 & 6.097996 & -3.276324 & -3.284781 \\
\hline 1 & 8.288899 & 3.623230 & -1.743333 \\
\hline 1 & 3.864399 & -2.200233 & -3.498254 \\
\hline
\end{tabular}




\begin{tabular}{|c|c|c|c|}
\hline . & -2.429808 & -3.921701 & -5.101993 \\
\hline & -0.117296 & -3.187276 & -4.770765 \\
\hline 1 & -0.153460 & -7.391052 & 0.661836 \\
\hline & 7.012309 & 1.533798 & -1.739704 \\
\hline & -0.355721 & -5.267335 & -0.542349 \\
\hline & 1.685616 & -2.406172 & -3.253162 \\
\hline & 7.573387 & 5.549230 & -0.331156 \\
\hline & 9.131423 & -0.889380 & 1.190988 \\
\hline & -4.667644 & -4.448635 & -4.182400 \\
\hline 1 & -0.529599 & -7.494573 & 3.121637 \\
\hline 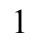 & 9.758032 & -2.792785 & -0.291455 \\
\hline & 2.199598 & 2.031048 & 1.717267 \\
\hline 1 & -3.759560 & -0.911553 & 1.961319 \\
\hline & -3.112886 & 0.331789 & -2.076444 \\
\hline 1 & -7.053748 & -0.764436 & -0.756066 \\
\hline 1 & -3.318816 & 2.121421 & 0.931912 \\
\hline 1 & 0.174682 & 0.426228 & 2.738527 \\
\hline 1 & -1.720009 & 3.842088 & 4.514396 \\
\hline 1 & 1.925482 & -3.978952 & -0.078881 \\
\hline 1 & 3.310975 & -3.200796 & -0.846751 \\
\hline 1 & 5.109558 & -2.922747 & 0.589468 \\
\hline 1 & 1.147649 & -2.450448 & 2.159865 \\
\hline 1 & 6.057028 & -2.402332 & 2.811721 \\
\hline 1 & 2.080395 & -1.962628 & 4.395457 \\
\hline 1 & 4.549820 & -1.906089 & 4.727673 \\
\hline 1 & -1.385060 & 1.642159 & -1.768272 \\
\hline 1 & 1.487204 & 2.309266 & -3.050818 \\
\hline 1 & 1.080840 & 0.623875 & -3.671385 \\
\hline 1 & -2.765579 & 3.140708 & -2.121081 \\
\hline 1 & -3.406762 & 5.515982 & -2.287805 \\
\hline 1 & -1.990666 & 7.108496 & -3.570485 \\
\hline 1 & 0.060530 & 6.283273 & -4.705390 \\
\hline 1 & 0.677610 & 3.894066 & -4.571444 \\
\hline 1 & -2.570574 & 1.154772 & -3.969007 \\
\hline 1 & -1.373049 & 1.300333 & -5.250239 \\
\hline 1 & -1.258380 & -0.043339 & -4.078321 \\
\hline 1 & 0.875214 & 3.012173 & 6.773708 \\
\hline 1 & 0.340795 & 4.119081 & 5.499687 \\
\hline 1 & -0.848442 & 3.159321 & 6.410834 \\
\hline 1 & 2.442632 & 1.906587 & 5.316628 \\
\hline 1 & 1.969327 & 1.158129 & 3.788889 \\
\hline 1 & 1.996414 & 2.929902 & 3.937385 \\
\hline 1 & 0.686935 & 0.562747 & 6.543264 \\
\hline 1 & -1.016902 & 0.649370 & 6.044215 \\
\hline 1 & 0.168318 & -0.185925 & 5.020960 \\
\hline 1 & -5.404066 & 4.889462 & 1.567095 \\
\hline 1 & -4.143591 & 4.163100 & 0.558678 \\
\hline 1 & -5.199517 & 3.130155 & 1.549577 \\
\hline 1 & -5.273711 & 4.949503 & 3.997139 \\
\hline 1 & -5.004129 & 3.196687 & 4.098695 \\
\hline 1 & -3.878070 & 4.318682 & 4.884004 \\
\hline 1 & -3.747314 & 6.397142 & 2.570358 \\
\hline 1 & -2.320726 & 5.781792 & 3.422769 \\
\hline 1 & -2.413165 & 5.656001 & 1.657536 \\
\hline 1 & -7.529856 & 0.153097 & -4.121785 \\
\hline 1 & -7.463839 & -1.135188 & -2.911468 \\
\hline
\end{tabular}




$\begin{array}{rrrr}1 & -7.788388 & 0.543820 & -2.416326 \\ 1 & -5.308453 & -0.421796 & -5.029565 \\ 1 & -3.904193 & -0.626519 & -3.962872 \\ 1 & -5.247929 & -1.766529 & -3.867564 \\ 1 & -5.846479 & 1.913575 & -4.259815 \\ 1 & -5.946033 & 2.284663 & -2.524792 \\ 1 & -4.384708 & 1.899093 & -3.263288 \\ 1 & -6.504495 & -1.984496 & 3.885516 \\ 1 & -4.955487 & -2.332383 & 3.104593 \\ 1 & -5.383089 & -0.659504 & 3.533588 \\ 1 & -8.325857 & -0.844070 & 2.754952 \\ 1 & -7.288160 & 0.523129 & 2.300168 \\ 1 & -8.223726 & -0.336478 & 1.063803 \\ 1 & -7.681976 & -3.214268 & 2.102626 \\ 1 & -7.539494 & -2.741959 & 0.399956 \\ 1 & -6.169221 & -3.514227 & 1.219750\end{array}$

TS1 $_{I L-s i-} \psi_{-1 \mathrm{~s} 2 \mathrm{a3s} 4 \mathrm{~s}}$

Number of imaginary frequencies : 1

The smallest frequencies are : $-631.0610 \quad 13.1040 \quad 17.3420 \mathrm{~cm}(-1)$

Electronic energy : $\quad H F=-4360.8585457$

Zero-point correction $=\quad 1.452226($ Hartree/Particle $)$

Thermal correction to Energy $=\quad 1.537142$

Thermal correction to Enthalpy= $\quad 1.538086$

Thermal correction to Gibbs Free Energy $=\quad 1.333672$

Sum of electronic and zero-point Energies $=\quad-4359.406319$

Sum of electronic and thermal Energies $=\quad-4359.321404$

Sum of electronic and thermal Enthalpies $=\quad-4359.320460$

Sum of electronic and thermal Free Energies $=\quad-4359.524874$

Cartesian Coordinates

$\begin{array}{cccc}6 & -3.791184 & -0.032264 & -1.347755 \\ 6 & -3.288537 & -0.304480 & -0.079907 \\ 6 & -4.160416 & -0.757330 & 0.912391 \\ 6 & -5.517414 & -0.943410 & 0.665325 \\ 6 & -5.991724 & -0.654199 & -0.618436 \\ 6 & -5.153727 & -0.198037 & -1.637363 \\ 15 & -1.496467 & -0.066507 & 0.340159 \\ 6 & -1.634837 & 1.131799 & 1.734737 \\ 6 & -2.654627 & 2.086576 & 1.725275 \\ 6 & -2.723211 & 3.073231 & 2.705089 \\ 6 & -1.765138 & 3.053472 & 3.727868 \\ 6 & -0.735394 & 2.113375 & 3.770553 \\ 6 & -0.660246 & 1.180406 & 2.728815 \\ 6 & -3.809386 & 4.146638 & 2.700948 \\ 6 & -3.151931 & 5.528822 & 2.628400 \\ 6 & 0.239020 & 2.009243 & 4.944553 \\ 6 & -0.025264 & 0.682821 & 5.669327 \\ 6 & -6.411010 & -1.449606 & 1.795794 \\ 6 & -5.892165 & -2.810336 & 2.276385 \\ 6 & -5.680189 & 0.134636 & -3.032944 \\ 6 & -5.440466 & 1.621627 & -3.318734 \\ 45 & -0.012705 & 0.994535 & -1.185782 \\ 6 & 0.312446 & 2.757920 & -0.234738\end{array}$




\begin{tabular}{|c|c|c|c|}
\hline & 0.169297 & 3.803451 & 0.237481 \\
\hline & 1.807457 & -0.355429 & -0.879180 \\
\hline & 1.549184 & -2.016905 & -0.637576 \\
\hline 6 & 2.510760 & -3.018560 & -0.122111 \\
\hline & 3.074858 & -2.714201 & 1.240226 \\
\hline & 2.230319 & -2.439798 & 2.317295 \\
\hline & 2.752754 & -2.141418 & 3.568317 \\
\hline o & 4.133215 & -2.113587 & 3.762344 \\
\hline & 4.981054 & -2.397560 & 2.697224 \\
\hline 6 & 4.452530 & -2.700141 & 1.444489 \\
\hline 8 & 2.989951 & -0.217584 & -2.081867 \\
\hline 6 & 4.249710 & -0.740241 & -1.928789 \\
\hline 6 & 5.148192 & -0.185347 & -1.033031 \\
\hline 6 & 6.401865 & -0.849387 & -0.833312 \\
\hline 6 & 6.748594 & -1.966974 & -1.649976 \\
\hline 6 & 5.834812 & -2.413750 & -2.635702 \\
\hline 6 & 4.605973 & -1.825498 & -2.760017 \\
\hline 6 & 7.295791 & -0.481203 & 0.204496 \\
\hline 6 & 8.472280 & -1.161388 & 0.402467 \\
\hline 6 & 8.827716 & -2.242654 & -0.430864 \\
\hline 6 & 7.978568 & -2.634784 & -1.434164 \\
\hline 6 & 4.762244 & 1.027213 & -0.277646 \\
\hline 6 & 5.532925 & 2.234723 & -0.298796 \\
\hline 6 & 5.100065 & 3.354423 & 0.475163 \\
\hline 6 & 3.900716 & 3.265698 & 1.221802 \\
\hline 6 & 3.141012 & 2.128252 & 1.186520 \\
\hline 6 & 3.576220 & 1.022429 & 0.431484 \\
\hline 6 & 5.864355 & 4.546609 & 0.463547 \\
\hline 6 & 6.999561 & 4.651641 & -0.298303 \\
\hline 6 & 7.409955 & 3.561089 & -1.093329 \\
\hline 6 & 6.697576 & 2.387353 & -1.093816 \\
\hline 8 & 2.792468 & -0.105015 & 0.432245 \\
\hline 6 & 0.747586 & 1.579401 & -3.171702 \\
\hline 6 & -0.610345 & 1.915907 & -3.323102 \\
\hline 6 & -1.004410 & 3.366609 & -3.317071 \\
\hline 6 & -0.222859 & 4.290655 & -4.016133 \\
\hline 6 & -0.590591 & 5.631966 & -4.085728 \\
\hline 6 & -1.751599 & 6.072139 & -3.461805 \\
\hline 6 & -2.543451 & 5.161187 & -2.766539 \\
\hline 6 & -2.172536 & 3.824757 & -2.696114 \\
\hline 6 & -1.475939 & 1.042118 & -4.200057 \\
\hline 6 & -1.244672 & -1.687856 & 1.242357 \\
\hline 6 & -0.932172 & -2.839674 & 0.535702 \\
\hline 6 & -0.817143 & -4.099590 & 1.211250 \\
\hline 6 & -1.033840 & -4.168539 & 2.617519 \\
\hline 6 & -1.370173 & -2.982925 & 3.308418 \\
\hline 6 & -1.474004 & -1.790456 & 2.643411 \\
\hline 6 & -0.906212 & -5.408700 & 3.287309 \\
\hline 6 & -0.574152 & -6.547165 & 2.598795 \\
\hline 6 & -0.357150 & -6.487450 & 1.206021 \\
\hline 6 & -0.478038 & -5.298673 & 0.529889 \\
\hline 6 & -0.730058 & -2.847579 & -0.937458 \\
\hline 6 & 0.499756 & -2.521630 & -1.477101 \\
\hline 6 & 0.724072 & -2.642445 & -2.868943 \\
\hline 6 & -0.270341 & -3.070463 & -3.708002 \\
\hline 6 & -1.551703 & -3.387024 & -3.198504 \\
\hline
\end{tabular}




\begin{tabular}{|c|c|c|c|}
\hline & -1.789238 & -3.270777 & -1.796518 \\
\hline & -3.085176 & -3.573801 & -1.303430 \\
\hline & -4.082116 & -3.985993 & -2.151727 \\
\hline & -3.834247 & -4.130475 & -3.534635 \\
\hline & -2.597724 & -3.828646 & -4.045444 \\
\hline & -4.643103 & 4.037900 & 3.982248 \\
\hline & -4.753404 & 4.010061 & 1.507597 \\
\hline & 1.688814 & 2.029660 & 4.455048 \\
\hline & 0.063756 & 3.145149 & 5.949724 \\
\hline & -6.378191 & -0.446755 & 2.955392 \\
\hline & -7.863392 & -1.624372 & 1.359975 \\
\hline & -4.956850 & -0.725100 & -4.074418 \\
\hline & -7.178033 & -0.128944 & -3.174308 \\
\hline & 7.026667 & 0.338106 & 0.866389 \\
\hline & -1.745972 & -0.903274 & 3.208298 \\
\hline & 8.227040 & -3.479770 & -2.075236 \\
\hline & -1.075139 & -5.436171 & 4.362917 \\
\hline & 5.519767 & 5.385038 & 1.067648 \\
\hline & -3.286890 & -3.461736 & -0.238638 \\
\hline & -5.072800 & -4.200890 & -1.755953 \\
\hline & 3.576968 & 4.127707 & 1.803779 \\
\hline & -1.550847 & -3.029332 & 4.382068 \\
\hline & 6.115866 & -3.249844 & -3.274063 \\
\hline & 8.296090 & 3.652250 & -1.717669 \\
\hline & 3.882663 & -2.174566 & -3.493733 \\
\hline & -2.396943 & -3.923364 & -5.111850 \\
\hline & -0.090164 & -3.167772 & -4.777754 \\
\hline & -0.093562 & -7.392077 & 0.662782 \\
\hline & 7.021446 & 1.561487 & -1.721882 \\
\hline & -0.310990 & -5.271417 & -0.544795 \\
\hline & 1.705123 & -2.376167 & -3.257333 \\
\hline & 7.575288 & 5.574314 & -0.302900 \\
\hline & 9.133628 & -0.866459 & 1.214213 \\
\hline & -4.630487 & -4.470375 & -4.193677 \\
\hline & -0.475787 & -7.495702 & 3.121587 \\
\hline & 9.765142 & -2.769076 & -0.267159 \\
\hline & 2.201538 & 2.045136 & 1.726660 \\
\hline & -3.772476 & -0.965628 & 1.907587 \\
\hline & -3.097826 & 0.306526 & -2.118017 \\
\hline & -7.048145 & -0.790560 & -0.828769 \\
\hline & -3.394897 & 2.055063 & 0.928974 \\
\hline & 0.151242 & 0.448993 & 2.715836 \\
\hline & -1.834898 & 3.799514 & 4.516620 \\
\hline & 1.947319 & -3.961794 & -0.073946 \\
\hline & 3.333243 & -3.179686 & -0.836802 \\
\hline & 5.123899 & -2.901507 & 0.607990 \\
\hline & 1.154633 & -2.421689 & 2.157311 \\
\hline & 6.060697 & -2.374132 & 2.833033 \\
\hline & 2.076753 & -1.926744 & 4.395876 \\
\hline & 4.544450 & -1.870750 & 4.739958 \\
\hline & -1.382645 & 1.647082 & -1.761508 \\
\hline & 1.492629 & 2.357971 & -3.015930 \\
\hline & 1.111930 & 0.674276 & -3.656509 \\
\hline & -2.782085 & 3.126442 & -2.119597 \\
\hline 1 & -3.449832 & 5.495425 & -2.265738 \\
\hline & -2.038136 & 7.120273 & -3.512174 \\
\hline
\end{tabular}




$\begin{array}{lrrr}1 & 0.035943 & 6.333377 & -4.632717 \\ 1 & 0.680064 & 3.950339 & -4.520476 \\ 1 & -2.542225 & 1.163781 & -3.983546 \\ 1 & -1.333727 & 1.337369 & -5.250689 \\ 1 & -1.214418 & -0.017084 & -4.091736 \\ 1 & 0.790570 & 3.031104 & 6.763852 \\ 1 & 0.233708 & 4.127334 & 5.489956 \\ 1 & -0.935730 & 3.145363 & 6.403043 \\ 1 & 2.376772 & 1.961626 & 5.308466 \\ 1 & 1.919133 & 1.188889 & 3.788649 \\ 1 & 1.909995 & 2.962937 & 3.919666 \\ 1 & 0.646328 & 0.575561 & 6.532201 \\ 1 & -1.059534 & 0.634223 & 6.035960 \\ 1 & 0.137941 & -0.179742 & 5.009493 \\ 1 & -5.515265 & 4.798828 & 1.549354 \\ 1 & -4.221272 & 4.114547 & 0.552656 \\ 1 & -5.277972 & 3.044852 & 1.501961 \\ 1 & -5.430751 & 4.803470 & 3.992007 \\ 1 & -5.125976 & 3.054037 & 4.052926 \\ 1 & -4.035042 & 4.178181 & 4.884118 \\ 1 & -3.917815 & 6.316137 & 2.608808 \\ 1 & -2.500129 & 5.721560 & 3.488902 \\ 1 & -2.542015 & 5.622469 & 1.720093 \\ 1 & -7.495301 & 0.108522 & -4.197640 \\ 1 & -7.428985 & -1.181459 & -2.988953 \\ 1 & -7.774283 & 0.493422 & -2.494481 \\ 1 & -5.265462 & -0.437013 & -5.088795 \\ 1 & -3.865928 & -0.631812 & -4.013890 \\ 1 & -5.197481 & -1.786837 & -3.933063 \\ 1 & -5.831608 & 1.890292 & -4.309278 \\ 1 & -5.945781 & 2.249729 & -2.572656 \\ 1 & -4.376173 & 1.883488 & -3.303001 \\ 1 & -8.459884 & -1.992464 & 2.204342 \\ 1 & -8.310951 & -0.677821 & 1.030763 \\ 1 & -7.962339 & -2.353581 & 0.545319 \\ 1 & -6.993295 & -0.807259 & 3.791050 \\ 1 & -5.361825 & -0.283505 & 3.335540 \\ 1 & -6.775720 & 0.527125 & 2.640199 \\ 1 & -6.517641 & -3.191483 & 3.094984 \\ 1 & -5.917249 & -3.547039 & 1.462095 \\ 1 & -4.859390 & -2.758047 & 2.644165\end{array}$

TS1 $_{I L-s i-} \psi_{-1 \mathrm{~s} 2 \mathrm{~s} 3 \mathrm{a} 4 \mathrm{a}}$

Number of imaginary frequencies : 1

The smallest frequencies are : $-757.6375 \quad 12.2765 \quad 14.5268 \mathrm{~cm}(-1)$

Electronic energy : $\quad H F=-4360.8632628$

Zero-point correction $=\quad 1.451987($ Hartree/Particle $)$

Thermal correction to Energy= $\quad 1.536772$

Thermal correction to Enthalpy= $\quad 1.537716$

Thermal correction to Gibbs Free Energy= $\quad 1.333113$

Sum of electronic and zero-point Energies $=\quad-4359.411276$

Sum of electronic and thermal Energies $=\quad-4359.326491$

Sum of electronic and thermal Enthalpies $=\quad-4359.325547$

Sum of electronic and thermal Free Energies $=\quad-4359.530150$ 
Cartesian Coordinates

\begin{tabular}{|c|c|c|c|}
\hline 6 & -7.383099 & 0.372473 & -0.066761 \\
\hline 6 & -6.429373 & 0.860701 & -0.996295 \\
\hline 6 & -6.721902 & 2.080221 & -1.677450 \\
\hline 6 & -7.955734 & 2.733617 & -1.441156 \\
\hline 6 & -8.860685 & 2.228511 & -0.542754 \\
\hline 6 & -8.561337 & 1.041314 & 0.158022 \\
\hline 6 & -5.169414 & 0.212348 & -1.209236 \\
\hline 6 & -4.215619 & 0.876945 & -1.962865 \\
\hline 6 & -4.524106 & 2.054448 & -2.679825 \\
\hline 6 & -5.756495 & 2.633772 & -2.553587 \\
\hline 6 & -4.841247 & -1.104859 & -0.617833 \\
\hline 6 & -3.704103 & -1.224814 & 0.156744 \\
\hline 6 & -3.316637 & -2.437669 & 0.756286 \\
\hline 6 & -4.078190 & -3.558397 & 0.568802 \\
\hline 6 & -5.230901 & -3.515694 & -0.252416 \\
\hline 6 & -5.612970 & -2.285330 & -0.870537 \\
\hline 6 & -5.997089 & -4.682436 & -0.491523 \\
\hline 6 & -7.084086 & -4.656104 & -1.326824 \\
\hline 6 & -7.442270 & -3.452201 & -1.968442 \\
\hline 6 & -6.727935 & -2.300771 & -1.747064 \\
\hline 8 & -2.930805 & -0.117254 & 0.391287 \\
\hline 15 & -1.852451 & 0.343113 & -0.783715 \\
\hline 8 & -2.938669 & 0.390683 & -2.086188 \\
\hline 45 & 0.022560 & -0.961419 & -1.192474 \\
\hline 6 & -0.196449 & -2.780991 & -0.459399 \\
\hline 8 & -0.310716 & -3.917837 & -0.271018 \\
\hline 6 & 0.579229 & -1.044457 & -3.483837 \\
\hline 6 & -0.663894 & -1.650591 & -3.120213 \\
\hline 6 & 1.679810 & -1.931351 & -4.003660 \\
\hline 6 & 2.468658 & -1.536407 & -5.089738 \\
\hline 6 & 3.460686 & -2.369808 & -5.604875 \\
\hline 6 & 3.690749 & -3.619261 & -5.046015 \\
\hline 6 & 2.916327 & -4.026798 & -3.960085 \\
\hline 6 & 1.931266 & -3.196188 & -3.446904 \\
\hline 6 & 0.521551 & 0.351617 & -4.056963 \\
\hline 15 & 1.449712 & -0.100404 & 0.524958 \\
\hline 6 & 3.200237 & 0.337063 & 0.122126 \\
\hline 6 & 4.086588 & 0.605320 & 1.173517 \\
\hline 6 & 5.413085 & 0.938191 & 0.928777 \\
\hline 6 & 5.831481 & 1.004287 & -0.408608 \\
\hline 6 & 4.979713 & 0.747333 & -1.479717 \\
\hline 6 & 3.649300 & 0.405531 & -1.188991 \\
\hline 6 & 6.398261 & 1.256029 & 2.052186 \\
\hline 6 & 7.629373 & 0.350847 & 1.940673 \\
\hline 6 & 5.485931 & 0.756621 & -2.921219 \\
\hline 6 & 6.718695 & 1.645311 & -3.090263 \\
\hline 6 & 1.095486 & 1.289443 & 1.713368 \\
\hline 6 & 0.741633 & 2.541800 & 1.233877 \\
\hline 6 & 0.532105 & 3.630500 & 2.144388 \\
\hline 6 & 0.700192 & 3.421164 & 3.543883 \\
\hline 6 & 1.092074 & 2.141706 & 3.997145 \\
\hline 6 & 1.283157 & 1.114930 & 3.112431 \\
\hline 6 & 0.470278 & 4.487415 & 4.445664 \\
\hline 6 & 0.085363 & 5.721835 & 3.989647 \\
\hline
\end{tabular}




\begin{tabular}{|c|c|c|c|}
\hline 6 & -0.081607 & 5.938084 & 2.605676 \\
\hline 6 & 0.138833 & 4.923431 & 1.707361 \\
\hline & 0.594736 & 2.854154 & -0.214721 \\
\hline & 1.666518 & 3.498135 & -0.908276 \\
\hline & 1.462772 & 3.962242 & -2.241904 \\
\hline & 0.195924 & 3.786070 & -2.846028 \\
\hline & -0.807012 & 3.137965 & -2.176028 \\
\hline & -0.609562 & 2.649097 & -0.863047 \\
\hline & 2.526444 & 4.600579 & -2.926205 \\
\hline & 3.749171 & 4.765865 & -2.325988 \\
\hline & 3.957357 & 4.298610 & -1.009857 \\
\hline y & 2.941043 & 3.691074 & -0.315600 \\
\hline & -1.675060 & 1.939656 & -0.215412 \\
\hline & -2.743751 & 2.780990 & 0.372554 \\
\hline & -3.327589 & 2.278980 & 1.667388 \\
\hline & -2.507455 & 1.848688 & 2.711202 \\
\hline y & -3.056770 & 1.412370 & 3.909344 \\
\hline & -4.439884 & 1.397704 & 4.082459 \\
\hline & -5.264205 & 1.822986 & 3.046319 \\
\hline & -4.709315 & 2.261988 & 1.846648 \\
\hline & 1.686249 & -1.552563 & 1.630546 \\
\hline y & 0.718640 & -1.906868 & 2.573421 \\
\hline & 0.848532 & -3.070004 & 3.335238 \\
\hline 5 & 1.937433 & -3.910345 & 3.072785 \\
\hline ) & 2.889006 & -3.611288 & 2.095383 \\
\hline & 2.754529 & -2.411616 & 1.394343 \\
\hline & -0.168605 & -3.362718 & 4.438176 \\
\hline & -1.585385 & -3.382747 & 3.856555 \\
\hline 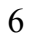 & 4.064147 & -4.525718 & 1.758542 \\
\hline$b$ & 5.378920 & -3.773174 & 1.996739 \\
\hline ) & 3.971063 & -4.935074 & 0.283035 \\
\hline b & 4.078773 & -5.794837 & 2.606106 \\
\hline b & -0.076018 & -2.259009 & 5.499178 \\
\hline & 0.082122 & -4.704492 & 5.122517 \\
\hline 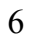 & 6.831120 & 2.722185 & 1.938981 \\
\hline & 5.782573 & 1.046849 & 3.434180 \\
\hline 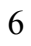 & 5.851616 & -0.683395 & -3.302517 \\
\hline & 4.409362 & 1.267017 & -3.879567 \\
\hline & -7.157164 & -0.531043 & 0.494383 \\
\hline 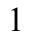 & 1.596740 & 0.147427 & 3.494416 \\
\hline & -8.161850 & 3.658252 & -1.978993 \\
\hline 1 & 0.602699 & 4.302137 & 5.510715 \\
\hline & -5.692285 & -5.607137 & -0.002951 \\
\hline 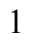 & 3.115195 & 3.326501 & 0.695506 \\
\hline & 4.932621 & 4.423525 & -0.543134 \\
\hline 1 & -3.792836 & -4.503242 & 1.029653 \\
\hline 1 & 1.242666 & 1.982335 & 5.064357 \\
\hline t & -6.001818 & 3.541609 & -3.102368 \\
\hline 1 & -8.289262 & -3.436405 & -2.650891 \\
\hline & -3.760670 & 2.474761 & -3.331138 \\
\hline 1 & 2.351879 & 4.953421 & -3.941861 \\
\hline & 0.038274 & 4.159689 & -3.856965 \\
\hline 1 & -0.386596 & 6.918461 & 2.246877 \\
\hline ] & -7.010589 & -1.385180 & -2.260070 \\
\hline & 0.008282 & 5.109805 & 0.643289 \\
\hline 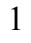 & -1.779970 & 2.983665 & -2.639239 \\
\hline
\end{tabular}




\begin{tabular}{|c|c|c|c|}
\hline . & -7.661130 & -5.560199 & -1.506944 \\
\hline & -9.268428 & 0.653792 & 0.888200 \\
\hline . & 4.561634 & 5.251937 & -2.862087 \\
\hline & -0.092777 & 6.534645 & 4.689841 \\
\hline & -9.800279 & 2.744665 & -0.359902 \\
\hline & -2.411140 & -2.446632 & 1.357635 \\
\hline & 3.725226 & 0.543121 & 2.196530 \\
\hline & 2.943240 & 0.195312 & -1.992258 \\
\hline & 6.866989 & 1.271196 & -0.611567 \\
\hline & 3.486385 & -2.149578 & 0.631253 \\
\hline & -0.144382 & -1.253551 & 2.722008 \\
\hline 1 & 2.042157 & -4.827909 & 3.643533 \\
\hline 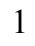 & -2.275311 & 3.759527 & 0.552306 \\
\hline & -3.554362 & 2.955267 & -0.351872 \\
\hline & -5.362647 & 2.577145 & 1.030974 \\
\hline & -1.430514 & 1.823568 & 2.561752 \\
\hline & -6.346208 & 1.805867 & 3.163452 \\
\hline 1 & -2.398372 & 1.079873 & 4.711079 \\
\hline 1 & -4.871688 & 1.050538 & 5.018852 \\
\hline 1 & 1.429102 & -1.179397 & -1.944762 \\
\hline 1 & -0.758829 & -2.734731 & -3.187717 \\
\hline 1 & -1.579444 & -1.108408 & -3.353893 \\
\hline & 1.360161 & -3.524294 & -2.579965 \\
\hline & 3.085851 & -4.999588 & -3.502364 \\
\hline 1 & 4.466685 & -4.269036 & -5.444350 \\
\hline 1 & 4.057561 & -2.028243 & -6.448789 \\
\hline 1 & 2.313549 & -0.565493 & -5.553343 \\
\hline 1 & 1.399716 & 0.958114 & -3.799127 \\
\hline 1 & 0.447884 & 0.318583 & -5.155598 \\
\hline 1 & -0.358859 & 0.878219 & -3.679876 \\
\hline & 4.814803 & 1.329731 & -4.898606 \\
\hline 1 & 3.549949 & 0.590783 & -3.922231 \\
\hline 1 & 4.051599 & 2.264533 & -3.587191 \\
\hline 1 & 7.002851 & 1.682899 & -4.149557 \\
\hline 1 & 6.519247 & 2.673748 & -2.758606 \\
\hline 1 & 7.590819 & 1.269707 & -2.541579 \\
\hline 1 & 6.208291 & -0.729889 & -4.341378 \\
\hline & 6.645623 & -1.072975 & -2.651436 \\
\hline 1 & 4.983845 & -1.351881 & -3.215774 \\
\hline 1 & 7.551174 & 2.971304 & 2.730277 \\
\hline 1 & 7.308747 & 2.937540 & 0.974952 \\
\hline 1 & 5.968463 & 3.393547 & 2.046653 \\
\hline 1 & 8.339197 & 0.575229 & 2.748072 \\
\hline 1 & 7.348993 & -0.707363 & 2.023180 \\
\hline 1 & 8.160355 & 0.483445 & 0.990622 \\
\hline 1 & 6.526851 & 1.274588 & 4.207615 \\
\hline 1 & 4.919340 & 1.704029 & 3.606284 \\
\hline 1 & 5.457826 & 0.008138 & 3.582013 \\
\hline 1 & -0.673994 & -4.866236 & 5.901038 \\
\hline 1 & 0.014159 & -5.542856 & 4.417283 \\
\hline 1 & 1.064795 & -4.742655 & 5.609766 \\
\hline 1 & -2.312546 & -3.657133 & 4.632497 \\
\hline 1 & -1.884893 & -2.401717 & 3.467833 \\
\hline 1 & -1.664708 & -4.115527 & 3.041393 \\
\hline 1 & -0.792292 & -2.445616 & 6.310951 \\
\hline 1 & 0.930023 & -2.218276 & 5.937664 \\
\hline
\end{tabular}


TS1 $_{I L-s i-} \psi_{-1 \mathrm{~s} 2 \mathrm{s3a4s}}$

\begin{tabular}{|c|c|}
\hline \multicolumn{2}{|l|}{ Number of imaginary frequencies : 1} \\
\hline Electronic energy : $\quad H F=-4360.863715$ & \\
\hline Zero-point correction $=$ & 0 (Hartree/Particle) \\
\hline Thermal correction to Energy= & 6877 \\
\hline Thermal correction to Enthalpy $=$ & 37821 \\
\hline Thermal correction to Gibbs Free Energy= & 1.335244 \\
\hline Sum of electronic and zero-point Energies= & -4359.411355 \\
\hline Sum of electronic and thermal Energies= & -4359.326838 \\
\hline Sum of electronic and thermal Enthalpies= & -4359.325894 \\
\hline Sum of electronic and thermal Free Energies= & -4359.528471 \\
\hline
\end{tabular}

Cartesian Coordinates

$\begin{array}{cccc}6 & 2.462194 & -1.777735 & -5.022812 \\ 6 & 1.682089 & -2.117787 & -3.912251 \\ 6 & 1.939159 & -3.352332 & -3.293537 \\ 6 & 2.920176 & -4.208008 & -3.772370 \\ 6 & 3.684177 & -3.856641 & -4.884896 \\ 6 & 3.448952 & -2.637069 & -5.504382 \\ 6 & 0.583995 & -1.206598 & -3.429885 \\ 6 & 0.526883 & 0.162039 & -4.065940 \\ 45 & 0.023872 & -1.009208 & -1.147925 \\ 6 & -0.660155 & -1.794331 & -3.038352 \\ 15 & 1.451989 & -0.057703 & 0.521761 \\ 6 & 1.090650 & 1.379790 & 1.650939 \\ 6 & 0.725835 & 2.607874 & 1.119393 \\ 6 & 0.506341 & 3.732104 & 1.982437 \\ 6 & 0.676435 & 3.584742 & 3.389448 \\ 6 & 1.078834 & 2.329150 & 3.896632 \\ 6 & 1.278711 & 1.266893 & 3.056410 \\ 6 & 0.437134 & 4.686744 & 4.244565 \\ 6 & 0.041049 & 5.896792 & 3.735839 \\ 6 & -0.127776 & 6.051744 & 2.343828 \\ 6 & 0.102047 & 5.001428 & 1.490019 \\ 6 & 0.580742 & 2.855378 & -0.341522 \\ 6 & 1.652042 & 3.471425 & -1.060231 \\ 6 & 1.456059 & 3.856885 & -2.419864 \\ 6 & 0.194461 & 3.640929 & -3.021824 \\ 6 & -0.810395 & 3.026913 & -2.323317 \\ 6 & -0.618606 & 2.610207 & -0.984980 \\ 6 & 2.521719 & 4.459904 & -3.132322 \\ 6 & 3.738403 & 4.667764 & -2.533230\end{array}$




\begin{tabular}{|c|c|c|c|}
\hline 6 & 3.937362 & 4.282388 & -1.189602 \\
\hline 6 & 2.919311 & 3.709962 & -0.468572 \\
\hline & -1.683731 & 1.928451 & -0.307610 \\
\hline & -2.753044 & 2.792253 & 0.244302 \\
\hline & -3.334292 & 2.345261 & 1.560260 \\
\hline & -2.512575 & 1.941775 & 2.613540 \\
\hline & -3.059053 & 1.556545 & 3.830095 \\
\hline & -4.441067 & 1.566827 & 4.012845 \\
\hline & -5.266977 & 1.965852 & 2.967601 \\
\hline 6 & -4.714720 & 2.353800 & 1.749156 \\
\hline & 1.712574 & -1.455256 & 1.691086 \\
\hline & 0.747273 & -1.780647 & 2.646786 \\
\hline U & 0.890708 & -2.906825 & 3.459605 \\
\hline & 1.990233 & -3.744470 & 3.235171 \\
\hline 6 & 2.939593 & -3.476839 & 2.246552 \\
\hline 6 & 2.794013 & -2.308301 & 1.496247 \\
\hline 6 & 4.119704 & -4.396751 & 1.943135 \\
\hline 6 & 4.022134 & -4.866201 & 0.485670 \\
\hline 6 & -0.123824 & -3.163576 & 4.573923 \\
\hline 6 & -0.031937 & -2.022665 & 5.594867 \\
\hline 6 & 3.196311 & 0.375206 & 0.088073 \\
\hline 6 & 4.072683 & 0.731167 & 1.113809 \\
\hline 6 & 5.401742 & 1.058426 & 0.855107 \\
\hline 6 & 5.834779 & 1.014336 & -0.473540 \\
\hline 6 & 4.988144 & 0.659429 & -1.527273 \\
\hline 6 & 3.658679 & 0.340737 & -1.223542 \\
\hline 6 & 6.312326 & 1.434118 & 2.022690 \\
\hline 6 & 5.700986 & 2.607946 & 2.797342 \\
\hline 6 & 5.501832 & 0.548610 & -2.961933 \\
\hline 6 & 5.788154 & -0.930861 & -3.248140 \\
\hline 5 & -1.856102 & 0.307988 & -0.803509 \\
\hline 8 & -2.930755 & -0.106035 & 0.392323 \\
\hline 6 & -3.695194 & -1.229253 & 0.207042 \\
\hline 6 & -4.832316 & -1.152531 & -0.572696 \\
\hline 6 & -5.594310 & -2.348774 & -0.774566 \\
\hline 6 & -5.201388 & -3.548695 & -0.105339 \\
\hline 6 & -4.049676 & -3.546005 & 0.718586 \\
\hline 6 & -3.298887 & -2.411357 & 0.859706 \\
\hline 6 & -6.710329 & -2.409953 & -1.647844 \\
\hline 6 & -7.414641 & -3.575775 & -1.820341 \\
\hline 6 & -7.044548 & -4.749034 & -1.130519 \\
\hline 6 & -5.956933 & -4.731198 & -0.295772 \\
\hline 6 & -5.171303 & 0.136787 & -1.216780 \\
\hline 6 & -4.223771 & 0.777544 & -1.998203 \\
\hline 6 & -4.541196 & 1.926498 & -2.756471 \\
\hline 6 & -5.776663 & 2.503121 & -2.647172 \\
\hline 6 & -6.736378 & 1.976798 & -1.748160 \\
\hline 6 & -6.434135 & 0.785175 & -1.023361 \\
\hline 6 & -7.973011 & 2.631419 & -1.530554 \\
\hline 6 & -8.870995 & 2.154692 & -0.609782 \\
\hline 6 & -8.561777 & 0.996038 & 0.133265 \\
\hline 6 & -7.380601 & 0.326235 & -0.071882 \\
\hline 8 & -2.944142 & 0.293595 & -2.105490 \\
\hline 6 & -0.188277 & -2.790758 & -0.324764 \\
\hline 8 & -0.287681 & -3.919706 & -0.086758 \\
\hline 6 & 4.144753 & -5.630519 & 2.841218 \\
\hline
\end{tabular}




\begin{tabular}{|c|c|c|c|}
\hline 6 & 5.431521 & -3.628801 & 2.145456 \\
\hline 6 & 0.131973 & -4.479826 & 5.304455 \\
\hline 6 & -1.541956 & -3.207780 & 3.997054 \\
\hline 6 & 4.457560 & 1.059863 & -3.956157 \\
\hline 6 & 6.785349 & 1.349677 & -3.180323 \\
\hline 6 & 7.707635 & 1.849525 & 1.563973 \\
\hline 6 & 6.452483 & 0.223725 & 2.953448 \\
\hline 1 & -7.146329 & -0.553931 & 0.521915 \\
\hline 1 & 1.599673 & 0.319028 & 3.479137 \\
\hline 1 & -8.187019 & 3.534433 & -2.101051 \\
\hline 1 & 0.571331 & 4.548634 & 5.316528 \\
\hline 1 & -5.643854 & -5.632215 & 0.230355 \\
\hline 1 & 3.086211 & 3.409546 & 0.564858 \\
\hline 1 & 4.909076 & 4.436998 & -0.724221 \\
\hline 1 & -3.756877 & -4.467753 & 1.219915 \\
\hline 1 & 1.230493 & 2.216529 & 4.969633 \\
\hline 1 & -6.028523 & 3.389481 & -3.227221 \\
\hline 1 & -8.262852 & -3.595644 & -2.501170 \\
\hline 1 & -3.781842 & 2.328987 & -3.423768 \\
\hline 1 & 2.353396 & 4.751489 & -4.168235 \\
\hline 1 & 0.042181 & 3.955808 & -4.053368 \\
\hline 1 & -0.441473 & 7.013006 & 1.943342 \\
\hline 1 & -7.002188 & -1.518102 & -2.196498 \\
\hline 1 & -0.029250 & 5.140455 & 0.418851 \\
\hline 1 & -1.779553 & 2.841918 & -2.783228 \\
\hline 1 & -7.613396 & -5.665000 & -1.272779 \\
\hline 1 & -9.263386 & 0.631895 & 0.880554 \\
\hline 1 & 4.552594 & 5.125980 & -3.090663 \\
\hline 1 & -0.144710 & 6.737285 & 4.400470 \\
\hline 1 & -9.812791 & 2.671927 & -0.441898 \\
\hline 1 & -2.395063 & -2.386549 & 1.462869 \\
\hline 1 & 3.711974 & 0.748924 & 2.141266 \\
\hline 1 & 2.962070 & 0.060553 & -2.013780 \\
\hline 1 & 6.867514 & 1.267926 & -0.694594 \\
\hline 1 & 3.525509 & -2.069629 & 0.725105 \\
\hline 1 & -0.124888 & -1.133135 & 2.763899 \\
\hline 1 & 2.103896 & -4.635984 & 3.844176 \\
\hline 1 & -2.286553 & 3.778709 & 0.381439 \\
\hline 1 & -3.564316 & 2.933466 & -0.486636 \\
\hline 1 & -5.369769 & 2.649597 & 0.927643 \\
\hline 1 & -1.437233 & 1.896495 & 2.457181 \\
\hline 1 & -6.348299 & 1.966880 & 3.091764 \\
\hline 1 & -2.399563 & 1.243731 & 4.638892 \\
\hline 1 & -4.870774 & 1.259037 & 4.963856 \\
\hline 1 & 1.427468 & -1.272583 & -1.892774 \\
\hline 1 & -0.754642 & -2.880550 & -3.052982 \\
\hline 1 & -1.575470 & -1.264886 & -3.300416 \\
\hline 1 & 1.375428 & -3.635848 & -2.406281 \\
\hline 1 & 3.094608 & -5.156012 & -3.267016 \\
\hline 1 & 4.456758 & -4.525865 & -5.256662 \\
\hline 1 & 4.038771 & -2.338536 & -6.369344 \\
\hline 1 & 2.304386 & -0.830714 & -5.532659 \\
\hline 1 & 1.405583 & 0.778896 & -3.835370 \\
\hline 1 & 0.452633 & 0.080153 & -5.161953 \\
\hline 1 & -0.352452 & 0.705930 & -3.711733 \\
\hline 1 & 4.865138 & 1.032999 & -4.976099 \\
\hline
\end{tabular}




$\begin{array}{rrrr}1 & 3.557975 & 0.436409 & -3.958347 \\ 1 & 4.159559 & 2.093188 & -3.728344 \\ 1 & 7.075175 & 1.297820 & -4.237447 \\ 1 & 6.649550 & 2.408886 & -2.921694 \\ 1 & 7.630340 & 0.959605 & -2.599776 \\ 1 & 6.153020 & -1.062997 & -4.276700 \\ 1 & 6.552070 & -1.321491 & -2.562469 \\ 1 & 4.883972 & -1.543896 & -3.132646 \\ 1 & 6.331258 & 2.863238 & 3.659869 \\ 1 & 5.626893 & 3.501184 & 2.162851 \\ 1 & 4.695566 & 2.385572 & 3.177130 \\ 1 & 7.082660 & 0.474783 & 3.817523 \\ 1 & 5.481387 & -0.118054 & 3.334060 \\ 1 & 6.919435 & -0.620378 & 2.428578 \\ 1 & 8.314750 & 2.128031 & 2.434634 \\ 1 & 8.231427 & 1.035035 & 1.047464 \\ 1 & 7.675087 & 2.718123 & 0.892765 \\ 1 & -0.623771 & -4.617596 & 6.087941 \\ 1 & 0.067757 & -5.342246 & 4.628480 \\ 1 & 1.114463 & -4.497326 & 5.793196 \\ 1 & -2.266380 & -3.454589 & 4.784678 \\ 1 & -1.844559 & -2.242322 & 3.573225 \\ 1 & -1.622227 & -3.970604 & 3.210032 \\ 1 & -0.745857 & -2.182256 & 6.414459 \\ 1 & 0.974954 & -1.963550 & 6.029237 \\ 1 & -0.260105 & -1.049664 & 5.140038 \\ 1 & 5.007628 & -6.257271 & 2.582657 \\ 1 & 4.238642 & -5.365144 & 3.902332 \\ 1 & 3.243752 & -6.245083 & 2.717956 \\ 1 & 6.290210 & -4.271603 & 1.908579 \\ 1 & 5.494176 & -2.740225 & 1.503468 \\ 1 & 5.534533 & -3.297013 & 3.187299 \\ 1 & 4.849096 & -5.549465 & 0.248373 \\ 1 & 3.078127 & -5.398787 & 0.308373 \\ 1 & 4.068819 & -4.029673 & -0.222886\end{array}$

TS1 $_{I L-s i-}-\psi_{-1 \mathrm{~s} 23334 a}$

Number of imaginary frequencies : 1

The smallest frequencies are : $-643.5683 \quad 14.8789 \quad 18.8561 \mathrm{~cm}(-1)$

Electronic energy : $\quad H F=-4360.8589436$

Zero-point correction $=\quad 1.452516($ Hartree/Particle $)$

Thermal correction to Energy $=\quad 1.537390$

Thermal correction to Enthalpy= $\quad 1.538334$

Thermal correction to Gibbs Free Energy $=\quad 1.334021$

Sum of electronic and zero-point Energies $=\quad-4359.406428$

Sum of electronic and thermal Energies $=\quad-4359.321554$

Sum of electronic and thermal Enthalpies $=\quad-4359.320610$

Sum of electronic and thermal Free Energies $=\quad-4359.524923$

Cartesian Coordinates

$\begin{array}{llll}6 & -0.100781 & 4.311745 & -3.987350 \\ 6 & -0.933828 & 3.406733 & -3.323932 \\ 6 & -2.100036 & 3.898311 & -2.725790 \\ 6 & -2.419422 & 5.248422 & -2.781922\end{array}$




\begin{tabular}{|c|c|c|c|}
\hline 6 & -1.576835 & 6.140083 & -3.441732 \\
\hline 6 & -0.417326 & 5.666739 & -4.043688 \\
\hline 6 & -0.597095 & 1.942350 & -3.335429 \\
\hline 6 & -1.504313 & 1.099794 & -4.200873 \\
\hline 45 & -0.011568 & 1.005652 & -1.191277 \\
\hline 6 & 0.321004 & 2.773597 & -0.244406 \\
\hline 8 & 0.145855 & 3.783744 & 0.290216 \\
\hline 15 & -1.488548 & -0.029496 & 0.349375 \\
\hline 6 & -1.566458 & 1.137532 & 1.774698 \\
\hline 6 & -2.559494 & 2.111017 & 1.811540 \\
\hline 6 & -2.606963 & 3.062332 & 2.833131 \\
\hline 6 & -1.643293 & 2.991048 & 3.841123 \\
\hline 6 & -0.625094 & 2.029611 & 3.832832 \\
\hline 6 & -0.577187 & 1.134531 & 2.761685 \\
\hline 6 & -3.719080 & 4.108787 & 2.814030 \\
\hline 6 & 0.355600 & 1.864213 & 4.995494 \\
\hline 6 & -3.294312 & -0.217519 & -0.043329 \\
\hline 6 & -4.165431 & -0.664839 & 0.961166 \\
\hline 6 & -5.518881 & -0.849459 & 0.715998 \\
\hline 6 & -5.995688 & -0.558009 & -0.571891 \\
\hline 6 & -5.168187 & -0.100864 & -1.591339 \\
\hline 6 & -3.799524 & 0.057574 & -1.305707 \\
\hline 6 & -5.700118 & 0.238445 & -2.982961 \\
\hline 6 & -6.476176 & -1.387641 & 1.777262 \\
\hline 6 & -1.265225 & -1.675197 & 1.216376 \\
\hline 6 & -0.979050 & -2.820682 & 0.488827 \\
\hline 6 & -0.888895 & -4.094929 & 1.142311 \\
\hline 6 & -1.105800 & -4.184250 & 2.547372 \\
\hline 6 & -1.417805 & -3.004460 & 3.259328 \\
\hline 6 & -1.495980 & -1.798671 & 2.615756 \\
\hline 6 & -1.003501 & -5.43 & 3.195362 \\
\hline 6 & -0.695377 & -6.571225 & 2.486942 \\
\hline 6 & -0.477476 & -6.491487 & 1.095414 \\
\hline 6 & -0.574080 & -5.288768 & 0.440220 \\
\hline 6 & -0.774281 & -2.811128 & -0.984019 \\
\hline 6 & 0.464685 & -2.504933 & -1.513727 \\
\hline 6 & 0.697478 & -2.623361 & -2.904219 \\
\hline 6 & -0.298547 & -3.028934 & -3.752550 \\
\hline 6 & -1.589465 & -3.323288 & -3.253764 \\
\hline 6 & -1.835404 & -3.208222 & -1.853056 \\
\hline 6 & -3.140651 & -3.489224 & -1.371335 \\
\hline 6 & -4.137622 & -3.883994 & -2.227837 \\
\hline 6 & -3.881559 & -4.028054 & -3.609360 \\
\hline 6 & -2.636897 & -3.743367 & -4.110090 \\
\hline 7 & 1.516924 & -2.024893 & -0.662902 \\
\hline 6 & 2.471034 & -3.045983 & -0.172532 \\
\hline 6 & 3.047801 & -2.774104 & 1.191127 \\
\hline 6 & 2.213558 & -2.530825 & 2.283666 \\
\hline 6 & 2.748951 & -2.269513 & 3.537657 \\
\hline 6 & 4.131185 & -2.246864 & 3.718832 \\
\hline 6 & 4.968637 & -2.499468 & 2.637838 \\
\hline 6 & 4.427465 & -2.766124 & 1.382430 \\
\hline 6 & 0.746629 & 1.551331 & -3.192569 \\
\hline 15 & 1.791217 & -0.362838 & -0.881033 \\
\hline 8 & 2.977380 & -0.222630 & -2.078952 \\
\hline 6 & 4.235828 & -0.748871 & -1.929629 \\
\hline
\end{tabular}




\begin{tabular}{|c|c|c|c|}
\hline & 5.133863 & -0.205278 & -1.026510 \\
\hline & 6.388272 & -0.870625 & -0.836149 \\
\hline & 6.735942 & -1.976971 & -1.667671 \\
\hline & 5.821614 & -2.412602 & -2.657920 \\
\hline & 4.592256 & -1.823803 & -2.774128 \\
\hline & 7.283043 & -0.514288 & 0.205010 \\
\hline & 8.461702 & -1.193893 & 0.391645 \\
\hline & 8.818107 & -2.263407 & -0.456280 \\
\hline & 7.967746 & -2.644824 & -1.462657 \\
\hline & 4.748132 & 0.999978 & -0.259328 \\
\hline & 3.559813 & 0.992230 & 0.446125 \\
\hline & 3.121344 & 2.095681 & 1.202753 \\
\hline & 3.882730 & 3.231655 & 1.248277 \\
\hline & 5.086309 & 3.322361 & 0.508889 \\
\hline & 5.520360 & 2.206891 & -0.270389 \\
\hline & 5.852622 & 4.513347 & 0.508157 \\
\hline & 6.990652 & 4.621719 & -0.248939 \\
\hline & 7.401602 & 3.536131 & -1.050394 \\
\hline & 6.687439 & 2.363545 & -1.061130 \\
\hline & 2.773373 & -0.134429 & 0.436614 \\
\hline & 7.013215 & 0.295676 & 0.877997 \\
\hline & -1.749424 & -0.916913 & 3.197632 \\
\hline & 8.216822 & -3.480957 & -2.115009 \\
\hline & -1.172518 & -5.480805 & 4.270475 \\
\hline & 5.507112 & 5.348200 & 1.116658 \\
\hline & -3.348864 & -3.376285 & -0.308228 \\
\hline & -5.134660 & -4.084360 & -1.839791 \\
\hline & 3.556406 & 4.091514 & 1.831986 \\
\hline & -1.599817 & -3.065847 & 4.332029 \\
\hline & 6.102976 & -3.240327 & -3.306961 \\
\hline & 8.289650 & 3.630208 & -1.671580 \\
\hline & 3.868610 & -2.163847 & -3.511818 \\
\hline 1 & -2.429987 & -3.837028 & -5.175417 \\
\hline & -0.112392 & -3.125739 & -4.821330 \\
\hline & -0.232328 & -7.391562 & 0.536218 \\
\hline & 7.012016 & 1.541856 & -1.694198 \\
\hline & -0.406060 & -5.246634 & -0.633827 \\
\hline 1 & 1.686713 & -2.374727 & -3.283703 \\
\hline & 7.567962 & 5.543404 & -0.245207 \\
\hline ] & 9.123978 & -0.907872 & 1.205804 \\
\hline & -4.678206 & -4.352781 & -4.275513 \\
\hline 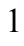 & -0.616470 & -7.530569 & 2.993038 \\
\hline 1 & 9.757203 & -2.789508 & -0.301383 \\
\hline & 2.177585 & 2.012683 & 1.735590 \\
\hline 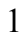 & -3.767915 & -0.870193 & 1.950456 \\
\hline & -3.108429 & 0.390684 & -2.080691 \\
\hline 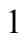 & -7.054947 & -0.700911 & -0.773741 \\
\hline & -3.312390 & 2.128825 & 1.023577 \\
\hline & 0.216606 & 0.384971 & 2.716280 \\
\hline 1 & -1.687484 & 3.701689 & 4.660763 \\
\hline 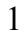 & 1.897071 & -3.983431 & -0.137433 \\
\hline 1 & 3.286931 & -3.203870 & -0.895411 \\
\hline ] & 5.090122 & -2.944873 & 0.534060 \\
\hline 1 & 1.135840 & -2.511212 & 2.135898 \\
\hline & 6.049515 & -2.481103 & 2.764016 \\
\hline . & 2.082032 & -2.081532 & 4.378710 \\
\hline
\end{tabular}




\begin{tabular}{|c|c|c|c|}
\hline 1 & 4.551707 & -2.033299 & 4.699318 \\
\hline & -1.367332 & 1.682311 & -1.783003 \\
\hline & 1.523899 & 2.298337 & -3.042069 \\
\hline & 1.070299 & 0.630306 & -3.676172 \\
\hline & -2.748300 & 3.213485 & -2.176316 \\
\hline & -3.324332 & 5.608157 & -2.296433 \\
\hline & -1.822745 & 7.198949 & -3.481267 \\
\hline & 0.248375 & 6.352823 & -4.563156 \\
\hline & 0.801492 & 3.946937 & -4.475089 \\
\hline & -2.562987 & 1.259572 & -3.971560 \\
\hline & -1.363838 & 1.387435 & -5.253626 \\
\hline & -1.278954 & 0.032203 & -4.092982 \\
\hline & -3.644132 & 5.063729 & 4.002324 \\
\hline & -3.611346 & 4.936107 & 1.527779 \\
\hline & -5.079998 & 3.402248 & 2.853383 \\
\hline & 0.215069 & 2.968274 & 6.041056 \\
\hline ) & 0.063352 & 0.520384 & 5.676419 \\
\hline & 1.802809 & 1.867605 & 4.498270 \\
\hline 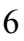 & -7.200254 & -0.015949 & -3.115278 \\
\hline & -5.453366 & 1.724572 & -3.265802 \\
\hline & -4.988464 & -0.621161 & -4.032243 \\
\hline 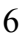 & -5.794387 & -1.564778 & 3.132428 \\
\hline ) & -7.654775 & -0.426395 & 1.961587 \\
\hline ) & -6.997130 & -2.755876 & 1.321999 \\
\hline ] & 0.948940 & 2.811308 & 6.841663 \\
\hline 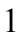 & 0.399815 & 3.962290 & 5.613543 \\
\hline 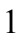 & -0.778479 & 2.973355 & 6.507114 \\
\hline & 2.492482 & 1.736920 & 5.343022 \\
\hline & 2.006560 & 1.056732 & 3.787822 \\
\hline 1 & 2.048827 & 2.819888 & 4.009556 \\
\hline & 0.737976 & 0.365801 & 6.529802 \\
\hline 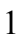 & -0.969399 & 0.485001 & 6.049046 \\
\hline t & 0.198756 & -0.321638 & 4.984799 \\
\hline 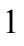 & -4.459792 & 5.795201 & 3.939176 \\
\hline ] & -3.749128 & 4.537767 & 4.960201 \\
\hline 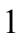 & -2.700610 & 5.624130 & 4.018380 \\
\hline 1 & -5.894214 & 4.139389 & 2.833837 \\
\hline & -5.220672 & 2.729101 & 1.997844 \\
\hline 1 & -5.185749 & 2.805294 & 3.769154 \\
\hline 1 & -4.399985 & 5.700459 & 1.497939 \\
\hline ] & -2.640575 & 5.444784 & 1.465843 \\
\hline 1 & -3.716455 & 4.313967 & 0.630673 \\
\hline ] & -7.523614 & 0.228985 & -4.134917 \\
\hline 1 & -7.455382 & -1.068249 & -2.934093 \\
\hline ] & -7.787888 & 0.605767 & -2.427363 \\
\hline 1 & -5.299861 & -0.324577 & -5.043290 \\
\hline 1 & -3.896609 & -0.536362 & -3.977339 \\
\hline 1 & -5.237045 & -1.681539 & -3.896256 \\
\hline 1 & -5.840508 & 1.996054 & -4.257096 \\
\hline 1 & -5.958325 & 2.353721 & -2.520726 \\
\hline 1 & -4.387772 & 1.980920 & -3.247028 \\
\hline 1 & -6.520865 & -1.940047 & 3.864177 \\
\hline 1 & -4.969463 & -2.288476 & 3.088115 \\
\hline 1 & -5.397171 & -0.615441 & 3.516291 \\
\hline 1 & -7.686564 & -3.172814 & 2.068685 \\
\hline 1 & -7.536123 & -2.693852 & 0.368481 \\
\hline
\end{tabular}




$\begin{array}{llll}1 & -6.168200 & -3.465292 & 1.193619 \\ 1 & -8.343197 & -0.812549 & 2.725194 \\ 1 & -7.308416 & 0.562611 & 2.289071 \\ 1 & -8.231724 & -0.291049 & 1.039093\end{array}$

TS1 $_{I L-s i-} \psi_{-1 \mathrm{~s} 2 \mathrm{~s} 3 \mathrm{~s} 4 \mathrm{~s}}$

Number of imaginary frequencies : 1

The smallest frequencies are : $-650.9132 \quad 16.5913 \quad 18.0323 \mathrm{~cm}(-1)$

Electronic energy : $\quad H F=-4360.8590835$

Zero-point correction $=\quad 1.452949($ Hartree/Particle $)$

Thermal correction to Energy $=\quad 1.537608$

Thermal correction to Enthalpy= $\quad 1.538553$

Thermal correction to Gibbs Free Energy= $\quad 1.335181$

Sum of electronic and zero-point Energies $=\quad-4359.406134$

Sum of electronic and thermal Energies $=\quad-4359.321475$

Sum of electronic and thermal Enthalpies $=\quad-4359.320531$

Sum of electronic and thermal Free Energies $=\quad-4359.523903$

\section{Cartesian Coordinates}

$\begin{array}{cccc}6 & -0.089838 & 4.390877 & -3.884410 \\ 6 & -0.919800 & 3.462028 & -3.250751 \\ 6 & -2.099286 & 3.926803 & -2.656962 \\ 6 & -2.434772 & 5.273770 & -2.689306 \\ 6 & -1.595023 & 6.189309 & -3.319464 \\ 6 & -0.422286 & 5.742876 & -3.916269 \\ 6 & -0.564254 & 2.002437 & -3.289151 \\ 6 & -1.447532 & 1.168464 & -4.186977 \\ 45 & 0.001601 & 1.023472 & -1.162901 \\ 6 & 0.337005 & 2.769202 & -0.181367 \\ 8 & 0.189960 & 3.794329 & 0.332438 \\ 15 & -1.500721 & -0.038746 & 0.341976 \\ 6 & -1.628600 & 1.131710 & 1.761362 \\ 6 & -2.648433 & 2.077457 & 1.793323 \\ 6 & -2.712649 & 3.041186 & 2.803185 \\ 6 & -1.746257 & 3.002308 & 3.810189 \\ 6 & -0.709384 & 2.060874 & 3.813234 \\ 6 & -0.640989 & 1.158895 & 2.749848 \\ 6 & -3.847413 & 4.062818 & 2.774766 \\ 6 & 0.274137 & 1.928500 & 4.977493 \\ 6 & -3.293387 & -0.253190 & -0.092373 \\ 6 & -4.173194 & -0.735847 & 0.879313 \\ 6 & -5.525421 & -0.929783 & 0.611002 \\ 6 & -5.988495 & -0.608266 & -0.669048 \\ 6 & -5.143938 & -0.117170 & -1.665848 \\ 6 & -3.785465 & 0.049348 & -1.357999 \\ 6 & -5.658308 & 0.241616 & -3.059561 \\ 6 & -6.424073 & -1.498712 & 1.707434 \\ 6 & -1.268381 & -1.676270 & 1.221789 \\ 6 & -0.968083 & -2.822929 & 0.501739 \\ 6 & -0.869290 & -4.092565 & 1.161576 \\ 6 & -1.088102 & -4.176331 & 2.566661 \\ 6 & -1.409857 & -2.995191 & 3.271956 \\ 6 & -1.499501 & -1.793598 & 2.621439 \\ 6 & -0.977064 & -5.426375 & 3.220962\end{array}$




\begin{tabular}{|c|c|c|c|}
\hline 6 & -0.659439 & -6.560345 & 2.518412 \\
\hline 6 & -0.440501 & -6.485998 & 1.126627 \\
\hline 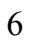 & -0.545124 & -5.287327 & 0.465447 \\
\hline 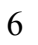 & -0.763810 & -2.814652 & -0.970883 \\
\hline f & 0.470368 & -2.496037 & -1.504445 \\
\hline 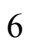 & 0.694803 & -2.600319 & -2.897660 \\
\hline 6 & -0.303549 & -3.005423 & -3.743514 \\
\hline 6 & -1.588805 & -3.315356 & -3.239604 \\
\hline 6 & -1.826223 & -3.214885 & -1.836537 \\
\hline 6 & -3.125096 & -3.511244 & -1.347851 \\
\hline 6 & -4.125433 & -3.904657 & -2.200814 \\
\hline 6 & -3.878018 & -4.034297 & -3.585353 \\
\hline 6 & -2.638732 & -3.736511 & -4.092190 \\
\hline 7 & 1.524252 & -2.015956 & -0.655832 \\
\hline 6 & 2.472651 & -3.037294 & -0.154674 \\
\hline 6 & 3.046423 & -2.755397 & 1.208320 \\
\hline 6 & 2.209724 & -2.490657 & 2.293934 \\
\hline 6 & 2.741752 & -2.211811 & 3.545493 \\
\hline 6 & 4.123461 & -2.194251 & 3.731395 \\
\hline 6 & 4.963384 & -2.469544 & 2.657779 \\
\hline 6 & 4.425396 & -2.752589 & 1.404544 \\
\hline 6 & 0.782591 & 1.624715 & -3.136305 \\
\hline 15 & 1.801974 & -0.354561 & -0.874286 \\
\hline 8 & 2.984361 & -0.217314 & -2.077516 \\
\hline 6 & 4.239881 & -0.751997 & -1.931900 \\
\hline 6 & 5.144672 & -0.213065 & -1.032648 \\
\hline 6 & 6.394435 & -0.887418 & -0.843599 \\
\hline 6 & 6.731563 & -1.998668 & -1.672922 \\
\hline 6 & 5.811471 & -2.429524 & -2.659850 \\
\hline 6 & 4.586334 & -1.831594 & -2.774638 \\
\hline 6 & 7.294259 & -0.535486 & 0.194763 \\
\hline 6 & 8.467774 & -1.223999 & 0.380810 \\
\hline 6 & 8.813997 & -2.298392 & -0.465167 \\
\hline 6 & 7.958575 & -2.675620 & -1.468822 \\
\hline 6 & 4.769414 & 0.994111 & -0.263501 \\
\hline 6 & 3.584728 & 0.990702 & 0.447883 \\
\hline 6 & 3.158496 & 2.092539 & 1.213740 \\
\hline 6 & 3.927408 & 3.223322 & 1.260741 \\
\hline 6 & 5.126907 & 3.310278 & 0.514160 \\
\hline 6 & 5.549344 & 2.195872 & -0.273054 \\
\hline 6 & 5.901149 & 4.496086 & 0.515017 \\
\hline 6 & 7.035910 & 4.600469 & -0.247585 \\
\hline 6 & 7.435636 & 3.515925 & -1.056166 \\
\hline 6 & 6.713568 & 2.348194 & -1.068766 \\
\hline 8 & 2.792247 & -0.130916 & 0.438244 \\
\hline 1 & 7.032402 & 0.278340 & 0.866216 \\
\hline 1 & -1.762247 & -0.910811 & 3.197403 \\
\hline 1 & 8.199769 & -3.515318 & -2.119568 \\
\hline 1 & -1.147273 & -5.465116 & 4.296022 \\
\hline 1 & 5.564622 & 5.330265 & 1.129449 \\
\hline 1 & -3.325035 & -3.410093 & -0.281856 \\
\hline 1 & -5.117729 & -4.116229 & -1.806665 \\
\hline 1 & 3.610846 & 4.081745 & 1.851916 \\
\hline 1 & -1.591686 & -3.052465 & 4.344884 \\
\hline 1 & 6.084825 & -3.260999 & -3.307535 \\
\hline 1 & 8.321277 & 3.606992 & -1.681220 \\
\hline
\end{tabular}




\begin{tabular}{|c|c|c|c|}
\hline 1 & 3.858820 & -2.168275 & -3.509992 \\
\hline & -2.438712 & -3.819387 & -5.159732 \\
\hline & -0.123191 & -3.090117 & -4.814316 \\
\hline & -0.188406 & -7.387127 & 0.572236 \\
\hline & 7.029419 & 1.526975 & -1.706908 \\
\hline & -0.376963 & -5.248707 & -0.608720 \\
\hline & 1.679173 & -2.339772 & -3.281465 \\
\hline & 7.619369 & 5.518272 & -0.242482 \\
\hline & 9.133949 & -0.941174 & 1.192908 \\
\hline & -4.676771 & -4.359222 & -4.248894 \\
\hline & -0.574007 & -7.516591 & 3.029289 \\
\hline 1 & 9.749247 & -2.831470 & -0.310845 \\
\hline & 2.218013 & 2.012062 & 1.752213 \\
\hline & -3.793990 & -0.970691 & 1.872077 \\
\hline & -3.086552 & 0.409711 & -2.113488 \\
\hline & -7.040778 & -0.750286 & -0.895318 \\
\hline & -3.405847 & 2.067259 & 1.009658 \\
\hline & 0.170432 & 0.428243 & 2.710389 \\
\hline & -1.802707 & 3.722920 & 4.620271 \\
\hline & 1.895014 & -3.972246 & -0.113926 \\
\hline & 3.289979 & -3.203565 & -0.874118 \\
\hline & 5.090248 & -2.946825 & 0.561223 \\
\hline & 1.132892 & -2.465409 & 2.141293 \\
\hline & 6.043917 & -2.454754 & 2.787507 \\
\hline 1 & 2.072514 & -2.005126 & 4.380412 \\
\hline & 4.541796 & -1.966771 & 4.709688 \\
\hline 1 & -1.352382 & 1.708857 & -1.745139 \\
\hline & 1.548787 & 2.377949 & -2.960530 \\
\hline & 1.123907 & 0.719411 & -3.637193 \\
\hline 1 & -2.746088 & 3.222529 & -2.130673 \\
\hline & -3.350680 & 5.612570 & -2.209329 \\
\hline 1 & -1.853634 & 7.245675 & -3.340107 \\
\hline & 0.241341 & 6.447824 & -4.412628 \\
\hline & 0.822775 & 4.047301 & -4.368385 \\
\hline & -2.511600 & 1.309345 & -3.970913 \\
\hline ] & -1.294999 & 1.482129 & -5.230679 \\
\hline 1 & -1.209923 & 0.101657 & -4.100080 \\
\hline & -3.756657 & 5.068778 & 3.919079 \\
\hline 6 & -3.801803 & 4.837928 & 1.452381 \\
\hline ) & -5.188105 & 3.326668 & 2.887948 \\
\hline 6 & 0.116057 & 3.045773 & 6.006380 \\
\hline 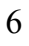 & 0.004757 & 0.590137 & 5.678195 \\
\hline 6 & 1.720568 & 1.947652 & 4.478387 \\
\hline 6 & -7.154263 & -0.021885 & -3.219848 \\
\hline & -5.419264 & 1.733616 & -3.316972 \\
\hline 6 & -4.923718 & -0.598144 & -4.109430 \\
\hline ) & -7.870688 & -1.666304 & 1.249726 \\
\hline 6 & -5.894565 & -2.878005 & 2.119533 \\
\hline 5 & -6.413609 & -0.558370 & 2.917950 \\
\hline t & 0.851845 & 2.912214 & 6.809449 \\
\hline 1 & 0.285472 & 4.035947 & 5.563887 \\
\hline t & -0.877630 & 3.042379 & 6.472106 \\
\hline 1 & 2.413829 & 1.856676 & 5.325352 \\
\hline & 1.939986 & 1.119189 & 3.793299 \\
\hline & 1.945455 & 2.890034 & 3.960759 \\
\hline & 0.683165 & 0.458887 & 6.532468 \\
\hline
\end{tabular}




$\begin{array}{rrrr}1 & -1.026613 & 0.543271 & 6.053279 \\ 1 & 0.153221 & -0.259510 & 4.998475 \\ 1 & -4.586543 & 5.783327 & 3.848401 \\ 1 & -3.826794 & 4.583224 & 4.901038 \\ 1 & -2.822411 & 5.643852 & 3.884760 \\ 1 & -6.022069 & 4.040892 & 2.850616 \\ 1 & -5.330458 & 2.603524 & 2.073953 \\ 1 & -5.253840 & 2.778020 & 3.837130 \\ 1 & -4.589627 & 5.603375 & 1.432486 \\ 1 & -2.833968 & 5.338753 & 1.319672 \\ 1 & -3.956639 & 4.182534 & 0.586723 \\ 1 & -7.463418 & 0.238894 & -4.239995 \\ 1 & -7.404215 & -1.079064 & -3.061727 \\ 1 & -7.757712 & 0.582848 & -2.530518 \\ 1 & -5.225012 & -0.294030 & -5.121340 \\ 1 & -3.833765 & -0.502128 & -4.038113 \\ 1 & -5.161730 & -1.662886 & -3.987752 \\ 1 & -5.791800 & 2.016399 & -4.310734 \\ 1 & -5.942470 & 2.347764 & -2.571869 \\ 1 & -4.356564 & 1.999132 & -3.275881 \\ 1 & -8.471779 & -2.077155 & 2.070795 \\ 1 & -8.323304 & -0.709739 & 0.958478 \\ 1 & -7.955228 & -2.359273 & 0.402446 \\ 1 & -7.021481 & -0.976349 & 3.731855 \\ 1 & -5.401568 & -0.392114 & 3.308351 \\ 1 & -6.832538 & 0.421834 & 2.653957 \\ 1 & -6.521143 & -3.307806 & 2.912818 \\ 1 & -5.908273 & -3.571044 & 1.267132 \\ 1 & -4.864372 & -2.834824 & 2.496190\end{array}$

TS1 $_{\text {IL-re- }} \psi_{-1 \mathbf{a} 2 a 3 a 4 a}$

Number of imaginary frequencies : 1

The smallest frequencies are : - $665.9911 \quad 12.7668 \quad 17.9847 \mathrm{~cm}(-1)$

Electronic energy : $\quad H F=-4360.8595761$

Zero-point correction $=\quad 1.451151$ (Hartree/Particle)

Thermal correction to Energy= $\quad 1.536369$

Thermal correction to Enthalpy= $\quad 1.537314$

Thermal correction to Gibbs Free Energy $=\quad 1.331638$

Sum of electronic and zero-point Energies $=\quad-4359.408425$

Sum of electronic and thermal Energies $=\quad-4359.323207$

Sum of electronic and thermal Enthalpies $=\quad-4359.322263$

Sum of electronic and thermal Free Energies $=\quad-4359.527939$

Cartesian Coordinates

$\begin{array}{cccc}6 & -3.748883 & -0.664451 & -1.078001 \\ 6 & -3.322637 & 0.076566 & 0.021008 \\ 6 & -4.242789 & 0.462088 & 1.001162 \\ 6 & -5.587013 & 0.114499 & 0.905080 \\ 6 & -5.981818 & -0.628124 & -0.212460 \\ 6 & -5.096309 & -1.022627 & -1.215831 \\ 15 & -1.550227 & 0.555340 & 0.213518 \\ 6 & -1.331726 & 0.112872 & 2.012702 \\ 6 & -1.053704 & -1.192326 & 2.385474\end{array}$




\begin{tabular}{|c|c|c|c|}
\hline 6 & -0.950772 & -1.544420 & 3.773228 \\
\hline 6 & -1.148085 & -0.545076 & 4.769280 \\
\hline 6 & -1.455130 & 0.771157 & 4.358017 \\
\hline 6 & -1.541291 & 1.086938 & 3.029224 \\
\hline 6 & -1.026165 & -0.883215 & 6.138022 \\
\hline 6 & -0.716749 & -2.163225 & 6.519831 \\
\hline 6 & -0.519451 & -3.158907 & 5.540311 \\
\hline 6 & -0.636345 & -2.860553 & 4.205093 \\
\hline 6 & -0.878848 & -2.306709 & 1.415347 \\
\hline 6 & 0.362695 & -2.580869 & 0.871933 \\
\hline 6 & 0.579572 & -3.782601 & 0.156775 \\
\hline 6 & -0.439929 & -4.673769 & -0.047353 \\
\hline 6 & -1.746864 & -4.385594 & 0.411677 \\
\hline 6 & -1.969068 & -3.196295 & 1.166179 \\
\hline 6 & -3.278731 & -2.935150 & 1.645805 \\
\hline 6 & -4.313919 & -3.792151 & 1.369068 \\
\hline 6 & -4.091056 & -4.966662 & 0.616913 \\
\hline 6 & -2.833963 & -5.254798 & 0.149957 \\
\hline 7 & 1.449626 & -1.658495 & 1.033257 \\
\hline 6 & 2.452462 & -2.018350 & 2.064356 \\
\hline 6 & 3.032707 & -0.856119 & 2.826412 \\
\hline 6 & 4.411960 & -0.743921 & 2.989457 \\
\hline 6 & 4.957517 & 0.325592 & 3.695439 \\
\hline 6 & 4.126284 & 1.292776 & 4.250527 \\
\hline 6 & 2.745217 & 1.181090 & 4.097492 \\
\hline 6 & 2.205182 & 0.115526 & 3.390073 \\
\hline 6 & -6.611853 & 0.492900 & 1.972374 \\
\hline 6 & -5.998532 & 1.341917 & 3.083855 \\
\hline 6 & -5.629137 & -1.815067 & -2.407821 \\
\hline 6 & -4.530883 & -2.150299 & -3.409705 \\
\hline 45 & 0.009309 & 0.193567 & -1.576527 \\
\hline 6 & 0.951606 & -0.354344 & -3.452210 \\
\hline 6 & -0.404850 & -0.478334 & -3.855096 \\
\hline 6 & -0.946348 & 0.608893 & -4.759131 \\
\hline 15 & 1.743735 & -0.728647 & -0.363026 \\
\hline 8 & 2.817582 & 0.310030 & 0.364111 \\
\hline 6 & 3.671856 & 1.031982 & -0.427402 \\
\hline 6 & 4.804223 & 0.425562 & -0.936941 \\
\hline 6 & 5.651996 & 1.192443 & -1.800955 \\
\hline 6 & 5.353962 & 2.570541 & -2.029647 \\
\hline 6 & 4.213220 & 3.149002 & -1.422921 \\
\hline 6 & 3.377231 & 2.391919 & -0.648694 \\
\hline 6 & 6.194277 & 3.334708 & -2.875319 \\
\hline 6 & 7.275931 & 2.766479 & -3.497466 \\
\hline 6 & 7.553335 & 1.397299 & -3.302913 \\
\hline 6 & 6.765159 & 0.632612 & -2.478846 \\
\hline 6 & 5.069026 & -0.994718 & -0.613877 \\
\hline 6 & 4.095094 & -1.947854 & -0.858869 \\
\hline 6 & 4.354613 & -3.329201 & -0.714219 \\
\hline 6 & 5.555045 & -3.754888 & -0.216105 \\
\hline 6 & 6.534951 & -2.817174 & 0.192168 \\
\hline 6 & 6.292501 & -1.424303 & -0.003253 \\
\hline 6 & 7.255967 & -0.506409 & 0.488117 \\
\hline 6 & 8.398630 & -0.943187 & 1.112114 \\
\hline 6 & 8.651005 & -2.321990 & 1.269899 \\
\hline 6 & 7.733699 & -3.236248 & 0.818604 \\
\hline
\end{tabular}




\begin{tabular}{|c|c|c|c|}
\hline & 2.849847 & -1.591561 & -1.309917 \\
\hline & 0.436597 & 2.135209 & -1.952185 \\
\hline 8 & 0.560316 & 3.098645 & -2.582140 \\
\hline & -0.971843 & -1.844671 & -4.056502 \\
\hline & -1.594800 & -2.166876 & -5.270431 \\
\hline 6 & -2.030835 & -3.461870 & -5.536921 \\
\hline 6 & -1.861191 & -4.467827 & -4.594074 \\
\hline 6 & -1.258482 & -4.158278 & -3.376719 \\
\hline 6 & -0.830228 & -2.865258 & -3.107369 \\
\hline 6 & -1.643647 & 2.391481 & 0.289648 \\
\hline 6 & -2.658213 & 3.086462 & -0.361836 \\
\hline 6 & -2.629445 & 4.481707 & -0.446140 \\
\hline 6 & -1.567425 & 5.152968 & 0.163448 \\
\hline 6 & -0.540397 & 4.485560 & 0.840703 \\
\hline 6 & -0.582017 & 3.093229 & 0.866685 \\
\hline 6 & -3.719548 & 5.276596 & -1.163252 \\
\hline 6 & -4.793761 & 4.372620 & -1.764717 \\
\hline 6 & 0.600770 & 5.271545 & 1.484694 \\
\hline 6 & 1.462189 & 4.391719 & 2.391339 \\
\hline 6 & -4.399303 & 6.223524 & -0.168034 \\
\hline 6 & -3.091061 & 6.091487 & -2.298534 \\
\hline 6 & 0.045204 & 6.413737 & 2.341657 \\
\hline 6 & 1.483271 & 5.852858 & 0.374896 \\
\hline 6 & -7.175851 & -0.784756 & 2.604140 \\
\hline 6 & -7.752838 & 1.293145 & 1.335727 \\
\hline 6 & -6.240318 & -3.132322 & -1.919105 \\
\hline 6 & -6.700055 & -0.988855 & -3.129076 \\
\hline 1 & 7.065656 & 0.559736 & 0.392107 \\
\hline 1 & -1.785069 & 2.108011 & 2.750272 \\
\hline 1 & 7.902667 & -4.304592 & 0.948279 \\
\hline 1 & -1.179206 & -0.100877 & 6.880116 \\
\hline 1 & 5.952469 & 4.385741 & -3.028420 \\
\hline 1 & -3.460913 & -2.025345 & 2.215597 \\
\hline 1 & -5.317828 & -3.561136 & 1.721912 \\
\hline 1 & 3.999654 & 4.202185 & -1.601319 \\
\hline 1 & -1.623556 & 1.534857 & 5.116324 \\
\hline 1 & 5.762647 & -4.817725 & -0.102574 \\
\hline 1 & 8.396586 & 0.939945 & -3.815801 \\
\hline 1 & 3.581895 & -4.032069 & -1.017563 \\
\hline 1 & -2.648309 & -6.158451 & -0.429811 \\
\hline 1 & -0.261852 & -5.607202 & -0.580597 \\
\hline 1 & -0.273853 & -4.173451 & 5.845718 \\
\hline 1 & 6.986944 & -0.423704 & -2.350240 \\
\hline 1 & -0.483175 & -3.643625 & 3.465222 \\
\hline 1 & 1.580646 & -3.981333 & -0.220409 \\
\hline 1 & 7.911238 & 3.362171 & -4.148875 \\
\hline 1 & 9.113587 & -0.217472 & 1.493511 \\
\hline 1 & -4.920392 & -5.639077 & 0.407472 \\
\hline 1 & -0.621068 & -2.413995 & 7.573704 \\
\hline 1 & 9.563207 & -2.654584 & 1.759705 \\
\hline 1 & 2.480132 & 2.805636 & -0.192119 \\
\hline 1 & -3.895745 & 1.043043 & 1.851668 \\
\hline 1 & -3.008227 & -0.964064 & -1.819619 \\
\hline 1 & -7.030550 & -0.912355 & -0.303539 \\
\hline 1 & -3.470394 & 2.527067 & -0.819746 \\
\hline 1 & 0.217127 & 2.530358 & 1.349160 \\
\hline
\end{tabular}




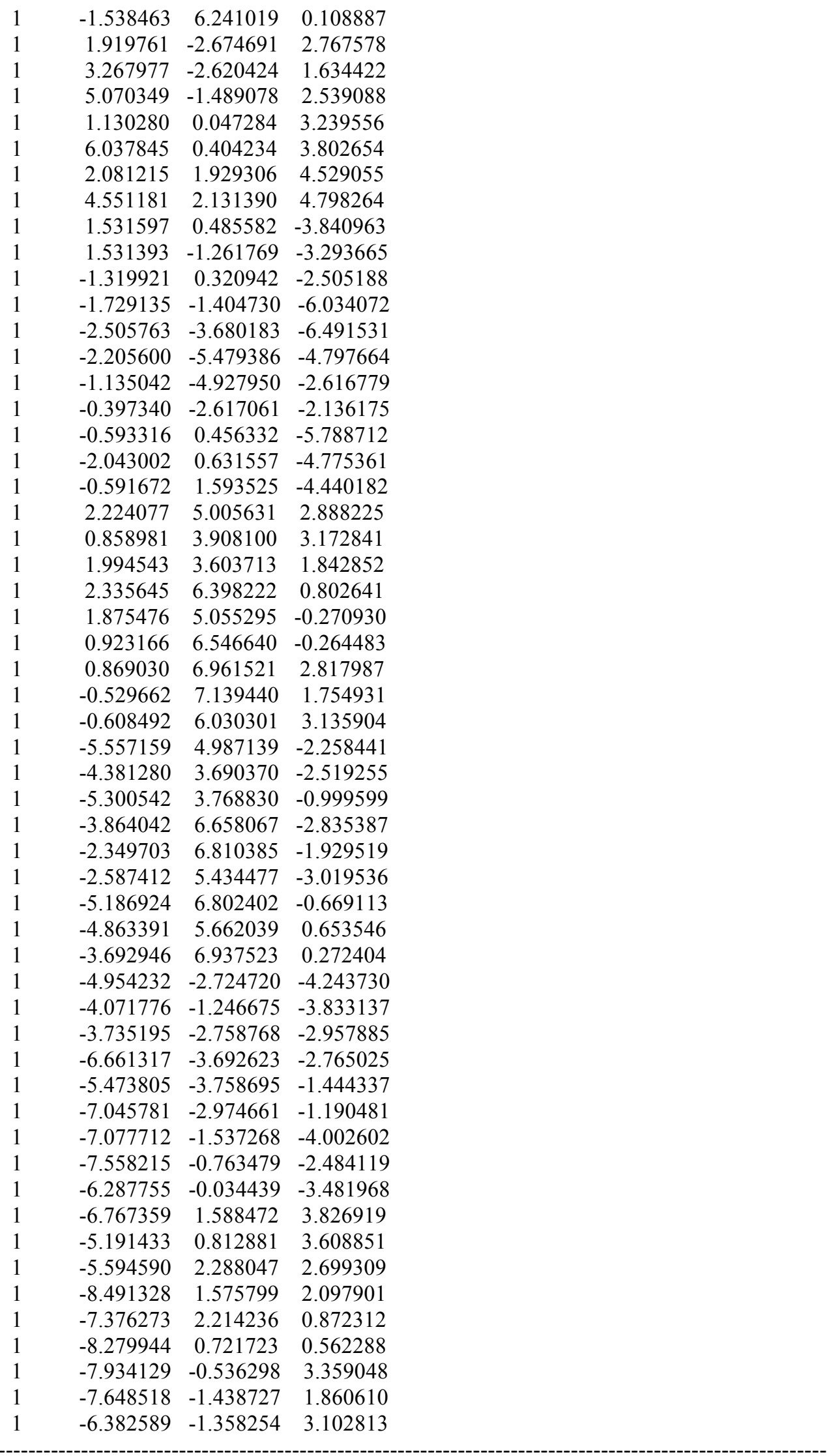


TS1 $1_{\text {IL-re- }} \psi_{\text {-1a2a3a4s }}$

Number of imaginary frequencies : 1

The smallest frequencies are : - $672.1014 \quad 13.8916 \quad 17.0822 \mathrm{~cm}(-1)$

Electronic energy : $\quad \mathrm{HF}=-4360.8603557$

Zero-point correction $=\quad 1.451177$ (Hartree/Particle)

Thermal correction to Energy $=\quad 1.536407$

Thermal correction to Enthalpy= $\quad 1.537351$

Thermal correction to Gibbs Free Energy $=\quad 1.331470$

Sum of electronic and zero-point Energies $=\quad-4359.409179$

Sum of electronic and thermal Energies $=\quad-4359.323949$

Sum of electronic and thermal Enthalpies $=\quad-4359.323005$

Sum of electronic and thermal Free Energies $=\quad-4359.528885$

Cartesian Coordinates

$\begin{array}{cccc}6 & 6.754050 & 0.647730 & -2.503144 \\ 6 & 5.635303 & 1.207558 & -1.834494 \\ 6 & 5.320504 & 2.577257 & -2.089976 \\ 6 & 6.150098 & 3.334320 & -2.952478 \\ 6 & 7.237302 & 2.766698 & -3.565405 \\ 6 & 7.531577 & 1.405165 & -3.343844 \\ 6 & 4.798293 & 0.448153 & -0.953562 \\ 6 & 3.659475 & 1.051334 & -0.454474 \\ 6 & 3.348897 & 2.403278 & -0.701234 \\ 6 & 4.174493 & 3.154501 & -1.492071 \\ 6 & 5.081869 & -0.961176 & -0.599209 \\ 6 & 4.119923 & -1.932187 & -0.820223 \\ 6 & 4.396425 & -3.306348 & -0.642707 \\ 6 & 5.602829 & -3.705110 & -0.136787 \\ 6 & 6.571663 & -2.745801 & 0.246983 \\ 6 & 6.311380 & -1.361039 & 0.019481 \\ 6 & 7.776504 & -3.135037 & 0.880880 \\ 6 & 8.683018 & -2.199044 & 1.308937 \\ 6 & 8.412945 & -0.827583 & 1.119480 \\ 6 & 7.263762 & -0.419962 & 0.487665 \\ 8 & 2.869879 & -1.602555 & -1.278333 \\ 15 & 1.754064 & -0.731652 & -0.351386 \\ 8 & 2.815426 & 0.335194 & 0.352970 \\ 45 & 0.012837 & 0.147887 & -1.586177 \\ 6 & 0.426846 & 2.080569 & -2.009274 \\ 8 & 0.549563 & 3.030826 & -2.659086 \\ 6 & -0.401046 & -0.585613 & -3.847073 \\ 6 & 0.955065 & -0.438909 & -3.451196 \\ 6 & -0.956244 & -1.961504 & -4.010391 \\ 6 & -1.586014 & -2.320644 & -5.210238 \\ 6 & -2.010124 & -3.626669 & -5.439913 \\ 6 & -1.821396 & -4.606459 & -4.473326 \\ 6 & -1.211897 & -4.259739 & -3.269441 \\ 6 & -0.795555 & -2.955896 & -3.036879 \\ 6 & -0.955831 & 0.473221 & -4.776189 \\ 15 & -1.548462 & 0.530527 & 0.192551 \\ 6 & -1.679206 & 2.366241 & 0.227542 \\ 6 & -2.718418 & 3.030000 & -0.418078 \\ 6 & -2.719651 & 4.424145 & -0.524715 \\ 6 & -1.667398 & 5.127208 & 0.066015 \\ & & & \\ 6 & & & \end{array}$




\begin{tabular}{|c|c|c|c|}
\hline 6 & -0.617178 & 4.491879 & 0.738620 \\
\hline 6 & -0.625252 & 3.099200 & 0.778970 \\
\hline & -3.842395 & 5.184162 & -1.229602 \\
\hline & -4.587780 & 6.044762 & -0.203215 \\
\hline & 0.513689 & 5.309149 & 1.361874 \\
\hline & -0.054269 & 6.470865 & 2.183587 \\
\hline & -1.324565 & 0.137126 & 2.003243 \\
\hline & -1.035635 & -1.155985 & 2.408019 \\
\hline & -0.928900 & -1.473995 & 3.803477 \\
\hline & -1.132140 & -0.452697 & 4.775363 \\
\hline & -1.447455 & 0.851276 & 4.332459 \\
\hline & -1.538653 & 1.134088 & 2.996297 \\
\hline & -1.007669 & -0.757544 & 6.151718 \\
\hline f & -0.690670 & -2.026362 & 6.563585 \\
\hline & -0.487472 & -3.043761 & 5.607870 \\
\hline & -0.606121 & -2.777689 & 4.266051 \\
\hline$b$ & -0.853012 & -2.291796 & 1.465280 \\
\hline & 0.390502 & -2.568848 & 0.927919 \\
\hline 6 & 0.616810 & -3.786589 & 0.243323 \\
\hline & -0.395980 & -4.690608 & 0.062635 \\
\hline & -1.705431 & -4.400883 & 0.513876 \\
\hline ) & -1.936644 & -3.195128 & 1.238948 \\
\hline 6 & -3.248149 & -2.931351 & 1.712349 \\
\hline 5 & -4.276983 & -3.802092 & 1.455674 \\
\hline$b$ & -4.045839 & -4.992351 & 0.731237 \\
\hline b & -2.786310 & -5.283661 & 0.272801 \\
\hline t & 1.469127 & -1.632827 & 1.065628 \\
\hline 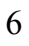 & 2.476154 & -1.958843 & 2.103856 \\
\hline 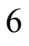 & 3.049170 & -0.774223 & 2.836774 \\
\hline 6 & 4.427588 & -0.654575 & 3.002397 \\
\hline 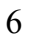 & 4.968224 & 0.433992 & 3.682580 \\
\hline 6 & 4.132809 & 1.413790 & 4.208205 \\
\hline 6 & 2.752650 & 1.295750 & 4.051612 \\
\hline 6 & 2.217276 & 0.210503 & 3.370942 \\
\hline b & -3.312896 & 0.022341 & 0.010633 \\
\hline & -4.232870 & 0.433718 & 0.971088 \\
\hline 6 & -5.583305 & 0.092915 & 0.882748 \\
\hline & -5.981232 & -0.674931 & -0.210413 \\
\hline 6 & -5.086702 & -1.103597 & -1.200088 \\
\hline 6 & -3.741166 & -0.749628 & -1.070819 \\
\hline 6 & -6.542152 & 0.588081 & 1.962771 \\
\hline 6 & -6.047714 & 0.130298 & 3.340243 \\
\hline ) & -5.620375 & -1.924411 & -2.372304 \\
\hline 6 & -6.252610 & -3.219143 & -1.851216 \\
\hline 6 & -6.674868 & -1.106275 & -3.126381 \\
\hline 6 & -4.519293 & -2.303165 & -3.355402 \\
\hline 6 & -6.590387 & 2.120277 & 1.917322 \\
\hline 6 & -7.959859 & 0.056806 & 1.770892 \\
\hline 6 & -3.253793 & 6.084404 & -2.320857 \\
\hline 6 & -4.852480 & 4.245481 & -1.886419 \\
\hline 6 & 1.392880 & 5.866167 & 0.237125 \\
\hline 5 & 1.381664 & 4.465027 & 2.296194 \\
\hline 1 & 7.059742 & 0.641130 & 0.367312 \\
\hline 1 & -1.788553 & 2.146788 & 2.693525 \\
\hline 1 & 7.958990 & -4.197844 & 1.035225 \\
\hline 1 & -1.165428 & 0.041143 & 6.875184 \\
\hline
\end{tabular}




\begin{tabular}{|c|c|c|c|}
\hline & 5.895466 & 4.379142 & -3.125977 \\
\hline & -3.437339 & -2.010155 & 2.261555 \\
\hline 1 & -5.282647 & -3.569320 & 1.801755 \\
\hline & 3.948594 & 4.201586 & -1.690186 \\
\hline & -1.618628 & 1.632430 & 5.072190 \\
\hline & 5.823791 & -4.762214 & 0.002109 \\
\hline & 8.379359 & 0.947765 & -3.849167 \\
\hline & 3.631484 & -4.025533 & -0.927053 \\
\hline & -2.594299 & -6.199687 & -0.285068 \\
\hline & -0.210721 & -5.636206 & -0.446212 \\
\hline 1 & -0.235897 & -4.049359 & 5.937101 \\
\hline & 6.988627 & -0.403117 & -2.354290 \\
\hline & -0.448471 & -3.577159 & 3.544857 \\
\hline & 1.619336 & -3.987016 & -0.129447 \\
\hline & 7.864248 & 3.356773 & -4.229893 \\
\hline & 9.119199 & -0.084181 & 1.482601 \\
\hline & -4.870457 & -5.675182 & 0.537441 \\
\hline & -0.593571 & -2.251613 & 7.623083 \\
\hline & 9.600142 & -2.508574 & 1.804658 \\
\hline & 2.448298 & 2.815837 & -0.250424 \\
\hline & -3.893065 & 1.041627 & 1.808375 \\
\hline & -3.000455 & -1.070987 & -1.803144 \\
\hline ] & -7.028946 & -0.953480 & -0.306276 \\
\hline & -3.526498 & 2.447363 & -0.853652 \\
\hline ] & 0.192111 & 2.560044 & 1.258270 \\
\hline & -1.665989 & 6.214932 & 0.001278 \\
\hline 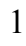 & 1.949704 & -2.602171 & 2.823633 \\
\hline 1 & 3.295831 & -2.564833 & 1.687293 \\
\hline & 5.089563 & -1.409420 & 2.573820 \\
\hline 1 & 1.143061 & 0.137088 & 3.216687 \\
\hline & 6.047916 & 0.517576 & 3.792230 \\
\hline 1 & 2.085953 & 2.054604 & 4.459727 \\
\hline & 4.553630 & 2.267454 & 4.735440 \\
\hline & 1.526274 & 0.396183 & -3.862550 \\
\hline 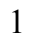 & 1.543655 & -1.336585 & -3.271169 \\
\hline & -1.319786 & 0.240545 & -2.514440 \\
\hline 1 & -1.735125 & -1.579213 & -5.991374 \\
\hline & -2.490913 & -3.874259 & -6.384391 \\
\hline 1 & -2.156577 & -5.626453 & -4.648239 \\
\hline ] & -1.073909 & -5.008257 & -2.491138 \\
\hline ] & -0.357565 & -2.677342 & -2.076282 \\
\hline 1 & -0.606694 & 0.296562 & -5.803281 \\
\hline 1 & -2.052729 & 0.486518 & -4.787238 \\
\hline 1 & -0.607862 & 1.468671 & -4.484526 \\
\hline & 2.132474 & 5.102108 & 2.780597 \\
\hline 1 & 0.780156 & 3.994261 & 3.086845 \\
\hline 1 & 1.928116 & 3.670259 & 1.772028 \\
\hline 1 & 2.236561 & 6.435994 & 0.650022 \\
\hline 1 & 1.797976 & 5.053525 & -0.381376 \\
\hline 1 & 0.825584 & 6.531501 & -0.425753 \\
\hline 1 & 0.763479 & 7.037493 & 2.648056 \\
\hline 1 & -0.630478 & 7.176359 & 1.574048 \\
\hline 1 & -0.709344 & 6.105729 & 2.985301 \\
\hline 1 & -5.634671 & 4.834905 & -2.381282 \\
\hline 1 & -4.384548 & 3.608912 & -2.648642 \\
\hline 1 & -5.347092 & 3.592701 & -1.154609 \\
\hline
\end{tabular}




$\begin{array}{rrrr}1 & -4.056625 & 6.621122 & -2.844137 \\ 1 & -2.566680 & 6.836289 & -1.914899 \\ 1 & -2.701291 & 5.492537 & -3.062033 \\ 1 & -5.398730 & 6.604277 & -0.689068 \\ 1 & -5.032061 & 5.419708 & 0.582972 \\ 1 & -3.925090 & 6.771763 & 0.281995 \\ 1 & -4.944018 & -2.897449 & -4.174709 \\ 1 & -4.045287 & -1.419163 & -3.803124 \\ 1 & -3.734083 & -2.907277 & -2.880041 \\ 1 & -6.664466 & -3.802850 & -2.685731 \\ 1 & -5.500207 & -3.836726 & -1.343355 \\ 1 & -7.069333 & -3.030738 & -1.143142 \\ 1 & -7.051120 & -1.674393 & -3.987876 \\ 1 & -7.536084 & -0.853720 & -2.495940 \\ 1 & -6.247993 & -0.166863 & -3.501601 \\ 1 & -8.605670 & 0.425401 & 2.577901 \\ 1 & -8.399776 & 0.389611 & 0.822099 \\ 1 & -7.990630 & -1.040644 & 1.798265 \\ 1 & -7.241201 & 2.508933 & 2.712340 \\ 1 & -5.596303 & 2.567814 & 2.047142 \\ 1 & -6.988685 & 2.468399 & 0.955132 \\ 1 & -6.718121 & 0.501390 & 4.127121 \\ 1 & -6.030243 & -0.965865 & 3.406038 \\ 1 & -5.037799 & 0.493636 & 3.569138\end{array}$

$\operatorname{TS1}_{\text {IL-re- }} \psi_{-1 \mathbf{a} 2 a 3 s 4 a}$

Number of imaginary frequencies : 1

The smallest frequencies are : $-662.9225 \quad 14.2532 \quad 19.5416 \mathrm{~cm}(-1)$

Electronic energy : $\quad H F=-4360.8631861$

Zero-point correction $=\quad 1.451761$ (Hartree/Particle)

Thermal correction to Energy= $\quad 1.536641$

Thermal correction to Enthalpy= $\quad 1.537586$

Thermal correction to Gibbs Free Energy $=\quad 1.333685$

Sum of electronic and zero-point Energies $=\quad-4359.411425$

Sum of electronic and thermal Energies $=\quad-4359.326545$

Sum of electronic and thermal Enthalpies $=\quad-4359.325601$

Sum of electronic and thermal Free Energies $=\quad-4359.529501$

Cartesian Coordinates

$\begin{array}{cccc}6 & -4.228348 & 0.618779 & 0.978690 \\ 6 & -3.310948 & 0.170021 & 0.017500 \\ 6 & -3.755520 & -0.592023 & -1.052322 \\ 6 & -5.116535 & -0.913165 & -1.193515 \\ 6 & -5.996787 & -0.456713 & -0.218224 \\ 6 & -5.579169 & 0.314382 & 0.879244 \\ 15 & -1.521502 & 0.577620 & 0.206689 \\ 45 & -0.014279 & 0.147118 & -1.597247 \\ 6 & 0.445339 & 2.055829 & -2.051830 \\ 8 & 0.528720 & 3.044788 & -2.646973 \\ 6 & -5.570516 & -1.745826 & -2.389463 \\ 6 & -6.596939 & 0.760950 & 1.926642 \\ 6 & -1.535023 & 2.416464 & 0.292569 \\ 6 & -2.522631 & 3.154080 & -0.353613 \\ 6 & -2.444853 & 4.548015 & -0.424511\end{array}$




\begin{tabular}{|c|c|c|c|}
\hline 6 & -1.360728 & 5.174959 & 0.192452 \\
\hline 6 & -0.358581 & 4.465042 & 0.864088 \\
\hline f & -0.447892 & 3.074093 & 0.875587 \\
\hline 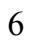 & 0.795601 & 5.210887 & 1.533117 \\
\hline$b$ & -3.504386 & 5.387237 & -1.136407 \\
\hline 6 & -1.318029 & 0.117992 & 2.002027 \\
\hline 6 & -1.063890 & -1.192722 & 2.371900 \\
\hline 5 & -0.968224 & -1.547007 & 3.760948 \\
\hline 6 & -1.156275 & -0.546389 & 4.757692 \\
\hline 6 & -1.443471 & 0.774776 & 4.347722 \\
\hline 6 & -1.516669 & 1.093729 & 3.019371 \\
\hline 6 & -1.040882 & -0.886060 & 6.126556 \\
\hline 6 & -0.743881 & -2.168793 & 6.508985 \\
\hline 6 & -0.552163 & -3.165101 & 5.529310 \\
\hline 6 & -0.664363 & -2.865462 & 4.193912 \\
\hline 6 & -0.900341 & -2.317053 & 1.409687 \\
\hline 6 & 0.340040 & -2.615646 & 0.877155 \\
\hline 6 & 0.552807 & -3.842063 & 0.203111 \\
\hline 6 & -0.465736 & -4.744016 & 0.044560 \\
\hline 6 & -1.770478 & -4.439742 & 0.500744 \\
\hline 6 & -1.994017 & -3.210713 & 1.188527 \\
\hline 6 & -3.305208 & -2.922343 & 1.647139 \\
\hline 6 & -4.337091 & -3.800449 & 1.429371 \\
\hline 6 & -4.109369 & -5.022428 & 0.759079 \\
\hline 6 & -2.853170 & -5.331191 & 0.302996 \\
\hline 7 & 1.434097 & -1.698926 & 1.018627 \\
\hline 15 & 1.716156 & -0.774365 & -0.383876 \\
\hline 8 & 2.792567 & 0.267088 & 0.335417 \\
\hline 6 & 3.632168 & 0.994738 & -0.466678 \\
\hline 6 & 4.763637 & 0.396855 & -0.988191 \\
\hline 6 & 5.596319 & 1.171373 & -1.860462 \\
\hline 6 & 5.284831 & 2.547289 & -2.085188 \\
\hline 6 & 4.143567 & 3.115352 & -1.469663 \\
\hline 6 & 3.321087 & 2.350771 & -0.688622 \\
\hline 6 & 6.706926 & 0.621277 & -2.550339 \\
\hline 6 & 7.481025 & 1.393156 & -3.381097 \\
\hline 6 & 7.191078 & 2.760357 & -3.571071 \\
\hline 6 & 6.110857 & 3.319100 & -2.937924 \\
\hline 6 & 5.041821 & -1.022074 & -0.669342 \\
\hline 6 & 4.070453 & -1.981750 & -0.899659 \\
\hline 6 & 4.339145 & -3.361358 & -0.754707 \\
\hline 6 & 5.549168 & -3.779534 & -0.273878 \\
\hline 6 & 6.530080 & -2.835520 & 0.117161 \\
\hline 6 & 6.276365 & -1.444284 & -0.075702 \\
\hline 6 & 7.740693 & -3.247010 & 0.725578 \\
\hline 6 & 8.659087 & -2.327059 & 1.162845 \\
\hline 6 & 8.395478 & -0.949920 & 1.009124 \\
\hline 6 & 7.240937 & -0.520442 & 0.402092 \\
\hline 8 & 2.817354 & -1.635572 & -1.336339 \\
\hline 6 & 2.451421 & -2.067012 & 2.033379 \\
\hline 6 & 3.042384 & -0.912352 & 2.798657 \\
\hline 6 & 4.424156 & -0.792622 & 2.933124 \\
\hline 6 & 4.977414 & 0.267181 & 3.647669 \\
\hline 6 & 4.151670 & 1.215965 & 4.241542 \\
\hline 6 & 2.768384 & 1.095673 & 4.118489 \\
\hline 6 & 2.220764 & 0.041128 & 3.400342 \\
\hline
\end{tabular}




\begin{tabular}{|c|c|c|c|}
\hline & 0.827136 & -0.541320 & -3.481055 \\
\hline & -0.546588 & -0.601790 & -3.837762 \\
\hline & -1.205971 & -1.935306 & -3.952958 \\
\hline & -0.976651 & -2.962684 & -3.026031 \\
\hline & -1.481855 & -4.238286 & -3.229812 \\
\hline & -2.259394 & -4.523473 & -4.351337 \\
\hline & -2.528167 & -3.508678 & -5.260007 \\
\hline & -2.007814 & -2.231224 & -5.064113 \\
\hline & -1.044151 & 0.478682 & -4.774381 \\
\hline & 7.042464 & 0.544456 & 0.309109 \\
\hline & -1.738403 & 2.119924 & 2.740526 \\
\hline & 7.918002 & -4.314263 & 0.853060 \\
\hline & -1.187958 & -0.102215 & 6.868282 \\
\hline & 5.858928 & 4.368241 & -3.087641 \\
\hline & -3.490456 & -1.979940 & 2.160506 \\
\hline & -5.339906 & -3.548475 & 1.769071 \\
\hline & 3.916437 & 4.165865 & -1.648310 \\
\hline & -1.603423 & 1.539661 & 5.106688 \\
\hline & 5.764029 & -4.840933 & -0.160358 \\
\hline & 8.322728 & 0.942998 & -3.902813 \\
\hline & 3.564122 & -4.068377 & -1.042611 \\
\hline & -2.665488 & -6.267443 & -0.221915 \\
\hline & -0.288710 & -5.699926 & -0.447835 \\
\hline & -0.314164 & -4.181566 & 5.834353 \\
\hline & 6.938333 & -0.433413 & -2.425515 \\
\hline & -0.513652 & -3.650308 & 3.455531 \\
\hline & 1.552832 & -4.054949 & -0.170535 \\
\hline & 7.815353 & 3.361914 & -4.227737 \\
\hline & 9.111159 & -0.219626 & 1.380280 \\
\hline 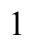 & -4.934730 & -5.712659 & 0.598019 \\
\hline & -0.652742 & -2.420754 & 7.562975 \\
\hline 1 & 9.580611 & -2.653882 & 1.638888 \\
\hline & 2.420510 & 2.754719 & -0.229490 \\
\hline & -3.868310 & 1.217587 & 1.811245 \\
\hline ] & -3.024226 & -0.948078 & -1.780600 \\
\hline & -7.053301 & -0.702565 & -0.303201 \\
\hline 1 & -3.353883 & 2.628459 & -0.817048 \\
\hline & 0.329322 & 2.479478 & 1.356352 \\
\hline 1 & -1.294566 & 6.262017 & 0.151441 \\
\hline & 1.927515 & -2.728455 & 2.738451 \\
\hline ] & 3.260739 & -2.665840 & 1.588158 \\
\hline ] & 5.077694 & -1.524987 & 2.455766 \\
\hline 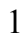 & 1.143325 & -0.035019 & 3.275140 \\
\hline 1 & 6.059245 & 0.352123 & 3.732517 \\
\hline & 2.108852 & 1.828001 & 4.582735 \\
\hline 1 & 4.582479 & 2.046316 & 4.797237 \\
\hline & 1.439933 & 0.245904 & -3.924112 \\
\hline 1 & 1.362241 & -1.470800 & -3.296254 \\
\hline 1 & -1.353698 & 0.297909 & -2.508468 \\
\hline & -2.219537 & -1.463749 & -5.804697 \\
\hline 1 & -3.144677 & -3.705460 & -6.135170 \\
\hline & -2.666263 & -5.521214 & -4.500910 \\
\hline 1 & -1.284184 & -5.012269 & -2.489918 \\
\hline & -0.407463 & -2.735361 & -2.122848 \\
\hline 1 & -0.766257 & 0.236362 & -5.809809 \\
\hline 1 & -2.132736 & 0.607146 & -4.736040 \\
\hline
\end{tabular}




$\begin{array}{lrrr}1 & -0.584513 & 1.441587 & -4.532021 \\ 6 & 1.644377 & 4.287650 & 2.407380 \\ 6 & 1.687439 & 5.826838 & 0.449813 \\ 6 & 0.250497 & 6.325677 & 2.432708 \\ 6 & -4.617942 & 4.528256 & -1.731852 \\ 6 & -4.140085 & 6.362811 & -0.139794 \\ 6 & -2.846159 & 6.173869 & -2.274723 \\ 6 & -7.084609 & -1.937969 & -2.427640 \\ 6 & -5.149087 & -1.044215 & -3.685152 \\ 6 & -4.916100 & -3.128278 & -2.311078 \\ 6 & -5.976120 & 1.678545 & 2.978082 \\ 6 & -7.745577 & 1.520239 & 1.254866 \\ 6 & -7.150916 & -0.478424 & 2.638566 \\ 1 & 2.537471 & 6.354340 & 0.903728 \\ 1 & 2.085926 & 5.052605 & -0.220176 \\ 1 & 1.135389 & 6.545261 & -0.168911 \\ 1 & 1.079063 & 6.847817 & 2.929275 \\ 1 & -0.318764 & 7.077269 & 1.873560 \\ 1 & -0.406681 & 5.918161 & 3.211966 \\ 1 & 2.416662 & 4.871890 & 2.923799 \\ 1 & 1.033799 & 3.788353 & 3.173014 \\ 1 & 2.163125 & 3.510211 & 1.831684 \\ 1 & -5.359584 & 5.173442 & -2.219586 \\ 1 & -4.238265 & 3.831084 & -2.489927 \\ 1 & -5.142803 & 3.944208 & -0.963288 \\ 1 & -3.595929 & 6.772833 & -2.809366 \\ 1 & -2.073211 & 6.860024 & -1.908032 \\ 1 & -2.373434 & 5.495307 & -2.996769 \\ 1 & -4.905393 & 6.972695 & -0.638600 \\ 1 & -4.623416 & 5.822254 & 0.684757 \\ 1 & -3.403549 & 7.048321 & 0.296445 \\ 1 & -5.467187 & -1.631634 & -4.557793 \\ 1 & -5.606171 & -0.048421 & -3.759673 \\ 1 & -4.060658 & -0.927456 & -3.751300 \\ 1 & -5.207382 & -3.738184 & -3.177510 \\ 1 & -3.820021 & -3.069545 & -2.297556 \\ 1 & -5.232867 & -3.654041 & -1.400745 \\ 1 & -7.355981 & -2.529589 & -3.311128 \\ 1 & -7.453119 & -2.480128 & -1.546966 \\ 1 & -7.620765 & -0.982069 & -2.494010 \\ 1 & -7.890617 & -0.188349 & 3.397143 \\ 1 & -7.642100 & -1.167408 & 1.940182 \\ 1 & -6.346319 & -1.028823 & 3.145054 \\ 1 & -8.472757 & 1.851779 & 2.008072 \\ 1 & -7.375429 & 2.409776 & 0.728761 \\ 1 & -8.285702 & 0.902359 & 0.527983 \\ 1 & -6.743864 & 1.985022 & 3.699687 \\ 1 & -5.176665 & 1.179866 & 3.542454 \\ 1 & -5.560613 & 2.590381 & 2.528035\end{array}$

TS1 $_{I L-r e-}-\Psi_{-1 \mathrm{a} 2 \mathrm{a} 3 \mathrm{~s} 4 \mathrm{~s}}$

Number of imaginary frequencies : 1

The smallest frequencies are : -670.9756 $15.2610 \quad 19.4237 \mathrm{~cm}(-1)$

Electronic energy :

Zero-point correction $=$

$\mathrm{HF}=-4360.8636454$

1.452025 (Hartree/Particle) 
Thermal correction to Energy= $\quad 1.536816$

Thermal correction to Enthalpy= $\quad 1.537760$

Thermal correction to Gibbs Free Energy $=1.334133$

Sum of electronic and zero-point Energies $=\quad-4359.411621$

Sum of electronic and thermal Energies $=\quad-4359.326829$

Sum of electronic and thermal Enthalpies $=\quad-4359.325885$

Sum of electronic and thermal Free Energies $=\quad-4359.529512$

Cartesian Coordinates

$\begin{array}{ccccc}6 & -4.218540 & 0.567864 & 0.961675 \\ 6 & -3.301788 & 0.119053 & 0.009360 \\ 6 & -3.748716 & -0.648779 & -1.060085 \\ 6 & -5.107629 & -0.961121 & -1.202657 \\ 6 & -5.996543 & -0.490664 & -0.234002 \\ 6 & -5.575046 & 0.272478 & 0.860800 \\ 15 & -1.518897 & 0.553576 & 0.194069 \\ 45 & -0.011014 & 0.117911 & -1.604818 \\ 6 & 0.426312 & 2.026064 & -2.084598 \\ 8 & 0.486123 & 3.010903 & -2.689315 \\ 6 & -5.562206 & -1.806139 & -2.390393 \\ 6 & -6.523199 & 0.788159 & 1.941108 \\ 6 & -1.566245 & 2.392887 & 0.253294 \\ 6 & -2.577867 & 3.106851 & -0.382039 \\ 6 & -2.525453 & 4.501261 & -0.466755 \\ 6 & -1.447374 & 5.153366 & 0.135024 \\ 6 & -0.422812 & 4.467242 & 0.797954 \\ 6 & -0.483311 & 3.074900 & 0.815479 \\ 6 & 0.724786 & 5.237310 & 1.451027 \\ 6 & -3.617387 & 5.313804 & -1.160635 \\ 6 & -1.309144 & 0.130556 & 1.998559 \\ 6 & -1.045763 & -1.171365 & 2.391583 \\ 6 & -0.945508 & -1.501631 & 3.785756 \\ 6 & -1.137211 & -0.485450 & 4.765528 \\ 6 & -1.431520 & 0.826997 & 4.333156 \\ 6 & -1.510171 & 1.122726 & 2.999421 \\ 6 & -1.018275 & -0.801670 & 6.139719 \\ 6 & -0.715064 & -2.076595 & 6.542922 \\ 6 & -0.520070 & -3.088343 & 5.579779 \\ 6 & -0.635050 & -2.811396 & 4.239764 \\ 6 & -0.879719 & -2.308417 & 1.446103 \\ 6 & 0.359106 & -2.605273 & 0.909070 \\ 6 & 0.573440 & -3.836512 & 0.244440 \\ 6 & -0.442754 & -4.743395 & 0.098301 \\ 6 & -1.746186 & -4.441372 & 0.559957 \\ 6 & -1.970507 & -3.208919 & 1.241220 \\ 6 & -3.278832 & -2.923768 & 1.710492 \\ 6 & -4.307823 & -3.808540 & 1.506850 \\ 6 & -4.080495 & -5.032243 & 0.839440 \\ 6 & -2.826652 & -5.338276 & 0.375073 \\ 7 & 1.448601 & -1.680714 & 1.035609 \\ 15 & 1.725021 & -0.774279 & -0.379639 \\ 8 & 2.791894 & 0.287115 & 0.325146 \\ 6 & 3.624514 & 1.010712 & -0.487695 \\ 6 & 4.760830 & 0.414897 & -1.001041 \\ 6 & 5.586276 & 1.183469 & -1.885264 \\ & & & \\ 6\end{array}$




\begin{tabular}{|c|c|c|c|}
\hline 6 & 5.262520 & 2.553091 & -2.130420 \\
\hline 6 & 4.117119 & 3.120631 & -1.522028 \\
\hline & 3.301873 & 2.360878 & -0.728749 \\
\hline & 6.701546 & 0.632924 & -2.567207 \\
\hline & 7.468299 & 1.398971 & -3.410073 \\
\hline & 7.165896 & 2.760417 & -3.620809 \\
\hline & 6.081157 & 3.319133 & -2.995403 \\
\hline & 5.051811 & -0.996391 & -0.659730 \\
\hline & 4.089320 & -1.968340 & -0.875172 \\
\hline & 4.369318 & -3.342939 & -0.706561 \\
\hline & 5.582357 & -3.742519 & -0.217578 \\
\hline & 6.554704 & -2.783580 & 0.158323 \\
\hline & 6.289254 & -1.397970 & -0.057945 \\
\hline b & 7.767812 & -3.174359 & 0.775286 \\
\hline & 8.677628 & -2.239303 & 1.198345 \\
\hline & 8.402496 & -0.867251 & 1.020979 \\
\hline b & 7.245103 & -0.458007 & 0.405368 \\
\hline & 2.833643 & -1.640179 & -1.318513 \\
\hline D & 2.467903 & -2.026674 & 2.055971 \\
\hline & 3.053381 & -0.857588 & 2.803664 \\
\hline & 4.434408 & -0.735762 & 2.944498 \\
\hline y & 4.983687 & 0.334788 & 3.646069 \\
\hline 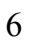 & 4.154693 & 1.292992 & 4.219758 \\
\hline 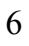 & 2.772193 & 1.171744 & 4.089008 \\
\hline 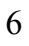 & 2.228466 & 0.106206 & 3.384245 \\
\hline b & 0.836035 & -0.587885 & -3.480749 \\
\hline ) & -0.536493 & -0.659955 & -3.838629 \\
\hline & -1.189093 & -1.998015 & -3.938085 \\
\hline 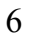 & -0.964839 & -3.008894 & -2.992156 \\
\hline ) & -1.465618 & -4.288928 & -3.178608 \\
\hline 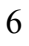 & -2.233274 & -4.594743 & -4.301457 \\
\hline 6 & -2.496207 & -3.596276 & -5.229783 \\
\hline 6 & -1.980154 & -2.314600 & -5.051233 \\
\hline 6 & -1.039810 & 0.406823 & -4.787552 \\
\hline & 7.037486 & 0.603399 & 0.294349 \\
\hline & -1.737910 & 2.142757 & 2.703732 \\
\hline 1 & 7.953982 & -4.237746 & 0.921029 \\
\hline & -1.168109 & -0.006339 & 6.868559 \\
\hline 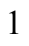 & 5.819894 & 4.363628 & -3.160797 \\
\hline | & -3.464031 & -1.980609 & 2.223262 \\
\hline . & -5.308075 & -3.563324 & 1.858958 \\
\hline 1 & 3.881298 & 4.166600 & -1.715520 \\
\hline & -1.593130 & 1.604268 & 5.079091 \\
\hline 1 & 5.806114 & -4.799901 & -0.085394 \\
\hline & 8.313775 & 0.948498 & -3.925380 \\
\hline 1 & 3.600069 & -4.061129 & -0.982134 \\
\hline l & -2.639237 & -6.276766 & -0.145910 \\
\hline 1 & -0.264635 & -5.702244 & -0.387984 \\
\hline 1 & -0.277344 & -4.098536 & 5.901485 \\
\hline 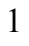 & 6.942351 & -0.417670 & -2.426710 \\
\hline 1 & -0.482149 & -3.607471 & 3.513892 \\
\hline ( & 1.572157 & -4.048407 & -0.133489 \\
\hline 1 & 7.784383 & 3.357426 & -4.287028 \\
\hline 1 & 9.111343 & -0.124684 & 1.380734 \\
\hline 1 & -4.904074 & -5.726973 & 0.689105 \\
\hline 1 & -0.621620 & -2.310580 & 7.600850 \\
\hline
\end{tabular}




\begin{tabular}{|c|c|c|c|}
\hline 1 & 9.601169 & -2.550115 & 1.681175 \\
\hline 1 & 2.399135 & 2.764740 & -0.273633 \\
\hline 1 & -3.864861 & 1.166409 & 1.799981 \\
\hline & -3.016789 & -1.011804 & -1.784135 \\
\hline 1 & -7.052012 & -0.727375 & -0.331109 \\
\hline & -3.407792 & 2.563045 & -0.826581 \\
\hline 1 & 0.311722 & 2.497395 & 1.288198 \\
\hline 1 & -1.404761 & 6.241375 & 0.089796 \\
\hline 1 & 1.947943 & -2.680066 & 2.771390 \\
\hline 1 & 3.280361 & -2.628025 & 1.619882 \\
\hline 1 & 5.090812 & -1.475438 & 2.482412 \\
\hline 1 & 1.151801 & 0.029398 & 3.252573 \\
\hline 1 & 6.064994 & 0.420694 & 3.736314 \\
\hline 1 & 2.110127 & 1.911902 & 4.536938 \\
\hline 1 & 4.582342 & 2.131567 & 4.765438 \\
\hline 1 & 1.445347 & 0.197851 & -3.931127 \\
\hline 1 & 1.375671 & -1.512235 & -3.283587 \\
\hline 1 & -1.352625 & 0.246267 & -2.517184 \\
\hline 1 & -2.187343 & -1.559927 & -5.806131 \\
\hline 1 & -3.104778 & -3.809381 & -6.106657 \\
\hline 1 & -2.637127 & -5.595682 & -4.437224 \\
\hline 1 & -1.272664 & -5.049715 & -2.424041 \\
\hline 1 & -0.404294 & -2.763944 & -2.088074 \\
\hline 1 & -0.758416 & 0.155235 & -5.819804 \\
\hline 1 & -2.129336 & 0.527895 & -4.752283 \\
\hline 1 & -0.587266 & 1.375515 & -4.555309 \\
\hline 6 & 1.584972 & 4.338474 & 2.339871 \\
\hline 6 & 1.607924 & 5.843009 & 0.354983 \\
\hline 6 & 0.171380 & & \\
\hline 6 & -4.665562 & 4.423834 & -1.825360 \\
\hline 6 & -4.326250 & 6.191745 & -0.123334 \\
\hline 6 & -2.992628 & 6.200325 & -2.242853 \\
\hline 6 & -7.077676 & -1.986400 & -2.437214 \\
\hline 6 & -5.126354 & -1.129276 & -3.694207 \\
\hline 6 & -4.920066 & -3.192884 & -2.285163 \\
\hline 6 & -7.965049 & 0.341289 & 1.715394 \\
\hline 6 & -6.068996 & 0.254680 & 3.305547 \\
\hline 6 & -6.492894 & 2.321371 & 1.949171 \\
\hline 1 & 2.448881 & 6.394164 & 0.797605 \\
\hline 1 & 2.020147 & 5.060395 & -0.296737 \\
\hline 1 & 1.044486 & 6.537987 & -0.280053 \\
\hline 1 & 0.996269 & 6.897316 & 2.821423 \\
\hline 1 & -0.400973 & 7.102424 & 1.760961 \\
\hline 1 & -0.484281 & 5.963546 & 3.117233 \\
\hline 1 & 2.350547 & 4.940445 & 2.845768 \\
\hline 1 & 0.980791 & 3.844820 & 3.114201 \\
\hline 1 & 2.113075 & 3.557617 & 1.777457 \\
\hline 1 & -5.425793 & 5.049534 & -2.309850 \\
\hline 1 & -4.225394 & 3.779158 & -2.597293 \\
\hline 1 & -5.182634 & 3.781783 & -1.099368 \\
\hline 1 & -3.772822 & 6.772391 & -2.762946 \\
\hline 1 & -2.277609 & 6.921079 & -1.828565 \\
\hline 1 & -2.461390 & 5.593614 & -2.987664 \\
\hline 1 & -5.112099 & 6.791926 & -0.601836 \\
\hline 1 & -4.797543 & 5.575114 & 0.653839 \\
\hline 1 & -3.633593 & 6.883050 & 0.372145 \\
\hline
\end{tabular}




$\begin{array}{lrrr}1 & -5.442156 & -1.728690 & -4.559507 \\ 1 & -5.575796 & -0.131670 & -3.788497 \\ 1 & -4.036840 & -1.021108 & -3.753874 \\ 1 & -5.210535 & -3.815453 & -3.142931 \\ 1 & -3.823668 & -3.141860 & -2.265124 \\ 1 & -5.246677 & -3.700253 & -1.367686 \\ 1 & -7.346893 & -2.587643 & -3.314887 \\ 1 & -7.458059 & -2.513519 & -1.552600 \\ 1 & -7.605525 & -1.027269 & -2.521031 \\ 1 & -7.129046 & 2.714273 & 2.753910 \\ 1 & -5.479585 & 2.715830 & 2.099679 \\ 1 & -6.867459 & 2.722439 & 0.998056 \\ 1 & -6.736676 & 0.616159 & 4.099312 \\ 1 & -6.090726 & -0.843646 & 3.321672 \\ 1 & -5.049162 & 0.569696 & 3.561217 \\ 1 & -8.602837 & 0.731573 & 2.518634 \\ 1 & -8.368146 & 0.715885 & 0.765763 \\ 1 & -8.060070 & -0.752484 & 1.721600\end{array}$

TS1 $1_{\text {IL-re- }} \psi_{\text {-1a2s3a4a }}$

Number of imaginary frequencies : 1

The smallest frequencies are : $-646.1163 \quad 10.9798 \quad 18.1322 \mathrm{~cm}(-1)$

Electronic energy : $\quad H F=-4360.8606212$

Zero-point correction $=\quad 1.451271$ (Hartree/Particle)

Thermal correction to Energy= $\quad 1.536394$

Thermal correction to Enthalpy= $\quad 1.537338$

Thermal correction to Gibbs Free Energy $=\quad 1.332008$

Sum of electronic and zero-point Energies $=\quad-4359.409350$

Sum of electronic and thermal Energies $=\quad-4359.324227$

Sum of electronic and thermal Enthalpies $=\quad-4359.323283$

Sum of electronic and thermal Free Energies= $\quad-4359.528614$

Cartesian Coordinates

$\begin{array}{cccc}6 & -0.532434 & 3.070720 & 0.956431 \\ 6 & -1.593007 & 2.392599 & 0.340261 \\ 6 & -2.559051 & 3.113554 & -0.343596 \\ 6 & -2.495191 & 4.511660 & -0.429083 \\ 6 & -1.448149 & 5.158059 & 0.220688 \\ 6 & -0.460118 & 4.458429 & 0.934848 \\ 15 & -1.549147 & 0.556800 & 0.235065 \\ 45 & -0.011639 & 0.197327 & -1.570166 \\ 6 & 0.422733 & 2.141818 & -1.933753 \\ 8 & 0.554244 & 3.102175 & -2.566621 \\ 6 & -3.568124 & 5.244708 & -1.230026 \\ 6 & -3.500750 & 4.783492 & -2.691189 \\ 6 & 0.660048 & 5.223669 & 1.638148 \\ 6 & 0.063236 & 6.297252 & 2.555126 \\ 6 & -3.335827 & 0.131201 & 0.041120 \\ 6 & -3.778993 & -0.622563 & -1.042502 \\ 6 & -5.134308 & -0.952845 & -1.173666 \\ 6 & -6.011378 & -0.515796 & -0.180354 \\ 6 & -5.599881 & 0.241600 & 0.921301 \\ 6 & -4.247572 & 0.559713 & 1.011423 \\ 6 & -6.611444 & 0.651844 & 1.989788\end{array}$




\begin{tabular}{|c|c|c|c|}
\hline 6 & -7.794512 & 1.383163 & 1.347835 \\
\hline 6 & -5.684860 & -1.753150 & -2.352490 \\
\hline p & -6.705946 & -0.896889 & -3.110189 \\
\hline & -1.335148 & 0.074166 & 2.022654 \\
\hline y & -1.056156 & -1.238053 & 2.368241 \\
\hline ( & -0.953447 & -1.617424 & 3.749218 \\
\hline 6 & -1.152897 & -0.638263 & 4.764903 \\
\hline 6 & -1.462439 & 0.685558 & 4.380259 \\
\hline 6 & -1.548049 & 1.027533 & 3.058154 \\
\hline 6 & -1.029695 & -1.002893 & 6.126625 \\
\hline 6 & -0.716649 & -2.289307 & 6.483246 \\
\hline 6 & -0.516742 & -3.264886 & 5.484376 \\
\hline 6 & -0.635088 & -2.940714 & 4.155286 \\
\hline 6 & -0.881167 & -2.336070 & 1.378974 \\
\hline 6 & 0.360557 & -2.607627 & 0.835154 \\
\hline 6 & 0.577967 & -3.805145 & 0.112943 \\
\hline 6 & -0.440490 & -4.696781 & -0.094555 \\
\hline 6 & -1.748122 & -4.410413 & 0.363808 \\
\hline 6 & -1.972101 & -3.221765 & 1.118885 \\
\hline 6 & -3.284016 & -2.957130 & 1.589644 \\
\hline 6 & -4.319168 & -3.812442 & 1.307693 \\
\hline 6 & -4.093861 & -4.988563 & 0.558772 \\
\hline 6 & -2.834670 & -5.279212 & 0.098959 \\
\hline 7 & 1.448254 & -1.688052 & 1.004644 \\
\hline 15 & 1.733246 & -0.736394 & -0.379033 \\
\hline 8 & 2.837243 & -1.581278 & -1.344638 \\
\hline 6 & 4.086121 & -1.940039 & -0.905468 \\
\hline 6 & 5.057874 & -0.987536 & -0.649279 \\
\hline 6 & 6.285212 & -1.422363 & -0.050403 \\
\hline 6 & 6.533176 & -2.817229 & 0.122376 \\
\hline 6 & 5.554937 & -3.751831 & -0.296963 \\
\hline 6 & 4.351092 & -3.322604 & -0.783725 \\
\hline 6 & 7.247233 & -0.508720 & 0.451672 \\
\hline 6 & 8.393523 & -0.951085 & 1.064961 \\
\hline 6 & 8.651180 & -2.331297 & 1.200488 \\
\hline 6 & 7.735581 & -3.241722 & 0.738075 \\
\hline 6 & 4.786804 & 0.436573 & -0.949478 \\
\hline 6 & 3.653911 & 1.030493 & -0.426669 \\
\hline 6 & 3.348924 & 2.390773 & -0.631572 \\
\hline 6 & 4.177157 & 3.161507 & -1.400795 \\
\hline 6 & 5.319762 & 2.597719 & -2.017645 \\
\hline 6 & 5.627038 & 1.218855 & -1.806914 \\
\hline 6 & 6.152127 & 3.377200 & -2.857169 \\
\hline 6 & 7.234880 & 2.823196 & -3.490147 \\
\hline 6 & 7.521393 & 1.453469 & -3.313241 \\
\hline 6 & 6.740921 & 0.674010 & -2.495682 \\
\hline 8 & 2.808087 & 0.292889 & 0.360041 \\
\hline 6 & 2.458507 & -2.067094 & 2.021590 \\
\hline 6 & 3.037777 & -0.921115 & 2.808375 \\
\hline 6 & 4.417409 & -0.804721 & 2.965069 \\
\hline 6 & 4.961116 & 0.247183 & 3.698395 \\
\hline 6 & 4.127648 & 1.191687 & 4.288229 \\
\hline 6 & 2.746277 & 1.075456 & 4.141607 \\
\hline 6 & 2.208198 & 0.028137 & 3.405930 \\
\hline 6 & -5.997252 & 1.577057 & 3.038308 \\
\hline 6 & -7.117279 & -0.609552 & 2.699358 \\
\hline
\end{tabular}




\begin{tabular}{|c|c|c|c|}
\hline & -4.588719 & -2.164336 & -3.327927 \\
\hline & -6.364574 & -3.028434 & -1.843446 \\
\hline & 0.907487 & -0.326272 & -3.463836 \\
\hline & -0.454904 & -0.445335 & -3.848802 \\
\hline & -1.024432 & -1.808897 & -4.061302 \\
\hline & -1.688310 & -2.106623 & -5.259618 \\
\hline & -2.127927 & -3.397461 & -5.540587 \\
\hline & -1.919238 & -4.424097 & -4.628679 \\
\hline & -1.274217 & -4.139521 & -3.426711 \\
\hline & -0.844156 & -2.850791 & -3.141841 \\
\hline & -1.011276 & 0.654567 & -4.728577 \\
\hline & -4.949859 & 4.916103 & -0.651967 \\
\hline & -3.387428 & 6.759833 & -1.199636 \\
\hline & 1.550266 & 5.893011 & 0.585929 \\
\hline & 1.526277 & 4.307453 & 2.502097 \\
\hline & 7.052936 & 0.558108 & 0.372578 \\
\hline & -1.790383 & 2.054393 & 2.799268 \\
\hline & 7.908776 & -4.311349 & 0.850500 \\
\hline & -1.184624 & -0.235836 & 6.884083 \\
\hline & 5.903334 & 4.428488 & -2.996732 \\
\hline & -3.467989 & -2.045909 & 2.156467 \\
\hline & -5.324710 & -3.576632 & 1.652110 \\
\hline & 3.955526 & 4.214676 & -1.568789 \\
\hline & -1.632009 & 1.433557 & 5.153802 \\
\hline & 5.766557 & -4.815593 & -0.200848 \\
\hline & 8.365589 & 1.007600 & -3.834609 \\
\hline & 3.579318 & -4.023301 & -1.094565 \\
\hline & -2.647664 & -6.182886 & -0.480336 \\
\hline & -0.261591 & -5.628164 & -0.631089 \\
\hline & -0.267857 & -4.284448 & 5.769715 \\
\hline & 6.969651 & -0.382382 & -2.380494 \\
\hline & -0.479378 & -3.708806 & 3.400450 \\
\hline & 1.579478 & -4.000988 & -0.264876 \\
\hline & 7.864089 & 3.430633 & -4.136615 \\
\hline & 9.107329 & -0.228865 & 1.455051 \\
\hline & -4.923006 & -5.660136 & 0.345778 \\
\hline & -0.619987 & -2.560404 & 7.531976 \\
\hline & 9.566186 & -2.668208 & 1.682039 \\
\hline & 2.448901 & 2.792625 & -0.169838 \\
\hline & -3.887620 & 1.151230 & 1.849253 \\
\hline & -3.045232 & -0.955972 & -1.776532 \\
\hline & -7.066407 & -0.776737 & -0.267267 \\
\hline & -3.367663 & 2.579754 & -0.841530 \\
\hline & 0.237215 & 2.488873 & 1.463019 \\
\hline & -1.385446 & 6.243172 & 0.174811 \\
\hline & 1.932258 & -2.741780 & 2.712256 \\
\hline & 3.274089 & -2.655618 & 1.573947 \\
\hline & 5.077099 & -1.533177 & 2.490132 \\
\hline & 1.132711 & -0.044058 & 3.261890 \\
\hline & 6.041655 & 0.329664 & 3.800395 \\
\hline & 2.080589 & 1.804895 & 4.601703 \\
\hline & 4.551029 & 2.016113 & 4.858245 \\
\hline & 1.481386 & 0.518954 & -3.849849 \\
\hline & 1.490527 & -1.234987 & -3.325491 \\
\hline 1 & -1.351451 & 0.337081 & -2.480137 \\
\hline & -1.853078 & -1.328102 & -6.000310 \\
\hline
\end{tabular}




$\begin{array}{rrrr}1 & -2.635780 & -3.596050 & -6.482464 \\ 1 & -2.264850 & -5.432850 & -4.843799 \\ 1 & -1.117867 & -4.926676 & -2.691197 \\ 1 & -0.378038 & -2.624594 & -2.180777 \\ 1 & -0.678999 & 0.514591 & -5.766872 \\ 1 & -2.108229 & 0.679507 & -4.722525 \\ 1 & -0.649243 & 1.634657 & -4.403821 \\ 1 & -5.024770 & -2.745373 & -4.150747 \\ 1 & -4.083724 & -1.295766 & -3.771905 \\ 1 & -3.825653 & -2.791929 & -2.846705 \\ 1 & -6.792707 & -3.590842 & -2.684279 \\ 1 & -5.636873 & -3.676375 & -1.337650 \\ 1 & -7.177810 & -2.817647 & -1.137552 \\ 1 & -7.099182 & -1.449767 & -3.974024 \\ 1 & -7.559154 & -0.614271 & -2.481308 \\ 1 & -6.244313 & 0.027073 & -3.482339 \\ 1 & -6.761382 & 1.860944 & 3.772863 \\ 1 & -5.178568 & 1.093731 & 3.588190 \\ 1 & -5.609542 & 2.500814 & 2.587983 \\ 1 & -8.519080 & 1.679506 & 2.117961 \\ 1 & -7.463221 & 2.292300 & 0.829799 \\ 1 & -8.327141 & 0.758982 & 0.620734 \\ 1 & -7.857293 & -0.347913 & 3.467933 \\ 1 & -7.593803 & -1.309643 & 2.001387 \\ 1 & -6.290754 & -1.137365 & 3.194076 \\ 1 & -4.183727 & 7.238001 & -1.784005 \\ 1 & -3.443544 & 7.159606 & -0.178705 \\ 1 & -2.429487 & 7.067165 & -1.638398 \\ 1 & -4.264436 & 5.297842 & -3.290469 \\ 1 & -2.518204 & 5.008259 & -3.126307 \\ 1 & -3.668848 & 3.704052 & -2.791810 \\ 1 & -5.735208 & 5.419873 & -1.231457 \\ 1 & -5.162439 & 3.839597 & -0.670496 \\ 1 & -5.029256 & 5.254051 & 0.389820 \\ 1 & 2.281522 & 4.902045 & 3.031663 \\ 1 & 0.925132 & 3.781181 & 3.257061 \\ 1 & 2.065093 & 3.551826 & 1.915082 \\ 1 & 0.865165 & 6.837384 & 3.075838 \\ 1 & -0.526098 & 7.039611 & 2.004539 \\ 1 & -0.589188 & 5.847767 & 3.315105 \\ 1 & 2.374656 & 6.435931 & 1.067902 \\ 1 & 1.985199 & 5.146291 & -0.092479 \\ 1 & 0.989443 & 6.608794 & -0.027619\end{array}$

\section{$\mathrm{TS1}_{\text {IL-re-}-} \boldsymbol{\psi}_{-1 \mathrm{a} 2 \mathrm{2s} 3 \mathrm{a} 4 \mathrm{~s}}$}

Number of imaginary frequencies : 1

The smallest frequencies are : - $650.6430 \quad 12.6428 \quad 20.1024 \mathrm{~cm}(-1)$

Electronic energy : $\quad \mathrm{HF}=-4360.8610877$

Zero-point correction $=\quad 1.451632($ Hartree/Particle $)$

Thermal correction to Energy $=\quad 1.536580$

Thermal correction to Enthalpy= $\quad 1.537524$

Thermal correction to Gibbs Free Energy= $\quad 1.332909$

Sum of electronic and zero-point Energies $=\quad-4359.409456$

Sum of electronic and thermal Energies $=\quad-4359.324508$

Sum of electronic and thermal Enthalpies $=\quad-4359.323564$ 
Cartesian Coordinates

\begin{tabular}{|c|c|c|c|}
\hline 6 & -0.824819 & -2.885962 & -3.116558 \\
\hline 6 & -1.003585 & -1.856226 & -4.049697 \\
\hline 6 & -1.657785 & -2.171268 & -5.248897 \\
\hline 6 & -2.089667 & -3.467412 & -5.517075 \\
\hline 6 & -1.882654 & -4.481989 & -4.591271 \\
\hline 6 & -1.247094 & -4.180056 & -3.388603 \\
\hline 6 & -0.441953 & -0.487733 & -3.849049 \\
\hline 6 & -1.003332 & 0.601090 & -4.739052 \\
\hline 45 & -0.006013 & 0.177874 & -1.572898 \\
\hline 6 & 0.918520 & -0.357710 & -3.461863 \\
\hline 15 & -1.543880 & 0.536826 & 0.228457 \\
\hline 6 & -1.329429 & 0.081006 & 2.023628 \\
\hline 6 & -1.048562 & -1.225913 & 2.386567 \\
\hline 6 & -0.949526 & -1.589059 & 3.771804 \\
\hline 6 & -1.154611 & -0.598548 & 4.775101 \\
\hline 6 & -1.462900 & 0.720475 & 4.373454 \\
\hline 6 & -1.545049 & 1.046792 & 3.046912 \\
\hline 6 & -1.037556 & -0.947477 & 6.141507 \\
\hline 6 & -0.725100 & -2.229479 & 6.514082 \\
\hline 6 & -0.519137 & -3.216164 & 5.527319 \\
\hline 6 & -0.631274 & -2.907287 & 4.194087 \\
\hline 6 & -0.869440 & -2.332486 & 1.408597 \\
\hline 6 & 0.372511 & -2.600852 & 0.863352 \\
\hline 6 & 0.593291 & -3.801245 & 0.147057 \\
\hline 6 & -0.422904 & -4.696611 & -0.055735 \\
\hline 6 & -1.731150 & -4.412538 & 0.402462 \\
\hline 6 & -1.957834 & -3.223126 & 1.155603 \\
\hline 6 & -3.269586 & -2.962223 & 1.629879 \\
\hline 6 & -4.301933 & -3.821829 & 1.350508 \\
\hline 6 & -4.074905 & -4.996804 & 0.600359 \\
\hline 6 & -2.815556 & -5.284439 & 0.139195 \\
\hline 7 & 1.455705 & -1.674010 & 1.023762 \\
\hline 6 & 2.464720 & -2.034255 & 2.048849 \\
\hline 6 & 3.039973 & -0.874127 & 2.817791 \\
\hline 6 & 4.419307 & -0.749131 & 2.970773 \\
\hline 6 & 4.959290 & 0.316992 & 3.686074 \\
\hline 6 & 4.122420 & 1.267581 & 4.261079 \\
\hline 6 & 2.741396 & 1.142851 & 4.118336 \\
\hline 6 & 2.206913 & 0.081088 & 3.400971 \\
\hline 6 & -1.619306 & 2.372706 & 0.314432 \\
\hline 6 & -0.563871 & 3.073127 & 0.914297 \\
\hline 6 & -0.517414 & 4.461778 & 0.885869 \\
\hline 6 & -1.524797 & 5.139236 & 0.177214 \\
\hline 6 & -2.567310 & 4.470405 & -0.457212 \\
\hline 6 & -2.607098 & 3.072142 & -0.360563 \\
\hline 6 & -3.656592 & 5.177974 & -1.259036 \\
\hline 6 & -3.513488 & 6.697037 & -1.225400 \\
\hline 6 & 0.595671 & 5.249931 & 1.575326 \\
\hline 6 & 1.474209 & 4.355927 & 2.450342 \\
\hline 6 & -3.323487 & 0.085796 & 0.034216 \\
\hline 6 & -3.767116 & -0.668854 & -1.053409 \\
\hline 6 & -5.119762 & -0.992733 & -1.187581 \\
\hline
\end{tabular}




\begin{tabular}{|c|c|c|c|}
\hline 6 & -6.006659 & -0.551062 & -0.196889 \\
\hline & -5.592963 & 0.195598 & 0.905031 \\
\hline & -4.235754 & 0.508715 & 0.997318 \\
\hline & -5.668668 & -1.792718 & -2.367222 \\
\hline & -6.360206 & -3.061662 & -1.858038 \\
\hline & -6.537460 & 0.671257 & 2.006193 \\
\hline & -6.071030 & 0.097748 & 3.349997 \\
\hline & 0.404953 & 2.122838 & -1.956390 \\
\hline & 0.509314 & 3.083712 & -2.593530 \\
\hline & 1.739571 & -0.736907 & -0.370226 \\
\hline & 2.807500 & 0.307131 & 0.358273 \\
\hline & 3.647483 & 1.041622 & -0.437472 \\
\hline & 4.784196 & 0.449495 & -0.954131 \\
\hline & 5.618160 & 1.227477 & -1.821550 \\
\hline & 5.300858 & 2.601538 & -2.048497 \\
\hline & 4.154744 & 3.164580 & -1.437462 \\
\hline & 3.332579 & 2.397171 & -0.658448 \\
\hline & 6.735486 & 0.682483 & -2.504616 \\
\hline & 7.509892 & 1.457760 & -3.331859 \\
\hline & 7.213468 & 2.823201 & -3.524828 \\
\hline & 6.127160 & 3.376932 & -2.897737 \\
\hline & 5.065794 & -0.968772 & -0.635987 \\
\hline & 4.100546 & -1.931378 & -0.878771 \\
\hline & 4.374286 & -3.310283 & -0.737089 \\
\hline 6 & 5.581273 & -3.724696 & -0.245268 \\
\hline & 6.553811 & -2.777865 & 0.159598 \\
\hline 6 & 6.296375 & -1.387164 & -0.032062 \\
\hline f & 7.759641 & -3.185877 & 0.779729 \\
\hline 6 & 8.669696 & -2.263175 & 1.228535 \\
\hline 5 & 8.402578 & -0.886701 & 1.074560 \\
\hline 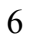 & 7.252630 & -0.460420 & 0.456687 \\
\hline 8 & 2.849575 & -1.587130 & -1.323622 \\
\hline 6 & -3.571340 & 4.719896 & -2.720409 \\
\hline 6 & -5.032665 & 4.814323 & -0.688259 \\
\hline 5 & -0.010073 & 6.330209 & 2.478506 \\
\hline 6 & 1.475991 & 5.913992 & 0.511586 \\
\hline 6 & -6.516780 & 2.202989 & 2.065782 \\
\hline 6 & -7.977097 & 0.221457 & 1.773381 \\
\hline 6 & -6.678848 & -0.932347 & -3.134839 \\
\hline 5 & -4.569380 & -2.215635 & -3.333966 \\
\hline 1 & 7.051022 & 0.603905 & 0.363548 \\
\hline 1 & -1.786971 & 2.070314 & 2.775188 \\
\hline 1 & 7.940009 & -4.252679 & 0.906676 \\
\hline 1 & -1.196881 & -0.171988 & 6.889409 \\
\hline & 5.870812 & 4.424706 & -3.049520 \\
\hline 1 & -3.456492 & -2.052061 & 2.198421 \\
\hline 1 & -5.307287 & -3.591565 & 1.698617 \\
\hline 1 & 3.925354 & 4.214128 & -1.617298 \\
\hline 1 & -1.634754 & 1.477673 & 5.137478 \\
\hline 1 & 5.799839 & -4.785558 & -0.133837 \\
\hline 1 & 8.356931 & 1.011781 & -3.848499 \\
\hline 1 & 3.606550 & -4.020244 & -1.036779 \\
\hline 1 & -2.626806 & -6.188033 & -0.439645 \\
\hline 1 & -0.241644 & -5.629705 & -0.588504 \\
\hline 1 & -0.270537 & -4.232120 & 5.825491 \\
\hline & 6.971758 & -0.370844 & -2.377296 \\
\hline
\end{tabular}




\begin{tabular}{|c|c|c|c|}
\hline ] & -0.471234 & -3.683486 & 3.448465 \\
\hline & 1.594922 & -3.995625 & -0.231310 \\
\hline & 7.837982 & 3.427419 & -4.178819 \\
\hline 1 & 9.111861 & -0.154474 & 1.454105 \\
\hline 1 & -4.902562 & -5.670688 & 0.388973 \\
\hline & -0.633468 & -2.488655 & 7.566274 \\
\hline & 9.587460 & -2.587369 & 1.713563 \\
\hline & 2.430194 & 2.798330 & -0.200770 \\
\hline & -3.883748 & 1.097533 & 1.843189 \\
\hline & -3.031871 & -1.002750 & -1.785684 \\
\hline & -7.060315 & -0.804151 & -0.297279 \\
\hline & -3.413672 & 2.521797 & -0.843695 \\
\hline & 0.221140 & 2.507493 & 1.415903 \\
\hline & -1.481650 & 6.224904 & 0.123538 \\
\hline & 1.938524 & -2.698906 & 2.749208 \\
\hline & 3.282522 & -2.628012 & 1.612060 \\
\hline & 5.081705 & -1.482030 & 2.506494 \\
\hline & 1.131482 & 0.001974 & 3.259816 \\
\hline & 6.039540 & 0.405810 & 3.785696 \\
\hline & 2.073233 & 1.877130 & 4.567013 \\
\hline & 4.542863 & 2.103186 & 4.816802 \\
\hline & 1.488843 & 0.487407 & -3.853393 \\
\hline & 1.506022 & -1.261982 & -3.313509 \\
\hline ] & -1.346673 & 0.299790 & -2.485232 \\
\hline & -1.820987 & -1.402397 & -5.999983 \\
\hline 1 & -2.590221 & -3.679734 & -6.459863 \\
\hline & -2.222469 & -5.494772 & -4.796469 \\
\hline & -1.092552 & -4.957410 & -2.642299 \\
\hline ] & -0.366841 & -2.645134 & -2.155134 \\
\hline & -0.668041 & 0.454230 & -5.775421 \\
\hline 1 & -2.100392 & 0.619172 & -4.735471 \\
\hline & -0.648075 & 1.586224 & -4.422284 \\
\hline 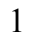 & -5.004115 & -2.797377 & -4.156997 \\
\hline & -4.055703 & -1.352638 & -3.778672 \\
\hline 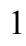 & -3.813874 & -2.846092 & -2.844693 \\
\hline ] & -6.777601 & -3.629446 & -2.700652 \\
\hline & -5.642028 & -3.707708 & -1.336259 \\
\hline 1 & -7.183283 & -2.842652 & -1.166600 \\
\hline & -7.069987 & -1.485736 & -3.999318 \\
\hline 1 & -7.534527 & -0.641957 & -2.513027 \\
\hline & -6.208190 & -0.012981 & -3.507235 \\
\hline 1 & -8.613545 & 0.580625 & 2.591971 \\
\hline ] & -8.387302 & 0.623776 & 0.838152 \\
\hline & -8.064174 & -0.872685 & 1.744833 \\
\hline 1 & -7.165045 & 2.563390 & 2.875999 \\
\hline & -5.508290 & 2.597083 & 2.244846 \\
\hline 1 & -6.881007 & 2.635945 & 1.124554 \\
\hline 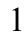 & -6.729404 & 0.438844 & 4.160376 \\
\hline 1 & -6.096815 & -1.000222 & 3.336109 \\
\hline 1 & -5.047397 & 0.402558 & 3.603584 \\
\hline 1 & -4.321146 & 7.156728 & -1.808976 \\
\hline 1 & -3.579441 & 7.092776 & -0.203458 \\
\hline 1 & -2.563572 & 7.029253 & -1.663414 \\
\hline 1 & -4.344560 & 5.215751 & -3.323088 \\
\hline & -2.592363 & 4.968362 & -3.150818 \\
\hline 1 & -3.712474 & 3.636517 & -2.821240 \\
\hline
\end{tabular}




$\begin{array}{lrrr}1 & -5.826343 & 5.306526 & -1.266368 \\ 1 & -5.222848 & 3.733547 & -0.717971 \\ 1 & -5.123137 & 5.140881 & 0.356382 \\ 1 & 2.222831 & 4.966912 & 2.970545 \\ 1 & 0.880619 & 3.832626 & 3.213343 \\ 1 & 2.021733 & 3.598962 & 1.873216 \\ 1 & 0.787559 & 6.882933 & 2.992493 \\ 1 & -0.604599 & 7.061490 & 1.918830 \\ 1 & -0.659240 & 5.885422 & 3.244013 \\ 1 & 2.290310 & 6.480005 & 0.984047 \\ 1 & 1.925193 & 5.161922 & -0.151414 \\ 1 & 0.903635 & 6.607862 & -0.116338\end{array}$

TS1 $1_{L-r e-} \psi_{-1}$ 1a2s334a

Number of imaginary frequencies : 1

The smallest frequencies are : - $641.0168 \quad 13.1941 \quad 15.2672 \mathrm{~cm}(-1)$

Electronic energy : $\quad \mathrm{HF}=-4360.864114$

Zero-point correction $=\quad 1.450921$ (Hartree/Particle)

Thermal correction to Energy= $\quad 1.536053$

Thermal correction to Enthalpy= $\quad 1.536997$

Thermal correction to Gibbs Free Energy= 1.331577

Sum of electronic and zero-point Energies $=\quad-4359.413193$

Sum of electronic and thermal Energies $=\quad-4359.328061$

Sum of electronic and thermal Enthalpies $=\quad-4359.327117$

Sum of electronic and thermal Free Energies $=\quad-4359.532537$

Cartesian Coordinates

$\begin{array}{cccc}6 & 7.257119 & -0.510113 & 0.363242 \\ 6 & 6.290744 & -1.425690 & -0.126871 \\ 6 & 6.550164 & -2.820106 & 0.032521 \\ 6 & 7.767624 & -3.241643 & 0.620071 \\ 6 & 8.687685 & -2.329142 & 1.069190 \\ 6 & 8.418580 & -0.949726 & 0.949428 \\ 6 & 5.050171 & -0.994017 & -0.701255 \\ 6 & 4.080582 & -1.951529 & -0.947561 \\ 6 & 4.354010 & -3.333116 & -0.833782 \\ 6 & 5.568550 & -3.758152 & -0.370751 \\ 6 & 4.764588 & 0.431747 & -0.980672 \\ 6 & 3.634826 & 1.011230 & -0.435071 \\ 6 & 3.321189 & 2.373345 & -0.611929 \\ 6 & 4.137597 & 3.162691 & -1.374511 \\ 6 & 5.274536 & 2.615140 & -2.016070 \\ 6 & 5.589414 & 1.233596 & -1.835440 \\ 6 & 6.092680 & 3.413301 & -2.851976 \\ 6 & 7.168226 & 2.875249 & -3.510545 \\ 6 & 7.461329 & 1.503363 & -3.363973 \\ 6 & 6.694995 & 0.705938 & -2.550283 \\ 8 & 2.799877 & 0.258075 & 0.347708 \\ 15 & 1.723645 & -0.765847 & -0.395354 \\ 8 & 2.824335 & -1.600310 & -1.371227 \\ 45 & -0.015322 & 0.177907 & -1.577520 \\ 6 & 0.450150 & 2.097040 & -1.986114 \\ 8 & 0.549273 & 3.088603 & -2.574380 \\ 6 & -0.563512 & -0.508942 & -3.830708\end{array}$




\begin{tabular}{|c|c|c|c|}
\hline & 0.813389 & -0.455897 & -3.484127 \\
\hline & -1.222072 & -1.839815 & -3.979242 \\
\hline & -0.985832 & -2.893597 & -3.084376 \\
\hline & -1.495554 & -4.162060 & -3.319558 \\
\hline & -2.284363 & -4.414279 & -4.441174 \\
\hline & -2.557630 & -3.374206 & -5.319248 \\
\hline & -2.032527 & -2.103937 & -5.092180 \\
\hline & -1.072509 & 0.596992 & -4.731257 \\
\hline & -1.514314 & 0.561644 & 0.243506 \\
\hline & -3.305878 & 0.168708 & 0.048583 \\
\hline & -3.755763 & -0.586898 & -1.023282 \\
\hline & -5.121539 & -0.883558 & -1.173224 \\
\hline & -6.000807 & -0.407293 & -0.206443 \\
\hline & -5.577108 & 0.357904 & 0.893027 \\
\hline & -4.221384 & 0.635150 & 1.002756 \\
\hline & -5.581854 & -1.705192 & -2.374250 \\
\hline & -5.152746 & -0.996023 & -3.663421 \\
\hline & -6.591784 & 0.825771 & 1.934155 \\
\hline & -5.962174 & 1.747143 & 2.977392 \\
\hline & -1.308132 & 0.060391 & 2.026053 \\
\hline & -1.051594 & -1.258955 & 2.362765 \\
\hline & -0.954408 & -1.646938 & 3.742479 \\
\hline & -1.143903 & -0.671316 & 4.763649 \\
\hline & -1.432362 & 0.659436 & 4.386747 \\
\hline & -1.506791 & 1.011084 & 3.066667 \\
\hline & -1.029208 & -1.045205 & 6.123682 \\
\hline 6 & -0.730912 & -2.336738 & 6.474109 \\
\hline & -0.536604 & -3.307995 & 5.470033 \\
\hline 6 & -0.648407 & -2.975165 & 4.142562 \\
\hline 5 & -0.888008 & -2.358348 & 1.371674 \\
\hline 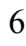 & 0.350586 & -2.637814 & 0.823978 \\
\hline 5 & 0.564059 & -3.843832 & 0.114567 \\
\hline 6 & -0.451476 & -4.745319 & -0.062863 \\
\hline 6 & -1.753885 & -4.460314 & 0.411557 \\
\hline 6 & -1.979263 & -3.251266 & 1.133861 \\
\hline 6 & -3.288680 & -2.984906 & 1.611706 \\
\hline 6 & -4.314367 & -3.868040 & 1.384300 \\
\hline ) & -4.084815 & -5.069221 & 0.678346 \\
\hline 6 & -2.831833 & -5.354013 & 0.199038 \\
\hline I & 1.443804 & -1.723535 & 0.985095 \\
\hline 6 & 2.459787 & -2.112098 & 1.993877 \\
\hline 5 & 3.052691 & -0.970162 & 2.776231 \\
\hline 6 & 4.434862 & -0.842037 & 2.896507 \\
\hline 6 & 4.988340 & 0.213950 & 3.616508 \\
\hline 6 & 4.162348 & 1.149847 & 4.230323 \\
\hline 6 & 2.778574 & 1.019417 & 4.123579 \\
\hline 6 & 2.230833 & -0.031699 & 3.400661 \\
\hline 6 & -1.531202 & 2.397792 & 0.361945 \\
\hline 6 & -0.455874 & 3.061436 & 0.968580 \\
\hline 6 & -0.374736 & 4.449414 & 0.961495 \\
\hline 6 & -1.371101 & 5.164197 & 0.274600 \\
\hline 6 & -2.433619 & 4.532467 & -0.364226 \\
\hline 6 & -2.502858 & 3.134135 & -0.297752 \\
\hline 6 & 0.767071 & 5.199459 & 1.646539 \\
\hline 6 & 1.658322 & 5.838132 & 0.575671 \\
\hline 6 & -3.519345 & 5.281452 & -1.132204 \\
\hline
\end{tabular}




\begin{tabular}{|c|c|c|c|}
\hline & -3.339471 & 6.795669 & -1.070113 \\
\hline & -4.892275 & 4.937863 & -0.541053 \\
\hline & -3.473459 & 4.852008 & -2.603817 \\
\hline & 0.204649 & 6.296546 & 2.557182 \\
\hline & 1.622951 & 4.275450 & 2.512778 \\
\hline & -7.097546 & -1.883451 & -2.415556 \\
\hline & -4.940801 & -3.094545 & -2.305453 \\
\hline & -7.732519 & 1.590702 & 1.255675 \\
\hline & -7.157887 & -0.402234 & 2.656307 \\
\hline & 7.055531 & 0.556070 & 0.295391 \\
\hline & -1.729284 & 2.043742 & 2.812846 \\
\hline & 7.949045 & -4.311008 & 0.721483 \\
\hline & -1.177926 & -0.280641 & 6.884897 \\
\hline & 5.838346 & 4.466105 & -2.968205 \\
\hline & -3.478969 & -2.056512 & 2.148683 \\
\hline & -5.315082 & -3.639224 & 1.745612 \\
\hline & 3.908466 & 4.217961 & -1.519115 \\
\hline & -1.592348 & 1.405010 & 5.164673 \\
\hline & 5.787011 & -4.821158 & -0.281856 \\
\hline & 8.299193 & 1.070201 & -3.905853 \\
\hline & 3.579014 & -4.036147 & -1.131432 \\
\hline & -2.642144 & -6.273791 & -0.353514 \\
\hline & -0.273646 & -5.686259 & -0.582930 \\
\hline & -0.296986 & -4.331303 & 5.749858 \\
\hline & 6.928390 & -0.351736 & -2.458934 \\
\hline & -0.495767 & -3.740747 & 3.384681 \\
\hline & 1.562615 & -4.041406 & -0.271020 \\
\hline & 7.786283 & 3.497059 & -4.154101 \\
\hline & 9.135755 & -0.225786 & 1.330031 \\
\hline I & -4.906334 & -5.761923 & 0.508671 \\
\hline & -0.640547 & -2.614898 & 7.521546 \\
\hline 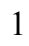 & 9.614756 & -2.663846 & 1.528687 \\
\hline & 2.423075 & 2.761422 & -0.134574 \\
\hline & -3.856469 & 1.231377 & 1.834863 \\
\hline & -3.025903 & -0.954578 & -1.747381 \\
\hline & -7.061185 & -0.632461 & -0.299974 \\
\hline 1 & -3.323117 & 2.612508 & -0.789121 \\
\hline & 0.316075 & 2.469156 & 1.460049 \\
\hline 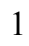 & -1.303459 & 6.249417 & 0.242963 \\
\hline & 1.933758 & -2.783714 & 2.687610 \\
\hline & 2.501838 & 6.366253 & 1.040864 \\
\hline 1 & 2.065904 & 5.076052 & -0.102963 \\
\hline & 1.104062 & 6.560915 & -0.035797 \\
\hline 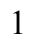 & 1.024587 & 6.823023 & 3.063467 \\
\hline & -0.372099 & 7.047352 & 2.004856 \\
\hline 1 & -0.450162 & 5.871187 & 3.328887 \\
\hline & 1.016393 & 3.762248 & 3.272373 \\
\hline & 2.149727 & 3.509413 & 1.928923 \\
\hline 1 & 2.388618 & 4.861976 & 3.036398 \\
\hline & -4.145904 & 7.286522 & -1.629501 \\
\hline 1 & -3.377721 & 7.171367 & -0.039229 \\
\hline & -2.389437 & 7.114008 & -1.518095 \\
\hline 1 & -5.110143 & 3.863337 & -0.595174 \\
\hline | & -4.952338 & 5.238971 & 0.513388 \\
\hline l & -5.685751 & 5.464058 & -1.088698 \\
\hline & -2.496932 & 5.085268 & -3.048054 \\
\hline
\end{tabular}




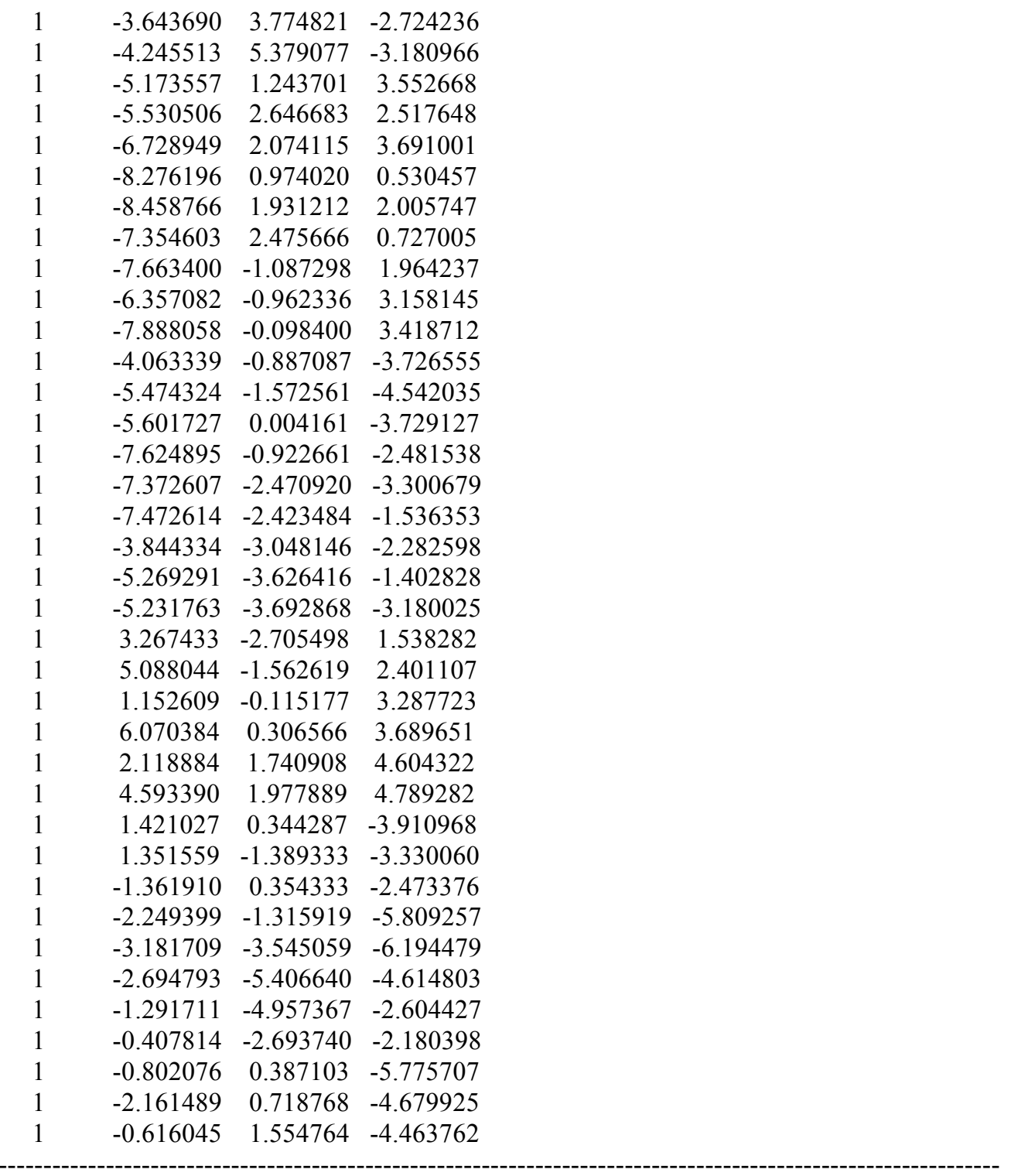

$\mathrm{TS1}_{\text {IL-re- }} \boldsymbol{\psi}_{-1 \mathrm{a} 25334 \mathrm{~s}}$

Number of imaginary frequencies : 1

The smallest frequencies are : $-643.5264 \quad 11.6622 \quad 19.4172 \mathrm{~cm}(-1)$

Electronic energy : $\quad H F=-4360.8643237$

Zero-point correction $=\quad 1.451674$ (Hartree/Particle)

Thermal correction to Energy= $\quad 1.536480$

Thermal correction to Enthalpy= $\quad 1.537425$

Thermal correction to Gibbs Free Energy $=\quad 1.333446$

Sum of electronic and zero-point Energies $=\quad-4359.412650$

Sum of electronic and thermal Energies $=\quad-4359.327843$

Sum of electronic and thermal Enthalpies $=\quad-4359.326899$

Sum of electronic and thermal Free Energies $=\quad-4359.530878$

Cartesian Coordinates

$\begin{array}{cccc}6 & -4.229212 & 0.530319 & 1.013513 \\ 6 & -3.309620 & 0.122397 & 0.045295\end{array}$




\begin{tabular}{|c|c|c|c|}
\hline 5 & -3.750457 & -0.608216 & -1.052410 \\
\hline 6 & -5.107044 & -0.925558 & -1.206213 \\
\hline 6 & -5.997741 & -0.503638 & -0.217186 \\
\hline 6 & -5.582497 & 0.221682 & 0.905656 \\
\hline 1 & -1.528330 & 0.555236 & 0.249160 \\
\hline & -0.013562 & 0.215084 & -1.570222 \\
\hline 6 & 0.440405 & 2.148653 & -1.935161 \\
\hline 3 & 0.534648 & 3.134520 & -2.534239 \\
\hline 5 & -5.557322 & -1.724267 & -2.427162 \\
\hline f & -6.533784 & 0.674691 & 2.011468 \\
\hline 5 & -1.589029 & 2.388978 & 0.376154 \\
\hline 6 & -2.572902 & 3.108669 & -0.283605 \\
\hline 6 & -2.523684 & 4.508037 & -0.353178 \\
\hline 6 & -1.475779 & 5.157158 & 0.292632 \\
\hline 6 & -0.471776 & 4.458832 & 0.985644 \\
\hline 6 & -0.527515 & 3.069999 & 0.987226 \\
\hline 6 & 0.648619 & 5.226389 & 1.686141 \\
\hline 6 & -3.608768 & 5.238271 & -1.140128 \\
\hline 6 & -1.314445 & 0.049445 & 2.029686 \\
\hline 6 & -1.040746 & -1.268566 & 2.357428 \\
\hline 6 & -0.937142 & -1.665647 & 3.733573 \\
\hline 6 & -1.134412 & -0.699430 & 4.761973 \\
\hline 6 & -1.438023 & 0.630705 & 4.394869 \\
\hline 6 & -1.521436 & 0.990621 & 3.077223 \\
\hline 6 & -1.013694 & -1.082328 & 6.118975 \\
\hline 6 & -0.703538 & -2.373843 & 6.459070 \\
\hline 6 & -0.503043 & -3.336145 & 5.447524 \\
\hline 6 & & -2.994306 & \\
\hline 6 & -0.871817 & -2.356847 & 1.355887 \\
\hline 6 & 0.366035 & -2.622514 & 0.799947 \\
\hline 6 & 0.580817 & -3.817319 & 0.072199 \\
\hline 6 & -0.433047 & -4.718056 & -0.118280 \\
\hline 6 & -1.735408 & -4.443919 & 0.362416 \\
\hline 6 & -1.960753 & -3.249472 & 1.108257 \\
\hline 6 & -3.268916 & -2.993575 & 1.594812 \\
\hline 6 & -4.295343 & -3.870660 & 1.348475 \\
\hline 6 & -4.066335 & -5.057180 & 0.617898 \\
\hline 6 & -2.813370 & -5.333413 & 0.133261 \\
\hline 7 & 1.455695 & -1.705012 & 0.969114 \\
\hline 15 & 1.733814 & -0.736816 & -0.404233 \\
\hline 8 & 2.810505 & 0.283848 & 0.342766 \\
\hline 6 & 3.654943 & 1.033382 & -0.433629 \\
\hline 6 & 4.784111 & 0.447322 & -0.973354 \\
\hline 6 & 5.620555 & 1.244222 & -1.821165 \\
\hline 6 & 5.316423 & 2.627956 & -2.002140 \\
\hline 6 & 4.179775 & 3.182735 & -1.366413 \\
\hline 6 & 3.353281 & 2.398659 & -0.608959 \\
\hline 6 & 6.727488 & 0.709736 & -2.528882 \\
\hline 6 & 7.504575 & 1.502819 & -3.336557 \\
\hline 6 & 7.221574 & 2.876764 & -3.483818 \\
\hline 6 & 6.145356 & 3.421446 & -2.831846 \\
\hline 6 & 5.057185 & -0.981502 & -0.697903 \\
\hline 6 & 4.083681 & -1.930467 & -0.960344 \\
\hline 6 & 4.348417 & -3.314578 & -0.857679 \\
\hline 6 & 5.555606 & -3.750720 & -0.385670 \\
\hline 6 & 6.537961 & -2.822112 & 0.037171 \\
\hline
\end{tabular}




\begin{tabular}{|c|c|c|c|}
\hline & 6.289233 & -1.424942 & -0.114747 \\
\hline & 7.745795 & -3.255570 & 0.635787 \\
\hline & 8.666275 & -2.352038 & 1.101915 \\
\hline & 8.407925 & -0.970026 & 0.987951 \\
\hline & 7.256267 & -0.518815 & 0.391408 \\
\hline & 2.832413 & -1.565812 & -1.388039 \\
\hline & 2.469382 & -2.092352 & 1.980098 \\
\hline & 3.053267 & -0.951073 & 2.770271 \\
\hline & 4.433978 & -0.828452 & 2.911360 \\
\hline & 4.980843 & 0.224095 & 3.641420 \\
\hline & 4.149559 & 1.162487 & 4.244022 \\
\hline & 2.767078 & 1.038803 & 4.114683 \\
\hline & 2.225888 & -0.009209 & 3.382359 \\
\hline ) & 0.829878 & -0.360350 & -3.484809 \\
\hline & -0.547576 & -0.427353 & -3.830010 \\
\hline & -1.185272 & -1.765695 & -4.005908 \\
\hline ) & -0.941141 & -2.831780 & -3.127844 \\
\hline & -1.433338 & -4.102314 & -3.388462 \\
\hline ) & -2.212445 & -4.344937 & -4.518852 \\
\hline & -2.492939 & -3.293496 & -5.380910 \\
\hline & -1.984659 & -2.021168 & -5.128837 \\
\hline & -1.073833 & 0.687082 & -4.710652 \\
\hline & 7.062230 & 0.549096 & 0.328315 \\
\hline & -1.757567 & 2.022022 & 2.831052 \\
\hline & 7.919336 & -4.326740 & 0.731800 \\
\hline & -1.168035 & -0.325026 & 6.886284 \\
\hline & 5.899031 & 4.476095 & -2.948599 \\
\hline & -3.457122 & -2.076939 & 2.152877 \\
\hline & -5.295955 & -3.647193 & 1.713461 \\
\hline . & 3.960675 & 4.239849 & -1.511043 \\
\hline & -1.603929 & 1.369109 & 5.178360 \\
\hline ] & 5.767187 & -4.815742 & -0.304731 \\
\hline & 8.343217 & 1.064620 & -3.873159 \\
\hline & 3.573828 & -4.010895 & -1.171389 \\
\hline & -2.624241 & -6.242161 & -0.437406 \\
\hline & -0.253782 & -5.649642 & -0.654398 \\
\hline 1 & -0.254924 & -4.359541 & 5.719489 \\
\hline & 6.953227 & -0.349557 & -2.436967 \\
\hline 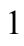 & -0.463002 & -3.752630 & 3.358448 \\
\hline ] & 1.578758 & -4.006404 & -0.318489 \\
\hline 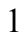 & 7.848044 & 3.494977 & -4.122684 \\
\hline 1 & 9.125679 & -0.253462 & 1.381208 \\
\hline & -4.888254 & -5.745816 & 0.433989 \\
\hline 1 & -0.608979 & -2.659023 & 7.504240 \\
\hline & 9.585652 & -2.695890 & 1.570028 \\
\hline 1 & 2.457051 & 2.793596 & -0.134168 \\
\hline l & -3.879607 & 1.102483 & 1.871933 \\
\hline 1 & -3.015412 & -0.938663 & -1.789111 \\
\hline 1 & -7.050725 & -0.747932 & -0.321486 \\
\hline 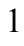 & -3.383539 & 2.573641 & -0.776930 \\
\hline 1 & 0.254250 & 2.489235 & 1.476649 \\
\hline ( & -1.425096 & 6.243394 & 0.260170 \\
\hline 1 & 1.944461 & -2.770122 & 2.668698 \\
\hline 1 & 3.281984 & -2.679590 & 1.525313 \\
\hline 1 & 5.091698 & -1.551375 & 2.425432 \\
\hline 1 & 1.149178 & -0.086855 & 3.251311 \\
\hline
\end{tabular}




\begin{tabular}{|c|c|c|c|}
\hline 1 & 6.062049 & 0.311993 & 3.731053 \\
\hline & 2.103101 & 1.762597 & 4.586000 \\
\hline & 4.575506 & 1.987615 & 4.811103 \\
\hline & 1.423917 & 0.458342 & -3.895873 \\
\hline & 1.382793 & -1.288659 & -3.353099 \\
\hline & -1.359339 & 0.399399 & -2.464104 \\
\hline & -2.207090 & -1.223672 & -5.833597 \\
\hline & -3.109498 & -3.456866 & -6.262861 \\
\hline & -2.609498 & -5.339155 & -4.711906 \\
\hline & -1.223440 & -4.907555 & -2.686482 \\
\hline & -0.371753 & -2.640869 & -2.216436 \\
\hline & -0.796208 & 0.503126 & -5.758097 \\
\hline & -2.165124 & 0.787207 & -4.660532 \\
\hline & -0.636483 & 1.647813 & -4.422552 \\
\hline & 1.527904 & 4.309391 & 2.536040 \\
\hline 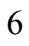 & 1.525789 & 5.909343 & 0.631781 \\
\hline 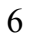 & 0.052722 & 6.289090 & 2.616201 \\
\hline & -3.455443 & 6.755358 & -1.076898 \\
\hline 6 & -3.527198 & 4.808014 & -2.610191 \\
\hline 5 & -4.986890 & 4.872367 & -0.575843 \\
\hline f & -7.072455 & -1.905821 & -2.483647 \\
\hline 6 & -5.122355 & -0.992988 & -3.701809 \\
\hline 6 & -4.913690 & -3.113596 & -2.376316 \\
\hline 6 & -7.973361 & 0.233432 & 1.761343 \\
\hline 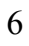 & -6.076267 & 0.070233 & 3.344794 \\
\hline 6 & -6.511570 & 2.204632 & 2.106575 \\
\hline 1 & 2.282369 & 4.905218 & 3.065324 \\
\hline 1 & 0.936078 & 3.772096 & 3.290612 \\
\hline 1 & 2.068375 & 3.562924 & 1.938964 \\
\hline 1 & 2.349708 & 6.455416 & 1.111011 \\
\hline 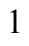 & 1.960861 & 5.170207 & -0.054730 \\
\hline I & 0.954683 & 6.624570 & 0.027116 \\
\hline 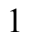 & 0.855606 & 6.829573 & 3.135030 \\
\hline 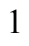 & -0.544921 & 7.032632 & 2.076324 \\
\hline I & -0.591252 & 5.829887 & 3.377572 \\
\hline 1 & -4.260515 & 7.231845 & -1.650489 \\
\hline 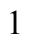 & -3.517651 & 7.131344 & -0.047279 \\
\hline & -2.503665 & 7.089625 & -1.509278 \\
\hline 1 & -4.297264 & 5.320647 & -3.202814 \\
\hline 1 & -2.546672 & 5.057891 & -3.036228 \\
\hline D & -3.675846 & 3.727682 & -2.731743 \\
\hline 1 & -5.777578 & 5.382554 & -1.142365 \\
\hline ] & -5.184691 & 3.793871 & -0.628941 \\
\hline 1 & -5.074288 & 5.176510 & 0.475795 \\
\hline & -7.339016 & -2.471628 & -3.385354 \\
\hline 1 & -7.453288 & -2.469160 & -1.621842 \\
\hline 1 & -7.602151 & -0.945139 & -2.529173 \\
\hline 1 & -5.438857 & -1.555304 & -4.591416 \\
\hline 1 & -5.572919 & 0.007339 & -3.753266 \\
\hline 1 & -4.033046 & -0.881383 & -3.759080 \\
\hline 1 & -5.195674 & -3.698424 & -3.262988 \\
\hline 1 & -3.817503 & -3.065049 & -2.342048 \\
\hline 1 & -5.248967 & -3.660506 & -1.485004 \\
\hline 1 & -7.165569 & 2.547261 & 2.919927 \\
\hline 1 & -5.503983 & 2.592873 & 2.302334 \\
\hline 1 & -6.868080 & 2.659909 & 1.172942 \\
\hline
\end{tabular}




$\begin{array}{rrrr}1 & -6.739804 & 0.392371 & 4.158758 \\ 1 & -6.101445 & -1.027128 & 3.305087 \\ 1 & -5.054176 & 0.368973 & 3.611362 \\ 1 & -8.612849 & 0.576986 & 2.584291 \\ 1 & -8.378068 & 0.656570 & 0.832977 \\ 1 & -8.063606 & -0.859494 & 1.709287\end{array}$

TS1 $_{I L-r e-} \psi_{-1 \text { s2aza4a }}$

Number of imaginary frequencies : 1

The smallest frequencies are : -663.6395 $11.6444 \quad 19.4857 \mathrm{~cm}(-1)$

Electronic energy : $\quad H F=-4360.8592978$

Zero-point correction $=\quad 1.451396$ (Hartree/Particle)

Thermal correction to Energy $=\quad 1.536521$

Thermal correction to Enthalpy= $\quad 1.537465$

Thermal correction to Gibbs Free Energy= $\quad 1.331787$

Sum of electronic and zero-point Energies $=\quad-4359.407902$

Sum of electronic and thermal Energies $=\quad-4359.322777$

Sum of electronic and thermal Enthalpies= $\quad-4359.321833$

Sum of electronic and thermal Free Energies $=\quad-4359.527511$

Cartesian Coordinates

$\begin{array}{cccc}6 & -1.403499 & -1.999511 & -5.323774 \\ 6 & -0.815988 & -1.699020 & -4.086749 \\ 6 & -0.658613 & -2.746564 & -3.169845 \\ 6 & -1.037449 & -4.042978 & -3.492752 \\ 6 & -1.603360 & -4.330144 & -4.732976 \\ 6 & -1.788656 & -3.298218 & -5.644254 \\ 6 & -0.300844 & -0.320383 & -3.830682 \\ 6 & -0.876358 & 0.777513 & -4.701223 \\ 45 & 0.067662 & 0.276008 & -1.537798 \\ 6 & 1.050452 & -0.163725 & -3.418567 \\ 15 & -1.535435 & 0.532806 & 0.224358 \\ 6 & -1.298326 & 0.096718 & 2.022749 \\ 6 & -0.979386 & -1.202241 & 2.389215 \\ 6 & -0.857759 & -1.555054 & 3.774511 \\ 6 & -1.074152 & -0.564639 & 4.775833 \\ 6 & -1.425467 & 0.742337 & 4.371550 \\ 6 & -1.534553 & 1.058390 & 3.044116 \\ 6 & -0.931171 & -0.904139 & 6.142357 \\ 6 & -0.584855 & -2.176743 & 6.516781 \\ 6 & -0.371133 & -3.164170 & 5.532051 \\ 6 & -0.507111 & -2.864470 & 4.199083 \\ 6 & -0.781116 & -2.305958 & 1.410290 \\ 6 & 0.464609 & -2.539362 & 0.856651 \\ 6 & 0.705857 & -3.715190 & 0.107236 \\ 6 & -0.295790 & -4.618829 & -0.124586 \\ 6 & -1.605797 & -4.373965 & 0.349216 \\ 6 & -1.850616 & -3.217764 & 1.147513 \\ 6 & -3.162682 & -3.011371 & 1.647984 \\ 6 & -4.178630 & -3.881544 & 1.341813 \\ 6 & -3.933742 & -5.019415 & 0.542356 \\ 6 & -2.672909 & -5.258685 & 0.058958 \\ 7 & 1.532767 & -1.598908 & 1.038634 \\ 6 & 2.534335 & -1.954135 & 2.071517\end{array}$




\begin{tabular}{|c|c|c|c|}
\hline 6 & 3.099633 & -0.787599 & 2.837470 \\
\hline 5 & 4.476423 & -0.666535 & 3.012712 \\
\hline f & 5.008687 & 0.408985 & 3.719583 \\
\hline f & 4.166161 & 1.373872 & 4.261522 \\
\hline & 2.787380 & 1.255312 & 4.092848 \\
\hline & 2.260591 & 0.182493 & 3.386723 \\
\hline & -3.267634 & -0.074061 & 0.021332 \\
\hline 6 & -4.200561 & 0.209942 & 1.022840 \\
\hline 6 & -5.523314 & -0.207167 & 0.916372 \\
\hline 6 & -5.886984 & -0.904991 & -0.239891 \\
\hline 6 & -4.987214 & -1.199399 & -1.265153 \\
\hline 6 & -3.659003 & -0.779580 & -1.112941 \\
\hline 6 & -5.485593 & -1.957582 & -2.493513 \\
\hline 6 & -6.599651 & -1.150618 & -3.169902 \\
\hline 6 & -6.554175 & 0.049189 & 2.013374 \\
\hline 6 & -7.734267 & 0.840527 & 1.440458 \\
\hline 6 & -1.802777 & 2.355700 & 0.283226 \\
\hline 6 & -2.870261 & 2.921864 & -0.416232 \\
\hline 6 & -3.017771 & 4.304215 & -0.505079 \\
\hline 6 & -2.081254 & 5.109843 & 0.155479 \\
\hline 6 & -1.002092 & 4.578166 & 0.861969 \\
\hline 6 & -0.860234 & 3.186384 & 0.885447 \\
\hline 6 & -4.173738 & 4.950359 & -1.266649 \\
\hline 6 & -3.618242 & 5.883461 & -2.347673 \\
\hline 6 & -0.015460 & 5.445040 & 1.642968 \\
\hline 6 & 1.423195 & 5.117336 & 1.232169 \\
\hline 6 & 0.435360 & 2.246150 & -1.826321 \\
\hline 8 & 0.513839 & 3.219214 & -2.449719 \\
\hline 15 & 1.820127 & -0.651324 & -0.346104 \\
\hline 8 & 2.931132 & -1.498275 & -1.304183 \\
\hline 6 & 4.181928 & -1.843722 & -0.858839 \\
\hline 6 & 5.146478 & -0.881159 & -0.612841 \\
\hline 6 & 6.375917 & -1.298258 & -0.006482 \\
\hline 6 & 6.634922 & -2.688796 & 0.183713 \\
\hline 6 & 5.665019 & -3.635962 & -0.226343 \\
\hline 6 & 4.458489 & -3.222436 & -0.720346 \\
\hline 6 & 7.329531 & -0.370403 & 0.485309 \\
\hline 6 & 8.478615 & -0.795744 & 1.105378 \\
\hline 6 & 8.747411 & -2.172096 & 1.258172 \\
\hline 6 & 7.839953 & -3.095765 & 0.806045 \\
\hline 6 & 4.863073 & 0.536464 & -0.930183 \\
\hline 6 & 3.726465 & 1.124984 & -0.410931 \\
\hline 6 & 3.407071 & 2.479037 & -0.627980 \\
\hline 6 & 4.222126 & 3.249790 & -1.410850 \\
\hline 6 & 5.367598 & 2.690289 & -2.027016 \\
\hline 6 & 5.691892 & 1.317679 & -1.799474 \\
\hline 6 & 6.187842 & 3.467534 & -2.880364 \\
\hline 6 & 7.274571 & 2.917458 & -3.510122 \\
\hline 6 & 7.578411 & 1.553930 & -3.315239 \\
\hline 6 & 6.810060 & 0.776664 & -2.484174 \\
\hline 8 & 2.888520 & 0.387778 & 0.385254 \\
\hline 6 & -4.377336 & -2.189167 & -3.513538 \\
\hline 6 & -6.027953 & -3.325961 & -2.067029 \\
\hline 6 & -5.969327 & 0.842295 & 3.180255 \\
\hline 6 & -7.052153 & -1.294766 & 2.557371 \\
\hline 6 & -0.245285 & 6.937272 & 1.416479 \\
\hline
\end{tabular}




\begin{tabular}{|c|c|c|c|}
\hline 6 & -0.189492 & 5.152348 & 3.138588 \\
\hline 6 & -5.068996 & 3.916608 & -1.947743 \\
\hline 6 & -5.036697 & 5.755473 & -0.288602 \\
\hline 1 & 7.126472 & 0.693700 & 0.392403 \\
\hline 1 & -1.824831 & 2.068876 & 2.771378 \\
\hline 1 & 8.021923 & -4.162450 & 0.931746 \\
\hline 1 & -1.098612 & -0.128776 & 6.888619 \\
\hline 1 & 5.926657 & 4.513996 & -3.033073 \\
\hline 1 & -3.367666 & -2.130544 & 2.255012 \\
\hline 1 & -5.185399 & -3.688934 & 1.709449 \\
\hline 1 & 3.988331 & 4.299030 & -1.586915 \\
\hline 1 & -1.613689 & 1.498551 & 5.132885 \\
\hline 1 & 5.885107 & -4.696812 & -0.117786 \\
\hline 1 & 8.426226 & 1.111123 & -3.833346 \\
\hline 1 & 3.694717 & -3.934262 & -1.025136 \\
\hline 1 & -2.467920 & -6.134619 & -0.555804 \\
\hline 1 & -0.100865 & -5.529853 & -0.689681 \\
\hline 1 & -0.097674 & -4.173229 & 5.831947 \\
\hline 1 & 7.051593 & -0.275309 & -2.354969 \\
\hline 1 & -0.341752 & -3.640523 & 3.454482 \\
\hline 1 & 1.709277 & -3.882002 & -0.278210 \\
\hline 1 & 7.894070 & 3.523332 & -4.167369 \\
\hline 1 & 9.186124 & -0.063016 & 1.487252 \\
\hline 1 & -4.748566 & -5.702187 & 0.310772 \\
\hline 1 & -0.473166 & -2.428399 & 7.568861 \\
\hline 1 & 9.664661 & -2.495473 & 4733 \\
\hline 1 & 2.509292 & 2.875172 & -0.160893 \\
\hline 1 & -3.879233 & 0.764252 & 1.900777 \\
\hline 1 & -2.908494 & -0.999424 & -1.872299 \\
\hline 1 & -6.919829 & -1.238900 & -0.342936 \\
\hline 1 & -3.585394 & 2.265427 & -0.904959 \\
\hline 1 & -0.004865 & 2.737763 & 1.396171 \\
\hline 1 & -2.205764 & 6.189770 & 084 \\
\hline 1 & 2.005169 & -2.617582 & 2.770853 \\
\hline 1 & 3.357150 & -2.547152 & 1.642515 \\
\hline 1 & 5.143391 & -1.408629 & 2.569815 \\
\hline 1 & 1.187959 & 0.108984 & 3.221702 \\
\hline 1 & 6.087536 & 0.494881 & 3.835687 \\
\hline 1 & 2.114849 & 2.003564 & 4.510759 \\
\hline 1 & 4.580954 & 2.217698 & 4.808980 \\
\hline 1 & 1.601164 & 0.710863 & -3.772690 \\
\hline 1 & 1.661645 & -1.055560 & -3.292462 \\
\hline 1 & -1.256257 & 0.396696 & -2.472184 \\
\hline 1 & -1.549097 & -1.215592 & -6.062875 \\
\hline 1 & -2.236287 & -3.498906 & -6.615755 \\
\hline 1 & -1.908144 & -5.345118 & -4.978434 \\
\hline 1 & -0.903782 & -4.834323 & -2.757398 \\
\hline 1 & -0.253801 & -2.519727 & -2.181387 \\
\hline 1 & -0.508132 & 0.676712 & -5.731769 \\
\hline 1 & -1.973056 & 0.756872 & -4.727702 \\
\hline 1 & -0.564180 & 1.762965 & -4.342759 \\
\hline 1 & 0.494742 & 7.514099 & 1.985367 \\
\hline 1 & -0.136837 & 7.211036 & 0.359001 \\
\hline 1 & -1.238389 & 7.258677 & 1.755579 \\
\hline 1 & 2.127784 & 5.771536 & 1.762844 \\
\hline 1 & 1.697558 & 4.083984 & 1.477775 \\
\hline
\end{tabular}




$\begin{array}{rrrr}1 & 1.569225 & 5.268023 & 0.153693 \\ 1 & 0.502381 & 5.764077 & 3.733560 \\ 1 & -1.212284 & 5.380903 & 3.466742 \\ 1 & 0.011155 & 4.098513 & 3.372996 \\ 1 & -5.880529 & 4.428262 & -2.480554 \\ 1 & -4.517007 & 3.317576 & -2.683843 \\ 1 & -5.531939 & 3.229115 & -1.226648 \\ 1 & -4.440009 & 6.347562 & -2.909679 \\ 1 & -3.009519 & 6.691640 & -1.925008 \\ 1 & -2.990487 & 5.329727 & -3.057890 \\ 1 & -5.876246 & 6.226538 & -0.817588 \\ 1 & -5.451252 & 5.105845 & 0.493782 \\ 1 & -4.466921 & 6.552138 & 0.204869 \\ 1 & -4.776899 & -2.740764 & -4.374315 \\ 1 & -3.963951 & -1.244581 & -3.892538 \\ 1 & -3.551559 & -2.781032 & -3.094842 \\ 1 & -6.421260 & -3.867328 & -2.938252 \\ 1 & -5.230085 & -3.933521 & -1.620121 \\ 1 & -6.839035 & -3.243707 & -1.332051 \\ 1 & -6.955785 & -1.673474 & -4.067803 \\ 1 & -7.463549 & -0.998766 & -2.511239 \\ 1 & -6.236000 & -0.161110 & -3.477025 \\ 1 & -6.740527 & 0.994614 & 3.945842 \\ 1 & -5.131688 & 0.314896 & 3.657846 \\ 1 & -5.615536 & 1.833108 & 2.866034 \\ 1 & -8.479255 & 1.035875 & 2.223422 \\ 1 & -7.400932 & 1.807809 & 1.041845 \\ 1 & -8.240508 & 0.302062 & 0.630255 \\ 1 & -7.810566 & -1.136200 & 3.336008 \\ 1 & -7.504740 & -1.916210 & 1.774354 \\ 1 & -6.225645 & -1.863659 & 3.005998\end{array}$

TS1 $1_{L-r e-} \psi_{-1 \mathbf{s} 2 a 3 a 4 s}$

Number of imaginary frequencies : 1

The smallest frequencies are : - $649.2328 \quad 13.2082 \quad 17.0098 \mathrm{~cm}(-1)$

Electronic energy : $\quad H F=-4360.860166$

Zero-point correction $=\quad 1.451291$ (Hartree/Particle)

Thermal correction to Energy= $\quad 1.536394$

Thermal correction to Enthalpy= $\quad 1.537338$

Thermal correction to Gibbs Free Energy $=\quad 1.332474$

Sum of electronic and zero-point Energies $=\quad-4359.408875$

Sum of electronic and thermal Energies $=\quad-4359.323772$

Sum of electronic and thermal Enthalpies $=\quad-4359.322828$

Sum of electronic and thermal Free Energies $=\quad-4359.527692$

Cartesian Coordinates

$\begin{array}{crrr}6 & -4.200542 & 0.179249 & 0.976936 \\ 6 & -3.254752 & -0.147304 & 0.009515 \\ 6 & -3.632614 & -0.905268 & -1.099567 \\ 6 & -4.955986 & -1.328337 & -1.250344 \\ 6 & -5.878804 & -0.980836 & -0.254923 \\ 6 & -5.529299 & -0.231375 & 0.867331 \\ 15 & -1.539043 & 0.506327 & 0.195592 \\ 45 & 0.073077 & 0.227167 & -1.555227\end{array}$




\begin{tabular}{|c|c|c|c|}
\hline 6 & 0.400001 & 2.187600 & -1.914374 \\
\hline 8 & 0.469279 & 3.153693 & -2.549396 \\
\hline b & -5.436198 & -2.143987 & -2.448763 \\
\hline 5 & -6.518939 & 0.173776 & 1.956776 \\
\hline$b$ & -1.861532 & 2.321986 & 0.194642 \\
\hline 6 & -2.977149 & 2.835739 & -0.468485 \\
\hline 6 & -3.172426 & 4.209930 & -0.588031 \\
\hline 6 & -2.228209 & 5.062169 & -0.001959 \\
\hline 6 & -1.101631 & 4.583330 & 0.668288 \\
\hline 6 & -0.919936 & 3.197559 & 0.731577 \\
\hline 6 & -0.104584 & 5.503700 & 1.370785 \\
\hline 6 & -4.385659 & 4.794331 & -1.309189 \\
\hline 6 & -1.297143 & 0.146270 & 2.010595 \\
\hline 6 & -0.960847 & -1.132543 & 2.428072 \\
\hline 6 & -0.836508 & -1.429787 & 3.825830 \\
\hline 6 & -1.067275 & -0.404321 & 4.787529 \\
\hline 6 & -1.431184 & 0.882258 & 4.331946 \\
\hline 6 & -1.543002 & 1.145217 & 2.992873 \\
\hline 6 & -0.924892 & -0.689748 & 6.166538 \\
\hline 6 & -0.565308 & -1.943094 & 6.590296 \\
\hline 6 & -0.336190 & -2.964717 & 5.644685 \\
\hline 6 & -0.470960 & -2.717686 & 4.300935 \\
\hline 6 & -0.743026 & -2.269000 & 1.492256 \\
\hline 6 & 0.506832 & -2.499458 & 0.946696 \\
\hline 6 & 0.769909 & -3.699970 & 0.245183 \\
\hline 6 & -0.214061 & -4.631561 & 0.051456 \\
\hline 6 & -1.528618 & -4.393115 & 0.515645 \\
\hline 6 & -1.794772 & -3.210993 & 1.267425 \\
\hline 6 & -3.108991 & -3.010741 & 1.764316 \\
\hline 6 & -4.106745 & -3.915615 & 1.501545 \\
\hline 6 & -3.842016 & -5.077831 & 0.744452 \\
\hline 6 & -2.578614 & -5.309505 & 0.263798 \\
\hline 7 & 1.557521 & -1.532846 & 1.091476 \\
\hline 15 & 1.834620 & -0.638105 & -0.330390 \\
\hline 8 & 2.890298 & 0.442598 & 0.358026 \\
\hline 6 & 3.720898 & 1.153711 & -0.469024 \\
\hline 6 & 4.868275 & 0.557039 & -0.953638 \\
\hline 6 & 5.695087 & 1.309617 & -1.849494 \\
\hline 6 & 5.355161 & 2.666468 & -2.140721 \\
\hline 6 & 4.195269 & 3.236711 & -1.562353 \\
\hline 6 & 3.383832 & 2.490860 & -0.752067 \\
\hline 6 & 6.827203 & 0.754179 & -2.498891 \\
\hline 6 & 7.594119 & 1.504521 & -3.355528 \\
\hline 6 & 7.274789 & 2.853990 & -3.613141 \\
\hline 6 & 6.174381 & 3.416478 & -3.019174 \\
\hline 6 & 5.163112 & -0.842696 & -0.573654 \\
\hline 6 & 4.210019 & -1.824311 & -0.787963 \\
\hline 6 & 4.500568 & -3.193358 & -0.593253 \\
\hline 6 & 5.706236 & -3.573549 & -0.071089 \\
\hline 6 & 6.661089 & -2.600162 & 0.311848 \\
\hline 6 & 6.390016 & -1.221530 & 0.061802 \\
\hline 6 & 7.862200 & -2.968118 & 0.965116 \\
\hline 6 & 8.754079 & -2.016685 & 1.390114 \\
\hline 6 & 8.473224 & -0.650708 & 1.176969 \\
\hline 6 & 7.327730 & -0.263820 & 0.525751 \\
\hline 8 & 2.958008 & -1.509674 & -1.252978 \\
\hline
\end{tabular}




\begin{tabular}{|c|c|c|c|}
\hline & 2.561763 & -1.827895 & 2.140620 \\
\hline & 3.103201 & -0.622107 & 2.862066 \\
\hline & 4.475963 & -0.481459 & 3.054056 \\
\hline & 4.988338 & 0.628998 & 3.720282 \\
\hline & 4.129542 & 1.610096 & 4.204455 \\
\hline & 2.754586 & 1.472420 & 4.019078 \\
\hline & 2.247548 & 0.364391 & 3.353880 \\
\hline & 1.067923 & -0.263076 & -3.418247 \\
\hline & -0.277370 & -0.474405 & -3.824724 \\
\hline & -0.756451 & -1.875489 & -4.020495 \\
\hline & -0.575128 & -2.875327 & -3.056063 \\
\hline & -0.921536 & -4.194280 & -3.317605 \\
\hline & -1.477735 & -4.552653 & -4.543441 \\
\hline & -1.686260 & -3.569057 & -5.502202 \\
\hline & -1.334011 & -2.247452 & -5.242620 \\
\hline & -0.880458 & 0.570555 & -4.740185 \\
\hline & 7.114510 & 0.793197 & 0.386342 \\
\hline & -1.841233 & 2.141741 & 2.680150 \\
\hline & 8.053525 & -4.026692 & 1.136934 \\
\hline & -1.103965 & 0.111457 & 6.882211 \\
\hline & 5.901227 & 4.451629 & -3.220362 \\
\hline & -3.328077 & -2.110850 & 2.336987 \\
\hline & -5.115096 & -3.732451 & 1.869231 \\
\hline & 3.947852 & 4.273274 & -1.788446 \\
\hline & -1.626515 & 1.665945 & 5.063171 \\
\hline & 5.936587 & -4.626733 & 0.081564 \\
\hline & 8.452951 & 1.050829 & -3.845317 \\
\hline & 3.748400 & -3.925655 & -0.877495 \\
\hline & -2.358215 & -6.204091 & -0.317898 \\
\hline & -0.000952 & -5.561849 & -0.474322 \\
\hline ] & -0.051684 & -3.958203 & 5.983777 \\
\hline & 7.080993 & -0.287803 & -2.321766 \\
\hline 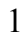 & -0.293769 & -3.519638 & 3.587088 \\
\hline & 1.775998 & -3.863966 & -0.133838 \\
\hline 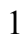 & 7.893400 & 3.438768 & -4.290028 \\
\hline & 9.168277 & 0.104713 & 1.536879 \\
\hline & -4.643260 & -5.786099 & 0.544290 \\
\hline 1 & -0.454754 & -2.153233 & 7.651577 \\
\hline & 9.668221 & -2.309678 & 1.901190 \\
\hline 1 & 2.475061 & 2.893761 & -0.313404 \\
\hline & -3.899614 & 0.780503 & 1.833718 \\
\hline 1 & -2.872054 & -1.154487 & -1.839382 \\
\hline ] & -6.908744 & -1.314356 & -0.366830 \\
\hline & -3.695227 & 2.145678 & -0.902644 \\
\hline 1 & -0.033621 & 2.787572 & 1.222137 \\
\hline & -2.384934 & 6.136417 & -0.073518 \\
\hline 1 & 2.042375 & -2.473404 & 2.863693 \\
\hline & 3.396987 & -2.422173 & 1.737751 \\
\hline 1 & 5.156134 & -1.236675 & 2.655685 \\
\hline 1 & 1.178465 & 0.275007 & 3.173500 \\
\hline & 6.064633 & 0.729237 & 3.848186 \\
\hline 1 & 2.069839 & 2.233095 & 4.392305 \\
\hline & 4.528562 & 2.481380 & 4.719671 \\
\hline 1 & 1.594709 & 0.610918 & -3.808291 \\
\hline & 1.703372 & -1.131522 & -3.254627 \\
\hline 1 & -1.254086 & 0.283004 & -2.491569 \\
\hline
\end{tabular}




\begin{tabular}{|c|c|c|c|}
\hline 1 & -1.497842 & -1.502610 & -6.017536 \\
\hline & -2.126917 & -3.825891 & -6.463616 \\
\hline & -1.757651 & -5.584991 & -4.741592 \\
\hline & -0.770630 & -4.946407 & -2.545135 \\
\hline & -0.178744 & -2.591381 & -2.079175 \\
\hline & -0.509788 & 0.435190 & -5.765969 \\
\hline & -1.976166 & 0.522378 & -4.764730 \\
\hline & -0.592478 & 1.577734 & -4.424156 \\
\hline & -5.296337 & 3.708897 & -1.881042 \\
\hline b & -5.208196 & 5.633168 & -0.324772 \\
\hline & -3.915041 & 5.681058 & -2.466811 \\
\hline 6 & -0.353056 & 6.977638 & 1.059209 \\
\hline & -0.241019 & 5.300483 & 2.884838 \\
\hline & 1.326911 & 5.162421 & 0.945377 \\
\hline & -4.314596 & -2.415372 & -3.444108 \\
\hline & -5.976403 & -3.494183 & -1.965829 \\
\hline & -6.546270 & -1.376862 & -3.176137 \\
\hline & -7.896854 & -0.449356 & 1.751820 \\
\hline 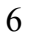 & -5.993971 & -0.277520 & 3.325256 \\
\hline & -6.670227 & 1.699822 & 1.944342 \\
\hline & 0.399064 & 7.593194 & 1.568470 \\
\hline & -0.279257 & 7.184994 & -0.016228 \\
\hline & -1.337512 & 7.314505 & 1.408063 \\
\hline & 2.036256 & 5.865996 & 1.401058 \\
\hline ] & 1.624284 & 4.156031 & 1.265333 \\
\hline & 1.438718 & 5.226117 & -0.145703 \\
\hline 1 & 0.465212 & 5.945276 & 3.425640 \\
\hline & -1.255526 & 5.548696 & 3.224225 \\
\hline & -0.034575 & 4.261710 & 3.174701 \\
\hline 1 & -6.159840 & 4.174869 & -2.372447 \\
\hline & -4.782111 & 3.094027 & -2.631281 \\
\hline 1 & -5.682207 & 3.038869 & -1.099619 \\
\hline & -4.778081 & 6.099112 & -3.002569 \\
\hline 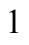 & -3.301615 & 6.521567 & -2.120626 \\
\hline 1 & -3.316382 & 5.104292 & -3.183631 \\
\hline I & -6.084514 & 6.062712 & -0.828807 \\
\hline 1 & -5.567279 & 5.017801 & 0.511026 \\
\hline & -4.628259 & 6.463571 & 0.095660 \\
\hline | & -4.700664 & -3.007994 & -4.283522 \\
\hline ] & -3.901712 & -1.486542 & -3.860549 \\
\hline 1 & -3.490766 & -2.982258 & -2.989171 \\
\hline ] & -6.347884 & -4.081501 & -2.816673 \\
\hline t & -5.182883 & -4.071790 & -1.473555 \\
\hline 1 & -6.803020 & -3.381706 & -1.252964 \\
\hline & -6.882939 & -1.940978 & -4.056402 \\
\hline 1 & -7.422715 & -1.205770 & -2.539432 \\
\hline & -6.186276 & -0.397987 & -3.519564 \\
\hline 1 & -8.565561 & -0.146042 & 2.567278 \\
\hline 1 & -8.360325 & -0.124674 & 0.811560 \\
\hline 1 & -7.851286 & -1.546753 & 1.752023 \\
\hline 1 & -7.345083 & 2.027189 & 2.746977 \\
\hline 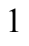 & -5.707901 & 2.209302 & 2.084886 \\
\hline 1 & -7.091484 & 2.042089 & 0.989441 \\
\hline & -6.694453 & 0.019428 & 4.117333 \\
\hline & -5.888003 & -1.370061 & 3.365548 \\
\hline & -5.017807 & 0.160777 & 3.569393 \\
\hline
\end{tabular}


TS1 $1_{L-r e-} \psi_{-1 \mathbf{1 s 2 a 3 s 4 a}}$

Number of imaginary frequencies : 1

The smallest frequencies are : -665.2512 $8.8188 \quad 15.1851 \mathrm{~cm}(-1)$

Electronic energy : $\quad H F=-4360.8625725$

Zero-point correction $=\quad 1.451731$ (Hartree/Particle)

Thermal correction to Energy $=\quad 1.536602$

Thermal correction to Enthalpy= $\quad 1.537546$

Thermal correction to Gibbs Free Energy $=\quad 1.332383$

Sum of electronic and zero-point Energies $=\quad-4359.410842$

Sum of electronic and thermal Energies $=\quad-4359.325971$

Sum of electronic and thermal Enthalpies $=\quad-4359.325026$

Sum of electronic and thermal Free Energies $=\quad-4359.530190$

Cartesian Coordinates

$\begin{array}{cccc}6 & -4.223944 & 0.285806 & 1.017799 \\ 6 & -3.273916 & -0.031119 & 0.036404 \\ 6 & -3.659068 & -0.742758 & -1.088745 \\ 6 & -4.995216 & -1.142452 & -1.265181 \\ 6 & -5.909200 & -0.816028 & -0.269459 \\ 6 & -5.549995 & -0.101235 & 0.885587 \\ 15 & -1.532095 & 0.537546 & 0.255200 \\ 45 & 0.044889 & 0.294481 & -1.525684 \\ 6 & 0.431764 & 2.255609 & -1.808245 \\ 8 & 0.485902 & 3.260142 & -2.381565 \\ 6 & -5.384470 & -1.919765 & -2.519146 \\ 6 & -6.604035 & 0.213063 & 1.945318 \\ 6 & -1.762693 & 2.363147 & 0.356465 \\ 6 & -2.800992 & 2.962635 & -0.359625 \\ 6 & -2.915607 & 4.348384 & -0.433412 \\ 6 & -1.979290 & 5.124209 & 0.262496 \\ 6 & -0.927483 & 4.559412 & 0.983935 \\ 6 & -0.815364 & 3.164080 & 0.990430 \\ 6 & 0.067909 & 5.396435 & 1.786255 \\ 6 & -4.033948 & 5.029601 & -1.219608 \\ 6 & -1.301910 & 0.044228 & 2.036597 \\ 6 & -0.982165 & -1.265287 & 2.362119 \\ 6 & -0.860842 & -1.658037 & 3.737807 \\ 6 & -1.083896 & -0.698484 & 4.767626 \\ 6 & -1.439733 & 0.618850 & 4.402539 \\ 6 & -1.544340 & 0.974527 & 3.085189 \\ 6 & -0.941352 & -1.076563 & 6.123989 \\ 6 & -0.587436 & -2.357085 & 6.462509 \\ 6 & -0.364585 & -3.313540 & 5.449888 \\ 6 & -0.500678 & -2.976351 & 4.125901 \\ 6 & -0.777712 & -2.347985 & 1.359223 \\ 6 & 0.472373 & -2.575196 & 0.813256 \\ 6 & 0.729193 & -3.756588 & 0.077460 \\ 6 & -0.255800 & -4.683566 & -0.132199 \\ 6 & -1.568652 & -4.453012 & 0.341197 \\ 6 & -1.836325 & -3.274496 & 1.098766 \\ 6 & -3.154661 & -3.071434 & 1.582535 \\ 6 & -4.150014 & -3.979438 & 1.319220 \\ 6 & -3.878354 & -5.148149 & 0.575010\end{array}$




$\begin{array}{cccc}6 & -2.614157 & -5.375812 & 0.094823 \\ 7 & 1.536173 & -1.631331 & 1.001413 \\ 15 & 1.806084 & -0.656506 & -0.368453 \\ 8 & 2.879193 & 0.368761 & 0.374782 \\ 6 & 3.708683 & 1.126874 & -0.410293 \\ 6 & 4.840015 & 0.553938 & -0.957615 \\ 6 & 5.658150 & 1.359567 & -1.814921 \\ 6 & 5.332030 & 2.738109 & -1.999412 \\ 6 & 4.194113 & 3.279825 & -1.354050 \\ 6 & 3.387252 & 2.486477 & -0.585474 \\ 6 & 6.767055 & 0.837775 & -2.528807 \\ 6 & 7.525447 & 1.637887 & -3.347304 \\ 6 & 7.220409 & 3.006555 & -3.499408 \\ 6 & 6.142121 & 3.538940 & -2.840545 \\ 6 & 5.131206 & -0.870387 & -0.680118 \\ 6 & 4.166986 & -1.830475 & -0.936947 \\ 6 & 4.451934 & -3.211088 & -0.838403 \\ 6 & 5.666515 & -3.631862 & -0.371149 \\ 6 & 6.637810 & -2.691286 & 0.050436 \\ 6 & 6.370561 & -1.297620 & -0.101079 \\ 6 & 7.853237 & -3.108737 & 0.644991 \\ 6 & 8.763400 & -2.192971 & 1.107554 \\ 6 & 8.486725 & -0.814401 & 0.993966 \\ 6 & 7.327337 & -0.378465 & 0.401054 \\ 8 & 2.908507 & -1.480574 & -1.356138 \\ 1 & -3.377358 & -2.168815 & 2.149934 \\ 1 & -5.158868 & -3.796440 & 1.684483 \\ 6 & 2.553927 & -2.007328 & 2.011818 \\ 6 & 3.117112 & -0.857315 & 2.803571 \\ 6 & 4.495247 & -0.714151 & 2.949163 \\ 1 & 5.892598 & 4.333686 & -1.497198 \\ 6 & 5.366102 & -4.694191 & -0.293817 \\ 6 & -1.209448 & -3.888632 \\ 6 & -1.83782 & 0.348926 & 3.677120 \\ 6 & 4.176170 & 1.278212 & 4.271206 \\ 6 & 2.796118 & 1.135600 & 4.134907 \\ 6 & -2.390366 & -6.269370 & -0.487239 \\ 6 & 2.273116 & 0.075507 & 3.406804 \\ 6 & 0.949842 & -0.179530 & -3.436827 \\ 6 & -0.420501 & -0.307513 & -3.797124 \\ 6 & -0.986156 & -1.672808 & -4.013746 \\ 6 & -0.697569 & -2.747509 & -3.159256 \\ 6 & -1.117237 & -4.035213 & -3.459651 \\ 6 & -1.865180 & -4.289284 & -4.608430 \\ 6 & -0.083607 & -4.328750 & 5.720608\end{array}$




\begin{tabular}{|c|c|c|c|}
\hline & 7.009170 & -0.217587 & -2.432936 \\
\hline ] & -0.326818 & -3.730115 & 3.360739 \\
\hline & 1.735279 & -3.913840 & -0.304905 \\
\hline & 7.831952 & 3.630566 & -4.147067 \\
\hline & 9.196634 & -0.088484 & 1.384287 \\
\hline & -4.675954 & -5.861399 & 0.378423 \\
\hline & -0.476141 & -2.638335 & 7.507096 \\
\hline & 9.688878 & -2.524593 & 1.572480 \\
\hline & 2.495062 & 2.868935 & -0.097062 \\
\hline 1 & -3.909048 & 0.849343 & 1.892108 \\
\hline 1 & -2.903071 & -0.995484 & -1.834879 \\
\hline 1 & -6.946770 & -1.123345 & -0.383513 \\
\hline 1 & -3.516778 & 2.328821 & -0.876779 \\
\hline 1 & 0.021195 & 2.689774 & 1.509197 \\
\hline 1 & -2.079737 & 6.206940 & 0.232479 \\
\hline 1 & 2.038877 & -2.695206 & 2.697935 \\
\hline & 3.376902 & -2.580070 & 1.556802 \\
\hline 1 & 5.165013 & -1.428413 & 2.466723 \\
\hline & 1.198058 & -0.018681 & 3.271062 \\
\hline & 6.103317 & 0.453253 & 3.769331 \\
\hline ] & 2.119640 & 1.854079 & 4.596313 \\
\hline 1 & 4.587977 & 2.112342 & 4.835518 \\
\hline 1 & 1.504530 & 0.679758 & -3.819635 \\
\hline & 1.548600 & -1.081534 & -3.325421 \\
\hline 1 & -1.291857 & 0.450378 & -2.433132 \\
\hline 1 & -2.015084 & -1.137309 & -5.839220 \\
\hline 1 & -2.789008 & -3.403960 & -6.339710 \\
\hline$\frac{1}{1}$ & -2.204680 & -5.297986 & -4.833533 \\
\hline 1 & -0.875554 & -4.846292 & -2.774647 \\
\hline 1 & -0.153437 & -2.550993 & -2.233658 \\
\hline 1 & -0.692562 & 0.653134 & -5.708009 \\
\hline 1 & -2.086383 & 0.845493 & -4.621605 \\
\hline 1 & -0.603862 & 1.773190 & -4.344704 \\
\hline 6 & -4.916302 & 4.025756 & -1.959531 \\
\hline 6 & -4.920955 & 5.822122 & -0.253004 \\
\hline 6 & -3.426130 & 5.980334 & -2.256215 \\
\hline 6 & -0.143880 & 6.896189 & 1.594001 \\
\hline 5 & -0.105987 & 5.073732 & 3.275502 \\
\hline 5 & 1.501167 & 5.060393 & 1.363560 \\
\hline 5 & -6.878723 & -2.226609 & -2.578425 \\
\hline 6 & -5.019524 & -1.095981 & -3.758815 \\
\hline 6 & -4.622602 & -3.248237 & -2.533948 \\
\hline 6 & -6.031473 & 1.025479 & 3.105198 \\
\hline 6 & -7.744152 & 1.020923 & 1.316352 \\
\hline 6 & -7.158538 & -1.098178 & 2.513043 \\
\hline 1 & 0.599046 & 7.450515 & 2.181171 \\
\hline 1 & -0.025014 & 7.194439 & 0.544314 \\
\hline 1 & -1.135637 & 7.220392 & 1.934430 \\
\hline 1 & 2.216054 & 5.699777 & 1.898550 \\
\hline 1 & 1.764773 & 4.020950 & 1.593919 \\
\hline 1 & 1.642061 & 5.222279 & 0.285804 \\
\hline 1 & 0.592640 & 5.667022 & 3.881114 \\
\hline 1 & -1.125824 & 5.304838 & 3.610861 \\
\hline 1 & 0.086527 & 4.014082 & 3.488816 \\
\hline 1 & -5.698296 & 4.561780 & -2.512216 \\
\hline 1 & -4.343504 & 3.434592 & -2.686341 \\
\hline
\end{tabular}




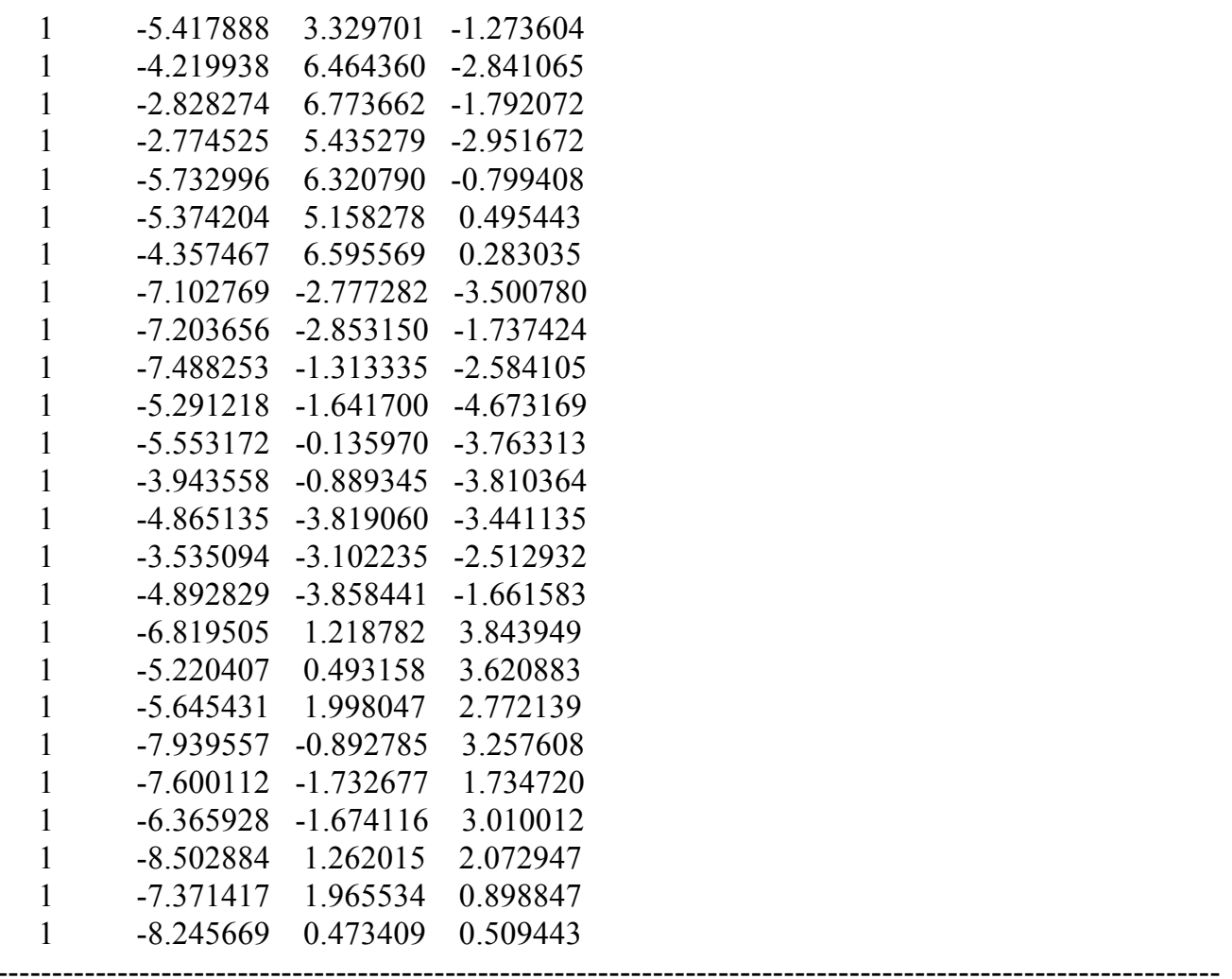

TS1 $_{I L-r e-} \psi_{-1 \mathrm{~s} 2 \mathrm{a} 3 \mathrm{~s} 4 \mathrm{~s}}$

Number of imaginary frequencies : 1

The smallest frequencies are : -697.0199 $16.0149 \quad 19.4497 \mathrm{~cm}(-1)$

Electronic energy : $\quad H F=-4360.863231$

Zero-point correction $=\quad 1.451558$ (Hartree/Particle)

Thermal correction to Energy= $\quad 1.536441$

Thermal correction to Enthalpy $=\quad 1.537385$

Thermal correction to Gibbs Free Energy= $\quad 1.333184$

Sum of electronic and zero-point Energies $=\quad-4359.411673$

Sum of electronic and thermal Energies $=\quad-4359.326790$

Sum of electronic and thermal Enthalpies= $\quad-4359.325846$

Sum of electronic and thermal Free Energies $=\quad-4359.530047$

Cartesian Coordinates

$\begin{array}{cccc}6 & -4.210991 & 0.296104 & 0.977070 \\ 6 & -3.260392 & -0.084338 & 0.028010 \\ 6 & -3.647604 & -0.866447 & -1.053661 \\ 6 & -4.983490 & -1.262029 & -1.210837 \\ 6 & -5.906870 & -0.861497 & -0.243412 \\ 6 & -5.545110 & -0.082550 & 0.862110 \\ 15 & -1.525910 & 0.517787 & 0.206753 \\ 45 & 0.039691 & 0.185986 & -1.573588 \\ 6 & 0.397089 & 2.125354 & -1.985930 \\ 8 & 0.439593 & 3.101290 & -2.607524 \\ 6 & -5.377942 & -2.115425 & -2.413713 \\ 6 & -6.542195 & 0.392538 & 1.917255 \\ 6 & -1.783903 & 2.342589 & 0.208572 \\ 6 & -2.869009 & 2.896001 & -0.473110\end{array}$




\begin{tabular}{|c|c|c|c|}
\hline 6 & -3.013678 & 4.276371 & -0.592243 \\
\hline & -2.053642 & 5.093991 & 0.017767 \\
\hline & -0.956713 & 4.574694 & 0.706895 \\
\hline & -0.821351 & 3.183257 & 0.763178 \\
\hline & 0.050989 & 5.456431 & 1.443420 \\
\hline & -4.187962 & 4.904663 & -1.340544 \\
\hline & -1.285552 & 0.149071 & 2.017974 \\
\hline & -0.953174 & -1.132138 & 2.429164 \\
\hline & -0.823890 & -1.433663 & 3.826579 \\
\hline & -1.049175 & -0.410280 & 4.791806 \\
\hline & -1.412391 & 0.878507 & 4.341562 \\
\hline & -1.527025 & 1.145822 & 3.003983 \\
\hline & -0.903899 & -0.699138 & 6.169687 \\
\hline & -0.545528 & -1.954074 & 6.589791 \\
\hline & -0.320023 & -2.973282 & 5.641015 \\
\hline & -0.458159 & -2.722924 & 4.298117 \\
\hline & -0.742615 & -2.272794 & 1.496099 \\
\hline & 0.505887 & -2.517657 & 0.954029 \\
\hline & 0.769261 & -3.740183 & 0.291381 \\
\hline & -0.208447 & -4.687312 & 0.144801 \\
\hline & -1.521677 & -4.439495 & 0.608615 \\
\hline 6 & -1.794290 & -3.221358 & 1.298401 \\
\hline ) & -3.112226 & -2.996772 & 1.773522 \\
\hline 2 & -4.104648 & -3.920616 & 1.560571 \\
\hline 6 & -3.829088 & -5.127955 & 0.882174 \\
\hline ) & -2.563955 & -5.378739 & 0.415975 \\
\hline 7 & 1.557220 & -1.547850 & 1.069229 \\
\hline & 1.814431 & -0.670135 & -0.367488 \\
\hline 3 & 2.872284 & 0.422286 & 0.297835 \\
\hline 6 & 3.704473 & 1.122396 & -0.536085 \\
\hline 6 & 4.849800 & 0.517456 & -1.015099 \\
\hline 6 & 5.682064 & 1.260564 & -1.913753 \\
\hline 6 & 5.349963 & 2.617611 & -2.212750 \\
\hline 6 & 4.191830 & 3.196851 & -1.639840 \\
\hline 6 & 3.374616 & 2.459634 & -0.827371 \\
\hline 6 & 6.812961 & 0.696020 & -2.557389 \\
\hline 6 & 7.586084 & 1.438038 & -3.415687 \\
\hline 5 & 7.274430 & 2.787785 & -3.681020 \\
\hline 6 & 6.175291 & 3.358869 & -3.092943 \\
\hline 6 & 5.138918 & -0.880435 & -0.624283 \\
\hline 6 & 4.183095 & -1.860903 & -0.831830 \\
\hline 6 & 4.470937 & -3.229419 & -0.629530 \\
\hline 6 & 5.674825 & -3.609157 & -0.102838 \\
\hline 6 & 6.630604 & -2.635447 & 0.276872 \\
\hline 6 & 6.363802 & -1.258055 & 0.015879 \\
\hline 6 & 7.828636 & -3.001545 & 0.936867 \\
\hline 6 & 8.721412 & -2.049185 & 1.357883 \\
\hline 6 & 8.445037 & -0.684114 & 1.133174 \\
\hline 6 & 7.302813 & -0.299255 & 0.475092 \\
\hline 8 & 2.930186 & -1.545819 & -1.295075 \\
\hline 6 & 2.572162 & -1.819721 & 2.114527 \\
\hline 6 & 3.107098 & -0.598615 & 2.815310 \\
\hline 6 & 4.479148 & -0.447455 & 3.004237 \\
\hline 6 & 4.986258 & 0.679373 & 3.646688 \\
\hline 6 & 4.123100 & 1.667217 & 4.109008 \\
\hline 6 & 2.748728 & 1.518786 & 3.927374 \\
\hline
\end{tabular}




\begin{tabular}{|c|c|c|c|}
\hline 6 & 2.246930 & 0.393545 & 3.287596 \\
\hline & 0.938999 & -0.407639 & -3.454378 \\
\hline & -0.430028 & -0.574270 & -3.802289 \\
\hline & -0.982420 & -1.956121 & -3.922940 \\
\hline & -0.699662 & -2.959192 & -2.984460 \\
\hline & -1.112619 & -4.268025 & -3.187301 \\
\hline & -1.848401 & -4.613117 & -4.319978 \\
\hline U & -2.166813 & -3.625772 & -5.242775 \\
\hline & -1.737745 & -2.314840 & -5.048014 \\
\hline & -1.014322 & 0.466015 & -4.735610 \\
\hline & 7.092752 & 0.757205 & 0.326903 \\
\hline 1 & -1.820583 & 2.144932 & 2.694837 \\
\hline 1 & 8.016726 & -4.059272 & 1.117247 \\
\hline & -1.079810 & 0.100828 & 6.887519 \\
\hline 1 & 5.907969 & 4.394365 & -3.300128 \\
\hline 1 & -3.337276 & -2.066177 & 2.292569 \\
\hline 1 & -5.114929 & -3.718299 & 1.911244 \\
\hline 1 & 3.950822 & 4.233562 & -1.871906 \\
\hline 1 & -1.603643 & 1.660281 & 5.075917 \\
\hline 1 & 5.902775 & -4.661923 & 0.056138 \\
\hline 1 & 8.443903 & 0.977443 & -3.900792 \\
\hline 1 & 3.718516 & -3.961834 & -0.912622 \\
\hline 1 & -2.337651 & -6.303430 & -0.114180 \\
\hline 1 & 0.008201 & -5.637580 & -0.342609 \\
\hline 1 & -0.035598 & -3.967971 & 5.976635 \\
\hline 1 & 7.060995 & -0.346308 & -2.374350 \\
\hline 1 & -0.282795 & -3.524131 & $3.58 ?$ \\
\hline 1 & 1.774452 & -3.911874 & -0.086595 \\
\hline 1 & 7.897883 & 3.365961 & -4.359133 \\
\hline 1 & 9.140898 & 0.072207 & 1.489620 \\
\hline 1 & -4.624497 & -5.853379 & 0.725004 \\
\hline 1 & -0.432966 & -2.166996 & 7.650305 \\
\hline 1 & 9.632945 & -2.340639 & 1.874470 \\
\hline 1 & 2.468399 & 2.870578 & -0.390879 \\
\hline 1 & -3.903852 & 0.916592 & 1.817843 \\
\hline 1 & -2.889279 & -1.168758 & -1.778744 \\
\hline 1 & -6.944647 & -1.161870 & -0.353774 \\
\hline 1 & -3.601292 & 2.231390 & -0.923325 \\
\hline 1 & 0.041841 & 2.742263 & 1.267331 \\
\hline 1 & -2.173615 & 6.173133 & -0.050624 \\
\hline 1 & 2.064688 & -2.461085 & 2.849649 \\
\hline 1 & 3.410332 & -2.410993 & 1.713267 \\
\hline 1 & 5.163015 & -1.207263 & 2.621161 \\
\hline 1 & 1.178371 & 0.295281 & 3.109237 \\
\hline 1 & 6.062108 & 0.787371 & 3.772182 \\
\hline 1 & 2.060234 & 2.284587 & 4.283727 \\
\hline 1 & 4.518226 & 2.551735 & 4.604269 \\
\hline 1 & 1.483761 & 0.429192 & -3.896624 \\
\hline 1 & 1.546918 & -1.294433 & -3.284203 \\
\hline 1 & -1.305963 & 0.265345 & -2.480993 \\
\hline 1 & -1.987446 & -1.568101 & -5.797935 \\
\hline 1 & -2.751538 & -3.870336 & -6.127617 \\
\hline 1 & -2.184794 & -5.637004 & -4.468150 \\
\hline 1 & -0.876736 & -5.021737 & -2.437692 \\
\hline 1 & -0.166453 & -2.685645 & -2.072039 \\
\hline 1 & -0.711520 & 0.256514 & -5.771256 \\
\hline
\end{tabular}




$\begin{array}{lrrr}1 & -2.110282 & 0.498315 & -4.701534 \\ 1 & -0.641468 & 1.463465 & -4.483436 \\ 6 & -5.121422 & 3.852920 & -1.938016 \\ 6 & -5.004599 & 5.771070 & -0.375306 \\ 6 & -3.657253 & 5.775597 & -2.484179 \\ 6 & -0.155300 & 6.941491 & 1.154765 \\ 6 & -0.118818 & 5.229649 & 2.951028 \\ 6 & 1.481960 & 5.087585 & 1.040646 \\ 6 & -6.882226 & -2.367370 & -2.488308 \\ 6 & -4.955085 & -1.405126 & -3.704294 \\ 6 & -4.672955 & -3.471187 & -2.307731 \\ 6 & -7.935219 & -0.196461 & 1.711605 \\ 6 & -6.057216 & -0.017989 & 3.312713 \\ 6 & -6.648301 & 1.920806 & 1.841919 \\ 1 & 0.601834 & 7.529021 & 1.689100 \\ 1 & -0.056053 & 7.166546 & 0.084949 \\ 1 & -1.137876 & 7.295878 & 1.491402 \\ 1 & 2.198721 & 5.759286 & 1.531603 \\ 1 & 1.746961 & 4.064995 & 1.337980 \\ 1 & 1.621067 & 5.181045 & -0.045262 \\ 1 & 0.586911 & 5.853818 & 3.516159 \\ 1 & -1.135633 & 5.488336 & 3.275417 \\ 1 & 0.066299 & 4.182973 & 3.227047 \\ 1 & -5.956063 & 4.349735 & -2.448685 \\ 1 & -4.610157 & 3.222974 & -2.677811 \\ 1 & -5.549032 & 3.194211 & -1.168990 \\ 1 & -4.491239 & 6.224128 & -3.040907 \\ 1 & -3.023759 & 6.593685 & -2.120880 \\ 1 & -3.061027 & 5.178861 & -3.186647 \\ 1 & -5.851566 & 6.232835 & -0.900550 \\ 1 & -5.407206 & 5.168152 & 0.449680 \\ 1 & -4.406236 & 6.579407 & 0.061977 \\ 1 & -7.107213 & -2.972313 & -3.375837 \\ 1 & -7.252508 & -2.920812 & -1.615608 \\ 1 & -7.453685 & -1.433625 & -2.572818 \\ 1 & -5.238337 & -2.005353 & -4.580234 \\ 1 & -5.443374 & -0.424961 & -3.790743 \\ 1 & -3.870331 & -1.253267 & -3.753247 \\ 1 & -4.907578 & -4.095173 & -3.181478 \\ 1 & -3.581531 & -3.368323 & -2.251153 \\ 1 & -5.002447 & -4.005596 & -1.406753 \\ 1 & -8.606859 & 0.157451 & 2.504000 \\ 1 & -8.374024 & 0.106654 & 0.752528 \\ 1 & -7.924054 & -1.293772 & 1.753331 \\ 1 & -7.330343 & 2.298848 & 2.615749 \\ 1 & -5.674424 & 2.407486 & 1.984076 \\ 1 & -7.039246 & 2.236660 & 0.865278 \\ 1 & -6.758530 & 0.339750 & 4.078531 \\ 1 & -5.994730 & -1.110755 & 3.401857 \\ 1 & -5.068879 & 0.392884 & 3.554418\end{array}$

TS1 $1_{L-r e-} \Psi_{-1 \mathbf{1 s} 23 a 4 a}$

Number of imaginary frequencies : 1

The smallest frequencies are : -656.4059 $5.3652 \quad 15.8375 \mathrm{~cm}(-1)$

Electronic energy : $\quad \mathrm{HF}=-4360.859915$ 
Zero-point correction $=$

Thermal correction to Energy=

Thermal correction to Enthalpy=

Thermal correction to Gibbs Free Energy=

Sum of electronic and zero-point Energies=

Sum of electronic and thermal Energies $=$

Sum of electronic and thermal Enthalpies=

Sum of electronic and thermal Free Energies=
1.451059 (Hartree/Particle)

1.536298

1.537242

1.330057

$-4359.408856$

$-4359.323617$

$-4359.322673$

$-4359.529858$

Cartesian Coordinates

\begin{tabular}{cccc}
6 & -3.670753 & -0.814296 & -1.069739 \\
6 & -3.288850 & -0.060576 & 0.036676 \\
6 & -4.238270 & 0.296762 & 0.999014 \\
6 & -5.570440 & -0.086847 & 0.876622 \\
6 & -5.921196 & -0.841746 & -0.247593 \\
6 & -5.004290 & -1.213476 & -1.231551 \\
15 & -1.547567 & 0.520190 & 0.245766 \\
6 & -1.316055 & 0.083422 & 2.043040 \\
6 & -0.977385 & -1.210213 & 2.408684 \\
6 & -0.848549 & -1.561229 & 3.793919 \\
6 & -1.078993 & -0.574050 & 4.795254 \\
6 & -1.454897 & 0.726458 & 4.391633 \\
6 & -1.570644 & 1.040651 & 3.064511 \\
6 & -0.925716 & -0.910192 & 6.161417 \\
6 & -0.556639 & -2.176556 & 6.535500 \\
6 & -0.330200 & -3.161053 & 5.550799 \\
6 & -0.475685 & -2.864541 & 4.218064 \\
6 & -0.770555 & -2.313325 & 1.431009 \\
6 & 0.476443 & -2.544717 & 0.880156 \\
6 & 0.725024 & -3.727800 & 0.144526 \\
6 & -0.269727 & -4.642501 & -0.073631 \\
6 & -1.581571 & -4.400350 & 0.396486 \\
6 & -1.834818 & -3.233062 & 1.175435 \\
6 & -3.148094 & -3.025789 & 1.671773 \\
6 & -4.156340 & -3.912562 & 1.388312 \\
6 & -3.902925 & -5.062671 & 0.608867 \\
6 & -2.641845 & -5.298815 & 0.124370 \\
7 & 1.538797 & -1.596175 & 1.053201 \\
6 & 2.553012 & -1.946632 & 2.075487 \\
6 & 3.114101 & -0.778884 & 2.842545 \\
6 & 4.491574 & -0.642532 & 3.001152 \\
6 & 5.019854 & 0.431420 & 3.713179 \\
6 & 4.172569 & 1.379148 & 4.277658 \\
6 & 2.793280 & 1.245326 & 4.125702 \\
6 & 2.270376 & 0.174615 & 3.413265 \\
6 & -6.624282 & 0.263972 & 1.925345 \\
6 & -6.065294 & 1.170254 & 3.020454 \\
6 & -5.493221 & -2.018543 & -2.433914 \\
6 & -4.362396 & -2.353337 & -3.398748 \\
45 & 0.048580 & 0.263604 & -1.523039 \\
6 & 1.024582 & -0.168942 & -3.410201 \\
6 & -0.326139 & -0.354288 & -3.811891 \\
6 & -0.930467 & 0.727684 & -4.682831 \\
15 & 1.811619 & -0.652146 & -0.337447 \\
8 & 2.880913 & 0.394118 & 0.383114 \\
& & & \\
\hline
\end{tabular}




\begin{tabular}{|c|c|c|c|}
\hline 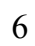 & 3.705633 & 1.133533 & -0.425084 \\
\hline 6 & 4.841395 & 0.550426 & -0.952045 \\
\hline 6 & 5.656524 & 1.332777 & -1.833169 \\
\hline & 5.319880 & 2.701593 & -2.065390 \\
\hline & 4.175910 & 3.255990 & -1.441865 \\
\hline & 3.373802 & 2.483711 & -0.647164 \\
\hline & 6.126416 & 3.480070 & -2.930588 \\
\hline & 7.211436 & 2.934530 & -3.567217 \\
\hline & 7.527339 & 1.574454 & -3.367388 \\
\hline 6 & 6.772465 & 0.796219 & -2.524948 \\
\hline 6 & 5.137429 & -0.863689 & -0.630981 \\
\hline & 4.177567 & -1.833890 & -0.865051 \\
\hline O & 4.466043 & -3.210136 & -0.725314 \\
\hline & 5.679820 & -3.613106 & -0.240430 \\
\hline 6 & 6.645914 & -2.657514 & 0.158929 \\
\hline U & 6.374902 & -1.269640 & -0.033638 \\
\hline 6 & 7.325246 & -0.332915 & 0.447415 \\
\hline 6 & 8.482512 & -0.747505 & 1.059554 \\
\hline 6 & 8.763079 & -2.121312 & 1.214408 \\
\hline 6 & 7.859043 & -3.053331 & 0.772605 \\
\hline 8 & 2.920120 & -1.499179 & -1.299391 \\
\hline 6 & 0.391200 & 2.234431 & -1.825717 \\
\hline 8 & 0.454311 & 3.216799 & -2.435637 \\
\hline 6 & -0.815759 & -1.743957 & -4.056201 \\
\hline 6 & -1.413840 & -2.061883 & -5.283782 \\
\hline 6 & -1.776616 & -3.369741 & -5.593522 \\
\hline 6 & -1.556832 & -4.393832 & -4.680995 \\
\hline 6 & -0.978683 & -4.089810 & -3.450410 \\
\hline 6 & -0.622802 & -2.784598 & -3.137990 \\
\hline 6 & -1.786746 & 2.346275 & 0.305356 \\
\hline 6 & -2.820452 & 2.925779 & -0.421973 \\
\hline 6 & -2.946882 & 4.313189 & -0.524493 \\
\hline 6 & -2.020741 & 5.108452 & 0.152613 \\
\hline 6 & -0.965041 & 4.559058 & 0.890650 \\
\hline 6 & -0.844215 & 3.169138 & 0.927579 \\
\hline 6 & -4.083750 & 4.886566 & -1.366928 \\
\hline 6 & -5.426220 & 4.358863 & -0.845901 \\
\hline 6 & 0.021865 & 5.419539 & 1.679403 \\
\hline 6 & 1.459109 & 5.082855 & 1.271241 \\
\hline 6 & -4.125472 & 6.411894 & -1.330912 \\
\hline 6 & -3.892283 & 4.445926 & -2.823342 \\
\hline 6 & -0.196231 & 6.913931 & 1.454213 \\
\hline 6 & -0.158598 & 5.128349 & 3.174394 \\
\hline 6 & -7.126578 & -1.027058 & 2.581613 \\
\hline 6 & -7.801371 & 0.989283 & 1.265221 \\
\hline 6 & -6.110274 & -3.337493 & -1.957354 \\
\hline 6 & -6.546992 & -1.203503 & -3.192213 \\
\hline 1 & 7.113131 & 0.729277 & 0.352677 \\
\hline 1 & -1.874961 & 2.047236 & 2.792028 \\
\hline 1 & 8.050118 & -4.118200 & 0.900166 \\
\hline 1 & -1.103879 & -0.137353 & 6.907814 \\
\hline 1 & 5.856007 & 4.523677 & -3.086755 \\
\hline 1 & -3.355789 & -2.134575 & 2.261674 \\
\hline 1 & -5.163150 & -3.724977 & 1.757608 \\
\hline 1 & 3.932848 & 4.302472 & -1.621819 \\
\hline 1 & -1.655147 & 1.479111 & 5.153397 \\
\hline
\end{tabular}




\begin{tabular}{|c|c|c|c|}
\hline & 5.908848 & -4.671957 & -0.130883 \\
\hline & 8.373734 & 1.135051 & -3.890686 \\
\hline & 3.705475 & -3.928776 & -1.021943 \\
\hline & -2.431421 & -6.182772 & -0.476953 \\
\hline & -0.067723 & -5.560814 & -0.624265 \\
\hline & -0.039342 & -4.165314 & 5.850441 \\
\hline & 7.023089 & -0.253164 & -2.392112 \\
\hline & -0.300443 & -3.638469 & 3.473452 \\
\hline & 1.729256 & -3.892565 & -0.239515 \\
\hline & 7.820326 & 3.541237 & -4.233545 \\
\hline & 9.187456 & -0.008230 & 1.433467 \\
\hline & -4.711605 & -5.758166 & 0.394102 \\
\hline & -0.437096 & -2.425499 & 7.587363 \\
\hline & 9.686708 & -2.436122 & 1.694481 \\
\hline & 2.478013 & 2.876083 & -0.173297 \\
\hline & -3.925156 & 0.892034 & 1.852620 \\
\hline & -2.908872 & -1.086956 & -1.800220 \\
\hline & -6.959586 & -1.154167 & -0.361268 \\
\hline & -3.533507 & 2.281692 & -0.934606 \\
\hline & -0.007768 & 2.710377 & 1.460419 \\
\hline & -2.119727 & 6.188380 & 0.101831 \\
\hline & 2.036178 & -2.618094 & 2.776396 \\
\hline & 3.377538 & -2.530023 & 1.637040 \\
\hline & 5.161508 & -1.371945 & 2.542002 \\
\hline ] & 1.196356 & 0.087799 & 3.263858 \\
\hline & 6.099049 & 0.529411 & 3.815778 \\
\hline & 2.117292 & 1.979636 & 4.562168 \\
\hline & 4.583980 & 2.221433 & 4.830015 \\
\hline & 1.555528 & 0.713942 & -3.773650 \\
\hline 1 & 1.654098 & -1.047869 & -3.283788 \\
\hline & -1.283087 & 0.361855 & -2.448297 \\
\hline l & -1.585875 & -1.284392 & -6.023989 \\
\hline ] & -2.233749 & -3.583802 & -6.557720 \\
\hline ] & -1.844129 & -5.415893 & -4.917999 \\
\hline 1 & -0.818040 & -4.875221 & -2.713955 \\
\hline & -0.209313 & -2.545653 & -2.156026 \\
\hline 1 & -0.568414 & 0.629912 & -5.715958 \\
\hline & -2.026688 & 0.684524 & -4.700412 \\
\hline 1 & -0.636946 & 1.721323 & -4.330750 \\
\hline ] & -4.755858 & -2.937575 & -4.240552 \\
\hline l & -3.894414 & -1.451575 & -3.816302 \\
\hline 1 & -3.577542 & -2.952739 & -2.916858 \\
\hline 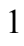 & -6.491090 & -3.909596 & -2.814311 \\
\hline 1 & -5.356695 & -3.951275 & -1.446807 \\
\hline & -6.946267 & -3.183425 & -1.263553 \\
\hline 1 & -6.897163 & -1.759801 & -4.072221 \\
\hline 1 & -7.423533 & -0.978057 & -2.572383 \\
\hline 1 & -6.129435 & -0.249392 & -3.539883 \\
\hline 1 & -6.855485 & 1.404015 & 3.745018 \\
\hline 1 & -5.244260 & 0.693293 & 3.572879 \\
\hline 1 & -5.696943 & 2.121321 & 2.612524 \\
\hline 1 & -8.559100 & 1.245299 & 2.017791 \\
\hline 1 & -7.472533 & 1.921489 & 0.787931 \\
\hline 1 & -8.292950 & 0.376961 & 0.500024 \\
\hline 1 & -7.903014 & -0.802174 & 3.325438 \\
\hline 1 & -7.558231 & -1.720146 & 1.848537 \\
\hline
\end{tabular}




$\begin{array}{rrrr}1 & -6.308925 & -1.547597 & 3.098616 \\ 1 & -4.960950 & 6.772442 & -1.944267 \\ 1 & -4.275454 & 6.794589 & -0.312911 \\ 1 & -3.207070 & 6.857117 & -1.734480 \\ 1 & -4.691755 & 4.854565 & -3.456465 \\ 1 & -2.930495 & 4.801606 & -3.215654 \\ 1 & -3.909620 & 3.353779 & -2.927172 \\ 1 & -6.253108 & 4.763668 & -1.444955 \\ 1 & -5.488496 & 3.264239 & -0.894766 \\ 1 & -5.587583 & 4.657205 & 0.198690 \\ 1 & 0.541501 & 7.483974 & 2.032881 \\ 1 & -0.073792 & 7.190695 & 0.399088 \\ 1 & -1.190952 & 7.240954 & 1.782992 \\ 1 & 0.534528 & 5.736880 & 3.771195 \\ 1 & -1.181112 & 5.362794 & 3.499274 \\ 1 & 0.035757 & 4.073953 & 3.410776 \\ 1 & 2.168194 & 5.732860 & 1.801163 \\ 1 & 1.725416 & 4.047687 & 1.517335 \\ 1 & 1.605688 & 5.230297 & 0.192218\end{array}$

$\operatorname{TS1}_{I L-r e-} \Psi-1 \mathrm{~s} 23324 \mathrm{~s}$

Number of imaginary frequencies : 1

The smallest frequencies are : -668.1576 $9.0944 \quad 14.5166 \mathrm{~cm}(-1)$

Electronic energy : $\quad H F=-4360.8605295$

Zero-point correction $=\quad 1.451298($ Hartree/Particle $)$

Thermal correction to Energy= $\quad 1.536381$

Thermal correction to Enthalpy= $\quad 1.537325$

Thermal correction to Gibbs Free Energy $=\quad 1.331260$

Sum of electronic and zero-point Energies $=\quad-4359.409231$

Sum of electronic and thermal Energies $=\quad-4359.324148$

Sum of electronic and thermal Enthalpies $=\quad-4359.323204$

Sum of electronic and thermal Free Energies $=\quad-4359.529269$

Cartesian Coordinates

$\begin{array}{llll}6 & 6.803853 & 0.848952 & -2.460572 \\ 6 & 5.688163 & 1.372471 & -1.758575 \\ 6 & 5.363830 & 2.750646 & -1.949174 \\ 6 & 6.181845 & 3.549502 & -2.784509 \\ 6 & 7.266316 & 3.015533 & -3.431814 \\ 6 & 7.570011 & 1.647232 & -3.273572 \\ 6 & 4.861442 & 0.568814 & -0.907993 \\ 6 & 3.726157 & 1.143386 & -0.370875 \\ 6 & 3.406932 & 2.502704 & -0.551274 \\ 6 & 4.220146 & 3.293919 & -1.315413 \\ 6 & 5.144645 & -0.856726 & -0.629163 \\ 6 & 4.178910 & -1.811593 & -0.899308 \\ 6 & 4.455203 & -3.193564 & -0.797333 \\ 6 & 5.662102 & -3.620563 & -0.315793 \\ 6 & 6.633460 & -2.685223 & 0.117131 \\ 6 & 6.375086 & -1.290103 & -0.036464 \\ 6 & 7.839895 & -3.109053 & 0.725335 \\ 6 & 8.749389 & -2.198027 & 1.198488 \\ 6 & 8.481292 & -0.817975 & 1.082096 \\ 6 & 7.330864 & -0.375896 & 0.476464\end{array}$




\begin{tabular}{|c|c|c|c|}
\hline 8 & 2.927938 & -1.453248 & -1.334362 \\
\hline & 1.818390 & -0.632276 & -0.351730 \\
\hline & 2.888278 & 0.385585 & 0.406060 \\
\hline & 0.062875 & 0.330692 & -1.510928 \\
\hline & 0.422292 & 2.313580 & -1.724350 \\
\hline & 0.504571 & 3.309729 & -2.309381 \\
\hline & -0.299472 & -0.186745 & -3.820496 \\
\hline & 1.051035 & -0.026958 & -3.405185 \\
\hline & -0.795677 & -1.563096 & -4.122340 \\
\hline & -1.382579 & -1.830627 & -5.367096 \\
\hline & -1.749724 & -3.123702 & -5.729304 \\
\hline & -1.545789 & -4.182562 & -4.853484 \\
\hline & -0.979797 & -3.928664 & -3.605904 \\
\hline & -0.619708 & -2.638093 & -3.241325 \\
\hline 6 & -0.890510 & 0.931729 & -4.653882 \\
\hline & -1.546036 & 0.517634 & 0.253068 \\
\hline 5 & -1.826641 & 2.335891 & 0.363046 \\
\hline 6 & -2.886913 & 2.912243 & -0.327949 \\
\hline & -3.032425 & 4.299648 & -0.407950 \\
\hline 6 & -2.101849 & 5.096796 & 0.260598 \\
\hline 6 & -1.024438 & 4.550398 & 0.969204 \\
\hline 6 & -0.884298 & 3.162246 & 0.980873 \\
\hline 6 & -4.191269 & 4.870399 & -1.222254 \\
\hline 6 & -0.027009 & 5.411465 & 1.743537 \\
\hline 6 & -1.311763 & 0.028984 & 2.037426 \\
\hline 6 & -0.986430 & -1.278672 & 2.365376 \\
\hline 6 & -0.868665 & -1.672552 & 3.739729 \\
\hline 6 & -1.095062 & -0.714103 & 4.769587 \\
\hline 6 & -1.448522 & 0.603579 & 4.403678 \\
\hline 6 & -1.553795 & 0.959397 & 3.085845 \\
\hline 6 & -0.958351 & -1.094972 & 6.125847 \\
\hline 6 & -0.608344 & -2.376953 & 6.462969 \\
\hline 6 & -0.383502 & -3.332537 & 5.449658 \\
\hline 6 & -0.513088 & -2.992494 & 4.125844 \\
\hline 6 & -0.778811 & -2.350954 & 1.353939 \\
\hline 6 & 0.468701 & -2.558955 & 0.794198 \\
\hline 6 & 0.718410 & -3.710590 & 0.010777 \\
\hline 6 & -0.275709 & -4.615904 & -0.245475 \\
\hline 6 & -1.586855 & -4.396404 & 0.237345 \\
\hline 6 & -1.841065 & -3.263460 & 1.065344 \\
\hline 6 & -3.153694 & -3.081227 & 1.573344 \\
\hline 6 & -4.160019 & -3.957900 & 1.253986 \\
\hline 6 & -3.905657 & -5.074159 & 0.427228 \\
\hline 6 & -2.645092 & -5.286085 & -0.069608 \\
\hline 7 & 1.531210 & -1.618338 & 1.005955 \\
\hline 6 & 2.528905 & -1.996912 & 2.034573 \\
\hline 6 & 3.089801 & -0.846259 & 2.827011 \\
\hline 6 & 4.466397 & -0.717562 & 2.997711 \\
\hline 6 & 4.993474 & 0.348923 & 3.721887 \\
\hline 6 & 4.145822 & 1.296407 & 4.286307 \\
\hline 6 & 2.767029 & 1.168563 & 4.124482 \\
\hline 6 & 2.245513 & 0.104610 & 3.401170 \\
\hline 6 & -3.274976 & -0.091025 & 0.025932 \\
\hline 6 & -4.215438 & 0.188792 & 1.013522 \\
\hline 6 & -5.543760 & -0.221921 & 0.894675 \\
\hline 6 & -5.901377 & -0.909450 & -0.264013 \\
\hline
\end{tabular}




\begin{tabular}{|c|c|c|c|}
\hline & -4.986177 & -1.202309 & -1.283638 \\
\hline & -3.660650 & -0.790919 & -1.118603 \\
\hline & -6.523471 & 0.104895 & 2.019341 \\
\hline & -5.981490 & -0.440168 & 3.346610 \\
\hline & -5.478706 & -1.944631 & -2.524017 \\
\hline & -6.064480 & -3.301143 & -2.117568 \\
\hline & -6.558892 & -1.107224 & -3.218339 \\
\hline & -4.356628 & -2.199530 & -3.522735 \\
\hline & -6.681729 & 1.626646 & 2.117401 \\
\hline & -7.901370 & -0.508448 & 1.786097 \\
\hline & 7.128903 & 0.690444 & 0.410697 \\
\hline & -1.843005 & 1.977639 & 2.841964 \\
\hline & 8.021427 & -4.178782 & 0.822683 \\
\hline & -1.133765 & -0.344018 & 6.894838 \\
\hline & 5.920748 & 4.599696 & -2.909185 \\
\hline & -3.364738 & -2.215902 & 2.200079 \\
\hline & -5.167483 & -3.786993 & 1.630090 \\
\hline & 3.986096 & 4.347500 & -1.463141 \\
\hline & -1.640825 & 1.335822 & 5.187038 \\
\hline & 5.881592 & -4.684067 & -0.235829 \\
\hline & 8.415925 & 1.217694 & -3.805755 \\
\hline & 3.691743 & -3.897408 & -1.120600 \\
\hline & -2.433246 & -6.143683 & -0.707494 \\
\hline & -0.073217 & -5.509829 & -0.834661 \\
\hline & -0.106440 & -4.348891 & 5.720097 \\
\hline & 7.045018 & -0.206188 & -2.359724 \\
\hline & -0.339176 & -3.744136 & 3.358549 \\
\hline & 1.722635 & -3.858408 & -0.380022 \\
\hline & 7.884020 & 3.638047 & -4.075054 \\
\hline 1 & 9.190688 & -0.095870 & 1.480342 \\
\hline & -4.713235 & -5.761846 & 0.185069 \\
\hline ] & -0.501964 & -2.660442 & 7.507462 \\
\hline & 9.667835 & -2.534523 & 1.673747 \\
\hline & 2.510242 & 2.885790 & -0.072009 \\
\hline & -3.908940 & 0.743967 & 1.899069 \\
\hline & -2.904294 & -1.009243 & -1.872553 \\
\hline ] & -6.932093 & -1.236518 & -0.386557 \\
\hline & -3.604101 & 2.267331 & -0.833525 \\
\hline 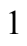 & -0.028873 & 2.706629 & 1.485258 \\
\hline & -2.213373 & 6.176139 & 0.225001 \\
\hline & 1.996835 & -2.675578 & 2.716975 \\
\hline 1 & 3.353409 & -2.580131 & 1.595389 \\
\hline & 5.136919 & -1.445264 & 2.536775 \\
\hline ] & 1.172446 & 0.024036 & 3.242215 \\
\hline & 6.072187 & 0.441913 & 3.833665 \\
\hline 1 & 2.090538 & 1.902453 & 4.561072 \\
\hline & 4.556687 & 2.133614 & 4.846739 \\
\hline 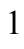 & 1.589325 & 0.866946 & -3.729042 \\
\hline 1 & 1.674452 & -0.914519 & -3.312697 \\
\hline & -1.261351 & 0.474138 & -2.442217 \\
\hline 1 & -1.541856 & -1.024456 & -6.078982 \\
\hline ( & -2.197719 & -3.298709 & -6.705583 \\
\hline 1 & -1.836556 & -5.193173 & -5.131824 \\
\hline ] & -0.832740 & -4.741861 & -2.897116 \\
\hline 1 & -0.215688 & -2.436302 & -2.247194 \\
\hline 1 & -0.522663 & 0.869618 & -5.687671 \\
\hline
\end{tabular}




$\begin{array}{lrrr}1 & -1.986917 & 0.897731 & -4.679484 \\ 1 & -0.591421 & 1.909029 & -4.263157 \\ 1 & -4.751021 & -2.741750 & -4.391775 \\ 1 & -3.913051 & -1.265315 & -3.892986 \\ 1 & -3.553078 & -2.809471 & -3.087401 \\ 1 & -6.439265 & -3.832598 & -3.002888 \\ 1 & -5.295833 & -3.926905 & -1.645095 \\ 1 & -6.899284 & -3.202934 & -1.412372 \\ 1 & -6.912144 & -1.618397 & -4.124116 \\ 1 & -7.430021 & -0.935071 & -2.574405 \\ 1 & -6.163896 & -0.126920 & -3.516094 \\ 1 & -8.562053 & -0.264497 & 2.627612 \\ 1 & -8.376055 & -0.121682 & 0.875309 \\ 1 & -7.852459 & -1.603105 & 1.710616 \\ 1 & -7.359194 & 1.890267 & 2.941007 \\ 1 & -5.722615 & 2.128977 & 2.297729 \\ 1 & -7.102429 & 2.036236 & 1.189302 \\ 1 & -6.670326 & -0.197069 & 4.166794 \\ 1 & -5.878203 & -1.533107 & 3.310210 \\ 1 & -5.000434 & -0.021705 & 3.605874 \\ 6 & -0.255406 & 6.905661 & 1.527769 \\ 6 & -0.176605 & 5.113428 & 3.240422 \\ 6 & 1.403245 & 5.080528 & 1.305719 \\ 6 & -4.236581 & 6.395582 & -1.183628 \\ 6 & -4.034917 & 4.432921 & -2.683983 \\ 6 & -5.518978 & 4.338672 & -0.669143 \\ 1 & -5.086676 & 6.754225 & -1.777709 \\ 1 & -4.365346 & 6.777001 & -0.162297 \\ 1 & -3.328493 & 6.843753 & -1.606893 \\ 1 & -4.848509 & 4.845224 & -3.296365 \\ 1 & -3.082241 & 4.788112 & -3.098385 \\ 1 & -4.057604 & 3.341217 & -2.791315 \\ 1 & -6.360399 & 4.731031 & -1.256219 \\ 1 & -5.573912 & 3.242577 & -0.703157 \\ 1 & -5.660958 & 4.649313 & 0.374528 \\ 1 & 0.491021 & 7.476965 & 2.093870 \\ 1 & -0.154916 & 7.186043 & 0.471264 \\ 1 & -1.244795 & 7.227560 & 1.877045 \\ 1 & 0.526828 & 5.721360 & 3.825700 \\ 1 & -1.192892 & 5.343387 & 3.587153 \\ 1 & 0.026134 & 4.058544 & 3.468010 \\ 1 & 2.119421 & 5.743975 & 1.808735 \\ 1 & 1.683371 & 4.051510 & 1.562799 \\ 1 & 1.521812 & 5.210842 & 0.221129\end{array}$

TS1 $_{I L-r e-} \Psi-1 \mathbf{1 5 2 3 3 4 a}$

Number of imaginary frequencies : 1

The smallest frequencies are : - $671.3809 \quad 14.3134 \quad 16.4543 \mathrm{~cm}(-1)$

Electronic energy : $\quad \mathrm{HF}=-4360.8633534$

Zero-point correction $=\quad 1.451774($ Hartree/Particle)

Thermal correction to Energy $=\quad 1.536559$

Thermal correction to Enthalpy= $\quad 1.537503$

Thermal correction to Gibbs Free Energy= 1.333632

Sum of electronic and zero-point Energies $=\quad-4359.411579$

Sum of electronic and thermal Energies $=\quad-4359.326794$ 
Sum of electronic and thermal Enthalpies=

Sum of electronic and thermal Free Energies=
$-4359.325850$

$-4359.529721$

Cartesian Coordinates

\begin{tabular}{|c|c|c|c|}
\hline 6 & -4.235007 & 0.378831 & 1.000990 \\
\hline 6 & -3.284108 & 0.007021 & 0.039774 \\
\hline 6 & -3.674842 & -0.740609 & -1.060365 \\
\hline 6 & -5.016712 & -1.123373 & -1.231078 \\
\hline 6 & -5.930901 & -0.744172 & -0.254168 \\
\hline 6 & -5.567351 & 0.010476 & 0.873814 \\
\hline 15 & -1.530921 & 0.544989 & 0.249137 \\
\hline 45 & 0.029676 & 0.252709 & -1.542806 \\
\hline 6 & 0.414484 & 2.202469 & -1.880081 \\
\hline 8 & 0.471856 & 3.205279 & -2.455607 \\
\hline 6 & -5.413996 & -1.939064 & -2.458165 \\
\hline 6 & -6.619819 & 0.373941 & 1.919502 \\
\hline 6 & -1.718720 & 2.375204 & 0.322390 \\
\hline 6 & -2.737914 & 2.991106 & -0.394994 \\
\hline 6 & -2.818293 & 4.382446 & -0.491100 \\
\hline 6 & -1.865426 & 5.144124 & 0.187805 \\
\hline 6 & -0.826578 & 4.556702 & 0.920685 \\
\hline 6 & -0.748570 & 3.163694 & 0.945994 \\
\hline 6 & 0.186316 & 5.378514 & 1.717394 \\
\hline 6 & -3.937572 & 4.994813 & -1.329269 \\
\hline 6 & -1.305511 & 0.091218 & 2.041514 \\
\hline 6 & -0.984101 & -1.208830 & 2.398745 \\
\hline 6 & -0.859568 & -1.568712 & 3.783108 \\
\hline 6 & -1.079986 & -0.584391 & 4.789610 \\
\hline 6 & -1.438238 & 0.723411 & 4.393233 \\
\hline 6 & -1.547927 & 1.046526 & 3.068008 \\
\hline 6 & -0.935849 & -0.929851 & 6.154393 \\
\hline 6 & -0.583376 & -2.202523 & 6.522951 \\
\hline 6 & -0.363780 & -3.183483 & 5.533407 \\
\hline 6 & -0.501310 & -2.878029 & 4.201797 \\
\hline 6 & -0.787031 & -2.314246 & 1.420704 \\
\hline 6 & 0.458437 & -2.559451 & 0.872476 \\
\hline 6 & 0.703979 & -3.760542 & 0.165117 \\
\hline 6 & -0.287881 & -4.686437 & -0.017423 \\
\hline 6 & -1.598031 & -4.434922 & 0.453109 \\
\hline 6 & -1.853176 & -3.237704 & 1.184818 \\
\hline 6 & -3.168747 & -3.008689 & 1.664113 \\
\hline 6 & -4.174958 & -3.909476 & 1.419365 \\
\hline 6 & -3.916070 & -5.097337 & 0.701133 \\
\hline 6 & -2.654352 & -5.350633 & 0.226933 \\
\hline 7 & 1.524526 & -1.612027 & 1.026009 \\
\hline 15 & 1.791147 & -0.677748 & -0.372893 \\
\hline 8 & 2.869146 & 0.365401 & 0.336985 \\
\hline 6 & 3.704207 & 1.094276 & -0.468747 \\
\hline 6 & 4.835072 & 0.498723 & -0.991982 \\
\hline 6 & 5.664122 & 1.274331 & -1.866143 \\
\hline 6 & 5.346767 & 2.648747 & -2.093301 \\
\hline 6 & 4.206867 & 3.215196 & -1.472968 \\
\hline 6 & 3.390722 & 2.449258 & -0.686678 \\
\hline 6 & 6.776482 & 0.726573 & -2.554887 \\
\hline 6 & 7.546155 & 1.498929 & -3.389321 \\
\hline
\end{tabular}




\begin{tabular}{|c|c|c|c|}
\hline & 7.249490 & 2.864081 & -3.583723 \\
\hline & 6.168262 & 3.420700 & -2.950211 \\
\hline & 5.113623 & -0.918768 & -0.670228 \\
\hline & 4.143575 & -1.878854 & -0.905134 \\
\hline & 4.417664 & -3.257939 & -0.764533 \\
\hline & 5.625458 & -3.673678 & -0.275491 \\
\hline & 6.599934 & -2.728158 & 0.127180 \\
\hline & 6.344790 & -1.337696 & -0.068370 \\
\hline & 7.806239 & -3.136456 & 0.746212 \\
\hline & 8.718573 & -2.213882 & 1.190615 \\
\hline & 8.453709 & -0.837307 & 1.032844 \\
\hline & 7.303673 & -0.410877 & 0.415321 \\
\hline & 2.889548 & -1.531525 & -1.340443 \\
\hline & 2.539858 & -1.945767 & 2.053207 \\
\hline & 3.099276 & -0.763876 & 2.800186 \\
\hline & 4.476537 & -0.623924 & 2.957244 \\
\hline & 5.004551 & 0.470046 & 3.638348 \\
\hline & 4.157246 & 1.435107 & 4.172531 \\
\hline & 2.777924 & 1.296232 & 4.024973 \\
\hline & 2.255125 & 0.204238 & 3.345406 \\
\hline & 0.919082 & -0.277636 & -3.447391 \\
\hline & -0.452464 & -0.412891 & -3.799159 \\
\hline & -1.023457 & -1.781575 & -3.973401 \\
\hline & -0.744518 & -2.828332 & -3.082156 \\
\hline & -1.174964 & -4.122068 & -3.338077 \\
\hline & -1.925491 & -4.409134 & -4.477198 \\
\hline & -2.240916 & -3.378638 & -5.352514 \\
\hline & -1.794084 & -2.082539 & -5.105100 \\
\hline & -1.024266 & 0.671591 & -4.689218 \\
\hline & 7.103656 & 0.653479 & 0.318823 \\
\hline & -1.836796 & 2.058933 & 2.800248 \\
\hline & 7.985153 & -4.203186 & 0.875844 \\
\hline 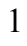 & -1.107526 & -0.158956 & 6.904296 \\
\hline & 5.912531 & 4.468571 & -3.102525 \\
\hline & -3.379165 & -2.092102 & 2.212952 \\
\hline & -5.182175 & -3.704266 & 1.777365 \\
\hline & 3.977930 & 4.265756 & -1.648192 \\
\hline ] & -1.629536 & 1.474105 & 5.159205 \\
\hline & 5.843070 & -4.734878 & -0.165390 \\
\hline 1 & 8.389426 & 1.050940 & -3.910386 \\
\hline & 3.651593 & -3.968733 & -1.065659 \\
\hline 1 & -2.441432 & -6.259633 & -0.334941 \\
\hline & -0.085072 & -5.621454 & -0.538988 \\
\hline & -0.084513 & -4.192485 & 5.828085 \\
\hline 1 & 7.012530 & -0.326692 & -2.425953 \\
\hline & -0.330476 & -3.650330 & 3.454555 \\
\hline 1 & 1.707140 & -3.933419 & -0.217887 \\
\hline & 7.870014 & 3.466064 & -4.243574 \\
\hline 1 & 9.165073 & -0.105268 & 1.408826 \\
\hline 1 & -4.721810 & -5.805292 & 0.518875 \\
\hline & -0.471161 & -2.458830 & 7.573850 \\
\hline 1 & 9.636668 & -2.538189 & 1.674952 \\
\hline & 2.498715 & 2.850737 & -0.213811 \\
\hline 1 & -3.916357 & 0.971358 & 1.854479 \\
\hline & -2.918123 & -1.033871 & -1.790871 \\
\hline 1 & -6.972460 & -1.039372 & -0.363143 \\
\hline
\end{tabular}




\begin{tabular}{|c|c|c|c|}
\hline & -3.474438 & 2.372753 & -0.906445 \\
\hline & 0.074117 & 2.675397 & 1.473598 \\
\hline 1 & -1.929365 & 6.226991 & 0.142247 \\
\hline & 2.024582 & -2.607792 & 2.764135 \\
\hline & 3.365351 & -2.533979 & 1.622626 \\
\hline & 5.146888 & -1.365344 & 2.518369 \\
\hline & 1.181131 & 0.114007 & 3.197276 \\
\hline & 6.083689 & 0.570556 & 3.739294 \\
\hline & 2.101959 & 2.043126 & 4.440124 \\
\hline & 4.568471 & 2.293992 & 4.698823 \\
\hline 1 & 1.473802 & 0.569289 & -3.856739 \\
\hline & 1.516345 & -1.177652 & -3.313167 \\
\hline & -1.313757 & 0.388495 & -2.445813 \\
\hline 1 & -2.042193 & -1.301537 & -5.819752 \\
\hline $\mathbf{1}$ & -2.836944 & -3.577237 & -6.241343 \\
\hline ] & -2.274896 & -5.421774 & -4.666684 \\
\hline & -0.940490 & -4.910955 & -2.625225 \\
\hline 1 & -0.199979 & -2.603765 & -2.163162 \\
\hline & -0.727144 & 0.498767 & -5.733296 \\
\hline 1 & -2.119575 & 0.718316 & -4.650606 \\
\hline ] & -0.637204 & 1.653276 & -4.398978 \\
\hline 6 & 0.019223 & 6.880703 & 1.500953 \\
\hline 0 & -0.009634 & 5.083559 & 3.209782 \\
\hline 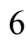 & 1.612841 & 4.995565 & 1.312136 \\
\hline 0 & -3.905977 & 6.520700 & -1.320910 \\
\hline 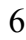 & -3.787699 & 4.520157 & -2.780156 \\
\hline 6 & -5.295280 & 4.540585 & -0.779456 \\
\hline 0 & -6.918575 & -2.187342 & -2.535044 \\
\hline 6 & -4.994337 & -1.186279 & -3.725581 \\
\hline 6 & -4.709180 & -3.297711 & -2.398223 \\
\hline 6 & -6.055794 & 1.281090 & 3.011335 \\
\hline 6 & -7.790951 & 1.105490 & 1.255598 \\
\hline 6 & -7.131644 & -0.910661 & 2.581115 \\
\hline 1 & 0.772657 & 7.422281 & 2.086692 \\
\hline 1 & 0.155707 & 7.159841 & 0.448152 \\
\hline 1 & -0.965611 & 7.238497 & 1.827376 \\
\hline 1 & 2.341363 & 5.627752 & 1.837219 \\
\hline 1 & 1.849107 & 3.954685 & 1.565975 \\
\hline 1 & 1.763028 & 5.130426 & 0.231668 \\
\hline 1 & 0.706432 & 5.658433 & 3.812896 \\
\hline 1 & -1.022502 & 5.357018 & 3.534446 \\
\hline 1 & 0.140150 & 4.019853 & 3.437720 \\
\hline 1 & -4.729162 & 6.909918 & -1.933379 \\
\hline 1 & -4.028340 & 6.928718 & -0.309144 \\
\hline 1 & -2.970769 & 6.913515 & -1.740104 \\
\hline 1 & -4.565519 & 4.969216 & -3.412782 \\
\hline 1 & -2.808536 & 4.807201 & -3.185970 \\
\hline 1 & -3.876282 & 3.430005 & -2.867692 \\
\hline 1 & -6.111390 & 4.959347 & -1.383765 \\
\hline 1 & -5.401685 & 3.448169 & -0.789814 \\
\hline 1 & -5.432884 & 4.879862 & 0.255943 \\
\hline 1 & -7.148023 & -2.764432 & -3.439756 \\
\hline 1 & -7.285388 & -2.766936 & -1.677885 \\
\hline 1 & -7.489234 & -1.250752 & -2.587966 \\
\hline 1 & -4.946411 & -3.892516 & -3.291306 \\
\hline 1 & -3.617381 & -3.198392 & -2.341799 \\
\hline
\end{tabular}




$\begin{array}{lrrr}1 & -5.035885 & -3.861454 & -1.514227 \\ 1 & -5.279379 & -1.757209 & -4.620276 \\ 1 & -5.483246 & -0.204065 & -3.778535 \\ 1 & -3.909611 & -1.032204 & -3.771658 \\ 1 & -7.903481 & -0.676655 & 3.326928 \\ 1 & -7.572100 & -1.601733 & 1.851639 \\ 1 & -6.316541 & -1.437326 & 3.095921 \\ 1 & -8.545243 & 1.373747 & 2.007365 \\ 1 & -7.453711 & 2.031307 & 0.771669 \\ 1 & -8.289465 & 0.492670 & 0.495466 \\ 1 & -6.845654 & 1.524504 & 3.733087 \\ 1 & -5.239731 & 0.800611 & 3.567912 \\ 1 & -5.679380 & 2.227148 & 2.599212\end{array}$

$\mathrm{TS1}_{I L-r e-} \psi_{-1 \mathrm{~s} 2 \mathrm{~s} 3 \mathrm{~s} 4 \mathrm{~s}}$

Number of imaginary frequencies : 1

The smallest frequencies are : $-677.7744 \quad 14.9416 \quad 19.1404 \mathrm{~cm}(-1)$

Electronic energy : $\quad H F=-4360.8636907$

Zero-point correction $=\quad 1.452245$ (Hartree/Particle)

Thermal correction to Energy $=\quad 1.536794$

Thermal correction to Enthalpy $=\quad 1.537738$

Thermal correction to Gibbs Free Energy $=\quad 1.335075$

Sum of electronic and zero-point Energies $=\quad-4359.411445$

Sum of electronic and thermal Energies $=\quad-4359.326897$

Sum of electronic and thermal Enthalpies= $\quad-4359.325953$

Sum of electronic and thermal Free Energies $=\quad-4359.528615$

Cartesian Coordinates

$\begin{array}{cccc}6 & -4.222436 & 0.319670 & 0.979080 \\ 6 & -3.272130 & -0.012905 & 0.011846 \\ 6 & -3.656876 & -0.753034 & -1.100016 \\ 6 & -4.988253 & -1.158680 & -1.266303 \\ 6 & -5.908780 & -0.818379 & -0.273697 \\ 6 & -5.549854 & -0.082239 & 0.861639 \\ 15 & -1.529677 & 0.553022 & 0.230261 \\ 45 & 0.042199 & 0.272398 & -1.550205 \\ 6 & 0.426687 & 2.223282 & -1.879155 \\ 8 & 0.491565 & 3.223362 & -2.458743 \\ 6 & -5.376982 & -1.968005 & -2.500814 \\ 6 & -6.536942 & 0.293492 & 1.965128 \\ 6 & -1.745673 & 2.380161 & 0.302340 \\ 6 & -2.783657 & 2.982914 & -0.399078 \\ 6 & -2.881851 & 4.373529 & -0.494795 \\ 6 & -1.929914 & 5.146805 & 0.172085 \\ 6 & -0.874873 & 4.572557 & 0.892555 \\ 6 & -0.778251 & 3.180958 & 0.914745 \\ 6 & 0.132994 & 5.406578 & 1.683106 \\ 6 & -4.018294 & 4.972768 & -1.319735 \\ 6 & -1.312778 & 0.103793 & 2.025599 \\ 6 & -1.001633 & -1.197387 & 2.387721 \\ 6 & -0.887976 & -1.555406 & 3.773403 \\ 6 & -1.109849 & -0.568257 & 4.776717 \\ 6 & -1.454554 & 0.741538 & 4.375382 \\ 6 & -1.553105 & 1.063037 & 3.048682\end{array}$




\begin{tabular}{|c|c|c|c|}
\hline 6 & -0.979019 & -0.912518 & 6.143198 \\
\hline 5 & -0.637287 & -2.186705 & 6.516369 \\
\hline p & -0.414842 & -3.170207 & 5.529915 \\
\hline & -0.539844 & -2.865982 & 4.196844 \\
\hline f & -0.802175 & -2.304197 & 1.412097 \\
\hline & 0.444901 & -2.546625 & 0.865866 \\
\hline 6 & 0.693814 & -3.747127 & 0.158622 \\
\hline 6 & -0.295402 & -4.675923 & -0.023233 \\
\hline 6 & -1.606046 & -4.429114 & 0.448446 \\
\hline 6 & -1.865648 & -3.231362 & 1.177776 \\
\hline 6 & -3.181573 & -3.007944 & 1.659148 \\
\hline 6 & -4.182572 & -3.916318 & 1.420811 \\
\hline 6 & -3.919052 & -5.105348 & 0.706190 \\
\hline 6 & -2.657305 & -5.352091 & 0.228466 \\
\hline 7 & 1.510516 & -1.599374 & 1.025680 \\
\hline 15 & 1.791964 & -0.666985 & -0.371509 \\
\hline 8 & 2.868897 & 0.372434 & 0.345430 \\
\hline 6 & 3.712161 & 1.097767 & -0.454854 \\
\hline 6 & 4.844051 & 0.496886 & -0.969733 \\
\hline 6 & 5.681919 & 1.267394 & -1.839772 \\
\hline 6 & 5.371132 & 2.642447 & -2.071900 \\
\hline 6 & 4.229748 & 3.214705 & -1.459647 \\
\hline 6 & 3.405758 & 2.453920 & -0.676385 \\
\hline 6 & 6.796796 & 0.713952 & -2.519871 \\
\hline 6 & 7.574717 & 1.481590 & -3.350965 \\
\hline 6 & 7.284258 & 2.847349 & -3.550441 \\
\hline 6 & 6.201013 & 3.409409 & -2.925246 \\
\hline 6 & 5.114670 & -0.921110 & -0.643349 \\
\hline 6 & 4.142817 & -1.877879 & -0.884108 \\
\hline 6 & 4.410265 & -3.257664 & -0.738208 \\
\hline 6 & 5.612431 & -3.677054 & -0.238503 \\
\hline 6 & 6.587379 & -2.734494 & 0.169864 \\
\hline 6 & 6.339335 & -1.343458 & -0.030780 \\
\hline 6 & 7.787072 & -3.146178 & 0.799400 \\
\hline 6 & 8.699560 & -2.226226 & 1.248872 \\
\hline 6 & 8.441573 & -0.848971 & 1.085748 \\
\hline 6 & 7.298160 & -0.419357 & 0.458236 \\
\hline 8 & 2.893727 & -1.526172 & -1.330294 \\
\hline 6 & 2.514946 & -1.930730 & 2.064420 \\
\hline 6 & 3.071648 & -0.745753 & 2.808532 \\
\hline 6 & 4.447590 & -0.612058 & 2.981157 \\
\hline 6 & 4.973749 & 0.487320 & 3.654970 \\
\hline 6 & 4.125746 & 1.464179 & 4.166092 \\
\hline 6 & 2.747471 & 1.331279 & 4.003492 \\
\hline 6 & 2.226570 & 0.233583 & 3.331838 \\
\hline 6 & 0.947176 & -0.244801 & -3.450438 \\
\hline 6 & -0.422065 & -0.387037 & -3.809185 \\
\hline 6 & -0.982658 & -1.759008 & -3.991922 \\
\hline 6 & -0.699487 & -2.808265 & -3.104715 \\
\hline 6 & -1.114983 & -4.104756 & -3.371162 \\
\hline 6 & -1.853577 & -4.392761 & -4.517853 \\
\hline 6 & -2.175742 & -3.359836 & -5.387818 \\
\hline 6 & -1.745069 & -2.060415 & -5.129089 \\
\hline 6 & -0.995661 & 0.696877 & -4.698888 \\
\hline 1 & 7.103299 & 0.645576 & 0.357563 \\
\hline 1 & -1.832996 & 2.076861 & 2.777198 \\
\hline
\end{tabular}




\begin{tabular}{|c|c|c|c|}
\hline ] & 7.960675 & -4.213309 & 0.932909 \\
\hline & -1.152006 & -0.139422 & 6.890520 \\
\hline & 5.950217 & 4.457883 & -3.081552 \\
\hline & -3.398235 & -2.090143 & 2.204413 \\
\hline & -5.190093 & -3.715988 & 1.781247 \\
\hline & 4.006614 & 4.265835 & -1.638624 \\
\hline & -1.644624 & 1.495324 & 5.138634 \\
\hline & 5.824936 & -4.738855 & -0.124290 \\
\hline & 8.419754 & 1.029377 & -3.865485 \\
\hline & 3.643917 & -3.966211 & -1.043818 \\
\hline & -2.440487 & -6.261099 & -0.331912 \\
\hline & -0.089599 & -5.611148 & -0.543240 \\
\hline & -0.143273 & -4.180238 & 5.828238 \\
\hline & 7.028083 & -0.339878 & -2.386951 \\
\hline & -0.366787 & -3.640190 & 3.452114 \\
\hline & 1.697564 & -3.917965 & -0.223694 \\
\hline & 7.911260 & 3.445531 & -4.207618 \\
\hline & 9.152989 & -0.118934 & 1.465500 \\
\hline & -4.720982 & -5.818905 & 0.529315 \\
\hline & -0.535340 & -2.442242 & 7.568498 \\
\hline ] & 9.612479 & -2.553087 & 1.741212 \\
\hline & 2.512720 & 2.860260 & -0.209288 \\
\hline 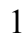 & -3.917736 & 0.906789 & 1.844420 \\
\hline ] & -2.899084 & -1.021563 & -1.838684 \\
\hline & -6.941043 & -1.136728 & -0.385173 \\
\hline$\frac{1}{1}$ & -3.520482 & 2.355821 & -0.899162 \\
\hline & 0.056579 & 2.702724 & 1.432445 \\
\hline 1 & -2.007600 & 6.228807 & 0.127255 \\
\hline & 1.990615 & -2.586563 & 2.774472 \\
\hline 1 & 3.342286 & -2.524528 & 1.644817 \\
\hline 1 & 5.118995 & -1.362411 & 2.559457 \\
\hline & 1.154154 & 0.148788 & 3.169658 \\
\hline 1 & 6.052184 & 0.582900 & 3.767560 \\
\hline - & 2.070879 & 2.087570 & 4.400329 \\
\hline 1 & 4.535620 & 2.327605 & 4.685967 \\
\hline 1 & 1.498704 & 0.606851 & -3.854272 \\
\hline 1 & 1.549264 & -1.141659 & -3.316715 \\
\hline 1 & -1.297669 & 0.407537 & -2.458598 \\
\hline | & -1.997085 & -1.277624 & -5.840376 \\
\hline 1 & -2.764388 & -3.559062 & -6.281407 \\
\hline 1 & -2.190494 & -5.407900 & -4.716326 \\
\hline 1 & -0.878684 & -4.895346 & -2.660762 \\
\hline 1 & -0.163971 & -2.583718 & -2.180482 \\
\hline 1 & -0.691977 & 0.529257 & -5.741939 \\
\hline 1 & -2.091392 & 0.737006 & -4.665815 \\
\hline 1 & -0.616172 & 1.679939 & -4.403382 \\
\hline 6 & -0.045932 & 6.906139 & 1.457979 \\
\hline 6 & -0.061324 & 5.118116 & 3.177075 \\
\hline 6 & 1.563194 & 5.033019 & 1.282024 \\
\hline 6 & -4.007643 & 6.498969 & -1.306563 \\
\hline 6 & -3.877010 & 4.505211 & -2.773726 \\
\hline 6 & -5.364437 & 4.498741 & -0.758535 \\
\hline 6 & -6.872993 & -2.267491 & -2.559987 \\
\hline 6 & -5.001438 & -1.183555 & -3.762763 \\
\hline 6 & -4.623794 & -3.301426 & -2.472775 \\
\hline 6 & -7.926862 & -0.291055 & 1.728104 \\
\hline
\end{tabular}




$\begin{array}{lrrr}6 & -6.023540 & -0.237579 & 3.309481 \\ 6 & -6.661428 & 1.820101 & 2.031279 \\ 1 & 0.706368 & 7.456973 & 2.036492 \\ 1 & 0.083103 & 7.179186 & 0.402646 \\ 1 & -1.031604 & 7.259089 & 1.787080 \\ 1 & 2.285149 & 5.670162 & 1.810151 \\ 1 & 1.806382 & 3.993809 & 1.536334 \\ 1 & 1.716841 & 5.170351 & 0.202574 \\ 1 & 0.647774 & 5.704132 & 3.777698 \\ 1 & -1.077481 & 5.382165 & 3.499234 \\ 1 & 0.100077 & 4.057298 & 3.410717 \\ 1 & -4.842100 & 6.878656 & -1.909683 \\ 1 & -4.125748 & 6.901937 & -0.292260 \\ 1 & -3.082219 & 6.906221 & -1.733616 \\ 1 & -4.671729 & 4.940333 & -3.395007 \\ 1 & -2.909508 & 4.813767 & -3.191152 \\ 1 & -3.944127 & 3.413734 & -2.863575 \\ 1 & -6.191306 & 4.910891 & -1.352718 \\ 1 & -5.458221 & 3.404948 & -0.773343 \\ 1 & -5.495484 & 4.831296 & 0.279860 \\ 1 & -7.095457 & -2.838526 & -3.470278 \\ 1 & -7.206476 & -2.872230 & -1.706574 \\ 1 & -7.477189 & -1.351179 & -2.590393 \\ 1 & -4.867994 & -3.899193 & -3.362062 \\ 1 & -3.535486 & -3.161224 & -2.453103 \\ 1 & -4.899700 & -3.882009 & -1.582111 \\ 1 & -5.273165 & -1.754314 & -4.661708 \\ 1 & -5.528782 & -0.220715 & -3.798463 \\ 1 & -3.924009 & -0.985746 & -3.816549 \\ 1 & -7.345088 & 2.114799 & 2.839078 \\ 1 & -5.694385 & 2.304696 & 2.217214 \\ 1 & -7.058814 & 2.221359 & 1.089354 \\ 1 & -6.716592 & 0.035235 & 4.116776 \\ 1 & -5.942702 & -1.333013 & 3.293581 \\ 1 & -5.036445 & 0.164519 & 3.571747 \\ 1 & -8.592409 & -0.007143 & 2.553108 \\ 1 & -8.378456 & 0.082367 & 0.800163 \\ 1 & -7.906379 & -1.388185 & 1.683650\end{array}$

TS1 $_{I B I-} \psi-1$ a2a3a4a

Number of imaginary frequencies : 1

The smallest frequencies are : - $687.7941 \quad 15.0044 \quad 18.1342 \mathrm{~cm}(-1)$

Electronic energy : $\quad H F=-4360.8642385$

Zero-point correction $=\quad 1.451664($ Hartree/Particle $)$

Thermal correction to Energy= $\quad 1.536728$

Thermal correction to Enthalpy $=\quad 1.537673$

Thermal correction to Gibbs Free Energy $=\quad 1.333509$

Sum of electronic and zero-point Energies $=\quad-4359.412575$

Sum of electronic and thermal Energies $=\quad-4359.327510$

Sum of electronic and thermal Enthalpies $=\quad-4359.326566$

Sum of electronic and thermal Free Energies $=\quad-4359.530730$

Cartesian Coordinates 


\begin{tabular}{|c|c|c|c|}
\hline 5 & -2.632733 & 2.646743 & 1.312156 \\
\hline 6 & -1.727735 & 1.596287 & 1.443914 \\
\hline 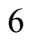 & -0.702677 & 1.681171 & 2.390906 \\
\hline 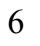 & -0.603009 & 2.777492 & 3.246647 \\
\hline ) & -1.520191 & 3.820626 & 3.076997 \\
\hline 6 & -2.534413 & 3.784198 & 2.118340 \\
\hline 15 & -1.724868 & 0.184749 & 0.263137 \\
\hline & -0.069083 & 0.849725 & -1.393667 \\
\hline o & 0.498218 & 2.608324 & -0.615501 \\
\hline 8 & 0.565427 & 3.737995 & -0.379379 \\
\hline 6 & 0.464399 & 2.869367 & 4.336677 \\
\hline 6 & 1.448050 & 3.990850 & 3.987573 \\
\hline 6 & -3.496698 & 4.962836 & 1.983962 \\
\hline 6 & -4.546439 & 4.731873 & 0.899029 \\
\hline 6 & -1.733872 & -1.278987 & 1.425828 \\
\hline 6 & -1.547305 & -2.561865 & 0.931910 \\
\hline 6 & -1.618486 & -3.693606 & 1.808423 \\
\hline 6 & -1.892286 & -3.495398 & 3.192561 \\
\hline 6 & -2.098177 & -2.178695 & 3.662095 \\
\hline 6 & -2.020824 & -1.110798 & 2.808831 \\
\hline 6 & -1.412026 & -5.022690 & 1.352901 \\
\hline 6 & -1.468606 & -6.085278 & 2.220710 \\
\hline 6 & -1.740165 & -5.880782 & 3.589993 \\
\hline 6 & -1.948305 & -4.610436 & 4.062469 \\
\hline 6 & -1.300836 & -2.824902 & -0.511349 \\
\hline 6 & -2.377889 & -3.248094 & -1.349122 \\
\hline 6 & -2.122589 & -3.554468 & -2.719378 \\
\hline 6 & -0.801998 & -3.451891 & -3.214966 \\
\hline 6 & 0.215932 & -3.044141 & -2.394747 \\
\hline 6 & -0.029072 & -2.709562 & -1.041875 \\
\hline 6 & -3.709571 & -3.356367 & -0.870590 \\
\hline 6 & -4.726645 & -3.754672 & -1.701270 \\
\hline 6 & -4.467482 & -4.072339 & -3.053028 \\
\hline 6 & -3.193014 & -3.968687 & -3.549781 \\
\hline 7 & 1.051725 & -2.208620 & -0.241537 \\
\hline 15 & 1.565412 & -0.671796 & -0.758578 \\
\hline 8 & 2.483382 & -0.312673 & 0.583668 \\
\hline 6 & 3.409167 & 0.695374 & 0.465294 \\
\hline 6 & 4.625005 & 0.449862 & -0.147853 \\
\hline 6 & 5.518425 & 1.553866 & -0.342610 \\
\hline 6 & 5.177102 & 2.834765 & 0.188762 \\
\hline 6 & 3.953901 & 2.998885 & 0.881055 \\
\hline 6 & 3.079125 & 1.954565 & 1.004145 \\
\hline 6 & 6.712581 & 1.447049 & -1.100861 \\
\hline 6 & 7.532561 & 2.531838 & -1.292883 \\
\hline 6 & 7.208934 & 3.786791 & -0.735483 \\
\hline 6 & 6.051559 & 3.930201 & -0.013524 \\
\hline 6 & 4.923970 & -0.911705 & -0.645161 \\
\hline 6 & 4.020736 & -1.534179 & -1.487219 \\
\hline 6 & 4.290428 & -2.785714 & -2.082915 \\
\hline 6 & 5.443670 & -3.454124 & -1.777562 \\
\hline 6 & 6.357115 & -2.917164 & -0.837083 \\
\hline 6 & 6.093347 & -1.641680 & -0.252515 \\
\hline 6 & 7.506092 & -3.643317 & -0.440479 \\
\hline 6 & 8.354828 & -3.152653 & 0.518604 \\
\hline 6 & 8.078018 & -1.910277 & 1.126145 \\
\hline
\end{tabular}




\begin{tabular}{|c|c|c|c|}
\hline 6 & 6.980743 & -1.174386 & 0.750158 \\
\hline & 2.844507 & -0.920206 & -1.832034 \\
\hline & -3.494460 & 0.107172 & -0.269687 \\
\hline & -3.827745 & 0.099373 & -1.621294 \\
\hline & -5.164892 & 0.039500 & -2.033219 \\
\hline & -6.146720 & -0.019285 & -1.043532 \\
\hline & -5.847827 & -0.028383 & 0.323747 \\
\hline & -4.506506 & 0.034123 & 0.691619 \\
\hline & -5.573335 & 0.084624 & -3.505483 \\
\hline & -6.966620 & -0.158669 & 1.355354 \\
\hline & 1.854102 & -3.236321 & 0.462239 \\
\hline & 2.427122 & -2.814746 & 1.788672 \\
\hline & 3.789122 & -2.957277 & 2.043916 \\
\hline & 4.325312 & -2.576854 & 3.271900 \\
\hline & 3.498261 & -2.061557 & 4.263968 \\
\hline & 2.131806 & -1.931439 & 4.020255 \\
\hline 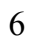 & 1.603028 & -2.300624 & 2.791001 \\
\hline & -0.863926 & 1.171881 & -3.498019 \\
\hline ) & 0.559765 & 1.199505 & -3.516514 \\
\hline ) & 1.310638 & 2.487443 & -3.580581 \\
\hline & 2.659529 & 2.519861 & -3.190267 \\
\hline & 3.376305 & 3.706943 & -3.178758 \\
\hline 6 & 2.768939 & 4.898025 & -3.576012 \\
\hline & 1.442753 & 4.878580 & -3.990573 \\
\hline 6 & 0.722973 & 3.685980 & -3.998156 \\
\hline b & 1.231675 & 0.038993 & -4.216045 \\
\hline f & -0.196539 & 3.177740 & 5.685108 \\
\hline 6 & 1.240010 & 1.561921 & 4.492015 \\
\hline b & -4.229575 & 5.182166 & 3.311935 \\
\hline 6 & -2.702697 & 6.222107 & 1.620210 \\
\hline 1 & 6.770170 & -0.227476 & 1.241082 \\
\hline & -2.186740 & -0.112345 & 3.204056 \\
\hline 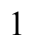 & 7.692106 & -4.610002 & -0.906966 \\
\hline & -2.157002 & -4.434669 & 5.116900 \\
\hline 1 & 5.773042 & 4.895538 & 0.407186 \\
\hline & -3.922935 & -3.101079 & 0.166152 \\
\hline 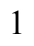 & -5.743506 & -3.818371 & -1.318193 \\
\hline 1 & 3.703188 & 3.979483 & 1.284144 \\
\hline & -2.321878 & -2.024181 & 4.717052 \\
\hline 1 & 5.663877 & -4.417381 & -2.234879 \\
\hline & 8.437332 & 2.422911 & -1.886855 \\
\hline 1 & 3.563392 & -3.190983 & -2.784052 \\
\hline & -2.979387 & -4.206186 & -4.591210 \\
\hline 1 & -0.609092 & -3.699636 & -4.258048 \\
\hline 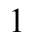 & -1.304399 & -7.094229 & 1.849241 \\
\hline & 6.971594 & 0.491321 & -1.549078 \\
\hline 1 & -1.205993 & -5.197078 & 0.298829 \\
\hline & 1.232671 & -2.948902 & -2.772933 \\
\hline 1 & 7.869547 & 4.636687 & -0.890504 \\
\hline l & 8.736655 & -1.532886 & 1.905306 \\
\hline 1 & -5.281841 & -4.396685 & -3.697791 \\
\hline 1 & -1.782401 & -6.731473 & 4.266217 \\
\hline 1 & 9.230375 & -3.722197 & 0.821446 \\
\hline 1 & 2.115960 & 2.066355 & 1.497980 \\
\hline 1 & -4.234190 & 0.036843 & 1.743756 \\
\hline 1 & -3.020961 & 0.126552 & -2.349443 \\
\hline
\end{tabular}




\begin{tabular}{|c|c|c|c|}
\hline . & -7.192213 & -0.064608 & -1.347301 \\
\hline . & -3.415325 & 2.576215 & 0.560727 \\
\hline & 0.013140 & 0.861960 & 2.465394 \\
\hline . & -1.440816 & 4.696432 & 3.720817 \\
\hline & 1.164930 & -4.077258 & 0.627133 \\
\hline & 2.232376 & 4.067406 & 4.752878 \\
\hline & 1.934890 & 3.800823 & 3.021460 \\
\hline 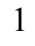 & 0.947537 & 4.964641 & 3.919712 \\
\hline & 0.564099 & 3.228891 & 6.475515 \\
\hline 1 & -0.729388 & 4.135774 & 5.682170 \\
\hline 1 & -0.916021 & 2.395067 & 5.959243 \\
\hline & 0.566665 & 0.721225 & 4.712081 \\
\hline & 1.822548 & 1.301164 & 3.598830 \\
\hline ] & 1.950212 & 1.647559 & 5.324513 \\
\hline ] & -5.221904 & 5.595329 & 0.850548 \\
\hline 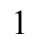 & -4.093126 & 4.612087 & -0.093290 \\
\hline & -5.160286 & 3.843686 & 1.103131 \\
\hline 1 & -4.811235 & 4.293154 & 3.590264 \\
\hline & -3.539422 & 5.401046 & 4.135565 \\
\hline 1 & -4.924703 & 6.028553 & 3.228878 \\
\hline & -1.971116 & 6.485057 & 2.393776 \\
\hline & -2.155056 & 6.080375 & 0.679259 \\
\hline ] & -3.379057 & 7.078928 & 1.496863 \\
\hline & 2.659395 & -3.615201 & -0.186919 \\
\hline 1 & 4.444947 & -3.351751 & 1.266534 \\
\hline 1 & 0.542969 & -2.162816 & 2.588496 \\
\hline 1 & 5.394794 & -2.679859 & 3.445915 \\
\hline 1 & 1.471333 & -1.537362 & 4.791774 \\
\hline & 3.914090 & -1.762744 & 5.224028 \\
\hline 1 & -1.334157 & 1.637862 & -2.001345 \\
\hline . & -1.348371 & 0.262689 & -3.858118 \\
\hline 1 & -1.430686 & 2.075771 & -3.730374 \\
\hline 1 & -0.312382 & 3.695245 & -4.334804 \\
\hline 1 & 0.959379 & 5.797931 & -4.315991 \\
\hline 1 & 3.328415 & 5.830836 & -3.565189 \\
\hline 1 & 4.413567 & 3.703857 & -2.844902 \\
\hline 1 & 3.131673 & 1.602198 & -2.837421 \\
\hline 1 & 1.265499 & 0.248637 & -5.296567 \\
\hline 1 & 2.256744 & -0.133468 & -3.883406 \\
\hline 1 & 0.666307 & -0.888804 & -4.068628 \\
\hline 6 & -4.382443 & -0.117266 & -4.439813 \\
\hline 6 & -6.600467 & -1.008273 & -3.815501 \\
\hline 6 & -6.191877 & 1.456760 & -3.797577 \\
\hline 6 & -6.460260 & 0.054740 & 2.781236 \\
\hline 6 & -8.068689 & 0.870546 & 1.087447 \\
\hline 6 & -7.553469 & -1.571604 & 1.255678 \\
\hline 1 & -4.728783 & -0.130668 & -5.481037 \\
\hline 1 & -3.649396 & 0.694676 & -4.353455 \\
\hline 1 & -3.870549 & -1.070246 & -4.243487 \\
\hline 1 & -6.490365 & 1.527892 & -4.852549 \\
\hline 1 & -7.082480 & 1.638941 & -3.183051 \\
\hline 1 & -5.474829 & 2.262708 & -3.592498 \\
\hline 1 & -6.869141 & -0.980346 & -4.879832 \\
\hline 1 & -6.189387 & -2.001847 & -3.594658 \\
\hline 1 & -7.528902 & -0.890672 & -3.244562 \\
\hline 1 & -8.354640 & -1.711172 & 1.994345 \\
\hline
\end{tabular}


TS1 $_{\text {IBI- }}-\psi_{-1 \text { 1a2aza4s }}$

Number of imaginary frequencies : 1

The smallest frequencies are : $-692.3870 \quad 14.0709 \quad 16.2424 \mathrm{~cm}(-1)$

Electronic energy : $\quad H F=-4360.8648553$

Zero-point correction $=\quad 1.451842($ Hartree/Particle $)$

Thermal correction to Energy= $\quad 1.536856$

Thermal correction to Enthalpy $=\quad 1.537800$

Thermal correction to Gibbs Free Energy= 1.333482

Sum of electronic and zero-point Energies $=\quad-4359.413013$

Sum of electronic and thermal Energies $=\quad-4359.327999$

Sum of electronic and thermal Enthalpies $=\quad-4359.327055$

Sum of electronic and thermal Free Energies $=\quad-4359.531373$

Cartesian Coordinates

$\begin{array}{cccc}6 & -2.711066 & 2.584997 & 1.306141 \\ 6 & -1.776902 & 1.559923 & 1.434820 \\ 6 & -0.754949 & 1.670888 & 2.382042 \\ 6 & -0.687285 & 2.765673 & 3.242646 \\ 6 & -1.632183 & 3.784283 & 3.075062 \\ 6 & -2.642740 & 3.723892 & 2.113495 \\ 15 & -1.731308 & 0.151491 & 0.250672 \\ 45 & -0.072882 & 0.856276 & -1.386812 \\ 6 & 0.477928 & 2.608975 & -0.584234 \\ 8 & 0.532720 & 3.736798 & -0.336075 \\ 6 & 0.381603 & 2.884129 & 4.328386 \\ 6 & 1.345298 & 4.017595 & 3.961904 \\ 6 & -3.639121 & 4.874415 & 1.981891 \\ 6 & -4.647866 & 4.640207 & 0.859175 \\ 6 & -1.717890 & -1.315638 & 1.408346 \\ 6 & -1.512735 & -2.592859 & 0.907100 \\ 6 & -1.566757 & -3.730475 & 1.776520 \\ 6 & -1.839840 & -3.544362 & 3.162478 \\ 6 & -2.061513 & -2.233345 & 3.640480 \\ 6 & -2.001943 & -1.159187 & 2.793203 \\ 6 & -1.344468 & -5.053839 & 1.311875 \\ 6 & -1.384800 & -6.122409 & 2.173104 \\ 6 & -1.655052 & -5.929929 & 3.544456 \\ 6 & -1.878632 & -4.665482 & 4.025543 \\ 6 & -1.262956 & -2.841791 & -0.537967 \\ 6 & -2.335356 & -3.266689 & -1.380295 \\ 6 & -2.077366 & -3.553503 & -2.754178 \\ 6 & -0.757870 & -3.431709 & -3.248249 \\ 6 & 0.256007 & -3.023069 & -2.423301 \\ 6 & 0.007608 & -2.706654 & -1.066715 \\ 6 & -3.665606 & -3.393213 & -0.902382\end{array}$




\begin{tabular}{|c|c|c|c|}
\hline 6 & -4.679282 & -3.789246 & -1.738005 \\
\hline 6 & -4.417300 & -4.088875 & -3.093384 \\
\hline & -3.144094 & -3.967910 & -3.589230 \\
\hline 7 & 1.081768 & -2.200483 & -0.260909 \\
\hline 15 & 1.577726 & -0.653137 & -0.763024 \\
\hline & 2.487490 & -0.295120 & 0.584745 \\
\hline & 3.409220 & 0.717808 & 0.479894 \\
\hline & 4.629003 & 0.484122 & -0.130096 \\
\hline 6 & 5.518395 & 1.594087 & -0.308556 \\
\hline & 5.169883 & 2.867401 & 0.236272 \\
\hline & 3.943173 & 3.018589 & 0.925351 \\
\hline 6 & 3.071863 & 1.969509 & 1.031501 \\
\hline 6 & 6.716041 & 1.500654 & -1.063071 \\
\hline 6 & 7.532600 & 2.590698 & -1.239089 \\
\hline 6 & 7.201861 & 3.837810 & -0.668433 \\
\hline 6 & 6.041060 & 3.968414 & 0.050420 \\
\hline 6 & 4.936657 & -0.870655 & -0.640658 \\
\hline 6 & 4.039824 & -1.488100 & -1.493154 \\
\hline 6 & 4.316990 & -2.732215 & -2.100664 \\
\hline 6 & 5.471746 & -3.398936 & -1.797382 \\
\hline 6 & 6.379201 & -2.867719 & -0.847932 \\
\hline 6 & 6.107946 & -1.599366 & -0.251331 \\
\hline 6 & 7.529600 & -3.593280 & -0.454365 \\
\hline 6 & 8.372833 & -3.108945 & 0.512730 \\
\hline 6 & 8.088984 & -1.873696 & 1.131463 \\
\hline 6 & 6.990125 & -1.138439 & 0.758878 \\
\hline 8 & 2.862130 & -0.875202 & -1.835571 \\
\hline 6 & -3.496494 & 0.047099 & -0.292086 \\
\hline 6 & -3.832888 & 0.084916 & -1.646362 \\
\hline 6 & -5.167095 & 0.039177 & -2.055903 \\
\hline 6 & -6.155301 & -0.053048 & -1.067310 \\
\hline 6 & -5.852875 & -0.114766 & 0.292635 \\
\hline 6 & -4.506282 & -0.064188 & 0.659641 \\
\hline 6 & -5.581409 & 0.124840 & -3.524291 \\
\hline 6 & -6.911142 & -0.231474 & 1.387211 \\
\hline 6 & 1.889402 & -3.222913 & 0.444396 \\
\hline 6 & 2.455694 & -2.800334 & 1.773592 \\
\hline 6 & 3.816346 & -2.944595 & 2.035000 \\
\hline 6 & 4.347685 & -2.564906 & 3.265370 \\
\hline 6 & 3.517304 & -2.047266 & 4.253306 \\
\hline 6 & 2.152325 & -1.913785 & 4.003013 \\
\hline 6 & 1.628252 & -2.283658 & 2.771968 \\
\hline 6 & -0.861475 & 1.208424 & -3.490115 \\
\hline 6 & 0.561740 & 1.237049 & -3.503785 \\
\hline 6 & 1.311178 & 2.526812 & -3.548500 \\
\hline 6 & 2.657392 & 2.557508 & -3.148952 \\
\hline 6 & 3.371924 & 3.745661 & -3.121118 \\
\hline 6 & 2.765088 & 4.939772 & -3.510078 \\
\hline 6 & 1.441794 & 4.922240 & -3.933828 \\
\hline 6 & 0.724327 & 3.728443 & -3.958319 \\
\hline 6 & 1.237354 & 0.086208 & -4.215687 \\
\hline 6 & -0.276642 & 3.195554 & 5.677188 \\
\hline 6 & 1.178067 & 1.590001 & 4.492802 \\
\hline 6 & -4.415407 & 5.029791 & 3.294110 \\
\hline 6 & -2.881771 & 6.171307 & 1.677340 \\
\hline 1 & 6.774154 & -0.197305 & 1.258456 \\
\hline
\end{tabular}




\begin{tabular}{|c|c|c|c|}
\hline & -2.179802 & -0.165195 & 3.194410 \\
\hline & 7.721308 & -4.554411 & -0.929925 \\
\hline & -2.086779 & -4.499178 & 5.081601 \\
\hline & 5.757171 & 4.927713 & 0.481251 \\
\hline & -3.879601 & -3.153073 & 0.138018 \\
\hline & -5.695669 & -3.864732 & -1.356262 \\
\hline & 3.687082 & 3.993044 & 1.339744 \\
\hline & -2.283164 & -2.088078 & 4.697182 \\
\hline & 5.697810 & -4.356560 & -2.263571 \\
\hline & 8.440151 & 2.492220 & -1.830640 \\
\hline & 3.594651 & -3.133420 & -2.808900 \\
\hline & -2.928663 & -4.191557 & -4.633323 \\
\hline & -0.562439 & -3.665017 & -4.294203 \\
\hline & -1.208782 & -7.126870 & 1.794944 \\
\hline & 6.980488 & 0.551165 & -1.521266 \\
\hline & -1.139527 & -5.218643 & 0.256049 \\
\hline & 1.271396 & -2.912356 & -2.800855 \\
\hline & 7.859789 & 4.691977 & -0.810838 \\
\hline & 8.743393 & -1.501395 & 1.916613 \\
\hline & -5.228847 & -4.413479 & -3.741532 \\
\hline & -1.684158 & -6.785312 & 4.215446 \\
\hline & 9.249524 & -3.678035 & 0.813105 \\
\hline & 2.105930 & 2.071445 & 1.521901 \\
\hline & -4.241009 & -0.101194 & 1.715434 \\
\hline ] & -3.026759 & 0.142266 & -2.373334 \\
\hline & -7.197834 & -0.078633 & -1.377819 \\
\hline & -3.493196 & 2.494600 & 0.556254 \\
\hline & -0.015254 & 0.872824 & 2.452247 \\
\hline & -1.577743 & 4.659941 & 3.721789 \\
\hline 1 & 1.206154 & -4.069428 & 0.605547 \\
\hline & 2.139249 & 4.108099 & 4.715710 \\
\hline 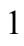 & 1.819576 & 3.831869 & 2.988643 \\
\hline ] & 0.829466 & 4.983693 & 3.897794 \\
\hline ] & 0.487766 & 3.268276 & 6.462231 \\
\hline 1 & -0.825637 & 4.144309 & 5.667775 \\
\hline 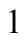 & -0.980831 & 2.403680 & 5.964247 \\
\hline 1 & 0.518171 & 0.739608 & 4.716807 \\
\hline & 1.766610 & 1.332851 & 3.602567 \\
\hline 1 & 1.885403 & 1.692529 & 5.325761 \\
\hline 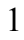 & -5.346657 & 5.484908 & 0.810237 \\
\hline l & -4.159272 & 4.558365 & -0.120333 \\
\hline 1 & -5.241317 & 3.729787 & 1.020839 \\
\hline 1 & -4.980883 & 4.117324 & 3.526094 \\
\hline 1 & -3.752984 & 5.237490 & 4.143210 \\
\hline & -5.130480 & 5.860258 & 3.219242 \\
\hline 1 & -2.182764 & 6.440366 & 2.478278 \\
\hline 1 & -2.303914 & 6.078063 & 0.748678 \\
\hline 1 & -3.586474 & 7.005574 & 1.558586 \\
\hline 1 & 2.699644 & -3.594614 & -0.202730 \\
\hline 1 & 4.475137 & -3.339708 & 1.260412 \\
\hline 1 & 0.569727 & -2.142083 & 2.564202 \\
\hline ( & 5.416162 & -2.669850 & 3.444255 \\
\hline 1 & 1.489253 & -1.516313 & 4.770614 \\
\hline 1 & 3.929520 & -1.748633 & 5.214960 \\
\hline ] & -1.342853 & 1.643146 & -1.985957 \\
\hline & -1.345902 & 0.305725 & -3.865862 \\
\hline
\end{tabular}




\begin{tabular}{|c|c|c|c|}
\hline & -1.426704 & 2.116783 & -3.707691 \\
\hline & -0.308567 & 3.739179 & -4.302357 \\
\hline & .958930 & 5.843938 & -4.253295 \\
\hline & 3.322918 & 5.873319 & -3.486160 \\
\hline & 4.407096 & 3.741160 & -2.780930 \\
\hline & 3.128690 & 1.637123 & -2.802054 \\
\hline & 1.274461 & 0.309411 & -5.293380 \\
\hline & 2.261661 & -0.088880 & -3.881958 \\
\hline & 0.672575 & -0.843990 & -4.081705 \\
\hline & -4.381967 & 0.047088 & -4.465263 \\
\hline & -6.529731 & -1.024673 & -3.878922 \\
\hline & -6.294387 & 1.461471 & -3.759631 \\
\hline & -8.327345 & -0.312136 & 0.824081 \\
\hline & -6.651510 & -1.501708 & 2.20600 \\
\hline & -6.829016 & 6590 & \\
\hline & -4.727001 & & \\
\hline & 0101 & 0.89 & \\
\hline & -3.811979 & -0.880579 & -4. \\
\hline & -6.592338 & 1.557160 & -4.812728 \\
\hline & -7.199423 & 1.554970 & -3.146572 \\
\hline & -5.636670 & 2.305911 & -3.514982 \\
\hline & -6.819986 & -0.961661 & -4.93623 \\
\hline & -6.040728 & -1.994272 & -3.71781 \\
\hline & -7.2 & -1.0 & -3.28617 \\
\hline & -7.5 & 0.91 & 3.12 \\
\hline & -5.832307 & 1.117547 & 2.745499 \\
\hline & -7.059961 & 1.913782 & 1.74371 \\
\hline & -7.402281 & -1.604016 & 3.00116 \\
\hline & -6.710305 & -2.394874 & 1.56950 \\
\hline & -5.662828 & -1.498161 & 2.68228 \\
\hline & -9.047529 & -0.399897 & 1.64738 \\
\hline & -8.593534 & 0.585155 & 0.250725 \\
\hline & -8.460707 & -1.187604 & 0.17523 \\
\hline
\end{tabular}

TS1 $_{I B I-} \Psi_{-122 a 354 a}$

Number of imaginary frequencies : 1

The smallest frequencies are : -687.2146 $11.1853 \quad 14.9816 \mathrm{~cm}(-1)$

Electronic energy : $\quad H F=-4360.8637592$

Zero-point correction $=\quad 1.451802($ Hartree/Particle $)$

Thermal correction to Energy $=\quad 1.536838$

Thermal correction to Enthalpy= $\quad 1.537783$

Thermal correction to Gibbs Free Energy $=\quad 1.333333$

Sum of electronic and zero-point Energies $=\quad-4359.411957$

Sum of electronic and thermal Energies $=\quad-4359.326921$

Sum of electronic and thermal Enthalpies $=\quad-4359.325977$

Sum of electronic and thermal Free Energies $=\quad-4359.530426$

Cartesian Coordinates

$\begin{array}{llll}6 & -2.654594 & 2.624798 & 1.360769 \\ 6 & -1.745691 & 1.576067 & 1.477644 \\ 6 & -0.721590 & 1.649489 & 2.426489 \\ 6 & -0.624969 & 2.734423 & 3.296914 \\ 6 & -1.544275 & 3.777806 & 3.140489 \\ 6 & -2.559110 & 3.751611 & 2.182241\end{array}$




\begin{tabular}{|c|c|c|c|}
\hline & -1.739152 & 0.186807 & 0.271233 \\
\hline & -0.081442 & 0.879368 & -1.373024 \\
\hline 6 & 0.494712 & 2.620844 & -0.566669 \\
\hline 8 & 0.563278 & 3.744130 & -0.301536 \\
\hline 6 & 0.442593 & 2.814990 & 4.387614 \\
\hline 6 & 1.422901 & 3.943784 & 4.052772 \\
\hline 6 & -3.519605 & 4.933104 & 2.059316 \\
\hline 6 & -4.587585 & 4.701546 & 0.992362 \\
\hline 6 & -1.745264 & -1.299653 & 1.401094 \\
\hline 6 & -1.555656 & -2.570796 & 0.879587 \\
\hline 6 & -1.626130 & -3.721145 & 1.732766 \\
\hline 6 & -1.906175 & -3.553021 & 3.119708 \\
\hline 6 & -2.115795 & -2.246959 & 3.616718 \\
\hline 6 & -2.035362 & -1.161051 & 2.786930 \\
\hline 6 & -1.410155 & -5.039902 & 1.251843 \\
\hline 6 & -1.465678 & -6.120188 & 2.097502 \\
\hline 6 & -1.745784 & -5.945407 & 3.469069 \\
\hline 6 & -1.961864 & -4.685898 & 3.966277 \\
\hline 6 & -1.297435 & -2.814185 & -0.565564 \\
\hline 6 & -2.360866 & -3.252632 & -1.413259 \\
\hline 6 & -2.080815 & -3.605219 & -2.767389 \\
\hline 6 & -0.754028 & -3.501142 & -3.246303 \\
\hline 6 & 0.246924 & -3.058283 & -2.423284 \\
\hline 6 & -0.018905 & -2.701955 & -1.080060 \\
\hline 6 & -3.700925 & -3.347934 & -0.955963 \\
\hline 6 & -4.699792 & -3.789685 & -1.787113 \\
\hline 6 & -4.412030 & -4.171840 & -3.115789 \\
\hline 6 & -3.131184 & -4.071683 & -3.595445 \\
\hline 7 & 1.052028 & -2.198871 & -0.267261 \\
\hline 15 & 1.553948 & -0.650293 & -0.758202 \\
\hline 8 & 2.467976 & -0.309529 & \\
\hline 6 & 3.397612 & 0.697100 & 0.493960 \\
\hline 6 & 4.615957 & 0.458275 & -0.116849 \\
\hline 6 & 5.514339 & 1.562351 & -0.287610 \\
\hline 6 & 5.173754 & 2.835563 & 0.262308 \\
\hline 6 & 3.947176 & 2.991961 & 0.950310 \\
\hline 6 & 3.069049 & 1.948155 & 1.052234 \\
\hline 6 & 6.713240 & 1.463997 & -1.039590 \\
\hline 6 & 7.538190 & 2.548912 & -1.208137 \\
\hline 6 & 7.214603 & 3.795867 & -0.633105 \\
\hline 6 & 6.052693 & 3.931449 & 0.082989 \\
\hline 6 & 4.912180 & -0.893169 & -0.642349 \\
\hline 6 & 4.011402 & -1.491590 & -1.504178 \\
\hline 6 & 4.283582 & -2.725750 & -2.134140 \\
\hline 6 & 5.434235 & -3.404066 & -1.841127 \\
\hline 6 & 6.342637 & -2.895391 & -0.880322 \\
\hline 6 & 6.078195 & -1.635521 & -0.263358 \\
\hline 6 & 7.487347 & -3.634801 & -0.496317 \\
\hline 6 & 8.330930 & -3.172297 & 0.481197 \\
\hline 6 & 8.053454 & -1.945696 & 1.119864 \\
\hline 6 & 6.960727 & -1.196835 & 0.756303 \\
\hline 8 & 2.836188 & -0.867583 & -1.835032 \\
\hline 6 & -3.508978 & 0.120216 & -0.260801 \\
\hline 6 & -3.840730 & 0.224596 & -1.602815 \\
\hline 6 & -5.177773 & 0.190786 & -2.028946 \\
\hline 6 & -6.161620 & 0.020155 & -1.061087 \\
\hline
\end{tabular}




\begin{tabular}{|c|c|c|c|}
\hline & -5.862683 & -0.101229 & 0.306638 \\
\hline & -4.528096 & -0.043217 & 0.688033 \\
\hline & -5.490509 & 0.363340 & -3.513701 \\
\hline & -6.989006 & -0.324341 & 1.314075 \\
\hline & 1.863387 & -3.229570 & 0.422574 \\
\hline & 2.420295 & -2.827305 & 1.761936 \\
\hline & 3.780690 & -2.965446 & 2.028212 \\
\hline & 4.301955 & -2.604725 & 3.268501 \\
\hline & 3.461688 & -2.113727 & 4.261791 \\
\hline & 2.096903 & -1.988178 & 4.006756 \\
\hline & 1.582978 & -2.337549 & 2.765474 \\
\hline & -0.874585 & 1.252379 & -3.473616 \\
\hline & 0.548563 & 1.245565 & -3.495662 \\
\hline b & 1.325518 & 2.518633 & -3.557137 \\
\hline & 2.667285 & 2.530625 & -3.142395 \\
\hline & 3.407232 & 3.703521 & -3.137821 \\
\hline b & 2.831519 & 4.899428 & -3.566897 \\
\hline & 1.512742 & 4.899670 & -4.004634 \\
\hline D & 0.769476 & 3.721276 & -4.005259 \\
\hline & 1.192707 & 0.076252 & -4.206990 \\
\hline & -0.218466 & 3.103931 & 5.740253 \\
\hline & 1.221606 & 1.507629 & 4.525221 \\
\hline & -4.230660 & 5.164319 & 3.397006 \\
\hline & -2.724438 & 6.186229 & 1.676886 \\
\hline 1 & 6.749625 & -0.262351 & 1.270332 \\
\hline & -2.203143 & -0.170966 & 3.202033 \\
\hline & 7.674233 & -4.589054 & -0.987379 \\
\hline & -2.175964 & -4.532364 & 5.023087 \\
\hline & 5.774296 & 4.890795 & 0.517273 \\
\hline 1 & -3.933006 & -3.057698 & 0.067515 \\
\hline & -5.722371 & -3.846326 & -1.419544 \\
\hline ] & 3.697077 & 3.966332 & 1.368574 \\
\hline & -2.343283 & -2.115298 & 4.673960 \\
\hline & 5.656152 & -4.354063 & -2.324557 \\
\hline 1 & 8.446768 & 2.446489 & -1.797441 \\
\hline & 3.561837 & -3.110163 & -2.852110 \\
\hline ] & -2.897082 & -4.349181 & -4.622420 \\
\hline & -0.542574 & -3.778159 & -4.278285 \\
\hline 1 & -1.293810 & -7.120355 & 1.706291 \\
\hline ] & 6.972250 & 0.515042 & -1.501838 \\
\hline & -1.196310 & -5.192871 & 0.196061 \\
\hline 1 & 1.267706 & -2.961642 & -2.789671 \\
\hline & 7.878885 & 4.646010 & -0.769955 \\
\hline 1 & 8.708055 & -1.590975 & 1.912945 \\
\hline & -5.211168 & -4.533343 & -3.759536 \\
\hline 1 & -1.787410 & -6.809977 & 4.127494 \\
\hline 1 & 9.202991 & -3.752107 & 0.774448 \\
\hline 1 & 2.104273 & 2.054529 & 1.544027 \\
\hline 1 & -4.261331 & -0.120682 & 1.738797 \\
\hline 1 & -3.037987 & 0.331622 & -2.329594 \\
\hline 1 & -7.204726 & -0.021722 & -1.367676 \\
\hline 1 & -3.437147 & 2.561708 & 0.608417 \\
\hline 1 & -0.003711 & 0.831034 & 2.489460 \\
\hline 1 & -1.465502 & 4.646011 & 3.794535 \\
\hline 1 & 1.184015 & -4.082461 & 0.565345 \\
\hline 1 & 2.208554 & 4.011214 & 4.817571 \\
\hline
\end{tabular}




\begin{tabular}{|c|c|c|c|}
\hline & 1.908094 & 3.769257 & 3.082911 \\
\hline ] & 0.919900 & 4.917237 & 4.000082 \\
\hline 1 & 0.542473 & 3.147242 & 6.530838 \\
\hline & -0.754252 & 4.060297 & 5.749883 \\
\hline & -0.935409 & 2.315638 & 6.004713 \\
\hline 1 & 0.550150 & 0.662527 & 4.733907 \\
\hline [ & 1.804390 & 1.260263 & 3.628381 \\
\hline 1 & 1.931792 & 1.583636 & 5.358652 \\
\hline & -5.263759 & 5.565037 & 0.955064 \\
\hline 1 & -4.150998 & 4.582667 & -0.007497 \\
\hline 1 & -5.197168 & 3.812975 & 1.206897 \\
\hline 1 & -4.812009 & 4.280364 & 3.690843 \\
\hline 1 & -3.527807 & 5.386781 & 4.208751 \\
\hline 1 & -4.923170 & 6.013222 & 3.318266 \\
\hline 1 & -1.978774 & 6.447377 & 2.437592 \\
\hline ] & -2.192708 & 6.037011 & 0.727982 \\
\hline & -3.397550 & 7.046634 & 1.560493 \\
\hline 1 & 2.678507 & -3.585329 & -0.227208 \\
\hline & 4.446826 & -3.340844 & 1.250133 \\
\hline 1 & 0.524174 & -2.203011 & 2.555017 \\
\hline & 5.370302 & -2.703989 & 3.451317 \\
\hline 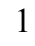 & 1.426029 & -1.612968 & 4.778742 \\
\hline$\frac{1}{1}$ & 3.865945 & -1.830249 & 5.231401 \\
\hline & -1.338125 & 1.691599 & -1.968320 \\
\hline 1 & -1.383211 & 0.362450 & -3.848222 \\
\hline 1 & -1.415515 & 2.176054 & -3.688288 \\
\hline 1 & -0.258028 & 3.744636 & -4.364442 \\
\hline 1 & 1.053984 & 5.822559 & -4.354620 \\
\hline 1 & 3.409657 & 5.820831 & -3.561491 \\
\hline 1 & 4.437905 & 3.686321 & -2.784760 \\
\hline & 3.114030 & 1.608848 & -2.767389 \\
\hline 1 & 1.221224 & 0.290644 & -5.286752 \\
\hline 1 & 2.217638 & -0.114510 & -3.883723 \\
\hline 1 & 0.612446 & -0.842306 & -4.058374 \\
\hline 6 & -6.988766 & 0.321017 & -3.803111 \\
\hline 6 & -4.952954 & 1.719547 & -3.985408 \\
\hline 6 & -4.820836 & -0.757661 & -4.313092 \\
\hline 6 & -6.485986 & -0.296935 & 2.756172 \\
\hline 6 & -8.057105 & 0.763836 & 1.165720 \\
\hline 5 & -7.619131 & -1.697000 & 1.051368 \\
\hline 1 & -7.159279 & 0.448164 & -4.879633 \\
\hline 1 & -7.435416 & -0.638591 & -3.511837 \\
\hline 1 & -7.530596 & 1.124764 & -3.287942 \\
\hline 1 & -5.009028 & -0.625998 & -5.387395 \\
\hline 1 & -3.733950 & -0.783122 & -4.164101 \\
\hline 1 & -5.214133 & -1.737076 & -4.013321 \\
\hline 1 & -5.175961 & 1.869011 & -5.050442 \\
\hline 1 & -5.412829 & 2.542917 & -3.423528 \\
\hline 1 & -3.865408 & 1.795839 & -3.860763 \\
\hline 1 & -8.431213 & -1.891017 & 1.765253 \\
\hline 1 & -8.039238 & -1.767463 & 0.040236 \\
\hline 1 & -6.872961 & -2.495665 & 1.161294 \\
\hline 1 & -8.861676 & 0.606849 & 1.896453 \\
\hline 1 & -7.632056 & 1.760607 & 1.341051 \\
\hline 1 & -8.516104 & 0.766181 & 0.170124 \\
\hline 1 & -7.328918 & -0.442613 & 3.443390 \\
\hline
\end{tabular}


TS1 $_{I B 1-} \Psi-1 \mathrm{a} 2 \mathrm{a} 3 \mathrm{~s} 4 \mathrm{~s}$

\begin{tabular}{|c|c|}
\hline \\
\hline \multirow{2}{*}{\multicolumn{2}{|c|}{$\begin{array}{l}\text { The smallest frequencies are : }-684.6833 \\
\text { Electronic energy : } \quad \mathrm{HF}=-4360.8641472\end{array}$}} \\
\hline & \\
\hline & 51344 (Hartree/Particle) \\
\hline Thermal correction to Energy $=$ & 1.536636 \\
\hline Thermal correction to Enthalpy= & 1.537580 \\
\hline Thermal correction to Gibbs Free Energy= & $=1.331725$ \\
\hline Sum of electronic and zero-point Energies= & -4359.412803 \\
\hline Sum of electronic and thermal Energies= & -4359.327511 \\
\hline Sum of electronic and thermal Enthalpies= & -4359.326567 \\
\hline Sum of electronic and thermal Free Energie & -4359.532422 \\
\hline
\end{tabular}

Cartesian Coordinates

$\begin{array}{cccc}6 & -2.767151 & 2.564215 & 1.310666 \\ 6 & -1.808661 & 1.561732 & 1.431063 \\ 6 & -0.794512 & 1.684720 & 2.384683 \\ 6 & -0.756575 & 2.770243 & 3.258534 \\ 6 & -1.724700 & 3.768472 & 3.097989 \\ 6 & -2.730983 & 3.693676 & 2.132780 \\ 15 & -1.733575 & 0.160887 & 0.241015 \\ 45 & -0.061790 & 0.876352 & -1.369612 \\ 6 & 0.501222 & 2.619450 & -0.542754 \\ 8 & 0.523693 & 3.747931 & -0.291460 \\ 6 & 0.304646 & 2.896786 & 4.350971 \\ 6 & 1.268556 & 4.029966 & 3.984608 \\ 6 & -3.766103 & 4.811278 & 2.018930 \\ 6 & -4.759286 & 4.565963 & 0.884314 \\ 6 & -1.734194 & -1.308673 & 1.393978 \\ 6 & -1.537675 & -2.584304 & 0.886623 \\ 6 & -1.605631 & -3.726553 & 1.749319 \\ 6 & -1.885775 & -3.545716 & 3.134576 \\ 6 & -2.098354 & -2.235469 & 3.618768 \\ 6 & -2.023810 & -1.156968 & 2.778198 \\ 6 & -1.390080 & -5.049274 & 1.279396 \\ 6 & -1.445067 & -6.121959 & 2.134626 \\ 6 & -1.723836 & -5.934759 & 3.505003 \\ 6 & -1.940109 & -4.671091 & 3.991318 \\ 6 & -1.279581 & -2.830284 & -0.557190 \\ 6 & -2.344666 & -3.262992 & -1.404696 \\ 6 & -2.069186 & -3.592975 & -2.765237 \\ 6 & -0.744912 & -3.474711 & -3.248376 \\ 6 & 0.258419 & -3.038851 & -2.424062 \\ 6 & -0.003579 & -2.703249 & -1.074657 \\ 6 & -3.682739 & -3.364471 & -0.942917 \\ 6 & -4.685238 & -3.789831 & -1.778024 \\ 6 & -4.402304 & -4.151781 & -3.113432 \\ 6 & -3.122861 & -4.046400 & -3.596223 \\ 7 & 1.064831 & -2.200017 & -0.258487 \\ 15 & 1.571280 & -0.653619 & -0.749275 \\ 8 & 2.475875 & -0.304160 & 0.604128\end{array}$




\begin{tabular}{|c|c|c|c|}
\hline 6 & 3.399699 & 0.707943 & 0.508756 \\
\hline 6 & 4.618322 & 0.477537 & -0.104503 \\
\hline & 5.505069 & 1.589307 & -0.283475 \\
\hline & 5.156468 & 2.860632 & 0.265561 \\
\hline & 3.935204 & 3.006248 & 0.965635 \\
\hline & 3.065628 & 1.955692 & 1.071741 \\
\hline & 6.699422 & 1.499228 & -1.043660 \\
\hline & 7.512268 & 2.591688 & -1.222971 \\
\hline & 7.180121 & 3.837751 & -0.650649 \\
\hline & 6.022874 & 3.964445 & 0.074785 \\
\hline & 4.928648 & -0.875766 & -0.617555 \\
\hline & 4.034840 & -1.493372 & -1.472703 \\
\hline & 4.314154 & -2.737119 & -2.079902 \\
\hline f & 5.468695 & -3.402989 & -1.773824 \\
\hline & 6.373720 & -2.871102 & -0.822402 \\
\hline & 6.099955 & -1.603200 & -0.225962 \\
\hline b & 7.524463 & -3.595209 & -0.427186 \\
\hline & 8.365802 & -3.109671 & 0.540953 \\
\hline D & 8.079617 & -1.874739 & 1.159210 \\
\hline & 6.980204 & -1.140970 & 0.785307 \\
\hline & 2.858181 & -0.879830 & -1.818144 \\
\hline ) & -3.487585 & 0.054563 & -0.330001 \\
\hline 6 & -3.801657 & 0.178439 & -1.678224 \\
\hline 5 & -5.129804 & 0.156333 & -2.119977 \\
\hline$b$ & -6.134435 & -0.021671 & -1.166947 \\
\hline 6 & -5.851502 & -0.172094 & 0.196027 \\
\hline ) & -4.517494 & -0.125325 & 0.594438 \\
\hline & -5.423810 & 0.356802 & -3.605574 \\
\hline 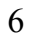 & -6.929360 & -0.377076 & 1.258578 \\
\hline 6 & 1.865096 & -3.228459 & 0.447125 \\
\hline 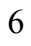 & 2.429002 & -2.810075 & 1.778610 \\
\hline 6 & 3.788962 & -2.956234 & 2.042306 \\
\hline 6 & 4.319034 & -2.575424 & 3.272846 \\
\hline 6 & 3.487974 & -2.054053 & 4.258260 \\
\hline b & 2.123492 & -1.919018 & 4.005893 \\
\hline & 1.600511 & -2.291310 & 2.775081 \\
\hline 6 & -0.811287 & 1.332706 & -3.473244 \\
\hline ) & 0.608914 & 1.277680 & -3.479026 \\
\hline 6 & 1.430942 & 2.523466 & -3.482637 \\
\hline 6 & 2.777212 & 2.467781 & -3.085573 \\
\hline 6 & 3.553142 & 3.614988 & -3.010547 \\
\hline 6 & 3.011257 & 4.853210 & -3.354289 \\
\hline 6 & 1.689987 & 4.921224 & -3.780089 \\
\hline 6 & 0.909996 & 3.769597 & -3.847238 \\
\hline 6 & 1.221318 & 0.100325 & -4.205127 \\
\hline 6 & -0.361006 & 3.213386 & 5.694843 \\
\hline 6 & 1.102225 & 1.604349 & 4.526346 \\
\hline 6 & -4.555722 & 4.904979 & 3.329123 \\
\hline 6 & -3.055787 & 6.142063 & 1.750802 \\
\hline 1 & 6.762533 & -0.199969 & 1.284401 \\
\hline 1 & -2.197347 & -0.163853 & 3.183281 \\
\hline I & 7.718143 & -4.556058 & -0.902504 \\
\hline 1 & -2.153925 & -4.508513 & 5.046826 \\
\hline 1 & 5.738338 & 4.922777 & 0.507323 \\
\hline 1 & -3.911892 & -3.084423 & 0.084543 \\
\hline 1 & -5.706887 & -3.848030 & -1.407969 \\
\hline
\end{tabular}




\begin{tabular}{|c|c|c|c|}
\hline 1 & 3.680811 & 3.978376 & 1.386431 \\
\hline & -2.325433 & -2.094305 & 4.674868 \\
\hline & 5.696610 & -4.360319 & -2.239707 \\
\hline 1 & 8.417485 & 2.496045 & -1.818540 \\
\hline & 3.594036 & -3.138744 & -2.790156 \\
\hline 1 & -2.893522 & -4.306951 & -4.628742 \\
\hline 1 & -0.537266 & -3.734021 & -4.285768 \\
\hline 1 & -1.274067 & -7.125710 & 1.752305 \\
\hline 1 & 6.964232 & 0.550516 & -1.503357 \\
\hline 1 & -1.178132 & -5.210648 & 0.224441 \\
\hline 1 & 1.276436 & -2.929305 & -2.794976 \\
\hline 1 & 7.834377 & 4.694149 & -0.796536 \\
\hline 1 & 8.732688 & -1.501479 & 1.945013 \\
\hline 1 & -5.204265 & -4.501448 & -3.760245 \\
\hline 1 & -1.764833 & -6.793483 & 4.171074 \\
\hline 1 & 9.242873 & -3.677574 & 0.842455 \\
\hline 1 & 2.102772 & 2.054582 & 1.568688 \\
\hline 1 & -4.272331 & -0.222143 & 1.651469 \\
\hline 1 & -2.987051 & 0.295851 & -2.390419 \\
\hline 1 & -7.170455 & -0.045032 & -1.492131 \\
\hline 1 & -3.543932 & 2.463232 & 0.556875 \\
\hline 1 & -0.039264 & 0.900920 & 2.450897 \\
\hline 1 & -1.695130 & 4.637210 & 3.755544 \\
\hline 1 & 1.176627 & -4.071513 & 0.604131 \\
\hline 1 & 2.052390 & 4.132069 & 4.747529 \\
\hline 1 & 1.756483 & 3.835433 & 3.019795 \\
\hline 1 & 0.748926 & & 3.903065 \\
\hline 1 & 0.399034 & 3.285459 & 6.484095 \\
\hline 1 & -0.906318 & & 5.680703 \\
\hline 1 & -1.069528 & 2.424595 & 5.979657 \\
\hline 1 & 0.441420 & 0.752947 & 4.744133 \\
\hline 1 & 1.701304 & 1.346876 & 3.643245 \\
\hline 1 & 1.800291 & 1.709995 & 5.366650 \\
\hline 1 & -5.487048 & 5.386543 & 0.848944 \\
\hline 1 & -4.262434 & 4.522386 & -0.093624 \\
\hline 1 & -5.321471 & 3.631819 & \\
\hline 1 & -5.088064 & 3.966160 & 3.533109 \\
\hline 1 & -3.905872 & 5.114756 & 4.187425 \\
\hline 1 & -5.300581 & 5.710240 & 3.270440 \\
\hline 1 & -2.374856 & 6.419251 & 2.564438 \\
\hline 1 & -2.466593 & 6.092148 & 0.825915 \\
\hline 1 & -3.790309 & 6.951865 & 1.644210 \\
\hline 1 & 2.675535 & -3.602877 & -0.198267 \\
\hline 1 & 4.448353 & -3.352848 & 1.269019 \\
\hline 1 & 0.542392 & -2.148817 & 2.565560 \\
\hline 1 & 5.387049 & -2.681819 & 3.453640 \\
\hline 1 & 1.460225 & -1.518251 & 4.771590 \\
\hline 1 & 3.899342 & -1.753754 & 5.219751 \\
\hline 1 & -1.314134 & 1.705774 & -1.946692 \\
\hline 1 & -1.345392 & 0.474745 & -3.884081 \\
\hline 1 & -1.319557 & 2.281294 & -3.653363 \\
\hline 1 & -0.120222 & 3.850071 & -4.189760 \\
\hline 1 & 1.257277 & 5.878270 & -4.065636 \\
\hline 1 & 3.617462 & 5.754445 & -3.294152 \\
\hline 1 & 4.585442 & 3.543552 & -2.668106 \\
\hline 1 & 3.199978 & 1.511756 & -2.775515 \\
\hline
\end{tabular}




$\begin{array}{lrrr}1 & 1.285589 & 0.338847 & -5.278209 \\ 1 & 2.228583 & -0.144249 & -3.863231 \\ 1 & 0.598789 & -0.795607 & -4.093453 \\ 6 & -6.917589 & 0.311762 & -3.917478 \\ 6 & -4.888027 & 1.726211 & -4.040346 \\ 6 & -4.736447 & -0.742714 & -4.419939 \\ 6 & -8.331841 & -0.457762 & 0.661828 \\ 6 & -6.656131 & -1.687208 & 2.007400 \\ 6 & -6.895645 & 0.797356 & 2.244228 \\ 1 & -7.072806 & 0.456089 & -4.994164 \\ 1 & -7.363695 & -0.654533 & -3.648405 \\ 1 & -7.471489 & 1.104286 & -3.397868 \\ 1 & -4.917528 & -0.592786 & -5.493093 \\ 1 & -3.650533 & -0.759098 & -4.263094 \\ 1 & -5.121040 & -1.732114 & -4.141625 \\ 1 & -5.099395 & 1.899569 & -5.104199 \\ 1 & -5.358343 & 2.533999 & -3.464412 \\ 1 & -3.802541 & 1.804620 & -3.900265 \\ 1 & -7.633564 & 0.648917 & 3.044328 \\ 1 & -5.911032 & 0.915370 & 2.714888 \\ 1 & -7.136917 & 1.740128 & 1.735087 \\ 1 & -7.418892 & -1.852591 & 2.780238 \\ 1 & -6.683116 & -2.542714 & 1.318947 \\ 1 & -5.676670 & -1.690862 & 2.502497 \\ 1 & -9.065499 & -0.611724 & 1.463251 \\ 1 & -8.609547 & 0.465161 & 0.136726 \\ 1 & -8.430257 & -1.296034 & -0.040289\end{array}$

TS1 $_{I B I-} \psi_{-1 \mathbf{a} 2 s 3 a 4 a}$

Number of imaginary frequencies : 1

The smallest frequencies are : - $699.709920 .884623 .7702 \mathrm{~cm}(-1)$

Electronic energy : $\quad H F=-4360.8652463$

Zero-point correction $=\quad 1.452631($ Hartree/Particle $)$

Thermal correction to Energy $=\quad 1.537300$

Thermal correction to Enthalpy= $\quad 1.538244$

Thermal correction to Gibbs Free Energy $=\quad 1.336234$

Sum of electronic and zero-point Energies $=\quad-4359.412615$

Sum of electronic and thermal Energies $=\quad-4359.327946$

Sum of electronic and thermal Enthalpies $=\quad-4359.327002$

Sum of electronic and thermal Free Energies $=\quad-4359.529012$

\begin{tabular}{cccc}
\multicolumn{4}{c}{ Cartesian Coordinates } \\
\hline $6 \ldots \ldots \ldots \ldots \ldots \ldots \ldots \ldots \ldots \ldots \ldots \ldots \ldots \ldots \ldots \ldots \ldots \ldots \ldots \ldots \ldots \ldots \ldots$ \\
6 & -2.646774 & 2.612962 & 1.373688 \\
6 & -1.735655 & 1.573287 & 1.485551 \\
6 & -0.709258 & 1.654974 & 2.437090 \\
6 & -0.624206 & 2.735465 & 3.307983 \\
6 & -1.557296 & 3.775623 & 3.160176 \\
6 & -2.565409 & 3.741352 & 2.201768 \\
15 & -1.719491 & 0.187137 & 0.277623 \\
45 & -0.061521 & 0.895797 & -1.357663 \\
6 & 0.515891 & 2.627699 & -0.530405 \\
8 & 0.589135 & 3.749533 & -0.260791 \\
6 & 0.450984 & 2.827736 & 4.389996 \\
6 & 1.423943 & 3.956914 & 4.034381
\end{tabular}




\begin{tabular}{|c|c|c|c|}
\hline 6 & -3.582817 & 4.863682 & 2.013705 \\
\hline 6 & -3.386490 & 6.002788 & 3.010251 \\
\hline f & -1.717545 & -1.300062 & 1.408621 \\
\hline & -1.533315 & -2.569691 & 0.881467 \\
\hline$b$ & -1.602135 & -3.723681 & 1.727622 \\
\hline f & -1.867140 & -3.561578 & 3.118175 \\
\hline 6 & -2.067325 & -2.257009 & 3.623205 \\
\hline 5 & -1.994526 & -1.166980 & 2.797510 \\
\hline 6 & -1.403369 & -5.040363 & 1.234487 \\
\hline 6 & -1.457652 & -6.125778 & 2.073540 \\
\hline 6 & -1.719140 & -5.957264 & 3.449747 \\
\hline 6 & -1.920494 & -4.699599 & 3.957972 \\
\hline 6 & -1.295158 & -2.794697 & -0.569279 \\
\hline 6 & -2.381528 & -3.174585 & -1.414774 \\
\hline 6 & -2.136719 & -3.439716 & -2.795275 \\
\hline 6 & -0.815809 & -3.346153 & -3.291906 \\
\hline 6 & 0.210962 & -2.976169 & -2.464449 \\
\hline 6 & -0.024659 & -2.676306 & -1.101936 \\
\hline 6 & -3.714173 & -3.264558 & -0.935394 \\
\hline 6 & -4.745190 & -3.595277 & -1.777970 \\
\hline 6 & -4.496248 & -3.878070 & -3.139597 \\
\hline 6 & -3.219494 & -3.799402 & -3.635112 \\
\hline 7 & 1.061501 & -2.199350 & -0.294456 \\
\hline 15 & 1.571655 & -0.647884 & -0.769290 \\
\hline 8 & 2.492198 & -0.326163 & 0.580239 \\
\hline 6 & 3.425719 & 0.677233 & 0.490167 \\
\hline 6 & 4.639689 & 0.440109 & -0.129966 \\
\hline 6 & 5.541760 & 1.542283 & -0.292420 \\
\hline 6 & 5.211049 & 2.809614 & 0.276899 \\
\hline 6 & 3.989627 & 2.962789 & 0.974852 \\
\hline 6 & 3.106211 & 1.922509 & 1.066332 \\
\hline 6 & 6.734588 & 1.448284 & -1.054440 \\
\hline 6 & 7.563168 & 2.531618 & -1.215033 \\
\hline 6 & 7.249953 & 3.772124 & -0.620609 \\
\hline 6 & 6.094212 & 3.903492 & 0.106226 \\
\hline 6 & 4.927798 & -0.908282 & -0.668041 \\
\hline 6 & 4.019793 & -1.497236 & -1.528820 \\
\hline 6 & 4.279799 & -2.731469 & -2.163495 \\
\hline 6 & 5.428241 & -3.417625 & -1.879851 \\
\hline 6 & 6.345933 & -2.917125 & -0.923512 \\
\hline 6 & 6.091804 & -1.658748 & -0.299149 \\
\hline 6 & 7.489643 & -3.663684 & -0.550563 \\
\hline 6 & 8.342059 & -3.209659 & 0.423330 \\
\hline 6 & 8.074526 & -1.984845 & 1.069681 \\
\hline 6 & 6.982873 & -1.229283 & 0.716934 \\
\hline 8 & 2.847934 & -0.863599 & -1.852808 \\
\hline 6 & -3.490279 & 0.107768 & -0.254315 \\
\hline 6 & -3.827479 & 0.155696 & -1.603613 \\
\hline 6 & -5.164060 & 0.078648 & -2.016565 \\
\hline 6 & -6.139914 & -0.057679 & -1.029419 \\
\hline 6 & -5.836932 & -0.121726 & 0.336177 \\
\hline 6 & -4.497944 & -0.032687 & 0.705348 \\
\hline 6 & -5.575388 & 0.202350 & -3.483872 \\
\hline 6 & -6.168904 & 1.599587 & -3.700629 \\
\hline 6 & -6.953905 & -0.327546 & 1.357382 \\
\hline 6 & -8.019989 & 0.761255 & 1.201418 \\
\hline
\end{tabular}




\begin{tabular}{|c|c|c|c|}
\hline 6 & -4.389737 & 0.028333 & -4.431082 \\
\hline 6 & -6.624844 & -0.850879 & -3.850908 \\
\hline 6 & -6.437860 & -0.281150 & 2.794360 \\
\hline 6 & -7.587588 & -1.703472 & 1.120409 \\
\hline 6 & 1.860223 & -3.242389 & 0.390518 \\
\hline 6 & 2.432058 & -2.848598 & 1.726309 \\
\hline 6 & 3.792317 & -3.005490 & 1.982580 \\
\hline 6 & 4.328229 & -2.648740 & 3.217836 \\
\hline 6 & 3.502963 & -2.141985 & 4.215696 \\
\hline 6 & 2.138253 & -1.996812 & 3.970592 \\
\hline 6 & 1.609766 & -2.343622 & 2.734801 \\
\hline 6 & -0.859999 & 1.286218 & -3.449684 \\
\hline 6 & 0.563604 & 1.301842 & -3.472441 \\
\hline 6 & 1.324873 & 2.584962 & -3.505643 \\
\hline 6 & 2.671378 & 2.599028 & -3.106349 \\
\hline 6 & 3.397683 & 3.779742 & -3.070192 \\
\hline 6 & 2.802439 & 4.982666 & -3.450242 \\
\hline 6 & 1.478953 & 4.981576 & -3.873621 \\
\hline 6 & 0.749659 & 3.795073 & -3.906600 \\
\hline 6 & 1.223450 & 0.156309 & -4.207443 \\
\hline 6 & -0.196533 & 3.126242 & 5.746961 \\
\hline 6 & 1.236550 & 1.524426 & 4.531678 \\
\hline 6 & -4.999032 & 4.304859 & 2.198757 \\
\hline 6 & -3.439682 & 5.435201 & 0.598204 \\
\hline 1 & 6.779504 & -0.296394 & 1.236863 \\
\hline 1 & -2.157877 & -0.178793 & 3.218996 \\
\hline 1 & 7.668646 & -4.616578 & -1.047188 \\
\hline 1 & -2.121819 & -4.551955 & 5.018124 \\
\hline 1 & 5.823835 & 4.858196 & 0.555584 \\
\hline 1 & -3.915903 & -3.046296 & 0.111885 \\
\hline 1 & -5.763840 & -3.635877 & -1.395672 \\
\hline 1 & 3.747196 & 3.932459 & 1.408301 \\
\hline 1 & -2.283578 & -2.129691 & 4.683348 \\
\hline 1 & 5.641448 & -4.367618 & -2.367226 \\
\hline 1 & 8.466656 & 2.433075 & -1.812759 \\
\hline 1 & 3.549834 & -3.109382 & -2.876667 \\
\hline 1 & -3.014562 & -4.010966 & -4.683880 \\
\hline 1 & -0.630317 & -3.565100 & -4.342761 \\
\hline 1 & -1.299716 & -7.124949 & 1.674001 \\
\hline 1 & 6.985501 & 0.504101 & -1.530863 \\
\hline 1 & -1.205216 & -5.186229 & 0.174590 \\
\hline 1 & 1.227373 & -2.882678 & -2.843975 \\
\hline 1 & 7.917272 & 4.620898 & -0.751041 \\
\hline 1 & 8.735957 & -1.636939 & 1.860105 \\
\hline 1 & -5.320316 & -4.157231 & -3.793204 \\
\hline 1 & -1.759278 & -6.825795 & 4.103044 \\
\hline 1 & 9.213386 & -3.794873 & 0.707910 \\
\hline 1 & 2.144324 & 2.027103 & 1.564030 \\
\hline 1 & -4.223678 & -0.069424 & 1.756498 \\
\hline 1 & -3.023862 & 0.238046 & -2.331189 \\
\hline 1 & -7.185009 & -0.119128 & -1.332107 \\
\hline 1 & -3.430075 & 2.554452 & 0.618871 \\
\hline 1 & 0.016688 & 0.843394 & 2.495808 \\
\hline 1 & -1.483111 & 4.636158 & 3.821368 \\
\hline 1 & 1.169003 & -4.085085 & 0.537002 \\
\hline 1 & 2.222334 & 4.029147 & 4.785464 \\
\hline
\end{tabular}




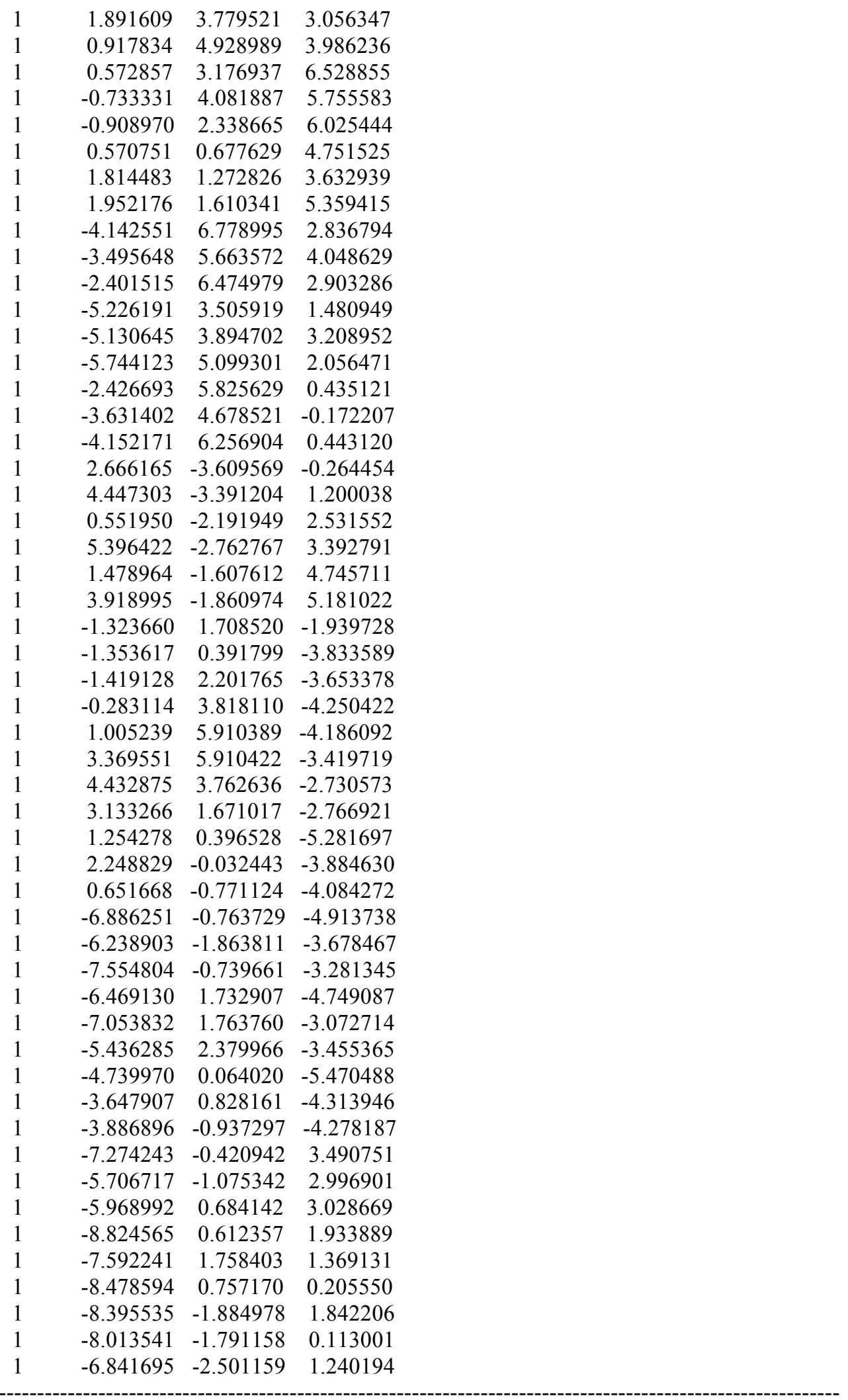

TS1 $_{I B I-}-\psi_{-122 s 3 a 4 s}$

Number of imaginary frequencies : 1 
The smallest frequencies are : -699.8118 $18.6979 \quad 18.9331 \mathrm{~cm}(-1)$ Electronic energy : $\quad H F=-4360.8656639$

Zero-point correction $=\quad 1.453036($ Hartree/Particle $)$

Thermal correction to Energy $=\quad 1.537605$

Thermal correction to Enthalpy= $\quad 1.538549$

Thermal correction to Gibbs Free Energy= 1.336153

Sum of electronic and zero-point Energies $=\quad-4359.412628$

Sum of electronic and thermal Energies $=\quad-4359.328059$

Sum of electronic and thermal Enthalpies $=\quad-4359.327115$

Sum of electronic and thermal Free Energies $=\quad-4359.529511$

Cartesian Coordinates

$\begin{array}{cccc}6 & -3.214301 & -3.767004 & -3.694730 \\ 6 & -2.137314 & -3.407914 & -2.847382 \\ 6 & -2.388282 & -3.159588 & -1.464759 \\ 6 & -3.721524 & -3.266150 & -0.989921 \\ 6 & -4.745526 & -3.603765 & -1.838434 \\ 6 & -4.490755 & -3.867161 & -3.202803 \\ 6 & -1.306061 & -2.786069 & -0.611098 \\ 6 & -0.033649 & -2.659466 & -1.137372 \\ 6 & 0.207741 & -2.941248 & -2.502637 \\ 6 & -0.815201 & -3.302863 & -3.338357 \\ 6 & -1.548793 & -2.578785 & 0.841486 \\ 6 & -1.727857 & -1.315062 & 1.384417 \\ 6 & -2.004981 & -1.198262 & 2.774768 \\ 6 & -2.086121 & -2.298729 & 3.586099 \\ 6 & -1.894095 & -3.597874 & 3.064206 \\ 6 & -1.625403 & -3.743088 & 1.672524 \\ 6 & -1.956523 & -4.746560 & 3.888770 \\ 6 & -1.760004 & -5.998439 & 3.364677 \\ 6 & -1.493903 & -6.150125 & 1.987334 \\ 6 & -1.431026 & -5.054139 & 1.162903 \\ 15 & -1.724774 & 0.185714 & 0.271507 \\ 6 & -3.495042 & 0.114068 & -0.264187 \\ 6 & -3.839248 & 0.216679 & -1.612969 \\ 6 & -5.173964 & 0.158097 & -2.020273 \\ 6 & -6.153397 & -0.023076 & -1.035595 \\ 6 & -5.842974 & -0.148630 & 0.318469 \\ 6 & -4.497775 & -0.067858 & 0.684778 \\ 6 & -5.594668 & 0.324252 & -3.480254 \\ 6 & -6.889537 & -0.398526 & 1.402290 \\ 7 & 1.049654 & -2.194547 & -0.319073 \\ 6 & 1.841885 & -3.247803 & 0.357815 \\ 6 & 2.411594 & -2.869400 & 1.698904 \\ 6 & 3.770969 & -3.031607 & 1.956535 \\ 6 & 4.304931 & -2.687818 & 3.196287 \\ 6 & 3.478603 & -2.188885 & 4.197201 \\ 6 & 2.114701 & -2.038768 & 3.950679 \\ 6 & 1.588144 & -2.372719 & 2.710529 \\ 45 & -0.061224 & 0.914092 & -1.349315 \\ 6 & -0.851957 & 1.344402 & -3.437744 \\ 6 & 0.571259 & 1.343549 & -3.458644 \\ 6 & 1.219721 & 0.200398 & -4.207440 \\ 6 & 1.346602 & 2.618673 & -3.474456 \\ 6 & 2.692860 & 2.613805 & -3.074060\end{array}$




\begin{tabular}{|c|c|c|c|}
\hline 6 & 3.431694 & 3.786270 & -3.024703 \\
\hline 6 & 2.849754 & 4.999659 & -3.391651 \\
\hline & 1.526699 & 5.017451 & -3.816055 \\
\hline & 0.784834 & 3.839327 & -3.862471 \\
\hline & 1.566693 & -0.640001 & -0.774856 \\
\hline 8 & 2.845268 & -0.848130 & -1.857566 \\
\hline 6 & 4.014074 & -1.489451 & -1.537064 \\
\hline 6 & 4.921467 & -0.913062 & -0.667200 \\
\hline & 6.081962 & -1.671184 & -0.303154 \\
\hline & 6.333923 & -2.923590 & -0.940186 \\
\hline 6 & 5.417338 & -3.410710 & -1.904481 \\
\hline & 4.271989 & -2.717671 & -2.184174 \\
\hline 6 & 7.474233 & -3.677636 & -0.571941 \\
\hline 6 & 8.325316 & -3.236560 & 0.409091 \\
\hline 6 & 8.059895 & -2.017682 & 1.067492 \\
\hline & 6.971600 & -1.255052 & 0.719659 \\
\hline & 4.636526 & 0.430348 & -0.115300 \\
\hline 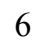 & 3.421690 & 0.665058 & 0.503965 \\
\hline & 3.104924 & 1.905291 & 1.092363 \\
\hline & 3.992435 & 2.943253 & 1.014820 \\
\hline & 5.215357 & 2.793166 & 0.318811 \\
\hline & 5.543061 & 1.530867 & -0.263220 \\
\hline & 6.103160 & 3.885476 & 0.162587 \\
\hline & 7.260727 & 3.757501 & -0.561931 \\
\hline 6 & 7.571107 & 2.522323 & -1.168837 \\
\hline b & 6.737832 & 1.440550 & -1.022669 \\
\hline 8 & 2.484364 & -0.335993 & 0.580496 \\
\hline 5 & 0.522337 & 2.635180 & -0.503807 \\
\hline & 0.598011 & 3.755327 & -0.227889 \\
\hline 6 & -1.745543 & 1.559414 & 1.494482 \\
\hline 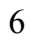 & -0.719929 & 1.632790 & 2.447589 \\
\hline 6 & -0.633223 & 2.707798 & 3.325084 \\
\hline 6 & -1.561336 & 3.752742 & 3.180147 \\
\hline 6 & -2.568161 & 3.727382 & 2.220105 \\
\hline 5 & -2.655169 & 2.601388 & 1.388900 \\
\hline 6 & 0.439429 & 2.789965 & 4.410388 \\
\hline 6 & -0.211527 & 3.078981 & 5.767762 \\
\hline 6 & -3.571809 & 4.861740 & 2.028377 \\
\hline 6 & -4.996994 & 4.318390 & 2.184806 \\
\hline 6 & 1.222412 & 1.484279 & 4.543837 \\
\hline 6 & 1.415398 & 3.920229 & 4.066584 \\
\hline 6 & -3.400093 & 5.444564 & 0.620593 \\
\hline 6 & -3.378550 & 5.989467 & 3.038412 \\
\hline 1 & 6.769899 & -0.326773 & 1.248403 \\
\hline & -2.161234 & -0.214312 & 3.208726 \\
\hline 1 & 7.651726 & -4.625820 & -1.078026 \\
\hline 1 & -2.160575 & -4.611763 & 4.950106 \\
\hline 1 & 5.834986 & 4.836312 & 0.621376 \\
\hline 1 & -3.928767 & -3.055895 & 0.058461 \\
\hline 1 & -5.764790 & -3.661691 & -1.460745 \\
\hline 1 & 3.752117 & 3.909081 & 1.457894 \\
\hline 1 & -2.302421 & -2.184028 & 4.647679 \\
\hline 1 & 5.628866 & -4.356105 & -2.401426 \\
\hline 1 & 8.476094 & 2.426803 & -1.764787 \\
\hline 1 & 3.543056 & -3.085610 & -2.903573 \\
\hline 1 & -3.004576 & -3.963721 & -4.745388 \\
\hline
\end{tabular}




\begin{tabular}{|c|c|c|c|}
\hline 1 & -0.625398 & -3.508742 & -4.391091 \\
\hline 1 & -1.339205 & -7.144726 & 1.575335 \\
\hline 1 & 6.986500 & 0.500674 & -1.508675 \\
\hline & -1.229245 & -5.187065 & 0.102015 \\
\hline 1 & 1.225416 & -2.841163 & -2.877055 \\
\hline & 7.931674 & 4.605073 & -0.681076 \\
\hline 1 & 8.720288 & -1.680109 & 1.863237 \\
\hline 1 & -5.310586 & -4.148157 & -3.860860 \\
\hline 1 & -1.807068 & -6.875303 & 4.006260 \\
\hline 1 & 9.193986 & -3.827504 & 0.689916 \\
\hline 1 & 2.141894 & 2.008003 & 1.588184 \\
\hline 1 & -4.227076 & -0.154009 & 1.736469 \\
\hline 1 & -3.038573 & 0.330770 & -2.339260 \\
\hline 1 & -7.195903 & -0.069219 & -1.343282 \\
\hline 1 & -3.438541 & 2.548987 & 0.633512 \\
\hline 1 & 0.004666 & 0.819609 & 2.501378 \\
\hline 1 & -1.483714 & 4.610427 & 3.844632 \\
\hline 1 & 1.146530 & -4.088806 & 0.493960 \\
\hline 1 & 2.211794 & 3.984822 & 4.820467 \\
\hline 1 & 1.885537 & 3.749977 & 3.088482 \\
\hline 1 & 0.911393 & 4.893680 & 4.024981 \\
\hline 1 & 0.555681 & 3.122972 & 6.552207 \\
\hline 1 & -0.747375 & 4.035111 & 5.781853 \\
\hline 1 & -0.925623 & 2.290149 & 6.038329 \\
\hline 1 & 0.554564 & 0.636994 & 4.755436 \\
\hline 1 & 1.801905 & 1.238845 & 3.644375 \\
\hline 1 & 1.936205 & 1.562456 & 5.373907 \\
\hline 1 & -4.125903 & 6.773607 & 2.862851 \\
\hline 1 & -3.503625 & 5.640965 & 4.071932 \\
\hline 1 & -2.388674 & 6.454862 & 2.948527 \\
\hline 1 & -5.221490 & 3.529213 & 1.455691 \\
\hline 1 & -5.150653 & 3.900431 & 3.188636 \\
\hline 1 & -5.729535 & 5.123558 & 2.037695 \\
\hline 1 & -2.382445 & 5.831041 & 0.478987 \\
\hline 1 & -3.581919 & 4.694908 & -0.159141 \\
\hline 1 & -4.105224 & 6.271572 & 0.459896 \\
\hline 1 & 2.648035 & -3.611880 & -0.298613 \\
\hline 1 & 4.426809 & -3.410946 & 1.171626 \\
\hline 1 & 0.531096 & -2.216873 & 2.506550 \\
\hline 1 & 5.372469 & -2.805885 & 3.372522 \\
\hline 1 & 1.454533 & -1.655739 & 4.728126 \\
\hline 1 & 3.893189 & -1.917935 & 5.166019 \\
\hline 1 & -1.319284 & 1.740901 & -1.920434 \\
\hline 1 & -1.356944 & 0.462763 & -3.835633 \\
\hline 1 & -1.398938 & 2.270375 & -3.625779 \\
\hline 1 & -0.247066 & 3.877450 & -4.207455 \\
\hline 1 & 1.063249 & 5.954632 & -4.118777 \\
\hline 1 & 3.426935 & 5.920784 & -3.350752 \\
\hline 1 & 4.466596 & 3.754671 & -2.685494 \\
\hline 1 & 3.144804 & 1.677150 & -2.745059 \\
\hline 1 & 1.258812 & 0.455992 & -5.277868 \\
\hline 1 & 2.241136 & -0.006367 & -3.882881 \\
\hline 1 & 0.635439 & -0.721223 & -4.100832 \\
\hline 6 & -4.401950 & 0.266045 & -4.431758 \\
\hline 6 & -6.572621 & -0.780581 & -3.891612 \\
\hline 6 & -6.276917 & 1.687938 & -3.639739 \\
\hline
\end{tabular}




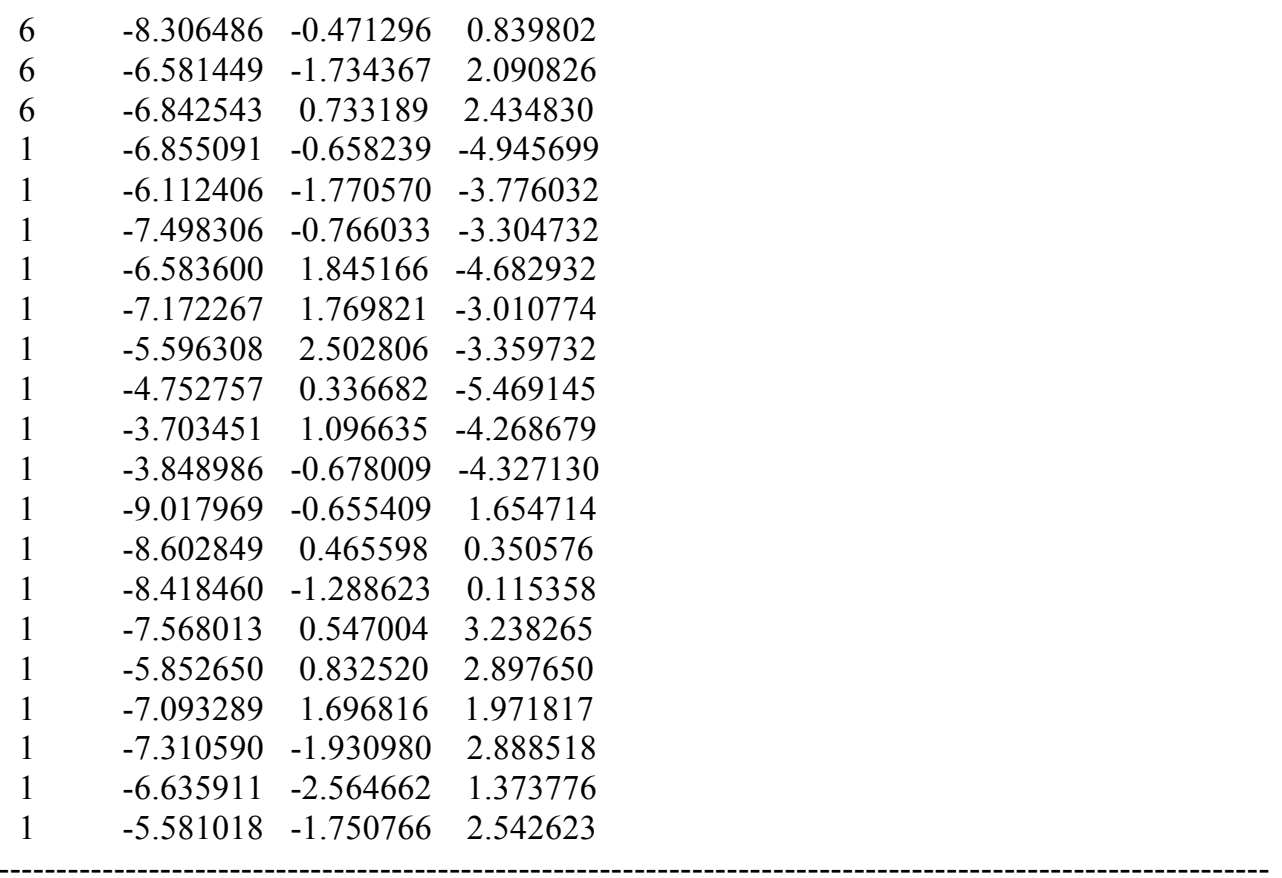

$\operatorname{TS1}_{I B 1-} \psi-1 \mathrm{a} 2 \mathrm{~s} 3 \mathrm{~s} 4 \mathrm{a}$

Number of imaginary frequencies : 1

The smallest frequencies are : $-689.4805 \quad 16.8468 \quad 22.2534 \mathrm{~cm}(-1)$

Electronic energy : $\quad \mathrm{HF}=-4360.8646358$

Zero-point correction $=\quad 1.451731($ Hartree/Particle $)$

Thermal correction to Energy $=\quad 1.536856$

Thermal correction to Enthalpy= $\quad 1.537800$

Thermal correction to Gibbs Free Energy= $\quad 1.333382$

Sum of electronic and zero-point Energies= $\quad-4359.412905$

Sum of electronic and thermal Energies $=\quad-4359.327780$

Sum of electronic and thermal Enthalpies $=\quad-4359.326835$

Sum of electronic and thermal Free Energies $=\quad-4359.531253$

\begin{tabular}{cccc}
\multicolumn{4}{c}{ Cartesian Coordinates } \\
\hline 6 & 6.967363 & -1.202832 & 0.743894 \\
6 & 6.078960 & -1.646464 & -0.268484 \\
6 & 6.339491 & -2.909664 & -0.880314 \\
6 & 7.486540 & -3.647085 & -0.499501 \\
6 & 8.336261 & -3.179398 & 0.470207 \\
6 & 8.062466 & -1.949699 & 1.104432 \\
6 & 4.911611 & -0.905178 & -0.645697 \\
6 & 4.007068 & -1.506780 & -1.501048 \\
6 & 4.273736 & -2.745613 & -2.123991 \\
6 & 5.425073 & -3.423528 & -1.832639 \\
6 & 4.617460 & 0.446471 & -0.119302 \\
6 & 3.404648 & 0.683714 & 0.502680 \\
6 & 3.085005 & 1.930514 & 1.075613 \\
6 & 3.963894 & 2.973548 & 0.972659 \\
6 & 5.180158 & 2.821580 & 0.265329 \\
6 & 5.513677 & 1.551216 & -0.295191 \\
6 & 6.055919 & 3.918601 & 0.078275 \\
6 & 7.207822 & 3.786648 & -0.654684
\end{tabular}




\begin{tabular}{|c|c|c|c|}
\hline 6 & 7.524794 & 2.542033 & -1.238617 \\
\hline 6 & 6.702735 & 1.455877 & -1.062986 \\
\hline 8 & 2.472330 & -0.320666 & 0.597268 \\
\hline 15 & 1.554677 & -0.652479 & -0.751612 \\
\hline 8 & 2.832351 & -0.881567 & -1.831797 \\
\hline 45 & -0.070505 & 0.893518 & -1.351980 \\
\hline 6 & 0.519770 & 2.626315 & -0.524512 \\
\hline 8 & 0.571693 & 3.753509 & -0.272270 \\
\hline 6 & 0.584077 & 1.299969 & -3.465559 \\
\hline 6 & -0.835499 & 1.367305 & -3.446967 \\
\hline 6 & 1.180383 & 0.120808 & -4.202046 \\
\hline 6 & 1.416761 & 2.538682 & -3.470642 \\
\hline 15 & -1.736215 & 0.182533 & 0.269774 \\
\hline 6 & -3.500293 & 0.113663 & -0.276937 \\
\hline 6 & -3.818007 & 0.217538 & -1.622168 \\
\hline 6 & -5.151346 & 0.192246 & -2.060489 \\
\hline 6 & -6.145226 & 0.029610 & -1.101285 \\
\hline 6 & -5.859814 & -0.092898 & 0.269458 \\
\hline 6 & -4.528489 & -0.042370 & 0.663079 \\
\hline 6 & -5.449723 & 0.374454 & -3.547013 \\
\hline 6 & -4.922871 & 1.742486 & -3.996720 \\
\hline 6 & -6.996206 & -0.309730 & 1.266940 \\
\hline 6 & -6.505076 & -0.292153 & 2.713390 \\
\hline 6 & -1.748751 & -1.301208 & 1.402899 \\
\hline 6 & -1.564093 & -2.573650 & 0.882653 \\
\hline 6 & -1.638678 & -3.722731 & 1.736501 \\
\hline 6 & -1.917143 & -3.551988 & 3.123493 \\
\hline 6 & -2.121776 & -2.244711 & 3.619306 \\
\hline 6 & -2.038088 & -1.159703 & 2.788455 \\
\hline 6 & -1.977240 & -4.684030 & 3.970964 \\
\hline 6 & -1.767147 & -5.944832 & 3.474516 \\
\hline 6 & -1.488975 & -6.122082 & 2.102787 \\
\hline 6 & -1.429174 & -5.042746 & 1.256261 \\
\hline 6 & -1.308800 & -2.813597 & -0.563389 \\
\hline 6 & -0.032593 & -2.691881 & -1.081755 \\
\hline 6 & 0.228367 & -3.029181 & -2.430985 \\
\hline 6 & -0.774982 & -3.466246 & -3.254244 \\
\hline 6 & -2.098819 & -3.583289 & -2.769994 \\
\hline 6 & -2.374446 & -3.246564 & -1.410968 \\
\hline 6 & -3.712622 & -3.348921 & -0.949865 \\
\hline 6 & -4.713909 & -3.783639 & -1.781849 \\
\hline 6 & -4.429930 & -4.152856 & -3.114963 \\
\hline 6 & -3.151039 & -4.044697 & -3.598415 \\
\hline 7 & 1.040131 & -2.197733 & -0.265767 \\
\hline 6 & 1.841465 & -3.234994 & 0.426196 \\
\hline 6 & 2.406095 & -2.831726 & 1.761962 \\
\hline 6 & 3.767264 & -2.973148 & 2.022242 \\
\hline 6 & 4.296405 & -2.603871 & 3.256655 \\
\hline 6 & 3.463222 & -2.100553 & 4.249806 \\
\hline 6 & 2.097441 & -1.972446 & 4.001209 \\
\hline 6 & 1.575612 & -2.331448 & 2.766000 \\
\hline 6 & -1.760277 & 1.572465 & 1.473944 \\
\hline 6 & -0.739141 & 1.659557 & 2.430618 \\
\hline 6 & -0.660187 & 2.744022 & 3.297147 \\
\hline 6 & -1.594226 & 3.782059 & 3.140107 \\
\hline 6 & -2.598181 & 3.741388 & 2.177637 \\
\hline
\end{tabular}




\begin{tabular}{|c|c|c|c|}
\hline 6 & -2.673484 & 2.609292 & 1.354062 \\
\hline 6 & 0.409217 & 2.842826 & 4.384287 \\
\hline 6 & 1.382929 & 3.971302 & 4.028408 \\
\hline 6 & -3.616865 & 4.860775 & 1.979522 \\
\hline 6 & -3.431980 & 6.001708 & 2.976174 \\
\hline 6 & -5.033240 & 4.298576 & 2.152799 \\
\hline 6 & -3.462850 & 5.430609 & 0.564440 \\
\hline 6 & -0.245633 & 3.147318 & 5.736411 \\
\hline 6 & 1.194974 & 1.540824 & 4.536383 \\
\hline 6 & -6.944069 & 0.316948 & -3.853277 \\
\hline 6 & -4.758076 & -0.728882 & -4.352130 \\
\hline 6 & -8.053781 & 0.787979 & 1.114177 \\
\hline 6 & -7.635313 & -1.676221 & 0.993819 \\
\hline 1 & 6.759344 & -0.265867 & 1.254601 \\
\hline 1 & -2.203272 & -0.168747 & 3.202509 \\
\hline 1 & 7.670348 & -4.603892 & -0.986745 \\
\hline 1 & -2.189949 & -4.528797 & 5.027809 \\
\hline 1 & 5.782912 & 4.876188 & 0.519808 \\
\hline 1 & -3.941706 & -3.068323 & 0.076930 \\
\hline 1 & -5.735058 & -3.844581 & -1.411011 \\
\hline 1 & 3.720597 & 3.944902 & 1.401769 \\
\hline 1 & -2.348552 & -2.111314 & 4.676474 \\
\hline 1 & 5.643142 & -4.377035 & -2.310897 \\
\hline 1 & 8.425583 & 2.442498 & -1.840230 \\
\hline 1 & 3.546958 & -3.134097 & -2.834656 \\
\hline 1 & -2.920409 & -4.310635 & -4.629254 \\
\hline 1 & -0.567592 & -3.729060 & -4.290774 \\
\hline 1 & -1.322100 & -7.123385 & 1.712320 \\
\hline 1 & 6.956001 & 0.508367 & -1.531568 \\
\hline 1 & -1.217184 & -5.197010 & 0.200285 \\
\hline 1 & 1.246425 & -2.921595 & -2.802036 \\
\hline 1 & 7.869381 & 4.637911 & -0.797674 \\
\hline 1 & 8.721880 & -1.590895 & 1.891667 \\
\hline 1 & -5.230527 & -4.509860 & -3.759410 \\
\hline 1 & -1.812163 & -6.808684 & 4.133659 \\
\hline 1 & 9.210274 & -3.757551 & 0.760904 \\
\hline 1 & 2.125578 & 2.034455 & 1.578017 \\
\hline 1 & -4.271650 & -0.118469 & 1.716472 \\
\hline 1 & -3.005897 & 0.319787 & -2.339768 \\
\hline 1 & -7.185794 & -0.005481 & -1.417399 \\
\hline 1 & -3.452987 & 2.546233 & 0.595618 \\
\hline 1 & -0.012757 & 0.848964 & 2.496870 \\
\hline 1 & -1.524571 & 4.645777 & 3.797637 \\
\hline 1 & 1.152992 & -4.079659 & 0.573963 \\
\hline 1 & 2.176881 & 4.048370 & 4.783712 \\
\hline 1 & 1.856527 & 3.789662 & 3.054004 \\
\hline 1 & 0.875949 & 4.942512 & 3.972421 \\
\hline 1 & 0.519593 & 3.202188 & 6.522104 \\
\hline 1 & -0.783005 & 4.102671 & 5.737685 \\
\hline 1 & -0.959097 & 2.360662 & 6.014831 \\
\hline 1 & 0.528322 & 0.694725 & 4.756317 \\
\hline 1 & 1.777915 & 1.285246 & 3.642008 \\
\hline 1 & 1.906047 & 1.630927 & 5.367612 \\
\hline 1 & -4.189173 & 6.775270 & 2.795972 \\
\hline 1 & -3.547908 & 5.663233 & 4.014065 \\
\hline 1 & -2.447802 & 6.477085 & 2.876211 \\
\hline
\end{tabular}




\begin{tabular}{|c|c|c|c|}
\hline & -5.252764 & 3.500232 & 1.432040 \\
\hline & -5.172104 & 3.886544 & 3.161268 \\
\hline & -5.778961 & 5.091508 & 2.005509 \\
\hline & -2.449933 & 5.824821 & 0.410274 \\
\hline & -3.644173 & 4.671675 & -0.206391 \\
\hline & -4.177090 & 6.249233 & 0.40121 \\
\hline & -5.784395 & -1.095748 & 2.916103 \\
\hline & 32501 & 0.665361 & 971242 \\
\hline & -7.354808 & -0.436354 & 3.392531 \\
\hline & -8.501007 & 0.801602 & 0.113313 \\
\hline & -8.867921 & 0.632253 & 1.834515 \\
\hline & -7.622916 & 1.780095 & 1.302630 \\
\hline & -8.045912 & -1.740717 & -0.021585 \\
\hline & -6.896787 & -2.481249 & 1.109435 \\
\hline & -8.455860 & -1.865604 & 1.6 \\
\hline & 38450 & & -3 \\
\hline & -5.131876 & 1.900750 & -5.063366 \\
\hline & -5.401754 & 2.553751 & -3.433006 \\
\hline & -7.500909 & 1.111631 & -3.340125 \\
\hline & -7.104302 & 0.448353 & -4.930861 \\
\hline & -7.382592 & -0.649338 & -3.571677 \\
\hline & -3.671596 & -0.738411 & -4.198785 \\
\hline & 37186 & -1.717162 & \\
\hline & -4.942807 & -0.5 & -5.426124 \\
\hline & 2.651054 & -3.60 & -0.224522 \\
\hline & 4.428055 & -3.356259 & 1.243344 \\
\hline & 0.516249 & -2.194373 & 2.559929 \\
\hline & 5.365368 & -2.705299 & 3.434687 \\
\hline & 1.432138 & -1.587155 & 4.773095 \\
\hline & 3.873865 & -1.809470 & $5.21445 \mathrm{c}$ \\
\hline & -1.320221 & & -1.91 \\
\hline & 688 & 0.51 & -3.857 \\
\hline & -1.337116 & 2.321257 & -3.617311 \\
\hline & 0.904957 & 3.789905 & -3.830374 \\
\hline & 1.695382 & 4.934558 & -3.764427 \\
\hline & 3.017948 & 4.854185 & -3.344852 \\
\hline & 3.550642 & 3.610619 & -3.005898 \\
\hline & 2.764299 & 2.470497 & -3.079842 \\
\hline & -0.126048 & 3.879878 & -4.168175 \\
\hline & 1.269782 & 5.895912 & -4.046167 \\
\hline & 3.632178 & 5.750040 & -3.285749 \\
\hline & 4.583851 & 3.529182 & -2.668441 \\
\hline & 3.179843 & 1.509911 & -2.77423 \\
\hline & 1.237751 & 0.363559 & -5.274582 \\
\hline & 2.188499 & -0.133378 & -3.869643 \\
\hline & 0.551432 & -0.770480 & -4.08906 \\
\hline
\end{tabular}

TS1 $_{I B 1-} \psi_{-1 \mathrm{a} 23354 \mathrm{~s}}$

Number of imaginary frequencies : 1

The smallest frequencies are : $-700.1811 \quad 18.773921 .5880 \mathrm{~cm}(-1)$

Electronic energy : $\quad H F=-4360.8649085$

Zero-point correction $=\quad 1.452908($ Hartree/Particle $)$

Thermal correction to Energy $=\quad 1.537495$

Thermal correction to Enthalpy= $\quad 1.538440$

Thermal correction to Gibbs Free Energy $=\quad 1.336075$ 
Sum of electronic and zero-point Energies= Sum of electronic and thermal Energies= Sum of electronic and thermal Enthalpies= Sum of electronic and thermal Free Energies=
$-4359.412001$

$-4359.327413$

$-4359.326469$

$-4359.528833$

Cartesian Coordinates

\begin{tabular}{|c|c|c|c|}
\hline 6 & 0.666991 & 3.828792 & -3.907409 \\
\hline 6 & 1.271686 & 2.629559 & -3.516590 \\
\hline 6 & 2.624864 & 2.668556 & -3.142014 \\
\hline 6 & 3.331031 & 3.861884 & -3.127525 \\
\hline 6 & 2.707456 & 5.053162 & -3.498019 \\
\hline 6 & 1.374965 & 5.028255 & -3.890936 \\
\hline 6 & 0.535745 & 1.332421 & -3.471677 \\
\hline 6 & 1.210832 & 0.196942 & -4.208325 \\
\hline 45 & -0.071501 & 0.924345 & -1.355499 \\
\hline 6 & -0.888612 & 1.292365 & -3.442347 \\
\hline 15 & -1.719385 & 0.191647 & 0.282695 \\
\hline 6 & -3.489667 & 0.116635 & -0.247925 \\
\hline 6 & -3.836324 & 0.266093 & -1.585839 \\
\hline 6 & -5.171238 & 0.207392 & -2.002190 \\
\hline 6 & -6.149256 & -0.027825 & -1.033913 \\
\hline 6 & -5.835425 & -0.192053 & 0.320537 \\
\hline 6 & -4.495618 & -0.107775 & 0.693847 \\
\hline 6 & -5.505468 & 0.436411 & -3.475144 \\
\hline 6 & -4.693921 & -0.517760 & -4.355993 \\
\hline 6 & -6.885223 & -0.469276 & 1.395552 \\
\hline 6 & -6.568174 & -1.806566 & 2.076665 \\
\hline 6 & -1.742767 & 1.557128 & 1.513215 \\
\hline 6 & -2.662920 & 2.590709 & 1.418352 \\
\hline 6 & -2.582866 & 3.711740 & 2.256564 \\
\hline 6 & -1.571059 & 3.741068 & 3.211291 \\
\hline 6 & -0.632574 & 2.703996 & 3.345564 \\
\hline 6 & -0.713784 & 1.633288 & 2.462231 \\
\hline 6 & 0.445123 & 2.789697 & 4.425548 \\
\hline 6 & 1.236195 & 1.488121 & 4.551547 \\
\hline 6 & -3.599641 & 4.836212 & 2.077345 \\
\hline 6 & -3.438632 & 5.431410 & 0.673432 \\
\hline 6 & -1.716816 & -1.316538 & 1.382496 \\
\hline 6 & -1.543439 & -2.575620 & 0.827709 \\
\hline 6 & -1.617064 & -3.746390 & 1.651209 \\
\hline 6 & -1.881333 & -3.611210 & 3.044832 \\
\hline 6 & -2.069301 & -2.315929 & 3.577676 \\
\hline 6 & -1.987646 & -1.209440 & 2.774900 \\
\hline 6 & -1.941796 & -4.765441 & 3.861764 \\
\hline 6 & -1.745722 & -6.013698 & 3.329081 \\
\hline 6 & -1.481321 & -6.155542 & 1.950449 \\
\hline 6 & -1.421309 & -5.054093 & 1.133156 \\
\hline 6 & -1.300554 & -2.781108 & -0.625628 \\
\hline 6 & -2.374916 & -3.187260 & -1.475352 \\
\hline 6 & -2.110196 & -3.498039 & -2.842687 \\
\hline 6 & -0.787193 & -3.386838 & -3.330952 \\
\hline 6 & 0.224645 & -2.975741 & -2.504509 \\
\hline 6 & -0.026336 & -2.658669 & -1.148606 \\
\hline 6 & -3.171490 & -3.932828 & -3.673728 \\
\hline 6 & -4.448725 & -4.038560 & -3.184692 \\
\hline
\end{tabular}




\begin{tabular}{|c|c|c|c|}
\hline 6 & -4.721269 & -3.694472 & -1.842377 \\
\hline 6 & -3.710839 & -3.287006 & -1.007476 \\
\hline 7 & 1.053652 & -2.182576 & -0.331843 \\
\hline 6 & 1.856404 & -3.232151 & 0.339125 \\
\hline 6 & 2.419585 & -2.859585 & 1.684769 \\
\hline 6 & 3.777275 & -3.025720 & 1.948844 \\
\hline 6 & 4.304676 & -2.692740 & 3.194413 \\
\hline 6 & 3.473768 & -2.200012 & 4.194513 \\
\hline 6 & 2.111717 & -2.044877 & 3.941050 \\
\hline 6 & 1.591624 & -2.368661 & 2.695514 \\
\hline 6 & 0.499909 & 2.649385 & -0.516543 \\
\hline 8 & 0.577778 & 3.764067 & -0.220043 \\
\hline 15 & 1.561112 & -0.623710 & -0.783644 \\
\hline 8 & 2.844278 & -0.817382 & -1.863153 \\
\hline 6 & 4.013740 & -1.458716 & -1.545142 \\
\hline 6 & 4.916759 & -0.890739 & -0.664880 \\
\hline 6 & 6.075444 & -1.652893 & -0.302928 \\
\hline 6 & 6.332148 & -2.897488 & -0.953139 \\
\hline 6 & 5.422314 & -3.373140 & -1.929445 \\
\hline 6 & 4.277666 & -2.678053 & -2.206675 \\
\hline 6 & 6.957949 & -1.249077 & 0.730958 \\
\hline 6 & 8.043608 & -2.015976 & 1.077598 \\
\hline 6 & 8.313802 & -3.226892 & 0.406542 \\
\hline 6 & 7.469922 & -3.655905 & -0.585995 \\
\hline 6 & 4.630518 & 0.448557 & -0.103794 \\
\hline 6 & 3.410264 & 0.682066 & 0.505344 \\
\hline 6 & 3.087830 & 1.921149 & 1.093069 \\
\hline 6 & 3.979247 & 2.956880 & 1.032792 \\
\hline 6 & 5.213073 & 2.805535 & 0.356980 \\
\hline 6 & 5.542972 & 1.546962 & -0.231686 \\
\hline 6 & 6.110969 & 3.893324 & 0.229059 \\
\hline 6 & 7.279527 & 3.765818 & -0.477290 \\
\hline 6 & 7.590931 & 2.536002 & -1.094328 \\
\hline 6 & 6.748578 & 1.458195 & -0.974058 \\
\hline 8 & 2.473666 & -0.319909 & 0.574754 \\
\hline 6 & 1.412331 & 3.926510 & 4.078716 \\
\hline 6 & -0.199796 & 3.071661 & 5.787250 \\
\hline 6 & -3.415266 & 5.957967 & 3.095632 \\
\hline 6 & -5.018129 & 4.275398 & 2.234373 \\
\hline 6 & -5.155709 & 1.884366 & -3.838073 \\
\hline 6 & -6.984561 & 0.209314 & -3.778041 \\
\hline 6 & -6.856793 & 0.655279 & 2.437143 \\
\hline 6 & -8.298462 & -0.553359 & 0.825007 \\
\hline 1 & 6.752450 & -0.327178 & 1.269304 \\
\hline 1 & -2.140039 & -0.228310 & 3.216532 \\
\hline 1 & 7.651086 & -4.597929 & -1.102180 \\
\hline 1 & -2.143272 & -4.637597 & 4.924447 \\
\hline 1 & 5.841356 & 4.840557 & 0.694434 \\
\hline 1 & -3.930147 & -3.022823 & 0.026160 \\
\hline 1 & -5.741037 & -3.754036 & -1.467093 \\
\hline 1 & 3.735929 & 3.920969 & 1.478032 \\
\hline 1 & -2.281664 & -2.209268 & 4.640885 \\
\hline 1 & 5.638131 & -4.311686 & -2.437390 \\
\hline 1 & 8.504564 & 2.441349 & -1.677107 \\
\hline 1 & 3.553773 & -3.037223 & -2.935538 \\
\hline 1 & -2.949663 & -4.180016 & -4.711152 \\
\hline
\end{tabular}




\begin{tabular}{|c|c|c|c|}
\hline & -0.587315 & -3.633217 & -4.372998 \\
\hline & -1.325348 & -7.147014 & 1.531446 \\
\hline & 6.999144 & 0.522606 & -1.467153 \\
\hline & -1.219841 & -5.180924 & 0.071507 \\
\hline & 1.243160 & -2.875302 & -2.876543 \\
\hline & 7.958345 & 4.609880 & -0.574872 \\
\hline & 8.698089 & -1.688105 & 1.882232 \\
\hline & -5.256539 & -4.374611 & -3.831333 \\
\hline & -1.790956 & -6.894918 & 3.964798 \\
\hline & 9.180358 & -3.821314 & 0.686553 \\
\hline & 2.119027 & 2.023803 & 1.577650 \\
\hline & -4.226167 & -0.223786 & 1.742968 \\
\hline & -3.043075 & 0.424210 & -2.313045 \\
\hline & -7.189382 & -0.087580 & -1.340674 \\
\hline & -3.449310 & 2.535629 & 0.666326 \\
\hline & 0.017240 & 0.825380 & 2.508842 \\
\hline & -1.498073 & 4.595451 & 3.880532 \\
\hline & 1.169874 & -4.081629 & 0.467526 \\
\hline & 2.213640 & 3.993189 & 4.827193 \\
\hline & 1.876302 & 3.761963 & 3.096701 \\
\hline & 0.902359 & 4.897132 & 4.043826 \\
\hline & 0.571680 & 3.117985 & 6.567347 \\
\hline 1 & -0.740832 & 4.024749 & 5.807002 \\
\hline & -0.907851 & 2.278142 & 6.059958 \\
\hline 1 & 0.573713 & 0.636502 & 4.762537 \\
\hline & 1.814085 & 1.249070 & 3.649341 \\
\hline & 1.952348 & 1.567505 & 5.379438 \\
\hline 1 & -4.172246 & 6.734875 & 2.929445 \\
\hline & -3.532063 & 5.599701 & 4.126784 \\
\hline I & -2.431150 & 6.435412 & 3.005575 \\
\hline & -5.237096 & 3.490210 & 1.499220 \\
\hline 1 & -5.163254 & 3.846256 & 3.234754 \\
\hline 1 & -5.760190 & 5.073637 & 2.097552 \\
\hline & -2.424312 & 5.826019 & 0.530072 \\
\hline 1 & -3.618462 & 4.686407 & -0.111243 \\
\hline & -4.150503 & 6.254414 & 0.522231 \\
\hline 1 & 2.667390 & -3.583135 & -0.318289 \\
\hline 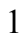 & 4.437023 & -3.400561 & 1.165042 \\
\hline 1 & 0.536207 & -2.209080 & 2.486724 \\
\hline ] & 5.370902 & -2.814543 & 3.375883 \\
\hline & 1.447673 & -1.666450 & 4.717468 \\
\hline 1 & 3.883338 & -1.937595 & 5.167798 \\
\hline & -1.334910 & 1.735029 & -1.943912 \\
\hline 1 & -1.366226 & 0.384239 & -3.814745 \\
\hline 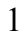 & -1.462485 & 2.195048 & -3.661249 \\
\hline 1 & -0.372600 & 3.833455 & -4.230629 \\
\hline 1 & 0.878061 & 5.947815 & -4.194565 \\
\hline 1 & 3.259653 & 5.990218 & -3.483527 \\
\hline 1 & 4.374903 & 3.865236 & -2.817176 \\
\hline 1 & 3.109436 & 1.750274 & -2.807823 \\
\hline 1 & 1.232843 & 0.436648 & -5.282910 \\
\hline 1 & 2.240444 & 0.024360 & -3.890064 \\
\hline 1 & 0.654231 & -0.739267 & -4.081925 \\
\hline 1 & -4.926467 & -0.344645 & -5.415465 \\
\hline 1 & -3.612017 & -0.384951 & -4.230707 \\
\hline 1 & -4.926942 & -1.563868 & -4.120145 \\
\hline
\end{tabular}


$\mathrm{TS1}_{I B 1-} \psi-1 \mathrm{~s} 2 \mathrm{a} 3 \mathrm{a} 4 \mathrm{a}$

Number of imaginary frequencies : 1

The smallest frequencies are : $-696.4125 \quad 10.3007 \quad 20.8971 \mathrm{~cm}(-1)$

Electronic energy : $\quad H F=-4360.8635198$

Zero-point correction $=\quad 1.451776($ Hartree/Particle)

Thermal correction to Energy $=\quad 1.536770$

Thermal correction to Enthalpy= $\quad 1.537715$

Thermal correction to Gibbs Free Energy= $\quad 1.333441$

Sum of electronic and zero-point Energies= $\quad-4359.411743$

Sum of electronic and thermal Energies $=\quad-4359.326749$

Sum of electronic and thermal Enthalpies $=\quad-4359.325805$

Sum of electronic and thermal Free Energies= $\quad-4359.530079$

Cartesian Coordinates

$\begin{array}{cccc}6 & -3.703988 & -3.295048 & -1.016527 \\ 6 & -2.353322 & -3.230843 & -1.447914 \\ 6 & -2.058131 & -3.553803 & -2.805835 \\ 6 & -3.109687 & -3.947295 & -3.670083 \\ 6 & -4.404069 & -4.007727 & -3.219062 \\ 6 & -4.702338 & -3.668476 & -1.880648 \\ 6 & -0.718305 & -3.484131 & -3.253750 \\ 6 & 0.277454 & -3.081487 & -2.403603 \\ 6 & -0.007179 & -2.728846 & -1.062815 \\ 6 & -1.296889 & -2.827201 & -0.575453 \\ 6 & -1.592889 & -2.575811 & 0.859339 \\ 6 & -1.792684 & -1.299952 & 1.362617 \\ 6 & -2.159641 & -1.154496 & 2.730167 \\ 6 & -2.289975 & -2.234640 & 3.560575 \\ 6 & -2.062428 & -3.545198 & 3.083025 \\ 6 & -1.715339 & -3.721957 & 1.712985 \\ 6 & -2.173130 & -4.673792 & 3.929602 \\ 6 & -1.948136 & -5.937806 & 3.447342 \\ 6 & -1.603287 & -6.121260 & 2.091921 \\ 6 & -1.492999 & -5.044612 & 1.246622 \\ 15 & -1.707381 & 0.191064 & 0.233870 \\ 45 & -0.053698 & 0.827245 & -1.438863 \\ 6 & 0.499216 & 2.597105 & -0.683132 \\ 8 & 0.549958 & 3.729842 & -0.457353\end{array}$




\begin{tabular}{|c|c|c|c|}
\hline 7 & 1.046273 & -2.215301 & -0.233812 \\
\hline 6 & 1.815312 & -3.223887 & 0.532623 \\
\hline 5 & 2.366682 & -2.740411 & 1.847169 \\
\hline 6 & 3.733489 & -2.812656 & 2.105917 \\
\hline 6 & 4.256493 & -2.335231 & 3.305120 \\
\hline 6 & 3.410559 & -1.793586 & 4.266559 \\
\hline 6 & 2.038049 & -1.745202 & 4.026469 \\
\hline 6 & 1.520991 & -2.212587 & 2.824985 \\
\hline 6 & -1.635048 & 1.554433 & 1.468699 \\
\hline 6 & -0.667866 & 1.519719 & 2.471975 \\
\hline 6 & -0.571967 & 2.544594 & 3.420671 \\
\hline 6 & -1.414299 & 3.647029 & 3.272010 \\
\hline 6 & -2.356988 & 3.740089 & 2.239570 \\
\hline 6 & -2.466703 & 2.670250 & 1.353225 \\
\hline 6 & -3.237562 & 4.983362 & 2.129263 \\
\hline 6 & -2.348986 & 6.205524 & 1.874344 \\
\hline 6 & 0.339019 & 2.360156 & 4.635824 \\
\hline 6 & 0.406166 & 3.608780 & 5.511994 \\
\hline 6 & -3.482090 & 0.208446 & -0.295394 \\
\hline 6 & -3.811327 & 0.170857 & -1.647810 \\
\hline 6 & -5.147897 & 0.141882 & -2.065270 \\
\hline 6 & -6.134882 & 0.139580 & -1.079784 \\
\hline 6 & -5.841699 & 0.163895 & 0.288618 \\
\hline 6 & -4.500921 & 0.199357 & 0.661944 \\
\hline 6 & -5.548237 & 0.157478 & -3.540453 \\
\hline 6 & -6.600183 & -0.918043 & -3.827616 \\
\hline 6 & -6.971548 & 0.109684 & 1.314892 \\
\hline 6 & -7.662011 & -1.255125 & 1.209991 \\
\hline 6 & -4.357984 & -0.097433 & -4.462412 \\
\hline 6 & -6.132239 & 1.535725 & -3.872214 \\
\hline 6 & -6.458470 & 0.280543 & 2.743657 \\
\hline 6 & -7.991395 & 1.219982 & 1.042773 \\
\hline 6 & -4.244079 & 4.877468 & 0.985823 \\
\hline 6 & -4.021601 & 5.182814 & 3.431033 \\
\hline 6 & 1.766593 & 2.001601 & 4.216901 \\
\hline 6 & -0.241017 & 1.214800 & 5.477422 \\
\hline 6 & -0.836558 & 1.110598 & -3.552676 \\
\hline 6 & 0.587185 & 1.153091 & -3.559132 \\
\hline 6 & 1.324704 & 2.447924 & -3.636143 \\
\hline 6 & 0.725863 & 3.635634 & -4.068656 \\
\hline 6 & 1.433053 & 4.835660 & -4.071608 \\
\hline 6 & 2.757625 & 4.873531 & -3.652986 \\
\hline 6 & 3.375959 & 3.693357 & -3.240732 \\
\hline 6 & 2.671721 & 2.498759 & -3.241497 \\
\hline 15 & 1.574340 & -0.689732 & -0.772650 \\
\hline 8 & 2.476393 & -0.300101 & 0.570450 \\
\hline 6 & 3.390365 & 0.717727 & 0.434501 \\
\hline 6 & 4.611794 & 0.474508 & -0.167870 \\
\hline 6 & 5.490631 & 1.585825 & -0.384519 \\
\hline 6 & 5.131230 & 2.872862 & 0.119683 \\
\hline 6 & 3.903449 & 3.035485 & 0.804494 \\
\hline 6 & 3.040278 & 1.983351 & 0.942851 \\
\hline 6 & 5.993481 & 3.974297 & -0.101970 \\
\hline 6 & 7.154545 & 3.830525 & -0.818061 \\
\hline 6 & 7.495051 & 2.568855 & -1.349895 \\
\hline 6 & 6.687944 & 1.478200 & -1.137679 \\
\hline
\end{tabular}




\begin{tabular}{|c|c|c|c|}
\hline & 4.932734 & -0.896850 & -0.621476 \\
\hline & 4.041981 & -1.560548 & -1.445336 \\
\hline & 4.327422 & -2.834128 & -1.984561 \\
\hline & 5.485567 & -3.477697 & -1.644704 \\
\hline & 6.389870 & -2.891757 & -0.725028 \\
\hline & 6.108284 & -1.596467 & -0.194627 \\
\hline & 7.547844 & -3.585228 & -0.296870 \\
\hline & 8.388420 & -3.043555 & 0.641608 \\
\hline & 8.093429 & -1.781144 & 1.197165 \\
\hline & 6.986901 & -1.076701 & 0.789950 \\
\hline & 2.865373 & -0.967991 & -1.825997 \\
\hline & 1.278855 & -0.010302 & -4.233609 \\
\hline & 6.763210 & -0.113101 & 1.240832 \\
\hline & -2.341574 & -0.162473 & 3.134984 \\
\hline & 7.747771 & -4.567773 & -0.722641 \\
\hline & -2.438206 & -4.514051 & 4.973845 \\
\hline & 5.702287 & 4.944503 & 0.298502 \\
\hline & -3.947243 & -3.023220 & 0.009445 \\
\hline & -5.733796 & -3.692516 & -1.533524 \\
\hline & 3.638204 & 4.019813 & 1.188123 \\
\hline & -2.571612 & -2.093516 & 4.603726 \\
\hline & 5.717488 & -4.457180 & -2.059893 \\
\hline & 8.402556 & 2.459320 & -1.939577 \\
\hline & 3.609789 & -3.276446 & -2.672817 \\
\hline & -2.866444 & -4.199976 & -4.701334 \\
\hline & -0.493074 & -3.746181 & -4.286815 \\
\hline & -1.425484 & -7.124959 & 1.712598 \\
\hline & 6.959478 & 0.516577 & -1.565643 \\
\hline & -1.230515 & -5.203602 & 0.202747 \\
\hline ] & 1.306637 & -3.003154 & -2.750229 \\
\hline & 7.805234 & 4.685197 & -0.988263 \\
\hline & 8.745652 & -1.362833 & 1.960647 \\
\hline & -5.204292 & -4.311643 & -3.890901 \\
\hline & -2.033380 & -6.799361 & 4.105472 \\
\hline 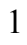 & 9.271269 & -3.588005 & 0.968419 \\
\hline & 2.076530 & 2.090531 & 1.434177 \\
\hline 1 & -4.235345 & 0.225013 & 1.714973 \\
\hline & -3.002511 & 0.148005 & -2.373533 \\
\hline 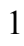 & -7.180137 & 0.117609 & -1.387378 \\
\hline 1 & -3.202465 & 2.694837 & 0.552984 \\
\hline & -0.008886 & 0.651138 & 2.546110 \\
\hline 1 & -1.347813 & 4.463583 & 3.987743 \\
\hline & 1.109058 & -4.045621 & 0.720198 \\
\hline 1 & 2.379088 & 1.799519 & 5.106829 \\
\hline & 1.814705 & 1.105093 & 3.585812 \\
\hline 1 & 2.237489 & 2.829489 & 3.670430 \\
\hline | & 0.373692 & 1.043492 & 6.372278 \\
\hline 1 & -1.262853 & 1.448251 & 5.806301 \\
\hline 1 & -0.280659 & 0.275526 & 4.909916 \\
\hline 1 & 0.783639 & 4.477139 & 4.956594 \\
\hline 1 & -0.571125 & 3.870162 & 5.937362 \\
\hline l & 1.089184 & 3.431380 & 6.352422 \\
\hline 1 & -4.866161 & 5.780966 & 0.957241 \\
\hline 1 & -3.749671 & 4.787257 & 0.010211 \\
\hline 1 & -4.915866 & 4.016492 & 1.109259 \\
\hline 1 & -4.665065 & 4.317816 & 3.639512 \\
\hline
\end{tabular}




\begin{tabular}{|c|c|c|c|}
\hline & 3.363490 & 5.326620 & 4.2 \\
\hline & -4.663622 & 6.070730 & 3.355400 \\
\hline & -1.638255 & 6.373917 & 692465 \\
\hline & -1.769982 & 6.081070 & 950139 \\
\hline & -2.962634 & 7.111212 & 77405 \\
\hline & 2.629379 & -3.642039 & -0.080643 \\
\hline & 4.403545 & -3.221765 & 1.3486 \\
\hline & 0.453335 & -2.139369 & 2.623723 \\
\hline & 5.329556 & -2.382185 & 3.480838 \\
\hline & 1.366938 & -1.334469 & 4.780701 \\
\hline & 3.815412 & -1.413017 & 5.202248 \\
\hline & -1.314819 & 1.604064 & -2.067450 \\
\hline & -1.308660 & 0.190677 & -3.901688 \\
\hline & -1.410499 & 2.004545 & -3.805157 \\
\hline & -0.308522 & 3.630631 & \\
\hline & 0.9 & 5.74 & \\
\hline & 3.307288 & 5.8122 & -3.6 \\
\hline & 4.411975 & 3.704737 & -2.9 \\
\hline & 3.152572 & 1.590755 & -2.875422 \\
\hline & 1.325186 & 0.184868 & -5.316360 \\
\hline & 2.300937 & -0.16 & -3.88 \\
\hline & 0.720 & -0.94 & -4.08 \\
\hline & -6.42 & 1.5 & -4.9 \\
\hline & -7.0 & 1.75 & -3.2 \\
\hline & -5.397108 & 2.329547 & -3.684921 \\
\hline & -6.868502 & -0.905728 & -4.892329 \\
\hline & -6.212044 & -1.916187 & -3.587182 \\
\hline & -7.525417 & -0.767702 & -3.259123 \\
\hline & -4.699580 & -0.132021 & -5.504714 \\
\hline & -3.607341 & 0.700019 & -4.394397 \\
\hline & -3.868303 & -1.055643 & -4.236941 \\
\hline & & -1.333 & 1.943385 \\
\hline & -8.0 & -1.41 & 0.213991 \\
\hline & -6.950249 & -2.068547 & 1.404915 \\
\hline & -8.798984 & 1.182483 & 1.785939 \\
\hline & -7.523053 & 2.210851 & 1.103698 \\
\hline & -8.454514 & 1.129467 & 0.053177 \\
\hline & -7.303057 & 0.262149 & 3.443886 \\
\hline & -5.773402 & -0.526407 & 3.036528 \\
\hline & -5.936441 & 1.238298 & 2.876865 \\
\hline
\end{tabular}

$\mathrm{TS1}_{I B 1-} \psi_{-1 \mathrm{~s} 2 \mathrm{a3a4s}}$

Number of imaginary frequencies : 1

The smallest frequencies are : $-692.9312 \quad 17.7117 \quad 21.8441 \mathrm{~cm}(-1)$

Electronic energy : $\quad H F=-4360.8639673$

Zero-point correction $=\quad 1.452051$ (Hartree/Particle)

Thermal correction to Energy= $\quad 1.537116$

Thermal correction to Enthalpy= $\quad 1.538060$

Thermal correction to Gibbs Free Energy $=\quad 1.333203$

Sum of electronic and zero-point Energies $=\quad-4359.411916$

Sum of electronic and thermal Energies $=\quad-4359.326852$

Sum of electronic and thermal Enthalpies $=\quad-4359.325907$

Sum of electronic and thermal Free Energies $=\quad-4359.530764$

Cartesian Coordinates 


$\begin{array}{cccc}6 & 0.893936 & 3.906882 & -3.740206 \\ 6 & 1.447218 & 2.673909 & -3.379349 \\ 6 & 2.787798 & 2.654119 & -2.961259 \\ 6 & 3.528931 & 3.822426 & -2.865707 \\ 6 & 2.955479 & 5.047004 & -3.207585 \\ 6 & 1.638540 & 5.080556 & -3.650625 \\ 6 & 0.667902 & 1.401567 & -3.407988 \\ 6 & 1.326956 & 0.268484 & -4.163252 \\ 45 & -0.009224 & 0.929251 & -1.320728 \\ 6 & -0.754162 & 1.403986 & -3.415983 \\ 15 & -1.705695 & 0.149636 & 0.240997 \\ 6 & -3.441968 & -0.023651 & -0.378383 \\ 6 & -3.733864 & 0.062602 & -1.740361 \\ 6 & -5.050751 & -0.019043 & -2.198631 \\ 6 & -6.066355 & -0.200506 & -1.251258 \\ 6 & -5.807065 & -0.311205 & 0.114552 \\ 6 & -4.477678 & -0.218423 & 0.530844 \\ 6 & -5.418504 & 0.125468 & -3.674874 \\ 6 & -6.894268 & -0.525464 & 1.164955 \\ 6 & -1.890067 & 1.554025 & 1.420879 \\ 6 & -2.871652 & 2.524306 & 1.205160 \\ 6 & -2.963441 & 3.648679 & 2.021303 \\ 6 & -2.063970 & 3.762014 & 3.089010 \\ 6 & -1.070831 & 2.813540 & 3.334407 \\ 6 & -0.973836 & 1.726775 & 2.456563 \\ 6 & -0.140865 & 2.887409 & 4.545178 \\ 6 & -0.334289 & 4.169463 & 5.351357 \\ 6 & -4.014337 & 4.733453 & 1.793338 \\ 6 & -3.310822 & 6.068919 & 1.528559 \\ 6 & -1.693887 & -1.326207 & 1.389428 \\ 6 & -1.458815 & -2.595790 & 0.879754 \\ 6 & -1.509043 & -3.742315 & 1.736849 \\ 6 & -1.808335 & -3.575514 & 3.119935 \\ 6 & -2.065182 & -2.273862 & 3.605327 \\ 6 & -2.011883 & -1.190567 & 2.768787 \\ 6 & -1.843002 & -4.706500 & 3.970395 \\ 6 & -1.591577 & -5.961905 & 3.479559 \\ 6 & -1.296578 & -6.135381 & 2.110617 \\ 6 & -1.259289 & -5.057130 & 1.261664 \\ 6 & -1.190342 & -2.822611 & -0.565732 \\ 6 & -2.249159 & -3.252316 & -1.423491 \\ 6 & -1.979890 & -3.492063 & -2.804004 \\ 6 & -0.663074 & -3.320420 & -3.289756 \\ 6 & 0.339090 & -2.915824 & -2.448917 \\ 6 & 0.080257 & -2.647887 & -1.083451 \\ 6 & -3.032930 & -3.907005 & -3.656008 \\ 6 & -4.304166 & -4.075216 & -3.169456 \\ 6 & -4.578561 & -3.821114 & -1.807403 \\ 6 & -3.578438 & -3.424432 & -0.955793 \\ 6 & 1.141288 & -2.147032 & -0.257612 \\ 6 & 1.928952 & -3.167015 & 0.473509 \\ 6 & 2.471926 & -2.727847 & 1.807144 \\ & 3.826833 & -2.872575 & 2.095621 \\ 6.339210 & -2.461362 & 3.323940 \\ 6 & 3.496087 & -1.906898 & 4.280683 \\ 6\end{array}$




\begin{tabular}{|c|c|c|c|}
\hline 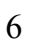 & 2.136513 & -1.770875 & 4.003050 \\
\hline 5 & 1.630746 & -2.178750 & 2.776416 \\
\hline & 0.511809 & 2.646534 & -0.426727 \\
\hline & 0.523244 & 3.764765 & -0.132092 \\
\hline & 1.644474 & -0.595762 & -0.737542 \\
\hline & 2.925622 & -0.812143 & -1.817671 \\
\hline & 4.092032 & -1.449893 & -1.480211 \\
\hline & 4.998891 & -0.854827 & -0.622238 \\
\hline & 6.155746 & -1.606912 & -0.235423 \\
\hline 6 & 6.404395 & -2.876696 & -0.838595 \\
\hline 6 & 5.488636 & -3.385460 & -1.792215 \\
\hline & 4.346882 & -2.695669 & -2.093836 \\
\hline 6 & 7.043598 & -1.167974 & 0.779531 \\
\hline 6 & 8.127316 & -1.925957 & 1.150866 \\
\hline 6 & 8.390027 & -3.162437 & 0.525004 \\
\hline 6 & 7.540535 & -3.625420 & -0.447138 \\
\hline & 4.711274 & 0.499672 & -0.099969 \\
\hline 6 & 3.494819 & 0.742769 & 0.511870 \\
\hline & 3.171844 & 1.991143 & 1.077059 \\
\hline & 4.054210 & 3.031768 & 0.981782 \\
\hline & 5.278702 & 2.873111 & 0.289772 \\
\hline 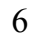 & 5.612930 & 1.601146 & -0.267741 \\
\hline 6 & 6.161816 & 3.965923 & 0.112195 \\
\hline & 7.320120 & 3.829145 & -0.609891 \\
\hline 6 & 7.637186 & 2.583497 & -1.191451 \\
\hline & 6.809005 & 1.500888 & -1.023716 \\
\hline & 2.560897 & -0.259835 & 0.608503 \\
\hline & 1.322252 & 2.822403 & 4.099056 \\
\hline 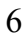 & -0.440019 & 1.694121 & 5.461471 \\
\hline 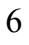 & -4.914247 & 4.423369 & 0.598528 \\
\hline & -4.902714 & 4.861633 & 3.035582 \\
\hline ] & 6.843954 & -0.225344 & 1.283176 \\
\hline ] & -2.226473 & -0.205942 & 3.174959 \\
\hline . & 7.715784 & -4.587077 & -0.927971 \\
\hline L & -2.071309 & -4.555068 & 5.024500 \\
\hline & 5.889222 & 4.924509 & 0.551915 \\
\hline l & -3.803325 & -3.220397 & 0.090037 \\
\hline & -5.594363 & -3.934612 & -1.433612 \\
\hline L & 3.809269 & 4.004030 & 1.407450 \\
\hline L & -2.311483 & -2.141729 & 4.658473 \\
\hline L & 5.697389 & -4.344724 & -2.263092 \\
\hline l & 8.543159 & 2.480398 & -1.784617 \\
\hline 1 & 3.618946 & -3.081400 & -2.804674 \\
\hline 1 & -2.807976 & -4.094082 & -4.705301 \\
\hline & -0.459364 & -3.515376 & -4.341980 \\
\hline 1 & -1.099093 & -7.132876 & 1.724716 \\
\hline 1 & 7.062415 & 0.552133 & -1.489843 \\
\hline 1 & -1.035692 & -5.206980 & 0.207414 \\
\hline 1 & 1.351677 & -2.770409 & -2.821284 \\
\hline 1 & 7.986974 & 4.677441 & -0.745796 \\
\hline 1 & 8.786524 & -1.570667 & 1.939862 \\
\hline 1 & -5.104701 & -4.402106 & -3.829988 \\
\hline 1 & -1.617996 & -6.824930 & 4.140801 \\
\hline 1 & 9.255391 & -3.749320 & 0.823977 \\
\hline 1 & 2.210110 & 2.095896 & 1.570419 \\
\hline 1 & -4.247910 & -0.287039 & 1.593431 \\
\hline
\end{tabular}




\begin{tabular}{|c|c|c|c|}
\hline & -2.907277 & 0.188106 & -2.434975 \\
\hline & -7.095746 & -0.257495 & -1.599075 \\
\hline & -3.566584 & 2.393866 & 0.380017 \\
\hline & -0.186179 & 0.982342 & 2.598837 \\
\hline & -2.148494 & 4.624062 & 3.746910 \\
\hline & 1.238765 & -4.008544 & 0.630249 \\
\hline & 1.989409 & 2.937474 & 4.963756 \\
\hline & 1.568752 & 1.862294 & 3.629369 \\
\hline & 1.551634 & 3.625470 & 3.384760 \\
\hline 1 & 0.210250 & 1.716326 & 6.346727 \\
\hline 1 & -1.482785 & 1.714414 & 5.805829 \\
\hline & -0.273970 & 0.736898 & 4.949373 \\
\hline & -0.124452 & 5.064816 & 4.751969 \\
\hline 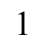 & -1.351619 & 4.255010 & 5.754119 \\
\hline & 0.355396 & 4.174446 & 6.204767 \\
\hline ] & -5.664558 & 5.216360 & 0.487003 \\
\hline & -4.347178 & 4.372094 & -0.340006 \\
\hline 1 & -5.452151 & 3.472816 & 0.722608 \\
\hline & -5.426104 & 3.919159 & 3.244261 \\
\hline 1 & -4.328811 & 5.130539 & 3.930498 \\
\hline & -5.660647 & 5.641788 & 2.882734 \\
\hline & -2.692864 & 6.383716 & 2.378111 \\
\hline 1 & -2.657249 & 5.999434 & 0.649318 \\
\hline & -4.049653 & 6.860510 & 1.343264 \\
\hline 1 & 2.749850 & -3.549130 & -0.153863 \\
\hline 1 & 4.497225 & -3.288181 & 1.341601 \\
\hline 1 & 0.577460 & -2.035253 & 2.545276 \\
\hline 1 & 5.403630 & -2.567664 & 3.524604 \\
\hline 1 & 1.464014 & -1.342848 & 4.745986 \\
\hline 1 & 3.894954 & -1.580468 & 5.238893 \\
\hline & -1.272838 & 1.744465 & -1.892436 \\
\hline 1 & -1.249567 & 0.533359 & -3.847989 \\
\hline 1 & -1.298426 & 2.334608 & -3.587042 \\
\hline 1 & -0.133245 & 3.958113 & -4.097379 \\
\hline 1 & 1.182171 & 6.027344 & -3.933562 \\
\hline 1 & 3.534028 & 5.965127 & -3.132178 \\
\hline 1 & 4.558467 & 3.777762 & -2.510275 \\
\hline & 3.232875 & 1.707884 & -2.651534 \\
\hline 1 & 1.378905 & 0.537484 & -5.229791 \\
\hline 1 & 2.344130 & 0.057836 & -3.828861 \\
\hline 1 & 0.742384 & -0.655179 & -4.074793 \\
\hline 6 & -4.187700 & 0.116794 & -4.578241 \\
\hline 6 & -6.329150 & -1.023947 & -4.116942 \\
\hline 6 & -6.151921 & 1.457775 & -3.869041 \\
\hline 6 & -8.283008 & -0.664942 & 0.547767 \\
\hline 6 & -6.592465 & -1.807360 & 1.950788 \\
\hline 6 & -6.912087 & 0.672222 & 2.122105 \\
\hline 1 & -4.500012 & 0.174006 & -5.628691 \\
\hline 1 & -3.531590 & 0.975658 & -4.387414 \\
\hline 1 & -3.600822 & -0.804040 & -4.452278 \\
\hline 1 & -6.582555 & -0.916022 & -5.180061 \\
\hline 1 & -5.825722 & -1.990448 & -3.983578 \\
\hline 1 & -7.272425 & -1.053400 & -3.559145 \\
\hline 1 & -6.416780 & 1.600698 & -4.925638 \\
\hline 1 & -7.078218 & 1.501759 & -3.282736 \\
\hline 1 & -5.521209 & 2.301808 & -3.559859 \\
\hline
\end{tabular}




$\begin{array}{rrrr}1 & -9.025022 & -0.825573 & 1.340116 \\ 1 & -8.581267 & 0.237017 & -0.001967 \\ 1 & -8.341582 & -1.520904 & -0.137428 \\ 1 & -7.657549 & 0.520015 & 2.914461 \\ 1 & -5.938789 & 0.830875 & 2.603808 \\ 1 & -7.173644 & 1.595111 & 1.587027 \\ 1 & -7.358319 & -1.972586 & 2.720566 \\ 1 & -6.590170 & -2.681609 & 1.286012 \\ 1 & -5.618396 & -1.771298 & 2.455181\end{array}$

$\mathrm{TS1}_{I B 1-} \psi-1 \mathrm{~s} 2 \mathrm{a3s} 4 \mathrm{a}$

Number of imaginary frequencies : 1

The smallest frequencies are : - $693.8221 \quad 14.687424 .3996 \mathrm{~cm}(-1)$

Electronic energy :

$\mathrm{HF}=-4360.8630249$

Zero-point correction $=$

1.452947 (Hartree/Particle)

Thermal correction to Energy= $\quad 1.537553$

Thermal correction to Enthalpy= $\quad 1.538497$

Thermal correction to Gibbs Free Energy $=\quad 1.336325$

Sum of electronic and zero-point Energies $=\quad-4359.410077$

Sum of electronic and thermal Energies $=\quad-4359.325472$

Sum of electronic and thermal Enthalpies $=\quad-4359.324528$

Sum of electronic and thermal Free Energies $=\quad-4359.526700$

Cartesian Coordinates

$\begin{array}{cccc}6 & -0.715445 & 1.470976 & 2.539646 \\ 6 & -1.676038 & 1.513750 & 1.530694 \\ 6 & -2.522984 & 2.619451 & 1.431301 \\ 6 & -2.431679 & 3.674309 & 2.336932 \\ 6 & -1.498241 & 3.570726 & 3.376982 \\ 6 & -0.643258 & 2.476072 & 3.511444 \\ 15 & -1.720000 & 0.189897 & 0.253643 \\ 6 & -3.494691 & 0.202353 & -0.274713 \\ 6 & -3.821220 & 0.354751 & -1.613479 \\ 6 & -5.156790 & 0.355773 & -2.045425 \\ 6 & -6.146120 & 0.158927 & -1.088900 \\ 6 & -5.853362 & -0.015002 & 0.274509 \\ 6 & -4.520520 & 0.022387 & 0.664315 \\ 6 & -5.459075 & 0.581884 & -3.524977 \\ 6 & -6.956782 & 0.590468 & -3.819803 \\ 6 & -6.986712 & -0.275110 & 1.264822 \\ 6 & -6.489564 & -0.347102 & 2.707273 \\ 6 & -3.319858 & 4.913147 & 2.237900 \\ 6 & -4.293703 & 4.834245 & 1.064018 \\ 6 & 0.260074 & 2.278571 & 4.730239 \\ 6 & 1.696784 & 1.952500 & 4.316135 \\ 45 & -0.062619 & 0.908657 & -1.386828 \\ 6 & -0.846845 & 1.307407 & -3.485800 \\ 6 & 0.576748 & 1.308169 & -3.498588 \\ 6 & 1.231930 & 0.153474 & -4.223321 \\ 15 & 1.566764 & -0.632708 & -0.785360 \\ 7 & 1.051184 & -2.184028 & -0.315410 \\ 6 & 1.823223 & -3.216115 & 0.416444 \\ 6 & 2.362639 & -2.782508 & 1.753546 \\ 6 & 3.723436 & -2.889844 & 2.030877\end{array}$




\begin{tabular}{|c|c|c|c|}
\hline & 4.235836 & -2.463290 & 3.253835 \\
\hline & 3.385849 & -1.933732 & 4.218419 \\
\hline & 2.018811 & -1.848109 & 3.957745 \\
\hline & 1.512455 & -2.268633 & 2.734806 \\
\hline & 2.857766 & -0.849859 & -1.852318 \\
\hline U & 4.024616 & -1.481251 & -1.504882 \\
\hline & 4.922101 & -0.881062 & -0.640407 \\
\hline & 6.083549 & -1.624638 & -0.249774 \\
\hline & 6.344765 & -2.891797 & -0.853268 \\
\hline & 5.436750 & -3.406946 & -1.810685 \\
\hline & 4.291839 & -2.724893 & -2.117662 \\
\hline & 6.966090 & -1.179160 & 0.767178 \\
\hline & 8.056396 & -1.927441 & 1.138985 \\
\hline & 8.331083 & -3.161060 & 0.512722 \\
\hline & 7.486755 & -3.630951 & -0.460463 \\
\hline & 4.622221 & 0.470499 & -0.117464 \\
\hline & 5.521290 & 1.575686 & -0.274695 \\
\hline & 5.177809 & 2.844508 & 0.283912 \\
\hline & 3.946663 & 2.996084 & 0.965210 \\
\hline & 3.065913 & 1.952782 & 1.049654 \\
\hline & 3.399496 & 0.706374 & 0.485695 \\
\hline 6 & 6.059387 & 3.940669 & 0.119373 \\
\hline & 7.225404 & 3.810005 & -0.591052 \\
\hline & 7.551332 & 2.567856 & -1.175200 \\
\hline & 6.724403 & 1.482400 & -1.020517 \\
\hline 8 & 2.469311 & -0.301378 & 0.571861 \\
\hline & 0.497365 & 2.638495 & -0.551269 \\
\hline & 0.542088 & 3.758255 & -0.266048 \\
\hline & 1.348968 & 2.584832 & -3.535607 \\
\hline & 0.789330 & 3.794441 & -3.959724 \\
\hline 6 & 1.530734 & 4.973799 & -3.939618 \\
\hline & 2.850856 & 4.967743 & -3.505937 \\
\hline & 3.429599 & 3.765556 & -3.099517 \\
\hline & 2.691363 & 2.591929 & -3.122966 \\
\hline 6 & -1.783317 & -1.344368 & 1.319829 \\
\hline 6 & -1.585440 & -2.595281 & 0.756850 \\
\hline & -1.694792 & -3.778804 & 1.560016 \\
\hline 6 & -2.023942 & -3.664908 & 2.941385 \\
\hline & -2.248524 & -2.377853 & 3.480375 \\
\hline 6 & -2.132612 & -1.261068 & 2.697283 \\
\hline & -2.118812 & -4.830518 & 3.738233 \\
\hline 6 & -1.895604 & -6.070847 & 3.197302 \\
\hline 6 & -1.568590 & -6.192128 & 1.830411 \\
\hline 6 & -1.473781 & -5.078666 & 1.032577 \\
\hline 6 & -1.289899 & -2.787847 & -0.687692 \\
\hline & -2.338448 & -3.180520 & -1.574709 \\
\hline 6 & -2.029742 & -3.497213 & -2.931049 \\
\hline & -0.688664 & -3.403826 & -3.370711 \\
\hline 6 & 0.296960 & -2.996550 & -2.510965 \\
\hline 6 & 0.002413 & -2.673058 & -1.165361 \\
\hline 6 & -3.067922 & -3.913520 & -3.800595 \\
\hline 6 & -4.363430 & -3.995901 & -3.358253 \\
\hline & -4.678727 & -3.645515 & -2.026962 \\
\hline 6 & -3.692915 & -3.254285 & -1.156075 \\
\hline & -4.882280 & 1.936652 & -3.952833 \\
\hline & -4.817104 & -0.532684 & -4.356101 \\
\hline
\end{tabular}




\begin{tabular}{|c|c|c|c|}
\hline 6 & -8.031348 & 0.842073 & 1.179274 \\
\hline 6 & -7.641762 & -1.617321 & 0.918435 \\
\hline & -4.139789 & 5.067559 & 3.523489 \\
\hline & -2.435387 & 6.149225 & 2.042450 \\
\hline & -0.306190 & 1.103979 & 5.540225 \\
\hline & 0.298546 & 3.508018 & 5.634777 \\
\hline & 6.758195 & -0.238397 & 1.270914 \\
\hline & -2.313738 & -0.288076 & 3.146439 \\
\hline & 7.671146 & -4.590571 & -0.941934 \\
\hline 1 & -2.369855 & -4.718302 & 4.792068 \\
\hline & 5.779577 & 4.896655 & 0.560178 \\
\hline 1 & -3.947770 & -2.982016 & -0.132735 \\
\hline & -5.712675 & -3.684067 & -1.689797 \\
\hline & 3.694411 & 3.965836 & 1.392317 \\
\hline & -2.516748 & -2.284758 & 4.532445 \\
\hline & 5.654849 & -4.364011 & -2.281780 \\
\hline & 8.463186 & 2.469799 & -1.760184 \\
\hline & 3.572184 & -3.114538 & -2.834722 \\
\hline & -2.812781 & -4.165755 & -4.829047 \\
\hline & -0.454022 & -3.654445 & -4.404496 \\
\hline & -1.392065 & -7.177111 & 1.404326 \\
\hline & 6.984767 & 0.536813 & -1.489111 \\
\hline & -1.224065 & -5.190805 & -0.020422 \\
\hline & 1.326254 & -2.899439 & -2.851968 \\
\hline & 7.891304 & 4.660665 & -0.716518 \\
\hline & 8.711602 & -1.566458 & 1.928739 \\
\hline 1 & -5.153152 & -4.317773 & -4.033846 \\
\hline & -1.968151 & -6.961226 & \\
\hline & 9.201458 & -3.740213 & 0.812207 \\
\hline 1 & 2.101359 & 2.052272 & 1.541186 \\
\hline & -4.261478 & -0.094172 & 1.713060 \\
\hline D & -3.015772 & 0.468239 & -2.335804 \\
\hline & -7.188484 & 0.136905 & -1.400366 \\
\hline 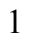 & -3.252562 & 2.649888 & 0.625290 \\
\hline 1 & -0.041575 & 0.612578 & 2.597251 \\
\hline 1 & -1.448591 & 4.373852 & 4.109117 \\
\hline 1 & 1.121477 & -4.049346 & 0.566616 \\
\hline & 2.308825 & 1.756904 & 5.207676 \\
\hline 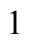 & 1.765314 & 1.060535 & 3.680621 \\
\hline & 2.153484 & 2.793194 & 3.777148 \\
\hline 1 & 0.303724 & 0.923380 & 6.436442 \\
\hline & -1.335044 & 1.310653 & 5.864926 \\
\hline 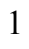 & -0.322673 & 0.177272 & 4.950926 \\
\hline l & 0.669054 & 4.394088 & 5.103168 \\
\hline & -0.687470 & 3.745350 & 6.054036 \\
\hline 1 & 0.974414 & 3.322198 & 6.479123 \\
\hline & -4.913956 & 5.739202 & 1.039535 \\
\hline 1 & -3.770919 & 4.767559 & 0.101174 \\
\hline l & -4.969819 & 3.972422 & 1.146381 \\
\hline 1 & -4.782714 & 4.192650 & 3.687103 \\
\hline 1 & -3.505022 & 5.186928 & 4.409575 \\
\hline & -4.785316 & 5.953866 & 3.458853 \\
\hline 1 & -1.748684 & 6.300976 & 2.883976 \\
\hline & -1.830343 & 6.056056 & 1.131273 \\
\hline & -3.054439 & 7.052131 & 1.950822 \\
\hline & 2.644244 & -3.605044 & -0.206868 \\
\hline
\end{tabular}




$\begin{array}{rrrr}1 & 4.398052 & -3.286940 & 1.271073 \\ 1 & 0.450584 & -2.166150 & 2.518674 \\ 1 & 5.304866 & -2.537539 & 3.444247 \\ 1 & 1.343499 & -1.445287 & 4.712931 \\ 1 & 3.783193 & -1.590942 & 5.171715 \\ 1 & -1.312894 & 1.732685 & -1.979623 \\ 1 & -1.347939 & 0.418273 & -3.872371 \\ 1 & -1.392089 & 2.229937 & -3.695156 \\ 1 & -0.239377 & 3.822674 & -4.315067 \\ 1 & 1.069556 & 5.902183 & -4.271431 \\ 1 & 3.427628 & 5.889815 & -3.486166 \\ 1 & 4.461390 & 3.743703 & -2.750215 \\ 1 & 3.140883 & 1.664562 & -2.765706 \\ 1 & 1.274908 & 0.389328 & -5.298111 \\ 1 & 2.252680 & -0.043115 & -3.890342 \\ 1 & 0.650575 & -0.768121 & -4.101474 \\ 1 & -5.099863 & 2.126685 & -5.012478 \\ 1 & -5.318889 & 2.754267 & -3.364451 \\ 1 & -3.793042 & 1.978316 & -3.825556 \\ 1 & -4.991233 & -0.359328 & -5.426877 \\ 1 & -3.732874 & -0.596109 & -4.198788 \\ 1 & -5.242522 & -1.509746 & -4.093805 \\ 1 & -7.119842 & 0.759013 & -4.891789 \\ 1 & -7.430906 & -0.365799 & -3.563117 \\ 1 & -7.478122 & 1.390611 & -3.278456 \\ 1 & -8.458284 & -1.839327 & 1.618925 \\ 1 & -8.061628 & -1.619614 & -0.095132 \\ 1 & -6.910036 & -2.434376 & 0.979940 \\ 1 & -8.845495 & 0.655830 & 1.892400 \\ 1 & -7.586658 & 1.815947 & 1.421764 \\ 1 & -8.480518 & 0.918645 & 0.182072 \\ 1 & -7.337430 & -0.526882 & 3.380247 \\ 1 & -5.772982 & -1.165721 & 2.857543 \\ 1 & -6.010623 & 0.589795 & 3.021623\end{array}$

$\operatorname{TS1}_{I B 1-} \psi_{-1 \mathrm{~s} 2 \mathrm{a} 3 \mathrm{~s} 4 \mathrm{~s}}$

Number of imaginary frequencies : 1

The smallest frequencies are : -693.8821 $4.87399 .5932 \mathrm{~cm}(-1)$

Electronic energy : $\quad H F=-4360.8633533$

Zero-point correction $=\quad 1.452005$ (Hartree/Particle)

Thermal correction to Energy= $\quad 1.537170$

Thermal correction to Enthalpy= $\quad 1.538114$

Thermal correction to Gibbs Free Energy $=\quad 1.330394$

Sum of electronic and zero-point Energies $=\quad-4359.411349$

Sum of electronic and thermal Energies $=\quad-4359.326183$

Sum of electronic and thermal Enthalpies $=\quad-4359.325239$

Sum of electronic and thermal Free Energies $=\quad-4359.532959$

Cartesian Coordinates

$\begin{array}{rrrr}6 & 6.717151 & 1.541635 & -1.010894 \\ 6 & 5.510179 & 1.626314 & -0.270289 \\ 6 & 5.151323 & 2.894290 & 0.280470 \\ 6 & 6.022352 & 3.998621 & 0.114058 \\ 6 & 7.192386 & 3.876404 & -0.591229\end{array}$




$\begin{array}{cccc}6 & 7.533369 & 2.634806 & -1.167925 \\ 6 & 4.622551 & 0.512383 & -0.110239 \\ 6 & 3.394792 & 0.738260 & 0.486337 \\ 6 & 3.045624 & 1.984292 & 1.041741 \\ 6 & 3.915811 & 3.036355 & 0.955896 \\ 6 & 4.940753 & -0.839852 & -0.620219 \\ 6 & 4.054473 & -1.459105 & -1.482762 \\ 6 & 4.338342 & -2.706643 & -2.080020 \\ 6 & 5.488794 & -3.372908 & -1.759265 \\ 6 & 6.386315 & -2.837248 & -0.803202 \\ 6 & 6.108308 & -1.566144 & -0.215558 \\ 6 & 7.534044 & -3.559589 & -0.395783 \\ 6 & 8.368204 & -3.069455 & 0.576084 \\ 6 & 8.077203 & -1.831690 & 1.186458 \\ 6 & 6.980839 & -1.099627 & 0.800702 \\ 8 & 2.882695 & -0.844077 & -1.842938 \\ 15 & 1.583909 & -0.630201 & -0.784803 \\ 8 & 2.475566 & -0.279359 & 0.574739 \\ 45 & -0.059571 & 0.891321 & -1.401671 \\ 6 & 0.470850 & 2.628874 & -0.561676 \\ 8 & 0.490932 & 3.747772 & -0.270323 \\ 6 & 0.590702 & 1.292960 & -3.508772 \\ 6 & -0.832786 & 1.271893 & -3.507635 \\ 6 & 1.344984 & 2.580262 & -3.541192 \\ 6 & 0.768631 & 3.783929 & -3.960076 \\ 6 & 1.492924 & 4.973649 & -3.932974 \\ 6 & 2.812489 & 4.984469 & -3.497625 \\ 6 & 3.408003 & 3.788643 & -3.096993 \\ 6 & 2.686622 & 2.604715 & -3.126913 \\ 6 & -3.018757 & -3.964685 & -3.791382 \\ 6 & -4.632929 & -3.700713 & -2.019251 \\ 6 & -3.649885 & -3.300308 & -1.149744 \\ 6 & 1.080513 & -2.183340 & -0.307145 \\ 6 & -1.857237 & -3.202308 & 0.438044 \\ 6 & -0.642756 & -3.432641 & -3.361392 \\ 6 & -1.722076 & 0.145093 & -4.224459 \\ 6 & -1.779563 & -1.361362 & 1.307644 \\ 6 & -1.557897 & -2.614259 & 0.758879 \\ 6 & -1.252680 & -2.813376 & -0.682132 \\ 6 & -1.658792 & -3.792243 & 1.570786 \\ 6 & -2.002950 & -3.671047 & 2.947732 \\ 6 & -2.250048 & -2.382458 & 3.472990 \\ 6 & -2.142650 & -1.270856 & 2.681097 \\ 6 & -2.089367 & -4.830953 & 3.753791 \\ 6 & -1.844260 & -6.072630 & 3.225575 \\ 6 & -1.502938 & -6.201119 & 1.862831 \\ 6 & 4.249075 & -2.396351 & 3.278245\end{array}$




\begin{tabular}{|c|c|c|c|}
\hline 6 & 3.388836 & -1.869442 & 4.235147 \\
\hline 6 & 2.021788 & -1.803674 & 3.968934 \\
\hline & 1.525177 & -2.240514 & 2.747685 \\
\hline & -3.490228 & 0.158035 & -0.321646 \\
\hline & -3.811897 & 0.293259 & -1.667541 \\
\hline & -5.142548 & 0.299707 & -2.102115 \\
\hline & -6.144394 & 0.131560 & -1.145084 \\
\hline & -5.856608 & -0.023709 & 0.216149 \\
\hline & -4.519812 & 0.001312 & 0.608075 \\
\hline & -5.440304 & 0.508113 & -3.586000 \\
\hline & -4.865555 & 1.859359 & -4.027459 \\
\hline O & -6.933280 & -0.217603 & 1.282416 \\
\hline & -6.866063 & 0.938911 & 2.287008 \\
\hline ס & -1.713981 & 1.492879 & 1.500664 \\
\hline & -2.593658 & 2.573321 & 1.410228 \\
\hline & -2.532585 & 3.623786 & 2.323769 \\
\hline f & -1.596104 & 3.540365 & 3.362608 \\
\hline & -0.708916 & 2.470448 & 3.488415 \\
\hline b & -0.752110 & 1.471133 & 2.509172 \\
\hline & -3.464935 & 4.830761 & 2.239882 \\
\hline 2 & -4.312858 & 4.914303 & 3.513879 \\
\hline כ & 0.197045 & 2.289787 & 4.707813 \\
\hline 6 & -0.349584 & 1.105315 & 5.516927 \\
\hline 0 & -4.793347 & -0.613668 & -4.403226 \\
\hline y & -6.936868 & 0.510037 & -3.887800 \\
\hline 6 & -6.688604 & -1.546662 & 2.007334 \\
\hline 6 & -8.341142 & -0.253153 & 0.693673 \\
\hline ר & 0.212471 & 3.519850 & 5.612335 \\
\hline 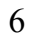 & 1.639847 & 1.989539 & 4.295676 \\
\hline 6 & -2.628887 & 6.106683 & 2.094440 \\
\hline 6 & -4.413624 & 4.745670 & 1.046262 \\
\hline ] & 6.760292 & -0.155615 & 1.292799 \\
\hline 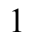 & -2.339023 & -0.296544 & 3.120768 \\
\hline 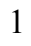 & 7.731213 & -4.522656 & -0.865138 \\
\hline I & -2.352083 & -4.713312 & 4.804172 \\
\hline 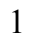 & 5.731014 & 4.953841 & 0.549011 \\
\hline 1 & -3.905205 & -3.026515 & -0.126951 \\
\hline & -5.666432 & -3.742530 & -1.681154 \\
\hline 1 & 3.651484 & 4.005587 & 1.376818 \\
\hline 1 & -2.528369 & -2.283797 & 4.521924 \\
\hline 1 & 5.719275 & -4.332973 & -2.218229 \\
\hline 1 & 8.448479 & 2.543316 & -1.748872 \\
\hline 1 & 3.626333 & -3.111900 & -2.796094 \\
\hline 1 & -2.761932 & -4.219038 & -4.818901 \\
\hline 1 & -0.404170 & -3.684557 & -4.393969 \\
\hline 1 & -1.309350 & -7.187236 & 1.446916 \\
\hline 1 & 6.989146 & 0.596307 & -1.473410 \\
\hline 1 & -1.155196 & -5.210718 & 0.006497 \\
\hline 1 & 1.366973 & -2.907739 & -2.842112 \\
\hline 1 & 7.850020 & 4.733231 & -0.718326 \\
\hline 1 & 8.724477 & -1.454553 & 1.975209 \\
\hline 1 & -5.100956 & -4.383943 & -4.024033 \\
\hline 1 & -1.910641 & -6.958639 & 3.852694 \\
\hline 1 & 9.243084 & -3.635801 & 0.886729 \\
\hline 1 & 2.077607 & 2.076845 & 1.527443 \\
\hline 1 & -4.273273 & -0.102861 & 1.663644 \\
\hline
\end{tabular}




\begin{tabular}{|c|c|c|c|}
\hline & -3.002205 & 0.392222 & -2.387239 \\
\hline & -7.182362 & 0.124910 & -1.464705 \\
\hline & -3.326338 & 2.587608 & 0.606778 \\
\hline & -0.055928 & 0.630384 & 2.562376 \\
\hline & -1.571493 & 4.338151 & 4.101906 \\
\hline & 1.162041 & -4.040311 & 0.591792 \\
\hline & 2.254509 & 1.807315 & 5.188225 \\
\hline & 1.725921 & 1.097409 & 3.662563 \\
\hline & 2.081209 & 2.837547 & 3.755358 \\
\hline & 0.263090 & 0.933955 & 6.413118 \\
\hline & -1.381785 & 1.294806 & 5.841633 \\
\hline & -0.350821 & 0.179212 & 4.926489 \\
\hline & 0.563302 & 4.413304 & 5.079737 \\
\hline & -0.776993 & 3.736931 & 6.034388 \\
\hline & 0.893960 & 3.347820 & 6.455074 \\
\hline & -5.068353 & 5.626244 & 1.032748 \\
\hline & -3.870821 & 4.722945 & 0.092535 \\
\hline & -5.056899 & 3.856301 & 1.094498 \\
\hline & -4.929178 & 4.013245 & 3.633834 \\
\hline & -3.697779 & 5.021884 & 4.415389 \\
\hline & -4.986581 & 5.780612 & 3.467505 \\
\hline & -1.964610 & 6.265223 & 2.952443 \\
\hline 1 & -2.004266 & 6.064015 & 1.192681 \\
\hline & -3.283631 & 6.985145 & 2.014655 \\
\hline ] & 2.685178 & -3.589315 & -0.177223 \\
\hline & 4.428610 & -3.236036 & 1.303755 \\
\hline 1 & 0.462827 & -2.153023 & 2.526776 \\
\hline 1 & 5.318237 & -2.455471 & 3.473163 \\
\hline & 1.338733 & -1.403639 & 4.718490 \\
\hline ] & 3.778192 & -1.513456 & 5.186907 \\
\hline & -1.316042 & 1.697136 & -2.005714 \\
\hline 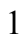 & -1.317870 & 0.374533 & -3.895664 \\
\hline ] & -1.389434 & 2.185821 & -3.724402 \\
\hline | & -0.260263 & 3.799493 & -4.315611 \\
\hline ] & 1.018707 & 5.896986 & -4.260462 \\
\hline | & 3.375950 & 5.914603 & -3.472562 \\
\hline 1 & 4.439761 & 3.779644 & -2.747020 \\
\hline & 3.149127 & 1.682736 & -2.772389 \\
\hline 1 & 1.314691 & 0.377386 & -5.299827 \\
\hline ] & 2.288233 & -0.036366 & -3.883486 \\
\hline l & 0.698027 & -0.784062 & -4.102962 \\
\hline 1 & -5.077931 & 2.036018 & -5.090523 \\
\hline 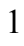 & -5.307890 & 2.682631 & -3.451449 \\
\hline 1 & -3.777300 & 1.905574 & -3.894336 \\
\hline 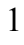 & -4.967173 & -0.453777 & -5.476153 \\
\hline 1 & -3.709092 & -0.671523 & -4.244401 \\
\hline 1 & -5.215800 & -1.588792 & -4.129577 \\
\hline 1 & -7.094596 & 0.661838 & -4.963108 \\
\hline 1 & -7.411347 & -0.442555 & -3.618525 \\
\hline 1 & -7.462248 & 1.317942 & -3.362262 \\
\hline 1 & -7.448345 & -1.704408 & 2.784772 \\
\hline 1 & -6.744603 & -2.389216 & 1.304838 \\
\hline 1 & -5.704390 & -1.584219 & 2.491451 \\
\hline 1 & -7.603971 & 0.794608 & 3.087825 \\
\hline 1 & -5.877306 & 1.026480 & 2.755231 \\
\hline 1 & -7.086519 & 1.895312 & 1.794732 \\
\hline
\end{tabular}


$\mathrm{TS1}_{I B 1-} \Psi-1 \mathrm{~s} 2 \mathrm{~s} 3 \mathrm{a} 4 \mathrm{a}$

Number of imaginary frequencies : 1

The smallest frequencies are : - $691.4484 \quad 11.5953 \quad 22.2241 \mathrm{~cm}(-1)$

Electronic energy : $\quad H F=-4360.8641683$

Zero-point correction $=\quad 1.452101($ Hartree/Particle $)$

Thermal correction to Energy= $\quad 1.537001$

Thermal correction to Enthalpy= $\quad 1.537945$

Thermal correction to Gibbs Free Energy $=\quad 1.333850$

Sum of electronic and zero-point Energies $=\quad-4359.412067$

Sum of electronic and thermal Energies $=\quad-4359.327167$

Sum of electronic and thermal Enthalpies $=\quad-4359.326223$

Sum of electronic and thermal Free Energies $=\quad-4359.530319$

Cartesian Coordinates

$\begin{array}{cccc}6 & -2.477198 & 2.643194 & 1.373296 \\ 6 & -1.642825 & 1.536720 & 1.488514 \\ 6 & -0.679603 & 1.508800 & 2.501420 \\ 6 & -0.603156 & 2.528653 & 3.451787 \\ 6 & -1.457647 & 3.629043 & 3.304109 \\ 6 & -2.386324 & 3.715526 & 2.265148 \\ 15 & -1.700790 & 0.182685 & 0.243827 \\ 45 & -0.051802 & 0.853514 & -1.416491 \\ 6 & 0.493708 & 2.607224 & -0.620017 \\ 8 & 0.529231 & 3.733052 & -0.360069 \\ 6 & 0.300460 & 2.351249 & 4.674259 \\ 6 & 1.734215 & 2.004946 & 4.266435 \\ 6 & -3.322182 & 4.907824 & 2.078746 \\ 6 & -3.108680 & 5.990130 & 3.133572 \\ 6 & -1.775441 & -1.319916 & 1.356364 \\ 6 & -1.571426 & -2.588854 & 0.837138 \\ 6 & -1.685296 & -3.745219 & 1.677616 \\ 6 & -2.026518 & -3.586236 & 3.051400 \\ 6 & -2.258411 & -2.282477 & 3.545307 \\ 6 & -2.137493 & -1.191947 & 2.726938 \\ 6 & -1.459870 & -5.061189 & 1.194198 \\ 6 & -1.560927 & -6.148285 & 2.027061 \\ 6 & -1.899210 & -5.982495 & 3.386462 \\ 6 & -2.127497 & -4.725390 & 3.884979 \\ 6 & -1.280099 & -2.820839 & -0.602060 \\ 6 & -2.338732 & -3.217452 & -1.475146 \\ 6 & -2.048859 & -3.517230 & -2.839549 \\ 6 & -0.712297 & -3.430544 & -3.293962 \\ 6 & 0.285759 & -3.036409 & -2.442611 \\ 6 & 0.006874 & -2.708920 & -1.094192 \\ 6 & -3.686698 & -3.297227 & -1.037766 \\ 6 & -4.686940 & -3.664570 & -1.902253 \\ 6 & -4.393641 & -3.981480 & -3.247216 \\ 6 & -3.102262 & -3.904857 & -3.704169 \\ 7 & 1.062516 & -2.203954 & -0.263131 \\ 15 & 1.582815 & -0.668165 & -0.779694\end{array}$




\begin{tabular}{|c|c|c|c|}
\hline 8 & 2.488022 & -0.300094 & 0.566383 \\
\hline 6 & 3.406511 & 0.715240 & 0.450386 \\
\hline & 4.626576 & 0.478631 & -0.157783 \\
\hline & 5.510681 & 1.590105 & -0.350648 \\
\hline y & 5.158461 & 2.867442 & 0.182527 \\
\hline & 3.932574 & 3.020918 & 0.872923 \\
\hline D & 3.063894 & 1.970531 & 0.988742 \\
\hline & 6.706639 & 1.493095 & -1.107339 \\
\hline$b$ & 7.519129 & 2.584025 & -1.296217 \\
\hline b & 7.185697 & 3.835292 & -0.736161 \\
\hline 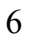 & 6.026179 & 3.969071 & -0.015647 \\
\hline 6 & 4.940888 & -0.884789 & -0.639905 \\
\hline f & 4.045331 & -1.527421 & -1.475339 \\
\hline$b$ & 4.321721 & -2.791680 & -2.040086 \\
\hline 6 & 5.476678 & -3.448398 & -1.715575 \\
\hline$b$ & 6.386398 & -2.885468 & -0.787054 \\
\hline 6 & 6.113877 & -1.599343 & -0.230140 \\
\hline$b$ & 7.541490 & -3.594283 & -0.376615 \\
\hline$b$ & 8.388118 & -3.076660 & 0.569872 \\
\hline b & 8.102134 & -1.824084 & 1.151683 \\
\hline b & 6.998635 & -1.104918 & 0.762112 \\
\hline 5 & 2.871084 & -0.921554 & -1.841668 \\
\hline 6 & -3.475547 & 0.195014 & -0.285284 \\
\hline 6 & -3.803538 & 0.173528 & -1.638205 \\
\hline 6 & -5.139839 & 0.146236 & -2.057044 \\
\hline 6 & -6.127125 & 0.125496 & -1.072164 \\
\hline 6 & -5.834910 & 0.128708 & 0.296836 \\
\hline 6 & -4.494643 & 0.168389 & 0.671771 \\
\hline 6 & -5.539455 & 0.180269 & -3.532117 \\
\hline 5 & -6.966261 & 0.047881 & 1.319751 \\
\hline 6 & 1.831939 & -3.217145 & 0.497288 \\
\hline 5 & 2.379617 & -2.741825 & 1.816592 \\
\hline 5 & 3.745533 & -2.815958 & 2.079463 \\
\hline 6 & 4.265250 & -2.339829 & 3.280696 \\
\hline 5 & 3.417184 & -1.797155 & 4.239742 \\
\hline 6 & 2.045323 & -1.748011 & 3.995907 \\
\hline 5 & 1.531664 & -2.215113 & 2.792964 \\
\hline 5 & -0.839234 & 1.178983 & -3.523333 \\
\hline 6 & 0.584353 & 1.224519 & -3.531158 \\
\hline 6 & 1.319149 & 2.522212 & -3.581904 \\
\hline 6 & 2.666490 & 2.567320 & -3.187746 \\
\hline 6 & 3.368528 & 3.762879 & -3.162669 \\
\hline 6 & 2.747371 & 4.950433 & -3.549120 \\
\hline 6 & 1.422480 & 4.918807 & -3.967216 \\
\hline 6 & 0.717637 & 3.717504 & -3.988977 \\
\hline 6 & 1.277241 & 0.076140 & -4.229757 \\
\hline 6 & -0.274921 & 1.200236 & 5.511337 \\
\hline 6 & 0.351436 & 3.599711 & 5.552116 \\
\hline 6 & -4.776266 & 4.428803 & 2.173652 \\
\hline 6 & -3.077073 & 5.531098 & 0.699466 \\
\hline 1 & 6.782280 & -0.149130 & 1.232825 \\
\hline 1 & -2.323219 & -0.205501 & 3.143665 \\
\hline 1 & 7.734175 & -4.569165 & -0.822884 \\
\hline 1 & -2.387812 & -4.579379 & 4.932413 \\
\hline 1 & 5.740532 & 4.931613 & 0.406748 \\
\hline 1 & -3.926473 & -3.042303 & -0.006640 \\
\hline
\end{tabular}




\begin{tabular}{|c|c|c|c|}
\hline & -5.716495 & -3.701936 & -1.550629 \\
\hline & 3.673844 & 3.997384 & 1.280389 \\
\hline 1 & -2.536038 & -2.155010 & 4.591290 \\
\hline 1 & 5.702023 & -4.420942 & -2.150210 \\
\hline & 8.425414 & 2.483010 & -1.889289 \\
\hline & 3.600050 & -3.216253 & -2.735165 \\
\hline & -2.862827 & -4.139881 & -4.740483 \\
\hline & -0.491350 & -3.673593 & -4.332590 \\
\hline & -1.380873 & -7.146612 & 1.634858 \\
\hline & 6.972693 & 0.539760 & -1.556800 \\
\hline & -1.202421 & -5.206377 & 0.147082 \\
\hline & 1.312269 & -2.945727 & -2.793939 \\
\hline & 7.840619 & 4.690169 & -0.888047 \\
\hline & 8.759103 & -1.424998 & 1.921378 \\
\hline & -5.195371 & -4.281077 & -3.919191 \\
\hline & -1.976916 & -6.852207 & 4.034727 \\
\hline & 9.268772 & -3.632646 & 0.882895 \\
\hline & 2.102320 & 2.070695 & 1.486166 \\
\hline & -4.229880 & 0.182811 & 1.725416 \\
\hline & -2.993558 & 0.161925 & -2.363005 \\
\hline & -7.172153 & 0.103241 & -1.380588 \\
\hline & -3.210820 & 2.671642 & 0.568248 \\
\hline ] & -0.011971 & 0.646805 & 2.575671 \\
\hline & -1.401460 & 4.437706 & 4.026556 \\
\hline ] & 1.127142 & -4.041919 & 0.676738 \\
\hline & 2.343560 & 1.815977 & 5.161394 \\
\hline | & 1.794996 & 1.103817 & 3.643396 \\
\hline 1 & 2.199735 & 2.833457 & 3.716254 \\
\hline & 0.333968 & 1.034863 & 6.411261 \\
\hline 1 & -1.301727 & 1.423204 & 5.831929 \\
\hline & -0.300475 & 0.260775 & 4.943145 \\
\hline ] & 0.724586 & 4.472037 & 5.000061 \\
\hline 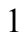 & -0.630242 & 3.852473 & 5.972371 \\
\hline . & 1.031043 & 3.427105 & 6.396312 \\
\hline 1 & -3.800627 & 6.822414 & 2.952437 \\
\hline 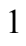 & -3.300697 & 5.619380 & 4.148828 \\
\hline l & -2.089740 & 6.396769 & 3.102005 \\
\hline & -5.008982 & 3.662316 & 1.422946 \\
\hline l & -4.983112 & 4.001762 & 3.164182 \\
\hline ] & -5.466917 & 5.268571 & 2.017188 \\
\hline 1 & -2.038552 & 5.873071 & 0.603001 \\
\hline 1 & -3.269033 & 4.821706 & -0.114616 \\
\hline 1 & -3.736644 & 6.396643 & 0.549209 \\
\hline 1 & 2.648409 & -3.628564 & -0.117427 \\
\hline . & 4.417727 & -3.223807 & 1.323297 \\
\hline 1 & 0.465019 & -2.140469 & 2.588430 \\
\hline 1 & 5.337774 & -2.387595 & 3.459509 \\
\hline 1 & 1.372094 & -1.336634 & 4.748161 \\
\hline 1 & 3.819840 & -1.416421 & 5.176326 \\
\hline 1 & -1.315390 & 1.640834 & -2.027995 \\
\hline 1 & -1.310025 & 0.265260 & -3.890057 \\
\hline 1 & -1.415539 & 2.076659 & -3.756522 \\
\hline 1 & -0.317172 & 3.717606 & -4.327391 \\
\hline 1 & 0.928396 & 5.835417 & -4.284150 \\
\hline 1 & 3.295256 & 5.889905 & -3.527382 \\
\hline 1 & 4.405051 & 3.769072 & -2.826601 \\
\hline
\end{tabular}




$\begin{array}{rrrr}1 & 3.148944 & 1.652254 & -2.842004 \\ 1 & 1.320995 & 0.292406 & -5.308608 \\ 1 & 2.300416 & -0.086571 & -3.886404 \\ 1 & 0.721158 & -0.858979 & -4.093890 \\ 6 & -6.452912 & 0.151304 & 2.754700 \\ 6 & -7.969882 & 1.182698 & 1.092070 \\ 6 & -7.675518 & -1.301930 & 1.158991 \\ 6 & -4.348205 & -0.059007 & -4.457023 \\ 6 & -6.588271 & -0.894373 & -3.833709 \\ 6 & -6.127197 & 1.561094 & -3.845991 \\ 1 & -6.854435 & -0.870656 & -4.898771 \\ 1 & -6.198264 & -1.894248 & -3.603630 \\ 1 & -7.515040 & -0.752600 & -3.265502 \\ 1 & -6.419471 & 1.623754 & -4.903215 \\ 1 & -7.016400 & 1.771005 & -3.238312 \\ 1 & -5.393845 & 2.354315 & -3.649304 \\ 1 & -4.689442 & -0.079690 & -5.499813 \\ 1 & -3.599399 & 0.739102 & -4.377607 \\ 1 & -3.856441 & -1.019283 & -4.245136 \\ 1 & -8.485806 & -1.401255 & 1.894027 \\ 1 & -8.114889 & -1.416770 & 0.160280 \\ 1 & -6.973287 & -2.132408 & 1.312268 \\ 1 & -8.784133 & 1.121336 & 1.826423 \\ 1 & -7.489873 & 2.163833 & 1.203331 \\ 1 & -8.424871 & 1.144250 & 0.095223 \\ 1 & -7.298129 & 0.105509 & 3.452928 \\ 1 & -5.771779 & -0.671649 & 3.009971 \\ 1 & -5.926547 & 1.099213 & 2.932636\end{array}$

$\operatorname{TS1}_{1 B 1-\vee-15233 a 4 \mathrm{~s}}$

Number of imaginary frequencies : 1

The smallest frequencies are : $-702.3567 \quad 12.8183 \quad 21.8241 \mathrm{~cm}(-1)$

Electronic energy : $\quad H F=-4360.8644936$

Zero-point correction $=\quad 1.452575($ Hartree/Particle $)$

Thermal correction to Energy= $\quad 1.537290$

Thermal correction to Enthalpy= $\quad 1.538234$

Thermal correction to Gibbs Free Energy $=\quad 1.334692$

Sum of electronic and zero-point Energies $=\quad-4359.411919$

Sum of electronic and thermal Energies $=\quad-4359.327203$

Sum of electronic and thermal Enthalpies $=\quad-4359.326259$

Sum of electronic and thermal Free Energies $=\quad-4359.529801$

Cartesian Coordinates

$\begin{array}{cccc}6 & -2.543545 & 2.607325 & 1.404139 \\ 6 & -1.694247 & 1.511089 & 1.509507 \\ 6 & -0.732371 & 1.485042 & 2.523408 \\ 6 & -0.671872 & 2.494566 & 3.485437 \\ 6 & -1.537162 & 3.587426 & 3.344929 \\ 6 & -2.462741 & 3.674872 & 2.303044 \\ 15 & -1.717882 & 0.176915 & 0.242601 \\ 45 & -0.055838 & 0.914777 & -1.380169 \\ 6 & 0.492552 & 2.636470 & -0.519807 \\ 8 & 0.530802 & 3.751364 & -0.215766 \\ 6 & 0.232542 & 2.315250 & 4.706818\end{array}$




\begin{tabular}{|c|c|c|c|}
\hline 6 & 1.671728 & 1.996474 & 4.295587 \\
\hline 6 & -3.387728 & 4.874516 & 2.107591 \\
\hline & -3.217494 & 5.928818 & 3.198127 \\
\hline & -1.773040 & -1.346535 & 1.327120 \\
\hline & -1.558529 & -2.601475 & 0.779195 \\
\hline & -1.657016 & -3.777397 & 1.592995 \\
\hline & -1.989120 & -3.653100 & 2.972609 \\
\hline & -2.229119 & -2.363005 & 3.497543 \\
\hline & -2.126369 & -1.252808 & 2.702762 \\
\hline & -1.425355 & -5.079593 & 1.076686 \\
\hline & -1.509957 & -6.186468 & 1.884745 \\
\hline & -1.837272 & -6.055091 & 3.250743 \\
\hline & -2.072514 & -4.812053 & 3.780372 \\
\hline & -1.271657 & -2.794248 & -0.666313 \\
\hline & -2.331944 & -3.172186 & -1.545006 \\
\hline & -2.047714 & -3.426748 & -2.919627 \\
\hline & -0.714691 & -3.314534 & -3.378375 \\
\hline & 0.285396 & -2.940245 & -2.520422 \\
\hline & 0.011985 & -2.657552 & -1.160935 \\
\hline & -3.676136 & -3.277548 & -1.101586 \\
\hline & -4.678047 & -3.628854 & -1.970558 \\
\hline & -4.390078 & -3.901448 & -3.326431 \\
\hline & -3.102955 & -3.797045 & -3.789444 \\
\hline & 1.068629 & -2.172530 & -0.319344 \\
\hline 15 & 1.583133 & -0.620796 & -0.790656 \\
\hline 8 & 2.490505 & -0.291243 & 0.563321 \\
\hline 6 & 3.425474 & 0.711746 & 0.475905 \\
\hline 0 & 4.645644 & 0.470624 & -0.130307 \\
\hline 6 & 5.550434 & 1.571315 & -0.285894 \\
\hline 6 & 5.216152 & 2.839843 & 0.279065 \\
\hline 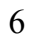 & 3.987260 & 2.996336 & 0.963415 \\
\hline 6 & 3.100100 & 1.958272 & 1.044190 \\
\hline 6 & 6.750528 & 1.474047 & -1.035968 \\
\hline 6 & 7.583172 & 2.555338 & -1.189099 \\
\hline 6 & 7.266717 & 3.796789 & -0.598304 \\
\hline 6 & 6.103979 & 3.931265 & 0.116811 \\
\hline 6 & 4.936960 & -0.881962 & -0.655544 \\
\hline 5 & 4.033865 & -1.476605 & -1.517992 \\
\hline 6 & 4.290519 & -2.722760 & -2.129963 \\
\hline 6 & 5.431474 & -3.412397 & -1.825024 \\
\hline 6 & 6.345357 & -2.902458 & -0.870350 \\
\hline 6 & 6.094254 & -1.633078 & -0.267186 \\
\hline 6 & 7.482870 & -3.649317 & -0.479159 \\
\hline 6 & 8.332134 & -3.184852 & 0.492381 \\
\hline 6 & 8.067208 & -1.948973 & 1.118440 \\
\hline 6 & 6.981643 & -1.193198 & 0.747934 \\
\hline p & 2.870164 & -0.837687 & -1.862433 \\
\hline 6 & -3.489970 & 0.169953 & -0.298024 \\
\hline 6 & -3.818527 & 0.231858 & -1.653826 \\
\hline 6 & -5.150525 & 0.206524 & -2.072914 \\
\hline 6 & -6.145185 & 0.099841 & -1.092887 \\
\hline 6 & -5.852063 & 0.013519 & 0.267832 \\
\hline 6 & -4.507956 & 0.058870 & 0.645754 \\
\hline 6 & -5.554751 & 0.322592 & -3.542297 \\
\hline 6 & -6.918456 & -0.159679 & 1.347420 \\
\hline 6 & 1.837151 & -3.201932 & 0.419658 \\
\hline
\end{tabular}




\begin{tabular}{|c|c|c|c|}
\hline & 2.378757 & -2.762589 & 1.754144 \\
\hline & 3.738931 & -2.876615 & 2.032043 \\
\hline & 4.254023 & -2.446364 & 3.252605 \\
\hline & 3.407516 & -1.905787 & 4.214130 \\
\hline & 2.041157 & -1.812307 & 3.952650 \\
\hline & 1.532113 & -2.236934 & 2.732252 \\
\hline & -0.838279 & 1.331857 & -3.474983 \\
\hline & 0.584827 & 1.346378 & -3.485347 \\
\hline & 1.347382 & 2.629016 & -3.500497 \\
\hline & 2.693688 & 2.636451 & -3.100873 \\
\hline & 3.424052 & 3.814508 & -3.057537 \\
\hline & 2.832540 & 5.021801 & -3.428792 \\
\hline & 1.508033 & 5.028155 & -3.849245 \\
\hline & 0.774965 & 3.844281 & -3.890265 \\
\hline & 1.251144 & 0.206761 & -4.223818 \\
\hline & -0.324222 & 1.144125 & 5.528317 \\
\hline ) & 0.262783 & 3.553160 & 5.600358 \\
\hline & -4.847792 & 4.406533 & 2.121327 \\
\hline & -3.073184 & 5.529108 & 0.756901 \\
\hline & 6.781171 & -0.250726 & 1.251468 \\
\hline & -2.318020 & -0.277529 & 3.142386 \\
\hline & 7.659606 & -4.610568 & -0.960255 \\
\hline & -2.325265 & -4.692770 & 4.833015 \\
\hline & 5.831444 & 4.886972 & 0.562769 \\
\hline 1 & -3.910530 & -3.053650 & -0.061765 \\
\hline & -5.704946 & -3.688493 & -1.614684 \\
\hline & 3.741553 & 3.965932 & 1.394692 \\
\hline & -2.499025 & -2.261954 & 4.548447 \\
\hline & 5.641717 & -4.371677 & -2.295199 \\
\hline 1 & 8.492329 & 2.454486 & -1.777791 \\
\hline & 3.565504 & -3.108230 & -2.843900 \\
\hline ] & -2.868205 & -3.998020 & -4.833949 \\
\hline & -0.497674 & -3.523176 & -4.425308 \\
\hline & -1.325440 & -7.173929 & 1.467901 \\
\hline & 7.003674 & 0.528797 & -1.509253 \\
\hline & -1.176689 & -5.197759 & 0.024058 \\
\hline 1 & 1.308781 & -2.830578 & -2.875044 \\
\hline & 7.937283 & 4.643982 & -0.722368 \\
\hline 1 & 8.726216 & -1.592222 & 1.906951 \\
\hline ] & -5.193098 & -4.189934 & -4.001717 \\
\hline & -1.901376 & -6.940282 & 3.879256 \\
\hline 1 & 9.198865 & -3.770006 & 0.790767 \\
\hline & 2.136471 & 2.062020 & 1.535931 \\
\hline 1 & -4.251836 & 0.002064 & 1.702705 \\
\hline & -3.007943 & 0.288121 & -2.375711 \\
\hline 1 & -7.185559 & 0.080770 & -1.410635 \\
\hline l & -3.274001 & 2.635381 & 0.596025 \\
\hline 1 & -0.050174 & 0.633671 & 2.586445 \\
\hline 1 & -1.490255 & 4.390843 & 4.073849 \\
\hline ] & 1.133538 & -4.032570 & 0.574949 \\
\hline 1 & 2.285803 & 1.814679 & 5.188625 \\
\hline 1 & 1.747245 & 1.099006 & 3.668665 \\
\hline 1 & 2.120997 & 2.835888 & 3.748367 \\
\hline 1 & 0.285691 & 0.978331 & 6.427400 \\
\hline 1 & -1.355439 & 1.344768 & 5.849448 \\
\hline 1 & -0.331485 & 0.211359 & 4.948415 \\
\hline
\end{tabular}




$\begin{array}{rrrr}1 & 0.625713 & 4.437413 & 5.060601 \\ 1 & -0.724126 & 3.786919 & 6.019382 \\ 1 & 0.941435 & 3.379785 & 6.445143 \\ 1 & -3.902350 & 6.765665 & 3.011088 \\ 1 & -3.449371 & 5.530751 & 4.194638 \\ 1 & -2.199005 & 6.337092 & 3.218304 \\ 1 & -5.056431 & 3.673142 & 1.331819 \\ 1 & -5.102351 & 3.943340 & 3.083970 \\ 1 & -5.522483 & 5.259611 & 1.967559 \\ 1 & -2.033625 & 5.880064 & 0.724700 \\ 1 & -3.213599 & 4.833725 & -0.079854 \\ 1 & -3.730067 & 6.393449 & 0.588285 \\ 1 & 2.656958 & -3.596741 & -0.201467 \\ 1 & 4.411212 & -3.281694 & 1.274284 \\ 1 & 0.471130 & -2.127951 & 2.515056 \\ 1 & 5.322575 & -2.526267 & 3.443410 \\ 1 & 1.368445 & -1.400101 & 4.705115 \\ 1 & 3.807147 & -1.560465 & 5.165528 \\ 1 & -1.317116 & 1.729936 & -1.960785 \\ 1 & -1.330414 & 0.443557 & -3.874005 \\ 1 & -1.394218 & 2.251162 & -3.669333 \\ 1 & -0.257837 & 3.873364 & -4.233402 \\ 1 & 1.036783 & 5.960754 & -4.154037 \\ 1 & 3.402928 & 5.947362 & -3.393256 \\ 1 & 4.459729 & 3.791529 & -2.719943 \\ 1 & 3.153386 & 1.704775 & -2.768869 \\ 1 & 1.298395 & 0.458971 & -5.294699 \\ 1 & 2.271139 & 0.010073 & -3.888685 \\ 1 & 0.674370 & -0.719775 & -4.119397 \\ 6 & -8.332916 & -0.174153 & 0.774093 \\ 6 & -6.680511 & -1.494598 & 2.064329 \\ 6 & -6.825574 & 0.989739 & 2.356890 \\ 6 & -4.352850 & 0.228382 & -4.478555 \\ 6 & -6.529972 & -0.796278 & -3.921609 \\ 6 & -6.233039 & 1.680171 & -3.759954 \\ 1 & -6.812107 & -0.706404 & -4.979137 \\ 1 & -6.067002 & -1.781116 & -3.776669 \\ 1 & -7.455352 & -0.767147 & -3.334655 \\ 1 & -6.526468 & 1.798667 & -4.812010 \\ 1 & -7.136264 & 1.787702 & -3.146422 \\ 1 & -5.554487 & 2.504036 & -3.502459 \\ 1 & -4.692481 & 0.267028 & -5.521332 \\ 1 & -3.652706 & 1.060982 & -4.333727 \\ 1 & -3.805199 & -0.714209 & -4.338590 \\ 1 & -7.568923 & 0.856966 & 3.154580 \\ 1 & -5.837900 & 1.049547 & 2.830981 \\ 1 & -7.022131 & 1.954641 & 1.870876 \\ 1 & -7.429259 & -1.645066 & 2.853879 \\ 1 & -6.758751 & -2.333833 & 1.359957 \\ 1 & -5.688110 & -1.545052 & 2.530758 \\ 1 & -9.059117 & -0.297643 & 1.587455 \\ 1 & -8.575362 & 0.762959 & 0.256360 \\ 1 & -8.483266 & -1.004458 & 0.071992\end{array}$

TS1 $_{I B 1-} \psi-1 \mathrm{~s} 2 \mathrm{~s} 3 \mathrm{~s} 4 \mathrm{a}$ 
Number of imaginary frequencies : 1

The smallest frequencies are : -686.0270 $9.0816 \quad 23.6817 \mathrm{~cm}(-1)$

Electronic energy :

$\mathrm{HF}=-4360.8636538$

Zero-point correction $=$

1.452461 (Hartree/Particle)

Thermal correction to Energy=

1.537346

Thermal correction to Enthalpy=

1.538291

Thermal correction to Gibbs Free Energy=

Sum of electronic and zero-point Energies=

1.334031

Sum of electronic and thermal Energies $=$

$-4359.411193$

Sum of electronic and thermal Enthalpies=

$-4359.326307$

Sum of electronic and thermal Free Energies=

$-4359.325363$

$-4359.529623$

Cartesian Coordinates

$\begin{array}{cccc}6 & -2.520835 & 2.631571 & 1.395568 \\ 6 & -1.679480 & 1.530110 & 1.505974 \\ 6 & -0.721284 & 1.498772 & 2.523405 \\ 6 & -0.656479 & 2.510681 & 3.482916 \\ 6 & -1.514532 & 3.608703 & 3.337605 \\ 6 & -2.437525 & 3.699143 & 2.293901 \\ 15 & -1.720756 & 0.191927 & 0.244170 \\ 45 & -0.052018 & 0.887401 & -1.383244 \\ 6 & 0.516159 & 2.621308 & -0.549185 \\ 8 & 0.534152 & 3.745184 & -0.277961 \\ 6 & 0.239883 & 2.327825 & 4.709822 \\ 6 & 1.677813 & 1.991319 & 4.308641 \\ 6 & -3.368645 & 4.894612 & 2.104198 \\ 6 & -3.172962 & 5.964529 & 3.175014 \\ 6 & -1.801864 & -1.326318 & 1.331546 \\ 6 & -1.605058 & -2.586713 & 0.789796 \\ 6 & -1.727719 & -3.757070 & 1.610203 \\ 6 & -2.070250 & -3.619903 & 2.986160 \\ 6 & -2.293275 & -2.323537 & 3.503013 \\ 6 & -2.163445 & -1.219638 & 2.704134 \\ 6 & -1.507856 & -5.066177 & 1.105540 \\ 6 & -1.616951 & -6.166550 & 1.919615 \\ 6 & -1.957861 & -6.022242 & 3.280816 \\ 6 & -2.179868 & -4.772466 & 3.800016 \\ 6 & -1.301097 & -2.801725 & -0.649733 \\ 6 & -2.346354 & -3.204099 & -1.536196 \\ 6 & -2.031981 & -3.541746 & -2.886067 \\ 6 & -0.689125 & -3.453994 & -3.321697 \\ 6 & 0.293237 & -3.035197 & -2.463692 \\ 6 & -0.006475 & -2.695546 & -1.123095 \\ 6 & -3.703034 & -3.267511 & -1.123123 \\ 6 & -4.685609 & -3.671718 & -1.991728 \\ 6 & -4.364282 & -4.046144 & -3.315025 \\ 6 & -3.066717 & -3.972030 & -3.752957 \\ 7 & 1.039446 & -2.199264 & -0.273803 \\ 15 & 1.566581 & -0.658911 & -0.764688 \\ 8 & 2.472270 & -0.313249 & 0.586805 \\ 6 & 3.399371 & 0.696128 & 0.486197 \\ 6 & 4.616738 & 0.459848 & -0.127279 \\ 6 & 5.505519 & 1.568889 & -0.311587 \\ 6 & 5.160725 & 2.842612 & 0.234556 \\ 6 & 3.940796 & 2.993211 & 0.936113\end{array}$




\begin{tabular}{|c|c|c|c|}
\hline 6 & 3.067794 & 1.945625 & 1.044631 \\
\hline 6 & 6.698001 & 1.473479 & -1.074067 \\
\hline & 7.513351 & 2.563251 & -1.258304 \\
\hline & 7.185418 & 3.811689 & -0.688598 \\
\hline & 6.029898 & 3.943399 & 0.038835 \\
\hline & 4.922938 & -0.898541 & -0.628268 \\
\hline & 4.024106 & -1.522928 & -1.473636 \\
\hline & 4.293304 & -2.779952 & -2.057517 \\
\hline & 5.443898 & -3.448496 & -1.741875 \\
\hline & 6.356379 & -2.905186 & -0.804492 \\
\hline & 6.091647 & -1.625806 & -0.228807 \\
\hline 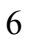 & 7.507095 & -3.626944 & -0.404631 \\
\hline & 8.356890 & -3.128192 & 0.549156 \\
\hline & 8.078748 & -1.882336 & 1.148977 \\
\hline & 6.979653 & -1.150783 & 0.769973 \\
\hline & 2.853780 & -0.903450 & -1.830671 \\
\hline ) & -3.490352 & 0.211120 & -0.299152 \\
\hline & -3.801603 & 0.324264 & -1.645652 \\
\hline & -5.132946 & 0.328440 & -2.090417 \\
\hline & -6.133629 & 0.174779 & -1.137374 \\
\hline 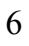 & -5.856703 & 0.045376 & 0.234254 \\
\hline y & -4.526824 & 0.077493 & 0.635582 \\
\hline ) & -5.420595 & 0.523316 & -3.577555 \\
\hline 5 & -7.001187 & -0.167957 & 1.223339 \\
\hline ) & 1.802400 & -3.223938 & 0.478354 \\
\hline & 2.344565 & -2.762199 & 1.804582 \\
\hline & 3.709227 & -2.838889 & 2.072945 \\
\hline ) & 4.224234 & -2.366420 & 3.277573 \\
\hline 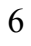 & 3.372588 & -1.824965 & 4.234231 \\
\hline$b$ & 2.001646 & -1.774944 & 3.985359 \\
\hline ) & 1.492661 & -2.239115 & 2.779361 \\
\hline 6 & -0.801479 & 1.340029 & -3.487915 \\
\hline b & 0.619362 & 1.287276 & -3.490830 \\
\hline & 1.440228 & 2.533678 & -3.490541 \\
\hline b & 2.787918 & 2.475689 & -3.098585 \\
\hline & 3.563452 & 3.622702 & -3.017073 \\
\hline 6 & 3.019626 & 4.863214 & -3.349255 \\
\hline f & 1.696796 & 4.933775 & -3.769907 \\
\hline 6 & 0.917185 & 3.782310 & -3.843501 \\
\hline 6 & 1.234798 & 0.111434 & -4.216390 \\
\hline & -0.335281 & 1.167307 & 5.533777 \\
\hline 6 & 0.279166 & 3.569536 & 5.597793 \\
\hline & -4.825565 & 4.419663 & 2.164389 \\
\hline 6 & -3.094137 & 5.531868 & 0.736668 \\
\hline & 6.769517 & -0.200044 & 1.253651 \\
\hline 1 & -2.343183 & -0.239040 & 3.137023 \\
\hline 1 & 7.693917 & -4.596352 & -0.865093 \\
\hline 1 & -2.441322 & -4.642541 & 4.849273 \\
\hline 1 & 5.748817 & 4.903805 & 0.469083 \\
\hline ] & -3.962115 & -2.978444 & -0.105572 \\
\hline 1 & -5.720928 & -3.700390 & -1.658214 \\
\hline ( & 3.689028 & 3.966068 & 1.356354 \\
\hline 1 & -2.571096 & -2.212775 & 4.550849 \\
\hline 1 & 5.663683 & -4.415678 & -2.191043 \\
\hline 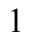 & 8.417026 & 2.463702 & -1.855583 \\
\hline & 3.570642 & -3.190305 & -2.759903 \\
\hline
\end{tabular}




\begin{tabular}{|c|c|c|c|}
\hline & -2.807350 & -4.241342 & -4.776016 \\
\hline & -0.450395 & -3.718849 & -4.350967 \\
\hline & -1.441124 & -7.158964 & 1.510843 \\
\hline & 6.959053 & 0.522648 & -1.531715 \\
\hline & -1.247650 & -5.195988 & 0.057125 \\
\hline & 1.323857 & -2.942279 & -2.801874 \\
\hline & 7.841568 & 4.666039 & -0.838129 \\
\hline & 8.738457 & -1.498301 & 1.923970 \\
\hline & -5.151073 & -4.378915 & -3.988785 \\
\hline & -2.041993 & -6.902414 & 3.913986 \\
\hline & 9.234195 & -3.693931 & 0.854005 \\
\hline & 2.109870 & 2.044398 & 1.548905 \\
\hline & -4.278534 & -0.002958 & 1.690401 \\
\hline & -2.986102 & 0.406084 & -2.361475 \\
\hline & -7.172986 & 0.155610 & -1.458441 \\
\hline & -3.249776 & 2.661823 & 0.586196 \\
\hline & -0.048185 & 0.640462 & 2.592462 \\
\hline & -1.465284 & 4.412827 & 4.065591 \\
\hline & 1.093560 & -4.047781 & 0.645400 \\
\hline & 2.283182 & 1.801603 & 5.206103 \\
\hline & 1.746861 & 1.093243 & 3.681981 \\
\hline & 2.141692 & 2.825133 & 3.765026 \\
\hline & 0.268408 & 0.997589 & 6.436370 \\
\hline & -1.365277 & 1.382555 & 5.849285 \\
\hline [ & -0.352323 & 0.232380 & 4.957725 \\
\hline & 0.652558 & 4.447725 & 5.055276 \\
\hline 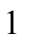 & -0.706666 & 3.814875 & 6.012665 \\
\hline 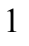 & 0.953286 & 3.393266 & 6.445621 \\
\hline 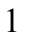 & -3.860562 & 6.799761 & 2.990931 \\
\hline ] & -3.383625 & 5.582130 & 4.182257 \\
\hline & -2.153304 & 6.370455 & 3.166627 \\
\hline 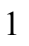 & -5.049918 & 3.672876 & 1.392099 \\
\hline & -5.051232 & 3.969589 & 3.140420 \\
\hline L & -5.509777 & 5.266116 & 2.016138 \\
\hline 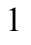 & -2.053005 & 5.872255 & 0.665109 \\
\hline l & -3.270440 & 4.830379 & -0.087963 \\
\hline l & -3.748821 & 6.400310 & 0.581474 \\
\hline & 2.620417 & -3.631411 & -0.137007 \\
\hline L & 4.384225 & -3.243965 & 1.317776 \\
\hline 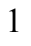 & 0.426977 & -2.163009 & 2.570508 \\
\hline L & 5.296018 & -2.415105 & 3.460647 \\
\hline 1 & 1.325673 & -1.364908 & 4.735977 \\
\hline 1 & 3.771781 & -1.446042 & 5.173037 \\
\hline 1 & -1.292462 & 1.731080 & -1.966332 \\
\hline 1 & -1.333269 & 0.478800 & -3.895143 \\
\hline 1 & -1.311314 & 2.286424 & -3.675807 \\
\hline . & -0.114574 & 3.865157 & -4.180834 \\
\hline 1 & 1.262508 & 5.892815 & -4.046236 \\
\hline 1 & 3.625496 & 5.764325 & -3.284061 \\
\hline 1 & 4.596905 & 3.549320 & -2.678540 \\
\hline 1 & 3.212283 & 1.517698 & -2.796587 \\
\hline 1 & 1.300894 & 0.351269 & -5.289057 \\
\hline 1 & 2.241636 & -0.132343 & -3.872831 \\
\hline & 0.613513 & -0.785529 & -4.106884 \\
\hline 0 & -6.523444 & -0.137321 & 2.673981 \\
\hline b & -8.066311 & 0.919867 & 1.053485 \\
\hline
\end{tabular}




$\begin{array}{rrrr}6 & -7.627655 & -1.541148 & 0.953473 \\ 6 & -6.915138 & 0.519967 & -3.888280 \\ 6 & -4.845257 & 1.872847 & -4.023691 \\ 6 & -4.766066 & -0.602379 & -4.382850 \\ 1 & -5.050922 & 2.042319 & -5.089208 \\ 1 & -5.292879 & 2.699040 & -3.456002 \\ 1 & -3.758018 & 1.921519 & -3.883833 \\ 1 & -4.925947 & -0.445011 & -5.458273 \\ 1 & -3.684071 & -0.663921 & -4.210447 \\ 1 & -5.195510 & -1.574927 & -4.111996 \\ 1 & -7.067154 & 0.661852 & -4.965748 \\ 1 & -7.389007 & -0.431312 & -3.613089 \\ 1 & -7.444785 & 1.331479 & -3.372629 \\ 1 & -8.454068 & -1.731816 & 1.651727 \\ 1 & -8.027044 & -1.615120 & -0.065799 \\ 1 & -6.884420 & -2.339762 & 1.082370 \\ 1 & -8.883109 & 0.763989 & 1.770711 \\ 1 & -7.645646 & 1.917556 & 1.234285 \\ 1 & -8.508982 & 0.919555 & 0.050538 \\ 1 & -7.379678 & -0.272773 & 3.346720 \\ 1 & -5.807622 & -0.941350 & 2.891122 \\ 1 & -6.050158 & 0.821071 & 2.926838\end{array}$

TS1 $_{I B 1-} \psi_{-1 \mathrm{~s} 2 \mathrm{~s} 3 \mathrm{~s} 4 \mathrm{~s}}$

Number of imaginary frequencies : 1

The smallest frequencies are : -699.2962 $10.6266 \quad 19.5656 \mathrm{~cm}(-1)$

Electronic energy : $\quad H F=-4360.8638283$

Zero-point correction $=\quad 1.452269($ Hartree/Particle $)$

Thermal correction to Energy $=\quad 1.537246$

Thermal correction to Enthalpy= $\quad 1.538191$

Thermal correction to Gibbs Free Energy $=\quad 1.333077$

Sum of electronic and zero-point Energies $=\quad-4359.411559$

Sum of electronic and thermal Energies $=\quad-4359.326582$

Sum of electronic and thermal Enthalpies $=\quad-4359.325638$

Sum of electronic and thermal Free Energies $=\quad-4359.530752$

Cartesian Coordinates

$\begin{array}{cccc}6 & 0.852261 & 3.897616 & -3.806213 \\ 6 & 1.396762 & 2.658061 & -3.453568 \\ 6 & 2.745452 & 2.622133 & -3.063372 \\ 6 & 3.503141 & 3.781378 & -2.989386 \\ 6 & 2.938767 & 5.012636 & -3.321355 \\ 6 & 1.613007 & 5.062022 & -3.735255 \\ 6 & 0.599764 & 1.396342 & -3.462926 \\ 6 & 1.232293 & 0.245905 & -4.214449 \\ 45 & -0.052538 & 0.943914 & -1.363407 \\ 6 & -0.822380 & 1.424317 & -3.452338 \\ 15 & -1.724125 & 0.187631 & 0.239382 \\ 6 & -3.488617 & 0.167709 & -0.320194 \\ 6 & -3.802731 & 0.325209 & -1.665358 \\ 6 & -5.130234 & 0.315478 & -2.109693 \\ 6 & -6.135423 & 0.105360 & -1.164444 \\ 6 & -5.854047 & -0.079583 & 0.194559 \\ 6 & -4.521297 & -0.030906 & 0.598219\end{array}$




\begin{tabular}{|c|c|c|c|}
\hline 6 & -5.421121 & 0.542246 & -3.592195 \\
\hline 6 & -4.753005 & -0.559595 & -4.419789 \\
\hline y & -6.931593 & -0.358165 & 1.240880 \\
\hline 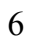 & -6.663091 & -1.730442 & 1.871819 \\
\hline$b$ & -1.733571 & 1.515506 & 1.512206 \\
\hline p & -2.614523 & 2.586983 & 1.418390 \\
\hline 5 & -2.561409 & 3.648776 & 2.326387 \\
\hline 6 & -1.629497 & 3.579751 & 3.363758 \\
\hline 6 & -0.734449 & 2.509492 & 3.493994 \\
\hline 6 & -0.770375 & 1.505982 & 2.525068 \\
\hline 6 & 0.175598 & 2.345506 & 4.713107 \\
\hline 6 & 0.190755 & 3.585533 & 5.604215 \\
\hline 6 & -3.533712 & 4.813795 & 2.153286 \\
\hline 6 & -3.286357 & 5.474927 & 0.792183 \\
\hline 6 & -1.778346 & -1.340141 & 1.315396 \\
\hline 6 & -1.565564 & -2.591558 & 0.759295 \\
\hline 6 & -1.666697 & -3.772366 & 1.566499 \\
\hline 6 & -2.001257 & -3.655534 & 2.946291 \\
\hline 6 & -2.239102 & -2.368315 & 3.479033 \\
\hline 6 & -2.132271 & -1.253552 & 2.691314 \\
\hline 6 & -2.088380 & -4.818752 & 3.747571 \\
\hline 6 & -1.853225 & -6.059188 & 3.212059 \\
\hline 6 & -1.521711 & -6.183256 & 1.846401 \\
\hline 6 & -1.433960 & -5.072246 & 1.044431 \\
\hline 6 & -1.271299 & -2.781020 & -0.685464 \\
\hline 6 & -2.321448 & -3.180281 & -1.567230 \\
\hline 6 & -2.019928 & -3.480919 & -2.928651 \\
\hline 6 & -0.684500 & -3.360711 & -3.379075 \\
\hline 6 & 0.302782 & -2.948401 & -2.523484 \\
\hline 6 & 0.016024 & -2.645276 & -1.171193 \\
\hline 6 & -3.059478 & -3.906723 & -3.791845 \\
\hline 6 & -4.349912 & -4.010230 & -3.338853 \\
\hline 6 & -4.658968 & -3.671815 & -2.002982 \\
\hline 6 & -3.670985 & -3.274519 & -1.137458 \\
\hline 7 & 1.066136 & -2.155965 & -0.323238 \\
\hline 6 & 1.833353 & -3.185647 & 0.417281 \\
\hline 6 & 2.368937 & -2.748384 & 1.754947 \\
\hline 6 & 3.726423 & -2.871623 & 2.041932 \\
\hline 6 & 4.236144 & -2.445231 & 3.266129 \\
\hline 6 & 3.387169 & -1.898096 & 4.221701 \\
\hline 6 & 2.023465 & -1.793870 & 3.950478 \\
\hline 6 & 1.519639 & -2.215910 & 2.727052 \\
\hline 6 & 0.507062 & 2.650204 & -0.477705 \\
\hline 8 & 0.539281 & 3.761426 & -0.159612 \\
\hline 15 & 1.581209 & -0.602946 & -0.786843 \\
\hline 8 & 2.869034 & -0.814315 & -1.859705 \\
\hline 6 & 4.027987 & -1.464453 & -1.518825 \\
\hline 6 & 4.933001 & -0.884084 & -0.648701 \\
\hline 6 & 6.082603 & -1.647746 & -0.262008 \\
\hline 6 & 6.325999 & -2.914069 & -0.874468 \\
\hline 6 & 5.412435 & -3.408047 & -1.837758 \\
\hline 6 & 4.278067 & -2.706881 & -2.141153 \\
\hline 6 & 6.968543 & -1.223664 & 0.761020 \\
\hline 6 & 8.045288 & -1.992039 & 1.131325 \\
\hline 6 & 8.302772 & -3.225075 & 0.496528 \\
\hline 6 & 7.454944 & -3.673952 & -0.483613 \\
\hline
\end{tabular}




\begin{tabular}{|c|c|c|c|}
\hline 6 & 4.651758 & 0.466737 & -0.113784 \\
\hline 6 & 3.431890 & 0.714133 & 0.490312 \\
\hline & 3.116918 & 1.958734 & 1.068425 \\
\hline & 4.014100 & 2.988952 & 1.000290 \\
\hline & 5.243713 & 2.826673 & 0.318520 \\
\hline & 5.567553 & 1.560054 & -0.256659 \\
\hline & 6.143441 & 3.910326 & 0.170509 \\
\hline & 7.308467 & 3.770319 & -0.539814 \\
\hline & 7.615172 & 2.531074 & -1.140373 \\
\hline & 6.770357 & 1.457258 & -1.001743 \\
\hline & 2.487972 & -0.280932 & 0.569016 \\
\hline & 1.617965 & 2.045934 & 4.298913 \\
\hline & -0.362964 & 1.167933 & 5.537508 \\
\hline & -3.370509 & 5.878134 & 3.235037 \\
\hline & -4.973652 & 4.289576 & 2.212775 \\
\hline & -6.915532 & 0.527462 & -3.904083 \\
\hline y & -4.862344 & 1.907047 & -4.011620 \\
\hline & -8.336271 & -0.377392 & 0.643955 \\
\hline & -6.889792 & 0.723398 & 2.326026 \\
\hline & 6.773736 & -0.283489 & 1.270996 \\
\hline & -2.323968 & -0.280341 & 3.135521 \\
\hline & 7.625996 & -4.632723 & -0.971652 \\
\hline & -2.343499 & -4.704586 & 4.800211 \\
\hline & 5.878558 & 4.864655 & 0.623977 \\
\hline & -3.920117 & -3.012726 & -0.109704 \\
\hline & -5.689263 & -3.724606 & -1.656767 \\
\hline & 3.776426 & 3.956861 & 1.439755 \\
\hline & -2.510686 & -2.273353 & 4.530056 \\
\hline & 5.617174 & -4.364691 & -2.315654 \\
\hline 1 & 8.526427 & 2.426050 & -1.725081 \\
\hline & 3.553562 & -3.081052 & -2.861489 \\
\hline 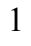 & -2.810113 & -4.147724 & -4.824423 \\
\hline & -0.455122 & -3.596674 & -4.417481 \\
\hline & -1.336238 & -7.168507 & 1.424776 \\
\hline & 7.016118 & 0.513881 & -1.482556 \\
\hline & -1.181418 & -5.185835 & -0.007757 \\
\hline ] & 1.327245 & -2.832606 & -2.872882 \\
\hline & 7.988436 & 4.611581 & -0.652571 \\
\hline 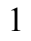 & 8.703016 & -1.647580 & 1.926336 \\
\hline 1 & -5.140734 & -4.338853 & -4.009932 \\
\hline & -1.920029 & -6.947703 & 3.835576 \\
\hline 1 & 9.162613 & -3.820337 & 0.794844 \\
\hline & 2.152420 & 2.067411 & 1.556633 \\
\hline 1 & -4.280195 & -0.159124 & 1.652516 \\
\hline & -2.988727 & 0.450775 & -2.376280 \\
\hline 1 & -7.170470 & 0.081782 & -1.492564 \\
\hline 1 & -3.349740 & 2.599794 & 0.614305 \\
\hline 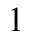 & -0.067162 & 0.671257 & 2.580514 \\
\hline 1 & -1.601874 & 4.378808 & 4.098456 \\
\hline & 1.129877 & -4.017179 & 0.568372 \\
\hline 1 & 2.238459 & 1.882763 & 5.190965 \\
\hline t & 1.705424 & 1.143413 & 3.680750 \\
\hline 1 & 2.051014 & 2.887410 & 3.741771 \\
\hline ] & 0.252602 & 1.009935 & 6.434143 \\
\hline & -1.395302 & 1.355842 & 5.862664 \\
\hline & -0.361153 & 0.235134 & 4.957461 \\
\hline
\end{tabular}




$\begin{array}{rrrr}1 & 0.535851 & 4.474466 & 5.060443 \\ 1 & -0.797282 & 3.803976 & 6.028737 \\ 1 & 0.876807 & 3.424964 & 6.445531 \\ 1 & -4.087399 & 6.690995 & 3.062889 \\ 1 & -3.563362 & 5.477726 & 4.238862 \\ 1 & -2.365662 & 6.319474 & 3.226996 \\ 1 & -5.175042 & 3.541739 & 1.434777 \\ 1 & -5.181454 & 3.824527 & 3.185707 \\ 1 & -5.685680 & 5.114327 & 2.073159 \\ 1 & -2.260031 & 5.857808 & 0.724390 \\ 1 & -3.434968 & 4.775255 & -0.039397 \\ 1 & -3.975250 & 6.318151 & 0.646108 \\ 1 & 2.655916 & -3.578923 & -0.201142 \\ 1 & 4.401183 & -3.281022 & 1.288631 \\ 1 & 0.461178 & -2.098432 & 2.502254 \\ 1 & 5.302692 & -2.533158 & 3.464403 \\ 1 & 1.348637 & -1.375974 & 4.697926 \\ 1 & 3.782911 & -1.556057 & 5.175872 \\ 1 & -1.303077 & 1.787063 & -1.928224 \\ 1 & -1.340785 & 0.561139 & -3.872511 \\ 1 & -1.348535 & 2.364870 & -3.624855 \\ 1 & -0.180953 & 3.962527 & -4.142893 \\ 1 & 1.162375 & 6.013671 & -4.010898 \\ 1 & 3.531082 & 5.923040 & -3.261524 \\ 1 & 4.540018 & 3.724856 & -2.659708 \\ 1 & 3.185570 & 1.671173 & -2.761000 \\ 1 & -5.286813 & 0.507179 & -5.282784 \\ 1 & -7.109260 & 1.712408 & 1.902088 \\ 1 & -9.069623 & -0.581763 & 1.434282 \\ 1 & -8.602853 & 0.585620 & 0.189646 \\ 1 & 0.628785 & -0.664674 & -4.118336 \\ 1 & -5.072152 & 2.095886 & -5.073081 \\ 1 & -5.16372 & -1.160060 & -0.117593\end{array}$

$\operatorname{TS1}_{I B 2-} \psi_{-1 \mathbf{a} 2 \mathrm{a} 3 \mathrm{a} 4 \mathrm{a}}$

Number of imaginary frequencies : 1

The smallest frequencies are : $-479.6604 \quad 14.496720 .8506 \mathrm{~cm}(-1)$ Electronic energy : $\quad \mathrm{HF}=-4360.8647568$

Zero-point correction $=\quad 1.453286($ Hartree/Particle) Thermal correction to Energy $=\quad 1.537742$ 
Thermal correction to Enthalpy=

Thermal correction to Gibbs Free Energy=

Sum of electronic and zero-point Energies=

Sum of electronic and thermal Energies=

Sum of electronic and thermal Enthalpies=

Sum of electronic and thermal Free Energies=

\author{
1.538686 \\ 1.336801 \\ $-4359.411471$ \\ $-4359.327015$ \\ $-4359.326071$ \\ $-4359.527956$
}

Cartesian Coordinates

\begin{tabular}{|c|c|c|c|}
\hline 6 & -4.483159 & 0.434125 & 0.588191 \\
\hline 6 & -3.457117 & -0.024795 & -0.243467 \\
\hline 6 & -3.735392 & -0.966141 & -1.229402 \\
\hline 6 & -5.033287 & -1.462639 & -1.405552 \\
\hline 6 & -6.033426 & -0.969507 & -0.567623 \\
\hline 6 & -5.788999 & -0.023687 & 0.436491 \\
\hline 15 & -1.727123 & 0.563335 & 0.010671 \\
\hline 45 & -0.172743 & 0.015903 & -1.694367 \\
\hline 6 & 0.510408 & 2.022043 & -2.091781 \\
\hline 8 & 0.117162 & 2.830107 & -2.828435 \\
\hline 6 & -5.376330 & -2.459579 & -2.513050 \\
\hline 6 & -6.025778 & -1.690094 & -3.669295 \\
\hline 6 & -6.925724 & 0.437008 & 1.347238 \\
\hline 6 & -8.096482 & 0.962323 & 0.510187 \\
\hline 6 & -1.879745 & 2.392691 & -0.025291 \\
\hline 6 & -2.897560 & 3.024711 & -0.734119 \\
\hline 6 & -2.915625 & 4.415244 & -0.872861 \\
\hline 6 & -1.895941 & 5.147111 & -0.260435 \\
\hline 6 & -0.866203 & 4.545091 & 0.470237 \\
\hline 6 & -0.857756 & 3.153135 & 0.549462 \\
\hline 6 & 0.222085 & 5.400317 & 1.117887 \\
\hline 6 & -0.404666 & 6.557339 & 1.903214 \\
\hline 6 & -4.007822 & 5.142400 & -1.655034 \\
\hline 6 & -4.738986 & 6.120546 & -0.728869 \\
\hline 6 & -1.589251 & 0.225808 & 1.840558 \\
\hline 6 & -1.281183 & -1.046556 & 2.297742 \\
\hline 6 & -1.236127 & -1.317695 & 3.706015 \\
\hline 6 & -1.522733 & -0.275102 & 4.633684 \\
\hline 6 & -1.858431 & 1.003702 & 4.135347 \\
\hline 6 & -1.888636 & 1.244215 & 2.788219 \\
\hline 6 & -1.462585 & -0.535959 & 6.023368 \\
\hline 6 & -1.129025 & -1.781355 & 6.490266 \\
\hline 6 & -0.842501 & -2.818920 & 5.578540 \\
\hline 6 & -0.897041 & -2.595697 & 4.224833 \\
\hline 6 & -1.017587 & -2.198352 & 1.393025 \\
\hline 6 & 0.251015 & -2.430734 & 0.891783 \\
\hline 6 & 0.543409 & -3.629648 & 0.202712 \\
\hline 6 & -0.425469 & -4.574058 & -0.002816 \\
\hline 6 & -1.750117 & -4.349403 & 0.435307 \\
\hline 6 & -2.053574 & -3.151950 & 1.148279 \\
\hline 6 & -3.387524 & -2.948383 & 1.586739 \\
\hline 6 & -4.365921 & -3.873764 & 1.322234 \\
\hline 6 & -4.060196 & -5.062330 & 0.622600 \\
\hline 6 & -2.779093 & -5.292190 & 0.189298 \\
\hline 7 & 1.293610 & -1.458756 & 1.066193 \\
\hline 15 & 1.628769 & -0.601607 & -0.358160 \\
\hline 8 & 2.541170 & 0.583044 & 0.354806 \\
\hline
\end{tabular}




\begin{tabular}{|c|c|c|c|}
\hline 6 & 3.493795 & 1.272671 & -0.352187 \\
\hline 6 & 4.695191 & 0.673821 & -0.684452 \\
\hline & 5.627299 & 1.426160 & -1.475615 \\
\hline & 5.345902 & 2.791267 & -1.784821 \\
\hline & 4.136171 & 3.372166 & -1.333972 \\
\hline & 3.219303 & 2.623101 & -0.650882 \\
\hline & 6.815537 & 0.860083 & -2.004312 \\
\hline & 7.685923 & 1.607333 & -2.759414 \\
\hline & 7.421103 & 2.965436 & -3.029907 \\
\hline & 6.269933 & 3.538852 & -2.553921 \\
\hline & 4.965576 & -0.724764 & -0.279481 \\
\hline & 4.054895 & -1.716018 & -0.595571 \\
\hline & 4.333434 & -3.085772 & -0.395818 \\
\hline f & 5.487744 & -3.460723 & 0.234998 \\
\hline & 6.390530 & -2.481939 & 0.719193 \\
\hline & 6.132540 & -1.101496 & 0.462719 \\
\hline$b$ & 7.527372 & -2.849684 & 1.479369 \\
\hline & 8.367151 & -1.897915 & 1.998768 \\
\hline 6 & 8.097295 & -0.531314 & 1.775896 \\
\hline & 7.015346 & -0.143816 & 1.024146 \\
\hline & 2.852129 & -1.404222 & -1.175926 \\
\hline ) & 2.220333 & -1.702823 & 2.195304 \\
\hline 6 & 2.715916 & -0.471007 & 2.906773 \\
\hline 5 & 4.072560 & -0.330984 & 3.191152 \\
\hline$b$ & 4.545207 & 0.791428 & 3.867429 \\
\hline 6 & 3.660912 & 1.784102 & 4.274321 \\
\hline ) & 2.300785 & 1.645739 & 4.000447 \\
\hline ) & 1.834234 & 0.529503 & 3.319821 \\
\hline ) & 1.076843 & 4.592501 & 2.094720 \\
\hline 6 & 1.123662 & 5.967144 & 0.015526 \\
\hline ) & -3.370109 & 5.913884 & -2.815211 \\
\hline 6 & -5.041679 & 4.179227 & -2.234366 \\
\hline 6 & -0.630326 & -0.372601 & -3.847072 \\
\hline 6 & 0.630331 & -0.955103 & -3.583957 \\
\hline 6 & 0.733869 & -2.436477 & -3.451307 \\
\hline ? & 1.967739 & -3.088667 & -3.592770 \\
\hline 6 & 2.062077 & -4.477504 & -3.565062 \\
\hline 5 & 0.924266 & -5.261752 & -3.408057 \\
\hline 6 & -0.308597 & -4.630660 & -3.253136 \\
\hline 6 & -0.396658 & -3.246629 & -3.258284 \\
\hline 6 & 1.857806 & -0.232617 & -4.095556 \\
\hline 6 & -4.132365 & -3.173628 & -3.041139 \\
\hline 5 & -6.350930 & -3.529797 & -2.013867 \\
\hline 6 & -6.486460 & 1.551823 & 2.294936 \\
\hline 6 & -7.396553 & -0.753239 & 2.191156 \\
\hline 1 & 6.808116 & 0.913450 & 0.877855 \\
\hline 1 & -2.152911 & 2.238839 & 2.440109 \\
\hline 1 & 7.709884 & -3.909070 & 1.655567 \\
\hline 1 & -1.684108 & 0.277483 & 6.712906 \\
\hline 1 & 6.036362 & 4.580863 & -2.769039 \\
\hline 1 & -3.635079 & -2.031216 & 2.118704 \\
\hline 1 & -5.387391 & -3.689543 & 1.650926 \\
\hline 1 & 3.937095 & 4.418567 & -1.561500 \\
\hline 1 & -2.094367 & 1.799293 & 4.841162 \\
\hline 1 & 5.715335 & -4.513097 & 0.396978 \\
\hline 1 & 8.585319 & 1.143292 & -3.158248 \\
\hline
\end{tabular}




\begin{tabular}{|c|c|c|c|}
\hline 1 & 3.614536 & -3.814308 & -0.766189 \\
\hline & -2.527913 & -6.203672 & -0.352471 \\
\hline & -0.192443 & -5.497226 & -0.530470 \\
\hline ] & -0.577205 & -3.805510 & 5.951574 \\
\hline & 7.031779 & -0.188659 & -1.818830 \\
\hline 1 & -0.675871 & -3.409222 & 3.536817 \\
\hline & 1.556542 & -3.783043 & -0.164398 \\
\hline & 8.120858 & 3.548004 & -3.624653 \\
\hline & 8.749722 & 0.224206 & 2.208171 \\
\hline & -4.843515 & -5.792559 & 0.429355 \\
\hline 1 & -1.082665 & -1.972532 & 7.559847 \\
\hline 1 & 9.231128 & -2.191192 & 2.590518 \\
\hline 1 & 2.265214 & 3.030611 & -0.327012 \\
\hline 1 & -4.249655 & 1.163453 & 1.359912 \\
\hline 1 & -2.907610 & -1.318681 & -1.843260 \\
\hline 1 & -7.050292 & -1.339296 & -0.694090 \\
\hline 1 & -3.676176 & 2.418937 & -1.191280 \\
\hline 1 & -0.055242 & 2.638236 & 1.078838 \\
\hline 1 & -1.902912 & 6.232438 & -0.357622 \\
\hline 1 & 1.649337 & -2.316090 & 2.907535 \\
\hline 1 & 1.948324 & 6.548959 & 0.449834 \\
\hline 1 & 1.555472 & 5.161349 & -0.593684 \\
\hline 1 & 0.565327 & 6.625027 & -0.662253 \\
\hline 1 & 0.381688 & 7.157242 & 2.380062 \\
\hline 1 & -0.986246 & 7.233820 & 1.266261 \\
\hline 1 & -1.070011 & 6.184509 & 2.692873 \\
\hline 1 & 0.457253 & 4.114913 & 2.867394 \\
\hline 1 & 1.663546 & 3.806766 & 1.602051 \\
\hline 1 & 1.791511 & 5.254853 & 2.599701 \\
\hline 1 & -5.814015 & 4.745870 & -2.769659 \\
\hline 1 & -4.594012 & 3.476660 & -2.948800 \\
\hline 1 & -5.543910 & 3.595499 & -1.450552 \\
\hline 1 & -5.208911 & 5.590534 & 0.110131 \\
\hline 1 & -4.065658 & 6.878056 & -0.310148 \\
\hline 1 & -5.528968 & 6.648731 & -1.279744 \\
\hline 1 & -2.660883 & 6.673089 & -2.464196 \\
\hline 1 & -2.826076 & 5.234473 & -3.484118 \\
\hline 1 & -4.142599 & 6.427456 & -3.403673 \\
\hline 1 & 3.078375 & -2.315795 & 1.877855 \\
\hline 1 & 4.773796 & -1.100831 & 2.865169 \\
\hline 1 & 0.778779 & 0.443398 & 3.070282 \\
\hline 1 & 5.610805 & 0.888205 & 4.067169 \\
\hline 1 & 1.596089 & 2.414788 & 4.315085 \\
\hline 1 & 4.027384 & 2.663241 & 4.800278 \\
\hline 1 & -1.477409 & 0.444649 & -2.529459 \\
\hline 1 & -1.501194 & -1.001997 & -4.025450 \\
\hline 1 & -0.651416 & 0.560168 & -4.410398 \\
\hline 1 & -1.369119 & -2.787446 & -3.096565 \\
\hline 1 & -1.215765 & -5.219369 & -3.115163 \\
\hline 1 & 0.995721 & -6.347528 & -3.405086 \\
\hline 1 & 3.037513 & -4.946806 & -3.683564 \\
\hline 1 & 2.874611 & -2.504461 & -3.725713 \\
\hline 1 & 2.172522 & -0.647367 & -5.065760 \\
\hline 1 & 1.648024 & 0.831174 & -4.244977 \\
\hline 1 & 2.710709 & -0.308422 & -3.411711 \\
\hline 1 & -4.424567 & -3.941203 & -3.769140 \\
\hline
\end{tabular}




$\begin{array}{lrrr}1 & -3.454041 & -2.484515 & -3.560557 \\ 1 & -3.574859 & -3.667296 & -2.231389 \\ 1 & -6.552057 & -4.252878 & -2.815015 \\ 1 & -5.929221 & -4.077205 & -1.160945 \\ 1 & -7.318185 & -3.113994 & -1.708696 \\ 1 & -6.276208 & -2.371156 & -4.494324 \\ 1 & -6.949700 & -1.190856 & -3.350325 \\ 1 & -5.347068 & -0.919795 & -4.059024 \\ 1 & -7.335981 & 1.864827 & 2.914826 \\ 1 & -5.687630 & 1.226779 & 2.974918 \\ 1 & -6.131386 & 2.435638 & 1.747297 \\ 1 & -8.215703 & -0.451871 & 2.858134 \\ 1 & -7.761597 & -1.578356 & 1.566664 \\ 1 & -6.578370 & -1.138628 & 2.814682 \\ 1 & -8.907159 & 1.304742 & 1.167077 \\ 1 & -7.787341 & 1.810801 & -0.113770 \\ 1 & -8.515254 & 0.195615 & -0.152086\end{array}$

$\mathrm{TS1}_{\mathrm{IB2}-} \psi_{-1 \mathrm{a} 2 \mathrm{a} 3 \mathrm{a} 4 \mathrm{~s}}$

Number of imaginary frequencies : 1

The smallest frequencies are : -479.8162 $12.0361 \quad 15.1802 \mathrm{~cm}(-1)$

Electronic energy : $\quad H F=-4360.8653946$

Zero-point correction $=\quad 1.452102($ Hartree/Particle $)$

Thermal correction to Energy= $\quad 1.537150$

Thermal correction to Enthalpy= $\quad 1.538094$

Thermal correction to Gibbs Free Energy $=1.332995$

Sum of electronic and zero-point Energies $=\quad-4359.413292$

Sum of electronic and thermal Energies $=\quad-4359.328244$

Sum of electronic and thermal Enthalpies $=\quad-4359.327300$

Sum of electronic and thermal Free Energies $=\quad-4359.532400$

\section{Cartesian Coordinates}

$\begin{array}{cccc}6 & -3.067455 & 2.929184 & -0.676062 \\ 6 & -1.989196 & 2.355786 & -0.006024 \\ 6 & -0.992500 & 3.174483 & 0.529302 \\ 6 & -1.083212 & 4.564202 & 0.452823 \\ 6 & -2.172630 & 5.106111 & -0.236700 \\ 6 & -3.169734 & 4.315598 & -0.813730 \\ 15 & -1.733695 & 0.536436 & 0.021618 \\ 6 & -1.554883 & 0.190320 & 1.845635 \\ 6 & -1.218515 & -1.081997 & 2.283985 \\ 6 & -1.133115 & -1.363881 & 3.687372 \\ 6 & -1.407104 & -0.332323 & 4.631045 \\ 6 & -1.773082 & 0.945551 & 4.152588 \\ 6 & -1.841787 & 1.197072 & 2.808545 \\ 6 & -1.299992 & -0.602086 & 6.016281 \\ 6 & -0.933127 & -1.845434 & 6.463025 \\ 6 & -0.661188 & -2.872689 & 5.535128 \\ 6 & -0.761656 & -2.640961 & 4.185627 \\ 6 & -0.960486 & -2.219387 & 1.359360 \\ 6 & 0.300035 & -2.427125 & 0.827897 \\ 6 & 0.591001 & -3.604520 & 0.102545 \\ 6 & -0.371864 & -4.554354 & -0.105396 \\ 6 & -1.687850 & -4.357799 & 0.370676\end{array}$




\begin{tabular}{|c|c|c|c|}
\hline 6 & -1.989527 & -3.181758 & 1.119594 \\
\hline 6 & -3.314452 & -3.007678 & 1.596856 \\
\hline 6 & -4.285527 & -3.941700 & 1.335433 \\
\hline 6 & -3.981710 & -5.108721 & 0.599751 \\
\hline 6 & -2.709402 & -5.309333 & 0.127853 \\
\hline 7 & 1.339176 & -1.453715 & 1.013709 \\
\hline 6 & 2.295141 & -1.740263 & 2.106842 \\
\hline 6 & 2.779033 & -0.548909 & 2.891990 \\
\hline 6 & 4.122714 & -0.461191 & 3.251411 \\
\hline 6 & 4.583852 & 0.600771 & 4.026187 \\
\hline 6 & 3.702006 & 1.587585 & 4.451785 \\
\hline 6 & 2.357257 & 1.507409 & 4.092858 \\
\hline 6 & 1.901565 & 0.449643 & 3.318074 \\
\hline 6 & -0.006387 & 5.471522 & 1.046769 \\
\hline 6 & 0.859638 & 4.733707 & 2.069429 \\
\hline 6 & -4.337330 & 4.976535 & -1.544703 \\
\hline 6 & -3.803512 & 5.847241 & -2.687040 \\
\hline 45 & -0.170200 & 0.069768 & -1.697339 \\
\hline 6 & -0.613881 & -0.261194 & -3.859456 \\
\hline 6 & 0.659001 & -0.823880 & -3.609613 \\
\hline 6 & 1.871058 & -0.060249 & -4.095999 \\
\hline 15 & 1.650421 & -0.549334 & -0.386447 \\
\hline 8 & 2.880665 & -1.307897 & -1.234595 \\
\hline 6 & 4.088865 & -1.626675 & -0.669412 \\
\hline 6 & 4.986094 & -0.637195 & -0.312123 \\
\hline 6 & 6.155221 & -1.029015 & 0.418796 \\
\hline 6 & 6.431481 & -2.415516 & 0.617634 \\
\hline 6 & 5.543437 & -3.385366 & 0.089920 \\
\hline 6 & 4.385186 & -2.999865 & -0.526972 \\
\hline 6 & 7.571671 & -2.799301 & 1.364642 \\
\hline 6 & 8.397965 & -1.858569 & 1.924426 \\
\hline 6 & 8.110502 & -0.487627 & 1.757400 \\
\hline 6 & 7.024188 & -0.083694 & 1.020784 \\
\hline 6 & 4.702237 & 0.772124 & -0.668520 \\
\hline 6 & 3.493232 & 1.346773 & -0.322238 \\
\hline 6 & 3.203338 & 2.702365 & -0.579208 \\
\hline 6 & 4.112741 & 3.483512 & -1.235468 \\
\hline 6 & 5.331511 & 2.931420 & -1.698679 \\
\hline 6 & 5.628360 & 1.560635 & -1.431313 \\
\hline 6 & 6.825678 & 1.026117 & -1.972214 \\
\hline 6 & 7.690065 & 1.807291 & -2.699317 \\
\hline 6 & 7.409882 & 3.169960 & -2.927842 \\
\hline 6 & 6.249731 & 3.714037 & -2.439309 \\
\hline 8 & 2.547564 & 0.625280 & 0.361645 \\
\hline 6 & 0.474586 & 2.104704 & -2.035883 \\
\hline 8 & 0.081372 & 2.904641 & -2.781624 \\
\hline 6 & 0.789226 & -2.306526 & -3.522304 \\
\hline 6 & 2.029229 & -2.935025 & -3.709078 \\
\hline 6 & 2.145035 & -4.322538 & -3.722017 \\
\hline 6 & 1.023248 & -5.128611 & -3.560096 \\
\hline 6 & -0.215174 & -4.521504 & -3.360252 \\
\hline 6 & -0.324090 & -3.139349 & -3.325399 \\
\hline 6 & -3.438429 & -0.124363 & -0.217477 \\
\hline 6 & -3.709190 & -1.049465 & -1.225305 \\
\hline 6 & -4.995491 & -1.567858 & -1.395842 \\
\hline 6 & -6.003042 & -1.115357 & -0.534958 \\
\hline
\end{tabular}




\begin{tabular}{|c|c|c|c|}
\hline 5 & -5.762561 & -0.198209 & 0.489533 \\
\hline & -4.460838 & 0.283454 & 0.635134 \\
\hline & -5.329705 & -2.549741 & -2.519413 \\
\hline & -6.841606 & 0.290443 & 1.453116 \\
\hline & 0.887196 & 5.973300 & -0.093273 \\
\hline & -0.637198 & 6.671712 & 1.759506 \\
\hline & -5.300133 & 3.952142 & -2.141437 \\
\hline & -5.122119 & 5.849662 & -0.559201 \\
\hline & 6.801885 & 0.975768 & 0.919283 \\
\hline & -2.128205 & 2.190688 & 2.475330 \\
\hline & 7.768139 & -3.862563 & 1.497176 \\
\hline & -1.511455 & 0.203184 & 6.718458 \\
\hline & 6.004512 & 4.759478 & -2.622366 \\
\hline & -3.562981 & -2.105773 & 2.154761 \\
\hline & -5.301283 & -3.780919 & 1.692893 \\
\hline & 3.901054 & 4.534137 & -1.430267 \\
\hline & -1.999169 & 1.732507 & 4.871203 \\
\hline & 5.784905 & -4.440439 & 0.208390 \\
\hline & 8.596849 & 1.367047 & -3.108283 \\
\hline & 3.674695 & -3.721468 & -0.926201 \\
\hline & -2.459623 & -6.203418 & -0.442893 \\
\hline & -0.140563 & -5.461286 & -0.661275 \\
\hline & -0.370582 & -3.857953 & 5.892536 \\
\hline & 7.054055 & -0.025086 & -1.817945 \\
\hline & -0.551825 & -3.446284 & 3.484495 \\
\hline & 1.597738 & -3.736502 & -0.290425 \\
\hline & 8.105036 & 3.779562 & -3.500473 \\
\hline & 8.752012 & 0.257941 & 2.222047 \\
\hline & -4.759515 & -5.845608 & 0.409564 \\
\hline 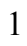 & -0.849430 & -2.043131 & 7.529154 \\
\hline & 9.265024 & -2.164544 & 2.505166 \\
\hline 1 & 2.243862 & 3.087902 & -0.244954 \\
\hline & -4.239428 & 0.998113 & 1.427396 \\
\hline & -2.881557 & -1.366833 & -1.858433 \\
\hline 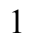 & -7.011002 & -1.500984 & -0.667064 \\
\hline | & -3.827993 & 2.280943 & -1.103666 \\
\hline 1 & -0.141541 & 2.706976 & 1.025523 \\
\hline & -2.248075 & 6.188980 & -0.329138 \\
\hline 1 & 1.755689 & -2.411739 & 2.790713 \\
\hline ] & 1.703796 & 6.595835 & 0.298244 \\
\hline 1 & 1.329559 & 5.133134 & -0.645869 \\
\hline 1 & 0.317461 & 6.573589 & -0.813954 \\
\hline 1 & 0.147463 & 7.296017 & 2.206482 \\
\hline 1 & -1.210363 & 7.313937 & 1.081089 \\
\hline & -1.309117 & 6.347021 & 2.564668 \\
\hline 1 & 0.248425 & 4.285880 & 2.865744 \\
\hline & 1.464255 & 3.935555 & 1.620873 \\
\hline 1 & 1.559709 & 5.436773 & 2.538158 \\
\hline 1 & -6.123427 & 4.472096 & -2.647420 \\
\hline l & -4.807955 & 3.311132 & -2.884118 \\
\hline 1 & -5.742999 & 3.304651 & -1.372198 \\
\hline & -5.527680 & 5.245315 & 0.263414 \\
\hline 1 & -4.498640 & 6.637459 & -0.119221 \\
\hline & -5.964976 & 6.337240 & -1.067589 \\
\hline 1 & -3.151665 & 6.651811 & -2.326352 \\
\hline 1 & -3.226768 & 5.245884 & -3.401462 \\
\hline
\end{tabular}




$\begin{array}{rrrr}1 & -4.636067 & 6.315218 & -3.229615 \\ 1 & 3.159903 & -2.311803 & 1.736104 \\ 1 & 4.823019 & -1.225876 & 2.910504 \\ 1 & 0.859927 & 0.406976 & 3.007073 \\ 1 & 5.638463 & 0.656380 & 4.289516 \\ 1 & 1.655818 & 2.274976 & 4.417481 \\ 1 & 4.059581 & 2.419291 & 5.055281 \\ 1 & -1.489676 & 0.485684 & -2.516795 \\ 1 & -1.468684 & -0.904733 & -4.062323 \\ 1 & -0.654697 & 0.688471 & -4.392426 \\ 1 & -1.298771 & -2.699741 & -3.125323 \\ 1 & -1.109537 & -5.128165 & -3.216136 \\ 1 & 1.111353 & -6.212776 & -3.587962 \\ 1 & 3.124516 & -4.773133 & -3.874708 \\ 1 & 2.924009 & -2.333362 & -3.846035 \\ 1 & 2.193512 & -0.434999 & -5.079874 \\ 1 & 1.639407 & 1.003187 & -4.210210 \\ 1 & 2.725528 & -0.141225 & -3.415224 \\ 6 & -4.079379 & -3.249039 & -3.052847 \\ 6 & -6.298631 & -3.634278 & -2.039753 \\ 6 & -5.980821 & -1.767380 & -3.665978 \\ 6 & -8.192960 & -0.372566 & 1.201407 \\ 6 & -6.418089 & -0.028534 & 2.892138 \\ 6 & -7.011352 & 1.805828 & 1.291135 \\ 1 & -4.364667 & -4.010843 & -3.789641 \\ 1 & -3.403975 & -2.550972 & -3.563558 \\ 1 & -3.521508 & -3.746952 & -2.245904 \\ 1 & -6.484502 & -4.351464 & -2.849809 \\ 1 & -5.880010 & -4.185917 & -1.187826 \\ 1 & -7.273278 & -3.231701 & -1.740583 \\ 1 & -6.227251 & -2.437995 & -4.500747 \\ 1 & -6.907510 & -1.276778 & -3.341445 \\ 1 & -5.304834 & -0.988716 & -4.043771 \\ 1 & -8.929573 & 0.005055 & 1.921779 \\ 1 & --8.577163 & -0.156200 & 0.196307 \\ 1 & -8.141538 & -1.462683 & 1.323315 \\ 1 & -7.177192 & 0.328645 & 3.601021 \\ 1 & -6.307664 & -1.111703 & 3.036477 \\ 1 & -5.464567 & 0.441762 & 3.163874 \\ 1 & -7.752024 & 2.188422 & 2.006374 \\ 1 & -6.071556 & 2.347534 & 1.460725 \\ 1 & -7.361766 & 2.052259 & 0.279909\end{array}$

TS1 $_{\text {IB2 } 2}-\psi_{-1 \text { 1a2a3s4a }}$

Number of imaginary frequencies : 1

The smallest frequencies are : -486.5266 $14.0408 \quad 15.4945 \mathrm{~cm}(-1)$

Electronic energy : $\quad H F=-4360.8642595$

Zero-point correction $=\quad 1.452098($ Hartree/Particle $)$

Thermal correction to Energy= $\quad 1.537115$

Thermal correction to Enthalpy= $\quad 1.538059$

Thermal correction to Gibbs Free Energy= 1.333763

Sum of electronic and zero-point Energies $=\quad-4359.412161$

Sum of electronic and thermal Energies $=\quad-4359.327145$

Sum of electronic and thermal Enthalpies $=\quad-4359.326200$

Sum of electronic and thermal Free Energies $=\quad-4359.530496$ 
Cartesian Coordinates

$\begin{array}{cccc}0 & -4.473061 & 0.350156 & 0.610187 \\ 6 & -3.433798 & -0.066340 & -0.232872 \\ 6 & -3.702903 & -0.950470 & -1.265533 \\ 6 & -5.002358 & -1.429955 & -1.492128 \\ 6 & -6.011259 & -0.991338 & -0.640836 \\ 6 & -5.774590 & -0.095880 & 0.416598 \\ 15 & -1.716423 & 0.550213 & 0.026532 \\ 45 & -0.159758 & 0.058109 & -1.694325 \\ 6 & 0.528447 & 2.042391 & -2.080023 \\ 8 & 0.155568 & 2.882790 & -2.790847 \\ 6 & -5.247180 & -2.393324 & -2.651218 \\ 6 & -4.425324 & -3.667668 & -2.431446 \\ 6 & -6.923384 & 0.328018 & 1.330008 \\ 6 & -8.100145 & 0.849176 & 0.498762 \\ 6 & -1.924384 & 2.375380 & 0.003628 \\ 6 & -2.997388 & 2.976751 & -0.648564 \\ 6 & -3.077979 & 4.366346 & -0.763969 \\ 6 & -2.056993 & 5.130673 & -0.194157 \\ 6 & -0.970445 & 4.559818 & 0.476939 \\ 6 & -0.907198 & 3.167839 & 0.540195 \\ 6 & 0.128175 & 5.440749 & 1.070725 \\ 6 & -0.474240 & 6.639422 & 1.810281 \\ 6 & -4.249222 & 5.055775 & -1.461477 \\ 6 & -4.965648 & 5.973550 & -0.464517 \\ 6 & -1.562694 & 0.201866 & 1.851526 \\ 6 & -1.244480 & -1.072253 & 2.297724 \\ 6 & -1.178497 & -1.348327 & 3.704649 \\ 6 & -1.458040 & -0.310892 & 4.640440 \\ 6 & -1.804933 & 0.968740 & 4.152519 \\ 6 & -1.852089 & 1.215376 & 2.807007 \\ 6 & -1.377229 & -0.576232 & 6.028371 \\ 6 & -1.028173 & -1.820526 & 6.486393 \\ 6 & -0.746016 & -2.852412 & 5.566880 \\ 6 & -0.821678 & -2.625208 & 4.214937 \\ 6 & -0.978429 & -2.220463 & 1.387714 \\ 6 & 0.287679 & -2.434722 & 0.872301 \\ 6 & 0.594716 & -3.634183 & 0.189827 \\ 6 & -0.350179 & -4.610719 & 0.029423 \\ 6 & -1.667885 & -4.418150 & 0.503678 \\ 6 & -1.994483 & -3.204760 & 1.178709 \\ 6 & -3.325082 & -3.028417 & 1.638594 \\ 6 & -4.270937 & -4.006551 & 1.455678 \\ 6 & -3.935839 & -5.218215 & 0.812491 \\ 6 & -2.663442 & -5.412401 & 0.338644 \\ 7 & 1.321331 & -1.452571 & 1.043364 \\ 15 & 1.644531 & -0.580191 & -0.373492 \\ 8 & 2.552852 & 0.600796 & 0.349639 \\ 6 & 3.495404 & 1.308781 & -0.351620 \\ 6 & 4.697955 & 0.724322 & -0.704966 \\ 6 & 5.617292 & 1.498209 & -1.490285 \\ 6 & 5.324656 & 2.867463 & -1.769656 \\ 6 & 4.116153 & 3.432307 & -1.295094 \\ & 3.210400 & 2.663420 & -0.619415 \\ & & & \\ 6\end{array}$




\begin{tabular}{|c|c|c|c|}
\hline 6 & 6.803716 & 0.949931 & -2.041251 \\
\hline 6 & 7.662322 & 1.717066 & -2.789821 \\
\hline & 7.386847 & 3.078582 & -3.030739 \\
\hline & 6.236943 & 3.635549 & -2.532546 \\
\hline & 4.985042 & -0.677010 & -0.320846 \\
\hline & 4.079724 & -1.673295 & -0.636328 \\
\hline & 4.370643 & -3.042357 & -0.450099 \\
\hline & 5.534422 & -3.413628 & 0.165023 \\
\hline & 6.434956 & -2.431727 & 0.646960 \\
\hline & 6.163033 & -1.051152 & 0.405718 \\
\hline & 7.582775 & -2.797370 & 1.391430 \\
\hline & 8.420606 & -1.843809 & 1.910616 \\
\hline & 8.137296 & -0.477525 & 1.703368 \\
\hline ) & 7.044256 & -0.091834 & 0.966833 \\
\hline & 2.869249 & -1.367777 & -1.204025 \\
\hline & 2.259497 & -1.702973 & 2.162504 \\
\hline b & 2.757736 & -0.475234 & 2.878718 \\
\hline & 4.116189 & -0.331719 & 3.151877 \\
\hline D & 4.590268 & 0.788965 & 3.830146 \\
\hline & 3.705765 & 1.776303 & 4.249263 \\
\hline & 2.343793 & 1.634465 & 3.986119 \\
\hline & 1.875828 & 0.519417 & 3.304822 \\
\hline ) & 0.992598 & 4.672784 & 2.072230 \\
\hline ) & 1.014286 & 5.947129 & -0.073162 \\
\hline ) & -3.727268 & 5.886093 & -2.638607 \\
\hline b & -5.270234 & 4.054866 & -1.999597 \\
\hline ) & -0.646487 & -0.315062 & -3.848519 \\
\hline & 0.594169 & -0.939759 & -3.593068 \\
\hline 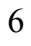 & 0.649632 & -2.422919 & -3.450368 \\
\hline 6 & 1.868684 & -3.108619 & -3.556770 \\
\hline ) & 1.926576 & -4.499015 & -3.509897 \\
\hline 6 & 0.766464 & -5.251484 & -3.363610 \\
\hline 6 & -0.453447 & -4.587851 & -3.244437 \\
\hline 6 & -0.506782 & -3.202416 & -3.278066 \\
\hline b & 1.841987 & -0.257197 & -4.109244 \\
\hline & -6.714873 & -2.792204 & -2.779762 \\
\hline 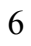 & -4.820492 & -1.725643 & -3.963348 \\
\hline & -6.507174 & 1.434141 & 2.298317 \\
\hline 6 & -7.377650 & -0.885204 & 2.149664 \\
\hline . & 6.827510 & 0.965083 & 0.832692 \\
\hline | & -2.123740 & 2.210519 & 2.466177 \\
\hline 1 & 7.775361 & -3.856858 & 1.555916 \\
\hline & -1.594566 & 0.233576 & 6.723506 \\
\hline 1 & 5.995351 & 4.680173 & -2.724825 \\
\hline & -3.594487 & -2.095435 & 2.131377 \\
\hline 1 & -5.287669 & -3.846916 & 1.809376 \\
\hline 1 & 3.908507 & 4.482178 & -1.498056 \\
\hline 1 & -2.034474 & 1.760253 & 4.864979 \\
\hline 1 & 5.771516 & -4.465460 & 0.316533 \\
\hline 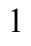 & 8.560537 & 1.266460 & -3.206346 \\
\hline 1 & 3.652149 & -3.773269 & -0.816683 \\
\hline ] & -2.393116 & -6.335089 & -0.174246 \\
\hline 1 & -0.103391 & -5.539693 & -0.481718 \\
\hline 1 & -0.467140 & -3.838059 & 5.932457 \\
\hline 1 & 7.027755 & -0.100881 & -1.877910 \\
\hline 1 & -0.603176 & -3.435082 & 3.521841 \\
\hline
\end{tabular}




\begin{tabular}{|c|c|c|c|}
\hline ] & 1.603862 & -3.766450 & -0.195729 \\
\hline & 8.077358 & 3.677036 & -3.620491 \\
\hline & 8.788183 & 0.279299 & 2.135671 \\
\hline 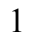 & -4.692573 & -5.989152 & 0.683047 \\
\hline & -0.965449 & -2.015009 & 7.554548 \\
\hline & 9.293185 & -2.135586 & 2.490364 \\
\hline & 2.258209 & 3.058771 & -0.275415 \\
\hline & -4.248934 & 1.043290 & 1.417436 \\
\hline & -2.872558 & -1.271241 & -1.895748 \\
\hline & -7.025986 & -1.352894 & -0.793881 \\
\hline & -3.773376 & 2.347835 & -1.076167 \\
\hline & -0.064151 & 2.677766 & 1.028945 \\
\hline & -2.112051 & 6.215796 & -0.274685 \\
\hline & 1.696401 & -2.323128 & 2.875030 \\
\hline & 1.856230 & 6.535323 & 0.317747 \\
\hline & 1.419727 & 5.110317 & -0.657880 \\
\hline & 0.448083 & 6.582856 & -0.765731 \\
\hline & 0.326228 & 7.246702 & 2.252776 \\
\hline ] & -1.049678 & 7.298640 & 1.150324 \\
\hline & -1.137279 & 6.312631 & 2.621945 \\
\hline & 0.379168 & 4.221588 & 2.865207 \\
\hline & 1.580970 & 3.871810 & 1.607386 \\
\hline & 1.707475 & 5.356489 & 2.547437 \\
\hline 1 & -6.101879 & 4.594323 & -2.470239 \\
\hline & -4.835328 & 3.392698 & -2.759252 \\
\hline$\frac{1}{1}$ & -5.692028 & 3.426595 & -1.202262 \\
\hline & -5.355423 & 5.399696 & 0.386800 \\
\hline ] & -4.302183 & 6.751011 & -0.067068 \\
\hline ] & -5.812857 & 6.476819 & -0.949771 \\
\hline & -3.030997 & 6.668985 & -2.314691 \\
\hline 1 & -3.200844 & 5.251073 & -3.362720 \\
\hline & -4.560604 & 6.377905 & -3.158592 \\
\hline 1 & 3.116012 & -2.311338 & 1.832430 \\
\hline & 4.817635 & -1.096893 & 2.815429 \\
\hline 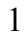 & 0.818975 & 0.429673 & 3.063332 \\
\hline ] & 5.657126 & 0.888717 & 4.021468 \\
\hline 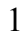 & 1.638917 & 2.398619 & 4.311736 \\
\hline 1 & 4.073543 & 2.654118 & 4.776494 \\
\hline & -1.461303 & 0.509395 & -2.523452 \\
\hline 1 & -1.538073 & -0.914624 & -4.024771 \\
\hline ] & -0.639674 & 0.619466 & -4.409246 \\
\hline 1 & -1.474444 & -2.716968 & -3.165506 \\
\hline ] & -1.377020 & -5.152397 & -3.114122 \\
\hline 1 & 0.809968 & -6.338565 & -3.341133 \\
\hline 1 & 2.891524 & -4.994786 & -3.604624 \\
\hline & 2.792621 & -2.548826 & -3.676793 \\
\hline 1 & 2.142646 & -0.686291 & -5.077779 \\
\hline l & 1.662416 & 0.811600 & -4.263812 \\
\hline 1 & 2.693403 & -0.353505 & -3.426927 \\
\hline 1 & -6.835225 & -3.486576 & -3.620911 \\
\hline 1 & -7.080941 & -3.302310 & -1.879265 \\
\hline 1 & -7.361289 & -1.926139 & -2.973110 \\
\hline 1 & -4.997287 & -2.401576 & -4.810636 \\
\hline 1 & -5.389026 & -0.803283 & -4.139808 \\
\hline & -3.754872 & -1.463996 & -3.966333 \\
\hline 1 & -4.524406 & -4.342037 & -3.293501 \\
\hline
\end{tabular}




$\begin{array}{rrrr}1 & -3.358090 & -3.453271 & -2.289179 \\ 1 & -4.767880 & -4.201712 & -1.536114 \\ 1 & -7.367606 & 1.732043 & 2.910542 \\ 1 & -5.715241 & 1.108264 & 2.985562 \\ 1 & -6.151336 & 2.327559 & 1.766005 \\ 1 & -8.210734 & -0.612728 & 2.811884 \\ 1 & -7.716297 & -1.707876 & 1.507274 \\ 1 & -6.558099 & -1.262223 & 2.776560 \\ 1 & -8.914158 & 1.174775 & 1.159932 \\ 1 & -7.800675 & 1.708382 & -0.115324 \\ 1 & -8.511618 & 0.085901 & -0.171819\end{array}$

$\mathrm{TS1}_{I B 2-} \psi_{-1 \mathrm{a} 2 \mathrm{2} 3 \mathrm{~s} 4 \mathrm{~s}}$

Number of imaginary frequencies : 1

The smallest frequencies are : -486.2206 $13.1943 \quad 16.6287 \mathrm{~cm}(-1)$

Electronic energy : $\quad H F=-4360.8647535$

Zero-point correction $=\quad 1.452201($ Hartree/Particle $)$

Thermal correction to Energy= $\quad 1.537174$

Thermal correction to Enthalpy= $\quad 1.538118$

Thermal correction to Gibbs Free Energy= 1.333592

Sum of electronic and zero-point Energies $=\quad-4359.412553$

Sum of electronic and thermal Energies $=\quad-4359.327580$

Sum of electronic and thermal Enthalpies $=\quad-4359.326636$

Sum of electronic and thermal Free Energies $=\quad-4359.531161$

\section{Cartesian Coordinates}

$\begin{array}{cccc}6 & -4.449216 & 0.311958 & 0.638742 \\ 6 & -3.424114 & -0.078792 & -0.223544 \\ 6 & -3.708660 & -0.942615 & -1.273960 \\ 6 & -5.008439 & -1.413624 & -1.492732 \\ 6 & -6.010698 & -1.000164 & -0.612691 \\ 6 & -5.755586 & -0.137835 & 0.460998 \\ 15 & -1.708982 & 0.544597 & 0.030204 \\ 45 & -0.158963 & 0.062399 & -1.698290 \\ 6 & 0.535283 & 2.044204 & -2.078495 \\ 8 & 0.165472 & 2.889617 & -2.784991 \\ 6 & -5.271804 & -2.345465 & -2.674183 \\ 6 & -4.457313 & -3.630871 & -2.495466 \\ 6 & -6.836721 & 0.339707 & 1.428836 \\ 6 & -6.434039 & -0.017342 & 2.864691 \\ 6 & -1.928155 & 2.368824 & 0.010847 \\ 6 & -2.999793 & 2.967014 & -0.647609 \\ 6 & -3.076898 & 4.356287 & -0.773372 \\ 6 & -2.063325 & 5.123159 & -0.193505 \\ 6 & -0.980986 & 4.555646 & 0.486758 \\ 6 & -0.914210 & 3.163942 & 0.549497 \\ 6 & 0.112765 & 5.439177 & 1.085454 \\ 6 & -0.495692 & 6.638048 & 1.819599 \\ 6 & -4.237028 & 5.044810 & -1.490586 \\ 6 & -5.010760 & 5.906639 & -0.486529 \\ 6 & -1.552231 & 0.190734 & 1.854248 \\ 6 & -1.242667 & -1.087814 & 2.293792 \\ 6 & -1.181085 & -1.373182 & 3.698660 \\ 6 & -1.456392 & -0.340109 & 4.640356\end{array}$




\begin{tabular}{|c|c|c|c|}
\hline 6 & -1.792858 & 0.944963 & 4.159541 \\
\hline 6 & -1.835271 & 1.200523 & 2.815284 \\
\hline f & -1.381356 & -0.615255 & 6.026714 \\
\hline 6 & -1.042105 & -1.864993 & 6.477215 \\
\hline 6 & -0.764351 & -2.892720 & 5.551620 \\
\hline 5 & -0.834507 & -2.655942 & 4.201058 \\
\hline 6 & -0.986176 & -2.229956 & 1.374178 \\
\hline 6 & 0.275556 & -2.444373 & 0.847786 \\
\hline 6 & 0.569545 & -3.634049 & 0.142907 \\
\hline 6 & -0.384306 & -4.600124 & -0.028085 \\
\hline 6 & -1.697659 & -4.406129 & 0.457288 \\
\hline 6 & -2.011019 & -3.202889 & 1.156249 \\
\hline 6 & -3.337691 & -3.024094 & 1.627127 \\
\hline 6 & -4.292522 & -3.990281 & 1.428299 \\
\hline 6 & -3.971138 & -5.191936 & 0.759807 \\
\hline 6 & -2.702294 & -5.388611 & 0.277716 \\
\hline 7 & 1.315857 & -1.470682 & 1.027287 \\
\hline 15 & 1.643932 & -0.586285 & -0.380527 \\
\hline 8 & 2.548472 & 0.589943 & 0.354843 \\
\hline 6 & 3.491428 & 1.306056 & -0.337421 \\
\hline 6 & 4.695541 & 0.727052 & -0.694942 \\
\hline 6 & 5.614633 & 1.510527 & -1.470987 \\
\hline 6 & 5.320894 & 2.882495 & -1.735328 \\
\hline 6 & 4.111142 & 3.440706 & -1.256133 \\
\hline 6 & 3.205019 & 2.663182 & -0.590911 \\
\hline 6 & 6.802014 & 0.969567 & -2.027100 \\
\hline 6 & 7.660550 & 1.745865 & -2.766240 \\
\hline 6 & 7.384146 & 3.109832 & -2.991809 \\
\hline 6 & 6.233245 & 3.660059 & -2.488462 \\
\hline 6 & 4.985568 & -0.676604 & -0.321612 \\
\hline 6 & 4.081828 & -1.672379 & -0.643009 \\
\hline 6 & 4.372522 & -3.041937 & -0.460722 \\
\hline 6 & 5.536660 & -3.415137 & 0.152515 \\
\hline 6 & 6.437475 & -2.434820 & 0.637042 \\
\hline 6 & 6.164197 & -1.053278 & 0.402853 \\
\hline 6 & 7.586233 & -2.803390 & 1.378620 \\
\hline 6 & 8.423813 & -1.851884 & 1.901915 \\
\hline 6 & 8.138993 & -0.484834 & 1.702139 \\
\hline 6 & 7.044906 & -0.096250 & 0.968660 \\
\hline 8 & 2.872701 & -1.365087 & -1.213038 \\
\hline 6 & 2.247696 & -1.731588 & 2.149373 \\
\hline 6 & 2.753648 & -0.510054 & 2.870926 \\
\hline 6 & 4.112853 & -0.375373 & 3.144525 \\
\hline 6 & 4.593652 & 0.740776 & 3.825655 \\
\hline 6 & 3.715275 & 1.732707 & 4.246621 \\
\hline 6 & 2.352434 & 1.599643 & 3.983251 \\
\hline 6 & 1.877801 & 0.488658 & 3.300127 \\
\hline 6 & 0.973363 & 4.674393 & 2.092765 \\
\hline 6 & 1.004270 & 5.944788 & -0.054676 \\
\hline 6 & -3.694804 & 5.932027 & -2.616082 \\
\hline 6 & -5.211889 & 4.043253 & -2.106397 \\
\hline 6 & -0.655807 & -0.299196 & -3.853115 \\
\hline 6 & 0.580396 & -0.934874 & -3.604414 \\
\hline 6 & 0.626839 & -2.419007 & -3.467859 \\
\hline 6 & 1.845420 & -3.108721 & -3.554149 \\
\hline 6 & 1.897812 & -4.499431 & -3.512181 \\
\hline
\end{tabular}




\begin{tabular}{|c|c|c|c|}
\hline & 0.732479 & -5.248639 & -3.392386 \\
\hline & -0.487082 & -4.581232 & -3.293405 \\
\hline & -0.535105 & -3.195430 & -3.321131 \\
\hline & 1.832407 & -0.258817 & -4.119524 \\
\hline & -4.853047 & -1.647550 & -3.973284 \\
\hline & -6.743547 & -2.730696 & -2.800037 \\
\hline & -6.983209 & 1.861079 & 1.300249 \\
\hline & -8.195524 & -0.296464 & 1.148516 \\
\hline & 6.827248 & 0.961200 & 0.840563 \\
\hline & -2.099496 & 2.199559 & 2.480102 \\
\hline & 7.779696 & -3.863561 & 1.537570 \\
\hline & -1.595188 & 0.191296 & 6.726692 \\
\hline & 5.990846 & 4.706557 & -2.669185 \\
\hline & -3.597873 & -2.098688 & 2.139635 \\
\hline & -5.306555 & -3.829863 & 1.789193 \\
\hline & 3.902843 & 4.492572 & -1.447746 \\
\hline & -2.018025 & 1.733733 & 4.876421 \\
\hline & 5.773813 & -4.467440 & 0.300623 \\
\hline & 8.559483 & 1.300797 & -3.187147 \\
\hline & 3.653545 & -3.771959 & -0.828049 \\
\hline & -2.442151 & -6.303772 & -0.253619 \\
\hline & -0.148074 & -5.521455 & -0.557545 \\
\hline & -0.493421 & -3.882692 & 5.911445 \\
\hline & 7.026765 & -0.082836 & -1.875278 \\
\hline & -0.620051 & -3.462373 & 3.502698 \\
\hline & 1.573653 & -3.766889 & -0.255960 \\
\hline & 8.074665 & 3.715564 & -3.574075 \\
\hline & 8.789467 & 0.270362 & 2.137903 \\
\hline & -4.735483 & -5.953225 & 0.618493 \\
\hline ] & -0.983826 & -2.067054 & 7.544214 \\
\hline & 9.297197 & -2.145909 & 2.479313 \\
\hline D & 2.251601 & 3.053574 & -0.244339 \\
\hline & -4.221840 & 0.989714 & 1.461159 \\
\hline & -2.886526 & -1.249993 & -1.921409 \\
\hline & -7.024798 & -1.358016 & -0.765761 \\
\hline & -3.774450 & 2.336135 & -1.076096 \\
\hline 1 & -0.070527 & 2.676060 & 1.039332 \\
\hline & -2.119684 & 6.207964 & -0.275736 \\
\hline | & 1.676980 & -2.349278 & 2.857948 \\
\hline & 1.844245 & 6.533509 & 0.339682 \\
\hline & 1.412475 & 5.107451 & -0.636768 \\
\hline & 0.441223 & 6.579707 & -0.750530 \\
\hline & 0.301325 & 7.248022 & 2.264595 \\
\hline 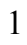 & -1.069460 & 7.294552 & 1.155508 \\
\hline & -1.161829 & 6.311507 & 2.628827 \\
\hline | & 0.357307 & 4.224118 & 2.884212 \\
\hline & 1.565059 & 3.873292 & 1.632343 \\
\hline ] & 1.685051 & 5.360280 & 2.569594 \\
\hline 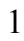 & -6.026733 & 4.582500 & -2.605839 \\
\hline ] & -4.726129 & 3.407520 & -2.857865 \\
\hline 1 & -5.665745 & 3.389684 & -1.348855 \\
\hline & -5.422699 & 5.289934 & 0.323717 \\
\hline 1 & -4.377155 & 6.677358 & -0.030960 \\
\hline & -5.848260 & 6.414663 & -0.983707 \\
\hline l & -3.036377 & 6.724277 & -2.240408 \\
\hline & -3.123058 & 5.339119 & -3.341499 \\
\hline
\end{tabular}




$\begin{array}{rrrr}1 & -4.522942 & 6.417492 & -3.149948 \\ 1 & 3.100185 & -2.345968 & 1.819990 \\ 1 & 4.809896 & -1.143728 & 2.806169 \\ 1 & 0.820479 & 0.405701 & 3.058756 \\ 1 & 5.661063 & 0.833517 & 4.017353 \\ 1 & 1.652149 & 2.367486 & 4.310168 \\ 1 & 4.088436 & 2.607223 & 4.775545 \\ 1 & -1.461417 & 0.521806 & -2.521682 \\ 1 & -1.553434 & -0.889778 & -4.028597 \\ 1 & -0.643165 & 0.637790 & -4.409583 \\ 1 & -1.503542 & -2.708132 & -3.225270 \\ 1 & -1.414924 & -5.142997 & -3.183013 \\ 1 & 0.771811 & -6.335970 & -3.374557 \\ 1 & 2.862703 & -4.998110 & -3.591052 \\ 1 & 2.773286 & -2.551578 & -3.653970 \\ 1 & 2.132622 & -0.690994 & -5.086822 \\ 1 & 1.657882 & 0.810562 & -4.275930 \\ 1 & 2.682445 & -0.357427 & -3.435866 \\ 1 & -6.876107 & -3.400771 & -3.658909 \\ 1 & -7.105578 & -3.263042 & -1.910946 \\ 1 & -7.385589 & -1.855293 & -2.963504 \\ 1 & -4.561593 & -4.278091 & -3.377539 \\ 1 & -3.388943 & -3.426542 & -2.347274 \\ 1 & -4.802239 & -4.189925 & -1.616604 \\ 1 & -5.046401 & -2.298950 & -4.836065 \\ 1 & -5.414125 & -0.715250 & -4.118757 \\ 1 & -3.784942 & -1.396280 & -3.981921 \\ 1 & -7.194919 & 0.337479 & 3.572850 \\ 1 & -6.343862 & -1.104907 & 2.987274 \\ 1 & -5.476052 & 0.430727 & 3.157345 \\ 1 & -7.725364 & 2.238449 & 2.016833 \\ 1 & -6.036816 & 2.384363 & 1.490282 \\ 1 & -7.319883 & 2.135301 & 0.291495 \\ 1 & -8.931606 & 0.070140 & 1.875151 \\ 1 & -8.570248 & -0.044489 & 0.148237 \\ 1 & -8.160766 & -1.390310 & 1.237636\end{array}$

$\mathrm{TS1}_{I B 2-} \psi_{-1 \mathrm{a} 253 \mathrm{ata}}$

Number of imaginary frequencies : 1

The smallest frequencies are : -479.3173 $13.0478 \quad 17.4371 \mathrm{~cm}(-1)$

Electronic energy : $\quad H F=-4360.8654936$

Zero-point correction $=\quad 1.452046($ Hartree/Particle $)$

Thermal correction to Energy= $\quad 1.537120$

Thermal correction to Enthalpy= $\quad 1.538065$

Thermal correction to Gibbs Free Energy $=\quad 1.333164$

Sum of electronic and zero-point Energies $=\quad-4359.413447$

Sum of electronic and thermal Energies $=\quad-4359.328373$

Sum of electronic and thermal Enthalpies $=\quad-4359.327429$

Sum of electronic and thermal Free Energies $=\quad-4359.532330$

Cartesian Coordinates

$\begin{array}{llll}6 & -0.895478 & 3.170194 & 0.578124 \\ 6 & -1.914317 & 2.391548 & 0.013612 \\ 6 & -2.945317 & 3.010190 & -0.677177\end{array}$




\begin{tabular}{|c|c|c|c|}
\hline 6 & -2.992018 & 4.404646 & -0.811022 \\
\hline & -1.981555 & 5.151982 & -0.212240 \\
\hline 6 & -0.927923 & 4.558168 & 0.501338 \\
\hline 15 & -1.730662 & 0.565506 & 0.028277 \\
\hline 4 & -0.182503 & 0.068207 & -1.696356 \\
\hline & 1.633216 & -0.567161 & -0.387760 \\
\hline 8 & 2.855290 & -1.344374 & -1.231505 \\
\hline b & 4.056437 & -1.677708 & -0.659618 \\
\hline 6 & 4.967029 & -0.699316 & -0.305434 \\
\hline & 6.127716 & -1.103228 & 0.432184 \\
\hline & 6.381786 & -2.492185 & 0.643036 \\
\hline 0 & 5.481684 & -3.452394 & 0.118180 \\
\hline 6 & 4.332101 & -3.054050 & -0.506800 \\
\hline 0 & 7.009207 & -0.166663 & 1.029887 \\
\hline 6 & 8.086166 & -0.581411 & 1.774158 \\
\hline 6 & 8.351457 & -1.955289 & 1.953485 \\
\hline 6 & 7.512901 & -2.887530 & 1.397828 \\
\hline 6 & 4.704457 & 0.711287 & -0.672495 \\
\hline 6 & 3.501959 & 1.304592 & -0.335236 \\
\hline 6 & 3.230388 & 2.661258 & -0.605558 \\
\hline 6 & 4.152641 & 3.425295 & -1.264083 \\
\hline 6 & 5.366116 & 2.853790 & -1.717366 \\
\hline 6 & 5.643858 & 1.481378 & -1.437801 \\
\hline 6 & 6.297414 & 3.618236 & -2.460669 \\
\hline 6 & 7.452183 & 3.055057 & -2.940173 \\
\hline 6 & 7.713521 & 1.690715 & -2.699539 \\
\hline 6 & 6.836109 & 0.926827 & -1.969681 \\
\hline 8 & 2.544026 & 0.602135 & 0.351253 \\
\hline 6 & -4.141965 & 5.029864 & -1.596950 \\
\hline 6 & -4.091441 & 4.528787 & -3.045044 \\
\hline 6 & 0.169566 & 5.424101 & 1.117926 \\
\hline 6 & 1.025484 & 4.637985 & 2.112143 \\
\hline 6 & -3.456040 & -0.038050 & -0.216730 \\
\hline 6 & -3.736472 & -0.968973 & -1.211356 \\
\hline 6 & -5.032853 & -1.472379 & -1.381454 \\
\hline 6 & -6.028576 & -0.995275 & -0.529324 \\
\hline 6 & -5.781226 & -0.060360 & 0.484644 \\
\hline 6 & -4.477500 & 0.404773 & 0.629681 \\
\hline 6 & -6.913279 & 0.380578 & 1.410733 \\
\hline 6 & -7.388893 & -0.829336 & 2.223409 \\
\hline 6 & -5.376480 & -2.461446 & -2.495797 \\
\hline 6 & -4.133297 & -3.179951 & -3.020513 \\
\hline 6 & -1.567330 & 0.205305 & 1.850319 \\
\hline 6 & -1.247851 & -1.072344 & 2.284816 \\
\hline 6 & -1.175857 & -1.361188 & 3.688029 \\
\hline 6 & -1.446633 & -0.330935 & 4.634072 \\
\hline 6 & -1.795944 & 0.952958 & 4.158809 \\
\hline 6 & -1.852267 & 1.210830 & 2.815674 \\
\hline 6 & -1.356403 & -0.608505 & 6.018912 \\
\hline 6 & -1.008812 & -1.858256 & 6.463360 \\
\hline 6 & -0.739131 & -2.883955 & 5.533230 \\
\hline 6 & -0.823218 & -2.644564 & 4.183854 \\
\hline 6 & -0.994310 & -2.209468 & 1.358388 \\
\hline 6 & 0.267775 & -2.427867 & 0.834660 \\
\hline 6 & 0.554475 & -3.611170 & 0.117044 \\
\hline 6 & -0.413159 & -4.556069 & -0.091261 \\
\hline
\end{tabular}




\begin{tabular}{|c|c|c|c|}
\hline 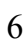 & -1.731010 & -4.347398 & 0.374172 \\
\hline & -2.029291 & -3.164340 & 1.113367 \\
\hline & -3.357004 & -2.977056 & 1.577390 \\
\hline & -4.333315 & -3.905471 & 1.315272 \\
\hline & -4.032201 & -5.080206 & 0.590857 \\
\hline & -2.757706 & -5.293228 & 0.130390 \\
\hline & 1.311792 & -1.459232 & 1.018426 \\
\hline & 2.257218 & -1.736366 & 2.123309 \\
\hline & 2.748138 & -0.533659 & 2.886622 \\
\hline & 4.094702 & -0.438573 & 3.232402 \\
\hline & 4.563187 & 0.641200 & 3.977673 \\
\hline & 3.685755 & 1.638639 & 4.387341 \\
\hline & 2.337228 & 1.549482 & 4.044639 \\
\hline & 1.874240 & 0.473608 & 3.299615 \\
\hline & -6.466477 & 1.466064 & 2.388313 \\
\hline & -8.081858 & 0.934323 & 0.589274 \\
\hline & -6.015979 & -1.682593 & -3.651205 \\
\hline & -6.359304 & -3.529021 & -2.007061 \\
\hline & 0.483420 & 2.096914 & -2.044026 \\
\hline & 0.081730 & 2.908418 & -2.772295 \\
\hline & -0.637042 & -0.265050 & -3.856378 \\
\hline 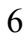 & 0.630536 & -0.840056 & -3.607896 \\
\hline & -4.074161 & 6.554507 & -1.617896 \\
\hline & -5.475775 & 4.620204 & -0.960289 \\
\hline 0 & 1.065537 & 5.946897 & -0.011241 \\
\hline & -0.436586 & 6.611448 & 1.872948 \\
\hline 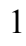 & 6.803882 & 0.895288 & 0.918771 \\
\hline ] & -2.126008 & 2.208911 & 2.485027 \\
\hline & 7.692376 & -3.952591 & 1.539636 \\
\hline 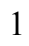 & -1.565616 & 0.195878 & 6.722777 \\
\hline & 6.066591 & 4.665248 & -2.653238 \\
\hline 1 & -3.602489 & -2.069520 & 2.126787 \\
\hline & -5.350244 & -3.734155 & 1.664258 \\
\hline & 3.955276 & 4.476839 & -1.468688 \\
\hline & -2.020092 & 1.738746 & 4.879265 \\
\hline & 5.707128 & -4.510081 & 0.244660 \\
\hline 1 & 8.615994 & 1.235272 & -3.101335 \\
\hline & 3.614134 & -3.767910 & -0.906501 \\
\hline 1 & -2.510039 & -6.193180 & -0.431931 \\
\hline & -0.184254 & -5.467864 & -0.640146 \\
\hline | & -0.463393 & -3.874217 & 5.888581 \\
\hline 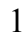 & 7.050136 & -0.126032 & -1.806394 \\
\hline & -0.615184 & -3.449027 & 3.481211 \\
\hline 1 & 1.562484 & -3.752338 & -0.268978 \\
\hline & 8.157496 & 3.650782 & -3.514983 \\
\hline 1 & 8.737527 & 0.157841 & 2.235184 \\
\hline & -4.813926 & -5.812604 & 0.399413 \\
\hline 1 & -0.938623 & -2.062006 & 7.529324 \\
\hline 1 & 9.211269 & -2.269991 & 2.540297 \\
\hline 1 & 2.273790 & 3.060533 & -0.279415 \\
\hline 1 & -4.241841 & 1.127456 & 1.407176 \\
\hline & -2.911856 & -1.308498 & -1.836994 \\
\hline 1 & -7.044280 & -1.369825 & -0.650801 \\
\hline & -3.722375 & 2.398328 & -1.133549 \\
\hline 1 & -0.076927 & 2.666474 & 1.093081 \\
\hline 1 & -2.002585 & 6.235580 & -0.300853 \\
\hline
\end{tabular}




\begin{tabular}{|c|c|c|c|}
\hline ] & 1.705634 & -2.388799 & 2.815895 \\
\hline & 1.910413 & 6.519549 & 0.395919 \\
\hline & 1.466371 & 5.118693 & -0.611459 \\
\hline 1 & 0.509116 & 6.602359 & -0.693107 \\
\hline 1 & 0.361414 & 7.214916 & 2.325190 \\
\hline & -1.013875 & 7.277000 & 1.220988 \\
\hline & -1.099748 & 6.271311 & 2.679053 \\
\hline & 0.407281 & 4.182404 & 2.898934 \\
\hline & 1.608698 & 3.837859 & 1.639150 \\
\hline & 1.743931 & 5.311117 & 2.596884 \\
\hline & -4.923211 & 6.953146 & -2.187448 \\
\hline & -4.125890 & 6.982176 & -0.608152 \\
\hline & -3.157338 & 6.917342 & -2.099921 \\
\hline & -5.612378 & 3.530941 & -0.950706 \\
\hline & -5.541442 & 4.976265 & 0.076466 \\
\hline & -6.315159 & 5.053930 & -1.520448 \\
\hline [ & -3.138265 & 4.797708 & -3.518423 \\
\hline & -4.197296 & 3.439069 & -3.107753 \\
\hline ] & -4.903775 & 4.977063 & -3.633238 \\
\hline & -5.664474 & 1.118780 & 3.053515 \\
\hline ] & -6.112612 & 2.365213 & 1.865762 \\
\hline & -7.311991 & 1.762084 & 3.021895 \\
\hline & -8.500013 & 0.189305 & -0.097814 \\
\hline 1 & -8.893032 & 1.258001 & 1.255003 \\
\hline & -7.769521 & 1.801533 & -0.006989 \\
\hline$\frac{1}{1}$ & -7.756851 & -1.637491 & 1.578810 \\
\hline & -6.572363 & -1.232801 & 2.837645 \\
\hline ] & -8.207063 & -0.541645 & 2.897539 \\
\hline ] & -5.330882 & -0.914388 & -4.034011 \\
\hline & -6.266468 & -2.358037 & -4.480828 \\
\hline 1 & -6.938495 & -1.179630 & -3.333822 \\
\hline & -7.327665 & -3.110464 & -1.709288 \\
\hline 1 & -6.556586 & -4.249267 & -2.811656 \\
\hline ] & -5.947036 & -4.080331 & -1.151904 \\
\hline 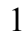 & -3.451732 & -2.494604 & -3.540270 \\
\hline ] & -3.579424 & -3.673083 & -2.208056 \\
\hline & -4.426413 & -3.948157 & -3.747482 \\
\hline 1 & 3.118482 & -2.323623 & 1.768914 \\
\hline & 4.791869 & -1.210889 & 2.902647 \\
\hline 1 & 0.829575 & 0.424212 & 2.999823 \\
\hline ] & 5.620268 & 0.702440 & 4.229693 \\
\hline 1 & 1.638697 & 2.324270 & 4.358640 \\
\hline 1 & 4.049240 & 2.484791 & 4.966734 \\
\hline 5 & 1.849277 & -0.090916 & -4.100226 \\
\hline 6 & 0.746284 & -2.323531 & -3.514416 \\
\hline & -1.497840 & 0.500256 & -2.513971 \\
\hline 1 & -1.499589 & -0.900175 & -4.053204 \\
\hline 1 & -0.669554 & 0.682907 & -4.393092 \\
\hline 6 & -0.374959 & -3.144870 & -3.314297 \\
\hline 6 & -0.279029 & -4.528118 & -3.343674 \\
\hline 6 & 0.953693 & -5.147716 & -3.540446 \\
\hline 6 & 2.083188 & -4.352999 & -3.704880 \\
\hline 6 & 1.980358 & -2.964451 & -3.698102 \\
\hline 1 & -1.345392 & -2.695043 & -3.116044 \\
\hline 1 & -1.179255 & -5.125524 & -3.197912 \\
\hline 1 & 1.031498 & -6.232793 & -3.563796 \\
\hline
\end{tabular}




$\begin{array}{llrr}1 & 3.058454 & -4.813456 & -3.855129 \\ 1 & 2.880702 & -2.371711 & -3.837311 \\ 1 & 2.166615 & -0.473955 & -5.082570 \\ 1 & 1.627681 & 0.974164 & -4.219602 \\ 1 & 2.704043 & -0.176460 & -3.420309\end{array}$

TS1 $_{I B 2-} \Psi_{-1 \text { a2s3a4s }}$

\begin{tabular}{|c|c|}
\hline \multicolumn{2}{|l|}{ Number of imaginary frequencies $: 1$} \\
\hline \multirow{2}{*}{\multicolumn{2}{|c|}{ The smallest frequencies are : - $\begin{array}{llll}480.3566 & 13.0357 & 19.7833 \mathrm{~cm}(-1)\end{array}$}} \\
\hline & \\
\hline Zero-point correction $=\quad 1.4521$ & 70 (Hartree/Particle) \\
\hline Thermal correction to Energy= & 37099 \\
\hline Thermal correction to Enthalpy= & 38043 \\
\hline Thermal correction to Gibbs Free Energy= & 1.333576 \\
\hline Sum of electronic and zero-point Energies $=$ & -4359.413620 \\
\hline Sum of electronic and thermal Energies= & -4359.328692 \\
\hline Sum of electronic and thermal Enthalpies $=$ & -4359.327747 \\
\hline Sum of electronic and thermal Free Energies $=$ & -4359.532214 \\
\hline
\end{tabular}

Cartesian Coordinates

$\begin{array}{cccc}6 & 6.994873 & -0.167494 & 1.033824 \\ 6 & 6.115968 & -1.105145 & 0.434080 \\ 6 & 6.372221 & -2.493834 & 0.644102 \\ 6 & 7.502999 & -2.887786 & 1.400133 \\ 6 & 8.339079 & -1.954487 & 1.957758 \\ 6 & 8.071566 & -0.580936 & 1.779213 \\ 6 & 4.955676 & -0.702654 & -0.304929 \\ 6 & 4.047330 & -1.682434 & -0.660943 \\ 6 & 4.324760 & -3.058420 & -0.508317 \\ 6 & 5.474307 & -3.455253 & 0.117672 \\ 6 & 4.691766 & 0.707534 & -0.672928 \\ 6 & 3.487763 & 1.299556 & -0.338372 \\ 6 & 3.213772 & 2.655162 & -0.612779 \\ 6 & 4.136647 & 3.419010 & -1.270891 \\ 6 & 5.352318 & 2.849038 & -1.719889 \\ 6 & 5.631658 & 1.477619 & -1.437592 \\ 6 & 6.284004 & 3.614046 & -2.462163 \\ 6 & 7.440783 & 3.052119 & -2.938212 \\ 6 & 7.703726 & 1.688508 & -2.695116 \\ 6 & 6.825920 & 0.924197 & -1.966189 \\ 8 & 2.530797 & 0.595775 & 0.348708 \\ 15 & 1.622649 & -0.575063 & -0.391579 \\ 8 & 2.846260 & -1.350829 & -1.234156 \\ 45 & -0.191681 & 0.059626 & -1.701755 \\ 6 & 0.476803 & 2.082310 & -2.062850 \\ 8 & 0.079069 & 2.889803 & -2.797740 \\ 6 & 0.614052 & -0.858757 & -3.613004 \\ 6 & -0.652104 & -0.280835 & -3.860561 \\ 6 & 0.726332 & -2.342331 & -3.516515 \\ 6 & 1.960428 & -2.985535 & -3.692225 \\ 6 & 2.060646 & -4.374278 & -3.699215 \\ 6 & 0.928547 & -5.166895 & -3.542833 \\ 6 & -0.304191 & -4.544992 & -3.353555 \\ 6 & -0.397771 & -3.161604 & -3.324038\end{array}$




\begin{tabular}{|c|c|c|c|}
\hline 5 & 1.834382 & -0.114657 & -4.109288 \\
\hline 15 & -1.731609 & 0.566254 & 0.026976 \\
\hline 6 & -3.460392 & -0.027649 & -0.219845 \\
\hline 6 & -3.750945 & -0.948175 & -1.226726 \\
\hline 6 & -5.046141 & -1.443776 & -1.394478 \\
\hline 6 & -6.042171 & -0.977428 & -0.527881 \\
\hline 6 & -5.782926 & -0.065115 & 0.496675 \\
\hline 6 & -4.473679 & 0.400014 & 0.634632 \\
\hline 6 & -5.399059 & -2.420577 & -2.516744 \\
\hline 6 & -6.847210 & 0.417809 & 1.480201 \\
\hline 6 & -1.573312 & 0.201112 & 1.848547 \\
\hline 6 & -1.259053 & -1.078597 & 2.280006 \\
\hline 6 & -1.192725 & -1.372960 & 3.682295 \\
\hline 6 & -1.464609 & -0.345712 & 4.631122 \\
\hline 6 & -1.808385 & 0.940873 & 4.159121 \\
\hline 6 & -1.858896 & 1.203839 & 2.816619 \\
\hline 6 & -1.380230 & -0.628585 & 6.015230 \\
\hline 6 & -1.037356 & -1.880862 & 6.456207 \\
\hline 6 & -0.766644 & -2.903703 & 5.523238 \\
\hline 6 & -0.845073 & -2.659066 & 4.174466 \\
\hline 6 & -1.007866 & -2.213085 & 1.350395 \\
\hline 6 & 0.254090 & -2.434631 & 0.827968 \\
\hline 6 & 0.537759 & -3.617867 & 0.109153 \\
\hline 6 & -0.433658 & -4.557995 & -0.103396 \\
\hline 6 & -1.752131 & -4.343967 & 0.358091 \\
\hline 6 & -2.046870 & -3.161985 & \\
\hline 6 & -3.374798 & -2.969019 & 1.561858 \\
\hline 6 & -4.354763 & -3.891519 & 1.292530 \\
\hline 6 & -4.057715 & -5.064958 & 0.564359 \\
\hline 6 & -2.782904 & -5.283441 & 0.107439 \\
\hline 7 & 1.300292 & -1.468594 & 1.013569 \\
\hline 6 & 2.246262 & -1.751109 & 2.116492 \\
\hline 6 & 2.738272 & -0.553295 & 2.886740 \\
\hline 6 & 4.084291 & -0.464395 & 3.236570 \\
\hline 6 & 4.553751 & 0.607861 & 3.991951 \\
\hline 6 & 3.677724 & 1.603712 & 4.408367 \\
\hline 6 & 2.329929 & 1.520983 & 4.061379 \\
\hline 6 & 1.866002 & 0.452994 & 3.305563 \\
\hline 6 & -1.900929 & 2.393658 & 0.015503 \\
\hline 6 & -0.883668 & 3.162598 & 0.596730 \\
\hline 6 & -0.904412 & 4.550859 & 0.523051 \\
\hline 6 & -1.937738 & 5.153895 & -0.212450 \\
\hline 6 & -2.942449 & 4.416200 & -0.832386 \\
\hline 6 & -2.915195 & 3.021490 & -0.691731 \\
\hline 6 & 0.176406 & 5.411610 & 1.175586 \\
\hline 6 & 1.077438 & 5.987036 & 0.077057 \\
\hline 6 & -4.053696 & 5.051765 & -1.664526 \\
\hline 6 & -3.990349 & 6.576757 & -1.656437 \\
\hline 6 & -5.419568 & 4.628022 & -1.112123 \\
\hline 6 & -3.919785 & 4.573846 & -3.115364 \\
\hline 6 & -0.459438 & 6.562806 & 1.962281 \\
\hline 6 & 1.034872 & 4.607538 & 2.152403 \\
\hline 1 & 6.787750 & 0.894167 & 0.923316 \\
\hline 1 & -2.128145 & 2.204022 & 2.488710 \\
\hline 1 & 7.684186 & -3.952639 & 1.541312 \\
\hline 1 & -1.590172 & 0.173646 & 6.721313 \\
\hline
\end{tabular}




\begin{tabular}{|c|c|c|c|}
\hline 1 & 6.051827 & 4.660385 & -2.656761 \\
\hline & -3.617842 & -2.061897 & 2.114027 \\
\hline & -5.372280 & -3.716812 & 1.638004 \\
\hline & 3.938250 & 4.469562 & -1.479131 \\
\hline & -2.032880 & 1.724729 & 4.881574 \\
\hline & 5.701238 & -4.512633 & 0.244042 \\
\hline & 8.607796 & 1.233945 & -3.094303 \\
\hline & 3.607797 & -3.773131 & -0.908367 \\
\hline & -2.538250 & -6.182897 & -0.456950 \\
\hline & -0.207444 & -5.469796 & -0.653322 \\
\hline & -0.494644 & -3.895959 & 5.875891 \\
\hline & 7.041316 & -0.128088 & -1.801136 \\
\hline & -0.636333 & -3.461362 & 3.469560 \\
\hline & 1.546318 & -3.762505 & -0.274305 \\
\hline & 8.146458 & 3.648191 & -3.512215 \\
\hline & 8.720924 & 0.159096 & 2.241812 \\
\hline & -4.842859 & -5.792300 & 0.367722 \\
\hline & -0.971710 & -2.088809 & 7.521647 \\
\hline & 9.198640 & -2.268130 & 2.545501 \\
\hline & 2.255330 & 3.054473 & -0.291176 \\
\hline & -4.237827 & 1.107364 & 1.429413 \\
\hline & -2.930481 & -1.282384 & -1.860282 \\
\hline & -7.056047 & -1.349036 & -0.654610 \\
\hline & -3.689871 & 2.415907 & -1.161085 \\
\hline & -0.077256 & 2.651413 & 1.123274 \\
\hline & -1.945838 & 6.237736 & -0.301844 \\
\hline & 1.695000 & -2.407056 & 2.806009 \\
\hline & 1.894027 & 6.577265 & 0.515284 \\
\hline & 1.520715 & 5.185182 & -0.529264 \\
\hline & 0.516261 & 6.639274 & -0.603752 \\
\hline & 0.321969 & 7.164360 & 2.445134 \\
\hline 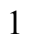 & -1.040858 & 7.239187 & 1.325195 \\
\hline & -1.126674 & 6.183993 & 2.747506 \\
\hline & 0.417621 & 4.127391 & 2.925183 \\
\hline & 1.625648 & 3.824525 & 1.660146 \\
\hline & 1.745919 & 5.273824 & 2.657105 \\
\hline & -4.814569 & 6.982744 & -2.256478 \\
\hline & -4.088036 & 6.986212 & -0.642482 \\
\hline 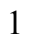 & -3.054855 & 6.951316 & -2.091176 \\
\hline & -5.554914 & 3.539137 & -1.133560 \\
\hline 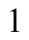 & -5.545501 & 4.962060 & -0.073586 \\
\hline 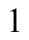 & -6.226206 & 5.072889 & -1.710281 \\
\hline & -2.946079 & 4.861175 & -3.532734 \\
\hline 1 & -4.007121 & 3.483600 & -3.197823 \\
\hline & -4.704980 & 5.021167 & -3.740172 \\
\hline 1 & 3.107119 & -2.336833 & 1.758811 \\
\hline l & 4.780219 & -1.235997 & 2.902547 \\
\hline 1 & 0.821825 & 0.408596 & 3.003118 \\
\hline 1 & 5.610370 & 0.664245 & 4.246952 \\
\hline$\frac{1}{1}$ & 1.632639 & 2.294481 & 4.381143 \\
\hline 1 & 4.041718 & 2.443687 & 4.996358 \\
\hline 1 & -1.507244 & 0.493276 & -2.518350 \\
\hline 1 & -1.517315 & -0.913844 & -4.052645 \\
\hline 1 & -0.683243 & 0.665450 & -4.400223 \\
\hline 1 & -1.369012 & -2.710422 & -3.133154 \\
\hline 1 & -1.206625 & -5.140580 & -3.214344 \\
\hline
\end{tabular}




$\begin{array}{lrrr}1 & 1.004343 & -6.252098 & -3.566700 \\ 1 & 3.035961 & -4.836505 & -3.843633 \\ 1 & 2.862740 & -2.394384 & -3.825433 \\ 1 & 2.150276 & -0.503293 & -5.089885 \\ 1 & 1.615367 & 0.950356 & -4.233632 \\ 1 & 2.689331 & -0.199093 & -3.429436 \\ 6 & -4.159975 & -3.131961 & -3.060664 \\ 6 & -6.375031 & -3.496085 & -2.031083 \\ 6 & -6.051198 & -1.630233 & -3.657181 \\ 6 & -8.216803 & -0.198038 & 1.207495 \\ 6 & -6.426558 & 0.029065 & 2.903083 \\ 6 & -6.979327 & 1.941892 & 1.383746 \\ 1 & -4.458418 & -3.886827 & -3.799353 \\ 1 & -3.479181 & -2.439107 & -3.571693 \\ 1 & -3.603464 & -3.640592 & -2.259277 \\ 1 & -6.577510 & -4.207765 & -2.842030 \\ 1 & -5.953420 & -4.056083 & -1.186150 \\ 1 & -7.341745 & -3.083737 & -1.719769 \\ 1 & -6.311812 & -2.297371 & -4.490390 \\ 1 & -6.969874 & -1.130121 & -3.324485 \\ 1 & -5.370103 & -0.858633 & -4.040163 \\ 1 & -7.168106 & 0.384682 & 3.631194 \\ 1 & -6.352875 & -1.061983 & 3.005104 \\ 1 & -5.454818 & 0.455316 & 3.183491 \\ 1 & -7.719282 & 2.307899 & 2.108264 \\ 1 & -6.030885 & 2.454139 & 1.589106 \\ 1 & -7.311694 & 2.243664 & 0.381590 \\ 1 & -8.942348 & 0.176831 & 1.940401 \\ 1 & -8.593614 & 0.062430 & 0.209997 \\ 1 & -8.198209 & -1.292457 & 1.293954\end{array}$

TS1 $_{I B 2}-\psi_{-1225354 a}$

Number of imaginary frequencies : 1

The smallest frequencies are : $-488.8144 \quad 14.8248 \quad 18.2467 \mathrm{~cm}(-1)$

Electronic energy : $\quad H F=-4360.8649691$

Zero-point correction $=\quad 1.452256($ Hartree/Particle $)$

Thermal correction to Energy= $\quad 1.537070$

Thermal correction to Enthalpy= $\quad 1.538014$

Thermal correction to Gibbs Free Energy= 1.334456

Sum of electronic and zero-point Energies $=\quad-4359.412713$

Sum of electronic and thermal Energies $=\quad-4359.327899$

Sum of electronic and thermal Enthalpies $=\quad-4359.326955$

Sum of electronic and thermal Free Energies= $\quad-4359.530513$

Cartesian Coordinates

$\begin{array}{cccc}6 & 1.838407 & -2.972003 & -3.704132 \\ 6 & 0.619047 & -2.296106 & -3.550292 \\ 6 & -0.530965 & -3.085624 & -3.383099 \\ 6 & -0.471530 & -4.471150 & -3.398846 \\ 6 & 0.748503 & -5.124896 & -3.562707 \\ 6 & 1.902692 & -4.362809 & -3.706347 \\ 6 & 0.554001 & -0.809386 & -3.640406 \\ 6 & 1.794048 & -0.101316 & -4.140260 \\ 45 & -0.195591 & 0.117687 & -1.704016\end{array}$




\begin{tabular}{|c|c|c|c|}
\hline & -0.692436 & -0.185338 & -3.866188 \\
\hline & -1.719877 & 0.596432 & 0.047134 \\
\hline & -1.564386 & 0.189449 & 1.858384 \\
\hline & -1.265583 & -1.102480 & 2.263338 \\
\hline & -1.196494 & -1.422914 & 3.660557 \\
\hline & -1.456106 & -0.410892 & 4.629179 \\
\hline & -1.789930 & 0.887416 & 4.182773 \\
\hline & -1.838284 & 1.176510 & 2.845886 \\
\hline & -1.369073 & -0.719076 & 6.007690 \\
\hline & -1.033190 & -1.981208 & 6.425164 \\
\hline & -0.772696 & -2.988690 & 5.472894 \\
\hline & -0.855238 & -2.719713 & 4.128974 \\
\hline & -1.025072 & -2.225080 & 1.315586 \\
\hline & 0.234111 & -2.445087 & 0.786194 \\
\hline & 0.516726 & -3.628711 & 0.066969 \\
\hline 6 & -0.448393 & -4.579654 & -0.124684 \\
\hline 6 & -1.762030 & -4.374831 & 0.355841 \\
\hline & -2.061709 & -3.180595 & 1.076489 \\
\hline 6 & -3.387897 & -2.992779 & 1.545196 \\
\hline 6 & -4.356297 & -3.939765 & 1.320607 \\
\hline 6 & -4.048733 & -5.132619 & 0.630282 \\
\hline 6 & -2.779888 & -5.338838 & 0.152004 \\
\hline 7 & 1.285321 & -1.485674 & 0.979409 \\
\hline 6 & 2.235583 & -1.794850 & 2.072208 \\
\hline 6 & 2.739331 & -0.615147 & 2.862191 \\
\hline 6 & 4.087698 & -0.540938 & 3.206257 \\
\hline 6 & 4.567885 & 0.514946 & 3.977821 \\
\hline 6 & 3.700261 & 1.508248 & 4.417259 \\
\hline 6 & 2.349934 & 1.439241 & 4.077300 \\
\hline 6 & 1.875462 & 0.388075 & 3.304715 \\
\hline 6 & -1.868268 & 2.425134 & 0.074855 \\
\hline 6 & -0.839749 & 3.173495 & 0.663087 \\
\hline 6 & -0.848210 & 4.562957 & 0.614922 \\
\hline 6 & -1.880752 & 5.188803 & -0.102750 \\
\hline 6 & -2.897132 & 4.471784 & -0.727529 \\
\hline 6 & -2.882260 & 3.074693 & -0.611871 \\
\hline 6 & -4.014555 & 5.131362 & -1.531907 \\
\hline 6 & -5.372389 & 4.750931 & -0.929896 \\
\hline 6 & 0.243229 & 5.402671 & 1.277239 \\
\hline 6 & -0.379499 & 6.542391 & 2.090846 \\
\hline 6 & -3.452821 & 0.025547 & -0.213817 \\
\hline 6 & -3.750893 & -0.805245 & -1.282481 \\
\hline 6 & -5.053782 & -1.282488 & -1.491917 \\
\hline 6 & -6.031233 & -0.916405 & -0.572712 \\
\hline 6 & -5.764074 & -0.080599 & 0.525583 \\
\hline 6 & -4.465856 & 0.388325 & 0.684441 \\
\hline 6 & -5.330882 & -2.176340 & -2.698328 \\
\hline 6 & -4.503207 & -3.459817 & -2.577086 \\
\hline 6 & -6.877033 & 0.245312 & 1.520025 \\
\hline 6 & -8.077121 & 0.856526 & 0.789859 \\
\hline 15 & 1.611779 & -0.572212 & -0.411911 \\
\hline 8 & 2.532507 & 0.577027 & 0.346369 \\
\hline 6 & 3.494998 & 1.284114 & -0.329341 \\
\hline 6 & 4.692444 & 0.686859 & -0.678082 \\
\hline 6 & 5.639043 & 1.462877 & -1.428386 \\
\hline 6 & 5.373625 & 2.842738 & -1.681397 \\
\hline
\end{tabular}




\begin{tabular}{|c|c|c|c|}
\hline 5 & 4.164362 & 3.415620 & -1.219112 \\
\hline & 3.234257 & 2.647658 & -0.576126 \\
\hline & 6.826648 & 0.908294 & -1.970566 \\
\hline & 7.711517 & 1.678662 & -2.684395 \\
\hline & 7.462899 & 3.049941 & -2.897612 \\
\hline & 6.312542 & 3.613636 & -2.408305 \\
\hline & 4.944372 & -0.732199 & -0.336774 \\
\hline 6 & 4.026360 & -1.697550 & -0.706957 \\
\hline 6 & 4.292318 & -3.078386 & -0.579585 \\
\hline 6 & 5.439962 & -3.496392 & 0.035979 \\
\hline 6 & 6.347594 & -2.552276 & 0.576983 \\
\hline 6 & 6.103052 & -1.157967 & 0.391871 \\
\hline 6 & 7.476427 & -2.969250 & 1.323506 \\
\hline 6 & 8.321795 & -2.053222 & 1.895628 \\
\hline 6 & 8.065989 & -0.674491 & 1.741728 \\
\hline 6 & 6.991494 & -0.238719 & 1.005973 \\
\hline 8 & 2.826309 & -1.345631 & -1.270319 \\
\hline 6 & 0.502888 & 2.118832 & -2.042417 \\
\hline 8 & 0.116316 & 2.962795 & -2.741626 \\
\hline 6 & -3.941350 & 4.639044 & -2.982428 \\
\hline 6 & -3.908214 & 6.653938 & -1.540052 \\
\hline 6 & 1.101421 & 4.573141 & 2.232634 \\
\hline 6 & 1.142019 & 5.993962 & 0.185439 \\
\hline 6 & -7.309709 & -1.048882 & 2.219089 \\
\hline 6 & -6.421326 & 1.233201 & 2.592044 \\
\hline 6 & -6.801743 & -2.568584 & -2.808982 \\
\hline 6 & -4.940178 & -1.431410 & -3.980130 \\
\hline 1 & 6.793545 & 0.826485 & 0.914742 \\
\hline 1 & -2.099005 & 2.184942 & 2.536904 \\
\hline 1 & 7.648629 & -4.037949 & 1.445607 \\
\hline 1 & -1.570255 & 0.072242 & 6.728481 \\
\hline 1 & 6.091129 & 4.666240 & -2.580299 \\
\hline 1 & -3.637997 & -2.073965 & 2.074450 \\
\hline 1 & -5.370655 & -3.768645 & 1.676395 \\
\hline 1 & 3.976578 & 4.472217 & -1.405317 \\
\hline 1 & -2.006825 & 1.659087 & 4.920440 \\
\hline 1 & 5.657875 & -4.557795 & 0.143010 \\
\hline 1 & 8.610214 & 1.223202 & -3.094552 \\
\hline 1 & 3.567632 & -3.779722 & -0.989398 \\
\hline 1 & -2.529929 & -6.247457 & -0.395174 \\
\hline 1 & -0.220978 & -5.495989 & -0.666761 \\
\hline 1 & -0.505255 & -3.988776 & 5.806419 \\
\hline 1 & 7.031158 & -0.149447 & -1.828071 \\
\hline 1 & -0.653906 & -3.511081 & 3.409746 \\
\hline 1 & 1.523262 & -3.768603 & -0.323210 \\
\hline 1 & 8.174254 & 3.650711 & -3.459584 \\
\hline 1 & 8.722647 & 0.051680 & 2.215839 \\
\hline 1 & -4.823500 & -5.879260 & 0.469148 \\
\hline 1 & -0.964718 & -2.208457 & 7.486478 \\
\hline 1 & 9.179711 & -2.384659 & 2.475960 \\
\hline 1 & 2.280971 & 3.050772 & -0.243944 \\
\hline 1 & -4.220266 & 1.041610 & 1.518242 \\
\hline 1 & -2.939346 & -1.091424 & -1.952560 \\
\hline 1 & -7.045127 & -1.290083 & -0.701933 \\
\hline 1 & -3.666682 & 2.484799 & -1.084966 \\
\hline 1 & -0.035014 & 2.644655 & 1.174767 \\
\hline
\end{tabular}




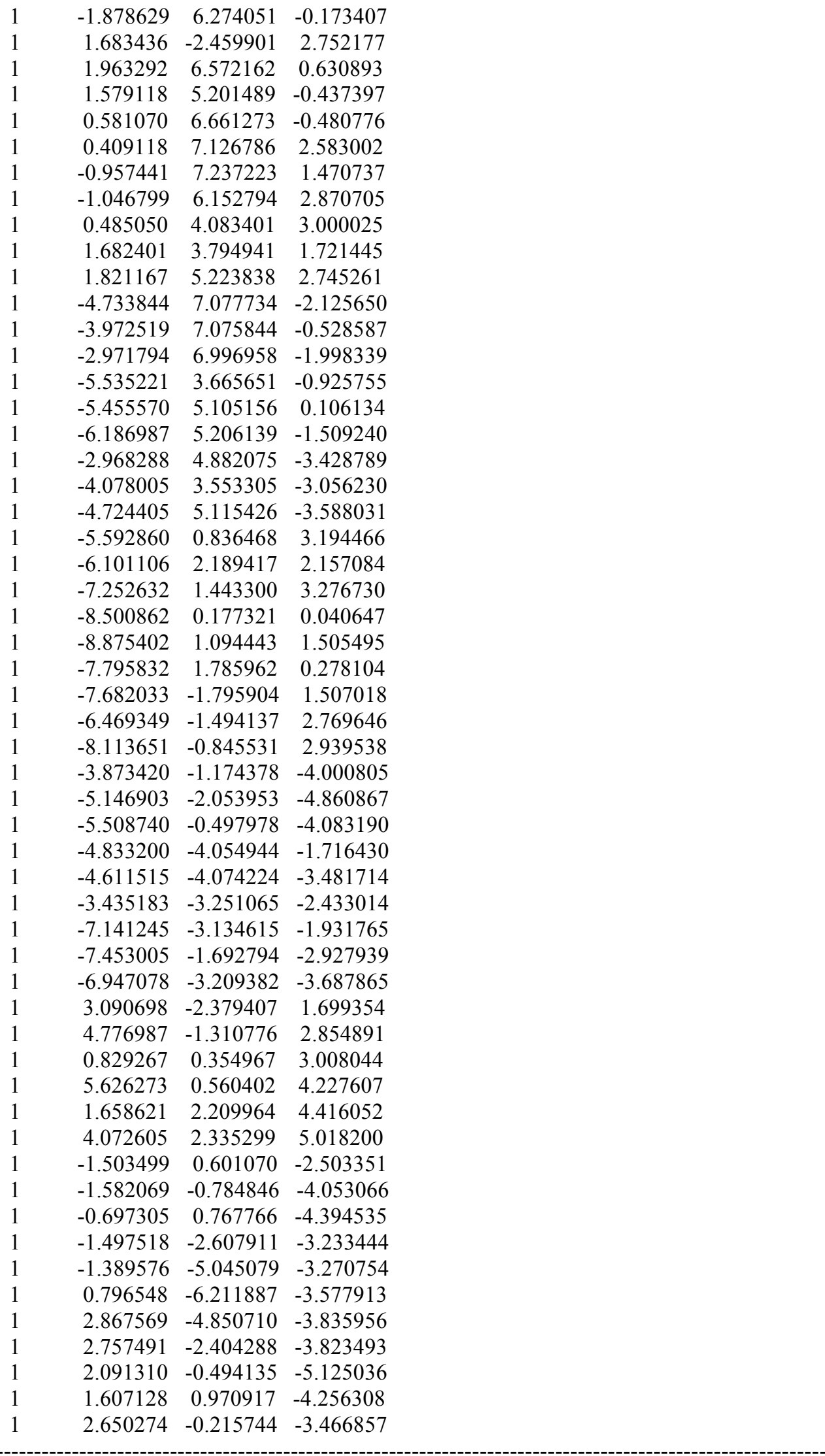


$\operatorname{TS1}_{1 B 2-} \psi_{-1225354 s}$

Number of imaginary frequencies : 1

The smallest frequencies are : $-489.7980 \quad 14.8133 \quad 18.1911 \mathrm{~cm}(-1)$

Electronic energy : $\quad H F=-4360.8651995$

Zero-point correction $=\quad 1.452320($ Hartree/Particle $)$

Thermal correction to Energy= $\quad 1.536992$

Thermal correction to Enthalpy= $\quad 1.537936$

Thermal correction to Gibbs Free Energy $=\quad 1.334990$

Sum of electronic and zero-point Energies $=\quad-4359.412879$

Sum of electronic and thermal Energies $=\quad-4359.328208$

Sum of electronic and thermal Enthalpies $=\quad-4359.327264$

Sum of electronic and thermal Free Energies $=\quad-4359.530209$

Cartesian Coordinates

$\begin{array}{cccc}6 & 7.014220 & -0.204066 & 0.991080 \\ 6 & 6.129014 & -1.125527 & 0.375452 \\ 6 & 6.385967 & -2.519072 & 0.549208 \\ 6 & 7.523685 & -2.932598 & 1.284078 \\ 6 & 8.365759 & -2.014322 & 1.857324 \\ 6 & 8.097209 & -0.636696 & 1.716268 \\ 6 & 4.960867 & -0.703811 & -0.341155 \\ 6 & 4.048363 & -1.674402 & -0.711064 \\ 6 & 4.324606 & -3.053862 & -0.592024 \\ 6 & 5.480301 & -3.466918 & 0.011556 \\ 6 & 4.693176 & 0.717645 & -0.660048 \\ 6 & 3.494278 & 1.299814 & -0.290771 \\ 6 & 3.228164 & 2.669198 & -0.495097 \\ 6 & 4.150859 & 3.458965 & -1.121755 \\ 6 & 5.357668 & 2.902812 & -1.610661 \\ 6 & 5.630045 & 1.517528 & -1.397483 \\ 6 & 6.287777 & 3.696491 & -2.324127 \\ 6 & 7.435798 & 3.150413 & -2.838398 \\ 6 & 7.690886 & 1.774763 & -2.664735 \\ 6 & 6.814949 & 0.982194 & -1.964253 \\ 8 & 2.536402 & 0.573350 & 0.368499 \\ 15 & 1.624208 & -0.572025 & -0.404457 \\ 8 & 2.843417 & -1.329492 & -1.268331 \\ 45 & -0.180353 & 0.125646 & -1.696785 \\ 6 & 0.527266 & 2.123612 & -2.020999 \\ 8 & 0.161074 & 2.968126 & -2.730587 \\ 6 & 0.569385 & -0.793944 & -3.637884 \\ 6 & -0.676693 & -0.167992 & -3.860408 \\ 6 & 0.634659 & -2.281121 & -3.555103 \\ 6 & 1.855120 & -2.955344 & -3.708177 \\ 6 & 1.920525 & -4.346047 & -3.718093 \\ 6 & 0.766428 & -5.109830 & -3.582997 \\ 6 & -0.454657 & -4.457924 & -3.419772 \\ 6 & -0.515270 & -3.072541 & -3.396489 \\ 6 & 1.809392 & -0.084154 & -4.135383 \\ 15 & -1.713788 & 0.578348 & 0.051742 \\ 6 & -3.436182 & -0.024441 & -0.205368 \\ 6 & -3.737465 & -0.840213 & -1.289108 \\ 6 & -5.037420 & -1.313510 & -1.500258 \\ 6 & -6.019496 & -0.963292 & -0.571556\end{array}$




\begin{tabular}{|c|c|c|c|}
\hline 6 & -5.746974 & -0.152761 & 0.537967 \\
\hline 6 & -4.444537 & 0.315754 & 0.697658 \\
\hline & -5.317880 & -2.193782 & -2.716510 \\
\hline & -6.800697 & 0.223423 & 1.578673 \\
\hline & -1.546489 & 0.173648 & 1.862524 \\
\hline & -1.249194 & -1.119970 & 2.264744 \\
\hline & -1.177906 & -1.442681 & 3.661051 \\
\hline & -1.430069 & -0.430877 & 4.632004 \\
\hline & -1.755424 & 0.870389 & 4.188604 \\
\hline & -1.808481 & 1.161337 & 2.852037 \\
\hline & -1.342397 & -0.742056 & 6.009923 \\
\hline ) & -1.012982 & -2.006714 & 6.424538 \\
\hline & -0.759360 & -3.013911 & 5.469928 \\
\hline y & -0.842486 & -2.742133 & 4.126698 \\
\hline & -1.011246 & -2.240369 & 1.313370 \\
\hline b & 0.246603 & -2.456411 & 0.778754 \\
\hline ) & 0.527917 & -3.634175 & 0.049825 \\
\hline 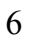 & -0.437944 & -4.583187 & -0.147901 \\
\hline b & -1.749862 & -4.383024 & 0.339178 \\
\hline ) & -2.047332 & -3.195739 & 1.072181 \\
\hline$b$ & -3.370877 & -3.013918 & 1.550246 \\
\hline ) & -4.339752 & -3.959024 & 1.320163 \\
\hline ) & -4.035152 & -5.144247 & 0.615514 \\
\hline ) & -2.768201 & -5.345407 & 0.130124 \\
\hline y & 1.298142 & -1.499159 & 0.977422 \\
\hline ) & 2.242200 & -1.811925 & 2.075211 \\
\hline b & 2.758568 & -0.628760 & 2.851266 \\
\hline 6 & 4.113524 & -0.543248 & \\
\hline 6 & 4.606584 & 0.530275 & 3.902061 \\
\hline$b$ & 3.745059 & 1.529588 & 4.339756 \\
\hline 6 & 2.386730 & 1.446755 & 4.035474 \\
\hline 6 & 1.899564 & 0.376978 & 3.297605 \\
\hline ) & -1.915593 & 2.403182 & 0.074439 \\
\hline 6 & -0.901008 & 3.185871 & 0.639706 \\
\hline 6 & -0.954111 & 4.574806 & 0.588473 \\
\hline 6 & -2.020497 & 5.166109 & -0.108075 \\
\hline 6 & -3.024375 & 4.414403 & -0.712896 \\
\hline 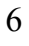 & -2.962846 & 3.019349 & -0.594624 \\
\hline 6 & 0.141448 & 5.442087 & 1.207015 \\
\hline 5 & 1.048199 & 5.950116 & 0.079634 \\
\hline 6 & -4.170694 & 5.034887 & -1.507956 \\
\hline 5 & -4.140955 & 6.560657 & -1.480999 \\
\hline 6 & -5.512716 & 4.572811 & -0.928145 \\
\hline 6 & -4.061689 & 4.578210 & -2.968001 \\
\hline 5 & -0.462697 & 6.639571 & 1.947098 \\
\hline 6 & 0.985810 & 4.660329 & 2.215142 \\
\hline t & 6.807868 & 0.860266 & 0.909543 \\
\hline 1 & -2.065174 & 2.171407 & 2.545216 \\
\hline 1 & 7.705069 & -4.000806 & 1.396841 \\
\hline 1 & -1.537855 & 0.049109 & 6.732441 \\
\hline 1 & 6.061440 & 4.752598 & -2.465542 \\
\hline 1 & -3.617728 & -2.101009 & 2.091242 \\
\hline 1 & -5.353132 & -3.792163 & 1.681013 \\
\hline & 3.958722 & 4.520220 & -1.274582 \\
\hline 1 & -1.963370 & 1.642721 & 4.928151 \\
\hline & 5.706353 & -4.527284 & 0.111703 \\
\hline
\end{tabular}




\begin{tabular}{|c|c|c|c|}
\hline 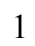 & 8.587429 & 1.333852 & -3.095021 \\
\hline . & 3.600790 & -3.758059 & -0.998555 \\
\hline & -2.520282 & -6.248466 & -0.427104 \\
\hline & -0.212124 & -5.494694 & -0.698681 \\
\hline & -0.496849 & -4.016031 & 5.801247 \\
\hline & 7.023914 & -0.078409 & -1.852148 \\
\hline & -0.646702 & -3.533213 & 3.405666 \\
\hline & 1.533908 & -3.770614 & -0.343359 \\
\hline & 8.140187 & 3.768763 & -3.389973 \\
\hline 1 & 8.751008 & 0.091313 & 2.191545 \\
\hline & -4.810520 & -5.889256 & 0.449801 \\
\hline & -0.944130 & -2.236325 & 7.485312 \\
\hline & 9.230467 & -2.343199 & 2.428955 \\
\hline & 2.276763 & 3.058636 & -0.142062 \\
\hline & -4.203936 & 0.956339 & 1.545815 \\
\hline 1 & -2.927575 & -1.112437 & -1.966979 \\
\hline 1 & -7.031109 & -1.332841 & -0.712841 \\
\hline 1 & -3.736619 & 2.404668 & -1.052816 \\
\hline 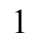 & -0.069017 & 2.684612 & 1.135303 \\
\hline 1 & -2.055100 & 6.250305 & -0.182197 \\
\hline 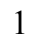 & 1.679839 & -2.463602 & 2.759689 \\
\hline 1 & 1.891568 & 6.524818 & 0.487255 \\
\hline 1 & 1.451070 & 5.114625 & -0.509005 \\
\hline ( & 0.498368 & 6.599559 & -0.613340 \\
\hline 1 & 0.335718 & 7.237700 & 2.405486 \\
\hline 1 & -1.024325 & 7.307601 & 1.284199 \\
\hline 1 & -1.139266 & 6.311353 & 2.746949 \\
\hline 1 & 0.358475 & 4.214190 & 3.000048 \\
\hline & 1.570378 & 3.854096 & 1.754487 \\
\hline 1 & 1.702595 & 5.334755 & 2.700486 \\
\hline 1 & -4.989272 & 6.955826 & -2.054037 \\
\hline 1 & -4.221153 & 6.954672 & -0.459434 \\
\hline 1 & -3.225506 & 6.961560 & -1.934565 \\
\hline 1 & -5.625052 & 3.481474 & -0.961035 \\
\hline 1 & -5.620256 & 4.888852 & 0.118020 \\
\hline 1 & -6.343490 & 5.007785 & -1.499979 \\
\hline 1 & -3.099567 & 4.881069 & -3.401275 \\
\hline 1 & -4.139429 & 3.488178 & -3.063376 \\
\hline 1 & -4.864538 & 5.024944 & -3.570369 \\
\hline 1 & 3.090535 & -2.409959 & 1.707644 \\
\hline 1 & 4.798511 & -1.315919 & 2.810205 \\
\hline 1 & 0.846962 & 0.333143 & 3.026636 \\
\hline 1 & 5.670824 & 0.585260 & 4.123525 \\
\hline 1 & 1.699680 & 2.222177 & 4.372244 \\
\hline 1 & 4.128077 & 2.371854 & 4.912201 \\
\hline 1 & -1.486717 & 0.614380 & -2.495756 \\
\hline 1 & -1.567078 & -0.765631 & -4.049567 \\
\hline 1 & -0.680716 & 0.787141 & -4.385054 \\
\hline 1 & -1.482923 & -2.596699 & -3.247996 \\
\hline 1 & -1.372723 & -5.033208 & -3.297932 \\
\hline 1 & 0.815361 & -6.196673 & -3.604261 \\
\hline 1 & 2.886255 & -4.832447 & -3.846967 \\
\hline 1 & 2.774209 & -2.386346 & -3.821147 \\
\hline 1 & 2.106262 & -0.473595 & -5.121607 \\
\hline 1 & 1.622691 & 0.988429 & -4.247677 \\
\hline 1 & 2.665717 & -0.201206 & -3.462697 \\
\hline
\end{tabular}




$\begin{array}{lrrr}6 & -6.790412 & -2.578390 & -2.834100 \\ 6 & -4.921812 & -1.440741 & -3.991873 \\ 6 & -4.496682 & -3.482406 & -2.603987 \\ 6 & -8.159578 & -0.407670 & 1.287515 \\ 6 & -6.340650 & -0.257954 & 2.960522 \\ 6 & -6.973193 & 1.746482 & 1.601422 \\ 1 & -6.936458 & -3.208656 & -3.720471 \\ 1 & -7.134747 & -3.152727 & -1.964327 \\ 1 & -7.437803 & -1.698689 & -2.944844 \\ 1 & -5.131156 & -2.054913 & -4.877860 \\ 1 & -5.485146 & -0.503374 & -4.087929 \\ 1 & -3.853753 & -1.189332 & -4.009903 \\ 1 & -4.609984 & -4.091852 & -3.511396 \\ 1 & -3.427360 & -3.279285 & -2.460779 \\ 1 & -4.827862 & -4.079979 & -1.745284 \\ 1 & -7.080196 & 0.015162 & 3.725375 \\ 1 & -6.228043 & -1.350358 & 2.978443 \\ 1 & -5.379359 & 0.180100 & 3.258534 \\ 1 & -7.714182 & 2.036236 & 2.358808 \\ 1 & -6.035128 & 2.263949 & 1.838895 \\ 1 & -7.323097 & 2.116672 & 0.628577 \\ 1 & -8.875391 & -0.118118 & 2.067194 \\ 1 & -8.570549 & -0.075169 & 0.325646 \\ 1 & -8.107969 & -1.504567 & 1.279261\end{array}$

TS1 $_{\text {IB2 } 2}-\psi_{-1 \text { s2a3a4a }}$

Number of imaginary frequencies : 1

The smallest frequencies are : $-484.8107 \quad 16.1545 \quad 21.6726 \mathrm{~cm}(-1)$

Electronic energy : $\quad H F=-4360.8641546$

Zero-point correction $=\quad 1.451916($ Hartree/Particle $)$

Thermal correction to Energy= $\quad 1.536813$

Thermal correction to Enthalpy $=\quad 1.537757$

Thermal correction to Gibbs Free Energy= 1.334994

Sum of electronic and zero-point Energies $=\quad-4359.412238$

Sum of electronic and thermal Energies $=\quad-4359.327341$

Sum of electronic and thermal Enthalpies = $\quad-4359.326397$

Sum of electronic and thermal Free Energies $=\quad-4359.529160$

Cartesian Coordinates

$\begin{array}{cccc}6 & -3.160830 & 2.794600 & -0.834048 \\ 6 & -2.071987 & 2.314987 & -0.103527 \\ 6 & -1.160273 & 3.216949 & 0.440989 \\ 6 & -1.353772 & 4.598410 & 0.327973 \\ 6 & -2.457200 & 5.042484 & -0.400393 \\ 6 & -3.366612 & 4.162224 & -1.000396 \\ 15 & -1.732356 & 0.510145 & 0.004345 \\ 6 & -1.577812 & 0.247838 & 1.845793 \\ 6 & -1.193427 & -0.987915 & 2.346025 \\ 6 & -1.118722 & -1.203328 & 3.761867 \\ 6 & -1.449778 & -0.143356 & 4.654750 \\ 6 & -1.865855 & 1.093734 & 4.114261 \\ 6 & -1.928850 & 1.279916 & 2.759498 \\ 6 & -1.356773 & -0.348156 & 6.052037 \\ 6 & -0.949763 & -1.555312 & 6.559115\end{array}$




\begin{tabular}{|c|c|c|c|}
\hline 6 & -0.621206 & -2.610315 & 5.681955 \\
\hline 6 & -0.706412 & -2.441665 & 4.322037 \\
\hline 6 & -0.885111 & -2.155131 & 1.475665 \\
\hline 6 & 0.384827 & -2.342536 & 0.958713 \\
\hline 6 & 0.712084 & -3.534882 & 0.273660 \\
\hline 6 & -0.220229 & -4.520173 & 0.093902 \\
\hline 6 & -1.542734 & -4.349045 & 0.560232 \\
\hline 6 & -1.882419 & -3.157894 & 1.267339 \\
\hline 6 & -3.214737 & -3.008125 & 1.732301 \\
\hline 6 & -4.156736 & -3.977987 & 1.497836 \\
\hline 6 & -3.814224 & -5.160150 & 0.804554 \\
\hline 6 & -2.534127 & -5.338488 & 0.345440 \\
\hline 7 & 1.392252 & -1.329593 & 1.107316 \\
\hline 6 & 2.325949 & -1.506756 & 2.242967 \\
\hline 6 & 2.765844 & -0.235291 & 2.920744 \\
\hline 6 & 4.109232 & -0.042947 & 3.236305 \\
\hline 6 & 4.527543 & 1.119089 & 3.881140 \\
\hline 6 & 3.603090 & 2.100822 & 4.219735 \\
\hline 6 & 2.256852 & 1.912196 & 3.910089 \\
\hline 6 & 1.843948 & 0.753887 & 3.266238 \\
\hline 6 & -0.402982 & 5.554457 & 1.046645 \\
\hline 6 & -0.644446 & 7.013023 & 0.664502 \\
\hline 6 & -4.555171 & 4.716452 & -1.783411 \\
\hline 6 & -4.046279 & 5.565448 & -2.953016 \\
\hline 45 & -0.117601 & 0.042439 & -1.677736 \\
\hline 6 & -0.517285 & -0.346510 & -3.836344 \\
\hline 6 & 0.762524 & -0.876562 & -3.549680 \\
\hline 6 & 1.968376 & -0.100940 & -4.031986 \\
\hline 15 & 1.710730 & -0.509264 & -0.342878 \\
\hline 8 & 2.940448 & -1.328055 & -1.138535 \\
\hline 6 & 4.145426 & -1.613783 & -0.548975 \\
\hline 6 & 5.046303 & -0.605823 & -0.257788 \\
\hline 6 & 6.210242 & -0.947073 & 0.504708 \\
\hline 6 & 6.480044 & -2.316101 & 0.805421 \\
\hline 6 & 5.593807 & -3.317968 & 0.338225 \\
\hline 6 & 4.440826 & -2.974409 & -0.312948 \\
\hline 6 & 7.614150 & -2.647786 & 1.585930 \\
\hline 6 & 8.439916 & -1.671110 & 2.081342 \\
\hline 6 & 8.158741 & -0.314914 & 1.812783 \\
\hline 6 & 7.079054 & 0.037416 & 1.040844 \\
\hline 6 & 4.767684 & 0.773777 & -0.716152 \\
\hline 6 & 3.560031 & 1.373256 & -0.411566 \\
\hline 6 & 3.268399 & 2.703611 & -0.772284 \\
\hline 6 & 4.173808 & 3.429475 & -1.494655 \\
\hline 6 & 5.391578 & 2.843816 & -1.916932 \\
\hline 6 & 5.692010 & 1.499716 & -1.540023 \\
\hline 6 & 6.889896 & 0.925205 & -2.037007 \\
\hline 6 & 7.751180 & 1.646399 & -2.827046 \\
\hline 6 & 7.467045 & 2.985224 & -3.165907 \\
\hline 6 & 6.306686 & 3.565099 & -2.721039 \\
\hline 8 & 2.615644 & 0.704164 & 0.326876 \\
\hline 6 & 0.451868 & 2.095364 & -2.029897 \\
\hline 8 & 0.058113 & 2.870165 & -2.801545 \\
\hline 6 & 0.918534 & -2.353948 & -3.421169 \\
\hline 6 & 2.167988 & -2.967521 & -3.591997 \\
\hline 6 & 2.307058 & -4.352392 & -3.555662 \\
\hline
\end{tabular}




\begin{tabular}{|c|c|c|c|}
\hline & 1.199667 & -5.170747 & -3.359652 \\
\hline & -0.048406 & -4.578101 & -3.177107 \\
\hline & -0.180129 & -3.197544 & -3.191322 \\
\hline & -3.408441 & -0.224423 & -0.237271 \\
\hline & -3.618941 & -1.203847 & -1.202668 \\
\hline & -4.887694 & -1.768415 & -1.388749 \\
\hline & -5.928972 & -1.300107 & -0.586687 \\
\hline & -5.753721 & -0.313355 & 0.391453 \\
\hline & -4.472680 & 0.203837 & 0.561870 \\
\hline & -5.165380 & -2.805187 & -2.477787 \\
\hline & -6.932411 & 0.131640 & 1.255902 \\
\hline & 1.051895 & 5.210084 & 0.715702 \\
\hline & -0.621512 & 5.408349 & 2.557690 \\
\hline & -5.441187 & 3.608452 & -2.349201 \\
\hline & -5.414883 & 5.584062 & -0.857352 \\
\hline & 6.861518 & 1.087220 & 0.859349 \\
\hline & -2.261955 & 2.241579 & 2.379572 \\
\hline & 7.806585 & -3.699106 & 1.796568 \\
\hline & -1.613112 & 0.477509 & 6.714449 \\
\hline & 6.059055 & 4.591792 & -2.987941 \\
\hline & -3.491090 & -2.097720 & 2.261295 \\
\hline & -5.178233 & -3.835757 & 1.846008 \\
\hline & 3.959545 & 4.461000 & -1.771290 \\
\hline & -2.140118 & 1.901069 & 4.792593 \\
\hline & 5.832922 & -4.363023 & 0.528498 \\
\hline & 8.658674 & 1.176570 & -3.199910 \\
\hline & 3.738297 & -3.723329 & -0.673065 \\
\hline & -2.254601 & -6.243368 & -0.193664 \\
\hline & 0.040967 & -5.436454 & -0.432728 \\
\hline ] & -0.299295 & -3.566893 & 6.087166 \\
\hline & 7.121264 & -0.109352 & -1.797382 \\
\hline & -0.453655 & -3.267843 & 3.660456 \\
\hline & 1.721314 & -3.650134 & -0.115505 \\
\hline & 8.159867 & 3.547447 & -3.787753 \\
\hline 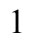 & 8.800432 & 0.460277 & 2.225837 \\
\hline & -4.568496 & -5.926393 & 0.636889 \\
\hline 1 & -0.878275 & -1.703354 & 7.634133 \\
\hline & 9.301929 & -1.936673 & 2.688851 \\
\hline 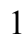 & 2.313377 & 3.114911 & -0.462132 \\
\hline 1 & -4.291140 & 0.967788 & 1.313839 \\
\hline & -2.763893 & -1.526323 & -1.795922 \\
\hline 1 & -6.924553 & -1.719249 & -0.726256 \\
\hline & -3.850105 & 2.081772 & -1.278367 \\
\hline 1 & -0.292266 & 2.831956 & 0.981543 \\
\hline & -2.623600 & 6.111923 & -0.510473 \\
\hline 1 & 1.780258 & -2.124946 & 2.970666 \\
\hline l & 1.729343 & 5.945141 & 1.170273 \\
\hline 1 & 1.341451 & 4.226186 & 1.105336 \\
\hline 1 & 1.221117 & 5.217943 & -0.370403 \\
\hline & 0.050141 & 6.078807 & 3.111294 \\
\hline 1 & -1.654695 & 5.660605 & 2.831516 \\
\hline & -0.424992 & 4.382605 & 2.896970 \\
\hline 1 & -0.517552 & 7.178588 & -0.413243 \\
\hline 1 & -1.647029 & 7.355758 & 0.949958 \\
\hline 1 & 0.076955 & 7.654199 & 1.186455 \\
\hline 1 & -6.289462 & 4.052577 & -2.885199 \\
\hline
\end{tabular}




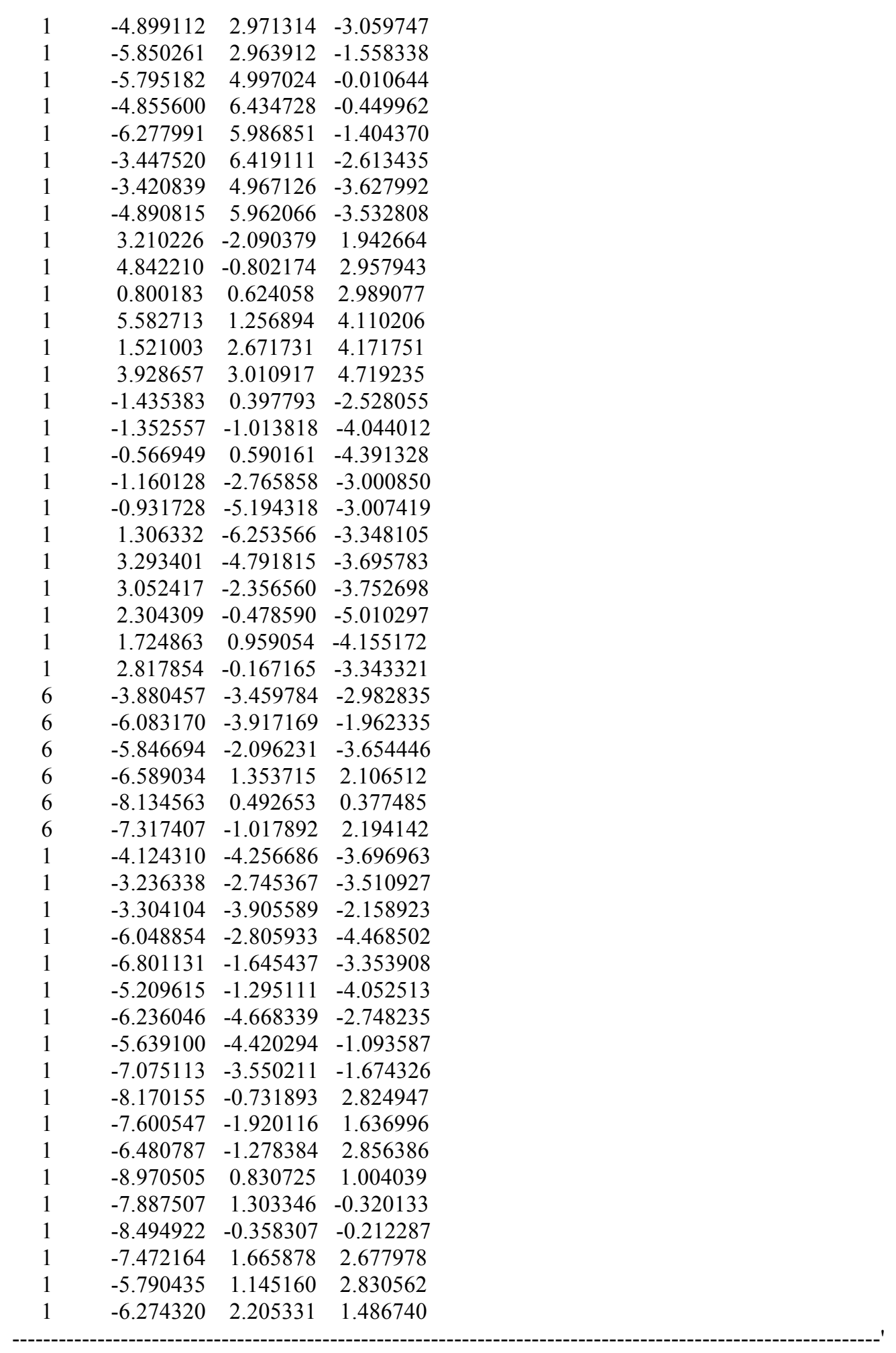

TS1 $_{1 B 2-} \psi_{-1 \mathrm{~s} 2 a 3 a 4 s}$

Number of imaginary frequencies : 1

The smallest frequencies are : $-484.7770 \quad 13.2562 \quad 16.4658 \mathrm{~cm}(-1)$

Electronic energy :

$\mathrm{HF}=-4360.8648427$

Zero-point correction $=\quad 1.451573($ Hartree/Particle $)$ 


$\begin{array}{lc}\text { Thermal correction to Energy= } & 1.536713 \\ \text { Thermal correction to Enthalpy= } & 1.537657 \\ \text { Thermal correction to Gibbs Free Energy= } & 1.332558 \\ \text { Sum of electronic and zero-point Energies }= & -4359.413270 \\ \text { Sum of electronic and thermal Energies }= & -4359.328130 \\ \text { Sum of electronic and thermal Enthalpies }= & -4359.327185 \\ \text { Sum of electronic and thermal Free Energies }= & -4359.532285\end{array}$

Cartesian Coordinates

$\begin{array}{cccc}6 & -3.177823 & 2.772704 & -0.835665 \\ 6 & -2.084552 & 2.303697 & -0.104213 \\ 6 & -1.180070 & 3.214267 & 0.437717 \\ 6 & -1.385430 & 4.593837 & 0.322876 \\ 6 & -2.490678 & 5.027297 & -0.409168 \\ 6 & -3.391191 & 4.138280 & -1.010228 \\ 15 & -1.726458 & 0.502119 & 0.002577 \\ 6 & -1.570798 & 0.236937 & 1.843839 \\ 6 & -1.192111 & -1.001988 & 2.340604 \\ 6 & -1.123377 & -1.223871 & 3.755507 \\ 6 & -1.455280 & -0.167088 & 4.651774 \\ 6 & -1.863337 & 1.074218 & 4.114965 \\ 6 & -1.919437 & 1.266924 & 2.760581 \\ 6 & -1.370663 & -0.379195 & 6.048538 \\ 6 & -0.970910 & -1.590389 & 6.551662 \\ 6 & -0.641059 & -2.642145 & 5.671006 \\ 6 & -0.718076 & -2.466377 & 4.311542 \\ 6 & -0.885784 & -2.165384 & 1.464900 \\ 6 & 0.383381 & -2.350350 & 0.944815 \\ 6 & 0.707466 & -3.537085 & 0.248741 \\ 6 & -0.228134 & -4.517403 & 0.058946 \\ 6 & -1.550155 & -4.347147 & 0.527020 \\ 6 & -1.886008 & -3.163518 & 1.248555 \\ 6 & -3.217973 & -3.014798 & 1.715738 \\ 6 & -4.163045 & -3.978366 & 1.467515 \\ 6 & -3.824773 & -5.152554 & 0.758849 \\ 6 & -2.544902 & -5.330164 & 0.298941 \\ 7 & 1.391997 & -1.339465 & 1.099109 \\ 6 & 2.319952 & -1.517680 & 2.239438 \\ 6 & 2.759540 & -0.246390 & 2.917954 \\ 6 & 4.102384 & -0.054034 & 3.235609 \\ 6 & 4.520231 & 1.110060 & 3.877142 \\ 6 & 3.596029 & 2.094067 & 4.209724 \\ 6 & 2.250180 & 1.905223 & 3.898410 \\ 6 & 1.837733 & 0.744445 & 3.258910 \\ 6 & -0.443192 & 5.558022 & 1.041934 \\ 6 & -0.696950 & 7.014321 & 0.659394 \\ 6 & -4.575614 & 4.681475 & -1.807506 \\ 6 & -4.060120 & 5.543587 & -2.964745 \\ 45 & -0.113550 & 0.047109 & -1.680927 \\ 6 & -0.513275 & -0.330034 & -3.842113 \\ 6 & 0.764991 & -0.864532 & -3.557551 \\ 6 & 1.972813 & -0.088965 & -4.035074 \\ 15 & 1.712294 & -0.510613 & -0.345828 \\ 8 & 2.945518 & -1.321302 & -1.143782 \\ 6 & 4.148777 & -1.608204 & -0.551086\end{array}$




\begin{tabular}{|c|c|c|c|}
\hline 6 & 5.047381 & -0.600369 & -0.252574 \\
\hline & 6.209961 & -0.944036 & 0.511068 \\
\hline & 6.480452 & -2.314192 & 0.806095 \\
\hline & 5.595842 & -3.314841 & 0.333284 \\
\hline & 4.444138 & -2.969472 & -0.319174 \\
\hline & 7.613391 & -2.648469 & 1.587220 \\
\hline & 8.437348 & -1.673396 & 2.088712 \\
\hline & 8.155271 & -0.316239 & 1.826087 \\
\hline & 7.076685 & 0.038688 & 1.053806 \\
\hline & 4.767487 & 0.781761 & -0.702846 \\
\hline & 3.558099 & 1.377261 & -0.397039 \\
\hline & 3.266181 & 2.710583 & -0.746671 \\
\hline & 4.173142 & 3.443789 & -1.459608 \\
\hline & 5.392679 & 2.862951 & -1.883602 \\
\hline & 5.693182 & 1.515755 & -1.517946 \\
\hline & 6.892889 & 0.946688 & -2.016794 \\
\hline & 7.755823 & 1.675645 & -2.797852 \\
\hline & 7.471594 & 3.017259 & -3.125350 \\
\hline & 6.309486 & 3.592168 & -2.678575 \\
\hline & 2.612272 & 0.701246 & 0.332741 \\
\hline & 0.456574 & 2.101265 & -2.022099 \\
\hline & 0.062598 & 2.880251 & -2.789368 \\
\hline & 0.917810 & -2.342812 & -3.435766 \\
\hline & 2.167682 & -2.957059 & -3.601465 \\
\hline & 2.304920 & -4.342267 & -3.572075 \\
\hline & 1.195213 & -5.160309 & -3.388435 \\
\hline & -0.053376 & -4.567122 & -3.211256 \\
\hline f & -0.183579 & -3.186367 & -3.218618 \\
\hline ) & -3.398050 & -0.242049 & -0.236463 \\
\hline 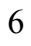 & -3.621720 & -1.203718 & -1.221510 \\
\hline & -4.890141 & -1.761762 & -1.402883 \\
\hline & -5.927338 & -1.314600 & -0.574400 \\
\hline 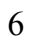 & -5.733454 & -0.362628 & 0.427675 \\
\hline & -4.448811 & 0.159374 & 0.583816 \\
\hline 6 & -5.180011 & -2.776665 & -2.509395 \\
\hline & -6.846265 & 0.129883 & 1.350345 \\
\hline 5 & 1.015084 & 5.225873 & 0.712850 \\
\hline b & -0.661486 & 5.410103 & 2.552928 \\
\hline & -5.437478 & 3.565447 & -2.394498 \\
\hline 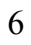 & -5.461054 & 5.532802 & -0.890839 \\
\hline & 6.858401 & 1.089147 & 0.877112 \\
\hline ] & -2.247000 & 2.231552 & 2.383447 \\
\hline & 7.806323 & -3.700589 & 1.793360 \\
\hline . & -1.627635 & 0.444014 & 6.713734 \\
\hline ] & 6.061772 & 4.621024 & -2.936932 \\
\hline & -3.493294 & -2.109337 & 2.254784 \\
\hline 1 & -5.185024 & -3.837413 & 1.814952 \\
\hline & 3.958791 & 4.477608 & -1.727491 \\
\hline & -2.136671 & 1.879832 & 4.795714 \\
\hline & 5.835134 & -4.360442 & 0.520304 \\
\hline 1 & 8.664673 & 1.209868 & -3.172494 \\
\hline 1 & 3.742446 & -3.717271 & -0.683264 \\
\hline 1 & -2.268250 & -6.229301 & -0.251145 \\
\hline 1 & 0.030038 & -5.428747 & -0.477615 \\
\hline & -0.324673 & -3.601828 & 6.073218 \\
\hline & 7.124288 & -0.089905 & -1.786191 \\
\hline
\end{tabular}




\begin{tabular}{|c|c|c|c|}
\hline & -0.464606 & -3.289955 & 3.647023 \\
\hline & 1.716380 & -3.651673 & -0.141567 \\
\hline & 8.165709 & 3.585648 & -3.740107 \\
\hline & 8.795336 & 0.457643 & 2.244090 \\
\hline & -4.582024 & -5.913445 & 0.580597 \\
\hline & -0.906062 & -1.744186 & 7.626292 \\
\hline & 9.298498 & -1.940987 & 2.696552 \\
\hline & 2.309605 & 3.118106 & -0.435974 \\
\hline & -4.265556 & 0.906777 & 1.355029 \\
\hline & -2.773858 & -1.513750 & -1.831283 \\
\hline & -6.921918 & -1.730417 & -0.717056 \\
\hline & -3.863209 & 2.053687 & -1.276632 \\
\hline & -0.307886 & 2.837770 & 0.977575 \\
\hline & -2.665059 & 6.095121 & -0.522877 \\
\hline & 1.769473 & -2.134241 & 2.964904 \\
\hline & 1.685493 & 5.965745 & 1.170056 \\
\hline & 1.311695 & 4.243820 & 1.101842 \\
\hline & 1.186976 & 5.236729 & -0.372745 \\
\hline & 0.002814 & 6.087613 & 3.106806 \\
\hline & -1.697380 & 5.651907 & 2.825938 \\
\hline & -0.454511 & 4.386548 & 2.892851 \\
\hline & -0.570049 & 7.180899 & -0.418184 \\
\hline & -1.703028 & 7.347936 & 0.943369 \\
\hline & 0.017929 & 7.661994 & 1.182291 \\
\hline & -6.280912 & 4.002263 & -2.944059 \\
\hline & -4.874119 & 2.938830 & -3.097895 \\
\hline & -5.853856 & 2.912264 & -1.614694 \\
\hline 1 & -5.856989 & 4.933691 & -0.059949 \\
\hline & -4.915886 & 6.381958 & -0.461514 \\
\hline & -6.314434 & 5.936516 & -1.452313 \\
\hline & -3.481595 & 6.405971 & -2.612517 \\
\hline & -3.413647 & 4.958265 & -3.631255 \\
\hline & -4.901162 & 5.928499 & -3.557335 \\
\hline & 3.204313 & -2.103382 & 1.943281 \\
\hline 1 & 4.835576 & -0.814361 & 2.960813 \\
\hline 1 & 0.794540 & 0.614509 & 2.980029 \\
\hline 1 & 5.574998 & 1.247892 & 4.108034 \\
\hline & 1.514378 & 2.666726 & 4.154726 \\
\hline & 3.921525 & 3.006038 & 4.705842 \\
\hline & -1.431897 & 0.406560 & -2.529017 \\
\hline 1 & -1.350212 & -0.993980 & -4.053585 \\
\hline 1 & -0.560626 & 0.609926 & -4.391671 \\
\hline 1 & -1.164891 & -2.755131 & -3.033614 \\
\hline 1 & -0.938499 & -5.183420 & -3.051738 \\
\hline 1 & 1.300398 & -6.243315 & -3.382436 \\
\hline 1 & 3.291669 & -4.782065 & -3.708102 \\
\hline & 3.053808 & -2.346272 & -3.753059 \\
\hline 1 & 2.309651 & -0.463584 & -5.014237 \\
\hline 1 & 1.731099 & 0.971869 & -4.154609 \\
\hline 1 & 2.821111 & -0.158803 & -3.345441 \\
\hline 6 & -3.901997 & -3.433049 & -3.030213 \\
\hline 6 & -6.103807 & -3.891828 & -2.011456 \\
\hline 6 & -5.860426 & -2.041738 & -3.670463 \\
\hline 6 & -8.172714 & -0.580529 & 1.095824 \\
\hline 0 & -6.446544 & -0.119586 & 2.809720 \\
\hline 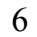 & -7.053583 & 1.632887 & 1.127142 \\
\hline
\end{tabular}




$\begin{array}{lrrr}1 & -4.154961 & -4.212605 & -3.760165 \\ 1 & -3.250763 & -2.715127 & -3.544679 \\ 1 & -3.329708 & -3.901802 & -2.216068 \\ 1 & -6.075340 & -2.735963 & -4.494565 \\ 1 & -6.808253 & -1.585729 & -3.356961 \\ 1 & -5.216950 & -1.241316 & -4.059459 \\ 1 & -6.261058 & -4.629010 & -2.809616 \\ 1 & -5.661596 & -4.411818 & -1.151659 \\ 1 & -7.093661 & -3.524748 & -1.716624 \\ 1 & -7.822639 & 2.020646 & 1.808893 \\ 1 & -6.132849 & 2.204935 & 1.301124 \\ 1 & -7.383780 & 1.832845 & 0.098829 \\ 1 & -7.228925 & 0.248338 & 3.487108 \\ 1 & -6.314464 & -1.192517 & 3.002602 \\ 1 & -5.510442 & 0.384670 & 3.081301 \\ 1 & -8.934475 & -0.200319 & 1.788150 \\ 1 & -8.542405 & -0.409990 & 0.076569 \\ 1 & -8.092432 & -1.663919 & 1.256233\end{array}$

$\mathrm{TS1}_{I B 2-} \psi_{-1 \mathrm{~s} 2 \mathrm{a} 3 \mathrm{~s} 4 \mathrm{a}}$

Number of imaginary frequencies : 1

The smallest frequencies are : -476.8797 $16.8602 \quad 19.3735 \mathrm{~cm}(-1)$

Electronic energy : $\quad H F=-4360.8637265$

Zero-point correction $=\quad 1.452423($ Hartree/Particle)

Thermal correction to Energy $=\quad 1.537233$

Thermal correction to Enthalpy= $\quad 1.538177$

Thermal correction to Gibbs Free Energy $=\quad 1.335554$

Sum of electronic and zero-point Energies $=\quad-4359.411303$

Sum of electronic and thermal Energies $=\quad-4359.326493$

Sum of electronic and thermal Enthalpies $=\quad-4359.325549$

Sum of electronic and thermal Free Energies $=\quad-4359.528173$

Cartesian Coordinates

$\begin{array}{cccc}6 & -3.156047 & 2.833032 & -0.775667 \\ 6 & -2.061274 & 2.340802 & -0.063172 \\ 6 & -1.141718 & 3.232023 & 0.485856 \\ 6 & -1.332809 & 4.615338 & 0.394835 \\ 6 & -2.440744 & 5.072543 & -0.318876 \\ 6 & -3.358021 & 4.203499 & -0.922971 \\ 15 & -1.727923 & 0.533579 & 0.015996 \\ 6 & -1.571301 & 0.242400 & 1.851138 \\ 6 & -1.189511 & -1.001580 & 2.331845 \\ 6 & -1.104564 & -1.234599 & 3.745066 \\ 6 & -1.428353 & -0.185588 & 4.653452 \\ 6 & -1.846267 & 1.058954 & 4.131819 \\ 6 & -1.914878 & 1.263397 & 2.780105 \\ 6 & -1.325419 & -0.407110 & 6.047519 \\ 6 & -0.913892 & -1.619801 & 6.537301 \\ 6 & -0.590401 & -2.663477 & 5.645002 \\ 6 & -0.686375 & -2.478849 & 4.287888 \\ 6 & -0.889385 & -2.163625 & 1.450888 \\ 6 & 0.378404 & -2.354853 & 0.931191 \\ 6 & 0.707468 & -3.557080 & 0.263605 \\ 6 & -0.215026 & -4.557730 & 0.122808\end{array}$




\begin{tabular}{|c|c|c|c|}
\hline 6 & -1.532573 & -4.391371 & 0.606571 \\
\hline 6 & -1.881784 & -3.176878 & 1.267776 \\
\hline 6 & -3.212579 & -3.024678 & 1.735587 \\
\hline 6 & -4.138256 & -4.024663 & 1.569185 \\
\hline 6 & -3.779904 & -5.237380 & 0.940617 \\
\hline 6 & -2.506000 & -5.410374 & 0.462542 \\
\hline 7 & 1.388789 & -1.344372 & 1.076820 \\
\hline 6 & 2.332605 & -1.537372 & 2.202037 \\
\hline 6 & 2.779013 & -0.276261 & 2.894679 \\
\hline 6 & 4.124153 & -0.093437 & 3.208347 \\
\hline 6 & 4.549129 & 1.057839 & 3.867919 \\
\hline 6 & 3.629580 & 2.038224 & 4.223367 \\
\hline 6 & 2.281602 & 1.859093 & 3.915495 \\
\hline 6 & 1.862101 & 0.711582 & 3.256847 \\
\hline 6 & -0.376317 & 5.559218 & 1.122145 \\
\hline 6 & -0.614860 & 7.023438 & 0.760147 \\
\hline 6 & -4.551403 & 4.771600 & -1.688411 \\
\hline 6 & -4.050067 & 5.636719 & -2.849415 \\
\hline 45 & -0.126670 & 0.087603 & -1.683286 \\
\hline 6 & -0.574192 & -0.295426 & -3.840251 \\
\hline 6 & 0.686088 & -0.874351 & -3.570540 \\
\hline 6 & 1.913546 & -0.146361 & -4.072422 \\
\hline 15 & 1.699449 & -0.507140 & -0.364812 \\
\hline 8 & 2.922332 & -1.317908 & -1.179643 \\
\hline 6 & 4.129690 & -1.615876 & -0.600828 \\
\hline 6 & 5.035186 & -0.615505 & -0.297805 \\
\hline 6 & 6.201155 & -0.973427 & 0.454330 \\
\hline 6 & 6.467943 & -2.348143 & 0.731010 \\
\hline 6 & 5.576312 & -3.339377 & 0.251724 \\
\hline 6 & 4.421433 & -2.981087 & -0.387936 \\
\hline 6 & 7.604372 & -2.696599 & 1.500798 \\
\hline 6 & 8.435334 & -1.731110 & 2.009131 \\
\hline 6 & 8.156981 & -0.369716 & 1.765132 \\
\hline 6 & 7.075194 & -0.000980 & 1.003912 \\
\hline 6 & 4.760220 & 0.773059 & -0.731183 \\
\hline 6 & 3.555922 & 1.371836 & -0.412134 \\
\hline 6 & 3.269730 & 2.710779 & -0.744643 \\
\hline 6 & 4.178476 & 3.448165 & -1.450974 \\
\hline 6 & 5.393085 & 2.865939 & -1.886968 \\
\hline 6 & 5.686663 & 1.512019 & -1.541029 \\
\hline 6 & 6.880741 & 0.942594 & -2.052780 \\
\hline 6 & 7.745262 & 1.677059 & -2.826894 \\
\hline 6 & 7.468230 & 3.024857 & -3.134459 \\
\hline 6 & 6.311335 & 3.600515 & -2.675325 \\
\hline 8 & 2.611392 & 0.693661 & 0.316998 \\
\hline 6 & 0.472463 & 2.108659 & -2.035896 \\
\hline 8 & 0.076932 & 2.927391 & -2.759957 \\
\hline 6 & 0.790136 & -2.355100 & -3.427412 \\
\hline 6 & 2.024657 & -3.005953 & -3.566513 \\
\hline 6 & 2.124835 & -4.393496 & -3.509692 \\
\hline 6 & 0.992474 & -5.177652 & -3.320059 \\
\hline 6 & -0.242594 & -4.548929 & -3.171331 \\
\hline 6 & -0.337755 & -3.165891 & -3.215075 \\
\hline 6 & -3.403234 & -0.193708 & -0.242195 \\
\hline 6 & -3.615049 & -1.103310 & -1.266305 \\
\hline 6 & -4.884016 & -1.658145 & -1.495059 \\
\hline
\end{tabular}




\begin{tabular}{|c|c|c|c|}
\hline ) & -5.919741 & -1.272855 & -0.650571 \\
\hline & -5.741832 & -0.353204 & 0.397458 \\
\hline & -4.470141 & 0.172174 & 0.590100 \\
\hline & -5.067978 & -2.645046 & -2.645481 \\
\hline & -6.922152 & 0.014804 & 1.295227 \\
\hline & 1.076643 & 5.215570 & 0.782321 \\
\hline & -0.590558 & 5.393805 & 2.631885 \\
\hline 0 & -5.443696 & 3.673371 & -2.263288 \\
\hline U & -5.402183 & 5.627109 & -0.743010 \\
\hline 1 & 6.859865 & 1.052455 & 0.841927 \\
\hline 1 & -2.247550 & 2.230617 & 2.414142 \\
\hline 1 & 7.794181 & -3.751957 & 1.692801 \\
\hline 1 & -1.577175 & 0.410587 & 6.721458 \\
\hline 1 & 6.068934 & 4.634333 & -2.918454 \\
\hline 1 & -3.498329 & -2.092477 & 2.219988 \\
\hline 1 & -5.156753 & -3.881367 & 1.925320 \\
\hline 1 & 3.969225 & 4.486556 & -1.704803 \\
\hline 1 & -2.115956 & 1.857479 & 4.822286 \\
\hline 1 & 5.812670 & -4.388179 & 0.423977 \\
\hline 1 & 8.649639 & 1.210856 & -3.211704 \\
\hline 1 & 3.713625 & -3.721151 & -0.756083 \\
\hline 1 & -2.218445 & -6.333186 & -0.040818 \\
\hline 1 & 0.050331 & -5.486496 & -0.379641 \\
\hline 1 & -0.263656 & -3.624310 & 6.036048 \\
\hline 1 & 7.106460 & -0.098645 & -1.838221 \\
\hline 1 & -0.436318 & -3.297101 & 3.615540 \\
\hline 1 & 1.715183 & -3.672073 & -0.129285 \\
\hline 1 & 8.163542 & 3.597526 & -3.743870 \\
\hline 1 & 8.802551 & 0.396435 & 2.188866 \\
\hline 1 & -4.520270 & -6.026324 & 0.825433 \\
\hline 1 & -0.834388 & -1.780667 & 7.609916 \\
\hline 1 & 9.299141 & -2.009559 & 2.608261 \\
\hline 1 & 2.317099 & 3.120210 & -0.424431 \\
\hline 1 & -4.291530 & 0.888826 & 1.388098 \\
\hline 1 & -2.764115 & -1.383801 & -1.888493 \\
\hline 1 & -6.911371 & -1.693790 & -0.803906 \\
\hline 1 & -3.851563 & 2.127932 & -1.222681 \\
\hline 1 & -0.270454 & 2.836296 & 1.013519 \\
\hline 1 & -2.604718 & 6.143900 & -0.412602 \\
\hline 1 & 1.792790 & -2.165468 & 2.925593 \\
\hline 1 & 1.757174 & 5.943518 & 1.243673 \\
\hline 1 & 1.365468 & 4.226460 & 1.158982 \\
\hline 1 & 1.242404 & 5.236385 & -0.304099 \\
\hline 1 & 0.084867 & 6.054816 & 3.192251 \\
\hline 1 & -1.622110 & 5.645520 & 2.912284 \\
\hline 1 & -0.396251 & 4.363024 & 2.956815 \\
\hline 1 & -0.492026 & 7.202684 & -0.315871 \\
\hline 1 & -1.615083 & 7.365659 & 1.054360 \\
\hline 1 & 0.110744 & 7.655437 & 1.287453 \\
\hline 1 & -6.296949 & 4.126477 & -2.783666 \\
\hline 1 & -4.908682 & 3.047970 & -2.989445 \\
\hline 1 & -5.844924 & 3.016027 & -1.479088 \\
\hline 1 & -5.777856 & 5.028179 & 0.097323 \\
\hline 1 & -4.837616 & 6.470057 & -0.326915 \\
\hline 1 & -6.268119 & 6.040409 & -1.277597 \\
\hline 1 & -3.448857 & 6.485516 & -2.502027 \\
\hline
\end{tabular}




\begin{tabular}{|c|c|c|c|}
\hline & -3.429273 & 5.047759 & -3.536793 \\
\hline & -4.898395 & 6.041569 & -3.417868 \\
\hline & 3.213919 & -2.117362 & 1.886701 \\
\hline & 4.853209 & -0.851619 & 2.917219 \\
\hline & 0.817140 & 0.589689 & 2.980674 \\
\hline & 5.605622 & 1.188375 & 4.095249 \\
\hline & 1.549525 & 2.617657 & 4.190253 \\
\hline & 3.960259 & 2.939944 & 4.734559 \\
\hline & -1.440826 & 0.486196 & -2.518915 \\
\hline & -1.439615 & -0.928133 & -4.031386 \\
\hline & -0.595254 & 0.641417 & -4.396808 \\
\hline & -1.314027 & -2.705964 & -3.071279 \\
\hline & -1.143449 & -5.140617 & -3.006649 \\
\hline & 1.068655 & -6.262661 & -3.287905 \\
\hline & 3.100775 & -4.861690 & -3.628348 \\
\hline & 2.928105 & -2.421225 & -3.718502 \\
\hline & 2.228056 & -0.548088 & -5.048286 \\
\hline & 1.703357 & 0.919560 & -4.207334 \\
\hline & 2.766341 & -0.231213 & -3.390280 \\
\hline 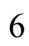 & -6.514884 & -3.107599 & -2.794764 \\
\hline 5 & -4.646735 & -1.978880 & -3.960150 \\
\hline 6 & -4.197099 & -3.879683 & -2.391758 \\
\hline & -6.570598 & 1.132094 & 2.275750 \\
\hline 6 & -8.106092 & 0.488679 & 0.445571 \\
\hline 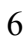 & -7.340112 & -1.219644 & 2.102377 \\
\hline & -6.589993 & -3.816170 & -3.629356 \\
\hline 1 & -6.875463 & -3.623144 & -1.895262 \\
\hline & -7.193901 & -2.272156 & -3.009933 \\
\hline 1 & -4.233628 & -4.560797 & -3.253575 \\
\hline & -3.147535 & -3.613679 & -2.210407 \\
\hline 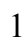 & -4.546842 & -4.425663 & -1.506445 \\
\hline | & -4.787336 & -2.671868 & -4.800333 \\
\hline & -5.245839 & -1.080319 & -4.157004 \\
\hline 1 & -3.591262 & -1.679567 & -3.953967 \\
\hline & -8.203051 & -0.986681 & 2.741045 \\
\hline 1 & -7.622140 & -2.057840 & 1.452811 \\
\hline & -6.522386 & -1.556427 & 2.753836 \\
\hline 1 & -8.946847 & 0.770289 & 1.093454 \\
\hline 1 & -7.835669 & 1.366605 & -0.155237 \\
\hline 1 & -8.468185 & -0.286856 & -0.239480 \\
\hline 1 & -7.449830 & 1.380369 & 2.883429 \\
\hline 1 & -5.768063 & 0.840220 & 2.966203 \\
\hline 1 & -6.257257 & 2.047069 & 1.754088 \\
\hline
\end{tabular}

$\mathrm{TS1}_{I B 2-} \psi_{-1 \mathrm{~s} 2 \mathrm{a} 3 \mathrm{~s} 4 \mathrm{~s}}$

\footnotetext{
Number of imaginary frequencies : 1

The smallest frequencies are : - $477.2381 \quad 16.5665 \quad 19.0762 \mathrm{~cm}(-1)$

Electronic energy : $\quad \mathrm{HF}=-4360.8643004$

Zero-point correction $=\quad 1.451856($ Hartree/Particle $)$

Thermal correction to Energy= $\quad 1.536875$

Thermal correction to Enthalpy= $\quad 1.537819$

Thermal correction to Gibbs Free Energy= $\quad 1.333891$

Sum of electronic and zero-point Energies $=\quad-4359.412445$

Sum of electronic and thermal Energies $=\quad-4359.327425$

Sum of electronic and thermal Enthalpies $=\quad-4359.326481$
} 
Cartesian Coordinates

\begin{tabular}{|c|c|c|c|}
\hline 6 & -3.184258 & 2.809823 & -0.777532 \\
\hline 6 & -2.076087 & 2.330602 & -0.076591 \\
\hline 6 & -1.160005 & 3.232267 & 0.461131 \\
\hline 6 & -1.366236 & 4.613259 & 0.370372 \\
\hline 6 & -2.484434 & 5.057585 & -0.335689 \\
\hline 6 & -3.397869 & 4.178096 & -0.930671 \\
\hline 15 & -1.718948 & 0.527774 & 0.004646 \\
\hline 6 & -1.567062 & 0.240451 & 1.841495 \\
\hline 6 & -1.195782 & -1.005568 & 2.325197 \\
\hline 6 & -1.123269 & -1.239768 & 3.738651 \\
\hline 6 & -1.450132 & -0.189977 & 4.644960 \\
\hline 6 & -1.854291 & 1.057794 & 4.120409 \\
\hline 6 & -1.909112 & 1.263899 & 2.768092 \\
\hline 6 & -1.363148 & -0.413924 & 6.039769 \\
\hline 6 & -0.963839 & -1.629631 & 6.532056 \\
\hline 6 & -0.636277 & -2.673798 & 5.641702 \\
\hline 6 & -0.716763 & -2.486803 & 4.283952 \\
\hline 6 & -0.895165 & -2.166710 & 1.443871 \\
\hline 6 & 0.372715 & -2.354676 & 0.922545 \\
\hline 6 & 0.700946 & -3.552437 & 0.246825 \\
\hline 6 & -0.223310 & -4.550129 & 0.096992 \\
\hline 6 & -1.540950 & -4.386062 & 0.581139 \\
\hline 6 & -1.888761 & -3.177700 & 1.254414 \\
\hline 6 & -3.219748 & -3.028921 & 1.723910 \\
\hline 6 & -4.145869 & -4.026484 & 1.546017 \\
\hline 6 & -3.789761 & -5.232335 & 0.903303 \\
\hline 6 & -2.515713 & -5.401915 & 0.424613 \\
\hline 7 & 1.383393 & -1.345133 & 1.073601 \\
\hline 6 & 2.317623 & -1.535681 & 2.207436 \\
\hline 6 & 2.762956 & -0.272347 & 2.896856 \\
\hline 6 & 4.107057 & -0.088550 & 3.214206 \\
\hline 6 & 4.530622 & 1.067365 & 3.866655 \\
\hline 6 & 3.610887 & 2.051644 & 4.210633 \\
\hline 6 & 2.263780 & 1.871255 & 3.899621 \\
\hline 6 & 1.845637 & 0.718666 & 3.249178 \\
\hline 6 & -0.414901 & 5.568000 & 1.090410 \\
\hline 6 & -0.663037 & 7.028121 & 0.718366 \\
\hline 6 & -4.597837 & 4.733139 & -1.695760 \\
\hline 6 & -4.106185 & 5.615228 & -2.848328 \\
\hline 45 & -0.115892 & 0.090554 & -1.691281 \\
\hline 6 & -0.556883 & -0.287758 & -3.850632 \\
\hline 6 & 0.702155 & -0.867910 & -3.577932 \\
\hline 6 & 1.931847 & -0.139262 & -4.073320 \\
\hline 15 & 1.702908 & -0.506296 & -0.365312 \\
\hline 8 & 2.930715 & -1.315088 & -1.174214 \\
\hline 6 & 4.134648 & -1.612388 & -0.587812 \\
\hline 6 & 5.038029 & -0.611463 & -0.280315 \\
\hline 6 & 6.200461 & -0.968862 & 0.477612 \\
\hline 6 & 6.465943 & -2.343405 & 0.756522 \\
\hline 6 & 5.576487 & -3.335077 & 0.274138 \\
\hline 6 & 4.424922 & -2.977355 & -0.371765 \\
\hline 6 & 7.598768 & -2.691393 & 1.531823 \\
\hline
\end{tabular}




\begin{tabular}{|c|c|c|c|}
\hline ) & 8.427447 & -1.725634 & 2.043326 \\
\hline & 8.150261 & -0.364417 & 1.797107 \\
\hline & 7.071979 & 0.003862 & 1.030697 \\
\hline & 4.763952 & 0.777374 & -0.713689 \\
\hline & 3.557643 & 1.374736 & -0.399401 \\
\hline & 3.272233 & 2.714366 & -0.729955 \\
\hline & 4.184046 & 3.453960 & -1.429985 \\
\hline & 5.401048 & 2.873350 & -1.861528 \\
\hline & 5.693743 & 1.518748 & -1.517395 \\
\hline & 6.890532 & 0.951116 & -2.024772 \\
\hline & 7.758312 & 1.687812 & -2.793089 \\
\hline & 7.482000 & 3.036179 & -3.098832 \\
\hline & 6.322665 & 3.610204 & -2.643810 \\
\hline & 2.609779 & 0.694346 & 0.323040 \\
\hline & 0.479594 & 2.114586 & -2.040262 \\
\hline & 0.080107 & 2.932665 & -2.762850 \\
\hline & 0.805118 & -2.349057 & -3.438120 \\
\hline & 2.040770 & -2.999411 & -3.569676 \\
\hline & 2.140684 & -4.387079 & -3.516124 \\
\hline & 1.006901 & -5.171857 & -3.337694 \\
\hline & -0.229313 & -4.543660 & -3.196669 \\
\hline & -0.324323 & -3.160496 & -3.236897 \\
\hline 6 & -3.385940 & -0.216129 & -0.253439 \\
\hline & -3.609783 & -1.103805 & -1.298883 \\
\hline 6 & -4.876689 & -1.656593 & -1.520630 \\
\hline 5 & -5.907160 & -1.302573 & -0.647324 \\
\hline & -5.712271 & -0.419502 & 0.421984 \\
\hline 6 & -4.438735 & 0.115281 & 0.600127 \\
\hline & -5.073005 & -2.621416 & -2.688338 \\
\hline 6 & -6.824958 & -0.010577 & 1.385505 \\
\hline 0 & 1.040844 & 5.231935 & 0.754568 \\
\hline 6 & -0.628194 & 5.410964 & 2.601271 \\
\hline 6 & -5.469965 & 3.626082 & -2.284643 \\
\hline & -5.465464 & 5.567537 & -0.747076 \\
\hline 1 & 6.857598 & 1.057210 & 0.866982 \\
\hline & -2.232564 & 2.233360 & 2.400003 \\
\hline 1 & 7.787572 & -3.746639 & 1.725434 \\
\hline 1 & -1.617263 & 0.404280 & 6.712193 \\
\hline & 6.080913 & 4.644466 & -2.885695 \\
\hline 1 & -3.508348 & -2.100484 & 2.215252 \\
\hline & -5.165213 & -3.885558 & 1.901010 \\
\hline 1 & 3.975416 & 4.492854 & -1.682272 \\
\hline & -2.124182 & 1.857864 & 4.809013 \\
\hline 1 & 5.811804 & -4.383700 & 0.448884 \\
\hline ] & 8.664728 & 1.222970 & -3.174729 \\
\hline l & 3.718735 & -3.717680 & -0.742536 \\
\hline 1 & -2.229046 & -6.319346 & -0.088991 \\
\hline & 0.041016 & -5.475147 & -0.412780 \\
\hline 1 & -0.318672 & -3.636826 & 6.034871 \\
\hline ] & 7.115705 & -0.090527 & -1.811546 \\
\hline 1 & -0.463921 & -3.305253 & 3.612927 \\
\hline 1 & 1.709030 & -3.666274 & -0.145509 \\
\hline 1 & 8.179875 & 3.610612 & -3.703633 \\
\hline 1 & 8.793905 & 0.402009 & 2.223269 \\
\hline 1 & -4.531577 & -6.018395 & 0.778366 \\
\hline & -0.896851 & -1.792534 & 7.605216 \\
\hline
\end{tabular}




\begin{tabular}{|c|c|c|c|}
\hline 1 & 9.288458 & -2.003718 & 2.646631 \\
\hline & 2.317500 & 3.122296 & -0.413855 \\
\hline & -4.259829 & 0.813731 & 1.416935 \\
\hline & -2.766429 & -1.366047 & -1.938938 \\
\hline 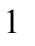 & -6.895375 & -1.726250 & -0.801812 \\
\hline 1 & -3.880134 & 2.097330 & -1.212699 \\
\hline 1 & -0.279334 & 2.846242 & 0.980349 \\
\hline 1 & -2.659434 & 6.127099 & -0.431070 \\
\hline 1 & 1.769898 & -2.158449 & 2.929646 \\
\hline 1 & 1.715265 & 5.966399 & 1.214538 \\
\hline 1 & 1.335468 & 4.246621 & 1.136707 \\
\hline 1 & 1.209845 & 5.249461 & -0.331327 \\
\hline 1 & 0.040668 & 6.082235 & 3.157295 \\
\hline 1 & -1.662204 & 5.654577 & 2.879742 \\
\hline 1 & -0.423712 & 4.384235 & 2.933026 \\
\hline 1 & -0.544798 & 7.199870 & -0.359382 \\
\hline 1 & -1.664217 & 7.366845 & 1.013295 \\
\hline 1 & 0.060465 & 7.668321 & 1.238611 \\
\hline 1 & -6.324135 & 4.071170 & -2.810482 \\
\hline 1 & -4.919375 & 3.012369 & -3.009219 \\
\hline 1 & -5.870790 & 2.959126 & -1.508457 \\
\hline 1 & -5.841189 & 4.954414 & 0.083065 \\
\hline 1 & -4.912984 & 6.411368 & -0.316600 \\
\hline 1 & -6.332079 & 5.977428 & -1.283223 \\
\hline 1 & -3.523142 & 6.473374 & -2.493238 \\
\hline 1 & -3.470971 & 5.042378 & -3.536157 \\
\hline 1 & -4.959111 & 6.007560 & -3.418622 \\
\hline 1 & 3.199344 & -2.120136 & 1.901367 \\
\hline 1 & 4.836678 & -0.849126 & 2.930810 \\
\hline 1 & 0.801566 & 0.595596 & 2.970540 \\
\hline 1 & 5.586330 & 1.198634 & 4.097144 \\
\hline 1 & 1.531378 & 2.632899 & 4.165082 \\
\hline 1 & 3.940782 & 2.957238 & 4.715446 \\
\hline 1 & -1.429014 & 0.488203 & -2.529715 \\
\hline 1 & -1.421845 & -0.919683 & -4.046271 \\
\hline 1 & -0.575824 & 0.650462 & -4.404905 \\
\hline 1 & -1.301835 & -2.701066 & -3.099460 \\
\hline 1 & -1.131411 & -5.135815 & -3.040734 \\
\hline 1 & 1.082893 & -6.256956 & -3.308270 \\
\hline 1 & 3.117547 & -4.854860 & -3.628599 \\
\hline 1 & 2.945251 & -2.414146 & -3.713152 \\
\hline 1 & 2.250525 & -0.539468 & -5.048458 \\
\hline 1 & 1.722479 & 0.926953 & -4.207234 \\
\hline 1 & 2.781678 & -0.225294 & -3.387702 \\
\hline 6 & -6.523292 & -3.073192 & -2.839439 \\
\hline 6 & -4.653504 & -1.938458 & -3.994871 \\
\hline 6 & -4.207991 & -3.864730 & -2.457150 \\
\hline 6 & -8.129868 & -0.756752 & 1.120988 \\
\hline 6 & -6.393475 & -0.309575 & 2.826534 \\
\hline 6 & -7.088760 & 1.492247 & 1.231179 \\
\hline 1 & -6.606643 & -3.762017 & -3.689647 \\
\hline 1 & -6.881995 & -3.607706 & -1.950461 \\
\hline 1 & -7.199206 & -2.229600 & -3.031359 \\
\hline 1 & -4.255335 & -4.535726 & -3.326335 \\
\hline 1 & -3.155872 & -3.605316 & -2.281276 \\
\hline 1 & -4.553756 & -4.418444 & -1.575063 \\
\hline
\end{tabular}




$\begin{array}{rrrr}1 & -4.803908 & -2.617641 & -4.844588 \\ 1 & -5.246884 & -1.032670 & -4.175137 \\ 1 & -3.595749 & -1.647383 & -3.990521 \\ 1 & -7.852437 & 1.825566 & 1.947047 \\ 1 & -6.182777 & 2.088076 & 1.403705 \\ 1 & -7.451935 & 1.721874 & 0.220451 \\ 1 & -7.184002 & -0.013520 & 3.529150 \\ 1 & -6.206199 & -1.382882 & 2.965689 \\ 1 & -5.480330 & 0.227188 & 3.113674 \\ 1 & -8.890180 & -0.439839 & 1.846046 \\ 1 & -8.527818 & -0.550069 & 0.119315 \\ 1 & -8.006556 & -1.843097 & 1.224849\end{array}$

TS1 $1_{I B 2-}-\psi_{-1 s 2 s 3 a 4 a}$

Number of imaginary frequencies : 1

The smallest frequencies are : -471.6573 $16.5314 \quad 18.4206 \mathrm{~cm}(-1)$

Electronic energy : $\quad H F=-4360.8646084$

Zero-point correction $=\quad 1.452432($ Hartree/Particle $)$

Thermal correction to Energy= $\quad 1.537267$

Thermal correction to Enthalpy= $\quad 1.538211$

Thermal correction to Gibbs Free Energy $=\quad 1.334969$

Sum of electronic and zero-point Energies $=\quad-4359.412177$

Sum of electronic and thermal Energies $=\quad-4359.327341$

Sum of electronic and thermal Enthalpies $=\quad-4359.326397$

Sum of electronic and thermal Free Energies $=\quad-4359.529640$

Cartesian Coordinates

$\begin{array}{cccc}6 & -3.065882 & 2.869433 & -0.784735 \\ 6 & -2.029613 & 2.353377 & -0.013547 \\ 6 & -1.120195 & 3.229510 & 0.583932 \\ 6 & -1.279982 & 4.612564 & 0.483783 \\ 6 & -2.336039 & 5.097145 & -0.296731 \\ 6 & -3.226209 & 4.246623 & -0.955146 \\ 15 & -1.729862 & 0.540169 & 0.040537 \\ 6 & -1.573437 & 0.207050 & 1.868592 \\ 6 & -1.208580 & -1.053517 & 2.318840 \\ 6 & -1.135745 & -1.325002 & 3.725212 \\ 6 & -1.450179 & -0.295902 & 4.659173 \\ 6 & -1.848428 & 0.967638 & 4.168244 \\ 6 & -1.909078 & 1.207912 & 2.821893 \\ 6 & -1.359237 & -0.556538 & 6.047309 \\ 6 & -0.969915 & -1.788567 & 6.506318 \\ 6 & -0.657619 & -2.813143 & 5.588244 \\ 6 & -0.741266 & -2.590185 & 4.236052 \\ 6 & -0.920314 & -2.191622 & 1.403528 \\ 6 & 0.345635 & -2.379503 & 0.876659 \\ 6 & 0.654779 & -3.552837 & 0.151758 \\ 6 & -0.291628 & -4.518330 & -0.058603 \\ 6 & -1.610653 & -4.344353 & 0.416203 \\ 6 & -1.932526 & -3.172325 & 1.162848 \\ 6 & -3.262101 & -3.019633 & 1.634732 \\ 6 & -4.217097 & -3.969624 & 1.371944 \\ 6 & -3.892097 & -5.133191 & 0.639915 \\ 6 & -2.615415 & -5.313293 & 0.172091\end{array}$




\begin{tabular}{|c|c|c|c|}
\hline 7 & 1.368665 & -1.388029 & 1.057747 \\
\hline 6 & 2.299766 & -1.616947 & 2.186311 \\
\hline 6 & 2.759139 & -0.375409 & 2.905305 \\
\hline 6 & 4.106501 & -0.209492 & 3.218287 \\
\hline 6 & 4.542709 & 0.926164 & 3.897344 \\
\hline 6 & 3.632271 & 1.907327 & 4.273544 \\
\hline 6 & 2.281807 & 1.744464 & 3.967371 \\
\hline 6 & 1.851193 & 0.612607 & 3.289363 \\
\hline 6 & -0.335755 & 5.536900 & 1.251668 \\
\hline 6 & -0.563511 & 7.010121 & 0.921002 \\
\hline 6 & -4.350572 & 4.751709 & -1.856514 \\
\hline 6 & -4.104647 & 4.244254 & -3.282856 \\
\hline 45 & -0.133423 & 0.111287 & -1.666438 \\
\hline 6 & -0.554348 & -0.199707 & -3.834625 \\
\hline 6 & 0.721824 & -0.752543 & -3.577296 \\
\hline 6 & 1.931487 & 0.027306 & -4.042451 \\
\hline 15 & 1.691454 & -0.514098 & -0.359831 \\
\hline 8 & 2.911189 & -1.309059 & -1.193635 \\
\hline 6 & 4.111794 & -1.634641 & -0.615940 \\
\hline 6 & 5.025004 & -0.651291 & -0.282261 \\
\hline 6 & 6.184781 & -1.039704 & 0.464150 \\
\hline 6 & 6.436469 & -2.423718 & 0.706799 \\
\hline 6 & 5.536694 & -3.393452 & 0.199606 \\
\hline 6 & 4.388431 & -3.007616 & -0.435993 \\
\hline 6 & 7.566405 & -2.803271 & 1.471427 \\
\hline 6 & 8.405524 & -1.859662 & 2.006599 \\
\hline 6 & 8.142350 & -0.489727 & 1.795878 \\
\hline 6 & 7.067065 & -0.090588 & 1.040797 \\
\hline 6 & 4.763602 & 0.750383 & -0.680252 \\
\hline 6 & 3.562853 & 1.350831 & -0.350590 \\
\hline 6 & 3.288456 & 2.699768 & -0.651117 \\
\hline 6 & 4.205084 & 3.446426 & -1.337289 \\
\hline 6 & 5.416431 & 2.865241 & -1.783703 \\
\hline 6 & 5.698477 & 1.501275 & -1.469114 \\
\hline 6 & 6.889565 & 0.934817 & -1.991108 \\
\hline 6 & 7.761994 & 1.680596 & -2.745240 \\
\hline 6 & 7.496211 & 3.037359 & -3.021720 \\
\hline 6 & 6.342529 & 3.611010 & -2.552129 \\
\hline 8 & 2.609130 & 0.661957 & 0.356501 \\
\hline 6 & 0.472770 & 2.160561 & -1.949309 \\
\hline 8 & 0.097753 & 2.972881 & -2.691106 \\
\hline 6 & 0.863228 & -2.234916 & -3.501374 \\
\hline 6 & 2.103497 & -2.855163 & -3.710531 \\
\hline 6 & 2.228149 & -4.241823 & -3.723308 \\
\hline 6 & 1.114437 & -5.054801 & -3.541228 \\
\hline 6 & -0.124655 & -4.455787 & -3.322003 \\
\hline 6 & -0.241423 & -3.074268 & -3.285579 \\
\hline 6 & -3.423403 & -0.148909 & -0.213956 \\
\hline 6 & -3.659826 & -1.090508 & -1.209635 \\
\hline 6 & -4.937974 & -1.634562 & -1.395677 \\
\hline 6 & -5.959449 & -1.188414 & -0.557194 \\
\hline 6 & -5.755231 & -0.247001 & 0.460187 \\
\hline 6 & -4.469352 & 0.260564 & 0.619424 \\
\hline 6 & -5.238524 & -2.630160 & -2.516569 \\
\hline 6 & -6.912981 & 0.157146 & 1.371724 \\
\hline 6 & 1.120476 & 5.194973 & 0.923230 \\
\hline
\end{tabular}




\begin{tabular}{|c|c|c|c|}
\hline 6 & -0.572437 & 5.339175 & 2.754048 \\
\hline 6 & -4.426515 & 6.275633 & -1.899350 \\
\hline 6 & -5.696817 & 4.220998 & -1.350277 \\
\hline 1 & 6.863380 & 0.968822 & 0.904523 \\
\hline 1 & -2.228899 & 2.188292 & 2.479671 \\
\hline 1 & 7.744472 & -3.865052 & 1.637515 \\
\hline 1 & -1.602689 & 0.246341 & 6.741782 \\
\hline 1 & 6.108839 & 4.652131 & -2.771456 \\
\hline 1 & -3.526490 & -2.121494 & 2.190498 \\
\hline 1 & -5.236011 & -3.826834 & 1.727697 \\
\hline 1 & 4.004962 & 4.492211 & -1.567029 \\
\hline 1 & -2.110520 & 1.751509 & 4.878145 \\
\hline 1 & 5.761686 & -4.448578 & 0.346650 \\
\hline 1 & 8.663833 & 1.216540 & -3.138523 \\
\hline 1 & 3.675316 & -3.731047 & -0.826131 \\
\hline 1 & -2.348875 & -6.203677 & -0.396873 \\
\hline 1 & -0.044044 & -5.420524 & -0.615297 \\
\hline 1 & -0.349391 & -3.789420 & 5.955372 \\
\hline 1 & 7.106586 & -0.112920 & -1.800748 \\
\hline 1 & -0.500711 & -3.393474 & 3.542543 \\
\hline 1 & 1.662165 & -3.670170 & -0.241774 \\
\hline 1 & 8.197611 & 3.618790 & -3.615662 \\
\hline 1 & 8.794517 & 0.258763 & 2.240568 \\
\hline 1 & -4.657076 & -5.883253 & 0.449259 \\
\hline 1 & -0.899929 & -1.979439 & 7.574671 \\
\hline 1 & 9.264243 & -2.162034 & 2.601438 \\
\hline 1 & 2.337470 & 3.108393 & -0.324788 \\
\hline 1 & -4.267061 & 0.993578 & 1.396458 \\
\hline 1 & -2.817165 & -1.403204 & 5548 \\
\hline 1 & -6.961289 & -1.594225 & -0.692229 \\
\hline 1 & -3.751091 & 2.182545 & -1.279492 \\
\hline 1 & -0.281916 & 2.818035 & 1.151202 \\
\hline 1 & -2.461908 & 6.170447 & -0.400380 \\
\hline 1 & 1.744614 & -2.250237 & 2.893579 \\
\hline 1 & 1.799160 & 5.907960 & 1.410223 \\
\hline 1 & 1.398384 & 4.194803 & 1.278214 \\
\hline 1 & 1.298931 & 5.240160 & -0.160532 \\
\hline 1 & 0.095574 & 5.987116 & 3.338052 \\
\hline 1 & -1.607430 & 5.587229 & 3.024888 \\
\hline 1 & -0.384345 & 4.301895 & 3.061227 \\
\hline 1 & -0.406490 & 7.218700 & -0.145206 \\
\hline 1 & -1.573052 & 7.344034 & 1.192304 \\
\hline 1 & 0.145396 & 7.627388 & 1.487088 \\
\hline 1 & -5.251714 & 6.584804 & -2.553306 \\
\hline 1 & -4.614472 & 6.706019 & -0.907009 \\
\hline 1 & -3.506921 & 6.721174 & -2.299779 \\
\hline 1 & -5.728248 & 3.124102 & -1.331939 \\
\hline 1 & -5.903744 & 4.580265 & -0.333375 \\
\hline 1 & -6.511530 & 4.564175 & -2.002149 \\
\hline 1 & -3.134403 & 4.593615 & -3.659613 \\
\hline 1 & -4.104769 & 3.148476 & -3.334380 \\
\hline 1 & -4.887598 & 4.610751 & -3.960806 \\
\hline 1 & 3.174680 & -2.204184 & 1.866224 \\
\hline 1 & 4.828654 & -0.967789 & 2.910893 \\
\hline 1 & 0.804525 & 0.504737 & 3.013931 \\
\hline 1 & 5.600853 & 1.044083 & 4.123851 \\
\hline
\end{tabular}




\begin{tabular}{|c|c|c|c|}
\hline & 1.556665 & 2.504023 & 4.257697 \\
\hline & 3.971937 & 2.797058 & 4.799639 \\
\hline & -1.450838 & 0.519531 & \\
\hline & .399346 & -0.850790 & -4.054737 \\
\hline & .599151 & 0.755960 & -4.356781 \\
\hline & -1.213597 & -2.638639 & -3.065268 \\
\hline & -1.012275 & -5.068657 & -3.163069 \\
\hline & 1.209271 & -6.138428 & -3.568663 \\
\hline & 3.207721 & -4.686451 & -3.8916 \\
\hline & 2.991929 & -2.248192 & \\
\hline & 2.255782 & -0.318913 & -5.036170 \\
\hline & 1.697549 & 1.093187 & -4.126350 \\
\hline & 2.785433 & -0.071506 & -3.363354 \\
\hline & -3.969603 & -3.315125 & -3.023380 \\
\hline & -6.201189 & -3.723498 & -2.044938 \\
\hline & -5.879377 & -1.865022 & -3 . \\
\hline & -6.51 & & \\
\hline & & & \\
\hline & -7.3 & -1.0 & \\
\hline & -6.362899 & -4.450320 & -2.851549 \\
\hline & -5.790936 & -4.261599 & -1.180328 \\
\hline & -7.186493 & -3.331435 & -1.76728 \\
\hline & -6.098112 & -2.544295 & -4.51598 \\
\hline & -6.819962 & -1.387464 & -3.377216 \\
\hline & -5.208449 & -1.077730 & -4.049776 \\
\hline & -4.2 & -4.09 & -3.7548 \\
\hline & -3.2 & -2.6 & -3.532736 \\
\hline & -3.415005 & -3.793846 & -2.202999 \\
\hline & -8.915330 & 0.976281 & 1.191252 \\
\hline & -7.789632 & 1.562667 & -0.048936 \\
\hline & -8.469828 & -0.070242 & -0.162747 \\
\hline & -8.207963 & -0.806666 & 2.829951 \\
\hline & -7.698948 & -1.886306 & 1.519416 \\
\hline & -6.553024 & -1.448332 & 2.802909 \\
\hline & -7.373072 & 1.514099 & 2.987281 \\
\hline & & 0.922281 & 3.033342 \\
\hline & -6.180366 & 2.162570 & 1.84946 \\
\hline
\end{tabular}

$\mathrm{TS1}_{I B 2-} \psi_{-1 \mathrm{~s} 2 \mathrm{~s} 3 \mathrm{a} 4 \mathrm{~s}}$

Number of imaginary frequencies : 1

The smallest frequencies are : -471.4698 $16.7462 \quad 21.1613 \mathrm{~cm}(-1)$

Electronic energy : $\quad H F=-4360.8649514$

Zero-point correction $=\quad 1.452337$ (Hartree/Particle)

Thermal correction to Energy= $\quad 1.537139$

Thermal correction to Enthalpy $=\quad 1.538084$

Thermal correction to Gibbs Free Energy= $\quad 1.335164$

Sum of electronic and zero-point Energies $=\quad-4359.412615$

Sum of electronic and thermal Energies $=\quad-4359.327812$

Sum of electronic and thermal Enthalpies $=\quad-4359.326868$

Sum of electronic and thermal Free Energies $=\quad-4359.529787$

Cartesian Coordinates

$\begin{array}{llll}6 & -3.088077 & 2.853524 & -0.781631 \\ 6 & -2.047485 & 2.342674 & -0.012225\end{array}$




\begin{tabular}{|c|c|c|c|}
\hline 6 & -1.140850 & 3.223393 & 0.582731 \\
\hline 6 & -1.306289 & 4.605637 & 0.481547 \\
\hline 6 & -2.363084 & 5.084840 & -0.301208 \\
\hline 6 & -3.250402 & 4.229957 & -0.958124 \\
\hline 15 & -1.732032 & 0.531681 & 0.039832 \\
\hline 6 & -1.575641 & 0.197468 & 1.868031 \\
\hline 6 & -1.210311 & -1.063469 & 2.316757 \\
\hline 6 & -1.139543 & -1.337977 & 3.722442 \\
\hline 6 & -1.456208 & -0.311162 & 4.658052 \\
\hline 6 & -1.852934 & 0.953490 & 4.168955 \\
\hline 6 & -1.911531 & 1.196626 & 2.822842 \\
\hline 6 & -1.368248 & -0.574988 & 6.045797 \\
\hline 6 & -0.979707 & -1.808022 & 6.502682 \\
\hline 6 & -0.665082 & -2.830378 & 5.582866 \\
\hline 6 & -0.745767 & -2.604276 & 4.231048 \\
\hline 6 & -0.921309 & -2.198660 & 1.398583 \\
\hline 6 & 0.344033 & -2.382878 & 0.868806 \\
\hline 6 & 0.652195 & -3.551993 & 0.136781 \\
\hline 6 & -0.294666 & -4.516172 & -0.077782 \\
\hline 6 & -1.612959 & -4.345338 & 0.400214 \\
\hline 6 & -1.933808 & -3.178105 & 1.154748 \\
\hline 6 & -3.262998 & -3.027264 & 1.628806 \\
\hline 6 & -4.218368 & -3.975222 & 1.359987 \\
\hline 6 & -3.894327 & -5.134524 & 0.620815 \\
\hline 6 & -2.618106 & -5.312500 & 0.151011 \\
\hline 7 & 1.366573 & -1.391476 & \\
\hline 6 & 2.294155 & -1.619866 & 2.184880 \\
\hline 6 & 2.751442 & -0.377779 & 2.904324 \\
\hline 6 & 4.098274 & -0.209735 & 3.218325 \\
\hline 6 & 4.532474 & 0.927866 & 3.895480 \\
\hline 6 & 3.620616 & 1.908892 & 4.268579 \\
\hline 6 & 2.270574 & 1.743688 & 3.961746 \\
\hline 6 & 1.841969 & 0.609734 & \\
\hline 6 & -0.365755 & 5.534203 & 1.248887 \\
\hline 6 & -0.599011 & 7.006183 & 0.916631 \\
\hline 6 & -4.366295 & 4.730394 & -1.872914 \\
\hline 6 & -4.090023 & 4.235065 & -3.298106 \\
\hline 45 & -0.133848 & 0.114565 & -1.667232 \\
\hline 6 & -0.549524 & -0.183972 & -3.837886 \\
\hline 6 & 0.726239 & -0.737688 & -3.580279 \\
\hline 6 & 1.936694 & 0.044940 & -4.038610 \\
\hline 15 & 1.690445 & -0.512710 & -0.361149 \\
\hline 8 & 2.912481 & -1.303384 & -1.195450 \\
\hline 6 & 4.112104 & -1.629146 & -0.615661 \\
\hline 6 & 5.023786 & -0.645599 & -0.278411 \\
\hline 6 & 6.182690 & -1.034416 & 0.469185 \\
\hline 6 & 6.434975 & -2.418656 & 0.709932 \\
\hline 6 & 5.536571 & -3.388203 & 0.199989 \\
\hline 6 & 4.389145 & -3.002170 & -0.437002 \\
\hline 6 & 7.563992 & -2.798717 & 1.475675 \\
\hline 6 & 8.401626 & -1.855446 & 2.013740 \\
\hline 6 & 8.137803 & -0.485344 & 1.804932 \\
\hline 6 & 7.063408 & -0.085679 & 1.048862 \\
\hline 6 & 4.761355 & 0.756891 & -0.672886 \\
\hline 6 & 3.559506 & 1.354996 & -0.342890 \\
\hline 6 & 3.284586 & 2.704979 & -0.638273 \\
\hline
\end{tabular}




\begin{tabular}{|c|c|c|c|}
\hline & 4.201583 & 3.455014 & -1.320252 \\
\hline & 5.413968 & 2.876481 & -1.767342 \\
\hline & 5.696684 & 1.511560 & -1.457538 \\
\hline & 6.889026 & 0.948049 & -1.979852 \\
\hline & 7.761948 & 1.697376 & -2.729875 \\
\hline & 7.495422 & 3.054948 & -3.001648 \\
\hline & 6.340584 & 3.625894 & -2.531588 \\
\hline & 2.605243 & 0.662583 & 0.359876 \\
\hline & 0.467994 & 2.168786 & -1.937725 \\
\hline & 0.093282 & 2.980137 & -2.680708 \\
\hline & 0.867673 & -2.220381 & -3.510964 \\
\hline & 2.108524 & -2.839568 & -3.719731 \\
\hline & 2.233514 & -4.226141 & -3.737204 \\
\hline & 1.119577 & -5.039989 & -3.560383 \\
\hline & -0.120186 & -4.442063 & -3.341966 \\
\hline & -0.237380 & -3.060693 & -3.300993 \\
\hline & -3.419520 & -0.170427 & -0.217230 \\
\hline & -3.657932 & -1.106459 & -1.223110 \\
\hline & -4.932003 & -1.650372 & -1.408116 \\
\hline & -5.958221 & -1.218176 & -0.558803 \\
\hline & -5.749423 & -0.293269 & 0.465800 \\
\hline & -4.461168 & 0.221116 & 0.620024 \\
\hline & -5.235151 & -2.636024 & -2.537239 \\
\hline & -6.849118 & 0.166426 & 1.421171 \\
\hline & 1.092135 & 5.197485 & 0.922145 \\
\hline & -0.602399 & 5.336981 & 2.751386 \\
\hline & -4.456417 & 6.253825 & -1.906830 \\
\hline & -5.716287 & 4.183648 & -1.395634 \\
\hline & 6.859289 & 0.973823 & 0.914014 \\
\hline & -2.230254 & 2.177874 & 2.482187 \\
\hline & 7.742516 & -3.860656 & 1.640254 \\
\hline & -1.613407 & 0.226186 & 6.741623 \\
\hline & 6.106393 & 4.667654 & -2.747313 \\
\hline & -3.527607 & -2.131720 & 2.189472 \\
\hline 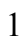 & -5.237861 & -3.833174 & 1.714262 \\
\hline & 4.000971 & 4.501587 & -1.545976 \\
\hline & -2.115476 & 1.736132 & 4.880049 \\
\hline & 5.761887 & -4.443396 & 0.346053 \\
\hline & 8.664774 & 1.235573 & -3.123546 \\
\hline . & 3.676997 & -3.725531 & -0.828981 \\
\hline & -2.352412 & -6.199465 & -0.423669 \\
\hline . & -0.047936 & -5.414941 & -0.640356 \\
\hline & -0.357466 & -3.807443 & 5.948410 \\
\hline 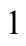 & 7.106604 & -0.100204 & -1.792972 \\
\hline & -0.503670 & -3.405824 & 3.536079 \\
\hline & 1.658794 & -3.666949 & -0.259475 \\
\hline 1 & 8.197212 & 3.639197 & -3.592350 \\
\hline & 8.788755 & 0.262858 & 2.251883 \\
\hline & -4.659795 & -5.883080 & 0.426242 \\
\hline & -0.912102 & -2.001444 & 7.570727 \\
\hline 1 & 9.259634 & -2.158221 & 2.609396 \\
\hline & 2.332845 & 3.111623 & -0.311636 \\
\hline 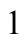 & -4.265521 & 0.947361 & 1.408446 \\
\hline 1 & -2.816166 & -1.410631 & -1.844397 \\
\hline 1 & -6.955799 & -1.626700 & -0.700198 \\
\hline 1 & -3.772485 & 2.163789 & -1.273804 \\
\hline
\end{tabular}




\begin{tabular}{|c|c|c|c|}
\hline & -0.299678 & 2.816103 & 1.148657 \\
\hline & -2.491602 & 6.157474 & -0.408454 \\
\hline 1 & 1.736737 & -2.252724 & 2.890738 \\
\hline & 1.767423 & 5.912751 & 1.410522 \\
\hline & 1.373158 & 4.198219 & 1.277323 \\
\hline & 1.272308 & 5.243925 & -0.161241 \\
\hline & 0.061463 & 5.989539 & 3.334970 \\
\hline & -1.638964 & 5.579395 & 3.021312 \\
\hline & -0.408206 & 4.301232 & 3.060076 \\
\hline 1 & -0.441918 & 7.214354 & -0.149640 \\
\hline 1 & -1.610133 & 7.336260 & 1.186741 \\
\hline & 0.106948 & 7.626832 & 1.482693 \\
\hline 1 & -5.276132 & 6.559209 & -2.569410 \\
\hline L & -4.661194 & 6.675535 & -0.914068 \\
\hline & -3.536542 & 6.711310 & -2.292773 \\
\hline ] & -5.739176 & 3.086287 & -1.386480 \\
\hline & -5.945583 & 4.532769 & -0.380005 \\
\hline 1 & -6.521274 & 4.525363 & -2.060256 \\
\hline & -3.119100 & 4.600707 & -3.657111 \\
\hline 1 & -4.072999 & 3.139584 & -3.354956 \\
\hline & -4.867107 & 4.594274 & -3.986691 \\
\hline & 3.169961 & -2.207320 & 1.867581 \\
\hline 1 & 4.821709 & -0.967581 & 2.912830 \\
\hline & 0.795707 & 0.500118 & 3.009969 \\
\hline 1 & 5.590268 & 1.047485 & 4.122721 \\
\hline & 1.544194 & 2.503118 & 4.249462 \\
\hline 1 & 3.958862 & 2.800254 & 4.792824 \\
\hline ] & -1.451874 & 0.523247 & -2.493132 \\
\hline & -1.393459 & -0.834312 & -4.064158 \\
\hline 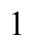 & -0.593648 & 0.774830 & -4.354286 \\
\hline & -1.210220 & -2.626085 & -3.081491 \\
\hline 1 & -1.008040 & -5.055638 & -3.187093 \\
\hline 1 & 1.214753 & -6.123491 & -3.591327 \\
\hline 1 & 3.213534 & -4.669976 & -3.904995 \\
\hline 1 & 2.997108 & -2.231792 & -3.868874 \\
\hline 1 & 2.261819 & -0.294374 & -5.034442 \\
\hline 1 & 1.703274 & 1.111500 & -4.115124 \\
\hline 1 & 2.789903 & -0.059053 & -3.359423 \\
\hline 6 & -3.966991 & -3.312986 & -3.056672 \\
\hline 6 & -6.192134 & -3.737588 & -2.072737 \\
\hline 6 & -5.884107 & -1.861493 & -3.690489 \\
\hline 6 & -8.181701 & -0.527925 & 1.154258 \\
\hline 6 & -6.432386 & -0.146279 & 2.863665 \\
\hline 6 & -7.053932 & 1.677638 & 1.265854 \\
\hline ] & -6.353180 & -4.457372 & -2.885789 \\
\hline 1 & -5.776738 & -4.282379 & -1.214821 \\
\hline 1 & -7.178244 & -3.352646 & -1.788239 \\
\hline 1 & -6.108108 & -2.533814 & -4.530203 \\
\hline 1 & -6.823120 & -1.387292 & -3.377169 \\
\hline 1 & -5.216078 & -1.070659 & -4.057348 \\
\hline 1 & -4.230498 & -4.077816 & -3.798419 \\
\hline 1 & -3.296784 & -2.603002 & -3.557514 \\
\hline 1 & -3.410991 & -3.803306 & -2.243906 \\
\hline 1 & -7.201782 & 0.202100 & 3.565857 \\
\hline 1 & -6.309945 & -1.227402 & 3.011935 \\
\hline 1 & -5.487203 & 0.336833 & 3.142179 \\
\hline
\end{tabular}


$\mathrm{TS1}_{\mathrm{IB2}-} \psi_{-1 \mathrm{~s} 2 \mathrm{2} 334 \mathrm{a}}$

Number of imaginary frequencies : 1

The smallest frequencies are : $-475.0678 \quad 15.7127 \quad 19.0005 \mathrm{~cm}(-1)$

Electronic energy : $\quad H F=-4360.8641822$

Zero-point correction $=\quad 1.452315($ Hartree/Particle $)$

Thermal correction to Energy= $\quad 1.537262$

Thermal correction to Enthalpy= $\quad 1.538206$

Thermal correction to Gibbs Free Energy $=\quad 1.334325$

Sum of electronic and zero-point Energies $=\quad-4359.411867$

Sum of electronic and thermal Energies $=\quad-4359.326920$

Sum of electronic and thermal Enthalpies $=\quad-4359.325976$

Sum of electronic and thermal Free Energies $=\quad-4359.529857$

Cartesian Coordinates

$\begin{array}{cccc}6 & -3.099098 & 2.877274 & -0.743333 \\ 6 & -2.033156 & 2.363006 & -0.012904 \\ 6 & -1.111471 & 3.240712 & 0.563652 \\ 6 & -1.284118 & 4.623083 & 0.480378 \\ 6 & -2.370780 & 5.106227 & -0.258682 \\ 6 & -3.278480 & 4.255327 & -0.891456 \\ 15 & -1.722927 & 0.550793 & 0.037468 \\ 6 & -1.564465 & 0.222090 & 1.865078 \\ 6 & -1.196305 & -1.035955 & 2.319083 \\ 6 & -1.114504 & -1.299471 & 3.727117 \\ 6 & -1.428265 & -0.266700 & 4.657307 \\ 6 & -1.832682 & 0.993110 & 4.162080 \\ 6 & -1.897926 & 1.226987 & 2.814940 \\ 6 & -1.329135 & -0.518946 & 6.046458 \\ 6 & -0.930761 & -1.746069 & 6.510599 \\ 6 & -0.616987 & -2.773726 & 5.596559 \\ 6 & -0.709491 & -2.559192 & 4.243603 \\ 6 & -0.907403 & -2.182108 & 1.413518 \\ 6 & 0.358322 & -2.372991 & 0.888414 \\ 6 & 0.677553 & -3.563961 & 0.196364 \\ 6 & -0.253620 & -4.553223 & 0.034228 \\ 6 & -1.570119 & -4.384856 & 0.520034 \\ 6 & -1.908621 & -3.182693 & 1.208908 \\ 6 & -3.237835 & -3.030310 & 1.681185 \\ 6 & -4.172524 & -4.017551 & 1.490322 \\ 6 & -3.825627 & -5.217535 & 0.831777 \\ 6 & -2.552934 & -5.390861 & 0.350774 \\ 7 & 1.376830 & -1.373764 & 1.052527 \\ 6 & 2.319203 & -1.594758 & 2.173751 \\ 6 & 2.776946 & -0.349889 & 2.887972 \\ 6 & 4.124622 & -0.181182 & 3.198354 \\ 6 & 4.559975 & 0.955393 & 3.876349 \\ 6 & 3.648326 & 1.934885 & 4.253978\end{array}$




\begin{tabular}{|c|c|c|c|}
\hline 6 & 2.297732 & 1.769624 & 3.949730 \\
\hline 6 & 1.867917 & 0.636865 & 3.272587 \\
\hline 6 & -0.328561 & 5.549805 & 1.231420 \\
\hline 6 & -0.555996 & 7.021742 & 0.894020 \\
\hline 6 & -4.449957 & 4.760941 & -1.730611 \\
\hline 6 & -4.276507 & 4.272937 & -3.173836 \\
\hline 45 & -0.132276 & 0.125448 & -1.676536 \\
\hline 6 & -0.594152 & -0.217520 & -3.838893 \\
\hline 6 & 0.662329 & -0.811524 & -3.586346 \\
\hline 6 & 1.893529 & -0.085289 & -4.081458 \\
\hline 15 & 1.691331 & -0.510028 & -0.372508 \\
\hline 8 & 2.908031 & -1.310802 & -1.205977 \\
\hline 6 & 4.113501 & -1.629085 & -0.633978 \\
\hline 6 & 5.026132 & -0.641544 & -0.310985 \\
\hline 6 & 6.190421 & -1.023327 & 0.432110 \\
\hline 6 & 6.447399 & -2.405404 & 0.680170 \\
\hline 6 & 5.548004 & -3.380138 & 0.182049 \\
\hline 6 & 4.395234 & -3.000405 & -0.448970 \\
\hline 6 & 7.581989 & -2.778092 & 1.441272 \\
\hline 6 & 8.420650 & -1.829541 & 1.968347 \\
\hline 6 & 8.152175 & -0.461406 & 1.752738 \\
\hline 6 & 7.072360 & -0.068986 & 1.000621 \\
\hline 6 & 4.760361 & 0.757812 & -0.714510 \\
\hline 6 & 3.560332 & 1.357837 & -0.381786 \\
\hline 6 & 3.283061 & 2.705680 & -0.684661 \\
\hline 6 & 4.196767 & 3.452129 & -1.374936 \\
\hline 6 & 5.407228 & 2.871267 & -1.824160 \\
\hline 6 & 5.691571 & 1.508032 & -1.508370 \\
\hline 6 & 6.881484 & 0.941895 & -2.033309 \\
\hline 6 & 7.750898 & 1.687354 & -2.791237 \\
\hline 6 & 7.483054 & 3.043480 & -3.068 \\
\hline 6 & 6.330260 & 3.616737 & -2.596563 \\
\hline 8 & 2.611423 & 0.670485 & 0.332800 \\
\hline 6 & 0.491392 & 2.138821 & -1.999742 \\
\hline 8 & 0.116048 & 2.975308 & -2.714222 \\
\hline 6 & 0.754595 & -2.295193 & -3.469313 \\
\hline 6 & 1.983942 & -2.953258 & -3.619293 \\
\hline 6 & 2.072810 & -4.342389 & -3.587770 \\
\hline 6 & 0.933764 & -5.120567 & -3.414007 \\
\hline 6 & -0.296369 & -4.484606 & -3.255119 \\
\hline 6 & -0.380114 & -3.100324 & -3.272982 \\
\hline 6 & -3.409908 & -0.148522 & -0.223207 \\
\hline 6 & -3.643375 & -1.029062 & -1.267477 \\
\hline 6 & -4.920612 & -1.565837 & -1.493926 \\
\hline 6 & -5.939733 & -1.200206 & -0.620722 \\
\hline 6 & -5.738362 & -0.313450 & 0.451432 \\
\hline 6 & -4.461905 & 0.204094 & 0.633586 \\
\hline 6 & -5.129875 & -2.512515 & -2.673470 \\
\hline 6 & -6.900756 & 0.031152 & 1.381568 \\
\hline 6 & 1.124197 & 5.203772 & 0.892841 \\
\hline 6 & -0.552445 & 5.360634 & 2.736934 \\
\hline 6 & -4.540688 & 6.284544 & -1.750128 \\
\hline 6 & -5.763392 & 4.211697 & -1.161084 \\
\hline 1 & 6.864737 & 0.989176 & 0.860826 \\
\hline 1 & -2.220580 & 2.205075 & 2.468959 \\
\hline 1 & 7.763983 & -3.838566 & 1.611388 \\
\hline
\end{tabular}




\begin{tabular}{|c|c|c|c|}
\hline & -1.573077 & 0.286793 & 6.737442 \\
\hline & 6.094908 & 4.657307 & -2.816714 \\
\hline 1 & -3.515537 & -2.107451 & 2.187857 \\
\hline & -5.189764 & -3.873885 & 1.849607 \\
\hline & 3.994817 & 4.497261 & -1.605991 \\
\hline 1 & -2.094497 & 1.779518 & 4.869259 \\
\hline & 5.776746 & -4.433919 & 0.332881 \\
\hline & 8.651910 & 1.223532 & -3.186682 \\
\hline & 3.681480 & -3.727552 & -0.831127 \\
\hline & -2.273319 & -6.304192 & -0.173871 \\
\hline 1 & 0.003721 & -5.473716 & -0.487176 \\
\hline & -0.300448 & -3.745950 & 5.967351 \\
\hline & 7.100010 & -0.105386 & -1.842172 \\
\hline & -0.466915 & -3.365395 & 3.554197 \\
\hline & 1.684698 & -3.679692 & -0.197811 \\
\hline & 8.182102 & 3.624713 & -3.665707 \\
\hline & 8.803844 & 0.291103 & 2.191323 \\
\hline & -4.573369 & -5.996330 & 0.697059 \\
\hline & -0.854175 & -1.930518 & 7.579628 \\
\hline & 9.282975 & -2.126601 & 2.560637 \\
\hline & 2.333314 & 3.114498 & -0.354922 \\
\hline & -4.266756 & 0.900066 & 1.445966 \\
\hline ] & -2.803457 & -1.301220 & -1.908140 \\
\hline & -6.936364 & -1.611342 & -0.769402 \\
\hline 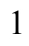 & -3.797048 & 2.189482 & -1.218771 \\
\hline & -0.255596 & 2.829226 & 1.104315 \\
\hline 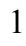 & -2.509398 & 6.179384 & -0.345840 \\
\hline 1 & 1.773940 & -2.230494 & 2.886476 \\
\hline & 1.807381 & 5.919377 & 1.369467 \\
\hline 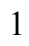 & 1.404860 & 4.206172 & 1.252683 \\
\hline & 1.294065 & 5.241792 & -0.192541 \\
\hline 1 & 0.123001 & 6.008843 & 3.312067 \\
\hline ] & -1.584216 & 5.613474 & 3.015570 \\
\hline | & -0.365707 & 4.323768 & 3.046438 \\
\hline 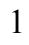 & -0.418380 & 7.221263 & -0.176602 \\
\hline | & -1.558349 & 7.363175 & 1.181877 \\
\hline 1 & 0.166102 & 7.639850 & 1.442240 \\
\hline & -5.400460 & 6.594471 & -2.357531 \\
\hline 1 & -4.681573 & 6.700875 & -0.744112 \\
\hline ] & -3.646190 & 6.743084 & -2.190766 \\
\hline 1 & -5.784771 & 3.114335 & -1.160457 \\
\hline 1 & -5.917187 & 4.551524 & -0.128224 \\
\hline 1 & -6.614844 & 4.558902 & -1.761864 \\
\hline 1 & -3.334584 & 4.641424 & -3.600459 \\
\hline & -4.264090 & 3.178036 & -3.238455 \\
\hline 1 & -5.101350 & 4.635269 & -3.802570 \\
\hline l & 3.195076 & -2.177160 & 1.847769 \\
\hline 1 & 4.847518 & -0.938485 & 2.890228 \\
\hline 1 & 0.821005 & 0.526862 & 2.998862 \\
\hline 1 & 5.618307 & 1.075292 & 4.100974 \\
\hline 1 & 1.571785 & 2.527861 & 4.241328 \\
\hline 1 & 3.987133 & 2.825231 & 4.779584 \\
\hline 1 & -1.444723 & 0.554194 & -2.499503 \\
\hline 1 & -1.466396 & -0.839436 & -4.034234 \\
\hline 1 & -0.610760 & 0.728996 & -4.378966 \\
\hline 1 & -1.352881 & -2.634758 & -3.123952 \\
\hline
\end{tabular}




$\begin{array}{lrrr}1 & -1.202573 & -5.071575 & -3.103452 \\ 1 & 1.000997 & -6.206585 & -3.401979 \\ 1 & 3.045000 & -4.816343 & -3.714127 \\ 1 & 2.892036 & -2.373084 & -3.760500 \\ 1 & 2.202749 & -0.476112 & -5.063399 \\ 1 & 1.690226 & 0.983649 & -4.201709 \\ 1 & 2.747538 & -0.184868 & -3.402868 \\ 6 & -6.581681 & -2.962369 & -2.812667 \\ 6 & -4.727244 & -1.802605 & -3.971178 \\ 6 & -4.260970 & -3.759115 & -2.479302 \\ 6 & -6.511668 & 1.075841 & 2.425717 \\ 6 & -8.076492 & 0.586924 & 0.571222 \\ 6 & -7.347147 & -1.236449 & 2.118868 \\ 1 & -4.886077 & -2.464657 & -4.832805 \\ 1 & -5.324094 & -0.894361 & -4.126076 \\ 1 & -3.669833 & -1.509459 & -3.971781 \\ 1 & -4.319782 & -4.411120 & -3.362026 \\ 1 & -3.206421 & -3.503806 & -2.313223 \\ 1 & -4.594505 & -4.332129 & -1.605061 \\ 1 & -6.675997 & -3.640351 & -3.670373 \\ 1 & -6.928460 & -3.507776 & -1.925414 \\ 1 & -7.259927 & -2.116465 & -2.985198 \\ 1 & -8.908209 & 0.845541 & 1.240124 \\ 1 & -7.787074 & 1.495728 & 0.027829 \\ 1 & -8.457688 & -0.134280 & -0.161275 \\ 1 & -8.201184 & -1.017371 & 2.774123 \\ 1 & -7.653826 & -2.029959 & 1.426054 \\ 1 & -6.536075 & -1.629132 & 2.746848 \\ 1 & -7.374740 & 1.295682 & 3.066694 \\ 1 & -5.699627 & 0.726190 & 3.077546 \\ 1 & -6.195498 & 2.019243 & 1.960564\end{array}$

$\operatorname{TS1}_{1 B 2-} \psi_{-1 \mathrm{~s} 2 s 334 \mathrm{~s}}$

Number of imaginary frequencies : 1

The smallest frequencies are : -471.4158 $15.987320 .1815 \mathrm{~cm}(-1)$

Electronic energy : $\quad H F=-4360.8644509$

Zero-point correction $=\quad 1.452569($ Hartree/Particle $)$

Thermal correction to Energy= $\quad 1.537274$

Thermal correction to Enthalpy= $\quad 1.538218$

Thermal correction to Gibbs Free Energy $=\quad 1.335985$

Sum of electronic and zero-point Energies $=\quad-4359.411882$

Sum of electronic and thermal Energies $=\quad-4359.327177$

Sum of electronic and thermal Enthalpies $=\quad-4359.326233$

Sum of electronic and thermal Free Energies $=\quad-4359.528466$

Cartesian Coordinates

$\begin{array}{cccc}6 & -3.116770 & 2.876283 & -0.726938 \\ 6 & -2.042161 & 2.367023 & -0.005800 \\ 6 & -1.120759 & 3.248374 & 0.565585 \\ 6 & -1.299991 & 4.629874 & 0.484572 \\ 6 & -2.391223 & 5.108329 & -0.251082 \\ 6 & -3.299074 & 4.253632 & -0.878830 \\ 15 & -1.716981 & 0.557415 & 0.034381 \\ 6 & -1.563587 & 0.218377 & 1.860988\end{array}$




\begin{tabular}{|c|c|c|c|}
\hline 6 & -1.210265 & -1.046105 & 2.309239 \\
\hline 6 & -1.140817 & -1.320206 & 3.715597 \\
\hline f & -1.451143 & -0.291079 & 4.650873 \\
\hline 6 & -1.835703 & 0.977176 & 4.161640 \\
\hline 6 & -1.888846 & 1.221324 & 2.815548 \\
\hline f & -1.367421 & -0.555114 & 6.038908 \\
\hline 6 & -0.987320 & -1.790270 & 6.496873 \\
\hline 6 & -0.676396 & -2.814267 & 5.577641 \\
\hline 6 & -0.753881 & -2.588211 & 4.225672 \\
\hline 6 & -0.926360 & -2.186276 & 1.395136 \\
\hline 6 & 0.338040 & -2.376064 & 0.865784 \\
\hline 6 & 0.649014 & -3.558030 & 0.154902 \\
\hline 6 & -0.290733 & -4.535668 & -0.028046 \\
\hline 6 & -1.606528 & -4.365612 & 0.458969 \\
\hline 6 & -1.934519 & -3.176418 & 1.174903 \\
\hline 6 & -3.262314 & -3.023423 & 1.651282 \\
\hline 6 & -4.206979 & -3.994997 & 1.431923 \\
\hline 6 & -3.872194 & -5.181058 & 0.742748 \\
\hline 6 & -2.599415 & -5.356758 & 0.262688 \\
\hline 7 & 1.362017 & -1.384365 & 1.041946 \\
\hline 6 & 2.292699 & -1.614166 & 2.171350 \\
\hline 6 & 2.755819 & -0.373239 & 2.889069 \\
\hline 6 & 4.103445 & -0.211813 & 3.203146 \\
\hline 6 & 4.543527 & 0.924609 & 3.878460 \\
\hline 6 & 3.636873 & 1.911306 & 4.249305 \\
\hline 6 & 2.286041 & 1.752779 & 3.942327 \\
\hline 6 & 1.851505 & 0.619699 & 3.268962 \\
\hline 6 & -0.345991 & 5.560008 & 1.233532 \\
\hline 6 & -0.575011 & 7.030527 & 0.891039 \\
\hline 6 & -4.467985 & 4.754432 & -1.724691 \\
\hline 6 & -4.278272 & 4.270084 & -3.167314 \\
\hline 45 & -0.124688 & 0.147923 & -1.680198 \\
\hline 6 & -0.572554 & -0.161408 & -3.849879 \\
\hline 6 & 0.680866 & -0.761951 & -3.597208 \\
\hline 6 & 1.917049 & -0.031956 & -4.073874 \\
\hline 15 & 1.688736 & -0.509213 & -0.373799 \\
\hline 8 & 2.905736 & -1.307711 & -1.208468 \\
\hline 6 & 4.105240 & -1.638171 & -0.630838 \\
\hline 6 & 5.021742 & -0.658663 & -0.294759 \\
\hline 6 & 6.179525 & -1.053221 & 0.451853 \\
\hline 6 & 6.425923 & -2.438691 & 0.691754 \\
\hline 6 & 5.523100 & -3.404087 & 0.181806 \\
\hline 6 & 4.376743 & -3.012497 & -0.453634 \\
\hline 6 & 7.553579 & -2.824215 & 1.456769 \\
\hline 6 & 8.395568 & -1.885002 & 1.995089 \\
\hline 6 & 8.137680 & -0.513657 & 1.787157 \\
\hline 6 & 7.064791 & -0.108802 & 1.031710 \\
\hline 6 & 4.765854 & 0.745660 & -0.687446 \\
\hline 6 & 3.567502 & 1.349454 & -0.355193 \\
\hline 6 & 3.300087 & 2.702147 & -0.645214 \\
\hline 6 & 4.221566 & 3.449751 & -1.323816 \\
\hline 6 & 5.430505 & 2.865906 & -1.773336 \\
\hline 6 & 5.705371 & 1.498038 & -1.469264 \\
\hline 6 & 6.894602 & 0.929919 & -1.993596 \\
\hline 6 & 7.772001 & 1.677367 & -2.740255 \\
\hline 6 & 7.513282 & 3.037563 & -3.006409 \\
\hline
\end{tabular}




\begin{tabular}{|c|c|c|c|}
\hline 6 & 6.361604 & 3.613143 & -2.534257 \\
\hline 8 & 2.610393 & 0.660576 & 0.346609 \\
\hline & 0.502283 & 2.169250 & -1.974438 \\
\hline & 0.127719 & 3.007187 & -2.687689 \\
\hline & 0.767971 & -2.247303 & -3.499829 \\
\hline & 1.995950 & -2.907421 & -3.651427 \\
\hline & 2.080407 & -4.297050 & -3.634953 \\
\hline & 0.937981 & -5.073466 & -3.475631 \\
\hline & -0.291089 & -4.435407 & -3.317043 \\
\hline & -0.370481 & -3.050763 & -3.319727 \\
\hline & -3.397836 & -0.151896 & -0.236231 \\
\hline & -3.642083 & -0.986620 & -1.320052 \\
\hline & -4.914223 & -1.524228 & -1.547847 \\
\hline & -5.926640 & -1.218116 & -0.636249 \\
\hline & -5.709875 & -0.394133 & 0.475374 \\
\hline & -4.434981 & 0.139468 & 0.651113 \\
\hline & -5.132658 & -2.430304 & -2.757933 \\
\hline & -6.796076 & -0.069485 & 1.500098 \\
\hline & 1.108384 & 5.215347 & 0.900016 \\
\hline & -0.572066 & 5.374803 & 2.739310 \\
\hline & -4.566236 & 6.277635 & -1.742395 \\
\hline & -5.784080 & 4.197772 & -1.169122 \\
\hline & 6.865379 & 0.951681 & 0.897681 \\
\hline & -2.198591 & 2.205125 & 2.474245 \\
\hline & 7.727415 & -3.887032 & 1.620682 \\
\hline & -1.608427 & 0.248007 & 6.733944 \\
\hline & 6.133459 & 4.657118 & -2.745696 \\
\hline & -3.534160 & -2.110183 & 2.178935 \\
\hline & -5.225146 & -3.847065 & 1.788764 \\
\hline 1 & 4.027085 & 4.498420 & -1.545067 \\
\hline & -2.092402 & 1.761935 & 4.872512 \\
\hline & 5.744050 & -4.460340 & 0.326848 \\
\hline & 8.672297 & 1.212071 & -3.135601 \\
\hline & 3.660763 & -3.732299 & -0.845402 \\
\hline 1 & -2.327998 & -6.259962 & -0.283362 \\
\hline & -0.040442 & -5.447659 & -0.567493 \\
\hline ] & -0.374041 & -3.792770 & 5.943724 \\
\hline & 7.106141 & -0.120315 & -1.810900 \\
\hline . & -0.513999 & -3.391472 & 3.531949 \\
\hline 1 & 1.655904 & -3.675315 & -0.239489 \\
\hline & 8.218559 & 3.620250 & -3.594495 \\
\hline 1 & 8.792024 & 0.231439 & 2.234338 \\
\hline & -4.628383 & -5.947248 & 0.585148 \\
\hline 1 & -0.922807 & -1.983901 & 7.565070 \\
\hline & 9.252422 & -2.191907 & 2.590292 \\
\hline 1 & 2.351284 & 3.113523 & -0.315705 \\
\hline 1 & -4.239476 & 0.794283 & 1.500078 \\
\hline 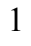 & -2.809829 & -1.221051 & -1.984972 \\
\hline 1 & -6.916506 & -1.637401 & -0.791397 \\
\hline & -3.815917 & 2.185476 & -1.196366 \\
\hline 1 & -0.259485 & 2.840135 & 1.100044 \\
\hline 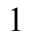 & -2.532919 & 6.180937 & -0.340321 \\
\hline 1 & 1.735515 & -2.245095 & 2.879100 \\
\hline 1 & 1.788426 & 5.932142 & 1.379345 \\
\hline 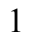 & 1.389264 & 4.218419 & 1.261695 \\
\hline & 1.283265 & 5.253768 & -0.184452 \\
\hline
\end{tabular}




\begin{tabular}{|c|c|c|c|}
\hline ] & 0.099104 & 6.028202 & 3.313579 \\
\hline & -1.605572 & 5.623464 & 3.015201 \\
\hline & -0.380435 & 4.339816 & 3.052493 \\
\hline 1 & -0.438043 & 7.226202 & -0.180372 \\
\hline 1 & -1.577620 & 7.371927 & 1.177978 \\
\hline & 0.146543 & 7.651458 & 1.436770 \\
\hline & -5.424101 & 6.584173 & -2.354199 \\
\hline & -4.714807 & 6.691557 & -0.736468 \\
\hline & -3.671775 & 6.741553 & -2.177417 \\
\hline & -5.802820 & 3.100263 & -1.169203 \\
\hline & -5.950020 & 4.535823 & -0.137594 \\
\hline & -6.630365 & 4.543088 & -1.778307 \\
\hline & -3.334147 & 4.644054 & -3.584287 \\
\hline & -4.259415 & 3.175287 & -3.233253 \\
\hline & -5.098941 & 4.629302 & -3.803299 \\
\hline & 3.165878 & -2.204439 & 1.852075 \\
\hline & 4.822948 & -0.973981 & 2.899147 \\
\hline & 0.804797 & 0.515100 & 2.992728 \\
\hline ] & 5.601888 & 1.038928 & 4.105827 \\
\hline & 1.563722 & 2.516842 & 4.228052 \\
\hline ] & 3.979767 & 2.801902 & 4.771828 \\
\hline & -1.432863 & 0.590315 & -2.503354 \\
\hline & -1.444370 & -0.778881 & -4.060610 \\
\hline ] & -0.583794 & 0.793014 & -4.375912 \\
\hline & -1.342543 & -2.583673 & -3.170999 \\
\hline 1 & -1.200087 & -5.020970 & -3.177073 \\
\hline & 1.001793 & -6.159763 & -3.474994 \\
\hline & 3.051770 & -4.772674 & -3.761322 \\
\hline ] & 2.906477 & -2.328622 & -3.781843 \\
\hline & 2.232033 & -0.409919 & -5.059007 \\
\hline 1 & 1.716991 & 1.038982 & -4.180848 \\
\hline ] & 2.766231 & -0.142570 & -3.391039 \\
\hline 6 & -6.588693 & -2.862026 & -2.912270 \\
\hline 0 & -4.722340 & -1.689163 & -4.035476 \\
\hline 6 & -4.275693 & -3.690211 & -2.596210 \\
\hline 6 & -8.110030 & -0.787557 & 1.204472 \\
\hline 6 & -6.324812 & -0.501313 & 2.894686 \\
\hline 6 & -7.061095 & 1.440298 & 1.497463 \\
\hline & -4.888247 & -2.325582 & -4.914857 \\
\hline 1 & -5.309892 & -0.770986 & -4.165527 \\
\hline ] & -3.662070 & -1.407017 & -4.029676 \\
\hline 1 & -4.340635 & -4.319928 & -3.494577 \\
\hline l & -3.219179 & -3.447577 & -2.423353 \\
\hline 1 & -4.614361 & -4.281106 & -1.735648 \\
\hline 1 & -6.690009 & -3.503597 & -3.796783 \\
\hline & -6.939483 & -3.440598 & -2.048089 \\
\hline 1 & -7.259725 & -2.003806 & -3.048316 \\
\hline 1 & -7.089904 & -0.262060 & 3.645588 \\
\hline 1 & -6.147000 & -1.584918 & 2.932907 \\
\hline 1 & -5.396641 & -0.000232 & 3.198599 \\
\hline 1 & -7.823068 & 1.696816 & 2.245993 \\
\hline 1 & -6.156885 & 2.016814 & 1.730216 \\
\hline 1 & -7.426565 & 1.772570 & 0.516668 \\
\hline 1 & -8.849625 & -0.536013 & 1.975089 \\
\hline 1 & -8.532959 & -0.491449 & 0.235951 \\
\hline 1 & -7.988501 & -1.879021 & 1.207880 \\
\hline
\end{tabular}


TS1 $1_{\text {IL-si-v-12a2a3a4a }}$

Number of imaginary frequencies : 1

The smallest frequencies are : -598.5547 $17.5692 \quad 20.2719 \mathrm{~cm}(-1)$

Electronic energy : $\quad H F=-4360.8714378$

Zero-point correction $=\quad 1.452740($ Hartree/Particle $)$

Thermal correction to Energy= $\quad 1.537090$

Thermal correction to Enthalpy= $\quad 1.538034$

Thermal correction to Gibbs Free Energy= $\quad 1.337073$

Sum of electronic and zero-point Energies $=\quad-4359.418698$

Sum of electronic and thermal Energies $=\quad-4359.334348$

Sum of electronic and thermal Enthalpies $=\quad-4359.333404$

Sum of electronic and thermal Free Energies $=\quad-4359.534365$

Cartesian Coordinates

$\begin{array}{cccc}6 & -1.038004 & 2.554765 & 1.381204 \\ 6 & -2.028111 & 1.769672 & 0.779832 \\ 6 & -3.344782 & 2.231200 & 0.760116 \\ 6 & -3.704926 & 3.417546 & 1.402540 \\ 6 & -2.695105 & 4.156847 & 2.022721 \\ 6 & -1.354002 & 3.762354 & 2.009143 \\ 15 & -1.512114 & 0.130098 & 0.103218 \\ 45 & 0.118563 & 0.316749 & -1.849635 \\ 15 & 1.994785 & -0.473201 & -0.821665 \\ 8 & 3.188204 & -0.870559 & -1.940967 \\ 6 & 4.417897 & -1.336752 & -1.541730 \\ 6 & 5.322658 & -0.504282 & -0.907217 \\ 6 & 6.516384 & -1.092644 & -0.372776 \\ 6 & 6.806288 & -2.464631 & -0.637603 \\ 6 & 5.907141 & -3.225135 & -1.424700 \\ 6 & 4.729167 & -2.678569 & -1.852947 \\ 6 & 7.397470 & -0.379524 & 0.480080 \\ 6 & 8.507342 & -0.983219 & 1.018294 \\ 6 & 8.808097 & -2.329925 & 0.725673 \\ 6 & 7.970574 & -3.051934 & -0.085563 \\ 6 & 5.021211 & 0.937633 & -0.762880 \\ 6 & 3.825126 & 1.337241 & -0.198900 \\ 6 & 3.519898 & 2.690433 & 0.043838 \\ 6 & 4.401203 & 3.666873 & -0.327199 \\ 6 & 5.606018 & 3.330186 & -0.991053 \\ 6 & 5.916403 & 1.957031 & -1.230543 \\ 6 & 6.496733 & 4.335714 & -1.437776 \\ 6 & 7.641717 & 4.013398 & -2.120240 \\ 6 & 7.933474 & 2.661042 & -2.392684 \\ 6 & 7.096165 & 1.662286 & -1.960159 \\ 8 & 2.911657 & 0.409237 & 0.230842 \\ 6 & -5.164559 & 3.854367 & 1.536467 \\ 6 & -0.302681 & 4.587286 & 2.751255 \\ 6 & -0.566469 & 6.087776 & 2.597404 \\ 6 & -3.125028 & -0.643466 & -0.357668 \\ 6 & -4.142046 & -0.827949 & 0.584746 \\ 6 & -5.362492 & -1.394544 & 0.224631 \\ 6 & -5.521365 & -1.808900 & -1.104379 \\ & & & \\ 6\end{array}$




\begin{tabular}{|c|c|c|c|}
\hline 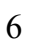 & -4.520669 & -1.663919 & -2.066092 \\
\hline & -3.321650 & -1.062823 & -1.668618 \\
\hline & -4.758294 & -2.093659 & -3.513093 \\
\hline & -5.682264 & -1.066196 & -4.177125 \\
\hline & -6.499564 & -1.588784 & 1.228007 \\
\hline & -6.741206 & -3.086937 & 1.440389 \\
\hline & -1.198667 & -0.788418 & 1.704989 \\
\hline & -0.713643 & -2.091509 & 1.683132 \\
\hline & -0.484605 & -2.798430 & 2.908617 \\
\hline & -0.751975 & -2.159015 & 4.153701 \\
\hline & -1.262248 & -0.842242 & 4.142932 \\
\hline & -1.477185 & -0.183135 & 2.961994 \\
\hline & -0.500732 & -2.847576 & 5.364522 \\
\hline & -0.003695 & -4.125420 & 5.356112 \\
\hline & 0.257116 & -4.768516 & 4.127521 \\
\hline & 0.019546 & -4.126121 & 2.937578 \\
\hline & -0.451645 & -2.830955 & 0.415769 \\
\hline & -1.439306 & -3.734971 & -0.088804 \\
\hline & -1.170899 & -4.483423 & -1.273398 \\
\hline & 0.084831 & -4.345669 & -1.906624 \\
\hline & 1.024594 & -3.493251 & -1.396566 \\
\hline & 0.759445 & -2.717931 & -0.243621 \\
\hline & -2.156792 & -5.363690 & -1.782566 \\
\hline & -3.361168 & -5.512842 & -1.143966 \\
\hline & -3.631995 & -4.774405 & 0.028024 \\
\hline & -2.696926 & -3.912596 & 0.544494 \\
\hline 7 & 1.768544 & -1.800191 & 0.211120 \\
\hline & 2.764609 & -2.357689 & 1.155465 \\
\hline & 3.222494 & -1.431608 & 2.252571 \\
\hline 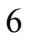 & 2.316815 & -0.647387 & 2.969040 \\
\hline & 2.753431 & 0.176778 & 3.997157 \\
\hline 5 & 4.107114 & 0.231983 & 4.326362 \\
\hline 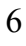 & 5.015982 & -0.544514 & 3.616601 \\
\hline 5 & 4.574180 & -1.371377 & 2.585939 \\
\hline 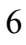 & -3.453706 & -2.165662 & -4.307461 \\
\hline & -5.415026 & -3.475463 & -3.572104 \\
\hline 5 & -6.181831 & -0.964660 & 2.585534 \\
\hline 6 & -7.782454 & -0.940241 & 0.696849 \\
\hline 6 & 0.170290 & -0.951434 & -3.257170 \\
\hline & 0.274043 & -1.581763 & -4.229107 \\
\hline & -0.328256 & 2.474866 & -2.721179 \\
\hline 6 & 0.630412 & 2.478065 & -1.683059 \\
\hline 6 & 0.159051 & 2.522531 & -4.156473 \\
\hline 6 & -1.646381 & 3.176254 & -2.519060 \\
\hline 6 & -1.717309 & 4.334878 & -1.742043 \\
\hline 6 & -2.906697 & 5.049601 & -1.635456 \\
\hline 6 & -4.043679 & 4.623808 & -2.313754 \\
\hline 6 & -3.987628 & 3.467928 & -3.089689 \\
\hline 6 & -2.799588 & 2.756760 & -3.191635 \\
\hline 6 & -0.359933 & 4.207104 & 4.235319 \\
\hline 6 & 1.107711 & 4.313062 & 2.232115 \\
\hline & 7.167137 & 0.652259 & 0.733770 \\
\hline 1 & -1.884124 & 0.824463 & 2.993621 \\
\hline & 8.176341 & -4.098122 & -0.308646 \\
\hline 1 & -0.708808 & -2.336089 & 6.303398 \\
\hline 1 & 6.243220 & 5.375579 & -1.235250 \\
\hline
\end{tabular}




\begin{tabular}{|c|c|c|c|}
\hline & -2.921998 & -3.343886 & 1.445431 \\
\hline & -4.592472 & -4.891741 & 0.525896 \\
\hline & 4.178572 & 4.715383 & -0.132798 \\
\hline & -1.487629 & -0.356511 & 5.091918 \\
\hline & 6.156140 & -4.258774 & -1.659072 \\
\hline & 8.827382 & 2.405394 & -2.957256 \\
\hline & 4.016638 & -3.251529 & -2.441650 \\
\hline & -1.931604 & -5.924622 & -2.688871 \\
\hline & 0.288997 & -4.923393 & -2.806861 \\
\hline & 0.646536 & -5.784083 & 4.123377 \\
\hline 1 & 7.331058 & 0.626673 & -2.191095 \\
\hline & 0.218127 & -4.639547 & 1.998949 \\
\hline & 1.989155 & -3.374433 & -1.884667 \\
\hline ] & 8.316084 & 4.794591 & -2.463233 \\
\hline & 9.157547 & -0.417879 & 1.682311 \\
\hline 1 & -4.109463 & -6.198254 & -1.536627 \\
\hline & 0.189626 & -4.645827 & 6.291285 \\
\hline 1 & 9.693746 & -2.793919 & 1.153420 \\
\hline & 2.576807 & 2.917641 & 0.531973 \\
\hline & -3.971672 & -0.509357 & 1.611042 \\
\hline & -2.503491 & -0.926574 & -2.372691 \\
\hline & -6.468005 & -2.263454 & -1.396741 \\
\hline$\frac{1}{1}$ & -4.103572 & 1.636508 & 0.261239 \\
\hline & -0.013689 & 2.176342 & 1.401367 \\
\hline ] & -2.966289 & 5.071863 & 2.546851 \\
\hline & 2.270616 & -3.226649 & 1.613551 \\
\hline & 3.640298 & -2.751503 & 0.616572 \\
\hline 1 & 5.295396 & -1.964884 & 2.021100 \\
\hline & 1.266513 & -0.658507 & 2.685294 \\
\hline 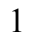 & 6.077140 & -0.503782 & 3.855824 \\
\hline & 2.029505 & 0.781785 & 4.542254 \\
\hline 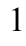 & 4.450431 & 0.881757 & 5.128726 \\
\hline$\frac{1}{1}$ & 0.357547 & 2.888785 & -0.711124 \\
\hline & 1.682774 & 2.552330 & -1.955883 \\
\hline 1 & -2.762593 & 1.852571 & -3.800564 \\
\hline & -4.872331 & 3.121574 & -3.620246 \\
\hline 1 & -4.971929 & 5.188336 & -2.240757 \\
\hline & -2.937905 & 5.947209 & -1.019179 \\
\hline ] & -0.829273 & 4.687804 & -1.219928 \\
\hline 1 & -0.525358 & 2.010296 & -4.842775 \\
\hline & 0.226372 & 3.570685 & -4.481632 \\
\hline 1 & 1.144920 & 2.055853 & -4.250411 \\
\hline & -1.081342 & 1.026262 & -2.700185 \\
\hline 1 & 1.828708 & 4.960758 & 2.747181 \\
\hline & 1.425884 & 3.278527 & 2.411932 \\
\hline 1 & 1.181854 & 4.521914 & 1.154761 \\
\hline 1 & 0.247997 & 6.660324 & 3.059499 \\
\hline 1 & -0.622508 & 6.373301 & 1.537819 \\
\hline 1 & -1.495442 & 6.407792 & 3.082893 \\
\hline 1 & 0.387092 & 4.770375 & 4.811485 \\
\hline 1 & -1.348499 & 4.417198 & 4.663632 \\
\hline 1 & -0.157105 & 3.135219 & 4.370600 \\
\hline 1 & -3.658132 & -2.536124 & -5.320122 \\
\hline 1 & -2.971210 & -1.185126 & -4.415353 \\
\hline 1 & -2.731015 & -2.847591 & -3.837811 \\
\hline 1 & -5.863688 & -1.330436 & -5.228077 \\
\hline
\end{tabular}




$\begin{array}{lrrr}1 & -6.653938 & -1.011342 & -3.669568 \\ 1 & -5.235906 & -0.062911 & -4.150531 \\ 1 & -5.555045 & -3.779761 & -4.617830 \\ 1 & -4.784718 & -4.227586 & -3.079476 \\ 1 & -6.402460 & -3.495254 & -3.095922 \\ 1 & -7.030114 & -1.109088 & 3.266418 \\ 1 & -5.301876 & -1.423845 & 3.055862 \\ 1 & -6.000843 & 0.117114 & 2.506553 \\ 1 & -8.597320 & -1.061552 & 1.423092 \\ 1 & -7.641335 & 0.135064 & 0.524592 \\ 1 & -8.115493 & -1.387967 & -0.246925 \\ 1 & -7.577674 & -3.246601 & 2.134460 \\ 1 & -6.982909 & -3.600662 & 0.501303 \\ 1 & -5.851982 & -3.567107 & 1.871781 \\ 6 & -6.096976 & 3.023536 & 0.656788 \\ 6 & -5.585701 & 3.661068 & 2.998870 \\ 6 & -5.341889 & 5.326972 & 1.156356 \\ 1 & -7.126737 & 3.388874 & 0.760206 \\ 1 & -5.819794 & 3.086803 & -0.403731 \\ 1 & -6.095815 & 1.962863 & 0.944588 \\ 1 & -6.641264 & 3.933539 & 3.134920 \\ 1 & -5.462485 & 2.613952 & 3.306979 \\ 1 & -4.988804 & 4.280639 & 3.679911 \\ 1 & -6.389860 & 5.626129 & 1.291578 \\ 1 & -4.730652 & 5.997648 & 1.771965 \\ 1 & -5.075474 & 5.495734 & 0.105932\end{array}$

TS1 $_{\text {ILL-si }} \psi_{\text {-1a2a3a4s }}$

Number of imaginary frequencies : 1

The smallest frequencies are : $-568.8770 \quad 18.8153 \quad 20.9843 \mathrm{~cm}(-1)$

Electronic energy : $\quad H F=-4360.8718103$

Zero-point correction $=\quad 1.453475$ (Hartree/Particle)

Thermal correction to Energy $=\quad 1.537610$

Thermal correction to Enthalpy= $\quad 1.538554$

Thermal correction to Gibbs Free Energy= $\quad 1.338045$

Sum of electronic and zero-point Energies $=\quad-4359.418335$

Sum of electronic and thermal Energies $=\quad-4359.334200$

Sum of electronic and thermal Enthalpies= $\quad-4359.333256$

Sum of electronic and thermal Free Energies $=\quad-4359.533766$

Cartesian Coordinates

$\begin{array}{lrrr}6 & -2.183762 & -5.294534 & -1.964268 \\ 6 & -1.189598 & -4.440046 & -1.427592 \\ 6 & -1.447083 & -3.736926 & -0.213456 \\ 6 & -2.698287 & -3.938948 & 0.424355 \\ 6 & -3.642098 & -4.773365 & -0.120010 \\ 6 & -3.385938 & -5.461392 & -1.325434 \\ 6 & -0.455426 & -2.852288 & 0.317314 \\ 6 & 0.752142 & -2.718824 & -0.344599 \\ 6 & 1.008598 & -3.452513 & -1.526502 \\ 6 & 0.063030 & -4.282546 & -2.062677 \\ 6 & -0.712172 & -2.155676 & 1.610254 \\ 6 & -1.192810 & -0.852314 & 1.679742 \\ 6 & -1.472387 & -0.292877 & 2.957264\end{array}$




\begin{tabular}{|c|c|c|c|}
\hline 6 & -1.265739 & -0.996534 & 4.113733 \\
\hline 6 & -0.758127 & -2.313925 & 4.077024 \\
\hline 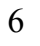 & -0.486564 & -2.906777 & 2.809996 \\
\hline & -0.511056 & -3.046685 & 5.262464 \\
\hline ) & -0.013049 & -4.323031 & 5.208667 \\
\hline & 0.252850 & -4.919917 & 3.958086 \\
\hline 6 & 0.018612 & -4.234284 & 2.791888 \\
\hline & -1.511724 & 0.125192 & 0.114827 \\
\hline 4 & 0.104405 & 0.380789 & -1.823021 \\
\hline 6 & -0.310469 & 2.570387 & -2.621243 \\
\hline 6 & 0.184626 & 2.655418 & -4.052327 \\
\hline 7 & 1.766608 & -1.822331 & 0.139092 \\
\hline 6 & 2.766334 & -2.418655 & 1.055109 \\
\hline 6 & 3.228423 & -1.539407 & 2.188116 \\
\hline 6 & 2.324469 & -0.787794 & 2.940620 \\
\hline 6 & 2.763315 & -0.010867 & 4.003717 \\
\hline 6 & 4.117916 & 0.030240 & 4.331613 \\
\hline 6 & 5.025314 & -0.712896 & 3.585217 \\
\hline 6 & 4.580937 & -1.493236 & 2.519909 \\
\hline 6 & -3.125084 & -0.635993 & -0.361644 \\
\hline 6 & -3.348665 & -0.972196 & -1.695480 \\
\hline 6 & -4.543363 & -1.567732 & -2.099673 \\
\hline 6 & -5.512589 & -1.820101 & -1.120623 \\
\hline 6 & -5.319183 & -1.506034 & 0.226550 \\
\hline 6 & -4.109580 & -0.907131 & 0.586178 \\
\hline 6 & -6.358899 & -1.790877 & 1.311272 \\
\hline 6 & -4.818723 & -1.886920 & -3.568286 \\
\hline 6 & -2.034603 & 1.742062 & \\
\hline 6 & -3.346757 & 2.213448 & 0.789702 \\
\hline 6 & -3.712470 & 3.399635 & 1.431445 \\
\hline 6 & -2.714640 & 4.116292 & 2.095478 \\
\hline 6 & -1.377650 & 3.706347 & 2.118225 \\
\hline 6 & -1.054297 & 2.508879 & 1.476456 \\
\hline 6 & -5.171589 & 3.852142 & 1.514106 \\
\hline 6 & -6.052147 & 3.143800 & 0.485536 \\
\hline 6 & -0.340615 & 4.506345 & 2.906721 \\
\hline 6 & -0.584049 & 6.012438 & 2.775002 \\
\hline 6 & -5.692931 & 3.510758 & 2.915792 \\
\hline 6 & -5.306470 & 5.360799 & 1.289218 \\
\hline 6 & -0.445993 & 4.100084 & 4.381263 \\
\hline 6 & 1.082114 & 4.227705 & 2.423876 \\
\hline 6 & 0.132441 & -0.825909 & -3.286878 \\
\hline 8 & 0.215307 & -1.406896 & -4.290953 \\
\hline 15 & 1.984110 & -0.451506 & -0.836023 \\
\hline 8 & 2.899345 & 0.389378 & 0.252086 \\
\hline 6 & 3.813425 & 1.335047 & -0.133779 \\
\hline 6 & 5.007070 & 0.963199 & -0.721478 \\
\hline 6 & 5.899316 & 2.004203 & -1.145430 \\
\hline 6 & 5.590197 & 3.364325 & -0.839236 \\
\hline 6 & 4.390357 & 3.668143 & -0.150831 \\
\hline 6 & 3.511351 & 2.674801 & 0.178302 \\
\hline 6 & 6.477258 & 4.390486 & -1.244478 \\
\hline 6 & 7.617405 & 4.101461 & -1.949566 \\
\hline 6 & 7.908034 & 2.763600 & -2.286874 \\
\hline 6 & 7.074480 & 1.744873 & -1.895667 \\
\hline 6 & 5.311105 & -0.469916 & -0.932918 \\
\hline
\end{tabular}




\begin{tabular}{|c|c|c|c|}
\hline ) & 4.406495 & -1.275015 & -1.601560 \\
\hline & 4.719006 & -2.600833 & -1.973954 \\
\hline & 5.900633 & -3.162618 & -1.576449 \\
\hline & 6.801541 & -2.435692 & -0.760060 \\
\hline & 6.508685 & -1.078378 & -0.430789 \\
\hline & 7.970589 & -3.043616 & -0.241528 \\
\hline & 8.809525 & -2.356971 & 0.598495 \\
\hline & 8.505195 & -1.026655 & 0.955516 \\
\hline & 7.390665 & -0.402743 & 0.451062 \\
\hline 8 & 3.174972 & -0.792966 & -1.975506 \\
\hline 6 & 0.643554 & 2.528037 & -1.579430 \\
\hline 6 & -1.617919 & 3.288289 & -2.407704 \\
\hline 6 & -2.775978 & 2.905228 & -3.094287 \\
\hline 6 & -3.945969 & 3.645688 & -2.992835 \\
\hline 6 & -3.979531 & 4.792426 & -2.201703 \\
\hline 6 & -2.839278 & 5.178563 & -1.505942 \\
\hline 6 & -1.667597 & 4.434967 & -1.612013 \\
\hline 1 & 7.156937 & 0.614735 & 0.754478 \\
\hline 1 & -1.874942 & 0.714803 & 3.023451 \\
\hline 1 & 8.178972 & -4.077479 & -0.513992 \\
\hline 1 & -0.722521 & -2.570027 & 6.218773 \\
\hline 1 & 6.224710 & 5.419448 & -0.991360 \\
\hline 1 & -2.910354 & -3.412054 & 1.352897 \\
\hline 1 & -4.598848 & -4.909158 & 0.382215 \\
\hline 1 & 4.169608 & 4.705932 & 0.096142 \\
\hline 1 & -1.493536 & -0.546064 & 5.079371 \\
\hline 1 & 6.151649 & -4.183790 & -1.858495 \\
\hline 1 & 8.798194 & 2.535428 & -2.868871 \\
\hline 1 & 4.004617 & -3.148402 & -2.584326 \\
\hline 1 & -1.967964 & -5.820031 & -2.893763 \\
\hline 1 & 0.260243 & -4.826588 & -2.985144 \\
\hline 1 & 0.643802 & -5.934095 & 3.917701 \\
\hline 1 & 7.308582 & 0.721349 & -2.176230 \\
\hline 1 & 0.221327 & -4.712539 & 1.835747 \\
\hline 1 & 1.971273 & -3.318936 & -2.014915 \\
\hline 1 & 8.288772 & 4.898509 & -2.260440 \\
\hline 1 & 9.156207 & -0.490737 & 1.642725 \\
\hline 1 & -4.141670 & -6.124028 & -1.742111 \\
\hline 1 & 0.177707 & -4.877327 & 6.124687 \\
\hline 1 & 9.698982 & -2.837008 & 0.999789 \\
\hline 1 & 2.571739 & 2.877681 & 0.683914 \\
\hline 1 & -3.927102 & -0.650170 & 1.629992 \\
\hline 1 & -2.551319 & -0.772626 & -2.408154 \\
\hline 1 & -6.448212 & -2.285043 & -1.423638 \\
\hline 1 & -4.097705 & 1.628977 & 0.265781 \\
\hline 1 & -0.032544 & 2.124595 & 1.510533 \\
\hline 1 & -2.990214 & 5.027759 & 2.622652 \\
\hline 1 & 2.273735 & -3.305615 & 1.478906 \\
\hline 1 & 3.639701 & -2.789911 & 0.497010 \\
\hline 1 & 5.300835 & -2.061162 & 1.927771 \\
\hline 1 & 1.273783 & -0.786614 & 2.658425 \\
\hline 1 & 6.087212 & -0.682104 & 3.822750 \\
\hline 1 & 2.040556 & 0.568488 & 4.577260 \\
\hline 1 & 4.462937 & 0.643425 & 5.161576 \\
\hline 1 & 0.372437 & 2.908948 & -0.594577 \\
\hline 1 & 1.697714 & 2.598812 & -1.846229 \\
\hline
\end{tabular}




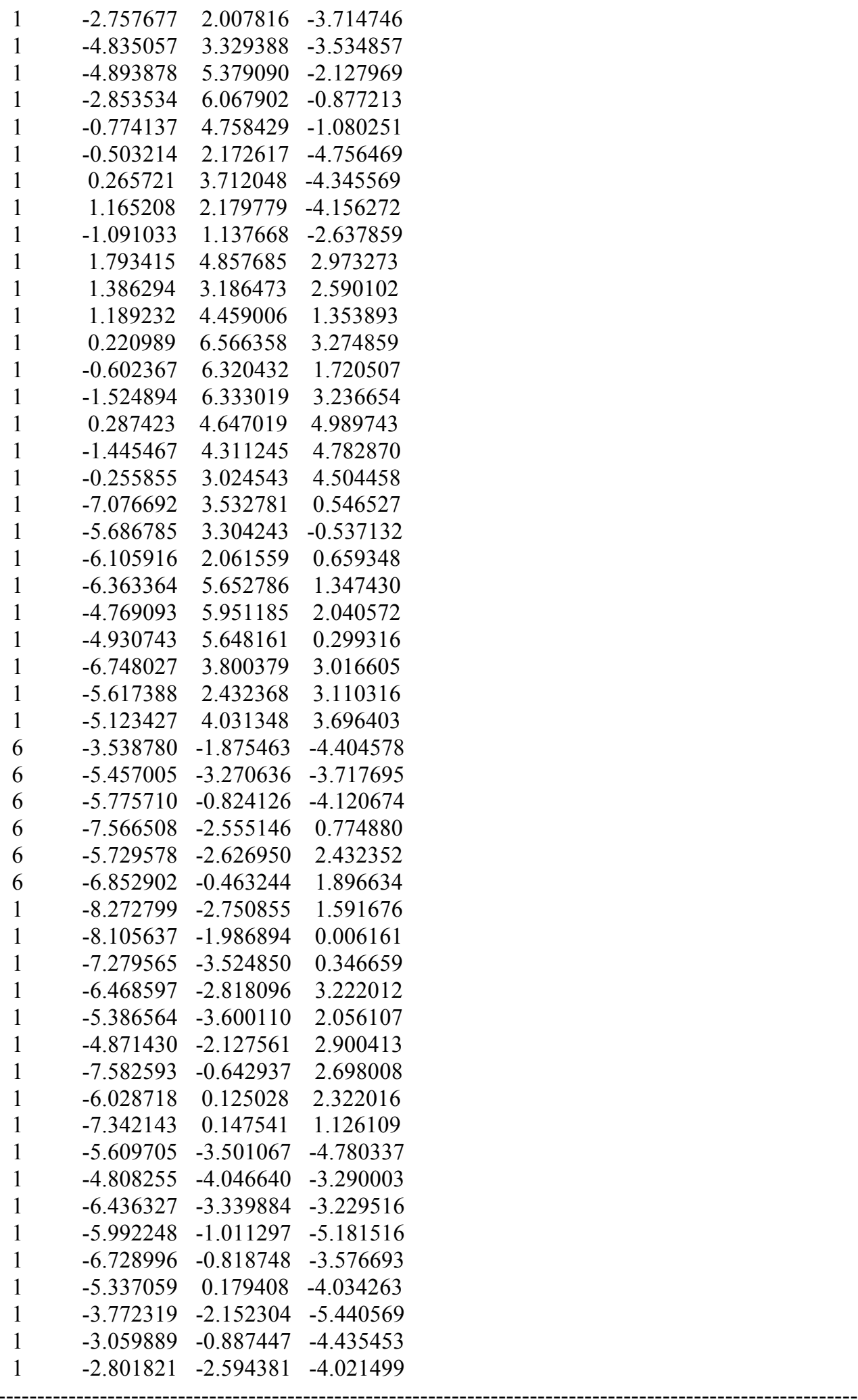

TS1 $_{\text {IIL-si- }} \psi_{-1 \text { a2a3s4a }}$

Number of imaginary frequencies : 1

The smallest frequencies are : $-584.7110 \quad 18.5672 \quad 20.5284 \mathrm{~cm}(-1)$ 
Electronic energy : $\quad H F=-4360.8712275$

$\begin{array}{lc}\text { Zero-point correction= } & 1.452796 \text { (Hartree/Particle) } \\ \text { Thermal correction to Energy= } & 1.537111 \\ \text { Thermal correction to Enthalpy= } & 1.538056 \\ \text { Thermal correction to Gibbs Free Energy= } & 1.336656 \\ \text { Sum of electronic and zero-point Energies }= & -4359.418431 \\ \text { Sum of electronic and thermal Energies }= & -4359.334116 \\ \text { Sum of electronic and thermal Enthalpies }= & -4359.333172 \\ \text { Sum of electronic and thermal Free Energies }= & -4359.534572\end{array}$

Cartesian Coordinates

$\begin{array}{cccc}6 & -3.315831 & -1.006578 & -1.711868 \\ 6 & -3.121168 & -0.638470 & -0.389853 \\ 6 & -4.146620 & -0.856409 & 0.541097 \\ 6 & -5.362177 & -1.409099 & 0.154491 \\ 6 & -5.519070 & -1.775495 & -1.193498 \\ 6 & -4.518211 & -1.585179 & -2.141221 \\ 15 & -1.516300 & 0.130291 & 0.101012 \\ 6 & -2.042306 & 1.761677 & 0.787869 \\ 6 & -3.362093 & 2.213350 & 0.776968 \\ 6 & -3.728216 & 3.391124 & 1.431859 \\ 6 & -2.721547 & 4.131544 & 2.055807 \\ 6 & -1.377806 & 3.745835 & 2.035263 \\ 6 & -1.055747 & 2.546994 & 1.394435 \\ 6 & -5.191240 & 3.811669 & 1.578589 \\ 6 & -0.330402 & 4.568260 & 2.785625 \\ 6 & -6.505449 & -1.637127 & 1.143352 \\ 6 & -4.655367 & -1.995031 & -3.605459 \\ 45 & 0.107859 & 0.333710 & -1.836598 \\ 6 & 0.118679 & -0.934488 & -3.244630 \\ 8 & 0.159110 & -1.566642 & -4.220350 \\ 15 & 1.985605 & -0.460930 & -0.817990 \\ 7 & 1.761621 & -1.787949 & 0.214666 \\ 6 & 2.766386 & -2.343316 & 1.151739 \\ 6 & 3.216026 & -1.421476 & 2.255454 \\ 6 & 2.302509 & -0.654232 & 2.980131 \\ 6 & 2.731446 & 0.164750 & 4.015715 \\ 6 & 4.085009 & 0.232304 & 4.343190 \\ 6 & 5.001626 & -0.526936 & 3.624624 \\ 6 & 4.567505 & -1.349228 & 2.587178 \\ 8 & 3.178016 & -0.856623 & -1.939632 \\ 6 & 4.412596 & -1.314450 & -1.546031 \\ 6 & 5.313407 & -0.478250 & -0.910706 \\ 6 & 6.511596 & -1.060834 & -0.379837 \\ 6 & 6.810653 & -2.429627 & -0.650622 \\ 6 & 5.916614 & -3.192197 & -1.441485 \\ 6 & 4.734272 & -2.651957 & -1.865668 \\ 6 & 7.388431 & -0.345248 & 0.475377 \\ 6 & 8.502603 & -0.943740 & 1.010531 \\ 6 & 8.812274 & -2.287144 & 0.712074 \\ 6 & 7.979222 & -3.011367 & -0.101791 \\ 6 & 5.005316 & 0.961790 & -0.762233 \\ 6 & 5.894535 & 1.986157 & -1.230347 \\ 6 & 5.579019 & 3.357269 & -0.986032 \\ 6 & 4.375813 & 3.686779 & -0.315589\end{array}$




\begin{tabular}{|c|c|c|c|}
\hline 6 & 3.500130 & 2.705611 & 0.056368 \\
\hline 6 & 3.809681 & 1.354565 & -0.192882 \\
\hline & 6.463383 & 4.367845 & -1.434022 \\
\hline & 7.607023 & 4.052261 & -2.121889 \\
\hline & 7.903967 & 2.701870 & -2.398424 \\
\hline & 7.072911 & 1.698334 & -1.964913 \\
\hline & 2.902006 & 0.420641 & 0.235822 \\
\hline & 0.628361 & 2.497334 & -1.679675 \\
\hline & -0.344731 & 2.497099 & -2.703456 \\
\hline & -1.659272 & 3.200147 & -2.483675 \\
\hline & -2.821448 & 2.782392 & -3.141849 \\
\hline & -4.006990 & 3.495336 & -3.025175 \\
\hline & -4.052178 & 4.651055 & -2.248312 \\
\hline f & -2.906751 & 5.074094 & -1.582731 \\
\hline & -1.719714 & 4.357568 & -1.703947 \\
\hline & 0.120408 & 2.537852 & -4.146248 \\
\hline b & -1.217498 & -0.800602 & 1.697087 \\
\hline & -0.726939 & -2.101310 & 1.671988 \\
\hline D & -0.496329 & -2.810050 & 2.897179 \\
\hline & -0.773733 & -2.176604 & 4.143124 \\
\hline & -1.294771 & -0.864087 & 4.134588 \\
\hline ) & -1.506931 & -0.202014 & 2.955114 \\
\hline 6 & -0.519517 & -2.864964 & 5.353414 \\
\hline ) & -0.007749 & -4.136916 & 5.344336 \\
\hline 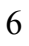 & 0.265550 & -4.773525 & 4.115228 \\
\hline$b$ & 0.024175 & -4.131590 & 2.925770 \\
\hline 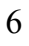 & -0.449808 & -2.841708 & 0.408000 \\
\hline & 0.765734 & -2.719780 & -0.241658 \\
\hline 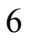 & 1.061519 & -3.519294 & -1.370417 \\
\hline 6 & 0.158051 & -4.426550 & -1.850411 \\
\hline 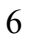 & -1.096267 & -4.585614 & -1.218804 \\
\hline 6 & -1.409500 & -3.785417 & -0.079885 \\
\hline 6 & -2.673331 & -3.970742 & 0.538762 \\
\hline 6 & -3.564179 & -4.899977 & 0.061258 \\
\hline 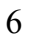 & -3.242075 & -5.700537 & -1.055327 \\
\hline & -2.034005 & -5.539520 & -1.683357 \\
\hline ] & 7.151198 & 0.683869 & 0.733555 \\
\hline & -1.920398 & 0.802816 & 2.988519 \\
\hline 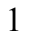 & 8.191934 & -4.055182 & -0.329445 \\
\hline ] & -0.736438 & -2.357461 & 6.292460 \\
\hline 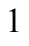 & 6.206021 & 5.406088 & -1.228038 \\
\hline 1 & -2.937799 & -3.357098 & 1.398902 \\
\hline & -4.528742 & -5.023412 & 0.549740 \\
\hline 1 & 4.149937 & 4.733738 & -0.116691 \\
\hline & -1.529093 & -0.383678 & 5.084115 \\
\hline 1 & 6.173244 & -4.222451 & -1.682430 \\
\hline 1 & 8.796882 & 2.451580 & -2.966954 \\
\hline 1 & 4.026693 & -3.226481 & -2.458674 \\
\hline 1 & -1.772069 & -6.140497 & -2.553380 \\
\hline ] & 0.391988 & -5.036800 & -2.721398 \\
\hline 1 & 0.668103 & -5.783957 & 4.109961 \\
\hline I & 7.311619 & 0.664284 & -2.198969 \\
\hline 1 & 0.233953 & -4.641161 & 1.987501 \\
\hline 1 & 2.026464 & -3.386851 & -1.853949 \\
\hline 1 & 8.276415 & 4.837301 & -2.465852 \\
\hline 1 & 9.149360 & -0.376829 & 1.676571 \\
\hline
\end{tabular}




\begin{tabular}{|c|c|c|c|}
\hline 1 & -3.955529 & -6.437577 & -1.417413 \\
\hline & 0.188366 & -4.657081 & 6.279062 \\
\hline & 9.701274 & -2.746935 & 1.137400 \\
\hline & 2.558675 & 2.927446 & 0.550248 \\
\hline & -3.982631 & -0.570462 & 1.578046 \\
\hline & -2.499727 & -0.847028 & -2.415160 \\
\hline & -6.462493 & -2.222958 & -1.500900 \\
\hline & -4.118625 & 1.617020 & 0.276425 \\
\hline & -0.028855 & 2.175467 & 1.406571 \\
\hline & -2.997246 & 5.039724 & 2.589371 \\
\hline & 2.282497 & -3.220962 & 1.603975 \\
\hline 1 & 3.645038 & -2.723740 & 0.608031 \\
\hline 1 & 5.294220 & -1.929525 & 2.015704 \\
\hline & 1.251966 & -0.675132 & 2.697548 \\
\hline & 6.062790 & -0.476040 & 3.862031 \\
\hline & 2.001614 & 0.756304 & 4.567642 \\
\hline & 4.422187 & 0.878355 & 5.151147 \\
\hline & 0.371622 & 2.909540 & -0.703898 \\
\hline & 1.677198 & 2.565016 & -1.967271 \\
\hline & -2.795613 & 1.876658 & -3.748981 \\
\hline & -4.898523 & 3.149892 & -3.544849 \\
\hline & -4.978457 & 5.217321 & -2.164177 \\
\hline & -2.929492 & 5.971453 & -0.965862 \\
\hline & -0.825278 & 4.708462 & -1.191482 \\
\hline ] & -0.574897 & 2.022271 & -4.819229 \\
\hline & 0.182745 & 3.584342 & -4.477644 \\
\hline & 1.104394 & 2.070074 & -4.253195 \\
\hline & -1.100550 & 1.048056 & -2.669235 \\
\hline & -0.391147 & 4.177007 & 4.266726 \\
\hline b & 1.082387 & 4.301328 & 2.269103 \\
\hline ) & -0.597252 & 6.069128 & 2.641923 \\
\hline b & -6.117770 & 2.993900 & 0.680507 \\
\hline 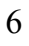 & -5.606635 & 3.577567 & 3.036728 \\
\hline 5 & -5.382925 & 5.291244 & 1.235529 \\
\hline 6 & -3.671271 & -3.134196 & -3.892415 \\
\hline 6 & -6.063975 & -2.476157 & -3.943091 \\
\hline 6 & -4.323421 & -0.805962 & -4.515212 \\
\hline 6 & -7.787806 & -0.979178 & 0.622246 \\
\hline 6 & -6.740825 & -3.141844 & 1.311033 \\
\hline f & -6.200947 & -1.050518 & 2.520451 \\
\hline 1 & 0.211851 & 6.640193 & 3.115200 \\
\hline 1 & -0.645121 & 6.363137 & 1.584325 \\
\hline 1 & -1.531132 & 6.382652 & 3.122151 \\
\hline 1 & 1.799897 & 4.946934 & 2.791583 \\
\hline 1 & 1.402742 & 3.266200 & 2.441755 \\
\hline 1 & 1.159764 & 4.518825 & 1.193685 \\
\hline ] & 0.353991 & 4.736466 & 4.849025 \\
\hline 1 & -1.380934 & 4.383085 & 4.694157 \\
\hline 1 & -0.187993 & 3.104233 & 4.394237 \\
\hline 1 & -7.149920 & 3.350420 & 0.790645 \\
\hline 1 & -5.839576 & 3.081293 & -0.378010 \\
\hline 1 & -6.110382 & 1.927372 & 0.945483 \\
\hline 1 & -6.434269 & 5.576037 & 1.375206 \\
\hline & -4.780728 & 5.952612 & 1.869743 \\
\hline & -5.115467 & 5.489071 & 0.190419 \\
\hline & -6.664370 & 3.836301 & 3.182629 \\
\hline
\end{tabular}




\begin{tabular}{rrrr}
1 & -5.472338 & 2.524060 & 3.317416 \\
1 & -5.013168 & 4.185263 & 3.731409 \\
1 & -3.749991 & -3.453863 & -4.940786 \\
1 & -2.631778 & -2.829799 & -3.711964 \\
1 & -3.879111 & -4.000004 & -3.249950 \\
1 & -4.464260 & -1.084562 & -5.568111 \\
1 & -4.975228 & 0.052037 & -4.300972 \\
1 & -3.280069 & -0.480785 & -4.403562 \\
1 & -6.119066 & -2.741667 & -5.006362 \\
1 & -6.340250 & -3.370541 & -3.369841 \\
1 & -6.818197 & -1.700443 & -3.755547 \\
1 & -7.589455 & -3.325744 & 1.984074 \\
1 & -6.958892 & -3.632771 & 0.354250 \\
1 & -5.856442 & -3.626137 & 1.747251 \\
1 & -7.053237 & -1.219281 & 3.190708 \\
1 & -5.321938 & -1.518187 & 2.984089 \\
1 & -6.025570 & 0.034052 & 2.473473 \\
1 & -8.605771 & -1.122257 & 1.340980 \\
1 & -7.649454 & 0.100848 & 0.479444 \\
1 & -8.114992 & -1.402608 & -0.334601 \\
\hline
\end{tabular}

TS1 $_{\text {IIL-si- }} \psi_{-1 \text { a2a3s4s }}$

Number of imaginary frequencies : 1

The smallest frequencies are : $-557.7308 \quad 15.0326 \quad 17.5629 \mathrm{~cm}(-1)$

Electronic energy : $\quad H F=-4360.8714941$

Zero-point correction $=\quad 1.453229($ Hartree/Particle $)$

Thermal correction to Energy $=\quad 1.537485$

Thermal correction to Enthalpy $=\quad 1.538430$

Thermal correction to Gibbs Free Energy= 1.336911

Sum of electronic and zero-point Energies $=\quad-4359.418265$

Sum of electronic and thermal Energies $=\quad-4359.334009$

Sum of electronic and thermal Enthalpies $=\quad-4359.333065$

Sum of electronic and thermal Free Energies $=\quad-4359.534583$

\begin{tabular}{crrr}
\multicolumn{4}{c}{ Cartesian Coordinates } \\
\hline $6 \ldots \ldots \ldots \ldots \ldots \ldots \ldots \ldots \ldots \ldots \ldots \ldots \ldots \ldots \ldots$ \\
6 & -2.065450 & -5.476908 & -1.868530 \\
6 & -1.118875 & -4.546804 & -1.374283 \\
6 & -1.422684 & -3.785904 & -0.206646 \\
6 & -2.683152 & -3.987674 & 0.412701 \\
6 & -3.582648 & -4.892605 & -0.093397 \\
6 & -3.272158 & -5.652508 & -1.241216 \\
6 & -0.459601 & -2.859855 & 0.308477 \\
6 & 0.754932 & -2.722903 & -0.339787 \\
6 & 1.044639 & -3.487921 & -1.493970 \\
6 & 0.135138 & -4.373431 & -2.002819 \\
6 & -0.733310 & -2.157906 & 1.595823 \\
6 & -1.215143 & -0.855150 & 1.666058 \\
6 & -1.506406 & -0.299852 & 2.943105 \\
6 & -1.308935 & -1.005997 & 4.099266 \\
6 & -0.796185 & -2.321347 & 4.063218 \\
6 & -0.512266 & -2.910251 & 2.797034 \\
6 & -0.555215 & -3.054479 & 5.249649 \\
6 & -0.048754 & -4.327528 & 5.198504 \\
6 & 0.232670 & -4.919728 & 3.949275
\end{tabular}




\begin{tabular}{|c|c|c|c|}
\hline 6 & 0.003392 & -4.233989 & 2.782105 \\
\hline & -1.519476 & 0.133319 & 0.106211 \\
\hline & 0.092764 & 0.386408 & -1.826759 \\
\hline 6 & -0.307547 & 2.584676 & -2.622831 \\
\hline 6 & 0.184262 & 2.664296 & -4.055040 \\
\hline 7 & 1.755101 & -1.809940 & 0.143754 \\
\hline 6 & 2.757051 & -2.395711 & 1.064824 \\
\hline 6 & 3.205799 & -1.509452 & 2.197629 \\
\hline 6 & 2.291652 & -0.762879 & 2.942787 \\
\hline 6 & 2.718650 & 0.021301 & 4.005521 \\
\hline 6 & 4.071237 & 0.074807 & 4.340011 \\
\hline 6 & 4.988578 & -0.663577 & 3.600993 \\
\hline 6 & 4.556144 & -1.451346 & 2.536284 \\
\hline 6 & -3.134884 & -0.612122 & -0.383710 \\
\hline 6 & -3.366883 & -0.913525 & -1.719732 \\
\hline 6 & -4.568055 & -1.492776 & -2.138371 \\
\hline 6 & -5.530272 & -1.776915 & -1.166662 \\
\hline 6 & -5.328468 & -1.493732 & 0.190629 \\
\hline 6 & -4.121053 & -0.903733 & 0.560801 \\
\hline 6 & -6.367822 & -1.798013 & 1.270655 \\
\hline 6 & -4.745291 & -1.835248 & -3.616000 \\
\hline 6 & -4.397625 & -0.621556 & -4.486380 \\
\hline 6 & -2.037663 & 1.747831 & 0.834391 \\
\hline 6 & -3.349205 & 2.221087 & 0.790142 \\
\hline 6 & -3.713592 & 3.403376 & 1.439189 \\
\hline 6 & -2.714899 & 4.114603 & 2.108207 \\
\hline 6 & -1.378557 & 3.702497 & 2.129163 \\
\hline 6 & -1.056642 & 2.508241 & 1.480520 \\
\hline 6 & -5.172257 & 3.856897 & 1.523260 \\
\hline 6 & -5.305265 & 5.366604 & 1.304222 \\
\hline 6 & -0.339333 & 4.493295 & 2.923951 \\
\hline 6 & -0.594577 & 6.000008 & 2.829042 \\
\hline 6 & -6.053392 & 3.153496 & 0.491646 \\
\hline 6 & -5.694407 & 3.509981 & 2.923269 \\
\hline 6 & -0.426021 & 4.052080 & 4.389653 \\
\hline 6 & 1.080289 & 4.235919 & 2.420166 \\
\hline 6 & -3.798942 & -2.989568 & -3.964988 \\
\hline 6 & -6.173365 & -2.262999 & -3.945701 \\
\hline 6 & 0.060737 & -0.846212 & -3.266855 \\
\hline 8 & 0.066467 & -1.454392 & -4.258349 \\
\hline 15 & 1.974052 & -0.446625 & -0.841053 \\
\hline 8 & 2.885003 & 0.401975 & 0.244739 \\
\hline 6 & 3.801120 & 1.345820 & -0.140430 \\
\hline 6 & 4.995966 & 0.971395 & -0.723687 \\
\hline 6 & 5.890596 & 2.010289 & -1.147985 \\
\hline 6 & 5.582734 & 3.371402 & -0.845251 \\
\hline 6 & 4.382093 & 3.678018 & -0.159483 \\
\hline 6 & 3.500837 & 2.686618 & 0.169325 \\
\hline 6 & 6.471948 & 4.395538 & -1.250797 \\
\hline 6 & 7.613243 & 4.103488 & -1.952810 \\
\hline 6 & 7.902911 & 2.764518 & -2.286462 \\
\hline 6 & 7.067159 & 1.747725 & -1.894841 \\
\hline 6 & 5.301057 & -0.462351 & -0.928331 \\
\hline 6 & 4.400560 & -1.271537 & -1.597728 \\
\hline 6 & 4.719845 & -2.596500 & -1.967781 \\
\hline 6 & 5.900820 & -3.154546 & -1.563150 \\
\hline
\end{tabular}




\begin{tabular}{|c|c|c|c|}
\hline ) & 6.794997 & -2.424420 & -0.742328 \\
\hline & 6.497425 & -1.066911 & -0.418407 \\
\hline 6 & 7.962323 & -3.028612 & -0.215614 \\
\hline & 8.795150 & -2.337994 & 0.627200 \\
\hline & 8.486317 & -1.007216 & 0.978579 \\
\hline & 7.373513 & -0.386841 & 0.466020 \\
\hline & 3.167880 & -0.795364 & -1.975841 \\
\hline & 0.646671 & 2.530867 & -1.582659 \\
\hline & -1.609894 & 3.310065 & -2.404300 \\
\hline 6 & -2.769077 & 2.940076 & -3.095810 \\
\hline 6 & -3.936430 & 3.683211 & -2.985919 \\
\hline & -3.965938 & 4.820646 & -2.181361 \\
\hline 6 & -2.823679 & 5.195518 & -1.482745 \\
\hline & -1.654666 & 4.448817 & -1.596971 \\
\hline 1 & 7.136439 & 0.631183 & 0.764990 \\
\hline 1 & -1.911579 & 0.706778 & 3.008678 \\
\hline 1 & 8.174293 & -4.062788 & -0.484118 \\
\hline 1 & -0.777639 & -2.580217 & 6.204658 \\
\hline 1 & 6.220209 & 5.425375 & -1.000449 \\
\hline 1 & -2.937629 & -3.406998 & 1.297772 \\
\hline 1 & -4.546664 & -5.026744 & 0.395125 \\
\hline 1 & 4.162471 & 4.716468 & 0.085694 \\
\hline 1 & -1.546672 & -0.558895 & 5.064054 \\
\hline 1 & 6.156418 & -4.175093 & -1.843324 \\
\hline 1 & 8.794081 & 2.533909 & -2.865944 \\
\hline 1 & 4.012015 & -3.146439 & -2.583455 \\
\hline 1 & -1.811685 & -6.048239 & -2.760619 \\
\hline 1 & 0.364213 & -4.954824 & -2.894574 \\
\hline 1 & 0.632034 & -5.930676 & 3.910260 \\
\hline 1 & 7.300572 & 0.723282 & -2.172596 \\
\hline 1 & 0.219532 & -4.710165 & 1.827931 \\
\hline 1 & 2.010150 & -3.346025 & -1.973832 \\
\hline 1 & 8.286298 & 4.898985 & -2.264007 \\
\hline 1 & 9.132521 & -0.467996 & 1.667741 \\
\hline 1 & -3.992273 & -6.370715 & -1.627200 \\
\hline 1 & 0.137547 & -4.882138 & 6.115244 \\
\hline 1 & 9.683242 & -2.815147 & 1.034905 \\
\hline 1 & 2.560716 & 2.892294 & 0.672742 \\
\hline 1 & -3.937726 & -0.666693 & 1.609014 \\
\hline 1 & -2.573755 & -0.707190 & -2.436850 \\
\hline 1 & -6.464667 & -2.239282 & -1.472238 \\
\hline 1 & -4.100757 & 1.641047 & 0.262172 \\
\hline 1 & -0.036101 & 2.120522 & 1.515064 \\
\hline 1 & -2.989605 & 5.023242 & 2.640566 \\
\hline 1 & 2.270652 & -3.286042 & 1.488738 \\
\hline 1 & 3.636073 & -2.760593 & 0.511321 \\
\hline 1 & 5.283543 & -2.015548 & 1.949831 \\
\hline 1 & 1.242391 & -0.771704 & 2.655193 \\
\hline 1 & 6.049056 & -0.623051 & 3.843514 \\
\hline 1 & 1.988348 & 0.597668 & 4.572787 \\
\hline 1 & 4.406976 & 0.693979 & 5.169332 \\
\hline 1 & 0.380199 & 2.910484 & -0.595909 \\
\hline 1 & 1.700969 & 2.592845 & -1.850951 \\
\hline 1 & -2.754148 & 2.050200 & -3.726468 \\
\hline 1 & -4.826299 & 3.375734 & -3.531804 \\
\hline 1 & -4.878190 & 5.409654 & -2.100474 \\
\hline
\end{tabular}




\begin{tabular}{|c|c|c|c|}
\hline & -2.834455 & 6.078501 & -0.845047 \\
\hline & -0.760121 & 4.764809 & -1.062769 \\
\hline & -0.508174 & 2.184263 & -4.756528 \\
\hline & 0.270830 & 3.719936 & -4.350466 \\
\hline & 1.161421 & 2.182219 & -4.160747 \\
\hline & -1.100787 & 1.151375 & -2.635394 \\
\hline & 0.213007 & 6.548171 & 3.331035 \\
\hline & -0.628129 & 6.331093 & 1.781860 \\
\hline & -1.531563 & 6.303818 & 3.309585 \\
\hline & 1.792702 & 4.860415 & 2.974362 \\
\hline & 1.394097 & 3.194330 & 2.563324 \\
\hline & 1.174772 & 4.488113 & 1.353758 \\
\hline & 0.309405 & 4.590295 & 5.003472 \\
\hline & -1.423105 & 4.245704 & 4.805922 \\
\hline & -0.226279 & 2.975312 & 4.485013 \\
\hline & -7.077159 & 3.544430 & 0.553130 \\
\hline & -5.687001 & 3.316674 & -0.530252 \\
\hline & -6.109739 & 2.070667 & 0.661833 \\
\hline 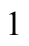 & -6.361684 & 5.659919 & 1.364443 \\
\hline ] & -4.766283 & 5.953252 & 2.057356 \\
\hline 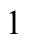 & -4.929982 & 5.657308 & 0.315050 \\
\hline ] & -6.749242 & 3.800216 & 3.024991 \\
\hline & -5.620061 & 2.430564 & 3.112757 \\
\hline ] & -5.124690 & 4.026450 & 3.706440 \\
\hline ] & -3.899581 & -3.262158 & -5.024824 \\
\hline 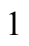 & -2.748594 & -2.724228 & -3.783585 \\
\hline I & -4.025675 & -3.875571 & -3.357245 \\
\hline & -4.573020 & -0.853985 & -5.545183 \\
\hline 1 & -5.015903 & 0.247238 & -4.222231 \\
\hline & -3.342284 & -0.332862 & -4.390552 \\
\hline 1 & -6.259451 & -2.466119 & -5.020635 \\
\hline 1 & -6.460238 & -3.182201 & -3.419231 \\
\hline ] & -6.902771 & -1.481246 & -3.695442 \\
\hline 6 & -7.567359 & -2.568651 & 0.724979 \\
\hline 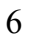 & -5.734397 & -2.638042 & 2.386416 \\
\hline 6 & -6.874438 & -0.478986 & 1.865080 \\
\hline 1 & -8.271055 & -2.782394 & 1.539505 \\
\hline 1 & -8.113475 & -1.997161 & -0.036283 \\
\hline 1 & -7.269520 & -3.529517 & 0.284448 \\
\hline 1 & -6.475363 & -2.846840 & 3.169751 \\
\hline 1 & -5.376649 & -3.602393 & 2.001267 \\
\hline 1 & -4.885256 & -2.133459 & 2.865209 \\
\hline 1 & -7.606876 & -0.670388 & 2.661279 \\
\hline 1 & -6.056004 & 0.111242 & 2.299053 \\
\hline 1 & -7.363841 & 0.135042 & 1.097176 \\
\hline
\end{tabular}

$\mathrm{TS1}_{\text {IIL-si- }} \psi_{-1 \mathrm{a} 253 \mathrm{a} 4 \mathrm{~s}}$

Number of imaginary frequencies : 1

The smallest frequencies are : $-554.5837 \quad 16.9772 \quad 19.5542 \mathrm{~cm}(-1)$

Electronic energy : $\quad H F=-4360.8674346$

Zero-point correction $=\quad 1.453494$ (Hartree/Particle)

Thermal correction to Energy $=\quad 1.537726$

Thermal correction to Enthalpy $=\quad 1.538670$

Thermal correction to Gibbs Free Energy= $\quad 1.337316$

Sum of electronic and zero-point Energies $=\quad-4359.413941$ 
Sum of electronic and thermal Energies= Sum of electronic and thermal Enthalpies= Sum of electronic and thermal Free Energies=
$-4359.329709$

$-4359.328765$

$-4359.530119$

Cartesian Coordinates

\begin{tabular}{|c|c|c|c|}
\hline 6 & -1.016326 & 2.432914 & 1.674951 \\
\hline 6 & -2.022054 & 1.738186 & 0.986582 \\
\hline 6 & -3.306072 & 2.274385 & 0.940353 \\
\hline 6 & -3.626243 & 3.455060 & 1.624010 \\
\hline 6 & -2.606433 & 4.105617 & 2.314972 \\
\hline 6 & -1.290348 & 3.622863 & 2.347692 \\
\hline 15 & -1.558138 & 0.149600 & 0.170444 \\
\hline 45 & 0.061206 & 0.465197 & -1.769625 \\
\hline 15 & 1.954893 & -0.430264 & -0.844651 \\
\hline 8 & 3.135278 & -0.728011 & -2.009524 \\
\hline 6 & 4.363772 & -1.243725 & -1.670919 \\
\hline 6 & 5.281743 & -0.486430 & -0.966000 \\
\hline 6 & 6.472497 & -1.135010 & -0.499569 \\
\hline 6 & 6.746873 & -2.477451 & -0.897833 \\
\hline 6 & 5.836287 & -3.147800 & -1.750987 \\
\hline 6 & 4.661275 & -2.550839 & -2.116004 \\
\hline 6 & 7.365021 & -0.515922 & 0.412505 \\
\hline 6 & 8.470723 & -1.179730 & 0.884217 \\
\hline 6 & 8.755935 & -2.494968 & 0.461244 \\
\hline 6 & 7.907558 & -3.126744 & -0.411797 \\
\hline 6 & 5.001063 & 0.938015 & -0.680316 \\
\hline 6 & 3.817811 & 1.299555 & -0.066366 \\
\hline 6 & 3.546813 & 2.626247 & 0.322133 \\
\hline 6 & 4.443533 & 3.618754 & 0.042928 \\
\hline 6 & 5.631854 & 3.330439 & -0.671570 \\
\hline 6 & 5.910689 & 1.983482 & -1.054655 \\
\hline 6 & 6.535906 & 4.359763 & -1.028058 \\
\hline 6 & 7.664049 & 4.087608 & -1.758723 \\
\hline 6 & 7.925022 & 2.764836 & -2.171144 \\
\hline 6 & 7.074642 & 1.742567 & -1.828151 \\
\hline 8 & 2.884597 & 0.355088 & 0.273524 \\
\hline 6 & -5.076263 & 3.941506 & 1.648251 \\
\hline 6 & -5.538456 & 4.304207 & 0.235334 \\
\hline 6 & -0.227015 & 4.330781 & 3.188091 \\
\hline 6 & -0.459636 & 5.842767 & 3.241727 \\
\hline 6 & -3.200321 & -0.549493 & -0.309278 \\
\hline 6 & -4.158647 & -0.894358 & 0.643668 \\
\hline 6 & -5.359709 & -1.507541 & 0.276225 \\
\hline 6 & -5.581543 & -1.734395 & -1.084603 \\
\hline 6 & -4.647485 & -1.391699 & -2.069885 \\
\hline 6 & -3.453369 & -0.800393 & -1.656658 \\
\hline 6 & -4.948912 & -1.629115 & -3.548840 \\
\hline 6 & -5.924859 & -0.545984 & -4.022280 \\
\hline 6 & -6.349834 & -1.932551 & 1.363785 \\
\hline 6 & -7.550430 & -2.685593 & 0.795568 \\
\hline 6 & -1.228928 & -0.929608 & 1.663247 \\
\hline 6 & -0.759310 & -2.229766 & 1.506347 \\
\hline 6 & -0.539369 & -3.059757 & 2.654899 \\
\hline 6 & -0.801109 & -2.548525 & 3.959008 \\
\hline 6 & -1.291421 & -1.229811 & 4.083899 \\
\hline
\end{tabular}




\begin{tabular}{|c|c|c|c|}
\hline 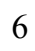 & -1.495566 & -0.451327 & 2.976033 \\
\hline & -0.563167 & -3.361482 & 5.093094 \\
\hline & -0.083219 & -4.638305 & 4.954464 \\
\hline & 0.173932 & -5.154337 & 3.666872 \\
\hline & -0.051136 & -4.389802 & 2.548976 \\
\hline & -0.503722 & -2.850960 & 0.174201 \\
\hline & -1.498498 & -3.695392 & -0.413159 \\
\hline 6 & -1.234231 & -4.341852 & -1.657301 \\
\hline 6 & 0.030809 & -4.177489 & -2.264333 \\
\hline 6 & 0.979822 & -3.390032 & -1.673874 \\
\hline 6 & 0.715013 & -2.706157 & -0.464595 \\
\hline 6 & -2.230237 & -5.158344 & -2.247081 \\
\hline 6 & -3.439255 & -5.347733 & -1.628326 \\
\hline 6 & -3.700513 & -4.719779 & -0.391867 \\
\hline 6 & -2.756705 & -3.920129 & 0.201997 \\
\hline 7 & 1.734711 & -1.843736 & 0.067618 \\
\hline 6 & 2.733021 & -2.491226 & 0.948394 \\
\hline 6 & 3.201876 & -1.667476 & 2.119510 \\
\hline 6 & 2.301719 & -0.949126 & 2.908348 \\
\hline 6 & 2.746433 & -0.217790 & 4.000968 \\
\hline 6 & 4.102842 & -0.190669 & 4.323026 \\
\hline 6 & 5.005911 & -0.903019 & 3.542028 \\
\hline 6 & 4.555676 & -1.638203 & 2.447546 \\
\hline 6 & -5.578740 & -3.007621 & -3.766692 \\
\hline 6 & -3.684250 & -1.556981 & -4.405509 \\
\hline 6 & -6.868631 & -0.694197 & 2.100420 \\
\hline 6 & -5.651207 & -2.849117 & 2.376315 \\
\hline 6 & -0.020214 & -0.734648 & -3.236307 \\
\hline 8 & -0.016829 & -1.297152 & -4.253815 \\
\hline 6 & -0.185731 & 2.678694 & -2.523961 \\
\hline 6 & 0.644088 & 2.563340 & -1.383410 \\
\hline 6 & 0.466643 & 2.759707 & -3.890696 \\
\hline 6 & -1.460802 & 3.476595 & -2.444129 \\
\hline 6 & -1.530021 & 4.608701 & -1.628296 \\
\hline 6 & -2.644844 & 5.441095 & -1.660426 \\
\hline 6 & -3.702090 & 5.163226 & -2.519350 \\
\hline 6 & -3.654149 & 4.027320 & -3.323547 \\
\hline 6 & -2.545085 & 3.193078 & -3.282405 \\
\hline 6 & -5.267374 & 5.166453 & 2.538941 \\
\hline 6 & -5.964837 & 2.815363 & 2.187234 \\
\hline 6 & -0.291910 & 3.761450 & 4.610430 \\
\hline 6 & 1.176865 & 4.101434 & 2.629356 \\
\hline 1 & 7.146280 & 0.488797 & 0.765413 \\
\hline 1 & -1.886742 & 0.554524 & 3.107679 \\
\hline 1 & 8.101882 & -4.148202 & -0.736314 \\
\hline 1 & -0.767539 & -2.946319 & 6.079141 \\
\hline 1 & 6.306182 & 5.377993 & -0.716555 \\
\hline 1 & -2.973053 & -3.438461 & 1.153717 \\
\hline 1 & -4.662978 & -4.874185 & 0.093384 \\
\hline 1 & 4.244765 & 4.645125 & 0.349157 \\
\hline 1 & -1.509091 & -0.840055 & 5.077841 \\
\hline 1 & 6.074315 & -4.155167 & -2.088528 \\
\hline 1 & 8.805693 & 2.551441 & -2.772864 \\
\hline 1 & 3.941957 & -3.055826 & -2.756370 \\
\hline 1 & -2.008622 & -5.639271 & -3.198958 \\
\hline 1 & 0.234487 & -4.681863 & -3.207634 \\
\hline
\end{tabular}




\begin{tabular}{|c|c|c|c|}
\hline ] & 0.551403 & -6.168679 & 3.558729 \\
\hline & 7.286509 & 0.731387 & -2.165576 \\
\hline & 0.145732 & -4.807059 & 1.563558 \\
\hline 1 & 1.953023 & -3.250877 & -2.138377 \\
\hline & 8.348564 & 4.887257 & -2.031946 \\
\hline & 9.129739 & -0.687805 & 1.596246 \\
\hline & -4.196102 & -5.982916 & -2.083902 \\
\hline & 0.100083 & -5.254613 & 5.831557 \\
\hline & 9.638580 & -3.006953 & 0.837270 \\
\hline & 2.613554 & 2.820391 & 0.842037 \\
\hline & -3.953928 & -0.702304 & 1.697696 \\
\hline & -2.675788 & -0.546872 & -2.373845 \\
\hline & -6.509793 & -2.209637 & -1.393326 \\
\hline & -4.083577 & 1.745057 & 0.391989 \\
\hline & -0.015756 & 1.994782 & 1.712825 \\
\hline & -2.838805 & 5.015768 & 2.860080 \\
\hline [ & 2.236294 & -3.394306 & 1.331258 \\
\hline & 3.602866 & -2.839700 & 0.370437 \\
\hline ] & 5.271919 & -2.182591 & 1.829637 \\
\hline & 1.249329 & -0.936732 & 2.632285 \\
\hline ] & 6.068940 & -0.883420 & 3.775757 \\
\hline & 2.026942 & 0.335748 & 4.603284 \\
\hline & 4.452541 & 0.387259 & 5.176000 \\
\hline 1 & 0.281829 & 2.933365 & -0.423392 \\
\hline & 1.721660 & 2.613697 & -1.536096 \\
\hline$\frac{1}{1}$ & -2.514440 & 2.303280 & -3.913521 \\
\hline & -4.483255 & 3.792722 & -3.987720 \\
\hline ] & -4.568820 & 5.820482 & -2.553054 \\
\hline ] & -2.680240 & 6.315891 & -1.012646 \\
\hline & -0.694112 & 4.849806 & -0.972975 \\
\hline 1 & -0.156351 & 2.308195 & -4.671569 \\
\hline & 0.615002 & 3.815714 & -4.161210 \\
\hline 1 & 1.435094 & 2.249096 & -3.894868 \\
\hline & -1.083133 & 1.299210 & -2.575259 \\
\hline 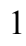 & 0.370481 & 6.327053 & 3.771646 \\
\hline ] & -0.513911 & 6.274315 & 2.233071 \\
\hline & -1.377308 & 6.112723 & 3.776951 \\
\hline 1 & 1.911757 & 4.672395 & 3.211465 \\
\hline & 1.480103 & 3.048819 & 2.683465 \\
\hline 1 & 1.245107 & 4.434431 & 1.583184 \\
\hline ] & 0.462148 & 4.234445 & 5.254689 \\
\hline 1 & -1.278749 & 3.931739 & 5.060312 \\
\hline ] & -0.106816 & 2.678172 & 4.608574 \\
\hline 1 & -6.322370 & 5.468325 & 2.525735 \\
\hline 1 & -4.996285 & 4.963202 & 3.583219 \\
\hline & -4.677979 & 6.024479 & 2.190269 \\
\hline 1 & -6.605311 & 4.567155 & 0.241884 \\
\hline l & -4.977616 & 5.163708 & -0.151720 \\
\hline 1 & -5.398339 & 3.475867 & -0.470137 \\
\hline 1 & -7.009214 & 3.149436 & 2.255211 \\
\hline 1 & -5.941295 & 1.935763 & 1.533378 \\
\hline 1 & -5.643155 & 2.500294 & 3.189210 \\
\hline 1 & -5.745751 & -3.177957 & -4.838451 \\
\hline 1 & -4.917682 & -3.801550 & -3.394182 \\
\hline & -6.550560 & -3.112933 & -3.269943 \\
\hline 1 & -6.162586 & -0.677002 & -5.087023 \\
\hline
\end{tabular}




$\begin{array}{llrl}1 & -6.866368 & -0.578380 & -3.458847 \\ 1 & -5.491393 & 0.454793 & -3.890655 \\ 1 & -3.931506 & -1.790575 & -5.448950 \\ 1 & -3.228042 & -0.558212 & -4.399302 \\ 1 & -2.924728 & -2.276344 & -4.069144 \\ 1 & -6.351731 & -3.133842 & 3.172965 \\ 1 & -5.296156 & -3.773446 & 1.901772 \\ 1 & -4.789194 & -2.366174 & 2.855031 \\ 1 & -8.216817 & -2.987439 & 1.613483 \\ 1 & -8.138302 & -2.063828 & 0.108135 \\ 1 & -7.250000 & -3.596953 & 0.261788 \\ 1 & -7.587415 & -0.985440 & 2.878506 \\ 1 & -6.056115 & -0.142861 & 2.589499 \\ 1 & -7.377835 & -0.008193 & 1.410526\end{array}$

TS1 $_{\text {IIL-si- }} \psi_{-1 \mathrm{a} 2 \mathrm{2} 334 \mathrm{~s}}$

Number of imaginary frequencies : 1

The smallest frequencies are : $-556.8769 \quad 15.1610 \quad 18.5363 \mathrm{~cm}(-1)$

Electronic energy : $\quad H F=-4360.8673537$

Zero-point correction $=\quad 1.453413($ Hartree/Particle $)$

Thermal correction to Energy= $\quad 1.537718$

Thermal correction to Enthalpy= $\quad 1.538662$

Thermal correction to Gibbs Free Energy= $\quad 1.336402$

Sum of electronic and zero-point Energies $=\quad-4359.413941$

Sum of electronic and thermal Energies $=\quad-4359.329636$

Sum of electronic and thermal Enthalpies $=\quad-4359.328691$

Sum of electronic and thermal Free Energies $=\quad-4359.530952$

\begin{tabular}{cccc}
\multicolumn{4}{c}{ Cartesian Coordinates } \\
\hline 6 & -1.534216 & 4.575807 & -1.653078 \\
6 & -1.452088 & 3.451594 & -2.478573 \\
6 & -2.527439 & 3.170986 & -3.328996 \\
6 & -3.643835 & 3.995200 & -3.367237 \\
6 & -3.707038 & 5.120621 & -2.549377 \\
6 & -2.655831 & 5.399253 & -1.683245 \\
6 & -0.177919 & 2.651997 & -2.549446 \\
6 & 0.643814 & 2.538084 & -1.403660 \\
45 & 0.049380 & 0.438580 & -1.768640 \\
15 & 1.952917 & -0.439074 & -0.841460 \\
8 & 3.135499 & -0.722879 & -2.008065 \\
6 & 4.371150 & -1.223314 & -1.673528 \\
6 & 5.281083 & -0.457424 & -0.967445 \\
6 & 6.479056 & -1.094308 & -0.503040 \\
6 & 6.768682 & -2.432205 & -0.905903 \\
6 & 5.866599 & -3.109252 & -1.762860 \\
6 & 4.684772 & -2.524340 & -2.125389 \\
6 & 7.364335 & -0.468472 & 0.411598 \\
6 & 8.476980 & -1.121656 & 0.881788 \\
6 & 8.777125 & -2.432089 & 0.454228 \\
6 & 7.936388 & -3.070129 & -0.421641 \\
6 & 4.986216 & 0.963567 & -0.678781 \\
6 & 3.799777 & 1.313194 & -0.064105 \\
6 & 3.518414 & 2.636148 & 0.329837 \\
6 & 4.406492 & 3.637200 & 0.054335
\end{tabular}




\begin{tabular}{|c|c|c|c|}
\hline 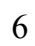 & 5.596713 & 3.361520 & -0.662100 \\
\hline & 5.886659 & 2.018285 & -1.050120 \\
\hline & 6.491734 & 4.399780 & -1.015347 \\
\hline & 7.621823 & 4.139891 & -1.747460 \\
\hline & 7.893784 & 2.820901 & -2.164684 \\
\hline & 7.052302 & 1.790179 & -1.824988 \\
\hline & 2.873909 & 0.361217 & 0.273657 \\
\hline & -1.566071 & 0.158677 & 0.160842 \\
\hline & -2.028529 & 1.746530 & 0.979534 \\
\hline 6 & -3.314464 & 2.278344 & 0.933040 \\
\hline & -3.639633 & 3.457241 & 1.616771 \\
\hline & -2.622680 & 4.111108 & 2.308816 \\
\hline & -1.304799 & 3.633155 & 2.341763 \\
\hline & -1.025460 & 2.444736 & 1.668150 \\
\hline & -5.091594 & 3.937855 & 1.638219 \\
\hline U & -5.976636 & 2.809308 & 2.178503 \\
\hline 6 & -0.245098 & 4.345176 & 3.183236 \\
\hline 6 & 1.160516 & 4.116483 & 2.628929 \\
\hline 6 & -1.232253 & -0.925623 & 1.645238 \\
\hline 6 & -0.762981 & -2.224556 & 1.481283 \\
\hline 6 & -0.545214 & -3.059935 & 2.628401 \\
\hline 6 & -0.810270 & -2.554181 & 3.934151 \\
\hline 6 & -1.298785 & -1.235309 & 4.064503 \\
\hline 6 & -1.499441 & -0.452138 & 2.959863 \\
\hline 6 & -0.052828 & -4.388736 & 2.520947 \\
\hline 6 & 0.169609 & -5.156539 & 3.637256 \\
\hline 6 & -0.093500 & -4.646277 & 4.925656 \\
\hline 6 & -0.575544 & -3.370563 & 5.066353 \\
\hline 6 & -0.487592 & -2.854550 & 0.155679 \\
\hline 6 & -1.459517 & -3.728270 & -0.428423 \\
\hline 6 & -1.133565 & -4.467734 & -1.605010 \\
\hline 6 & 0.155663 & -4.335085 & -2.167428 \\
\hline 6 & 1.069235 & -3.490009 & -1.600923 \\
\hline 6 & 0.752333 & -2.736124 & -0.447266 \\
\hline 6 & -2.749938 & -3.904593 & 0.132997 \\
\hline 6 & -3.663188 & -4.750789 & -0.444290 \\
\hline 6 & -3.333219 & -5.485781 & -1.602975 \\
\hline 6 & -2.093469 & -5.342423 & -2.170266 \\
\hline 7 & 1.753981 & -1.849781 & 0.080108 \\
\hline 6 & 2.766802 & -2.478292 & 0.958320 \\
\hline 6 & 3.218963 & -1.644924 & 2.129202 \\
\hline 6 & 2.304800 & -0.939984 & 2.913991 \\
\hline 6 & 2.734706 & -0.198257 & 4.005582 \\
\hline 6 & 4.089751 & -0.147629 & 4.330850 \\
\hline 6 & 5.006504 & -0.847045 & 3.554130 \\
\hline 6 & 4.571225 & -1.592322 & 2.460424 \\
\hline 6 & -3.215353 & -0.528737 & -0.308723 \\
\hline 6 & -3.503925 & -0.728770 & -1.653704 \\
\hline 6 & -4.718222 & -1.288272 & -2.061401 \\
\hline 6 & -5.626618 & -1.670165 & -1.071183 \\
\hline 6 & -5.368826 & -1.490575 & 0.293900 \\
\hline 6 & -4.159585 & -0.895345 & 0.653901 \\
\hline 6 & -4.963513 & -1.541650 & -3.547422 \\
\hline 6 & -6.431824 & -1.833916 & -3.851476 \\
\hline 6 & -6.339803 & -1.940570 & 1.388712 \\
\hline 6 & -5.623910 & -2.879725 & 2.367762 \\
\hline
\end{tabular}




\begin{tabular}{|c|c|c|c|}
\hline & -0.100590 & -0.824363 & -3.173876 \\
\hline & -0.180562 & -1.451535 & -4.149434 \\
\hline & 0.481256 & 2.716059 & -3.913173 \\
\hline & -7.549849 & -2.681999 & 0.825086 \\
\hline & -6.846163 & -0.718314 & 2.160522 \\
\hline & -4.540886 & -0.326476 & -4.380821 \\
\hline & -4.125447 & -2.754452 & -3.967357 \\
\hline U & -5.552883 & 4.296380 & 0.223769 \\
\hline 6 & -5.288438 & 5.163742 & 2.526352 \\
\hline 6 & -0.480423 & 5.856949 & 3.231493 \\
\hline 6 & -0.312898 & 3.779813 & 4.607020 \\
\hline 1 & 7.134402 & 0.532610 & 0.767720 \\
\hline 1 & -1.889305 & 0.553811 & 3.094503 \\
\hline 1 & 8.142401 & -4.088121 & -0.749824 \\
\hline 1 & -0.783695 & -2.958360 & 6.052855 \\
\hline 1 & 6.253447 & 5.414892 & -0.700122 \\
\hline 1 & -3.018371 & -3.345638 & 1.027258 \\
\hline 1 & -4.653215 & -4.859269 & -0.003914 \\
\hline 1 & 4.199505 & 4.660670 & 0.364881 \\
\hline 1 & -1.517139 & -0.849664 & 5.059880 \\
\hline 1 & 6.116989 & -4.111865 & -2.105576 \\
\hline 1 & 8.775961 & 2.617102 & -2.767529 \\
\hline 1 & 3.971635 & -3.032972 & -2.769857 \\
\hline 1 & -1.822112 & -5.898037 & -3.066936 \\
\hline 1 & 0.405168 & -4.908129 & -3.059025 \\
\hline 1 & 0.550004 & -6.169510 & 3.526480 \\
\hline 1 & 7.272718 & 0.782120 & -2.166227 \\
\hline 1 & 0.150893 & -4.804244 & 1.536253 \\
\hline 1 & 2.061647 & -3.374603 & -2.030025 \\
\hline 1 & 8.299405 & 4.946287 & -2.018117 \\
\hline 1 & 9.130038 & -0.624961 & 1.595992 \\
\hline 1 & -4.064579 & -6.159687 & -2.044111 \\
\hline 1 & 0.087431 & -5.265356 & 5.801281 \\
\hline 1 & 9.665196 & -2.935553 & 0.828977 \\
\hline 1 & 2.583920 & 2.820213 & 0.851028 \\
\hline 1 & -3.933812 & -0.732444 & 1.708370 \\
\hline 1 & -2.742856 & -0.463111 & -2.385810 \\
\hline 1 & -6.562703 & -2.132858 & -1.371028 \\
\hline 1 & -4.089982 & 1.746875 & 0.384103 \\
\hline 1 & -0.023324 & 2.010314 & 1.706481 \\
\hline 1 & -2.858621 & 5.019958 & 2.854625 \\
\hline 1 & 2.288027 & -3.391221 & 1.341153 \\
\hline 1 & 3.643327 & -2.809136 & 0.380096 \\
\hline 1 & 5.297794 & -2.126494 & 1.845719 \\
\hline 1 & 1.253010 & -0.945980 & 2.635248 \\
\hline 1 & 6.068625 & -0.809034 & 3.789891 \\
\hline 1 & 2.004711 & 0.345171 & 4.604515 \\
\hline 1 & 4.427605 & 0.438575 & 5.182948 \\
\hline 1 & 0.279764 & 2.916632 & -0.447590 \\
\hline 1 & 1.722440 & 2.580307 & -1.551551 \\
\hline 1 & -2.484127 & 2.290066 & -3.971267 \\
\hline 1 & -4.466353 & 3.760389 & -4.039749 \\
\hline 1 & -4.579762 & 5.770092 & -2.579605 \\
\hline 1 & -2.701497 & 6.267520 & -1.027327 \\
\hline 1 & -0.704015 & 4.818448 & -0.991278 \\
\hline 1 & -0.138721 & 2.258353 & -4.692572 \\
\hline
\end{tabular}




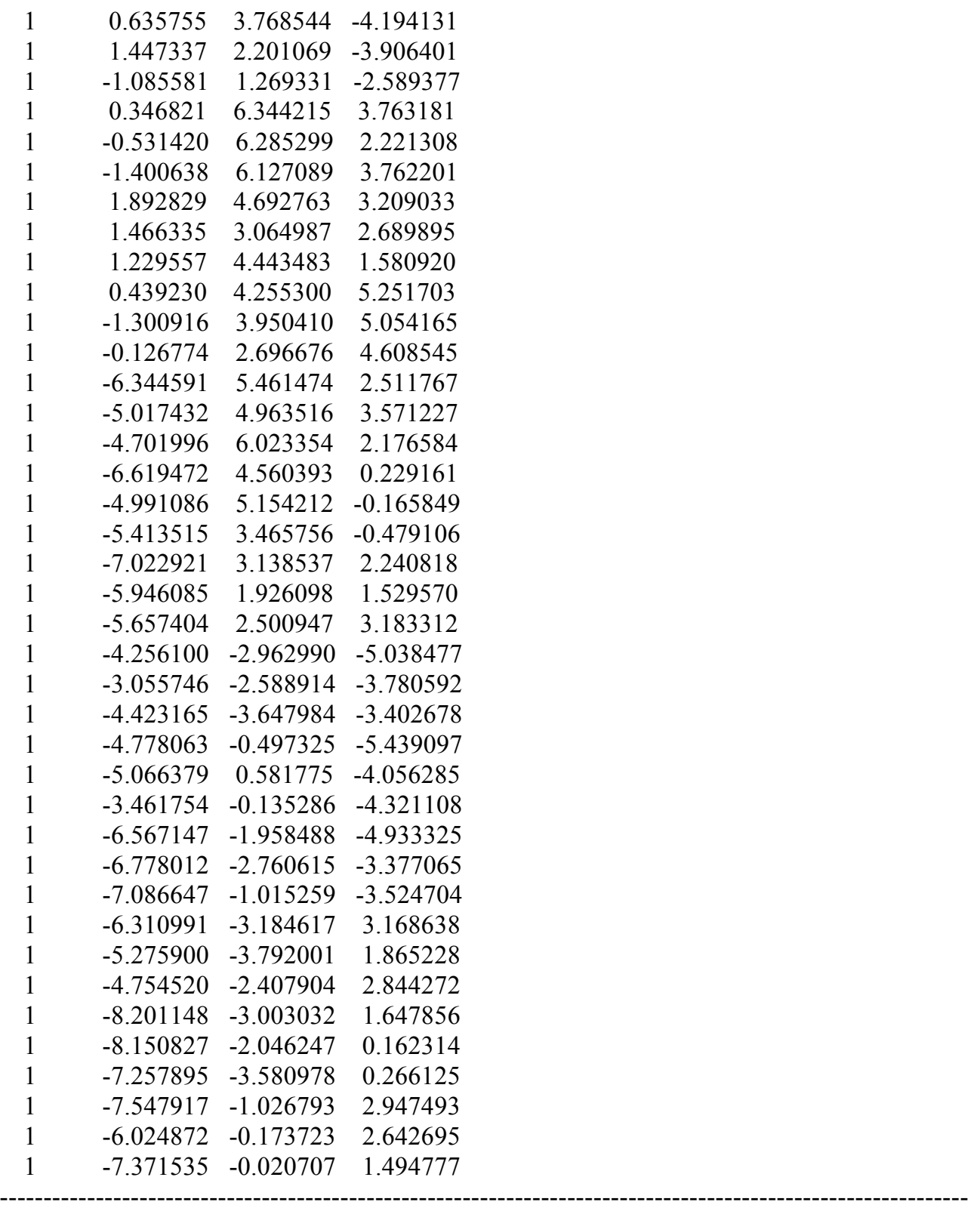

TS1 $_{\text {IIL-si- }} \psi_{-1 \mathrm{~s} 2 \mathrm{aza} a \mathrm{a}}$

Number of imaginary frequencies : 1

The smallest frequencies are : -565.3572 $19.146920 .5034 \mathrm{~cm}(-1)$

Electronic energy : $\quad H F=-4360.8729153$

Zero-point correction $=\quad 1.455312($ Hartree/Particle $)$

Thermal correction to Energy= $\quad 1.539109$

Thermal correction to Enthalpy $=\quad 1.540053$

Thermal correction to Gibbs Free Energy $=\quad 1.340462$

Sum of electronic and zero-point Energies $=\quad-4359.417603$

Sum of electronic and thermal Energies $=\quad-4359.333806$

Sum of electronic and thermal Enthalpies $=\quad-4359.332862$

Sum of electronic and thermal Free Energies $=\quad-4359.532454$

Cartesian Coordinates 


\begin{tabular}{|c|c|c|c|}
\hline 6 & -2.289028 & -5.364696 & -1.781934 \\
\hline 5 & -1.279854 & -4.508785 & -1.276877 \\
\hline p & -1.537857 & -3.730943 & -0.108727 \\
\hline & -2.806865 & -3.856946 & 0.514056 \\
\hline ) & -3.764623 & -4.695777 & 0.001338 \\
\hline & -3.504822 & -5.462310 & -1.155274 \\
\hline f & -0.524838 & -2.855754 & 0.394029 \\
\hline 5 & 0.695621 & -2.794701 & -0.254237 \\
\hline 6 & 0.951490 & -3.599873 & -1.388038 \\
\hline 6 & -0.010631 & -4.429972 & -1.893755 \\
\hline 6 & -0.758778 & -2.104299 & 1.660017 \\
\hline 6 & -1.210870 & -0.789735 & 1.689113 \\
\hline 6 & -1.439050 & -0.172411 & 2.950667 \\
\hline 6 & -1.212471 & -0.833305 & 4.128215 \\
\hline 6 & -0.738097 & -2.163445 & 4.131179 \\
\hline 6 & -0.518938 & -2.813679 & 2.882377 \\
\hline 6 & -0.466013 & -2.850995 & 5.337982 \\
\hline 6 & 0.005018 & -4.138591 & 5.322357 \\
\hline 6 & 0.216139 & -4.793104 & 4.090338 \\
\hline 6 & -0.042774 & -4.151832 & 2.904216 \\
\hline 15 & -1.535828 & 0.136669 & 0.095002 \\
\hline 45 & 0.066624 & 0.234978 & -1.846678 \\
\hline 6 & -0.350124 & 2.371856 & -2.774486 \\
\hline 6 & 0.069721 & 2.372488 & -4.231776 \\
\hline 7 & 1.726039 & -1.898297 & 0.195001 \\
\hline 6 & 2.747926 & -2.494617 & 1.085718 \\
\hline 6 & 3.253038 & -1.613657 & 2.198973 \\
\hline 6 & 2.382113 & -0.845466 & \\
\hline 6 & 2.861329 & -0.083468 & 4.030469 \\
\hline 6 & 4.223537 & -0.072036 & 4.327622 \\
\hline 6 & 5.098778 & -0.827172 & 3.555644 \\
\hline 6 & 4.614197 & -1.592964 & 2.497265 \\
\hline 6 & -3.187650 & -0.568908 & -0.330876 \\
\hline 6 & -3.423455 & -0.981212 & -1.638580 \\
\hline 6 & -4.654179 & -1.526761 & -2.015586 \\
\hline 6 & -5.641293 & -1.636113 & -1.034740 \\
\hline 6 & -5.439623 & -1.238526 & 0.293029 \\
\hline 6 & -4.192738 & -0.715319 & 0.630272 \\
\hline 6 & -6.557107 & -1.412146 & 1.321387 \\
\hline 6 & -6.836920 & -2.906218 & 1.517118 \\
\hline 6 & -4.942049 & -1.950031 & -3.455140 \\
\hline 6 & -5.927237 & -0.949681 & -4.070474 \\
\hline 6 & -1.950658 & 1.797417 & 0.783414 \\
\hline 6 & -3.240543 & 2.329372 & 0.852774 \\
\hline 6 & -3.486549 & 3.534625 & 1.510659 \\
\hline 6 & -2.399253 & 4.211981 & 2.077208 \\
\hline 6 & -1.092096 & 3.731579 & 1.993520 \\
\hline 6 & -0.887328 & 2.522459 & 1.321176 \\
\hline 6 & -4.904685 & 4.067534 & 1.720232 \\
\hline 6 & -5.938785 & 3.291922 & 0.906187 \\
\hline 6 & 0.099556 & 4.482101 & 2.586156 \\
\hline 6 & 1.026208 & 3.509496 & 3.325364 \\
\hline 6 & -5.255873 & 3.922094 & 3.206357 \\
\hline 6 & -5.004101 & 5.543890 & 1.325522 \\
\hline 6 & 0.860885 & 5.165555 & 1.443767 \\
\hline 6 & -0.328376 & 5.559293 & 3.582066 \\
\hline
\end{tabular}




\begin{tabular}{|c|c|c|c|}
\hline 6 & -7.832105 & -0.717497 & 0.831845 \\
\hline 6 & -6.186913 & -0.820026 & 2.679842 \\
\hline 5 & -5.555608 & -3.352664 & -3.491529 \\
\hline & -3.674905 & -1.974041 & -4.309623 \\
\hline ) & 0.105394 & -1.043936 & -3.250587 \\
\hline 5 & 0.196080 & -1.674882 & -4.223607 \\
\hline 1 & 1.934311 & -0.551087 & -0.812726 \\
\hline 3 & 2.845605 & 0.318227 & 0.259442 \\
\hline 6 & 3.735372 & 1.276386 & -0.150291 \\
\hline 6 & 4.925674 & 0.921195 & -0.756095 \\
\hline 6 & 5.782980 & 1.974922 & -1.219102 \\
\hline 6 & 5.447940 & 3.333024 & -0.932027 \\
\hline 6 & 4.259838 & 3.621677 & -0.217099 \\
\hline 6 & 3.413483 & 2.614279 & 0.152317 \\
\hline 6 & 6.299237 & 4.372151 & -1.378809 \\
\hline 6 & 7.429040 & 4.096739 & -2.105594 \\
\hline 6 & 7.744936 & 2.759716 & -2.422926 \\
\hline 6 & 6.946596 & 1.729069 & -1.991586 \\
\hline 6 & 5.263893 & -0.508221 & -0.943988 \\
\hline 6 & 4.369282 & -1.351777 & -1.576771 \\
\hline 6 & 4.705904 & -2.678727 & -1.922734 \\
\hline 6 & 5.909460 & -3.200822 & -1.537905 \\
\hline 6 & 6.809088 & -2.430729 & -0.760417 \\
\hline 6 & 6.487319 & -1.074507 & -0.453766 \\
\hline 6 & 8.005605 & -2.996094 & -0.256717 \\
\hline 6 & 8.845402 & -2.269106 & 0.547764 \\
\hline 6 & 8.513586 & -0.939924 & 3017 \\
\hline 6 & 7.370738 & -0.357088 & 2615 \\
\hline 8 & 3.122859 & -0.909088 & -1.946329 \\
\hline 6 & 0.653879 & 2.379725 & -1.778864 \\
\hline 6 & -1.633113 & 3.119326 & -2.522233 \\
\hline 6 & -2.844615 & 2.710672 & -3.089936 \\
\hline 6 & -3.999927 & 3.464429 & -2.928800 \\
\hline 6 & -3.963088 & 4.650150 & -2.198072 \\
\hline 6 & -2.765556 & 5.066309 & -1.626198 \\
\hline 6 & -1.609725 & 4.309560 & -1.792423 \\
\hline 1 & 7.117455 & 0.660480 & 0.679474 \\
\hline 1 & -1.811445 & 0.848213 & 2.988491 \\
\hline 1 & 8.235021 & -4.029982 & -0.511744 \\
\hline 1 & -0.635558 & -2.330426 & 6.279626 \\
\hline 1 & 6.027420 & 5.399526 & -1.139625 \\
\hline 1 & -3.021306 & -3.265545 & 1.402992 \\
\hline 1 & -4.734516 & -4.772313 & 0.489028 \\
\hline 1 & 4.023134 & 4.658946 & 0.016762 \\
\hline 1 & -1.396852 & -0.337608 & 5.080828 \\
\hline 1 & 6.180668 & -4.222082 & -1.800249 \\
\hline 1 & 8.626636 & 2.541467 & -3.021371 \\
\hline 1 & 3.990479 & -3.257385 & -2.502690 \\
\hline 1 & -2.072581 & -5.949512 & -2.675094 \\
\hline 1 & 0.184507 & -5.032769 & -2.779387 \\
\hline 1 & 0.584531 & -5.816456 & 4.080414 \\
\hline 1 & 7.199501 & 0.705994 & -2.257043 \\
\hline 1 & 0.119268 & -4.673870 & 1.963335 \\
\hline 1 & 1.927344 & -3.523116 & -1.863886 \\
\hline 1 & 8.072863 & 4.903390 & -2.448341 \\
\hline 1 & 9.165797 & -0.371606 & 1.542504 \\
\hline
\end{tabular}




\begin{tabular}{|c|c|c|c|}
\hline 1 & -4.271239 & -6.128705 & -1.545730 \\
\hline & 0.215935 & -4.657863 & 6.254362 \\
\hline & 9.756770 & -2.716323 & 0.937657 \\
\hline & 2.485131 & 2.806211 & 0.685075 \\
\hline & -3.990288 & -0.407871 & 1.654237 \\
\hline & -2.610756 & -0.882456 & -2.355350 \\
\hline & -6.609911 & -2.053352 & -1.311313 \\
\hline & -4.063000 & 1.777820 & 0.406930 \\
\hline & 0.114322 & 2.091093 & 1.265396 \\
\hline & -2.588848 & 5.138840 & 2.614472 \\
\hline & 2.260587 & -3.373673 & 1.531965 \\
\hline & 3.601154 & -2.878453 & 0.505323 \\
\hline & 5.309107 & -2.172111 & 1.886256 \\
\hline 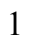 & 1.325244 & -0.820727 & 2.715960 \\
\hline & 6.166154 & -0.818108 & 3.768515 \\
\hline & 2.164744 & 0.508376 & 4.622989 \\
\hline & 4.599455 & 0.528702 & 5.153270 \\
\hline & 0.424587 & 2.827102 & -0.811397 \\
\hline 1 & 1.696753 & 2.417156 & -2.093950 \\
\hline & -2.881697 & 1.780834 & -3.659252 \\
\hline & -4.932748 & 3.127065 & -3.376068 \\
\hline & -4.866631 & 5.245954 & -2.078064 \\
\hline & -2.723365 & 5.988411 & -1.047948 \\
\hline & -0.671563 & 4.654349 & -1.362435 \\
\hline 1 & -0.665289 & 1.870566 & -4.872346 \\
\hline & 0.158006 & 3.410340 & -4.583302 \\
\hline I & 1.033519 & 1.869325 & -4.361506 \\
\hline & -1.125355 & 0.952163 & -2.705549 \\
\hline & 0.560964 & 6.036925 & 4.012435 \\
\hline ] & -0.921305 & 6.351881 & 3.109128 \\
\hline 1 & -0.914567 & 5.138486 & 4.409463 \\
\hline 1 & 1.857863 & 4.056182 & 3.788812 \\
\hline D & 0.481449 & 2.981548 & 4.120404 \\
\hline 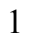 & 1.467096 & 2.748582 & 2.669769 \\
\hline ] & 1.766387 & 5.660767 & 1.822178 \\
\hline 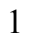 & 1.161700 & 4.451394 & 0.665305 \\
\hline 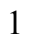 & 0.232772 & 5.928724 & 0.963595 \\
\hline & -6.934508 & 3.725971 & 1.063138 \\
\hline 1 & -5.719931 & 3.326846 & -0.169350 \\
\hline ] & -5.991177 & 2.236452 & 1.207485 \\
\hline 1 & -6.019951 & 5.916127 & 1.514155 \\
\hline 1 & -4.314249 & 6.178416 & 1.894403 \\
\hline$\frac{1}{1}$ & -4.787271 & 5.680011 & 0.258863 \\
\hline 1 & -6.281991 & 4.266285 & 3.394971 \\
\hline 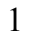 & -5.185192 & 2.873335 & 3.524757 \\
\hline 1 & -4.582944 & 4.509342 & 3.843602 \\
\hline 1 & -3.917617 & -2.329742 & -5.319075 \\
\hline 1 & -3.223210 & -0.978616 & -4.418174 \\
\hline 1 & -2.914735 & -2.647205 & -3.890019 \\
\hline 1 & -6.145026 & -1.214277 & -5.114310 \\
\hline 1 & -6.878155 & -0.928017 & -3.523144 \\
\hline 1 & -5.512226 & 0.067204 & -4.057445 \\
\hline 1 & -5.758833 & -3.648155 & -4.529543 \\
\hline 1 & -4.868121 & -4.089591 & -3.055708 \\
\hline 1 & -6.504577 & -3.410865 & -2.945104 \\
\hline 1 & -7.658758 & -3.053052 & 2.231243 \\
\hline
\end{tabular}


TS1 $_{\text {IIL-si- }} \psi_{-1 \mathrm{~s} 2 \mathrm{a3a4s}}$

$\begin{array}{lcc}\text { Number of imaginary frequencies : } 1 & \\ \text { The smallest frequencies are : }-542.3195 & 14.5284 & 18.7239 \mathrm{~cm}(-1) \\ \text { Electronic energy : HF=-4360.8732133 } & \\ \text { Zero-point correction= } & 1.452633 & \text { (Hartree/Particle) } \\ \text { Thermal correction to Energy= } & 1.537129 \\ \text { Thermal correction to Enthalpy= } & 1.538073 \\ \text { Thermal correction to Gibbs Free Energy= } & 1.335580 \\ \text { Sum of electronic and zero-point Energies }= & -4359.420580 \\ \text { Sum of electronic and thermal Energies }= & -4359.336084 \\ \text { Sum of electronic and thermal Enthalpies }= & -4359.335140 \\ \text { Sum of electronic and thermal Free Energies }= & -4359.537633\end{array}$

Cartesian Coordinates

$\begin{array}{rrrr}6 & -2.343332 & -5.263465 & -1.981828 \\ 6 & -1.320631 & -4.445490 & -1.441876 \\ 6 & -1.562834 & -3.724142 & -0.235125 \\ 6 & -2.826441 & -3.871388 & 0.392895 \\ 6 & -3.797991 & -4.670754 & -0.154937 \\ 6 & -3.556756 & -5.377862 & -1.352736 \\ 6 & -0.542523 & -2.876334 & 0.298659 \\ 6 & 0.673090 & -2.791053 & -0.355677 \\ 6 & 0.916662 & -3.547349 & -1.525529 \\ 6 & -0.054511 & -4.347054 & -2.062520 \\ 6 & -0.771797 & -2.170601 & 1.591517 \\ 6 & -1.211891 & -0.853692 & 1.665528 \\ 6 & -1.445614 & -0.280730 & 2.946524 \\ 6 & -1.239645 & -0.987762 & 4.100948 \\ 6 & -0.778545 & -2.322002 & 4.058898 \\ 6 & -0.549883 & -2.926568 & 2.789062 \\ 6 & -0.530104 & -3.058033 & 5.241966 \\ 6 & -0.072423 & -4.349203 & 5.183192 \\ 6 & 0.149597 & -4.958042 & 3.929874 \\ 6 & -0.086396 & -4.269117 & 2.765910 \\ 15 & -1.531783 & 0.129443 & 0.104944 \\ 45 & 0.058785 & 0.302426 & -1.836966 \\ 6 & -0.318432 & 2.473708 & -2.695971 \\ 6 & 0.089603 & 2.503110 & -4.156707 \\ 7 & 1.708330 & -1.916500 & 0.123933 \\ 6 & 2.717149 & -2.544280 & 1.008332 \\ 6 & 3.225067 & -1.693818 & 2.143743 \\ 6 & 2.355433 & -0.945618 & 2.939430 \\ 6 & 2.837127 & -0.205462 & 4.010370 \\ 6 & 4.200568 & -0.197158 & 4.302079 \\ 6 & 5.073973 & -0.935393 & 3.511917\end{array}$




\begin{tabular}{|c|c|c|c|}
\hline 6 & 4.586763 & -1.679650 & 2.439476 \\
\hline 6 & -3.186977 & -0.558893 & -0.334074 \\
\hline 6 & -3.448436 & -0.894744 & -1.661985 \\
\hline & -4.674132 & -1.440677 & -2.041072 \\
\hline & -5.634307 & -1.648627 & -1.042254 \\
\hline & -5.402576 & -1.337436 & 0.298964 \\
\hline & -4.163226 & -0.785399 & 0.633322 \\
\hline & -6.426914 & -1.584086 & 1.406589 \\
\hline & -5.811415 & -2.477055 & 2.491177 \\
\hline 6 & -4.992214 & -1.762878 & -3.500303 \\
\hline 6 & -1.944959 & 1.769461 & 0.838938 \\
\hline 6 & -3.230100 & 2.314162 & 0.887565 \\
\hline 6 & -3.473592 & 3.523290 & 1.541782 \\
\hline 6 & -2.391064 & 4.177164 & 2.142709 \\
\hline 6 & -1.089264 & 3.675891 & 2.092157 \\
\hline 6 & -0.884344 & 2.472276 & 1.411875 \\
\hline 6 & -4.890220 & 4.075248 & 1.712513 \\
\hline 6 & -5.882533 & 3.424484 & 0.749684 \\
\hline 6 & 0.095289 & 4.409634 & 2.719587 \\
\hline 6 & 1.019141 & 3.418359 & 3.436580 \\
\hline 6 & -5.344525 & 3.783373 & 3.148234 \\
\hline 6 & -4.931679 & 5.587801 & 1.477125 \\
\hline 6 & 0.865362 & 5.130701 & 1.606388 \\
\hline 6 & -0.346136 & 5.453441 & 3.745328 \\
\hline 6 & -6.828508 & -0.243043 & 2.030531 \\
\hline 6 & -7.690212 & -2.268337 & 0.891260 \\
\hline 6 & 0.066346 & -0.921602 & -3.292868 \\
\hline 8 & 0.136614 & -1.513065 & -4.291845 \\
\hline 15 & 1.923300 & -0.536175 & -0.836146 \\
\hline 8 & 2.834106 & 0.294412 & 0.266425 \\
\hline 6 & 3.733092 & 1.259353 & -0.104688 \\
\hline 6 & 4.923023 & 0.917782 & -0.719063 \\
\hline 6 & 5.789552 & 1.981039 & -1.140991 \\
\hline 6 & 5.463939 & 3.330257 & -0.805408 \\
\hline 6 & 4.275953 & 3.601498 & -0.083454 \\
\hline 6 & 3.420860 & 2.587792 & 0.246296 \\
\hline 6 & 6.324442 & 4.378370 & -1.211750 \\
\hline 6 & 7.454280 & 4.120819 & -1.944983 \\
\hline 6 & 7.761067 & 2.793855 & -2.309890 \\
\hline 6 & 6.953707 & 1.754328 & -1.918564 \\
\hline 6 & 5.252592 & -0.506395 & -0.954938 \\
\hline 6 & 4.354591 & -1.321916 & -1.618649 \\
\hline 6 & 4.681461 & -2.640528 & -2.003378 \\
\hline 6 & 5.878624 & -3.184574 & -1.628859 \\
\hline 6 & 6.780901 & -2.446479 & -0.823861 \\
\hline 6 & 6.469023 & -1.097599 & -0.477132 \\
\hline 6 & 7.969436 & -3.037654 & -0.331025 \\
\hline 6 & 8.810434 & -2.343431 & 0.500695 \\
\hline 6 & 8.488106 & -1.022335 & 0.875131 \\
\hline 6 & 7.353502 & -0.414538 & 0.396115 \\
\hline 8 & 3.113520 & -0.856124 & -1.979074 \\
\hline 6 & 0.696191 & 2.430394 & -1.712052 \\
\hline 6 & -1.579142 & 3.250064 & -2.418978 \\
\hline 6 & -2.805361 & 2.885906 & -2.985752 \\
\hline 6 & -3.932865 & 3.679630 & -2.820294 \\
\hline 6 & -3.854143 & 4.859779 & -2.083448 \\
\hline
\end{tabular}




\begin{tabular}{|c|c|c|c|}
\hline 6 & -2.643482 & 5.228844 & -1.507375 \\
\hline 6 & -1.514784 & 4.433572 & -1.680726 \\
\hline & 7.107012 & 0.595682 & 0.713154 \\
\hline & -1.807568 & 0.741912 & 3.017571 \\
\hline & 8.191446 & -4.065168 & -0.616432 \\
\hline & -0.707083 & -2.571828 & 6.200475 \\
\hline & 6.059881 & 5.398436 & -0.935977 \\
\hline & -3.024810 & -3.328516 & 1.315362 \\
\hline & -4.764483 & -4.763836 & 0.338239 \\
\hline & 4.046279 & 4.631142 & 0.188084 \\
\hline & -1.429972 & -0.526515 & 5.069555 \\
\hline 1 & 6.142273 & -4.199819 & -1.920639 \\
\hline & 8.643011 & 2.590761 & -2.913300 \\
\hline & 3.964258 & -3.195200 & -2.604139 \\
\hline & -2.140138 & -5.805124 & -2.904804 \\
\hline & 0.130833 & -4.909096 & -2.976578 \\
\hline & 0.508367 & -5.983889 & 3.885626 \\
\hline & 7.199713 & 0.739541 & -2.220124 \\
\hline & 0.083343 & -4.756361 & 1.807910 \\
\hline & 1.889683 & -3.455122 & -2.004471 \\
\hline & 8.105215 & 4.934416 & -2.256394 \\
\hline & 9.141025 & -0.480602 & 1.555946 \\
\hline & -4.333921 & -6.013757 & -1.771707 \\
\hline & 0.120092 & -4.905945 & 6.097362 \\
\hline & 9.715294 & -2.810708 & 0.882111 \\
\hline & 2.491717 & 2.767974 & 0.781858 \\
\hline & -3.949690 & -0.534871 & 1.672932 \\
\hline & -2.655188 & -0.734703 & -2.389247 \\
\hline & -6.594301 & -2.075367 & -1.325138 \\
\hline 1 & -4.050378 & 1.773528 & 0.422849 \\
\hline & 0.113181 & 2.030308 & 1.367514 \\
\hline & -2.577808 & 5.104617 & 2.678901 \\
\hline & 2.216024 & -3.426979 & 1.431429 \\
\hline & 3.569538 & -2.925005 & 0.424678 \\
\hline 1 & 5.280257 & -2.245942 & 1.815135 \\
\hline & 1.297417 & -0.918245 & 2.686877 \\
\hline 1 & 6.141976 & -0.930174 & 3.721613 \\
\hline & 2.141387 & 0.371063 & 4.618741 \\
\hline 1 & 4.578599 & 0.386957 & 5.138614 \\
\hline & 0.497259 & 2.860391 & -0.729984 \\
\hline 1 & 1.735255 & 2.450145 & -2.041261 \\
\hline 1 & -2.874841 & 1.961517 & -3.561051 \\
\hline & -4.877336 & 3.377215 & -3.268620 \\
\hline 1 & -4.735782 & 5.486786 & -1.960016 \\
\hline & -2.568402 & 6.145888 & -0.924427 \\
\hline 1 & -0.563173 & 4.744746 & -1.254268 \\
\hline & -0.662929 & 2.036618 & -4.803748 \\
\hline 1 & 0.199967 & 3.547740 & -4.480961 \\
\hline & 1.039853 & 1.980753 & -4.308477 \\
\hline 1 & -1.129120 & 1.071692 & -2.656732 \\
\hline 1 & 0.537206 & 5.914371 & 4.205283 \\
\hline & -0.930475 & 6.263382 & 3.291670 \\
\hline 1 & -0.945745 & 5.005382 & 4.548404 \\
\hline & 1.840566 & 3.954537 & 3.929448 \\
\hline & 0.468318 & 2.858992 & 4.205540 \\
\hline & 1.474743 & 2.684486 & 2.760647 \\
\hline
\end{tabular}




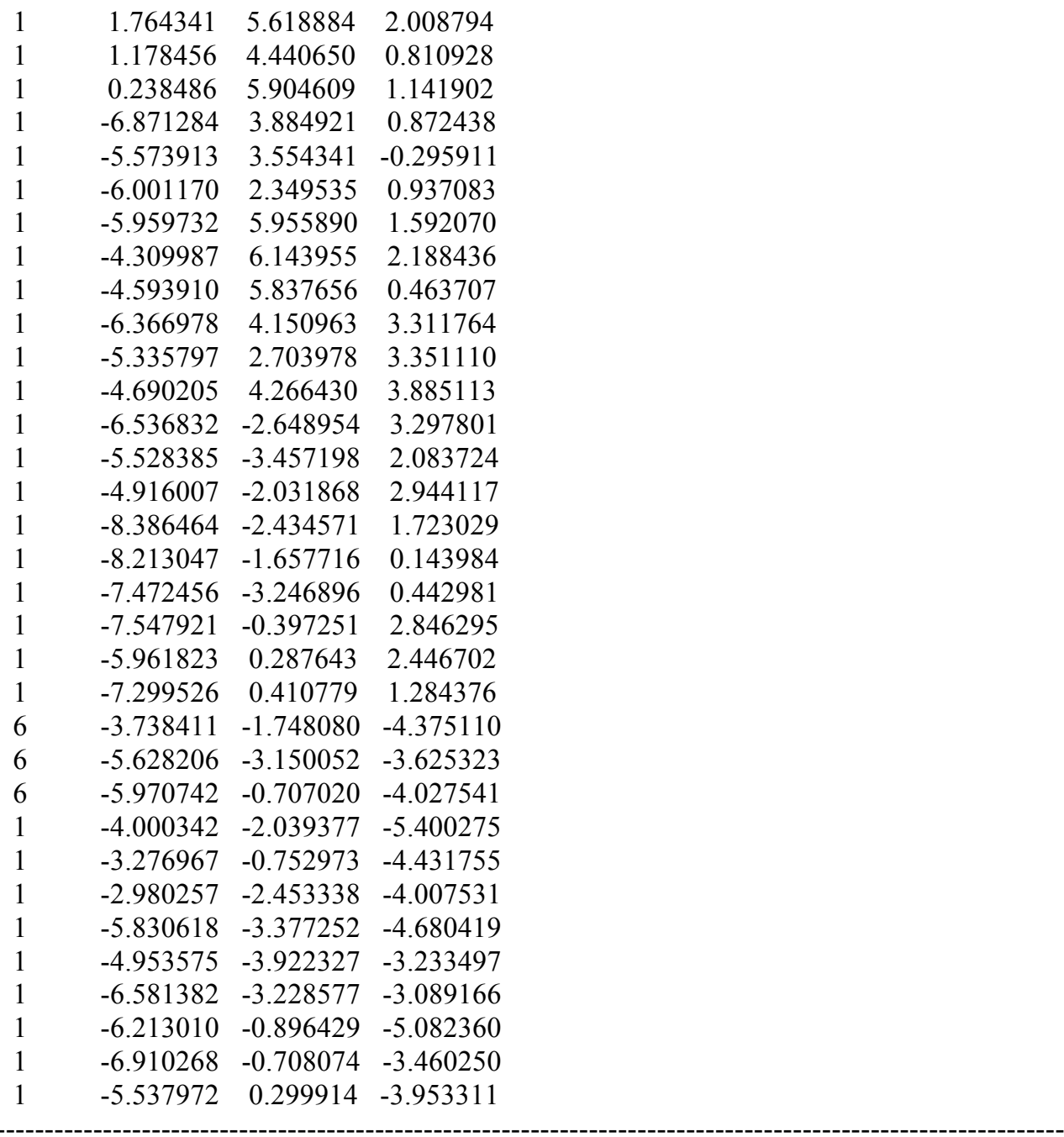

$\mathrm{TS1}_{\text {IIL-si- }} \psi_{-1 \mathrm{~s} 2 \mathrm{a3s4a}}$

Number of imaginary frequencies : 1

The smallest frequencies are : $-545.8084 \quad 18.1837 \quad 18.5819 \mathrm{~cm}(-1)$

Electronic energy : $\quad H F=-4360.8726625$

Zero-point correction $=\quad 1.452401($ Hartree/Particle $)$

Thermal correction to Energy $=\quad 1.536855$

Thermal correction to Enthalpy $=\quad 1.537800$

Thermal correction to Gibbs Free Energy= 1.335696

Sum of electronic and zero-point Energies $=\quad-4359.420262$

Sum of electronic and thermal Energies $=\quad-4359.335807$

Sum of electronic and thermal Enthalpies $=\quad-4359.334863$

Sum of electronic and thermal Free Energies $=\quad-4359.536966$

Cartesian Coordinates

\begin{tabular}{|c|c|c|c|}
\hline 6 & -4.200743 & -0.722395 & 0.602223 \\
\hline 6 & -3.190168 & -0.550190 & -0.354186 \\
\hline 6 & -3.428379 & -0.922626 & -1.668128 \\
\hline 6 & -4.662420 & -1.454389 & -2.066020 \\
\hline 6 & -5.646314 & -1.602804 & -1.093247 \\
\hline 6 & & & \\
\hline
\end{tabular}




\begin{tabular}{|c|c|c|c|}
\hline 15 & -1.539218 & 0.143883 & 0.090098 \\
\hline 6 & -1.231418 & -0.799120 & 1.676036 \\
\hline 6 & -0.781126 & -2.113762 & 1.639987 \\
\hline 6 & -0.550489 & -2.831545 & 2.860499 \\
\hline 6 & -0.783838 & -2.191142 & 4.111862 \\
\hline 6 & -1.260775 & -0.861956 & 4.114537 \\
\hline 6 & -1.473527 & -0.191772 & 2.940008 \\
\hline 6 & -0.521781 & -2.885770 & 5.316798 \\
\hline 6 & -0.044454 & -4.170923 & 5.297631 \\
\hline 6 & 0.183891 & -4.814980 & 4.063337 \\
\hline 6 & -0.066216 & -4.167047 & 2.878893 \\
\hline 6 & -0.530105 & -2.864743 & 0.376328 \\
\hline 6 & -1.520264 & -3.771477 & -0.118118 \\
\hline 6 & -1.217201 & -4.605119 & -1.236055 \\
\hline 6 & 0.058112 & -4.516219 & -1.839446 \\
\hline 6 & 0.989064 & -3.637464 & -1.357955 \\
\hline 6 & 0.699949 & -2.803063 & -0.253441 \\
\hline 6 & -2.186603 & -5.525022 & -1.704456 \\
\hline 6 & -3.414590 & -5.619084 & -1.101979 \\
\hline 6 & -3.726403 & -4.783289 & -0.008115 \\
\hline 6 & -2.803859 & -3.888872 & 0.475471 \\
\hline 7 & 1.717193 & -1.893562 & 0.200083 \\
\hline 6 & 2.743455 & -2.484028 & 1.090577 \\
\hline 6 & 3.234985 & -1.602730 & 2.209395 \\
\hline 6 & 2.352024 & -0.850319 & 2.985878 \\
\hline 6 & 2.818662 & -0.087551 & 4.047674 \\
\hline 6 & 4.179980 & -0.059022 & 4.347690 \\
\hline 6 & 5.067021 & -0.798639 & 3.574049 \\
\hline 6 & 4.595022 & -1.565912 & 2.511134 \\
\hline 6 & -4.850045 & -1.871314 & -3.522775 \\
\hline 6 & -6.561118 & -1.442016 & 1.270537 \\
\hline 45 & 0.059555 & 0.244069 & -1.840138 \\
\hline 6 & 0.082652 & -1.031211 & -3.249517 \\
\hline 8 & 0.149773 & -1.657843 & -4.227346 \\
\hline 6 & -0.356555 & 2.382412 & -2.778239 \\
\hline 6 & 0.031511 & 2.365280 & -4.244704 \\
\hline 6 & -1.628371 & 3.143162 & -2.506653 \\
\hline 6 & -1.590767 & 4.326013 & -1.765570 \\
\hline 6 & -2.738869 & 5.090701 & -1.583008 \\
\hline 6 & -3.943545 & 4.690880 & -2.151237 \\
\hline 6 & -3.995073 & 3.512486 & -2.892817 \\
\hline 6 & -2.847658 & 2.750171 & -3.069063 \\
\hline 6 & 0.672586 & 2.385075 & -1.808016 \\
\hline 15 & 1.923367 & -0.546704 & -0.807778 \\
\hline 8 & 2.831412 & 0.324018 & 0.265885 \\
\hline 6 & 3.718111 & 1.285294 & -0.143313 \\
\hline 6 & 4.909963 & 0.933591 & -0.747872 \\
\hline 6 & 5.764979 & 1.989629 & -1.209727 \\
\hline 6 & 5.425207 & 3.346798 & -0.923759 \\
\hline 6 & 4.235022 & 3.632096 & -0.210939 \\
\hline 6 & 3.391455 & 2.622206 & 0.158019 \\
\hline 6 & 6.930947 & 1.747007 & -1.979692 \\
\hline 6 & 7.727011 & 2.779943 & -2.409736 \\
\hline 6 & 7.406289 & 4.116118 & -2.093667 \\
\hline 6 & 6.274172 & 4.388367 & -1.369316 \\
\hline 6 & 5.252181 & -0.494781 & -0.936081 \\
\hline
\end{tabular}




\begin{tabular}{|c|c|c|c|}
\hline 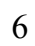 & 4.361406 & -1.340269 & -1.571787 \\
\hline & 4.704399 & -2.664697 & -1.921336 \\
\hline & 5.908645 & -3.183590 & -1.534272 \\
\hline & 6.802949 & -2.412696 & -0.751476 \\
\hline & 6.476067 & -1.058023 & -0.443548 \\
\hline & 7.999366 & -2.975393 & -0.244537 \\
\hline & 8.834115 & -2.247322 & 0.564197 \\
\hline 6 & 8.497272 & -0.919647 & 0.900463 \\
\hline 6 & 7.354527 & -0.339276 & 0.406919 \\
\hline 8 & 3.113157 & -0.902299 & -1.940974 \\
\hline 6 & -1.952604 & 1.799282 & 0.791717 \\
\hline 6 & -3.243919 & 2.326493 & 0.867386 \\
\hline 6 & -3.492496 & 3.525876 & 1.534671 \\
\hline 6 & -2.405967 & 4.202107 & 2.103827 \\
\hline 6 & -1.097381 & 3.726395 & 2.014098 \\
\hline 6 & -0.889924 & 2.521496 & 1.334644 \\
\hline 6 & -4.912320 & 4.050380 & 1.752558 \\
\hline 6 & -5.262648 & 3.877675 & 3.235953 \\
\hline 6 & 0.092773 & 4.480733 & 2.604758 \\
\hline 6 & 0.845776 & 5.171149 & 1.460905 \\
\hline 6 & -5.017014 & 5.532735 & 1.383317 \\
\hline 6 & -5.943247 & 3.285023 & 0.924859 \\
\hline 6 & -0.336043 & 5.553598 & 3.605001 \\
\hline 6 & 1.026578 & 3.510889 & 3.338160 \\
\hline 1 & 7.097104 & 0.676992 & 0.694770 \\
\hline 1 & -1.846555 & 0.828410 & 2.981741 \\
\hline 1 & 8.232781 & -4.008146 & -0.500525 \\
\hline 1 & -0.703478 & -2.372035 & 6.259929 \\
\hline 1 & 5.998776 & 5.415001 & -1.131065 \\
\hline 1 & -3.057077 & -3.249521 & 1.320106 \\
\hline 1 & -4.708042 & -4.850727 & 0.457217 \\
\hline 1 & 3.994820 & 4.668814 & 0.021781 \\
\hline 1 & -1.456718 & -0.374353 & 5.068985 \\
\hline 1 & 6.184407 & -4.203003 & -1.799025 \\
\hline 1 & 8.610710 & 2.564173 & -3.006124 \\
\hline 1 & 3.993557 & -3.243894 & -2.506286 \\
\hline 1 & -1.932877 & -6.154666 & -2.556360 \\
\hline 1 & 0.284780 & -5.155960 & -2.690965 \\
\hline 1 & 0.558921 & -5.835877 & 4.049879 \\
\hline 1 & 7.187660 & 0.724603 & -2.244082 \\
\hline 1 & 0.110572 & -4.682535 & 1.937087 \\
\hline 1 & 1.971060 & -3.556106 & -1.820195 \\
\hline 1 & 8.048353 & 4.924596 & -2.435396 \\
\hline 1 & 9.145486 & -0.350576 & 1.563234 \\
\hline 1 & -4.152489 & -6.329738 & -1.468060 \\
\hline 1 & 0.159068 & -4.695656 & 6.228213 \\
\hline 1 & 9.745339 & -2.692553 & 0.956683 \\
\hline 1 & 2.461568 & 2.811358 & 0.689006 \\
\hline 1 & -4.000964 & -0.438369 & 1.633428 \\
\hline 1 & -2.619629 & -0.806607 & -2.388754 \\
\hline 1 & -6.612350 & -2.017727 & -1.374985 \\
\hline 1 & -4.065474 & 1.775146 & 0.419587 \\
\hline 1 & 0.113282 & 2.094645 & 1.270096 \\
\hline 1 & -2.597083 & 5.125084 & 2.647145 \\
\hline 1 & 2.263905 & -3.370264 & 1.531047 \\
\hline 1 & 3.602641 & -2.855973 & 0.511361 \\
\hline
\end{tabular}




\begin{tabular}{|c|c|c|c|}
\hline 1 & 5.298649 & -2.133585 & 1.899398 \\
\hline & 1.295482 & -0.839346 & 2.725888 \\
\hline 1 & 6.133758 & -0.776352 & 3.789321 \\
\hline 1 & 2.112907 & 0.491248 & 4.642110 \\
\hline 1 & 4.545935 & 0.542633 & 5.177136 \\
\hline 1 & 0.482604 & 2.845079 & -0.837896 \\
\hline 1 & 1.706845 & 2.404900 & -2.152065 \\
\hline 1 & -2.898101 & 1.825962 & -3.646141 \\
\hline 1 & -4.933723 & 3.187687 & -3.337370 \\
\hline 1 & -4.840974 & 5.293518 & -2.020018 \\
\hline 1 & -2.684510 & 6.006690 & -0.996108 \\
\hline 1 & -0.646874 & 4.659321 & -1.338736 \\
\hline 1 & -0.722231 & 1.865057 & -4.864736 \\
\hline 1 & 0.122931 & 3.398742 & -4.607744 \\
\hline 1 & 0.987014 & 1.850802 & -4.390382 \\
\hline 1 & -1.134158 & 0.973789 & -2.689299 \\
\hline 1 & 0.552945 & 6.033322 & 4.033747 \\
\hline 1 & -0.933464 & 6.345315 & 3.136288 \\
\hline 1 & -0.917819 & 5.128305 & 4.433200 \\
\hline 1 & 1.860225 & 4.059612 & 3.795558 \\
\hline 1 & 0.487998 & 2.983039 & 4.137374 \\
\hline 1 & 1.464278 & 2.750098 & 2.680494 \\
\hline 1 & 1.750246 & 5.670210 & 1.836647 \\
\hline 1 & 1.147052 & 4.460816 & 0.679067 \\
\hline 1 & 0.211500 & 5.932327 & 0.985542 \\
\hline 1 & -6.940091 & 3.714910 & 1.086039 \\
\hline 1 & -5.721754 & 3.336452 & -0.149480 \\
\hline 1 & -5.994642 & 2.225066 & 1.209979 \\
\hline 1 & -4.800326 & 5.687756 & 0.319231 \\
\hline 1 & -6.034350 & 5.897858 & 1.577565 \\
\hline 1 & -4.329986 & 6.160180 & 1.963260 \\
\hline 1 & -6.289978 & 4.214662 & 3.430875 \\
\hline 1 & -5.187897 & 2.823667 & 3.535666 \\
\hline 1 & -4.591429 & 4.456124 & 3.883011 \\
\hline 6 & -6.290309 & -2.272966 & -3.830165 \\
\hline 6 & -4.466274 & -0.716143 & -4.455261 \\
\hline 6 & -3.940064 & -3.069814 & -3.810588 \\
\hline 6 & -6.201339 & -0.869309 & 2.640130 \\
\hline 6 & -7.843184 & -0.753647 & 0.790279 \\
\hline 6 & -6.824615 & -2.942331 & 1.439410 \\
\hline 1 & -7.038101 & -1.017481 & 3.334348 \\
\hline 1 & -5.322296 & -1.362346 & 3.076569 \\
\hline 1 & -5.997661 & 0.210067 & 2.591762 \\
\hline 1 & -7.648223 & -3.111017 & 2.146657 \\
\hline 1 & -7.095513 & -3.420770 & 0.489809 \\
\hline 1 & -5.934308 & -3.450817 & 1.834780 \\
\hline 1 & -8.642420 & -0.880746 & 1.532681 \\
\hline 1 & -7.684998 & 0.323223 & 0.645771 \\
\hline 1 & -8.208339 & -1.166690 & -0.157396 \\
\hline 1 & -6.381364 & -2.540292 & -4.890522 \\
\hline 1 & -6.605933 & -3.147573 & -3.246914 \\
\hline 1 & -6.995252 & -1.454603 & -3.632384 \\
\hline 1 & -4.047074 & -3.391513 & -4.855808 \\
\hline 1 & -2.882809 & -2.827665 & -3.639633 \\
\hline 1 & -4.194064 & -3.916869 & -3.160182 \\
\hline 1 & -4.635912 & -1.003378 & -5.501523 \\
\hline
\end{tabular}




$\begin{array}{ccccc}1 & -5.069961 & 0.177882 & -4.247956 \\ 1 & -3.405638 & -0.444260 & -4.363006\end{array}$

TS1 $_{I I L-s i-} \Psi_{-1 \mathrm{~s} 2 \mathrm{a} 3 \mathrm{~s} 4 \mathrm{~s}}$

Number of imaginary frequencies : 1

The smallest frequencies are : -537.7606 $17.4688 \quad 18.9127 \mathrm{~cm}(-1)$

Electronic energy : $\quad H F=-4360.8728264$

Zero-point correction $=\quad 1.452530($ Hartree/Particle $)$

Thermal correction to Energy $=\quad 1.537031$

Thermal correction to Enthalpy= $\quad 1.537975$

Thermal correction to Gibbs Free Energy= 1.335535

Sum of electronic and zero-point Energies $=\quad-4359.420297$

Sum of electronic and thermal Energies $=\quad-4359.335795$

Sum of electronic and thermal Enthalpies $=\quad-4359.334851$

Sum of electronic and thermal Free Energies $=\quad-4359.537292$

Cartesian Coordinates

$\begin{array}{cccc}6 & -3.455853 & -0.841849 & -1.680922 \\ 6 & -3.194353 & -0.536991 & -0.351059 \\ 6 & -4.176724 & -0.784913 & 0.609591 \\ 6 & -5.410670 & -1.331752 & 0.259519 \\ 6 & -5.643254 & -1.614305 & -1.092796 \\ 6 & -4.684142 & -1.374583 & -2.079620 \\ 15 & -1.540188 & 0.143260 & 0.102076 \\ 6 & -1.954885 & 1.780488 & 0.840387 \\ 6 & -3.243359 & 2.315767 & 0.901237 \\ 6 & -3.488093 & 3.527393 & 1.549908 \\ 6 & -2.404140 & 4.191061 & 2.137240 \\ 6 & -1.099606 & 3.697801 & 2.076633 \\ 6 & -0.893222 & 2.491863 & 1.400825 \\ 6 & -4.907094 & 4.071435 & 1.725592 \\ 6 & 0.086639 & 4.446152 & 2.683248 \\ 6 & -6.440168 & -1.600403 & 1.357629 \\ 6 & -4.893603 & -1.719610 & -3.552404 \\ 45 & 0.047022 & 0.304664 & -1.832381 \\ 6 & -0.028538 & -0.945338 & -3.263271 \\ 8 & -0.063664 & -1.553095 & -4.254531 \\ 15 & 1.914296 & -0.533332 & -0.833672 \\ 7 & 1.701934 & -1.904569 & 0.139249 \\ 6 & 2.714275 & -2.517802 & 1.030306 \\ 6 & 3.203196 & -1.657508 & 2.166494 \\ 6 & 2.318082 & -0.916522 & 2.951557 \\ 6 & 2.782041 & -0.167044 & 4.023904 \\ 6 & 4.142703 & -0.142016 & 4.327363 \\ 6 & 5.031364 & -0.873237 & 3.547662 \\ 6 & 4.562025 & -1.626913 & 2.473874 \\ 8 & 3.107024 & -0.860250 & -1.972544 \\ 6 & 4.347526 & -1.324157 & -1.607635 \\ 6 & 5.243105 & -0.507594 & -0.941786 \\ 6 & 6.456259 & -1.098842 & -0.455579 \\ 6 & 6.769010 & -2.448746 & -0.797333 \\ 6 & 5.871874 & -3.186913 & -1.607900 \\ 6 & 4.677273 & -2.642766 & -1.990370 \\ 6 & 7.336351 & -0.414472 & 0.421211\end{array}$




\begin{tabular}{|c|c|c|c|}
\hline 6 & 8.467362 & -1.022327 & 0.908537 \\
\hline 6 & 8.790600 & -2.344649 & 0.539194 \\
\hline 6 & 7.953945 & -3.039976 & -0.295937 \\
\hline 6 & 4.916378 & 0.918609 & -0.714125 \\
\hline 6 & 5.789026 & 1.976668 & -1.136885 \\
\hline 6 & 5.467491 & 3.328581 & -0.808443 \\
\hline 6 & 4.277528 & 3.607504 & -0.092695 \\
\hline 6 & 3.416978 & 2.598663 & 0.237458 \\
\hline 6 & 3.724920 & 1.267409 & -0.106972 \\
\hline 6 & 6.333974 & 4.371504 & -1.215339 \\
\hline 6 & 7.466092 & 4.106331 & -1.942354 \\
\hline 6 & 7.769127 & 2.776613 & -2.300214 \\
\hline 6 & 6.955769 & 1.742020 & -1.908160 \\
\hline 8 & 2.820606 & 0.308013 & 0.264681 \\
\hline 6 & 0.715231 & 2.424869 & -1.727125 \\
\hline 6 & -0.306206 & 2.479902 & -2.702445 \\
\hline 6 & -1.553880 & 3.272845 & -2.414516 \\
\hline 6 & -2.788265 & 2.925525 & -2.973829 \\
\hline 6 & -3.906289 & 3.728806 & -2.792125 \\
\hline 6 & -3.809765 & 4.902069 & -2.046368 \\
\hline 6 & -2.590130 & 5.255541 & -1.479441 \\
\hline 6 & -1.470539 & 4.450904 & -1.669326 \\
\hline 6 & 0.086341 & 2.492244 & -4.167350 \\
\hline 6 & -1.234093 & -0.850858 & 1.655890 \\
\hline 6 & -0.795346 & -2.167838 & 1.578414 \\
\hline 6 & -0.584220 & -2.928669 & 2.776327 \\
\hline 6 & -0.828502 & -2.330223 & 4.046284 \\
\hline 6 & -1.289110 & -0.995866 & 4.090032 \\
\hline 6 & -1.479597 & -0.282883 & 2.936999 \\
\hline 6 & -0.594834 & -3.071008 & 5.229384 \\
\hline 6 & -0.133891 & -4.360978 & 5.171522 \\
\hline 6 & 0.107617 & -4.962759 & 3.918585 \\
\hline 6 & -0.114777 & -4.269459 & 2.754498 \\
\hline 6 & -0.545235 & -2.878505 & 0.290970 \\
\hline 6 & 0.682467 & -2.797127 & -0.340872 \\
\hline 6 & 0.965386 & -3.592405 & -1.475714 \\
\hline 6 & 0.030441 & -4.450167 & -1.986628 \\
\hline 6 & -1.243432 & -4.556726 & -1.383195 \\
\hline 6 & -1.537637 & -3.766153 & -0.232377 \\
\hline 6 & -2.817285 & -3.904022 & 0.364313 \\
\hline 6 & -3.746867 & -4.773566 & -0.149326 \\
\hline 6 & -3.446120 & -5.564140 & -1.279244 \\
\hline 6 & -2.220330 & -5.451719 & -1.883510 \\
\hline 1 & 7.088812 & 0.596590 & 0.734779 \\
\hline 1 & -1.839394 & 0.740569 & 3.008112 \\
\hline 1 & 8.176688 & -4.068332 & -0.577734 \\
\hline 1 & -0.785353 & -2.588860 & 6.187351 \\
\hline 1 & 6.072260 & 5.393764 & -0.945025 \\
\hline 1 & -3.062508 & -3.298352 & 1.235193 \\
\hline 1 & -4.726723 & -4.855484 & 0.319055 \\
\hline 1 & 4.050590 & 4.639084 & 0.173763 \\
\hline 1 & -1.489993 & -0.539410 & 5.058739 \\
\hline 1 & 6.137295 & -4.202193 & -1.897934 \\
\hline 1 & 8.652970 & 2.567372 & -2.898723 \\
\hline 1 & 3.965046 & -3.197348 & -2.597031 \\
\hline 1 & -1.974291 & -6.047689 & -2.761478 \\
\hline
\end{tabular}




\begin{tabular}{|c|c|c|c|}
\hline 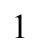 & 0.252353 & -5.057750 & -2.862589 \\
\hline 1 & 0.470981 & -5.986988 & 3.874216 \\
\hline 1 & 7.199092 & 0.725028 & -2.204365 \\
\hline 1 & 0.071410 & -4.753093 & 1.797786 \\
\hline & 1.946151 & -3.497293 & -1.937611 \\
\hline 1 & 8.121718 & 4.915973 & -2.254226 \\
\hline & 9.116663 & -0.479693 & 1.592086 \\
\hline 1 & -4.189503 & -6.255338 & -1.670596 \\
\hline & 0.047217 & -4.921452 & 6.085738 \\
\hline 1 & 9.692649 & -2.811930 & 0.927204 \\
\hline 1 & 2.486123 & 2.785323 & 0.767701 \\
\hline 1 & -3.967676 & -0.552618 & 1.654248 \\
\hline 1 & -2.664216 & -0.674056 & -2.409467 \\
\hline 1 & -6.599453 & -2.041250 & -1.382070 \\
\hline 1 & -4.064883 & 1.767342 & 0.448114 \\
\hline 1 & 0.107098 & 2.057101 & 1.346740 \\
\hline 1 & -2.591111 & 5.121242 & 2.668467 \\
\hline 1 & 2.222037 & -3.405795 & 1.452786 \\
\hline 1 & 3.575212 & -2.888959 & 0.453137 \\
\hline 1 & 5.266970 & -2.188117 & 1.857883 \\
\hline 1 & 1.262060 & -0.902931 & 2.689763 \\
\hline 1 & 6.097374 & -0.855197 & 3.766909 \\
\hline 1 & 2.074593 & 0.403496 & 4.624287 \\
\hline 1 & 4.506684 & 0.449441 & 5.164968 \\
\hline 1 & 0.530119 & 2.861200 & -0.745223 \\
\hline 1 & 1.751885 & 2.426901 & -2.064503 \\
\hline 1 & -2.873269 & 2.005559 & -3.553789 \\
\hline 1 & -4.857454 & 3.438281 & -3.234500 \\
\hline 1 & -4.684260 & 5.536292 & -1.909455 \\
\hline 1 & -2.501234 & 6.167135 & -0.889816 \\
\hline 1 & -0.512401 & 4.749687 & -1.248280 \\
\hline 1 & -0.680053 & 2.031526 & -4.802320 \\
\hline 1 & 0.207841 & 3.532590 & -4.501308 \\
\hline 1 & 1.026970 & 1.954655 & -4.325305 \\
\hline 1 & -1.137170 & 1.084686 & -2.644886 \\
\hline 6 & -5.359658 & 3.764397 & 3.158589 \\
\hline 6 & -4.955951 & 5.585714 & 1.503084 \\
\hline 6 & -5.896319 & 3.424777 & 0.756343 \\
\hline 6 & 0.845097 & 5.152308 & 1.552425 \\
\hline 6 & -0.350491 & 5.505465 & 3.694717 \\
\hline 6 & 1.019949 & 3.470506 & 3.408940 \\
\hline 6 & -4.005253 & -2.919115 & -3.901088 \\
\hline 6 & -6.343652 & -2.083268 & -3.862776 \\
\hline 6 & -4.500462 & -0.530405 & -4.437369 \\
\hline 6 & -6.866483 & -0.267884 & 1.983849 \\
\hline 6 & -7.688545 & -2.301475 & 0.828390 \\
\hline 6 & -5.822121 & -2.490013 & 2.443445 \\
\hline 1 & 0.534813 & 5.976833 & 4.140074 \\
\hline 1 & -0.940969 & 6.305700 & 3.231803 \\
\hline 1 & -0.942362 & 5.069151 & 4.509897 \\
\hline 1 & 1.846970 & 4.016957 & 3.880768 \\
\hline 1 & 0.478972 & 2.927060 & 4.195963 \\
\hline 1 & 1.467417 & 2.723295 & 2.742415 \\
\hline 1 & 1.740902 & 5.656942 & 1.941393 \\
\hline 1 & 1.162184 & 4.450222 & 0.769148 \\
\hline 1 & 0.208789 & 5.911191 & 1.075750 \\
\hline
\end{tabular}




$\begin{array}{llll}1 & -6.886324 & 3.882071 & 0.880685 \\ 1 & -5.586198 & 3.562903 & -0.287842 \\ 1 & -6.013682 & 2.348211 & 0.935513 \\ 1 & -5.986884 & 5.946575 & 1.614774 \\ 1 & -4.342637 & 6.139546 & 2.223374 \\ 1 & -4.613420 & 5.846773 & 0.493873 \\ 1 & -6.383338 & 4.126556 & 3.326569 \\ 1 & -5.346573 & 2.683052 & 3.350893 \\ 1 & -4.706102 & 4.242849 & 3.899214 \\ 1 & -6.451773 & -2.289683 & -4.935084 \\ 1 & -6.666369 & -2.984630 & -3.326340 \\ 1 & -7.033286 & -1.267022 & -3.609830 \\ 1 & -4.700643 & -0.762822 & -5.491749 \\ 1 & -5.074477 & 0.368545 & -4.173552 \\ 1 & -3.431232 & -0.290442 & -4.358874 \\ 1 & -4.125167 & -3.192117 & -4.958815 \\ 1 & -2.942291 & -2.703656 & -3.726587 \\ 1 & -4.270276 & -3.790027 & -3.287249 \\ 1 & -6.552985 & -2.677144 & 3.241709 \\ 1 & -5.520666 & -3.463505 & 2.033189 \\ 1 & -4.937942 & -2.035023 & 2.908427 \\ 1 & -8.386306 & -2.487751 & 1.654658 \\ 1 & -8.218455 & -1.693674 & 0.083926 \\ 1 & -7.450092 & -3.271709 & 0.372508 \\ 1 & -7.595446 & -0.434942 & 2.788599 \\ 1 & -6.011522 & 0.270069 & 2.414992 \\ 1 & -7.334153 & 0.385126 & 1.234821\end{array}$

TS1 $_{I I L-s i-} \psi_{-1 \mathrm{~s} 2 \mathrm{~s} 3 \mathrm{a} 4 \mathrm{~s}}$

Number of imaginary frequencies : 1

The smallest frequencies are : $-559.5809 \quad 8.2097 \quad 18.5728 \mathrm{~cm}(-1)$

Electronic energy : $\quad H F=-4360.8693668$

Zero-point correction $=\quad 1.454336($ Hartree/Particle $)$

Thermal correction to Energy= $\quad 1.538142$

Thermal correction to Enthalpy= $\quad 1.539086$

Thermal correction to Gibbs Free Energy= $\quad 1.338475$

Sum of electronic and zero-point Energies $=\quad-4359.415031$

Sum of electronic and thermal Energies $=\quad-4359.331225$

Sum of electronic and thermal Enthalpies $=\quad-4359.330281$

Sum of electronic and thermal Free Energies $=\quad-4359.530891$

Cartesian Coordinates

$\begin{array}{cccc}6 & -0.842469 & 2.444016 & 1.554100 \\ 6 & -1.924916 & 1.790862 & 0.954895 \\ 6 & -3.188257 & 2.374531 & 1.019473 \\ 6 & -3.399983 & 3.565519 & 1.726403 \\ 6 & -2.298272 & 4.178175 & 2.325939 \\ 6 & -1.008893 & 3.637412 & 2.255620 \\ 15 & -1.560897 & 0.174187 & 0.146814 \\ 45 & 0.042013 & 0.382341 & -1.799771 \\ 15 & 1.904303 & -0.520471 & -0.831085 \\ 8 & 3.088118 & -0.820561 & -1.987932 \\ 6 & 4.322993 & -1.318901 & -1.649936 \\ 6 & 5.235209 & -0.541770 & -0.960707\end{array}$




\begin{tabular}{|c|c|c|c|}
\hline & 6.443307 & -1.167290 & -0.506645 \\
\hline & 6.734392 & -2.507707 & -0.900564 \\
\hline & 5.820255 & -3.202263 & -1.730578 \\
\hline & 4.630741 & -2.627336 & -2.083265 \\
\hline & 7.337792 & -0.528738 & 0.389729 \\
\hline & 8.461956 & -1.170690 & 0.848186 \\
\hline & 8.763911 & -2.482857 & 0.427831 \\
\hline & 7.913240 & -3.134125 & -0.428508 \\
\hline & 4.929845 & 0.877318 & -0.670914 \\
\hline & 3.750333 & 1.216469 & -0.035529 \\
\hline & 3.472296 & 2.535140 & 0.377013 \\
\hline & 4.346538 & 3.543699 & 0.084153 \\
\hline & 5.521480 & 3.279616 & -0.661112 \\
\hline & 5.815216 & 1.939401 & -1.055830 \\
\hline & 6.399881 & 4.326017 & -1.031752 \\
\hline & 7.517437 & 4.076140 & -1.786168 \\
\hline & 7.793174 & 2.759507 & -2.208615 \\
\hline & 6.967815 & 1.721289 & -1.852800 \\
\hline & 2.829597 & 0.257939 & 0.298211 \\
\hline & -4.822284 & 4.108018 & 1.882085 \\
\hline & -5.370250 & 4.545459 & 0.522531 \\
\hline & 0.196629 & 4.328469 & 2.891914 \\
\hline & -0.211633 & 5.348406 & 3.955179 \\
\hline & -3.238898 & -0.461431 & -0.288446 \\
\hline & -4.193625 & -0.751064 & 0.686108 \\
\hline & -5.423126 & -1.322269 & 0.347424 \\
\hline & -5.682509 & -1.557114 & -1.003489 \\
\hline 6 & -4.747358 & -1.282140 & -2.008510 \\
\hline & -3.522502 & -0.735160 & -1.625008 \\
\hline 6 & -5.071939 & -1.548719 & -3.477822 \\
\hline 5 & -5.939697 & -0.396831 & -3.997058 \\
\hline & -6.400493 & -1.708172 & 1.461054 \\
\hline 6 & -7.649322 & -2.403826 & 0.924884 \\
\hline ) & -1.243955 & -0.894939 & 1.649805 \\
\hline 6 & -0.833627 & -2.216471 & 1.506160 \\
\hline 0 & -0.635844 & -3.039017 & 2.664315 \\
\hline 6 & -0.856006 & -2.496931 & 3.963828 \\
\hline 5 & -1.278844 & -1.153966 & 4.075331 \\
\hline 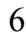 & -1.462863 & -0.383052 & 2.958787 \\
\hline 6 & -0.637389 & -3.302015 & 5.107354 \\
\hline 6 & -0.215131 & -4.600442 & 4.982640 \\
\hline 6 & 0.001157 & -5.146685 & 3.699986 \\
\hline 5 & -0.206274 & -4.390336 & 2.573131 \\
\hline 6 & -0.601490 & -2.864392 & 0.182149 \\
\hline 6 & -1.621788 & -3.677907 & -0.404490 \\
\hline 6 & -1.367948 & -4.348870 & -1.638157 \\
\hline 6 & -0.088620 & -4.247368 & -2.229607 \\
\hline 6 & 0.883747 & -3.488231 & -1.639733 \\
\hline 6 & 0.627363 & -2.772874 & -0.447299 \\
\hline 6 & -2.391244 & -5.126122 & -2.234472 \\
\hline 6 & -3.616532 & -5.254669 & -1.632318 \\
\hline 6 & -3.867017 & -4.604878 & -0.404543 \\
\hline 6 & -2.895649 & -3.845767 & 0.197565 \\
\hline 7 & 1.667100 & -1.931360 & 0.079639 \\
\hline 6 & 2.662899 & -2.609795 & 0.941118 \\
\hline 6 & 3.186646 & -1.801727 & 2.099393 \\
\hline
\end{tabular}




\begin{tabular}{|c|c|c|c|}
\hline & 2.324956 & -1.084114 & 2.930944 \\
\hline & 2.819990 & -0.369179 & 4.012903 \\
\hline & 4.188644 & -0.356042 & 4.279466 \\
\hline & 5.053244 & -1.068214 & 3.456332 \\
\hline & 4.552404 & -1.789185 & 2.374441 \\
\hline & -5.832915 & -2.866605 & -3.647416 \\
\hline & -3.803690 & -1.634186 & -4.329094 \\
\hline & -6.841282 & -0.454869 & 2.222257 \\
\hline & -5.710273 & -2.664180 & 2.442993 \\
\hline & -0.035175 & -0.839687 & -3.252653 \\
\hline & -0.021104 & -1.413808 & -4.263824 \\
\hline & -0.196891 & 2.569154 & -2.639707 \\
\hline & 0.704089 & 2.481892 & -1.551409 \\
\hline & 0.365666 & 2.593195 & -4.047946 \\
\hline & -1.451259 & 3.391632 & -2.498955 \\
\hline & -1.432335 & 4.565412 & -1.742284 \\
\hline & -2.542024 & 5.404032 & -1.701864 \\
\hline & -3.684367 & 5.088766 & -2.428525 \\
\hline & -3.719150 & 3.917419 & -3.181000 \\
\hline & -2.612215 & 3.079610 & -3.214929 \\
\hline & -4.892384 & 5.303539 & 2.829398 \\
\hline & -5.719295 & 3.001480 & 2.446726 \\
\hline & 1.109298 & 3.300149 & 3.569304 \\
\hline & 0.965673 & 5.068857 & 1.790534 \\
\hline & 7.107071 & 0.473693 & 0.741351 \\
\hline & -1.797205 & 0.644306 & 3.081316 \\
\hline & 8.119702 & -4.154292 & -0.749588 \\
\hline & -0.808152 & -2.862745 & 6.089353 \\
\hline & 6.159172 & 5.338857 & -0.711133 \\
\hline & -3.104780 & -3.344419 & 1.140862 \\
\hline & -4.842888 & -4.709663 & 0.067612 \\
\hline & 4.141779 & 4.564976 & 0.402808 \\
\hline & -1.458242 & -0.738704 & 5.066519 \\
\hline & 6.068269 & -4.210059 & -2.059538 \\
\hline & 8.665330 & 2.563676 & -2.828425 \\
\hline & 3.905942 & -3.149697 & -2.703614 \\
\hline & -2.177635 & -5.626420 & -3.178141 \\
\hline & 0.105635 & -4.774104 & -3.162588 \\
\hline & 0.333214 & -6.177892 & 3.602889 \\
\hline & 7.190036 & 0.715131 & -2.198483 \\
\hline & -0.040782 & -4.830984 & 1.592200 \\
\hline & 1.868266 & -3.394166 & -2.093509 \\
\hline & 8.182326 & 4.888511 & -2.070065 \\
\hline & 9.122562 & -0.663458 & 1.547915 \\
\hline & -4.394407 & -5.859736 & -2.093532 \\
\hline & -0.045746 & -5.210506 & 5.866862 \\
\hline & 9.660785 & -2.977455 & 0.793289 \\
\hline & 2.553593 & 2.712676 & 0.930224 \\
\hline & -3.962353 & -0.554382 & 1.733832 \\
\hline & -2.744093 & -0.534830 & -2.358381 \\
\hline & -6.635040 & -1.997659 & -1.287390 \\
\hline & -4.030174 & 1.871832 & 0.545985 \\
\hline & 0.139487 & 1.969488 & 1.502392 \\
\hline & -2.448695 & 5.096809 & 2.884572 \\
\hline & 2.143080 & -3.493876 & 1.337647 \\
\hline & 3.507183 & -2.989155 & 0.344654 \\
\hline
\end{tabular}




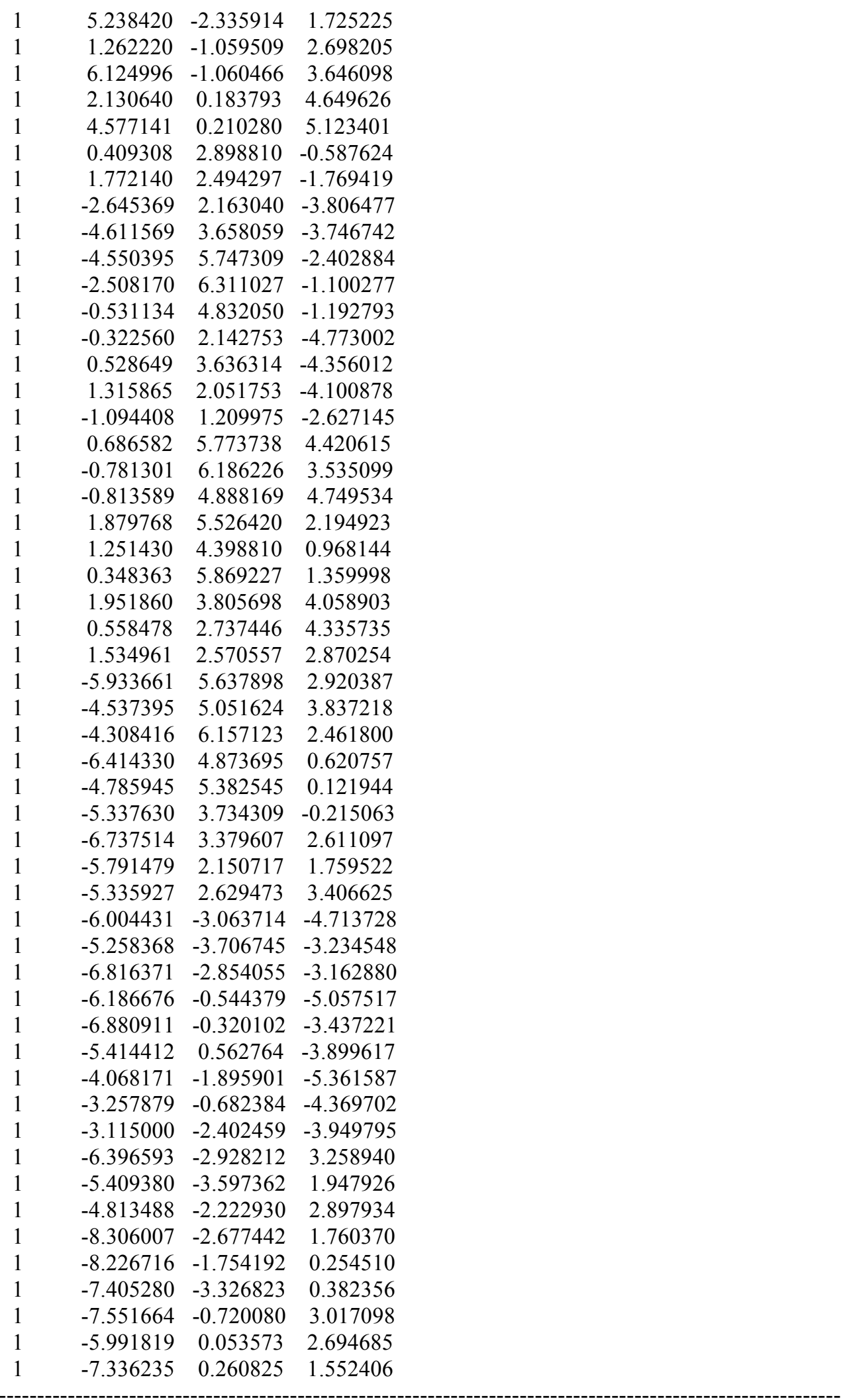

$\mathrm{TS1}_{\text {IIL-si }-\Psi-1 \mathrm{~s} 23354 \mathrm{~s}}$

Number of imaginary frequencies : 1 
The smallest frequencies are : $-553.3127 \quad 9.6745 \quad 17.5576 \mathrm{~cm}(-1)$ Electronic energy : $\quad H F=-4360.8689855$

Zero-point correction $=\quad 1.452466($ Hartree/Particle $)$

Thermal correction to Energy $=\quad 1.537077$

Thermal correction to Enthalpy= $\quad 1.538022$

Thermal correction to Gibbs Free Energy $=\quad 1.333675$

Sum of electronic and zero-point Energies $=\quad-4359.416519$

Sum of electronic and thermal Energies $=\quad-4359.331908$

Sum of electronic and thermal Enthalpies $=\quad-4359.330964$

Sum of electronic and thermal Free Energies $=\quad-4359.535310$

Cartesian Coordinates

$\begin{array}{crrr}6 & -4.202995 & -0.779705 & 0.688338 \\ 6 & -3.259325 & -0.453740 & -0.288835 \\ 6 & -3.568239 & -0.661702 & -1.628109 \\ 6 & -4.802255 & -1.190304 & -2.016343 \\ 6 & -5.711876 & -1.530500 & -1.011798 \\ 6 & -5.433892 & -1.340952 & 0.347935 \\ 15 & -1.580133 & 0.172306 & 0.150659 \\ 6 & -1.253984 & -0.913889 & 1.635370 \\ 6 & -0.824544 & -2.226857 & 1.474653 \\ 6 & -0.614265 & -3.059843 & 2.625149 \\ 6 & -0.844341 & -2.536306 & 3.930591 \\ 6 & -1.289690 & -1.201978 & 4.057819 \\ 6 & -1.482883 & -0.421197 & 2.950353 \\ 6 & -0.610769 & -3.348304 & 5.066115 \\ 6 & -0.163201 & -4.636915 & 4.929167 \\ 6 & 0.064168 & -5.165119 & 3.641223 \\ 6 & -0.158070 & -4.401849 & 2.521747 \\ 6 & -0.574767 & -2.873806 & 0.152013 \\ 6 & -1.572042 & -3.723705 & -0.423410 \\ 6 & -1.265995 & -4.486288 & -1.590578 \\ 6 & 0.026865 & -4.397049 & -2.154544 \\ 6 & 0.964912 & -3.572069 & -1.597821 \\ 6 & 0.668102 & -2.798975 & -0.451525 \\ 6 & -2.249763 & -5.342277 & -2.143454 \\ 6 & -3.491903 & -5.446492 & -1.572555 \\ 6 & -3.802025 & -4.687842 & -0.423474 \\ 6 & -2.866548 & -3.857156 & 0.140683 \\ 7 & 1.693629 & -1.937307 & 0.070088 \\ 6 & 2.716903 & -2.598297 & 0.912394 \\ 6 & 3.211978 & -1.798059 & 2.089201 \\ 6 & 2.329205 & -1.103210 & 2.918002 \\ 6 & 2.799128 & -0.397820 & 4.017272 \\ 6 & 4.163201 & -0.372935 & 4.305471 \\ 6 & 5.048830 & -1.061832 & 3.484834 \\ 6 & 4.573575 & -1.771405 & 2.383926 \\ 6 & -5.067165 & -1.460028 & -3.496160 \\ 6 & -4.259170 & -2.696563 & -3.906119 \\ 6 & -6.401416 & -1.751081 & 1.460514 \\ 6 & -6.842466 & -0.508911 & 2.240843 \\ 45 & 0.019657 & 0.381710 & -1.777081 \\ 6 & -0.161048 & -0.887081 & -3.178732 \\ 8 & -0.267705 & -1.509439 & -4.154998 \\ 6 & -0.166798 & 2.578792 & -2.610966\end{array}$




\begin{tabular}{|c|c|c|c|}
\hline & 0.430554 & 2.596299 & -4.004575 \\
\hline & -1.411441 & 3.419178 & -2.492474 \\
\hline & -1.423604 & 4.547614 & -1.669522 \\
\hline & -2.524549 & 5.398366 & -1.645302 \\
\hline & -3.625813 & 5.142660 & -2.454295 \\
\hline & -3.631497 & 4.014407 & -3.270522 \\
\hline & -2.534684 & 3.163258 & -3.286797 \\
\hline & 0.706753 & 2.465916 & -1.502576 \\
\hline & 1.897168 & -0.516325 & -0.831825 \\
\hline & 2.818229 & 0.261182 & 0.301964 \\
\hline & 3.733340 & 1.230380 & -0.012190 \\
\hline & 4.910049 & 0.914584 & -0.664623 \\
\hline & 5.785160 & 1.992372 & -1.029656 \\
\hline & 5.488049 & 3.319571 & -0.595524 \\
\hline & 4.320047 & 3.556114 & 0.169954 \\
\hline & 3.453836 & 2.535242 & 0.442254 \\
\hline & 6.930698 & 1.803074 & -1.843973 \\
\hline & 7.746188 & 2.855601 & -2.180115 \\
\hline & 7.467320 & 4.158503 & -1.719244 \\
\hline & 6.356320 & 4.380926 & -0.946677 \\
\hline & 5.227069 & -0.494982 & -0.987767 \\
\hline & 4.319947 & -1.267968 & -1.688116 \\
\hline & 4.642486 & -2.560405 & -2.157056 \\
\hline & 5.840093 & -3.129815 & -1.823420 \\
\hline & 6.748724 & -2.445220 & -0.979156 \\
\hline & 6.444464 & -1.117236 & -0.553773 \\
\hline 6 & 7.936990 & -3.068559 & -0.527127 \\
\hline & 8.785213 & -2.426712 & 0.338697 \\
\hline 6 & 8.471121 & -1.127469 & 0.789129 \\
\hline 6 & 7.337377 & -0.488317 & 0.350971 \\
\hline & 3.074879 & -0.781258 & -2.003478 \\
\hline 5 & -1.950729 & 1.779094 & 0.972317 \\
\hline 6 & -3.213977 & 2.364382 & 1.014197 \\
\hline 6 & -3.434818 & 3.560680 & 1.708510 \\
\hline 6 & -2.344211 & 4.169668 & 2.331735 \\
\hline 6 & -1.055335 & 3.623427 & 2.289589 \\
\hline 6 & -0.877430 & 2.431229 & 1.589047 \\
\hline 6 & -4.857036 & 4.114401 & 1.817414 \\
\hline 6 & -4.938885 & 5.343866 & 2.718886 \\
\hline 6 & 0.139192 & 4.309311 & 2.952269 \\
\hline 6 & 1.038953 & 3.276527 & 3.640431 \\
\hline 6 & -5.768005 & 3.030673 & 2.405212 \\
\hline 6 & -5.380139 & 4.504971 & 0.432859 \\
\hline 6 & 0.930801 & 5.053513 & 1.869830 \\
\hline 6 & -0.287926 & 5.324124 & 4.013061 \\
\hline 6 & -6.544114 & -1.725054 & -3.782359 \\
\hline 6 & -4.626125 & -0.266971 & -4.351585 \\
\hline 6 & -5.701913 & -2.719446 & 2.423181 \\
\hline 6 & -7.650491 & -2.443581 & 0.920922 \\
\hline 1 & 7.097657 & 0.503429 & 0.725998 \\
\hline 1 & -1.832699 & 0.599633 & 3.083476 \\
\hline 1 & 8.153140 & -4.078906 & -0.872033 \\
\hline 1 & -0.790233 & -2.921775 & 6.052186 \\
\hline 1 & 6.113149 & 5.383201 & -0.596111 \\
\hline 1 & -3.118388 & -3.278432 & 1.027247 \\
\hline 1 & -4.793878 & -4.764431 & 0.019785 \\
\hline
\end{tabular}




\begin{tabular}{|c|c|c|c|}
\hline ] & 4.114579 & 4.566693 & 0.520558 \\
\hline & -1.477159 & -0.801671 & 5.053648 \\
\hline & 6.099511 & -4.125402 & -2.179634 \\
\hline 1 & 8.612700 & 2.681964 & -2.814286 \\
\hline 1 & 3.921931 & -3.072317 & -2.790688 \\
\hline & -1.994452 & -5.916639 & -3.032994 \\
\hline & 0.258924 & -4.987178 & -3.039695 \\
\hline & 0.416968 & -6.188349 & 3.533336 \\
\hline & 7.154972 & 0.808285 & -2.219802 \\
\hline & 0.018435 & -4.831339 & 1.537824 \\
\hline & 1.960640 & -3.487721 & -2.028902 \\
\hline & 8.124371 & 4.982324 & -1.987986 \\
\hline & 9.130051 & -0.628208 & 1.496116 \\
\hline & -4.240627 & -6.107438 & -2.004081 \\
\hline & 0.018068 & -5.252310 & 5.807320 \\
\hline & 9.689546 & -2.919134 & 0.688377 \\
\hline [ & 2.540244 & 2.690916 & 1.010868 \\
\hline & -3.959206 & -0.614501 & 1.738503 \\
\hline 1 & -2.805716 & -0.431604 & -2.370692 \\
\hline & -6.664737 & -1.968393 & -1.295575 \\
\hline ] & -4.048892 & 1.861896 & 0.528194 \\
\hline & 0.105266 & 1.956597 & 1.551570 \\
\hline & -2.502440 & 5.091340 & 2.883318 \\
\hline 1 & 2.232535 & -3.511013 & 1.288490 \\
\hline & 3.574344 & -2.931099 & 0.307197 \\
\hline$\frac{1}{1}$ & 5.276003 & -2.298543 & 1.735834 \\
\hline & 1.269976 & -1.088270 & 2.669276 \\
\hline ] & 6.117047 & -1.044473 & 3.693242 \\
\hline ] & 2.093620 & 0.138660 & 4.650575 \\
\hline & 4.532001 & 0.184989 & 5.163706 \\
\hline 1 & 0.399830 & 2.879304 & -0.541102 \\
\hline & 1.778914 & 2.468862 & -1.699934 \\
\hline 1 & -2.546512 & 2.279264 & -3.926112 \\
\hline 1 & -4.492938 & 3.797840 & -3.898950 \\
\hline 1 & -4.483370 & 5.812615 & -2.442113 \\
\hline 1 & -2.515039 & 6.268804 & -0.990565 \\
\hline 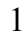 & -0.557600 & 4.767668 & -1.047661 \\
\hline 1 & -0.241377 & 2.150701 & -4.747244 \\
\hline & 0.612390 & 3.637196 & -4.310156 \\
\hline 1 & 1.375659 & 2.044269 & -4.031664 \\
\hline ] & -1.097044 & 1.232958 & -2.607476 \\
\hline 1 & 0.601700 & 5.743585 & 4.499843 \\
\hline 1 & -0.845779 & 6.166525 & 3.586355 \\
\hline 1 & -0.907969 & 4.861084 & 4.791706 \\
\hline 1 & 1.835394 & 5.511133 & 2.295020 \\
\hline & 1.235606 & 4.384744 & 1.053236 \\
\hline 1 & 0.322330 & 5.854211 & 1.427328 \\
\hline l & 1.871450 & 3.779527 & 4.149483 \\
\hline 1 & 0.475101 & 2.707220 & 4.392376 \\
\hline 1 & 1.478820 & 2.552683 & 2.944152 \\
\hline 1 & -5.978522 & 5.691242 & 2.771628 \\
\hline 1 & -4.611985 & 5.126191 & 3.744015 \\
\hline 1 & -4.336377 & 6.177154 & 2.334370 \\
\hline 1 & -6.425902 & 4.835071 & 0.502730 \\
\hline & -4.790798 & 5.327142 & 0.007580 \\
\hline 1 & -5.336346 & 3.668530 & -0.275566 \\
\hline
\end{tabular}




$\begin{array}{lrrr}1 & -6.788909 & 3.415704 & 2.532700 \\ 1 & -5.825950 & 2.152463 & 1.751241 \\ 1 & -5.405931 & 2.696973 & 3.387130 \\ 1 & -4.405172 & -2.917572 & -4.972748 \\ 1 & -3.184149 & -2.551892 & -3.732612 \\ 1 & -4.570620 & -3.575211 & -3.325886 \\ 1 & -4.873511 & -0.450035 & -5.405482 \\ 1 & -5.132432 & 0.656183 & -4.038828 \\ 1 & -3.542834 & -0.096628 & -4.301623 \\ 1 & -6.692243 & -1.864018 & -4.860764 \\ 1 & -6.905595 & -2.636260 & -3.289656 \\ 1 & -7.178086 & -0.887228 & -3.463118 \\ 1 & -6.384437 & -3.004627 & 3.235182 \\ 1 & -5.394298 & -3.640187 & 1.909084 \\ 1 & -4.807708 & -2.280513 & 2.885178 \\ 1 & -8.300632 & -2.734019 & 1.755879 \\ 1 & -8.235263 & -1.786287 & 0.264700 \\ 1 & -7.405192 & -3.356445 & 0.361955 \\ 1 & -7.541143 & -0.787385 & 3.041577 \\ 1 & -5.990983 & 0.002581 & 2.707009 \\ 1 & -7.351226 & 0.209046 & 1.583831\end{array}$

TS1 $1_{\text {IL-re- }} \psi_{-1 \mathbf{a} 2 a 3 a 4 a}$

Number of imaginary frequencies : 1

The smallest frequencies are : $-607.8420 \quad 13.7022 \quad 17.0308 \mathrm{~cm}(-1)$

Electronic energy : $\quad H F=-4360.8705721$

Zero-point correction $=\quad 1.451710($ Hartree/Particle $)$

Thermal correction to Energy $=\quad 1.536603$

Thermal correction to Enthalpy= $\quad 1.537547$

Thermal correction to Gibbs Free Energy $=\quad 1.332992$

Sum of electronic and zero-point Energies $=\quad-4359.418862$

Sum of electronic and thermal Energies $=\quad-4359.333969$

Sum of electronic and thermal Enthalpies $=\quad-4359.333025$

Sum of electronic and thermal Free Energies $=\quad-4359.537580$

Cartesian Coordinates

$\begin{array}{cccc}6 & 3.617493 & 0.153348 & -1.254159 \\ 6 & 3.195476 & 0.371092 & 0.052021 \\ 6 & 4.116126 & 0.777600 & 1.021537 \\ 6 & 5.458011 & 0.966867 & 0.698126 \\ 6 & 5.849122 & 0.732891 & -0.627048 \\ 6 & 4.954561 & 0.331090 & -1.619559 \\ 15 & 1.423531 & 0.090389 & 0.482604 \\ 45 & 0.098767 & -0.688891 & -1.329071 \\ 6 & 0.211838 & -3.080336 & -1.684254 \\ 6 & 0.108447 & -3.849596 & -0.386777 \\ 6 & 6.485822 & 1.447489 & 1.721852 \\ 6 & 6.999259 & 2.830541 & 1.305077 \\ 6 & 5.436058 & 0.061595 & -3.043358 \\ 6 & 6.299401 & 1.223919 & -3.543012 \\ 6 & 1.533918 & -1.290965 & 1.691272 \\ 6 & 2.603128 & -2.184450 & 1.645129 \\ 6 & 2.628789 & -3.318595 & 2.460428\end{array}$




\begin{tabular}{|c|c|c|c|}
\hline 6 & 1.568432 & -3.506943 & 3.350217 \\
\hline 6 & 0.491456 & -2.616576 & 3.441354 \\
\hline & 0.473013 & -1.527399 & 2.569635 \\
\hline & -0.619532 & -2.861314 & 4.462372 \\
\hline & -1.418287 & -4.102307 & 4.050600 \\
\hline & 3.763059 & -4.340505 & 2.392028 \\
\hline & 4.420110 & -4.477999 & 3.769557 \\
\hline & 1.125474 & 1.569610 & 1.567197 \\
\hline & 0.824917 & 2.794306 & 0.988391 \\
\hline 6 & 0.671385 & 3.959960 & 1.807628 \\
\hline 6 & 0.832563 & 3.854822 & 3.219835 \\
\hline 6 & 1.149157 & 2.594859 & 3.776541 \\
\hline 6 & 1.288916 & 1.491698 & 2.977327 \\
\hline 6 & 0.669723 & 5.003840 & 4.030082 \\
\hline 6 & 0.357486 & 6.217474 & 3.473111 \\
\hline 6 & 0.196216 & 6.329069 & 2.075855 \\
\hline 6 & 0.351093 & 5.232351 & 1.264678 \\
\hline 6 & 0.693404 & 2.977209 & -0.483563 \\
\hline 6 & -0.508135 & 2.735311 & -1.125838 \\
\hline 6 & -0.661507 & 3.042878 & -2.497429 \\
\hline 6 & 0.389233 & 3.521526 & -3.232445 \\
\hline 6 & 1.655174 & 3.711632 & -2.631898 \\
\hline 6 & 1.805997 & 3.466094 & -1.234908 \\
\hline 6 & 3.079814 & 3.680283 & -0.646458 \\
\hline 6 & 4.147666 & 4.087996 & -1.405857 \\
\hline 6 & 3.995548 & 4.324582 & -2.789789 \\
\hline 6 & 2.773716 & 4.143742 & -3.386303 \\
\hline 7 & -1.596889 & 2.111065 & -0.426810 \\
\hline 15 & -1.780235 & 0.476346 & -0.857544 \\
\hline 8 & -2.808536 & 0.070577 & 0.385569 \\
\hline 6 & -3.547527 & -1.076425 & 0.254319 \\
\hline 6 & -4.695574 & -1.069059 & -0.516453 \\
\hline 6 & -5.404665 & -2.303232 & -0.683951 \\
\hline 6 & -4.962789 & -3.466600 & 0.017355 \\
\hline 6 & -3.813240 & -3.391002 & 0.840185 \\
\hline 6 & -3.106432 & -2.223645 & 0.943843 \\
\hline 6 & -6.514766 & -2.437120 & -1.556934 \\
\hline 6 & -7.167916 & -3.636501 & -1.697255 \\
\hline 6 & -6.750387 & -4.773220 & -0.973919 \\
\hline 6 & -5.666097 & -4.685681 & -0.139456 \\
\hline 6 & -5.101079 & 0.184643 & -1.191941 \\
\hline 6 & -4.187685 & 0.850075 & -1.991821 \\
\hline 6 & -4.550665 & 1.981528 & -2.755414 \\
\hline 6 & -5.812266 & 2.499988 & -2.656748 \\
\hline 6 & -6.753595 & 1.933162 & -1.762736 \\
\hline 6 & -6.396017 & 0.772061 & -1.013581 \\
\hline 6 & -8.026847 & 2.522589 & -1.572271 \\
\hline 6 & -8.910344 & 2.013477 & -0.655037 \\
\hline 6 & -8.546404 & 0.889422 & 0.115411 \\
\hline 6 & -7.326632 & 0.283060 & -0.061446 \\
\hline 8 & -2.899059 & 0.399970 & -2.119654 \\
\hline 6 & -2.633455 & 3.019225 & 0.114040 \\
\hline 6 & -3.252686 & 2.570659 & 1.411159 \\
\hline 6 & -4.635483 & 2.456305 & 1.531872 \\
\hline 6 & -5.208734 & 2.012432 & 2.721272 \\
\hline 6 & -4.401405 & 1.685361 & 3.805520 \\
\hline
\end{tabular}




\begin{tabular}{|c|c|c|c|}
\hline 6 & -3.017427 & 1.815305 & 3.696546 \\
\hline 6 & -2.450354 & 2.255028 & 2.508491 \\
\hline & -0.010094 & -3.091825 & 5.849959 \\
\hline & -1.574416 & -1.672306 & 4.570963 \\
\hline & 3.191293 & -5.696188 & 1.962220 \\
\hline & 4.846270 & -3.940125 & 1.391179 \\
\hline & 0.541460 & -0.043676 & -3.173638 \\
\hline & 0.354061 & 0.223357 & -4.286087 \\
\hline & -0.954323 & -2.438054 & -2.167057 \\
\hline & 1.304428 & -3.517695 & -2.618992 \\
\hline & 1.244760 & -3.294462 & -3.999057 \\
\hline y & 2.298443 & -3.659454 & -4.831453 \\
\hline & 3.438785 & -4.259226 & -4.307348 \\
\hline & 3.509393 & -4.497721 & -2.937338 \\
\hline & 2.456878 & -4.131238 & -2.107533 \\
\hline & 5.891218 & 1.566450 & 3.123710 \\
\hline & 7.661338 & 0.466900 & 1.785891 \\
\hline & 6.261021 & -1.230098 & -3.041902 \\
\hline & 4.270026 & -0.107066 & -4.017558 \\
\hline & -7.052788 & -0.570890 & 0.553447 \\
\hline & 1.541447 & 0.538322 & 3.434432 \\
\hline & -8.281982 & 3.402280 & -2.161931 \\
\hline & 0.795423 & 4.900300 & 5.106982 \\
\hline & -5.316354 & -5.557256 & 0.412460 \\
\hline & 3.209372 & 3.491865 & 0.418216 \\
\hline & 5.122479 & 4.224758 & -0.940800 \\
\hline & -3.485452 & -4.285998 & 1.368875 \\
\hline & 1.285327 & 2.514649 & 4.854422 \\
\hline & -6.101682 & 3.369726 & -3.244340 \\
\hline 1 & -8.012269 & -3.711693 & -2.378870 \\
\hline & -3.804883 & 2.414930 & -3.418500 \\
\hline 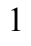 & 2.640672 & 4.325827 & -4.451878 \\
\hline & 0.273568 & 3.730147 & -4.294943 \\
\hline & -0.051232 & 7.293285 & 1.637718 \\
\hline 1 & -6.843027 & -1.574843 & -2.131371 \\
\hline & 0.227988 & 5.334741 & 0.188499 \\
\hline ] & -1.626809 & 2.851525 & -2.963194 \\
\hline & -7.279807 & -5.715970 & -1.090676 \\
\hline 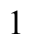 & -9.236361 & 0.501263 & 0.861502 \\
\hline 1 & 4.849176 & 4.654322 & -3.378555 \\
\hline & 0.232441 & 7.094544 & 4.103854 \\
\hline 1 & -9.881895 & 2.480012 & -0.509821 \\
\hline & -2.196298 & -2.149074 & 1.537489 \\
\hline ] & 3.772467 & 0.945463 & 2.039884 \\
\hline & 2.873797 & -0.149897 & -1.986798 \\
\hline 1 & 6.896917 & 0.875906 & -0.892213 \\
\hline 1 & 3.416119 & -1.994981 & 0.948258 \\
\hline 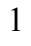 & -0.364475 & -0.828262 & 2.579339 \\
\hline 1 & 1.580244 & -4.380328 & 4.002310 \\
\hline & -2.121350 & 3.979264 & 0.273047 \\
\hline 1 & -3.424183 & 3.205294 & -0.629905 \\
\hline ] & -5.272536 & 2.689374 & 0.676992 \\
\hline 1 & -1.369205 & 2.320832 & 2.410834 \\
\hline 1 & -6.290328 & 1.912944 & 2.791838 \\
\hline ] & -2.373210 & 1.570562 & 4.540411 \\
\hline & -4.847031 & 1.331924 & 4.733149 \\
\hline
\end{tabular}




\begin{tabular}{|c|c|c|c|}
\hline & 1.293077 & -1.792108 & -1.303496 \\
\hline & -1.872079 & -2.582864 & -1.595561 \\
\hline & -1.117431 & -2.289781 & -3.232820 \\
\hline & 0.049087 & -4.927623 & -0.599365 \\
\hline & 0.961025 & -3.692052 & 0.283360 \\
\hline & -0.795207 & -3.556037 & 0.1569 \\
\hline & 2.541120 & -4.310705 & -1.036364 \\
\hline & 4.391501 & -4.969996 & 508005 \\
\hline & 4.263105 & -4.540328 & -4.958936 \\
\hline & 2.223023 & -3.470783 & -5.90033 \\
\hline & 0.366135 & -2.830423 & -4.44007 \\
\hline & 5.640368 & -4.697343 & 1.387402 \\
\hline & 4.459615 & -3.864595 & 0.365251 \\
\hline & 5.308581 & -2.977395 & 1.647602 \\
\hline & 3.990775 & -6.446731 & 1.8 \\
\hline & 2.440030 & -6.068954 & \\
\hline & 2.713359 & -5.628993 & 0.9 \\
\hline & 5.239233 & -5.208520 & \\
\hline & 4.838307 & -3.519192 & 4.102450 \\
\hline & 3.712214 & -4.817872 & 4.534923 \\
\hline & -2.313939 & -1.863692 & 5.35886 \\
\hline & -1.038324 & -0.749202 & 4.83314 \\
\hline & 3897 & -1.484941 & \\
\hline & .806193 & -3.247188 & 6.5 \\
\hline & 0.64 & -3.9 & 5.8 \\
\hline & 0.583028 & -2.225006 & $6.1 \mathrm{C}$ \\
\hline & -2.227443 & -4.295715 & 4.76784 \\
\hline & -1.871341 & -3.967956 & 3.05872 \\
\hline & -0.784792 & -4.997196 & 4.00835 \\
\hline & 4.658478 & -0.248728 & -5.034409 \\
\hline & 3.649895 & -0.984276 & -3.784331 \\
\hline & & 0.780190 & -4.025696 \\
\hline & 6.60 & -1.46 & -4.0 \\
\hline & 7.143136 & -1.147891 & -2.39376 \\
\hline & 5.656935 & -2.075022 & -2.68367 \\
\hline & 6.621538 & 1.038339 & -4.57631 \\
\hline & 5.731191 & 2.164190 & -3.52736 \\
\hline & 7.205286 & 1.366142 & -2.941494 \\
\hline & 8.397747 & 0.804221 & 2.527596 \\
\hline & 7.322889 & -0.535560 & 2.078255 \\
\hline & 8.181480 & & 0.824860 \\
\hline & 7.758685 & 3.188013 & 2.013853 \\
\hline & 7.454262 & 2.817296 & 0.306751 \\
\hline & 6.180661 & 3.563340 & 1.294275 \\
\hline & 6.663221 & 1.909378 & 3.824127 \\
\hline & 5.067491 & 2.292369 & 3.162727 \\
\hline & 5.515068 & 0.602348 & 3.490830 \\
\hline
\end{tabular}

TS1 $_{\text {IIL-re- }} \psi_{\text {-1a2a3a4s }}$

Number of imaginary frequencies : 1

The smallest frequencies are : $-616.6830 \quad 14.5195 \quad 21.0917 \mathrm{~cm}(-1)$

Electronic energy : $\quad H F=-4360.871332$

Zero-point correction $=\quad 1.451630($ Hartree/Particle $)$

Thermal correction to Energy= $\quad 1.536475$

Thermal correction to Enthalpy= $\quad 1.537419$ 
Thermal correction to Gibbs Free Energy= Sum of electronic and zero-point Energies= Sum of electronic and thermal Energies= Sum of electronic and thermal Enthalpies= Sum of electronic and thermal Free Energies=

\author{
1.333481 \\ $-4359.419702$ \\ $-4359.334857$ \\ $-4359.333913$ \\ $-4359.537851$
}

Cartesian Coordinates

\begin{tabular}{|c|c|c|c|}
\hline 6 & -2.491151 & 4.080858 & -2.076308 \\
\hline 6 & -1.314879 & 3.504497 & -2.576655 \\
\hline 6 & -1.223700 & 3.308426 & -3.959081 \\
\hline 6 & -2.268748 & 3.666407 & -4.805284 \\
\hline 6 & -3.431857 & 4.231463 & -4.292819 \\
\hline 6 & -3.534796 & 4.440903 & -2.920133 \\
\hline 6 & -0.229816 & 3.075244 & -1.629498 \\
\hline 6 & 0.960374 & 2.470363 & -2.101481 \\
\hline 45 & -0.067331 & 0.680638 & -1.312616 \\
\hline 6 & -0.470295 & 0.057136 & -3.171944 \\
\hline 8 & -0.275999 & -0.200501 & -4.285319 \\
\hline 15 & -1.404123 & -0.148099 & 0.460810 \\
\hline 6 & -1.075935 & -1.612621 & 1.555180 \\
\hline 6 & -0.741401 & -2.830522 & 0.979593 \\
\hline 6 & -0.563032 & -3.990066 & 1.801331 \\
\hline 6 & -0.733740 & -3.886456 & 3.212694 \\
\hline 6 & -1.084938 & -2.634178 & 3.765537 \\
\hline 6 & -1.248555 & -1.535943 & 2.963690 \\
\hline 6 & -0.544202 & -5.029364 & 4.025901 \\
\hline 6 & -0.197099 & -6.235168 & 3.472810 \\
\hline 6 & -0.027127 & -6.345280 & 2.076331 \\
\hline 6 & -0.207633 & -5.254752 & 1.262254 \\
\hline 6 & -0.602474 & -3.009238 & -0.492557 \\
\hline 6 & 0.593144 & -2.736767 & -1.133532 \\
\hline 6 & 0.752026 & -3.025652 & -2.508625 \\
\hline 6 & -0.289590 & -3.517654 & -3.247839 \\
\hline 6 & -1.549552 & -3.744477 & -2.647204 \\
\hline 6 & -1.704045 & -3.517759 & -1.247390 \\
\hline 6 & -2.971421 & -3.768325 & -0.659355 \\
\hline 6 & -4.030135 & -4.194508 & -1.421310 \\
\hline 6 & -3.875019 & -4.411749 & -2.808065 \\
\hline 6 & -2.659119 & -4.194360 & -3.404588 \\
\hline 7 & 1.670615 & -2.100889 & -0.428061 \\
\hline 6 & 2.728452 & -2.997902 & 0.090151 \\
\hline 6 & 3.345100 & -2.564780 & 1.393849 \\
\hline 6 & 4.726531 & -2.434226 & 1.513477 \\
\hline 6 & 5.297316 & -2.011172 & 2.711673 \\
\hline 6 & 4.488743 & -1.720244 & 3.805141 \\
\hline 6 & 3.106132 & -1.863597 & 3.696096 \\
\hline 6 & 2.541797 & -2.284050 & 2.499855 \\
\hline 6 & -3.151986 & -0.494796 & -0.012808 \\
\hline 6 & -3.565130 & -0.280253 & -1.325338 \\
\hline 6 & -4.900182 & -0.438894 & -1.692211 \\
\hline 6 & -5.812627 & -0.821315 & -0.700218 \\
\hline 6 & -5.427670 & -1.057357 & 0.621356 \\
\hline 6 & -4.079743 & -0.887432 & 0.946192 \\
\hline 6 & -6.406891 & -1.456409 & 1.723716 \\
\hline 6 & -5.374874 & -0.160452 & -3.116083 \\
\hline
\end{tabular}




\begin{tabular}{|c|c|c|c|}
\hline 6 & -4.204832 & 0.027664 & -4.081758 \\
\hline 6 & -1.639370 & 1.233135 & 1.656004 \\
\hline 6 & -2.762308 & 2.054512 & 1.557275 \\
\hline 6 & -2.897894 & 3.191150 & 2.354912 \\
\hline 6 & -1.889283 & 3.461892 & 3.283706 \\
\hline 6 & -0.757768 & 2.649593 & 3.424585 \\
\hline 6 & -0.632685 & 1.552645 & 2.569768 \\
\hline 6 & -4.107021 & 4.117397 & 2.232466 \\
\hline 6 & -5.081564 & 3.657451 & 1.147929 \\
\hline 6 & 0.314354 & 2.994642 & 4.458791 \\
\hline 6 & 1.284221 & 1.835397 & 4.692011 \\
\hline 15 & 1.825917 & -0.457830 & -0.835578 \\
\hline 8 & 2.950923 & -0.341851 & -2.088279 \\
\hline 6 & 4.252026 & -0.752741 & -1.957028 \\
\hline 6 & 5.140432 & -0.069227 & -1.144321 \\
\hline 6 & 6.453309 & -0.617509 & -0.969871 \\
\hline 6 & 6.850545 & -1.755458 & -1.734832 \\
\hline 6 & 5.929760 & -2.341823 & -2.637605 \\
\hline 6 & 4.652409 & -1.863117 & -2.732522 \\
\hline 6 & 7.366079 & -0.112150 & -0.009021 \\
\hline 6 & 8.605548 & -0.679477 & 0.160416 \\
\hline 6 & 9.008059 & -1.779034 & -0.625955 \\
\hline 6 & 8.142696 & -2.304417 & -1.551207 \\
\hline 6 & 4.693131 & 1.159061 & -0.448210 \\
\hline 6 & 3.544218 & 1.115579 & 0.320138 \\
\hline 6 & 3.071488 & 2.231253 & 1.040389 \\
\hline 6 & 3.744784 & 3.420511 & 0.965675 \\
\hline 6 & 4.889621 & 3.549912 & 0.142559 \\
\hline 6 & 5.364985 & 2.417332 & -0.586711 \\
\hline 6 & 5.556526 & 4.792581 & 0.014212 \\
\hline 6 & 6.636085 & 4.932120 & -0.819384 \\
\hline 6 & 7.085914 & 3.825876 & -1.570071 \\
\hline 6 & 6.469148 & 2.604526 & -1.457228 \\
\hline 8 & 2.838430 & -0.054539 & 0.420076 \\
\hline 6 & -0.168525 & 3.820416 & -0.315346 \\
\hline 6 & -6.225831 & -1.325495 & -3.630429 \\
\hline 6 & -6.209323 & 1.125354 & -3.106936 \\
\hline 6 & 1.105578 & 4.209311 & 3.961439 \\
\hline 6 & -0.331267 & 3.331324 & 5.807157 \\
\hline 6 & -4.862008 & 4.148037 & 3.565648 \\
\hline 6 & -3.632204 & 5.531394 & 1.880632 \\
\hline 1 & 7.064049 & 0.723521 & 0.617592 \\
\hline 1 & -1.528883 & -0.588641 & 3.417123 \\
\hline 1 & 8.427653 & -3.166378 & -2.153231 \\
\hline 1 & -0.677628 & -4.927122 & 5.102024 \\
\hline 1 & 5.182321 & 5.640144 & 0.587118 \\
\hline 1 & -3.105023 & -3.593067 & 0.406980 \\
\hline 1 & -5.000658 & -4.358342 & -0.956252 \\
\hline 1 & 3.394163 & 4.291891 & 1.518588 \\
\hline 1 & -1.228395 & -2.555686 & 4.842608 \\
\hline 1 & 6.248161 & -3.194695 & -3.234856 \\
\hline 1 & 7.926214 & 3.942362 & -2.250869 \\
\hline 1 & 3.920370 & -2.311741 & -3.400800 \\
\hline 1 & -2.523867 & -4.361342 & -4.472367 \\
\hline 1 & -0.171454 & -3.711034 & -4.312957 \\
\hline 1 & 0.247493 & -7.303568 & 1.641403 \\
\hline
\end{tabular}




\begin{tabular}{|c|c|c|c|}
\hline 1 & 6.821869 & 1.766066 & -2.052101 \\
\hline 1 & -0.078025 & -5.355684 & 0.186684 \\
\hline & 1.712758 & -2.807828 & -2.972986 \\
\hline | & 7.137004 & 5.892717 & -0.914345 \\
\hline & 9.281365 & -0.279078 & 0.912956 \\
\hline & -4.721422 & -4.755521 & -3.399186 \\
\hline & -0.051264 & -7.107353 & 4.105861 \\
\hline & 9.994869 & -2.214288 & -0.486668 \\
\hline 1 & 2.167113 & 2.113663 & 1.636527 \\
\hline & -3.751309 & -1.044346 & 1.973638 \\
\hline 1 & -2.814432 & 0.015982 & -2.053574 \\
\hline 1 & -6.859767 & -0.937063 & -0.973278 \\
\hline 1 & -3.532919 & 1.807494 & 0.832206 \\
\hline 1 & 0.250801 & 0.915247 & 2.620219 \\
\hline 1 & -1.988443 & 4.339466 & 3.922098 \\
\hline 1 & 2.237073 & -3.971356 & 0.231995 \\
\hline 1 & 3.518966 & -3.152776 & -0.661332 \\
\hline 1 & 5.364264 & -2.638743 & 51857 \\
\hline 1 & 1.461573 & -2.363448 & 2.402282 \\
\hline 1 & 6.377721 & -1.899829 & 2.782197 \\
\hline 1 & 2.460939 & -1.645169 & 4.546161 \\
\hline 1 & 4.932519 & -1.383412 & 4.739776 \\
\hline 1 & -1.283841 & 1.759261 & -1.293548 \\
\hline 1 & 1.863533 & 2.625416 & 9783 \\
\hline 1 & 1.146261 & & 56197 \\
\hline 1 & -0.128774 & 4.903521 & -0.504966 \\
\hline 1 & -1.031001 & 3.630375 & 3158 \\
\hline 1 & 0.730847 & 3.534244 & 0.239631 \\
\hline 1 & -2.601848 & 4.235170 & -1.003428 \\
\hline 1 & -4.435528 & 4.884780 & 9024 \\
\hline 1 & -4.249097 & 4.507580 & 55350 \\
\hline 1 & -2.168476 & & \\
\hline 1 & -0.326844 & 2.871062 & -4.390698 \\
\hline 1 & -5.931221 & 4.350227 & 1.100254 \\
\hline 1 & -4.617388 & 3.633137 & 0.151712 \\
\hline 1 & -5.482997 & 2.654983 & 1.352045 \\
\hline 1 & -4.490477 & 6.209958 & 1.784475 \\
\hline 1 & -2.966103 & 5.946499 & 2.646624 \\
\hline 1 & -3.087334 & 5.541179 & 0.926021 \\
\hline 1 & -5.732611 & 4.814199 & 3.497511 \\
\hline 1 & -5.223010 & 3.145853 & 3.832756 \\
\hline 1 & -4.232240 & 4.507650 & 4.388452 \\
\hline 1 & 1.984236 & 2.097427 & 5.495344 \\
\hline 1 & 0.754462 & 0.921367 & 4.994747 \\
\hline 1 & 1.889764 & 1.596410 & 3.808526 \\
\hline 1 & 0.446415 & 3.545120 & 6.551981 \\
\hline 1 & -0.980511 & 4.212832 & 5.756951 \\
\hline 1 & -0.932449 & 2.491427 & 6.178729 \\
\hline 1 & 1.891282 & 4.480770 & 4.679682 \\
\hline 1 & 1.586721 & 3.995003 & 2.996999 \\
\hline 1 & 0.456943 & 5.083396 & 3.821788 \\
\hline 1 & -4.588772 & 0.179681 & -5.098812 \\
\hline 1 & -3.592367 & 0.906047 & -3.832746 \\
\hline 1 & -3.548911 & -0.854441 & -4.098104 \\
\hline 1 & -6.545093 & 1.375368 & -4.122955 \\
\hline . & -7.099445 & 1.028719 & -2.472025 \\
\hline
\end{tabular}




$\begin{array}{lrrr}1 & -5.615259 & 1.968802 & -2.728179 \\ 1 & -6.548562 & -1.132237 & -4.662205 \\ 1 & -5.647878 & -2.259877 & -3.624151 \\ 1 & -7.130212 & -1.483812 & -3.030738 \\ 6 & -7.808573 & -1.731833 & 1.186098 \\ 6 & -5.912269 & -2.727787 & 2.423756 \\ 6 & -6.495661 & -0.312821 & 2.742119 \\ 1 & -8.467388 & -2.037808 & 2.008692 \\ 1 & -8.255579 & -0.842489 & 0.723975 \\ 1 & -7.806782 & -2.541041 & 0.443644 \\ 1 & -6.603754 & -3.011205 & 3.228629 \\ 1 & -5.858114 & -3.568238 & 1.718827 \\ 1 & -4.918559 & -2.604566 & 2.872838 \\ 1 & -7.158697 & -0.586755 & 3.574182 \\ 1 & -5.513308 & -0.060670 & 3.162725 \\ 1 & -6.900654 & 0.594597 & 2.274025\end{array}$

TS1 $_{\text {ILL-re- }} \psi_{-1 \text { a22a3s4a }}$

Number of imaginary frequencies : 1

The smallest frequencies are : $-627.4869 \quad 17.2216 \quad 17.9739 \mathrm{~cm}(-1)$

Electronic energy : $\quad \mathrm{HF}=-4360.8707405$

Zero-point correction $=\quad 1.451560($ Hartree/Particle $)$

Thermal correction to Energy $=\quad 1.536587$

Thermal correction to Enthalpy= $\quad 1.537531$

Thermal correction to Gibbs Free Energy= $\quad 1.332292$

Sum of electronic and zero-point Energies $=\quad-4359.419180$

Sum of electronic and thermal Energies $=\quad-4359.334153$

Sum of electronic and thermal Enthalpies $=\quad-4359.333209$

Sum of electronic and thermal Free Energies $=\quad-4359.538449$

Cartesian Coordinates

$\begin{array}{cccc}6 & -2.661383 & 2.160687 & 1.600388 \\ 6 & -1.585877 & 1.275911 & 1.672247 \\ 6 & -0.544207 & 1.526621 & 2.569417 \\ 6 & -0.592711 & 2.616815 & 3.439384 \\ 6 & -1.677658 & 3.494202 & 3.326570 \\ 6 & -2.715006 & 3.295969 & 2.412108 \\ 15 & -1.448910 & -0.104205 & 0.463131 \\ 6 & -1.117138 & -1.581555 & 1.539545 \\ 6 & -0.778899 & -2.796964 & 0.961363 \\ 6 & -0.576168 & -3.953097 & 1.785234 \\ 6 & -0.738251 & -3.850284 & 3.197456 \\ 6 & -1.108413 & -2.603837 & 3.751081 \\ 6 & -1.287552 & -1.508641 & 2.949515 \\ 6 & -0.519517 & -4.986326 & 4.012563 \\ 6 & -0.149665 & -6.185985 & 3.460817 \\ 6 & 0.014424 & -6.295431 & 2.063936 \\ 6 & -0.195859 & -5.211419 & 1.248077 \\ 6 & -0.637892 & -2.990217 & -0.509216 \\ 6 & 0.557665 & -2.715632 & -1.148071 \\ 6 & 0.741855 & -3.055272 & -2.508329 \\ 6 & -0.265898 & -3.634026 & -3.230528 \\ 6 & -1.520197 & -3.890734 & -2.630014 \\ 6 & -1.711632 & -3.572384 & -1.252297\end{array}$




\begin{tabular}{|c|c|c|c|}
\hline 6 & -2.975841 & -3.845876 & -0.668316 \\
\hline 6 & -3.988472 & -4.403808 & -1.408310 \\
\hline 6 & -3.791531 & -4.726928 & -2.768369 \\
\hline & -2.583115 & -4.471716 & -3.364161 \\
\hline & 1.624747 & -2.056804 & -0.448084 \\
\hline 6 & 2.710761 & -2.934489 & 0.042075 \\
\hline 6 & 3.309879 & -2.551374 & 1.370157 \\
\hline 6 & 4.693679 & -2.534082 & 1.532648 \\
\hline 6 & 5.259535 & -2.219534 & 2.766325 \\
\hline 6 & 4.444190 & -1.921193 & 3.852365 \\
\hline 6 & 3.058895 & -1.936320 & 3.695821 \\
\hline 6 & 2.498755 & -2.247030 & 2.464363 \\
\hline 6 & 0.496027 & 2.876900 & 4.480326 \\
\hline 6 & -0.141086 & 3.104575 & 5.855849 \\
\hline 6 & -3.858133 & 4.305943 & 2.321268 \\
\hline 6 & -3.287676 & 5.684432 & 1.969167 \\
\hline & -0.140306 & 0.707624 & -1.346992 \\
\hline 6 & -0.626771 & 0.026159 & -3.153446 \\
\hline 8 & -0.551060 & -0.307856 & -4.260861 \\
\hline 15 & 1.765679 & -0.417041 & -0.868322 \\
\hline 8 & 2.881048 & -0.300849 & -2.130912 \\
\hline 6 & 4.176566 & -0.735908 & -2.018204 \\
\hline 6 & 5.081352 & -0.088003 & -1.195013 \\
\hline 6 & 6.376786 & -0.676113 & -1.022510 \\
\hline 6 & 6.746470 & -1.811776 & -1.803837 \\
\hline 6 & 5.818567 & -2.348927 & -2.729845 \\
\hline 6 & 4.555165 & -1.834049 & -2.822095 \\
\hline 6 & 8.017929 & -2.405301 & -1.614737 \\
\hline 6 & 8.888044 & -1.923555 & -0.670098 \\
\hline 6 & 8.513050 & -0.822904 & 0.128275 \\
\hline 6 & 7.294820 & -0.212941 & -0.045933 \\
\hline 6 & 4.670137 & 1.149472 & -0.493671 \\
\hline 6 & 3.520742 & 1.138322 & 0.274149 \\
\hline 6 & 3.077018 & 2.268871 & 0.988952 \\
\hline 6 & 3.780341 & 3.440139 & 0.910089 \\
\hline 6 & 4.930782 & 3.535723 & 0.090511 \\
\hline 6 & 5.377203 & 2.388220 & -0.633661 \\
\hline 6 & 6.490183 & 2.542006 & -1.499531 \\
\hline 6 & 7.141055 & 3.745479 & -1.613138 \\
\hline 6 & 6.718302 & 4.866346 & -0.868445 \\
\hline 6 & 5.631673 & 4.759332 & -0.039267 \\
\hline 8 & 2.786908 & -0.014027 & 0.382279 \\
\hline 6 & 0.903231 & 2.465245 & -2.171860 \\
\hline 6 & -0.244847 & 3.114554 & -1.654293 \\
\hline 6 & -1.331200 & 3.606591 & -2.565974 \\
\hline 6 & -1.346261 & 3.313169 & -3.935539 \\
\hline 6 & -2.377884 & 3.759411 & -4.753618 \\
\hline 6 & -3.424080 & 4.511494 & -4.226912 \\
\hline 6 & -3.425474 & 4.809353 & -2.868097 \\
\hline 6 & -2.394572 & 4.360220 & -2.050485 \\
\hline 6 & -0.111107 & 3.840454 & -0.336196 \\
\hline 6 & -3.217989 & -0.413494 & 0.044229 \\
\hline 6 & -4.127932 & -0.844901 & 1.018397 \\
\hline 6 & -5.468738 & -1.037030 & 0.706344 \\
\hline 6 & -5.883471 & -0.780449 & -0.612962 \\
\hline 6 & -5.004068 & -0.350644 & -1.601136 \\
\hline
\end{tabular}




\begin{tabular}{|c|c|c|c|}
\hline 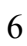 & -3.659970 & -0.176024 & -1.246155 \\
\hline & -6.482633 & -1.535285 & 1.734880 \\
\hline & -5.410499 & -0.098073 & -3.049355 \\
\hline & -4.707916 & -1.130584 & -3.939106 \\
\hline & -6.916899 & -0.221645 & -3.259261 \\
\hline & -4.977631 & 1.312173 & -3.469304 \\
\hline & 1.462070 & 1.699400 & 4.609992 \\
\hline & 1.288778 & 4.125388 & 4.079342 \\
\hline & -4.883857 & 3.927671 & 1.253662 \\
\hline & -4.581461 & 4.384658 & 3.669962 \\
\hline & 7.011385 & 0.621722 & 0.590625 \\
\hline & -1.576188 & -0.564629 & 3.404466 \\
\hline & 8.282466 & -3.265958 & -2.227880 \\
\hline & -0.647990 & -4.883410 & 5.089222 \\
\hline & 5.278478 & 5.618658 & 0.529386 \\
\hline & -3.143991 & -3.590761 & 0.377299 \\
\hline & -4.954108 & -4.595392 & -0.944617 \\
\hline & 3.450149 & 4.322666 & 1.457771 \\
\hline & -1.250014 & -2.526791 & 4.828479 \\
\hline & 6.118931 & -3.194598 & -3.346327 \\
\hline & 7.987765 & 3.836303 & -2.289890 \\
\hline & 3.818429 & -2.245882 & -3.508237 \\
\hline & -2.417920 & -4.708757 & -4.414294 \\
\hline & -0.123634 & -3.885252 & -4.280452 \\
\hline & 0.308842 & -7.247983 & 1.629374 \\
\hline & 6.822976 & 1.692191 & -2.089719 \\
\hline & -0.068370 & -5.314184 & 0.172467 \\
\hline & 1.700045 & -2.824747 & -2.970965 \\
\hline & 7.245945 & 5.812443 & -0.964203 \\
\hline 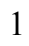 & 9.192841 & -0.456599 & 0.894451 \\
\hline & -4.601870 & -5.172651 & -3.341184 \\
\hline 1 & 0.019672 & -7.052959 & 4.095181 \\
\hline & 9.858076 & -2.393590 & -0.525929 \\
\hline | & 2.167802 & 2.178571 & 1.582064 \\
\hline 1 & -3.772453 & -1.025661 & 2.030246 \\
\hline & -2.933638 & 0.147260 & -1.989044 \\
\hline 1 & -6.931863 & -0.930971 & -0.864347 \\
\hline & -3.455872 & 1.965400 & 0.883947 \\
\hline 1 & 0.302357 & 0.839220 & 2.595692 \\
\hline & -1.714550 & 4.366509 & 3.979099 \\
\hline ] & 2.254861 & -3.930808 & 0.137553 \\
\hline 1 & 3.511241 & -3.031803 & -0.707941 \\
\hline | & 5.340017 & -2.754408 & 0.680934 \\
\hline 1 & 1.419477 & -2.224990 & 2.331319 \\
\hline & 6.342551 & -2.202377 & 2.872700 \\
\hline 1 & 2.408085 & -1.704177 & 4.537981 \\
\hline & 4.884335 & -1.673467 & 4.816094 \\
\hline 1 & -1.337323 & 1.813327 & -1.322564 \\
\hline 1 & 1.832101 & 2.586909 & -1.613317 \\
\hline 1 & 1.047476 & 2.350006 & -3.244483 \\
\hline 1 & -0.017018 & 4.922194 & -0.515715 \\
\hline & -0.967334 & 3.689726 & 0.330921 \\
\hline 1 & 0.784690 & 3.501633 & 0.192713 \\
\hline & -2.426470 & 4.593849 & -0.987545 \\
\hline 1 & -4.235557 & 5.393440 & -2.434644 \\
\hline 1 & -4.230473 & 4.858819 & -4.868985 \\
\hline
\end{tabular}




$\begin{array}{rrrr}1 & -2.360940 & 3.515692 & -5.813913 \\ 1 & -0.543109 & 2.728847 & -4.377788 \\ 1 & -5.682130 & 4.680196 & 1.228883 \\ 1 & -4.440660 & 3.883175 & 0.248807 \\ 1 & -5.353855 & 2.955994 & 1.457483 \\ 1 & -4.096948 & 6.421391 & 1.877973 \\ 1 & -2.590813 & 6.050614 & 2.732650 \\ 1 & -2.746490 & 5.657713 & 1.012732 \\ 1 & -5.403030 & 5.111983 & 3.621212 \\ 1 & -5.007870 & 3.410810 & 3.943591 \\ 1 & -3.911181 & 4.695530 & 4.480415 \\ 1 & 2.181259 & 1.899224 & 5.414279 \\ 1 & 0.932084 & 0.769293 & 4.858891 \\ 1 & 2.044392 & 1.522153 & 3.696826 \\ 1 & 0.639679 & 3.271824 & 6.609554 \\ 1 & -0.802948 & 3.978180 & 5.872927 \\ 1 & -0.729413 & 2.231712 & 6.167271 \\ 1 & 2.083053 & 4.329225 & 4.810208 \\ 1 & 1.760608 & 3.993578 & 3.095768 \\ 1 & 0.646649 & 5.013309 & 4.023231 \\ 1 & -7.162100 & -0.017619 & -4.309223 \\ 1 & -7.282741 & -1.230888 & -3.029424 \\ 1 & -7.475811 & 0.495551 & -2.643554 \\ 1 & -4.976840 & -0.974681 & -4.992967 \\ 1 & -3.614858 & -1.062530 & -3.858482 \\ 1 & -4.997455 & -2.152004 & -3.657360 \\ 1 & -5.295278 & 1.515775 & -4.501314 \\ 1 & -5.428277 & 2.074286 & -2.819179 \\ 1 & -3.887543 & 1.444851 & -3.433926 \\ 6 & -5.867437 & -1.691798 & 3.124131 \\ 6 & -7.648136 & -0.546064 & 1.839822 \\ 6 & -7.011545 & -2.905074 & 1.295323 \\ 1 & -7.762662 & -3.273541 & 2.007250 \\ 1 & -7.480080 & -2.868737 & 0.304175 \\ 1 & -6.196591 & -3.640598 & 1.256107 \\ 1 & -8.373942 & -0.890829 & 2.588559 \\ 1 & -7.294125 & 0.447547 & 2.144736 \\ 1 & -8.184496 & -0.432731 & 0.890246 \\ 1 & -6.629944 & -2.051677 & 3.826458 \\ 1 & -5.044842 & -2.419989 & 3.131605 \\ 1 & -5.483914 & -0.738823 & 3.511645\end{array}$

TS1 $_{\text {IIL-re- }} \Psi-1$ a2a3s4s

Number of imaginary frequencies : 1

The smallest frequencies are : $-634.8035 \quad 12.5505 \quad 17.0778 \mathrm{~cm}(-1)$

Electronic energy : $\quad H F=-4360.8712859$

Zero-point correction $=\quad 1.451310($ Hartree/Particle $)$

Thermal correction to Energy $=\quad 1.536433$

Thermal correction to Enthalpy= $\quad 1.537378$

Thermal correction to Gibbs Free Energy $=\quad 1.331082$

Sum of electronic and zero-point Energies $=\quad-4359.419976$

Sum of electronic and thermal Energies $=\quad-4359.334853$

Sum of electronic and thermal Enthalpies $=\quad-4359.333908$

Sum of electronic and thermal Free Energies $=\quad-4359.540204$ 
Cartesian Coordinates

\begin{tabular}{|c|c|c|c|}
\hline 6 & 7.327954 & -0.092408 & 0.005695 \\
\hline 6 & 6.423531 & -0.572600 & -0.975402 \\
\hline 6 & 6.819030 & -1.700781 & -1.755203 \\
\hline 6 & 8.101190 & -2.269008 & -1.560995 \\
\hline 6 & 8.957689 & -1.770551 & -0.612700 \\
\hline 6 & 8.557258 & -0.678298 & 0.184861 \\
\hline 6 & 5.118023 & -0.009047 & -1.155104 \\
\hline 6 & 4.230548 & -0.674050 & -1.983623 \\
\hline 6 & 4.634025 & -1.765434 & -2.784574 \\
\hline 6 & 5.906430 & -2.256116 & -2.685539 \\
\hline 6 & 4.679523 & 1.219055 & -0.453471 \\
\hline 6 & 3.527687 & 1.182905 & 0.309700 \\
\hline 6 & 3.063227 & 2.299302 & 1.033690 \\
\hline 6 & 3.744677 & 3.484044 & 0.963661 \\
\hline 6 & 4.893395 & 3.606416 & 0.144821 \\
\hline 6 & 5.363174 & 2.471646 & -0.584844 \\
\hline 6 & 5.570744 & 4.843952 & 0.022090 \\
\hline 6 & 6.655972 & 4.976283 & -0.805340 \\
\hline 6 & 7.101666 & 3.867611 & -1.554861 \\
\hline 6 & 6.474406 & 2.651068 & -1.447879 \\
\hline 8 & 2.814324 & 0.017758 & 0.406201 \\
\hline 15 & 1.807033 & -0.400280 & -0.850109 \\
\hline 8 & 2.928006 & -0.263116 & -2.105428 \\
\hline 45 & -0.113773 & 0.690310 & -1.345641 \\
\hline 6 & 0.901805 & 2.487557 & -2.123114 \\
\hline 6 & -0.269513 & 3.100607 & -1.611889 \\
\hline 6 & -0.172077 & 3.804161 & -0.278556 \\
\hline 6 & -1.350159 & 3.587242 & -2.533598 \\
\hline 6 & -1.345989 & 3.303336 & -3.905323 \\
\hline 6 & -2.373314 & 3.743818 & -4.731761 \\
\hline 6 & -3.434225 & 4.480032 & -4.211606 \\
\hline 6 & -3.455196 & 4.766953 & -2.850722 \\
\hline 6 & -2.428527 & 4.323176 & -2.024669 \\
\hline 6 & -0.573396 & 0.014429 & -3.156014 \\
\hline 8 & -0.529435 & -0.330261 & -4.261702 \\
\hline 15 & -1.435742 & -0.157456 & 0.433454 \\
\hline 6 & -3.183525 & -0.522192 & -0.024240 \\
\hline 6 & -4.101095 & -0.933128 & 0.942651 \\
\hline 6 & -5.447904 & -1.108966 & 0.631336 \\
\hline 6 & -5.856027 & -0.861514 & -0.686646 \\
\hline 6 & -4.960177 & -0.454439 & -1.677230 \\
\hline 6 & -3.618199 & -0.294036 & -1.321863 \\
\hline 6 & -6.414525 & -1.513297 & 1.743433 \\
\hline 6 & -5.362003 & -0.191615 & -3.125200 \\
\hline 6 & -1.084016 & -1.616530 & 1.528080 \\
\hline 6 & -0.709911 & -2.827077 & 0.961689 \\
\hline 6 & -0.489856 & -3.972942 & 1.794390 \\
\hline 6 & -0.669582 & -3.865173 & 3.204145 \\
\hline 6 & -1.074704 & -2.624461 & 3.745691 \\
\hline 6 & -1.270898 & -1.538388 & 2.935376 \\
\hline 6 & -0.433325 & -4.990938 & 4.028604 \\
\hline 6 & -0.030282 & -6.185013 & 3.488213 \\
\hline 6 & 0.150196 & -6.299463 & 2.093642 \\
\hline 6 & -0.076336 & -5.225596 & 1.268808 \\
\hline
\end{tabular}




\begin{tabular}{|c|c|c|c|}
\hline ) & -0.551772 & -3.021661 & -0.506848 \\
\hline & 0.642075 & -2.721295 & -1.137502 \\
\hline & 0.840231 & -3.051054 & -2.498253 \\
\hline & -0.153559 & -3.642240 & -3.229614 \\
\hline & -1.406991 & -3.922938 & -2.638131 \\
\hline & -1.610906 & -3.619024 & -1.258883 \\
\hline & -2.874216 & -3.915726 & -0.684076 \\
\hline 6 & -3.875034 & -4.479897 & -1.435289 \\
\hline 6 & -3.665975 & -4.787836 & -2.797163 \\
\hline 6 & -2.457437 & -4.512304 & -3.383410 \\
\hline 7 & 1.691350 & -2.041993 & -0.430362 \\
\hline 6 & 2.787736 & -2.897820 & 0.074903 \\
\hline 6 & 3.366805 & -2.494808 & 1.406057 \\
\hline 6 & 4.748236 & -2.455804 & 1.583386 \\
\hline 6 & 5.295806 & -2.115203 & 2.818391 \\
\hline 6 & 4.464251 & -1.812002 & 3.890732 \\
\hline 6 & 3.081081 & -1.848500 & 3.719180 \\
\hline 6 & 2.539324 & -2.185963 & 2.486662 \\
\hline 6 & -1.675822 & 1.221619 & 1.630474 \\
\hline 6 & -0.671103 & 1.540243 & 2.546824 \\
\hline 6 & -0.800900 & 2.633039 & 3.406038 \\
\hline 6 & -1.935549 & 3.441430 & 3.267993 \\
\hline 6 & -2.940511 & 3.173719 & 2.334306 \\
\hline 6 & -2.799870 & 2.041287 & 1.530983 \\
\hline 6 & 0.268917 & 2.978275 & 4.442533 \\
\hline 6 & -4.153209 & 4.096043 & 2.216576 \\
\hline 1 & 7.025657 & 0.736218 & 0.641476 \\
\hline 1 & -1.586062 & -0.598522 & 3.381079 \\
\hline 1 & 8.385285 & -3.123869 & -2.173474 \\
\hline 1 & -0.575440 & -4.884540 & 5.103223 \\
\hline 1 & 5.200356 & 5.693476 & 0.594544 \\
\hline 1 & -3.051687 & -3.672053 & 0.362742 \\
\hline 1 & -4.841214 & -4.686606 & -0.979798 \\
\hline 1 & 3.398613 & 4.356306 & 1.518010 \\
\hline 1 & -1.229022 & -2.544078 & 4.821121 \\
\hline 1 & 6.226046 & -3.096080 & -3.300153 \\
\hline 1 & 7.947238 & 3.978361 & -2.230065 \\
\hline 1 & 3.908853 & -2.191217 & -3.474524 \\
\hline 1 & -2.282956 & -4.738127 & -4.434523 \\
\hline 1 & -0.001275 & -3.884402 & -4.280263 \\
\hline 1 & 0.470293 & -7.247879 & 1.668308 \\
\hline 1 & 6.824322 & 1.810664 & -2.041683 \\
\hline 1 & 0.063539 & -5.331603 & 0.195023 \\
\hline 1 & 1.797043 & -2.801808 & -2.953964 \\
\hline 1 & 7.164963 & 5.933046 & -0.895948 \\
\hline 1 & 9.226303 & -0.299060 & 0.954209 \\
\hline 1 & -4.467227 & -5.238618 & -3.378772 \\
\hline 1 & 0.152559 & -7.043893 & 4.129807 \\
\hline 1 & 9.936406 & -2.221046 & -0.464909 \\
\hline 1 & 2.157072 & 2.185942 & 1.627859 \\
\hline 1 & -3.760419 & -1.095826 & 1.964907 \\
\hline 1 & -2.886744 & 0.022111 & -2.062819 \\
\hline 1 & -6.903714 & -0.989724 & -0.944455 \\
\hline 1 & -3.566505 & 1.797236 & 0.800381 \\
\hline 1 & 0.214396 & 0.905323 & 2.595409 \\
\hline 1 & -2.039558 & 4.314161 & 3.912283 \\
\hline
\end{tabular}




\begin{tabular}{|c|c|c|c|}
\hline 1 & 2.348773 & -3.901557 & 0.171856 \\
\hline & 3.596778 & -2.984588 & -0.667373 \\
\hline & 5.406959 & -2.677222 & 0.741512 \\
\hline & 1.461528 & -2.177465 & 2.340189 \\
\hline & 6.377251 & -2.080366 & 2.936028 \\
\hline & 2.417497 & -1.610865 & 4.549609 \\
\hline & 4.890178 & -1.542947 & 4.855140 \\
\hline & -1.329798 & 1.776711 & -1.333200 \\
\hline & 1.818923 & 2.619086 & -1.547615 \\
\hline & 1.064768 & 2.395944 & -3.195257 \\
\hline & -0.095702 & 4.890602 & -0.435957 \\
\hline & -1.036343 & 3.623806 & 0.370770 \\
\hline & 0.721575 & 3.471949 & 0.258181 \\
\hline & -2.474651 & 4.546269 & -0.960228 \\
\hline & -4.276952 & 5.338185 & -2.421862 \\
\hline & -4.236993 & 4.822892 & -4.860575 \\
\hline & -2.341800 & 3.507967 & -5.793518 \\
\hline & -0.531567 & 2.730421 & -4.342109 \\
\hline & -5.107692 & 3.656795 & 1.105913 \\
\hline & -4.925693 & 4.091084 & 3.540100 \\
\hline & -3.684791 & 5.521892 & 1.907160 \\
\hline & 1.248865 & 1.825406 & 4.664102 \\
\hline ) & 1.049364 & 4.204450 & 3.956273 \\
\hline & -0.379118 & 3.296770 & 5.794163 \\
\hline 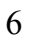 & -6.867596 & -0.313586 & -3.342147 \\
\hline$b$ & -4.928632 & 1.224691 & -3.525770 \\
\hline 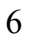 & -4.656291 & -1.212972 & -4.025207 \\
\hline & -7.817239 & -1.809747 & 1.219249 \\
\hline & -5.900062 & -2.771016 & 2.453209 \\
\hline b & -6.509412 & -0.360875 & 2.751352 \\
\hline & -5.961820 & 4.344455 & 1.062977 \\
\hline ] & -4.626298 & 3.663343 & 0.117314 \\
\hline t & -5.505273 & 2.646508 & 1.277893 \\
\hline 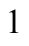 & -4.547525 & 6.194748 & 1.811219 \\
\hline ] & -3.036459 & 5.925767 & 2.694017 \\
\hline 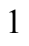 & -3.123341 & 5.558698 & 0.963067 \\
\hline ] & -5.798772 & 4.754486 & 3.476446 \\
\hline & -5.285008 & 3.081774 & 3.780364 \\
\hline 1 & -4.307622 & 4.434379 & 4.378695 \\
\hline 1 & 1.948503 & 2.085895 & 5.468216 \\
\hline | & 0.726208 & 0.904952 & 4.959953 \\
\hline 1 & 1.854041 & 1.599715 & 3.776875 \\
\hline d & 0.396824 & 3.511554 & 6.540531 \\
\hline 1 & -1.037053 & 4.172254 & 5.751835 \\
\hline 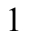 & -0.971899 & 2.447787 & 6.158490 \\
\hline 1 & 1.831863 & 4.476790 & 4.677673 \\
\hline 1 & 1.533795 & 4.003344 & 2.990593 \\
\hline 1 & 0.393054 & 5.073843 & 3.823513 \\
\hline 1 & -7.107716 & -0.106133 & -4.392624 \\
\hline 1 & -7.235108 & -1.323208 & -3.116569 \\
\hline 1 & -7.429252 & 0.402326 & -2.727510 \\
\hline 1 & -4.925525 & -1.046804 & -5.077421 \\
\hline 1 & -3.563471 & -1.142807 & -3.944254 \\
\hline 1 & -4.942804 & -2.238398 & -3.754756 \\
\hline 1 & -5.248967 & 1.445614 & -4.553442 \\
\hline 1 & -5.375588 & 1.976675 & -2.860937 \\
\hline
\end{tabular}




$\begin{array}{lrrr}1 & -3.838170 & 1.354985 & -3.490220 \\ 1 & -8.464543 & -2.119307 & 2.049619 \\ 1 & -8.280102 & -0.928811 & 0.756807 \\ 1 & -7.812552 & -2.622675 & 0.481202 \\ 1 & -6.587069 & -3.059216 & 3.260175 \\ 1 & -5.830753 & -3.615419 & 1.754896 \\ 1 & -4.908830 & -2.628412 & 2.901701 \\ 1 & -7.170837 & -0.630225 & 3.586271 \\ 1 & -5.527706 & -0.100846 & 3.168302 \\ 1 & -6.918162 & 0.540614 & 2.274829\end{array}$

TS1 $_{\text {IIL-re- }} \Psi_{-1 \mathbf{a} 2 \mathrm{~s} 3 \mathrm{a} 4 \mathrm{a}}$

Number of imaginary frequencies : 1

The smallest frequencies are : $-593.0643 \quad 15.3903 \quad 18.5781 \mathrm{~cm}(-1)$

Electronic energy : $\quad H F=-4360.8710602$

Zero-point correction $=\quad 1.451351$ (Hartree/Particle)

Thermal correction to Energy $=\quad 1.536374$

Thermal correction to Enthalpy= $\quad 1.537318$

Thermal correction to Gibbs Free Energy $=\quad 1.332860$

Sum of electronic and zero-point Energies $=\quad-4359.419709$

Sum of electronic and thermal Energies $=\quad-4359.334686$

Sum of electronic and thermal Enthalpies $=\quad-4359.333742$

Sum of electronic and thermal Free Energies $=\quad-4359.538200$

Cartesian Coordinates

$\begin{array}{cccc}6 & -6.491619 & -2.457799 & -1.482675 \\ 6 & -5.372522 & -2.310029 & -0.623500 \\ 6 & -4.912481 & -3.466350 & 0.077513 \\ 6 & -5.607550 & -4.691989 & -0.064330 \\ 6 & -6.701293 & -4.792688 & -0.884817 \\ 6 & -7.136582 & -3.663199 & -1.608951 \\ 6 & -4.670881 & -1.069296 & -0.471198 \\ 6 & -3.514832 & -1.062380 & 0.287349 \\ 6 & -3.053882 & -2.203970 & 0.973462 \\ 6 & -3.752018 & -3.377760 & 0.883319 \\ 6 & -5.089469 & 0.173246 & -1.158657 \\ 6 & -4.186665 & 0.831699 & -1.976146 \\ 6 & -4.565081 & 1.946033 & -2.757386 \\ 6 & -5.830253 & 2.455714 & -2.659203 \\ 6 & -6.760585 & 1.897669 & -1.748170 \\ 6 & -6.387672 & 0.753425 & -0.980857 \\ 6 & -8.037765 & 2.479010 & -1.559563 \\ 6 & -8.910498 & 1.978434 & -0.627390 \\ 6 & -8.531255 & 0.871796 & 0.160682 \\ 6 & -7.307200 & 0.273310 & -0.013536 \\ 8 & -2.896033 & 0.389276 & -2.108251 \\ 15 & -1.763403 & 0.474894 & -0.858472 \\ 8 & -2.782398 & 0.092639 & 0.400648 \\ 45 & 0.097661 & -0.714080 & -1.338720 \\ 15 & 1.442514 & 0.051876 & 0.462400 \\ 6 & 1.491691 & -1.274925 & 1.734209 \\ 6 & 2.545900 & -2.175838 & 1.809471 \\ 6 & 2.531666 & -3.234625 & 2.729844 \\ 6 & 1.438942 & -3.339568 & 3.585264\end{array}$




\begin{tabular}{|c|c|c|c|}
\hline & 0.365381 & -2.432711 & 3.551087 \\
\hline & 0.391876 & -1.427992 & 2.590353 \\
\hline & 3.725678 & -4.186153 & 2.778865 \\
\hline & -0.775639 & -2.568567 & 4.559272 \\
\hline & 0.174727 & -3.103435 & -1.691542 \\
\hline & 0.152913 & -3.875227 & -0.390161 \\
\hline & 1.196858 & -3.559127 & -2.696458 \\
\hline & 2.463508 & -3.976167 & -2.263538 \\
\hline & 3.436056 & -4.383886 & -3.166567 \\
\hline & 3.168294 & -4.381073 & -4.534016 \\
\hline & 1.915051 & -3.976512 & -4.978140 \\
\hline & 0.938810 & -3.574805 & -4.069885 \\
\hline & -1.014180 & -2.462686 & -2.109414 \\
\hline & 0.513675 & -0.100080 & -3.203708 \\
\hline & 0.325116 & 0.132069 & -4.323456 \\
\hline & 1.187027 & 1.572620 & 1.503737 \\
\hline & 0.892910 & 2.785552 & 0.898503 \\
\hline & 0.762999 & 3.973685 & 1.690499 \\
\hline & 0.942196 & 3.902515 & 3.102556 \\
\hline & 1.255546 & 2.654034 & 3.686296 \\
\hline & 1.372331 & 1.530282 & 2.913163 \\
\hline & 0.797033 & 5.071784 & 3.886651 \\
\hline & 0.484607 & 6.273535 & 3.304554 \\
\hline & 0.305864 & 6.351915 & 1.907320 \\
\hline & 0.443660 & 5.234738 & 1.121127 \\
\hline & 0.738228 & 2.939000 & -0.574441 \\
\hline & -0.480479 & 2.712911 & -1.188486 \\
\hline & -0.658849 & 3.009444 & -2.559507 \\
\hline & 0.383003 & 3.465375 & -3.320971 \\
\hline & 1.664799 & 3.638618 & -2.749486 \\
\hline & 1.843431 & 3.397913 & -1.354812 \\
\hline & 3.134037 & 3.590463 & -0.796542 \\
\hline & 4.190162 & 3.980121 & -1.581446 \\
\hline & 4.009501 & 4.216629 & -2.962030 \\
\hline & 2.772296 & 4.050733 & -3.530562 \\
\hline & -1.564401 & 2.115130 & -0.458981 \\
\hline & -2.601692 & 3.042715 & 0.046396 \\
\hline & -3.213539 & 2.645245 & 1.363529 \\
\hline & -4.594123 & 2.512560 & 1.492114 \\
\hline & -5.158866 & 2.118887 & 2.702847 \\
\hline & -4.345548 & 1.865621 & 3.802375 \\
\hline & -2.964686 & 2.018393 & 3.685864 \\
\hline & -2.405224 & 2.402491 & 2.474814 \\
\hline & 3.225453 & 0.264304 & 0.034344 \\
\hline & 3.635563 & 0.050124 & -1.276886 \\
\hline & 4.973588 & 0.206420 & -1.649398 \\
\hline & 5.881879 & 0.581678 & -0.658763 \\
\hline & 5.505360 & 0.805460 & 0.672377 \\
\hline & 4.161188 & 0.641609 & 1.001990 \\
\hline & 5.444404 & -0.045794 & -3.080325 \\
\hline & 6.548931 & 1.255223 & 1.694585 \\
\hline & -7.022021 & -0.567794 & 0.613845 \\
\hline & 1.618374 & 0.586176 & 3.392472 \\
\hline & -8.304827 & 3.345636 & -2.163108 \\
\hline & 0.936401 & 4.993625 & 4.964025 \\
\hline & -5.243732 & -5.557831 & 0.487491 \\
\hline
\end{tabular}




\begin{tabular}{|c|c|c|c|}
\hline ] & 3.285539 & 3.399536 & 0.264604 \\
\hline & 5.177981 & 4.100569 & -1.140168 \\
\hline & -3.407466 & -4.268800 & 1.408242 \\
\hline 1 & 1.406366 & 2.599377 & 4.763846 \\
\hline 1 & -6.131026 & 3.311950 & -3.260735 \\
\hline & -7.988343 & -3.748704 & -2.280034 \\
\hline & -3.827392 & 2.372799 & -3.433629 \\
\hline & 2.618253 & 4.230352 & -4.593693 \\
\hline & 0.247482 & 3.669169 & -4.382042 \\
\hline & 0.058349 & 7.306623 & 1.448839 \\
\hline & -6.834272 & -1.601600 & -2.057660 \\
\hline & 0.306313 & 5.312061 & 0.044562 \\
\hline & -1.637183 & 2.830398 & -3.002536 \\
\hline & -7.224477 & -5.740250 & -0.990168 \\
\hline & -9.212813 & 0.490837 & 0.918113 \\
\hline 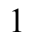 & 4.854171 & 4.532498 & -3.571015 \\
\hline ] & 0.372768 & 7.166428 & 3.915280 \\
\hline & -9.885323 & 2.438662 & -0.484046 \\
\hline 1 & -2.135253 & -2.121138 & 1.552587 \\
\hline & 3.827964 & 0.806079 & 2.024287 \\
\hline ] & 2.880093 & -0.227361 & -2.008051 \\
\hline ] & 6.929876 & 0.709791 & -0.931011 \\
\hline - & 3.398259 & -2.053357 & 1.141414 \\
\hline$\frac{1}{1}$ & -0.433025 & -0.718580 & 2.512325 \\
\hline & 1.414171 & -4.142449 & 4.318513 \\
\hline 1 & -2.091273 & 4.009480 & 0.164608 \\
\hline & -3.395097 & 3.195640 & -0.702307 \\
\hline ] & -5.235728 & 2.692238 & 0.628018 \\
\hline ] & -1.325650 & 2.489679 & 2.374055 \\
\hline & -6.238398 & 2.003085 & 2.779450 \\
\hline 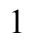 & -2.316749 & 1.835218 & 4.542296 \\
\hline & -4.783971 & 1.553360 & 4.748101 \\
\hline 1 & 1.280409 & -1.829103 & -1.334467 \\
\hline[ & -1.900894 & -2.600728 & -1.490245 \\
\hline 1 & -1.226265 & -2.299394 & -3.164131 \\
\hline 1 & 0.068647 & -4.951325 & -0.601609 \\
\hline ] & 1.053465 & -3.729000 & 0.217383 \\
\hline 1 & -0.705087 & -3.573909 & 0.220892 \\
\hline 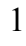 & 2.696151 & -3.961243 & -1.199062 \\
\hline 1 & 4.409682 & -4.703716 & -2.799400 \\
\hline ] & 3.929559 & -4.695614 & -5.244314 \\
\hline 1 & 1.686466 & -3.976374 & -6.041827 \\
\hline 1 & -0.038762 & -3.280498 & -4.444122 \\
\hline 6 & 3.543426 & -5.298499 & 3.807836 \\
\hline 6 & 3.927545 & -4.835200 & 1.404858 \\
\hline 6 & 4.982040 & -3.388313 & 3.149025 \\
\hline 6 & -1.721356 & -1.367917 & 4.522380 \\
\hline 6 & -1.572131 & -3.840999 & 4.254139 \\
\hline 6 & -0.205145 & -2.659603 & 5.979637 \\
\hline 1 & -2.483414 & -1.471870 & 5.305497 \\
\hline 1 & -1.181510 & -0.427729 & 4.703770 \\
\hline 1 & -2.254873 & -1.269117 & 3.568352 \\
\hline 1 & -1.022458 & -2.732279 & 6.709311 \\
\hline 1 & 0.436628 & -3.537003 & 6.120565 \\
\hline 1 & 0.386862 & -1.767946 & 6.224073 \\
\hline 1 & -2.403826 & -3.954490 & 4.962572 \\
\hline
\end{tabular}




$\begin{array}{rrrr}1 & -1.994553 & -3.807394 & 3.240686 \\ 1 & -0.945752 & -4.739064 & 4.324404 \\ 1 & 4.422694 & -5.954933 & 3.800874 \\ 1 & 3.438439 & -4.903292 & 4.826382 \\ 1 & 2.666061 & -5.920318 & 3.587340 \\ 1 & 5.859694 & -4.048148 & 3.180548 \\ 1 & 5.188918 & -2.591321 & 2.423055 \\ 1 & 4.873139 & -2.921126 & 4.136694 \\ 1 & 4.772642 & -5.536034 & 1.435950 \\ 1 & 3.035481 & -5.397910 & 1.097230 \\ 1 & 4.148486 & -4.091879 & 0.628202 \\ 6 & 4.272304 & -0.235064 & -4.043073 \\ 6 & 6.275900 & 1.139516 & -3.579978 \\ 6 & 6.300757 & -1.316397 & -3.100314 \\ 6 & 5.972418 & 1.345309 & 3.106266 \\ 6 & 7.721148 & 0.269055 & 1.722419 \\ 6 & 7.064978 & 2.644500 & 1.301983 \\ 1 & 4.653811 & -0.371716 & -5.063156 \\ 1 & 3.669158 & -1.121974 & -3.801668 \\ 1 & 3.609703 & 0.642184 & -4.048362 \\ 1 & 6.646670 & -1.531407 & -4.120710 \\ 1 & 7.185563 & -1.222237 & -2.457696 \\ 1 & 5.720196 & -2.180556 & -2.750449 \\ 1 & 6.603340 & 0.962872 & -4.613256 \\ 1 & 5.681355 & 2.062782 & -3.564563 \\ 1 & 7.176394 & 1.306787 & -2.976572 \\ 1 & 6.757487 & 1.658951 & 3.805861 \\ 1 & 5.159776 & 2.081038 & 3.175034 \\ 1 & 5.586631 & 0.377285 & 3.453545 \\ 1 & 7.833486 & 2.983252 & 2.010178 \\ 1 & 7.509726 & 2.649147 & 0.298932 \\ 1 & 6.250297 & 3.381281 & 1.314244 \\ 1 & 8.466849 & 0.587537 & 2.463096 \\ 1 & 7.384250 & -0.739116 & 1.996481 \\ 1 & 8.231015 & 0.199367 & 0.754275\end{array}$

TS1 $_{\text {IIL-re- }} \psi_{-1 \mathrm{a} 2 \mathrm{~s} 3 \mathrm{a} 4 \mathrm{~s}}$

Number of imaginary frequencies : 1

The smallest frequencies are : -604.6929 $16.0185 \quad 19.6995 \mathrm{~cm}(-1)$

Electronic energy : $\quad H F=-4360.8711855$

Zero-point correction $=\quad 1.451660($ Hartree/Particle $)$

Thermal correction to Energy $=\quad 1.536461$

Thermal correction to Enthalpy= $\quad 1.537405$

Thermal correction to Gibbs Free Energy $=\quad 1.334008$

Sum of electronic and zero-point Energies $=\quad-4359.419526$

Sum of electronic and thermal Energies= $\quad-4359.334724$

Sum of electronic and thermal Enthalpies $=\quad-4359.333780$

Sum of electronic and thermal Free Energies $=\quad-4359.537178$

Cartesian Coordinates

$\begin{array}{cccc}6 & -7.331508 & 0.079782 & 0.013656 \\ 6 & -6.433907 & 0.557883 & -0.975054 \\ 6 & -6.844428 & 1.670654 & -1.768976 \\ 6 & -8.134399 & 2.223681 & -1.582665\end{array}$




\begin{tabular}{|c|c|c|c|}
\hline 6 & -8.984481 & 1.726250 & -0.628196 \\
\hline 6 & -8.568936 & 0.651027 & 0.184741 \\
\hline p & -5.121750 & 0.008555 & -1.148930 \\
\hline & -4.243345 & 0.669627 & -1.990750 \\
\hline f & -4.660651 & 1.749008 & -2.801049 \\
\hline & -5.938358 & 2.226667 & -2.705136 \\
\hline 6 & -4.665099 & -1.202818 & -0.431003 \\
\hline 5 & -3.506076 & -1.142394 & 0.320167 \\
\hline 6 & -3.012483 & -2.249141 & 1.039495 \\
\hline 6 & -3.678749 & -3.443677 & 0.987507 \\
\hline 6 & -4.839064 & -3.587871 & 0.189333 \\
\hline 6 & -5.333385 & -2.465772 & -0.543404 \\
\hline 6 & -5.500915 & -4.835656 & 0.086280 \\
\hline 6 & -6.594147 & -4.990216 & -0.726478 \\
\hline 6 & -7.063112 & -3.894695 & -1.481126 \\
\hline 6 & -6.451385 & -2.668828 & -1.392562 \\
\hline 8 & -2.806769 & 0.034690 & 0.395521 \\
\hline 15 & -1.804014 & 0.415218 & -0.877208 \\
\hline 8 & -2.940416 & 0.263006 & -2.118982 \\
\hline 45 & 0.099648 & -0.716675 & -1.344982 \\
\hline 6 & -0.931358 & -2.532200 & -2.078755 \\
\hline 6 & 0.257045 & -3.131487 & -1.596795 \\
\hline 6 & 0.208070 & -3.831917 & -0.257967 \\
\hline 6 & 1.330294 & -3.591758 & -2.541753 \\
\hline 6 & 1.260802 & -3.372984 & -3.922833 \\
\hline 6 & 2.299886 & -3.760038 & -4.763065 \\
\hline 6 & 3.435861 & -4.376575 & -4.247292 \\
\hline 6 & 3.515016 & -4.610391 & -2.877438 \\
\hline 6 & 2.476697 & -4.221441 & -2.039111 \\
\hline 6 & 0.550307 & -0.115027 & -3.193184 \\
\hline 8 & 0.462518 & 0.163236 & -4.314315 \\
\hline 15 & 1.429663 & 0.150001 & 0.417187 \\
\hline 6 & 3.204053 & 0.401279 & -0.021863 \\
\hline 6 & 4.109964 & 0.904953 & 0.907395 \\
\hline 6 & 5.470103 & 1.016340 & 0.605775 \\
\hline 6 & 5.893177 & 0.597746 & -0.658334 \\
\hline 6 & 5.006635 & 0.094754 & -1.618960 \\
\hline 6 & 3.657293 & 0.008608 & -1.279104 \\
\hline 6 & 6.420804 & 1.564814 & 1.669602 \\
\hline 6 & 5.514192 & -0.404515 & -2.970330 \\
\hline 6 & 1.126930 & 1.681004 & 1.426295 \\
\hline 6 & 0.784911 & 2.869643 & 0.798053 \\
\hline 6 & 0.618453 & 4.068260 & 1.567698 \\
\hline 6 & 0.814660 & 4.032951 & 2.978936 \\
\hline 6 & 1.177117 & 2.808991 & 3.585346 \\
\hline 6 & 1.324436 & 1.673776 & 2.834532 \\
\hline 6 & 0.634377 & 5.211717 & 3.741326 \\
\hline 6 & 0.270333 & 6.388650 & 3.139287 \\
\hline 6 & 0.073087 & 6.431514 & 1.743027 \\
\hline 6 & 0.244648 & 5.304718 & 0.977506 \\
\hline 6 & 0.604623 & 2.988349 & -0.675619 \\
\hline 6 & -0.611667 & 2.697451 & -1.266475 \\
\hline 6 & -0.828145 & 2.971190 & -2.636928 \\
\hline 6 & 0.174384 & 3.474795 & -3.420256 \\
\hline 6 & 1.455909 & 3.715532 & -2.873621 \\
\hline 6 & 1.672261 & 3.491729 & -1.481142 \\
\hline
\end{tabular}




\begin{tabular}{|c|c|c|c|}
\hline 6 & 2.961634 & 3.752478 & -0.949352 \\
\hline 6 & 3.981694 & 4.189162 & -1.757243 \\
\hline 6 & 3.764809 & 4.404890 & -3.135514 \\
\hline 6 & 2.526609 & 4.175964 & -3.678110 \\
\hline 7 & -1.659710 & 2.068838 & -0.511315 \\
\hline 6 & -2.728186 & 2.966355 & -0.016387 \\
\hline 6 & -3.310659 & 2.582160 & 1.317877 \\
\hline 6 & -4.685753 & 2.418505 & 1.468291 \\
\hline 6 & -5.225524 & 2.046304 & 2.697006 \\
\hline 6 & -4.392682 & 1.845803 & 3.792749 \\
\hline 6 & -3.017309 & 2.026493 & 3.653109 \\
\hline 6 & -2.482437 & 2.388779 & 2.424242 \\
\hline 6 & 1.562888 & -1.152081 & 1.712736 \\
\hline 6 & 0.490587 & -1.347408 & 2.594179 \\
\hline 6 & 0.534334 & -2.340217 & 3.567424 \\
\hline 6 & 1.653415 & -3.190660 & 3.591369 \\
\hline 6 & 2.721927 & -3.043011 & 2.711579 \\
\hline 6 & 2.662477 & -1.998999 & 1.776739 \\
\hline 6 & -0.583404 & -2.526656 & 4.593483 \\
\hline 6 & 3.958820 & -3.939663 & 2.736812 \\
\hline 1 & -7.018911 & -0.737317 & 0.659438 \\
\hline 1 & 1.608561 & 0.748783 & 3.329732 \\
\hline 1 & -8.429831 & 3.066394 & -2.206485 \\
\hline 1 & 0.788075 & 5.160345 & \\
\hline 1 & -5.111527 & -5.674758 & 0.661513 \\
\hline 1 & 3.141289 & 3.579025 & 0.110604 \\
\hline 1 & 4.968796 & 4.365888 & -1.334816 \\
\hline 1 & -3.308533 & -4.308817 & 1.537464 \\
\hline 1 & 1.339538 & 2.782214 & 4.662232 \\
\hline 1 & -6.267853 & 3.056321 & -3.328497 \\
\hline 1 & -7.914491 & -4.023159 & \\
\hline 1 & -3.942892 & 2.175686 & -3.498168 \\
\hline 1 & 2.343860 & 4.338227 & -4.739493 \\
\hline 1 & 0.009323 & 3.664393 & -4.479727 \\
\hline 1 & -0.216288 & 7.366448 & 1.268608 \\
\hline 1 & -6.819651 & -1.839454 & -1.990598 \\
\hline 1 & 0.091371 & 5.355446 & -0.098459 \\
\hline 1 & -1.804414 & 2.741381 & -3.059878 \\
\hline 1 & -7.091273 & -5.954476 & -0.802211 \\
\hline 1 & -9.233057 & 0.272819 & 0.958865 \\
\hline 1 & 4.582751 & 4.751363 & -3.763349 \\
\hline 1 & 0.131152 & 7.288943 & 3.733290 \\
\hline 1 & -9.969475 & 2.164938 & -0.486872 \\
\hline 1 & -2.094958 & -2.121989 & 1.612762 \\
\hline 1 & 3.753521 & 1.206599 & 1.891985 \\
\hline 1 & 2.926486 & -0.368631 & -1.989272 \\
\hline 1 & 6.949727 & 0.664002 & -0.909417 \\
\hline 1 & 3.491069 & -1.849786 & 1.084407 \\
\hline 1 & -0.368658 & -0.678501 & 2.527137 \\
\hline 1 & 1.683765 & -3.983819 & 4.334910 \\
\hline 1 & -2.255682 & 3.955442 & 0.071342 \\
\hline 1 & -3.534913 & 3.069454 & -0.759374 \\
\hline 1 & -5.342372 & 2.557236 & 0.607854 \\
\hline 1 & -1.406544 & 2.498242 & 2.306338 \\
\hline 1 & -6.300789 & 1.905865 & 2.790581 \\
\hline 1 & -2.354419 & 1.884634 & 4.505767 \\
\hline
\end{tabular}




\begin{tabular}{|c|c|c|c|}
\hline 1 & -4.811575 & 1.551697 & 4.752980 \\
\hline 1 & 1.309912 & -1.804176 & -1.310357 \\
\hline 1 & -1.832017 & -2.662999 & -1.477843 \\
\hline 1 & -1.121190 & -2.438466 & -3.146179 \\
\hline 1 & 0.162432 & -4.920665 & -0.409358 \\
\hline 1 & 1.078389 & -3.621711 & 0.374766 \\
\hline 1 & -0.682644 & -3.525077 & 0.299314 \\
\hline 1 & 2.567051 & -4.399476 & -0.970021 \\
\hline 1 & 4.392740 & -5.094814 & -2.451796 \\
\hline 1 & 4.250496 & -4.671039 & -4.905403 \\
\hline 1 & 2.218543 & -3.572542 & -5.831773 \\
\hline 1 & 0.387321 & -2.893806 & -4.357947 \\
\hline 6 & -1.575672 & -1.363930 & 4.578757 \\
\hline 6 & -1.334241 & -3.827287 & 4.290328 \\
\hline 6 & 0.009766 & -2.605632 & 6.005060 \\
\hline 6 & 3.881745 & -5.009055 & 3.822924 \\
\hline 6 & 4.110729 & -4.642205 & 1.382491 \\
\hline 6 & 5.200579 & -3.078134 & 2.995810 \\
\hline 6 & 4.372063 & -0.601785 & -3.968211 \\
\hline 6 & 6.508975 & 0.587047 & -3.579749 \\
\hline 6 & 6.206975 & -1.755000 & -2.751781 \\
\hline 6 & 7.846625 & 1.726887 & 1.149140 \\
\hline 6 & 5.932270 & 2.938453 & 2.145048 \\
\hline 6 & 6.451266 & 0.599042 & 2.860218 \\
\hline 1 & -2.319178 & -1.500971 & 5.374319 \\
\hline 1 & -1.069063 & -0.404655 & \\
\hline 1 & -2.128848 & -1.283036 & 3.634453 \\
\hline 1 & -2.148415 & -3.979949 & \\
\hline 1 & -1.774981 & -3.802761 & 3.284306 \\
\hline 1 & -0.670858 & -4.699716 & 4.341716 \\
\hline 1 & -0.794471 & -2.712594 & 6.744920 \\
\hline 1 & 0.684192 & -3.460308 & 6.131928 \\
\hline 1 & 0.572402 & -1.694769 & 6.247828 \\
\hline 1 & 4.792017 & -5.621443 & 3.802994 \\
\hline 1 & 3.803398 & -4.570228 & 4.825952 \\
\hline 1 & 3.028815 & -5.683914 & 3.674552 \\
\hline 1 & 6.102506 & -3.704864 & 3.016064 \\
\hline 1 & 5.341152 & -2.317839 & 2.216462 \\
\hline 1 & 5.125954 & -2.559612 & 3.960914 \\
\hline 1 & 4.987666 & -5.303689 & 1.392732 \\
\hline 1 & 3.228111 & -5.257631 & 1.157626 \\
\hline 1 & 4.248479 & -3.925476 & 0.561800 \\
\hline 1 & 4.780830 & -0.906420 & -4.940732 \\
\hline 1 & 3.673583 & -1.390173 & -3.656254 \\
\hline 1 & 3.798639 & 0.324149 & -4.116129 \\
\hline 1 & 6.839375 & 0.230559 & -4.564325 \\
\hline 1 & 6.048418 & 1.574288 & -3.714959 \\
\hline 1 & 7.408057 & 0.714985 & -2.965170 \\
\hline 1 & 6.554476 & -2.170914 & -3.708185 \\
\hline 1 & 7.075427 & -1.661030 & -2.086784 \\
\hline 1 & 5.511890 & -2.477898 & -2.302232 \\
\hline 1 & 7.101191 & 0.990205 & 3.654961 \\
\hline 1 & 5.452736 & 0.443954 & 3.288960 \\
\hline 1 & 6.841709 & -0.382313 & 2.558743 \\
\hline 1 & 6.603405 & 3.331180 & 2.920759 \\
\hline 1 & 5.920593 & 3.660181 & 1.317676 \\
\hline
\end{tabular}




$\begin{array}{llll}1 & 4.922091 & 2.902180 & 2.572862 \\ 1 & 8.483749 & 2.142512 & 1.940121 \\ 1 & 8.286440 & 0.767972 & 0.846703 \\ 1 & 7.892912 & 2.411924 & 0.292397\end{array}$

TS1 $1_{\text {ILL-re- }} \psi_{-1 \mathrm{a} 25354 a}$

Number of imaginary frequencies : 1

The smallest frequencies are : -609.8066 $10.0989 \quad 16.2934 \mathrm{~cm}(-1)$

Electronic energy : $\quad H F=-4360.8712151$

Zero-point correction $=\quad 1.450877$ (Hartree/Particle)

Thermal correction to Energy= $\quad 1.536143$

Thermal correction to Enthalpy= $\quad 1.537087$

Thermal correction to Gibbs Free Energy $=\quad 1.330165$

Sum of electronic and zero-point Energies $=\quad-4359.420338$

Sum of electronic and thermal Energies $=\quad-4359.335072$

Sum of electronic and thermal Enthalpies $=\quad-4359.334128$

Sum of electronic and thermal Free Energies $=\quad-4359.541050$

Cartesian Coordinates

$\begin{array}{cccc}6 & -3.653761 & -0.123332 & -1.291507 \\ 6 & -3.241332 & -0.304396 & 0.017921 \\ 6 & -4.181607 & -0.658528 & 0.995200 \\ 6 & -5.521780 & -0.830893 & 0.667102 \\ 6 & -5.903491 & -0.639647 & -0.673254 \\ 6 & -4.995033 & -0.282964 & -1.664173 \\ 15 & -1.463968 & -0.051567 & 0.443411 \\ 45 & -0.116850 & 0.669974 & -1.376347 \\ 6 & -0.182466 & 3.071207 & -1.749562 \\ 6 & -0.094903 & 3.825674 & -0.443517 \\ 6 & -6.564847 & -1.255920 & 1.700053 \\ 6 & -5.367849 & -0.091629 & -3.130947 \\ 6 & -4.905374 & 1.292156 & -3.604517 \\ 6 & -1.548464 & 1.326415 & 1.658363 \\ 6 & -2.611760 & 2.219782 & 1.663308 \\ 6 & -2.634620 & 3.316364 & 2.537486 \\ 6 & -1.564224 & 3.473567 & 3.413247 \\ 6 & -0.478408 & 2.581461 & 3.444211 \\ 6 & -0.472315 & 1.530695 & 2.533070 \\ 6 & 0.645230 & 2.787058 & 4.459879 \\ 6 & 1.442464 & 4.039373 & 4.080884 \\ 6 & -3.836023 & 4.259094 & 2.505777 \\ 6 & -5.109525 & 3.461294 & 2.811681 \\ 6 & -1.187688 & -1.526683 & 1.541211 \\ 6 & -0.858592 & -2.755568 & 0.988438 \\ 6 & -0.703899 & -3.905823 & 1.832466 \\ 6 & -0.904201 & -3.781984 & 3.238025 \\ 6 & -1.260620 & -2.520334 & 3.765629 \\ 6 & -1.393826 & -1.432679 & 2.945672 \\ 6 & -0.735241 & -4.911990 & 4.073151 \\ 6 & -0.376210 & -6.126538 & 3.547501 \\ 6 & -0.172783 & -6.256921 & 2.157730 \\ 6 & -0.334838 & -5.179101 & 1.322734 \\ 6 & -0.681624 & -2.981550 & -0.473340 \\ 6 & 0.540376 & -2.763606 & -1.083184\end{array}$




\begin{tabular}{|c|c|c|c|}
\hline & 0.759279 & -3.169218 & -2.419922 \\
\hline & -0.238768 & -3.756443 & -3.149154 \\
\hline & -1.521174 & -3.945243 & -2.583902 \\
\hline & -1.749087 & -3.556794 & -1.230082 \\
\hline & -3.043140 & -3.752688 & -0.681877 \\
\hline & -4.050541 & -4.306278 & -1.432041 \\
\hline & -3.816322 & -4.705118 & -2.766149 \\
\hline & -2.578578 & -4.523632 & -3.328053 \\
\hline & 1.599811 & -2.097699 & -0.378181 \\
\hline & 1.764381 & -0.472139 & -0.844500 \\
\hline & 2.781081 & -0.036567 & 0.398923 \\
\hline & 3.518326 & 1.110376 & 0.258494 \\
\hline & 4.671075 & 1.097210 & -0.504681 \\
\hline & 5.377044 & 2.331751 & -0.683747 \\
\hline & 4.926721 & 3.502506 & -0.000422 \\
\hline & 3.773416 & 3.432859 & 0.817526 \\
\hline & 3.071160 & 2.263973 & 0.933009 \\
\hline & 6.491803 & 2.458513 & -1.551825 \\
\hline & 7.140855 & 3.658624 & -1.704402 \\
\hline & 6.714530 & 4.803214 & -0.998901 \\
\hline & 5.625839 & 4.722345 & -0.169546 \\
\hline & 5.086497 & -0.161587 & -1.163824 \\
\hline & 4.184256 & -0.839602 & -1.965668 \\
\hline & 4.566101 & -1.966333 & -2.727505 \\
\hline & 5.831193 & -2.473376 & -2.617132 \\
\hline & 6.758036 & -1.899330 & -1.712689 \\
\hline 6 & 6.384107 & -0.738806 & -0.970780 \\
\hline & 8.032903 & -2.479663 & -1.506350 \\
\hline 6 & 8.901924 & -1.962989 & -0.579601 \\
\hline 5 & 8.521389 & -0.839853 & 0.184153 \\
\hline & 7.300229 & -0.241262 & -0.009006 \\
\hline 3 & 2.890230 & -0.407626 & -2.101639 \\
\hline o & 2.661741 & -2.975042 & 0.166679 \\
\hline 6 & 3.247616 & -2.525526 & 1.478816 \\
\hline 6 & 4.625769 & -2.383889 & 1.624216 \\
\hline 6 & 5.171119 & -1.955799 & 2.832117 \\
\hline 6 & 4.340639 & -1.675805 & 3.912151 \\
\hline 5 & 2.961891 & -1.833640 & 3.778221 \\
\hline 5 & 2.421634 & -2.252040 & 2.569733 \\
\hline 6 & 0.055655 & 2.971835 & 5.863035 \\
\hline 6 & 1.593537 & 1.589406 & 4.516610 \\
\hline 6 & -3.721299 & 5.385548 & 3.529345 \\
\hline 6 & -3.953593 & 4.889715 & 1.113232 \\
\hline 6 & -0.578016 & -0.065112 & -3.166462 \\
\hline & -0.494863 & -0.471009 & -4.249213 \\
\hline 6 & 0.978385 & 2.399063 & -2.204633 \\
\hline 6 & -1.222969 & 3.549841 & -2.720019 \\
\hline 6 & -1.233434 & 3.147190 & -4.062346 \\
\hline 6 & -2.227137 & 3.579537 & -4.932578 \\
\hline 6 & -3.240170 & 4.424543 & -4.486789 \\
\hline 6 & -3.244088 & 4.832214 & -3.157366 \\
\hline 6 & -2.248887 & 4.399298 & -2.287535 \\
\hline 6 & -4.662413 & -1.171511 & -3.959812 \\
\hline 6 & -6.871146 & -0.204238 & -3.367466 \\
\hline 1 & 7.013849 & 0.612472 & 0.600474 \\
\hline 1 & -1.670613 & -0.476797 & 3.382729 \\
\hline
\end{tabular}




\begin{tabular}{|c|c|c|c|}
\hline & 8.300789 & -3.358789 & -2.091143 \\
\hline & -0.893123 & -4.792097 & 5.144149 \\
\hline & 5.269386 & 5.599679 & 0.368756 \\
\hline & -3.236465 & -3.440395 & 0.343395 \\
\hline & -5.039919 & -4.434962 & -0.997281 \\
\hline & 3.438898 & 4.333413 & 1.332326 \\
\hline & -1.429235 & -2.425687 & 4.837742 \\
\hline & 6.134119 & -3.339727 & -3.202878 \\
\hline & 7.988835 & 3.728150 & -2.382096 \\
\hline & 3.831778 & -2.403957 & -3.400164 \\
\hline & -2.385948 & -4.817406 & -4.358978 \\
\hline & -0.067457 & -4.061609 & -4.180261 \\
\hline & 0.113669 & -7.220903 & 1.743547 \\
\hline & 6.826933 & 1.590197 & -2.112970 \\
\hline & -0.176811 & -5.299459 & 0.253020 \\
\hline & 1.738606 & -2.986061 & -2.858565 \\
\hline & 7.240738 & 5.746500 & -1.125347 \\
\hline & 9.199745 & -0.445883 & 0.937796 \\
\hline & -4.622063 & -5.148404 & -3.347349 \\
\hline & -0.245653 & -6.988856 & 4.197166 \\
\hline & 9.874669 & -2.422875 & -0.421755 \\
\hline & 2.158970 & 2.192651 & 1.524261 \\
\hline ] & -3.850393 & -0.792509 & 2.022450 \\
\hline & -2.905578 & 0.140907 & -2.036210 \\
\hline ] & -6.949469 & -0.781265 & -0.938967 \\
\hline & -3.440281 & 2.063115 & 0.972652 \\
\hline & 0.359395 & 0.825279 & 2.511070 \\
\hline & -1.567094 & 4.308825 & 4.109790 \\
\hline & 2.181421 & -3.954138 & 0.307013 \\
\hline l & 3.469018 & -3.125148 & -0.567284 \\
\hline & 5.280896 & -2.586321 & 0.775255 \\
\hline l & 1.343852 & -2.343161 & 2.454023 \\
\hline ] & 6.249053 & -1.834262 & 2.921282 \\
\hline ] & 2.300475 & -1.628504 & 4.619161 \\
\hline 1 & 4.763907 & -1.337604 & 4.855814 \\
\hline 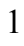 & -1.294771 & 1.799534 & -1.410951 \\
\hline l & 1.885511 & 2.523527 & -1.611957 \\
\hline & 1.161723 & 2.260196 & -3.268590 \\
\hline 1 & 0.043307 & 4.899003 & -0.642150 \\
\hline ] & -0.989696 & 3.715845 & 0.180704 \\
\hline l & 0.760375 & 3.473784 & 0.141242 \\
\hline 1 & -2.279160 & 4.727334 & -1.251495 \\
\hline ] & -4.026883 & 5.491891 & -2.786770 \\
\hline 1 & -4.018324 & 4.758770 & -5.169435 \\
\hline & -2.208704 & 3.249302 & -5.969200 \\
\hline 1 & -0.459152 & 2.483847 & -4.439976 \\
\hline 1 & 2.343074 & 1.746427 & 5.302788 \\
\hline 1 & 1.050584 & 0.663096 & 4.751497 \\
\hline 1 & 2.141924 & 1.428659 & 3.579679 \\
\hline 1 & 0.863304 & 3.092555 & 6.597025 \\
\hline 1 & -0.586075 & 3.857293 & 5.937110 \\
\hline 1 & -0.540738 & 2.098770 & 6.158350 \\
\hline 1 & 2.265209 & 4.202775 & 4.790076 \\
\hline 1 & 1.876492 & 3.941336 & 3.076408 \\
\hline 1 & 0.811711 & 4.937210 & 4.084292 \\
\hline 1 & -7.090948 & -0.046525 & -4.430945 \\
\hline
\end{tabular}




$\begin{array}{rrrr}1 & -7.256284 & -1.196580 & -3.098659 \\ 1 & -7.432805 & 0.548700 & -2.798716 \\ 1 & -4.906744 & -1.057371 & -5.024922 \\ 1 & -3.570691 & -1.113907 & -3.858848 \\ 1 & -4.971866 & -2.176318 & -3.641828 \\ 1 & -5.188056 & 1.448034 & -4.654911 \\ 1 & -5.366902 & 2.090516 & -3.008117 \\ 1 & -3.815481 & 1.415780 & -3.540604 \\ 1 & -4.606701 & 6.030819 & 3.468189 \\ 1 & -3.664520 & 5.002929 & 4.556579 \\ 1 & -2.841935 & 6.016896 & 3.347467 \\ 1 & -5.988333 & 4.119700 & 2.786005 \\ 1 & -5.275969 & 2.655310 & 2.085583 \\ 1 & -5.056224 & 3.006220 & 3.809600 \\ 1 & -4.813268 & 5.572489 & 1.074947 \\ 1 & -3.052191 & 5.470796 & 0.871724 \\ 1 & -4.094207 & 4.135891 & 0.327940 \\ 6 & -5.998705 & -1.270175 & 3.118782 \\ 6 & -7.758273 & -0.295329 & 1.678751 \\ 6 & -7.046422 & -2.671706 & 1.362812 \\ 1 & -6.785219 & -1.560570 & 3.826748 \\ 1 & -5.176908 & -1.990096 & 3.230391 \\ 1 & -5.629546 & -0.280238 & 3.419681 \\ 1 & -7.810936 & -2.999884 & 2.080278 \\ 1 & -7.484123 & -2.726852 & 0.358217 \\ 1 & -6.213911 & -3.386920 & 1.406929 \\ 1 & -8.499498 & -0.596958 & 2.430848 \\ 1 & -7.444217 & 0.731044 & 1.909075 \\ 1 & -8.266952 & -0.279741 & 0.707828\end{array}$

TS1 $1_{\text {IIL-re- }} \psi_{-1 \mathrm{a} 2 \mathrm{~s} 3 \mathrm{~s} 4 \mathrm{~s}}$

Number of imaginary frequencies : 1

The smallest frequencies are : -631.3926 $14.3169 \quad 19.1636 \mathrm{~cm}(-1)$

Electronic energy : $\quad H F=-4360.8713979$

Zero-point correction $=\quad 1.451223($ Hartree/Particle $)$

Thermal correction to Energy $=\quad 1.536236$

Thermal correction to Enthalpy= $\quad 1.537180$

Thermal correction to Gibbs Free Energy $=\quad 1.332304$

Sum of electronic and zero-point Energies $=\quad-4359.420175$

Sum of electronic and thermal Energies $=\quad-4359.335162$

Sum of electronic and thermal Enthalpies $=\quad-4359.334218$

Sum of electronic and thermal Free Energies $=\quad-4359.539094$

Cartesian Coordinates

$\begin{array}{cccc}6 & -2.625156 & 2.196229 & 1.677196 \\ 6 & -1.558885 & 1.305735 & 1.670325 \\ 6 & -0.487227 & 1.506255 & 2.551380 \\ 6 & -0.500648 & 2.550117 & 3.470495 \\ 6 & -1.587011 & 3.441374 & 3.438719 \\ 6 & -2.652475 & 3.289286 & 2.555875 \\ 15 & -1.461582 & -0.056968 & 0.438005 \\ 6 & -1.185124 & -1.548394 & 1.514060 \\ 6 & -0.865821 & -2.771654 & 0.942791 \\ 6 & -0.719167 & -3.935363 & 1.769566\end{array}$




\begin{tabular}{|c|c|c|c|}
\hline 6 & -0.918551 & -3.831375 & 3.176807 \\
\hline 6 & -1.261843 & -2.574416 & 3.723749 \\
\hline 6 & -1.385984 & -1.473274 & 2.920260 \\
\hline 6 & -0.760366 & -4.975613 & 3.994545 \\
\hline & -0.412414 & -6.185135 & 3.450305 \\
\hline ] & -0.208918 & -6.295746 & 2.058767 \\
\hline 6 & -0.360591 & -5.203753 & 1.240496 \\
\hline D & -0.690860 & -2.976177 & -0.522399 \\
\hline 6 & 0.530152 & -2.747313 & -1.130062 \\
\hline 0 & 0.746485 & -3.127004 & -2.474848 \\
\hline 0 & -0.253496 & -3.698665 & -3.213726 \\
\hline 6 & -1.534441 & -3.899487 & -2.649334 \\
\hline 6 & -1.758775 & -3.539773 & -1.287020 \\
\hline 6 & -3.049064 & -3.753849 & -0.737273 \\
\hline 6 & -4.056987 & -4.295980 & -1.494898 \\
\hline 6 & -3.827996 & -4.661990 & -2.839240 \\
\hline 6 & -2.593225 & -4.463987 & -3.402105 \\
\hline 7 & 1.591174 & -2.096589 & -0.413558 \\
\hline 6 & 2.646225 & -2.986994 & 0.123365 \\
\hline 6 & 3.233470 & -2.553339 & 1.440190 \\
\hline 6 & 4.611782 & -2.415625 & 1.587055 \\
\hline 6 & 5.157485 & -1.996681 & 2.798022 \\
\hline 6 & 4.327080 & -1.721465 & 3.879314 \\
\hline 6 & 2.948097 & -1.876332 & 3.744061 \\
\hline 6 & 2.407662 & -2.286666 & 2.532979 \\
\hline 6 & 0.616984 & 2.751301 & 4.493674 \\
\hline 6 & 0.019182 & 2.925019 & 5.894753 \\
\hline 6 & -3.844070 & 4.244463 & 2.511384 \\
\hline 45 & -0.115312 & 0.690458 & -1.370516 \\
\hline 6 & -0.594866 & -0.016018 & -3.166218 \\
\hline 8 & -0.546627 & -0.410611 & -4.255043 \\
\hline 15 & 1.762035 & -0.464581 & -0.852552 \\
\hline 8 & 2.892012 & -0.383370 & -2.104877 \\
\hline 6 & 4.184463 & -0.820846 & -1.970877 \\
\hline 6 & 5.085935 & -0.156406 & -1.156940 \\
\hline 6 & 6.382211 & -0.738509 & -0.969456 \\
\hline 6 & 6.755800 & -1.889032 & -1.726869 \\
\hline 6 & 5.829700 & -2.448857 & -2.640942 \\
\hline 6 & 4.565824 & -1.937829 & -2.747126 \\
\hline 6 & 8.029351 & -2.474255 & -1.526199 \\
\hline 6 & 8.897487 & -1.971840 & -0.590795 \\
\hline 6 & 8.517287 & -0.858810 & 0.187765 \\
\hline 6 & 7.297337 & -0.255817 & 0.000789 \\
\hline 6 & 4.671020 & 1.092695 & -0.479271 \\
\hline 6 & 3.516489 & 1.095809 & 0.281493 \\
\hline 6 & 3.071328 & 2.238850 & 0.975216 \\
\hline 6 & 3.777195 & 3.407495 & 0.881455 \\
\hline 6 & 4.931941 & 3.488301 & 0.066603 \\
\hline 6 & 5.380309 & 2.328356 & -0.636098 \\
\hline 6 & 6.496829 & 2.467131 & -1.500072 \\
\hline 6 & 7.149245 & 3.668000 & -1.630901 \\
\hline 6 & 6.724751 & 4.801366 & -0.906399 \\
\hline 6 & 5.634514 & 4.709030 & -0.080267 \\
\hline 8 & 2.775947 & -0.051337 & 0.400663 \\
\hline 6 & 0.991519 & 2.426766 & -2.171822 \\
\hline 6 & -0.167745 & 3.098801 & -1.713186 \\
\hline
\end{tabular}




\begin{tabular}{|c|c|c|c|}
\hline 6 & -1.199516 & 3.595774 & -2.683388 \\
\hline 6 & -1.210992 & 3.200737 & -4.028059 \\
\hline & -2.196996 & 3.649514 & -4.898555 \\
\hline & -3.200984 & 4.504432 & -4.450951 \\
\hline & -3.202981 & 4.905867 & -3.119714 \\
\hline & -2.215436 & 4.456201 & -2.249496 \\
\hline & -0.083806 & 3.833653 & -0.395738 \\
\hline & -3.238238 & -0.310800 & 0.008602 \\
\hline & -4.165730 & -0.717383 & 0.969216 \\
\hline & -5.510573 & -0.894247 & 0.645352 \\
\hline & -5.908313 & -0.639084 & -0.674245 \\
\hline & -5.005487 & -0.226694 & -1.656322 \\
\hline & -3.665502 & -0.073923 & -1.291068 \\
\hline & -6.479125 & -1.358138 & 1.733606 \\
\hline & -6.539278 & -0.302399 & 2.843532 \\
\hline & -5.393633 & 0.013474 & -3.112446 \\
\hline & -4.727131 & -1.066018 & -3.973969 \\
\hline & -7.894434 & -1.572359 & 1.203112 \\
\hline & -5.989489 & -2.686830 & 2.322858 \\
\hline & -6.902609 & -0.055172 & -3.331053 \\
\hline & -4.903986 & 1.395401 & -3.563287 \\
\hline & 1.568080 & 1.555801 & 4.547491 \\
\hline & 1.413575 & 4.008018 & 4.127862 \\
\hline & 7.011031 & 0.589595 & 0.621791 \\
\hline & -1.653551 & -0.521535 & 3.371801 \\
\hline & 8.296887 & -3.345653 & -2.122609 \\
\hline & -0.917344 & -4.870892 & 5.067236 \\
\hline & 5.279486 & 5.577914 & 0.472488 \\
\hline & -3.238599 & -3.463606 & 0.295446 \\
\hline & -5.043636 & -4.438763 & -1.058462 \\
\hline & 3.444553 & 4.299700 & 1.411728 \\
\hline & -1.427348 & -2.494217 & 4.797525 \\
\hline & 6.132197 & -3.307668 & -3.237898 \\
\hline & 7.998492 & 3.746969 & -2.305959 \\
\hline & 3.832339 & -2.364925 & -3.427361 \\
\hline & -2.403926 & -4.734138 & -4.440082 \\
\hline ] & -0.084625 & -3.982656 & -4.251261 \\
\hline & 0.069317 & -7.255709 & 1.629893 \\
\hline & 6.830830 & 1.607716 & -2.075431 \\
\hline & -0.202538 & -5.308714 & 0.169194 \\
\hline & 1.724869 & -2.935600 & -2.911980 \\
\hline ] & 7.253633 & 5.745301 & -1.015681 \\
\hline & 9.194871 & -0.476334 & 0.947996 \\
\hline 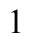 & -4.634905 & -5.094558 & -3.426829 \\
\hline & -0.290245 & -7.058624 & 4.086535 \\
\hline t & 9.869207 & -2.435427 & -0.437551 \\
\hline 1 & 2.158049 & 2.160185 & 1.563764 \\
\hline & -3.831593 & -0.898780 & 1.990524 \\
\hline | & -2.925276 & 0.226729 & -2.029714 \\
\hline & -6.952852 & -0.771175 & -0.942263 \\
\hline 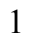 & -3.449565 & 2.043144 & 0.980766 \\
\hline & 0.347083 & 0.803794 & 2.527026 \\
\hline 1 & -1.593679 & 4.273006 & 4.139546 \\
\hline ] & 2.158815 & -3.964008 & 0.253521 \\
\hline & 3.452898 & -3.135054 & -0.611808 \\
\hline & 5.266874 & -2.612666 & 0.736832 \\
\hline
\end{tabular}




$\begin{array}{rrrr}1 & 1.329752 & -2.374887 & 2.416492 \\ 1 & 6.235619 & -1.877834 & 2.888444 \\ 1 & 2.286699 & -1.675384 & 4.586020 \\ 1 & 4.750632 & -1.389624 & 4.825095 \\ 1 & -1.285348 & 1.829670 & -1.390024 \\ 1 & 1.896315 & 2.537534 & -1.572831 \\ 1 & 1.178982 & 2.301310 & -3.236657 \\ 1 & 0.060841 & 4.909242 & -0.576871 \\ 1 & -0.982804 & 3.718114 & 0.221510 \\ 1 & 0.766518 & 3.468608 & 0.188084 \\ 1 & -2.243592 & 4.780127 & -1.212279 \\ 1 & -3.978195 & 5.573709 & -2.747808 \\ 1 & -3.973292 & 4.851473 & -5.133840 \\ 1 & -2.179759 & 3.324504 & -5.936836 \\ 1 & -0.443420 & 2.530732 & -4.407422 \\ 1 & 2.312850 & 1.709796 & 5.338751 \\ 1 & 1.026760 & 0.626309 & 4.773299 \\ 1 & 2.121899 & 1.403234 & 3.612425 \\ 1 & 0.822406 & 3.043545 & 6.633948 \\ 1 & -0.625979 & 3.807776 & 5.971050 \\ 1 & -0.575941 & 2.048111 & 6.181094 \\ 1 & 2.231806 & 4.168246 & 4.842978 \\ 1 & 1.853657 & 3.918146 & 3.125226 \\ 1 & 0.780960 & 4.904511 & 4.133809 \\ 1 & -7.132586 & 0.142879 & -4.385611 \\ 1 & -7.309513 & -1.045866 & -3.090272 \\ 1 & -7.437602 & 0.691710 & -2.729543 \\ 1 & -4.979608 & -0.922210 & -5.033650 \\ 1 & -3.633086 & -1.041402 & -3.882405 \\ 1 & -5.061524 & -2.068239 & -3.673575 \\ 1 & -5.205010 & 1.582914 & -4.603306 \\ 1 & -5.331456 & 2.191404 & -2.938902 \\ 1 & -3.810238 & 1.490396 & -3.521765 \\ 1 & -8.545495 & -1.915296 & 2.017270 \\ 1 & -8.329358 & -0.647036 & 0.804207 \\ 1 & -7.924441 & -2.334794 & 0.413713 \\ 1 & -6.670070 & -3.025109 & 3.115931 \\ 1 & -5.957889 & -3.469403 & 1.553087 \\ 1 & -4.985972 & -2.608171 & 2.760691 \\ 1 & -7.208583 & -0.632186 & 3.649852 \\ 1 & -2.755541 & 5.605986 & 1.081253 \\ 1 & -4.020763 & 5.492800 & 0.941299 \\ 1 & -5.552773 & -0.112435 & 3.285021 \\ 1 & -6.922827 & 0.651194 & 2.456879 \\ 6 & -3.753326 & 5.339802 & 3.570522 \\ 6 & -3.903613 & 4.914367 & 1.133459 \\ 6 & -5.138656 & 3.457307 & 2.745930 \\ 1 & -4.632248 & 5.993052 & 3.501426 \\ 1 & -3.729769 & 4.926358 & 4.587143 \\ 1 & -2.865363 & 5.970476 & 3.434358 \\ 1 & -6.004723 & 4.132222 & 2.717498 \\ 1 & -5.295584 & 2.684952 & 1.982225 \\ 1 & -5.126481 & 2.963073 & 3.726302 \\ 1 & -324701\end{array}$

TS1 $_{\text {IIL-re- }} \psi_{-1 \mathrm{~s} 2 \mathrm{a3a} a \mathrm{a}}$ 
Number of imaginary frequencies : 1

The smallest frequencies are : -615.5140 $16.7265 \quad 19.3783 \mathrm{~cm}(-1)$

Electronic energy : $\quad H F=-4360.8696418$

Zero-point correction $=\quad 1.451798($ Hartree/Particle $)$

Thermal correction to Energy $=\quad 1.536666$

Thermal correction to Enthalpy= $\quad 1.537611$

Thermal correction to Gibbs Free Energy= $\quad 1.333474$

Sum of electronic and zero-point Energies $=\quad-4359.417844$

Sum of electronic and thermal Energies $=\quad-4359.332975$

Sum of electronic and thermal Enthalpies $=\quad-4359.332031$

Sum of electronic and thermal Free Energies $=\quad-4359.536168$

Cartesian Coordinates

$\begin{array}{cccc}6 & -6.557956 & -2.670180 & -1.413873 \\ 6 & -5.440884 & -2.490249 & -0.558613 \\ 6 & -4.971212 & -3.622320 & 0.175567 \\ 6 & -5.655056 & -4.857436 & 0.064926 \\ 6 & -6.746298 & -4.990069 & -0.754553 \\ 6 & -7.191773 & -3.884363 & -1.508594 \\ 6 & -4.751468 & -1.239958 & -0.438321 \\ 6 & -3.601293 & -1.201611 & 0.325700 \\ 6 & -3.132241 & -2.316147 & 1.047359 \\ 6 & -3.816083 & -3.500045 & 0.985608 \\ 6 & -5.175215 & -0.016389 & -1.155580 \\ 6 & -4.275708 & 0.625593 & -1.989866 \\ 6 & -4.664440 & 1.711487 & -2.805568 \\ 6 & -5.930349 & 2.220882 & -2.713825 \\ 6 & -6.851643 & 1.690900 & -1.777314 \\ 6 & -6.472704 & 0.566364 & -0.984584 \\ 6 & -8.125391 & 2.279862 & -1.589120 \\ 6 & -8.988332 & 1.804738 & -0.634582 \\ 6 & -8.603818 & 0.715591 & 0.175141 \\ 6 & -7.383427 & 0.109795 & 0.001998 \\ 8 & -2.977694 & 0.196051 & -2.098190 \\ 15 & -1.864779 & 0.345108 & -0.833704 \\ 8 & -2.889094 & -0.033583 & 0.417655 \\ 45 & 0.046709 & -0.781493 & -1.261613 \\ 15 & 1.405410 & 0.137142 & 0.459538 \\ 6 & 1.701038 & -1.189467 & 1.705325 \\ 6 & 2.842527 & -1.990315 & 1.599796 \\ 6 & 3.059076 & -3.056434 & 2.467754 \\ 6 & 2.113270 & -3.285823 & 3.476011 \\ 6 & 0.963589 & -2.506554 & 3.613607 \\ 6 & 0.753265 & -1.477978 & 2.685264 \\ 6 & 4.289887 & -3.953727 & 2.349903 \\ 6 & -0.021918 & -2.695615 & 4.766934 \\ 6 & 0.263197 & -3.186276 & -1.478350 \\ 6 & 0.231696 & -3.872035 & -0.131246 \\ 6 & 1.350618 & -3.631231 & -2.415918 \\ 6 & 2.541941 & -4.163670 & -1.902664 \\ 6 & 3.588410 & -4.531860 & -2.739746 \\ 6 & 3.473668 & -4.373876 & -4.118304 \\ 6 & 2.295969 & -3.851921 & -4.643259 \\ 6 & 1.247963 & -3.486694 & -3.804002 \\ & & & \\ 6\end{array}$




\begin{tabular}{|c|c|c|c|}
\hline 6 & -0.943588 & -2.627873 & -1.965642 \\
\hline 6 & 0.404298 & -0.217792 & -3.149983 \\
\hline & 0.164986 & -0.000267 & -4.263217 \\
\hline & 1.073513 & 1.636323 & 1.507345 \\
\hline b & 0.686071 & 2.823560 & 0.900902 \\
\hline & 0.470127 & 3.998784 & 1.691056 \\
\hline & 0.657150 & 3.943252 & 3.102997 \\
\hline & 1.076916 & 2.726386 & 3.685933 \\
\hline ( & 1.279267 & 1.613797 & 2.913541 \\
\hline & 0.421425 & 5.098400 & 3.886049 \\
\hline & 0.016070 & 6.271399 & 3.302800 \\
\hline f & -0.166373 & 6.335257 & 1.905119 \\
\hline & 0.057503 & 5.231546 & 1.119909 \\
\hline y & 0.527445 & 2.954111 & -0.574314 \\
\hline & -0.666904 & 2.633339 & -1.194564 \\
\hline b & -0.842794 & 2.870970 & -2.577363 \\
\hline ) & 0.180509 & 3.359523 & -3.343047 \\
\hline ) & 1.438125 & 3.639687 & -2.761527 \\
\hline b & 1.608911 & 3.467315 & -1.355563 \\
\hline ) & 2.872862 & 3.777842 & -0.788964 \\
\hline$b$ & 3.912114 & 4.206868 & -1.575869 \\
\hline & 3.741148 & 4.367201 & -2.968317 \\
\hline ) & 2.527894 & 4.092229 & -3.545197 \\
\hline T & -1.726002 & 1.997645 & -0.460842 \\
\hline b & -2.803863 & 2.881309 & 0.033795 \\
\hline ) & -3.377902 & 2.505510 & 1.375030 \\
\hline 6 & -4.758188 & 2.465026 & 1.560112 \\
\hline 6 & -5.297829 & 2.136320 & 2.801749 \\
\hline 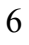 & -4.459491 & 1.846874 & 3.872819 \\
\hline f & -3.077321 & 1.886113 & 3.693750 \\
\hline 6 & -2.543509 & 2.212451 & 2.454784 \\
\hline 6 & 3.135060 & 0.501795 & -0.074081 \\
\hline ) & 3.521075 & 0.214371 & -1.377614 \\
\hline 6 & 4.841669 & 0.399867 & -1.795495 \\
\hline 6 & 5.755324 & 0.888730 & -0.860740 \\
\hline 6 & 5.399635 & 1.194923 & 0.459370 \\
\hline 6 & 4.073902 & 0.991898 & 0.836904 \\
\hline 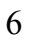 & 5.294059 & 0.033438 & -3.207108 \\
\hline 6 & 6.445499 & 1.756881 & 1.421482 \\
\hline ] & -7.092306 & -0.716166 & 0.646461 \\
\hline 1 & 1.616694 & 0.697218 & 3.390404 \\
\hline 1 & -8.397536 & 3.131762 & -2.211101 \\
\hline 1 & 0.567694 & 5.032583 & 4.963339 \\
\hline 1 & -5.285144 & -5.704961 & 0.640689 \\
\hline & 3.020126 & 3.650261 & 0.282403 \\
\hline 1 & 4.878762 & 4.423256 & -1.124571 \\
\hline 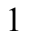 & -3.467823 & -4.371148 & 1.539755 \\
\hline 1 & 1.243445 & 2.686226 & 4.761938 \\
\hline 1 & -6.237567 & 3.057248 & -3.339577 \\
\hline 1 & -8.042495 & -3.995576 & -2.177222 \\
\hline 1 & -3.934831 & 2.119782 & -3.501230 \\
\hline 1 & 2.378625 & 4.215289 & -4.617068 \\
\hline 1 & 0.048917 & 3.511410 & -4.413284 \\
\hline & -0.485481 & 7.268122 & 1.445952 \\
\hline 1 & -6.907930 & -1.831920 & -2.010695 \\
\hline & -0.082718 & 5.296914 & 0.042873 \\
\hline
\end{tabular}




\begin{tabular}{|c|c|c|c|}
\hline & -1.800892 & 2.615853 & -3.025982 \\
\hline & -7.260520 & -5.944947 & -0.835164 \\
\hline & -9.278097 & 0.354955 & 0.948851 \\
\hline | & 4.573021 & 4.712407 & -3.578742 \\
\hline & -0.165512 & 7.153361 & 3.912654 \\
\hline & -9.960135 & 2.271378 & -0.491254 \\
\hline & -2.224479 & -2.202017 & 1.635234 \\
\hline ] & 3.758944 & 1.207194 & 1.855564 \\
\hline & 2.765599 & -0.159600 & -2.064023 \\
\hline 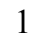 & 6.790395 & 1.038826 & -1.168948 \\
\hline 1 & 3.565452 & -1.772550 & 0.818454 \\
\hline 1 & -0.149863 & -0.864712 & 2.746187 \\
\hline 1 & 2.289313 & -4.099535 & 4.176210 \\
\hline 1 & -2.347928 & 3.879210 & 0.110580 \\
\hline 1 & -3.616740 & 2.967752 & -0.704472 \\
\hline 1 & -5.421883 & 2.673147 & 0.718685 \\
\hline 1 & -1.466500 & 2.205400 & 2.302234 \\
\hline 1 & -6.378515 & 2.098689 & 2.925397 \\
\hline 1 & -2.408298 & 1.661504 & 4.523627 \\
\hline 1 & -4.879643 & 1.586511 & 4.842134 \\
\hline ] & 1.286098 & -1.833712 & -1.221491 \\
\hline 1 & -1.839556 & -2.776801 & -1.361775 \\
\hline 1 & -1.138915 & -2.551166 & -3.033498 \\
\hline & 0.199967 & -4.962778 & -0.272137 \\
\hline 1 & 1.104310 & -3.645838 & 0.491697 \\
\hline 1 & -0.658849 & -3.569891 & 0.430141 \\
\hline 1 & 2.662970 & -4.274753 & -0.825663 \\
\hline 1 & 4.500539 & -4.941519 & -2.308576 \\
\hline 1 & 4.293222 & -4.655698 & -4.775580 \\
\hline 1 & 2.186694 & -3.724804 & -5.718289 \\
\hline 1 & 0.339922 & -3.083613 & -4.245380 \\
\hline 6 & 0.305800 & -3.919057 & 5.619924 \\
\hline 6 & 0.038753 & -1.454031 & 5.665617 \\
\hline 6 & -1.446821 & -2.857787 & 4.231641 \\
\hline 5 & 5.208844 & -3.527607 & 1.205167 \\
\hline 6 & 5.095438 & -3.893032 & 3.652155 \\
\hline 5 & 3.842160 & -5.396769 & 2.092793 \\
\hline 1 & -0.434435 & -4.014495 & 6.424130 \\
\hline 1 & 0.278138 & -4.846679 & 5.033469 \\
\hline 1 & 1.292250 & -3.840392 & 6.094251 \\
\hline 1 & -2.143163 & -3.058843 & 5.056477 \\
\hline 1 & -1.803058 & -1.952850 & 3.725162 \\
\hline 1 & -1.507653 & -3.697805 & 3.525326 \\
\hline 1 & -0.653804 & -1.558173 & 6.511941 \\
\hline 1 & 1.049731 & -1.311094 & 6.069945 \\
\hline 1 & -0.237280 & -0.542724 & 5.119294 \\
\hline 1 & 6.080443 & -4.193130 & 1.166388 \\
\hline 1 & 4.707438 & -3.582422 & 0.228805 \\
\hline 1 & 5.580436 & -2.501332 & 1.332238 \\
\hline 1 & 5.985564 & -4.532406 & 3.580713 \\
\hline 1 & 5.431049 & -2.867859 & 3.857112 \\
\hline 1 & 4.512511 & -4.233594 & 4.516327 \\
\hline 1 & 4.714542 & -6.058073 & 2.002972 \\
\hline 1 & 3.213624 & -5.784225 & 2.903726 \\
\hline 1 & 3.265632 & -5.472225 & 1.159888 \\
\hline 6 & 4.113898 & -0.299834 & -4.119442 \\
\hline
\end{tabular}




$\begin{array}{rrrr}6 & 6.062038 & 1.198882 & -3.837266 \\ 6 & 6.201969 & -1.198667 & -3.121683 \\ 6 & 5.894483 & 1.929743 & 2.835558 \\ 6 & 7.651662 & 0.814973 & 1.496049 \\ 6 & 6.902137 & 3.130723 & 0.917907 \\ 1 & 4.482870 & -0.529024 & -5.127678 \\ 1 & 3.549641 & -1.178051 & -3.774647 \\ 1 & 3.417028 & 0.545795 & -4.202185 \\ 1 & 6.532459 & -1.504826 & -4.123999 \\ 1 & 7.096569 & -1.005565 & -2.515847 \\ 1 & 5.664393 & -2.043553 & -2.669273 \\ 1 & 6.372689 & 0.940500 & -4.858478 \\ 1 & 5.429774 & 2.095415 & -3.890424 \\ 1 & 6.968209 & 1.457969 & -3.276397 \\ 1 & 6.679422 & 2.327154 & 3.491429 \\ 1 & 5.052668 & 2.634916 & 2.868234 \\ 1 & 5.558044 & 0.975109 & 3.261699 \\ 1 & 8.402058 & 1.213187 & 2.192131 \\ 1 & 7.353439 & -0.178925 & 1.854694 \\ 1 & 8.142055 & 0.686453 & 0.523898 \\ 1 & 7.676716 & 3.544443 & 1.578021 \\ 1 & 7.318321 & 3.079752 & -0.096069 \\ 1 & 6.061747 & 3.838514 & 0.903026\end{array}$

TS1 $1_{\text {ILL-re- }} \psi_{-1 \mathrm{~s} 2 \mathrm{aza} a \mathrm{~s}}$

Number of imaginary frequencies : 1

The smallest frequencies are : -613.7786 $16.352120 .2579 \mathrm{~cm}(-1)$

Electronic energy : $\quad \mathrm{HF}=-4360.8705464$

Zero-point correction $=\quad 1.451990($ Hartree/Particle $)$

Thermal correction to Energy= $\quad 1.536693$

Thermal correction to Enthalpy= $\quad 1.537637$

Thermal correction to Gibbs Free Energy= $\quad 1.334557$

Sum of electronic and zero-point Energies $=\quad-4359.418557$

Sum of electronic and thermal Energies $=\quad-4359.333853$

Sum of electronic and thermal Enthalpies $=\quad-4359.332909$

Sum of electronic and thermal Free Energies $=\quad-4359.535989$

Cartesian Coordinates

$\begin{array}{cccc}6 & 4.068011 & 1.008928 & 0.793947 \\ 6 & 3.125873 & 0.524357 & -0.107293 \\ 6 & 3.508027 & 0.238684 & -1.416218 \\ 6 & 4.825156 & 0.418525 & -1.834933 \\ 6 & 5.750761 & 0.902991 & -0.901500 \\ 6 & 5.397340 & 1.210192 & 0.413851 \\ 15 & 1.404616 & 0.150496 & 0.444590 \\ 6 & 1.073432 & 1.650350 & 1.492433 \\ 6 & 0.673730 & 2.833782 & 0.886830 \\ 6 & 0.457872 & 4.009928 & 1.675416 \\ 6 & 0.659102 & 3.959822 & 3.085505 \\ 6 & 1.091449 & 2.747026 & 3.667718 \\ 6 & 1.291986 & 1.632990 & 2.896781 \\ 6 & 0.424132 & 5.115915 & 3.867368 \\ 6 & 0.005821 & 6.284556 & 3.284521 \\ 6 & -0.190655 & 6.343045 & 1.888505\end{array}$




\begin{tabular}{|c|c|c|c|}
\hline 6 & 0.032460 & 5.238364 & 1.104453 \\
\hline 6 & 0.508907 & 2.960005 & -0.587729 \\
\hline 6 & -0.684025 & 2.630673 & -1.205931 \\
\hline 6 & -0.860785 & 2.862548 & -2.589732 \\
\hline 6 & 0.160132 & 3.353850 & -3.357274 \\
\hline 6 & 1.416469 & 3.642994 & -2.777006 \\
\hline 6 & 1.587565 & 3.475966 & -1.370739 \\
\hline 6 & 2.849669 & 3.792501 & -0.803669 \\
\hline 6 & 3.887892 & 4.224071 & -1.590252 \\
\hline 6 & 3.716869 & 4.380229 & -2.983304 \\
\hline 6 & 2.505203 & 4.098282 & -3.560690 \\
\hline 7 & -1.739140 & 1.992078 & -0.468909 \\
\hline 6 & -2.818024 & 2.872178 & 0.030125 \\
\hline 6 & -3.383323 & 2.495947 & 1.375019 \\
\hline 6 & -4.762417 & 2.445601 & 1.566369 \\
\hline 6 & -5.294065 & 2.113622 & 2.810569 \\
\hline 6 & -4.448849 & 1.830818 & 3.878022 \\
\hline 6 & -3.067825 & 1.880553 & 3.692882 \\
\hline 6 & -2.542016 & 2.210345 & 2.451416 \\
\hline 6 & 5.273710 & 0.052130 & -3.247683 \\
\hline 6 & 6.192277 & -1.172331 & -3.164896 \\
\hline 6 & 6.393160 & 1.719506 & 1.454201 \\
\hline 45 & 0.043006 & -0.781313 & -1.265428 \\
\hline 6 & 0.385111 & -0.223593 & -3.158621 \\
\hline 8 & 0.137852 & -0.011963 & -4.271267 \\
\hline 15 & -1.871615 & 0.337308 & -0.833870 \\
\hline 8 & -2.888545 & -0.041276 & 0.423410 \\
\hline 6 & -3.595128 & -1.213262 & 0.339607 \\
\hline 6 & -4.748357 & -1.260787 & -0.419305 \\
\hline 6 & -5.431670 & -2.515222 & -0.531295 \\
\hline 6 & -4.953109 & -3.641633 & 0.205908 \\
\hline 6 & -3.795197 & -3.509875 & 1.010511 \\
\hline 6 & -3.117226 & -2.322212 & 1.064088 \\
\hline 6 & -5.630997 & -4.880751 & 0.103489 \\
\hline 6 & -6.724739 & -5.022699 & -0.711074 \\
\hline 6 & -7.178873 & -3.922705 & -1.468271 \\
\hline 6 & -6.551097 & -2.704806 & -1.381364 \\
\hline 6 & -5.181443 & -0.042405 & -1.139936 \\
\hline 6 & -4.288466 & 0.601035 & -1.980102 \\
\hline 6 & -4.685125 & 1.682504 & -2.797738 \\
\hline 6 & -5.953315 & 2.185683 & -2.703523 \\
\hline 6 & -6.868820 & 1.653846 & -1.762403 \\
\hline 6 & -6.481391 & 0.534253 & -0.966690 \\
\hline 6 & -7.386640 & 0.076641 & 0.024450 \\
\hline 6 & -8.609860 & 0.676349 & 0.198876 \\
\hline 6 & -9.002822 & 1.760179 & -0.613923 \\
\hline 6 & -8.145252 & 2.236461 & -1.572682 \\
\hline 8 & -2.988963 & 0.177116 & -2.092502 \\
\hline 6 & 0.276764 & -3.185328 & -1.471734 \\
\hline 6 & -0.937131 & -2.639059 & -1.955168 \\
\hline 6 & 1.726176 & -1.166491 & 1.694291 \\
\hline 6 & 2.879549 & -1.950984 & 1.593063 \\
\hline 6 & 3.110250 & -3.010314 & 2.465962 \\
\hline 6 & 2.169348 & -3.245919 & 3.477385 \\
\hline 6 & 1.009627 & -2.481168 & 3.612319 \\
\hline 6 & 0.783917 & -1.462004 & 2.677385 \\
\hline
\end{tabular}




\begin{tabular}{|c|c|c|c|}
\hline 6 & 4.349270 & -3.896054 & 2.347516 \\
\hline 6 & 3.913074 & -5.345664 & 2.107658 \\
\hline 6 & 0.029100 & -2.675304 & 4.768953 \\
\hline 6 & -1.396468 & -2.849476 & 4.239039 \\
\hline 6 & 5.254726 & -3.472662 & 1.191250 \\
\hline 6 & 5.164889 & -3.814387 & 3.642210 \\
\hline 6 & 0.369039 & -3.894186 & 5.623717 \\
\hline 6 & 0.083493 & -1.430922 & 5.664218 \\
\hline 6 & 0.257786 & -3.864229 & -0.120767 \\
\hline 6 & 1.362436 & -3.627627 & -2.412745 \\
\hline 6 & 2.561322 & -4.146158 & -1.902835 \\
\hline 6 & 3.605579 & -4.511983 & -2.743686 \\
\hline 6 & 3.481134 & -4.365102 & -4.122626 \\
\hline 6 & 2.296020 & -3.856734 & -4.644148 \\
\hline 6 & 1.250075 & -3.494193 & -3.801136 \\
\hline 6 & 4.092129 & -0.292823 & -4.153696 \\
\hline 6 & 6.029375 & 1.222499 & -3.883801 \\
\hline 1 & -7.089125 & -0.745227 & 0.671230 \\
\hline 1 & 1.637590 & 0.719393 & 3.373469 \\
\hline 1 & -8.423869 & 3.084492 & -2.197071 \\
\hline 1 & 0.581395 & 5.054408 & 4.943362 \\
\hline 1 & -5.254358 & -5.723763 & 0.681523 \\
\hline 1 & 2.995267 & 3.667289 & 0.268017 \\
\hline 1 & 4.853910 & 4.443682 & -1.13 \\
\hline 1 & -3.440060 & -4.376604 & 1.567140 \\
\hline 1 & 1.268160 & 2.710885 & 4.742249 \\
\hline 1 & -6.266895 & 3.018443 & -3.330925 \\
\hline 1 & -8.031540 & -4.041339 & -2.13 \\
\hline 1 & -3.959469 & 2.092161 & -3.496747 \\
\hline 1 & 2.356871 & 4.217828 & -4.633096 \\
\hline 1 & 0.027403 & 3.501064 & -4.42 \\
\hline 1 & -0.519982 & 7.272529 & 1.429714 \\
\hline 1 & -6.907735 & -1.871120 & -1.980629 \\
\hline 1 & -0.118403 & 5.299462 & 0.028583 \\
\hline 1 & -1.817495 & 2.600816 & -3.037725 \\
\hline 1 & -7.234293 & -5.980578 & -0.785423 \\
\hline 1 & -9.279842 & 0.314984 & 0.975974 \\
\hline 1 & 4.547558 & 4.727254 & -3.594318 \\
\hline 1 & -0.175188 & 7.167285 & 3.893431 \\
\hline 1 & -9.976819 & 2.221910 & -0.469602 \\
\hline 1 & -2.207462 & -2.200768 & 1.647409 \\
\hline 1 & 3.766138 & 1.219168 & 1.819893 \\
\hline 1 & 2.749124 & -0.132084 & -2.100318 \\
\hline 1 & 6.783170 & 1.040855 & -1.217343 \\
\hline 1 & 3.599582 & -1.727975 & 0.810466 \\
\hline 1 & -0.127173 & -0.860366 & 2.735793 \\
\hline 1 & 2.357015 & -4.053494 & 4.181686 \\
\hline 1 & -2.366013 & 3.872147 & 0.102562 \\
\hline 1 & -3.635160 & 2.953571 & -0.703991 \\
\hline 1 & -5.431327 & 2.648071 & 0.727720 \\
\hline 1 & -1.465680 & 2.211344 & 2.294198 \\
\hline 1 & -6.373873 & 2.068154 & 2.939148 \\
\hline 1 & -2.393336 & 1.661465 & 4.519814 \\
\hline 1 & -4.862763 & 1.567640 & 4.849255 \\
\hline 1 & 1.289185 & -1.825880 & -1.227368 \\
\hline 1 & -1.828612 & -2.791711 & -1.345656 \\
\hline
\end{tabular}




\begin{tabular}{|c|c|c|c|}
\hline 1 & -1.138645 & -2.568859 & -3.022305 \\
\hline & 0.233843 & -4.955877 & -0.255852 \\
\hline & 1.131990 & -3.628113 & 0.496375 \\
\hline & -0.632306 & -3.566146 & 0.443595 \\
\hline 1 & 2.690185 & -4.247875 & -0.825786 \\
\hline & 4.523709 & -4.910925 & -2.315 \\
\hline 1 & 4.299017 & -4.644943 & -4.782 \\
\hline 1 & 2.179134 & -3.738454 & -5.719 \\
\hline 1 & 0.335906 & -3.102251 & -4.239 \\
\hline 1 & -0.368671 & -3.994366 & 6.429 \\
\hline 1 & 0.348222 & -4.823109 & 5.03905 \\
\hline 1 & 1.355669 & -3.806160 & 6.09598 \\
\hline 1 & -2.088036 & -3.054430 & 5.06690 \\
\hline 1 & -1.761938 & -1.948218 & 3.73250 \\
\hline 1 & -1.453667 & -3.691165 & 3.534 \\
\hline 1 & -0.605572 & -1.537811 & 6.513 \\
\hline 1 & 1.094707 & -1.279578 & \\
\hline 1 & -0.200826 & -0.523164 & 5.11619 \\
\hline 1 & 6.131998 & -4.130609 & \\
\hline 1 & 4.746022 & -3.539519 & 0.219416 \\
\hline 1 & 5.618254 & -2.442056 & 1.307486 \\
\hline 1 & 6.057708 & -4.450161 & 3.571994 \\
\hline 1 & 5.497256 & -2.784820 & 3.830259 \\
\hline 1 & 4.590492 & -4.146178 & 4.515485 \\
\hline 1 & 4.790916 & -5.998930 & 2.012581 \\
\hline 1 & 3.298389 & -5.732888 & \\
\hline 1 & 3.325890 & -5.434129 & 1.182541 \\
\hline 1 & 4.458561 & -0.524956 & -5.16220 \\
\hline 1 & 3.534415 & -1.172547 & -3.80189 \\
\hline 1 & 3.389906 & 0.548240 & -4.23820 \\
\hline 1 & 6.518920 & -1.477904 & -4.16864 \\
\hline 1 & 7.089557 & -0.970904 & -2.56590 \\
\hline 1 & 5.664498 & -2.020516 & -2.70721 \\
\hline 1 & 6.338092 & 0.964390 & -4.905709 \\
\hline 1 & 5.389198 & 2.113383 & -3.936484 \\
\hline 1 & 6.935259 & 1.490480 & -3.326703 \\
\hline 6 & 7.758373 & 2.033503 & 0.848013 \\
\hline 6 & 5.860105 & 3.002470 & 2.103331 \\
\hline 6 & 6.576838 & 0.641573 & 2.529662 \\
\hline 1 & 8.429453 & 2.419552 & 1.625776 \\
\hline 1 & 8.234890 & 1.142655 & 0.419664 \\
\hline 1 & 7.687271 & 2.796985 & 0.061647 \\
\hline 1 & 6.573780 & 3.373673 & 2.851030 \\
\hline 1 & 5.718880 & 3.793079 & 1.353994 \\
\hline 1 & 4.900348 & 2.851869 & 2.613833 \\
\hline 1 & 7.254474 & 0.994411 & 3.319226 \\
\hline 1 & 5.623786 & 0.367276 & 3.000813 \\
\hline 1 & 7.009682 & -0.270689 & 2.09715 \\
\hline
\end{tabular}

TS1 $1_{I L-r e-}-\psi_{-1 \mathbf{s} 2 \mathbf{a} 3 \mathrm{~s} 4 \mathrm{a}}$

Number of imaginary frequencies : 1

The smallest frequencies are : $-615.7885 \quad 17.8058 \quad 18.8645 \mathrm{~cm}(-1)$

Electronic energy : $\quad H F=-4360.8700745$

Zero-point correction $=\quad 1.452255$ (Hartree/Particle)

Thermal correction to Energy= $\quad 1.537063$ 
Thermal correction to Enthalpy=

Thermal correction to Gibbs Free Energy=

Sum of electronic and zero-point Energies=

Sum of electronic and thermal Energies=

Sum of electronic and thermal Enthalpies=

Sum of electronic and thermal Free Energies=

\author{
1.538007 \\ 1.333557 \\ $-4359.417820$ \\ $-4359.333011$ \\ $-4359.332067$ \\ $-4359.536518$
}

Cartesian Coordinates

\begin{tabular}{|c|c|c|c|}
\hline 6 & 6.565423 & 2.610856 & -1.415032 \\
\hline 6 & 5.451913 & 2.441855 & -0.552916 \\
\hline 6 & 5.004475 & 3.575851 & 0.192090 \\
\hline 6 & 5.705175 & 4.801652 & 0.083902 \\
\hline 6 & 6.792061 & 4.923686 & -0.742989 \\
\hline 6 & 7.216009 & 3.816359 & -1.507013 \\
\hline 6 & 4.745292 & 1.200863 & -0.435740 \\
\hline 6 & 3.598073 & 1.175469 & 0.333400 \\
\hline 6 & 3.154168 & 2.290598 & 1.069874 \\
\hline 6 & 3.855122 & 3.464428 & 1.011811 \\
\hline 6 & 5.153239 & -0.026380 & -1.155839 \\
\hline 6 & 4.246439 & -0.658787 & -1.989252 \\
\hline 6 & 4.623550 & -1.747510 & -2.806696 \\
\hline 6 & 5.883673 & -2.271091 & -2.715137 \\
\hline 6 & 6.810428 & -1.752579 & -1.777578 \\
\hline 6 & 6.444275 & -0.623747 & -0.985070 \\
\hline 6 & 8.076904 & -2.356765 & -1.588467 \\
\hline 6 & 8.944668 & -1.892107 & -0.633134 \\
\hline 6 & 8.572628 & -0.798365 & 0.176193 \\
\hline 6 & 7.359830 & -0.177859 & 0.001969 \\
\hline 8 & 2.952227 & -0.216753 & -2.095172 \\
\hline 15 & 1.839950 & -0.355083 & -0.829735 \\
\hline 8 & 2.865892 & 0.020200 & 0.421335 \\
\hline 45 & -0.062228 & 0.787287 & -1.271060 \\
\hline 15 & -1.426576 & -0.114992 & 0.457076 \\
\hline 6 & -1.713421 & 1.225690 & 1.688470 \\
\hline 6 & -2.832608 & 2.053113 & 1.550693 \\
\hline 6 & -3.036656 & 3.136139 & 2.400592 \\
\hline 6 & -2.106012 & 3.351758 & 3.426494 \\
\hline 6 & -0.978770 & 2.546764 & 3.594699 \\
\hline 6 & -0.776112 & 1.503663 & 2.681015 \\
\hline 6 & -4.240368 & 4.064795 & 2.250301 \\
\hline 6 & -0.006564 & 2.727257 & 4.760515 \\
\hline 6 & -0.211545 & 3.205790 & -1.463199 \\
\hline 6 & -0.147071 & 3.861639 & -0.103335 \\
\hline 6 & -1.271634 & 3.723025 & -2.392426 \\
\hline 6 & -2.358525 & 4.448333 & -1.886122 \\
\hline 6 & -3.368967 & 4.913593 & -2.720615 \\
\hline 6 & -3.322172 & 4.661019 & -4.087546 \\
\hline 6 & -2.251446 & 3.937786 & -4.605735 \\
\hline 6 & -1.240984 & 3.474972 & -3.770861 \\
\hline 6 & 0.971405 & 2.608704 & -1.966281 \\
\hline 6 & -0.456196 & 0.201545 & -3.135432 \\
\hline 8 & -0.281307 & -0.070458 & -4.248931 \\
\hline 6 & -1.103211 & -1.609657 & 1.513378 \\
\hline 6 & -0.726391 & -2.803981 & 0.914559 \\
\hline 6 & -0.522375 & -3.976409 & 1.713456 \\
\hline
\end{tabular}




\begin{tabular}{|c|c|c|c|}
\hline & -0.716745 & -3.911561 & 3.123956 \\
\hline & -1.129795 & -2.688141 & 3.697848 \\
\hline & -1.316491 & -1.577973 & 2.918435 \\
\hline & -0.494235 & -5.063530 & 3.915437 \\
\hline & -0.092821 & -6.242684 & 3.342039 \\
\hline & 0.099231 & -6.315465 & 1.946186 \\
\hline & -0.112741 & -5.215141 & 1.152918 \\
\hline & -0.561968 & -2.953033 & -0.558602 \\
\hline & 0.637022 & -2.646133 & -1.176247 \\
\hline & 0.829418 & -2.923830 & -2.549411 \\
\hline & -0.176181 & -3.460011 & -3.306387 \\
\hline & -1.435150 & -3.740652 & -2.727544 \\
\hline & -1.630842 & -3.500262 & -1.334736 \\
\hline & -2.900462 & -3.796426 & -0.773726 \\
\hline & -3.917863 & -4.288818 & -1.552355 \\
\hline & -3.716844 & -4.533740 & -2.928185 \\
\hline & -2.499863 & -4.265435 & -3.500120 \\
\hline & 1.693514 & -2.003251 & -0.445711 \\
\hline & 2.769318 & -2.886383 & 0.056240 \\
\hline & 3.339336 & -2.507642 & 1.398337 \\
\hline & 4.719114 & -2.472563 & 1.588376 \\
\hline & 5.255512 & -2.140721 & 2.830588 \\
\hline & 4.414513 & -1.842769 & 3.897253 \\
\hline & 3.032856 & -1.877042 & 3.713257 \\
\hline & 2.502246 & -2.206609 & 2.473775 \\
\hline & -3.156133 & -0.486008 & -0.069180 \\
\hline & -3.545302 & -0.208838 & -1.367902 \\
\hline f & -4.864607 & -0.413796 & -1.792995 \\
\hline & -5.769144 & -0.930584 & -0.871441 \\
\hline 6 & -5.407508 & -1.228622 & 0.454640 \\
\hline & -4.093780 & -0.991070 & \\
\hline 6 & -5.223557 & -0.064975 & -3.233755 \\
\hline & -6.446836 & -1.810169 & 1.412062 \\
\hline & 7.078184 & 0.651300 & 0.646471 \\
\hline 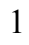 & -1.649128 & -0.656212 & 3.388441 \\
\hline & 8.339286 & -3.211837 & -2.210291 \\
\hline 1 & -0.646947 & -4.989964 & 4.991333 \\
\hline & 5.351916 & 5.650738 & 0.667741 \\
\hline & -3.069791 & -3.608266 & 0.285590 \\
\hline 1 & -4.889540 & -4.492293 & -1.105872 \\
\hline & 3.525308 & 4.335645 & 1.576928 \\
\hline 1 & -1.302968 & -2.640761 & 4.772506 \\
\hline & 6.181798 & -3.110080 & -3.341762 \\
\hline 1 & 8.063280 & 3.919494 & -2.181289 \\
\hline & 3.890124 & -2.146393 & -3.503760 \\
\hline 1 & -2.330476 & -4.443682 & -4.561224 \\
\hline | & -0.028385 & -3.656471 & -4.367179 \\
\hline & 0.416516 & -7.252621 & 1.494565 \\
\hline 1 & 6.898632 & 1.771464 & -2.019822 \\
\hline & 0.035725 & -5.288566 & 0.077524 \\
\hline 1 & 1.790217 & -2.674347 & -2.995236 \\
\hline 1 & 7.319393 & 5.871546 & -0.821629 \\
\hline 1 & 9.250510 & -0.445847 & 0.950484 \\
\hline 1 & -4.530381 & -4.931332 & -3.531285 \\
\hline 1 & 0.078815 & -7.122204 & 3.958256 \\
\hline 1 & 9.910601 & -2.370494 & -0.488906 \\
\hline
\end{tabular}




\begin{tabular}{|c|c|c|c|}
\hline & 2.251095 & 2.184786 & 1.666474 \\
\hline ] & -3.779821 & -1.196746 & 1.861691 \\
\hline & -2.799503 & 0.177880 & -2.059695 \\
\hline | & -6.797422 & -1.110019 & -1.180705 \\
\hline & -3.544564 & 1.844579 & 0.756099 \\
\hline & 0.113171 & 0.873110 & 2.762899 \\
\hline & -2.275824 & 4.177009 & 4.114631 \\
\hline 1 & 2.311811 & -3.883421 & 0.134378 \\
\hline & 3.584787 & -2.976360 & -0.678672 \\
\hline 1 & 5.385086 & -2.687484 & 0.750451 \\
\hline 1 & 1.425888 & -2.195704 & 2.317215 \\
\hline 1 & 6.335909 & -2.107206 & 2.958007 \\
\hline 1 & 2.361579 & -1.645423 & 4.539436 \\
\hline 1 & 4.832236 & -1.579729 & 4.866887 \\
\hline 1 & -1.276256 & 1.877556 & -1.238182 \\
\hline 1 & 1.875294 & 2.720389 & -1.366039 \\
\hline & 1.158447 & 2.552244 & -3.037136 \\
\hline 1 & -0.048736 & 4.951273 & -0.220855 \\
\hline & -1.036050 & 3.673675 & 0.509553 \\
\hline 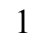 & 0.720794 & 3.496188 & 0.454470 \\
\hline ] & -2.426010 & 4.646898 & -0.817918 \\
\hline 1 & -4.198186 & 5.475147 & -2.293336 \\
\hline 1 & -4.112212 & 5.020962 & -4.742850 \\
\hline . & -2.199146 & 3.729148 & -5.672334 \\
\hline 1 & -0.419248 & 2.911655 & -4.206157 \\
\hline 6 & -0.332414 & 3.955634 & 5.606973 \\
\hline 6 & -0.090198 & 1.488438 & 5.661161 \\
\hline 5 & 1.426242 & 2.873717 & 4.241699 \\
\hline 6 & -5.120008 & 3.685483 & 1.059180 \\
\hline 6 & -5.098623 & 3.993253 & 3.517991 \\
\hline 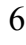 & -3.752502 & 5.502973 & 2.044794 \\
\hline 1 & 0.395934 & 4.042781 & 6.422877 \\
\hline 1 & -0.283553 & 4.882265 & 5.020337 \\
\hline 1 & -1.327547 & 3.889687 & 6.064909 \\
\hline 1 & 2.115492 & 3.066978 & 5.074369 \\
\hline 1 & 1.777333 & 1.964473 & 3.739229 \\
\hline 1 & 1.504735 & 3.712738 & 3.535909 \\
\hline 1 & 0.591610 & 1.588964 & 6.516567 \\
\hline 1 & -1.107702 & 1.355186 & 6.052182 \\
\hline 1 & 0.185715 & 0.573097 & 5.121476 \\
\hline 1 & -5.964199 & 4.383175 & 0.989848 \\
\hline 1 & -4.572722 & 3.731193 & 0.107048 \\
\hline 1 & -5.536366 & 2.673765 & 1.157919 \\
\hline 1 & -5.970110 & 4.655681 & 3.428344 \\
\hline 1 & -5.465474 & 2.971702 & 3.684131 \\
\hline 1 & -4.540509 & 4.298159 & 4.411550 \\
\hline 1 & -4.606695 & 6.182522 & 1.922978 \\
\hline 1 & -3.160258 & 5.864855 & 2.893767 \\
\hline 1 & -3.126875 & 5.584102 & 1.144516 \\
\hline 6 & -6.712073 & -0.241409 & -3.519577 \\
\hline 6 & -4.846914 & 1.396725 & -3.511513 \\
\hline 6 & -4.435118 & -0.979309 & -4.178142 \\
\hline 6 & -5.888073 & -2.009933 & 2.819565 \\
\hline 6 & -7.650948 & -0.867350 & 1.508853 \\
\hline 6 & -6.908254 & -3.175245 & 0.889479 \\
\hline 1 & -6.923870 & 0.032282 & -4.560932 \\
\hline
\end{tabular}




$\begin{array}{lrrr}1 & -7.035979 & -1.281520 & -3.383358 \\ 1 & -7.331056 & 0.398803 & -2.877068 \\ 1 & -4.665361 & -0.737955 & -5.225053 \\ 1 & -3.351005 & -0.872036 & -4.040423 \\ 1 & -4.687213 & -2.034213 & -4.004448 \\ 1 & -5.145300 & 1.679176 & -4.530740 \\ 1 & -5.349899 & 2.076451 & -2.809755 \\ 1 & -3.765532 & 1.573992 & -3.429459 \\ 1 & -6.665573 & -2.433094 & 3.468171 \\ 1 & -5.036970 & -2.704951 & 2.831222 \\ 1 & -5.562293 & -1.062568 & 3.268879 \\ 1 & -8.397431 & -1.273051 & 2.204907 \\ 1 & -7.347110 & 0.120950 & 1.878118 \\ 1 & -8.146658 & -0.724972 & 0.541265 \\ 1 & -7.682972 & -3.595090 & 1.545494 \\ 1 & -7.326873 & -3.111054 & -0.122537 \\ 1 & -6.070185 & -3.885534 & 0.864868\end{array}$

TS1 $_{\text {IIL-re- }} \psi_{-1 \mathrm{~s} 2 \mathrm{a} 3 \mathrm{~s} 4 \mathrm{~s}}$

Number of imaginary frequencies : 1

The smallest frequencies are : $-617.1147 \quad 16.8624 \quad 19.7585 \mathrm{~cm}(-1)$

Electronic energy : $\quad H F=-4360.8705947$

Zero-point correction $=\quad 1.452611($ Hartree/Particle $)$

Thermal correction to Energy= $\quad 1.537162$

Thermal correction to Enthalpy= $\quad 1.538106$

Thermal correction to Gibbs Free Energy= $\quad 1.335241$

Sum of electronic and zero-point Energies $=\quad-4359.417984$

Sum of electronic and thermal Energies $=\quad-4359.333433$

Sum of electronic and thermal Enthalpies = $\quad-4359.332489$

Sum of electronic and thermal Free Energies $=\quad-4359.535354$

Cartesian Coordinates

$\begin{array}{cccc}6 & -6.556409 & -2.661726 & -1.378928 \\ 6 & -5.440113 & -2.479760 & -0.523111 \\ 6 & -4.979722 & -3.606763 & 0.224678 \\ 6 & -5.671164 & -4.838602 & 0.125676 \\ 6 & -6.761091 & -4.973189 & -0.695243 \\ 6 & -7.197618 & -3.872869 & -1.462269 \\ 6 & -4.743078 & -1.232499 & -0.415136 \\ 6 & -3.591703 & -1.194679 & 0.347332 \\ 6 & -3.135128 & -2.302805 & 1.086769 \\ 6 & -3.827068 & -3.482398 & 1.037935 \\ 6 & -5.165378 & -0.011214 & -1.137299 \\ 6 & -4.268375 & 0.626521 & -1.977259 \\ 6 & -4.657905 & 1.710613 & -2.794861 \\ 6 & -5.922016 & 2.223580 & -2.699025 \\ 6 & -6.840215 & 1.698933 & -1.756499 \\ 6 & -6.460765 & 0.575631 & -0.962245 \\ 6 & -8.111418 & 2.292107 & -1.564330 \\ 6 & -8.971339 & 1.822455 & -0.604415 \\ 6 & -8.586129 & 0.734764 & 0.206895 \\ 6 & -7.368385 & 0.124773 & 0.029886 \\ 8 & -2.971447 & 0.194385 & -2.090101 \\ 15 & -1.852915 & 0.345870 & -0.832323\end{array}$




\begin{tabular}{|c|c|c|c|}
\hline 3 & -2.868462 & -0.033352 & 0.425957 \\
\hline 45 & 0.055697 & -0.781834 & -1.280055 \\
\hline 15 & 1.418766 & 0.136417 & 0.438033 \\
\hline 6 & 1.738913 & -1.195528 & 1.670058 \\
\hline 6 & 2.876488 & -1.996725 & 1.532064 \\
\hline 6 & 3.110303 & -3.070190 & 2.385867 \\
\hline 6 & 2.189146 & -3.303680 & 3.416466 \\
\hline 6 & 1.043231 & -2.524909 & 3.584919 \\
\hline 6 & 0.812858 & -1.490366 & 2.667995 \\
\hline 6 & 4.336016 & -3.968041 & 2.228945 \\
\hline 6 & 0.079602 & -2.722556 & 4.755086 \\
\hline 6 & 0.234453 & -3.201023 & -1.456080 \\
\hline 6 & 0.182484 & -3.847371 & -0.090971 \\
\hline 6 & 1.298332 & -3.714495 & -2.383499 \\
\hline 6 & 2.405404 & -4.403978 & -1.870268 \\
\hline 6 & 3.418636 & -4.866289 & -2.702701 \\
\hline 6 & 3.355564 & -4.645010 & -4.074541 \\
\hline 6 & 2.265145 & -3.957259 & -4.599489 \\
\hline 6 & 1.250862 & -3.498718 & -3.766591 \\
\hline 6 & -0.957616 & -2.622223 & -1.959055 \\
\hline 6 & 0.434011 & -0.200194 & -3.148324 \\
\hline 8 & 0.258878 & 0.070397 & -4.262084 \\
\hline 6 & 1.092931 & 1.627636 & 1.498923 \\
\hline 6 & 0.699979 & 2.818390 & 0.903901 \\
\hline 6 & 0.492171 & 3.989199 & 1.703712 \\
\hline 6 & 0.699536 & 3.926686 & 3.112357 \\
\hline 6 & 1.128337 & 2.707053 & 3.682767 \\
\hline 6 & 1.317876 & 1.598012 & 2.902196 \\
\hline 6 & 0.473765 & 5.077062 & 3.905208 \\
\hline 6 & 0.056928 & 6.252236 & 3.334628 \\
\hline 6 & -0.147693 & 6.322691 & 1.940414 \\
\hline 6 & 0.067224 & 5.223885 & 1.145860 \\
\hline 6 & 0.530070 & 2.964969 & -0.568344 \\
\hline 6 & -0.665820 & 2.646672 & -1.186015 \\
\hline 6 & -0.857555 & 2.919295 & -2.560440 \\
\hline 6 & 0.145570 & 3.461124 & -3.317240 \\
\hline 6 & 1.402052 & 3.752065 & -2.737610 \\
\hline 6 & 1.596507 & 3.516577 & -1.344191 \\
\hline 6 & 2.864040 & 3.816971 & -0.780981 \\
\hline 6 & 3.881526 & 4.311203 & -1.557995 \\
\hline 6 & 3.681560 & 4.553782 & -2.934546 \\
\hline 6 & 2.466451 & 4.280176 & -3.508553 \\
\hline 7 & -1.717132 & 1.996469 & -0.454394 \\
\hline 6 & -2.795436 & 2.872001 & 0.055739 \\
\hline 6 & -3.354637 & 2.487390 & 1.400882 \\
\hline 6 & -4.732946 & 2.440297 & 1.598651 \\
\hline 6 & -5.259535 & 2.098557 & 2.842387 \\
\hline 6 & -4.410118 & 1.802357 & 3.902860 \\
\hline 6 & -3.029848 & 1.849336 & 3.711386 \\
\hline 6 & -2.509117 & 2.189440 & 2.470599 \\
\hline 6 & 3.139787 & 0.519912 & -0.106608 \\
\hline 6 & 3.523604 & 0.253607 & -1.412965 \\
\hline 6 & 4.840537 & 0.449373 & -1.837546 \\
\hline 6 & 5.760160 & 0.945659 & -0.911698 \\
\hline 6 & 5.403143 & 1.233634 & 0.412728 \\
\hline 6 & 4.083708 & 1.005182 & 0.798440 \\
\hline
\end{tabular}




\begin{tabular}{|c|c|c|c|}
\hline 6 & 5.195357 & 0.107002 & -3.281712 \\
\hline 6 & 6.395743 & 1.754959 & 1.451088 \\
\hline 1 & -7.076904 & -0.699890 & 0.675802 \\
\hline 1 & 1.661244 & 0.679095 & 3.369952 \\
\hline 1 & -8.383879 & 3.142871 & -2.187712 \\
\hline 1 & 0.636470 & 5.005605 & 4.979777 \\
\hline 1 & -5.308178 & -5.682110 & 0.711649 \\
\hline 1 & 3.031582 & 3.629919 & 0.278525 \\
\hline 1 & 4.852543 & 4.516048 & -1.110117 \\
\hline 1 & -3.487451 & -4.348062 & 1.605780 \\
\hline 1 & 1.310572 & 2.661450 & 4.756016 \\
\hline 1 & -6.230052 & 3.058631 & -3.326110 \\
\hline 1 & -8.047228 & -3.985951 & -2.131990 \\
\hline 1 & -3.930344 & 2.113982 & -3.495464 \\
\hline 1 & 2.299207 & 4.455717 & -4.570466 \\
\hline 1 & -0.002231 & 3.653372 & -4.378816 \\
\hline 1 & -0.476994 & 7.256837 & 1.491182 \\
\hline 1 & -6.899286 & -1.827915 & -1.986014 \\
\hline 1 & -0.090671 & 5.295170 & 0.071636 \\
\hline 1 & -1.815455 & 2.660933 & -3.007745 \\
\hline 1 & -7.281170 & -5.925596 & -0.766897 \\
\hline 1 & -9.257757 & 0.378499 & 0.984920 \\
\hline 1 & 4.494595 & 4.953081 & -3.537220 \\
\hline 1 & -0.117160 & 7.130563 & 3.951859 \\
\hline 1 & -9.941115 & 2.292346 & -0.458052 \\
\hline 1 & -2.229986 & -2.187026 & 1.678484 \\
\hline 1 & 3.783775 & 1.198844 & 1.828130 \\
\hline 1 & 2.772474 & -0.121947 & -2.104990 \\
\hline 1 & 6.786942 & 1.110495 & -1.226595 \\
\hline 1 & 3.579872 & -1.776023 & 0.733724 \\
\hline 1 & -0.089308 & -0.8787 & 2.752062 \\
\hline 1 & 2.381137 & -4.121558 & 4.107682 \\
\hline 1 & -2.344228 & 3.872017 & 0.131963 \\
\hline 1 & -3.616007 & 2.956969 & -0.674099 \\
\hline 1 & -5.405405 & 2.652654 & 0.765291 \\
\hline 1 & -1.433651 & 2.187897 & 2.308109 \\
\hline 1 & -6.338867 & 2.055603 & 2.975850 \\
\hline 1 & -2.351823 & & 4.532470 \\
\hline 1 & -4.820208 & 1.530922 & 4.873435 \\
\hline 1 & 1.280370 & -1.860342 & -1.248445 \\
\hline 1 & -1.858125 & -2.740478 & -1.354968 \\
\hline 1 & -1.148679 & -2.573397 & -3.029493 \\
\hline 1 & 0.099162 & -4.939035 & -0.200464 \\
\hline 1 & 1.070346 & -3.642908 & 0.518261 \\
\hline 1 & -0.689121 & -3.489615 & 0.466053 \\
\hline 1 & 2.487058 & -4.575240 & -0.798163 \\
\hline 1 & 4.263329 & -5.400031 & -2.269957 \\
\hline 1 & 4.148089 & -5.002148 & -4.728373 \\
\hline 1 & 2.199471 & -3.774212 & -5.670033 \\
\hline 1 & 0.411362 & -2.967462 & -4.208170 \\
\hline 6 & 0.427194 & -3.948851 & 5.595985 \\
\hline 6 & 0.151126 & -1.485065 & 5.658736 \\
\hline 6 & -1.354337 & -2.887091 & 4.244337 \\
\hline 6 & 5.195190 & -3.568192 & 1.029250 \\
\hline 6 & 5.202983 & -3.871952 & 3.488975 \\
\hline 6 & 3.882000 & -5.418199 & 2.029763 \\
\hline
\end{tabular}




$\begin{array}{lrrr}1 & -0.297538 & -4.050866 & 6.413378 \\ 1 & 0.391910 & -4.873727 & 5.005644 \\ 1 & 1.422157 & -3.868788 & 6.051976 \\ 1 & -2.035277 & -3.092451 & 5.080925 \\ 1 & -1.721806 & -1.981180 & 3.747402 \\ 1 & -1.426282 & -3.724891 & 3.536453 \\ 1 & -0.523247 & -1.597976 & 6.518498 \\ 1 & 1.169221 & -1.337929 & 6.043202 \\ 1 & -0.142018 & -0.572423 & 5.123437 \\ 1 & 6.063102 & -4.236174 & 0.960417 \\ 1 & 4.643592 & -3.641541 & 0.081209 \\ 1 & 5.574807 & -2.540415 & 1.115871 \\ 1 & 6.086272 & -4.518122 & 3.396069 \\ 1 & 5.552015 & -2.842552 & 3.645481 \\ 1 & 4.658240 & -4.182012 & 4.389014 \\ 1 & 4.751162 & -6.076415 & 1.897053 \\ 1 & 3.309899 & -5.793993 & 2.886548 \\ 1 & 3.246452 & -5.515155 & 1.137829 \\ 6 & 6.682159 & 0.288907 & -3.573792 \\ 6 & 4.823089 & -1.355356 & -3.561801 \\ 6 & 4.401156 & 1.019933 & -4.222416 \\ 6 & 7.752998 & 2.094848 & 0.840619 \\ 6 & 5.844618 & 3.026174 & 2.108519 \\ 6 & 6.603142 & 0.676441 & 2.521518 \\ 1 & 6.888939 & 0.022155 & -4.617973 \\ 1 & 7.004444 & 1.328837 & -3.432447 \\ 1 & 7.306423 & -0.354103 & -2.939293 \\ 1 & 4.632572 & 0.783410 & -5.270172 \\ 1 & 3.317810 & 0.905496 & -4.085172 \\ 1 & 4.647121 & 2.075641 & -4.045441 \\ 1 & 5.112872 & -1.632770 & -4.584964 \\ 1 & 5.336940 & -2.034569 & -2.867602 \\ 1 & 3.743448 & -1.538065 & -3.468950 \\ 1 & 8.420124 & 2.489436 & 1.617517 \\ 1 & 8.243341 & 1.214254 & 0.406744 \\ 1 & 7.666196 & 2.860351 & 0.057793 \\ 1 & 6.553078 & 3.402846 & 2.858533 \\ 1 & 5.692413 & 3.819928 & 1.364607 \\ 1 & 4.887016 & 2.858849 & 2.617954 \\ 1 & 7.287382 & 1.034095 & 3.303180 \\ 1 & 5.658877 & 0.392696 & 3.004196 \\ 1 & 7.038167 & -0.231097 & 2.081801\end{array}$

TS1 $_{\text {IIL-re- }}-\psi_{-1 \mathrm{~s} 2 \mathrm{~s} 3 \mathrm{a} 4 \mathrm{a}}$

Number of imaginary frequencies : 1

The smallest frequencies are : $-600.2133 \quad 16.6745 \quad 20.2507 \mathrm{~cm}(-1)$

Electronic energy : $\quad H F=-4360.8697162$

Zero-point correction $=\quad 1.451526($ Hartree/Particle $)$

Thermal correction to Energy $=\quad 1.536450$

Thermal correction to Enthalpy= $\quad 1.537395$

Thermal correction to Gibbs Free Energy= 1.333102

Sum of electronic and zero-point Energies $=\quad-4359.418190$

Sum of electronic and thermal Energies $=\quad-4359.333266$

Sum of electronic and thermal Enthalpies $=\quad-4359.332322$

Sum of electronic and thermal Free Energies $=\quad-4359.536614$ 
Cartesian Coordinates

\begin{tabular}{cccc}
\cline { 1 - 1 } & 1.101845 & -3.484768 & -3.892281 \\
6 & 1.243314 & -3.654877 & -2.510482 \\
6 & 2.431316 & -4.231912 & -2.042765 \\
6 & 3.439857 & -4.619714 & -2.916322 \\
6 & 3.287007 & -4.438791 & -4.288097 \\
6 & 2.110913 & -3.871717 & -4.768655 \\
6 & 0.199661 & -3.198153 & -1.529937 \\
6 & 0.199660 & -3.897807 & -0.188837 \\
45 & 0.025639 & -0.799310 & -1.276807 \\
6 & -1.014245 & -2.618911 & -1.973005 \\
15 & 1.417767 & 0.077108 & 0.437854 \\
6 & 1.721462 & -1.254904 & 1.677632 \\
6 & 2.884912 & -2.019254 & 1.621433 \\
6 & 3.127364 & -3.045199 & 2.539055 \\
6 & 2.167067 & -3.283898 & 3.523597 \\
6 & 0.978526 & -2.546157 & 3.602285 \\
6 & 0.757859 & -1.551941 & 2.646273 \\
6 & 4.443668 & -3.816468 & 2.450925 \\
6 & 4.544711 & -4.508170 & 1.086665 \\
6 & -0.020189 & -2.737293 & 4.744665 \\
6 & 0.044569 & -1.500444 & 5.649878 \\
6 & 3.140723 & 0.447473 & -0.113767 \\
6 & 4.098898 & 0.900423 & 0.796601 \\
6 & 5.417336 & 1.117737 & 0.400790 \\
6 & 5.743479 & 0.872730 & -0.939531 \\
6 & 4.809061 & 0.427124 & -1.875550 \\
6 & 3.498745 & 0.217277 & -1.436692 \\
6 & 5.229844 & 0.143727 & -3.315916 \\
6 & 6.485411 & 1.627755 & 1.367663 \\
6 & 1.105661 & 1.564549 & 1.511403 \\
6 & 0.721227 & 2.764327 & 0.928181 \\
6 & 0.517199 & 3.927347 & 1.739819 \\
6 & 0.714078 & 3.847425 & 3.149187 \\
6 & 1.131575 & 2.618727 & 3.708166 \\
6 & 1.321752 & 1.518233 & 2.915706 \\
6 & 0.488124 & 4.989725 & 3.953590 \\
6 & 0.082241 & 6.173815 & 3.393597 \\
6 & -0.110443 & 6.261875 & 1.998620 \\
6 & 0.104276 & 5.171072 & 1.193075 \\
6 & 0.550558 & 2.925090 & -0.542672 \\
6 & -0.652574 & 2.628454 & -1.157444 \\
6 & -0.843149 & 2.903544 & -2.531243 \\
6 & 0.171481 & 3.414707 & -3.293765 \\
6 & 1.435084 & 3.679821 & -2.717954 \\
6 & 1.624097 & 3.458459 & -1.321276 \\
6 & 2.893935 & 3.752808 & -0.759378 \\
6 & 3.920073 & 4.220435 & -1.541508 \\
6 & 3.729256 & 4.435573 & -2.924076 \\
6 & 2.511564 & 4.171149 & -3.496707 \\
7 & -1.711421 & 1.990735 & -0.425261 \\
6 & -1.866206 & 0.344269 & -0.815134 \\
6 & -2.986527 & 0.216283 & -2.074598 \\
& -4.279891 & 0.657584 & -1.958177 \\
6 & & & \\
6 & & &
\end{tabular}




\begin{tabular}{|c|c|c|c|}
\hline 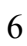 & -5.182143 & 0.019585 & -1.123851 \\
\hline & -6.472626 & 0.614968 & -0.943137 \\
\hline & -6.843510 & 1.748037 & -1.727464 \\
\hline & -5.921476 & 2.273447 & -2.665855 \\
\hline & -4.661369 & 1.751578 & -2.766486 \\
\hline & -7.383206 & 0.162463 & 0.045457 \\
\hline & -8.595990 & 0.780545 & 0.228526 \\
\hline & -8.972824 & 1.878506 & -0.572845 \\
\hline & -8.109770 & 2.349685 & -1.529237 \\
\hline & -4.768625 & -1.213743 & -0.417340 \\
\hline & -3.614076 & -1.195208 & 0.340792 \\
\hline & -3.154959 & -2.320830 & 1.051454 \\
\hline & -3.852901 & -3.496012 & 0.984735 \\
\hline & -5.013626 & -3.598256 & 0.179929 \\
\hline & -5.473473 & -2.455045 & -0.543158 \\
\hline & -5.712668 & -4.824269 & 0.063622 \\
\hline & -6.809877 & -4.937650 & -0.750777 \\
\hline & -7.246182 & -3.820964 & -1.493877 \\
\hline & -6.597281 & -2.615210 & -1.393537 \\
\hline & -2.885991 & -0.037423 & 0.438855 \\
\hline & -2.782426 & 2.877772 & 0.079194 \\
\hline & -3.353695 & 2.498358 & 1.420507 \\
\hline & -4.733532 & 2.467684 & 1.611006 \\
\hline & -5.270544 & 2.139279 & 2.853870 \\
\hline & -4.430060 & 1.840284 & 3.920613 \\
\hline & -3.048385 & 1.869423 & 3.735994 \\
\hline 6 & -2.517141 & 2.195262 & 2.495778 \\
\hline ? & 0.349891 & -0.208141 & -3.163916 \\
\hline & 0.075547 & 0.045394 & -4.261448 \\
\hline 5 & 5.610698 & -2.832051 & 2.603717 \\
\hline 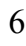 & 4.570642 & -4.882012 & 3.536230 \\
\hline 6 & -1.443028 & -2.884025 & 4.200696 \\
\hline & 0.290005 & -3.968821 & 5.593252 \\
\hline & -7.097726 & -0.670159 & 0.683854 \\
\hline 1 & 1.655979 & 0.592082 & 3.375991 \\
\hline & -8.375801 & 3.208092 & -2.144885 \\
\hline 1 & 0.642008 & 4.904830 & 5.028498 \\
\hline & -5.349784 & -5.680504 & 0.630882 \\
\hline 1 & 3.055188 & 3.583439 & 0.304087 \\
\hline ] & 4.891327 & 4.425336 & -1.094708 \\
\hline | & -3.511944 & -4.375184 & 1.530637 \\
\hline l & 1.305315 & 2.559015 & 4.782181 \\
\hline & -6.223177 & 3.116223 & -3.285661 \\
\hline 1 & -8.101873 & -3.916829 & -2.158525 \\
\hline & -3.930924 & 2.155642 & -3.463766 \\
\hline 1 & 2.348302 & 4.333977 & -4.561215 \\
\hline & 0.026901 & 3.601491 & -4.356773 \\
\hline 1 & -0.430230 & 7.203292 & 1.557755 \\
\hline 1 & -6.940619 & -1.768263 & -1.981882 \\
\hline & -0.044114 & 5.255702 & 0.118484 \\
\hline 1 & -1.807426 & 2.664134 & -2.975327 \\
\hline & -7.335912 & -5.885687 & -0.835765 \\
\hline 1 & -9.270064 & 0.422840 & 1.003772 \\
\hline | & 4.550022 & 4.812931 & -3.530556 \\
\hline 1 & -0.092036 & 7.045757 & 4.019770 \\
\hline 1 & -9.938659 & 2.354940 & -0.421623 \\
\hline
\end{tabular}




\begin{tabular}{|c|c|c|c|}
\hline . & -2.242462 & -2.222058 & 1.634389 \\
\hline & 3.805961 & 1.070382 & 1.830369 \\
\hline 1 & 2.728705 & -0.127950 & -2.122026 \\
\hline 1 & 6.771189 & 1.038636 & -1.264209 \\
\hline 1 & 3.625366 & -1.802220 & 0.852773 \\
\hline 1 & -0.161202 & -0.960296 & 2.674874 \\
\hline 1 & 2.351101 & -4.061728 & 4.258168 \\
\hline . & -2.320068 & 3.872346 & 0.160282 \\
\hline 1 & -3.597654 & 2.973899 & -0.655169 \\
\hline 1 & -5.399117 & 2.684509 & 0.773280 \\
\hline 1 & -1.440822 & 2.181679 & 2.339025 \\
\hline & -6.350958 & 2.109889 & 2.982038 \\
\hline & -2.377791 & 1.637906 & 4.562624 \\
\hline ] & -4.848208 & 1.580585 & 4.890971 \\
\hline ] & 1.251513 & -1.872619 & -1.273425 \\
\hline ] & -1.893012 & -2.762860 & -1.343666 \\
\hline & -1.240801 & -2.527792 & -3.033528 \\
\hline 1 & 0.138563 & -4.985694 & -0.339543 \\
\hline 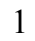 & 1.097550 & -3.696808 & 0.407657 \\
\hline 1 & -0.665224 & -3.582813 & 0.404875 \\
\hline 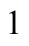 & 2.579938 & -4.365565 & -0.973374 \\
\hline 1 & 4.351040 & -5.063871 & -2.518292 \\
\hline ] & 4.075117 & -4.738205 & -4.975307 \\
\hline & 1.972250 & -3.726554 & -5.837997 \\
\hline 1 & 0.194397 & -3.046431 & -4.299992 \\
\hline 1 & -0.469460 & -4.073609 & 6.378165 \\
\hline 1 & 0.279617 & -4.890238 & 4.996546 \\
\hline 1 & 1.263320 & -3.893882 & 6.094083 \\
\hline . & -2.144660 & -3.085851 & 5.020709 \\
\hline 1 & -1.790308 & -1.972015 & 3.701151 \\
\hline 1 & -1.507950 & -3.717834 & 3.487421 \\
\hline 1 & -0.654936 & -1.601363 & 6.490984 \\
\hline 1 & 1.054030 & -1.367946 & 6.061710 \\
\hline 1 & -0.218595 & -0.585317 & 5.103336 \\
\hline 1 & 5.529834 & -5.404136 & 3.429606 \\
\hline 1 & 4.544178 & -4.447792 & 4.543984 \\
\hline 1 & 3.776332 & -5.636371 & 3.465614 \\
\hline 1 & 6.570463 & -3.362742 & 2.540544 \\
\hline 1 & 5.601967 & -2.064768 & 1.818414 \\
\hline 1 & 5.568980 & -2.320215 & 3.574510 \\
\hline 1 & 5.483198 & -5.074535 & 1.014736 \\
\hline 1 & 3.714418 & -5.213808 & 0.942171 \\
\hline 1 & 4.530424 & -3.787196 & 0.259106 \\
\hline 6 & 4.033638 & -0.182680 & -4.209061 \\
\hline 6 & 5.939559 & 1.365999 & -3.906020 \\
\hline 6 & 6.181156 & -1.058096 & -3.325131 \\
\hline 6 & 5.965792 & 1.725529 & 2.800686 \\
\hline 6 & 7.691343 & 0.682447 & 1.367102 \\
\hline 6 & 6.933368 & 3.025568 & 0.926303 \\
\hline 1 & 4.378499 & -0.353980 & -5.236945 \\
\hline 1 & 3.505343 & -1.091414 & -3.887379 \\
\hline 1 & 3.309560 & 0.643449 & -4.233210 \\
\hline 1 & 6.497330 & -1.289663 & -4.351549 \\
\hline 1 & 7.084042 & -0.869640 & -2.730419 \\
\hline 1 & 5.686378 & -1.948695 & -2.914442 \\
\hline 1 & 6.234537 & 1.168445 & -4.945364 \\
\hline
\end{tabular}




$\begin{array}{lrrr}1 & 5.274421 & 2.240092 & -3.900992 \\ 1 & 6.848655 & 1.630859 & -3.352401 \\ 1 & 6.766514 & 2.084318 & 3.459727 \\ 1 & 5.128062 & 2.430519 & 2.889758 \\ 1 & 5.634546 & 0.749648 & 3.181260 \\ 1 & 8.458765 & 1.046716 & 2.063218 \\ 1 & 7.400838 & -0.327872 & 1.685888 \\ 1 & 8.158072 & 0.600192 & 0.378449 \\ 1 & 7.720795 & 3.405369 & 1.591587 \\ 1 & 7.330262 & 3.026068 & -0.096726 \\ 1 & 6.093357 & 3.732941 & 0.963374\end{array}$

TS1 $_{\text {IIL-re- }} \Psi_{\text {-1s2s3a4s }}$

Number of imaginary frequencies : 1

The smallest frequencies are : $-598.5726 \quad 17.0763 \quad 18.5825 \mathrm{~cm}(-1)$

Electronic energy : $\quad H F=-4360.8700015$

Zero-point correction $=\quad 1.451041($ Hartree/Particle $)$

Thermal correction to Energy= $\quad 1.536139$

Thermal correction to Enthalpy= $\quad 1.537083$

Thermal correction to Gibbs Free Energy $=\quad 1.332060$

Sum of electronic and zero-point Energies $=\quad-4359.418961$

Sum of electronic and thermal Energies $=\quad-4359.333863$

Sum of electronic and thermal Enthalpies $=\quad-4359.332919$

Sum of electronic and thermal Free Energies $=\quad-4359.537941$

Cartesian Coordinates

$\begin{array}{cccc}6 & -1.041842 & 3.556764 & -3.862394 \\ 6 & -1.175315 & 3.731094 & -2.480139 \\ 6 & -2.336900 & 4.358114 & -2.011490 \\ 6 & -3.327486 & 4.791810 & -2.884073 \\ 6 & -3.182766 & 4.607158 & -4.255915 \\ 6 & -2.033173 & 3.988748 & -4.737670 \\ 6 & -0.149338 & 3.234728 & -1.500550 \\ 6 & -0.137017 & 3.918309 & -0.151126 \\ 45 & -0.025453 & 0.830984 & -1.269178 \\ 6 & 1.052467 & 2.632702 & -1.947124 \\ 15 & -1.425625 & -0.043659 & 0.441011 \\ 6 & -1.710984 & 1.278418 & 1.694851 \\ 6 & -2.860392 & 2.064196 & 1.642900 \\ 6 & -3.076518 & 3.098233 & 2.558745 \\ 6 & -2.110912 & 3.313171 & 3.543679 \\ 6 & -0.940287 & 2.547292 & 3.623312 \\ 6 & -0.740556 & 1.552623 & 2.663590 \\ 6 & -4.365935 & 3.913412 & 2.464235 \\ 6 & -4.433548 & 4.608892 & 1.099873 \\ 6 & 0.058798 & 2.709787 & 4.769909 \\ 6 & -0.054246 & 1.479555 & 5.679476 \\ 6 & -3.151204 & -0.399755 & -0.114120 \\ 6 & -4.096028 & -0.903265 & 0.775179 \\ 6 & -5.403663 & -1.177183 & 0.364976 \\ 6 & -5.737139 & -0.906745 & -0.963697 \\ 6 & -4.813421 & -0.390215 & -1.881681 \\ 6 & -3.515134 & -0.147744 & -1.435557 \\ 6 & -5.241718 & -0.081114 & -3.314558\end{array}$




$\begin{array}{llll}6 & -6.389316 & -1.762716 & 1.375654 \\ 6 & -1.132811 & -1.549084 & 1.496999 \\ 6 & -0.772646 & -2.750695 & 0.901705 \\ 6 & -0.595608 & -3.926620 & 1.701353 \\ 6 & -0.797717 & -3.858959 & 3.110498 \\ 6 & -1.187867 & -2.627131 & 3.682106 \\ 6 & -1.349426 & -1.513549 & 2.901543 \\ 6 & -0.603638 & -5.015864 & 3.902338 \\ 6 & -0.222899 & -6.202478 & 3.330296 \\ 6 & -0.023491 & -6.278053 & 1.935542 \\ 6 & -0.207756 & -5.172919 & 1.142118 \\ 6 & -0.606789 & -2.900489 & -0.570797 \\ 6 & 0.596474 & -2.609161 & -1.187550 \\ 6 & 0.776234 & -2.866659 & -2.566378 \\ 6 & -0.247857 & -3.356231 & -3.330497 \\ 6 & -1.510443 & -3.618562 & -2.751021 \\ 6 & -1.688658 & -3.415313 & -1.350387 \\ 6 & -2.956970 & -3.706260 & -0.784146 \\ 6 & -3.992841 & -4.152629 & -1.565460 \\ 6 & -3.812759 & -4.352020 & -2.951704 \\ 6 & -2.596344 & -4.090268 & -3.528868 \\ 7 & 1.666498 & -1.995630 & -0.451300 \\ 15 & 1.847855 & -0.348602 & -0.824496 \\ 8 & 2.969397 & -0.225847 & -2.083093 \\ 6 & 4.254535 & -0.692012 & -1.969377 \\ 6 & 5.167429 & -0.075413 & -1.130636 \\ 6 & 6.447191 & -0.694277 & -0.953010 \\ 6 & 6.798186 & -1.829826 & -1.742751 \\ 6 & 5.866937 & -2.334207 & -2.683593 \\ 6 & 4.378902 & -1.899393 & 3.898000 \\ 6 & 2.997000 & -1.909402 & 3.713015 \\ 6 & 2.461724 & -2.226769 & 2.472418 \\ 6 & -0.367272 & 0.262576 & -3.160379 \\ 6 & -0.616444 & -1.789075 & -2.782514 \\ 6 & 7.365163 & -0.262867 & 0.038158 \\ 6 & -5.568279 & 2.972805 & 2.609758 \\ 6 & 8.566783 & -0.902995 & 0.218539 \\ 6 & 8.924393 & -2.003605 & -0.587982 \\ 6 & 8.053498 & -2.454868 & -1.546921 \\ 6 & 4.774765 & 1.157909 & -0.412215 \\ 6 & 3.621950 & 1.149922 & 0.348781 \\ 6 & 3.185606 & 2.273565 & 1.076825 \\ 6 & 3.903685 & 3.437158 & 1.022622 \\ 6 & 5.062747 & 3.529974 & 0.214246 \\ 6 & 5.500451 & 2.388307 & -0.524807 \\ 6 & 5.782349 & 4.745171 & 0.110841 \\ 6 & 6.878800 & 4.849873 & -0.705756 \\ 6 & -4.461793 & 4.983126 & 3.548772\end{array}$




\begin{tabular}{|c|c|c|c|}
\hline 6 & 1.489776 & 2.805964 & 4.235783 \\
\hline 6 & -0.214546 & 3.954831 & 5.611528 \\
\hline & 7.094015 & 0.571159 & 0.680841 \\
\hline & -1.663663 & -0.585327 & 3.371583 \\
\hline & 8.304548 & -3.314904 & -2.166585 \\
\hline & -0.761436 & -4.940101 & 4.977351 \\
\hline & 5.436083 & 5.600307 & 0.690022 \\
\hline & -3.109610 & -3.549136 & 0.282280 \\
\hline & -4.964542 & -4.350366 & -1.114918 \\
\hline & 3.580565 & 4.314840 & 1.581624 \\
\hline & -1.363363 & -2.575352 & 4.756246 \\
\hline & 6.153533 & -3.179700 & -3.306876 \\
\hline & 8.148842 & 3.824706 & -2.130240 \\
\hline 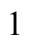 & 3.879426 & -2.177671 & -3.481776 \\
\hline & -2.441709 & -4.240547 & -4.596519 \\
\hline & -0.111021 & -3.528732 & -4.396942 \\
\hline & 0.277152 & -7.221225 & 1.485046 \\
\hline & 6.951086 & 1.694341 & -1.976767 \\
\hline & -0.054568 & -5.247994 & 0.067515 \\
\hline & 1.740447 & -2.630789 & -3.012462 \\
\hline & 7.420879 & 5.789690 & -0.780539 \\
\hline & 9.246707 & -0.561043 & 0.995767 \\
\hline & -4.640657 & -4.713733 & -3.558038 \\
\hline & -0.073373 & -7.085870 & 3.946722 \\
\hline & 9.881531 & -2.497859 & -0.438705 \\
\hline & 2.274290 & 2.182780 & 1.662960 \\
\hline 1 & -3.808190 & -1.091836 & 1.809455 \\
\hline & -2.753485 & 0.234065 & -2.110837 \\
\hline & -6.751063 & -1.106169 & -1.305154 \\
\hline & -3.606257 & 1.862679 & 0.874900 \\
\hline & 0.167072 & 0.943692 & 2.687811 \\
\hline 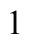 & -2.276032 & 4.095965 & 4.277447 \\
\hline & 2.233868 & -3.890285 & 0.132869 \\
\hline & 3.531780 & -3.018005 & -0.679233 \\
\hline ] & 5.337225 & -2.752065 & 0.749557 \\
\hline & 1.385740 & -2.197278 & 2.315539 \\
\hline 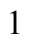 & 6.296077 & -2.195145 & 2.960079 \\
\hline & 2.329178 & -1.668052 & 4.539180 \\
\hline 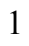 & 4.800396 & -1.646385 & 4.868675 \\
\hline 1 & -1.229428 & 1.931848 & -1.254745 \\
\hline | & 1.932739 & 2.751362 & -1.314825 \\
\hline 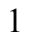 & 1.279537 & 2.549985 & -3.008277 \\
\hline & -0.046456 & 5.005995 & -0.287928 \\
\hline 1 & -1.043036 & 3.733489 & 0.438506 \\
\hline & 0.716406 & 3.573379 & 0.442403 \\
\hline 1 & -2.478236 & 4.497608 & -0.942222 \\
\hline 1 & -4.217879 & 5.275270 & -2.484873 \\
\hline 1 & -3.956308 & 4.943236 & -4.942686 \\
\hline 1 & -1.901085 & 3.839745 & -5.807314 \\
\hline 1 & -0.153825 & 3.081633 & -4.271596 \\
\hline 1 & 0.545156 & 4.039367 & 6.398636 \\
\hline 1 & -0.173100 & 4.872343 & 5.010153 \\
\hline 1 & -1.191323 & 3.913604 & 6.109432 \\
\hline 1 & 2.191150 & 2.986819 & 5.060878 \\
\hline 1 & 1.812118 & 1.881784 & 3.741189 \\
\hline 1 & 1.588059 & 3.635655 & 3.521586 \\
\hline
\end{tabular}




$\begin{array}{rrrr}1 & 0.643194 & 1.559331 & 6.524572 \\ 1 & -1.070147 & 1.383748 & 6.085521 \\ 1 & 0.180070 & 0.553736 & 5.137782 \\ 1 & -5.402231 & 5.537328 & 3.436994 \\ 1 & -4.455467 & 4.548617 & 4.556759 \\ 1 & -3.642184 & 5.710257 & 3.482239 \\ 1 & -6.506905 & 3.539022 & 2.538343 \\ 1 & -5.583878 & 2.201724 & 1.828578 \\ 1 & -5.553120 & 2.463719 & 3.582611 \\ 1 & -5.353032 & 5.204753 & 1.020659 \\ 1 & -3.580301 & 5.288447 & 0.963929 \\ 1 & -4.434329 & 3.888470 & 0.271793 \\ 6 & -4.052693 & 0.289056 & -4.200365 \\ 6 & -5.928798 & -1.300275 & -3.937329 \\ 6 & -6.217897 & 1.100437 & -3.291921 \\ 6 & -7.733625 & -2.110976 & 0.742048 \\ 6 & -5.805608 & -3.046265 & 1.979882 \\ 6 & -6.632940 & -0.743085 & 2.493859 \\ 1 & -4.401255 & 0.474963 & -5.224450 \\ 1 & -3.541841 & 1.201200 & -3.860404 \\ 1 & -3.313304 & -0.523078 & -4.241184 \\ 1 & -6.540469 & 1.352482 & -4.311475 \\ 1 & -7.115621 & 0.876584 & -2.701582 \\ 1 & -5.742687 & 1.990220 & -2.857426 \\ 1 & -6.227462 & -1.079172 & -4.970845 \\ 1 & -5.247676 & -2.161651 & -3.956445 \\ 1 & -6.833227 & -1.596957 & -3.392477 \\ 1 & -8.398007 & -2.546112 & 1.499332 \\ 1 & -8.238614 & -1.226005 & 0.334342 \\ 1 & -7.625407 & -2.847501 & -0.065550 \\ 1 & -6.506295 & -3.474952 & 2.709049 \\ 1 & -5.627581 & -3.803031 & 1.203441 \\ 1 & -4.855232 & -2.872847 & 2.501318 \\ 1 & -7.317680 & -1.155837 & 3.247352 \\ 1 & -5.702092 & -0.464549 & 3.004169 \\ 1 & -7.084230 & 0.175615 & 2.095467\end{array}$

$T_{S} 1_{I I L-r e-} \Psi-15233 s 4 a$

Number of imaginary frequencies : 1

The smallest frequencies are : -595.6759 $16.7472 \quad 20.2934 \mathrm{~cm}(-1)$

Electronic energy : $\quad H F=-4360.8701853$

Zero-point correction $=\quad 1.451958($ Hartree/Particle $)$

Thermal correction to Energy= $\quad 1.536806$

Thermal correction to Enthalpy= $\quad 1.537750$

Thermal correction to Gibbs Free Energy= $\quad 1.332955$

Sum of electronic and zero-point Energies $=\quad-4359.418227$

Sum of electronic and thermal Energies $=\quad-4359.333379$

Sum of electronic and thermal Enthalpies $=\quad-4359.332435$

Sum of electronic and thermal Free Energies $=\quad-4359.537230$

Cartesian Coordinates

$\begin{array}{llll}6 & -6.598849 & -2.576802 & -1.395451 \\ 6 & -5.477841 & -2.425136 & -0.539831 \\ 6 & -5.034928 & -3.569774 & 0.191589\end{array}$




\begin{tabular}{|c|c|c|c|}
\hline 6 & -5.746634 & -4.788596 & 0.076904 \\
\hline 6 & -6.840360 & -4.893782 & -0.743269 \\
\hline 6 & -7.260382 & -3.775832 & -1.493820 \\
\hline 6 & -4.760040 & -1.191050 & -0.416299 \\
\hline 6 & -3.608524 & -1.182217 & 0.346610 \\
\hline 6 & -3.168606 & -2.308086 & 1.068812 \\
\hline 6 & -3.878996 & -3.475745 & 1.004265 \\
\hline 6 & -5.160537 & 0.044574 & -1.126360 \\
\hline 6 & -4.252267 & 0.673456 & -1.960860 \\
\hline 6 & -4.624385 & 1.767722 & -2.773184 \\
\hline 6 & -5.879452 & 2.301756 & -2.673102 \\
\hline 6 & -6.805433 & 1.788094 & -1.732059 \\
\hline 6 & -6.445383 & 0.652444 & -0.946511 \\
\hline 6 & -8.064954 & 2.403446 & -1.532821 \\
\hline 6 & -8.931401 & 1.942957 & -0.574272 \\
\hline 6 & -8.565226 & 0.841937 & 0.227848 \\
\hline 6 & -7.359547 & 0.210517 & 0.043594 \\
\hline 8 & -2.961537 & 0.222908 & -2.072844 \\
\hline 15 & -1.844622 & 0.346392 & -0.810496 \\
\hline 8 & -2.866071 & -0.033864 & 0.442364 \\
\hline 45 & 0.042035 & -0.806697 & -1.279369 \\
\hline 15 & 1.433430 & 0.065227 & 0.440557 \\
\hline 6 & 1.730543 & -1.270814 & 1.675716 \\
\hline 6 & 2.882006 & -2.050933 & 1.599295 \\
\hline 6 & 3.120313 & -3.087245 & 2.505916 \\
\hline 6 & 2.168599 & -3.319172 & 3.500667 \\
\hline 6 & 0.991593 & -2.565536 & 3.598869 \\
\hline 6 & 0.773982 & -1.560657 & 2.653201 \\
\hline 6 & 4.417900 & -3.885221 & 2.386736 \\
\hline 6 & 0.000165 & -2.755042 & 4.747843 \\
\hline 6 & 0.170293 & -3.217970 & -1.510665 \\
\hline 6 & 0.137965 & -3.884695 & -0.154602 \\
\hline 6 & 1.198383 & -3.733655 & -2.476091 \\
\hline 6 & 2.266953 & -4.511249 & -2.013293 \\
\hline 6 & 3.254416 & -4.971775 & -2.877501 \\
\hline 6 & 3.199461 & -4.664595 & -4.232613 \\
\hline 6 & 2.142606 & -3.893379 & -4.709167 \\
\hline 6 & 1.156479 & -3.433553 & -3.844226 \\
\hline 6 & -1.021516 & -2.609481 & -1.976841 \\
\hline 6 & 0.388366 & -0.188325 & -3.146086 \\
\hline 8 & 0.145589 & 0.112438 & -4.239533 \\
\hline 6 & 1.128310 & 1.554102 & 1.512475 \\
\hline 6 & 0.751887 & 2.756369 & 0.929764 \\
\hline 6 & 0.558047 & 3.920891 & 1.743190 \\
\hline 6 & 0.762690 & 3.840091 & 3.151399 \\
\hline 6 & 1.174989 & 2.608865 & 3.708639 \\
\hline 6 & 1.351374 & 1.506662 & 2.915746 \\
\hline 6 & 0.549025 & 4.983563 & 3.957453 \\
\hline 6 & 0.145743 & 6.169940 & 3.400563 \\
\hline 6 & -0.057195 & 6.258514 & 2.007179 \\
\hline 6 & 0.146365 & 5.166748 & 1.199999 \\
\hline 6 & 0.572252 & 2.926039 & -0.539363 \\
\hline 6 & -0.637117 & 2.638336 & -1.145803 \\
\hline 6 & -0.849798 & 2.949778 & -2.508541 \\
\hline 6 & 0.144812 & 3.504127 & -3.266996 \\
\hline 6 & 1.412908 & 3.768500 & -2.700568 \\
\hline
\end{tabular}




\begin{tabular}{|c|c|c|c|}
\hline 6 & 1.631126 & 3.488203 & -1.318608 \\
\hline & 2.909598 & 3.768679 & -0.769744 \\
\hline & 3.913123 & 4.288036 & -1.548825 \\
\hline & 3.689054 & 4.574947 & -2.913072 \\
\hline & 2.464141 & 4.319177 & -3.473741 \\
\hline & -1.688653 & 1.990225 & -0.412513 \\
\hline & -2.761184 & 2.872486 & 0.098722 \\
\hline & -3.323758 & 2.490601 & 1.442908 \\
\hline & -4.702531 & 2.458186 & 1.640765 \\
\hline & -5.232496 & 2.127381 & 2.885969 \\
\hline & -4.385965 & 1.827923 & 3.947810 \\
\hline & -3.005343 & 1.859356 & 3.755870 \\
\hline & -2.480989 & 2.187471 & 2.513279 \\
\hline & 3.155413 & 0.436346 & -0.109280 \\
\hline & 3.516000 & 0.190273 & -1.422509 \\
\hline & 4.824769 & 0.409715 & -1.872804 \\
\hline & 5.750448 & 0.901489 & -0.958519 \\
\hline & 5.419572 & 1.162937 & 0.383420 \\
\hline & 4.113421 & 0.919095 & 0.791993 \\
\hline & 5.150837 & 0.099967 & -3.330494 \\
\hline & 6.479039 & 1.720285 & 1.333204 \\
\hline & -7.082009 & -0.624109 & 0.682837 \\
\hline & 1.681961 & 0.578402 & 3.374217 \\
\hline & -8.322785 & 3.263749 & -2.149307 \\
\hline & 0.709785 & 4.897384 & 5.031253 \\
\hline & -5.396445 & -5.646036 & 0.650274 \\
\hline & 3.096163 & 3.548852 & 0.280545 \\
\hline I & 4.891128 & 4.480887 & -1.111512 \\
\hline & -3.552064 & -4.354799 & 1.558869 \\
\hline 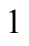 & 1.355025 & 2.548533 & 4.781560 \\
\hline & -6.173803 & 3.145424 & -3.295213 \\
\hline & -8.113413 & -3.865437 & -2.162745 \\
\hline 1 & -3.891043 & 2.162016 & -3.473048 \\
\hline & 2.277634 & 4.528740 & -4.526229 \\
\hline 1 & -0.019497 & 3.729238 & -4.319619 \\
\hline & -0.376295 & 7.201184 & 1.568509 \\
\hline 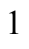 & -6.929500 & -1.728910 & -1.989699 \\
\hline 1 & -0.010850 & 5.253011 & 0.126807 \\
\hline & -1.818404 & 2.714038 & -2.944901 \\
\hline ] & -7.376233 & -5.836405 & -0.826917 \\
\hline & -9.241896 & 0.492485 & 1.004585 \\
\hline 1 & 4.491404 & 4.994113 & -3.516509 \\
\hline & -0.019168 & 7.042837 & 4.027931 \\
\hline 1 & -9.891711 & 2.430098 & -0.422024 \\
\hline 1 & -2.260653 & -2.215242 & 1.659748 \\
\hline ] & 3.822089 & 1.098493 & 1.824459 \\
\hline 1 & 2.754654 & -0.179547 & -2.106721 \\
\hline 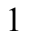 & 6.770853 & 1.091336 & -1.286952 \\
\hline 1 & 3.614759 & -1.839779 & 0.821462 \\
\hline 1 & -0.136732 & -0.956984 & 2.695863 \\
\hline 1 & 2.350223 & -4.105198 & 4.227113 \\
\hline 1 & -2.302249 & 3.868778 & 0.177652 \\
\hline 1 & -3.580997 & 2.966024 & -0.630794 \\
\hline 1 & -5.372802 & 2.675767 & 0.806982 \\
\hline 1 & -1.405420 & 2.176129 & 2.351054 \\
\hline & -6.312207 & 2.096429 & 3.019720 \\
\hline
\end{tabular}




\begin{tabular}{|c|c|c|c|}
\hline . & -2.330113 & 1.628102 & 4.578771 \\
\hline & -4.798545 & 1.566321 & 4.920029 \\
\hline & 1.252670 & -1.904434 & -1.281589 \\
\hline & -1.911308 & -2.722186 & -1.356388 \\
\hline & -1.232851 & -2.540640 & -3.042562 \\
\hline & 0.018516 & -4.971416 & -0.277284 \\
\hline & 1.048650 & -3.714450 & 0.432816 \\
\hline & -0.707277 & -3.511133 & 0.432158 \\
\hline & 2.339544 & -4.756594 & -0.956680 \\
\hline & 4.071931 & -5.572455 & -2.481693 \\
\hline & 3.971187 & -5.020376 & -4.911616 \\
\hline 1 & 2.083024 & -3.643360 & -5.766485 \\
\hline & 0.346439 & -2.831088 & -4.247832 \\
\hline & 0.309470 & -3.991323 & 5.589755 \\
\hline & 0.077196 & -1.522248 & 5.657473 \\
\hline & -1.427020 & -2.892606 & 4.212531 \\
\hline 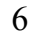 & 4.558271 & -4.939541 & 3.481284 \\
\hline U & 4.454549 & -4.595642 & 1.028799 \\
\hline 6 & 5.611323 & -2.926451 & 2.488103 \\
\hline 6 & 6.627570 & 0.310811 & -3.651870 \\
\hline 6 & 4.792809 & -1.361288 & -3.632268 \\
\hline 6 & 4.321784 & 1.020730 & -4.232408 \\
\hline & 5.961728 & 1.843612 & 2.765055 \\
\hline 6 & 7.705504 & 0.801942 & 1.351787 \\
\hline 6 & 6.894703 & 3.116455 & 0.856245 \\
\hline 1 & -0.443710 & -4.093515 & 6.381045 \\
\hline 1 & 0.287430 & -4.910794 & 4.990382 \\
\hline 1 & 1.287826 & -3.924440 & 6.081870 \\
\hline 1 & -2.124916 & -3.090596 & 5.036701 \\
\hline 1 & -1.771417 & -1.978058 & 3.715570 \\
\hline 1 & -1.501822 & -3.725479 & 3.499070 \\
\hline 1 & -0.616900 & -1.623218 & 6.503021 \\
\hline 1 & 1.090096 & -1.396337 & 6.062799 \\
\hline 1 & -0.185349 & -0.603526 & 5.116783 \\
\hline 1 & 5.504390 & -5.480319 & 3.352856 \\
\hline 1 & 4.569152 & -4.492130 & 4.483630 \\
\hline 1 & 3.749405 & -5.680638 & 3.444051 \\
\hline 1 & 6.555350 & -3.481731 & 2.404295 \\
\hline 1 & 5.599651 & -2.169651 & 1.693226 \\
\hline 1 & 5.611991 & -2.400692 & 3.452290 \\
\hline 1 & 5.384626 & -5.170679 & 0.922754 \\
\hline 1 & 3.613168 & -5.296728 & 0.933547 \\
\hline 1 & 4.404555 & -3.886892 & 0.192154 \\
\hline 1 & 6.815792 & 0.065508 & -4.704764 \\
\hline 1 & 6.935642 & 1.353503 & -3.499213 \\
\hline 1 & 7.275740 & -0.332507 & -3.042107 \\
\hline 1 & 4.536478 & 0.814577 & -5.290048 \\
\hline 1 & 3.243546 & 0.883303 & -4.077552 \\
\hline 1 & 4.553016 & 2.075519 & -4.032885 \\
\hline 1 & 5.046533 & -1.608467 & -4.672530 \\
\hline 1 & 5.345853 & -2.047360 & -2.976508 \\
\hline 1 & 3.720927 & -1.565153 & -3.502164 \\
\hline 1 & 6.755485 & 2.239074 & 3.411429 \\
\hline 1 & 5.107146 & 2.530266 & 2.838327 \\
\hline 1 & 5.655638 & 0.870678 & 3.172487 \\
\hline 1 & 8.466398 & 1.198943 & 2.037125 \\
\hline
\end{tabular}




\begin{tabular}{|c|c|c|}
\hline & 7.437467 & -0.206406 \\
\hline & 8.171490 & 0.706654 \\
\hline & 7.674517 & 3.529836 \\
\hline & 7.290401 & 3.101204 \\
\hline & 6.038767 & 3.805405 \\
\hline
\end{tabular}

TS1 $_{\text {IIL-re- }}-\psi_{-1223354 s}$

Number of imaginary frequencies : 1

The smallest frequencies are : -599.9721 $17.9677 \quad 21.6585 \mathrm{~cm}(-1)$

Electronic energy : $\quad H F=-4360.8703268$

Zero-point correction $=\quad 1.452003($ Hartree/Particle)

Thermal correction to Energy= $\quad 1.536869$

Thermal correction to Enthalpy= $\quad 1.537813$

Thermal correction to Gibbs Free Energy= 1.333234

Sum of electronic and zero-point Energies $=\quad-4359.418324$

Sum of electronic and thermal Energies $=\quad-4359.333458$

Sum of electronic and thermal Enthalpies $=\quad-4359.332514$

Sum of electronic and thermal Free Energies $=\quad-4359.537093$

Cartesian Coordinates

$\begin{array}{cccc}6 & 6.610060 & 2.542603 & -1.393757 \\ 6 & 5.486574 & 2.400599 & -0.539718 \\ 6 & 5.051746 & 3.549265 & 0.190243 \\ 6 & 5.773028 & 4.762397 & 0.075285 \\ 6 & 6.868678 & 4.858263 & -0.743476 \\ 6 & 7.281134 & 3.736313 & -1.492250 \\ 6 & 4.758788 & 1.172432 & -0.416212 \\ 6 & 3.606766 & 1.173090 & 0.345902 \\ 6 & 3.175247 & 2.302632 & 1.067395 \\ 6 & 3.894493 & 3.464788 & 1.002122 \\ 6 & 5.149703 & -0.066562 & -1.125780 \\ 6 & 4.237364 & -0.687111 & -1.962039 \\ 6 & 4.601678 & -1.783515 & -2.775064 \\ 6 & 5.851577 & -2.329167 & -2.672157 \\ 6 & 6.779670 & -1.825111 & -1.727971 \\ 6 & 6.428443 & -0.686148 & -0.943263 \\ 6 & 8.032545 & -2.452732 & -1.524978 \\ 6 & 8.900447 & -2.000877 & -0.563616 \\ 6 & 8.542767 & -0.896233 & 0.237403 \\ 6 & 7.344012 & -0.252921 & 0.049387 \\ 8 & 2.950261 & -0.226236 & -2.073804 \\ 15 & 1.832932 & -0.346007 & -0.811200 \\ 8 & 2.855307 & 0.030709 & 0.441948 \\ 45 & -0.049621 & 0.813490 & -1.282201 \\ 15 & -1.442417 & -0.051500 & 0.439616 \\ 6 & -1.728692 & 1.281405 & 1.681046 \\ 6 & -2.870774 & 2.075879 & 1.605937 \\ 6 & -3.087828 & 3.123936 & 2.505052 \\ 6 & -2.130675 & 3.344330 & 3.497264 \\ 6 & -0.967019 & 2.570693 & 3.599031 \\ 6 & -0.765936 & 1.560339 & 2.655791 \\ 6 & -4.362967 & 3.956509 & 2.376142 \\ 6 & 0.025383 & 2.745831 & 4.749565 \\ 6 & -0.159217 & 3.225697 & -1.516069\end{array}$




\begin{tabular}{|c|c|c|c|}
\hline & -0.124493 & 3.891957 & -0.159859 \\
\hline & -1.178948 & 3.750690 & -2.485226 \\
\hline & -2.230746 & 4.554671 & -2.029859 \\
\hline & -3.210117 & 5.025841 & -2.897701 \\
\hline & -3.163438 & 4.703106 & -4.249366 \\
\hline & -2.122838 & 3.905701 & -4.718860 \\
\hline & -1.144980 & 3.435435 & -3.850322 \\
\hline & 1.028432 & 2.607435 & -1.979976 \\
\hline & -0.409559 & 0.189682 & -3.143528 \\
\hline & -0.188195 & -0.122616 & -4.238138 \\
\hline & -1.146103 & -1.549157 & 1.501765 \\
\hline & -0.778526 & -2.751602 & 0.913688 \\
\hline & -0.600198 & -3.922593 & 1.722115 \\
\hline & -0.814296 & -3.848460 & 3.129253 \\
\hline & -1.215243 & -2.615903 & 3.691743 \\
\hline & -1.374174 & -1.507118 & 2.904395 \\
\hline & -0.619680 & -4.999147 & 3.929883 \\
\hline & -0.224825 & -6.186379 & 3.368941 \\
\hline & -0.010638 & -6.268067 & 1.976867 \\
\hline & -0.195981 & -5.169242 & 1.174902 \\
\hline & -0.590461 & -2.918185 & -0.555052 \\
\hline & 0.624627 & -2.635722 & -1.152206 \\
\hline & 0.849115 & -2.953705 & -2.511596 \\
\hline & -0.139041 & -3.510394 & -3.276629 \\
\hline 6 & -1.412747 & -3.769600 & -2.720545 \\
\hline & -1.643139 & -3.481267 & -1.342192 \\
\hline & -2.925958 & -3.758478 & -0.802708 \\
\hline & -3.922482 & -4.283554 & -1.586951 \\
\hline & -3.686921 & -4.577834 & -2.947709 \\
\hline & -2.457431 & -4.324475 & -3.499526 \\
\hline & 1.672178 & -1.989043 & -0.412330 \\
\hline 5 & 2.739605 & -2.873058 & 0.106375 \\
\hline ) & 3.294020 & -2.490908 & 1.453780 \\
\hline & 4.671570 & -2.460457 & 1.660194 \\
\hline ( & 5.194307 & -2.129291 & 2.908317 \\
\hline & 4.341641 & -1.827307 & 3.964527 \\
\hline 5 & 2.962210 & -1.856756 & 3.763910 \\
\hline & 2.444927 & -2.185177 & 2.518433 \\
\hline & -3.167060 & -0.416069 & -0.107079 \\
\hline 6 & -3.535987 & -0.157790 & -1.419470 \\
\hline & -4.839654 & -0.387821 & -1.866731 \\
\hline 6 & -5.760364 & -0.913243 & -0.958280 \\
\hline & -5.419574 & -1.192925 & 0.372397 \\
\hline 6 & -4.115048 & -0.922581 & 0.783280 \\
\hline 6 & -5.172439 & -0.075391 & -3.322859 \\
\hline & -6.411724 & -1.765608 & 1.385013 \\
\hline 1 & 7.072625 & 0.584366 & 0.687793 \\
\hline & -1.695744 & -0.577441 & 3.366422 \\
\hline 1 & 8.283908 & -3.315417 & -2.140810 \\
\hline & -0.787937 & -4.917661 & 5.002894 \\
\hline & 5.428779 & 5.623080 & 0.647392 \\
\hline & -3.119878 & -3.532538 & 0.244764 \\
\hline 1 & -4.904863 & -4.474120 & -1.158158 \\
\hline 1 & 3.574112 & 4.346821 & 1.555822 \\
\hline 1 & -1.399822 & -2.559804 & 4.764118 \\
\hline 1 & 6.139876 & -3.174900 & -3.294306 \\
\hline
\end{tabular}




\begin{tabular}{|c|c|c|c|}
\hline & 8.135906 & 3.818583 & -2.159897 \\
\hline & 3.866773 & -2.170240 & -3.477642 \\
\hline & -2.262075 & -4.540167 & -4.549140 \\
\hline & 0.034943 & -3.741914 & -4.326284 \\
\hline & 0.302794 & -7.211078 & 1.534851 \\
\hline & 6.934963 & 1.691535 & -1.986649 \\
\hline & -0.029822 & -5.250786 & 0.102718 \\
\hline & 1.823086 & -2.723684 & -2.938863 \\
\hline & 7.411991 & 5.796605 & -0.827266 \\
\hline & 9.220506 & -0.553463 & 1.016178 \\
\hline & -4.483898 & -5.000816 & -3.555573 \\
\hline & -0.074749 & -7.064959 & 3.992069 \\
\hline & 9.855387 & -2.497530 & -0.408380 \\
\hline & 2.266952 & 2.216683 & 1.658803 \\
\hline & -3.827182 & -1.111687 & 1.817323 \\
\hline & -2.779890 & 0.226243 & -2.101471 \\
\hline & -6.774553 & -1.112056 & -1.293682 \\
\hline & -3.608848 & 1.871651 & 0.831047 \\
\hline & 0.136691 & 0.944773 & 2.697067 \\
\hline & -2.296751 & 4.138454 & 4.218563 \\
\hline & 2.277869 & -3.868256 & 0.183183 \\
\hline & 3.564076 & -2.969106 & -0.617556 \\
\hline 1 & 5.346586 & -2.680010 & 0.830774 \\
\hline & 1.370284 & -2.172319 & 2.349746 \\
\hline 1 & 6.273230 & -2.099848 & 3.048656 \\
\hline & 2.282227 & -1.622915 & 4.582179 \\
\hline 1 & 4.748498 & -1.565295 & 4.939035 \\
\hline & -1.251762 & 1.921627 & -1.286440 \\
\hline & 1.918045 & 2.713484 & -1.358108 \\
\hline 1 & 1.240724 & 2.537643 & -3.045447 \\
\hline & 0.008606 & 4.977257 & -0.281316 \\
\hline ] & -1.040020 & 3.732113 & 0.423014 \\
\hline & 0.713437 & 3.508156 & 0.430772 \\
\hline & -2.296227 & 4.813743 & -0.976318 \\
\hline 1 & -4.014524 & 5.647224 & -2.507040 \\
\hline & -3.928640 & 5.067219 & -4.931306 \\
\hline 1 & -2.069788 & 3.643210 & -5.773500 \\
\hline & -0.347973 & 2.812276 & -4.248435 \\
\hline 6 & -0.252928 & 3.999736 & 5.576271 \\
\hline 6 & -0.091146 & 1.525923 & 5.672246 \\
\hline 5 & 1.458752 & 2.838050 & 4.221004 \\
\hline 6 & -4.482159 & 5.015904 & 3.468473 \\
\hline 5 & -4.368275 & 4.666565 & 1.017551 \\
\hline 6 & -5.584529 & 3.034258 & 2.468079 \\
\hline j & -6.654685 & -0.263459 & -3.633718 \\
\hline 6 & -4.793127 & 1.377887 & -3.636537 \\
\hline 6 & -4.363442 & -1.014371 & -4.224642 \\
\hline 6 & -7.744634 & -2.144019 & 0.744148 \\
\hline 6 & -5.827010 & -3.026973 & 2.032692 \\
\hline 5 & -6.679018 & -0.717594 & 2.471469 \\
\hline 1 & 0.501378 & 4.091859 & 6.367718 \\
\hline 1 & -0.206384 & 4.911010 & 4.965859 \\
\hline 1 & -1.233293 & 3.964301 & 6.067561 \\
\hline 1 & 2.156821 & 3.024215 & 5.047757 \\
\hline 1 & 1.782682 & 1.910700 & 3.733477 \\
\hline 1 & 1.560274 & 3.663118 & 3.501800 \\
\hline
\end{tabular}




$\begin{array}{rrrr}1 & 0.602683 & 1.615348 & 6.519323 \\ 1 & -1.108735 & 1.434468 & 6.074975 \\ 1 & 0.145495 & 0.594114 & 5.142144 \\ 1 & -5.413258 & 5.580612 & 3.333408 \\ 1 & -4.510686 & 4.570555 & 4.471407 \\ 1 & -3.654645 & 5.736261 & 3.435086 \\ 1 & -6.510258 & 3.618350 & 2.376382 \\ 1 & -5.590220 & 2.276686 & 1.673879 \\ 1 & -5.609343 & 2.509879 & 3.432475 \\ 1 & -5.281621 & 5.266280 & 0.902771 \\ 1 & -3.507655 & 5.344865 & 0.929699 \\ 1 & -4.329729 & 3.955483 & 0.182219 \\ 1 & -6.847311 & -0.009422 & -4.683758 \\ 1 & -6.977535 & -1.302186 & -3.485472 \\ 1 & -7.288609 & 0.385411 & -3.014883 \\ 1 & -4.581334 & -0.812480 & -5.282541 \\ 1 & -3.282622 & -0.890996 & -4.075407 \\ 1 & -4.608479 & -2.064499 & -4.016343 \\ 1 & -5.056032 & 1.624087 & -4.674681 \\ 1 & -5.324952 & 2.077468 & -2.977497 \\ 1 & -3.716276 & 1.563513 & -3.522393 \\ 1 & -8.410087 & -2.575973 & 1.502363 \\ 1 & -8.259308 & -1.274282 & 0.316505 \\ 1 & -7.617092 & -2.892242 & -0.049744 \\ 1 & -6.531451 & -3.435019 & 2.770014 \\ 1 & -5.641822 & -3.807406 & 1.282349 \\ 1 & -4.880953 & -2.835235 & 2.555055 \\ 1 & -7.369125 & -1.115280 & 3.228265 \\ 1 & -5.756165 & -0.415967 & 2.983281 \\ 1 & -7.133908 & 0.184969 & 2.041626\end{array}$

$\mathrm{TS1}_{\text {IIBI-}}-\psi_{-1 \mathrm{a} 2 \mathrm{a} 3 \mathrm{a} 4 \mathrm{a}}$

Number of imaginary frequencies : 1

The smallest frequencies are : -584.8699 $16.4472 \quad 18.3795 \mathrm{~cm}(-1)$

Electronic energy : $\quad H F=-4360.8644155$

Zero-point correction $=\quad 1.451529($ Hartree/Particle $)$

Thermal correction to Energy= $\quad 1.536664$

Thermal correction to Enthalpy= $\quad 1.537608$

Thermal correction to Gibbs Free Energy $=\quad 1.332505$

Sum of electronic and zero-point Energies $=\quad-4359.412886$

Sum of electronic and thermal Energies $=\quad-4359.327752$

Sum of electronic and thermal Enthalpies $=\quad-4359.326808$

Sum of electronic and thermal Free Energies $=\quad-4359.531911$

Cartesian Coordinates

$\begin{array}{llll}6 & 1.827257 & -1.469761 & 2.975673 \\ 6 & 2.640023 & -2.252670 & 2.153721 \\ 6 & 3.991008 & -2.379288 & 2.471463 \\ 6 & 4.524234 & -1.737914 & 3.587480 \\ 6 & 3.706863 & -0.964606 & 4.403319 \\ 6 & 2.354000 & -0.835018 & 4.092300 \\ 6 & 2.083263 & -3.006466 & 0.972701 \\ 7 & 1.136460 & -2.270999 & 0.102688\end{array}$




\begin{tabular}{|c|c|c|c|}
\hline & 1.480417 & -0.892761 & -0.827400 \\
\hline 8 & 2.672469 & -1.283751 & -1.942375 \\
\hline 6 & 3.862049 & -1.857721 & -1.566398 \\
\hline 6 & 4.804377 & -1.143926 & -0.848353 \\
\hline 6 & 5.950548 & -1.858861 & -0.361024 \\
\hline 6 & 6.156933 & -3.216092 & -0.752044 \\
\hline 6 & 5.219944 & -3.844622 & -1.608864 \\
\hline 6 & 4.085257 & -3.185526 & -1.992195 \\
\hline 6 & 6.860238 & -1.291770 & 0.567785 \\
\hline 6 & 7.920064 & -2.015075 & 1.057534 \\
\hline 6 & 8.140434 & -3.343791 & 0.639170 \\
\hline 6 & 7.272152 & -3.928033 & -0.247072 \\
\hline 6 & 4.592924 & 0.292302 & -0.554492 \\
\hline 6 & 3.413016 & 0.718900 & 0.028445 \\
\hline 6 & 3.211627 & 2.047954 & 0.450610 \\
\hline 6 & 4.203313 & 2.971499 & 0.276055 \\
\hline 6 & 5.395511 & 2.625196 & -0.404879 \\
\hline 6 & 5.583527 & 1.285253 & -0.857959 \\
\hline 6 & 6.383203 & 3.601120 & -0.681392 \\
\hline 6 & 7.507219 & 3.286190 & -1.401779 \\
\hline 6 & 7.680084 & 1.971818 & -1.882845 \\
\hline 6 & 6.747195 & 0.999621 & -1.616643 \\
\hline 8 & 2.419356 & -0.173987 & 0.325935 \\
\hline 6 & 0.014823 & -3.031081 & -0.369968 \\
\hline 6 & -1.226899 & -2.887355 & 0.218073 \\
\hline 6 & -2.346138 & -3.586309 & -0.330388 \\
\hline 6 & -2.154684 & -4.454538 & -1.446016 \\
\hline 6 & -0.853306 & -4.620472 & -1.975546 \\
\hline 6 & 0.199923 & -3.918131 & -1.454965 \\
\hline 6 & -3.655318 & -3.443397 & 0.197579 \\
\hline 6 & -4.714528 & -4.121728 & -0.351038 \\
\hline 6 & -4.520717 & -4.985988 & -1.451287 \\
\hline 6 & -3.267565 & -5.144899 & -1.98 \\
\hline 6 & -1.394165 & -2.147700 & 1.499848 \\
\hline 6 & -1.612151 & -0.780119 & 1.587117 \\
\hline 6 & -1.858881 & -0.208322 & 2.867852 \\
\hline 6 & -1.846903 & -0.959880 & 4.010783 \\
\hline 6 & -1.583441 & -2.346951 & 3.959327 \\
\hline 6 & -1.365714 & -2.953329 & 2.689268 \\
\hline 6 & -1.526670 & -3.133158 & 5.134104 \\
\hline 6 & -1.262069 & -4.477173 & 5.067412 \\
\hline 6 & -1.047164 & -5.086920 & 3.814171 \\
\hline 6 & -1.101675 & -4.348420 & 2.657530 \\
\hline 15 & -1.704450 & 0.277238 & 0.047866 \\
\hline 6 & -1.639665 & 1.973269 & 0.758919 \\
\hline 6 & -2.526814 & 2.959770 & 0.329337 \\
\hline 6 & -2.417854 & 4.275735 & 0.787797 \\
\hline 6 & -1.419781 & 4.560883 & 1.723466 \\
\hline 6 & -0.517808 & 3.594388 & 2.183731 \\
\hline 6 & -0.612315 & 2.311081 & 1.645439 \\
\hline 6 & -3.365309 & 5.380551 & 0.322813 \\
\hline 6 & -2.554766 & 6.518408 & -0.306868 \\
\hline 6 & 0.507025 & 3.955417 & 3.260116 \\
\hline 6 & 1.251517 & 2.725252 & 3.778491 \\
\hline 45 & -0.317332 & 0.032969 & -1.895605 \\
\hline 6 & -0.534934 & -1.326394 & -3.274013 \\
\hline
\end{tabular}




\begin{tabular}{|c|c|c|c|}
\hline & -0.648753 & -1.979646 & -4.226334 \\
\hline & 0.756348 & 1.877896 & -3.160898 \\
\hline 6 & -0.628772 & 1.834122 & -3.400633 \\
\hline & 1.316916 & 3.030343 & -2.415725 \\
\hline & 0.559498 & 3.751354 & -1.476662 \\
\hline & 1.032193 & 4.928901 & -0.918207 \\
\hline & 2.286177 & 5.428727 & -1.269744 \\
\hline U & 3.063284 & 4.712685 & -2.171912 \\
\hline U & 2.591880 & 3.527351 & -2.729124 \\
\hline 6 & 1.672791 & 1.183212 & -4.137752 \\
\hline 6 & -3.512484 & 0.067919 & -0.299721 \\
\hline 6 & -3.929092 & -0.412406 & -1.536353 \\
\hline 6 & -5.284306 & -0.614225 & -1.818875 \\
\hline 6 & -6.204799 & -0.327496 & -0.811268 \\
\hline 6 & -5.821790 & 0.136569 & 0.454125 \\
\hline 6 & -4.463197 & 0.331259 & 0.691000 \\
\hline 6 & -5.757883 & -1.096335 & -3.189415 \\
\hline 6 & -6.879875 & 0.376423 & 1.529303 \\
\hline 6 & 1.527746 & 4.949607 & 2.697245 \\
\hline 6 & -0.212461 & 4.593133 & 4.454723 \\
\hline 6 & -4.152091 & 5.915888 & 1.524028 \\
\hline 6 & -4.365747 & 4.881867 & -0.717604 \\
\hline 1 & 6.691781 & -0.277233 & 0.919316 \\
\hline 1 & -2.061840 & 0.855862 & 2.949238 \\
\hline 1 & 7.413480 & -4.959506 & -0.567484 \\
\hline 1 & -1.694027 & -2.643961 & 6.092747 \\
\hline 1 & 6.217967 & 4.616008 & -0.320034 \\
\hline 1 & -3.817191 & -2.770313 & 1.038128 \\
\hline 1 & -5.713595 & -3.984761 & 0.059395 \\
\hline 1 & 4.069942 & 3.997842 & 0.614862 \\
\hline 1 & -2.038842 & -0.494228 & 4.976942 \\
\hline 1 & 5.403701 & -4.868370 & -1.930763 \\
\hline 1 & 8.557543 & 1.725958 & -2.476937 \\
\hline 1 & 3.335673 & -3.651827 & -2.628008 \\
\hline 1 & -3.104426 & -5.805649 & -2.836989 \\
\hline 1 & -0.709472 & -5.296529 & -2.817006 \\
\hline 1 & -0.837673 & -6.152947 & 3.762679 \\
\hline 1 & 6.892061 & -0.004940 & -2.005899 \\
\hline 1 & -0.935781 & -4.839372 & 1.700920 \\
\hline 1 & 1.200489 & -4.014909 & -1.874668 \\
\hline 1 & 8.255713 & 4.045935 & -1.614656 \\
\hline 1 & 8.591998 & -1.559386 & 1.781563 \\
\hline 1 & -5.368420 & -5.521994 & -1.873447 \\
\hline 1 & -1.216409 & -5.073901 & 5.975457 \\
\hline 1 & 8.987316 & -3.903333 & 1.029453 \\
\hline 1 & 2.255856 & 2.300868 & 0.906745 \\
\hline 1 & -4.129789 & 0.696439 & 1.658801 \\
\hline 1 & -3.166277 & -0.645528 & -2.274796 \\
\hline 1 & -7.266109 & -0.476220 & -1.011341 \\
\hline 1 & -3.312743 & 2.688629 & -0.372184 \\
\hline 1 & 0.094286 & 1.537995 & 1.949081 \\
\hline 1 & -1.344402 & 5.575645 & 2.115138 \\
\hline 1 & 1.512464 & -3.867184 & 1.350175 \\
\hline 1 & 2.914470 & -3.426464 & 0.386112 \\
\hline 1 & 4.641353 & -2.976905 & 1.830434 \\
\hline 1 & 0.780448 & -1.334099 & 2.712649 \\
\hline
\end{tabular}




\begin{tabular}{|c|c|c|c|}
\hline 1 & 5.584788 & -1.840748 & 3.809615 \\
\hline 1 & 1.701690 & -0.233400 & 4.724246 \\
\hline 1 & 4.119687 & -0.461307 & 5.275113 \\
\hline & -1.565635 & 0.780315 & -2.624755 \\
\hline 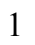 & 3.229627 & 2.992723 & -3.428751 \\
\hline & 4.052947 & 5.072111 & -2.449645 \\
\hline 1 & 2.653588 & 6.355359 & -0.832228 \\
\hline 1 & 0.410203 & 5.453379 & -0.192625 \\
\hline 1 & -0.417620 & 3.380271 & -1.170924 \\
\hline 1 & 2.564630 & 0.769178 & -3.652162 \\
\hline 1 & 1.160021 & 0.356938 & -4.639073 \\
\hline 1 & 2.013699 & 1.883102 & -4.916653 \\
\hline 1 & -1.249051 & 2.662301 & -3.054448 \\
\hline 1 & -0.961145 & 1.401613 & -4.345667 \\
\hline 1 & -5.019683 & 5.707308 & -1.025638 \\
\hline 1 & -3.863554 & 4.504695 & -1.618715 \\
\hline 1 & -5.007771 & 4.082336 & -0.323603 \\
\hline 1 & -3.224796 & 7.315565 & -0.656146 \\
\hline 1 & -1.851027 & 6.968520 & 0.404480 \\
\hline 1 & -1.977830 & 6.159825 & -1.170124 \\
\hline 1 & -4.839576 & 6.711584 & 1.206882 \\
\hline 1 & -4.748080 & 5.119352 & 1.988892 \\
\hline 1 & -3.493638 & 6.333872 & 2.295186 \\
\hline 1 & 0.510491 & 4.837503 & 5.244348 \\
\hline 1 & -0.730400 & 5.521377 & 4.186514 \\
\hline 1 & -0.955912 & 3.905320 & 4.878674 \\
\hline 1 & 2.277393 & 5.199371 & 3.460362 \\
\hline 1 & 2.052064 & 4.531765 & 1.826964 \\
\hline 1 & 1.051892 & 5.886665 & 2.379381 \\
\hline 1 & 1.921629 & 3.016032 & 4.597531 \\
\hline 1 & 0.553949 & 1.970470 & 4.168974 \\
\hline 1 & 1.872360 & 2.246925 & 3.010309 \\
\hline 6 & -4.595220 & -1.575805 & -4.057570 \\
\hline 6 & -6.741580 & -2.260807 & -3.042041 \\
\hline 6 & -6.454832 & 0.067206 & -3.903789 \\
\hline 6 & -6.277268 & 0.933136 & 2.817759 \\
\hline 6 & -7.925215 & 1.376049 & 1.024497 \\
\hline 6 & -7.562180 & -0.956890 & 1.856693 \\
\hline 1 & -4.979589 & -1.955338 & -5.012851 \\
\hline 1 & -3.889147 & -0.767188 & -4.291067 \\
\hline 1 & -4.037443 & -2.389831 & -3.572734 \\
\hline 1 & -7.072628 & -2.600861 & -4.032367 \\
\hline 1 & -6.265620 & -3.109803 & -2.534123 \\
\hline 1 & -7.639667 & -1.985691 & -2.476249 \\
\hline 1 & -6.800070 & -0.243476 & -4.899443 \\
\hline 1 & -7.327347 & 0.423946 & -3.341851 \\
\hline 1 & -5.770279 & 0.916081 & -4.032103 \\
\hline 1 & -7.072381 & 1.095716 & 3.556287 \\
\hline 1 & -5.549142 & 0.243359 & 3.265523 \\
\hline 1 & -5.778176 & 1.897520 & 2.651348 \\
\hline 1 & -8.686204 & 1.552826 & 1.796405 \\
\hline 1 & -7.461428 & 2.340818 & 0.780604 \\
\hline 1 & -8.444687 & 1.018637 & 0.127503 \\
\hline 1 & -8.321859 & -0.819159 & 2.638103 \\
\hline 1 & -8.060086 & -1.388896 & 0.979807 \\
\hline 1 & -6.830230 & -1.690570 & 2.220715 \\
\hline
\end{tabular}


$\mathrm{TS1}_{\text {IIB1- }} \psi_{-1 \mathrm{a} 2 \mathrm{aza} 4 \mathrm{~s}}$

Number of imaginary frequencies : 1

The smallest frequencies are : -580.9126 $16.2202 \quad 16.9663 \mathrm{~cm}(-1)$

Electronic energy : $\quad \mathrm{HF}=-4360.865017$

Zero-point correction $=\quad 1.451246($ Hartree/Particle $)$

Thermal correction to Energy $=\quad 1.536502$

Thermal correction to Enthalpy= $\quad 1.537446$

Thermal correction to Gibbs Free Energy $=\quad 1.331509$

Sum of electronic and zero-point Energies $=\quad-4359.413771$

Sum of electronic and thermal Energies $=\quad-4359.328515$

Sum of electronic and thermal Enthalpies $=\quad-4359.327571$

Sum of electronic and thermal Free Energies $=\quad-4359.533508$

Cartesian Coordinates

\begin{tabular}{|c|c|c|c|}
\hline 6 & 1.833422 & -1.458585 & 2.964463 \\
\hline 6 & 2.640347 & -2.251416 & 2.146223 \\
\hline 6 & 3.991320 & -2.382520 & 2.461935 \\
\hline 6 & 4.530401 & -1.735138 & 3.571729 \\
\hline 6 & 3.719057 & -0.950900 & 4.383056 \\
\hline 6 & 2.366059 & -0.817101 & 4.074278 \\
\hline 6 & 2.077689 & -3.008001 & 0.969697 \\
\hline 7 & 1.133502 & -2.271542 & 0.097448 \\
\hline 15 & 1.478410 & -0.892058 & -0.830220 \\
\hline 8 & 2.670671 & -1.281160 & -1.945056 \\
\hline 6 & 3.859408 & -1.856473 & -1.568220 \\
\hline 6 & 4.802175 & -1.143202 & -0.850236 \\
\hline 6 & 5.948335 & -1.858747 & -0.363675 \\
\hline 6 & 6.1535 & -3.216 & -0.754603 \\
\hline 6 & 5.215136 & -3.844726 & -1.609875 \\
\hline 6 & 4.080709 & -3.184936 & -1.992754 \\
\hline 6 & 6.859253 & -1.292123 & 0.564203 \\
\hline 6 & 7.919190 & -2.015944 & 1.052965 \\
\hline 6 & 8.138365 & -3.344862 & 0.634654 \\
\hline 6 & 7.26 & -3.928737 & -0.250587 \\
\hline 6 & 4.590668 & 0.292808 & -0.554867 \\
\hline 6 & 3.410724 & 0.718463 & 0.028836 \\
\hline 6 & 3.209512 & 2.046766 & 0.453545 \\
\hline 6 & 4.200837 & 2.970865 & 0.279950 \\
\hline 6 & 5.392801 & 2.625782 & -0.402037 \\
\hline 6 & 5.580951 & 1.286459 & -0.857054 \\
\hline 6 & 6.380282 & 3.602237 & -0.677454 \\
\hline 6 & 7.504141 & 3.288507 & -1.398604 \\
\hline 6 & 7.677083 & 1.974857 & -1.881598 \\
\hline 6 & 6.744443 & 1.002126 & -1.616497 \\
\hline 8 & 2.416672 & -0.174479 & 0.324198 \\
\hline 6 & 0.008260 & -3.028767 & -0.371445 \\
\hline 6 & -1.229058 & -2.887463 & 0.226326 \\
\hline 6 & -2.352908 & -3.584217 & -0.314997 \\
\hline 6 & -2.170299 & -4.448405 & -1.435197 \\
\hline 6 & -0.873231 & -4.611325 & -1.976405 \\
\hline 6 & 0.184475 & -3.911058 & -1.461816 \\
\hline 6 & -3.657823 & -3.441880 & 0.224079 \\
\hline 6 & -4.721200 & -4.118174 & -0.318865 \\
\hline
\end{tabular}




\begin{tabular}{|c|c|c|c|}
\hline 6 & -4.536421 & -4.979010 & -1.423343 \\
\hline 6 & -3.287581 & -5.136884 & -1.969119 \\
\hline 6 & -1.389640 & -2.147882 & 1.508401 \\
\hline 6 & -1.611307 & -0.780827 & 1.592798 \\
\hline & -1.856105 & -0.207141 & 2.872844 \\
\hline b & -1.837742 & -0.956469 & 4.017460 \\
\hline 6 & -1.570432 & -2.342811 & 3.968301 \\
\hline 6 & -1.355518 & -2.951172 & 2.698798 \\
\hline 6 & -1.507947 & -3.126690 & 5.144365 \\
\hline 6 & -1.241000 & -4.470294 & 5.079121 \\
\hline 6 & -1.029581 & -5.082107 & 3.826223 \\
\hline 6 & -1.089505 & -4.345883 & 2.668443 \\
\hline 15 & -1.706414 & 0.271295 & 0.049946 \\
\hline 6 & -1.660680 & 1.969445 & 0.758330 \\
\hline 6 & -2.557457 & 2.948725 & 0.331619 \\
\hline 6 & -2.450925 & 4.267887 & 0.782535 \\
\hline 6 & -1.452009 & 4.561057 & 1.714789 \\
\hline 6 & -0.544481 & 3.600519 & 2.176699 \\
\hline 6 & -0.631883 & 2.316228 & 1.639985 \\
\hline 6 & -3.395598 & 5.370369 & 0.306197 \\
\hline 6 & -2.579684 & 6.495351 & -0.340078 \\
\hline 6 & 0.479495 & 3.968965 & 3.251472 \\
\hline 6 & 1.228387 & 2.743284 & 3.774221 \\
\hline 45 & -0.319871 & 0.039055 & -1.893247 \\
\hline 6 & -0.557983 & -1.317033 & -3.271750 \\
\hline 8 & -0.703681 & -1.961077 & -4.226030 \\
\hline 6 & 0.754221 & 1.884472 & -3.153916 \\
\hline 6 & -0.630396 & 1.840185 & -3.395875 \\
\hline 6 & 1.311910 & 3.037204 & -2.407103 \\
\hline 6 & 0.551707 & 3.756318 & -1.468733 \\
\hline 6 & 1.021263 & 4.934935 & -0.909951 \\
\hline 6 & 2.274985 & 5.437020 & -1.259281 \\
\hline 6 & 3.054965 & 4.722446 & -2.160111 \\
\hline 6 & 2.586427 & 3.536484 & -2.718425 \\
\hline 6 & 1.672617 & 1.191300 & -4.130072 \\
\hline 6 & -3.509467 & 0.043598 & -0.309219 \\
\hline 6 & -3.918622 & -0.440837 & -1.551029 \\
\hline 6 & -5.267494 & -0.646074 & -1.841050 \\
\hline 6 & -6.203116 & -0.357919 & -0.839999 \\
\hline 6 & -5.827834 & 0.103819 & 0.422997 \\
\hline 6 & -4.466427 & 0.300281 & 0.668670 \\
\hline 6 & -5.727734 & -1.133056 & -3.214377 \\
\hline 6 & -6.824548 & 0.376058 & 1.547339 \\
\hline 6 & 1.497445 & 4.963941 & 2.685230 \\
\hline 6 & -0.242026 & 4.608706 & 4.443741 \\
\hline 6 & -4.177446 & 5.928024 & 1.500236 \\
\hline 6 & -4.399040 & 4.862568 & -0.726750 \\
\hline 1 & 6.691907 & -0.277386 & 0.915651 \\
\hline 1 & -2.061962 & 0.856552 & 2.952542 \\
\hline 1 & 7.409184 & -4.960363 & -0.570936 \\
\hline 1 & -1.673151 & -2.636182 & 6.102699 \\
\hline 1 & 6.215052 & 4.616565 & -0.314540 \\
\hline 1 & -3.813398 & -2.771223 & 1.068336 \\
\hline 1 & -5.717047 & -3.981559 & 0.099126 \\
\hline 1 & 4.067312 & 3.996614 & 0.620479 \\
\hline 1 & -2.027915 & -0.489358 & 4.983263 \\
\hline
\end{tabular}




\begin{tabular}{|c|c|c|c|}
\hline & 5.397649 & -4.868964 & -1.930919 \\
\hline & 8.554408 & 1.729991 & -2.476296 \\
\hline & 3.329869 & -3.651162 & -2.627156 \\
\hline & -3.131624 & -5.794847 & -2.822985 \\
\hline & -0.736380 & -5.283718 & -2.821959 \\
\hline & -0.818701 & -6.147921 & 3.776034 \\
\hline & 6.889338 & -0.001858 & -2.007230 \\
\hline & -0.926746 & -4.838122 & 1.711934 \\
\hline & 1.181551 & -4.005906 & -1.890334 \\
\hline & 8.252459 & 4.048675 & -1.610586 \\
\hline & 8.592151 & -1.560482 & 1.776186 \\
\hline & -5.387636 & -5.513489 & -1.840350 \\
\hline & -1.191088 & -5.065294 & 5.988070 \\
\hline & 8.985323 & -3.904825 & 1.024168 \\
\hline & 2.254017 & 2.298923 & 0.910575 \\
\hline & -4.147512 & 0.662038 & 1.645633 \\
\hline & -3.149936 & -0.672207 & -2.283535 \\
\hline & -7.259461 & -0.506619 & -1.054195 \\
\hline & -3.346891 & 2.670610 & -0.363405 \\
\hline & 0.082246 & 1.548953 & 1.940917 \\
\hline & -1.379492 & 5.578027 & 2.101235 \\
\hline & 1.502931 & -3.864132 & 1.351696 \\
\hline 1 & 2.905695 & -3.434836 & 0.383405 \\
\hline & 4.637438 & -2.987615 & 1.823621 \\
\hline 1 & 0.786860 & -1.319113 & 2.702542 \\
\hline & 5.590977 & -1.841527 & 3.792041 \\
\hline ] & 1.718065 & -0.206926 & 4.702497 \\
\hline & 4.136665 & -0.442383 & 5.249523 \\
\hline & -1.569890 & 0.789527 & -2.615054 \\
\hline I & 3.225999 & 3.003201 & -3.417439 \\
\hline & 4.044413 & 5.083725 & -2.436200 \\
\hline l & 2.639839 & 6.364338 & -0.821068 \\
\hline ] & 0.397198 & 5.458555 & -0.185555 \\
\hline | & -0.425042 & 3.383023 & -1.163995 \\
\hline ] & 2.564581 & 0.778351 & -3.643857 \\
\hline | & 1.161281 & 0.364267 & -4.631729 \\
\hline 1 & 2.013209 & 1.891794 & -4.908583 \\
\hline & -1.251607 & 2.668194 & -3.050928 \\
\hline 1 & -0.961670 & 1.406417 & -4.340696 \\
\hline ] & -5.053368 & 5.685355 & -1.040991 \\
\hline l & -3.899135 & 4.476628 & -1.625409 \\
\hline 1 & -5.040058 & 4.066709 & -0.323778 \\
\hline 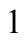 & -3.246284 & 7.289459 & -0.702682 \\
\hline 1 & -1.875530 & 6.953764 & 0.365593 \\
\hline & -2.002494 & 6.121430 & -1.196650 \\
\hline 1 & -4.858958 & 6.724370 & 1.172080 \\
\hline 1 & -4.779608 & 5.143478 & 1.977037 \\
\hline 1 & -3.515522 & 6.352260 & 2.264964 \\
\hline 1 & 0.480010 & 4.857235 & 5.232894 \\
\hline 1 & -0.762023 & 5.534886 & 4.172446 \\
\hline 1 & -0.984045 & 3.920497 & 4.869568 \\
\hline ] & 2.243405 & 5.222277 & 3.449114 \\
\hline 1 & 2.026423 & 4.542162 & 1.819703 \\
\hline ] & 1.018524 & 5.896596 & 2.359045 \\
\hline 1 & 1.897971 & 3.039663 & 4.591682 \\
\hline 1 & 0.534101 & 1.987236 & 4.168061 \\
\hline
\end{tabular}




$\begin{array}{lrrr}1 & 1.850578 & 2.264489 & 3.007440 \\ 6 & -4.560792 & -1.648670 & -4.056312 \\ 6 & -6.739629 & -2.274200 & -3.073480 \\ 6 & -6.385939 & 0.037683 & -3.953242 \\ 6 & -8.267803 & 0.110882 & 1.127570 \\ 6 & -6.496531 & -0.542645 & 2.731005 \\ 6 & -6.717165 & 1.841450 & 1.984408 \\ 1 & -4.936687 & -2.027044 & -5.015465 \\ 1 & -3.826889 & -0.863520 & -4.283444 \\ 1 & -4.034132 & -2.472127 & -3.553098 \\ 1 & -7.046399 & -2.628541 & -4.066548 \\ 1 & -6.297869 & -3.121811 & -2.532942 \\ 1 & -7.649708 & -1.969101 & -2.543595 \\ 1 & -6.726107 & -0.277427 & -4.949282 \\ 1 & -7.256278 & 0.423352 & -3.407198 \\ 1 & -5.678392 & 0.867234 & -4.083418 \\ 1 & -8.941297 & 0.323973 & 1.967397 \\ 1 & -8.575755 & 0.749464 & 0.289590 \\ 1 & -8.425089 & -0.936299 & 0.838283 \\ 1 & -7.201274 & -0.372714 & 3.556276 \\ 1 & -6.569485 & -1.598155 & 2.435138 \\ 1 & -5.483035 & -0.377955 & 3.118462 \\ 1 & -7.396210 & 2.041610 & 2.824284 \\ 1 & -5.702030 & 2.106795 & 2.305990 \\ 1 & -6.992346 & 2.514562 & 1.161599\end{array}$

TS1 $_{I I B I-} \psi_{-1 \text { a2a3s4a }}$

Number of imaginary frequencies : 1

The smallest frequencies are : -564.8856 $17.6673 \quad 19.6206 \mathrm{~cm}(-1)$

Electronic energy : $\quad H F=-4360.8642994$

Zero-point correction $=\quad 1.451610($ Hartree/Particle $)$

Thermal correction to Energy= $\quad 1.536728$

Thermal correction to Enthalpy= $\quad 1.537672$

Thermal correction to Gibbs Free Energy $=\quad 1.332906$

Sum of electronic and zero-point Energies $=\quad-4359.412690$

Sum of electronic and thermal Energies $=\quad-4359.327572$

Sum of electronic and thermal Enthalpies $=\quad-4359.326627$

Sum of electronic and thermal Free Energies $=\quad-4359.531393$

Cartesian Coordinates

$\begin{array}{cccc}6 & 1.828236 & -1.483946 & 2.977763 \\ 6 & 2.659711 & -2.236078 & 2.146036 \\ 6 & 4.008798 & -2.354274 & 2.475923 \\ 6 & 4.520813 & -1.737260 & 3.615197 \\ 6 & 3.684174 & -0.997250 & 4.442517 \\ 6 & 2.333918 & -0.874560 & 4.118319 \\ 6 & 2.125540 & -2.975849 & 0.945898 \\ 7 & 1.161628 & -2.246501 & 0.089510 \\ 15 & 1.487820 & -0.856534 & -0.830479 \\ 8 & 2.672392 & -1.230713 & -1.960100 \\ 6 & 3.865224 & -1.808278 & -1.599860 \\ 6 & 4.809617 & -1.105020 & -0.874022 \\ 6 & 5.955621 & -1.827229 & -0.397667 \\ 6 & 6.161102 & -3.178933 & -0.807925\end{array}$




\begin{tabular}{|c|c|c|c|}
\hline 6 & 5.224958 & -3.793198 & -1.675912 \\
\hline & 4.090254 & -3.128124 & -2.048915 \\
\hline & 6.866700 & -1.273040 & 0.537693 \\
\hline & 7.926404 & -2.003635 & 1.016548 \\
\hline & 8.145365 & -3.326797 & 0.580133 \\
\hline & 7.276166 & -3.898476 & -0.313323 \\
\hline & 4.602280 & 0.329000 & -0.567447 \\
\hline & 3.424157 & 0.754357 & 0.019880 \\
\hline & 3.224128 & 2.083682 & 0.442175 \\
\hline 6 & 4.217284 & 3.006089 & 0.269418 \\
\hline & 5.409382 & 2.659776 & -0.411326 \\
\hline & 5.594412 & 1.321052 & -0.869056 \\
\hline & 6.399368 & 3.634546 & -0.683731 \\
\hline & 7.523049 & 3.319688 & -1.404583 \\
\hline & 7.692837 & 2.006815 & -1.890778 \\
\hline & 6.757471 & 1.035832 & -1.628906 \\
\hline & 2.432946 & -0.141260 & 0.320718 \\
\hline U & 0.053603 & -3.023992 & -0.388153 \\
\hline 6 & -1.194181 & -2.898867 & 0.191344 \\
\hline & -2.295543 & -3.627832 & -0.355286 \\
\hline & -2.075528 & -4.527761 & -1.439785 \\
\hline 6 & -0.768841 & -4.667946 & -1.964075 \\
\hline 6 & 0.262471 & -3.921827 & -1.460382 \\
\hline 6 & -3.613138 & -3.493705 & 0.154140 \\
\hline 6 & -4.650169 & -4.222789 & -0.371511 \\
\hline 6 & -4.423474 & -5.133601 & -1.426752 \\
\hline 6 & -3.164589 & -5.274890 & -1.952507 \\
\hline 6 & -1.381379 & -2.172323 & 1.478201 \\
\hline 6 & -1.623547 & -0.810485 & 1.580743 \\
\hline 6 & -1.885366 & -0.256648 & 2.866527 \\
\hline 6 & -1.866560 & -1.020591 & 4.000754 \\
\hline 6 & -1.577592 & -2.402062 & 3.934833 \\
\hline 6 & -1.343013 & -2.990663 & 2.659361 \\
\hline 6 & -1.510160 & -3.198862 & 5.101765 \\
\hline 6 & -1.218428 & -4.536580 & 5.022762 \\
\hline 6 & -0.985671 & -5.128729 & 3.764405 \\
\hline 6 & -1.050179 & -4.379702 & 2.615020 \\
\hline 5 & -1.721349 & 0.263675 & 0.056228 \\
\hline 6 & -1.669981 & 1.948957 & 0.791717 \\
\hline 6 & -2.566057 & 2.936439 & 0.384239 \\
\hline 6 & -2.460299 & 4.245495 & 0.863094 \\
\hline 6 & -1.456386 & 4.521769 & 1.795346 \\
\hline 6 & -0.546355 & 3.552778 & 2.234484 \\
\hline 6 & -0.639207 & 2.277745 & 1.677432 \\
\hline 6 & -3.417031 & 5.352325 & 0.422922 \\
\hline 6 & -2.616857 & 6.502167 & -0.198192 \\
\hline 6 & 0.487645 & 3.900778 & 3.306458 \\
\hline 6 & 1.237113 & 2.663844 & 3.801021 \\
\hline 45 & -0.332130 & 0.072468 & -1.872104 \\
\hline 6 & -0.663010 & -1.298858 & -3.221841 \\
\hline 8 & -1.014423 & -1.918369 & -4.138518 \\
\hline 6 & 0.733771 & 1.904977 & -3.141153 \\
\hline 6 & -0.651656 & 1.865878 & -3.375331 \\
\hline 6 & 1.294774 & 3.053473 & -2.389396 \\
\hline 6 & 0.546005 & 3.747601 & -1.423576 \\
\hline 6 & 1.013695 & 4.922415 & -0.855034 \\
\hline
\end{tabular}




\begin{tabular}{|c|c|c|c|}
\hline & 2.253910 & 5.444496 & -1.222964 \\
\hline & 3.023257 & 4.753575 & -2.151094 \\
\hline & 2.556908 & 3.571132 & -2.718895 \\
\hline & 1.645210 & 1.214312 & -4.125285 \\
\hline & -3.525283 & 0.049433 & -0.306882 \\
\hline & -3.937460 & -0.363475 & -1.564014 \\
\hline & -5.294345 & -0.535440 & -1.873160 \\
\hline & -6.220520 & -0.315839 & -0.859610 \\
\hline & -5.840572 & 0.083985 & 0.433777 \\
\hline & -4.487042 & 0.267948 & 0.689271 \\
\hline & -5.677140 & -0.950691 & -3.291716 \\
\hline & -6.906503 & 0.280253 & 1.510074 \\
\hline & 1.503733 & 4.902219 & 2.748025 \\
\hline & -0.221222 & 4.522981 & 4.515416 \\
\hline & -4.194906 & 5.866617 & 1.639055 \\
\hline & -4.425470 & 4.863739 & -0.614589 \\
\hline & 6.698724 & -0.263443 & 0.903558 \\
\hline & -2.105411 & 0.803301 & 2.958165 \\
\hline & 7.416778 & -4.925501 & -0.648039 \\
\hline & -1.691139 & -2.722779 & 6.064524 \\
\hline & 6.236191 & 4.648475 & -0.318820 \\
\hline & -3.798055 & -2.790752 & 0.965091 \\
\hline & -5.655350 & -4.091329 & 0.024588 \\
\hline & 4.084048 & 4.031977 & 0.609479 \\
\hline & -2.071255 & -0.569548 & 4.971182 \\
\hline & 5.409209 & -4.811240 & -2.015156 \\
\hline & 8.569954 & 1.761112 & -2.485446 \\
\hline & 3.342011 & -3.583778 & -2.693868 \\
\hline & -2.978386 & -5.963862 & -2.775340 \\
\hline 1 & -0.602876 & -5.363756 & -2.785130 \\
\hline & -0.754214 & -6.189669 & 3.702886 \\
\hline & 6.900309 & 0.032650 & -2.022314 \\
\hline & -0.869698 & -4.858052 & 1.654669 \\
\hline & 1.266927 & -4.003080 & -1.873317 \\
\hline 1 & 8.273539 & 4.078399 & -1.614092 \\
\hline & 8.599252 & -1.558418 & 1.746204 \\
\hline ] & -5.252536 & -5.711961 & -1.829152 \\
\hline & -1.164655 & -5.141533 & 5.924903 \\
\hline 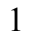 & 8.992131 & -3.892135 & 0.962240 \\
\hline 1 & 2.267609 & 2.342011 & 0.893210 \\
\hline & -4.160889 & 0.591619 & 1.674057 \\
\hline ] & -3.178763 & -0.562244 & -2.318235 \\
\hline & -7.279720 & -0.453923 & -1.068589 \\
\hline 1 & -3.355353 & 2.672113 & -0.316176 \\
\hline & 0.074397 & 1.503787 & 1.961795 \\
\hline t & -1.383006 & 5.531127 & 2.201127 \\
\hline 1 & 1.576396 & -3.858890 & 1.303187 \\
\hline 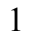 & 2.968780 & -3.362159 & 0.354046 \\
\hline 1 & 4.673222 & -2.928038 & 1.827629 \\
\hline & 0.782749 & -1.354578 & 2.706172 \\
\hline 1 & 5.579544 & -1.833918 & 3.848811 \\
\hline & 1.667277 & -0.298222 & 4.758713 \\
\hline 1 & 4.080238 & -0.514227 & 5.333346 \\
\hline 1 & -1.590184 & 0.819242 & -2.573738 \\
\hline & 3.186929 & 3.055981 & -3.439930 \\
\hline & 4.002279 & 5.131483 & -2.441631 \\
\hline
\end{tabular}




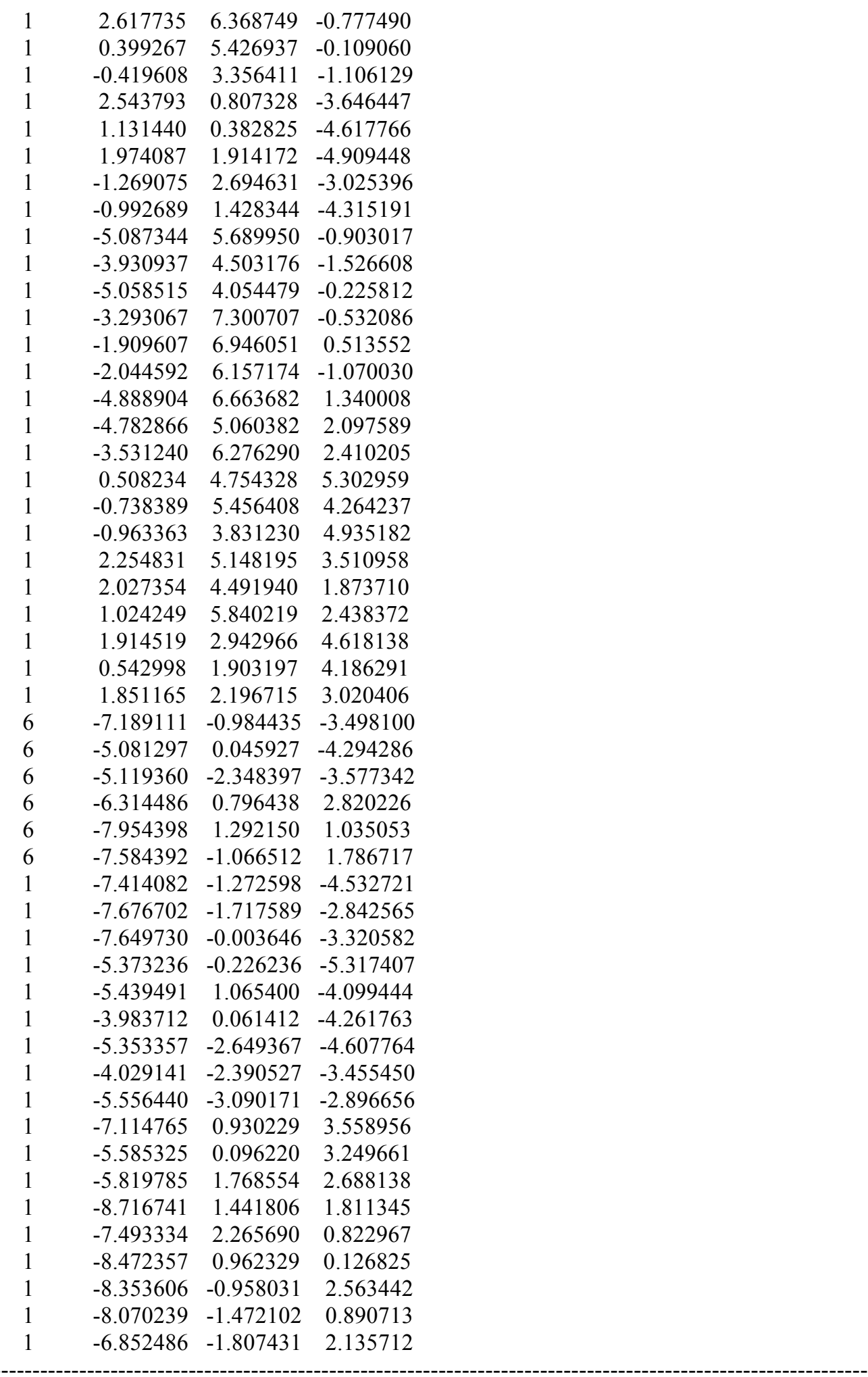

TS1 $_{\text {IIB1- }} \psi_{-1 \mathrm{a} 2 \mathrm{a} 3 \mathrm{~s} 4 \mathrm{~s}}$

Number of imaginary frequencies : 1

The smallest frequencies are : $-564.1984 \quad 17.2863 \quad 17.9466 \mathrm{~cm}(-1)$

Electronic energy : $\quad H F=-4360.8647196$ 
Zero-point correction $=$

Thermal correction to Energy=

Thermal correction to Enthalpy=

Thermal correction to Gibbs Free Energy=

Sum of electronic and zero-point Energies=

Sum of electronic and thermal Energies=

Sum of electronic and thermal Enthalpies=

Sum of electronic and thermal Free Energies=
1.451524 (Hartree/Particle)

1.536661

1.537605

1.332798

$-4359.413196$

$-4359.328059$

$-4359.327114$

$-4359.531921$

Cartesian Coordinates

$\begin{array}{cccc}6 & 1.834472 & -1.485790 & 2.966097 \\ 6 & 2.664785 & -2.240639 & 2.135651 \\ 6 & 4.013672 & -2.360135 & 2.465710 \\ 6 & 4.526627 & -1.741676 & 3.603816 \\ 6 & 3.691204 & -0.998532 & 4.429515 \\ 6 & 2.341114 & -0.874395 & 4.105072 \\ 6 & 2.129750 & -2.980037 & 0.935583 \\ 7 & 1.166028 & -2.249911 & 0.079663 \\ 15 & 1.489693 & -0.855502 & -0.834265 \\ 8 & 2.676808 & -1.221990 & -1.963615 \\ 6 & 3.871066 & -1.796447 & -1.602923 \\ 6 & 4.812126 & -1.091460 & -0.874404 \\ 6 & 5.960336 & -1.810583 & -0.398746 \\ 6 & 6.171127 & -3.160557 & -0.811985 \\ 6 & 5.237660 & -3.776495 & -1.681670 \\ 6 & 4.100816 & -3.114744 & -2.054077 \\ 6 & 6.868550 & -1.255260 & 0.538676 \\ 6 & 7.930646 & -1.982986 & 1.016611 \\ 6 & 8.154915 & -3.304268 & 0.577209 \\ 6 & 7.288519 & -3.877077 & -0.318260 \\ 6 & 4.598837 & 0.340962 & -0.564145 \\ 6 & 3.418405 & 0.759584 & 0.023320 \\ 6 & 3.211823 & 2.086907 & 0.448684 \\ 6 & 4.200716 & 3.014423 & 0.278692 \\ 6 & 5.395001 & 2.675118 & -0.401788 \\ 6 & 5.586698 & 1.338230 & -0.862354 \\ 6 & 6.380837 & 3.654995 & -0.671081 \\ 6 & 7.506620 & 3.346881 & -1.391538 \\ 6 & 7.682915 & 2.035903 & -1.880495 \\ 6 & 6.751797 & 1.060053 & -1.621697 \\ 8 & 2.431059 & -0.141411 & 0.320483 \\ 6 & 0.057563 & -3.026123 & -0.399269 \\ 6 & -1.188522 & -2.905591 & 0.184810 \\ 6 & -2.291747 & -3.630710 & -0.362559 \\ 6 & -2.075493 & -4.521726 & -1.455096 \\ 6 & -0.770308 & -4.658423 & -1.984225 \\ 6 & 0.263089 & -3.916641 & -1.478158 \\ 6 & -3.607158 & -3.500713 & 0.153814 \\ 6 & -4.646230 & -4.224282 & -0.375352 \\ 6 & -4.423912 & -5.125151 & -1.440038 \\ 6 & -3.166797 & -5.263368 & -1.970910 \\ 6 & -1.373410 & -2.184043 & 1.474308 \\ 6 & -1.619874 & -0.823259 & 1.579329 \\ 6 & -1.879317 & -0.272523 & 2.866752 \\ 6 & -1.855297 & -1.038770 & 3.999590\end{array}$




\begin{tabular}{|c|c|c|c|}
\hline 6 & -1.563671 & -2.419450 & 3.930417 \\
\hline 6 & -1.330665 & -3.004849 & 2.653251 \\
\hline & -1.492216 & -3.218664 & 5.095468 \\
\hline & -1.198620 & -4.555724 & 5.012730 \\
\hline & -0.967995 & -5.144837 & 3.752498 \\
\hline & -1.036115 & -4.393385 & 2.604945 \\
\hline & -1.722595 & 0.251529 & 0.055427 \\
\hline 6 & -1.691993 & 1.936452 & 0.794068 \\
\hline 6 & -2.600814 & 2.915164 & 0.393217 \\
\hline 6 & -2.501968 & 4.226031 & 0.869403 \\
\hline 6 & -1.498047 & 4.510283 & 1.799287 \\
\hline 6 & -0.579030 & 3.548696 & 2.236112 \\
\hline 6 & -0.660808 & 2.274351 & 1.676022 \\
\hline 6 & -3.461946 & 5.328008 & 0.423877 \\
\hline 6 & -2.663140 & 6.477797 & -0.199503 \\
\hline 6 & 0.453411 & 3.903438 & 3.307487 \\
\hline 6 & 1.211033 & 2.671342 & 3.801951 \\
\hline & -0.331452 & 0.074525 & -1.871882 \\
\hline & -0.657667 & -1.290372 & -3.228518 \\
\hline 8 & -1.004230 & -1.907322 & -4.148751 \\
\hline 6 & 0.728056 & 1.919730 & -3.131665 \\
\hline 6 & -0.656291 & 1.873203 & -3.369355 \\
\hline 6 & 1.280515 & 3.068968 & -2.375083 \\
\hline & 0.524745 & 3.758189 & -1.411027 \\
\hline 6 & 0.984995 & 4.934463 & -0.839426 \\
\hline & 2.224301 & 5.462679 & -1.201682 \\
\hline 6 & 3.000537 & 4.776402 & -2.127489 \\
\hline & 2.541677 & 3.592663 & -2.698590 \\
\hline & 1.645919 & 1.236047 & -4.114739 \\
\hline & -3.522983 & 0.022721 & -0.316339 \\
\hline 0 & -3.934059 & -0.372336 & -1.583762 \\
\hline 6 & -5.286329 & -0.539840 & -1.896764 \\
\hline o & -6.221995 & -0.336203 & -0.881173 \\
\hline 6 & -5.843419 & 0.036117 & 0.415012 \\
\hline 5 & -4.486057 & 0.218844 & 0.674655 \\
\hline 6 & -5.666373 & -0.939255 & -3.321113 \\
\hline 6 & -6.843722 & 0.247518 & 1.550037 \\
\hline o & 1.463432 & 4.910883 & 2.748970 \\
\hline 6 & -0.258899 & 4.521385 & 4.516596 \\
\hline 6 & -4.244558 & 5.845653 & 1.635331 \\
\hline 6 & -4.465250 & 4.832717 & -0.615353 \\
\hline 1 & 6.696472 & -0.247205 & 0.906844 \\
\hline & -2.101339 & 0.786736 & 2.960948 \\
\hline 1 & 7.433202 & -4.902785 & -0.655269 \\
\hline 1 & -1.671876 & -2.745103 & 6.059702 \\
\hline 1 & 6.212742 & 4.667322 & -0.303977 \\
\hline 1 & -3.788791 & -2.805469 & 0.972692 \\
\hline 1 & -5.649943 & -4.097582 & 0.025930 \\
\hline 1 & 4.062342 & 4.038912 & 0.620950 \\
\hline 1 & -2.057997 & -0.590001 & 4.971490 \\
\hline 1 & 5.425693 & -4.793271 & -2.022638 \\
\hline 1 & 8.561734 & 1.795569 & -2.474844 \\
\hline 1 & 3.354341 & -3.571886 & -2.700025 \\
\hline 1 & -2.983999 & -5.945339 & -2.800308 \\
\hline 1 & -0.607269 & -5.347701 & -2.811358 \\
\hline 1 & -0.735456 & -6.205373 & 3.688152 \\
\hline
\end{tabular}




\begin{tabular}{|c|c|c|c|}
\hline [ & 6.899581 & 0.058426 & -2.017225 \\
\hline & -0.857598 & -4.869180 & 1.642940 \\
\hline & 1.266171 & -3.994731 & -1.895169 \\
\hline & 8.253811 & 4.109506 & -1.598612 \\
\hline & 8.601254 & -1.536967 & 1.747839 \\
\hline & -5.254868 & -5.699074 & -1.844863 \\
\hline & -1.141914 & -5.162639 & 5.913369 \\
\hline & 9.003528 & -3.867330 & 0.958581 \\
\hline & 2.253882 & 2.339557 & 0.899863 \\
\hline & -4.170193 & 0.524475 & 1.671381 \\
\hline & -3.173201 & -0.558936 & -2.338720 \\
\hline & -7.277330 & -0.468920 & -1.101983 \\
\hline & -3.392741 & 2.643781 & -0.301678 \\
\hline & 0.062153 & 1.507304 & 1.955498 \\
\hline & -1.431268 & 5.520455 & 2.204194 \\
\hline & 1.580365 & -3.863010 & 1.292655 \\
\hline & 2.972584 & -3.366374 & 0.343132 \\
\hline & 4.677416 & -2.935665 & 1.818262 \\
\hline & 0.789388 & -1.355005 & 2.693783 \\
\hline & 5.585258 & -1.839357 & 3.837386 \\
\hline & 1.675437 & -0.295112 & 4.743829 \\
\hline & 4.088133 & -0.514000 & 5.319137 \\
\hline 1 & -1.591546 & 0.821290 & -2.569768 \\
\hline & 3.176930 & 3.081230 & -3.417690 \\
\hline & 3.979006 & 5.159042 & -2.413670 \\
\hline ] & 2.581943 & 6.388083 & -0.753602 \\
\hline & 0.365509 & 5.435492 & -0.095335 \\
\hline 1 & -0.440444 & 3.362402 & -1.097448 \\
\hline & 2.544783 & 0.831629 & -3.634281 \\
\hline 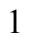 & 1.137454 & 0.403676 & -4.611217 \\
\hline 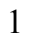 & 1.974139 & 1.939595 & -4.895857 \\
\hline & -1.279527 & 2.697283 & -3.018664 \\
\hline l & -0.992757 & 1.435640 & -4.310797 \\
\hline & -5.129995 & 5.655305 & -0.907530 \\
\hline 1 & -3.966311 & 4.472117 & -1.524994 \\
\hline & -5.095650 & 4.021421 & -0.226616 \\
\hline 1 & -3.340994 & 7.271989 & -0.540380 \\
\hline 1 & -1.960820 & 6.928116 & 0.513087 \\
\hline & -2.085670 & 6.130732 & -1.067094 \\
\hline 1 & -4.937047 & 6.642090 & 1.331184 \\
\hline 1 & -4.834994 & 5.041235 & 2.093638 \\
\hline 1 & -3.583885 & 6.257370 & 2.407982 \\
\hline 1 & 0.469213 & 4.756098 & 5.304375 \\
\hline 1 & -0.780742 & 5.452290 & 4.265795 \\
\hline 1 & -0.997574 & 3.825679 & 4.935950 \\
\hline 1 & 2.210336 & 5.164801 & 3.513433 \\
\hline 1 & 1.992830 & 4.501826 & 1.877576 \\
\hline & 0.977776 & 5.844275 & 2.434946 \\
\hline 1 & 1.887010 & 2.955151 & 4.618622 \\
\hline 1 & 0.522327 & 1.906094 & 4.187800 \\
\hline 1 & 1.827847 & 2.208192 & 3.021150 \\
\hline 6 & -7.177808 & -0.968424 & -3.533096 \\
\hline 6 & -5.066004 & 0.065179 & -4.313088 \\
\hline 6 & -5.110107 & -2.335391 & -3.618071 \\
\hline 6 & -8.283750 & -0.020711 & 1.120554 \\
\hline 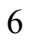 & -6.505305 & -0.709438 & 2.699757 \\
\hline
\end{tabular}




$\begin{array}{rrrr}6 & -6.755506 & 1.697533 & 2.040869 \\ 1 & -7.399228 & -1.244973 & -4.571670 \\ 1 & -7.669304 & -1.707741 & -2.887570 \\ 1 & -7.637823 & 0.011028 & -3.346917 \\ 1 & -5.356478 & -0.196367 & -5.339415 \\ 1 & -5.421787 & 1.083743 & -4.109213 \\ 1 & -3.968492 & 0.077424 & -4.278084 \\ 1 & -5.344224 & -2.628334 & -4.650822 \\ 1 & -4.019965 & -2.378690 & -3.495968 \\ 1 & -5.547609 & -3.082191 & -2.943049 \\ 1 & -8.958092 & 0.140358 & 1.971232 \\ 1 & -8.605546 & 0.652605 & 0.315715 \\ 1 & -8.424597 & -1.055058 & 0.780895 \\ 1 & -7.211516 & -0.575910 & 3.530410 \\ 1 & -6.568155 & -1.754544 & 2.367300 \\ 1 & -5.493734 & -0.548658 & 3.093661 \\ 1 & -7.438709 & 1.859208 & 2.885716 \\ 1 & -5.743843 & 1.963285 & 2.373221 \\ 1 & -7.036962 & 2.396403 & 1.241904\end{array}$

TS1 $_{I I B I-} \psi_{-1 \mathbf{a} 2 s 3 a 4 a}$

Number of imaginary frequencies : 1

The smallest frequencies are : -580.7673 $18.376920 .3461 \mathrm{~cm}(-1)$

Electronic energy : $\quad H F=-4360.8658762$

Zero-point correction $=\quad 1.452187($ Hartree/Particle $)$

Thermal correction to Energy= $\quad 1.537017$

Thermal correction to Enthalpy= $\quad 1.537961$

Thermal correction to Gibbs Free Energy $=1.334566$

Sum of electronic and zero-point Energies $=\quad-4359.413689$

Sum of electronic and thermal Energies $=\quad-4359.328859$

Sum of electronic and thermal Enthalpies $=\quad-4359.327915$

Sum of electronic and thermal Free Energies $=\quad-4359.531310$

Cartesian Coordinates

$\begin{array}{cccc}6 & -2.468058 & 2.999082 & 0.376052 \\ 6 & -1.610709 & 1.991050 & 0.798274 \\ 6 & -0.576147 & 2.300362 & 1.693201 \\ 6 & -0.454589 & 3.573926 & 2.238223 \\ 6 & -1.333590 & 4.570879 & 1.782200 \\ 6 & -2.328300 & 4.314707 & 0.841840 \\ 15 & -1.700574 & 0.308123 & 0.063547 \\ 6 & -1.623300 & -0.772778 & 1.585900 \\ 6 & -1.426192 & -2.141561 & 1.476255 \\ 6 & -1.416755 & -2.967635 & 2.652023 \\ 6 & -1.629318 & -2.378496 & 3.931173 \\ 6 & -1.868633 & -0.987934 & 4.005121 \\ 6 & -1.864691 & -0.217573 & 2.874835 \\ 6 & -1.591198 & -3.184856 & 5.092897 \\ 6 & -1.349776 & -4.532035 & 5.004965 \\ 6 & -1.140247 & -5.124710 & 3.742682 \\ 6 & -1.176746 & -4.366433 & 2.598142 \\ 6 & -1.261959 & -2.863456 & 0.183863 \\ 6 & -0.016742 & -3.026524 & -0.391612 \\ 6 & 0.163315 & -3.909611 & -1.480788\end{array}$




\begin{tabular}{|c|c|c|c|}
\hline 6 & -0.897995 & -4.586058 & -2.019281 \\
\hline 6 & -2.203129 & -4.390855 & -1.509163 \\
\hline & -2.389992 & -3.527991 & -0.388652 \\
\hline & -3.704187 & -3.349158 & 0.114963 \\
\hline & -4.773295 & -3.986556 & -0.462906 \\
\hline & -4.584185 & -4.847253 & -1.566756 \\
\hline & -3.325663 & -5.042190 & -2.077453 \\
\hline & 1.111784 & -2.284895 & 0.094013 \\
\hline & 2.052138 & -3.041059 & 0.953257 \\
\hline & 2.615220 & -2.306491 & 2.143243 \\
\hline & 3.967952 & -2.435826 & 2.452329 \\
\hline & 4.505631 & -1.809967 & 3.574946 \\
\hline ס & 3.690875 & -1.050653 & 4.406514 \\
\hline & 2.336091 & -0.919451 & 4.104793 \\
\hline & 1.805033 & -1.538021 & 2.981088 \\
\hline U & 0.579629 & 3.907122 & 3.313916 \\
\hline & -3.272478 & 5.390052 & 0.309598 \\
\hline & -0.315836 & 0.061827 & -1.879006 \\
\hline O & -0.574495 & -1.285649 & -3.262215 \\
\hline & -0.740255 & -1.918410 & -4.220805 \\
\hline & 1.470369 & -0.899662 & -0.820835 \\
\hline & 2.655981 & -1.293064 & -1.941781 \\
\hline 6 & 3.842805 & -1.877825 & -1.574085 \\
\hline 5 & 4.792872 & -1.175489 & -0.854808 \\
\hline 6 & 5.936748 & -1.901089 & -0.378258 \\
\hline 6 & 6.132720 & -3.256699 & -0.780309 \\
\hline 6 & 5.187593 & -3.873007 & -1.637085 \\
\hline 6 & 4.055429 & -3.203902 & -2.010376 \\
\hline 6 & 7.246068 & -3.979192 & -0.286222 \\
\hline 6 & 8.122332 & -3.406836 & 0.599860 \\
\hline 6 & 7.912217 & -2.080006 & 1.029251 \\
\hline 6 & 6.854480 & -1.346531 & 0.550277 \\
\hline 6 & 4.590772 & 0.259928 & -0.550367 \\
\hline 6 & 3.415139 & 0.688265 & 0.039846 \\
\hline 6 & 3.218562 & 2.017008 & 0.464929 \\
\hline 6 & 4.211426 & 2.938664 & 0.286868 \\
\hline 6 & 5.400403 & 2.590279 & -0.398515 \\
\hline 6 & 5.583321 & 1.250599 & -0.854810 \\
\hline 6 & 6.743527 & 0.963346 & -1.618210 \\
\hline 6 & 7.678277 & 1.933454 & -1.885455 \\
\hline 6 & 7.510894 & 3.247203 & -1.400732 \\
\hline 6 & 6.390165 & 3.563860 & -0.676016 \\
\hline 8 & 2.420265 & -0.203832 & 0.337986 \\
\hline 6 & 0.786655 & 1.905865 & -3.126165 \\
\hline 6 & -0.595409 & 1.867254 & -3.384055 \\
\hline 6 & -3.510853 & 0.120813 & -0.284697 \\
\hline 6 & -3.927832 & -0.353489 & -1.522755 \\
\hline 6 & -5.281794 & -0.569856 & -1.801609 \\
\hline 6 & -6.199944 & -0.309628 & -0.785226 \\
\hline 6 & -5.816837 & 0.152358 & 0.481771 \\
\hline 6 & -4.460562 & 0.370024 & 0.711619 \\
\hline 6 & -5.749600 & -1.019144 & -3.185674 \\
\hline 6 & -6.871521 & 0.353259 & 1.568727 \\
\hline 6 & 1.712695 & 1.207165 & -4.090899 \\
\hline 6 & 1.339212 & 3.058788 & -2.376014 \\
\hline 6 & 2.615122 & 3.558925 & -2.679401 \\
\hline
\end{tabular}




\begin{tabular}{|c|c|c|c|}
\hline 6 & 3.078742 & 4.745955 & -2.119101 \\
\hline 6 & 2.292770 & 5.460486 & -1.223367 \\
\hline & 1.037279 & 4.957446 & -0.881372 \\
\hline & 0.572734 & 3.777871 & -1.442611 \\
\hline & 6.694129 & -0.333569 & 0.910125 \\
\hline & -2.051192 & 0.848278 & 2.972799 \\
\hline & 7.379472 & -5.009076 & -0.615058 \\
\hline & -1.753970 & -2.708663 & 6.058862 \\
\hline 1 & 6.229695 & 4.578199 & -0.311052 \\
\hline & -3.862340 & -2.679171 & 0.958670 \\
\hline 1 & -5.775630 & -3.820622 & -0.071492 \\
\hline 1 & 4.081649 & 3.965095 & 0.626984 \\
\hline 1 & -2.055314 & -0.535348 & 4.978487 \\
\hline 1 & 5.363177 & -4.895542 & -1.967285 \\
\hline 1 & 8.553040 & 1.686393 & -2.483010 \\
\hline 1 & 3.299973 & -3.660737 & -2.646122 \\
\hline 1 & -3.166234 & -5.700094 & -2.930716 \\
\hline 1 & -0.757966 & -5.259150 & -2.863740 \\
\hline 1 & -0.949148 & -6.193256 & 3.674426 \\
\hline 1 & 6.884249 & -0.040742 & -2.010155 \\
\hline 1 & -1.015151 & -4.844397 & 1.634200 \\
\hline 1 & 1.166749 & -4.024123 & -1.889072 \\
\hline 1 & 8.261081 & 4.005144 & -1.614083 \\
\hline 1 & 8.590658 & -1.633904 & 1.753176 \\
\hline 1 & -5.439417 & -5.352116 & -2.011694 \\
\hline 1 & -1.318308 & -5.144362 & 5.903191 \\
\hline 1 & 8.967761 & -3.974378 & 0.981649 \\
\hline 1 & 2.265817 & 2.270553 & 0.926852 \\
\hline 1 & -4.127141 & 0.735523 & 1.679485 \\
\hline 1 & -3.165807 & -0.575234 & -2.265456 \\
\hline 1 & -7.259424 & -0.477035 & -0.978240 \\
\hline 1 & -3.260140 & 2.759307 & -0.333740 \\
\hline 1 & 0.112336 & 1.509136 & 1.991179 \\
\hline 1 & -1.226536 & 5.577366 & 2.181684 \\
\hline 1 & 1.473040 & -3.900908 & 19829 \\
\hline 1 & 2.879556 & -3.460771 & 0.361201 \\
\hline 1 & 4.615938 & -3.023051 & 1.799445 \\
\hline 1 & 0.756531 & -1.401200 & 2.725569 \\
\hline 1 & 5.567439 & -1.914126 & 3.790375 \\
\hline 1 & 1.685945 & -0.329174 & 4.749474 \\
\hline 1 & 4.107210 & -0.559713 & 5.283681 \\
\hline 1 & -1.549124 & 0.835108 & -2.607245 \\
\hline 1 & 3.259698 & 3.025860 & -3.374106 \\
\hline 1 & 4.069564 & 5.107946 & -2.389310 \\
\hline 1 & 2.654330 & 6.388403 & -0.783861 \\
\hline 1 & 0.408776 & 5.479892 & -0.159434 \\
\hline 1 & -0.404768 & 3.402839 & -1.142813 \\
\hline 1 & 2.599866 & 0.794841 & -3.595646 \\
\hline 1 & 1.204489 & 0.378569 & -4.593309 \\
\hline 1 & 2.060975 & 1.903908 & -4.869397 \\
\hline 1 & -1.213765 & 2.702028 & -3.050838 \\
\hline 1 & -0.917467 & 1.433501 & -4.332092 \\
\hline 6 & -0.124299 & 4.544932 & 4.517752 \\
\hline 6 & 1.305751 & 2.659804 & 3.816880 \\
\hline 6 & 1.613620 & 4.890471 & 2.755583 \\
\hline 6 & -2.992438 & 6.763861 & 0.912706 \\
\hline
\end{tabular}




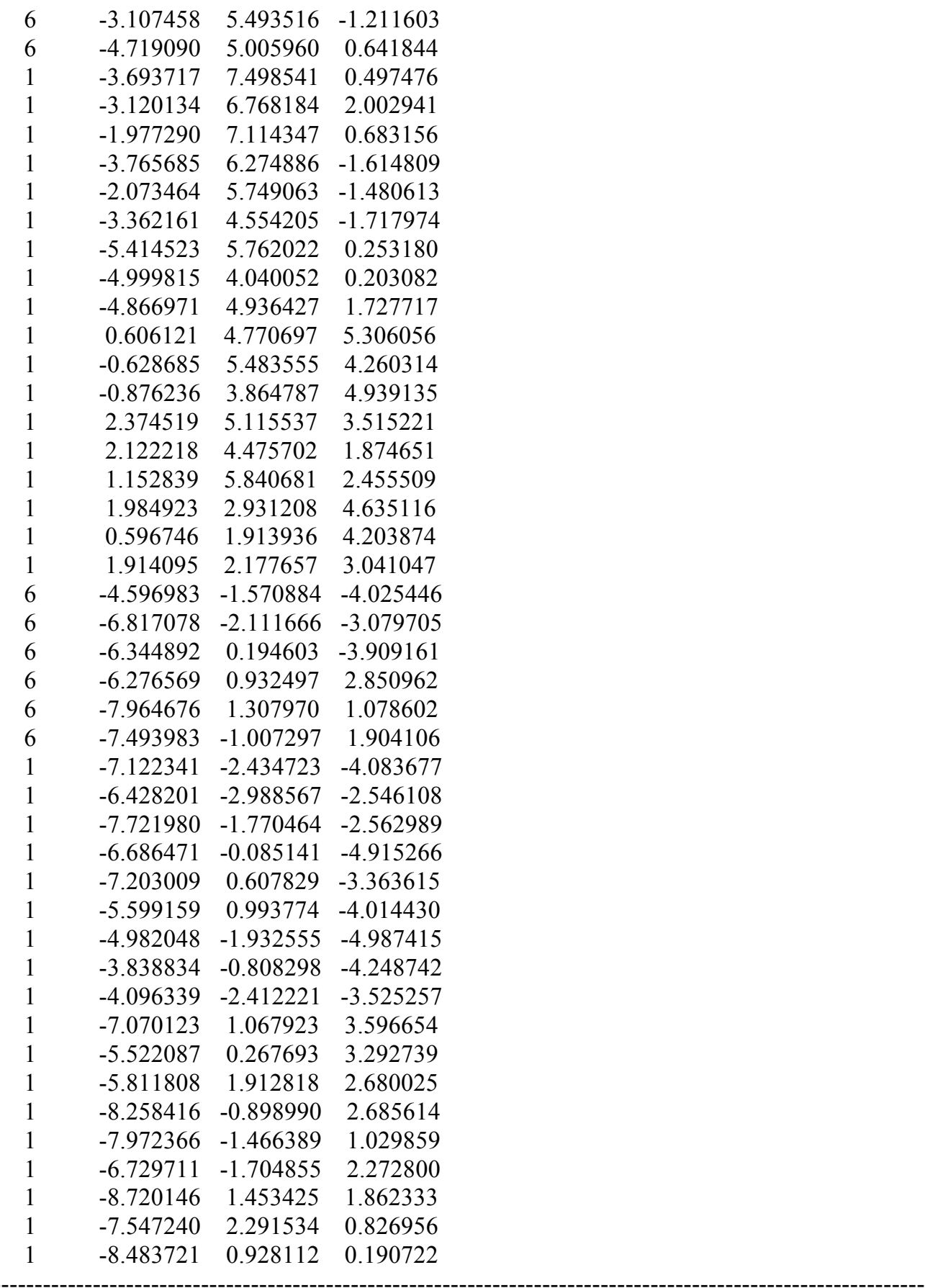

$\mathrm{TS1}_{\text {IIBI- }} \psi_{-1 \mathrm{a} 2 \mathrm{2s} 3 \mathrm{a} 4 \mathrm{~s}}$

Number of imaginary frequencies : 1

The smallest frequencies are : $-574.9104 \quad 17.9414 \quad 19.5652 \mathrm{~cm}(-1)$

Electronic energy : $\quad H F=-4360.8660483$

Zero-point correction $=\quad 1.452182$ (Hartree/Particle)

Thermal correction to Energy= $\quad 1.537007$

Thermal correction to Enthalpy $=\quad 1.537951$

Thermal correction to Gibbs Free Energy= $\quad 1.334292$

Sum of electronic and zero-point Energies $=\quad-4359.413866$

Sum of electronic and thermal Energies $=\quad-4359.329041$ 
Sum of electronic and thermal Enthalpies=

Sum of electronic and thermal Free Energies=
$-4359.328097$

$-4359.531756$

Cartesian Coordinates

\begin{tabular}{|c|c|c|c|}
\hline 6 & -2.508596 & 2.954110 & 0.390539 \\
\hline 6 & -1.640819 & 1.952292 & 0.806718 \\
\hline 6 & -0.608762 & 2.267547 & 1.702489 \\
\hline 6 & -0.500424 & 3.538946 & 2.255248 \\
\hline 6 & -1.386242 & 4.530843 & 1.801115 \\
\hline 6 & -2.377286 & 4.270171 & 0.857993 \\
\hline 15 & -1.711536 & 0.271514 & 0.062468 \\
\hline 6 & -1.623108 & -0.815448 & 1.580454 \\
\hline 6 & -1.403431 & -2.180683 & 1.467934 \\
\hline 6 & -1.377807 & -3.009191 & 2.641285 \\
\hline 6 & -1.597888 & -2.427034 & 3.922232 \\
\hline 6 & -1.860591 & -1.040975 & 3.999833 \\
\hline 6 & -1.871222 & -0.267729 & 2.871307 \\
\hline 6 & -1.543287 & -3.235589 & 5.081766 \\
\hline 6 & -1.279325 & -4.578229 & 4.989562 \\
\hline 6 & -1.062986 & -5.164157 & 3.725217 \\
\hline 6 & -1.114943 & -4.403584 & 2.582868 \\
\hline 6 & -1.234982 & -2.892734 & 0.171204 \\
\hline 6 & 0.006517 & -3.024843 & -0.420096 \\
\hline 6 & 0.188189 & -3.883403 & -1.528400 \\
\hline 6 & -0.868470 & -4.564056 & -2.070322 \\
\hline 6 & -2.169800 & -4.404927 & -1.538637 \\
\hline 6 & -2.357160 & -3.571218 & -0.396324 \\
\hline 6 & -3.665687 & -3.437428 & 0.135826 \\
\hline 6 & -4.728698 & -4.088915 & -0.437620 \\
\hline 6 & -4.539899 & -4.915703 & -1.567008 \\
\hline 6 & -3.286771 & -5.067599 & -2.104476 \\
\hline 7 & 1.129497 & -2.276647 & 0.068586 \\
\hline 6 & 2.077234 & -3.030797 & 0.921392 \\
\hline 6 & 2.633314 & -2.303606 & 2.119302 \\
\hline 6 & 3.985191 & -2.432646 & 2.432405 \\
\hline 6 & 4.516855 & -1.817549 & 3.563799 \\
\hline 6 & 3.696942 & -1.069385 & 4.400361 \\
\hline 6 & 2.343199 & -0.937786 & 4.094291 \\
\hline 6 & 1.818211 & -1.545411 & 2.961760 \\
\hline 6 & 0.525987 & 3.874088 & 3.337925 \\
\hline 6 & -3.321850 & 5.342086 & 0.318940 \\
\hline 45 & -0.323191 & 0.070665 & -1.882219 \\
\hline 6 & -0.547659 & -1.259059 & -3.290198 \\
\hline 8 & -0.673069 & -1.881125 & -4.261713 \\
\hline 15 & 1.474265 & -0.880014 & -0.833824 \\
\hline 8 & 2.663298 & -1.250523 & -1.959182 \\
\hline 6 & 3.853373 & -1.832593 & -1.597613 \\
\hline 6 & 4.798153 & -1.133544 & -0.868285 \\
\hline 6 & 5.945413 & -1.858412 & -0.398710 \\
\hline 6 & 6.149918 & -3.207800 & -0.817226 \\
\hline 6 & 5.210049 & -3.818784 & -1.683515 \\
\hline 6 & 4.074648 & -3.151677 & -2.050397 \\
\hline 6 & 7.266699 & -3.929952 & -0.330373 \\
\hline 6 & 8.138340 & -3.363574 & 0.564045 \\
\hline 6 & 7.919902 & -2.043392 & 1.009459 \\
\hline
\end{tabular}




\begin{tabular}{|c|c|c|c|}
\hline & 6.858618 & -1.310175 & 0.538034 \\
\hline & 4.587764 & 0.297339 & -0.548272 \\
\hline & 3.408888 & 0.713325 & 0.044380 \\
\hline & 3.205151 & 2.036400 & 0.483731 \\
\hline & 4.193616 & 2.964947 & 0.317152 \\
\hline & 5.385335 & 2.630063 & -0.370184 \\
\hline & 5.575791 & 1.296225 & -0.840359 \\
\hline & 6.738715 & 1.023068 & -1.604829 \\
\hline & 7.668941 & 2.000685 & -1.860122 \\
\hline & 7.494019 & 3.308378 & -1.361963 \\
\hline & 6.370506 & 3.611633 & -0.635883 \\
\hline & 2.418219 & -0.186871 & 0.331484 \\
\hline & 0.757861 & 1.936985 & -3.109385 \\
\hline & -0.625156 & 1.892800 & -3.360948 \\
\hline & -3.520543 & 0.079132 & -0.292110 \\
\hline & -3.943495 & -0.358466 & -1.546851 \\
\hline & -5.296610 & -0.541881 & -1.832126 \\
\hline & -6.220885 & -0.289864 & -0.811080 \\
\hline & -5.832399 & 0.123593 & 0.464596 \\
\hline & -4.467928 & 0.310331 & 0.702083 \\
\hline & -5.776776 & -0.972628 & -3.217301 \\
\hline & -6.817680 & 0.339360 & 1.611786 \\
\hline & 1.684202 & 1.258203 & -4.088079 \\
\hline & 1.307449 & 3.083199 & -2.346563 \\
\hline & 2.581600 & 3.590322 & -2.645852 \\
\hline & 3.041851 & 4.773591 & -2.074997 \\
\hline & 2.253704 & 5.477762 & -1.173085 \\
\hline & 1.000232 & 4.967262 & -0.834950 \\
\hline & 0.539369 & 3.790787 & -1.405640 \\
\hline & 6.691771 & -0.302882 & 0.910571 \\
\hline & -2.072697 & 0.795059 & 2.972486 \\
\hline & 7.406503 & -4.954881 & -0.671800 \\
\hline & -1.712000 & -2.764923 & 6.049396 \\
\hline & 6.204018 & 4.621303 & -0.260796 \\
\hline . & -3.824643 & -2.792191 & 0.999110 \\
\hline & -5.727382 & -3.959637 & -0.024383 \\
\hline & 4.058128 & 3.986894 & 0.668308 \\
\hline & -2.052715 & -0.593820 & 4.974631 \\
\hline & 5.392215 & -4.835994 & -2.026351 \\
\hline . & 8.545940 & 1.764384 & -2.458754 \\
\hline & 3.323100 & -3.604862 & -2.693307 \\
\hline | & -3.127005 & -5.700811 & -2.976159 \\
\hline & -0.727560 & -5.215667 & -2.931334 \\
\hline 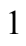 & -0.854529 & -6.229238 & 3.653746 \\
\hline & 6.885163 & 0.024058 & -2.007437 \\
\hline & -0.948555 & -4.875770 & 1.616897 \\
\hline 1 & 1.188572 & -3.973860 & -1.949796 \\
\hline & 8.240581 & 4.072396 & -1.566156 \\
\hline & 8.594646 & -1.602382 & 1.739927 \\
\hline & -5.391124 & -5.429834 & -2.008843 \\
\hline 1 & -1.235465 & -5.192324 & 5.886052 \\
\hline & 8.986511 & -3.930894 & 0.940045 \\
\hline 1 & 2.250598 & 2.280576 & 0.946951 \\
\hline & -4.138669 & 0.636741 & 1.687922 \\
\hline 1 & -3.182866 & -0.572263 & -2.293304 \\
\hline 1 & -7.279456 & -0.428163 & -1.021104 \\
\hline
\end{tabular}




\begin{tabular}{|c|c|c|c|}
\hline 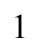 & -3.300372 & 2.710296 & -0.318267 \\
\hline 1 & 0.088366 & 1.481974 & 1.995214 \\
\hline 1 & -1.287252 & 5.536912 & 2.203837 \\
\hline 1 & 1.507309 & -3.900310 & 1.279333 \\
\hline & 2.908770 & -3.436347 & 0.325379 \\
\hline 1 & 4.637159 & -3.011737 & 1.776255 \\
\hline 1 & 0.770635 & -1.407833 & 2.702987 \\
\hline 1 & 5.578023 & -1.921540 & 3.782458 \\
\hline & 1.689134 & -0.355544 & 4.742300 \\
\hline 1 & 4.108525 & -0.587154 & 5.284567 \\
\hline 1 & -1.568677 & 0.834825 & -2.597809 \\
\hline 1 & 3.227577 & 3.066010 & -3.345811 \\
\hline 1 & 4.031508 & 5.140997 & -2.342150 \\
\hline 1 & 2.612012 & 6.403256 & -0.725883 \\
\hline 1 & 0.369887 & 5.481674 & -0.109025 \\
\hline 1 & -0.436875 & 3.410176 & -1.108275 \\
\hline 1 & 2.574318 & 0.841717 & -3.601610 \\
\hline 1 & 1.178403 & 0.435932 & -4.602854 \\
\hline 1 & 2.027268 & 1.969118 & -4.855940 \\
\hline 1 & -1.248354 & 2.717211 & -3.011482 \\
\hline 1 & -0.949326 & 1.470277 & -4.313366 \\
\hline 6 & -0.190849 & 4.493376 & 4.543830 \\
\hline 6 & 1.263717 & 2.630492 & 3.833242 \\
\hline 6 & 1.552295 & 4.873345 & 2.794039 \\
\hline 6 & -3.049005 & 6.717467 & 0.921736 \\
\hline 6 & -3.146359 & 5.443488 & -1.201431 \\
\hline 6 & -4.770117 & 4.955738 & 0.640577 \\
\hline 1 & -3.750109 & 7.449650 & 0.501795 \\
\hline 1 & -3.182784 & 6.722718 & 2.011235 \\
\hline 1 & -2.033600 & 7.070567 & 0.697370 \\
\hline 1 & -3.800057 & 6.226010 & -1.609736 \\
\hline 1 & -2.110048 & 5.696064 & -1.464347 \\
\hline 1 & -3.400707 & 4.504164 & -1.708134 \\
\hline 1 & -5.464011 & 5.704290 & 0.234834 \\
\hline 1 & -5.042414 & 3.982483 & 0.212177 \\
\hline 1 & -4.929136 & 4.900305 & 1.725674 \\
\hline 1 & 0.532958 & 4.719884 & 5.338023 \\
\hline 1 & -0.704051 & 5.428720 & 4.291890 \\
\hline 1 & -0.937614 & 3.801439 & 4.955137 \\
\hline 1 & 2.306305 & 5.099787 & 3.560102 \\
\hline 1 & 2.070381 & 4.471568 & 1.912645 \\
\hline 1 & 1.083296 & 5.821290 & 2.499662 \\
\hline 1 & 1.937003 & 2.902599 & 4.656101 \\
\hline 1 & 0.561802 & 1.873572 & 4.211633 \\
\hline 1 & 1.880044 & 2.161565 & 3.055597 \\
\hline 6 & -4.619520 & -1.424207 & -4.107196 \\
\hline 6 & -6.763355 & -2.139016 & -3.107814 \\
\hline 6 & -6.473533 & 0.217433 & -3.886875 \\
\hline 6 & -8.266949 & 0.115395 & 1.188428 \\
\hline 6 & -6.488922 & -0.654122 & 2.733259 \\
\hline 6 & -6.692886 & 1.771704 & 2.142444 \\
\hline 1 & -7.091094 & -2.449527 & -4.108870 \\
\hline 1 & -6.290733 & -3.003136 & -2.622738 \\
\hline 1 & -7.663272 & -1.878597 & -2.538070 \\
\hline 1 & -6.827393 & -0.058134 & -4.889801 \\
\hline 1 & -7.340606 & 0.558870 & -3.307288 \\
\hline
\end{tabular}




$\begin{array}{rrrr}1 & -5.785825 & 1.066969 & -3.991893 \\ 1 & -5.008784 & -1.761438 & -5.076265 \\ 1 & -3.907414 & -0.612356 & -4.309249 \\ 1 & -4.067569 & -2.261725 & -3.657233 \\ 1 & -8.931603 & 0.284546 & 2.045094 \\ 1 & -8.573749 & 0.806744 & 0.392875 \\ 1 & -8.438893 & -0.910655 & 0.838363 \\ 1 & -7.380171 & 1.926963 & 2.985082 \\ 1 & -5.679376 & 1.998094 & 2.496223 \\ 1 & -6.946272 & 2.502319 & 1.362718 \\ 1 & -7.176906 & -0.520663 & 3.579186 \\ 1 & -6.586975 & -1.688739 & 2.377529 \\ 1 & -5.465737 & -0.529739 & 3.110718\end{array}$

TS1 $_{\text {IIB1- }} \psi_{-1 \mathrm{a} 2 \mathrm{~s} 3 \mathrm{~s} 4 \mathrm{a}}$

$\begin{array}{lcc}\text { Number of imaginary frequencies : } & \\ \text { The smallest frequencies are }:-569.3044 & 14.3032 & 17.3636 \mathrm{~cm}(-1) \\ \text { Electronic energy : } \mathrm{HF}=-4360.8656677 & \\ \text { Zero-point correction= } & 1.452027 \text { (Hartree/Particle) } \\ \text { Thermal correction to Energy= } & 1.536909 \\ \text { Thermal correction to Enthalpy= } & 1.537853 \\ \text { Thermal correction to Gibbs Free Energy= } & 1.334008 \\ \text { Sum of electronic and zero-point Energies= } & -4359.413641 \\ \text { Sum of electronic and thermal Energies= } & -4359.328759 \\ \text { Sum of electronic and thermal Enthalpies= } & -4359.327815 \\ \text { Sum of electronic and thermal Free Energies }= & -4359.531660\end{array}$

Cartesian Coordinates

$\begin{array}{cccc}6 & -2.549472 & 2.923498 & 0.447344 \\ 6 & -1.660531 & 1.928861 & 0.833458 \\ 6 & -0.623285 & 2.242812 & 1.723525 \\ 6 & -0.526699 & 3.505341 & 2.297948 \\ 6 & -1.438601 & 4.489419 & 1.879291 \\ 6 & -2.439628 & 4.229707 & 0.946665 \\ 15 & -1.716134 & 0.256943 & 0.069793 \\ 6 & -1.621513 & -0.839769 & 1.578027 \\ 6 & -1.380261 & -2.200074 & 1.456346 \\ 6 & -1.344451 & -3.035491 & 2.625477 \\ 6 & -1.579628 & -2.464739 & 3.909015 \\ 6 & -1.866508 & -1.083844 & 3.994505 \\ 6 & -1.883513 & -0.303812 & 2.871274 \\ 6 & -1.514686 & -3.278420 & 5.064392 \\ 6 & -1.225032 & -4.615318 & 4.966379 \\ 6 & -0.992012 & -5.189821 & 3.699914 \\ 6 & -1.053994 & -4.424274 & 2.561346 \\ 6 & -1.191648 & -2.906703 & 0.158808 \\ 6 & 0.057161 & -3.024232 & -0.420264 \\ 6 & 0.265904 & -3.903113 & -1.508030 \\ 6 & -0.767055 & -4.634422 & -2.029762 \\ 6 & -2.074890 & -4.498541 & -1.507268 \\ 6 & -2.294082 & -3.621359 & -0.404089 \\ 6 & -3.612454 & -3.493095 & 0.105062 \\ 6 & -4.651204 & -4.205320 & -0.439885 \\ 6 & -4.425917 & -5.092433 & -1.515331 \\ 6 & & & \\ 6 & & & \end{array}$




\begin{tabular}{|c|c|c|c|}
\hline 6 & -3.166019 & -5.228531 & -2.040026 \\
\hline 7 & 1.165033 & -2.254328 & 0.070502 \\
\hline & 2.128594 & -2.995138 & 0.917457 \\
\hline & 2.664162 & -2.269122 & 2.125329 \\
\hline & 4.015554 & -2.382057 & 2.447592 \\
\hline & 4.528227 & -1.776817 & 3.592845 \\
\hline & 3.689922 & -1.054664 & 4.434208 \\
\hline & 2.337249 & -0.937966 & 4.118133 \\
\hline & 1.830982 & -1.535030 & 2.971303 \\
\hline & 0.517115 & 3.839082 & 3.364252 \\
\hline & -3.422656 & 5.290027 & 0.456549 \\
\hline 45 & -0.325674 & 0.088636 & -1.863118 \\
\hline 6 & -0.622528 & -1.252414 & -3.245994 \\
\hline & -0.917594 & -1.863442 & -4.187760 \\
\hline 15 & 1.492124 & -0.854034 & -0.833216 \\
\hline 8 & 2.676125 & -1.216409 & -1.968091 \\
\hline 6 & 3.871443 & -1.793038 & -1.614742 \\
\hline 6 & 4.815376 & -1.092127 & -0.885882 \\
\hline 6 & 5.965684 & -1.813158 & -0.418826 \\
\hline 6 & 6.175430 & -3.160688 & -0.840465 \\
\hline 6 & 5.238803 & -3.771924 & -1.710068 \\
\hline 6 & 4.100254 & -3.108574 & -2.074378 \\
\hline 6 & 7.295286 & -3.879252 & -0.355360 \\
\hline 6 & 8.165007 & -3.310845 & 0.539679 \\
\hline 6 & 7.941786 & -1.992196 & 0.987450 \\
\hline 6 & 6.877340 & -1.262419 & 0.517970 \\
\hline 6 & 4.602319 & 0.338860 & -0.569570 \\
\hline 6 & 3.422755 & 0.753663 & 0.022287 \\
\hline 6 & 3.211265 & 2.081223 & 0.443617 \\
\hline 6 & 4.195518 & 3.012665 & 0.267517 \\
\hline 6 & 5.389854 & 2.676335 & -0.414016 \\
\hline 6 & 5.585849 & 1.339018 & -0.871945 \\
\hline 6 & 6.750406 & 1.063723 & -1.633255 \\
\hline 6 & 7.677449 & 2.042450 & -1.895613 \\
\hline 6 & 7.497572 & 3.353603 & -1.408387 \\
\hline 6 & 6.371983 & 3.659146 & -0.686633 \\
\hline 8 & 2.440402 & -0.152138 & 0.323582 \\
\hline 6 & 0.726655 & 1.972972 & -3.094844 \\
\hline 6 & -0.653964 & 1.904173 & -3.347840 \\
\hline 6 & -3.521480 & 0.057395 & -0.296423 \\
\hline 6 & -3.935090 & -0.326209 & -1.562507 \\
\hline 6 & -5.292905 & -0.481861 & -1.876591 \\
\hline 6 & -6.217763 & -0.285613 & -0.856945 \\
\hline 6 & -5.836346 & 0.078464 & 0.446506 \\
\hline 6 & -4.482409 & 0.257153 & 0.704702 \\
\hline 6 & -5.679663 & -0.863911 & -3.303603 \\
\hline 6 & -6.901516 & 0.240029 & 1.529575 \\
\hline 6 & 1.665872 & 1.304305 & -4.068198 \\
\hline 6 & 1.253461 & 3.127488 & -2.328894 \\
\hline 6 & 2.515025 & 3.662769 & -2.630865 \\
\hline 6 & 2.953480 & 4.851310 & -2.053864 \\
\hline 6 & 2.156333 & 5.531030 & -1.141124 \\
\hline 6 & 0.915453 & 4.992171 & -0.800220 \\
\hline 6 & 0.474858 & 3.811739 & -1.378978 \\
\hline 1 & 6.706108 & -0.256538 & 0.892502 \\
\hline 1 & -2.102328 & 0.755019 & 2.977901 \\
\hline
\end{tabular}




\begin{tabular}{|c|c|c|c|}
\hline ] & 7.439252 & -4.902951 & -0.698728 \\
\hline & -1.695871 & -2.816042 & 6.033766 \\
\hline & 6.201891 & 4.671187 & -0.319792 \\
\hline 1 & -3.796757 & -2.807089 & 0.930538 \\
\hline 1 & -5.657140 & -4.078183 & -0.044211 \\
\hline & 4.053175 & 4.037543 & 0.606900 \\
\hline 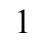 & -2.071200 & -0.646457 & 4.971137 \\
\hline & 5.425976 & -4.786543 & -2.057850 \\
\hline & 8.556018 & 1.804191 & -2.491155 \\
\hline & 3.351910 & -3.562377 & -2.720505 \\
\hline & -2.980531 & -5.899816 & -2.877514 \\
\hline 1 & -0.601512 & -5.313907 & -2.864459 \\
\hline 1 & -0.762452 & -6.250204 & 3.623443 \\
\hline & 6.901184 & 0.061942 & -2.027189 \\
\hline & -0.873726 & -4.888856 & 1.594186 \\
\hline & 1.271028 & -3.980089 & -1.920129 \\
\hline & 8.241960 & 4.118349 & -1.617703 \\
\hline & 8.615212 & -1.549920 & 1.718355 \\
\hline 1 & -5.256632 & -5.656993 & -1.933589 \\
\hline & -1.173163 & -5.233287 & 5.859774 \\
\hline ] & 9.015566 & -3.875452 & 0.914369 \\
\hline & 2.253774 & 2.330409 & 0.897445 \\
\hline & -4.155530 & 0.559292 & 1.696182 \\
\hline$\frac{1}{1}$ & -3.176217 & -0.514227 & -2.319503 \\
\hline & -7.277563 & -0.414835 & -1.068743 \\
\hline$\frac{1}{1}$ & -3.344260 & 2.680727 & -0.258333 \\
\hline & 0.088662 & 1.462041 & 1.992704 \\
\hline ] & -1.353332 & 5.488240 & 2.302507 \\
\hline$\frac{1}{1}$ & 1.578273 & -3.881431 & 1.264828 \\
\hline & 2.971010 & -3.375341 & 0.320480 \\
\hline 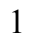 & 4.681087 & -2.942416 & 1.788802 \\
\hline & 0.783291 & -1.409839 & 2.706410 \\
\hline 1 & 5.588672 & -1.868846 & 3.820419 \\
\hline 1 & 1.669184 & -0.375948 & 4.769678 \\
\hline 1 & 4.086513 & -0.581116 & 5.329881 \\
\hline 1 & -1.580537 & 0.845047 & -2.563549 \\
\hline 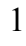 & 3.167593 & 3.156556 & -3.338177 \\
\hline 1 & 3.933010 & 5.242521 & -2.324503 \\
\hline & 2.497982 & 6.459956 & -0.688099 \\
\hline 1 & 0.278817 & 5.488238 & -0.067114 \\
\hline ] & -0.492833 & 3.410055 & -1.080844 \\
\hline 1 & 2.563165 & 0.907548 & -3.578534 \\
\hline 1 & 1.174095 & 0.468443 & -4.575336 \\
\hline 1 & 1.996266 & 2.015231 & -4.841663 \\
\hline 1 & -1.291894 & 2.719066 & -3.002537 \\
\hline l & -0.972262 & 1.467843 & -4.296111 \\
\hline 6 & -0.179785 & 4.436792 & 4.592416 \\
\hline 6 & 1.278122 & 2.597496 & 3.828134 \\
\hline 6 & 1.521107 & 4.855511 & 2.810052 \\
\hline 6 & -3.182220 & 6.652380 & 1.101174 \\
\hline 6 & -3.272948 & 5.446448 & -1.061693 \\
\hline 6 & -4.853735 & 4.849021 & 0.787099 \\
\hline 1 & -3.911832 & 7.376104 & 0.716669 \\
\hline 1 & -3.297957 & 6.617362 & 2.192169 \\
\hline 1 & -2.181861 & 7.043980 & 0.873183 \\
\hline 1 & -3.957315 & 6.219824 & -1.435814 \\
\hline
\end{tabular}




$\begin{array}{rrrr}1 & -2.249036 & 5.741496 & -1.329154 \\ 1 & -3.502868 & 4.516116 & -1.595604 \\ 1 & -5.576024 & 5.592827 & 0.424581 \\ 1 & -5.107444 & 3.886876 & 0.323656 \\ 1 & -4.991144 & 4.744647 & 1.871615 \\ 1 & 0.558583 & 4.659696 & 5.374089 \\ 1 & -0.705799 & 5.370911 & 4.363549 \\ 1 & -0.911819 & 3.733293 & 5.010489 \\ 1 & 2.289011 & 5.081399 & 3.562400 \\ 1 & 2.024500 & 4.469427 & 1.913131 \\ 1 & 1.035669 & 5.801549 & 2.536595 \\ 1 & 1.963184 & 2.865143 & 4.642729 \\ 1 & 0.591787 & 1.826741 & 4.207184 \\ 1 & 1.885593 & 2.147076 & 3.032748 \\ 6 & -7.189819 & -0.827462 & -3.524089 \\ 6 & -5.030954 & 0.112303 & -4.293293 \\ 6 & -5.182813 & -2.282874 & -3.596138 \\ 6 & -6.307100 & 0.700079 & 2.859378 \\ 6 & -7.943780 & 1.274900 & 1.093527 \\ 6 & -7.587642 & -1.112373 & 1.754437 \\ 1 & -5.333361 & -0.134175 & -5.319778 \\ 1 & -5.338088 & 1.146461 & -4.088900 \\ 1 & -3.934118 & 0.071984 & -4.259538 \\ 1 & -5.412408 & -2.562734 & -4.633500 \\ 1 & -4.098252 & -2.376772 & -3.454464 \\ 1 & -5.666348 & -3.009626 & -2.930823 \\ 1 & -7.416897 & -1.084834 & -4.566343 \\ 1 & -7.716011 & -1.551824 & -2.889145 \\ 1 & -7.608645 & 0.168768 & -3.329850 \\ 1 & -7.106564 & 0.804828 & 3.603682 \\ 1 & -5.579494 & -0.019730 & 3.258035 \\ 1 & -5.809971 & 1.675309 & 2.768303 \\ 1 & -8.358353 & -1.027552 & 2.532602 \\ 1 & -8.073580 & -1.482516 & 0.843305 \\ 1 & -6.861246 & -1.869787 & 2.078703 \\ 1 & -8.708691 & 1.394857 & 1.872483 \\ 1 & -7.478863 & 2.255085 & 0.924496 \\ 1 & -8.458974 & 0.985112 & 0.170101\end{array}$

$\mathrm{TS1}_{I I B I-} \psi_{-1 \mathrm{a} 23334 \mathrm{~s}}$

Number of imaginary frequencies : 1

The smallest frequencies are : -571.0515 $16.7964 \quad 18.1227 \mathrm{~cm}(-1)$

Electronic energy : $\quad \mathrm{HF}=-4360.8657751$

Zero-point correction $=\quad 1.452063($ Hartree/Particle)

Thermal correction to Energy= $\quad 1.536909$

Thermal correction to Enthalpy= $\quad 1.537854$

Thermal correction to Gibbs Free Energy $=\quad 1.334162$

Sum of electronic and zero-point Energies $=\quad-4359.413712$

Sum of electronic and thermal Energies $=\quad-4359.328866$

Sum of electronic and thermal Enthalpies $=\quad-4359.327922$

Sum of electronic and thermal Free Energies $=\quad-4359.531613$

Cartesian Coordinates

$\begin{array}{llll}6 & -2.554881 & 2.923315 & 0.445412\end{array}$ 


\begin{tabular}{|c|c|c|c|}
\hline 6 & -1.673939 & 1.925046 & 0.841217 \\
\hline 6 & -0.640312 & 2.237147 & 1.736102 \\
\hline 6 & -0.540426 & 3.501287 & 2.306264 \\
\hline 6 & -1.439106 & 4.490815 & 1.872114 \\
\hline 6 & -2.433754 & 4.233562 & 0.931797 \\
\hline 15 & -1.725556 & 0.255126 & 0.072279 \\
\hline 6 & -1.628219 & -0.850628 & 1.573359 \\
\hline 6 & -1.386646 & -2.210069 & 1.442644 \\
\hline 6 & -1.348145 & -3.052990 & 2.606149 \\
\hline 6 & -1.580180 & -2.490493 & 3.893896 \\
\hline 6 & -1.866494 & -1.110155 & 3.988926 \\
\hline 6 & -1.886697 & -0.322953 & 2.870574 \\
\hline 6 & -1.512101 & -3.311487 & 5.043922 \\
\hline 6 & -1.222805 & -4.647690 & 4.936559 \\
\hline 6 & -0.993407 & -5.214179 & 3.665817 \\
\hline 6 & -1.058305 & -4.441443 & 2.532323 \\
\hline 6 & -1.200672 & -2.907492 & 0.139874 \\
\hline 6 & 0.047023 & -3.020647 & -0.442438 \\
\hline 6 & 0.253187 & -3.889188 & -1.538874 \\
\hline 6 & -0.781490 & -4.614066 & -2.066001 \\
\hline 6 & -2.088113 & -4.482645 & -1.539378 \\
\hline 6 & -2.304670 & -3.616673 & -0.426736 \\
\hline 6 & -3.621352 & -3.495200 & 0.088638 \\
\hline 6 & -4.660621 & -4.203496 & -0.460554 \\
\hline 6 & -4.438571 & -5.077580 & -1.547157 \\
\hline 6 & -3.180321 & -5.207040 & -2.077308 \\
\hline 7 & 1.156136 & -2.255880 & 0.053249 \\
\hline 6 & 2.119697 & -3.003977 & 0.893669 \\
\hline 6 & 2.656682 & -2.288175 & 2.107046 \\
\hline 6 & 4.006787 & -2.410455 & 2.431131 \\
\hline 6 & & -1.814898 & \\
\hline 6 & 3.684727 & -1.092696 & 4.424765 \\
\hline 6 & 2.333398 & -0.966200 & 4.106597 \\
\hline 6 & 1.826044 & -1.554203 & 2.955617 \\
\hline 6 & 0.493693 & 3.830722 & 3.383375 \\
\hline 6 & -3.390322 & 5.303798 & 0.411143 \\
\hline 45 & -0.335248 & 0.103903 & -1.859810 \\
\hline 6 & -0.640488 & -1.230270 & -3.249934 \\
\hline 8 & -0.944936 & -1.831701 & -4.194789 \\
\hline 15 & 1.482521 & -0.846922 & -0.837169 \\
\hline 8 & 2.666480 & -1.197087 & -1.975220 \\
\hline 6 & 3.860412 & -1.780242 & -1.628206 \\
\hline 6 & 4.805275 & -1.090149 & -0.890296 \\
\hline 6 & 5.954231 & -1.819553 & -0.432518 \\
\hline 6 & 6.161214 & -3.162063 & -0.871510 \\
\hline 6 & 5.223326 & -3.760533 & -1.748564 \\
\hline 6 & 4.086215 & -3.090290 & -2.104362 \\
\hline 6 & 7.280117 & -3.888963 & -0.396650 \\
\hline 6 & 8.151742 & -3.333767 & 0.504732 \\
\hline 6 & 7.931263 & -2.020655 & 0.969690 \\
\hline 6 & 6.867758 & -1.282881 & 0.510709 \\
\hline 6 & 4.595166 & 0.337555 & -0.556719 \\
\hline 6 & 3.416140 & 0.748825 & 0.038903 \\
\hline 6 & 3.209039 & 2.071374 & 0.478301 \\
\hline 6 & 4.196369 & 3.001812 & 0.315042 \\
\hline 6 & 5.389935 & 2.670527 & -0.370467 \\
\hline
\end{tabular}




\begin{tabular}{|c|c|c|c|}
\hline 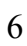 & 5.582029 & 1.338624 & -0.845456 \\
\hline & 6.745896 & 1.069805 & -1.610140 \\
\hline & 7.675811 & 2.049116 & -1.859896 \\
\hline & 7.499606 & 3.354478 & -1.356148 \\
\hline & 6.374827 & 3.653941 & -0.630577 \\
\hline & 2.429821 & -0.156514 & 0.327332 \\
\hline & 0.729005 & 1.978220 & -3.082685 \\
\hline & -0.653384 & 1.927407 & -3.331139 \\
\hline & -3.530682 & 0.054605 & -0.295430 \\
\hline & -3.949573 & -0.306716 & -1.570510 \\
\hline & -5.304609 & -0.454578 & -1.882073 \\
\hline & -6.233640 & -0.276191 & -0.855638 \\
\hline & -5.847523 & 0.058013 & 0.448784 \\
\hline & -4.488148 & 0.233204 & 0.704876 \\
\hline & -5.696887 & -0.819751 & -3.312485 \\
\hline & -6.842244 & 0.223417 & 1.597067 \\
\hline & 1.657312 & 1.310019 & -4.066720 \\
\hline & 1.270729 & 3.121894 & -2.310291 \\
\hline & 2.535256 & 3.648693 & -2.614775 \\
\hline & 2.985652 & 4.830346 & -2.032704 \\
\hline & 2.197071 & 5.512061 & -1.114072 \\
\hline & 0.953649 & 4.980877 & -0.770493 \\
\hline & 0.502227 & 3.806102 & -1.352319 \\
\hline & 6.698916 & -0.281678 & 0.898579 \\
\hline & -2.104218 & 0.735389 & 2.984246 \\
\hline & 7.421791 & -4.908380 & -0.753468 \\
\hline & -1.690617 & -2.855327 & 6.016724 \\
\hline & 6.207138 & 4.661875 & -0.251466 \\
\hline & -3.803788 & -2.819121 & 0.923279 \\
\hline 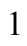 & -5.665259 & -4.084299 & -0.059574 \\
\hline & 4.057469 & 4.022751 & 0.667592 \\
\hline 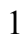 & -2.067754 & -0.678921 & 4.969004 \\
\hline & 5.408422 & -4.771004 & -2.109270 \\
\hline & 8.553681 & 1.815938 & -2.458481 \\
\hline 1 & 3.336893 & -3.533975 & -2.756357 \\
\hline & -2.996948 & -5.868849 & -2.922752 \\
\hline 1 & -0.618348 & -5.285041 & -2.908018 \\
\hline & -0.764377 & -6.274122 & 3.581992 \\
\hline 1 & 6.893724 & 0.072844 & -2.017159 \\
\hline ] & -0.881015 & -4.899803 & 1.561647 \\
\hline 1 & 1.257537 & -3.962851 & -1.953445 \\
\hline 1 & 8.246067 & 4.119769 & -1.555890 \\
\hline & 8.606176 & -1.589051 & 1.705586 \\
\hline 1 & -5.270381 & -5.637922 & -1.968843 \\
\hline & -1.168514 & -5.271370 & 5.825828 \\
\hline 1 & 9.001685 & -3.904654 & 0.871219 \\
\hline & 2.252077 & 2.317507 & 0.935027 \\
\hline 1 & -4.166035 & 0.511821 & 1.707526 \\
\hline 1 & -3.192242 & -0.483171 & -2.331745 \\
\hline & -7.290564 & -0.398240 & -1.074928 \\
\hline 1 & -3.347892 & 2.682108 & -0.262931 \\
\hline & 0.067587 & 1.454723 & 2.010774 \\
\hline 1 & -1.347301 & 5.492276 & 2.287854 \\
\hline & 1.569096 & -3.892744 & 1.234220 \\
\hline 1 & 2.961450 & -3.379935 & 0.293087 \\
\hline 1 & 4.670580 & -2.970408 & 1.770228 \\
\hline
\end{tabular}




\begin{tabular}{|c|c|c|c|}
\hline 1 & 0.779836 & -1.421458 & 2.688689 \\
\hline & 5.579963 & -1.914295 & 3.810233 \\
\hline & 1.667515 & -0.403219 & 4.759534 \\
\hline & 4.082328 & -0.626365 & 5.323769 \\
\hline & -1.590154 & 0.866529 & -2.552083 \\
\hline & 3.180464 & 3.141684 & -3.328172 \\
\hline & 3.967314 & 5.215042 & -2.304897 \\
\hline & 2.547550 & 6.436323 & -0.658242 \\
\hline & 0.323378 & 5.478369 & -0.032963 \\
\hline & -0.466569 & 3.408782 & -1.051532 \\
\hline & 2.552903 & 0.900583 & -3.584497 \\
\hline & 1.155470 & 0.483579 & -4.579407 \\
\hline & 1.990522 & 2.025173 & -4.835031 \\
\hline & -1.281390 & 2.745971 & -2.976453 \\
\hline & -0.980275 & 1.501400 & -4.281211 \\
\hline & -0.215601 & 4.429492 & 4.603916 \\
\hline ) & 1.245725 & 2.586342 & 3.854607 \\
\hline & 1.507728 & 4.844191 & 2.842433 \\
\hline ) & -3.137131 & 6.670545 & 1.041446 \\
\hline & -3.209390 & 5.436058 & -1.106297 \\
\hline & -4.835022 & 4.893757 & 0.719387 \\
\hline 1 & -3.848525 & 7.400958 & 0.635969 \\
\hline 1 & -3.270783 & 6.651886 & 2.130823 \\
\hline & -2.126979 & 7.042759 & 0.824415 \\
\hline 1 & -3.870362 & 6.218324 & -1.503318 \\
\hline | & -2.174725 & 5.705403 & -1.359152 \\
\hline 1 & -3.449663 & 4.503096 & -1.631370 \\
\hline 1 & -5.536191 & 5.641496 & 0.324829 \\
\hline 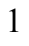 & -5.094380 & 3.925519 & 0.272000 \\
\hline ] & -4.997640 & 4.816151 & 1.802578 \\
\hline & 0.514263 & 4.651073 & 5.393938 \\
\hline 1 & -0.737537 & 5.364366 & 4.368734 \\
\hline 1 & -0.953612 & 3.727306 & 5.013603 \\
\hline 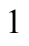 & 2.267434 & 5.066011 & 3.604223 \\
\hline 1 & 2.020325 & 4.457353 & 1.951082 \\
\hline 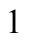 & 1.029396 & 5.792569 & 2.564858 \\
\hline 1 & 1.923922 & 2.851961 & 4.675566 \\
\hline & 0.553190 & 1.818180 & 4.227615 \\
\hline 1 & 1.859079 & 2.133757 & 3.064889 \\
\hline 5 & -7.206826 & -0.766029 & -3.532357 \\
\hline 6 & -5.039446 & 0.156061 & -4.296645 \\
\hline 6 & -5.215380 & -2.242315 & -3.614490 \\
\hline 6 & -8.286468 & -0.004674 & 1.158504 \\
\hline 6 & -6.510841 & -0.797195 & 2.692884 \\
\hline 6 & -6.736698 & 1.641413 & 2.169498 \\
\hline 1 & -5.345678 & -0.080498 & -5.324331 \\
\hline 1 & -5.335827 & 1.191932 & -4.085054 \\
\hline 1 & -3.943107 & 0.104789 & -4.264935 \\
\hline 1 & -5.450098 & -2.514501 & -4.652793 \\
\hline 1 & -4.131597 & -2.347795 & -3.474729 \\
\hline 1 & -5.705311 & -2.967313 & -2.951763 \\
\hline 1 & -7.436036 & -1.009074 & -4.577603 \\
\hline 1 & -7.740660 & -1.492445 & -2.906326 \\
\hline 1 & -7.616050 & 0.231888 & -3.326690 \\
\hline 1 & -8.956642 & 0.119899 & 2.018494 \\
\hline 1 & -8.601506 & 0.713535 & 0.390533 \\
\hline
\end{tabular}


$\mathrm{TS}_{\text {IIBI- }} \psi_{-1 \mathrm{~s} 2 \mathrm{a3a} 4 \mathrm{~s}}$

Number of imaginary frequencies : 1

The smallest frequencies are : $-587.6822 \quad 16.9860 \quad 22.6446 \mathrm{~cm}(-1)$

Electronic energy : $\quad H F=-4360.8647735$

Zero-point correction $=\quad 1.452702$ (Hartree/Particle)

Thermal correction to Energy $=\quad 1.537310$

Thermal correction to Enthalpy $=\quad 1.538254$

Thermal correction to Gibbs Free Energy $=\quad 1.335951$

Sum of electronic and zero-point Energies=

Sum of electronic and thermal Energies=

$-4359.412072$

Sum of electronic and thermal Enthalpies=

$-4359.327464$

Sum of electronic and thermal Free Energies=

$-4359.326520$

$-4359.528823$

Cartesian Coordinates

$\begin{array}{cccc}6 & 6.845302 & 0.993202 & -1.642977 \\ 6 & 5.694474 & 1.300857 & -0.873416 \\ 6 & 5.545413 & 2.639935 & -0.402827 \\ 6 & 6.555963 & 3.592892 & -0.676635 \\ 6 & 7.665942 & 3.256890 & -1.409294 \\ 6 & 7.801309 & 1.943757 & -1.905578 \\ 6 & 4.681349 & 0.330217 & -0.572846 \\ 6 & 3.514091 & 0.783105 & 0.016139 \\ 6 & 3.354039 & 2.108922 & 0.461284 \\ 6 & 4.369688 & 3.007174 & 0.295525 \\ 6 & 4.861096 & -1.110479 & -0.863385 \\ 6 & 3.901637 & -1.807147 & -1.575435 \\ 6 & 4.093344 & -3.143215 & -1.989617 \\ 6 & 5.210864 & -3.826864 & -1.598467 \\ 6 & 6.162781 & -3.213875 & -0.747185 \\ 6 & 5.991324 & -1.847519 & -0.371201 \\ 6 & 7.260912 & -3.948140 & -0.236374 \\ 6 & 8.146016 & -3.376052 & 0.640859 \\ 6 & 7.960786 & -2.037262 & 1.043926 \\ 6 & 6.918567 & -1.292844 & 0.548328 \\ 8 & 2.724507 & -1.206383 & -1.952293 \\ 15 & 1.545471 & -0.811844 & -0.824466 \\ 8 & 2.492824 & -0.077749 & 0.307587 \\ 45 & -0.277505 & 0.102602 & -1.859403 \\ 6 & -0.632614 & 1.951436 & -3.273004 \\ 6 & 0.753553 & 2.000344 & -3.038154 \\ 6 & 1.671390 & 1.365842 & -4.054937 \\ 6 & 1.303692 & 3.129428 & -2.248606 \\ 6 & 2.564962 & 3.662083 & -2.557778 \\ 6 & 3.023438 & 4.831214 & -1.957872 \\ 6 & 2.246725 & 5.495287 & -1.016345 \\ 6 & 1.007483 & 4.957649 & -0.667853\end{array}$




\begin{tabular}{|c|c|c|c|}
\hline 5 & 0.546909 & 3.795612 & -1.269168 \\
\hline 6 & -0.453887 & -1.230018 & -3.268778 \\
\hline 8 & -0.522929 & -1.883910 & -4.224806 \\
\hline 15 & -1.698652 & 0.226788 & 0.076791 \\
\hline 6 & -1.572173 & -0.798533 & 1.633449 \\
\hline 6 & -1.295894 & -2.156874 & 1.559922 \\
\hline 6 & -1.226364 & -2.947450 & 2.755949 \\
\hline 6 & -1.464120 & -2.337906 & 4.021063 \\
\hline 6 & -1.791578 & -0.964249 & 4.058823 \\
\hline 6 & -1.843897 & -0.225856 & 2.907611 \\
\hline 6 & -1.372548 & -3.111017 & 5.202536 \\
\hline 6 & -1.055046 & -4.443950 & 5.146417 \\
\hline 6 & -0.819566 & -5.056368 & 3.897826 \\
\hline 6 & -0.907132 & -4.331166 & 2.734997 \\
\hline 6 & -1.119489 & -2.891640 & 0.277222 \\
\hline 6 & 0.112763 & -2.971761 & -0.343209 \\
\hline 6 & 0.304247 & -3.825048 & -1.453513 \\
\hline 6 & -0.733176 & -4.555472 & -1.966542 \\
\hline 6 & -2.024090 & -4.461074 & -1.396887 \\
\hline 6 & -2.221760 & -3.629568 & -0.254570 \\
\hline 6 & -3.521391 & -3.558371 & 0.311491 \\
\hline 6 & -4.563829 & -4.270067 & -0.226553 \\
\hline 6 & -4.362292 & -5.100302 & -1.351313 \\
\hline 6 & -3.119135 & -5.189327 & -1.924086 \\
\hline 7 & 1.216899 & -2.182427 & 0.122742 \\
\hline 6 & 2.147567 & -2.872229 & 1.046699 \\
\hline 6 & 2.689992 & -2.049094 & 2.187596 \\
\hline 6 & 1.866001 & -1.215883 & 2.946979 \\
\hline 6 & 2.383367 & -0.479624 & 4.003291 \\
\hline 6 & 3.737721 & -0.567328 & 4.324252 \\
\hline 6 & 4.563824 & -1.399853 & 3.578514 \\
\hline 6 & 4.040834 & -2.135865 & 2.516898 \\
\hline 6 & -1.808976 & & 0.743117 \\
\hline 6 & -2.749300 & 2.829010 & 0.208449 \\
\hline 6 & -2.783608 & 4.164494 & 0.601615 \\
\hline 6 & -1.888252 & 4.579709 & 1.597366 \\
\hline 6 & -0.937462 & 3.723504 & 2.153961 \\
\hline 6 & -0.876260 & 2.411503 & 1.666972 \\
\hline 6 & -3.747560 & 5.172139 & -0.020803 \\
\hline 6 & -2.932120 & 6.275686 & -0.704809 \\
\hline 6 & -0.028819 & 4.146914 & 3.309222 \\
\hline 6 & -0.159652 & 5.632146 & 3.640063 \\
\hline 6 & -3.476117 & -0.124230 & -0.316140 \\
\hline 6 & -4.462593 & 0.092194 & 0.641583 \\
\hline 6 & -5.811444 & -0.143603 & 0.363924 \\
\hline 6 & -6.142528 & -0.605581 & -0.910793 \\
\hline 6 & -5.175081 & -0.857939 & -1.891642 \\
\hline 6 & -3.839580 & -0.615314 & -1.569537 \\
\hline 6 & -6.843224 & 0.112860 & 1.460112 \\
\hline 6 & -6.799420 & 1.592299 & 1.860472 \\
\hline 6 & -5.595283 & -1.340238 & -3.279094 \\
\hline 6 & -6.537384 & -2.542818 & -3.168520 \\
\hline 6 & -4.637546 & 5.787596 & 1.063682 \\
\hline 6 & -4.652450 & 4.531771 & -1.071042 \\
\hline 6 & 1.439111 & 3.859738 & 2.984101 \\
\hline 6 & -0.430734 & 3.342953 & 4.552876 \\
\hline
\end{tabular}




\begin{tabular}{|c|c|c|c|}
\hline 6 & -6.318231 & -0.195048 & -3.997620 \\
\hline 6 & -4.395007 & -1.763969 & -4.124246 \\
\hline 6 & -6.511294 & -0.760371 & 2.676345 \\
\hline 6 & -8.265067 & -0.217762 & 1.014433 \\
\hline 1 & 6.778565 & -0.269898 & 0.887678 \\
\hline 1 & -2.105016 & 0.826307 & 2.977427 \\
\hline 1 & 7.375411 & -4.986338 & -0.545561 \\
\hline 1 & -1.557135 & -2.620899 & 6.157499 \\
\hline 1 & 6.421445 & 4.607211 & -0.301307 \\
\hline 1 & -3.691179 & -2.913453 & 1.172507 \\
\hline 1 & -5.557007 & -4.187278 & 0.210815 \\
\hline 1 & 4.266764 & 4.030076 & 0.653997 \\
\hline 1 & -2.004055 & -0.497245 & 5.020239 \\
\hline 1 & 5.369611 & -4.857450 & -1.911652 \\
\hline 1 & 8.667980 & 1.681798 & -2.508566 \\
\hline 1 & 3.336900 & -3.596727 & -2.626445 \\
\hline 1 & -2.949770 & -5.822531 & -2.794007 \\
\hline 1 & -0.583821 & -5.203334 & -2.828959 \\
\hline 1 & -0.568471 & -6.113765 & 3.855449 \\
\hline 1 & 6.961059 & -0.010656 & -2.043900 \\
\hline 1 & -0.726366 & -4.822435 & 1.781120 \\
\hline 1 & 1.296327 & -3.872718 & -1.899160 \\
\hline 1 & 8.432413 & 3.999246 & -1.619620 \\
\hline 1 & 8.646575 & -1.590190 & \\
\hline 1 & -5.196298 & -5.665827 & -1.762078 \\
\hline 1 & -0.983664 & -5.030460 & 6.059435 \\
\hline 1 & 8.979439 & -3.952345 & 1.035710 \\
\hline 1 & 2.408356 & 2.382016 & 0.922531 \\
\hline 1 & -4.179144 & 0.465188 & 1.625234 \\
\hline 1 & -3.047155 & -0.811920 & -2.287258 \\
\hline 1 & -7.188625 & -0.780644 & 3878 \\
\hline 1 & -3.456843 & 2.460069 & -0.530430 \\
\hline 1 & -0.109643 & 1.729005 & 2.042251 \\
\hline 1 & -1.937071 & 5.611306 & 1.939242 \\
\hline 1 & 1.565969 & -3.707900 & 1.463161 \\
\hline 1 & 2.984870 & -3.326685 & 0.494431 \\
\hline 1 & 4.700420 & -2.771555 & 1.923655 \\
\hline 1 & 0.818598 & -1.114055 & \\
\hline 1 & 5.624839 & -1.472756 & 3.810405 \\
\hline 1 & 1.722257 & 0.168041 & 4.578970 \\
\hline 1 & 4.145098 & 0.014145 & 5.148664 \\
\hline 1 & -1.557297 & 0.830755 & -2.549281 \\
\hline 1 & 3.202437 & 3.168958 & -3.287547 \\
\hline 1 & 4.003189 & 5.219311 & -2.232430 \\
\hline 1 & 2.602328 & 6.412256 & -0.550524 \\
\hline 1 & 0.382377 & 5.444515 & 0.081271 \\
\hline 1 & -0.420541 & 3.394523 & -0.970432 \\
\hline 1 & 2.573636 & 0.942371 & -3.597214 \\
\hline 1 & 1.165517 & 0.556288 & -4.589366 \\
\hline 1 & 1.994790 & 2.105665 & -4.803803 \\
\hline 1 & -1.262119 & 2.752149 & -2.880752 \\
\hline 1 & -0.965594 & 1.560922 & -4.235768 \\
\hline 1 & -5.333052 & 5.287878 & -1.482044 \\
\hline 1 & -4.077229 & 4.116895 & -1.909689 \\
\hline 1 & -5.268178 & 3.725963 & -0.648738 \\
\hline 1 & -3.599339 & 6.999853 & -1.191673 \\
\hline
\end{tabular}




$\begin{array}{rrrr}1 & -2.307037 & 6.828153 & 0.007751 \\ 1 & -2.269477 & 5.853730 & -1.473146 \\ 1 & -5.326145 & 6.519451 & 0.620501 \\ 1 & -5.240054 & 5.016683 & 1.562126 \\ 1 & -4.055520 & 6.307928 & 1.833740 \\ 1 & 0.189279 & 3.630237 & 5.413109 \\ 1 & -1.481515 & 3.523888 & 4.816319 \\ 1 & -0.304143 & 2.263480 & 4.395931 \\ 1 & 2.082755 & 4.215985 & 3.799472 \\ 1 & 1.635028 & 2.786858 & 2.867813 \\ 1 & 1.747541 & 4.368496 & 2.059857 \\ 1 & 0.512943 & 5.883563 & 4.469649 \\ 1 & 0.115383 & 6.269779 & 2.789235 \\ 1 & -1.176769 & 5.898245 & 3.954533 \\ 1 & -4.741305 & -2.135829 & -5.096991 \\ 1 & -3.707030 & -0.930642 & -4.321303 \\ 1 & -3.825524 & -2.570066 & -3.640413 \\ 1 & -6.824822 & -2.889336 & -4.170214 \\ 1 & -6.044664 & -3.375685 & -2.649717 \\ 1 & -7.462054 & -2.305764 & -2.628984 \\ 1 & -6.628014 & -0.506978 & -5.004497 \\ 1 & -7.216730 & 0.123175 & -3.453889 \\ 1 & -5.662510 & 0.679793 & -4.100406 \\ 1 & -7.238574 & -0.588230 & 3.481294 \\ 1 & -6.546312 & -1.825954 & 2.412495 \\ 1 & -5.512608 & -0.552561 & 3.081006 \\ 1 & -7.503225 & 1.789112 & 2.680598 \\ 1 & -5.800990 & 1.902919 & 2.194026 \\ 1 & -7.081114 & 2.233114 & 1.014280 \\ 1 & -8.965044 & -0.019293 & 1.836004 \\ 1 & -8.580952 & 0.394319 & 0.159857 \\ 1 & -8.372436 & -1.275025 & 0.738980\end{array}$

TS1 $_{\text {IIBI- }} \psi_{-1 \mathrm{~s} 2 a 3 s 4 a}$

Number of imaginary frequencies : 1

The smallest frequencies are : -574.5216 $14.8090 \quad 17.6142 \mathrm{~cm}(-1)$

Electronic energy : $\quad \mathrm{HF}=-4360.863836$

Zero-point correction $=\quad 1.452244($ Hartree/Particle $)$

Thermal correction to Energy $=\quad 1.537140$

Thermal correction to Enthalpy $=\quad 1.538085$

Thermal correction to Gibbs Free Energy $=\quad 1.333503$

Sum of electronic and zero-point Energies $=\quad-4359.411592$

Sum of electronic and thermal Energies $=\quad-4359.326696$

Sum of electronic and thermal Enthalpies $=\quad-4359.325751$

Sum of electronic and thermal Free Energies $=\quad-4359.530333$

Cartesian Coordinates

$\begin{array}{llll}6 & 2.574132 & 3.676435 & -2.553988 \\ 6 & 1.317727 & 3.134127 & -2.242644 \\ 6 & 0.559942 & 3.789197 & -1.256736 \\ 6 & 1.013640 & 4.952465 & -0.652444 \\ 6 & 2.247711 & 5.500339 & -1.003211 \\ 6 & 3.026362 & 4.845918 & -1.950071 \\ 6 & 0.772154 & 2.004866 & -3.035278\end{array}$




$\begin{array}{cccc}6 & 1.695240 & 1.364731 & -4.043623 \\ 45 & -0.271738 & 0.117206 & -1.852654 \\ 6 & -0.501745 & -1.222677 & -3.247311 \\ 8 & -0.684014 & -1.863603 & -4.197509 \\ 15 & -1.698881 & 0.238846 & 0.069922 \\ 6 & -1.811866 & 1.948888 & 0.746957 \\ 6 & -2.768369 & 2.831389 & 0.233727 \\ 6 & -2.821000 & 4.158736 & 0.651314 \\ 6 & -1.915186 & 4.574014 & 1.637379 \\ 6 & -0.944805 & 3.723835 & 2.169460 \\ 6 & -0.875756 & 2.417779 & 1.668061 \\ 6 & -3.836481 & 5.150424 & 0.087853 \\ 6 & -4.728372 & 5.672369 & 1.219553 \\ 6 & -0.030516 & 4.144027 & 3.321539 \\ 6 & 1.436058 & 3.855636 & 2.991852 \\ 6 & -3.476642 & -0.105139 & -0.328012 \\ 6 & -4.467569 & 0.060085 & 0.649221 \\ 6 & -5.807008 & -0.172936 & 0.361684 \\ 6 & -6.141999 & -0.570034 & -0.944909 \\ 6 & -5.184445 & -0.744992 & -1.938204 \\ 6 & -3.842553 & -0.522749 & -1.597184 \\ 6 & -5.518644 & -1.162727 & -3.368226 \\ 6 & -4.918600 & -2.545295 & -3.640633 \\ 6 & -6.899680 & -0.043263 & 1.421408 \\ 6 & -8.018401 & 0.878401 & 0.925414 \\ 6 & -1.575382 & -0.795960 & 1.618793 \\ 6 & -1.299724 & -2.153957 & 1.537808 \\ 6 & -1.235032 & -2.950458 & 2.731395 \\ 6 & -1.478148 & -2.346970 & 3.998547 \\ 6 & -1.803640 & -0.973019 & 4.042565 \\ 6 & -1.849801 & -0.228936 & 2.895183 \\ 6 & -1.392387 & -3.125140 & 5.177114 \\ 6 & -1.074161 & -4.457685 & 5.117152 \\ 6 & -0.831341 & -5.063964 & 3.867123 \\ 6 & -0.913473 & -4.333782 & 2.706977 \\ 6 & -1.108269 & -2.889303 & 0.256432 \\ 6 & 0.133143 & -2.971854 & -0.345412 \\ 6 & 0.348978 & -3.850122 & -1.432029 \\ 6 & -0.668839 & -4.618169 & -1.929248 \\ 6 & -1.967528 & -4.528869 & -1.376085 \\ 6 & -2.195185 & -3.652200 & -0.273749 \\ 6 & -3.505619 & -3.575816 & 0.266123 \\ 6 & -4.525838 & -4.336510 & -0.247961 \\ 6 & -4.289671 & -5.223645 & -1.320839 \\ 6 & -3.038799 & -5.309349 & -1.876235 \\ 7 & 1.228280 & -2.172425 & 0.125422 \\ 6 & 2.165688 & -2.859770 & 1.044582 \\ 6 & 2.695948 & -2.039186 & 2.192909 \\ 6 & 1.861725 & -1.216251 & 2.952121 \\ 6 & 2.368177 & -0.482192 & 4.015308 \\ 6 & 3.721226 & -0.562298 & 4.343455 \\ 6 & 4.557460 & -1.384634 & 3.597629 \\ 6 & 4.045691 & -2.118098 & 2.528886 \\ 6 & -0.612065 & 1.959478 & -3.278922 \\ 15 & 1.554041 & -0.799720 & -0.819546 \\ 8 & 2.732598 & -1.190722 & -1.949353\end{array}$




\begin{tabular}{|c|c|c|c|}
\hline 6 & 3.911139 & -1.790744 & -1.576226 \\
\hline & 4.870132 & -1.095541 & -0.862152 \\
\hline & 6.001086 & -1.833015 & -0.372492 \\
\hline & 6.174164 & -3.197852 & -0.753237 \\
\hline & 5.223698 & -3.808341 & -1.607940 \\
\hline & 4.105507 & -3.124462 & -1.996829 \\
\hline & 6.927884 & -1.280151 & 0.548573 \\
\hline & 7.970957 & -2.025023 & 1.041641 \\
\hline & 8.157555 & -3.362325 & 0.634265 \\
\hline & 7.273089 & -3.932569 & -0.244815 \\
\hline & 4.689773 & 0.344418 & -0.568809 \\
\hline & 3.522458 & 0.795757 & 0.021107 \\
\hline & 3.361759 & 2.121398 & 0.466730 \\
\hline & 4.376355 & 3.020685 & 0.300528 \\
\hline & 5.551871 & 2.654905 & -0.398894 \\
\hline & 5.701924 & 1.316042 & -0.869722 \\
\hline y & 6.561086 & 3.609017 & -0.673486 \\
\hline & 7.670940 & 3.274236 & -1.406909 \\
\hline b & 7.807428 & 1.961239 & -1.903206 \\
\hline & 6.852596 & 1.009647 & -1.640015 \\
\hline & 2.502379 & -0.066710 & 0.312908 \\
\hline & -4.920493 & -0.149352 & -4.352542 \\
\hline & -7.023163 & -1.233240 & -3.615569 \\
\hline & -7.472143 & -1.436450 & 1.706423 \\
\hline & -6.365225 & 0.531847 & 2.732010 \\
\hline 6 & -4.737771 & 4.516491 & -0.969503 \\
\hline ) & -3.089815 & 6.319555 & -0.563186 \\
\hline & -0.429969 & 3.337446 & 4.564357 \\
\hline & -0.157977 & 5.628728 & 3.656273 \\
\hline & 6.786730 & -0.258380 & 0.891066 \\
\hline & -2.109850 & 0.823301 & 2.968886 \\
\hline 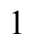 & 7.388850 & -4.969578 & -0.557508 \\
\hline & -1.581641 & -2.638832 & 6.133116 \\
\hline & 6.425634 & 4.623195 & -0.298114 \\
\hline & -3.700178 & -2.892730 & 1.091764 \\
\hline & -5.525482 & -4.254915 & 0.174138 \\
\hline 1 & 4.272460 & 4.043258 & 0.659655 \\
\hline & -2.019101 & -0.510713 & 5.005570 \\
\hline 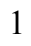 & 5.384187 & -4.837207 & -1.925867 \\
\hline & 8.674007 & 1.700185 & -2.506722 \\
\hline | & 3.350449 & -3.575718 & -2.636804 \\
\hline 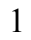 & -2.844597 & -5.979603 & -2.712598 \\
\hline & -0.497168 & -5.296388 & -2.763716 \\
\hline 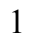 & -0.578504 & -6.120797 & 3.821118 \\
\hline & 6.969219 & 0.005965 & -2.041110 \\
\hline 1 & -0.725731 & -4.821773 & 1.752824 \\
\hline & 1.347531 & -3.897427 & -1.862917 \\
\hline 1 & 8.436423 & 4.017446 & -1.617820 \\
\hline 1 & 8.656370 & -1.579552 & 1.759371 \\
\hline ] & -5.105091 & -5.828516 & -1.711973 \\
\hline 1 & -1.007213 & -5.048134 & 6.027963 \\
\hline ] & 8.991635 & -3.938969 & 1.027210 \\
\hline 1 & 2.416691 & 2.393460 & 0.929689 \\
\hline & -4.176060 & 0.387297 & 1.643796 \\
\hline 1 & -3.058756 & -0.679276 & -2.335634 \\
\hline 1 & -7.189617 & -0.747611 & -1.180178 \\
\hline
\end{tabular}




\begin{tabular}{|c|c|c|c|}
\hline 1 & -3.478895 & 2.462627 & -0.502223 \\
\hline 1 & -0.101119 & 1.737481 & 2.030784 \\
\hline 1 & -1.975472 & 5.599430 & 1.995951 \\
\hline & 1.593570 & -3.705673 & 1.453131 \\
\hline 1 & 3.009275 & -3.300117 & 0.490626 \\
\hline & 4.712955 & -2.745432 & 1.935285 \\
\hline 1 & 0.815035 & -1.120853 & 2.672669 \\
\hline 1 & 5.617454 & -1.451499 & 3.835999 \\
\hline 1 & 1.699602 & 0.157659 & 4.591011 \\
\hline 1 & 4.119785 & 0.017249 & 5.173518 \\
\hline 1 & -1.545827 & 0.852180 & -2.541088 \\
\hline 1 & 3.211928 & 3.190913 & -3.288644 \\
\hline 1 & 4.002547 & 5.242207 & -2.225635 \\
\hline 1 & 2.598400 & 6.417818 & -0.534653 \\
\hline 1 & 0.387792 & 5.432295 & 0.100783 \\
\hline 1 & -0.402694 & 3.377970 & -0.956270 \\
\hline 1 & 2.597634 & 0.949220 & -3.579269 \\
\hline 1 & 1.193327 & 0.547883 & -4.571074 \\
\hline 1 & 2.017718 & 2.098816 & -4.798528 \\
\hline 1 & -1.241479 & 2.764140 & -2.895011 \\
\hline 1 & -0.942485 & 1.560930 & -4.239553 \\
\hline 1 & -5.450425 & 5.263062 & -1.341912 \\
\hline 1 & -4.163904 & 4.150930 & -1.831546 \\
\hline 1 & -5.319443 & 3.676694 & -0.565502 \\
\hline 1 & -3.802171 & 7.045411 & -0.978146 \\
\hline 1 & -2.450904 & 6.852906 & 0.151433 \\
\hline 1 & -2.450886 & 5.965982 & -1.383864 \\
\hline 1 & -5.465289 & 6.385594 & 0.826455 \\
\hline 1 & -5.276117 & 4.848678 & 1.696734 \\
\hline 1 & -4.153182 & 6.187169 & 1.998407 \\
\hline 1 & 0.194023 & 3.620156 & 5.423261 \\
\hline 1 & -1.479321 & 3.520428 & 4.832014 \\
\hline 1 & -0.307360 & 2.258166 & 4.402951 \\
\hline 1 & 2.082048 & 4.208698 & 3.806718 \\
\hline 1 & 1.630448 & 2.782828 & 2.872526 \\
\hline 1 & 1.743353 & 4.366462 & 2.068369 \\
\hline 1 & 0.522864 & 5.878589 & 4.479588 \\
\hline 1 & 0.108304 & 6.267663 & 2.803640 \\
\hline 1 & -1.171543 & 5.894776 & 3.981909 \\
\hline 1 & -7.213113 & -1.518060 & -4.658122 \\
\hline 1 & -7.509213 & -1.984268 & -2.979498 \\
\hline 1 & -7.513041 & -0.266001 & -3.442206 \\
\hline 1 & -5.118154 & -2.852150 & -4.676565 \\
\hline 1 & -3.831234 & -2.558109 & -3.490102 \\
\hline 1 & -5.352183 & -3.298041 & -2.969928 \\
\hline 1 & -5.180117 & -0.424787 & -5.383468 \\
\hline 1 & -5.306962 & 0.861061 & -4.164269 \\
\hline 1 & -3.824611 & -0.108923 & -4.292452 \\
\hline 1 & -8.259087 & -1.381985 & 2.471039 \\
\hline 1 & -7.908978 & -1.890386 & 0.808126 \\
\hline 1 & -6.686794 & -2.110359 & 2.075291 \\
\hline 1 & -8.797593 & 0.974606 & 1.693315 \\
\hline 1 & -7.633891 & 1.883545 & 0.708230 \\
\hline 1 & -8.501572 & 0.501122 & 0.016613 \\
\hline 1 & -7.185498 & 0.626847 & 3.454643 \\
\hline 1 & -5.601738 & -0.113114 & 3.187345 \\
\hline
\end{tabular}


TS1 $_{I I B 1-} \Psi-1 \mathrm{~s} 2 \mathrm{a} 3 \mathrm{~s} 4 \mathrm{~s}$

Number of imaginary frequencies : 1

The smallest frequencies are : $-580.9791 \quad 17.1470 \quad 19.1851 \mathrm{~cm}(-1)$

Electronic energy : $\quad H F=-4360.8643822$

Zero-point correction $=\quad 1.452734($ Hartree/Particle $)$

Thermal correction to Energy= $\quad 1.537364$

Thermal correction to Enthalpy= $\quad 1.538309$

Thermal correction to Gibbs Free Energy $=\quad 1.335588$

Sum of electronic and zero-point Energies $=\quad-4359.411648$

Sum of electronic and thermal Energies $=\quad-4359.327018$

Sum of electronic and thermal Enthalpies $=\quad-4359.326074$

Sum of electronic and thermal Free Energies $=\quad-4359.528795$

\begin{tabular}{|c|c|c|c|}
\hline & \multicolumn{3}{|c|}{ Cartesian Coordinates } \\
\hline 6 & -4.446679 & -0.055404 & 0.642796 \\
\hline 6 & -3.454878 & -0.191425 & -0.329275 \\
\hline 6 & -3.820227 & -0.574815 & -1.613332 \\
\hline 6 & -5.158735 & -0.779351 & -1.962767 \\
\hline 6 & -6.126347 & -0.620718 & -0.968972 \\
\hline 6 & -5.791177 & -0.269364 & 0.344917 \\
\hline 15 & -1.687601 & 0.191956 & 0.079263 \\
\hline 45 & -0.260057 & 0.113988 & -1.838869 \\
\hline 6 & -0.639936 & 1.958162 & -3.256480 \\
\hline 6 & 0.740722 & 2.042020 & -3.005479 \\
\hline 6 & 1.686574 & 1.429320 & -4.009789 \\
\hline 6 & -5.491581 & -1.161623 & -3.403462 \\
\hline 6 & -6.825378 & -0.098269 & 1.455880 \\
\hline 6 & -1.538945 & -0.847244 & 1.621984 \\
\hline 6 & -1.244316 & -2.200340 & 1.528488 \\
\hline 6 & -1.166719 & -3.007370 & 2.713482 \\
\hline 6 & -1.412127 & -2.418088 & 3.986884 \\
\hline 6 & -1.752741 & -1.048267 & 4.044515 \\
\hline 6 & -1.812812 & -0.294160 & 2.904078 \\
\hline 6 & -0.831203 & -4.387034 & 2.674362 \\
\hline 6 & -0.736524 & -5.126875 & 3.827312 \\
\hline 6 & -0.979964 & -4.534434 & 5.083945 \\
\hline 6 & -1.312563 & -3.206101 & 5.157816 \\
\hline 6 & -1.053578 & -2.918819 & 0.238091 \\
\hline 6 & 0.184162 & -2.986618 & -0.372971 \\
\hline 6 & 0.395221 & -3.846650 & -1.475051 \\
\hline 6 & -0.625157 & -4.606068 & -1.980751 \\
\hline 6 & -1.921496 & -4.526915 & -1.420076 \\
\hline 6 & -2.142389 & -3.673761 & -0.298422 \\
\hline 6 & -2.998029 & -5.291856 & -1.932924 \\
\hline 6 & -4.247295 & -5.210515 & -1.372816 \\
\hline 6 & -4.475758 & -4.347193 & -0.278949 \\
\hline 6 & -3.449390 & -3.605908 & 0.250418 \\
\hline 7 & 1.277916 & -2.186620 & 0.100591 \\
\hline 15 & 1.579466 & -0.790709 & -0.818499 \\
\hline 8 & 2.515335 & -0.062607 & 0.327898 \\
\hline 6 & 3.506486 & 0.835736 & 0.042907 \\
\hline 6 & 4.683926 & 0.425882 & -0.556568 \\
\hline
\end{tabular}




\begin{tabular}{|c|c|c|c|}
\hline 6 & 5.666283 & 1.429805 & -0.849521 \\
\hline 6 & 5.476842 & 2.759379 & -0.366426 \\
\hline & 4.291887 & 3.083830 & 0.337582 \\
\hline & 3.305944 & 2.152205 & 0.499927 \\
\hline & 6.824217 & 1.164829 & -1.624307 \\
\hline & 7.750159 & 2.146563 & -1.879486 \\
\hline & 7.575846 & 3.450086 & -1.370349 \\
\hline & 6.457573 & 3.745223 & -0.632859 \\
\hline & 4.902138 & -1.004341 & -0.871638 \\
\hline & 3.959947 & -1.713746 & -1.594075 \\
\hline & 4.188553 & -3.035022 & -2.036494 \\
\hline f & 5.326681 & -3.694012 & -1.662783 \\
\hline & 6.263507 & -3.071434 & -0.801642 \\
\hline f & 6.054222 & -1.718512 & -0.397522 \\
\hline & 7.383619 & -3.783942 & -0.308163 \\
\hline & 8.254412 & -3.204278 & 0.578502 \\
\hline$b$ & 8.032424 & -1.879480 & 1.008462 \\
\hline & 6.967904 & -1.155535 & 0.530216 \\
\hline 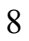 & 2.764771 & -1.139731 & -1.954827 \\
\hline & 2.237526 & -2.884219 & 0.988910 \\
\hline & 2.765996 & -2.091476 & 2.157525 \\
\hline ) & 1.926382 & -1.305344 & 2.948682 \\
\hline 6 & 2.429442 & -0.603821 & 4.035247 \\
\hline 5 & 3.785183 & -0.678223 & 4.352842 \\
\hline$b$ & 4.627669 & -1.461843 & 3.573109 \\
\hline 6 & 4.118737 & -2.164739 & 2.482844 \\
\hline ) & -1.862341 & 1.894740 & 0.763190 \\
\hline ) & -2.859854 & 2.737533 & 0.262162 \\
\hline ) & -2.964240 & 4.060470 & 0.682486 \\
\hline 6 & -2.067749 & 4.512168 & 1.660881 \\
\hline ) & -1.057645 & 3.701990 & 2.181849 \\
\hline 6 & -0.938801 & 2.400347 & 1.677562 \\
\hline 6 & -4.021203 & 5.008080 & 0.120534 \\
\hline 6 & -4.903358 & 4.331914 & -0.927518 \\
\hline 6 & -0.152376 & 4.158833 & 3.327089 \\
\hline ? & -0.536979 & 3.363208 & 4.581601 \\
\hline 6 & -3.320354 & 6.198584 & -0.542851 \\
\hline 6 & -4.925775 & 5.505169 & 1.253105 \\
\hline 6 & -0.310271 & 5.645739 & 3.638796 \\
\hline 6 & 1.320258 & 3.895694 & 3.003043 \\
\hline 6 & -0.471402 & -1.214703 & -3.246652 \\
\hline 8 & -0.658415 & -1.847590 & -4.201337 \\
\hline 6 & 1.250167 & 3.182355 & -2.205577 \\
\hline 6 & 0.466457 & 3.819499 & -1.227741 \\
\hline 5 & 0.885488 & 4.994236 & -0.620633 \\
\hline 6 & 2.110266 & 5.570887 & -0.958033 \\
\hline 6 & 2.914827 & 4.934021 & -1.895126 \\
\hline 6 & 2.496853 & 3.753873 & -2.502998 \\
\hline 1 & 6.799371 & -0.143803 & 0.889689 \\
\hline 1 & -2.085730 & 0.753999 & 2.987531 \\
\hline 1 & 7.526978 & -4.812052 & -0.638323 \\
\hline W & -1.503008 & -2.731005 & 6.119188 \\
\hline 1 & 6.293250 & 4.751244 & -0.247298 \\
\hline 1 & -3.636778 & -2.940918 & 1.092519 \\
\hline 1 & -5.473958 & -4.266312 & 0.147101 \\
\hline 1 & 4.158125 & 4.099927 & 0.705131 \\
\hline
\end{tabular}




\begin{tabular}{|c|c|c|c|}
\hline & -1.968986 & -0.597351 & 5.012721 \\
\hline & 5.513776 & -4.713135 & -1.997145 \\
\hline & 8.623251 & 1.916855 & -2.486353 \\
\hline & 3.441763 & -3.496937 & -2.678625 \\
\hline & -2.810221 & -5.944583 & -2.784481 \\
\hline & -0.457989 & -5.266934 & -2.829935 \\
\hline & -0.473124 & -6.180610 & 3.770678 \\
\hline & 6.969708 & 0.168945 & -2.035158 \\
\hline & -0.642945 & -4.863658 & 1.714532 \\
\hline & 1.391047 & -3.883899 & -1.913744 \\
\hline & 8.318958 & 4.217460 & -1.574608 \\
\hline & 8.707311 & -1.427300 & 1.731949 \\
\hline & -5.067215 & -5.801310 & -1.775950 \\
\hline & -0.902444 & -5.132474 & 5.988951 \\
\hline & 9.105074 & -3.764253 & 0.959851 \\
\hline & 2.356032 & 2.389304 & 0.972272 \\
\hline & -4.165071 & 0.243071 & 1.652048 \\
\hline & -3.034846 & -0.713280 & -2.353619 \\
\hline & -7.171759 & -0.775046 & -1.220636 \\
\hline & -3.561644 & 2.342066 & -0.467789 \\
\hline & -0.133646 & 1.752223 & 2.032723 \\
\hline & -2.166926 & 5.534107 & 2.020875 \\
\hline & 1.685988 & -3.752742 & 1.377157 \\
\hline & 3.082846 & -3.291564 & 0.413282 \\
\hline ] & 4.790298 & -2.763523 & 1.865131 \\
\hline & 0.877556 & -1.214699 & 2.676630 \\
\hline . & 5.689955 & -1.522911 & 3.802513 \\
\hline . & 1.756452 & 0.004527 & 4.638575 \\
\hline . & 4.181088 & -0.124012 & 5.201293 \\
\hline ] & -1.547818 & 0.831221 & -2.519707 \\
\hline & 3.153686 & 3.283126 & -3.230505 \\
\hline ] & 3.883857 & 5.353659 & -2.161125 \\
\hline & 2.433252 & 6.497372 & -0.487350 \\
\hline 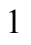 & 0.239852 & 5.460491 & 0.124192 \\
\hline 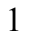 & -0.489311 & 3.385517 & -0.936532 \\
\hline . & 2.595596 & 1.033206 & -3.541408 \\
\hline 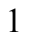 & 1.208424 & 0.602799 & -4.544292 \\
\hline & 1.996436 & 2.174602 & -4.759012 \\
\hline ] & -1.294028 & 2.743704 & -2.874023 \\
\hline & -0.954383 & 1.552812 & -4.219559 \\
\hline L & -5.654014 & 5.044246 & -1.292294 \\
\hline . & -4.322071 & 3.992982 & -1.795628 \\
\hline 1 & -5.440146 & 3.464566 & -0.518029 \\
\hline L & -4.059143 & 6.888180 & -0.973142 \\
\hline 1 & -2.709624 & 6.767826 & 0.168778 \\
\hline 1 & -2.659834 & 5.860055 & -1.352891 \\
\hline & -5.683977 & 6.195876 & 0.860226 \\
\hline 1 & -5.448554 & 4.666836 & 1.732995 \\
\hline 1 & -4.364072 & 6.038234 & 2.029568 \\
\hline 1 & 0.081002 & 3.669984 & 5.436592 \\
\hline 1 & -1.589739 & 3.530206 & 4.846096 \\
\hline 1 & -0.393515 & 2.284343 & 4.435473 \\
\hline & 1.958159 & 4.279940 & 3.810010 \\
\hline & 1.538073 & 2.825173 & 2.907015 \\
\hline 1 & 1.615957 & 4.392673 & 2.068452 \\
\hline 1 & 0.368473 & 5.923283 & 4.454928 \\
\hline
\end{tabular}




$\begin{array}{lrrr}1 & -0.061854 & 6.276349 & 2.774584 \\ 1 & -1.327558 & 5.895776 & 3.965210 \\ 6 & -6.995696 & -1.210921 & -3.659510 \\ 6 & -4.879175 & -0.133667 & -4.363619 \\ 6 & -4.905219 & -2.544778 & -3.702683 \\ 6 & -8.244702 & -0.409385 & 0.988368 \\ 6 & -6.486366 & -1.049639 & 2.609985 \\ 6 & -6.793472 & 1.351771 & 1.954769 \\ 1 & -7.182921 & -1.472318 & -4.708688 \\ 1 & -7.493486 & -1.969214 & -3.041518 \\ 1 & -7.476376 & -0.242225 & -3.469396 \\ 1 & -5.141060 & -0.381120 & -5.401069 \\ 1 & -5.252519 & 0.877101 & -4.151527 \\ 1 & -3.783082 & -0.108843 & -4.301608 \\ 1 & -5.105185 & -2.828786 & -4.745084 \\ 1 & -3.818472 & -2.571815 & -3.549104 \\ 1 & -5.348219 & -3.306494 & -3.048127 \\ 1 & -7.499474 & 1.488760 & 2.785196 \\ 1 & -5.797168 & 1.645657 & 2.309437 \\ 1 & -7.079591 & 2.045275 & 1.152255 \\ 1 & -7.219832 & -0.945216 & 3.420930 \\ 1 & -6.503538 & -2.094405 & 2.271059 \\ 1 & -5.492833 & -0.856066 & 3.034191 \\ 1 & -8.945074 & -0.279810 & 1.823237 \\ 1 & -8.568933 & 0.261362 & 0.182393 \\ 1 & -8.341724 & -1.444133 & 0.634627\end{array}$

$\operatorname{TS1}_{\text {IIBI- }} \psi_{-1 \mathbf{1 s 2} 23 a 4 a}$

Number of imaginary frequencies : 1

The smallest frequencies are : $-571.3152 \quad 18.2568 \quad 21.6423 \mathrm{~cm}(-1)$

Electronic energy : $\quad H F=-4360.8647465$

Zero-point correction $=\quad 1.452605$ (Hartree/Particle)

Thermal correction to Energy= $\quad 1.537239$

Thermal correction to Enthalpy= $\quad 1.538183$

Thermal correction to Gibbs Free Energy= $\quad 1.335643$

Sum of electronic and zero-point Energies $=\quad-4359.412142$

Sum of electronic and thermal Energies $=\quad-4359.327508$

Sum of electronic and thermal Enthalpies= $\quad-4359.326564$

Sum of electronic and thermal Free Energies $=\quad-4359.529104$

Cartesian Coordinates

$\begin{array}{cccc}\ldots & 1.855397 & -1.252670 & 2.962773 \\ 6 & 2.680493 & -2.076649 & 2.194736 \\ 6 & 4.032764 & -2.160743 & 2.518920 \\ 6 & 4.556318 & -1.430692 & 3.584232 \\ 6 & 3.729077 & -0.607751 & 4.339341 \\ 6 & 2.373351 & -0.523140 & 4.023573 \\ 6 & 2.136817 & -2.892093 & 1.049232 \\ 7 & 1.207484 & -2.192991 & 0.131035 \\ 15 & 1.546712 & -0.825542 & -0.816053 \\ 8 & 2.724927 & -1.229415 & -1.942409 \\ 6 & 3.902838 & -1.828204 & -1.565271 \\ 6 & 4.863386 & -1.129406 & -0.856465 \\ 6 & 5.994468 & -1.864421 & -0.363833\end{array}$




$\begin{array}{cccc}6 & 6.166531 & -3.231516 & -0.736637 \\ 6 & 5.214063 & -3.846972 & -1.585533 \\ 6 & 4.095499 & -3.165065 & -1.976814 \\ 6 & 6.921865 & -1.306569 & 0.553525 \\ 6 & 7.965012 & -2.048977 & 1.050218 \\ 6 & 8.150914 & -3.388631 & 0.650273 \\ 6 & 7.265641 & -3.963629 & -0.224929 \\ 6 & 4.683287 & 0.312202 & -0.571264 \\ 6 & 3.515316 & 0.765343 & 0.015641 \\ 6 & 3.347443 & 2.094796 & 0.445837 \\ 6 & 4.359013 & 2.996140 & 0.271025 \\ 6 & 5.536970 & 2.626908 & -0.422509 \\ 6 & 5.692651 & 1.283979 & -0.880176 \\ 6 & 6.543788 & 3.581572 & -0.704300 \\ 6 & 7.655876 & 3.243495 & -1.432736 \\ 6 & 7.797387 & 1.926419 & -1.916760 \\ 6 & 6.845365 & 0.974177 & -1.646061 \\ 8 & 2.499744 & -0.099613 & 0.316314 \\ 6 & 0.103015 & -2.976438 & -0.343199 \\ 6 & -1.136506 & -2.881609 & 0.260237 \\ 6 & -2.236274 & -3.615910 & -0.282636 \\ 6 & -2.028733 & -4.458317 & -1.415327 \\ 6 & -0.730908 & -4.566871 & -1.966271 \\ 6 & 0.303887 & -3.839193 & -1.444633 \\ 6 & -3.542487 & -3.533544 & 0.265582 \\ 6 & -4.582352 & -4.243291 & -0.280297 \\ 6 & -0.056401 & 5.605715 & 3.685543 \\ 6 & -4.371430 & -5.082452 & -1.396614 \\ 6 & -0.264792 & 0.103708 & -1.857731 \\ 6 & -3.121243 & -5.183477 & -1.951796 \\ 6 & -1.319888 & -2.145800 & 1.542428 \\ 6 & -0.542097 & -1.838905 & -3.257422 \\ 6 & -1.581200 & -0.784479 & 1.622220 \\ 6 & -0.793760 & 1.996644 & -3.212667 \\ 6 & -0.586211 & 1.936579 & -3.301364 \\ 6 & -1.854161 & -0.215538 & 2.898145 \\ 6 & -2.317524 & 3.143700 & -2.251515 \\ 6 & -1.818080 & -0.959976 & 4.045726 \\ 6 & -1.507264 & -2.337326 & 4.002544 \\ 6 & -1.267742 & -2.943002 & 2.735820 \\ 6 & -1.433001 & -3.117063 & 5.180854 \\ 6 & -1.130311 & -4.453253 & 5.120737 \\ 6 & -0.892401 & -5.061911 & 3.870889 \\ 6 & -0.963260 & -4.330093 & 2.711022 \\ 6 & -1.691196 & 0.256713 & 0.074508 \\ 6 & -1.766391 & 1.967370 & 0.755998 \\ 6 & -537186 & 3.818420 & -1.296293\end{array}$




\begin{tabular}{|c|c|c|c|}
\hline & 0.976642 & 4.993483 & -0.703818 \\
\hline & 2.217910 & 5.536060 & -1.037446 \\
\hline & 3.016440 & 4.865593 & -1.956028 \\
\hline & 2.578996 & 3.683632 & -2.546387 \\
\hline & 1.738087 & 1.349836 & -4.017530 \\
\hline & -3.477835 & -0.057455 & -0.312492 \\
\hline & -3.845768 & -0.550240 & -1.559337 \\
\hline & -5.186046 & -0.799571 & -1.874862 \\
\hline & -6.141013 & -0.547403 & -0.889920 \\
\hline & -5.806313 & -0.074700 & 0.385974 \\
\hline & -4.461307 & 0.166924 & 0.655864 \\
\hline & -5.609159 & -1.292330 & -3.258071 \\
\hline & -6.898308 & 0.125742 & 1.435903 \\
\hline & 1.514611 & 3.821755 & 3.002755 \\
\hline & -0.350681 & 3.312870 & 4.582199 \\
\hline & -5.131268 & 4.683840 & 0.286751 \\
\hline & -3.568691 & 6.594576 & 0.543481 \\
\hline & 6.781230 & -0.282724 & 0.890020 \\
\hline & -2.102801 & 0.839449 & 2.972441 \\
\hline & 7.380769 & -5.002506 & -0.531586 \\
\hline & -1.618845 & -2.629218 & 6.136745 \\
\hline & 6.404926 & 4.598616 & -0.338112 \\
\hline & -3.717373 & -2.882873 & 1.120654 \\
\hline & -5.580524 & -4.154021 & 0.144725 \\
\hline & 4.251976 & 4.022414 & 0.618322 \\
\hline & -2.030875 & -0.495273 & 5.008168 \\
\hline & 5.373251 & -4.878082 & -1.896773 \\
\hline & 8.665786 & 1.662647 & -2.516454 \\
\hline & 3.338690 & -3.620701 & -2.611697 \\
\hline - & -2.944098 & -5.824201 & -2.814621 \\
\hline & -0.574277 & -5.222794 & -2.821250 \\
\hline & -0.652525 & -6.121763 & 3.824888 \\
\hline & 6.965961 & -0.032966 & -2.037197 \\
\hline & -0.780062 & -4.819391 & 1.756668 \\
\hline 1 & 1.301911 & -3.897149 & -1.875532 \\
\hline & 8.419423 & 3.987121 & -1.649110 \\
\hline 1 & 8.651065 & -1.599663 & 1.764939 \\
\hline & -5.203559 & -5.645430 & -1.814586 \\
\hline & -1.072275 & -5.044923 & 6.031365 \\
\hline 1 & 8.985055 & -3.963307 & 1.045954 \\
\hline & 2.401122 & 2.365263 & 0.906888 \\
\hline 1 & -4.165450 & 0.544425 & 1.631244 \\
\hline & -3.057202 & -0.749052 & -2.280872 \\
\hline 1 & -7.191085 & -0.732204 & -1.117510 \\
\hline & -3.402542 & 2.533932 & -0.512980 \\
\hline 1 & -0.071864 & 1.713978 & 2.059044 \\
\hline | & -1.838961 & 5.622585 & 2.006725 \\
\hline & 1.553483 & -3.729189 & 1.460138 \\
\hline 1 & 2.973240 & -3.344612 & 0.494099 \\
\hline & 4.692774 & -2.789298 & 1.918573 \\
\hline 1 & 0.806480 & -1.153361 & 2.692910 \\
\hline l & 5.618378 & -1.500970 & 3.812079 \\
\hline 1 & 1.711719 & 0.116216 & 4.607585 \\
\hline 1 & 4.136594 & -0.031354 & 5.167238 \\
\hline 1 & -1.525880 & 0.850027 & -2.565617 \\
\hline 1 & 3.233669 & 3.185884 & -3.257676 \\
\hline
\end{tabular}




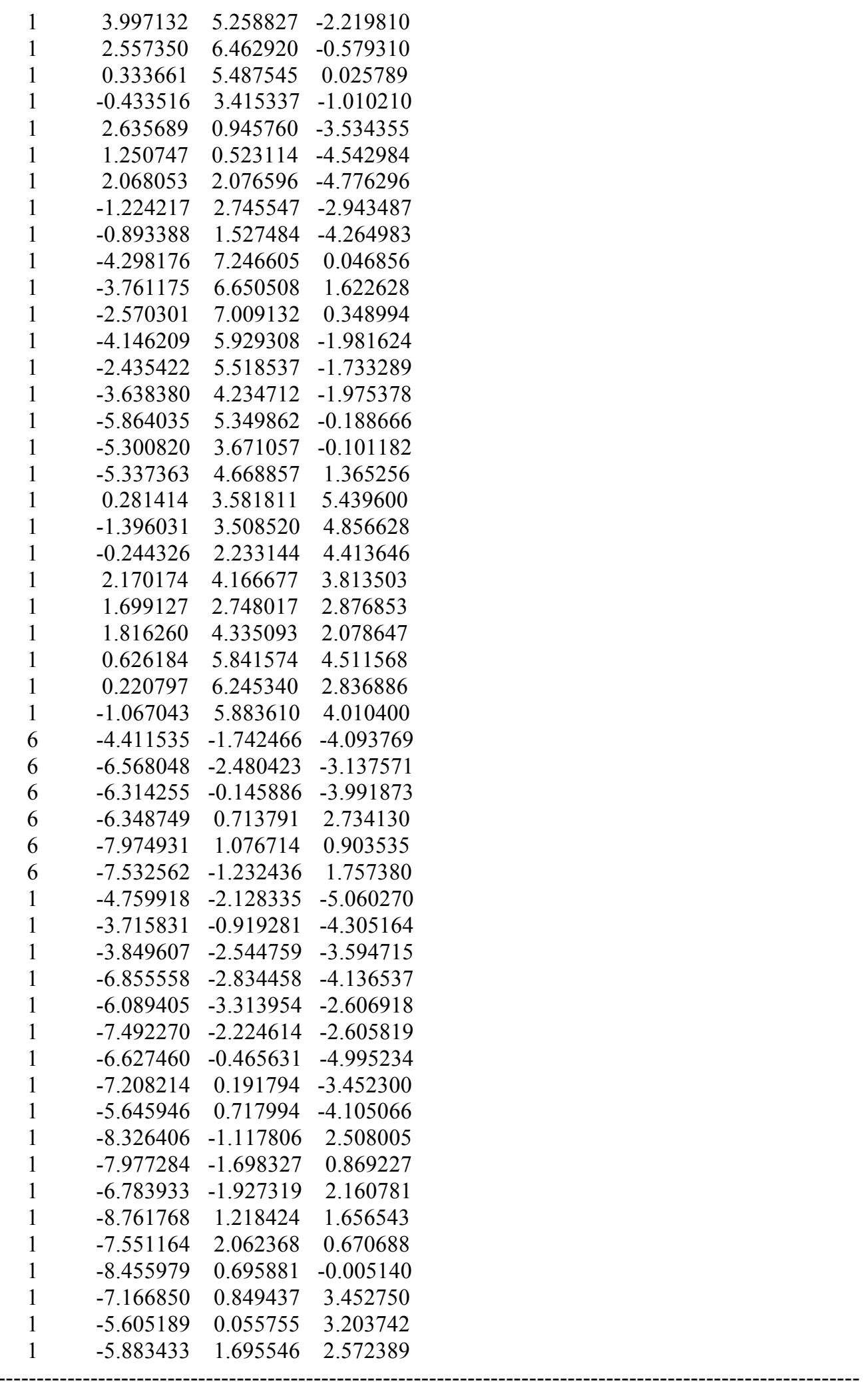

TS1 $_{I I B 1-} \psi_{-1 \mathrm{~s} 2 \mathrm{s3a} 4 \mathrm{~s}}$

Number of imaginary frequencies : 1

The smallest frequencies are : $-567.0824 \quad 18.2480 \quad 20.2069 \mathrm{~cm}(-1)$ 
Electronic energy : $\quad H F=-4360.8651838$

Zero-point correction $=\quad 1.453579($ Hartree/Particle $)$

Thermal correction to Energy= $\quad 1.537824$

Thermal correction to Enthalpy $=\quad 1.538768$

Thermal correction to Gibbs Free Energy $=\quad 1.337619$

Sum of electronic and zero-point Energies $=\quad-4359.411605$

Sum of electronic and thermal Energies $=\quad-4359.327360$

Sum of electronic and thermal Enthalpies $=\quad-4359.326416$

Sum of electronic and thermal Free Energies $=\quad-4359.527565$

Cartesian Coordinates

$\begin{array}{cccc}6 & -4.443657 & 0.128001 & 0.648129 \\ 6 & -3.465638 & -0.042335 & -0.328626 \\ 6 & -3.836656 & -0.505897 & -1.589485 \\ 6 & -5.169641 & -0.781743 & -1.896982 \\ 6 & -6.125944 & -0.590797 & -0.892679 \\ 6 & -5.787905 & -0.148781 & 0.388092 \\ 15 & -1.682111 & 0.281123 & 0.066108 \\ 45 & -0.253215 & 0.118498 & -1.860727 \\ 6 & -0.548623 & 1.954805 & -3.308305 \\ 6 & 0.828541 & 2.006848 & -3.025331 \\ 6 & 1.780196 & 1.354384 & -3.998708 \\ 6 & -5.592391 & -1.222962 & -3.297846 \\ 6 & -6.592963 & -2.380269 & -3.232333 \\ 6 & -6.805780 & 0.014832 & 1.515280 \\ 6 & -6.803373 & 1.467461 & 2.004195 \\ 6 & -1.583664 & -0.762077 & 1.612828 \\ 6 & -1.346073 & -2.126541 & 1.525237 \\ 6 & -1.316478 & -2.934259 & 2.711989 \\ 6 & -1.551044 & -2.333539 & 3.981910 \\ 6 & -1.832285 & -0.950115 & 4.033911 \\ 6 & -1.848872 & -0.196406 & 2.891630 \\ 6 & -1.042219 & -4.327384 & 2.677502 \\ 6 & -0.995339 & -5.069814 & 3.831822 \\ 6 & -1.227800 & -4.466025 & 5.085091 \\ 6 & -1.501320 & -3.124000 & 5.154331 \\ 6 & -1.169869 & -2.854825 & 0.238704 \\ 6 & 0.072063 & -2.972438 & -0.355981 \\ 6 & 0.262916 & -3.838662 & -1.456309 \\ 6 & -0.784064 & -4.542696 & -1.986784 \\ 6 & -2.085022 & -4.401578 & -1.450322 \\ 6 & -2.283226 & -3.556726 & -0.317972 \\ 6 & -3.191018 & -5.093839 & -2.002560 \\ 6 & -4.445268 & -4.954243 & -1.465239 \\ 6 & -4.647437 & -4.106771 & -0.353342 \\ 6 & -3.594379 & -3.432356 & 0.211147 \\ 7 & 1.186106 & -2.205312 & 0.123341 \\ 15 & 1.541444 & -0.835351 & -0.814809 \\ 8 & 2.495438 & -0.122300 & 0.324964 \\ 6 & 3.516253 & 0.738787 & 0.030456 \\ 6 & 4.683851 & 0.280791 & -0.553678 \\ 6 & 5.699681 & 1.247892 & -0.855919 \\ 6 & 5.549323 & 2.590540 & -0.395437 \\ 6 & 4.370587 & 2.964583 & 0.294255 \\ 6 & 3.353534 & 2.068297 & 0.463146 \\ & & & \\ 6\end{array}$




\begin{tabular}{|c|c|c|c|}
\hline 6 & 6.853820 & 0.933882 & -1.617950 \\
\hline 6 & 7.812159 & 1.881602 & -1.882105 \\
\hline & 7.675852 & 3.198125 & -1.395092 \\
\hline & 6.562568 & 3.540386 & -0.670460 \\
\hline & 4.856053 & -1.161063 & -0.843562 \\
\hline & 3.893110 & -1.851085 & -1.557798 \\
\hline & 4.076697 & -3.188319 & -1.971984 \\
\hline & 5.189303 & -3.879238 & -1.579606 \\
\hline & 6.144060 & -3.272717 & -0.726832 \\
\hline & 5.980640 & -1.905532 & -0.350178 \\
\hline & 7.236701 & -4.014161 & -0.214651 \\
\hline 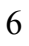 & 8.123703 & -3.448243 & 0.664664 \\
\hline & 7.946113 & -2.108654 & 1.068508 \\
\hline f & 6.909514 & -1.357315 & 0.571545 \\
\hline & 2.721193 & -1.242526 & -1.937803 \\
\hline & 2.104888 & -2.916049 & 1.043541 \\
\hline$b$ & 2.652935 & -2.106885 & 2.191627 \\
\hline & 1.832572 & -1.276989 & 2.958284 \\
\hline 6 & 2.354792 & -0.546600 & 4.016332 \\
\hline & 3.710076 & -0.638152 & 4.332172 \\
\hline ) & 4.532227 & -1.468902 & 3.580005 \\
\hline$b$ & 4.004522 & -2.198528 & 2.516412 \\
\hline 6 & -1.764447 & 1.988886 & 0.751534 \\
\hline 6 & -2.689090 & 2.894910 & 0.235458 \\
\hline$b$ & -2.704739 & 4.230536 & 0.646165 \\
\hline 6 & -1.805746 & 4.620000 & 1.641549 \\
\hline 6 & -0.862129 & 3.738221 & 2.183390 \\
\hline ) & -0.819857 & 2.435830 & 1.681396 \\
\hline 6 & -3.699091 & 5.193479 & 0.001458 \\
\hline 6 & -3.550909 & 6.619953 & 0.523950 \\
\hline 6 & 0.057543 & 4.139996 & 3.337884 \\
\hline 6 & -0.352017 & 3.336789 & 4.579257 \\
\hline 6 & -3.461333 & 5.217861 & -1.513404 \\
\hline 6 & -5.128288 & 4.718985 & 0.287879 \\
\hline 6 & -0.044772 & 5.625826 & 3.676753 \\
\hline 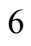 & 1.518425 & 3.831086 & 3.002360 \\
\hline 6 & -0.452594 & -1.215823 & -3.264011 \\
\hline 2 & -0.536132 & -1.868382 & -4.219789 \\
\hline 6 & 1.348956 & 3.150420 & -2.237103 \\
\hline 6 & 0.561104 & 3.827059 & -1.289453 \\
\hline 6 & 0.998483 & 5.000650 & -0.692549 \\
\hline 6 & 2.245522 & 5.538386 & -1.012289 \\
\hline 6 & 3.051383 & 4.865215 & -1.922483 \\
\hline 6 & 2.615531 & 3.685464 & -2.518325 \\
\hline 6 & -4.398498 & -1.685333 & -4.133255 \\
\hline 6 & -6.250029 & -0.029987 & -4.001758 \\
\hline 6 & -8.224650 & -0.337890 & 1.077062 \\
\hline 6 & -6.419538 & -0.916498 & 2.671206 \\
\hline 1 & 6.775276 & -0.333626 & 0.911141 \\
\hline 1 & -2.077611 & 0.862530 & 2.972237 \\
\hline 1 & 7.345284 & -5.052839 & -0.524368 \\
\hline 1 & -1.682787 & -2.639903 & 6.112961 \\
\hline 1 & 6.427875 & 4.557154 & -0.301978 \\
\hline 1 & -3.764852 & -2.771030 & 1.060050 \\
\hline 1 & -5.649664 & -3.983644 & 0.053628 \\
\hline 1 & 4.267872 & 3.990621 & 0.643575 \\
\hline
\end{tabular}




\begin{tabular}{|c|c|c|c|}
\hline & -2.038868 & -0.488084 & 4.999003 \\
\hline & 5.341788 & -4.910733 & -1.892921 \\
\hline & 8.681618 & 1.614675 & -2.478857 \\
\hline & 3.317764 & -3.636685 & -2.609542 \\
\hline & -3.021401 & -5.739464 & -2.863236 \\
\hline & -0.634983 & -5.201838 & -2.840652 \\
\hline & -0.778963 & -6.134389 & 3.778756 \\
\hline & 6.970544 & -0.072866 & -2.011255 \\
\hline & -0.864112 & -4.812979 & 1.720281 \\
\hline & 1.262843 & -3.918843 & -1.879405 \\
\hline & 8.444429 & 3.938081 & -1.606170 \\
\hline & 8.633430 & -1.666502 & 1.786475 \\
\hline & -5.287847 & -5.492361 & -1.895015 \\
\hline & -1.188887 & -5.066187 & 5.991173 \\
\hline & 8.952745 & -4.030047 & 1.060643 \\
\hline & 2.407260 & 2.342365 & 0.922326 \\
\hline & -4.154364 & 0.479256 & 1.638067 \\
\hline & -3.050999 & -0.665239 & -2.323573 \\
\hline & -7.169190 & -0.799792 & -1.119103 \\
\hline 1 & -3.408387 & 2.552012 & -0.508341 \\
\hline & -0.064335 & 1.736208 & 2.047373 \\
\hline & -1.833923 & 5.643652 & 2.001853 \\
\hline & 1.511622 & -3.747943 & 1.450601 \\
\hline & 2.938860 & -3.375596 & 0.490435 \\
\hline & 4.661052 & -2.831943 & 1.917368 \\
\hline & 0.784529 & -1.171943 & 2.687857 \\
\hline 1 & 5.593853 & -1.545001 & 3.808001 \\
\hline 1 & 1.697052 & 0.099609 & 4.597360 \\
\hline & 4.121272 & -0.061181 & 5.157847 \\
\hline & -1.502607 & 0.880888 & -2.573347 \\
\hline & 3.275569 & 3.185372 & -3.223025 \\
\hline & 4.036364 & 5.254795 & -2.175529 \\
\hline & 2.583407 & 6.463836 & -0.550186 \\
\hline 1 & 0.349550 & 5.497235 & 0.029960 \\
\hline 1 & -0.413900 & 3.427153 & -1.013211 \\
\hline 1 & 2.670961 & 0.946994 & -3.505762 \\
\hline 1 & 1.294753 & 0.528936 & -4.527985 \\
\hline & 2.121311 & 2.078559 & -4.755024 \\
\hline & -1.185734 & 2.768433 & -2.959037 \\
\hline 1 & -0.848543 & 1.544691 & -4.273748 \\
\hline 1 & -4.276209 & 7.273318 & 0.022953 \\
\hline 1 & -3.741196 & 6.686795 & 1.602853 \\
\hline 1 & -2.549568 & 7.024885 & 0.324314 \\
\hline 1 & -4.137205 & 5.937818 & -1.994242 \\
\hline 1 & -2.429295 & 5.516015 & -1.744214 \\
\hline 1 & -3.641939 & 4.239395 & -1.975185 \\
\hline 1 & -5.856527 & 5.381867 & -0.198854 \\
\hline 1 & -5.305456 & 3.699896 & -0.080349 \\
\hline 1 & -5.333535 & 4.724973 & 1.366600 \\
\hline 1 & 0.278212 & 3.607154 & 5.437589 \\
\hline 1 & -1.397661 & 3.535894 & 4.850014 \\
\hline 1 & -0.247179 & 2.256157 & 4.414852 \\
\hline 1 & 2.174121 & 4.178687 & 3.811841 \\
\hline 1 & 1.698032 & 2.755416 & 2.885352 \\
\hline 1 & 1.824318 & 4.336074 & 2.075100 \\
\hline 1 & 0.638273 & 5.860773 & 4.502649 \\
\hline
\end{tabular}




$\begin{array}{rrrr}1 & 0.235977 & 6.261796 & 2.826545 \\ 1 & -1.054270 & 5.909432 & 4.000191 \\ 1 & -4.748133 & -2.046501 & -5.108846 \\ 1 & -3.684046 & -0.874260 & -4.327326 \\ 1 & -3.857094 & -2.507219 & -3.643336 \\ 1 & -6.561768 & -0.305679 & -5.018634 \\ 1 & -7.138324 & 0.318729 & -3.459595 \\ 1 & -5.551857 & 0.814210 & -4.078296 \\ 1 & -6.865340 & -2.696873 & -4.247827 \\ 1 & -6.157646 & -3.244420 & -2.713719 \\ 1 & -7.523308 & -2.107622 & -2.720179 \\ 1 & -7.510696 & 1.591992 & 2.835338 \\ 1 & -5.814465 & 1.784181 & 2.358901 \\ 1 & -7.104861 & 2.151791 & 1.199975 \\ 1 & -7.127762 & -0.808381 & 3.503928 \\ 1 & -6.437319 & -1.966327 & 2.348859 \\ 1 & -5.414312 & -0.705892 & 3.058546 \\ 1 & -8.914233 & -0.210515 & 1.921146 \\ 1 & -8.577013 & 0.310562 & 0.264474 \\ 1 & -8.302849 & -1.381038 & 0.743581\end{array}$

$\operatorname{TS1}_{I I B 1-} \psi_{-1225354 a}$

Number of imaginary frequencies : 1

The smallest frequencies are : -567.9558 $12.4190 \quad 17.1723 \mathrm{~cm}(-1)$

Electronic energy : $\quad H F=-4360.8645313$

Zero-point correction $=\quad 1.452701$ (Hartree/Particle)

Thermal correction to Energy $=\quad 1.537337$

Thermal correction to Enthalpy= $\quad 1.538281$

Thermal correction to Gibbs Free Energy $=\quad 1.334984$

Sum of electronic and zero-point Energies $=\quad-4359.411831$

Sum of electronic and thermal Energies $=\quad-4359.327194$

Sum of electronic and thermal Enthalpies $=\quad-4359.326250$

Sum of electronic and thermal Free Energies $=\quad-4359.529548$

\begin{tabular}{cccc}
\multicolumn{4}{c}{ Cartesian Coordinates } \\
\hline $6 \ldots \ldots \ldots \ldots \ldots \ldots \ldots \ldots \ldots \ldots \ldots \ldots$ \\
6 & -4.469123 & 0.069792 & 0.658113 \\
6 & -3.477536 & -0.079531 & -0.321166 \\
6 & -3.843317 & -0.477441 & -1.596823 \\
6 & -5.184425 & -0.699805 & -1.940835 \\
6 & -6.142000 & -0.544122 & -0.944456 \\
6 & -5.807836 & -0.165142 & 0.367841 \\
6 & -1.697738 & 0.245667 & 0.086246 \\
6 & -0.269445 & 0.146190 & -1.822862 \\
6 & -0.612345 & 1.987629 & -3.259496 \\
6 & 0.765443 & 2.065008 & -2.991638 \\
6 & 1.719758 & 1.434844 & -3.976720 \\
6 & -5.517014 & -1.093353 & -3.378111 \\
6 & -4.878215 & -2.449800 & -3.691974 \\
6 & -6.900056 & -0.061960 & 1.431003 \\
6 & -8.024186 & 0.863680 & 0.955271 \\
6 & -1.575188 & -0.827222 & 1.607629 \\
6 & -1.293909 & -2.181981 & 1.497038 \\
6 & -1.231346 & -3.004380 & 2.673716 \\
6 & -1.480518 & -2.429198 & 3.952884
\end{tabular}




\begin{tabular}{|c|c|c|c|}
\hline 6 & -1.812555 & -1.057960 & 4.025904 \\
\hline 5 & -1.857062 & -0.289443 & 2.895085 \\
\hline b & -0.902633 & -4.385280 & 2.621456 \\
\hline & -0.817250 & -5.138664 & 3.766427 \\
\hline f & -1.064101 & -4.559734 & 5.028412 \\
\hline 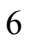 & -1.390911 & -3.230745 & 5.115264 \\
\hline 6 & -1.096231 & -2.894974 & 0.203274 \\
\hline 6 & 0.149433 & -2.981111 & -0.388943 \\
\hline 6 & 0.370828 & -3.855539 & -1.477875 \\
\hline 6 & -0.645822 & -4.617619 & -1.986317 \\
\hline 6 & -1.949270 & -4.522879 & -1.444993 \\
\hline 6 & -2.182581 & -3.648650 & -0.341935 \\
\hline 6 & -3.019722 & -5.296856 & -1.956631 \\
\hline 6 & -4.275160 & -5.206831 & -1.412197 \\
\hline 6 & -4.516216 & -4.323218 & -0.337506 \\
\hline 6 & -3.496876 & -3.568924 & 0.187833 \\
\hline 7 & 1.246617 & -2.192724 & 0.095397 \\
\hline 15 & 1.559539 & -0.790455 & -0.810030 \\
\hline 8 & 2.514048 & -0.090039 & 0.339514 \\
\hline 6 & 3.506845 & 0.805152 & 0.045308 \\
\hline 6 & 4.679890 & 0.386174 & -0.556387 \\
\hline 6 & 5.669475 & 1.382280 & -0.852009 \\
\hline 6 & 5.488058 & 2.714735 & -0.373700 \\
\hline 6 & 4.303453 & 3.050470 & 0.325274 \\
\hline 6 & 3.311490 & 2.125740 & 0.491616 \\
\hline 6 & 6.825995 & 1.107852 & -1.625701 \\
\hline 6 & 7.758460 & 2.082835 & -1.882998 \\
\hline 6 & 7.592376 & 3.388940 & -1.377687 \\
\hline 6 & 6.475361 & 3.693490 & -0.642211 \\
\hline 6 & 4.880417 & -1.043587 & -0.884941 \\
\hline 6 & 3.927019 & -1.731958 & -1.613366 \\
\hline 6 & 4.141532 & -3.046830 & -2.081037 \\
\hline 6 & 5.275212 & -3.722874 & -1.724807 \\
\hline 6 & 6.221396 & -3.124804 & -0.856482 \\
\hline 6 & 6.026809 & -1.777158 & -0.427708 \\
\hline 6 & 7.337635 & -3.855839 & -0.381617 \\
\hline 6 & 8.218360 & -3.299355 & 0.510038 \\
\hline 6 & 8.010439 & -1.980570 & 0.964771 \\
\hline 6 & 6.950000 & -1.238792 & 0.505285 \\
\hline 8 & 2.734773 & -1.142497 & -1.956924 \\
\hline 6 & 2.215337 & -2.913894 & 0.954026 \\
\hline 6 & 2.739445 & -2.160473 & 2.149830 \\
\hline 6 & 1.897904 & -1.397169 & 2.960525 \\
\hline 6 & 2.394284 & -0.746476 & 4.081471 \\
\hline 6 & 3.745161 & -0.847187 & 4.411729 \\
\hline 6 & 4.590506 & -1.604690 & 3.609308 \\
\hline 6 & 4.088379 & -2.257400 & 2.485391 \\
\hline 6 & -1.804242 & 1.939888 & 0.802690 \\
\hline 6 & -2.744704 & 2.838360 & 0.302284 \\
\hline 6 & -2.788822 & 4.164299 & 0.740073 \\
\hline 6 & -1.894167 & 4.554747 & 1.738764 \\
\hline 6 & -0.932270 & 3.681829 & 2.262362 \\
\hline 6 & -0.868424 & 2.387902 & 1.740694 \\
\hline 6 & -3.812863 & 5.113533 & 0.122939 \\
\hline 6 & -3.705560 & 6.529723 & 0.682043 \\
\hline 6 & -0.018355 & 4.081281 & 3.422027 \\
\hline
\end{tabular}




\begin{tabular}{|c|c|c|c|}
\hline 6 & -0.424153 & 3.262943 & 4.654657 \\
\hline 6 & -3.581201 & 5.183280 & -1.391406 \\
\hline 6 & -5.226010 & 4.587986 & 0.401639 \\
\hline 6 & -0.134246 & 5.562903 & 3.774853 \\
\hline 6 & 1.445663 & 3.789173 & 3.085510 \\
\hline 6 & -0.536995 & -1.189082 & -3.215630 \\
\hline 8 & -0.809934 & -1.822207 & -4.149394 \\
\hline 6 & 1.271214 & 3.208900 & -2.194633 \\
\hline 6 & 0.481374 & 3.855903 & -1.227852 \\
\hline 6 & 0.902598 & 5.029654 & -0.619743 \\
\hline 6 & 2.134296 & 5.597122 & -0.947667 \\
\hline 6 & 2.942466 & 4.953043 & -1.876721 \\
\hline 6 & 2.523255 & 3.772775 & -2.483549 \\
\hline 6 & -7.020531 & -1.203056 & -3.616439 \\
\hline 6 & -4.956881 & -0.036847 & -4.339185 \\
\hline 6 & -6.367088 & 0.487711 & 2.753066 \\
\hline 6 & -7.465115 & -1.463854 & 1.687482 \\
\hline 1 & 6.791640 & -0.232457 & 0.884272 \\
\hline 1 & -2.121064 & 0.760074 & 2.990845 \\
\hline 1 & 7.470129 & -4.879146 & -0.730732 \\
\hline 1 & -1.583903 & -2.765315 & 6.080846 \\
\hline 1 & 6.317144 & 4.701680 & -0.259804 \\
\hline 1 & -3.695157 & -2.888396 & 1.014806 \\
\hline 1 & -5.518865 & -4.239234 & 0.077028 \\
\hline 1 & 4.175032 & 4.070141 & 0.684542 \\
\hline 1 & -2.033326 & -0.617896 & \\
\hline 1 & 5.451441 & -4.737223 & -2.078979 \\
\hline 1 & 8.630402 & 1.845701 & -2.488652 \\
\hline 1 & 3.387060 & -3.489607 & -2.727715 \\
\hline 1 & -2.821449 & -5.965174 & -2.793583 \\
\hline 1 & -0.469979 & -5.294670 & -2.820867 \\
\hline 1 & -0.558261 & -6.192844 & 3.698770 \\
\hline 1 & 6.965578 & 0.110103 & -2.033882 \\
\hline 1 & -0.711066 & -4.853027 & 1.657988 \\
\hline 1 & 1.373693 & -3.906394 & -1.898988 \\
\hline 1 & 8.340683 & 4.150820 & -1.583519 \\
\hline 1 & 8.693108 & -1.547556 & 1.692642 \\
\hline 1 & -5.089923 & -5.806452 & -1.812617 \\
\hline 1 & -0.993677 & -5.168224 & 5.927005 \\
\hline 1 & 9.066214 & -3.873298 & 0.876590 \\
\hline 1 & 2.361460 & 2.372189 & 0.958592 \\
\hline 1 & -4.178573 & 0.383779 & 1.657246 \\
\hline 1 & -3.060432 & -0.621116 & -2.338585 \\
\hline 1 & -7.188837 & -0.724189 & -1.181337 \\
\hline 1 & -3.457604 & 2.496404 & -0.447867 \\
\hline 1 & -0.102375 & 1.695246 & 2.097556 \\
\hline 1 & -1.942335 & 5.570807 & 2.118017 \\
\hline 1 & 1.673183 & -3.800720 & 1.312540 \\
\hline 1 & 3.062734 & -3.293589 & 0.363511 \\
\hline 1 & 4.761141 & -2.837643 & 1.851440 \\
\hline 1 & 0.853035 & -1.287382 & 2.679984 \\
\hline 1 & 5.649391 & -1.684313 & 3.848833 \\
\hline 1 & 1.720429 & -0.156057 & 4.701165 \\
\hline 1 & 4.135497 & -0.332947 & 5.287470 \\
\hline 1 & -1.535773 & 0.894420 & -2.512362 \\
\hline 1 & 3.183961 & 3.295253 & -3.203196 \\
\hline
\end{tabular}




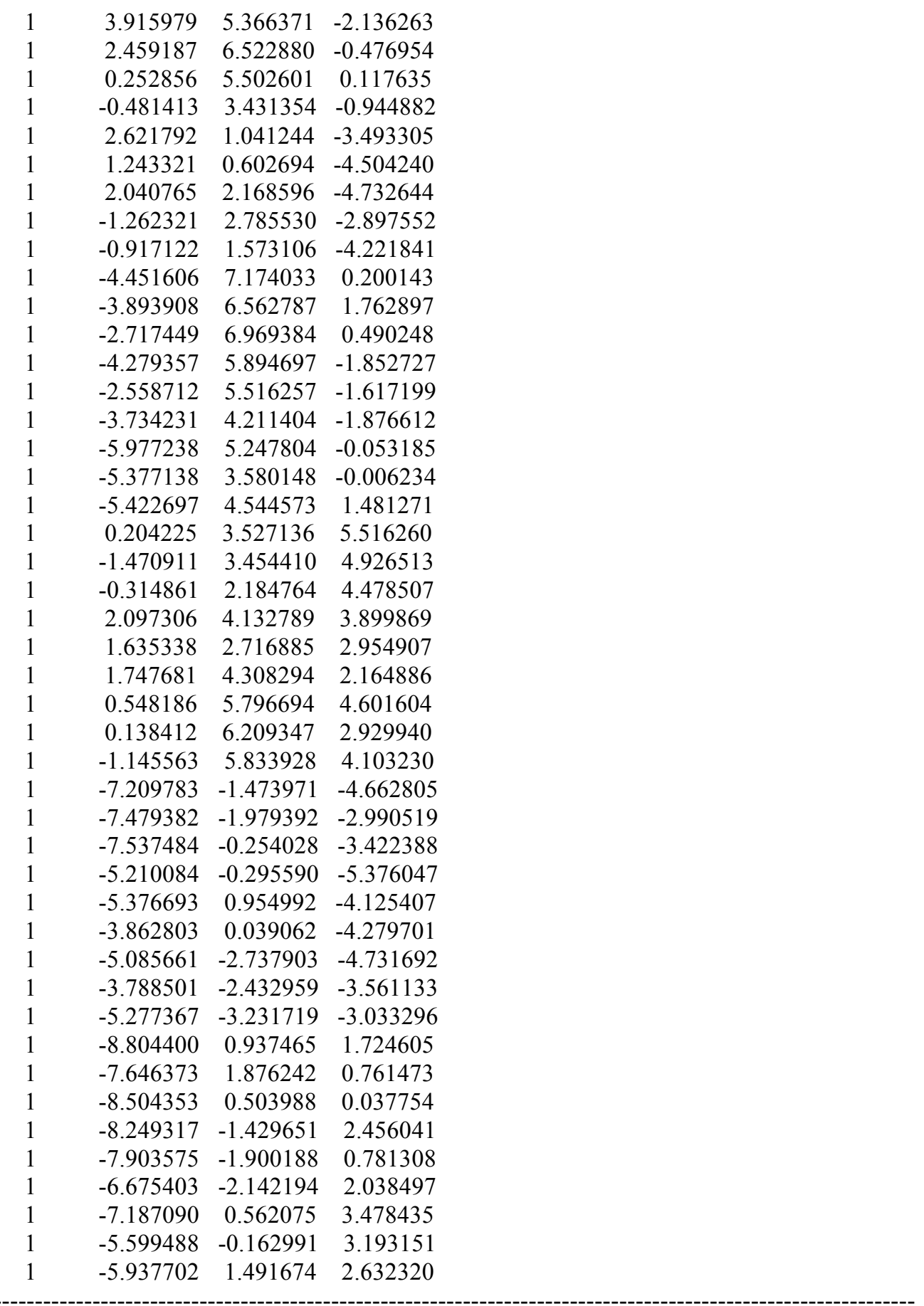

$\operatorname{TS1}_{\text {IIB1- }} \Psi-1 \mathrm{~s} 2 \mathrm{~s} 3 \mathrm{~s} 4 \mathrm{~s}$

Number of imaginary frequencies : 1

The smallest frequencies are : $-564.3755 \quad 16.0963 \quad 18.2057 \mathrm{~cm}(-1)$

Electronic energy : $\quad H F=-4360.8647583$

Zero-point correction $=\quad 1.453003$ (Hartree/Particle)

Thermal correction to Energy $=\quad 1.537458$

Thermal correction to Enthalpy= $\quad 1.538402$

Thermal correction to Gibbs Free Energy= 1.336230

Sum of electronic and zero-point Energies $=\quad-4359.411755$ 
Sum of electronic and thermal Energies= Sum of electronic and thermal Enthalpies= Sum of electronic and thermal Free Energies=
$-4359.327301$

$-4359.326356$

$-4359.528528$

Cartesian Coordinates

$\begin{array}{cccc}6 & 1.894307 & -1.407381 & 2.946476 \\ 6 & 2.734508 & -2.172431 & 2.135906 \\ 6 & 4.083479 & -2.270487 & 2.470799 \\ 6 & 4.587290 & -1.615961 & 3.592980 \\ 6 & 3.743651 & -0.855333 & 4.394168 \\ 6 & 2.392473 & -0.754255 & 4.065133 \\ 6 & 2.209070 & -2.923738 & 0.939229 \\ 7 & 1.244921 & -2.198194 & 0.079244 \\ 15 & 1.558053 & -0.789395 & -0.815735 \\ 8 & 2.735626 & -1.132035 & -1.962610 \\ 6 & 3.926925 & -1.724052 & -1.619798 \\ 6 & 4.879024 & -1.040132 & -0.885485 \\ 6 & 6.025155 & -1.776370 & -0.431627 \\ 6 & 6.220200 & -3.121428 & -0.868338 \\ 6 & 5.274795 & -3.714519 & -1.740870 \\ 6 & 4.141650 & -3.036291 & -2.094527 \\ 6 & 6.947859 & -1.243664 & 0.505135 \\ 6 & 8.008122 & -1.988118 & 0.960696 \\ 6 & 8.216383 & -3.304145 & 0.498257 \\ 6 & 7.336174 & -3.855281 & -0.397190 \\ 6 & 4.677339 & 0.387230 & -0.546992 \\ 6 & 3.503246 & 0.801329 & 0.056134 \\ 6 & 3.307606 & 2.117843 & 0.514265 \\ 6 & 4.299511 & 3.044156 & 0.356713 \\ 6 & 5.484888 & 2.714423 & -0.343915 \\ 6 & 5.667133 & 1.385950 & -0.832954 \\ 6 & 6.472351 & 3.695504 & -0.603312 \\ 6 & 7.590265 & 3.397179 & -1.339959 \\ 6 & 7.757211 & 2.095284 & -1.855702 \\ 6 & 6.824630 & 1.118074 & -1.607493 \\ 8 & 2.509221 & -0.095402 & 0.340159 \\ 6 & 0.147084 & -2.981139 & -0.412232 \\ 6 & -1.098122 & -2.898819 & 0.181284 \\ 6 & -2.187793 & -3.639038 & -0.374809 \\ 6 & -1.959452 & -4.494312 & -1.493593 \\ 6 & -0.655903 & -4.588599 & -2.034806 \\ 6 & 0.364904 & -3.840927 & -1.513354 \\ 6 & -3.501641 & -3.559175 & 0.155704 \\ 6 & -4.526812 & -4.292246 & -0.387872 \\ 6 & -4.291883 & -5.155449 & -1.480321 \\ 6 & -3.035770 & -5.247807 & -2.022962 \\ 6 & -1.293730 & -2.197577 & 1.481485 \\ 6 & -1.572864 & -0.843243 & 1.602501 \\ 6 & -1.849395 & -0.314337 & 2.894533 \\ 6 & -1.805550 & -1.091953 & 4.019385 \\ 6 & -1.479623 & -2.463963 & 3.935078 \\ 6 & -1.232823 & -3.029611 & 2.651188 \\ 6 & -1.393586 & -3.275575 & 5.090772 \\ 6 & -1.072922 & -4.605227 & 4.992736 \\ 6 & -0.828581 & -5.174777 & 3.725950\end{array}$




\begin{tabular}{|c|c|c|c|}
\hline 5 & -0.910229 & -4.411533 & 2.587307 \\
\hline 15 & -1.700694 & 0.238603 & 0.088049 \\
\hline 6 & -1.818067 & 1.929680 & 0.811460 \\
\hline 6 & -2.761166 & 2.825966 & 0.311258 \\
\hline 6 & -2.801794 & 4.154530 & 0.742107 \\
\hline 6 & -1.909039 & 4.546299 & 1.742124 \\
\hline 6 & -0.949407 & 3.673806 & 2.270456 \\
\hline 6 & -0.882723 & 2.379955 & 1.748924 \\
\hline 6 & -3.815031 & 5.107673 & 0.112449 \\
\hline 6 & -3.576464 & 5.162239 & -1.401518 \\
\hline 6 & -0.038573 & 4.074739 & 3.432076 \\
\hline 6 & -0.160404 & 5.555354 & 3.787115 \\
\hline 45 & -0.271707 & 0.154737 & -1.821996 \\
\hline 6 & -0.548351 & -1.171326 & -3.223593 \\
\hline 8 & -0.830876 & -1.782300 & -4.169216 \\
\hline 6 & 0.773380 & 2.068861 & -2.977377 \\
\hline 6 & -0.603049 & 1.996388 & -3.254228 \\
\hline 6 & 1.275829 & 3.210000 & -2.174064 \\
\hline 6 & 0.481419 & 3.853413 & -1.208684 \\
\hline 6 & 0.899067 & 5.026155 & -0.596172 \\
\hline 6 & 2.132013 & 5.595041 & -0.916803 \\
\hline 6 & 2.944885 & 4.953824 & -1.843692 \\
\hline 6 & 2.528774 & 3.775244 & -2.455931 \\
\hline 6 & 1.732028 & 1.442523 & -3.960691 \\
\hline 6 & -3.479572 & -0.093211 & -0.320268 \\
\hline 6 & -3.854006 & -0.453761 & -1.608519 \\
\hline 6 & -5.193108 & -0.668033 & -1.949312 \\
\hline 6 & -6.151376 & -0.550567 & -0.941015 \\
\hline 6 & -5.807596 & -0.222500 & 0.376954 \\
\hline 6 & -4.464140 & 0.015677 & 0.663391 \\
\hline 6 & -5.535106 & -1.019850 & -3.395689 \\
\hline 6 & -6.831439 & -0.123155 & 1.507007 \\
\hline 6 & 1.427031 & 3.788205 & 3.097603 \\
\hline 6 & -0.443208 & 3.253191 & 4.662992 \\
\hline 6 & -5.234556 & 4.599547 & 0.388927 \\
\hline 6 & -3.696297 & 6.527876 & 0.659106 \\
\hline 1 & 6.789540 & -0.239567 & 0.889974 \\
\hline 1 & -2.109369 & 0.735362 & 2.998488 \\
\hline 1 & 7.468873 & -4.876458 & -0.752414 \\
\hline 1 & -1.584598 & -2.817420 & 6.060209 \\
\hline 1 & 6.313481 & 4.700526 & -0.212920 \\
\hline 1 & -3.694426 & -2.893585 & 0.996456 \\
\hline 1 & -5.530128 & -4.205652 & 0.024489 \\
\hline 1 & 4.170711 & 4.060752 & 0.724537 \\
\hline 1 & -2.022537 & -0.658667 & 4.995415 \\
\hline 1 & 5.451246 & -4.726861 & -2.100615 \\
\hline 1 & 8.629915 & 1.863263 & -2.462241 \\
\hline 1 & 3.388006 & -3.475237 & -2.744751 \\
\hline 1 & -2.841967 & -5.901153 & -2.872674 \\
\hline 1 & -0.483640 & -5.252060 & -2.880939 \\
\hline 1 & -0.574711 & -6.229609 & 3.649568 \\
\hline 1 & 6.964814 & 0.123699 & -2.023628 \\
\hline 1 & -0.721151 & -4.871889 & 1.619783 \\
\hline 1 & 1.367542 & -3.890104 & -1.935156 \\
\hline 1 & 8.338643 & 4.160868 & -1.538712 \\
\hline 1 & 8.690415 & -1.559352 & 1.691432 \\
\hline
\end{tabular}




\begin{tabular}{|c|c|c|c|}
\hline & -5.111732 & -5.737977 & -1.895343 \\
\hline & -1.005500 & -5.221591 & 5.886176 \\
\hline & 9.064083 & -3.880209 & 0.861824 \\
\hline & 2.357246 & 2.359424 & 0.983158 \\
\hline & -4.176200 & 0.295085 & 1.676291 \\
\hline | & -3.074951 & -0.573396 & -2.358472 \\
\hline & -7.196097 & -0.722725 & -1.183836 \\
\hline & -3.474943 & 2.481814 & -0.437211 \\
\hline & -0.113907 & 1.689566 & 2.104323 \\
\hline 1 & -1.955886 & 5.563769 & 2.117685 \\
\hline 1 & 1.662478 & -3.808354 & 1.296459 \\
\hline 1 & 3.055861 & -3.306468 & 0.349675 \\
\hline 1 & 4.755245 & -2.852409 & 1.837337 \\
\hline 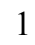 & 0.849313 & -1.296788 & 2.666749 \\
\hline & 5.646224 & -1.696449 & 3.831939 \\
\hline ] & 1.719735 & -0.161827 & 4.684193 \\
\hline & 4.135444 & -0.339200 & 5.268144 \\
\hline 1 & -1.537240 & 0.908711 & -2.505397 \\
\hline & 3.192554 & 3.300336 & -3.174487 \\
\hline 1 & 3.919306 & 5.368411 & -2.097735 \\
\hline & 2.454109 & 6.519801 & -0.442217 \\
\hline & 0.245536 & 5.497162 & 0.139019 \\
\hline$\frac{1}{1}$ & -0.482231 & 3.427045 & -0.930931 \\
\hline & 2.632253 & 1.047765 & -3.474988 \\
\hline 1 & 1.258149 & 0.611954 & -4.493041 \\
\hline 1 & 2.055365 & 2.179179 & -4.712766 \\
\hline 1 & -1.252407 & 2.796150 & -2.895602 \\
\hline 1 & -0.903817 & 1.583413 & -4.218507 \\
\hline 1 & -4.434155 & 7.174795 & 0.168165 \\
\hline 1 & -3.889006 & 6.572692 & 1.738744 \\
\hline & -2.703203 & 6.956352 & 0.467624 \\
\hline 1 & -4.265804 & 5.876848 & -1.871120 \\
\hline 1 & -2.550119 & 5.483929 & -1.626184 \\
\hline 1 & -3.737697 & 4.188092 & -1.879452 \\
\hline 1 & -5.975822 & 5.258338 & -0.083502 \\
\hline 1 & -5.391912 & 3.585435 & -0.001512 \\
\hline 1 & -5.439126 & 4.578324 & 1.467587 \\
\hline & 0.182258 & 3.519325 & 5.526109 \\
\hline 1 & -1.491319 & 3.439666 & 4.933053 \\
\hline 1 & -0.328461 & 2.175662 & 4.486113 \\
\hline 1 & 2.076117 & 4.133306 & 3.913386 \\
\hline 1 & 1.620423 & 2.716646 & 2.966549 \\
\hline 1 & 1.728768 & 4.309047 & 2.177871 \\
\hline 1 & 0.518428 & 5.789825 & 4.616631 \\
\hline 1 & 0.113165 & 6.204348 & 2.944471 \\
\hline 1 & -1.173802 & 5.822421 & 4.112337 \\
\hline 6 & -7.040474 & -1.112072 & -3.630987 \\
\hline 6 & -4.971104 & 0.055986 & -4.332752 \\
\hline 6 & -4.907690 & -2.372536 & -3.746816 \\
\hline 6 & -8.247563 & -0.454746 & 1.043700 \\
\hline 6 & -6.451214 & -1.112301 & 2.615884 \\
\hline 6 & -6.834685 & 1.302320 & 2.070493 \\
\hline 1 & -7.235382 & -1.351471 & -4.683997 \\
\hline 1 & -7.503013 & -1.902633 & -3.026078 \\
\hline 1 & -7.550037 & -0.165447 & -3.407805 \\
\hline 1 & -5.234732 & -0.172446 & -5.374135 \\
\hline
\end{tabular}




$\begin{array}{rrrr}1 & -5.378668 & 1.046216 & -4.089634 \\ 1 & -3.875887 & 0.118827 & -4.280071 \\ 1 & -5.123769 & -2.634418 & -4.791763 \\ 1 & -3.817083 & -2.364043 & -3.622794 \\ 1 & -5.307108 & -3.167641 & -3.104166 \\ 1 & -7.539749 & 1.380837 & 2.909236 \\ 1 & -5.845342 & 1.605168 & 2.436255 \\ 1 & -7.142358 & 2.025212 & 1.303111 \\ 1 & -7.172119 & -1.056216 & 3.442764 \\ 1 & -6.452670 & -2.143183 & 2.237189 \\ 1 & -5.454524 & -0.913150 & 3.029994 \\ 1 & -8.940120 & -0.380122 & 1.891670 \\ 1 & -8.600821 & 0.239532 & 0.270456 \\ 1 & -8.320445 & -1.476273 & 0.648142\end{array}$

$\mathrm{TS1}_{\text {IIB2}}-\psi_{-1 \mathrm{a} 2 \mathrm{a3a} 4 \mathrm{a}}$

Number of imaginary frequencies : 1

The smallest frequencies are : $-624.4187 \quad 17.8704 \quad 21.9291 \mathrm{~cm}(-1)$

Electronic energy : $\quad H F=-4360.8678322$

Zero-point correction $=\quad 1.452663$ (Hartree/Particle)

Thermal correction to Energy $=\quad 1.537311$

Thermal correction to Enthalpy= $\quad 1.538255$

Thermal correction to Gibbs Free Energy $=\quad 1.336094$

Sum of electronic and zero-point Energies $=\quad-4359.415169$

Sum of electronic and thermal Energies $=\quad-4359.330522$

Sum of electronic and thermal Enthalpies $=\quad-4359.329577$

Sum of electronic and thermal Free Energies $=\quad-4359.531739$

\begin{tabular}{cccc}
\multicolumn{4}{c}{ Cartesian Coordinates } \\
$\ldots 6$ & 1.702532 & 1.532445 & -4.406343 \\
6 & 1.794627 & 2.169846 & -3.156220 \\
6 & 3.073579 & 2.553884 & -2.727035 \\
6 & 4.206622 & 2.291049 & -3.491649 \\
6 & 4.095262 & 1.643101 & -4.715221 \\
6 & 2.830060 & 1.271414 & -5.168959 \\
6 & 0.604460 & 2.452086 & -2.314276 \\
6 & 0.798005 & 3.347252 & -1.116718 \\
45 & -0.314470 & 0.325553 & -1.726438 \\
6 & -0.700835 & 2.355837 & -2.833703 \\
15 & -1.853996 & 0.365057 & 0.157710 \\
6 & -3.598930 & -0.040268 & -0.300568 \\
6 & -3.867692 & -0.547422 & -1.566230 \\
6 & -5.167963 & -0.892620 & -1.949051 \\
6 & -6.184390 & -0.717918 & -1.010571 \\
6 & -5.949910 & -0.219728 & 0.278914 \\
6 & -4.641252 & 0.115412 & 0.618190 \\
6 & -5.480191 & -1.378030 & -3.363962 \\
6 & -7.108091 & -0.091843 & 1.266798 \\
6 & -1.727293 & -0.648538 & 1.715982 \\
6 & -1.404093 & -1.996158 & 1.641598 \\
6 & -1.331040 & -2.788299 & 2.835406 \\
6 & -1.600137 & -2.186922 & 4.098475 \\
6 & -1.961716 & -0.821398 & 4.136883
\end{tabular}




\begin{tabular}{|c|c|c|c|}
\hline 6 & -2.023868 & -0.081940 & 2.987012 \\
\hline 6 & -0.977146 & -4.163428 & 2.815245 \\
\hline 6 & -0.883552 & -4.888410 & 3.977724 \\
\hline 6 & -1.146950 & -4.283914 & 5.224637 \\
\hline 6 & -1.500219 & -2.959861 & 5.279396 \\
\hline 6 & -1.174733 & -2.714182 & 0.356859 \\
\hline 6 & 0.077417 & -2.764024 & -0.227704 \\
\hline 6 & 0.320589 & -3.608086 & -1.334878 \\
\hline 6 & -0.684803 & -4.356663 & -1.883689 \\
\hline 6 & -1.994466 & -4.290087 & -1.355340 \\
\hline 6 & -2.245490 & -3.467079 & -0.217273 \\
\hline 6 & -3.056858 & -5.037427 & -1.921292 \\
\hline 6 & -4.321542 & -4.968387 & -1.395211 \\
\hline 6 & -4.578428 & -4.138847 & -0.281185 \\
\hline 6 & -3.566719 & -3.415868 & 0.299167 \\
\hline 7 & 1.157417 & -1.963145 & 0.277942 \\
\hline 15 & 1.490091 & -0.563870 & -0.628795 \\
\hline 8 & 2.391104 & 0.157062 & 0.561907 \\
\hline 6 & 3.503224 & 0.933100 & 0.408089 \\
\hline 6 & 4.663791 & 0.460666 & -0.178497 \\
\hline 6 & 5.727046 & 1.400072 & -0.400890 \\
\hline 6 & 5.633124 & 2.720189 & 0.133778 \\
\hline 6 & 4.481077 & 3.087806 & 0.871813 \\
\hline 6 & 3.435132 & 2.217227 & 0.991073 \\
\hline 6 & 6.865899 & 1.084440 & -1.185336 \\
\hline 6 & 7.857307 & 2.009449 & -1.406476 \\
\hline 6 & 7.772752 & 3.302597 & -0.850298 \\
\hline 6 & 6.678307 & 3.646033 & -0.097729 \\
\hline 6 & 4.795468 & -0.958787 & -0.579901 \\
\hline 6 & 3.842399 & -1.556498 & -1.381880 \\
\hline 6 & 4.007718 & -2.854647 & -1.911156 \\
\hline 6 & 5.086668 & -3.609128 & -1.543650 \\
\hline 6 & 6.023639 & -3.108233 & -0.606383 \\
\hline 6 & 5.883465 & -1.774511 & -0.117081 \\
\hline 6 & 7.080105 & -3.921504 & -0.129189 \\
\hline 6 & 7.954729 & -3.458457 & 0.820116 \\
\hline 6 & 7.800181 & -2.153554 & 1.332598 \\
\hline 6 & 6.798840 & -1.333476 & 0.873304 \\
\hline 8 & 2.695869 & -0.891069 & -1.736358 \\
\hline 6 & 2.082347 & -2.662829 & 1.198995 \\
\hline 6 & 2.572284 & -1.877814 & 2.389891 \\
\hline 6 & 1.724800 & -1.051637 & 3.131335 \\
\hline 6 & 2.197732 & -0.366726 & 4.242054 \\
\hline 6 & 3.530157 & -0.496349 & 4.633726 \\
\hline 6 & 4.379361 & -1.320198 & 3.904712 \\
\hline 6 & 3.900589 & -2.006692 & 2.790317 \\
\hline 6 & -1.998488 & 2.077025 & 0.807641 \\
\hline 6 & -1.032408 & 2.557770 & 1.695130 \\
\hline 6 & -1.027112 & 3.889429 & 2.109274 \\
\hline 6 & -1.978436 & 4.748284 & 1.548179 \\
\hline 6 & -2.936683 & 4.311740 & 0.628738 \\
\hline 6 & -2.940439 & 2.959780 & 0.277998 \\
\hline 6 & 0.020820 & 4.419353 & 3.088077 \\
\hline 6 & 0.676547 & 3.290966 & 3.886601 \\
\hline 6 & -3.925754 & 5.308733 & 0.028039 \\
\hline 6 & -3.145006 & 6.354101 & -0.776358 \\
\hline
\end{tabular}




\begin{tabular}{|c|c|c|c|}
\hline 6 & -0.293700 & -0.960358 & -3.158566 \\
\hline 8 & -0.221337 & -1.636820 & -4.099983 \\
\hline & -4.713331 & 6.001865 & 1.144510 \\
\hline & -4.927627 & 4.636383 & -0.908892 \\
\hline U & 1.100399 & 5.158935 & 2.288800 \\
\hline & -0.610466 & 5.386183 & 4.094432 \\
\hline & 6.680799 & -0.339705 & 1.297270 \\
\hline & -2.310031 & 0.964377 & 3.051870 \\
\hline & 7.171813 & -4.932228 & -0.524887 \\
\hline & -1.707264 & -2.476785 & 6.233385 \\
\hline & 6.585306 & 4.645116 & 0.326351 \\
\hline & -3.780429 & -2.771844 & 1.150767 \\
\hline & -5.589587 & -4.069160 & 0.115956 \\
\hline & 4.431551 & 4.078694 & 1.321607 \\
\hline & -2.193622 & -0.364402 & 5.098372 \\
\hline & 5.226552 & -4.611199 & -1.945527 \\
\hline & 8.712141 & 1.742921 & -2.024057 \\
\hline & 3.264369 & -3.216306 & -2.618337 \\
\hline & -2.844883 & -5.666916 & -2.784607 \\
\hline & -0.494505 & -4.998086 & -2.742814 \\
\hline & -0.605872 & -5.939197 & 3.936553 \\
\hline & 6.939562 & 0.099171 & -1.638191 \\
\hline & -0.775179 & -4.647658 & 1.861898 \\
\hline & 1.328292 & -3.638004 & -1.745965 \\
\hline & 8.566233 & 4.024465 & -1.028895 \\
\hline & 8.477124 & -1.793525 & 2.104284 \\
\hline & -5.130275 & -5.548075 & -1.835818 \\
\hline & -1.069245 & -4.869704 & \\
\hline & 8.756214 & -4.095380 & 1.186939 \\
\hline & 2.528120 & 2.478035 & 1.533085 \\
\hline & -4.417880 & 0.508802 & 1.607270 \\
\hline & -3.029683 & -0.683778 & -2.245727 \\
\hline & -7.205475 & -0.979167 & -1.288392 \\
\hline & -3.680736 & 2.577968 & -0.421297 \\
\hline & -0.277647 & 1.866452 & 2.070660 \\
\hline & -1.969548 & 5.799699 & 1.832606 \\
\hline & 1.515818 & -3.531242 & 1.565602 \\
\hline & 2.946410 & -3.072353 & 0.653133 \\
\hline & 4.577343 & -2.639585 & 2.213959 \\
\hline & 0.696195 & -0.916913 & 2.803492 \\
\hline 1 & 5.423847 & -1.426491 & 4.192907 \\
\hline & 1.518644 & 0.274406 & 4.803451 \\
\hline & 3.902126 & 0.045529 & 5.500777 \\
\hline ] & -1.550945 & 1.032693 & -2.525579 \\
\hline & -1.467477 & 2.977119 & -2.364075 \\
\hline 1 & -0.850554 & 2.207253 & -3.902572 \\
\hline & -5.627428 & 5.386136 & -1.298828 \\
\hline 1 & -4.435296 & 4.168721 & -1.772044 \\
\hline & -5.519352 & 3.865797 & -0.395693 \\
\hline 1 & -3.832339 & 7.069103 & -1.248484 \\
\hline 1 & -2.452478 & 6.924344 & -0.145433 \\
\hline & -2.554800 & 5.875009 & -1.569814 \\
\hline 1 & -5.427046 & 6.719195 & 0.717661 \\
\hline & -5.281089 & 5.271650 & 1.735922 \\
\hline & -4.062362 & 6.555133 & 1.831767 \\
\hline & 0.146313 & 5.721912 & 4.815359 \\
\hline
\end{tabular}




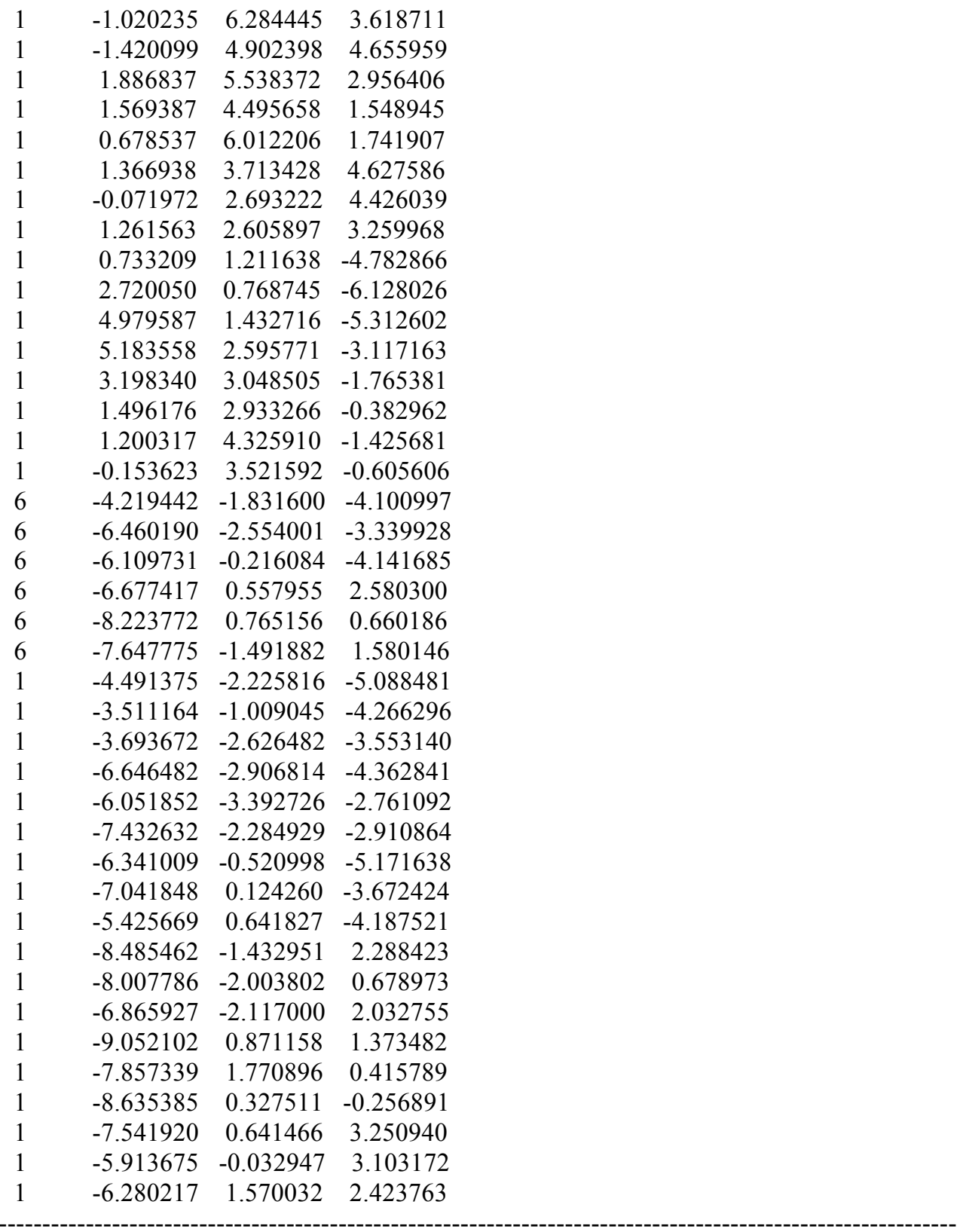

TS1 $_{\text {II } B 2-} \psi_{-1 \mathrm{a} 2 \mathrm{a} 3 \mathrm{a} 4 \mathrm{~s}}$

Number of imaginary frequencies : 1

The smallest frequencies are : $-630.5667 \quad 14.1555 \quad 17.5704 \mathrm{~cm}(-1)$

Electronic energy : $\quad H F=-4360.8683753$

Zero-point correction $=\quad 1.451834($ Hartree/Particle $)$

Thermal correction to Energy $=\quad 1.536904$

Thermal correction to Enthalpy= $\quad 1.537848$

Thermal correction to Gibbs Free Energy= $\quad 1.332812$

Sum of electronic and zero-point Energies= $\quad-4359.416542$

Sum of electronic and thermal Energies $=\quad-4359.331471$

Sum of electronic and thermal Enthalpies $=\quad-4359.330527$

Sum of electronic and thermal Free Energies $=\quad-4359.535563$

Cartesian Coordinates 


\begin{tabular}{cccc}
0 & -3.011792 & 2.916227 & 0.295835 \\
6 & -2.046165 & 2.053105 & 0.816374 \\
6 & -1.086535 & 2.553258 & 1.699680 \\
6 & -1.110974 & 3.882388 & 2.122185 \\
6 & -2.083836 & 4.721259 & 1.568899 \\
6 & -3.033306 & 4.267482 & 0.648118 \\
15 & -1.859240 & 0.348669 & 0.155214 \\
6 & -1.715376 & -0.674466 & 1.705642 \\
6 & -1.375400 & -2.017412 & 1.619254 \\
6 & -1.287164 & -2.817923 & 2.806027 \\
6 & -1.558079 & -2.229836 & 4.074909 \\
6 & -1.939014 & -0.870018 & 4.125462 \\
6 & -2.014617 & -0.121924 & 2.981904 \\
6 & -1.437467 & -3.009084 & 5.249687 \\
6 & -1.063106 & -4.326767 & 5.183119 \\
6 & -0.800178 & -4.918817 & 3.930105 \\
6 & -0.913743 & -4.187556 & 2.773421 \\
6 & -1.149489 & -2.721194 & 0.326137 \\
6 & 0.096303 & -2.754249 & -0.273050 \\
6 & 0.331213 & -3.577427 & -1.397647 \\
6 & -0.676048 & -4.323369 & -1.946778 \\
6 & -1.978976 & -4.276952 & -1.399596 \\
6 & -2.221326 & -3.474782 & -0.245097 \\
6 & -3.535449 & -3.443899 & 0.291014 \\
6 & -4.548528 & -4.167994 & -0.285102 \\
6 & -4.299806 & -4.977721 & -1.415473 \\
6 & -3.042802 & -5.024867 & -1.962130 \\
7 & 1.179710 & -1.960019 & 0.236256 \\
6 & 2.119253 & -2.678326 & 1.128177 \\
6 & 2.607755 & -1.933301 & 2.345106 \\
6 & 1.759837 & -1.135967 & 3.116564 \\
6 & 2.229552 & -0.500706 & 4.257826 \\
6 & 3.560236 & -0.649063 & 4.648271 \\
6 & 4.411191 & -1.441193 & 3.886838 \\
6 & 3.934861 & -2.080089 & 2.743653 \\
6 & -0.065373 & 4.429850 & 3.094053 \\
6 & -0.693205 & 5.423270 & 4.075830 \\
6 & -4.043350 & 5.246581 & 0.053054 \\
6 & -4.986710 & 4.572802 & -0.941113 \\
45 & -0.313513 & 0.353308 & -1.722184 \\
6 & -0.247925 & -0.903180 & -3.179026 \\
8 & -0.127580 & -1.564897 & -4.126002 \\
6 & 0.579120 & 2.497890 & -2.265822 \\
6 & -0.725128 & 2.402721 & -2.788354 \\
6 & 1.771412 & 2.237656 & -3.110863 \\
6 & 1.681862 & 1.645140 & -4.382926 \\
6 & 2.812166 & 1.403340 & -5.147746 \\
6 & 4.076834 & 1.751231 & -4.674148 \\
6 & 4.185650 & 2.353949 & -3.427381 \\
6 & 3.050155 & 2.595371 & -2.659698 \\
6 & 0.767102 & 3.369417 & -1.049950 \\
6 & 1.498507 & -0.536359 & -0.636780 \\
6 & 2.709186 & -0.817278 & -1.752027 \\
& 3.857509 & -1.489093 & -1.415974 \\
6 & 4.805678 & -0.911009 & -0.594422 \\
\hline
\end{tabular}




\begin{tabular}{|c|c|c|c|}
\hline 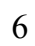 & 5.896504 & -1.734548 & -0.153529 \\
\hline & 6.043010 & -3.053058 & -0.680579 \\
\hline 6 & 5.109281 & -3.531440 & -1.632890 \\
\hline & 4.027722 & -2.771321 & -1.981115 \\
\hline & 6.808243 & -1.317441 & 0.850285 \\
\hline & 7.812566 & -2.145711 & 1.287852 \\
\hline & 7.973353 & -3.435029 & 0.738911 \\
\hline & 7.102226 & -3.874909 & -0.224628 \\
\hline & 4.665185 & 0.496094 & -0.154488 \\
\hline 6 & 3.500489 & 0.947333 & 0.440864 \\
\hline 6 & 3.422127 & 2.218376 & 1.050843 \\
\hline & 4.461050 & 3.099679 & 0.949999 \\
\hline O & 5.617023 & 2.756019 & 0.206632 \\
\hline & 5.722457 & 1.447431 & -0.353566 \\
\hline U & 6.654767 & 3.694845 & -0.005495 \\
\hline 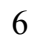 & 7.753051 & 3.375177 & -0.762860 \\
\hline 6 & 7.849437 & 2.093557 & -1.343193 \\
\hline 6 & 6.865440 & 1.156282 & -1.141485 \\
\hline 8 & 2.392338 & 0.161450 & 0.573976 \\
\hline 6 & -3.594030 & -0.086989 & -0.311764 \\
\hline 6 & -3.861813 & -0.577287 & -1.588770 \\
\hline 6 & -5.157097 & -0.924839 & -1.972512 \\
\hline 6 & -6.179955 & -0.779240 & -1.027354 \\
\hline 6 & -5.943923 & -0.309860 & 0.266799 \\
\hline 6 & -4.633694 & 0.037478 & 0.605352 \\
\hline 6 & -5.473665 & -1.386385 & -3.394483 \\
\hline 6 & -6.152891 & -0.228726 & -4.136174 \\
\hline 6 & -7.040796 & -0.160790 & 1.318998 \\
\hline 6 & -6.670388 & -0.987089 & 2.556797 \\
\hline 6 & -4.210433 & -1.780112 & -4.160489 \\
\hline 6 & -6.413315 & -2.595152 & -3.384565 \\
\hline 6 & -8.399137 & -0.645039 & 0.818866 \\
\hline 6 & -7.171440 & 1.317454 & 1.704853 \\
\hline 6 & 0.581973 & 3.315056 & 3.918121 \\
\hline 6 & 1.022429 & 5.145075 & 2.283448 \\
\hline 6 & -3.285727 & 6.353946 & -0.687915 \\
\hline 6 & -4.891284 & 5.859639 & 1.172166 \\
\hline 1 & 6.684344 & -0.336613 & 1.301967 \\
\hline 1 & -2.313476 & 0.920195 & 3.056199 \\
\hline 1 & 7.199095 & -4.873734 & -0.648322 \\
\hline 1 & -1.645191 & -2.535989 & 6.208512 \\
\hline 1 & 6.552691 & 4.684790 & 0.437505 \\
\hline 1 & -3.741662 & -2.813894 & 1.155352 \\
\hline 1 & -5.554624 & -4.113339 & 0.127183 \\
\hline 1 & 4.402636 & 4.080984 & 1.419291 \\
\hline 1 & -2.172731 & -0.423746 & 5.091549 \\
\hline 1 & 5.253818 & -4.521664 & -2.061629 \\
\hline 1 & 8.707522 & 1.845848 & -1.964101 \\
\hline 1 & 3.285219 & -3.115857 & -2.697841 \\
\hline 1 & -2.837909 & -5.638844 & -2.838244 \\
\hline 1 & -0.492315 & -4.948098 & -2.819545 \\
\hline 1 & -0.506674 & -5.964884 & 3.879774 \\
\hline 1 & 6.947999 & 0.180682 & -1.613219 \\
\hline 1 & -0.712006 & -4.661827 & 1.814999 \\
\hline 1 & 1.333821 & -3.592029 & -1.822415 \\
\hline 1 & 8.540559 & 4.107034 & -0.926581 \\
\hline
\end{tabular}




\begin{tabular}{|c|c|c|c|}
\hline & 8.486707 & -1.804848 & 2.070608 \\
\hline & -5.108887 & -5.559698 & -1.852355 \\
\hline$\frac{1}{1}$ & -0.968703 & -4.917273 & 6.091481 \\
\hline & 8.776956 & -4.078689 & 1.088955 \\
\hline & 2.513727 & 2.459931 & 1.599516 \\
\hline & -4.419942 & 0.417202 & 1.604457 \\
\hline & -3.023592 & -0.693357 & -2.271841 \\
\hline & -7.195817 & -1.042409 & -1.314567 \\
\hline & -3.747418 & 2.520744 & -0.400999 \\
\hline & -0.309102 & 1.880604 & 2.062831 \\
\hline & -2.098977 & 5.771109 & 1.858630 \\
\hline & 1.566718 & -3.566177 & 1.467830 \\
\hline & 2.984403 & -3.057879 & 0.563521 \\
\hline & 4.612337 & -2.690362 & 2.144323 \\
\hline & 0.733167 & -0.986067 & 2.790155 \\
\hline ] & 5.454859 & -1.560436 & 4.172978 \\
\hline & 1.549451 & 0.115207 & 4.845255 \\
\hline & 3.929796 & -0.145787 & 5.539283 \\
\hline & -1.556201 & 1.061128 & -2.513838 \\
\hline & -1.497576 & 3.006633 & -2.305729 \\
\hline & -0.873454 & 2.275988 & -3.860191 \\
\hline & -5.697401 & 5.311418 & -1.332575 \\
\hline ] & -4.446753 & 4.149881 & -1.798904 \\
\hline & -5.570087 & 3.767548 & -0.475120 \\
\hline l & -3.990615 & 7.052588 & -1.158460 \\
\hline & -2.641091 & 6.934669 & -0.017402 \\
\hline 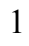 & -2.649452 & 5.929826 & -1.477186 \\
\hline 1 & -5.614953 & 6.573246 & 0.755771 \\
\hline & -5.452930 & 5.082386 & 1.707343 \\
\hline 1 & -4.281246 & 6.397638 & 1.907637 \\
\hline & 0.061717 & 5.762846 & 4.796750 \\
\hline 1 & -1.085576 & 6.318003 & 3.579154 \\
\hline ] & -1.514238 & 4.961568 & 4.639390 \\
\hline | & 1.799539 & 5.548359 & 2.948060 \\
\hline 1 & 1.502948 & 4.458560 & 1.572671 \\
\hline | & 0.605321 & 5.978538 & 1.703091 \\
\hline 1 & 1.269278 & 3.749105 & 4.655193 \\
\hline & -0.169930 & 2.727008 & 4.463103 \\
\hline 1 & 1.169111 & 2.619428 & 3.305786 \\
\hline ] & 0.712838 & 1.344737 & -4.776533 \\
\hline 1 & 2.704796 & 0.935693 & -6.124648 \\
\hline 1 & 4.963116 & 1.557390 & -5.274262 \\
\hline 1 & 5.162301 & 2.639739 & -3.037468 \\
\hline 1 & 3.171755 & 3.056111 & -1.681088 \\
\hline & 1.446680 & 2.933663 & -0.310856 \\
\hline 1 & 1.187814 & 4.347320 & -1.335696 \\
\hline 1 & -0.189895 & 3.548888 & -0.551003 \\
\hline 1 & -4.483891 & -2.153524 & -5.155613 \\
\hline 1 & -3.530780 & -0.930735 & -4.309912 \\
\hline 1 & -3.653577 & -2.574564 & -3.643335 \\
\hline 1 & -6.609881 & -2.925071 & -4.413249 \\
\hline 1 & -5.963627 & -3.434917 & -2.839148 \\
\hline 1 & -7.384150 & -2.372082 & -2.926589 \\
\hline 1 & -6.389984 & -0.518578 & -5.169140 \\
\hline 1 & -7.088591 & 0.070477 & -3.646679 \\
\hline 1 & -5.497984 & 0.652001 & -4.172804 \\
\hline
\end{tabular}




$\begin{array}{rrrr}1 & -7.447767 & -0.891391 & 3.326854 \\ 1 & -6.577327 & -2.051337 & 2.301774 \\ 1 & -5.719899 & -0.670394 & 3.004421 \\ 1 & -7.922484 & 1.443282 & 2.496616 \\ 1 & -6.225011 & 1.736106 & 2.070984 \\ 1 & -7.488734 & 1.917345 & 0.841621 \\ 1 & -9.148185 & -0.528308 & 1.612355 \\ 1 & -8.748101 & -0.069194 & -0.047860 \\ 1 & -8.376486 & -1.706828 & 0.539943\end{array}$

TS1 $_{\text {II } B 2-} \psi-1$ a2a3s4a

Number of imaginary frequencies : 1

The smallest frequencies are : $-615.6575 \quad 14.2028 \quad 20.4109 \mathrm{~cm}(-1)$

Electronic energy : $\quad H F=-4360.8672527$

Zero-point correction $=\quad 1.453887($ Hartree/Particle $)$

Thermal correction to Energy= $\quad 1.538457$

Thermal correction to Enthalpy= $\quad 1.539401$

Thermal correction to Gibbs Free Energy $=\quad 1.336405$

Sum of electronic and zero-point Energies $=\quad-4359.413366$

Sum of electronic and thermal Energies $=\quad-4359.328796$

Sum of electronic and thermal Enthalpies $=\quad-4359.327852$

Sum of electronic and thermal Free Energies $=\quad-4359.530848$

Cartesian Coordinates

$\begin{array}{cccc}6 & -4.642248 & 0.021483 & 0.653975 \\ 6 & -3.614319 & -0.010817 & -0.298578 \\ 6 & -3.906821 & -0.376052 & -1.602660 \\ 6 & -5.212854 & -0.696934 & -1.998358 \\ 6 & -6.205834 & -0.672488 & -1.025221 \\ 6 & -5.945729 & -0.317207 & 0.310691 \\ 15 & -1.867640 & 0.378044 & 0.165727 \\ 45 & -0.332502 & 0.397808 & -1.721938 \\ 15 & 1.476271 & -0.528490 & -0.661382 \\ 8 & 2.370712 & 0.161631 & 0.552947 \\ 6 & 3.499095 & 0.920985 & 0.439900 \\ 6 & 4.656619 & 0.455448 & -0.157953 \\ 6 & 5.734124 & 1.387884 & -0.337859 \\ 6 & 5.656144 & 2.687548 & 0.246832 \\ 6 & 4.505985 & 3.041441 & 0.994464 \\ 6 & 3.447525 & 2.181724 & 1.074188 \\ 6 & 6.871705 & 1.087284 & -1.130035 \\ 6 & 7.875624 & 2.007046 & -1.313385 \\ 6 & 7.806117 & 3.279105 & -0.708747 \\ 6 & 6.713999 & 3.607721 & 0.053764 \\ 6 & 4.775176 & -0.947248 & -0.617008 \\ 6 & 3.823662 & -1.500503 & -1.451686 \\ 6 & 3.983023 & -2.775623 & -2.036311 \\ 6 & 5.051794 & -3.555396 & -1.691457 \\ 6 & 5.983473 & -3.105025 & -0.723734 \\ 6 & 5.851007 & -1.792207 & -0.179625 \\ 6 & 7.026893 & -3.948279 & -0.270215 \\ 6 & 7.895484 & -3.534376 & 0.707026 \\ 6 & 7.748422 & -2.250394 & 1.272248 \\ 6 & 6.760154 & -1.401769 & 0.837256\end{array}$




\begin{tabular}{|c|c|c|c|}
\hline 8 & 2.683319 & -0.810876 & -1.780482 \\
\hline 6 & -5.470650 & -1.044109 & -3.462252 \\
\hline & -5.063812 & 0.146813 & -4.339031 \\
\hline & -7.082310 & -0.331782 & 1.331463 \\
\hline & -7.626035 & -1.759251 & 1.458245 \\
\hline 0 & -2.008296 & 2.069737 & 0.869336 \\
\hline 6 & -2.937207 & 2.974453 & 0.352408 \\
\hline 6 & -2.918597 & 4.318887 & 0.732038 \\
\hline 6 & -1.967560 & 4.721279 & 1.674241 \\
\hline 6 & -1.031528 & 3.839347 & 2.224710 \\
\hline 6 & -1.045505 & 2.518772 & 1.776909 \\
\hline 6 & 0.010751 & 4.336522 & 3.226463 \\
\hline 6 & 1.096889 & 5.100106 & 2.459335 \\
\hline 6 & -3.881453 & 5.347501 & 0.141363 \\
\hline 6 & -4.701636 & 5.996445 & 1.260994 \\
\hline 6 & -1.736339 & -0.689956 & 1.685754 \\
\hline 6 & -1.411823 & -2.034107 & 1.565468 \\
\hline 6 & -1.333952 & -2.864816 & 2.733748 \\
\hline 6 & -1.603658 & -2.305777 & 4.016084 \\
\hline 6 & -1.969790 & -0.943471 & 4.099818 \\
\hline 6 & -2.032668 & -0.166559 & 2.975342 \\
\hline 6 & -1.498902 & -3.115157 & 5.171879 \\
\hline 6 & -1.139387 & -4.435015 & 5.075065 \\
\hline 6 & -0.874400 & -4.997976 & 3.809368 \\
\hline 6 & -0.973111 & -4.237299 & 2.670397 \\
\hline 6 & -1.179776 & -2.716651 & 0.261166 \\
\hline 6 & 0.073184 & -2.749800 & -0.322335 \\
\hline 6 & 0.325946 & -3.581424 & -1.437421 \\
\hline 6 & -0.666064 & -4.351285 & -1.981227 \\
\hline 6 & -1.975087 & -4.311146 & -1.447662 \\
\hline 6 & -2.240743 & -3.480708 & -0.318380 \\
\hline 6 & -3.562547 & -3.450123 & 0.197497 \\
\hline 6 & -4.557582 & -4.210519 & -0.364680 \\
\hline 6 & -4.282109 & -5.057020 & -1.460652 \\
\hline 6 & -3.019106 & -5.097448 & -1.993691 \\
\hline 7 & 1.151450 & -1.957044 & 0.201206 \\
\hline 6 & 2.089299 & -2.679819 & 1.090851 \\
\hline 6 & 2.566045 & -1.942327 & 2.317114 \\
\hline 6 & 1.715584 & -1.136876 & 3.077126 \\
\hline 6 & 2.177839 & -0.501337 & 4.221413 \\
\hline 6 & 3.502372 & -0.660552 & 4.628316 \\
\hline 6 & 4.354614 & -1.464263 & 3.880492 \\
\hline 6 & 3.886417 & -2.101256 & 2.732929 \\
\hline 6 & -0.627722 & 5.271848 & 4.257924 \\
\hline 6 & 0.660764 & 3.183099 & 3.993027 \\
\hline 6 & -4.854152 & 4.725062 & -0.857961 \\
\hline 6 & -3.069747 & 6.422677 & -0.590208 \\
\hline 6 & -0.337467 & -0.839310 & -3.194162 \\
\hline 8 & -0.295028 & -1.481147 & -4.161556 \\
\hline 6 & 0.613658 & 2.531254 & -2.221802 \\
\hline 6 & 0.818663 & 3.368252 & -0.984824 \\
\hline 6 & -0.690626 & 2.477064 & -2.748025 \\
\hline 6 & 1.801243 & 2.264457 & -3.072211 \\
\hline 6 & 1.701257 & 1.682707 & -4.348457 \\
\hline 6 & 2.825910 & 1.435313 & -5.119603 \\
\hline 6 & 4.095906 & 1.765331 & -4.647130 \\
\hline
\end{tabular}




\begin{tabular}{|c|c|c|c|}
\hline & 4.215322 & 2.355944 & -3.395621 \\
\hline & 3.085019 & 2.603821 & -2.622069 \\
\hline & -4.635785 & -2.267069 & -3.854580 \\
\hline & -6.938732 & -1.360583 & -3.733140 \\
\hline & -8.205009 & 0.604789 & 0.872645 \\
\hline & -6.622329 & 0.122478 & 2.715239 \\
\hline & 6.647026 & -0.425113 & 1.300572 \\
\hline & -2.321369 & 0.876345 & 3.074781 \\
\hline & 7.113416 & -4.942381 & -0.707051 \\
\hline & -1.706820 & -2.663239 & 6.140793 \\
\hline & 6.632474 & 4.591051 & 0.515370 \\
\hline & -3.789017 & -2.795310 & 1.038090 \\
\hline & -5.567931 & -4.158188 & 0.036399 \\
\hline & 4.467468 & 4.014175 & 1.483215 \\
\hline & -2.202969 & -0.519208 & 5.075874 \\
\hline & 5.187003 & -4.540467 & -2.134920 \\
\hline & 8.728727 & 1.752999 & -1.938586 \\
\hline & 3.242841 & -3.099572 & -2.764803 \\
\hline & -2.794763 & -5.733283 & -2.849201 \\
\hline & -0.464867 & -4.994246 & -2.836725 \\
\hline & -0.591160 & -6.045409 & 3.734440 \\
\hline & 6.933750 & 0.119595 & -1.620827 \\
\hline & -0.768912 & -4.691203 & 1.702725 \\
\hline & 1.333668 & -3.593927 & -1.849753 \\
\hline & 8.609223 & 3.996994 & -0.857978 \\
\hline & 8.420405 & -1.930010 & 2.065431 \\
\hline & -5.076809 & -5.664674 & -1.888310 \\
\hline & -1.057616 & -5.049037 & 5.968936 \\
\hline & 8.686549 & -4.194365 & 1.055213 \\
\hline 1 & 2.541069 & 2.435093 & 1.620507 \\
\hline & -4.405447 & 0.311850 & 1.674954 \\
\hline 1 & -3.088580 & -0.420498 & -2.319873 \\
\hline & -7.225740 & -0.934753 & -1.300827 \\
\hline & -3.675417 & 2.617316 & -0.362887 \\
\hline & -0.299144 & 1.811683 & 2.139291 \\
\hline & -1.950929 & 5.765247 & 1.984517 \\
\hline 1 & 1.541173 & -3.574221 & 1.418837 \\
\hline & 2.960087 & -3.049478 & 0.528414 \\
\hline 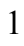 & 4.565380 & -2.719078 & 2.143176 \\
\hline & 0.693678 & -0.979968 & 2.738630 \\
\hline & 5.393688 & -1.592646 & 4.179284 \\
\hline 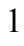 & 1.497172 & 0.125781 & 4.796628 \\
\hline & 3.866186 & -0.156596 & 5.521299 \\
\hline 1 & -1.560003 & 1.148576 & -2.493690 \\
\hline & -1.450369 & 3.086126 & -2.252411 \\
\hline 1 & -0.841364 & 2.373256 & -3.822011 \\
\hline & -5.526192 & 5.499808 & -1.247990 \\
\hline 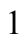 & -4.334188 & 4.280341 & -1.716967 \\
\hline 1 & -5.478425 & 3.948164 & -0.397013 \\
\hline 1 & -3.738947 & 7.160577 & -1.053002 \\
\hline 1 & -2.396549 & 6.962969 & 0.086160 \\
\hline ] & -2.455512 & 5.976229 & -1.384351 \\
\hline 1 & -5.394914 & 6.739139 & 0.843802 \\
\hline ] & -5.294642 & 5.245269 & 1.798812 \\
\hline 1 & -4.069244 & 6.510892 & 1.994359 \\
\hline 1 & 0.123360 & 5.583642 & 4.995417 \\
\hline
\end{tabular}




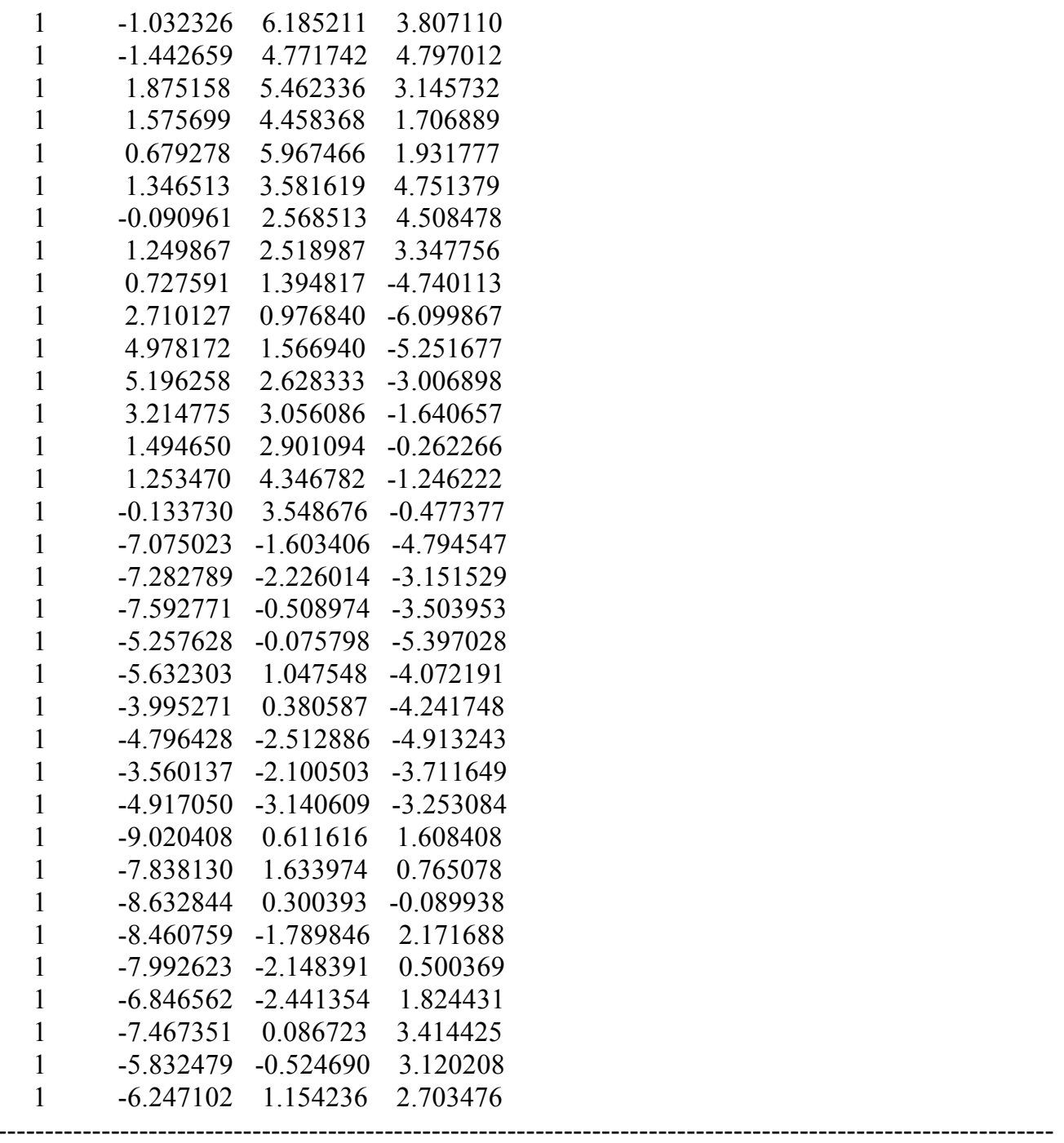

TS1 $_{\text {IIB2- }} \psi-1$ a2a3s4s

Number of imaginary frequencies : 1

The smallest frequencies are : $-617.7227 \quad 12.4554 \quad 14.8048 \mathrm{~cm}(-1)$

Electronic energy : $\quad H F=-4360.8675873$

Zero-point correction $=\quad 1.452327($ Hartree/Particle $)$

Thermal correction to Energy $=\quad 1.537436$

Thermal correction to Enthalpy= $\quad 1.538380$

Thermal correction to Gibbs Free Energy= $\quad 1.332240$

Sum of electronic and zero-point Energies= $\quad-4359.415261$

Sum of electronic and thermal Energies $=\quad-4359.330151$

Sum of electronic and thermal Enthalpies $=\quad-4359.329207$

Sum of electronic and thermal Free Energies $=\quad-4359.535348$

Cartesian Coordinates

$\begin{array}{llll}6 & 1.669496 & 1.694340 & -4.338056 \\ 6 & 1.756177 & 2.285487 & -3.065130 \\ 6 & 3.032048 & 2.655693 & -2.616749 \\ 6 & 4.167777 & 2.428278 & -3.388702 \\ 6 & 4.061839 & 1.828130 & -4.636880\end{array}$




\begin{tabular}{|c|c|c|c|}
\hline 5 & 2.799500 & 1.467785 & -5.107701 \\
\hline & 0.562708 & 2.531850 & -2.217134 \\
\hline 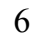 & 0.746252 & 3.383117 & -0.986409 \\
\hline 4 & -0.332103 & 0.376928 & -1.705471 \\
\hline J & -0.740073 & 2.442920 & -2.741910 \\
\hline & -1.867660 & 0.321838 & 0.171555 \\
\hline 5 & -3.601750 & -0.092998 & -0.313539 \\
\hline 6 & -3.877257 & -0.485381 & -1.617359 \\
\hline 6 & -5.180532 & -0.773363 & -2.033112 \\
\hline & -6.203030 & -0.683058 & -1.086691 \\
\hline ) & -5.957838 & -0.314257 & 0.242986 \\
\hline 6 & -4.646104 & -0.016083 & 0.608791 \\
\hline o & -5.416200 & -1.152511 & -3.493572 \\
\hline 6 & -7.057921 & -0.214828 & 1.298606 \\
\hline 5 & -1.724449 & -0.738356 & 1.695277 \\
\hline 6 & -1.370996 & -2.075894 & 1.581473 \\
\hline 6 & -1.275893 & -2.898574 & 2.753491 \\
\hline 6 & -1.556695 & -2.338923 & 4.033177 \\
\hline 6 & -1.953420 & -0.984855 & 4.110128 \\
\hline 6 & -2.033070 & -0.215009 & 2.981726 \\
\hline 6 & -0.884223 & -4.262547 & 2.696298 \\
\hline 6 & -0.763730 & -5.014120 & 3.839177 \\
\hline 6 & -1.037710 & -4.450146 & 5.102569 \\
\hline 6 & -1.429191 & -3.138974 & 5.193173 \\
\hline 6 & -1.130017 & -2.757966 & 0.278618 \\
\hline 6 & 0.118729 & -2.766626 & -0.314797 \\
\hline 6 & 0.375749 & -3.586335 & 37894 \\
\hline 6 & -0.607363 & -4.370431 & \\
\hline 6 & -1.910986 & -4.360512 & -1.429250 \\
\hline 6 & -2.180956 & -3.541648 & -0.292771 \\
\hline 6 & -2.944453 & -5.166703 & -1.966297 \\
\hline 6 & -4.200611 & -5.158390 & -1.415908 \\
\hline 6 & -4.479982 & -4.326352 & -0.309918 \\
\hline 6 & -3.495586 & -3.545104 & 0.241883 \\
\hline 7 & 1.188318 & -1.961856 & 0.207042 \\
\hline 15 & 1.492141 & -0.523871 & -0.647587 \\
\hline 8 & 2.383667 & 0.163057 & 0.571282 \\
\hline 6 & 3.484605 & 0.960583 & 0.448707 \\
\hline 6 & 4.650736 & 0.528933 & -0.157804 \\
\hline 6 & 5.700134 & 1.491459 & -0.343844 \\
\hline 6 & 5.587083 & 2.788957 & 0.239980 \\
\hline 6 & 4.430877 & 3.110779 & 0.992691 \\
\hline 6 & 3.398512 & 2.220343 & 1.080827 \\
\hline 6 & 6.842120 & 1.222911 & -1.141120 \\
\hline 6 & 7.818612 & 2.170758 & -1.329341 \\
\hline 6 & 7.715084 & 3.441001 & -0.725689 \\
\hline 6 & 6.617188 & 3.739113 & 0.040933 \\
\hline 6 & 4.802030 & -0.869548 & -0.620447 \\
\hline 6 & 3.858013 & -1.444469 & -1.449238 \\
\hline 6 & 4.042846 & -2.714952 & -2.036243 \\
\hline 6 & 5.133195 & -3.468781 & -1.701855 \\
\hline 6 & 6.062233 & -2.996689 & -0.741955 \\
\hline 6 & 5.901773 & -1.688707 & -0.193510 \\
\hline 6 & 7.130422 & -3.814489 & -0.299832 \\
\hline 6 & 7.996963 & -3.381193 & 0.670793 \\
\hline 6 & 7.822337 & -2.102933 & 1.241038 \\
\hline
\end{tabular}




\begin{tabular}{|c|c|c|c|}
\hline 6 & 6.809252 & -1.278326 & 0.817015 \\
\hline 8 & 2.700236 & -0.781548 & -1.771388 \\
\hline & 2.143439 & -2.680769 & 1.081986 \\
\hline & 2.617315 & -1.952092 & 2.314383 \\
\hline & 1.755095 & -1.184303 & 3.099596 \\
\hline & 2.212258 & -0.566147 & 4.255407 \\
\hline & 3.544440 & -0.700718 & 4.645627 \\
\hline & 4.409774 & -1.462670 & 3.869618 \\
\hline & 3.945726 & -2.086074 & 2.713038 \\
\hline & -2.056379 & 2.011444 & 0.869877 \\
\hline & -1.100281 & 2.493973 & 1.767061 \\
\hline f & -1.126009 & 3.815356 & 2.212946 \\
\hline & -2.094910 & 4.665419 & 1.669253 \\
\hline f & -3.040723 & 4.229461 & 0.736509 \\
\hline & -3.019536 & 2.884364 & 0.361238 \\
\hline & -0.089226 & 4.346465 & 3.203079 \\
\hline$b$ & 0.579207 & 3.216138 & 3.987743 \\
\hline & -4.047249 & 5.220227 & 0.154677 \\
\hline D & -3.285547 & 6.345173 & -0.555059 \\
\hline & -0.332949 & -0.853302 & -3.184634 \\
\hline & -0.321834 & -1.481717 & -4.161671 \\
\hline ) & -4.905215 & 5.806633 & 1.280496 \\
\hline f & -4.981279 & 4.568028 & -0.862533 \\
\hline ) & 0.985016 & 5.109698 & 2.418834 \\
\hline 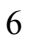 & -0.736649 & 5.292048 & 4.219215 \\
\hline & 6.674835 & -0.306450 & 1.284792 \\
\hline 1 & -2.342154 & 0.822554 & 3.075728 \\
\hline & 7.237917 & -4.805097 & -0.739936 \\
\hline & -1.645195 & -2.686819 & 6.160218 \\
\hline 1 & 6.509366 & 4.720271 & 0.501769 \\
\hline & -3.721827 & -2.907659 & 1.095528 \\
\hline ] & -5.483945 & -4.304362 & 0.109223 \\
\hline & 4.366810 & 4.083253 & 1.479306 \\
\hline & -2.195081 & -0.560858 & 5.084240 \\
\hline I & 5.288231 & -4.449855 & -2.147655 \\
\hline & 8.676065 & 1.940588 & -1.957837 \\
\hline 1 & 3.304024 & -3.056438 & -2.758010 \\
\hline & -2.717484 & -5.792540 & -2.828469 \\
\hline 1 & -0.402757 & -5.003554 & -2.839601 \\
\hline 1 & -0.455890 & -6.054935 & 3.769431 \\
\hline ] & 6.929252 & 0.257012 & -1.631621 \\
\hline ] & -0.672898 & -4.716705 & 1.730279 \\
\hline 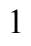 & 1.379504 & -3.577502 & -1.860270 \\
\hline 1 & 8.496660 & 4.181416 & -0.879066 \\
\hline ] & 8.492772 & -1.767879 & 2.029463 \\
\hline 1 & -4.986763 & -5.782132 & -1.836140 \\
\hline 1 & -0.937862 & -5.056732 & 5.999674 \\
\hline 1 & 8.807534 & -4.021816 & 1.010174 \\
\hline 1 & 2.488908 & 2.446816 & 1.634071 \\
\hline 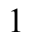 & -4.430295 & 0.292130 & 1.631755 \\
\hline 1 & -3.045200 & -0.566243 & -2.315221 \\
\hline 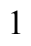 & -7.221835 & -0.908075 & -1.389140 \\
\hline 1 & -3.753218 & 2.502092 & -0.345036 \\
\hline ] & -0.326525 & 1.813059 & 2.122853 \\
\hline 1 & -2.109497 & 5.710208 & 1.977030 \\
\hline 1 & 1.610316 & -3.586833 & 1.403562 \\
\hline
\end{tabular}




\begin{tabular}{|c|c|c|c|}
\hline ] & 3.015638 & -3.030477 & 0.509052 \\
\hline & 4.633699 & -2.673546 & 2.103054 \\
\hline & 0.726622 & -1.044721 & 2.773892 \\
\hline 1 & 5.454972 & -1.570430 & 4.154827 \\
\hline 1 & 1.520984 & 0.025088 & 4.854959 \\
\hline & 3.903952 & -0.210103 & 5.547736 \\
\hline & -1.579261 & 1.098525 & -2.470612 \\
\hline & -1.513598 & 3.039332 & -2.251838 \\
\hline & -0.889070 & 2.324980 & -3.814785 \\
\hline & -5.688509 & 5.315116 & -1.244148 \\
\hline & -4.433255 & 4.164458 & -1.724479 \\
\hline & -5.568956 & 3.752681 & -0.420220 \\
\hline & -3.988005 & 7.052934 & -1.015497 \\
\hline & -2.647357 & 6.912158 & 0.133142 \\
\hline & -2.642219 & 5.940018 & -1.348576 \\
\hline & -5.625736 & 6.529536 & 0.874748 \\
\hline ] & -5.470726 & 5.016794 & 1.792560 \\
\hline & -4.301318 & 6.327409 & 2.033274 \\
\hline ] & 0.012185 & 5.624416 & 4.949879 \\
\hline & -1.151630 & 6.193511 & 3.754148 \\
\hline ] & -1.545259 & 4.792485 & 4.768274 \\
\hline & 1.755299 & 5.502428 & 3.097479 \\
\hline & 1.477002 & 4.457127 & 1.684241 \\
\hline 1 & 0.553409 & 5.956048 & 1.868707 \\
\hline & 1.258272 & 3.637334 & 4.739753 \\
\hline$\frac{1}{1}$ & -0.161125 & 2.595351 & 4.511828 \\
\hline & 1.179489 & 2.553958 & 3.351367 \\
\hline ] & 0.702516 & 1.383473 & -4.728587 \\
\hline ] & 2.694022 & 1.001598 & -6.085499 \\
\hline & 4.948453 & 1.645792 & -5.240116 \\
\hline 1 & 5.142430 & 2.723768 & -3.001140 \\
\hline & 3.151577 & 3.115853 & -1.637618 \\
\hline 1 & 1.431045 & 2.938493 & -0.257959 \\
\hline ] & 1.158148 & 4.369635 & -1.254786 \\
\hline ] & -0.211116 & 3.544836 & -0.481764 \\
\hline 5 & -6.889543 & -1.410749 & -3.796948 \\
\hline 6 & -4.933036 & -0.010081 & -4.396046 \\
\hline 6 & -4.626971 & -2.422991 & -3.824976 \\
\hline 6 & -8.423735 & -0.634642 & 0.761587 \\
\hline 6 & -6.712242 & -1.129281 & 2.480275 \\
\hline 6 & -7.159927 & 1.236363 & 1.783438 \\
\hline 1 & -7.007381 & -1.678214 & -4.854662 \\
\hline 1 & -7.289940 & -2.241981 & -3.201983 \\
\hline 1 & -7.509812 & -0.524034 & -3.611238 \\
\hline 1 & -5.112142 & -0.257199 & -5.451207 \\
\hline & -5.465193 & 0.923789 & -4.171504 \\
\hline 1 & -3.857231 & 0.177638 & -4.280115 \\
\hline 1 & -4.773842 & -2.698205 & -4.878445 \\
\hline 1 & -3.548747 & -2.295590 & -3.661456 \\
\hline 1 & -4.958919 & -3.262884 & -3.201444 \\
\hline 1 & -7.920358 & 1.326439 & 2.571138 \\
\hline 1 & -6.209739 & 1.603809 & 2.191797 \\
\hline 1 & -7.448160 & 1.902525 & 0.959429 \\
\hline 1 & -7.492771 & -1.069654 & 3.250807 \\
\hline 1 & -6.638080 & -2.175703 & 2.155165 \\
\hline 1 & -5.758807 & -0.861734 & 2.953276 \\
\hline
\end{tabular}




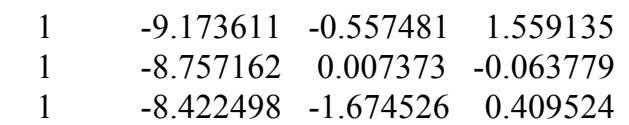

TS1 $_{\text {IIB2- }} \psi_{-1 \mathrm{a} 2 \mathrm{~s} 3 \mathrm{a} 4 \mathrm{a}}$

Number of imaginary frequencies : 1

The smallest frequencies are : -753.2070 $9.4983 \quad 18.6390 \mathrm{~cm}(-1)$

Electronic energy : $\quad H F=-4360.8655407$

Zero-point correction $=\quad 1.452419($ Hartree/Particle $)$

Thermal correction to Energy $=\quad 1.537238$

Thermal correction to Enthalpy= $\quad 1.538182$

Thermal correction to Gibbs Free Energy $=\quad 1.333638$

Sum of electronic and zero-point Energies $=\quad-4359.413122$

Sum of electronic and thermal Energies $=\quad-4359.328303$

Sum of electronic and thermal Enthalpies $=\quad-4359.327359$

Sum of electronic and thermal Free Energies $=\quad-4359.531903$

Cartesian Coordinates

$\begin{array}{cccc}6 & 0.662595 & 3.032627 & 4.288992 \\ 6 & -0.563070 & 2.800643 & 3.641999 \\ 6 & -1.687031 & 2.590216 & 4.453540 \\ 6 & -1.589833 & 2.593702 & 5.840951 \\ 6 & -0.366834 & 2.818773 & 6.462161 \\ 6 & 0.759415 & 3.041458 & 5.673141 \\ 6 & -0.683659 & 2.750344 & 2.159456 \\ 6 & -2.058709 & 3.004156 & 1.588063 \\ 45 & 0.262230 & 0.861646 & 1.364823 \\ 15 & 1.725076 & 0.203345 & -0.436167 \\ 6 & 1.925095 & 1.735847 & -1.428990 \\ 6 & 0.883369 & 2.133423 & -2.275346 \\ 6 & 0.945929 & 3.332113 & -2.980792 \\ 6 & 2.050814 & 4.168868 & -2.756779 \\ 6 & 3.083380 & 3.821815 & -1.888135 \\ 6 & 3.009946 & 2.582244 & -1.240455 \\ 6 & -0.185236 & 3.756385 & -3.917505 \\ 6 & 0.342806 & 4.562151 & -5.107893 \\ 6 & 4.283347 & 4.724647 & -1.611091 \\ 6 & 5.578279 & 3.976213 & -1.949527 \\ 15 & -1.612152 & -0.273066 & 0.635968 \\ 7 & -1.337589 & -1.869924 & 0.110018 \\ 6 & -2.313826 & -2.761873 & -0.559813 \\ 6 & -2.872089 & -2.296251 & -1.881136 \\ 6 & -4.218419 & -2.515143 & -2.165702 \\ 6 & -4.761527 & -2.124712 & -3.388401 \\ 6 & -3.960443 & -1.508152 & -4.341948 \\ 6 & -2.611467 & -1.287419 & -4.064898 \\ 6 & -2.073799 & -1.680659 & -2.847438 \\ 8 & -2.609333 & 0.094167 & -0.643993 \\ 6 & -3.659297 & 0.961568 & -0.616216 \\ 6 & -4.800609 & 0.696027 & 0.118657 \\ 6 & -5.811433 & 1.713795 & 0.173040 \\ 6 & -5.678955 & 2.885145 & -0.633263 \\ 6 & -4.538641 & 3.036891 & -1.459480 \\ 6 & -3.543692 & 2.101205 & -1.438168\end{array}$




\begin{tabular}{|c|c|c|c|}
\hline 6 & -6.936490 & 1.627290 & 1.032502 \\
\hline & -7.883585 & 2.621266 & 1.068424 \\
\hline & -7.766069 & 3.758346 & 0.242996 \\
\hline & -6.680857 & 3.883684 & -0.586267 \\
\hline & -4.935936 & -0.584717 & 0.850814 \\
\hline & -6.058038 & -1.457997 & 0.653592 \\
\hline & -6.189787 & -2.630755 & 1.456502 \\
\hline 0 & -5.210088 & -2.916199 & 2.438933 \\
\hline b & -4.102067 & -2.124578 & 2.558928 \\
\hline 6 & -3.946247 & -0.995824 & 1.725224 \\
\hline 6 & -7.281574 & -3.506551 & 1.241756 \\
\hline 6 & -8.199026 & -3.261846 & 0.252269 \\
\hline 6 & -8.054177 & -2.122706 & -0.567006 \\
\hline 6 & -7.018313 & -1.242918 & -0.368388 \\
\hline 8 & -2.773862 & -0.293153 & 1.855439 \\
\hline 6 & 0.424957 & 3.118340 & 1.338045 \\
\hline 6 & 0.505657 & -0.061775 & 3.095549 \\
\hline 8 & 0.435648 & -0.461970 & 4.179100 \\
\hline 6 & 3.455239 & -0.189710 & 0.064306 \\
\hline 6 & 4.453348 & -0.390409 & -0.893432 \\
\hline 6 & 5.764000 & -0.670580 & -0.511133 \\
\hline 6 & 6.040228 & -0.750209 & 0.860200 \\
\hline 6 & 5.067274 & -0.559598 & 1.842183 \\
\hline 6 & 3.765580 & -0.278848 & 1.416790 \\
\hline 6 & 6.875362 & -0.925182 & -1.528803 \\
\hline 6 & 8.077642 & -0.020870 & -1.238964 \\
\hline 6 & 5.437784 & -0.608124 & 3.323544 \\
\hline 6 & 6.239393 & -1.874662 & 3.635962 \\
\hline 6 & 1.455586 & -1.095732 & -1.736074 \\
\hline 6 & 1.101321 & -2.381427 & -1.351538 \\
\hline 6 & 0.913258 & -3.401579 & -2.338020 \\
\hline 6 & 1.094698 & -3.089214 & -3.716622 \\
\hline 6 & 1.480517 & -1.777137 & -4.072767 \\
\hline 6 & 1.653536 & -0.813550 & -3.115196 \\
\hline 6 & 0.876848 & -4.089121 & -4.694274 \\
\hline 6 & 0.494104 & -5.354786 & -4.330937 \\
\hline 6 & 0.319304 & -5.672757 & -2.967389 \\
\hline 6 & 0.527574 & -4.724476 & -1.996831 \\
\hline 6 & 0.944363 & -2.762922 & 0.078996 \\
\hline 6 & -0.246992 & -2.549839 & 0.749501 \\
\hline 6 & -0.413375 & -3.008855 & 2.076089 \\
\hline 6 & 0.608434 & -3.631250 & 2.740161 \\
\hline 6 & 1.856502 & -3.831379 & 2.106409 \\
\hline 6 & 2.025712 & -3.408357 & 0.755013 \\
\hline 6 & 3.282334 & -3.628522 & 0.132658 \\
\hline 6 & 4.311586 & -4.228252 & 0.813903 \\
\hline 6 & 4.137561 & -4.652577 & 2.149404 \\
\hline 6 & 2.936335 & -4.452868 & 2.781002 \\
\hline 6 & 7.308457 & -2.392671 & -1.434354 \\
\hline 6 & 6.418147 & -0.655230 & -2.960938 \\
\hline 6 & 4.201324 & -0.602346 & 4.221994 \\
\hline 6 & 6.286942 & 0.624160 & 3.655693 \\
\hline 6 & -0.928388 & 2.543461 & -4.484839 \\
\hline 6 & -1.163286 & 4.630888 & -3.123389 \\
\hline 6 & 4.286192 & 5.104913 & -0.125486 \\
\hline 6 & 4.249628 & 6.010653 & -2.432391 \\
\hline
\end{tabular}




\begin{tabular}{|c|c|c|c|}
\hline 1 & 0.220059 & 3.424820 & 0.310695 \\
\hline & -6.906881 & -0.384045 & -1.025368 \\
\hline & 1.953074 & 0.186266 & -3.419142 \\
\hline 1 & -7.366031 & -4.390030 & 1.873340 \\
\hline 1 & 1.016209 & -3.828197 & -5.742493 \\
\hline 1 & -6.562386 & 4.762661 & -1.218686 \\
\hline 1 & 3.428950 & -3.301268 & -0.895193 \\
\hline 1 & 5.271389 & -4.377468 & 0.322959 \\
\hline & -4.457053 & 3.920436 & -2.091383 \\
\hline & 1.637985 & -1.542007 & -5.124855 \\
\hline 1 & -5.341689 & -3.788380 & 3.077152 \\
\hline 1 & -8.730041 & 2.531801 & 1.745733 \\
\hline 1 & -3.328342 & -2.326685 & 3.296108 \\
\hline 1 & 2.788413 & -4.771729 & 3.812014 \\
\hline 1 & 0.479473 & -3.966101 & 3.768299 \\
\hline 1 & 0.019050 & -6.678799 & -2.683452 \\
\hline & -7.038627 & 0.764124 & 1.685049 \\
\hline & 0.395505 & -4.984614 & -0.948430 \\
\hline 1 & -1.372215 & -2.836308 & 2.561145 \\
\hline 1 & -8.526509 & 4.535084 & 0.275703 \\
\hline 1 & -8.766125 & -1.941611 & -1.369328 \\
\hline 1 & 4.958831 & -5.136053 & 2.674470 \\
\hline 1 & 0.324636 & -6.115430 & -5.089456 \\
\hline 1 & -9.027543 & -3.947044 & 0.088846 \\
\hline 1 & -2.638555 & 2.200616 & -2.033774 \\
\hline 1 & 4.195667 & -0.317284 & -1.948108 \\
\hline 1 & 2.962687 & -0.129763 & 2.134529 \\
\hline 1 & 7.061692 & -0.968320 & 1.171973 \\
\hline 1 & 3.808490 & 2.279233 & -0.564718 \\
\hline 1 & 0.020883 & 1.474501 & -2.384595 \\
\hline 1 & 2.098329 & 5.120774 & -3.278642 \\
\hline 1 & -1.769681 & -3.702727 & -0.727811 \\
\hline 1 & -2.024808 & 4.915296 & -3.744196 \\
\hline 1 & -1.537442 & 4.106187 & -2.233424 \\
\hline 1 & -0.675788 & 5.552026 & -2.778062 \\
\hline 1 & -0.482228 & 4.793403 & -5.793851 \\
\hline 1 & 0.784750 & 5.519705 & -4.809635 \\
\hline 1 & 1.099316 & 3.998719 & -5.669371 \\
\hline 1 & -0.240427 & 1.861853 & -5.004709 \\
\hline 1 & -1.454357 & 1.960126 & -3.719734 \\
\hline 1 & -1.685017 & 2.874993 & -5.207165 \\
\hline 1 & 5.133919 & 6.618191 & -2.202196 \\
\hline 1 & 4.260558 & 5.808769 & -3.511337 \\
\hline 1 & 3.365875 & 6.620434 & -2.204765 \\
\hline 1 & 5.692982 & 3.056450 & -1.361621 \\
\hline 1 & 5.605011 & 3.699013 & -3.011697 \\
\hline 1 & 6.450110 & 4.611715 & -1.743791 \\
\hline 1 & 3.367509 & 5.644505 & 0.140933 \\
\hline 1 & 4.357447 & 4.223883 & 0.524573 \\
\hline 1 & 5.140285 & 5.757045 & 0.102171 \\
\hline 1 & 5.595915 & -1.316712 & -3.264271 \\
\hline 1 & 6.088662 & 0.384061 & -3.095290 \\
\hline 1 & 7.250905 & -0.831352 & -3.653444 \\
\hline 1 & 8.512413 & -0.205575 & -0.249639 \\
\hline 1 & 8.868718 & -0.194249 & -1.980606 \\
\hline 1 & 7.796324 & 1.039199 & -1.288811 \\
\hline
\end{tabular}




$\begin{array}{rrrr}1 & 7.665949 & -2.651032 & -0.429598 \\ 1 & 6.471922 & -3.062064 & -1.677338 \\ 1 & 8.121661 & -2.600050 & -2.143283 \\ 1 & 5.732014 & 1.549464 & 3.451687 \\ 1 & 6.566103 & 0.623819 & 4.718247 \\ 1 & 7.211621 & 0.651356 & 3.065214 \\ 1 & 7.185168 & -1.921797 & 3.082882 \\ 1 & 6.486052 & -1.910128 & 4.705443 \\ 1 & 5.657309 & -2.772509 & 3.390457 \\ 1 & 3.615608 & 0.322200 & 4.124148 \\ 1 & 3.537268 & -1.450398 & 4.003511 \\ 1 & 4.508918 & -0.680079 & 5.272580 \\ 1 & -3.145121 & -3.009134 & 0.118607 \\ 1 & -4.858508 & -2.981572 & -1.414928 \\ 1 & -1.032101 & -1.468881 & -2.616600 \\ 1 & -5.818426 & -2.295727 & -3.585719 \\ 1 & -1.971504 & -0.801789 & -4.801005 \\ 1 & -4.383210 & -1.195065 & -5.294339 \\ 1 & 1.264411 & 3.668198 & 1.768510 \\ 1 & -2.656142 & 2.404336 & 3.996370 \\ 1 & -2.482356 & 2.419735 & 6.439003 \\ 1 & -0.290004 & 2.822542 & 7.547113 \\ 1 & 1.725807 & 3.221818 & 6.140403 \\ 1 & 1.567477 & 3.187630 & 3.704003 \\ 1 & -2.804470 & 2.259243 & 1.884325 \\ 1 & -2.429475 & 3.986152 & 1.921388 \\ 1 & -2.017204 & 3.018477 & 0.493100 \\ 1 & 1.487222 & 1.909115 & 1.546653\end{array}$

TS1 $_{\text {IIB2- }} \psi_{-1 \mathrm{a} 2 s 3 a 4 \mathrm{~s}}$

Number of imaginary frequencies : 1

The smallest frequencies are : $-631.4153 \quad 11.8668 \quad 18.1359 \mathrm{~cm}(-1)$

Electronic energy : $\quad H F=-4360.8689157$

Zero-point correction $=\quad 1.452900($ Hartree/Particle $)$

Thermal correction to Energy $=\quad 1.537615$

Thermal correction to Enthalpy= $\quad 1.538559$

Thermal correction to Gibbs Free Energy $=\quad 1.334758$

Sum of electronic and zero-point Energies $=\quad-4359.416015$

Sum of electronic and thermal Energies $=\quad-4359.331301$

Sum of electronic and thermal Enthalpies $=\quad-4359.330357$

Sum of electronic and thermal Free Energies $=\quad-4359.534158$

Cartesian Coordinates

$\begin{array}{cccc}6 & -1.077710 & 2.604920 & 1.642491 \\ 6 & -2.048973 & 2.090119 & 0.774068 \\ 6 & -3.038638 & 2.937174 & 0.287168 \\ 6 & -3.088527 & 4.286974 & 0.661552 \\ 6 & -2.129122 & 4.753621 & 1.558183 \\ 6 & -1.120416 & 3.925815 & 2.076492 \\ 15 & -1.853622 & 0.380641 & 0.125767 \\ 6 & -3.589994 & -0.063221 & -0.332947 \\ 6 & -3.864152 & -0.538252 & -1.614122 \\ 6 & -5.157001 & -0.907578 & -1.986975 \\ 6 & -6.170287 & -0.798308 & -1.027169\end{array}$




\begin{tabular}{|c|c|c|c|}
\hline 6 & -5.927948 & -0.345795 & 0.272222 \\
\hline 6 & -4.621612 & 0.027289 & 0.598622 \\
\hline & -5.479243 & -1.360103 & -3.410714 \\
\hline & -7.010059 & -0.271052 & 1.348447 \\
\hline & -4.187138 & 5.174798 & 0.080062 \\
\hline & -4.127306 & 6.603444 & 0.614105 \\
\hline & -0.063087 & 4.483932 & 3.029855 \\
\hline 6 & -0.671446 & 5.499134 & 4.001571 \\
\hline & -0.293460 & 0.350473 & -1.739145 \\
\hline & 1.508210 & -0.538315 & -0.634229 \\
\hline & 2.397991 & 0.169327 & 0.573270 \\
\hline & 3.509011 & 0.950721 & 0.438134 \\
\hline & 4.675897 & 0.490176 & -0.145821 \\
\hline & 5.736944 & 1.436759 & -0.347848 \\
\hline & 5.632206 & 2.750757 & 0.199810 \\
\hline & 4.472787 & 3.104949 & 0.932809 \\
\hline & 3.430678 & 2.227696 & 1.035492 \\
\hline & 6.883565 & 1.135180 & -1.126480 \\
\hline & 7.871854 & 2.067447 & -1.330399 \\
\hline & 7.776219 & 3.354419 & -0.761843 \\
\hline & 6.674297 & 3.684338 & -0.014198 \\
\hline & 4.814973 & -0.922212 & -0.569403 \\
\hline & 3.868898 & -1.508618 & -1.387697 \\
\hline & 4.039034 & -2.797957 & -1.936466 \\
\hline & 5.117931 & -3.555716 & -1.575511 \\
\hline & 6.048980 & -3.067572 & -0.625601 \\
\hline & 5.902505 & -1.742783 & -0.114393 \\
\hline & 7.105116 & -3.885905 & -0.156392 \\
\hline & 7.973410 & -3.436510 & 0.805268 \\
\hline & 7.812418 & -2.140905 & 1.338988 \\
\hline & 6.811017 & -1.316093 & 0.888372 \\
\hline & 2.723377 & -0.839575 & -1.738389 \\
\hline o & 0.626906 & 2.477222 & -2.309942 \\
\hline & 0.799135 & 3.368909 & -1.106493 \\
\hline 5 & 1.829946 & 2.205213 & -3.136015 \\
\hline & 3.100900 & 2.586078 & -2.681779 \\
\hline 6 & 4.245932 & 2.338294 & -3.433279 \\
\hline ) & 4.155117 & 1.705567 & -4.666458 \\
\hline & 2.898428 & 1.334214 & -5.143465 \\
\hline 0 & 1.758748 & 1.582251 & -4.394675 \\
\hline & -0.670399 & 2.378912 & -2.849318 \\
\hline 6 & -0.242843 & -0.927501 & -3.178433 \\
\hline & -0.141028 & -1.595613 & -4.123052 \\
\hline 5 & -1.711016 & -0.626979 & 1.687671 \\
\hline 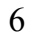 & -1.380501 & -1.973538 & 1.616601 \\
\hline 6 & -1.295772 & -2.760305 & 2.812797 \\
\hline 5 & -1.559835 & -2.155594 & 4.075300 \\
\hline 6 & -1.929483 & -0.792360 & 4.110681 \\
\hline 6 & -2.001657 & -0.057223 & 2.958382 \\
\hline 6 & -1.441733 & -2.921695 & 5.259022 \\
\hline 6 & -1.076264 & -4.242463 & 5.207321 \\
\hline 6 & -0.820414 & -4.851076 & 3.960739 \\
\hline 6 & -0.931864 & -4.132845 & 2.795739 \\
\hline 6 & -1.155311 & -2.694480 & 0.332570 \\
\hline 6 & 0.093955 & -2.743310 & -0.257870 \\
\hline 6 & 0.330382 & -3.578122 & -1.373474 \\
\hline
\end{tabular}




\begin{tabular}{|c|c|c|c|}
\hline & -0.679641 & -4.318672 & -1.924709 \\
\hline & -1.985669 & -4.257139 & -1.386588 \\
\hline & -2.228322 & -3.446644 & -0.237930 \\
\hline & -3.543769 & -3.406560 & 0.293519 \\
\hline & -4.559553 & -4.125515 & -0.284456 \\
\hline & -4.311772 & -4.940062 & -1.411548 \\
\hline & -3.052338 & -4.999121 & -1.951508 \\
\hline & 1.178627 & -1.951128 & 0.251211 \\
\hline & 2.107878 & -2.666741 & 1.155790 \\
\hline & 2.599620 & -1.910518 & 2.364543 \\
\hline & 3.923934 & -2.065065 & 2.769450 \\
\hline & 4.402720 & -1.416299 & 3.906103 \\
\hline & 3.557332 & -0.605453 & 4.653832 \\
\hline & 2.229633 & -0.448530 & 4.256622 \\
\hline & 1.757248 & -1.094384 & 3.122486 \\
\hline & -4.036373 & 5.227295 & -1.444798 \\
\hline & -5.558969 & 4.588448 & 0.433338 \\
\hline & 0.583215 & 3.377563 & 3.866581 \\
\hline & 1.022088 & 5.177786 & 2.197434 \\
\hline & 6.687056 & -0.330272 & 1.328913 \\
\hline & -2.292428 & 0.987932 & 3.021286 \\
\hline & 7.201800 & -4.889620 & -0.568407 \\
\hline & -1.643832 & -2.435720 & 6.212604 \\
\hline & 6.572779 & 4.678489 & 0.419422 \\
\hline & -3.747747 & -2.773442 & 1.156014 \\
\hline & -5.566883 & -4.064170 & 0.124340 \\
\hline & 4.414358 & 4.090749 & 1.392593 \\
\hline & -2.156563 & -0.332484 & 5.071967 \\
\hline & 5.262202 & -4.551160 & -1.992057 \\
\hline & 8.732802 & 1.811593 & -1.943996 \\
\hline & 3.298631 & -3.149987 & -2.651655 \\
\hline & -2.847565 & -5.618720 & -2.823687 \\
\hline & -0.495627 & -4.950823 & -2.792057 \\
\hline & -0.534149 & -5.899646 & 3.922091 \\
\hline & 6.965771 & 0.155163 & -1.589072 \\
\hline & -0.735847 & -4.619950 & 1.842618 \\
\hline & 1.335805 & -3.604610 & -1.790912 \\
\hline & 8.567173 & 4.082230 & -0.927025 \\
\hline & 8.484034 & -1.792209 & 2.120474 \\
\hline & -5.122995 & -5.517753 & -1.850166 \\
\hline & -0.983368 & -4.822725 & 6.122413 \\
\hline & 8.774743 & -4.077351 & 1.165552 \\
\hline & 2.520084 & 2.476510 & 1.577235 \\
\hline & -4.402061 & 0.388286 & 1.603526 \\
\hline & -3.032266 & -0.629811 & -2.308808 \\
\hline & -7.182886 & -1.082348 & -1.305052 \\
\hline & -3.786260 & 2.539999 & -0.399377 \\
\hline & -0.281685 & 1.942861 & 1.984613 \\
\hline & -2.159328 & 5.794688 & 1.868547 \\
\hline & 1.544938 & -3.544542 & 1.504926 \\
\hline & 2.971352 & -3.062385 & 0.599451 \\
\hline & 4.597358 & -2.689858 & 2.180559 \\
\hline & 0.733481 & -0.937493 & 2.790230 \\
\hline & 5.444017 & -1.542404 & 4.197787 \\
\hline 1 & 1.554171 & 0.182812 & 4.832940 \\
\hline & 3.928985 & -0.094128 & 5.539382 \\
\hline
\end{tabular}




$\begin{array}{rrrr}1 & -1.522060 & 1.059251 & -2.551033 \\ 1 & -1.443500 & 2.999094 & -2.391007 \\ 1 & -0.803876 & 2.231779 & -3.920492 \\ 1 & -4.939001 & 7.195528 & 0.172985 \\ 1 & -4.249038 & 6.639694 & 1.704416 \\ 1 & -3.182771 & 7.099480 & 0.356039 \\ 1 & -4.800250 & 5.884151 & -1.882348 \\ 1 & -3.050217 & 5.618995 & -1.728462 \\ 1 & -4.151563 & 4.237184 & -1.902822 \\ 1 & -6.359420 & 5.210769 & 0.010943 \\ 1 & -5.685819 & 3.572027 & 0.039292 \\ 1 & -5.700967 & 4.546679 & 1.521327 \\ 1 & 0.091659 & 5.832841 & 4.716474 \\ 1 & -1.047736 & 6.395892 & 3.496150 \\ 1 & -1.499075 & 5.059193 & 4.572911 \\ 1 & 1.803420 & 5.594828 & 2.848538 \\ 1 & 1.497633 & 4.474306 & 1.500163 \\ 1 & 0.602802 & 5.998718 & 1.600785 \\ 1 & 1.282462 & 3.817502 & 4.588835 \\ 1 & -0.168582 & 2.806091 & 4.428992 \\ 1 & 1.157266 & 2.665694 & 3.260834 \\ 1 & 0.796270 & 1.263572 & -4.790081 \\ 1 & 2.804671 & 0.842999 & -6.110150 \\ 1 & 5.048977 & 1.506792 & -5.253555 \\ 1 & 5.215979 & 2.642504 & -3.041047 \\ 1 & 3.209433 & 3.069374 & -1.712496 \\ 1 & 1.475086 & 2.948687 & -0.355122 \\ 1 & 1.216572 & 4.344928 & -1.403437 \\ 1 & -0.163599 & 3.550054 & -0.618577 \\ 6 & -4.219082 & -1.757534 & -4.180163 \\ 6 & -6.425245 & -2.564028 & -3.407204 \\ 6 & -6.153909 & -0.194820 & -4.144450 \\ 6 & -8.374140 & -0.725893 & 0.836666 \\ 6 & -6.615107 & -1.180523 & 2.518776 \\ 6 & -7.142621 & 1.172255 & 1.846812 \\ 1 & -4.495977 & -2.129795 & -5.174757 \\ 1 & -3.537330 & -0.910425 & -4.331836 \\ 1 & -3.663976 & -2.553698 & -3.663773 \\ 1 & -6.616322 & -2.892077 & -4.437442 \\ 1 & -5.983965 & -3.406798 & -2.859281 \\ 1 & -7.398572 & -2.336289 & -2.956923 \\ 1 & -6.396746 & -0.477885 & -5.177979 \\ 1 & -7.085832 & 0.107017 & -3.649179 \\ 1 & -5.493915 & 0.682211 & -4.179171 \\ 1 & -7.903098 & 1.235077 & 2.636931 \\ 1 & -6.201703 & 1.555500 & 2.261075 \\ 1 & -7.447851 & 1.842245 & 1.032065 \\ 1 & -7.366291 & -1.123181 & 3.318258 \\ 1 & -6.550015 & -2.228208 & 2.195509 \\ 1 & -5.645236 & -0.905090 & 2.952855 \\ 1 & -9.112494 & -0.661471 & 1.645953 \\ 1 & -8.734565 & -0.097232 & 0.012172 \\ 1 & -8.354699 & -1.768178 & 0.491857\end{array}$

TS1 $_{\text {IIB2- }} \Psi-1$ a2s3s4a 
Number of imaginary frequencies : 1

The smallest frequencies are : -617.5379 $5.9982 \quad 18.3451 \mathrm{~cm}(-1)$

Electronic energy :

$\mathrm{HF}=-4360.8680832$

Zero-point correction $=$

1.452622 (Hartree/Particle)

Thermal correction to Energy=

1.537489

Thermal correction to Enthalpy=

1.538434

Thermal correction to Gibbs Free Energy $=$

Sum of electronic and zero-point Energies=

Sum of electronic and thermal Energies $=$

1.333535

Sum of electronic and thermal Enthalpies=

$-4359.415461$

$-4359.330594$

Sum of electronic and thermal Free Energies=

$-4359.329650$

$-4359.534548$

Cartesian Coordinates

$\begin{array}{cccc}6 & 1.762289 & -1.107669 & 3.127213 \\ 6 & 2.613617 & -1.905362 & 2.359933 \\ 6 & 3.941246 & -2.045005 & 2.759357 \\ 6 & 4.414753 & -1.398954 & 3.899666 \\ 6 & 3.560397 & -0.606723 & 4.657186 \\ 6 & 2.229274 & -0.465330 & 4.265674 \\ 6 & 2.128299 & -2.657989 & 1.146575 \\ 7 & 1.186146 & -1.946601 & 0.251376 \\ 6 & 0.111747 & -2.752710 & -0.258155 \\ 6 & -1.141146 & -2.713388 & 0.325187 \\ 6 & -2.196251 & -3.500942 & -0.232950 \\ 6 & -1.925557 & -4.356928 & -1.341782 \\ 6 & -0.617394 & -4.398252 & -1.877760 \\ 6 & 0.369577 & -3.608395 & -1.353773 \\ 6 & -3.516377 & -3.471042 & 0.286794 \\ 6 & -4.506023 & -4.254153 & -0.253172 \\ 6 & -4.225521 & -5.124802 & -1.328777 \\ 6 & -2.963526 & -5.166782 & -1.864388 \\ 6 & -1.376417 & -1.994941 & 1.609659 \\ 6 & -1.711219 & -0.650069 & 1.690176 \\ 6 & -2.002192 & -0.088109 & 2.964417 \\ 6 & -1.925371 & -0.828920 & 4.112363 \\ 6 & -1.549308 & -2.190160 & 4.068754 \\ 6 & -1.285072 & -2.787460 & 2.802612 \\ 6 & -1.424091 & -2.960549 & 5.248954 \\ 6 & -1.049936 & -4.278586 & 5.191170 \\ 6 & -0.791662 & -4.879573 & 3.941516 \\ 6 & -0.910493 & -4.157254 & 2.779755 \\ 15 & -1.853057 & 0.370113 & 0.138418 \\ 45 & -0.301249 & 0.359501 & -1.725911 \\ 6 & -0.275706 & -0.910958 & -3.171019 \\ 8 & -0.223481 & -1.572259 & -4.124620 \\ 6 & -2.049997 & 2.072082 & 0.803747 \\ 6 & -1.073899 & 2.585008 & 1.667765 \\ 6 & -1.126800 & 3.899036 & 2.121566 \\ 6 & -2.152811 & 4.721956 & 1.629357 \\ 6 & -3.117959 & 4.256195 & 0.738660 \\ 6 & -3.054669 & 2.914012 & 0.341384 \\ 6 & -4.248798 & 5.129135 & 0.199163 \\ 6 & -5.594982 & 4.528864 & 0.623394 \\ 6 & -0.064416 & 4.454538 & 3.070916 \\ 6 & 1.009532 & 5.162169 & 2.235640\end{array}$




$\begin{array}{cccc}6 & -3.586698 & -0.064016 & -0.334252 \\ 6 & -3.856551 & -0.473214 & -1.629847 \\ 6 & -5.155530 & -0.808074 & -2.037268 \\ 6 & -6.166229 & -0.748382 & -1.084006 \\ 6 & -5.930268 & -0.345026 & 0.242881 \\ 6 & -4.631248 & -0.000559 & 0.598770 \\ 6 & -5.387274 & -1.202663 & -3.493472 \\ 6 & -4.542971 & -2.434858 & -3.832917 \\ 6 & -7.083228 & -0.324798 & 1.245420 \\ 6 & -8.233857 & 0.533378 & 0.709490 \\ 6 & -6.849870 & -1.530964 & -3.779631 \\ 6 & -4.967236 & -0.038999 & -4.400342 \\ 6 & -6.661458 & 0.245600 & 2.597995 \\ 6 & -7.577091 & -1.759350 & 1.465094 \\ 6 & -4.180566 & 6.561996 & 0.720555 \\ 6 & -4.168895 & 5.173681 & -1.331171 \\ 6 & -0.668737 & 5.457999 & 4.057230 \\ 6 & 0.593756 & 3.343478 & 3.892328 \\ 15 & 1.506203 & -0.530069 & -0.631387 \\ 8 & 2.717882 & -0.824566 & -1.741510 \\ 6 & 3.871043 & -1.484324 & -1.398524 \\ 6 & 4.816137 & -0.892959 & -0.582532 \\ 6 & 5.912001 & -1.706474 & -0.134681 \\ 6 & 6.067326 & -3.028125 & -0.651324 \\ 6 & 5.136878 & -3.519984 & -1.599887 \\ 6 & 4.050334 & -2.769790 & -1.953576 \\ 6 & 6.820778 & -1.276182 & 0.866380 \\ 6 & 7.830284 & -2.094656 & 1.310414 \\ 6 & 7.999799 & -3.386904 & 0.771229 \\ 6 & 7.131725 & -3.839660 & -0.189034 \\ 6 & 4.129095 & 1.735106 & -4.670721 \\ 6 & 2.871394 & 1.357418 & -5.140224 \\ 6 & 1.734759 & 1.602087 & -4.385853 \\ 6 & 6.690531 & -0.293020 & 1.311081 \\ 6 & -2.297461 & 0.955381 & 3.033133 \\ 6 & 3.668728 & 0.516973 & -0.153661 \\ 6 & 3.501681 & 0.966880 & 0.438027 \\ 6 & 3.417431 & 2.241029 & 1.040515 \\ 6 & 4.452719 & 3.126127 & 0.935537 \\ 6 & 5.610796 & 2.782937 & 0.195248 \\ 6 & 5.721960 & 1.471777 & -0.357817 \\ 6 & 6.645322 & 3.724551 & -0.020464 \\ 6 & 7.745926 & 3.404949 & -0.774498 \\ 6 & 7.847694 & 2.120764 & -1.348279 \\ 6 & 6.866737 & 1.181025 & -1.143208 \\ 1 & 2.397647 & 0.176171 & 0.576193 \\ 6 & -1.6270357 & 2.497527 & -2.297520 \\ 6 & -3.5390398 & -4.841018 & -0.605171 \\ 6 & -2.396339 & -2.829403 \\ 6 & & -2.479656 & 6.204892 \\ 6 & & -3.716376 & 0.417318 \\ 6 & -2.800471 & 1.114098\end{array}$




\begin{tabular}{|c|c|c|c|}
\hline & -5.515237 & -4.202330 & 0.150978 \\
\hline & 4.389704 & 4.109774 & 1.399340 \\
\hline ] & -2.153221 & -0.375431 & 5.076482 \\
\hline 1 & 5.287818 & -4.512416 & -2.021227 \\
\hline & 8.707442 & 1.873081 & -1.966893 \\
\hline & 3.310128 & -3.125216 & -2.667223 \\
\hline & -2.735628 & -5.821985 & -2.704230 \\
\hline & -0.412547 & -5.059832 & -2.718072 \\
\hline 1 & -0.497164 & -5.925652 & 3.897603 \\
\hline 1 & 6.953189 & 0.203428 & -1.610111 \\
\hline 1 & -0.710954 & -4.639646 & 1.824994 \\
\hline & 1.376978 & -3.622682 & -1.767285 \\
\hline & 8.531082 & 4.138775 & -0.940703 \\
\hline & 8.501843 & -1.743517 & 2.090856 \\
\hline & -5.015420 & -5.750766 & -1.738577 \\
\hline 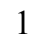 & -0.951348 & -4.862108 & 6.103590 \\
\hline & 8.807531 & -4.022608 & 1.126278 \\
\hline & 2.507913 & 2.481423 & 1.587831 \\
\hline & -4.412066 & 0.326191 & 1.612892 \\
\hline & -3.026236 & -0.538014 & -2.331472 \\
\hline ] & -7.180902 & -1.019335 & -1.370125 \\
\hline & -3.806449 & 2.517839 & -0.340815 \\
\hline$\frac{1}{1}$ & -0.267729 & 1.925675 & 1.991527 \\
\hline & -2.193305 & 5.757479 & 1.956718 \\
\hline l & 1.578417 & -3.545912 & 1.490365 \\
\hline ] & 2.995067 & -3.037559 & 0.584410 \\
\hline & 4.621280 & -2.655268 & 2.162890 \\
\hline$\frac{1}{1}$ & 0.735272 & -0.962857 & 2.799483 \\
\hline & 5.458797 & -1.512672 & 4.186662 \\
\hline$\frac{1}{1}$ & 1.546928 & 0.150989 & 4.850030 \\
\hline & 3.927669 & -0.097836 & 5.545961 \\
\hline ] & -1.536482 & 1.066098 & -2.524390 \\
\hline 1 & -1.463775 & 3.009756 & -2.365284 \\
\hline 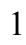 & -0.828903 & 2.245989 & -3.899307 \\
\hline 1 & -5.012117 & 7.145280 & 0.305393 \\
\hline 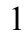 & -4.263129 & 6.605665 & 1.814209 \\
\hline 1 & -3.248996 & 7.061867 & 0.425165 \\
\hline ] & -4.964631 & 5.812698 & -1.737122 \\
\hline 1 & -3.204793 & 5.583631 & -1.661025 \\
\hline 1 & -4.283639 & 4.179421 & -1.780143 \\
\hline 1 & -6.423840 & 5.132895 & 0.230121 \\
\hline 1 & -5.725406 & 3.503168 & 0.254576 \\
\hline | & -5.684431 & 4.503013 & 1.717540 \\
\hline 1 & 0.098739 & 5.786501 & 4.769849 \\
\hline 1 & -1.050668 & 6.359003 & 3.563717 \\
\hline 1 & -1.490988 & 5.010121 & 4.630163 \\
\hline 1 & 1.795443 & 5.575755 & 2.883470 \\
\hline 1 & 1.481148 & 4.469034 & 1.525646 \\
\hline 1 & 0.580236 & 5.987652 & 1.652490 \\
\hline 1 & 1.296733 & 3.779882 & 4.613126 \\
\hline 1 & -0.151623 & 2.764134 & 4.455226 \\
\hline 1 & 1.165943 & 2.638894 & 3.276338 \\
\hline 1 & 0.771482 & 1.278161 & -4.775029 \\
\hline 1 & 2.774616 & 0.863626 & -6.105295 \\
\hline 1 & 5.020618 & 1.538618 & -5.262134 \\
\hline 1 & 5.194850 & 2.679758 & -3.052954 \\
\hline
\end{tabular}




$\begin{array}{rrrr}1 & 3.193478 & 3.101462 & -1.714707 \\ 1 & 1.467804 & 2.960671 & -0.345282 \\ 1 & 1.196570 & 4.362387 & -1.382970 \\ 1 & -0.175295 & 3.555878 & -0.595016 \\ 1 & -6.966792 & -1.809616 & -4.834529 \\ 1 & -7.203202 & -2.376944 & -3.175444 \\ 1 & -7.509221 & -0.673476 & -3.591166 \\ 1 & -5.144037 & -0.294623 & -5.453837 \\ 1 & -5.540338 & 0.869071 & -4.170815 \\ 1 & -3.900434 & 0.198838 & -4.293551 \\ 1 & -4.683602 & -2.712716 & -4.886492 \\ 1 & -3.470502 & -2.260544 & -3.675550 \\ 1 & -4.833137 & -3.290991 & -3.210834 \\ 1 & -9.058716 & 0.556911 & 1.434139 \\ 1 & -7.907347 & 1.567348 & 0.537352 \\ 1 & -8.638217 & 0.148829 & -0.234149 \\ 1 & -8.417797 & -1.772632 & 2.172118 \\ 1 & -7.918877 & -2.223411 & 0.531541 \\ 1 & -6.777749 & -2.387278 & 1.881825 \\ 1 & -7.521626 & 0.253440 & 3.279330 \\ 1 & -5.873365 & -0.354246 & 3.072458 \\ 1 & -6.298766 & 1.278807 & 2.509703\end{array}$

$\mathrm{TS1}_{\text {IIB2- }}-\psi_{-1 \mathrm{a} 2 \mathrm{~s} 334 \mathrm{~s}}$

Number of imaginary frequencies : 1

The smallest frequencies are : $-622.4962 \quad 16.2106 \quad 20.3640 \mathrm{~cm}(-1)$

Electronic energy : $\quad H F=-4360.8681433$

Zero-point correction $=\quad 1.452438($ Hartree/Particle $)$

Thermal correction to Energy= $\quad 1.537329$

Thermal correction to Enthalpy= $\quad 1.538273$

Thermal correction to Gibbs Free Energy= 1.333998

Sum of electronic and zero-point Energies $=\quad-4359.415705$

Sum of electronic and thermal Energies $=\quad-4359.330814$

Sum of electronic and thermal Enthalpies $=\quad-4359.329870$

Sum of electronic and thermal Free Energies $=\quad-4359.534145$

Cartesian Coordinates

$\begin{array}{cccc}6 & -1.088330 & 2.497809 & 1.761047 \\ 6 & -2.047043 & 2.018992 & 0.858269 \\ 6 & -3.010010 & 2.893202 & 0.366272 \\ 6 & -3.044294 & 4.236552 & 0.764330 \\ 6 & -2.099331 & 4.667697 & 1.692658 \\ 6 & -1.119285 & 3.810654 & 2.219546 \\ 15 & -1.866425 & 0.330068 & 0.154329 \\ 6 & -3.608844 & -0.070236 & -0.317032 \\ 6 & -3.902248 & -0.448617 & -1.621202 \\ 6 & -5.212285 & -0.728997 & -2.022081 \\ 6 & -6.220887 & -0.651725 & -1.059737 \\ 6 & -5.957059 & -0.301034 & 0.271388 \\ 6 & -4.640985 & -0.001608 & 0.620269 \\ 6 & -5.470133 & -1.101428 & -3.480662 \\ 6 & -7.038885 & -0.239973 & 1.349104 \\ 6 & -4.103207 & 5.157214 & 0.161473 \\ 6 & -4.035599 & 6.573707 & 0.726092\end{array}$




\begin{tabular}{|c|c|c|c|}
\hline 6 & -0.083083 & 4.337215 & 3.212739 \\
\hline 6 & -0.732495 & 5.266790 & 4.242628 \\
\hline 45 & -0.322313 & 0.366261 & -1.717397 \\
\hline & 1.499852 & -0.521681 & -0.646116 \\
\hline 8 & 2.386244 & 0.167481 & 0.575137 \\
\hline 6 & 3.483521 & 0.970498 & 0.452417 \\
\hline & 4.653908 & 0.541623 & -0.147874 \\
\hline & 5.700463 & 1.507685 & -0.332149 \\
\hline & 5.579377 & 2.806756 & 0.246564 \\
\hline 6 & 4.417713 & 3.127190 & 0.991334 \\
\hline 6 & 3.388494 & 2.232930 & 1.077622 \\
\hline & 6.847449 & 1.241103 & -1.122937 \\
\hline 6 & 7.821266 & 2.192059 & -1.309205 \\
\hline 6 & 7.709946 & 3.463761 & -0.710056 \\
\hline 6 & 6.606972 & 3.760157 & 0.049858 \\
\hline 6 & 4.811255 & -0.856678 & -0.608994 \\
\hline 6 & 3.871534 & -1.433863 & -1.441074 \\
\hline 6 & 4.062221 & -2.702838 & -2.029388 \\
\hline 6 & 5.154017 & -3.453505 & -1.692701 \\
\hline 6 & 6.078579 & -2.979551 & -0.729346 \\
\hline 6 & 5.912398 & -1.672695 & -0.179839 \\
\hline 6 & 7.148055 & -3.794443 & -0.285012 \\
\hline 6 & 8.010477 & -3.359396 & 0.688501 \\
\hline 6 & 7.830304 & -2.082244 & 1.259508 \\
\hline 6 & 6.815778 & -1.260395 & 0.833543 \\
\hline$\gamma$ & 2.712708 & -0.774397 & -1.765868 \\
\hline 6 & 0.561664 & 2.522778 & -2.235960 \\
\hline 6 & 0.731463 & 3.381746 & -1.008316 \\
\hline 6 & 1.761890 & 2.281876 & -3.075757 \\
\hline 6 & 3.032858 & 2.659008 & -2.619058 \\
\hline 6 & 4.174991 & 2.436399 & -3.382780 \\
\hline 6 & 4.080770 & 1.834016 & -4.630868 \\
\hline 6 & 2.823594 & 1.466811 & -5.109993 \\
\hline 6 & 1.687053 & 1.688915 & -4.348657 \\
\hline 6 & -0.737521 & 2.422066 & -2.768592 \\
\hline 6 & -0.284089 & -0.868606 & -3.192006 \\
\hline 8 & -0.229702 & -1.500735 & -4.165073 \\
\hline 6 & -1.722343 & -0.733177 & 1.675970 \\
\hline 6 & -1.370071 & -2.070981 & 1.560697 \\
\hline 6 & -1.276920 & -2.895011 & 2.732234 \\
\hline 6 & -1.558248 & -2.336558 & 4.012355 \\
\hline 6 & -1.952760 & -0.981978 & 4.090734 \\
\hline 6 & -2.030420 & -0.210892 & 2.963102 \\
\hline 6 & -1.431752 & -3.137586 & 5.171784 \\
\hline 6 & -1.040435 & -4.448728 & 5.080433 \\
\hline 6 & -0.765712 & -5.011599 & 3.816753 \\
\hline 6 & -0.885304 & -4.259026 & 2.674441 \\
\hline 6 & -1.122616 & -2.752960 & 0.258686 \\
\hline 6 & 0.131987 & -2.766763 & -0.322148 \\
\hline 6 & 0.399060 & -3.590169 & -1.439897 \\
\hline 6 & -0.581148 & -4.370755 & -1.989553 \\
\hline 6 & -1.890945 & -4.353346 & -1.456683 \\
\hline 6 & -2.170308 & -3.533354 & -0.322958 \\
\hline 6 & -3.490846 & -3.532519 & 0.197680 \\
\hline 6 & -4.471028 & -4.311469 & -0.365114 \\
\hline 6 & -4.183187 & -5.142193 & -1.469900 \\
\hline
\end{tabular}




\begin{tabular}{|c|c|c|c|}
\hline 6 & -2.921612 & -5.154076 & -2.007066 \\
\hline & 1.197610 & -1.961149 & 0.206303 \\
\hline & 2.152499 & -2.681062 & 1.080328 \\
\hline & 2.621948 & -1.954814 & 2.315702 \\
\hline & 3.949317 & -2.088313 & 2.717936 \\
\hline & 4.409212 & -1.467242 & 3.877392 \\
\hline & 3.540712 & -0.708002 & 4.652530 \\
\hline & 2.209703 & -0.573493 & 4.258369 \\
\hline & 1.756590 & -1.189454 & 3.099775 \\
\hline & -3.888846 & 5.235998 & -1.354929 \\
\hline & -5.499290 & 4.592996 & 0.449611 \\
\hline & 0.594759 & 3.202573 & 3.982860 \\
\hline & 0.983183 & 5.116801 & 2.433689 \\
\hline & 6.676891 & -0.289380 & 1.301847 \\
\hline & -2.337617 & 0.827134 & 3.058459 \\
\hline & 7.259918 & -4.784245 & -0.725845 \\
\hline & -1.648126 & -2.686061 & 6.139035 \\
\hline & 6.493049 & 4.742366 & 0.506963 \\
\hline & -3.725090 & -2.894620 & 1.049239 \\
\hline & -5.479302 & -4.289829 & 0.043091 \\
\hline & 4.347302 & 4.101502 & 1.473371 \\
\hline & -2.193617 & -0.558451 & 5.065244 \\
\hline & 5.313746 & -4.433409 & -2.139419 \\
\hline & 8.682749 & 1.963144 & -1.932628 \\
\hline & 3.326578 & -3.045373 & -2.753951 \\
\hline & -2.687365 & -5.779674 & -2.867419 \\
\hline & -0.369846 & -5.005740 & -2.848563 \\
\hline & -0.457947 & -6.052384 & 3.746239 \\
\hline & 6.940893 & 0.273997 & -1.609842 \\
\hline & -0.673299 & -4.712617 & 1.708312 \\
\hline & 1.407477 & -3.585695 & -1.851105 \\
\hline & 8.489559 & 4.206597 & -0.861711 \\
\hline & 8.497555 & -1.745903 & 2.050084 \\
\hline & -4.967284 & -5.762409 & -1.899147 \\
\hline & -0.941189 & -5.056048 & 5.977105 \\
\hline & 8.822099 & -3.997821 & 1.029512 \\
\hline & 2.474900 & 2.457652 & 1.624861 \\
\hline & -4.410964 & 0.293489 & 1.643907 \\
\hline . & -3.079126 & -0.528317 & -2.330019 \\
\hline & -7.243601 & -0.874789 & -1.350108 \\
\hline . & -3.746928 & 2.523683 & -0.346795 \\
\hline & -0.313774 & 1.814370 & 2.109982 \\
\hline & -2.115703 & 5.703883 & 2.020756 \\
\hline 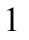 & 1.620107 & -3.589260 & 1.397359 \\
\hline & 3.026784 & -3.026978 & 0.508250 \\
\hline l & 4.639642 & -2.673750 & 2.108651 \\
\hline & 0.729018 & -1.050021 & 2.771084 \\
\hline . & 5.453600 & -1.574675 & 4.165637 \\
\hline & 1.516224 & 0.015797 & 4.857248 \\
\hline & 3.896947 & -0.219196 & 5.556915 \\
\hline & -1.565660 & 1.076011 & -2.501389 \\
\hline & -1.517690 & 3.014581 & -2.285557 \\
\hline & -0.878528 & 2.300693 & -3.842191 \\
\hline & -4.819602 & 7.190263 & 0.268683 \\
\hline 1 & -4.195398 & 6.590760 & 1.811974 \\
\hline 1 & -3.073290 & 7.056073 & 0.511517 \\
\hline
\end{tabular}




$\begin{array}{lrrr}1 & -4.620757 & 5.916430 & -1.810764 \\ 1 & -2.883963 & 5.613104 & -1.589390 \\ 1 & -4.003347 & 4.257004 & -1.837064 \\ 1 & -6.269515 & 5.237105 & 0.004347 \\ 1 & -5.630818 & 3.584327 & 0.037280 \\ 1 & -5.685923 & 4.539943 & 1.530330 \\ 1 & 0.016412 & 5.590995 & 4.976891 \\ 1 & -1.150669 & 6.173618 & 3.791173 \\ 1 & -1.538695 & 4.757088 & 4.785947 \\ 1 & 1.754045 & 5.505837 & 3.113805 \\ 1 & 1.475450 & 4.476473 & 1.688628 \\ 1 & 0.544013 & 5.967516 & 1.896503 \\ 1 & 1.276454 & 3.619565 & 4.734835 \\ 1 & -0.140333 & 2.574287 & 4.505359 \\ 1 & 1.193876 & 2.548177 & 3.337610 \\ 1 & 0.724357 & 1.373318 & -4.745882 \\ 1 & 2.727236 & 0.998918 & -6.087918 \\ 1 & 4.972464 & 1.655335 & -5.227668 \\ 1 & 5.145459 & 2.737271 & -2.988945 \\ 1 & 3.143335 & 3.120733 & -1.639597 \\ 1 & 1.411224 & 2.943928 & -0.270986 \\ 1 & 1.142306 & 4.368058 & -1.278885 \\ 1 & -0.231332 & 3.542903 & -0.513618 \\ 6 & -6.953473 & -1.308258 & -3.775251 \\ 6 & -4.953355 & 0.018674 & -4.392346 \\ 6 & -4.728373 & -2.400942 & -3.809100 \\ 6 & -8.414325 & -0.637588 & 0.820055 \\ 6 & -6.671157 & -1.201145 & 2.486393 \\ 6 & -7.132101 & 1.187982 & 1.898635 \\ 1 & -7.087173 & -1.564036 & -4.833953 \\ 1 & -7.378199 & -2.130185 & -3.184467 \\ 1 & -7.542059 & -0.402557 & -3.578281 \\ 1 & -5.151850 & -0.226394 & -5.444435 \\ 1 & -5.448989 & 0.972386 & -4.167594 \\ 1 & -3.870453 & 0.167977 & -4.289274 \\ 1 & -4.883758 & -2.673772 & -4.861984 \\ 1 & -3.646777 & -2.311532 & -3.642847 \\ 1 & -5.090622 & -3.226631 & -3.183357 \\ 1 & -7.887697 & 1.243648 & 2.694072 \\ 1 & -6.178712 & 1.530636 & 2.320221 \\ 1 & -7.421678 & 1.893935 & 1.108797 \\ 1 & -7.434661 & -1.170023 & 3.275480 \\ 1 & -6.607018 & -2.234353 & 2.120199 \\ 1 & -5.706701 & -0.953108 & 2.947808 \\ 1 & -9.150601 & -0.587413 & 1.632261 \\ 1 & -8.759185 & 0.034452 & 0.023696 \\ 1 & -8.422570 & -1.664319 & 0.431478\end{array}$

$\mathrm{TS1}_{\text {IIB2- }} \psi_{-1 \mathrm{~s} 2 \mathrm{a3a} a \mathrm{a}}$

Number of imaginary frequencies : 1

The smallest frequencies are : -615.8766 $9.1335 \quad 19.8714 \mathrm{~cm}(-1)$

Electronic energy : $\quad H F=-4360.8665113$

Zero-point correction $=\quad 1.452931($ Hartree/Particle $)$

Thermal correction to Energy= $\quad 1.537675$

Thermal correction to Enthalpy= $\quad 1.538619$ 
Thermal correction to Gibbs Free Energy= Sum of electronic and zero-point Energies= Sum of electronic and thermal Energies= Sum of electronic and thermal Enthalpies= Sum of electronic and thermal Free Energies=

\author{
1.334829 \\ $-4359.413580$ \\ $-4359.328837$ \\ $-4359.327893$ \\ $-4359.531683$
}

Cartesian Coordinates

\begin{tabular}{|c|c|c|c|}
\hline 6 & 1.646236 & 1.889900 & -4.315288 \\
\hline 6 & 1.744247 & 2.436498 & -3.023467 \\
\hline 6 & 3.020367 & 2.818711 & -2.585117 \\
\hline 6 & 4.147429 & 2.632532 & -3.380251 \\
\hline 6 & 4.031271 & 2.070883 & -4.645416 \\
\hline 6 & 2.767514 & 1.708361 & -5.109580 \\
\hline 6 & 0.562371 & 2.615983 & -2.143305 \\
\hline 6 & 0.744836 & 3.431165 & -0.888203 \\
\hline 45 & -0.243740 & 0.406909 & -1.682680 \\
\hline 6 & -0.746230 & 2.493032 & -2.645673 \\
\hline 15 & -1.832249 & 0.296784 & 0.183679 \\
\hline 6 & -3.536042 & -0.187724 & -0.351075 \\
\hline 6 & -3.735224 & -0.684655 & -1.634368 \\
\hline 6 & -5.013207 & -1.026465 & -2.088184 \\
\hline 6 & -6.080390 & -0.853173 & -1.206365 \\
\hline 6 & -5.916462 & -0.369033 & 0.098198 \\
\hline 6 & -4.625298 & -0.049601 & 0.513944 \\
\hline 6 & -5.256726 & -1.511057 & -3.516566 \\
\hline 6 & -7.127614 & -0.234991 & 1.020142 \\
\hline 6 & -1.706968 & -0.729978 & 1.735735 \\
\hline 6 & -1.331711 & -2.064857 & 1.654277 \\
\hline 6 & -1.238455 & -2.862692 & 2.841687 \\
\hline 6 & -1.540034 & -2.282493 & 4.107456 \\
\hline 6 & -1.959607 & -0.934146 & 4.152433 \\
\hline 6 & -2.043390 & -0.188940 & 3.007214 \\
\hline 6 & -0.833618 & -4.223402 & 2.812238 \\
\hline 6 & -0.720193 & -4.954060 & 3.969207 \\
\hline 6 & -1.014501 & -4.370129 & 5.219279 \\
\hline 6 & -1.418804 & -3.061207 & 5.282720 \\
\hline 6 & -1.076859 & -2.752512 & 0.358147 \\
\hline 6 & 0.163411 & -2.713407 & -0.252147 \\
\hline 6 & 0.414674 & -3.478686 & -1.413998 \\
\hline 6 & -0.566368 & -4.244869 & -1.981233 \\
\hline 6 & -1.858137 & -4.289051 & -1.408574 \\
\hline 6 & -2.118855 & -3.542280 & -0.221603 \\
\hline 6 & -2.892094 & -5.070294 & -1.981102 \\
\hline 6 & -4.138098 & -5.113876 & -1.409458 \\
\hline 6 & -4.405136 & -4.364632 & -0.242522 \\
\hline 6 & -3.421541 & -3.604563 & 0.338901 \\
\hline 7 & 1.223483 & -1.903270 & 0.281168 \\
\hline 15 & 1.570188 & -0.498733 & -0.613287 \\
\hline 8 & 2.487752 & 0.198379 & 0.578456 \\
\hline 6 & 3.588880 & 0.988414 & 0.407848 \\
\hline 6 & 4.741183 & 0.528303 & -0.202747 \\
\hline 6 & 5.791641 & 1.477424 & -0.442075 \\
\hline 6 & 5.699378 & 2.792600 & 0.105149 \\
\hline 6 & 4.560623 & 3.144939 & 0.870922 \\
\hline 6 & 3.523149 & 2.266226 & 1.002732 \\
\hline
\end{tabular}




\begin{tabular}{|c|c|c|c|}
\hline & 6.916664 & 1.174431 & -1.250508 \\
\hline & 7.897907 & 2.107254 & -1.483634 \\
\hline & 7.816317 & 3.395367 & -0.915294 \\
\hline & 6.734467 & 3.726523 & -0.139060 \\
\hline & 4.879606 & -0.891514 & -0.596775 \\
\hline & 3.921506 & -1.501872 & -1.382770 \\
\hline & 4.095654 & -2.800995 & -1.907189 \\
\hline & 5.184538 & -3.543693 & -1.544694 \\
\hline & 6.125001 & -3.030328 & -0.617957 \\
\hline & 5.978027 & -1.694464 & -0.136987 \\
\hline & 7.192220 & -3.831416 & -0.144201 \\
\hline & 8.070369 & -3.354434 & 0.794862 \\
\hline & 7.908739 & -2.047594 & 1.300191 \\
\hline & 6.897283 & -1.238679 & 0.843244 \\
\hline & 2.767220 & -0.845355 & -1.729121 \\
\hline & 2.147810 & -2.605570 & 1.202014 \\
\hline & 2.620775 & -1.821568 & 2.399676 \\
\hline & 1.749511 & -1.027674 & 3.147991 \\
\hline & 2.204089 & -0.329234 & 4.257715 \\
\hline & 3.542237 & -0.414641 & 4.641845 \\
\hline & 4.415406 & -1.206948 & 3.905556 \\
\hline & 3.954777 & -1.906846 & 2.792010 \\
\hline & -2.119696 & 1.991634 & 0.842548 \\
\hline & -1.249962 & 2.544562 & 1.781295 \\
\hline 6 & -1.393688 & 3.866393 & 2.218849 \\
\hline 0 & -2.393939 & 4.641797 & 1.630978 \\
\hline & -3.253203 & 4.135554 & 0.646503 \\
\hline 6 & -3.108386 & 2.801359 & 0.273550 \\
\hline & -0.496447 & 4.390178 & 3.339888 \\
\hline 6 & -0.675317 & 5.887511 & 3.578563 \\
\hline 5 & -4.308730 & 5.041925 & 0.016511 \\
\hline 6 & -3.608628 & 6.204411 & -0.695791 \\
\hline 6 & -0.126414 & -0.785067 & -3.184028 \\
\hline & 0.031537 & -1.414167 & -4.147410 \\
\hline 6 & -5.237693 & 5.591118 & 1.104365 \\
\hline 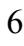 & -5.166738 & 4.301821 & -1.007951 \\
\hline 6 & 0.977208 & 4.132363 & 3.010679 \\
\hline 5 & -0.854899 & 3.648422 & 4.633841 \\
\hline & 6.774302 & -0.242919 & 1.261305 \\
\hline 1 & -2.381317 & 0.841337 & 3.077919 \\
\hline & 7.289320 & -4.843991 & -0.533838 \\
\hline 1 & -1.651362 & -2.594958 & 6.239181 \\
\hline & 6.644163 & 4.721566 & 0.295003 \\
\hline 1 & -3.641836 & -3.024923 & 1.233347 \\
\hline 1 & -5.400825 & -4.387953 & 0.195793 \\
\hline & 4.513032 & 4.130717 & 1.331878 \\
\hline 1 & -2.222355 & -0.494874 & 5.114399 \\
\hline & 5.329597 & -4.546326 & -1.943300 \\
\hline 1 & 8.742360 & 1.850936 & -2.119534 \\
\hline 1 & 3.353673 & -3.175835 & -2.608424 \\
\hline 1 & -2.672543 & -5.637237 & -2.884993 \\
\hline 1 & -0.368411 & -4.822040 & -2.883195 \\
\hline 1 & -0.402937 & -5.993281 & 3.921668 \\
\hline 1 & 6.987475 & 0.192467 & -1.711109 \\
\hline 1 & -0.609919 & -4.690864 & 1.855396 \\
\hline 1 & 1.408172 & -3.429933 & -1.854301 \\
\hline
\end{tabular}




\begin{tabular}{|c|c|c|c|}
\hline & 8.602172 & 4.123267 & -1.102974 \\
\hline ] & 8.588803 & -1.677009 & 2.064076 \\
\hline 1 & -4.923725 & -5.721792 & -1.853616 \\
\hline & -0.920712 & -4.960314 & 6.127908 \\
\hline & 8.880174 & -3.982004 & 1.159526 \\
\hline 1 & 2.627540 & 2.512696 & 1.566301 \\
\hline & -4.455299 & 0.333853 & 1.517533 \\
\hline | & -2.863342 & -0.802365 & -2.274020 \\
\hline & -7.085222 & -1.105683 & -1.544773 \\
\hline 1 & -3.770298 & 2.370137 & -0.473070 \\
\hline 1 & -0.447637 & 1.925550 & 2.189336 \\
\hline 1 & -2.513176 & 5.677772 & 1.940504 \\
\hline 1 & 1.586449 & -3.481343 & 1.557949 \\
\hline ] & 3.018849 & -3.002784 & 0.657813 \\
\hline & 4.649650 & -2.512425 & 2.207827 \\
\hline ] & 0.716405 & -0.927294 & 2.822986 \\
\hline & 5.464807 & -1.277048 & 4.186907 \\
\hline 1 & 1.506627 & 0.287827 & 4.823897 \\
\hline & 3.900800 & 0.137872 & 5.507840 \\
\hline 1 & -1.510469 & 1.094404 & -2.450512 \\
\hline & -1.534905 & 3.032791 & -2.115630 \\
\hline 1 & -0.910079 & 2.416756 & -3.720053 \\
\hline$\frac{1}{1}$ & -5.925540 & 4.982404 & -1.413980 \\
\hline & -4.571430 & 3.932501 & -1.853854 \\
\hline 1 & -5.692476 & 3.444989 & -0.563478 \\
\hline 1 & -4.346110 & 6.856101 & -1.183475 \\
\hline 1 & -3.026735 & 6.822389 & -0.001399 \\
\hline 1 & -2.920982 & 5.832708 & -1.467798 \\
\hline 1 & -5.997735 & 6.247720 & 0.660089 \\
\hline 1 & -5.757833 & 4.776088 & 1.624516 \\
\hline & -4.696100 & 6.176234 & 1.857085 \\
\hline 1 & -0.231511 & 4.002874 & 5.466158 \\
\hline 1 & -1.906395 & 3.814233 & 4.903001 \\
\hline 1 & -0.699095 & 2.565548 & 4.537416 \\
\hline 1 & 1.621183 & 4.563821 & 3.788587 \\
\hline 1 & 1.201295 & 3.059263 & 2.968422 \\
\hline 1 & 1.257399 & 4.584094 & 2.048297 \\
\hline | & 0.000912 & 6.216350 & 4.377561 \\
\hline 1 & -0.439757 & 6.474911 & 2.681573 \\
\hline 1 & -1.695991 & 6.136687 & 3.895103 \\
\hline 1 & 0.678812 & 1.574498 & -4.700941 \\
\hline 1 & 2.653915 & 1.273223 & -6.100660 \\
\hline 1 & 4.911455 & 1.919611 & -5.266329 \\
\hline 1 & 5.124081 & 2.926382 & -2.996351 \\
\hline 1 & 3.148428 & 3.246547 & -1.592871 \\
\hline 1 & 1.440814 & 2.975354 & -0.177464 \\
\hline 1 & 1.140989 & 4.430235 & -1.131590 \\
\hline 1 & -0.210957 & 3.566285 & -0.373176 \\
\hline 6 & -3.953122 & -1.881236 & -4.223687 \\
\hline 6 & -6.164559 & -2.744056 & -3.523052 \\
\hline 6 & -5.933259 & -0.382532 & -4.303705 \\
\hline 6 & -6.776103 & 0.464585 & 2.331762 \\
\hline 6 & -8.229199 & 0.579463 & 0.334212 \\
\hline 6 & -7.658783 & -1.635251 & 1.346733 \\
\hline 1 & -4.174660 & -2.270745 & -5.225533 \\
\hline 1 & -3.288627 & -1.016536 & -4.352163 \\
\hline
\end{tabular}


$\mathrm{TS}_{\text {IIB2-}} \psi_{-1 \mathrm{~s} 2 \mathrm{2a} a 4 \mathrm{~s}}$

Number of imaginary frequencies : 1

The smallest frequencies are : -609.2904 $13.7993 \quad 19.2016 \mathrm{~cm}(-1)$

Electronic energy : $\quad H F=-4360.8673325$

Zero-point correction $=\quad 1.453040($ Hartree/Particle $)$

Thermal correction to Energy= $\quad 1.537814$

Thermal correction to Enthalpy= $\quad 1.538758$

Thermal correction to Gibbs Free Energy $=\quad 1.334712$

Sum of electronic and zero-point Energies $=\quad-4359.414292$

Sum of electronic and thermal Energies $=\quad-4359.329519$

Sum of electronic and thermal Enthalpies $=\quad-4359.328575$

Sum of electronic and thermal Free Energies $=\quad-4359.532620$

\section{Cartesian Coordinates}

$\begin{array}{cccc}6 & 1.645235 & 2.021856 & -4.294642 \\ 6 & 1.756357 & 2.507066 & -2.979544 \\ 6 & 3.039823 & 2.850204 & -2.530161 \\ 6 & 4.160343 & 2.688208 & -3.339497 \\ 6 & 4.030461 & 2.193011 & -4.630911 \\ 6 & 2.760006 & 1.867205 & -5.103970 \\ 6 & 0.581130 & 2.665564 & -2.086328 \\ 6 & 0.779453 & 3.436939 & -0.806253 \\ 45 & -0.235675 & 0.452749 & -1.682695 \\ 6 & -0.731934 & 2.568444 & -2.582692 \\ 15 & -1.829170 & 0.293999 & 0.174306 \\ 6 & -3.522964 & -0.205366 & -0.374668 \\ 6 & -3.726643 & -0.663276 & -1.675511 \\ 6 & -5.000592 & -1.007378 & -2.127615 \\ 6 & -6.068327 & -0.890164 & -1.228155 \\ 6 & -5.895733 & -0.457717 & 0.088098 \\ 6 & -4.603455 & -0.119975 & 0.497873 \\ 6 & -5.250927 & -1.439659 & -3.571668 \\ 6 & -7.042379 & -0.327614 & 1.088489 \\ 6 & -1.709184 & -0.751372 & 1.714899 \\ 6 & -1.331381 & -2.084149 & 1.616615 \\ 6 & -1.244922 & -2.899771 & 2.792072 \\ 6 & -1.559227 & -2.340121 & 4.063893\end{array}$




\begin{tabular}{|c|c|c|c|}
\hline 6 & -1.983215 & -0.993766 & 4.125699 \\
\hline 6 & -2.057646 & -0.230622 & 2.991534 \\
\hline & -0.837310 & -4.259101 & 2.744635 \\
\hline & -0.732681 & -5.007611 & 3.890939 \\
\hline & -1.039377 & -4.443952 & 5.147323 \\
\hline & -1.446962 & -3.136980 & 5.227827 \\
\hline & -1.074797 & -2.749780 & 0.309872 \\
\hline & 0.163372 & -2.694364 & -0.303329 \\
\hline & 0.411427 & -3.433392 & -1.482762 \\
\hline & -0.571079 & -4.187350 & -2.063798 \\
\hline & -1.860678 & -4.247169 & -1.487476 \\
\hline & -2.117648 & -3.528988 & -0.282427 \\
\hline & -2.895774 & -5.016449 & -2.073915 \\
\hline & -4.138553 & -5.077377 & -1.496773 \\
\hline & -4.401813 & -4.356244 & -0.311513 \\
\hline & -3.417954 & -3.606338 & 0.282300 \\
\hline & 1.222922 & -1.893290 & 0.244878 \\
\hline & 1.573628 & -0.475497 & -0.626883 \\
\hline 8 & 2.485015 & 0.207029 & 0.578945 \\
\hline 6 & 3.599698 & 0.983062 & 0.433886 \\
\hline 6 & 4.749435 & 0.521209 & -0.180289 \\
\hline 6 & 5.811952 & 1.462080 & -0.397992 \\
\hline 6 & 5.735062 & 2.766438 & 0.176358 \\
\hline 6 & 4.599217 & 3.115958 & 0.947583 \\
\hline 6 & 3.549727 & 2.248507 & 1.057316 \\
\hline 0 & 6.933999 & 1.162494 & -1.211748 \\
\hline 6 & 7.926409 & 2.088318 & -1.424855 \\
\hline 0 & 7.860096 & 3.364913 & -0.829302 \\
\hline & 6.781633 & 3.692667 & -0.046939 \\
\hline 6 & 4.876913 & -0.892491 & -0.598900 \\
\hline f & 3.920296 & -1.480071 & -1.403411 \\
\hline 6 & 4.088599 & -2.770346 & -1.951098 \\
\hline 6 & 5.168523 & -3.528507 & -1.593436 \\
\hline 6 & 6.104754 & -3.040634 & -0.648828 \\
\hline 6 & 5.964565 & -1.712845 & -0.144467 \\
\hline 6 & 7.161016 & -3.859176 & -0.180502 \\
\hline 6 & 8.034406 & -3.406937 & 0.775188 \\
\hline 6 & 7.879000 & -2.108266 & 1.303238 \\
\hline 6 & 6.878519 & -1.282620 & 0.852037 \\
\hline 8 & 2.773328 & -0.807363 & -1.744117 \\
\hline 6 & 2.139504 & -2.607289 & 1.164121 \\
\hline 6 & 2.605142 & -1.840680 & 2.375932 \\
\hline 6 & 1.732870 & -1.049077 & 3.125648 \\
\hline 6 & 2.181183 & -0.371469 & 4.250777 \\
\hline 6 & 3.513731 & -0.475913 & 4.649497 \\
\hline 6 & 4.387908 & -1.265514 & 3.911536 \\
\hline 6 & 3.933656 & -1.944210 & 2.782334 \\
\hline 6 & -2.144951 & 1.976112 & 0.852694 \\
\hline 6 & -1.285477 & 2.523810 & 1.803523 \\
\hline 6 & -1.443186 & 3.837521 & 2.259372 \\
\hline 6 & -2.444887 & 4.613087 & 1.674468 \\
\hline 6 & -3.292013 & 4.114243 & 0.675488 \\
\hline 6 & -3.138155 & 2.784498 & 0.288991 \\
\hline 6 & -0.554370 & 4.351350 & 3.391420 \\
\hline U & -0.748767 & 5.842592 & 3.654083 \\
\hline 6 & -4.343484 & 5.025805 & 0.045523 \\
\hline
\end{tabular}




\begin{tabular}{|c|c|c|c|}
\hline 6 & -3.642206 & 6.219992 & -0.611401 \\
\hline 6 & -0.123134 & -0.705354 & -3.211575 \\
\hline & 0.031876 & -1.315658 & -4.187369 \\
\hline & -5.307822 & 5.527802 & 1.125316 \\
\hline & -5.162784 & 4.309043 & -1.025987 \\
\hline & 0.922550 & 4.112867 & 3.061234 \\
\hline & -0.908476 & 3.584642 & 4.671936 \\
\hline & 6.759547 & -0.293675 & 1.287111 \\
\hline & -2.398027 & 0.797847 & 3.075553 \\
\hline 1 & 7.253444 & -4.865236 & -0.587735 \\
\hline & -1.689302 & -2.686530 & 6.189390 \\
\hline 1 & 6.702777 & 4.679387 & 0.407850 \\
\hline & -3.635585 & -3.047167 & 1.190817 \\
\hline & -5.395594 & -4.392286 & 0.130174 \\
\hline & 4.563374 & 4.091683 & 1.430210 \\
\hline & -2.255989 & -0.570107 & 5.091870 \\
\hline & 5.309377 & -4.524502 & -2.009805 \\
\hline & 8.767992 & 1.835420 & -2.065913 \\
\hline & 3.350231 & -3.125965 & -2.665952 \\
\hline & -2.679398 & -5.561536 & -2.991922 \\
\hline & -0.375675 & -4.743337 & -2.979534 \\
\hline & -0.412943 & -6.045375 & 3.830003 \\
\hline & 6.993153 & 0.190079 & -1.693702 \\
\hline & -0.604424 & -4.710811 & 1.782412 \\
\hline & 1.403162 & -3.374396 & -1.925385 \\
\hline & 8.654788 & 4.087098 & -1.001219 \\
\hline & 8.554946 & -1.757602 & 2.080065 \\
\hline & -4.924723 & -5.677143 & -1.950899 \\
\hline & -0.952673 & -5.048253 & 6.047333 \\
\hline 1 & 8.835573 & -4.047977 & 1.135489 \\
\hline & 2.656305 & 2.496331 & 1.623781 \\
\hline & -4.439215 & 0.233189 & 1.515823 \\
\hline & -2.857998 & -0.746465 & -2.324795 \\
\hline & -7.068426 & -1.145971 & -1.572266 \\
\hline & -3.793974 & 2.358000 & -0.466267 \\
\hline & -0.478514 & 1.907106 & 2.205616 \\
\hline & -2.574822 & 5.643808 & 1.997083 \\
\hline & 1.574861 & -3.486819 & 1.505115 \\
\hline & 3.013964 & -2.998989 & 0.621587 \\
\hline & 4.629317 & -2.548532 & 2.197859 \\
\hline 1 & 0.704112 & -0.934960 & 2.791216 \\
\hline & 5.433362 & -1.349988 & 4.203493 \\
\hline & 1.483093 & 0.244368 & 4.817562 \\
\hline & 3.867293 & 0.060179 & 5.527782 \\
\hline & -1.499525 & 1.166391 & -2.432858 \\
\hline 1 & -1.513215 & 3.095206 & -2.029172 \\
\hline & -0.904846 & 2.527781 & -3.657513 \\
\hline 1 & -5.912544 & 4.996534 & -1.437250 \\
\hline & -4.537152 & 3.967626 & -1.861721 \\
\hline 1 & -5.697691 & 3.437706 & -0.623613 \\
\hline 1 & -4.376809 & 6.874538 & -1.099524 \\
\hline & -3.089720 & 6.826464 & 0.116222 \\
\hline 1 & -2.928296 & 5.882489 & -1.375162 \\
\hline & -6.060713 & 6.194540 & 0.683834 \\
\hline & -5.836082 & 4.690588 & 1.600453 \\
\hline & -4.791650 & 6.088373 & 1.913880 \\
\hline
\end{tabular}




$\begin{array}{rrrr}1 & -0.291071 & 3.931834 & 5.511715 \\ 1 & -1.962351 & 3.735034 & 4.940936 \\ 1 & -0.740994 & 2.505142 & 4.558343 \\ 1 & 1.560127 & 4.531975 & 3.851082 \\ 1 & 1.155166 & 3.042695 & 2.994793 \\ 1 & 1.202268 & 4.588548 & 2.110498 \\ 1 & -0.078874 & 6.164895 & 4.461045 \\ 1 & -0.515640 & 6.446938 & 2.767782 \\ 1 & -1.773209 & 6.076243 & 3.970377 \\ 1 & 0.672180 & 1.736521 & -4.689657 \\ 1 & 2.635821 & 1.482138 & -6.114325 \\ 1 & 4.905199 & 2.062701 & -5.264106 \\ 1 & 5.142775 & 2.949237 & -2.946533 \\ 1 & 3.178724 & 3.226852 & -1.518705 \\ 1 & 1.473680 & 2.949346 & -0.115466 \\ 1 & 1.186731 & 4.438656 & -1.018546 \\ 1 & -0.172042 & 3.565914 & -0.281687 \\ 6 & -3.948286 & -1.750615 & -4.308623 \\ 6 & -6.129120 & -2.692950 & -3.619350 \\ 6 & -5.963643 & -0.296919 & -4.304656 \\ 6 & -8.377199 & -0.788781 & 0.509902 \\ 6 & -6.739984 & -1.183113 & 2.324695 \\ 6 & -7.180725 & 1.142137 & 1.503580 \\ 1 & -4.173538 & -2.098772 & -5.324778 \\ 1 & -3.301005 & -0.868682 & -4.404319 \\ 1 & -3.374452 & -2.539416 & -3.801666 \\ 1 & -6.289391 & -3.000444 & -4.661326 \\ 1 & -5.648231 & -3.525354 & -3.089295 \\ 1 & -7.117816 & -2.531629 & -3.173402 \\ 1 & -6.155199 & -0.570947 & -5.351250 \\ 1 & -6.927323 & -0.055003 & -3.838669 \\ 1 & -5.351353 & 0.614618 & -4.299837 \\ 1 & -7.975974 & 1.257308 & 2.252701 \\ 1 & -6.252824 & 1.537490 & 1.936897 \\ 1 & -7.439485 & 1.768499 & 0.639214 \\ 1 & -7.551977 & -1.093275 & 3.058903 \\ 1 & -6.646901 & -2.242981 & 2.053166 \\ 1 & -5.808417 & -0.886271 & 2.823052 \\ 1 & -9.164214 & -0.685759 & 1.267754 \\ 1 & -8.678538 & -0.188994 & -0.358478 \\ 1 & -8.347567 & -1.843333 & 0.205823\end{array}$

$\operatorname{TS1}_{I I B 2-} \psi_{-1 \mathrm{1s2} 2354 a}$

Number of imaginary frequencies : 1

The smallest frequencies are : $-595.6193 \quad 13.5603 \quad 15.9642 \mathrm{~cm}(-1)$

Electronic energy : $\quad \mathrm{HF}=-4360.866092$

Zero-point correction $=\quad 1.452536($ Hartree/Particle $)$

Thermal correction to Energy $=\quad 1.537431$

Thermal correction to Enthalpy= $\quad 1.538375$

Thermal correction to Gibbs Free Energy $=\quad 1.333526$

Sum of electronic and zero-point Energies $=\quad-4359.413556$

Sum of electronic and thermal Energies $=\quad-4359.328661$

Sum of electronic and thermal Enthalpies $=\quad-4359.327717$

Sum of electronic and thermal Free Energies $=\quad-4359.532566$ 
Cartesian Coordinates

\begin{tabular}{|c|c|c|c|}
\hline 6 & 1.745720 & 1.942284 & -4.223363 \\
\hline 6 & 1.834283 & 2.468629 & -2.922265 \\
\hline 6 & 3.109407 & 2.825339 & -2.460665 \\
\hline 6 & 4.244615 & 2.638290 & -3.244762 \\
\hline 6 & 4.137498 & 2.096091 & -4.518870 \\
\hline 6 & 2.875055 & 1.756692 & -5.004786 \\
\hline 6 & 0.640407 & 2.664700 & -2.060523 \\
\hline 6 & 0.826759 & 3.430491 & -0.775340 \\
\hline 45 & -0.265183 & 0.492674 & -1.681230 \\
\hline 6 & -0.660796 & 2.616719 & -2.595449 \\
\hline 15 & -1.849819 & 0.346341 & 0.174336 \\
\hline 6 & -3.559396 & -0.127709 & -0.348054 \\
\hline 6 & -3.813201 & -0.435806 & -1.674391 \\
\hline 6 & -5.100009 & -0.781107 & -2.111384 \\
\hline 6 & -6.111383 & -0.842248 & -1.158715 \\
\hline 6 & -5.886831 & -0.556625 & 0.199788 \\
\hline 6 & -4.603816 & -0.187436 & 0.584517 \\
\hline 6 & -5.318935 & -1.059577 & -3.595897 \\
\hline 6 & -7.037352 & -0.673893 & 1.198255 \\
\hline 6 & -1.713491 & -0.730860 & 1.687799 \\
\hline 6 & -1.348182 & -2.063775 & 1.554901 \\
\hline 6 & -1.255200 & -2.905402 & 2.712763 \\
\hline 6 & -1.550320 & -2.370490 & 3.999914 \\
\hline 6 & -1.963240 & -1.022564 & 4.095926 \\
\hline 6 & -2.042779 & -0.234777 & 2.979560 \\
\hline 6 & -0.855964 & -4.266101 & 2.633830 \\
\hline 6 & -0.741131 & -5.037800 & 3.763690 \\
\hline 6 & -1.028705 & -4.498246 & 5.034824 \\
\hline 6 & -1.428205 & -3.191151 & 5.146200 \\
\hline 6 & -1.097914 & -2.714446 & 0.238293 \\
\hline 6 & 0.150216 & -2.687512 & -0.356048 \\
\hline 6 & 0.411205 & -3.457907 & -1.512364 \\
\hline 6 & -0.568095 & -4.220402 & -2.087920 \\
\hline 6 & -1.870595 & -4.244415 & -1.538349 \\
\hline 6 & -2.143115 & -3.484653 & -0.362168 \\
\hline 6 & -2.900579 & -5.025185 & -2.117537 \\
\hline 6 & -4.158375 & -5.046103 & -1.571377 \\
\hline 6 & -4.439777 & -4.275371 & -0.422489 \\
\hline 6 & -3.457218 & -3.524130 & 0.173066 \\
\hline 7 & 1.213598 & -1.893824 & 0.196136 \\
\hline 15 & 1.546238 & -0.455004 & -0.646539 \\
\hline 8 & 2.452265 & 0.216165 & 0.568953 \\
\hline 6 & 3.589423 & 0.964673 & 0.459522 \\
\hline 6 & 4.735677 & 0.496966 & -0.156683 \\
\hline 6 & 5.822214 & 1.419809 & -0.330800 \\
\hline 6 & 5.769085 & 2.706276 & 0.284834 \\
\hline 6 & 4.631131 & 3.057693 & 1.051925 \\
\hline 6 & 3.560862 & 2.211923 & 1.120401 \\
\hline 6 & 6.945414 & 1.122282 & -1.144069 \\
\hline 6 & 7.960247 & 2.031822 & -1.317604 \\
\hline 6 & 7.917220 & 3.289048 & -0.680214 \\
\hline 6 & 6.838601 & 3.615114 & 0.102564 \\
\hline 6 & 4.839198 & -0.901589 & -0.629332 \\
\hline 6 & 3.880715 & -1.439269 & -1.465820 \\
\hline
\end{tabular}




\begin{tabular}{|c|c|c|c|}
\hline 6 & 4.035343 & -2.706276 & -2.069379 \\
\hline & 5.100575 & -3.496051 & -1.735646 \\
\hline & 6.033847 & -3.065094 & -0.760779 \\
\hline & 5.909159 & -1.758273 & -0.201052 \\
\hline & 7.072523 & -3.919609 & -0.317481 \\
\hline & 7.942713 & -3.522445 & 0.665264 \\
\hline & 7.802270 & -2.244929 & 1.246715 \\
\hline & 6.819475 & -1.385139 & 0.821366 \\
\hline & 2.742173 & -0.738755 & -1.779296 \\
\hline & 2.139538 & -2.622255 & 1.093338 \\
\hline & 2.597430 & -1.886498 & 2.327375 \\
\hline & 1.729893 & -1.091835 & 3.079333 \\
\hline & 2.174211 & -0.446795 & 4.225129 \\
\hline & 3.497292 & -0.588405 & 4.643405 \\
\hline & 4.366288 & -1.382276 & 3.903939 \\
\hline & 3.916261 & -2.027505 & 2.753694 \\
\hline y & -2.132276 & 2.022253 & 0.879673 \\
\hline & -1.265597 & 2.545420 & 1.837647 \\
\hline D & -1.395988 & 3.860556 & 2.297934 \\
\hline & -2.375134 & 4.661384 & 1.709246 \\
\hline & -3.228372 & 4.186266 & 0.703387 \\
\hline & -3.105012 & 2.854128 & 0.314620 \\
\hline & -0.501847 & 4.349886 & 3.436751 \\
\hline 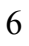 & -0.669458 & 5.842573 & 3.709454 \\
\hline & -4.253218 & 5.126386 & 0.071317 \\
\hline b & -3.519615 & 6.308074 & -0.572686 \\
\hline & -0.214041 & -0.676690 & -3.205137 \\
\hline & -0.122703 & -1.283637 & -4.191430 \\
\hline & -5.212571 & 5.643594 & 1.148494 \\
\hline 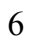 & -5.082675 & 4.437108 & -1.010705 \\
\hline & 0.971381 & 4.086126 & 3.109543 \\
\hline f & -0.873362 & 3.580901 & 4.710939 \\
\hline & 6.711573 & -0.413366 & 1.296160 \\
\hline & -2.376195 & 0.793801 & 3.087873 \\
\hline & 7.154005 & -4.908649 & -0.766605 \\
\hline & -1.655665 & -2.758318 & 6.119423 \\
\hline 1 & 6.777201 & 4.587884 & 0.589019 \\
\hline & -3.687527 & -2.933090 & 1.058595 \\
\hline 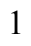 & -5.445177 & -4.277347 & -0.005478 \\
\hline & 4.610254 & 4.018610 & 1.564294 \\
\hline & -2.222016 & -0.617912 & 5.074000 \\
\hline 1 & 5.231573 & -4.474604 & -2.194551 \\
\hline & 8.801568 & 1.781397 & -1.959995 \\
\hline 1 & 3.297368 & -3.015846 & -2.806221 \\
\hline & -2.670528 & -5.604578 & -3.010834 \\
\hline 1 & -0.360803 & -4.810323 & -2.979435 \\
\hline & -0.427780 & -6.075751 & 3.677661 \\
\hline ] & 6.986571 & 0.166567 & -1.659945 \\
\hline 1 & -0.636278 & -4.700977 & 1.660797 \\
\hline 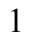 & 1.414234 & -3.426798 & -1.932898 \\
\hline 1 & 8.729495 & 3.998333 & -0.820858 \\
\hline ( & 8.475412 & -1.938508 & 2.044390 \\
\hline 1 & -4.943375 & -5.646890 & -2.025744 \\
\hline ] & -0.933509 & -5.120805 & 5.921419 \\
\hline 1 & 8.730019 & -4.190953 & 1.005614 \\
\hline 1 & 2.664727 & 2.464996 & 1.679933 \\
\hline
\end{tabular}




\begin{tabular}{|c|c|c|c|}
\hline & -4.395716 & 0.054857 & 1.624040 \\
\hline & -2.981771 & -0.408733 & -2.377285 \\
\hline & -7.116810 & -1.121893 & -1.468371 \\
\hline & -3.765848 & 2.443289 & -0.445469 \\
\hline & -0.474395 & 1.909322 & 2.240646 \\
\hline & -2.482064 & 5.693940 & 2.034341 \\
\hline & 1.586048 & -3.518064 & 1.409506 \\
\hline & 3.018063 & -2.988028 & 0.539813 \\
\hline & 4.608205 & -2.635683 & 2.168959 \\
\hline & 0.709119 & -0.949128 & 2.731523 \\
\hline & 5.404998 & -1.495369 & 4.210111 \\
\hline & 1.480598 & 0.173936 & 4.792256 \\
\hline & 3.847823 & -0.077291 & 5.537646 \\
\hline & -1.500145 & 1.253515 & -2.433372 \\
\hline & -1.435365 & 3.179583 & -2.068839 \\
\hline & -0.802925 & 2.574504 & -3.674855 \\
\hline & -5.803784 & 5.149845 & -1.430227 \\
\hline & -4.459528 & 4.074820 & -1.839707 \\
\hline & -5.652362 & 3.585287 & -0.615941 \\
\hline & -4.235866 & 6.984996 & -1.057578 \\
\hline & -2.954711 & 6.894408 & 0.161873 \\
\hline & -2.811617 & 5.959584 & -1.336971 \\
\hline & -5.952200 & 6.324292 & 0.705854 \\
\hline & -5.755934 & 4.814638 & 1.620447 \\
\hline [ & -4.689076 & 6.193699 & 1.939594 \\
\hline & -0.253440 & 3.912622 & 5.555104 \\
\hline 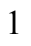 & -1.925610 & 3.746410 & 4.977188 \\
\hline & -0.722499 & 2.499576 & 4.591030 \\
\hline & 1.614683 & 4.490974 & 3.902267 \\
\hline 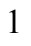 & 1.184387 & 3.012010 & 3.040628 \\
\hline & 1.262177 & 4.559265 & 2.160828 \\
\hline ] & 0.002235 & 6.146213 & 4.522142 \\
\hline & -0.420340 & 6.449008 & 2.828953 \\
\hline . & -1.691016 & 6.093010 & 4.022231 \\
\hline & 0.778946 & 1.646160 & -4.625647 \\
\hline & 2.769294 & 1.337482 & -6.003586 \\
\hline 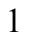 & 5.023432 & 1.943559 & -5.131276 \\
\hline & 5.219366 & 2.917855 & -2.846354 \\
\hline 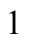 & 3.230011 & 3.237979 & -1.461039 \\
\hline & 1.502472 & 2.930173 & -0.075062 \\
\hline | & 1.250986 & 4.427495 & -0.976549 \\
\hline . & -0.132109 & 3.570298 & -0.266840 \\
\hline 5 & -6.771960 & -1.400579 & -3.914200 \\
\hline 5 & -4.927854 & 0.184900 & -4.402876 \\
\hline 5 & -4.442195 & -2.238765 & -4.029198 \\
\hline 5 & -6.608409 & -0.312948 & 2.619012 \\
\hline 6 & -8.175224 & 0.267031 & 0.788969 \\
\hline 6 & -7.549218 & -2.118585 & 1.214335 \\
\hline 1 & -6.880171 & -1.590522 & -4.989528 \\
\hline 1 & -7.103957 & -2.304313 & -3.386369 \\
\hline 1 & -7.453563 & -0.579815 & -3.654819 \\
\hline 1 & -5.099772 & 0.013798 & -5.474144 \\
\hline 1 & -5.522109 & 1.056912 & -4.098532 \\
\hline & -3.867095 & 0.438791 & -4.275813 \\
\hline 1 & -4.580545 & -2.442494 & -5.099900 \\
\hline 1 & -3.374388 & -2.045370 & -3.862761 \\
\hline
\end{tabular}


TS1 $_{\text {IIB2- }} \psi_{-1 \mathrm{~s} 2 \mathrm{a} 3 \mathrm{~s} 4 \mathrm{~s}}$

$\begin{array}{lcc}\text { Number of imaginary frequencies : } 1 & & \\ \text { The smallest frequencies are : }-608.1233 & 14.8767 & 18.8832 \mathrm{~cm}(-1) \\ \text { Electronic energy : HF }=-4360.8665962 & \\ \text { Zero-point correction= } & 1.452693 \text { (Hartree/Particle) } \\ \text { Thermal correction to Energy= } & 1.537498 \\ \text { Thermal correction to Enthalpy= } & 1.538443 \\ \text { Thermal correction to Gibbs Free Energy= } & 1.334367 \\ \text { Sum of electronic and zero-point Energies }= & -4359.413903 \\ \text { Sum of electronic and thermal Energies }= & -4359.329098 \\ \text { Sum of electronic and thermal Enthalpies }= & -4359.328154 \\ \text { Sum of electronic and thermal Free Energies }= & -4359.532229\end{array}$

Cartesian Coordinates

$\begin{array}{cccc}6 & 1.777431 & 1.869510 & -4.250604 \\ 6 & 1.844350 & 2.427478 & -2.961532 \\ 6 & 3.109465 & 2.811789 & -2.494479 \\ 6 & 4.256114 & 2.618535 & -3.259835 \\ 6 & 4.170765 & 2.044211 & -4.521472 \\ 6 & 2.918134 & 1.679114 & -5.014069 \\ 6 & 0.639172 & 2.630186 & -2.117633 \\ 6 & 0.805060 & 3.425700 & -0.847847 \\ 45 & -0.254963 & 0.459537 & -1.704811 \\ 6 & -0.655570 & 2.560977 & -2.666439 \\ 15 & -1.847922 & 0.355534 & 0.147808 \\ 6 & -3.549267 & -0.143185 & -0.377612 \\ 6 & -3.781017 & -0.546361 & -1.687125 \\ 6 & -5.051475 & -0.947511 & -2.110396 \\ 6 & -6.084702 & -0.942238 & -1.169699 \\ 6 & -5.885675 & -0.546103 & 0.158557 \\ 6 & -4.604762 & -0.144356 & 0.534430 \\ 6 & -5.269945 & -1.319487 & -3.575698 \\ 6 & -7.001109 & -0.532877 & 1.203131 \\ 6 & -1.722760 & -0.686179 & 1.688859 \\ 6 & -1.358707 & -2.022904 & 1.593375 \\ 6 & -1.273241 & -2.833738 & 2.773558 \\ 6 & -1.575852 & -2.265401 & 4.044377 \\ 6 & -1.987081 & -0.915028 & 4.102526 \\ 6 & -2.058791 & -0.156516 & 2.965425 \\ 6 & -0.875053 & -4.196313 & 2.732656 \\ 6 & -0.768959 & -4.938761 & 3.882840 \\ 6 & -1.064713 & -4.366218 & 5.137671 \\ 6 & -1.462846 & -3.056070 & 5.212374\end{array}$




\begin{tabular}{|c|c|c|c|}
\hline ) & -1.104325 & -2.707783 & 0.295118 \\
\hline & 0.143083 & -2.689294 & -0.301264 \\
\hline & 0.404914 & -3.483492 & -1.441250 \\
\hline & -0.571212 & -4.264670 & -1.996883 \\
\hline & -1.871548 & -4.285791 & -1.442076 \\
\hline & -2.146099 & -3.499173 & -0.284180 \\
\hline & -2.898105 & -5.087621 & -1.998768 \\
\hline & -4.156515 & -5.098562 & -1.452766 \\
\hline & -4.438992 & -4.302350 & -0.321765 \\
\hline 0 & -3.459009 & -3.532369 & 0.253973 \\
\hline & 1.206141 & -1.883697 & 0.234487 \\
\hline 5 & 1.548593 & -0.466248 & -0.639861 \\
\hline & 2.449722 & 0.229515 & 0.564276 \\
\hline & 3.587539 & 0.974998 & 0.442891 \\
\hline & 4.736365 & 0.493050 & -0.157726 \\
\hline & 5.823794 & 1.411907 & -0.346956 \\
\hline 6 & 5.768412 & 2.711042 & 0.241386 \\
\hline 6 & 4.627125 & 3.079549 & 0.995432 \\
\hline 6 & 3.555795 & 2.236284 & 1.076327 \\
\hline 6 & 6.950513 & 1.097098 & -1.148783 \\
\hline 6 & 7.966672 & 2.002317 & -1.336401 \\
\hline 6 & 7.921462 & 3.272508 & -0.725423 \\
\hline 6 & 6.839358 & 3.615330 & 0.045312 \\
\hline 6 & 4.841511 & -0.916452 & -0.596771 \\
\hline 6 & 3.885049 & -1.473772 & -1.422662 \\
\hline 6 & 4.038136 & -2.756200 & -1.992762 \\
\hline 6 & 5.100566 & -3.539335 & -1.635742 \\
\hline 6 & 6.032485 & -3.084748 & -0.670329 \\
\hline 6 & 5.909307 & -1.763668 & -0.144529 \\
\hline 6 & 7.068432 & -3.929689 & -0.203187 \\
\hline 6 & 7.937679 & -3.509242 & 0.770663 \\
\hline 6 & 7.799043 & -2.216816 & 1.318586 \\
\hline 6 & 6.818769 & -1.366218 & 0.869496 \\
\hline 8 & 2.749573 & -0.779089 & -1.759269 \\
\hline 6 & 2.123647 & -2.592548 & 1.156275 \\
\hline 6 & 2.581915 & -1.825481 & 2.370963 \\
\hline 6 & 1.714798 & -1.009975 & 3.100962 \\
\hline 6 & 2.158908 & -0.335243 & 4.229517 \\
\hline 6 & 3.481432 & -0.467736 & 4.652728 \\
\hline 6 & 4.349867 & -1.282367 & 3.935555 \\
\hline 6 & 3.900119 & -1.957005 & 2.802067 \\
\hline 6 & -2.147210 & 2.043630 & 0.815375 \\
\hline 6 & -1.285052 & 2.593952 & 1.762096 \\
\hline 6 & -1.427658 & 3.916328 & 2.196986 \\
\hline 6 & -2.415436 & 4.695723 & 1.593743 \\
\hline 6 & -3.263745 & 4.193037 & 0.597407 \\
\hline 6 & -3.128306 & 2.854740 & 0.234868 \\
\hline 6 & -0.537713 & 4.436178 & 3.325489 \\
\hline 6 & -0.709672 & 5.934964 & 3.559532 \\
\hline 6 & -4.297362 & 5.110615 & -0.052785 \\
\hline 6 & -3.576493 & 6.294520 & -0.706920 \\
\hline 6 & -0.172962 & -0.746976 & -3.196716 \\
\hline 8 & -0.041467 & -1.384600 & -4.158907 \\
\hline 6 & -5.271491 & 5.624722 & 1.012369 \\
\hline 6 & -5.108486 & 4.396876 & -1.132418 \\
\hline 6 & 0.937400 & 4.167656 & 3.010418 \\
\hline
\end{tabular}




\begin{tabular}{|c|c|c|c|}
\hline 6 & -0.911798 & 3.698465 & 4.617351 \\
\hline 1 & 6.712534 & -0.382039 & 1.318382 \\
\hline & -2.390280 & 0.875106 & 3.045701 \\
\hline & 7.148498 & -4.930168 & -0.626472 \\
\hline & -1.696251 & -2.597765 & 6.172445 \\
\hline & 6.776231 & 4.598103 & 0.510985 \\
\hline & -3.691961 & -2.916696 & 1.121986 \\
\hline & -5.444447 & -4.296895 & 0.095229 \\
\hline & 4.604745 & 4.051240 & 1.486962 \\
\hline & -2.250734 & -0.484422 & 5.068156 \\
\hline & 5.230508 & -4.529843 & -2.068498 \\
\hline 1 & 8.810797 & 1.738232 & -1.969573 \\
\hline & 3.301510 & -3.083039 & -2.723498 \\
\hline & -2.666113 & -5.688135 & -2.877559 \\
\hline & -0.362433 & -4.872925 & -2.875638 \\
\hline & -0.456246 & -5.978892 & 3.825855 \\
\hline & 6.993695 & 0.130671 & -1.644188 \\
\hline & -0.649510 & -4.656452 & 1.772698 \\
\hline & 1.406334 & -3.455183 & -1.865712 \\
\hline & 8.734838 & 3.978289 & -0.876919 \\
\hline & 8.471672 & -1.891149 & 2.109043 \\
\hline & -4.940132 & -5.711734 & -1.892813 \\
\hline & -0.976743 & -4.965737 & 6.040748 \\
\hline & 8.722852 & -4.170491 & 1.129650 \\
\hline ] & 2.656941 & 2.501116 & 1.626263 \\
\hline & -4.421464 & 0.174520 & 1.560390 \\
\hline 1 & -2.938003 & -0.552841 & -2.376241 \\
\hline & -7.076387 & -1.261798 & -1.476992 \\
\hline & -3.785055 & 2.423934 & -0.517482 \\
\hline & -0.487396 & 1.973170 & 2.176048 \\
\hline & -2.533093 & 5.733213 & 1.898850 \\
\hline ] & 1.562589 & -3.475360 & 1.495026 \\
\hline & 3.001690 & -2.980077 & 0.616851 \\
\hline 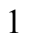 & 4.591759 & -2.581376 & 2.234259 \\
\hline 1 & 0.694539 & -0.875267 & 2.748547 \\
\hline I & 5.388009 & -1.388965 & 4.245938 \\
\hline 1 & 1.465294 & 0.300823 & 4.779441 \\
\hline & 3.831815 & 0.066231 & 5.533580 \\
\hline ] & -1.489645 & 1.197722 & -2.482614 \\
\hline & -1.439467 & 3.131327 & -2.161855 \\
\hline ] & -0.784117 & 2.494857 & -3.746280 \\
\hline 1 & -5.837298 & 5.093543 & -1.565374 \\
\hline & -4.473242 & 4.034671 & -1.952107 \\
\hline 1 & -5.668011 & 3.541209 & -0.731579 \\
\hline & -4.298635 & 6.952784 & -1.208406 \\
\hline 1 & -3.027567 & 6.900801 & 0.023467 \\
\hline & -2.856234 & 5.945246 & -1.459484 \\
\hline 1 & -6.016531 & 6.292080 & 0.558575 \\
\hline l & -5.807701 & 4.791987 & 1.486462 \\
\hline 1 & -4.760714 & 6.187534 & 1.802917 \\
\hline 1 & -0.296344 & 4.053645 & 5.455245 \\
\hline . & -1.965648 & 3.867113 & 4.875185 \\
\hline 1 & -0.756717 & 2.614932 & 4.525401 \\
\hline & 1.576413 & 4.594064 & 3.795215 \\
\hline & 1.153755 & 3.092735 & 2.969552 \\
\hline & 1.230991 & 4.617725 & 2.051435 \\
\hline
\end{tabular}




$\begin{array}{lrrr}1 & -0.039290 & 6.261665 & 4.364321 \\ 1 & -0.462019 & 6.518934 & 2.663563 \\ 1 & -1.731982 & 6.190781 & 3.865393 \\ 1 & 0.819022 & 1.551946 & -4.656624 \\ 1 & 2.829204 & 1.234948 & -6.003687 \\ 1 & 5.065809 & 1.886661 & -5.119182 \\ 1 & 5.222728 & 2.918388 & -2.856396 \\ 1 & 3.213129 & 3.252006 & -1.505030 \\ 1 & 1.477451 & 2.947292 & -0.128813 \\ 1 & 1.222374 & 4.421880 & -1.066797 \\ 1 & -0.160426 & 3.568180 & -0.353198 \\ 6 & -6.600173 & -2.037290 & -3.794911 \\ 6 & -5.262503 & -0.028991 & -4.404359 \\ 6 & -4.153305 & -2.241627 & -4.074560 \\ 6 & -8.319824 & -1.073114 & 0.656037 \\ 6 & -6.595914 & -1.402162 & 2.399840 \\ 6 & -7.231114 & 0.908758 & 1.672262 \\ 1 & -6.691927 & -2.333954 & -4.847405 \\ 1 & -6.669184 & -2.947579 & -3.183211 \\ 1 & -7.463178 & -1.400043 & -3.563793 \\ 1 & -5.425419 & -0.251922 & -5.467845 \\ 1 & -6.054002 & 0.657359 & -4.074954 \\ 1 & -4.302474 & 0.497030 & -4.314223 \\ 1 & -4.336250 & -2.516794 & -5.121906 \\ 1 & -3.162251 & -1.773314 & -4.031378 \\ 1 & -4.111741 & -3.163449 & -3.480140 \\ 1 & -8.010189 & 0.941892 & 2.446014 \\ 1 & -6.321502 & 1.355641 & 2.093450 \\ 1 & -7.557592 & 1.542831 & 0.837526 \\ 1 & -7.382978 & -1.386311 & 3.165859 \\ 1 & -6.447676 & -2.446436 & 2.093889 \\ 1 & -5.667063 & -1.060370 & 2.873830 \\ 1 & -9.081923 & -1.055977 & 1.445465 \\ 1 & -8.698883 & -0.468579 & -0.177860 \\ 1 & -8.224200 & -2.111686 & 0.312214\end{array}$

$\mathrm{TS1}_{\text {IIB2-}}-\psi_{-1 \mathrm{~s} 2 \mathrm{s3} 34 \mathrm{a}}$

Number of imaginary frequencies : 1

The smallest frequencies are : $-633.5688 \quad 15.2467 \quad 18.1355 \mathrm{~cm}(-1)$

Electronic energy : $\quad \mathrm{HF}=-4360.8672471$

Zero-point correction $=\quad 1.452855($ Hartree/Particle $)$

Thermal correction to Energy $=\quad 1.537477$

Thermal correction to Enthalpy= $\quad 1.538421$

Thermal correction to Gibbs Free Energy $=\quad 1.335501$

Sum of electronic and zero-point Energies $=\quad-4359.414393$

Sum of electronic and thermal Energies $=\quad-4359.329770$

Sum of electronic and thermal Enthalpies $=\quad-4359.328826$

Sum of electronic and thermal Free Energies $=\quad-4359.531746$

Cartesian Coordinates

$\begin{array}{llll}6 & 6.958786 & 1.103754 & -1.208345 \\ 6 & 5.824702 & 1.450678 & -0.430094 \\ 6 & 5.751225 & 2.781487 & 0.080597 \\ 6 & 6.811376 & 3.686259 & -0.165326\end{array}$




\begin{tabular}{|c|c|c|c|}
\hline 6 & 7.900749 & 3.312566 & -0.910874 \\
\hline 6 & 7.964456 & 2.009186 & -1.445266 \\
\hline 6 & 4.747872 & 0.530441 & -0.189875 \\
\hline 6 & 3.592220 & 1.032975 & 0.380545 \\
\hline 6 & 3.543656 & 2.328443 & 0.938884 \\
\hline 6 & 4.603583 & 3.179683 & 0.809540 \\
\hline 6 & 4.866856 & -0.898656 & -0.556379 \\
\hline 6 & 3.912302 & -1.504786 & -1.349511 \\
\hline 6 & 4.071547 & -2.813572 & -1.853569 \\
\hline 6 & 5.141183 & -3.569784 & -1.462321 \\
\hline 6 & 6.074944 & -3.058784 & -0.527212 \\
\hline 6 & 5.944693 & -1.713145 & -0.068887 \\
\hline 6 & 7.119977 & -3.871243 & -0.023911 \\
\hline 6 & 7.992303 & -3.395069 & 0.920936 \\
\hline 6 & 7.848031 & -2.076939 & 1.401874 \\
\hline 6 & 6.858384 & -1.257662 & 0.916763 \\
\hline 8 & 2.770696 & -0.837072 & -1.717039 \\
\hline 15 & 1.566308 & -0.490804 & -0.612626 \\
\hline 8 & 2.461437 & 0.285246 & 0.546227 \\
\hline 45 & -0.254369 & 0.340725 & -1.728198 \\
\hline 15 & -1.844310 & 0.363591 & 0.125135 \\
\hline 6 & -2.142197 & 2.085510 & 0.703419 \\
\hline 6 & -3.153075 & 2.854847 & 0.130956 \\
\hline 6 & -3.322806 & 4.201435 & 0.466165 \\
\hline 6 & -2.455046 & 4.758656 & 1.407241 \\
\hline 6 & & 4.020413 & 1.995123 \\
\hline 6 & -1.260244 & 2.690366 & 1.604566 \\
\hline 6 & -4.463006 & 4.984154 & -0.182734 \\
\hline 6 & -4.520439 & 6.434281 & 0.289954 \\
\hline 6 & -0.513675 & 4.604035 & 3.080210 \\
\hline 6 & -0.679407 & 6.114884 & 3.228970 \\
\hline 6 & 0.630972 & 2.469915 & -2.295257 \\
\hline 6 & 0.800737 & 3.353804 & -1.085105 \\
\hline 6 & 1.831128 & 2.213897 & -3.132097 \\
\hline 6 & 3.099939 & 2.619576 & -2.694364 \\
\hline 6 & 4.242187 & 2.378574 & -3.452747 \\
\hline 6 & 4.149291 & 1.730727 & -4.677700 \\
\hline 6 & 2.893533 & 1.338269 & -5.140323 \\
\hline 6 & 1.756983 & 1.578393 & -4.384387 \\
\hline 6 & -0.667997 & 2.361107 & -2.829835 \\
\hline 6 & -0.166395 & -0.968358 & -3.134266 \\
\hline 8 & -0.020207 & -1.663800 & -4.053128 \\
\hline 6 & -1.699079 & -0.582903 & 1.724550 \\
\hline 6 & -1.313228 & -1.916906 & 1.707166 \\
\hline 6 & -1.205784 & -2.653242 & 2.933223 \\
\hline 6 & -1.501943 & -2.011753 & 4.170447 \\
\hline 6 & -1.934777 & -0.667012 & 4.150510 \\
\hline 6 & -2.032814 & 0.018312 & 2.969723 \\
\hline 6 & -1.360620 & -2.726898 & 5.383244 \\
\hline 6 & -0.942570 & -4.032904 & 5.383925 \\
\hline 6 & -0.656669 & -4.678557 & 4.162758 \\
\hline 6 & -0.790012 & -4.010621 & 2.970480 \\
\hline 6 & -1.059650 & -2.677454 & 0.451238 \\
\hline 6 & 0.188700 & -2.702830 & -0.142992 \\
\hline 6 & 0.447423 & -3.564709 & -1.232918 \\
\hline 6 & -0.538533 & -4.355742 & -1.756368 \\
\hline
\end{tabular}




\begin{tabular}{|c|c|c|c|}
\hline & -1.842656 & -4.324658 & -1.211749 \\
\hline & -2.107916 & -3.486379 & -0.088464 \\
\hline & -3.422101 & -3.476326 & 0.447880 \\
\hline & -4.414751 & -4.248084 & -0.101469 \\
\hline & -4.143277 & -5.089838 & -1.202658 \\
\hline & -2.884570 & -5.122234 & -1.746397 \\
\hline & 1.251847 & -1.864651 & 0.338182 \\
\hline & 2.181986 & -2.512912 & 1.290770 \\
\hline & 2.640102 & -1.673695 & 2.457007 \\
\hline & 1.768776 & -0.827794 & 3.146480 \\
\hline & 2.213223 & -0.085533 & 4.231451 \\
\hline & 3.540342 & -0.178531 & 4.650860 \\
\hline & 4.412975 & -1.022722 & 3.974365 \\
\hline & 3.962699 & -1.765912 & 2.884705 \\
\hline & -3.550125 & -0.166035 & -0.361873 \\
\hline & -4.619730 & -0.044957 & 0.530904 \\
\hline & -5.903249 & -0.449764 & 0.171360 \\
\hline & -6.083614 & -0.985667 & -1.111310 \\
\hline & -5.038628 & -1.130090 & -2.023722 \\
\hline 6 & -3.765351 & -0.710682 & -1.622361 \\
\hline 6 & -7.085676 & -0.367702 & 1.135994 \\
\hline 6 & -8.261503 & 0.362319 & 0.478738 \\
\hline 6 & -5.296999 & -1.661221 & -3.433231 \\
\hline 6 & -5.952170 & -0.544283 & -4.254596 \\
\hline 6 & -6.230797 & -2.873957 & -3.402783 \\
\hline 6 & -4.002351 & -2.082112 & -4.129637 \\
\hline 6 & -7.516237 & -1.791674 & 1.507613 \\
\hline 6 & -6.729233 & 0.375231 & 2.422322 \\
\hline 6 & -4.280551 & 4.984809 & -1.704771 \\
\hline 6 & -5.796539 & 4.312437 & 0.168333 \\
\hline 6 & -0.875437 & 3.943238 & 4.416659 \\
\hline 6 & 0.957642 & 4.315232 & 2.768061 \\
\hline 1 & 6.748308 & -0.252359 & 1.315091 \\
\hline 1 & -2.379937 & 1.047790 & 2.990319 \\
\hline 1 & 7.204460 & -4.891576 & -0.395856 \\
\hline 1 & -1.588544 & -2.213651 & 6.316492 \\
\hline 1 & 6.734045 & 4.693800 & 0.241565 \\
\hline 1 & -3.645201 & -2.826130 & 1.292018 \\
\hline 1 & -5.420777 & -4.210537 & 0.312886 \\
\hline 1 & 4.567590 & 4.178447 & 1.242668 \\
\hline 1 & -2.194421 & -0.181812 & 5.091058 \\
\hline 1 & 5.275876 & -4.580377 & -1.844132 \\
\hline 1 & 8.814769 & 1.718750 & -2.058308 \\
\hline 1 & 3.332590 & -3.182038 & -2.561990 \\
\hline 1 & -2.661250 & -5.761987 & -2.599311 \\
\hline 1 & -0.335576 & -5.008622 & -2.603912 \\
\hline 1 & -0.330383 & -5.716064 & 4.165635 \\
\hline 1 & 7.017212 & 0.109937 & -1.644451 \\
\hline 1 & -0.572759 & -4.526279 & 2.037155 \\
\hline 1 & 1.452049 & -3.573772 & -1.651237 \\
\hline 1 & 8.705908 & 4.018425 & -1.100969 \\
\hline 1 & 8.523964 & -1.705915 & 2.169219 \\
\hline 1 & -4.935471 & -5.709514 & -1.618154 \\
\hline 1 & -0.832130 & -4.573588 & 6.321038 \\
\hline 1 & 8.784583 & -4.031370 & 1.308283 \\
\hline 1 & 2.640318 & 2.610631 & 1.472426 \\
\hline
\end{tabular}




\begin{tabular}{|c|c|c|c|}
\hline & -4.438722 & 0.380664 & 1.515437 \\
\hline & -2.907136 & -0.815957 & -2.282431 \\
\hline & -7.083759 & -1.304397 & -1.404185 \\
\hline & -3.829514 & 2.390845 & -0.586430 \\
\hline & -0.440333 & 2.097970 & 2.017327 \\
\hline & -2.587211 & 5.797769 & 1.692385 \\
\hline & 1.631299 & -3.380861 & 1.681795 \\
\hline & 3.060267 & -2.921275 & 0.767136 \\
\hline & 4.657403 & -2.414078 & 2.348232 \\
\hline & 0.744774 & -0.723439 & 2.794993 \\
\hline & 5.454413 & -1.099988 & 4.282374 \\
\hline & 1.515997 & 0.571114 & 4.751649 \\
\hline & 3.890531 & 0.408245 & 5.497528 \\
\hline & -1.496030 & 1.013796 & -2.550905 \\
\hline & -1.448571 & 2.963538 & -2.360193 \\
\hline & -0.803873 & 2.223739 & -3.901968 \\
\hline & 0.795729 & 1.240453 & -4.766618 \\
\hline & 2.798426 & 0.835364 & -6.100847 \\
\hline & 5.040867 & 1.536130 & -5.269647 \\
\hline & 5.211496 & 2.699243 & -3.072019 \\
\hline & 3.209967 & 3.115428 & -1.731967 \\
\hline & 1.478068 & 2.931631 & -0.336167 \\
\hline & 1.212326 & 4.333861 & -1.375850 \\
\hline & -0.163489 & 3.525973 & -0.597013 \\
\hline 1 & 0.011999 & 6.487153 & 3.995299 \\
\hline & -0.455276 & 6.644111 & 2.293702 \\
\hline 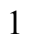 & -1.692371 & 6.391957 & 3.546630 \\
\hline & -0.252854 & 4.346702 & 5.227094 \\
\hline & -1.927165 & 4.126052 & 4.673696 \\
\hline ] & -0.720381 & 2.856190 & 4.385112 \\
\hline & 1.604760 & 4.789833 & 3.517703 \\
\hline 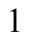 & 1.173362 & 3.240040 & 2.794988 \\
\hline & 1.241983 & 4.702504 & 1.779302 \\
\hline 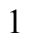 & -5.352711 & 6.950278 & -0.205111 \\
\hline & -4.687077 & 6.507505 & 1.372381 \\
\hline & -3.601534 & 6.982425 & 0.044994 \\
\hline 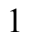 & -5.085160 & 5.557378 & -2.185464 \\
\hline & -3.324046 & 5.445601 & -1.985488 \\
\hline 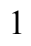 & -4.303243 & 3.971041 & -2.123216 \\
\hline & -6.631428 & 4.851694 & -0.299329 \\
\hline 1 & -5.836124 & 3.271889 & -0.179054 \\
\hline 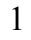 & -5.960541 & 4.310620 & 1.254134 \\
\hline | & -4.235330 & -2.513350 & -5.111606 \\
\hline L & -3.326052 & -1.234870 & -4.302602 \\
\hline & -3.456788 & -2.839348 & -3.548659 \\
\hline 1 & -6.147118 & -0.883451 & -5.281358 \\
\hline & -6.907588 & -0.229236 & -3.815474 \\
\hline 1 & -5.300662 & 0.338239 & -4.307197 \\
\hline 1 & -6.373892 & -3.261738 & -4.419940 \\
\hline 1 & -5.806606 & -3.679119 & -2.788805 \\
\hline 1 & -7.224829 & -2.632030 & -3.008517 \\
\hline & -7.612714 & 0.435101 & 3.070419 \\
\hline & -5.941777 & -0.136962 & 2.990902 \\
\hline & -6.393600 & 1.401855 & 2.220838 \\
\hline 1 & -8.366836 & -1.768456 & 2.202553 \\
\hline 1 & -7.820610 & -2.369608 & 0.625815 \\
\hline
\end{tabular}


TS1 $_{\text {II } B 2-} \psi_{-1 \mathrm{~s} 2 \mathrm{~s} 3 \mathrm{a} 4 \mathrm{~s}}$

\begin{tabular}{lcc}
\hline Number of imaginary frequencies : 1 & & \\
The smallest frequencies are $:-602.8469$ & 13.3289 & $20.5723 \mathrm{~cm}(-1)$ \\
Electronic energy : HF $\quad$ HF & & \\
Zero-point correction= & 1.453213 & (Hartree/Particle) \\
Thermal correction to Energy= & 1.537766 \\
Thermal correction to Enthalpy= & 1.538710 \\
Thermal correction to Gibbs Free Energy $=$ & 1.335534 \\
Sum of electronic and zero-point Energies $=$ & -4359.414342 \\
Sum of electronic and thermal Energies $=$ & -4359.329789 \\
Sum of electronic and thermal Enthalpies $=$ & -4359.328845 \\
Sum of electronic and thermal Free Energies $=$ & -4359.532021
\end{tabular}

Cartesian Coordinates

$\begin{array}{crrr}6 & 3.122792 & 2.723832 & -2.556221 \\ 6 & 1.830684 & 2.420972 & -3.008214 \\ 6 & 1.709336 & 1.912413 & -4.313333 \\ 6 & 2.822851 & 1.692130 & -5.108532 \\ 6 & 4.101802 & 1.980139 & -4.633116 \\ 6 & 4.241809 & 2.503393 & -3.354038 \\ 6 & 0.655108 & 2.642843 & -2.127956 \\ 6 & -0.658169 & 2.596746 & -2.634217 \\ 45 & -0.246453 & 0.490305 & -1.696825 \\ 6 & -0.171285 & -0.701946 & -3.206871 \\ 8 & -0.041303 & -1.332050 & -4.173683 \\ 15 & -1.840051 & 0.355302 & 0.147196 \\ 6 & -1.687155 & -0.660401 & 1.704564 \\ 6 & -1.299315 & -1.992288 & 1.627415 \\ 6 & -1.183411 & -2.781280 & 2.818268 \\ 6 & -1.475323 & -2.196379 & 4.084005 \\ 6 & -1.911708 & -0.853420 & 4.125352 \\ 6 & -2.015444 & -0.115770 & 2.976640 \\ 6 & -1.326093 & -2.964545 & 5.262947 \\ 6 & -0.905989 & -4.268568 & 5.202805 \\ 6 & -0.625827 & -4.858813 & 3.952381 \\ 6 & -0.766163 & -4.138244 & 2.792112 \\ 6 & -1.060229 & -2.685216 & 0.331049 \\ 6 & 0.170372 & -2.646579 & -0.298178 \\ 6 & 0.402043 & -3.406575 & -1.467488 \\ 6 & -0.592237 & -4.161518 & -2.026967 \\ 6 & -1.875164 & -4.205172 & -1.434409 \\ 6 & -2.111739 & -3.473213 & -0.233312 \\ 6 & -3.401022 & -3.546364 & 0.356114 \\ 6 & -4.397413 & -4.296330 & -0.216375 \\ 6 & -4.156863 & -5.024966 & -1.401713 \\ 6 & -2.922695 & -4.974413 & -1.998262 \\ 7 & 1.242192 & -1.845673 & 0.226006 \\ 6 & 2.164714 & -2.557225 & 1.139857 \\ 6 & 2.631159 & -1.800991 & 2.358367\end{array}$




\begin{tabular}{|c|c|c|c|}
\hline 6 & 1.778238 & -0.973924 & 3.092266 \\
\hline 6 & 2.231300 & -0.320943 & 4.230088 \\
\hline 6 & 3.548996 & -0.484281 & 4.657095 \\
\hline 6 & 4.404012 & -1.308346 & 3.934737 \\
\hline 6 & 3.944948 & -1.962568 & 2.793214 \\
\hline 6 & -3.529518 & -0.186416 & -0.377697 \\
\hline 6 & -4.584920 & -0.202126 & 0.529892 \\
\hline 6 & -5.862698 & -0.620749 & 0.149737 \\
\hline 6 & -6.052903 & -1.002888 & -1.179847 \\
\hline 6 & -5.013982 & -1.001020 & -2.119467 \\
\hline 6 & -3.749074 & -0.597759 & -1.691376 \\
\hline 6 & -5.286026 & -1.380987 & -3.574145 \\
\hline 6 & -6.079286 & -2.687910 & -3.656391 \\
\hline 6 & -6.973391 & -0.651046 & 1.198723 \\
\hline 6 & -6.542836 & -1.542619 & 2.370344 \\
\hline 6 & -2.181572 & 2.043535 & 0.797282 \\
\hline 6 & -3.210233 & 2.814481 & 0.258485 \\
\hline 6 & -3.399783 & 4.145925 & 0.641593 \\
\hline 6 & -2.540963 & 4.681570 & 1.603758 \\
\hline 6 & -1.493426 & 3.939090 & 2.164125 \\
\hline 6 & -1.309365 & 2.629404 & 1.719641 \\
\hline 6 & -4.541058 & 4.941945 & 0.009872 \\
\hline 6 & -4.319762 & 5.029912 & -1.504539 \\
\hline 6 & -0.590848 & 4.494309 & 3.266367 \\
\hline 6 & -0.883799 & 3.729925 & 4.563852 \\
\hline 15 & 1.571600 & -0.422519 & -0.643646 \\
\hline 8 & 2.774462 & -0.716947 & -1.766241 \\
\hline 6 & 3.904252 & -1.424101 & -1.435575 \\
\hline 6 & 4.862097 & -0.882238 & -0.601330 \\
\hline 6 & 5.923193 & -1.740279 & -0.154766 \\
\hline 6 & 6.037296 & -3.058000 & -0.690651 \\
\hline 6 & 5.103944 & -3.497469 & -1.661676 \\
\hline 6 & 4.048537 & -2.703200 & -2.015377 \\
\hline 6 & 6.834380 & -1.356700 & 0.862942 \\
\hline 6 & 7.807225 & -2.218040 & 1.307557 \\
\hline 6 & 7.936012 & -3.507960 & 0.751219 \\
\hline 6 & 7.065454 & -3.914630 & -0.227335 \\
\hline 6 & 4.765640 & 0.524296 & -0.151629 \\
\hline 6 & 3.617916 & 1.011743 & 0.446876 \\
\hline 6 & 3.593481 & 2.273472 & 1.080844 \\
\hline 6 & 4.667912 & 3.112483 & 0.997183 \\
\hline 6 & 5.807345 & 2.738471 & 0.243255 \\
\hline 6 & 5.858258 & 1.437244 & -0.340522 \\
\hline 6 & 6.880480 & 3.639123 & 0.043406 \\
\hline 6 & 7.961214 & 3.290626 & -0.726517 \\
\hline 6 & 8.003295 & 2.017633 & -1.331630 \\
\hline 6 & 6.984778 & 1.115707 & -1.140350 \\
\hline 8 & 2.472637 & 0.276646 & 0.561003 \\
\hline 6 & -3.992238 & -1.569130 & -4.365935 \\
\hline 6 & -6.098070 & -0.255947 & -4.226531 \\
\hline 6 & -8.282955 & -1.204711 & 0.643629 \\
\hline 6 & -7.229584 & 0.771195 & 1.709381 \\
\hline 6 & 0.883412 & 4.299444 & 2.899632 \\
\hline 6 & -0.825681 & 5.981287 & 3.520871 \\
\hline 6 & -5.871573 & 4.231761 & 0.286462 \\
\hline 6 & -4.637249 & 6.362795 & 0.559510 \\
\hline
\end{tabular}




\begin{tabular}{|c|c|c|c|}
\hline 6 & 0.872407 & 3.428882 & -0.859639 \\
\hline 1 & 6.735231 & -0.374643 & 1.318084 \\
\hline & -2.365403 & 0.910725 & 3.044970 \\
\hline & 7.138585 & -4.912641 & -0.657645 \\
\hline 1 & -1.549749 & -2.493983 & 6.219439 \\
\hline & 6.819982 & 4.623870 & 0.505214 \\
\hline & -3.599767 & -2.982900 & 1.266503 \\
\hline & -5.384248 & -4.324919 & 0.242592 \\
\hline & 4.649625 & 4.085256 & 1.486478 \\
\hline & -2.168605 & -0.411098 & 5.087514 \\
\hline & 5.226822 & -4.485817 & -2.101418 \\
\hline 1 & 8.846996 & 1.748442 & -1.963220 \\
\hline & 3.311014 & -3.019531 & -2.749760 \\
\hline & -2.722909 & -5.528655 & -2.914548 \\
\hline & -0.411372 & -4.731252 & -2.937222 \\
\hline & -0.298190 & -5.894924 & 3.907406 \\
\hline & 7.026386 & 0.146952 & -1.631212 \\
\hline & -0.554001 & -4.610490 & 1.834940 \\
\hline & 1.391030 & -3.362570 & -1.919034 \\
\hline & 8.776142 & 3.993837 & -0.881505 \\
\hline & 8.481362 & -1.903168 & 2.101103 \\
\hline & -4.953035 & -5.623384 & -1.839848 \\
\hline & -0.789558 & -4.850377 & 6.114211 \\
\hline & 8.715062 & -4.178056 & 1.107130 \\
\hline & 2.696591 & 2.543650 & 1.631623 \\
\hline & -4.408942 & 0.116518 & 1.557396 \\
\hline 1 & -2.898104 & -0.604800 & -2.368648 \\
\hline & -7.043516 & -1.317764 & -1.501388 \\
\hline & -3.879233 & 2.365924 & -0.475813 \\
\hline 1 & -0.475441 & 2.038030 & 2.105405 \\
\hline & -2.689749 & 5.706849 & 1.928008 \\
\hline ] & 1.607105 & -3.443108 & 1.476407 \\
\hline & 3.039316 & -2.941131 & 0.592708 \\
\hline & 4.625875 & -2.596158 & 2.222698 \\
\hline & 0.762289 & -0.813737 & 2.737750 \\
\hline . & 5.438734 & -1.438651 & 4.247525 \\
\hline & 1.548768 & 0.323720 & 4.783433 \\
\hline & 3.906181 & 0.033270 & 5.544994 \\
\hline 1 & -1.490672 & 1.233414 & -2.454218 \\
\hline & -1.416837 & 3.168453 & -2.095443 \\
\hline 1 & -0.825090 & 2.545734 & -3.709560 \\
\hline 1 & 0.728223 & 1.656982 & -4.708903 \\
\hline & 2.691140 & 1.287969 & -6.110448 \\
\hline ] & 4.975112 & 1.802009 & -5.256623 \\
\hline & 5.230242 & 2.742910 & -2.962507 \\
\hline 1 & 3.267963 & 3.121050 & -1.553449 \\
\hline & 1.552491 & 2.934754 & -0.159867 \\
\hline 1 & 1.305876 & 4.416494 & -1.086277 \\
\hline 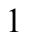 & -0.077384 & 3.589508 & -0.339313 \\
\hline 1 & -0.141025 & 6.333258 & 4.302700 \\
\hline 1 & -0.640637 & 6.583934 & 2.622238 \\
\hline & -1.846443 & 6.185379 & 3.867957 \\
\hline 1 & -0.263246 & 4.113861 & 5.385157 \\
\hline & -1.936897 & 3.838878 & 4.855169 \\
\hline & -0.672491 & 2.657058 & 4.459595 \\
\hline & 1.528182 & 4.733567 & 3.675447 \\
\hline
\end{tabular}




$\begin{array}{rrrr}1 & 1.140537 & 3.235940 & 2.824473 \\ 1 & 1.128771 & 4.782843 & 1.943746 \\ 1 & -5.470668 & 6.888307 & 0.076511 \\ 1 & -4.825370 & 6.373147 & 1.640886 \\ 1 & -3.726148 & 6.942363 & 0.362737 \\ 1 & -5.126518 & 5.607579 & -1.975584 \\ 1 & -3.368888 & 5.528820 & -1.734466 \\ 1 & -4.304722 & 4.039415 & -1.975878 \\ 1 & -6.702152 & 4.788695 & -0.167904 \\ 1 & -5.889755 & 3.214477 & -0.125322 \\ 1 & -6.062002 & 4.160614 & 1.365503 \\ 1 & -4.229139 & -1.883322 & -5.390435 \\ 1 & -3.407326 & -0.642368 & -4.438131 \\ 1 & -3.352020 & -2.341121 & -3.916551 \\ 1 & -6.306312 & -0.491628 & -5.279265 \\ 1 & -7.059560 & -0.103922 & -3.719625 \\ 1 & -5.548849 & 0.694392 & -4.194410 \\ 1 & -6.254755 & -2.955805 & -4.706819 \\ 1 & -5.524685 & -3.508727 & -3.183751 \\ 1 & -7.060386 & -2.617524 & -3.171887 \\ 1 & -9.042625 & -1.221516 & 1.435360 \\ 1 & -8.676704 & -0.588422 & -0.174771 \\ 1 & -8.166972 & -2.232764 & 0.275076 \\ 1 & -7.328217 & -1.568249 & 3.137806 \\ 1 & -6.368323 & -2.573882 & 2.035078 \\ 1 & -5.622078 & -1.189142 & 2.851973 \\ 1 & -8.003312 & 0.765294 & 2.489201 \\ 1 & -6.326132 & 1.223172 & 2.138171 \\ 1 & -7.575937 & 1.422797 & 0.896068\end{array}$

TS1 $_{\text {IIB2- }}-\psi_{-1 \mathrm{~s} 2 s 334 a}$

Number of imaginary frequencies : 1

The smallest frequencies are : $-595.2354 \quad 11.8480 \quad 18.5870 \mathrm{~cm}(-1)$

Electronic energy : $\quad \mathrm{HF}=-4360.866597$

Zero-point correction $=\quad 1.452655($ Hartree/Particle $)$

Thermal correction to Energy= $\quad 1.537434$

Thermal correction to Enthalpy= $\quad 1.538378$

Thermal correction to Gibbs Free Energy $=\quad 1.334129$

Sum of electronic and zero-point Energies $=\quad-4359.413942$

Sum of electronic and thermal Energies $=\quad-4359.329163$

Sum of electronic and thermal Enthalpies $=\quad-4359.328219$

Sum of electronic and thermal Free Energies $=\quad-4359.532468$

Cartesian Coordinates

$\begin{array}{cccc}6 & -4.590888 & -0.224262 & 0.532040 \\ 6 & -3.526756 & -0.187932 & -0.379342 \\ 6 & -3.746398 & -0.550775 & -1.697903 \\ 6 & -5.018385 & -0.932222 & -2.148230 \\ 6 & -6.049968 & -0.969348 & -1.215951 \\ 6 & -5.862206 & -0.620116 & 0.133171 \\ 15 & -1.842016 & 0.354883 & 0.156312 \\ 45 & -0.241598 & 0.505663 & -1.678047 \\ 15 & 1.571922 & -0.422403 & -0.631950 \\ 8 & 2.478012 & 0.263396 & 0.575748\end{array}$




\begin{tabular}{|c|c|c|c|}
\hline 6 & 3.613251 & 1.013197 & 0.457536 \\
\hline 6 & 4.759428 & 0.542904 & -0.157326 \\
\hline 6 & 5.843268 & 1.466958 & -0.341735 \\
\hline 6 & 5.788422 & 2.758236 & 0.263371 \\
\hline 6 & 4.651643 & 3.113511 & 1.030329 \\
\hline 6 & 3.583370 & 2.266064 & 1.108223 \\
\hline 6 & 6.965457 & 1.165491 & -1.154981 \\
\hline 6 & 7.977893 & 2.075857 & -1.337834 \\
\hline 6 & 7.933334 & 3.338090 & -0.710520 \\
\hline 6 & 6.855471 & 3.668055 & 0.071649 \\
\hline 6 & 4.866602 & -0.859419 & -0.618281 \\
\hline 6 & 3.908004 & -1.407200 & -1.447917 \\
\hline 6 & 4.064303 & -2.678810 & -2.041202 \\
\hline 6 & 5.132335 & -3.462842 & -1.703182 \\
\hline 6 & 6.066718 & -3.020805 & -0.734290 \\
\hline 6 & 5.940171 & -1.709449 & -0.185550 \\
\hline 6 & 7.107992 & -3.869245 & -0.285542 \\
\hline 6 & 7.979257 & -3.461756 & 0.692032 \\
\hline 6 & 7.837472 & -2.179375 & 1.262323 \\
\hline 6 & 6.852013 & -1.325404 & 0.831418 \\
\hline 8 & 2.766506 & -0.713153 & -1.764267 \\
\hline 6 & -5.203212 & -1.266803 & -3.626035 \\
\hline 6 & -7.031291 & -0.715190 & 1.112726 \\
\hline 6 & -2.203041 & 2.034026 & 0.818533 \\
\hline 6 & -3.243630 & 2.789925 & 0.282009 \\
\hline 6 & -3.464716 & 4.111542 & 0.679018 \\
\hline 6 & -2.619684 & 4.656945 & 1.647849 \\
\hline 6 & -1.556979 & 3.931942 & 2.201998 \\
\hline 6 & -1.345161 & 2.629211 & 1.748072 \\
\hline 6 & -0.653240 & 4.502225 & 3.295323 \\
\hline 6 & 0.811288 & 4.435502 & 2.852218 \\
\hline 6 & -4.632036 & 4.879656 & 0.061696 \\
\hline 6 & -5.938864 & 4.132532 & 0.355898 \\
\hline 6 & -1.687051 & -0.676234 & 1.700647 \\
\hline 6 & -1.304385 & -2.008151 & 1.605054 \\
\hline 6 & -1.183727 & -2.810333 & 2.787623 \\
\hline 6 & -1.465248 & -2.237351 & 4.061363 \\
\hline 6 & -1.897370 & -0.893654 & 4.120094 \\
\hline 6 & -2.006192 & -0.143854 & 2.980136 \\
\hline 6 & -1.307003 & -3.016120 & 5.232156 \\
\hline 6 & -0.886628 & -4.319230 & 5.157358 \\
\hline 6 & -0.615120 & -4.897430 & 3.899574 \\
\hline 6 & -0.764672 & -4.166664 & 2.746835 \\
\hline 6 & -1.057175 & -2.693732 & 0.305313 \\
\hline 6 & 0.183417 & -2.660057 & -0.304459 \\
\hline 6 & 0.443355 & -3.448292 & -1.449176 \\
\hline 6 & -0.527160 & -4.244996 & -1.992060 \\
\hline 6 & -1.818732 & -4.289383 & -1.418117 \\
\hline 6 & -2.092884 & -3.503210 & -0.259764 \\
\hline 6 & -3.397019 & -3.562325 & 0.297421 \\
\hline 6 & -4.366380 & -4.360895 & -0.256672 \\
\hline 6 & -4.081931 & -5.158326 & -1.386257 \\
\hline 6 & -2.835039 & -5.116440 & -1.955619 \\
\hline 7 & 1.246026 & -1.851977 & 0.228034 \\
\hline 6 & 2.183714 & -2.571665 & 1.120565 \\
\hline 6 & 2.650703 & -1.833120 & 2.349263 \\
\hline
\end{tabular}




\begin{tabular}{|c|c|c|c|}
\hline & 1.791864 & -1.034205 & 3.106696 \\
\hline & 2.244563 & -0.400663 & 4.255726 \\
\hline & 3.567937 & -0.553465 & 4.668556 \\
\hline & 4.429482 & -1.347777 & 3.920991 \\
\hline & 3.970415 & -1.984498 & 2.769808 \\
\hline & -0.984197 & 5.954276 & 3.630554 \\
\hline & -0.828469 & 3.663443 & 4.567617 \\
\hline & -4.757672 & 6.296465 & 0.615466 \\
\hline & -4.432871 & 4.975210 & -1.455369 \\
\hline & -0.179413 & -0.679348 & -3.189995 \\
\hline & -0.089249 & -1.300134 & -4.167737 \\
\hline & 0.652878 & 2.677321 & -2.077458 \\
\hline & 0.831419 & 3.459357 & -0.801229 \\
\hline & -0.647741 & 2.616132 & -2.613283 \\
\hline & 1.849851 & 2.483109 & -2.935236 \\
\hline & 1.769062 & 1.940997 & -4.230243 \\
\hline & 2.901859 & 1.756959 & -5.006970 \\
\hline & 4.160055 & 2.113664 & -4.522333 \\
\hline & 4.259172 & 2.672590 & -3.254818 \\
\hline & 3.120439 & 2.858406 & -2.475661 \\
\hline & 6.742816 & -0.349923 & 1.298222 \\
\hline & -2.353636 & 0.882538 & 3.061191 \\
\hline & 7.190543 & -4.862077 & -0.726021 \\
\hline & -1.523188 & -2.553908 & 6.194432 \\
\hline & 6.792784 & 4.644583 & 0.550330 \\
\hline & -3.630665 & -2.949956 & 1.167160 \\
\hline & -5.362979 & -4.381115 & 0.180405 \\
\hline & 4.630471 & 4.078530 & 1.534523 \\
\hline & -2.146304 & -0.460694 & 5.088561 \\
\hline & 5.264695 & -4.445179 & -2.153512 \\
\hline & 8.818511 & 1.822099 & -1.979838 \\
\hline & 3.324662 & -2.996808 & -2.772854 \\
\hline 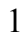 & -2.603287 & -5.714860 & -2.835907 \\
\hline & -0.320015 & -4.851716 & -2.872296 \\
\hline 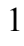 & -0.286177 & -5.932547 & 3.842572 \\
\hline & 7.007878 & 0.205691 & -1.663097 \\
\hline & -0.557105 & -4.630872 & 1.784754 \\
\hline 1 & 1.439638 & -3.405817 & -1.884785 \\
\hline & 8.743783 & 4.047983 & -0.858423 \\
\hline 1 & 8.511515 & -1.864509 & 2.055947 \\
\hline & -4.855961 & -5.796233 & -1.807606 \\
\hline 1 & -0.762660 & -4.909077 & 6.062579 \\
\hline ] & 8.768462 & -4.125753 & 1.036805 \\
\hline 1 & 2.688635 & 2.519977 & 1.670349 \\
\hline 1 & -4.409297 & 0.068027 & 1.563958 \\
\hline & -2.899984 & -0.535884 & -2.383227 \\
\hline 1 & -7.043789 & -1.275537 & -1.537102 \\
\hline & -3.901010 & 2.335109 & -0.458581 \\
\hline 1 & -0.499480 & 2.051666 & 2.130442 \\
\hline 1 & -2.792589 & 5.674853 & 1.983377 \\
\hline & 1.637492 & -3.468773 & 1.445442 \\
\hline 1 & 3.058200 & -2.936510 & 0.560358 \\
\hline 1 & 4.655631 & -2.595637 & 2.180254 \\
\hline 1 & 0.771238 & -0.880171 & 2.762719 \\
\hline & 5.468700 & -1.468972 & 4.222331 \\
\hline 1 & 1.557520 & 0.220891 & 4.829207 \\
\hline
\end{tabular}




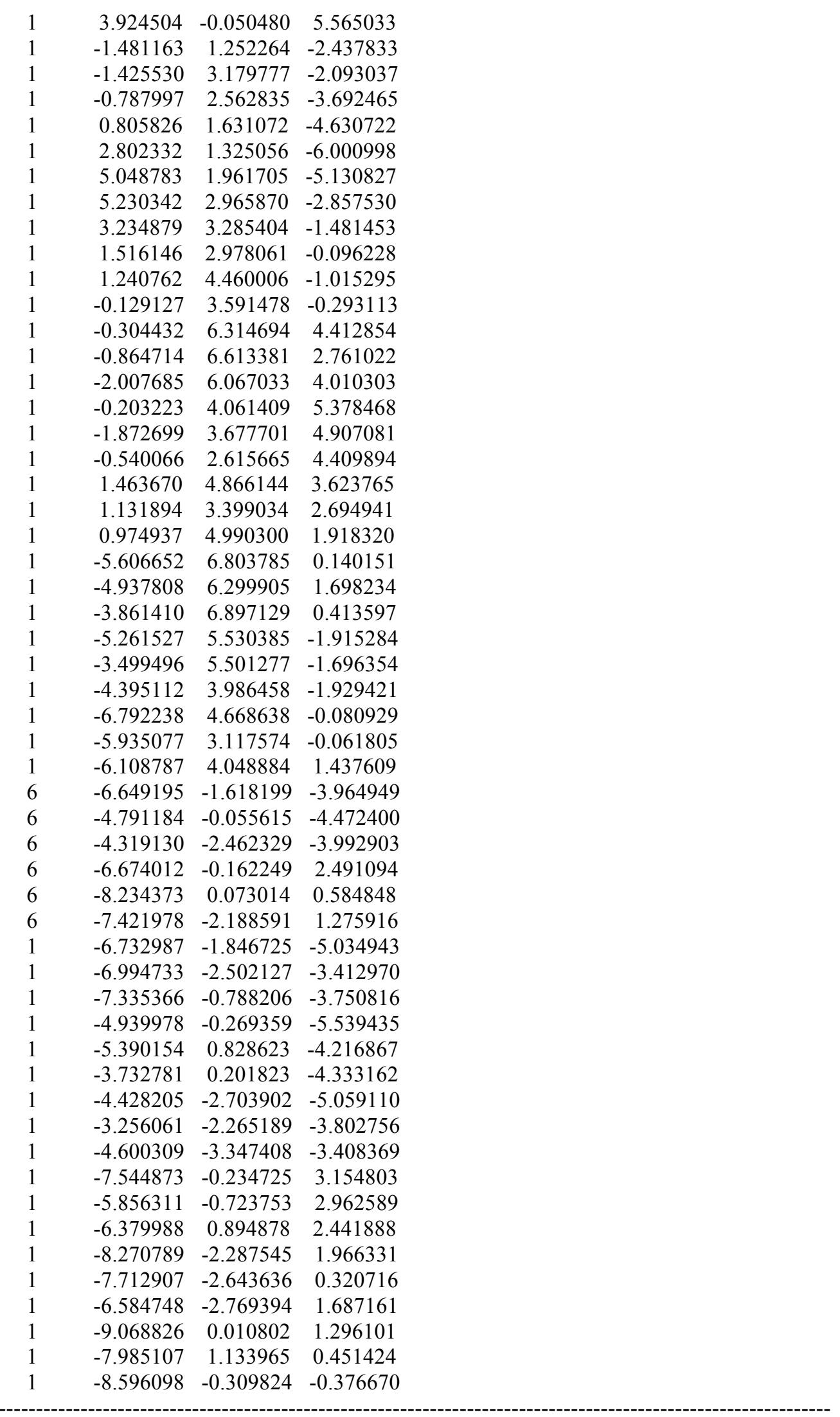

TS1 $_{I I B 2-} \psi_{-1 \mathrm{~s} 2 \mathrm{~s} 3 \mathrm{~s} 4 \mathrm{~s}}$ 
Number of imaginary frequencies : 1

The smallest frequencies are : -598.6574 $13.5178 \quad 19.4459 \mathrm{~cm}(-1)$

Electronic energy : $\quad H F=-4360.8669725$

Zero-point correction $=\quad 1.453266($ Hartree/Particle $)$

Thermal correction to Energy $=\quad 1.537814$

Thermal correction to Enthalpy= $\quad 1.538758$

Thermal correction to Gibbs Free Energy= 1.335491

Sum of electronic and zero-point Energies $=\quad-4359.413706$

Sum of electronic and thermal Energies $=\quad-4359.329159$

Sum of electronic and thermal Enthalpies $=\quad-4359.328214$

Sum of electronic and thermal Free Energies $=\quad-4359.531481$

Cartesian Coordinates

\begin{tabular}{|c|c|c|c|}
\hline 6 & -4.582073 & -0.224914 & 0.522193 \\
\hline 6 & -3.534118 & -0.162967 & -0.397572 \\
\hline 6 & -3.773736 & -0.502499 & -1.722780 \\
\hline 6 & -5.047318 & -0.883334 & -2.157369 \\
\hline 6 & -6.070065 & -0.947656 & -1.209023 \\
\hline 6 & -5.859181 & -0.630435 & 0.139443 \\
\hline 15 & -1.842729 & 0.360489 & 0.136716 \\
\hline 45 & -0.238213 & 0.495606 & -1.695806 \\
\hline 15 & 1.567586 & -0.434372 & -0.638967 \\
\hline 8 & 2.465933 & 0.252491 & 0.573477 \\
\hline 6 & 3.603575 & 0.999987 & 0.462691 \\
\hline 6 & 4.753468 & 0.526289 & -0.142567 \\
\hline 6 & 5.840133 & & -0.321260 \\
\hline 6 & & & \\
\hline 6 & & & \\
\hline 6 & 3.5708 & 2.254 & 0298 \\
\hline 6 & 6.967443 & 1.143189 & -1.126067 \\
\hline 6 & 7.982522 & 2.051615 & -1.303880 \\
\hline 6 & 7.935663 & 3.315209 & -0.679495 \\
\hline 6 & 6.852975 & 3.648 & 0.094560 \\
\hline 6 & 3 & -0.877 & -0.600391 \\
\hline 6 & 3.90 & -1.42 & -1.4 \\
\hline 6 & & -2.6 & 577 \\
\hline 6 & 5.127521 & -3.483299 & -1.678174 \\
\hline 6 & 6.056102 & -3.041658 & -0.703531 \\
\hline 6 & 5.929052 & -1.728854 & -0.158372 \\
\hline 6 & 7.092255 & -3.891638 & -0.245971 \\
\hline 6 & 7.957925 & -3.484127 & 0.736550 \\
\hline 6 & 7.815493 & -2.200166 & 1.303139 \\
\hline 6 & 6.834990 & -1.344746 & 0.863858 \\
\hline 8 & 2.769642 & -0.727409 & -1.762567 \\
\hline 6 & -5.254351 & -1.199460 & -3.636870 \\
\hline 6 & -6.957436 & -0.712163 & 1.199270 \\
\hline 6 & -2.185470 & 2.038468 & 0.811952 \\
\hline 6 & -3.213008 & 2.814497 & 0.278818 \\
\hline 6 & -3.407150 & 4.140123 & 0.678433 \\
\hline 6 & -2.553528 & 4.664768 & 1.651243 \\
\hline 6 & & & 2.207321 \\
\hline 6 & -1.319621 & 2.612600 & 1.747553 \\
\hline 6 & -0.610262 & 4.459920 & 3.319709 \\
\hline 6 & 0.865884 & 4.269903 & 2.957472 \\
\hline
\end{tabular}




\begin{tabular}{|c|c|c|c|}
\hline 6 & -4.549697 & 4.939272 & 0.053305 \\
\hline & -5.879083 & 4.227282 & 0.331498 \\
\hline & -1.703835 & -0.682105 & 1.675632 \\
\hline & -1.330163 & -2.015945 & 1.575441 \\
\hline & -1.232773 & -2.828528 & 2.753308 \\
\hline & -1.531533 & -2.264582 & 4.027027 \\
\hline & -1.951631 & -0.917223 & 4.090304 \\
\hline & -2.035177 & -0.156888 & 2.955148 \\
\hline & -1.403027 & -3.055734 & 5.193129 \\
\hline & -0.994238 & -4.362271 & 5.113743 \\
\hline & -0.703630 & -4.930925 & 3.855915 \\
\hline & -0.824415 & -4.187971 & 2.707577 \\
\hline & -1.074204 & -2.695481 & 0.274589 \\
\hline ) & 0.172648 & -2.668337 & -0.322519 \\
\hline & 0.438292 & -3.456714 & -1.465783 \\
\hline & -0.533516 & -4.242492 & -2.022332 \\
\hline b & -1.832579 & -4.275062 & -1.464800 \\
\hline & -2.111176 & -3.492755 & -0.305036 \\
\hline D & -3.421884 & -3.540179 & 0.237500 \\
\hline & -4.394549 & -4.322522 & -0.333638 \\
\hline & -4.107045 & -5.115425 & -1.465669 \\
\hline & -2.852654 & -5.085993 & -2.019598 \\
\hline & 1.233304 & -1.863395 & 0.218971 \\
\hline 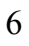 & 2.159034 & -2.580772 & 1.125532 \\
\hline ) & 2.613706 & -1.834410 & 2.354307 \\
\hline b & 1.746650 & -1.029766 & 3.096236 \\
\hline 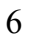 & 2.188252 & -0.381428 & 4.241251 \\
\hline & 3.508326 & -0.527438 & 4.667248 \\
\hline 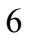 & 4.377289 & -1.329551 & 3.936745 \\
\hline 6 & 3.929689 & -1.979378 & 2.788220 \\
\hline 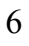 & -0.846818 & 5.944044 & 3.589054 \\
\hline 6 & -0.908753 & 3.681738 & 4.607652 \\
\hline 6 & -4.643872 & 6.357536 & 0.609844 \\
\hline 6 & -4.333461 & 5.034406 & -1.461493 \\
\hline 6 & -0.161046 & -0.694148 & -3.203146 \\
\hline & -0.045938 & -1.319706 & -4.175142 \\
\hline 6 & 0.663109 & 2.664282 & -2.093029 \\
\hline 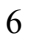 & 0.836541 & 3.445632 & -0.815705 \\
\hline 6 & -0.634505 & 2.605779 & -2.636287 \\
\hline 6 & 1.864450 & 2.467139 & -2.943677 \\
\hline 5 & 1.790032 & 1.925026 & -4.239026 \\
\hline 6 & 2.926909 & 1.739216 & -5.009316 \\
\hline 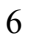 & 4.182901 & 2.093641 & -4.517431 \\
\hline 6 & 4.275803 & 2.651983 & -3.249160 \\
\hline ) & 3.133030 & 2.839882 & -2.476625 \\
\hline 1 & 6.725399 & -0.367655 & 1.327226 \\
\hline 1 & -2.373759 & 0.872183 & 3.039492 \\
\hline 1 & 7.175399 & -4.885577 & -0.683824 \\
\hline 1 & -1.632839 & -2.600522 & 6.155543 \\
\hline ] & 6.788511 & 4.626173 & 0.570693 \\
\hline 1 & -3.659027 & -2.929937 & 1.107975 \\
\hline t & -5.398056 & -4.329638 & 0.088673 \\
\hline 1 & 4.617838 & 4.065997 & 1.540286 \\
\hline ] & -2.211720 & -0.490040 & 5.058418 \\
\hline 1 & 5.260612 & -4.466865 & -2.125592 \\
\hline 1 & 8.827100 & 1.795237 & -1.939613 \\
\hline
\end{tabular}




\begin{tabular}{|c|c|c|c|}
\hline 1 & 3.329156 & -3.014281 & -2.762334 \\
\hline 1 & -2.617935 & -5.682026 & -2.900676 \\
\hline & -0.321979 & -4.847625 & -2.902600 \\
\hline & -0.382989 & -5.968432 & 3.795223 \\
\hline 1 & 7.011824 & 0.182306 & -1.631971 \\
\hline & -0.602517 & -4.645071 & 1.745311 \\
\hline 1 & 1.439465 & -3.422154 & -1.890554 \\
\hline 1 & 8.748219 & 4.023531 & -0.823319 \\
\hline 1 & 8.485185 & -1.885169 & 2.100383 \\
\hline 1 & -4.884152 & -5.739974 & -1.901099 \\
\hline 1 & -0.893869 & -4.962009 & 6.015381 \\
\hline 1 & 8.743215 & -4.149269 & 1.087998 \\
\hline 1 & 2.672028 & 2.512710 & 1.663809 \\
\hline 1 & -4.394083 & 0.046196 & 1.560967 \\
\hline 1 & -2.938224 & -0.471173 & -2.420834 \\
\hline 1 & -7.062250 & -1.256473 & -1.525624 \\
\hline 1 & -3.877888 & 2.374918 & -0.464498 \\
\hline 1 & -0.487297 & 2.016965 & 2.130373 \\
\hline 1 & -2.705119 & 5.685788 & 1.987527 \\
\hline 1 & 1.606926 & -3.474774 & 1.449045 \\
\hline 1 & 3.039054 & -2.950468 & 0.577131 \\
\hline 1 & 4.621232 & -2.595300 & 2.211159 \\
\hline 1 & 0.728592 & -0.882868 & 2.741979 \\
\hline 1 & 5.413892 & -1.445933 & 4.248776 \\
\hline 1 & 1.495085 & 0.245971 & 4.801194 \\
\hline 1 & 3.856573 & -0.013340 & 5.560685 \\
\hline 1 & -1.470601 & 1.244244 & -2.466320 \\
\hline 1 & -1.413220 & 3.171233 & -2.119772 \\
\hline 1 & -0.769157 & 2.553314 & -3.716203 \\
\hline 1 & 0.828536 & 1.616694 & -4.644911 \\
\hline 1 & 2.832335 & 1.307538 & -6.003922 \\
\hline 1 & 5.074813 & 1.940226 & -5.120878 \\
\hline 1 & 5.245243 & 2.943192 & -2.846152 \\
\hline 1 & 3.242533 & 3.266639 & -1.481778 \\
\hline 1 & 1.514529 & 2.959909 & -0.107382 \\
\hline 1 & 1.251921 & 4.444508 & -1.026323 \\
\hline 1 & -0.126248 & 3.581883 & -0.313248 \\
\hline 1 & -0.165872 & 6.287838 & 4.377724 \\
\hline 1 & -0.657950 & 6.556292 & 2.697719 \\
\hline 1 & -1.869212 & 6.144097 & 3.933653 \\
\hline 1 & -0.290941 & 4.056118 & 5.435412 \\
\hline 1 & -1.962829 & 3.788532 & 4.896158 \\
\hline 1 & -0.698047 & 2.609864 & 4.492588 \\
\hline 1 & 1.506692 & 4.699685 & 3.738966 \\
\hline 1 & 1.125758 & 3.207566 & 2.875800 \\
\hline 1 & 1.113984 & 4.760861 & 2.006124 \\
\hline 1 & -5.477077 & 6.886475 & 0.130223 \\
\hline 1 & -4.831147 & 6.362976 & 1.691386 \\
\hline 1 & -3.732021 & 6.936679 & 0.415181 \\
\hline 1 & -5.140523 & 5.616505 & -1.926478 \\
\hline 1 & -3.382266 & 5.532387 & -1.692136 \\
\hline 1 & -4.322413 & 4.046641 & -1.938818 \\
\hline 1 & -6.711145 & 4.784134 & -0.120305 \\
\hline 1 & -5.897405 & 3.209941 & -0.080594 \\
\hline 1 & -6.066976 & 4.154681 & 1.410866 \\
\hline 6 & -6.694572 & -1.595408 & -3.950769 \\
\hline
\end{tabular}




\begin{tabular}{|c|c|c|c|}
\hline & 4.902688 & 0.039388 & -4.469923 \\
\hline & .339208 & -2.357966 & -4.045677 \\
\hline & 269923 & -1.252942 & 0.638050 \\
\hline & .508508 & -1.645179 & 2.331077 \\
\hline & -7.216554 & 0.687694 & 1.767783 \\
\hline & -6.792774 & -1.812327 & -5.021960 \\
\hline & -6.998107 & -2.497708 & -3.403660 \\
\hline & -7.404332 & -0.792524 & \\
\hline & -5.056795 & -0.163381 & -5.538429 \\
\hline & -5.532889 & 0.894904 & -4.192812 \\
\hline & -3.853787 & 0.336495 & -4.338165 \\
\hline & -4.479625 & -2.596744 & -5.108837 \\
\hline & -3.277960 & -2.120692 & -3.89647 \\
\hline & -4.562437 & -3.257575 & -3.45791 \\
\hline & -9.018827 & -1.309339 & 1.438198 \\
\hline & -8.679645 & -0.606470 & -0.14853 \\
\hline & & -2.263712 & 0.225265 \\
\hline & -7.985613 & 0.647764 & \\
\hline & -6.311776 & 1.124973 & 2.208831 \\
\hline & -7.569509 & 1.370184 & 0.983114 \\
\hline & -7.284850 & -1.704309 & 3.10592 \\
\hline & -6.332935 & -2.662808 & 1.95642 \\
\hline & & & \\
\hline
\end{tabular}

\section{Cartesian coordinates for 4,5 , TS2, 6, 7, TS3, 8, TS4, and 9}

\section{$4_{R}$}

Number of imaginary frequencies : 0

The smallest frequencies are : $16.3702 \quad 21.3623 \quad 22.6439 \mathrm{~cm}(-1)$

Electronic energy : $\quad H F=-4360.9184114$

Zero-point correction $=\quad 1.457713($ Hartree/Particle $)$

Thermal correction to Energy= $\quad 1.542397$

Thermal correction to Enthalpy= $\quad 1.543341$

Thermal correction to Gibbs Free Energy $=\quad 1.339814$

Sum of electronic and zero-point Energies $=\quad-4359.460699$

Sum of electronic and thermal Energies $=\quad-4359.376015$

Sum of electronic and thermal Enthalpies $=\quad-4359.375070$

Sum of electronic and thermal Free Energies $=\quad-4359.578597$

\begin{tabular}{cccc}
\multicolumn{4}{c}{ Cartesian Coordinates } \\
\hline $6 \ldots \ldots \ldots \ldots \ldots \ldots \ldots \ldots \ldots \ldots \ldots \ldots \ldots \ldots \ldots \ldots \ldots$ \\
6 & -2.451771 & 2.542341 & 1.347822 \\
6 & -1.505309 & 1.527088 & 1.341651 \\
6 & -0.376664 & 1.624090 & 2.168410 \\
6 & -0.210308 & 2.700359 & 3.031729 \\
6 & -1.166384 & 3.729513 & 2.984733 \\
6 & -2.281914 & 3.680220 & 2.153321 \\
15 & -1.591093 & 0.078296 & 0.221401 \\
6 & -1.600870 & -1.327954 & 1.451482 \\
6 & -1.516278 & -2.643539 & 1.019995 \\
6 & -1.610715 & -3.721153 & 1.959036 \\
6 & -1.814450 & -3.435865 & 3.339788 \\
6 & -1.921516 & -2.086796 & 3.744686
\end{tabular}




\begin{tabular}{|c|c|c|c|}
\hline 6 & -1.816351 & -1.070123 & 2.833063 \\
\hline 6 & -1.899713 & -4.500011 & 4.269666 \\
\hline 6 & -1.784660 & -5.802732 & 3.857811 \\
\hline 6 & -1.578807 & -6.092915 & 2.492196 \\
\hline 6 & -1.496596 & -5.081704 & 1.567635 \\
\hline 6 & -1.340959 & -2.996863 & -0.413210 \\
\hline 6 & -0.083651 & -2.961086 & -0.985673 \\
\hline 6 & 0.112616 & -3.370767 & -2.326317 \\
\hline 6 & -0.940628 & -3.813658 & -3.080643 \\
\hline 6 & -2.244442 & -3.865920 & -2.533970 \\
\hline 6 & -2.454521 & -3.446071 & -1.187046 \\
\hline 6 & -3.773514 & -3.478260 & -0.665897 \\
\hline 6 & -4.821763 & -3.921075 & -1.432930 \\
\hline 6 & -4.606086 & -4.365935 & -2.755851 \\
\hline 6 & -3.345281 & -4.330149 & -3.295119 \\
\hline 7 & 1.036935 & -2.486675 & -0.219747 \\
\hline 15 & 1.578614 & -1.004160 & -0.823389 \\
\hline 8 & 2.768191 & -1.358032 & -1.962515 \\
\hline 6 & 4.008712 & -1.842771 & -1.623517 \\
\hline 6 & 4.916563 & -1.058500 & -0.932359 \\
\hline 6 & 6.156497 & -1.651413 & -0.532117 \\
\hline 6 & 6.474051 & -2.977565 & -0.954185 \\
\hline 6 & 5.545426 & -3.693349 & -1.749386 \\
\hline 6 & 4.331597 & -3.144578 & -2.063051 \\
\hline 6 & 7.071469 & -0.987893 & 0.324534 \\
\hline 6 & 8.241514 & -1.590561 & 0.716170 \\
\hline 6 & 8.564319 & -2.890799 & 0.273849 \\
\hline 6 & 7.693485 & -3.567326 & -0.541934 \\
\hline 6 & 4.547341 & 0.334260 & -0.594069 \\
\hline 6 & 3.359440 & 0.560207 & 0.072762 \\
\hline 6 & 2.894774 & 1.851459 & 0.387793 \\
\hline 6 & 3.642819 & 2.939684 & 0.029150 \\
\hline 6 & 4.855721 & 2.780122 & -0.682279 \\
\hline 6 & 5.313133 & 1.469520 & -1.016277 \\
\hline 6 & 5.604566 & 3.907497 & -1.098208 \\
\hline 6 & 6.751189 & 3.760380 & -1.835493 \\
\hline 6 & 7.186864 & 2.468875 & -2.198628 \\
\hline 6 & 6.487595 & 1.355433 & -1.802610 \\
\hline 8 & 2.566192 & -0.522765 & 0.414082 \\
\hline 6 & 0.954752 & 2.783448 & 4.017828 \\
\hline 6 & 1.766014 & 1.488617 & 4.053794 \\
\hline 6 & -3.315587 & 4.802848 & 2.080984 \\
\hline 6 & -2.986415 & 5.957406 & 3.023252 \\
\hline 6 & -3.338992 & -0.021673 & -0.331598 \\
\hline 6 & -3.617153 & -0.153289 & -1.692154 \\
\hline 6 & -4.931677 & -0.264201 & -2.146439 \\
\hline 6 & -5.954826 & -0.272860 & -1.189839 \\
\hline 6 & -5.707360 & -0.183424 & 0.181639 \\
\hline 6 & -4.379809 & -0.044215 & 0.593774 \\
\hline 6 & -6.807876 & -0.256103 & 1.239245 \\
\hline 6 & -8.196060 & -0.414749 & 0.625062 \\
\hline 6 & -5.262416 & -0.289351 & -3.638405 \\
\hline 6 & -4.055086 & -0.693688 & -4.485058 \\
\hline 45 & 0.071115 & 0.557657 & -1.528438 \\
\hline 6 & 1.280656 & 1.165209 & -2.807622 \\
\hline 8 & 2.063803 & 1.514269 & -3.583830 \\
\hline
\end{tabular}




\begin{tabular}{|c|c|c|c|}
\hline 6 & -6.550237 & -1.463125 & 2.149985 \\
\hline 6 & -6.799424 & 1.025074 & 2.079908 \\
\hline 6 & -6.398021 & -1.269334 & -3.943836 \\
\hline 6 & -5.698019 & 1.122915 & -4.048039 \\
\hline 6 & 1.835093 & -3.540925 & 0.444840 \\
\hline 6 & 2.566032 & -3.079004 & 1.674125 \\
\hline 6 & 1.865683 & -2.529234 & 2.749494 \\
\hline 6 & 2.544061 & -2.096819 & 3.881293 \\
\hline 6 & 3.932961 & -2.200062 & 3.949207 \\
\hline 6 & 4.635107 & -2.755439 & 2.885143 \\
\hline 6 & 3.950824 & -3.197982 & 1.756371 \\
\hline 6 & 0.409741 & 3.027726 & 5.430138 \\
\hline 6 & 1.883940 & 3.937780 & 3.628338 \\
\hline 6 & -3.365509 & 5.354572 & 0.651148 \\
\hline 6 & -4.694923 & 4.250032 & 2.455398 \\
\hline 1 & 6.827647 & 0.007661 & 0.686699 \\
\hline 1 & -1.906761 & -0.045026 & 3.181423 \\
\hline 1 & 7.918828 & -4.578112 & -0.879763 \\
\hline 1 & -2.055273 & -4.258836 & 5.320271 \\
\hline 1 & 5.236806 & 4.896390 & -0.826046 \\
\hline 1 & -3.949943 & -3.126807 & 0.349827 \\
\hline 1 & -5.830200 & -3.925015 & -1.023559 \\
\hline 1 & 3.285601 & 3.945800 & 0.246896 \\
\hline 1 & -2.090242 & -1.864542 & 4.797632 \\
\hline 1 & 5.804452 & -4.695382 & -2.087719 \\
\hline 1 & 8.082004 & 2.353090 & -2.805812 \\
\hline 1 & 3.594915 & -3.687173 & -2.652473 \\
\hline 1 & -3.167064 & -4.656917 & -4.318795 \\
\hline 1 & -0.789372 & -4.131140 & -4.111140 \\
\hline 1 & -1.486180 & -7.127365 & 2.169543 \\
\hline 1 & 6.830383 & 0.369236 & -2.104602 \\
\hline 1 & -1.342240 & -5.322340 & 0.517941 \\
\hline 1 & 1.116417 & -3.317066 & -2.744361 \\
\hline 1 & 7.316529 & 4.633259 & -2.153880 \\
\hline 1 & 8.922388 & -1.062681 & 1.380210 \\
\hline 1 & -5.444342 & -4.728701 & -3.347200 \\
\hline 1 & -1.849067 & -6.613867 & 4.579312 \\
\hline 1 & 9.495516 & -3.355875 & 0.588987 \\
\hline 1 & 1.934829 & 1.957246 & 0.890221 \\
\hline 1 & -4.151657 & 0.036611 & 1.656637 \\
\hline 1 & -2.780231 & -0.174916 & -2.387042 \\
\hline 1 & -6.983325 & -0.359300 & -1.532038 \\
\hline 1 & -3.329139 & 2.460440 & 0.707369 \\
\hline 1 & 0.353452 & 0.814205 & 2.153987 \\
\hline 1 & -1.026879 & 4.592639 & 3.632323 \\
\hline 1 & 1.111346 & -4.320639 & 0.723806 \\
\hline 6 & -1.232698 & 2.153393 & -2.166949 \\
\hline 1 & 2.541145 & -4.002319 & -0.264085 \\
\hline 1 & 4.503497 & -3.620845 & 0.916686 \\
\hline 1 & 0.783078 & -2.423534 & 2.683031 \\
\hline 1 & 5.719801 & -2.840355 & 2.923863 \\
\hline 1 & 1.986546 & -1.673433 & 4.715482 \\
\hline 1 & 4.463622 & -1.850713 & 4.832519 \\
\hline 1 & 2.544945 & 1.559996 & 4.823726 \\
\hline 1 & 1.132382 & 0.624404 & 4.299200 \\
\hline 1 & 2.272921 & 1.277149 & 3.103257 \\
\hline
\end{tabular}




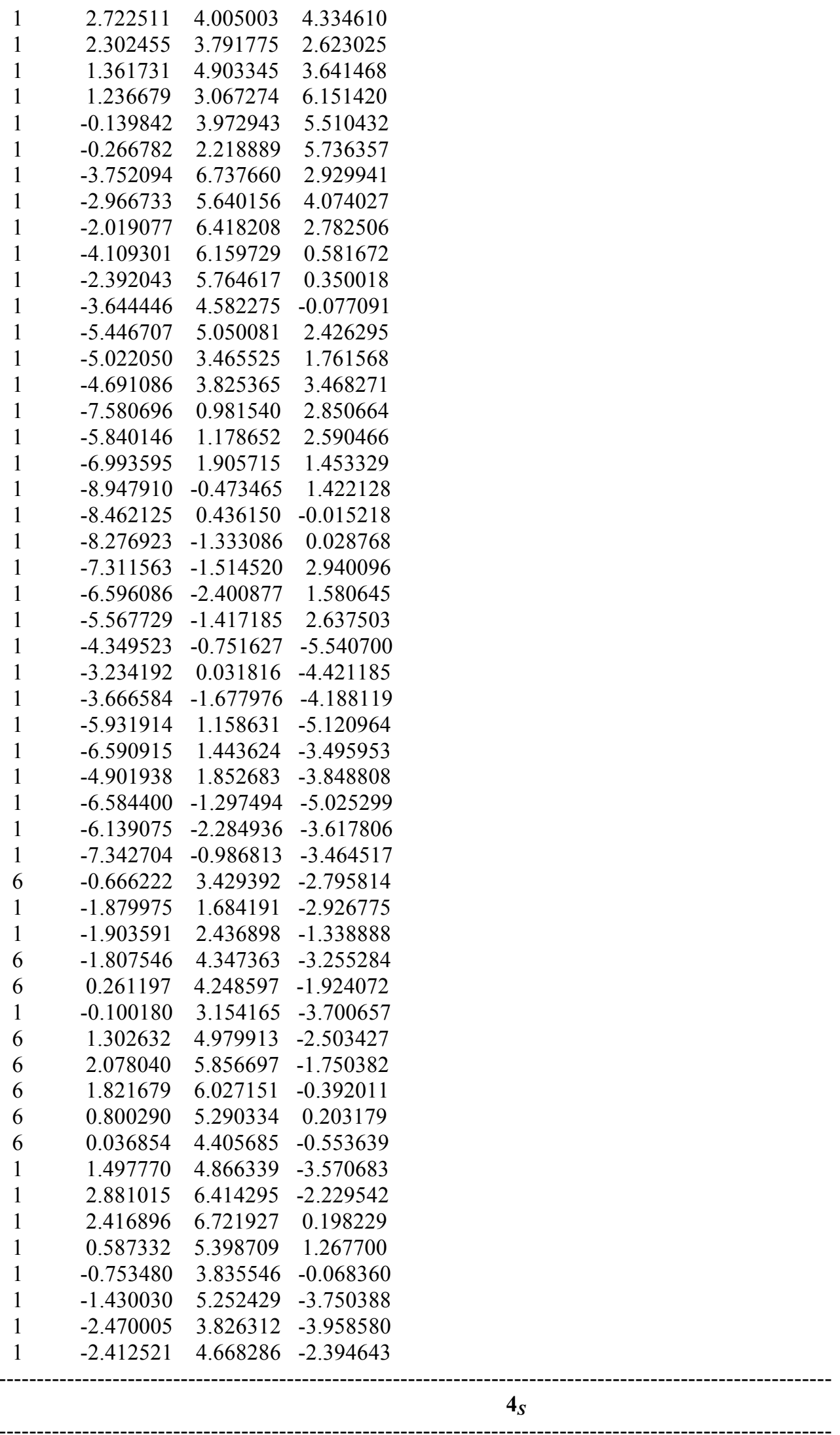

Number of imaginary frequencies : 0 
The smallest frequencies are : $16.0098 \quad 17.5545 \quad 20.4772 \mathrm{~cm}(-1)$ Electronic energy : $\quad \mathrm{HF}=-4360.911977$

$\begin{array}{lc}\text { Zero-point correction }= & 1.458203 \text { (Hartree/Particle) } \\ \text { Thermal correction to Energy= } & 1.542643 \\ \text { Thermal correction to Enthalpy= } & 1.543587 \\ \text { Thermal correction to Gibbs Free Energy= } & 1.340456 \\ \text { Sum of electronic and zero-point Energies }= & -4359.453774 \\ \text { Sum of electronic and thermal Energies }= & -4359.369334 \\ \text { Sum of electronic and thermal Enthalpies }= & -4359.368390 \\ \text { Sum of electronic and thermal Free Energies }= & -4359.571521\end{array}$

Cartesian Coordinates

$\begin{array}{cccc}6 & 7.026559 & -1.123446 & 0.336993 \\ 6 & 6.101233 & -1.727129 & -0.552139 \\ 6 & 6.388977 & -3.041215 & -1.028996 \\ 6 & 7.590355 & -3.678499 & -0.634794 \\ 6 & 8.472483 & -3.058932 & 0.213541 \\ 6 & 8.179166 & -1.770785 & 0.708587 \\ 6 & 4.879101 & -1.086728 & -0.933676 \\ 6 & 3.955653 & -1.818158 & -1.661681 \\ 6 & 4.250182 & -3.107303 & -2.155557 \\ 6 & 5.448587 & -3.698361 & -1.860037 \\ 6 & 4.547083 & 0.301948 & -0.544691 \\ 6 & 3.358199 & 0.537587 & 0.116852 \\ 6 & 2.923077 & 1.829871 & 0.468832 \\ 6 & 3.704801 & 2.908238 & 0.155746 \\ 6 & 4.921032 & 2.740014 & -0.547744 \\ 6 & 5.347693 & 1.429660 & -0.920753 \\ 6 & 5.703573 & 3.860684 & -0.917185 \\ 6 & 6.853677 & 3.708497 & -1.647882 \\ 6 & 7.258734 & 2.418919 & -2.051111 \\ 6 & 6.526516 & 1.311576 & -1.700032 \\ 8 & 2.534642 & -0.534653 & 0.416522 \\ 15 & 1.542071 & -0.946132 & -0.842813 \\ 8 & 2.728543 & -1.289913 & -1.986850 \\ 45 & 0.056876 & 0.668300 & -1.473132 \\ 15 & -1.605335 & 0.085162 & 0.271494 \\ 6 & -1.614849 & -1.337564 & 1.483584 \\ 6 & -1.541555 & -2.648773 & 1.034884 \\ 6 & -1.615279 & -3.737196 & 1.961577 \\ 6 & -1.789634 & -3.468799 & 3.349680 \\ 6 & -1.893071 & -2.125078 & 3.772271 \\ 6 & -1.806453 & -1.096720 & 2.871421 \\ 6 & -1.853354 & -4.544744 & 4.267720 \\ 6 & -1.745632 & -5.841927 & 3.836917 \\ 6 & -1.570122 & -6.115139 & 2.463398 \\ 6 & -1.509681 & -5.092340 & 1.550133 \\ 6 & -1.414339 & -2.965964 & -0.410756 \\ 6 & -0.187385 & -2.864287 & -1.039038 \\ 6 & -0.053049 & -3.178012 & -2.413368 \\ 6 & -1.134300 & -3.599167 & -3.140653 \\ 6 & -2.403690 & -3.736909 & -2.530482 \\ 6 & -2.553245 & -3.409111 & -1.150997 \\ 6 & -3.840370 & -3.523408 & -0.564580 \\ 6 & -4.913264 & -3.958873 & -1.301485\end{array}$




\begin{tabular}{|c|c|c|c|}
\hline & -4.757304 & -4.305988 & -2.661101 \\
\hline & -3.529464 & -4.188719 & -3.262108 \\
\hline & 0.962646 & -2.435158 & -0.291149 \\
\hline & 1.746519 & -3.530872 & 0.321669 \\
\hline & 2.513120 & -3.143947 & 1.555467 \\
\hline & 1.864077 & -2.570334 & 2.650553 \\
\hline & 2.580646 & -2.224905 & 3.788533 \\
\hline & 3.956730 & -2.443496 & 3.845860 \\
\hline & 4.607687 & -3.019530 & 2.760916 \\
\hline & 3.884812 & -3.372077 & 1.624395 \\
\hline & -1.310000 & 2.264529 & -1.978858 \\
\hline 6 & -0.897309 & 3.588664 & -2.641477 \\
\hline 6 & -0.653504 & 3.435987 & -4.136845 \\
\hline & 0.180724 & 4.351578 & -1.894703 \\
\hline 6 & 0.044148 & 4.550865 & -0.513854 \\
\hline 6 & 0.926962 & 5.355228 & 0.195286 \\
\hline 6 & 1.997963 & 5.965850 & -0.456723 \\
\hline 6 & 2.175218 & 5.741668 & -1.817164 \\
\hline 6 & 1.273760 & 4.948362 & -2.526398 \\
\hline 6 & -3.338118 & -0.009608 & -0.321330 \\
\hline 6 & -3.573573 & -0.122142 & -1.692060 \\
\hline 6 & -4.872573 & -0.189930 & -2.193502 \\
\hline 6 & -5.926580 & -0.202635 & -1.270128 \\
\hline 6 & -5.722073 & -0.148472 & 0.110394 \\
\hline 6 & -4.407333 & -0.032762 & 0.569570 \\
\hline 6 & -5.146578 & -0.164696 & -3.696940 \\
\hline 6 & -6.297250 & -1.102337 & -4.069670 \\
\hline 6 & -6.856550 & -0.236616 & 1.129899 \\
\hline 6 & -6.633773 & -1.467228 & 2.018160 \\
\hline 6 & -1.528997 & 1.520771 & 1.411204 \\
\hline 6 & -2.477152 & 2.534582 & 1.429230 \\
\hline 6 & -2.298914 & 3.674329 & 2.229981 \\
\hline 6 & -1.174605 & 3.725566 & 3.049373 \\
\hline 6 & -0.220617 & 2.694387 & 3.090894 \\
\hline 6 & -0.393734 & 1.618367 & 2.228473 \\
\hline 6 & -3.333260 & 4.797011 & 2.165584 \\
\hline 6 & -3.377109 & 5.365564 & 0.742174 \\
\hline 6 & 0.949306 & 2.774324 & 4.071250 \\
\hline 6 & 0.411558 & 3.020991 & 5.486066 \\
\hline 6 & -5.523771 & 1.271066 & -4.083490 \\
\hline 6 & -3.920534 & -0.580730 & -4.511739 \\
\hline 6 & -6.867697 & 1.023907 & 2.001012 \\
\hline 6 & -8.225077 & -0.372590 & 0.468111 \\
\hline 6 & 1.879740 & 3.925920 & 3.677383 \\
\hline 6 & 1.756482 & 1.476665 & 4.105175 \\
\hline 6 & -4.714603 & 4.241078 & 2.528159 \\
\hline 6 & -3.007705 & 5.941197 & 3.121588 \\
\hline 1 & -1.789816 & 4.243447 & -2.565955 \\
\hline 1 & 6.804803 & -0.138476 & 0.740084 \\
\hline 1 & -1.894043 & -0.076397 & 3.233748 \\
\hline 1 & 7.792775 & -4.679391 & -1.014156 \\
\hline 1 & -1.986460 & -4.317203 & 5.324424 \\
\hline 1 & 5.358455 & 4.849213 & -0.615296 \\
\hline 1 & -3.972478 & -3.251314 & 0.481440 \\
\hline 1 & -5.894383 & -4.033585 & -0.836718 \\
\hline 1 & 3.374181 & 3.915917 & 0.403350 \\
\hline
\end{tabular}




\begin{tabular}{|c|c|c|c|}
\hline & -2.044198 & -1.915763 & 4.830506 \\
\hline & 5.685290 & -4.690457 & -2.241219 \\
\hline ] & 8.156566 & 2.300037 & -2.653704 \\
\hline 1 & 3.504168 & -3.606097 & -2.771109 \\
\hline & -3.396941 & -4.440485 & -4.313787 \\
\hline & -1.030746 & -3.838732 & -4.197946 \\
\hline & -1.483900 & -7.145418 & 2.126000 \\
\hline & 6.846415 & 0.327630 & -2.032726 \\
\hline & -1.380185 & -5.318135 & 0.493696 \\
\hline & 0.925094 & -3.070771 & -2.879239 \\
\hline & 7.445066 & 4.576354 & -1.930677 \\
\hline & 8.868568 & -1.288558 & 1.398066 \\
\hline & -5.614083 & -4.660439 & -3.230395 \\
\hline I & -1.792980 & -6.662108 & 4.549451 \\
\hline & 9.389812 & -3.560126 & 0.513695 \\
\hline & 1.958888 & 1.945194 & 0.961344 \\
\hline & -4.211857 & 0.029425 & 1.640458 \\
\hline & -2.715085 & -0.151122 & -2.359375 \\
\hline & -6.945062 & -0.261985 & -1.646483 \\
\hline & -3.361934 & 2.451154 & 0.799324 \\
\hline & 0.338294 & 0.810446 & 2.205420 \\
\hline & -1.027868 & 4.589894 & 3.693605 \\
\hline$\frac{1}{1}$ & 1.010719 & -4.305052 & 0.583257 \\
\hline & 2.427360 & -3.980818 & -0.418612 \\
\hline ] & 4.398401 & -3.811008 & 0.768368 \\
\hline & 0.794470 & -2.370003 & 2.592345 \\
\hline & 5.682734 & -3.189659 & 2.787566 \\
\hline 1 & 2.062745 & -1.781912 & 4.637795 \\
\hline & 4.517210 & -2.164239 & 4.735618 \\
\hline 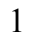 & 2.540599 & 1.548396 & 4.869538 \\
\hline & 1.120565 & 0.616492 & 4.358847 \\
\hline 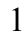 & 2.255900 & 1.259940 & 3.151820 \\
\hline$\frac{1}{1}$ & 2.720907 & 3.993071 & 4.380566 \\
\hline & 2.294163 & 3.776801 & 2.670959 \\
\hline 1 & 1.358951 & 4.892243 & 3.690015 \\
\hline & 1.241828 & 3.057129 & 6.203707 \\
\hline 1 & -0.133724 & 3.968479 & 5.568651 \\
\hline & -0.267014 & 2.215096 & 5.795408 \\
\hline ] & -3.772490 & 6.722966 & 3.033712 \\
\hline 1 & -2.991619 & 5.612273 & 4.168860 \\
\hline & -2.039287 & 6.403779 & 2.888884 \\
\hline 1 & -4.134579 & 6.158025 & 0.673912 \\
\hline & -2.408295 & 5.798314 & 0.458865 \\
\hline 1 & -3.631453 & 4.598285 & -0.000250 \\
\hline & -5.463592 & 5.044072 & 2.513362 \\
\hline 1 & -5.044793 & 3.471333 & 1.819519 \\
\hline 1 & -4.713476 & 3.797427 & 3.532817 \\
\hline 1 & -7.674263 & 0.967684 & 2.744447 \\
\hline 1 & -5.924900 & 1.158046 & 2.546635 \\
\hline 1 & -7.035195 & 1.920715 & 1.389832 \\
\hline 1 & -9.002612 & -0.443391 & 1.239129 \\
\hline 1 & -8.465407 & 0.493643 & -0.161692 \\
\hline 1 & -8.291688 & -1.277333 & -0.150327 \\
\hline 1 & -7.428433 & -1.543188 & 2.772678 \\
\hline 1 & -6.648705 & -2.388372 & 1.420548 \\
\hline 1 & -5.673621 & -1.430885 & 2.548986 \\
\hline
\end{tabular}




$\begin{array}{rrrr}1 & -4.176245 & -0.589580 & -5.578977 \\ 1 & -3.076614 & 0.111418 & -4.390987 \\ 1 & -3.577967 & -1.588491 & -4.237879 \\ 1 & -5.733001 & 1.337458 & -5.160075 \\ 1 & -6.417109 & 1.610623 & -3.543473 \\ 1 & -4.707069 & 1.968697 & -3.853010 \\ 1 & -6.436675 & -1.108354 & -5.158513 \\ 1 & -6.084297 & -2.129876 & -3.747778 \\ 1 & --7.252657 & -0.796751 & -3.627141 \\ 1 & -2.058388 & 1.801411 & -2.640130 \\ 1 & -1.845007 & 2.508508 & -1.051235 \\ 1 & 1.430172 & 4.800349 & -3.592967 \\ 1 & 3.016270 & 6.193786 & -2.340184 \\ 1 & 2.690605 & 6.601305 & 0.093251 \\ 1 & 0.771511 & 5.502928 & 1.265048 \\ 1 & -0.782272 & 4.077928 & 0.015972 \\ 1 & -0.521326 & 4.405977 & -4.632858 \\ 1 & 0.222593 & 2.820680 & -4.370086 \\ 1 & -1.523048 & 2.950399 & -4.598914 \\ 6 & 1.322417 & 1.352040 & -2.651537 \\ 8 & 2.189649 & 1.718116 & -3.324705 \\ ----------------------------------------------------------------- \\ \end{array}$

Number of imaginary frequencies : 0

The smallest frequencies are : $16.1187 \quad 22.3540 \quad 22.8691 \mathrm{~cm}(-1)$

Electronic energy : $\quad H F=-4360.9037249$

Zero-point correction $=\quad 1.456996($ Hartree/Particle $)$

Thermal correction to Energy $=\quad 1.541857$

Thermal correction to Enthalpy= $\quad 1.542801$

Thermal correction to Gibbs Free Energy= $\quad 1.339029$

Sum of electronic and zero-point Energies $=\quad-4359.446729$

Sum of electronic and thermal Energies $=\quad-4359.361868$

Sum of electronic and thermal Enthalpies $=\quad-4359.360923$

Sum of electronic and thermal Free Energies $=\quad-4359.564696$

Cartesian Coordinates

$\begin{array}{cccc}6 & -4.361290 & 0.037320 & 0.465868 \\ 6 & -3.273109 & 0.084061 & -0.400399 \\ 6 & -3.461937 & -0.119156 & -1.768408 \\ 6 & -4.739266 & -0.320166 & -2.293045 \\ 6 & -5.812574 & -0.357600 & -1.392932 \\ 6 & -5.651296 & -0.198851 & -0.014893 \\ 15 & -1.559299 & 0.264206 & 0.224632 \\ 6 & -1.508600 & 1.718476 & 1.341946 \\ 6 & -2.520847 & 2.660379 & 1.458672 \\ 6 & -2.358491 & 3.793037 & 2.271570 \\ 6 & -1.187159 & 3.898137 & 3.017028 \\ 6 & -0.175414 & 2.924368 & 2.979798 \\ 6 & -0.334618 & 1.863297 & 2.095204 \\ 6 & -3.439939 & 4.870815 & 2.271983 \\ 6 & -3.174632 & 5.961389 & 3.306667 \\ 6 & 1.048024 & 3.050656 & 3.887445 \\ 6 & 1.849692 & 1.749812 & 3.937936 \\ 6 & -4.976079 & -0.404253 & -3.801553\end{array}$




\begin{tabular}{|c|c|c|c|}
\hline & -3.741364 & -0.914029 & -4.546615 \\
\hline & -6.802096 & -0.311945 & 0.983034 \\
\hline & -8.141664 & -0.573369 & 0.300231 \\
\hline & 0.166110 & 0.695209 & -1.572695 \\
\hline & 1.546489 & -0.924114 & -0.752286 \\
\hline & 0.941596 & -2.351634 & -0.086760 \\
\hline & 1.701073 & -3.403782 & 0.626506 \\
\hline & 2.464906 & -2.928182 & 1.830305 \\
\hline & 3.830477 & -3.181785 & 1.931511 \\
\hline & 4.552653 & -2.756944 & 3.043319 \\
\hline & 3.906368 & -2.083026 & 4.073276 \\
\hline U & 2.536650 & -1.837768 & 3.984435 \\
\hline & 1.820850 & -2.252940 & 2.869024 \\
\hline & -0.789138 & 2.555700 & -2.454700 \\
\hline & -2.193034 & 2.923028 & -1.980752 \\
\hline & -0.914348 & 2.397303 & -3.970526 \\
\hline 6 & 0.163455 & 3.648539 & -2.048421 \\
\hline & 1.452547 & 1.021138 & -2.877585 \\
\hline & 2.296322 & 1.181344 & -3.651401 \\
\hline & 2.536946 & -0.409905 & 0.468627 \\
\hline & 3.388773 & 0.610437 & 0.073140 \\
\hline & 4.572121 & 0.286610 & -0.560307 \\
\hline & 5.395315 & 1.355791 & -1.044568 \\
\hline & 4.995689 & 2.705250 & -0.803986 \\
\hline & 3.789595 & 2.966090 & -0.112384 \\
\hline & 2.985831 & 1.939783 & 0.302610 \\
\hline & 5.791017 & 3.768405 & -1.295699 \\
\hline & 6.933576 & 3.521281 & -2.012067 \\
\hline 6 & 7.317986 & 2.189940 & -2.275531 \\
\hline ) & 6.569825 & 1.137374 & -1.808665 \\
\hline & 4.876938 & -1.139002 & -0.814870 \\
\hline 6 & 3.942395 & -1.914972 & -1.478514 \\
\hline & 4.212685 & -3.247669 & -1.856737 \\
\hline 6 & 5.398897 & -3.832435 & -1.505034 \\
\hline 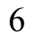 & 6.348826 & -3.122176 & -0.730176 \\
\hline & 6.084961 & -1.766053 & -0.371710 \\
\hline 6 & 7.018399 & -1.103809 & 0.465395 \\
\hline & 8.156725 & -1.737899 & 0.898877 \\
\hline 6 & 8.426944 & -3.069786 & 0.520032 \\
\hline 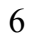 & 7.536406 & -3.744762 & -0.275689 \\
\hline & 2.727886 & -1.391813 & -1.855084 \\
\hline 6 & -0.180874 & -2.837890 & -0.843759 \\
\hline & -1.431825 & -2.867833 & -0.259636 \\
\hline 5 & -2.545712 & -3.369739 & -0.999888 \\
\hline 6 & -2.340389 & -3.849689 & -2.326476 \\
\hline 6 & -1.044965 & -3.786397 & -2.893285 \\
\hline 6 & 0.008519 & -3.291697 & -2.171776 \\
\hline 6 & -3.439124 & -4.372893 & -3.051515 \\
\hline 6 & -4.694871 & -4.403352 & -2.499330 \\
\hline 6 & -4.906683 & -3.895187 & -1.199105 \\
\hline 6 & -3.858906 & -3.399377 & -0.464722 \\
\hline 6 & -1.592431 & -2.441974 & 1.152747 \\
\hline 6 & -1.611997 & -1.102596 & 1.516104 \\
\hline 6 & -1.816662 & -0.772373 & 2.884231 \\
\hline 6 & -1.973546 & -1.737739 & 3.843249 \\
\hline 0 & -1.924715 & -3.107626 & 3.507589 \\
\hline
\end{tabular}




\begin{tabular}{|c|c|c|c|}
\hline & -1.729353 & -3.468037 & 2.143639 \\
\hline & -1.663877 & -4.850568 & 1.822619 \\
\hline & -1.790373 & -5.809686 & 2.796270 \\
\hline & -1.994313 & -5.443963 & 4.144090 \\
\hline & -2.058908 & -4.119006 & 4.489293 \\
\hline & -3.471078 & 5.520440 & 0.882917 \\
\hline & -4.807630 & 4.249171 & 2.572206 \\
\hline & 0.605608 & 3.372598 & 5.319610 \\
\hline & 1.951155 & 4.177357 & 3.375245 \\
\hline & -6.143352 & -1.334470 & -4.140668 \\
\hline & -5.306322 & 1.006834 & -4.305387 \\
\hline & -6.519275 & -1.478174 & 1.938999 \\
\hline & -6.917690 & 0.987766 & 1.786687 \\
\hline & 6.813918 & -0.083215 & 0.779132 \\
\hline & -1.866397 & 0.268607 & 3.187786 \\
\hline & 7.721114 & -4.778638 & -0.564853 \\
\hline & -2.209493 & -3.818608 & 5.525230 \\
\hline & 5.459187 & 4.788524 & -1.103675 \\
\hline & -4.031745 & -3.005855 & 0.535995 \\
\hline & -5.910873 & -3.894866 & -0.778100 \\
\hline & 3.479298 & 3.998849 & 0.039301 \\
\hline & -2.137353 & -1.454937 & 4.882346 \\
\hline & 5.618367 & -4.858362 & -1.796381 \\
\hline & 8.211596 & 1.993758 & -2.864021 \\
\hline 1 & 3.459041 & -3.784593 & -2.429669 \\
\hline & -3.264846 & -4.745599 & -4.060263 \\
\hline 1 & -0.900136 & -4.140422 & -3.912912 \\
\hline & -1.733841 & -6.861816 & 2.526748 \\
\hline & 6.873130 & 0.119031 & -2.037164 \\
\hline 1 & -1.510170 & -5.150640 & 0.788402 \\
\hline & 1.005698 & -3.236611 & -2.604920 \\
\hline 1 & 7.534738 & 4.344728 & -2.390708 \\
\hline & 8.852747 & -1.210294 & 1.547206 \\
\hline ] & -5.531285 & -4.810284 & -3.063793 \\
\hline ] & -2.095377 & -6.215001 & 4.904319 \\
\hline & 9.333365 & -3.559844 & 0.867841 \\
\hline 1 & 2.026436 & 2.127845 & 0.781102 \\
\hline & -4.198866 & 0.163080 & 1.536593 \\
\hline 1 & -2.580861 & -0.107698 & -2.411905 \\
\hline ] & -6.812088 & -0.524941 & -1.785962 \\
\hline 1 & -3.441864 & 2.530125 & 0.892073 \\
\hline 1 & 0.435762 & 1.095099 & 2.013663 \\
\hline & -1.051425 & 4.758596 & 3.668060 \\
\hline 1 & 0.947315 & -4.138770 & 0.944760 \\
\hline 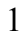 & 2.379821 & -3.926072 & -0.066346 \\
\hline 1 & 4.339936 & -3.701763 & 1.119736 \\
\hline 1 & 0.756821 & -2.030461 & 2.785320 \\
\hline 1 & 5.623107 & -2.949101 & 3.093564 \\
\hline 1 & 2.023197 & -1.317392 & 4.791336 \\
\hline 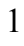 & 4.465269 & -1.748589 & 4.944786 \\
\hline 1 & 2.664081 & 1.842506 & 4.667751 \\
\hline 1 & 1.216768 & 0.905329 & 4.245956 \\
\hline 1 & 2.310884 & 1.491515 & 2.976156 \\
\hline ] & 2.837231 & 4.278618 & 4.016521 \\
\hline 1 & 2.297299 & 3.976169 & 2.352296 \\
\hline 1 & 1.428381 & 5.142731 & 3.372085 \\
\hline
\end{tabular}




$\begin{array}{lrrr}1 & 1.482225 & 3.423868 & 5.978503 \\ 1 & 0.086676 & 4.335079 & 5.396125 \\ 1 & -0.065000 & 2.596221 & 5.710806 \\ 1 & -3.987341 & 6.697888 & 3.280433 \\ 1 & -3.126484 & 5.557454 & 4.326490 \\ 1 & -2.240947 & 6.501632 & 3.104908 \\ 1 & -4.217810 & 6.325551 & 0.853225 \\ 1 & -2.493773 & 5.951986 & 0.627345 \\ 1 & -3.728908 & 4.796929 & 0.099182 \\ 1 & -5.580370 & 5.028888 & 2.592405 \\ 1 & -5.106190 & 3.517078 & 1.811995 \\ 1 & -4.810504 & 3.745509 & 3.548122 \\ 1 & -7.742975 & 0.919185 & 2.508118 \\ 1 & -6.003136 & 1.206636 & 2.352868 \\ 1 & -7.117977 & 1.840906 & 1.125028 \\ 1 & -8.932479 & -0.653386 & 1.056677 \\ 1 & -8.420661 & 0.239623 & -0.382380 \\ 1 & -8.135085 & -1.512958 & -0.268087 \\ 1 & -7.323589 & -1.567929 & 2.681678 \\ 1 & -6.462067 & -2.428802 & 1.391033 \\ 1 & -5.575128 & -1.351568 & 2.485222 \\ 1 & -3.965702 & -0.995382 & -5.617841 \\ 1 & -2.880511 & -0.240586 & -4.451467 \\ 1 & -3.437122 & -1.905717 & -4.186479 \\ 1 & -5.498402 & 0.993205 & -5.387114 \\ 1 & -6.198207 & 1.409229 & -3.807454 \\ 1 & -4.476024 & 1.701833 & -4.119524 \\ 1 & -6.236072 & -1.429273 & -5.230182 \\ 1 & -5.984524 & -2.338503 & -3.725077 \\ 1 & -7.105419 & -0.958516 & -3.772586 \\ 6 & 1.093096 & 4.225256 & -2.931048 \\ 6 & 1.943986 & 5.253868 & -2.534532 \\ 6 & 1.886002 & 5.767619 & -1.243475 \\ 6 & 0.969135 & 5.216435 & -0.349064 \\ 6 & 0.134415 & 4.179174 & -0.743157 \\ 1 & 1.159225 & 3.869291 & -3.956565 \\ 1 & 2.649073 & 5.666925 & -3.254484 \\ 1 & 2.539846 & 6.583315 & -0.939677 \\ 1 & 0.894448 & 5.597645 & 0.670368 \\ 1 & -0.564260 & 3.771007 & -0.015272 \\ 1 & -2.481580 & 3.901997 & -2.408280 \\ 1 & -2.939039 & 2.194873 & -2.316750 \\ 1 & -2.290182 & 3.015627 & -0.897843 \\ 1 & -1.335502 & 3.313947 & -4.426630 \\ 1 & 0.017422 & 2.176108 & -4.500334 \\ 1 & -1.605525 & 1.577214 & -4.207912\end{array}$

\section{$\mathbf{5}_{R}$}

Number of imaginary frequencies : 0

The smallest frequencies are : $14.1255 \quad 17.6951 \quad 18.8344 \mathrm{~cm}(-1)$

Electronic energy : $\quad \mathrm{HF}=-4474.1903351$

Zero-point correction $=\quad 1.466883($ Hartree/Particle $)$

Thermal correction to Energy= $\quad 1.554012$

Thermal correction to Enthalpy= $\quad 1.554956$ 
Thermal correction to Gibbs Free Energy= Sum of electronic and zero-point Energies= Sum of electronic and thermal Energies= Sum of electronic and thermal Enthalpies= Sum of electronic and thermal Free Energies=

\author{
1.344979 \\ $-4472.723452$ \\ $-4472.636323$ \\ $-4472.635379$ \\ $-4472.845356$
}

Cartesian Coordinates

\begin{tabular}{|c|c|c|c|}
\hline 6 & 5.801818 & 5.035284 & -0.060016 \\
\hline 6 & 5.090716 & 3.815033 & 0.041930 \\
\hline 6 & 5.494263 & 2.696929 & -0.749810 \\
\hline 6 & 6.572838 & 2.878077 & -1.652816 \\
\hline 6 & 7.233674 & 4.078391 & -1.738314 \\
\hline 6 & 6.856032 & 5.168050 & -0.926274 \\
\hline 6 & 4.781223 & 1.458507 & -0.633848 \\
\hline 6 & 3.658976 & 1.432838 & 0.171283 \\
\hline 6 & 3.256068 & 2.532793 & 0.950591 \\
\hline 6 & 3.971127 & 3.697200 & 0.899737 \\
\hline 6 & 5.177000 & 0.242447 & -1.380179 \\
\hline 6 & 4.258506 & -0.394648 & -2.194659 \\
\hline 6 & 4.620894 & -1.464658 & -3.040811 \\
\hline 6 & 5.890916 & -1.970118 & -2.995947 \\
\hline 6 & 6.837738 & -1.451931 & -2.078597 \\
\hline 6 & 6.481958 & -0.339245 & -1.259104 \\
\hline 6 & 8.116258 & -2.043757 & -1.935672 \\
\hline 6 & 9.006167 & -1.581799 & -0.999793 \\
\hline 6 & 8.646156 & -0.503105 & -0.165374 \\
\hline 6 & 7.421640 & 0.104878 & -0.294414 \\
\hline 8 & 2.947579 & 0.026773 & -2.244166 \\
\hline 15 & 1.915924 & -0.175831 & -0.941757 \\
\hline 8 & 2.916537 & 0.279112 & 0.282707 \\
\hline 7 & 1.857535 & -1.819556 & -0.546899 \\
\hline 6 & 0.817936 & -2.551654 & -1.218036 \\
\hline 6 & -0.258302 & -3.055705 & -0.509424 \\
\hline 6 & -1.280370 & -3.776057 & -1.201497 \\
\hline 6 & -1.167040 & -3.978679 & -2.608680 \\
\hline 6 & -0.049592 & -3.449193 & -3.293634 \\
\hline 6 & 0.915510 & -2.755363 & -2.614212 \\
\hline 6 & -2.170212 & -4.712930 & -3.288066 \\
\hline 6 & -3.247043 & -5.222842 & -2.608731 \\
\hline 6 & -3.379473 & -4.996051 & -1.220911 \\
\hline 6 & -2.420511 & -4.295596 & -0.534115 \\
\hline 6 & -0.347799 & -2.925133 & 0.969507 \\
\hline 6 & -0.854538 & -1.783177 & 1.572073 \\
\hline 6 & -1.009919 & -1.760415 & 2.985550 \\
\hline 6 & -0.640597 & -2.825259 & 3.762848 \\
\hline 6 & -0.087552 & -3.988808 & 3.181155 \\
\hline 6 & 0.050062 & -4.048040 & 1.764265 \\
\hline 6 & 0.602601 & -5.224970 & 1.194630 \\
\hline 6 & 1.008068 & -6.270751 & 1.986566 \\
\hline 6 & 0.874140 & -6.202903 & 3.389337 \\
\hline 6 & 0.334128 & -5.084485 & 3.971156 \\
\hline 15 & -1.373304 & -0.336216 & 0.518221 \\
\hline 6 & -1.597843 & 0.978388 & 1.778148 \\
\hline 6 & -0.504280 & 1.281462 & 2.603994 \\
\hline 6 & -0.560882 & 2.327858 & 3.515727 \\
\hline
\end{tabular}




\begin{tabular}{|c|c|c|c|}
\hline 6 & -1.709003 & 3.137692 & 3.514189 \\
\hline 6 & -2.791821 & 2.895687 & 2.675275 \\
\hline & -2.731692 & 1.778390 & 1.827507 \\
\hline & 0.577428 & 2.620172 & 4.492189 \\
\hline & 0.024158 & 2.707563 & 5.919304 \\
\hline & -4.017386 & 3.803726 & 2.614845 \\
\hline & -5.289891 & 2.985760 & 2.860005 \\
\hline & 3.004859 & -2.639057 & -0.082910 \\
\hline & 3.618394 & -2.249531 & 1.236305 \\
\hline & 2.831387 & -1.977000 & 2.356598 \\
\hline & 3.420135 & -1.693234 & 3.581658 \\
\hline & 4.808352 & -1.669276 & 3.705622 \\
\hline & 5.599348 & -1.929473 & 2.592550 \\
\hline & 5.005921 & -2.219274 & 1.366001 \\
\hline 45 & -0.090997 & 0.937906 & -1.310719 \\
\hline 6 & -0.267707 & 0.308498 & -3.118837 \\
\hline & -0.378071 & -0.000001 & -4.229325 \\
\hline & 0.310308 & 2.583770 & -0.393800 \\
\hline & 0.351996 & 3.623633 & 0.111544 \\
\hline & -3.085881 & -0.858056 & 0.085494 \\
\hline & -4.001666 & -1.188409 & 1.079508 \\
\hline & -5.296421 & -1.608251 & 0.761056 \\
\hline 6 & -5.649962 & -1.653964 & -0.587518 \\
\hline & -4.753365 & -1.333797 & -1.616569 \\
\hline 6 & -3.459331 & -0.957532 & -1.257093 \\
\hline 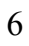 & -6.238343 & -2.015015 & 1.891995 \\
\hline & -6.437542 & -0.833792 & 2.848066 \\
\hline 6 & -5.223022 & -1.366953 & -3.071161 \\
\hline o & -6.295303 & -0.290130 & -3.275582 \\
\hline 6 & -7.609152 & -2.450987 & 1.382198 \\
\hline 5 & -5.614227 & -3.192584 & 2.651640 \\
\hline 6 & -4.080055 & -1.099715 & -4.048534 \\
\hline 6 & -5.824467 & -2.734864 & -3.407615 \\
\hline 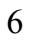 & -3.964082 & 4.923220 & 3.651201 \\
\hline 5 & -4.079977 & 4.439743 & 1.220230 \\
\hline 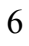 & 1.640647 & 1.522064 & 4.478667 \\
\hline 6 & 1.235055 & 3.954155 & 4.124357 \\
\hline 1 & 7.150586 & 0.921372 & 0.370031 \\
\hline & -1.428525 & -0.878952 & 3.464252 \\
\hline 1 & 8.370014 & -2.886803 & -2.577033 \\
\hline & 0.223045 & -5.016829 & 5.052465 \\
\hline 1 & 5.481233 & 5.871677 & 0.559616 \\
\hline & -2.537391 & -4.119833 & 0.534039 \\
\hline 1 & -4.251068 & -5.378035 & -0.693464 \\
\hline ] & 3.672825 & 4.556108 & 1.499307 \\
\hline & -0.769597 & -2.788004 & 4.843964 \\
\hline 1 & 6.181564 & -2.795469 & -3.643582 \\
\hline & 8.052330 & 4.191448 & -2.445519 \\
\hline 1 & 3.871997 & -1.866586 & -3.719107 \\
\hline 1 & -2.063767 & -4.866220 & -4.361182 \\
\hline 1 & 0.029521 & -3.599046 & -4.369215 \\
\hline 1 & 1.433518 & -7.160837 & 1.528746 \\
\hline 1 & 6.869398 & 2.054523 & -2.296876 \\
\hline 1 & 0.704190 & -5.294959 & 0.113374 \\
\hline 1 & 1.772194 & -2.337350 & -3.139414 \\
\hline 1 & 7.391359 & 6.111729 & -1.000414 \\
\hline
\end{tabular}




\begin{tabular}{|c|c|c|c|}
\hline ] & 9.342810 & -0.152529 & 0.592845 \\
\hline & -4.007707 & -5.793390 & -3.137598 \\
\hline & 1.198993 & -7.038734 & 4.004558 \\
\hline ] & 9.981363 & -2.050452 & -0.890648 \\
\hline & 2.370119 & 2.425201 & 1.572761 \\
\hline & -3.699290 & -1.123737 & 2.124533 \\
\hline & -2.711745 & -0.737971 & -2.016640 \\
\hline & -6.660945 & -1.953401 & -0.856488 \\
\hline & -3.579248 & 1.546092 & 1.183394 \\
\hline & 0.391335 & 0.663439 & 2.534183 \\
\hline & -1.745426 & 3.985121 & 4.195205 \\
\hline & 2.604713 & -3.658877 & 0.008356 \\
\hline & -1.935760 & 2.031709 & -1.688352 \\
\hline & 3.787266 & -2.682803 & -0.855466 \\
\hline & 5.633345 & -2.415419 & 0.494610 \\
\hline & 1.748179 & -1.961129 & 2.253992 \\
\hline & 6.684385 & -1.903820 & 2.672482 \\
\hline & 2.788303 & -1.491352 & 4.445730 \\
\hline ] & 5.269157 & -1.443628 & 4.664988 \\
\hline & 2.403253 & 1.733955 & 5.238813 \\
\hline ] & 1.203643 & 0.539345 & 4.707269 \\
\hline & 2.161614 & 1.441871 & 3.515653 \\
\hline & 0.839548 & 2.900946 & 6.629012 \\
\hline ] & -0.708683 & 3.514307 & 6.035691 \\
\hline & -0.462119 & 1.767677 & 6.211755 \\
\hline$\frac{1}{1}$ & 2.070189 & 4.171943 & 4.804009 \\
\hline & 1.626596 & 3.932854 & 3.098490 \\
\hline ] & 0.525035 & 4.787937 & 4.186655 \\
\hline ] & -4.871431 & 5.535719 & 3.577061 \\
\hline & -3.912026 & 4.533291 & 4.676355 \\
\hline 1 & -3.107042 & 5.589899 & 3.491315 \\
\hline & -4.913594 & 5.152696 & 1.157958 \\
\hline 1 & -3.150712 & 4.980525 & 0.993376 \\
\hline & -4.228548 & 3.692049 & 0.430614 \\
\hline 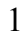 & -6.174088 & 3.634486 & 2.801267 \\
\hline ] & -5.418617 & 2.188515 & 2.117159 \\
\hline & -5.276681 & 2.521293 & 3.855242 \\
\hline 1 & -7.087802 & -1.124869 & 3.684048 \\
\hline & -5.490806 & -0.477089 & 3.273009 \\
\hline 1 & -6.911451 & 0.011537 & 2.331759 \\
\hline ] & -8.244200 & -2.736878 & 2.230198 \\
\hline 1 & -8.123105 & -1.643447 & 0.844943 \\
\hline 1 & -7.541215 & -3.319881 & 0.714752 \\
\hline 1 & -6.268751 & -3.508186 & 3.475341 \\
\hline 1 & -5.470164 & -4.053434 & 1.984675 \\
\hline & -4.636348 & -2.938738 & 3.080865 \\
\hline 1 & -4.456101 & -1.147489 & -5.078256 \\
\hline 1 & -3.638205 & -0.102250 & -3.911101 \\
\hline 1 & -3.279148 & -1.845950 & -3.950936 \\
\hline 1 & -6.655235 & -0.304142 & -4.313203 \\
\hline 1 & -7.160911 & -0.444107 & -2.619488 \\
\hline 1 & -5.895340 & 0.712659 & -3.074811 \\
\hline 1 & -6.158184 & -2.752144 & -4.453820 \\
\hline 1 & -5.081128 & -3.530832 & -3.274243 \\
\hline 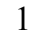 & -6.693892 & -2.974553 & -2.783608 \\
\hline 6 & -1.920161 & 3.367307 & -2.424068 \\
\hline
\end{tabular}


$5_{S}$

Number of imaginary frequencies : 0

The smallest frequencies are : $11.8620 \quad 18.4190 \quad 22.7393 \mathrm{~cm}(-1)$

Electronic energy : $\quad H F=-4474.1931805$

Zero-point correction $=\quad 1.467034($ Hartree/Particle $)$

Thermal correction to Energy $=\quad 1.553826$

Thermal correction to Enthalpy= $\quad 1.554770$

Thermal correction to Gibbs Free Energy $=\quad 1.346542$

Sum of electronic and zero-point Energies $=\quad-4472.726146$

Sum of electronic and thermal Energies $=\quad-4472.639354$

Sum of electronic and thermal Enthalpies $=\quad-4472.638410$

Sum of electronic and thermal Free Energies $=\quad-4472.846638$

Cartesian Coordinates

$\begin{array}{cccc}6 & -4.421285 & 3.212605 & -3.353792 \\ 6 & -3.301098 & 3.744668 & -2.710213 \\ 6 & -3.472587 & 4.906503 & -1.949636 \\ 6 & -4.719562 & 5.514341 & -1.839358 \\ 6 & -5.827090 & 4.968774 & -2.483544 \\ 6 & -5.672516 & 3.812505 & -3.242152 \\ 6 & -1.968726 & 3.024658 & -2.777144 \\ 1 & -2.015131 & 2.355663 & -3.652740 \\ 6 & -1.850971 & 2.157858 & -1.517097 \\ 45 & -0.053026 & 0.951749 & -1.218498 \\ 6 & 0.469452 & 2.561164 & -0.304037 \\ 8 & 0.600297 & 3.595579 & 0.197793 \\ 15 & 1.939221 & -0.204988 & -0.904285 \\ 7 & 1.870300 & -1.849140 & -0.516619 \\ 6 & 2.989201 & -2.684650 & -0.009095 \\ 6 & 3.628372 & -2.218591 & 1.272503 \\ 6 & 2.855174 & -1.894412 & 2.388737 \\ 6 & 3.454440 & -1.469414 & 3.566018 \\ 6 & 4.842560 & -1.364227 & 3.647623 \\ 6 & 5.620428 & -1.691525 & 2.542971 \\ 6 & 5.014788 & -2.117374 & 1.362451\end{array}$




\begin{tabular}{|c|c|c|c|}
\hline 8 & 2.950992 & 0.003463 & -2.218176 \\
\hline 6 & 4.253765 & -0.444850 & -2.189347 \\
\hline & 5.197517 & 0.180907 & -1.395728 \\
\hline & 6.498000 & -0.418196 & -1.304149 \\
\hline & 6.818106 & -1.539873 & -2.126518 \\
\hline & 5.840726 & -2.053855 & -3.013340 \\
\hline & 4.577566 & -1.530003 & -3.031312 \\
\hline 6 & 7.467343 & 0.012659 & -0.362674 \\
\hline 6 & 8.686208 & -0.611909 & -0.262107 \\
\hline 6 & 9.011727 & -1.696273 & -1.103178 \\
\hline 6 & 8.091865 & -2.148503 & -2.014214 \\
\hline & 4.835011 & 1.388872 & -0.618933 \\
\hline & 5.575039 & 2.613498 & -0.709193 \\
\hline f & 5.238671 & 3.702411 & 0.151594 \\
\hline & 4.161819 & 3.568330 & 1.060648 \\
\hline 6 & 3.414141 & 2.424296 & 1.084414 \\
\hline O & 3.739090 & 1.359607 & 0.222894 \\
\hline 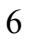 & 6.619428 & 2.807816 & -1.648737 \\
\hline & 7.308739 & 3.993608 & -1.709240 \\
\hline 6 & 6.996112 & 5.053760 & -0.833040 \\
\hline & 5.977692 & 4.907507 & 0.073115 \\
\hline & 2.957523 & 0.233248 & 0.308850 \\
\hline 6 & 0.806171 & -2.562977 & -1.166156 \\
\hline ) & -0.248518 & -3.081526 & -0.433094 \\
\hline 6 & -1.296521 & -3.779983 & -1.110209 \\
\hline 6 & -1.247435 & -3.917501 & -2.529137 \\
\hline 6 & -0.158549 & -3.362438 & -3.239153 \\
\hline 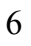 & 0.844207 & -2.711509 & -2.572348 \\
\hline 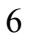 & -2.288093 & -4.607497 & -3.198165 \\
\hline 5 & -3.338633 & -5.142761 & -2.497451 \\
\hline 6 & -3.402545 & -4.989897 & -1.095357 \\
\hline 6 & -2.409381 & -4.327053 & -0.419279 \\
\hline f & -0.303911 & -2.943250 & 1.048610 \\
\hline & -0.773796 & -1.780601 & 1.646152 \\
\hline 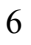 & -0.878834 & -1.722410 & 3.061708 \\
\hline 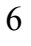 & -0.511326 & -2.782077 & 3.848130 \\
\hline 5 & -0.013111 & -3.972641 & 3.272205 \\
\hline ) & 0.084746 & -4.061009 & 1.852826 \\
\hline 6 & 0.581917 & -5.265955 & 1.289992 \\
\hline 6 & 0.968135 & -6.313071 & 2.089280 \\
\hline 6 & 0.872052 & -6.217635 & 3.493966 \\
\hline 6 & 0.389293 & -5.070589 & 4.069815 \\
\hline 15 & -1.321148 & -0.363203 & 0.571789 \\
\hline 6 & -2.994724 & -0.963638 & 0.099813 \\
\hline 6 & -3.906381 & -1.360515 & 1.072135 \\
\hline 6 & -5.197153 & -1.770686 & 0.728116 \\
\hline 6 & -5.544988 & -1.751994 & -0.623156 \\
\hline 6 & -4.651940 & -1.355969 & -1.628034 \\
\hline 6 & -3.364012 & -0.985309 & -1.245044 \\
\hline 6 & -6.163003 & -2.173550 & 1.840281 \\
\hline 6 & -5.537343 & -3.285591 & 2.690049 \\
\hline 6 & -5.129635 & -1.240722 & -3.074065 \\
\hline 6 & -5.774460 & -2.544466 & -3.550186 \\
\hline 6 & -1.682320 & 0.971079 & 1.775734 \\
\hline 6 & -0.663160 & 1.411810 & 2.624577 \\
\hline 6 & -0.837082 & 2.531275 & 3.437331 \\
\hline
\end{tabular}




\begin{tabular}{|c|c|c|c|}
\hline & -2.030925 & 3.251423 & 3.305915 \\
\hline & -3.049663 & 2.866621 & 2.430646 \\
\hline & -2.870954 & 1.693517 & 1.695231 \\
\hline & 0.257742 & 3.007926 & 4.391571 \\
\hline & 0.948881 & 4.228169 & 3.772513 \\
\hline & -4.313840 & 3.713040 & 2.288603 \\
\hline & -3.924989 & 5.159122 & 1.963252 \\
\hline & -0.338831 & 3.397957 & 5.747550 \\
\hline & 1.305621 & 1.923224 & 4.647095 \\
\hline & -5.221107 & 3.209282 & 1.165339 \\
\hline & -5.100467 & 3.674203 & 3.602707 \\
\hline & -0.244224 & 0.433584 & -3.058612 \\
\hline & -0.299485 & 0.230177 & -4.198065 \\
\hline & -6.439760 & -0.948923 & 2.721391 \\
\hline & -7.494479 & -2.685981 & 1.298368 \\
\hline & -6.162902 & -0.107268 & -3.133541 \\
\hline b & -3.986940 & -0.900752 & -4.029664 \\
\hline & 7.224533 & 0.831992 & 0.308834 \\
\hline & -1.265034 & -0.821717 & 3.532390 \\
\hline & 8.317161 & -2.997218 & -2.658742 \\
\hline & 0.308896 & -4.981396 & 5.152252 \\
\hline & 5.708183 & 5.721741 & 0.744427 \\
\hline | & -2.474593 & -4.204849 & 0.660461 \\
\hline & -4.250238 & -5.397857 & -0.548988 \\
\hline 1 & 3.923815 & 4.398676 & 1.724094 \\
\hline & -0.603399 & -2.720920 & 4.931903 \\
\hline 1 & 6.102960 & -2.890080 & -3.659182 \\
\hline & 8.099289 & 4.118434 & -2.445844 \\
\hline & 3.806152 & -1.927581 & -3.686621 \\
\hline 1 & -2.232317 & -4.705294 & -4.281814 \\
\hline & -0.129788 & -3.461133 & -4.323291 \\
\hline ] & 1.349036 & -7.225620 & 1.636488 \\
\hline & 6.865048 & 2.006325 & -2.340618 \\
\hline | & 0.653615 & -5.356029 & 0.207874 \\
\hline ] & 1.682528 & -2.280022 & -3.115794 \\
\hline & 7.553270 & 5.986033 & -0.888186 \\
\hline 1 & 9.405271 & -0.269587 & 0.478799 \\
\hline & -4.128877 & -5.678523 & -3.019180 \\
\hline 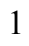 & 1.180995 & -7.055136 & 4.115063 \\
\hline 1 & 9.982957 & -2.177767 & -1.017263 \\
\hline 1 & 2.563863 & 2.303968 & 1.752622 \\
\hline 1 & -3.611664 & -1.333239 & 2.121172 \\
\hline ] & -2.622664 & -0.689433 & -1.985219 \\
\hline 1 & -6.553693 & -2.041204 & -0.911813 \\
\hline 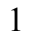 & -3.650834 & 1.352171 & 1.020601 \\
\hline 1 & 0.278747 & 0.861296 & 2.642562 \\
\hline 1 & -2.167246 & 4.153381 & 3.901838 \\
\hline 1 & 2.549541 & -3.678578 & 0.153581 \\
\hline 1 & 3.760581 & -2.809143 & -0.784562 \\
\hline 1 & 5.630806 & -2.353489 & 0.493069 \\
\hline 1 & 1.771225 & -1.935951 & 2.313081 \\
\hline 1 & 6.704506 & -1.606866 & 2.589504 \\
\hline 1 & 2.831561 & -1.217286 & 4.423416 \\
\hline ] & 5.313378 & -1.025385 & 4.568045 \\
\hline 1 & 2.025720 & 2.274287 & 5.396930 \\
\hline 1 & 0.846224 & 1.000213 & 5.028412 \\
\hline
\end{tabular}




$\begin{array}{rrrr}1 & 1.881429 & 1.665541 & 3.749026 \\ 1 & 0.460442 & 3.709140 & 6.432969 \\ 1 & -1.042759 & 4.234634 & 5.673862 \\ 1 & -0.867051 & 2.552490 & 6.207222 \\ 1 & 1.769823 & 4.576164 & 4.415053 \\ 1 & 1.360077 & 3.988233 & 2.782654 \\ 1 & 0.246858 & 5.060997 & 3.638905 \\ 1 & -6.009563 & 4.286021 & 3.524798 \\ 1 & -5.404493 & 2.647185 & 3.846503 \\ 1 & -4.510529 & 4.057498 & 4.444765 \\ 1 & -4.826036 & 5.771904 & 1.823504 \\ 1 & -3.331799 & 5.622856 & 2.760303 \\ 1 & -3.337594 & 5.202706 & 1.035785 \\ 1 & -6.092295 & 3.870525 & 1.071237 \\ 1 & -4.708179 & 3.204308 & 0.192482 \\ 1 & -5.598219 & 2.196159 & 1.360490 \\ 1 & -7.103825 & -1.215812 & 3.554814 \\ 1 & -5.517816 & -0.531278 & 3.147175 \\ 1 & -6.929106 & -0.155333 & 2.141207 \\ 1 & -8.143338 & -2.983263 & 2.131919 \\ 1 & -8.029833 & -1.917366 & 0.726535 \\ 1 & -7.360380 & -3.564311 & 0.653090 \\ 1 & -6.220791 & -3.576443 & 3.499015 \\ 1 & -5.336528 & -4.177623 & 2.082184 \\ 1 & -4.590621 & -2.979534 & 3.152355 \\ 1 & -4.370013 & -0.839775 & -5.056357 \\ 1 & -3.526822 & 0.069370 & -3.794345 \\ 1 & -3.196670 & -1.665090 & -4.012575 \\ 1 & -6.467014 & 0.085255 & -4.172509 \\ 1 & -7.066301 & -0.350048 & -2.560105 \\ 1 & -5.746036 & 0.821140 & -2.719793 \\ 1 & -6.151148 & -2.425459 & -4.575092 \\ 1 & -5.045371 & -3.365383 & -3.549397 \\ 1 & -6.622799 & -2.845233 & -2.923734 \\ 1 & -2.690214 & 1.448324 & -1.502521 \\ 1 & -1.974249 & 2.800357 & -0.631585 \\ 6 & -0.800094 & 3.978800 & -2.986078 \\ 1 & -2.617235 & 5.342845 & -1.433548 \\ 1 & -4.825967 & 6.419379 & -1.243252 \\ 1 & -6.802358 & 5.442868 & -2.396182 \\ 1 & -6.527726 & 3.373660 & -3.754169 \\ 1 & -4.305424 & 2.309504 & -3.956421 \\ 1 & -0.969115 & 4.626604 & -3.855302 \\ 1 & -0.640214 & 4.625970 & -2.114067 \\ 1 & 0.129481 & 3.419988 & -3.145642\end{array}$

\section{$5_{B}$}

Number of imaginary frequencies : 0

The smallest frequencies are : $11.9518 \quad 17.9173 \quad 22.8365 \mathrm{~cm}(-1)$

Electronic energy : $\quad H F=-4474.1780321$

Zero-point correction $=\quad 1.465862($ Hartree/Particle $)$

Thermal correction to Energy= $\quad 1.553044$

Thermal correction to Enthalpy= $\quad 1.553988$

Thermal correction to Gibbs Free Energy= $\quad 1.345170$

Sum of electronic and zero-point Energies $=\quad-4472.712170$ 
Sum of electronic and thermal Energies= Sum of electronic and thermal Enthalpies= Sum of electronic and thermal Free Energies=
$-4472.624988$

$-4472.624044$

$-4472.832862$

Cartesian Coordinates

$\begin{array}{cccc}6 & -7.211479 & -1.066935 & -0.219990 \\ 6 & -6.261510 & -1.247946 & 0.816806 \\ 6 & -6.513199 & -2.272704 & 1.777924 \\ 6 & -7.706707 & -3.030108 & 1.698589 \\ 6 & -8.613552 & -2.815300 & 0.692285 \\ 6 & -8.352395 & -1.829641 & -0.281554 \\ 6 & -5.039912 & -0.493502 & 0.883127 \\ 6 & -4.090010 & -0.899451 & 1.801187 \\ 6 & -4.344008 & -1.895457 & 2.768321 \\ 6 & -5.541555 & -2.554037 & 2.769802 \\ 6 & -4.771725 & 0.634874 & -0.040375 \\ 6 & -3.622873 & 0.655170 & -0.810794 \\ 6 & -3.409687 & 1.600517 & -1.832775 \\ 6 & -4.324388 & 2.593127 & -2.043126 \\ 6 & -5.458675 & 2.708958 & -1.203920 \\ 6 & -5.679864 & 1.737682 & -0.181041 \\ 6 & -6.366903 & 3.784657 & -1.353314 \\ 6 & -7.440139 & 3.925199 & -0.511485 \\ 6 & -7.639814 & 2.991022 & 0.525902 \\ 6 & -6.786695 & 1.926999 & 0.684748 \\ 8 & -2.656828 & -0.308032 & -0.669118 \\ 15 & -1.704244 & -0.434863 & 0.667419 \\ 8 & -2.854325 & -0.296519 & 1.859989 \\ 45 & -0.116271 & 1.211304 & 1.205641 \\ 6 & 0.190008 & 0.467876 & 2.969577 \\ 8 & 0.208629 & 0.076385 & 4.057544 \\ 6 & 1.099363 & 3.027149 & 1.860406 \\ 6 & 1.137330 & 4.080359 & 0.753662 \\ 6 & 0.485413 & 3.576777 & 3.128746 \\ 6 & 2.543624 & 2.601389 & 2.085215 \\ 6 & -1.387070 & 2.527964 & 0.687921 \\ 8 & -2.207263 & 3.336617 & 0.552374 \\ 15 & 1.648472 & 0.189324 & -0.462752 \\ 6 & 3.305723 & -0.382974 & 0.109563 \\ 6 & 4.290848 & -0.746280 & -0.806499 \\ 6 & 5.548647 & -1.181595 & -0.381721 \\ 6 & 5.796318 & -1.206881 & 0.992681 \\ 6 & 4.827163 & -0.860658 & 1.942765 \\ 6 & 3.569944 & -0.476650 & 1.474992 \\ 6 & 6.573495 & -1.627117 & -1.425384 \\ 6 & 5.981013 & -2.763401 & -2.268562 \\ 6 & 5.170431 & -0.816601 & 3.432067 \\ 6 & 6.069183 & -1.985877 & 3.841016 \\ 6 & 1.275133 & -1.213531 & -1.639101 \\ 6 & 1.038683 & -2.495069 & -1.147869 \\ 6 & 0.803811 & -3.578208 & -2.053453 \\ 6 & 0.812895 & -3.338299 & -3.459077 \\ 6 & 1.063866 & -2.029061 & -3.923434 \\ 6 & 1.282135 & -1.002599 & -3.042784 \\ 6 & 0.528554 & -4.398877 & -4.353265\end{array}$




\begin{tabular}{|c|c|c|c|}
\hline & 0.235162 & -5.653186 & -3.884699 \\
\hline & 0.226841 & -5.899236 & -2.494932 \\
\hline & 0.509681 & -4.893697 & -1.604915 \\
\hline & 1.013188 & -2.794355 & 0.313282 \\
\hline & -0.120913 & -2.533268 & 1.062265 \\
\hline & -0.169573 & -2.828028 & 2.444897 \\
\hline & 0.895065 & -3.417432 & 3.069427 \\
\hline & 2.050582 & -3.767998 & 2.331276 \\
\hline & 2.121708 & -3.444312 & 0.943328 \\
\hline & 3.285294 & -3.820534 & 0.225716 \\
\hline & 4.307643 & -4.496444 & 0.843641 \\
\hline & 4.229529 & -4.824061 & 2.213698 \\
\hline & 3.127200 & -4.455478 & 2.942492 \\
\hline & -1.314347 & -2.054399 & 0.423469 \\
\hline & -2.286906 & -3.138215 & 0.110345 \\
\hline & -3.077968 & -3.012788 & -1.165707 \\
\hline & -4.444046 & -3.284820 & -1.155095 \\
\hline & -5.189660 & -3.235297 & -2.331697 \\
\hline & -4.572315 & -2.915590 & -3.534849 \\
\hline & -3.204451 & -2.644889 & -3.553667 \\
\hline & -2.466749 & -2.694927 & -2.379763 \\
\hline & 2.044771 & 1.584449 & -1.598706 \\
\hline & 0.981171 & 2.140738 & -2.314992 \\
\hline & 1.152408 & 3.266065 & -3.118665 \\
\hline & 2.415743 & 3.868700 & -3.138314 \\
\hline & 3.489925 & 3.370486 & -2.399780 \\
\hline & 3.291074 & 2.205451 & -1.648938 \\
\hline & -0.044850 & 3.839564 & -3.874120 \\
\hline & -1.038595 & 4.403318 & -2.851520 \\
\hline & 4.833061 & 4.094953 & -2.319268 \\
\hline & 4.954799 & 4.711894 & -0.920116 \\
\hline & 5.989559 & 3.116719 & -2.541074 \\
\hline & 4.956837 & 5.214442 & -3.350682 \\
\hline & 0.349930 & 4.966065 & -4.826663 \\
\hline & -0.720927 & 2.741002 & -4.701594 \\
\hline & 6.917175 & -0.450317 & -2.343872 \\
\hline & 7.866942 & -2.132654 & -0.791891 \\
\hline & 5.913556 & 0.498115 & 3.703338 \\
\hline & 3.917943 & -0.857332 & 4.309386 \\
\hline & -7.016335 & -0.329204 & -0.993434 \\
\hline & 1.478455 & -0.007399 & -3.434070 \\
\hline & -7.879345 & -3.800745 & 2.448792 \\
\hline & 0.536492 & -4.190296 & -5.422320 \\
\hline & -6.182666 & 4.508040 & -2.146503 \\
\hline & 3.359681 & -3.569404 & -0.830720 \\
\hline & 5.188217 & -4.785749 & 0.272136 \\
\hline & -4.174105 & 3.325208 & -2.835634 \\
\hline & 1.077529 & -1.844366 & -4.996965 \\
\hline & -5.753405 & -3.320085 & 3.513739 \\
\hline & -8.473431 & 3.117973 & 1.212925 \\
\hline & -3.568913 & -2.107756 & 3.501616 \\
\hline & 3.055158 & -4.692004 & 4.003737 \\
\hline & 0.859620 & -3.646955 & 4.133499 \\
\hline & -0.004858 & -6.895922 & -2.126459 \\
\hline & -6.947862 & 1.225149 & 1.498687 \\
\hline & 0.504714 & -5.102226 & -0.537031 \\
\hline
\end{tabular}




\begin{tabular}{|c|c|c|c|}
\hline & -1.079529 & -2.584424 & 2.992468 \\
\hline & -8.126805 & 4.760055 & -0.630254 \\
\hline & -9.056699 & -1.676530 & -1.096274 \\
\hline & 5.045424 & -5.364556 & 2.688899 \\
\hline & 0.007240 & -6.459229 & -4.578122 \\
\hline & -9.522992 & -3.408825 & 0.635465 \\
\hline & -2.506309 & 1.507915 & -2.432940 \\
\hline & 4.069230 & -0.697439 & -1.873367 \\
\hline & 2.768354 & -0.240638 & 2.171087 \\
\hline & 6.778077 & -1.516902 & 1.342539 \\
\hline & 4.114687 & 1.794870 & -1.067747 \\
\hline & -0.006981 & 1.685734 & -2.220988 \\
\hline & 2.559561 & 4.762545 & -3.737444 \\
\hline & -1.677026 & -4.050341 & 0.049013 \\
\hline & -2.975449 & -3.284989 & 0.956418 \\
\hline & -4.939594 & -3.527460 & -0.214077 \\
\hline & -1.408191 & -2.450833 & -2.391350 \\
\hline & -6.257978 & -3.440411 & -2.297464 \\
\hline & -2.704978 & -2.392236 & -4.487966 \\
\hline & -5.152378 & -2.873069 & -4.454318 \\
\hline & -1.611748 & 3.138874 & -5.206653 \\
\hline & -0.040863 & 2.353833 & -5.472232 \\
\hline 1 & -1.042852 & 1.888792 & -4.090288 \\
\hline & -0.535618 & 5.308480 & -5.376879 \\
\hline ] & 0.755304 & 5.835031 & -4.293317 \\
\hline & 1.092602 & 4.637293 & -5.565297 \\
\hline ] & -1.903345 & 4.848979 & -3.363052 \\
\hline & -1.411474 & 3.631864 & -2.165954 \\
\hline & -0.567986 & 5.187176 & -2.243098 \\
\hline l & 5.949639 & 5.675363 & -3.274188 \\
\hline & 4.842496 & 4.840269 & -4.376530 \\
\hline l & 4.219089 & 6.010404 & -3.189890 \\
\hline ] & 5.906958 & 5.250711 & -0.818616 \\
\hline | & 4.139179 & 5.422967 & -0.733799 \\
\hline ] & 4.915334 & 3.946714 & -0.134169 \\
\hline 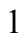 & 6.948622 & 3.649296 & -2.492230 \\
\hline 1 & 6.017485 & 2.327449 & -1.780364 \\
\hline & 5.920327 & 2.635787 & -3.525936 \\
\hline 1 & 7.631301 & -0.764047 & -3.117365 \\
\hline ] & 6.028928 & -0.054324 & -2.852540 \\
\hline 1 & 7.375324 & 0.370430 & -1.776092 \\
\hline 1 & 8.562695 & -2.452019 & -1.578037 \\
\hline 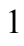 & 8.370515 & -1.352559 & -0.206614 \\
\hline 1 & 7.692309 & -2.995491 & -0.135394 \\
\hline & 6.706000 & -3.092728 & -3.025028 \\
\hline 1 & 5.731910 & -3.631575 & -1.643407 \\
\hline 1 & 5.068417 & -2.459495 & -2.797493 \\
\hline 1 & 4.208895 & -0.895264 & 5.366737 \\
\hline 1 & 3.287911 & 0.033141 & 4.185203 \\
\hline 1 & 3.300072 & -1.740601 & 4.095231 \\
\hline 1 & 6.169760 & 0.583225 & 4.768425 \\
\hline 1 & 6.845045 & 0.556823 & 3.125527 \\
\hline 1 & 5.296630 & 1.365384 & 3.432139 \\
\hline 1 & 6.263360 & -1.945774 & 4.920754 \\
\hline 1 & 5.592074 & -2.948052 & 3.615776 \\
\hline 1 & 7.044739 & -1.964106 & 3.341185 \\
\hline
\end{tabular}




$\begin{array}{rrrr}1 & 1.739298 & 4.942794 & 1.091669 \\ 1 & 1.615640 & 3.680693 & -0.146719 \\ 1 & 0.161351 & 4.471540 & 0.453399 \\ 1 & 3.133399 & 3.457145 & 2.461929 \\ 1 & 2.666067 & 1.782675 & 2.800398 \\ 1 & 3.009413 & 2.281418 & 1.145852 \\ 6 & 0.949629 & 3.198497 & 4.400025 \\ 6 & 0.394542 & 3.706576 & 5.569094 \\ 6 & -0.645457 & 4.627607 & 5.515751 \\ 6 & -1.109639 & 5.035890 & 4.269721 \\ 6 & -0.550848 & 4.525458 & 3.104059 \\ 1 & 1.761743 & 2.481027 & 4.491447 \\ 1 & 0.786968 & 3.379731 & 6.530589 \\ 1 & -1.081082 & 5.027108 & 6.429004 \\ 1 & -1.914567 & 5.765332 & 4.198167 \\ 1 & -0.943234 & 4.875857 & 2.153725\end{array}$

\section{$\mathrm{TS}_{R}$}

Number of imaginary frequencies : 1

The smallest frequencies are : -246.0869 $17.5102 \quad 17.7372 \mathrm{~cm}(-1)$

Electronic energy : $\quad \mathrm{HF}=-4474.170648$

Zero-point correction $=\quad 1.465556($ Hartree/Particle $)$

Thermal correction to Energy $=\quad 1.552150$

Thermal correction to Enthalpy= $\quad 1.553095$

Thermal correction to Gibbs Free Energy $=\quad 1.344241$

Sum of electronic and zero-point Energies $=\quad-4472.705093$

Sum of electronic and thermal Energies $=\quad-4472.618498$

Sum of electronic and thermal Enthalpies $=\quad-4472.617553$

Sum of electronic and thermal Free Energies $=\quad-4472.826407$

Cartesian Coordinates

$\begin{array}{cccc}6 & -0.869954 & 1.695654 & 2.526892 \\ 6 & -1.884821 & 1.597077 & 1.564319 \\ 6 & -2.771931 & 2.651455 & 1.397302 \\ 6 & -2.678229 & 3.808000 & 2.185287 \\ 6 & -1.685378 & 3.856427 & 3.159947 \\ 6 & -0.774574 & 2.805256 & 3.357932 \\ 15 & -1.852544 & 0.182128 & 0.398337 \\ 6 & -3.598787 & 0.048842 & -0.171219 \\ 6 & -3.864933 & -0.041607 & -1.537019 \\ 6 & -5.170275 & -0.197743 & -2.008253 \\ 6 & -6.200217 & -0.261900 & -1.062451 \\ 6 & -5.967297 & -0.189978 & 0.313269 \\ 6 & -4.648403 & -0.027315 & 0.741374 \\ 6 & -5.483661 & -0.237934 & -3.503889 \\ 6 & -6.594005 & -1.246851 & -3.811079 \\ 6 & -7.073568 & -0.326703 & 1.357692 \\ 6 & -6.804098 & -1.583931 & 2.194314 \\ 6 & -3.658547 & 4.950993 & 1.933004 \\ 6 & -3.452105 & 5.476522 & 0.507159 \\ 6 & 0.292511 & 2.914423 & 4.445993 \\ 6 & 1.281812 & 4.021944 & 4.067856 \\ 45 & -0.087016 & 0.774616 & -1.256545\end{array}$




\begin{tabular}{|c|c|c|c|}
\hline & 0.484982 & 2.413062 & -0.337189 \\
\hline & 0.661060 & 3.499584 & 0.010632 \\
\hline & -1.034359 & 1.954324 & -3.064423 \\
\hline & -0.162910 & 2.464372 & -4.218496 \\
\hline & 0.015099 & 1.621812 & -4.904528 \\
\hline & 1.184723 & 2.936983 & -3.722050 \\
\hline & 1.404123 & 4.249190 & -3.298730 \\
\hline & 2.632734 & 4.633167 & -2.765765 \\
\hline & 3.663566 & 3.706925 & -2.640654 \\
\hline & 3.459669 & 2.396192 & -3.064274 \\
\hline & 2.233974 & 2.020438 & -3.601598 \\
\hline & -0.567300 & 0.060240 & -2.911385 \\
\hline & -0.758846 & -0.571260 & -3.885142 \\
\hline & 1.617223 & -0.620132 & -0.654543 \\
\hline & 1.168951 & -2.178154 & -0.149444 \\
\hline & 2.070329 & -3.165372 & 0.490455 \\
\hline & 2.599106 & -2.770515 & 1.844269 \\
\hline & 1.742714 & -2.305379 & 2.843751 \\
\hline & 2.235823 & -1.973781 & 4.098580 \\
\hline & 3.597479 & -2.095416 & 4.371849 \\
\hline & 4.457447 & -2.554841 & 3.380214 \\
\hline & 3.958638 & -2.892636 & 2.124334 \\
\hline 8 & 2.806113 & -0.779599 & -1.835545 \\
\hline & 4.011209 & -1.396119 & -1.598369 \\
\hline & 4.945015 & -0.834016 & -0.745791 \\
\hline & 6.118862 & -1.597822 & -0.437589 \\
\hline & 6.361542 & -2.827658 & -1.119425 \\
\hline & 5.428463 & -3.282038 & -2.083802 \\
\hline & 4.269273 & -2.591227 & -2.305531 \\
\hline & 7.510447 & -3.589813 & -0.797429 \\
\hline & 8.377233 & -3.179223 & 0.183228 \\
\hline & 8.121157 & -1.983169 & 0.885616 \\
\hline & 7.026821 & -1.211349 & 0.580622 \\
\hline & 4.692711 & 0.495345 & -0.144582 \\
\hline & 3.515422 & 0.722795 & 0.541393 \\
\hline & 3.242769 & 1.937901 & 1.198050 \\
\hline & 4.144989 & 2.963239 & 1.129846 \\
\hline & 5.333847 & 2.824023 & 0.374649 \\
\hline & 5.608408 & 1.588629 & -0.286280 \\
\hline & 6.762546 & 1.516846 & -1.107953 \\
\hline & 7.607884 & 2.590932 & -1.241559 \\
\hline & 7.354161 & 3.796500 & -0.553883 \\
\hline & 6.236989 & 3.905922 & 0.234631 \\
\hline & 2.579440 & -0.276998 & 0.645008 \\
\hline & 0.079351 & -2.731408 & -0.908234 \\
\hline & -1.192797 & -2.810829 & -0.369418 \\
\hline & -2.264490 & -3.308892 & -1.174888 \\
\hline & -1.997472 & -3.758618 & -2.502300 \\
\hline & -0.673041 & -3.709019 & -2.993158 \\
\hline & 0.332768 & -3.197281 & -2.219545 \\
\hline & -3.062483 & -4.252688 & -3.295324 \\
\hline & -4.343506 & -4.293097 & -2.805838 \\
\hline & -4.613303 & -3.837893 & -1.496190 \\
\hline & -3.600244 & -3.367784 & -0.698666 \\
\hline & -1.452921 & -2.530379 & 1.070206 \\
\hline & -1.781399 & -1.273166 & 1.554412 \\
\hline
\end{tabular}




\begin{tabular}{|c|c|c|c|}
\hline & -2.106753 & -1.126247 & 2.931792 \\
\hline & -2.072778 & -2.187854 & 3.794292 \\
\hline & -1.714986 & -3.477125 & 3.338188 \\
\hline & -1.416447 & -3.657762 & 1.956718 \\
\hline & -1.067266 & -4.961661 & 1.515823 \\
\hline & -0.999282 & -6.012883 & 2.396896 \\
\hline & -1.286791 & -5.823610 & 3.764580 \\
\hline & -1.640979 & -4.579969 & 4.221532 \\
\hline & -5.943216 & 1.160370 & -3.934233 \\
\hline & -4.260506 & -0.627440 & -4.334470 \\
\hline & -7.083150 & 0.904554 & 2.270263 \\
\hline & -8.457930 & -0.461712 & 0.729073 \\
\hline & -5.095736 & 4.438682 & 2.084122 \\
\hline & -3.462687 & 6.113134 & 2.902840 \\
\hline & -0.362888 & 3.257053 & 5.788481 \\
\hline & 1.062757 & 1.606796 & 4.628160 \\
\hline & -0.940503 & 3.525703 & -4.993508 \\
\hline & 6.832311 & -0.301949 & 1.143604 \\
\hline & -2.383562 & -0.147032 & 3.313947 \\
\hline & 7.681032 & -4.520280 & -1.337521 \\
\hline & -1.868418 & -4.416427 & 5.274055 \\
\hline & 6.012632 & 4.835160 & 0.756880 \\
\hline & -3.821561 & -3.014730 & 0.307573 \\
\hline & -5.633957 & -3.854080 & -1.117592 \\
\hline 1 & 3.947146 & 3.909340 & 1.631756 \\
\hline & -2.323308 & -2.051808 & 4.845763 \\
\hline & 5.637089 & -4.203162 & -2.625470 \\
\hline & 8.479101 & 2.512190 & -1.887938 \\
\hline & 3.528733 & -2.933651 & -3.024814 \\
\hline 1 & -2.840404 & -4.595088 & -4.305130 \\
\hline & -0.472272 & -4.058977 & -4.004301 \\
\hline ] & -0.724134 & -7.001371 & 2.036212 \\
\hline & 6.967908 & 0.598727 & -1.652929 \\
\hline & -0.848811 & -5.127508 & 0.462663 \\
\hline & 1.349278 & -3.128559 & -2.602164 \\
\hline & 8.036238 & 4.636661 & -0.661178 \\
\hline ] & 8.793965 & -1.670897 & 1.681242 \\
\hline & -5.154263 & -4.672451 & -3.424650 \\
\hline 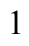 & -1.228127 & -6.664595 & 4.451600 \\
\hline 1 & 9.251265 & -3.777365 & 0.429955 \\
\hline . & 2.305886 & 2.028867 & 1.744798 \\
\hline 1 & -4.431364 & 0.030717 & 1.807730 \\
\hline & -3.025349 & -0.016995 & -2.227809 \\
\hline 1 & -7.222174 & -0.378459 & -1.414727 \\
\hline & -3.545047 & 2.579607 & 0.632552 \\
\hline 1 & -0.158705 & 0.875051 & 2.623988 \\
\hline l & -1.603841 & 4.738896 & 3.790615 \\
\hline 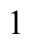 & 1.467040 & -4.078176 & 0.598424 \\
\hline 1 & 2.909378 & -3.425420 & -0.173264 \\
\hline 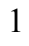 & 4.640158 & -3.238537 & 1.345053 \\
\hline 1 & 0.685948 & -2.175027 & 2.618716 \\
\hline ] & 5.523786 & -2.646246 & 3.578116 \\
\hline 1 & 1.550404 & -1.617059 & 4.866504 \\
\hline ] & 3.985157 & -1.829606 & 5.353086 \\
\hline 1 & 1.777510 & 1.709233 & 5.454598 \\
\hline 1 & 0.388158 & 0.773191 & 4.870103 \\
\hline
\end{tabular}




$\begin{array}{rrrr}1 & 1.639723 & 1.323533 & 3.737883 \\ 1 & 0.400546 & 3.324747 & 6.574922 \\ 1 & -0.892397 & 4.216495 & 5.764022 \\ 1 & -1.083648 & 2.483410 & 6.083809 \\ 1 & 2.077551 & 4.101960 & 4.820936 \\ 1 & 1.750825 & 3.815501 & 3.096121 \\ 1 & 0.789327 & 4.999316 & 3.993551 \\ 1 & -4.194632 & 6.901394 & 2.686052 \\ 1 & -3.609157 & 5.805888 & 3.946543 \\ 1 & -2.464283 & 6.559709 & 2.811576 \\ 1 & -4.135843 & 6.312038 & 0.304178 \\ 1 & -2.423835 & 5.835566 & 0.368014 \\ 1 & -3.639715 & 4.702222 & -0.246770 \\ 1 & -5.810938 & 5.250397 & 1.894441 \\ 1 & -5.321943 & 3.627112 & 1.380811 \\ 1 & -5.274387 & 4.060943 & 3.099644 \\ 1 & -7.862613 & 0.803086 & 3.037412 \\ 1 & -6.126817 & 1.046895 & 2.788442 \\ 1 & -7.292656 & 1.817075 & 1.696543 \\ 1 & -9.214126 & -0.551610 & 1.519134 \\ 1 & -8.719698 & 0.414064 & 0.121242 \\ 1 & -8.537260 & -1.355763 & 0.097263 \\ 1 & -7.579731 & -1.707887 & 2.962242 \\ 1 & -6.809268 & -2.481559 & 1.561082 \\ 1 & -5.832549 & -1.543536 & 2.703708 \\ 1 & -4.548387 & -0.724405 & -5.389086 \\ 1 & -3.462788 & 0.124158 & -4.291737 \\ 1 & -3.835675 & -1.587355 & -4.009510 \\ 1 & -6.177728 & 1.176410 & -5.007534 \\ 1 & -6.840996 & 1.475237 & -3.386561 \\ 1 & -5.158265 & 1.906159 & -3.747801 \\ 1 & -6.760399 & -1.297873 & -4.894838 \\ 1 & -6.319808 & -2.251812 & -3.463472 \\ 1 & -7.553062 & -0.976980 & -3.353697 \\ 1 & -2.038677 & 1.683013 & -3.415706 \\ 1 & -1.186592 & 2.755683 & -2.327629 \\ 1 & 2.080389 & 0.988999 & -3.918065 \\ 1 & 4.257376 & 1.660404 & -2.965229 \\ 1 & 4.623078 & 4.001057 & -2.216429 \\ 1 & 2.781589 & 5.661825 & -2.442733 \\ 1 & 0.605562 & 4.986147 & -3.377588 \\ 1 & -1.842351 & 3.087256 & -5.438130 \\ 1 & -1.268714 & 4.347622 & -4.343699 \\ 1 & -0.338286 & 3.955563 & -5.803376\end{array}$

$\mathrm{TS}_{S}$

Number of imaginary frequencies : 1

The smallest frequencies are : $-242.6342 \quad 14.4268 \quad 17.5878 \mathrm{~cm}(-1)$

Electronic energy : $\quad H F=-4474.1673597$

Zero-point correction $=\quad 1.465801($ Hartree/Particle $)$

Thermal correction to Energy= $\quad 1.552222$

Thermal correction to Enthalpy $=\quad 1.553166$

Thermal correction to Gibbs Free Energy $=\quad 1.344993$

Sum of electronic and zero-point Energies $=\quad-4472.701559$

Sum of electronic and thermal Energies $=\quad-4472.615138$ 
Sum of electronic and thermal Enthalpies=

Sum of electronic and thermal Free Energies=
$-4472.614193$

$-4472.822367$

Cartesian Coordinates

$\begin{array}{ccccc}6 & 1.435599 & 4.227698 & -3.416540 \\ 6 & 1.202605 & 2.889984 & -3.738099 \\ 6 & 2.242476 & 1.973678 & -3.545990 \\ 6 & 3.470707 & 2.383999 & -3.040042 \\ 6 & 3.688401 & 3.721898 & -2.719860 \\ 6 & 2.665860 & 4.645321 & -2.913145 \\ 6 & -0.156532 & 2.452031 & -4.235967 \\ 1 & -0.685050 & 3.374315 & -4.537567 \\ 6 & -1.038822 & 1.859272 & -3.124832 \\ 45 & -0.081236 & 0.727120 & -1.289833 \\ 6 & -0.612844 & -0.073156 & -2.889369 \\ 8 & -0.886047 & -0.774915 & -3.791647 \\ 15 & 1.607302 & -0.652756 & -0.622668 \\ 8 & 2.797944 & -0.850836 & -1.795120 \\ 6 & 3.996842 & -1.470643 & -1.535591 \\ 6 & 4.934977 & -0.890137 & -0.700216 \\ 6 & 6.102533 & -1.655278 & -0.369808 \\ 6 & 6.332134 & -2.908405 & -1.013125 \\ 6 & 5.393137 & -3.385678 & -1.960442 \\ 6 & 4.240786 & -2.690918 & -2.203103 \\ 6 & 7.017713 & -1.246000 & 0.632985 \\ 6 & 8.105932 & -2.018118 & 0.958759 \\ 6 & 8.348810 & -3.237964 & 0.293732 \\ 6 & 7.474908 & -3.670837 & -0.670766 \\ 6 & 4.693532 & 0.457713 & -0.135340 \\ 6 & 3.516977 & 0.715440 & 0.541882 \\ 6 & 3.261345 & 1.944852 & 1.178829 \\ 6 & 4.176405 & 2.956879 & 1.092994 \\ 6 & 5.361348 & 2.789771 & 0.336881 \\ 6 & 5.621130 & 1.538223 & -0.299535 \\ 6 & 6.276113 & 3.858518 & 0.174166 \\ 6 & 7.390375 & 3.721520 & -0.614213 \\ 6 & 7.628974 & 2.500193 & -1.278960 \\ 6 & 6.772583 & 1.438035 & -1.122126 \\ 8 & 2.566658 & -0.266570 & 0.666662 \\ 7 & 1.158119 & -2.188529 & -0.056430 \\ 6 & 0.055135 & -2.769809 & -0.772841 \\ 6 & -1.210221 & -2.813388 & -0.213487 \\ 6 & -2.294465 & -3.351353 & -0.975408 \\ 6 & -2.047160 & -3.873206 & -2.279828 \\ 6 & -0.729588 & -3.854330 & -2.791563 \\ 6 & 0.288797 & -3.305245 & -2.061007 \\ 6 & -3.124915 & -4.406151 & -3.029376 \\ 6 & -4.398983 & -4.414804 & -2.520298 \\ 6 & -4.649113 & -3.888410 & -1.233663 \\ 6 & -3.623213 & -3.380814 & -0.476806 \\ 6 & -1.451165 & -2.451031 & 1.211492 \\ 6 & -1.771591 & -1.167058 & 1.626900 \\ 6 & -2.078871 & -0.939990 & 2.997386 \\ 6 & -2.035218 & -1.950581 & 3.919117 \\ 6 & -1.688162 & -3.265271 & 3.532860\end{array}$




\begin{tabular}{|c|c|c|c|}
\hline & -1.408815 & -3.525798 & 2.160120 \\
\hline & -1.073496 & -4.854627 & 1.788908 \\
\hline & -1.000541 & -5.855095 & 2.726712 \\
\hline & -1.268189 & -5.586813 & 4.085330 \\
\hline & -1.608371 & -4.316959 & 4.476072 \\
\hline & -1.854834 & 0.214824 & 0.386097 \\
\hline & -3.600936 & 0.035674 & -0.173582 \\
\hline & -3.875163 & -0.123608 & -1.531491 \\
\hline & -5.183149 & -0.307089 & -1.985509 \\
\hline 6 & -6.207971 & -0.319001 & -1.032079 \\
\hline & -5.967082 & -0.173197 & 0.336342 \\
\hline & -4.645365 & 0.007163 & 0.747477 \\
\hline & -5.505331 & -0.432094 & -3.474643 \\
\hline & -5.963404 & 0.940782 & -3.981066 \\
\hline & -7.067734 & -0.240657 & 1.393148 \\
\hline & -7.074865 & 1.053017 & 2.215127 \\
\hline & 2.052915 & -3.148358 & 0.632579 \\
\hline & 2.591153 & -2.686019 & 1.961410 \\
\hline & 1.747697 & -2.136127 & 2.928519 \\
\hline & 2.250429 & -1.732722 & 4.158025 \\
\hline & 3.609182 & -1.869835 & 4.439198 \\
\hline & 4.456006 & -2.414980 & 3.480496 \\
\hline 6 & 3.947587 & -2.821715 & 2.248896 \\
\hline & 0.521591 & 2.390541 & -0.430239 \\
\hline 3 & 0.728545 & 3.479739 & -0.109974 \\
\hline & -1.910276 & 1.706815 & 1.450340 \\
\hline & -2.795728 & 2.740517 & 1.174894 \\
\hline & -2.722693 & 3.959655 & 1.862318 \\
\hline & -1.752761 & 4.094616 & 2.851986 \\
\hline & -0.843111 & 3.068523 & 3.156923 \\
\hline & -0.916632 & 1.890990 & 2.421593 \\
\hline 6 & -3.697650 & 5.073275 & 1.487828 \\
\hline & -5.137480 & 4.574141 & 1.656705 \\
\hline & 0.218295 & 3.282880 & 4.234563 \\
\hline & -0.426749 & 3.812106 & 5.519730 \\
\hline & -3.520534 & 6.319330 & 2.351149 \\
\hline 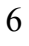 & -3.462288 & 5.466820 & 0.024317 \\
\hline & 0.950310 & 1.987441 & 4.586939 \\
\hline 6 & 1.238346 & 4.304974 & 3.719845 \\
\hline 6 & -8.454325 & -0.423362 & 0.781959 \\
\hline & -6.791659 & -1.434232 & 2.316370 \\
\hline 6 & -4.288611 & -0.874120 & -4.288388 \\
\hline & -6.619993 & -1.454230 & -3.717021 \\
\hline | & 6.834360 & -0.317368 & 1.167507 \\
\hline & -2.351070 & 0.059938 & 3.325336 \\
\hline 1 & 7.634934 & -4.619179 & -1.182246 \\
\hline 1 & -1.820676 & -4.093225 & 5.520644 \\
\hline & 6.063215 & 4.800206 & 0.678562 \\
\hline 1 & -3.827327 & -2.974833 & 0.512863 \\
\hline & -5.664345 & -3.882453 & -0.840452 \\
\hline 1 & 3.993010 & 3.913159 & 1.581099 \\
\hline & -2.271513 & -1.753906 & 4.964292 \\
\hline 1 & 5.592054 & -4.326067 & -2.471794 \\
\hline & 8.497410 & 2.399322 & -1.926003 \\
\hline 1 & 3.494884 & -3.047943 & -2.909768 \\
\hline 1 & -2.918639 & -4.804171 & -4.022017 \\
\hline
\end{tabular}




\begin{tabular}{|c|c|c|c|}
\hline 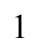 & -0.544645 & -4.259247 & -3.785091 \\
\hline & -0.736895 & -6.864380 & 2.418921 \\
\hline 1 & 6.966501 & 0.507593 & -1.650154 \\
\hline & -0.870164 & -5.080469 & 0.743887 \\
\hline & 1.300814 & -3.260858 & -2.459790 \\
\hline & 8.081458 & 4.551824 & -0.739215 \\
\hline & 8.784320 & -1.686956 & 1.741919 \\
\hline & -5.219368 & -4.824877 & -3.105958 \\
\hline & -1.205611 & -6.388282 & 4.817748 \\
\hline 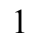 & 9.218222 & -3.836091 & 0.556308 \\
\hline & 2.326747 & 2.055918 & 1.726075 \\
\hline & -4.422260 & 0.121163 & 1.808027 \\
\hline & -3.042109 & -0.132314 & -2.229851 \\
\hline & -7.231905 & -0.453535 & -1.371919 \\
\hline & -3.549951 & 2.602071 & 0.400506 \\
\hline 1 & -0.199510 & 1.087816 & 2.595659 \\
\hline 1 & -1.685593 & 5.028556 & 3.405153 \\
\hline 1 & 1.441415 & -4.048234 & 0.791326 \\
\hline 1 & 2.886727 & -3.451005 & -0.020039 \\
\hline 1 & 4.620556 & -3.230511 & 1.492812 \\
\hline & 0.694986 & -1.992309 & 2.693555 \\
\hline 1 & 5.519995 & -2.519154 & 3.684520 \\
\hline 1 & 1.575096 & -1.307319 & 4.899375 \\
\hline & 4.004936 & -1.548629 & 5.400433 \\
\hline 1 & 1.650363 & 2.171150 & 5.411803 \\
\hline 1 & 0.249248 & 1.204726 & 4.910019 \\
\hline 1 & 1.539241 & 1.586512 & 3.751644 \\
\hline 1 & 0.339112 & 3.957044 & 6.293178 \\
\hline 1 & -0.927125 & 4.776232 & 5.373489 \\
\hline 1 & -1.169382 & 3.103779 & 5.909513 \\
\hline 1 & 2.049526 & 4.443365 & 4.447791 \\
\hline 1 & 1.679752 & 3.974276 & 2.769660 \\
\hline 1 & 0.774726 & 5.283090 & 3.540898 \\
\hline 1 & -4.246291 & 7.084303 & 2.047444 \\
\hline 1 & -3.690118 & 6.108962 & 3.415159 \\
\hline 1 & -2.519470 & 6.755599 & 2.241061 \\
\hline 1 & -4.139233 & 6.281429 & -0.266986 \\
\hline 1 & -2.430289 & 5.810236 & -0.126852 \\
\hline 1 & -3.638014 & 4.627623 & -0.660025 \\
\hline 1 & -5.848982 & 5.362482 & 1.376186 \\
\hline 1 & -5.348594 & 3.698517 & 1.029450 \\
\hline 1 & -5.337010 & 4.294019 & 2.699599 \\
\hline 1 & -7.848447 & 1.005593 & 2.993391 \\
\hline 1 & -6.114543 & 1.234115 & 2.713735 \\
\hline 1 & -7.290412 & 1.921572 & 1.578671 \\
\hline 1 & -9.206513 & -0.465281 & 1.579781 \\
\hline 1 & -8.723971 & 0.409390 & 0.119583 \\
\hline 1 & -8.530965 & -1.357105 & 0.209898 \\
\hline 1 & -7.564645 & -1.505281 & 3.093573 \\
\hline 1 & -6.795930 & -2.374837 & 1.748988 \\
\hline 1 & -5.819079 & -1.354856 & 2.819239 \\
\hline 1 & -4.582687 & -1.025298 & -5.334948 \\
\hline 1 & -3.482611 & -0.129840 & -4.289316 \\
\hline 1 & -3.871920 & -1.819574 & -3.913679 \\
\hline 1 & -6.202640 & 0.897679 & -5.052568 \\
\hline 1 & -6.857824 & 1.288301 & -3.447760 \\
\hline
\end{tabular}




\begin{tabular}{|c|c|c|c|}
\hline & -5.175541 & 1.693329 & -3.839631 \\
\hline & -6.793359 & -1.565969 & -4.795137 \\
\hline & -6.345952 & -2.439142 & -3.315041 \\
\hline & -7.575646 & -1.156435 & -3.269916 \\
\hline & -2.015485 & 1.553525 & -3.522289 \\
\hline & -1.261977 & 2.632826 & -2.377952 \\
\hline & -0.0 & 1.584259 & -5.487804 \\
\hline & 2.090 & 0.918548 & -3.766 \\
\hline & 4.2 & 1.6 & 271 \\
\hline & 4.651628 & 4.036356 & -2.319294 \\
\hline & 2.822751 & 5.694567 & -2.670536 \\
\hline & 0.634353 & 4.954053 & -3.560174 \\
\hline & 0.429797 & 2.138294 & -6.293902 \\
\hline & 0.497821 & 0.660964 & -5.323672 \\
\hline & & 1.29 & -5.8371 \\
\hline
\end{tabular}

\section{$\mathrm{TS}_{B}$}

Number of imaginary frequencies : 1

The smallest frequencies are : - $152.1794 \quad 16.2705 \quad 19.0364 \mathrm{~cm}(-1)$

Electronic energy :

$\mathrm{HF}=-4474.1581637$

Zero-point correction $=$

1.464921 (Hartree/Particle)

Thermal correction to Energy=

1.551540

Thermal correction to Enthalpy=

1.552484

Thermal correction to Gibbs Free Energy=

Sum of electronic and zero-point Energies=

Sum of electronic and thermal Energies=

1.344963

$-4472.693243$

Sum of electronic and thermal Enthalpies=

$-4472.606624$

Sum of electronic and thermal Free Energies=

$-4472.605679$

$-4472.813201$

Cartesian Coordinates

$\begin{array}{lrrr}6 & -1.662981 & -4.064855 & 4.628437 \\ 6 & -1.731338 & -3.068888 & 3.625759 \\ 6 & -1.405590 & -3.397204 & 2.278318 \\ 6 & -1.038437 & -4.739430 & 1.992525 \\ 6 & -0.977314 & -5.684960 & 2.986584 \\ 6 & -1.289523 & -5.347688 & 4.319796 \\ 6 & -1.432869 & -2.375077 & 1.269905 \\ 6 & -1.779861 & -1.075608 & 1.604907 \\ 6 & -2.137319 & -0.784239 & 2.952084 \\ 6 & -2.110539 & -1.741727 & 3.928344 \\ 6 & -1.131166 & -2.829652 & -0.115777 \\ 6 & 0.165419 & -2.850383 & -0.596934 \\ 6 & 0.470879 & -3.519989 & -1.805371 \\ 6 & -0.508998 & -4.134253 & -2.536868 \\ 6 & -1.857336 & -4.080996 & -2.114816 \\ 6 & -2.176167 & -3.428238 & -0.887311 \\ 6 & -2.895258 & -4.666583 & -2.879758 \\ 6 & -4.198714 & -4.613153 & -2.453587 \\ 6 & -4.517289 & -3.970557 & -1.237290 \\ 6 & -3.532212 & -3.399490 & -0.470303 \\ 7 & 1.226790 & -2.204930 & 0.128393 \\ 6 & 2.133845 & -3.110393 & 0.871232 \\ 6 & 2.659124 & -2.551217 & 2.166479\end{array}$




\begin{tabular}{|c|c|c|c|}
\hline 6 & 1.792176 & -2.003244 & 3.113276 \\
\hline 6 & 2.283609 & -1.497278 & 4.309400 \\
\hline & 3.652041 & -1.527544 & 4.574393 \\
\hline & 4.521367 & -2.073172 & 3.635861 \\
\hline 6 & 4.024838 & -2.584730 & 2.439828 \\
\hline 5 & -1.861500 & 0.263598 & 0.311759 \\
\hline+ & -0.072499 & 0.635970 & -1.271908 \\
\hline 5 & 1.678503 & -0.714629 & -0.557532 \\
\hline & 2.642585 & -0.217186 & 0.696269 \\
\hline 6 & 3.522549 & 0.809387 & 0.449903 \\
\hline & 4.697827 & 0.557344 & -0.230918 \\
\hline 6 & 5.538708 & 1.671152 & -0.553978 \\
\hline 6 & 5.196707 & 2.971020 & -0.072923 \\
\hline & 4.022688 & 3.144043 & 0.698022 \\
\hline & 3.192137 & 2.085716 & 0.943578 \\
\hline & 6.680852 & 1.549389 & -1.386530 \\
\hline & 7.450862 & 2.642246 & -1.700844 \\
\hline 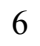 & 7.128335 & 3.919430 & -1.194765 \\
\hline 6 & 6.020780 & 4.075027 & -0.400659 \\
\hline 6 & 5.020792 & -0.828804 & -0.638291 \\
\hline 6 & 4.111668 & -1.560795 & -1.381575 \\
\hline 6 & 4.428489 & -2.836990 & -1.899031 \\
\hline 6 & 5.625069 & -3.424256 & -1.593871 \\
\hline 6 & 6.542472 & -2.777677 & -0.729416 \\
\hline 6 & 6.237800 & -1.472618 & -0.238041 \\
\hline 6 & 7.737016 & -3.417378 & -0.319546 \\
\hline 6 & 8.592116 & -2.814224 & 0.567217 \\
\hline 6 & 8.276983 & -1.540343 & 1.083972 \\
\hline 6 & 7.135772 & -0.885890 & 0.689562 \\
\hline 8 & 2.879199 & -1.047914 & -1.698625 \\
\hline 6 & -1.931336 & 1.779953 & 1.340573 \\
\hline 6 & -0.942666 & 1.988029 & 2.311510 \\
\hline 6 & -0.876391 & 3.181390 & 3.020270 \\
\hline 6 & -1.794259 & 4.193147 & 2.693707 \\
\hline 6 & -2.764181 & 4.030807 & 1.708222 \\
\hline 6 & -2.825871 & 2.798391 & 1.043446 \\
\hline 6 & -3.744093 & 5.130750 & 1.307821 \\
\hline 6 & -3.573664 & 6.396872 & 2.142801 \\
\hline 6 & 0.184974 & 3.426672 & 4.090918 \\
\hline 6 & 0.945090 & 2.149468 & 4.448803 \\
\hline 6 & -3.607476 & 0.053543 & -0.241015 \\
\hline 6 & -3.894566 & -0.146904 & -1.590076 \\
\hline 6 & -5.202639 & -0.382155 & -2.020794 \\
\hline 6 & -6.216786 & -0.383441 & -1.056578 \\
\hline 6 & -5.964365 & -0.183140 & 0.302823 \\
\hline 6 & -4.641622 & 0.031266 & 0.692751 \\
\hline 6 & -5.534066 & -0.585578 & -3.499193 \\
\hline 6 & -4.312554 & -1.045519 & -4.295314 \\
\hline 6 & -7.053437 & -0.223854 & 1.372923 \\
\hline 6 & -8.442887 & -0.452260 & 0.783993 \\
\hline 6 & -6.626177 & -1.643671 & -3.681182 \\
\hline 6 & -6.027123 & 0.748134 & -4.071930 \\
\hline 6 & -6.751379 & -1.371564 & 2.344504 \\
\hline 6 & -7.071783 & 1.104061 & 2.138400 \\
\hline 6 & -3.507324 & 5.491395 & -0.164049 \\
\hline o & -5.181820 & 4.629149 & 1.485791 \\
\hline
\end{tabular}




\begin{tabular}{|c|c|c|c|}
\hline & 1.183699 & 4.461995 & 3.562022 \\
\hline & -0.466212 & 3.954974 & 5.373203 \\
\hline & 0.374825 & 2.369220 & -0.463949 \\
\hline & 0.525080 & 3.473468 & -0.162707 \\
\hline & -0.257930 & 1.575151 & -3.636151 \\
\hline & 1.096871 & 2.220723 & -3.504207 \\
\hline & -0.638747 & -0.349086 & -2.764582 \\
\hline & -1.017391 & -1.176552 & -3.504114 \\
\hline & 6.899563 & 0.088689 & 1.109213 \\
\hline & -2.434706 & 0.225732 & 3.220079 \\
\hline & 7.953407 & -4.409004 & -0.715085 \\
\hline & -1.910951 & -3.786934 & 5.651908 \\
\hline & 5.744110 & 5.056120 & -0.016677 \\
\hline & -3.792947 & -2.895357 & 0.459772 \\
\hline & -5.553372 & -3.924011 & -0.906688 \\
\hline & 3.775928 & 4.138859 & 1.066584 \\
\hline & -2.383582 & -1.491479 & 4.952810 \\
\hline & 5.877744 & -4.406030 & -1.991106 \\
\hline & 8.315407 & 2.522235 & -2.349977 \\
\hline & 3.700875 & -3.325395 & -2.543627 \\
\hline & -2.634763 & -5.158023 & -3.816192 \\
\hline & -0.268886 & -4.646527 & -3.467207 \\
\hline & -0.688165 & -6.704631 & 2.742615 \\
\hline & 6.936762 & 0.574388 & -1.794213 \\
\hline & -0.799260 & -5.021999 & 0.969298 \\
\hline & 1.506306 & -3.530977 & -2.140666 \\
\hline & 7.750325 & 4.775662 & -1.445351 \\
\hline & 8.941953 & -1.072576 & 1.806650 \\
\hline & -4.989089 & -5.064095 & -3.050282 \\
\hline ] & -1.235289 & -6.106138 & 5.097282 \\
\hline & 9.502401 & -3.318666 & 0.882728 \\
\hline D & 2.269814 & 2.196741 & 1.510484 \\
\hline & -4.411092 & 0.176697 & 1.747497 \\
\hline & -3.072389 & -0.143631 & -2.300835 \\
\hline & -7.240759 & -0.554050 & -1.379604 \\
\hline & -3.575857 & 2.638852 & 0.268970 \\
\hline 1 & -0.221155 & 1.192598 & 2.502059 \\
\hline & -1.733933 & 5.139226 & 3.226977 \\
\hline | & 1.535722 & -4.007835 & 1.085478 \\
\hline & 2.975573 & -3.438098 & 0.241365 \\
\hline & 4.711971 & -2.993243 & 1.696611 \\
\hline & 0.727903 & -1.948979 & 2.889911 \\
\hline & 5.592594 & -2.094506 & 3.826770 \\
\hline 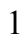 & 1.593057 & -1.075068 & 5.038216 \\
\hline & 4.037639 & -1.124250 & 5.508474 \\
\hline 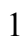 & 1.647839 & 2.353164 & 5.266692 \\
\hline & 0.262556 & 1.354940 & 4.782597 \\
\hline l & 1.535309 & 1.756035 & 3.610564 \\
\hline 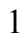 & 0.298886 & 4.123096 & 6.142689 \\
\hline ] & -0.987091 & 4.907030 & 5.218855 \\
\hline 1 & -1.192910 & 3.235335 & 5.772227 \\
\hline & 1.987803 & 4.631603 & 4.290975 \\
\hline 1 & 1.638232 & 4.120279 & 2.621926 \\
\hline & 0.700479 & 5.426311 & 3.361556 \\
\hline 1 & -4.303499 & 7.150821 & 1.821895 \\
\hline 1 & -3.742273 & 6.209767 & 3.211291 \\
\hline
\end{tabular}




$\begin{array}{lrrr}1 & -2.574937 & 6.835924 & 2.022852 \\ 1 & -4.179682 & 6.303436 & -0.472517 \\ 1 & -2.473190 & 5.825246 & -0.322953 \\ 1 & -3.688166 & 4.638980 & -0.830447 \\ 1 & -5.896333 & 5.405649 & 1.181013 \\ 1 & -5.386394 & 3.735248 & 0.882619 \\ 1 & -5.383535 & 4.377546 & 2.535513 \\ 1 & -7.836808 & 1.079401 & 2.926090 \\ 1 & -6.109119 & 1.320080 & 2.618500 \\ 1 & -7.306346 & 1.940978 & 1.467273 \\ 1 & -9.186624 & -0.470836 & 1.590561 \\ 1 & -8.730918 & 0.347801 & 0.089811 \\ 1 & -8.511545 & -1.410463 & 0.252910 \\ 1 & -7.517454 & -1.420739 & 3.130123 \\ 1 & -6.744160 & -2.336176 & 1.819326 \\ 1 & -5.777015 & -1.254748 & 2.836093 \\ 1 & -4.604691 & -1.244957 & -5.334278 \\ 1 & -3.518282 & -0.289132 & -4.326269 \\ 1 & -3.882095 & -1.967472 & -3.879423 \\ 1 & -6.275572 & 0.643239 & -5.137021 \\ 1 & -6.924522 & 1.102268 & -3.547977 \\ 1 & -5.256779 & 1.525495 & -3.978928 \\ 1 & -6.801728 & -1.815535 & -4.750983 \\ 1 & -6.326547 & -2.599246 & -3.230325 \\ 1 & -7.586491 & -1.345153 & -3.244755 \\ 6 & -0.364594 & 0.979374 & -5.035490 \\ 6 & -1.456125 & 2.483802 & -3.419742 \\ 6 & 2.264981 & 1.453725 & -3.641033 \\ 6 & 3.522229 & 2.025672 & -3.525163 \\ 6 & 3.655193 & 3.395811 & -3.296069 \\ 6 & 2.509871 & 4.176223 & -3.202474 \\ 6 & 1.245766 & 3.599372 & -3.314933 \\ 1 & 2.180396 & 0.376489 & -3.777541 \\ 1 & 4.408458 & 1.395649 & -3.598153 \\ 1 & 4.642344 & 3.842657 & -3.190525 \\ 1 & 2.592750 & 5.249361 & -3.038674 \\ 1 & 0.367447 & 4.234866 & -3.221565 \\ 1 & -0.226623 & 1.805124 & -5.751896 \\ 1 & 0.401242 & 0.227672 & -5.249063 \\ 1 & -1.345175 & 0.528350 & -5.222925 \\ 1 & -1.429963 & 3.042086 & -2.477108 \\ 1 & -2.382820 & 1.894384 & -3.417222\end{array}$

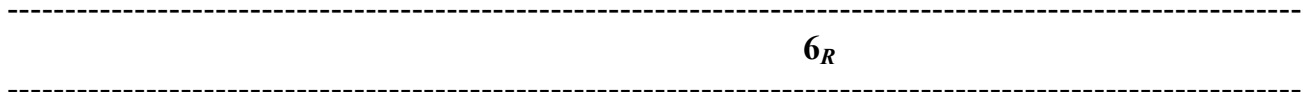

Number of imaginary frequencies : 0

The smallest frequencies are : $12.0704 \quad 16.0492 \quad 18.5254 \mathrm{~cm}(-1)$

Electronic energy : $\quad H F=-4474.1968468$

Zero-point correction $=\quad 1.468069$ (Hartree/Particle)

Thermal correction to Energy= $\quad 1.554898$

Thermal correction to Enthalpy= $\quad 1.555843$

Thermal correction to Gibbs Free Energy= $\quad 1.345906$

Sum of electronic and zero-point Energies $=\quad-4472.728778$

Sum of electronic and thermal Energies $=\quad-4472.641949$ 
Sum of electronic and thermal Enthalpies=

Sum of electronic and thermal Free Energies=
$-4472.641004$

$-4472.850941$

Cartesian Coordinates

$\begin{array}{cccc}6 & -5.228080 & 2.448547 & 0.161917 \\ 6 & -3.870235 & 2.739798 & 0.261795 \\ 6 & -3.293536 & 2.869706 & 1.526762 \\ 6 & -4.064069 & 2.698302 & 2.669224 \\ 6 & -5.418952 & 2.385761 & 2.562458 \\ 6 & -6.000724 & 2.264754 & 1.305230 \\ 6 & -3.035733 & 2.908786 & -0.976668 \\ 7 & -1.908971 & 1.954423 & -1.075057 \\ 15 & -1.986736 & 0.269328 & -1.094809 \\ 45 & -0.063619 & -1.003511 & -0.954207 \\ 6 & 1.347198 & -2.477800 & -0.495587 \\ 6 & 2.372510 & -2.937808 & -1.523612 \\ 6 & 3.433185 & -3.910421 & -1.010500 \\ 6 & -0.787719 & 2.400315 & -1.858819 \\ 6 & 0.313672 & 2.950068 & -1.232181 \\ 6 & 1.429072 & 3.375493 & -2.016500 \\ 6 & 1.384337 & 3.224404 & -3.434405 \\ 6 & 0.241370 & 2.644825 & -4.033751 \\ 6 & -0.818568 & 2.244909 & -3.265202 \\ 6 & 2.586042 & 3.952963 & -1.433027 \\ 6 & 3.629771 & 4.378053 & -2.215719 \\ 6 & 3.572970 & 4.248870 & -3.620998 \\ 6 & 2.478129 & 3.673729 & -4.214279 \\ 6 & 0.304463 & 3.187910 & 0.235149 \\ 6 & 0.627038 & 2.198708 & 1.151973 \\ 6 & 0.668978 & 2.532637 & 2.534330 \\ 6 & 0.366817 & 3.790856 & 2.980066 \\ 6 & -0.002521 & 4.809288 & 2.073733 \\ 6 & -0.027464 & 4.509048 & 0.681615 \\ 6 & -0.347432 & 6.108596 & 2.517219 \\ 6 & -0.708196 & 7.082739 & 1.622354 \\ 6 & -0.734305 & 6.793609 & 0.241608 \\ 6 & -0.399706 & 5.543652 & -0.217361 \\ 15 & 1.128345 & 0.483427 & 0.603484 \\ 6 & 2.904236 & 0.785586 & 0.251009 \\ 6 & 3.414146 & 0.515982 & -1.021680 \\ 6 & 4.752533 & 0.777948 & -1.323504 \\ 6 & 5.553312 & 1.330413 & -0.314944 \\ 6 & 5.066731 & 1.638265 & 0.955822 \\ 6 & 3.725971 & 1.351231 & 1.222330 \\ 6 & 5.374571 & 0.441323 & -2.678457 \\ 6 & 6.312121 & -0.756898 & -2.495855 \\ 6 & 5.914125 & 2.297051 & 2.042587 \\ 6 & 5.929130 & 1.411916 & 3.293446 \\ 6 & 1.081232 & -0.423593 & 2.192963 \\ 6 & 2.146179 & -1.182665 & 2.651185 \\ 6 & 2.027733 & -1.964263 & 3.808701 \\ 6 & 0.821274 & -1.923434 & 4.501901 \\ 6 & -0.272239 & -1.153388 & 4.073316 \\ 6 & -0.133209 & -0.434569 & 2.892021 \\ 6 & -1.550189 & -1.106414 & 4.910392\end{array}$




\begin{tabular}{|c|c|c|c|}
\hline 6 & -2.184035 & -2.499305 & 4.974384 \\
\hline 6 & 3.193708 & -2.858148 & 4.226495 \\
\hline & 4.494521 & -2.049490 & 4.269069 \\
\hline & -3.006462 & -0.160385 & -2.362402 \\
\hline & -4.371714 & -0.002808 & -2.320441 \\
\hline & -5.146068 & -0.739891 & -1.441230 \\
\hline & -6.550689 & -0.467889 & -1.384497 \\
\hline & -7.128126 & 0.454934 & -2.307638 \\
\hline & -6.296241 & 1.101320 & -3.254729 \\
\hline & -4.943987 & 0.891107 & -3.250338 \\
\hline & -8.514684 & 0.733996 & -2.240162 \\
\hline 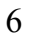 & -9.307148 & 0.145993 & -1.287427 \\
\hline & -8.735235 & -0.744815 & -0.354937 \\
\hline & -7.395705 & -1.043575 & -0.401682 \\
\hline & -4.488944 & -1.736226 & -0.565182 \\
\hline ) & -3.432765 & -1.331919 & 0.226686 \\
\hline & -2.726555 & -2.215442 & 1.066544 \\
\hline & -3.085047 & -3.536189 & 1.103932 \\
\hline o & -4.133756 & -4.024805 & 0.287867 \\
\hline & -4.838465 & -3.126031 & -0.569292 \\
\hline 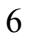 & -5.827944 & -3.664914 & -1.430047 \\
\hline & -6.128283 & -5.004584 & -1.414532 \\
\hline & -5.455677 & -5.882906 & -0.539879 \\
\hline & -4.475558 & -5.398942 & 0.287920 \\
\hline & -3.018693 & -0.009805 & 0.167638 \\
\hline & -2.574951 & -0.129728 & 4.335154 \\
\hline f & -1.206413 & -0.640047 & 6.330174 \\
\hline ) & 2.984735 & -3.485369 & 5.602864 \\
\hline & 3.328637 & -3.987092 & 3.196897 \\
\hline ) & 7.357912 & 2.521539 & 1.601530 \\
\hline & 5.302691 & 3.660664 & 2.388165 \\
\hline 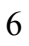 & 4.319555 & 0.082287 & -3.723271 \\
\hline & 6.180739 & 1.627099 & -3.216821 \\
\hline 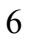 & -0.774082 & -2.305367 & -2.100612 \\
\hline 5 & -1.253575 & -3.102851 & -2.787261 \\
\hline & 1.249914 & -3.049891 & 0.575269 \\
\hline 1 & -6.966253 & -1.719220 & 0.333760 \\
\hline & 0.946584 & 1.774714 & 3.261023 \\
\hline & -8.935618 & 1.436665 & -2.958280 \\
\hline & -0.322553 & 6.314458 & 3.586392 \\
\hline . & -3.929893 & -6.065339 & 0.954546 \\
\hline & 2.641648 & 4.048492 & -0.349652 \\
\hline 1 & 4.513324 & 4.810903 & -1.750935 \\
\hline 1 & -2.541381 & -4.233186 & 1.740206 \\
\hline & 0.407219 & 4.020590 & 4.044057 \\
\hline 1 & -6.747591 & 1.785297 & -3.971665 \\
\hline & -6.887757 & -5.392878 & -2.089531 \\
\hline 1 & -4.285790 & 1.395629 & -3.954896 \\
\hline . & 2.426345 & 3.557826 & -5.296000 \\
\hline & 0.217635 & 2.526596 & -5.115847 \\
\hline 1 & -1.019830 & 7.569084 & -0.465403 \\
\hline & -6.346765 & -3.004833 & -2.120182 \\
\hline 1 & -0.418818 & 5.339046 & -1.285833 \\
\hline & -1.698486 & 1.791966 & -3.718748 \\
\hline & -5.705696 & -6.941287 & -0.535170 \\
\hline & -9.359768 & -1.194417 & 0.413801 \\
\hline
\end{tabular}




\begin{tabular}{|c|c|c|c|}
\hline 1 & 4.403906 & 4.599878 & -4.229435 \\
\hline & -0.974695 & 8.077502 & 1.971724 \\
\hline & -10.370073 & 0.371301 & -1.241500 \\
\hline 1 & -1.893127 & -1.834142 & 1.654446 \\
\hline & 3.311682 & 1.577749 & 2.204199 \\
\hline 1 & 2.734295 & 0.112765 & -1.771936 \\
\hline 1 & 6.598430 & 1.530779 & -0.539582 \\
\hline 1 & 3.073784 & -1.199263 & 2.080178 \\
\hline 1 & -0.964125 & 0.159164 & 2.510057 \\
\hline 1 & 0.715131 & -2.508159 & 5.413432 \\
\hline 1 & -2.573641 & 3.906528 & -0.974737 \\
\hline 1 & -3.673115 & 2.853472 & -1.873940 \\
\hline 1 & -5.681642 & 2.342413 & -0.823927 \\
\hline 1 & -2.228967 & 3.090180 & 1.608512 \\
\hline 1 & -7.056754 & 2.018729 & 1.205578 \\
\hline 1 & -3.605197 & 2.805910 & 3.650786 \\
\hline 1 & -6.017431 & 2.240570 & 3.459480 \\
\hline 1 & -3.457904 & -0.090223 & 4.986357 \\
\hline 1 & -2.166008 & 0.888435 & 4.265995 \\
\hline 1 & -2.923310 & -0.421460 & 3.335058 \\
\hline 1 & -3.088306 & -2.478109 & 5.597651 \\
\hline 1 & -2.474646 & -2.846722 & 3.974207 \\
\hline 1 & -1.500528 & -3.242022 & 5.403692 \\
\hline 1 & -2.117858 & -0.577334 & 6.939732 \\
\hline 1 & -0.517226 & -1.324649 & 6.838714 \\
\hline 1 & -0.740835 & 0.354388 & 6.315291 \\
\hline 1 & 3.860359 & -4.091469 & 5.867753 \\
\hline 1 & 2.858584 & -2.725083 & 6.385150 \\
\hline 1 & 2.112586 & -4.150821 & 5.625101 \\
\hline 1 & 4.175662 & -4.638948 & 3.452489 \\
\hline 1 & 2.418801 & -4.600256 & 3.167375 \\
\hline 1 & 3.484130 & -3.597791 & 2.183107 \\
\hline 1 & 5.330922 & -2.695178 & 4.568205 \\
\hline 1 & 4.749432 & -1.617076 & 3.293550 \\
\hline 1 & 4.426355 & -1.228871 & 4.995777 \\
\hline 1 & 6.513620 & 1.889589 & \\
\hline 1 & 4.920472 & 1.230007 & 3.685315 \\
\hline 1 & 6.386495 & 0.437158 & 3.078326 \\
\hline 1 & 7.922745 & 2.992601 & 2.415849 \\
\hline 1 & 7.862480 & 1.578790 & 1.353548 \\
\hline 1 & 7.422861 & 3.186459 & 0.730550 \\
\hline 1 & 5.885633 & 4.152325 & 3.178675 \\
\hline 1 & 5.300418 & 4.321326 & 1.510862 \\
\hline 1 & 4.267171 & 3.574908 & 2.741984 \\
\hline 1 & 4.809448 & -0.120568 & -4.684360 \\
\hline 1 & 3.757154 & -0.822184 & -3.456618 \\
\hline 1 & 3.603529 & 0.902651 & -3.875332 \\
\hline 1 & 6.770886 & -1.032967 & -3.455003 \\
\hline 1 & 7.121064 & -0.533513 & -1.788394 \\
\hline 1 & 5.767257 & -1.632267 & -2.118871 \\
\hline 1 & 6.606577 & 1.373221 & -4.196593 \\
\hline 1 & 5.542549 & 2.510501 & -3.342655 \\
\hline 1 & 7.017025 & 1.901098 & -2.562879 \\
\hline 1 & 1.782399 & -3.385589 & -2.342202 \\
\hline 1 & 2.836264 & -2.042339 & -1.961099 \\
\hline 6 & 4.613611 & -4.048698 & -1.945327 \\
\hline
\end{tabular}




\begin{tabular}{|c|c|c|c|}
\hline & 3.812886 & -3.502244 & -0.058320 \\
\hline & 2.846888 & -5.295294 & -0.731013 \\
\hline & 5.883036 & -4.302307 & -1.416874 \\
\hline & 6.985520 & -4.480833 & -2.244990 \\
\hline & 6.836780 & -4.412440 & -3.627791 \\
\hline & 5.578678 & -4.167360 & -4.16894 \\
\hline & 4.477610 & -3.990672 & -3.33519 \\
\hline & 6.001018 & -4.354526 & -0.33372 \\
\hline & 7.964709 & -4.671082 & -1.809827 \\
\hline & 7.697192 & -4.548459 & -4.27952 \\
\hline & 5.450417 & -4.113700 & -5.24836 \\
\hline & 3.4995 & -3.803096 & -3.776 \\
\hline & 3.610545 & -5.970971 & -0.32505 \\
\hline & 2.021119 & -5.231947 & -0.01640 \\
\hline & & -5.142 & -1.00 \\
\hline
\end{tabular}

$\mathbf{6}_{S}$

Number of imaginary frequencies : 0

The smallest frequencies are : $10.4150 \quad 16.2042 \quad 19.7874 \mathrm{~cm}(-1)$

Electronic energy : $\quad \mathrm{HF}=-4474.1982517$

Zero-point correction $=\quad 1.468567($ Hartree/Particle $)$

Thermal correction to Energy $=\quad 1.555127$

Thermal correction to Enthalpy= $\quad 1.556071$

Thermal correction to Gibbs Free Energy $=\quad 1.346202$

Sum of electronic and zero-point Energies $=\quad-4472.729685$

Sum of electronic and thermal Energies $=\quad-4472.643125$

Sum of electronic and thermal Enthalpies $=\quad-4472.642181$

Sum of electronic and thermal Free Energies $=\quad-4472.852050$

Cartesian Coordinates

$\begin{array}{cccc}6 & -5.322469 & 2.291387 & 0.108083 \\ 6 & -3.983364 & 2.655513 & 0.223203 \\ 6 & -3.440687 & 2.863327 & 1.492704 \\ 6 & -4.227797 & 2.698993 & 2.625394 \\ 6 & -5.561979 & 2.312220 & 2.504047 \\ 6 & -6.109480 & 2.111243 & 1.241721 \\ 6 & -3.136166 & 2.825921 & -1.005993 \\ 7 & -1.956449 & 1.932433 & -1.053482 \\ 15 & -1.969428 & 0.243535 & -1.066899 \\ 45 & -0.007513 & -0.963958 & -0.898640 \\ 6 & 1.457498 & -2.365421 & -0.395777 \\ 6 & 2.529667 & -2.805548 & -1.389008 \\ 6 & 3.681343 & -3.591170 & -0.770166 \\ 6 & -0.861214 & 2.423458 & -1.848117 \\ 6 & 0.226698 & 3.009245 & -1.232627 \\ 6 & 1.304850 & 3.503048 & -2.030176 \\ 6 & 1.225774 & 3.403023 & -3.450613 \\ 6 & 0.099985 & 2.779267 & -4.039195 \\ 6 & -0.915459 & 2.298135 & -3.257322 \\ 6 & 2.453847 & 4.106439 & -1.456723 \\ 6 & 3.453002 & 4.610751 & -2.250507 \\ 6 & 3.354013 & 4.544412 & -3.657727 \\ 6 & 2.270225 & 3.940105 & -4.242437 \\ 6 & 0.239199 & 3.247456 & 0.234165\end{array}$




\begin{tabular}{|c|c|c|c|}
\hline 6 & 0.653933 & 2.291922 & 1.148767 \\
\hline & 0.751550 & 2.654491 & 2.521555 \\
\hline & 0.394312 & 3.898459 & 2.964598 \\
\hline & -0.090619 & 4.873798 & 2.065106 \\
\hline & -0.155573 & 4.552216 & 0.679232 \\
\hline & -0.509399 & 6.150975 & 2.509034 \\
\hline & -0.979880 & 7.083441 & 1.620579 \\
\hline & -1.042882 & 6.774453 & 0.245512 \\
\hline & -0.636590 & 5.546134 & -0.214106 \\
\hline & 1.156478 & 0.569358 & 0.626921 \\
\hline & 2.907466 & 0.844288 & 0.154411 \\
\hline & 3.267885 & 0.629527 & -1.180131 \\
\hline & 4.570081 & 0.871505 & -1.618969 \\
\hline & 5.495269 & 1.341227 & -0.676687 \\
\hline & 5.160919 & 1.592679 & 0.654395 \\
\hline 6 & 3.845474 & 1.338388 & 1.055518 \\
\hline & 5.017483 & 0.612432 & -3.058864 \\
\hline 6 & 5.942557 & -0.609671 & -3.087382 \\
\hline & 6.149306 & 2.161918 & 1.669568 \\
\hline 6 & 6.245824 & 1.221112 & 2.874872 \\
\hline 6 & 1.129984 & -0.277857 & 2.251535 \\
\hline 6 & 2.205324 & -0.970370 & 2.786300 \\
\hline & 2.073478 & -1.699168 & 3.976767 \\
\hline 6 & 0.842944 & -1.665183 & 4.627274 \\
\hline 6 & -0.257254 & -0.949250 & 4.130052 \\
\hline 6 & -0.103152 & -0.288009 & 2.917270 \\
\hline 6 & -1.559484 & -0.898153 & 4.928239 \\
\hline 6 & -2.157216 & -2.303675 & 5.043095 \\
\hline 6 & 3.235822 & -2.558342 & 4.471604 \\
\hline 6 & 4.567312 & -1.812473 & 4.342011 \\
\hline 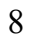 & -2.968794 & -0.225654 & -2.336461 \\
\hline 6 & -4.341475 & -0.160008 & -2.296198 \\
\hline 6 & -5.070156 & -0.939582 & -1.414468 \\
\hline 6 & -6.489631 & -0.756581 & -1.364196 \\
\hline 6 & -7.122824 & 0.116867 & -2.298510 \\
\hline 6 & -6.331026 & 0.804768 & -3.250797 \\
\hline 6 & -4.968004 & 0.684031 & -3.238137 \\
\hline 6 & -8.524646 & 0.306455 & -2.237310 \\
\hline 6 & -9.280211 & -0.320256 & -1.279217 \\
\hline 6 & -8.654516 & -1.161170 & -0.335110 \\
\hline 6 & -7.298529 & -1.374124 & -0.376346 \\
\hline 6 & -4.357691 & -1.886379 & -0.527087 \\
\hline 6 & -3.332946 & -1.410728 & 0.265533 \\
\hline 6 & -2.579146 & -2.239686 & 1.118944 \\
\hline 6 & -2.856247 & -3.579161 & 1.170172 \\
\hline 6 & -3.868189 & -4.139488 & 0.353898 \\
\hline 6 & -4.622433 & -3.295207 & -0.516754 \\
\hline 6 & -5.573529 & -3.903172 & -1.374615 \\
\hline 6 & -5.791300 & -5.258578 & -1.344620 \\
\hline 6 & -5.069769 & -6.084173 & -0.457565 \\
\hline 6 & -4.124883 & -5.531863 & 0.368248 \\
\hline 8 & -2.996761 & -0.066815 & 0.192071 \\
\hline 6 & -2.592688 & 0.020390 & 4.278272 \\
\hline 6 & -1.268916 & -0.353774 & 6.332093 \\
\hline 6 & 3.071664 & -2.971017 & 5.933692 \\
\hline 6 & 3.282128 & -3.824928 & 3.607465 \\
\hline
\end{tabular}




\begin{tabular}{|c|c|c|c|}
\hline & 7.549985 & 2.335669 & 1.088546 \\
\hline & 5.647240 & 3.536162 & 2.129247 \\
\hline & 3.832528 & 0.352636 & -3.988183 \\
\hline & 5.786452 & 1.818988 & -3.607129 \\
\hline & -0.645841 & -2.294127 & -2.058363 \\
\hline & -1.087778 & -3.102648 & -2.756851 \\
\hline & 1.349634 & -2.942708 & 0.671187 \\
\hline & -6.828528 & -2.012707 & 0.367356 \\
\hline & 1.113117 & 1.927859 & 3.243169 \\
\hline & -8.988106 & 0.971926 & -2.964616 \\
\hline & -0.452813 & 6.373348 & 3.573727 \\
\hline & -3.542051 & -6.155870 & 1.044412 \\
\hline & 2.541163 & 4.158920 & -0.372545 \\
\hline & 4.330667 & 5.062284 & -1.792573 \\
\hline & -2.275483 & -4.234718 & 1.817405 \\
\hline & 0.476880 & 4.149408 & 4.021293 \\
\hline & -6.822810 & 1.448919 & -3.977941 \\
\hline & -6.522954 & -5.700222 & -2.017546 \\
\hline & -4.340858 & 1.222397 & -3.945918 \\
\hline & 2.190569 & 3.865453 & -5.326151 \\
\hline & 0.053660 & 2.693146 & -5.123668 \\
\hline & -1.414351 & 7.516956 & -0.456944 \\
\hline & -6.129254 & -3.283639 & -2.073713 \\
\hline & -0.685743 & 5.326872 & -1.278841 \\
\hline & -1.781299 & 1.811316 & -3.703129 \\
\hline & -5.254519 & -7.155774 & -0.441824 \\
\hline & -9.250613 & -1.640725 & 0.438067 \\
\hline & 4.146816 & 4.961754 & -4.275017 \\
\hline & -1.304246 & 8.060551 & 1.970850 \\
\hline & -10.355515 & -0.163116 & -1.237993 \\
\hline & -1.776122 & -1.800205 & 1.707431 \\
\hline & 3.547013 & 1.532216 & 2.085687 \\
\hline & 2.496654 & 0.274602 & -1.864351 \\
\hline & 6.516830 & 1.522479 & -1.003362 \\
\hline 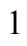 & 3.156980 & -0.961916 & 2.259756 \\
\hline & -0.937961 & 0.263340 & 2.483822 \\
\hline | & 0.723722 & -2.209663 & 5.561838 \\
\hline ] & -2.729120 & 3.846948 & -1.031003 \\
\hline & -3.753598 & 2.705702 & -1.910909 \\
\hline 1 & -5.747740 & 2.125125 & -0.881990 \\
\hline & -2.389964 & 3.141693 & 1.586637 \\
\hline 1 & -7.148110 & 1.803652 & 1.130551 \\
\hline & -3.797911 & 2.867868 & 3.611221 \\
\hline 1 & -6.171557 & 2.171517 & 3.394295 \\
\hline 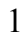 & -3.494362 & 0.067432 & 4.902898 \\
\hline & -2.209017 & 1.044954 & 4.169686 \\
\hline 1 & -2.906190 & -0.328388 & 3.284933 \\
\hline & -3.079442 & -2.277278 & 5.639195 \\
\hline l & -2.409411 & -2.707394 & 4.053725 \\
\hline & -1.467188 & -3.005317 & 5.527534 \\
\hline 1 & -2.198783 & -0.288489 & 6.912868 \\
\hline 1 & -0.575427 & -0.992047 & 6.892404 \\
\hline 1 & -0.831727 & 0.652253 & 6.280461 \\
\hline 1 & 3.952182 & -3.540712 & 6.256637 \\
\hline & 2.975976 & -2.099926 & 6.595459 \\
\hline & 2.199289 & -3.617589 & 6.088442 \\
\hline
\end{tabular}




\begin{tabular}{|c|c|c|c|}
\hline & 4.117113 & -4.470069 & 3.914833 \\
\hline & 2.350743 & -4.397835 & 3.705048 \\
\hline & 3.401540 & -3.582966 & 2.543836 \\
\hline & 5.382558 & -2.431349 & 4.739003 \\
\hline & 4.820668 & -1.581158 & 3.299883 \\
\hline & 4.554039 & -0.871097 & 4.907909 \\
\hline & 6.954398 & 1.618007 & 3.614159 \\
\hline & 5.279422 & 1.094437 & 3.378878 \\
\hline & 6.597488 & 0.226838 & 2.567951 \\
\hline & 8.219882 & 2.739194 & 1.858185 \\
\hline & 7.974809 & 1.381899 & 0.749932 \\
\hline & 7.561696 & 3.037429 & 0.244616 \\
\hline & 6.339951 & 3.972406 & 2.861559 \\
\hline & 5.571968 & 4.227746 & 1.279434 \\
\hline & 4.657262 & 3.479240 & 2.599275 \\
\hline & 4.196045 & 0.193112 & -5.011367 \\
\hline & 3.261347 & -0.541896 & -3.705061 \\
\hline & 3.139479 & 1.205755 & -4.007374 \\
\hline & 6.302613 & -0.791214 & -4.109562 \\
\hline & 6.820590 & -0.470208 & -2.443578 \\
\hline & 5.425447 & -1.518284 & -2.753598 \\
\hline & 6.071112 & 1.635765 & -4.651751 \\
\hline & 5.169268 & 2.725390 & -3.577354 \\
\hline & 6.710334 & 2.016390 & -3.050750 \\
\hline & 1.980657 & -3.418657 & -2.124285 \\
\hline & 2.886516 & -1.926541 & -1.944284 \\
\hline & 4.500911 & -4.381692 & -1.766864 \\
\hline & 4.588517 & -2.660573 & 0.040108 \\
\hline & 3.234075 & -4.309448 & -0.06622 \\
\hline & 5.287177 & -5.441667 & -1.301134 \\
\hline & 6.091034 & -6.177648 & -2.16210 \\
\hline & 6.127111 & -5.866876 & -3.51959 \\
\hline & 5.349742 & -4.819834 & -3.999607 \\
\hline & 4.542893 & -4.086507 & -3.131420 \\
\hline & 5.257027 & -5.692229 & -0.239926 \\
\hline & 6.687842 & -7.001069 & -1.774482 \\
\hline & 6.753651 & -6.441968 & -4.19797 \\
\hline & 5.363648 & -4.570796 & -5.05893 \\
\hline & 3.935853 & -3.275865 & -3.53223 \\
\hline 1 & 5.373545 & -3.219134 & 0.565598 \\
\hline 1 & 5.080023 & -1.921383 & -0.607804 \\
\hline & 4.004794 & -2.110034 & 0.788127 \\
\hline
\end{tabular}

\section{$6_{B}$}

Number of imaginary frequencies : 0

The smallest frequencies are : $16.6582 \quad 16.9292 \quad 20.8454 \mathrm{~cm}(-1)$

Electronic energy : $\quad H F=-4474.1929732$

Zero-point correction $=\quad 1.467227($ Hartree/Particle $)$

Thermal correction to Energy= $\quad 1.554083$

Thermal correction to Enthalpy= $\quad 1.555027$

Thermal correction to Gibbs Free Energy= $\quad 1.347017$

Sum of electronic and zero-point Energies $=\quad-4472.725746$

Sum of electronic and thermal Energies $=\quad-4472.638890$

Sum of electronic and thermal Enthalpies $=\quad-4472.637946$ 
Cartesian Coordinates

\begin{tabular}{|c|c|c|c|}
\hline 6 & -4.907864 & 2.610732 & 1.027925 \\
\hline 6 & -3.525370 & 2.767497 & 1.083597 \\
\hline 6 & -2.869137 & 2.589887 & 2.303023 \\
\hline 6 & -3.588686 & 2.254300 & 3.442795 \\
\hline 6 & -4.970218 & 2.078487 & 3.375797 \\
\hline 6 & -5.629694 & 2.260190 & 2.165242 \\
\hline 6 & -2.756761 & 3.140362 & -0.152510 \\
\hline 7 & -1.712338 & 2.164376 & -0.540949 \\
\hline 15 & -1.937310 & 0.540768 & -0.938890 \\
\hline 45 & -0.146067 & -0.875139 & -1.295918 \\
\hline 6 & 1.130110 & -2.553513 & -1.368036 \\
\hline 6 & 1.922073 & -3.049915 & -2.623942 \\
\hline 6 & 3.390733 & -3.308620 & -2.288555 \\
\hline 6 & -0.622358 & 2.723977 & -1.296596 \\
\hline 6 & 0.557402 & 3.048276 & -0.657294 \\
\hline 6 & 1.626943 & 3.630696 & -1.404454 \\
\hline 6 & 1.445747 & 3.891707 & -2.794877 \\
\hline 6 & 0.227760 & 3.523738 & -3.414788 \\
\hline 6 & -0.778891 & 2.949393 & -2.685423 \\
\hline 6 & 2.865651 & 3.975236 & -0.805715 \\
\hline 6 & 3.859106 & 4.573082 & -1.540463 \\
\hline 6 & 3.664413 & 4.863382 & -2.908697 \\
\hline 6 & 2.485280 & 4.522167 & -3.521870 \\
\hline 6 & 0.675329 & 2.910401 & 0.816877 \\
\hline 6 & 0.985931 & 1.708948 & 1.433898 \\
\hline 6 & 1.165044 & 1.693070 & 2.846025 \\
\hline 6 & 0.994832 & 2.817474 & 3.606894 \\
\hline 6 & 0.626668 & 4.044299 & 3.011672 \\
\hline 6 & 0.473294 & 4.096809 & 1.596991 \\
\hline 6 & 0.403891 & 5.208195 & 3.785652 \\
\hline 6 & 0.036896 & 6.387397 & 3.189933 \\
\hline 6 & -0.117767 & 6.447851 & 1.788954 \\
\hline 6 & 0.098853 & 5.336090 & 1.012910 \\
\hline 15 & 1.299796 & 0.142298 & 0.454225 \\
\hline 6 & 3.066327 & 0.424005 & 0.035787 \\
\hline 6 & 3.449879 & 0.528734 & -1.303647 \\
\hline 6 & 4.772662 & 0.818894 & -1.649512 \\
\hline 6 & 5.691063 & 1.002564 & -0.608437 \\
\hline 6 & 5.333713 & 0.935295 & 0.739377 \\
\hline 6 & 4.003508 & 0.640773 & 1.043768 \\
\hline 6 & 5.239875 & 0.881975 & -3.104929 \\
\hline 6 & 5.836878 & -0.479756 & -3.478765 \\
\hline 6 & 6.307448 & 1.238197 & 1.876401 \\
\hline 6 & 6.304606 & 0.094237 & 2.894996 \\
\hline 6 & 1.253818 & -1.085674 & 1.816204 \\
\hline 6 & 2.291349 & -1.953575 & 2.117658 \\
\hline 6 & 2.153285 & -2.924737 & 3.120455 \\
\hline 6 & 0.965549 & -2.947347 & 3.844246 \\
\hline 6 & -0.089972 & -2.055479 & 3.594315 \\
\hline 6 & 0.056750 & -1.157736 & 2.543375 \\
\hline 6 & -1.329133 & -2.075910 & 4.489080 \\
\hline 6 & -2.075049 & -3.402878 & 4.321007 \\
\hline
\end{tabular}




\begin{tabular}{|c|c|c|c|}
\hline & 3.277785 & -3.933767 & 3.342647 \\
\hline & 4.622139 & -3.214290 & 3.490058 \\
\hline & -3.075882 & 0.501233 & -2.176005 \\
\hline & -4.416492 & 0.743000 & -1.989893 \\
\hline & -5.200960 & -0.111816 & -1.234529 \\
\hline & -6.568160 & 0.248840 & -1.006824 \\
\hline & -7.114621 & 1.393573 & -1.660926 \\
\hline & -6.287123 & 2.167229 & -2.511538 \\
\hline & -4.961896 & 1.860030 & -2.658341 \\
\hline & -8.462318 & 1.756499 & -1.422777 \\
\hline & -9.244458 & 1.035948 & -0.556474 \\
\hline & -8.700090 & -0.079378 & 0.114203 \\
\hline & -7.399509 & -0.462442 & -0.104404 \\
\hline & -4.590874 & -1.334216 & -0.665442 \\
\hline & -3.441734 & -1.216932 & 0.090812 \\
\hline & -2.762820 & -2.330307 & 0.624405 \\
\hline & -3.250210 & -3.588409 & 0.393191 \\
\hline & -4.406866 & -3.781747 & -0.399801 \\
\hline & -5.080600 & -2.650898 & -0.953181 \\
\hline & -6.182253 & -2.888974 & -1.813611 \\
\hline & -6.615976 & -4.165310 & -2.074799 \\
\hline & -5.971974 & -5.277482 & -1.494161 \\
\hline & -4.886322 & -5.084420 & -0.679347 \\
\hline & -2.905866 & 0.044645 & 0.307577 \\
\hline & -2.285272 & -0.929373 & 4.166822 \\
\hline & -0.896674 & -1.919242 & 5.952276 \\
\hline & 3.060392 & -4.782878 & 4.592958 \\
\hline & 3.331437 & -4.866210 & 2.126552 \\
\hline & 7.739582 & 1.429777 & 1.384253 \\
\hline & 5.855785 & 2.533712 & 2.562744 \\
\hline & 4.090676 & 1.192717 & -4.063241 \\
\hline & 6.304826 & 1.965668 & -3.301900 \\
\hline & -1.158888 & -1.800438 & -2.571237 \\
\hline & -1.854171 & -2.337340 & -3.324107 \\
\hline & 1.085170 & -3.298368 & -0.407070 \\
\hline & -6.989153 & -1.314133 & 0.432314 \\
\hline & 1.442217 & 0.767975 & 3.342676 \\
\hline & -8.860427 & 2.630349 & -1.937095 \\
\hline & 0.526511 & 5.143116 & 4.865870 \\
\hline & -4.361216 & -5.931516 & -0.240100 \\
\hline & 3.025636 & 3.750251 & 0.247886 \\
\hline & 4.805937 & 4.822414 & -1.065219 \\
\hline & -2.730852 & -4.458730 & 0.791720 \\
\hline & 1.138783 & 2.776268 & 4.685816 \\
\hline & -6.717493 & 3.023741 & -3.027925 \\
\hline & -7.460472 & -4.319419 & -2.742824 \\
\hline & -4.304238 & 2.456109 & -3.287779 \\
\hline & 2.328944 & 4.724511 & -4.580610 \\
\hline & 0.102637 & 3.710949 & -4.480100 \\
\hline & -0.409028 & 7.384497 & 1.319230 \\
\hline & -6.682880 & -2.045008 & -2.280738 \\
\hline & -0.019153 & 5.402728 & -0.066625 \\
\hline & -1.716264 & 2.660059 & -3.157661 \\
\hline & -6.328071 & -6.282953 & -1.706169 \\
\hline & -9.313861 & -0.637511 & 0.817699 \\
\hline & 4.456760 & 5.346626 & -3.476415 \\
\hline
\end{tabular}




\begin{tabular}{|c|c|c|c|}
\hline 1 & -0.136845 & 7.276061 & 3.792172 \\
\hline 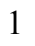 & -10.276862 & 1.326644 & -0.376769 \\
\hline 1 & -1.850138 & -2.168475 & 1.193950 \\
\hline & 3.688798 & 0.595332 & 2.085624 \\
\hline 1 & 2.687632 & 0.389125 & -2.070208 \\
\hline 1 & 6.725333 & 1.218239 & -0.862947 \\
\hline 1 & 3.223484 & -1.888962 & 1.560186 \\
\hline 1 & -0.746377 & -0.460523 & 2.301979 \\
\hline 1 & 0.847278 & -3.674997 & 4.644230 \\
\hline 1 & -2.220284 & 4.083757 & 0.023907 \\
\hline 1 & -3.451224 & 3.320193 & -0.988787 \\
\hline 1 & -5.423150 & 2.746024 & 0.076773 \\
\hline 1 & -1.785790 & 2.704840 & 2.349522 \\
\hline 1 & -6.707824 & 2.124140 & 2.096279 \\
\hline 1 & -3.068640 & 2.125434 & 4.390567 \\
\hline 1 & -5.528886 & 1.804354 & 4.268370 \\
\hline 1 & -3.132001 & -0.941831 & 4.865632 \\
\hline 1 & -1.788008 & 0.046159 & 4.263165 \\
\hline 1 & -2.703337 & -0.994834 & 3.153711 \\
\hline 1 & -2.949501 & -3.434767 & 4.985075 \\
\hline 1 & -2.432677 & -3.527290 & 3.290529 \\
\hline 1 & -1.440115 & -4.263866 & 4.563587 \\
\hline 1 & -1.778520 & -1.899531 & 6.606546 \\
\hline 1 & -0.255896 & -2.741930 & 6.290827 \\
\hline 1 & -0.345201 & -0.981121 & 6.100478 \\
\hline 1 & 3.905670 & -5.470664 & 4.721546 \\
\hline 1 & 2.991547 & -4.167939 & 5.500231 \\
\hline 1 & 2.153367 & -5.396225 & 4.522674 \\
\hline 1 & 4.141610 & -5.599524 & 2.242030 \\
\hline 1 & 2.386883 & -5.413598 & 2.013333 \\
\hline 1 & 3.500042 & -4.315961 & 1.192205 \\
\hline 1 & 5.424528 & -3.945307 & 3.655863 \\
\hline 1 & 4.888163 & -2.639617 & 2.593863 \\
\hline 1 & 4.612709 & -2.523837 & 4.344365 \\
\hline 1 & 6.989079 & 0.321410 & 3.723185 \\
\hline 1 & 5.311147 & -0.077054 & 3.327853 \\
\hline 1 & 6.637339 & -0.845167 & 2.433041 \\
\hline 1 & 8.400041 & 1.623550 & 2.238833 \\
\hline 1 & 8.117536 & 0.536647 & 0.869753 \\
\hline 1 & 7.830983 & 2.284452 & 0.701998 \\
\hline 1 & 6.528091 & 2.784125 & 3.394640 \\
\hline 1 & 5.866287 & 3.373322 & 1.854546 \\
\hline 1 & 4.837741 & 2.451895 & 2.965667 \\
\hline 1 & 4.483089 & 1.300365 & -5.082647 \\
\hline 1 & 3.343271 & 0.392542 & -4.095660 \\
\hline 1 & 3.583863 & 2.129271 & -3.796278 \\
\hline 1 & 6.185821 & -0.469882 & -4.520867 \\
\hline 1 & 6.691545 & -0.731600 & -2.837390 \\
\hline 1 & 5.093578 & -1.282427 & -3.373983 \\
\hline 1 & 6.564883 & 2.037106 & -4.365884 \\
\hline 1 & 5.936751 & 2.948592 & -2.977680 \\
\hline 1 & 7.235478 & 1.753447 & -2.762786 \\
\hline 6 & 1.231065 & -4.363028 & -3.020735 \\
\hline 6 & 1.831130 & -2.060253 & -3.782968 \\
\hline 6 & 4.191808 & -4.025320 & -3.187054 \\
\hline 6 & 5.537332 & -4.263683 & -2.929181 \\
\hline
\end{tabular}




$\begin{array}{llll}6 & 6.124047 & -3.785463 & -1.760884 \\ 6 & 5.348160 & -3.060237 & -0.864658 \\ 6 & 4.001087 & -2.825688 & -1.129159 \\ 1 & 3.758233 & -4.409529 & -4.109426 \\ 1 & 6.129325 & -4.830073 & -3.645543 \\ 1 & 7.175293 & -3.974892 & -1.554689 \\ 1 & 5.786965 & -2.670647 & 0.054079 \\ 1 & 3.413611 & -2.263124 & -0.411357 \\ 1 & 1.604798 & -4.734327 & -3.982677 \\ 1 & 1.388532 & -5.130661 & -2.256433 \\ 1 & 0.149436 & -4.211582 & -3.132044 \\ 1 & 2.529246 & -2.330120 & -4.585741 \\ 1 & 0.824873 & -2.038031 & -4.217966 \\ 1 & 2.063558 & -1.041986 & -3.450434\end{array}$

\section{$7_{R}$}

Number of imaginary frequencies : 0

The smallest frequencies are : $15.3471 \quad 18.1629 \quad 21.7803 \mathrm{~cm}(-1)$

Electronic energy : $\quad \mathrm{HF}=-4587.4777371$

Zero-point correction $=\quad 1.476697$ (Hartree/Particle)

Thermal correction to Energy $=\quad 1.565685$

Thermal correction to Enthalpy= $\quad 1.566629$

Thermal correction to Gibbs Free Energy $=\quad 1.353133$

Sum of electronic and zero-point Energies $=\quad-4586.001040$

Sum of electronic and thermal Energies $=\quad-4585.912052$

Sum of electronic and thermal Enthalpies $=\quad-4585.911108$

Sum of electronic and thermal Free Energies $=\quad-4586.124604$

\begin{tabular}{cccc}
\multicolumn{4}{c}{ Cartesian Coordinates } \\
\hline $6 \ldots \ldots \ldots \ldots \ldots \ldots \ldots \ldots \ldots \ldots \ldots \ldots \ldots$ \\
6 & -3.565866 & -0.382044 & -1.014642 \\
6 & -3.050528 & -1.072850 & 0.081360 \\
6 & -3.863037 & -1.957367 & 0.781770 \\
6 & -5.196300 & -2.160754 & 0.410005 \\
6 & -5.692497 & -1.418260 & -0.662094 \\
6 & -4.900062 & -0.516325 & -1.386444 \\
15 & -1.293242 & -0.803876 & 0.548148 \\
6 & -1.320125 & -0.415653 & 2.340454 \\
6 & -2.421670 & 0.170644 & 2.945299 \\
6 & -2.367941 & 0.604384 & 4.277159 \\
6 & -1.194088 & 0.385073 & 4.990943 \\
6 & -0.068137 & -0.228439 & 4.416735 \\
6 & -0.136295 & -0.586087 & 3.074528 \\
6 & -3.583626 & 1.318346 & 4.861798 \\
6 & -3.789605 & 2.628484 & 4.090642 \\
6 & 1.177221 & -0.477965 & 5.266915 \\
6 & 1.802254 & 0.864626 & 5.659926 \\
6 & -6.028947 & -3.182747 & 1.181650 \\
6 & -5.359285 & -4.557950 & 1.067861 \\
6 & -5.518591 & 0.342606 & -2.487659 \\
6 & -6.288576 & -0.522423 & -3.488144 \\
6 & -0.708349 & -2.574703 & 0.558378 \\
6 & -0.320931 & -3.181207 & -0.627178 \\
6 & 0.089425 & -4.553326 & -0.640454
\end{tabular}




\begin{tabular}{|c|c|c|c|}
\hline 6 & 0.090964 & -5.297488 & 0.574631 \\
\hline 6 & -0.335070 & -4.660044 & 1.762179 \\
\hline 6 & -0.720300 & -3.346073 & 1.753558 \\
\hline 6 & 0.518446 & -5.206666 & -1.825624 \\
\hline 6 & 0.934795 & -6.514745 & -1.800849 \\
\hline 6 & 0.937392 & -7.244424 & -0.593318 \\
\hline 6 & 0.521169 & -6.645603 & 0.568207 \\
\hline 6 & -0.357661 & -2.464790 & -1.930209 \\
\hline 6 & 0.694870 & -1.665272 & -2.338985 \\
\hline 6 & 0.691285 & -1.073508 & -3.622684 \\
\hline 6 & -0.364844 & -1.251038 & -4.475643 \\
\hline 6 & -1.472995 & -2.038274 & -4.088271 \\
\hline 6 & -1.469463 & -2.662279 & -2.805510 \\
\hline 6 & -2.580886 & -2.229982 & -4.950731 \\
\hline 6 & -3.644533 & -3.004601 & -4.564090 \\
\hline 6 & -3.651174 & -3.611640 & -3.288603 \\
\hline 6 & -2.590516 & -3.449767 & -2.433874 \\
\hline 7 & 1.801770 & -1.406103 & -1.457009 \\
\hline 15 & 1.844036 & 0.184943 & -0.893188 \\
\hline 8 & 2.934041 & -0.043603 & 0.317752 \\
\hline 6 & 3.644945 & 1.035072 & 0.797063 \\
\hline 6 & 4.705086 & 1.542711 & 0.070951 \\
\hline 6 & 5.384550 & 2.692562 & 0.592066 \\
\hline 6 & 5.014736 & 3.205643 & 1.872641 \\
\hline 6 & 3.956870 & 2.598147 & 2.590079 \\
\hline 6 & 3.270533 & 1.543288 & 2.054306 \\
\hline 6 & 6.393463 & 3.378529 & -0.131133 \\
\hline 6 & 7.023275 & 4.477972 & 0.397135 \\
\hline 6 & 6.681612 & 4.957392 & 1.678923 \\
\hline 6 & 5.692698 & 4.333883 & 2.394975 \\
\hline 6 & 5.058458 & 0.936370 & -1.232935 \\
\hline 6 & 4.084851 & 0.785990 & -2.203571 \\
\hline 6 & 4.387693 & 0.350070 & -3.511137 \\
\hline 6 & 5.663886 & -0.028569 & -3.825099 \\
\hline 6 & 6.678153 & -0.022802 & -2.836409 \\
\hline 6 & 6.377128 & 0.456565 & -1.526186 \\
\hline 6 & 7.973305 & -0.522125 & -3.116491 \\
\hline 6 & 8.932639 & -0.581404 & -2.138316 \\
\hline 6 & 8.628603 & -0.140976 & -0.833205 \\
\hline 6 & 7.388431 & 0.368662 & -0.536100 \\
\hline 8 & 2.771120 & 1.106191 & -1.938100 \\
\hline 6 & 2.961484 & -2.321978 & -1.600835 \\
\hline 6 & 3.699503 & -2.648810 & -0.330270 \\
\hline 6 & 5.088524 & -2.548203 & -0.285361 \\
\hline 6 & 5.787735 & -2.882500 & 0.872107 \\
\hline 6 & 5.102568 & -3.331350 & 1.995516 \\
\hline 6 & 3.713328 & -3.439531 & 1.954947 \\
\hline 6 & 3.018415 & -3.095369 & 0.803466 \\
\hline 45 & -0.214193 & 1.295283 & -0.494802 \\
\hline 6 & 0.284077 & 2.310614 & 1.082980 \\
\hline 8 & 0.380113 & 2.998719 & 2.007099 \\
\hline 6 & -0.397169 & 1.726235 & -2.365168 \\
\hline 8 & -0.444803 & 2.086470 & -3.464643 \\
\hline 6 & -1.971547 & 2.411028 & -0.145539 \\
\hline 6 & -2.103628 & 3.743240 & -0.866313 \\
\hline 6 & -3.195819 & 4.668931 & -0.321211 \\
\hline
\end{tabular}




\begin{tabular}{|c|c|c|c|}
\hline & 0.791630 & -1.245772 & 6.536883 \\
\hline & 2.220236 & -1.314163 & 4.526373 \\
\hline & -4.828658 & 0.437000 & 4.711307 \\
\hline & -3.411521 & 1.651180 & 6.341173 \\
\hline & -6.109466 & -2.773424 & 2.656532 \\
\hline & -7.451728 & -3.302887 & 0.642381 \\
\hline & -6.479761 & 1.341128 & -1.830818 \\
\hline & -4.462555 & 1.132345 & -3.259891 \\
\hline & -2.837211 & 2.031539 & 0.611020 \\
\hline & 7.162809 & 0.686323 & 0.478868 \\
\hline & -1.040754 & -2.884973 & 2.684144 \\
\hline & 8.184248 & -0.873770 & -4.125522 \\
\hline & 0.517329 & -7.195271 & 1.508413 \\
\hline & 5.398633 & 4.702131 & 3.376926 \\
\hline & -2.609252 & -3.912464 & -1.448423 \\
\hline & -4.508967 & -4.205480 & -2.979457 \\
\hline & 3.678724 & 3.001601 & 3.562848 \\
\hline & -0.353140 & -5.232326 & 2.689000 \\
\hline & 5.910140 & -0.365024 & -4.830760 \\
\hline & 7.788634 & 4.988724 & -0.182906 \\
\hline & 3.589077 & 0.329780 & -4.249244 \\
\hline & -2.565981 & -1.750442 & -5.928537 \\
\hline & -0.369312 & -0.779522 & -5.457101 \\
\hline & 1.263250 & -6.994095 & -2.720199 \\
\hline & 6.660815 & 3.033421 & -1.126227 \\
\hline ] & 0.515724 & -4.658457 & -2.765629 \\
\hline & 1.539262 & -0.454563 & -3.909793 \\
\hline 1 & 7.191727 & 5.826535 & 2.087498 \\
\hline & 9.382137 & -0.210567 & -0.051827 \\
\hline & -4.487363 & -3.151212 & -5.236418 \\
\hline & 1.269707 & -8.279865 & -0.588016 \\
\hline & 9.920793 & -0.976788 & -2.361287 \\
\hline $\mathbf{l}$ & 2.427917 & 1.081910 & 2.565680 \\
\hline & -3.452759 & -2.501150 & 1.632794 \\
\hline 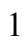 & -2.897410 & 0.280658 & -1.559561 \\
\hline & -6.736125 & -1.536592 & -0.946860 \\
\hline 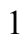 & -3.322628 & 0.332505 & 2.359081 \\
\hline 1 & 0.728673 & -1.032994 & 2.582616 \\
\hline & -1.140366 & 0.698204 & 6.031517 \\
\hline D & 2.538803 & -3.249651 & -2.012166 \\
\hline & 3.667273 & -1.939716 & -2.353952 \\
\hline 1 & 5.632198 & -2.190382 & -1.161211 \\
\hline & 1.931497 & -3.142193 & 0.786826 \\
\hline 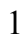 & 6.871420 & -2.783895 & 0.891451 \\
\hline 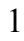 & 3.164063 & -3.793329 & 2.826568 \\
\hline ] & 5.646267 & -3.594105 & 2.900536 \\
\hline 1 & 3.066560 & -1.527841 & 5.191840 \\
\hline & 1.800825 & -2.276220 & 4.199028 \\
\hline 1 & 2.627963 & -0.808100 & 3.641687 \\
\hline & 1.684382 & -1.441975 & 7.145709 \\
\hline 1 & 0.083898 & -0.691327 & 7.163812 \\
\hline 1 & 0.333594 & -2.212169 & 6.288568 \\
\hline 1 & 2.719304 & 0.707026 & 6.243864 \\
\hline 1 & 2.061317 & 1.455485 & 4.770919 \\
\hline & 1.117597 & 1.469227 & 6.267417 \\
\hline & -4.314277 & 2.151603 & 6.713869 \\
\hline
\end{tabular}




$\begin{array}{rrrr}1 & -3.258503 & 0.750045 & 6.949736 \\ 1 & -2.567327 & 2.331075 & 6.513698 \\ 1 & -4.656046 & 3.175988 & 4.486723 \\ 1 & -2.905692 & 3.274091 & 4.184832 \\ 1 & -3.958132 & 2.451317 & 3.020928 \\ 1 & -5.708869 & 0.950394 & 5.121193 \\ 1 & -5.043934 & 0.201846 & 3.661505 \\ 1 & -4.708831 & -0.511619 & 5.251765 \\ 1 & -6.686075 & -3.513803 & 3.227610 \\ 1 & -5.118169 & -2.695225 & 3.120469 \\ 1 & -6.607105 & -1.800848 & 2.766166 \\ 1 & -8.003514 & -4.054785 & 1.220755 \\ 1 & -8.003150 & -2.357319 & 0.723658 \\ 1 & -7.465696 & -3.620667 & -0.408420 \\ 1 & -5.935102 & -5.311990 & 1.621532 \\ 1 & -5.302820 & -4.878898 & 0.019012 \\ 1 & -4.337734 & -4.557858 & 1.469499 \\ 1 & -4.943963 & 1.715417 & -4.055427 \\ 1 & -3.926949 & 1.844267 & -2.615198 \\ 1 & -3.720766 & 0.470873 & -3.729506 \\ 1 & -6.918230 & 2.008458 & -2.586026 \\ 1 & -7.303570 & 0.833680 & -1.313302 \\ 1 & -5.953143 & 1.958201 & -1.089754 \\ 1 & -6.734832 & 0.107819 & -4.269081 \\ 1 & -5.618040 & -1.242698 & -3.974468 \\ 1 & -7.104256 & -1.083496 & -3.016755 \\ 1 & -1.123929 & 4.245388 & -0.876615 \\ 1 & -2.307959 & 3.490993 & -1.920413 \\ 6 & -3.479542 & 5.754144 & -1.333968 \\ 1 & -4.108995 & 4.063579 & -0.215106 \\ 6 & -2.816939 & 5.202054 & 1.055625 \\ 6 & -4.537246 & 5.606921 & -2.235354 \\ 6 & -4.790280 & 6.566546 & -3.209878 \\ 6 & -3.982605 & 7.696011 & -3.302112 \\ 6 & -2.922535 & 7.853453 & -2.414374 \\ 6 & -2.673728 & 6.891073 & -1.440809 \\ 1 & -5.173735 & 4.723518 & -2.164975 \\ 1 & -5.623313 & 6.433325 & -3.897352 \\ 1 & -4.178216 & 8.449932 & -4.061569 \\ 1 & -2.283688 & 8.732075 & -2.479256 \\ 1 & -1.838805 & 7.027667 & -0.754522 \\ 1 & -3.554228 & 5.925839 & 1.422374 \\ 1 & -2.757043 & 4.382230 & 1.780327 \\ 1 & -1.837225 & 5.698372 & 1.041689\end{array}$

$7_{S}\left(\right.$ denoted as $\left.7_{\mathrm{s}-\mathrm{C} 1-\psi-1 \mathbf{a} 2 \mathbf{s} 3 \mathrm{a} 4 \mathrm{~s}}\right)$

Number of imaginary frequencies : 0

The smallest frequencies are : 8.0851 $11.7692 \quad 14.9850 \mathrm{~cm}(-1)$

Electronic energy : $\quad H F=-4587.4757933$

Zero-point correction $=\quad 1.476359($ Hartree/Particle $)$

Thermal correction to Energy= $\quad 1.565713$

Thermal correction to Enthalpy= $\quad 1.566657$

Thermal correction to Gibbs Free Energy= $\quad 1.349472$

Sum of electronic and zero-point Energies $=\quad-4585.999434$

Sum of electronic and thermal Energies $=\quad-4585.910080$ 
Sum of electronic and thermal Enthalpies=

Sum of electronic and thermal Free Energies=
$-4585.909136$

$-4586.126321$

Cartesian Coordinates

\begin{tabular}{|c|c|c|c|}
\hline 6 & -3.541513 & -0.461935 & -1.246699 \\
\hline 6 & -3.130996 & -0.939436 & -0.001079 \\
\hline 6 & -4.006862 & -1.697804 & 0.767832 \\
\hline 6 & -5.298242 & -1.990232 & 0.315932 \\
\hline 6 & -5.685512 & -1.472853 & -0.919864 \\
\hline 6 & -4.829662 & -0.701719 & -1.719355 \\
\hline 15 & -1.408804 & -0.613822 & 0.559639 \\
\hline 6 & -1.557850 & 0.101227 & 2.243048 \\
\hline 6 & -2.706278 & 0.735380 & 2.691995 \\
\hline 6 & -2.733048 & 1.382929 & 3.935216 \\
\hline 6 & -1.590836 & 1.325539 & 4.728468 \\
\hline 6 & -0.421586 & 0.664089 & 4.318248 \\
\hline 6 & -0.410955 & 0.093028 & 3.050544 \\
\hline 6 & -3.997095 & 2.135744 & 4.344283 \\
\hline 6 & -4.205956 & 3.304176 & 3.372663 \\
\hline 6 & 0.792246 & 0.606676 & 5.245693 \\
\hline 6 & 1.433617 & 1.996669 & 5.318263 \\
\hline 6 & -6.200194 & -2.871047 & 1.178672 \\
\hline 6 & -5.528767 & -4.236319 & 1.374147 \\
\hline 6 & -5.335694 & -0.123322 & -3.040638 \\
\hline 6 & -5.912394 & -1.228085 & -3.931136 \\
\hline 6 & -0.893131 & -2.363696 & 0.960817 \\
\hline 6 & -0.460214 & -3.211934 & -0.048280 \\
\hline 6 & -0.088125 & -4.563230 & 0.248685 \\
\hline 6 & -0.175999 & -5.037461 & 1.589181 \\
\hline 6 & -0.651972 & -4.160428 & 2.589825 \\
\hline 6 & -0.995641 & -2.870287 & 2.286003 \\
\hline 6 & 0.389136 & -5.457088 & -0.745924 \\
\hline 6 & 0.768497 & -6.736831 & -0.424096 \\
\hline 6 & 0.682967 & -7.197870 & 0.906491 \\
\hline 6 & 0.217309 & -6.363216 & 1.889953 \\
\hline 6 & -0.414060 & -2.785412 & -1.472575 \\
\hline 6 & 0.673028 & -2.099541 & -1.983871 \\
\hline 6 & 0.737848 & -1.777932 & -3.359469 \\
\hline 6 & -0.271048 & -2.134904 & -4.213369 \\
\hline 6 & -1.398589 & -2.839854 & -3.733764 \\
\hline 6 & -1.478321 & -3.165227 & -2.347327 \\
\hline 6 & -2.440409 & -3.246111 & -4.603595 \\
\hline 6 & -3.520974 & -3.942147 & -4.125520 \\
\hline 6 & -3.621176 & -4.233591 & -2.747339 \\
\hline 6 & -2.625730 & -3.858605 & -1.881015 \\
\hline 7 & 1.756896 & -1.704407 & -1.125124 \\
\hline 15 & 1.856231 & -0.037961 & -0.881493 \\
\hline 8 & 2.894824 & -0.079409 & 0.392840 \\
\hline 6 & 3.682893 & 1.010098 & 0.686655 \\
\hline 6 & 4.786745 & 1.297718 & -0.092768 \\
\hline 6 & 5.549733 & 2.467134 & 0.231804 \\
\hline 6 & 5.216206 & 3.222495 & 1.396783 \\
\hline 6 & 4.112328 & 2.829826 & 2.191167 \\
\hline 6 & 3.346629 & 1.754723 & 1.832412 \\
\hline 6 & 6.612387 & 2.936040 & -0.581964 \\
\hline
\end{tabular}




$\begin{array}{cccc}6 & 7.324622 & 4.059311 & -0.242501 \\ 6 & 7.016954 & 4.779871 & 0.930314 \\ 6 & 5.979172 & 4.369050 & 1.726627 \\ 6 & 5.114436 & 0.437872 & -1.252873 \\ 6 & 4.151035 & 0.179168 & -2.210803 \\ 6 & 4.446931 & -0.503898 & -3.409583 \\ 6 & 5.697414 & -1.020708 & -3.608646 \\ 6 & 6.688236 & -0.909630 & -2.602593 \\ 6 & 6.398574 & -0.180016 & -1.410803 \\ 6 & 7.946638 & -1.543118 & -2.745028 \\ 6 & 8.878759 & -1.493106 & -1.740349 \\ 6 & 8.582942 & -0.803108 & -0.546060 \\ 6 & 7.380186 & -0.159556 & -0.387445 \\ 8 & 2.857545 & 0.625214 & -2.045987 \\ 6 & 2.872833 & -2.678057 & -1.016484 \\ 6 & 3.535271 & -2.777742 & 0.332251 \\ 6 & 4.926009 & -2.774545 & 0.421413 \\ 6 & 5.559788 & -2.905853 & 1.654897 \\ 6 & 4.806059 & -3.049260 & 2.814129 \\ 6 & 3.414523 & -3.056853 & 2.731435 \\ 6 & 2.785429 & -2.917666 & 1.501659 \\ 45 & -0.148066 & 1.219768 & -0.760221 \\ 6 & 0.413973 & 2.461269 & 0.616137 \\ 8 & 0.575836 & 3.300663 & 1.394799 \\ 6 & -0.305182 & 1.321670 & -2.680009 \\ 1 & -0.343662 & 1.475538 & -3.827148 \\ 1 & 5.938859 & -1.548168 & -4.529925 \\ 1 & -2.129628 & 4.400824 & -0.889355 \\ 6 & -1.848113 & 2.464947 & -0.634997 \\ 6 & -1.830115 & 3.765323 & -1.423378 \\ 6 & -3.038787 & 4.677750 & -1.188287 \\ 1 & -0.217193 & -1.882315 & -5.271223 \\ 6 & 0.364215 & 0.176144 & 6.653166 \\ 1 & 1.135335 & -7.402526 & -1.202043 \\ 1 & 6.854912 & 2.402345 & -1.496946 \\ 1 & 0.833913 & -0.401394 & 4.759503 \\ 6 & -5.207688 & 1.198073 & 4.276711 \\ 6 & -3.909823 & 2.697466 & 5.760752 \\ 6 & -6.412568 & -2.208192 & 2.544198 \\ 6 & -7.567800 & -3.103676 & 0.542613 \\ 6 & -6.438461 & 0.899619 & -2.742338 \\ 6 & -4.222419 & 0.575658 & -3.819078 \\ 8 & -2.769542 & 2.197502 & 0.102884 \\ 1 & 7.158784 & 0.351026 & 0.546484 \\ 1 & -1.356866 & -2.221855 & 3.079709 \\ 1 & 8.150179 & -2.086115 & -3.667067 \\ 1 & 1.606597 & -1.232760 & -3.723108\end{array}$




\begin{tabular}{|c|c|c|c|}
\hline ] & 7.592259 & 5.665268 & 1.190485 \\
\hline & 9.312543 & -0.784663 & 0.260507 \\
\hline & -4.310985 & -4.259265 & -4.803049 \\
\hline 1 & 0.986364 & -8.214010 & 1.147370 \\
\hline 1 & 9.837953 & -1.992308 & -1.855508 \\
\hline & 2.470487 & 1.453586 & 2.403818 \\
\hline & -3.677346 & -2.077145 & 1.735254 \\
\hline & -2.824265 & 0.105341 & -1.837125 \\
\hline & -6.691930 & -1.671889 & -1.283025 \\
\hline & -3.583580 & 0.758362 & 2.050163 \\
\hline & 0.488091 & -0.400830 & 2.678409 \\
\hline & -1.597631 & 1.808619 & 5.702931 \\
\hline & 2.423825 & -3.651190 & -1.260956 \\
\hline & 3.632237 & -2.483815 & -1.789099 \\
\hline & 5.524508 & -2.653657 & -0.483115 \\
\hline & 1.699104 & -2.883406 & 1.445670 \\
\hline & 6.646884 & -2.888110 & 1.703964 \\
\hline & 2.811683 & -3.169093 & 3.631836 \\
\hline ] & 5.298115 & -3.151594 & 3.779041 \\
\hline & 2.648104 & -0.478478 & 5.491482 \\
\hline ] & 1.393762 & -1.401679 & 4.638819 \\
\hline & 2.292111 & -0.120733 & 3.802708 \\
\hline & 1.241888 & 0.113007 & 7.310031 \\
\hline ] & -0.336200 & 0.881150 & 7.115187 \\
\hline & -0.115501 & -0.811210 & 6.635274 \\
\hline 1 & 2.334785 & 1.975897 & 5.946592 \\
\hline & 1.719720 & 2.353284 & 4.319441 \\
\hline 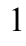 & 0.742920 & 2.736061 & 5.742751 \\
\hline ] & -4.844645 & 3.215409 & 6.010043 \\
\hline & -3.761275 & 1.906397 & 6.507672 \\
\hline 1 & -3.095924 & 3.426440 & 5.864590 \\
\hline & -5.108316 & 3.869865 & 3.643141 \\
\hline 1 & -3.350727 & 3.992607 & 3.401361 \\
\hline & -4.317906 & 2.961127 & 2.337086 \\
\hline ] & -6.119665 & 1.733859 & 4.572673 \\
\hline ] & -5.370798 & 0.806642 & 3.264961 \\
\hline & -5.083617 & 0.342480 & 4.954030 \\
\hline 1 & -7.051131 & -2.837330 & 3.179097 \\
\hline & -5.467245 & -2.050353 & 3.078503 \\
\hline 1 & -6.902934 & -1.231983 & 2.434224 \\
\hline ] & -8.173073 & -3.748320 & 1.192514 \\
\hline 1 & -8.120691 & -2.165795 & 0.402977 \\
\hline ] & -7.485442 & -3.603207 & -0.431722 \\
\hline 1 & -6.147169 & -4.878168 & 2.016370 \\
\hline 1 & -5.400388 & -4.750251 & 0.411929 \\
\hline & -4.539225 & -4.152126 & 1.841993 \\
\hline 1 & -4.621210 & 0.968958 & -4.762964 \\
\hline l & -3.801235 & 1.425200 & -3.262600 \\
\hline 1 & -3.403559 & -0.115977 & -4.064147 \\
\hline 1 & -6.803925 & 1.350540 & -3.675178 \\
\hline 1 & -7.295761 & 0.437647 & -2.236828 \\
\hline 1 & -6.067858 & 1.707532 & -2.098706 \\
\hline 1 & -6.300289 & -0.794882 & -4.863114 \\
\hline 1 & -5.141482 & -1.963849 & -4.193334 \\
\hline & -6.740570 & -1.762920 & -3.450237 \\
\hline 1 & -0.889693 & 4.277170 & -1.168316 \\
\hline
\end{tabular}




$\begin{array}{lrrr}1 & -1.730267 & 3.517127 & -2.492295 \\ 6 & -2.748406 & 6.072247 & -1.692692 \\ 6 & -4.304721 & 4.087045 & -1.801873 \\ 1 & -3.189779 & 4.740571 & -0.099837 \\ 6 & -2.545972 & 7.121460 & -0.793951 \\ 6 & -2.259539 & 8.407235 & -1.243061 \\ 6 & -2.167975 & 8.665129 & -2.606656 \\ 6 & -2.362578 & 7.627525 & -3.514570 \\ 6 & -2.649826 & 6.344835 & -3.061002 \\ 1 & -2.617693 & 6.923002 & 0.275470 \\ 1 & -2.108974 & 9.209979 & -0.523867 \\ 1 & -1.945022 & 9.669065 & -2.961493 \\ 1 & -2.290468 & 7.818624 & -4.583520 \\ 1 & -2.799364 & 5.542547 & -3.783516 \\ 1 & -5.169738 & 4.741000 & -1.639425 \\ 1 & -4.198728 & 3.940685 & -2.886098 \\ 1 & -4.518540 & 3.113578 & -1.346317\end{array}$

Cartesian Coordinates for Intermediate $7_{\mathrm{S}}$

$7_{\mathrm{s}-\mathrm{C} 2}-\psi-1 \mathrm{a} 2 \mathrm{s3a4s}$

Number of imaginary frequencies : 0

The smallest frequencies are : $14.4062 \quad 18.768421 .7414 \mathrm{~cm}(-1)$

Electronic energy : $\quad H F=-4587.4768984$

Zero-point correction $=\quad 1.476511$ (Hartree/Particle)

Thermal correction to Energy= $\quad 1.565515$

Thermal correction to Enthalpy= $\quad 1.566459$

Thermal correction to Gibbs Free Energy $=\quad 1.352657$

Sum of electronic and zero-point Energies $=\quad-4586.000388$

Sum of electronic and thermal Energies $=\quad-4585.911384$

Sum of electronic and thermal Enthalpies $=\quad-4585.910440$

Sum of electronic and thermal Free Energies $=\quad-4586.124242$

Cartesian Coordinates

$\begin{array}{cccc}6 & 4.986662 & -4.320391 & -1.546503 \\ 6 & 4.143957 & -4.104966 & -2.637602 \\ 6 & 4.718555 & -3.657190 & -3.832159 \\ 6 & 6.087067 & -3.436277 & -3.937172 \\ 6 & 6.915591 & -3.659082 & -2.840278 \\ 6 & 6.357448 & -4.097508 & -1.643640 \\ 6 & 2.655133 & -4.383685 & -2.599216 \\ 6 & 2.201728 & -5.269538 & -1.445938 \\ 6 & 1.788443 & -3.117754 & -2.686871 \\ 6 & 1.780415 & -2.213270 & -1.462989 \\ 8 & 2.698989 & -2.211515 & -0.677528 \\ 45 & 0.047085 & -1.023731 & -1.197134 \\ 6 & 0.220669 & -0.513459 & -3.051298 \\ 8 & 0.262796 & -0.313781 & -4.191369 \\ 15 & 1.214238 & 0.349034 & 0.622014 \\ 6 & 0.596336 & 1.870329 & 1.508832 \\ 6 & 0.153802 & 2.967196 & 0.781993\end{array}$




$\begin{array}{lrrr}6 & -0.263154 & 4.161319 & 1.454376 \\ 6 & -0.215078 & 4.220635 & 2.877232 \\ 6 & 0.256405 & 3.094334 & 3.588408 \\ 6 & 0.645163 & 1.959756 & 2.927189 \\ 6 & -0.643019 & 5.393551 & 3.543853 \\ 6 & -1.104823 & 6.474187 & 2.837252 \\ 6 & -1.153316 & 6.423652 & 1.428037 \\ 6 & -0.740890 & 5.300751 & 0.754347 \\ 6 & 0.131896 & 2.978106 & -0.706275 \\ 6 & -0.941004 & 2.458027 & -1.408088 \\ 6 & -0.998263 & 2.561665 & -2.816965 \\ 6 & 0.018455 & 3.149969 & -3.520063 \\ 6 & 1.144524 & 3.674264 & -2.845640 \\ 6 & 1.200038 & 3.601491 & -1.421973 \\ 6 & 2.334777 & 4.140520 & -0.762100 \\ 6 & 3.354919 & 4.717797 & -1.474999 \\ 6 & 3.288186 & 4.804363 & -2.882756 \\ 6 & 2.208335 & 4.287863 & -3.551838 \\ 7 & -2.009594 & 1.787824 & -0.717875 \\ 6 & -3.167306 & 2.635599 & -0.338401 \\ 6 & -3.828017 & 2.297606 & 0.972528 \\ 6 & -5.216669 & 2.209754 & 1.046197 \\ 6 & -5.847537 & 1.919941 & 2.254169 \\ 6 & -5.093140 & 1.718372 & 3.404127 \\ 6 & -3.703587 & 1.810024 & 3.338334 \\ 6 & -3.077998 & 2.096123 & 2.132819 \\ 6 & 2.908802 & 0.931629 & 0.210372 \\ 6 & 3.390155 & 0.754301 & -1.085460 \\ 6 & 4.668141 & 1.174761 & -1.440187 \\ 6 & 5.431060 & 1.840225 & -0.470150 \\ 6 & 4.969696 & 2.059586 & 0.828239 \\ 6 & 3.700705 & 1.572971 & 1.157521 \\ 6 & 5.775417 & 2.801328 & 1.893794 \\ 6 & 7.103360 & 3.328184 & 1.356861 \\ 6 & 5.267903 & 0.868006 & -2.810072 \\ 6 & 4.268915 & 0.164634 & -3.728088 \\ 6 & 1.454693 & -0.818650 & 2.015446 \\ 6 & 2.676108 & -1.425390 & 2.264030 \\ 6 & 2.807944 & -2.400243 & 3.261109 \\ 6 & 1.688410 & -2.704480 & 4.031182 \\ 6 & 0.444111 & -2.084567 & 3.828471 \\ 6 & 0.336677 & -1.172710 & 2.782978 \\ 6 & 4.163440 & -3.079624 & 3.442519 \\ 6 & 4.170262 & -4.071290 & 4.602212 \\ 6 & -0.752012 & -2.435891 & 4.714052 \\ 6 & -1.843908 & -1.366491 & 4.638894 \\ 6 & 4.508610 & -3.836644 & 2.153546 \\ 6 & 5.239443 & -2.020807 & 3.709071 \\ 6 & -1.329268 & -3.780842 & 4.258615 \\ 6 & -0.327830 & -2.547441 & 6.182285 \\ 6 & 4.964652 & 3.997922 & 2.407403 \\ 6 & 6.069891 & 1.851726 & 3.060440 \\ 6 & 5.729383 & 2.153716 & -3.499985\end{array}$




$\begin{array}{cccc}6 & 6.464212 & -0.068095 & -2.605540 \\ 15 & -2.016463 & 0.117722 & -0.956404 \\ 8 & -2.990180 & -0.248162 & -2.265990 \\ 6 & -4.301403 & 0.171773 & -2.313474 \\ 6 & -5.251316 & -0.380844 & -1.474101 \\ 6 & -6.560446 & 0.204935 & -1.469160 \\ 6 & -6.880261 & 1.224895 & -2.415217 \\ 6 & -5.896252 & 1.649349 & -3.341381 \\ 6 & -4.625904 & 1.146956 & -3.280105 \\ 6 & -7.540867 & -0.137512 & -0.503436 \\ 6 & -8.769163 & 0.476733 & -0.491240 \\ 6 & -9.092996 & 1.459442 & -1.450053 \\ 6 & -8.163513 & 1.823236 & -2.390363 \\ 6 & -4.886876 & -1.514004 & -0.592814 \\ 6 & -3.792841 & -1.411743 & 0.245461 \\ 6 & -3.434329 & -2.425508 & 1.153637 \\ 6 & -4.163717 & -3.580960 & 1.203968 \\ 6 & -5.249839 & -3.782868 & 0.319019 \\ 6 & -5.606854 & -2.754112 & -0.604842 \\ 6 & -5.971600 & -5.001243 & 0.320042 \\ 6 & -6.990889 & -5.219128 & -0.570642 \\ 6 & -7.320899 & -4.221376 & -1.511151 \\ 6 & -6.649615 & -3.023962 & -1.527668 \\ 8 & -3.043681 & -0.259085 & 0.271554 \\ 6 & -0.507794 & -2.673779 & -0.352804 \\ 8 & -0.685307 & -3.736373 & 0.068643 \\ 1 & -7.298897 & -0.877537 & 0.255376 \\ 1 & 1.007749 & 1.112481 & 3.503185 \\ 1 & -8.388707 & 2.592959 & -3.127371 \\ 1 & -0.600125 & 5.415584 & 4.631928 \\ 1 & -5.686879 & -5.767860 & 1.039452 \\ 1 & 2.399229 & 4.071420 & 0.322607 \\ 1 & 4.224854 & 5.114238 & -0.954116 \\ 1 & -3.901112 & -4.370014 & 1.907305 \\ 1 & 0.307771 & 3.141822 & 4.675606 \\ 1 & -6.160123 & 2.401005 & -4.083379 \\ 1 & -8.110903 & -4.404142 & -2.236114 \\ 1 & -3.847979 & 1.472474 & -3.966784 \\ 1 & 2.147249 & 4.341497 & -4.637995 \\ 1 & -0.023794 & 3.214997 & -4.606157 \\ 1 & -1.517889 & 7.283564 & 0.870768 \\ 1 & -6.909736 & -2.272464 & -2.268314 \\ 1 & -0.776869 & 5.279988 & -0.333052 \\ 1 & -1.864085 & 2.148361 & -3.330791 \\ 1 & -7.534516 & -6.160922 & -0.564646 \\ 1 & -9.497272 & 0.206324 & 0.270295 \\ 1 & 4.099687 & 5.275300 & -3.433552 \\ 1 & -1.434250 & 7.370167 & 3.358166 \\ 1 & -10.071672 & 1.933133 & -1.432707 \\ 1 & -2.571844 & -2.258341 & 1.795966 \\ 1 & 3.319423 & 1.707878 & 2.170008 \\ 1 & 2.743417 & 0.265404 & -1.809204 \\ 1 & 6.425310 & 2.188724 & -0.744144\end{array}$




$\begin{array}{rrrr}1 & 3.533527 & -1.154976 & 1.652838 \\ 1 & -0.618899 & -0.689825 & 2.569745 \\ 1 & 1.776230 & -3.446700 & 4.820990 \\ 1 & -2.763112 & 3.656302 & -0.279250 \\ 1 & -3.919693 & 2.644362 & -1.141940 \\ 1 & -5.815528 & 2.351975 & 0.145004 \\ 1 & -1.992051 & 2.126692 & 2.077553 \\ 1 & -6.932685 & 1.843705 & 2.287909 \\ 1 & -3.099387 & 1.656308 & 4.231585 \\ 1 & -5.583202 & 1.488008 & 4.347774 \\ 1 & -2.646607 & -1.603627 & 5.348666 \\ 1 & -1.446643 & -0.374550 & 4.897279 \\ 1 & -2.309179 & -1.290923 & 3.648221 \\ 1 & -1.205251 & -2.752558 & 6.809519 \\ 1 & 0.387150 & -3.359532 & 6.356056 \\ 1 & 0.128943 & -1.614048 & 6.536225 \\ 1 & -2.218366 & -4.042585 & 4.849228 \\ 1 & -1.616762 & -3.752330 & 3.198962 \\ 1 & -0.595649 & -4.588850 & 4.374513 \\ 1 & 5.163649 & -4.529299 & 4.690406 \\ 1 & 3.945843 & -3.584070 & 5.560272 \\ 1 & 3.448032 & -4.883997 & 4.451768 \\ 1 & 5.493215 & -4.318079 & 2.241898 \\ 1 & 3.764102 & -4.619747 & 1.953817 \\ 1 & 4.532917 & -3.170565 & 1.281923 \\ 1 & 6.217863 & -2.499838 & 3.849363 \\ 1 & 5.335389 & -1.313919 & 2.875481 \\ 1 & 5.011645 & -1.444544 & 4.615796 \\ 1 & 6.636702 & 2.371850 & 3.844702 \\ 1 & 5.150266 & 1.463467 & 3.516445 \\ 1 & 6.666537 & 0.993003 & 2.725571 \\ 1 & 7.634900 & 3.868771 & 2.150307 \\ 1 & 7.760247 & 2.517869 & 1.015717 \\ 1 & 6.957554 & 4.026935 & 0.522050 \\ 1 & 5.521688 & 4.526053 & 3.193196 \\ 1 & 4.766769 & 4.716117 & 1.600076 \\ 1 & 3.997707 & 3.699018 & 2.832558 \\ 1 & 4.739479 & -0.039089 & -4.699225 \\ 1 & 3.950747 & -0.802602 & -3.312346 \\ 1 & 3.375476 & 0.778794 & -3.911138 \\ 1 & 6.913690 & -0.333413 & -3.572733 \\ 1 & 7.245825 & 0.395054 & -1.990240 \\ 1 & 6.148330 & -0.997836 & -2.112029 \\ 1 & 6.214314 & 1.917746 & -4.457042 \\ 1 & 4.875631 & 2.812643 & -3.704356 \\ 1 & 6.450986 & 2.716655 & -2.894598 \\ 1 & 0.751477 & -3.391807 & -2.923530 \\ 1 & 2.120450 & -2.473792 & -3.519432 \\ 1 & 2.433556 & -4.930301 & -3.531008 \\ 1 & 4.078153 & -3.492127 & -4.700587 \\ 1 & 6.508492 & -3.091818 & -4.880106 \\ 1 & 7.987208 & -3.486754 & -2.917702 \\ 1 & 6.990235 & -4.271215 & -0.774699 \\ & & & \end{array}$


$7_{\mathrm{s}-\mathrm{C} 3-}-1 \mathrm{a} 2 \mathrm{~s} 3 \mathrm{a} 4 \mathrm{~s}$

Number of imaginary frequencies : 0

The smallest frequencies are : $14.6836 \quad 18.1888 \quad 21.6668 \mathrm{~cm}(-1)$

Electronic energy : $\quad H F=-4587.4777836$

Zero-point correction=

Thermal correction to Energy=

Thermal correction to Enthalpy=

Thermal correction to Gibbs Free Energy=

Sum of electronic and zero-point Energies=

Sum of electronic and thermal Energies=

Sum of electronic and thermal Enthalpies=

Sum of electronic and thermal Free Energies=
1.476577 (Hartree/Particle)

1.565642

1.566586

1.353130

$-4586.001206$

$-4585.912142$

$-4585.911197$

$-4586.124654$

Cartesian Coordinates

$\begin{array}{cccc}6 & -0.381219 & 0.873907 & 2.712135 \\ 6 & -1.499395 & 0.691800 & 1.883473 \\ 6 & -2.600354 & 1.521957 & 2.025477 \\ 6 & -2.608718 & 2.552326 & 2.975606 \\ 6 & -1.505779 & 2.678549 & 3.813763 \\ 6 & -0.386659 & 1.834891 & 3.717262 \\ 15 & -1.381449 & -0.516886 & 0.510919 \\ 45 & -0.155099 & 0.822779 & -1.326102 \\ 6 & -1.887973 & 1.955841 & -1.762365 \\ 8 & -2.834397 & 2.014322 & -1.011240 \\ 6 & -3.812358 & 3.487427 & 3.026057 \\ 6 & -3.694609 & 4.531731 & 4.132456 \\ 6 & 0.765099 & 1.991390 & 4.709648 \\ 6 & 1.821092 & 0.899827 & 4.542811 \\ 6 & -3.115942 & -0.965034 & 0.101809 \\ 6 & -4.009396 & -1.384839 & 1.080974 \\ 6 & -5.321906 & -1.741399 & 0.754983 \\ 6 & -5.713574 & -1.627829 & -0.579359 \\ 6 & -4.838157 & -1.204622 & -1.589378 \\ 6 & -3.527956 & -0.904393 & -1.229170 \\ 6 & -5.348142 & -1.012336 & -3.015988 \\ 6 & -4.218674 & -0.675199 & -3.988333 \\ 6 & -6.243247 & -2.251336 & 1.861084 \\ 6 & -7.643632 & -2.579018 & 1.350390 \\ 6 & -0.878253 & -2.061906 & 1.426737 \\ 6 & -0.419274 & -3.165324 & 0.724065 \\ 6 & -0.067198 & -4.370810 & 1.413121 \\ 6 & -0.202489 & -4.433889 & 2.829979 \\ 6 & -0.702388 & -3.303374 & 3.515915 \\ 6 & -1.026736 & -2.158748 & 2.838249 \\ 6 & 0.169321 & -5.614442 & 3.515896 \\ 6 & 0.659618 & -6.698370 & 2.833408\end{array}$




$\begin{array}{cccc}6 & 0.793128 & -6.644110 & 1.430046 \\ 6 & 0.435579 & -5.513610 & 0.737494 \\ 6 & -0.323999 & -3.169752 & -0.759726 \\ 6 & 0.785497 & -2.661393 & -1.410800 \\ 6 & 0.905093 & -2.768986 & -2.815330 \\ 6 & -0.085578 & -3.347922 & -3.562705 \\ 6 & -1.250057 & -3.852891 & -2.940708 \\ 6 & -1.371549 & -3.771438 & -1.521816 \\ 6 & -2.549531 & -4.278443 & -0.913427 \\ 6 & -3.545951 & -4.840551 & -1.670030 \\ 6 & -3.414420 & -4.936411 & -3.073271 \\ 6 & -2.293681 & -4.446329 & -3.693574 \\ 7 & 1.827167 & -2.001751 & -0.669384 \\ 15 & 1.884598 & -0.334249 & -0.933258 \\ 8 & 2.938698 & -0.028921 & -2.197619 \\ 6 & 4.259273 & -0.418356 & -2.146605 \\ 6 & 5.144407 & 0.196907 & -1.280368 \\ 6 & 6.467767 & -0.344449 & -1.176820 \\ 6 & 6.872667 & -1.391669 & -2.057418 \\ 6 & 5.953750 & -1.889839 & -3.013615 \\ 6 & 4.667113 & -1.426854 & -3.045776 \\ 6 & 7.379000 & 0.071744 & -0.173259 \\ 6 & 8.623431 & -0.498913 & -0.065011 \\ 6 & 9.032292 & -1.509823 & -0.959629 \\ 6 & 8.170456 & -1.944759 & -1.933915 \\ 6 & 4.693699 & 1.349584 & -0.468135 \\ 6 & 3.558554 & 1.235942 & 0.311102 \\ 6 & 3.094782 & 2.276833 & 1.135868 \\ 6 & 3.765582 & 3.468464 & 1.163717 \\ 6 & 4.898642 & 3.675121 & 0.341363 \\ 6 & 5.361216 & 2.617947 & -0.500065 \\ 6 & 5.563657 & 4.925391 & 0.320651 \\ 6 & 6.628319 & 5.144986 & -0.514580 \\ 6 & 7.063942 & 4.116299 & -1.375603 \\ 6 & 6.449253 & 2.888884 & -1.368756 \\ 8 & 2.856699 & 0.050141 & 0.338238 \\ 6 & 2.961209 & -2.867146 & -0.257560 \\ 6 & 3.581959 & -2.550293 & 1.076919 \\ 6 & 4.965794 & -2.432809 & 1.191242 \\ 6 & 5.557798 & -2.184013 & 2.427388 \\ 6 & 4.769347 & -2.059579 & 3.565670 \\ 6 & 3.384892 & -2.182203 & 3.458846 \\ 6 & 2.796986 & -2.418904 & 2.223619 \\ 6 & -6.045863 & -2.277968 & -3.519811 \\ 6 & -6.343443 & 0.154266 & -3.015793 \\ 6 & -5.639412 & -3.530995 & 2.453387 \\ 6 & -6.372118 & -1.189702 & 2.959209 \\ 6 & -0.146021 & 0.281775 & -3.176029 \\ 8 & -0.083689 & 0.056356 & -4.310782 \\ 6 & 0.225150 & 2.459967 & -0.346391 \\ 8 & 0.280658 & 3.476552 & 0.199268 \\ 6 & -1.896923 & 2.727405 & -3.072515 \\ 6 & -3.002704 & 3.767510 & -3.213499\end{array}$




$\begin{array}{rrrr}6 & -3.006396 & 4.319859 & -4.638780 \\ 6 & -2.913800 & 4.908606 & -2.224060 \\ 6 & -4.088494 & 5.501095 & -1.753431 \\ 6 & -4.048754 & 6.600574 & -0.903325 \\ 6 & -2.824152 & 7.125874 & -0.498800 \\ 6 & -1.646976 & 6.544296 & -0.957787 \\ 6 & -1.692879 & 5.450481 & -1.818240 \\ 6 & -3.918143 & 4.215132 & 1.679990 \\ 6 & -5.089303 & 2.673988 & 3.267007 \\ 6 & 1.426700 & 3.359840 & 4.518618 \\ 6 & 0.223090 & 1.890218 & 6.140732 \\ 1 & 7.070580 & 0.835359 & 0.536250 \\ 1 & -1.404621 & -1.305826 & 3.395907 \\ 1 & 8.461898 & -2.737633 & -2.621510 \\ 1 & 0.060161 & -5.640471 & 4.599226 \\ 1 & 5.198457 & 5.713803 & 0.977441 \\ 1 & -2.666024 & -4.197534 & 0.166053 \\ 1 & -4.449344 & -5.207003 & -1.186594 \\ 1 & 3.418518 & 4.282736 & 1.798559 \\ 1 & -0.826330 & -3.356016 & 4.596994 \\ 1 & 6.280215 & -2.666455 & -3.703076 \\ 1 & 7.891192 & 4.298826 & -2.057847 \\ 1 & 3.939025 & -1.814994 & -3.754340 \\ 1 & -2.183176 & -4.506724 & -4.775431 \\ 1 & 0.005868 & -3.415232 & -4.645638 \\ 1 & 1.180014 & -7.506653 & 0.892277 \\ 1 & 6.790852 & 2.112891 & -2.048564 \\ 1 & 0.537318 & -5.488757 & -0.345721 \\ 1 & 1.794527 & -2.361808 & -3.292756 \\ 1 & 7.127860 & 6.110846 & -0.526389 \\ 1 & 9.297986 & -0.171558 & 0.722996 \\ 1 & -4.207484 & -5.395815 & -3.659692 \\ 1 & 0.945924 & -7.600212 & 3.369496 \\ 1 & 10.022705 & -1.948984 & -0.866192 \\ 1 & 2.198058 & 2.103615 & 1.728128 \\ 1 & -3.679279 & -1.439913 & 2.118217 \\ 1 & -2.798559 & -0.596855 & -1.975313 \\ 1 & -6.739288 & -1.870033 & -0.850953 \\ 1 & -3.448615 & 1.390541 & 1.358905 \\ 1 & 0.485977 & 0.226308 & 2.577339 \\ 1 & -1.503373 & 3.457216 & 4.573634 \\ 1 & 2.540892 & -3.882119 & -0.217148 \\ 1 & 3.739130 & -2.882772 & -1.035804 \\ 1 & 5.590874 & -2.523463 & 0.301453 \\ 1 & 1.714259 & -2.478062 & 2.135750 \\ 1 & 6.639322 & -2.082887 & 2.493694 \\ 1 & 2.755625 & -2.092447 & 4.343371 \\ 1 & 5.229162 & -1.866965 & 4.532736 \\ 1 & 2.599230 & 1.014609 & 5.308520 \\ 1 & 1.381960 & -0.100987 & 4.661288 \\ 1 & 2.323051 & 0.931869 & 3.567013 \\ 1 & 1.043962 & 1.985425 & 6.864234 \\ 1 & -0.507417 & 2.675216 & 6.368120\end{array}$




$\begin{array}{rrrr}1 & -0.264055 & 0.920671 & 6.308545 \\ 1 & 2.271472 & 3.478985 & 5.210619 \\ 1 & 1.806865 & 3.477066 & 3.494923 \\ 1 & 0.723645 & 4.181076 & 4.703877 \\ 1 & -4.584112 & 5.174520 & 4.128356 \\ 1 & -3.625188 & 4.072443 & 5.127577 \\ 1 & -2.821331 & 5.181258 & 3.990194 \\ 1 & -4.758589 & 4.923327 & 1.696077 \\ 1 & -3.000619 & 4.778496 & 1.458608 \\ 1 & -4.077862 & 3.520156 & 0.846698 \\ 1 & -5.963929 & 3.338140 & 3.291737 \\ 1 & -5.260173 & 1.932976 & 2.475896 \\ 1 & -5.043903 & 2.138516 & 4.225059 \\ 1 & -7.017745 & -1.555351 & 3.769209 \\ 1 & -5.402637 & -0.928381 & 3.401496 \\ 1 & -6.817646 & -0.267824 & 2.562743 \\ 1 & -8.263149 & -2.943097 & 2.179844 \\ 1 & -8.143656 & -1.697208 & 0.929426 \\ 1 & -7.627428 & -3.364651 & 0.583861 \\ 1 & -6.276075 & -3.919266 & 3.260064 \\ 1 & -5.552367 & -4.312083 & 1.685782 \\ 1 & -4.637667 & -3.362487 & 2.869753 \\ 1 & -4.623204 & -0.571281 & -5.003167 \\ 1 & -3.728729 & 0.275668 & -3.732713 \\ 1 & -3.450756 & -1.461329 & -4.012480 \\ 1 & -6.709346 & 0.348738 & -4.033404 \\ 1 & -7.213852 & -0.052633 & -2.380706 \\ 1 & -5.867708 & 1.070293 & -2.640578 \\ 1 & -6.424682 & -2.121951 & -4.538903 \\ 1 & -5.346327 & -3.123615 & -3.542981 \\ 1 & -6.900087 & -2.562332 & -2.893799 \\ 1 & -0.897230 & 3.151353 & -3.245036 \\ 1 & -2.013696 & 1.968979 & -3.864628 \\ 1 & -0.758008 & 5.016125 & -2.171298 \\ 1 & -0.682815 & 6.941216 & -0.645997 \\ 1 & -2.788594 & 7.981083 & 0.172737 \\ 1 & -4.977340 & 7.043424 & -0.547652 \\ 1 & -5.048851 & 5.085899 & -2.061460 \\ 1 & -3.813206 & 5.049415 & -4.778449 \\ 1 & -2.058086 & 4.829254 & -4.858859 \\ 1 & -3.138561 & 3.518711 & -5.377411 \\ 1 & -3.961480 & 3.257277 & -3.035385\end{array}$

\section{$7_{B}$}

Number of imaginary frequencies : 0

The smallest frequencies are : $16.2284 \quad 19.9745 \quad 23.1005 \mathrm{~cm}(-1)$

Electronic energy : $\quad H F=-4587.4736352$

Zero-point correction $=\quad 1.476932($ Hartree/Particle $)$

Thermal correction to Energy= $\quad 1.565569$

Thermal correction to Enthalpy= $\quad 1.566513$

Thermal correction to Gibbs Free Energy= 1.355629

Sum of electronic and zero-point Energies $=\quad-4585.996703$ 
Sum of electronic and thermal Energies= Sum of electronic and thermal Enthalpies= Sum of electronic and thermal Free Energies=
$-4585.908066$

$-4585.907122$

$-4586.118006$

Cartesian Coordinates

\begin{tabular}{|c|c|c|c|}
\hline 6 & -0.302059 & 5.706471 & 3.200388 \\
\hline 6 & 0.104884 & 4.470628 & 2.643529 \\
\hline 6 & 0.063180 & 4.287442 & 1.230993 \\
\hline 6 & -0.380416 & 5.371603 & 0.428875 \\
\hline 6 & -0.772685 & 6.558118 & 0.997376 \\
\hline 6 & -0.734929 & 6.730373 & 2.397277 \\
\hline 6 & 0.447254 & 3.027152 & 0.671795 \\
\hline 6 & 0.854362 & 1.986968 & 1.496560 \\
\hline 6 & 0.914972 & 2.204881 & 2.900073 \\
\hline 6 & 0.553197 & 3.403687 & 3.454687 \\
\hline 6 & 0.450074 & 2.890337 & -0.810176 \\
\hline 6 & -0.604100 & 2.296036 & -1.482410 \\
\hline 6 & -0.599146 & 2.211789 & -2.893790 \\
\hline 6 & 0.448945 & 2.702615 & -3.625094 \\
\hline 6 & 1.543459 & 3.321513 & -2.980030 \\
\hline 6 & 1.547179 & 3.421814 & -1.557271 \\
\hline 6 & 2.632238 & 3.847677 & -3.718098 \\
\hline 6 & 3.685157 & 4.451264 & -3.079791 \\
\hline 6 & 3.701666 & 4.535988 & -1.670478 \\
\hline 6 & 2.661819 & 4.036031 & -0.928229 \\
\hline 7 & -1.721747 & 1.750667 & -0.760355 \\
\hline 6 & -2.832711 & 2.698954 & -0.490055 \\
\hline 6 & -3.523571 & 2.552171 & 0.840498 \\
\hline 6 & -2.799914 & 2.402980 & 2.025012 \\
\hline 6 & -3.453512 & 2.310556 & 3.246044 \\
\hline 6 & -4.845446 & 2.362582 & 3.303481 \\
\hline 6 & -5.573822 & 2.508724 & 2.128501 \\
\hline 6 & -4.914804 & 2.603949 & 0.904746 \\
\hline 15 & 1.376201 & 0.365199 & 0.739114 \\
\hline 45 & 0.109832 & -1.243897 & -0.922213 \\
\hline 15 & -1.854862 & 0.069906 & -0.853754 \\
\hline 8 & -2.944068 & -0.102615 & 0.365534 \\
\hline 6 & -3.810363 & -1.168693 & 0.420099 \\
\hline 6 & -4.875956 & -1.252669 & -0.455501 \\
\hline 6 & -5.714748 & -2.413446 & -0.378272 \\
\hline 6 & -5.499429 & -3.368391 & 0.661148 \\
\hline 6 & -4.439745 & -3.174148 & 1.579514 \\
\hline 6 & -3.599621 & -2.103312 & 1.451061 \\
\hline 6 & -6.741826 & -2.677690 & -1.319946 \\
\hline 6 & -7.528594 & -3.798165 & -1.217354 \\
\hline 6 & -7.336275 & -4.719374 & -0.166901 \\
\hline 6 & -6.337037 & -4.506688 & 0.747623 \\
\hline 6 & -5.100719 & -0.182560 & -1.454441 \\
\hline 6 & -4.078447 & 0.195557 & -2.305072 \\
\hline 6 & -4.279937 & 1.097124 & -3.371723 \\
\hline 6 & -5.494151 & 1.707109 & -3.526761 \\
\hline 6 & -6.539828 & 1.469683 & -2.601212 \\
\hline 6 & -6.346572 & 0.521025 & -1.552517 \\
\hline 6 & -7.760130 & 2.183893 & -2.680665 \\
\hline 6 & -8.746764 & 2.000232 & -1.746119 \\
\hline
\end{tabular}




\begin{tabular}{|c|c|c|c|}
\hline 6 & -8.545641 & 1.090123 & -0.687378 \\
\hline 6 & -7.381925 & 0.366949 & -0.595564 \\
\hline 8 & -2.814504 & -0.334987 & -2.162082 \\
\hline 6 & 1.537403 & -0.721556 & 2.207721 \\
\hline 6 & 0.429071 & -0.877217 & 3.046101 \\
\hline 6 & 0.442134 & -1.795185 & 4.095266 \\
\hline 6 & 1.570507 & -2.611453 & 4.229104 \\
\hline 6 & 2.677676 & -2.510214 & 3.382879 \\
\hline 6 & 2.656974 & -1.530898 & 2.387364 \\
\hline 6 & 3.871632 & -3.445215 & 3.568760 \\
\hline 6 & 4.517754 & -3.178796 & 4.932454 \\
\hline 6 & -0.742272 & -1.950946 & 5.048597 \\
\hline 6 & -1.456303 & -3.272152 & 4.743305 \\
\hline 6 & 3.096056 & 0.790254 & 0.261712 \\
\hline 6 & 3.496321 & 0.561394 & -1.053552 \\
\hline 6 & 4.799647 & 0.825782 & -1.462139 \\
\hline 6 & 5.679279 & 1.378353 & -0.520178 \\
\hline 6 & 5.301446 & 1.649705 & 0.795733 \\
\hline 6 & 3.992579 & 1.334274 & 1.174298 \\
\hline 6 & 5.297681 & 0.431623 & -2.850997 \\
\hline 6 & 6.065506 & 1.579707 & -3.510116 \\
\hline 6 & 6.252934 & 2.234337 & 1.838401 \\
\hline 6 & 5.629317 & 3.484976 & 2.468842 \\
\hline 6 & 6.225213 & -0.778218 & -2.684922 \\
\hline 6 & 4.152377 & 0.028807 & -3.780659 \\
\hline 6 & 6.503082 & 1.183944 & 2.927244 \\
\hline 6 & 7.599326 & 2.633019 & 1.239594 \\
\hline 6 & 3.399377 & -4.901394 & 3.501079 \\
\hline 6 & 4.932220 & -3.244462 & 2.487551 \\
\hline 6 & -0.256453 & -1.963168 & 6.501821 \\
\hline 6 & -1.743527 & -0.803888 & \\
\hline 6 & 0.315078 & -0.968012 & -2.817835 \\
\hline 8 & 0.353573 & -0.865305 & -3.970344 \\
\hline 6 & 1.785838 & -2.540854 & -1.064581 \\
\hline 6 & 1.729805 & -3.929915 & -1.796656 \\
\hline 6 & -0.499820 & -2.591038 & 0.322703 \\
\hline 8 & -0.753506 & -3.452444 & 1.051446 \\
\hline 8 & 2.828137 & -2.226561 & -0.540927 \\
\hline 1 & -7.232829 & -0.312591 & 0.239757 \\
\hline 1 & 1.258698 & 1.404666 & 3.550424 \\
\hline 1 & -7.889552 & 2.896221 & -3.494466 \\
\hline 1 & -0.266098 & 5.824164 & 4.282516 \\
\hline 1 & -6.159320 & -5.217037 & 1.553948 \\
\hline 1 & 2.691864 & 4.093241 & 0.158568 \\
\hline 1 & 4.551451 & 4.996390 & -1.171184 \\
\hline 1 & -4.291003 & -3.901473 & 2.376308 \\
\hline 1 & 0.609382 & 3.550486 & 4.532750 \\
\hline 1 & -5.663719 & 2.404153 & -4.345677 \\
\hline 1 & -8.303554 & -3.979872 & -1.958624 \\
\hline 1 & -3.456497 & 1.283666 & -4.057417 \\
\hline 1 & 2.611613 & 3.763306 & -4.804018 \\
\hline 1 & 0.452768 & 2.621863 & -4.711092 \\
\hline 1 & -1.112694 & 7.373892 & 0.363475 \\
\hline 1 & -6.896583 & -1.986018 & -2.143805 \\
\hline 1 & -0.406877 & 5.255422 & -0.652857 \\
\hline 1 & -1.444415 & 1.734488 & -3.385296 \\
\hline
\end{tabular}




\begin{tabular}{|c|c|c|c|}
\hline & -7.970058 & -5.600050 & -0.094156 \\
\hline ] & -9.317441 & 0.964059 & 0.068681 \\
\hline 1 & 4.513398 & 4.859481 & -3.655183 \\
\hline & -1.048656 & 7.675409 & 2.834445 \\
\hline 1 & -9.675805 & 2.561727 & -1.810127 \\
\hline 1 & -2.758408 & -1.945241 & 2.122138 \\
\hline 1 & 3.670076 & 1.505747 & 2.201607 \\
\hline 1 & 2.769887 & 0.140224 & -1.744536 \\
\hline & 6.701921 & 1.588149 & -0.827575 \\
\hline 1 & 3.498482 & -1.415802 & 1.711895 \\
\hline 1 & -0.451742 & -0.257139 & 2.874550 \\
\hline 1 & 1.585134 & -3.358533 & 5.022492 \\
\hline 1 & -2.372174 & 3.695712 & -0.540371 \\
\hline 1 & -3.576904 & 2.661553 & -1.300209 \\
\hline 1 & -5.494061 & 2.705771 & -0.014610 \\
\hline 1 & -1.715741 & 2.324573 & 1.979221 \\
\hline 1 & -6.661359 & 2.542108 & 2.157156 \\
\hline 1 & -2.869937 & 2.195465 & 4.158687 \\
\hline 1 & -5.357928 & 2.284814 & 4.260009 \\
\hline 1 & -2.538583 & -0.911561 & 5.657320 \\
\hline 1 & -1.260925 & 0.170963 & 5.068457 \\
\hline 1 & -2.231051 & -0.779972 & 3.925097 \\
\hline 1 & -1.111995 & -2.052588 & 7.184373 \\
\hline 1 & 0.415764 & -2.802751 & 6.713175 \\
\hline 1 & 0.276623 & -1.035742 & 6.748517 \\
\hline 1 & -2.333268 & -3.397809 & 5.393415 \\
\hline 1 & -1.793632 & -3.305644 & 3.698559 \\
\hline 1 & -0.794257 & -4.133112 & 4.899144 \\
\hline 1 & 7.163577 & 1.586677 & 3.707459 \\
\hline 1 & 5.571273 & 0.861464 & 3.410261 \\
\hline 1 & 6.984898 & 0.293194 & 2.503247 \\
\hline 1 & 8.234822 & 3.073528 & 2.018408 \\
\hline 1 & 8.139180 & 1.771002 & 0.827585 \\
\hline 1 & 7.484935 & 3.379969 & 0.442729 \\
\hline 1 & 6.302628 & 3.903817 & 3.228885 \\
\hline 1 & 5.453933 & 4.260303 & 1.711532 \\
\hline 1 & 4.669836 & 3.276527 & 2.958137 \\
\hline 1 & 4.550626 & -0.201356 & -4.777357 \\
\hline 1 & 3.629301 & -0.872435 & -3.429826 \\
\hline 1 & 3.413949 & 0.836122 & -3.891426 \\
\hline 1 & 6.596766 & -1.116024 & -3.662949 \\
\hline 1 & 7.093154 & -0.540760 & -2.056206 \\
\hline 1 & 5.682421 & -1.613797 & -2.220653 \\
\hline 1 & 6.430078 & 1.269581 & -4.498671 \\
\hline 1 & 5.417743 & 2.455719 & -3.646762 \\
\hline 1 & 6.939417 & 1.892935 & -2.926209 \\
\hline 1 & 5.750416 & -3.960496 & 2.637095 \\
\hline 1 & 4.525124 & -3.401811 & 1.479074 \\
\hline 1 & 5.367701 & -2.237125 & 2.519204 \\
\hline 1 & 4.251903 & -5.583556 & 3.621058 \\
\hline 1 & 2.672693 & -5.141864 & 4.286217 \\
\hline 1 & 2.927568 & -5.114279 & 2.532580 \\
\hline 1 & 5.380695 & -3.841408 & 5.084766 \\
\hline 1 & 4.871946 & -2.141454 & 5.000989 \\
\hline 1 & 3.817134 & -3.348548 & 5.759460 \\
\hline 6 & 2.770892 & -3.864140 & -2.912800 \\
\hline
\end{tabular}




$\begin{array}{lrrr}6 & 2.100364 & -4.964775 & -0.724402 \\ 6 & 0.355387 & -4.306624 & -2.337962 \\ 6 & 4.131379 & -4.074542 & -2.654128 \\ 6 & 5.077274 & -4.006858 & -3.670789 \\ 6 & 4.692655 & -3.707030 & -4.973998 \\ 6 & 3.349804 & -3.474175 & -5.245077 \\ 6 & 2.403365 & -3.553442 & -4.227186 \\ 1 & 4.465578 & -4.293966 & -1.643473 \\ 1 & 6.125873 & -4.183993 & -3.437871 \\ 1 & 5.433914 & -3.653029 & -5.768652 \\ 1 & 3.028156 & -3.232819 & -6.256193 \\ 1 & 1.360373 & -3.374065 & -4.474538 \\ 1 & 0.431779 & -5.243320 & -2.905750 \\ 1 & -0.355163 & -4.472166 & -1.521222 \\ 1 & -0.088041 & -3.547559 & -2.988703 \\ 1 & 2.160010 & -5.965943 & -1.169646 \\ 1 & 3.052188 & -4.740214 & -0.235277 \\ 1 & 1.329029 & -4.987424 & 0.056338\end{array}$

$\mathrm{TS}_{R}$

Number of imaginary frequencies : 1

The smallest frequencies are : - $805.0085 \quad 11.5307 \quad 17.6866 \mathrm{~cm}(-1)$

Electronic energy : $\quad H F=-4475.3553718$

Zero-point correction $=\quad 1.480981$ (Hartree/Particle)

Thermal correction to Energy $=\quad 1.568589$

Thermal correction to Enthalpy= $\quad 1.569533$

Thermal correction to Gibbs Free Energy $=\quad 1.357455$

Sum of electronic and zero-point Energies $=\quad-4473.874391$

Sum of electronic and thermal Energies $=\quad-4473.786783$

Sum of electronic and thermal Enthalpies $=\quad-4473.785838$

Sum of electronic and thermal Free Energies $=\quad-4473.997917$

\begin{tabular}{cccc}
\multicolumn{4}{c}{ Cartesian Coordinates } \\
\hline 6 & 6.363279 & 3.459169 & 0.418651 \\
6 & 5.368797 & 2.628846 & 0.996010 \\
6 & 5.003394 & 2.871522 & 2.355036 \\
6 & 5.671022 & 3.882574 & 3.088042 \\
6 & 6.646099 & 4.650122 & 2.505440 \\
6 & 6.983508 & 4.440116 & 1.152076 \\
6 & 4.697699 & 1.597101 & 0.260885 \\
6 & 3.653554 & 0.935383 & 0.878894 \\
6 & 3.282625 & 1.179409 & 2.214632 \\
6 & 3.959270 & 2.119110 & 2.943049 \\
6 & 5.034623 & 1.279265 & -1.145724 \\
6 & 4.043533 & 1.323891 & -2.109805 \\
6 & 4.327813 & 1.182683 & -3.485374 \\
6 & 5.601786 & 0.898815 & -3.893791 \\
6 & 6.634099 & 0.707224 & -2.942747 \\
6 & 6.352051 & 0.891460 & -1.555847 \\
6 & 7.928807 & 0.296791 & -3.343006 \\
6 & 8.906604 & 0.043675 & -2.415125 \\
6 & 8.622057 & 0.191749 & -1.041444 \\
6 & 7.382175 & 0.609014 & -0.623262
\end{tabular}




\begin{tabular}{|c|c|c|c|}
\hline & 2.733201 & 1.561094 & -1.763855 \\
\hline 15 & 1.828294 & 0.434754 & -0.915104 \\
\hline & 2.954709 & -0.034545 & 0.194914 \\
\hline 45 & -0.227919 & 1.408165 & -0.255746 \\
\hline & -1.266782 & -0.756353 & 0.466007 \\
\hline & -3.063063 & -0.982758 & 0.127828 \\
\hline & -3.775619 & -2.007894 & 0.741644 \\
\hline & -5.130457 & -2.214875 & 0.464670 \\
\hline & -5.753279 & -1.334942 & -0.420294 \\
\hline & -5.067462 & -0.288210 & -1.051075 \\
\hline & -3.708004 & -0.145692 & -0.783161 \\
\hline & -5.844901 & -3.398433 & 1.113347 \\
\hline 6 & -5.175434 & -4.693717 & 0.636904 \\
\hline 6 & -5.826939 & 0.684974 & -1.951179 \\
\hline 6 & -6.586578 & -0.068766 & -3.045909 \\
\hline 6 & -1.976876 & 2.444964 & 0.202935 \\
\hline 8 & -2.754383 & 2.077549 & 1.058685 \\
\hline 6 & -2.233394 & 3.736832 & -0.565704 \\
\hline 6 & -3.385257 & 4.585308 & -0.020623 \\
\hline 1 & -4.219588 & 3.900396 & 0.192793 \\
\hline 6 & -3.853802 & 5.572899 & -1.063350 \\
\hline 6 & -5.098902 & 5.417581 & -1.676687 \\
\hline 6 & -5.532116 & 6.302508 & -2.659883 \\
\hline 6 & -4.721528 & 7.363878 & -3.048360 \\
\hline 6 & -3.477075 & 7.531180 & -2.446156 \\
\hline 6 & -3.049089 & 6.644369 & -1.464330 \\
\hline 6 & 0.282170 & 2.171801 & 1.463562 \\
\hline 8 & 0.363166 & 2.714252 & 2.479541 \\
\hline 6 & -0.679297 & -2.484941 & 0.082149 \\
\hline 6 & -0.347136 & -2.848404 & -1.213965 \\
\hline 6 & 0.044431 & -4.195303 & -1.511788 \\
\hline 6 & 0.088211 & -5.162315 & -0.466657 \\
\hline 6 & -0.281521 & -4.768208 & 0.839257 \\
\hline 6 & -0.652221 & -3.477274 & 1.102055 \\
\hline 6 & 0.506951 & -6.483532 & -0.751441 \\
\hline 6 & 0.870308 & -6.846036 & -2.023212 \\
\hline 6 & 0.822776 & -5.896154 & -3.064880 \\
\hline 6 & 0.416978 & -4.607597 & -2.818231 \\
\hline 6 & -0.420761 & -1.899720 & -2.357418 \\
\hline 6 & 0.643357 & -1.076296 & -2.674772 \\
\hline 6 & 0.607109 & -0.269728 & -3.836062 \\
\hline 6 & -0.490657 & -0.265649 & -4.654359 \\
\hline 6 & -1.612998 & -1.071746 & -4.350878 \\
\hline 6 & -1.578303 & -1.906455 & -3.194419 \\
\hline 6 & -2.709723 & -2.713930 & -2.907306 \\
\hline 6 & -3.808921 & -2.700400 & -3.727840 \\
\hline 6 & -3.834432 & -1.884096 & -4.880318 \\
\hline 6 & -2.761869 & -1.083071 & -5.179568 \\
\hline 7 & 1.788849 & -0.999405 & -1.808585 \\
\hline 6 & 2.954161 & -1.831540 & -2.193789 \\
\hline 6 & 3.729145 & -2.435848 & -1.052413 \\
\hline 6 & 5.118996 & -2.338779 & -1.025787 \\
\hline 6 & 5.853156 & -2.928467 & 0.000777 \\
\hline 6 & 5.202478 & -3.631721 & 1.008271 \\
\hline 6 & 3.812612 & -3.736024 & 0.984998 \\
\hline 6 & 3.082984 & -3.137017 & -0.033167 \\
\hline
\end{tabular}




\begin{tabular}{|c|c|c|c|}
\hline 6 & -1.132181 & -0.718121 & 2.294311 \\
\hline 6 & 0.088183 & -1.076194 & 2.887665 \\
\hline & 0.258717 & -1.009421 & 4.266333 \\
\hline & -0.794271 & -0.483292 & 5.033661 \\
\hline & -1.997047 & -0.072574 & 4.467364 \\
\hline & -2.158802 & -0.220392 & 3.082669 \\
\hline & 1.520900 & -1.520971 & 4.961157 \\
\hline & 2.235981 & -0.363956 & 5.664754 \\
\hline & -3.144632 & 0.525429 & 5.276624 \\
\hline & -3.411777 & 1.950110 & 4.776393 \\
\hline & -4.404876 & -0.326777 & 5.084661 \\
\hline & -2.835108 & 0.589886 & 6.769574 \\
\hline & 1.129819 & -2.578319 & 6.001373 \\
\hline & 2.490837 & -2.180386 & 3.981513 \\
\hline & -5.741232 & -3.297664 & 2.639089 \\
\hline & -7.323845 & -3.462836 & 0.741480 \\
\hline & -6.825097 & 1.464989 & -1.086668 \\
\hline & -4.893429 & 1.687994 & -2.625597 \\
\hline & -0.695450 & 1.557213 & -1.847714 \\
\hline & 7.171707 & 0.700363 & 0.439494 \\
\hline & -0.927098 & -3.207681 & 2.118383 \\
\hline & 8.124779 & 0.170545 & -4.407062 \\
\hline & 0.536934 & -7.204603 & 0.064246 \\
\hline & 5.380388 & 4.043683 & 4.125293 \\
\hline & -2.705915 & -3.336630 & -2.013663 \\
\hline & -4.673839 & -3.313885 & -3.482800 \\
\hline & 3.683497 & 2.319876 & 3.977556 \\
\hline & -0.266854 & -5.509447 & 1.637462 \\
\hline & 5.832949 & 0.788524 & -4.951969 \\
\hline 1 & 7.738077 & 5.066373 & 0.681349 \\
\hline & 3.513927 & 1.307673 & -4.196396 \\
\hline & -2.771300 & -0.445151 & -6.062326 \\
\hline & -0.516815 & 0.359706 & -5.545401 \\
\hline & 1.107578 & -6.187936 & -4.073166 \\
\hline 1 & 6.627765 & 3.321600 & -0.626333 \\
\hline & 0.379821 & -3.888906 & -3.634413 \\
\hline ] & 1.468614 & 0.358913 & -4.054494 \\
\hline & 7.148401 & 5.427656 & 3.076170 \\
\hline 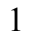 & 9.390576 & -0.031899 & -0.304911 \\
\hline 1 & -4.709365 & -1.890658 & -5.527295 \\
\hline & 1.194187 & -7.862840 & -2.232550 \\
\hline 1 & 9.894477 & -0.281657 & -2.732777 \\
\hline & 2.453784 & 0.611066 & 2.634214 \\
\hline ] & -3.265948 & -2.666580 & 1.444066 \\
\hline & -3.123984 & 0.630082 & -1.271578 \\
\hline t & -6.813886 & -1.459851 & -0.629460 \\
\hline 1 & -3.086092 & 0.092045 & 2.608197 \\
\hline t & 0.894947 & -1.444089 & 2.252528 \\
\hline 1 & -0.659755 & -0.401537 & 6.110217 \\
\hline & 2.535544 & -2.643955 & -2.804704 \\
\hline 1 & 3.637619 & -1.269806 & -2.848747 \\
\hline & 5.635913 & -1.782039 & -1.809320 \\
\hline 1 & 1.996278 & -3.186170 & -0.028804 \\
\hline 1 & 6.936847 & -2.828548 & 0.010629 \\
\hline & 3.290093 & -4.286498 & 1.766516 \\
\hline & 5.773119 & -4.094773 & 1.810573 \\
\hline
\end{tabular}




$\begin{array}{rrrr}1 & 3.355959 & -2.579504 & 4.526688 \\ 1 & 2.015751 & -3.018192 & 3.451568 \\ 1 & 2.879511 & -1.483763 & 3.227289 \\ 1 & 2.027673 & -2.975408 & 6.493787 \\ 1 & 0.477372 & -2.173995 & 6.784068 \\ 1 & 0.604366 & -3.418420 & 5.528193 \\ 1 & 3.139878 & -0.724695 & 6.174038 \\ 1 & 2.539940 & 0.408601 & 4.946167 \\ 1 & 1.596791 & 0.114400 & 6.416803 \\ 1 & -3.689205 & 1.024150 & 7.304546 \\ 1 & -2.652260 & -0.405533 & 7.195504 \\ 1 & -1.961834 & 1.220790 & 6.979989 \\ 1 & -4.222844 & 2.412478 & 5.355582 \\ 1 & -2.515750 & 2.575966 & 4.883714 \\ 1 & -3.700263 & 1.967351 & 3.718923 \\ 1 & -5.244223 & 0.102956 & 5.648058 \\ 1 & -4.707587 & -0.384340 & 4.031281 \\ 1 & -4.245760 & -1.352274 & 5.444421 \\ 1 & -6.237019 & -4.156082 & 3.112225 \\ 1 & -4.700532 & -3.285232 & 2.985972 \\ 1 & -6.226677 & -2.383593 & 3.005689 \\ 1 & -7.790262 & -4.326738 & 1.231780 \\ 1 & -7.867770 & -2.566608 & 1.066842 \\ 1 & -7.470907 & -3.581235 & -0.339866 \\ 1 & -5.665915 & -5.568616 & 1.085043 \\ 1 & -5.244854 & -4.788884 & -0.455345 \\ 1 & -4.111769 & -4.732580 & 0.905178 \\ 1 & -5.469339 & 2.346736 & -3.288463 \\ 1 & -4.386825 & 2.328752 & -1.889717 \\ 1 & -4.126621 & 1.187871 & -3.234838 \\ 1 & -7.369193 & 2.199813 & -1.696558 \\ 1 & -7.567905 & 0.804334 & -0.622374 \\ 1 & -6.307674 & 2.000566 & -0.279094 \\ 1 & -7.147759 & 0.638156 & -3.671784 \\ 1 & -5.890937 & -0.616814 & -3.694257 \\ 1 & -7.307296 & -0.787451 & -2.637574 \\ 1 & -0.073928 & 2.454955 & -1.503403 \\ 6 & -2.983311 & 5.262106 & 1.286944 \\ 1 & -1.295315 & 4.311226 & -0.592149 \\ 1 & -2.414267 & 3.434396 & -1.610615 \\ 1 & -2.072209 & 6.786825 & -1.002473 \\ 1 & -2.835325 & 8.358475 & -2.742987 \\ 1 & -5.056873 & 8.058553 & -3.815507 \\ 1 & -6.507879 & 6.163029 & -3.121315 \\ 1 & -5.737341 & 4.586869 & -1.372983 \\ 1 & -2.693479 & 4.516205 & 2.036273 \\ 1 & -2.127605 & 5.934672 & 1.140004 \\ 1 & -3.806785 & 5.857142 & 1.698910\end{array}$

\section{$\mathrm{TS3}_{S}$}

Number of imaginary frequencies : 1

The smallest frequencies are : -833.3189 $12.3539 \quad 14.9913 \mathrm{~cm}(-1)$

Electronic energy :

$\mathrm{HF}=-4475.352466$

Zero-point correction $=$

1.480711 (Hartree/Particle)

Thermal correction to Energy=

1.568336 
Thermal correction to Enthalpy=

Thermal correction to Gibbs Free Energy=

Sum of electronic and zero-point Energies=

Sum of electronic and thermal Energies=

Sum of electronic and thermal Enthalpies=

Sum of electronic and thermal Free Energies=
1.569281

1.356673

$-4473.871755$

$-4473.784130$

$-4473.783185$

$-4473.995793$

Cartesian Coordinates

\begin{tabular}{cccc}
6 & -2.796244 & 6.443264 & -3.076345 \\
6 & -2.476345 & 6.147567 & -1.748888 \\
6 & -1.721435 & 7.078755 & -1.029309 \\
6 & -1.302817 & 8.269878 & -1.611105 \\
6 & -1.632790 & 8.553566 & -2.933428 \\
6 & -2.380066 & 7.634889 & -3.663271 \\
6 & -2.866082 & 4.839438 & -1.097164 \\
6 & -4.234442 & 4.326436 & -1.523647 \\
6 & -1.768560 & 3.800871 & -1.361172 \\
6 & -1.795964 & 2.567723 & -0.464548 \\
8 & -2.677995 & 2.414801 & 0.352999 \\
45 & -0.154045 & 1.283657 & -0.609243 \\
6 & 0.376252 & 2.333280 & 0.943799 \\
8 & 0.485829 & 3.068081 & 1.828081 \\
15 & -1.446911 & -0.573778 & 0.502198 \\
6 & -0.988677 & -2.382249 & 0.609299 \\
6 & -0.613912 & -3.091842 & -0.522187 \\
6 & -0.309346 & -4.490328 & -0.433568 \\
6 & -0.404763 & -5.153314 & 0.823393 \\
6 & -0.819997 & -4.411404 & 1.952005 \\
6 & -1.100709 & -3.075938 & 1.846929 \\
6 & -0.077894 & -6.526876 & 0.918300 \\
6 & 0.330973 & -7.229273 & -0.186254 \\
6 & 0.424522 & -6.580866 & -1.435519 \\
6 & 0.109097 & -5.250003 & -1.557793 \\
6 & -0.556594 & -2.472291 & -1.873473 \\
6 & 0.572725 & -1.804231 & -2.308348 \\
6 & 0.652285 & -1.308908 & -3.630733 \\
6 & -0.386118 & -1.476224 & -4.506859 \\
6 & -1.559396 & -2.156008 & -4.104334 \\
6 & -1.651387 & -2.658539 & -2.772479 \\
6 & -2.834877 & -3.340146 & -2.386651 \\
6 & -3.857555 & -3.533816 & -3.280272 \\
6 & -3.753106 & -3.056271 & -4.605491 \\
6 & -2.633428 & -2.370973 & -5.002729 \\
7 & 1.680951 & -1.591163 & -1.417934 \\
6 & 2.767276 & -2.598105 & -1.495354 \\
6 & 3.455867 & -2.913123 & -0.194065 \\
6 & 4.847158 & -2.902606 & -0.120283 \\
6 & 5.497611 & -3.222761 & 1.069196 \\
6 & 4.760129 & -3.565935 & 2.196797 \\
6 & 3.367959 & -3.584024 & 2.127674 \\
6 & 2.722536 & -3.254761 & 0.943558 \\
6 & -1.448578 & -0.100622 & 2.275685 \\
6 & -0.286726 & -0.339179 & 3.025912 \\
6 & -0.195566 & 0.065410 & 4.352946 \\
6 & -1.267281 & 0.797456 & 4.890893 \\
& & & \\
\hline
\end{tabular}




\begin{tabular}{|c|c|c|c|}
\hline 6 & -2.415862 & 1.084018 & 4.160211 \\
\hline 6 & -2.500180 & 0.599690 & 2.846900 \\
\hline 5 & -3.575264 & 1.908974 & 4.713307 \\
\hline & -4.875345 & 1.102559 & 4.617194 \\
\hline f & 1.015943 & -0.260887 & 5.225907 \\
\hline & 0.552131 & -0.937462 & 6.521442 \\
\hline 6 & -3.222311 & -0.756488 & 0.048193 \\
\hline 6 & -4.073922 & -1.538231 & 0.822517 \\
\hline 6 & -5.410722 & -1.730143 & 0.460157 \\
\hline 6 & -5.865808 & -1.098937 & -0.697230 \\
\hline 6 & -5.034453 & -0.311492 & -1.505535 \\
\hline 6 & -3.703572 & -0.160057 & -1.118264 \\
\hline 6 & -6.287433 & -2.636880 & 1.321626 \\
\hline 6 & -6.327106 & -2.106542 & 2.759021 \\
\hline 6 & -5.609105 & 0.378621 & -2.742374 \\
\hline 6 & -6.597742 & 1.460398 & -2.291505 \\
\hline 15 & 1.824566 & 0.018079 & -0.926003 \\
\hline 8 & 2.842704 & 0.815229 & -1.993476 \\
\hline 6 & 4.135632 & 0.396011 & -2.209060 \\
\hline 6 & 5.098736 & 0.528015 & -1.224321 \\
\hline 6 & 6.386389 & -0.055410 & -1.462239 \\
\hline 6 & 6.681564 & -0.617496 & -2.740402 \\
\hline 6 & 5.690898 & -0.600564 & -3.752451 \\
\hline 6 & 4.437674 & -0.121272 & -3.487391 \\
\hline 6 & 7.366931 & -0.166479 & -0.443927 \\
\hline 6 & 8.574105 & -0.775405 & -0.685369 \\
\hline 6 & 8.875076 & -1.297998 & -1.960552 \\
\hline 6 & 7.943845 & -1.218927 & -2.964270 \\
\hline 6 & 4.760251 & 1.218956 & 0.040842 \\
\hline 6 & 3.651044 & 0.816167 & 0.759530 \\
\hline 6 & 3.277568 & 1.410043 & 1.978771 \\
\hline 6 & 4.017462 & 2.442407 & 2.484810 \\
\hline 6 & 5.133323 & 2.941862 & 1.772423 \\
\hline 6 & 5.502982 & 2.344512 & 0.528574 \\
\hline 6 & 5.871011 & 4.046029 & 2.263852 \\
\hline 6 & 6.919989 & 4.567634 & 1.552024 \\
\hline 6 & 7.264929 & 4.005578 & 0.305238 \\
\hline 6 & 6.577216 & 2.926409 & -0.192278 \\
\hline 8 & 2.883708 & -0.235367 & 0.312233 \\
\hline 6 & -3.367064 & 2.304753 & 6.172494 \\
\hline 6 & -3.708278 & 3.189087 & 3.879503 \\
\hline 6 & 1.982773 & -1.219204 & 4.531046 \\
\hline 6 & 1.758635 & 1.033689 & 5.572671 \\
\hline 6 & -4.515072 & 1.030081 & -3.586680 \\
\hline 6 & -6.350262 & -0.625694 & -3.629592 \\
\hline 6 & -7.722160 & -2.721658 & 0.807822 \\
\hline 6 & -5.692518 & -4.050506 & 1.313140 \\
\hline 1 & -0.544134 & 1.157967 & -2.229806 \\
\hline 1 & 7.141535 & 0.212808 & 0.549674 \\
\hline 1 & -1.414400 & -2.535734 & 2.735993 \\
\hline 1 & 8.150581 & -1.633613 & -3.950048 \\
\hline 1 & -0.155697 & -7.011678 & 1.890415 \\
\hline 1 & 5.574804 & 4.479332 & 3.218236 \\
\hline 1 & -2.930229 & -3.701086 & -1.363656 \\
\hline 1 & -4.761808 & -4.049857 & -2.963433 \\
\hline 1 & 3.740676 & 2.911332 & 3.428222 \\
\hline
\end{tabular}




\begin{tabular}{|c|c|c|c|}
\hline 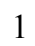 & -0.914424 & -4.919359 & 2.911082 \\
\hline . & 5.934919 & -1.000712 & -4.735132 \\
\hline & 8.079856 & 4.435627 & -0.272752 \\
\hline & 3.656132 & -0.121246 & -4.243844 \\
\hline & -2.544717 & -1.988418 & -6.018667 \\
\hline & -0.320240 & -1.096539 & -5.525274 \\
\hline & 0.746720 & -7.141122 & -2.310260 \\
\hline & 6.849881 & 2.516113 & -1.160991 \\
\hline & 0.180160 & -4.766642 & -2.530162 \\
\hline 1 & 1.557479 & -0.785460 & -3.932297 \\
\hline & 7.476073 & 5.419558 & 1.936204 \\
\hline & 9.302966 & -0.860599 & 0.117603 \\
\hline & -4.567282 & -3.227196 & -5.306822 \\
\hline & 0.583331 & -8.283853 & -0.103646 \\
\hline & 9.837119 & -1.772374 & -2.139551 \\
\hline 1 & 2.392242 & 1.030386 & 2.484495 \\
\hline 1 & -3.689410 & -2.015063 & 1.723781 \\
\hline 1 & -3.009439 & 0.427378 & -1.715624 \\
\hline 1 & -6.906829 & -1.221013 & -0.989109 \\
\hline 1 & -3.387285 & 0.799941 & 2.251516 \\
\hline 1 & 0.538246 & -0.879457 & 2.560102 \\
\hline 1 & -1.191417 & 1.147756 & 5.918023 \\
\hline 1 & 2.281986 & -3.511314 & -1.868421 \\
\hline 1 & 3.513780 & -2.310227 & -2.251214 \\
\hline 1 & 5.432064 & -2.625723 & -0.999098 \\
\hline 1 & 1.635571 & -3.231080 & 0.902075 \\
\hline 1 & 6.584814 & -3.196297 & 1.110324 \\
\hline 1 & 2.777773 & -3.854425 & 3.002384 \\
\hline 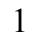 & 5.265707 & -3.816590 & 3.127034 \\
\hline 1 & 2.802282 & -1.480316 & 5.213009 \\
\hline 1 & 1.479749 & -2.152457 & 4.239827 \\
\hline 1 & 2.440419 & -0.790238 & 3.630249 \\
\hline 1 & 1.418903 & -1.190904 & 7.146437 \\
\hline 1 & -0.104710 & -0.294781 & 7.119059 \\
\hline 1 & 0.007646 & -1.866411 & 6.306995 \\
\hline 1 & 2.650158 & 0.816692 & 6.176700 \\
\hline 1 & 2.082507 & 1.560098 & 4.664689 \\
\hline 1 & 1.125868 & 1.723787 & 6.144250 \\
\hline 1 & -4.227970 & 2.888694 & 6.521975 \\
\hline 1 & -3.275869 & 1.428295 & 6.827625 \\
\hline 1 & -2.473852 & 2.928694 & 6.304996 \\
\hline 1 & -4.531667 & 3.809165 & 4.260080 \\
\hline 1 & -2.785019 & 3.781807 & 3.925649 \\
\hline 1 & -3.907292 & 2.972405 & 2.823525 \\
\hline 1 & -5.717708 & 1.691829 & 5.004057 \\
\hline 1 & -5.113418 & 0.827252 & 3.582080 \\
\hline 1 & -4.810985 & 0.177798 & 5.206261 \\
\hline 1 & -6.937867 & -2.764894 & 3.391558 \\
\hline 1 & -5.328299 & -2.048877 & 3.209238 \\
\hline 1 & -6.768066 & -1.101587 & 2.793885 \\
\hline 1 & -8.309260 & -3.380590 & 1.460017 \\
\hline 1 & -8.214295 & -1.740479 & 0.802693 \\
\hline 1 & -7.771423 & -3.136735 & -0.207209 \\
\hline 1 & -6.296567 & -4.725751 & 1.934376 \\
\hline 1 & -5.670746 & -4.458131 & 0.293389 \\
\hline 1 & -4.665304 & -4.070033 & 1.699557 \\
\hline
\end{tabular}




$\begin{array}{lrrr}1 & -4.961906 & 1.505292 & -4.469612 \\ 1 & -3.974700 & 1.809587 & -3.032815 \\ 1 & -3.781900 & 0.289385 & -3.937750 \\ 1 & -6.997700 & 1.999934 & -3.161267 \\ 1 & -7.446956 & 1.029337 & -1.746412 \\ 1 & -6.115470 & 2.191443 & -1.631379 \\ 1 & -6.759677 & -0.115682 & -4.512035 \\ 1 & -5.671932 & -1.414138 & -3.977735 \\ 1 & -7.190289 & -1.103904 & -3.111878 \\ 1 & 0.149477 & 2.032739 & -2.031906 \\ 1 & -2.892121 & 5.006819 & -0.009243 \\ 1 & -0.771693 & 4.249353 & -1.235853 \\ 1 & -1.794406 & 3.459195 & -2.410032 \\ 1 & -1.462184 & 6.859289 & 0.007136 \\ 1 & -0.720009 & 8.980859 & -1.028812 \\ 1 & -1.309642 & 9.485697 & -3.391913 \\ 1 & -2.642833 & 7.846143 & -4.698106 \\ 1 & -3.380253 & 5.735714 & -3.663297 \\ 1 & -5.015191 & 5.081262 & -1.369890 \\ 1 & -4.251688 & 4.040499 & -2.584822 \\ 1 & -4.490654 & 3.438356 & -0.936207\end{array}$

\section{$\mathrm{TS3}_{B}$}

Number of imaginary frequencies : 1

The smallest frequencies are : -915.1382 $14.1802 \quad 15.9708 \mathrm{~cm}(-1)$

Electronic energy : $\quad \mathrm{HF}=-4475.3503233$

Zero-point correction $=\quad 1.481113($ Hartree/Particle)

Thermal correction to Energy $=\quad 1.568248$

Thermal correction to Enthalpy= $\quad 1.569192$

Thermal correction to Gibbs Free Energy $=\quad 1.360490$

Sum of electronic and zero-point Energies $=\quad-4473.869210$

Sum of electronic and thermal Energies $=\quad-4473.782075$

Sum of electronic and thermal Enthalpies $=\quad-4473.781131$

Sum of electronic and thermal Free Energies $=\quad-4473.989833$

Cartesian Coordinates

$\begin{array}{cccc}6 & -2.556938 & 2.092105 & 1.862125 \\ 6 & -1.595814 & 1.093735 & 1.806148 \\ 6 & -0.509917 & 1.128233 & 2.693618 \\ 6 & -0.421744 & 2.109028 & 3.676556 \\ 6 & -1.394795 & 3.122265 & 3.684379 \\ 6 & -2.452454 & 3.144762 & 2.780188 \\ 15 & -1.610804 & -0.131914 & 0.442850 \\ 6 & -1.325182 & -1.733380 & 1.356613 \\ 6 & -1.017231 & -2.891639 & 0.658743 \\ 6 & -0.860397 & -4.136553 & 1.351518 \\ 6 & -1.031475 & -4.180866 & 2.765159 \\ 6 & -1.371993 & -2.989726 & 3.445491 \\ 6 & -1.513270 & -1.810364 & 2.764994 \\ 6 & -0.851367 & -5.403448 & 3.454446 \\ 6 & -0.513202 & -6.547679 & 2.778215 \\ 6 & -0.345763 & -6.512591 & 1.377994 \\ 6 & -0.518579 & -5.341278 & 0.682554\end{array}$




\begin{tabular}{|c|c|c|c|}
\hline 6 & -0.880851 & -2.921274 & -0.822639 \\
\hline 6 & 0.320067 & -2.615961 & -1.435372 \\
\hline 6 & 0.472315 & -2.763381 & -2.833511 \\
\hline 6 & -0.568089 & -3.194667 & -3.612130 \\
\hline 6 & -1.820615 & -3.498136 & -3.028819 \\
\hline 6 & -1.983080 & -3.361441 & -1.618217 \\
\hline 6 & -3.248636 & -3.660178 & -1.050645 \\
\hline 6 & -4.290482 & -4.082486 & -1.837002 \\
\hline 6 & -4.119594 & -4.240951 & -3.230129 \\
\hline 6 & -2.911969 & -3.948695 & -3.811834 \\
\hline 7 & 1.427760 & -2.125339 & -0.661865 \\
\hline 6 & 2.411423 & -3.147503 & -0.229682 \\
\hline 6 & 3.015241 & -2.950260 & 1.136399 \\
\hline 6 & 4.388647 & -3.106896 & 1.312004 \\
\hline 6 & 4.964768 & -2.974437 & 2.573474 \\
\hline 6 & 4.169037 & -2.686901 & 3.676375 \\
\hline 6 & 2.794027 & -2.530419 & 3.508152 \\
\hline 6 & 2.223629 & -2.658644 & 2.248905 \\
\hline 6 & 0.689472 & 2.113867 & 4.726034 \\
\hline 6 & 1.547929 & 0.850590 & 4.660965 \\
\hline 6 & -3.477289 & 4.273148 & 2.712737 \\
\hline 6 & -3.263218 & 5.323291 & 3.798888 \\
\hline 45 & -0.126054 & 0.938374 & -1.323983 \\
\hline 6 & 0.473069 & 2.501643 & -0.325466 \\
\hline 8 & 0.714437 & 3.458539 & 0.271371 \\
\hline 15 & 1.714460 & -0.473200 & -0.876180 \\
\hline 8 & 2.697277 & -0.272595 & 0.431391 \\
\hline 6 & 3.600023 & 0.765238 & 0.461368 \\
\hline 6 & 4.769156 & 0.690080 & -0.271866 \\
\hline 6 & 5.659892 & 1.813579 & -0.228994 \\
\hline 6 & 5.362043 & 2.911538 & 0.634659 \\
\hline 6 & 4.172963 & 2.896125 & 1.401951 \\
\hline 6 & 3.297733 & 1.850485 & \\
\hline 6 & 6.246477 & 4.015730 & 0.691747 \\
\hline 6 & 7.372604 & 4.060124 & -0.088845 \\
\hline 6 & 7.650345 & 2.996916 & -0.972822 \\
\hline 6 & 6.818976 & 1.906357 & -1.041551 \\
\hline 6 & 5.028190 & -0.494790 & -1.122127 \\
\hline 6 & 4.074615 & -0.897049 & -2.040483 \\
\hline 6 & 4.325928 & -1.913393 & -2.987513 \\
\hline 6 & 5.504793 & -2.605271 & -2.950260 \\
\hline 6 & 6.462168 & -2.335586 & -1.941875 \\
\hline 6 & 6.225708 & -1.276107 & -1.015336 \\
\hline 6 & 7.162399 & -1.087003 & 0.032462 \\
\hline 6 & 8.275289 & -1.884639 & 0.141249 \\
\hline 6 & 8.521999 & -2.908851 & -0.796923 \\
\hline 6 & 7.630053 & -3.126171 & -1.815849 \\
\hline 8 & 2.845548 & -0.281333 & -2.098575 \\
\hline 6 & -1.659833 & 2.234107 & -1.943298 \\
\hline 6 & -1.580917 & 3.088078 & -3.266925 \\
\hline 6 & -2.990859 & 3.590477 & -3.601494 \\
\hline 6 & -3.393671 & -0.365046 & 0.046198 \\
\hline 6 & -4.316053 & -0.645197 & 1.049644 \\
\hline 6 & -5.661946 & -0.875055 & 0.750789 \\
\hline 6 & -6.054638 & -0.788414 & -0.585539 \\
\hline 6 & -5.152016 & -0.507381 & -1.619504 \\
\hline
\end{tabular}




\begin{tabular}{|c|c|c|c|}
\hline & -3.813799 & -0.316224 & -1.281889 \\
\hline & -6.620303 & -1.232601 & 1.885003 \\
\hline & -8.052442 & -1.436428 & 1.397909 \\
\hline & -5.650354 & -0.327079 & -3.052614 \\
\hline & -4.503499 & -0.347195 & -4.062574 \\
\hline & -6.147607 & -2.538909 & 2.534828 \\
\hline & -6.628347 & -0.111082 & 2.929557 \\
\hline & -6.639921 & -1.427752 & -3.443263 \\
\hline & -6.350127 & 1.035049 & -3.139339 \\
\hline & -2.608369 & 2.423969 & -1.212068 \\
\hline & 1.588001 & 3.336849 & 4.515361 \\
\hline & 0.072396 & 2.177503 & 6.128345 \\
\hline & -3.345564 & 4.956632 & 1.344634 \\
\hline & -4.892005 & 3.704276 & 2.866547 \\
\hline & -0.454398 & 0.039060 & -2.696052 \\
\hline & 6.973890 & -0.316766 & 0.776231 \\
\hline & -1.774583 & -0.912583 & 3.318882 \\
\hline & 7.794348 & -3.923866 & -2.539176 \\
\hline & -0.984402 & -5.413141 & 4.535390 \\
\hline & 6.001805 & 4.836820 & 1.364244 \\
\hline & -3.393090 & -3.531904 & 0.021080 \\
\hline & -5.258606 & -4.288426 & -1.384049 \\
\hline & 3.953161 & 3.742725 & 2.051260 \\
\hline & -1.521821 & -3.023515 & 4.524064 \\
\hline & 5.710177 & -3.390904 & -3.675445 \\
\hline & 8.528805 & 3.043181 & -1.612665 \\
\hline & 3.566573 & -2.120398 & -3.738105 \\
\hline & -2.769514 & -4.058199 & -4.886113 \\
\hline & -0.446480 & -3.308440 & -4.688232 \\
\hline - & -0.080064 & -7.422311 & 0.844394 \\
\hline & 7.043798 & 1.103600 & -1.738685 \\
\hline & -0.392208 & -5.332995 & -0.398211 \\
\hline & 1.435323 & -2.515863 & -3.276042 \\
\hline & 8.042461 & 4.915469 & -0.040357 \\
\hline 1 & 8.969892 & -1.728675 & 0.963699 \\
\hline & -4.950519 & -4.589242 & -3.840423 \\
\hline 1 & -0.373334 & -7.482208 & 3.316487 \\
\hline & 9.410035 & -3.529333 & -0.702352 \\
\hline 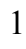 & 2.357976 & 1.829159 & 1.853318 \\
\hline 1 & -3.981249 & -0.692871 & 2.085551 \\
\hline & -3.068698 & -0.113258 & -2.047141 \\
\hline 1 & -7.102313 & -0.939244 & -0.836244 \\
\hline & -3.374028 & 2.074903 & 1.145726 \\
\hline 1 & 0.255686 & 0.354844 & 2.621509 \\
\hline & -1.311002 & 3.917081 & 4.422842 \\
\hline 1 & 1.855146 & -4.095942 & -0.233527 \\
\hline | & 3.213790 & -3.255743 & -0.975471 \\
\hline 1 & 5.021256 & -3.324094 & 0.449674 \\
\hline 1 & 1.155865 & -2.498059 & 2.115456 \\
\hline & 6.041097 & -3.089627 & 2.686352 \\
\hline 1 & 2.158345 & -2.305345 & 4.363737 \\
\hline 1 & 4.616010 & -2.580725 & 4.662585 \\
\hline 1 & 2.291079 & 0.865278 & 5.468438 \\
\hline l & 0.937053 & -0.055054 & 4.785309 \\
\hline 1 & 2.100958 & 0.755774 & 3.717460 \\
\hline 1 & 0.863104 & 2.165782 & 6.890473 \\
\hline
\end{tabular}




\begin{tabular}{|c|c|c|c|}
\hline & -0.519611 & 3.086676 & 6.284869 \\
\hline & -0.583295 & 1.315625 & 6.309058 \\
\hline & 2.409102 & 3.342649 & 5.245122 \\
\hline & 2.026884 & 3.335920 & 3.508592 \\
\hline & 1.031225 & 4.275033 & 4.629316 \\
\hline & -4.024125 & 6.108863 & 3.707642 \\
\hline & -3.350639 & 4.895872 & 4.806542 \\
\hline & -2.281706 & 5.807111 & 3.713511 \\
\hline & -4.053495 & 5.793275 & 1.263664 \\
\hline & -2.329038 & 5.351331 & 1.207737 \\
\hline & -3.543002 & 4.260752 & 0.519415 \\
\hline & -5.635043 & 4.510751 & 2.803269 \\
\hline & -5.130710 & 2.973604 & 2.083820 \\
\hline & -5.014150 & 3.207496 & 3.838382 \\
\hline & -7.287256 & -0.374936 & 3.767929 \\
\hline & -5.629963 & 0.083734 & 3.340762 \\
\hline & -6.997491 & 0.826124 & 2.493079 \\
\hline & -8.698773 & -1.689178 & 2.248031 \\
\hline & -8.460424 & -0.529628 & 0.932973 \\
\hline & -8.126838 & -2.257997 & 0.673603 \\
\hline & -6.816448 & -2.823517 & 3.358510 \\
\hline 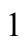 & -6.144255 & -3.358333 & 1.803101 \\
\hline & -5.132140 & -2.455234 & 2.942640 \\
\hline & -4.904812 & -0.257116 & -5.080174 \\
\hline & -3.809562 & 0.491128 & -3.912552 \\
\hline & -3.928322 & -1.282501 & -4.005705 \\
\hline 1 & -6.694727 & 1.229148 & -4.164721 \\
\hline & -7.222611 & 1.081822 & -2.475132 \\
\hline ] & -5.666909 & 1.844291 & -2.847616 \\
\hline ] & -6.967658 & -1.285492 & -4.481591 \\
\hline 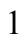 & -6.174569 & -2.419250 & -3.368090 \\
\hline 1 & -7.541286 & -1.426554 & -2.819246 \\
\hline & 0.297607 & 0.827730 & -2.891181 \\
\hline 6 & -0.662834 & 4.258940 & -2.924131 \\
\hline 5 & -1.102706 & 2.292271 & -4.474626 \\
\hline 1 & -2.945649 & 4.305481 & -4.432155 \\
\hline 1 & -3.625873 & 2.749941 & -3.913223 \\
\hline . & -3.484011 & 4.067998 & -2.751728 \\
\hline 1 & -1.159187 & 2.920076 & -5.373456 \\
\hline 1 & -0.077541 & 1.923467 & -4.390737 \\
\hline 1 & -1.750490 & 1.419490 & -4.633404 \\
\hline 6 & 0.638975 & 4.368024 & -3.421917 \\
\hline 6 & 1.457120 & 5.437800 & -3.067664 \\
\hline 6 & 0.993389 & 6.421511 & -2.202340 \\
\hline 6 & -0.296643 & 6.322009 & -1.689335 \\
\hline 6 & -1.109438 & 5.252694 & -2.043707 \\
\hline 1 & 1.032369 & 3.611723 & -4.096181 \\
\hline 1 & 2.465120 & 5.497292 & -3.473120 \\
\hline 1 & 1.632921 & 7.257073 & -1.926224 \\
\hline 1 & -0.673569 & 7.079209 & -1.004358 \\
\hline & -2.107623 & 5.184212 & -1.615986 \\
\hline
\end{tabular}

\section{$\mathbf{8}_{R}$}

Number of imaginary frequencies : 0 
The smallest frequencies are : $16.4276 \quad 18.443720 .8187 \mathrm{~cm}(-1)$ Electronic energy : $\quad H F=-4475.3659786$

Zero-point correction $=\quad 1.483930($ Hartree/Particle $)$

Thermal correction to Energy $=\quad 1.571430$

Thermal correction to Enthalpy= $\quad 1.572374$

Thermal correction to Gibbs Free Energy= $\quad 1.361210$

Sum of electronic and zero-point Energies $=\quad-4473.882049$

Sum of electronic and thermal Energies $=\quad-4473.794549$

Sum of electronic and thermal Enthalpies $=\quad-4473.793605$

Sum of electronic and thermal Free Energies $=\quad-4474.004768$

Cartesian Coordinates

\begin{tabular}{cccc}
6 & 0.143118 & -2.333197 & 2.173196 \\
6 & -1.079727 & -1.736687 & 1.829811 \\
6 & -2.108166 & -1.692733 & 2.758829 \\
6 & -1.947423 & -2.245008 & 4.037425 \\
6 & -0.740826 & -2.872251 & 4.331701 \\
6 & 0.316081 & -2.946224 & 3.409078 \\
15 & -1.214323 & -0.860929 & 0.224108 \\
45 & -0.115005 & 1.329788 & 0.637787 \\
15 & 1.882086 & 0.863041 & -0.531486 \\
8 & 3.069269 & -0.075616 & 0.128617 \\
6 & 3.816358 & 0.433639 & 1.165203 \\
6 & 4.827713 & 1.338836 & 0.905609 \\
6 & 5.549649 & 1.877515 & 2.020567 \\
6 & 5.269052 & 1.396252 & 3.335901 \\
6 & 4.257499 & 0.424559 & 3.523984 \\
6 & 3.530789 & -0.036489 & 2.460659 \\
6 & 6.513631 & 2.908907 & 1.883376 \\
6 & 7.184009 & 3.404327 & 2.974117 \\
6 & 6.930831 & 2.898781 & 4.266306 \\
6 & 5.987593 & 1.919049 & 4.438589 \\
6 & 5.078124 & 1.770990 & -0.488567 \\
6 & 4.032283 & 2.267264 & -1.246489 \\
6 & 4.233977 & 2.830803 & -2.524929 \\
6 & 5.478540 & 2.809689 & -3.091759 \\
6 & 6.562703 & 2.192732 & -2.420596 \\
6 & 6.365101 & 1.662865 & -1.110254 \\
6 & 7.827078 & 2.059720 & -3.044243 \\
6 & 8.855514 & 1.401448 & -2.419833 \\
6 & 8.654299 & 0.846343 & -1.138759 \\
6 & 7.446013 & 0.977847 & -0.499254 \\
8 & 2.747696 & 2.277838 & -0.754149 \\
6 & -3.093503 & -2.122702 & 5.037852 \\
6 & -2.791091 & -2.820636 & 6.361001 \\
6 & 1.604024 & -3.679354 & 3.782828 \\
6 & 2.541598 & -3.840488 & 2.586132 \\
6 & -3.013508 & -0.883757 & -0.169648 \\
6 & -3.700262 & -2.094398 & -0.128382 \\
6 & -5.048640 & -2.175731 & -0.481914 \\
6 & -5.688824 & -0.997006 & -0.863938 \\
6 & -5.030834 & 0.238223 & -0.921271 \\
6 & -3.678146 & 0.274713 & -0.575840 \\
6 & -5.817326 & 1.498036 & -1.286341 \\
6 & -4.897981 & 2.677453 & -1.595672 \\
\hline
\end{tabular}




\begin{tabular}{|c|c|c|c|}
\hline 6 & -5.736252 & -3.538997 & -0.467354 \\
\hline & -7.211511 & -3.453097 & -0.849397 \\
\hline & -0.643109 & -2.167425 & -0.979853 \\
\hline & -0.363351 & -1.849559 & -2.300193 \\
\hline & 0.008519 & -2.877143 & -3.229105 \\
\hline & 0.081130 & -4.230628 & -2.791352 \\
\hline & -0.239117 & -4.526670 & -1.447405 \\
\hline & -0.589064 & -3.531219 & -0.575786 \\
\hline & 0.479258 & -5.240958 & -3.698536 \\
\hline & 0.796589 & -4.932983 & -4.996568 \\
\hline & 0.721844 & -3.595847 & -5.439563 \\
\hline y & 0.334287 & -2.595731 & -4.582244 \\
\hline & -0.468235 & -0.467872 & -2.840288 \\
\hline & 0.595148 & 0.410096 & -2.758763 \\
\hline & 0.537398 & 1.672795 & -3.392246 \\
\hline & -0.576032 & 2.057919 & -4.088752 \\
\hline y & -1.695472 & 1.197932 & -4.181128 \\
\hline & -1.645526 & -0.080957 & -3.551077 \\
\hline & -2.776805 & -0.930901 & -3.652537 \\
\hline & -3.890999 & -0.538316 & -4.350125 \\
\hline & -3.929656 & 0.719836 & -4.991286 \\
\hline & -2.856942 & 1.570136 & -4.901680 \\
\hline T & 1.772792 & 0.058715 & -2.013186 \\
\hline 5 & 2.899448 & -0.483431 & -2.812086 \\
\hline 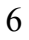 & 3.721252 & -1.549719 & -2.137091 \\
\hline & 5.111281 & -1.455238 & -2.123663 \\
\hline & 5.884792 & -2.447259 & -1.525242 \\
\hline & 5.273474 & -3.550412 & -0.940090 \\
\hline & 3.883334 & -3.654278 & -0.956192 \\
\hline 6 & 3.115266 & -2.659863 & -1.546373 \\
\hline ) & -6.707327 & 1.262899 & -2.510155 \\
\hline 6 & -6.703872 & 1.866454 & -0.090423 \\
\hline b & -5.033756 & -4.454013 & -1.478240 \\
\hline & -5.641740 & -4.152080 & 0.934208 \\
\hline & 0.424247 & 0.957550 & 2.497608 \\
\hline & 0.583859 & 0.892545 & 3.633626 \\
\hline 6 & -1.855663 & 2.101383 & 1.504379 \\
\hline f & -2.283913 & 3.510891 & 1.113283 \\
\hline 6 & -3.548408 & 4.008657 & 1.809410 \\
\hline 5 & -2.504307 & 1.465905 & 2.312550 \\
\hline & -3.340522 & -0.636145 & 5.321103 \\
\hline 6 & -4.361779 & -2.749305 & 4.446313 \\
\hline & 2.328977 & -2.897930 & 4.883552 \\
\hline 6 & 1.272531 & -5.085002 & 4.297508 \\
\hline & -0.712073 & 1.959141 & -0.717249 \\
\hline 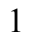 & 7.300243 & 0.528385 & 0.479915 \\
\hline 1 & -0.827699 & -3.796050 & 0.450431 \\
\hline 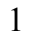 & 7.957803 & 2.479427 & -4.040856 \\
\hline 1 & 0.531613 & -6.267894 & -3.339769 \\
\hline & 5.761790 & 1.531247 & 5.431036 \\
\hline 1 & -2.758678 & -1.899621 & -3.155077 \\
\hline ( & -4.754774 & -1.198741 & -4.402454 \\
\hline 1 & 4.048635 & 0.068672 & 4.532202 \\
\hline ] & -0.203191 & -5.562507 & -1.112166 \\
\hline & 5.646059 & 3.242800 & -4.076493 \\
\hline & 7.912846 & 4.200608 & 2.840352 \\
\hline
\end{tabular}




\begin{tabular}{|c|c|c|c|}
\hline ] & 3.383977 & 3.281306 & -3.032827 \\
\hline & -2.877724 & 2.546257 & -5.384282 \\
\hline & -0.617838 & 3.030655 & -4.576133 \\
\hline ] & 0.971019 & -3.355526 & -6.470554 \\
\hline & 6.712620 & 3.320903 & 0.897695 \\
\hline & 0.275989 & -1.570585 & -4.942099 \\
\hline & 1.397081 & 2.333622 & -3.302018 \\
\hline & 7.472312 & 3.297124 & 5.121119 \\
\hline & 9.462656 & 0.303730 & -0.653664 \\
\hline & -4.815266 & 1.014173 & -5.550926 \\
\hline & 1.105807 & -5.714909 & -5.686171 \\
\hline & 9.819284 & 1.295134 & -2.912136 \\
\hline & 2.724748 & -0.758507 & 2.581207 \\
\hline & -3.173568 & -2.997867 & 0.176527 \\
\hline & -3.114635 & 1.203778 & -0.629751 \\
\hline & -6.743359 & -1.034613 & -1.127352 \\
\hline & -3.039375 & -1.194886 & 2.497600 \\
\hline & 0.952680 & -2.335347 & 1.442388 \\
\hline ] & -0.605379 & -3.324269 & 5.311773 \\
\hline & 2.429454 & -0.913426 & -3.708273 \\
\hline & 3.557229 & 0.326679 & -3.162398 \\
\hline & 5.597279 & -0.586213 & -2.570603 \\
\hline & 2.029670 & -2.724800 & -1.526040 \\
\hline 1 & 6.968521 & -2.348300 & -1.513168 \\
\hline & 3.391027 & -4.515485 & -0.506060 \\
\hline$\frac{1}{1}$ & 5.875385 & -4.326077 & -0.471311 \\
\hline & 3.420762 & -4.427673 & 2.880609 \\
\hline 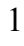 & 2.047592 & -4.370030 & 1.759081 \\
\hline ] & 2.912724 & -2.883657 & 2.197224 \\
\hline & 2.197004 & -5.622459 & 4.547208 \\
\hline 1 & 0.652555 & -5.066575 & 5.201207 \\
\hline & 0.738726 & -5.668076 & 3.535669 \\
\hline 1 & 3.273846 & -3.391304 & 5.148996 \\
\hline & 2.558881 & -1.875287 & 4.555270 \\
\hline ] & 1.721630 & -2.822243 & 5.793960 \\
\hline ] & -3.646397 & -2.709402 & 7.039387 \\
\hline & -2.614127 & -3.896217 & 6.228540 \\
\hline 1 & -1.917245 & -2.386292 & 6.863514 \\
\hline & -4.151433 & -0.516824 & 6.052729 \\
\hline 1 & -2.438354 & -0.162464 & 5.730307 \\
\hline & -3.617239 & -0.083955 & 4.415707 \\
\hline 1 & -5.199280 & -2.647278 & 5.149575 \\
\hline ] & -4.660499 & -2.270090 & 3.505246 \\
\hline & -4.216291 & -3.819714 & 4.247626 \\
\hline 1 & -6.109820 & -5.145656 & 0.947647 \\
\hline & -4.603586 & -4.270554 & 1.269127 \\
\hline 1 & -6.160431 & -3.525306 & 1.671597 \\
\hline ] & -7.658861 & -4.454663 & -0.819159 \\
\hline 1 & -7.778526 & -2.818397 & -0.156234 \\
\hline 1 & -7.350715 & -3.062966 & -1.865965 \\
\hline 1 & -5.500173 & -5.448539 & -1.485687 \\
\hline 1 & -5.105225 & -4.039584 & -2.493096 \\
\hline 1 & -3.968411 & -4.585543 & -1.248546 \\
\hline 1 & -5.493281 & 3.551907 & -1.890519 \\
\hline & -4.312374 & 2.979451 & -0.720074 \\
\hline 1 & -4.202723 & 2.444750 & -2.415317 \\
\hline
\end{tabular}




$\begin{array}{lrrr}1 & -7.275373 & 2.780554 & -0.303182 \\ 1 & -7.417947 & 1.066164 & 0.143091 \\ 1 & -6.095648 & 2.043260 & 0.807402 \\ 1 & -7.245946 & 2.186564 & -2.759487 \\ 1 & -6.107288 & 0.976386 & -3.383062 \\ 1 & -7.462073 & 0.485079 & -2.344793 \\ 1 & 0.404143 & 2.824020 & 0.777321 \\ 6 & -4.284734 & 5.080117 & 1.037525 \\ 1 & -4.227334 & 3.146076 & 1.904754 \\ 6 & -3.226142 & 4.499584 & 3.221051 \\ 1 & -1.422658 & 4.167382 & 1.312819 \\ 1 & -2.377736 & 3.507551 & 0.016891 \\ 6 & -5.672410 & 5.186720 & 1.174358 \\ 6 & -6.392271 & 6.159449 & 0.490473 \\ 6 & -5.731305 & 7.054198 & -0.347363 \\ 6 & -4.349454 & 6.970447 & -0.482003 \\ 6 & -3.633138 & 5.994969 & 0.207075 \\ 1 & -6.193211 & 4.482001 & 1.824408 \\ 1 & -7.473019 & 6.215715 & 0.606900 \\ 1 & -6.290408 & 7.813448 & -0.890136 \\ 1 & -3.822030 & 7.668305 & -1.129536 \\ 1 & -2.551535 & 5.943662 & 0.088257 \\ 1 & -4.134252 & 4.796069 & 3.760161 \\ 1 & -2.725100 & 3.715888 & 3.800773 \\ 1 & -2.563926 & 5.375667 & 3.177842\end{array}$

$\mathbf{8}_{S}$

Number of imaginary frequencies : 0

The smallest frequencies are : $12.7282 \quad 14.7962 \quad 17.5593 \mathrm{~cm}(-1)$

Electronic energy : $\quad \mathrm{HF}=-4475.3623926$

Zero-point correction $=\quad 1.483625($ Hartree/Particle $)$

Thermal correction to Energy= $\quad 1.571223$

Thermal correction to Enthalpy $=\quad 1.572167$

Thermal correction to Gibbs Free Energy $=\quad 1.359837$

Sum of electronic and zero-point Energies $=\quad-4473.878768$

Sum of electronic and thermal Energies $=\quad-4473.791169$

Sum of electronic and thermal Enthalpies $=\quad-4473.790225$

Sum of electronic and thermal Free Energies $=\quad-4474.002555$

Cartesian Coordinates

$\begin{array}{cccc}6 & -0.317634 & -0.374113 & 3.076970 \\ 6 & -1.464237 & -0.105296 & 2.313953 \\ 6 & -2.513733 & 0.604249 & 2.876171 \\ 6 & -2.443562 & 1.064531 & 4.199069 \\ 6 & -1.313660 & 0.742644 & 4.944891 \\ 6 & -0.244324 & 0.002229 & 4.413130 \\ 15 & -1.445961 & -0.543343 & 0.533258 \\ 45 & -0.110650 & 1.290490 & -0.521275 \\ 15 & 1.834069 & 0.013782 & -0.919858 \\ 8 & 2.902978 & -0.284343 & 0.302790 \\ 6 & 3.669485 & 0.751723 & 0.783273 \\ 6 & 4.768469 & 1.190435 & 0.069152 \\ 6 & 5.504482 & 2.305798 & 0.588370 \\ 6 & 5.142851 & 2.855900 & 1.855988\end{array}$




\begin{tabular}{|c|c|c|c|}
\hline 6 & 4.042572 & 2.317022 & 2.564073 \\
\hline 6 & 3.307709 & 1.294638 & 2.030218 \\
\hline & 6.563779 & 2.923673 & -0.124309 \\
\hline & 7.245425 & 3.992984 & 0.401771 \\
\hline & 6.908748 & 4.508528 & 1.670816 \\
\hline & 5.873898 & 3.951200 & 2.376394 \\
\hline & 5.105088 & 0.543084 & -1.219598 \\
\hline & 4.136467 & 0.429584 & -2.200913 \\
\hline & 4.431483 & -0.053571 & -3.493965 \\
\hline & 5.686873 & -0.513805 & -3.781437 \\
\hline & 6.686834 & -0.544882 & -2.778815 \\
\hline & 6.396632 & -0.020645 & -1.483341 \\
\hline & 7.954549 & -1.123618 & -3.030148 \\
\hline b & 8.896456 & -1.216819 & -2.037763 \\
\hline & 8.600600 & -0.733361 & -0.746145 \\
\hline & 7.387838 & -0.147569 & -0.477183 \\
\hline & 2.843937 & 0.839063 & -1.967219 \\
\hline & -3.595378 & 1.904666 & 4.744179 \\
\hline D & -3.405487 & 2.273163 & 6.212945 \\
\hline & 0.948424 & -0.360493 & 5.297708 \\
\hline & 1.889800 & -1.352786 & 4.614398 \\
\hline y & -3.211607 & -0.727118 & 0.047812 \\
\hline 6 & -4.044460 & -1.561761 & 0.787247 \\
\hline 5 & -5.364308 & -1.800052 & 0.395234 \\
\hline 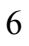 & -5.826093 & -1.154233 & -0.751430 \\
\hline$b$ & -5.016348 & -0.307708 & -1.520088 \\
\hline 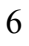 & -3.697601 & -0.113371 & -1.107439 \\
\hline & -5.604159 & 0.415646 & -2.732399 \\
\hline ) & -4.515061 & 1.063294 & -3.586383 \\
\hline 6 & -6.208750 & -2.781624 & 1.204942 \\
\hline ) & -7.629023 & -2.915362 & 0.662352 \\
\hline 6 & -0.985916 & -2.352977 & 0.597483 \\
\hline 6 & -0.630525 & -3.045580 & -0.550235 \\
\hline 5 & -0.342846 & -4.449824 & -0.489463 \\
\hline b & -0.439039 & -5.135877 & 0.754713 \\
\hline & -0.840189 & -4.411096 & 1.899142 \\
\hline 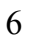 & -1.103333 & -3.070360 & 1.821367 \\
\hline ) & -0.126368 & -6.514314 & 0.822511 \\
\hline 6 & 0.270144 & -7.199804 & -0.296958 \\
\hline 6 & 0.363916 & -6.528896 & -1.534177 \\
\hline 6 & 0.061506 & -5.192811 & -1.630046 \\
\hline 6 & -0.580232 & -2.410767 & -1.894133 \\
\hline 5 & 0.557339 & -1.765426 & -2.339122 \\
\hline 6 & 0.638600 & -1.276942 & -3.663623 \\
\hline 6 & -0.406390 & -1.434386 & -4.533337 \\
\hline 6 & -1.590803 & -2.087791 & -4.119080 \\
\hline 6 & -1.686581 & -2.576358 & -2.782605 \\
\hline 6 & -2.883786 & -3.224408 & -2.381702 \\
\hline 6 & -3.917133 & -3.400297 & -3.266407 \\
\hline 5 & -3.806676 & -2.944482 & -4.598927 \\
\hline 6 & -2.673377 & -2.290530 & -5.010092 \\
\hline 7 & 1.676482 & -1.578091 & -1.456955 \\
\hline 6 & 2.757627 & -2.588542 & -1.570318 \\
\hline 6 & 3.461338 & -2.930131 & -0.283656 \\
\hline 6 & 4.852521 & -2.898107 & -0.217150 \\
\hline 6 & 5.514252 & -3.226550 & 0.963753 \\
\hline
\end{tabular}




\begin{tabular}{|c|c|c|c|}
\hline & 4.788472 & -3.599517 & 2.089521 \\
\hline & 3.396429 & -3.641964 & 2.026983 \\
\hline 6 & 2.740036 & -3.306558 & 0.850654 \\
\hline & -6.389622 & -0.546950 & -3.627463 \\
\hline & -6.561599 & 1.504382 & -2.232166 \\
\hline & -5.543616 & -4.162798 & 1.146660 \\
\hline & -6.296909 & -2.315063 & 2.661943 \\
\hline & 0.360117 & 2.206580 & 1.150877 \\
\hline & 0.466219 & 2.917048 & 2.049676 \\
\hline U & -1.735765 & 2.613229 & -0.457118 \\
\hline & -1.669353 & 3.852136 & -1.338495 \\
\hline 6 & -2.793494 & 4.871889 & -1.134946 \\
\hline . & -2.654655 & 2.454780 & 0.321193 \\
\hline & -3.683216 & 3.199920 & 3.927672 \\
\hline 6 & -4.910367 & 1.128195 & 4.611932 \\
\hline 6 & 1.730933 & 0.910300 & 5.643916 \\
\hline 6 & 0.453491 & -1.015902 & 6.592463 \\
\hline 1 & -0.672924 & 0.945920 & -1.997872 \\
\hline 1 & 7.167213 & 0.201512 & 0.528488 \\
\hline 1 & -1.409369 & -2.546336 & 2.722250 \\
\hline 1 & 8.157313 & -1.508762 & -4.028624 \\
\hline 1 & -0.204365 & -7.016309 & 1.785805 \\
\hline 1 & 5.584077 & 4.348461 & 3.348256 \\
\hline 1 & -2.983272 & -3.569791 & -1.353401 \\
\hline 1 & -4.833823 & -3.886606 & -2.937131 \\
\hline 1 & 3.774247 & 2.746713 & 3.528461 \\
\hline 1 & -0.937892 & -4.935970 & 2.848679 \\
\hline 1 & 5.926258 & -0.886485 & -4.775966 \\
\hline 1 & 8.048578 & 4.451899 & -0.170428 \\
\hline 1 & 3.642652 & -0.040987 & -4.242794 \\
\hline 1 & -2.581336 & -1.922318 & -6.031004 \\
\hline 1 & -0.339676 & -1.062733 & -5.554592 \\
\hline 1 & 0.676202 & -7.075713 & -2.420911 \\
\hline 1 & 6.828709 & 2.550095 & -1.109795 \\
\hline 1 & 0.132942 & -4.692606 & -2.593782 \\
\hline 1 & 1.550619 & -0.769673 & -3.972251 \\
\hline 1 & 7.459687 & 5.353294 & 2.077499 \\
\hline 1 & 9.338036 & -0.830556 & 0.047590 \\
\hline 1 & -4.627508 & -3.105839 & -5.294803 \\
\hline 1 & 0.512455 & -8.258167 & -0.235566 \\
\hline 1 & 9.863043 & -1.672861 & -2.238568 \\
\hline 1 & 2.437055 & 0.883066 & 2.537264 \\
\hline 1 & -3.655418 & -2.051961 & 1.679204 \\
\hline 1 & -3.019081 & 0.513422 & -1.680639 \\
\hline 1 & -6.854969 & -1.313862 & -1.066413 \\
\hline 1 & -3.384747 & 0.831093 & 2.266002 \\
\hline 1 & 0.508435 & -0.914481 & 2.613065 \\
\hline 1 & -1.250630 & 1.072918 & 5.979434 \\
\hline 1 & 2.262338 & -3.491415 & -1.955086 \\
\hline 1 & 3.495281 & -2.288077 & -2.329955 \\
\hline 1 & 5.427852 & -2.594502 & -1.093384 \\
\hline 1 & 1.652972 & -3.303996 & 0.812538 \\
\hline 1 & 6.601071 & -3.182948 & 1.000236 \\
\hline 1 & 2.816022 & -3.936938 & 2.900288 \\
\hline 1 & 5.303323 & -3.855107 & 3.013292 \\
\hline 1 & 2.690870 & -1.640900 & 5.307104 \\
\hline
\end{tabular}




$\begin{array}{lrrr}1 & 1.358832 & -2.268293 & 4.316906 \\ 1 & 2.374953 & -0.940612 & 3.720112 \\ 1 & 1.307615 & -1.299585 & 7.221598 \\ 1 & -0.180943 & -0.347977 & 7.186470 \\ 1 & -0.124252 & -1.924500 & 6.378111 \\ 1 & 2.599236 & 0.669172 & 6.272103 \\ 1 & 2.097218 & 1.408400 & 4.735956 \\ 1 & 1.11076 & 1.632620 & 6.189295 \\ 1 & -4.260230 & 2.869621 & 6.556191 \\ 1 & -3.344771 & 1.384724 & 6.855351 \\ 1 & -2.502021 & 2.875895 & 6.371527 \\ 1 & -4.500696 & 3.832172 & 4.300707 \\ 1 & -2.748356 & 3.771239 & 4.002450 \\ 1 & -3.864466 & 3.004240 & 2.864189 \\ 1 & -5.746292 & 1.729646 & 4.993732 \\ 1 & -5.136972 & 0.873858 & 3.569097 \\ 1 & -4.876509 & 0.192986 & 5.186605 \\ 1 & -6.883008 & -3.029322 & 3.255871 \\ 1 & -5.309530 & -2.226719 & 3.132089 \\ 1 & -6.789059 & -1.336261 & 2.730605 \\ 1 & -8.193291 & -3.628258 & 1.276838 \\ 1 & -8.169078 & -1.960047 & 0.686808 \\ 1 & -7.640501 & -3.291191 & -0.369115 \\ 1 & -6.117845 & -4.891730 & 1.734667 \\ 1 & -5.495227 & -4.527984 & 0.111645 \\ 1 & -4.519037 & -4.147091 & 1.540880 \\ 1 & -4.969512 & 1.553729 & -4.457021 \\ 1 & -3.956485 & 1.830693 & -3.035190 \\ 1 & -3.796190 & 0.317338 & -3.954916 \\ 1 & -6.965451 & 2.078248 & -3.077897 \\ 1 & -7.408620 & 1.071716 & -1.684462 \\ 1 & -6.054338 & 2.205202 & -1.558576 \\ 1 & -6.797856 & -0.003250 & -4.489889 \\ 1 & -5.742896 & -1.346746 & -4.007136 \\ 1 & -7.237184 & -1.010155 & -3.108468 \\ 1 & 0.585173 & 2.422529 & -1.379126 \\ 6 & -2.433925 & 6.197269 & -1.765823 \\ 6 & -4.130596 & 4.346474 & -1.646891 \\ 1 & -2.890767 & 5.033031 & -0.050673 \\ 1 & -0.688415 & 4.311955 & -1.145924 \\ 1 & -1.613090 & 3.501990 & -2.382302 \\ 6 & -2.269416 & 7.339664 & -0.980579 \\ 6 & -1.938037 & 8.564350 & -1.553690 \\ 6 & -1.762839 & 8.665837 & -2.929494 \\ 6 & -1.920964 & 7.534044 & -3.725380 \\ 6 & -2.253660 & 6.313882 & -3.148274 \\ 1 & -2.405386 & 7.264279 & 0.098266 \\ 1 & -1.816301 & 9.441684 & -0.921386 \\ 1 & -1.502997 & 9.621059 & -3.380753 \\ 1 & -1.783495 & 7.602400 & -4.802820 \\ 1 & -2.373392 & 5.435714 & -3.782875 \\ 1 & -4.938714 & 5.065525 & -1.465269 \\ 1 & -4.099455 & 4.151062 & -2.728187 \\ 1 & -4.378406 & 3.410376 & -1.134650\end{array}$


$\mathbf{8}_{B}$

Number of imaginary frequencies : 0

The smallest frequencies are : $15.1612 \quad 17.3846 \quad 20.7160 \mathrm{~cm}(-1)$

Electronic energy : $\quad \mathrm{HF}=-4475.3609921$

Zero-point correction $=\quad 1.484321$ (Hartree/Particle)

Thermal correction to Energy= $\quad 1.571165$

Thermal correction to Enthalpy $=\quad 1.572109$

Thermal correction to Gibbs Free Energy= 1.364853

Sum of electronic and zero-point Energies $=\quad-4473.876671$

Sum of electronic and thermal Energies $=\quad-4473.789827$

Sum of electronic and thermal Enthalpies= $\quad-4473.788883$

Sum of electronic and thermal Free Energies $=\quad-4473.996139$

Cartesian Coordinates

$\begin{array}{cccc}\ldots \ldots \ldots \ldots \ldots \ldots \ldots \ldots \ldots \ldots \ldots \ldots \\ 6 & 4.148627 & -3.063402 & -4.244639 \\ 6 & 3.346246 & -3.463681 & -3.172975 \\ 6 & 3.907804 & -4.353341 & -2.246070 \\ 6 & 5.210751 & -4.813604 & -2.379411 \\ 6 & 5.995925 & -4.402009 & -3.454001 \\ 6 & 5.456060 & -3.526694 & -4.386860 \\ 6 & 1.912677 & -2.971110 & -2.992106 \\ 6 & 1.804965 & -2.153803 & -1.652374 \\ 45 & 0.069102 & -1.083061 & -1.098982 \\ 15 & -1.938122 & 0.129018 & -0.943703 \\ 8 & -3.005929 & -0.131623 & 0.288749 \\ 6 & -3.731679 & -1.299918 & 0.305937 \\ 6 & -4.828011 & -1.438978 & -0.523190 \\ 6 & -5.536351 & -2.684879 & -0.497680 \\ 6 & -5.147468 & -3.693291 & 0.436386 \\ 6 & -4.045253 & -3.464013 & 1.294330 \\ 6 & -3.337723 & -2.295639 & 1.219375 \\ 6 & -6.594300 & -2.983619 & -1.394059 \\ 6 & -7.249792 & -4.188658 & -1.342888 \\ 6 & -6.887266 & -5.166467 & -0.393413 \\ 6 & -5.852946 & -4.920838 & 0.472207 \\ 6 & -5.180789 & -0.343792 & -1.455903 \\ 6 & -4.218977 & 0.150138 & -2.318919 \\ 6 & -4.530698 & 1.069450 & -3.343573 \\ 6 & -5.795154 & 1.581596 & -3.439069 \\ 6 & -6.786878 & 1.224258 & -2.492648 \\ 6 & -6.481706 & 0.256398 & -1.488889 \\ 6 & -8.063126 & 1.837519 & -2.504936 \\ 6 & -8.998768 & 1.537188 & -1.548110 \\ 6 & -8.689618 & 0.604896 & -0.535614 \\ 6 & -7.468329 & -0.022617 & -0.509538 \\ 8 & -2.913880 & -0.278821 & -2.241199 \\ 6 & 1.437616 & -2.143348 & -4.181655 \\ 8 & 2.712773 & -2.204402 & -0.851062 \\ 6 & -0.279924 & -2.625494 & 0.069818 \\ 8 & -0.352641 & -3.634818 & 0.617243 \\ 6 & 1.327185 & 0.263725 & 0.621234 \\ 6 & 1.444673 & -0.875621 & 2.053104 \\ 6 & 2.567902 & -1.668332 & 2.233591 \\ 6 & 2.595910 & -2.665020 & 3.217655\end{array}$




\begin{tabular}{|c|c|c|c|}
\hline 6 & 1.482010 & -2.803283 & 4.041116 \\
\hline 6 & 0.337533 & -2.000640 & 3.900568 \\
\hline & 0.321011 & -1.066599 & 2.870256 \\
\hline & 3.837060 & -3.547162 & 3.322678 \\
\hline & 5.066836 & -2.670149 & 3.586157 \\
\hline & -0.849121 & -2.186381 & 4.846145 \\
\hline & -0.367484 & -2.193308 & 6.301388 \\
\hline & 0.736307 & 1.843907 & 1.414790 \\
\hline & 0.335920 & 2.918351 & 0.634174 \\
\hline & -0.052211 & 4.154024 & 1.247732 \\
\hline & -0.016304 & 4.277371 & 2.666501 \\
\hline & 0.430446 & 3.177383 & 3.432293 \\
\hline & 0.792502 & 2.004005 & 2.827369 \\
\hline f & -0.437851 & 5.482742 & 3.276202 \\
\hline & -0.880132 & 6.535612 & 2.517144 \\
\hline 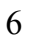 & -0.910330 & 6.424379 & 1.111159 \\
\hline$b$ & -0.503261 & 5.268310 & 0.492273 \\
\hline 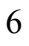 & 0.320302 & 2.870391 & -0.852662 \\
\hline 6 & -0.784735 & 2.399528 & -1.535689 \\
\hline f & -0.845201 & 2.469771 & -2.946574 \\
\hline ) & 0.197316 & 2.986899 & -3.667000 \\
\hline$b$ & 1.357104 & 3.460546 & -3.009744 \\
\hline$b$ & 1.421408 & 3.410987 & -1.585568 \\
\hline 5 & 2.590129 & 3.895462 & -0.943047 \\
\hline 6 & 3.634871 & 4.402453 & -1.673796 \\
\hline 6 & 3.562048 & 4.464652 & -3.082833 \\
\hline 6 & 2.448625 & 3.998827 & -3.734680 \\
\hline 1 & -1.884815 & 1.812073 & -0.820084 \\
\hline 6 & -3.039371 & 2.705949 & -0.560289 \\
\hline 6 & -3.718503 & 2.538764 & 0.774576 \\
\hline 6 & -5.110314 & 2.562004 & 0.848329 \\
\hline 6 & -5.758215 & 2.471402 & 2.078255 \\
\hline 6 & -5.018579 & 2.355226 & 3.249673 \\
\hline 6 & -3.626682 & 2.324195 & 3.182176 \\
\hline 6 & -2.984159 & 2.412890 & 1.954937 \\
\hline ? & 3.066906 & 0.753464 & 0.284374 \\
\hline 6 & 3.594439 & 0.648351 & -1.001023 \\
\hline 6 & 4.915971 & 1.006732 & -1.262503 \\
\hline 6 & 5.679090 & 1.518143 & -0.204951 \\
\hline 6 & 5.168561 & 1.672745 & 1.084295 \\
\hline 6 & 3.851837 & 1.265853 & 1.313588 \\
\hline 6 & 5.557936 & 0.769431 & -2.628164 \\
\hline 6 & 6.461305 & -0.465383 & -2.514026 \\
\hline 6 & 5.964087 & 2.283436 & 2.235953 \\
\hline 6 & 6.061596 & 1.278618 & 3.389224 \\
\hline 6 & 7.380666 & 2.673694 & 1.823293 \\
\hline 6 & 5.242322 & 3.548648 & 2.716676 \\
\hline 6 & 4.511058 & 0.497928 & -3.706320 \\
\hline 6 & 6.394944 & 1.973203 & -3.067506 \\
\hline 6 & 3.729767 & -4.573813 & 4.446341 \\
\hline 6 & 4.023100 & -4.296259 & 1.996446 \\
\hline 6 & -1.874846 & -1.060392 & 4.709929 \\
\hline 6 & -1.532705 & -3.522869 & 4.536723 \\
\hline 1 & 0.521017 & -0.100544 & -2.295096 \\
\hline 1 & -7.236220 & -0.723371 & 0.288673 \\
\hline 1 & 1.131459 & 1.178198 & 3.446735 \\
\hline
\end{tabular}




\begin{tabular}{|c|c|c|c|}
\hline & -8.278367 & 2.566803 & -3.284829 \\
\hline & -0.407228 & 5.552032 & 4.362662 \\
\hline & -5.543694 & -5.671467 & 1.198316 \\
\hline & 2.661451 & 3.840862 & 0.142553 \\
\hline & 4.530931 & 4.753768 & -1.165305 \\
\hline & -3.754476 & -4.241789 & 1.999495 \\
\hline & 0.479853 & 3.274827 & 4.516148 \\
\hline & -6.048058 & 2.291259 & -4.224996 \\
\hline & -8.053035 & -4.393276 & -2.047196 \\
\hline & -3.745365 & 1.347892 & -4.042630 \\
\hline & 2.382374 & 4.036188 & -4.821198 \\
\hline & 0.150810 & 3.036897 & -4.753700 \\
\hline & -1.257249 & 7.263277 & 0.512206 \\
\hline & -6.880313 & -2.248105 & -2.141081 \\
\hline & -0.527258 & 5.200777 & -0.593486 \\
\hline & -1.736081 & 2.091907 & -3.444645 \\
\hline & -7.418381 & -6.114824 & -0.360653 \\
\hline & -9.423380 & 0.385208 & 0.236676 \\
\hline & 4.392711 & 4.881412 & -3.648881 \\
\hline & -1.207376 & 7.456524 & 2.994042 \\
\hline & -9.971913 & 2.022366 & -1.560175 \\
\hline & -2.467229 & -2.107776 & 1.845794 \\
\hline 1 & 3.430018 & 1.357767 & 2.314164 \\
\hline & 2.953687 & 0.270153 & -1.792352 \\
\hline ] & 6.710987 & 1.802081 & -0.399827 \\
\hline & 3.421006 & -1.535597 & 1.572550 \\
\hline 1 & -0.565934 & -0.454650 & 2.700515 \\
\hline & 1.491262 & -3.561005 & 4.821523 \\
\hline & -2.628639 & 3.724323 & -0.619389 \\
\hline ] & -3.785217 & 2.626863 & -1.365568 \\
\hline & -5.698378 & 2.638878 & -0.068131 \\
\hline 1 & -1.899587 & 2.353710 & 1.900782 \\
\hline ] & -6.845843 & 2.483973 & 2.115026 \\
\hline | & -3.034975 & 2.228688 & 4.091765 \\
\hline 1 & -5.522659 & 2.282117 & 4.211038 \\
\hline 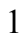 & -2.671444 & -1.194764 & 5.452527 \\
\hline 1 & -1.417313 & -0.076223 & 4.884075 \\
\hline & -2.358635 & -1.035614 & 3.724970 \\
\hline 1 & -1.223680 & -2.300258 & 6.980476 \\
\hline ] & 0.319918 & -3.020215 & 6.513461 \\
\hline l & 0.146652 & -1.256212 & 6.551730 \\
\hline 1 & -2.403770 & -3.671574 & 5.189523 \\
\hline 1 & -1.877622 & -3.559720 & 3.494201 \\
\hline 1 & -0.850683 & -4.369084 & 4.686266 \\
\hline 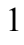 & 4.644375 & -5.179169 & 4.481301 \\
\hline 1 & 3.613248 & -4.097765 & 5.428881 \\
\hline 1 & 2.887551 & -5.261199 & 4.294201 \\
\hline 1 & 4.895096 & -4.962440 & 2.053617 \\
\hline 1 & 3.140018 & -4.908468 & 1.767600 \\
\hline 1 & 4.178860 & -3.610757 & 1.154710 \\
\hline 1 & 5.970559 & -3.291186 & 3.649910 \\
\hline 1 & 5.226016 & -1.935090 & 2.786882 \\
\hline 1 & 4.965432 & -2.121329 & 4.532125 \\
\hline 1 & 6.611718 & 1.717727 & 4.232544 \\
\hline 1 & 5.074744 & 0.974387 & 3.760186 \\
\hline 1 & 6.594259 & 0.371483 & 3.074583 \\
\hline
\end{tabular}




$\begin{array}{rrrr}1 & 7.907924 & 3.111048 & 2.680605 \\ 1 & 7.963345 & 1.806813 & 1.486019 \\ 1 & 7.381501 & 3.422373 & 1.020256 \\ 1 & 5.787971 & 4.009829 & 3.551149 \\ 1 & 5.173546 & 4.289046 & 1.908065 \\ 1 & 4.221314 & 3.338946 & 3.060992 \\ 1 & 5.005873 & 0.355452 & -4.676636 \\ 1 & 3.950285 & -0.420877 & -3.494162 \\ 1 & 3.798372 & 1.329796 & -3.804643 \\ 1 & 6.939051 & -0.684080 & -3.479498 \\ 1 & 7.254903 & -0.315344 & -1.770568 \\ 1 & 5.880096 & -1.349186 & -2.216194 \\ 1 & 6.819672 & 1.789919 & -4.063431 \\ 1 & 5.779245 & 2.880708 & -3.123731 \\ 1 & 7.235490 & 2.173295 & -2.392366 \\ 1 & -0.630315 & -1.830383 & -2.294022 \\ 1 & 1.546801 & -2.720423 & -5.108571 \\ 1 & 0.382141 & -1.881635 & -4.070897 \\ 1 & 1.992053 & -1.201385 & -4.290516 \\ 1 & 3.317841 & -4.686836 & -1.395847 \\ 1 & 5.615253 & -5.498920 & -1.636588 \\ 1 & 7.016463 & -4.763488 & -3.561612 \\ 1 & 6.051145 & -3.194448 & -5.235890 \\ 1 & 3.759949 & -2.377021 & -4.992901 \\ 6 & 0.974735 & -4.180551 & -2.847687 \\ 1 & -0.068474 & -3.857866 & -2.749449 \\ 1 & 1.053207 & -4.812005 & -3.741687 \\ 1 & 1.220074 & -4.799355 & -1.976486\end{array}$

\section{$\mathrm{TS}_{R}$}

Number of imaginary frequencies : 1

The smallest frequencies are : - $637.2151 \quad 11.9581 \quad 13.8473 \mathrm{~cm}(-1)$

Electronic energy : $\quad H F=-4475.3442673$

Zero-point correction $=\quad 1.482903($ Hartree/Particle $)$

Thermal correction to Energy $=\quad 1.569840$

Thermal correction to Enthalpy= $\quad 1.570784$

Thermal correction to Gibbs Free Energy= $\quad 1.359785$

Sum of electronic and zero-point Energies $=\quad-4473.861365$

Sum of electronic and thermal Energies $=\quad-4473.774427$

Sum of electronic and thermal Enthalpies $=\quad-4473.773483$

Sum of electronic and thermal Free Energies $=\quad-4473.984482$

\section{Cartesian Coordinates}

$\begin{array}{ccccc}6 & 2.680284 & -3.364975 & 0.656273 \\ 6 & 3.393712 & -2.974409 & -0.478473 \\ 6 & & 4.784645 & -3.050739 & -0.457424 \\ 6 & 5.453981 & -3.505885 & 0.676524 \\ 6 & & 4.735611 & -3.895154 & 1.801239 \\ 6 & & 3.343289 & -3.822979 & 1.786166 \\ 6 & & 2.685511 & -2.507189 & -1.722776 \\ 7 & & 1.631275 & -1.484571 & -1.517866 \\ 15 & 1.823320 & 0.057058 & -0.848993 \\ 45 & -0.031850 & 1.328416 & -0.227350\end{array}$




\begin{tabular}{|c|c|c|c|}
\hline 6 & -1.653495 & 2.675141 & -0.715068 \\
\hline 8 & -2.764079 & 2.475053 & -0.257760 \\
\hline & 0.501457 & -1.575781 & -2.398943 \\
\hline & -0.619187 & -2.291825 & -2.021158 \\
\hline & -1.729501 & -2.385774 & -2.916239 \\
\hline & -1.662748 & -1.737609 & -4.184882 \\
\hline & -0.503532 & -1.001246 & -4.524925 \\
\hline & 0.550124 & -0.924128 & -3.654263 \\
\hline & -2.909058 & -3.100327 & -2.583047 \\
\hline & -3.955745 & -3.180837 & -3.466388 \\
\hline & -3.874088 & -2.564496 & -4.734426 \\
\hline & -2.754926 & -1.850457 & -5.079964 \\
\hline & -0.652534 & -3.038131 & -0.734632 \\
\hline & -0.994193 & -2.432069 & 0.466697 \\
\hline & -1.098506 & -3.236221 & 1.635110 \\
\hline & -0.831556 & -4.578960 & 1.609909 \\
\hline & -0.443131 & -5.216724 & 0.410900 \\
\hline & -0.365040 & -4.440929 & -0.781409 \\
\hline & -0.130225 & -6.596411 & 0.371092 \\
\hline & 0.248146 & -7.196500 & -0.802257 \\
\hline & 0.322606 & -6.436113 & -1.988501 \\
\hline & 0.020056 & -5.097153 & -1.980379 \\
\hline & -1.462582 & -0.624910 & 0.515422 \\
\hline & -1.624644 & -0.291973 & 2.310960 \\
\hline 6 & -2.710040 & 0.418619 & 2.802457 \\
\hline & -2.754722 & 0.827133 & 4.143292 \\
\hline 6 & -1.694535 & 0.468841 & 4.971528 \\
\hline 0 & -0.584955 & -0.256099 & 4.506138 \\
\hline 6 & -0.550429 & -0.596380 & 3.158811 \\
\hline 5 & -3.947530 & 1.651464 & 4.620756 \\
\hline f & -3.882057 & 1.962702 & 6.113395 \\
\hline 6 & 0.546449 & -0.624547 & 5.465206 \\
\hline 6 & 1.532813 & -1.610609 & 4.839595 \\
\hline 6 & -3.197616 & -0.778124 & -0.076055 \\
\hline 6 & -3.619067 & -0.072861 & -1.200857 \\
\hline 6 & -4.941622 & -0.138241 & -1.634438 \\
\hline 6 & -5.813411 & -0.993018 & -0.947816 \\
\hline b & -5.408661 & -1.758945 & 0.147215 \\
\hline 6 & -4.089150 & -1.619209 & 0.584867 \\
\hline 6 & -6.328691 & -2.737889 & 0.874612 \\
\hline 6 & -7.721983 & -2.797350 & 0.254512 \\
\hline 6 & -5.462461 & 0.770397 & -2.747550 \\
\hline 6 & -4.327413 & 1.391955 & -3.561873 \\
\hline 6 & -3.966524 & 2.978013 & 3.851829 \\
\hline 5 & -5.246808 & 0.885028 & 4.347997 \\
\hline 6 & 1.307223 & 0.647161 & 5.854313 \\
\hline 6 & -0.025980 & -1.279814 & 6.726881 \\
\hline 6 & -5.718399 & -4.143419 & 0.805769 \\
\hline 6 & -6.474021 & -2.315366 & 2.340710 \\
\hline 6 & -6.375888 & 0.008256 & -3.709876 \\
\hline 6 & -6.255472 & 1.907712 & -2.090708 \\
\hline 8 & 2.892621 & -0.378911 & 0.336214 \\
\hline 6 & 3.676229 & 0.592325 & 0.908968 \\
\hline 6 & 4.791351 & 1.057463 & 0.237890 \\
\hline U & 5.554341 & 2.106826 & 0.846015 \\
\hline 6 & 5.197699 & 2.567062 & 2.150137 \\
\hline
\end{tabular}




\begin{tabular}{|c|c|c|c|}
\hline 6 & 4.077917 & 2.004142 & 2.808325 \\
\hline 6 & 3.319559 & 1.045248 & 2.193995 \\
\hline & 5.955780 & 3.597613 & 2.757436 \\
\hline & 7.011840 & 4.177404 & 2.102967 \\
\hline & 7.344250 & 3.751636 & 0.800005 \\
\hline & 6.636410 & 2.746422 & 0.189096 \\
\hline & 5.115615 & 0.504134 & -1.097233 \\
\hline & 4.148330 & 0.499719 & -2.086820 \\
\hline & 4.433430 & 0.104491 & -3.411842 \\
\hline & 5.674064 & -0.374205 & -3.730994 \\
\hline & 6.668030 & -0.513549 & -2.731488 \\
\hline & 6.388973 & -0.080290 & -1.400291 \\
\hline & 7.372070 & -0.314498 & -0.405244 \\
\hline & 8.566268 & -0.917502 & -0.715658 \\
\hline & 8.851082 & -1.312630 & -2.039350 \\
\hline & 7.916848 & -1.113711 & -3.023463 \\
\hline & 2.869753 & 0.933678 & -1.826552 \\
\hline & 0.231337 & 2.079002 & 1.524041 \\
\hline & 0.346555 & 2.698112 & 2.492432 \\
\hline & -1.306555 & 4.026390 & -1.323515 \\
\hline & -2.135935 & 5.183252 & -0.762699 \\
\hline & -1.053766 & 1.685461 & -1.576376 \\
\hline & 7.159514 & -0.034360 & 0.623402 \\
\hline & -1.402489 & -2.781629 & 2.573668 \\
\hline & 8.111225 & -1.428696 & -4.047916 \\
\hline & -0.192977 & -7.168693 & 1.295587 \\
\hline & 5.670398 & 3.926735 & 3.755820 \\
\hline & -2.985340 & -3.570933 & -1.603843 \\
\hline & -4.859319 & -3.719920 & -3.188268 \\
\hline 1 & 3.815758 & 2.363834 & 3.802418 \\
\hline & -0.918007 & -5.172862 & 2.519030 \\
\hline ] & 5.905289 & -0.679639 & -4.750099 \\
\hline & 8.165353 & 4.229323 & 0.270111 \\
\hline & 3.647269 & 0.194790 & -4.158472 \\
\hline & -2.683859 & -1.358879 & -6.049486 \\
\hline & -0.461960 & -0.502256 & -5.491884 \\
\hline ] & 0.620267 & -6.916192 & -2.917874 \\
\hline & 6.898431 & 2.440633 & -0.820376 \\
\hline 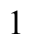 & 0.075585 & -4.526112 & -2.904899 \\
\hline 1 & 1.444787 & -0.358722 & -3.908349 \\
\hline . & 7.583551 & 4.971580 & 2.577382 \\
\hline 1 & 9.297201 & -1.097401 & 0.069576 \\
\hline & -4.704062 & -2.652373 & -5.432574 \\
\hline 1 & 0.491013 & -8.256297 & -0.823080 \\
\hline & 9.803038 & -1.783911 & -2.272415 \\
\hline 1 & 2.436331 & 0.617206 & 2.665281 \\
\hline 1 & -3.750989 & -2.178873 & 1.456886 \\
\hline 1 & -2.899814 & 0.549682 & -1.721651 \\
\hline 1 & -6.847068 & -1.056982 & -1.280912 \\
\hline ] & -3.522043 & 0.679731 & 2.125444 \\
\hline 1 & 0.311785 & -1.121921 & 2.747181 \\
\hline ( & -1.718097 & 0.764125 & 6.018094 \\
\hline 1 & 2.168642 & -3.364351 & -2.177902 \\
\hline ] & 3.424166 & -2.160093 & -2.461837 \\
\hline 1 & 5.356261 & -2.731329 & -1.330714 \\
\hline 1 & 1.596757 & -3.269966 & 0.660141 \\
\hline
\end{tabular}




\begin{tabular}{|c|c|c|c|}
\hline$\frac{1}{1}$ & 6.541614 & -3.544591 & 0.677993 \\
\hline & 2.767209 & -4.122650 & 2.660646 \\
\hline & 5.256486 & -4.248168 & 2.688846 \\
\hline 1 & 2.290301 & -1.895495 & 5.580604 \\
\hline 1 & 1.029768 & -2.528919 & 4.505029 \\
\hline & 2.069024 & -1.186317 & 3.980692 \\
\hline & 0.788851 & -1.567524 & 7.404251 \\
\hline & -0.690630 & -0.609961 & 7.284358 \\
\hline & -0.593890 & -2.185828 & 6.478300 \\
\hline I & 2.125195 & 0.410055 & 6.548241 \\
\hline & 1.742444 & 1.129249 & 4.968275 \\
\hline & 0.652680 & 1.380412 & 6.341367 \\
\hline & -4.763586 & 2.546718 & 6.406488 \\
\hline & -3.874000 & 1.049768 & 6.723328 \\
\hline & -2.996064 & 2.557296 & 6.370533 \\
\hline 1 & -4.810420 & 3.599336 & 4.181396 \\
\hline I & -3.040151 & 3.541457 & 4.025239 \\
\hline & -4.066111 & 2.824553 & 2.770319 \\
\hline 1 & -6.112335 & 1.476406 & 4.675706 \\
\hline & -5.381438 & 0.666387 & 3.281161 \\
\hline l & -5.263497 & -0.068519 & 4.892577 \\
\hline & -7.110470 & -3.027776 & 2.883065 \\
\hline - & -5.506801 & -2.273086 & 2.857353 \\
\hline 1 & -6.937290 & -1.322844 & 2.417885 \\
\hline & -8.338827 & -3.522505 & 0.800361 \\
\hline l & -8.235233 & -1.828352 & 0.302847 \\
\hline & -7.688579 & -3.117297 & -0.795320 \\
\hline 1 & -6.356661 & -4.864556 & 1.334235 \\
\hline$\frac{1}{1}$ & -5.624384 & -4.478172 & -0.235989 \\
\hline & -4.719966 & -4.188305 & 1.259562 \\
\hline 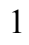 & -4.747593 & 1.997133 & -4.375527 \\
\hline & -3.703574 & 2.058285 & -2.951114 \\
\hline 1 & -3.680605 & 0.625501 & -4.011915 \\
\hline ] & -6.643606 & 2.596745 & -2.853922 \\
\hline 1 & -7.109010 & 1.525144 & -1.516484 \\
\hline 1 & -5.617196 & 2.479865 & -1.404483 \\
\hline 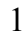 & -6.719245 & 0.676815 & -4.510402 \\
\hline 1 & -5.842196 & -0.831048 & -4.173924 \\
\hline & -7.271438 & -0.388408 & -3.217058 \\
\hline 1 & 0.897769 & 2.511535 & -0.682985 \\
\hline 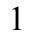 & -0.232042 & 4.231256 & -1.222363 \\
\hline 1 & -1.485766 & 3.911933 & -2.406521 \\
\hline 5 & -1.973757 & 6.395190 & -1.650782 \\
\hline 1 & -3.190816 & 4.873998 & -0.810648 \\
\hline 6 & -1.777272 & 5.430413 & 0.697651 \\
\hline 6 & -2.845902 & 6.579265 & -2.727753 \\
\hline 6 & -2.700685 & 7.655784 & -3.595191 \\
\hline 6 & -1.671540 & 8.573093 & -3.400568 \\
\hline 6 & -0.793244 & 8.399973 & -2.335771 \\
\hline 6 & -0.942927 & 7.320182 & -1.469873 \\
\hline 1 & -3.652159 & 5.860058 & -2.880932 \\
\hline 1 & -3.395562 & 7.782018 & -4.423196 \\
\hline 1 & -1.555977 & 9.418957 & -4.074867 \\
\hline 1 & 0.015118 & 9.110798 & -2.175537 \\
\hline 1 & -0.247094 & 7.199000 & -0.640806 \\
\hline 1 & -2.298286 & 6.307309 & 1.099793 \\
\hline
\end{tabular}


$\mathbf{T S 4}_{S}$ (same as $\mathbf{T S 4} 4_{S-\mathrm{Cl}}$ )

$\begin{array}{lcc}\text { Number of imaginary frequencies : } 1 & & \\ \text { The smallest frequencies are : }-702.3195 & 14.1416 & 15.3499 \mathrm{~cm}(-1) \\ \text { Electronic energy : HF=-4475.3435953 } & \\ \text { Zero-point correction= } & 1.482786 \text { (Hartree/Particle) } \\ \text { Thermal correction to Energy= } & 1.569793 \\ \text { Thermal correction to Enthalpy= } & 1.570737 \\ \text { Thermal correction to Gibbs Free Energy= } & 1.360016 \\ \text { Sum of electronic and zero-point Energies }= & -4473.860809 \\ \text { Sum of electronic and thermal Energies }= & -4473.773802 \\ \text { Sum of electronic and thermal Enthalpies }= & -4473.772858 \\ \text { Sum of electronic and thermal Free Energies }= & -4473.983579\end{array}$

Cartesian Coordinates

\begin{tabular}{cccc}
\hline 6 & 2.328310 & -3.559060 & 0.827241 \\
6 & 3.094229 & -3.254794 & -0.299459 \\
6 & 4.477689 & -3.404926 & -0.233116 \\
6 & 5.088741 & -3.847261 & 0.938015 \\
6 & 4.318274 & -4.150551 & 2.054927 \\
6 & 2.932934 & -4.005670 & 1.994026 \\
6 & 2.446173 & -2.798979 & -1.580115 \\
7 & 1.467627 & -1.692616 & -1.444410 \\
15 & 1.770346 & -0.135143 & -0.858595 \\
45 & 0.020274 & 1.314739 & -0.316252 \\
6 & -1.430528 & 2.828069 & -0.877541 \\
8 & -2.583997 & 2.778819 & -0.495603 \\
6 & 0.345477 & -1.747607 & -2.338510 \\
6 & -0.843111 & -2.325129 & -1.932590 \\
6 & -1.942841 & -2.385397 & -2.844825 \\
6 & -1.785489 & -1.872814 & -4.166262 \\
6 & -0.555278 & -1.279831 & -4.536125 \\
6 & 0.479551 & -1.214516 & -3.642507 \\
6 & -3.195751 & -2.942905 & -2.481312 \\
6 & -4.225459 & -3.009752 & -3.386138 \\
6 & -4.054409 & -2.536150 & -4.705387 \\
6 & -2.861297 & -1.973523 & -5.082452 \\
6 & -0.955775 & -2.996592 & -0.609488 \\
6 & -1.243436 & -2.310921 & 0.562347 \\
6 & -1.428278 & -3.051911 & 1.762584 \\
6 & -1.291229 & -4.413383 & 1.796544 \\
6 & -0.957895 & -5.135367 & 0.629336 \\
6 & -0.797979 & -4.422072 & -0.593115 \\
6 & -0.775722 & -6.538612 & 0.652877 \\
6 & -0.443450 & -7.222266 & -0.488223 \\
6 & -0.284557 & -6.523827 & -1.703526 \\
6 & -0.461285 & -5.163274 & -1.756794 \\
15 & -1.547572 & -0.467976 & 0.542534 \\
6 & -1.654562 & -0.061654 & 2.327794 \\
6 & -2.674453 & 0.741947 & 2.815944 \\
6 & -2.669366 & 1.181353 & 4.148296 \\
6 & -1.631061 & 0.756914 & 4.972437
\end{tabular}




\begin{tabular}{|c|c|c|c|}
\hline 6 & -0.588725 & -0.064471 & 4.511412 \\
\hline 6 & -0.596495 & -0.431610 & 3.170848 \\
\hline & -3.789375 & 2.103524 & 4.623292 \\
\hline & -3.676569 & 2.442961 & 6.107104 \\
\hline & 0.507719 & -0.519454 & 5.474031 \\
\hline & 1.427624 & -1.565665 & 4.845613 \\
\hline 0 & -3.290429 & -0.495105 & -0.036027 \\
\hline & -3.632322 & 0.166857 & -1.211086 \\
\hline & -4.942949 & 0.156006 & -1.686733 \\
\hline & -5.891755 & -0.581568 & -0.969408 \\
\hline 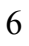 & -5.572468 & -1.291968 & 0.190938 \\
\hline y & -4.257024 & -1.223397 & 0.654328 \\
\hline & -6.579078 & -2.154944 & 0.949635 \\
\hline y & -7.969713 & -2.118022 & 0.321797 \\
\hline & -5.350088 & 1.008920 & -2.887234 \\
\hline b & -4.158445 & 1.325136 & -3.792333 \\
\hline & -3.723664 & 3.411074 & 3.825029 \\
\hline & -5.146128 & 1.429106 & 4.388882 \\
\hline b & 1.353266 & 0.688587 & 5.888755 \\
\hline ) & -0.124571 & -1.147869 & 6.721133 \\
\hline$b$ & -6.090498 & -3.608869 & 0.938496 \\
\hline ) & -6.694643 & -1.664847 & 2.397114 \\
\hline ) & -6.413406 & 0.316127 & -3.742335 \\
\hline ) & -5.915598 & 2.326435 & -2.341073 \\
\hline 5 & 2.797323 & -0.587386 & 0.357124 \\
\hline ) & 3.632271 & 0.359885 & 0.896335 \\
\hline 6 & 4.785481 & 0.717723 & 0.224130 \\
\hline 6 & 5.604679 & 1.746098 & \\
\hline 6 & 5.254462 & 2.294536 & 2.066220 \\
\hline$b$ & 4.089054 & 1.839969 & 2.728532 \\
\hline 6 & 3.281380 & 0.900908 & 2.148307 \\
\hline 6 & 6.065404 & 3.304801 & 2.637610 \\
\hline ) & 7.168294 & 3.782597 & 1.978091 \\
\hline 6 & 7.497237 & 3.269886 & 0.705875 \\
\hline 6 & 6.738081 & 2.280961 & 0.130691 \\
\hline 6 & 5.091572 & 0.073402 & -1.074233 \\
\hline 6 & 4.139486 & 0.074850 & -2.078996 \\
\hline 6 & 4.417223 & -0.412757 & -3.374533 \\
\hline 6 & 5.627990 & -0.988344 & -3.644764 \\
\hline 5 & 6.597077 & -1.133799 & -2.622079 \\
\hline 6 & 6.328232 & -0.608020 & -1.322429 \\
\hline 5 & 7.280157 & -0.847890 & -0.298758 \\
\hline 6 & 8.436881 & -1.542799 & -0.553989 \\
\hline 6 & 8.714279 & -2.029573 & -1.848412 \\
\hline 5 & 7.808612 & -1.827873 & -2.858248 \\
\hline 8 & 2.889010 & 0.606166 & -1.868665 \\
\hline f & 0.328120 & 2.129471 & 1.400314 \\
\hline 8 & 0.460081 & 2.813143 & 2.322086 \\
\hline 6 & -0.816600 & 4.139009 & -1.349323 \\
\hline 6 & -1.823396 & 5.238519 & -1.708411 \\
\hline 1 & -0.939887 & 1.727104 & -1.692563 \\
\hline 1 & 7.072376 & -0.495550 & 0.708465 \\
\hline 1 & -1.690540 & -2.532142 & 2.679336 \\
\hline & 7.996212 & -2.212331 & -3.860047 \\
\hline 1 & -0.901267 & -7.061272 & 1.600004 \\
\hline & 5.782929 & 3.702526 & 3.611520 \\
\hline
\end{tabular}




\begin{tabular}{|c|c|c|c|}
\hline & -3.343113 & -3.300057 & -1.462722 \\
\hline & -5.185447 & -3.423928 & -3.083151 \\
\hline & 3.832935 & 2.269624 & 3.696001 \\
\hline & -1.439424 & -4.956720 & 2.728978 \\
\hline & 5.852495 & -1.365780 & -4.641000 \\
\hline & 8.357284 & 3.666495 & 0.170902 \\
\hline & 3.649078 & -0.313139 & -4.138578 \\
\hline & -2.720888 & -1.588563 & -6.091705 \\
\hline & -0.445111 & -0.881435 & -5.543545 \\
\hline 1 & -0.021569 & -7.068768 & -2.607245 \\
\hline 1 & 6.999169 & 1.907044 & -0.855840 \\
\hline & -0.339829 & -4.642122 & -2.704104 \\
\hline & 1.429064 & -0.759408 & -3.918152 \\
\hline & 7.780688 & 4.562734 & 2.424169 \\
\hline & 9.143692 & -1.724181 & 0.252708 \\
\hline & -4.874139 & -2.609958 & -5.417134 \\
\hline & -0.300590 & -8.299955 & -0.460775 \\
\hline & 9.637057 & -2.572783 & -2.038203 \\
\hline & 2.362947 & 0.556272 & 2.620699 \\
\hline & -3.978803 & -1.752836 & 1.565570 \\
\hline & -2.855660 & 0.705709 & -1.743415 \\
\hline & -6.917081 & -0.601630 & -1.330957 \\
\hline 1 & -3.477598 & 1.047376 & 2.146540 \\
\hline & 0.213069 & -1.037890 & 2.763037 \\
\hline & -1.619479 & 1.071622 & 6.013644 \\
\hline & 1.874830 & -3.637050 & -2.004682 \\
\hline & 3.221841 & -2.543742 & -2.318649 \\
\hline ] & 5.089806 & -3.153554 & -1.101032 \\
\hline & 1.251766 & -3.407861 & 0.793457 \\
\hline 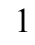 & 6.172190 & -3.943799 & 0.974218 \\
\hline & 2.316521 & -4.239354 & 2.861210 \\
\hline 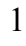 & 4.793497 & -4.494168 & 2.971348 \\
\hline ] & 2.160013 & -1.907457 & 5.587792 \\
\hline & 0.864589 & -2.444956 & 4.501949 \\
\hline 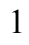 & 1.995235 & -1.172015 & 3.992305 \\
\hline & 0.659443 & -1.498368 & 7.405380 \\
\hline 1 & -0.749801 & -0.439651 & 7.276790 \\
\hline & -0.749757 & -2.009971 & 6.454398 \\
\hline 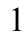 & 2.142327 & 0.383288 & 6.589331 \\
\hline 1 & 1.835902 & 1.147887 & 5.015405 \\
\hline & 0.749029 & 1.462113 & 6.378239 \\
\hline 1 & -4.507687 & 3.097412 & 6.398593 \\
\hline & -3.726209 & 1.546608 & 6.739189 \\
\hline 1 & -2.745055 & 2.976148 & 6.336549 \\
\hline & -4.508598 & 4.102823 & 4.159852 \\
\hline 1 & -2.752520 & 3.904350 & 3.963104 \\
\hline 1 & -3.861719 & 3.244451 & 2.750241 \\
\hline 1 & -5.960323 & 2.092014 & 4.711209 \\
\hline 1 & -5.313506 & 1.191464 & 3.330653 \\
\hline 1 & -5.225143 & 0.494704 & 4.960590 \\
\hline 1 & -7.398388 & -2.294153 & 2.958659 \\
\hline 1 & -5.732105 & -1.693649 & 2.923039 \\
\hline 1 & -7.063976 & -0.631386 & 2.432636 \\
\hline 1 & -8.652371 & -2.754621 & 0.898859 \\
\hline 1 & -8.390863 & -1.104405 & 0.316233 \\
\hline 1 & -7.963323 & -2.493846 & -0.709771 \\
\hline
\end{tabular}




$\begin{array}{lrrr}1 & -6.790262 & -4.254057 & 1.487011 \\ 1 & -6.017813 & -3.988096 & -0.089908 \\ 1 & -5.101794 & -3.718458 & 1.402494 \\ 1 & -4.494409 & 1.924412 & -4.648880 \\ 1 & -3.382476 & 1.906102 & -3.279384 \\ 1 & -3.695705 & 0.407322 & -4.179070 \\ 1 & -6.182454 & 3.005552 & -3.163373 \\ 1 & -6.816598 & 2.153334 & -1.737837 \\ 1 & -5.178009 & 2.829590 & -1.701470 \\ 1 & -6.647335 & 0.934529 & -4.618837 \\ 1 & -6.058148 & -0.659695 & -4.101877 \\ 1 & -7.355286 & 0.160200 & -3.202967 \\ 1 & 1.054554 & 2.386790 & -0.823421 \\ 1 & -0.165754 & 4.483293 & -0.529537 \\ 1 & -0.143234 & 3.939960 & -2.195765 \\ 6 & -1.073297 & 6.536834 & -1.904359 \\ 6 & -2.676343 & 4.846460 & -2.910273 \\ 1 & -2.490343 & 5.366424 & -0.843173 \\ 6 & -0.914097 & 7.417607 & -0.831262 \\ 6 & -0.192035 & 8.597910 & -0.970982 \\ 6 & 0.388682 & 8.919166 & -2.194348 \\ 6 & 0.242918 & 8.048958 & -3.270305 \\ 6 & -0.480140 & 6.868939 & -3.125210 \\ 1 & -1.369246 & 7.168289 & 0.127937 \\ 1 & -0.085882 & 9.270928 & -0.122453 \\ 1 & 0.952023 & 9.842805 & -2.308598 \\ 1 & 0.694951 & 8.289792 & -4.230587 \\ 1 & -0.584192 & 6.197362 & -3.976647 \\ 1 & -3.266150 & 5.695254 & -3.276287 \\ 1 & -2.061761 & 4.479754 & -3.744673 \\ 1 & -3.372557 & 4.045699 & -2.639451\end{array}$

\section{$\mathrm{TS4}_{S-\mathrm{C} 2}$}

Number of imaginary frequencies : 1

The smallest frequencies are : -682.2044 $15.3875 \quad 18.8602 \mathrm{~cm}(-1)$

Electronic energy : $\quad H F=-4475.3442425$

Zero-point correction $=\quad 1.482624$ (Hartree/Particle)

Thermal correction to Energy $=\quad 1.569447$

Thermal correction to Enthalpy $=\quad 1.570391$

Thermal correction to Gibbs Free Energy= $\quad 1.361259$

Sum of electronic and zero-point Energies $=\quad-4473.861618$

Sum of electronic and thermal Energies $=\quad-4473.774795$

Sum of electronic and thermal Enthalpies $=\quad-4473.773851$

Sum of electronic and thermal Free Energies $=\quad-4473.982984$

\section{Cartesian Coordinates}

$\begin{array}{lrll}6 & -0.634902 & 5.625265 & 3.217898 \\ 6 & -0.205808 & 4.409246 & 2.634176 \\ 6 & -0.218529 & 4.263886 & 1.216958 \\ 6 & -0.659570 & 5.365806 & 0.437375 \\ 6 & -1.074129 & 6.532072 & 1.031032 \\ 6 & -1.063155 & 6.666569 & 2.435603 \\ 6 & 0.191583 & 3.024114 & 0.628056\end{array}$




$\begin{array}{rrrr}6 & 0.588433 & 1.963952 & 1.432670 \\ 6 & 0.620545 & 2.145736 & 2.842496 \\ 6 & 0.236951 & 3.324907 & 3.423489 \\ 6 & 0.225769 & 2.940892 & -0.857139 \\ 6 & -0.809892 & 2.354235 & -1.561801 \\ 6 & -0.781907 & 2.308119 & -2.976210 \\ 6 & 0.256926 & 2.861238 & -3.675218 \\ 6 & 1.320818 & 3.498468 & -2.994693 \\ 6 & 1.314678 & 3.533655 & -1.569165 \\ 6 & 2.385962 & 4.110095 & -3.700114 \\ 6 & 3.407012 & 4.731844 & -3.027470 \\ 6 & 3.420236 & 4.740893 & -1.615580 \\ 6 & 2.402041 & 4.157630 & -0.904467 \\ 7 & -1.926537 & 1.780730 & -0.865438 \\ 6 & -3.060411 & 2.701054 & -0.604830 \\ 6 & -3.802590 & 2.469523 & 0.684576 \\ 6 & -3.121576 & 2.353206 & 1.897681 \\ 6 & -3.815902 & 2.154922 & 3.082771 \\ 6 & -5.207377 & 2.066877 & 3.073591 \\ 6 & -5.893483 & 2.183709 & 1.870136 \\ 6 & -5.193141 & 2.386952 & 0.683087 \\ 15 & 1.218341 & 0.380906 & 0.669984 \\ 45 & -0.027123 & -1.165247 & -0.903209 \\ 6 & -0.245619 & -2.618032 & 0.340951 \\ 8 & -0.312562 & -3.604203 & 0.939132 \\ 6 & 1.442062 & -0.722958 & 2.116425 \\ 6 & 0.359667 & -0.953060 & 2.976934 \\ 6 & 0.444530 & -1.895097 & 3.995578 \\ 6 & 1.620206 & -2.658711 & 4.087944 \\ 6 & 2.692333 & -2.486548 & 3.216781 \\ 6 & 2.592061 & -1.488948 & 2.237199 \\ 6 & 3.951474 & -3.348049 & 3.252027 \\ 6 & 3.936317 & -4.359736 & 4.394186 \\ 6 & -0.709857 & -2.145029 & 4.965128 \\ 6 & -1.786115 & -1.063278 & 4.870596 \\ 6 & 2.923064 & 0.930198 & 0.255601 \\ 6 & 3.399724 & 0.823691 & -1.049403 \\ 6 & 4.711111 & 1.173203 & -1.362631 \\ 6 & 5.508952 & 1.704884 & -0.340406 \\ 6 & 5.045790 & 1.873570 & 0.964986 \\ 6 & 3.742241 & 1.457542 & 1.249306 \\ 6 & 5.310464 & 0.903240 & -2.741828 \\ 6 & 4.248430 & 0.488881 & -3.759855 \\ 6 & 5.886812 & 2.480842 & 2.086260 \\ 6 & 7.266219 & 2.922541 & 1.605315 \\ 6 & 6.032505 & 2.140869 & -3.279463 \\ 6 & 6.310758 & -0.251070 & -2.606220 \\ 6 & 5.163461 & 3.710805 & 2.648490 \\ 6 & 6.076470 & 1.445462 & 3.200630 \\ 6 & 4.050090 & -4.115241 & 1.926533 \\ 6 & 5.185818 & -2.454297 & 3.417104 \\ 6 & -1.347961 & -3.499648 & 4.638223 \\ 6 & -0.195326 & -2.161650 & 6.408445\end{array}$




$\begin{array}{lrrr}15 & -1.985783 & 0.094350 & -0.985303 \\ 8 & -3.098163 & -0.144209 & 0.217415 \\ 6 & -3.797539 & -1.324725 & 0.249814 \\ 6 & -4.860847 & -1.517330 & -0.612469 \\ 6 & -5.530875 & -2.783848 & -0.581805 \\ 6 & -5.147283 & -3.756248 & 0.391851 \\ 6 & -4.091747 & -3.467897 & 1.290234 \\ 6 & -3.416852 & -2.280804 & 1.211082 \\ 6 & -6.542827 & -3.138237 & -1.510396 \\ 6 & -7.160382 & -4.363092 & -1.455438 \\ 6 & -6.803588 & -5.306100 & -0.469200 \\ 6 & -5.813175 & -5.005516 & 0.429997 \\ 6 & -5.225692 & -0.441847 & -1.563242 \\ 6 & -4.256071 & 0.093672 & -2.392661 \\ 6 & -4.565968 & 1.035123 & -3.397835 \\ 6 & -5.843124 & 1.507799 & -3.522641 \\ 6 & -6.853731 & 1.089410 & -2.622828 \\ 6 & -6.545238 & 0.115107 & -1.626192 \\ 6 & -8.151288 & 1.654499 & -2.669484 \\ 6 & -9.107009 & 1.303341 & -1.750767 \\ 6 & -8.795233 & 0.369066 & -0.741024 \\ 6 & -7.552185 & -0.212134 & -0.682743 \\ 8 & -2.945767 & -0.313243 & -2.301719 \\ 6 & 1.743630 & -2.051651 & -1.795689 \\ 6 & 1.538174 & -3.065142 & -2.910671 \\ 6 & 2.414869 & -4.320015 & -2.822577 \\ 8 & 2.812274 & -1.958191 & -1.223433 \\ 1 & 1.053542 & -0.854479 & -2.217622 \\ 1 & -7.320910 & -0.914826 & 0.113783 \\ 1 & 0.964358 & 1.336136 & 3.479978 \\ 1 & -8.366987 & 2.388800 & -3.444591 \\ 1 & -0.620157 & 5.712228 & 4.303378 \\ 1 & -5.509259 & -5.728113 & 1.186258 \\ 1 & 2.428265 & 4.159525 & 0.184062 \\ 1 & 4.249362 & 5.208931 & -1.089043 \\ 1 & -3.809843 & -4.215932 & 2.030095 \\ 1 & 0.271772 & 3.440481 & 4.506067 \\ 1 & -6.093347 & 2.231659 & -4.296474 \\ 1 & -7.928339 & -4.610586 & -2.184944 \\ 1 & -3.768502 & 1.365150 & -4.059742 \\ 1 & 2.370952 & 4.081800 & -4.789000 \\ 1 & 0.273537 & 2.828239 & -4.763593 \\ 1 & -1.412059 & 7.361054 & 0.413455 \\ 1 & -6.822175 & -2.429669 & -2.285704 \\ 1 & -0.667594 & 5.279913 & -0.647264 \\ 1 & -1.604589 & 1.818372 & -3.493780 \\ 1 & -7.304220 & -6.270860 & -0.433841 \\ 1 & -9.544791 & 0.110191 & 0.003543 \\ 1 & 4.214047 & 5.210471 & -3.578285 \\ 1 & -1.394721 & 7.595990 & 2.892567 \\ 1 & -10.097340 & 1.750947 & -1.789362 \\ 1 & -2.582168 & -2.045563 & 1.869093 \\ 1 & 3.361774 & 1.542914 & 2.267048\end{array}$




\begin{tabular}{|c|c|c|c|}
\hline & 2.735835 & 0.433462 & -1.813751 \\
\hline & 6.533512 & 1.985884 & -0.576500 \\
\hline & 3.409292 & -1.330659 & 1.535658 \\
\hline & -0.556414 & -0.378354 & 2.836075 \\
\hline & 1.684037 & -3.420317 & 4.861979 \\
\hline & -2.616594 & 3.706428 & -0.583206 \\
\hline & -3.768789 & 2.692051 & -1.447572 \\
\hline & -5.737349 & 2.460295 & -0.260107 \\
\hline & -2.034243 & 2.379000 & 1.900655 \\
\hline & -6.978903 & 2.107819 & 1.846607 \\
\hline & -3.264559 & 2.064965 & 4.017800 \\
\hline & -5.752209 & 1.904096 & 4.001188 \\
\hline & -2.558531 & -1.243897 & 5.628713 \\
\hline & -1.370132 & -0.061481 & 5.048518 \\
\hline & -2.291651 & -1.051697 & 3.896034 \\
\hline & -1.030349 & -2.318253 & 7.104027 \\
\hline & 0.530127 & -2.963603 & 6.586983 \\
\hline 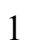 & 0.285769 & -1.209524 & 6.667596 \\
\hline 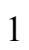 & -2.186259 & -3.705149 & 5.318034 \\
\hline D & -1.731150 & -3.514605 & 3.608591 \\
\hline | & -0.627063 & -4.321176 & 4.732639 \\
\hline 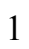 & 4.863256 & -4.946876 & 4.378916 \\
\hline 1 & 3.872664 & -3.870364 & 5.374990 \\
\hline 1 & 3.100559 & -5.065745 & 4.306148 \\
\hline 1 & 4.943651 & -4.755113 & 1.919190 \\
\hline 1 & 3.169119 & -4.756409 & 1.784008 \\
\hline 1 & 4.114249 & -3.437443 & 1.065627 \\
\hline 1 & 6.098765 & -3.064873 & 3.425286 \\
\hline 1 & 5.281590 & -1.727504 & 2.600459 \\
\hline 1 & 5.144270 & -1.896187 & 4.362039 \\
\hline 1 & 6.653959 & 1.876258 & 4.029981 \\
\hline 1 & 5.119248 & 1.095296 & 3.607521 \\
\hline 1 & 6.621961 & 0.567733 & 2.829483 \\
\hline 1 & 7.824898 & 3.364794 & 2.439979 \\
\hline 1 & 7.857794 & 2.080028 & 1.224659 \\
\hline 1 & 7.199332 & 3.681018 & 0.814191 \\
\hline 1 & 5.745375 & 4.156894 & 3.466421 \\
\hline 1 & 5.033011 & 4.476196 & 1.871811 \\
\hline | & 4.168771 & 3.466650 & 3.043085 \\
\hline | & 4.719269 & 0.332690 & -4.739462 \\
\hline | & 3.766573 & -0.457626 & -3.478400 \\
\hline & 3.471826 & 1.258445 & -3.877363 \\
\hline & 6.767707 & -0.476071 & -3.580114 \\
\hline & 7.118567 & -0.008360 & -1.903864 \\
\hline & 5.811786 & -1.162659 & -2.249320 \\
\hline & 6.460938 & 1.927180 & -4.267965 \\
\hline & 5.337275 & 2.983622 & -3.384607 \\
\hline & 6.857283 & 2.460929 & -2.631347 \\
\hline & -0.832147 & -2.096853 & -1.882584 \\
\hline & 0.480320 & -3.352904 & -2.948385 \\
\hline & 1.724534 & -2.512006 & -3.846252 \\
\hline & 2.076702 & -4.955304 & -3.658793 \\
\hline & 3.886694 & -4.057455 & -3.080745 \\
\hline
\end{tabular}




$\begin{array}{llll}6 & 2.117386 & -5.079109 & -1.536221 \\ 6 & 4.277320 & -3.522441 & -4.313507 \\ 6 & 5.616873 & -3.314892 & -4.620786 \\ 6 & 6.604476 & -3.647676 & -3.697258 \\ 6 & 6.232328 & -4.178894 & -2.467221 \\ 6 & 4.888297 & -4.380459 & -2.163502 \\ 1 & 3.514992 & -3.275897 & -5.053937 \\ 1 & 5.890819 & -2.896751 & -5.587961 \\ 1 & 7.654677 & -3.489011 & -3.933713 \\ 1 & 6.992788 & -4.439951 & -1.733693 \\ 1 & 4.620475 & -4.793787 & -1.193096 \\ 1 & 2.648353 & -6.037648 & -1.496679 \\ 1 & 2.396136 & -4.497548 & -0.648532 \\ 1 & 1.043398 & -5.292451 & -1.467578\end{array}$

\section{$\mathrm{TS}_{S-\mathrm{C} 3}$}

Number of imaginary frequencies : 1

The smallest frequencies are : $-683.2792 \quad 15.4950 \quad 16.4568 \mathrm{~cm}(-1)$

Electronic energy : $\quad \mathrm{HF}=-4475.3454275$

Zero-point correction $=\quad 1.483815($ Hartree/Particle $)$

Thermal correction to Energy $=\quad 1.570250$

Thermal correction to Enthalpy= $\quad 1.571194$

Thermal correction to Gibbs Free Energy $=\quad 1.363867$

Sum of electronic and zero-point Energies $=\quad-4473.861613$

Sum of electronic and thermal Energies $=\quad-4473.775178$

Sum of electronic and thermal Enthalpies $=\quad-4473.774234$

Sum of electronic and thermal Free Energies $=\quad-4473.981560$

Cartesian Coordinates

$\begin{array}{cccc}6 & -0.482657 & 0.873656 & 2.813563 \\ 6 & -1.547793 & 0.726796 & 1.914056 \\ 6 & -2.633905 & 1.586727 & 1.987055 \\ 6 & -2.692120 & 2.591582 & 2.963126 \\ 6 & -1.644792 & 2.677118 & 3.876436 \\ 6 & -0.531406 & 1.822297 & 3.828883 \\ 15 & -1.376532 & -0.432407 & 0.504326 \\ 6 & -3.104511 & -0.899909 & 0.082787 \\ 6 & -3.524920 & -0.900569 & -1.245205 \\ 6 & -4.842047 & -1.206158 & -1.580146 \\ 6 & -5.710530 & -1.579964 & -0.547002 \\ 6 & -5.305890 & -1.645722 & 0.787481 \\ 6 & -3.990995 & -1.280415 & 1.086538 \\ 6 & -5.364226 & -1.038622 & -3.006613 \\ 6 & -6.229528 & -2.229641 & -3.425505 \\ 6 & -6.218170 & -2.108853 & 1.921146 \\ 6 & -5.609378 & -3.363419 & 2.559930 \\ 6 & -3.865532 & 3.566241 & 2.942858 \\ 6 & -3.757042 & 4.415660 & 1.669516 \\ 6 & 0.586408 & 1.967168 & 4.860605 \\ 6 & 1.301762 & 3.304373 & 4.641952 \\ 45 & -0.026041 & 0.940702 & -1.122570\end{array}$




$\begin{array}{lrrr}15 & 1.908485 & -0.349342 & -0.972636 \\ 7 & 1.818859 & -2.028841 & -0.785532 \\ 6 & 2.916379 & -2.952216 & -0.407465 \\ 6 & 3.565273 & -2.667380 & 0.921050 \\ 6 & 2.795604 & -2.528716 & 2.077289 \\ 6 & 3.396640 & -2.266724 & 3.300458 \\ 6 & 4.782015 & -2.135889 & 3.386391 \\ 6 & 5.556468 & -2.278628 & 2.240489 \\ 6 & 4.949797 & -2.546647 & 1.015365 \\ 6 & -1.693676 & 1.771402 & -2.247910 \\ 8 & -2.812778 & 1.780810 & -1.773457 \\ 6 & -1.347505 & 2.648910 & -3.440409 \\ 6 & -2.444630 & 3.627789 & -3.839274 \\ 6 & 0.123859 & 2.510179 & -0.008370 \\ 8 & 0.156391 & 3.547738 & 0.497305 \\ 8 & 2.983498 & -0.012867 & -2.219274 \\ 6 & 4.301584 & -0.400033 & -2.164177 \\ 6 & 5.185222 & 0.204465 & -1.286855 \\ 6 & 6.516481 & -0.321099 & -1.204833 \\ 6 & 6.929660 & -1.344202 & -2.110075 \\ 6 & 6.007258 & -1.843367 & -3.062284 \\ 6 & 4.715240 & -1.394769 & -3.076622 \\ 6 & 8.239355 & -1.874276 & -2.014716 \\ 6 & 9.107192 & -1.440720 & -1.045170 \\ 6 & 8.690524 & -0.456911 & -0.124452 \\ 6 & 7.433176 & 0.089786 & -0.203676 \\ 6 & 4.717682 & 1.315901 & -0.426902 \\ 6 & 3.590113 & 1.132314 & 0.351205 \\ 6 & 3.103314 & 2.118757 & 1.230301 \\ 6 & 3.741758 & 3.325899 & 1.310715 \\ 6 & 4.864421 & 3.602565 & 0.494035 \\ 6 & 5.353965 & 2.599485 & -0.397621 \\ 6 & 6.434623 & 2.940112 & -1.250910 \\ 6 & 7.016430 & 4.182559 & -1.198986 \\ 6 & 6.552786 & 5.157729 & -0.291376 \\ 6 & 5.494957 & 4.869995 & 0.531876 \\ 8 & 2.921739 & -0.066060 & 0.305746 \\ 6 & 0.747492 & -2.610965 & -1.542784 \\ 6 & -0.380433 & -3.088312 & -0.901024 \\ 6 & -1.442983 & -3.652504 & -1.672813 \\ 6 & -1.315131 & -3.726848 & -3.091547 \\ 6 & -0.144540 & -3.225069 & -3.707536 \\ 6 & 0.858254 & -2.679667 & -2.951779 \\ 6 & -2.358256 & -4.310060 & -3.852475 \\ 6 & -3.489529 & -4.788273 & -3.241466 \\ 6 & -3.632214 & -4.687487 & -1.840008 \\ 6 & -2.633935 & -4.139686 & -1.074833 \\ 6 & -0.461047 & -3.118294 & 0.584446 \\ 6 & -0.862436 & -2.022667 & 1.335586 \\ 6 & -1.000602 & -2.169258 & 2.743765 \\ 6 & -0.712186 & -3.348543 & 3.376054 \\ 6 & -0.266236 & -4.471067 & 2.643456 \\ 6 & -0.148299 & -4.360889 & 1.228068\end{array}$




$\begin{array}{rrrc}6 & 0.299201 & -5.499025 & 0.506166 \\ 6 & 0.617945 & -6.667346 & 1.153151 \\ 6 & 0.500134 & -6.767556 & 2.555399 \\ 6 & 0.064787 & -5.689643 & 3.282447 \\ 6 & -6.208387 & 0.241660 & -3.051467 \\ 6 & -4.229349 & -0.896328 & -4.020980 \\ 6 & -6.337660 & -1.001577 & 2.974235 \\ 6 & -7.622561 & -2.457159 & 1.435745 \\ 6 & -5.191185 & 2.796685 & 2.941788 \\ 6 & -3.865661 & 4.504910 & 4.146708 \\ 6 & -0.002455 & 1.932569 & 6.275313 \\ 6 & 1.613673 & 0.839837 & 4.759394 \\ 1 & -1.038485 & 0.509595 & -2.451933 \\ 1 & 7.119929 & 0.831931 & 0.526082 \\ 1 & -1.346631 & -1.329162 & 3.338963 \\ 1 & 8.536157 & -2.647968 & -2.721724 \\ 1 & -0.031191 & -5.749008 & 4.365661 \\ 1 & 5.109292 & 5.616379 & 1.225181 \\ 1 & -2.758398 & -4.059695 & 0.004012 \\ 1 & -4.544980 & -5.040133 & -1.363573 \\ 1 & 3.378174 & 4.100362 & 1.985272 \\ 1 & -0.826504 & -3.434824 & 4.455929 \\ 1 & 6.336897 & -2.607445 & -3.764285 \\ 1 & 7.839266 & 4.418942 & -1.870015 \\ 1 & 3.983145 & -1.784153 & -3.781115 \\ 1 & -2.240518 & -4.370123 & -4.933662 \\ 1 & -0.054188 & -3.283047 & -4.791132 \\ 1 & 0.962529 & -7.524558 & 0.579331 \\ 1 & 6.797348 & 2.205481 & -1.965158 \\ 1 & 0.390710 & -5.440828 & -0.576474 \\ 1 & 1.759833 & -2.286543 & -3.418664 \\ 1 & 7.025752 & 6.136411 & -0.257399 \\ 1 & 9.369187 & -0.131781 & 0.660994 \\ 1 & -4.282580 & -5.239318 & -3.834464 \\ 1 & 0.755584 & -7.699218 & 3.054781 \\ 1 & 10.107682 & -1.861050 & -0.974969 \\ 1 & 2.217743 & 1.892013 & 1.822594 \\ 1 & -3.653587 & -1.292247 & 2.122548 \\ 1 & -2.809615 & -0.634761 & -2.015794 \\ 1 & -6.741621 & -1.821538 & -0.796437 \\ 1 & -3.434039 & 1.493367 & 1.254216 \\ 1 & 0.382237 & 0.217009 & 2.719887 \\ 1 & -1.679831 & 3.439193 & 4.652027 \\ 1 & 2.454252 & -3.949257 & -0.372810 \\ 1 & 3.682852 & -2.988524 & -1.197150 \\ 1 & 5.562732 & -2.639144 & 0.117180 \\ 1 & 1.712180 & -2.589279 & 2.004399 \\ 1 & 6.638385 & -2.173050 & 2.292255 \\ 1 & 2.777335 & -2.162368 & 4.190613 \\ 1 & 5.253977 & -1.922586 & 4.343238 \\ 1 & 2.363550 & 0.950677 & 5.552981 \\ 1 & 1.142881 & -0.146224 & 4.880451 \\ 1 & 2.154977 & 0.841033 & 3.803995\end{array}$




$\begin{array}{rrrr}1 & 0.799455 & 2.021917 & 7.020189 \\ 1 & -0.708492 & 2.751335 & 6.455975 \\ 1 & -0.530399 & 0.987825 & 6.459576 \\ 1 & 2.120359 & 3.426104 & 5.364287 \\ 1 & 1.729144 & 3.359782 & 3.631305 \\ 1 & 0.619680 & 4.155291 & 4.759359 \\ 1 & -4.738151 & 5.168526 & 4.095589 \\ 1 & -3.922764 & 3.955485 & 5.095826 \\ 1 & -2.973472 & 5.143471 & 4.170799 \\ 1 & -4.593046 & 5.126770 & 1.612151 \\ 1 & -2.820417 & 4.988891 & 1.664563 \\ 1 & -3.769688 & 3.805854 & 0.756781 \\ 1 & -6.034904 & 3.498811 & 2.907442 \\ 1 & -5.287244 & 2.126609 & 2.078340 \\ 1 & -5.294238 & 2.190047 & 3.851687 \\ 1 & -6.971399 & -1.333440 & 3.807870 \\ 1 & -5.363279 & -0.717247 & 3.391106 \\ 1 & -6.791301 & -0.099199 & 2.543209 \\ 1 & -8.234688 & -2.788251 & 2.284274 \\ 1 & -8.127046 & -1.593169 & 0.984267 \\ 1 & -7.611823 & -3.272448 & 0.700728 \\ 1 & -6.243137 & -3.724133 & 3.381566 \\ 1 & -5.519671 & -4.171646 & 1.821203 \\ 1 & -4.607796 & -3.175954 & 2.967995 \\ 1 & -4.647648 & -0.825524 & -5.033313 \\ 1 & -3.638017 & 0.012982 & -3.848841 \\ 1 & -3.551287 & -1.760829 & -3.996529 \\ 1 & -6.594471 & 0.411959 & -4.066293 \\ 1 & -7.066672 & 0.184064 & -2.369862 \\ 1 & -5.607446 & 1.113991 & -2.761336 \\ 1 & -6.580569 & -2.094988 & -4.457271 \\ 1 & -5.657036 & -3.165261 & -3.381773 \\ 1 & -7.119545 & -2.346454 & -2.795867 \\ 1 & 0.857400 & 1.760812 & -2.136473 \\ 1 & -0.393594 & 3.160847 & -3.250867 \\ 1 & -1.131032 & 1.964035 & -4.276548 \\ 6 & -2.077563 & 4.307778 & -5.157989 \\ 1 & -3.368797 & 3.049220 & -3.990865 \\ 6 & -2.740667 & 4.677471 & -2.790206 \\ 1 & -2.866400 & 4.999039 & -5.478225 \\ 1 & -1.151692 & 4.889521 & -5.051302 \\ 1 & -1.922286 & 3.572436 & -5.957802 \\ 6 & -4.058810 & 5.083130 & -2.569774 \\ 6 & -4.356230 & 6.104566 & -1.674328 \\ 6 & -3.332846 & 6.740409 & -0.976441 \\ 6 & -2.015778 & 6.341219 & -1.180784 \\ 6 & -1.723525 & 5.321514 & -2.082046 \\ 1 & -4.862356 & 4.581851 & -3.110672 \\ 1 & -5.391400 & 6.401541 & -1.516364 \\ 1 & -3.561804 & 7.535877 & -0.270015 \\ 1 & -1.207312 & 6.822362 & -0.633444 \\ 1 & -0.684459 & 5.027486 & -2.231732\end{array}$


$\mathrm{TS4}_{B}$

Number of imaginary frequencies : 1

The smallest frequencies are : -592.6217 $13.9187 \quad 17.2973 \mathrm{~cm}(-1)$

Electronic energy : $\quad H F=-4475.3411824$

Zero-point correction $=\quad 1.482406($ Hartree/Particle $)$

Thermal correction to Energy= $\quad 1.569115$

Thermal correction to Enthalpy= $\quad 1.570059$

Thermal correction to Gibbs Free Energy= $\quad 1.362411$

Sum of electronic and zero-point Energies $=\quad-4473.858777$

Sum of electronic and thermal Energies $=\quad-4473.772067$

Sum of electronic and thermal Enthalpies $=\quad-4473.771123$

Sum of electronic and thermal Free Energies $=\quad-4473.978772$

Cartesian Coordinates

$\begin{array}{cccc}6 & -2.836784 & 1.765181 & 1.785674 \\ 6 & -1.740718 & 0.918557 & 1.872464 \\ 6 & -0.741799 & 1.169263 & 2.823361 \\ 6 & -0.860732 & 2.221117 & 3.724423 \\ 6 & -1.979565 & 3.061858 & 3.608370 \\ 6 & -2.964402 & 2.865488 & 2.643567 \\ 15 & -1.441415 & -0.316312 & 0.551675 \\ 6 & -3.123646 & -0.843182 & 0.031622 \\ 6 & -4.051688 & -1.240132 & 0.990120 \\ 6 & -5.330958 & -1.662411 & 0.622808 \\ 6 & -5.657974 & -1.637906 & -0.734843 \\ 6 & -4.747902 & -1.241397 & -1.723021 \\ 6 & -3.465637 & -0.873533 & -1.317405 \\ 6 & -6.298141 & -2.121196 & 1.712439 \\ 6 & -7.633082 & -2.594944 & 1.144341 \\ 6 & -5.187239 & -1.079704 & -3.178089 \\ 6 & -6.218592 & -2.134085 & -3.584412 \\ 6 & 0.213837 & 2.511049 & 4.771475 \\ 6 & 0.985833 & 3.765683 & 4.347588 \\ 6 & -4.133226 & 3.823292 & 2.432978 \\ 6 & -4.197714 & 4.909119 & 3.503704 \\ 45 & 0.031203 & 1.063553 & -1.005409 \\ 15 & 1.897578 & -0.331310 & -0.874858 \\ 8 & 2.947702 & -0.069780 & 0.376678 \\ 6 & 3.702840 & 1.075002 & 0.389945 \\ 6 & 4.814242 & 1.173831 & -0.426004 \\ 6 & 5.539344 & 2.409449 & -0.426644 \\ 6 & 5.160986 & 3.444701 & 0.481665 \\ 6 & 4.058488 & 3.246629 & 1.347932 \\ 6 & 3.330353 & 2.089766 & 1.292767 \\ 6 & 6.605345 & 2.669869 & -1.325134 \\ 6 & 7.277026 & 3.866943 & -1.302174 \\ 6 & 6.923892 & 4.872825 & -0.378653 \\ 6 & 5.883544 & 4.662691 & 0.489498 \\ 6 & 5.187564 & 0.024418 & -1.282531 \\ 6 & 4.248272 & -0.535270 & -2.130565 \\ 6 & 4.577189 & -1.561834 & -3.041976 \\ 6 & 5.837582 & -2.092381 & -3.049097 \\ 6 & 6.809395 & -1.644854 & -2.120959\end{array}$




\begin{tabular}{|c|c|c|c|}
\hline 6 & 6.484048 & -0.585397 & -1.221126 \\
\hline 6 & 7.447533 & -0.224506 & -0.244961 \\
\hline & 8.666181 & -0.854828 & -0.183029 \\
\hline & 8.996951 & -1.875468 & -1.098600 \\
\hline & 8.082325 & -2.260489 & -2.045126 \\
\hline & 2.954157 & -0.071753 & -2.154256 \\
\hline & -1.632515 & 1.962329 & -2.062081 \\
\hline & -2.726014 & 1.866427 & -1.535264 \\
\hline & 0.274030 & 2.549553 & 0.183665 \\
\hline & 0.388668 & 3.561495 & 0.730343 \\
\hline & -1.487264 & 2.958815 & -3.254615 \\
\hline & -2.161312 & 4.258222 & -2.829139 \\
\hline & 1.739096 & -1.997841 & -0.633852 \\
\hline כ) & 0.643942 & -2.582601 & -1.354528 \\
\hline & 0.728735 & -2.694396 & -2.763207 \\
\hline & -0.271247 & -3.294413 & -3.480392 \\
\hline b & -1.412911 & -3.812242 & -2.824701 \\
\hline & -1.527680 & -3.671631 & -1.410639 \\
\hline כ) & -0.468910 & -3.048180 & -0.678026 \\
\hline & -2.700877 & -4.157231 & -0.777653 \\
\hline & -3.694093 & -4.763387 & -1.505262 \\
\hline o & -3.559813 & -4.936468 & -2.900060 \\
\hline 2 & -2.443709 & -4.465692 & -3.543890 \\
\hline 0 & -0.547247 & -2.994706 & 0.807224 \\
\hline$b$ & -0.954286 & -1.855160 & 1.488962 \\
\hline 6 & -1.109980 & -1.918738 & 2.900803 \\
\hline & -0.829542 & -3.059420 & 3.604327 \\
\hline & -0.372416 & -4.221610 & 2.944549 \\
\hline & -0.241221 & -4.195900 & 1.526149 \\
\hline 6 & 0.215300 & -5.373989 & 0.877785 \\
\hline & 0.534076 & -6.499523 & 1.596184 \\
\hline 6 & 0.405205 & -6.515541 & 3.001135 \\
\hline 6 & -0.041994 & -5.398435 & 3.658247 \\
\hline 6 & 2.811947 & -2.942456 & -0.232455 \\
\hline 6 & 3.472649 & -2.649462 & 1.088624 \\
\hline & 2.713677 & -2.427429 & 2.238817 \\
\hline 6 & 3.329932 & -2.159303 & 3.452994 \\
\hline 6 & 4.720797 & -2.107737 & 3.536236 \\
\hline 6 & 5.484504 & -2.331101 & 2.395976 \\
\hline 6 & 4.861902 & -2.603236 & 1.179804 \\
\hline 6 & -4.011576 & -1.180816 & -4.151038 \\
\hline 6 & -5.810808 & 0.316984 & -3.308519 \\
\hline 6 & -5.671626 & -3.287896 & 2.486086 \\
\hline 6 & -6.570206 & -0.958257 & 2.673330 \\
\hline 6 & -3.952027 & 4.500005 & 1.067511 \\
\hline 6 & -5.457635 & 3.052617 & 2.455691 \\
\hline 6 & 1.205079 & 1.356027 & 4.916130 \\
\hline 6 & -0.426410 & 2.746657 & 6.143238 \\
\hline 1 & -0.938420 & 0.753953 & -2.411686 \\
\hline 6 & -0.064501 & 3.237575 & -3.727867 \\
\hline 6 & -2.214869 & 2.258530 & -4.408317 \\
\hline 1 & 0.981896 & 1.889015 & -1.929996 \\
\hline 1 & 3.807419 & -1.908692 & -3.727932 \\
\hline 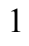 & 6.102555 & -2.883226 & -3.748889 \\
\hline 1 & 8.311567 & -3.060314 & -2.748200 \\
\hline 1 & 9.968014 & -2.361722 & -1.042505 \\
\hline
\end{tabular}




\begin{tabular}{|c|c|c|c|}
\hline 1 & 9.381306 & -0.566656 & 0.584286 \\
\hline 1 & 7.201791 & 0.547913 & 0.479347 \\
\hline 1 & 6.882488 & 1.910344 & -2.051615 \\
\hline 1 & 8.085485 & 4.043231 & -2.008221 \\
\hline 1 & 7.467681 & 5.814516 & -0.367297 \\
\hline 1 & 5.583332 & 5.435239 & 1.196267 \\
\hline 1 & 3.785029 & 4.039296 & 2.043158 \\
\hline 1 & 2.462901 & 1.919725 & 1.928856 \\
\hline 1 & 5.467036 & -2.756276 & 0.284358 \\
\hline 1 & 6.570769 & -2.283940 & 2.443871 \\
\hline 1 & 5.205401 & -1.890503 & 4.485845 \\
\hline 1 & 2.717911 & -1.987028 & 4.337464 \\
\hline 1 & 1.628923 & -2.424505 & 2.165671 \\
\hline 1 & 2.323185 & -3.925449 & -0.176706 \\
\hline 1 & 3.576434 & -3.017602 & -1.021228 \\
\hline 1 & 1.915867 & 1.579108 & 5.721809 \\
\hline 1 & 1.796407 & 1.186212 & 4.006589 \\
\hline 1 & 0.697583 & 0.414477 & 5.169008 \\
\hline 1 & -0.147481 & -5.393516 & 4.742193 \\
\hline 1 & 0.661293 & -7.414042 & 3.557657 \\
\hline 1 & 0.887180 & -7.387941 & 1.077676 \\
\hline 1 & 0.313960 & -5.380959 & -0.205752 \\
\hline 1 & -0.958861 & -3.083272 & 4.685693 \\
\hline 1 & -1.469554 & -1.046931 & 3.439711 \\
\hline 1 & -2.818233 & -4.024699 & 0.296821 \\
\hline 1 & -4.594917 & -5.113267 & -1.004806 \\
\hline 1 & -4.346824 & -5.435469 & -3.461689 \\
\hline 1 & -2.332572 & -4.577899 & -4.621859 \\
\hline 1 & -0.197311 & -3.390311 & -4.562629 \\
\hline 1 & 1.612877 & -2.298818 & -3.259892 \\
\hline 1 & -8.160772 & -1.793696 & 0.611258 \\
\hline 1 & -8.284927 & -2.929183 & 1.961495 \\
\hline 1 & -7.506469 & -3.441120 & 0.455933 \\
\hline 1 & -7.042645 & -0.118556 & 2.147020 \\
\hline 1 & -7.246497 & -1.276175 & 3.478580 \\
\hline 1 & -5.650018 & -0.583895 & 3.139455 \\
\hline 1 & -6.344172 & -3.619991 & 3.288633 \\
\hline 1 & -4.712839 & -3.017892 & 2.946856 \\
\hline 1 & -5.491347 & -4.145066 & 1.823531 \\
\hline 1 & -6.659783 & -1.932367 & -1.037475 \\
\hline 1 & -7.161812 & -2.039459 & -3.033440 \\
\hline 1 & -5.829621 & -3.149302 & -3.426138 \\
\hline 1 & -6.461503 & -2.027010 & -4.649609 \\
\hline 1 & -6.702933 & 0.414172 & -2.675859 \\
\hline 1 & -5.098544 & 1.093506 & -2.995227 \\
\hline 1 & -6.108045 & 0.514331 & -4.348389 \\
\hline 1 & -4.377540 & -1.077946 & -5.181073 \\
\hline 1 & -3.503120 & -2.150232 & -4.066078 \\
\hline 1 & -3.267423 & -0.390391 & -3.996220 \\
\hline 1 & 1.451045 & 3.624119 & 3.362331 \\
\hline 1 & 0.328131 & 4.640893 & 4.276092 \\
\hline 1 & 1.778388 & 3.995374 & 5.073157 \\
\hline 1 & 0.351779 & 2.934702 & 6.894724 \\
\hline 1 & -1.099323 & 3.611728 & 6.150242 \\
\hline 1 & -1.002736 & 1.870017 & 6.466494 \\
\hline 1 & -2.065948 & 3.907316 & 4.287453 \\
\hline
\end{tabular}




$\begin{array}{rrrr}1 & -3.304581 & 5.546951 & 3.496645 \\ 1 & -4.312004 & 4.486716 & 4.510950 \\ 1 & -5.062453 & 5.558651 & 3.317618 \\ 1 & -5.614524 & 2.566142 & 3.427794 \\ 1 & -6.297130 & 3.739241 & 2.282523 \\ 1 & -5.501970 & 2.277833 & 1.680395 \\ 1 & -4.767193 & 5.213065 & 0.880628 \\ 1 & -3.001926 & 5.050653 & 1.031022 \\ 1 & -3.938819 & 3.777490 & 0.241049 \\ 1 & -3.575089 & 1.597729 & 1.003716 \\ 1 & -3.773106 & -1.219324 & 2.043638 \\ 1 & 0.142014 & 0.530878 & 2.839864 \\ 1 & -2.718759 & -0.575401 & -2.048017 \\ 6 & -3.412023 & 4.653571 & -3.306759 \\ 6 & -3.999092 & 5.843716 & -2.884028 \\ 6 & -3.349470 & 6.661724 & -1.967313 \\ 6 & -2.108253 & 6.273434 & -1.470836 \\ 6 & -1.525328 & 5.085926 & -1.896559 \\ 1 & -3.946713 & 4.031012 & -4.020300 \\ 1 & -4.972842 & 6.129852 & -3.276951 \\ 1 & -3.807293 & 7.592145 & -1.638064 \\ 1 & -1.589393 & 6.896354 & -0.744563 \\ 1 & -0.557855 & 4.797539 & -1.488723 \\ 1 & -0.103226 & 3.914256 & -4.591842 \\ 1 & 0.561516 & 3.712137 & -2.965403 \\ 1 & 0.435937 & 2.314636 & -4.045613 \\ 1 & -2.231031 & 2.900141 & -5.298612 \\ 1 & -1.681785 & 1.336086 & -4.675983 \\ 1 & -3.244964 & 1.987940 & -4.150626\end{array}$

\section{$9_{R}$}

Number of imaginary frequencies : 0

The smallest frequencies are : $38.2386 \quad 63.0682 \quad 101.0586 \mathrm{~cm}(-1)$

Electronic energy : $\quad \mathrm{HF}=-463.1932287$

Zero-point correction $=\quad 0.193631$ (Hartree/Particle)

Thermal correction to Energy=

Thermal correction to Enthalpy=

0.204140

0.205084

Thermal correction to Gibbs Free Energy=

Sum of electronic and zero-point Energies=

Sum of electronic and thermal Energies=

0.156760

$-462.999598$

Sum of electronic and thermal Enthalpies=

$-462.989089$

$-462.988145$

Sum of electronic and thermal Free Energies=

$-463.036468$

Cartesian Coordinates

$\begin{array}{rrrr}6 & 0.908360 & -0.186989 & 0.353773 \\ 6 & 1.680298 & 0.475879 & -0.785130 \\ 6 & 3.133214 & 0.118026 & -0.852205 \\ 8 & 3.686930 & -0.696784 & -0.151062 \\ 1 & 3.699933 & 0.662238 & -1.645461 \\ 1 & 1.610448 & 1.575139 & -0.741890 \\ 1 & 1.254594 & 0.210794 & -1.767001 \\ 6 & -0.583687 & -0.119213 & 0.123812 \\ 1 & 1.200460 & -1.247283 & 0.366904\end{array}$




$\begin{array}{lrrr}6 & 1.291310 & 0.421601 & 1.701053 \\ 6 & -1.347640 & -1.284823 & 0.059790 \\ 6 & -2.723593 & -1.230177 & -0.141531 \\ 6 & -3.359074 & -0.002062 & -0.283997 \\ 6 & -2.609308 & 1.169241 & -0.221646 \\ 6 & -1.235820 & 1.109257 & -0.018550 \\ 1 & -0.852918 & -2.249421 & 0.170671 \\ 1 & -3.299864 & -2.151785 & -0.187681 \\ 1 & -4.434041 & 0.043799 & -0.442770 \\ 1 & -3.097943 & 2.135087 & -0.331553 \\ 1 & -0.661012 & 2.034108 & 0.031264 \\ 1 & 0.755223 & -0.070628 & 2.520206 \\ 1 & 2.365524 & 0.317684 & 1.887178 \\ 1 & 1.039787 & 1.490098 & 1.736422\end{array}$

9s

Number of imaginary frequencies : 0

The smallest frequencies are : $38.2392 \quad 63.0690 \quad 101.0592 \mathrm{~cm}(-1)$

Electronic energy : $\quad \mathrm{HF}=-463.1932287$

Zero-point correction $=\quad 0.193631$ (Hartree/Particle)

Thermal correction to Energy $=\quad 0.204140$

Thermal correction to Enthalpy $=\quad 0.205084$

Thermal correction to Gibbs Free Energy $=\quad 0.156760$

Sum of electronic and zero-point Energies $=\quad-462.999598$

Sum of electronic and thermal Energies $=\quad-462.989089$

Sum of electronic and thermal Enthalpies $=\quad-462.988145$

Sum of electronic and thermal Free Energies $=\quad-463.036468$

\begin{tabular}{rrrr}
\multicolumn{4}{c}{ Cartesian Coordinates } \\
\hline 6 & -0.908361 & -0.186983 & 0.353778 \\
6 & -1.680297 & 0.475877 & -0.785131 \\
6 & -3.133212 & 0.118019 & -0.852207 \\
8 & -3.686924 & -0.696796 & -0.151065 \\
1 & -3.699935 & 0.662229 & -1.645460 \\
1 & -1.254591 & 0.210785 & -1.766999 \\
1 & -1.610447 & 1.575136 & -0.741897 \\
6 & 0.583686 & -0.119212 & 0.123816 \\
6 & -1.291311 & 0.421624 & 1.701050 \\
1 & -1.200465 & -1.247276 & 0.366918 \\
6 & 1.347638 & -1.284823 & 0.059803 \\
6 & 2.723590 & -1.230180 & -0.141521 \\
6 & 3.359072 & -0.002067 & -0.284001 \\
6 & 2.609307 & 1.169238 & -0.221658 \\
6 & 1.235820 & 1.109256 & -0.018558 \\
1 & 0.852916 & -2.249420 & 0.170697 \\
1 & 3.299862 & -2.151788 & -0.187664 \\
1 & 4.434039 & 0.043792 & -0.442778 \\
1 & 3.097943 & 2.135082 & -0.331575 \\
1 & 0.661012 & 2.034108 & 0.031251 \\
1 & -0.755229 & -0.070600 & 2.520210 \\
1 & -1.039782 & 1.490119 & 1.736408 \\
1 & -2.365526 & 0.317713 & 1.887174
\end{tabular}


$9_{B}$

Number of imaginary frequencies : 0

The smallest frequencies are : $42.898969 .5881 \quad 123.5881 \mathrm{~cm}(-1)$

Electronic energy : $\quad \mathrm{HF}=-463.1938959$

Zero-point correction $=\quad 0.193336($ Hartree/Particle $)$

Thermal correction to Energy $=\quad 0.203840$

Thermal correction to Enthalpy= $\quad 0.204784$

Thermal correction to Gibbs Free Energy $=0.157179$

Sum of electronic and zero-point Energies= $\quad-463.000559$

Sum of electronic and thermal Energies $=\quad-462.990056$

Sum of electronic and thermal Enthalpies $=\quad-462.989112$

Sum of electronic and thermal Free Energies $=\quad-463.036717$

Cartesian Coordinates

$\begin{array}{rrrr}6 & -1.820270 & 0.576319 & 0.891432 \\ 8 & -2.580539 & 1.482149 & 0.648855 \\ 1 & -1.497951 & 0.355393 & 1.939746 \\ 6 & -1.212509 & -0.364304 & -0.133439 \\ 6 & -1.729161 & -0.042259 & -1.531338 \\ 6 & 0.297703 & -0.163436 & -0.050743 \\ 6 & -1.652432 & -1.770100 & 0.276843 \\ 1 & -1.282503 & -0.724845 & -2.263640 \\ 1 & -2.819166 & -0.150327 & -1.573817 \\ 1 & -1.488277 & 0.983502 & -1.827907 \\ 6 & 1.195800 & -1.210121 & 0.160106 \\ 6 & 2.566802 & -0.972101 & 0.208925 \\ 6 & 3.061908 & 0.317429 & 0.059271 \\ 6 & 2.176311 & 1.372086 & -0.141374 \\ 6 & 0.809813 & 1.131354 & -0.195697 \\ 1 & 0.832258 & -2.227291 & 0.284457 \\ 1 & 3.250159 & -1.803297 & 0.368494 \\ 1 & 4.132826 & 0.501596 & 0.101432 \\ 1 & 2.550885 & 2.386912 & -0.254228 \\ 1 & 0.124458 & 1.964364 & -0.352684 \\ 1 & -1.261245 & -2.520138 & -0.420526 \\ 1 & -1.314333 & -2.031052 & 1.287443 \\ 1 & -2.746592 & -1.841205 & 0.256474\end{array}$

7. Cartesian coordinates for additional substrate

$\left(\right.$ para- $\left.\mathrm{CH}_{3}\right) \mathrm{TS} 1_{\text {ILL-si- }} \psi_{-1 \mathrm{~s} 2 \mathrm{a} 3 \mathrm{a} a \mathrm{a}}$

Number of imaginary frequencies : 1

The smallest frequencies are : $-529.0493 \quad 18.2752 \quad 19.1765 \mathrm{~cm}(-1)$

Electronic energy : $\quad \mathrm{HF}=-4400.160639$

Zero-point correction $=\quad 1.479592($ Hartree/Particle $)$

Thermal correction to Energy= $\quad 1.565862$

Thermal correction to Enthalpy= $\quad 1.566806$

Thermal correction to Gibbs Free Energy $=\quad 1.360774$

Sum of electronic and zero-point Energies $=\quad-4398.681047$

Sum of electronic and thermal Energies $=\quad-4398.594777$

Sum of electronic and thermal Enthalpies $=\quad-4398.593833$

Sum of electronic and thermal Free Energies $=\quad-4398.799865$ 
Cartesian Coordinates

$\begin{array}{cccc}6 & -2.998456 & 2.714815 & -2.788284 \\ 6 & -1.793498 & 3.184543 & -2.253037 \\ 6 & -1.809490 & 4.413316 & -1.591525 \\ 6 & -2.984290 & 5.153013 & -1.483150 \\ 6 & -4.181238 & 4.692197 & -2.030289 \\ 6 & -4.169047 & 3.449126 & -2.672974 \\ 6 & -0.477340 & 2.502138 & -2.521689 \\ 6 & -0.051435 & 2.609378 & -3.973992 \\ 6 & -5.449319 & 5.487020 & -1.939630 \\ 45 & 0.060893 & 0.330066 & -1.759943 \\ 6 & 0.521280 & 2.501311 & -1.521360 \\ 15 & -1.475970 & -0.036226 & 0.190691 \\ 6 & -1.059402 & -1.069848 & 1.693561 \\ 6 & -0.532834 & -2.347636 & 1.542842 \\ 6 & -0.217959 & -3.140998 & 2.694257 \\ 6 & -0.437577 & -2.611190 & 3.998530 \\ 6 & -0.989556 & -1.316709 & 4.119452 \\ 6 & -1.288482 & -0.575338 & 3.007808 \\ 6 & -0.090661 & -3.379126 & 5.135672 \\ 6 & 0.452675 & -4.630685 & 4.999232 \\ 6 & 0.664247 & -5.166811 & 3.711498 \\ 6 & 0.334533 & -4.445569 & 2.590708 \\ 6 & -0.299608 & -2.976977 & 0.212284 \\ 6 & -1.282615 & -3.863040 & -0.328910 \\ 6 & -1.025286 & -4.532807 & -1.561759 \\ 6 & 0.214960 & -4.332859 & -2.210173 \\ 6 & 1.148643 & -3.491635 & -1.669771 \\ 6 & 0.891985 & -2.795256 & -0.466100 \\ 6 & -2.004678 & -5.403620 & -2.099381 \\ 6 & -3.190745 & -5.615983 & -1.444265 \\ 6 & -3.450408 & -4.954225 & -0.224785 \\ 6 & -2.520682 & -4.105172 & 0.321217 \\ 7 & 1.892201 & -1.888490 & 0.028226 \\ 6 & 2.968550 & -2.504351 & 0.838258 \\ 6 & 3.454398 & -1.704189 & 2.019632 \\ 6 & 2.566009 & -1.051860 & 2.876615 \\ 6 & 3.032217 & -0.366404 & 3.990109 \\ 6 & 4.398438 & -0.316326 & 4.263681 \\ 6 & 5.290584 & -0.956390 & 3.411345 \\ 6 & 4.819150 & -1.646500 & 2.296548 \\ 6 & -1.952440 & 1.542113 & 1.012778 \\ 6 & -0.920434 & 2.259797 & 1.618407 \\ 6 & -1.172399 & 3.418339 & 2.358582 \\ 6 & -2.492864 & 3.864892 & 2.431647 \\ 6 & -3.547484 & 3.197758 & 1.797124 \\ 6 & -3.257444 & 2.032399 & 1.086377 \\ 6 & -4.978834 & 3.706465 & 1.965970 \\ 6 & -5.051299 & 5.209777 & 1.680345 \\ 6 & -0.017608 & 4.164929 & 3.025097 \\ 6 & -0.498580 & 5.120264 & 4.117193 \\ 6 & -3.101098 & -0.788193 & -0.259057 \\ 6 & -4.080693 & -1.049819 & 0.703686 \\ & -5.308837 & -1.604573 & 0.347429\end{array}$




\begin{tabular}{|c|c|c|c|}
\hline 6 & -5.515485 & -1.915860 & -1.002289 \\
\hline & -4.553178 & -1.689210 & -1.987490 \\
\hline & -3.342053 & -1.115097 & -1.589891 \\
\hline & -6.398288 & -1.904465 & 1.376742 \\
\hline & -7.724020 & -1.271535 & 0.941461 \\
\hline & -4.850760 & -2.019867 & -3.449392 \\
\hline & -5.900865 & -1.032352 & -3.971127 \\
\hline & 1.998978 & -0.443852 & -0.852355 \\
\hline & 2.900922 & 0.364678 & 0.273210 \\
\hline 6 & 3.732312 & 1.399866 & -0.065938 \\
\hline & 4.921506 & 1.161723 & -0.728822 \\
\hline & 5.718498 & 2.294602 & -1.104026 \\
\hline U & 5.329079 & 3.601253 & -0.679463 \\
\hline & 4.146390 & 3.763289 & 0.082588 \\
\hline 6 & 3.356460 & 2.685895 & 0.370033 \\
\hline 6 & 6.873624 & 2.180203 & -1.918762 \\
\hline 6 & 7.614602 & 3.284397 & -2.261501 \\
\hline 6 & 7.246055 & 4.567935 & -1.808815 \\
\hline 6 & 6.121675 & 4.717901 & -1.038241 \\
\hline 6 & 5.316865 & -0.225780 & -1.062938 \\
\hline 6 & 4.442824 & -1.044322 & -1.754833 \\
\hline 6 & 4.826759 & -2.315130 & -2.235669 \\
\hline 6 & 6.059754 & -2.818784 & -1.926468 \\
\hline 6 & 6.943846 & -2.088684 & -1.094354 \\
\hline 6 & 6.573957 & -0.782409 & -0.653894 \\
\hline 6 & 8.172221 & -2.648426 & -0.666790 \\
\hline 6 & 8.998386 & -1.966266 & 0.189476 \\
\hline 6 & 8.620938 & -0.689278 & 0.654545 \\
\hline 6 & 7.445937 & -0.111148 & 0.240719 \\
\hline 8 & 3.167663 & -0.627147 & -2.047459 \\
\hline 6 & 0.131380 & -0.815426 & -3.275879 \\
\hline 8 & 0.226411 & -1.349487 & -4.304770 \\
\hline 6 & -5.953804 & 2.992350 & 1.030469 \\
\hline 6 & -5.423065 & 3.451631 & 3.411228 \\
\hline 6 & 0.957090 & 3.175158 & 3.673797 \\
\hline 6 & 0.708891 & 4.987832 & 1.954225 \\
\hline 6 & -5.392440 & -3.446511 & -3.579163 \\
\hline 6 & -3.603326 & -1.914434 & -4.325949 \\
\hline 6 & -6.576096 & -3.422263 & 1.493970 \\
\hline 6 & -6.047334 & -1.360150 & 2.759974 \\
\hline 1 & 7.158038 & 0.863471 & 0.626754 \\
\hline 1 & -1.719046 & 0.414040 & 3.139710 \\
\hline 1 & 8.437758 & -3.643083 & -1.022641 \\
\hline 1 & -0.261686 & -2.949771 & 6.122012 \\
\hline 1 & 5.809146 & 5.703215 & -0.694530 \\
\hline 1 & -2.734417 & -3.595830 & 1.259538 \\
\hline 1 & -4.396708 & -5.120816 & 0.286296 \\
\hline 1 & 3.868639 & 4.760818 & 0.420869 \\
\hline 1 & -1.175649 & -0.914126 & 5.114682 \\
\hline 1 & 6.367160 & -3.797382 & -2.291512 \\
\hline 1 & 8.491246 & 3.167226 & -2.894655 \\
\hline 1 & 4.122401 & -2.865405 & -2.855522 \\
\hline 1 & -1.789625 & -5.906481 & -3.041456 \\
\hline 1 & 0.411330 & -4.853389 & -3.146273 \\
\hline 1 & 1.089882 & -6.162172 & 3.605894 \\
\hline 1 & 7.166322 & 1.201010 & -2.288349 \\
\hline
\end{tabular}




\begin{tabular}{|c|c|c|c|}
\hline 1 & 0.497859 & -4.877451 & 1.605391 \\
\hline 1 & 2.102591 & -3.324745 & -2.166802 \\
\hline & 7.844797 & 5.433494 & -2.082656 \\
\hline & 9.263381 & -0.158848 & 1.353983 \\
\hline & -3.933760 & -6.292795 & -1.861124 \\
\hline & 0.720512 & -5.212069 & 5.878453 \\
\hline & 9.934437 & -2.410028 & 0.520369 \\
\hline & 2.435456 & 2.781145 & 0.939791 \\
\hline & -3.874263 & -0.806371 & 1.743941 \\
\hline & -2.548067 & -0.924883 & -2.309159 \\
\hline & -6.468587 & -2.357385 & -1.293899 \\
\hline & -4.054599 & 1.481372 & 0.596026 \\
\hline & 0.095001 & 1.864924 & 1.548123 \\
\hline & -2.717688 & 4.758470 & 3.009884 \\
\hline & 2.542010 & -3.447092 & 1.210486 \\
\hline & 3.826368 & -2.784071 & 0.207125 \\
\hline & 5.526764 & -2.135878 & 1.624818 \\
\hline & 1.503591 & -1.054411 & 2.641539 \\
\hline & 6.360727 & -0.916120 & 3.606068 \\
\hline & 2.321696 & 0.133359 & 4.647245 \\
\hline & 4.763833 & 0.224864 & 5.134011 \\
\hline & 0.266823 & 2.879040 & -0.530525 \\
\hline 1 & 1.561575 & 2.615022 & -1.827105 \\
\hline & -3.013714 & 1.755277 & -3.307176 \\
\hline & -5.094543 & 3.062078 & -3.100398 \\
\hline 1 & -2.964047 & 6.118082 & -0.976155 \\
\hline & -0.882408 & 4.808535 & -1.178531 \\
\hline 1 & -0.758341 & 2.107811 & -4.646180 \\
\hline & -0.019423 & 3.669713 & -4.263663 \\
\hline 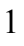 & 0.938418 & 2.167073 & -4.127475 \\
\hline ] & -1.196580 & 1.042236 & -2.518925 \\
\hline & 0.366194 & 5.589861 & 4.602994 \\
\hline 1 & -1.120809 & 5.930961 & 3.718849 \\
\hline & -1.072880 & 4.595306 & 4.891746 \\
\hline 1 & 1.754685 & 3.718045 & 4.197522 \\
\hline & 0.439413 & 2.540804 & 4.406738 \\
\hline 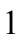 & 1.443593 & 2.510027 & 2.950313 \\
\hline 1 & 1.594455 & 5.482926 & 2.377542 \\
\hline & 1.034523 & 4.363449 & 1.111220 \\
\hline 1 & 0.046647 & 5.764263 & 1.547842 \\
\hline & -6.956725 & 3.425506 & 1.138620 \\
\hline 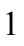 & -5.651996 & 3.085577 & -0.021725 \\
\hline & -6.036150 & 1.921076 & 1.261317 \\
\hline 1 & -4.661104 & 5.437753 & 0.680840 \\
\hline 1 & -6.092521 & 5.556409 & 1.730622 \\
\hline 1 & -4.478952 & 5.798858 & 2.406572 \\
\hline 1 & -6.454593 & 3.797929 & 3.563869 \\
\hline 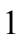 & -5.385120 & 2.380990 & 3.652045 \\
\hline 1 & -4.782742 & 3.977093 & 4.130783 \\
\hline 1 & -6.867524 & -1.567830 & 3.458659 \\
\hline 1 & -5.141745 & -1.826036 & 3.170558 \\
\hline 1 & -5.892556 & -0.271990 & 2.742561 \\
\hline 1 & -7.373845 & -3.662175 & 2.210252 \\
\hline 1 & -6.840983 & -3.879264 & 0.531997 \\
\hline 1 & -5.651099 & -3.895538 & 1.851424 \\
\hline & -8.502167 & -1.469336 & 1.690680 \\
\hline
\end{tabular}




\begin{tabular}{|c|c|c|c|}
\hline & -7.627520 & -0.183095 & 0.834604 \\
\hline & -8.082981 & -1.669804 & -0.014801 \\
\hline & -3.850245 & -2.203821 & 355380 \\
\hline & -3.202506 & -0.892361 & -4.365284 \\
\hline & -2.802540 & -2.578951 & -3.973389 \\
\hline & -5.603173 & -3.674682 & -4.632597 \\
\hline & -4.658571 & -4.176251 & -3.212887 \\
\hline & -6.325049 & -3.594917 & -3.021494 \\
\hline & -6.129833 & -1.234734 & .026425 \\
\hline & -6.838152 & -1.100334 & -3.404369 \\
\hline & -5.540306 & 0.002190 & -3.895004 \\
\hline & -5.267133 & 6.499368 & -1.561967 \\
\hline & -5.933687 & 5.576796 & -2.919822 \\
\hline & -6.177894 & 5.010711 & $-1.26808 \mathrm{~s}$ \\
\hline
\end{tabular}

$\left(\right.$ para-CH $\left.\mathrm{CH}_{3}\right) \mathrm{TS}_{\mathrm{IIL-si}-} \psi_{-1 \mathrm{~s} 2 \mathrm{a3a4}}$

Number of imaginary frequencies : 1

The smallest frequencies are : $-431.9807 \quad 16.3562 \quad 16.8729 \mathrm{~cm}(-1)$

Electronic energy : $\quad H F=-4400.1612519$

Zero-point correction $=\quad 1.482721($ Hartree/Particle $)$

Thermal correction to Energy= $\quad 1.568570$

Thermal correction to Enthalpy= $\quad 1.569515$

Thermal correction to Gibbs Free Energy $=\quad 1.364118$

Sum of electronic and zero-point Energies $=\quad-4398.678531$

Sum of electronic and thermal Energies $=\quad-4398.592682$

Sum of electronic and thermal Enthalpies $=\quad-4398.591737$

Sum of electronic and thermal Free Energies $=\quad-4398.797134$

\begin{tabular}{cccc} 
& \multicolumn{3}{c}{ Cartesian Coordinates } \\
\hline 6 & 7.442692 & -0.074213 & 0.227818 \\
6 & 6.578616 & -0.711960 & -0.698406 \\
6 & 6.966961 & -1.988242 & -1.205646 \\
6 & 8.205470 & -2.549692 & -0.810710 \\
6 & 9.023782 & -1.899530 & 0.077460 \\
6 & 8.627848 & -0.654309 & 0.608641 \\
6 & 5.310671 & -0.155678 & -1.073366 \\
6 & 4.446481 & -0.951184 & -1.802991 \\
6 & 4.845684 & -2.192884 & -2.344074 \\
6 & 6.088159 & -2.691588 & -2.066073 \\
6 & 4.895430 & 1.204769 & -0.660936 \\
6 & 3.707390 & 1.388221 & 0.021297 \\
6 & 3.321628 & 2.641614 & 0.538169 \\
6 & 4.098350 & 3.743065 & 0.311948 \\
6 & 5.275640 & 3.641658 & -0.468961 \\
6 & 5.675757 & 2.367660 & -0.974331 \\
6 & 6.051468 & 4.787139 & -0.768714 \\
6 & 7.168756 & 4.696817 & -1.558780 \\
6 & 7.547767 & 3.446878 & -2.089950 \\
6 & 6.823802 & 2.315290 & -1.805093 \\
8 & 2.887287 & 0.325673 & 0.296903 \\
15 & 1.993511 & -0.424008 & -0.876000 \\
8 & 3.166252 & -0.535090 & -2.075209 \\
45 & 0.049036 & 0.405000 & -1.737718 \\
6 & 0.071970 & -0.685433 & -3.293108
\end{tabular}




\begin{tabular}{|c|c|c|c|}
\hline & 0.134798 & -1.184304 & -4.341791 \\
\hline & -0.418498 & 2.625824 & -2.384928 \\
\hline & 0.532696 & 2.542698 & -1.342970 \\
\hline & -1.727993 & 3.328065 & -2.137118 \\
\hline & -2.926412 & 2.912216 & -2.728409 \\
\hline & -4.078402 & 3.677609 & -2.626381 \\
\hline & -4.078007 & 4.896569 & -1.938487 \\
\hline & -2.888135 & 5.303249 & -1.336290 \\
\hline & -1.731627 & 4.533937 & -1.434333 \\
\hline & -5.325690 & 5.724118 & -1.860267 \\
\hline & 0.072676 & 2.803724 & -3.809009 \\
\hline & -1.459843 & -0.071225 & 0.208936 \\
\hline & -3.086159 & -0.809050 & -0.249560 \\
\hline & -4.024577 & -1.154733 & 0.718842 \\
\hline & -5.254105 & -1.717392 & 0.368685 \\
\hline & -5.511696 & -1.921221 & -0.988553 \\
\hline & -4.587184 & -1.594758 & -1.989481 \\
\hline & -3.370861 & -1.037825 & -1.594640 \\
\hline & -6.242683 & -2.080904 & 1.476114 \\
\hline & -7.495407 & -2.766452 & 0.937596 \\
\hline & -4.935872 & -1.806990 & -3.461827 \\
\hline & -3.710911 & -1.667122 & -4.365235 \\
\hline & -1.031527 & -1.174379 & 1.656932 \\
\hline & -0.514280 & -2.446485 & 1.438782 \\
\hline & -0.201558 & -3.299405 & 2.547031 \\
\hline & -0.417148 & -2.836487 & 3.877326 \\
\hline 6 & -0.961557 & -1.546997 & 4.066331 \\
\hline & -1.255830 & -0.746845 & 2.994766 \\
\hline 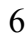 & -0.072454 & -3.663856 & 4.972585 \\
\hline 5 & 0.464765 & -4.909211 & 4.770793 \\
\hline & 0.671766 & -5.379314 & 3.456690 \\
\hline 6 & 0.343637 & -4.599608 & 2.375203 \\
\hline o & -0.299471 & -3.007176 & 0.074647 \\
\hline 6 & -1.297525 & -3.852703 & -0.502316 \\
\hline 6 & -1.070210 & -4.440803 & -1.781604 \\
\hline 6 & 0.159673 & -4.207483 & -2.439200 \\
\hline 6 & 1.110186 & -3.409240 & -1.863427 \\
\hline 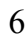 & 0.880354 & -2.789140 & -0.613401 \\
\hline 6 & -2.071081 & -5.261694 & -2.357520 \\
\hline 6 & -3.250297 & -5.499109 & -1.698094 \\
\hline 6 & -3.478982 & -4.918281 & -0.431804 \\
\hline 6 & -2.525926 & -4.124047 & 0.154180 \\
\hline 7 & 1.891632 & -1.917417 & -0.081152 \\
\hline 6 & 2.970137 & -2.587028 & 0.682683 \\
\hline 5 & 3.484846 & -1.853626 & 1.894317 \\
\hline 6 & 2.616403 & -1.267634 & 2.817246 \\
\hline 5 & 3.109660 & -0.646932 & 3.956579 \\
\hline 5 & 4.483395 & -0.593459 & 4.189224 \\
\hline 6 & 5.355549 & -1.166250 & 3.270972 \\
\hline 6 & 4.856675 & -1.794300 & 2.131667 \\
\hline 6 & -1.957747 & 1.467911 & 1.087728 \\
\hline 6 & -0.955956 & 2.166636 & 1.764232 \\
\hline 6 & -1.243526 & 3.312667 & 2.509961 \\
\hline 6 & -2.564771 & 3.766718 & 2.517280 \\
\hline 6 & -3.586024 & 3.119340 & 1.812841 \\
\hline 6 & -3.261752 & 1.964946 & 1.098396 \\
\hline
\end{tabular}




\begin{tabular}{|c|c|c|c|}
\hline 6 & -0.126766 & 4.052074 & 3.246672 \\
\hline 6 & 0.864019 & 3.061843 & 3.868066 \\
\hline 6 & -5.027374 & 3.619174 & 1.912351 \\
\hline 6 & -5.083185 & 5.144481 & 1.788131 \\
\hline 6 & -5.915652 & 3.016521 & 0.823463 \\
\hline 6 & -5.592202 & 3.210612 & 3.278065 \\
\hline 6 & 0.605474 & 4.947379 & 2.239538 \\
\hline 6 & -0.665278 & 4.935055 & 4.372917 \\
\hline 6 & -6.668158 & -0.802449 & 2.207751 \\
\hline 6 & -5.573211 & -3.032347 & 2.475514 \\
\hline 6 & -5.970703 & -0.753551 & -3.873490 \\
\hline 6 & -5.519986 & -3.204717 & -3.685535 \\
\hline 1 & 7.140984 & 0.874225 & 0.665027 \\
\hline 1 & -1.679792 & 0.237332 & 3.176945 \\
\hline 1 & 8.485039 & -3.520824 & -1.217116 \\
\hline 1 & -0.240039 & -3.285414 & 5.980142 \\
\hline 1 & 5.731282 & 5.746616 & -0.364509 \\
\hline 1 & -2.712707 & -3.679341 & 1.130047 \\
\hline 1 & -4.420570 & -5.104520 & 0.082881 \\
\hline 1 & 3.812460 & 4.714643 & 0.712976 \\
\hline 1 & -1.145088 & -1.196136 & 5.081445 \\
\hline 1 & 6.407157 & -3.647769 & -2.477585 \\
\hline 1 & 8.418844 & 3.378138 & -2.737739 \\
\hline 1 & 4.144530 & -2.726793 & -2.981730 \\
\hline 1 & -1.879964 & -5.703806 & -3.334568 \\
\hline 1 & 0.333306 & -4.665260 & -3.411883 \\
\hline 1 & 1.092708 & -6.369830 & 3.299427 \\
\hline 1 & 7.124200 & 1.362870 & -2.233838 \\
\hline 1 & 0.502784 & -4.979996 & 1.368192 \\
\hline 1 & 2.054263 & -3.212711 & -2.368956 \\
\hline 1 & 7.753920 & 5.584465 & -1.787548 \\
\hline 1 & 9.263885 & -0.150713 & 1.333338 \\
\hline 1 & -4.010611 & -6.135119 & -2.146808 \\
\hline 1 & 0.731209 & -5.536344 & 5.618402 \\
\hline 1 & 9.967551 & -2.345115 & 0.383006 \\
\hline 1 & 2.401513 & 2.692993 & 1.115782 \\
\hline 1 & -3.791627 & -0.982110 & 1.769978 \\
\hline 1 & -2.601272 & -0.781156 & -2.319819 \\
\hline 1 & -6.464791 & -2.354901 & -1.283888 \\
\hline 1 & -4.033456 & 1.428493 & 0.553438 \\
\hline 1 & 0.059982 & 1.767091 & 1.740829 \\
\hline 1 & -2.814259 & 4.651749 & 3.098039 \\
\hline 1 & 2.534631 & -3.540740 & 1.014378 \\
\hline 1 & 3.814513 & -2.846858 & 0.025597 \\
\hline 1 & 5.548290 & -2.232457 & 1.409958 \\
\hline 1 & 1.547306 & -1.271228 & 2.615863 \\
\hline 1 & 6.431171 & -1.121244 & 3.431329 \\
\hline 1 & 2.414508 & -0.199821 & 4.666005 \\
\hline 1 & 4.869763 & -0.101766 & 5.079631 \\
\hline 1 & 0.241735 & 2.860369 & -0.340267 \\
\hline 1 & 1.586567 & 2.657729 & -1.596844 \\
\hline 1 & -2.951583 & 1.972027 & -3.281667 \\
\hline 1 & -4.999586 & 3.333981 & -3.098063 \\
\hline 1 & -2.858462 & 6.249035 & -0.794461 \\
\hline 1 & -0.805383 & 4.890857 & -0.986498 \\
\hline 1 & -0.614235 & 2.354841 & -4.536756 \\
\hline
\end{tabular}




$\begin{array}{lrrr}1 & 0.136033 & 3.877716 & -4.037972 \\ 1 & 1.059610 & 2.349649 & -3.945974 \\ 1 & -1.188097 & 1.174443 & -2.469763 \\ 1 & -5.135268 & 6.702643 & -1.405591 \\ 1 & -5.753510 & 5.894682 & -2.856059 \\ 1 & -6.102501 & 5.227008 & -1.262345 \\ 1 & 0.171728 & 5.397033 & 4.911667 \\ 1 & -1.294340 & 5.752436 & 4.000295 \\ 1 & -1.251116 & 4.354732 & 5.097666 \\ 1 & 1.455673 & 5.455562 & 2.716458 \\ 1 & 0.986540 & 4.369667 & 1.386133 \\ 1 & -0.070099 & 5.715940 & 1.840253 \\ 1 & 1.615162 & 3.601916 & 4.458747 \\ 1 & 0.352211 & 2.353883 & 4.534661 \\ 1 & 1.409018 & 2.474698 & 3.119366 \\ 1 & -6.627450 & 3.562494 & 3.387680 \\ 1 & -5.592462 & 2.118290 & 3.393057 \\ 1 & -5.002575 & 3.634270 & 4.101100 \\ 1 & -6.921626 & 3.452407 & 0.883146 \\ 1 & -5.513999 & 3.211591 & -0.180427 \\ 1 & -6.028498 & 1.929649 & 0.934622 \\ 1 & -6.127170 & 5.484553 & 1.761891 \\ 1 & -4.602006 & 5.650817 & 2.633267 \\ 1 & -4.588329 & 5.479506 & 0.868279 \\ 1 & -3.993047 & -1.877650 & -5.404712 \\ 1 & -3.287780 & -0.653902 & -4.345473 \\ 1 & -2.917069 & -2.372060 & -4.082618 \\ 1 & -5.745606 & -3.352137 & -4.750080 \\ 1 & -4.803679 & -3.978001 & -3.378547 \\ 1 & -6.452348 & -3.366953 & -3.131825 \\ 1 & -6.237081 & -0.867035 & -4.933383 \\ 1 & -6.892021 & -0.838767 & -3.283311 \\ 1 & -5.575049 & 0.260925 & -3.729107 \\ 1 & -8.164638 & -3.021036 & 1.769197 \\ 1 & -8.057140 & -2.118755 & 0.252376 \\ 1 & -7.255083 & -3.698983 & 0.409480 \\ 1 & -6.274394 & -3.292748 & 3.279847 \\ 1 & -5.264496 & -3.966302 & 1.986459 \\ 1 & -4.684325 & -2.591493 & 2.944878 \\ 1 & -7.362122 & -1.038505 & 3.026016 \\ 1 & -5.806543 & -0.278190 & 2.642194 \\ 1 & -176151 & -0.108870 & 1.523988\end{array}$

$($ para-CH$) T S 1_{I L L-s i}-\psi_{-1 \mathrm{~s} 2 \mathrm{a} 3 \mathrm{~s} 4 \mathrm{a}}$

Number of imaginary frequencies : 1

The smallest frequencies are : $-534.0361 \quad 18.1478 \quad 19.1166 \mathrm{~cm}(-1)$

Electronic energy : $\quad H F=-4400.1603472$

Zero-point correction $=\quad 1.479633($ Hartree/Particle $)$

Thermal correction to Energy= $\quad 1.565874$

Thermal correction to Enthalpy= $\quad 1.566819$

Thermal correction to Gibbs Free Energy= $\quad 1.360870$

Sum of electronic and zero-point Energies $=\quad-4398.680714$

Sum of electronic and thermal Energies $=\quad-4398.594473$

Sum of electronic and thermal Enthalpies $=\quad-4398.593529$

Sum of electronic and thermal Free Energies= $\quad-4398.799478$ 
Cartesian Coordinates

\begin{tabular}{cccc}
\hline 6 & -0.927513 & 2.255071 & 1.644774 \\
6 & -1.955001 & 1.539554 & 1.028726 \\
6 & -3.260390 & 2.029544 & 1.092260 \\
6 & -3.556362 & 3.194380 & 1.801146 \\
6 & -2.506760 & 3.860847 & 2.444519 \\
6 & -1.185731 & 3.413339 & 2.383195 \\
15 & -1.480101 & -0.029645 & 0.191209 \\
45 & 0.034522 & 0.340451 & -1.752653 \\
15 & 1.986536 & -0.434300 & -0.864013 \\
8 & 3.156913 & -0.599757 & -2.060410 \\
6 & 4.432938 & -1.016203 & -1.769781 \\
6 & 5.302778 & -0.203445 & -1.065991 \\
6 & 6.558488 & -0.762860 & -0.656246 \\
6 & 6.932130 & -2.064198 & -1.107972 \\
6 & 6.054146 & -2.785659 & -1.953981 \\
6 & 4.822086 & -2.280251 & -2.264202 \\
6 & 7.424610 & -0.100091 & 0.250411 \\
6 & 8.597195 & -0.681893 & 0.665905 \\
6 & 8.978297 & -1.953935 & 0.190274 \\
6 & 8.158122 & -2.627635 & -0.678358 \\
6 & 4.905698 & 1.180705 & -0.720288 \\
6 & 3.714379 & 1.413656 & -0.059413 \\
6 & 3.340829 & 2.695640 & 0.390922 \\
6 & 4.133932 & 3.774471 & 0.118334 \\
6 & 5.318164 & 3.618786 & -0.642711 \\
6 & 5.705906 & 2.316188 & -1.080782 \\
6 & 6.114115 & 4.737620 & -0.986783 \\
6 & 7.240542 & 4.593803 & -1.755605 \\
6 & 7.607769 & 3.314578 & -2.221297 \\
6 & 6.863320 & 2.208276 & -1.893092 \\
8 & 2.879557 & 0.377489 & 0.266702 \\
6 & -4.990521 & 3.697321 & 1.961777 \\
6 & -5.950681 & 3.001927 & 0.997265 \\
6 & -0.037133 & 4.162516 & 3.057714 \\
6 & 0.943726 & 3.175706 & 3.701137 \\
6 & -3.108134 & -0.769490 & -0.262744 \\
6 & -4.073656 & -1.074383 & 0.706542 \\
6 & -5.296586 & -1.632033 & 0.350575 \\
6 & -5.529498 & -1.893458 & -1.010816 \\
6 & -4.592205 & -1.606466 & -1.998356 \\
6 & -3.374362 & -1.042245 & -1.595493 \\
6 & -4.810313 & -1.908972 & -3.478942 \\
6 & -3.854982 & -3.032154 & -3.895321 \\
6 & -6.362611 & -1.987237 & 1.386668 \\
6 & -6.539502 & -3.509131 & 1.428328 \\
6 & -1.067107 & -1.088377 & 1.673945 \\
6 & -0.541772 & -2.364081 & 1.503695 \\
6 & -0.232352 & -3.175170 & 2.645926 \\
6 & -0.461792 & -2.666443 & 3.957035 \\
6 & -1.013662 & -1.373697 & 4.095837 \\
6 & -1.302810 & -0.613947 & 2.994490 \\
6 & -0.124540 & -3.451943 & 5.084978 \\
6 & 0.420927 & -4.700846 & 4.934176
\end{tabular}




\begin{tabular}{|c|c|c|c|}
\hline 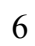 & 0.645604 & -5.215238 & 3.640033 \\
\hline & 0.324885 & -4.476804 & 2.527766 \\
\hline 6 & -0.292736 & -2.984729 & 0.170423 \\
\hline & -1.250099 & -3.900481 & -0.369540 \\
\hline & -0.938500 & -4.629453 & -1.556011 \\
\hline & 0.312660 & -4.426673 & -2.182571 \\
\hline & 1.208925 & -3.534222 & -1.661239 \\
\hline & 0.911139 & -2.804491 & -0.487022 \\
\hline & -1.873662 & -5.561634 & -2.068399 \\
\hline 6 & -3.076637 & -5.765817 & -1.442868 \\
\hline & -3.397455 & -5.033175 & -0.279795 \\
\hline & -2.507404 & -4.130749 & 0.247147 \\
\hline 7 & 1.894857 & -1.882605 & 0.012077 \\
\hline & 2.977361 & -2.488780 & 0.821818 \\
\hline U & 3.446047 & -1.690569 & 2.011311 \\
\hline 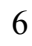 & 2.544617 & -1.055155 & 2.867344 \\
\hline 6 & 2.996063 & -0.371694 & 3.988136 \\
\hline 6 & 4.359939 & -0.306952 & 4.270285 \\
\hline 6 & 5.264790 & -0.930791 & 3.419295 \\
\hline 6 & 4.808280 & -1.618950 & 2.297121 \\
\hline 6 & -6.239816 & -2.355029 & -3.775312 \\
\hline 6 & -4.514820 & -0.662159 & -4.320956 \\
\hline 6 & -5.980838 & -1.518630 & 2.789291 \\
\hline 6 & -7.696717 & -1.331734 & 1.014450 \\
\hline 6 & -0.004897 & -0.855950 & -3.227212 \\
\hline 8 & -0.040348 & -1.441464 & -4.231669 \\
\hline 6 & -0.467713 & 2.528533 & -2.507906 \\
\hline 6 & 0.524569 & 2.504943 & -1.503437 \\
\hline 6 & -0.038604 & 2.627699 & -3.959097 \\
\hline 6 & -1.775594 & 3.226274 & -2.239380 \\
\hline 6 & -1.775947 & 4.448617 & -1.565598 \\
\hline 6 & -2.939546 & 5.205391 & -1.453524 \\
\hline 6 & -4.140409 & 4.769495 & -2.011825 \\
\hline 6 & -4.143936 & 3.533628 & -2.668472 \\
\hline 6 & -2.985367 & 2.780570 & -2.785110 \\
\hline 6 & -5.453127 & 3.409371 & 3.394982 \\
\hline 6 & -5.062198 & 5.206067 & 1.707775 \\
\hline 6 & 0.686084 & 4.997647 & 1.994084 \\
\hline 6 & -0.527029 & 5.106910 & 4.155492 \\
\hline 1 & 7.133331 & 0.870042 & 0.645167 \\
\hline 1 & -1.731797 & 0.374487 & 3.138419 \\
\hline 1 & 8.426531 & -3.618447 & -1.042675 \\
\hline 1 & -0.304734 & -3.037899 & 6.076216 \\
\hline 1 & 5.802702 & 5.719723 & -0.633066 \\
\hline 1 & -2.767453 & -3.572536 & 1.145414 \\
\hline 1 & -4.359641 & -5.187613 & 0.204879 \\
\hline 1 & 3.857589 & 4.768425 & 0.468087 \\
\hline 1 & -1.206468 & -0.987651 & 5.096301 \\
\hline 1 & 6.365275 & -3.759303 & -2.328956 \\
\hline 1 & 8.486170 & 3.202464 & -2.852934 \\
\hline 1 & 4.122454 & -2.823427 & -2.895494 \\
\hline 1 & -1.614103 & -6.111253 & -2.972320 \\
\hline 1 & 0.547242 & -4.987607 & -3.085911 \\
\hline 1 & 1.074671 & -6.207731 & 3.522050 \\
\hline 1 & 7.155095 & 1.232575 & -2.272517 \\
\hline 1 & 0.500349 & -4.893938 & 1.538246 \\
\hline
\end{tabular}




\begin{tabular}{|c|c|c|c|}
\hline & 2.171408 & -3.364432 & -2.140554 \\
\hline & 7.841945 & 5.461042 & -2.018051 \\
\hline ] & 9.234702 & -0.158489 & 1.375088 \\
\hline 1 & -3.788097 & -6.485020 & -1.843040 \\
\hline & 0.681082 & -5.295927 & 5.806500 \\
\hline & 9.912306 & -2.400725 & 0.522849 \\
\hline & 2.418647 & 2.786481 & 0.959572 \\
\hline & -3.852605 & -0.863739 & 1.750864 \\
\hline & -2.601054 & -0.820177 & -2.329442 \\
\hline & -6.480634 & -2.339248 & -1.296107 \\
\hline 1 & -4.053459 & 1.478097 & 0.595661 \\
\hline & 0.088327 & 1.859995 & 1.581940 \\
\hline & -2.735661 & 4.754749 & 3.020538 \\
\hline & 2.562577 & -3.440192 & 1.185453 \\
\hline & 3.841986 & -2.752138 & 0.193003 \\
\hline ] & 5.525515 & -2.095544 & 1.626459 \\
\hline & 1.483659 & -1.069813 & 2.626142 \\
\hline & 6.333251 & -0.879197 & 3.620730 \\
\hline & 2.275735 & 0.114530 & 4.644691 \\
\hline & 4.713515 & 0.232790 & 5.146375 \\
\hline & 0.271562 & 2.878978 & -0.510643 \\
\hline & 1.567969 & 2.603362 & -1.803996 \\
\hline$\frac{1}{1}$ & -3.016860 & 1.825836 & -3.312657 \\
\hline & -5.073634 & 3.167390 & -3.105091 \\
\hline 6 & -5.396952 & 5.582091 & -1.918587 \\
\hline & -2.906810 & 6.163901 & -0.934788 \\
\hline 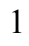 & -0.844144 & 4.826965 & -1.147874 \\
\hline 1 & -0.751718 & 2.134446 & -4.630878 \\
\hline & 0.007570 & 3.687075 & -4.251055 \\
\hline 1 & 0.944837 & 2.170986 & -4.110903 \\
\hline & -1.221019 & 1.064701 & -2.496737 \\
\hline 1 & 0.333475 & 5.577455 & 4.647903 \\
\hline ] & -1.152154 & 5.917464 & 3.761523 \\
\hline | & -1.101253 & 4.572830 & 4.923829 \\
\hline 1 & 1.734265 & 3.721080 & 4.232866 \\
\hline 1 & 0.429235 & 2.529859 & 4.426225 \\
\hline 1 & 1.439325 & 2.522299 & 2.973212 \\
\hline & 1.566000 & 5.497768 & 2.423258 \\
\hline 1 & 1.020161 & 4.380341 & 1.149097 \\
\hline ] & 0.018745 & 5.770771 & 1.589678 \\
\hline 1 & -6.954394 & 3.435704 & 1.094664 \\
\hline 1 & -5.628420 & 3.112317 & -0.046894 \\
\hline 1 & -6.040094 & 1.927097 & 1.206924 \\
\hline 1 & -4.650061 & 5.455909 & 0.722448 \\
\hline & -6.105405 & 5.548430 & 1.741853 \\
\hline 1 & -4.508852 & 5.781353 & 2.459389 \\
\hline 1 & -6.486179 & 3.753340 & 3.542814 \\
\hline 1 & -5.419425 & 2.333141 & 3.610614 \\
\hline 1 & -4.820807 & 3.917088 & 4.134161 \\
\hline 1 & -6.782138 & -1.772181 & 3.494736 \\
\hline 1 & -5.062060 & -2.000183 & 3.150197 \\
\hline 1 & -5.833742 & -0.430399 & 2.830771 \\
\hline 1 & -7.325184 & -3.785114 & 2.144927 \\
\hline 1 & -6.821157 & -3.917444 & 0.449568 \\
\hline 1 & -5.609049 & -3.999855 & 1.746292 \\
\hline 1 & -8.459241 & -1.569256 & 1.768259 \\
\hline
\end{tabular}




$\begin{array}{llll}1 & -7.600407 & -0.239073 & 0.964986 \\ 1 & -8.075796 & -1.677405 & 0.045556 \\ 1 & -6.355617 & -2.533503 & -4.851808 \\ 1 & -6.492543 & -3.291998 & -3.262603 \\ 1 & -6.975395 & -1.593665 & -3.483685 \\ 1 & -3.987004 & -3.273570 & -4.959160 \\ 1 & -2.804363 & -2.751868 & -3.742565 \\ 1 & -4.043789 & -3.940607 & -3.308763 \\ 1 & -4.704167 & -0.870044 & -5.382488 \\ 1 & -5.154731 & 0.178986 & -4.021664 \\ 1 & -3.466697 & -0.343740 & -4.236748 \\ 1 & -5.205120 & 6.580216 & -1.509245 \\ 1 & -5.863589 & 5.707764 & -2.903513 \\ 1 & -6.144004 & 5.098307 & -1.273265\end{array}$

$\left(\right.$ para $\left._{-} \mathrm{CH}_{3}\right) \mathrm{TS1}_{\mathrm{II} L-s i-} \psi_{-1 \mathrm{~s} 2 \mathrm{a} 3 \mathrm{~s} 4 \mathrm{~s}}$

Number of imaginary frequencies : 1

The smallest frequencies are : -541.7816 $14.1954 \quad 16.7250 \mathrm{~cm}(-1)$

Electronic energy : $\quad \mathrm{HF}=-4400.1607917$

Zero-point correction $=\quad 1.479979($ Hartree/Particle $)$

Thermal correction to Energy $=\quad 1.566232$

Thermal correction to Enthalpy $=\quad 1.567176$

Thermal correction to Gibbs Free Energy $=\quad 1.359954$

Sum of electronic and zero-point Energies $=\quad-4398.680813$

Sum of electronic and thermal Energies $=\quad-4398.594560$

Sum of electronic and thermal Enthalpies $=\quad-4398.593616$

Sum of electronic and thermal Free Energies $=\quad-4398.800838$

\begin{tabular}{cccc}
\multicolumn{4}{c}{ Cartesian Coordinates } \\
\hline 6 & -4.046151 & -1.153775 & 0.693472 \\
6 & -3.101033 & -0.796391 & -0.269453 \\
6 & -3.385101 & -1.015421 & -1.612005 \\
6 & -4.601564 & -1.571399 & -2.017896 \\
6 & -5.524844 & -1.916678 & -1.028396 \\
6 & -5.267879 & -1.720994 & 0.334829 \\
15 & -1.478531 & -0.058094 & 0.203699 \\
6 & -1.058262 & -1.158253 & 1.653685 \\
6 & -0.520693 & -2.423047 & 1.444677 \\
6 & -0.200870 & -3.264628 & 2.561325 \\
6 & -0.436349 & -2.799124 & 3.887271 \\
6 & -1.007762 & -1.519752 & 4.065466 \\
6 & -1.303859 & -0.729336 & 2.987748 \\
6 & -0.082019 & -3.611476 & 4.990584 \\
6 & 0.485529 & -4.845268 & 4.801495 \\
6 & 0.713561 & -5.318154 & 3.492146 \\
6 & 0.375410 & -4.553427 & 2.403022 \\
6 & -0.279896 & -3.003075 & 0.092086 \\
6 & -1.244049 & -3.900895 & -0.465375 \\
6 & -0.951765 & -4.584954 & -1.682584 \\
6 & 0.290696 & -4.359589 & -2.319245 \\
6 & 1.195769 & -3.487676 & -1.778116 \\
6 & 0.915092 & -2.800265 & -0.574215 \\
6 & -1.898339 & -5.493918 & -2.215451 \\
6 & -3.093334 & -5.717247 & -1.580510
\end{tabular}




$\begin{array}{cccc}6 & -3.393215 & -5.030221 & -0.384516 \\ 6 & -2.490991 & -4.152826 & 0.162929 \\ 7 & 1.905697 & -1.897480 & -0.054409 \\ 6 & 3.003664 & -2.535997 & 0.708752 \\ 6 & 3.488899 & -1.797112 & 1.929489 \\ 6 & 2.599542 & -1.223220 & 2.839904 \\ 6 & 3.067375 & -0.609318 & 3.993732 \\ 6 & 4.435881 & -0.550461 & 4.253476 \\ 6 & 5.329008 & -1.108828 & 3.346448 \\ 6 & 4.856063 & -1.729071 & 2.192007 \\ 6 & -4.841203 & -1.817497 & -3.506099 \\ 6 & -6.260443 & -2.097811 & 1.434777 \\ 45 & 0.027652 & 0.390827 & -1.727839 \\ 6 & -0.031323 & -0.761546 & -3.236816 \\ 8 & -0.080876 & -1.315678 & -4.258198 \\ 6 & -0.439226 & 2.607018 & -2.406484 \\ 6 & 0.036319 & 2.756721 & -3.838689 \\ 6 & -1.745252 & 3.314204 & -2.155510 \\ 6 & -1.743184 & 4.523702 & -1.459007 \\ 6 & -2.897893 & 5.295555 & -1.359052 \\ 6 & -4.091365 & 4.887918 & -1.953293 \\ 6 & -4.097614 & 3.664510 & -2.633333 \\ 6 & -2.947859 & 2.896118 & -2.737085 \\ 6 & 0.520792 & 2.535028 & -1.372582 \\ 15 & 1.984714 & -0.414636 & -0.871357 \\ 8 & 2.874893 & 0.357226 & 0.289882 \\ 6 & 3.692265 & 1.418746 & 0.003104 \\ 6 & 4.881483 & 1.231038 & -0.675674 \\ 6 & 5.660690 & 2.392486 & -0.997812 \\ 6 & 5.257684 & 3.670247 & -0.504271 \\ 6 & -3.284455 & 1.976522 & 1.090583 \\ 6 & -2.587494 & 3.134143 & 1.800586 \\ 6 & -1.262980 & 3.785799 & 2.498702 \\ 6 & 4.078474 & 3.776839 & 0.273053 \\ 6 & -0.976209 & 2.184606 & 1.748012 \\ 6 & -3.01633 & 2.490040 \\ 6 & 3.303360 & 2.676142 & 0.507737 \\ 6 & -5.048253 & 3.635122 & 1.901814 \\ 6 & -0.145744 & 4.077531 & 3.220958 \\ 6 & 7.810667 & 2.334890 & -1.825551 \\ 6 & 7.152126 & 4.718825 & -1.598136 \\ 6 & 6.032649 & 4.814223 & -0.811778 \\ 6 & 5.298653 & -0.131724 & -1.078716 \\ 6 & 4.437058 & -0.932065 & -1.806221 \\ 6 & 4.841822 & -2.171203 & -2.348907 \\ 6 & 6.085282 & -2.666181 & -2.068780 \\ 6 & 7.125378 & 0.907473 & 0.657525\end{array}$




\begin{tabular}{|c|c|c|c|}
\hline & -1.745094 & 0.248876 & 3.161105 \\
\hline ] & 8.482269 & -3.488592 & -1.213372 \\
\hline & -0.266068 & -3.230116 & 5.994169 \\
\hline & 5.710181 & 5.776578 & -0.416326 \\
\hline & -2.732063 & -3.630816 & 1.087303 \\
\hline & -4.349974 & -5.198731 & 0.107684 \\
\hline & 3.790078 & 4.751392 & 0.664982 \\
\hline & -1.208098 & -1.168320 & 5.077173 \\
\hline & 6.408767 & -3.620552 & -2.480975 \\
\hline 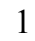 & 8.406709 & 3.391979 & -2.763002 \\
\hline 1 & 4.143445 & -2.704293 & -2.990396 \\
\hline & -1.655139 & -6.009695 & -3.143567 \\
\hline & 0.511310 & -4.885898 & -3.246632 \\
\hline ] & 1.159105 & -6.299275 & 3.344016 \\
\hline & 7.113459 & 1.379466 & -2.245783 \\
\hline 1 & 0.553259 & -4.938022 & 1.400765 \\
\hline 1 & 2.151301 & -3.299922 & -2.265280 \\
\hline & 7.736808 & 5.605278 & -1.832686 \\
\hline & 9.251861 & -0.109320 & 1.327746 \\
\hline ] & -3.813733 & -6.417314 & -1.998247 \\
\hline ] & 0.759919 & -5.460593 & 5.655196 \\
\hline & 9.961441 & -2.304318 & 0.383523 \\
\hline$\frac{1}{1}$ & 2.382437 & 2.730957 & 1.083643 \\
\hline & -3.819359 & -0.984617 & 1.746460 \\
\hline ] & -2.623377 & -0.756848 & -2.346175 \\
\hline ] & -6.472081 & -2.358026 & -1.325009 \\
\hline & -4.057109 & 1.436053 & 0.550672 \\
\hline 1 & 0.040042 & 1.786025 & 1.722672 \\
\hline & -2.833313 & 4.673564 & 3.075639 \\
\hline 1 & 2.599314 & -3.506418 & 1.031580 \\
\hline & 3.859401 & -2.763471 & 0.054662 \\
\hline ] & 5.563744 & -2.155820 & 1.478940 \\
\hline 1 & 1.534335 & -1.234190 & 2.618714 \\
\hline 1 & 6.400763 & -1.059461 & 3.529912 \\
\hline 1 & 2.356373 & -0.172856 & 4.693907 \\
\hline & 4.802200 & -0.065456 & 5.155928 \\
\hline 1 & 0.240248 & 2.868101 & -0.371846 \\
\hline 1 & 1.572714 & 2.642091 & -1.637934 \\
\hline 1 & -2.980009 & 1.951309 & -3.282466 \\
\hline 1 & -5.022140 & 3.319784 & -3.097771 \\
\hline 6 & -5.336480 & 5.719480 & -1.876371 \\
\hline 1 & -2.863760 & 6.244025 & -0.822188 \\
\hline 1 & -0.814391 & 4.881648 & -1.017386 \\
\hline 1 & -0.659403 & 2.294868 & -4.549772 \\
\hline 1 & 0.098471 & 3.826018 & -4.089290 \\
\hline 1 & 1.020670 & 2.297946 & -3.977813 \\
\hline 1 & -1.217063 & 1.153639 & -2.450242 \\
\hline 6 & -6.275930 & -2.248699 & -3.800356 \\
\hline 6 & -4.553079 & -0.541647 & -4.306043 \\
\hline 6 & -3.894650 & -2.927578 & -3.975635 \\
\hline 6 & -7.493697 & -2.811933 & 0.887996 \\
\hline 6 & -6.718510 & -0.823019 & 2.152728 \\
\hline 6 & -5.584722 & -3.030089 & 2.447790 \\
\hline 6 & 0.850239 & 3.091499 & 3.840475 \\
\hline 6 & 0.579666 & 4.973457 & 2.209504 \\
\hline 6 & -0.682879 & 4.960680 & 4.347811 \\
\hline
\end{tabular}




$\begin{array}{lrrr}6 & -5.103483 & 5.159408 & 1.765217 \\ 6 & -5.940791 & 3.024508 & 0.820917 \\ 6 & -5.607530 & 3.238719 & 3.273302 \\ 1 & -5.144887 & 6.694805 & -1.415367 \\ 1 & -5.758314 & 5.897392 & -2.873490 \\ 1 & -6.118377 & 5.221818 & -1.285647 \\ 1 & 0.154726 & 5.425389 & 4.883272 \\ 1 & -1.315187 & 5.776087 & 3.976379 \\ 1 & -1.264881 & 4.379753 & 5.075162 \\ 1 & 1.429397 & 5.486073 & 2.682493 \\ 1 & 0.960420 & 4.395183 & 1.356369 \\ 1 & -0.100229 & 5.738309 & 1.810380 \\ 1 & 1.601866 & 3.634692 & 4.427642 \\ 1 & 0.342273 & 2.383531 & 4.509886 \\ 1 & 1.394273 & 2.504637 & 3.090882 \\ 1 & -6.643152 & 3.589356 & 3.383171 \\ 1 & -5.604418 & 2.147834 & 3.399663 \\ 1 & -5.015970 & 3.672002 & 4.089894 \\ 1 & -6.946779 & 3.460136 & 0.881978 \\ 1 & -5.543586 & 3.213649 & -0.185992 \\ 1 & -6.052398 & 1.938276 & 0.939605 \\ 1 & -6.147280 & 5.499985 & 1.737949 \\ 1 & -4.620422 & 5.672331 & 2.605267 \\ 1 & -4.609947 & 5.486219 & 0.841821 \\ 1 & -6.406997 & -2.382150 & -4.881632 \\ 1 & -6.524134 & -3.205718 & -3.323922 \\ 1 & -7.005670 & -1.498822 & -3.467322 \\ 1 & -4.043433 & -3.132825 & -5.044899 \\ 1 & -2.841637 & -2.652820 & -3.829316 \\ 1 & -4.074834 & -3.855528 & -3.417104 \\ 1 & -4.761088 & -0.708611 & -5.371291 \\ 1 & -5.182717 & 0.291105 & -3.964276 \\ 1 & -3.502149 & -0.231869 & -4.227386 \\ 1 & -8.163495 & -3.079241 & 1.715138 \\ 1 & -8.064837 & -2.178981 & 0.196916 \\ 1 & -7.228174 & -3.740102 & 0.364177 \\ 1 & -6.290550 & -3.299035 & 3.245210 \\ 1 & -5.252771 & -3.960851 & 1.968055 \\ 1 & -4.710070 & -2.570347 & 2.925528 \\ 1 & -7.418122 & -1.066160 & 2.964120 \\ 1 & -5.871284 & -0.281019 & 2.593516 \\ & -7.230367 & -0.143403 & 1.457984\end{array}$

$\left(\right.$ para- $\left.\mathrm{CH}_{3}\right) \mathrm{TS} 1_{\mathrm{IIL}-\mathrm{re}-} \psi_{-1 \mathrm{a} 2 \mathrm{~s} 3 \mathrm{a} 4 \mathrm{a}}$

Number of imaginary frequencies : 1

The smallest frequencies are : $-619.1846 \quad 14.2982 \quad 21.0115 \mathrm{~cm}(-1)$

Electronic energy : $\quad H F=-4400.1592954$

Zero-point correction $=\quad 1.478733$ (Hartree/Particle)

Thermal correction to Energy= $\quad 1.565476$

Thermal correction to Enthalpy= $\quad 1.566420$

Thermal correction to Gibbs Free Energy= $\quad 1.357969$

Sum of electronic and zero-point Energies $=\quad-4398.680562$

Sum of electronic and thermal Energies $=\quad-4398.593819$

Sum of electronic and thermal Enthalpies $=\quad-4398.592875$ 
Cartesian Coordinates

\begin{tabular}{|c|c|c|c|}
\hline 6 & -6.425178 & -2.860090 & -1.334930 \\
\hline 6 & -5.342276 & -2.566471 & -0.466998 \\
\hline 6 & -4.859467 & -3.613053 & 0.376686 \\
\hline 6 & -5.498884 & -4.876579 & 0.356910 \\
\hline 6 & -6.558888 & -5.118556 & -0.478294 \\
\hline 6 & -7.015589 & -4.099450 & -1.339986 \\
\hline 6 & -4.697623 & -1.287023 & -0.438777 \\
\hline 6 & -3.569406 & -1.141026 & 0.346357 \\
\hline 6 & -3.086938 & -2.173352 & 1.175482 \\
\hline 6 & -3.733174 & -3.379743 & 1.202124 \\
\hline 6 & -5.146939 & -0.146143 & -1.267976 \\
\hline 6 & -4.249176 & 0.461289 & -2.129363 \\
\hline 6 & -4.651073 & 1.474907 & -3.027642 \\
\hline 6 & -5.936477 & 1.941791 & -3.006738 \\
\hline 6 & -6.867243 & 1.442124 & -2.062757 \\
\hline 6 & -6.470027 & 0.396152 & -1.176960 \\
\hline 6 & -8.168809 & 1.989690 & -1.957464 \\
\hline 6 & -9.043678 & 1.550647 & -0.996530 \\
\hline 6 & -8.642530 & 0.542766 & -0.095050 \\
\hline 6 & -7.393520 & -0.021362 & -0.184798 \\
\hline 8 & -2.939355 & 0.060266 & -2.184590 \\
\hline 15 & -1.847942 & 0.330003 & -0.921936 \\
\hline 8 & -2.891979 & 0.051689 & 0.343889 \\
\hline 45 & 0.081318 & -0.816773 & -1.188324 \\
\hline 15 & 1.336611 & 0.225871 & 0.530796 \\
\hline 6 & 1.441032 & -0.943410 & 1.947933 \\
\hline 6 & 2.555286 & -1.750296 & 2.140343 \\
\hline 6 & 2.601883 & -2.688617 & 3.181436 \\
\hline 6 & 1.498891 & -2.781169 & 4.025530 \\
\hline 6 & 0.360487 & -1.971810 & 3.868331 \\
\hline 6 & 0.337176 & -1.079920 & 2.801054 \\
\hline 6 & 3.867640 & -3.524502 & 3.363944 \\
\hline 6 & 3.765626 & -4.488139 & 4.543014 \\
\hline 6 & -0.803219 & -2.091801 & 4.852714 \\
\hline 6 & -0.281939 & -2.058411 & 6.294068 \\
\hline 6 & 0.321070 & -3.235208 & -1.213098 \\
\hline 6 & 0.235743 & -3.824257 & 0.176998 \\
\hline 6 & 1.461902 & -3.712606 & -2.065307 \\
\hline 6 & 2.641984 & -4.163410 & -1.463057 \\
\hline 6 & 3.755882 & -4.507876 & -2.219077 \\
\hline 6 & 3.737555 & -4.420406 & -3.611813 \\
\hline 6 & 2.551781 & -3.995952 & -4.216535 \\
\hline 6 & 1.436719 & -3.650965 & -3.463605 \\
\hline 6 & 4.940469 & -4.759168 & -4.438071 \\
\hline 6 & -0.865698 & -2.726565 & -1.793313 \\
\hline 6 & 0.562332 & -0.409314 & -3.089873 \\
\hline 8 & 0.414629 & -0.284870 & -4.232933 \\
\hline 6 & 0.978935 & 1.840971 & 1.380793 \\
\hline 6 & 0.654107 & 2.959597 & 0.627199 \\
\hline 6 & 0.447412 & 4.225076 & 1.268300 \\
\hline 6 & 0.582998 & 4.328512 & 2.683293 \\
\hline 6 & 0.927036 & 3.172580 & 3.420150 \\
\hline
\end{tabular}




\begin{tabular}{|c|c|c|c|}
\hline 6 & 1.116151 & 1.971856 & 2.789963 \\
\hline & 0.367365 & 5.575156 & 3.317944 \\
\hline & 0.027226 & 6.685292 & 2.588307 \\
\hline & -0.110085 & 6.590399 & 1.187356 \\
\hline & 0.096434 & 5.395154 & 0.544101 \\
\hline & 0.542369 & 2.929522 & -0.857432 \\
\hline & -0.643575 & 2.570229 & -1.472596 \\
\hline & -0.790214 & 2.692757 & -2.873782 \\
\hline & 0.254648 & 3.103522 & -3.656366 \\
\hline ) & 1.508906 & 3.402262 & -3.075982 \\
\hline & 1.651641 & 3.342928 & -1.657659 \\
\hline & 2.914424 & 3.661711 & -1.093395 \\
\hline y & 3.978987 & 3.996124 & -1.892583 \\
\hline & 3.834559 & 4.049521 & -3.296470 \\
\hline & 2.623419 & 3.762403 & -3.872580 \\
\hline & -1.723862 & 2.012649 & -0.706724 \\
\hline , & -2.806639 & 2.949922 & -0.332384 \\
\hline & -3.446259 & 2.675114 & 1.002637 \\
\hline 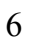 & -4.827316 & 2.528115 & 1.109321 \\
\hline 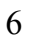 & -5.417609 & 2.249989 & 2.339843 \\
\hline & -4.630018 & 2.127258 & 3.479552 \\
\hline$b$ & -3.249164 & 2.292914 & 3.382265 \\
\hline & -2.664033 & 2.562446 & 2.152701 \\
\hline & 3.116251 & 0.481814 & 0.113318 \\
\hline 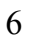 & 3.583744 & 0.109770 & -1.141593 \\
\hline ) & 4.928394 & 0.270136 & -1.488417 \\
\hline 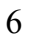 & 5.784142 & 0.812902 & -0.529130 \\
\hline 6 & 5.347776 & 1.201157 & 0.745008 \\
\hline b & 3.999351 & 1.027633 & 1.049227 \\
\hline 6 & 5.457855 & -0.155473 & -2.856860 \\
\hline ) & 6.302130 & 0.965518 & -3.470945 \\
\hline & 6.334292 & 1.822917 & 1.732804 \\
\hline 6 & 7.517282 & 0.875961 & 1.958784 \\
\hline ) & 6.845602 & 3.150112 & 1.160830 \\
\hline 6 & 5.691557 & 2.109461 & 3.088544 \\
\hline ) & 4.325019 & -0.476746 & -3.831908 \\
\hline 6 & 6.318807 & -1.411839 & -2.682593 \\
\hline 6 & 4.132685 & -4.345256 & 2.096746 \\
\hline & 5.053754 & -2.584028 & 3.612062 \\
\hline 6 & -1.802054 & -0.943658 & 4.702350 \\
\hline 6 & -1.527868 & -3.419967 & 4.611471 \\
\hline 1 & -7.091789 & -0.783756 & 0.529116 \\
\hline & 1.386066 & 1.102791 & 3.384446 \\
\hline 1 & -8.453611 & 2.781234 & -2.649581 \\
\hline 1 & 0.475001 & 5.630656 & 4.400368 \\
\hline 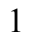 & -5.119129 & -5.656590 & 1.015714 \\
\hline 1 & 3.040011 & 3.611796 & -0.012808 \\
\hline ] & 4.946007 & 4.216131 & -1.443586 \\
\hline 1 & -3.372857 & -4.186998 & 1.839743 \\
\hline 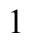 & 1.043442 & 3.251731 & 4.500419 \\
\hline 1 & -6.254260 & 2.721083 & -3.697528 \\
\hline 1 & -7.840098 & -4.298964 & -2.020872 \\
\hline 1 & -3.914684 & 1.862583 & -3.728363 \\
\hline 1 & 2.496313 & 3.801719 & -4.953562 \\
\hline 1 & 0.145047 & 3.171339 & -4.737646 \\
\hline . & -0.380298 & 7.472830 & 0.611810 \\
\hline
\end{tabular}




\begin{tabular}{|c|c|c|c|}
\hline & -6.782304 & -2.090517 & -2.014234 \\
\hline & -0.009609 & 5.338896 & -0.537334 \\
\hline & -1.744296 & 2.415110 & -3.318517 \\
\hline & -7.039109 & -6.094236 & -0.488843 \\
\hline & -9.326871 & 0.212366 & 0.683300 \\
\hline & 4.685563 & 4.322795 & -3.917086 \\
\hline & -0.138518 & 7.638547 & 3.084894 \\
\hline & -10.037644 & 1.984875 & -0.918303 \\
\hline & -2.195077 & -1.982133 & 1.770661 \\
\hline & 3.621459 & 1.314083 & 2.028103 \\
\hline & 2.869850 & -0.297710 & -1.852714 \\
\hline & 6.836835 & 0.945323 & -0.780395 \\
\hline & 3.409045 & -1.646999 & 1.471250 \\
\hline & -0.533908 & -0.445138 & 2.632906 \\
\hline & 1.518497 & -3.494891 & 4.845880 \\
\hline & -2.332697 & 3.941921 & -0.305361 \\
\hline & -3.581080 & 2.993280 & -1.114470 \\
\hline & -5.448971 & 2.605193 & 0.215817 \\
\hline & -1.583506 & 2.656155 & 2.069203 \\
\hline & -6.496572 & 2.120979 & 2.401056 \\
\hline & -2.621381 & 2.210618 & 4.268700 \\
\hline & -5.088590 & 1.905686 & 4.441157 \\
\hline & 1.309838 & -1.856912 & -0.971972 \\
\hline & -1.786753 & -2.837319 & -1.220083 \\
\hline & -1.011829 & -2.728959 & -2.871666 \\
\hline & 0.238133 & -4.922465 & 0.114147 \\
\hline & 1.066701 & -3.528207 & 0.828138 \\
\hline & -0.691214 & -3.508382 & 0.666937 \\
\hline & 2.705141 & -4.219536 & -0.378222 \\
\hline 1 & 4.663072 & -4.842626 & -1.714171 \\
\hline & 2.505264 & -3.923676 & -5.303323 \\
\hline & 0.539624 & -3.321124 & -3.981773 \\
\hline & -2.576767 & -1.023417 & 5.475633 \\
\hline & -1.308755 & 0.031382 & 4.821236 \\
\hline 1 & -2.317786 & -0.943293 & 3.733652 \\
\hline & -1.122887 & -2.111207 & 6.997979 \\
\hline 1 & 0.384255 & -2.898314 & 6.522253 \\
\hline & 0.267280 & -1.129194 & 6.494148 \\
\hline l & -2.376658 & -3.527774 & 5.300357 \\
\hline ] & -1.916114 & -3.475868 & 3.585279 \\
\hline & -0.860033 & -4.277496 & 4.762009 \\
\hline l & 4.746654 & -0.738969 & -4.811507 \\
\hline & 3.716901 & -1.331097 & -3.502103 \\
\hline 1 & 3.658316 & 0.386051 & -3.973621 \\
\hline & 6.651251 & 0.671429 & -4.469756 \\
\hline 1 & 5.709901 & 1.885246 & -3.574828 \\
\hline l & 7.191068 & 1.200656 & -2.873192 \\
\hline 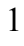 & 6.732176 & -1.732714 & -3.649147 \\
\hline 1 & 7.159820 & -1.237505 & -1.998989 \\
\hline & 5.718205 & -2.238827 & -2.277306 \\
\hline 1 & 4.697430 & -5.060581 & 4.632975 \\
\hline & 3.612334 & -3.960261 & 5.493048 \\
\hline 1 & 2.947988 & -5.209059 & 4.413687 \\
\hline ] & 5.980125 & -3.160810 & 3.737589 \\
\hline 1 & 5.204757 & -1.887056 & 2.777339 \\
\hline 1 & 4.898893 & -1.988427 & 4.521459 \\
\hline
\end{tabular}




$\begin{array}{rrrr}1 & 5.032040 & -4.962790 & 2.224289 \\ 1 & 3.291062 & -5.018160 & 1.880727 \\ 1 & 4.295635 & -3.705464 & 1.219729 \\ 1 & 6.436076 & 2.550241 & 3.763504 \\ 1 & 4.858738 & 2.821354 & 3.008945 \\ 1 & 5.314279 & 1.194629 & 3.565122 \\ 1 & 7.574202 & 3.607590 & 1.843981 \\ 1 & 7.336714 & 3.018087 & 0.188666 \\ 1 & 6.018638 & 3.860900 & 1.027625 \\ 1 & 8.227600 & 1.317735 & 2.670492 \\ 1 & 7.180577 & -0.084192 & 2.371723 \\ 1 & 8.066799 & 0.667110 & 1.033298 \\ 1 & 5.831032 & -4.901209 & -3.815584 \\ 1 & 4.789148 & -5.681800 & -5.013909 \\ 1 & 5.159393 & -3.964815 & -5.162948\end{array}$

$\left(\right.$ para- $\left.\mathrm{CH}_{3}\right) \mathrm{TS1}_{\mathrm{IIL-re}-} \Psi_{-1 \mathrm{a} 2 \mathrm{~s} 3 \mathrm{a} 4 \mathrm{~s}}$

Number of imaginary frequencies : 1

The smallest frequencies are : - $603.5647 \quad 15.4308 \quad 19.3672 \mathrm{~cm}(-1)$

Electronic energy : $\quad H F=-4400.1595821$

Zero-point correction $=\quad 1.479033($ Hartree/Particle $)$

Thermal correction to Energy= $\quad 1.565502$

Thermal correction to Enthalpy= $\quad 1.566446$

Thermal correction to Gibbs Free Energy $=\quad 1.359154$

Sum of electronic and zero-point Energies $=\quad-4398.680549$

Sum of electronic and thermal Energies $=\quad-4398.594080$

Sum of electronic and thermal Enthalpies $=\quad-4398.593136$

Sum of electronic and thermal Free Energies $=\quad-4398.800428$

Cartesian Coordinates

$\begin{array}{cccc}6 & 3.982335 & 1.113302 & 0.970536 \\ 6 & 3.109689 & 0.492592 & 0.081515 \\ 6 & 3.586311 & 0.042308 & -1.148471 \\ 6 & 4.924920 & 0.199986 & -1.503439 \\ 6 & 5.776118 & 0.830324 & -0.586415 \\ 6 & 5.329356 & 1.301472 & 0.650053 \\ 15 & 1.335247 & 0.236563 & 0.518868 \\ 6 & 0.978533 & 1.866933 & 1.339920 \\ 6 & 0.660165 & 2.973553 & 0.566129 \\ 6 & 0.460292 & 4.251571 & 1.183983 \\ 6 & 0.596478 & 4.379881 & 2.596803 \\ 6 & 0.931440 & 3.235174 & 3.354834 \\ 6 & 1.113433 & 2.021892 & 2.746740 \\ 6 & 0.389547 & 5.639312 & 3.208791 \\ 6 & 0.057102 & 6.738259 & 2.459081 \\ 6 & -0.081328 & 6.618679 & 1.060095 \\ 6 & 0.116820 & 5.410553 & 0.438634 \\ 6 & 0.550984 & 2.914438 & -0.917529 \\ 6 & -0.632187 & 2.537403 & -1.527688 \\ 6 & -0.773742 & 2.626948 & -2.931927 \\ 6 & 0.272579 & 3.023697 & -3.720082 \\ 6 & 1.523543 & 3.341829 & -3.142654 \\ 6 & 1.661264 & 3.314630 & -1.723064 \\ 6 & 2.920639 & 3.651046 & -1.161257\end{array}$




\begin{tabular}{|c|c|c|c|}
\hline 6 & 3.986614 & 3.972719 & -1.963539 \\
\hline 6 & 3.847742 & 3.993493 & -3.368852 \\
\hline & 2.639781 & 3.689005 & -3.942704 \\
\hline & -1.714552 & 1.997376 & -0.751994 \\
\hline & -2.788425 & 2.948703 & -0.386547 \\
\hline 6 & -3.431260 & 2.693625 & 0.950806 \\
\hline 6 & -4.813002 & 2.553441 & 1.057518 \\
\hline & -5.405694 & 2.291430 & 2.290505 \\
\hline & -4.619753 & 2.177706 & 3.432277 \\
\hline & -3.238102 & 2.336852 & 3.334816 \\
\hline & -2.650818 & 2.591146 & 2.103075 \\
\hline & 5.465520 & -0.323664 & -2.833079 \\
\hline & 6.321095 & -1.566367 & -2.560890 \\
\hline 6 & 6.233737 & 2.016823 & 1.652895 \\
\hline 6 & 7.644589 & 2.233248 & 1.112467 \\
\hline 45 & 0.078471 & -0.849864 & -1.171413 \\
\hline 6 & 0.564384 & -0.484344 & -3.080310 \\
\hline 8 & 0.414095 & -0.382702 & -4.225228 \\
\hline 15 & -1.846095 & 0.311317 & -0.932882 \\
\hline 8 & -2.889127 & 0.062294 & 0.339815 \\
\hline 6 & -3.570719 & -1.127506 & 0.368449 \\
\hline 6 & -4.700173 & -1.286855 & -0.412403 \\
\hline 6 & -5.348823 & -2.564656 & -0.411728 \\
\hline & -4.868139 & -3.593857 & 0.454430 \\
\hline 6 & -3.740310 & -3.346159 & 1.273545 \\
\hline 6 & -3.090615 & -2.142637 & 1.219746 \\
\hline 6 & -5.511141 & -4.855657 & 0.463249 \\
\hline 6 & -6.573029 & -5.112880 & -0.364926 \\
\hline b & -7.028019 & -4.111613 & -1.248182 \\
\hline 6 & -6.433917 & -2.874210 & -1.271390 \\
\hline$b$ & -5.146931 & -0.161371 & -1.263906 \\
\hline 6 & -4.248035 & 0.427396 & -2.137114 \\
\hline 6 & -4.646360 & 1.428053 & -3.051191 \\
\hline 6 & -5.930189 & 1.899609 & -3.037714 \\
\hline 6 & -6.862590 & 1.418150 & -2.085967 \\
\hline 5 & -6.468344 & 0.386502 & -1.182168 \\
\hline 6 & -7.393321 & -0.011037 & -0.183178 \\
\hline 6 & -8.641159 & 0.557249 & -0.104197 \\
\hline 6 & -9.039520 & 1.550156 & -1.023336 \\
\hline 6 & -8.162898 & 1.970615 & -1.990929 \\
\hline 8 & -2.940025 & 0.019368 & -2.187657 \\
\hline 6 & 0.307364 & -3.267951 & -1.138505 \\
\hline 6 & -0.873680 & -2.768666 & -1.737629 \\
\hline 6 & 1.458101 & -0.902841 & 1.958945 \\
\hline 6 & 2.582492 & -1.692154 & 2.165741 \\
\hline 6 & 2.639270 & -2.612217 & 3.222717 \\
\hline 6 & 1.538893 & -2.699334 & 4.071095 \\
\hline 6 & 0.392200 & -1.904220 & 3.901758 \\
\hline 6 & 0.357038 & -1.034715 & 2.816482 \\
\hline 6 & 3.908243 & -3.442111 & 3.411600 \\
\hline 6 & 5.100822 & -2.498876 & 3.611034 \\
\hline 6 & -0.769101 & -2.016484 & 4.890074 \\
\hline 6 & -1.492576 & -3.347779 & 4.663154 \\
\hline 6 & 3.823329 & -4.370291 & 4.620094 \\
\hline 6 & 4.151419 & -4.299909 & 2.164624 \\
\hline 6 & -0.245905 & -1.968426 & 6.330252 \\
\hline
\end{tabular}




\begin{tabular}{|c|c|c|c|}
\hline & -1.770263 & -0.871568 & 4.729799 \\
\hline & 0.212640 & -3.817734 & 0.267052 \\
\hline & 1.448401 & -3.777315 & -1.971447 \\
\hline & 2.618499 & -4.227513 & -1.349575 \\
\hline & 3.731023 & -4.610935 & -2.088438 \\
\hline & 3.722032 & -4.563547 & -3.483182 \\
\hline & 2.547185 & -4.136467 & -4.106985 \\
\hline & 1.433170 & -3.752709 & -3.371082 \\
\hline & 4.924146 & -4.948762 & -4.290035 \\
\hline & 6.319357 & 0.746531 & -3.519429 \\
\hline & 4.340670 & -0.713193 & -3.792620 \\
\hline & 6.330776 & 1.180889 & 2.934113 \\
\hline & 5.639830 & 3.390866 & 1.988064 \\
\hline & -7.093749 & -0.761155 & 0.544512 \\
\hline & 1.378409 & 1.162248 & 3.356864 \\
\hline & -8.445273 & 2.750848 & -2.696760 \\
\hline & 0.497594 & 5.713575 & 4.290044 \\
\hline & -5.132678 & -5.622030 & 1.138578 \\
\hline & 3.042394 & 3.625820 & -0.079308 \\
\hline & 4.951018 & 4.207214 & -1.515500 \\
\hline & -3.381547 & -4.140643 & 1.927890 \\
\hline & 1.046746 & 3.333083 & 4.433677 \\
\hline & -6.245518 & 2.668696 & -3.740957 \\
\hline & -7.854196 & -4.323586 & -1.923252 \\
\hline & -3.908545 & 1.802042 & -3.757876 \\
\hline & 2.516989 & 3.702814 & -5.024814 \\
\hline & 0.166323 & 3.065803 & -4.803009 \\
\hline & -0.345799 & 7.492289 & 0.468658 \\
\hline & -6.790013 & -2.118851 & -1.966989 \\
\hline & 0.010049 & 5.335098 & -0.641555 \\
\hline & -1.725170 & 2.335505 & -3.373619 \\
\hline & -7.056131 & -6.087123 & -0.353345 \\
\hline & -9.326616 & 0.242102 & 0.679474 \\
\hline & 4.700483 & 4.254324 & -3.992317 \\
\hline & -0.101861 & 7.701580 & 2.938143 \\
\hline & -10.032579 & 1.987855 & -0.953401 \\
\hline & -2.196562 & -1.941026 & 1.808258 \\
\hline & 3.606910 & 1.461933 & 1.932655 \\
\hline & 2.879768 & -0.423777 & -1.830032 \\
\hline & 6.822881 & 0.960920 & -0.853319 \\
\hline & 3.434396 & -1.592360 & 1.493612 \\
\hline & -0.521379 & -0.413115 & 2.636858 \\
\hline & 1.567273 & -3.397804 & 4.904170 \\
\hline & -2.304435 & 3.936026 & -0.368755 \\
\hline & -3.562110 & 2.992595 & -1.169412 \\
\hline & -5.433286 & 2.622307 & 0.162344 \\
\hline & -1.569828 & 2.679340 & 2.019872 \\
\hline & -6.485242 & 2.167612 & 2.352147 \\
\hline & -2.611606 & 2.261331 & 4.222784 \\
\hline & -5.080304 & 1.968360 & 4.395671 \\
\hline & 1.305134 & -1.886326 & -0.929689 \\
\hline & -1.798275 & -2.860522 & -1.167046 \\
\hline & -1.013705 & -2.797338 & -2.816462 \\
\hline & 0.209214 & -4.917302 & 0.235163 \\
\hline & 1.042336 & -3.508074 & 0.913782 \\
\hline & -0.715076 & -3.483312 & 0.742567 \\
\hline
\end{tabular}




$\begin{array}{lrrr}1 & 2.674230 & -4.253877 & -0.263401 \\ 1 & 4.629968 & -4.945577 & -1.568840 \\ 1 & 2.508665 & -4.092694 & -5.195617 \\ 1 & 0.544835 & -3.422655 & -3.904071 \\ 1 & -2.542538 & -0.943930 & 5.506211 \\ 1 & -1.278210 & 0.105588 & 4.836017 \\ 1 & -2.288959 & -0.883181 & 3.762748 \\ 1 & -1.086264 & -2.011348 & 7.035493 \\ 1 & 0.417988 & -2.807696 & 6.567268 \\ 1 & 0.305969 & -1.038566 & 6.519688 \\ 1 & -2.335857 & -3.452844 & 5.359222 \\ 1 & -1.888923 & -3.410773 & 3.640506 \\ 1 & -0.821555 & -4.202803 & 4.813895 \\ 1 & 4.769709 & -1.044450 & -4.747815 \\ 1 & 3.729284 & -1.542224 & -3.409483 \\ 1 & 3.675191 & 0.136621 & -4.000353 \\ 1 & 6.668129 & 0.382872 & -4.495121 \\ 1 & 5.734412 & 1.660939 & -3.686573 \\ 1 & 7.208994 & 1.015104 & -2.937198 \\ 1 & 6.747837 & -1.952046 & -3.497548 \\ 1 & 7.152620 & -1.346381 & -1.878850 \\ 1 & 5.713117 & -2.363659 & -2.109640 \\ 1 & 4.757269 & -4.938476 & 4.714695 \\ 1 & 3.681267 & -3.814199 & 5.555714 \\ 1 & 3.005785 & -5.096357 & 4.523233 \\ 1 & 6.024928 & -3.076068 & 3.750574 \\ 1 & 5.248961 & -1.837862 & 2.747213 \\ 1 & 4.959140 & -1.866161 & 4.497274 \\ 1 & 5.053185 & -4.913598 & 2.294171 \\ 1 & 3.306239 & -4.978472 & 1.982634 \\ 1 & 4.298259 & -3.684437 & 1.267369 \\ 1 & 8.249080 & 2.765158 & 1.858077 \\ 1 & 8.152703 & 1.284856 & 0.895671 \\ 1 & 7.641204 & 2.838739 & 0.196209 \\ 1 & 6.276207 & 3.912949 & 2.715332 \\ 1 & 5.572603 & 4.020839 & 1.090691 \\ 1 & 4.634216 & 3.319792 & 2.422408 \\ 1 & 6.951279 & 1.694200 & 3.681363 \\ 1 & 5.345388 & 1.002277 & 3.383159 \\ 1 & 6.788436 & 0.203370 & 2.730759 \\ 1 & 5.812787 & -5.064025 & -3.659250 \\ 1 & 4.769339 & -5.898670 & -4.818762 \\ 1 & 5.149441 & -4.193648 & -5.053774\end{array}$

$\left(\right.$ para- $\left.\mathrm{CH}_{3}\right) \mathrm{TS} 1_{\mathrm{IL}-\text {-re- }} \Psi_{-1 \mathrm{a} 25354 a}$

Number of imaginary frequencies : 1

The smallest frequencies are : -610.5183 $18.2587 \quad 19.1041 \mathrm{~cm}(-1)$

Electronic energy : $\quad \mathrm{HF}=-4400.1588698$

Zero-point correction $=\quad 1.479163($ Hartree/Particle)

Thermal correction to Energy $=\quad 1.565860$

Thermal correction to Enthalpy= $\quad 1.566804$

Thermal correction to Gibbs Free Energy $=\quad 1.358254$

Sum of electronic and zero-point Energies $=\quad-4398.679707$

Sum of electronic and thermal Energies $=\quad-4398.593010$

Sum of electronic and thermal Enthalpies $=\quad-4398.592066$ 
Cartesian Coordinates

\begin{tabular}{|c|c|c|c|}
\hline 6 & 2.401441 & -4.576417 & -1.387191 \\
\hline 6 & 1.375602 & -3.842966 & -1.991354 \\
\hline 6 & 1.441342 & -3.671496 & -3.381646 \\
\hline 6 & 2.483336 & -4.210848 & -4.121778 \\
\hline 6 & 3.507200 & -4.945248 & -3.514416 \\
\hline 6 & 3.443705 & -5.117186 & -2.133282 \\
\hline 6 & 0.282013 & -3.243551 & -1.158526 \\
\hline 6 & -0.876443 & -2.698785 & -1.764254 \\
\hline 45 & 0.120786 & -0.811221 & -1.196662 \\
\hline 6 & 0.592988 & -0.369972 & -3.079394 \\
\hline 8 & 0.483317 & -0.175269 & -4.217240 \\
\hline 15 & 1.386506 & 0.252696 & 0.509135 \\
\hline 6 & 1.029560 & 1.874142 & 1.347691 \\
\hline 6 & 0.674881 & 2.985868 & 0.597432 \\
\hline 6 & 0.456095 & 4.249069 & 1.242351 \\
\hline 6 & 0.620427 & 4.358593 & 2.653715 \\
\hline 6 & 1.005311 & 3.212047 & 3.384791 \\
\hline 6 & 1.199203 & 2.013509 & 2.753072 \\
\hline 6 & 0.389984 & 5.600371 & 3.292526 \\
\hline 6 & 0.005260 & 6.700947 & 2.570715 \\
\hline 6 & -0.163376 & 6.600035 & 1.173842 \\
\hline 6 & 0.058476 & 5.409608 & 0.526592 \\
\hline 6 & 0.533070 & 2.965461 & -0.885394 \\
\hline 6 & -0.659395 & 2.594957 & -1.479772 \\
\hline 6 & -0.849916 & 2.758124 & -2.871489 \\
\hline 6 & 0.149519 & 3.251062 & -3.665550 \\
\hline 6 & 1.404604 & 3.588506 & -3.108333 \\
\hline 6 & 1.601577 & 3.450303 & -1.701886 \\
\hline 6 & 2.867385 & 3.797024 & -1.162159 \\
\hline 6 & 3.876383 & 4.257577 & -1.970852 \\
\hline 6 & 3.673182 & 4.405281 & -3.360331 \\
\hline 6 & 2.463510 & 4.073677 & -3.914393 \\
\hline 7 & -1.714962 & 2.012365 & -0.699051 \\
\hline 6 & -2.818400 & 2.927518 & -0.325653 \\
\hline 6 & -3.432747 & 2.662591 & 1.023086 \\
\hline 6 & -4.808644 & 2.485148 & 1.149540 \\
\hline 6 & -5.377898 & 2.217605 & 2.392033 \\
\hline 6 & -4.574369 & 2.136939 & 3.524442 \\
\hline 6 & -3.199235 & 2.334341 & 3.407225 \\
\hline 6 & -2.634613 & 2.592350 & 2.165421 \\
\hline 6 & 3.165556 & 0.503740 & 0.089960 \\
\hline 6 & 3.625259 & 0.122261 & -1.159377 \\
\hline 6 & 4.972958 & 0.264113 & -1.516364 \\
\hline 6 & 5.836165 & 0.816890 & -0.575740 \\
\hline 6 & 5.404720 & 1.218471 & 0.701324 \\
\hline 6 & 4.060973 & 1.052215 & 1.017933 \\
\hline 6 & 6.398262 & 1.852824 & 1.674148 \\
\hline 6 & 6.849968 & 3.204450 & 1.109024 \\
\hline 6 & 5.401860 & -0.142648 & -2.923019 \\
\hline 6 & 6.917255 & -0.103216 & -3.101607 \\
\hline 6 & 1.486723 & -0.907389 & 1.933820 \\
\hline 6 & 2.583943 & -1.741419 & 2.105793 \\
\hline
\end{tabular}




$\begin{array}{cccc}6 & 2.628649 & -2.676626 & 3.149753 \\ 6 & 1.542549 & -2.736776 & 4.018391 \\ 6 & 0.421039 & -1.900887 & 3.880286 \\ 6 & 0.396718 & -1.014162 & 2.808883 \\ 6 & 3.868087 & -3.557195 & 3.298063 \\ 6 & 4.026793 & -4.427157 & 2.045820 \\ 6 & -0.723076 & -1.987566 & 4.890683 \\ 6 & -1.713160 & -0.832415 & 4.737228 \\ 15 & -1.814173 & 0.328762 & -0.907757 \\ 8 & -2.900677 & 0.031276 & -2.167334 \\ 6 & -4.213539 & 0.424370 & -2.125807 \\ 6 & -5.110423 & -0.167247 & -1.252871 \\ 6 & -6.435010 & 0.374053 & -1.174779 \\ 6 & -6.834626 & 1.398050 & -2.084691 \\ 6 & -5.906097 & 1.874682 & -3.042662 \\ 6 & -4.619377 & 1.411231 & -3.051936 \\ 6 & -7.356734 & -0.020553 & -0.171570 \\ 6 & -8.605997 & 0.544720 & -0.093354 \\ 6 & -9.009724 & 1.529856 & -1.018471 \\ 6 & -8.136785 & 1.946308 & -1.991117 \\ 6 & -4.663869 & -1.290539 & -0.398722 \\ 6 & -3.539420 & -1.131220 & 0.389257 \\ 6 & -3.073495 & -2.141768 & 1.253351 \\ 6 & -3.724065 & -3.344644 & 1.305004 \\ 6 & -4.842016 & -3.595231 & 0.473485 \\ 1 & -3.373514 & -4.135848 & 1.967632 \\ 1 & 1.147306 & 3.296418 & 4.461595 \\ 6 & -6.226898 & 2.633660 & -3.754333 \\ 6 & -7.804647 & -4.329996 & -1.931502 \\ 1 & -3.885780 & 1.779399 & -3.765942 \\ 6 & -6.539043 & -2.568171 & -0.400280 \\ 6 & -6.985053 & -4.116637 & -1.248894 \\ 1 & -2.294053 & 4.174748 & -4.985535 \\ 6 & -6.390439 & -2.879432 & -1.269338 \\ 6 & -2.852248 & 0.054525 & 0.363492 \\ 1 & -7.000416 & 3.367308 & -4.737889 \\ 6 & -0.470310 & 7.474069 & 0.603937 \\ 6 & 0.163821 & -3.762670 & 0.254917 \\ 6 & 5.783020 & 2.094836 & 3.051327 \\ 6 & 7.619187 & 0.945209 & 1.855454 \\ 6 & 4.918769 & -1.565872 & -3.230459 \\ 6 & 4.768741 & 0.833863 & -3.921106 \\ 6 & -1.466890 & -3.312369 & 4.693602 \\ 6 & -0.168905 & -1.928704 & 6.318908 \\ 6 & 5.105224 & -2.664631 & 3.454672 \\ 1 & -6.739641 & -2.125854 & -1.970332\end{array}$




\begin{tabular}{|c|c|c|c|}
\hline & -0.073310 & 5.351097 & -0.551874 \\
\hline & -1.807626 & 2.464567 & -3.297343 \\
\hline & -7.022456 & -6.090017 & -0.349733 \\
\hline & -9.288631 & 0.232862 & 0.694088 \\
\hline & 4.480649 & 4.775348 & -3.988537 \\
\hline & -0.172452 & 7.650429 & 3.070364 \\
\hline & -10.004028 & 1.964801 & -0.949231 \\
\hline & -2.188615 & -1.936125 & 1.854048 \\
\hline & 3.692099 & 1.343274 & 1.998749 \\
\hline & 2.908811 & -0.288201 & -1.868154 \\
\hline & 6.885817 & 0.948234 & -0.832622 \\
\hline & 3.423346 & -1.665272 & 1.414919 \\
\hline & -0.462706 & -0.360393 & 2.654992 \\
\hline & 1.561662 & -3.446450 & 4.842435 \\
\hline & -2.371133 & 3.932060 & -0.324788 \\
\hline & -3.605052 & 2.935418 & -1.096341 \\
\hline & -5.441799 & 2.529479 & 0.261932 \\
\hline & -1.557917 & 2.712993 & 2.067191 \\
\hline & -6.452670 & 2.063655 & 2.468264 \\
\hline & -2.559778 & 2.286008 & 4.287779 \\
\hline & -5.016031 & 1.923779 & 4.495819 \\
\hline & 1.337858 & -1.881354 & -1.005070 \\
\hline & -1.805566 & -2.755756 & -1.196081 \\
\hline & -1.014521 & -2.746062 & -2.843088 \\
\hline & 0.060749 & -4.858064 & 0.241211 \\
\hline & 1.031997 & -3.518359 & 0.878655 \\
\hline & -0.721102 & -3.341786 & 0.741121 \\
\hline & 2.397027 & -4.730006 & -0.310977 \\
\hline & 4.225383 & -5.683287 & -1.625673 \\
\hline & 4.630446 & -5.510088 & -4.329402 \\
\hline & 2.507691 & -4.057254 & -5.201000 \\
\hline & 0.671508 & -3.104378 & -3.899814 \\
\hline & -2.474460 & -0.889768 & 5.525734 \\
\hline & -1.208653 & 0.139906 & 4.828641 \\
\hline 1 & -2.246102 & -0.845031 & 3.777906 \\
\hline & -0.993431 & -1.963299 & 7.043163 \\
\hline ] & 0.499312 & -2.766829 & 6.547684 \\
\hline & 0.389050 & -0.998428 & 6.487441 \\
\hline 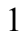 & -2.303335 & -3.394879 & 5.400815 \\
\hline ] & -1.875567 & -3.386765 & 3.676503 \\
\hline & -0.806531 & -4.174466 & 4.850567 \\
\hline 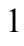 & 7.178616 & -0.429164 & -4.116363 \\
\hline & 7.322086 & 0.908887 & -2.972373 \\
\hline 1 & 7.429115 & -0.771182 & -2.396182 \\
\hline & 5.039544 & 0.561213 & -4.950549 \\
\hline 1 & 3.672808 & 0.838242 & -3.849801 \\
\hline 1 & 5.114749 & 1.859223 & -3.734851 \\
\hline 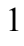 & 5.242844 & -1.862117 & -4.237831 \\
\hline 1 & 5.332227 & -2.288150 & -2.513659 \\
\hline & 3.824557 & -1.656078 & -3.203253 \\
\hline 1 & 4.701890 & -5.082578 & 4.576938 \\
\hline & 3.698667 & -3.915422 & 5.449828 \\
\hline 1 & 2.940592 & -5.173269 & 4.445095 \\
\hline ] & 6.008579 & -3.279819 & 3.563486 \\
\hline 1 & 5.252328 & -2.010094 & 2.586183 \\
\hline 1 & 5.019741 & -2.027354 & 4.344814 \\
\hline
\end{tabular}




$\begin{array}{rrrr}1 & 4.915968 & -5.066151 & 2.134329 \\ 1 & 3.153921 & -5.082152 & 1.913495 \\ 1 & 4.141268 & -3.822999 & 1.136635 \\ 1 & 6.534826 & 2.534743 & 3.718748 \\ 1 & 4.935251 & 2.791886 & 3.010095 \\ 1 & 5.435799 & 1.160330 & 3.512278 \\ 1 & 7.580997 & 3.677065 & 1.779233 \\ 1 & 7.318376 & 3.100441 & 0.122349 \\ 1 & 5.996454 & 3.888157 & 1.006295 \\ 1 & 8.326971 & 1.399829 & 2.561482 \\ 1 & 7.328329 & -0.034770 & 2.255844 \\ 1 & 8.158410 & 0.776455 & 0.916046 \\ 1 & 5.336784 & -6.071785 & -3.708634 \\ 1 & 4.260895 & -6.186732 & -5.110485 \\ 1 & 5.193398 & -4.717192 & -4.839137\end{array}$

$\left(\right.$ para- $\left.\mathrm{CH}_{3}\right) \mathrm{TS1}_{\mathrm{IL}-\mathrm{re}-} \psi_{-1 \mathrm{a} 2 \mathrm{2} 33 \mathrm{~s} 4 \mathrm{~s}}$

Number of imaginary frequencies : 1

The smallest frequencies are : -605.0982 $17.7327 \quad 18.1030 \mathrm{~cm}(-1)$

Electronic energy : $\quad H F=-4400.1591658$

Zero-point correction $=\quad 1.478937$ (Hartree/Particle)

Thermal correction to Energy $=\quad 1.565673$

Thermal correction to Enthalpy= $\quad 1.566617$

Thermal correction to Gibbs Free Energy $=\quad 1.357557$

Sum of electronic and zero-point Energies $=\quad-4398.680228$

Sum of electronic and thermal Energies $=\quad-4398.593493$

Sum of electronic and thermal Enthalpies $=\quad-4398.592549$

Sum of electronic and thermal Free Energies $=\quad-4398.801608$

Cartesian Coordinates

$\begin{array}{cccc}6 & 4.046023 & 1.079128 & 0.988697 \\ 6 & 3.159018 & 0.507542 & 0.075538 \\ 6 & 3.629718 & 0.098562 & -1.165270 \\ 6 & 4.975886 & 0.236809 & -1.514622 \\ 6 & 5.838246 & 0.813606 & -0.579574 \\ 6 & 5.395257 & 1.245475 & 0.677727 \\ 15 & 1.381380 & 0.254175 & 0.500758 \\ 6 & 1.025235 & 1.879929 & 1.331615 \\ 6 & 0.674647 & 2.988633 & 0.574952 \\ 6 & 0.462860 & 4.256968 & 1.211753 \\ 6 & 0.630313 & 4.374969 & 2.621991 \\ 6 & 1.008056 & 3.230677 & 3.360198 \\ 6 & 1.194279 & 2.026743 & 2.736180 \\ 6 & 0.409405 & 5.622640 & 3.252712 \\ 6 & 0.030918 & 6.720768 & 2.523976 \\ 6 & -0.141094 & 6.611373 & 1.128116 \\ 6 & 0.071506 & 5.415081 & 0.488670 \\ 6 & 0.534827 & 2.958166 & -0.907633 \\ 6 & -0.655883 & 2.582729 & -1.502267 \\ 6 & -0.840385 & 2.731247 & -2.896526 \\ 6 & 0.163136 & 3.214122 & -3.691855 \\ 6 & 1.416581 & 3.555887 & -3.133416 \\ 6 & 1.606927 & 3.433242 & -1.724801 \\ 6 & 2.870573 & 3.783156 & -1.182663\end{array}$




\begin{tabular}{|c|c|c|c|}
\hline 6 & 3.885144 & 4.230803 & -1.991475 \\
\hline 6 & 3.689049 & 4.362829 & -3.383591 \\
\hline & 2.480763 & 4.029077 & -3.939705 \\
\hline & -1.714129 & 2.008621 & -0.718886 \\
\hline & -2.811360 & 2.931183 & -0.345859 \\
\hline & -3.427449 & 2.670356 & 1.002863 \\
\hline & -4.803994 & 2.498311 & 1.129252 \\
\hline$b$ & -5.374203 & 2.232678 & 2.371794 \\
\hline 6 & -4.570869 & 2.148052 & 3.504016 \\
\hline 6 & -3.194919 & 2.339964 & 3.386842 \\
\hline 6 & -2.629548 & 2.596789 & 2.145208 \\
\hline 6 & 5.416550 & -0.187597 & -2.913063 \\
\hline 6 & 4.921226 & -1.607068 & -3.217283 \\
\hline & 6.317214 & 1.890763 & 1.712228 \\
\hline 5 & 7.748169 & 2.046317 & 1.204043 \\
\hline & 0.114918 & -0.820575 & -1.195300 \\
\hline 0 & 0.602206 & -0.398907 & -3.079512 \\
\hline 8 & 0.510474 & -0.209624 & -4.219661 \\
\hline 15 & -1.817591 & 0.323928 & -0.914967 \\
\hline 0 & -2.851898 & 0.058667 & 0.361344 \\
\hline 6 & -3.537682 & -1.127613 & 0.397178 \\
\hline 5 & -4.665163 & -1.292877 & -0.385347 \\
\hline 6 & -5.313311 & -2.571168 & -0.376403 \\
\hline 6 & -4.837290 & -3.592311 & 0.501786 \\
\hline 6 & -3.715948 & -3.335698 & 1.326844 \\
\hline 6 & -3.066660 & -2.132630 & 1.265045 \\
\hline 6 & -5.479275 & -4.854515 & 0.518169 \\
\hline 6 & -6.536268 & -5.119910 & -0.313633 \\
\hline 6 & -6.987056 & -4.126713 & -1.208057 \\
\hline 6 & -6.393766 & -2.889071 & -1.238917 \\
\hline 6 & -5.115898 & -0.174430 & -1.243710 \\
\hline 6 & -4.222149 & 0.414309 & -2.121894 \\
\hline 6 & -4.630894 & 1.399402 & -3.048586 \\
\hline 6 & -5.917984 & 1.861805 & -3.037277 \\
\hline 6 & -6.843941 & 1.386329 & -2.076297 \\
\hline 6 & -6.440781 & 0.365983 & -1.163827 \\
\hline 6 & -7.359772 & -0.026307 & -0.157195 \\
\hline 6 & -8.609898 & 0.536999 & -0.078542 \\
\hline 6 & -9.017200 & 1.518139 & -1.006322 \\
\hline 6 & -8.146812 & 1.932796 & -1.981996 \\
\hline 8 & -2.909763 & 0.019850 & -2.167897 \\
\hline 6 & 0.276737 & -3.253096 & -1.151726 \\
\hline 6 & -0.886693 & -2.710792 & -1.749483 \\
\hline 6 & 1.495057 & -0.897716 & 1.931782 \\
\hline 6 & 2.598932 & -1.722894 & 2.105959 \\
\hline 6 & 2.649346 & -2.657510 & 3.150344 \\
\hline 6 & 1.565155 & -2.721204 & 4.021196 \\
\hline 6 & 0.439540 & -1.890676 & 3.884130 \\
\hline 6 & 0.407386 & -1.008300 & 2.809288 \\
\hline 6 & 3.890823 & -3.536235 & 3.294719 \\
\hline 6 & 5.127514 & -2.643105 & 3.449678 \\
\hline 6 & -0.701422 & -1.979449 & 4.897962 \\
\hline 6 & -1.444834 & -3.304553 & 4.701547 \\
\hline 6 & 3.814371 & -4.460399 & 4.506901 \\
\hline 6 & 4.048174 & -4.403620 & 2.040465 \\
\hline 6 & -0.143351 & -1.921708 & 6.324700 \\
\hline
\end{tabular}




\begin{tabular}{|c|c|c|c|}
\hline & -1.692972 & -0.824957 & 4.748711 \\
\hline & 0.169753 & -3.773181 & 0.262711 \\
\hline & 1.368518 & -3.844953 & -1.992301 \\
\hline & 2.422897 & -4.542333 & -1.388672 \\
\hline & 3.473306 & -5.056391 & -2.136752 \\
\hline & 3.518650 & -4.897986 & -3.523313 \\
\hline & 2.466381 & -4.209127 & -4.128353 \\
\hline & 1.412906 & -3.692843 & -3.382865 \\
\hline & 4.661778 & -5.444203 & -4.323495 \\
\hline & 4.804065 & 0.788745 & -3.924649 \\
\hline & 6.934325 & -0.166379 & -3.075793 \\
\hline & 6.350477 & 1.021770 & 2.974678 \\
\hline & 5.786545 & 3.285133 & 2.067718 \\
\hline & -7.053576 & -0.767478 & 0.576896 \\
\hline & 1.487099 & 1.166369 & 3.332262 \\
\hline & -8.436149 & 2.704025 & -2.694848 \\
\hline & 0.543305 & 5.688094 & 4.331639 \\
\hline & -5.104042 & -5.614457 & 1.202487 \\
\hline & 3.034244 & 3.676143 & -0.111115 \\
\hline & 4.851466 & 4.479818 & -1.557130 \\
\hline & -3.361708 & -4.122736 & 1.992515 \\
\hline & 1.150319 & 3.321032 & 4.436480 \\
\hline & -6.241059 & 2.619027 & -3.749790 \\
\hline & -7.809342 & -4.345198 & -1.885786 \\
\hline & -3.899325 & 1.767534 & -3.764691 \\
\hline & 2.316958 & 4.118454 & -5.012762 \\
\hline & 0.018592 & 3.318228 & -4.766069 \\
\hline & -0.443202 & 7.483542 & 0.552801 \\
\hline 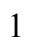 & -6.746766 & -2.140341 & -1.943198 \\
\hline & -0.062485 & 5.349843 & -0.589135 \\
\hline 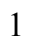 & -1.796174 & 2.433192 & -3.323645 \\
\hline ] & -7.018679 & -6.094400 & -0.296252 \\
\hline & -9.290384 & 0.226770 & 0.711399 \\
\hline ] & 4.500753 & 4.722630 & -4.012317 \\
\hline & -0.139313 & 7.674901 & 3.017327 \\
\hline 1 & -10.012156 & 1.951534 & -0.936709 \\
\hline & -2.178626 & -1.923149 & 1.859724 \\
\hline 1 & 3.677257 & 1.396314 & 1.964124 \\
\hline 1 & 2.918357 & -0.328118 & -1.869535 \\
\hline - & 6.887104 & 0.934662 & -0.836107 \\
\hline 1 & 3.438114 & -1.642293 & 1.415258 \\
\hline l & -0.456666 & -0.360752 & 2.654380 \\
\hline 1 & 1.588933 & -3.429762 & 4.846060 \\
\hline ] & -2.357272 & 3.932674 & -0.344992 \\
\hline 1 & -3.597675 & 2.944186 & -1.116887 \\
\hline 1 & -5.437005 & 2.544963 & 0.241638 \\
\hline 1 & -1.552338 & 2.712647 & 2.046850 \\
\hline 1 & -6.449571 & 2.083165 & 2.448285 \\
\hline 1 & -2.555563 & 2.288356 & 4.267273 \\
\hline 1 & -5.013337 & 1.936075 & 4.475279 \\
\hline 1 & 1.328851 & -1.892351 & -0.992774 \\
\hline 1 & -1.811374 & -2.767326 & -1.174355 \\
\hline & -1.032895 & -2.757584 & -2.827148 \\
\hline 1 & 0.078572 & -4.869600 & 0.249960 \\
\hline & 1.037212 & -3.519232 & 0.883706 \\
\hline 1 & -0.717962 & -3.361545 & 0.752088 \\
\hline
\end{tabular}




$\begin{array}{lrrr}1 & 2.434091 & -4.678033 & -0.310068 \\ 1 & 4.281492 & -5.586117 & -1.630399 \\ 1 & 2.475337 & -4.065506 & -5.208705 \\ 1 & 0.622147 & -3.155294 & -3.900908 \\ 1 & -2.451520 & -0.883766 & 5.539736 \\ 1 & -1.188934 & 0.147666 & 4.839409 \\ 1 & -2.229141 & -0.837145 & 3.791216 \\ 1 & -0.965990 & -1.956576 & 7.051062 \\ 1 & 0.525153 & -2.760192 & 6.551280 \\ 1 & 0.415310 & -0.991719 & 6.492470 \\ 1 & -2.277756 & -3.389517 & 5.412627 \\ 1 & -1.858461 & -3.376820 & 3.686306 \\ 1 & -0.782745 & -4.166272 & 4.853245 \\ 1 & 7.202434 & -0.506274 & -4.084189 \\ 1 & 7.349814 & 0.842138 & -2.953473 \\ 1 & 7.431356 & -0.832272 & -2.357850 \\ 1 & 5.085466 & 0.508717 & -4.949323 \\ 1 & 3.707333 & 0.800679 & -3.865777 \\ 1 & 5.154235 & 1.813176 & -3.739598 \\ 1 & 5.266534 & -1.921041 & -4.212436 \\ 1 & 5.303489 & -2.327905 & -2.481495 \\ 1 & 3.825519 & -1.680357 & -3.218144 \\ 1 & 4.729445 & -5.062771 & 4.569028 \\ 1 & 3.726027 & -3.899006 & 5.446041 \\ 1 & 2.968011 & -5.156142 & 4.440332 \\ 1 & 6.031986 & -3.258217 & 3.550179 \\ 1 & 5.269416 & -1.982340 & 2.584729 \\ 1 & 5.046257 & -2.011517 & 4.344312 \\ 1 & 4.937069 & -5.043251 & 2.127274 \\ 1 & 3.175031 & -5.057999 & 1.907018 \\ 1 & 4.163034 & -3.797110 & 1.132757 \\ 1 & 8.365608 & 2.518284 & 1.978871 \\ 1 & 8.206904 & 1.078607 & 0.963763 \\ 1 & 7.798534 & 2.682059 & 0.310438 \\ 1 & 6.430373 & 3.757211 & 2.822218 \\ 1 & 5.774219 & 3.936529 & 1.183557 \\ 1 & 4.767518 & 3.255110 & 2.474732 \\ 1 & 6.982707 & 1.486591 & 3.743495 \\ 1 & 5.350699 & 0.880236 & 3.404505 \\ 1 & 6.763288 & 0.027971 & 2.754848 \\ 1 & 4.622904 & -6.539645 & -4.388975 \\ 1 & 4.659096 & -5.053818 & -5.347201 \\ 1 & 5.626627 & -5.186690 & -3.868689\end{array}$

$\left(\right.$ para- $\left.\mathrm{CF}_{3}\right) \mathrm{TS1}_{\mathrm{IIL}-\mathrm{si}-}-\psi_{-1 \mathrm{~s} 2 \mathrm{a} 3 \mathrm{a} 4 \mathrm{a}}$

Number of imaginary frequencies : 1

The smallest frequencies are : $-528.3336 \quad 17.6637 \quad 18.5927 \mathrm{~cm}(-1)$

Electronic energy : $\quad H F=-4697.811878$

Zero-point correction $=\quad 1.458338$ (Hartree/Particle)

Thermal correction to Energy= $\quad 1.546219$

Thermal correction to Enthalpy $=\quad 1.547163$

Thermal correction to Gibbs Free Energy= $\quad 1.337324$

Sum of electronic and zero-point Energies $=\quad-4696.353540$

Sum of electronic and thermal Energies $=\quad-4696.265659$ 
Sum of electronic and thermal Enthalpies=

Sum of electronic and thermal Free Energies=
$-4696.264715$

$-4696.474554$

Cartesian Coordinates

\begin{tabular}{|c|c|c|c|}
\hline 6 & 7.612273 & 0.691229 & 0.137163 \\
\hline 6 & 6.812773 & -0.009211 & -0.801624 \\
\hline 6 & 7.340753 & -1.209036 & -1.366270 \\
\hline 6 & 8.644422 & -1.633414 & -1.012228 \\
\hline 6 & 9.395990 & -0.925167 & -0.109816 \\
\hline 6 & 8.864824 & 0.242069 & 0.477432 \\
\hline 6 & 5.482105 & 0.406940 & -1.139330 \\
\hline 6 & 4.706180 & -0.458257 & -1.888475 \\
\hline 6 & 5.241664 & -1.618365 & -2.489290 \\
\hline 6 & 6.539047 & -1.975372 & -2.247614 \\
\hline 6 & 4.918495 & 1.697151 & -0.680367 \\
\hline 6 & 3.725771 & 1.723782 & 0.017099 \\
\hline 6 & 3.201211 & 2.909933 & 0.568177 \\
\hline 6 & 3.839840 & 4.100467 & 0.364165 \\
\hline 6 & 5.012697 & 4.157087 & -0.427722 \\
\hline 6 & 5.553735 & 2.951616 & -0.969368 \\
\hline 6 & 5.646037 & 5.392881 & -0.702474 \\
\hline 6 & 6.757860 & 5.454480 & -1.502842 \\
\hline 6 & 7.274381 & 4.271589 & -2.070217 \\
\hline 6 & 6.690775 & 3.056022 & -1.810410 \\
\hline 8 & 3.038362 & 0.567026 & 0.277607 \\
\hline 15 & 2.231659 & -0.271096 & -0.896950 \\
\hline 8 & 3.378373 & -0.192851 & -2.122159 \\
\hline 45 & 0.176832 & 0.307860 & -1.697910 \\
\hline 6 & 0.326032 & -0.718473 & -3.292089 \\
\hline 8 & 0.441625 & -1.171388 & -4.356560 \\
\hline 6 & -0.623343 & 2.448369 & -2.248487 \\
\hline 6 & 0.384910 & 2.476501 & -1.257229 \\
\hline 6 & -2.006123 & 2.942336 & -1.906692 \\
\hline 6 & -3.152161 & 2.403446 & -2.505593 \\
\hline 6 & -4.397134 & 2.976330 & -2.306462 \\
\hline 6 & -4.517770 & 4.111283 & -1.503471 \\
\hline 6 & -3.395363 & 4.649987 & -0.886632 \\
\hline 6 & -2.149282 & 4.066555 & -1.091757 \\
\hline 6 & -5.868513 & 4.733908 & -1.358787 \\
\hline 9 & -6.367507 & 5.106391 & -2.546939 \\
\hline 6 & -0.243101 & 2.751538 & -3.685853 \\
\hline 9 & -6.761323 & 3.879409 & -0.830788 \\
\hline 9 & -5.854134 & 5.819803 & -0.576728 \\
\hline 15 & -1.231879 & -0.418055 & 0.248520 \\
\hline 6 & -2.762931 & -1.333917 & -0.223900 \\
\hline 6 & -3.663516 & -1.794178 & 0.741397 \\
\hline 6 & -4.836430 & -2.451776 & 0.375875 \\
\hline 6 & -5.064487 & -2.664776 & -0.989455 \\
\hline 6 & -4.175378 & -2.243913 & -1.979542 \\
\hline 6 & -3.021281 & -1.568627 & -1.570584 \\
\hline 6 & -5.843763 & -2.956022 & 1.408573 \\
\hline 6 & -5.486180 & -2.510894 & 2.825459 \\
\hline 6 & -4.495746 & -2.475924 & -3.455315 \\
\hline 6 & -3.304887 & -2.155368 & -4.357794 \\
\hline 6 & -0.637144 & -1.508046 & 1.646987 \\
\hline
\end{tabular}




\begin{tabular}{|c|c|c|c|}
\hline 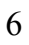 & 0.043292 & -2.688883 & 1.373356 \\
\hline & 0.487857 & -3.531821 & 2.443950 \\
\hline & 0.238156 & -3.149541 & 3.793732 \\
\hline ) & -0.469337 & -1.951957 & 4.039208 \\
\hline & -0.890534 & -1.161742 & 3.003500 \\
\hline & 0.708319 & -3.963192 & 4.852008 \\
\hline & 1.400634 & -5.118691 & 4.595748 \\
\hline & 1.644268 & -5.509214 & 3.262077 \\
\hline & 1.197488 & -4.740983 & 2.215504 \\
\hline & 0.318922 & -3.164036 & -0.012187 \\
\hline & -0.557615 & -4.117045 & -0.618839 \\
\hline & -0.248721 & -4.634706 & -1.912164 \\
\hline & 0.942407 & -4.225680 & -2.553748 \\
\hline & 1.774925 & -3.322700 & -1.951766 \\
\hline & 1.459679 & -2.771030 & -0.688785 \\
\hline & -1.127321 & -5.566117 & -2.517939 \\
\hline & -2.263526 & -5.981659 & -1.872142 \\
\hline & -2.572617 & -5.473392 & -0.591568 \\
\hline & -1.740449 & -4.570258 & 0.021068 \\
\hline & 2.343881 & -1.781245 & -0.135350 \\
\hline & 3.520233 & -2.310451 & 0.592579 \\
\hline & 3.929202 & -1.559371 & 1.833329 \\
\hline & 2.986503 & -1.099823 & 2.754339 \\
\hline & 3.389216 & -0.461797 & 3.919692 \\
\hline & 4.744616 & -0.266558 & 4.181664 \\
\hline 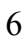 & 5.689802 & -0.714097 & 3.265985 \\
\hline & 5.282340 & -1.357378 & 2.099175 \\
\hline 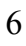 & -1.886762 & 1.007423 & 1.215235 \\
\hline & -0.945352 & 1.807751 & 1.862666 \\
\hline 5 & -1.332436 & 2.841499 & 2.721640 \\
\hline 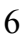 & -2.699626 & 3.070277 & 2.885932 \\
\hline & -3.672720 & 2.313273 & 2.221002 \\
\hline 5 & -3.244832 & 1.289862 & 1.375409 \\
\hline & -0.269473 & 3.687801 & 3.420681 \\
\hline 6 & 0.298356 & 4.687469 & 2.405391 \\
\hline 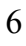 & -5.154926 & 2.551222 & 2.508197 \\
\hline 5 & -5.450554 & 2.091130 & 3.941156 \\
\hline 5 & -5.500063 & 4.037438 & 2.380583 \\
\hline & -6.055996 & 1.760461 & 1.560608 \\
\hline 6 & -0.838569 & 4.476999 & 4.599241 \\
\hline 6 & 0.854784 & 2.796163 & 3.960600 \\
\hline 6 & -7.242133 & -2.419845 & 1.084040 \\
\hline 6 & -5.867880 & -4.488252 & 1.381066 \\
\hline 6 & -5.666725 & -1.568734 & -3.850009 \\
\hline 6 & -4.882695 & -3.937729 & -3.697092 \\
\hline & 7.208276 & 1.579795 & 0.615840 \\
\hline 1 & -1.440439 & -0.251930 & 3.230787 \\
\hline & 9.029913 & -2.547079 & -1.462982 \\
\hline 1 & 0.510199 & -3.647020 & 5.875322 \\
\hline & 5.220424 & 6.297489 & -0.269957 \\
\hline 1 & -1.992239 & -4.178913 & 1.005396 \\
\hline 1 & -3.480272 & -5.800833 & -0.087882 \\
\hline 1 & 3.445344 & 5.021027 & 0.792677 \\
\hline 1 & -0.677654 & -1.664940 & 5.069435 \\
\hline 1 & 6.963758 & -2.867054 & -2.705734 \\
\hline 1 & 8.140509 & 4.321803 & -2.726285 \\
\hline
\end{tabular}




\begin{tabular}{|c|c|c|c|}
\hline 1 & 4.599428 & -2.202227 & -3.144736 \\
\hline 1 & -0.874707 & -5.949722 & -3.505627 \\
\hline 1 & 1.180254 & -4.633063 & -3.535125 \\
\hline & 2.188776 & -6.429127 & 3.061270 \\
\hline 1 & 7.095852 & 2.157178 & -2.267512 \\
\hline & 1.388068 & -5.060686 & 1.192957 \\
\hline 1 & 2.687890 & -2.991732 & -2.443128 \\
\hline 1 & 7.233304 & 6.409998 & -1.711627 \\
\hline 1 & 9.450233 & 0.789554 & 1.212858 \\
\hline 1 & -2.928860 & -6.702878 & -2.342351 \\
\hline 1 & 1.761760 & -5.735830 & 5.415185 \\
\hline 1 & 10.391893 & -1.265767 & 0.163654 \\
\hline 1 & 2.289752 & 2.838415 & 1.156302 \\
\hline 1 & -3.442595 & -1.619329 & 1.792288 \\
\hline 1 & -2.286638 & -1.224229 & -2.295520 \\
\hline 1 & -5.974591 & -3.184219 & -1.290008 \\
\hline 1 & -3.974570 & 0.680025 & 0.851935 \\
\hline 1 & 0.112633 & 1.571043 & 1.732015 \\
\hline 1 & -3.026976 & 3.854367 & 3.565448 \\
\hline 1 & 3.239810 & -3.333088 & 0.883379 \\
\hline 1 & 4.387291 & -2.410236 & -0.078342 \\
\hline 1 & 6.029251 & -1.696020 & 1.378857 \\
\hline 1 & 1.927997 & -1.214866 & 2.530304 \\
\hline 1 & 6.750914 & -0.557924 & 3.451455 \\
\hline 1 & 2.637555 & -0.112714 & 4.626492 \\
\hline 1 & 5.059836 & 0.238008 & 5.092644 \\
\hline 1 & 0.107772 & 2.720527 & -0.230949 \\
\hline 1 & 1.396713 & 2.747746 & -1.558633 \\
\hline 1 & -3.061253 & 1.522153 & -3.140975 \\
\hline 1 & -5.280482 & 2.554021 & -2.782369 \\
\hline 1 & -3.486602 & 5.532950 & -0.259192 \\
\hline 1 & -1.267361 & 4.508403 & -0.632817 \\
\hline 1 & -0.892357 & 2.232296 & -4.40 \\
\hline 1 & -0.346585 & 3.830600 & -3.870800 \\
\hline 1 & 0.790718 & 2.452613 & -3.886458 \\
\hline 1 & -1.179624 & 0.918951 & -2.370062 \\
\hline 1 & -0.031262 & 5.028623 & 5.097396 \\
\hline 1 & -1.585319 & 5.216294 & 4.284752 \\
\hline 1 & -1.301346 & 3.816901 & 5.344430 \\
\hline 1 & 1.587843 & 3.402141 & 4.508790 \\
\hline 1 & 0.455545 & 2.039494 & 4.650152 \\
\hline 1 & 1.402407 & 2.265560 & 3.172455 \\
\hline 1 & 1.121641 & 5.266347 & 2.847475 \\
\hline 1 & 0.678233 & 4.185020 & 1.505325 \\
\hline 1 & -0.478260 & 5.394410 & 2.082935 \\
\hline 1 & -7.107669 & 1.998005 & 1.765618 \\
\hline 1 & -5.859904 & 2.001793 & 0.509056 \\
\hline 1 & -5.933758 & 0.675608 & 1.690393 \\
\hline 1 & -5.234292 & 4.426283 & 1.391589 \\
\hline 1 & -6.578102 & 4.190741 & 2.522014 \\
\hline 1 & -4.983327 & 4.649241 & 3.129667 \\
\hline 1 & -6.513196 & 2.236600 & 4.179145 \\
\hline 1 & -5.217441 & 1.024985 & 4.066861 \\
\hline 1 & -4.861958 & 2.650598 & 4.679031 \\
\hline 1 & -6.246888 & -2.871516 & 3.529163 \\
\hline 1 & -4.518742 & -2.913246 & 3.154288 \\
\hline
\end{tabular}




$\begin{array}{llll}1 & -5.447129 & -1.415141 & 2.912029 \\ 1 & -6.602042 & -4.874957 & 2.101019 \\ 1 & -6.135375 & -4.875606 & 0.389746 \\ 1 & -4.884838 & -4.898160 & 1.651224 \\ 1 & -7.964607 & -2.767646 & 1.834417 \\ 1 & -7.255653 & -1.321736 & 1.086623 \\ 1 & -7.601615 & -2.755490 & 0.104173 \\ 1 & -3.561428 & -2.380151 & -5.400873 \\ 1 & -3.020245 & -1.095131 & -4.317003 \\ 1 & -2.422994 & -2.754163 & -4.092426 \\ 1 & -5.103228 & -4.098726 & -4.760787 \\ 1 & -4.060817 & -4.608351 & -3.413865 \\ 1 & -5.773338 & -4.236314 & -3.131533 \\ 1 & -5.915358 & -1.699307 & -4.912133 \\ 1 & -6.567421 & -1.790463 & -3.263713 \\ 1 & -5.416240 & -0.511610 & -3.687450\end{array}$

$\left(\right.$ para-CF $\left.{ }_{3}\right) \mathrm{TS1}_{\mathrm{IIL}-\mathrm{si}-} \psi_{-1 \mathrm{~s} 2 \mathrm{aza} 4 \mathrm{~s}}$

Number of imaginary frequencies : 1

The smallest frequencies are : $-506.6672 \quad 13.9855 \quad 18.6402 \mathrm{~cm}(-1)$

Electronic energy : $\quad \mathrm{HF}=-4697.8123816$

Zero-point correction $=\quad 1.458435($ Hartree/Particle $)$

Thermal correction to Energy $=\quad 1.546264$

Thermal correction to Enthalpy= $\quad 1.547208$

Thermal correction to Gibbs Free Energy $=\quad 1.336854$

Sum of electronic and zero-point Energies $=\quad-4696.353947$

Sum of electronic and thermal Energies $=\quad-4696.266118$

Sum of electronic and thermal Enthalpies $=\quad-4696.265173$

Sum of electronic and thermal Free Energies $=\quad-4696.475527$

Cartesian Coordinates

$\begin{array}{cccc}6 & -3.259892 & 1.229983 & 1.361435 \\ 6 & -1.898807 & 0.938594 & 1.255330 \\ 6 & -0.976145 & 1.735755 & 1.934034 \\ 6 & -1.388908 & 2.776880 & 2.770312 \\ 6 & -2.760268 & 3.021676 & 2.873601 \\ 6 & -3.712637 & 2.271023 & 2.173568 \\ 15 & -1.225706 & -0.455352 & 0.259170 \\ 6 & -0.611420 & -1.585035 & 1.615265 \\ 6 & 0.064833 & -2.757564 & 1.297433 \\ 6 & 0.506923 & -3.640212 & 2.336616 \\ 6 & 0.253036 & -3.309967 & 3.699328 \\ 6 & -0.447764 & -2.118311 & 3.989112 \\ 6 & -0.860899 & -1.285978 & 2.983291 \\ 6 & 0.713107 & -4.167768 & 4.726812 \\ 6 & 1.400855 & -5.315826 & 4.428365 \\ 6 & 1.651017 & -5.653416 & 3.081480 \\ 6 & 1.213650 & -4.841869 & 2.064040 \\ 6 & 0.334232 & -3.184230 & -0.105440 \\ 6 & -0.542808 & -4.117231 & -0.741971 \\ 6 & -0.247177 & -4.576746 & -2.060033 \\ 6 & 0.937845 & -4.139724 & -2.694110 \\ 6 & 1.773972 & -3.260680 & -2.062352 \\ 6 & 1.467031 & -2.759499 & -0.776506\end{array}$




$\begin{array}{cccc}6 & -1.134025 & -5.477570 & -2.699346 \\ 6 & -2.267127 & -5.917105 & -2.063939 \\ 6 & -2.560957 & -5.468391 & -0.757770 \\ 6 & -1.718430 & -4.599834 & -0.110826 \\ 7 & 2.346187 & -1.780954 & -0.196819 \\ 6 & 3.518510 & -2.319657 & 0.530763 \\ 6 & 3.916121 & -1.585020 & 1.785348 \\ 6 & 2.965827 & -1.155729 & 2.713614 \\ 6 & 3.356960 & -0.515347 & 3.881479 \\ 6 & 4.708419 & -0.291519 & 4.141479 \\ 6 & 5.661101 & -0.714641 & 3.222094 \\ 6 & 5.265114 & -1.358109 & 2.051300 \\ 6 & -0.350469 & 3.631354 & 3.496036 \\ 6 & 0.776051 & 2.754576 & 4.054272 \\ 6 & -5.204932 & 2.531097 & 2.382574 \\ 6 & -6.059356 & 1.837818 & 1.321286 \\ 45 & 0.150359 & 0.342363 & -1.676001 \\ 6 & 0.380607 & 2.488259 & -1.162389 \\ 6 & -0.625496 & 2.501707 & -2.156795 \\ 6 & -0.239558 & 2.841734 & -3.584594 \\ 15 & 2.216880 & -0.249331 & -0.910177 \\ 8 & 3.358064 & -0.118135 & -2.134868 \\ 6 & 4.687475 & -0.386657 & -1.912775 \\ 6 & 5.461214 & 0.457738 & -1.138484 \\ 6 & 6.795192 & 0.037839 & -0.818349 \\ 6 & -4.472528 & 4.265760 & -1.411741 \\ 6 & -3.333554 & 4.777456 & -0.801980 \\ 6 & -2.106319 & 4.153673 & -1.000166 \\ 6 & -5.797411 & 4.947614 & -1.293176 \\ 6 & -5.326449 & -1.141792 & -1.421101 \\ 6 & 6.525111 & -1.884710 & -2.322547 \\ 6 & 5.225583 & -1.525867 & -2.549828 \\ 6 & 8.633226 & -1.570936 & -1.084604 \\ 6 & -3.26345 & 6.000535 & -0.467570 \\ 6 & 9.384957 & -0.887215 & -0.163646 \\ 6 & -6.226347 & 5.392388 & -2.484168 \\ 6 & 8.850773 & 0.259160 & 0.460786 \\ 6 & -6.752011 & 4.119654 & -0.837845 \\ 6 & 7.595082 & 0.712899 & 0.138644 \\ 6 & 4.892483 & 1.727677 & -0.631702 \\ 6 & 3.704438 & 1.723818 & 0.074580 \\ 6 & 3.187239 & 2.884761 & 0.683839 \\ 6 & 3.822798 & 4.083639 & 0.523330 \\ 6 & 4.985484 & 4.175525 & -0.280315 \\ 6 & 5.522491 & 2.994073 & -0.875813 \\ 6 & 6.650748 & 3.133943 & -1.723430 \\ 6 & 7.229496 & 4.360113 & -1.940874 \\ 6 & 5.716818 & 5.518361 & -1.321510 \\ 6 & 5.4363 & 5.422584 & -0.512320 \\ 6 & 0.559190 & 0.289319 \\ 6 & -0.654576 & -3.292559 \\ 6 & -1.379085 & -0.214427\end{array}$




\begin{tabular}{|c|c|c|c|}
\hline 6 & -3.586835 & -1.931248 & 0.752013 \\
\hline 6 & -4.733771 & -2.645314 & 0.398202 \\
\hline 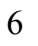 & -5.019844 & -2.781112 & -0.961790 \\
\hline & -4.198299 & -2.244520 & -1.962054 \\
\hline 0 & -3.058638 & -1.545866 & -1.563724 \\
\hline & -5.598949 & -3.247745 & 1.504716 \\
\hline 0 & -4.746881 & -4.180108 & 2.375395 \\
\hline & -4.574478 & -2.392665 & -3.435385 \\
\hline 0 & -4.985020 & -3.834108 & -3.750028 \\
\hline 6 & -6.771148 & -4.054944 & 0.953713 \\
\hline b & -6.159568 & -2.119367 & 2.377880 \\
\hline 6 & -3.412525 & -2.031174 & -4.360790 \\
\hline 6 & -5.749612 & -1.453800 & -3.731994 \\
\hline 6 & 0.224733 & 4.642198 & 2.495740 \\
\hline 6 & -0.954852 & 4.407685 & 4.665903 \\
\hline 6 & -5.603070 & 1.979887 & 3.757242 \\
\hline 6 & -5.510737 & 4.031060 & 2.344531 \\
\hline 1 & 7.188771 & 1.584360 & 0.646018 \\
\hline 1 & -1.403164 & -0.380011 & 3.242323 \\
\hline 1 & 9.020969 & -2.468767 & -1.564280 \\
\hline 1 & 0.511204 & -3.891188 & 5.760798 \\
\hline 1 & 5.191536 & 6.308374 & -0.039380 \\
\hline 1 & -1.957782 & -4.257439 & 0.894460 \\
\hline 1 & -3.466869 & -5.814381 & -0.262219 \\
\hline 1 & 3.433657 & 4.984431 & 0.996470 \\
\hline 1 & -0.655879 & -1.869388 & 5.029227 \\
\hline 1 & 6.952122 & -2.760301 & -2.808679 \\
\hline 1 & 8.088518 & 4.438274 & -2.603494 \\
\hline 1 & 4.584510 & -2.091969 & -3.221710 \\
\hline 1 & -0.891239 & -5.816643 & -3.705591 \\
\hline 1 & 1.166964 & -4.504260 & -3.694225 \\
\hline 1 & 2.193194 & -6.566746 & 2.847111 \\
\hline 1 & 7.052208 & 2.254560 & -2.220014 \\
\hline 1 & 1.408673 & -5.121093 & 1.030593 \\
\hline 1 & 2.681241 & -2.906536 & -2.548012 \\
\hline 1 & 7.188291 & 6.482498 & -1.497041 \\
\hline 1 & 9.436329 & 0.786504 & 1.210665 \\
\hline 1 & -2.939903 & -6.612979 & -2.560985 \\
\hline 1 & 1.753996 & -5.967252 & 5.224397 \\
\hline 1 & 10.383269 & -1.231585 & 0.095903 \\
\hline 1 & 2.284267 & 2.786136 & 1.281323 \\
\hline 1 & -3.335877 & -1.805143 & 1.805407 \\
\hline 1 & -2.368934 & -1.130961 & -2.295690 \\
\hline 1 & -5.910787 & -3.330006 & -1.259718 \\
\hline 1 & -3.972788 & 0.619309 & 0.814660 \\
\hline 1 & 0.084843 & 1.496632 & 1.836323 \\
\hline 1 & -3.105630 & 3.819175 & 3.527699 \\
\hline 1 & 3.237956 & -3.347764 & 0.801814 \\
\hline 1 & 4.390314 & -2.404713 & -0.136317 \\
\hline 1 & 6.017762 & -1.674966 & 1.327126 \\
\hline 1 & 1.909225 & -1.292947 & 2.493045 \\
\hline 1 & 6.719097 & -0.538405 & 3.407429 \\
\hline 1 & 2.599109 & -0.185973 & 4.591283 \\
\hline 1 & 5.014607 & 0.215231 & 5.054328 \\
\hline 1 & 0.101689 & 2.708859 & -0.131093 \\
\hline 1 & 1.395264 & 2.760962 & -1.453245 \\
\hline
\end{tabular}




$\begin{array}{lrrr}1 & -3.096347 & 1.610890 & -3.014758 \\ 1 & -5.283337 & 2.712000 & -2.666905 \\ 1 & -3.397527 & 5.671739 & -0.187169 \\ 1 & -1.209598 & 4.575839 & -0.550831 \\ 1 & -0.896009 & 2.351950 & -4.313989 \\ 1 & -0.328838 & 3.927129 & -3.738300 \\ 1 & 0.790224 & 2.534936 & -3.793953 \\ 1 & -1.209962 & 0.981814 & -2.312809 \\ 1 & -0.164191 & 4.959421 & 5.189989 \\ 1 & -1.696907 & 5.145909 & 4.338370 \\ 1 & -1.433914 & 3.738579 & 5.392502 \\ 1 & 1.489104 & 3.369798 & 4.618221 \\ 1 & 0.376818 & 1.988683 & 4.733615 \\ 1 & 1.346567 & 2.236534 & 3.274101 \\ 1 & 1.015595 & 5.245878 & 2.963203 \\ 1 & 0.650740 & 4.145639 & 1.612943 \\ 1 & -0.559625 & 5.325345 & 2.141414 \\ 1 & -7.115088 & 2.099155 & 1.468754 \\ 1 & -5.780125 & 2.146112 & 0.306093 \\ 1 & -5.983396 & 0.743833 & 1.380610 \\ 1 & -5.152381 & 4.488658 & 1.416015 \\ 1 & -6.594281 & 4.197891 & 2.403890 \\ 1 & -5.054645 & 4.571337 & 3.182673 \\ 1 & -6.672299 & 2.150045 & 3.944351 \\ 1 & -5.416771 & 0.899186 & 3.817362 \\ 1 & -5.038569 & 2.462437 & 4.565304 \\ 1 & -7.354088 & -4.474286 & 1.783503 \\ 1 & -7.451879 & -3.435757 & 0.355611 \\ 1 & -6.433244 & -4.894118 & 0.331011 \\ 1 & -5.357605 & -4.609991 & 3.180780 \\ 1 & -4.340575 & -5.013251 & 1.785479 \\ 1 & -3.901842 & -3.660566 & 2.845696 \\ 1 & -6.764793 & -2.530816 & 3.197114 \\ 1 & -5.359067 & -1.516073 & 2.825923 \\ 1 & -6.800192 & -1.448480 & 1.789793 \\ 1 & -3.704861 & -2.202607 & -5.404559 \\ 1 & -3.120030 & -0.975690 & -4.278407 \\ 1 & -2.525848 & -2.645962 & -4.153785 \\ 1 & -5.221222 & -3.932895 & -4.817809 \\ 1 & -4.168881 & -4.530630 & -3.515742 \\ 1 & -5.873803 & -4.151984 & -3.192409 \\ 1 & -6.042602 & -1.521892 & -4.788730 \\ 1 & -6.627629 & -1.701055 & -3.121746 \\ 1 & -5.480553 & -0.409856 & -3.520056\end{array}$

$\left.(\text { para-CF})_{3}\right) \mathrm{TS1}_{\mathrm{IIL}-\mathrm{si}-} \psi_{\text {-1s2a3s4a }}$

Number of imaginary frequencies : 1

The smallest frequencies are : $-536.7127 \quad 15.8730 \quad 18.3290 \mathrm{~cm}(-1)$

Electronic energy : $\quad \mathrm{HF}=-4697.8116823$

Zero-point correction $=\quad 1.458073$ (Hartree/Particle)

Thermal correction to Energy= $\quad 1.546060$

Thermal correction to Enthalpy= $\quad 1.547004$

Thermal correction to Gibbs Free Energy= $\quad 1.336379$

Sum of electronic and zero-point Energies $=\quad-4696.353609$

Sum of electronic and thermal Energies $=\quad-4696.265623$ 
Sum of electronic and thermal Enthalpies=

Sum of electronic and thermal Free Energies=
$-4696.264678$

$-4696.475303$

Cartesian Coordinates

$\begin{array}{ccccc}6 & -3.168266 & 2.421348 & -2.471842 \\ 6 & -2.013031 & 2.957794 & -1.888668 \\ 6 & -2.140517 & 4.089764 & -1.081755 \\ 6 & -3.379739 & 4.686400 & -0.873364 \\ 6 & -4.510853 & 4.153141 & -1.478963 \\ 6 & -4.406319 & 3.008052 & -2.269691 \\ 6 & -0.636599 & 2.454965 & -2.241474 \\ 6 & -0.271682 & 2.741786 & -3.685926 \\ 6 & -5.854576 & 4.790113 & -1.332075 \\ 45 & 0.155526 & 0.309338 & -1.687005 \\ 6 & 0.381026 & 2.481694 & -1.261447 \\ 15 & -1.244851 & -0.413416 & 0.254476 \\ 6 & -0.657325 & -1.516411 & 1.643076 \\ 6 & 0.021064 & -2.697176 & 1.364191 \\ 6 & 0.463232 & -3.544476 & 2.433979 \\ 6 & 0.206453 & -3.169413 & 3.784619 \\ 6 & -0.501539 & -1.972913 & 4.033658 \\ 6 & -0.915328 & -1.176235 & 3.000310 \\ 6 & 0.672188 & -3.986931 & 4.841875 \\ 6 & 1.369717 & -5.138978 & 4.584726 \\ 6 & 1.623899 & -5.520880 & 3.250699 \\ 6 & 1.180588 & -4.749338 & 2.205042 \\ 6 & 0.314967 & -3.172860 & -0.018576 \\ 6 & -0.524010 & -4.163176 & -0.621181 \\ 6 & -0.149053 & -4.738375 & -1.872416 \\ 6 & 1.045362 & -4.314800 & -2.498316 \\ 6 & 1.826708 & -3.356152 & -1.914560 \\ 6 & 1.464790 & -2.775431 & -0.677116 \\ 6 & -0.963313 & -5.741149 & -2.452535 \\ 6 & -2.111077 & -6.160297 & -1.830736 \\ 6 & -2.496444 & -5.581437 & -0.602269 \\ 6 & -1.721886 & -4.615291 & -0.009346 \\ 7 & 2.329051 & -1.770713 & -0.118635 \\ 6 & 3.509917 & -2.288278 & 0.611392 \\ 6 & 3.906591 & -1.531960 & 1.852687 \\ 6 & 2.955508 & -1.088164 & 2.772716 \\ 6 & 3.346826 & -0.443587 & 3.938394 \\ 6 & 4.698622 & -0.225795 & 4.201298 \\ 6 & 5.651892 & -0.658026 & 3.286592 \\ 6 & 5.256051 & -1.308308 & 2.119718 \\ 6 & -1.901760 & 1.007716 & 1.224395 \\ 6 & -0.961130 & 1.811948 & 1.868285 \\ 6 & -1.349919 & 2.850859 & 2.719869 \\ 6 & -2.717391 & 3.081643 & 2.878162 \\ 6 & -3.689659 & 2.320129 & 2.217511 \\ 6 & -3.260559 & 1.288066 & 1.382377 \\ 6 & -5.171717 & 2.563872 & 2.501036 \\ 6 & -5.514155 & 4.049824 & 2.364836 \\ 6 & -0.290195 & 3.705185 & 3.413726 \\ 6 & -0.860287 & 4.491520 & 4.593897 \\ 6 & -2.770616 & -1.327420 & -0.233226\end{array}$




\begin{tabular}{|c|c|c|c|}
\hline & -3.661344 & -1.837475 & 0.721668 \\
\hline & -4.809108 & -2.522467 & 0.338357 \\
\hline & -5.039856 & -2.702598 & -1.036481 \\
\hline & -4.175044 & -2.213004 & -2.010174 \\
\hline & -3.033070 & -1.524305 & -1.579892 \\
\hline & -5.792555 & -3.098927 & 1.356487 \\
\hline & -7.212980 & -2.611704 & 1.051243 \\
\hline & -4.389176 & -2.419080 & -3.507526 \\
\hline & -4.290369 & -1.076583 & -4.242252 \\
\hline & 2.214492 & -0.266446 & -0.890760 \\
\hline & 3.015243 & 0.580910 & 0.281744 \\
\hline & 3.694219 & 1.741786 & 0.017301 \\
\hline & 4.887360 & 1.720648 & -0.679369 \\
\hline & 5.513371 & 2.978185 & -0.975183 \\
\hline & 4.963448 & 4.182344 & -0.439818 \\
\hline & 3.791172 & 4.120961 & 0.352598 \\
\hline & 3.161061 & 2.926915 & 0.562466 \\
\hline & 6.649309 & 3.086565 & -1.817193 \\
\hline & 7.223392 & 4.305138 & -2.084072 \\
\hline & 6.698033 & 5.487141 & -1.523010 \\
\hline & 5.587158 & 5.421431 & -0.721633 \\
\hline & 5.461894 & 0.432616 & -1.130324 \\
\hline & 4.695293 & -0.443425 & -1.876550 \\
\hline & 5.244905 & -1.599181 & -2.473404 \\
\hline & 6.545127 & -1.942613 & -2.227311 \\
\hline & 7.336985 & -1.166031 & -1.346137 \\
\hline & 6.795713 & 0.030795 & -0.787778 \\
\hline & 8.643963 & -1.575945 & -0.987421 \\
\hline & 9.386349 & -0.856436 & -0.086337 \\
\hline & 8.842131 & 0.307816 & 0.494914 \\
\hline & 7.586017 & 0.7430 & 0.149905 \\
\hline & 3.365101 & -0.192985 & -2.113248 \\
\hline 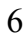 & 0.242582 & -0.744567 & -3.267406 \\
\hline & 0.269210 & -1.222549 & -4.327175 \\
\hline 6 & -6.072883 & 1.772077 & 1.554937 \\
\hline & -5.470044 & 2.113139 & 3.936400 \\
\hline 6 & 0.842490 & 2.822609 & 3.950495 \\
\hline & 0.264877 & 4.708051 & 2.394468 \\
\hline 6 & -3.299724 & -3.359777 & -4.033120 \\
\hline 6 & -5.751506 & -3.033009 & -3.819424 \\
\hline 6 & -5.755482 & -4.629484 & 1.282284 \\
\hline 6 & -5.448344 & -2.686786 & 2.786364 \\
\hline & 7.172196 & 1.629533 & 0.624010 \\
\hline 1 & -1.463621 & -0.265843 & 3.229162 \\
\hline & 9.039634 & -2.487515 & -1.433589 \\
\hline 1 & 0.467052 & -3.675682 & 5.865315 \\
\hline 1 & 5.154747 & 6.325134 & -0.293989 \\
\hline 1 & -2.029424 & -4.178752 & 0.939796 \\
\hline 1 & -3.415212 & -5.907798 & -0.118781 \\
\hline & 3.390097 & 5.041041 & 0.776028 \\
\hline 1 & -0.714388 & -1.691489 & 5.064472 \\
\hline 1 & 6.979922 & -2.830932 & -2.682523 \\
\hline 1 & 8.088735 & 4.358386 & -2.740933 \\
\hline 1 & 4.611760 & -2.190626 & -3.130743 \\
\hline 1 & -0.656031 & -6.169888 & -3.405589 \\
\hline 1 & 1.328605 & -4.760224 & -3.450655 \\
\hline
\end{tabular}




\begin{tabular}{|c|c|c|c|}
\hline . & 2.174389 & -6.436881 & 3.048317 \\
\hline & 7.061064 & 2.188349 & -2.269539 \\
\hline 1 & 1.381744 & -5.063743 & 1.182931 \\
\hline & 2.743289 & -3.016184 & -2.392341 \\
\hline & 7.165912 & 6.445130 & -1.737477 \\
\hline & 9.420387 & 0.864200 & 1.229315 \\
\hline & -2.730026 & -6.932128 & -2.283170 \\
\hline & 1.727697 & -5.759016 & 5.403346 \\
\hline & 10.385037 & -1.185823 & 0.190702 \\
\hline 1 & 2.248454 & 2.852495 & 1.148566 \\
\hline 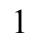 & -3.443139 & -1.684586 & 1.776621 \\
\hline 1 & -2.315390 & -1.141979 & -2.304460 \\
\hline 1 & -5.930211 & -3.247873 & -1.343880 \\
\hline 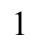 & -3.988772 & 0.671432 & 0.863971 \\
\hline 1 & 0.097094 & 1.575856 & 1.738477 \\
\hline 1 & -3.045780 & 3.871958 & 3.549830 \\
\hline 1 & 3.238953 & -3.313805 & 0.901413 \\
\hline 1 & 4.379853 & -2.378842 & -0.057160 \\
\hline 1 & 6.008960 & -1.635446 & 1.400337 \\
\hline 1 & 1.899199 & -1.221697 & 2.548162 \\
\hline 1 & 6.710175 & -0.484461 & 3.472857 \\
\hline 1 & 2.589111 & -0.107283 & 4.644834 \\
\hline 1 & 5.004685 & 0.284129 & 5.112421 \\
\hline 1 & 0.117135 & 2.730912 & -0.232735 \\
\hline 1 & 1.391872 & 2.742321 & -1.574901 \\
\hline 1 & -3.091971 & 1.531371 & -3.097252 \\
\hline 1 & -5.296929 & 2.588831 & -2.734904 \\
\hline 1 & -3.458969 & 5.575622 & -0.253086 \\
\hline 1 & -1.251359 & 4.527879 & -0.633104 \\
\hline 1 & -0.931797 & 2.217655 & -4.387725 \\
\hline 1 & -0.373417 & 3.819254 & -3.881158 \\
\hline 1 & 0.758331 & 2.436279 & -3.895686 \\
\hline 1 & -1.205723 & 0.920257 & -2.344809 \\
\hline 1 & -0.054490 & 5.047284 & 5.089896 \\
\hline 1 & -1.611608 & 5.227115 & 4.281751 \\
\hline 1 & -1.317690 & 3.828905 & 5.340158 \\
\hline 1 & 1.572660 & 3.434367 & 4.496068 \\
\hline 1 & 0.450378 & 2.063683 & 4.641693 \\
\hline 1 & 1.391900 & 2.295136 & 3.161560 \\
\hline 1 & 1.084539 & 5.295316 & 2.832186 \\
\hline 1 & 0.645521 & 4.207314 & 1.493709 \\
\hline 1 & -0.519730 & 5.407068 & 2.073811 \\
\hline 1 & -7.124277 & 2.014537 & 1.755333 \\
\hline 1 & -5.871851 & 2.008988 & 0.503225 \\
\hline 1 & -5.955064 & 0.687883 & 1.689439 \\
\hline 1 & -5.261892 & 4.427411 & 1.368339 \\
\hline 1 & -6.590123 & 4.206390 & 2.518306 \\
\hline 1 & -4.987020 & 4.667599 & 3.101523 \\
\hline 1 & -6.533724 & 2.257295 & 4.170488 \\
\hline 1 & -5.233696 & 1.049116 & 4.071291 \\
\hline 1 & -4.885140 & 2.680268 & 4.671388 \\
\hline 1 & -6.190457 & -3.101982 & 3.479784 \\
\hline 1 & -4.463683 & -3.059726 & 3.098814 \\
\hline 1 & -5.453963 & -1.594546 & 2.909598 \\
\hline 1 & -6.479172 & -5.065880 & 1.984245 \\
\hline 1 & -5.999815 & -4.997733 & 0.277840 \\
\hline
\end{tabular}




$\begin{array}{llll}1 & -4.759158 & -5.007832 & 1.550425 \\ 1 & -7.917230 & -3.008206 & 1.794733 \\ 1 & -7.271889 & -1.515861 & 1.082022 \\ 1 & -7.562671 & -2.936497 & 0.064284 \\ 1 & -5.868903 & -3.140544 & -4.905111 \\ 1 & -5.861134 & -4.033058 & -3.380666 \\ 1 & -6.576393 & -2.405575 & -3.456618 \\ 1 & -3.421642 & -3.523198 & -5.112873 \\ 1 & -2.293621 & -2.952511 & -3.866041 \\ 1 & -3.350077 & -4.333542 & -3.528670 \\ 1 & -4.485574 & -1.217062 & -5.313690 \\ 1 & -5.026164 & -0.358155 & -3.855601 \\ 1 & -3.291176 & -0.628453 & -4.152547 \\ 9 & -6.748020 & 3.954927 & -0.774435 \\ 9 & -5.821790 & 5.892350 & -0.573875 \\ 9 & -6.365068 & 5.140745 & -2.521840\end{array}$

$\left(\right.$ para $\left.-\mathrm{CF}_{3}\right) \mathrm{TS1}_{\mathrm{IIL-si}-} \psi_{-1 \mathrm{~s} 2 \mathrm{a} 3 \mathrm{~s} 4 \mathrm{~s}}$

Number of imaginary frequencies : 1

The smallest frequencies are : $-505.7268 \quad 15.3575 \quad 17.0541 \mathrm{~cm}(-1)$

Electronic energy : $\quad H F=-4697.811984$

Zero-point correction $=\quad 1.457986($ Hartree/Particle $)$

Thermal correction to Energy $=\quad 1.546036$

Thermal correction to Enthalpy= $\quad 1.546980$

Thermal correction to Gibbs Free Energy= $\quad 1.335680$

Sum of electronic and zero-point Energies $=\quad-4696.353998$

Sum of electronic and thermal Energies $=\quad-4696.265948$

Sum of electronic and thermal Enthalpies $=\quad-4696.265004$

Sum of electronic and thermal Free Energies $=\quad-4696.476304$

Cartesian Coordinates

$\begin{array}{cccc}6 & -2.115578 & 4.154189 & -1.003364 \\ 6 & -2.009360 & 3.018931 & -1.809002 \\ 6 & -3.173587 & 2.505475 & -2.394037 \\ 6 & -4.399114 & 3.121010 & -2.200004 \\ 6 & -4.481994 & 4.270229 & -1.412293 \\ 6 & -3.341760 & 4.779439 & -0.802812 \\ 6 & -0.639419 & 2.500414 & -2.164325 \\ 6 & -0.259885 & 2.828628 & -3.596388 \\ 6 & -5.805648 & 4.954299 & -1.293349 \\ 45 & 0.135457 & 0.339563 & -1.675131 \\ 6 & 0.370311 & 2.488937 & -1.174389 \\ 15 & -1.233175 & -0.455603 & 0.253364 \\ 6 & -0.620462 & -1.587792 & 1.605824 \\ 6 & 0.060375 & -2.757301 & 1.287685 \\ 6 & 0.504696 & -3.638184 & 2.329089 \\ 6 & 0.241796 & -3.311476 & 3.691037 \\ 6 & -0.466863 & -2.124207 & 3.979623 \\ 6 & -0.877346 & -1.291741 & 2.973271 \\ 6 & 0.702556 & -4.166383 & 4.720586 \\ 6 & 1.402076 & -5.308137 & 4.425703 \\ 6 & 1.664280 & -5.641022 & 3.080126 \\ 6 & 1.225518 & -4.832701 & 2.060666 \\ 6 & 0.348119 & -3.189531 & -0.110727\end{array}$




\begin{tabular}{|c|c|c|c|}
\hline 6 & -0.488772 & -4.166572 & -0.736923 \\
\hline 6 & -0.125647 & -4.695271 & -2.011661 \\
\hline f & 1.060850 & -4.244683 & -2.634063 \\
\hline & 1.842526 & -3.301200 & -2.025871 \\
\hline$b$ & 1.488454 & -2.762562 & -0.767235 \\
\hline f & -0.944551 & -5.678736 & -2.618174 \\
\hline 6 & -2.084465 & -6.123081 & -1.999175 \\
\hline 5 & -2.456187 & -5.591432 & -0.745370 \\
\hline 6 & -1.676580 & -4.645998 & -0.126996 \\
\hline 7 & 2.346014 & -1.765196 & -0.187049 \\
\hline 6 & 3.524909 & -2.286637 & 0.543360 \\
\hline 6 & 3.906456 & -1.547269 & 1.800036 \\
\hline 6 & 2.946295 & -1.132103 & 2.724537 \\
\hline 6 & 3.323994 & -0.488416 & 3.895067 \\
\hline 6 & 4.671151 & -0.246632 & 4.161211 \\
\hline 6 & 5.633452 & -0.655501 & 3.245355 \\
\hline 6 & 5.251144 & -1.302675 & 2.072135 \\
\hline 6 & -2.755296 & -1.379801 & -0.223759 \\
\hline 6 & -3.077254 & -1.525505 & -1.567752 \\
\hline 6 & -4.224937 & -2.215133 & -1.969770 \\
\hline 6 & -5.032562 & -2.775920 & -0.977118 \\
\hline 6 & -4.730635 & -2.660837 & 0.385787 \\
\hline 6 & -3.586866 & -1.948673 & 0.741740 \\
\hline 6 & -5.587210 & -3.280429 & 1.489795 \\
\hline 6 & -6.741540 & -4.110614 & 0.935121 \\
\hline 6 & -4.508143 & -2.378606 & -3.461761 \\
\hline 6 & -5.912149 & -2.915371 & -3.731563 \\
\hline 6 & -1.912084 & 0.935443 & 1.249166 \\
\hline 6 & -3.274647 & 1.220960 & 1.351976 \\
\hline 6 & -3.733475 & 2.260780 & 2.162216 \\
\hline 6 & -2.785751 & 3.015818 & 2.863707 \\
\hline 6 & -1.413119 & 2.776598 & 2.764007 \\
\hline 6 & -0.993997 & 1.736617 & 1.929482 \\
\hline 6 & -5.227256 & 2.515006 & 2.367351 \\
\hline 6 & -5.627731 & 1.957484 & 3.738792 \\
\hline 6 & -0.380937 & 3.637868 & 3.490625 \\
\hline 6 & 0.183217 & 4.656687 & 2.492096 \\
\hline 6 & 0.204383 & -0.680838 & -3.278815 \\
\hline 8 & 0.222738 & -1.136071 & -4.348371 \\
\hline 15 & 2.206638 & -0.239789 & -0.910543 \\
\hline 8 & 3.351768 & -0.106870 & -2.131871 \\
\hline 6 & 4.683338 & -0.360648 & -1.905652 \\
\hline 6 & 5.446730 & 0.491942 & -1.129887 \\
\hline 6 & 6.783138 & 0.084342 & -0.803861 \\
\hline 6 & 7.327919 & -1.090644 & -1.403656 \\
\hline 6 & 6.537675 & -1.840277 & -2.309238 \\
\hline 6 & 5.235805 & -1.493634 & -2.541813 \\
\hline 6 & 7.572762 & 0.767268 & 0.156105 \\
\hline 6 & 8.831115 & 0.325165 & 0.483934 \\
\hline 6 & 9.378563 & -0.816545 & -0.137626 \\
\hline 6 & 8.637131 & -1.507597 & -1.061415 \\
\hline 6 & 4.866745 & 1.759284 & -0.629590 \\
\hline 6 & 3.675540 & 1.749811 & 0.071134 \\
\hline 6 & 3.146621 & 2.910060 & 0.671827 \\
\hline 6 & 3.774889 & 4.112528 & 0.509633 \\
\hline 6 & 4.941411 & 4.209077 & -0.287874 \\
\hline
\end{tabular}




\begin{tabular}{|c|c|c|c|}
\hline & 5.489250 & 3.029018 & -0.876096 \\
\hline & 5.562326 & 5.459478 & -0.521591 \\
\hline & 6.668653 & 5.559682 & -1.325803 \\
\hline & 7.191957 & 4.402611 & -1.938404 \\
\hline & 6.620450 & 3.173381 & -1.719027 \\
\hline & 2.995276 & 0.580766 & 0.288057 \\
\hline & -6.170941 & -2.161374 & 2.359928 \\
\hline & -4.721693 & -4.196979 & 2.364003 \\
\hline & -4.368901 & -1.032555 & -4.182927 \\
\hline & -3.488809 & -3.365762 & -4.040985 \\
\hline & -0.990405 & 4.405515 & 4.663695 \\
\hline & 0.754134 & 2.769814 & 4.045063 \\
\hline & -5.537911 & 4.014145 & 2.334170 \\
\hline & -6.075782 & 1.822090 & 1.300987 \\
\hline & 7.156249 & 1.635127 & 0.661395 \\
\hline & -1.424139 & -0.388057 & 3.230704 \\
\hline & 9.035195 & -2.402010 & -1.539032 \\
\hline & 0.492155 & -3.891970 & 5.753455 \\
\hline & 5.131757 & 6.344165 & -0.054146 \\
\hline & -1.973420 & -4.245270 & 0.840801 \\
\hline & -3.371250 & -5.935556 & -0.265589 \\
\hline & 3.376769 & 5.012860 & 0.976143 \\
\hline & -0.681459 & -1.878290 & 5.019122 \\
\hline & 6.975268 & -2.710968 & -2.794779 \\
\hline & 8.053517 & 4.484038 & -2.597323 \\
\hline & 4.603966 & -2.064254 & -3.218502 \\
\hline & -0.647290 & -6.072225 & -3.589402 \\
\hline & 1.337084 & -4.655469 & -3.603844 \\
\hline & 2.217357 & -6.548317 & 2.847808 \\
\hline & 7.030224 & 2.294946 & -2.210428 \\
\hline & 1.432246 & -5.109788 & 1.028945 \\
\hline & 2.752584 & -2.939612 & -2.500428 \\
\hline & 7.134476 & 6.526310 & -1.502712 \\
\hline & 9.408530 & 0.858194 & 1.236113 \\
\hline ] & -2.707208 & -6.878829 & -2.473145 \\
\hline & 1.755962 & -5.957282 & 5.223260 \\
\hline 1 & 10.378901 & -1.151523 & 0.126397 \\
\hline & 2.239066 & 2.808947 & 1.262042 \\
\hline & -3.331320 & -1.833167 & 1.795122 \\
\hline ] & -2.397110 & -1.104154 & -2.307027 \\
\hline & -5.921487 & -3.326464 & -1.272244 \\
\hline 1 & -3.983792 & 0.607219 & 0.803591 \\
\hline & 0.068154 & 1.501693 & 1.834217 \\
\hline | & -3.135856 & 3.812822 & 3.515848 \\
\hline & 3.259447 & -3.319403 & 0.812228 \\
\hline 1 & 4.400300 & -2.357438 & -0.120621 \\
\hline | & 6.011042 & -1.609068 & 1.351073 \\
\hline & 1.892548 & -1.284313 & 2.499773 \\
\hline 1 & 6.688212 & -0.465366 & 3.435493 \\
\hline & 2.558941 & -0.171048 & 4.602548 \\
\hline 1 & 4.966440 & 0.262764 & 5.076180 \\
\hline & 0.096828 & 2.712984 & -0.142227 \\
\hline 1 & 1.384259 & 2.757668 & -1.471114 \\
\hline 1 & -3.113101 & 1.613131 & -3.017618 \\
\hline 1 & -5.296199 & 2.719365 & -2.668505 \\
\hline 1 & -3.403799 & 5.672909 & -0.186561 \\
\hline
\end{tabular}




$\begin{array}{lrrr}1 & -1.217689 & 4.575263 & -0.555386 \\ 1 & -0.919943 & 2.333010 & -4.318599 \\ 1 & -0.349883 & 3.912724 & -3.758798 \\ 1 & 0.768706 & 2.519572 & -3.808044 \\ 1 & -1.228758 & 0.976827 & -2.303973 \\ 1 & -0.203687 & 4.961980 & 5.188717 \\ 1 & -1.738811 & 5.138776 & 4.339560 \\ 1 & -1.463210 & 3.730065 & 5.388518 \\ 1 & 1.461119 & 3.389666 & 4.611556 \\ 1 & 0.362021 & 1.997571 & 4.721354 \\ 1 & 1.330063 & 2.260248 & 3.263283 \\ 1 & 0.970315 & 5.265499 & 2.959247 \\ 1 & 0.610731 & 4.166261 & 1.606576 \\ 1 & -0.607862 & 5.334242 & 2.141882 \\ 1 & -7.132692 & 2.080830 & 1.444409 \\ 1 & -5.792533 & 2.132619 & 0.287561 \\ 1 & -5.997842 & 0.728062 & 1.358587 \\ 1 & -5.174299 & 4.477941 & 1.410764 \\ 1 & -6.622355 & 4.177153 & 2.386874 \\ 1 & -5.089691 & 4.551560 & 3.178394 \\ 1 & -6.698113 & 2.123275 & 3.923255 \\ 1 & -5.437821 & 0.877242 & 3.795570 \\ 1 & -5.067277 & 2.439123 & 4.550211 \\ 1 & -7.314673 & -4.546990 & 1.762965 \\ 1 & -7.436531 & -3.504658 & 0.340032 \\ 1 & -6.383940 & -4.938628 & 0.308323 \\ 1 & -5.328173 & -4.639207 & 3.165875 \\ 1 & -4.296718 & -5.021605 & 1.775310 \\ 1 & -3.888677 & -3.662938 & 2.839369 \\ 1 & -6.230574 & 5.406521 & -2.483015 \\ 1 & -6.763202 & 4.125826 & -0.845084\end{array}$

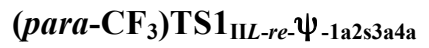

Number of imaginary frequencies : 1

The smallest frequencies are : $-598.3381 \quad 18.0809 \quad 19.4541 \mathrm{~cm}(-1)$

Electronic energy : $\quad H F=-4697.8114917$

Zero-point correction $=\quad 1.457438($ Hartree/Particle $)$

Thermal correction to Energy= $\quad 1.545618$

Thermal correction to Enthalpy $=\quad 1.546562$

Thermal correction to Gibbs Free Energy $=\quad 1.335728$ 
Sum of electronic and zero-point Energies= Sum of electronic and thermal Energies= Sum of electronic and thermal Enthalpies= Sum of electronic and thermal Free Energies=
$-4696.354054$

$-4696.265874$

$-4696.264930$

$-4696.475763$

Cartesian Coordinates

\begin{tabular}{|c|c|c|c|}
\hline 6 & 3.554625 & 2.033158 & 0.890561 \\
\hline 6 & 2.829835 & 1.068238 & 0.185724 \\
\hline 6 & 3.452020 & 0.334780 & -0.818282 \\
\hline 6 & 4.796472 & 0.543997 & -1.139229 \\
\hline 6 & 5.491048 & 1.513694 & -0.415369 \\
\hline 6 & 4.896336 & 2.273348 & 0.601008 \\
\hline 15 & 1.057324 & 0.740702 & 0.579225 \\
\hline 6 & 0.447703 & 2.482431 & 0.796483 \\
\hline 6 & 0.074777 & 3.224569 & -0.314322 \\
\hline 6 & -0.326755 & 4.592252 & -0.165386 \\
\hline 6 & -0.338027 & 5.185882 & 1.130353 \\
\hline 6 & 0.057851 & 4.405025 & 2.240021 \\
\hline 6 & 0.435635 & 3.098961 & 2.077642 \\
\hline 6 & -0.748191 & 6.532228 & 1.279760 \\
\hline 6 & -1.136355 & 7.272767 & 0.192790 \\
\hline 6 & -1.127684 & 6.691828 & -1.092929 \\
\hline 6 & -0.731752 & 5.389082 & -1.268729 \\
\hline 6 & 0.105961 & 2.676853 & -1.698332 \\
\hline 6 & -0.972774 & 1.977114 & -2.209435 \\
\hline 6 & -0.999345 & 1.597409 & -3.571287 \\
\hline 6 & 0.066456 & 1.849219 & -4.392194 \\
\hline 6 & 1.223516 & 2.486223 & -3.887598 \\
\hline 6 & 1.236439 & 2.934583 & -2.533385 \\
\hline 6 & 2.399909 & 3.589150 & -2.050890 \\
\hline 6 & 3.496628 & 3.761895 & -2.857795 \\
\hline 6 & 3.483050 & 3.308547 & -4.195299 \\
\hline 6 & 2.367396 & 2.689259 & -4.698124 \\
\hline 7 & -2.053555 & 1.571373 & -1.353320 \\
\hline 6 & -3.266024 & 2.420881 & -1.382944 \\
\hline 6 & -3.986763 & 2.542453 & -0.066549 \\
\hline 6 & -5.348268 & 2.261532 & 0.023330 \\
\hline 6 & -6.013194 & 2.359096 & 1.243262 \\
\hline 6 & -5.322172 & 2.750170 & 2.385185 \\
\hline 6 & -3.963052 & 3.048481 & 2.298070 \\
\hline 6 & -3.302383 & 2.942354 & 1.081864 \\
\hline 6 & 5.499475 & -0.279649 & -2.216579 \\
\hline 6 & 6.450731 & -1.266516 & -1.529848 \\
\hline 6 & 5.699844 & 3.360865 & 1.312935 \\
\hline 6 & 4.925026 & 3.983539 & 2.472961 \\
\hline 45 & 0.082155 & -0.991384 & -0.725903 \\
\hline 6 & 0.690104 & -1.183846 & -2.629268 \\
\hline 8 & 0.616151 & -1.483084 & -3.746463 \\
\hline 15 & -1.974310 & -0.083288 & -0.972286 \\
\hline 8 & -3.097683 & -0.050339 & 0.253109 \\
\hline 6 & -3.651917 & -1.247171 & 0.630750 \\
\hline 6 & -4.684068 & -1.786139 & -0.113848 \\
\hline 6 & -5.199154 & -3.066567 & 0.271447 \\
\hline 6 & -4.696853 & -3.698727 & 1.449604 \\
\hline 6 & -3.672928 & -3.068172 & 2.197169 \\
\hline
\end{tabular}




\begin{tabular}{|c|c|c|c|}
\hline & -3.145322 & -1.873165 & 1.787247 \\
\hline & -5.211245 & -4.959786 & 1.838104 \\
\hline & -6.165899 & -5.595108 & 1.086478 \\
\hline & -6.636662 & -4.992320 & -0.098690 \\
\hline & -6.167357 & -3.764667 & -0.494880 \\
\hline & -5.155594 & -1.065204 & -1.317972 \\
\hline & -4.238911 & -0.694113 & -2.286905 \\
\hline & -4.643269 & -0.125628 & -3.515259 \\
\hline & -5.962785 & 0.154770 & -3.740369 \\
\hline & -6.930144 & -0.085935 & -2.733804 \\
\hline & -6.526058 & -0.692290 & -1.506597 \\
\hline & -7.499031 & -0.838330 & -0.485013 \\
\hline & -8.797657 & -0.435279 & -0.679074 \\
\hline & -9.200892 & 0.128145 & -1.907714 \\
\hline & -8.281010 & 0.299610 & -2.910553 \\
\hline & -2.896831 & -0.918024 & -2.114375 \\
\hline & 0.564914 & -3.205701 & 0.089747 \\
\hline U & -0.609013 & -3.085126 & -0.696438 \\
\hline 6 & 1.146201 & 0.139889 & 2.314908 \\
\hline 6 & 2.316993 & -0.393434 & 2.838023 \\
\hline 6 & 2.358206 & -0.917805 & 4.138127 \\
\hline 6 & 1.191835 & -0.871217 & 4.896473 \\
\hline 6 & -0.005035 & -0.321062 & 4.405724 \\
\hline 6 & -0.017609 & 0.148213 & 3.096267 \\
\hline 6 & 3.681131 & -1.467056 & 4.669067 \\
\hline 6 & 4.721093 & -0.339686 & 4.673830 \\
\hline 6 & -1.238695 & -0.253795 & 5.306284 \\
\hline 6 & -1.787583 & -1.669145 & 5.512974 \\
\hline 6 & 3.558868 & -2.012001 & 6.089471 \\
\hline 6 & 4.170999 & -2.602714 & 3.763119 \\
\hline 6 & -0.864644 & 0.344117 & 6.667624 \\
\hline 6 & -2.336677 & 0.626230 & 4.708018 \\
\hline 6 & 0.411698 & -3.312366 & 1.590767 \\
\hline 6 & 1.823459 & -3.786293 & -0.489857 \\
\hline 6 & 2.983190 & -3.854359 & 0.293010 \\
\hline 6 & 4.196328 & -4.263866 & -0.242702 \\
\hline 6 & 4.270889 & -4.625275 & -1.583944 \\
\hline 6 & 3.120640 & -4.604292 & -2.369909 \\
\hline 6 & 1.912333 & -4.194006 & -1.827546 \\
\hline 6 & 5.563567 & -5.000443 & -2.227676 \\
\hline 9 & 5.845258 & -4.192349 & -3.265893 \\
\hline 9 & 6.599730 & -4.933021 & -1.384665 \\
\hline 9 & 5.534231 & -6.246856 & -2.724643 \\
\hline 6 & 6.296500 & 0.633827 & -3.152717 \\
\hline 6 & 4.506845 & -1.070550 & -3.068962 \\
\hline 6 & 7.003956 & 2.782897 & 1.870958 \\
\hline 6 & 6.025713 & 4.470329 & 0.306028 \\
\hline 1 & -7.199077 & -1.252700 & 0.474452 \\
\hline 1 & 0.740999 & 2.527020 & 2.950225 \\
\hline 1 & -8.569089 & 0.751464 & -3.858943 \\
\hline 1 & -0.751011 & 6.964896 & 2.279262 \\
\hline 1 & -4.820578 & -5.418545 & 2.745575 \\
\hline 1 & 2.425950 & 3.932059 & -1.017517 \\
\hline 1 & 4.387358 & 4.248593 & -2.463981 \\
\hline 1 & -3.292843 & -3.563962 & 3.090299 \\
\hline 1 & 0.060947 & 4.859854 & 3.229883 \\
\hline
\end{tabular}




\begin{tabular}{|c|c|c|c|}
\hline 1 & -6.283464 & 0.590310 & -4.685343 \\
\hline & -7.374878 & -5.507794 & -0.708995 \\
\hline & -3.881565 & 0.072284 & -4.266262 \\
\hline & 2.341051 & 2.338033 & -5.728727 \\
\hline 1 & 0.052635 & 1.529829 & -5.433081 \\
\hline & -1.435730 & 7.282400 & -1.952710 \\
\hline 1 & -6.532606 & -3.321098 & -1.417266 \\
\hline & -0.727033 & 4.956540 & -2.267066 \\
\hline 1 & -1.873835 & 1.064952 & -3.940871 \\
\hline 1 & -6.550348 & -6.565381 & 1.392108 \\
\hline 1 & -9.520989 & -0.546979 & 0.125708 \\
\hline 1 & 4.357880 & 3.457043 & -4.824959 \\
\hline 1 & -1.452075 & 8.305969 & 0.317424 \\
\hline 1 & -10.233772 & 0.436918 & -2.050611 \\
\hline 1 & -2.329200 & -1.386552 & 2.320257 \\
\hline 1 & 3.057521 & 2.600319 & 1.674463 \\
\hline 1 & 2.858575 & -0.396428 & -1.360933 \\
\hline 1 & 6.539359 & 1.693997 & -0.653596 \\
\hline 1 & 3.218800 & -0.398221 & 2.226418 \\
\hline 1 & -0.930339 & 0.565148 & 2.668233 \\
\hline 1 & 1.204423 & -1.260125 & 5.911977 \\
\hline 1 & -2.912908 & 3.415619 & -1.691254 \\
\hline 1 & -3.968523 & 2.082555 & -2.160763 \\
\hline 1 & -5.891948 & 1.938028 & -0.865922 \\
\hline 1 & -2.234892 & 3.141598 & 1.019775 \\
\hline 1 & -7.073601 & & 1.297384 \\
\hline 1 & -3.411946 & 3.365744 & 3.182507 \\
\hline 1 & -5.838921 & 2.823973 & 3.339927 \\
\hline 1 & 1.391840 & -1.729891 & -0.107763 \\
\hline 1 & -1.563366 & -3.120938 & -0.169774 \\
\hline 1 & -0.651533 & -3.474469 & -1.711821 \\
\hline 1 & 0.541086 & -4.358299 & 1.905688 \\
\hline 1 & 1.135345 & -2.705125 & \\
\hline 1 & -0.588254 & -2.983825 & 1.892582 \\
\hline 1 & 2.949485 & -3.545984 & 1.335373 \\
\hline 1 & 5.086660 & -4.286640 & 0.381403 \\
\hline 1 & 3.176216 & -4.895728 & -3.417244 \\
\hline 1 & 1.032875 & -4.180964 & -2.464921 \\
\hline 1 & -3.171289 & 0.710078 & 5.415671 \\
\hline 1 & -1.965884 & 1.640745 & 4.504792 \\
\hline 1 & -2.749883 & 0.226216 & 3.773324 \\
\hline 1 & -1.758459 & 0.423729 & 7.300242 \\
\hline 1 & -0.135995 & -0.267192 & 7.212313 \\
\hline 1 & -0.441374 & 1.350590 & 6.553880 \\
\hline 1 & -2.681139 & -1.647623 & 6.151437 \\
\hline 1 & -2.068571 & -2.126732 & 4.554564 \\
\hline 1 & -1.047753 & -2.323925 & 5.990474 \\
\hline 1 & 5.046947 & -1.614963 & -3.853876 \\
\hline 1 & 3.956797 & -1.821096 & -2.483818 \\
\hline 1 & 3.775939 & -0.407586 & -3.553989 \\
\hline 1 & 6.770384 & 0.039320 & -3.944900 \\
\hline 1 & 5.638296 & 1.373167 & -3.629727 \\
\hline 1 & 7.095634 & 1.176688 & -2.633718 \\
\hline 1 & 6.964955 & -1.891903 & -2.271775 \\
\hline 1 & 7.213898 & -0.746483 & -0.936474 \\
\hline 1 & 5.894650 & -1.930984 & -0.853212 \\
\hline
\end{tabular}




$\begin{array}{rrrr}1 & 4.531485 & -2.395741 & 6.422174 \\ 1 & 3.252287 & -1.235867 & 6.802371 \\ 1 & 2.840093 & -2.839476 & 6.150561 \\ 1 & 5.686713 & -0.711126 & 5.042705 \\ 1 & 4.883329 & 0.073239 & 3.669752 \\ 1 & 4.402798 & 0.483845 & 5.326572 \\ 1 & 5.113567 & -3.015855 & 4.146727 \\ 1 & 3.436813 & -3.419888 & 3.726478 \\ 1 & 4.357397 & -2.260340 & 2.736732 \\ 1 & 5.544565 & 4.744627 & 2.963820 \\ 1 & 4.003671 & 4.478907 & 2.137652 \\ 1 & 4.654926 & 3.235761 & 3.231089 \\ 1 & 6.610388 & 5.266853 & 0.786257 \\ 1 & 6.608972 & 4.093084 & -0.543497 \\ 1 & 5.104563 & 4.921082 & -0.089003 \\ 1 & 7.573651 & 3.565852 & 2.389026 \\ 1 & 6.804246 & 1.979379 & 2.591671 \\ 1 & 7.650671 & 2.374793 & 1.085484\end{array}$

$\left(\right.$ para- $\left.\mathrm{CF}_{3}\right) \mathrm{TS1}_{\mathrm{IIL-re}-} \Psi_{-1 \mathrm{a} 2 \mathrm{2s} 3 \mathrm{ats}}$

Number of imaginary frequencies : 1

The smallest frequencies are : $-585.7243 \quad 16.2800 \quad 18.7169 \mathrm{~cm}(-1)$

Electronic energy : $\quad H F=-4697.8118058$

Zero-point correction $=\quad 1.457842($ Hartree/Particle)

Thermal correction to Energy= $\quad 1.545911$

Thermal correction to Enthalpy= $\quad 1.546855$

Thermal correction to Gibbs Free Energy= $\quad 1.335694$

Sum of electronic and zero-point Energies $=\quad-4696.353964$

Sum of electronic and thermal Energies $=\quad-4696.265895$

Sum of electronic and thermal Enthalpies $=\quad-4696.264951$

Sum of electronic and thermal Free Energies $=\quad-4696.476112$

Cartesian Coordinates

$\begin{array}{cccc}6 & 1.973507 & -4.130676 & -1.878285 \\ 6 & 1.866806 & -3.760250 & -0.530670 \\ 6 & 3.014935 & -3.856170 & 0.265956 \\ 6 & 4.232626 & -4.262071 & -0.262333 \\ 6 & 4.324783 & -4.590030 & -1.610977 \\ 6 & 3.186484 & -4.537431 & -2.413182 \\ 6 & 0.602267 & -3.193468 & 0.049132 \\ 6 & 0.446346 & -3.309379 & 1.549125 \\ 6 & 5.621068 & -4.984757 & -2.236420 \\ 9 & 5.594834 & -6.248901 & -2.687731 \\ 45 & 0.094792 & -0.976638 & -0.743841 \\ 6 & -0.571552 & -3.077903 & -0.738122 \\ 15 & 1.056519 & 0.751824 & 0.575804 \\ 6 & 1.149658 & 0.144421 & 2.309210 \\ 6 & 2.320065 & -0.395860 & 2.827458 \\ 6 & 2.359617 & -0.933193 & 4.122732 \\ 6 & 1.193368 & -0.889367 & 4.881534 \\ 6 & -0.002381 & -0.332791 & 4.395816 \\ 6 & -0.014101 & 0.147766 & 3.090569 \\ 6 & 3.674801 & -1.510111 & 4.644589 \\ 6 & 4.111453 & -2.677738 & 3.752157\end{array}$




\begin{tabular}{|c|c|c|c|}
\hline 6 & -1.236141 & -0.273604 & 5.296595 \\
\hline 6 & -2.339106 & 0.601432 & 4.700374 \\
\hline f & 2.827069 & 1.093118 & 0.186393 \\
\hline & 3.549290 & 2.046450 & 0.899155 \\
\hline f & 4.894468 & 2.296760 & 0.613509 \\
\hline & 5.493221 & 1.552748 & -0.406320 \\
\hline 6 & 4.794135 & 0.590260 & -1.145518 \\
\hline 5 & 3.453392 & 0.374906 & -0.830383 \\
\hline 6 & 5.495696 & -0.219118 & -2.234568 \\
\hline 6 & 4.498173 & -0.986508 & -3.102615 \\
\hline 6 & 5.634401 & 3.365384 & 1.417720 \\
\hline 6 & 4.900195 & 4.705707 & 1.287854 \\
\hline 6 & 0.437948 & 2.489463 & 0.793958 \\
\hline 6 & 0.060914 & 3.229828 & -0.316663 \\
\hline 6 & -0.339900 & 4.597971 & -0.167364 \\
\hline 6 & -0.344787 & 5.193347 & 1.127636 \\
\hline 6 & 0.052463 & 4.412961 & 2.237120 \\
\hline 6 & 0.428184 & 3.106305 & 2.074866 \\
\hline 6 & -0.751941 & 6.540598 & 1.277268 \\
\hline 6 & -1.144012 & 7.280424 & 0.191279 \\
\hline 6 & -1.143109 & 6.697454 & -1.093516 \\
\hline 6 & -0.750085 & 5.393880 & -1.269558 \\
\hline 6 & 0.083500 & 2.683696 & -1.701849 \\
\hline 6 & -0.999058 & 1.986053 & -2.207433 \\
\hline 6 & -1.041302 & 1.620801 & -3.572870 \\
\hline 6 & 0.012948 & 1.885287 & -4.404593 \\
\hline 6 & 1.175716 & 2.516995 & -3.906480 \\
\hline 6 & 1.204675 & 2.948839 & -2.547122 \\
\hline 6 & 2.374640 & 3.594364 & -2.069130 \\
\hline 6 & 3.462793 & 3.774896 & -2.885895 \\
\hline 6 & 3.433003 & 3.339649 & -4.229096 \\
\hline 6 & 2.310210 & 2.729630 & -4.727553 \\
\hline 7 & -2.069019 & 1.569370 & -1.343128 \\
\hline 15 & -1.973750 & -0.086710 & -0.970771 \\
\hline 8 & -2.893673 & -0.923262 & -2.113784 \\
\hline 6 & -4.237712 & -0.707450 & -2.281529 \\
\hline 6 & -5.149110 & -1.087303 & -1.311034 \\
\hline 6 & -6.522130 & -0.720743 & -1.493398 \\
\hline 6 & -6.933607 & -0.111584 & -2.716784 \\
\hline 6 & -5.971262 & 0.136713 & -3.726258 \\
\hline 6 & -4.649567 & -0.137775 & -3.506855 \\
\hline 6 & -7.490551 & -0.875128 & -0.468691 \\
\hline 6 & -8.791601 & -0.476830 & -0.656203 \\
\hline 6 & -9.202000 & 0.089546 & -1.881128 \\
\hline 6 & -8.286821 & 0.268679 & -2.886897 \\
\hline 6 & -4.669692 & -1.811696 & -0.112200 \\
\hline 6 & -3.637734 & -1.271519 & 0.631733 \\
\hline 6 & -3.123524 & -1.901580 & 1.782581 \\
\hline 6 & -3.643124 & -3.101823 & 2.187382 \\
\hline 6 & -4.666654 & -3.733351 & 1.440135 \\
\hline 6 & -5.176687 & -3.097002 & 0.267593 \\
\hline 6 & -5.173326 & -4.999108 & 1.823468 \\
\hline 6 & -6.127770 & -5.634867 & 1.071946 \\
\hline 6 & -6.606017 & -5.027776 & -0.108007 \\
\hline 6 & -6.144313 & -3.795597 & -0.499017 \\
\hline 8 & -3.091909 & -0.068926 & 0.259711 \\
\hline
\end{tabular}




\begin{tabular}{|c|c|c|c|}
\hline 6 & -3.288538 & 2.409160 & -1.362171 \\
\hline & -3.999352 & 2.522491 & -0.039814 \\
\hline & -5.356714 & 2.226176 & 0.061843 \\
\hline & -6.011634 & 2.314370 & 1.287842 \\
\hline & -5.314973 & 2.712491 & 2.423933 \\
\hline & -3.960494 & 3.027573 & 2.324717 \\
\hline & -3.309478 & 2.929996 & 1.102550 \\
\hline & 6.435579 & -1.226897 & -1.562586 \\
\hline & 6.303324 & 0.702840 & -3.152984 \\
\hline & 7.069025 & 3.566243 & 0.937116 \\
\hline & 5.675171 & 2.951505 & 2.893237 \\
\hline & 0.696174 & -1.139999 & -2.651433 \\
\hline & 0.625973 & -1.432207 & -3.770693 \\
\hline & 4.753296 & -0.420775 & 4.608809 \\
\hline & 3.559334 & -2.023203 & 6.077379 \\
\hline & -1.777210 & -1.692445 & 5.500435 \\
\hline & -0.864129 & 0.323572 & 6.658781 \\
\hline & 5.909025 & -4.215604 & -3.301072 \\
\hline & 6.652604 & -4.887436 & -1.390206 \\
\hline & -7.185020 & -1.291883 & 0.487994 \\
\hline & 0.733199 & 2.533645 & 2.947181 \\
\hline & -8.580534 & 0.722823 & -3.832458 \\
\hline & -0.749353 & 6.974438 & 2.276258 \\
\hline & -4.776932 & -5.461043 & 2.726835 \\
\hline & 2.412106 & 3.921456 & -1.031087 \\
\hline & 4.360555 & 4.251114 & -2.495725 \\
\hline & -3.257255 & -3.600519 & 3.076408 \\
\hline & 0.057444 & 4.868515 & 3.226642 \\
\hline 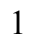 & -6.297603 & 0.573421 & -4.668749 \\
\hline I & -7.344033 & -5.543445 & -0.718388 \\
\hline & -3.891884 & 0.065571 & -4.260408 \\
\hline 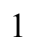 & 2.271760 & 2.391516 & -5.762151 \\
\hline & -0.013654 & 1.578677 & -5.449073 \\
\hline 1 & -1.454957 & 7.287102 & -1.952560 \\
\hline & -6.515326 & -3.348645 & -1.417454 \\
\hline 1 & -0.751949 & 4.960232 & -2.267400 \\
\hline ] & -1.919372 & 1.090747 & -3.937279 \\
\hline & -6.506309 & -6.608706 & 1.373598 \\
\hline 1 & -9.511363 & -0.594669 & 0.150898 \\
\hline & 4.301287 & 3.493910 & -4.866401 \\
\hline 1 & -1.457189 & 8.314383 & 0.316024 \\
\hline & -10.236726 & 0.394481 & -2.018800 \\
\hline 1 & -2.308827 & -1.413118 & 2.316046 \\
\hline 1 & 3.056817 & 2.606717 & 1.694125 \\
\hline I & 2.860538 & -0.348912 & -1.383029 \\
\hline 1 & 6.541209 & 1.727815 & -0.640740 \\
\hline & 3.220653 & -0.401394 & 2.213579 \\
\hline 1 & -0.926637 & 0.568024 & 2.665605 \\
\hline | & 1.204501 & -1.287352 & 5.893508 \\
\hline 1 & -2.945474 & 3.407174 & -1.671303 \\
\hline 1 & -3.994526 & 2.066681 & -2.134961 \\
\hline 1 & -5.904681 & 1.897729 & -0.822939 \\
\hline 1 & -2.245163 & 3.142892 & 1.030634 \\
\hline 1 & -7.068625 & 2.062570 & 1.351163 \\
\hline 1 & -3.405474 & 3.351555 & 3.204242 \\
\hline 1 & -5.823796 & 2.779139 & 3.383457 \\
\hline
\end{tabular}




$\begin{array}{rrrr}1 & 1.415764 & -1.707916 & -0.140945 \\ 1 & -1.526248 & -3.128112 & -0.213324 \\ 1 & -0.608364 & -3.461203 & -1.756104 \\ 1 & 0.581967 & -4.355935 & 1.859760 \\ 1 & 1.164523 & -2.699767 & 2.110021 \\ 1 & -0.556316 & -2.988565 & 1.849997 \\ 1 & 2.968050 & -3.577615 & 1.315886 \\ 1 & 5.112947 & -4.308783 & 0.374502 \\ 1 & 3.255030 & -4.801393 & -3.467028 \\ 1 & 1.104791 & -4.090697 & -2.529230 \\ 1 & -3.174252 & 0.679034 & 5.408143 \\ 1 & -1.974295 & 1.618563 & 4.499480 \\ 1 & -2.749903 & 0.201185 & 3.764683 \\ 1 & -1.756982 & 0.393816 & 7.293901 \\ 1 & -0.128602 & -0.282996 & 7.199556 \\ 1 & -0.449727 & 1.333935 & 6.546808 \\ 1 & -2.674038 & -1.676505 & 6.134432 \\ 1 & -2.050289 & -2.151666 & 4.540487 \\ 1 & -1.035973 & -2.342840 & 5.981673 \\ 1 & 5.034321 & -1.525165 & -3.894566 \\ 1 & 3.934734 & -1.735787 & -2.529130 \\ 1 & 3.776653 & -0.308319 & -3.580597 \\ 1 & 6.773041 & 0.117443 & -3.954397 \\ 1 & 5.653228 & 1.456460 & -3.618786 \\ 1 & 7.106724 & 1.228825 & -2.623408 \\ 1 & 6.944405 & -1.845460 & -2.314083 \\ 1 & 7.203288 & -0.724843 & -0.959585 \\ 1 & 5.871333 & -1.897034 & -0.898443 \\ 1 & 4.528016 & -2.420684 & 6.405349 \\ 1 & 3.277884 & -1.225937 & 6.777313 \\ 1 & 2.825123 & -2.834469 & 6.165483 \\ 1 & 5.708341 & -0.816985 & 4.979410 \\ 1 & 4.922063 & -0.044048 & 3.591800 \\ 1 & 4.472427 & 0.432514 & 5.240295 \\ 1 & 5.046912 & -3.114949 & 4.126571 \\ 1 & 3.350524 & -3.470890 & 3.741877 \\ 1 & 4.289825 & -2.356514 & 2.717542 \\ 1 & 7.552327 & 4.350362 & 1.533547 \\ 1 & 7.670993 & 2.654863 & 1.044944 \\ 1 & 7.106506 & 3.881044 & -0.114221 \\ 1 & 5.411022 & 5.477251 & 1.879716 \\ 1 & 4.881437 & 5.044620 & 0.243546 \\ 1 & 3.862187 & 4.652780 & 1.640897 \\ 1 & 6.180535 & 3.720731 & 3.492951 \\ 1 & 4.669142 & 2.811363 & 3.309100 \\ 1 & 6.225064 & 2.009425 & 3.021447\end{array}$

$\left(\right.$ para- $\left.-\mathrm{CF}_{3}\right) \mathrm{TS}_{\mathrm{IIL-re-}-} \psi_{-1 \mathrm{a} 233 s 4 a}$

Number of imaginary frequencies : 1

The smallest frequencies are : $-636.0037 \quad 14.3740 \quad 17.6318 \mathrm{~cm}(-1)$

Electronic energy : $\quad H F=-4697.8104597$

Zero-point correction $=\quad 1.457158($ Hartree/Particle $)$

Thermal correction to Energy $=\quad 1.545652$

Thermal correction to Enthalpy= $\quad 1.546596$

Thermal correction to Gibbs Free Energy= $\quad 1.333699$ 
Sum of electronic and zero-point Energies= Sum of electronic and thermal Energies= Sum of electronic and thermal Enthalpies= Sum of electronic and thermal Free Energies=
$-4696.353301$

$-4696.264808$

$-4696.263864$

$-4696.476761$

Cartesian Coordinates

\begin{tabular}{|c|c|c|c|}
\hline 6 & -6.030299 & -3.867988 & -0.595958 \\
\hline 6 & -5.080809 & -3.173103 & 0.196412 \\
\hline 6 & -4.566218 & -3.832938 & 1.353846 \\
\hline 6 & -5.050503 & -5.118571 & 1.697727 \\
\hline 6 & -5.988084 & -5.750696 & 0.922395 \\
\hline 6 & -6.470798 & -5.119324 & -0.242828 \\
\hline 6 & -4.596491 & -1.867550 & -0.143363 \\
\hline 6 & -3.576818 & -1.331765 & 0.620701 \\
\hline 6 & -3.062585 & -1.982612 & 1.759775 \\
\hline 6 & -3.561441 & -3.203052 & 2.127193 \\
\hline 6 & -5.091542 & -1.115583 & -1.318679 \\
\hline 6 & -4.191510 & -0.682350 & -2.277103 \\
\hline 6 & -4.618612 & -0.087528 & -3.485273 \\
\hline 6 & -5.946642 & 0.161904 & -3.695157 \\
\hline 6 & -6.900777 & -0.138203 & -2.692048 \\
\hline 6 & -6.473364 & -0.775775 & -1.488669 \\
\hline 6 & -8.262328 & 0.217347 & -2.848417 \\
\hline 6 & -9.171066 & -0.012247 & -1.847149 \\
\hline 6 & -8.745714 & -0.607014 & -0.640923 \\
\hline 6 & -7.436027 & -0.982953 & -0.467861 \\
\hline 8 & -2.841795 & -0.866987 & -2.117423 \\
\hline 15 & -1.939565 & -0.048137 & -0.949173 \\
\hline 8 & -3.047175 & -0.110799 & 0.289978 \\
\hline 45 & 0.154909 & -0.904822 & -0.805570 \\
\hline 15 & 1.107594 & 0.776653 & 0.577282 \\
\hline 6 & 1.210979 & 0.093154 & 2.280597 \\
\hline 6 & 2.381122 & -0.478526 & 2.762630 \\
\hline 6 & 2.431306 & -1.062713 & 4.037181 \\
\hline 6 & 1.275408 & -1.035983 & 4.812288 \\
\hline 6 & 0.078179 & -0.454243 & 4.360489 \\
\hline 6 & 0.055683 & 0.073790 & 3.074143 \\
\hline 6 & 3.747979 & -1.668922 & 4.519227 \\
\hline 6 & 3.647457 & -2.235158 & 5.933009 \\
\hline 6 & -1.143774 & -0.415061 & 5.278207 \\
\hline 6 & -0.750947 & 0.151297 & 6.647685 \\
\hline 6 & 0.633786 & -3.156296 & -0.065165 \\
\hline 6 & 0.453452 & -3.289193 & 1.428985 \\
\hline 6 & 1.874178 & -3.776715 & -0.635951 \\
\hline 6 & 2.936327 & -4.124180 & 0.207356 \\
\hline 6 & 4.112213 & -4.669018 & -0.292888 \\
\hline 6 & 4.247610 & -4.881051 & -1.659060 \\
\hline 6 & 3.203129 & -4.540080 & -2.518347 \\
\hline 6 & 2.033823 & -3.996221 & -2.013148 \\
\hline 6 & 5.497811 & -5.444715 & -2.246519 \\
\hline 6 & -0.525904 & -2.996471 & -0.866209 \\
\hline 6 & 0.746642 & -0.916961 & -2.707407 \\
\hline 8 & 0.753277 & -1.045840 & -3.859098 \\
\hline 6 & 0.460413 & 2.491472 & 0.885056 \\
\hline 6 & 0.019286 & 3.273147 & -0.172109 \\
\hline
\end{tabular}




\begin{tabular}{|c|c|c|c|}
\hline 6 & -0.434105 & 4.613852 & 0.065290 \\
\hline & -0.415890 & 5.140786 & 1.389354 \\
\hline & 0.064608 & 4.326110 & 2.439433 \\
\hline & 0.485097 & 3.047148 & 2.194394 \\
\hline & -0.884087 & 6.454818 & 1.628132 \\
\hline & -1.359373 & 7.228587 & 0.600660 \\
\hline & -1.381948 & 6.714223 & -0.712575 \\
\hline & -0.929437 & 5.444629 & -0.974628 \\
\hline & 0.014891 & 2.815403 & -1.589689 \\
\hline & -1.059208 & 2.116091 & -2.108842 \\
\hline & -1.147744 & 1.860661 & -3.496979 \\
\hline & -0.161428 & 2.271913 & -4.352010 \\
\hline & 0.982659 & 2.939205 & -3.856037 \\
\hline & 1.076044 & 3.216216 & -2.459530 \\
\hline & 2.234872 & 3.880169 & -1.979814 \\
\hline & 3.238385 & 4.254817 & -2.838232 \\
\hline ) & 3.133197 & 3.996752 & -4.222490 \\
\hline & 2.030572 & 3.348416 & -4.716847 \\
\hline 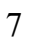 & -2.092917 & 1.616924 & -1.244932 \\
\hline 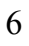 & -3.347384 & 2.405713 & -1.221522 \\
\hline & -4.041486 & 2.456618 & 0.113452 \\
\hline$b$ & -5.387427 & 2.114309 & 0.223136 \\
\hline & -6.029347 & 2.148131 & 1.458486 \\
\hline & -5.331766 & 2.539017 & 2.596610 \\
\hline & -3.989802 & 2.901750 & 2.489651 \\
\hline 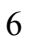 & -3.350801 & 2.856391 & 1.257938 \\
\hline & 2.868641 & 1.164069 & 0.192143 \\
\hline f & 3.471765 & 0.548970 & -0.892154 \\
\hline b & 4.805878 & 0.809124 & -1.232408 \\
\hline 6 & 5.509173 & 1.712087 & -0.442385 \\
\hline & 4.931254 & 2.353467 & 0.668291 \\
\hline & 3.604711 & 2.067151 & 0.971493 \\
\hline 6 & 5.386470 & 0.129547 & -2.468620 \\
\hline & 4.640317 & 0.650580 & -3.703031 \\
\hline 6 & 5.749592 & 3.365355 & 1.468750 \\
\hline 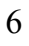 & 7.065450 & 2.734042 & 1.934627 \\
\hline 6 & 6.052121 & 4.573418 & 0.574645 \\
\hline 6 & 5.002340 & 3.863777 & 2.704208 \\
\hline & 6.874540 & 0.418296 & -2.644764 \\
\hline 6 & 5.199681 & -1.389549 & -2.369311 \\
\hline 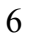 & 4.159964 & -2.806657 & 3.577527 \\
\hline 6 & 4.833887 & -0.586072 & 4.513513 \\
\hline 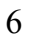 & -2.253883 & 0.474109 & 4.717066 \\
\hline 6 & -1.684408 & -1.837243 & 5.457982 \\
\hline | & -7.119341 & -1.422054 & 0.475124 \\
\hline ] & 0.849780 & 2.446637 & 3.023691 \\
\hline 1 & -8.567891 & 0.693916 & -3.779064 \\
\hline & -0.861933 & 6.834612 & 2.648681 \\
\hline 1 & -4.650694 & -5.598238 & 2.590260 \\
\hline . & 2.328549 & 4.078337 & -0.912998 \\
\hline 1 & 4.125067 & 4.751537 & -2.449016 \\
\hline 1 & -3.173216 & -3.718248 & 3.005649 \\
\hline 1 & 0.096191 & 4.732088 & 3.449812 \\
\hline 1 & -6.285004 & 0.618468 & -4.623882 \\
\hline 1 & -7.195148 & -5.631253 & -0.872416 \\
\hline . & -3.866931 & 0.155151 & -4.233432 \\
\hline
\end{tabular}




\begin{tabular}{|c|c|c|c|}
\hline ] & 1.941060 & 3.132529 & -5.780638 \\
\hline & -0.231245 & 2.069241 & -5.419585 \\
\hline & -1.760050 & 7.329770 & -1.525609 \\
\hline & -6.404358 & -3.402044 & -1.503688 \\
\hline & -0.951649 & 5.066038 & -1.994408 \\
\hline & -2.015100 & 1.314972 & -3.864405 \\
\hline & -6.349682 & -6.739744 & 1.193778 \\
\hline & -9.460923 & -0.764937 & 0.163349 \\
\hline & 3.933360 & 4.304849 & -4.892091 \\
\hline & -1.720286 & 8.236157 & 0.793624 \\
\hline & -10.212432 & 0.274347 & -1.973636 \\
\hline & -2.262394 & -1.493182 & 2.313908 \\
\hline & 3.125156 & 2.542658 & 1.824103 \\
\hline & 2.878448 & -0.138708 & -1.491023 \\
\hline & 6.544037 & 1.940360 & -0.690894 \\
\hline & 3.273496 & -0.470689 & 2.136748 \\
\hline & -0.858651 & 0.515814 & 2.676406 \\
\hline & 1.295997 & -1.468611 & 5.809945 \\
\hline & -3.051016 & 3.424651 & -1.509352 \\
\hline & -4.051063 & 2.054989 & -1.992446 \\
\hline & -5.936166 & 1.793785 & -0.663980 \\
\hline & -2.295462 & 3.107873 & 1.177672 \\
\hline & -7.076556 & 1.859221 & 1.526856 \\
\hline & -3.435199 & 3.222250 & 3.370654 \\
\hline & -5.830301 & 2.563958 & 3.563486 \\
\hline ] & 1.485897 & -1.677826 & -0.262010 \\
\hline & -1.488115 & -3.042937 & -0.355285 \\
\hline ] & -0.554454 & -3.366377 & -1.889460 \\
\hline & 0.517046 & -4.347971 & 1.721661 \\
\hline & 1.205589 & -2.737880 & 2.005858 \\
\hline & -0.529605 & -2.911820 & 1.725480 \\
\hline & 2.854269 & -3.960496 & 1.278500 \\
\hline ] & 4.922008 & -4.927554 & 0.384844 \\
\hline & 3.310114 & -4.702488 & -3.589433 \\
\hline 1 & 1.238004 & -3.737302 & -2.706153 \\
\hline & -3.080231 & 0.534866 & 5.436825 \\
\hline & -1.892174 & 1.495991 & 4.535207 \\
\hline ] & -2.676562 & 0.096223 & 3.777355 \\
\hline & -1.632821 & 0.203485 & 7.299724 \\
\hline 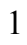 & -0.003585 & -0.465197 & 7.160328 \\
\hline & -0.341636 & 1.165438 & 6.552087 \\
\hline 1 & -2.573358 & -1.833720 & 6.103145 \\
\hline & -1.970098 & -2.275230 & 4.491806 \\
\hline 1 & -0.938798 & -2.499284 & 5.915927 \\
\hline ] & 7.250216 & -0.107046 & -3.531787 \\
\hline | & 7.071533 & 1.488246 & -2.791909 \\
\hline 1 & 7.462703 & 0.074797 & -1.783509 \\
\hline & 5.031774 & 0.177960 & -4.614377 \\
\hline 1 & 3.563958 & 0.438463 & -3.650549 \\
\hline l & 4.756205 & 1.738473 & -3.802186 \\
\hline 1 & 5.622981 & -1.880746 & -3.255105 \\
\hline 1 & 5.707461 & -1.795128 & -1.483720 \\
\hline 1 & 4.140831 & -1.678282 & -2.311878 \\
\hline 1 & 4.616974 & -2.652649 & 6.232448 \\
\hline 1 & 3.382051 & -1.462944 & 6.666556 \\
\hline 1 & 2.907506 & -3.043177 & 6.000228 \\
\hline
\end{tabular}




$\begin{array}{lrrr}1 & 5.793096 & -1.005443 & 4.845600 \\ 1 & 4.986081 & -0.157663 & 3.514611 \\ 1 & 4.570491 & 0.235633 & 5.192564 \\ 1 & 5.103402 & -3.257845 & 3.913500 \\ 1 & 3.395794 & -3.597035 & 3.564111 \\ 1 & 4.309049 & -2.456425 & 2.548030 \\ 1 & 5.633245 & 4.572121 & 3.255810 \\ 1 & 4.073265 & 4.388139 & 2.443214 \\ 1 & 4.750627 & 3.041706 & 3.387852 \\ 1 & 6.648129 & 5.317138 & 1.121008 \\ 1 & 6.614367 & 4.288217 & -0.323268 \\ 1 & 5.121980 & 5.058988 & 0.249258 \\ 1 & 7.648794 & 3.460198 & 2.516385 \\ 1 & 6.880135 & 1.861414 & 2.574512 \\ 1 & 7.692627 & 2.407098 & 1.097026 \\ 9 & 6.392793 & -5.793349 & -1.315702 \\ 9 & 5.249675 & -6.535492 & -2.988833 \\ 9 & 6.102284 & -4.567395 & -3.065870\end{array}$

$\left(\right.$ para- $\left.\mathrm{CF}_{3}\right) \mathrm{TS}_{\mathrm{ILL}-\mathrm{re}-} \Psi_{-1 \mathrm{a} 2 \mathrm{2} 33 \mathrm{~s} 4 \mathrm{~s}}$

Number of imaginary frequencies : 1

The smallest frequencies are : -629.4138 $8.9167 \quad 13.8041 \mathrm{~cm}(-1)$

Electronic energy : $\quad \mathrm{HF}=-4697.810475$

Zero-point correction $=\quad 1.456527($ Hartree/Particle $)$

Thermal correction to Energy= $\quad 1.545221$

Thermal correction to Enthalpy= $\quad 1.546165$

Thermal correction to Gibbs Free Energy= 1.330953

Sum of electronic and zero-point Energies $=\quad-4696.353948$

Sum of electronic and thermal Energies $=\quad-4696.265254$

Sum of electronic and thermal Enthalpies $=\quad-4696.264310$

Sum of electronic and thermal Free Energies $=\quad-4696.479522$

Cartesian Coordinates

$\begin{array}{cccc}6 & -3.346826 & 2.840304 & 1.226997 \\ 6 & -4.041451 & 2.442271 & 0.084145 \\ 6 & -5.389362 & 2.108855 & 0.196459 \\ 6 & -6.029904 & 2.151500 & 1.432374 \\ 6 & -5.328549 & 2.540913 & 2.568557 \\ 6 & -3.983868 & 2.892851 & 2.459343 \\ 6 & -3.349899 & 2.382803 & -1.251896 \\ 7 & -2.098501 & 1.589779 & -1.275548 \\ 15 & -1.939379 & -0.069079 & -0.952532 \\ 8 & -3.039078 & -0.116283 & 0.294200 \\ 6 & -3.566417 & -1.331940 & 0.646806 \\ 6 & -4.588460 & -1.880653 & -0.104872 \\ 6 & -5.068922 & -3.181838 & 0.255762 \\ 6 & -4.549531 & -3.823419 & 1.421340 \\ 6 & -3.543734 & -3.179776 & 2.182099 \\ 6 & -3.048001 & -1.964248 & 1.794454 \\ 6 & -6.018903 & -3.890330 & -0.523817 \\ 6 & -6.455109 & -5.137543 & -0.151607 \\ 6 & -5.967829 & -5.750676 & 1.021476 \\ 6 & -5.029942 & -5.105019 & 1.785257 \\ 6 & -5.090460 & -1.146564 & -1.288422\end{array}$




\begin{tabular}{|c|c|c|c|}
\hline 6 & -4.195798 & -0.726323 & -2.257480 \\
\hline 6 & -4.629564 & -0.147090 & -3.470707 \\
\hline 6 & -5.958915 & 0.098629 & -3.676875 \\
\hline 6 & -6.907768 & -0.189818 & -2.665418 \\
\hline 6 & -6.473575 & -0.810959 & -1.455901 \\
\hline 6 & -8.270688 & 0.161371 & -2.819919 \\
\hline 6 & -9.174364 & -0.057242 & -1.811642 \\
\hline 6 & -8.742388 & -0.636079 & -0.600019 \\
\hline 6 & -7.431185 & -1.007201 & -0.428228 \\
\hline 8 & -2.845164 & -0.909596 & -2.102069 \\
\hline 6 & -1.066497 & 2.077139 & -2.148282 \\
\hline 6 & -0.001703 & 2.799439 & -1.641746 \\
\hline 6 & 1.061371 & 3.186441 & -2.514820 \\
\hline 6 & 0.984981 & 2.862168 & -3.902035 \\
\hline 6 & -0.150625 & 2.173177 & -4.387432 \\
\hline 6 & -1.142888 & 1.782781 & -3.529334 \\
\hline 6 & 2.207725 & 3.878669 & -2.046062 \\
\hline 6 & 3.219593 & 4.228346 & -2.904836 \\
\hline 6 & 3.135290 & 3.916493 & -4.279336 \\
\hline 6 & 2.041605 & 3.245998 & -4.763963 \\
\hline 6 & -0.005753 & 3.282782 & -0.233096 \\
\hline 6 & 0.432222 & 2.517780 & 0.837789 \\
\hline 6 & 0.445156 & 3.092153 & 2.138999 \\
\hline 6 & 0.023397 & 4.375123 & 2.361645 \\
\hline 6 & -0.449405 & 5.174308 & 1.296328 \\
\hline 6 & -0.462106 & 4.625975 & -0.019077 \\
\hline 6 & -0.916146 & 6.492995 & 1.511348 \\
\hline 6 & -1.385270 & 7.250396 & 0.468992 \\
\hline 6 & -1.403377 & 6.714257 & -0.835711 \\
\hline 6 & -0.952006 & 5.439840 & -1.074782 \\
\hline 15 & 1.098595 & 0.805854 & 0.552988 \\
\hline 6 & 2.853168 & 1.211773 & 0.155305 \\
\hline 6 & 3.558285 & 2.169715 & 0.885349 \\
\hline 6 & 4.890642 & 2.462109 & 0.594501 \\
\hline 6 & 5.506967 & 1.754785 & -0.446608 \\
\hline 6 & 4.830072 & 0.787421 & -1.192656 \\
\hline 6 & 3.492186 & 0.536064 & -0.876444 \\
\hline 6 & 5.608417 & 3.534124 & 1.414813 \\
\hline 6 & 5.669500 & 3.094958 & 2.882314 \\
\hline 6 & 5.458284 & 0.039566 & -2.365854 \\
\hline 6 & 4.806577 & 0.541039 & -3.660005 \\
\hline 45 & 0.158303 & -0.914266 & -0.797122 \\
\hline 6 & 0.745939 & -0.967866 & -2.700018 \\
\hline 8 & 0.740678 & -1.131796 & -3.847268 \\
\hline 6 & 0.645085 & -3.154025 & -0.020453 \\
\hline 6 & -0.522494 & -3.006610 & -0.812432 \\
\hline 6 & 1.876449 & -3.790193 & -0.592810 \\
\hline 6 & 2.025072 & -4.024676 & -1.968765 \\
\hline 6 & 3.188292 & -4.578223 & -2.477093 \\
\hline 6 & 4.238424 & -4.913271 & -1.622322 \\
\hline 6 & 4.110942 & -4.693939 & -0.256582 \\
\hline 6 & 2.941083 & -4.138738 & 0.246683 \\
\hline 6 & 5.472993 & -5.509697 & -2.210604 \\
\hline 9 & 6.441662 & -5.687903 & -1.305622 \\
\hline 6 & 0.479747 & -3.257809 & 1.477665 \\
\hline 9 & 5.227737 & -6.706270 & -2.769079 \\
\hline
\end{tabular}




\begin{tabular}{|c|c|c|c|}
\hline & 5.979766 & -4.740667 & -3.188878 \\
\hline 6 & 1.231199 & 0.147243 & 2.265022 \\
\hline 6 & 2.414544 & -0.406056 & 2.737126 \\
\hline & 2.486447 & -0.984642 & 4.013081 \\
\hline & 1.340202 & -0.964879 & 4.802832 \\
\hline 6 & 0.131431 & -0.398011 & 4.363135 \\
\hline 6 & 0.086384 & 0.120018 & 3.073133 \\
\hline 6 & 3.810803 & -1.590013 & 4.475567 \\
\hline 6 & 3.742775 & -2.119974 & 5.905194 \\
\hline 6 & -1.079364 & -0.366923 & 5.295861 \\
\hline 6 & -0.675192 & 0.208371 & 6.658242 \\
\hline 6 & 4.184594 & -2.754100 & 3.550106 \\
\hline 6 & 4.909689 & -0.522776 & 4.411593 \\
\hline 6 & -2.205493 & 0.508521 & 4.745233 \\
\hline 6 & -1.604465 & -1.793423 & 5.487461 \\
\hline 6 & 6.964575 & 0.268210 & -2.462558 \\
\hline 6 & 5.212623 & -1.467997 & -2.226535 \\
\hline 6 & 4.838570 & 4.856765 & 1.312888 \\
\hline 6 & 7.035124 & 3.781227 & 0.931700 \\
\hline 1 & -7.109475 & -1.434218 & 0.518576 \\
\hline 1 & 0.804369 & 2.504182 & 2.979615 \\
\hline 1 & -8.581498 & 0.625352 & -3.755167 \\
\hline 1 & -0.898073 & 6.889496 & 2.525604 \\
\hline 1 & -4.626722 & -5.570808 & 2.683598 \\
\hline 1 & 2.287202 & 4.113005 & -0.985266 \\
\hline 1 & 4.100026 & 4.740522 & -2.521375 \\
\hline 1 & -3.151709 & -3.680987 & 3.066941 \\
\hline 1 & 0.047486 & 4.795932 & 3.366158 \\
\hline 1 & -6.302359 & 0.542938 & -4.609666 \\
\hline 1 & -7.179633 & -5.660507 & -0.771856 \\
\hline 1 & -3.882361 & 0.086509 & -4.226030 \\
\hline 1 & 1.966459 & 2.991873 & -5.820388 \\
\hline 1 & -0.208952 & 1.936211 & -5.448640 \\
\hline 1 & -1.777075 & 7.316891 & -1.660367 \\
\hline 1 & -6.396539 & -3.438529 & -1.437203 \\
\hline 1 & -0.970299 & 5.044327 & -2.088183 \\
\hline 1 & -2.002700 & 1.219919 & -3.888320 \\
\hline 1 & -6.326157 & -6.736621 & 1.308106 \\
\hline 1 & -9.453685 & -0.785520 & 0.209334 \\
\hline 1 & 3.943260 & 4.202733 & -4.949214 \\
\hline 1 & -1.745076 & 8.261721 & 0.643521 \\
\hline 1 & -10.216876 & 0.225698 & -1.936942 \\
\hline 1 & -2.246508 & -1.465166 & 2.337964 \\
\hline 1 & 3.058364 & 2.696605 & 1.697968 \\
\hline 1 & 2.919473 & -0.192522 & -1.446637 \\
\hline 1 & 6.545599 & 1.968921 & -0.682745 \\
\hline 1 & 3.298854 & -0.393670 & 2.100006 \\
\hline 1 & -0.838254 & 0.547440 & 2.683469 \\
\hline 1 & 1.377617 & -1.392249 & 5.802273 \\
\hline 1 & -3.050594 & 3.399562 & -1.544704 \\
\hline 1 & -4.056325 & 2.031239 & -2.020006 \\
\hline 1 & -5.941028 & 1.788097 & -0.688824 \\
\hline 1 & -2.289143 & 3.081219 & 1.145991 \\
\hline 1 & -7.079023 & 1.870200 & 1.502866 \\
\hline 1 & -3.425391 & 3.209885 & 3.339204 \\
\hline 1 & -5.825987 & 2.572197 & 3.535795 \\
\hline
\end{tabular}




$\begin{array}{rrrr}1 & 1.493051 & -1.677942 & -0.242982 \\ 1 & -1.479308 & -3.042658 & -0.290611 \\ 1 & -0.562173 & -3.395810 & -1.828204 \\ 1 & 0.542651 & -4.310792 & 1.790629 \\ 1 & 1.239463 & -2.697207 & 2.035495 \\ 1 & -0.499184 & -2.871567 & 1.775919 \\ 1 & 2.866102 & -3.967119 & 1.316910 \\ 1 & 4.924115 & -4.950317 & 0.417945 \\ 1 & 3.287338 & -4.748026 & -3.547703 \\ 1 & 1.225858 & -3.769136 & -2.658898 \\ 1 & -3.023412 & 0.562819 & 5.474999 \\ 1 & -1.857438 & 1.533671 & 4.555390 \\ 1 & -2.635369 & 0.122208 & 3.812245 \\ 1 & -1.549462 & 0.255036 & 7.320838 \\ 1 & 0.084035 & -0.399272 & 7.163947 \\ 1 & -0.276293 & 1.225785 & 6.553911 \\ 1 & -2.482746 & -1.796120 & 6.147095 \\ 1 & -1.902363 & -2.235694 & 4.526904 \\ 1 & -0.845839 & -2.448136 & 5.934395 \\ 1 & 7.372478 & -0.311742 & -3.299974 \\ 1 & 7.212050 & 1.321478 & -2.647111 \\ 1 & 7.486106 & -0.053627 & -1.551434 \\ 1 & 5.213486 & 0.004786 & -4.528378 \\ 1 & 3.717966 & 0.396136 & -3.651359 \\ 1 & 4.994420 & 1.613821 & -3.799761 \\ 1 & 5.691666 & -2.006577 & -3.054478 \\ 1 & 5.632061 & -1.851395 & -1.286285 \\ 1 & 4.144465 & -1.724462 & -2.248598 \\ 1 & 4.717534 & -2.534594 & 6.191263 \\ 1 & 3.498255 & -1.327810 & 6.624657 \\ 1 & 3.001735 & -2.922736 & 6.011570 \\ 1 & 5.869307 & -0.944347 & 4.739666 \\ 1 & 5.048748 & -0.135248 & 3.394374 \\ 1 & 4.671731 & 0.326750 & 5.065257 \\ 1 & 5.133793 & -3.206204 & 3.868407 \\ 1 & 3.413382 & -3.537264 & 3.577748 \\ 1 & 4.305366 & -2.427673 & 2.508809 \\ 1 & 7.501579 & 4.563787 & 1.543448 \\ 1 & 7.659996 & 2.883024 & 1.016943 \\ 1 & 7.060713 & 4.120042 & -0.112274 \\ 1 & 5.336157 & 5.633421 & 1.909360 \\ 1 & 4.798286 & 5.208346 & 0.273318 \\ 1 & 3.806658 & 4.771447 & 1.677293 \\ 1 & 6.153800 & 3.868686 & 3.493536 \\ 1 & 6.249647 & 2.168853 & 2.990526\end{array}$

(para-F)TS1 $1_{\mathrm{IIL-si}-} \psi_{-1 \mathrm{~s} 2 \mathrm{a} 3 \mathrm{a} 4 \mathrm{a}}$

Number of imaginary frequencies : 1

The smallest frequencies are : $-551.4445 \quad 17.0653 \quad 18.9886 \mathrm{~cm}(-1)$

Electronic energy : $\quad \mathrm{HF}=-4460.084358$

Zero-point correction $=\quad 1.445109($ Hartree/Particle $)$

Thermal correction to Energy $=\quad 1.530192$

Thermal correction to Enthalpy $=\quad 1.531136$ 
Thermal correction to Gibbs Free Energy= Sum of electronic and zero-point Energies= Sum of electronic and thermal Energies= Sum of electronic and thermal Enthalpies= Sum of electronic and thermal Free Energies=
1.328016

$-4458.639249$

$-4458.554166$

$-4458.553222$

$-4458.756342$

Cartesian Coordinates

\begin{tabular}{|c|c|c|c|}
\hline 6 & -3.262741 & 2.122585 & 0.950661 \\
\hline 6 & -1.951934 & 1.649429 & 0.847657 \\
\hline 6 & -0.913993 & 2.397861 & 1.402622 \\
\hline 6 & -1.163008 & 3.569580 & 2.124675 \\
\hline 6 & -2.488950 & 3.987161 & 2.244553 \\
\hline 6 & -3.553708 & 3.286643 & 1.662722 \\
\hline 15 & -1.473092 & 0.024562 & 0.114879 \\
\hline 6 & -1.106315 & -0.923125 & 1.688244 \\
\hline 6 & -0.609700 & -2.220292 & 1.628284 \\
\hline 6 & -0.337559 & -2.947116 & 2.833519 \\
\hline 6 & -0.570895 & -2.331796 & 4.097297 \\
\hline 6 & -1.091800 & -1.019562 & 4.125782 \\
\hline 6 & -1.348685 & -0.341801 & 2.964229 \\
\hline 6 & -0.267061 & -3.034761 & 5.287476 \\
\hline 6 & 0.248794 & -4.304294 & 5.241319 \\
\hline 6 & 0.474648 & -4.924520 & 3.994261 \\
\hline 6 & 0.185544 & -4.267738 & 2.823682 \\
\hline 6 & -0.362257 & -2.934928 & 0.343905 \\
\hline 6 & -1.350229 & -3.834019 & -0.166484 \\
\hline 6 & -1.080156 & -4.576478 & -1.354472 \\
\hline 6 & 0.176231 & -4.435397 & -1.986767 \\
\hline 6 & 1.114864 & -3.582445 & -1.474365 \\
\hline 6 & 0.846798 & -2.814893 & -0.317350 \\
\hline 6 & -2.064772 & -5.457559 & -1.864854 \\
\hline 6 & -3.269090 & -5.609764 & -1.226696 \\
\hline 6 & -3.541867 & -4.875673 & -0.052236 \\
\hline 6 & -2.606776 & -4.015998 & 0.467666 \\
\hline 7 & 1.852686 & -1.897832 & 0.145723 \\
\hline 6 & 2.896946 & -2.483047 & 1.018090 \\
\hline 6 & 3.382696 & -1.613311 & 2.148679 \\
\hline 6 & 2.493895 & -0.893111 & 2.949080 \\
\hline 6 & 2.957129 & -0.141589 & 4.020313 \\
\hline 6 & 4.320759 & -0.092769 & 4.307015 \\
\hline 6 & 5.213341 & -0.800490 & 3.510406 \\
\hline 6 & 4.744764 & -1.556217 & 2.437642 \\
\hline 6 & 0.002452 & 4.341813 & 2.740893 \\
\hline 6 & 0.955141 & 3.376734 & 3.456684 \\
\hline 6 & -4.992943 & 3.732561 & 1.928173 \\
\hline 6 & -6.003475 & 2.988747 & 1.056097 \\
\hline 45 & 0.121932 & 0.236847 & -1.832569 \\
\hline 6 & 0.644658 & 2.400608 & -1.741746 \\
\hline 6 & -0.386342 & 2.376335 & -2.710642 \\
\hline 6 & -0.001046 & 2.424307 & -4.177053 \\
\hline 15 & 2.013910 & -0.518688 & -0.825549 \\
\hline 8 & 3.205572 & -0.804800 & -1.974988 \\
\hline 6 & 4.471127 & -1.208609 & -1.625613 \\
\hline 6 & 5.339577 & -0.345933 & -0.982216 \\
\hline 6 & 6.588309 & -0.876560 & -0.515702 \\
\hline
\end{tabular}




\begin{tabular}{|c|c|c|c|}
\hline ) & 6.956036 & -2.213635 & -0.853261 \\
\hline & 6.077687 & -3.000288 & -1.638297 \\
\hline & 4.852566 & -2.514835 & -2.002163 \\
\hline & 8.176463 & -2.745952 & -0.371375 \\
\hline & 8.996972 & -2.006068 & 0.441141 \\
\hline U & 8.621041 & -0.696698 & 0.806132 \\
\hline & 7.453751 & -0.145264 & 0.337296 \\
\hline & 4.946502 & 1.063123 & -0.751877 \\
\hline & 3.750524 & 1.352348 & -0.122389 \\
\hline & 3.380061 & 2.665912 & 0.226981 \\
\hline & 4.180897 & 3.717238 & -0.120693 \\
\hline & 5.369694 & 3.498162 & -0.858954 \\
\hline 6 & 5.755078 & 2.164002 & -1.191803 \\
\hline & 6.917868 & 1.988666 & -1.984643 \\
\hline & 7.668901 & 3.063305 & -2.392919 \\
\hline & 7.303856 & 4.376192 & -2.030746 \\
\hline & 6.173257 & 4.583752 & -1.282999 \\
\hline & 2.903200 & 0.347960 & 0.266067 \\
\hline & 0.201162 & -0.997410 & -3.275741 \\
\hline & 0.308031 & -1.594159 & -4.268322 \\
\hline & -1.693113 & 3.061861 & -2.408203 \\
\hline 6 & -2.898402 & 2.604824 & -2.953221 \\
\hline & -4.093633 & 3.271742 & -2.725338 \\
\hline & -4.069307 & 4.418567 & -1.948028 \\
\hline & -2.900099 & 4.912416 & -1.398321 \\
\hline 6 & -1.713469 & 4.227720 & -1.639085 \\
\hline & -5.226351 & 5.060672 & -1.710348 \\
\hline 6 & -3.098188 & -0.733197 & -0.322460 \\
\hline & -4.097914 & -0.928635 & 0.635578 \\
\hline & -5.330096 & -1.478500 & 0.287175 \\
\hline 6 & -5.519980 & -1.856380 & -1.048087 \\
\hline & -4.535520 & -1.702389 & -2.025617 \\
\hline 6 & -3.321202 & -1.128618 & -1.637564 \\
\hline & -6.446173 & -1.694184 & 1.309151 \\
\hline 6 & -6.701149 & -3.196488 & 1.472701 \\
\hline 6 & -4.811454 & -2.107754 & -3.472675 \\
\hline & -5.376628 & -3.529650 & -3.533307 \\
\hline 6 & -6.090454 & -1.124189 & 2.680924 \\
\hline & -7.730822 & -1.009658 & 0.830417 \\
\hline 6 & -3.545609 & -2.073557 & -4.328534 \\
\hline & -5.831776 & -1.131403 & -4.068851 \\
\hline 6 & 0.746811 & 5.078980 & 1.621213 \\
\hline & -0.461478 & 5.377880 & 3.763646 \\
\hline 6 & -5.311491 & 3.429004 & 3.398054 \\
\hline & -5.172536 & 5.232098 & 1.674754 \\
\hline 1 & 7.167933 & 0.857037 & 0.646325 \\
\hline 1 & -1.757712 & 0.663283 & 3.027750 \\
\hline 1 & 8.440290 & -3.765481 & -0.649837 \\
\hline 1 & -0.448649 & -2.540459 & 6.240969 \\
\hline 1 & 5.864275 & 5.591707 & -1.008817 \\
\hline 1 & -2.830288 & -3.451403 & 1.371605 \\
\hline 1 & -4.503836 & -4.993118 & 0.442915 \\
\hline 1 & 3.907860 & 4.736557 & 0.149527 \\
\hline 1 & -1.288058 & -0.551279 & 5.089843 \\
\hline & 6.383096 & -4.005714 & -1.923323 \\
\hline & 8.551085 & 2.899117 & -3.007677 \\
\hline
\end{tabular}




\begin{tabular}{|c|c|c|c|}
\hline 1 & 4.153300 & -3.107659 & -2.587399 \\
\hline 1 & -1.839327 & -6.016258 & -2.772390 \\
\hline 1 & 0.380896 & -5.009920 & -2.888884 \\
\hline & 0.878528 & -5.933860 & 3.960198 \\
\hline 1 & 7.208083 & 0.985097 & -2.284436 \\
\hline & 0.358940 & -4.763922 & 1.870895 \\
\hline 1 & 2.080194 & -3.459223 & -1.961819 \\
\hline 1 & 7.910695 & 5.218176 & -2.355494 \\
\hline 1 & 9.258679 & -0.118585 & 1.471327 \\
\hline 1 & -4.016684 & -6.294169 & -1.622403 \\
\hline 1 & 0.483878 & -4.835605 & 6.160667 \\
\hline 1 & 9.927104 & -2.428170 & 0.814349 \\
\hline 1 & 2.453244 & 2.802406 & 0.779440 \\
\hline 1 & -3.904642 & -0.635630 & 1.665539 \\
\hline 1 & -2.510978 & -0.993495 & -2.351266 \\
\hline 1 & -6.477290 & -2.292999 & -1.333978 \\
\hline 1 & -4.066570 & 1.550507 & 0.497243 \\
\hline 1 & 0.104445 & 2.011851 & 1.320187 \\
\hline 1 & -2.711627 & 4.879462 & 2.825448 \\
\hline 1 & 2.438149 & -3.385642 & 1.447026 \\
\hline 1 & 3.757408 & -2.828494 & 0.424618 \\
\hline 1 & 5.452646 & -2.098634 & 1.808113 \\
\hline 1 & 1.434431 & -0.897037 & 2.700930 \\
\hline 1 & 6.281513 & -0.762392 & 3.715810 \\
\hline 1 & 2.246816 & 0.412237 & 4.633011 \\
\hline 1 & 4.684006 & 0.499847 & 5.144114 \\
\hline 1 & 0.437568 & 2.825522 & -0.759384 \\
\hline 1 & 1.676104 & 2.476078 & -2.086547 \\
\hline 1 & -2.904276 & 1.694911 & -3.554153 \\
\hline 1 & -5.037190 & 2.917853 & -3.132173 \\
\hline 1 & -2.922304 & 5.815473 & -0.792804 \\
\hline 1 & -0.783915 & 4.613350 & -1.226066 \\
\hline 1 & -0.736923 & 1.918299 & -4.813594 \\
\hline 1 & 0.052560 & 3.472194 & -4.504459 \\
\hline 1 & 0.972207 & 1.950012 & -4.340180 \\
\hline 1 & -1.095997 & 0.936267 & -2.670951 \\
\hline 1 & 0.411266 & 5.873967 & 4.206806 \\
\hline 1 & -1.081079 & 6.161602 & 3.310430 \\
\hline 1 & -1.032765 & 4.916688 & 4.579793 \\
\hline 1 & 1.767348 & 3.934187 & 3.941334 \\
\hline 1 & 0.422210 & 2.808588 & 4.231773 \\
\hline 1 & 1.421922 & 2.649885 & 2.780492 \\
\hline 1 & 1.632677 & 5.594860 & 2.017849 \\
\hline 1 & 1.077833 & 4.394686 & 0.828376 \\
\hline 1 & 0.096504 & 5.832342 & 1.155277 \\
\hline 1 & -7.015110 & 3.354266 & 1.273853 \\
\hline 1 & -5.815800 & 3.149575 & -0.013305 \\
\hline 1 & -6.000085 & 1.906582 & 1.247206 \\
\hline 1 & -5.017346 & 5.477044 & 0.617480 \\
\hline 1 & -6.195973 & 5.532799 & 1.935962 \\
\hline 1 & -4.492248 & 5.846436 & 2.276062 \\
\hline 1 & -6.350093 & 3.700531 & 3.631392 \\
\hline 1 & -5.182422 & 2.359461 & 3.613673 \\
\hline 1 & -4.656911 & 3.987079 & 4.079302 \\
\hline 1 & -6.923524 & -1.287830 & 3.376091 \\
\hline 1 & -5.203470 & -1.607455 & 3.112100 \\
\hline
\end{tabular}




$\begin{array}{llrl}1 & -5.902988 & -0.041500 & 2.638800 \\ 1 & -7.525091 & -3.371777 & 2.177869 \\ 1 & -6.967596 & -3.674126 & 0.521404 \\ 1 & -5.809087 & -3.701637 & 1.868417 \\ 1 & -8.532252 & -1.147539 & 1.568522 \\ 1 & -7.577387 & 0.069513 & 0.696498 \\ 1 & -8.089215 & -1.416249 & -0.122722 \\ 1 & -3.778392 & -2.414774 & -5.345274 \\ 1 & -3.124291 & -1.062611 & -4.415703 \\ 1 & -2.764900 & -2.732207 & -3.923793 \\ 1 & -5.573435 & -3.813187 & -4.575872 \\ 1 & -4.661824 & -4.249215 & -3.113199 \\ 1 & -6.320600 & -3.630869 & -2.984364 \\ 1 & -6.044418 & -1.386963 & -5.115960 \\ 1 & -6.780984 & -1.150015 & -3.518502 \\ 1 & -5.451468 & -0.101351 & -4.041891\end{array}$

(para-F)TS1 $1_{\mathrm{IIL-si}-} \psi_{-1 \mathrm{~s} 2 \mathrm{aza} a \mathrm{~s}}$

Number of imaginary frequencies : 1

The smallest frequencies are : $-514.1353 \quad 12.1482 \quad 18.1750 \mathrm{~cm}(-1)$

Electronic energy : $\quad \mathrm{HF}=-4460.0844941$

Zero-point correction $=\quad 1.444965($ Hartree/Particle $)$

Thermal correction to Energy $=\quad 1.530241$

Thermal correction to Enthalpy= $\quad 1.531185$

Thermal correction to Gibbs Free Energy $=\quad 1.326287$

Sum of electronic and zero-point Energies $=\quad-4458.639529$

Sum of electronic and thermal Energies $=\quad-4458.554253$

Sum of electronic and thermal Enthalpies $=\quad-4458.553309$

Sum of electronic and thermal Free Energies $=\quad-4458.758207$

\section{Cartesian Coordinates}

\begin{tabular}{|c|c|c|c|}
\hline 6 & 7.446531 & -0.138658 & 0.327138 \\
\hline 6 & 6.584305 & -0.835304 & -0.557433 \\
\hline 6 & 6.964789 & -2.149140 & -0.964952 \\
\hline 6 & 8.193273 & -2.691892 & -0.516481 \\
\hline 6 & 9.010046 & -1.985330 & 0.328867 \\
\hline 6 & 8.622291 & -0.700238 & 0.761495 \\
\hline 6 & 5.327313 & -0.295567 & -0.989886 \\
\hline 6 & 4.465153 & -1.133551 & -1.673063 \\
\hline 6 & 4.858184 & -2.414921 & -2.117101 \\
\hline 6 & 6.090437 & -2.904724 & -1.784358 \\
\hline 6 & 4.918930 & 1.094982 & -0.684893 \\
\hline 6 & 3.722747 & 1.336198 & -0.035637 \\
\hline 6 & 3.335893 & 2.626209 & 0.378008 \\
\hline 6 & 4.120876 & 3.704033 & 0.078982 \\
\hline 6 & 5.309357 & 3.537750 & -0.673180 \\
\hline 6 & 5.710743 & 2.227012 & -1.073211 \\
\hline 6 & 6.095845 & 4.653925 & -1.046714 \\
\hline 6 & 7.225313 & 4.498972 & -1.808795 \\
\hline 6 & 7.605898 & 3.210607 & -2.237244 \\
\hline 6 & 6.871360 & 2.106971 & -1.879293 \\
\hline 8 & 2.890652 & 0.301312 & 0.305078 \\
\hline 15 & 2.002701 & -0.508879 & -0.830967 \\
\hline 8 & 3.193839 & -0.726254 & -1.995996 \\
\hline
\end{tabular}




\begin{tabular}{|c|c|c|c|}
\hline 45 & 0.111977 & 0.297351 & -1.802223 \\
\hline 6 & 0.154213 & -0.893524 & -3.287412 \\
\hline 8 & 0.233213 & -1.457951 & -4.301059 \\
\hline 6 & -0.342584 & 2.471491 & -2.627766 \\
\hline 6 & 0.693707 & 2.441823 & -1.664203 \\
\hline 6 & -1.623719 & 3.194311 & -2.304180 \\
\hline 6 & -2.838277 & 2.818036 & -2.889151 \\
\hline 6 & -4.005967 & 3.528580 & -2.649447 \\
\hline 6 & -3.944751 & 4.634990 & -1.817062 \\
\hline 6 & -2.766138 & 5.044344 & -1.220677 \\
\hline 6 & -1.607014 & 4.319767 & -1.477498 \\
\hline 9 & -5.074955 & 5.319213 & -1.569162 \\
\hline 6 & 0.039734 & 2.541394 & -4.094257 \\
\hline 15 & -1.474632 & 0.024747 & 0.123620 \\
\hline 6 & -3.103069 & -0.720093 & -0.324322 \\
\hline 6 & -4.058548 & -1.023076 & 0.643293 \\
\hline 6 & -5.277867 & -1.613247 & 0.300493 \\
\hline 6 & -5.510752 & -1.884002 & -1.049503 \\
\hline 6 & -4.570710 & -1.599860 & -2.048753 \\
\hline 6 & -3.364619 & -1.017361 & -1.661012 \\
\hline 6 & -6.280457 & -1.941927 & 1.407405 \\
\hline 6 & -7.518573 & -2.661265 & 0.878696 \\
\hline 6 & -4.888303 & -1.881931 & -3.516282 \\
\hline 6 & -3.639427 & -1.808368 & -4.395324 \\
\hline 6 & -1.098803 & -0.994775 & 1.647471 \\
\hline 6 & -0.617670 & -2.294583 & \\
\hline 6 & -0.355377 & -3.076806 & 2.700944 \\
\hline 6 & -0.584921 & -2.516785 & 3.99 \\
\hline 6 & -1.088718 & -1.200445 & 4.078155 \\
\hline 6 & -1.332811 & -0.467469 & 2.947700 \\
\hline 6 & -0.288911 & -3.274868 & 5.149059 \\
\hline 6 & 0.215961 & -4.545450 & 5.04 \\
\hline 6 & 0.437784 & -5.111187 & \\
\hline 6 & 0.156002 & -4.400540 & 2.633108 \\
\hline 6 & -0.375047 & -2.957688 & 0.214897 \\
\hline 6 & -1.370345 & -3.822486 & -0.338450 \\
\hline 6 & -1.110365 & -4.505517 & -1.563613 \\
\hline 6 & 0.150765 & -4.354320 & -2.184365 \\
\hline 6 & 1.099773 & -3.541507 & -1.627936 \\
\hline 6 & 0.836362 & -2.823505 & -0.43 \\
\hline 6 & -2.109819 & -5.339989 & -2.121701 \\
\hline 6 & -3.317264 & -5.506856 & -1.492641 \\
\hline 6 & -3.574624 & -4.839804 & -0.275623 \\
\hline 6 & -2.625384 & -4.026453 & 0.290248 \\
\hline 7 & 1.849929 & -1.937485 & 0.065193 \\
\hline 6 & 2.903926 & -2.574969 & 0.887770 \\
\hline 6 & 3.400015 & -1.781702 & 2.069128 \\
\hline 6 & 2.517988 & -1.122863 & 2.927530 \\
\hline 6 & 2.989653 & -0.453252 & 4.048269 \\
\hline 6 & 4.355424 & -0.424768 & 4.326748 \\
\hline 6 & 5.241735 & -1.069207 & 3.471589 \\
\hline 6 & 4.764665 & -1.743951 & 2.349797 \\
\hline 6 & -1.960772 & 1.620192 & 0.912815 \\
\hline 6 & -0.931957 & 2.365753 & 1.490245 \\
\hline 6 & -1.197254 & 3.525895 & 2.224267 \\
\hline 6 & -2.526204 & 3.941702 & 2.323586 \\
\hline
\end{tabular}




\begin{tabular}{|c|c|c|c|}
\hline 5 & -3.579338 & 3.250718 & 1.711587 \\
\hline & -3.274379 & 2.086853 & 1.003202 \\
\hline & -0.046357 & 4.304221 & 2.860216 \\
\hline & 0.699253 & 5.060196 & 1.753583 \\
\hline & -5.025752 & 3.698950 & 1.934703 \\
\hline & -5.441399 & 3.247843 & 3.340842 \\
\hline & -5.170323 & 5.220256 & 1.836713 \\
\hline & -5.988706 & 3.078717 & 0.922425 \\
\hline & -0.531478 & 5.325818 & 3.887905 \\
\hline & 0.913216 & 3.346348 & 3.575097 \\
\hline & -6.733544 & -0.641712 & 2.080741 \\
\hline & -5.619135 & -2.843980 & 2.456900 \\
\hline & -5.893814 & -0.832451 & -4.003783 \\
\hline & -5.492122 & -3.278968 & -3.684384 \\
\hline & 7.152105 & 0.843888 & 0.687147 \\
\hline & -1.725955 & 0.540570 & 3.053716 \\
\hline & 8.466544 & -3.693040 & -0.847505 \\
\hline & -0.466752 & -2.821673 & 6.123472 \\
\hline & 5.774408 & 5.642710 & -0.721667 \\
\hline & -2.835614 & -3.515896 & 1.228171 \\
\hline & -4.536350 & -4.973554 & 0.217427 \\
\hline & 3.834194 & 4.705261 & 0.398357 \\
\hline & -1.279061 & -0.773113 & 5.062174 \\
\hline & 6.404961 & -3.891242 & -2.120952 \\
\hline & 8.486809 & 3.089165 & -2.863614 \\
\hline & 4.161063 & -2.985841 & -2.726345 \\
\hline & -1.893508 & -5.851824 & -3.058587 \\
\hline & 0.349619 & -4.886863 & -3.113116 \\
\hline & 0.833508 & -6.121263 & 3.694749 \\
\hline 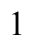 & 7.173214 & 1.123640 & -2.230112 \\
\hline & 0.327125 & -4.855572 & 1.659651 \\
\hline 1 & 2.069072 & -3.407742 & -2.105174 \\
\hline & 7.819091 & 5.364123 & -2.094450 \\
\hline | & 9.257450 & -0.149562 & 1.451859 \\
\hline ] & -4.076483 & -6.154749 & -1.925886 \\
\hline | & 0.446031 & -5.118974 & 5.941715 \\
\hline 1 & 9.946551 & -2.415664 & 0.675802 \\
\hline & 2.406901 & 2.725082 & 0.934810 \\
\hline 1 & -3.844139 & -0.802311 & 1.689531 \\
\hline ] & -2.585524 & -0.799426 & -2.388714 \\
\hline 1 & -6.454776 & -2.340331 & -1.339557 \\
\hline 1 & -4.069674 & 1.515299 & 0.532591 \\
\hline 1 & 0.090358 & 1.989420 & 1.407758 \\
\hline 1 & -2.757416 & 4.829698 & 2.906605 \\
\hline & 2.450198 & -3.504128 & 1.261939 \\
\hline 1 & 3.759007 & -2.880072 & 0.265211 \\
\hline 1 & 5.467681 & -2.238781 & 1.677182 \\
\hline 1 & 1.456685 & -1.110018 & 2.688784 \\
\hline 1 & 6.311597 & -1.044862 & 3.670071 \\
\hline 1 & 2.284335 & 0.052824 & 4.706153 \\
\hline 1 & 4.725354 & 0.103685 & 5.202964 \\
\hline 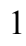 & 0.506061 & 2.849826 & -0.670758 \\
\hline 1 & 1.724508 & 2.498488 & -2.014586 \\
\hline | & -2.873298 & 1.940435 & -3.535593 \\
\hline 1 & -4.956490 & 3.238962 & -3.089913 \\
\hline 1 & -2.759377 & 5.916763 & -0.571153 \\
\hline
\end{tabular}




$\begin{array}{lrrr}1 & -0.668587 & 4.643683 & -1.032843 \\ 1 & -0.710250 & 2.069048 & -4.740053 \\ 1 & 0.120585 & 3.594365 & -4.398833 \\ 1 & 0.999512 & 2.044921 & -4.270574 \\ 1 & -1.092548 & 1.058605 & -2.610985 \\ 1 & 0.331691 & 5.822081 & 4.349234 \\ 1 & -1.149402 & 6.111110 & 3.435166 \\ 1 & -1.111321 & 4.851763 & 4.690481 \\ 1 & 1.719598 & 3.910342 & 4.061791 \\ 1 & 0.387546 & 2.769298 & 4.348364 \\ 1 & 1.386733 & 2.627341 & 2.896154 \\ 1 & 1.566491 & 5.595162 & 2.165744 \\ 1 & 1.058598 & 4.384457 & 0.965268 \\ 1 & 0.039845 & 5.800726 & 1.279272 \\ 1 & -7.005392 & 3.445989 & 1.112278 \\ 1 & -5.724532 & 3.350551 & -0.107278 \\ 1 & -6.022971 & 1.984218 & 0.990811 \\ 1 & -4.895842 & 5.582072 & 0.838795 \\ 1 & -6.216362 & 5.503070 & 2.013870 \\ 1 & -4.565581 & 5.755214 & 2.578013 \\ 1 & -6.482160 & 3.533432 & 3.546602 \\ 1 & -5.362402 & 2.156784 & 3.442394 \\ 1 & -4.806934 & 3.701730 & 4.112998 \\ 1 & -8.198656 & -2.885770 & 1.710164 \\ 1 & -8.074568 & -2.047876 & 0.158156 \\ 1 & -7.264452 & -3.613660 & 0.394648 \\ 1 & -6.328771 & -3.067593 & 3.264847 \\ 1 & -5.304513 & -3.800388 & 2.017477 \\ 1 & -4.736417 & -2.379190 & 2.914995 \\ 1 & -7.438031 & -0.855286 & 2.896123 \\ 1 & -5.887126 & -0.088374 & 2.509122 \\ 1 & -7.239431 & 0.016841 & 1.362080 \\ 1 & -3.899784 & -2.070242 & -5.428782 \\ 1 & -3.199844 & -0.801929 & -4.419917 \\ 1 & -2.863841 & -2.508127 & -4.055140 \\ 1 & -5.687497 & -3.478937 & -4.746255 \\ 1 & -4.801259 & -4.047735 & -3.313987 \\ 1 & -6.444600 & -3.394709 & -3.153710 \\ 1 & -6.139704 & -0.993806 & -5.062408 \\ 1 & -6.828634 & -0.871835 & -3.430098 \\ 1 & -5.482257 & 0.180978 & -3.900558\end{array}$

(para-F)TS1 $1_{\mathrm{IIL}-\mathrm{si}-}-\psi_{-1 \mathrm{1} 2 \mathrm{2a3s4a}}$

Number of imaginary frequencies : 1

The smallest frequencies are : $-538.2164 \quad 16.3685 \quad 18.2727 \mathrm{~cm}(-1)$

Electronic energy : $\quad \mathrm{HF}=-4460.084049$

Zero-point correction $=\quad 1.444525($ Hartree/Particle $)$

Thermal correction to Energy= $\quad 1.529868$

Thermal correction to Enthalpy= $\quad 1.530812$

Thermal correction to Gibbs Free Energy $=\quad 1.326245$

Sum of electronic and zero-point Energies $=\quad-4458.639524$

Sum of electronic and thermal Energies $=\quad-4458.554181$

Sum of electronic and thermal Enthalpies $=\quad-4458.553237$

Sum of electronic and thermal Free Energies $=\quad-4458.757804$ 
Cartesian Coordinates

\begin{tabular}{|c|c|c|c|}
\hline 6 & -0.913440 & 2.415283 & 1.398047 \\
\hline 6 & -1.957757 & 1.667065 & 0.854773 \\
\hline 6 & -3.268065 & 2.136640 & 0.977327 \\
\hline 6 & -3.550359 & 3.302397 & 1.690580 \\
\hline 6 & -2.478566 & 4.004821 & 2.256561 \\
\hline 6 & -1.153994 & 3.588135 & 2.121234 \\
\hline 15 & -1.485251 & 0.044851 & 0.112181 \\
\hline 45 & 0.109735 & 0.247489 & -1.828559 \\
\hline 15 & 1.999720 & -0.505540 & -0.817332 \\
\hline 8 & 3.191473 & -0.802881 & -1.964811 \\
\hline 6 & 4.456365 & -1.206499 & -1.613058 \\
\hline 6 & 5.324744 & -0.343758 & -0.969491 \\
\hline 6 & 6.569841 & -0.875856 & -0.495616 \\
\hline 6 & 6.936253 & -2.214624 & -0.827665 \\
\hline 6 & 6.060978 & -3.000252 & -1.617194 \\
\hline 6 & 4.838456 & -2.513157 & -1.987741 \\
\hline 6 & 7.432204 & -0.143833 & 0.359924 \\
\hline 6 & 8.595366 & -0.696768 & 0.837100 \\
\hline 6 & 8.970111 & -2.008160 & 0.477979 \\
\hline 6 & 8.152665 & -2.748418 & -0.337305 \\
\hline 6 & 4.936116 & 1.068192 & -0.750560 \\
\hline 6 & 3.738508 & 1.366232 & -0.128780 \\
\hline 6 & 3.367057 & 2.684465 & 0.200810 \\
\hline 6 & 4.170947 & 3.730504 & -0.155789 \\
\hline 6 & 5.364235 & 3.500926 & -0.883455 \\
\hline 6 & 5.749410 & 2.162372 & -1.198436 \\
\hline 6 & 6.172245 & 4.580249 & -1.315050 \\
\hline 6 & 7.307202 & 4.362215 & -2.053111 \\
\hline 6 & 7.672350 & 3.044618 & -2.397632 \\
\hline 6 & 6.916866 & 1.975996 & -1.981879 \\
\hline 8 & 2.890136 & 0.366137 & 0.269524 \\
\hline 6 & -4.984882 & 3.753602 & 1.971382 \\
\hline 6 & -6.007811 & 3.005868 & 1.117693 \\
\hline 6 & 0.017909 & 4.367614 & 2.715814 \\
\hline 6 & 0.984556 & 3.411186 & 3.423769 \\
\hline 6 & -3.107607 & -0.703216 & -0.348387 \\
\hline 6 & -4.110526 & -0.941781 & 0.601758 \\
\hline 6 & -5.328221 & -1.503608 & 0.233639 \\
\hline 6 & -5.514074 & -1.846235 & -1.116751 \\
\hline 6 & -4.536900 & -1.631808 & -2.084029 \\
\hline 6 & -3.328801 & -1.054186 & -1.671432 \\
\hline 6 & -4.699037 & -2.034605 & -3.547857 \\
\hline 6 & -3.745204 & -3.197585 & -3.840652 \\
\hline 6 & -6.440829 & -1.774906 & 1.245961 \\
\hline 6 & -6.654694 & -3.287194 & 1.371188 \\
\hline 6 & -1.136382 & -0.916927 & 1.679307 \\
\hline 6 & -0.641387 & -2.214417 & 1.617662 \\
\hline 6 & -0.376597 & -2.944271 & 2.824063 \\
\hline 6 & -0.622738 & -2.333778 & 4.087823 \\
\hline 6 & -1.145443 & -1.022345 & 4.116612 \\
\hline 6 & -1.389760 & -0.340008 & 2.955382 \\
\hline 6 & -0.327520 & -3.038770 & 5.278970 \\
\hline 6 & 0.194879 & -4.305594 & 5.234565 \\
\hline 6 & 0.436857 & -4.920132 & 3.987878 \\
\hline
\end{tabular}




\begin{tabular}{|c|c|c|c|}
\hline 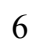 & 0.155126 & -4.261749 & 2.816371 \\
\hline & -0.374388 & -2.933074 & 0.338691 \\
\hline O & -1.332974 & -3.871400 & -0.159743 \\
\hline & -1.010233 & -4.672368 & -1.295738 \\
\hline & 0.250939 & -4.516905 & -1.915604 \\
\hline & 1.151637 & -3.609835 & -1.429065 \\
\hline & 0.844589 & -2.810345 & -0.303673 \\
\hline & -1.944996 & -5.626546 & -1.766026 \\
\hline & -3.157853 & -5.786223 & -1.146798 \\
\hline 6 & -3.489580 & -4.983479 & -0.034328 \\
\hline & -2.601349 & -4.055249 & 0.449853 \\
\hline & 1.835114 & -1.877884 & 0.162807 \\
\hline & 2.882406 & -2.456351 & 1.036981 \\
\hline & 3.355734 & -1.584326 & 2.170594 \\
\hline & 2.456209 & -0.875635 & 2.968929 \\
\hline & 2.907293 & -0.123450 & 4.044947 \\
\hline 6 & 4.269133 & -0.061626 & 4.337493 \\
\hline 6 & 5.172340 & -0.757540 & 3.542287 \\
\hline 6 & 4.715952 & -1.514884 & 2.465524 \\
\hline 6 & -6.121987 & -2.482195 & -3.872065 \\
\hline 6 & -4.348749 & -0.856483 & -4.464672 \\
\hline 6 & -6.103337 & -1.230599 & 2.632451 \\
\hline 6 & -7.743974 & -1.115988 & 0.781212 \\
\hline 6 & 0.178119 & -0.994539 & -3.264104 \\
\hline 8 & 0.258494 & -1.598580 & -4.254986 \\
\hline 6 & -0.412552 & 2.384235 & -2.714068 \\
\hline 6 & 0.630125 & 2.416670 & -1.759389 \\
\hline 6 & -0.049462 & 2.422571 & -4.186275 \\
\hline 6 & -1.718823 & 3.063470 & -2.396263 \\
\hline 6 & -1.730054 & 4.243928 & -1.649427 \\
\hline 6 & -2.915759 & 4.924197 & -1.392452 \\
\hline 6 & -4.093329 & 4.412125 & -1.906699 \\
\hline 6 & -4.126812 & 3.250948 & -2.661820 \\
\hline 6 & -2.932578 & 2.587555 & -2.904898 \\
\hline 6 & -5.285502 & 3.463683 & 3.447723 \\
\hline 6 & -5.163077 & 5.251817 & 1.708453 \\
\hline 6 & 0.741829 & 5.101212 & 1.580250 \\
\hline 6 & -0.433678 & 5.408780 & 3.739034 \\
\hline 1 & 7.146606 & 0.859924 & 0.664582 \\
\hline 1 & -1.797688 & 0.665480 & 3.018634 \\
\hline 1 & 8.415847 & -3.769280 & -0.611466 \\
\hline 1 & -0.520328 & -2.547526 & 6.231844 \\
\hline 1 & 5.863202 & 5.591804 & -1.054600 \\
\hline 1 & -2.870920 & -3.441081 & 1.308170 \\
\hline 1 & -4.458662 & -5.104908 & 0.445943 \\
\hline 1 & 3.896925 & 4.753500 & 0.099095 \\
\hline 1 & -1.351251 & -0.558039 & 5.080559 \\
\hline 1 & 6.366470 & -4.006152 & -1.900446 \\
\hline 1 & 8.558281 & 2.871841 & -3.004605 \\
\hline 1 & 4.142094 & -3.104482 & -2.577866 \\
\hline 1 & -1.676676 & -6.229877 & -2.632384 \\
\hline 1 & 0.492064 & -5.130441 & -2.782291 \\
\hline 1 & 0.847748 & -5.926659 & 3.954402 \\
\hline 1 & 7.207521 & 0.968570 & -2.267965 \\
\hline 1 & 0.342597 & -4.755086 & 1.864823 \\
\hline 1 & 2.123187 & -3.480449 & -1.902217 \\
\hline
\end{tabular}




\begin{tabular}{|c|c|c|c|}
\hline 1 & 7.917552 & 5.199380 & -2.383689 \\
\hline 1 & 9.230616 & -0.118401 & 1.504356 \\
\hline . & -3.868478 & -6.523797 & -1.513455 \\
\hline & 0.423676 & -4.838421 & 6.154614 \\
\hline & 9.896987 & -2.431472 & 0.857858 \\
\hline & 2.436689 & 2.829434 & 0.744758 \\
\hline & -3.924018 & -0.675996 & 1.640356 \\
\hline & -2.524640 & -0.888155 & -2.387426 \\
\hline & -6.460151 & -2.298209 & -1.409333 \\
\hline 1 & -4.077245 & 1.560761 & 0.537768 \\
\hline & 0.104041 & 2.029996 & 1.301863 \\
\hline 1 & -2.694511 & 4.899422 & 2.836415 \\
\hline 1 & 2.429492 & -3.363360 & 1.463022 \\
\hline & 3.747902 & -2.793702 & 0.446307 \\
\hline 1 & 5.431529 & -2.049329 & 1.837965 \\
\hline & 1.397875 & -0.890447 & 2.716217 \\
\hline 1 & 6.239331 & -0.709024 & 3.751885 \\
\hline & 2.188682 & 0.420313 & 4.656804 \\
\hline 1 & 4.622729 & 0.531813 & 5.178132 \\
\hline 1 & 0.434096 & 2.844086 & -0.775740 \\
\hline & 1.657655 & 2.488483 & -2.116334 \\
\hline & -2.948446 & 1.664821 & -3.485742 \\
\hline 1 & -5.077250 & 2.883192 & -3.039573 \\
\hline & -5.249391 & 5.051641 & -1.656910 \\
\hline 1 & -2.930948 & 5.838454 & -0.803787 \\
\hline 1 & -0.792816 & 4.645163 & -1.269966 \\
\hline 1 & -0.793890 & 1.909468 & -4.807402 \\
\hline 1 & -0.004447 & 3.468104 & -4.522335 \\
\hline & 0.922727 & 1.950117 & -4.360856 \\
\hline 1 & -1.114398 & 0.934806 & -2.664500 \\
\hline 1 & 0.444486 & 5.907963 & 4.167798 \\
\hline 1 & -1.059984 & 6.189581 & 3.290012 \\
\hline 1 & -0.993461 & 4.951473 & 4.565267 \\
\hline 1 & 1.809709 & 3.973554 & 3.880069 \\
\hline 1 & 0.467810 & 2.858881 & 4.220781 \\
\hline 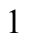 & 1.432356 & 2.671075 & 2.749457 \\
\hline 1 & 1.630752 & 5.624126 & 1.960505 \\
\hline 1 & 1.064199 & 4.414157 & 0.786257 \\
\hline 1 & 0.080564 & 5.848389 & 1.119768 \\
\hline 1 & -7.015821 & 3.375498 & 1.344856 \\
\hline l & -5.832885 & 3.157666 & 0.044724 \\
\hline 1 & -6.004228 & 1.925605 & 1.317958 \\
\hline 1 & -5.015926 & 5.488898 & 0.648334 \\
\hline 1 & -6.183585 & 5.556735 & 1.976206 \\
\hline 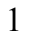 & -4.476389 & 5.868652 & 2.299813 \\
\hline 1 & -6.320966 & 3.738086 & 3.691391 \\
\hline 1 & -5.153952 & 2.396495 & 3.672232 \\
\hline 1 & -4.622392 & 4.027936 & 4.115469 \\
\hline 1 & -6.934811 & -1.429453 & 3.320290 \\
\hline 1 & -5.207259 & -1.704302 & 3.055537 \\
\hline 1 & -5.939288 & -0.143706 & 2.616402 \\
\hline 1 & -7.475751 & -3.502644 & 2.068554 \\
\hline 1 & -6.904315 & -3.748155 & 0.407257 \\
\hline 1 & -5.749802 & -3.776424 & 1.757412 \\
\hline 1 & -8.540901 & -1.291977 & 1.516043 \\
\hline 1 & -7.621848 & -0.030625 & 0.669561 \\
\hline
\end{tabular}




$\begin{array}{llll}1 & -8.091559 & -1.512408 & -0.180057 \\ 1 & -6.196565 & -2.735085 & -4.937226 \\ 1 & -6.410074 & -3.377037 & -3.305642 \\ 1 & -6.856895 & -1.692845 & -3.665745 \\ 1 & -3.833631 & -3.513255 & -4.889436 \\ 1 & -2.698469 & -2.919754 & -3.659314 \\ 1 & -3.973427 & -4.058992 & -3.199413 \\ 1 & -4.492646 & -1.140859 & -5.515512 \\ 1 & -4.991952 & 0.010229 & -4.259665 \\ 1 & -3.301125 & -0.542331 & -4.356880\end{array}$

(para-F)TS1 $1_{I L L-s i-} \psi_{-1 \mathrm{~s} 2 \mathrm{a} 3 \mathrm{~s} 4 \mathrm{~s}}$

Number of imaginary frequencies $\cdot 1$

The smallest frequencies are : $-513.2522 \quad 14.9175 \quad 17.0225 \mathrm{~cm}(-1)$

Electronic energy : $\quad H F=-4460.0841483$

Zero-point correction $=\quad 1.444456($ Hartree/Particle $)$

Thermal correction to Energy= $\quad 1.529944$

Thermal correction to Enthalpy $=\quad 1.530888$

Thermal correction to Gibbs Free Energy= $\quad 1.325203$

Sum of electronic and zero-point Energies $=\quad-4458.639692$

Sum of electronic and thermal Energies $=\quad-4458.554204$

Sum of electronic and thermal Enthalpies $=\quad-4458.553260$

Sum of electronic and thermal Free Energies $=\quad-4458.758945$

Cartesian Coordinates

$\begin{array}{cccc}\ldots & -2.827580 & 2.821483 & -2.919209 \\ 6 & -1.613554 & 3.195628 & -2.332224 \\ 6 & -1.595091 & 4.323207 & -1.508655 \\ 6 & -2.752624 & 5.052276 & -1.257235 \\ 6 & -3.931242 & 4.644585 & -1.855092 \\ 6 & -3.993798 & 3.535709 & -2.684109 \\ 6 & -0.335199 & 2.466196 & -2.650883 \\ 6 & 0.047873 & 2.524117 & -4.117534 \\ 9 & -5.059518 & 5.334324 & -1.613729 \\ 45 & 0.104917 & 0.292015 & -1.814850 \\ 6 & 0.699078 & 2.434328 & -1.685873 \\ 15 & -1.486559 & 0.037685 & 0.112525 \\ 6 & -1.139992 & -0.971269 & 1.650533 \\ 6 & -0.658483 & -2.272215 & 1.552887 \\ 6 & -0.409203 & -3.039892 & 2.739198 \\ 6 & -0.659189 & -2.465537 & 4.019059 \\ 6 & -1.166134 & -1.149148 & 4.083732 \\ 6 & -1.393245 & -0.429409 & 2.941666 \\ 6 & -0.383403 & -3.210165 & 5.190683 \\ 6 & 0.124104 & -4.481326 & 5.112135 \\ 6 & 0.370869 & -5.059889 & 3.849362 \\ 6 & 0.107976 & -4.362626 & 2.696161 \\ 6 & -0.395985 & -2.958265 & 0.254592 \\ 6 & -1.361376 & -3.875608 & -0.269248 \\ 6 & -1.052432 & -4.636959 & -1.435689 \\ 6 & 0.207662 & -4.470878 & -2.054967 \\ 6 & 1.118546 & -3.589943 & -1.539892 \\ 6 & 0.821931 & -2.825242 & -0.387646 \\ 6 & -1.999646 & -5.563522 & -1.935638\end{array}$




\begin{tabular}{|c|c|c|c|}
\hline 6 & -3.209672 & -5.736443 & -1.314025 \\
\hline 6 & -3.524177 & -4.976930 & -0.166913 \\
\hline & -2.624646 & -4.075061 & 0.344496 \\
\hline & 1.820008 & -1.914727 & 0.103957 \\
\hline & 2.859123 & -2.519837 & 0.969922 \\
\hline & 3.334325 & -1.673392 & 2.122373 \\
\hline f & 2.435213 & -0.982648 & 2.937020 \\
\hline o & 2.887813 & -0.243372 & 4.021177 \\
\hline 6 & 4.250962 & -0.178150 & 4.307194 \\
\hline 6 & 5.153325 & -0.859216 & 3.498379 \\
\hline 6 & 4.695305 & -1.603113 & 2.412858 \\
\hline 6 & -1.963553 & 1.641110 & 0.889058 \\
\hline 6 & -0.927116 & 2.374361 & 1.467693 \\
\hline 6 & -1.179883 & 3.533805 & 2.206974 \\
\hline 6 & -2.504218 & 3.962763 & 2.307574 \\
\hline 6 & -3.565097 & 3.283979 & 1.694537 \\
\hline 6 & -3.273190 & 2.118535 & 0.982764 \\
\hline 6 & -5.004899 & 3.750617 & 1.922289 \\
\hline 6 & -5.135158 & 5.270846 & 1.791623 \\
\hline 6 & -0.020325 & 4.292656 & 2.850320 \\
\hline 6 & -0.493953 & 5.312035 & 3.885498 \\
\hline 6 & -3.112491 & -0.696384 & -0.355664 \\
\hline 6 & -4.084104 & -1.003753 & 0.598873 \\
\hline 6 & -5.295388 & -1.592173 & 0.236227 \\
\hline 6 & -5.516245 & -1.852870 & -1.122195 \\
\hline 6 & -4.567897 & -1.554129 & -2.103443 \\
\hline 6 & -3.360970 & -0.982372 & -1.692831 \\
\hline 6 & -6.316732 & -1.923422 & 1.325323 \\
\hline 6 & -6.807848 & -0.620400 & 1.965273 \\
\hline 6 & -4.765661 & -1.883357 & -3.581611 \\
\hline 6 & -4.384197 & -0.680347 & -4.452586 \\
\hline 15 & 1.987335 & -0.511936 & -0.829779 \\
\hline 8 & 2.876029 & 0.319596 & 0.288768 \\
\hline 6 & 3.727453 & 1.333937 & -0.061638 \\
\hline 6 & 4.921726 & 1.064261 & -0.703107 \\
\hline 6 & 5.732110 & 2.178213 & -1.105070 \\
\hline 6 & 5.351932 & 3.500260 & -0.721950 \\
\hline 6 & 4.167148 & 3.695219 & 0.029492 \\
\hline 6 & 3.363726 & 2.634478 & 0.340296 \\
\hline 6 & 6.891616 & 2.028751 & -1.907837 \\
\hline 6 & 7.644151 & 3.115599 & -2.279509 \\
\hline 6 & 7.284035 & 4.415380 & -1.868360 \\
\hline 6 & 6.156785 & 4.598506 & -1.109188 \\
\hline 6 & 5.313772 & -0.337086 & -0.978570 \\
\hline 6 & 4.445248 & -1.180309 & -1.647091 \\
\hline 6 & 4.826127 & -2.475191 & -2.061353 \\
\hline 6 & 6.050596 & -2.972065 & -1.710824 \\
\hline 6 & 6.928974 & -2.209081 & -0.902781 \\
\hline 6 & 6.562045 & -0.881969 & -0.527109 \\
\hline 6 & 8.148951 & -2.755802 & -0.436155 \\
\hline 6 & 8.969766 & -2.040222 & 0.397511 \\
\hline 6 & 8.594424 & -0.741790 & 0.800271 \\
\hline 6 & 7.427743 & -0.176266 & 0.347039 \\
\hline 8 & 3.180025 & -0.766338 & -1.985445 \\
\hline 6 & 0.126386 & -0.917647 & -3.282733 \\
\hline 8 & 0.179210 & -1.498403 & -4.289118 \\
\hline
\end{tabular}




\begin{tabular}{|c|c|c|c|}
\hline 6 & -5.984283 & 3.118202 & 0.933940 \\
\hline 6 & -5.410283 & 3.336219 & 3.342686 \\
\hline 6 & 0.923443 & 3.314961 & 3.560294 \\
\hline 6 & 0.736667 & 5.047730 & 1.751207 \\
\hline 6 & -3.859510 & -3.067027 & -3.937821 \\
\hline 6 & -6.209263 & -2.262769 & -3.904150 \\
\hline 6 & -5.665791 & -2.796488 & 2.404935 \\
\hline 6 & -7.528542 & -2.674922 & 0.780101 \\
\hline 1 & 7.142444 & 0.816855 & 0.684790 \\
\hline 1 & -1.789312 & 0.578900 & 3.031299 \\
\hline 1 & 8.412161 & -3.767082 & -0.743707 \\
\hline 1 & -0.579196 & -2.745852 & 6.156375 \\
\hline 1 & 5.851509 & 5.596555 & -0.797108 \\
\hline 1 & -2.880513 & -3.494612 & 1.229294 \\
\hline 1 & -4.490927 & -5.108997 & 0.316798 \\
\hline 1 & 3.899534 & 4.704675 & 0.339219 \\
\hline 1 & -1.373168 & -0.711374 & 5.059758 \\
\hline 1 & 6.355738 & -3.968873 & -2.024960 \\
\hline 1 & 8.523590 & 2.971724 & -2.903205 \\
\hline 1 & 4.127312 & -3.049208 & -2.665621 \\
\hline 1 & -1.743081 & -6.135708 & -2.826328 \\
\hline 1 & 0.439981 & -5.055016 & -2.944090 \\
\hline 1 & 0.770727 & -6.069572 & 3.788517 \\
\hline 1 & 7.177867 & 1.036012 & -2.245146 \\
\hline 1 & 0.298992 & -4.829040 & 1.731896 \\
\hline 1 & 2.089874 & -3.452777 & -2.011572 \\
\hline 1 & 7.891911 & 5.267054 & -2.164644 \\
\hline 1 & 9.232146 & -0.183553 & 1.482152 \\
\hline 1 & -3.929674 & -6.452628 & -1.704058 \\
\hline 1 & 0.337956 & -5.044781 & 6.017398 \\
\hline 1 & 9.899522 & -2.473508 & 0.758617 \\
\hline 1 & 2.438888 & 2.753308 & 0.900358 \\
\hline 1 & -3.884446 & -0.785777 & 1.648537 \\
\hline 1 & -2.574423 & -0.769442 & -2.415716 \\
\hline 1 & -6.454647 & -2.311046 & -1.421636 \\
\hline 1 & -4.074611 & 1.553835 & 0.513493 \\
\hline 1 & 0.090914 & 1.986479 & 1.385474 \\
\hline 1 & -2.726554 & 4.851048 & 2.893840 \\
\hline 1 & 2.397441 & -3.432051 & 1.374731 \\
\hline 1 & 3.724408 & -2.850282 & 0.374757 \\
\hline 1 & 5.410532 & -2.124581 & 1.774120 \\
\hline 1 & 1.375810 & -0.998827 & 2.689551 \\
\hline 1 & 6.220941 & -0.809337 & 3.704294 \\
\hline 1 & 2.169147 & 0.287240 & 4.644569 \\
\hline 1 & 4.606043 & 0.405529 & 5.154013 \\
\hline 1 & 0.512298 & 2.847113 & -0.694148 \\
\hline 1 & 1.730826 & 2.482596 & -2.034706 \\
\hline 1 & -2.864152 & 1.941962 & -3.562470 \\
\hline 1 & -4.943757 & 3.246975 & -3.126583 \\
\hline 1 & -2.744398 & 5.926903 & -0.610769 \\
\hline 1 & -0.656721 & 4.645547 & -1.062976 \\
\hline 1 & -0.704117 & 2.050177 & -4.759992 \\
\hline 1 & 0.132950 & 3.574539 & -4.429720 \\
\hline 1 & 1.005506 & 2.022291 & -4.290210 \\
\hline 1 & -1.095760 & 1.054011 & -2.627292 \\
\hline 1 & 0.374719 & 5.795474 & 4.350173 \\
\hline
\end{tabular}




$\begin{array}{rrrr}1 & -1.103367 & 6.107170 & 3.438423 \\ 1 & -1.078952 & 4.838589 & 4.684707 \\ 1 & 1.736730 & 3.862853 & 4.053994 \\ 1 & 0.384370 & 2.742411 & 4.327780 \\ 1 & 1.388370 & 2.591960 & 2.879067 \\ 1 & 1.618801 & 5.555918 & 2.165643 \\ 1 & 1.074584 & 4.376198 & 0.950216 \\ 1 & 0.091857 & 5.810406 & 1.292328 \\ 1 & -6.995279 & 3.499649 & 1.126144 \\ 1 & -5.728673 & 3.365286 & -0.104404 \\ 1 & -6.028305 & 2.025707 & 1.026707 \\ 1 & -4.880941 & 5.605605 & 0.779177 \\ 1 & -6.173565 & 5.569896 & 1.986483 \\ 1 & -4.506472 & 5.815812 & 2.505137 \\ 1 & -6.448456 & 3.630138 & 3.549524 \\ 1 & -5.332950 & 2.248242 & 3.472087 \\ 1 & -4.768783 & 3.807545 & 4.098334 \\ 1 & -8.218587 & -2.907822 & 1.600997 \\ 1 & -8.085572 & -2.081144 & 0.044213 \\ 1 & -7.242620 & -3.624950 & 0.309196 \\ 1 & -6.391645 & -3.021855 & 3.197802 \\ 1 & -5.322365 & -3.752432 & 1.987050 \\ 1 & -4.803918 & -2.309240 & 2.878802 \\ 1 & -7.533708 & -0.830955 & 2.762557 \\ 1 & -5.980542 & -0.051035 & 2.409593 \\ 1 & -7.299382 & 0.020933 & 1.221567 \\ 1 & -6.310417 & -2.450404 & -4.980583 \\ 1 & -6.520687 & -3.179140 & -3.387034 \\ 1 & -6.911819 & -1.461928 & -3.638005 \\ 1 & -3.969588 & -3.331675 & -4.998722 \\ 1 & -2.801409 & -2.836587 & -3.754367 \\ 1 & -4.114664 & -3.947461 & -3.333405 \\ 1 & -4.574923 & -0.905674 & -5.510183 \\ 1 & -4.973731 & 0.207086 & -4.183968 \\ 1 & -3.318977 & -0.425882 & -4.365867\end{array}$

$\left(\right.$ para-F)TS1 $1_{I L-r e-} \psi_{-1 \mathbf{a} 2 s 3 a 4 a}$

Number of imaginary frequencies : 1

The smallest frequencies are : -605.2537 $16.2945 \quad 21.0738 \mathrm{~cm}(-1)$

Electronic energy : $\quad H F=-4460.0804402$

Zero-point correction $=\quad 1.443776($ Hartree/Particle $)$

Thermal correction to Energy $=\quad 1.529514$

Thermal correction to Enthalpy= $\quad 1.530458$

Thermal correction to Gibbs Free Energy $=\quad 1.324484$

Sum of electronic and zero-point Energies $=\quad-4458.636664$

Sum of electronic and thermal Energies $=\quad-4458.550927$

Sum of electronic and thermal Enthalpies $=\quad-4458.549982$

Sum of electronic and thermal Free Energies $=\quad-4458.755956$

Cartesian Coordinates

\begin{tabular}{|c|c|c|}
\hline & -6.436986 & -2.828247 \\
\hline & -5.348923 & -2.525595 \\
\hline & -4.874409 & -3.557622 \\
\hline
\end{tabular}




\begin{tabular}{|c|c|c|c|}
\hline 6 & -5.525977 & -4.815020 & 0.490263 \\
\hline 5 & -6.590423 & -5.065436 & -0.336691 \\
\hline 6 & -7.039377 & -4.061602 & -1.220093 \\
\hline 6 & -4.691789 & -1.252003 & -0.388382 \\
\hline 6 & -3.559034 & -1.100701 & 0.389337 \\
\hline & -3.086375 & -2.117980 & 1.242133 \\
\hline 6 & -3.744271 & -3.316868 & 1.297907 \\
\hline ) & -5.135933 & -0.123694 & -1.237388 \\
\hline 6 & -4.238226 & 0.461998 & -2.113523 \\
\hline 6 & -4.635740 & 1.464343 & -3.026000 \\
\hline 6 & -5.917441 & 1.941429 & -3.007070 \\
\hline 6 & -6.847942 & 1.463898 & -2.051501 \\
\hline 6 & -6.454734 & 0.429910 & -1.149982 \\
\hline 6 & -8.145168 & 2.022550 & -1.950598 \\
\hline 6 & -9.019562 & 1.605726 & -0.979442 \\
\hline 6 & -8.622063 & 0.610262 & -0.062725 \\
\hline 6 & -7.377370 & 0.035937 & -0.147451 \\
\hline 8 & -2.931714 & 0.048437 & -2.169053 \\
\hline 15 & -1.832453 & 0.336235 & -0.919443 \\
\hline 8 & -2.866712 & 0.082953 & 0.358784 \\
\hline 45 & 0.086806 & -0.832192 & -1.188089 \\
\hline 15 & 1.355530 & 0.236304 & 0.513062 \\
\hline 6 & 1.461495 & -0.919202 & 1.941044 \\
\hline 6 & 2.567729 & -1.739272 & 2.123058 \\
\hline 6 & 2.619156 & -2.668506 & 3.171781 \\
\hline 6 & 1.528423 & -2.739406 & 4.033932 \\
\hline 6 & 0.398024 & -1.917035 & 3.886825 \\
\hline 6 & 0.369077 & -1.034373 & 2.811904 \\
\hline 6 & 3.877077 & -3.518890 & 3.340897 \\
\hline 6 & 3.784849 & -4.466801 & 4.533433 \\
\hline 6 & -0.750877 & -2.012386 & 4.891152 \\
\hline 6 & -0.205147 & -1.964020 & 6.323001 \\
\hline 6 & 0.263855 & -3.250660 & -1.166126 \\
\hline 6 & 0.179857 & -3.804420 & 0.238355 \\
\hline 6 & 1.360835 & -3.806993 & -2.027314 \\
\hline 6 & 2.510543 & -4.342093 & -1.431183 \\
\hline 6 & 3.571014 & -4.822656 & -2.188127 \\
\hline 6 & 3.479984 & -4.763457 & -3.569097 \\
\hline 6 & 2.361736 & -4.246755 & -4.201286 \\
\hline 6 & 1.310012 & -3.771803 & -3.425948 \\
\hline 9 & 4.497129 & -5.223097 & -4.311615 \\
\hline 6 & -0.916356 & -2.720456 & -1.741216 \\
\hline 6 & 0.554592 & -0.456500 & -3.096501 \\
\hline 8 & 0.405535 & -0.347675 & -4.241022 \\
\hline 6 & 1.005725 & 1.862471 & 1.345932 \\
\hline 6 & 0.686637 & 2.974806 & 0.580563 \\
\hline 6 & 0.491744 & 4.249202 & 1.207718 \\
\hline 6 & 0.634454 & 4.368116 & 2.620735 \\
\hline 6 & 0.973840 & 3.218551 & 3.369471 \\
\hline 6 & 1.151242 & 2.009139 & 2.752689 \\
\hline 6 & 0.430984 & 5.623578 & 3.241883 \\
\hline 6 & 0.095806 & 6.727679 & 2.500931 \\
\hline 6 & -0.048698 & 6.617497 & 1.101834 \\
\hline 6 & 0.146012 & 5.413363 & 0.471561 \\
\hline 6 & 0.569669 & 2.927038 & -0.903122 \\
\hline 6 & -0.618565 & 2.560674 & -1.509427 \\
\hline
\end{tabular}




\begin{tabular}{|c|c|c|c|}
\hline & -0.769271 & 2.661032 & -2.911843 \\
\hline & 0.273070 & 3.060827 & -3.703471 \\
\hline & 1.528053 & 3.371516 & -3.130990 \\
\hline & 1.675903 & 3.330268 & -1.712550 \\
\hline & 2.939791 & 3.658951 & -1.156604 \\
\hline & 3.999699 & 3.989733 & -1.963237 \\
\hline & 3.849640 & 4.027744 & -3.367081 \\
\hline & 2.638246 & 3.727144 & -3.935685 \\
\hline & -1.698132 & 2.019739 & -0.730965 \\
\hline & -2.769342 & 2.971379 & -0.357555 \\
\hline & -3.406487 & 2.714221 & 0.982046 \\
\hline & -4.788050 & 2.576733 & 1.094403 \\
\hline & -5.376165 & 2.315263 & 2.329696 \\
\hline & -4.585763 & 2.199333 & 3.468115 \\
\hline & -3.204194 & 2.355630 & 3.364991 \\
\hline & -2.621469 & 2.609304 & 2.130962 \\
\hline & 3.133671 & 0.489609 & 0.089882 \\
\hline & 3.593837 & 0.124961 & -1.169615 \\
\hline & 4.936920 & 0.286064 & -1.522303 \\
\hline & 5.797295 & 0.826154 & -0.565803 \\
\hline & 5.367870 & 1.207932 & 0.712586 \\
\hline & 4.021544 & 1.030769 & 1.023999 \\
\hline & 5.461713 & -0.139350 & -2.891917 \\
\hline & 6.270224 & 0.996031 & -3.526855 \\
\hline & 6.359718 & 1.824631 & 1.698023 \\
\hline & 7.542530 & 0.875179 & 1.914320 \\
\hline & 6.869443 & 3.153595 & 1.128778 \\
\hline & 5.723825 & 2.105651 & 3.058075 \\
\hline & 4.326811 & -0.497110 & -3.851647 \\
\hline & 6.357761 & -1.369933 & -2.714081 \\
\hline & 4.106060 & -4.358973 & 2.079221 \\
\hline & 5.080356 & -2.591968 & 3.555001 \\
\hline 6 & -1.741375 & -0.856952 & 4.741440 \\
\hline & -1.492441 & -3.336229 & 4.679835 \\
\hline 1 & -7.078453 & -0.716169 & 0.578437 \\
\hline & 1.418492 & 1.145709 & 3.356412 \\
\hline & -8.426800 & 2.804593 & -2.654696 \\
\hline & 0.544103 & 5.690746 & 4.323075 \\
\hline & -5.152163 & -5.583490 & 1.165782 \\
\hline & 3.069935 & 3.620186 & -0.076137 \\
\hline & 4.967706 & 4.217082 & -1.520171 \\
\hline & -3.390379 & -4.112967 & 1.952946 \\
\hline & 1.096407 & 3.309592 & 4.448110 \\
\hline & -6.232353 & 2.711930 & -3.708924 \\
\hline & -7.867607 & -4.268339 & -1.894253 \\
\hline 1 & -3.899610 & 1.834861 & -3.736185 \\
\hline & 2.507300 & 3.753092 & -5.016600 \\
\hline & 0.160078 & 3.113094 & -4.785247 \\
\hline & -0.315072 & 7.495156 & 0.517293 \\
\hline & -6.788743 & -2.070942 & -1.940217 \\
\hline & 0.034517 & 5.345367 & -0.608647 \\
\hline & -1.724692 & 2.376783 & -3.349416 \\
\hline 1 & -7.080269 & -6.036275 & -0.323973 \\
\hline & -9.305661 & 0.297909 & 0.723673 \\
\hline 1 & 4.697078 & 4.298212 & -3.993797 \\
\hline & -0.060475 & 7.687884 & 2.987060 \\
\hline
\end{tabular}




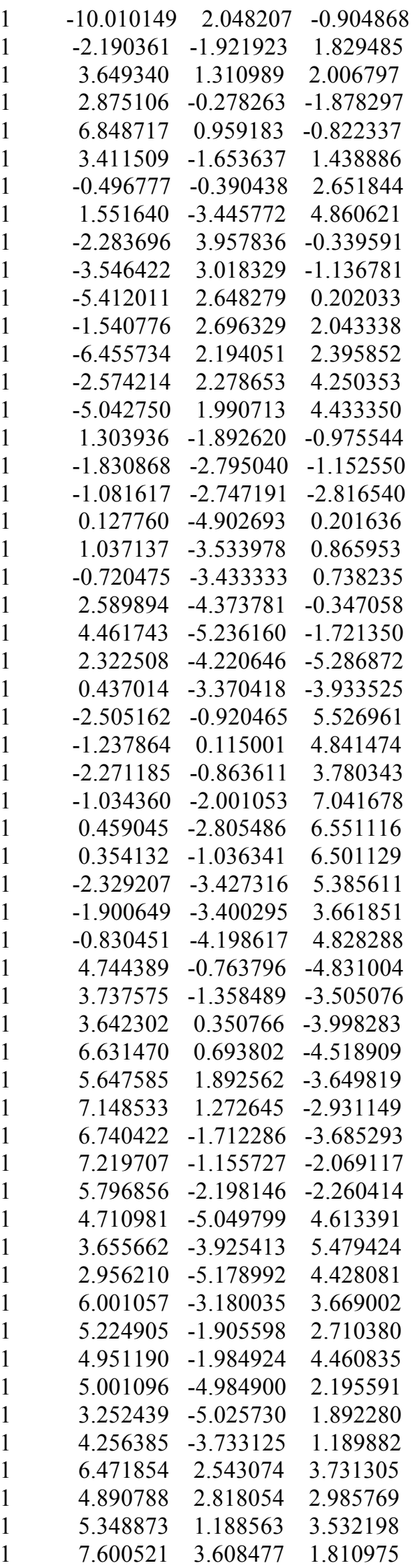




$\begin{array}{cccc}1 & 7.357368 & 3.024602 & 0.154621 \\ 1 & 6.042291 & 3.865148 & 1.000631 \\ 1 & 8.257349 & 1.313493 & 2.623638 \\ 1 & 7.206401 & -0.085766 & 2.325978 \\ 1 & 8.086672 & 0.668430 & 0.985206\end{array}$

(para-F)TS1 $1_{I L-r e-} \Psi_{-1 \mathbf{a} 2 s 3 a 4 s}$

Number of imaginary frequencies : 1

The smallest frequencies are : -594.0775 $15.0926 \quad 19.7651 \mathrm{~cm}(-1)$

Electronic energy : $\quad \mathrm{HF}=-4460.0807947$

Zero-point correction $=\quad 1.443402($ Hartree/Particle $)$

Thermal correction to Energy= $\quad 1.529184$

Thermal correction to Enthalpy= $\quad 1.530129$

Thermal correction to Gibbs Free Energy $=\quad 1.323471$

Sum of electronic and zero-point Energies $=\quad-4458.637393$

Sum of electronic and thermal Energies $=\quad-4458.551610$

Sum of electronic and thermal Enthalpies $=\quad-4458.550666$

Sum of electronic and thermal Free Energies $=\quad-4458.757323$

Cartesian Coordinates

$\begin{array}{cccc}6 & 3.977687 & 1.186519 & 0.937248 \\ 6 & 3.108442 & 0.528332 & 0.071838 \\ 6 & 3.593440 & 0.012188 & -1.127160 \\ 6 & 4.938420 & 0.131683 & -1.472970 \\ 6 & 5.785323 & 0.805946 & -0.584494 \\ 6 & 5.328607 & 1.349156 & 0.619208 \\ 15 & 1.334374 & 0.275791 & 0.510366 \\ 6 & 0.971335 & 1.917704 & 1.301211 \\ 6 & 0.654039 & 3.008392 & 0.504519 \\ 6 & 0.456434 & 4.298815 & 1.096281 \\ 6 & 0.592490 & 4.455203 & 2.506371 \\ 6 & 0.925004 & 3.325626 & 3.287828 \\ 6 & 1.105724 & 2.099909 & 2.704654 \\ 6 & 0.387957 & 5.727145 & 3.092719 \\ 6 & 0.058231 & 6.811425 & 2.320798 \\ 6 & -0.079578 & 6.664078 & 0.924374 \\ 6 & 0.116092 & 5.443263 & 0.327425 \\ 6 & 0.541492 & 2.917455 & -0.977511 \\ 6 & -0.644794 & 2.530321 & -1.575377 \\ 6 & -0.791415 & 2.589447 & -2.980601 \\ 6 & 0.255357 & 2.958782 & -3.781389 \\ 6 & 1.511364 & 3.277774 & -3.215679 \\ 6 & 1.651668 & 3.290358 & -1.796108 \\ 6 & 2.915240 & 3.630803 & -1.246852 \\ 6 & 3.985332 & 3.908493 & -2.060115 \\ 6 & 3.845963 & 3.881804 & -3.465108 \\ 6 & 2.631864 & 3.580646 & -4.027566 \\ 7 & -1.722567 & 2.002270 & -0.785252 \\ 6 & -2.795111 & 2.957196 & -0.424754 \\ 6 & -3.431971 & 2.713381 & 0.917484 \\ 6 & -4.812053 & 2.563198 & 1.030914 \\ 6 & -5.397464 & 2.305910 & 2.268459 \\ 6 & -4.605795 & 2.205758 & 3.407528 \\ 6 & -3.225825 & 2.375625 & 3.303294\end{array}$




\begin{tabular}{|c|c|c|c|}
\hline 5 & -2.646138 & 2.627072 & 2.067437 \\
\hline 6 & 5.483669 & -0.509358 & -2.747467 \\
\hline 6 & 6.234768 & -1.787192 & -2.353199 \\
\hline 6 & 6.231311 & 2.104823 & 1.594001 \\
\hline 6 & 7.638436 & 2.313262 & 1.040582 \\
\hline 45 & 0.080427 & -0.849085 & -1.161657 \\
\hline o & 0.572876 & -0.512816 & -3.074072 \\
\hline 8 & 0.433551 & -0.431279 & -4.221952 \\
\hline 15 & -1.846495 & 0.313175 & -0.933904 \\
\hline 8 & -2.879389 & 0.081395 & 0.349055 \\
\hline 6 & -3.556150 & -1.109872 & 0.407366 \\
\hline 6 & -4.687991 & -1.292571 & -0.365140 \\
\hline 6 & -5.329736 & -2.573547 & -0.332005 \\
\hline 6 & -4.840713 & -3.579293 & 0.556927 \\
\hline 6 & -3.711815 & -3.306544 & 1.366457 \\
\hline 6 & -3.068476 & -2.101494 & 1.281737 \\
\hline 6 & -5.476951 & -4.843780 & 0.598508 \\
\hline 6 & -6.540508 & -5.126312 & -0.219123 \\
\hline 6 & -7.003866 & -4.148922 & -1.124568 \\
\hline 6 & -6.416305 & -2.909369 & -1.179903 \\
\hline 6 & -5.145584 & -0.187414 & -1.237533 \\
\hline 6 & -4.254272 & 0.391055 & -2.125283 \\
\hline 6 & -4.661307 & 1.374520 & -3.053877 \\
\hline 6 & -5.948026 & 1.838247 & -3.043666 \\
\hline 6 & -6.874344 & 1.366302 & -2.081344 \\
\hline 6 & -6.470431 & 0.353227 & -1.160892 \\
\hline 6 & -7.390131 & -0.033014 & -0.152600 \\
\hline 6 & -8.641958 & 0.527381 & -0.080650 \\
\hline 6 & -9.049816 & 1.500976 & -1.016095 \\
\hline 6 & -8.178428 & 1.910941 & -1.992803 \\
\hline 8 & -2.944212 & -0.011905 & -2.175519 \\
\hline 6 & 0.294290 & -3.268155 & -1.105664 \\
\hline 6 & -0.889993 & -2.764977 & -1.694931 \\
\hline 6 & 1.470283 & -0.845971 & 1.963132 \\
\hline 6 & 2.594996 & -1.639087 & 2.153481 \\
\hline 6 & 2.667967 & -2.554729 & 3.212551 \\
\hline 6 & 1.582385 & -2.634601 & 4.080737 \\
\hline 6 & 0.435824 & -1.835852 & 3.928138 \\
\hline 6 & 0.384228 & -0.969505 & 2.840547 \\
\hline 6 & 3.939150 & -3.385700 & 3.381049 \\
\hline 6 & 5.134659 & -2.442945 & 3.564413 \\
\hline 6 & -0.708881 & -1.942234 & 4.936215 \\
\hline 6 & -1.434988 & -3.275325 & 4.728998 \\
\hline 6 & 3.870978 & -4.316148 & 4.588807 \\
\hline 6 & 4.165439 & -4.241240 & 2.129115 \\
\hline 6 & -0.161612 & -1.884580 & 6.367022 \\
\hline 6 & -1.714079 & -0.799671 & 4.785354 \\
\hline 6 & 0.212964 & -3.802507 & 0.306423 \\
\hline 6 & 1.416105 & -3.799160 & -1.950601 \\
\hline 6 & 2.571064 & -4.302986 & -1.337423 \\
\hline 6 & 3.670518 & -4.719043 & -2.077356 \\
\hline 6 & 3.614307 & -4.625254 & -3.458273 \\
\hline 6 & 2.484532 & -4.157808 & -4.108068 \\
\hline 6 & 1.393624 & -3.747372 & -3.349758 \\
\hline 9 & 4.680624 & -4.994819 & -4.182239 \\
\hline 6 & 6.434456 & 0.444196 & -3.475351 \\
\hline
\end{tabular}




\begin{tabular}{|c|c|c|c|}
\hline 6 & 4.363380 & -0.887379 & -3.717363 \\
\hline 6 & 6.338798 & 1.310960 & 2.900997 \\
\hline 6 & 5.631225 & 3.485338 & 1.888907 \\
\hline 1 & -7.083573 & -0.767750 & 0.587737 \\
\hline 1 & 1.370331 & 1.252135 & 3.331468 \\
\hline 1 & -8.468081 & 2.676613 & -2.711494 \\
\hline 1 & 0.495681 & 5.822975 & 4.172292 \\
\hline 1 & -5.092131 & -5.591595 & 1.290825 \\
\hline 1 & 3.037601 & 3.641584 & -0.164878 \\
\hline 1 & 4.953167 & 4.145350 & -1.620949 \\
\hline 1 & -3.347309 & -4.083177 & 2.038903 \\
\hline 1 & 1.039631 & 3.445456 & 4.364502 \\
\hline 1 & -6.270656 & 2.593600 & -3.758365 \\
\hline 1 & -7.831480 & -4.381281 & -1.791087 \\
\hline 1 & -3.928282 & 1.741647 & -3.769166 \\
\hline 1 & 2.507643 & 3.561126 & -5.109431 \\
\hline 1 & 0.146688 & 2.972961 & -4.864788 \\
\hline 1 & -0.341566 & 7.526290 & 0.315387 \\
\hline 1 & -6.779179 & -2.172876 & -1.892056 \\
\hline 1 & 0.009849 & 5.346118 & -0.751071 \\
\hline 1 & -1.746233 & 2.293507 & -3.411924 \\
\hline 1 & -7.018627 & -6.102365 & -0.182170 \\
\hline 1 & -9.323188 & 0.221112 & 0.710185 \\
\hline 1 & 4.702411 & 4.105822 & -4.097532 \\
\hline 1 & -0.098800 & 7.784565 & 2.780231 \\
\hline 1 & -10.045968 & 1.932399 & -0.951537 \\
\hline 1 & -2.173634 & -1.880991 & 1.862203 \\
\hline 1 & 3.596877 & 1.583599 & 1.878163 \\
\hline 1 & 2.889729 & -0.482036 & -1.790989 \\
\hline 1 & 6.836547 & 0.909732 & -0.844669 \\
\hline 1 & 3.434682 & -1.546562 & \\
\hline 1 & -0.495601 & -0.346175 & 2.673905 \\
\hline 1 & 1.622293 & -3.330147 & 4.915874 \\
\hline 1 & -2.310665 & 3.944417 & -0.418441 \\
\hline 1 & -3.571856 & 2.993333 & -1.205006 \\
\hline 1 & -5.436426 & 2.619344 & 0.137661 \\
\hline 1 & -1.566358 & 2.724329 & 1.979470 \\
\hline 1 & -6.475766 & 2.174554 & 2.335914 \\
\hline 1 & -2.594978 & 2.311217 & 4.189093 \\
\hline 1 & -5.060658 & 1.999097 & 4.374189 \\
\hline 1 & 1.306810 & -1.886329 & -0.910247 \\
\hline 1 & -1.806623 & -2.842834 & -1.109938 \\
\hline 1 & -1.048104 & -2.808474 & -2.770826 \\
\hline 1 & 0.184085 & -4.902090 & 0.286994 \\
\hline 1 & 1.060448 & -3.503714 & 0.935130 \\
\hline 1 & -0.698456 & -3.442797 & 0.794492 \\
\hline 1 & 2.626294 & -4.354166 & -0.252874 \\
\hline 1 & 4.568231 & -5.101078 & -1.596764 \\
\hline 1 & 2.471670 & -4.106459 & -5.193464 \\
\hline 1 & 0.516412 & -3.373976 & -3.871102 \\
\hline 1 & -2.474496 & -0.869861 & 5.573542 \\
\hline 1 & -1.222651 & 0.179013 & 4.879898 \\
\hline 1 & -2.246819 & -0.817169 & 3.826069 \\
\hline 1 & -0.989656 & -1.926201 & 7.086749 \\
\hline 1 & 0.509258 & -2.720192 & 6.597158 \\
\hline 1 & 0.390392 & -0.951940 & 6.541754 \\
\hline
\end{tabular}




\begin{tabular}{|c|c|c|c|}
\hline & -2.267276 & -3.376549 & \\
\hline & -1.847129 & -3.344379 & 712930 \\
\hline & -0.761397 & -4.129230 & 4.874389 \\
\hline & 4.795967 & -1.311624 & -4.63324 \\
\hline & 3.687296 & -1.647130 & -3.30257 \\
\hline & 3.760538 & -0.012598 & -3.9990 \\
\hline & 6.786847 & -0.014948 & -4.40834 \\
\hline & 5.925708 & 1.383772 & \\
\hline & 7.323032 & 0.690195 & -2.881713 \\
\hline & 6.592979 & -2.319518 & $-3.24561 \mathrm{~S}$ \\
\hline & 7.101518 & -1.566594 & -1.716392 \\
\hline & 5.573112 & -2.466952 & -1.797763 \\
\hline & 4.804501 & -4.887378 & 4.66756 \\
\hline & 3.745740 & -3.761259 & 5.527514 \\
\hline & 3.049492 & -5.039283 & 4.503 \\
\hline & 6.059896 & -3.02 & 3. \\
\hline & 5.27 & 32 & \\
\hline & 5.003999 & -1.807458 & 4.450254 \\
\hline & 5.070048 & -4.853465 & 2.244760 \\
\hline & 3.318907 & -4.921747 & 1.960303 \\
\hline & 4.298219 & -3.625094 & 1.229536 \\
\hline & 8.239277 & 2.881897 & 1.761620 \\
\hline & 8.156881 & 1.362577 & 0.862114 \\
\hline & 7.625718 & 2.879897 & 0.099866 \\
\hline & 6.268704 & 4.032379 & 2.596513 \\
\hline & 5.556683 & 4.087736 & 0.973 \\
\hline & 4.628436 & 3.423657 & 2.331068 \\
\hline & 6.957960 & 1.852407 & 3.629311 \\
\hline & 5.355741 & 1.140119 & 3.358171 \\
\hline & 6.803185 & 0.331008 & 2.72730 \\
\hline
\end{tabular}

(para-F)TS1 $1_{\text {IIL-re- }} \psi_{-1 \mathrm{a} 2 \mathrm{2} 33 \mathrm{~s} 4 \mathrm{a}}$

Number of imaginary frequencies : 1

The smallest frequencies are : -614.4141 $16.6477 \quad 19.0800 \mathrm{~cm}(-1)$

Electronic energy : $\quad \mathrm{HF}=-4460.0809634$

Zero-point correction $=\quad 1.444139($ Hartree/Particle $)$

Thermal correction to Energy= $\quad 1.529773$

Thermal correction to Enthalpy= $\quad 1.530717$

Thermal correction to Gibbs Free Energy= 1.324793

Sum of electronic and zero-point Energies $=\quad-4458.636824$

Sum of electronic and thermal Energies $=\quad-4458.551190$

Sum of electronic and thermal Enthalpies $=\quad-4458.550246$

Sum of electronic and thermal Free Energies $=\quad-4458.756171$

Cartesian Coordinates

$\begin{array}{cccc}6 & 2.401783 & -4.540939 & -1.470288 \\ 6 & 1.348703 & -3.824003 & -2.052354 \\ 6 & 1.380056 & -3.634377 & -3.441002 \\ 6 & 2.419524 & -4.130758 & -4.216633 \\ 6 & 3.445779 & -4.824665 & -3.596115 \\ 6 & 3.450636 & -5.045665 & -2.230188 \\ 6 & 0.266309 & -3.242462 & -1.192306 \\ 6 & -0.900086 & -2.688453 & -1.773744 \\ 45 & 0.113578 & -0.804692 & -1.210211\end{array}$




\begin{tabular}{|c|c|c|c|}
\hline 5 & 0.636690 & -0.356980 & -3.074012 \\
\hline 5 & 0.631487 & -0.122273 & -4.209096 \\
\hline 15 & 1.380083 & 0.239489 & 0.503887 \\
\hline 6 & 1.028807 & 1.857982 & 1.350011 \\
\hline 6 & 0.686685 & 2.976569 & 0.603977 \\
\hline 6 & 0.478638 & 4.238769 & 1.254342 \\
\hline 6 & 0.641475 & 4.340381 & 2.666499 \\
\hline 6 & 1.012051 & 3.186436 & 3.393316 \\
\hline 6 & 1.194877 & 1.988728 & 2.756633 \\
\hline 6 & 0.423170 & 5.581785 & 3.310286 \\
\hline 6 & 0.051290 & 6.689423 & 2.592571 \\
\hline 6 & -0.116493 & 6.596223 & 1.195039 \\
\hline 6 & 0.093825 & 5.406345 & 0.543021 \\
\hline 6 & 0.545361 & 2.964072 & -0.879028 \\
\hline 6 & -0.649665 & 2.603413 & -1.474108 \\
\hline 6 & -0.840229 & 2.772483 & -2.865167 \\
\hline 6 & 0.161760 & 3.262388 & -3.657892 \\
\hline 6 & 1.419931 & 3.588216 & -3.100528 \\
\hline 6 & 1.616655 & 3.444406 & -1.694580 \\
\hline 6 & 2.885197 & 3.779820 & -1.154457 \\
\hline 6 & 3.898552 & 4.231474 & -1.962769 \\
\hline 6 & 3.696140 & 4.383532 & -3.351899 \\
\hline 6 & 2.482697 & 4.065639 & -3.905913 \\
\hline 7 & -1.707818 & 2.024015 & -0.694337 \\
\hline 6 & -2.804338 & 2.944605 & -0.313488 \\
\hline 6 & -3.418546 & 2.675115 & 1.034383 \\
\hline 6 & -4.795059 & 2.503812 & 1.162186 \\
\hline 6 & -5.363331 & 2.231429 & 2.404131 \\
\hline 6 & -4.558163 & 2.139396 & 3.534485 \\
\hline 6 & -3.182288 & 2.330872 & 3.416061 \\
\hline 6 & -2.618877 & 2.594189 & 2.174898 \\
\hline 6 & 3.162424 & 0.487359 & 0.093444 \\
\hline 6 & 3.634935 & 0.097296 & -1.148287 \\
\hline 6 & 4.987125 & 0.233988 & -1.491226 \\
\hline 6 & 5.841831 & 0.789220 & -0.544690 \\
\hline 6 & 5.397982 & 1.197206 & 0.726086 \\
\hline 6 & 4.050344 & 1.036944 & 1.028296 \\
\hline 6 & 6.383146 & 1.829839 & 1.708521 \\
\hline 6 & 6.840885 & 3.182004 & 1.149632 \\
\hline 6 & 5.431580 & -0.197953 & -2.885285 \\
\hline 6 & 6.943148 & -0.098802 & -3.069641 \\
\hline 6 & 1.478845 & -0.930743 & 1.920586 \\
\hline 6 & 2.575182 & -1.767946 & 2.082347 \\
\hline 6 & 2.621516 & -2.712902 & 3.117091 \\
\hline 6 & 1.538124 & -2.779146 & 3.988616 \\
\hline 6 & 0.417958 & -1.939569 & 3.861741 \\
\hline 6 & 0.391486 & -1.043287 & 2.798139 \\
\hline 6 & 3.861480 & -3.594553 & 3.252957 \\
\hline 6 & 4.022964 & -4.445154 & 1.987632 \\
\hline 6 & -0.722382 & -2.032570 & 4.875886 \\
\hline 6 & -1.710490 & -0.873952 & 4.736734 \\
\hline 15 & -1.816099 & 0.342586 & -0.908988 \\
\hline 8 & -2.910425 & 0.055243 & -2.163884 \\
\hline 6 & -4.222045 & 0.452213 & -2.112111 \\
\hline 6 & -5.115728 & -0.143807 & -1.239017 \\
\hline 6 & -6.438968 & 0.399230 & -1.150550 \\
\hline
\end{tabular}




\begin{tabular}{|c|c|c|c|}
\hline & -6.840887 & 1.430776 & -2.050851 \\
\hline & -5.915739 & 1.913415 & -3.009086 \\
\hline & -4.629975 & 1.447572 & -3.028096 \\
\hline & -7.356845 & -0.001623 & -0.146334 \\
\hline & -8.604765 & 0.565156 & -0.058165 \\
\hline & -9.010829 & 1.558217 & -0.973734 \\
\hline & -8.141602 & 1.980653 & -1.947116 \\
\hline & -4.666369 & -1.273368 & -0.394772 \\
\hline & -3.537316 & -1.120898 & 0.388008 \\
\hline & -3.067279 & -2.138768 & 1.241440 \\
\hline & -3.719578 & -3.340993 & 1.288292 \\
\hline & -4.842936 & -3.584027 & 0.461841 \\
\hline & -5.317560 & -2.550097 & -0.401883 \\
\hline & -5.488179 & -4.844719 & 0.462236 \\
\hline & -6.546769 & -5.096276 & -0.371861 \\
\hline & -6.996036 & -4.090127 & -1.252451 \\
\hline & -6.399740 & -2.853659 & -1.267372 \\
\hline & -2.848468 & 0.064278 & 0.366074 \\
\hline & 0.168805 & -3.785043 & 0.214124 \\
\hline & 5.755625 & 2.068873 & 3.080701 \\
\hline & 7.602382 & 0.921788 & 1.899234 \\
\hline & 5.009612 & -1.651804 & -3.133389 \\
\hline & 4.754355 & 0.711359 & -3.917245 \\
\hline & -1.470013 & -3.353934 & 4.670404 \\
\hline & -0.162163 & -1.987489 & 6.302266 \\
\hline & 5.097044 & -2.702220 & 3.423435 \\
\hline & 3.781320 & -4.534501 & 4.452567 \\
\hline & -7.051502 & -0.752440 & 0.578247 \\
\hline & 1.482739 & 1.120823 & 3.344127 \\
\hline & -8.430116 & 2.760804 & -2.650510 \\
\hline & 0.553536 & 5.634726 & 4.390326 \\
\hline & -5.114252 & -5.614658 & 1.136017 \\
\hline & 3.053657 & 3.658765 & -0.085275 \\
\hline & 4.868330 & 4.471089 & -1.531182 \\
\hline & -3.366363 & -4.137553 & 1.943124 \\
\hline & 1.151830 & 3.264261 & 4.470898 \\
\hline ] & -6.238187 & 2.678905 & -3.712977 \\
\hline & -7.819607 & -4.297512 & -1.932095 \\
\hline & -3.898999 & 1.820859 & -3.742087 \\
\hline & 2.313737 & 4.170679 & -4.976726 \\
\hline & 0.012955 & 3.383412 & -4.729732 \\
\hline 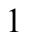 & -0.413626 & 7.475787 & 0.628467 \\
\hline & -6.751627 & -2.094588 & -1.961046 \\
\hline 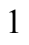 & -0.037310 & 5.353842 & -0.535806 \\
\hline & -1.799915 & 2.486171 & -3.291505 \\
\hline 1 & -7.031701 & -6.069647 & -0.366851 \\
\hline & -9.284473 & 0.248316 & 0.729810 \\
\hline & 4.506894 & 4.746717 & -3.979829 \\
\hline ! & -0.117105 & 7.638609 & 3.095973 \\
\hline & -10.004004 & 1.994395 & -0.896571 \\
\hline 1 & -2.177779 & -1.939742 & 1.837565 \\
\hline & 3.672151 & 1.333234 & 2.003867 \\
\hline 1 & 2.926698 & -0.317537 & -1.862889 \\
\hline . & 6.894348 & 0.916349 & -0.791430 \\
\hline 1 & 3.413487 & -1.686099 & 1.390893 \\
\hline 1 & -0.467515 & -0.386944 & 2.652957 \\
\hline
\end{tabular}




\begin{tabular}{|c|c|c|c|}
\hline 1 & 1.558544 & -3.496190 & 4.806245 \\
\hline 1 & -2.349629 & 3.945770 & -0.306770 \\
\hline & -3.591643 & 2.962951 & -1.083301 \\
\hline & -5.429808 & 2.557231 & 0.276247 \\
\hline & -1.541988 & 2.710881 & 2.075390 \\
\hline & -6.438740 & 2.082724 & 2.481594 \\
\hline & -2.541377 & 2.274155 & 4.295058 \\
\hline & -4.999189 & 1.922298 & 4.505270 \\
\hline & 1.316958 & -1.891644 & -1.020126 \\
\hline & -1.818978 & -2.746878 & -1.189832 \\
\hline & -1.058423 & -2.722701 & -2.849955 \\
\hline & 0.069248 & -4.880437 & 0.185431 \\
\hline & 1.042736 & -3.545628 & 0.831701 \\
\hline & -0.711497 & -3.374731 & 0.717848 \\
\hline & 2.417657 & -4.705248 & -0.396012 \\
\hline & 4.269309 & -5.596226 & -1.773508 \\
\hline & 4.462005 & -5.286952 & -4.338862 \\
\hline & 2.447902 & -3.978286 & -5.292213 \\
\hline & 0.584964 & -3.083676 & -3.936673 \\
\hline I & -2.469024 & -0.937352 & 5.527402 \\
\hline & -1.203541 & 0.096273 & 4.835933 \\
\hline ] & -2.246804 & -0.876272 & 3.779191 \\
\hline & -0.983830 & -2.026171 & 7.029529 \\
\hline & 0.504761 & -2.829298 & 6.521092 \\
\hline ] & 0.398922 & -1.060172 & 6.476547 \\
\hline & -2.302582 & -3.441377 & 5.381562 \\
\hline 1 & -1.884859 & -3.417711 & 3.655075 \\
\hline 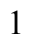 & -0.810641 & -4.218849 & 4.815535 \\
\hline | & 7.215210 & -0.435478 & -4.077943 \\
\hline 1 & 7.304184 & 0.932355 & -2.962022 \\
\hline & 7.485073 & -0.729936 & -2.352896 \\
\hline 1 & 5.047892 & 0.421068 & -4.935433 \\
\hline | & 3.659523 & 0.655882 & -3.854783 \\
\hline 1 & 5.041399 & 1.760041 & -3.762254 \\
\hline ] & 5.350563 & -1.985917 & -4.123330 \\
\hline 1 & 5.446768 & -2.323015 & -2.381800 \\
\hline 1 & 3.918899 & -1.781213 & -3.104682 \\
\hline 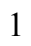 & 4.695047 & -5.139472 & 4.508442 \\
\hline 1 & 3.692422 & -3.985285 & 5.398794 \\
\hline . & 2.933508 & -5.227391 & 4.375438 \\
\hline 1 & 6.001556 & -3.317073 & 3.524076 \\
\hline t & 5.242931 & -2.035149 & 2.564284 \\
\hline 1 & 5.009640 & -2.077521 & 4.322275 \\
\hline 1 & 4.911315 & -5.086166 & 2.068700 \\
\hline 1 & 3.150039 & -5.097538 & 1.842602 \\
\hline 1 & 4.141762 & -3.826551 & 1.088476 \\
\hline 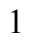 & 6.501882 & 2.505730 & 3.756286 \\
\hline 1 & 4.909244 & 2.767299 & 3.033988 \\
\hline 1 & 5.403003 & 1.133166 & 3.535310 \\
\hline 1 & 7.567589 & 3.651996 & 1.826348 \\
\hline 1 & 7.316721 & 3.079021 & 0.166384 \\
\hline 1 & 5.989584 & 3.867586 & 1.041788 \\
\hline 1 & 8.303313 & 1.375194 & 2.612795 \\
\hline 1 & 7.308144 & -0.059030 & 2.295011 \\
\hline 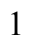 & 8.150692 & 0.755183 & 0.964744 \\
\hline
\end{tabular}


$\left(\right.$ para-F)TS1 $1_{\text {IL-re- }} \Psi_{-1 \mathrm{a} 2 \mathrm{2} 334 \mathrm{~s}}$

Number of imaginary frequencies : 1

The smallest frequencies are : -610.5676 $18.7023 \quad 21.0643 \mathrm{~cm}(-1)$

Electronic energy : $\quad H F=-4460.0811017$

Zero-point correction $=\quad 1.444283($ Hartree/Particle)

Thermal correction to Energy= $\quad 1.529787$

Thermal correction to Enthalpy= $\quad 1.530731$

Thermal correction to Gibbs Free Energy= $\quad 1.325569$

Sum of electronic and zero-point Energies $=\quad-4458.636818$

Sum of electronic and thermal Energies $=\quad-4458.551315$

Sum of electronic and thermal Enthalpies $=\quad-4458.550371$

Sum of electronic and thermal Free Energies $=\quad-4458.755533$

Cartesian Coordinates

$\begin{array}{cccc}6 & -6.401036 & -2.850834 & -1.240240 \\ 6 & -5.316898 & -2.541875 & -0.379103 \\ 6 & -4.842454 & -3.569579 & 0.492193 \\ 6 & -5.489680 & -4.829181 & 0.504009 \\ 6 & -6.550179 & -5.085816 & -0.326075 \\ 6 & -6.999324 & -4.086164 & -1.214100 \\ 6 & -4.663692 & -1.266117 & -0.383180 \\ 6 & -3.533367 & -1.109253 & 0.396919 \\ 6 & -3.063152 & -2.121160 & 1.257246 \\ 6 & -3.717186 & -3.321944 & 1.314642 \\ 6 & -5.112050 & -0.142145 & -1.235422 \\ 6 & -4.218033 & 0.446405 & -2.113314 \\ 6 & -4.624675 & 1.437084 & -3.034869 \\ 6 & -5.909761 & 1.904931 & -3.018358 \\ 6 & -6.835388 & 1.429410 & -2.057077 \\ 6 & -6.434440 & 0.403461 & -1.149962 \\ 6 & -8.135593 & 1.981205 & -1.957098 \\ 6 & -9.005263 & 1.566128 & -0.980967 \\ 6 & -8.599999 & 0.579120 & -0.058532 \\ 6 & -7.352573 & 0.010668 & -0.142780 \\ 8 & -2.907321 & 0.046019 & -2.164190 \\ 15 & -1.811130 & 0.341173 & -0.912938 \\ 8 & -2.842836 & 0.074724 & 0.365060 \\ 45 & 0.115681 & -0.813376 & -1.205712 \\ 15 & 1.383145 & 0.239464 & 0.502085 \\ 6 & 1.485901 & -0.920616 & 1.927398 \\ 6 & 2.580782 & -1.759780 & 2.091971 \\ 6 & 2.621238 & -2.706696 & 3.125653 \\ 6 & 1.538228 & -2.765740 & 3.998252 \\ 6 & 0.422612 & -1.920147 & 3.872069 \\ 6 & 0.398384 & -1.027339 & 2.805599 \\ 6 & 3.849002 & -3.606812 & 3.254693 \\ 6 & 3.764149 & -4.541334 & 4.458315 \\ 6 & -0.716285 & -2.004859 & 4.888512 \\ 6 & -0.152935 & -1.955709 & 6.313515 \\ 6 & 0.259219 & -3.251110 & -1.176800 \\ 6 & 0.164082 & -3.786496 & 0.232662 \\ 6 & 1.335621 & -3.841661 & -2.038259 \\ 6 & 2.383283 & -4.567439 & -1.457711 \\ 6 & 3.426696 & -5.081442 & -2.218976\end{array}$




\begin{tabular}{|c|c|c|c|}
\hline 6 & 3.421734 & -4.860764 & -3.584906 \\
\hline 6 & 2.400299 & -4.158544 & -4.204120 \\
\hline$b$ & 1.366263 & -3.653149 & -3.427114 \\
\hline$\theta$ & 4.432704 & -5.332091 & -4.329091 \\
\hline b & -0.906726 & -2.695504 & -1.757601 \\
\hline & 0.648641 & -0.377197 & -3.069294 \\
\hline & 0.658956 & -0.149234 & -4.205620 \\
\hline & 1.034361 & 1.865258 & 1.334987 \\
\hline & 0.698933 & 2.979482 & 0.579437 \\
\hline & 0.500236 & 4.248850 & 1.218846 \\
\hline & 0.665969 & 4.361945 & 2.629726 \\
\hline f & 1.026443 & 3.211329 & 3.366702 \\
\hline & 1.199571 & 2.006565 & 2.740564 \\
\hline f & 0.459368 & 5.610975 & 3.262621 \\
\hline & 0.095860 & 6.715169 & 2.535476 \\
\hline b & -0.075493 & 6.610533 & 1.139181 \\
\hline 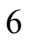 & 0.123491 & 5.413040 & 0.497658 \\
\hline f & 0.557458 & 2.955516 & -0.903321 \\
\hline b & -0.638428 & 2.594164 & -1.496057 \\
\hline 6 & -0.827742 & 2.753689 & -2.888483 \\
\hline$b$ & 0.176239 & 3.234308 & -3.684516 \\
\hline ) & 1.435551 & 3.559454 & -3.129204 \\
\hline b & 1.630596 & 3.425670 & -1.722159 \\
\hline 6 & 2.899793 & 3.759440 & -1.183280 \\
\hline 6 & 3.916530 & 4.199014 & -1.993899 \\
\hline ) & 3.716184 & 4.341191 & -3.384384 \\
\hline 6 & 2.501436 & 4.025565 & -3.937112 \\
\hline 1 & -1.698445 & 2.023889 & -0.712177 \\
\hline 6 & -2.789970 & 2.951819 & -0.334675 \\
\hline$b$ & -3.405011 & 2.690480 & 1.014354 \\
\hline 5 & -4.781705 & 2.521468 & 1.142924 \\
\hline 5 & -5.350130 & 2.254685 & 2.386049 \\
\hline 6 & -4.544840 & 2.165859 & 3.516573 \\
\hline 6 & -3.168733 & 2.355357 & 3.397316 \\
\hline 6 & -2.605281 & 2.613523 & 2.155119 \\
\hline 5 & 3.164925 & 0.486382 & 0.087607 \\
\hline 6 & 3.645307 & 0.067240 & -1.145851 \\
\hline 6 & 4.993893 & 0.203451 & -1.486782 \\
\hline 6 & 5.849319 & 0.788808 & -0.551216 \\
\hline 5 & 5.396998 & 1.230977 & 0.699350 \\
\hline 6 & 4.045909 & 1.065300 & 1.002545 \\
\hline 5 & 5.443620 & -0.244666 & -2.874646 \\
\hline 6 & 4.784431 & 0.668813 & -3.915282 \\
\hline 6 & 6.311881 & 1.887202 & 1.733575 \\
\hline 5 & 6.341913 & 1.028694 & 3.003185 \\
\hline 6 & 5.775958 & 3.282736 & 2.076397 \\
\hline 5 & 7.744805 & 2.043342 & 1.231006 \\
\hline 6 & 6.957715 & -0.165308 & -3.049529 \\
\hline 6 & 5.006227 & -1.694216 & -3.120412 \\
\hline 6 & 3.984135 & -4.464522 & 1.991021 \\
\hline 6 & 5.101835 & -2.736908 & 3.410023 \\
\hline 6 & -1.700556 & -0.843351 & 4.746231 \\
\hline 6 & -1.469173 & -3.324310 & 4.690188 \\
\hline & -7.047863 & -0.735203 & 0.587160 \\
\hline 1 & 1.480117 & 1.141340 & 3.335505 \\
\hline & -8.423339 & 2.756830 & -2.665795 \\
\hline
\end{tabular}




\begin{tabular}{|c|c|c|c|}
\hline 1 & 0.591923 & 5.672528 & 4.341932 \\
\hline & -5.115765 & -5.594174 & 1.183401 \\
\hline & 3.065875 & 3.644881 & -0.112891 \\
\hline & 4.887653 & 4.434100 & -1.562460 \\
\hline & -3.363917 & -4.114053 & 1.974864 \\
\hline 1 & 1.166059 & 3.297442 & 4.443669 \\
\hline & -6.231299 & 2.666574 & -3.726826 \\
\hline 1 & -7.824441 & -4.297746 & -1.890564 \\
\hline 1 & -3.893388 & 1.805169 & -3.751248 \\
\hline 1 & 2.334208 & 4.123130 & -5.008900 \\
\hline 1 & 0.028144 & 3.347794 & -4.757279 \\
\hline 1 & -0.366397 & 7.487325 & 0.565131 \\
\hline 1 & -6.753002 & -2.096991 & -1.939542 \\
\hline 1 & -0.010392 & 5.351799 & -0.580361 \\
\hline 1 & -1.788158 & 2.467286 & -3.313158 \\
\hline 1 & -7.036732 & -6.058288 & -0.312215 \\
\hline 1 & -9.279907 & 0.268418 & 0.731709 \\
\hline 1 & 4.529486 & 4.694713 & -4.014538 \\
\hline 1 & -0.063373 & 7.670365 & 3.030407 \\
\hline 1 & -9.998089 & 2.003623 & -0.906872 \\
\hline 1 & -2.171727 & -1.918942 & 1.849384 \\
\hline 1 & 3.670401 & 1.390720 & 1.972621 \\
\hline 1 & 2.940887 & -0.366750 & -1.852674 \\
\hline 1 & 6.899548 & 0.909102 & -0.802237 \\
\hline 1 & 3.419547 & -1.681988 & 1.400368 \\
\hline 1 & -0.459143 & -0.36 & 2.658043 \\
\hline 1 & 1.554859 & -3.482836 & 4.815897 \\
\hline 1 & -2.329663 & 3.950448 & -0.331748 \\
\hline 1 & -3.577334 & 2.971574 & -1.104422 \\
\hline 1 & -5.416373 & 2.571771 & 0.256729 \\
\hline 1 & -1.528281 & 2.728838 & 2.055008 \\
\hline 1 & -6.425712 & 2.107698 & 2.464363 \\
\hline 1 & -2.527818 & 2.301145 & 4.276471 \\
\hline 1 & -4.985980 & 1.952888 & 4.488219 \\
\hline 1 & 1.314092 & -1.905313 & -1.009342 \\
\hline 1 & -1.824118 & -2.747300 & -1.170879 \\
\hline 1 & -1.068146 & -2.734736 & -2.833181 \\
\hline 1 & 0.061366 & -4.881752 & 0.210247 \\
\hline 1 & 1.040485 & -3.545892 & 0.846328 \\
\hline 1 & -0.713734 & -3.371278 & 0.736797 \\
\hline 1 & 2.398698 & -4.732168 & -0.383638 \\
\hline 1 & 4.241097 & -5.639134 & -1.763342 \\
\hline 1 & 2.428154 & -4.006942 & -5.279831 \\
\hline 1 & 0.574797 & -3.096652 & -3.922015 \\
\hline 1 & -2.457965 & -0.900898 & 5.538411 \\
\hline 1 & -1.190103 & 0.125511 & 4.840697 \\
\hline 1 & -2.238389 & -0.847756 & 3.789542 \\
\hline 1 & -0.973161 & -1.988709 & 7.042690 \\
\hline 1 & 0.511761 & -2.798774 & 6.534275 \\
\hline 1 & 0.411536 & -1.029519 & 6.482793 \\
\hline 1 & -2.300889 & -3.405360 & 5.403092 \\
\hline 1 & -1.885994 & -3.390985 & 3.675863 \\
\hline 1 & -0.812979 & -4.191144 & 4.838158 \\
\hline 1 & 7.232591 & -0.517968 & -4.051596 \\
\hline 1 & 7.330758 & 0.862556 & -2.952847 \\
\hline 1 & 7.487232 & -0.793464 & -2.320970 \\
\hline
\end{tabular}




$\begin{array}{llll}1 & 5.083755 & 0.373257 & -4.930346 \\ 1 & 3.688581 & 0.623858 & -3.861028 \\ 1 & 5.079197 & 1.715709 & -3.760653 \\ 1 & 5.351676 & -2.036515 & -4.105966 \\ 1 & 5.428854 & -2.367530 & -2.362415 \\ 1 & 3.913966 & -1.811010 & -3.101103 \\ 1 & 4.669437 & -5.159278 & 4.509343 \\ 1 & 3.690466 & -3.987428 & 5.403126 \\ 1 & 2.905986 & -5.222307 & 4.390299 \\ 1 & 5.995667 & -3.368694 & 3.500986 \\ 1 & 5.250876 & -2.073017 & 2.548853 \\ 1 & 5.036329 & -2.110789 & 4.309636 \\ 1 & 4.862857 & -5.119476 & 2.065701 \\ 1 & 3.099442 & -5.103250 & 1.856919 \\ 1 & 4.103863 & -3.850310 & 1.088915 \\ 1 & 8.356782 & 2.523721 & 2.004977 \\ 1 & 8.208118 & 1.075343 & 1.000963 \\ 1 & 7.797423 & 2.671948 & 0.332482 \\ 1 & 6.414979 & 3.761300 & 2.830863 \\ 1 & 5.766689 & 3.928344 & 1.188039 \\ 1 & 4.755100 & 3.253177 & 2.478793 \\ 1 & 6.966974 & 1.503434 & 3.771764 \\ 1 & 5.340222 & 0.886255 & 3.428060 \\ 1 & 6.761938 & 0.035720 & 2.794392\end{array}$

$\left(\right.$ para-Cl)TS1 $1_{I I L-s i-} \psi_{-1 \mathrm{~s} 2 \mathrm{a} a \mathrm{ata}}$

Number of imaginary frequencies : 1

The smallest frequencies are : -534.7065 $16.2182 \quad 18.8914 \mathrm{~cm}(-1)$

Electronic energy : $\quad H F=-4820.441275$

Zero-point correction $=\quad 1.442612($ Hartree/Particle $)$

Thermal correction to Energy $=\quad 1.528684$

Thermal correction to Enthalpy $=\quad 1.529628$

Thermal correction to Gibbs Free Energy $=\quad 1.322156$

Sum of electronic and zero-point Energies $=\quad-4818.998663$

Sum of electronic and thermal Energies $=\quad-4818.912591$

Sum of electronic and thermal Enthalpies $=\quad-4818.911647$

Sum of electronic and thermal Free Energies $=\quad-4819.119119$

\begin{tabular}{rrrr}
\multicolumn{4}{c}{ Cartesian Coordinates } \\
\hline 6 & 7.505838 & 0.136596 & 0.187671 \\
6 & 6.653025 & -0.537367 & -0.723319 \\
6 & 7.068664 & -1.813032 & -1.210017 \\
6 & 8.320657 & -2.339586 & -0.809392 \\
6 & 9.126910 & -1.655439 & 0.064029 \\
6 & 8.705098 & -0.409917 & 0.574106 \\
6 & 5.372119 & -0.016414 & -1.104957 \\
6 & 4.525371 & -0.844430 & -1.818993 \\
6 & 4.953299 & -2.082596 & -2.345112 \\
6 & 6.206957 & -2.548454 & -2.060690 \\
6 & 4.926364 & 1.343440 & -0.723324 \\
6 & 3.733034 & 1.516183 & -0.047935 \\
6 & 3.312182 & 2.773074 & 0.429904
\end{tabular}




\begin{tabular}{|c|c|c|c|}
\hline 6 & 4.059701 & 3.887928 & 0.173147 \\
\hline 6 & 5.243267 & 3.794171 & -0.599078 \\
\hline b & 5.678140 & 2.516859 & -1.066601 \\
\hline 6 & 5.992079 & 4.950115 & -0.926335 \\
\hline ) & 7.116122 & 4.866181 & -1.707347 \\
\hline ) & 7.528881 & 3.612077 & -2.202225 \\
\hline b & 6.831505 & 2.470957 & -1.890440 \\
\hline 8 & 2.942172 & 0.441511 & 0.264098 \\
\hline 15 & 2.071664 & -0.378850 & -0.876169 \\
\hline 8 & 3.232086 & -0.467078 & -2.088432 \\
\hline 45 & 0.084168 & 0.330821 & -1.734051 \\
\hline 6 & 0.165148 & -0.790090 & -3.267030 \\
\hline 8 & 0.251384 & -1.305056 & -4.305843 \\
\hline 6 & -0.546395 & 2.489992 & -2.424779 \\
\hline 6 & 0.446708 & 2.504409 & -1.417295 \\
\hline 6 & -1.893910 & 3.103133 & -2.139053 \\
\hline 6 & -3.070021 & 2.595625 & -2.701254 \\
\hline 6 & -4.283189 & 3.255234 & -2.561993 \\
\hline 6 & -4.317384 & 4.451672 & -1.854503 \\
\hline 6 & -3.172893 & 4.978824 & -1.272416 \\
\hline 6 & -1.967640 & 4.299306 & -1.422080 \\
\hline 17 & -5.840034 & 5.313396 & -1.708496 \\
\hline 6 & -0.122451 & 2.673270 & -3.870166 \\
\hline 15 & -1.408690 & -0.171181 & 0.227951 \\
\hline 6 & -3.003610 & -0.981331 & -0.227303 \\
\hline 6 & -3.966840 & -1.300467 & 0.734777 \\
\hline 6 & -5.174549 & -1.894665 & 0.372600 \\
\hline 6 & & -2.192244 & -0.981531 \\
\hline 6 & -4.425552 & -1.912370 & -1.965904 \\
\hline 6 & -3.238622 & -1.292642 & -1.562856 \\
\hline 6 & -6.247320 & -2.253863 & 1.400301 \\
\hline 6 & -5.917912 & -1.709721 & 2.789141 \\
\hline 6 & -4.708938 & -2.234327 & -3.432695 \\
\hline 6 & -3.453050 & -2.113470 & -4.295650 \\
\hline 6 & -0.922755 & -1.225841 & 1.694317 \\
\hline 6 & -0.331010 & -2.468474 & 1.499156 \\
\hline 6 & 0.040700 & -3.276676 & 2.622904 \\
\hline 6 & -0.189816 & -2.796940 & 3.944532 \\
\hline 6 & -0.809279 & -1.538463 & 4.110391 \\
\hline 6 & -1.161900 & -0.782302 & 3.024817 \\
\hline 6 & 0.212759 & -3.577302 & 5.054453 \\
\hline 6 & 0.820446 & -4.793380 & 4.875206 \\
\hline 6 & 1.042130 & -5.280956 & 3.569994 \\
\hline 6 & 0.658892 & -4.546847 & 2.474803 \\
\hline 6 & -0.088900 & -3.047055 & 0.147229 \\
\hline 6 & -1.039533 & -3.961636 & -0.403940 \\
\hline 6 & -0.775847 & -4.580321 & -1.661868 \\
\hline 6 & 0.441596 & -4.303297 & -2.325173 \\
\hline 6 & 1.346456 & -3.437464 & -1.774345 \\
\hline 6 & 1.080858 & -2.790493 & -0.545363 \\
\hline 6 & -1.725613 & -5.477612 & -2.209506 \\
\hline 6 & -2.887905 & -5.764323 & -1.540038 \\
\hline 6 & -3.154168 & -5.152296 & -0.296099 \\
\hline 6 & -2.253535 & -4.278670 & 0.259437 \\
\hline 7 & 2.046202 & -1.852505 & -0.040200 \\
\hline 6 & 3.156160 & -2.438771 & 0.746535 \\
\hline
\end{tabular}




\begin{tabular}{|c|c|c|c|}
\hline 6 & 3.617868 & -1.648878 & 1.944537 \\
\hline 6 & 2.712468 & -1.055331 & 2.826349 \\
\hline & 3.162165 & -0.378432 & 3.951823 \\
\hline & 4.527961 & -0.279929 & 4.214174 \\
\hline & 5.436519 & -0.862125 & 3.338111 \\
\hline & 4.981926 & -1.542129 & 2.210170 \\
\hline & -1.945422 & 1.359824 & 1.100037 \\
\hline & -0.939186 & 2.105988 & 1.715407 \\
\hline & -1.233243 & 3.232120 & 2.488968 \\
\hline & -2.571883 & 3.615438 & 2.591230 \\
\hline & -3.603847 & 2.918796 & 1.950832 \\
\hline & -3.269754 & 1.789300 & 1.201874 \\
\hline & -0.104090 & 4.012504 & 3.161179 \\
\hline & 0.564648 & 4.903963 & 2.107375 \\
\hline & -5.057090 & 3.344163 & 2.159750 \\
\hline & -5.473966 & 2.957560 & 3.584029 \\
\hline & -5.210473 & 4.857987 & 1.987594 \\
\hline & -6.003297 & 2.653852 & 1.178134 \\
\hline & -0.612156 & 4.907502 & 4.291464 \\
\hline & 0.929125 & 3.051527 & 3.761010 \\
\hline & -7.601111 & -1.675638 & 0.975620 \\
\hline 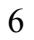 & -6.356535 & -3.779569 & 1.498465 \\
\hline & -5.762592 & -1.251100 & -3.955235 \\
\hline & -5.239556 & -3.663193 & -3.581006 \\
\hline & 7.183818 & 1.085668 & 0.608871 \\
\hline & -1.643336 & 0.178110 & 3.191049 \\
\hline & 8.620951 & -3.310925 & -1.200228 \\
\hline & 0.032253 & -3.186130 & 6.054881 \\
\hline & 5.645874 & 5.911886 & -0.549991 \\
\hline & -2.473424 & -3.806549 & 1.215547 \\
\hline & -4.083285 & -5.374968 & 0.225191 \\
\hline & 3.746535 & 4.862828 & 0.544767 \\
\hline & -1.004668 & -1.176280 & 5.119271 \\
\hline & 6.548872 & -3.501809 & -2.460116 \\
\hline 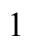 & 8.405283 & 3.547428 & -2.843201 \\
\hline & 4.265558 & -2.638480 & -2.978529 \\
\hline & -1.506328 & -5.941114 & -3.170615 \\
\hline 1 & 0.643632 & -4.784775 & -3.280773 \\
\hline & 1.517980 & -6.248993 & 3.430361 \\
\hline ] & 7.158238 & 1.515261 & -2.291631 \\
\hline & 0.830164 & -4.941638 & 1.475360 \\
\hline ] & 2.282685 & -3.212207 & -2.281810 \\
\hline & 7.680850 & 5.761471 & -1.956720 \\
\hline 1 & 9.332309 & 0.120811 & 1.287016 \\
\hline ] & -3.607095 & -6.461919 & -1.964349 \\
\hline ] & 1.130614 & -5.384191 & 5.733963 \\
\hline 1 & 10.081543 & -2.074011 & 0.373944 \\
\hline & 2.392047 & 2.814822 & 1.007481 \\
\hline 1 & -3.765353 & -1.069277 & 1.778854 \\
\hline & -2.456085 & -1.058820 & -2.281766 \\
\hline 1 & -6.310502 & -2.666100 & -1.276290 \\
\hline 1 & -4.048796 & 1.216734 & 0.707395 \\
\hline 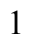 & 0.091521 & 1.757638 & 1.626693 \\
\hline 1 & -2.828777 & 4.480066 & 3.199006 \\
\hline 1 & 2.775493 & -3.409245 & 1.096695 \\
\hline & 4.019722 & -2.662910 & 0.101417 \\
\hline
\end{tabular}




$\begin{array}{lrrr}1 & 5.701761 & -1.983912 & 1.518720 \\ 1 & 1.648364 & -1.096147 & 2.601981 \\ 1 & 6.506114 & -0.783357 & 3.523898 \\ 1 & 2.439283 & 0.077500 & 4.627261 \\ 1 & 4.879952 & 0.254209 & 5.094322 \\ 1 & 0.171068 & 2.836599 & -0.415703 \\ 1 & 1.480852 & 2.680617 & -1.713886 \\ 1 & -3.035820 & 1.659723 & -3.260108 \\ 1 & -5.192524 & 2.853305 & -3.001575 \\ 1 & -3.216790 & 5.916036 & -0.722725 \\ 1 & -1.061414 & 4.719993 & -0.990060 \\ 1 & -0.800483 & 2.161874 & -4.564211 \\ 1 & -0.140837 & 3.743689 & -4.121620 \\ 1 & 0.888295 & 2.285645 & -4.033853 \\ 1 & -1.207787 & 1.007745 & -2.464266 \\ 1 & 0.236727 & 5.404187 & 4.778212 \\ 1 & -1.280769 & 5.698350 & 3.930267 \\ 1 & -1.145932 & 4.330035 & 5.057539 \\ 1 & 1.699926 & 3.615402 & 4.302266 \\ 1 & 0.454227 & 2.358221 & 4.469163 \\ 1 & 1.446437 & 2.446644 & 3.006312 \\ 1 & 1.433760 & 5.425190 & 2.533365 \\ 1 & 0.904902 & 4.324456 & 1.238281 \\ 1 & -0.139147 & 5.662730 & 1.739091 \\ 1 & -7.022912 & 3.035642 & 1.315071 \\ 1 & -5.713683 & 2.836848 & 0.135006 \\ 1 & -6.038495 & 1.566540 & 1.333592 \\ 1 & -4.855230 & 5.180976 & 1.001290 \\ 1 & -6.267567 & 5.142612 & 2.072649 \\ 1 & -4.660279 & 5.423505 & 2.748933 \\ 1 & -6.519513 & 3.239708 & 3.769416 \\ 1 & -5.381874 & 1.874028 & 3.739601 \\ 1 & -4.851683 & 3.456486 & 4.337732 \\ 1 & -6.728090 & -1.959172 & 3.485775 \\ 1 & -4.993560 & -2.141336 & 3.195016 \\ 1 & -5.809753 & -0.615389 & 2.783687 \\ 1 & -7.136193 & -4.064471 & 2.218149 \\ 1 & -6.610530 & -4.233623 & 0.532157 \\ 1 & -5.408054 & -4.217034 & 1.839910 \\ 1 & -8.366965 & -1.916973 & 1.724690 \\ 1 & -7.553967 & -0.582775 & 0.881797 \\ 1 & -7.945745 & -2.078286 & 0.015992 \\ 1 & -3.686101 & -2.400367 & -5.328983 \\ 1 & -3.060449 & -1.088052 & -4.326547 \\ 1 & -2.649768 & -2.772137 & -3.936173 \\ 1 & -5.426092 & -3.886285 & -4.639964 \\ 1 & -4.509649 & -4.390879 & -3.203147 \\ 1 & -6.183759 & -3.820181 & -3.046008 \\ 1 & -5.983479 & -1.448133 & -5.013254 \\ 1 & -6.702449 & -1.330211 & -3.394169 \\ 1 & -5.411342 & -0.214101 & -3.870198\end{array}$

$\left(\right.$ para-Cl)TS1 $1_{I I L-s i}-\Psi_{-1 \mathrm{~s} 2 a 3 a 4 s}$

Number of imaginary frequencies : 1

The smallest frequencies are : $-583.0778 \quad 13.5183 \quad 18.7706 \mathrm{~cm}(-1)$ 
Electronic energy : $\quad H F=-4820.441804$

Zero-point correction=

1.443622 (Hartree/Particle)

Thermal correction to Energy $=\quad 1.529090$

Thermal correction to Enthalpy= $\quad 1.530034$

Thermal correction to Gibbs Free Energy= $\quad 1.325511$

Sum of electronic and zero-point Energies $=\quad-4818.998182$

Sum of electronic and thermal Energies $=\quad-4818.912714$

Sum of electronic and thermal Enthalpies $=\quad-4818.911770$

Sum of electronic and thermal Free Energies $=\quad-4819.116293$

Cartesian Coordinates

\begin{tabular}{|c|c|c|c|}
\hline 6 & -3.290373 & 1.723822 & 1.211423 \\
\hline 6 & -1.964731 & 1.289440 & 1.161895 \\
\hline 6 & -0.982153 & 2.017130 & 1.836327 \\
\hline 6 & -1.306509 & 3.127382 & 2.619568 \\
\hline 6 & -2.646643 & 3.519678 & 2.663351 \\
\hline 6 & -3.651775 & 2.846251 & 1.960015 \\
\hline 15 & -1.401101 & -0.202147 & 0.241345 \\
\hline 6 & -0.909910 & -1.314202 & 1.662406 \\
\hline 6 & -0.320217 & -2.548408 & 1.415282 \\
\hline 6 & 0.053410 & -3.402133 & 2.503740 \\
\hline 6 & -0.176049 & -2.978102 & 3.844549 \\
\hline 6 & -0.798402 & -1.729069 & 4.063144 \\
\hline 6 & -1.150614 & -0.927946 & 3.010073 \\
\hline 6 & 0.232792 & -3.801660 & 4.920469 \\
\hline 6 & 0.845790 & -5.006276 & 4.689614 \\
\hline 6 & 1.065080 & -5.439425 & 3.364907 \\
\hline 6 & 0.674860 & -4.663216 & 2.301765 \\
\hline 6 & -0.088178 & -3.071449 & 0.039460 \\
\hline 6 & -1.049266 & -3.954420 & -0.544301 \\
\hline 6 & -0.804609 & -4.508837 & -1.835402 \\
\hline 6 & 0.408224 & -4.206982 & -2.496908 \\
\hline 6 & 1.324984 & -3.375209 & -1.913747 \\
\hline 6 & 1.075189 & -2.786884 & -0.652298 \\
\hline 6 & -1.769667 & -5.366805 & -2.418200 \\
\hline 6 & -2.928812 & -5.675280 & -1.752494 \\
\hline 6 & -3.173461 & -5.130000 & -0.473660 \\
\hline 6 & -2.256655 & -4.297918 & 0.117574 \\
\hline 7 & 2.049091 & -1.877583 & -0.112011 \\
\hline 6 & 3.173716 & -2.508339 & 0.617317 \\
\hline 6 & 3.659462 & -1.789516 & 1.849615 \\
\hline 6 & 2.770075 & -1.259428 & 2.786275 \\
\hline 6 & 3.239812 & -0.659352 & 3.946435 \\
\hline 6 & 4.610296 & -0.571641 & 4.187002 \\
\hline 6 & 5.503144 & -1.086656 & 3.254403 \\
\hline 6 & 5.028298 & -1.692210 & 2.092864 \\
\hline 6 & -0.210622 & 3.895935 & 3.357758 \\
\hline 6 & 0.833500 & 2.933887 & 3.934624 \\
\hline 6 & -5.109494 & 3.289407 & 2.091287 \\
\hline 6 & -5.992879 & 2.682297 & 1.000939 \\
\hline 45 & 0.080888 & 0.391106 & -1.690444 \\
\hline 6 & 0.452220 & 2.546202 & -1.275388 \\
\hline 6 & -0.529060 & 2.578445 & -2.294146 \\
\hline 6 & -0.086358 & 2.813374 & -3.726750 \\
\hline 15 & 2.069066 & -0.362543 & -0.870645 \\
\hline
\end{tabular}




\begin{tabular}{|c|c|c|c|}
\hline 8 & 3.221977 & -0.387202 & -2.092138 \\
\hline 6 & 4.525222 & -0.749386 & -1.852915 \\
\hline 6 & 5.364518 & 0.065763 & -1.115921 \\
\hline 6 & 6.663245 & -0.441406 & -0.778303 \\
\hline 6 & 7.099995 & -1.683890 & -1.328216 \\
\hline 6 & 6.239454 & -2.403162 & -2.193921 \\
\hline 6 & 4.970718 & -1.955072 & -2.436834 \\
\hline 6 & 8.371311 & -2.196465 & -0.973094 \\
\hline 6 & 9.176724 & -1.531973 & -0.083763 \\
\hline 6 & 8.734405 & -0.321373 & 0.488960 \\
\hline 6 & 7.515636 & 0.211884 & 0.147772 \\
\hline 6 & 4.893961 & 1.394958 & -0.662655 \\
\hline 6 & 3.709159 & 1.506073 & 0.040184 \\
\hline 6 & 3.268191 & 2.727178 & 0.587714 \\
\hline 6 & 3.986715 & 3.870257 & 0.376777 \\
\hline 6 & 5.157805 & 3.843043 & -0.419467 \\
\hline 6 & 5.612505 & 2.601517 & -0.959232 \\
\hline 6 & 6.750100 & 2.624479 & -1.805694 \\
\hline 6 & 7.414234 & 3.796279 & -2.072947 \\
\hline 6 & 6.982841 & 5.013700 & -1.507400 \\
\hline 6 & 5.873711 & 5.031393 & -0.701249 \\
\hline 8 & 2.946291 & 0.398730 & 0.305602 \\
\hline 6 & 0.165685 & -0.644273 & -3.283319 \\
\hline 8 & 0.257173 & -1.095229 & -4.351085 \\
\hline 6 & -1.870522 & 3.203212 & -2.005979 \\
\hline 6 & -3.051576 & 2.726163 & -2.583861 \\
\hline 6 & -4.249733 & 3.414965 & -2.455308 \\
\hline 6 & -4.263995 & 4.608262 & -1.741491 \\
\hline 6 & -3.115201 & 5.102015 & -1.138693 \\
\hline 6 & -1.925329 & 4.394041 & -1.278050 \\
\hline 17 & -5.764691 & 5.509761 & -1.615488 \\
\hline 6 & -2.987312 & -1.008961 & -0.242881 \\
\hline 6 & -3.912230 & -1.419073 & \\
\hline 6 & -5.107656 & -2.042337 & 0.346148 \\
\hline 6 & -5.346744 & -2.237930 & -1.015318 \\
\hline 6 & -4.434454 & -1.848213 & -2.004752 \\
\hline 6 & -3.251958 & -1.233625 & -1.593734 \\
\hline 6 & -6.078748 & -2.482727 & 1.440692 \\
\hline 6 & -5.360960 & -3.419971 & 2.419838 \\
\hline 6 & -4.763963 & -2.054838 & -3.482443 \\
\hline 6 & -5.242481 & -3.486936 & -3.737655 \\
\hline 6 & -7.291140 & -3.221850 & 0.881021 \\
\hline 6 & -6.574468 & -1.246392 & 2.199267 \\
\hline 6 & -3.552220 & -1.805578 & -4.380045 \\
\hline 6 & -5.873335 & -1.072050 & -3.874919 \\
\hline 6 & 0.462435 & 4.852332 & 2.365693 \\
\hline 6 & -0.767872 & 4.720937 & 4.518419 \\
\hline 6 & -5.636111 & 2.825705 & 3.455035 \\
\hline 6 & -5.227379 & 4.814572 & 2.008470 \\
\hline 1 & 7.179460 & 1.134044 & 0.615120 \\
\hline 1 & -1.633072 & 0.024238 & 3.215471 \\
\hline 1 & 8.687694 & -3.141763 & -1.412119 \\
\hline 1 & 0.053540 & -3.452867 & 5.936650 \\
\hline 1 & 5.513572 & 5.964415 & -0.269634 \\
\hline 1 & -2.457169 & -3.880671 & 1.102790 \\
\hline 1 & -4.098435 & -5.373934 & 0.047303 \\
\hline
\end{tabular}




\begin{tabular}{|c|c|c|c|}
\hline & 3.658772 & 4.817742 & 0.802457 \\
\hline & -0.994883 & -1.410276 & 5.086349 \\
\hline & 6.595307 & -3.330881 & -2.638945 \\
\hline & 8.278630 & 3.784962 & -2.733138 \\
\hline & 4.282801 & -2.500440 & -3.079250 \\
\hline & -1.566526 & -5.781177 & -3.404948 \\
\hline & 0.596398 & -4.640427 & -3.477993 \\
\hline & 1.544914 & -6.398752 & 3.184603 \\
\hline & 7.090614 & 1.697967 & -2.260392 \\
\hline & 0.844226 & -5.015719 & 1.286258 \\
\hline & 2.257133 & -3.129332 & -2.419951 \\
\hline & 7.521631 & 5.933663 & -1.721919 \\
\hline & 9.361596 & 0.192610 & 1.214027 \\
\hline & -3.661028 & -6.339975 & -2.206245 \\
\hline & 1.161797 & -5.630194 & 5.522418 \\
\hline & 10.146678 & -1.940101 & 0.190450 \\
\hline & 2.354186 & 2.719165 & 1.176723 \\
\hline & -3.694373 & -1.253097 & 1.768820 \\
\hline & -2.494345 & -0.931489 & -2.314129 \\
\hline & -6.273453 & -2.717617 & -1.323603 \\
\hline & -4.048141 & 1.166183 & 0.667281 \\
\hline & 0.050355 & 1.666569 & 1.781193 \\
\hline & -2.924281 & 4.376926 & 3.272190 \\
\hline & 2.799085 & -3.497238 & 0.918999 \\
\hline & 4.023878 & -2.695836 & -0.056448 \\
\hline & 5.735494 & -2.083829 & 1.359345 \\
\hline & 1.702257 & -1.290457 & 2.579544 \\
\hline & 6.575896 & -1.014037 & 3.423410 \\
\hline & 2.528770 & -0.255098 & 4.665836 \\
\hline 1 & 4.978331 & -0.097481 & 5.094494 \\
\hline & 0.167221 & 2.840748 & -0.264417 \\
\hline 1 & 1.490208 & 2.728266 & -1.553807 \\
\hline 1 & -3.033779 & 1.794605 & -3.150803 \\
\hline & -5.162790 & 3.038662 & -2.909914 \\
\hline ] & -3.143204 & 6.036985 & -0.584220 \\
\hline & -1.013504 & 4.791226 & -0.835659 \\
\hline 1 & -0.760419 & 2.333592 & -4.446940 \\
\hline & -0.095049 & 3.892727 & -3.937548 \\
\hline l & 0.923628 & 2.425216 & -3.894028 \\
\hline ] & -1.209314 & 1.108897 & -2.385777 \\
\hline & 0.057298 & 5.203582 & 5.057275 \\
\hline 1 & -1.438696 & 5.520092 & 4.180885 \\
\hline & -1.314806 & 4.094112 & 5.234740 \\
\hline 1 & 1.571969 & 3.490567 & 4.525783 \\
\hline & 0.363910 & 2.188858 & 4.591686 \\
\hline 1 & 1.389013 & 2.388259 & 3.162432 \\
\hline 1 & 1.296238 & 5.385672 & 2.843926 \\
\hline 1 & 0.855771 & 4.318513 & 1.489526 \\
\hline 1 & -0.254318 & 5.600292 & 2.000323 \\
\hline 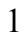 & -7.012846 & 3.077842 & 1.089197 \\
\hline 1 & -5.624693 & 2.926212 & -0.004795 \\
\hline 1 & -6.060122 & 1.589048 & 1.082658 \\
\hline 1 & -4.780753 & 5.198894 & 1.082860 \\
\hline 1 & -6.284678 & 5.110626 & 2.017479 \\
\hline 1 & -4.744555 & 5.318639 & 2.854038 \\
\hline 1 & -6.682738 & 3.132510 & 3.588111 \\
\hline
\end{tabular}




$\begin{array}{cccc}1 & -5.590493 & 1.732000 & 3.544351 \\ 1 & -5.051436 & 3.253598 & 4.279474 \\ 1 & -7.949677 & -3.528670 & 1.703400 \\ 1 & -7.883103 & -2.590151 & 0.206366 \\ 1 & -6.999869 & -4.129160 & 0.334844 \\ 1 & -6.050928 & -3.743946 & 3.210603 \\ 1 & -4.992506 & -4.319770 & 1.908251 \\ 1 & -4.503913 & -2.939226 & 2.908685 \\ 1 & -7.256626 & -1.538247 & 3.009322 \\ 1 & -5.743573 & -0.686577 & 2.648624 \\ 1 & -7.117457 & -0.566294 & 1.529238 \\ 1 & -3.817304 & -2.015557 & -5.424088 \\ 1 & -3.207201 & -0.763551 & -4.339375 \\ 1 & -2.706868 & -2.453934 & -4.110868 \\ 1 & -5.475361 & -3.621262 & -4.802406 \\ 1 & -4.463223 & -4.210869 & -3.465582 \\ 1 & -6.148713 & -3.736484 & -3.173286 \\ 1 & -6.125752 & -1.179212 & -4.938802 \\ 1 & -6.788519 & -1.240254 & -3.293234 \\ 1 & -5.556229 & -0.034285 & -3.703890\end{array}$

(para-Cl)TS1 $1_{I I L-s i-} \Psi_{-1 \mathrm{~s} 2 a 354 a}$

Number of imaginary frequencies : 1

The smallest frequencies are : $-508.7714 \quad 15.4981 \quad 18.2827 \mathrm{~cm}(-1)$

Electronic energy : $\quad H F=-4820.4410032$

Zero-point correction $=\quad 1.442577$ (Hartree/Particle)

Thermal correction to Energy $=\quad 1.528502$

Thermal correction to Enthalpy= $\quad 1.529446$

Thermal correction to Gibbs Free Energy $=\quad 1.323057$

Sum of electronic and zero-point Energies $=\quad-4818.998427$

Sum of electronic and thermal Energies $=\quad-4818.912501$

Sum of electronic and thermal Enthalpies $=\quad-4818.911557$

Sum of electronic and thermal Free Energies $=\quad-4819.117947$

Cartesian Coordinates

$\begin{array}{cccc}6 & -3.088996 & 2.596985 & -2.680520 \\ 6 & -1.904943 & 3.101869 & -2.132264 \\ 6 & -1.967997 & 4.297193 & -1.412657 \\ 6 & -3.169675 & 4.979593 & -1.247957 \\ 6 & -4.321608 & 4.456741 & -1.818870 \\ 6 & -4.298234 & 3.260660 & -2.527235 \\ 6 & -0.561570 & 2.487833 & -2.435029 \\ 6 & -0.161231 & 2.656341 & -3.888696 \\ 45 & 0.076351 & 0.326320 & -1.737921 \\ 6 & 0.445628 & 2.505551 & -1.443121 \\ 15 & -1.408939 & -0.163127 & 0.217623 \\ 6 & -0.936254 & -1.218768 & 1.685180 \\ 6 & -0.345030 & -2.461736 & 1.493338 \\ 6 & 0.021783 & -3.268561 & 2.620899 \\ 6 & -0.219196 & -2.788619 & 3.940639 \\ 6 & -0.841772 & -1.531068 & 4.101911 \\ 6 & -1.185287 & -0.774739 & 3.013835 \\ 6 & 0.177416 & -3.566536 & 5.054399 \\ 6 & 0.791539 & -4.780198 & 4.881253\end{array}$




\begin{tabular}{|c|c|c|c|}
\hline 6 & 1.026325 & -5.267017 & 3.578190 \\
\hline & 0.648111 & -4.535744 & 2.479339 \\
\hline & -0.087840 & -3.049931 & 0.147746 \\
\hline & -1.012815 & -3.999922 & -0.389221 \\
\hline & -0.694835 & -4.689863 & -1.596828 \\
\hline & 0.528585 & -4.411256 & -2.248756 \\
\hline & 1.394294 & -3.488312 & -1.728252 \\
\hline & 1.091439 & -2.799287 & -0.530916 \\
\hline & -1.595939 & -5.656872 & -2.105790 \\
\hline & -2.773711 & -5.929059 & -1.458371 \\
\hline & -3.102526 & -5.233792 & -0.274796 \\
\hline & -2.243482 & -4.300923 & 0.250326 \\
\hline & 2.043108 & -1.844623 & -0.030379 \\
\hline & 3.158838 & -2.417931 & 0.758653 \\
\hline & 3.608062 & -1.622918 & 1.957880 \\
\hline & 2.693406 & -1.036708 & 2.834899 \\
\hline & 3.132033 & -0.355589 & 3.962247 \\
\hline & 4.495705 & -0.245320 & 4.230954 \\
\hline & 5.413361 & -0.820166 & 3.359491 \\
\hline & 4.969942 & -1.504611 & 2.229814 \\
\hline & -1.956354 & 1.365054 & 1.087146 \\
\hline & -0.954899 & 2.118993 & 1.700544 \\
\hline 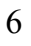 & -1.257411 & 3.237932 & 2.481427 \\
\hline & -2.599646 & 3.605295 & 2.593341 \\
\hline & -3.627330 & 2.900938 & 1.954244 \\
\hline & -3.284723 & 1.779434 & 1.197331 \\
\hline & -5.084322 & 3.307766 & 2.173648 \\
\hline 6 & -5.256042 & 4.822008 & 2.023000 \\
\hline 6 & -0.132951 & 4.026583 & 3.151578 \\
\hline 6 & -0.646018 & 4.924956 & 4.276733 \\
\hline 6 & -2.996669 & -0.975158 & -0.252978 \\
\hline 6 & -3.962385 & -1.320047 & 0.702602 \\
\hline 5 & -5.161398 & -1.916427 & 0.327442 \\
\hline 6 & -5.365365 & -2.184398 & -1.037304 \\
\hline 6 & -4.425263 & -1.862118 & -2.011045 \\
\hline 6 & -3.234973 & -1.254560 & -1.589791 \\
\hline 6 & -6.230767 & -2.305792 & 1.347534 \\
\hline 6 & -7.590260 & -1.732384 & 0.934185 \\
\hline 6 & -4.615814 & -2.154113 & -3.497626 \\
\hline 6 & -4.390311 & -0.876180 & -4.314885 \\
\hline 15 & 2.065122 & -0.377104 & -0.877093 \\
\hline 8 & 2.927739 & 0.452816 & 0.262586 \\
\hline 6 & 3.713808 & 1.530661 & -0.049926 \\
\hline 6 & 4.909319 & 1.362807 & -0.722641 \\
\hline 6 & 5.655248 & 2.539625 & -1.067327 \\
\hline 6 & 5.213004 & 3.815491 & -0.602798 \\
\hline 6 & 4.028253 & 3.904344 & 0.168211 \\
\hline 6 & 3.286241 & 2.786078 & 0.425847 \\
\hline 6 & 6.810059 & 2.498321 & -1.889377 \\
\hline 6 & 7.501779 & 3.642564 & -2.202351 \\
\hline 6 & 7.081689 & 4.895381 & -1.710415 \\
\hline 6 & 5.956129 & 4.974792 & -0.931127 \\
\hline 6 & 5.364839 & 0.004479 & -1.098079 \\
\hline 6 & 4.525911 & -0.832851 & -1.810545 \\
\hline 6 & 4.964865 & -2.069398 & -2.331557 \\
\hline 6 & 6.221319 & -2.524603 & -2.042603 \\
\hline
\end{tabular}




\begin{tabular}{|c|c|c|c|}
\hline & 7.075110 & -1.779807 & -1.192190 \\
\hline & 6.648614 & -0.505681 & -0.711006 \\
\hline & 8.330090 & -2.295515 & -0.786867 \\
\hline & 9.129044 & -1.602408 & 0.086154 \\
\hline & 8.696461 & -0.358559 & 0.591160 \\
\hline & 7.494074 & 0.177661 & 0.199919 \\
\hline & 3.230232 & -0.467264 & -2.084590 \\
\hline & 0.109521 & -0.827229 & -3.247654 \\
\hline & 0.122276 & -1.380626 & -4.270396 \\
\hline & -6.026601 & 2.619825 & 1.186400 \\
\hline & -5.490962 & 2.896698 & 3.594090 \\
\hline & 0.902918 & 3.072158 & 3.757114 \\
\hline & 0.533674 & 4.915932 & 2.094698 \\
\hline & -3.593833 & -3.210427 & -3.929545 \\
\hline & -6.014555 & -2.678438 & -3.811115 \\
\hline & -6.325081 & -3.833982 & 1.417417 \\
\hline & -5.906740 & -1.785097 & 2.746619 \\
\hline & 7.163874 & 1.125656 & 0.617201 \\
\hline & -1.668030 & 0.185686 & 3.176179 \\
\hline & 8.638617 & -3.265837 & -1.173842 \\
\hline & -0.012430 & -3.174896 & 6.052915 \\
\hline & 5.604341 & 5.935382 & -0.556942 \\
\hline & -2.507584 & -3.774077 & 1.165932 \\
\hline & -4.044892 & -5.442658 & 0.228048 \\
\hline & 3.710097 & 4.878210 & 0.538290 \\
\hline & -1.045573 & -1.169360 & 5.109275 \\
\hline & 6.571628 & -3.476504 & -2.438197 \\
\hline ] & 8.379443 & 3.581392 & -2.841943 \\
\hline & 4.283044 & -2.632522 & -2.964886 \\
\hline & -1.332182 & -6.176378 & -3.026222 \\
\hline & 0.767806 & -4.940645 & -3.169779 \\
\hline & 1.508773 & -6.232376 & 3.442701 \\
\hline 1 & 7.142466 & 1.543615 & -2.288271 \\
\hline & 0.831546 & -4.931070 & 1.482283 \\
\hline 1 & 2.335181 & -3.261631 & -2.226655 \\
\hline & 7.642043 & 5.793191 & -1.960604 \\
\hline | & 9.317809 & 0.179255 & 1.303894 \\
\hline 1 & -3.459450 & -6.673590 & -1.857073 \\
\hline & 1.097396 & -5.369063 & 5.742886 \\
\hline 1 & 10.086104 & -2.012637 & 0.399715 \\
\hline & 2.365709 & 2.823618 & 1.003031 \\
\hline 1 & -3.763865 & -1.105868 & 1.750855 \\
\hline & -2.461554 & -1.000599 & -2.313174 \\
\hline 1 & -6.296617 & -2.661458 & -1.336699 \\
\hline ] & -4.059949 & 1.200037 & 0.704570 \\
\hline & 0.079021 & 1.781793 & 1.605728 \\
\hline 1 & -2.862891 & 4.463210 & 3.207773 \\
\hline & 2.789097 & -3.392975 & 1.107778 \\
\hline 1 & 4.026633 & -2.632017 & 0.115925 \\
\hline & 5.696639 & -1.940727 & 1.541933 \\
\hline 1 & 1.630846 & -1.087334 & 2.605205 \\
\hline 1 & 6.481353 & -0.732129 & 3.550380 \\
\hline 1 & 2.402184 & 0.094315 & 4.634187 \\
\hline 1 & 4.838967 & 0.292173 & 5.112505 \\
\hline 1 & 0.185464 & 2.840927 & -0.438363 \\
\hline 1 & 1.476366 & 2.674720 & -1.755163 \\
\hline
\end{tabular}




\begin{tabular}{|c|c|c|c|}
\hline & -3.067782 & 1.660578 & -3.239264 \\
\hline & -5.213745 & 2.862761 & -2.957660 \\
\hline & -3.205095 & 5.915420 & -0.695325 \\
\hline & -1.056076 & 4.715523 & -0.990666 \\
\hline & -0.850720 & 2.137417 & -4.565890 \\
\hline & -0.184133 & 3.724014 & -4.151175 \\
\hline & 0.846522 & 2.266597 & -4.064938 \\
\hline & -1.222920 & 0.998239 & -2.457856 \\
\hline & 0.200122 & 5.428901 & 4.760781 \\
\hline & -1.318976 & 5.709944 & 3.910752 \\
\hline & -1.176589 & 4.348989 & 5.046157 \\
\hline & 1.673146 & 3.641214 & 4.293781 \\
\hline & 0.429845 & 2.383089 & 4.470542 \\
\hline & 1.420264 & 2.462875 & 3.006007 \\
\hline & 1.399506 & 5.443176 & 2.519904 \\
\hline & 0.878513 & 4.333868 & 1.229125 \\
\hline & -0.172959 & 5.669795 & 1.721710 \\
\hline & -7.049479 & 2.989861 & 1.331080 \\
\hline & -5.741997 & 2.818387 & 0.144694 \\
\hline & -6.050971 & 1.530390 & 1.328809 \\
\hline & -4.910937 & 5.163868 & 1.039304 \\
\hline & -6.315981 & 5.092562 & 2.118019 \\
\hline & -4.708670 & 5.383620 & 2.789253 \\
\hline & -6.538884 & 3.164198 & 3.787633 \\
\hline & -5.385807 & 1.812146 & 3.733852 \\
\hline & -4.871119 & 3.392131 & 4.352112 \\
\hline & -6.713945 & -2.056580 & 3.438463 \\
\hline & -4.977708 & -2.214777 & 3.144012 \\
\hline | & -5.810003 & -0.689782 & 2.762039 \\
\hline & -7.107023 & -4.139712 & 2.125983 \\
\hline & -6.566879 & -4.273617 & 0.441422 \\
\hline & -5.374539 & -4.267018 & 1.759112 \\
\hline & -8.352763 & -1.992969 & 1.680233 \\
\hline | & -7.552521 & -0.637667 & 0.859086 \\
\hline & -7.933146 & -2.121520 & -0.031589 \\
\hline & -6.110364 & -2.853603 & -4.890100 \\
\hline & -6.216990 & -3.632636 & -3.307924 \\
\hline . & -6.795510 & -1.963347 & -3.520551 \\
\hline & -3.701379 & -3.432316 & -5.000309 \\
\hline & -2.562175 & -2.875347 & -3.758289 \\
\hline & -3.736554 & -4.142367 & -3.367627 \\
\hline & -4.560742 & -1.074700 & -5.381412 \\
\hline & -5.081835 & -0.080978 & -4.004739 \\
\hline & -3.363247 & -0.497850 & -4.217055 \\
\hline & -5.839788 & 5.324406 & -1.660507 \\
\hline
\end{tabular}

(para-Cl)TS1 $1_{I L-s i-} \psi_{-1 \mathrm{~s} 2 \mathrm{a} 3 \mathrm{~s} 4 \mathrm{~s}}$

Number of imaginary frequencies : 1

The smallest frequencies are : $-572.3509 \quad 14.2903 \quad 18.0984 \mathrm{~cm}(-1)$

Electronic energy : $\quad H F=-4820.441239$

Zero-point correction $=\quad 1.442967($ Hartree/Particle)

Thermal correction to Energy= $\quad 1.528777$

Thermal correction to Enthalpy= $\quad 1.529721$

Thermal correction to Gibbs Free Energy $=\quad 1.322934$

Sum of electronic and zero-point Energies $=\quad-4818.998272$ 
Sum of electronic and thermal Energies= Sum of electronic and thermal Enthalpies= Sum of electronic and thermal Free Energies=
$-4818.912462$

$-4818.911518$

$-4819.118305$

Cartesian Coordinates

\begin{tabular}{|c|c|c|c|}
\hline 6 & -3.308538 & -1.147829 & -1.579335 \\
\hline 6 & -2.998799 & -0.982884 & -0.234660 \\
\hline 6 & -3.906529 & -1.417945 & 0.731646 \\
\hline 6 & -5.115605 & -2.012690 & 0.374996 \\
\hline 6 & -5.401791 & -2.149206 & -0.989317 \\
\hline 6 & -4.517360 & -1.721931 & -1.982703 \\
\hline 15 & -1.396372 & -0.203891 & 0.238324 \\
\hline 45 & 0.068075 & 0.373108 & -1.699502 \\
\hline 6 & 0.075130 & -0.712724 & -3.260955 \\
\hline 8 & 0.067248 & -1.204782 & -4.314380 \\
\hline 6 & -6.061665 & -2.482450 & 1.479440 \\
\hline 6 & -6.543590 & -1.264463 & 2.275932 \\
\hline 6 & -4.789031 & -1.906639 & -3.474316 \\
\hline 6 & -4.497848 & -0.606769 & -4.233872 \\
\hline 6 & -1.940299 & 1.285335 & 1.173895 \\
\hline 6 & -3.263011 & 1.727884 & 1.226658 \\
\hline 6 & -3.616175 & 2.852731 & 1.975220 \\
\hline 6 & -2.604954 & 3.520732 & 2.674347 \\
\hline 6 & -1.267403 & 3.118688 & 2.629491 \\
\hline 6 & -0.950994 & 2.005107 & 1.847532 \\
\hline 6 & -0.168226 & 3.887259 & 3.362797 \\
\hline 6 & -0.716269 & 4.682254 & 4.548915 \\
\hline 6 & -5.071350 & 3.302982 & 2.109867 \\
\hline 6 & -5.182767 & 4.828266 & 2.022807 \\
\hline 6 & -0.916494 & -1.339673 & 1.640457 \\
\hline 6 & -0.340150 & -2.575905 & 1.375447 \\
\hline 6 & 0.008197 & -3.454937 & 2.454213 \\
\hline 6 & -0.238234 & -3.054309 & 3.799431 \\
\hline 6 & -0.842476 & -1.799136 & 4.034032 \\
\hline 6 & -1.165684 & -0.972384 & 2.991970 \\
\hline 6 & 0.135507 & -3.906317 & 4.865924 \\
\hline 6 & 0.733083 & -5.116160 & 4.622712 \\
\hline 6 & 0.975049 & -5.523565 & 3.294005 \\
\hline 6 & 0.619250 & -4.719371 & 2.239551 \\
\hline 6 & -0.078835 & -3.084612 & -0.001850 \\
\hline 6 & -1.006576 & -3.991664 & -0.604612 \\
\hline 6 & -0.686382 & -4.604085 & -1.853079 \\
\hline 6 & 0.548958 & -4.304532 & -2.471824 \\
\hline 6 & 1.417840 & -3.424295 & -1.887211 \\
\hline 6 & 1.106982 & -2.802018 & -0.656014 \\
\hline 6 & -1.598688 & -5.517461 & -2.435904 \\
\hline 6 & -2.787389 & -5.813302 & -1.819700 \\
\hline 6 & -3.115628 & -5.197472 & -0.592546 \\
\hline 6 & -2.246506 & -4.317937 & 0.002938 \\
\hline 7 & 2.053396 & -1.871482 & -0.102874 \\
\hline 15 & 2.064700 & -0.364908 & -0.879740 \\
\hline 8 & 2.925337 & 0.418806 & 0.294333 \\
\hline 6 & 3.690888 & 1.523156 & 0.024363 \\
\hline 6 & 4.884590 & 1.405295 & -0.661925 \\
\hline 6 & 5.607414 & 2.608957 & -0.960882 \\
\hline
\end{tabular}




\begin{tabular}{|c|c|c|c|}
\hline 6 & 5.146501 & 3.855673 & -0.438813 \\
\hline 6 & 3.964114 & 3.891062 & 0.340200 \\
\hline 6 & 3.242384 & 2.750283 & 0.551984 \\
\hline 6 & 6.755984 & 2.623776 & -1.792627 \\
\hline 6 & 7.424469 & 3.792717 & -2.061637 \\
\hline 6 & 6.986630 & 5.015450 & -1.512852 \\
\hline 6 & 5.866755 & 5.041048 & -0.721918 \\
\hline 6 & 5.360617 & 0.073105 & -1.099773 \\
\hline 6 & 4.530718 & -0.747213 & -1.841964 \\
\hline 6 & 4.987973 & -1.951386 & -2.420270 \\
\hline 6 & 6.255068 & -2.395302 & -2.161851 \\
\hline 6 & 7.102728 & -1.672884 & -1.286312 \\
\hline 6 & 6.656724 & -0.429744 & -0.745679 \\
\hline 6 & 8.370709 & -2.181823 & -0.914411 \\
\hline 6 & 9.164055 & -1.512968 & -0.017617 \\
\hline 6 & 8.712559 & -0.301316 & 0.545735 \\
\hline 6 & 7.497078 & 0.228641 & 0.187979 \\
\hline 8 & 3.227494 & -0.392347 & -2.092861 \\
\hline 6 & 3.179227 & -2.478629 & 0.644685 \\
\hline 6 & 3.628130 & -1.742019 & 1.880057 \\
\hline 6 & 2.708951 & -1.227279 & 2.795777 \\
\hline 6 & 3.142481 & -0.598682 & 3.954979 \\
\hline 6 & 4.505937 & -0.468846 & 4.215911 \\
\hline 6 & 5.428069 & -0.972804 & 3.305897 \\
\hline 6 & 4.989546 & -1.606262 & 2.145009 \\
\hline 6 & 0.900285 & 2.931001 & 3.902886 \\
\hline 6 & 0.472309 & 4.871132 & 2.375837 \\
\hline 6 & -5.961212 & 2.696199 & 1.024504 \\
\hline 6 & -5.595111 & 2.845795 & 3.476849 \\
\hline 6 & 0.454329 & 2.529163 & -1.302771 \\
\hline 6 & -0.530852 & 2.563657 & -2.316678 \\
\hline 6 & -1.867607 & 3.195397 & -2.023494 \\
\hline 6 & -3.051541 & 2.727164 & -2.603023 \\
\hline 6 & -4.246885 & 3.418956 & -2.466142 \\
\hline 6 & -4.255605 & 4.606867 & -1.743186 \\
\hline 6 & -3.103634 & 5.092054 & -1.139604 \\
\hline 6 & -1.916584 & 4.380727 & -1.286182 \\
\hline 17 & -5.753083 & 5.511997 & -1.604895 \\
\hline 6 & -0.096669 & 2.781509 & -3.754115 \\
\hline 6 & -5.321880 & -3.440314 & 2.421850 \\
\hline 6 & -7.283316 & -3.213084 & 0.928733 \\
\hline 6 & -3.867174 & -3.011722 & -4.000910 \\
\hline 6 & -6.235846 & -2.305405 & -3.756301 \\
\hline 1 & 7.153254 & 1.151458 & 0.648512 \\
\hline 1 & -1.633625 & -0.015066 & 3.207529 \\
\hline 1 & 8.694118 & -3.127881 & -1.346633 \\
\hline 1 & -0.058665 & -3.575117 & 5.885221 \\
\hline 1 & 5.501297 & 5.978170 & -0.303874 \\
\hline 1 & -2.511162 & -3.848520 & 0.948915 \\
\hline 1 & -4.068405 & -5.422073 & -0.114830 \\
\hline 1 & 3.630384 & 4.843020 & 0.751188 \\
\hline 1 & -1.047353 & -1.496770 & 5.060526 \\
\hline 1 & 6.619205 & -3.321995 & -2.602277 \\
\hline 1 & 8.297466 & 3.774846 & -2.710272 \\
\hline 1 & 4.311222 & -2.498845 & -3.072469 \\
\hline 1 & -1.334088 & -5.977644 & -3.387054 \\
\hline
\end{tabular}




\begin{tabular}{|c|c|c|c|}
\hline & 0.792335 & -4.778817 & -3.421286 \\
\hline & 1.445951 & -6.485246 & 3.102937 \\
\hline & 7.101998 & 1.693180 & -2.234600 \\
\hline & 0.808836 & -5.053352 & 1.221425 \\
\hline & 2.365964 & -3.176922 & -2.361239 \\
\hline & 7.528836 & 5.933121 & -1.728574 \\
\hline & 9.329994 & 0.216243 & 1.276609 \\
\hline & -3.481688 & -6.515824 & -2.275831 \\
\hline & 1.021090 & -5.762574 & 5.448473 \\
\hline & 10.131356 & -1.918332 & 0.269730 \\
\hline & 2.318015 & 2.748284 & 1.124495 \\
\hline & -3.660468 & -1.289644 & 1.785929 \\
\hline & -2.572413 & -0.832327 & -2.317578 \\
\hline & -6.341114 & -2.608349 & -1.284517 \\
\hline & -4.025367 & 1.175742 & 0.683523 \\
\hline & 0.079430 & 1.647880 & 1.790420 \\
\hline & -2.874993 & 4.381858 & 3.281292 \\
\hline & 2.819446 & -3.473425 & 0.944537 \\
\hline & 4.043983 & -2.651756 & -0.014187 \\
\hline & 5.718802 & -1.989037 & 1.428740 \\
\hline & 1.646271 & -1.292301 & 2.571644 \\
\hline & 6.495533 & -0.868988 & 3.491698 \\
\hline & 2.408682 & -0.205300 & 4.657200 \\
\hline & 4.845757 & 0.028231 & 5.122154 \\
\hline & 0.176655 & 2.830642 & -0.291472 \\
\hline 1 & 1.492162 & 2.701910 & -1.587536 \\
\hline & -3.041186 & 1.799062 & -3.176098 \\
\hline 1 & -5.161997 & 3.048462 & -2.921765 \\
\hline & -3.126916 & 6.022983 & -0.578133 \\
\hline & -1.003481 & 4.770919 & -0.840222 \\
\hline 1 & -0.777922 & 2.297261 & -4.464529 \\
\hline & -0.101819 & 3.858596 & -3.976712 \\
\hline 1 & 0.909926 & 2.386049 & -3.924227 \\
\hline & -1.224711 & 1.089908 & -2.386212 \\
\hline 1 & 0.111935 & 5.162441 & 5.085314 \\
\hline 1 & -1.400439 & 5.481204 & 4.239197 \\
\hline & -1.245700 & 4.034439 & 5.259660 \\
\hline 1 & 1.639248 & 3.487626 & 4.493377 \\
\hline & 0.453486 & 2.164936 & 4.551767 \\
\hline 1 & 1.452272 & 2.411348 & 3.110947 \\
\hline & 1.305029 & 5.410981 & 2.848600 \\
\hline 1 & 0.859752 & 4.357273 & 1.485205 \\
\hline 1 & -0.262733 & 5.612028 & 2.032959 \\
\hline 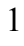 & -6.979406 & 3.095609 & 1.115858 \\
\hline 1 & -5.596325 & 2.936177 & 0.016525 \\
\hline & -6.031937 & 1.603280 & 1.109261 \\
\hline 1 & -4.739523 & 5.207254 & 1.093484 \\
\hline ] & -6.238600 & 5.129325 & 2.036641 \\
\hline 1 & -4.692743 & 5.332722 & 2.864003 \\
\hline 1 & -6.640117 & 3.156974 & 3.612441 \\
\hline 1 & -5.553184 & 1.752272 & 3.569326 \\
\hline 1 & -5.005973 & 3.274107 & 4.297878 \\
\hline 1 & -7.920582 & -3.545775 & 1.757833 \\
\hline 1 & -7.894961 & -2.567694 & 0.285449 \\
\hline & -6.999454 & -4.103610 & 0.351814 \\
\hline 1 & -5.994931 & -3.783294 & 3.219167 \\
\hline
\end{tabular}




$\begin{array}{lrrr}1 & -4.963479 & -4.327959 & 1.882647 \\ 1 & -4.454985 & -2.970577 & 2.904231 \\ 1 & -7.206556 & -1.575494 & 3.094817 \\ 1 & -5.703359 & -0.712173 & 2.717146 \\ 1 & -7.103294 & -0.571562 & 1.633263 \\ 1 & -6.392136 & -2.388072 & -4.839283 \\ 1 & -6.488278 & -3.279747 & -3.319220 \\ 1 & -6.947159 & -1.561968 & -3.372864 \\ 1 & -4.024147 & -3.164843 & -5.077714 \\ 1 & -2.808868 & -2.763622 & -3.844518 \\ 1 & -4.062657 & -3.960923 & -3.484958 \\ 1 & -4.738726 & -0.729559 & -5.298033 \\ 1 & -5.101817 & 0.224155 & -3.843637 \\ 1 & -3.438867 & -0.319903 & -4.175442\end{array}$

(para-CI)TS1 $1_{\mathrm{IL} L-r e-} \psi_{-1 \mathrm{a} 2 \mathrm{~s} 3 \mathrm{a} 4 \mathrm{a}}$

Number of imaginary frequencies : 1

The smallest frequencies are : -591.5355 $13.9296 \quad 15.4099 \mathrm{~cm}(-1)$

Electronic energy : $\quad H F=-4820.4400923$

Zero-point correction $=\quad 1.441736($ Hartree/Particle)

Thermal correction to Energy $=\quad 1.527997$

Thermal correction to Enthalpy= $\quad 1.528941$

Thermal correction to Gibbs Free Energy= $\quad 1.321066$

Sum of electronic and zero-point Energies $=\quad-4818.998357$

Sum of electronic and thermal Energies $=\quad-4818.912095$

Sum of electronic and thermal Enthalpies $=\quad-4818.911151$

Sum of electronic and thermal Free Energies $=\quad-4819.119026$

Cartesian Coordinates

$\begin{array}{cccc}6 & 3.894295 & 1.474172 & 0.936752 \\ 6 & 3.059427 & 0.709415 & 0.118027 \\ 6 & 3.582917 & 0.082395 & -1.008122 \\ 6 & 4.936845 & 0.196331 & -1.334467 \\ 6 & 5.743369 & 0.965468 & -0.493765 \\ 6 & 5.250600 & 1.614891 & 0.645252 \\ 15 & 1.278703 & 0.467340 & 0.536750 \\ 6 & 0.824570 & 2.185017 & 1.086082 \\ 6 & 0.444806 & 3.137612 & 0.151688 \\ 6 & 0.148607 & 4.477274 & 0.567783 \\ 6 & 0.252899 & 4.826829 & 1.945275 \\ 6 & 0.658202 & 3.837375 & 2.869322 \\ 6 & 0.931953 & 2.562183 & 2.453286 \\ 6 & -0.053774 & 6.145307 & 2.358646 \\ 6 & -0.452331 & 7.091418 & 1.449528 \\ 6 & -0.558215 & 6.752865 & 0.084053 \\ 6 & -0.263714 & 5.482690 & -0.346236 \\ 6 & 0.366495 & 2.850386 & -1.306913 \\ 6 & -0.777287 & 2.309570 & -1.865937 \\ 6 & -0.892898 & 2.171984 & -3.268669 \\ 6 & 0.141207 & 2.514917 & -4.096882 \\ 6 & 1.352316 & 3.010964 & -3.561844 \\ 6 & 1.461763 & 3.205958 & -2.152887 \\ 6 & 2.677913 & 3.724443 & -1.636895\end{array}$




\begin{tabular}{|c|c|c|c|}
\hline 6 & 3.729580 & 4.015504 & -2.469376 \\
\hline 6 & 3.619578 & 3.814837 & -3.862680 \\
\hline 6 & 2.454277 & 3.324945 & -4.394493 \\
\hline 7 & -1.843583 & 1.830366 & -1.029928 \\
\hline 6 & -2.985190 & 2.755159 & -0.843935 \\
\hline 6 & -3.644720 & 2.685392 & 0.507715 \\
\hline 6 & -5.018581 & 2.481547 & 0.614613 \\
\hline 6 & -5.628261 & 2.398545 & 1.864116 \\
\hline 6 & -4.868198 & 2.531668 & 3.021234 \\
\hline 6 & -3.495720 & 2.755310 & 2.920279 \\
\hline 6 & -2.890514 & 2.828067 & 1.673006 \\
\hline 6 & 5.534628 & -0.503168 & -2.553572 \\
\hline 6 & 6.517277 & -1.578126 & -2.076965 \\
\hline 6 & 6.180454 & 2.479368 & 1.496262 \\
\hline 6 & 5.509103 & 2.952287 & 2.784556 \\
\hline 45 & 0.110875 & -0.942715 & -0.980995 \\
\hline 6 & 0.608803 & -0.848882 & -2.918081 \\
\hline 8 & 0.468104 & -0.927897 & -4.065960 \\
\hline 15 & -1.876252 & 0.132934 & -0.951116 \\
\hline 8 & -2.942908 & 0.022272 & 0.321009 \\
\hline 6 & -3.579691 & -1.177138 & 0.513235 \\
\hline 6 & -4.677614 & -1.501436 & -0.261422 \\
\hline 6 & -5.277599 & -2.789900 & -0.078221 \\
\hline 6 & -4.788285 & -3.652201 & 0.949862 \\
\hline 6 & -3.694355 & -3.235976 & 1.746828 \\
\hline 6 & -3.087580 & -2.029750 & 1.521590 \\
\hline 6 & -5.386633 & -4.921884 & 1.139013 \\
\hline 6 & -6.411346 & -5.346971 & 0.333247 \\
\hline 6 & -6.871282 & -4.514884 & -0.708567 \\
\hline 6 & -6.320904 & -3.273340 & -0.908850 \\
\hline 6 & -5.137575 & -0.547544 & -1.295714 \\
\hline 6 & -4.232756 & -0.073176 & -2.230010 \\
\hline 6 & -4.638569 & 0.733161 & -3.316525 \\
\hline 6 & -5.940073 & 1.139599 & -3.421133 \\
\hline 6 & -6.884357 & 0.788671 & -2.425394 \\
\hline 6 & -6.482149 & -0.054411 & -1.346067 \\
\hline 6 & -7.425384 & -0.315808 & -0.319684 \\
\hline 6 & -8.695951 & 0.203198 & -0.374617 \\
\hline 6 & -9.100178 & 1.004970 & -1.462569 \\
\hline 6 & -8.207643 & 1.290697 & -2.463914 \\
\hline 8 & -2.906889 & -0.418530 & -2.170014 \\
\hline 6 & 0.436251 & -3.301216 & -0.563196 \\
\hline 6 & -0.757940 & -2.954320 & -1.241927 \\
\hline 6 & 1.377253 & -0.429162 & 2.140764 \\
\hline 6 & 2.510867 & -1.140272 & 2.512095 \\
\hline 6 & 2.545023 & -1.880640 & 3.703391 \\
\hline 6 & 1.414284 & -1.862627 & 4.514766 \\
\hline 6 & 0.257398 & -1.137792 & 4.178975 \\
\hline 6 & 0.244992 & -0.456455 & 2.966735 \\
\hline 6 & 3.823838 & -2.633353 & 4.066544 \\
\hline 6 & 4.976768 & -1.630173 & 4.192392 \\
\hline 6 & -0.932896 & -1.115750 & 5.138086 \\
\hline 6 & -1.586966 & -2.500994 & 5.162003 \\
\hline 6 & 3.700872 & -3.389760 & 5.386328 \\
\hline 6 & 4.155573 & -3.646581 & 2.964611 \\
\hline 6 & -0.458612 & -0.758563 & 6.551692 \\
\hline
\end{tabular}




\begin{tabular}{|c|c|c|c|}
\hline 6 & -1.978502 & -0.077112 & 4.730920 \\
\hline 6 & 0.346728 & -3.637806 & 0.908402 \\
\hline 6 & 1.600373 & -3.893490 & -1.304201 \\
\hline 6 & 2.780057 & -4.204346 & -0.614597 \\
\hline 6 & 3.908939 & -4.674644 & -1.272051 \\
\hline 6 & 3.862475 & -4.841051 & -2.650726 \\
\hline 6 & 2.706120 & -4.562100 & -3.365906 \\
\hline 6 & 1.586105 & -4.092768 & -2.689177 \\
\hline 17 & 5.292029 & -5.394947 & -3.503215 \\
\hline 6 & 6.271219 & 0.515034 & -3.429946 \\
\hline 6 & 4.463788 & -1.177177 & -3.410738 \\
\hline 6 & 7.439160 & 1.695106 & 1.880199 \\
\hline 6 & 6.582850 & 3.716316 & 0.684959 \\
\hline 1 & -7.123226 & -0.917613 & 0.534075 \\
\hline 1 & 1.246299 & 1.825886 & 3.188435 \\
\hline 1 & -8.495269 & 1.923815 & -3.302382 \\
\hline 1 & 0.031296 & 6.388971 & 3.416819 \\
\hline 1 & -5.003938 & -5.558809 & 1.935500 \\
\hline 1 & 2.776956 & 3.871928 & -0.562552 \\
\hline 1 & 4.660552 & 4.394993 & -2.052411 \\
\hline 1 & -3.325372 & -3.904373 & 2.524696 \\
\hline 1 & 0.752892 & 4.105563 & 3.920924 \\
\hline 1 & -6.261767 & 1.758816 & -4.256954 \\
\hline 1 & -7.665978 & -4.862480 & -1.364914 \\
\hline 1 & -3.893486 & 1.010060 & -4.059191 \\
\hline 1 & 2.354258 & 3.166074 & -5.467359 \\
\hline 1 & 0.056225 & 2.382607 & -5.174390 \\
\hline 1 & -0.874897 & 7.505587 & -0.634263 \\
\hline 1 & -6.679298 & -2.651084 & -1.724490 \\
\hline 1 & -0.346584 & 5.238285 & -1.403279 \\
\hline 1 & -1.812580 & 1.751 & -3.670867 \\
\hline 1 & -6.859913 & -6.326030 & 0.484574 \\
\hline 1 & -9.395831 & -0.002544 & \\
\hline 1 & 4.461175 & 4.050242 & -4.510589 \\
\hline 1 & -0.688228 & 8.101537 & 1.776247 \\
\hline 1 & -10.111339 & 1.403699 & -1.497019 \\
\hline 1 & -2.216768 & -1.704005 & 2.088944 \\
\hline 1 & 3.474225 & 1.956975 & 1.816294 \\
\hline 1 & 2.905996 & -0.492186 & -1.634695 \\
\hline 1 & 6.801434 & 1.069254 & -0.735504 \\
\hline 1 & 3.388016 & -1.120461 & 1.865480 \\
\hline 1 & -0.640032 & 0.100352 & 2.656240 \\
\hline 1 & 1.423600 & -2.417056 & 5.450463 \\
\hline 1 & -2.565415 & 3.761577 & -0.985516 \\
\hline 1 & -3.739930 & 2.616972 & -1.634272 \\
\hline 1 & -5.617667 & 2.359627 & -0.289289 \\
\hline 1 & -1.814547 & 2.967264 & 1.593056 \\
\hline 1 & -6.700153 & 2.220221 & 1.927554 \\
\hline 1 & -2.890211 & 2.870592 & 3.818550 \\
\hline 1 & -5.341273 & 2.463245 & 3.998764 \\
\hline 1 & 1.382554 & -1.871074 & -0.574160 \\
\hline 1 & -1.684132 & -3.000436 & -0.668407 \\
\hline 1 & -0.886795 & -3.163971 & -2.302066 \\
\hline 1 & 0.390550 & -4.728614 & 1.043967 \\
\hline 1 & 1.152051 & -3.196032 & 1.506821 \\
\hline 1 & -0.601051 & -3.277935 & 1.321988 \\
\hline
\end{tabular}




\begin{tabular}{|c|c|c|c|}
\hline & 2.832203 & -4.054555 & 0.461680 \\
\hline & 4.818145 & -4.902291 & -0.720000 \\
\hline & 2.682513 & -4.702240 & -4.443460 \\
\hline & 0.691191 & -3.876581 & -3.266552 \\
\hline & -2.776464 & -0.039342 & 5.483599 \\
\hline & -1.537388 & 0.927286 & 4.659923 \\
\hline & -2.457809 & -0.300868 & 3.7693 \\
\hline & -1.316565 & -0.712825 & 7.235366 \\
\hline & 0.243093 & -1.494979 & \\
\hline & 0.036352 & 0.221236 & 6.565659 \\
\hline & -2.452262 & -2.507655 & 5.83 \\
\hline & -1.938589 & -2.788173 & 4.161541 \\
\hline & -0.886638 & -3.273543 & 5.503640 \\
\hline & 4.935478 & -1.670391 & -4.271138 \\
\hline & 3.914224 & -1.951519 & -2.857694 \\
\hline & 3.736579 & -0.449100 & \\
\hline & 1764 & & \\
\hline & 5.588139 & 1.3 & -3.7 \\
\hline & 7.107980 & 0.991784 & -2.904940 \\
\hline & 6.976824 & -2.086611 & -2.935275 \\
\hline & 7.324862 & -1.154487 & -1.466561 \\
\hline & 5.999618 & -2.335998 & -1.47220 \\
\hline & 4.640923 & -3.913654 & 5.600217 \\
\hline & 3.50 & -2.7 & \\
\hline & 2.90 & $-4.1<$ & 5.35 \\
\hline & 5.910509 & -2.150119 & 4.44 \\
\hline & 5.144351 & -1.079994 & 3.257658 \\
\hline & 4.772863 & -0.894930 & 4.981940 \\
\hline & 5.062811 & -4.208536 & 3.224406 \\
\hline & 3.337936 & -4.370080 & 2.837134 \\
\hline & 4.338293 & -3.157960 & 1.998470 \\
\hline & 6.218120 & 3.548903 & 3.372307 \\
\hline & 4.634322 & 3.586552 & 2.588044 \\
\hline & 5.186147 & 2.108 & 3.409662 \\
\hline & 7.273461 & 4.347245 & 1.261123 \\
\hline & 7.079964 & 3.444785 & -0.254961 \\
\hline & 5.701146 & 4.322663 & 0.437095 \\
\hline & 8.095655 & 2.317085 & 2.503129 \\
\hline & 7.186428 & 0.793991 & 2.453909 \\
\hline & 8.021387 & 1.382772 & 1.005478 \\
\hline
\end{tabular}

(para-CI)TS1 $1_{\text {IL-re- }} \psi_{-1 \mathrm{a} 2 \mathrm{2s} 3 \mathrm{a} 4 \mathrm{~s}}$

Number of imaginary frequencies : 1

The smallest frequencies are : - $603.9645 \quad 15.5205 \quad 20.7687 \mathrm{~cm}(-1)$

Electronic energy : $\quad H F=-4820.4404689$

Zero-point correction $=\quad 1.442232($ Hartree/Particle)

Thermal correction to Energy $=\quad 1.528146$

Thermal correction to Enthalpy= $\quad 1.529090$

Thermal correction to Gibbs Free Energy= 1.322991

Sum of electronic and zero-point Energies $=\quad-4818.998237$

Sum of electronic and thermal Energies $=\quad-4818.912323$

Sum of electronic and thermal Enthalpies $=\quad-4818.911379$

Sum of electronic and thermal Free Energies $=\quad-4819.117478$

Cartesian Coordinates 


$\begin{array}{cccc}6 & 1.602917 & -4.078388 & -2.682258 \\ 6 & 1.596926 & -3.891893 & -1.295056 \\ 6 & 2.760281 & -4.226673 & -0.589463 \\ 6 & 3.891936 & -4.711162 & -1.231938 \\ 6 & 3.865600 & -4.866557 & -2.612034 \\ 6 & 2.726082 & -4.561436 & -3.343983 \\ 6 & 0.429604 & -3.292190 & -0.565996 \\ 6 & 0.334808 & -3.612804 & 0.908595 \\ 17 & 5.299625 & -5.440789 & -3.443069 \\ 45 & 0.094781 & -0.932263 & -1.001993 \\ 6 & -0.761875 & -2.952958 & -1.253405 \\ 15 & 1.253591 & 0.487442 & 0.508331 \\ 6 & 1.409836 & -0.407701 & 2.109360 \\ 6 & 2.570828 & -1.094029 & 2.442657 \\ 6 & 2.658331 & -1.840918 & 3.626122 \\ 6 & 1.551710 & -1.855087 & 4.470951 \\ 6 & 0.369259 & -1.154494 & 4.175531 \\ 6 & 0.304451 & -0.465070 & 2.968979 \\ 6 & 3.964135 & -2.568930 & 3.941475 \\ 6 & 4.266857 & -3.582950 & 2.831899 \\ 6 & -0.796141 & -1.170323 & 5.165155 \\ 6 & -1.855070 & -0.123130 & 4.818498 \\ 6 & 3.025741 & 0.758483 & 0.073692 \\ 6 & 3.833141 & 1.584509 & 0.849431 \\ 6 & 5.194951 & 1.733622 & 0.572715 \\ 6 & 5.724340 & 1.012055 & -0.499338 \\ 6 & 4.937766 & 0.178948 & -1.305939 \\ 6 & 3.581403 & 0.069787 & -1.003316 \\ 6 & 5.565627 & -0.611132 & -2.452627 \\ 6 & 4.507469 & -1.236705 & -3.361737 \\ 6 & 6.028994 & 2.662174 & 1.454204 \\ 6 & 5.387541 & 4.055041 & 1.487055 \\ 6 & 0.786238 & 2.200594 & 1.057921 \\ 6 & 0.414286 & 3.151636 & 0.118657 \\ 6 & 0.128029 & 4.495318 & 0.528237 \\ 6 & 0.235907 & 4.850775 & 1.903979 \\ 6 & 0.627988 & 3.861248 & 2.833592 \\ 6 & 0.890363 & 2.581329 & 2.424069 \\ 6 & -0.055621 & 6.174896 & 2.310421 \\ 6 & -0.443737 & 7.120514 & 1.396388 \\ 6 & -0.554997 & 6.775609 & 0.032843 \\ 6 & -0.275171 & 5.499996 & -0.390869 \\ 6 & 0.330437 & 2.857526 & -1.338314 \\ 6 & -0.816998 & 2.313050 & -1.886406 \\ 6 & -0.945334 & 2.173327 & -3.287819 \\ 6 & 0.081792 & 2.514903 & -4.125282 \\ 6 & 1.299743 & 3.005686 & -3.600974 \\ 6 & 1.420854 & 3.205837 & -2.193620 \\ 6 & 2.645520 & 3.715346 & -1.688941 \\ 6 & 3.695635 & 3.986418 & -2.529988 \\ 6 & 3.575654 & 3.776767 & -3.920931 \\ 6 & 2.399818 & 3.301668 & -4.442623 \\ 7 & -1.873644 & 1.831904 & -1.039443 \\ 8 & -1.899546 & 0.134474 & -0.956009 \\ & -2.943560 & -0.421597 & -2.161382 \\ & & & \\ 6 & & & \end{array}$




\begin{tabular}{|c|c|c|c|}
\hline 6 & -4.273383 & -0.088674 & -2.200964 \\
\hline 6 & -5.159881 & -0.576495 & -1.256195 \\
\hline 6 & -6.511544 & -0.101389 & -1.288011 \\
\hline 6 & -6.938637 & 0.739714 & -2.359122 \\
\hline 6 & -6.011027 & 1.108336 & -3.364088 \\
\hline 6 & -4.703155 & 0.718118 & -3.277759 \\
\hline 6 & -7.439075 & -0.380606 & -0.252055 \\
\hline 6 & -8.718043 & 0.118787 & -0.290842 \\
\hline 6 & -9.146636 & 0.918483 & -1.370889 \\
\hline 6 & -8.269639 & 1.222010 & -2.380749 \\
\hline 6 & -4.675084 & -1.522889 & -0.226561 \\
\hline 6 & -3.574598 & -1.180734 & 0.536970 \\
\hline 6 & -3.067881 & -2.019409 & 1.549828 \\
\hline 6 & -3.657081 & -3.232373 & 1.785272 \\
\hline 6 & -4.748394 & -3.668935 & 0.995648 \\
\hline 6 & -5.256474 & -2.818263 & -0.033068 \\
\hline 6 & -5.326506 & -4.946715 & 1.193315 \\
\hline 6 & -6.349688 & -5.390064 & 0.395504 \\
\hline 6 & -6.829032 & -4.568597 & -0.646025 \\
\hline 6 & -6.298581 & -3.319791 & -0.854451 \\
\hline 8 & -2.951323 & 0.022861 & 0.329202 \\
\hline 6 & -3.013767 & 2.755651 & -0.840477 \\
\hline 6 & -3.665455 & 2.676721 & 0.514 \\
\hline 6 & -5.037429 & 2.464525 & 0.627888 \\
\hline 6 & -5.639572 & 2.371231 & 1.880321 \\
\hline 6 & -4.873501 & 2.501580 & 3.033695 \\
\hline 6 & -3.502682 & 2.732861 & 2.926389 \\
\hline 6 & -2.905251 & 2.817221 & 1.676123 \\
\hline 6 & 6.420758 & -1.735896 & -1.857633 \\
\hline 6 & 6.443839 & 0.302249 & -3.31 \\
\hline 6 & 7.460441 & & \\
\hline 6 & 6.079264 & 2.093759 & 2.877101 \\
\hline 6 & 0.627078 & -0.830547 & -2.924069 \\
\hline 8 & 0.572179 & -0.889995 & -4.07 \\
\hline 6 & 5.106266 & -1.547923 & 4.009947 \\
\hline 6 & 3.907937 & -3.317712 & 5.270113 \\
\hline 6 & -1.445263 & -2.558092 & 5.155414 \\
\hline 6 & -0.292026 & -0.860397 & \\
\hline 1 & -7.118573 & -0.981509 & 0.595563 \\
\hline 1 & 1.197902 & 1.845339 & 3.162451 \\
\hline 1 & -8.576008 & 1.854102 & -3.213343 \\
\hline 1 & 0.032335 & 6.423127 & 3.367290 \\
\hline 1 & -4.929411 & -5.574952 & 1.989652 \\
\hline 1 & 2.756055 & 3.867735 & -0.616168 \\
\hline 1 & 4.634035 & 4.355302 & -2.120046 \\
\hline 1 & -3.275488 & -3.890999 & 2.565423 \\
\hline 1 & 0.721905 & 4.133193 & 3.884302 \\
\hline 1 & -6.350924 & 1.727447 & -4.192757 \\
\hline 1 & -7.622880 & -4.930336 & -1.295735 \\
\hline 1 & -3.971128 & 1.008419 & -4.028144 \\
\hline 1 & 2.290924 & 3.137120 & -5.513770 \\
\hline 1 & -0.012145 & 2.380665 & -5.201804 \\
\hline 1 & -0.864330 & 7.527846 & -0.689171 \\
\hline 1 & -6.671702 & -2.705699 & -1.669735 \\
\hline 1 & -0.362383 & 5.250632 & -1.446347 \\
\hline 1 & -1.869052 & 1.753024 & -3.681297 \\
\hline
\end{tabular}




\begin{tabular}{|c|c|c|c|}
\hline & -6.782439 & -6.375251 & 0.552954 \\
\hline & -9.405654 & -0.101090 & 0.522906 \\
\hline & 4.417042 & 3.994639 & -4.575105 \\
\hline & -0.667804 & 8.135033 & 1.717737 \\
\hline 1 & -10.164124 & 1.301711 & -1.392544 \\
\hline & -2.199953 & -1.678241 & 2.112458 \\
\hline 1 & 3.397448 & 2.118706 & 1.693338 \\
\hline 1 & 2.925056 & -0.553093 & -1.604802 \\
\hline 1 & 6.785050 & 1.102771 & -0.724191 \\
\hline 1 & 3.428000 & -1.051261 & 1.770935 \\
\hline 1 & -0.601273 & 0.075371 & 2.689898 \\
\hline 1 & 1.602240 & -2.416700 & 5.400930 \\
\hline 1 & -2.594317 & 3.762938 & -0.977192 \\
\hline 1 & -3.772931 & 2.622820 & -1.627649 \\
\hline 1 & -5.640864 & 2.343606 & -0.273217 \\
\hline 1 & -1.830637 & 2.963879 & 1.591281 \\
\hline 1 & -6.710131 & 2.187003 & 1.949010 \\
\hline 1 & -2.893179 & 2.848213 & 3.821780 \\
\hline 1 & -5.340761 & 2.425383 & 4.013407 \\
\hline 1 & 1.366241 & -1.861630 & -0.594639 \\
\hline 1 & -1.690808 & -2.995697 & -0.684029 \\
\hline 1 & -0.884063 & -3.171940 & -2.312412 \\
\hline 1 & 0.372597 & -4.702204 & 1.056677 \\
\hline 1 & 1.141220 & -3.168028 & 1.503709 \\
\hline 1 & -0.612102 & -3.243273 & 1.314457 \\
\hline 1 & 2.797540 & -4.087738 & \\
\hline 1 & 4.788060 & -4.958353 & -0.666674 \\
\hline 1 & 2.718325 & -4.691752 & -4.423033 \\
\hline 1 & 0.721764 & -3.841382 & -3.272675 \\
\hline 1 & -2.633746 & -0.113408 & 5.591735 \\
\hline 1 & -1.418049 & 0.884207 & 4.769244 \\
\hline 1 & -2.357349 & -0.318042 & 3.862523 \\
\hline 1 & -1.136210 & -0.834980 & \\
\hline 1 & 0.414769 & -1.611241 & 6.950741 \\
\hline 1 & 0.206067 & 0.117195 & 6.615679 \\
\hline 1 & -2.284934 & -2.596485 & 5.862580 \\
\hline 1 & -1.833789 & -2.803784 & 4.157477 \\
\hline 1 & -0.730109 & -3.341012 & 5.437741 \\
\hline 1 & 4.998065 & -1.764817 & -4.190208 \\
\hline 1 & 3.885159 & -1.973665 & -2.835820 \\
\hline 1 & 3.844989 & -0.473582 & -3.793427 \\
\hline 1 & 6.860262 & -0.262656 & -4.157926 \\
\hline 1 & 5.858904 & 1.138187 & -3.719758 \\
\hline 1 & 7.289891 & 0.721793 & -2.756143 \\
\hline 1 & 6.890016 & -2.328029 & -2.655263 \\
\hline 1 & 7.218486 & -1.342991 & -1.214052 \\
\hline 1 & 5.801781 & -2.414482 & -1.252608 \\
\hline 1 & 4.866483 & -3.819922 & 5.451407 \\
\hline 1 & 3.727217 & -2.640795 & 6.115026 \\
\hline 1 & 3.127096 & -4.089268 & 5.273635 \\
\hline 1 & 6.054875 & -2.051603 & 4.240107 \\
\hline 1 & 5.235375 & -1.014362 & 3.059226 \\
\hline 1 & 4.920571 & -0.800018 & 4.792173 \\
\hline 1 & 5.193849 & -4.127961 & 3.055809 \\
\hline 1 & 3.456851 & -4.320757 & 2.742129 \\
\hline 1 & 4.399894 & -3.094779 & 1.856906 \\
\hline
\end{tabular}




$\begin{array}{rrrr}1 & 8.012300 & 3.504850 & 1.597522 \\ 1 & 8.002411 & 1.860490 & 0.949522 \\ 1 & 7.490208 & 3.224013 & -0.071713 \\ 1 & 5.983880 & 4.732496 & 2.112968 \\ 1 & 5.336034 & 4.488669 & 0.479244 \\ 1 & 4.369575 & 4.041240 & 1.897667 \\ 1 & 6.643298 & 2.764921 & 3.539181 \\ 1 & 5.075673 & 1.967172 & 3.303261 \\ 1 & 6.574283 & 1.113331 & 2.890249\end{array}$

(para-CI)TS1 $1_{\text {IL-re- }-\Psi-1 \text { a2s3s4a }}$

Number of imaginary frequencies $: 1$

The smallest frequencies are : $-605.8431 \quad 12.2499 \quad 14.0911 \mathrm{~cm}(-1)$

Electronic energy :

$\mathrm{HF}=-4820.4403169$

Zero-point correction $=$

1.442207 (Hartree/Particle)

Thermal correction to Energy= $\quad 1.528399$

Thermal correction to Enthalpy= $\quad 1.529343$

Thermal correction to Gibbs Free Energy $=\quad 1.321472$

Sum of electronic and zero-point Energies $=\quad-4818.998110$

Sum of electronic and thermal Energies $=\quad-4818.911918$

Sum of electronic and thermal Enthalpies $=\quad-4818.910974$

Sum of electronic and thermal Free Energies $=\quad-4819.118845$

Cartesian Coordinates

$\begin{array}{cccc}6 & -6.302502 & -3.202700 & -1.058169 \\ 6 & -5.259431 & -2.759970 & -0.204826 \\ 6 & -4.774448 & -3.669295 & 0.784230 \\ 6 & -5.374848 & -4.945313 & 0.913781 \\ 6 & -6.398506 & -5.331313 & 0.087498 \\ 6 & -6.855437 & -4.451195 & -0.915634 \\ 6 & -4.655741 & -1.465878 & -0.327796 \\ 6 & -3.558707 & -1.180802 & 0.463486 \\ 6 & -3.074950 & -2.077569 & 1.436814 \\ 6 & -3.684106 & -3.291677 & 1.604374 \\ 6 & -5.114122 & -0.461817 & -1.314292 \\ 6 & -4.209698 & 0.059437 & -2.223682 \\ 6 & -4.616175 & 0.919361 & -3.268202 \\ 6 & -5.917331 & 1.332075 & -3.350223 \\ 6 & -6.860610 & 0.932892 & -2.371843 \\ 6 & -6.458148 & 0.035243 & -1.337712 \\ 6 & -8.182865 & 1.438978 & -2.382420 \\ 6 & -9.074158 & 1.104915 & -1.395005 \\ 6 & -8.669484 & 0.248447 & -0.349832 \\ 6 & -7.400073 & -0.275564 & -0.323941 \\ 8 & -2.883444 & -0.287828 & -2.181990 \\ 15 & -1.849951 & 0.196536 & -0.937681 \\ 8 & -2.915259 & 0.022640 & 0.328901 \\ 45 & 0.140114 & -0.882567 & -1.056505 \\ 15 & 1.302743 & 0.417306 & 0.560074 \\ 6 & 1.379752 & -0.565303 & 2.112229 \\ 6 & 2.496827 & -1.324978 & 2.433571 \\ 6 & 2.521243 & -2.129411 & 3.582608 \\ 6 & 1.396460 & -2.128901 & 4.402271 \\ 6 & 0.253870 & -1.362602 & 4.113678\end{array}$




\begin{tabular}{|c|c|c|c|}
\hline 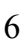 & 0.250857 & -0.613299 & 2.941949 \\
\hline & 3.780429 & -2.937456 & 3.889615 \\
\hline & 3.658775 & -3.739760 & 5.182193 \\
\hline & -0.930411 & -1.370945 & 5.080276 \\
\hline & -0.443141 & -1.079470 & 6.504466 \\
\hline & 0.406338 & -3.273113 & -0.738293 \\
\hline & 0.264059 & -3.654583 & 0.716733 \\
\hline & 1.554992 & -3.901789 & -1.470676 \\
\hline & 2.608823 & -4.485500 & -0.756261 \\
\hline & 3.709428 & -5.039324 & -1.398635 \\
\hline & 3.763304 & -5.010405 & -2.784862 \\
\hline & 2.736766 & -4.442212 & -3.528934 \\
\hline & 1.644730 & -3.893699 & -2.869133 \\
\hline & -0.755756 & -2.858127 & -1.437423 \\
\hline & 0.685851 & -0.620162 & -2.950857 \\
\hline & 0.686552 & -0.543980 & -4.107355 \\
\hline & 0.859757 & 2.108784 & 1.190924 \\
\hline & 0.494203 & 3.111394 & 0.305055 \\
\hline & 0.215139 & 4.432936 & 0.790231 \\
\hline & 0.330264 & 4.711823 & 2.183126 \\
\hline & 0.724760 & 3.672111 & 3.055279 \\
\hline 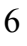 & 0.976749 & 2.415623 & 2.574885 \\
\hline ) & 0.042271 & 6.011201 & 2.664551 \\
\hline & -0.351887 & 7.006398 & 1.807575 \\
\hline 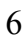 & -0.473032 & 6.737392 & 0.428188 \\
\hline & -0.195274 & 5.487630 & -0.067933 \\
\hline 6 & 0.395655 & 2.911896 & -1.167858 \\
\hline 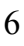 & -0.766105 & 2.427170 & -1.740529 \\
\hline 5 & -0.928835 & 2.424395 & -3.145273 \\
\hline 5 & 0.067363 & 2.872534 & -3.969436 \\
\hline & 1.293865 & 3.323547 & -3.428838 \\
\hline 5 & 1.464856 & 3.344558 & -2.012416 \\
\hline 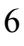 & 2.706364 & 3.792463 & -1.490655 \\
\hline 6 & 3.713981 & 4.205972 & -2.326161 \\
\hline 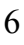 & 3.533250 & 4.202994 & -3.726538 \\
\hline 5 & 2.349896 & 3.764984 & -4.263312 \\
\hline 7 & -1.818230 & 1.894822 & -0.919674 \\
\hline 6 & -2.966065 & 2.801900 & -0.683612 \\
\hline 5 & -3.613275 & 2.668296 & 0.668895 \\
\hline 5 & -4.985418 & 2.454059 & 0.777506 \\
\hline 6 & -5.584417 & 2.312121 & 2.026772 \\
\hline 6 & -4.815240 & 2.396644 & 3.182489 \\
\hline 6 & -3.444638 & 2.631227 & 3.080589 \\
\hline 6 & -2.850014 & 2.762876 & 1.832986 \\
\hline 6 & 3.085571 & 0.688072 & 0.171032 \\
\hline 6 & 3.595105 & 0.210545 & -1.024702 \\
\hline 6 & 4.941392 & 0.388702 & -1.370111 \\
\hline 6 & 5.754715 & 1.068367 & -0.469602 \\
\hline 6 & 5.273821 & 1.564717 & 0.755734 \\
\hline 6 & 3.931761 & 1.365031 & 1.060141 \\
\hline 6 & 5.418636 & -0.125067 & -2.725232 \\
\hline 6 & 4.718065 & 0.683455 & -3.824123 \\
\hline 6 & 6.213413 & 2.334352 & 1.683145 \\
\hline 6 & 7.461606 & 1.499344 & 1.986246 \\
\hline 6 & 6.629719 & 3.639483 & 0.994969 \\
\hline 6 & 5.549363 & 2.688721 & 3.012364 \\
\hline
\end{tabular}




\begin{tabular}{|c|c|c|c|}
\hline 6 & 6.927231 & 0.021639 & -2.902651 \\
\hline 6 & 5.057473 & -1.607984 & -2.879395 \\
\hline & 4.048327 & -3.918179 & 2.741769 \\
\hline & 4.971129 & -1.981285 & 4.029092 \\
\hline & -1.968291 & -0.306318 & 4.725136 \\
\hline & -1.598024 & -2.749559 & 5.048918 \\
\hline & -7.097996 & -0.920528 & 0.497617 \\
\hline & 1.280482 & 1.638685 & 3.271532 \\
\hline & -8.470584 & 2.114226 & -3.187300 \\
\hline & 0.137394 & 6.200376 & 3.732928 \\
\hline & -4.994441 & -5.618278 & 1.681207 \\
\hline & 2.860215 & 3.789210 & -0.412272 \\
\hline & 4.662451 & 4.536556 & -1.907561 \\
\hline & -3.320129 & -3.995061 & 2.353136 \\
\hline & 0.826774 & 3.886057 & 4.118562 \\
\hline & -6.239461 & 1.992883 & -4.153366 \\
\hline & -7.649964 & -4.766691 & -1.588167 \\
\hline & -3.871895 & 1.232690 & -3.997385 \\
\hline & 2.199719 & 3.745863 & -5.341866 \\
\hline & -0.060346 & 2.864538 & -5.050750 \\
\hline & -0.788177 & 7.527983 & -0.248941 \\
\hline & -6.658949 & -2.542590 & -1.844485 \\
\hline & -0.291254 & 5.299429 & -1.135204 \\
\hline & -1.862251 & 2.041700 & -3.553966 \\
\hline & -6.848927 & -6.315494 & 0.192971 \\
\hline & -9.368011 & 0.003855 & 0.447374 \\
\hline & 4.338529 & 4.539043 & -4.376268 \\
\hline & -0.573687 & 8.001318 & 2.186581 \\
\hline & -10.084474 & 1.507048 & -1.407134 \\
\hline & -2.209389 & -1.776979 & 2.025936 \\
\hline & 3.525331 & 1.732420 & 1.999589 \\
\hline & 2.919175 & -0.301880 & -1.705888 \\
\hline & 6.801899 & 1.230349 & -0.718900 \\
\hline & 3.367794 & -1.293516 & 1.779000 \\
\hline & -0.623407 & -0.020837 & 2.668627 \\
\hline & 1.399246 & -2.732196 & 5.307308 \\
\hline & -2.554553 & 3.816664 & -0.784385 \\
\hline & -3.727087 & 2.692467 & -1.472236 \\
\hline & -5.591397 & 2.372044 & -0.126293 \\
\hline & -1.775743 & 2.912967 & 1.750271 \\
\hline & -6.655020 & 2.126378 & 2.090512 \\
\hline & -2.832581 & 2.710590 & 3.978291 \\
\hline & -5.279797 & 2.282313 & 4.159832 \\
\hline & 1.391580 & -1.872318 & -0.713901 \\
\hline & -1.699647 & -2.898372 & -0.893103 \\
\hline & -0.857487 & -3.035262 & -2.506512 \\
\hline & 0.230247 & -4.750089 & 0.813491 \\
\hline 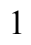 & 1.088061 & -3.290375 & 1.342007 \\
\hline & -0.664704 & -3.247669 & 1.127584 \\
\hline 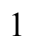 & 2.583991 & -4.505897 & 0.330403 \\
\hline & 4.518693 & -5.485157 & -0.825194 \\
\hline ] & 2.793183 & -4.424627 & -4.614324 \\
\hline & 0.854432 & -3.448343 & -3.467824 \\
\hline & -2.763849 & -0.296117 & 5.481192 \\
\hline & -1.518824 & 0.696464 & 4.698392 \\
\hline & -2.451511 & -0.482556 & 3.755665 \\
\hline
\end{tabular}




$\begin{array}{rrrr}1 & -1.295427 & -1.058947 & 7.196444 \\ 1 & 0.257513 & -1.837189 & 6.873817 \\ 1 & 0.058025 & -0.104189 & 6.557627 \\ 1 & -2.461103 & -2.775583 & 5.727797 \\ 1 & -1.955879 & -2.991246 & 4.038723 \\ 1 & -0.905220 & -3.543247 & 5.355398 \\ 1 & 7.225531 & -0.380467 & -3.879014 \\ 1 & 7.245020 & 1.071992 & -2.872304 \\ 1 & 7.484873 & -0.529247 & -2.133692 \\ 1 & 5.038429 & 0.337867 & -4.816693 \\ 1 & 3.625479 & 0.585574 & -3.771104 \\ 1 & 4.956651 & 1.752310 & -3.738232 \\ 1 & 5.405763 & -1.982770 & -3.851804 \\ 1 & 5.530146 & -2.214407 & -2.094969 \\ 1 & 3.973670 & -1.782037 & -2.833153 \\ 1 & 4.586895 & -4.297839 & 5.358534 \\ 1 & 3.494737 & -3.092138 & 6.053094 \\ 1 & 2.840209 & -4.469733 & 5.136851 \\ 1 & 5.890919 & -2.544199 & 4.237390 \\ 1 & 5.138600 & -1.394964 & 3.116623 \\ 1 & 4.811731 & -1.275461 & 4.854986 \\ 1 & 4.948302 & -4.512670 & 2.949463 \\ 1 & 3.206456 & -4.614825 & 2.619978 \\ 1 & 4.206750 & -3.401313 & 1.786539 \\ 1 & 6.263618 & 3.224625 & 3.650105 \\ 1 & 4.676442 & 3.341630 & 2.878506 \\ 1 & 5.225230 & 1.792183 & 3.558129 \\ 1 & 7.314067 & 4.211264 & 1.636477 \\ 1 & 7.140547 & 3.455569 & 0.041657 \\ 1 & 5.753054 & 4.269136 & 0.791198 \\ 1 & 8.132732 & 2.051852 & 2.657335 \\ 1 & 7.195480 & 0.555387 & 2.479237 \\ 1 & 8.031692 & 1.255174 & 1.082264 \\ 17 & 5.153587 & -5.691941 & -3.610464\end{array}$

$\left(\right.$ para-CI)TS1 $1_{I L-r e-} \psi_{-1 \mathrm{a} 23354 \mathrm{~s}}$

Number of imaginary frequencies : 1

The smallest frequencies are : $-610.3109 \quad 12.3309 \quad 19.6517 \mathrm{~cm}(-1)$

Electronic energy : $\quad H F=-4820.4404368$

Zero-point correction $=\quad 1.442324$ (Hartree/Particle)

Thermal correction to Energy= $\quad 1.528400$

Thermal correction to Enthalpy= $\quad 1.529344$

Thermal correction to Gibbs Free Energy $=\quad 1.321936$

Sum of electronic and zero-point Energies $=\quad-4818.998113$

Sum of electronic and thermal Energies $=\quad-4818.912037$

Sum of electronic and thermal Enthalpies $=\quad-4818.911093$

Sum of electronic and thermal Free Energies $=\quad-4819.118500$

Cartesian Coordinates

$\begin{array}{llll}6 & -2.840663 & 2.746679 & 1.830249 \\ 6 & -3.601739 & 2.679909 & 0.662652 \\ 6 & -4.977508 & 2.487062 & 0.764670 \\ 6 & -5.582629 & 2.340493 & 2.010588 \\ 6 & -4.815579 & 2.396341 & 3.169365\end{array}$




\begin{tabular}{|c|c|c|c|}
\hline 6 & -3.440625 & 2.607423 & 3.074340 \\
\hline 6 & -2.947881 & 2.816181 & -0.686665 \\
\hline 7 & -1.809359 & 1.898300 & -0.925395 \\
\hline 15 & -1.853766 & 0.200488 & -0.938040 \\
\hline 8 & -2.918962 & 0.039772 & 0.329491 \\
\hline 6 & -3.572659 & -1.156514 & 0.472104 \\
\hline 6 & -4.672003 & -1.437333 & -0.317664 \\
\hline 6 & -5.286657 & -2.725311 & -0.185231 \\
\hline 6 & -4.810257 & -3.631071 & 0.811380 \\
\hline 6 & -3.717487 & -3.256651 & 1.629937 \\
\hline 6 & -3.097618 & -2.049319 & 1.453501 \\
\hline 6 & -6.332948 & -3.165585 & -1.035859 \\
\hline 6 & -6.897023 & -4.407977 & -0.883895 \\
\hline 6 & -6.448620 & -5.284072 & 0.126565 \\
\hline 6 & -5.422093 & -4.900623 & 0.950501 \\
\hline 6 & -5.122521 & -0.434656 & -1.309701 \\
\hline 6 & -4.214148 & 0.076185 & -2.221210 \\
\hline 6 & -4.612916 & 0.936090 & -3.268575 \\
\hline 6 & -5.910791 & 1.358163 & -3.353386 \\
\hline 6 & -6.857812 & 0.968902 & -2.374705 \\
\hline 6 & -6.462939 & 0.072487 & -1.336408 \\
\hline 6 & -8.176420 & 1.484296 & -2.389269 \\
\hline 6 & -9.071558 & 1.160512 & -1.401978 \\
\hline 6 & -8.674314 & 0.305578 & -0.352724 \\
\hline 6 & -7.408602 & -0.227178 & -0.322756 \\
\hline 8 & -2.890693 & -0.281649 & -2.179393 \\
\hline 6 & -0.747239 & 2.422561 & -1.738341 \\
\hline 6 & 0.415366 & 2.894943 & -1.156602 \\
\hline 6 & 1.497905 & 3.310913 & -1.992087 \\
\hline 6 & 1.338271 & 3.291283 & -3.409824 \\
\hline 6 & 0.109386 & 2.858513 & -3.960098 \\
\hline 6 & -0.899028 & 2.422180 & -3.144177 \\
\hline 6 & 2.743881 & 3.733896 & -1.460060 \\
\hline 6 & 3.769463 & 4.117690 & -2.287550 \\
\hline 6 & 3.598798 & 4.121311 & -3.689323 \\
\hline 6 & 2.409289 & 3.712720 & -4.235643 \\
\hline 6 & 0.502181 & 3.092865 & 0.317116 \\
\hline 6 & 0.845178 & 2.083868 & 1.204744 \\
\hline 6 & 0.945076 & 2.384894 & 2.590968 \\
\hline 6 & 0.702910 & 3.643876 & 3.070813 \\
\hline 6 & 0.337807 & 4.691749 & 2.195736 \\
\hline 6 & 0.235976 & 4.417129 & 0.800950 \\
\hline 6 & 0.065455 & 5.995069 & 2.675790 \\
\hline 6 & -0.300558 & 6.998037 & 1.815499 \\
\hline 6 & -0.408948 & 6.733111 & 0.434158 \\
\hline 6 & -0.146477 & 5.479596 & -0.060578 \\
\hline 15 & 1.292309 & 0.397291 & 0.564403 \\
\hline 6 & 3.065170 & 0.696270 & 0.151040 \\
\hline 6 & 3.893416 & 1.425540 & 1.005455 \\
\hline 6 & 5.226682 & 1.672814 & 0.680594 \\
\hline 6 & 5.717901 & 1.151687 & -0.524235 \\
\hline 6 & 4.919269 & 0.403931 & -1.391919 \\
\hline 6 & 3.585156 & 0.193770 & -1.034222 \\
\hline 6 & 6.079808 & 2.499761 & 1.642221 \\
\hline 6 & 6.199569 & 1.757291 & 2.978188 \\
\hline 6 & 5.412083 & -0.145287 & -2.728401 \\
\hline
\end{tabular}




\begin{tabular}{|c|c|c|c|}
\hline 6 & 4.721323 & 0.632657 & -3.854812 \\
\hline 45 & 0.125391 & -0.896591 & -1.048839 \\
\hline 6 & 0.678034 & -0.642062 & -2.942410 \\
\hline 8 & 0.681436 & -0.563103 & -4.098658 \\
\hline 6 & 0.377962 & -3.287961 & -0.731776 \\
\hline 6 & -0.783407 & -2.867710 & -1.428894 \\
\hline 6 & 1.522257 & -3.922999 & -1.465650 \\
\hline 6 & 1.612410 & -3.913383 & -2.864044 \\
\hline 6 & 2.702538 & -4.465494 & -3.524110 \\
\hline 6 & 3.726598 & -5.038887 & -2.780490 \\
\hline 6 & 3.671779 & -5.069984 & -1.394368 \\
\hline 6 & 2.572935 & -4.512775 & -0.751924 \\
\hline 17 & 5.113434 & -5.726777 & -3.606507 \\
\hline 6 & 0.234927 & -3.667139 & 0.723906 \\
\hline 6 & 1.409604 & -0.592166 & 2.109775 \\
\hline 6 & 2.544242 & -1.336976 & 2.404934 \\
\hline 6 & 2.600432 & -2.152204 & 3.544956 \\
\hline 6 & 1.489909 & -2.175415 & 4.384328 \\
\hline 6 & 0.331943 & -1.422433 & 4.124178 \\
\hline 6 & 0.296732 & -0.663695 & 2.958930 \\
\hline 6 & 3.871476 & -2.954620 & 3.817751 \\
\hline 6 & 3.797436 & -3.737927 & 5.125672 \\
\hline 6 & -0.838134 & -1.457785 & 5.107523 \\
\hline 6 & -0.332611 & -1.219093 & 5038 \\
\hline 6 & 4.094408 & -3.951548 & 2.674340 \\
\hline 6 & 5.069469 & -2.000820 & 7602 \\
\hline 6 & -1.874630 & -0.375904 & 4.802921 \\
\hline 6 & -1.514463 & -2.830713 & 5934 \\
\hline 6 & 6.922552 & -0.000390 & -2.894506 \\
\hline 6 & 5.056505 & -1.632652 & -2.851745 \\
\hline 6 & 5.413296 & 3.861163 & 6781 \\
\hline 6 & 7.486564 & 2.750586 & 1.105560 \\
\hline 1 & -7.112266 & -0.870011 & 0.502521 \\
\hline 1 & 1.230686 & 1.602699 & 3.289274 \\
\hline 1 & -8.458099 & 2.158336 & -3.197292 \\
\hline 1 & 0.149916 & 6.180945 & 3.745637 \\
\hline 1 & -5.048331 & -5.570795 & 1.723615 \\
\hline 1 & 2.888836 & 3.729417 & -0.380306 \\
\hline 1 & 4.724219 & 4.419810 & -1.861050 \\
\hline 1 & -3.360593 & -3.957090 & 2.384805 \\
\hline 1 & 0.791899 & 3.853855 & 4.136075 \\
\hline 1 & -6.227330 & 2.018634 & -4.159022 \\
\hline 1 & -7.693848 & -4.721741 & -1.554517 \\
\hline 1 & -3.865261 & 1.240973 & -3.997824 \\
\hline 1 & 2.267182 & 3.698095 & -5.315365 \\
\hline 1 & -0.009663 & 2.851894 & -5.042409 \\
\hline 1 & -0.701977 & 7.530036 & -0.245474 \\
\hline 1 & -6.682895 & -2.508467 & -1.827599 \\
\hline 1 & -0.232463 & 5.294384 & -1.129195 \\
\hline 1 & -1.833462 & 2.049836 & -3.560274 \\
\hline 1 & -6.907910 & -6.263305 & 0.239579 \\
\hline 1 & -9.375590 & 0.069292 & 0.444577 \\
\hline 1 & 4.416923 & 4.438138 & -4.332736 \\
\hline 1 & -0.510006 & 7.996112 & 2.193234 \\
\hline 1 & -10.079013 & 1.569655 & -1.417284 \\
\hline 1 & -2.230070 & -1.751470 & 2.041250 \\
\hline
\end{tabular}




\begin{tabular}{|c|c|c|c|}
\hline & 3.487727 & 1.813720 & 1.939647 \\
\hline & 2.920820 & -0.358902 & -1.694890 \\
\hline 1 & 6.754808 & 1.335918 & -0.791395 \\
\hline 1 & 3.402682 & -1.286297 & 1.735237 \\
\hline & -0.590529 & -0.081024 & 2.706754 \\
\hline & 1.517260 & -2.788493 & 5.282209 \\
\hline & -2.525179 & 3.827159 & -0.779356 \\
\hline & -3.707172 & 2.720107 & -1.478772 \\
\hline & -5.582048 & 2.424694 & -0.141758 \\
\hline & -1.763695 & 2.877939 & 1.752841 \\
\hline & -6.656486 & 2.172852 & 2.069307 \\
\hline & -2.829721 & 2.663709 & 3.974535 \\
\hline & -5.285204 & 2.277504 & 4.143689 \\
\hline & 1.368313 & -1.896905 & -0.702815 \\
\hline & -1.726505 & -2.901804 & -0.882794 \\
\hline & -0.887800 & -3.044167 & -2.497835 \\
\hline & 0.191572 & -4.762128 & 0.822025 \\
\hline ] & 1.062980 & -3.309184 & 1.347691 \\
\hline & -0.690082 & -3.251343 & 1.134285 \\
\hline & 2.547081 & -4.535207 & 0.334507 \\
\hline & 4.478556 & -5.520384 & -0.820861 \\
\hline & 2.759197 & -4.447086 & -4.609457 \\
\hline$\frac{1}{1}$ & 0.823989 & -3.464523 & -3.462556 \\
\hline & -2.656816 & -0.384284 & 5.572604 \\
\hline l & -1.418011 & 0.623954 & 4.799132 \\
\hline & -2.375518 & -0.520208 & 3.837200 \\
\hline 1 & -1.177440 & -1.214024 & 7.236266 \\
\hline 1 & 0.363937 & -1.995327 & 6.871880 \\
\hline & 0.178453 & -0.250893 & 6.615872 \\
\hline 1 & -2.363438 & -2.879328 & 5.731214 \\
\hline & -1.894806 & -3.030329 & 4.024609 \\
\hline 1 & -0.819287 & -3.638866 & 5.295881 \\
\hline$\frac{1}{1}$ & 7.232018 & -0.434520 & -3.853559 \\
\hline 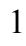 & 7.238602 & 1.050802 & -2.897221 \\
\hline 1 & 7.473075 & -0.523148 & -2.101166 \\
\hline 1 & 5.046376 & 0.257831 & -4.835270 \\
\hline 1 & 3.628152 & 0.539135 & -3.803659 \\
\hline | & 4.962390 & 1.702765 & -3.797827 \\
\hline 1 & 5.426612 & -2.029479 & -3.807077 \\
\hline ] & 5.512223 & -2.219811 & -2.042926 \\
\hline 1 & 3.972402 & -1.808791 & -2.826095 \\
\hline 1 & 4.734476 & -4.287641 & 5.279601 \\
\hline 1 & 3.657241 & -3.077746 & 5.991355 \\
\hline 1 & 2.983369 & -4.474235 & 5.118563 \\
\hline l & 5.992106 & -2.564320 & 4.091065 \\
\hline 1 & 5.213605 & -1.439457 & 2.965611 \\
\hline 1 & 4.939987 & -1.274177 & 4.710457 \\
\hline 1 & 5.002434 & -4.543054 & 2.853956 \\
\hline 1 & 3.248570 & -4.649533 & 2.595887 \\
\hline 1 & 4.213382 & -3.446498 & 1.706978 \\
\hline 1 & 8.052191 & 3.359338 & 1.822307 \\
\hline 1 & 8.044171 & 1.816943 & 0.957582 \\
\hline 1 & 7.468695 & 3.295370 & 0.152268 \\
\hline 1 & 6.011060 & 4.462213 & 2.575302 \\
\hline 1 & 5.328174 & 4.425369 & 0.938423 \\
\hline 1 & 4.405391 & 3.767927 & 2.301130 \\
\hline
\end{tabular}




$\begin{array}{llll}1 & 6.793111 & 2.345079 & 3.691667 \\ 1 & 5.218325 & 1.571370 & 3.433115 \\ 1 & 6.696534 & 0.787050 & 2.844951\end{array}$

Consolidated single point energies using the $\omega B$ 97XD functional $\left(\mathrm{E}_{\mathrm{sp}(\mathrm{wB} 97 \mathrm{XD})}\right)$, thermal and entropic corrections that incorporates the quasi-harmonic rigid-rotor approximation obtained from the frequency calculations at the M06 level of theory $\left(\mathrm{S}_{\mathrm{qh}(\mathrm{M} 06)}\right)$, the corresponding corrected Gibbs free energies $\left(\mathrm{G}_{\mathrm{qh}(\mathrm{wB} \text { B9XD) }}\right)$ of various reactans, intermediates, transition states, products and moieties.

\begin{tabular}{|c|c|c|c|c|c|c|}
\hline & \multicolumn{3}{|c|}{$1 C_{1}$} & \multicolumn{3}{|c|}{$1 C_{2}$} \\
\hline & $\mathbf{E}_{\mathrm{sp}(\mathrm{wB} 97 \mathrm{XD})}$ & $S_{\mathrm{qh}(\mathrm{M} 06)}$ & $\mathbf{G}_{\mathbf{q h}(\mathrm{wB} 97 \mathbf{X D})}$ & $\mathbf{E}_{\mathrm{sp}(\mathrm{wB} 97 \mathrm{XD})}$ & $\mathrm{S}_{\mathrm{qh}(\mathrm{M} 06)}$ & $\mathbf{G}_{\mathbf{q h}(\mathrm{wB} 97 \mathrm{XD})}$ \\
\hline$\psi$-1a2a3a4a & -4127.73796243 & 1.204939 & -4126.53302343 & -4127.73834446 & 1.205749 & -4126.53259546 \\
\hline$\psi$-1a2a3a4s & -4127.73850827 & 1.204936 & -4126.53357227 & -4127.73861006 & 1.206115 & -4126.53249506 \\
\hline$\psi-1 a 2 a 3 s 4 a$ & -4127.73767390 & 1.204993 & -4126.53268090 & -4127.73815598 & 1.206560 & -4126.53159598 \\
\hline$\psi-1 \mathrm{a} 2 \mathrm{a} 3 \mathrm{~s} 4 \mathrm{~s}$ & -4127.73792155 & 1.204674 & -4126.53324755 & -4127.73817629 & 1.205460 & -4126.53271629 \\
\hline$\psi-1 \mathrm{a} 2 \mathrm{~s} 3 \mathrm{a} 4 \mathrm{a}$ & -4127.73869643 & 1.205002 & -4126.53369443 & -4127.73901414 & 1.205756 & -4126.53325814 \\
\hline$\psi-1 \mathrm{a} 2 \mathrm{~s} 3 \mathrm{a} 4 \mathrm{~s}$ & -4127.73906492 & 1.205406 & -4126.53365892 & -4127.73916290 & 1.205690 & -4126.53347290 \\
\hline$\psi-1 a 2 s 3 s 4 a$ & -4127.73836276 & 1.204962 & -4126.53340076 & -4127.73881929 & 1.206994 & -4126.53182529 \\
\hline$\psi-1 \mathrm{a} 2 \mathrm{~s} 3 \mathrm{~s} 4 \mathrm{~s}$ & -4127.73853820 & 1.205188 & -4126.53335020 & -4127.73873881 & 1.206023 & -4126.53271581 \\
\hline$\psi$-1s2a3a4a & -4127.73616568 & 1.205478 & -4126.53068768 & -4127.73660894 & 1.206104 & -4126.53050494 \\
\hline$\psi-1 \mathrm{~s} 2 \mathrm{a} 3 \mathrm{a} 4 \mathrm{~s}$ & -4127.73674349 & 1.205114 & -4126.53162949 & -4127.73700053 & 1.207292 & -4126.52970853 \\
\hline$\psi-1 \mathrm{~s} 2 \mathrm{a} 3 \mathrm{~s} 4 \mathrm{a}$ & -4127.73582615 & 1.205464 & -4126.53036215 & -4127.73636585 & 1.206915 & -4126.52945085 \\
\hline$\psi-1 \mathrm{~s} 2 \mathrm{a} 3 \mathrm{~s} 4 \mathrm{~s}$ & -4127.73619905 & 1.205275 & -4126.53092405 & -4127.73642093 & 1.206262 & -4126.53015893 \\
\hline$\psi-1 \mathrm{~s} 2 \mathrm{~s} 3 \mathrm{a} 4 \mathrm{a}$ & -4127.73658138 & 1.205872 & -4126.53070938 & -4127.73694414 & 1.206360 & -4126.53058414 \\
\hline$\psi-1 \mathrm{~s} 2 \mathrm{~s} 3 \mathrm{a} 4 \mathrm{~s}$ & -4127.73665282 & 1.205967 & -4126.53068582 & -4127.73722737 & 1.206822 & -4126.53040537 \\
\hline$\psi-1 \mathrm{~s} 2 \mathrm{~s} 3 \mathrm{~s} 4 \mathrm{a}$ & -4127.73616498 & 1.206106 & -4126.53005898 & -4127.73669399 & 1.207018 & -4126.52967599 \\
\hline$\psi-1 \mathrm{~s} 2 \mathrm{~s} 3 \mathrm{~s} 4 \mathrm{~s}$ & -4127.73606822 & 1.205473 & -4126.53059522 & -4127.73681656 & 1.206491 & -4126.53032556 \\
\hline
\end{tabular}

\begin{tabular}{|c|c|c|c|c|c|c|}
\hline & \multicolumn{3}{|c|}{$1 \mathrm{C}_{3}$} & \multicolumn{3}{|c|}{$1 \mathrm{C}_{4}$} \\
\hline & $\mathbf{E}_{\mathrm{sp}(\mathrm{wB} 97 \mathrm{XD})}$ & $\mathrm{S}_{\mathrm{qh}(\mathrm{M} 06)}$ & $\mathbf{G}_{\text {qh(wB97XD) }}$ & $\mathbf{E}_{\text {sp(wB97XD) }}$ & $\mathrm{S}_{\mathrm{qh}(\mathrm{M} 06)}$ & $\mathbf{G}_{\text {qh(wB97XD) }}$ \\
\hline$\psi-1 a 2 a 3 a 4 a$ & -4127.73467621 & 1.205714 & -4126.52896221 & -4127.73786418 & 1.205357 & -4126.53250718 \\
\hline$\psi-1 \mathrm{a} 2 \mathrm{a} 3 \mathrm{a} 4 \mathrm{~s}$ & -4127.73496274 & 1.205492 & -4126.52947074 & -4127.73815598 & 1.205234 & -4126.53292198 \\
\hline$\psi-1 a 2 a 3 s 4 a$ & -4127.73436722 & 1.206214 & -4126.52815322 & -4127.73871012 & 1.204636 & -4126.53407412 \\
\hline$\psi$-1a2a3s4s & -4127.73483266 & 1.205419 & -4126.52941366 & -4127.73767924 & 1.205221 & -4126.53245824 \\
\hline$\psi-1 a 2 s 3 a 4 a$ & -4127.73515925 & 1.205050 & -4126.53010925 & -4127.73864371 & 1.205508 & -4126.53313571 \\
\hline$\psi$-1a2s3a4s & -4127.73548716 & 1.205952 & -4126.52953516 & -4127.73893831 & 1.206027 & -4126.53291131 \\
\hline$\psi-1 \mathrm{a} 2 \mathrm{~s} 3 \mathrm{~s} 4 \mathrm{a}$ & -4127.73484111 & 1.205736 & -4126.52910511 & -4127.73926384 & 1.206042 & -4126.53322184 \\
\hline$\psi-1 \mathrm{a} 2 \mathrm{~s} 3 \mathrm{~s} 4 \mathrm{~s}$ & -4127.73486254 & 1.206141 & -4126.52872154 & -4127.73929221 & 1.206642 & -4126.53265021 \\
\hline$\psi$-1s2a3a4a & -4127.73284693 & 1.206191 & -4126.52665593 & -4127.73664074 & 1.205988 & -4126.53065274 \\
\hline$\psi$-1s2a3a4s & -4127.73413066 & 1.206382 & -4126.52774866 & -4127.73698975 & 1.205932 & -4126.53105775 \\
\hline$\psi-1 \mathrm{~s} 2 \mathrm{a} 3 \mathrm{~s} 4 \mathrm{a}$ & -4127.73285756 & 1.206437 & -4126.52642056 & -4127.73786853 & 1.205993 & -4126.53187553 \\
\hline$\psi-1 \mathrm{~s} 2 \mathrm{a} 3 \mathrm{~s} 4 \mathrm{~s}$ & -4127.73374606 & 1.205588 & -4126.52815806 & -4127.73775642 & 1.205601 & -4126.53215542 \\
\hline
\end{tabular}




\begin{tabular}{|l|l|l|l|l|l|l|}
\hline$\psi-1 \mathbf{s} 2 s 3 a 4 a$ & -4127.73346138 & 1.205516 & -4126.52794538 & -4127.73712208 & 1.205959 & -4126.53116308 \\
\hline$\psi$-1s2s3a4s & -4127.73397572 & 1.206827 & -4126.52714872 & -4127.73714801 & 1.206089 & -4126.53105901 \\
\hline$\psi$-1s2s3s4a & -4127.73316603 & 1.205987 & -4126.52717903 & -4127.73786778 & 1.206185 & -4126.53168278 \\
\hline$\psi-1 \mathbf{s} 2 s 3 s 4 s$ & -4127.73357213 & 1.206379 & -4126.52719313 & -4127.73783301 & 1.206727 & -4126.53110601 \\
\hline
\end{tabular}

\begin{tabular}{|c|c|c|c|c|c|c|}
\hline & \multicolumn{3}{|c|}{$1 C_{5}$} & \multicolumn{3}{|c|}{$1 C_{6}$} \\
\hline & $\mathbf{E}_{\mathrm{sp}(\mathrm{wB} \text { 97XD) }}$ & $S_{\mathrm{qh}(\mathrm{M06})}$ & $G_{q h(w B 97 X D)}$ & $\mathbf{E}_{\mathrm{sp}(\mathrm{wB} 97 \mathrm{XD})}$ & $\mathrm{S}_{\mathrm{qh}(\mathrm{M06})}$ & $\mathbf{G}_{\mathrm{qh}(\mathrm{wB} 97 \times D)}$ \\
\hline$\psi-1 \mathrm{a} 2 \mathrm{a} 3 \mathrm{a} 4 \mathrm{a}$ & -4127.73926538 & 1.206420 & 4126.53284538 & 4127.73472240 & 1.205841 & -4126.52888140 \\
\hline$\psi-1 \mathrm{a} 2 \mathrm{a} 3 \mathrm{a} 4 \mathrm{~s}$ & -4127.73947056 & 206755 & -4126.53271556 & 4127.73521010 & 1.205414 & -4126.52979610 \\
\hline$\psi-1$ a2a3s4a & -4127.73995203 & 1.206757 & -4126.53319503 & -4127.73534215 & 1.206167 & -4126.52917515 \\
\hline$\psi-1 \mathrm{a} 2 \mathrm{a} 3 \mathrm{~s} 4 \mathrm{~s}$ & -4127.73885319 & 1.208660 & -4126.53019319 & -4127.73552208 & 1.207881 & -4126.52764108 \\
\hline$\psi-1 \mathrm{a} 2 \mathrm{s3a4a}$ & -4127.73786986 & .208155 & -4126.52971486 & -4127.73543534 & 1.206677 & -4126.52875834 \\
\hline$\psi-1 \mathrm{a} 2 \mathrm{~s} 3 \mathrm{a} 4 \mathrm{~s}$ & -4127.74007312 & 206518 & -4126.53355512 & -4127.73576742 & 1.206905 & -4126.52886242 \\
\hline$\psi-1 \mathrm{a} 2 \mathrm{~s} 3 \mathrm{~s} 4 \mathrm{a}$ & -4127.74067131 & & -4126.531 & -4127.73591278 & 1.207103 & -4126.52880978 \\
\hline$\psi-1 a 2 s 3 s 4 s$ & -4127.7393 & 1.209048 & -4126.53028797 & -4127.73600038 & 1.206592 & -4126.52940838 \\
\hline$\psi$-1s2a3a4a & -4127.73667615 & 1.206032 & -4126.53064415 & -4127.73250882 & 1.206217 & -4126.52629182 \\
\hline$\psi-1$ s2a3a4s & -4127.73697527 & 1.205953 & -4126.53102227 & -4127.73271454 & 1.206183 & -4126.52653154 \\
\hline$\psi-1$ s2a3s4a & -4127.73813263 & 1.208740 & -4126.52939263 & -4127.73311210 & 1.206794 & -4126.52631810 \\
\hline$\psi-1 \mathrm{~s} 2 \mathrm{a} 3 \mathrm{~s} 4 \mathrm{~s}$ & -4127.73804292 & 1.206564 & -4126.53147892 & -4127.73305608 & 1.206081 & -4126.52697508 \\
\hline$\psi-1 \mathrm{~s} 2 \mathrm{~s} 3 \mathrm{a} 4 \mathrm{a}$ & -4127.73764374 & 1.206928 & -4126.53071574 & -4127.73291435 & 1.206620 & -4126.52629435 \\
\hline$\psi-1 \mathrm{~s} 2 \mathrm{~s} 3 \mathrm{a} 4 \mathrm{~s}$ & -4127.73720898 & 1.206395 & -4126.53081398 & -4127.73304125 & 1.207015 & -4126.52602625 \\
\hline$\psi-1 \mathrm{~s} 2 \mathrm{~s} 3 \mathrm{~s} 4 \mathrm{a}$ & -4127.73832932 & 1.207609 & -4126.53072032 & -4127.73336546 & 1.206825 & -4126.52654046 \\
\hline$\psi-1 \mathrm{~s} 2 \mathrm{~s} 3 \mathrm{~s} 4 \mathrm{~s}$ & -4127.73821455 & 1.207338 & -4126.53087655 & -4127.73333737 & 1.207124 & -4126.52621337 \\
\hline
\end{tabular}

\begin{tabular}{|c|c|c|c|c|c|c|}
\hline & \multicolumn{3}{|c|}{$1 \mathrm{C}_{7}$} & \multicolumn{3}{|c|}{$1 \mathrm{C}_{8}$} \\
\hline & $E_{\mathrm{sp}(\mathrm{wB} 97 X D)}$ & $\mathrm{S}_{\mathrm{qh}(\mathrm{M} 06)}$ & $\mathbf{G}_{\mathbf{q h}(\text { wB97XD) }}$ & $\mathbf{E}_{\mathrm{sp}(\mathrm{wB} 97 \mathrm{XD})}$ & $\mathrm{S}_{\mathrm{qh}(\mathrm{M} 06)}$ & $\mathbf{G}_{\text {qh(wB97XD) }}$ \\
\hline$\psi-1 a 2 a 3 a 4 a$ & -4127.73332884 & 1.205042 & -4126.52828684 & -4127.73405992 & 1.208639 & -4126.52542092 \\
\hline$\psi$-1a2a3a4s & -4127.73360377 & 1.205130 & -4126.52847377 & -4127.73452394 & 1.205866 & -4126.52865794 \\
\hline$\psi-1 a 2 a 3 s 4 a$ & -4127.73348748 & 1.205250 & -4126.52823748 & -4127.73449085 & 1.206377 & -4126.52811385 \\
\hline$\psi-1 \mathrm{a} 2 \mathrm{a} 3 \mathrm{~s} 4 \mathrm{~s}$ & -4127.73352336 & 1.207793 & -4126.52573036 & -4127.73427270 & 1.205058 & -4126.52921470 \\
\hline$\psi$-1a2s3a4a & -4127.73393772 & 1.204774 & -4126.52916372 & -4127.73498918 & 1.206270 & -4126.52871918 \\
\hline$\psi-1 \mathrm{a} 2 \mathrm{~s} 3 \mathrm{a} 4 \mathrm{~s}$ & -4127.73426716 & 1.205607 & -4126.52866016 & -4127.73519428 & 1.205842 & -4126.52935228 \\
\hline$\psi$-1a2s3s4a & -4127.73428146 & 1.208036 & -4126.52624546 & -4127.73503679 & 1.206600 & -4126.52843679 \\
\hline$\psi-1 \mathrm{a} 2 \mathrm{~s} 3 \mathrm{~s} 4 \mathrm{~s}$ & -4127.73434826 & 1.205269 & -4126.52907926 & -4127.73498793 & 1.205662 & -4126.52932593 \\
\hline$\psi-1$ s2a3a4a & -4127.73212899 & 1.205302 & -4126.52682699 & -4127.73242215 & 1.207802 & -4126.52462015 \\
\hline$\psi$-1s2a3a4s & -4127.73247970 & 1.205081 & -4126.52739870 & -4127.73274301 & 1.209130 & -4126.52361301 \\
\hline$\psi-1 \mathrm{~s} 2 \mathrm{a} 3 \mathrm{~s} 4 \mathrm{a}$ & -4127.73024922 & 1.205264 & -4126.52498522 & -4127.73319074 & 1.204741 & -4126.52844974 \\
\hline$\psi-1 \mathrm{~s} 2 \mathrm{a} 3 \mathrm{~s} 4 \mathrm{~s}$ & -4127.73245928 & 1.205206 & -4126.52725328 & -4127.73318457 & 1.206190 & -4126.52699457 \\
\hline$\psi-1 \mathrm{~s} 2 \mathrm{~s} 3 \mathrm{a} 4 \mathrm{a}$ & -4127.73250367 & 1.204716 & -4126.52778767 & -4127.73323315 & 1.205244 & -4126.52798915 \\
\hline$\psi$-1s2s3a4s & -4127.73286539 & 1.205205 & -4126.52766039 & -4127.73352275 & 1.206294 & -4126.52722875 \\
\hline$\psi-1 \mathrm{~s} 2 \mathrm{~s} 3 \mathrm{~s} 4 \mathrm{a}$ & -4127.73258597 & 1.205477 & -4126.52710897 & -4127.73346265 & 1.205200 & -4126.52826265 \\
\hline$\psi-1 \mathrm{~s} 2 \mathrm{~s} 3 \mathrm{~s} 4 \mathrm{~s}$ & -4127.73244694 & 1.205163 & -4126.52728394 & -4127.73360121 & 1.206337 & -4126.52726421 \\
\hline
\end{tabular}




\begin{tabular}{|c|c|c|c|c|c|c|}
\hline & \multicolumn{3}{|c|}{$1 C_{9}$} & \multicolumn{3}{|c|}{$1 \mathrm{C}_{10}$} \\
\hline & $\mathbf{E}_{\text {sp(wB97XD) }}$ & $\mathbf{S}_{\mathrm{qh}(\mathrm{M} 06)}$ & $\mathbf{G}_{\mathrm{qh}(\mathrm{wB} 97 \mathrm{XD})}$ & $E_{\mathrm{sp}(w B 97 X D)}$ & $\mathrm{S}_{\mathrm{qh}(\mathrm{M} 06)}$ & $\mathbf{G}_{\mathbf{q h}(\mathrm{wB} 97 \times D)}$ \\
\hline$\psi-1 a 2 a 3 a 4 a$ & -4127.72965800 & 1.205846 & -4126.52381200 & -4127.73226371 & 1.205241 & -4126.52702271 \\
\hline$\psi-1 \mathrm{a} 2 \mathrm{a} 3 \mathrm{a} 4 \mathrm{~s}$ & -4127.72997260 & 1.206097 & -4126.52387560 & -4127.73248869 & 1.206254 & -4126.52623469 \\
\hline$\psi-1 \mathrm{a} 2 \mathrm{a} 3 \mathrm{~s} 4 \mathrm{a}$ & -4127.72969722 & 1.205976 & -4126.52372122 & -4127.73210417 & 1.206980 & -4126.52512417 \\
\hline$\psi-1 \mathrm{a} 2 \mathrm{a} 3 \mathrm{~s} 4 \mathrm{~s}$ & -4127.72987545 & 1.206241 & -4126.52363445 & -4127.73225504 & 1.206822 & -4126.52543304 \\
\hline$\psi-1 a 2 s 3 a 4 a$ & -4127.73013984 & 1.205899 & -4126.52424084 & -4127.73283884 & 1.206439 & -4126.52639984 \\
\hline$\psi-1 \mathrm{a} 2 \mathrm{~s} 3 \mathrm{a} 4 \mathrm{~s}$ & -4127.73041856 & 1.206543 & -4126.52387556 & -4127.73285912 & 1.205812 & -4126.52704712 \\
\hline$\psi-1 a 2 s 3 s 4 a$ & -4127.73033004 & 1.206252 & -4126.52407804 & -4127.73012288 & 1.206270 & -4126.52385288 \\
\hline$\psi-1 \mathrm{a} 2 \mathrm{~s} 3 \mathrm{~s} 4 \mathrm{~s}$ & -4127.73021080 & 1.210407 & -4126.51980380 & -4127.73146445 & 1.206592 & -4126.52487245 \\
\hline$\psi$-1s2a3a4a & -4127.72784428 & 1.206287 & -4126.52155728 & -4127.73129067 & 1.207928 & -4126.52336267 \\
\hline$\psi$-1s2a3a4s & -4127.72832364 & 1.206607 & -4126.52171664 & -4127.73162781 & 1.206706 & -4126.52492181 \\
\hline$\psi-1 \mathrm{~s} 2 \mathrm{a} 3 \mathrm{~s} 4 \mathrm{a}$ & -4127.72801287 & 1.207752 & -4126.52026087 & -4127.73122671 & 1.206385 & -4126.52484171 \\
\hline$\psi-1 \mathrm{~s} 2 \mathrm{a} 3 \mathrm{~s} 4 \mathrm{~s}$ & -4127.72834967 & 1.207414 & -4126.52093567 & -4127.73145051 & 1.206798 & -4126.52465251 \\
\hline$\psi$-1s2s3a4a & -4127.72825749 & 1.206101 & -4126.52215649 & -4127.73170977 & 1.208231 & -4126.52347877 \\
\hline$\psi-1 \mathrm{~s} 2 \mathrm{~s} 3 \mathrm{a} 4 \mathrm{~s}$ & -4127.72874221 & 1.206547 & -4126.52219521 & -4127.73199501 & 1.207019 & -4126.52497601 \\
\hline$\psi-1 \mathrm{~s} 2 \mathrm{~s} 3 \mathrm{~s} 4 \mathrm{a}$ & -4127.72839125 & 1.207082 & -4126.52130925 & -4127.73171082 & 1.206445 & -4126.52526582 \\
\hline$\psi-1 \mathrm{~s} 2 \mathrm{~s} 3 \mathrm{~s} 4 \mathrm{~s}$ & -4127.72865501 & 1.207610 & -4126.52104501 & -4127.73152336 & 1.206321 & -4126.52520236 \\
\hline
\end{tabular}

\begin{tabular}{|c|c|c|c|c|c|c|}
\hline & \multicolumn{3}{|c|}{$1 C_{11}$} & \multicolumn{3}{|c|}{$1 C_{12}$} \\
\hline & $\mathbf{E}_{\mathrm{sp}(\mathrm{wB} 97 \mathrm{XD})}$ & $\mathrm{S}_{\mathrm{qh}(\mathrm{M} 06)}$ & $G_{q h(w B 97 X D)}$ & $\mathbf{E}_{\text {sp(wB97XD) }}$ & $\mathrm{S}_{\mathrm{qh}(\mathrm{M} 06)}$ & $G_{q h(w B 97 X D)}$ \\
\hline$\psi$-1a2a3a4a & -4127.73148856 & 1.205058 & -4126.52643056 & -4127.72703191 & 1.206441 & -4126.52059091 \\
\hline$\psi-1 \mathrm{a} 2 \mathrm{a} 3 \mathrm{a} 4 \mathrm{~s}$ & -4127.73214903 & 1.204681 & -4126.52746803 & -4127.72732590 & 1.206093 & -4126.52123290 \\
\hline$\psi-1 \mathrm{a} 2 \mathrm{a} 3 \mathrm{~s} 4 \mathrm{a}$ & -4127.73172403 & 1.204963 & -4126.52676103 & -4127.72713357 & 1.206546 & -4126.52058757 \\
\hline$\psi-1 \mathrm{a} 2 \mathrm{a} 3 \mathrm{~s} 4 \mathrm{~s}$ & -4127.73196796 & 1.204618 & -4126.52734996 & -4127.72722781 & 1.205844 & -4126.52138381 \\
\hline$\psi$-1a2s3a4a & -4127.73224553 & 1.204943 & -4126.52730253 & -4127.72753334 & 1.205963 & -4126.52157034 \\
\hline$\psi-1 \mathrm{a} 2 \mathrm{~s} 3 \mathrm{a} 4 \mathrm{~s}$ & -4127.73235001 & 1.206714 & -4126.52563601 & -4127.72823479 & 1.210194 & -4126.51804079 \\
\hline$\psi-1 \mathrm{a} 2 \mathrm{~s} 3 \mathrm{~s} 4 \mathrm{a}$ & -4127.73233090 & 1.204951 & -4126.52737990 & -4127.72760382 & 1.20661 & -4126.52099382 \\
\hline$\psi-1 \mathrm{a} 2 \mathrm{~s} 3 \mathrm{~s} 4 \mathrm{~s}$ & -4127.73214150 & 1.205210 & -4126.52693150 & -4127.72776111 & 1.206465 & -4126.52129611 \\
\hline$\psi$-1s2a3a4a & -4127.72991601 & 1.205832 & -4126.52408401 & -4127.72593959 & 1.20659 & -4126.51934959 \\
\hline$\psi-1 \mathrm{~s} 2 \mathrm{a} 3 \mathrm{a} 4 \mathrm{~s}$ & -4127.73085659 & 1.204964 & -4126.52589259 & -4127.72626316 & 1.206804 & -4126.51945916 \\
\hline$\psi-1$ s2a3s4a & -4127.73039524 & 1.204693 & -4126.52570224 & -4127.72604060 & 1.206184 & -4126.51985660 \\
\hline$\psi-1 \mathrm{~s} 2 \mathrm{a} 3 \mathrm{~s} 4 \mathrm{~s}$ & -4127.73034143 & 1.205849 & -4126.52449243 & -4127.72573864 & 1.20611 & -4126.51962864 \\
\hline$\psi-1 \mathrm{~s} 2 \mathrm{~s} 3 \mathrm{a} 4 \mathrm{a}$ & -4127.73034544 & 1.206042 & -4126.52430344 & -4127.72639903 & 1.206385 & -4126.52001403 \\
\hline$\psi-1 \mathrm{~s} 2 \mathrm{~s} 3 \mathrm{a} 4 \mathrm{~s}$ & -4127.73098774 & 1.206467 & -4126.52452074 & -4127.72659400 & 1.206606 & -4126.51998800 \\
\hline$\psi-1 \mathrm{~s} 2 \mathrm{~s} 3 \mathrm{~s} 4 \mathrm{a}$ & -4127.73038401 & 1.205861 & -4126.52452301 & -4127.72645708 & 1.206297 & -4126.52016008 \\
\hline$\psi-1 \mathrm{~s} 2 \mathrm{~s} 3 \mathrm{~s} 4 \mathrm{~s}$ & -4127.73112923 & 1.205481 & -4126.52564823 & -4127.72629595 & 1.205823 & -4126.52047295 \\
\hline
\end{tabular}

\begin{tabular}{|l|c|c|c|c|c|c|}
\hline & \multicolumn{3}{|c|}{$\mathbf{2}_{\mathbf{C}}$} & \multicolumn{3}{c|}{$\mathbf{2}_{\mathbf{t}}$} \\
\hline & $\mathbf{E}_{\mathbf{s p}(\mathbf{w B 9 7 X D})}$ & $\mathbf{S}_{\mathbf{q h}(\mathbf{M 0 6})}$ & $\mathbf{G}_{\mathbf{q h}(\mathbf{w B} \text { 97XD) }}$ & $\mathbf{E}_{\mathbf{s p}(\mathbf{w B 9 7 X D})}$ & $\mathbf{S}_{\mathbf{q h}(\mathbf{M} 06)}$ & $\mathbf{G}_{\mathbf{q h}(\mathbf{w B 9 7} \mathbf{X D})}$ \\
\hline $\boldsymbol{\psi}$-1a2a3a4a & -4014.39830971 & 1.199252 & -4013.19905771 & -4014.40096236 & 1.198781 & -4013.20218136 \\
\hline $\boldsymbol{\psi - 1 a 2 a 3 a 4 s}$ & -4014.39867811 & 1.198938 & -4013.19974011 & -4014.40114199 & 1.198473 & -4013.20266899 \\
\hline
\end{tabular}




\begin{tabular}{|c|c|c|c|c|c|c|}
\hline$\psi-1 \mathrm{a} 2 \mathrm{a} 3 \mathrm{~s} 4 \mathrm{a}$ & -4014.39816315 & 1.198968 & -4013.19919515 & -4014.40072373 & 1.199344 & -4013.20137973 \\
\hline$\psi-1 \mathrm{a} 2 \mathrm{a} 3 \mathrm{~s} 4 \mathrm{~s}$ & -4014.39808168 & 1.198705 & -4013.19937668 & -4014.40082492 & 1.199562 & -4013.20126292 \\
\hline$\psi-1 \mathrm{a} 2 \mathrm{~s} 3 \mathrm{a} 4 \mathrm{a}$ & -4014.39879206 & 1.199478 & -4013.19931406 & -4014.40157199 & 1.199739 & -4013.20183299 \\
\hline$\psi-1 \mathrm{a} 2 \mathrm{~s} 3 \mathrm{a} 4 \mathrm{~s}$ & -4014.39912437 & 1.199001 & -4013.20012337 & -4014.40173224 & 1.201227 & -4013.20050524 \\
\hline$\psi-1 \mathrm{a} 2 \mathrm{~s} 3 \mathrm{~s} 4 \mathrm{a}$ & $-4014.39880819^{a}$ & $1.198788^{\mathrm{a}}$ & $-4013.20002019^{a}$ & -4014.40143488 & 1.198948 & -4013.20248688 \\
\hline$\psi-1 \mathrm{a} 2 \mathrm{~s} 3 \mathrm{~s} 4 \mathrm{~s}$ & $-4014.39912609^{a}$ & $1.198982^{\mathrm{a}}$ & $-4013.20014409^{a}$ & -4014.40124970 & 1.19944 & -4013.20180970 \\
\hline$\psi$-1s2a3a4a & -4014.39798289 & 1.198809 & -4013.19917389 & -4014.40033894 & 1.201182 & -4013.19915694 \\
\hline$\psi-1 \mathrm{~s} 2 \mathrm{a} 3 \mathrm{a} 4 \mathrm{~s}$ & -4014.39933231 & 1.199626 & -4013.19970631 & -4014.40065288 & 1.200424 & -4013.20022888 \\
\hline$\psi-1$ s2a3s4a & -4014.39746022 & 1.199533 & -4013.19792722 & -4014.39990983 & 1.199557 & -4013.20035283 \\
\hline$\psi-1 \mathrm{~s} 2 \mathrm{a} 3 \mathrm{~s} 4 \mathrm{~s}$ & -4014.39734503 & 1.199769 & -4013.19757603 & -4014.39989785 & 1.199456 & -4013.20044185 \\
\hline$\psi-1$ s2s3a4a & -4014.39809842 & 1.199755 & -4013.19834342 & -4014.40071146 & 1.200029 & -4013.20068246 \\
\hline$\psi-1 \mathrm{~s} 2 \mathrm{~s} 3 \mathrm{a} 4 \mathrm{~s}$ & -4014.39821295 & 1.199433 & -4013.19877995 & -4014.40086957 & 1.200132 & -4013.20073757 \\
\hline$\psi-1 \mathrm{~s} 2 \mathrm{~s} 3 \mathrm{~s} 4 \mathrm{a}$ & -4014.39763414 & 1.199992 & -4013.19764214 & -4014.40036705 & 1.199485 & -4013.20088205 \\
\hline$\psi-1 \mathrm{~s} 2 \mathrm{~s} 3 \mathrm{~s} 4 \mathrm{~s}$ & -4014.39745226 & 1.199849 & -4013.19760326 & -4014.40014118 & 1.200264 & -4013.19987718 \\
\hline
\end{tabular}

${ }^{\mathrm{a}} \psi_{3 \mathrm{~s}}$ dihedral converged to $\psi_{3 \mathrm{a}}$ during the geometry optimization.

\begin{tabular}{|c|c|c|c|c|c|c|}
\hline & \multicolumn{3}{|c|}{$3_{\mathrm{IA}-s i}$} & \multicolumn{3}{|c|}{$3_{\text {IB-re }}$} \\
\hline & $E_{s p(w B 97 X D)}$ & $\mathrm{S}_{\mathrm{qh}(\mathrm{M} 06)}$ & $\mathbf{G}_{\mathbf{q h}(\mathrm{wB} 97 \mathrm{XD})}$ & $\mathbf{E}_{\text {sp(wB97XD) }}$ & $\mathrm{S}_{\mathrm{qh}(\mathrm{M} 06)}$ & $\mathbf{G}_{\mathbf{q h}(\mathrm{wB} 97 \mathrm{XD})}$ \\
\hline$\psi-1 \mathbf{a} 2 \mathbf{a} 3 \mathbf{a} 4 \mathbf{a}$ & -4363.35609095 & 1.356328 & -4361.99976295 & -4363.34957584 & 1.356041 & -4361.99353484 \\
\hline$\psi$-1a2a3a4s & -4363.35648263 & 1.355865 & -4362.00061763 & -4363.34987264 & 1.359837 & -4361.99003564 \\
\hline$\psi$-1a2a3s4a & -4363.35634069 & 1.355125 & -4362.00121569 & -4363.35060458 & 1.357860 & -4361.99274458 \\
\hline$\psi$-1a2a3s4s & -4363.35635974 & 1.355661 & -4362.00069874 & -4363.35060673 & 1.357943 & -4361.99266373 \\
\hline$\psi$-1a2s3a4a & -4363.35684855 & 1.355792 & -4362.00105655 & -4363.35026712 & 1.356018 & -4361.99424912 \\
\hline$\psi$-1a2s3a4s & -4363.35723003 & 1.356212 & -4362.00101803 & -4363.35025731 & 1.355902 & -4361.99435531 \\
\hline$\psi-1 a 2 s 3 s 4 a$ & -4363.35709089 & 1.355546 & -4362.00154489 & -4363.35121313 & 1.359436 & -4361.99177713 \\
\hline$\psi-1 \mathrm{a} 2 \mathrm{~s} 3 \mathrm{~s} 4 \mathrm{~s}$ & -4363.35718406 & 1.356102 & -4362.00108206 & -4363.35110746 & 1.360521 & -4361.99058646 \\
\hline$\psi$-1s2a3a4a & -4363.35428651 & 1.354553 & -4361.99973351 & -4363.34770932 & 1.355679 & -4361.99203032 \\
\hline$\psi$-1s2a3a4s & -4363.35510757 & 1.355377 & -4361.99973057 & -4363.34812571 & 1.356531 & -4361.99159471 \\
\hline$\psi-1$ s2a3s4a & -4363.35481582 & 1.355736 & -4361.99907982 & -4363.34863909 & 1.356320 & -4361.99231909 \\
\hline$\psi-1 \mathrm{~s} 2 \mathrm{a} 3 \mathrm{~s} 4 \mathrm{~s}$ & -4363.35488523 & 1.355489 & -4361.99939623 & -4363.34848859 & 1.357391 & -4361.99109759 \\
\hline$\psi-1 \mathrm{~s} 2 \mathrm{~s} 3 \mathrm{a} 4 \mathrm{a}$ & -4363.35518271 & 1.356178 & -4361.99900471 & -4363.34818051 & 1.356735 & -4361.99144551 \\
\hline$\psi-1 \mathrm{~s} 2 \mathrm{~s} 3 \mathrm{a} 4 \mathrm{~s}$ & -4363.35400682 & 1.356054 & -4361.99795282 & -4363.34810083 & 1.357706 & -4361.99039483 \\
\hline$\psi-1 \mathrm{~s} 2 \mathrm{~s} 3 \mathrm{~s} 4 \mathrm{a}$ & -4363.35201563 & 1.355965 & -4361.99605063 & -4363.34890192 & 1.357591 & -4361.99131092 \\
\hline$\psi-1 \mathrm{~s} 2 \mathrm{~s} 3 \mathrm{~s} 4 \mathrm{~s}$ & -4363.35514508 & 1.356102 & -4361.99904308 & -4363.34904508 & 1.358323 & -4361.99072208 \\
\hline
\end{tabular}

\begin{tabular}{|c|c|c|c|c|c|c|}
\hline & \multicolumn{3}{|c|}{$\mathbf{3}_{\mathbf{I C}-\mathbf{r e}}$} & \multicolumn{3}{c|}{$\mathbf{3}_{\mathbf{I D}-\mathbf{s i}}$} \\
\hline & $\mathbf{E}_{\mathbf{s p ( w B 9 7 X D )}}$ & $\mathbf{S}_{\mathbf{q h}(\mathbf{M 0 6})}$ & $\mathbf{G}_{\mathbf{q h}(\mathbf{w B 9 7 X D})}$ & $\mathbf{E}_{\mathbf{s p}(\mathbf{w B 9 7 X D})}$ & $\mathbf{S}_{\mathbf{q h}(\mathbf{M 0 6})}$ & $\mathbf{G}_{\mathbf{q h}(\mathbf{w B 9 7 X D})}$ \\
\hline $\boldsymbol{\psi - 1 a 2 a 3 a 4 a}$ & -4363.33995797 & 1.356881 & -4361.98307697 & -4363.34074609 & 1.356315 & -4361.98443109 \\
\hline $\boldsymbol{\psi - 1 a 2 a 3 a 4 s}$ & -4363.34027158 & 1.357633 & -4361.98263858 & -4363.34077282 & 1.356002 & -4361.98477082 \\
\hline $\boldsymbol{\psi - 1 a 2 a 3 s 4 a}$ & -4363.34001545 & 1.356423 & -4361.98359245 & -4363.34059511 & 1.356037 & -4361.98455811 \\
\hline $\boldsymbol{\psi - 1 a 2 a 3 s 4 s}$ & -4363.34004845 & 1.356654 & -4361.98339445 & -4363.34049602 & 1.356132 & -4361.98436402 \\
\hline $\boldsymbol{\psi - 1 a 2 s 3 a 4 a}$ & -4363.34063041 & 1.357716 & -4361.98291441 & -4363.34101220 & 1.356123 & -4361.98488920 \\
\hline $\boldsymbol{\psi - 1 a 2 s 3 a 4 s}$ & -4363.34082784 & 1.357375 & -4361.98345284 & -4363.34085362 & 1.355815 & -4361.98503862 \\
\hline $\boldsymbol{\psi - 1 a 2 s 3 s 4 a}$ & -4363.34072891 & 1.356307 & -4361.98442191 & -4363.34100384 & 1.356327 & -4361.98467684 \\
\hline $\boldsymbol{\psi - 1 a 2 s 3 s 4 s}$ & -4363.34064788 & 1.356391 & -4361.98425688 & -4363.34082534 & 1.355691 & -4361.98513434 \\
\hline $\boldsymbol{\psi - 1 s 2 a 3 a 4 a}$ & -4363.33911998 & 1.362830 & -4361.97628998 & -4363.33985169 & 1.356492 & -4361.98335969 \\
\hline $\boldsymbol{\psi - 1 s 2 a 3 a 4 s}$ & -4363.33948755 & 1.362735 & -4361.97675255 & -4363.34035483 & 1.356272 & -4361.98408283 \\
\hline
\end{tabular}




\begin{tabular}{|c|l|l|l|l|l|l|}
\hline$\psi$-1s2a3s4a & -4363.33994902 & 1.357186 & -4361.98276302 & -4363.33987055 & 1.357758 & -4361.98211255 \\
\hline$\psi$-1s2a3s4s & -4363.34038654 & 1.356837 & -4361.98354954 & -4363.34000526 & 1.357106 & -4361.98289926 \\
\hline$\psi$-1s2s3a4a & -4363.33955052 & 1.357430 & -4361.98212052 & -4363.34041905 & 1.358292 & -4361.98212705 \\
\hline$\psi$-1s2s3a4s & -4363.33948651 & 1.362649 & -4361.97683751 & -4363.34056562 & 1.356927 & -4361.98363862 \\
\hline$\psi$-1s2s3s4a & -4363.34001489 & 1.357158 & -4361.98285689 & -4363.34033924 & 1.357498 & -4361.98284124 \\
\hline$\psi$-1s2s3s4s & -4363.33992234 & 1.356998 & -4361.98292434 & -4363.34031977 & 1.357011 & -4361.98330877 \\
\hline
\end{tabular}

\begin{tabular}{|c|c|c|c|c|c|c|}
\hline & \multicolumn{3}{|c|}{$3_{\text {IIA-si }}$} & \multicolumn{3}{|c|}{$3_{\text {IIB-re }}$} \\
\hline & $E_{\text {sp(wB97XD) }}$ & $S_{\text {qh(M06) }}$ & $\mathbf{G}_{\mathbf{q h}(\mathrm{wB97XD})}$ & $E_{\text {sp(wB97XD) }}$ & $\mathrm{S}_{\mathrm{qh}(\mathrm{M} 06)}$ & $\mathbf{G}_{\mathbf{q h}(\mathrm{wB97XD)})}$ \\
\hline$\psi-1 \mathrm{a} 2 \mathrm{a} 3 \mathrm{a} 4 \mathrm{a}$ & -4363.34553075 & 1.356944 & -4361.98858675 & -4363.34989767 & 1.360095 & -4361.98980267 \\
\hline$\psi-1 \mathrm{a} 2 \mathrm{a} 3 \mathrm{a} 4 \mathrm{~s}$ & -4363.34577802 & 1.356802 & -4361.98897602 & -4363.34818496 & 1.359871 & -4361.98831396 \\
\hline$\psi-1 \mathrm{a} 2 \mathrm{a} 3 \mathrm{~s} 4 \mathrm{a}$ & -4363.34517049 & 1.360920 & -4361.98425049 & -4363.35012317 & 1.359180 & -4361.99094317 \\
\hline$\psi$-1a2a3s4s & -4363.34529766 & 1.360179 & -4361.98511866 & -4363.35004842 & 1.358820 & -4361.99122842 \\
\hline$\psi-1 a 2 s 3 a 4 a$ & -4363.34544167 & 1.356432 & -4361.98900967 & -4363.35022515 & 1.357123 & -4361.99310215 \\
\hline$\psi$-1a2s3a4s & -4363.34556426 & 1.355528 & -4361.99003626 & -4363.34969007 & 1.356607 & -4361.99308307 \\
\hline$\psi-1 a 2 s 3 s 4 a$ & -4363.34540473 & 1.357337 & -4361.98806773 & -4363.34995494 & 1.359608 & -4361.99034694 \\
\hline$\psi-1 \mathrm{a} 2 \mathrm{~s} 3 \mathrm{~s} 4 \mathrm{~s}$ & -4363.34524393 & 1.357441 & -4361.98780293 & -4363.34954709 & 1.356391 & -4361.99315609 \\
\hline$\psi$-1s2a3a4a & -4363.34349569 & 1.356967 & -4361.98652869 & -4363.35140818 & 1.357126 & -4361.99428218 \\
\hline$\psi$-1s2a3a4s & -4363.34384217 & 1.356820 & -4361.98702217 & -4363.35203031 & 1.356955 & -4361.99507531 \\
\hline$\psi$-1s2a3s4a & -4363.34372081 & 1.356960 & -4361.98676081 & -4363.35159352 & 1.356496 & -4361.99509752 \\
\hline$\psi-1 \mathrm{~s} 2 \mathrm{a} 3 \mathrm{~s} 4 \mathrm{~s}$ & -4363.34371403 & 1.357054 & -4361.98666003 & -4363.35209820 & 1.357731 & -4361.99436720 \\
\hline$\psi-1 \mathrm{~s} 2 \mathrm{~s} 3 \mathrm{a} 4 \mathrm{a}$ & -4363.34364055 & 1.357019 & -4361.98662155 & -4363.35086838 & 1.356882 & -4361.99398638 \\
\hline$\psi-1 \mathrm{~s} 2 \mathrm{~s} 3 \mathrm{a} 4 \mathrm{~s}$ & -4363.34372169 & 1.357190 & -4361.98653169 & -4363.35015314 & 1.357879 & -4361.99227414 \\
\hline$\psi-1 \mathrm{~s} 2 \mathrm{~s} 3 \mathrm{~s} 4 \mathrm{a}$ & -4363.34378044 & 1.357932 & -4361.98584844 & -4363.35126573 & 1.357292 & -4361.99397373 \\
\hline$\psi-1 \mathrm{~s} 2 \mathrm{~s} 3 \mathrm{~s} 4 \mathrm{~s}$ & -4363.34352817 & 1.357687 & -4361.98584117 & -4363.35037119 & 1.357452 & -4361.99291919 \\
\hline
\end{tabular}

\begin{tabular}{|c|c|c|c|c|c|c|}
\hline & \multicolumn{3}{|c|}{$3_{\text {IIC-re }}$} & \multicolumn{3}{|c|}{$3_{\text {IID-si }}$} \\
\hline & $\mathbf{E}_{\text {sp(wB97XD) }}$ & $S_{\text {qh(M06) }}$ & $\mathbf{G}_{\text {qh(wB97XD) }}$ & $\mathbf{E}_{\text {sp(wB97XD) }}$ & $\mathrm{S}_{\mathrm{qh}(\mathrm{M} 06)}$ & $\mathbf{G}_{\mathbf{q h}(\mathrm{wB97XD)})}$ \\
\hline$\psi-1 a 2 a 3 a 4 a$ & -4363.35967645 & 1.355958 & -4362.00371845 & -4363.35569594 & 1.355857 & -4361.99983894 \\
\hline$\psi-1 \mathrm{a} 2 \mathrm{a} 3 \mathrm{a} 4 \mathrm{~s}$ & -4363.35997355 & 1.355951 & -4362.00402255 & -4363.35612398 & 1.355434 & -4362.00068998 \\
\hline$\psi-1 \mathrm{a} 2 \mathrm{a} 3 \mathrm{~s} 4 \mathrm{a}$ & -4363.35982422 & 1.355220 & -4362.00460422 & -4363.35625128 & 1.355815 & -4362.00043628 \\
\hline$\psi-1 \mathrm{a} 2 \mathrm{a} 3 \mathrm{~s} 4 \mathrm{~s}$ & -4363.35989033 & 1.356446 & -4362.00344433 & -4363.35590955 & 1.355683 & -4362.00022655 \\
\hline$\psi-1 a 2 s 3 a 4 a$ & -4363.36022462 & 1.355090 & -4362.00513462 & -4363.35690045 & 1.355579 & -4362.00132145 \\
\hline$\psi-1 \mathrm{a} 2 \mathrm{~s} 3 \mathrm{a} 4 \mathrm{~s}$ & -4363.36052564 & 1.355383 & -4362.00514264 & -4363.35724842 & 1.355443 & -4362.00180542 \\
\hline$\psi-1 a 2 s 3 s 4 a$ & -4363.36015331 & 1.355190 & -4362.00496331 & -4363.35716636 & 1.355750 & -4362.00141636 \\
\hline$\psi-1 \mathrm{a} 2 \mathrm{~s} 3 \mathrm{~s} 4 \mathrm{~s}$ & -4363.36032794 & 1.355673 & -4362.00465494 & -4363.35721694 & 1.355930 & -4362.00128694 \\
\hline$\psi-1$ s2a3a4a & -4363.35839768 & 1.356698 & -4362.00169968 & -4363.35519735 & 1.355162 & -4362.00003535 \\
\hline$\psi$-1s2a3a4s & -4363.35881727 & 1.356263 & -4362.00255427 & -4363.35534934 & 1.355704 & -4361.99964534 \\
\hline$\psi-1 \mathrm{~s} 2 \mathrm{a} 3 \mathrm{~s} 4 \mathrm{a}$ & -4363.35845622 & 1.356786 & -4362.00167022 & -4363.35526298 & 1.357846 & -4361.99741698 \\
\hline$\psi-1 \mathrm{~s} 2 \mathrm{a} 3 \mathrm{~s} 4 \mathrm{~s}$ & -4363.35857838 & 1.356103 & -4362.00247538 & -4363.35477805 & 1.357723 & -4361.99705505 \\
\hline$\psi-1 \mathrm{~s} 2 \mathrm{~s} 3 \mathrm{a} 4 \mathrm{a}$ & -4363.35881489 & 1.357044 & -4362.00177089 & -4363.35589959 & 1.355625 & -4362.00027459 \\
\hline$\psi-1 \mathrm{~s} 2 \mathrm{~s} 3 \mathrm{a} 4 \mathrm{~s}$ & -4363.35905571 & 1.357436 & -4362.00161971 & -4363.35535265 & 1.355466 & -4361.99988665 \\
\hline$\psi-1 \mathrm{~s} 2 \mathrm{~s} 3 \mathrm{~s} 4 \mathrm{a}$ & -4363.35890879 & 1.357204 & -4362.00170479 & -4363.35598970 & 1.356287 & -4361.99970270 \\
\hline$\psi-1 \mathrm{~s} 2 \mathrm{~s} 3 \mathrm{~s} 4 \mathrm{~s}$ & -4363.35883400 & 1.357135 & -4362.00169900 & -4363.35620296 & 1.357237 & -4361.99896596 \\
\hline
\end{tabular}

\begin{tabular}{|c|c|c|c|c|c|c|}
\hline & \multicolumn{3}{|c|}{ TS1 $_{\text {IL-si }}$} & \multicolumn{3}{c|}{ TS1 $_{\text {IL-re }}$} \\
\hline & $\mathbf{E}_{\mathbf{s p}(\mathbf{w B 9 7 X D})}$ & $\mathbf{S}_{\mathbf{q h}(\mathbf{M 0 6})}$ & $\mathbf{G}_{\mathbf{q h}(\mathbf{w B 9 7 X D})}$ & $\mathbf{E}_{\mathbf{s p}(\mathbf{w B 9 7 X D})}$ & $\mathbf{S}_{\mathbf{q h}(\mathbf{M 0 6})}$ & $\mathbf{G}_{\mathbf{q h}(\mathbf{w B 9 7 X D})}$ \\
\hline $\boldsymbol{\psi}$-1a2a3a4a & -4363.32083623 & 1.353436 & -4361.96740023 & -4363.32043157 & 1.352102 & -4361.96832957 \\
\hline
\end{tabular}




\begin{tabular}{|c|c|c|c|c|c|c|}
\hline$\psi-1 \mathrm{a} 2 \mathrm{a} 3 \mathrm{a} 4 \mathrm{~s}$ & -4363.32125250 & 1.353389 & -4361.96786350 & -4363.32073405 & 1.352249 & -4361.96848505 \\
\hline$\psi-1 \mathrm{a} 2 \mathrm{a} 3 \mathrm{~s} 4 \mathrm{a}$ & -4363.31996355 & 1.352458 & -4361.96750555 & -4363.32327309 & 1.352859 & -4361.97041409 \\
\hline$\psi-1 a 2 a 3 s 4 s$ & -4363.32076091 & 1.352668 & -4361.96809291 & -4363.32328744 & 1.353204 & -4361.97008344 \\
\hline$\psi-1 \mathrm{a} 2 \mathrm{~s} 3 \mathrm{a} 4 \mathrm{a}$ & -4363.32216120 & 1.352086 & -4361.97007520 & -4363.32067589 & 1.352226 & -4361.96844989 \\
\hline$\psi$-1a2s3a4s & -4363.32209733 & 1.352625 & -4361.96947233 & -4363.32108217 & 1.352829 & -4361.96825317 \\
\hline$\psi-1 \mathrm{a} 2 \mathrm{~s} 3 \mathrm{~s} 4 \mathrm{a}$ & -4363.32109606 & 1.352702 & -4361.96839406 & -4363.32384949 & 1.351948 & -4361.97190149 \\
\hline$\psi-1 \mathrm{a} 2 \mathrm{~s} 3 \mathrm{~s} 4 \mathrm{~s}$ & -4363.32089764 & 1.352569 & -4361.96832864 & -4363.32353509 & 1.352907 & -4361.97062809 \\
\hline$\psi$-1s2a3a4a & -4363.31890352 & 1.352673 & -4361.96623052 & -4363.31965824 & 1.352498 & -4361.96716024 \\
\hline$\psi$-1s2a3a4s & -4363.31962146 & 1.353806 & -4361.96581546 & -4363.32004675 & 1.352233 & -4361.96781375 \\
\hline$\psi-1 \mathrm{~s} 2 \mathrm{a} 3 \mathrm{~s} 4 \mathrm{a}$ & -4363.31805345 & & & -4363.32213254 & & -4361.96908654 \\
\hline$\psi-1 \mathrm{~s} 2 \mathrm{a} 3 \mathrm{~s} 4 \mathrm{~s}$ & -4363.31824679 & 1.353247 & -4361.96499979 & -4363.32201425 & 1.352772 & -4361.96924225 \\
\hline$\psi-1 \mathrm{~s} 2 \mathrm{~s} 3 \mathrm{a} 4 \mathrm{a}$ & -4363.32439880 & 1.353269 & -4361.97112980 & -4363.31978190 & 1.352146 & -4361.96763590 \\
\hline$\psi-1 \mathrm{~s} 2 \mathrm{~s} 3 \mathrm{a} 4 \mathrm{~s}$ & -4363.32462881 & 1.353808 & -4361.97082081 & -4363.32013673 & 1.352490 & -4361.96764673 \\
\hline$\psi-1 \mathrm{~s} 2 \mathrm{~s} 3 \mathrm{~s} 4 \mathrm{a}$ & -4363.31842125 & 1.353647 & -4361.96477425 & -4363.32240379 & 1.353085 & -4361.96931879 \\
\hline$\psi-1 \mathrm{~s} 2 \mathrm{~s} 3 \mathrm{~s} 4 \mathrm{~s}$ & -4363.31852385 & 1.354342 & -4361.96418185 & -4363.32270970 & 1.353698 & -4361.96901170 \\
\hline
\end{tabular}

\begin{tabular}{|c|c|c|c|c|c|c|}
\hline & \multicolumn{3}{|c|}{ TS1 $_{I B I}$} & \multicolumn{3}{|c|}{ TS1 $_{I B 2}$} \\
\hline & $\mathbf{E}_{\text {sp(wB97XD) }}$ & $S_{\text {qh(M06) }}$ & $\mathbf{G}_{\text {qh(wB97XD) }}$ & $\mathbf{E}_{\mathrm{sp}(\mathrm{wB} 97 \mathrm{XD})}$ & $S_{\text {qh(M06) }}$ & $\mathbf{G}_{\text {qh(wB97XD) }}$ \\
\hline$\psi-1 \mathbf{a} 2 \mathrm{a} 3 \mathrm{a} 4 \mathrm{a}$ & -4363.32449901 & 1.352599 & -4361.97190001 & -4363.31999644 & 1.354732 & -4361.96526444 \\
\hline$\psi$-1a2a3a4s & -4363.32497787 & 1.352992 & -4361.97198587 & -4363.32026138 & 1.353227 & -4361.96703438 \\
\hline$\psi$-1a2a3s4a & -4363.32443164 & 1.352924 & -4361.97150764 & -4363.32057826 & 1.353130 & -4361.96744826 \\
\hline$\psi-1 \mathrm{a} 2 \mathrm{a} 3 \mathrm{~s} 4 \mathrm{~s}$ & -4363.32484133 & 1.352372 & -4361.97246933 & -4363.32089897 & 1.353529 & -4361.96736997 \\
\hline$\psi-1 \mathrm{a} 2 \mathrm{~s} 3 \mathrm{a} 4 \mathrm{a}$ & -4363.32534395 & 1.353829 & -4361.97151495 & -4363.32051394 & 1.353216 & -4361.96729794 \\
\hline$\psi-1 \mathrm{a} 2 \mathrm{~s} 3 \mathrm{a} 4 \mathrm{~s}$ & -4363.32560313 & 1.354510 & -4361.97109313 & -4363.32074371 & 1.353628 & -4361.96711571 \\
\hline$\psi-1 \mathrm{a} 2 \mathrm{~s} 3 \mathrm{~s} 4 \mathrm{a}$ & -4363.32536038 & 1.352808 & -4361.97255238 & -4363.32087334 & 1.353642 & -4361.96723134 \\
\hline$\psi-1 \mathrm{a} 2 \mathrm{~s} 3 \mathrm{~s} 4 \mathrm{~s}$ & -4363.32513367 & 1.354332 & -4361.97080167 & -4363.32119745 & 1.354103 & -4361.96709445 \\
\hline$\psi$-1s2a3a4a & -4363.32313572 & 1.352808 & -4361.97032772 & -4363.31873281 & 1.352836 & -4361.96589681 \\
\hline$\psi-1 \mathrm{~s} 2 \mathrm{a} 3 \mathrm{a} 4 \mathrm{~s}$ & -4363.32351702 & 1.353237 & -4361.97028002 & -4363.31933632 & 1.352635 & -4361.96670132 \\
\hline$\psi-1$ s2a3s4a & -4363.32284634 & 1.354446 & -4361.96840034 & -4363.31972098 & 1.353468 & -4361.96625298 \\
\hline$\psi-1 \mathrm{~s} 2 \mathrm{a} 3 \mathrm{~s} 4 \mathrm{~s}$ & -4363.32289846 & 1.353302 & -4361.96959646 & -4363.31994904 & 1.352852 & -4361.96709704 \\
\hline$\psi-1 \mathrm{~s} 2 \mathrm{~s} 3 \mathrm{a} 4 \mathrm{a}$ & -4363.32352078 & 1.353216 & -4361.97030478 & -4363.31919700 & 1.353687 & -4361.96551000 \\
\hline$\psi-1 \mathrm{~s} 2 \mathrm{~s} 3 \mathrm{a} 4 \mathrm{~s}$ & -4363.32368902 & 1.354061 & -4361.96962802 & -4363.31940436 & 1.353688 & -4361.96571636 \\
\hline$\psi-1 \mathrm{~s} 2 \mathrm{~s} 3 \mathrm{~s} 4 \mathrm{a}$ & -4363.32328174 & 1.353698 & -4361.96958374 & -4363.32016399 & 1.353408 & -4361.96675599 \\
\hline$\psi-1 \mathrm{~s} 2 \mathrm{~s} 3 \mathrm{~s} 4 \mathrm{~s}$ & -4363.32322008 & 1.353565 & -4361.96965508 & -4363.32017390 & 1.353775 & -4361.96639890 \\
\hline
\end{tabular}

\begin{tabular}{|c|c|c|c|c|c|c|}
\hline & \multicolumn{3}{|c|}{ TS1 $_{\text {IIL-si }}$} & \multicolumn{3}{|c|}{ TS1 $1_{\text {IIL-re }}$} \\
\hline & $\mathbf{E}_{\text {sp(wB97XD) }}$ & $S_{\mathrm{qh}(\mathrm{M} 06)}$ & $\mathbf{G}_{\mathbf{q h}(\mathrm{wB} 97 \mathbf{X D})}$ & $\mathbf{E}_{\mathrm{sp}(\mathrm{wB} 97 \mathrm{XD})}$ & $\mathbf{S}_{\mathrm{qh}(\mathrm{M} 06)}$ & $\mathbf{G}_{\mathbf{q h}(\mathrm{wB} 97 \mathrm{XD})}$ \\
\hline$\psi-1 a 2 a 3 a 4 a$ & -4363.33382852 & 1.353873 & -4361.97995552 & -4363.33161954 & 1.352904 & -4361.97871554 \\
\hline$\psi$-1a2a3a4s & -4363.33436609 & 1.354917 & -4361.97944909 & -4363.33179291 & 1.352881 & -4361.97891191 \\
\hline$\psi-1 a 2 a 3 s 4 a$ & -4363.33427268 & 1.354111 & -4361.98016168 & -4363.33252402 & 1.352809 & -4361.97971502 \\
\hline$\psi-1 \mathrm{a} 2 \mathrm{a} 3 \mathrm{~s} 4 \mathrm{~s}$ & -4363.33445550 & 1.354581 & -4361.97987450 & -4363.33271579 & 1.352620 & -4361.98009579 \\
\hline$\psi-1 a 2 s 3 a 4 a$ & $-4363.33382852^{\mathrm{a}}$ & $1.353873^{\mathrm{a}}$ & $-4361.97995552^{a}$ & -4363.33175956 & 1.352368 & -4361.97939156 \\
\hline$\psi$-1a2s3a4s & -4363.32973384 & 1.354871 & -4361.97486284 & -4363.33238577 & 1.352943 & -4361.97944277 \\
\hline$\psi-1 \mathrm{a} 2 \mathrm{~s} 3 \mathrm{~s} 4 \mathrm{a}$ & $-4363.33427268^{\mathrm{a}}$ & $1.354111^{\mathrm{a}}$ & $-4361.98016168^{a}$ & -4363.33321225 & 1.352074 & -4361.98113825 \\
\hline$\psi-1 \mathrm{a} 2 \mathrm{~s} 3 \mathrm{~s} 4 \mathrm{~s}$ & -4363.32984862 & 1.354938 & -4361.97491062 & -4363.33334691 & 1.352442 & -4361.98090491 \\
\hline$\psi$-1s2a3a4a & -4363.33698392 & 1.357052 & -4361.97993192 & -4363.32979282 & 1.353085 & -4361.97670782 \\
\hline$\psi$-1s2a3a4s & -4363.33729430 & 1.353765 & -4361.98352930 & -4363.33006538 & 1.353269 & -4361.97679638 \\
\hline$\psi-1$ s2a3s4a & -4363.33723916 & 1.353633 & -4361.98360616 & -4363.33093230 & 1.353752 & -4361.97718030 \\
\hline
\end{tabular}




\begin{tabular}{|c|c|c|c|c|c|c|}
\hline$\psi$-1s2a3s4s & -4363.33727365 & 1.353643 & -4361.98363065 & -4363.33096098 & 1.354119 & -4361.97684198 \\
\hline$\psi$-1s2s3a4a & $-4363.33698392^{\mathrm{a}}$ & $1.357052^{\mathrm{a}}$ & $-4361.97993192^{\mathrm{a}}$ & -4363.32993174 & 1.352727 & -4361.97720474 \\
\hline$\psi$-1s2s3a4s & -4363.33303969 & 1.356317 & -4361.97672269 & -4363.33024483 & 1.352178 & -4361.97806683 \\
\hline$\psi$-1s2s3s4a & $-4363.33723916^{\mathrm{a}}$ & $1.353633^{\mathrm{a}}$ & $-4361.98360616^{\mathrm{a}}$ & -4363.33083947 & 1.353463 & -4361.97737647 \\
\hline$\psi$-1s2s3s4s & -4363.33285107 & 1.353862 & -4361.97898907 & -4363.33080451 & 1.353477 & -4361.97732751 \\
\hline
\end{tabular}

${ }^{\text {a }}$ Dihedral $\psi 2$ s converged to $\psi 2_{\text {s }}$ during optimization.

\begin{tabular}{|c|c|c|c|c|c|c|}
\hline & \multicolumn{3}{|c|}{$\mathrm{TS1}_{I I B 1}$} & \multicolumn{3}{|c|}{$\mathrm{TS1}_{\mathrm{IIB2}}$} \\
\hline & $\mathbf{E}_{\text {sp(wB97XD) }}$ & $\mathrm{S}_{\mathrm{qh}(\mathrm{M} 06)}$ & $\mathbf{G}_{\mathbf{q h}(\mathrm{wB} 97 \mathrm{XD})}$ & $\mathbf{E}_{\text {sp(wB97XD) }}$ & $\mathrm{S}_{\mathrm{qh}(\mathrm{M} 06)}$ & $\mathbf{G}_{\mathbf{q h}(\mathrm{wB} 97 \mathrm{XD})}$ \\
\hline$\psi-1 \mathrm{a} 2 \mathrm{a} 3 \mathrm{a} 4 \mathrm{a}$ & -4363.32615113 & 1.352478 & -4361.97367313 & -4363.33063940 & 1.353852 & -4361.97678740 \\
\hline$\psi$-1a2a3a4s & -4363.32645613 & 1.352221 & -4361.97423513 & -4363.33060798 & 1.352790 & -4361.97781798 \\
\hline$\psi-1 a 2 a 3 s 4 a$ & -4363.32609062 & 1.352591 & -4361.97349962 & -4363.33039411 & 1.355282 & -4361.97511211 \\
\hline$\psi-1 \mathrm{a} 2 \mathrm{a} 3 \mathrm{~s} 4 \mathrm{~s}$ & -4363.32612392 & 1.352457 & -4361.97366692 & -4363.33036249 & 1.353463 & -4361.97689949 \\
\hline$\psi-1 a 2 s 3 a 4 a$ & -4363.32670477 & 1.353381 & -4361.97332377 & -4363.32597437 & 1.353838 & -4361.97213637 \\
\hline$\psi$-1a2s3a4s & -4363.32689297 & 1.353518 & -4361.973 & -4363.33130539 & 1.354280 & -4361.97702539 \\
\hline$\psi-1 \mathrm{a} 2 \mathrm{~s} 3 \mathrm{~s} 4 \mathrm{a}$ & -4363.32645813 & 1.353236 & -4361.97322213 & -4363.33110369 & 1.353802 & -4361.97730169 \\
\hline$\psi-1 \mathrm{a} 2 \mathrm{~s} 3 \mathrm{~s} 4 \mathrm{~s}$ & -4363.32662057 & 1.353358 & -4361.97326257 & -4363.33105779 & 1.353590 & -4361.97746779 \\
\hline$\psi$-1s2a3a4a & $-4363.32496869^{a}$ & $1.353969^{\mathrm{a}}$ & $-4361.97099969^{\mathrm{a}}$ & -4363.32869317 & 1.354104 & -4361.97458917 \\
\hline$\psi$-1s2a3a4s & -4363.32502142 & 1.354048 & -4361.97097342 & -4363.32923400 & 1.354306 & -4361.97492800 \\
\hline$\psi$-1s2a3s4a & -4363.32486269 & 1.353555 & -4361.97130769 & -4363.32941828 & 1.353866 & -4361.97555228 \\
\hline$\psi-1 \mathrm{~s} 2 \mathrm{a} 3 \mathrm{~s} 4 \mathrm{~s}$ & -4363.32499634 & 1.354127 & -4361.97086934 & -4363.32923274 & 1.353913 & -4361.97531974 \\
\hline$\psi$-1s2s3a4a & -4363.32496869 & 1.353969 & -4361.97099969 & -4363.32982646 & 1.354130 & -4361.97569646 \\
\hline$\psi-1 \mathrm{~s} 2 \mathrm{~s} 3 \mathrm{a} 4 \mathrm{~s}$ & -4363.32514086 & 1.355374 & -4361.96976686 & -4363.32996144 & 1.354674 & -4361.97528744 \\
\hline$\psi-1 \mathrm{~s} 2 \mathrm{~s} 3 \mathrm{~s} 4 \mathrm{a}$ & -4363.32481855 & 1.354143 & -4361.97067555 & -4363.33001141 & 1.354007 & -4361.97600441 \\
\hline$\psi-1 \mathrm{~s} 2 \mathrm{~s} 3 \mathrm{~s} 4 \mathrm{~s}$ & -4363.32495098 & 1.354534 & -4361.97041698 & -4363.33007038 & 1.354822 & -4361.97524838 \\
\hline
\end{tabular}

${ }^{\mathrm{a}}$ Dihedral $\psi 22_{\mathrm{a}}$ converged to $\psi 22_{\text {s }}$ during optimization.

\begin{tabular}{|c|c|c|r|}
\hline & $\mathbf{E}_{\mathbf{s p}(\mathbf{w B 9} \mathbf{9 D D})}$ & $\mathbf{S}_{\mathbf{q h}(\mathbf{M 0 6})}$ & $\mathbf{G}_{\mathbf{q h}(\mathbf{w B} \mathbf{9} \mathbf{X D})}$ \\
\hline$\alpha$-methylstyrene & -348.933939910 & 0.128805 & -348.805134910 \\
\hline $\mathrm{CO}$ & -113.300680366 & -0.014075 & -113.314755366 \\
\hline $\mathrm{H}_{2}$ & -1.175630412 & -0.001365 & -1.176995412 \\
\hline
\end{tabular}

\section{Linear $S$ pathway}

\begin{tabular}{|c|c|c|c|}
\hline & \multicolumn{1}{|c|}{$\mathbf{E}_{\mathbf{s p}(\mathbf{w B 9 7 X D})}$} & $\mathbf{S}_{\mathbf{q h}(\mathbf{M 0 6})}$ & \multicolumn{1}{|c|}{$\mathbf{G}_{\mathbf{q h}(\mathbf{w B 9 7} \mathbf{X D})}$} \\
\hline $\mathbf{1}$ & -4127.73869643 & 1.205002 & -4126.53369443 \\
\hline $\mathbf{2}$ & -4014.40114199 & 1.198473 & -4013.20266899 \\
\hline $\mathbf{3}_{\boldsymbol{s} i}$ & -4363.35724842 & 1.355443 & -4362.00180542 \\
\hline $\mathbf{T S 1}_{\boldsymbol{s} i}$ & -4363.33729430 & 1.353765 & -4361.98352930 \\
\hline $\mathbf{4}_{\boldsymbol{S}}$ & -4363.36931413 & 1.359733 & -4362.00958113 \\
\hline $\mathbf{5}_{\boldsymbol{S}}$ & -4476.70362133 & 1.366135 & -4475.33748633 \\
\hline $\mathbf{T S 2}_{\boldsymbol{S}}$ & -4476.67483636 & 1.365923 & -4475.30891336 \\
\hline $\mathbf{6}_{\boldsymbol{S}}$ & -4476.70486839 & 1.368393 & -4475.33647539 \\
\hline $\mathbf{7}_{\boldsymbol{S}}$ & -4590.03575169 & 1.373609 & -4588.66214269 \\
\hline $\mathbf{T S 3}_{\boldsymbol{S}}$ & -4477.87446178 & 1.379675 & -4476.49478678 \\
\hline
\end{tabular}




\begin{tabular}{|c|r|r|r|}
\hline $\mathbf{8}_{\boldsymbol{S}}$ & -4477.88755566 & 1.382481 & -4476.50507466 \\
\hline $\mathbf{T S 4}_{\boldsymbol{S}}$ & -4477.86511943 & 1.382318 & -4476.48280143 \\
\hline $\mathbf{9}_{\boldsymbol{S}}$ & -463.483400059 & 0.158090 & -463.325310059 \\
\hline
\end{tabular}

\section{Linear $\boldsymbol{R}$ pathway}

\begin{tabular}{|c|l|l|l|}
\hline & $\mathbf{E}_{\mathbf{s p}(\mathbf{w B 9 7} \mathbf{X D})}$ & $\mathbf{S}_{\mathbf{q h}(\mathbf{M 0 6})}$ & $\mathbf{G}_{\mathbf{q h}(\mathbf{w B 9 7} \mathbf{X D})}$ \\
\hline $\mathbf{1}$ & -4127.73869643 & 1.205002 & -4126.53369443 \\
\hline $\mathbf{2}$ & -4014.40114199 & 1.198473 & -4013.20266899 \\
\hline $\mathbf{3}_{\boldsymbol{r}}$ & -4363.36022462 & 1.355090 & -4362.00513462 \\
\hline $\mathbf{T S 1}_{\boldsymbol{r}}$ & -4363.33321225 & 1.352074 & -4361.98113825 \\
\hline $\mathbf{4}_{\boldsymbol{R}}$ & -4363.37643717 & 1.358821 & -4362.01761617 \\
\hline $\mathbf{5}_{\boldsymbol{R}}$ & -4476.70088097 & 1.365963 & -4475.33491797 \\
\hline $\mathbf{T S} \mathbf{2}_{\boldsymbol{R}}$ & -4476.67834897 & 1.365504 & -4475.31284497 \\
\hline $\mathbf{6}_{\boldsymbol{R}}$ & -4476.70442351 & 1.367418 & -4475.33700551 \\
\hline $\mathbf{7}_{\boldsymbol{R}}$ & -4590.03688859 & 1.373993 & -4588.66289559 \\
\hline $\mathbf{T S 3}_{\boldsymbol{R}}$ & -4477.87772485 & 1.379882 & -4476.49784285 \\
\hline $\mathbf{8}_{\boldsymbol{R}}$ & -4477.89199900 & 1.382848 & -4476.50915100 \\
\hline $\mathbf{T S 4}_{\boldsymbol{R}}$ & -4477.86567546 & 1.382532 & -4476.48314346 \\
\hline $\mathbf{9}_{\boldsymbol{R}}$ & -463.48340006 & 0.158090 & -463.32531006 \\
\hline
\end{tabular}

\section{Branched pathway}

\begin{tabular}{|c|l|l|l|}
\hline & $\mathbf{E}_{\mathbf{s p}(\mathbf{w B 9 7} \mathbf{X D})}$ & $\mathbf{S}_{\mathbf{q h}(\mathbf{M 0 6})}$ & $\mathbf{G}_{\mathbf{q h}(\mathbf{w B 9} \mathbf{X D})}$ \\
\hline $\mathbf{1}$ & -4127.73869643 & 1.205002 & -4126.53369443 \\
\hline $\mathbf{2}$ & -4014.40114199 & 1.198473 & -4013.20266899 \\
\hline $\mathbf{3}_{\boldsymbol{r e}}$ & -4363.36022462 & 1.355090 & -4362.00513462 \\
\hline $\mathbf{T S 1}_{\boldsymbol{B}}$ & -4363.33060798 & 1.352790 & -4361.97781798 \\
\hline $\mathbf{4}_{\boldsymbol{B}}$ & -4363.36027631 & 1.358232 & -4362.00204431 \\
\hline $\mathbf{5}_{\boldsymbol{B}}$ & -4476.68543636 & 1.365159 & -4475.32027736 \\
\hline $\mathbf{T S 2}_{\boldsymbol{B}}$ & -4476.66283823 & 1.364875 & -4475.29796323 \\
\hline $\mathbf{6}_{\boldsymbol{B}}$ & -4476.69900637 & 1.366596 & -4475.33241037 \\
\hline $\mathbf{7}_{\boldsymbol{B}}$ & -4590.03173637 & 1.374941 & -4588.65679537 \\
\hline $\mathbf{T S 3}_{\boldsymbol{B}}$ & -4477.87218465 & 1.380953 & -4476.49123165 \\
\hline $\mathbf{8}_{\boldsymbol{B}}$ & -4477.88664740 & 1.384108 & -4476.50253940 \\
\hline $\mathbf{T S 4}_{\boldsymbol{B}}$ & -4477.86283436 & 1.382432 & -4476.48040236 \\
\hline $\mathbf{9}_{\boldsymbol{B}}$ & -463.48297168 & 0.158308 & -463.32466368 \\
\hline
\end{tabular}

Transition States for Additional Substrates

S2 (para-CH3)

\begin{tabular}{|c|c|c|c|}
\hline & $\mathbf{E}_{\mathbf{s p}(\mathbf{w B 9 7 X D})}$ & $\mathbf{S}_{\mathbf{q h}(\mathbf{M 0 6})}$ & $\mathbf{G}_{\mathbf{q h}(\mathbf{w B 9 7 X D})}$ \\
\hline & \multicolumn{3}{|c|}{ TS1 $_{\text {IIL-si }}$} \\
\hline$\psi$-1s2a3a4a & -4402.65372628 & 1.379172 & -4401.27455428 \\
\hline
\end{tabular}




\begin{tabular}{|c|c|c|c|}
\hline$\psi-1 s 2 a 3 a 4 s$ & -4402.65404788 & 1.382866 & -4401.27118188 \\
\hline$\psi-1 s 2 a 3 s 4 a$ & -4402.65400560 & 1.379158 & -4401.27484760 \\
\hline$\psi-1 s 2 a 3 s 4 s$ & -4402.65403919 & 1.379888 & -4401.27415119 \\
\hline & \multicolumn{3}{|c|}{ TS1 $_{\text {IIL-re }}$} \\
\hline$\psi-1 a 2 s 3 a 4 a$ & -4402.64936104 & 1.378313 & -4401.27104804 \\
\hline$\Psi-1 a 2 s 3 a 4 s$ & -4402.64969339 & 1.379095 & -4401.27059839 \\
\hline$\Psi-1 a 2 s 3 s 4 a$ & -4402.64962139 & 1.378922 & -4401.27069939 \\
\hline$\Psi-1 a 2 s 3 s 4 s$ & -4402.64983675 & 1.378782 & -4401.27105475 \\
\hline
\end{tabular}

S3 (para-CF3)

\begin{tabular}{|c|c|c|c|}
\hline & \multicolumn{3}{|c|}{ TS1 $_{\text {IIL-si }}$} \\
\hline$\psi-1 \mathrm{~s} 2 \mathrm{a} 3 \mathrm{a} 4 \mathrm{a}$ & -4700.38974127 & 1.356669 & -4699.03307227 \\
\hline$\psi-1 \mathrm{~s} 2 \mathrm{a} 3 \mathrm{a} 4 \mathrm{~s}$ & -4700.39006030 & 1.357039 & -4699.03302130 \\
\hline$\psi-1 s 2 a 3 s 4 a$ & -4700.38976832 & 1.356448 & -4699.03332032 \\
\hline \multirow[t]{2}{*}{$\psi-1 \mathrm{~s} 2 \mathrm{a} 3 \mathrm{~s} 4 \mathrm{~s}$} & -4700.39000870 & 1.356458 & -4699.03355070 \\
\hline & \multicolumn{3}{|c|}{ TS1 $_{\text {IIL-re }}$} \\
\hline$\psi-1 a 2 s 3 a 4 a$ & -4700.38640789 & 1.355728 & -4699.03067989 \\
\hline$\psi-1 \mathrm{a} 2 \mathrm{~s} 3 \mathrm{a} 4 \mathrm{~s}$ & -4700.38673742 & 1.356400 & -4699.03033742 \\
\hline$\psi-1 \mathrm{a} 2 \mathrm{~s} 3 \mathrm{~s} 4 \mathrm{a}$ & -4700.38646592 & 1.355422 & -4699.03104392 \\
\hline$\psi-1 \mathrm{a} 2 \mathrm{~s} 3 \mathrm{~s} 4 \mathrm{~s}$ & -4700.38665796 & 1.354851 & -4699.03180696 \\
\hline
\end{tabular}

S4 (para-F)

\begin{tabular}{|c|c|c|c|}
\hline & \multicolumn{3}{|c|}{ TS1 $_{\text {IIL-si }}$} \\
\hline$\psi-1 s 2 a 3 a 4 a$ & -4462.57685325 & 1.345880 & -4461.23097325 \\
\hline$\psi-1 s 2 a 3 a 4 s$ & -4462.57705588 & 1.345714 & -4461.23134188 \\
\hline$\psi-1 s 2 a 3 s 4 a$ & -4462.57699788 & 1.345222 & -4461.23177588 \\
\hline$\psi-1 s 2 a 3 s 4 s$ & -4462.57692497 & 1.345020 & -4461.23190497 \\
\hline & \multicolumn{3}{|c|}{ TS1 $_{\text {IIL-re }}$} \\
\hline$\psi-1 a 2 s 3 a 4 a$ & -4462.57037949 & 1.344272 & -4461.22610749 \\
\hline$\psi-1 a 2 s 3 a 4 s$ & -4462.57071236 & 1.344082 & -4461.22663036 \\
\hline$\psi-1 a 2 s 3 s 4 a$ & -4462.57178335 & 1.344871 & -4461.22691235 \\
\hline$\psi-1 a 2 s 3 s 4 s$ & -4462.57188208 & 1.345105 & -4461.22677708 \\
\hline
\end{tabular}

\begin{tabular}{|c|c|c|c|}
\multicolumn{4}{|c|}{ S5 (para-Cl) } \\
\hline & \multicolumn{3}{|c|}{ TS1 $_{\text {IIL-si }}$} \\
\hline$\psi-1 s 2 a 3 a 4 a$ & -4822.94507782 & 1.342736 & -4821.60234182 \\
\hline$\psi-1 s 2 a 3 a 4 s$ & -4822.94553168 & 1.344060 & -4821.60147168 \\
\hline$\psi-1 s 2 a 3 s 4 a$ & -4822.94555265 & 1.342588 & -4821.60296465 \\
\hline$\psi-1 s 2 a 3 s 4 s$ & -4822.94545543 & 1.343323 & -4821.60213243 \\
\hline & \multicolumn{3}{|c|}{ TS1 $_{\text {IIL-re }}$} \\
\hline$\psi-1 a 2 s 3 a 4 a$ & -4822.94162060 & 1.341744 & -4821.59987660 \\
\hline$\psi-1 a 2 s 3 a 4 s$ & -4822.94228658 & 1.342585 & -4821.59970158 \\
\hline$\psi-1 a 2 s 3 s 4 a$ & -4822.94250684 & 1.342296 & -4821.60021084 \\
\hline$\psi-1 a 2 s 3 s 4 s$ & -4822.94264262 & 1.342595 & -4821.60004762 \\
\hline
\end{tabular}

Fragments Used in the Activation Strain Analysis

\begin{tabular}{|c|c|c|}
\hline & Ecat-F1 & Esub-F2 \\
\hline & $\mathbf{E}_{\mathrm{sp}(\mathrm{wB} 97 \mathrm{XD})}$ & $\mathbf{E}_{\mathrm{sp}(\mathrm{wB} 97 \mathbf{X D})}$ \\
\hline
\end{tabular}




\begin{tabular}{|c|r|r|}
\hline TS1 $_{\text {IIL-si }}$ & -4014.36687868 & -348.907180598 \\
\hline TS1 $_{\text {IIL-re }}$ & -4014.35716929 & -348.911531908 \\
\hline TS1 $_{\text {IIB2 }}$ & -4014.36760333 & -348.913895893 \\
\hline
\end{tabular}

----End of Part-B---- 